*als View/Print Document Cover Sheet tok

This document was retrieved from the Boeing ISEARCH System.

Accession \#: D196050987

Document \#: SD-WM-ER-315

Title/Desc:

SUPPORTING DOCUMENT FOR THE SOUTHEAST QUADRANT HISTORICAL TANK CONTENT ESTIMATE REPORT FOR AP

TANK FARM [VOL I SEC 1 OF 2 PAGE 1 TO D-294] 
THIS DOCUMENT WAS TOO LARGE TO SCAN AS A SINGLE

DOCUMENT. IT REQUIRED BREAKING INTO SMALLER SECTIONS.

DOCUMENT NUMBER: $S D-\omega m-E R-315(\mathrm{Vol} I)$

SECTION: 1 OF 2

TITE: Southeast Quadrant Historidal Leak Osulent Estimate Reporl for APsank Torm

AUTHOR: LA Cadis / SP Coneor RECIPIENT:

DATE: $\quad 9 / 13 / 95$

KEYWORDS:

REFERENCES: 


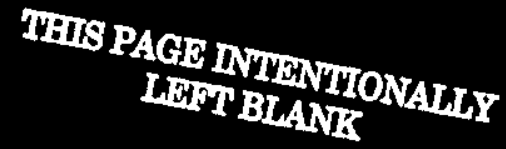




\begin{tabular}{|c|c|c|c|c|c|c|c|c|c|c|}
\hline \multicolumn{4}{|c|}{$\begin{array}{l}\text { 2. To: (Receiving organization) } \\
\text { TWRS }\end{array}$} & \multicolumn{3}{|c|}{$\begin{array}{l}\text { 3. From: (Originating Organization) } \\
\text { ICF-KH }\end{array}$} & \multicolumn{4}{|c|}{ 4. Related EDT NO.: } \\
\hline \multicolumn{4}{|c|}{$\begin{array}{l}\text { 5. Proj./Prog./Dept./Div.: } \\
\text { ER4945/E11730 }\end{array}$} & \multicolumn{3}{|c|}{$\begin{array}{l}\text { 6. Cog. Engr.: } \\
\text { L.A. Gaddis/S.D. Consort }\end{array}$} & \multicolumn{4}{|c|}{ 7. Purchase Order No.: } \\
\hline \multirow{2}{*}{\multicolumn{7}{|c|}{$\begin{array}{l}\text { 8. Originator Renarks: } \\
\text { This document provides Supporting Document is for the } \\
\text { Southeast Quadrant Historical Tank Content Estimate Report } \\
\text { for AP-Tank Farm located in the Hanford } 200 \text { East Area. For } \\
\text { external release }\end{array}$}} & \multicolumn{4}{|c|}{$\begin{array}{l}\text { 9. Equip./Component No.: } \\
\qquad N / A\end{array}$} \\
\hline & & & & & & & \multicolumn{4}{|c|}{$\begin{array}{r}\text { 10. System/Bldg./Facility: } \\
\text { Tank Farms }\end{array}$} \\
\hline \multirow{3}{*}{\multicolumn{7}{|c|}{ 11. Receiver Renarks: }} & \multicolumn{4}{|c|}{$\begin{array}{l}\text { 12. Major Assm. Dug. No.: } \\
\qquad N / A\end{array}$} \\
\hline & & & & & & & \multicolumn{4}{|c|}{$\begin{array}{l}\text { 13. Permit/Permit Application No.: } \\
N / A\end{array}$} \\
\hline & & & & & & & \multicolumn{4}{|c|}{$\begin{array}{l}\text { 14. Required Response Date: } \\
\text { N/A }\end{array}$} \\
\hline \multicolumn{3}{|l|}{15.} & \multicolumn{4}{|c|}{ DATA TRAKSMITTED } & \multirow{2}{*}{$\begin{array}{l}\text { (F) } \\
\text { Approval } \\
\text { Desig: } \\
\text { nator }\end{array}$} & \multirow{2}{*}{$\begin{array}{c}\text { (G) } \\
\text { Roason } \\
\text { for } \\
\text { Trane- } \\
\text { mittal }\end{array}$} & \multirow{2}{*}{$\begin{array}{c}\text { (H) } \\
\text { Origi- } \\
\text { nator } \\
\text { Dispo- } \\
\text { sition } \\
\end{array}$} & \multirow{2}{*}{$\begin{array}{c}\text { (I) } \\
\text { Rocoiv- } \\
\text { or } \\
\text { Dispo- } \\
\text { sition }\end{array}$} \\
\hline $\begin{array}{l}\text { (A) } \\
\text { Irem } \\
\text { No. }\end{array}$ & (B) Document/D & ing No. & $\begin{array}{l}\text { (C) } \\
\text { Shoot } \\
\text { No. }\end{array}$ & $\begin{array}{l}\text { (D) } \\
\text { Rov. } \\
\text { No. }\end{array}$ & (E) Titlo o & $\begin{array}{l}\text { iption of Data } \\
\text { itted }\end{array}$ & & & & \\
\hline 1 & WHC-SD-WM- & -315 & & 0 & $\begin{array}{l}\text { Support } \\
\text { for the } \\
\text { Quadran } \\
\text { Tank Cor } \\
\text { Report } \\
\text { Farm. ( } \\
\text { II) }\end{array}$ & $\begin{array}{l}\text { Document } \\
\text { theast } \\
\text { storical } \\
\text { E Estimate } \\
\text { AP-Tank } \\
\text { ne I and }\end{array}$ & $N / A$ & 1 & 1 & 1 \\
\hline \multicolumn{11}{|l|}{16.} \\
\hline \multicolumn{2}{|c|}{ Approval Designator $(F)$} & \multicolumn{4}{|c|}{ Reason for Tranemittal (G) } & \multicolumn{5}{|c|}{ Disposition (H) \& (I) } \\
\hline \multicolumn{2}{|c|}{$\begin{array}{l}\text { E, S, Q, D or N/A } \\
(000 \text { WHC-CM-3-5. } \\
\text { Soc. } 12.7 \text { ) }\end{array}$} & $\begin{array}{l}\text { 1. Appro: } \\
\text { 2. Aoloas: } \\
\text { 3. Inform }\end{array}$ & $\begin{array}{l}\text { 4. R } \\
\text { 5. Pc } \\
\text { 6. D }\end{array}$ & Recewipt A & ow. Roquired) & $\begin{array}{l}\text { 1. Approved } \\
\text { 2. Approved w/ec } \\
\text { 3. Disapproved w }\end{array}$ & $\begin{array}{l}\text { mont } \\
\text { omment }\end{array}$ & \multicolumn{3}{|c|}{$\begin{array}{l}\text { Reviewed no/comment } \\
\text { Reviewed w/comment } \\
\text { Receipt acknowledged }\end{array}$} \\
\hline
\end{tabular}

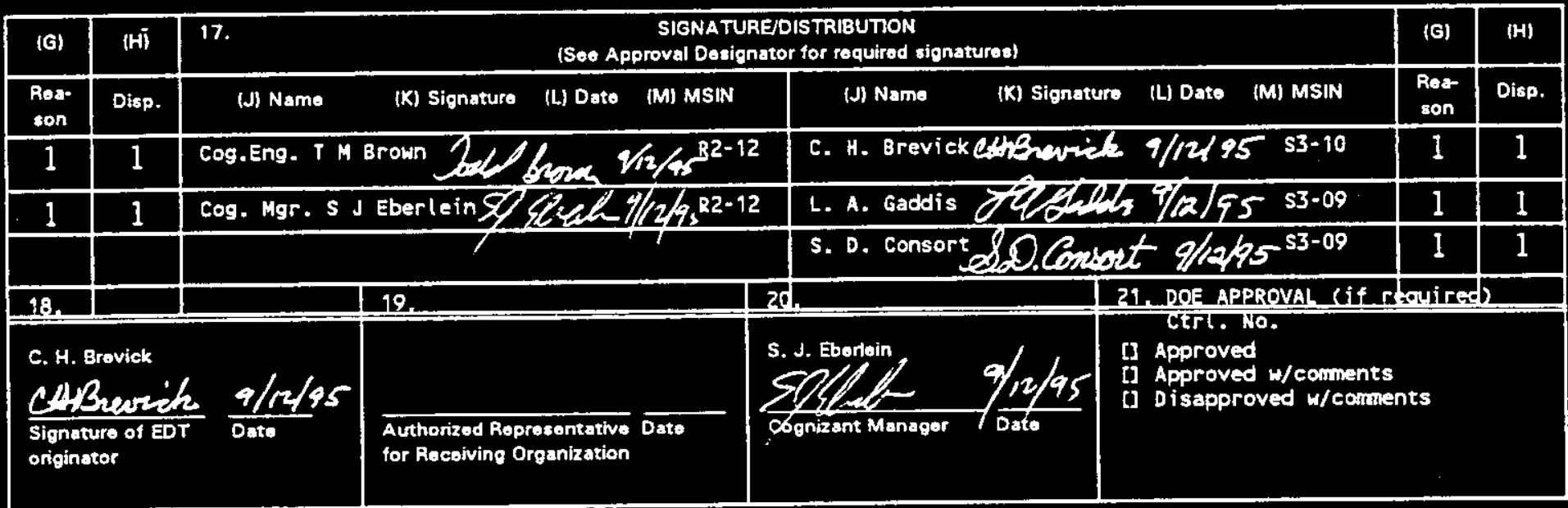

BD-7400-172-2 (04/94) GEF097 


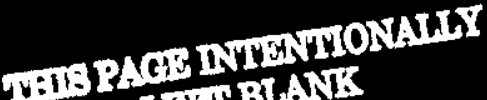 hHT BIANS}


2. Title

Supporting Document for the Southeast Quadrant Historical Tank Content Estimate Report for APTank Farm. (Volume I and II)

5. Key Words

Southeast Quadrant, Historical Tank Content Estimate, Historical Characterization, Tank Farms, Tank Waste Level, Tank Aerial Photos, In-Tank Photo, Tank Layer Model. Estimated Composite Inventories, Riser Locations, Tank Layering Model. Supernatant Mixing Model.
3. Number

WHC-SD-WM-ER-315

4. Rev No.

0

6. Author

Name: CHBrevick/LAGadd is

SDConsort

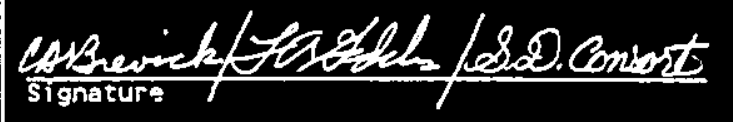

Organization/Charge code 5A400/E11730

Facilities and Technical Support

\section{Abstract}

Historical Tank Content Estimate of the Southeast Quadrant provides historical evaluations on a tank by tank bas is of the radioactive mixed wastes stored in the underground double-shell tanks of the Hanford 200 East and West Areas. This report summarizes historical information such as waste history, temperature profiles, psychrometric data, tank integrity, inventory estimates and tank level history on a tank by tank basis. Tank Farm aerial photos and in-tank photos of each tank are provided. A brief description of instrumentation methods used for waste tank surveillance are included. Components of the data management effort, such as Waste Status and Transaction Record Summary, Tank Layer Mode1, Supernatant Mixing Model, Defined Waste Types, and Inventory Estimates which generate these tank content estimates, are also given in this report.

8. RELEASE STAMP

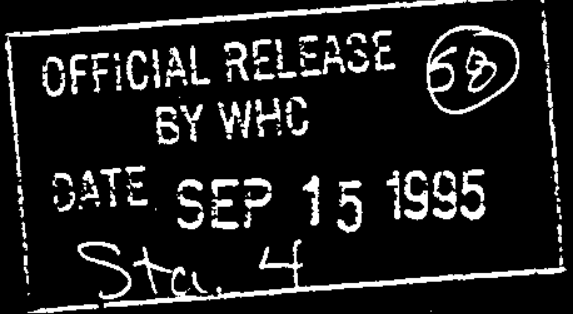




\section{PAGE INTENTIONALT}

IDIT BLANK 


\section{RELEASE AUTHORIZATION}

Document Number: WHC-SD-WM-ER-315, REV 0

Supporting Document for the Southeast Quadrant Historical Tank Content Estimate Report for AP-Tank Farm (Volume I and II)

Release Date: $\quad 9 / 14 / 95$

This document was reviewed following the procedures described in WHC-CM-3-4 and is:

APPROVED FOR PUBLIC RELEASE

WHC Information Release Administration Specialist:
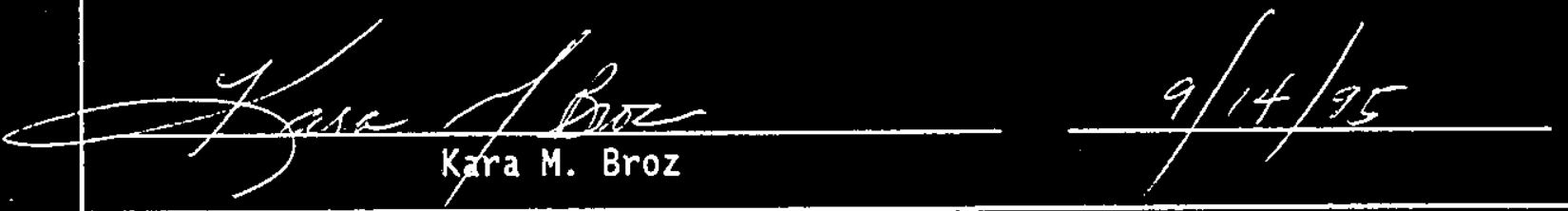

TRADEMARK DISCLAIMER. Reference herein to any specific commercial product, process, or service by trade name, trademark, manufacturer, or otherwise, does not necessarily constitute or imply its endorsement, recommendation, or favoring by the United States Government or any agency thereof or its contractors or subcontractors.

This report has been reproduced from the best available copy. Available in paper copy. Printed in the United States of America. To obtain copies of this report, contact:

Westinghouse Hanford Company - Document Control Services

P.0. Box 1970, Mailstop H6-08, Richland, WA 99352

Telephone: (509) 372-2420; Fax: (509) 376-4989 


\section{THIS PAGE NNTENTIONALWY}

LFIT BLANK 


\title{
SUPPORTING DOCUMENT FOR THE HISTORICAL TANK CONTENT ESTIMATE FOR
}

\section{AP TANK FARM}

\section{WORK ORDER E11730}

\author{
Prepared for
}

Westinghouse Hanford Company

September 1995

Prepared by

ICF Kaiser Hanford Company

Richland, Washington 


\section{THIS PAGE INTENTIONALLY}

\section{IWIT BLANK}




\title{
SUPPORTING DOCUMENT FOR THE HISTORICAL TANK CONTENT ESTIMATE
}

FOR

\section{AP TANK FARM}

Work Order E11729

\author{
Prepared by \\ ICF Kaiser Hanford Company \\ Richland, Washington
}

for

Westinghouse Hanford Company
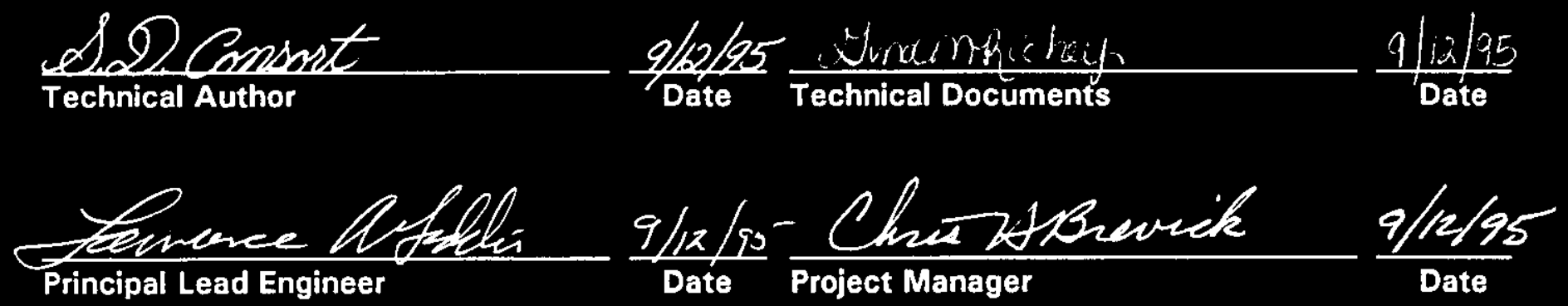

Westinghouse Hanford Company

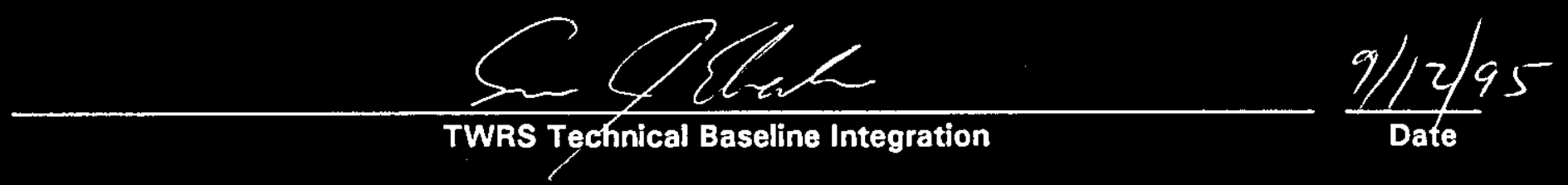


THIS PAGE INTENTYONALISY

LEFT BLANK 


\section{ACKANOWLEDGMENTS}

A project of the this magnitude would not be possible without the help of a significant number of persons and organizations.

ICF Kaiser Hanford Company would like to acknowledge the contributions made by our Los Alamos National Laboratory counterparts: Stephen F. Agnew, Kenn Jurgensen, Rob Corbin, Toma Duran, and Bonnie Young. Also, Todd Brown and Brett Simpson of Westinghouse Hanford Company are recognized for their contributions. 


\section{THIS PAGE INTENTIONALLY}

LFFT BLANK 


\section{INFORMATION FEEDBACK CARD}

\section{SUPPORTING DOCUMENT FOR THE HISTORICAL TANK CONTENT ESTMMATE FOR AP TANK FARM \\ E11730}

\section{COMMENTS AND CONTRIBUTIONS}

The reader is requested to utilize this card to comment on this working document, report any discrepancies, or contribute new information to improve the accuracy and content of the report. Please use the space provided below, add additional pages if necessary, and return to the address listed below.

Send Comments to: MS. S.J. Eberlein

Manager, TWRS Technical Baseline Integration

P.O. Box 1970, MSIN R2-12

Richland, WA 99352 


\section{TIS PAGE INTENTIONAUIY TFT BLANK}


1.0 Introduction..................... 1

1.1 Purpose . . . . . . . . . ............. 1

1.2 Scope . . . . . . . . . . . . . . . . . . . . . 1

1.3 Approach . . . . . . . . . . . . . . . . . . . 2

1.3.1 Surveillance Techniques for the Hanford Mixed Waste Storage Tanks . . . . . . . . . . : 2

1.3.2 Approach and Development of Supporting Information Contained in the Historical Tank Content Estimate and Supporting Documents . 8

1.3.3 Approach and Development of the Process-Based Historical Tank Content Estimate . . . . 11 1.3.4 Assumptions .. . . . . . . . . . 15

1.4 Historical Information Quality . . . . . . . . . . 17

1.5 Southeast Quadrant Background . . . . . . . . . . 21 1.5.1 Waste Generating Plants and Processes . . 21 1.5.2 Waste Management Operations . . . . . . 24 1.5.3 Miscellaneous Waste Sources and Equipment

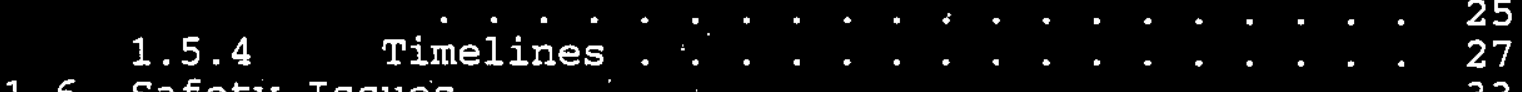

1.6 Safety Issues . .. . . . ............ . 33 1.6.1 Watch List Safety Issues . . . . . . . 33 1.6.2 Non-Watch List Safety Issues . . . . . . 34

1.7 Occurrences ................. 35

2.0 AP Tank Farm . . . . . . . . . . . . . . . . . . 36 2.0.1 AP Tank Farm History . . . . . . . . 36

2.0.1.1 AP Tank Farm Waste History . . . . . 36

2.0.1.2 AP Tank Farm Temperature History . . 36

2.0.1.3 AP Tank Farm Occurrences . . . . . 37

2.0.1.4 AP Tank Farm Ventilation . . . . . 37

2.0.1.5 AP Tank Farm Historical Sample Analysis Data............ . 38 2.0.2 Current Status of AP Tank Farm . . . . . 38 2.0.2.1 AP Tank Farm Inventory Estimates . . 38 2.0.2.2 Waste Volume Approximations AP Tank Farm

2.0.2.3 AP Tank Farm In-Tank Photographs. . 39

2.1 Tank 241-AP-101... . . . . . . . . . . . . . 40 2.1.1 241-AP-101 Tank History........ 40

2.1.1.1 Waste History of Tank 241-AP-101: 40

2.1.1.2 Temperature History of Tank 241-AP-101

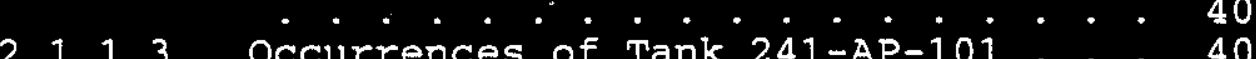

2.1.1.4 Psychrometrics of Tank 241 -AP-101: 40

2.1.1.5 Historical Sample Analysis Data for Tank 241-AP-101 :. . . . . . . . . . 41

2.1.2 Current status of Tank $241-\mathrm{AP}-101 . .41$

2.1.2.1 Inventory Estimate of Tank 241-AP-101

2.1.2.2 Waste volume Approximations for Tank 241-

$2.1 .2 .3 \quad$ In-Tank photograph of Tank $24 \dot{1}-\dot{A P}-101$ 
$-101 \cdot \cdot \cdot \cdot \cdot 41$

Tank 241-AP-102................... 42

2.2.1 241-AP-102 Tank History......... 42

2.2.1.1 Waste History of Tank 241-AP-102 . 42

2.2.1.2 Temperature History of Tank 241-AP-102

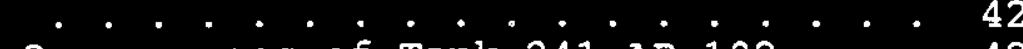

2.2.1.3 Occurrences of Tank 241-AP-102... 42

2.2.1.4 Psychrometrics of Tank 241-AP-102. 42

2.2.1.5 Historical Sample Analysis Data for Tank 241-AP-102 ............ 43

2.2.2 Current Status of Tank 241-AP-102 . . . 43

2.2.2.1 Inventory Estimate of Tank 241-AP-102

2.2.2.2 Waste volume Approximations for Tank 241-

2.2.2.3 In-Tank Photograph of Tank $24 \dot{1}$-AP-102

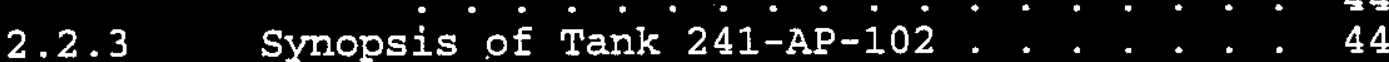

2.3 Tank 241-AP-103 ... . . . . . . . . . . . . . . . 45

2.3 .1 241-AP-103 Tank History . . . ... . 45

2.3.1.1 Waste History of Tank 241-AP-103. . 45

2.3.1.2 Temperature History of Tank 241-AP-103

2.3 .1 .3 Occurrences of Tank $241-\mathrm{AP}-103^{\circ} \cdot 45$

2.3.1.4 Psychrometrics of Tank 241-AP-103. 45

2.3.1.5 Historical Sample Analysis Data for Tank 241-AP-103 ......... . 46

2.3.2 Current Status of Tank 241-AP-103... . 46

2.3.2.1 Inventory Estimate of Tank 241-AP-103

2.3.2.2 Waste Volume Approximations for Tank 241AP-103 . . . . . . . . . . 46

2.3.2.3 In-Tank Photograph of Tank 241-AP-103

Symopsis of Tank $241-A \dot{P}-103 \cdot \cdot \cdot \cdot 47$

2.4 Tank 241-AP-104 ............... . . . . 48

2.4.1 241-AP-104 Tank History .. . . . . . . . 48

2.4.1.1 Waste History of Tank 241-AP-104. 48

2.4.1.2 Temperature History of Tank 241-AP-104

2.4 .1 .3 Occurrences of Tank $241-\mathrm{AP}-10 \dot{4} \cdot \cdot \cdot 48$

2.4.1.4 Psychrometrics of Tank 241-AP-104. 48

2.4.1.5 Historical Sample Analysis Data for Tank 241-AP-104 . . . . . : . . . . . . . . . 49

2.4.2 Current Status of Tank 241-AP-104... . 49

2.4.2.1 Inventory Estimate of Tank 241-AP-104

Waste Volume Approximations for Tank 241 Waste volume Approximations for Tank 2419

2.4.2.3 In-Tank Photograph of Tank 241-AP-104

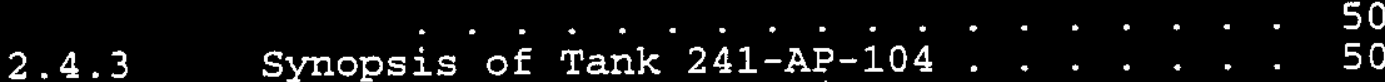

2.5 Tank 241-AP-105.............. 51 2.5.1 241-AP-105 Tank History. . . . . . . 51 
2.5.1.1 Waste History of Tank 241-AP-105 .. 51

2.5.1.2 Temperature fistory of Tank 241-AP-105

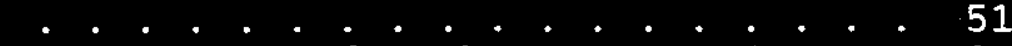

2.5.1.3 Occurrences of Tank 241-AP-105... . 51

2.5.1.4 Psychrometrics of Tank 241-AP-105. . 51

2.5.1.5 Historical Sample Analysis Data for Tank $241-\mathrm{AP}-105$. . . . . . . . . . . . 52

2.5.2 Current Status of Tank 241-AP-105. . . . 52

2.5.2.1 Inventory Estimate of Tank 241-AP-105

2.5.2.2 Waste Volume Approximations for Tank 241Ap-105... . . . . . . . . . . 52

2.5.2.3 In-Tank Photograph of Tank 241-AP-105

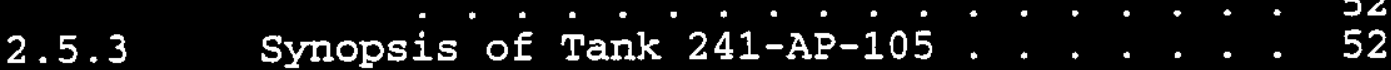

2.6 Tank 241-AP-106. . . . . . . . . . . . . . . . . . 53

2.6.1 241-AP-106 Tank History . . . . . . . . . 53

2.6.1.1 Waste History of Tank 241-AP-106. . 53

2.6.1.2 Temperature History of Tank 241-AP-106

2.6.1.3 Occurrences of Tank $241-\mathrm{AP}-106^{\circ} \cdot \dot{*} 53$

2.6 .1 .4 Psychrometrics of Tank 241-AP-106. 53

2.6.1.5 Historical Sample Analysis Data for Tank 241-AP-106 . . . . . . . . . . . . 54

2.6.2 Current Status of Tank 241-AP-106. . . . 54

2.6.2.1 Inventory Estimate of Tank 241-AP-106

2.6.2.2 Waste Volume Approximations for Tank $241-$

AP-106............... . 54

2.6.2.3 In-Tank Photograph of Tank 241-AP-106

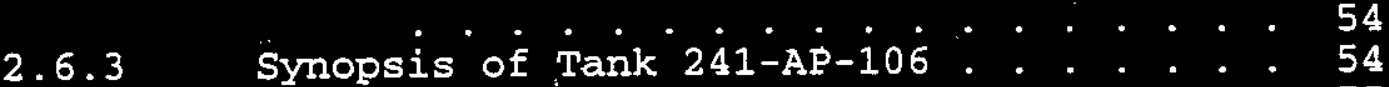

2.7 Tank 241-AP-107... . . . . . . . . . . . . . . . 55

2.7.1 241-AP-107 Tank History. . . . . . . . . 55

$2.7: 1.1$ Waste History of Tank $241-\mathrm{AP}-107$. . 55

2.7.1.2 Temperature History of Tank 241-AP-107

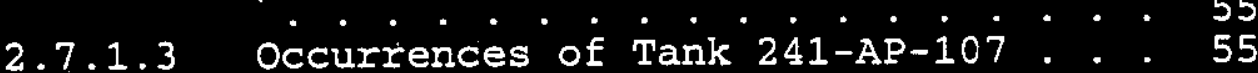

2.7 .1 .4 Psychrometrics of Tank 241-AP-107. 55

2.7.1.5 Historical Sample Analysis Data for Tank 241-AP-107 . . . . . . . . . . . . . . 56

2.7.2 Current Status of Tank.241-AP-107. . . j56

2.7.2.1 Inventory Estimate of Tank 241-AP-107

2.7.2.2 Waste Volume Approximations for Tank 241-

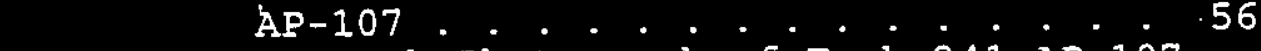

2.7.2.3 In-Tank Photograph of Tank 241-AP-107

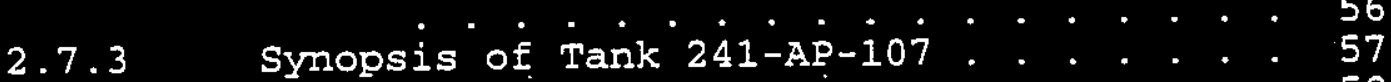

2.8 Tank 241-AP-108 . . . . . . . . . . . . . . . . . . 58

2.8.1 241-AP-108 Tank History . . . . . . . . . 58

2.8.1.1 Waste History of Tank 241-AP-108. 58

2.8.1.2 Temperature History of Tank 241-AP-108

2.8 .1 .3 occurrences of Tank $24 \dot{1-A P}-10 \dot{8} \cdot \cdot \cdot \cdot 58$ 
WHC-SD-WM-ER-315, Rev. 0

2.8.1.4 Psychrometrics of Tank 241-AP-108. 58

2.8.1.5 Historical Sample Analysis Data for Tank 241-AP-108 . . . . . . . . . . . . . . . 59

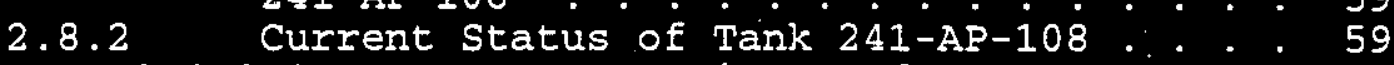

2.8.2.1 Inventory Estimate of Tank 241-AP-108

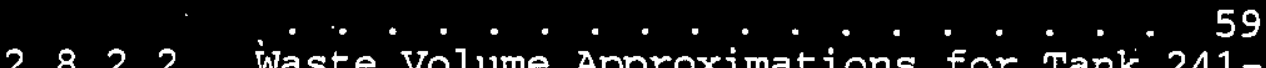

2.8.2.2 Waste Volume Approximations for Tank 241-

$2.8 .2 .3 \quad$ In-Tank Photograph of Tank 241 -AP-108

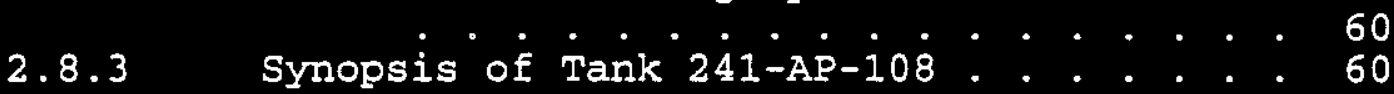




\section{APPENDICES}

Appendix A Appendix $B$ Appendix C Appendix D Appendix $\mathrm{E}$ Appendix $F$ Appendix G Appendix $F$ Appendix I Appendix $J$ Appendix $K$
Glossary

References

Tank Level Histories and Data

Temperature Graph's and Data

Surface Level Graphs and Data

Psychrometric Data

Riser Configuration and Tank Cross Section

Sampling Data

Tank Photographs

Inventory Estimates

Tank Layering Comparison and Data (Supernatant Mixing Mode1) 
THIIS PAGG INTFNHTONALIY
HGTT BLANK 


\section{TRADEMARKS}

Microsoft Excel is a registered trademark of Microsoft Corporation. ENRAF is a registered trademark of Delft Instruments. 


\section{THEIS PAGE INTENTIONAUKT}

LEFT BLANK 


\section{THIS PAGE INTIBNTIONALLY}

LEFT BLANK 


\subsection{Purpose}

The purpose of this historical characterization document is to present the synthesized summaries and evaluations of the historical records concerning the physical, radiological, and chemical composition of mixed wastes stored in underground double-shell tanks and the physical conditions of these tanks. The double-shell tanks are located on the Department of Energy Hanford Site, approximately 25 miles northwest of Richland, Washington. The document will be used to assist in characterizing the waste in the tanks after the current program of sampling and analyzing the tank wastes is completed. A historical review of the tanks may reveal anomalies or unusual contents that could be critical to characterization and post characterization activities.

This report was developed by reviewing the operating plant process histories, waste transfer data, and available physical and chemical data from numerous resources. These resources were generated by numerous contractors from 1945 to the present.

Waste characterization, the process of describing the character or quality of a waste, is required by Federal law (Resource Conservation and Recovery Act) and state law (Washington Administrative Code (WAC) 173-303, Dangerous Waste Regulations). Characterizing the waste is necessary for determining methods to safely retrieve, transport, and/or treat the wastes.

This document is not intended to be used as a total design basis document. Further field investigations of the information may be required before using this data for design purposes or safety analysis.

\subsection{Scope}

The scope of this document covers available information about the wastes contained in the double-shell tanks in the AP Tank Farm. Waste transfer and level data, tank physical information, temperature data, and sampling data have been compiled for this report. A summary of this information is contained in the "Historical Tank Content Estimate for the Southeast Quadrant of the Hanford 200 Areas" (WHC-SD-WM-ER-350). The southeast quadrant document contains all six of the double-shell tank farms. Five tank farms, AN, AP, AW, AY, and $A Z$, are located in the 200-East Area and are shown on the map in Figure 1. SY Tank Farm, located in the 200-West Area shown in Figure 2 , was included in the southeast quadrant because of its double-shell tank construction.

The six tank farms in the southeast quadrant contain 28 tanks: AN Tank Farm (seven 1,160,000-gallon tanks), AP Tank Farm (eight 1,160,000-gallon tanks), AW Tank Farm (six 1,160,000-galion tanks), AY Tank Farm (two 1,000,000-gallon tanks), AZ Tank Farm (two 1,000,000-gallon tanks), and SY Tank Farm (three 1,160,000-gallon tanks). Double-shell tanks are constructed of a primary carbon steel 
cylinder and base inside a secondary carbon steel liner. The secondary liner is encased in a reinforced concrete shell and both liners are covered by a reinforced concrete dome. Depending on the farm, the tops of the tanks are buried between six and eight feet below grade.

\subsection{Approach}

This report was compiled from work performed by ICF Kaiser Hanford Company, Los Alamos National Laboratory, and Westinghouse Hanford Company.

ICF Kaiser Hanford Company reviewed the historical records of the tanks in each of the four quadrants and incorporated the inventory estimates and models of tank layers being developed by Los Alamos National Laboratory into the report.

1.3.1 Surveillance Techniques for the Hanford Mixed Waste Storage Tanks

Surveillance information (e.g., temperatures, waste surface levels, psychrometric data) is used to determine the physical condition of the tanks and the waste: Tank integrity (i.e., whether assumed as sound, assumed leaker, assumed re-leaker) is investigated by monitoring waste levels and the leak detection pits. Waste temperatures can be used to calculate the heat load of heatgenerating radionuclides such as cesium $137\left({ }^{137} \mathrm{Cs}\right)$ and strontium 91 $\left({ }^{91} \mathrm{Sr}\right)$. The instrumentation and methods used for tank surveillance are described in the following sections.

Waste Surface Levels

Four types of liquid level devices are used to monitor liquid levels in the primary tank of a double-shell tank: a level indicating transmitter or Food Instrument Corporation gauge, a level indicator assembly or manual tape, a high-level detector (an FIC gauge in intrusion mode), and the ENRAF 854 ATG liquid level indicator/transmitter. A level indicator assembly or manual tape is used to measure liquid levels in the annulus.

The Food Instrument Corporation gauge is used as the primary liquid level monitoring device with the manual tape as a backup in the primary tank. The high-level detector is used to detect intrusions (i.e., any unexplained addition to the tank like rainwater) within the primary tank. The Food Instrument Corporation gauge consists of an electrode and steel tape that detects liquid on contact. The instruments are scanned hourly on the computer automated surveillance system (CASS). Daily at 7:00 a.m., the automatic FIC reading is loaded on the Surveillance Automated Computer System (SACS). Manual FIC readings are taken at any time of day and are manually entered into the SACS. 
WHC-SD-WM-ER-315. Rev. O
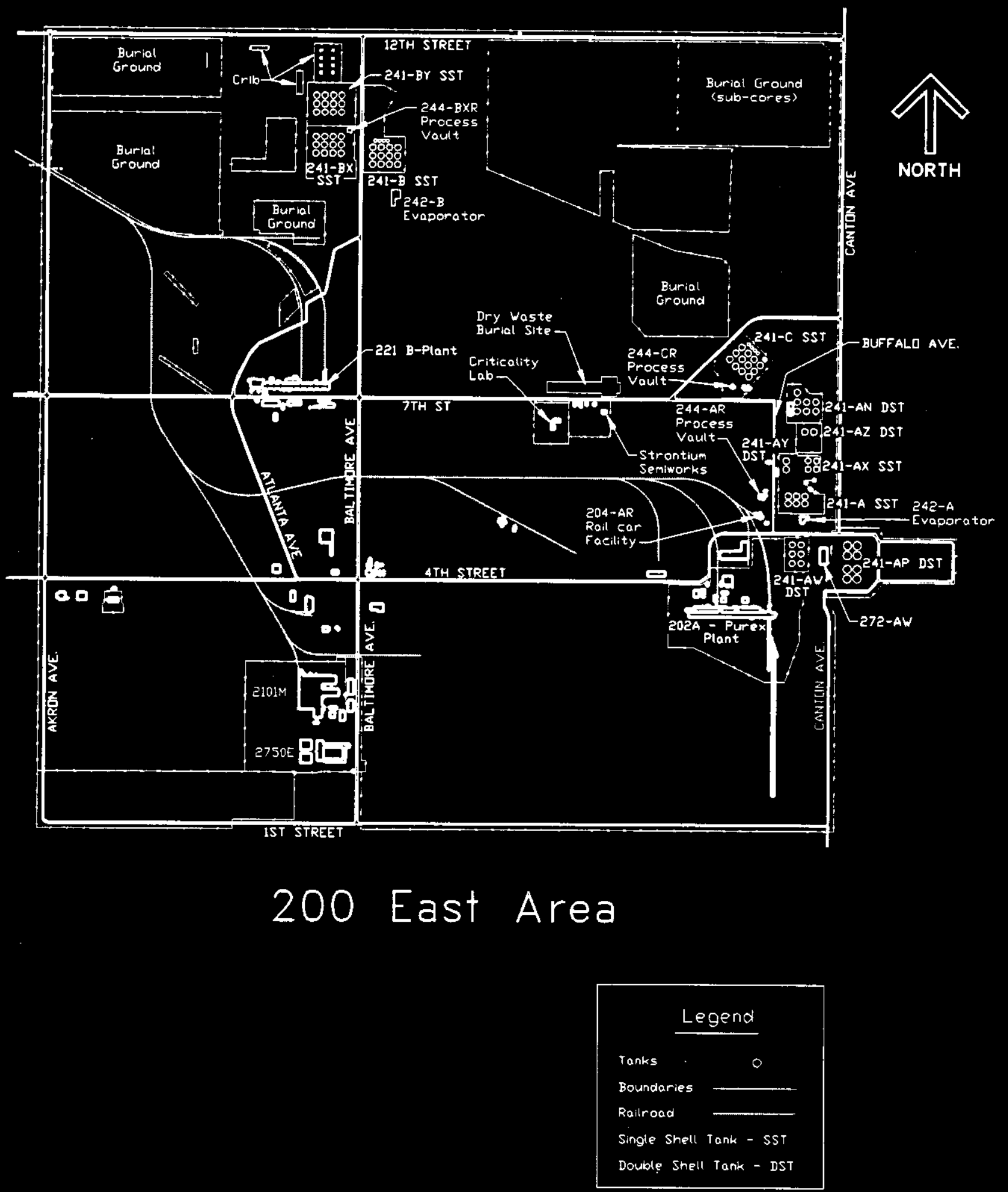

FIGURE 1

- 3 - 
WHC-SD-WM-ER-315, Rev. 0

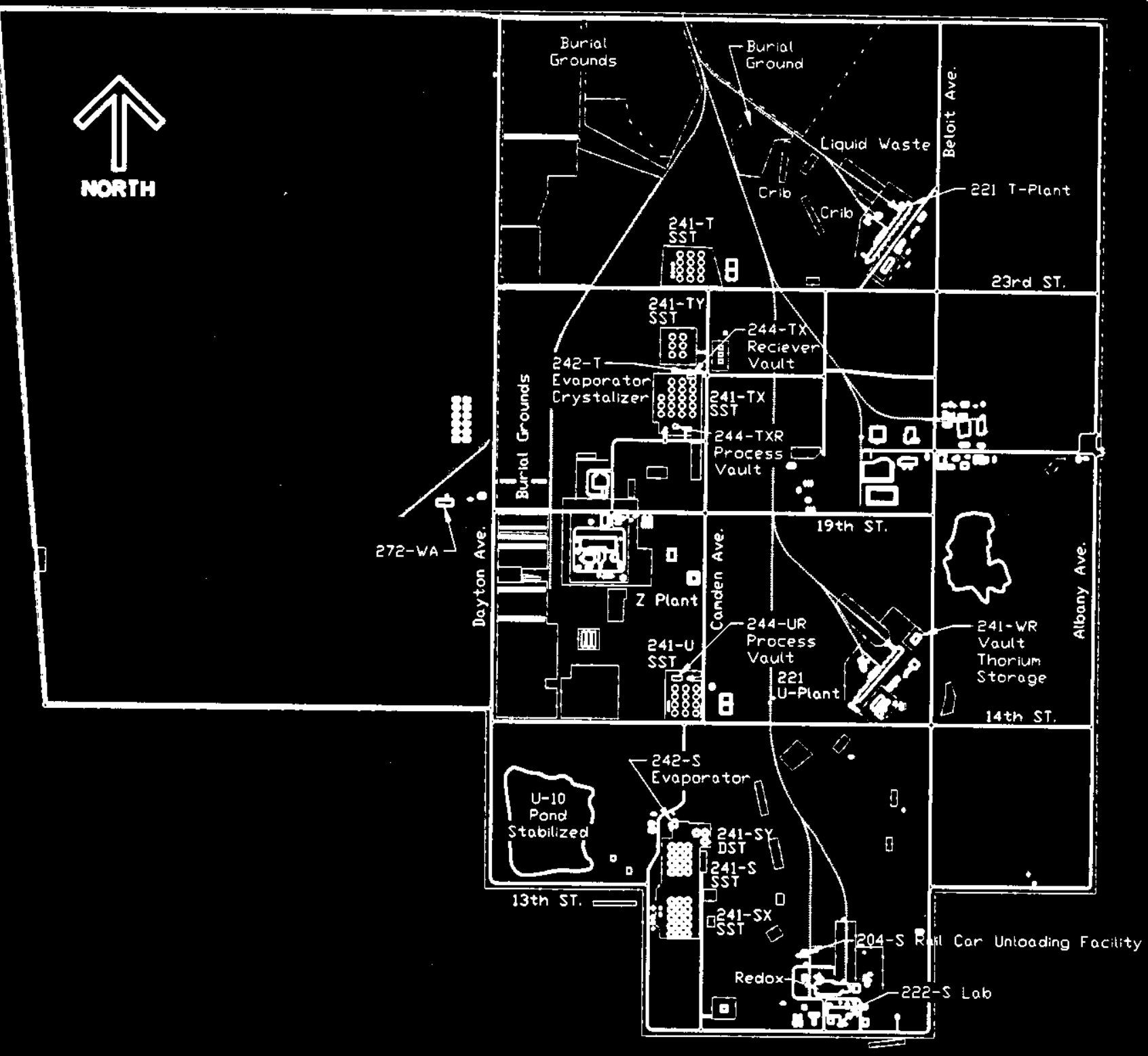

200 West Area

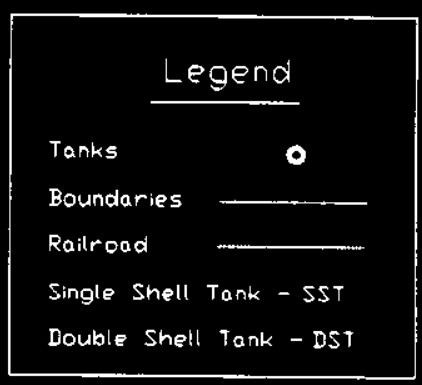

FIGURE 2 
The manual tape flake boxes are used as an access point for measuring liquid levels manually if the automatic system fails or does not exist in the primary tank. A hand crank on the flake box is used to lower the tape probe until liquid is contacted and a liquid level measurement can be obtained (this is similar to the FIC gauge). The flake boxes located over the annulus can be used to lower manual tapes into the annulus to measure any liquid (condensate or leakage from the primary tank) if present.

The ENRAF 854 Liquid Level Indicator/Transmitter has been installed on several tanks and will eventually replace the old level measurement devices. The ENRAF 854 ATG is a microprocessor controlled surface level gauge. Level detection is based on the principle of buoyancy of a non-floating polyethylene displacer. The displacer is attached to a stainless steel measuring wire. The measuring wire is attached to a measuring drum which is fixed to a riser of known elevation. The weight of the displacer is entered into the memory of a force transducer. A second weight of about 1015 grams less than the actual weight of the displacer is entered into the transducer as the control point. An electronic servomechanism turns the measuring drum causing the displacer to move. As the displacer is put in contact with the surface in question, the displacer will exert a smaller force on the transducer due to buoyancy. The displacer is continually lowered until the force exerted on the transducer is equal to the control point. By knowing the elevations of the riser and tank bottom, and the distance from the riser to the surface of the waste, the surface level of the waste can be determined. If the surface level changes, the displacer will. be raised or lowered by the measuring drum depending on the force exerted on the transducer relative to the control point. The measurements are recorded by the Tank Monitoring and Control system (TMACS). Daily at 12:00 a.m., the ENRAF reading is loaded on the SACS.

Sludge levels are determined with a sludge measurement device which consists of a "doughnut." shaped weight attached to a steel tape. The sludge measurement device is lowered into the tank until it stops. Several risers are used to determine the approximate levels of the sludge below, the liquid waste.

- Interstitial Liquid Level (Liquid Observation Wel1s)

The interstitial liquid is the liquid within and underneath the solid waste. Wells to monitor the levels of interstitial liquid were installed only in Tanks 241-SY-102 and 241-AW-103. Gamma, neutron, and acoustic probes may be lowered into the wells to determine the interstitial liquid levels.

Temperatures

The temperatures of the double-shell tanks are monitored with thermocouples. A typical double-shell tank contains approximately 100 thermocouples at a variety of locations including the structural concrete of the tank foundation, walls, and dome; the tank knuckle region; the insulating concrete in the base; the primary steel liner; and the tank waste and vapor space. The aging waste tanks (AY and AZ Tank Farms) have thermocouples in their airlift circulators. 
Thermocouples in the waste are attached to a fabricated assembly called a thermocouple tree. The number of thermocouples on a tree varies between tanks with 18 being the most prevalent. The thermocouples are spaced along the tree so that a temperature profile can be developed. Recently, multi-functional instrument trees (MIT) have been installed into a few of the watch list tanks (Tanks 241-SY-101 and 241-SY-103). These trees measure other properties, such as gas pressure and hydrogen concentration in the vapor space, in addition to the temperature. An MIT contains 22 thermocouples. Details on the status of the thermocouples as of April 1993 can be found in the "Thermocouple status, single shell and Double shell Waste Tanks" (Tran 1993).

The temperatures are recorded either manually on data sheets using a portable potentiometer or by one of several computerautomated systems. These systems include the computer automated surveillance system (CASS), the tank monitor and control system (TMACS), and the data acquisition and control system (DACS). These systems record other data besides temperatures. The surveillance analysis computer system (SACS) is the primary database for storing tank temperature data. The CASS and TMACS record temperatures on some single-shell tanks and double-shell tanks. The DACS only records temperatures in Tank 241-SY-101. In the past, the CASS has recorded temperatures of all double-shell tanks, but it presently records temperatures for the AP Tank farm only. The CASS stores temperature data on magnetic tape. The TMACS presently records temperatures for the $A N$ and SY Tank Farms, but it is planned to operate for all tank farms by September 1996. The TMACs transfers its daily temperature readings to the SACS. Operators manually record temperatures on data sheets for the AP, AW, AY, and AZ Tank Farms. For the AP and AW Tank Farms, the temperatures from data sheets are manually entered into the SACS. The SACS database does not presently store temperature data for the AY and AZ Tank Farms.

\section{Annulus}

The double-shell tank's are designed so that if a leak develops in the primary tank shell, the leak can be detected in the annulus, the space between the steel liners. The annulus monitoring equipment includes a conductivity probe to detect the presence of liquids, a continuous air monitor (CAM) to monitor the level of radiation, and thermocouples to detect an increase in temperature. Either the conductivity probe or the CAM can be inoperable for up to ten days. There is a forced ventilation system for each annulus to aid in heat transfer from the primary tank and containment of waste should a leak in the primary tank occur: A grid of drain channels in the basal concrete collect any leakage and direct it to the leak detection pit. Photographs were taken inside the annulus for inspection purposes.

Leak Detection Pits

Leak detections pits are located next to the tank. Three instruments are used in these pits: a liquid level detector, a thermocouple, and a radiation monitor. The liquid level detector used is a specific gravity dip tube. The dip tubes need to be checked for accuracy periodically; therefore, a 6-inch water level is maintained in the bottom of the 24-inch leak detection pit to allow 
for continuous checking. A thermocouple is placed at the bottom of the pit to detect an increase in temperature from any leaking heat generating radionuclides. A drywell next to the leak detection pit contains a radiation monitoring device to determine background radiation levels and detect leaks.

- Ventilation

The ventilation for the primary tank and the annulus is filtered through high-efficiency particulate air (HEPA) filters and monitored continuously for anomalies. AlI the double-shell tanks are actively ventilated to prevent radioactive releases to the environment. Because the ventilation creates negative pressure in the primary tank, the negative pressure is monitored in the primary tank to prevent the tank bottom from buckling. The annulus vacuum or negative pressure is also monitored for changes that indicate a tank leak or system problems.

Since differential pressure is related to air flow in the tank, differential pressure readings are taken at the exhaust fans, filters, and deentrainers. The vapor space and atmospheric pressures are monitored and compared. High differential pressures are caused by filter plugging or wet filters, while low differential pressure can be caused by filter seal problems or tank pressurization. Continuous monitoring exists for radiation levels at various locations along the ventilation system.

\section{Interior Tank Pressures}

The double shell tanks have certain limiting factors to maintain each tank for its full design life. One limiting factor is tank pressure. The safety limits for pressures vary with the design of the tank, but all double-shell tanks have an operating pressure between -4 and 0 inches of water gauge $(-0.14 \mathrm{psig}$ or $-1 \mathrm{kPa}$ gauge and 0 psig or $0 \mathrm{kPa}$ gauge). The pressure is recorded on continuous strip charts. Refer to the occurrence sections on tank farms and tanks in this document for details on the frequency and duration of the tanks pressurizing.

\section{Evaporation Rate (Psychrometrics)}

Psychrometric data are collected on all the double-shell tanks and high-heat load single-shell tanks. The data are obtained from air moving through the inside of each tank and the annulus. The schedule for obtaining psychrometric data is determined by Westinghouse Hanford Company Process Engineering. Presently, data are recorded about once a month.

The main reason for recording psychrometric data is to determine the rate of evaporation in the tanks. The evaporation rates can be used to account for loss of liquid from the tanks. The data collected in the annulus are used to quantify the moisture content of the air in the annulus. The psychrometric data can also be used to estimate the instantaneous rate of heat transfer into the ventilation systems of the tank and the annulus.

Five pieces of information are required to calculate the evaporation rates and heat exchange in the tank: the wet-bulb and 
dry-bulb temperatures of air entering the tank, the wet-bulb and arybulb temperatures of air leaving the tank, and the volumetric flow rate of air moving through the tank.

Dry-bulb temperatures are obtained using a standara thermometer. Wet-bulb temperatures are measured by a thermometer having the bulb covered by a cotton wick saturated with water. When the wet wick is placed in the air stream, some of the water in the wick is evaporated. As a result, the temperature of the water decreases. The heat loss from the water by evaporation equals the heat gain from the air, and the water temperature stabilizes. This stabilized temperature is the wet-bulb temperature.

Once the wet-bulb and dry-bulb temperatures are known, the properties (enthalpy, relative humidity, specific volume, and humidity ratio) of air at the tank entrance and exit can be determined. By comparing the properties of air at these points, the amount of water added and heat transferred to or from the air stream can be determined.

The raw data have always been measured and recorded in the field. However, methods of calculating the evaporation rate and heat transfer rate have differed. Evaporation rates and heat transfer rates were calculated using a program on a Hewlett Packard Calculator from the time data were first recorded (1977) to 1989 . From 1989 until the present, the raw data were entered into a computer spreadsheet (Microsoft ${ }^{\circ}$ Excel) and the evaporation rate and heat transfer rate were automatically calculated. The program was modified to represent more accurately the calculations made by the American Society of Heating, Refrigerating and Air-Conditioning Engineers, Inc. The main difference is that the density of water is now fit to a curve as a function of temperature to approximate a mass to volume conversion. These calculations have been documented and checked (Wood 1993).

\section{In-Tank Photography}

In-tank photography is another method used to determine the volume of waste. Photographs are used to resolve anomalous measurements inside the tanks and to assist in determining tank integrity. Photographs can also be used to determine sludge and liquid levels, provide a degree of corroboration with other waste monitoring techniques, and be a source of information about foreign objects contained in the tanks. The photographs can be compared to see if wastes in tanks with similar histories have similar characteristics. Currently, photographs are not used to determine waste levels in double-shell tanks because none are leaking.

1.3.2 Approach and Development of Supporting Information Contained in the Historical Tank Content Estimate and Supporting Documents

Extensive historical information was gathered for each tank. This document contains a comprehensive data set on each tank. The data are summarized in the Historical Tank Content Estimate. 
Tank Level Histories

The tank level histories graphically represent waste levels over the lifetime of a tank. They include information such as waste types, in-tank photograph dates, transfer data, and physical data. Information was gathered from various documents and the resulting data, commentary, and dates were delineated on each drawing.

The liquid and solids levels on the tank level history graphs are based on waste volumes. The levels are calculated from the volumes and depicted in height above the bottom of the tank (see Appendix C). The sources of waste volume information include the "Tank Farm Surveillance and Waste Status Summary Reports" (Hanlon 1989 to 1993) and "A History of the 200 Areas Tank Farms" (Anderson 1990). The following formula was used to convert the waste volumes (total gallons) to the respective waste surface levels (total inches) for all tanks in the double-shell tank farms:

FORMULA

$$
\frac{\text { Total Gallons }}{2750 \frac{\text { Gallons }}{\text { Inch }}}=\text { Total Inches }
$$

Temperature Data

The sources of the historical temperatures presented here are the computer automated surveillance system (CASS), the surveillance analysis computer system (SACS), and manually recorded data sheets. The data from these sources span from July 1981 to June 1995 for al1 double-shell tanks. The CASS stored daily temperature readings for all double-shell tank farms on magnetic tapes. This system stored temperatures for most tank thermocouples (e.g., those in the foundation, walls, primary liner, etc.); however, only data from thermocouples inside the tank are presented in this report. The CASS occasionally stored an error code in place of a daily temperature for any errors that the system was programmed to recognize. These codes were removed from the data set before any data were processed. The SACS stored internal temperature readings for all farms except the AY and AZ Tank Farms. This system stored temperature readings for some subset of the internal thermocouples for a given double-shell tank. The temperature data from the SACS are available through the PC SACS software on the Hanford Local Area Network. Daily temperature readings for the AZ Tank Farm were obtained from data sheets in late 1989 and early 1990.

The data from the CASS, the SACS, and the data sheets were imported into Microsoft ${ }^{\circ}$ Excel spreadsheets, then reviewed for suspect data points. Each data set was divided for this review into subsets of suspect and non-suspect temperatures. Because the full temperature data set was extremely large for each tank farm, each non-suspect subset was further reduced by extracting the weekly maximum temperature from the set. However, the full data set was presented for the two tanks in the AZ Tank Farm due to the large 
temperature fluctuations measured in these tanks. The integration of the weekly maximum temperature set and the suspect temperature set is tabulated in Appendix D. For thermocouples with large amounts of suspect data, weekly temperatures were extracted in the same manner. Temperature history plots were developed for most of the thermocouples. A plot was not created for a thermocouple with five or less data points available. A discontinuity in the temperature plot represents a hiatus of greater than three years between two sequential data points. A straight line is used to connect sequential data points spanning less than three years. Linear regressions were performed on the temperature plots within Microsoft Excel to quantify the temperature trend within an individual tank. Other statistics such as the mean, maximum, and minimum temperatures were also determined for each tank (see individual tank sections). The statistics are a function of the non-suspect temperature subset before being reduced to a set of weekly maximums.

The naming convention used for the thermocouples in this report follows that used by the thermocouple status report (Tran 1993). For example, according to Tran, the name of the topmost thermocouple on the tree in Tank 241-AP-101 is TE-101-AP-36. The computer automated surveillance system (CASS) generally uses this naming convention. The CASS excludes the name of the tank farm from the thermocouple name (e.g., TE-101-36) for some tank farms. The surveillance analysis computer system (SACS) uses a positive integer to identify the intank thermocouples (typically, 1 through 18 for an 18-element thermocouple tree). Tran also identifies thermocouples with a positive integer (e.g., TE-101-AP-36 is thermocouple 1). Using the two sets of thermocouple names provided by Tran, the data from the CASS were matched with the data from the SACS for each thermocouple.

Surface Level Plots

The data recorded from January 1, 1991 to June 30, 1995 for the waste surface levels were obtained from the Westinghouse Hanford Company surveillance analysis computer system (SACS). PC SACS is the user interface to the SACS database on a personal computer. The information was parsed in a spreadsheet (Microsoft Excel) and graphed to show waste level versus time. There is a separate graph for the data from each type of measuring instrument. However, data from the manual FIC and automatic FIC gauges are shown together on the graphs with the heading FIC. Current information on the waste surface levels is in Appendix $\mathrm{E}$.

- Tank Profiles and Other Drawings

The drawings in this report were compiled from several sources including as-built drawings and the "Double-Shell Underground waste Storage Tanks Riser Survey" (Anderson 1992). The risers and other penetrations through the tank dome serve several purposes including points of access for monitoring equipment and sampling the waste.

Photographs

The latest tank interior and tank farm aerial photographs were obtained from Pacific Northwest Laboratory and are in Appendix I. The tank farm aerial photographs were labeled to indicate the relative position of each tank in the farm. Larger equipment was 
labeled in the photographs. Photographs of tank interiors have been arranged to show the contents of each tank. The auxiliary equipment shown on each photographic montage was identified if possible and labeled.

- Historical Sample Anaiysis Data

The sampling data were obtained from the Westinghouse Hanford Company Tank Waste and Retrieval Group. Tables of analytical data were made for samples taken prior to september 1989. The information was compiled in the Microsoft ${ }^{\circ}$ Excel computer program to present the data as similarly as possible. The tables include physical data, chemical analysis data by component, and radiological analysis data by component. Other data included with the sample references are summarized as text. Tank historical sample analysis data are in Appendix $\mathrm{H}$.

\section{Psychrometric Plots}

The psychrometric data were obtained from both the manually recorded and electronic records of the Westinghouse Hanford Company Tank Farm Surveillance Group: All the data were entered into computer spreadsheets (Microsoft Excel) and the latest program (Wood 1993) was used to calculate evaporation rates and heat transfer rates. These quantities have been plotted against time on separate graphs (see Appendix F). Evaporation rate versus time was plotted in both gallons per week and inches per week for the tanks. Evaporation rate is used to show the amount of water removed from the tank. Heat transfer rates were plotted in $\mathrm{Btu} / \mathrm{hr}$.

The heat generated in the waste is dispersed into the environment through the ventilation systems of the tank and annulus, through the tank walls into the soil, and through other types of heat loss. The major portion of the heat exchanged with the environment occurs through the ventilation systems. By plotting the rate of heat exchange between the waste and the ventilation systems and reviewing the respective trends in conjunction with the flow rates and ambient temperatures, trends were calculated for the heat generation rate of the waste.

1.3.3 Approach and Development of the Process-Based Historical Tank Content Estimate

The historical characterization inventory estimates of tank contents using process-based historical data, presented in this report, were developed by Los Alamos National Laboratory (S.F. Agnew et al.). The historical estimates are the primary sources of data to characterize tank contents until the tank characterization reports are completed. These reports will incorporate the results of current core sampling and analyses with the historical inventory estimates to present the most current evaluation of waste constituents.

Generating tank content estimates based on historical information involves managing and evaluating large quantities of data. The system of generating the estimates is composed of several parts that are described in the following sections. 
Waste Status and Transaction Record Sumary

The waste status and transaction record summary (i.e., transaction summary) contains all known waste transfer records. Historically, when waste was pumped from plant (process) to tank or tank to tank, a record was kept of the volume and type of waste that was pumped. The transaction summary contains the recorded levels of the solids and supernatant.

The transaction summary is partitioned into four separate sections (i.e., quadrants). The development method of the database, the strategies of subsequent data sets, and the data for the southeast quadrant are in the "Waste Status and Transaction Record Summary for the Southeast Quadrant" (Agnew 1995).

Defined Waste Types

Early in the development of the historical tank content estimates, a need to compile the chemical compositions of all known waste types into one reference document was recognized. The information was compiled by Los Alamos National Laboratory. The Hanford defined wastes document was developed independently of the waste status and transaction record summary and the tank layer model. However, the Hanford defined waste list was used with the model to obtain the tank waste inventory estimates described below.

The Hanford defined wastes document contains the compositions and physical properties for all known types of Hanford waste that were sent to the double-shell and single-shell tanks. For more details on the methods used to develop these waste types, refer to "Hanford Defined Wastes: Chemical and Radionuclide Compositions" (Agnew 1994).

Tank Layer Model

The tank layer model is derived from the waste status and transaction record summary. The purpose of the model is to predict the waste types and solids volumes in each tank. The model is generated by reconciling the reported levels of solids from each tank in the transaction summary with the volume percent of solids expected for the primary additions of waste. The transaction summary contains records of the types of waste that entered each tank and the corresponding increase in the solids volume from each waste entry. Not all transactions that occurred were recorded in the transaction summary because records were lost and transfers were unrecorded or mis-recorded. However, the tank layer model is representative of the waste because two crucial pieces of information required for the inventory estimates are well represented; the primary waste additions and measurements of solids levels.

The tank layer model associates a solids volume percent with each waste type. The solids volume percent was derived by comparing the observed change in the solids. volume with the primary additions recorded in the waste status and transaction record summary. In waste types that lacked an adequate number of solids measurements to develop a solids volume percent, a nominal value was assigned based on similarity to other waste types that had adequate data. If the predicted volume percent solids was different from the measured 
volume, the difference was indicated as an unknown gain or loss of solids for the tank. The unknown losses and gains were designated unknown layers and were assigned to a best estimate defined waste type when possible for the composite inventory estimates. Layers that were not assigned a best estimate waste type were not included in the composite tank estimates. The unknown layers were depicted on the tank layer model graphs but the volume of the unknown layers was assigned to the supernatant volume and not included as a volume in the solids inventory estimate.

A layer "set" in the tank is assumed not to move or change volume. The layers are presented in chronological order from the bottom of the tank to the top. Lateral variations were not accounted for and only average layer thickness can be derived from the model.

There are two main sources for variations in the solids volume percent in a single waste type. First, there is an inherent variability in the process stream as the process evolves. Second, solids can be added to or removed from a tank by inadvertent entrainment during supernatant transfers. In addition to these sources of variation, there are a number of other minor sources of solids changes such as compaction, subsidence, and dissolution of soluble salts by later additions.

Diatomaceous earth and cement were added to immobilize residual supernatant in tanks where pumping the liquid was not feasible. The conversion factor in the tank layer model is $160 \mathrm{gal} / \mathrm{ton}$ for diatomaceous earth and 120 gal/ton for cement. Cement and diatomaceous earth are not included in the composite inventory estimates.

It was assumed that any solids that accumulated were saltcake or salt slurry from the time a tank became a "bottoms" receiver to the end of the evaporator campaign. Saltcake can be any one of seven different types depending on which evaporator campaign created it. For saltcake accumulation, it was assumed that all of the solids reported were saltcake. The results of the model are presented graphically with the inventory estimate in Appendix $\mathrm{K}$.

Supernatant Mixing Model

Los Alamos National Laboratory developed the supernatant mixing model that describes a "supernatant" portion of each tank as a combination of Hanford defined waste supernatants that have been concentrated by some factor (see Appendix K). The following fundamental criteria were used for this model:

1) Ideal mixing of the total volume of each tank throughout its history was assumed ( $i . e .$, no excluded volume for remaining solids layers).

2) Transactions were added to the waste status and transaction record summary to resolve unknown transactions for each guarter according to a set of rules. Therefore, an updated transaction summary was used that will be published later. 
3) Not all of the saltwell liquid sources for 1978 through 1995 are known. All unknown saltwell liquid is now assigned to water for this estimate.

Inventory Estimates

The end result of combining the information from the tank layer model, the supernatant mixing model, and the Hanford defined wastes document is the tank inventory estimate (see Figure 3). When the volumes and compositions of each waste type in a particular tank are determined from the various sources of information, the total chemical inventory in the tank is calculated.

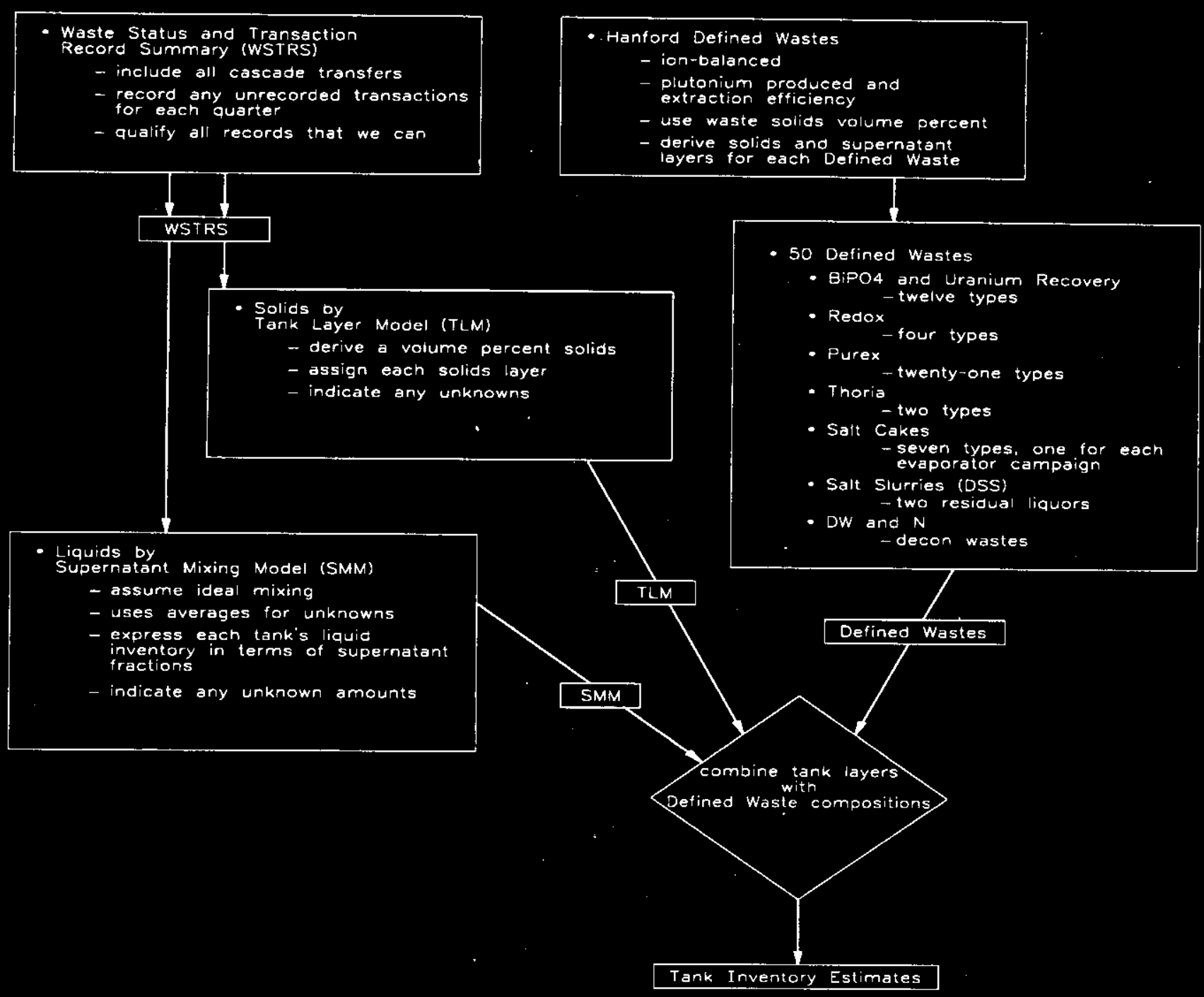

Figure 3. Schematic of Inventory Estimate strategy. 
The tank inventories used data from the Tank Farm Surveillance and Waste Status Summary. Reports (Hanlon 1993). The inventories represent the estimated contents of the tanks as of January 1, 1994. The results for individual tanks are presented in tabular form and tanks that have unassigned' waste layers are indicated.

The inventory estimates developed by Los Alamos National Laboratory were checked for accuracy against existing data from current sample analyses, thermal and physical models, and historical records. When the checking process reveals discrepancies between the estimates and the existing data, the models may be reevaluated. Thus, the process of generating estimates will be iterated and the associated errors will be reduced over time as knowledge of the tank waste operations increases.

\subsubsection{Assumptions}

The quality of the historical information available for the tanks often requires assumptions and judgements on the applicability and validity of the data. Some assumptions were used to interpret the data.

Temperature Data

All the temperature data obtained by ICF Kaiser Hanford Company were reviewed for suspect data points. Data that deviated from expected trends were marked as suspect and comments added accordingly. Suspect data points satisfied one of the following criteria: 1) a temperature less than $20^{\circ} \mathrm{F}, 2$ ) a large scatter in temperatures from one day to the next over a continuous period of time, 3) an individual temperature reading not following the trend of other temperatures recorded during the same period, 4) disagreement between an individual thermocouple reading and the reading of thermocouples located above and below it on the tree, or 5) disagreement between data recorded at the same time from the CASS, the SACS, and/or manually recorded data sheets. All temperatures less than $20^{\circ} \mathrm{F}$ were removed from the data set and do not appear in the tables in Appendix D. Temperatures that deviated more than 15 percent from the data trend were assumed to be suspect from criterion 3 above. A clear indication that the data acquisition system recording the temperatures was in error was that the suspect data points were repeated concurrently for other tanks in the same farm. This behavior occurred in data from the CASS.

The instrumentation allowing manual recording of temperatures on data sheets is assumed to be calibrated the same as the instrumentation used by the CASS and the tank monitor and control system.

- Sampling Data

When the sampling data were compiled, a sample was considered to be a solids sample if it contained greater than 308 solids.

Psychrometric Plots

Psychrometric data are collected randomly once a month. The data from air entering the tank would not always be collected at the 
same time as the data from air exiting the tank. Therefore, the calculated evaporation rate and heat transfer rates may not truly represent the monthly, weekly, or daily average. The data represent only one point per month. By combining all these points, a trend is assumed to exist for each tank.

The program used to calculate evaporation rates contains some assumptions. The liquid waste temperature is assumed to be the same temperature as the outlet $d r y-b u l b$ temperature in order to convert the mass of liquid in the air stream into a volume equivalent (gallons/week). Standing liquid is assumed to exist in order to convert the volume equivalent evaporation rate into a liquid level evaporation rate (inches/week).

\section{Inventory Estimates}

The result of combining the information from the tank layer model, the supernatant mixing model, and the Hanford defined wastes document is the tank inventory estimate. Waste stream compositions (Hanford defined wastes) were based on process flowsheet information and previous analytical data. The assumptions for the other precursors to the inventory estimates are described below.

\section{Tank Layer Model}

The formation of solids in the wastes varied by type and was determined individually by examination of the historical fill records. The volume of solids determined must conform to physically attainable systems $(e . g$. , waste volumes contained in the tanks do not exceed the tank capacity and negative void fractions are not possible).

The solids were assumed to be evenly distributed throughout the slurry during their transfer. In the tank layer model, the distribution of solids is chronological and volumetric, but does not attempt to predict the solids stratigraphy (configuration of layers). Localized mounding and pocketing in various areas of the tank may have occurred due to phenomena around the inlets and outlets (e.g., transfers through pumps removing or depositing sludge). These phenomena would have affected only a small fraction of the tank area and do not impact the overall results of this study. However, spatial variability within the physical configuration and process history is acknowledged. No method is currently available that would adequately model this discrepancy. An effort is underway to develop this concept further and incorporate it into the tank characterization process, but it is incomplete at this time.

Solids fxom a specifically designated waste type are assumed to be homogeneous. Variation and layering of the sludge due to differences in component concentration between batches, precipitate particle size, and particle density were quite likely. The effect of these factors on the distribution of components within the solids is unknown. However, the effect was assumed to be small and not detectable with the present sample collection and analysis process.

Solids were not assumed to be compacted; therefore, compaction was not accounted for in the study. Although compaction probably 
occurred in the history of the tanks because additional layers of solids formed atop each other, the degree of compression is unknown and, therefore, was not incorporated.

Density and other physical characteristics of the solids were determined from individual waste types using available information (e.g., process flowsheets), or were based on approximate physical analogues.

The capacity of each tank was assumed to never have been exceeded during transfers. Tank capacity is dependent on fill status, fill order, and tank type.

Tank transfers were assumed to be normally free of particulates. Therefore, the majority of solids were confined to their initial receiver tanks and were not sent to cribs or transferred extensively between tanks. This assumption was based on the following assumptions. The pumping equipment typically was not designed to transfer substantial quantities of solids (except when sluicing or specifically transferring solids). Monitors on the crib discharge lines would have indicated significant solids contamination, and the tank farm operating procedures would have prevented discharging solids to the ground.

\section{Supernatant Mixing Model}

Los Alamos National Laboratory developed the supernatant mixing model that describes a "supernatant" portion of each tank as a combination of fanford defined waste supernatants that have been concentrated by some factor. (see Appendix K). The following assumptions were used for this model:

1) Ideal mixing of the total volume of each tank throughout its history was assumed ( $i . e .$, no excluded volume for remaining solids layers).

2) Transactions were added to the waste status and transaction record summary to resolve unknown transactions for each quarter according to a set of rules. Therefore, an updated transaction summary was used that will be published later.

3) Not all of the saltwell. liquid sources for 1978 through 1995 are known. All unknown saltweli liquid is now assigned to water for this estimate.

\subsection{Historical Information Quality}

The quality of information compiled for this report varied greatly. Some data came from published documentation. Other data were retrieved electronically from various Westinghouse Hanford Company databases. The quality of data used in the estimates is described in the following section.

Waste (Level) Histories

The waste history narrative contains a general description of the waste types contained in a specific tank from receipt of the 
first waste transferred to the present. The current status of the tank is also presented. The history is not designed to be a detailed summary of every waste type and transaction for a tank. The waste types are general and are usually given only for transactions of sufficient volume to significantly impact the total volume of the tank (e.g., a transaction that increased the waste volume by 3,000 gallons in a tank originally containing a waste volume of 6,000 gallons would be considered significant. The same amount added to a tank containing 600,000 gallons of waste would not be considered significant.). For a more complete record of transactions and types of wastes involved, refer to the "Waste status and Transaction Record Summary for the Southeast Quadrant" (Agnew 1995).

The accuracy of the tank waste histories relies on the completeness of the Westinghouse Hanford Company document "A History of the 200 Area Tank Farms" (Anderson 1990); the monthly Westinghouse Hanford Company, Atlantic Richfield Hanford Company, and Rockwell Hanford Operations waste summary reports; and the "Waste Status and Transaction Record Summary for the Southeast Quadrant" (Agnew 1995).

Surface level readings may be affected by plummet (e.g., manual
tape) error. flushing water accumulation, irregularities, and gas generation. Crystalline wastes (i.e. saltcake) can build up on the end of the plummet gradually and contact the waste which indicates a false surface level increase. Significant level discrepancies occur when the buildup breaks off or when the measuring instrument plummet is flushed to remove the saltcake buildups. Flushing the Food Instrument Corporation gauge, manual tapes, or any other equipment may cause accumulated wash water to collect under the plummet which indicates a false increase in the overall volume of waste within the tank. Surface level readings are often difficult to obtain from tanks with a relatively dry saltcake surface. Some tanks have crystalline buildup on internal tank equipment (i.e., pumps, thermocouples, and other protruding equipment). As the supernatant is pumped from the tanks, the crystalline structure may remain attached to the equipment and be suspended above the liquid. Therefore, an accurate surface level measurement would be difficult if the breakup of the crystalline structure was inconsistent and a nonuniform waste surface was created. Steel tapes that are bent or warped from operation or those discarded on the waste surface are other sources of altered surface level readings.

\section{Temperature Data}

The quality of data from the computer automated surveillance system (CASS), surveillance analysis computer system (SACS), and manually recorded data sheets has not been verified. One exception is the subset of data from the SACS that had been reviewed. Engineering judgement formed the basis of removing suspect data from the original data set to produce a set of non-suspect data.

In the temperature tables of Appendix D, suspect data are labeled $S$, transcribed data are labeled $T$, and good data are labeled G. This quality indicator is used by the custodians of the SACS. The labels $S$ and $G$ both indicate that a review has taken place 
(either by the SACS custodians or during creation of this report). Only data already labeled transcribed ( $T$ ) and good (G) were retrieved from SACS. If interpreted as suspect during the compilation of this report, the data from SACS that originally were labeled $\mathrm{T}$ or $\mathrm{G}$ were relabeled with an $S$ or $S$ (G in SACS), respectively. Otherwise, the data retained the original label. Data from the CASS or the data sheets were labeled with a $T$ when retrieved for this report, assuming such data had never been reviewed previously. If interpreted as suspect during the compilation of this report, the data from the CASS and data sheets were relabeled with an $S$; otherwise, the label remained $T$.

Surface Level Data

Data from the surveillance analysis computer system (SACS) were obtained electronically from the Westinghouse Hanford Company surveillance group and were plotted. The data are actual surface levels recorded from the surveillance equipment. If the surveillance equipment in a particular tank riser was removed from service, the readings may show a level change when a new instrument and/or riser is used, especially if the waste surface shows severe heterogeneity.

The data used to produce the plots and the data obtained from the surveillance group have been verified as identical. However, errors in the data prior to the exchange of information could still exist. The SACS data were understood to be unverified prior to the exchange. Westinghouse Hanford Company qualified the data with $G, S$, T for good, suspect, and transcribed, respectively. The criteria for determining these gualities are unknown.

\section{Riser Configuration}

The riser configurations are based on the design drawings and engineering change notices dated before August 3, 1995. The riser configuration document (WHC-SD-RE-TI-093, Rev. 3) was used as a guide; however, the design drawings and engineering change notices take precedence. The tables in Appendix $G$ contain a riser number, diameter, and description of each riser and nozzle. A brief explanation and date of the pertaining engineering change notice, if applicable, is in parentheses, under the description and comments heading for each riser. Available risers are labeled spare and do not have any attached equipment; this includes observation ports.

\section{Photographs}

The photographic montages were made from the latest available photographs of the tank interiors and are in Appendix I. In some tanks, photographs have not been taken since the early 1970's. Some tanks had fogging problems in the vapor space which prevented use of the latest photographs. Sometimes, reviewing the older photographs was useful to determine changes in waste volume or equipment. Montages made from older photographs may not be indicative of current tank contents, especially if the tank had been pumped since the last time a photograph was taken.

Psychrometric Data

The psychrometric data obtained from Westinghouse Hanford Company came from sheets of manually recorded data before 1989 and 
computer files after 1989. Agreement between the raw data obtained from Westinghouse Hanford Company and the psychrometric plots has been checked. However, errors in the data prior to the exchange of information may still exist.

The raw data could contain exrors. The inlet (ambient) conditions could have been measured when the operators first entered the farm and not immediately before the outlet measurements of each tank were taken. The volumetric flow rate is measured using a pitot tube. As the flow rate decreases, the pitot tube measurements become less accurate. Minteer (1994) demonstrated a high degree of uncertainty in determining changes in moisture content as air passed through a primary tank and annulus. Even though Minteer (1994) discusses uncertainties with Tank 241-C-106 (a single-shell tank), some of the same principles and errors exist in the data obtained from the double-shell tanks. Dodd (1994) suggested that collecting psychrometric data should cease for tank annuli until more rigorous examinations determined whether collecting the data was worthwhile.

Other Iimitations, besides the fact that the calculated data represent only one recorded data point per month, should be considered before drawing any conclusions about the data. The heat transfer rate only expresses heat transferred to or from the air stream; it does not include other methods of heat transfer such as conduction through the soil or radiant heat transfer. Floating objects or a surface crust could exist in the tank which would change the surface area available for evaporation and possibly change the temperature of the liquid.

When the raw data varied from certain physical laws, the data were labeled suspect and were not included in the graphs. The data point was labeled suspect if the wet-bulb temperature was greater than the corresponding dry-bulb temperature or if the flow rate was extremely high compared to the rest of the data. A brief explanation of why the data point was labeled suspect is in the comment column of Appendix F. Data labeled as suspect may vary between the originally labeled data from Westinghouse Hanford Company and the data after review during the creation of the supporting documents.

Historical Sample Analysis Data

The historical sample analysis data refer only to information obtained before September 1989 (see Appendix H). The data (results from analyzing the samples and details about the samples) have not been validated by the characterization program. When validated, the data will be reissued in a later revision of the Historical Tank Content Estimate report and supporting documentation. The sample data often were retrieved from memoranda indicating the results of the sample analysis. The analytical methods, holding times, quality control information, and location at which the sample was taken were not provided. The scope of analysis frequently was quite narrow which limited the available data set. However, the data sets are the only available historical information on chemical characterization.

The data obtained from the sample analysis records vary widely in type, quality, and scope. The data range from a process control 
analysis (e.g., checking the plutonium separation process by analyzing a grab sample for a few selected components) to a total characterization suite performed on a core sample. There are no specific criteria for determining quantitative data quality for the information incorporated into the supporting Document. The information sources were assessed to determine if the samples were from the waste in the tank. The available data were assessed for suitability and consistency with this report and selected information was incorporated.

\subsection{Southeast Quadrant Background}

The double-shell tanks were built between 1968 and 1986 to provide interim storage for high-level nuclear wastes. Processes such as plutonium separation from spent nuclear fuel and uranium metal recovery generated the millions of gallons of mixed (radioactive and hazardous) waste stored within these tanks.

The most common waste stored in the tanks in the southeast quadrant is dilute waste. However, many tanks in the quadrant currently store wastes from the Plutonium Finishing Plant process and other onsite separation processes. Much of the waste is scheduled for concentration in the 242-A Evaporator.

\subsubsection{Waste Generating Plants and Processes}

Although not all of the processes listed below contributed waste directly to tanks in the southeast quadrant, the waste they generated could have been transferred indirectly tank-to-tank. The plants and processes that generated waste now contained in the single-shell and double-shell tanks are presented in chronological order. Typically, the name of the plant and the process are symonymous.

T Plant

T Plant was the first full-scale separations plant at Hanford. It was constructed between June 1943 and October 1944 and was used as a separations facility for irradiated production reactor fuel until 1956. T Plant was designed to separate plutonium from uranium and the bulk of fission products in irradiated fuel through the bismuth phosphate separations process. The 224 (Concentration) Building, adjacent to $T$ Plant, was part of this process. Since 1957, T Plant has been used as a facility for repair and decontamination of equipment. The facility was modified in 1978 to store pressurized water reactor (PWR) core II fuel assemblies. T Plant facilities have been used for repairing, testing, shipping, decontaminating, decommissioning, and storing contaminated tools and equipment from locations both onsite and offsite.

The following wastes were sent to the tank farms: metal waste (MW), decontamination waste (DW), first-cycle decontamination waste (1C), second-cycle decontamination waste (2C), and 224 waste.

B Plant

B Plant was constructed between August 1943 and February 1945 and was used for the bismuth phosphate process until 1952. B Plant 
was designed to use the same bismuth phosphate separations process that was used at $T$ Plant. This process used both bismuth phosphate and lanthanum fluoride. The 224 (Concentration) Building, adjacent to B Plant, was part of this process. In 1968, B Plant became a waste fractionation plant after it was modified to remove ${ }^{137} \mathrm{Cs}$ and ${ }^{90} \mathrm{Sr}$ from PUREX acid waste, high-level supernatant, and sludge from self-boiling liquid wastes.

The following B Plant wastes were sent to the tanks: B Plant high-level waste (B), B Plant flush waste water (BFSH), B Plant lowlevel waste (BL), complexant concentrate (CC or CCPL), complexed waste (CPLX), fission product waste (FP), metal waste (MW), REDOX ion exchange waste processed at B Plant (RIX), ion exchange waste (IX), strontium sludge (SRS), cell 5 tank 6 waste (5-6), first-cycle decontamination waste (1C), second-cycle decontamination waste (2C), and 224 waste.

\section{Bismuth Phosphate Process}

$B$ Plant and $T$ Plant used the bismuth phosphate extraction process to remove plutonium from uranium fuel elements. The 224 Buildings were also part of this process. The waste that resulted was a metal waste (MW) that still contained $90 \%$ of the fission products and $99 \%$ of the original uranium. The $M W$ was sent to specific tank cascades in the 200-East and 200-West Areas. Firstcycle decontamination waste (1C), second-cycle decontamination waste (2C), and 224 waste were also stored in other underground waste tanks in the 200 Areas.

Plutonium Finishing Plant

The Plutonium Finishing Plant (PFP) or $Z$ Plant, previously called Plutonium Recovery and Finishing Operations, began operating in late 1949 to process plutonium and prepare plutonium products. The PFP operations included plutonium handling, plutonium reclamation, plutonium conversion, decontamination, and decommissioning.

Waste from the Plutonium Reclamation Facility (near PFP) and $\mathbf{z}$ waste from the remote mechanical "C" Iine process were sent to tanks.

Uranium Oxide Plant

The 224-U Building was completed in 1944 as part of the U Plant complex. The building was converted to a uranium oxide (UO $\left.{ }_{3}\right)$ plant in 1951. The 224-UA Building was constructed in 1957 with six installed calciners. The $\mathrm{UO}_{3}$ plant was capable of handling the uranyl nitrate hexahydrate (UNH) stream from REDOX, U Plant, and PUREX. The $\mathrm{UO}_{3}$ plant was shut down in 1972 but restarted in 1984 with the feed lines from REDOX and the U PIant canyon disconnected. Since 1984, there have been 17 campaigns at the plant averaging eight days each. The plant can calcine UNH much faster than it is produced by the PUREX plant. Final deactivation was ordered for the plants in 1992. In April 1993, the $\mathrm{UO}_{3}$ plant resumed operations to convert 200,000 gallons of remaining UNH to uranium oxide powder. A final deactivation plan for the facility was prepared in the summer of 1993 and will be carried out in the next few years. 
REDOX

The Reduction and Oxidation extraction. (REDOX) plant was built between May 1950 and August 1951 and operated until July 1967. Uranium and plutonium were extracted from solid uranium metal slugs that were irradiated in the Hanford reactors or piles. Uranium and plutonium were extracted with methyl isobutyl ketone (hexone) solvent in a continuous solvent extraction process. The REDox process was the first at Hanford to recover both plutonium and uranium.

The following wastes were sent to the tank farms: REDOX process waste resulting from the dissolution of aluminum and zircaloy fuel element cladding, REDOX coating waste (CWR), REDOX waste (R), the supernatant portion of waste generated by the REDOX process, and REDOX supernatant (RSN). RSN is found above the sludge in the underground storage tanks that received waste from REDOX.

U Plant

U Plant (221-U) was built as one of three original bismuth phosphate process facilities, but it was not used for that purpose. $U$ Plant was modified extensively and used for the uranium recovery process, operating from 1952 to 1958. The main canyon at U Plant currently stores failed equipment.

Uranium in waste from the $\mathrm{BiPO}_{4}$ process initially was stored in the single-shell tanks. Later, the waste was sluiced, dissolved in nitric acid, and processed through a solvent extraction process to recover the uranium. The process yielded approximately 1.4 gallons of UR waste for every gallon of metal waste originally stored. The increase in waste volume made developing technology to reduce the volume of stored waste a priority. This need resulted in developing and implementing the ferrocyanide scavenging process.

The waste sent to the tanks from U Plant was called tributyl phosphate (TBP) even though it contained very little TBP. Therefore, to eliminate confusion, the waste will be referred to as uranium recovery (UR) waste in this report.

\section{Strontium Semiworks}

The Strontium or Hot Semiworks facility (i.e., C Plant) was built in 1949 as a hot pilot plant for the REDOx process but was converted to a pilot plant for the PUREX process. In 1960, the plant was reactivated and used as a process demonstration for the conversion of B Plant to a waste fractionation facility. The plant was used to recover ${ }^{90} \mathrm{Sr}$, promethium $147\left({ }^{147} \mathrm{Pm}\right)$, and ${ }^{144} \mathrm{Cs}$ from PUREX waste. The building and the site have been decontaminated and decommissioned.

Waste sent from the Strontium Semiworks to the tank farms was known as hot semiworks (HS), strontium semiworks (SSW), and fission product waste (FP).

PUREX

The process used in the Plutonium Uranium Extraction (PUREX) plant (i.e., A Plant) was an advanced solvent extraction method using tributyl phosphate in a paraffin hydrocarbon solvent to recover 
uranium and plutonium from nitric acid solutions of irradiated uranium. The PUREX plant, built between April 1953 and April 1955, operated until 1972 when it was closed for 11 years. Two thorium campaigns were conducted between 1966 and 1971. The irradiated waste was similar to the PUREX waste, but it contained thorium and uranium 233 rather than uranium and plutonium found in typical PUREX waste. The PUREX plant was restarted in November 1983 but was shut down in 1988 after a safety violation. It was shut down several times between 1988 and 1990 for various reasons. In October 1990, the plant was put on standby and was identified for closure in December 1992 by Secretary of Energy James Watkins.

The following PUREx wastes were sent to the tank farms: cladding (coating) waste (CW or CWP), organic wash waste (OWW). neutralized PUREX acid waste (P), low-level waste from PUREX (PL), PUREX sludge supernatant liquid (PSS), organic wash waste (CARB), cesium feed (CF), and inorganic wash waste (IWW).

\subsubsection{Waste Management Operations}

This section describes the different methods used to concentrate waste in the 200 Area. Evaporating, concentrating, and scavenging are all methods used to reduce liquid volumes or precipitate solids out of supernatant. The operations are presented in chronological order.

\section{2-T Evaporator}

The 242-T Evaporator was built in the early 1950's to reclaim nonboiling waste storage capacity in existing tanks. The evaporator was shut down in the summer of 1955 and modified for tributyl phosphate scavenging, although scavenging was never performed in this evaporator. The evaporator was restarted in 1965 and operated until 1976. Tank 241-TX-118 was the feed tank for this evaporator.

The following wastes were sent from the 242-T Evaporator to the tanks: slurry product from the evaporator bottoms (EB), evaporator feed (EF), and terminal liquor (TL).

\section{2-B Evaporator}

The 242-B Evaporator, south of the B Tank Farm, started operating in December 1951. It received feed waste until November 1954, was shutdown in September 1955, and was never reactivated. Tanks 241-B-106 and -105 were used as the feed tank and bottoms tank, respectively. The evaporator operated at atmospheric pressure.

\section{Ferrocyanide Scavenging}

Ferrocyanide scavenging was developed to reduce waste volumes. The ferrocyanide flowsheet was first tested in $U$ Plant in October 1953, and in-farm scavenging was completed in March 1956 and December 1957 for the 200-West and 200-East Areas, respectively. The ferrocyanide scavenging program was designed to remove fission products such as ${ }^{137} \mathrm{Cs}$ (half life $=28.6 \mathrm{yx}$ ) and ${ }^{90} \mathrm{Sr}$ (half life $=30.2$ yr) from the uranium recovery and $1 \mathrm{C}$ wastes as a precipitate. After the ${ }^{137} \mathrm{Cs}$ and ${ }^{90} \mathrm{Sr}$ precipitates settled, the clarified supernatant was sampled and disposed of in cribs. 
In-Tank Solidification

The primary function of the in-tank solidification systems was to concentrate the nonboiling waste directly inside of specially designed tanks. These tanks produced a saltcake slurry. Each system included a heat exchanger for water evaporation and a series of bottoms tanks. The first in-tank solidification unit (ITS \#1) operated with an airlift circulator through Tank 241-BY-102, and the second in-tank solidification unit (ITS \#2) operated with a similar circulator through Tank 241-BY-112. Tank 241-BY-112 also contained a $4,000 \mathrm{~kW}$ electric immersion heater. Tank 241-BY-101 contained an in-tank solidification prototype that was used for demonstration purposes only. In-tank solidification units 1 and 2 began operating in 1965 and 1968, respectively. In 1971, ITS \#1 became the cooler for ITS \#2. Both units were shut down in 1974 .

\section{RFDOX Concentrator}

The REDOX concentrator was used to reduce the volume of dilute 200-West Area tank farm wastes by removing water. The concentrator received tank farm wastes from July 28, 1967 until June 30, 1972. Cell $D$ in the REDOX process was the concentration and neutralization section of the plant.

\section{2-S Evaporator-Crystallizer}

The 242-S Evaporator-Crystallizer began operating in November 1973 and was shut down in 1981. It was designed as a reduced pressure (i.e., partial vacuum) evaporator-crystallizer. Aqueous salt wastes were converted in the evaporator to salt crystals for storage in underground tanks in the $S$ and $S X$ Tank Farms.

The following waste types were sent to the tanks from the 242-S Evaporator: noncomplexed waste concentrated until the solution was nearly saturated with sodium aluminate known as double-shell slurry feed (DSSF), slurry product from the evaporator bottoms (EB), evaporator feed (EF), Hanford defense residual liquor (HDRL), partially neutralized feed waste (PNF), and terminal liquor (TL).

\section{2-A Evaporator-Crystallizer}

The 242-A Evaporator-Crystallizer was constructed in 1976 and began operating in March 1977 with a design life of ten years. In 1987, design changes were developed to extend the facility's operating life by ten years. The evaporator was shut down in April 1989 because of regulatory issues, but was restarted in May 1994 after extensive modifications.

The following wastes were sent to the tanks: B Plant low-level evaporator bottoms (BLEB), double-shell slurry feed (DSSF), evaporator feed (EF or EVAP), evaporator bottoms (EB), and terminal liquor (TL).

\subsubsection{Miscellaneous Waste Sources and Equipment}

Wastes from various other souxces on the Hanford site have been added to the tanks. Some wastes are from the 300 Area, 100 Area production reactors, various laboratories, and catch tanks. Unique contents added to single-shell tanks included laboratory wastes; 
diatomaceous earth; Portland cement; shroud tubes; ceramic balls; experimental fuel elements; and relatively small amounts of enriched uranium, plutonium, cobalt; and natural uranium.

The following wastes were added to the tanks: Hanford defense residual liquor (HDRL), Hanford laboratory operations waste (HLO), water $\left(\mathrm{H}_{2} \mathrm{O}\right), \mathrm{N}$ Reactor waste (N). from phosphate decontamination process, and noncomplexed waste (NCPL).

\section{Critical Mass Lab}

The critical mass lab was used to develop data on the factors necessary to make a criticality or self-sustained nuclear reaction occur. Because plutonium was the key radioactive element on the Hanford site, the research was based on plutonium criticality safety in various fuel cycles. The facility was located next to the Strontium Semiworks and ran from the early 1950's to the early $1980^{\prime} \mathrm{s}$. The plutonium used in the lab was reprocessed in PUREX.

244-AR, -BXR, and -CR Process Vaults

The three process vaults in the northeast quadrant are the 244-AR Vault, the 244-BXR Vault, and the 244-CR Vault. These vaults were composed of several process vessels or tanks used to prepare the waste for treatment or storage. Specific wastes from tanks can be pumped temporarily to the vaults and later sent directly to desired tanks or processing facilities.

The AR Vault was designed and constructed between 1964 and 1968 and is located next to the $A$ and AX Tank Farms. The AR Vault has been on standby since 1978 .

The 244-BXR Vault is located south of the BX Tank Farm and was constructed between 1950 and 1951. The BXR Vault began operating in 1952 and became inactive in 1956. The waste in the vault was difficult to handle, so the vault was jetted with high-pressure steam in 1976. The vault was interimly isolated after 1976 and interimly stabilized in March 1985. Tank BXR-004 was removed in 1963.

The 244-CR Vault was constructed in 1952 and is located next to the C Tank Farm. Saltwell waste from the C Tank Farm is interimly stored in the CR Vault. The 244-BXR and -CR Vaults were originally constructed to aid in the recovery of uranium from bismuth phosphate metal waste. The CR Vault received waste from the strontium Semiworks Plant en route to the C Tank Farm.

- 204-AR and 204-S Railroad Car Facilities

The 204-AR rail car unloading facility was built in 1981 and replaced the 204-S rail car unloading facility. The facilities were built for unloading tank cars containing radioactive liquid waste. Liquids or slurries from the 1314-N Building in the 100 Area and the 340 Building in the 300 Area are unloaded at the 204-AR unloading facility. 


\subsubsection{Timelines}

Timelines are presented on the following pages that represent the different events that occurred during the lifetime of major Hanford plants and tank farms in the 200-East and 200-West Areas. The plants and associated processes were the main contributors to the waste currently contained in the tanks. The following is a list of abbreviations used in the timelines [Figures 4 ( $A$ and $B$ ) and 5 ( $A-C$ )] and their corresponding meanings. Abbreviations in parentheses are wastes that are listed in Appendix A.

PUREX: Plutonium uranium extraction process

$\mathrm{UO}_{3}: \quad$ Uranium trioxide

PFP: $\quad$ Plutonium Finishing Plant

RG-RB: Rubber glove - remote button line

RMA: Remote mechanical "A" line

RSE: Recuplex solvent extraction

RMC: $\quad$ Remote mechanical "C" line

PRF : Plutonium Reclamation Facility

PCB : Polychlorinated biphenyls

WESF : Waste Encapsulation and Storage Facility

ITS: $\quad$ In-tank solidification (units \#1 and \#2)

PWR: $\quad$ Pressurized water reactor

REDOX: Reduction oxidation process 



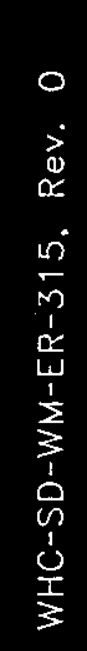

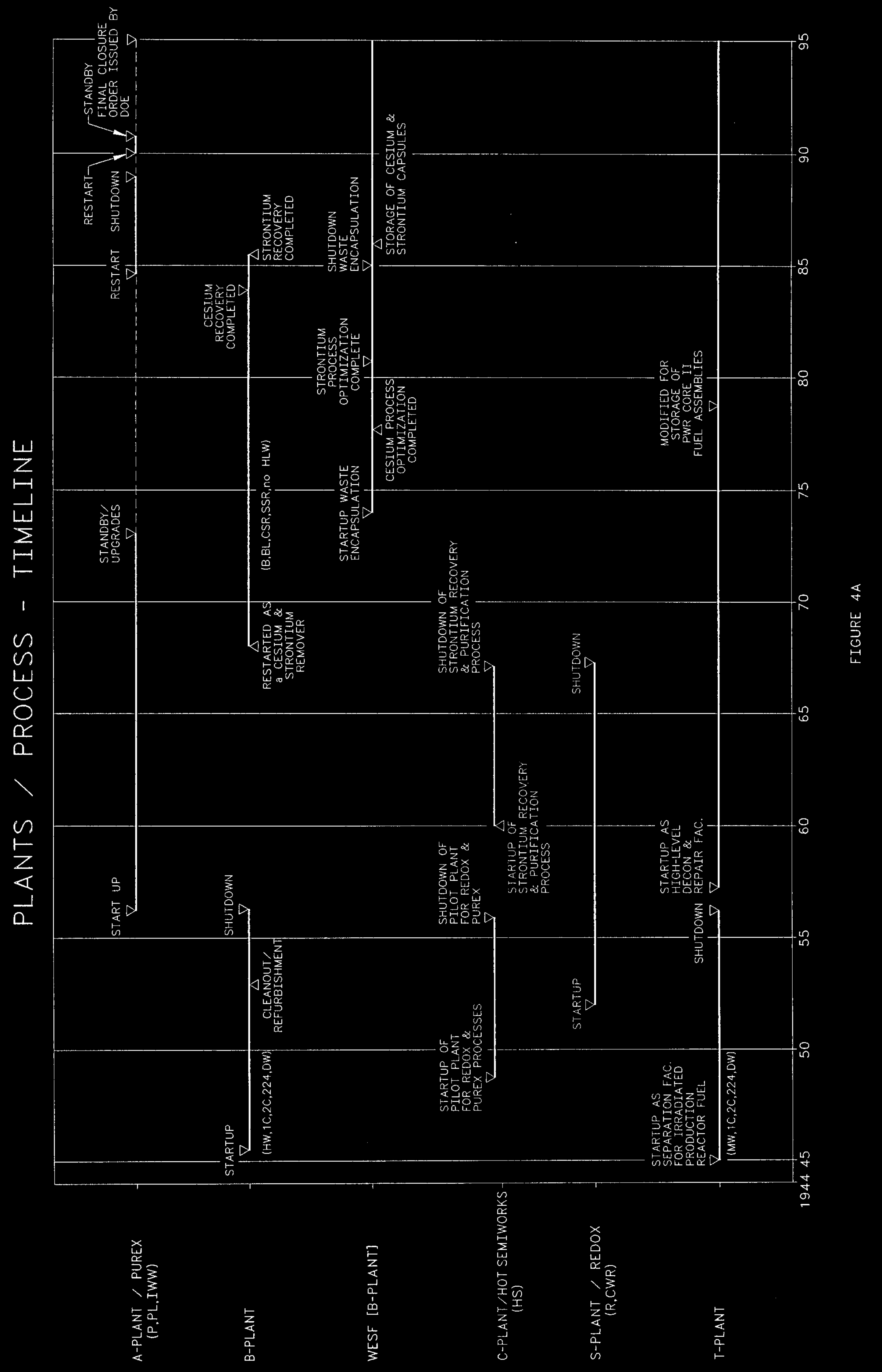


THES PAGE INHWNHONAKTY WFTBAIK 


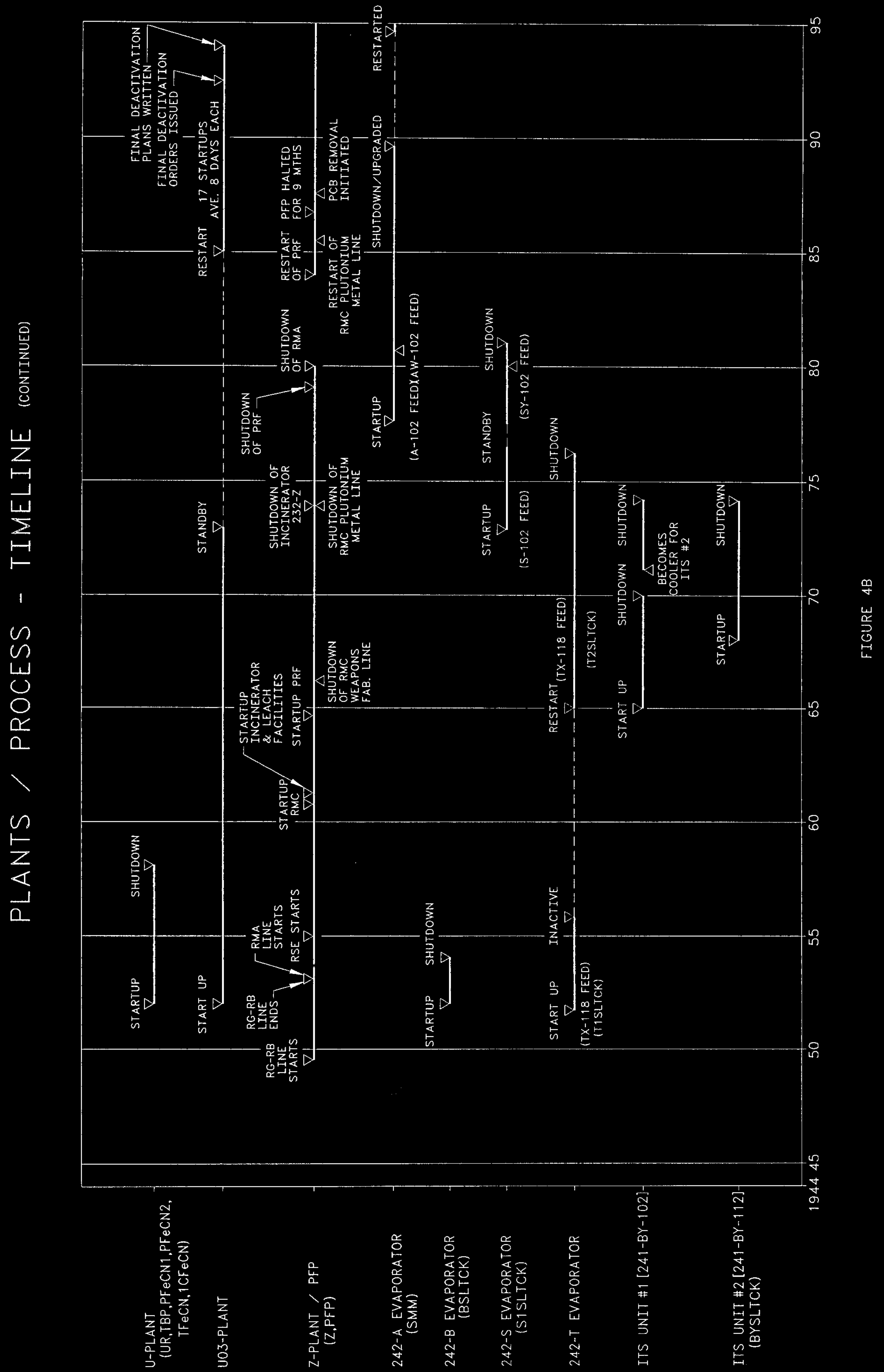


THE PAGS INYMHYONAWKY DFHBIALTK 


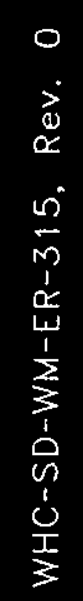

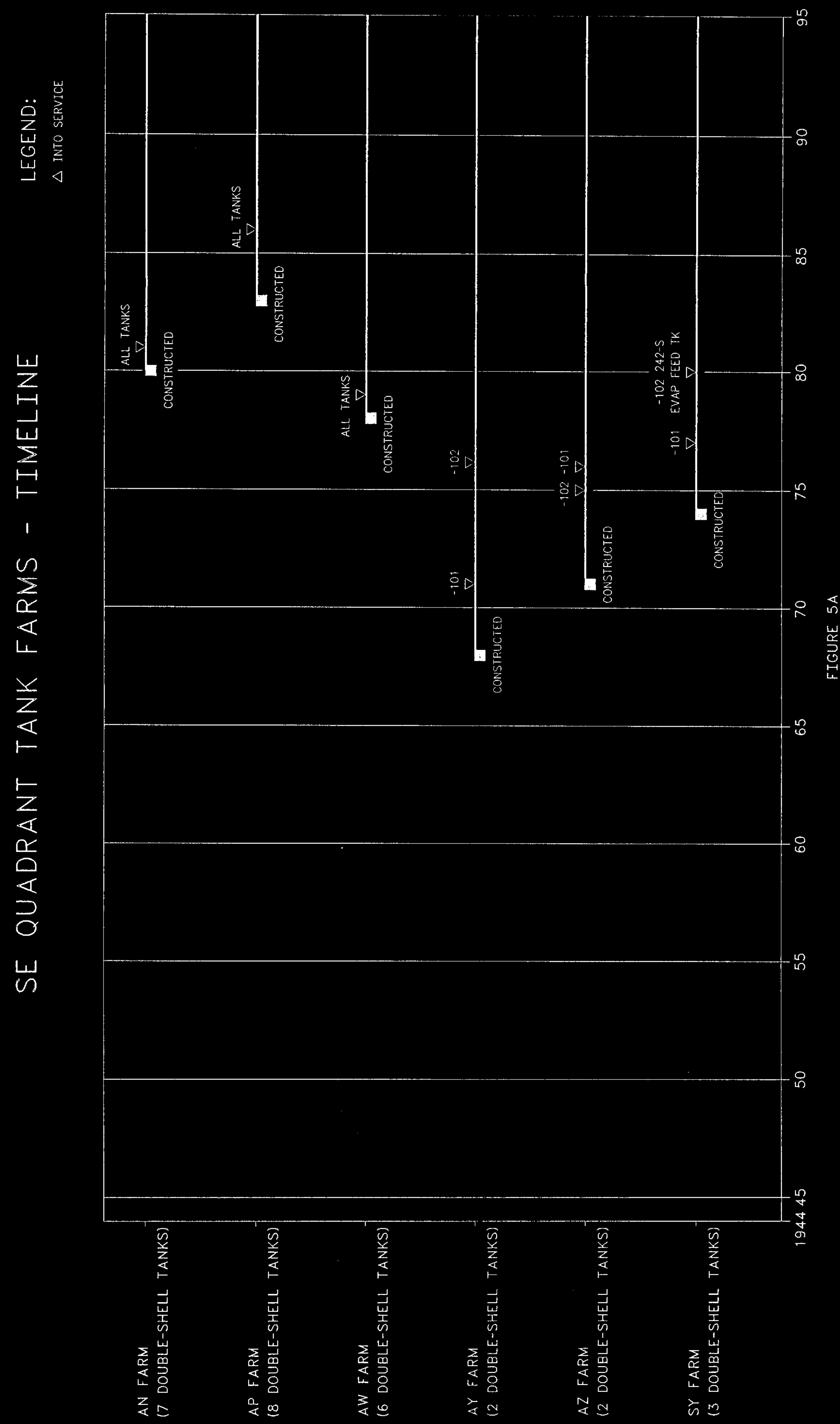




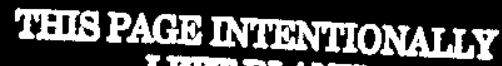
MFI IBLATK 


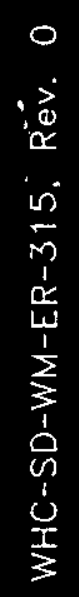

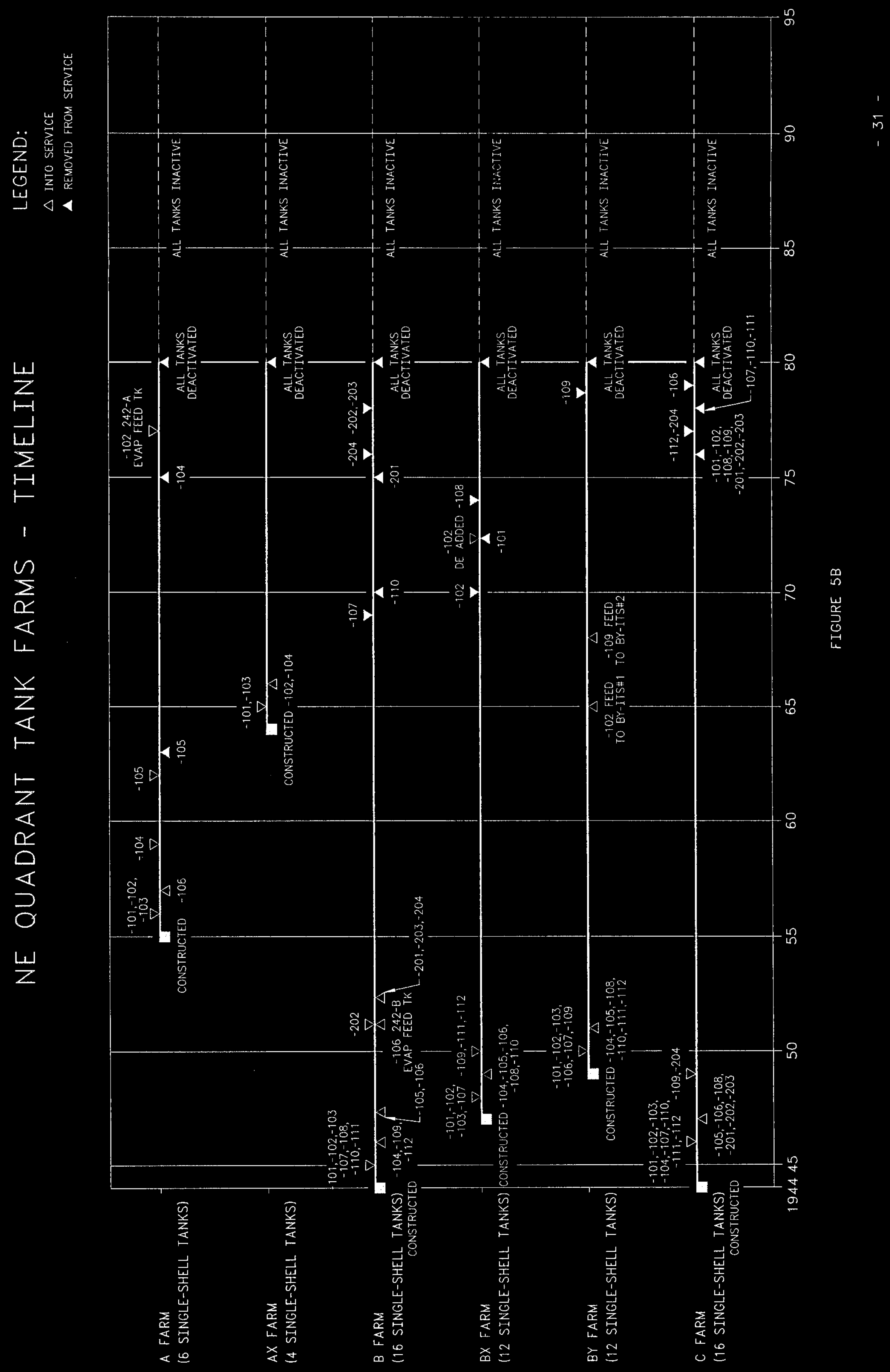




\section{TEIS PACD MYGHYHONAWT}

THIIBLANT: 


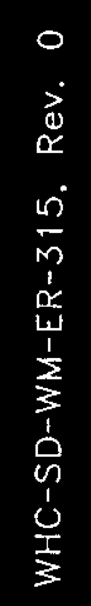

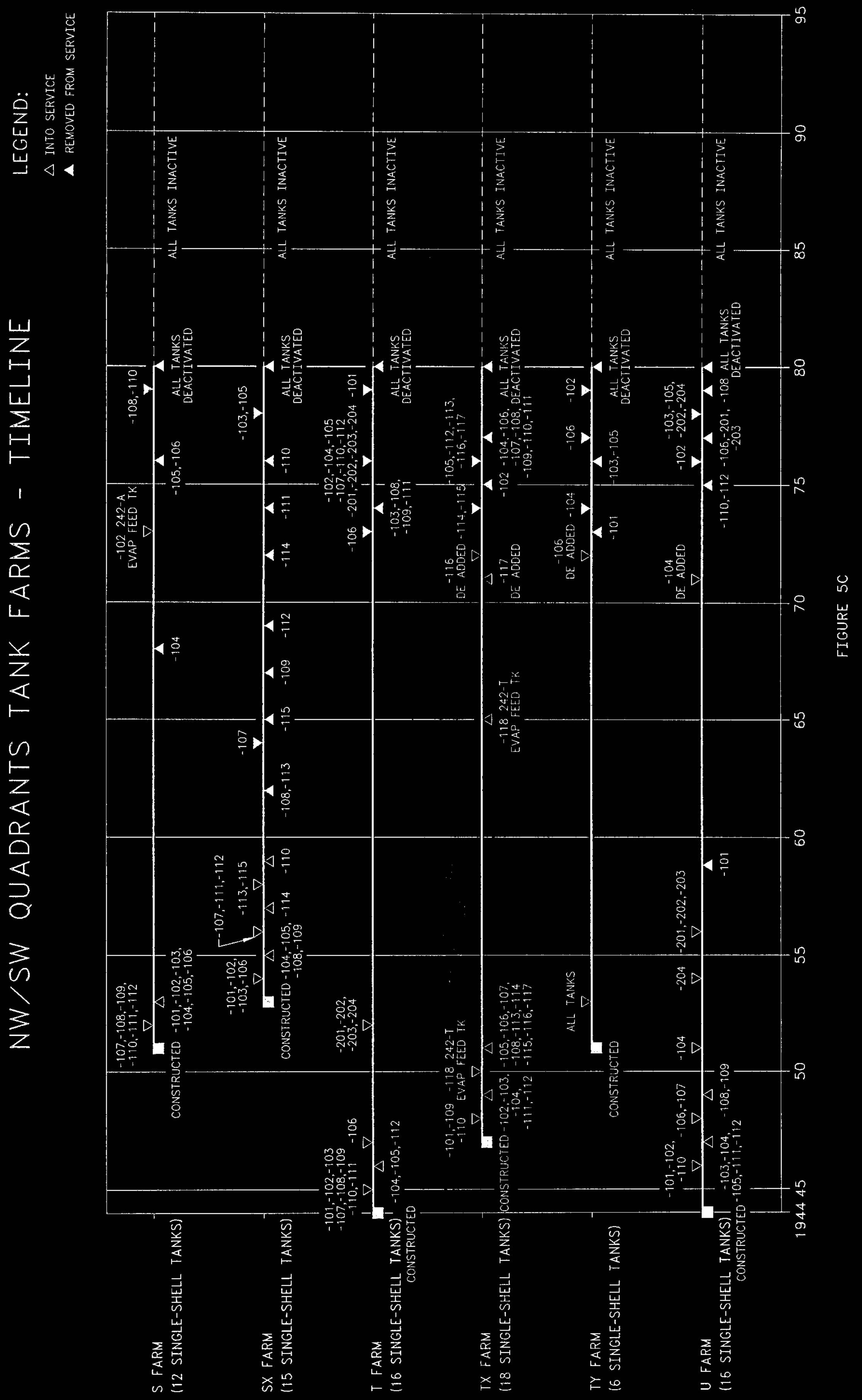


TEIS PACB INHFYHONAURY MFISBIAIT 
The safety issues that affect the tanks can be divided into two groups: watch list and non-watch list. The watch lists are a listing of all tanks that are believed to pose potential safety hazards to the environment and the public. Non-watch list issues are of concern because they may impact the environment.

1.6.1 Watch List Safety Issues

Safety issues for these tanks were identified as "issues/situations that contain most necessary conditions that could lead to worker (onsite) or offsite radiation exposure through an uncontrolled release of fission products" under Public Law 101-510, Section 3137, of the National Defense Authorization Act of Fiscal Year 1991 ( $i . e .$, the Wyden Amendment). As of November 1993, 45 single-shell tanks and 6 double-shell tanks are on a watch list. All 6 of the double-shell tanks are on the watch list for hydrogen or other flammable gas. The four watch list designations described in this report are ferrocyanide, hydrogen or other flammable gas, organic salts, and high-heat load. The following sections provide a general description and criteria of the different watch lists. For a more complete explanation, refer to the Hanford Site Tank Farm Facilities Interim Safety Basis (WHC 1993).

\section{Ferrocyanide}

A tank containing or believed to contain greater than 1,000 gram moles of ferrocyanide (dry basis) is on the ferrocyanide watch list. Tanks containing ferrocyanide are potentially explosive if ferrocyanide and nitrates combine.

Hydrogen or Other Flammable Gas

Tanks were placed on this watch list due to the potential of containing flammable gas rather than the verified presence of hazardous concentrations. Tanks on the watch list for hydrogen or other flammable gas have been identified as an unreviewed safety question due to the concern that burning flammable gas could result in a radiological release.

Organic Salts

Tanks containing or believed to contain more than the equivalent of $10 \%$ by weight of sodium acetate or 3. wt\% total organic carbon (TOC) on a dry basis received a watch list designation as in the "Tank Farm Surveillance and Waste Status Summary Report" (Hanlon 1993). Tanks containing organic salts could release waste if an uncontrolled increase in temperature or pressure results from oxidation by nitrates and nitrites.

High-Heat Load

Tanks with a heat load greater than 40,000 Btu/hr are designated as high-heat load tanks. Tank 241-C-106 is the only high-heat load tank on the high-heat load watch list. 
WHC-SD-WM-ER-315, Rev . 0

Any tank not listed on the high-heat load tank list or the highheat load watch list with a heat load less than $40,000 \mathrm{Btu} / \mathrm{hr}$ is considered a low-heat load tank by default.

The following table lists the tanks in the southeast quadrant that appeared on the watch list as of June 1995. A current listing is published monthly in the "Tank Farm Surveillance and Waste status Summary Report".

Southeast Quadrant Double-Shell Watch List Tanks

\begin{tabular}{|l|l|}
\hline DST Tank & Category \\
\hline $241-$ AN-103 & Hydrogen \\
\hline $241-$ AN-104 & Hydrogen \\
\hline $241-$ AN-105 & Hydrogen \\
\hline $241-A W-101$ & Hydrogen \\
\hline $241-S Y-101$ & Hydrogen \\
\hline $241-$ SY-103 & Hydrogen \\
\hline
\end{tabular}

1.6.2 Non-Watch List Safety Issues

Tank leaks are a safety hazard because of the potential to release chemicals and radioactive liquids to the ground. Corrosion is the main cause of tank leaks. Three other safety issues that do not require a watch list and continual monitoring under the Wyden Amendment include criticality, tank bumps, and toxic vapor releases.

Corrosion

Corrosion is a localized or general reduction in the thickness of the carbon steel liner. Localized reduction in the liner thickness is one of three types of corrosion: pitting corrosion, stress corrosion cracking, and crevice corrosion. General reduction in liner thickness may be uniform corrosion.

The tanks are equipped with corrosion (cathodic) protection. None of the double-shell tanks are assumed or confirmed leaking.

- Criticality

Criticality is a self-sustained, nuclear chain reaction. Studies have concluded that a nuclear criticality accident in the tank farms is probably not an imminent risk. However, knowledge of the inventory of fissile material and its distribution within the tanks is incomplete. Therefore, criticality remains a safety issue. The key radionuclide on the Hanford site that would be involved in a criticality accident is plutonium. To prevent a criticality, a maximum content of $125 \mathrm{~kg}$ of fissile material has been specified for the tanks. An unusual occurrence report involving nuclear criticality was filed in June 1991 for Tank 241-C-104. Later, an inventory of $56 \mathrm{~kg}$ was calculated which indicated a low probability 
for criticality. Criticality is considered an unreviewed safety question, but is not affected by the Wyden Amendment according to the Hanford Site Tank Farm Facilities Interim Safety Basis (WHC 1993).

\section{- Tank Bumps}

Tank bumps have been an issue at Hanford since 1953 with the last bump occurring in 1968. Tank bumps occur when heat from the hotter solid waste in the lower portion of the tank is suddenly transferred to a near boiling supernatant. Mixing can be achieved by "roll over" or sudden restart of airlift circulators which results in rapid fluid vaporization and a sudden internal tank pressurization that causes a bump. The three documented bumps that have occurred were due to airlift circulator failure followed by rapid startup of the airlift circulators which causes rapid mixing. Two key factors have been identified to control bumping: liquid and solid temperatures must be maintained as far away from the local boiling point as possible, and airlift circulators and ventilation systems must be operated as designed. A tank bump is extremely unlikely if airlift circulators and vent flowrates are maintained at 500 to 1,000 $\mathrm{ft}^{3} / \mathrm{min}$ per tank and $50 \mathrm{ft}^{3} / \mathrm{min}$ to the airlift circulators according to the Hanford Site Tank Farm Facilities Interim Safety Basis (WHC 1993).

\section{Toxic Vapor Releases}

Toxic vapor releases are a recently analyzed safety concern at Hanford. Odors have been released from the $C$ Tank Farm in the northeast quadrant and from Tank 241-SY-101 in the southeast quadrant. The entire issue of toxic gas releases at the tank farms has just begun to be understood according to the Hanford Site Tank Farm Facilities Interim Safety Basis (WHC 1993).

\subsection{Occurrences}

Over the years, unusual events that occurred at the Hanford Site have been documented by various reporting methods. Some of these reports include unusual occurrences reports, off normal reports, event fact sheets and occurrence reports. According to these reports, four unusual events affected all of the tank farms included in the southeastern quadrant. Occurrence reports RL--WHC-Tank Farm-1993-0072, RL--WHC-Tank Farm-1992-0028， RL--WHC-Tank Farm-1991-1031, and RL--WHC-Tank Farm-1991-1026 were written due to loss of power in the area. For more information on occurrences, refer to the Farm Information section for the specific tank. 
THIS PAGE INTENMONALLY

LFTP BLANK 


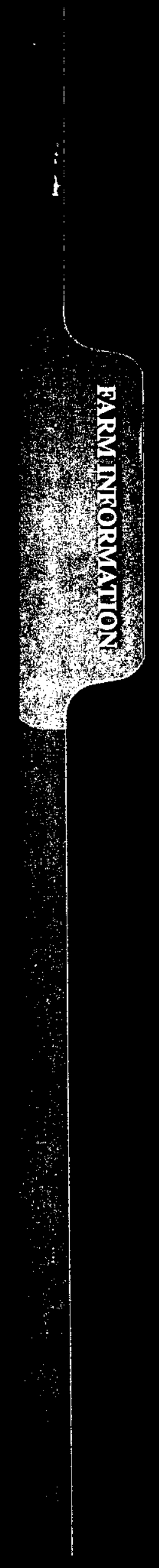


THIS PAGE INTENTIONAJLY IFIIT BLANK 


\subsubsection{AP Tank Farm History}

The AP Tank Farm is located south of 4 th street and east of Canton Avenue on the eastern side of the 200-East Area. The farm contains eight 75-foot diameter, 1,160,000-gallon capacity (1,140,000-gallon operating limit) double-shell tanks. The tanks are described as double-shell because of their two steel Iiners. The tanks were constructed with a primary steel liner (stress-relieved carbon steel), a secondary steel liner, and a reinforced concrete shelI with an insulating concrete foundation between the primary and secondary liners (see tank cross-section in Appendix G). These tanks were designed to hold concentrated supernatant. The maximum design temperature for liquid storage is $300^{\circ} \mathrm{F}$.' The tanks are all at the same elevation with no cascade lines between the tanks.

A color aerial photograph taken in 1993 of the AP Tank Farm shows the orientation of the tanks, leak detection pits, annulus ventilation equipment, air intake stations, and a valve pit (see Appendix I) .

\subsubsection{AP Tank Farm Waste History}

The tanks in the AP Tank Farm began receiving waste in July 1986. The first wastes consisted of non-complexed waste, Hanford Site facilities waste, and double-shell slurry waste. Low-level waste from PUREX was sent to Tanks 241-AP-101, $-102,-103,-107$, and -108 from 1987 to 1992. Tank 241-AP-104 received $N$ Reactor decontamination waste in 1987. Tanks 241-AN-102 and -104 were feed tanks for the grout facility. Presently, only Tanks 241-AN-101, -107 , and -108 are active, and they are dilute process feed tanks to the 242-A Evaporator.

\subsubsection{AP Tank Farm Temperature History}

The eight tanks within the AP Tank Farm each contain one thermocouple tree with 18 thermocouples at known elevations. The thermocouple elevations on the tree range from six inches to 38.5 feet above the bottom of the tank. Each tree was installed in riser 4 during construction of the tanks. A tree is constructed from a capped 10-inch stainless steel pipe and a set of guide tubes, one to contain each thermocouple. Each guide tube allows access to replace a failed thermocouple. It appears that all trees are operating normally; none have needed repair or replacement.

All of the AP tanks have a requirement for temperatures to be recorded weekly. Data were available from the computer automated surveillance system (CASS) and the surveillance analysis computer systen (SACS). The CASS is currently recording temperatures in the AP Tank Farm daily. Temperatures from manually recorded data sheets are currently entered weekly into the SACS database. See tables and graphs of temperature data in Appendix D. 
There are two sets of equipment that compile and display temperature data in the AP Tank Farm, one for Tanks 241-AP-101 through 241-AP-104 and one for Tanks 241-AP-105 through 241-AP-108. The thermocouple leads from each set of four tanks enter each set of equipment which compiles the data and displays the temperatures on $a$ monitor for manual recording on data sheets. The CASS receives the same data from this equipment that an operator would record on a data sheet. The temperature histories of Tanks 241-AP-105 through 241-AP-108 indicate a temperature rise on the same date in May 1994 that did not appear in the data from the other set of four tanks. An apparent malfunction between the point where the four leads from this set of tanks enter the compiling equipment and the final display of data probably caused this behavior. The lack of transfers to and from Tanks 241-AP-105 through 241-AP-108 eliminate transfers as the cause on the temperature rise (see surface level data in Appendix E). The reason behind the temperature increase is still under investigation. All temperature data for Tanks 241-AP-105 through 241-AP-108 after May 1994 are questionable.

\subsubsection{AP Tank Farm Occurrences.}

Six unusual events occurred at the AP Tank Farm with one specific to Tank 241-AP-104. Since 1986 when the tanks began operating, the following five occurrences affected all eight tanks. Four occurrence reports were written because equipment failures caused the tanks to reach atmospheric pressure (occurrence reports RL-WHC-Tank Farm-1993-0049, RL-WFC-Tank Farm-1992-0055, RL--WFC-Tank Farm-1992-0019, and RL-WHC-Tank Farm-1991-0226). According to off-normal report 86-55 issued in December 1986, the tanks were pressurized from a power failure causing loss of ventilation.

\subsubsection{AP Tank Farm Ventilation}

Each tank in the AP tank farm is designed to have 146.25 standard cubic feet per minute (SCFM) of air. travel through the tank and 1075 SCFM of air travel through the annulus. Air enters each tank by infiltrating around the pit cover.blocks and leaves via riser 7. The air ducts for all of the tanks are joined and one fan sends air into the primary exhaust system. Before the tank air is released to the atmosphere the air is heated and routed through a prefilter and two high-efficiency particulate air (HEPA) filters. Each annulus receives supplied air after passing through a heater, a prefilter, and a HEPA filter. Air. leaves each annulus through riser 9 and travels through the ducts to a common. AP Tank Farm heater and two HEPA filters before it is released to the atmosphere. Both the tank and annulus systems have a primary and a backup system. For more information, refer to drawing $\mathrm{H}-2-90514$, sheets 1 and 2 .

The tanks in AP Tank Farm have a minimum and maximum safety limit pressure of -12 inches of water gauge $(-0.43 \mathrm{psig}$ or $-2.99 \cdot \mathrm{kPa}$ gauge) and 6.0 inches of water gauge $(2.17 \mathrm{psig}$ or $14.93 \mathrm{kPa}$ gauge), respectively. The operating pressure is between -4 and 0 inches of water gauge $(-0.14 \mathrm{psig}$ or $-1 \mathrm{kPa}$ gauge and 0 psig or $0 \mathrm{kPa}$ gauge). Each tanks exhaust ventilation system may be inoperable (potentially 
WHC-SD-WM-ER-315, Rev. 0

pressurizing to 0 inches of water gauge) for less than 2 hours continuously or 40 hours. in 12 months without exceeding safety limits. These limits can be found in document WHC-SD-WM-OSR-016, Rev. OB (Heubach 1994).

The psychrometric data were prepared in two parts, the tank data and the annulus data. Positive number's represent moisture and heat leaving the tank and annulus, and negative. numbers represent moisture and heat entering the tank and annulus. Three plots were made for each of the tanks: evaporation in inches per week versus time, heat transfer rate to the ventilation system in BTU per hour versus time, and evaporation in gallons per week versus time. Two plots were made for each tank's annulus: heat transfer rate to the ventilation system versus time, and evaporation in gallons per week versus time. Evaporation in inches per week, though calculated, was not plotted because this data is significant only if standing water is present in the annulus. If there were insufficient raw data or the data point was labeled suspect, the point was not plotted on the graph. See Appendix $F$ for the tables and plots.

\subsubsection{AP Tank Farm Fistorical Sample Analysis Data}

Samples were drawn from three tanks (241-AP-102, -103, and -104) and analyzed. The samples were similar in composition, containing primarily sodium phosphate and radionuclides of strontium and cobalt. The samples were yellow to a yellowish-brown with little or no solids. The remaining tanks have no historical sampling data. No information on sampling procedures and methods were available for the tanks (see Appendix H).

\subsubsection{Current Status of AP Tank Farm}

All tanks in AP Farm are active, actively ventilated and categorized as sound. Currently, the total waste volume is $2,207,000$ gallons (Hanlon, June 1995).

\subsubsection{AP Tank Farm Inventory Estimates}

There are three inventory estimates for each tank in the AP Tank Farm created by Los Alamos National Laboratory (see Appendix J). The supernatant mixing model (SMM) composite estimate is based on the output of the supernatant mixing model. The tank layer model (TLM) solids composite estimate is based on the output of the tank layer model. The total inventory estimate is a combined volume weighted average of the SMM composite and the TLM composite inventory estimates.

\subsubsection{Waste Volume Approximations AP Tank Farm}

The waste volume approximations in each tank was modeled using two programs developed by Los Alamos National. Laboratory (IANL). One program, the supernatant mixing model (SMM), modeled the liquid (supernatant) portion of the tank. The other program, the tank layer model (TLM), modeled the solids portion of the tank. The models were run using data representing the estimated contents of each tank as of 
the inventory estimate date (January 1, 1994). Results from both models are in Appendix $K$.

The waste volume approximations have been modified in both programs and are presented on each level history sketch in Appendix $C$. There are two areas that have been modified. The first area concerns the concentrated supernatant solids (CS solids). The CS solids are determined by taking the difference between the measured solids volume and the solids volume predicted by the tank layer model. The concentrated supernatant solids are considered part of the total supernatant and not as a solid according to the tank layer model. The second area concerns the supernatant volume. The supernatant volume from IANL presented on the level history sketch was determined by taking the difference between the predicted supernatant mixing model volume and the CS solids volume. These modifications were necessary in order to reflect the actual supernatant and solids volumes that were measured within each tank.

\subsubsection{AP Tank Farm In-Tank Photographs}

Only the photograph of the AP Tank Farm is in Appendix I. There are no interior tank photographs available at this time. 
2.1 Tank 241-AP-101

2.1.1 241-AP-101 Tank History

2.1.1.1 Waste History of Tank 241-AP-101

The history of the primary additions is derived from the "Waste Status and Transaction Record Summary for the Southeast Quadrant" (Agnew 1995). Tank 241-AP-101 received zircaloy cladding waste from PUREX during the third quarter of 1987. The tank had an unknown transaction during the third quarter of 1987 . The tank received PUREX ammonia scrubber feed waste from the third quarter of 1988 until the fourth quarter of 1989.

The history of the waste types is derived from the Tank Farm Surveillance and Waste Status Summary Reports for January 1980 through March 1994. Tank 241-AP-101 began receiving non-complexed waste in July 1986 and continued to receive this waste until May 1990. From June 1990 until March 1994, the tank waste was designated as dilute non-complexed waste.

The tank is currently an active dilute receiver tank with waste being concentrated by the 242-A Evaporator. Sketch ES-TKS-E157, a graph representing the level history, and the historical waste level data used to generate the graph are in Appendix $C$.

\subsubsection{Temperature History of Tank 241-AP-101}

Temperatures from within Tank 241-AP-101 are recorded by 18 thermocouples. Non-suspect teinperature data recorded between April 1986 and March 1995 for al1 18 thermocouples were obtained from the computer automated surveillance system (CASS). Non-suspect temperature data taken between July 1989 and June 1995 for 12 of the thermocouples were obtained from the surveillance analysis computer system (SACS). However, not all of the 12 thermocouples have data covering that entire period. There were small breaks in the sequence of temperature data form each system. The average temperature during these periods was $60^{\circ} \mathrm{F}$ with a minimum of $48^{\circ} \mathrm{F}$, a maximum of $81^{\circ} \mathrm{F}$, and a standard deviation of $4.1^{\circ} \mathrm{F}$. Linear regressions were performed on the data for each thermocouple with a resulting average decreasing slope of $-0.0001^{\circ} \mathrm{F} / \mathrm{day}$. See tables and graphs of temperature data in Appendix D.

\subsubsection{Occurrences of Tank 241-AP-101}

No unusual events exclusively, affected Tank 241-AP-101.

\subsubsection{Psychrometrics of Tank 241-AP-101}

Psychrometric data have been recorded for twelve years from the tank and six years from the annulus. The apparent trend (according to the plot in Appendix F) of the evaporation rate from the tank has decreased from an average of 76.7 gallons per week in May 1988 to an average of 48.3 gallons per week in January 1995. Over that same period, the trend in the rates of heat exchange through the primary 
WHC-SD-WM-ER-315, Rev. 0

tank and annular ventilation has apparently decreased. A review of the psychrometric data from. Tank 241-AP-101 suggests an overal1 decrease in the rate of heat generation by the waste.

2.1.1.5 Historical Sample Analysis Data for Tank 241-AP-101

No sampling data are available for this tank.

2.1.2 Current status of Tank 241-AP-101

Tank 241-AP-101 was placed into service in October 1986 and currently stores approximately 78,000 gallons of waste (Hanlon, June 1995). The waste level in the tank is measured with a FIC gauge and a manual tape and has fluctuated between 385 inches and 54 inches. The increases were additions of waste fron Tank 241-AP-108 in October. 1994 and January 1995. The decreases came from transferring waste to Tank 241-AW-102 in October 1994. See Appendix E for details on waste surface level. This tank is active, actively ventilated, and categorized as sound. The waste is currently being concentrated by the 242-A Evaporator. A plan view in Appendix G depicts the riser locations as of August 3, 1995. Tank 241-AP-10.1 has 71 risers. Seven 4-inch risers (no. 15, no. 21, no. 24, three no. 27 's, and no. 28), four 12 -inch risers (no. 7 , two no. 10 's, and no. 12), two 24-inch risers (two no. 6's), and two 42-inch risers (two no. 5's) are available for use.

\subsubsection{Inventory Estimate of Tank 241-AP-101}

The three inventory estimates derived by the Los Alamos National Laboratory (LANL) predict the physical, chemical, and radiological constituents of the supernatant, the solids and the total volume for each tank. These estimates are presented in Appendix $\mathrm{J}$.

2.1.2.2 Waste Volume Approximations for Tank 241-AP-101

The summary of approximated waste volumes, as derived from. Los Alamos National Laboratory's supernatant mixing model (SMM) and tank layer model (TLM), are presented in Appendix.K. The modeled volume is from the tank waste contents corresponding to the inventory estimate date of January 1, 1994. A tank cross-section that depicts the waste volume on the inventory estimate date is in Appendix $G$. The total waste volume on the inventory estimate date was approximately $1,060,000$ gallons according to the Tank Farm Surveillance and Waste Summary Report (Hanlon 1993). The total waste volume was divided by the SMM and the TLM into the following subsets. The SMM predicted 1,060,000 gallons of supernatant. The TIM did not predict any solids within the tank.

2.1.2.3 In-Tank Photograph of Tank 241-AP-101

No interior tank photographs are available.

2.1.3 Synopsis of Tank 241-AP-101

(To be completed.) 
2.2 Tank 241-AP-102

2.2.1 241-AP-102 Tank History

2.2.1.1 Waste History of Tank 241-AP-102

The history of the primary additions is derived from the "Waste Status and Transaction Record Summary for the Southeast Quadrant" (Agnew 1995). Tank 241-AP-102 received PUREX ammonia scrubber feed waste during the third and fourth quarters of 1989. The tank received four unknown transactions between the first quarter of 1991 and the third quarter of 1992 .

The history of the waste types is derived from the Tank Farm Surveillance and Waste status Summary Reports for January 1980 through March 1994. Tank 241-AP-102 began receiving fanford facility waste in July 1986 and continued to receive this waste until May 1990. The tank waste was designated as dilute non-complexed waste from June 1990 until March 1994.

The tank is currently an inactive grout feed tank containing excess water from the grout facility. Sketch ES-TKS-E158, a graph representing the level history, and the historical waste level data used to generate the graph are in Appendix C.

\subsubsection{Temperature History of Tank 241-AP-102}

Temperatures from within Tank 241-AP-102 are recorded by 18 thermocouples. Non-suspect temperature data recorded between April 1986 and March 1995 for all 18 thermocouples were obtained from the computer automated surveillance system (CASS). Non-suspect temperature data taken between July 1989 and June 1995 for 12 of the thermocouples were obtained from the surveillance analysis computer system (SACS). However, not all of the 12 thermocouples have data covering that entire period. There were small breaks in the sequence of temperature data from each system. The average temperature during these periods was $67^{\circ} \mathrm{F}$ with a minimum of $30^{\circ} \mathrm{F}$, a maximum of $90^{\circ} \mathrm{F}$, and a standard deviation of $8.5^{\circ} \mathrm{F}$. Linear regressions were performed on the data for each thermocouple with a resulting average increasing slope of $0.0045^{\circ} \mathrm{F} / \mathrm{day}$. See Appendix D for graphs and tables of temperature data.

\subsubsection{Occurrences of Tank 241-AP-102}

No unusual events concerned this tank since it went into service.

\subsubsection{Psychrometrics of Tank 241-AP-102}

Psychrometric data have been recorded from this tank and annulus for six years. The apparent trend (according to the plot in Appendix F) of the evaporation rate from the tank has decreased from an average of 63.4 gallons per week in May 1988 to an average of 49.3 gallons per week in January 1995. Over that same period, the trend in the rates of heat exchange through the primary tank ventilation 


$$
\text { WHC-SS-WM-ER-315, ReV. } 0
$$

has decreased. The rates of heat exchange through the annular ventilation show an increasing trend. The majority of the changes in the rate of heat exchange can be attributed to changes in the average ventilation rates. A review of the psychrometric data in Tank 241-AP-102 suggests an increase in rate of heat generation by the waste.

\subsubsection{Historical Sample Analysis Data for Tank 241-AP-102}

A sample was drawn from Tank 241-AP-102 and analyzed on June 8, 1988 (see Appendix H). The sample was a phosphate and sulfate liquid waste sample. It was composed of primarily sodium and phosphorous cations and carbonate, phosphate and sulfate anions. The highest concentrations of radionuclides present were cobalt, cesium and strontium.

available.

Information on sampling procedures and methods were not

\subsubsection{Current Status of Tank 24:1-AP-102}

Tank 241-AP-102 was placed into service in October 1986 and currently stores approximately 1,099,000 gallons of waste (Hanlon, June 1995). The waste level in the tank is measured with a FIC gauge and a manual tape and has increased from about 50 inches in January 1991 to 400 inches in January 199.3. The increase was attributed to waste received from Tank 241-AN-106 between November 1992 and January 1993. See Appendix $\mathrm{E}$ for details on waste surface level. This tank is inactive at the present time. The tank is actively ventilated and categorized as sound. A plan view in Appendix G depicts the riser configuration as of August 3, 1995. Tank 241-AP-102 has 71 risers. Seven 4-inch risers (no. 15, no. 21, no. 24 , three no. 27 's, and no. 28), three 12-inch risers (no. 7, no. 10, and no. 12), two 24-inch risers (two no. $6^{\prime} \mathrm{s}$ ), and two 42-inch risers (two no. 5's) are available for use.

\subsubsection{Inventory Estimate of Tank 241-AP-102}

The three inventory estimates derived by the Los Alamos National Laboratory (LANL) predict the physical, chemical, and radiological constituents of the supernatant, the solids and the total volume for each tank. These estimates are presented in Appendix $\mathrm{J}$.

\subsubsection{Waste Volume Approximations for Tank 241-AP-102}

The summary of approximated waste volumes, as derived from Los Alamos National Laboratory's supernatant mixing model (SMM) and tank layer model (TLM), are presented in Appendix K. The modeled volume is from the tank waste contents corresponding to the inventory estimate date of January 1, 1994. A tank cross-section that depicts the waste volume on the inventory estimate date is in Appendix G. The total waste volume on the inventory estimate date was approximately 1,104,000 gallons according to the Tank Farm Surveillance and Waste Summary Report (Fanlon 1993). The total waste volume was divided by the SMM and the TLM into the following subsets. 


$$
\text { WHC-SD-WM-ER-315, Rev: } 0
$$

The SMM predicted 1,104,000 gallons of supernatant. The TLM did not predict any solids within the tank.

2.2.2.3 In-Tank Photograph of Tank 241-AP-102

No interior tank photographs are available.

2.2.3 Synopsis of Tank 241-AP-102

(To be completed.) 
2.3 Tank 241-AP-103

2.3.1 241-AP-103 Tank History

2.3.1.1 Waste History of Tank 241-AP-103

The history of the primary additions is derived from the "Waste Status and Transaction Record Summary for the Southeast Quadrant" (Agnew 1995). Tank 241-AP-103 received PUREX ammonia scrubber feed waste from the first quarter until the third quarter of 1988 .

The history of the waste types is derived from the Tank Farm Surveillance and Waste status Summary Reports for January 1980 through March 1994. Tank 241-AP-103 began receiving non-complexed waste in July 1986 and continued to receive.this waste until May 1990. From June 1990 until March 1994, the tank waste was designated as dilute non-complexed waste.

The tank is currently an inactive dilute receiver tank. Sketch ES-TKS-E159, a graph representing the level history, and the historical waste level data used to generate the graph are in Appendix C.

\subsubsection{Temperature History of Tank 241-AP-103}

Temperatures from within Tank 241-AP-103 are recorded by 18 thermocouples. Non-suspect temperature data recorded between April 1986 and March 1995 for all 18 thermocouples were obtained from the computer automated surveillance system (CASS). However, not all of the 18 thermocouples have data covering that entire period. Nonsuspect temperature data taken between July 1989 and June 1995 for 9 of the thermocouples were obtained from the surveillance analysis computer system (SACS). However, not all of the 9 thermocouples have data covering that entire period. There were small breaks in the sequence of temperature data from each system. The average temperature during these periods was $62^{\circ} \mathrm{F}$ with a minimum of $50^{\circ} \mathrm{F}$, a maximum of $76^{\circ} \mathrm{F}$, and a standard deviation of $5.1^{\circ} \mathrm{F}$. Inear regressions were performed on the data' from 17 of the 18 thermocouples with a resulting average decreasing slope of $-0.0020^{\circ} \mathrm{F} /$ day. One thermocouple's temperature data consistently disagreed with the trend of the rest and, subsequently, was not considered in the regressions. See tables and graphs of temperature data in Appendix D.

\section{3 .1 .3 Occurrences of Tank 241-AP-103}

No unusual events exclusively concerned Tank 241-AP-103.

\subsubsection{Psychrometrics of Tank 241-AP-103}

Psychrometric data have been recorded for six years from this tank and annulus. The apparent trend (according to the plot in Appendix F) of the evaporation rate from the tank has increased from an average of 60.7 gallons per week in February 1988 to an average of 
79.9 gallons per week in January 1995. Over that same period, the trend in the rates of heat exchange through the primary tank ventilation has increased. The rates of heat exchange in the annular ventilation show a decreasing trend. The majority of the changes in the rates of heat exchange can be attributed to changes in the average ventilation rates. A review of the psychrometric data in Tank 241-AP-103 suggests a decrease in the rate of heat generation by the waste.

\subsubsection{Historical Sample Analysis Data for Tank 241-AP-103}

One sample analysis is available for Tank 241-AP-103, reported on February 26, 1988 (see Appendix H). The sample was clear with very little light brown solids. The sample was comprised of mostly sodium, ammonia, nitrite, nitrate, and hydroxide according to the chemical analysis. The most predominant radionuclides were strontium, americium, and rhodium.

Information on sampling procedures and methods were not available.

\subsubsection{Current ștatus of Tank 241-AP-103}

Tank 241-AP-103 was placed in service in October 1986 and currently stores approximately 26,000 gallons of waste (Hanlon, June 1995). The large decrease in volume was due to transferring waste to Tank 241-AW-102. The waste level in the tank is measured with a FIC gauge and a manual tape and has increased from about 350 inches in January 1991 to 410 inches in August 1991. In June 1993, the surface level dropped to about 10 inches. See Appendix $E$ for details on waste surface level. This tank is active and the waste is currently being concentrated by the 242-A Evaporator: The tank is actively ventilated and categorized as sound. A plan view in Appendix G depicts the riser configuration as of August 3, 1995. Tank 241-AP-103 has 71 risers. Seven 4-inch risers (no. 15, no. 21, no. 24, three no. $27 \mathrm{~s}$, and no. 28), four 12-inch risers (no. 7, two no. $10^{\prime} \mathrm{s}$, and no. 12), two 24-inch risers (two no. 6's), and two 42inch risers (two no. $5^{\prime} \mathrm{s}$ ) are available for use.

\subsubsection{Inventory Estimate of Tank 241-AP-103}

The three inventory estimates derived by the Los Alamos National Laboratory (LANL) predict the physical, chemical, and radiological constituents of the supernatant, the solids and the total volume for each tank. These estimates are presented in Appendix $J$.

\subsubsection{Waste Volume Approximations for Tank 241-AP-103}

The summary of approximated waste volumes, as derived from Los Alamos National Laboratory's supernatant mixing model (SMM) and tank layer model (TLM), are presented in Appendix $K$. The modeled volume is from the tank waste contents corresponding to the inventory estimate date of January 1, 1994. A tank cross-section that depicts the waste volume on the inventory estimate date is in Appendix $G$. The total waste volume on the inventory estimate date was 
WHC-SD-WM-ER-315, Rev. 0

approximately 1,131,000 gallons according to the Tank Farm Surveillance and Waste Summary Report (Hanlon 1993). The total waste volume was divided by the SMM and the TLM into the following subsets. The SMM predicted 1,131,000 gallons of supernatant. The TLM did not predict any solids within the tank.

2.3.2.3 In-Tank Photograph of Tank 241-AP-103

No interior tank photographs are available.

$2.3 .3 \quad$ Synopsis of Tank 241-AP-103

(To be completed.) 
2.4 Tank 241-AP-104

2.4.1 241-AP-104 Tank History

2.4.1.1 Waste History of Tank 241-AP-104

The history of the primary additions is derived from the "Waste Status and Transaction Record. Summary for the Southeast Quadrant" (Agnew 1995). Tank 241-AP-104 received N Reactor waste from the first quarter until the third quarter of 1987. The tank had two unknown transactions between the fourth quarter of 1989 and the first quarter of 1992 .

The history of the waste types is derived from the Tank Farm Surveillance and Waste status Summary Reports for January 1980 through March 1994. Tank 241-AP-104 began receiving Hanford facility waste in July 1986 and continued to receive this waste until May 1990. From June 1990 until March 1994, the tank waste was designated as dilute non-complexed waste.

The tank is currently an inactive grout feed tank. Sketch ES-TKS-E160, a graph representing the level history, and the historical waste level data used to generate the graph are in Appendix C.

\subsubsection{Temperature History of Tank 241-AP-104}

Temperatures from within Tank 241-AP-104 are recorded by 18 thermocouples. Non-suspect temperature data recorded between April 1986 and March 1995 for ali 18 thermocouples were obtained from the computer automated surveiliance system (CASS). Non-suspect temperature data taken between July 1989 and June 1995 for 12 of the thermocouples were obtained from the surveillance analysis computer system (SACS). However, not all of the 12 thermocouples have data covering that entire period. There were small breaks in the sequence of temperature data from each system. The average temperature during these periods was $61^{\circ} \mathrm{F}$ with a minimum of $46^{\circ} \mathrm{F}$, a maximum of $78^{\circ} \mathrm{F}$, and a standard deviation of $6.1^{\circ} \mathrm{F}$. Linear regressions were performed on the data for each thermocouple with a resulting average decreasing slope of $-0.0019^{\circ} \mathrm{F} / \mathrm{day}$. See tables and graphs of temperature data in Appendix D.

\subsubsection{Occurrences of Tank 241-AP-104}

One unusual event affected only Tank 241-AP-104. Occurrence report RL--WHC-Tank Farm-1991-0085 was issued in January 1991 because a loose wire to the alarm panel that monitors Tank 241-AP-104 caused a loss of power.

\subsubsection{Psychrometrics of Tank 241-AP-104}

Psychrometric data have been recorded for seven years from this tank and annulus. The apparent trend (according to the plot in Appendix F) of the evaporation rate from the tank has decreased from an average of 71.1 gallons per week in April 1987 to an average of 
38.3 gallons per week in January 1995. Over that same period, the trend in the rates of heat exchange through the primary tank ventilation has decreased. The heat exchange rates in the annular ventilation show an increasing trend. The majority of the changes in the rate of heat exchange can be attributed to changes in the average ventilation rates. A review of the psychrometric data in Tank 241-AP-104 suggests an increase in the rate of heat generation by the waste.

\subsubsection{Historical Sample Analysis Data for Tank 241-AP-104}

A single sample is available for Tank 241-AP-104 (T-513) and was analyzed on August 5, 1987 (see Appendix H). The sample was a yellow brown liquid with almost no solids. The chemical analysis revealed a high concentration of sodium and phosphorous cations with phosphate and sulfate anions. Terbium and cobalt were the primary radionuclides found during the analysis.

available.

Information on sampling procedures and methods were not

\subsubsection{Current Status of Tank 241-AP-104}

Tank 241-AP-104 was placed into service in october 1986: and currently stores approximately 22,000 gallons of waste (Hanlon, June 1995). The waste level in.the tank is measured with a FIC gauge and a manual tape and has remained. constant at about 10 inches since January 1991. See Appendix E for details on waste surface level. This tank is inactive at the present time. The tank is actively ventilated and categorized as sound. A p.lan view in Appendix G depicts the riser configuration as of August 3, 1995: Tank 241-AP-104 has 71 risers. Six 4-inch risers (no. 15, no. 21, three no. 27 's, and no. 28), three 12-inch risers (no. 7, no. 10, and no. 12), two 24-inch risers (two no. $6^{\prime} s$ ), and two 42 -inch risers (two no. 5 's) are available for. use.

\subsubsection{Inventory Estimate of Tank 241-AP-104}

The three inventory estimates derived by the Los Alamos National Laboratory (LANL) predict the physical, chemical, and radiological constituents of the supernatant, the solids and the total volume for each tank. These estimates are presented in Appendix $\mathrm{J}$.

\subsubsection{Waste Volume Approximations for Tank 241-AP-104}

The summary of approximated waste volumes, as derived from Los Alamos National Laboratory's supernatant mixing model (SMM) and tank layer model (TLM), are presented in Appendix $K$. The modeled volume is from the tank waste contents corresponding to the inventory estimate date of January 1, 1994. A tank cross-section that depicts the waste volume on the inventory estimate date is in Appendix $G$ : The total waste volume on the inventory estimate date was approximately 18,000 gallons according to the Tank Farm Surveillance and Waste Summary Report (Hanlon 1993). The total waste volume was divided by the SMM and the TLM into the following subsets. The SMM 
WHC-SD-WM-ER-315, Rev. 0

predicted 18,000 gallons of supernatant. The TLM did not predict any solids within the tank.

2.4.2.3 In-Tank Photograph of Tank 241-AP-104

No interior tank photographs are available.

2.4.3 Synopsis of Tank 241-AP-104

(To be completed.) 
2.5 Tank 241-AP-105

2.5.1 241-AP-105 Tank History

2.5.1.1 Waste History of Tank 241-AP-105

Tank 241-AP-105 has no primary additions according to the "Waste Status and Transaction Record Summary for the Southeast Quadrant" (Agnew 1995).

The history of the waste types is derived from the Tank Farm Surveillance and Waste status Summary Reports for January 1980 through March 1994. Tank 241-AP-105 began receiving non-complexed waste in July 1986 and continued to receive this waste until June 1989. From July 1989 until March 1994, the tank waste was designated as double-shell slurry feed waste.

The tank is currently an inactive concentrated waste holding tank. Sketch ES-TKS-E161, a graph representing the level history, and the historical waste level data used to generate the graph are in Appendix C.

\subsubsection{Temperature History of Tank 241-AP-105}

Temperatures from within Tank 241-AP-105 are recorded by 18 thermocouples. Non-suspect temperature data recorded between April 1986 and March 1995 for all 18 thermocouples were obtained from the computer automated surveillance system (CASS). Non-suspect temperature data taken between July 1989 and June 1995 for 12 of the thermocouples were obtained from the surveillance analysis computer system (SACS). However, not all: of the 12 thermocouples have data covering that entire period. There were small breaks in the sequence of temperature data from each system. The average temperature during these periods was $74^{\circ} \mathrm{F}$ with a minimum of $53^{\circ} \mathrm{F}$, a maximum of $121^{\circ} \mathrm{F}$, and a standard deviation of $12.8^{\circ} \mathrm{F}$. Linear regressions were performed on the data for each thermocouple with a resulting average increasing slope of $0.0101^{\circ} \mathrm{F} / \mathrm{day}$. See tables and graphs of temperature data in Appendix D.

\section{5 .1 .3 Occurrences of Tank 241-AP-105}

No unusual events exclusively concerned Tank 241-AP-105 since it went into service.

\subsubsection{Psychrometrics of Tank 241-AP-105}

Psychrometric data have been recorded for six years from this tank and annulus. The apparent trend (according to the plot in Appendix F) of the evaporation rate from the tank has increased from an average of 80 gallons per week in February 1988 to an average of 95.5 gallons per week in January 1995. Over that same period, the trend in the rates of heat exchange through the primary tank and annular ventilation has apparently increased. A review of the psychrometric data in Tank 241-AP-105 suggests an overall increasé in the rate of heat generation by the waste. 
2.5.1.5 Historical Sample Analysis Data for Tank 241-AP-105

No sampling data are available for this tank.

2.5.2 Current status of Tank 241-AP-105

Tank 241-AP-105 entered service in October 1986 and currently stores 817,000 gallons of waste (fianlon, June 1995). The waste level in the tank is measured with a FIC gauge and a manual tape and has remained constant at about 300 inches since January 1991 . See Appendix $\mathrm{E}$ for details on waste surface level. This tank is inactive at the present time. The tank is actively ventilated and categorized as sound. A plan view in Appendix $G$ depicts the riser configuration as of August 3, 1995. Tank $241-A P-105$ has 71 risers. Seven 4-inch risers (no. 15, no. 21 , no. 24 , three no. $27^{\prime} \mathrm{s}$, and no. 28), four 12inch risers (no. 7, two no. $10^{\prime} \mathrm{s}$, and no. 12), two 24-inch risers (two no. 6's), and two 42-inch risers (two no. $5^{\prime}$ s) are available for use.

2.5.2.1 Inventory Estimate of Tank 241-AP-105

The three inventory estimates derived by the Los Alamos National Laboratory (LANL) predict the physical, chemical, and radiological constituents of the supernatant, the solids and the total volume for each tank. These estimates are presented in Appendix $J$.

\subsubsection{Waste Volume Approximations for Tank 241-AP-105}

The summary of approximated waste volumes, as derived from Los Alamos National Laboratory's supernatant mixing model (SMM) and tank layer model (TLM), are presented in Appendix $\mathrm{K}$. The modeled volume is from the tank waste contents corresponding to the inventory estimate date of January 1, 1994. A tank cross-section that depicts the waste volume on the inventory estimate date is in Appendix G. The total waste volume on the inventary estimate date was approximately 821,000 gallons according to the Tank Farm Surveillance and Waste Summary Report (Hanlon 1993). The total waste volume was divided by the SMM and the TLM into the following subsets. The SMM predicted 821,000 gallons of supernatant. The TLM did not predict any solids within the tank.

2.5.2.3 In-Tank Photograph of Tank 241-AP-105

No interior tank photographs are available.

2.5 .3 Symopsis of Tank 241-AP-105

(To be completed.) 
2.6 Tank 241-AP-106

2.6.1 241-AP-106 Tank History

2.6.1.1 Waste History of Tank 2.41-AP-106

Tank 241-AP-106 has no primary additions according to the "Waste Status and Transaction Record Summary for the Southeast Quadrant" (Agnew 1995).

The history of the waste types is derived from the Tank Farm Surveillance and Waste status Summary Reports for January 1980 through March 1994. Tank 241-AP-106 began receiving Hanford facility waste in July $1986^{\circ}$ and continued to receive this waste until September 1986. From October 1986 until May 1990, the tank received non-complexed waste. From June 1990 until March 1994, the tank waste was designated as dilute non-complexed waste.

The tank is currently an inactive dilute receiver tank. Sketch ES-TKS-E162, a graph representing the level history, and the historical waste level data used to generate the graph are in Appendix $\mathrm{C}$.

\subsubsection{Temperature History of Tank 241-AP-106}

Temperatures from within Tank 24i-AP-106 are recorded by 18 thermocouples. Non-suspect temperature data recorded between April 1986 and March 1995 for all 18 thermocouples were obtained from the computer automated surveillance system (CASS). Non-suspect temperature data taken between July 1989 and June 1995 for 12 of the thermocouples were obtained from the surveillance analysis computer system (SACS). However, not all of the 12 thermocouples have data covering that entire period. There were small breaks in the sequence of temperature data from each system. The average temperature during these periods was $70^{\circ} \mathrm{F}$ with a minimum of $52^{\circ} \mathrm{F}$, a maximum of $119^{\circ} \mathrm{F}$, and a standard deviation of $12.6^{\circ} \mathrm{F}$. Linear regressions were performed on the data for each thermocouple with a resulting average increasing slope of $0.0076^{\circ} \mathrm{F} / \mathrm{day}$. See tables and graphs of temperature data in Appendix D.

\section{6 .1 .3 Occurrences of Tank 241-AP-106}

No unusual events exclusively concerned this tank since it went into service.

\subsubsection{Psychrometrics of Tank 241-AP-106}

Psychrometric data have been recorded for six years from this tank and annulus. The apparent trend (according to the plot in Appendix F) of the evaporation rate from the tank has decreased from an average of 94.7 gallons per week in May 1988 to an average of 57 gallons per week in January 1995. Over that same period, the trend in the rates of heat exchange through the primary tank ventilation has decreased. The rates of heat exchange through the annular ventilation show an increasing trend. The majority of the changes in 
the rate of heat exchange can be attributed to changes in the average ventilation rates. A review of the psychrometric data in Tank 241-AP-106 suggests an increase in the rate of heat generation by the waste.

\subsubsection{Historical Sample Analysis Data for Tank 241-AP-106}

No sampling data are available for this tank.

\subsubsection{Current Status of Tank 241-AP-106}

Tank 241-AP-106 was placed in service in october 1986 and currently stores 108,000 gallons of waste (fanlon, June 1995). The waste level in the tank is measured with a FIC gauge and a manual tape and has remained constant at about 410 inches from January 1991 to April 1995. From April 1995 to May 1995, the waste level dropped to about 40 inches. See Appendix $\mathrm{E}$ for details on waste surface level. The tank is actively ventilated and categorized as sound. A plan view in Appendix G depicts the riser configuration as of August 3, 1995. Tank 241-AP-106 has 71 irisers. Seven 4-inch risers (no. 15, no. 21, no. 24 , three no. $27^{\prime} \mathrm{s}$, and no. 28), four 12-inch risers (no. 7 , two no. $10^{\prime} \mathrm{s}$, and no. 12), two 24 -inch risers (two no. $6^{\prime} \mathrm{s}$ ), and two 42-inch risers (two no. 5's) are available for use.

\subsubsection{Inventory Estimate of Tank 241-AP-106}

The three inventory estimates derived by the Los Alamos National Laboratory (LANL) predict the physical, chemical, and radiological constituents of the supernatant, the solids and the total volume for each tank. These estimates are presented in Appendix $\mathrm{J}$.

\subsubsection{Waste Volume Approximations for Tank 241-AP-106}

The summary of approximated waste volumes, as derived from Los Alamos National Laboratory's supernatant mixing model' (SMM) and tank layer model (TLM), are presented in Appendix $K$. The modeled volume is from the tank waste contents corresponding to the inventory estimate date of January 1, 1994.: A tank cross-section that depicts the waste volume on the inventory estimate date is in Appendix $G$. The total waste volume on the inventory estimate date was approximately $1,128,000$ gallons according to the Tank Farm Surveillance and Waste Sumary Report (Hanlon 1993). The total waste volume. was divided by the SMM and the TIM into the following subsets. The SMM predicted 1,128,000 gallons of supernatant. The TLM did not predict any solids within the tank.

2.6.2.3 In-Tank Photograph of Tank 241-AP-106

No interior tank photographs are available.

2.6.3 Synopsis of Tank 241-AP-106

(To be completed.) 
2.7 Tank 241-AP-107

2.7.1 241-AP-107 Tank History

2.7.1.1 Waste Fistory of Tank 241-AP-107

The history of the primary additions is derived from the "Waste Status and Transaction Record. Summary for the Southeast Quadrant" (Agnew 1995). Tank 241-AP-107 received PUREX ammonia scrubber feed waste and an unknown transaction during the first quarter of 1990.

The history of the waste types is derived from the Tank Farm Surveillance and Waste status Summary Reports for January 1980 through March 1994. Tank 241-AP-107 began receiving double-shel1 slurry feed waste in July 1986 and continued to receive this waste until September 1986. From October 1986 until May 1990, the tank received non-complexed waste. The tank waste was designated as dilute non-complexed waste from June 1990 until March 1994.

The tank is currently an active dilute receiver tank with waste being concentrated by the 242-A Evaporator. Sketch ES-TKS-E163, a graph representing the level history, and the historical waste level data used to generaţe the graph are in Appendix C.

2.7.1.2 Temperature History of Tank 241-AP-107

Temperatures from within Tank 241-AP-107 are recorded by 18 thermocouples. Non-suspect temperature data recorded between April 1986 and March 1995 for 16 of 18 thermocouples were obtained from the computer automated surveiliance system (CASS). However, not all of the 16 thermocouples have data covering that entire period. Nonsuspect temperature data taken between July 1989 and June 1995 for 13 of the thermocouples were obtained from the surveillance analysis computer system (SACS). However, not all of the 13 thermocouples have data covering that entire period. There were small breaks in the sequence of temperature data from each system. The average temperature during these periods was $65^{\circ} \mathrm{F}$ with a minimum of $47^{\circ} \mathrm{F}$, a maximum of $104^{\circ} \mathrm{F}$, and $a$. standard deviation of $12.3^{\circ} \mathrm{F}$. Linear regressions were performed on the data from 15 of the 18 thermocouples with a resulting average increasing slope of $0.0072^{\circ} \mathrm{F} / \mathrm{day}$. The temperature data from 3 of the 18 thermocouples consistently disagreed with the trend of the rest and, subsequently, were not considered in the regressions. See tables and graphs of temperature data in Appendix D.

\section{7 .1 .3 Occurrences of Tank 241-AP-107}

No unusual events concerned the tank since it went into service.

2.7.1.4 Psychrometrics of Tank 241-AP-107

Psychrometric data have been recorded from this tank for five years and from the annulus for six years. The apparent trend (according to the plot in Appendix F) of the evaporation rate from 
the tank has increased from an average of 122.3 gallons per week in December 1989 to an average of 184.6 gallons per week in January 1995. Over that same period, the trend in the rates of heat exchange through the primary tank and annular ventilation has apparently increased. A review of the psychrometric data in Tank 241-AP-107 suggests an overall increase in the rate of heat generation by the waste.

\subsubsection{Historical Sample Analysis Data for Tank 241-AP-107}

No sampling data are available for this tank.

\subsubsection{Current Status of Tank 241-AP-107}

Tank 241-AP-107 was placed in service in October 1986 and currently stores 28,000 gallons of waste (Hanlon, June 1995). The waste level in the tank is measured with a FIC gauge and a manual tape, and has decreased from 405 inches to 59 inches in October 1994. In March 1995, the waste level increased to about 380 inches and then decreased to about 10 inches in June 1995. The decrease was due to transferring waste to Tank 241-AW-102 on October 1994. See Appendix $\mathbf{E}$ for details on waste surface level. This tank is active and the waste is currently being concentrated by the $242-\mathrm{A}$ Evaporator. The tank is actively ventilated and categorized as sound. A plan view in Appendix $G$ depicts the riser configuration as of August 3, 1995. Tank 241-ÄP-107 has 71 risers. Seven 4-inch risers (no. 15 , no. 21 , no. 24 , three no. 27 's, and no. 28 ), three 12-inch risers (two no. 10's and no. 12), two 24-inch risers (two no. 6's), and three 42-inch risers (three no. 5's) are available for use.

\subsubsection{Inventory Estimate of Tank 241-AP-107}

The three inventory estimates derived by the Los Alamos National Laboratory (LANL) predict the physical, chemical, and radiological constituents of the supernatant, the solids and the total volume for each tank. These estimates are presented in Appendix J.

\subsubsection{Waste Volume Approximations for Tank 241-AP-107}

The summary of approximated waste volumes, as derived from Los Alamos National Laboratory's supernatant mixing model (SMM) and tank layer model (TLM), are presented in Appendix $\mathrm{K}$. The modeled volume is from the tank waste contents corresponding to the inventory estimate date of January 1, 1994. A tank cross-section that depicts the waste volume on the inventory estimate date is in Appendix $G$. The total waste volume on the inventory estimate date was approximately $1,108,000$ gallons according to the Tank Farm Surveillance and Waste Sumary Report (Hanlon 1993). The total waste volume was divided by the SMM and the TLM into the following subsets. The SMM predicted 1,108,000 gallons of supernatant. The TLM did not predict any solids within the tank.

2.7.2.3 In-Tank Photograph of Tank 241-AP-107

No interior tank photographs are available. 
2.7.3 Synopsis of Tank 241-AP-107

(To be completed.) . 
2.8 Tank 241-AP-108

2.8.1 241-AP-108 Tank History

2.8.1.1 Waste History of Tank 241-AP-10.8

The history of the primary additions is derived from the "Waste Status and Transaction Record Summary for the Southeast Quadrant" (Agnew 1995). Tank 241-AP-108 received PUREX ammonia scrubber feed waste during the first quarter of 1990. From the first quarter of 1991 until the second quarter of 1992, the tank received PUREX lowlevel waste. The tank had two unknown transactions between the fourth quarter of 1991 and the fourth quarter of 1992 . The tank received dilute non-complexed waste from the 200-East Area singleshell tanks during the first quarter of 1994.

The history of the waste types is derived from the Tank Farm Surveillance and Waste status Summary Reports for January 1980 through March 1994. Tank 241-AP-108 began receiving double-shell slurry feed waste in July 1986 and continued to receive this waste until September 1986. From October 1986 until May 1990, the tank received non-complexed waste. From June 1990 until March 1994, the tank was designated as containing dilute non-complexed waste.

The tank is currently an active dilute receiver tank with waste being concentrated by the 242-A Evaporator. Sketch ES-TKS-E164, a graph representing the level history, and the historical waste level data used to generate the graph are in Appendix C.

\subsubsection{Temperature History of Tank 241-AP-108}

Temperatures from within Tank 241-AP-108 are recorded by 18 thermocouples. Non-suspect temperature data recorded between April 1986 and March 1995 for all 18 thermocouples were obtained from the computer automated surveiliance system (CASS). Non-suspect temperature data taken between July 1989 and June 1995 for 12 of the thermocouples were obtained from the surveillance analysis computer system (SACS). However, not all of the 12 thermocouples have data covering that entire period. There were smalil breaks in the sequence of temperature data from each system. The average temperature during these periods was $69^{\circ} \mathrm{F}$ with a minimum of $50^{\circ} \mathrm{F}$, a maximum of $117^{\circ} \mathrm{F}$, and a standard deviation of $14.3^{\circ} \mathrm{F}$. Linear regressions were performed on the data for each thermocouple with a resulting average increasing slope of $0.0102^{\circ} \mathrm{F} / \mathrm{day}$. See tables and graphs of temperature data in Appendix $D$.

\subsubsection{Occurrences of Tank 241-AP-108}

No unusual events concerned the tank since it went into service.

\subsubsection{Psychrometrics of Tank 241-AP-108}

Psychrometric data have been recorded from this tank for five years and from the annulus for six years. The apparent trend (according to the plot in Appendix F) of the evaporation rate from 
the tank has increased from an average of 42.3 gallons per week in December 1989 to an average of 125.9 gallons per week in January 1995. Over that same period, the trend in the rates of heat exchange through the primary tank and annular ventilation has apparently increased. A review of the psychrometric data in Tank 241-AP-108 suggests an overall increase in the rate of heat generation by the waste.

\subsubsection{Historical Sample Analysis Data for Tank 241-AP-108}

No sampling data are available for this tank.

\subsubsection{Current Status of Tank 241-AP-108}

Tank 241-AP-108 entered service in October 1986 and currently stores 29,000 gallons of waste (Hanlon, June 1995). The waste level in the tank is measured with a FIC gauge and a manual tape and has increased from about 50 inches in January 1991 to 340 inches in November 1991. In February 1994, the surface level increased to about 410 inches. The waste level then decreased to 288 inches in January 1995. In 1995, the tank has decreased to about 10 inches, increased to about 360 inches, and then decreased to about 10 inches. The increase was attributed to waste received from Tank 241-AY-102 in November 1991. The decrease was because waste was transferred to Tanks 241-AP-101 and 241-AW-102 in October 1994. See Appendix E for details on waste surface level. This tank isiactive and the waste is currently being concentrated by the 242-A Evaporator. The tank is actively ventilated and categorized as sound. A plan view in Appendix $G$ depicts the riser configuration as of August $3,1995$. Tank 241-AP-108 has 71 risers, Seven 4 -inch risers (no. 15, no. 21, no. 24, three no. 27 's, and no. 28), four 12-inch risers, (no. 7, two no. 10 's, and no. 12), two 24-inch risers (two no. 6 's), and two 42inch risers (two no. 5's) are available for use.

\subsubsection{Inventory Estimate of Tank 241-AP-108}

The three inventory estimates derived by the Los Alamos National Laboratory (LANL) predict the physical, chemical, and radiological constituents of the supernatant, the solids and the total volume for each tank. These estimate's are presented in Appendix $\mathrm{J}$.

\subsubsection{Waste Volume Approximations for Tank 241-AP-108}

The summary of approximated waste volumes, as derived from Los Alamos National Laboratory's supernatant mixing model (SMM) and tank layer model (TLM), are presented in Appendix $K$. The modeled volume is from the tank waste contents corresponding to the inventory estimate date of January 1, 1994. A tank cross-section that depicts the waste volume on the inventory estimate date is in Appendix.G. The total waste volume on the inventory estimate date was approximately 899,000 gallons according to the Tank Farm Surveillance and Waste Summary Report(Hanlon 1993). The total waste volume was divided by the SMM and the TLM into the following subsets. The SMM predicted 899,000 gallons of supernatant. The TLM did not predict any solids within the tank. 
2.8.2.3 In-Tank Photograph of Tank 241-AP-108

No interior tank photographs are available.

2.8.3 Synopsis of Tank 241-AP-108

(To be completed.) 


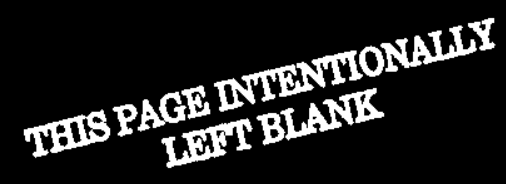


APPENDIX A

Glossary 



\section{GLOSSARY}
0.17
Transaction flag key-monthly volumes derived from semiannual reports.
0.33
Transaction flag key-monthly volumes derived from quarterly reports.
1
1AYIN
IAZIN
$1 \mathrm{C}$
1CEB
$1 \mathrm{CS}$
1st Generation Tank
$222-B$
$222-C$
$222-\mathrm{T}$
$222-U$
224
$224-F$
$231 \dot{z}$
242-A
$242-B$
$242-5$
Monthly report
See Waste Abbreviations (pg. A29-A40).
See Waste Abbreviations (pg. A29-A40).
See Waste Abbreviations and first- and second-cycle decontamination waste.
See Waste Abbreviations (pg. A29-A40).
See Waste Abbreviations (pg. A29-A40).
The original tank design found in Tank Farms B, C, T, U (excluding the 200 series tanks), and BX. These tanks have an operating capacity of 530,000 gallons, a 75-foot diameter, a 12-inch dish bottom, and a 4-foot knuckle. See Type II tanks.
See Facilities (pg. A41).
See Facilities (pg. A41).
See Facilities (pg. A41).
See Facilities (pg. A41).
See Waste Abbreviations (pg. A29-A40).
See Waste Abbreviations (pg. A29-A40).
See Waste Abbreviations (pg. A29-A40).
See Facilities (pg. A41).
See Facilities (pg. A41).
See Facilities (pg. A41). 
$242-T$

2AYIN

2AZIN

$2 \mathrm{C}$

2nd Generation Tank

2SYIN

3

3ANIN

3rd Generation Tank

4th Generation Tank

5

$5-6 \#$

5AWIN

5th Generation Tank

6

6AWIN

A Plant (PUREX)
See Facilities (pg. A41).

See Waste Abbreviations (pg. A29-A40).

See Waste Abbreviations (pg. A29-A40).

See Waste Abbreviations (pg. A29-A40)

and first- and second-cycle

decontamination waste.

Same as original tank design (1st generation or Type II) except the operating capacity was increased to 758,000 gallons. See Type III tanks.

See Waste Abbreviations (pg. A29-A40).

Quarterly report

See Waste Abbreviations (pg. A29-A40).

The first generation of the Type IV tanks, contained only in the SX Tank Farm. These tanks have a 1,000,000 gallon operating capacity, a 75-foot diameter, a 14.875-inch dish bottom, and no knuckle. See Type IV tanks.

The second generation of the Type IV tanks, found only in the A Tank Farm. These tanks are the same as the 3rd generation except they have a flat bottom. See Type IV tanks.

See Waste Abbreviations (pg. A29-A40).

Cell 5 tank 6 in B Plant

See Waste Abbreviations (pg. A29-A40).

The third generation of the Type IV tanks, found only in the AX Tank Farm. These tanks are the same as the 4 th generation with the addition of a grid of drain slots in the concrete beneath the bottom of primary steel liner.

Semiannual report

See Waste Abbreviations (pg. A29-A40).

See Facilities (pg. A41). 
A1 SLTCK

A2 SLTSLRY

Active Tank

Active Drywel1

ADD

ADJ

AGE, AGING

Aging Waste

Airlift Circulator

Annulus

AR Vault

AR

Assumed Leaker
See Waste Abbreviations (pg. A29-A40).

See Waste Abbreviations (pg. A29-A40).

A tank that contains more than 33,000 gallons of waste and/or is still involved in waste management operations.

Drywell in which radiation readings of greater than 50 counts/second are detected. The readings must be consistent as to depth and radiation level for repeated readings to be considered active.

Add primary waste from process.

Adjustment to waste amount; see CORR. Abbreviations for aging waste

High-level, first-cycle solvent extraction waste from the PUREX Plant sent to tanks to allow decay (aging) of the radionuclides, thus reducing the high temperature of the waste; see NCAW.

A device installed in tanks used to age wastes. The circulators promote mixing of the supernatant and prevent agglomeration of radionuclides. By maintaining motion within the body of liquid, the circulators minimize overheating and, consequently, minimize bumping.

A vessel space in the form of a ring; the space between concentric circular walls. The annulus of the double-shell tanks includes all of the space between the two steel liners.

A structure containing tanks and chemical processing equipment, used for waste processing or storage operations.

See Waste Abbreviations (pg. A29-A40).

A waste storage tank for which past surveillance data has indicated a loss of liquid attributed to a breach of integrity. 
WHC-SD-WM-ER-315, Rev. 0

Assumed Re-leaker

Assumed Leaking Tank

B

B860N

B SLTCK

B Plant (222-B)

Background Radiation

Baseline

BESH

BL

BLEB

BLIX

BLIXB

BNW

BPDCC

BPDCS

BPDCV

BPFPS

BPLCS
A designation that is applied when surveillance data indicate a new loss of liquid from a tank that has been declared an "assumed leaker."

In 1984, the designations of "suspect leaker," "questionable integrity," "confirmed leaker," "declared leaker," "borderline," and "dormant" were merged into one category called "assumed leaker."

See Waste Abbreviations (pg. A29-A40).

See Waste Abbreviations (pg. A29-A40).

See Waste Abbreviations (pg. A29-A40).

See Facilities (pg. A41).

Radioactivity from naturally occurring sources; primarily radiation from cosmogenic and naturally occurring radionuclides.

A reference; for example, a specified liquid level or radiation level against which new information is compared.

See Waste Abbreviations (pg. A29-A40).

See Waste Abbreviations (pg. A29-A40).

See Waste Abbreviations (pg. A29-A40).

See Waste Abbreviations (pg. A29-A40).

See Waste Abbreviations (pg. A29-A40).

Battelle Northwest Laboratory; also, see Waste Abbreviations.

See Waste Abbreviations (pg. A29-A40).

See Waste Abbreviations (pg. A29-A40).

See Waste Abbreviations (pg. A29-A40).

See Waste Abbreviations (pg. A29-A40).

See Waste Abbreviations (pg. A29-A40). 
WHC-SD-WM-ER-315, Rev. 0

BPLDC

BPLDN

Bumping, Tank Bump

BVCLN

BY SLTCK

C Plant (222-C)

Caisson

Calcine

CARB

CAS

Cascade

CASS

Catch Tanks
See Waste Abbreviations (pg. A29-A40).

See Waste Abbreviations (pg. A29-A40).

A tank bump occurs when solids overheat in the lower portion of the tank. The hot solids rapidly transfer heat to the liquid, some of which quickly vaporizes. The sudden pressurization caused by vapor generation is called a "bump."

See Waste Abbreviations (pg. A29-A40).

See Waste Abbreviations (pg. A29-A40).

See Facilities (pg. 38); see strontium Semiworks; see SSW in Waste Abbreviations (pg. 36).

An underground structure used to store high-level waste; typical designs include corrugated metal or concrete cylinders, 55-galion drums welded endto-end, and vertical steel pipes.

To heat a substance to a high temperature, but below its melting point, causing loss of volatile constituents such as moisture; refers also to the material produced by this process.

See Waste Abbreviations (pg. A29-A40) and organic wash waste.

Cascade; see SET and END.

Eleven of the single-shell tank farms (all except the AX Tank Farm) were equipped with overflow lines between tanks. The tanks were connected in series and were placed at different elevations creating a downhill gradient for liquids to flow from one tank to another. Thus, multiple tanks could be filled with one pump.

Computer automated surveillance system

Small capacity single-shell tanks associated with diversion boxes and diverter stations. The tanks are 
designed to receive any transfer line clean out, spills or leakage from the boxes, or leakage from the adjacent pipe encasement.

CAN

$\mathrm{CC}$

CCGL

CCGR

CCPL, CCPLX

$\operatorname{CCW}$

$\mathrm{CDF}$

$C E$

CELI 23

CEM

CF

CON

COND

Condensate

Conductivity Probe

Confirmed or Declared Leaker

COOL

CORR
Current acid waste; this is PUREX acid high-level waste.

See Waste Abbreviations (pg. A29-A40).

See Waste Abbreviations (pg. A29-A40).

See Waste Abbreviations (pg. A29-A40).

See Waste Abbreviations (pg. A29-A40).

Concentrated customer waste

Composition data file or transaction flag key-unit volume assumed to make stream active.

See Waste Abbreviations (pg. A29-A40).

Waste from Cell 23 at B Plant; Cell 23 contained an evaporator.

See Waste Abbreviations (pg. A29-A40).

See Waste Abbreviations (pg. A29-A40).

See Waste Abbreviations (pg. A29-A40).

Condensate; see EVAP, EB.

Liquid formed from cooling vapors

A device that completes an electrical circuit when contacted by a conductive material.

The designation of any underground waste storage tank where the data are considered sufficient to support a conclusion with 95 percent confidence that the tank has leaked.

Change in waste volume due to cooling; see CORR.

Correction to waste amount; see ADJ, LEAK, COOL.

$$
\text { A- } 6
$$


$\mathrm{CP}$

CPLX

CR Vault

Crib

Crust

CSFD

CSR

css

CST

CSWLE

CSWLW

CTW

$\mathrm{CW}$

CW/ZR1

$\mathrm{CW} / \mathrm{ZR} 2$

CWP

CWP2

CWR

CWR1
See Waste Abbreviations (pg. A29-A40).

See Waste Abbreviations (pg. A29-A40).

Facility located adjacent to C Tank Farm, used for scavenging campaign following uranium recovery. Ferrocyanide was added to tank supernatant in the CR Vault and the slurry was returned to C Tank Farm.

A linear excavation approximately 15 feet in depth with a perforated pipe along the bottom. The ditch was backfilled with broken rock, gravel, or other loose material and then covered by soil and a membrane with very low permeablity. The pipe distributed intermediate level liquid wastes along the crib.

A hard surface layer that has formed on top of the liquid in many waste.tanks that contain concentrated solutions.

See Waste Abbreviations (pg. A29-A40).

See Waste Abbreviations (pg. A29-A40).

See Waste Abbreviations (pg. A29-A40).

See Waste Abbreviations (pg. A29-A40).

See Waste Abbreviations (pg. A29-A40).

See Waste Abbreviations (pg. A29-A40).

Caustic waste for makeup

See Waste Abbreviations (pg. A29-A40).

See Waste Abbreviations (pg. A29-A40).

See Waste Abbreviations (pg. A29-A40).

See Waste Abbreviations (pg. A29-A40).

See Waste Abbreviations (pg. A29-A40).

See Waste Abbreviations (pg. A29-A40).

See Waste Abbreviations (pg. A29-A40). 
WHC-SD-WM-ER-315, Rev. 0

CWR/Al

$\operatorname{CX} 70$

D

DBP

DC

DCS

$\mathrm{DE}$

Deliquescent

Desiccant

Diatomaceous Earth

DIL

DILFD

Ditch

Diversion Box

DN

DN/PT

$\mathrm{DN} / \mathrm{PD}$
See Waste Abbreviations (pg. A29-A40).

See Waste Abbreviations (pg. A29-A40).

Transaction Flag Key-amount by

difference

Dibutyl phosphate

See Waste Abbreviations (pg. A29-A40).

Dilute caustic solutions

Diatomaceous earth; this was added to Tanks 241-BX-102, -SX-113, -TX-106, $-\mathrm{TY}-106$, and $-\mathrm{U}-104$ from 1970 to 1972 .

Also, see Waste Abbreviations (pg. A29A40).

Capable of absorbing moisture from the air and dissolving to become a liquid.

A drying agent such as diatomaceous earth.

Diatomite; a light friable siliceous material derived from diatom (algae) remains. It was added to selected underground waste storage tanks to absorb and, thereby, immobilize residual liquids (i.e. help stabilize the tank).

Dilution

\section{Dilute feed}

A linearly oriented excavation often used for the temporary diversion or disposal of process waste streams.

A below grade, concrete enclosure containing the remotely maintained jumpers and spare nozzles for the routing of waste solution to storage tank farms.

See Waste Abbreviations (pg. A29-A40). See Waste Abbreviations (pg. A29-A40). See Waste Abbreviations (pg. A29-A40). 
Double-Shell Tank

Double-Shell slurry

Double-Shell slurry Feed

Drywe 11

Drywell (in-tank)

DSS

DSSF

DST

DUMM， DUMMY

DW

DWBIX

E

EB

EDTA
The newer underground waste storage tanks consisting of a concrete shell and two concentric carbon steel liners with an annulus between the liners.

Waste that exceeds the sodium aluminate saturation boundary in the evaporator without exceeding receiver tank composition limits. It is considered a solid for reporting purposes.

Waste concentrated just before reaching the sodium aluminate saturation boundary in the evaporator without exceeding receiver tank composition limits. This form is not as concentrated as doubleshell siurry.

A steel casing, generally 6 inches in diameter, drilled into the ground to various depths and used to insert monitoring instruments for measuring the presence of radioactivity or moisture content.

A sealed casing within a tank that is attached to a riser. It is used to insert experimental equipment, such as neutron or acoustical probes, to determine the level of drainable interstitial liquor.

See Waste Abbreviations and double-shell slurry.

See Waste Abbreviations and double-shell slurry feed.

Double-shell tank

See Waste Abbreviations (pg. A29-A40).

See Waste Abbreviations (pg. A29-A40).

See Waste Abbreviations (pg. A29-A40).

Transaction flag key-waste transferred through evaporator.

See Waste Abbreviations (pg. A29-A40).

Ethylenediaminetetra-acetic acid 
WHC-SD-WM-ER-315, Rev. 0

EF

EFD

END

ENRAF

EV

EVAP

EVAPF

Evaporator Feed
See Waste Abbreviations (pg. A29-A40).

See Waste Abbreviations (pg. A29-A40).

Disconnect cascaded tanks; see CAS, SET.

Electronic surface level gauge that uses a weighted plummet connected to a scale for measuring waste surface level. At Hanford, they are electrically connected to a computer for data transmission, analysis, and reporting.

Evaporation

See Waste Abbreviations (pg. A29-A40).

See Waste Abbreviations (pg. A29-A40).

Any waste liquid that can be concentrated to form saltcake; e.g., aged waste, low heat waste, dilute interstitial liquor, and other radioactive waste solutions.

Evaporator-Crystallizer 242-A and 242-S waste concentration facilities that operate at a reduced pressure (vacuum) and are capable of producing a slurry containing about 30 volume percent solids at a specific gravity greater than 1.6 .

EVS

Partial neutralization in the $242-5$ Evaporator.

EVT

ED

FECN

Ferrocyanide

FIC gauge
HEDTA destruction in the $242-\mathrm{B}$ or $242-\mathrm{T}$ Evaporators.

See Waste Abbreviations (pg. A29-A40).

Ferrocyanide wastes created during a scavenging campaign from 1953-57. See SCAV, POO-P\#\#, TOO-T\#\#.

An ion composed of iron and cyanide with the chemical formula of $\mathrm{Fe}(\mathrm{CN})_{6}^{-4}$.

A Food Instrument Corporation (a.k.a. Food Industry Corporation) automatic liquid level gauge based on a conductivity probe. At Hanford, they are electrically connected to a computer 
for data transmission, analysis, and reporting. Local readings may also be obtained from a dial.

First- and Second-Cycle Waste containing $10 \%$ of the original Decontamination Wastes fission product activity and 28 of the products. By-product cake solution was mixed with product waste and neutralized with $50 \%$ caustic. The waste contained a mixture of suspended solids, iron, hydroxides, carbonate, phosphate, scavenger metals, chromium, sodium, and silicofluoride.

FLSH

FP

GA

Gamma Ray

GAS

GM Instrument

GROUP

Gunite

$\mathrm{H}_{2} \mathrm{O}$

HDRL

HEAT
See Waste Abbreviations (pg. A29-A40).

Fission product waste (see Waste Abbreviations, pg. 28-38)-produced at B Plant and Hot Semiworks in campaigns for cesium and strontium recovery during the $1960^{\prime} \mathrm{s}$. Cesium was removed from PUREX supernatant and strontium was removed from PUREX sludge; both were removed from acidic waste.

Gain to tank; any increase in volume

Electromagnetic radiation emitted by the nuclei of some radioactive substances during decay, similar to $x$-rays.

Slurry growth resulting from gas generation.

Instrument for detecting low-level beta and gamma radiation using a GeigerMueller tube.

A group of tanks where ITS was used to evaporate the supernatant phases. See ITS.

A.building material, consisting of a mixture of cement, sand, and water, that is sprayed onto a mold.

Water

See Waste Abbreviations (pg. A29-A40).

A tank correction; see CORR, COOL. 
WHC-SD-WM-ER-315, Rev. 0

HEDTA

Heel

Hexone

High-Heat Load

High-Level Waste

Historical Information

HLO

$\mathrm{HLW}$

HOT-SEMI

HS

HWVP

$I \& S$

Inactive Tank
N-hydroxyethylenediaminetri-acetic acid

The amount remaining in a vessel or container after most of the contents have been removed.

Methyl isobutyl ketone, an organic solvent used in the REDOX solvent extraction process. Also known as isobutyl methyl ketone, methyl isobutyl ketone, 4-methyl 2-pentanone, MIBK.

Heat load in tanks produced by radionuclides exceeds $40,000 \mathrm{Btu} / \mathrm{hr}$.

Waste from the fuel reprocessing operations in separations plants.

All information obtained from published and unpublished sources. Unpublished sources include photographs and electronically retrieved data (i.e. liquid observation well data, sampling results, temperatures, and surface level data).

Hanford Laboratory Operations; also, see Waste Abbreviations (pg. A29-A40).

See Waste Abbreviations (pg. A29-A40).

See HS, SSW.

Hot Semiworks (C Plant); a pilot facility with a variety of operations. See Waste Abbreviations (pg. A29-A40).

Dilute non-complexed waste from the vitrification plant.

Tank isolated and stabilized

A tank that has been removed from liquid-processing service, has been pumped to less than 33,000 gallons of waste, and will be, or is in the process of being, stabilized and interimly isolated. This includes all tanks not in active or active-restricted categories. Also included are inactive

- spare tanks that would be used if an active tank failed. 
INST

Interim Isolation

Interim Stabilization

Interstitial Liquor

Interstitial

Intrusion Mode

FIC Setting

Intrusion Prevention
Change in tank level due to change in instrumentation.

An administrative designation reflecting the completion of the physical effort required to minimize the addition of liquids into an inactive storage tank, process vault, sump, catch tank, or diversion box. (In June 1993, "interim isolation" was replaced by "intrusion prevention".)

A tank which contains less than 50,000 gallons of drainable interstitial liquid and less than 5,000 gallons of supernatant. If a jet pump was used to achieve interim stabilization, then the jet pump flowrate must have been at or below 0.05 gallons per minute before interim stabilization was completed.

The liquid that fills the interstices (voids) within the solid material in a waste tank.

The volume within a solid matrix made up of the crevices, cracks, and other voids.

The FIC probe is positioned a short distance above the waste surface. If the surface level of the waste in the tank increases, thereby touching the probe tip, a positive indication is received.

An administrative designation reflecting the completion of the physical effort required to minimize the addition of liquids into an inactive storage tank, process vault, catch tank, sump, or diversion box.

Tank is interimly isolated.

The In-Tank Solidification Program was used to reduce waste volume by evaporating the wastes from inside certain tanks in the BX and BY Farms. The process used both hot air (ITS-1) and an electric heater (ITS-2). 
WHC-SD-WM-ER-315, Rev . 0

IWW

IX

Jet Pump

Knuckle

I $222 \mathrm{~S}$

L3A4A

LaF

Lateral

Leak Detection Pit

LEAK

LETF

LIT

Level Adjustment

Level History

Liquid Observation Well (LOW)
See Waste Abbreviations (pg. A29-A40).

See Waste Abbreviations (pg. A29-A40).

A modified, commercially available, low capacity jet pump used as a saltwell pump.

Point where the side wall and the bottom curved surface of a tank meet.

See Waste Abbreviations (pg. A29-A40).

See Waste Abbreviations (pg. A29-A40).

See Waste Abbreviations (pg. A29-A40).

Horizontal drywell under all tanks in A Tank Farm and certain tanks in SX Tank Farm.

Collection point for any leakage from tanks in AX Tank Farm. The pits are equipped with radiation and liquid detection instruments.

Tank leak volume; see CORR.

Liquid effluent treatment facility at N Reactor.

Level indicating transmitter

Any update in the waste inventory lor tank level) in a tank. The adjustments usually result from surveillance observations or historical investigations.

A diagram representing the history of the waste surface level in a tank. The diagram also includes other related data.

Liquid observation wells are used to monitor the interstitial liquid level (ILL) in single-shell waste storage tanks. Three probes are used to monitor changes in the ILL: acoustic, gamma, and neutron. Each method can indicate intrusions or leaks by increases or decreases in the ILL. 
Liquid Level Best
Engineering Judgement
Line

LO

LUNC

LW

Metal Waste (MW)

MH

Mixed Waste

Molarity

Mole

MW

MWF

N

NCAW

NCPL, NCPLEX, NCPLX
A line representing the level of interstitial liquids on the history level plot, according to engineering judgement, between the time the liquid was initially pumped until Low readings were obtained.

Loss from tank; unexplained loss of volume, does not include transfers

See Waste Abbreviations (pg. A29-A40).

See Waste Abbreviations (pg. A29-A40).

Waste from extraction containing all the uranium, approximately $90 \%$ of the original fission product activity, and approximately $1 \%$ of the product. This waste was brought to the neutral point with $50 \%$ caustic and then treated with an excess of sodium carbonate. This procedure yielded almost completely soluble waste at a minimum total volume. The exact composition of the carbonate compounds was not known, but was assumed to be a uranium phosphate carbonate mixture.

Manhole

Waste containing both radioactive and hazardous (dangerous as defined in WAC 173-303) waste.

Number of gram molecular weights (moles) per liter of solution.

The amount of a substance with weight equal to the molecular weight in grams.

See Waste Abbreviations (pg. A29-A40) and metal waste.

See Waste Abbreviations (pg. A29-A40).

See Waste Abbreviations (pg. A29-A40). See Waste Abbreviations (pg. A29-A40).

See Waste Abbreviations (pg. A29-A40) and non-complexed. 
NCRW

Neutralized PUREX Acid Waste

Neutron Probe

NFAW

NHAW

NIT

Noise At The Bottom of Well-Drywell Probe

Non-Complexed

NPH

NRAW

NRP82

NRPO4

NRSO4

NTA

Nuclear reactor
See Waste Abbreviations (pg. A29-A40).

In 1956, the original plant neutralized all of the high-level waste and sent it to the A Tank Earm. As recovery of fission products began, a portion of the waste was treated to recover strontium and then neutralized. As of 1967, all of the high-level waste left PUREX as an acid solution for treatment at $B$ Plant. See $P$, NCAW, and IWW.

Probe equipped with a neutron source and detector. They are used in dry well monitoring to determine the moisture content of the soil as one way to detect leaks in underground waste storage tanks or pipelines.

See Waste Abbreviations (pg. A29-A40).

See Waste Abbreviations (pg. A29-A40).

$\mathrm{HNO}_{3} / \mathrm{KMNO}_{4}$ solution added during evaporator operation.

Erroneous reading caused by probe contacting foreign objects at the bottom of a well.

General waste term applied to all Hanford Site liquors not identified as complexed (containing organics).

Normal paraffin hydrocarbon

See Waste Abbreviations (pg. A29-A40).

See Waste Abbreviations (pg. A29-A40).

See Waste Abbreviations (pg. A29-A40).

See Waste Abbreviations (pg. A29-A40).

Nitrilotriacetic acid

A device for creating a controlled nuclear chain reaction using atomic fuel, as for the production of energy. 
WHC-SD-WM-ER-315, Rev . 0

Open Hole Saltwell

Organic Wash Waste (OWW)

Out-of-Service-Tank

OWW

$\mathbf{P}$

P1

P2

P3

PADFG

PADWG

Partial Interim Isolation
A well in which a pump is inserted in solid waste. It is frequently used to remove the liquid from tanks containing less than two feet of sludge.

The solvent used in PUREX was treated before reuse by washing with potassium permanganate and sodium carbonate, followed by dilute nitric acid and then a sodium carbonate wash.

A tank that does not meet the definition of an in-service tank. Before September 1988, these tanks were defined as inactive. (Note: All single-shell tanks are out of service.)

See Waste Abbreviations (pg. A29-A40) and organic wash waste.

See Waste Abbreviations (pg. A29-A40).

See Waste Abbreviations (pg. A29-A40).

See Waste Abbreviations (pg. A29-A40).

See Waste Abbreviations (pg. A29-A40).

See Waste Abbreviations (pg. A29-A40).

See Waste Abbreviations (pg. A29-A40).

The administrative designation for completion of the physical effort required for Interim Isolation; except for isolating the risers and piping that will be required for jet pumping or for other methods of stabilization.

PAS

See Waste Abbreviations (pg. A29-A40).

PASF

See Waste Abbreviations (pg. A29-A40).

PAW

See Waste Abbreviations (pg. A29-A40).

PD

PDBNG

PDBSU

PDBTG
See Waste Abbreviations (pg. A29-A40).

See Waste Abbreviations (pg. A29-A40).

See Waste Abbreviations (pg. A29-A40).

See Waste Abbreviations (pg. A29-A40). 
WHC-SD-WM-ER-315, Rev. 0

PDCSS

PDL 87

PDL89

PDNSG

PDS 87

PDS89

PDSLG

PDSUP

PEECN

PEeCN1

PFeCN2

PFM

PEMMS

PFP ( 2 Plant)

PFPGR

PEPNT

PEPPT

PEPSI

$\mathrm{pH}$

Pile

PL

PL1

PL2

PML89
See Waste Abbreviations (pg. A29-A40).

See Waste Abbreviations (pg. A29-A40).

See Waste Abbreviations (pg. A29-A40).

See Waste Abbreviations (pg. A29-A40).

See Waste Abbreviations (pg. A29-A40).

See Waste Abbreviations (pg. A29-A40).

See Waste Abbreviations (pg. A29-A40).

See Waste Abbreviations (pg. A29-A40).

See Waste Abbreviations (pg. A29-A40).

See Waste Abbreviations (pg. A29-A40).

See Waste Abbreviations (pg. A29-A40).

See Waste Abbreviations (pg. A29-A40).

See Waste Abbreviations (pg. A29-A40).

See facilities (pg. A41). Also,

Plutonium Finishing Plant waste (see $P R F$, and $Z, 224$, and PFP in Waste

Abbreviations, pg. 28-38).

See Waste Abbreviations (pg. A29-A40).

See Waste Abbreviations (pg. A29-A40).

See Waste Abbreviations (pg. A29-A40).

See Waste Abbreviations (pg. A29-A40).

A measure of the hydrogen ion concentration in solution.

An early term used to describe a nuclear reactor.

See Waste Abbreviations (pg. A29-A40).

See Waste Abbreviations (pg. A29-A40).

See Waste Abbreviations (pg. A29-A40).

See Waste Abbreviations (pg. A29-A40). 
WHC-SD-WM-ER-315，Rev. 0

PMS89

PN

PNE

POO-P\#\#

Portland Cement

PRF

Primary Addition

Primary Stabilization

Probe

PSI

PSS

PSSF

Psychrometry

PT

PUREX
See Waste Abbreviations (pg. A29-A40).

See Waste Abbreviations (pg. A29-A40).

See Waste Abbreviations (pg. A29-A40).

In-Plant scavenging with FeCN; see SCAV, TOO-T\#\#.

A hydraulic cement made by finely pulverizing a mixture of clay and limestone or similar materials.

Plutonium Reclamation Facility; a facility by PFP ( $\mathrm{Z}$ Plant) that generated a type of waste (also known as PRF) from a solvent based extraction process using $\mathrm{CCl}_{4} / \mathrm{TBP}$.

An addition of waste from a specific plant or process vault. Thesé additions are listed in the "Waste Status and Transaction Record Summary for the Southeast Quadrant."

The condition of an inactive waste storage tank after all liquid above the solids, other than isolated surface pockets, has been removed. Isolated surface pockets of liquid are those not pumpable by conventional techniques.

A device used to obtain information about the environment. In this report, an instrument package designed to be inserted in drywells, risers, or ports to measure waste characteristics.

See Waste Abbreviations (pg. A29-A40).

See Waste Abbreviations (pg. A29-A40).

See Waste Abbreviations (pg. A29-A40).

Determination of humidity or dew point from wet and dry bulb temperatures with the difference in the two used as a measure of moisture in the atmosphere. See Waste Abbreviations (pg. A29-A40). See Facilities (pg. A41). 
WHC-SD-WM-ER-315, Rev. 0

PX86S

PXBAW

PXBSG

PXFTE

PXIOW

PXMET

PXMSC

PXNAN

Questionable Integrity

$\mathbf{R}$

\section{R SLTCK}

R1

R2

Radiation Zone

Radiation

Readily Available

Risers

REC

REDOX

Removed From Service (Tanks)
See Waste Abbreviations (pg. A29-A40).

See Waste Abbreviations (pg. A29-A40).

See Waste Abbreviations (pg. A29-A40).

See Waste Abbreviations (pg. A29-A40).

See Waste Abbreviations (pg. A29-A40).

See Waste Abbreviations (pg. A29-A40).

See Waste Abbreviations (pg. A29-A40).

See Waste Abbreviations (pg. A29-A40).

A designation for any tank with a small decrease in liquid level or a radiation increase in an associated dry well for which the remaining data for the tank are insufficient to support a conclusion with $95 \%$ confidence that the tank is sound.

See Waste Abbreviations (pg. A29-A40).

See Waste Abbreviations (pg. A29-A40).

See Waste Abbreviations (pg. A29-A40).

See Waste Abbreviations (pg. A29-A40).

An area containing radioactive materials in sufficient quantities to require controlling entry of personnel into the area.

Particles and electromagnetic energy emitted by nuclear transformations that are capable of producing ions when interacting with matter.

Risers with easily removable or no attached apparatus.

Receive waste from another tank; see XFER.

See Facilities (pg. A41).

Any tank that is a confirmed leaker or is not intended for reuse. 
WHC-SD-WM-ER-315, Rev. 0

Rerun Drywell Data

RESD

RIX

RMA

RMC

RSN

RSS

$\operatorname{RTX}$

S

S Plant (REDOX)

S1 SLTCK

S2 SLTSLRY

Saltcake

Saltwel1

SCAV

Scavenged

Scintillation Monitor
Surveillance data which supersedes

"suspect data."

See Waste Abbreviations (pg. A29-A40).

See Waste Abbreviations (pg. A29-A40).

Remote mechanical "A" line; process used in $\mathrm{Z}$ Plant.

Remote mechanical "C" line; process used in $\mathrm{Z}$ Plant.

See Waste Abbreviations (pg. A29-A40).

See Waste Abbreviations (pg. A29-A40).

See Waste Abbreviations (pg. A29-A40).

Transaction Flag Key-partial

neutralization (PNF).

See Facilities (pg. A41).

See Waste Abbreviations (pg. A29-A40).

See Waste Abbreviations (pg. A29-A40).

Crystallized nitrate and other salts deposited in waste tanks, usually after measures are taken to remove moisture.

A hole drilled or sluiced into a saltcake and lined with a cylindrical screen to permit drainage and jet pumping of interstitial liquors.

Scavenging campaign with FECN on TBP, 1952-57. See TOO-T\#\#, POO-P\#\#.

Waste which has been treated with ferrocyanide to remove cesium from the supernatant by precipitating it into the sludge.

A radiation detection instrument based on the principle that light pulses are produced in some materials when they are exposed to radiation. 
Self Concentrating

Self-Evaporation

SET

SIX

SI

SL3SY

Sludge

Slugs

Sluicing, or sluiced

slurry

SMP

Solids Level Best Engineering Judgement Line

Sound Tank
Liquid, high-level radioactive waste whose decaying radionuclides heat the solution sufficiently to boil off the water, thus concentrating the waste.

A waste material in which moisture is lost as the liquid changes to a vapor and diffuses into the atmosphere.

Connect cascaded tanks together; see CAS and END.

See Waste Abbreviations (pg. A29-A40).

See Waste Abbreviations (pg. A29-A40).

See Waste Abbreviations (pg. A29-A40).

Mainly insoluble metal hydroxides and oxides precipitated from neutralized waste.

An early term for uranium fuel elements which had been machined or extruded into short cylinders which were then clad or encased in corrosion-resistant metals.

To wash with water. At Hanford, this has meant to dissolve or suspend waste in solution using a high pressure water stream.

Insoluble material suspended in water or aqueous solution.

Sludge measuring port

During the initial filling of certain single-shell tanks, only the liquid level was reported. To adjust for the abrupt increase in solids level when data for solids were added to the record, a sloped line was used to reflect solids volume between the initial fill and the time the solids data were recorded.

The classification of a waste storage tank for which surveillance data indicate no loss of liquid attributed to a breach of integrity. 
SPRG

SRR

SRS

SST

SSW

STAB

Stabilization

Static Tank

Strontium Semiworks

SU

Supernatant

Surveillance

Suspect Data

SV

SW RCR

SWLIQ
Sparge-transfer of water or volume

See Waste Abbreviations (pg. A29-A40).

See Waste Abbreviations (pg. A29-A40).

Single-shell tank

See Waste Abbreviations (pg. A29-A40).

Tank stabilized by removal of liquid. Both floating suction and saltwell jet pumps are used to remove liquid.

The removal or immobilization, as completely as possible, of the liquid contained in a radioactive waste storage tank by saltwell pumping, open hole saltwell pumping, adding diatomaceous earth, etc.

A tank with no significant change in liquid level or involvement in transfer operations during a stated period of time.

C Plant or Hot Semiworks; was a pilot plant for both REDOX and PUREX, then was reconfigured for strontium recovery.

See Waste Abbreviations (pg. A29-A40) and supernatant.

The liquid quantity defined by the difference between the measured liquid level and the measured average solids level in a waste storage tank.

Regular or systematic watch kept over someone or something.

Surveillance data which does not fall within the expected range and is superseded by "reruns."

Transaction Flag Key-amount by difference in solids.

Saltwell receiver

See Waste Abbreviations (pg. A29-A40). 
SWLQW

T Plant (222-T)

T1 SLTCK

T2 SLTCK

Tank Farm

TBP

TCO

Terminal Liquor

TFeCN

$\mathrm{TH}$

Thermocouple

Thermocouple Tree

Thermowell

THI
WHC-SD-WM-ER-315， Rev. 0

See Waste Abbreviations (pg. A29-A40).

See Facilities (pg. A41).

See Waste Abbreviations (pg. A29-A40).

See Waste Abbreviations (pg. A29-A40).

An area containing a number of aboveground or underground storage tanks for storing chemicals, petroleum products, or waste.

Tributyl phosphate, a solvent used in the PUREX solvent extraction process. Also, see Waste Abbreviations.

See Waste Abbreviations (pg. A29-A40):

The liquid product from the evaporationcrystallization process which, upon

further concentration, formed a solid that was unacceptable for storage in single-shell tanks. Terminal liquor was characterized by a caustic concentration of approximately $5.5 \mathrm{M}$ (the caustic molarity was lower if the aluminum salt saturation was reached first).

See Waste Abbreviations.

See Waste Abbreviations.

A probe for measuring temperature, consisting of two dissimilar metal wires joined at one end (hot junction) with the free ends joined to a measuring instrument. Changes in electrical potential due to temperature changes at the hot end are measured and calibrated to read out as temperature.

A group of thermocouples assembled in a pipe and inserted into a waste tank for measuring temperatures at regular vertical intervals.

A well in a waste tank which contains thermocouples.

See Waste Abbreviations (pg. A29-A40). 
Thorium

TK

TL

TMACS

TOO-T\#\#

TPLAL

TPLAN

TPLAS

TR

Trench

trFlag

TRUEX

Type I Tank

Type II Tank
A chemical element which is a fertile material. Fertile means that when it is subjected to radiation in a nuclear reactor, it will be converted, in this case, to ${ }^{233} \mathrm{U}$, a potential fuel.

Tank. However, TK-17-2 was an early name for B Plant.

See Waste Abbreviations (pg. A29-A40).

Tank monitor and control system

In-tank scavenging with FECN; see SCAV, POO-P\#\# .

See Waste Abbreviations (pg. A29-A40).

See Waste Abbreviations (pg. A29-A40).

See Waste Abbreviations (pg. A29-A40).

Transfer from tank

A deep furrow in the ground. At Hanford, they are used for the disposal of solid waste.

Transaction Flag Keys (used by Wtrac)-see $\mathrm{CDF}, \mathrm{D}, \mathrm{E}, \mathrm{S}, \mathrm{SV}, 1,3,6$, $0.17,0.33$.

Tributyl Phosphate(TBP) A chemical compound, an organic solvent, used in the PUREX solvent extraction process.

Transuranic extraction process

These are the 200 series tanks found in $B, C, T$, and U Tank Farms. They have an operating capacity of 55,000 gallons, a 20-foot diameter, a six-inch deep dish bottom, and a three-foot knuckle. Generation is not associated with Type I tanks.

These are the original (1st generation) tank designs, which are found in B, C, $T$, $U$ (excluding the 200 series tanks), and BX Tank Farms. See 1st Generation Tank. 
WHC-SD-WM-ER-315, Rev. 0

Type III Tank

Type IV Tank

Type V Tank

U Plant (222-U)

$\mathrm{U} 1 \mathrm{U} 2$

UNKN

UNKNOWN, UNK

UR

Watch Iist Tank
These are the 2nd Generation tank designs, which are found in BY, $S, T X$, and TY Tank Farms. See 2nd Generation Tank.

These are $3 r d, 4$ th, and 5 th generation tank designs found in SX, A, and AX Tank Farms, respectively. See $3 r d, 4$ th, and 5th Generation Tanks.

These are the first double shell tank designs, which are found in $A Y, A Z$; and SY Tank Farms.

See Facilities (pg. A41).

See Waste Abbreviations (pg. A29-A40).

Unknown waste origin sink

See Waste Abbreviations (pg. A29-A40).

Uranium recovery operation in 222-U, 1952-57. Created TBP (primary waste, also known as UR) and FECN (scavenging wastes). See TFeCN, PEeCN, POO-P\#\#, TOO-T\#\#, FeCN, and Waste Abbreviations (pg. A29-A40).

An underground storage tank requiring special safety precautions because the tank potentially could release highlevel radioactive waste if there are uncontrolled increases in temperature or pressure. Special restrictions have been placed on these tanks by "Safety Measures for Waste Tanks at Hanford Nuclear Reservation," Section 3137, National Defense Authorization Act for Fiscal Year 1991, November 5, 1990, Public Law 101-501 (also known as the Wyden Amendment).

WATER

Flush water from various sources

WTR

Water

WWTR

WVP

XFER
Waste water

Waste volume projections

Transfer of waste out of tank 
WHC-SD-WM-ER-315, Rev. 0

Z

Z Plant

ZAW

ZHIGH

ZLAB

ZLOW

ZPRFL

ZPRES

ZRM

ZRMCL

ZRMCS
See Waste Abbreviations (pg. A29-A40).

Plutonium Finishing Plant (PFP); see Facilities (pg. A41).

See Waste Abbreviations (pg. A29-A40).

See Waste Abbreviations (pg. A29-A40).

See Waste Abbreviations (pg. A29-A40).

See Waste Abbreviations (pg. A29-A40).

See Waste Abbreviations (pg. A29-A40).

See Waste Abbreviations (pg. A29-A40).

See Waste Abbreviations (pg. A29-A40).

See Waste'Abbreviations (pg. A29-A40).

See Waste Abbreviations (pg. A29-A40). 
WHC-SD-WM-ER-315, Rev. 0

This page left intentionally blank. 
WHC-SD-WM-ER-315, Rev. 0

\section{Waste Abbreviations}

$1 A Y I N$

1AZIN

$1 \mathrm{C}$

$1 \mathrm{Cl}$

$1 \mathrm{C} 2$

1CEB

$1 \mathrm{CS}$

1CEeCN

$224,224-\mathrm{E}$

2312

2AYIN

2AZIN

$2 \mathrm{C}$

$2 \mathrm{C} 1$

$2 \mathrm{C} 2$
Concentrated complexed waste from Tank 241-AY-101 inventory

Pre-February 1981 Tank 241-AZ-101 inventory

First-cycle decontamination waste from the $\mathrm{BiPO}_{4}$ process

First-cycle decontamination waste from the $\mathrm{BiPO}_{4}$ process, 1944-51 (LANL defined waste \#3)

First-cycle decontamination waste from the $\mathrm{BiPO}_{4}$ process, 1952-56 (LANL defined waste \#4)

First-cycle evaporator bottoms

First-cycle scavenging waste

First-cycle ferrocyanide scavenging waste (LANL defined waste \#12)

$\mathrm{LaF}_{3}$ finishing waste from $\mathrm{BiPO}_{4}$ process and uranium recovery (also known as 224-U waste) in the 224 buildings by $T$ Plant and $B$ Plant and the Plutonium Finishing Plant (LANL defined waste \#7)

Dilute phosphate waste from $231-\mathrm{Z}$ laboratories

Pre-February 1981 Tank 241-AY-102 inventory

Pre-February 1981 concentrated complexed waste from Tank 241-AZ-102 inventory

Second-cycle decontamination waste from the $\mathrm{BiPO}_{4}$ process

Second-cycle decontamination waste from the $\mathrm{BiPO}_{4}$ process, 1944-51 (LANL defined waste \#5)

Second-cycle decontamination waste from the $\mathrm{BiPO}_{4}$ process, 1952-56 (LANL defined waste \#6) 
WHC-SD-WM-ER-315, Rev. 0

\section{Faste Abbreviations}

2SYIN

3AWIN

$5-6$

5AWIN

6AWIN

A1 SITCK

A2 SLTSLRY

AGE, AGING

AR

B

B SLTCK

B860N

BFSH

BI

BLEB

BLIX

BLIXB

BNW
Pre-February 1981 Tank 241-SY-102 inventory

Pre-February 1981 Tank 241-AW-103 inventory

Waste from cell 5 tank 6 in B Plant

Pre-February 1981 Tank 241-AW-105 inventory

Concentrated phosphate waste from Tank 241-AW-106 inventory

Saltcake waste. generated from the 242-A Evaporator-crystallizer, 1977-80 (LANI defined waste \#45)

Salt slurry waste generated from the 242-A Evaporator-crystallizer, 1981-94 (IANL defined waste \#46)

Aging waste

"Washed" PUREX sludge from the 244-AR vault (IANL defined waste \#31)

B Plant high-level waste from $P A W$ processed through B Plant to extract strontium (LANL defined waste \#32)

Saltcake waste generated from the 242-B Evaporator, 1951-55 (LANL defined waste \#38)

Dilute non-complexed waste from B Plant cell drainage

B Plant flush waste water

B Plant low-level waste (LANL defined waste \#33)

B Plant low-level evaporator bottoms

B Plant low level ion exchange?

B Plant low level ion exchange bottoms? Battelle Northwest Laboratory waste 
WHC-SD-WM-ER-315, Rev. 0

\section{Faste Abbreviations}

BPDCC

BPDCS

BPDCV

BPFPS

BPLCS

BPLDC

BPLDN

BVCLN

BY SLTCK

CARB

$\mathrm{CC}$

CCGL

CCGR

CCPL, CCPLX

CCW

CE

CEM

CF
Dilute complexed waste from B Plant cesium processing

Dilute complexed waste from B Plant strontium processing

Dilute complexed waste from B Plant vessel clean-out

B Plant TRU solids from retrieved PFP solids

Dilute non-complexed waste from B Plant strontium processing

Dilute complexed waste from B Plant cesium processing

Dilute non-complexed waste from B Plant cesium processing

Dilute non-complexed waste from B Plant vessel clean-out

Saltcake waste generated from in-tank solidification units 1 and $2,1965-74$ (LANL defined waste \#42)

Organic wash waste from PUREX

Complexant concentrate waste or concentrated complexant; concentrated product from evaporating dilute complexed waste

B Plant high TRU solids from retrieved complexant concentrate

Dilute non-complexed waste from retrieved complexant concentrate

Complexant concentrate; See CPLX

Concentrated customer waste

Evaporator concentrate

Concrete; see CON

Cesium feed; see CSFD 
WHC-SD-WM-ER-3I5, Rev. 0

\section{Waste Abbreviations}

CON

$\mathrm{CP}$

CPLX

CSFD

CSR

Css, cs Solids

CST

CSWLE

$\operatorname{CSWLW}$

$\mathrm{CW}$

CW/ZR1

CW/ZR2

CWP

CWP2

CWR

CWR 1

CWR/Al

$\mathrm{cx} 70$
Concrete; see CEM

Concentrated phosphate waste from $N$ Reactor decontamination

Complexed waste

Cesium feed from B Plant

Waste (supernatant) from cesium recovery at B Plant (LANL defined waste \#35)

Concentrated supernatant solids

Caustic solution

Complexed saltwell liquid from the 200-East Area

Complexed saltwell liquid from the 200-West Area

Coating (cladding) waste

Coating (cladding) waste (PUREX), Zircaloy cladding (LANL defined waste \#23)

Coating (cladding) waste (PUREX), Zircaloy cladding (LANL defined waste \#49); see NCRW and $\mathrm{PD}$; also known as CWP / ZR2

Coating (cladding) waste (PUREX); see NCRW; (LANL defined waste \#21; CWP/Al 1956-60)

Cladding (coating) waste (PUREX), (LANL defined waste \#22, CWP/A1 1961-72)

Coating (cladding) waste (REDOX)

REDOX cladding (coating) waste, (LANL defined waste \#15; CWR/Al 1952-60)

Coating (cladding) waste (REDOX), (LANL defined waste \#16; 1961-67)

Dilute complexed mixture hot-semiworks TRU solids 


\section{Raste Abbreviations}

DC

DE

DN

$\mathrm{DN} / \mathrm{PD}$

$\mathrm{DN} / \mathrm{PT}$

DSS

DSSF

DDSSE

DUMM, DUMMY

DW

DWBIX

EB

EF, EVED

EFD

EVAP

EVAPF

FD

FLSH

FP

HDRL

HFW

HLO

HLW
Dilute complexed waste

Diatomaceous earth waste

Dilute non-complexed waste

DN with $P$ TRU solids

DN with PFP TRU solids

Double-shell slurry; same as salt slurry

Double-shell slurry feed; supernatant of salt slurry

Dilute double-shell slurry feed

Dummy waste

Decontamination waste (LANL defined waste \#36)

Decontamination waste and B Plant ion exchange

Evaporator bottoms

Evaporator feed

Evaporator feed dilute

Evaporator feed (post 1976)

Dilute non-complexed waste from

evaporator pad flush

Feed dilute

Flush water

Fission products waste

Hanford defense residual liquor

Hanford facility waste

Hanford laboratory operations waste

High-level waste; generic for all

Hanford tank wastes 
WHC-SD-WM-ER-315, Rev , 0

\section{Naste Abbreviations}

HS

IWW

IX

L222S

L3A4A

IaF

LUNC

$\dot{L} W$

MW

MW1

MW2

MWE

$\mathbf{N}$

NCAW

NCPL, NCPLEX, NCPLX

NCRW

NFAW

NHAW
Hot or Strontium Semiworks waste (LANL defined waste. \#28); see SSW

Inorganic wash waste (i.e., concentrated neutralized high-level waste from PUREX); see NCAW and $P$

Ion exchange waste

222-s laboratory, dilute non-complexed waste

Dilute non-complexed laboratory wastes from 300 and 400 Areas

Lanthanum fluoride $\left(\mathrm{LaF}_{3}\right)$ finishing waste generated in $\mathrm{PFP}$

Dilute non-complexed waste from UNC fuels fabrication?

Laboratory waste

Metal waste

Metal waste from $\mathrm{BiPO}_{4}, 1944-51$ (LANL defined waste \#1, same as MW)

Metal waste from $\mathrm{BiPO}_{4}, 1952-56$ (LANL defined waste \#2, same as $M W$ )

Metal waste feed

N Reactor decontamination waste (IANL defined waste \#37)

Neutralized current acid waste, primary HLW stream from PUREX process

Non-complexed waste

Neutralized cladding removal waste, same as CWP

Aging waste from PUREX/PFM high level waste

Aging waste from PUREX/PFM processing of $\mathrm{N}$ production reactor (NPR) fuel 
WHC-SD-WM-ER-315, Rev . 0

\section{Naste Abbreviations}

NRAW

NRP82

NRPO4

NRSO4

OWW

P

P1

P2

P3

PADFG

PADWG

PAS

PASF

PAW

PD

PDBNG

PDBSU
Aging waste from PUREX/PFM residue acid waste

Dilute non-complexed waste from FY82 100-N Area waste transfer

Dilute phosphate waste from 100-N Area

Dilute non-complexed waste from $100-\mathrm{N}$ Area

Organic wash waste from PUREX [includes LANL defined wastes \#24, 1956-62 (also known as (ARB); \#25, 1963-67, and \#26, 1968-72]

PUREX high-level waste or neutralized. PUREX acid waste

PUREX high-level waste, 1955-62 (LANL defined waste \#17)

PUREX high-level waste, 1963-67 (LANL defined waste \#18)

PUREX high-level waste, 1983-88 (LANL defined waste \#47)

PUREX ammonia destruction waste from fuels grade fuel

PUREX ammonia destruction waste from weapons grade fuel

PUREX acidified sludge

PUREX ammonia scrubber feed (LANL

defined waste \#50)

PUREX acidified waste

PUREX decladding waste

Decladding sludge (non-TRU) from B Plant processing

Dilute non-complexed waste from B Plant decladding waste 
WHC-SD-WM-ER-315, Rev. 0

\section{Faste Abbreviations}

PDBTG

PDCSS

PDL 87

PDL89

PDNSG

PDS 87

PDS89

PDSLG

PDSUP

PFeCN

PFeCN1

PFeCN2

PFM

PFMMS

PEPGR

PEPNT

PEPPT

PEPSL
B Plant aging waste solids from PUREX decladding waste

Dilute non-complexed PUREX decladding waste, FY1986 only

PUREX decladding supernatant, 1987

PUREX decladding supernatant, non-TRU, spent metathesis removed

Non-TRU decladding sludge from PUREX

PUREX decladding sludge, 1987

PUREX decladding sludge after FY89

PUREX decladding sludge

Dilute non-complexed waste, PUREX decladding waste

Ferrocyanide sludge produced by in-plant scavenging of waste from uranium recovery

Ferrocyanide sludge produced by in-plant scavenging (using $0.005 \mathrm{M}$ ferrocyanide) of waste from uranium recovery (LANL defined waste \#9)

Same as PFeCN1 except $0.0025 \mathrm{M}$ ferrocyanide used (IANL defined waste \#10)

Waste from the process facility modification of PUREX

Dilute non-complexed waste from shear/leach processing of NPR fuel

Dilute non-complexed waste from retrieved PFP solids

Non-TRU sludge from the PFP (Z Plant)

Dilute non-complexed waste from the PFP (with TRUEX)

TRU sludge from the PFP ( $\mathrm{Z}$ Plant) 
WHC-SD-WM-ER-315, Rev. 0

\section{Naste Abbreviations}

PL

PL1

PL2

PML89

PMS89

PN

PNF

PSL

PSS

PSSF

PT

PX86S

PXBAW

PXBSG

PXFTE

PXLOW

PXMET

PXMSC

PXNAW

R
PUREX low-level waste

PUREX low-level waste (LANL defined waste \#20)

PUREX low-level waste, 1983-88 (LANL defined waste \#48)

PUREX spent metathesis liquid after FY89

PUREX spent metathesis solids after FY89

PUREX neutralized cladding waste

Partially neutralized waste

PUREX sludge sluiced during recovery of strontium

PUREX sludge supernatant

PUREX sludge supernatant feed

TRU solids from 200-West Area

Dilute non-complexed waste from PUREX miscellaneous streams (NPR fuel) FY86

B Plant aging waste supernatant from retrieved aging waste

B Plant aging waste solids from retrieved aging waste

Dilute non-complexed waste from PUREX miscellaneous streams (FFTF)

PUREX low level waste

PUREX dilute non-complexed decladding; spent metathesis

Dilute non-complexed waste from PUREX miscellaneous streams (NPR fuel)

Aging waste from PUREX high level waste

REDOX waste 
WHC-SD-WM-ER-315, Rev. 0

\section{Waste Abbreviations}

R SITCK

R1

R2

RESD

RIX

RSN

RSS

RTX

S1 SLTCK

S2 SLTSLRY

SIX

SL

SL3SY

SRR

SRS

SRS

SSW

SU

SWLIQ
Saltcake waste from the REDOX

concentrator (LANL defined waste \#40)

REDOX waste, 1952-57 (LANL defined waste \#13, same as R)

REDOX waste, 1958-66 (IANL defined waste \#14, same as $R$ )

Residual evaporator liquor

REDOX ion exchange waste

REDOX supernatant

REDOX sludge supernatant

REDOX ion exchange

Saltcake waste generated from the 242-S Evaporator-crystallizer, 1973-76 (LANL defined waste \#43)

Salt slurry waste generated from the 242-S Evaporator-crystallizer, 1977-80

(LANL defined waste \#44)

PUREX ion exchange waste

Sludge

Double shell slurry from late FY80, Tank 241-SY-103 inventory

Waste sent to B Plant for strontium recovery from sluiced $P$ sludge (IANL defined waste \#34)

Strontium sludge

Strontium recovery supernatant

Strontium Semiworks waste

Supernatant

Dilute non-complexed waste from 200-East Area single shell tanks 
WHC-SD-WM-ER-315， Rev. 0

\section{Naste Abbreviations}

SWLQW

T1 SLTCK

T2 SLTCK

TBP

TCO

TFeCN

TH

TH66

TH70

THL

TL

TPLAL, TPLAN

TPLAS

$\mathrm{U} 1 \mathrm{U} 2$

UNKNOWN, UNK

UR

Z
Dilute non-complexed waste from 200-West Area single shell tanks

Saltcake waste generated from the 242-T Evaporator, 1951-55 (LANL defined waste \#39)

Saltcake waste generated from the 242-T Evaporator, 1965-55 (LANL defined waste \#41)

Tri-butyl phosphate; also known as uranium recovery waste (UR)

Dilute non-complexed waste from terminal cleanout

Ferrocyanide sludge produced by in-tank or in-farm scavenging (LANL defined waste \#11)

Thoria HLW or cladding waste

Thoria HLW or cladding waste (LANL defined waste \#29)

Thoria HLW or cladding waste (LANL defined waste \#30)

Thoria low-level waste

Terminal liquor

Dilute non-complexed waste from $T$ Plant

Sludge from T Plant operations

Dilute non-complexed waste from U1/U2 pumping of ground water

Unknown waste type (IANL defined waste)

Uranium recovery waste (LANL defined waste \#8); also known as tributyl phosphate (TBP) waste

Z Plant (PFP) waste (IANL defined waste \#27)

PUREX waste from zircaloy cladded fuel 
WHC-SD-WM-ER-315, Rev. 0

\section{Waste Abbreviations}

ZHIGH

Dilute non-complexed waste from the PFP (without TRUEX)

ZIAB

Dilute non-complexed waste from the PFP laboratories

ZLOW

Dilute non-complexed waste from before FY85 Z Plant operations

ZPREL

Dilute non-complexed waste from PRF processing

ZPRES

PFP TRU solids from PRF processing

ZRMCL

Dilute non-complexed waste from PFP RMC processing

ZRMCS

PFP TRU solids from PFP RMC processing 
. 


\section{Facilities}

A Plant (PUREX)

B Plant (222-B)

C Plant (222-C)

S Plant (REDOX)

T Plant (222-T)

U Plant (222-U)

PFP ( 2 Plant)

$242-A$

$242-B$

$242-S$

$242-T$
This facility contained the latest solvent extraction process for recovery of both plutonium and uranium.

One of the three original bismuthphosphate processing facilities, later converted to a waste fractionation plant.

Initially a pilot plant for REDOX, later a pilot plant for PUREX and B Plant waste partitioning.

This facility contained the original extraction process for recovery of both plutonium and uranium.

One of the three original bismuthphosphate processing facilities, later converted to a decontamination facility.

One of the three original bismuthphosphate processing facilities, later converted to a uranium recovery plant.

Plutonium Finishing Plant (234-5Z) where the final operations occurred for production of plutonium products.

A forced circulation vacuum evaporation system.

An evaporator that operates at atmospheric pressure; also referred to as an open air type evaporator.

A forced circulation vacuum evaporation system.

An evaporator that operates at atmospheric pressure; also referred to as an open air type evaporator. 
APPENDIX B

References 


$$
\text { - }
$$


WHC-SD-WM-ER-315, Rev . 0

\section{RFFIRTNCIS}

Agnew, S.F., April 1995, Waste Status and Transaction Record Summary for the Southeast Quadrant, WHC-SD-WM-TI-689, Rev. 0, Westinghouse Hanford Company, Richland, Washington.

Agnew, S.F., August 1994, Hanford Defined Wastes: Chemical and Radionuclide Compositions, LA-UR-94-2657, Los Alamos National Laboratory, Los Alamos, New Mexico.

Aguirre, H. Jr., March 22, 1994 with ECN's through October 24, 1994, Aging Waste Facility Interim Operational Safety Requirements, WHC-SD-WM-OSR-004, Rev. 1B, Westinghouse Hanford Company, Richland, Washington.

Allen, G.K., March 1976, Estimated Inventory of Chemicals Added to Underground Waste Tanks, 1944 through 1975, ARH-CD-610B, Atlantic Richfield Hanford Company, Richland, Washington.

Alstad, A.T., September 19, 1991, Riser Configuration Document for Single-Shell Waste Tanks, WHC-SD-RE-TI-053, Rev. 8, Westinghouse Hanford Company, Richland, Washington.

Anderson, A.A., 1992, Double-Shell Underground Waste Storage Tanks Riser Survey, WHC-SD-RE-TI-093, Rev. 3, Westinghouse Hanford Company, Richland, Washington.

Anderson, J.D., January 1972-December 1972, Waste Status Summary Chemical Processing Division, ARH-2456, Atlantic Richfield Hanford Company, Richland, Washington.

Anderson, J.D., January 1973-December 1973, Waste Status Summary Chemical Processing Division, ARH-2794, Atlantic Richfield Hanford Company, Richland, Washington.

Anderson, J.D., January 1974-December 1974a, Waste Status Summary Operations Division, ARH-CD-133, Atlantic Richfield Hanford Company, Richland, Washington.

Anderson, J.D., January 1974-December 1974b, Waste Status Summary Chemical Processing Division, ARH-CD-133, Atlantic Richfield Hanford Company, Richland, Washington.

Anderson, J.D., January 1975-December 1975, Waste Status Summary Production and Waste Management Division, ARH-CD-336, Atlantic Richfield Hanford Company, Richland, Washington.

Anderson, J.D., January 1976-September 1976, Waste Status Summary Production and Waste Management Division, ARH-C-702, Atlantic Richfield Hanford Company, Richland, Washington. 
WHC-SD-WM-ER-315, Rev. 0

Anderson, J.D., October 1976-May 1977, Waste Status Summary Production and Waste Management Division, ARH-CD-822, Atlantic Richfield Hanford Company, Richland, Washington.

Anderson, J.D., June 1990, A History of the 200 Areas Tank Farms, WHC-MR-0132, Westinghouse Hanford Company, Richland, Washington.

ARHCO, October 1967, Monthly Report, 200 Areas Operation, ARH-60 DEL, Atlantic Richfield Hanford Company, Richland, Washington.

ARHCO, June 1969, SX Tank Farm Air Cooling Safety Analysis, ARH-1291, Atlantic Richfield Hanford Company, Richland, Washington.

ARHCO, July 1972-December, Chemical Processing Division Waste Status Summary, Quarterly Reports, ARH-2456 C and D, Atlantic Richfield Hanford Company, Richland, Washington.

ARHCO, January 1973-December 1973, Chemical Processing Division Waste Status Summary, Quarterly Reports, ARH-2694 A, B, C, and D, Atlantic Richfield Hanford Company, Richland, Washington.

ARHCO, January 1973-September 1975, Operations Division Waste Status Summary, Quarterly Reports, ARH-CD-133 A, B, C, and D, Atlantic Richfield Hanford Company, Richland, Washington.

ARHCO, September 1976, Production and Waste Management Division Waste Status Summary, Monthly Reports, ARH-CD-702, Atlantic Richfield Hanford Company, Richland, Washington.

ARHCO, October 1976-June 1977, Production and Waste Management Division Waste Status Summary, Monthly Report, ARH-CD-822, Atlantic Richfield Hanford Company, Richland, Washington.

Babad, H., D.M. Camaioni, M.A. Lilga, W.D. Samuels, and D.M. Strachan, February 1993, Tank Waste Chemistry - A New Understanding of Waste Aging, WHC-SA-1694-FP, Westinghouse Hanford Company, Richland, Washington.

Boomer, R.D., June 1990, Functional Requirement Baseline for the Closure of Single-Shell Tanks, WHC-EP-0338, Westinghouse Hanford Company, Richland, Washington.

Borsheim, G.L., and B.C. Simpson, October 1991, An Assessment of the Inventories of the Ferrocyanide Watchlist Tanks, WHC-SD-WM-ER-133, Rev. 0, Westinghouse Hanford Company, Richland, Washington:

Borsheim, G.L., August 2, 1989, Single-Shell Tank Isolation Safety Analysis Report, SD-WM-SAR-006, Rev. 2, Westinghouse Hanford Company, Richland, Washington. 
Borshiem, G.L. and N.W. Kirch, March 1991, Summary of Single-Shell Tank Waste Stability, WHC-EP-0347, Westinghouse Hanford Company, Richland, Washington.

Boyles, V.C., June 1981, Safety Analysis Report: Stabilization of Single-Shell Waste Storage Tanks by Saltwell Jet Pumping, RHO-SD-WM-SAR-034, Rev. 0, Rockwell Hanford Operations, Richland, Washington.

Brevick, C.H., L.A. Gaddis, and W.W. Pickett, September 1995a, Supporting Document for the Southeast Quadrant Historical Tank Content Estimate Report for AN-Tank Farm, WHC-SD-WM-ER-314, Rev. 0, ICF Kaiser Hanford Company, Richland, Washington.

Brevick, C.H., L.A. Gaddis, and W.W. Pickett, September 1995b, Supporting Document for the Southeast Quadrant Historical Tank Content Estimate Report for AP-Tank Farm, WHC-SD-WM-ER-315, Rev. 0, ICF Kaiser Hanford Company, Richland, Washington.

Brevick, C.H., L.A. Gaddis, and W.W. Pickett, September 1995c, Supporting Document for the Southeast Quadrant Historical Tank Content Estimate Report for AW-Tank Farm, WHC-SD-WM-ER-316, Rev. 0, ICF Kaiser Hanford Company, Richland, Washington.

Brevick, C.H., L.A. Gaddis, and W.W. Pickett, September 1995d, Supporting Document for the Southeast Quadrant Historical Tank Content Estimate Report for AY-Tank Farm, WHC-SD-WM-ER-317, Rev. 0, ICF Kaiser Hanford Company, Richland, Washington.

Brevick, C.H., L.A. Gaddis, and W.W. Pickett, September 1995e, Supporting Document for the Southeast Quadrant Historical Tank Content Estimate Report for AZ-Tank Farm, WHC-SD-WM-ER-318, Rev. 0, ICF Kaiser Hanford Company, Richland, Washington.

Brevick, C.H., L.A. Gaddis, and W.W. Pickett, September 1995f, Supporting Document for the Southeast Quadrant Historical Tank Content Estimate Report for SY-Tank Farm, WHC-SD-WM-ER-319, Rev. 0, ICF Kaiser Hanford Company, Richland, Washington.

Brown, W.G., April 26, 1993, Hazard Identification and Evaluation for Non-Stabilized Single Shell Tanks, SD-WM-SAR-022, Rev. 0, Westinghouse Hanford Company, Richland, Washington.

Bussell, J.H., February 3, 1992, Engineering Evaluation of Thermocouples in FeCN Watchlist Tanks, WHC-SD-WM-ER-134, Rev OA, Westinghouse Hanford Company, Richland, Washington.

Cain, R.J., 1977, Isolation and Stabilization of Special Tanks, Internal Letter to J.C. Womack, Rockwell Hanford Company, Richland, Washington. 
WHC-SD-WM-ER-315， Rev. 0

Carpenter, G.K., April 1953-June 1953, Waste Status Summary Separations Section, HW-28043 etc., General Electric Company, Richland, Washington.

Carpenter, G.K., September 1953-May 1954, Waste Status Summary Separations Section, HW-29624 etc., General Electric Company, Richland, Washington.

Carter, G.J., April 1986-September 1986, Waste Status Summary, RHO-RE-SR-14, Rockwell Hanford Operations, Richland, Washington.

Cooley, C.R., and G.L. Richardson, July 17, 1963, Hot Semiworks Strontium-90 Recovery Program, HW-72666, General Electric Company, Richland, Washington.

Dodd, R.A., December 27, 1994, Collection of Psychrometric Data from the Annular Regions of Double-Shell Tanks, Internal Memorandum, Westinghouse Hanford Company, Richland, Washington.

ERDA, December 1975, Waste Management Operations, ERDA-1538, Energy Research and Development Administration, Richland, Washington.

Escobar, G.A., October 1986-January 1987, Waste Status Summary, RHO-RE-SR-14, Rockwell Hanford Operations, Richland, Washington.

Farley, W.G., May 20, 1992, Safety Assessment for Thermocouple Tree System Installation and Operation in Nonleaking Ferrocyanide Tanks, WHC-SD-WM-SAD-014, Rev. 1, Westinghouse Hanford Company, Richland, Washington.

GE Co., July 10, 1951, REDOX Technical Manual, HW-18700 DEL, General Electric Company, Richland, Washington.

Gerber, M.S., September 1992, Legend and Legacy: Fifty Years of Defense Production at the Hanford Site, WHC-MR-0293, Rev. 2, Westinghouse Hanford Company, Richland, Washington.

Gerber, M.S., September 1993a, Multiple Missions: The 300 Area in Hanford Site History, WHC-MR-0440, Westinghouse Hanford Company, Richland, Washington.

Gerber, M.S., November 1993b, A Brief History of the PUREX and $\mathrm{UO}_{3}$ Facilities, WHC-MR-0437, Westinghouse Hanford Company, Richland, Washington.

Gerber, M.S., April 1994, Pac-Men of Hanford Site Await Decommissioning, WHC-SA-2454FP, Westinghouse Hanford Company, Richland, Washington. 
WHC-SD-WM-ER-315， Rev . 0

Hanlon, B.M., November 1989-December 1990, Tank Farm Surveillance and Waste Status Summary Report, WHC-EP-0182, Westinghouse Hanford Company, Richland, Washington.

Hanlon, B.M., January 1991-December 1991, Tank Farm Surveillance and Waste Status Summary Report, WHC-EP-0182, Westinghouse Hanford Company, Richland, Washington.

Hanlon, B.M., January 1992-December 1992, Tank Farm Surveillance and Waste Status Summary Report, WHC-EP-0182, Westinghouse Hanford Company, Richland, Washington.

Hanlon, B.M., July 1993, Tank Farm Surveillance and Waste Status Summaxy Report for July 1993, WHC-EP-0182-64, Westinghouse Hanford Company, Richland, Washington.

Hanlon, B.M., January 1993-December, 1993, Tank Farm Surveillance and Waste Status Summary Report, WHC-EP-0182, Westinghouse Hanford Company, Richland, Washington.

Hanlon, B.M., June 1995, Tank Farm Surveillance and Waste Status Summary Report, WHC-EP-0182-87, Westinghouse Hanford Company, Richland, Washington.

Hatch, P., December 18, 1969, Limitations for Use of Underground Waste Tanks, ARH-951, Atlantic Richfield Hanford Company, Richland, Washington.

Heubach, E.C. II, March 22, 1994 with ECN's through April 3, 1995, Double Shell Tank Interim Operational Safety Requirements, WHC-SD-WM-OSR-016, Rev. OB, Westinghouse Hanford Company, Richland, Washington.

HEW, January 11, 1944, CVI, Specifications for Construction of Composite Storage Tanks Bldg. No. 241, Project 9536, CVI 73550, Hanford Engineer Works, Richland, Washington.

Jenkins, C., M. Danielson, B. Rarig, R. Campbell, T. Ambalam, C. Kenoyer, and L. Hall, February 1993, Engineering Evaluation of Alternatives Managing the Assumed Leak From SST 241-T-101, EEA-93-1, Kaiser Engineers Hanford, Richland, Washington.

Jungfleisch, F.M., March 1984, Preliminary Estimation of the Waste Inventories in Hanford Tanks Through 1980, SD-WM-TI-057, Rev.0, Rockwell Hanford Operations, Richland, Washington.

KEH, February 1993, EEP Managing the Assumed Leak From SST, KEEA-93-1, Kaiser Engineers Hanford, Richland, Washington. 
Kirkman, M.J., G.L. Ritter, and P.W. Smith, March 6, 1969, B Plant Production Schedule and In-Tank Solidification Alternatives, ARH-900, Atlantic Richfield Hanford Company, Richland, Washington.

Kreig, S.A., W.W. Jenkins, K.J. Leist, K.G. Squires, and J.F. Thompson, June 1990, Single-Shell Tank Waste Retrieval Study, WHC-EP-0352, UC-721, Westinghouse Hanford Company, Richland, Washington.

Lentz, J.E., April 1959-December 1959, Waste Status Summary Chemical Processing Department, HW-58579 etc., General Electric Company, Richland, Washington.

Lentz, J.E., January 1960-December 1960, Waste Status Summary Chemical Processing Department, HW-63896 etc., General Electric Company, Richland, Washington.

Liverman, J.L., December 1975, Final Environmental Statement, Waste Management Operations, Volumes 1 and 2, ERDA-1538, Energy Research and Development Administration, Richland, Washington.

Lucas, G.E., June 1989, Waste Types in Hanford Single-Shell Tanks, WHC-SD-ER-TI-001, Rev. 0, Westinghouse Hanford Company, Richland, Washington.

McBride, J.P., July 1953, Waste Status Summary - Separations Section, HW-29054, General Electric Company, Richland, Washington.

McCann, D.C., July 1981-October 1981, Waste Status Summary, RHO-CD-14, Rockwell Hanford Operations, Richland, Washington.

McCann, D.C., October 1981-December 1982, Waste Status Summary, RHO-RE-SR-14, Rockwell Hanford Operations, Richland, Washington.

McCann, D.C., January 1983-December 1983, Waste Status Summary, RHO-RE-SR-14, Rockwell Hanford Operations, Richland, Washington.

McCann, D.C., January 1984-February 1985, Waste Status Summary, RHO-RE-SR-14, Rockwell Hanford Operations, Richland, Washington.

Minteer, D.J., November 1994, Uncertainties in the Measured Net Mass Flow Rate of Water Leaving Waste Storage Tank 241-C-106 Via the Vent System, WHC-SD-WM-ER-383, Rev. 0, Westinghouse Hanford Company, Richland, Washington.

Morganthaler, A.C., February 1952-March 1953, Waste Status Summary - Separations Section, HW-27897 etc., General Electric Company, Richland, Washington.

Mudd, O.C., January 1979-December 1979, Waste Status Summary, RHO-CD-14, Rockwell Hanford Operations, Richland, Washington. 
WHC-SD-WM-ER-315, Rev. 0

Mudd, O.C., January 1980-December 1980, Waste Status Summary, RHO-CD-14, Rockwell Hanford Operations, Richland, Washington.

Mudd, O.C., January 1981-June 1981, Waste Status Summary, RHO-CD-14, Rockwell Hanford Operations, Richland, Washington.

Mulvey, C.A., June 1977-December 1977, Waste Status Summary, RHO-CD-14, Rockwell Hanford Operations, Richland, Washington.

Mulvey, C.A., January 1978-December 1978, Waste Status Summary, RHO-CD-14, Rockwell Hanford Operations, Richland, Washington.

Peterson, D.E., June 1954-May 1955, Waste Status Summary Separations Section, HW-32389 etc., General Electric Company, Richland, Washington.

Peterson, D.E., June 1955-May 1956, Waste Status Summary Separations Section, HW-38000 etc., General Electric Company, Richland, Washington.

Peterson, D.E., September 1956-December 1956, Waste Status Summary - Chemical Processing Department, HW-45738 etc., General Electric Company, Richland, Washington.

Peterson, D.E., June 1956-August 1956, Waste Status Summary Separations Section, HW-43895 etc., General Electric Company, Richland, Washington.

Pines, A.G., January 4, 1991, 244-AR Vault Safety Analysis Report, SD-WM-SAR-018, Rev. 0, Westinghouse Hanford Company, Richland, Washington.

Prosk, W. and D.A. Smith, January 1986, Tank Isolation Safety Analysis Report, SD-WM-SAR-006, Rev. 1, Rockwell Hanford Operations, Richland, Washington.

RHO, June 1977-October 1981, Waste Status Summary, Monthly Reports, RHO-CD-14, Rockwell Hanford Operations, Richland, Washington.

RHO, November 1981-June 1987, Waste Status Summary, Monthly Reports, RHO-RE-SR-014, Rockwell Hanford Operations, Richland, Washington.

Roberts, R.E., January 1957-December 1957, Waste Status Summary Chemical Processing Department, HW-48144 etc., General Electric Company, Richland, Washington.

Roberts, R.E., January 1958-October 1958, Waste Status Summary Chemical Processing Department, HW-54916 etc., General Electric Company, Richland, Washington. 
WHC-SD-WM-ER-315, Rev. 0

Roberts, R.E., January 1961-December 1961, Waste Status Summary Chemical Processing Department, HW-71610 etc., General Electric Company, Richland, Washington.

Roberts, R.E., January 1962-December 1962, Waste Status Summary Chemical Processing Department, HW-74647 etc., General Electric Company, Richland, Washington.

Roberts, R.E., January 1963-December 1963, Waste Status Summary Chemical Processing Department, HW-78279 etc., General Electric Company, Richland, Washington.

Roberts, R.E., January 1964-December 1964, Waste Status Summary Chemical Processing Department, HW-83308 etc., General Electric Company, Richland, Washington.

Roberts, R.E., January 1965-December 1965, Waste Status Summary Chemical Processing Department, HW-83906 etc., General Electric Company, Richland, Washington.

Roberts, R.E., October 1966-December 1966, Waste Status Summary Chemical Processing Division, ISO-674, Isochem Inc., Richland, Washington.

Roberts, R.E., January 1966-September 1966, Waste Status Summary Chemical Processing Department, HW-83906 etc., General Electric Company, Richland, Washington.

Rodenhizer, D.G., September 30, 1987, Hanford Waste Tank Sluicing History, SD-WM-TI-302, Westinghouse Hanford Company, Richland, Washington.

Rutherford, M.J., August 31, 1948, Additional Waste Storage Facilities 200-East Area, Specification No. HW-3783, General Electric Company, Richland, Washington.

Scaief, C.C. III, October 19, 1993, TMACS I/O Termination Point Listing, WHC-SD-WM-TI-594, Rev. 0, Westinghouse Hanford Company, Richland, Washington.

Smith, D.A., August 1986, SAR, Hazard Identification and Evaluation for Nonstabilized Single-Shell Tanks, SD-WM-SAR-022, Rev. 0, Rockwell Hanford Operations, Richland, Washington.

Stahl, S.M., November 22, 1993, Hanford Site Tank Farm Facilities Interim Safety Basis, Volume 1 and 2, WHC-SD-WM-ISB-001, Westinghouse Hanford Company, Richland, Washington. 
WHC-SD-WM-ER-315, Rev . 0

Stong, F.S., September 29, 1986, Drywell Van In-Tank Liquid Observation Well Surveillance Data Interpretation, RHO-SD-WM-TI-237, Rockwell Hanford Operations, Richland, Washington.

Thress, M.A., November 1958-February 1959, Waste Status Summary Chemical Processing Department, HW-58579 etc., General Electric Company, Richland, Washington.

Thurman, J.M., June 1987-December 1987, Tank Farm Surveillance and Waste Status Summary Report, Monthly Reports WHC-SP-0038, Westinghouse Hanford Company, Richland, Washington.

Thurman, J.M., December 1987-June 1993, Tank Farm Surveillance and Waste Status Summary Report, Monthly Reports, WHC-EP-0182-55, Westinghouse Hanford Company, Richland, Washington.

Thurman, J.M., February 1987-June 1987, Waste Status Summary, RHO-RE-SR-14, Rockwell Hanford Operations, Richland, Washington.

Thurman, J.M., July 1987-March 1988, Waste Status Summary, WHC-SP-0038, Westinghouse Hanford Company, Richland, Washington.

Thurman, J.M., April 1988-December 1988, Tank Farm Surveillance and Waste Status Summary Report, WHC-EP-0182, Westinghouse Hanford Company, Richland, Washington.

Thurman, J.M., January 1989-October 1989, Tank Farm Surveillance and Waste Status Summary Report, WHC-EP-0182, Westinghouse Hanford Company, Richland, Washington.

Tran, T.T., April 27, 1993, Thermocouple Status Single-Shell and Double-Shell Waste Tanks, WHC-SD-WM-TI-553, Rev. 0, Westinghouse Hanford Company, Richland, Washington.

U.S. DOE, December 1987, EIS, Disposal of Hanford Defense High Level, Transuranic, and Tank Wastes, DOE/EIS-0113, Volumes 1-5, Department of Energy, Richland, Washington.

Uebelacker, D.L., October 1968-December 1968, Waste Status Summary - Chemical Processing Division, ARH-1061, Atlantic Richfield Hanford Company, Richland, Washington.

Uebelacker, D.L., January 1969-December 1969, Waste Status Summary - Chemical Processing Division, ARH-1200, Atlantic Richfield Hanford Company, Richland, Washington.

Uebelacker, D.L., January 1970-December 1970, Waste Status Summary - Chemical Processing Division, ARH-1666, Atlantic Richfield Hanford Company, Richland, Washington. 
WHC-SD-WM-ER-315, Rev. 0

Vail, T.S., January 1985-March 1986, Waste Status Summary, RHO-RE-SR-14, Rockwell Hanford Operations, Richland, Washington.

Wagoner, J.D., March 18, 1993, Wyden Request, Document No. $9301156 \mathrm{~B}$ R3, U.S. Department of Energy, Richland, Washington.

Waite, J.L., April 1991, Tank Wastes Discharged Directly to the Soil at the Hanford Site, WHC-MR-0227, Westinghouse Hanford Company, Richland, Washington.

Welty, R.K., September 1988, Waste Storage Tank Status and Leak Detection Criteria, WHC-SD-WM-TI-356, Volumes 1 and 2, Westinghouse Hanford Company, Richland, Washington.

Welty, R.K. and N.J. Vermeulen, September 12, 1989 with ECN's through November 8, 1993, Waste storage Tank Status and Leak Detection Criteria, WHC-SD-WM-TI-357, Rev. 1K, Westinghouse Hanford Company, Richland, Washington.

WHC, September 1991, Failure to Continuously Man Control Room During Transfer Per Procedure, Occurrence Report RL-WHC-Tank Farm-1991-1019, Westinghouse Hanford Company, Richland, Washington.

WHC, July 1991, High Radiation Readings in 241-BY Drywells, Occurrence Report RL--WHC-Tank Farm-1991-1022, Westinghouse Hanford Company, Richland, Washington.

WHC, August 1993, Hanford Site Tank Farm Facilities Interim Safety Basis, WHC-SD-WM-ISB-001, Rev. 1, Volumes 1 and 2, Westinghouse Hanford Company, Richland, Washington.

WHC, March 1993, Hanford Watchlist Tank Surveillance Trends, Letter to the Honorable Ron Wyden, Westinghouse Hanford Company, Richland, Washington.

WHC, July 1993, Unauthorized Water Usage at 241-BY Tank Farm Results in Exceeding Raw Water Operational Limit, Occurrence Report RL--WHC-Tank Farm-1993-0050, Westinghouse Hanford Company, Richland, Washington.

Winters, W.I., L. Jensen, L.M. Sasaki, R.L. Weiss, J.F. Keller, A.J. Schmidt, and M.G. Woodruff, May 1989, Waste Characterization Plan for the Hanford Site Single-Shell Tanks, WHC-EP-0210, Westinghouse Hanford Company, Richland, Washington.

Wood, S.A., May 1993, Waste Tank Evaporation Water Loss Computer Program, WHC-SD-WM-CSWD-055, Rev. 0., Westinghouse Hanford Company, Richland, Washington. 


\section{APPENDIX C}

\section{Tank Level Histories and LOW Data}


L 


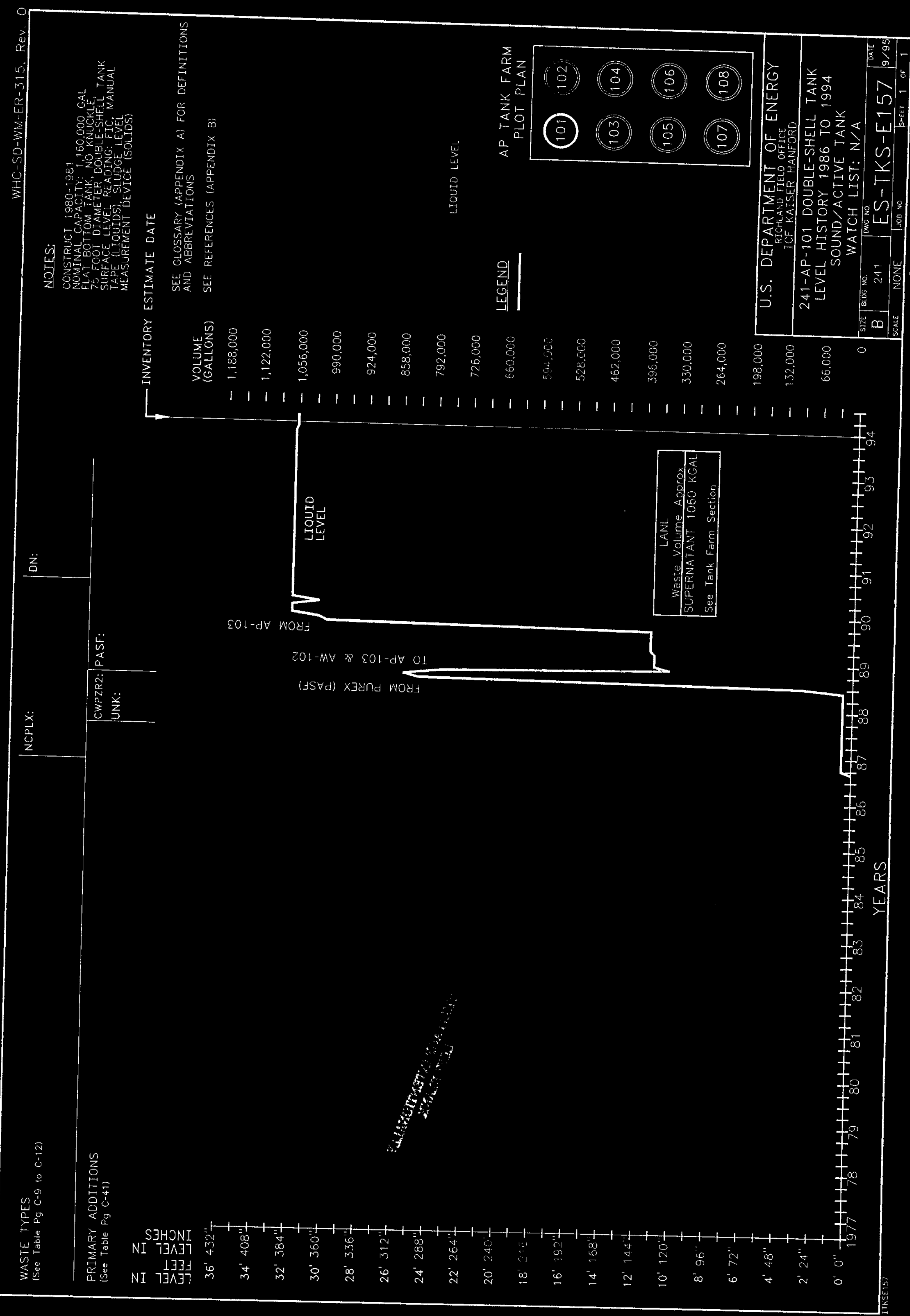


THIS PAGM INHENHONAWTY FHTBMNTK 


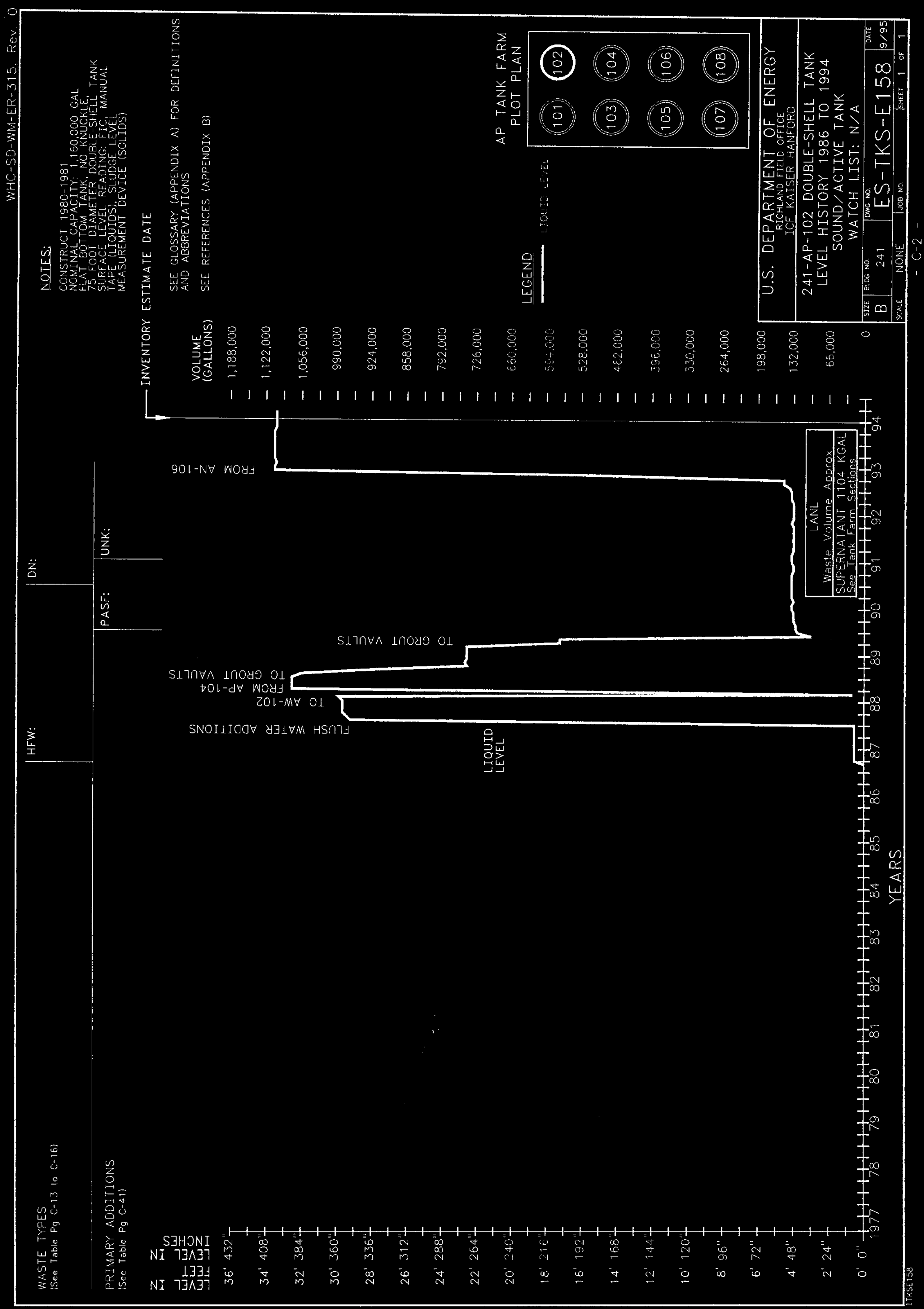


TEITS PAGO INTHYHONAWY MWSBMANT 


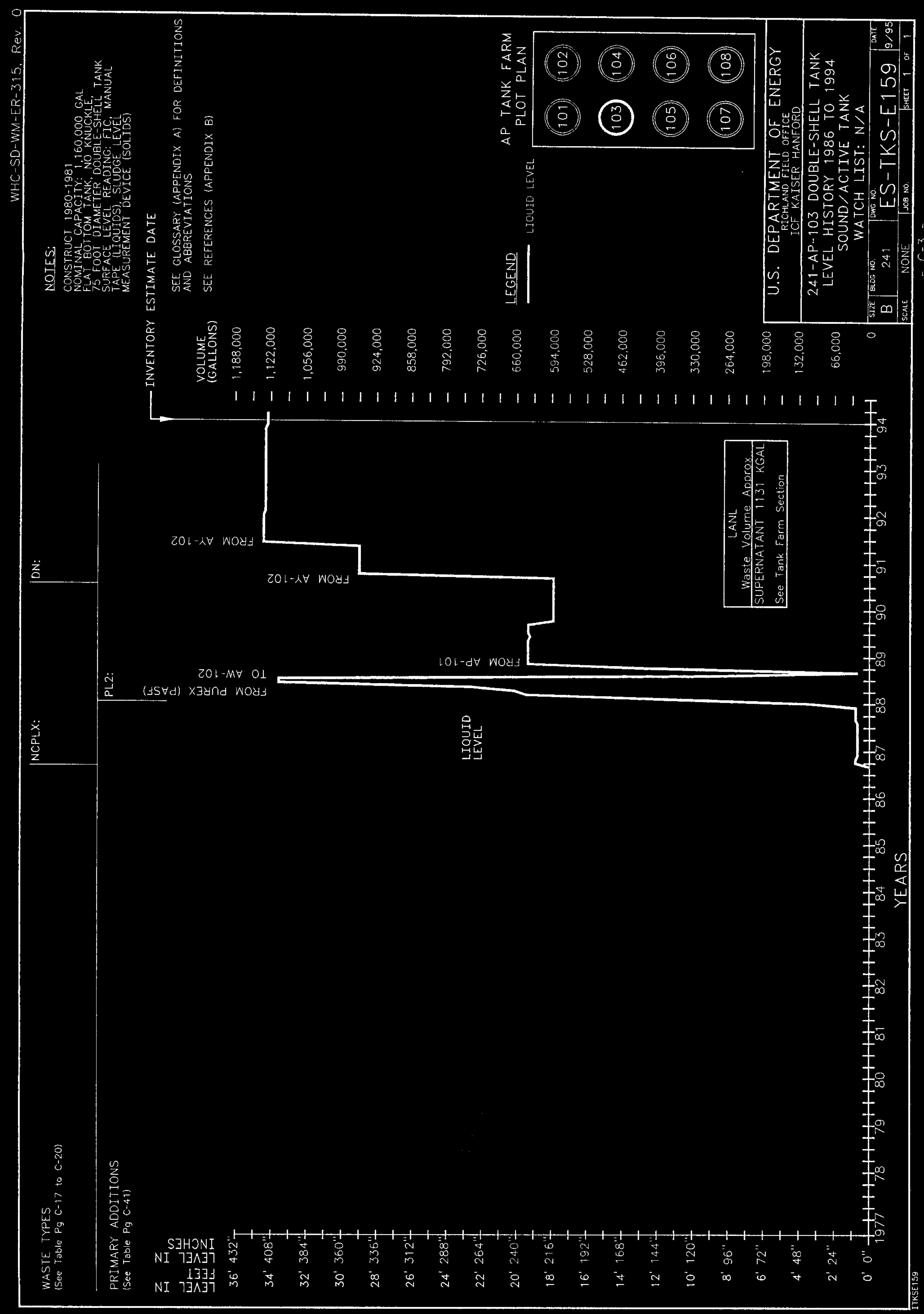


THEIS PAGE NYHANOMALEY FWOTBMANK 


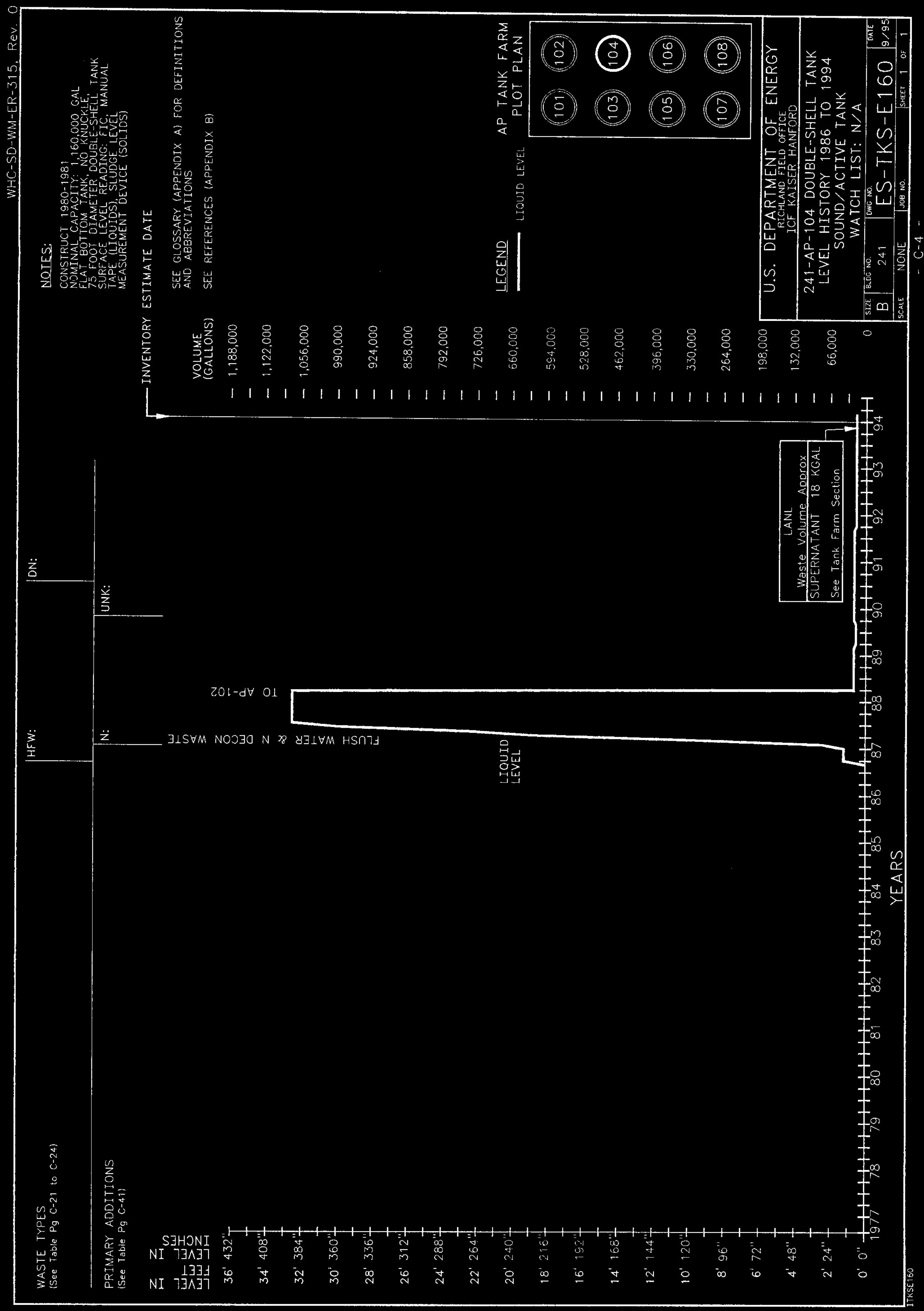




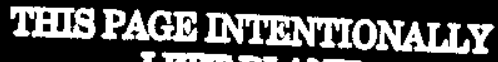
IGISBLANR 


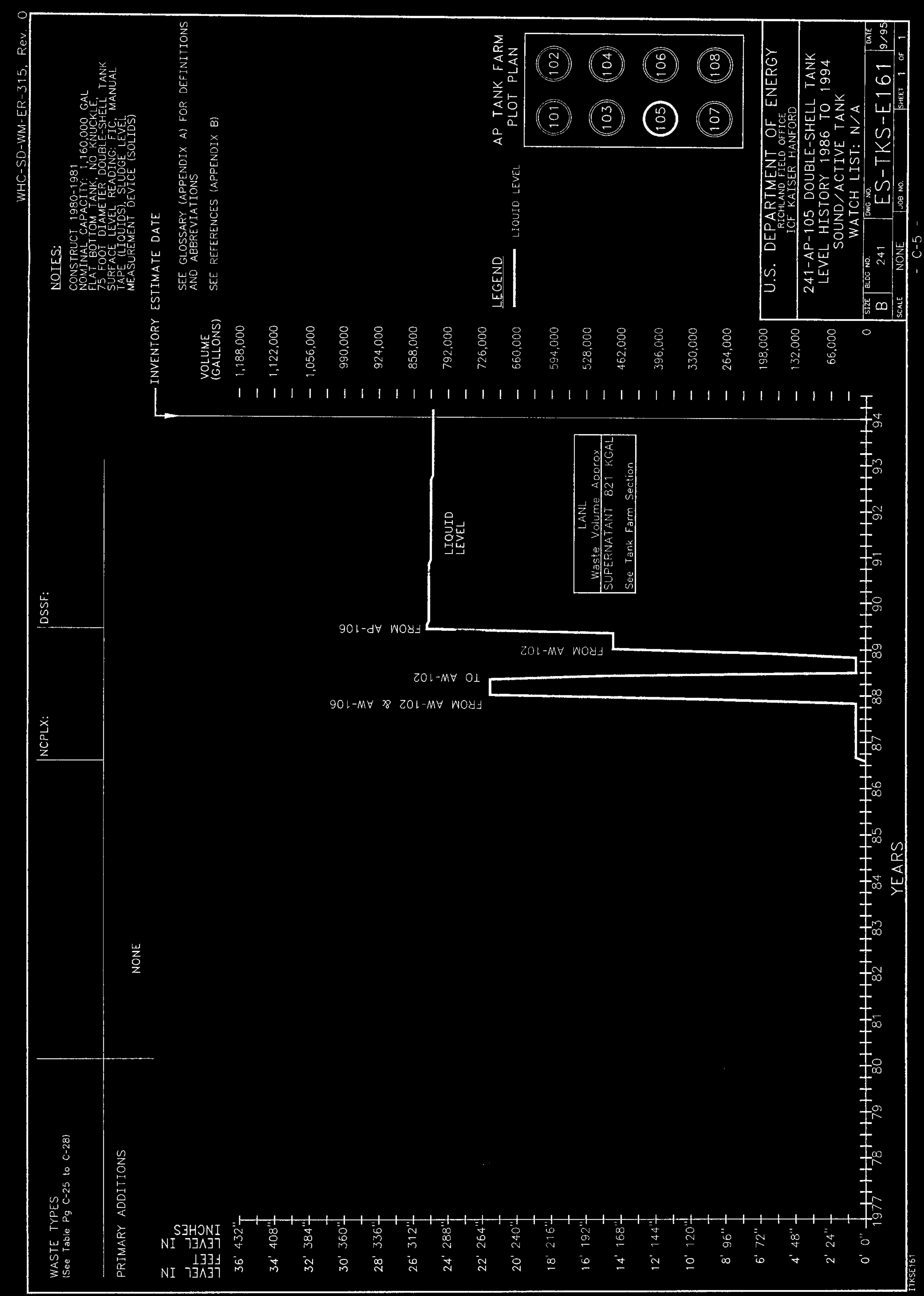


TEITS PAGE NNGHNHONATEY LESTBRIATR 


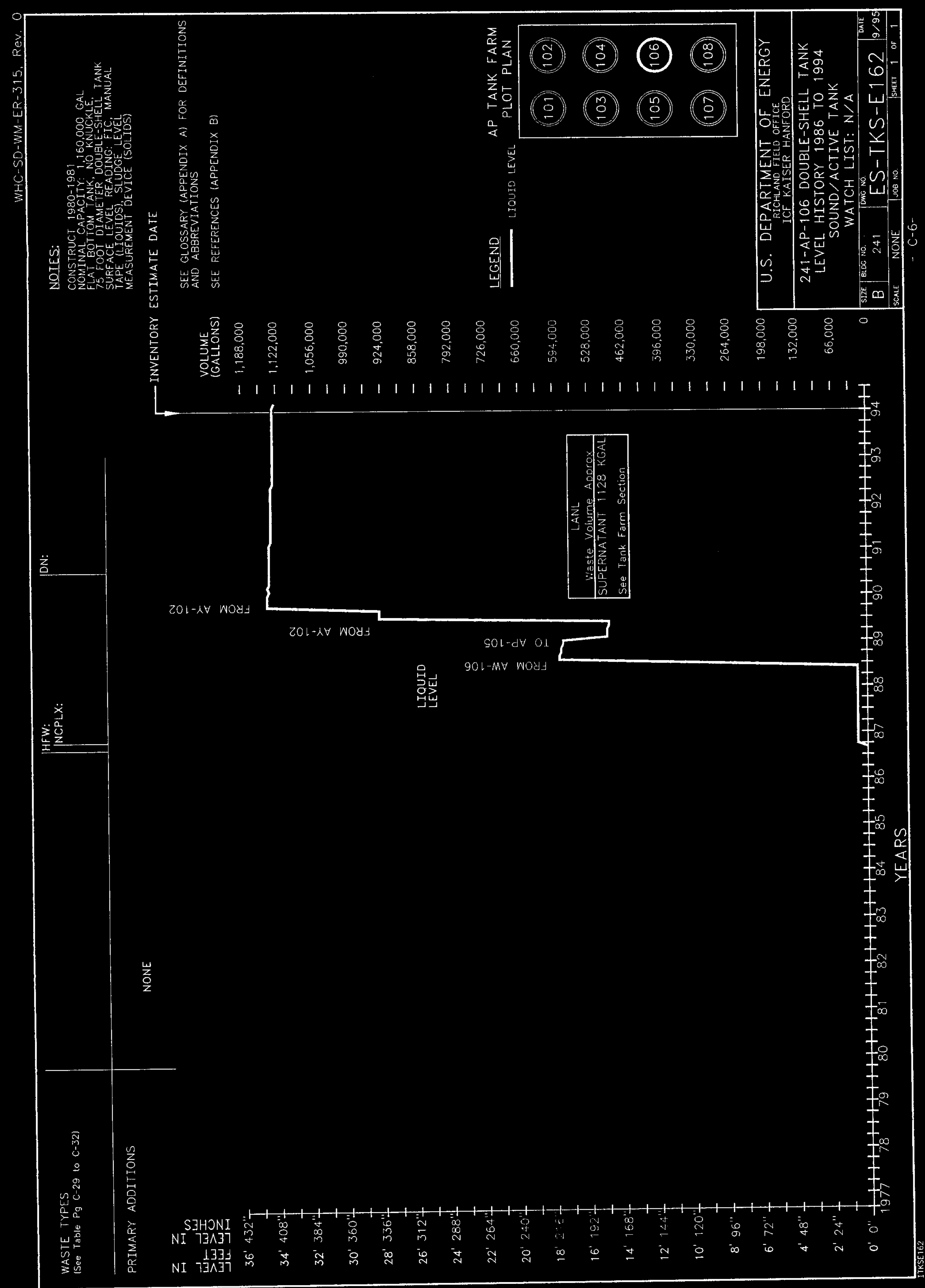


TETS PAGS NYMANONALT

FFITBIAIIK 


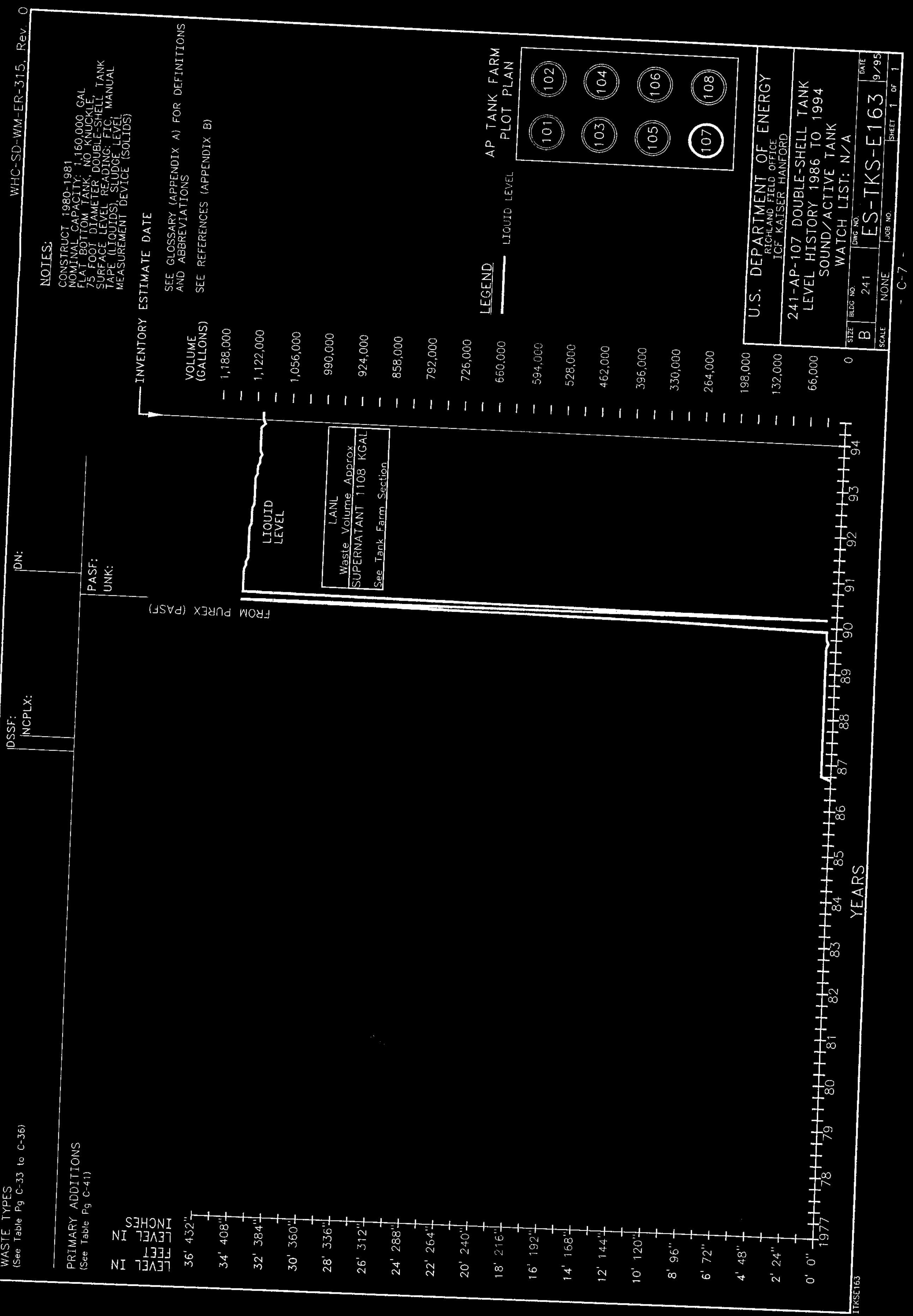


THITS PAGO INUFNTIONAWG THERTANT 


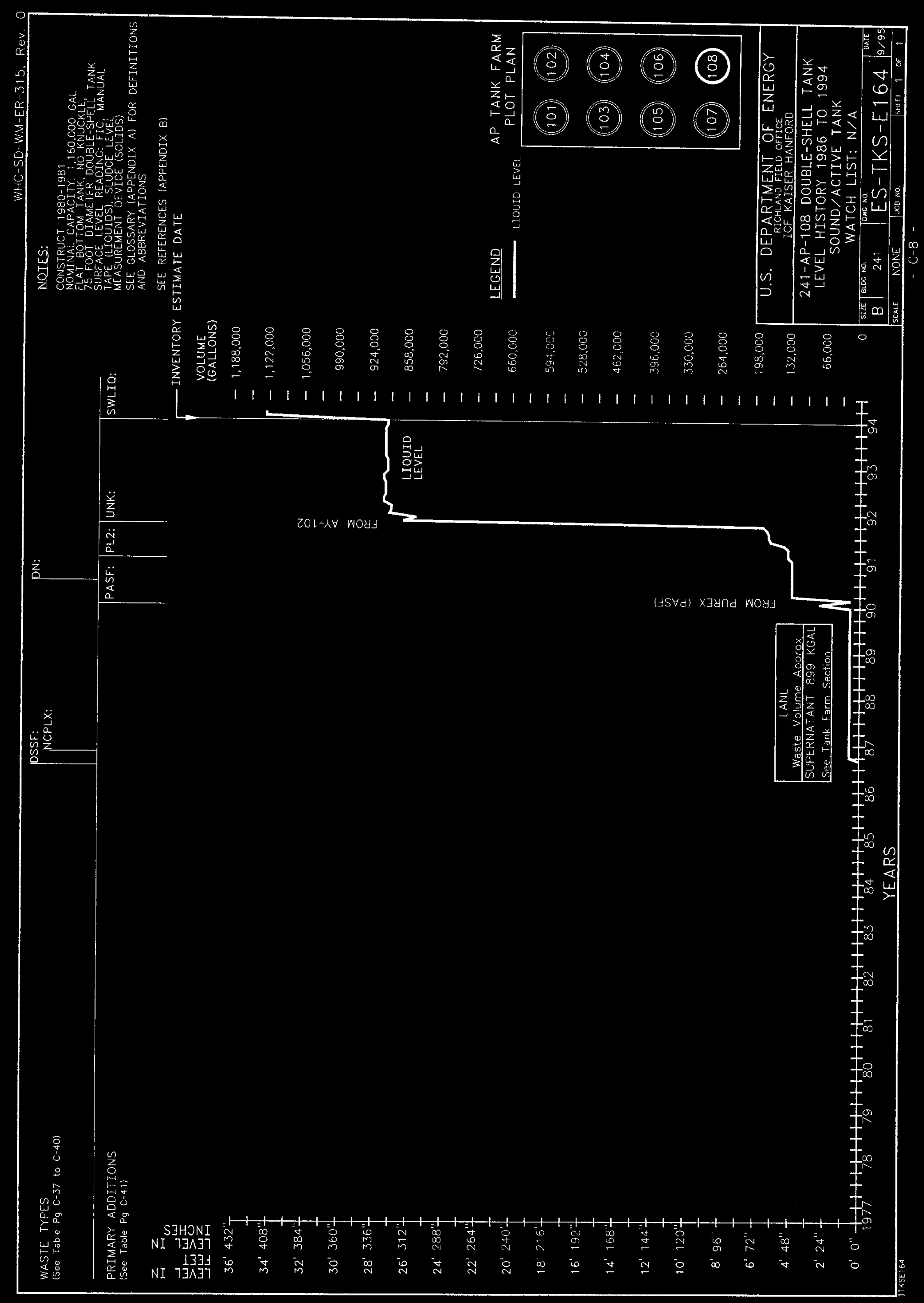


TEIS PAGB INTHNYONALLY

WH'TBLAMK 
TANK LEVEL HISTORY AND WASTE TYPES

241-AP-101

\begin{tabular}{|c|c|c|c|c|c|c|}
\hline Date & & Total Waste & DSS & Sludge & Salt & Total solids \\
\hline$(\mathrm{M} / \mathrm{Yr})$ & Waste Type & (K Gal) & (K Gal) & (K Gal) & (K Gal) & (K Gal) \\
\hline $1 / 80$ & & N/A & N/A & N/A & N/A & 0 \\
\hline $2 / 80$ & & N/A & N/A & N/A & N/A & 0 \\
\hline $3 / 80$ & & N/A & N/A & $\mathbf{N} / \mathbf{A}$ & $N / A$ & 0 \\
\hline $4 / 80$ & & N/A & $\mathbf{N} / \mathbf{A}$ & $N / A$ & N/A & 0 \\
\hline $5 / 80$ & & N/A & N/A & N/A & N/A & 0 \\
\hline $6 / 80$ & & $\bar{N} / \mathbf{A}$ & N/A & N/A & N/A & 0 \\
\hline $7 / 80$ & & $\mathbf{N} / \mathbf{A}$ & N/A & N/A & N/A & 0 \\
\hline $8 / 80$ & & N/A & N/A & N/A & N/A & 0 \\
\hline $9 / 80$ & & N/A & $N / A$ & N/A & $\mathrm{N} / \mathrm{A}$ & 0 \\
\hline $10 / 80$ & & N/A & N/A & N/A & N/A & 0 \\
\hline $11 / 80$ & & N/A & $\mathbf{N} / \mathbf{A}$ & $\mathbf{N} / \mathbf{A}$ & $\mathrm{N} / \mathrm{A}$ & 0 \\
\hline $12 / 80$ & & N/A & N/A & N/A & $\mathrm{N} / \mathrm{A}$ & 0 \\
\hline $1 / 81$ & & $\mathbf{N} / \mathbf{A}$ & N/A & N/A & N/A & 0 \\
\hline $2 / 81$ & & N/A & N/A & N/A & $\mathbf{N} / \mathbf{A}$ & 0 \\
\hline $3 / 81$ & & N/A & N/A & $\mathrm{N} / \mathrm{A}$ & N/A & 0 \\
\hline $4 / 81$ & & N/A & N/A & N/A & $\mathrm{N} / \mathrm{A}$ & 0 \\
\hline $5 / 81$ & & N/A & N/A & N/A & N/A & 0 \\
\hline $6 / 81$ & & $\bar{N} / \mathbf{A}$ & N/A & $\mathbf{N} / \mathbf{A}$ & $N / A$ & 0 \\
\hline $7 / 81$ & & $N / A$ & N/A & N/A & N/A & 0 \\
\hline $8 / 81$ & & N/A & N/A & N/A & N/A & 0 \\
\hline $9 / 81$ & & $\mathrm{~N} / \mathrm{A}$ & N/A & N/A & N/A & 0 \\
\hline $10 / 81$ & & N/A & N/A & N/A & $\mathrm{N} / \mathrm{A}$ & 0 \\
\hline $11 / 81$ & & N/A & N/A & $\mathrm{N} / \mathrm{A}$ & N/A & 0 \\
\hline $12 / 81$ & & $\mathrm{~N} / \mathrm{A}$ & N/A & $\mathbf{N} / \mathbf{A}$ & N/A & 0 \\
\hline $1 / 82$ & & N/A & N/A & $\mathrm{N} / \mathrm{A}$ & N/A & 0 \\
\hline $2 / 82$ & & N/A & N/A & N/A & N/A & 0 \\
\hline $3 / 82$ & & $\mathrm{~N} / \mathrm{A}$ & N/A & $\mathrm{N} / \mathrm{A}$ & N/A & 0 \\
\hline $4 / 82$ & & $\mathrm{~N} / \mathrm{A}$ & N/A & N/A & N/A & 0 \\
\hline $5 / 82$ & & $N / A$ & N/A & N/A & N/A & 0 \\
\hline $6 / 82$ & & N/A & N/A & N/A & $\mathrm{N} / \mathrm{A}$ & 0 \\
\hline $7 / 82$ & & N/A & N/A & $\mathbf{N} / \mathbf{A}$ & $N / A$ & 0 \\
\hline $8 / 82$ & & $\mathrm{~N} / \mathrm{A}$ & N/A & $\mathrm{N} / \mathrm{A}$ & $\mathrm{N} / \mathrm{A}$ & 0 \\
\hline $9 / 82$ & & $\mathrm{~N} / \mathrm{A}$ & N/A & N/A & N/A & 0 \\
\hline $10 / 82$ & & $\mathrm{~N} / \mathrm{A}$ & N/A & N/A & $\mathrm{N} / \mathrm{A}$ & 0 \\
\hline $11 / 82$ & & $\mathrm{~N} / \mathrm{A}$ & N/A & N/A & N/A & 0 \\
\hline $12 / 82$ & & $\mathrm{~N} / \mathrm{A}$ & N/A & N/A & $N / A$ & 0 \\
\hline $1 / 83$ & & $\mathrm{~N} / \mathrm{A}$ & N/A & N/A & N/A & 0 \\
\hline $2 / 83$ & & N/A & N/A & $\mathrm{N} / \mathrm{A}$ & N/A & 0 \\
\hline $3 / 83$ & & N/A & $\mathrm{N} / \mathrm{A}$ & $\mathrm{N} / \mathrm{A}$ & N/A & 0 \\
\hline $4 / 83$ & & N/A & $N / A$ & N/A & N/A & 0 \\
\hline $5 / 83$ & & N/A & N/A & N/A & N/A & 0 \\
\hline $6 / 83$ & & N/A & N/A & N/A & $\mathrm{N} / \mathrm{A}$ & 0 \\
\hline $7 / 83$ & & N/A & $\mathrm{N} / \mathrm{A}$ & N/A & N/A & 0 \\
\hline $8 / 83$ & & N/A & N/A & N/A & N/A & 0 \\
\hline
\end{tabular}

Data obtained from Tank Farm Surveillance and Waste Status Summary Report, WHC-EP-0182. 
WHC-SD-WM-ER-315, Rev. 0

TANK LEVEL HISTORY AND WASTE TYPES

241-AP-101

\begin{tabular}{|c|c|c|c|c|c|c|}
\hline Date & & Total Waste & DSS & Sludge & Salt & Total solids \\
\hline$(M / Y r)$ & Waste Type & (K Gal) & (K Gal) & (K Gal) & (K Gal) & (K Gal) \\
\hline $9 / 83$ & & N/A & $\overline{N / A}$ & $\mathbf{N} / \mathbf{A}$ & $\mathbf{N} / \mathbf{A}$ & 0 \\
\hline $10 / 83$ & & $\mathbf{N} / \mathbf{A}$ & N/A & $\mathbf{N} / \mathbf{A}$ & N/A & 0 \\
\hline $11 / 83$ & & N/A & $\overline{N / A}$ & N/A & $N / A$ & $\overline{0}$ \\
\hline $12 / 83$ & & N/A & $N / A$ & N/A & N/A & 0 \\
\hline $1 / 84$ & & N/A & $N / A$ & N/A & N/A & 0 \\
\hline $2 / 84$ & & $\mathrm{~N} / \mathrm{A}$ & $\mathbf{N} / \mathbf{A}$ & N/A & $\mathrm{N} / \mathrm{A}$ & 0 \\
\hline $3 / 84$ & & N/A & N/A & N/A & N/A & 0 \\
\hline $4 / 84$ & & $\mathbf{N} / \mathbf{A}$ & $\mathbf{N} / \mathbf{A}$ & N/A & N/A & $\overline{0}$ \\
\hline $5 / 84$ & & $\mathrm{~N} / \mathrm{A}$ & $N / A$ & N/A & N/A & 0 \\
\hline $6 / 84$ & & $\mathbf{N} / \mathbf{A}$ & N/A & N/A & N/A & 0 \\
\hline $7 / 84$ & & N/A & N/A & N/A & N/A & 0 \\
\hline $8 / 84$ & & N/A & N/A & N/A & $\mathbf{N} / \mathbf{A}$ & 0 \\
\hline $9 / 84$ & & N/A & N/A & N/A & N/A & 0 \\
\hline $10 / 84$ & & N/A & N/A & $N / A$ & N/A & 0 \\
\hline $11 / 84$ & & N/A & N/A & N/A & N/A & 0 \\
\hline $12 / 84$ & & N/A & $\mathbf{N} / \mathbf{A}$ & $\mathrm{N} / \mathrm{A}$ & N/A & 0 \\
\hline $1 / 85$ & & N/A & N/A & $\mathbf{N} / \mathbf{A}$ & N/A & 0 \\
\hline $2 / 85$ & & N/A & N/A & N/A & N/A & 0 \\
\hline $3 / 85$ & & N/A & N/A & $N / A$ & N/A & $\mathbf{Q}$ \\
\hline $4 / 85$ & & N/A & $\mathbf{N} / \mathbf{A}$ & $\mathbf{N} / \mathbf{A}$ & N/A & 0 \\
\hline $5 / 85$ & & $\mathrm{~N} / \mathrm{A}$ & N/A & $N / A$ & N/A & 0 \\
\hline $6 / 85$ & & $\mathrm{~N} / \mathrm{A}$ & N/A & N/A & N/A & 0 \\
\hline $7 / 85$ & & N/A & N/A & $N / A$ & N/A & 0 \\
\hline $8 / 85$ & & N/A & $\mathbf{N} / \mathbf{A}$ & $N / A$ & $\mathrm{~N} / \mathrm{A}$ & 0 \\
\hline $9 / 85$ & & N/A & $\mathbf{N} / \mathbf{A}$ & N/A & N/A & 0 \\
\hline $10 / 85$ & & N/A & $\mathbf{N} / \mathbf{A}$ & N/A & N/A & 0 \\
\hline $11 / 85$ & & N/A & N/A & N/A & N/A & 0 \\
\hline $12 / 85$ & & N/A & $\mathbf{N} / \mathbf{A}$ & N/A & N/A & 0 \\
\hline $1 / 86$ & & N/A & N/A & $N / A$ & $\mathrm{~N} / \mathrm{A}$ & 0 \\
\hline $2 / 86$ & & N/A & N/A & N/A & N/A & 0 \\
\hline $3 / 86$ & & N/A & N/A & $\mathrm{N} / \mathrm{A}$ & $N / A$ & 0 \\
\hline $4 / 86$ & & N/A & $N / A$ & $N / A$ & N/A & 0 \\
\hline $5 / 86$ & & $\mathrm{~N} / \mathrm{A}$ & $N / A$ & $N / A$ & N/A & 0 \\
\hline $6 / 86$ & & $\mathrm{~N} / \mathrm{A}$ & N/A & $\mathrm{N} / \mathrm{A}$ & N/A & 0 \\
\hline $7 / 86$ & NCPLX & 0 & 0 & 0 & 0 & 0 \\
\hline $8 / 86$ & NCPLX & 0 & 0 & 0 & 0 & 0 \\
\hline $9 / 86$ & NCPLX & 0 & 0 & 0 & 0 & 0 \\
\hline $10 / 86$ & NCPLX & 19 & 0 & 0 & 0 & 0 \\
\hline $11 / 86$ & NCPLX & 18 & 0 & 0 & 0 & 0 \\
\hline $12 / 86$ & NCPLX & 18 & 0 & 0 & 0 & 0 \\
\hline $1 / 87$ & NCPLX & 18 & 0 & 0 & 0 & 0 \\
\hline $2 / 87$ & NCPLX & 18 & 0 & 0 & 0 & 0 \\
\hline $3 / 87$ & NCPLX & 18 & 0 & 0 & 0 & 0 \\
\hline $4 / 87$ & NCPLX & 18 & 0 & 0 & 0 & 0 \\
\hline
\end{tabular}

Data obtained from Tank Farm Surveillance and Waste Status Summary Report, WHC-EP-0182. 
TANK LEVEL HISTORY AND WASTE TYPES 241-AP-101

\begin{tabular}{|c|c|c|c|c|c|c|}
\hline Date & & Total Waste. & DSS & Sludge & Salt & Total solids \\
\hline$\left(M / Y_{r}\right)$ & Waste Type & (K Gal) & (K Gal) & (K Gal) & (K Gal) & (K Gal) \\
\hline $5 / 87$ & NCPLX & 18 & $\overline{0}$ & 0 & $\overline{0}$ & $\overline{0}$ \\
\hline $6 / 87$ & NCPLX & 17 & 0 & 0 & 0 & 0 \\
\hline $7 / 87$ & NCPLX & 17 & 0 & 0 & 0 & 0 \\
\hline $8 / 87$ & NCPLX & 17 & 0 & $\overline{0}$ & 0 & 0 \\
\hline $9 / 87$ & NCPLX & 17 & 0 & 0 & 0 & 0 \\
\hline $10 / 87$ & NCPLX & 18 & 0 & 0 & 0 & 0 \\
\hline $11 / 87$ & NCPLX & 18 & 0 & 0 & 0 & 0 \\
\hline $12 / 87$ & NCPLX & 17 & 0 & $\overline{0}$ & $\overline{0}$ & 0 \\
\hline $1 / 88$ & NCPLX & 18 & 0 & 0 & 0 & 0 \\
\hline $2 / 88$ & NCPLX & 19 & $\overline{0}$ & 0 & 0 & 0 \\
\hline $3 / 88$ & NCPLX & 19 & 0 & 0 & 0 & $\overline{0}$ \\
\hline $4 / 88$ & NCPLX & 18 & 0 & 0 & 0 & 0 \\
\hline $5 / 88$ & NCPLX & 19 & 0 & 0 & 0 & $\overline{0}$ \\
\hline $6 / 88$ & NCPLX & 19 & 0 & 0 & 0 & 0 \\
\hline $7 / 88$ & NCPLX & 94 & 0 & 0 & 0 & 0 \\
\hline $8 / 88$ & NCPLX & 820 & 0 & 0 & 0 & 0 \\
\hline $9 / 88$ & NCPLX & 850 & 0 & $\overline{0}$ & 0 & $\overline{0}$ \\
\hline $10 / 88$ & NCPLX & 778 & 0 & 0 & 0 & 0 \\
\hline $11 / 88$ & NCPLX & 344 & 0 & 0 & 0 & 0 \\
\hline $12 / 88$ & NCPLX & 374 & 0 & 0 & 0 & 0 \\
\hline $1 / 89$ & NCPLX & 373 & 0 & 0 & 0 & 0 \\
\hline $2 / 89$ & NCPLX & 373 & $\overline{0}$ & 0 & 0 & 0 \\
\hline $3 / 89$ & NCPLX & 373 & 0 & 0 & 0. & 0 \\
\hline $4 / 89$ & $\overline{\text { NCPLX }}$ & 382 & 0 & 0 & 0 & 0 \\
\hline $5 / 89$ & NCPLX & 382 & 0 & 0 & 0 & 0 \\
\hline $6 / 89$ & NCPLX & 382 & 0 & 0 & 0 & 0 \\
\hline $7 / 89$ & NCPLX & 382 & 0 & 0 & 0 & 0 \\
\hline $8 / 89$ & NCPLX & 382 & 0 & 0 & 0 & 0 \\
\hline $9 / 89$ & NCPLX & 381 & 0 & 0 & 0 & 0 \\
\hline $10 / 89$ & NCPLX & 997 & 0 & 0 & 0 & 0 \\
\hline $11 / 89$ & NCPLX & 1012 & 0 & $\overline{0}$ & 0 & 0 \\
\hline $12 / 89$ & NCPLX & 1065 & 0 & .0 & 0 & 0 \\
\hline $1 / 90$ & NCPLX & 1065 & 0 & 0 & 0 & 0 \\
\hline $2 / 90$ & NCPLX & 1065 & 0 & 0 & 0 & 0 \\
\hline $3 / 90$ & NCPLX & 1012 & 0 & 0 & 0 & 0 \\
\hline $4 / 90$ & NCPLX & 1065 & 0 & 0 & 0 & 0 \\
\hline $5 / 90$ & NCPLX & 1064 & 0 & 0 & 0 & 0 \\
\hline $6 / 90$ & DN & 1064 & 0 & 0 & 0 & 0 \\
\hline $7 / 90$ & DN & 1064 & 0 & 0 & 0 & 0 \\
\hline $8 / 90$ & $\overline{D N}$ & 1064 & 0 & 0 & 0 & 0 \\
\hline $9 / 90$ & DN & 1064 & 0 & 0 & 0 & 0 \\
\hline $10 / 90$ & DN & 1064 & 0 & 0 & 0 & 0 \\
\hline $11 / 90$ & $\overline{\mathrm{DN}}$ & 1064 & 0 & 0 & 0 & 0 \\
\hline $12 / 90$ & DN & 1064 & 0 & 0 & 0 & 0 \\
\hline
\end{tabular}

Data obtained from Tank Farm Surveillance and Waste Status Summary Report, WHC-EP-0182. 
WHC-SD-WM-ER-315, Rev. 0

TANK LEVEL HISTORY AND WASTE TYPES

241-AP-101

\begin{tabular}{|c|c|c|c|c|c|c|}
\hline Date & & Total Waste & DSS & Sludge & Salt & Total solids \\
\hline$(\mathrm{M} / \mathrm{Yr})$ & Waste Type & (K Gal) & (K Gal) & (K Gal) & (K Gal) & (K Gal) \\
\hline $1 / 91$ & DN & 1063 & 0 & 0 & 0 & 0 \\
\hline $2 / 91$ & DN & 1063 & 0 & 0 & 0 & 0 \\
\hline $3 / 91$ & DN & 1063 & 0 & 0 & 0 & 0 \\
\hline $4 / 91$ & $\overline{D N}$ & 1063 & 0 & 0 & 0 & 0 \\
\hline $5 / 91$ & DN & 1063 & 0 & 0 & 0 & 0 \\
\hline $6 / 91$ & DN & 1063 & 0 & 0 & 0 & 0 \\
\hline $7 / 91$ & DN & 1063 & 0 & 0 & 0 & 0 \\
\hline $8 / 91$ & DN & 1063 & 0 & 0 & 0 & 0 \\
\hline $9 / 91$ & DN & 1063 & 0 & 0 & 0 & 0 \\
\hline $10 / 91$ & DN & 1063 & 0 & 0 & 0 & $\overline{0}$ \\
\hline $11 / 91$ & DN & 1063 & 0 & 0 & 0 & 0 \\
\hline $12 / 91$ & DN & 1062 & 0 & 0 & 0 & 0 \\
\hline $1 / 92$ & DN & 1062 & 0 & 0 & 0 & 0 \\
\hline $2 / 92$ & DN & 1062 & 0 & 0 & 0 & 0 \\
\hline $3 / 92$ & DN & 1062 & 0 & 0 & 0 & 0 \\
\hline $4 / 92$ & DN & 1062 & 0 & 0 & 0 & 0 \\
\hline $5 / 92$ & DN & 1062 & 0 & 0 & 0 & 0 \\
\hline $6 / 92$ & DN & 1062 & 0 & 0 & 0 & 0 \\
\hline $7 / 92$ & DN & 1062 & 0 & 0 & 0 & 0 \\
\hline $8 / 92$ & DN & 1062 & 0 & 0 & 0 & 0 \\
\hline $9 / 92$ & DN & 1062 & 0 & 0 & 0 & 0 \\
\hline $10 / 92$ & DN & 1062 & 0 & 0 & 0 & 0 \\
\hline $11 / 92$ & DN & 1062 & 0 & 0 & 0 & 0 \\
\hline $12 / 92$ & DN & 1061 & 0 & 0 & 0 & 0 \\
\hline $1 / 93$ & DN & 1061 & 0 & 0 & 0 & 0 \\
\hline $2 / 93$ & DN & 1061 & 0 & 0 & 0 & 0 \\
\hline $3 / 93$ & DN & 1061 & 0 & 0 & 0 & 0 \\
\hline $4 / 93$ & $\overline{D N}$ & 1061 & 0 & 0 & 0 & 0 \\
\hline $5 / 93$ & DN & 1061 & 0 & .0 & 0 & 0 \\
\hline $6 / 93$ & DN & 1061 & 0 & 0 & 0 & 0 \\
\hline $7 / 93$ & DN & 1061 & 0 & 0 & 0 & 0 \\
\hline $8 / 93$ & DN & 1061 & 0 & 0 & 0 & 0 \\
\hline $9 / 93$ & DN & 1061 & 0 & 0 & 0 & 0 \\
\hline $10 / 93$ & DN & 1061 & 0 & 0 & 0 & 0 \\
\hline $11 / 93$ & $\overline{D N}$ & 1061 & 0 & 0 & 0 & 0 \\
\hline $12 / 93$ & DN & 1060 & 0 & 0 & 0 & 0 \\
\hline $1 / 94$ & $\overline{\text { DN }}$ & 1060 & 0 & 0 & 0 & 0 \\
\hline $2 / 94$ & DN & .1060 & 0 & 0 & 0 & 0 \\
\hline $3 / 94$ & DN & 1060 & 0 & 0 & 0 & 0 \\
\hline
\end{tabular}

Data obtained from Tank Farm Surveillance and Waste Status Summary Report, WHC-EP-0182. 
WHC-SD-WM-ER-315, Rev. 0

TANK LEVEL HISTORY AND WASTE TYPES

241-AP-102

\begin{tabular}{|c|c|c|c|c|c|c|}
\hline Date & & Total Waste & DSS & Sludge & Salt & Total solids \\
\hline$(\mathrm{M} / \mathrm{Yr})$ & Waste Type & (K Gal) & (K Gal) & (K Gal) & (K Gal) & (K Gal) \\
\hline $1 / 80$ & & N/A & N/A & $N / A$ & N/A & 0 \\
\hline $2 / 80$ & & N/A & N/A & N/A & $\mathrm{N} / \mathrm{A}$ & 0 \\
\hline $3 / 80$ & & $\mathbf{N} / \mathbf{A}$ & N/A & $N / A$ & N/A & 0 \\
\hline $4 / 80$ & & $N / A$ & N/A & N/A & N/A & 0 \\
\hline $5 / 80$ & & $\mathbf{N} / \mathbf{A}$ & N/A & $\overline{N / A}$ & N/A & 0 \\
\hline $6 / 80$ & & N/A & $\mathbf{N} / \mathrm{A}$ & N/A & N/A & $\overline{0}$ \\
\hline $7 / 80$ & & $\mathbf{N} / \mathbf{A}$ & N/A & $\mathrm{N} / \mathrm{A}$ & N/A & 0 \\
\hline $8 / 80$ & & N/A & $\mathrm{N} / \mathrm{A}$ & $\mathbf{N} / \mathbf{A}$ & N/A & 0 \\
\hline $9 / 80$ & & N/A & $\bar{N} / \mathbf{A}$ & N/A & N/A & 0 \\
\hline $10 / 80$ & & $\mathrm{~N} / \mathrm{A}$ & N/A & N/A & N/A & 0 \\
\hline $11 / 80$ & & N/A & N/A & $\mathrm{N} / \mathrm{A}$ & $\mathbf{N} / \mathbf{A}$ & 0 \\
\hline $12 / 80$ & & $\mathbf{N} / \mathbf{A}$ & $N / A$ & N/A & N/A & 0 \\
\hline $1 / 81$ & & N/A & N/A & $N / A$ & N/A & 0 \\
\hline $2 / 81$ & & N/A & N/A & N/A & N/A & 0 \\
\hline $3 / 81$ & & N/A & N/A & N/A & $\mathrm{N} / \mathrm{A}$ & 0 \\
\hline $4 / 81$ & & $\bar{N} / \mathbf{A}$ & N/A & N/A & N/A & 0 \\
\hline $5 / 81$ & & N/A & N/A & $\mathbf{N} / \mathbf{A}$ & N/A & 0 \\
\hline $6 / 81$ & & N/A & N/A & N/A & N/A & 0 \\
\hline $7 / 81$ & & N/A & N/A & $\mathbf{N} / \mathbf{A}$ & N/A & 0 \\
\hline $8 / 81$ & & $\mathbf{N} / \mathbf{A}$. & $N / A$ & $N / A$ & $N / A$ & $\overline{0}$ \\
\hline $9 / 81$ & & N/A & $\mathrm{N} / \mathrm{A}$ & $\mathrm{N} / \mathrm{A}$ & $\mathrm{N} / \mathrm{A}$ & 0 \\
\hline $10 / 81$ & & $N / A$ & $\mathrm{~N} / \mathrm{A}$ & $N / A$ & $N / A$ & 0 \\
\hline $11 / 81$ & & N/A & $\mathrm{N} / \mathrm{A}$ & N/A & N/A & 0 \\
\hline $12 / 81$ & & N/A & $\mathbf{N} / \mathbf{A}$ & N/A & $N / A$ & 0 \\
\hline $1 / 82$ & & N/A & N/A & $N / A$ & $N / A$ & 0 \\
\hline $2 / 82$ & & $N / A$ & N/A & $N / A$ & N/A & 0 \\
\hline $3 / 82$ & & N/A & $\mathrm{N} / \mathrm{A}$ & $\mathbf{N} / \mathbf{A}$ & $N / A$ & 0 \\
\hline $4 / 82$ & & $\mathrm{~N} / \mathrm{A}$ & N/A & $\mathrm{N} / \mathrm{A}$ & N/A & 0 \\
\hline $5 / 82$ & & $\mathrm{~N} / \mathrm{A}$ & N/A & $\mathrm{N} / \mathrm{A}$ & N/A & 0 \\
\hline $6 / 82$ & & N/A & $\mathrm{N} / \mathrm{A}$ & $N / A$ & N/A & 0 \\
\hline $7 / 82$ & & N/A & N/A & N/A & $N / A$ & 0 \\
\hline $8 / 82$ & & N/A & N/A & $\mathrm{N} / \mathrm{A}$ & N/A & 0 \\
\hline $9 / 82$ & & N/A & $\mathrm{N} / \mathrm{A}$ & $\mathrm{N} / \mathrm{A}$ & N/A & 0 \\
\hline $10 / 82$ & & $\mathbf{N} / \mathbf{A}$ & $\mathrm{N} / \mathrm{A}$ & $N / A$ & N/A & 0 \\
\hline $11 / 82$ & & N/A & N/A & N/A & $N / A$ & 0 \\
\hline $12 / 82$ & & N/A & N/A & $N / A$ & N/A & 0 \\
\hline $1 / 83$ & & N/A & $N / A$ & $N / A$ & $N / A$ & 0 \\
\hline $2 / 83$ & & $N / A$ & $\mathrm{~N} / \mathrm{A}$ & N/A & $N / A$ & 0 \\
\hline $3 / 83$ & & N/A & $\mathbf{N} / \mathbf{A}$ & N/A & $N / A$ & 0 \\
\hline $4 / 83$ & & N/A & $\mathbf{N} / \mathrm{A}$ & $\mathrm{N} / \mathrm{A}$ & N/A & 0 \\
\hline $5 / 83$ & & N/A & N/A & $N / A$ & N/A & 0 \\
\hline $6 / 83$ & & N/A & $\mathrm{N} / \mathrm{A}$ & $\mathrm{N} / \mathrm{A}$ & N/A & 0 \\
\hline $7 / 83$ & & N/A & $N / A$ & N/A & N/A & 0 \\
\hline $8 / 83$ & & N/A & N/A & N/A & $\mathrm{N} / \mathrm{A}$ & 0 \\
\hline
\end{tabular}

Data obtained from Tank Farm Surveillance and Waste Status Summary Report, WHC-EP-0182. 
WHC-SD-WM-ER-315, Rev. 0

TANK LEVEL HISTORY AND WASTE TYPES

241-AP-102

\begin{tabular}{|c|c|c|c|c|c|c|}
\hline Date & & Total Waste & DSs & Sludge & Salt & Total solids \\
\hline$\left(M / Y_{r}\right)$ & Waste Type & (K Gal) & (K Gal) & (K Gal) & (K Gal) & (K Gal) \\
\hline $9 / 83$ & & $\mathbf{N} / \mathbf{A}$ & N/A & $\mathrm{N} / \mathrm{A}$ & N/A & 0 \\
\hline $10 / 83$ & & N/A & $\overline{N / A}$ & $N / A$ & N/A & 0 \\
\hline $11 / 83$ & & $\mathbf{N} / \mathbf{A}$ & N/A & N/A & N/A & 0 \\
\hline $12 / 83$ & & N/A & N/A & N/A & N/A & 0 \\
\hline $1 / 84$ & & N/A & N/A & $\mathbf{N} / \mathbf{A}$ & N/A & 0 \\
\hline $2 / 84$ & & N/A & N/A & $\mathrm{N} / \mathrm{A}$ & $\mathbf{N} / \mathbf{A}$ & 0 \\
\hline $3 / 84$ & & N/A & $\mathbf{N} / \mathbf{A}$ & $\mathbf{N} / \mathbf{A}$ & $\mathrm{N} / \mathrm{A}$ & 0 \\
\hline $4 / 84$ & & $\mathbf{N} / \mathbf{A}$ & N/A & $\mathbf{N} / \mathbf{A}$ & N/A & 0 \\
\hline $5 / 84$ & & $N / A$ & N/A & $N / A$ & N/A & 0 \\
\hline $6 / 84$ & & N/A & N/A & N/A & N/A & $\overline{0}$ \\
\hline $7 / 84$ & & $N / A$ & N/A & N/A & $N / A$ & 0 \\
\hline $8 / 84$ & & $\mathbf{N} / \mathbf{A}$ & N/A & N/A & N/A & 0 \\
\hline $9 / 84$ & & N/A & $N / A$ & N/A & N/A & $\overline{0}$ \\
\hline $10 / 84$ & & N/A & N/A & N/A & $\mathbf{N} / \mathbf{A}$ & 0 \\
\hline $11 / 84$ & & $\mathbf{N} / \mathbf{A}$ & $N / A$ & $\mathbf{N} / \mathbf{A}$ & N/A & 0 \\
\hline $12 / 84$ & & N/A & N/A & $\mathbf{N} / \mathbf{A}$ & N/A & 0 \\
\hline $1 / 85$ & & N/A & $\mathrm{N} / \mathrm{A}$ & N/A & N/A & 0 \\
\hline $2 / 85$ & & N/A & $\mathbf{N} / \mathbf{A}$ & $\mathbf{N} / \mathbf{A}$ & N/A & 0 \\
\hline $3 / 85$ & & N/A & $\mathrm{N} / \mathrm{A}$ & N/A & N/A & $\overline{0}$ \\
\hline $4 / 85$ & & N/A & N/A & $\mathrm{N} / \mathrm{A}$ & $N / A$ & 0 \\
\hline $5 / 85$ & & N/A & N/A & N/A & N/A & 0 \\
\hline $6 / 85$ & & $\mathbf{N} / \mathbf{A}$ & N/A & N/A & N/A & 0 \\
\hline $7 / 85$ & $\cdot$ & $N / A$ & $\mathrm{~N} / \mathrm{A}$ & $\mathbf{N} / \mathbf{A}$ & N/A & 0 \\
\hline $8 / 85$ & & $N / A$ & $N / A$ & $N / A$ & N/A & 0 \\
\hline $9 / 85$ & & $N / A$ & N/A & N/A & N/A & 0 \\
\hline $10 / 85$ & & N/A & $\mathrm{N} / \mathrm{A}$ & N/A & $\mathrm{N} / \mathrm{A}$ & 0 \\
\hline $11 / 85$ & & N/A & $N / A$ & N/A & $\mathbf{N} / \mathbf{A}$ & 0 \\
\hline $12 / 85$ & & N/A & N/A & N/A & $N / A$ & 0 \\
\hline $1 / 86$ & & N/A & $N / A$ & $\mathbf{N} / \mathbf{A}$ & $N / A$ & 0 \\
\hline $2 / 86$ & & N/A & N/A & $\mathrm{N} / \mathrm{A}$ & $\mathrm{N} / \mathrm{A}$ & 0 \\
\hline $3 / 86$ & & $N / A$ & $N / A$ & N/A & $N / A$ & 0 \\
\hline $4 / 86$ & & $N / A$ & $N / A$ & $\mathbf{N} / \mathbf{A}$ & $N / A$ & 0 \\
\hline $5 / 86$ & & N/A. & $\mathbf{N} / \mathbf{A}$ & N/A & N/A & 0 \\
\hline $6 / 86$ & & $\mathrm{~N} / \mathrm{A}$ & $N / A$ & $N / A$ & N/A & 0 \\
\hline $7 / 86$ & HFW & 0 & 0 & 0 & 0 & 0 \\
\hline $8 / 86$ & HFW & 0 & 0 & 0 & 0 & 0 \\
\hline $9 / 86$ & HFW & 0 & 0 & 0 & 0 & 0 \\
\hline $10 / 86$ & HFW & 18 & 0 & 0 & 0 & 0 \\
\hline $11 / 86$ & HFW & 19 & 0 & 0 & 0 & 0 \\
\hline $12 / 86$ & HFW & 17 & 0 & 0 & 0 & 0 \\
\hline $1 / 87$ & HFW & 17. & 0 & 0 & 0 & 0 \\
\hline $2 / 87$ & HFW & 17 & 0 & 0 & 0 & 0 \\
\hline $3 / 87$ & HFW & 17 & 0 & 0 & 0 & 0 \\
\hline $4 / 87$ & HFW & 17 & 0 & 0 & 0 & 0 \\
\hline
\end{tabular}

Data obtained from Tank Farm Surveillance and Waste Status Summary Report, WHC-EP-0182. 
TANK LEVEL HISTORY AND WASTE TYPES

241-AP-102

\begin{tabular}{|c|c|c|c|c|c|c|}
\hline Date & & Total Waste & DSS & Sludge & Salt & Total solids \\
\hline$(\mathrm{M} / \mathrm{Vr})$ & Waste Type & (K Gal) & (K Gal) & (K Gal) & (K Gal) & (K Gal) \\
\hline $5 / 87$ & HFW & 17 & 0 & 0 & 0 & 0 \\
\hline $6 / 87$ & HFW & 17 & 0 & 0 & 0 & 0 \\
\hline $7 / 87$ & HFW & 17 & 0 & 0 & 0 & 0 \\
\hline $8 / 87$ & HFW & 964 & 0 & 0 & 0 & 0 \\
\hline $9 / 87$ & HFW & 971 & 0 & 0 & 0 & 0 \\
\hline $10 / 87$ & HFW & 979 & 0 & 0 & 0 & $\overline{0}$ \\
\hline $11 / 87$ & HFW & 978 & 0 & 0 & 0 & 0 \\
\hline $12 / 87$ & HFW & 978 & 0 & 0 & 0 & 0 \\
\hline $1 / 88$ & HFW & 978 & 0 & 0 & 0 & 0 \\
\hline $2 / 88$ & HFW & 985 & 0 & 0 & 0 & 0 \\
\hline $3 / 88$ & HFW & 23 & 0 & 0 & 0 & 0 \\
\hline $4 / 88$ & HFW & 1076 & 0 & 0 & 0 & 0 \\
\hline $5 / 88$ & HFW & 1075 & 0 & 0 & 0 & 0 \\
\hline $6 / 88$ & HFW & 1076 & 0 & 0 & 0 & 0 \\
\hline $7 / 88$ & HFW & 1078 & 0 & 0 & 0 & 0 \\
\hline $8 / 88$ & HFW & 1056 & 0 & 0 & 0 & 0 \\
\hline $9 / 88$ & HFW & 924 & 0 & 0 & 0 & 0 \\
\hline $10 / 88$ & HFW & 745 & 0 & 0 & 0 & 0 \\
\hline $11 / 88$ & HFW & 747 & 0 & 0 & 0 & 0 \\
\hline $12 / 88$ & HFW & 746 & 0. & 0 & 0 & 0 \\
\hline $1 / 89$ & HFW & 746 & 0 & 0 & 0 & 0 \\
\hline $2 / 89$ & HFW & $.745^{\circ}$ & 0 & 0 & 0 & 0 \\
\hline $3 / 89$ & HFW & 745 & 0 & 0 & 0 & 0 \\
\hline $4 / 89$ & HFW & 569 & 0 & 0 & 0 & 0 \\
\hline $5 / 89$ & HFW & 570 & 0 & 0 & 0 & 0 \\
\hline $6 / 89$ & HFW & 99 & 0 & 0 & 0 & 0 \\
\hline $7 / 89$ & HFW & 123 & 0 & 0 & 0 & 0 \\
\hline $8 / 89$ & HFW & 126 & 0 & 0 & 0 & 0 \\
\hline $9 / 89$ & HFW & 130 & 0 & 0 & 0 & 0 \\
\hline $10 / 89$ & HFW & .133 & 0 & 0 & 0 & 0 \\
\hline $11 / 89$ & HFW & 133 & 0 & 0 & 0 & 0 \\
\hline $12 / 89$ & HFW & 133 & 0 & 0 & 0 & 0 \\
\hline $1 / 90$ & HFW & 134 & 0 & 0 & 0 & 0 \\
\hline $2 / 90$ & HFW & 134 & 0 & 0 & 0 & 0 \\
\hline $3 / 90$ & HFW & 133 & 0 & 0 & 0 & 0 \\
\hline $4 / 90$ & HFW & 134 & 0 & 0 & 0 & 0 \\
\hline $5 / 90$ & HFW & 134 & 0 & 0 & 0 & 0 \\
\hline $6 / 90$ & DN & 134 & 0 & 0 & 0 & 0 \\
\hline $7 / 90$ & DN & 134 & 0 & 0. & 0 & 0 \\
\hline $8 / 90$ & DN & 134 & 0 & 0 & 0 & 0 \\
\hline $9 / 90$ & DN & 134 & 0 & 0 & 0 & 0 \\
\hline $10 / 90$ & DN & 134 & 0 & 0 & 0 & 0 \\
\hline $11 / 90$ & DN & 133 & 0 & 0 & 0 & 0 \\
\hline $12 / 90$ & DN & 133 & 0 & 0 & 0 & 0 \\
\hline
\end{tabular}

Data obtained from Tank Farm Surveillance and Waste Status Summary Report, WHC-EP-0182. 
TANK LEVEL HISTORY AND WASTE TYPES

241-AP-102

\begin{tabular}{|c|c|c|c|c|c|c|}
\hline Date & & Total Waste & DSS & Sludge & Salt & Total solids \\
\hline$(\mathrm{M} / \mathrm{Yr})$ & Waste Type & (K Gal). & (K Gal) & (K Gal) & (K Gal) & (K Gal) \\
\hline $1 / 91$ & DN & 134 & 0 & 0 & 0 & $\overline{0}$ \\
\hline $2 / 91$ & DN & 134 & 0 & 0 & 0 & 0 \\
\hline $3 / 91$ & $\overline{\text { DN }}$ & 134 & 0 & 0 & 0 & 0 \\
\hline $4 / 91$ & $\overline{D N}$ & 133 & 0 & 0 & 0 & 0 \\
\hline $5 / 91$ & $\overline{D N}$ & 133 & 0 & 0 & 0 & 0 \\
\hline $6 / 91$ & $\overline{D N}$ & 133 & 0 & 0 & 0 & 0 \\
\hline $7 / 91$ & DN & 133 & $\overline{0}$ & 0 & 0 & 0 \\
\hline $8 / 91$ & DN & 133 & 0 & 0 & 0 & 0 \\
\hline $9 / 91$ & $\mathrm{DN}$ & 133 & 0 & 0 & 0 & 0 \\
\hline $10 / 91$ & DN & 133 & 0 & 0 & 0 & 0 \\
\hline $11 / 91$ & $\overline{\text { DN }}$ & 134 & 0 & 0 & 0 & 0 \\
\hline $12 / 91$ & DN & 133 & 0 & 0 & 0 & 0 \\
\hline $1 / 92$ & DN & 133 & 0 & 0 & 0 & 0 \\
\hline $2 / 92$ & DN & 133 & 0 & 0 & 0 & 0 \\
\hline $3 / 92$ & DN & 133 & 0 & 0 & 0 & 0 \\
\hline $4 / 92$ & DN & 133 & 0 & 0 & 0 & 0 \\
\hline $5 / 92$ & $\overline{\text { DN }}$ & 136 & $\overline{0}$ & 0 & 0 & 0 \\
\hline $6 / 92$ & DN & 136 & 0 & 0 & 0 & 0 \\
\hline $7 / 92$ & DN & 136 & 0 & 0 & 0 & 0 \\
\hline $8 / 92$ & DN & 137 & 0 & 0 & 0 & 0 \\
\hline $9 / 92$ & DN & 150 & 0 & 0 & 0 & 0 \\
\hline $10 / 92$ & DN & 149 & 0 & 0 & 0 & 0 \\
\hline $11 / 92$ & $\overline{D N}$ & 501 & 0 & 0 & 0 & 0 \\
\hline $12 / 92$ & DN & 1105 & 0 & 0 & 0 & 0 \\
\hline $1 / 93$ & DN & 1105 & 0 & 0 & 0 & 0 \\
\hline $2 / 93$ & DN & 1103 & 0 & 0 & 0 & 0 \\
\hline $3 / 93$ & DN & 1107 & 0 & 0 & 0 & 0 \\
\hline $4 / 93$ & DN & 1108 & 0 & 0 & 0 & 0 \\
\hline $5 / 93$ & DN & 1107 & 0 & 0 & 0 & 0 \\
\hline $6 / 93$ & DN & 1107 & 0 & 0 & 0 & 0 \\
\hline $7 / 93$ & DN & 1107 & 0 & 0 & 0 & 0 \\
\hline $8 / 93$ & DN & 1106 & 0 & 0 & 0 & 0 \\
\hline $9 / 93$ & DN & 1106. & 0 & 0 & 0 & 0 \\
\hline $10 / 93$ & DN & 1106 & 0. & 0 & 0 & 0 \\
\hline $11 / 93$ & DN & 1104 & 0 & 0 & 0 & 0 \\
\hline $12 / 93$ & DN & 1104 & 0 & 0 & 0 & 0 \\
\hline $1 / 94$ & $\overline{D N}$ & 1103 & 0 & 0 & 0 & 0 \\
\hline $2 / 94$ & DN & 1103 & 0 & 0 & 0 & 0 \\
\hline $3 / 94$ & $\overline{D N}$ & 1103 & 0 & 0 & 0 & 0 \\
\hline
\end{tabular}

Data obtained from Tank Farm Surveillance and Waste Status Summary Report, WHC-EP-0182. 
WHC-SD-WM-ER-315, Rev. 0

TANK LEVEL HISTORY AND WASTE TYPES

241-AP-103

\begin{tabular}{|c|c|c|c|c|c|c|}
\hline Date & & Total Waste & DSS & Sludge & Salt & Total solids \\
\hline$\left(\mathrm{M} / \mathrm{Yr}_{\mathrm{r}}\right)$ & Waste Type & (K Gal) & (K Gal) & (K Gal) & (K Gal) & (K Gal) \\
\hline $1 / 80$ & & N/A & N/A & N/A & N/A & 0 \\
\hline $2 / 80$ & & $\mathrm{~N} / \mathrm{A}$ & $\mathrm{N} / \mathrm{A}$ & N/A & N/A & 0 \\
\hline $3 / 80$ & & $\mathbf{N} / \mathbf{A}$ & N/A & $\mathrm{N} / \mathrm{A}$ & $N / A$ & 0 \\
\hline $4 / 80$ & & $\mathrm{~N} / \mathrm{A}$ & N/A & $\mathrm{N} / \mathrm{A}$ & N/A & 0 \\
\hline $5 / 80$ & & N/A & $\mathrm{N} / \mathrm{A}$ & $N / A$ & N/A & 0 \\
\hline $6 / 80$ & & N/A & N/A & N/A & N/A & 0 \\
\hline $7 / 80$ & & $\mathbf{N} / \mathbf{A}$ & N/A & N/A & N/A & 0 \\
\hline $8 / 80$ & & N/A & N/A & N/A & $N / A$ & 0 \\
\hline $9 / 80$ & & N/A & N/A & $N / A$ & N/A & 0 \\
\hline $10 / 80$ & & N/A & N/A & $N / A$ & N/A & 0 \\
\hline $11 / 80$ & & N/A & N/A & N/A & N/A & $\overline{0}$ \\
\hline $12 / 80$ & & $N / A$ & N/A & N/A & $\mathrm{N} / \mathrm{A}$ & 0 \\
\hline $1 / 81$ & & N/A & $\mathbf{N} / \mathbf{A}$ & N/A & $N / A$ & 0 \\
\hline $2 / 81$ & & N/A & $\mathbf{N} / \mathbf{A}$ & $\mathbf{N} / \mathbf{A}$ & $N / A$ & 0 \\
\hline $3 / 81$ & & N/A & N/A & N/A & $N / A$ & 0 \\
\hline $4 / 81$ & & N/A & N/A & N/A & N/A & 0 \\
\hline $5 / 81$ & & N/A & N/A & N/A & N/A & 0 \\
\hline $6 / 81$ & & $\mathrm{~N} / \mathrm{A}$ & N/A & $N / A$ & N/A & 0 \\
\hline $7 / 81$ & & N/A & $\mathrm{N} / \mathrm{A}$ & $N / A$ & N/A & 0 \\
\hline $8 / 81$ & & N/A & $N / A$ & N/A & N/A & 0 \\
\hline $9 / 81$ & & N/A & $\mathbf{N} / \mathbf{A}$ & N/A & $\bar{N} / \mathrm{A}$ & $\overline{0}$ \\
\hline $10 / 81$ & & N/A & $\mathrm{N} / \mathrm{A}$ & $\mathbf{N} / \mathbf{A}$ & N/A & 0 \\
\hline $11 / 81$ & & N/A & $N / A$ & $N / A$ & N/A & 0 \\
\hline $12 / 81$ & & N/A & N/A & N/A & N/A & 0 \\
\hline $1 / 82$ & & $N / A$ & N/A & N/A & $\mathbf{N} / \mathbf{A}$ & 0 \\
\hline $2 / 82$ & & $N / A$ & $N / A$ & N/A & N/A & 0 \\
\hline $3 / 82$ & & $\mathrm{~N} / \mathrm{A}$ & N/A & N/A & N/A & $\overline{0}$ \\
\hline $4 / 82$ & & $\mathbf{N} / \mathbf{A}$ & $\bar{N} / \mathbf{A}$ & $\bar{N} / \mathbf{A}$ & N/A & 0 \\
\hline $5 / 82$ & & N/A & N/A & N/A & N/A & 0 \\
\hline $6 / 82$ & & $N / A$ & N/A & $\mathrm{N} / \mathrm{A}$ & N/A & 0 \\
\hline $7 / 82$ & & $N / A$ & N/A & N/A & N/A & 0 \\
\hline $8 / 82$ & & $N / A$ & N/A & N/A & N/A & 0 \\
\hline $9 / 82$ & & $N / A$ & N/A & N/A & N/A & 0 \\
\hline $10 / 82$ & & $N / A$ & N/A & $\mathrm{N} / \mathrm{A}$ & N/A & 0 \\
\hline $11 / 82$ & & N/A & $\mathrm{N} / \mathrm{A}$ & $\mathrm{N} / \mathrm{A}$ & N/A & 0 \\
\hline $12 / 82$ & & N/A & N/A & N/A & $N / A$ & 0 \\
\hline $1 / 83$ & & N/A & N/A & N/A & N/A & 0 \\
\hline $2 / 83$ & & N/A & N/A & N/A & N/A & 0 \\
\hline $3 / 83$ & & N/A & N/A & N/A & N/A & 0 \\
\hline $4 / 83$ & & $\mathrm{~N} / \mathrm{A}$ & $\mathbf{N} / \mathbf{A}$ & N/A & $N / A$ & 0 \\
\hline $5 / 83$ & & N/A & N/A & $\mathrm{N} / \mathrm{A}$ & N/A & 0 \\
\hline $6 / 83$ & & $\mathrm{~N} / \mathrm{A}$ & N/A & N/A & N/A & 0 \\
\hline $7 / 83$ & & N/A & N/A & N/A & N/A & 0 \\
\hline $8 / 83$ & & $\mathrm{~N} / \mathrm{A}$ & N/A & N/A & N/A & 0 \\
\hline
\end{tabular}

Data obtained from Tank Farm Surveillance and Waste Status Summary Report, WHC-EP-0182. 
WHC-SD-WM-ER-315, Rev. 0

TANK LEVEL HISTORY AND WASTE TYPES

241-AP-103

\begin{tabular}{|c|c|c|c|c|c|c|}
\hline Date & & Total Waste & DSS & Sludge & Salt & Total solids \\
\hline$(\mathrm{M} / \mathrm{Yr})$ & Waste Type & (K Gal) & (K Gal) & (K Gal) & (K Gal) & (K Gal) \\
\hline $9 / 83$ & & N/A & N/A & N/A & N/A & 0 \\
\hline $10 / 83$ & & N/A & N/A & N/A & N/A & 0 \\
\hline $11 / 83$ & & N/A & N/A & N/A & N/A & 0 \\
\hline $12 / 83$ & & $N / A$ & N/A & N/A & $\mathrm{N} / \mathrm{A}$ & 0 \\
\hline $1 / 84$ & & $N / A$ & N/A & $\mathbf{N} / \mathbf{A}$ & N/A & 0 \\
\hline $2 / 84$ & & N/A & N/A & N/A & $\mathbf{N} / \mathbf{A}$ & 0 \\
\hline $3 / 84$ & & $N / A$ & N/A & N/A & $N / A$ & 0 \\
\hline $4 / 84$ & & $\mathbf{N} / \mathbf{A}$ & $\mathrm{N} / \mathrm{A}$ & $\mathrm{N} / \mathrm{A}$ & N/A & 0 \\
\hline $5 / 84$ & & N/A & $N / A$ & N/A & $\mathbf{N} / \mathbf{A}$ & 0 \\
\hline $6 / 84$ & & $\mathbf{N} / \mathbf{A}$ & N/A & $\mathbf{N} / \mathbf{A}$ & $N / A$ & 0 \\
\hline $7 / 84$ & & $\mathbf{N} / \mathbf{A}$ & $\mathbf{N} / \mathbf{A}$ & N/A & $\mathbf{N} / \mathbf{A}$ & 0 \\
\hline $8 / 84$ & & N/A & N/A & N/A & N/A & 0 \\
\hline $9 / 84$ & & N/A. & N/A & N/A & $N / A$ & 0 \\
\hline $10 / 84$ & & N/A & N/A & N/A & N/A & 0 \\
\hline $11 / 84$ & & N/A & N/A & $N / A$ & N/A & 0 \\
\hline $12 / 84$ & & N/A & N/A & $N / A$ & N/A & 0 \\
\hline $1 / 85$ & & N/A & N/A & N/A & $\mathrm{N} / \mathrm{A}$ & 0 \\
\hline $2 / 85$ & & N/A & N/A & N/A & $\mathbf{N} / \mathbf{A}$ & 0 \\
\hline $3 / 85$ & & $\mathbf{N} / \mathbf{A}$ & $\mathbf{N} / \mathbf{A}$ & N/A & $N / A$ & 0 \\
\hline $4 / 85$ & & $\mathbf{N} / \mathbf{A}$ & N/A & $N / A$ & $N / A$ & 0 \\
\hline $5 / 85$ & & $\mathbf{N} / \mathbf{A}$ & $\mathbf{N} / \mathbf{A}$ & N/A & $N / A$ & 0 \\
\hline $6 / 85$ & & $N / A$ & $\mathbf{N} / \mathbf{A}$ & $N / A$ & $N / A$ & 0 \\
\hline $7 / 85$ & & $N / A$ & N/A & $N / A$ & $\mathbf{N} / \mathbf{A}$ & 0 \\
\hline $8 / 85$ & & N/A & $\mathbf{N} / \mathbf{A}$ & N/A & $\mathbf{N} / \mathbf{A}$ & 0 \\
\hline $9 / 85$ & & $\mathbf{N} / \mathbf{A}$ & N/A & $N / A$ & $N / A$ & 0 \\
\hline $10 / 85$ & & $\mathrm{~N} / \mathrm{A}$ & N/A & $\mathrm{N} / \mathrm{A}$ & $\mathrm{N} / \mathrm{A}$ & 0 \\
\hline $11 / 85$ & & $N / A$ & $N / A$ & $\mathbf{N} / \mathrm{A}$ & N/A & 0 \\
\hline $12 / 85$ & & $N / A$ & $N / A$ & N/A & $N / A$ & 0 \\
\hline $1 / 86$ & & N/A & N/A & $\mathrm{N} / \mathrm{A}$ & $\mathrm{N} / \mathrm{A}$ & 0 \\
\hline $2 / 86$ & & $\mathrm{~N} / \mathrm{A}$ & $\mathbf{N} / \mathbf{A}$ & $\mathbf{N} / \mathbf{A}$ & $N / A$ & 0 \\
\hline $3 / 86$ & & $\mathbf{N} / \mathbf{A}$ & N/A & $\mathrm{N} / \mathrm{A}$ & N/A & 0 \\
\hline $4 / 86$ & & $N / A$ & $N / A$ & $N / A$ & $N / A$ & 0 \\
\hline $5 / 86$ & & $\mathbf{N} / \mathbf{A}$ & N/A & $\mathrm{N} / \mathrm{A}$ & $\mathrm{N} / \mathrm{A}$ & 0 \\
\hline $6 / 86$ & & $N / A$ & N/A & $\mathrm{N} / \mathrm{A}$ & $\mathrm{N} / \mathrm{A}$ & 0 \\
\hline $7 / 86$ & NCPLX & 0 & 0 & 0 & 0 & 0 \\
\hline $8 / 86$ & NCPLX & 0 & 0 & 0 & 0 & 0 \\
\hline $9 / 86$ & NCPLX & 0 & 0 & 0 & 0 & 0 \\
\hline $10 / 86$ & NCPLX & 24 & 0 & 0 & 0 & 0 \\
\hline $11 / 86$ & NCPLX & 24 & 0 & 0 & 0 & 0 \\
\hline $12 / 86$ & NCPLX & 23 & 0 & 0 & 0 & 0 \\
\hline $1 / 87$ & NCPLX & 23 & 0 & 0 & 0 & 0 \\
\hline $2 / 87$ & NCPLX & 23 & 0 & 0 & 0 & 0 \\
\hline $3 / 87$ & NCPLX & 23 & 0 & 0 & 0 & 0 \\
\hline $4 / 87$ & NCPLX & 23 & 0 & 0 & 0 & 0 \\
\hline
\end{tabular}

Data obtained from Tank Farm Surveillance and Waste Status Summary Report, WHC-EP-0182. 
TANK LEVEL HISTORY AND WASTE TYPES

241-AP-103

\begin{tabular}{|c|c|c|c|c|c|c|}
\hline Date & & Total Waste & DSS & Sludge & Salt & Total solids \\
\hline$\left(\mathrm{M} / \mathrm{Yr}_{\mathrm{r}}\right)$ & Waste Type & (K Gal) & (K Gal) & (K Gal) & (K Gal) & (K Gal) \\
\hline $5 / 87$ & NCPLX & 23 & 0 & 0 & 0 & 0 \\
\hline $6 / 87$ & NCPLX & 23 & 0 & 0 & 0 & 0 \\
\hline $7 / 87$ & NCPLX & 23 & 0 & 0 & 0 & 0 \\
\hline $8 / 87$ & NCPLX & 23 & 0 & 0 & 0 & 0 \\
\hline $9 / 87$ & NCPLX & 25 & 0 & 0 & 0 & 0 \\
\hline $10 / 87$ & NCPLX & 25 & 0 & 0 & 0 & 0 \\
\hline $11 / 87$ & NCPLX & 24 & 0 & 0 & $\overline{0}$ & 0 \\
\hline $12 / 87$ & NCPLX & 24 & 0 & 0 & 0 & 0 \\
\hline $1 / 88$ & NCPLX & 109 & 0 & 0 & 0 & 0 \\
\hline $2 / 88$ & NCPLX & 383 & 0 & 0 & 0 & 0 \\
\hline $3 / 88$ & NCPLX & 642 & 0 & 0 & 0 & 0 \\
\hline $4 / 88$ & NCPLX & 662 & 0 & 0 & 0 & 0 \\
\hline $5 / 88$ & NCPLX & 747 & 0 & 0 & 0 & 0 \\
\hline $6 / 88$ & NCPLX & 1107 & 0 & 0 & 0 & 0 \\
\hline$7 \longdiv { 8 8 }$ & NCPLX & 1107 & 0 & $\overline{0}$ & 0 & $\overline{0}$ \\
\hline $8 / 88$ & NCPLX & 303 & 0 & 0 & 0 & 0 \\
\hline $9 / 88$ & NCPLX & 22 & $\tilde{0}$ & 0 & 0 & 0 \\
\hline $10 / 88$ & NCPLX & 432 & 0 & 0 & 0 & 0 \\
\hline $11 / 88$ & NCPLX & 638 & 0 & 0 & 0 & 0 \\
\hline $12 / 88$ & NCPLX & 638 & 0 & 0 & 0 & 0 \\
\hline $1 / 89$ & NCPLX & 637 & 0 & 0 & 0 & 0 \\
\hline $2 / 89$ & NCPLX & 637 & 0 & 0 & 0 & 0 \\
\hline $3 / 89$ & NCPLX & 637 & 0 & 0 & 0 & 0 \\
\hline $4 / 89$ & NCPLX & 637 & 0 & 0 & 0 & 0 \\
\hline $5 / 89$ & NCPLX & 637 & 0. & 0 & 0 & 0 \\
\hline $6 / 89$ & NCPLX & 636 & 0 & 0 & 0 & 0 \\
\hline $7 / 89$ & NCPLX & 637 & 0 & 0 & 0 & 0 \\
\hline $8 / 89$ & NCPLX & 637 & 0 & 0 & 0 & 0 \\
\hline $9 / 89$ & NCPLX & 637 & 0 & 0 & 0 & 0 \\
\hline $10 / 89$ & NCPLX & 591 & 0 & 0 & 0 & 0 \\
\hline $11 / 89$ & NCPLX & 591 & 0 & 0 & 0 & 0 \\
\hline $12 / 89$ & NCPLX & 591 & 0 & 0 & 0 & 0 \\
\hline $1 / 90$ & NCPLX & 590 & 0. & 0 & 0 & 0 \\
\hline $2 / 90$ & NCPLXX & 590 & 0 & 0 & 0 & 0 \\
\hline $3 / 90$ & NCPLX & 591. & 0 & 0 & 0 & 0 \\
\hline $4 / 90$ & NCPLX & 590 & 0 & 0 & 0 & 0 \\
\hline $5 / 90$ & NCPLX & 590 & 0 & 0 & 0 & 0 \\
\hline $6 / 90$ & DN & 590 & 0 & 0 & 0 & 0 \\
\hline $7 / 90$ & DN & 590 & 0 & 0 & 0 & 0 \\
\hline $8 / 90$ & DN & 590 & 0 & 0 & 0 & 0 \\
\hline $9 / 90$ & DN & 590 & 0 & 0 & 0 & 0 \\
\hline $10 / 90$ & DN & 958 & 0 & 0 & 0 & 0 \\
\hline $11 / 90$ & DN & 957. & 0 & 0 & 0 & 0 \\
\hline $12 / 90$ & DN & 956 & 0 & 0 & 0 & 0 \\
\hline
\end{tabular}

Data obtained from Tank Farm Surveillance and Waste Status Summary Report, WHC-EP-0182. 
TANK LEVEL HISTORY AND WASTE TYPES

WHC-SD-WM-ER-315, Rev. 0 241-AP-103

\begin{tabular}{|c|c|c|c|c|c|c|}
\hline Date & & Total Waste & DSS & Sludge & Salt & Total solids \\
\hline$(\mathrm{M} / \mathrm{Yr})$ & Waste Type & (K Gal) & (K Gal) & (K Gal) & (K Gal) & (K Gal) \\
\hline $1 / 91$ & DN & 956 & 0 & 0 & 0 & 0 \\
\hline $2 / 91$ & $\mathrm{DN}$ & 956 & 0 & 0 & 0 & 0 \\
\hline $3 / 91$ & $\overline{D N}$ & 956 & 0 & $\overline{0}$ & 0 & 0 \\
\hline $4 / 91$ & DN & 956 & 0 & 0 & 0 & 0 \\
\hline $5 / 91$ & DN & 956 & 0 & 0 & 0 & 0 \\
\hline $6 / 91$ & DN & 1135 & 0 & 0 & 0 & 0 \\
\hline $7 / 91$ & DN & 1135 & 0 & 0 & 0 & 0 \\
\hline $8 / 91$ & DN & 1135 & 0 & 0 & 0 & 0 \\
\hline $9 / 91$ & DN & 1135 & 0 & 0 & 0 & 0 \\
\hline $10 / 91$ & $\overline{D N}$ & 1135 & 0 & 0 & 0 & 0 \\
\hline $11 / 91$ & $\overline{D N}$ & 1135 & 0 & 0 & 0 & 0 \\
\hline $12 / 91$ & $\overline{D N}$ & 1135 & 0 & 0 & 0 & 0 \\
\hline $1 / 92$ & DN & 1135 & 0 . & 0 & 0 & 0 \\
\hline $2 / 92$ & DN & 1134 & 0 & $\overline{0}$ & $\overline{0}$ & 0 \\
\hline $3 / 92$ & DN & 1134 & 0 & 0 & 0 & 0 \\
\hline $4 / 92$ & DN & 1134 & 0 & 0 & 0 & 0 \\
\hline $5 / 92$ & DN & 1134 & 0 & 0 & 0 & 0 \\
\hline $6 / 92$ & DN & 1134 & 0 & 0 & 0 & 0 \\
\hline $7 / 92$ & DN & 1134 & 0 & 0 & 0 & 0 \\
\hline $8 / 92$ & DN & 1134 & 0 & 0 & 0 & 0 \\
\hline $9 / 92$ & $\overline{\mathrm{DN}}$ & 1134 & 0 & 0 & 0 & 0 \\
\hline $10 / 92$ & DN & 1134 & 0 & 0 & 0 & 0 \\
\hline $11 / 92$ & $\overline{D N}$ & 1134 & 0 & $\overline{0}$ & 0 & 0 \\
\hline $12 / 92$ & DN & 1133 & 0 & 0 & 0 & $\overline{0}$ \\
\hline $1 / 93$ & DN & 1133 & 0 & 0 & 0 & 0 \\
\hline $2 / 93$ & $\overline{\text { DN }}$ & 1132 & 0 & 0 & 0 & 0 \\
\hline $3 / 93$ & DN & 1132 & 0 & 0 & 0 & 0 \\
\hline $4 / 93$ & DN & 1132 & 0 & 0 & 0 & 0 \\
\hline $5 / 93$ & DN & 1132 & 0 & 0 & 0 & 0 \\
\hline $6 / 93$ & DN & 1132 & 0 & 0 & 0 & 0 \\
\hline $7 / 93$ & DN & 1133 & 0 & 0 & 0 & 0 \\
\hline $8 / 93$ & $\overline{D N}$ & 1132 & 0 & 0 & 0 & 0 \\
\hline $9 / 93$ & DN & 1133 & 0 & 0 & 0 & 0 \\
\hline $10 / 93$ & $\overline{\text { DN }}$ & 1132 & 0 & 0 & 0 & 0 \\
\hline $11 / 93$ & $\overline{D N}$ & 1132 & 0 & 0 & 0 & 0 \\
\hline $12 / 93$ & DN & 1131 & 0 & 0 & 0 & 0 \\
\hline $1 / 94$ & $\overline{D N}$ & 1131 & 0 & 0 & 0 & 0 \\
\hline $2 / 94$ & DN & 1131 & 0 & 0 & 0 & 0 \\
\hline $3 / 94$ & $\overline{D N}$ & 1131 & 0 & 0 & 0 & 0 \\
\hline
\end{tabular}

Data obtained from Tank Farm Surveillance and Waste Status Summary Report, WHC-EP-0182. 
TANK LEVEL HISTORY AND WASTE TYPES 241-AP-104

\begin{tabular}{|c|c|c|c|c|c|c|}
\hline Date & & Total Waste & DSS & Sludge. & Salt & Total solids \\
\hline$\left(M / Y_{r}\right)$ & Waste Type & (K Gal) & (K Gal) & (K Gal) & (K Gal) & (K Gal) \\
\hline $1 / 80$ & & N/A & N/A & $\mathbf{N} / \mathbf{A}$ & N/A & 0 \\
\hline $2 / 80$ & & N/A & N/A & N/A & N/A & 0 \\
\hline $3 / 80$ & & N/A & N/A & $\mathrm{N} / \mathrm{A}$ & $\mathrm{N} / \mathrm{A}$ & 0 \\
\hline $4 / 80$ & & $N / A$ & N/A & N/A & $\mathrm{N} / \mathrm{A}$ & 0 \\
\hline $5 / 80$ & & $N / A$ & N/A & N/A & N/A & 0 \\
\hline $6 / 80$ & & $N / A$ & N/A & N/A & N/A & 0 \\
\hline $7 / 80$ & & N/A & N/A & N/A & N/A & 0 \\
\hline $8 / 80$ & & N/A & N/A & $N / A$ & $\mathrm{~N} / \mathrm{A}$ & 0 \\
\hline $9 / 80$ & & N/A & N/A & N/A & N/A & 0 \\
\hline $10 / 80$ & & N/A & N/A & N/A & N/A & 0 \\
\hline $11 / 80$ & & $\mathbf{N} / \mathbf{A}$ & N/A & $\mathbf{N} / \mathbf{A}$ & $N / A$ & 0 \\
\hline $12 / 80$ & & $N / A$ & N/A & N/A & $\mathbf{N} / \mathbf{A}$ & 0 \\
\hline $1 / 81$ & & $\mathbf{N} / \mathbf{A}$ & N/A & $\mathrm{N} / \mathrm{A}$ & N/A & 0 \\
\hline $2 / 81$ & & N/A & $\mathrm{N} / \mathrm{A}$ & N/A & N/A & 0 \\
\hline $3 / 81$ & & N/A & N/A & N/A & N/A & 0 \\
\hline $4 / 81$ & & $\mathrm{~N} / \mathrm{A}$ & N/A & $N / A$ & $\mathrm{~N} / \mathrm{A}$ & 0 \\
\hline $5 / 81$ & & N/A & N/A & N/A & N/A & 0 \\
\hline $6 / 81$ & & N/A & N/A & N/A & $\mathrm{N} / \mathrm{A}$ & 0 \\
\hline $7 / 81$ & & $N / A$ & $\mathrm{~N} / \mathrm{A}$ & $\mathrm{N} / \mathrm{A}$ & $N / A$ & 0 \\
\hline $8 / 81$ & & $\mathbf{N} / \mathbf{A}$ & N/A & N/A & N/A & 0 \\
\hline $9 / 81$ & & N/A & N/A & N/A & $N / A$ & 0 \\
\hline $10 / 81$ & & $\mathrm{~N} / \mathrm{A}$ & N/A & $\mathrm{N} / \mathrm{A}$ & $N / A$ & 0 \\
\hline $11 / 81$ & & N/A & N/A & $\mathbf{N} / \mathbf{A}$ & $N / A$ & 0 \\
\hline $12 / 81$ & . & $\mathbf{N} / \mathbf{A}$ & $\mathbf{N} / \mathrm{A}$ & N/A & $N / A$ & 0 \\
\hline $1 / 82$ & & $\mathbf{N} / \mathbf{A}$ & N/A & N/A & N/A & 0 \\
\hline $2 / 82$ & & N/A & N/A & $\mathrm{N} / \mathrm{A}$ & N/A & 0 \\
\hline $3 / 82$ & & $\mathrm{~N} / \mathrm{A}$ & N/A & $\mathrm{N} / \mathrm{A}$ & N/A & 0 \\
\hline $4 / 82$ & & N/A. & N/A & $\mathrm{N} / \mathrm{A}$ & N/A & 0 \\
\hline $5 / 82$ & & $\mathrm{~N} / \mathrm{A}$ & $\mathrm{N} / \mathrm{A}$ & N/A & $N / A$ & 0 \\
\hline $6 / 82$ & & $\mathrm{~N} / \mathrm{A}$ & $N / A$ & N/A & N/A & 0 \\
\hline $7 / 82$ & & N/A & N/A & N/A & $\mathrm{N} / \mathrm{A}$ & $\overline{0}$ \\
\hline $8 / 82$ & & N/A & N/A & N/A & N/A & $\overline{0}$ \\
\hline $9 / 82$ & & N/A & N/A & $N / A$ & $\mathrm{~N} / \mathrm{A}$ & 0 \\
\hline $10 / 82$ & & N/A & N/A & $N / A$ & $\mathrm{~N} / \mathrm{A}$ & 0 \\
\hline $11 / 82$ & & N/A & $\mathrm{N} / \mathrm{A}$ & N/A & $\mathrm{N} / \mathrm{A}$ & 0 \\
\hline $12 / 82$ & & N/A. & N/A & $N / A$ & N/A & 0 \\
\hline $1 / 83$ & & $N / A$ & N/A & N/A & $\mathrm{N} / \mathrm{A}$ & 0 \\
\hline $2 / 83$ & & $\mathbf{N} / \mathbf{A}$ & $N / A$ & N/A & $N / A$ & 0 \\
\hline $3 / 83$ & & N/A & N/A & $\mathrm{N} / \mathrm{A}$ & $\mathrm{N} / \mathrm{A}$ & 0 \\
\hline $4 / 83$ & & N/A & $\mathrm{N} / \mathrm{A}$ & $N / A$ & $N / A$ & 0 \\
\hline $5 / 83$ & & N/A & N/A & N/A & $N / A$ & 0 \\
\hline $6 / 83$ & & $N / A$ & N/A & $N / A$ & $N / A$ & 0 \\
\hline $7 / 83$ & & N/A & N/A & N/A & $\mathrm{N} / \mathrm{A}$ & 0 \\
\hline $8 / 83$ & & N/A & N/A & $N / A$ & N/A & 0 \\
\hline
\end{tabular}

Data obtained from Tank Farm Surveillance and Waste Status Summary Report, WHC-EP-0182. 
TANK LEVEL HISTORY AND WASTE TYPES

241-AP-104

\begin{tabular}{|c|c|c|c|c|c|c|}
\hline Date & & Total Waste & DSS & Sludge & Salt & Total solids \\
\hline$\left(\mathrm{M} / \mathrm{Yr}_{\mathrm{r}}\right)$ & Waste Type & (K Gal) & (K Gal) & (K Gal) & (K Gai) & (K Gal) \\
\hline $9 / 83$ & & N/A & N/A & N/A & $\mathrm{N} / \mathrm{A}$ & 0 \\
\hline $10 / 83$ & & N/A & $N / A$ & $N / A$ & N/A & 0 \\
\hline $11 / 83$ & & $\mathbf{N} / \mathbf{A}$ & N/A & N/A & N/A & 0 \\
\hline $12 / 83$ & & N/A & N/A & N/A & N/A & 0 \\
\hline $1 / 84$ & & N/A & N/A & N/A & N/A & 0 \\
\hline$\overline{2 / 84}$ & & N/A & N/A & N/A & $\mathbf{N} / \mathbf{A}$ & 0 \\
\hline $3 / 84$ & & $\bar{N} / \mathbf{A}$ & $\overline{N / A}$ & $\overline{N / A}$ & N/A & 0 \\
\hline $4 / 84$ & & N/A. & $\mathrm{N} / \mathrm{A}$ & $\mathrm{N} / \mathrm{A}$ & N/A & 0 \\
\hline $5 / 84$ & & $\overline{N / A}$ & N/A & N/A & $\overline{N / A}$ & 0 \\
\hline $6 / 84$ & & N/A & N/A & N/A & N/A & 0 \\
\hline $7 / 84$ & & N/A & N/A & N/A & N/A & 0 \\
\hline $8 / 84$ & & $\mathbf{N} / \mathbf{A}$ & N/A & N/A & N/A & 0 \\
\hline $9 / 84$ & & N/A & N/A & N/A & $\mathbf{N} / \mathbf{A}$ & 0 \\
\hline $10 / 84$ & & $\mathbf{N} / \mathbf{A}$ & N/A & N/A & $N / A$ & 0 \\
\hline $11 / 84$ & & $\mathbf{N} / \mathbf{A}$ & $\mathbf{N} / \mathbf{A}^{\prime}$ & $\bar{N} / \mathrm{A}$ & $N / A$ & 0 \\
\hline $12 / 84$ & & N/A & $\mathrm{N} / \mathrm{A}$ & $N / A$ & N/A & 0 \\
\hline $1 / 85$ & & $\mathrm{~N} / \mathrm{A}$ & $\bar{N} / \mathbf{A}$ & $\mathbf{N} / \mathbf{A}$ & N/A & 0 \\
\hline $2 / 85$ & & $\bar{N} / \mathrm{A}$ & $\mathrm{N} / \mathrm{A}$ & N/A & N/A & 0 \\
\hline $3 / 85$ & & $\mathrm{~N} / \mathrm{A}$ & N/A & N/A & N/A & 0 \\
\hline $4 / 85$ & & N/A & $N / A$ & N/A & N/A & 0 \\
\hline $5 / 85$ & & $\mathbf{N} / \mathbf{A}$ & $\mathbf{N} / \mathbf{A}$ & $\mathbf{N} / \mathbf{A}$ & N/A & 0 \\
\hline $6 / 85$ & & $\mathbf{N} / \mathbf{A}$ & $\mathbf{N} / \mathbf{A}$ & $\mathrm{N} / \mathrm{A}$ & $\mathrm{N} / \mathrm{A}$ & 0 \\
\hline $7 / 85$ & & N/A & $\mathrm{N} / \mathrm{A}$ & N/A & N/A & 0 \\
\hline $8 / 85$ & & $\mathbf{N} / \mathbf{A}$ & $\mathbf{N} / \mathbf{A}$ & $\mathbf{N} / \mathbf{A}$ & N/A & 0 \\
\hline $9 / 85$ & & N/A & $N / A$ & $\mathrm{~N} / \mathrm{A}$ & $\bar{N} / \mathbf{A}$ & 0 \\
\hline $10 / 85$ & & $\mathrm{~N} / \mathrm{A}$ & N/A & $\mathrm{N} / \mathrm{A}$ & $\mathrm{N} / \mathrm{A}$ & 0 \\
\hline $11 / 85$ & & N/A & $\mathrm{N} / \mathrm{A}$ & $N / A$ & $\mathbf{N} / \mathbf{A}$ & 0 \\
\hline $12 / 85$ & & $\mathrm{~N} / \mathrm{A}$ & N/A & N/A & $\mathrm{N} / \mathrm{A}$ & 0 \\
\hline $1 / 86$ & & $\overline{\text { N/A }}$ & $\bar{N} / \mathbf{A}$ & $N / A$ & $\bar{N} / \mathbf{A}$ & 0 \\
\hline $2 / 86$ & & N/A & $\mathbf{N} / \mathbf{A}$ & $N / A$ & $\mathrm{~N} / \mathrm{A}$ & 0 \\
\hline $3 / 86$ & & N/A & $\mathrm{N} / \mathrm{A}$ & N/A & N/A & 0 \\
\hline $4 / 86$ & & N/A & $\mathbf{N} / \mathbf{A}$ & $N / A$ & $N / A$ & 0 \\
\hline $5 / 86$ & & $N / A$ & N/A & $\mathbf{N} / \mathbf{A}$ & $\mathrm{N} / \mathrm{A}$ & 0 \\
\hline $6 / 86$ & & N/A & $\mathbf{N} / \mathbf{A}$ & $N / A$ & $N / A$ & 0 \\
\hline $7 / 86$ & HFW & 0 & 0 & 0 & 0 & 0 \\
\hline $8 / 86$ & HFW & 0 & 0 & 0 & 0 & $\overline{0}$ \\
\hline $9 / 86$ & HFW & 0 & 0 & 0 & 0 & $\overline{0}$ \\
\hline $10 / 86$ & HFW & 40 & 0 & 0 & 0 & 0 \\
\hline $11 / 86$ & HFW & 40 & 0 & 0 & 0 & 0 \\
\hline $12 / 86$ & HFW & 42 & 0 & 0 & 0 & 0 \\
\hline $1 / 87$ & HFW & 41 & 0 & 0 & 0 & 0 \\
\hline $2 / 87$ & HFW & 81 & 0 & 0 & 0 & 0 \\
\hline $\mathbf{3 / 8 7}$ & HFW & 309 & 0 & 0 & 0 & 0 \\
\hline $4 / 87$ & HFW & 620 & 0 & 0 & 0 & 0 \\
\hline
\end{tabular}

Data obtained from Tank Farm Surveillance and Waste Status Summary Report, WHC-EP-0182. 
TANK LEVEL HISTORY AND WASTE TYPES

241-AP-104

\begin{tabular}{|c|c|c|c|c|c|c|}
\hline Date & & Total Waste & DSS & Sludge & Salt & Total solids \\
\hline$(\mathrm{M} / \mathrm{Yr})$ & Waste Type & (K Gal) & (K Gal) & (K Gal) & (K Gal) & (K Gal) \\
\hline $5 / 87$ & HFW & $\overline{743}$ & 0 & 0 & $\overline{0}$ & 0 \\
\hline $6 / 87$ & HFW & 991 & 0 & 0 & 0 & 0 \\
\hline $7 / 87$ & HFW & 1075 & 0 & 0 & 0 & 0 \\
\hline $8 / 87$ & HFW & 1075 & 0 & 0 & 0 & $\mathbf{0}$ \\
\hline $9 / 87$ & HFW & 1074 & 0 & 0 & 0 & 0 \\
\hline $10 / 87$ & HFW & 1074 & 0 & $\overline{0}$ & 0 & 0 \\
\hline $11 / 87$ & HFW & 1073 & 0 & 0 & 0 & $\overline{0}$ \\
\hline $12 / 87$ & HFW & 1073 & 0 & 0 & 0 & 0 \\
\hline $1 / 88$ & HFW & 1072 & 0 & 0 & 0 & 0 \\
\hline $2 / 88$ & HFW & 1072 & 0 & 0 & 0 & $\overline{0}$ \\
\hline $3 / 88$ & HFW & 1072 & 0 & 0 & 0 & 0 \\
\hline $4 / 88$ & HFW & 22 & 0 & 0 & 0 & 0 \\
\hline $5 / 88$ & HFW & 22 & 0 & 0 & 0 & 0 \\
\hline $6 / 88$ & HFW & 22 & 0 & 0 & 0 & 0 \\
\hline $7 / 88$ & HFW & 22 & 0 & 0 & 0 & 0 \\
\hline $8 / 88$ & HFW & 22 & 0 & 0 & 0 & 0 \\
\hline $9 / 88$ & HFW & 22 & 0 & 0 & 0 & 0 \\
\hline $10 / 88$ & HFW & 22 & 0 & 0 & 0 & 0 \\
\hline $11 / 88$ & HFW & 21 & 0 & .0 & 0 & 0 \\
\hline $12 / 88$ & HFW & 21 & 0 & 0 & 0 & 0 \\
\hline $1 / 89$ & HFW & 21 & 0 & 0 & 0 & $\overline{0}$ \\
\hline $2 / 89$ & HFW & 21 & 0 & 0 & 0 & 0 \\
\hline $3 / 89$ & HFW & 21 & 0 & 0 & 0 & 0 \\
\hline $4 / 89$ & HFW & 20 & 0 & 0 & 0 & 0 \\
\hline $5 / 89$ & HFW & 20 & 0 & 0 & 0 & 0 \\
\hline $6 / 89$ & HFW & 20 & 0 & 0 & 0 & 0 \\
\hline $7 / 89$ & HFW & 20 & 0 & 0 & 0 & 0 \\
\hline $8 / 89$ & HFW & 20 & 0 & 0 & 0 & 0 \\
\hline $9 / 89$ & HFW & 19 & 0 & 0 & 0 & 0 \\
\hline $10 / 89$ & HFW & 23 & 0 & 0 & 0 & 0 \\
\hline $11 / 89$ & HFW & 23 & 0 & 0 & 0 & 0 \\
\hline $12 / 89$ & HFW & 22 & 0 & 0 & 0 & 0 \\
\hline $1 / 90$ & HFW & 22 & 0 & 0 & 0 & 0 \\
\hline $2 / 90$ & HFW & 22 & 0 & 0 & 0 & 0 \\
\hline $3 / 90$ & HFW & 23 & 0 & 0 & 0 & 0 \\
\hline $4 / 90$ & HFW & 22 & 0. & 0 & 0 & 0 \\
\hline $5 / 90$ & HFW & 22 & 0 & 0 & 0 & 0 \\
\hline $6 / 90$ & DN & 22 & 0 & 0 & 0 & 0 \\
\hline $7 / 90$ & DN & 22 & 0 & 0 & 0 & 0 \\
\hline $8 / 90$ & DN & 22 & 0 & 0 & 0 & 0 \\
\hline $9 / 90$ & DN & 22 & 0 & 0 & 0 & 0 \\
\hline $10 / 90$ & $\overline{D N}$ & 21 & 0 & 0 & 0 & 0 \\
\hline $11 / 90$ & DN & 21 & 0 & 0 & 0 & 0 \\
\hline $12 / 90$ & $\overline{\mathbf{D N}}$ & 21 & 0 & 0 & 0 & 0 \\
\hline
\end{tabular}

Data obtained from Tank Farm Surveillance and Waste Status Summary Report, WHC-EP-0182. 
TANK LEVEL HISTORY AND WASTE TYPES

241-AP-104

\begin{tabular}{|c|c|c|c|c|c|c|}
\hline Date & & Total Waste & DSS & Sludge & Salt & Total solids \\
\hline$(\mathrm{M} / \mathrm{Yr})$ & Waste Type & (K Gal) & (K Gal) & (K Gal) & (K Gal) & (K Gal) \\
\hline $1 / 91$ & DN & 21 & 0 & 0 & 0 & 0 \\
\hline $2 / 91$ & DN & 21 & 0 & 0 & 0 & 0 \\
\hline $3 / 91$ & DN & 21 & 0 & 0 & 0 & 0 \\
\hline $4 / 91$ & $\overline{D N}$ & 21 & 0 & 0 & 0 & 0 \\
\hline $5 / 91$ & $\overline{D N}$ & 21 & 0 & 0 & 0 & 0 \\
\hline$\overline{6 / 91}$ & $\overline{D N}$ & 21 & 0 & 0 & $\mathbf{0}$ & 0 \\
\hline $7 / 91$ & $\overline{\text { DN }}$ & 21 & 0 & 0 & 0 & 0 \\
\hline $8 / 91$ & $\overline{D N}$ & 21 & 0 & 0 & 0 & 0 \\
\hline $9 / 91$ & DN & 21 & 0 & 0 & $\overline{0}$ & 0 \\
\hline $10 / 91$ & DN & 20 & $\overline{0}$ & 0 & 0 & 0 \\
\hline $11 / 91$ & DN & 20 & 0 & 0 & 0 & 0 \\
\hline $12 / 91$ & DN & 20 & 0 & 0 & 0 & 0 \\
\hline $1 / 92$ & DN & 20 & 0 & 0 & 0 & 0 \\
\hline $2 / 92$ & DN & 20 & 0 & 0 & 0 & 0 \\
\hline $3 / 92$ & DN & 20 & 0. & 0 & 0 & 0 \\
\hline $4 / 92$ & DN & 20 & 0 & 0 & 0 & 0 \\
\hline $5 / 92$ & DN & 20 & 0 & 0 & 0 & $\overline{0}$ \\
\hline $6 / 92$ & DN & 20 & 0 & 0 & 0 & 0 \\
\hline $7 / 92$ & DN & 20 & $\mathbf{Q}$ & 0 & 0 & 0 \\
\hline $8 / 92$ & $\overline{\text { DN }}$ & 20 & 0 & 0 & 0 & 0 \\
\hline $9 / 92$ & DN & 20 & $\overline{0}$ & 0 & 0 & 0 \\
\hline $10 / 92$ & DN & 20 & 0 & 0 & 0 & 0 \\
\hline $11 / 92$ & $\overline{D N}$ & 20 & 0 & 0 & 0 & 0 \\
\hline $12 / 92$ & DN & 20 & 0 & 0 & 0 & $\overline{0}$ \\
\hline $1 / 93$ & DN & 19 & 0 & 0 & 0 & 0 \\
\hline $2 / 93$ & DN & 19 & 0 & 0 & 0 & 0 \\
\hline $3 / 93$ & $\overline{\text { DN }}$ & 19 & 0 & 0 & 0 & 0 \\
\hline $4 / 93$ & $\overline{\text { DN }}$ & 19 & 0 & 0 & 0 & 0 \\
\hline $5 / 93$ & DN & 19 & 0 & 0 & 0 & 0 \\
\hline $6 / 93$ & DN & 19 & 0 & 0 & 0 & 0 \\
\hline $7 / 93$ & $\overline{D N}$ & 19 & 0 & 0 & 0 & 0 \\
\hline $8 / 93$ & DN & 19 & 0 & 0 & 0 & 0 \\
\hline $9 / 93$ & DN & 19 & 0. & 0 & 0 & 0 \\
\hline $10 / 93$ & DN & 18 & 0 & 0 & 0 & 0 \\
\hline $11 / 93$ & $\overline{D N}$ & 18 & 0 & 0 & 0 & 0 \\
\hline $12 / 93$ & DN & 18 & 0 & 0 & 0 & 0 \\
\hline $1 / 94$ & DN & 18 & 0 & 0 & 0 & 0 \\
\hline $2 / 94$ & DN & 18 & 0 & 0 & 0 & 0 \\
\hline $3 / 94$ & DN & 18 & 0 & 0 & 0 & 0 \\
\hline
\end{tabular}

Data obtained from Tank Farm Surveillance and Waste Status Summary Report, WHC-EP-0182. 
WHC-SD-WM-ER-315, Rev. 0

TANK LEVEL HISTORY AND WASTE TYPES

241-AP-105

\begin{tabular}{|c|c|c|c|c|c|c|}
\hline Date & & Total Waste & DSS & Sludge & Salt & Total solids \\
\hline$(\mathrm{M} / \mathrm{Yr})$ & Waste Type & (K Gal) & (K Gal) & (K Gal) & (K Gal) & (K Gal) \\
\hline $1 / 80$ & & N/A & N/A & $\mathrm{N} / \mathrm{A}$ & N/A & 0 \\
\hline $2 / 80$ & & $\mathrm{~N} / \mathrm{A}$ & $N / A$ & $\mathrm{~N} / \mathrm{A}$ & N/A & 0 \\
\hline $3 / 80$ & & N/A & N/A & N/A & $N / A$ & 0 \\
\hline $4 / 80$ & & N/A & N/A & $\mathrm{N} / \mathrm{A}$ & $\mathrm{N} / \mathrm{A}$ & 0 \\
\hline $5 / 80$ & & N/A & $\mathrm{N} / \mathrm{A}$ & $N / A$ & N/A & 0 \\
\hline $6 / 80$ & & $N / A$ & $\mathbf{N} / \mathbf{A}$ & N/A & $\mathrm{N} / \mathrm{A}$ & 0 \\
\hline $7 / 80$ & & $\overline{N / A}$ & $N / A$ & N/A & N/A & 0 \\
\hline $8 / 80$ & & $\mathbf{N} / \mathbf{A}$ & $\mathrm{N} / \mathrm{A}$ & $\mathrm{N} / \mathrm{A}$ & N/A & 0 \\
\hline $9 / 80$ & & N/A & $N / A$ & $\mathrm{~N} / \mathrm{A}$ & N/A & 0 \\
\hline $10 / 80$ & & $\mathbf{N} / \mathbf{A}$ & N/A & $N / A$ & N/A & 0 \\
\hline $11 / 80$ & & $\mathbf{N} / \mathbf{A}$ & N/A & $N / A$ & N/A & 0 \\
\hline $12 / 80$ & & $\mathrm{~N} / \mathrm{A}$ & $\bar{N} / \mathbf{A}$ & $N / A$ & $\mathbf{N} / \mathbf{A}$ & 0 \\
\hline $1 / 81$ & & $N / A$ & N/A & N/A & N/A & 0 \\
\hline $2 / 81$ & & $N / A$ & $\mathrm{~N} / \mathrm{A}$ & $N / A$ & N/A & 0 \\
\hline $3 / 81$ & & N/A & $N / A$ & $N / A$ & $\mathbf{N} / \mathbf{A}$ & 0 \\
\hline $4 / 81$ & & N/A & N/A & $\bar{N} / \mathbf{A}$ & $N / A$ & 0 \\
\hline $5 / 81$ & & N/A & N/A & N/A & N/A & 0 \\
\hline $6 / 81$ & & $N / A$ & $N / A$ & $\mathrm{~N} / \mathrm{A}$ & N/A & 0 \\
\hline $7 / 81$ & & N/A & $N / A$ & $\mathrm{~N} / \mathrm{A}$ & $\bar{N} / \mathrm{A}$ & 0 \\
\hline $8 / 81$ & & N/A & N/A & $N / A$ & $\mathrm{~N} / \mathrm{A}$ & 0 \\
\hline $9 / 81$ & & $N / A$ & N/A & $\mathrm{N} / \mathrm{A}$ & N/A & 0 \\
\hline $10 / 81$ & & $N / A$ & N/A & $N / A$ & $\mathbf{N} / \mathbf{A}$ & 0 \\
\hline $11 / 81$ & & N/A. & $N / A$ & $\mathbf{N} / \mathbf{A}$ & $N / A$ & 0 \\
\hline $12 / 81$ & & N/A & $N / A$ & $\mathrm{~N} / \mathrm{A}$ & $\mathbf{N} / \mathbf{A}$ & 0 \\
\hline $1 / 82$ & & $N / A$ & $\mathrm{~N} / \mathrm{A}$ & N/A & $\mathrm{N} / \mathrm{A}$ & 0 \\
\hline $2 / 82$ & & $N / A$ & $\mathrm{~N} / \mathrm{A}$ & $N / A$ & N/A & 0 \\
\hline $3 / 82$ & & $N / A^{\circ}$ & $\mathrm{N} / \mathrm{A}$ & $\mathbf{N} / \mathbf{A}$ & N/A & 0 \\
\hline $4 / 82$ & & N/A & N/A & N/A & N/A & 0 \\
\hline $5 / 82$ & & $N / A$ & N/A & N/A & $N / A$ & 0 \\
\hline $6 / 82$ & & N/A & $\mathrm{N} / \mathrm{A}$ & N/A & N/A & 0 \\
\hline $7 / 82$ & & N/A. & $\mathrm{N} / \mathrm{A}$ & N/A & $N / A$ & 0 \\
\hline $8 / 82$ & & $N / A$ & $N / A$ & $N / A$ & N/A & 0 \\
\hline $9 / 82$ & & $N / A$ & N/A & N/A & $N / A$ & 0 \\
\hline $10 / 82$ & & N/A & N/A & $\mathrm{N} / \mathrm{A}$ & N/A & 0 \\
\hline $11 / 82$ & & N/A & N/A & $\mathrm{N} / \mathrm{A}$ & N/A & 0 \\
\hline $12 / 82$ & & N/A & N/A & $\mathrm{N} / \mathrm{A}$ & $\mathrm{N} / \mathrm{A}$ & 0 \\
\hline $1 / 83$ & & N/A & N/A & $N / A$ & N/A & 0 \\
\hline $2 / 83$ & & $N / A$ & $\mathrm{~N} / \mathrm{A}$ & $\mathrm{N} / \mathrm{A}$ & N/A & 0 \\
\hline $3 / 83$ & & N/A & N/A & N/A & N/A & 0 \\
\hline $4 / 83$ & & N/A & $N / A$ & $\mathrm{~N} / \mathrm{A}$ & N/A & 0 \\
\hline $5 / 83$ & & N/A & N/A & $N / A$ & N/A & 0 \\
\hline $6 / 83$ & & N/A & N/A & N/A & N/A & 0 \\
\hline $7 / 83$ & & N/A & $\mathbf{N} / \mathbf{A}$ & $\mathrm{N} / \mathrm{A}$ & N/A & 0 \\
\hline $8 / 83$ & & N/A & $\mathbf{N} / \mathbf{A}$ & $\mathbf{N} / \mathbf{A}$ & N/A & 0 \\
\hline
\end{tabular}

Data obtained from Tank Farm Surveillance and Waste Status Summary Report, WHC-EP-0182. 
WHC-SD-WM-ER-315, Rev. 0

TANK LEVEL HISTORY AND WASTE TYPES

241-AP-105

\begin{tabular}{|c|c|c|c|c|c|c|}
\hline Date & & Total Waste & DSS & Sludge & Salt & Total solids \\
\hline$\left(\mathrm{M} / \mathrm{Yr}_{\mathrm{r}}\right)$ & Waste Type & (K Gal) & (K Gal) & (K Gal) & (K Gal) & (K Gal) \\
\hline $9 / 83$ & & $\mathbf{N} / \mathbf{A}$ & N/A & $\mathrm{N} / \mathrm{A}$ & $\mathrm{N} / \mathrm{A}$ & 0 \\
\hline $10 / 83$ & & N/A & N/A & N/A & $\mathbf{N} / \mathbf{A}$ & 0 \\
\hline $11 / 83$ & & N/A & N/A & $\mathrm{N} / \mathrm{A}$ & N/A & 0 \\
\hline $12 / 83$ & & $\mathrm{~N} / \mathrm{A}$ & $N / A$ & N/A & N/A & 0 \\
\hline $1 / 84$ & & N/A & N/A & N/A & $\mathrm{N} / \mathrm{A}$ & 0 \\
\hline $2 / 84$ & & $\mathbf{N} / \mathbf{A}$ & N/A & $N / A$ & $\mathrm{~N} / \mathrm{A}$ & 0 \\
\hline $3 / 84$ & & $\mathbf{N} / \mathbf{A}$ & N/A & N/A & N/A & 0 \\
\hline $4 / 84$ & & N/A & N/A & N/A & N/A & 0 \\
\hline $5 / 84$ & & N/A & N/A & N/A & N/A & 0 \\
\hline $6 / 84$ & & N/A & N/A & N/A & $\mathrm{N} / \mathrm{A}$ & 0 \\
\hline $7 / 84$ & & N/A & N/A & $\mathbf{N} / \mathbf{A}$ & $\mathrm{N} / \mathrm{A}$ & 0 \\
\hline $8 / 84$ & & N/A & N/A & N/A & N/A & 0 \\
\hline $9 / 84$ & & $\mathbf{N} / \mathbf{A}$ & N/A & N/A & $\overline{N / A}$ & $\overline{0}$ \\
\hline $10 / 84$ & & $\mathbf{N} / \mathbf{A}$ & $N / A$ & N/A & $\mathbf{N} / \mathbf{A}$ & 0 \\
\hline $11 / 84$ & & N/A & N/A & N/A & N/A & 0 \\
\hline $12 / 84$ & & $N / A$ & N/A & N/A & N/A. & 0 \\
\hline $1 / 85$ & & N/A & N/A & N/A & N/A & 0 \\
\hline $2 / 85$ & & $\mathbf{N} / \mathbf{A}$ & $\mathrm{N} / \mathrm{A}$ & N/A & $\mathbf{N} / \mathbf{A}$ & $\overline{0}$ \\
\hline $3 / 85$ & & N/A & N/A & $N / A$ & $\mathrm{~N} / \mathrm{A}$ & 0 \\
\hline $4 / 85$ & & N/A & $N / A$ & N/A & $\mathbf{N} / \mathbf{A}$ & 0 \\
\hline $5 / 85$ & & N/A & $N / A$ & $\mathrm{~N} / \mathrm{A}$ & N/A & 0 \\
\hline $6 / 85$ & & N/A & N/A & N/A & N/A & 0 \\
\hline $7 / 85$ & & $\mathbf{N} / \mathbf{A}$ & N/A & N/A & N/A & 0 \\
\hline $8 / 85$ & & $N / A$ & N/A & N/A & N/A & 0 \\
\hline $9 / 85$ & & N/A & N/A & N/A & N/A & 0 \\
\hline $10 / 85$ & & $\mathbf{N} / \mathbf{A}$ & $\mathbf{N} / \mathbf{A}$ & $\mathrm{N} / \mathrm{A}$ & N/A & 0 \\
\hline $11 / 85$ & & N/A & $\mathrm{N} / \mathrm{A}$ & N/A & N/A & 0 \\
\hline $12 / 85$ & & $\mathrm{~N} / \mathrm{A}$ & N/A & $\mathrm{N} / \mathrm{A}$ & N/A & 0 \\
\hline $1 / 86$ & & $N / A$ & N/A & $N / A$ & N/A & 0 \\
\hline $2 / 86$ & & $\mathrm{~N} / \mathrm{A}$ & N/A & $N / A$ & N/A & 0 \\
\hline $3 / 86$ & & $\mathbf{N} / \mathbf{A}$ & $\mathrm{N} / \mathrm{A}$ & $\mathrm{N} / \mathrm{A}$ & $\mathrm{N} / \mathrm{A}$ & 0 \\
\hline $4 / 86$ & & N/A & N/A & $N / A$ & $\mathrm{~N} / \mathrm{A}$ & 0 \\
\hline $5 / 86$ & & $\mathbf{N} / \mathbf{A}$ & N/A & $N / A$ & N/A & $\overline{0}$ \\
\hline $6 / 86$ & & $\mathbf{N} / \mathbf{A}$ & N/A & $N / A$ & N/A & $\overline{0}$ \\
\hline $7 / 86$ & NCPLX & 0 & 0 & 0 & 0 & 0 \\
\hline $8 / 86$ & NCPLX & 0 & 0 & 0 & 0 & 0 \\
\hline $9 / 86$ & NCPLX & 0 & 0 & 0 & 0 & 0 \\
\hline $10 / 86$ & NCPLX & 17 & 0 & 0 & 0 & 0 \\
\hline $11 / 86$ & NCPLX & 17 & 0 & 0 & 0 & 0 \\
\hline $12 / 86$ & NCPLX & 17 & 0 & 0 & 0 & 0 \\
\hline $1 / 87$ & NCPLX & 18 & 0 & 0 & 0 & 0 \\
\hline $2 / 87$ & NCPLX & 18 & 0 & 0 & 0 & 0 \\
\hline $3 / 87$ & NCPLX & 18 & 0 & 0 & 0 & 0 \\
\hline $4 / 87$ & NCPLX & 17 & 0 & 0 & 0 & 0 \\
\hline
\end{tabular}

Data obtained from Tank Farm Surveillance and Waste Status Summary Report, WHC-EP-0182. 
TANK LEVEL HISTORY AND WASTE TYPES

241-AP-105

\begin{tabular}{|c|c|c|c|c|c|c|}
\hline Date & & Total Waste & DSS & Sludge & Salt & Total solids \\
\hline$(\mathrm{M} / \mathrm{Yr})$ & Waste Type & (K Gal) & (K Gal) & (K Gal) & (K Gal) & (K Gal) \\
\hline $5 / 87$ & NCPLX & 17 & 0 & 0 & 0 & 0 \\
\hline $6 / 87$ & NCPLX & 17 & 0 & 0 & 0 & 0 \\
\hline $7 / 87$ & NCPLX & 17 & 0 & 0 & 0 & 0 \\
\hline $8 / 87$ & NCPLX & 17 & 0 & 0 & 0 & 0 \\
\hline $9 / 87$ & NCPLX & 18 & 0 & 0 & 0 & 0 \\
\hline $10 / 87$ & NCPLX & 18 & 0 & 0 & 0 & 0 \\
\hline $11 / 87$ & NCPLX & 18 & 0 & 0 & 0 & 0 \\
\hline $12 / 87$ & NCPLXX & 18 & 0 & 0 & 0 & 0 \\
\hline $1 / 88$ & NCPLX & 305 & 0 & 0 & 0 & 0 \\
\hline $2 / 88$ & NCPLX & 713 & 0 & 0 & 0 & $\overline{0}$ \\
\hline $3 / 88$ & NCPLX & 712 & 0 & 0 & 0 & 0 \\
\hline $4 / 88$ & NCPLX & 711 & 0 & 0 & 0 & 0 \\
\hline $5 / 88$ & NCPLX & 711 & 0 & 0 & 0 & 0 \\
\hline $6 / 88$ & NCPLX & 711 & $\overline{0}$ & 0 & 0 & 0 \\
\hline $7 / 88$ & NCPLX & 493 & 0 & 0 & 0 & 0 \\
\hline $8 / 88$ & NCPLX & 19 & 0 & 0 & 0 & 0 \\
\hline $9 / 88$ & NCPLX & 19 & 0 & 0 & 0 & 0 \\
\hline $10 / 88$ & NCPLX & 19 & 0 & 0 & 0 & 0 \\
\hline $11 / 88$ & NCPLX & 19 & 0 & 0 & 0 & 0 \\
\hline $12 / 88$ & NCPLX & 18 & 0 & 0 & 0 & 0 \\
\hline $1 / 89$ & NCPLX & 179 & 0 & 0 & 0 & 0 \\
\hline $2 / 89$ & NCPLX & 476 & 0 & 0 & 0 & 0 \\
\hline $3 / 89$ & NCPLX & 476 & 0 & 0 & 0 & 0 \\
\hline $4 / 89$ & NCPLX & 477 & 0. & 0 & 0 & 0 \\
\hline $5 / 89$ & NCPLX & 476 & 0 & 0 & 0 & 0 \\
\hline $6 / 89$ & NCPLX & .476 & 0 & 0 & 0 & 0 \\
\hline $7 / 89$ & DSSF & 831 & 0 & 0 & 0 & 0 \\
\hline $8 / 89$ & DSSF & 831 & 0 & 0 & 0 & 0 \\
\hline $9 / 89$ & DSSF & 830 & 0 & 0 & 0 & 0 \\
\hline $10 / 89$ & DSSF & 830 & 0 & 0 & 0 & 0 \\
\hline $11 / 89$ & DSSF & 829 & 0 & 0 & 0 & 0 \\
\hline $12 / 89$ & DSSF & 829 & 0 & 0 & 0 & 0 \\
\hline $1 / 90$ & DSSF & 828 & 0 & 0 & 0 & 0 \\
\hline $2 / 90$ & DSSF & 828. & 0 & 0 & 0 & 0 \\
\hline $3 / 90$ & DSSF & 829 & 0 & 0 & 0 & 0 \\
\hline $4 / 90$ & DSSF & 827 & 0 & 0 & 0 & 0 \\
\hline $5 / 90$ & DSSF & 827 & 0 & 0 & 0 & 0 \\
\hline $6 / 90$ & DSSF & 827 & 0 & 0 & 0 & 0 \\
\hline $7 / 90$ & DSSF & 828 & 0 & 0 & 0 & 0 \\
\hline $8 / 90$ & DSSF & 828 & 0 & 0 & 0 & 0 \\
\hline $9 / 90$ & DSSF & 828 & 0 & 0 & 0 & 0 \\
\hline $10 / 90$ & DSSF & 827 & 0 & 0 & 0 & 0 \\
\hline $11 / 90$ & DSSF & 827 & 0 & 0 & 0 & 0 \\
\hline $12 / 90$ & DSSF & 827 & 0 & 0 & 0 & 0 \\
\hline
\end{tabular}

Data obtained from Tank Farm Surveillance and Waste Status Summary Report, WHC-EP-0182. 
TANK LEVEL HISTORY AND WASTE TYPES

241-AP-105

\begin{tabular}{|c|c|c|c|c|c|c|}
\hline Date & & Total Waste & DSS & Sludge & Salt & Total solids \\
\hline$(\mathrm{M} / \mathrm{Yr})$ & Waste Type & (K Gal) & (K Gal) & (K Gal) & (K Gal) & (K Gal) \\
\hline $1 / 91$ & DSSF & 826 & 0 & $\overline{0}$ & 0 & 0 \\
\hline $2 / 91$ & DSSF & 826 & 0 & 0 & 0 & 0 \\
\hline $3 / 91$ & DSSF & 826 & 0 & 0 & 0 & 0 \\
\hline $4 / 91$ & DSSF & 826 & 0 & 0 & 0 & 0 \\
\hline $5 / 91$ & DSSF & 826 & 0 & 0 & 0 & 0 \\
\hline $6 / 91$ & DSSF & 826 & 0 & 0 & 0 & 0 \\
\hline $7 / 91$ & DSSF & 826 & 0 & $\overline{0}$ & 0 & $\overline{0}$ \\
\hline $8 / 91$ & DSSF & 826 & 0 & 0 & 0 & 0 \\
\hline $9 / 91$ & DSSF & 826 & 0 & 0 & 0 & 0 \\
\hline $10 / 91$ & DSSF & 826 & 0 & 0 & 0 & 0 \\
\hline $11 / 91$ & DSSF & 826 & 0 & $\overline{0}$ & 0 & 0 \\
\hline $12 / 91$ & DSSF & 825 & 0 & 0 & 0 & 0 \\
\hline $1 / 92$ & DSSF & 825 & $\overline{0}$ & 0 & 0 & 0 \\
\hline $2 / 92$ & DSSF & 824 & 0 & 0 & 0 & 0 \\
\hline $3 / 92$ & DSSF & 824 & 0 & 0 & 0 & 0 \\
\hline $4 / 92$ & DSSF & 824 & 0 & 0 & 0 & 0 \\
\hline $5 / 92$ & DSSF & 824 & 0 & 0 & 0 & 0 \\
\hline $6 / 92$ & DSSF & 824 & 0 & 0 & 0 & 0 \\
\hline $7 / 92$ & DSSF & 824 & 0 & 0 & 0 & 0 \\
\hline $8 / 92$ & DSSF & 824 & 0 & 0 & 0 & 0 \\
\hline $9 / 92$ & DSSF & 824 & 0. & 0 & 0 & 0 \\
\hline $10 / 92$ & DSSF & 824 & 0 & 0 & 0 & 0 \\
\hline $11 / 92$ & DSSF & 823 & 0 & 0 & 0 & 0 \\
\hline $12 / 92$ & DSSF & 823 & 0 & 0 & 0 & 0 \\
\hline $1 / 93$ & DSSF & 823 & $\overline{0}$ & 0 & 0 & 0 \\
\hline $2 / 93$ & DSSF & 822 & 0 & 0 & 0 & 0 \\
\hline $3 / 93$ & DSSF & 821 & 0 & 0 & 0 & 0 \\
\hline $4 / 93$ & DSSF & 821 & 0 & 0 & 0 & 0 \\
\hline $5 / 93$ & DSSF & 821 & 0 & 0 & 0 & 0 \\
\hline $6 / 93$ & DSSF & 821 & 0 & 0 & 0 & 0 \\
\hline $7 / 93$ & DSSF & 821 & 0 & 0 & 0 & 0 \\
\hline $8 / 93$ & DSSF & 822 & $\overline{0}$ & 0 & $\overline{0}$ & 0 \\
\hline $9 / 93$ & DSSF & 822 & 0 & 0 & 0 & 0 \\
\hline $10 / 93$ & DSSF & 821 & 0 & 0 & 0 & 0 \\
\hline $11 / 93$ & DSSF & 821 & $\overline{0}$ & 0 & $\overline{0}$ & 0 \\
\hline $12 / 93$ & DSSF & 821 & 0 & 0 & $\overline{0}$ & 0 \\
\hline $1 / 94$ & DSSF & 820 & 0 & 0 & 0 & 0 \\
\hline $2 / 94$ & DSSF & 820 & 0 & 0 & 0 & 0 \\
\hline $3 / 94$ & DSSF & 820. & 0 & 0 & 0 & 0 \\
\hline
\end{tabular}

Data obtained from Tank Farm Surveillance and Waste Status Summary Report, WHC-EP-0182. 
TANK LEVEL HISTORY AND WASTE TYPES

WHC-SD-WM-ER-315, Rev. 0

241-AP-106

\begin{tabular}{|c|c|c|c|c|c|c|}
\hline Date & & Total Waste & DSS & Sludge & Salt & Total solids \\
\hline$\left(\mathrm{M} / \mathrm{Yr}_{\mathrm{r}}\right)$ & Waste Type & (K Gal) & (K Gal) & (K Gal) & (K Gal) & (K Gal) \\
\hline $1 / 80$ & & N/A & $N / A$ & N/A & $N / A$ & 0 \\
\hline $2 / 80$ & & N/A & N/A & N/A & N/A & 0 \\
\hline $3 / 80$ & & N/A. & N/A & N/A & N/A & 0 \\
\hline $4 / 80$ & & N/A & N/A & $\mathrm{N} / \mathrm{A}$ & N/A & 0 \\
\hline $5 / 80$ & & N/A & N/A & N/A & N/A & 0 \\
\hline $6 / 80$ & & N/A & $N / A$. & $N / A$ & N/A & 0 \\
\hline $7 / 80$ & & N/A & N/A & N/A & $N / A$ & 0 \\
\hline $8 / 80$ & & $N / A$ & N/A & N/A & N/A & 0 \\
\hline $9 / 80$ & & N/A & $\overline{N / A}$ & $N / A$ & N/A & 0 \\
\hline $10 / 80$ & & N/A & $\mathrm{N} / \mathrm{A}$ & $N / A$ & N/A & 0 \\
\hline $11 / 80$ & & N/A & N/A & $\mathrm{N} / \mathrm{A}$ & N/A & 0 \\
\hline $12 / 80$ & & N/A & N/A & N/A & $\mathrm{N} / \mathrm{A}$ & $\overline{0}$ \\
\hline $1 / 81$ & & N/A & N/A & $\mathbf{N} / \mathbf{A}$ & N/A & 0 \\
\hline $2 / 81$ & & N/A & $\mathbf{N} / \mathbf{A}$ & N/A & N/A & 0 \\
\hline $3 / 81$ & & N/A & $N / A$ & N/A & N/A & 0 \\
\hline $4 / 81$ & & N/A & N/A & N/A & $N / A$ & 0 \\
\hline $5 / 81$ & & N/A & N/A & N/A & N/A & 0 \\
\hline $6 / 81$ & & $\mathrm{~N} / \mathrm{A}$ & N/A & N/A & N/A & 0 \\
\hline $7 / 81$ & & $\mathrm{~N} / \mathrm{A}$ & $\mathrm{N} / \mathrm{A}$ & $N / A$ & N/A & 0 \\
\hline $8 / 81$ & & $\mathrm{~N} / \mathrm{A}$ & N/A & N/A & $N / A$ & 0 \\
\hline $9 / 81$ & & $N / A$ & N/A & N/A & N/A & 0 \\
\hline $10 / 81$ & & N/A & $\mathbf{N} / \mathbf{A}$ & $\mathrm{N} / \mathrm{A}$ & $N / A$ & 0 \\
\hline $11 / 81$ & & $N / A$ & N/A & $N / A$ & N/A & 0 \\
\hline $12 / 81$ & & $N / A$ & N/A & N/A & $N / A$ & 0 \\
\hline $1 / 82$ & & $N / A$ & N/A & N/A & N/A & 0 \\
\hline $2 / 82$ & & $\mathrm{~N} / \mathrm{A}$ & N/A & $\mathrm{N} / \mathrm{A}$ & N/A & 0 \\
\hline $3 / 82$ & & N/A & N/A & $N / A$ & $\mathrm{~N} / \mathrm{A}$ & 0 \\
\hline $4 / 82$ & & N/A & $\mathrm{N} / \mathrm{A}$ & N/A & $\mathbf{N} / \mathbf{A}$ & 0 \\
\hline $5 / 82$ & & N/A & N/A & $\mathrm{N} / \mathrm{A}$ & N/A & 0 \\
\hline $6 / 82$ & & $\mathbf{N} / \mathbf{A}$ & N/A & $\mathrm{N} / \mathrm{A}$ & N/A & 0 \\
\hline $7 / 82$ & & N/A & N/A & $\mathrm{N} / \mathrm{A}$ & N/A & 0 \\
\hline $8 / 82$ & & N/A & $N / A$ & N/A & $N / A$ & 0 \\
\hline $9 / 82$ & & $\mathrm{~N} / \mathrm{A}$ & $\mathrm{N} / \mathrm{A}$ & $\mathrm{N} / \mathrm{A}$ & N/A & 0 \\
\hline $10 / 82$ & & N/A & N/A & N/A & N/A & 0 \\
\hline $11 / 82$ & & N/A & N/A & N/A & $\mathrm{N} / \mathrm{A}$ & 0 \\
\hline $12 / 82$ & & N/A. & N/A & N/A & N/A & 0 \\
\hline $1 / 83$ & & N/A & $N / A$ & N/A & N/A & 0 \\
\hline $2 / 83$ & & N/A & $N / A$ & N/A & N/A & 0 \\
\hline $3 / 83$ & & $\mathrm{~N} / \mathrm{A}$ & $\mathrm{N} / \mathrm{A}$ & $N / A$ & N/A & 0 \\
\hline $4 / 83$ & & $\mathrm{~N} / \mathrm{A}$ & $\mathrm{N} / \mathrm{A}$ & N/A & $\mathrm{N} / \mathrm{A}$ & 0 \\
\hline $5 / 83$ & & N/A & $\mathrm{N} / \mathrm{A}$ & $N / A$ & N/A & 0 \\
\hline $6 / 83$ & & $\mathrm{~N} / \mathrm{A}$ & $\mathrm{N} / \mathrm{A}$ & $N / A$ & N/A & 0 \\
\hline $7 / 83$ & & N/A & $N / A$ & $N / A$ & N/A & 0 \\
\hline $8 / 83$ & & $N / A$ & N/A & N/A & N/A & 0 \\
\hline
\end{tabular}

Data obtained from Tank Farm Surveillance and Waste Status Summary Report, WHC-EP-0182. 
TANK LEVĘL HISTORY AND WASTE TYPES 241-AP-106

\begin{tabular}{|c|c|c|c|c|c|c|}
\hline Date & & Total Waste & DSS & Sludge & Salt & Total solids \\
\hline$\left(\mathrm{M} / \mathrm{Y}_{\mathrm{r}}\right)$ & Waste Type & (K Gal) & (K Gal) & (K Gal) & (K Gal) & (K Gal) \\
\hline $9 / 83$ & & N/A & N/A & N/A & N/A & 0 \\
\hline $10 / 83$ & & N/A & N/A & N/A & $N / A$ & 0 \\
\hline $11 / 83$ & & N/A & N/A & N/A & N/A & 0 \\
\hline $12 / 83$ & & $\mathrm{~N} / \mathrm{A}$ & N/A & $N / A$ & N/A & $\overline{0}$ \\
\hline $1 / 84$ & & N/A & N/A & N/A & N/A & 0 \\
\hline $2 / 84$ & & N/A & $N / A$ & N/A & N/A & 0 \\
\hline $3 / 84$ & & N/A & N/A & $N / A$ & N/A & 0 \\
\hline $4 / 84$ & & N/A & N/A & N/A & N/A & 0 \\
\hline $5 / 84$ & & $\mathbf{N} / \mathbf{A}$ & N/A & N/A & N/A & 0 \\
\hline $6 / 84$ & & N/A & N/A & N/A & N/A & 0 \\
\hline $7 / 84$ & & N/A & N/A & N/A & N/A & 0 \\
\hline $8 / 84$ & & N/A & N/A & N/A & N/A & $\overline{0}$ \\
\hline $9 / 84$ & & N/A & N/A & N/A & N/A & 0 \\
\hline $10 / 84$ & & N/A & $\mathbf{N} / \mathbf{A}$ & N/A & N/A & 0 \\
\hline $11 / 84$ & & N/A & N/A & N/A & N/A & 0 \\
\hline $12 / 84$ & & N/A & $\mathbf{N} / \mathbf{A}$ & N/A & $\mathrm{N} / \mathrm{A}$ & 0 \\
\hline $1 / 85$ & & $\mathrm{~N} / \mathrm{A}$ & $N / A$ & N/A & N/A & 0 \\
\hline $2 / 85$ & & $N / A$ & N/A & N/A & N/A & 0 \\
\hline $3 / 85$ & & N/A & N/A & $N / A$ & N/A & 0 \\
\hline $4 / 85$ & & N/A & $\mathrm{N} / \mathrm{A}$ & N/A & N/A & 0 \\
\hline $5 / 85$ & & $\mathrm{~N} / \mathrm{A}$ & $\mathbf{N} / \mathbf{A}$ & N/A & N/A & 0 \\
\hline $6 / 85$ & & N/A & N/A & N/A & $N / A$ & 0 \\
\hline $7 / 85$ & & N/A & N/A & N/A & $N / A$ & 0 \\
\hline $8 / 85$ & & $\mathbf{N} / \mathbf{A}$ & N/A & N/A & N/A & 0 \\
\hline $9 / 85$ & & N/A & N/A & $\mathbf{N} / \mathbf{A}$ & N/A & 0 \\
\hline $10 / 85$ & & N/A & N/A & $\mathrm{N} / \mathrm{A}$ & $N / A$ & 0 \\
\hline $11 / 85$ & & N/A & N/A & N/A & N/A & 0 \\
\hline $12 / 85$ & & N/A & N/A & N/A & $N / A$ & 0 \\
\hline $1 / 86$ & & N/A & N/A & N/A & $N / A$ & 0 \\
\hline $2 / 86$ & & N/A & $N / A$ & N/A & N/A & 0 \\
\hline $3 / 86$ & & $\mathrm{~N} / \mathrm{A}$ & N/A & N/A & N/A & 0 \\
\hline $4 / 86$ & & N/A & N/A & N/A & N/A & 0 \\
\hline $5 / 86$ & & N/A & $N / A$ & N/A & N/A & 0 \\
\hline $6 / 86$ & & N/A & $N / A$ & N/A & N/A & 0 \\
\hline $7 / 86$ & HFW & 0 & 0 & 0 & 0 & 0 \\
\hline $8 / 86$ & HFW & 0 & 0 & 0 & 0 & 0 \\
\hline $9 / 86$ & HFW & 0 & 0 & 0 & 0 & 0 \\
\hline $10 / 86$ & NCPLX & 18 & 0 & 0 & 0 & 0 \\
\hline $11 / 86$ & NCPLX & 19 & 0 & 0 & 0 & 0 \\
\hline $12 / 86$ & NCPLX & 19 & $0^{\circ}$ & 0 & 0 & 0 \\
\hline $1 / 87$ & NCPLX & 18 & 0 & 0 & 0 & 0 \\
\hline $2 / 87$ & NCPLX & 20 & 0 & 0 & 0 & 0 \\
\hline $3 / 87$ & NCPLX & 20 & 0 & 0 & 0 & 0 \\
\hline $4 / 87$ & NCPLX & 20 & 0 & 0 & 0 & 0 \\
\hline
\end{tabular}

Data obtained from Tank Farm Surveillance and Waste Status Summary Report, WHC-EP-0182. 
TANK LEVEL HISTORY AND WASTE TYPES 241-AP-106

\begin{tabular}{|c|c|c|c|c|c|c|}
\hline Date & & Total Waste & DSS & Sludge & Salt & Total solids \\
\hline$(\mathrm{M} / \mathrm{Yr})$ & Waste Type & (K Gal) & (K Gal) & (K Gal) & (K Gal) & (K Gal) \\
\hline $5 / 87$ & NCPLX & 18 & 0 & 0 & 0 & 0 \\
\hline $6 / 87$ & NCPLX & 18 & 0 & 0 & 0 & 0 \\
\hline $7 / 87$ & NCPLX & 17 & 0. & 0 & 0 & 0 \\
\hline $8 / 87$ & NCPLX & 17 & $\overline{0}$ & 0 & 0 & 0 \\
\hline $9 / 87$ & NCPLX & 18 & 0 & 0 & 0 & 0 \\
\hline $10 / 87$ & NCPLX & 17 & 0 & 0 & 0 & 0 \\
\hline $11 / 87$ & NCPLX & 17 & 0 & 0 & 0 & 0 \\
\hline $12 / 87$ & NCPLX & 18 & 0 & 0 & 0 & 0 \\
\hline $1 / 88$ & NCPLX & 18 & 0 & 0 & 0 & 0 \\
\hline $2 / 88$ & NCPLX & 18 & 0 & 0 & 0 & 0 \\
\hline $3 / 88$ & NCPLX & 19 & 0 & 0 & 0 & 0 \\
\hline $4 / 88$ & NCPLX & 19 & 0 & 0 & 0 & 0 \\
\hline $5 / 88$ & NCPLX & 19 & 0 & 0 & 0 & 0 \\
\hline $6 / 88$ & NCPLX & 18 & 0 & 0 & 0 & 0 \\
\hline $7 / 88$ & NCPLX & 295 & 0 & 0 & 0 & 0 \\
\hline $8 / 88$ & NCPLX & 583 & 0 & 0 & 0 & 0 \\
\hline $9 / 88$ & NCPLX & 583 & 0 & 0 & 0 & 0 \\
\hline $10 / 88$ & NCPLX & 579 & 0 & 0 & 0 & 0 \\
\hline $11 / 88$ & NCPLX & 578 & 0 & 0 & 0 & 0 \\
\hline $12 / 88$ & NCPLX & 576 & 0 & 0 & 0 & 0 \\
\hline $1 / 89$ & $\overline{\text { NCPLX }}$ & 575 & 0 & 0 & $\overline{0}$ & 0 \\
\hline $2 / 89$ & $\overline{N C P L X}$ & 490 & 0 & 0 & 0 & 0 \\
\hline $3 / 89$ & NCPLX & 490 & 0 & 0 & 0 & 0 \\
\hline $4 / 89$ & NCPLX & 490 & 0 & 0 & 0 & 0 \\
\hline $5 / 89$ & NCPLX & 489 & 0 & 0 & 0. & 0 \\
\hline $6 / 89$ & NCPLX & 489 & 0 & 0 & 0 & 0 \\
\hline $7 / 89$ & NCPLX & 927 & 0 & 0 & 0 & 0 \\
\hline $8 / 89$ & NCPLX & 926 & 0 & 0 & 0 & 0 \\
\hline $9 / 89$ & NCPLX & 926 & 0 & 0 & 0 & 0 \\
\hline $10 / 89$ & NCPLX & 1140 & 0 & 0 & 0 & 0 \\
\hline $11 / 89$ & NCPLX & 1139 & 0 & 0 & 0 & 0 \\
\hline $12 / 89$ & NCPLX & 1139 & 0 & 0 & 0 & $\overline{0}$ \\
\hline $1 / 90$ & NCPLX & 1139 & 0 & 0 & 0 & 0 \\
\hline $2 / 90$ & NCPLX & 1138 & 0 & 0 & 0 & 0 \\
\hline $3 / 90$ & NCPLX & 1139 & 0 & 0 & 0 & 0 \\
\hline $4 / 90$ & NCPLX & 1137 & 0 & 0 & 0 & 0 \\
\hline $5 / 90$ & NCPLX & 1137 & 0 & 0 & 0 & 0 \\
\hline $6 / 90$ & DN & 1137 & 0 & 0 & 0 & 0 \\
\hline $7 / 90$ & DN & 1137 & 0 & 0 & 0 & 0 \\
\hline $8 / 90$ & DN & 1137 & 0 & 0 & 0 & 0 \\
\hline $9 / 90$ & DN & 1137 & $\overline{0}$ & 0 & 0 & 0 \\
\hline $10 / 90$ & DN & 1136 & 0 & 0 & 0 & 0 \\
\hline $11 / 90$ & DN & 1136 & 0 & 0 & 0 & 0 \\
\hline $12 / 90$ & DN & 1136 & 0 & 0 & 0 & 0 \\
\hline
\end{tabular}

Data obtained from Tank Farm Surveillance and Waste Status Summary Report, WHC-EP-0182. 
TANK LEVEL HISTORY AND WASTE TYPES

241-AP-106

\begin{tabular}{|c|c|c|c|c|c|c|}
\hline Date & & Total Waste & DSS & Sludge & Salt & Total solids \\
\hline$\left(\mathrm{M} / \mathrm{Y}_{\mathrm{r}}\right)$ & Waste Type & (K Gal) & (K Gal) & (K Gal) & (K Gal) & (K Gal) \\
\hline $1 / 91$ & DN & 1135 & 0 & 0 & 0 & 0 \\
\hline $2 / 91$ & DN & 1135 & 0 & 0 & 0 & 0 \\
\hline $3 / 91$ & DN & 1134 & 0 & 0 & 0 & 0 \\
\hline $4 / 91$ & DN & 1134 & 0 & 0 & 0 & 0 \\
\hline $5 / 91$ & DN & 1134 & 0 & 0 & 0 & 0 \\
\hline $6 / 91$ & DN & 1134 & 0 & 0 & $\overline{0}$ & 0 \\
\hline $7 / 91$ & $\overline{D N}$ & 1134 & 0 & 0 & 0 & 0 \\
\hline $8 / 91$ & $\overline{D N}$ & 1134 & 0 & 0 & 0 & 0 \\
\hline $9 / 91$ & DN & 1134 & 0 & 0 & 0 & 0 \\
\hline $10 / 91$ & $\overline{D N}$ & 1133 & 0 & 0 & 0 & 0 \\
\hline $11 / 91$ & DN & 1133 & 0 & 0 & 0 & 0 \\
\hline $12 / 91$ & DN & 1133 & 0 & 0 & 0 & 0 \\
\hline $1 / 92$ & DN & 1132 & 0 & 0 & 0 & 0 \\
\hline $2 / 92$ & DN & 1132 & 0 & 0 & 0 & 0 \\
\hline $3 / 92$ & $\overline{D N}$ & 1132 & 0 & 0 & 0 & 0 \\
\hline $4 / 92$ & DN & 1132 & 0 & 0 & 0 & 0 \\
\hline $5 / 92$ & DN & 1132 & 0 & 0 & 0 & 0 \\
\hline $6 / 92$ & DN & 1131 & 0 & 0 & 0 & 0 \\
\hline $7 / 92$ & DN & 1131 & 0 & 0 & 0 & 0 \\
\hline $8 / 92$ & DN & 1131 & 0 & 0 & 0 & 0 \\
\hline $9 / 92$ & $\overline{D N}$ & 1131 & 0 & 0 & 0 & 0 \\
\hline $10 / 92$ & DN & 1131 & 0 & 0 & 0 & 0 \\
\hline $11 / 92$ & DN & 1131 & 0 & 0 & 0 & 0 \\
\hline $12 / 92$ & DN & 1130 & 0 & 0 & 0 & 0 \\
\hline $1 / 93$ & DN & 1130 & 0 & 0 & 0 & 0 \\
\hline $2 / 93$ & $\overline{\text { DN }}$ & 1130 & 0 & 0 & 0 & 0 \\
\hline $3 / 93$ & $\overline{D N}$ & 1129 & 0 & 0 & 0 & 0 \\
\hline $4 / 93$ & DN & 1129 & 0 & 0 & 0 & 0 \\
\hline $5 / 93$ & DN & 1129 & 0 & 0 & 0 & 0 \\
\hline $6 / 93$ & DN & 1129 & 0 & 0 & 0 & 0 \\
\hline $7 / 93$ & DN & 1129 & 0 & 0 & 0 & 0 \\
\hline $8 / 93$ & $\overline{D N}$ & 1129 & 0 & 0 & 0 & 0 \\
\hline $9 / 93$ & DN & 1129 & 0 & 0 & 0 & 0 \\
\hline $10 / 93$ & $\overline{D N}$ & 1129 & 0 & 0 & 0 & 0 \\
\hline $11 / 93$ & DN & 1128 & 0 & 0 & 0 & 0 \\
\hline $12 / 93$ & DN & 1128 & 0 & 0 & 0 & 0 \\
\hline $1 / 94$ & DN & 1128 & 0 & 0 & 0 & 0 \\
\hline $2 / 94$ & $\overline{\mathrm{DN}}$ & 1128 & 0 & 0 & 0 & 0 \\
\hline $3 / 94$ & DN & 1127 & 0 & 0 & 0 & 0 \\
\hline
\end{tabular}

Data obtained from Tank Farm Surveillance and Waste Status Summary Report, WHC-EP-0182. 
TANK LEVEL HISTORY AND WASTE TYPES

241-AP-107

\begin{tabular}{|c|c|c|c|c|c|c|}
\hline Date & & Total Waste & DSS & Sludge & Salt & Total solids \\
\hline$(\mathrm{M} / \mathrm{Yr})$ & Waste Type & (K Gal) & (K Gal) & (K Gal) & (K Gal) & (K Gal) \\
\hline $1 / 80$ & & $N / A$ & N/A & N/A & $\mathbf{N} / \mathbf{A}$ & 0 \\
\hline $2 / 80$ & & $N / A$ & $\mathrm{~N} / \mathrm{A}$ & $\mathrm{N} / \mathrm{A}$ & $\mathbf{N} / \mathbf{A}$ & 0 \\
\hline $3 / 80$ & & $N / A$ & $\mathrm{~N} / \mathrm{A}$ & N/A & N/A & $\overline{0}$ \\
\hline $4 / 80$ & & N/A & N/A & N/A & N/A & 0 \\
\hline $5 / 80$ & & N/A & N/A & N/A & N/A & 0 \\
\hline $6 / 80$ & & N/A & N/A & $\mathbf{N} / \mathbf{A}$ & N/A & 0 \\
\hline $7 / 80$ & & N/A & N/A & $\mathbf{N} / \mathbf{A}$ & $\mathrm{N} / \mathrm{A}$ & 0 \\
\hline $8 / 80$ & & $\mathbf{N} / \mathbf{A}$ & N/A & $\mathrm{N} / \mathrm{A}$ & N/A & 0 \\
\hline $9 / 80$ & & N/A & N/A & $\mathrm{N} / \mathrm{A}$ & N/A & 0 \\
\hline $10 / 80$ & & $\mathbf{N} / \mathbf{A}$ & $N / A$ & N/A & $\mathrm{N} / \mathrm{A}$ & 0 \\
\hline $11 / 80$ & & N/A & $\mathrm{N} / \mathrm{A}$ & $\mathbf{N} / \mathbf{A}$ & N/A & 0 \\
\hline $12 / 80$ & & $\mathbf{N} / \mathbf{A}$ & $\mathrm{N} / \mathrm{A}$ & N/A & $N / A$ & 0 \\
\hline $1 / 81$ & & N/A & N/A & N/A & N/A & 0 \\
\hline $2 / 81$ & & $\mathrm{~N} / \mathrm{A}$ & $\mathbf{N} / \mathbf{A}$ & $N / A$ & N/A & 0 \\
\hline $3 / 81$ & & $N / A$ & N/A & $N / A$ & N/A & 0 \\
\hline $4 / 81$ & & $\mathbf{N} / \mathbf{A}$ & N/A & $N / A$ & N/A & 0 \\
\hline $5 / 81$ & & N/A & $\mathbf{N} / \mathbf{A}$ & N/A & $\mathbf{N} / \mathbf{A}$ & 0 \\
\hline $6 / 81$ & & N/A & N/A & $N / A$ & $N / A$ & 0 \\
\hline $7 / 81$ & & $N / A$ & $N / A$ & N/A & $N / A$ & 0 \\
\hline $8 / 81$ & & $N / A$ & N/A & - N/A & $N / A$ & 0 \\
\hline $9 / 81$ & & N/A & $N / A$ & N/A & N/A & 0 \\
\hline $10 / 81$ & & $N / A$ & N/A & $N / A$ & N/A & 0 \\
\hline $11 / 81$ & & N/A & $N / A$ & $\mathrm{~N} / \mathrm{A}$ & $N / A$ & 0 \\
\hline $12 / 81$ & & $N / A$ & $N / A$ & $N / A$ & N/A & 0 \\
\hline $1 / 82$ & & $N / A$ & $\mathrm{~N} / \mathrm{A}$ & $\mathbf{N} / \mathbf{A}$ & N/A & 0 \\
\hline $2 / 82$ & & N/A & $\mathbf{N} / \mathbf{A}$ & N/A & $\mathbf{N} / \mathbf{A}$ & 0 \\
\hline $3 / 82$ & & N/A & N/A & $\mathrm{N} / \mathrm{A}$ & N/A & 0 \\
\hline $4 / 82$ & & N/A & $N / A$ & $N / A$ & $\mathrm{~N} / \mathrm{A}$ & 0 \\
\hline $5 / 82$ & & $\mathrm{~N} / \mathrm{A}$ & $\mathrm{N} / \mathrm{A}$ & $\mathrm{N} / \mathrm{A}$ & $\mathbf{N} / \mathbf{A}$ & 0 \\
\hline $6 / 82$ & & $N / A$ & $N / A$ & $\mathbf{N} / \mathbf{A}$ & N/A & 0 \\
\hline $7 / 82$ & & $\mathrm{~N} / \mathrm{A}$ & $N / A$ & N/A & $\mathrm{N} / \mathrm{A}$ & $\overline{0}$ \\
\hline $8 / 82$ & & $N / A$ & $N / A$ & N/A & N/A & 0 \\
\hline $9 / 82$ & & N/A & N/A & N/A & N/A & 0 \\
\hline $10 / 82$ & & $N / A$ & $N / A$ & N/A & N/A & 0 \\
\hline $11 / 82$ & & $\mathrm{~N} / \mathrm{A}$ & $N / A$ & N/A & $\mathbf{N} / \mathbf{A}$ & 0 \\
\hline $12 / 82$ & & N/A & $N / A$ & N/A & N/A & 0 \\
\hline $1 / 83$ & & N/A & N/A & $N / A$ & N/A & 0 \\
\hline $2 / 83$ & & N/A & N/A & N/A & N/A & 0 \\
\hline $3 / 83$ & & $\mathbf{N} / \mathbf{A}$. & $N / A$ & $\mathbf{N} / \mathbf{A}$ & $N / A$ & 0 \\
\hline $4 / 83$ & & N/A & N/A & $N / A$ & N/A & 0 \\
\hline $5 / 83$ & & $\mathrm{~N} / \mathrm{A}$ & N/A & N/A & N/A & 0 \\
\hline $6 / 83$ & & N/A & N/A & N/A & N/A & 0 \\
\hline $7 / 83$ & & N/A & $N / A$ & N/A & N/A & 0 \\
\hline $8 / 83$ & & N/A & $N / A$ & N/A & N/A & 0 \\
\hline
\end{tabular}

Data obtained from Tank Farm Surveillance and Waste Status Summary Report, WHC-EP-0182. 
WHC-SD-KM-ER-315, Rev. 0

TANK LEVEL HISTORY AND WASTE TYPES

241-AP-107

\begin{tabular}{|c|c|c|c|c|c|c|}
\hline Date & & Total Waste & DSS & Sludge. & Salt & Total solids \\
\hline$(\mathrm{M} / \mathrm{Yr})$ & Waste Type & (K Gal) & (K Gal) & (K Gal) & (K Gal) & (K Gal) \\
\hline $9 / 83$ & & $\mathrm{~N} / \mathrm{A}$ & N/A & N/A & $\mathrm{N} / \mathrm{A}$ & 0 \\
\hline $10 / 83$ & & $N / A$ & N/A & $\mathrm{N} / \mathrm{A}$ & N/A & 0 \\
\hline $11 / 83$ & & N/A. & N/A & $N / A$ & $N / A$ & 0 \\
\hline $12 / 83$ & & $\mathrm{~N} / \mathrm{A}$ & $N / A$ & N/A & N/A & 0 \\
\hline $1 / 84$ & & N/A & $N / A$ & N/A & $\mathbf{N} / \mathbf{A}$ & 0 \\
\hline $2 / 84$ & & N/A & $\mathrm{N} / \mathrm{A}$ & N/A & $N / A$ & 0 \\
\hline $3 / 84$ & & N/A & $\mathrm{N} / \mathrm{A}$ & N/A & $\mathrm{N} / \mathrm{A}$ & 0 \\
\hline $4 / 84$ & & N/A & $\mathrm{N} / \mathrm{A}$ & $\mathrm{N} / \mathrm{A}$ & N/A & 0 \\
\hline $5 / 84$ & & N/A & N/A & $\mathrm{N} / \mathrm{A}$ & N/A & 0 \\
\hline $6 / 84$ & & N/A & $\mathrm{N} / \mathrm{A}$ & $\mathbf{N} / \mathbf{A}$ & N/A & 0 \\
\hline $7 / 84$ & & N/A & N/A & $N / A$ & N/A & 0 \\
\hline $8 / 84$ & & N/A & N/A & $\mathbf{N} / \mathbf{A}$ & N/A & 0 \\
\hline $9 / 84$ & & $N / A$ & $\mathbf{N} / \mathbf{A}$ & N/A & N/A & 0 \\
\hline $10 / 84$ & & $\mathrm{~N} / \mathrm{A}$ & N/A & N/A & $N / A$ & 0 \\
\hline $11 / 84$ & & N/A & N/A & $N / A$ & $\mathrm{~N} / \mathrm{A}$ & 0 \\
\hline $12 / 84$ & & N/A & $N / A$ & N/A & N/A & 0 \\
\hline $1 / 85$ & & N/A & N/A & N/A & N/A & 0 \\
\hline $2 / 85$ & & N/A & $\mathbf{N} / \mathbf{A}$ & $\mathrm{N} / \mathrm{A}$ & N/A & 0 \\
\hline $3 / 85$ & & $\mathrm{~N} / \mathrm{A}$ & $N / A$ & $\mathrm{~N} / \mathrm{A}$ & $N / A$ & 0 \\
\hline $4 / 85$ & & $N / A$ & N/A & N/A & $N / A$ & 0 \\
\hline $5 / 85$ & & N/A & $N / A$ & N/A & $N / A$ & 0 \\
\hline $6 / 85$ & & $N / A$ & $\mathrm{~N} / \mathrm{A}$ & N/A & $N / A$ & 0 \\
\hline $7 / 85$ & & N/A & $N / A$ & $N / A$ & $N / A$ & 0 \\
\hline $8 / 85$ & & $\mathrm{~N} / \mathrm{A}$ & $\mathrm{N} / \mathrm{A}$ & $\mathbf{N} / \mathbf{A}$ & N/A & 0 \\
\hline $9 / 85$ & & N/A & $\mathrm{N} / \mathrm{A}$ & N/A & N/A & 0 \\
\hline $10 / 85$ & & $\mathrm{~N} / \mathrm{A}$ & $\mathrm{N} / \mathrm{A}$ & N/A & $N / A$ & 0 \\
\hline $11 / 85$ & & N/A & N/A & N/A & N/A & 0 \\
\hline $12 / 85$ & & N/A. & $N / A$ & N/A & N/A & 0 \\
\hline $1 / 86$ & & $N / A$ & N/A & $\mathrm{N} / \mathrm{A}$ & $N / A$ & 0 \\
\hline $2 / 86$ & & N/A & N/A & N/A & N/A & 0 \\
\hline $3 / 86$ & & N/A & $N / A$ & $\mathrm{~N} / \mathrm{A}$ & $N / A$ & 0 \\
\hline $4 / 86$ & & $N / A$ & N/A & $N / A$ & N/A & 0 \\
\hline $5 / 86$ & & $\mathrm{~N} / \mathrm{A}$ & $\mathrm{N} / \mathrm{A}$ & N/A & N/A & 0 \\
\hline $6 / 86$ & & $\mathrm{~N} / \mathrm{A}$ & N/A & $N / A$ & N/A & 0 \\
\hline $7 / 86$ & DSSF & 0 & 0 & 0 & 0 & 0 \\
\hline $8 / 86$ & DSSF & 0 & 0 & 0 & 0 & 0 \\
\hline $9 / 86$ & DSSF & 0 & 0 & 0 & 0 & 0 \\
\hline $10 / 86$ & NCPLX & 18 & 0 & 0 & 0 & 0 \\
\hline $11 / 86$ & NCPLX & 18 & 0 & 0 & 0 & 0 \\
\hline $12 / 86$ & NCPLX & 17 & 0 & 0 & 0 & 0 \\
\hline $1 / 87$ & NCPLX & 18 & 0 & 0 & 0 & 0 \\
\hline $2 / 87$ & NCPLX & 18 & 0 & 0 & 0 & 0 \\
\hline $3 / 87$ & NCPLX & 18 & 0 & 0 & 0 & 0 \\
\hline $4 / 87$ & NCPLX & 18 & 0 & 0 & 0 & 0 \\
\hline
\end{tabular}

Data obtained from Tank Farm Surveillance and Waste Status Summary Report, WHC-EP-0182. 
TANK LEVEL HISTORY AND WASTE TYPES

241-AP-107

\begin{tabular}{|c|c|c|c|c|c|c|}
\hline Date & & Total Waste & DSS & Sludge & Salt & Total solids \\
\hline$\left(M / Y_{r}\right)$ & Waste Type & (K Gal) & (K Gal) & (K Gal) & (K Gal) & (K Gal) \\
\hline $5 / 87$ & NCPLX & 18 & $\overline{0}$ & 0 & 0 & $\overline{0}$ \\
\hline $6 / 87$ & NCPLX & 18 & 0 & 0 & 0 & 0 \\
\hline $7 / 87$ & NCPLX & 18 & 0 & 0 & 0 & 0 \\
\hline $8 / 87$ & NCPLX & 17 & 0 & 0 & 0 & 0 \\
\hline $9 / 87$ & NCPLX & 18 & 0 & 0 & 0 & 0 \\
\hline $10 / 87$ & NCPLX & 17 & 0 & 0 & 0 & 0 \\
\hline $11 / 87$ & NCPLX & 17 & 0 & 0 & 0 & 0 \\
\hline $12 / 87$ & NCPLX & 17 & 0 & 0 & 0 & 0 \\
\hline $1 / 88$ & NCPLX & 18 & 0 & 0 & 0 & 0 \\
\hline $2 / 88$ & NCPLX & 18 & 0 & 0 & 0 & 0 \\
\hline $3 / 88$ & NCPLX & 18 & 0 & 0 & 0 & 0 \\
\hline $4 / 88$ & NCPLX & 18 & 0 & 0 & 0 & 0 \\
\hline $5 / 88$ & NCPLX & 18 & 0 & 0 & 0 & 0 \\
\hline $6 / 88$ & NCPLX & 18 & 0 & 0 & 0 & 0 \\
\hline $7 / 88$ & NCPLX & 18 & 0. & .0 & 0 & 0 \\
\hline $8 / 88$ & NCPLX & 19 & 0 & 0 & 0 & 0 \\
\hline $9 / 88$ & NCPLX & 19 & 0 & 0 & 0 & 0 \\
\hline $10 / 88$ & NCPLX & 18 & 0 & 0 & 0 & 0 \\
\hline $11 / 88$ & NCPLX & 20 & 0 & 0 & 0 & 0 \\
\hline $12 / 88$ & NCPLX & 19 & 0 & 0 & 0 & 0 \\
\hline $1 / 89$ & NCPLX & 18 & 0 & 0 & 0 & 0 \\
\hline $2 / 89$ & NCPLX & 18 & 0 & 0 & 0 & 0 \\
\hline $3 / 89$ & NCPLX & 18 & 0 & 0 & 0 & 0 \\
\hline $4 / 89$ & NCPLX & 18 & 0 & 0 & 0 & 0 \\
\hline $5 / 89$ & NCPLX & 18 & 0 & 0 & 0 & 0 \\
\hline $6 / 89$ & NCPLX & 18 & 0 & 0 & 0 & 0 \\
\hline $7 / 89$ & NCPLX & 18 & 0 & 0 & 0 & 0 \\
\hline $8 / 89$ & NCPLX & 18 & 0 & 0 & 0 & 0 \\
\hline $9 / 89$ & NCPLX & 14 & 0 & 0 & 0 & 0 \\
\hline $10 / 89$ & NCPLX & 18 & 0 & 0 & 0 & 0 \\
\hline $11 / 89$ & NCPLX & 18 & 0 & 0 & 0 & 0 \\
\hline $12 / 89$ & NCPLX & 19 & 0 & 0 & 0 & 0 \\
\hline $1 / 90$ & NCPLXX & 325 & 0 & 0 & 0 & 0 \\
\hline $2 / 90$ & NCPLX & 1139 & 0 & 0 & 0 & 0 \\
\hline $3 / 90$ & NCPLX & 18 & 0 & 0 & 0 & 0 \\
\hline $4 / 90$ & NCPLX & 1138 & 0 & 0 & 0 & 0 \\
\hline $5 / 90$ & NCPLX & 1137 & 0 & 0 & 0 & 0 \\
\hline $6 / 90$ & DN & 1137 & 0 & 0 & 0 & 0 \\
\hline $7 / 90$ & DN & 1136 & 0 & 0 & 0 & 0 \\
\hline $8 / 90$ & DN & 1136 & 0 & 0 & 0 & 0 \\
\hline $9 / 90$ & DN & 1136 & 0 & 0 & 0 & 0 \\
\hline $10 / 90$ & DN & 1135 & 0 & 0 & 0 & 0 \\
\hline $11 / 90$ & DN & 1133 & 0 & 0 & 0 & 0 \\
\hline $12 / 90$ & DN & 1132 & 0 & 0 & 0 & 0 \\
\hline
\end{tabular}

Data obtained from Tank Farm Surveillance and Waste Status Summary Report, WHC-EP-0182. 
TANK LEVEL HISTORY AND WASTE TYPES

WHC-SD-WM-ER-315, Rev. 0 241-AP-107

\begin{tabular}{|c|c|c|c|c|c|c|}
\hline Date & & Total Waste & DSS & Sludge & Salt & Total solids \\
\hline$(\mathrm{M} / \mathrm{Yr})$ & Waste Type & (K Gal) & (K Gal) & (K Gal) & (K Gal) & (K Gal) \\
\hline $1 / 91$ & $\overline{D N}$ & 1131 & 0 & 0 & 0 & 0 \\
\hline $2 / 91$ & DN & 1130 & 0 & 0 & 0 & 0 \\
\hline $3 / 91$ & DN & 1130 & 0 & 0 & 0 & 0 \\
\hline $4 / 91$ & DN & 1129 & 0 & 0 & 0 & 0 \\
\hline $5 / 91$ & DN & 1129 & 0 & 0 & 0 & 0 \\
\hline $6 / 91$ & DN & 1129 & 0 & 0 & 0 & $\overline{0}$ \\
\hline $7 / 91$ & DN & 1128 & 0 & 0 & 0 & 0 \\
\hline $8 / 91$ & DN & 1128 & 0 & 0 & 0 & 0 \\
\hline $9 / 91$ & DN & 1128 & 0 & 0 & 0 & 0 \\
\hline $10 / 91$ & DN & 1127 & 0 & 0 & 0 & 0 \\
\hline $11 / 91$ & DN & 1126 & 0 & 0 & 0 & 0 \\
\hline $12 / 91$ & DN & 1125 & 0 & 0 & 0 & 0 \\
\hline $1 / 92$ & DN & 1124 & 0 & 0 & 0 & 0 \\
\hline $2 / 92$ & DN & 1124 & 0 & 0 & 0 & 0 \\
\hline $3 / 92$ & $\overline{D N}$ & 1123 & 0 & 0 & 0 & 0 \\
\hline $4 / 92$ & $\overline{D N}$ & 1123 & 0 & 0 & 0 & 0 \\
\hline $5 / 92$ & DN & 1123 & 0 & 0 & 0 & 0 \\
\hline $6 / 92$ & DN & 1122 & 0 & 0 & 0 & 0 \\
\hline $7 / 92$ & DN & 1122 & 0 & 0 & 0 & 0 \\
\hline $8 / 92$ & $\overline{D N}$ & 1122 & 0 & 0 & 0 & 0 \\
\hline $9 / 92$ & DN & 1121 & 0 & 0 & 0 & 0 \\
\hline $10 / 92$ & DN & 1121 & 0 & 0 & 0 & 0 \\
\hline $11 / 92$ & $\overline{D N}$ & 1120 & 0 & 0 & 0 & 0 \\
\hline $12 / 92$ & DN & 1118 & 0 & 0 & 0 & 0 \\
\hline $1 / 93$ & DN & 1117 & 0 & 0 & 0 & 0 \\
\hline $2 / 93$ & $\overline{\text { DN }}$ & 1116 & 0 & 0 & 0 & 0 \\
\hline $3 / 93$ & DN & 1115 & 0 & 0 & 0 & 0 \\
\hline $4 / 93$ & DN & 1112 & 0 & 0 & 0 & 0 \\
\hline $5 / 93$ & DN & 1115 & 0 & 0 & 0 & 0 \\
\hline $6 / 93$ & $\overline{\text { DN }}$ & 1115 & 0 & 0 & 0 & 0 \\
\hline $7 / 93$ & $\overline{D N}$ & 1115 & 0 & 0 & $\overline{0}$ & 0 \\
\hline $8 / 93$ & DN & 1114 & 0 & 0 & 0 & 0 \\
\hline $9 / 93$ & $\overline{\text { DN }}$ & 1114 & 0 & 0 & 0 & 0 \\
\hline $10 / 93$ & $\overline{D N}$ & 1113 & 0 & 0 & 0 & 0 \\
\hline $11 / 93$ & DN & 1113 & 0 & 0 & 0 & 0 \\
\hline $12 / 93$ & $\overline{\text { DN }}$ & 1108 & 0. & 0 & 0 & 0 \\
\hline $1 / 94$ & $\overline{D N}$ & 1111 & 0 & 0 & 0 & 0 \\
\hline $2 / 94$ & DN & 1110 & 0 & 0 & 0 & 0 \\
\hline $3 / 94$ & $\overline{\text { DN }}$ & 1110 & 0 & 0 & 0 & 0 \\
\hline
\end{tabular}

Data obtained from Tank Farm Surveillance and Waste Status Summary Report, WHC-EP-0182. 
TANK LEVEL HISTORY AND WASTE TYPES

241-AP-108

\begin{tabular}{|c|c|c|c|c|c|c|}
\hline Date & & Total Waste & $\overline{D S S}$ & Sludge & Salt & Total solids \\
\hline$(\mathrm{M} / \mathrm{Yr})$ & Waste Type & (K Gal) & (K Gal) & (K Gal) & (K Gal) & (K Gal) \\
\hline $1 / 80$ & & $\mathrm{~N} / \mathrm{A}$ & $\mathrm{N} / \mathrm{A}$ & $\mathbf{N} / \mathbf{A}$ & N/A & 0 \\
\hline $2 / 80$ & & N/A & N/A & $\mathbf{N} / \mathbf{A}$ & N/A & 0 \\
\hline $3 / 80$ & & $N / A$ & N/A & N/A & $\mathrm{N} / \mathrm{A}$ & 0 \\
\hline $4 / 80$ & & N/A & N/A & $\mathrm{N} / \mathrm{A}$ & $\mathbf{N} / \mathbf{A}$ & $\overline{0}$ \\
\hline $5 / 80$ & & $\mathrm{~N} / \mathrm{A}$ & N/A & N/A & N/A & 0 \\
\hline $6 / 80$ & & $\mathbf{N} / \mathbf{A}$ & N/A & $\mathbf{N} / \mathbf{A}$ & N/A & 0 \\
\hline $7 / 80$ & & N/A & N/A & N/A & $\mathbf{N} / \mathbf{A}$ & 0 \\
\hline $8 / 80$ & & N/A & N/A & $\mathbf{N} / \mathbf{A}$ & $\mathrm{N} / \mathrm{A}$ & 0 \\
\hline $9 / 80$ & & $\mathbf{N} / \mathbf{A}$ & N/A & $N / A$ & $N / A$ & 0 \\
\hline $10 / 80$ & & N/A & $N / A$ & N/A & $N / A$ & 0 \\
\hline $11 / 80$ & & N/A & $\mathbf{N} / \mathbf{A}$ & N/A & N/A & 0 \\
\hline $12 / 80$ & & N/A & N/A & N/A & N/A & 0 \\
\hline $1 / 81$ & & $\mathbf{N} / \mathbf{A}$ & N/A & N/A & N/A & 0 \\
\hline $2 / 81$ & & N/A & $\mathbf{N} / \mathbf{A}$ & $\mathbf{N} / \mathbf{A}$ & N/A & 0 \\
\hline $3 / 81$ & & $\mathbf{N} / \mathbf{A}$ & N/A & N/A & N/A & $\overline{0}$ \\
\hline $4 / 81$ & & N/A & N/A & N/A & N/A & 0 \\
\hline $5 / 81$ & & $N / A$ & N/A & N/A & N/A & 0 \\
\hline $6 / 81$ & & N/A. & N/A & N/A & N/A & $\overline{0}$ \\
\hline $7 / 81$ & & N/A & $\mathrm{N} / \mathrm{A}$ & N/A & N/A & 0 \\
\hline $8 / 81$ & & N/A & $N / A$ & $N / A$ & $N / A$ & 0 \\
\hline $9 / 81$ & & $\mathbf{N} / \mathbf{A}$ & N/A & N/A & $N / A$ & 0 \\
\hline $10 / 81$ & & N/A & N/A & N/A & $\mathrm{N} / \mathrm{A}$ & 0 \\
\hline $11 / 81$ & $\therefore$ & N/A & N/A & N/A & $N / A$ & 0 \\
\hline $12 / 81$ & & N/A & N/A & $N / A$ & $N / A$ & 0 \\
\hline $1 / 82$ & & $N / A$ & N/A & N/A & N/A & 0 \\
\hline $2 / 82$ & & N/A & N/A & N/A & $\mathbf{N} / \mathbf{A}$ & 0 \\
\hline $3 / 82$ & & N/A & $N / A$ & N/A & N/A & 0 \\
\hline $4 / 82$ & & N/A & $N / A$ & N/A & $N / A$ & 0 \\
\hline $5 / 82$ & & N/A & N/A & N/A & $\mathbf{N} / \mathbf{A}$ & 0 \\
\hline $6 / 82$ & & N/A & N/A & N/A & N/A & 0 \\
\hline $7 / 82$ & & N/A & N/A & $\mathrm{N} / \mathrm{A}$ & N/A & 0 \\
\hline $8 / 82$ & & N/A & $\mathrm{N} / \mathrm{A}$ & N/A & $\mathbf{N} / \mathbf{A}$ & 0 \\
\hline $9 / 82$ & & N/A & $\mathbf{N} / \mathbf{A}$ & $N / A$ & N/A & 0 \\
\hline $10 / 82$ & & N/A & N/A & $\mathrm{N} / \mathrm{A}$ & N/A & 0 \\
\hline $11 / 82$ & & N/A & N/A & N/A & N/A & 0 \\
\hline $12 / 82$ & & N/A & N/A & $N / A$ & N/A & 0 \\
\hline $1 / 83$ & & N/A & $\mathbf{N} / \mathbf{A}$ & N/A & N/A & 0 \\
\hline $2 / 83$ & & N/A & N/A & N/A & N/A & 0 \\
\hline $3 / 83$ & & N/A & $\mathrm{N} / \mathrm{A}$ & N/A & N/A & 0 \\
\hline $4 / 83$ & & $N / A$ & $\mathrm{~N} / \mathrm{A}$ & N/A & N/A & 0 \\
\hline $5 / 83$ & & N/A & $\mathrm{N} / \mathrm{A}$ & $\mathbf{N} / \mathbf{A}$ & N/A & 0 \\
\hline $6 / 83$ & & N/A & N/A & N/A & $N / A$ & 0 \\
\hline $7 / 83$ & & $\mathrm{~N} / \mathrm{A}$ & $N / A$ & $\mathrm{~N} / \mathrm{A}$ & $\mathbf{N} / \mathbf{A}$ & 0 \\
\hline $8 / 83$ & & $\mathrm{~N} / \mathrm{A}$ & $\mathrm{N} / \mathrm{A}$ & N/A & $\mathrm{N} / \mathrm{A}$ & 0 \\
\hline
\end{tabular}

Data obtained from Tank Farm Surveillance and Waste Status Summary Report, WHC-EP-0182. 
TANK LEVEL. HISTORY AND WASTE TYPES

241-AP-108

\begin{tabular}{|c|c|c|c|c|c|c|}
\hline Date & & Total Waste & DSS & Sludge & Salt & Total solids \\
\hline$(\mathrm{M} / \mathrm{Yr})$ & Waste Type & (K Gal) & (K Gal) & (K Gal) & (K Gal) & (K Gal) \\
\hline $9 / 83$ & & $\mathbf{N} / \mathbf{A}$ & N/A & N/A & N/A & 0 \\
\hline $10 / 83$ & & $N / A$ & $N / A$ & $N / A$ & N/A & 0 \\
\hline $11 / 83$ & & $\mathrm{~N} / \mathrm{A}$ & N/A & $\mathrm{N} / \mathrm{A}$ & $\mathrm{N} / \mathrm{A}$ & 0 \\
\hline $12 / 83$ & & N/A & N/A & N/A & $\mathrm{N} / \mathrm{A}$ & 0 \\
\hline $1 / 84$ & & N/A & $\mathrm{N} / \mathrm{A}$ & N/A & $\mathrm{N} / \mathrm{A}$ & 0 \\
\hline $2 / 84$ & & $\mathrm{~N} / \mathrm{A}$ & $\mathrm{N} / \mathrm{A}$ & N/A & N/A & 0 \\
\hline $3 / 84$ & & N/A & N/A & N/A & $\mathrm{N} / \mathrm{A}$ & 0 \\
\hline $4 / 84$ & & N/A & N/A & N/A & $N / A$ & 0 \\
\hline $5 / 84$ & & N/A & N/A & $N / A$ & N/A & 0 \\
\hline $6 / 84$ & & N/A & $\mathbf{N} / \mathbf{A}$ & N/A & N/A & 0 \\
\hline $7 / 84$ & & N/A & N/A & N/A & N/A & 0 \\
\hline $8 / 84$ & & $\mathrm{~N} / \mathrm{A}$ & N/A & N/A & N/A & 0 \\
\hline $9 / 84$ & & N/A & $N / A$ & N/A & N/A & 0 \\
\hline $10 / 84$ & & N/A & N/A & N/A & N/A & 0 \\
\hline $1 \cdot 1 / 84$ & & N/A. & N/A & N/A & $N / A$ & 0 \\
\hline $12 / 84$ & & N/A & N/A & $\mathrm{N} / \mathrm{A}$ & N/A & 0 \\
\hline $1 / 85$ & & N/A & $\mathbf{N} / \mathbf{A}$ & $N / A$ & N/A & 0 \\
\hline $2 / 85$ & & N/A & N/A & N/A & $N / A$ & 0 \\
\hline $3 / 85$ & & $N / A$ & N/A & N/A & $N / A$ & 0 \\
\hline $4 / 85$ & & N/A & N/A & N/A & N/A & 0 \\
\hline $5 / 85$ & & $\mathbf{N} / \mathbf{A}$ & N/A & N/A & N/A & 0 \\
\hline $6 / 85$ & & N/A & $N / A$ & $N / A$ & $\mathrm{~N} / \mathrm{A}$ & 0 \\
\hline $7 / 85$ & & N/A & N/A & $N / A$ & N/A & 0 \\
\hline $8 / 85$ & & N/A & $\mathrm{N} / \mathrm{A}$ & N/A & N/A & 0 \\
\hline $9 / 85$ & & $\mathbf{N} / \mathbf{A}$ & $\mathbf{N} / \mathbf{A}$ & $N / A$ & N/A & 0 \\
\hline $10 / 85$ & & $\mathrm{~N} / \mathrm{A}$ & $N / A$ & $N / A$ & $\mathbf{N} / \mathbf{A}$ & 0 \\
\hline $11 / 85$ & & N/A & N/A & N/A & N/A & 0 \\
\hline $12 / 85$ & & N/A & $\mathrm{N} / \mathrm{A}$ & $N / A$ & N/A & 0 \\
\hline $1 / 86$ & & N/A & N/A & $\mathrm{N} / \mathrm{A}$ & N/A & 0 \\
\hline $2 / 86$ & & $N / A$ & N/A & N/A & N/A & 0 \\
\hline $3 / 86$ & & N/A & $\mathrm{N} / \mathrm{A}$ & N/A & $N / A$ & 0 \\
\hline $4 / 86$ & & N/A & $N / A$ & $N / A$ & N/A & 0 \\
\hline $5 / 86$ & & N/A & N/A & $N / A$ & N/A & 0 \\
\hline $6 / 86$ & & N/A & N/A & $N / A$ & N/A & 0 \\
\hline $7 / 86$ & DSSF & 0 & 0 & 0 & 0 & 0 \\
\hline $8 / 86$ & DSSF & 0 & 0 & 0 & 0 & 0 \\
\hline $9 / 86$ & DSSF & 0 & 0 & 0 & 0 & 0 \\
\hline $10 / 86$ & NCPLX & 18 & 0. & 0 & 0 & 0 \\
\hline $11 / 86$ & NCPLX & 18 & 0 & 0 & 0 & 0 \\
\hline $12 / 86$ & NCPLX & 17 & 0 & 0 & 0 & 0 \\
\hline $1 / 87$ & NCPLX & 18 & 0 & 0 & 0 & 0 \\
\hline $2 / 87$ & NCPLX & 18 & 0 & 0 & 0 & 0 \\
\hline $3 / 87$ & NCPLX & 18 & 0 & 0 & 0 & 0 \\
\hline $4 / 87$ & NCPLX & 18 & 0 & $\overline{0}$ & 0 & 0 \\
\hline
\end{tabular}

Data obtained from Tank Farm Surveillance and Waste Status Summary Report, WHC-EP-0182. 
WHC-SD-WM-ER-315, Rev. 0

TANK LEVEL HISTORY AND WASTE TYPES

241-AP-108

\begin{tabular}{|c|c|c|c|c|c|c|}
\hline Date & & Total Waste & DSS & Sludge & Salt & Total solids \\
\hline (M/Yr) & Waste Type & (K Gal) & (K Gal) & (K Gal) & (K Gal) & (K Gal) \\
\hline $5 / 87$ & NCPLX & 18 & 0 & 0 & 0 & 0 \\
\hline $6 / 87$ & NCPLX & 18 & 0 & 0 & 0 & 0 \\
\hline $7 / 87$ & NCPLX & 18 & 0 & 0 & 0 & 0 \\
\hline $8 / 87$ & NCPLX & 18 & 0 & 0 & 0 & 0 \\
\hline $9 / 87$ & NCPLX & 18 & 0 & 0 & 0 & 0 \\
\hline $10 / 87$ & NCPLX & 18 & 0 & 0 & 0 & 0 \\
\hline $11 / 87$ & NCPLX & 18 & 0 & 0 & 0 & 0 \\
\hline $12 / 87$ & NCPLX & 17 & 0 & 0 & 0 & 0 \\
\hline $1 / 88$ & NCPLX & 19 & 0 & 0 & 0 & 0 \\
\hline $2 / 88$ & NCPLX & 19 & 0 & 0 & 0 & 0 \\
\hline $3 / 88$ & NCPLX & 18 & 0 & 0 & 0 & 0 \\
\hline $4 / 88$ & NCPLX & 18 & 0 & 0 & 0 & 0 \\
\hline $5 / 88$ & NCPLX & 19 & 0 & 0 & 0 & 0 \\
\hline $6 / 88$ & NCPLX & 18 & 0 & 0 & 0 & 0 \\
\hline $7 / 88$ & NCPLX & 18 & 0 & 0 & 0 & 0 \\
\hline $8 / 88$ & NCPLX & 19 & 0 & 0 & 0 & 0 \\
\hline $9 / 88$ & NCPLX & 19 & 0 & 0 & 0 & 0 \\
\hline $10 / 88$ & NCPLX & 18 & 0 & 0 & 0 & 0 \\
\hline $11 / 88$ & NCPLX & 19 & 0 & 0 & 0 & 0 \\
\hline $12 / 88$ & NCPLX & 18 & 0 & 0 & 0 & 0 \\
\hline $1 / 89$ & NCPLX & 18 & 0 & 0 & 0 & 0 \\
\hline $2 / 89$ & NCPLX & 18 & 0 & 0 & 0 & 0 \\
\hline $3 / 89$ & NCPLX & 18 & 0 & 0 & 0 & 0 \\
\hline $4 / 89$ & NCPLX & 18 & 0 & 0 & 0 & 0 \\
\hline $5 / 89$ & NCPLX & 18 & 0 & 0 & 0 & 0 \\
\hline $6 / 89$ & NCPLX & 18 & 0 & 0 & 0 & 0 \\
\hline $7 / 89$ & NCPLX & 19 & 0 & 0 & 0 & 0 \\
\hline $8 / 89$ & NCPLX & 19 & 0 & 0 & 0 & 0 \\
\hline $9 / 89$ & NCPLX & 19 & 0 & 0 & 0 & 0 \\
\hline $10 / 89$ & NCPLX & 18 & 0 & 0 & 0 & 0 \\
\hline $11 / 89$ & NCPLX & 18 & 0 & 0 & 0 & 0 \\
\hline $12 / 89$ & NCPLX & 20 & 0 & 0 & 0 & 0 \\
\hline $1 / 90$ & NCPLX & 20 & 0 & 0 & 0 & 0 \\
\hline $2 / 90$ & NCPLX & 78 & 0 & 0 & 0 & 0 \\
\hline $3 / 90$ & NCPLX & 18 & 0 & 0 & 0 & 0 \\
\hline $4 / 90$ & NCPLX & 130 & 0 & 0 & 0 & 0 \\
\hline $5 / 90$ & NCPLX & 130 & 0 & 0 & 0 & 0 \\
\hline $6 / 90$ & DN & 130 & 0 & 0 & 0 & 0 \\
\hline $7 / 90$ & DN & 130 & 0 & 0 & 0 & 0 \\
\hline $8 / 90$ & DN & 130 & 0 & 0 & 0 & 0 \\
\hline $9 / 90$ & DN & 130 & 0 & 0 & 0 & 0 \\
\hline $10 / 90$ & DN & 129 & 0 & 0 & 0 & 0 \\
\hline $11 / 90$ & DN & 129 & 0 & 0 & 0 & 0 \\
\hline $12 / 90$ & DN & 128 & 0 & 0 & 0 & 0 \\
\hline & & & & & & \\
\hline
\end{tabular}

Data obtained from Tank Farm Surveillance and Waste Status Summary Report, WHC-EP-0182. 
TANK LEVEL HISTORY AND WASTE TYPES

241-AP-108

\begin{tabular}{|c|c|c|c|c|c|c|}
\hline Date & & Total Waste & DSS & Sludge & Salt & Total solids \\
\hline$(\mathrm{M} / \mathrm{Yr})$ & Waste Type & (K Gal) & (K Gal) & (K Gal) & (K Gal) & (K Gal) \\
\hline $1 / 91$ & DN & 128 & 0 & 0 & 0 & 0 \\
\hline $2 / 91$ & DN & 136 & 0 & 0 & 0 & 0 \\
\hline $3 / 91$ & DN & 136 & 0 & 0 & 0 & 0 \\
\hline $4 / 91$ & $\overline{\mathrm{DN}}$ & 136 & 0 & 0 & 0 & 0 \\
\hline $5 / 91$ & DN & 144 & 0 & 0 & 0 & 0 \\
\hline $6 / 91$ & DN & 168 & 0 & 0 & 0 & 0 \\
\hline $7 / 91$ & DN & 172 & 0 & 0 & 0 & 0 \\
\hline $8 / 91$ & DN & 172 & 0 & 0 & 0 & 0 \\
\hline $9 / 91$ & DN & 175 & 0 & 0 & 0 & 0 \\
\hline $10 / 91$ & DN & 185 & 0 & 0 & 0 & 0 \\
\hline $11 / 91$ & DN & 870 & 0 & 0 & 0 & 0 \\
\hline $12 / 91$ & DN & 844 & 0 & 0 & 0 & 0 \\
\hline $1 / 92$ & DN & 893 & 0 & 0 & 0 & 0 \\
\hline $2 / 02$ & DN & 892 & 0 & 0 & 0 & 0 \\
\hline $3 / 92$ & DN & 892 & 0. & 0 & 0 & 0 \\
\hline $4 / 92$ & DN & 907 & 0 & 0 & 0 & 0 \\
\hline $5 / 92$ & DN & 904 & 0 & 0 & 0 & 0 \\
\hline $6 / 92$ & DN & 903 & 0 & 0 & 0 & 0 \\
\hline $7 / 92$ & DN & 903 & 0 & 0 & 0 & 0 \\
\hline $8 / 92$ & DN & 903 & 0 & 0 & 0 & 0 \\
\hline $9 / 92$ & DN & 903 & 0 & 0 & 0 & 0 \\
\hline $10 / 92$ & DN & 904 & 0 & 0 & 0 & 0 \\
\hline $11 / 92$ & DN & 904 & 0 & 0 & 0 & 0 \\
\hline $12 / 92$ & $\overline{D N}$ & 900 & 0 & 0 & 0 & 0 \\
\hline $1 / 93$ & DN & 900 & 0 & 0 & 0 & 0 \\
\hline $2 / 93$ & DN & 899 & 0 & 0 & 0 & 0 \\
\hline $3 / 93$ & DN & 900 & 0 & 0 & $Q$ & 0 \\
\hline $4 / 93$ & DN & 901 & 0 & 0 & 0 & 0 \\
\hline $5 / 93$ & DN & 901 & 0 & 0 & 0 & 0 \\
\hline $6 / 93$ & $\overline{D N}$ & 901 & 0 & 0 & 0 & 0 \\
\hline $7 / 93$ & DN & 902 & 0 & 0 & 0 & 0 \\
\hline $8 / 93$ & DN & 902 & 0 & 0 & 0 & 0 \\
\hline $9 / 93$ & DN & 902 & 0 & 0 & 0 & 0 \\
\hline $10 / 93$ & DN & 902 & 0 & 0 & 0 & 0 \\
\hline $11 / 93$ & DN & 902 & 0 & 0 & 0 & 0 \\
\hline $12 / 93$ & DN & 899 & 0 & 0 & 0 & 0 \\
\hline $1 / 94$ & DN & 898 & 0 & 0 & 0 & 0 \\
\hline $2 / 94$ & DN & 1131 & 0 & 0 & 0 & 0 \\
\hline $3 / 94$ & DN & 1131 & 0 & 0 & 0 & 0 \\
\hline
\end{tabular}

Data obtained from Tank Farm Surveillance and Waste Status Summary Report, WHC-EP-0182. 
PRIMARY ADDITIONS

AP TANKS

\begin{tabular}{|l|l|r|r|r|r|}
\hline Tank & Waste & Total (Kgal) & \# Trans & Start Date & End Date \\
\hline AP-101 & CWIZR2 & 1 & 1 & 1987.5 & 1987.5 \\
\cline { 2 - 6 } & UNK & 19 & 1 & 1987.5 & 1987.5 \\
\cline { 2 - 6 } & PASF & 1929 & 9 & 1988.5 & 1989.75 \\
\hline AP-102 & PASF & 5 & 2 & 1989.5 & 1989.75 \\
\cline { 2 - 6 } & UNK & 10 & 4 & 1991 & 1992.5 \\
\hline AP-103 & PASF & 1068 & 7 & 1988 & 1988.5 \\
\hline AP-104 & N & 794 & 6 & 1987 & 1987.5 \\
\cline { 2 - 6 } & UNK & 4 & 2 & 1989.75 & 1992 \\
\hline AP-107 & PASF & 1115 & 3 & 1990 & 1990 \\
\cline { 2 - 6 } & UNK & 110 & 1 & 1990 & 1990 \\
\hline AP-108 & PASF & 92 & 1 & 1990 & 1990 \\
\cline { 2 - 6 } & PL2 & 4 & 9 & 1991 & 1992.25 \\
\cline { 2 - 6 } & UNK & 232 & 2 & 1991.75 & 1992.75 \\
\cline { 2 - 6 } & SWLIQ & 1994 & 1994 \\
\hline
\end{tabular}

Derived from Waste Status and Transaction Record Summary(WSTRS) For The Southeast Quadrant, WHC-SD-WM-TI-689, Rev. O(Agnew, S.F., March 1995) 


\section{APPENDIX D}

\section{Temperature Graphs and Data}


THIS PAGE INTENTIONALIY

LEITT BLANK 


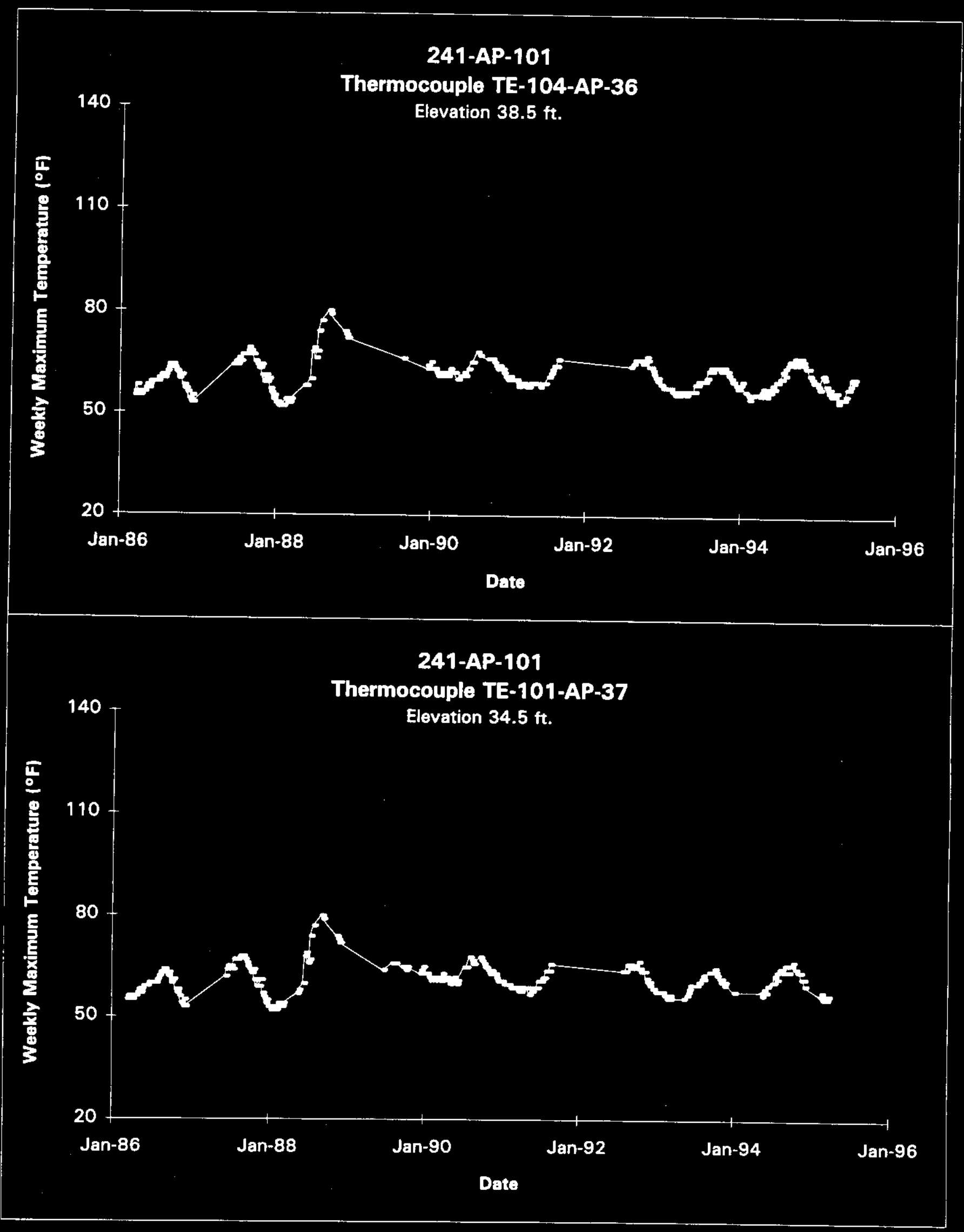




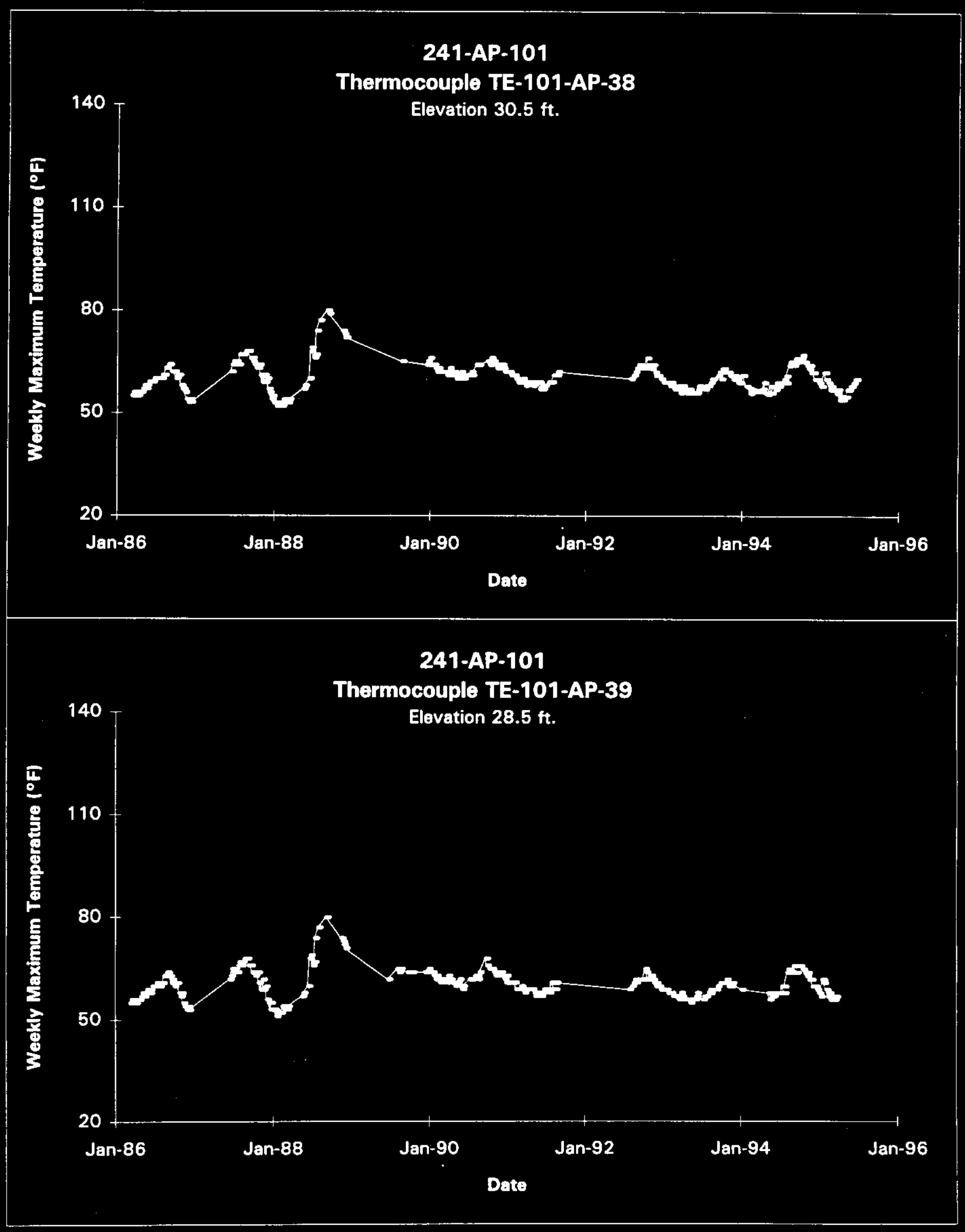




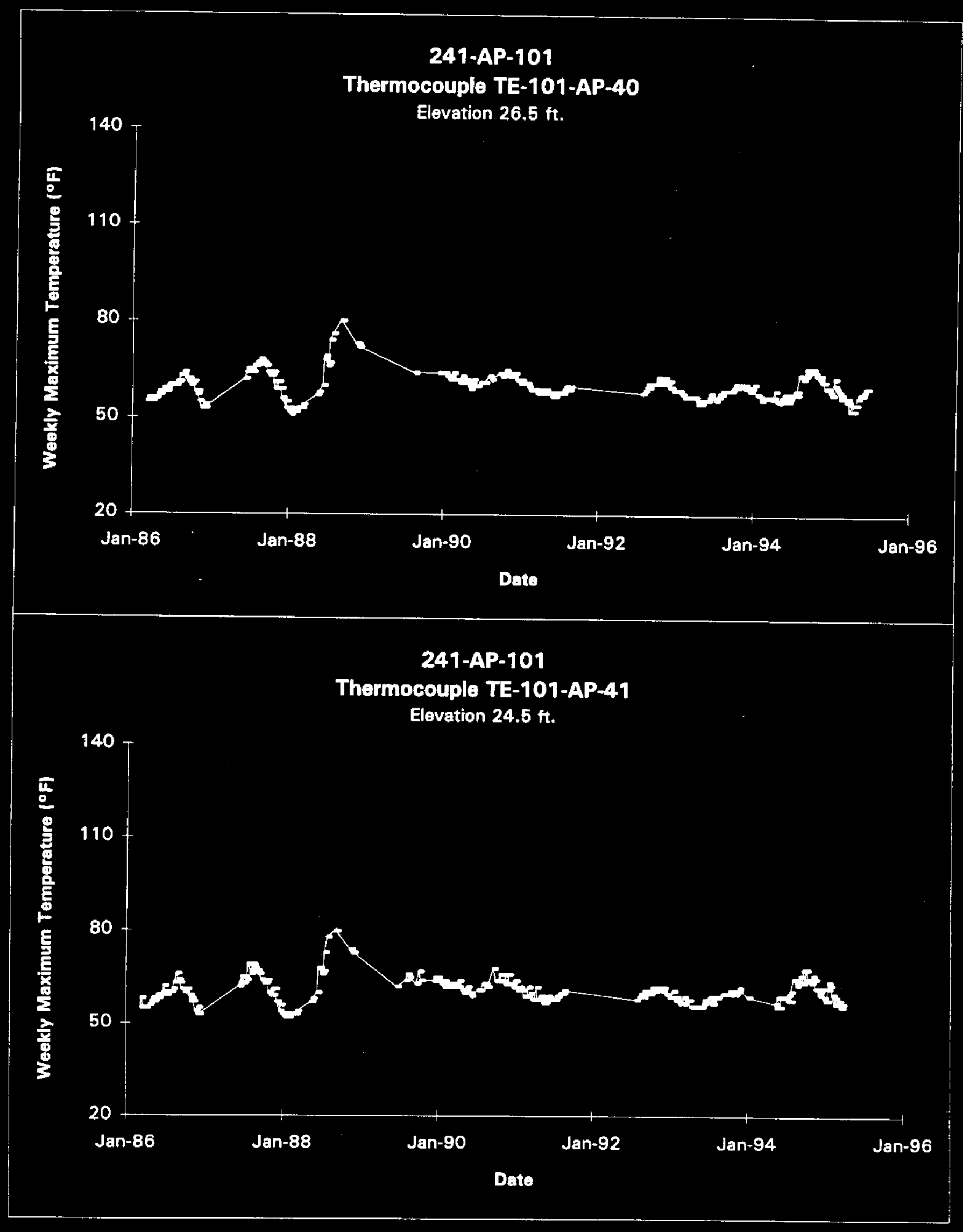

Date obtained from Computer Automated Surveillance Syatem (CASS), April 1995; and Surveillance Analytis Computer Syatem (SACS), June 1995. D-3 


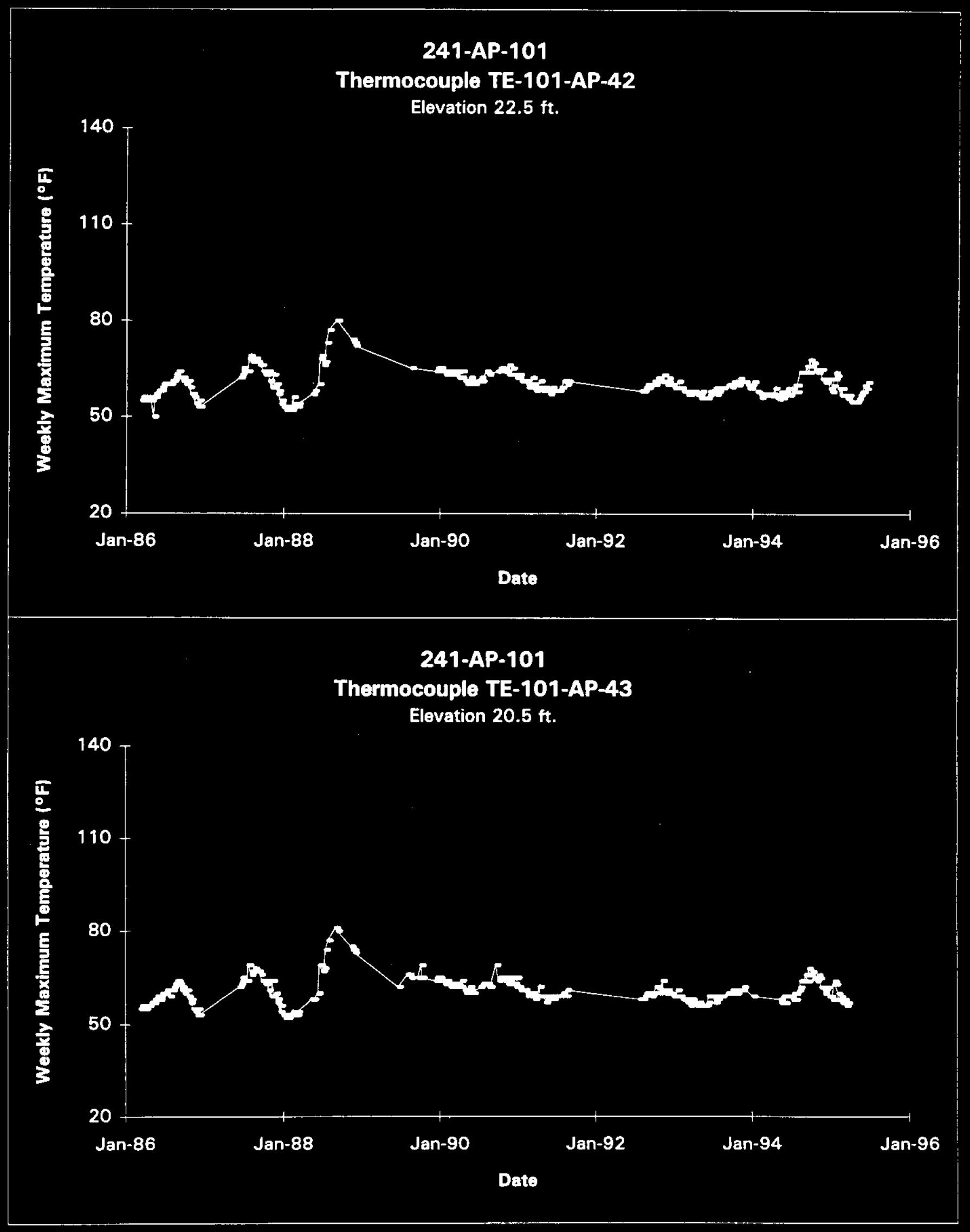

Data obtained from Computer Autometed Surveillence System (CASS), April 1995; and Surveillance Analysis Computer System (SACS). June 1995. 


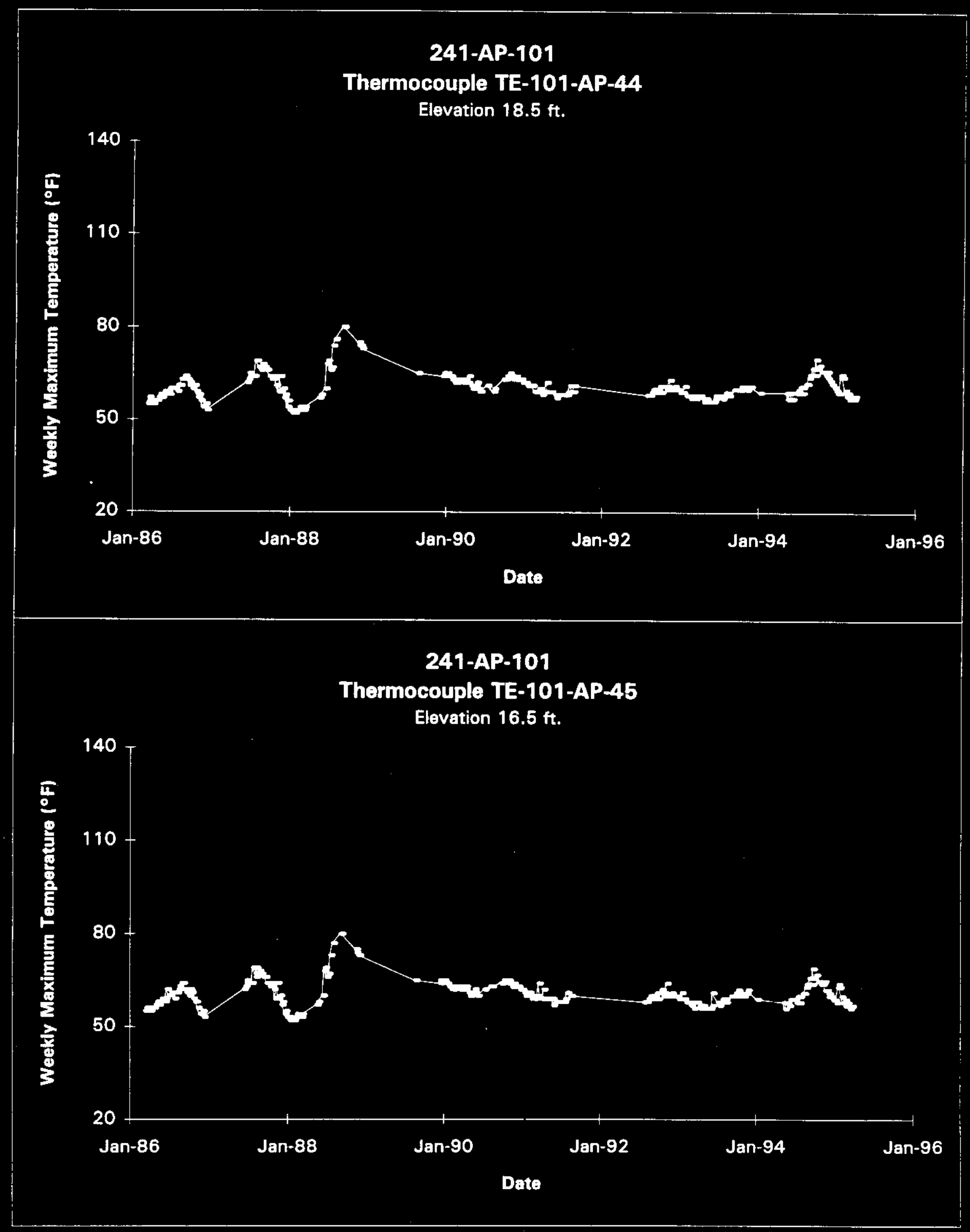




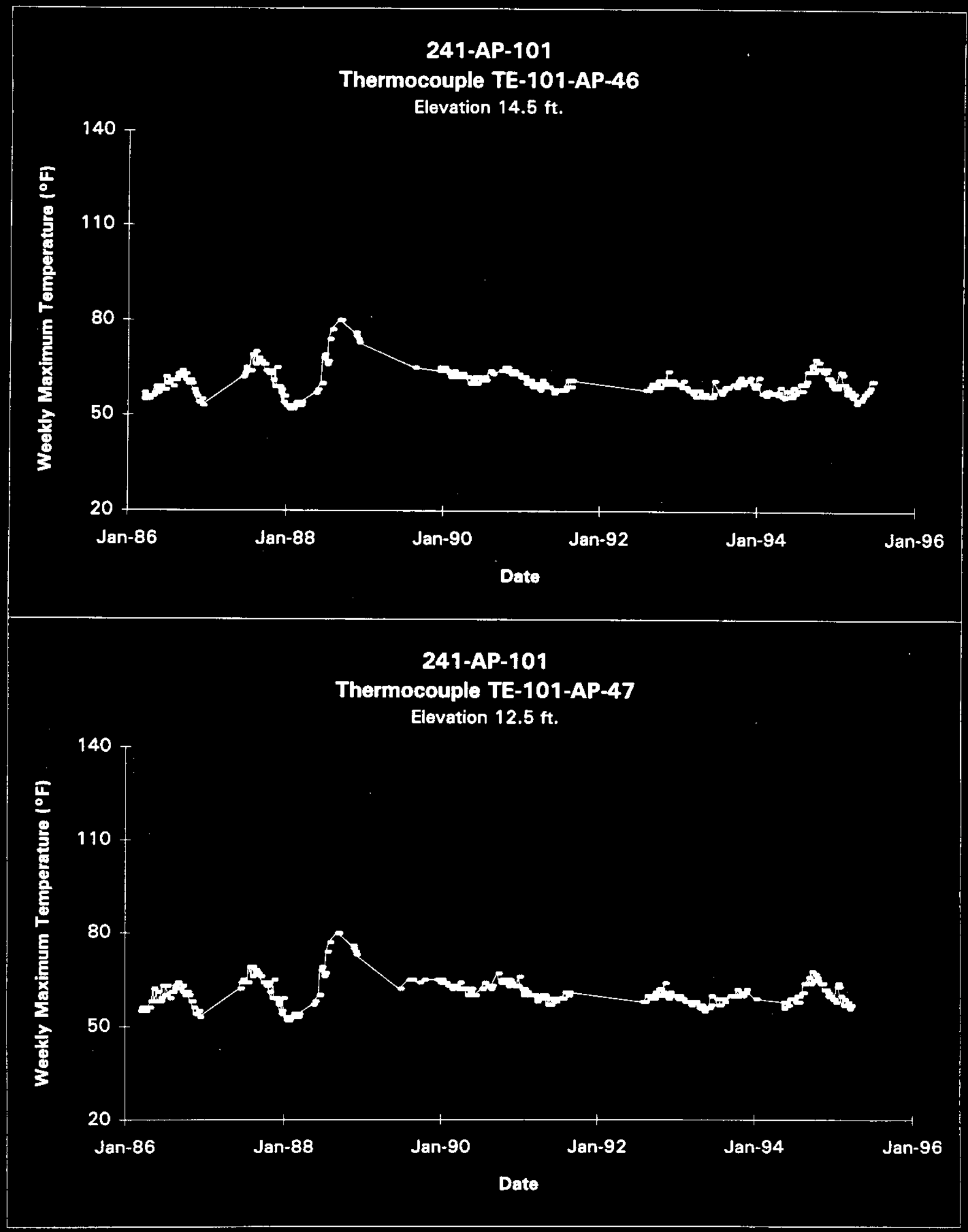

Data obtained from Computer Automated Surveillence Syatem (CASS), April 1995; and Surveillance Analysis Computer System (SACS), June 1995. D-6 


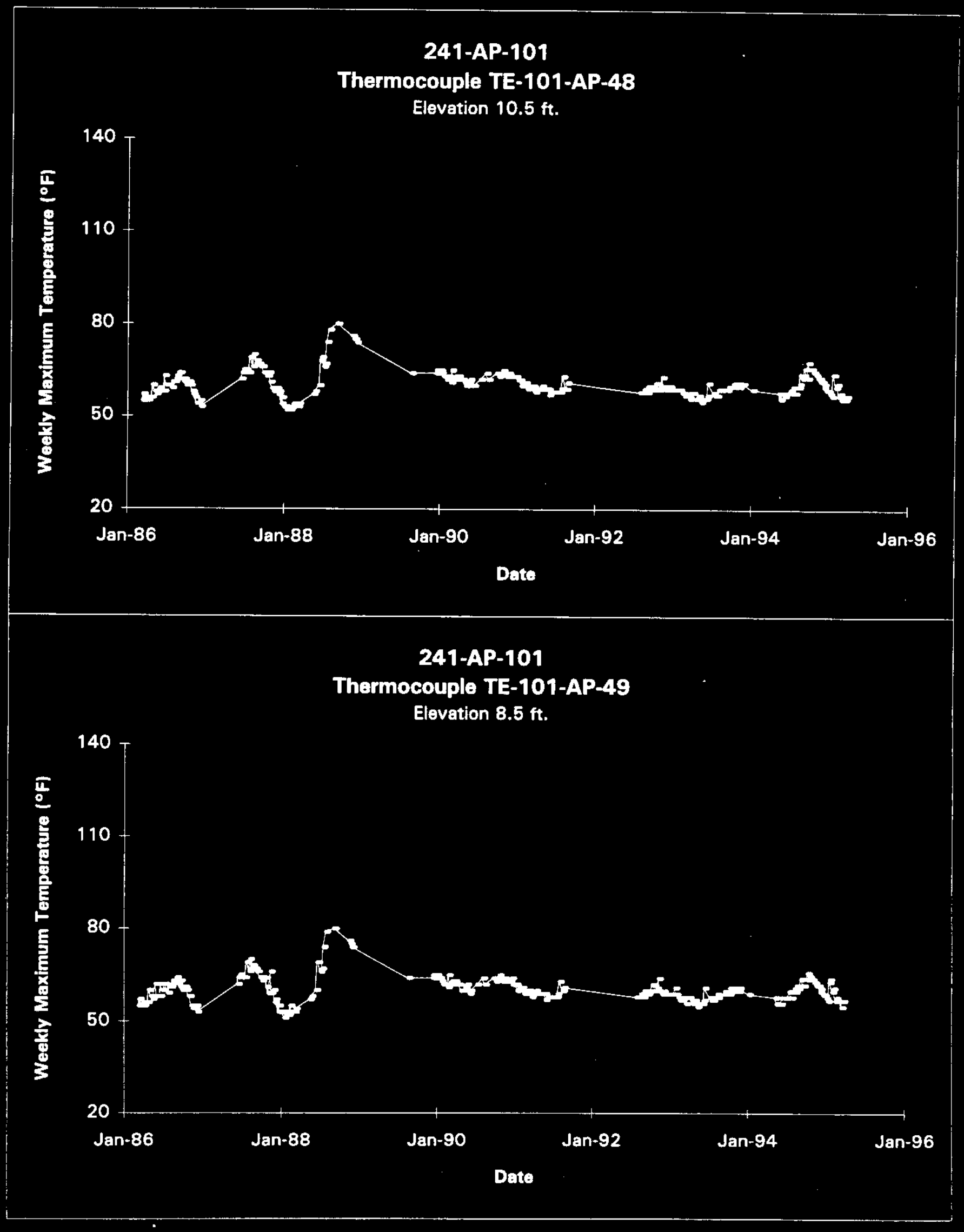

Data obtained from Computer Automatod Surveillance Syatem (CASS), April 1995; und Surveillance Analyais Computer System (SACS), June 1995. D-7 


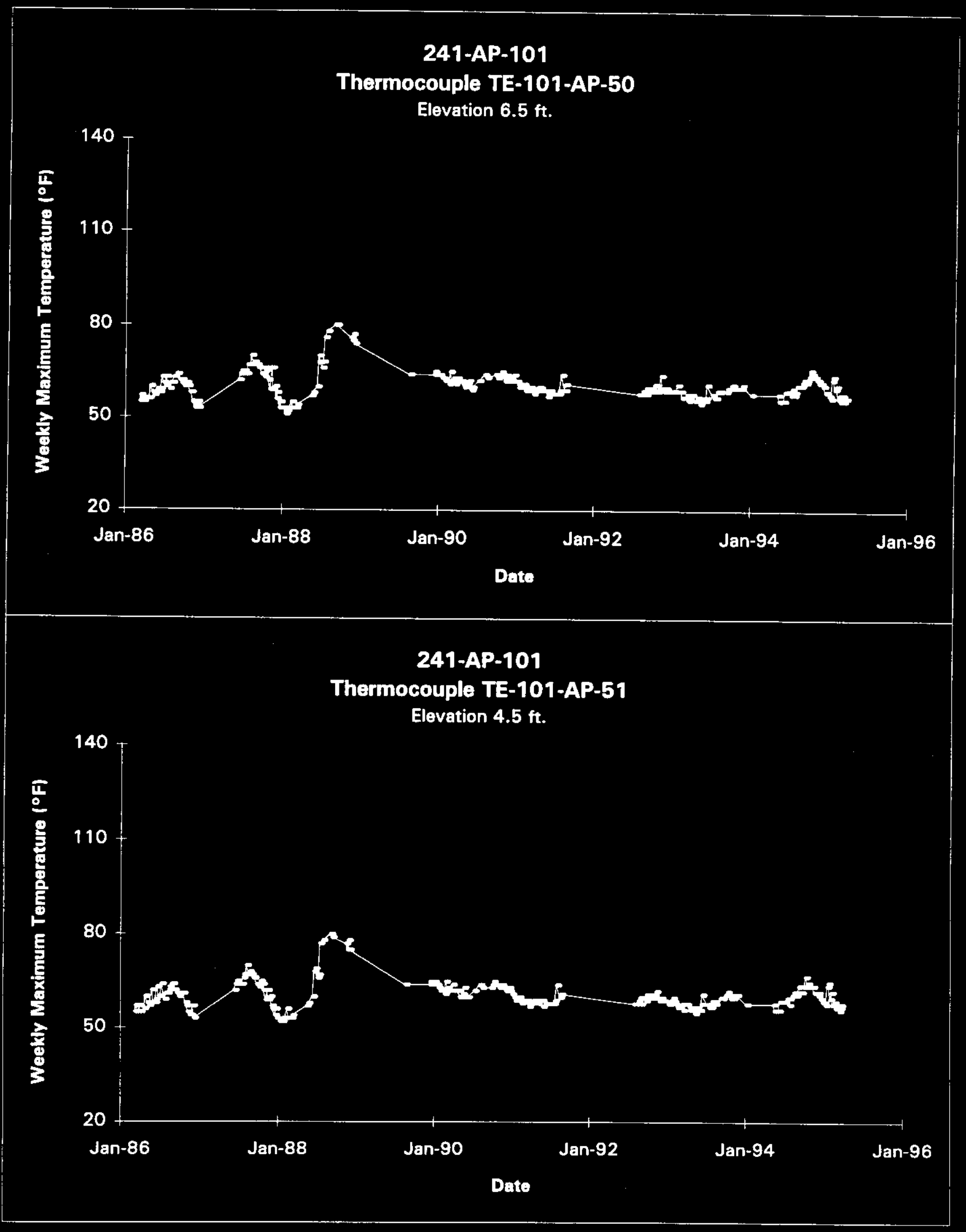

Data obtained from Computer Automated Surveillance Syatem (CASS), April 1995; and Surveillance Analyais Computer System (SACS), June 1995. D-8 


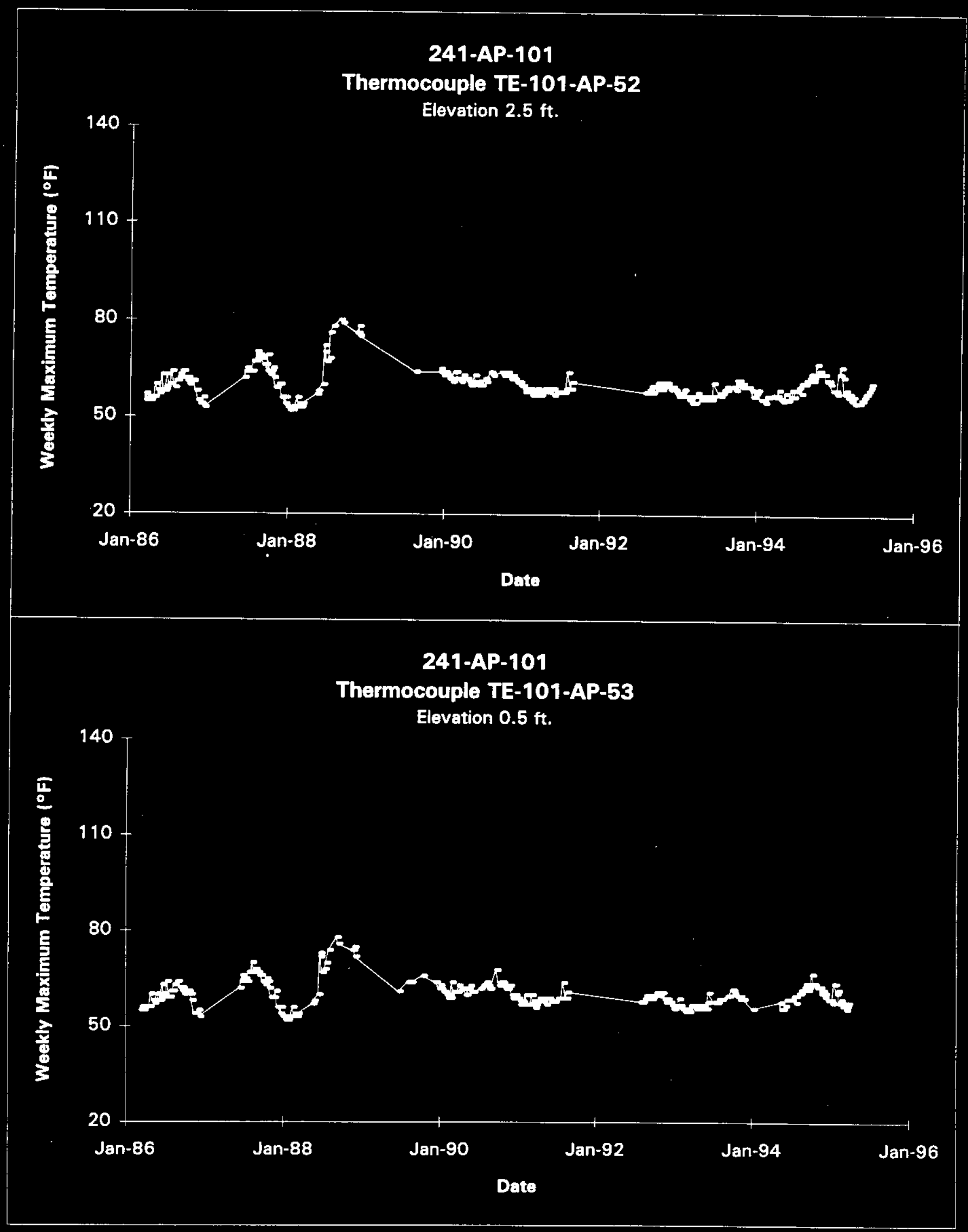

Date obtained from Computer Automated Surveillance System (CASS), April 1995; and Surveillance Analysis Computer Systom (SACS), June 1995. 


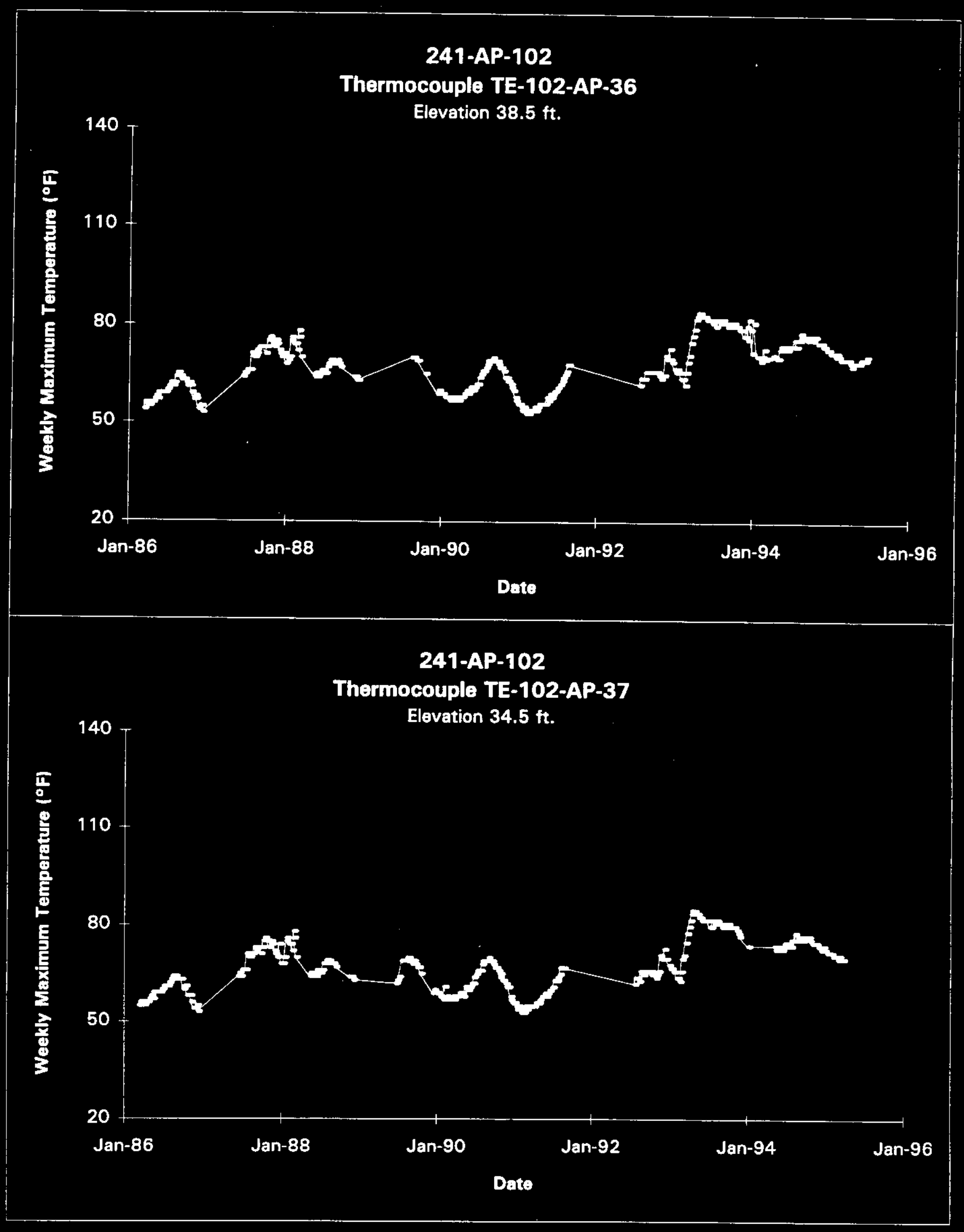

Data obtained from Computer Automated Surveillance Syatem (CASS), April 1995; and Surveillance Analyais Computer Syatem (SACS), June 1995. 


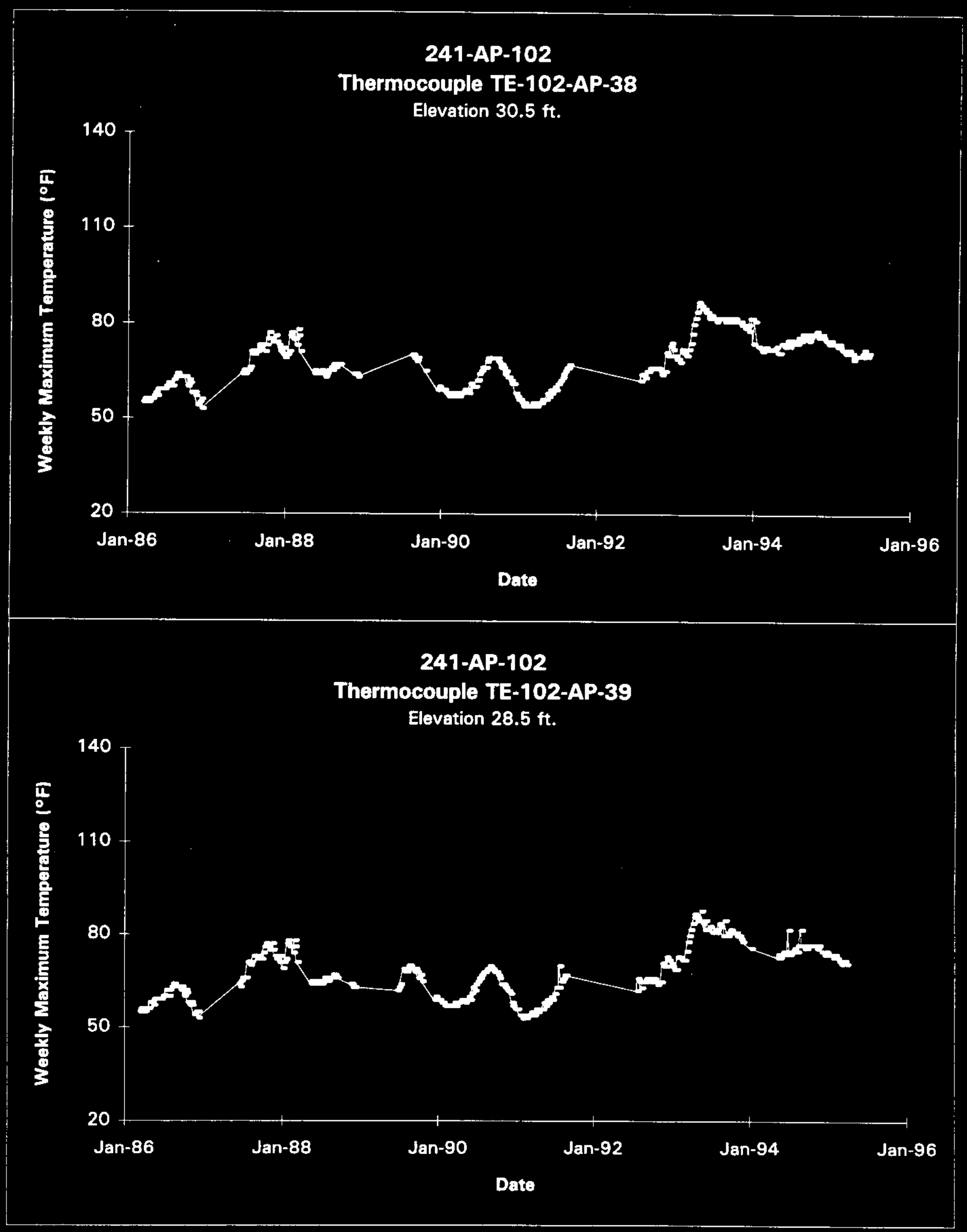

Data obtained from Computer Automated Surveillance Syatem (CASS), April 1995; and Surveillance Analysis Computer System (SACS), June 1995. 


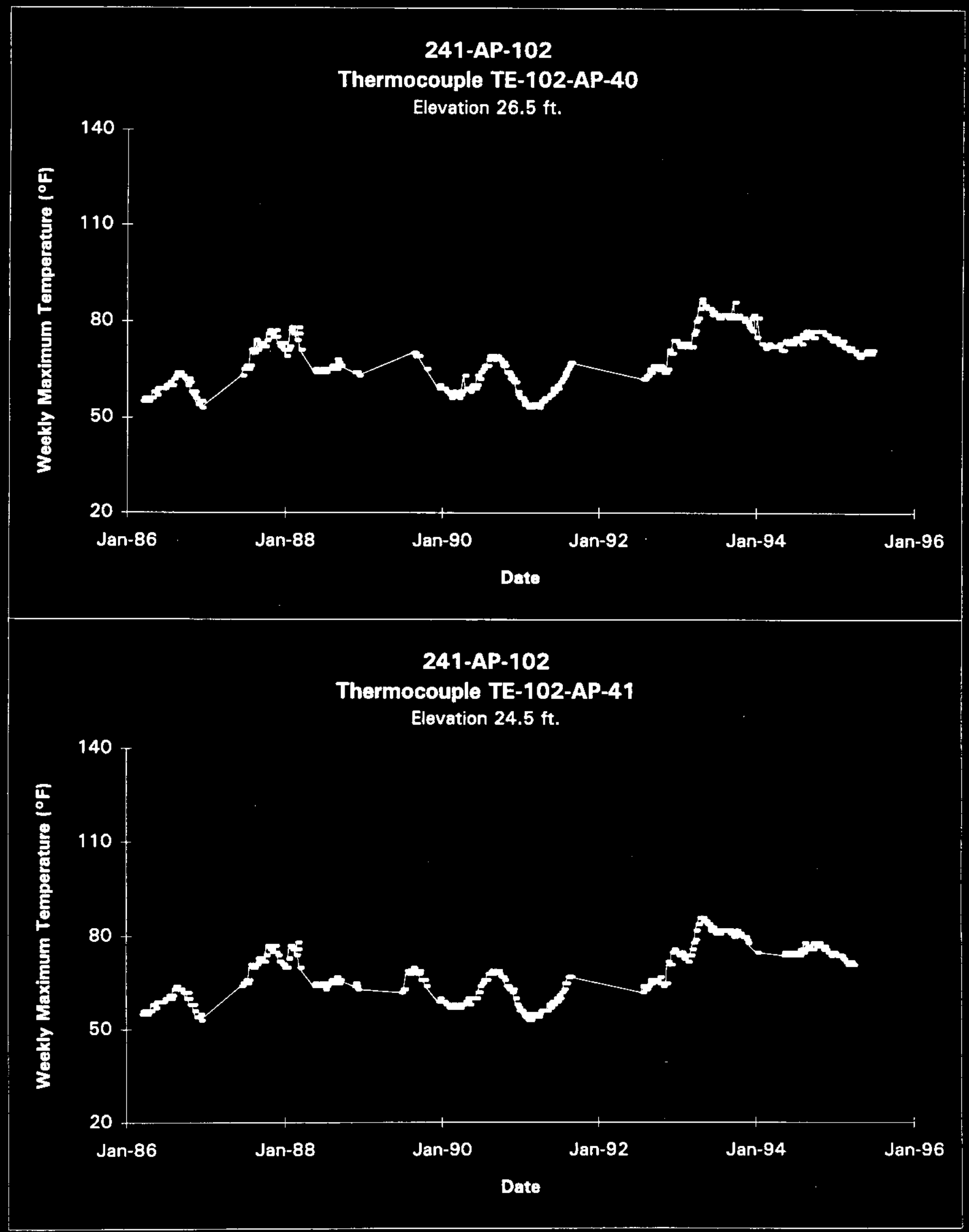

Data obtained from Computer Automated Surveillance Syatem (CASS), April 1995; and Survaillance Analysis Computer System (SACS), June 1995. D-12 


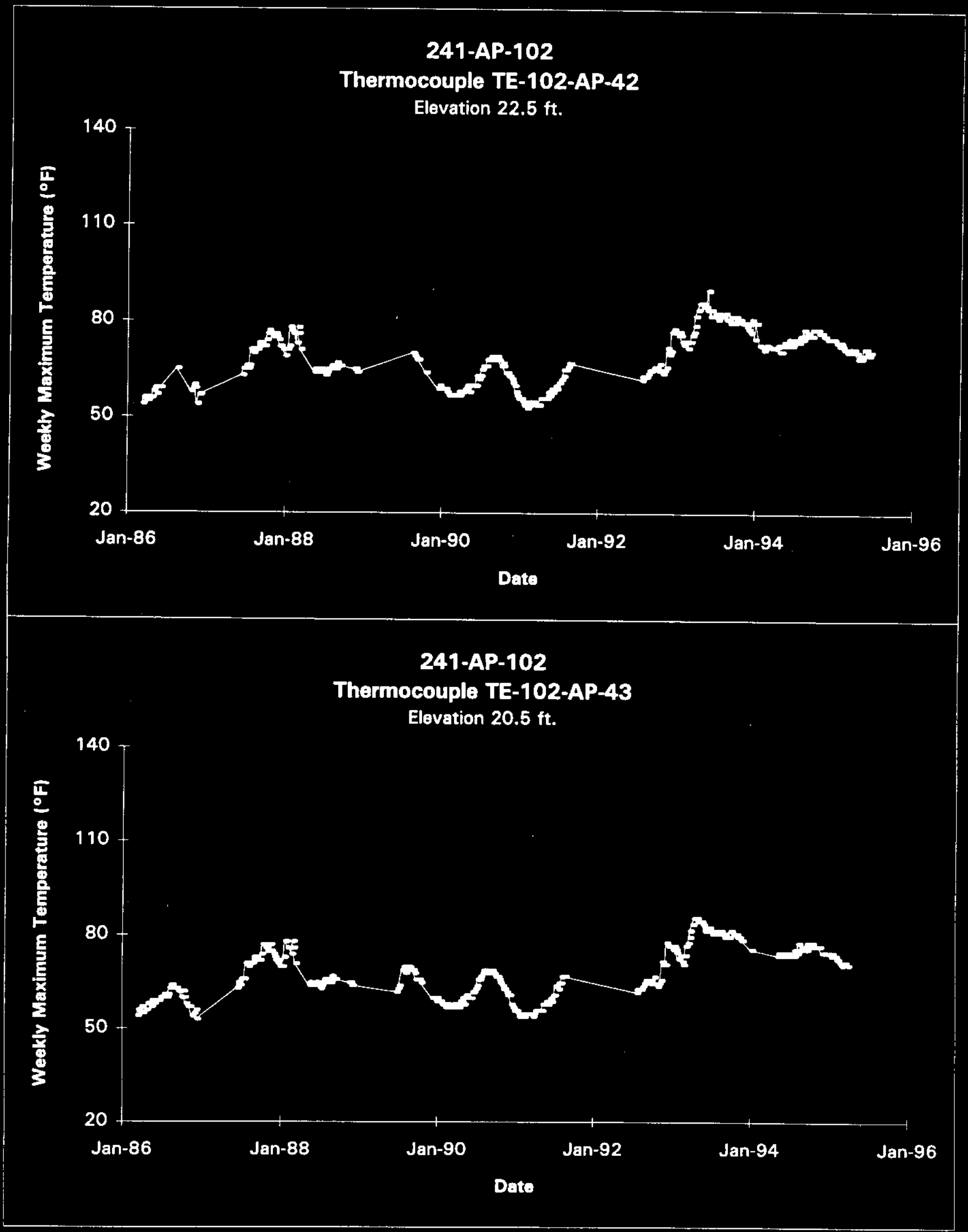




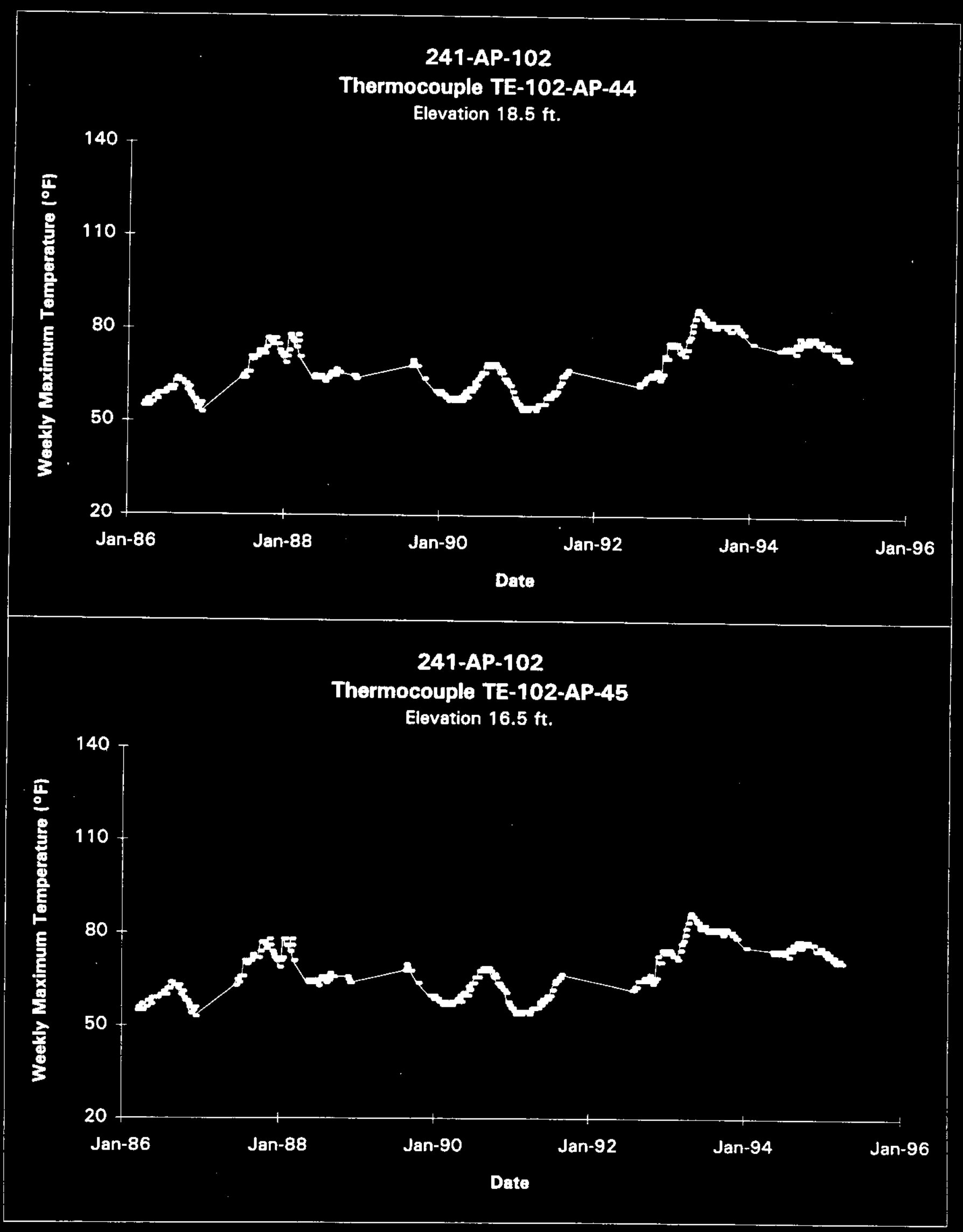

Data obteined from Computer Automated Survoillance System (CASS). April 1995; and Surveillance Analysis Computer System (SACS), June 1995. D-14 


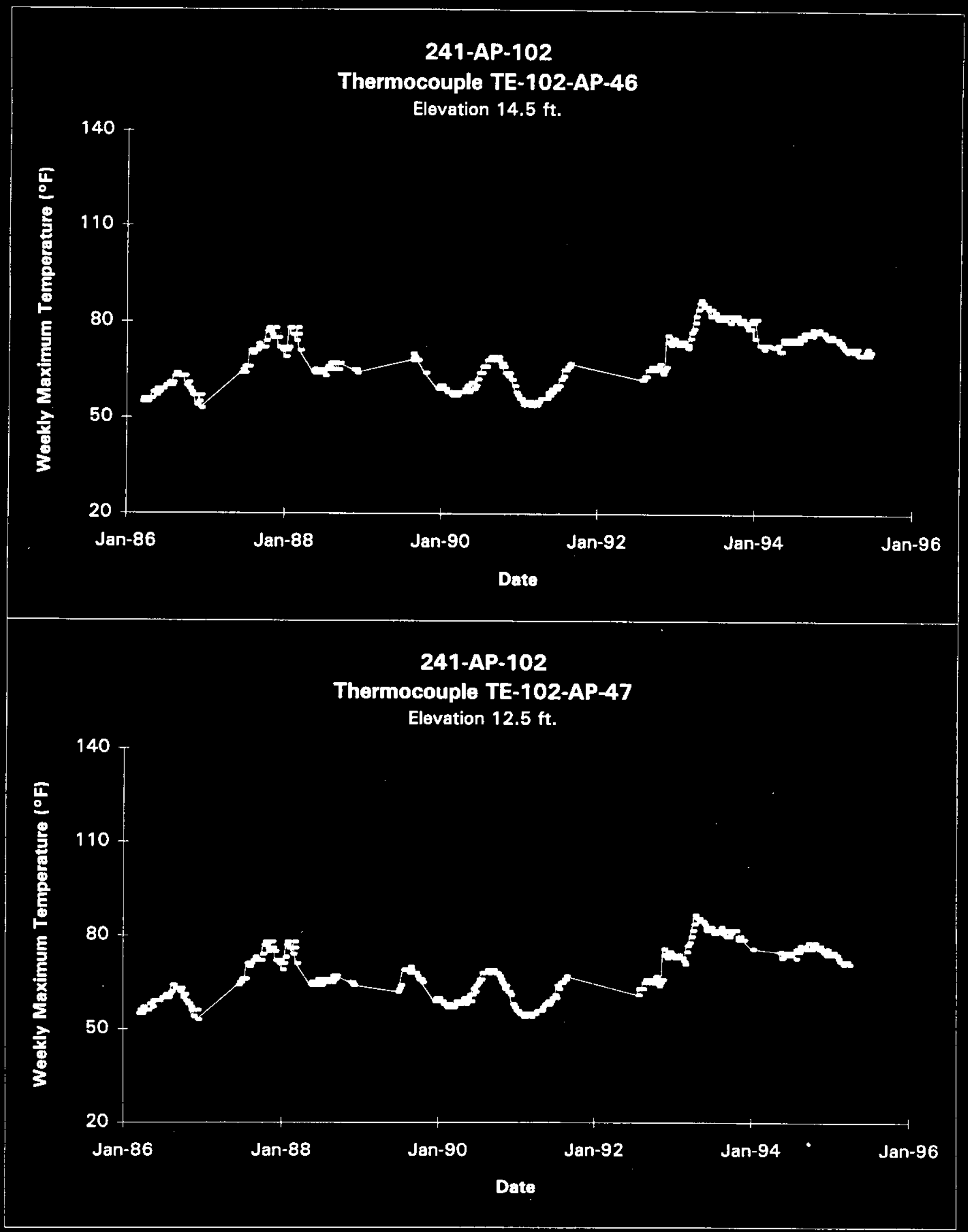




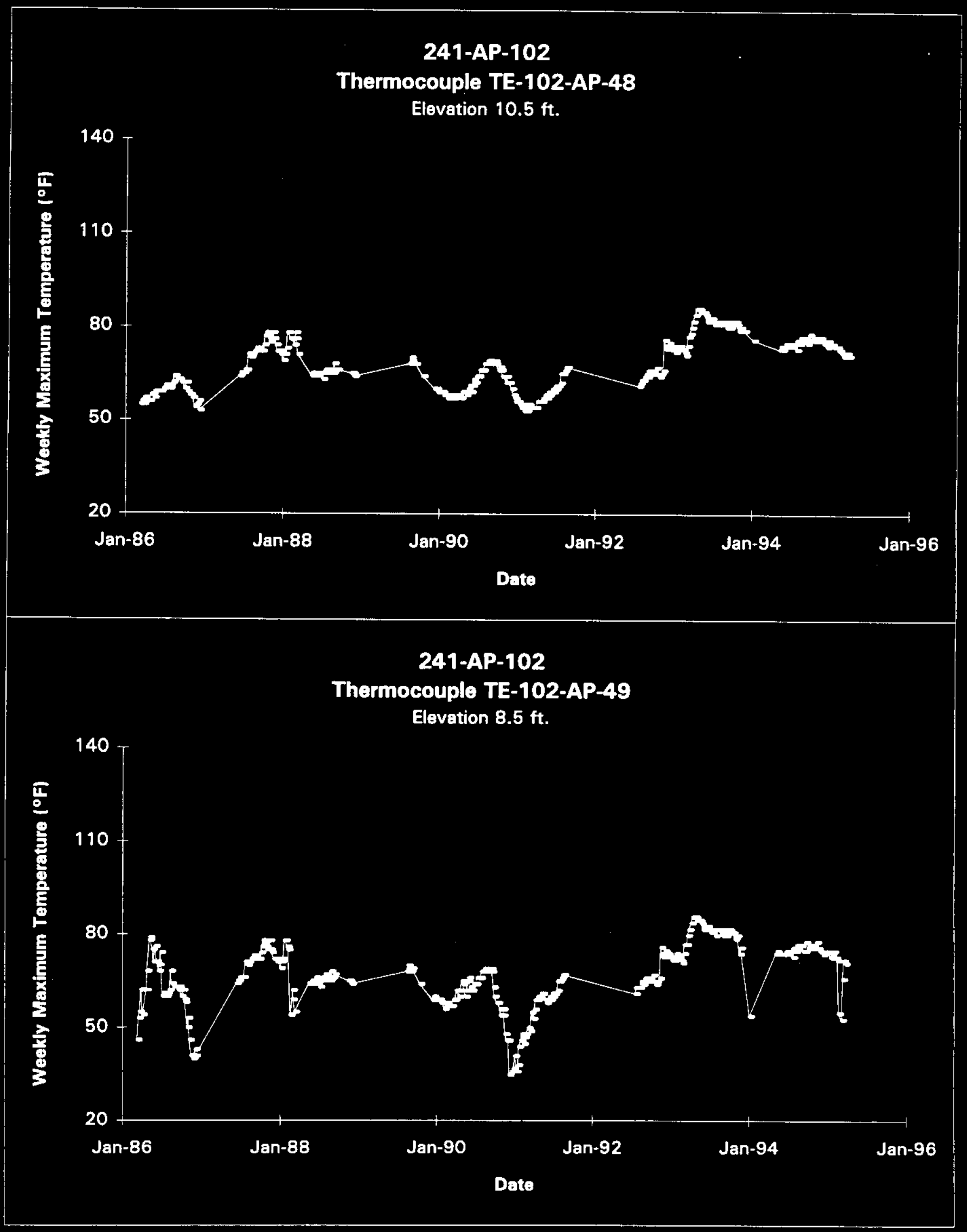

Data obtained from Computer Automated Survaillance System (CASS), April 1995; and Surveillance Analysis Computer System (SACS). June 1995. D-16 


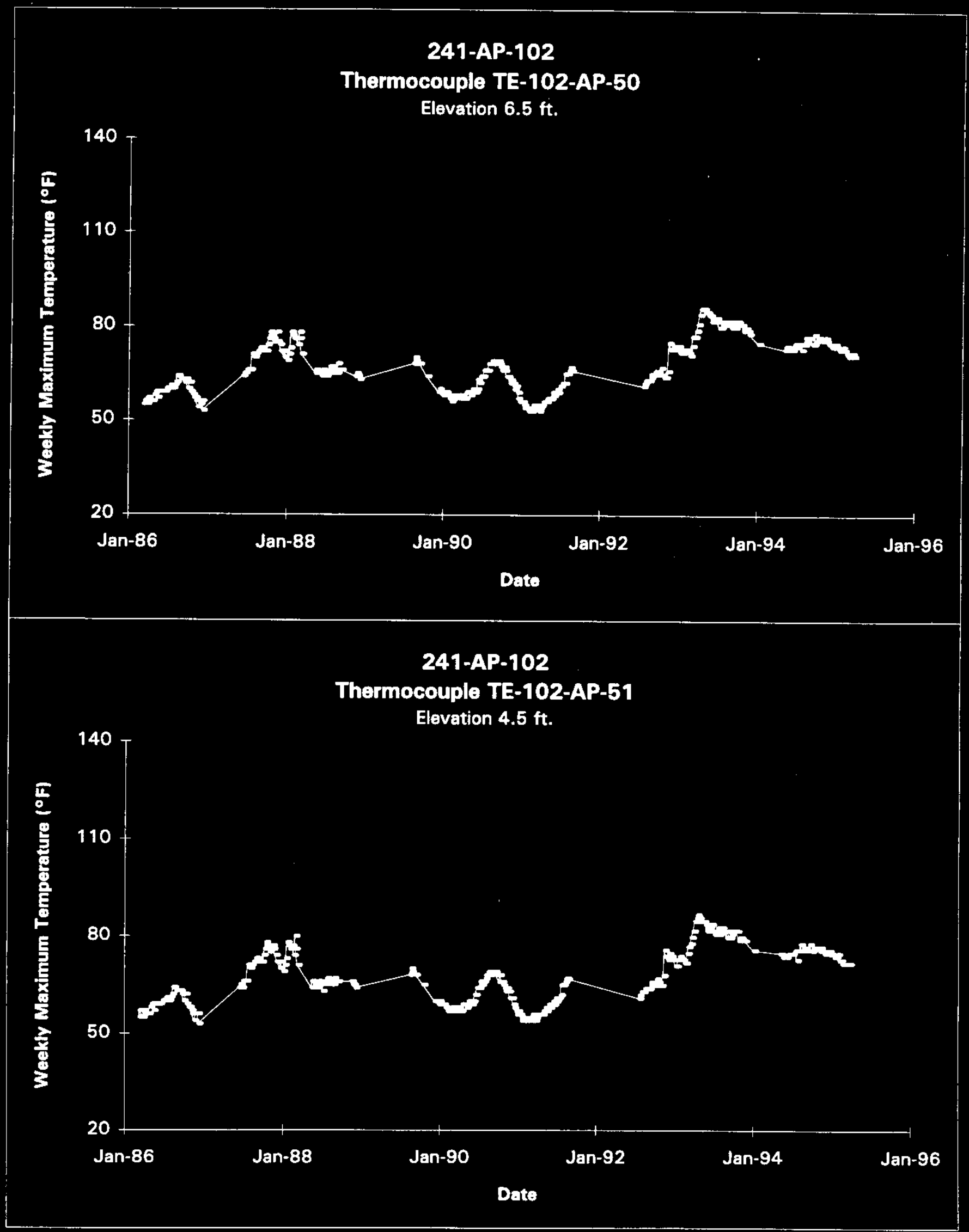

Data obtained from Computer Automated Surveillence Syatem (CASS), April 1995; and Survaillance Analyais Computer System (SACS), June 1995. $\mathrm{D}-17$ 


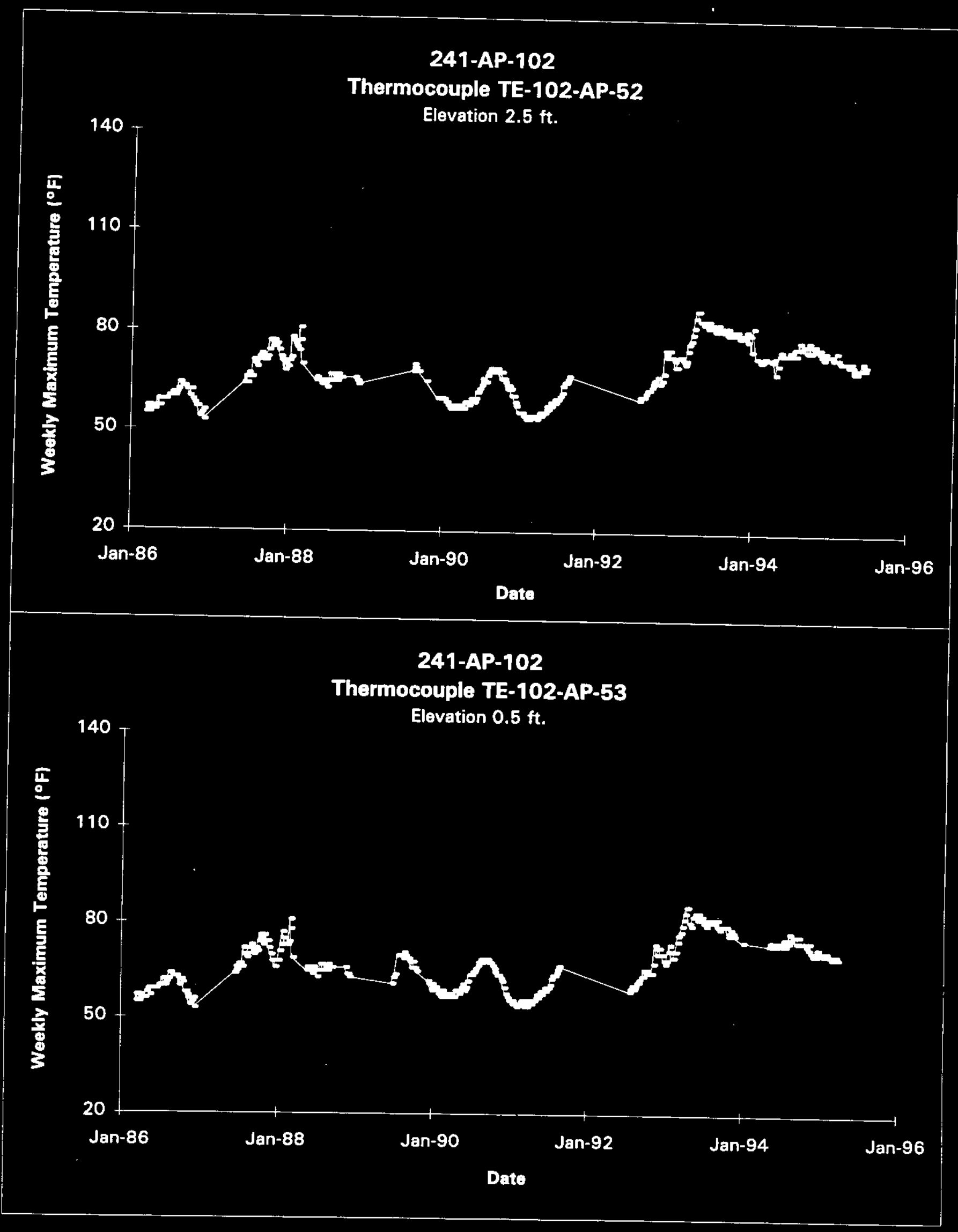




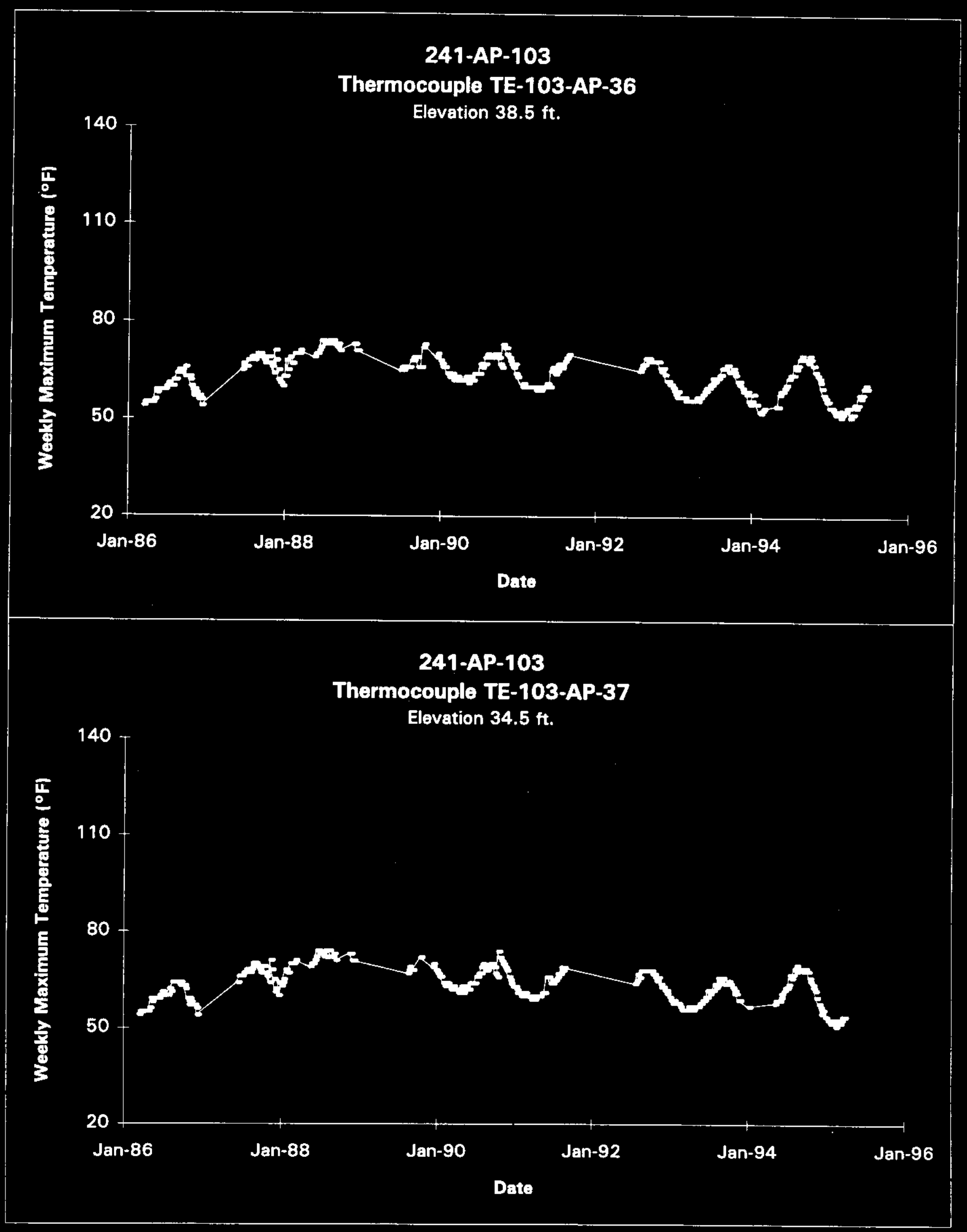

Data obtained from Computer Automated Surveillance System (CASS), April 1995; and Surveillance Analysis Computer System (SACS), June 1995. D-19 


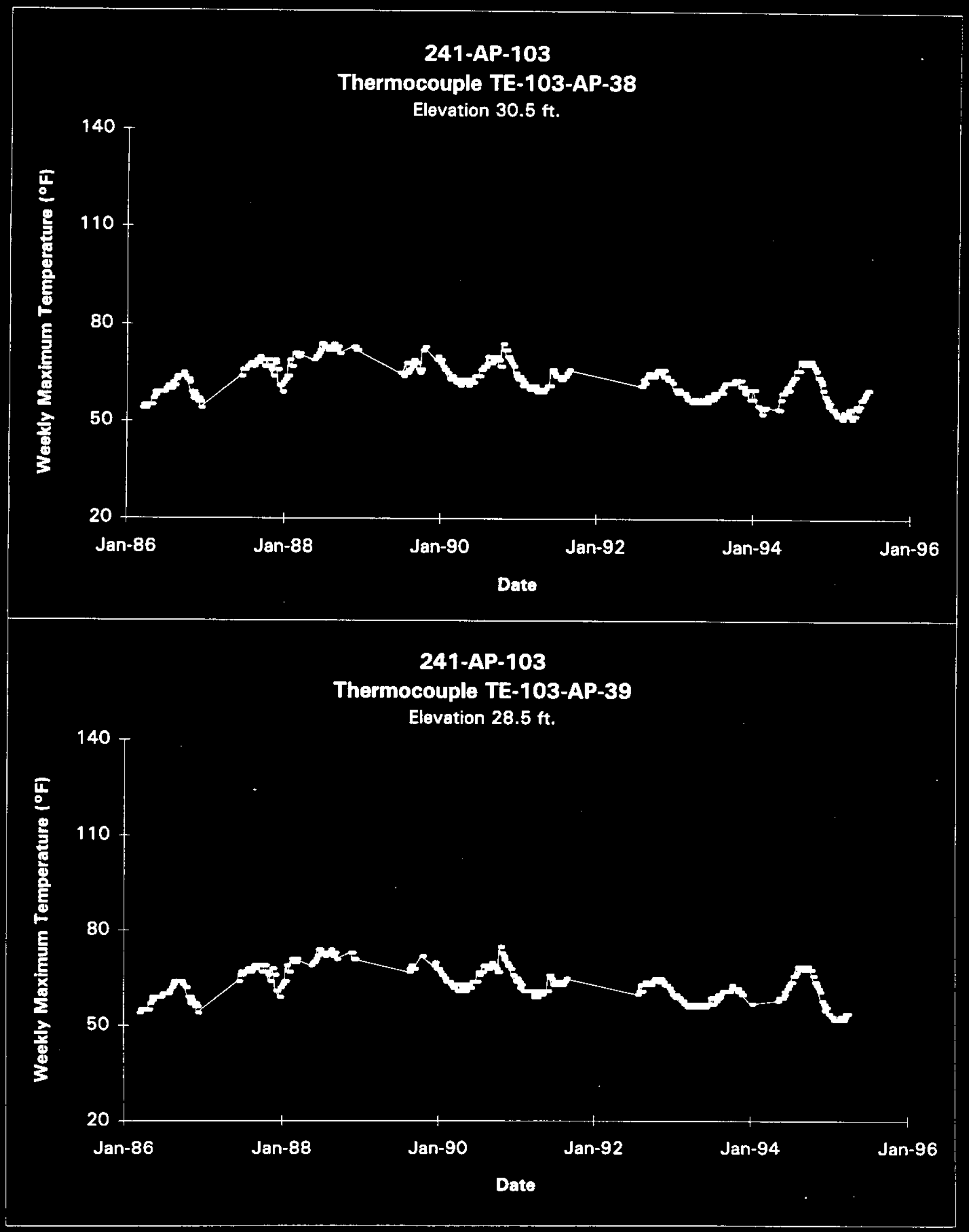

Data obtained from Computer Automated Survaillance Syatem (CASS), April 1995; end Surveillance Analysis Computer System (SACS), June 1995. 


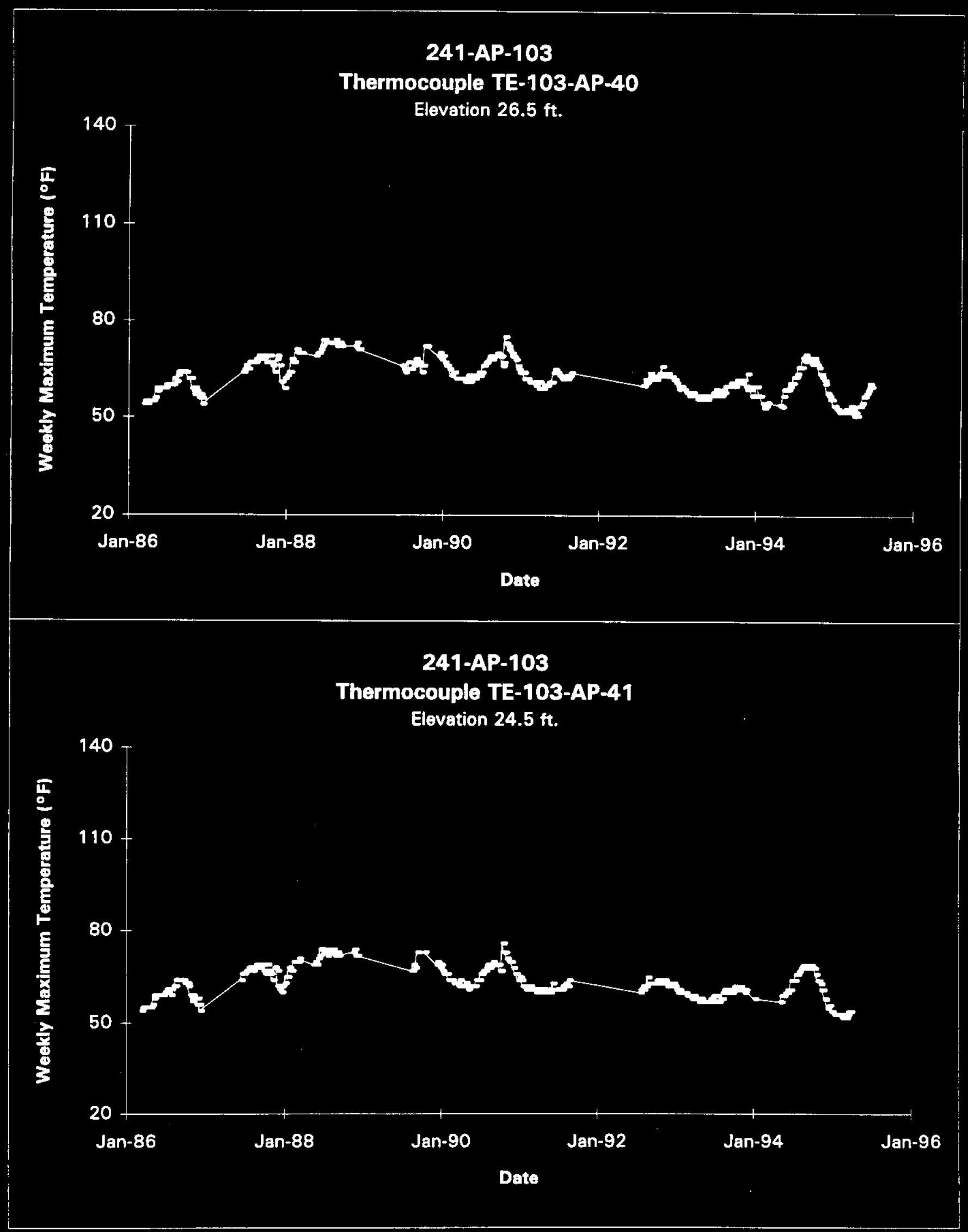




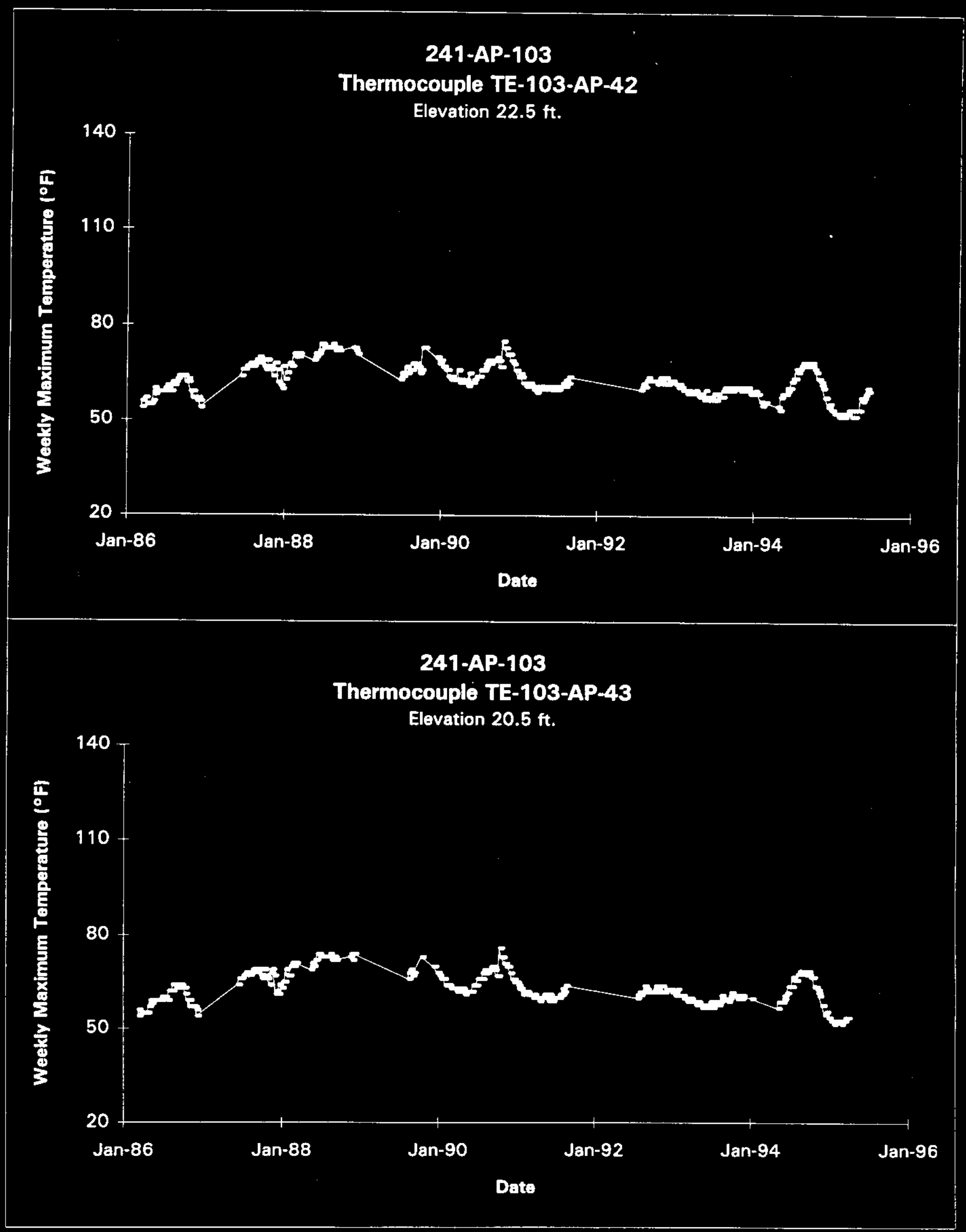

Data obtained from Computer Automated Surveillanee Syatem (CASS), April 1995; and Surveillance Analysis Computer System (SACS). June 1995. 


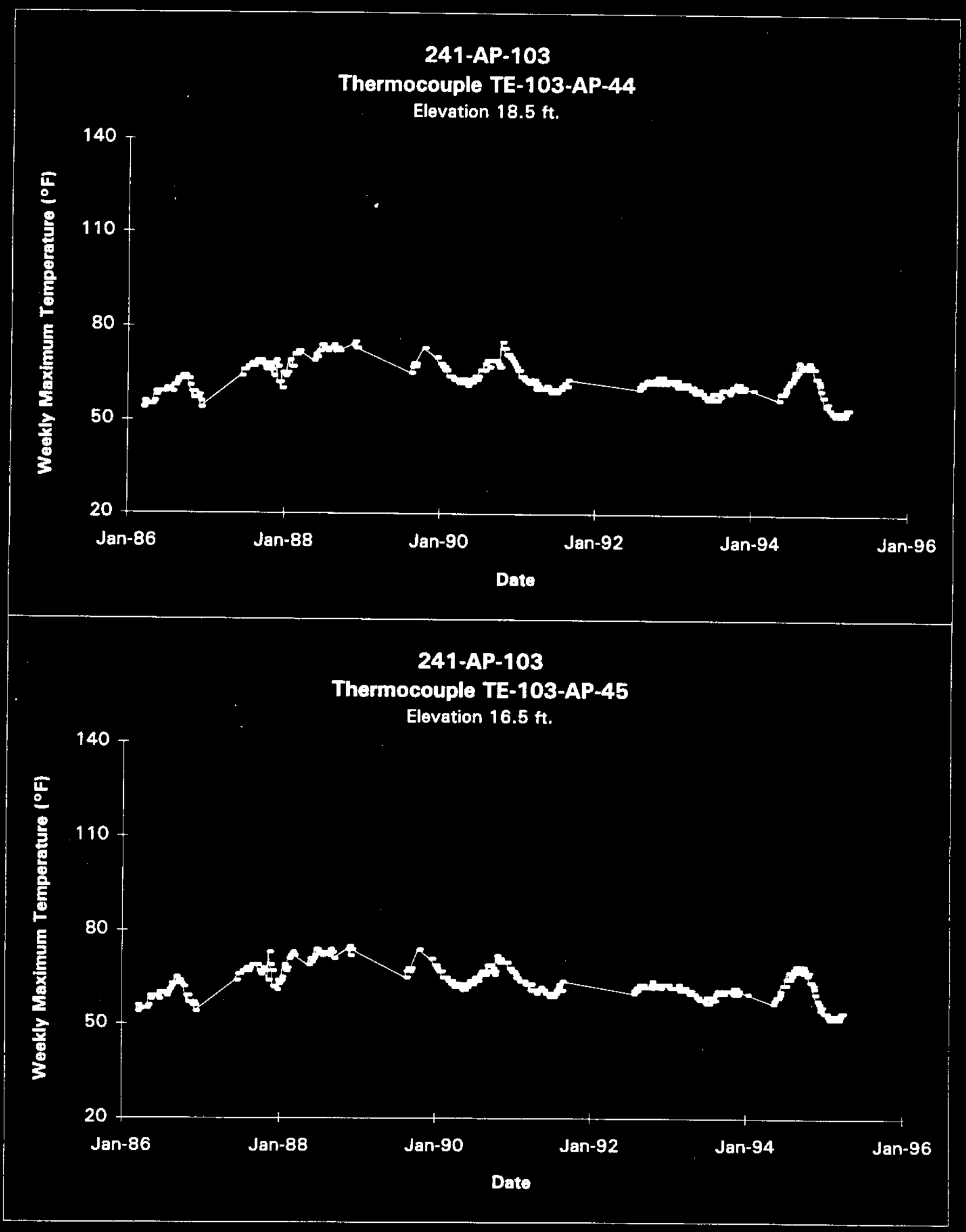




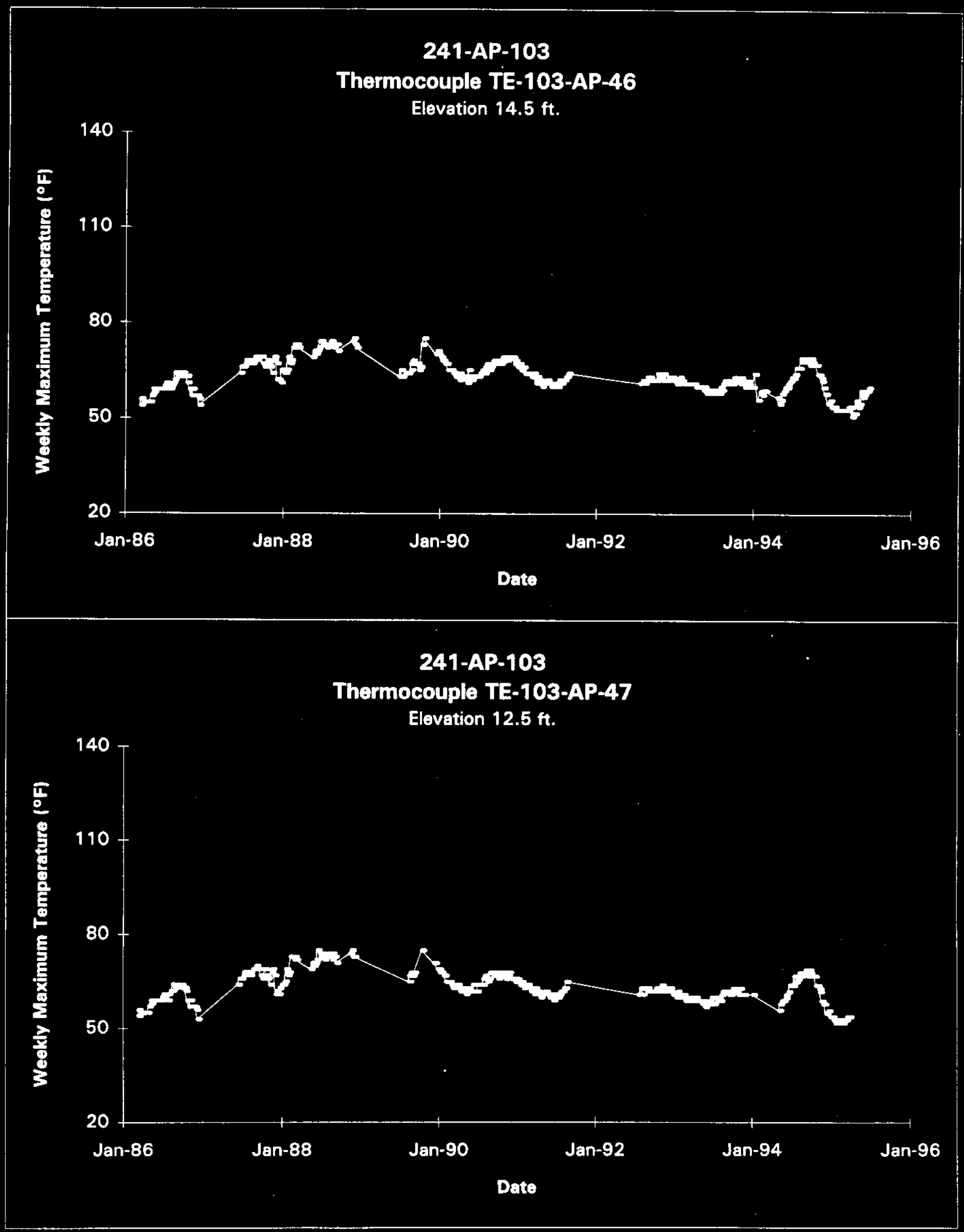

Data obtained from Computer Automated Surveillance Syatem (CASS), April 1995; and Surveillance Analysis Computer Syatem (SACS), June 1995. $\mathrm{D}-24$ 


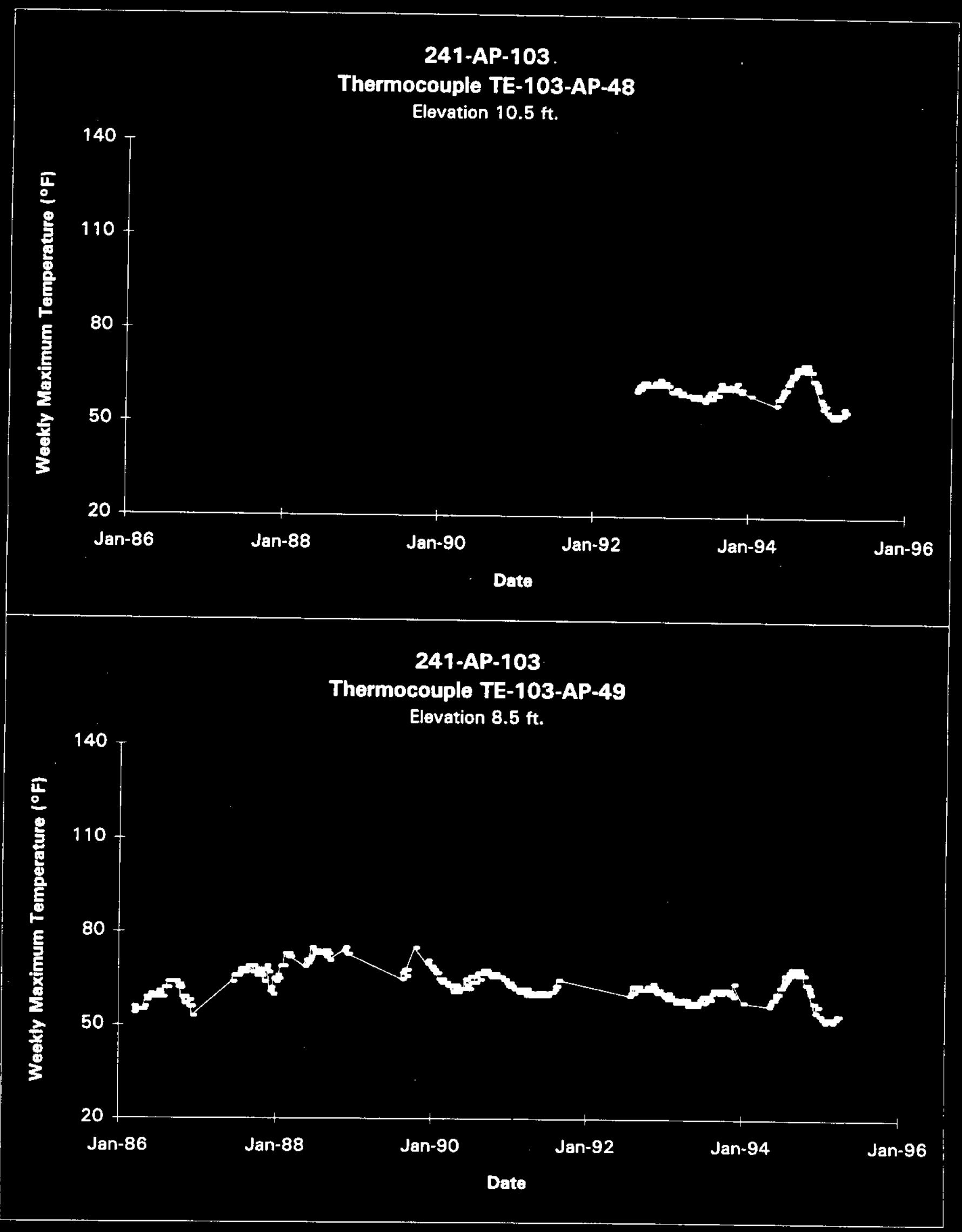

Data obtained from Computer Automated Surveillance Syatem (CASS), April 1995; and Survaillance Analysis Computer Syatem (SACS), June 1995. $D-25$ 


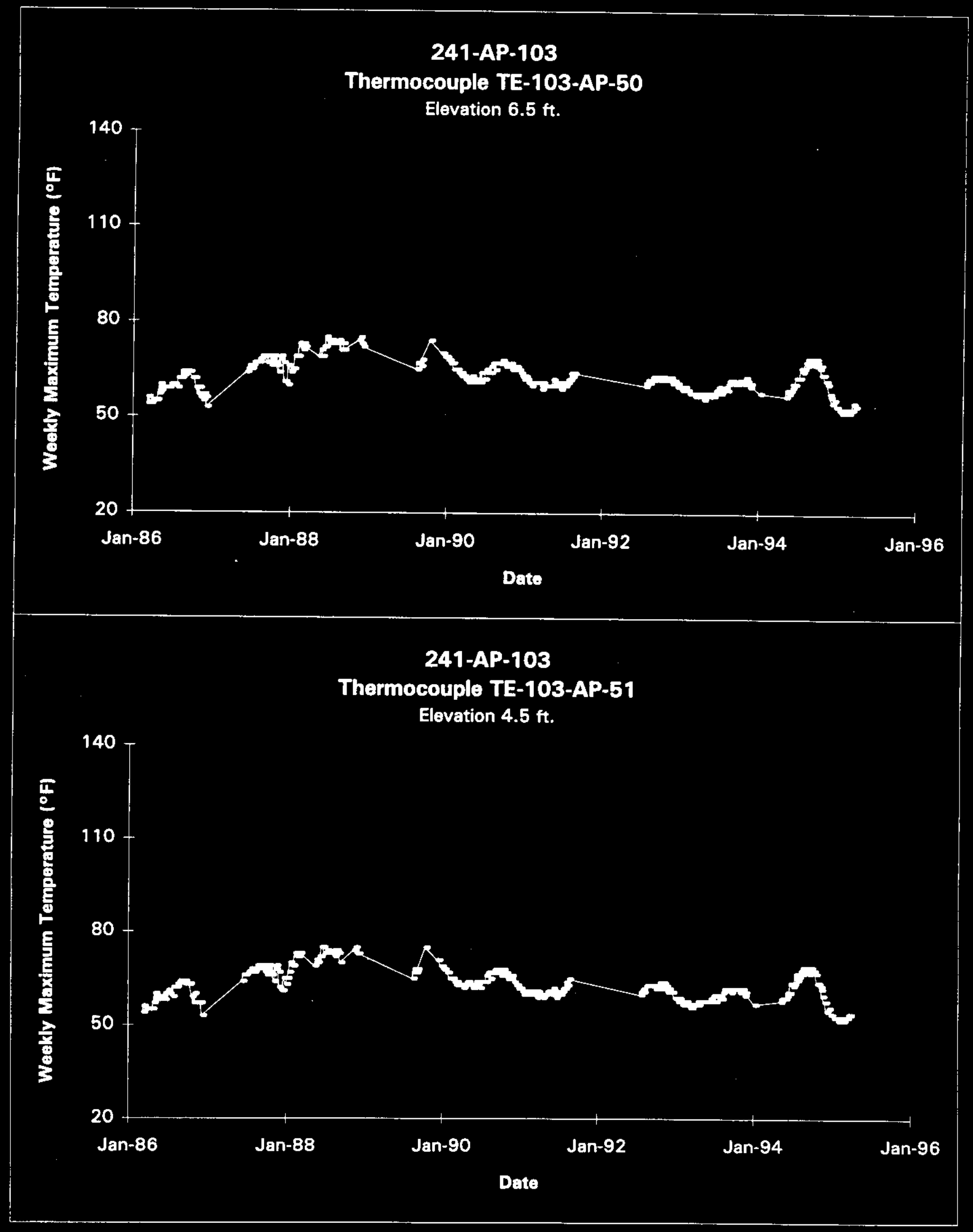

Data obtained from Computer Automated Surveillance Syatem (CASS), April 1995; and Surveillance Analyais Computer System (SACS), June 1995. D -26 


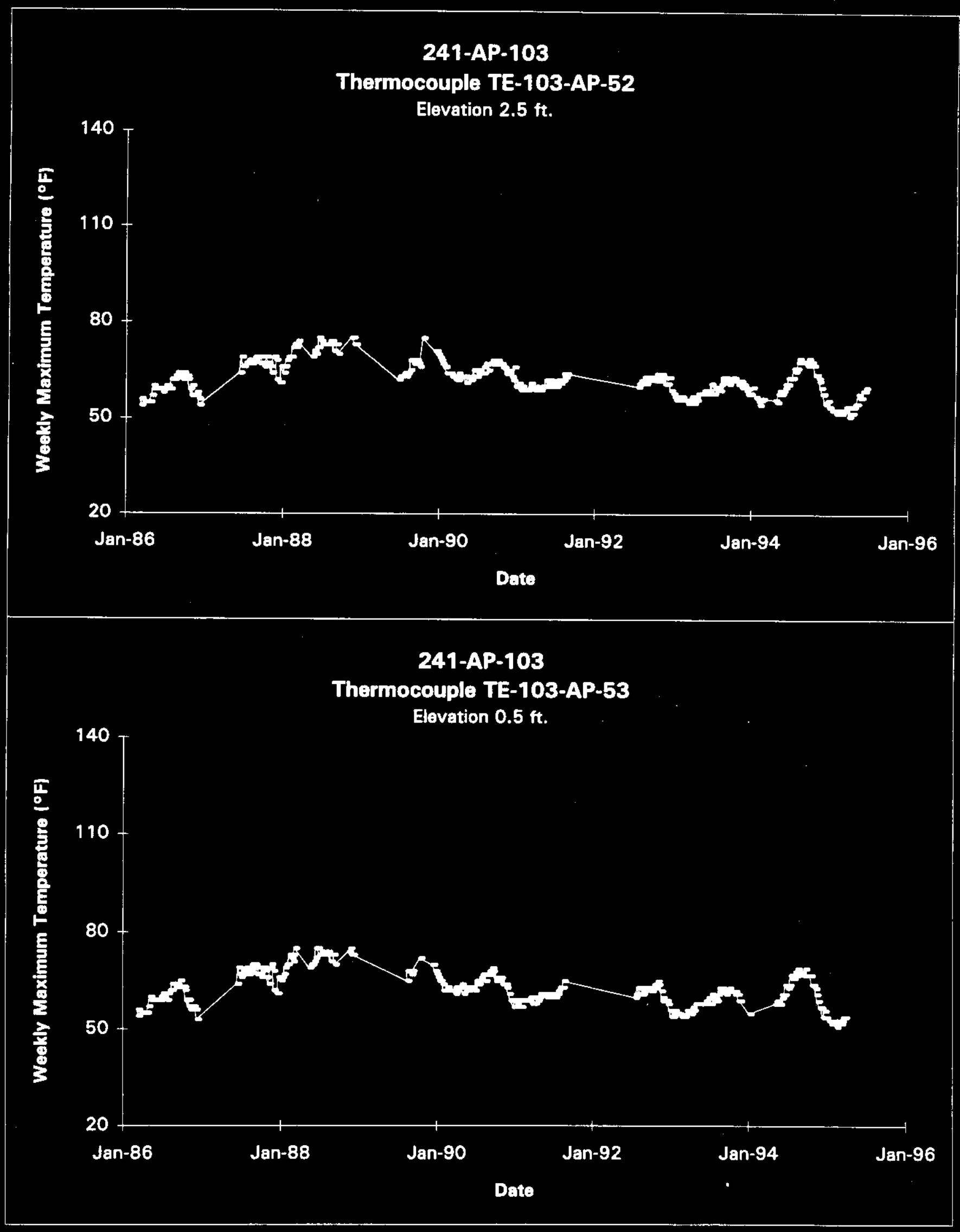

Date obtained from Computer Automated Sunveillance Syatem (CASS), April 1995; and Surveillence Analyais Computer Syatem (SACS), June 1995. 


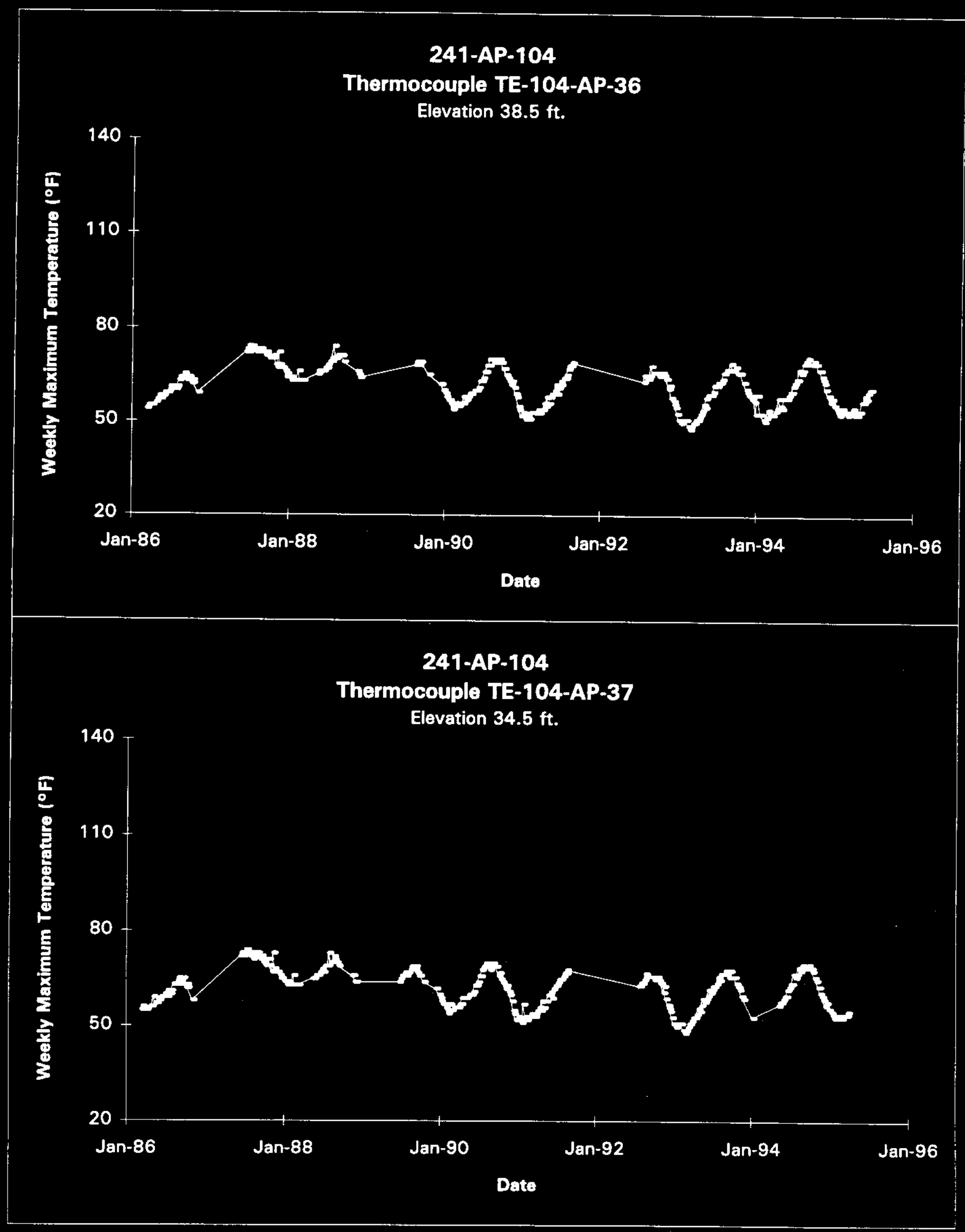

Data obtained from Computer Automated Survaillance Syatem (CASS), April 1995; and Surveillance Analyais Computer System (SACS), June 1995. D-28 


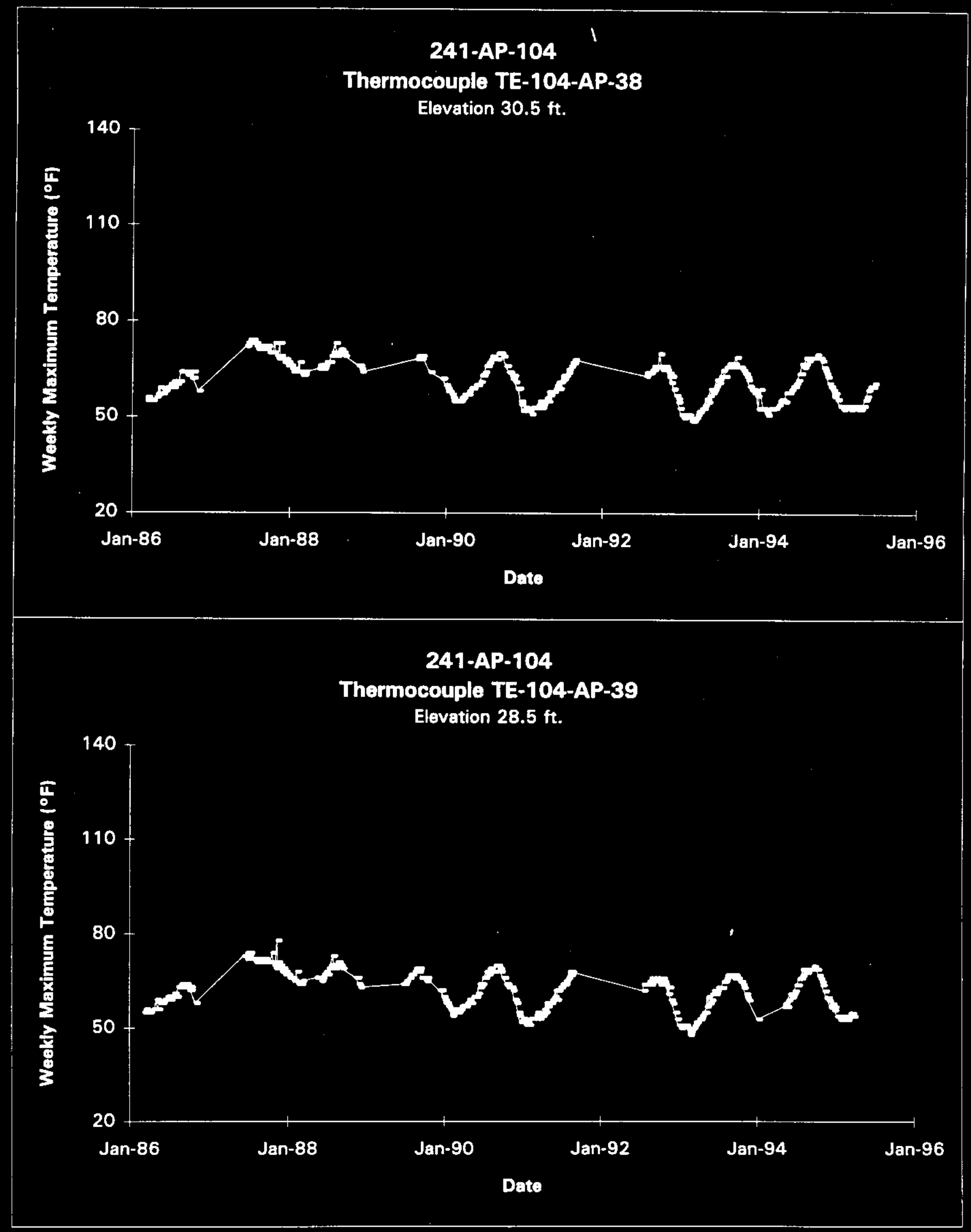




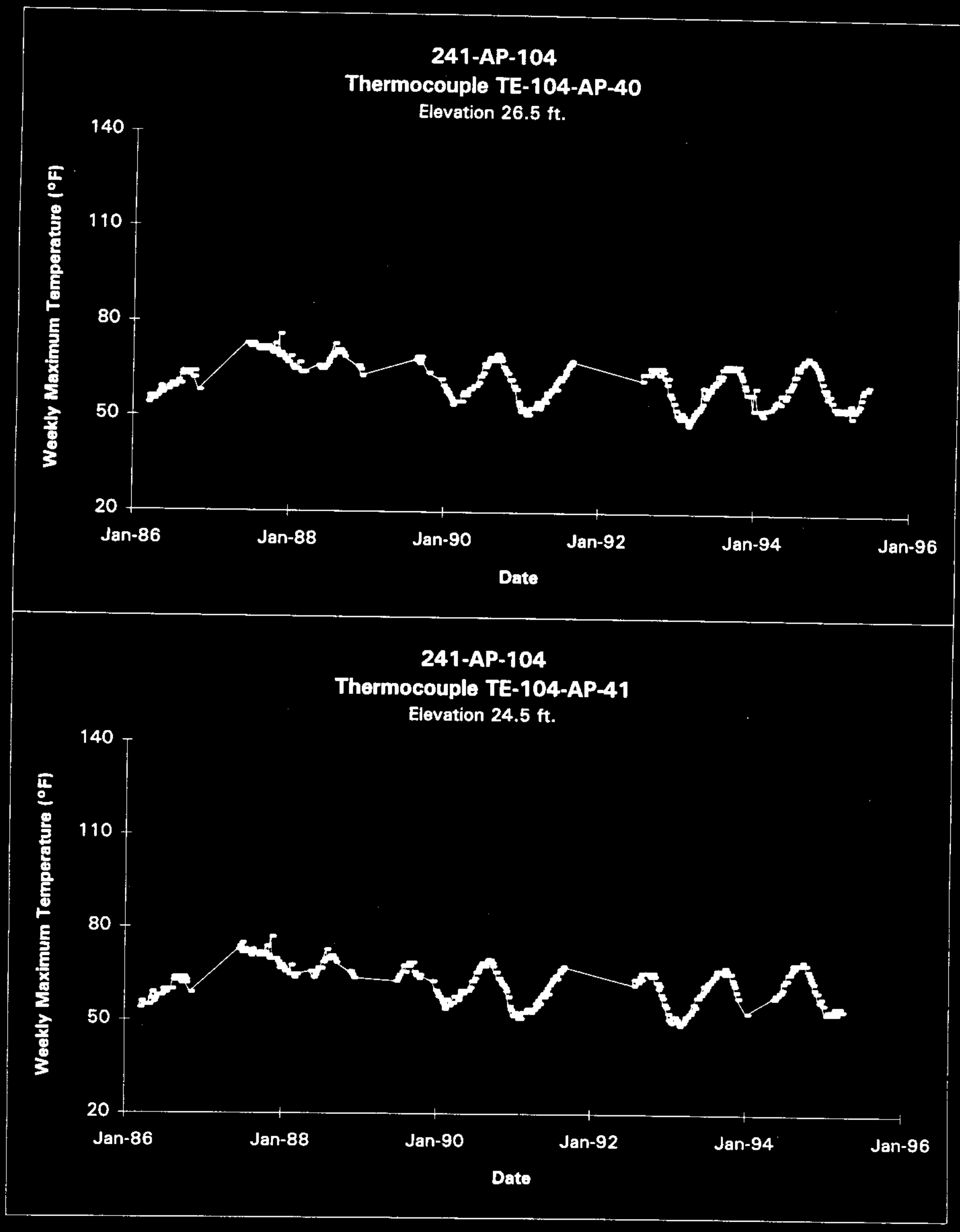

Data obtained from Computer Automated Survaillance Syatem (CASS), April 1995; and Survaillance Analyais Computer System (SACS), June 1995. $\mathrm{D}-30$ 


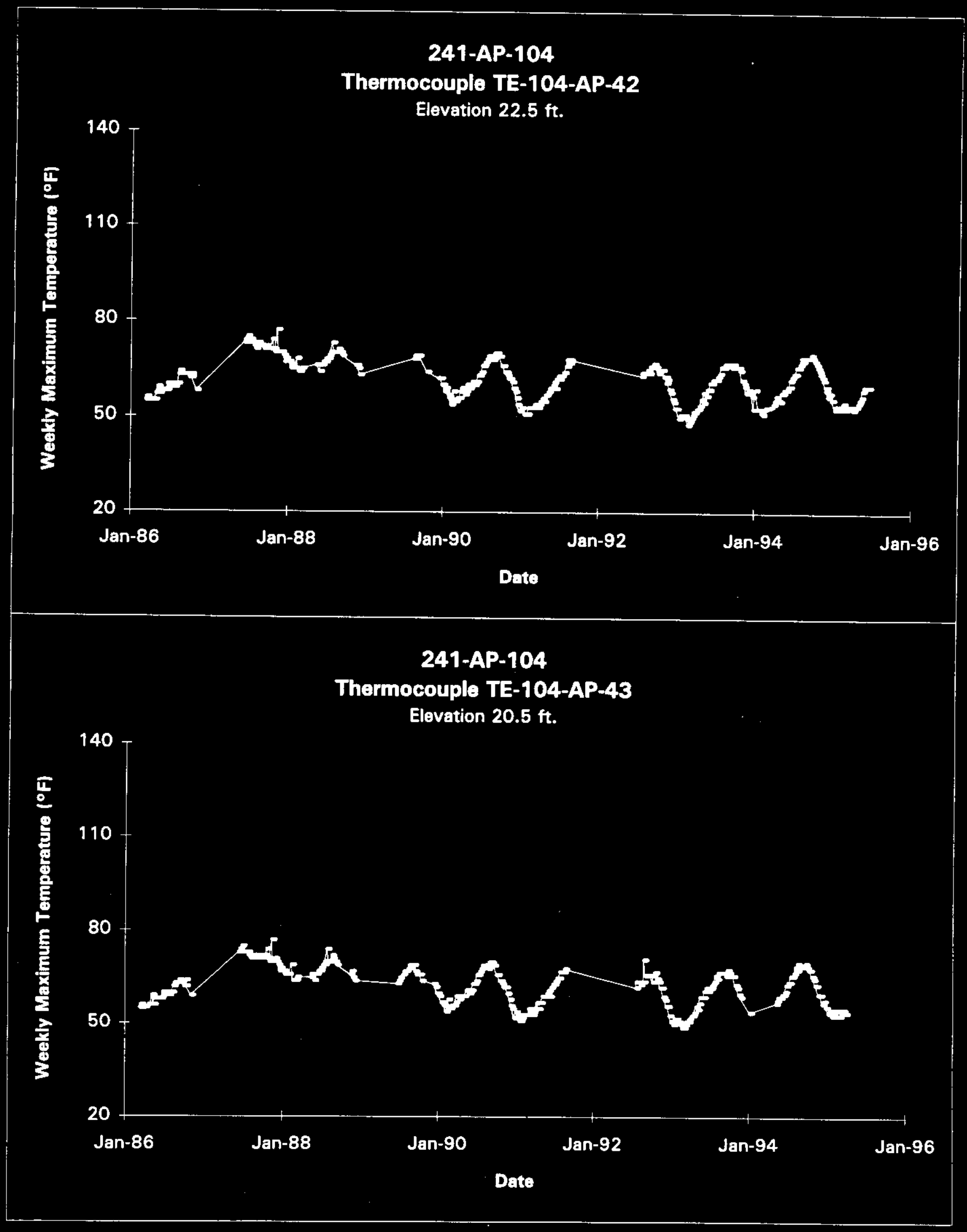




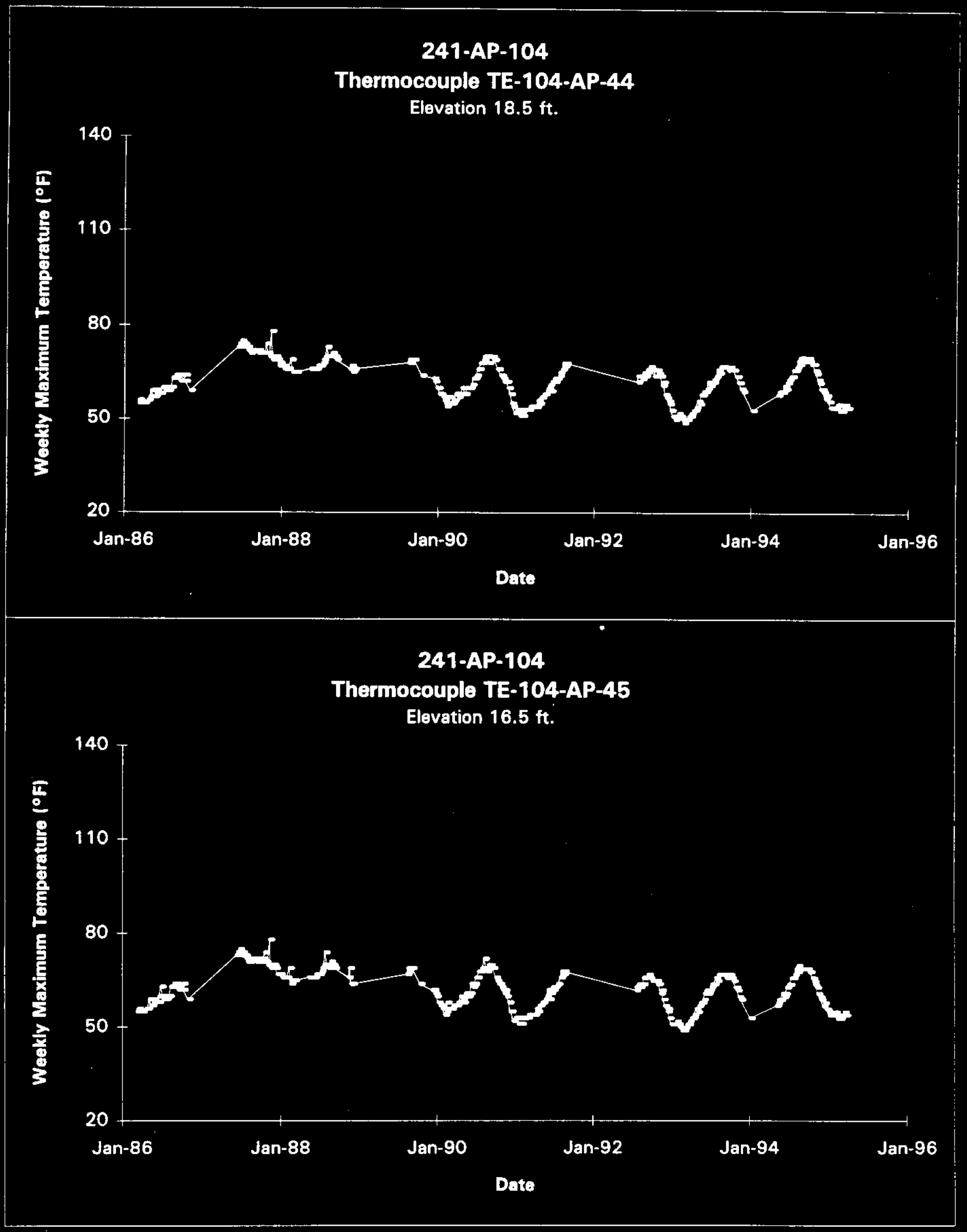

Date obteined from Computer Automated Surveillance System (CASS), April 1995; and Surveillance Analysis Computer Syatem (SACS). June 1995. 


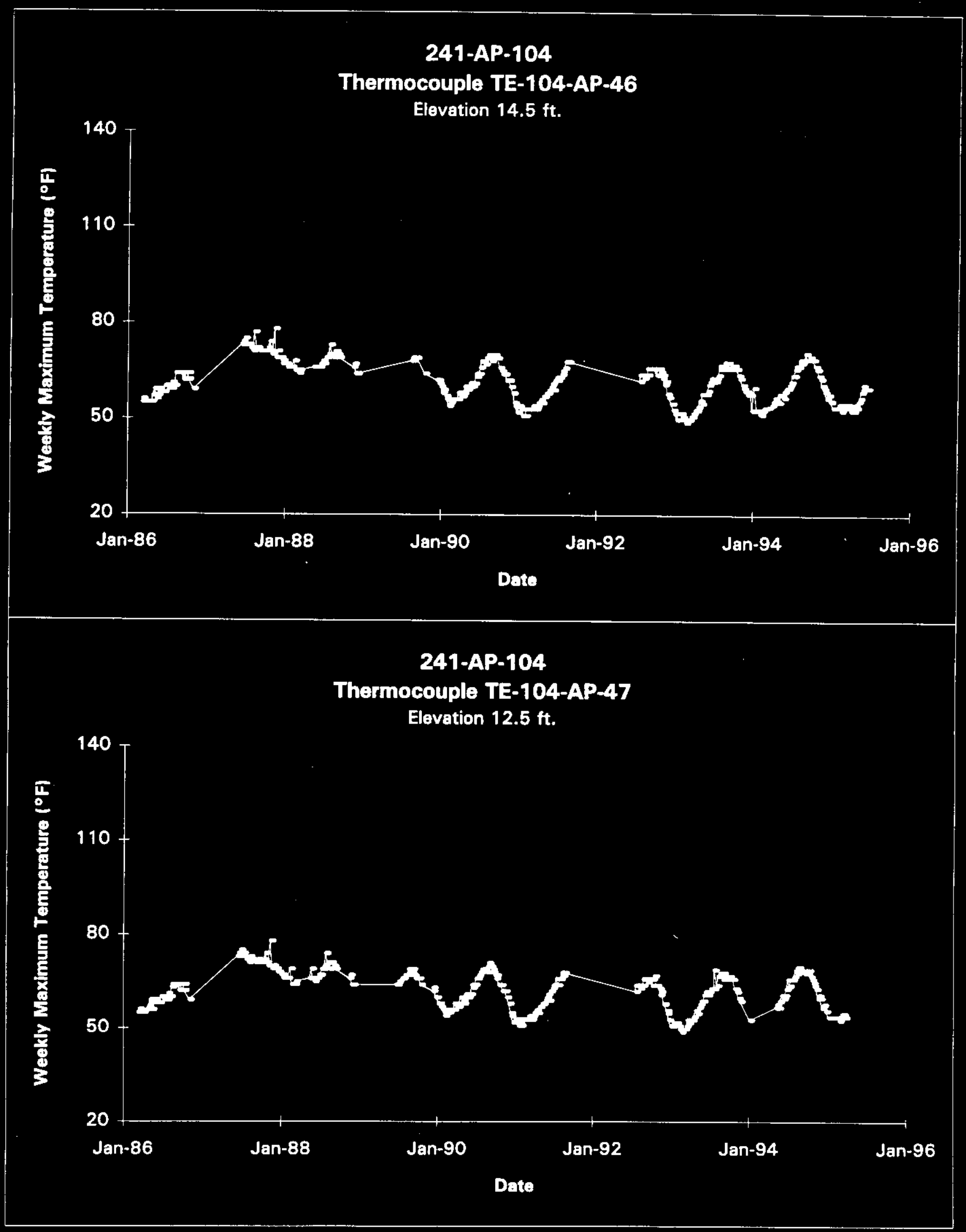




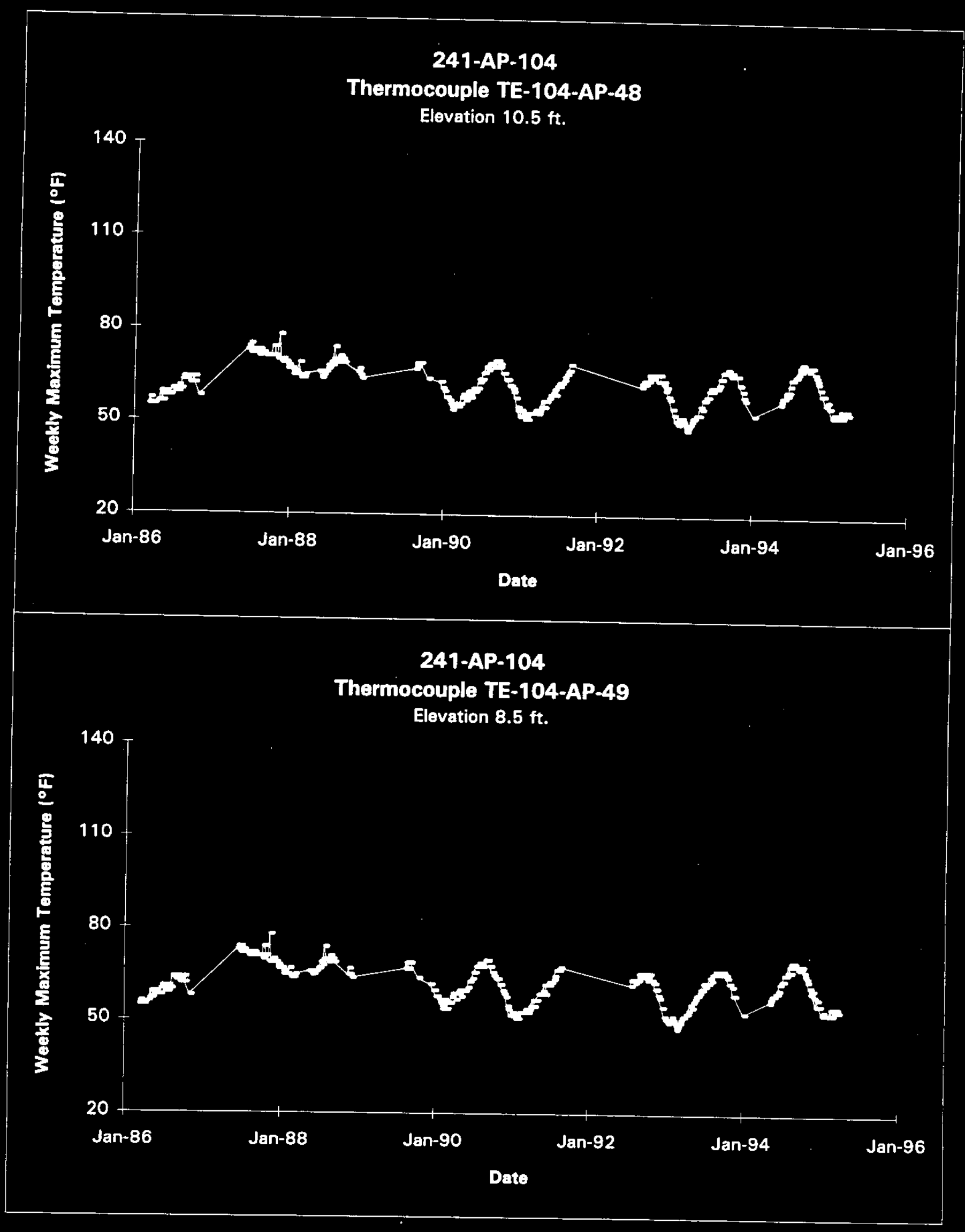

Dote obtained from Cornputer Automated Surveillance Syatem (CASS), April 1995; and Survaillance Analyais Computer System (SACS), June 1995. D-34 


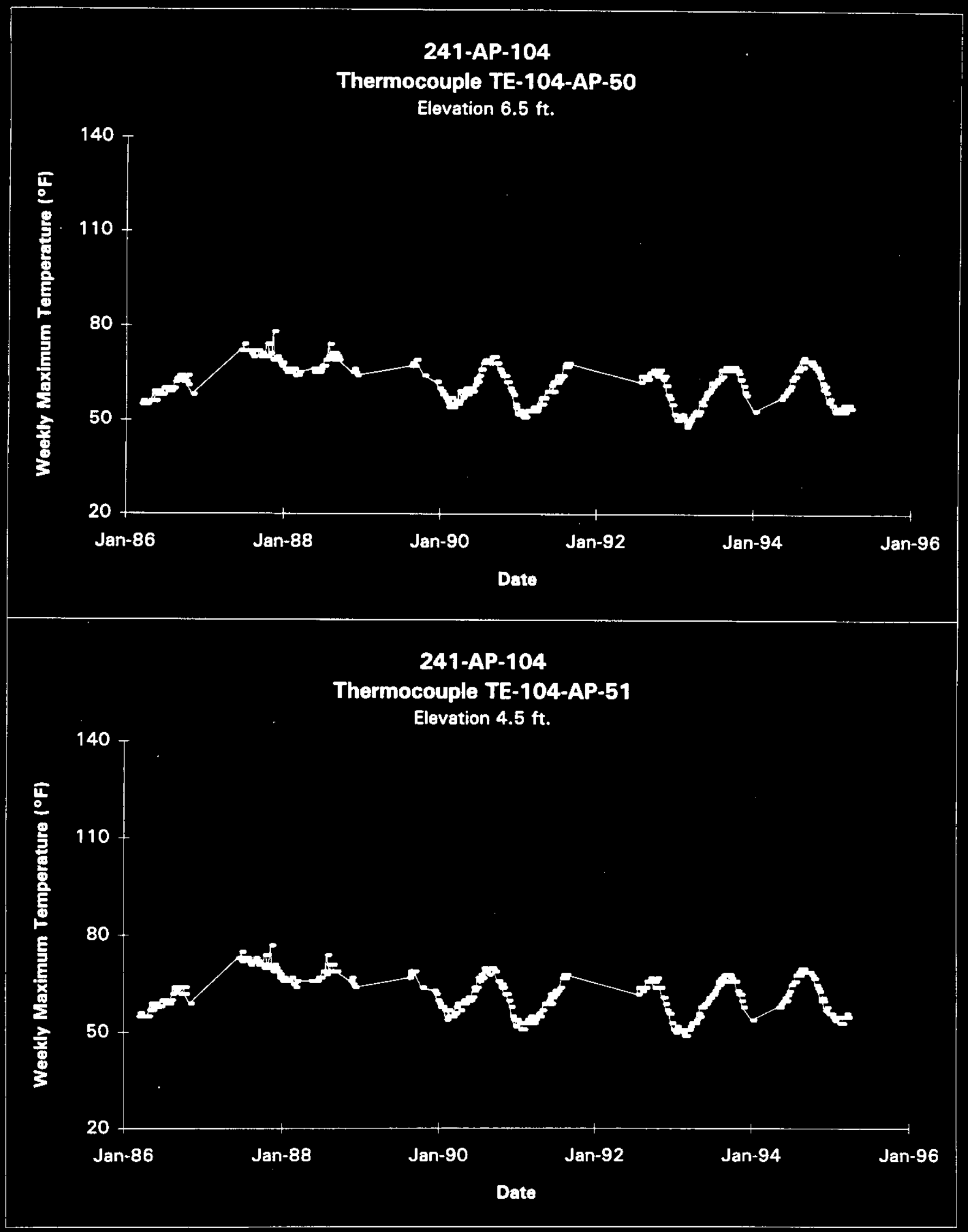

Data obtained from Computer Automated Surveillance Syatem (CASS), April 1995; and Surveillence Analysis Computer System (SACS), June 1995. 


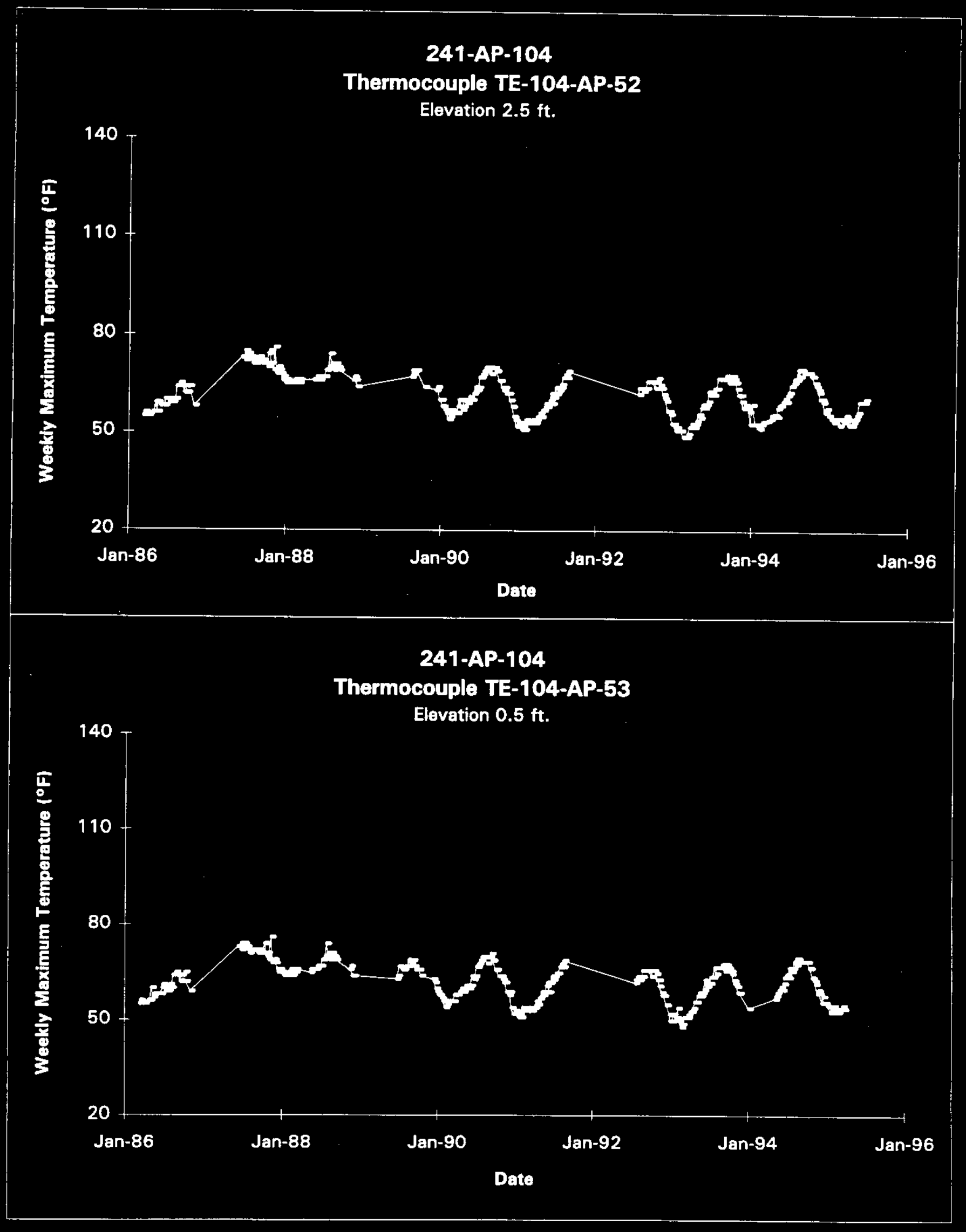

Data obtained from Computer Autometed Surveillance Syatem (CASS). April 1995; and Surveillenee Analyais Computer Syatem (SACS). June 1995. D-36 


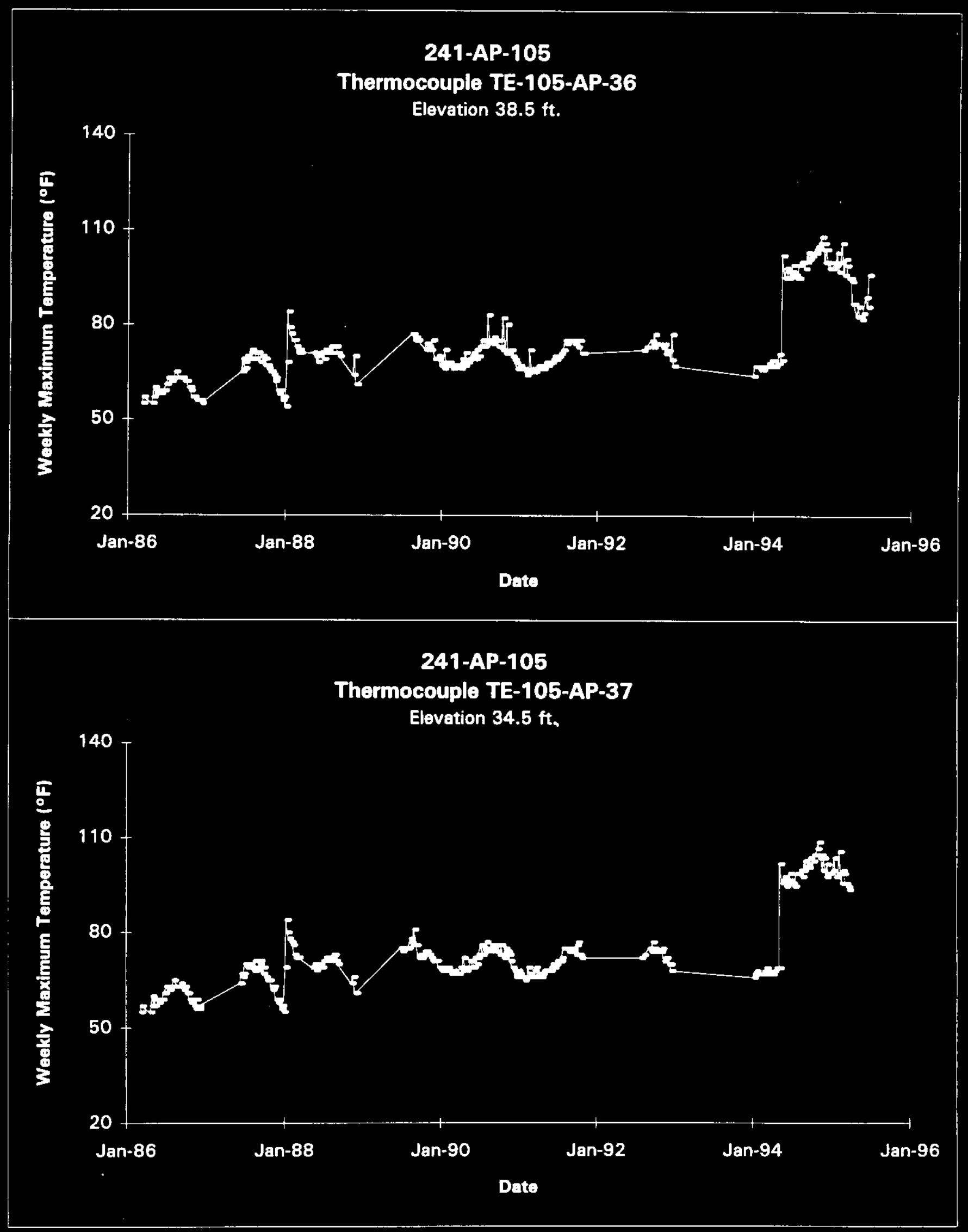

Data obtained from Computer Automated Surveillance Syatem (CASS), April 1995; and Surveillance Analyais Computer Syatem (SACS), June 1995. D-37 


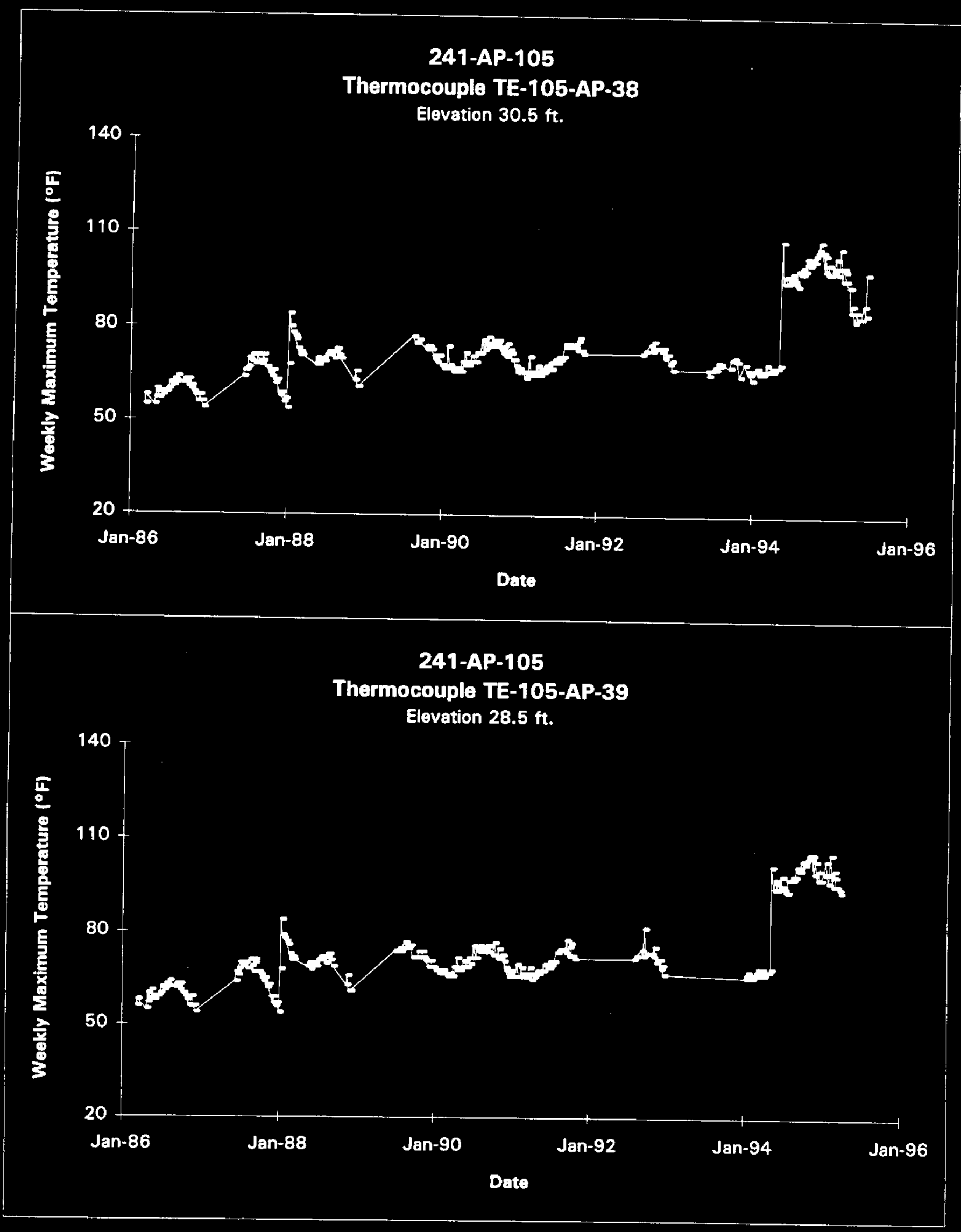

Data obtained from Computer Automated Surveillence Syatem (CASS), April 1995; and Survaillance Analyais Computer Syatem (SACS), June 1995.

D-38 


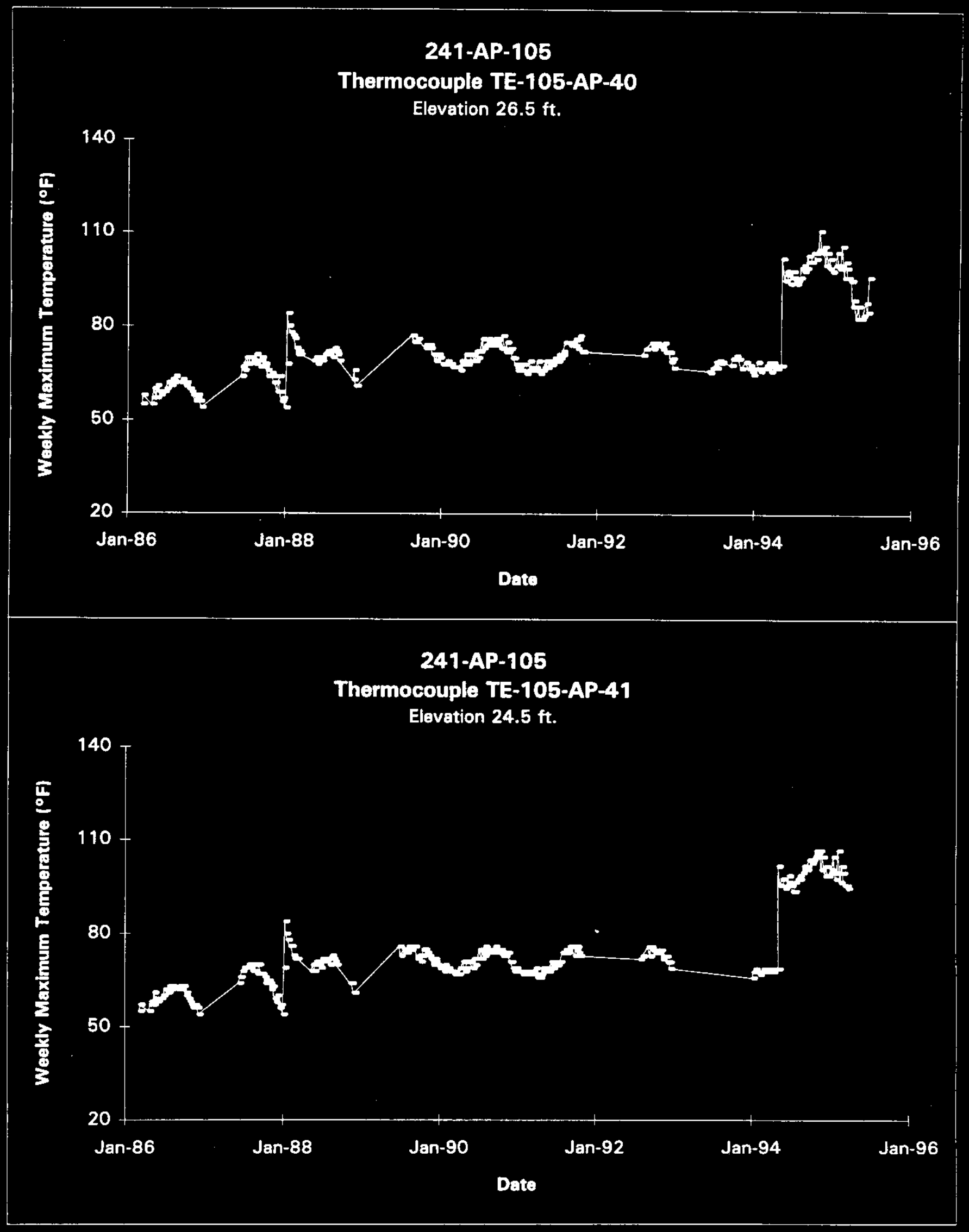




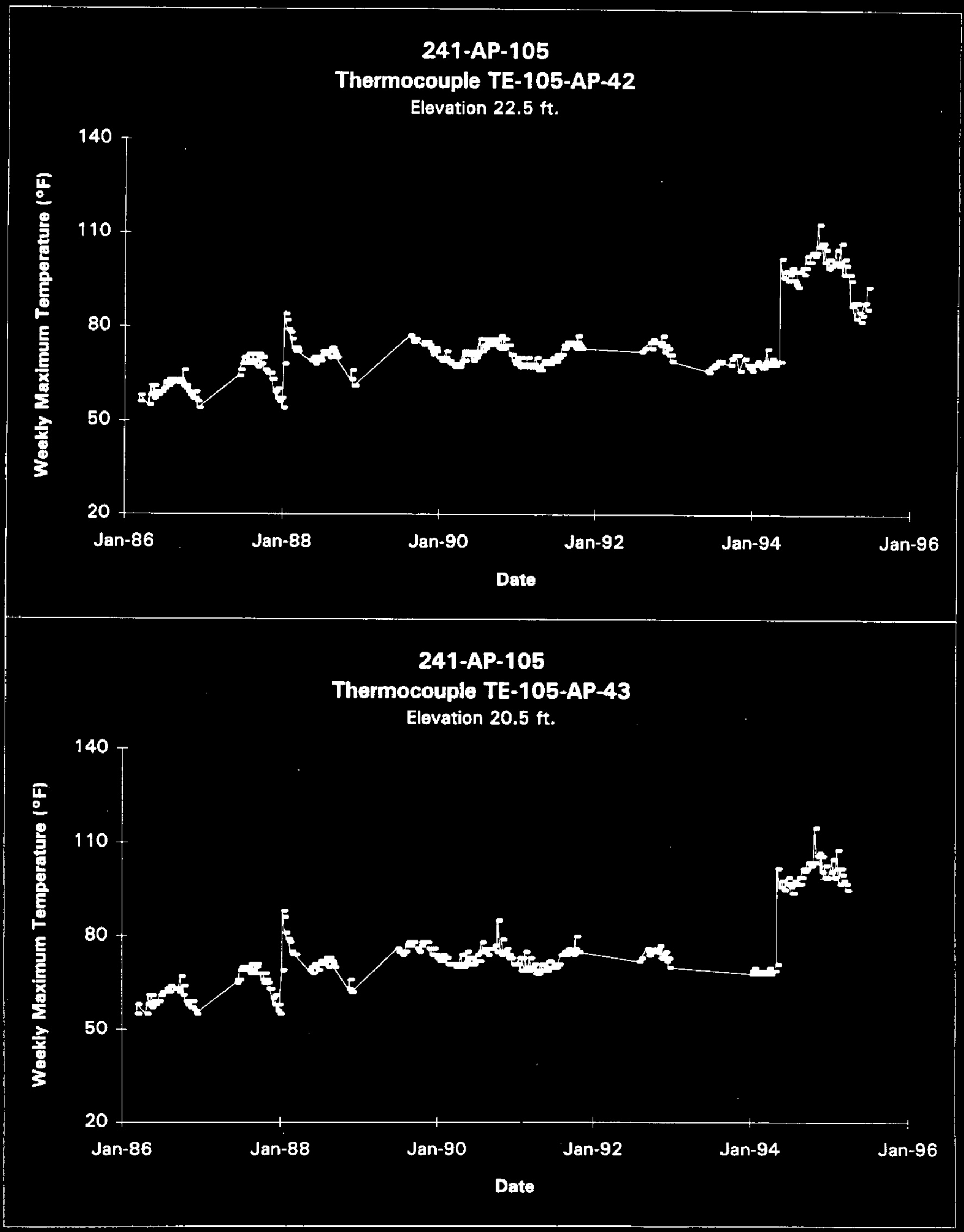

Data obtained from Computer Automated Surveillance Syatem (CASS), April 1995: and Survaillance Analysis Computer Syatem (SACS). June 1995. 


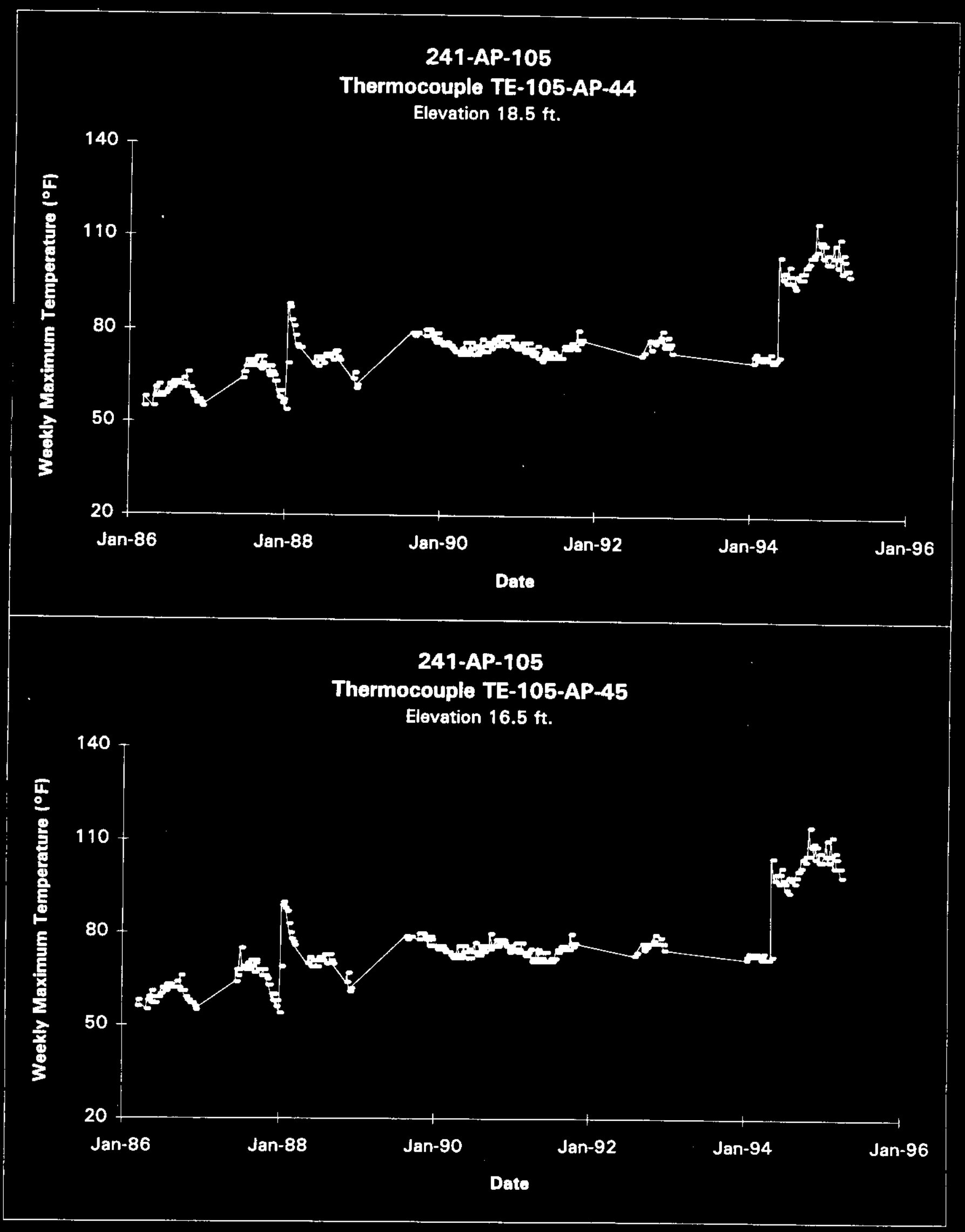




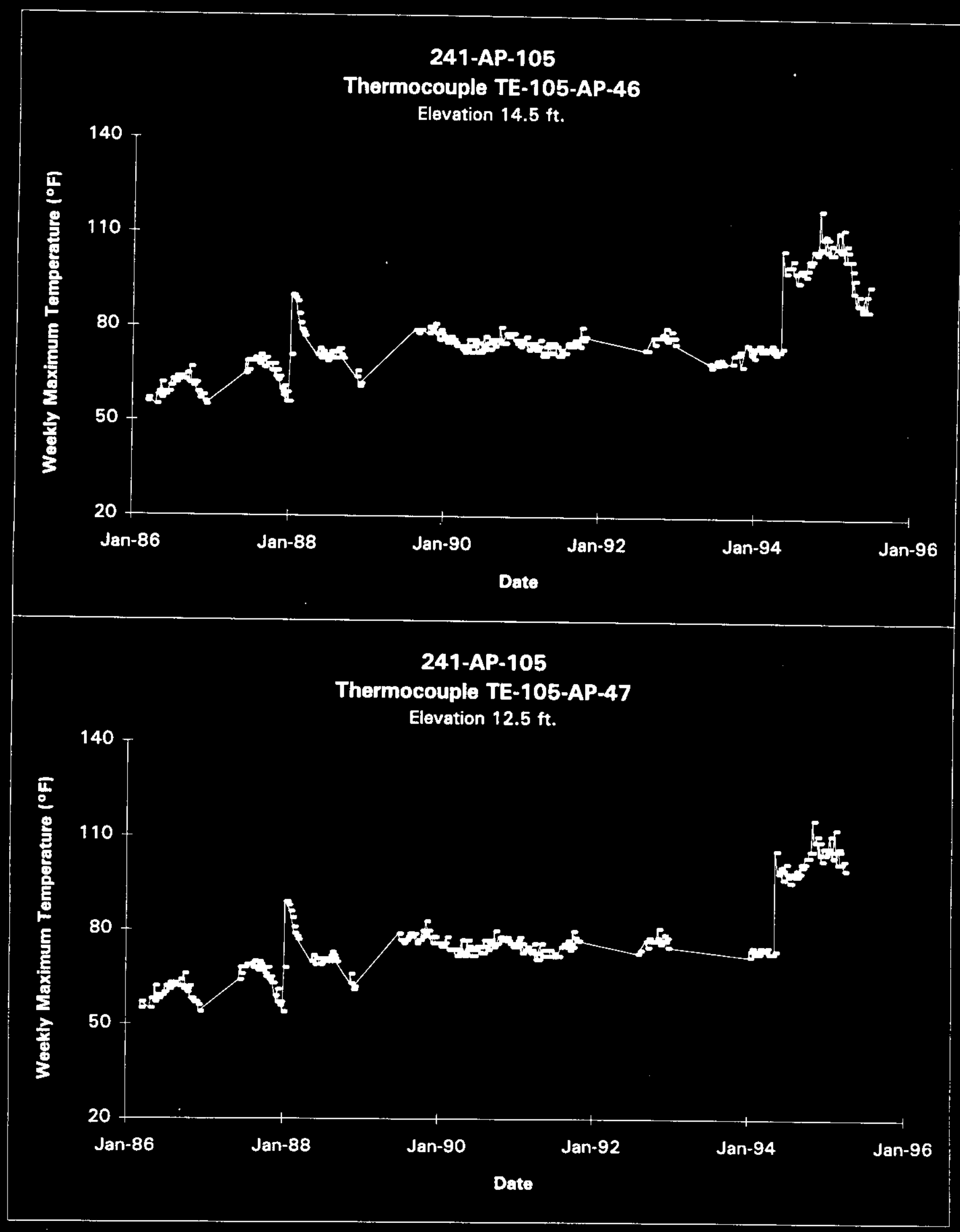

Data obtained from Computer Automnted Surveillance System (CASS), April 1995; and Surveillance Analysis Computer System (SACS), June 1995. $\mathrm{D}-42$ 


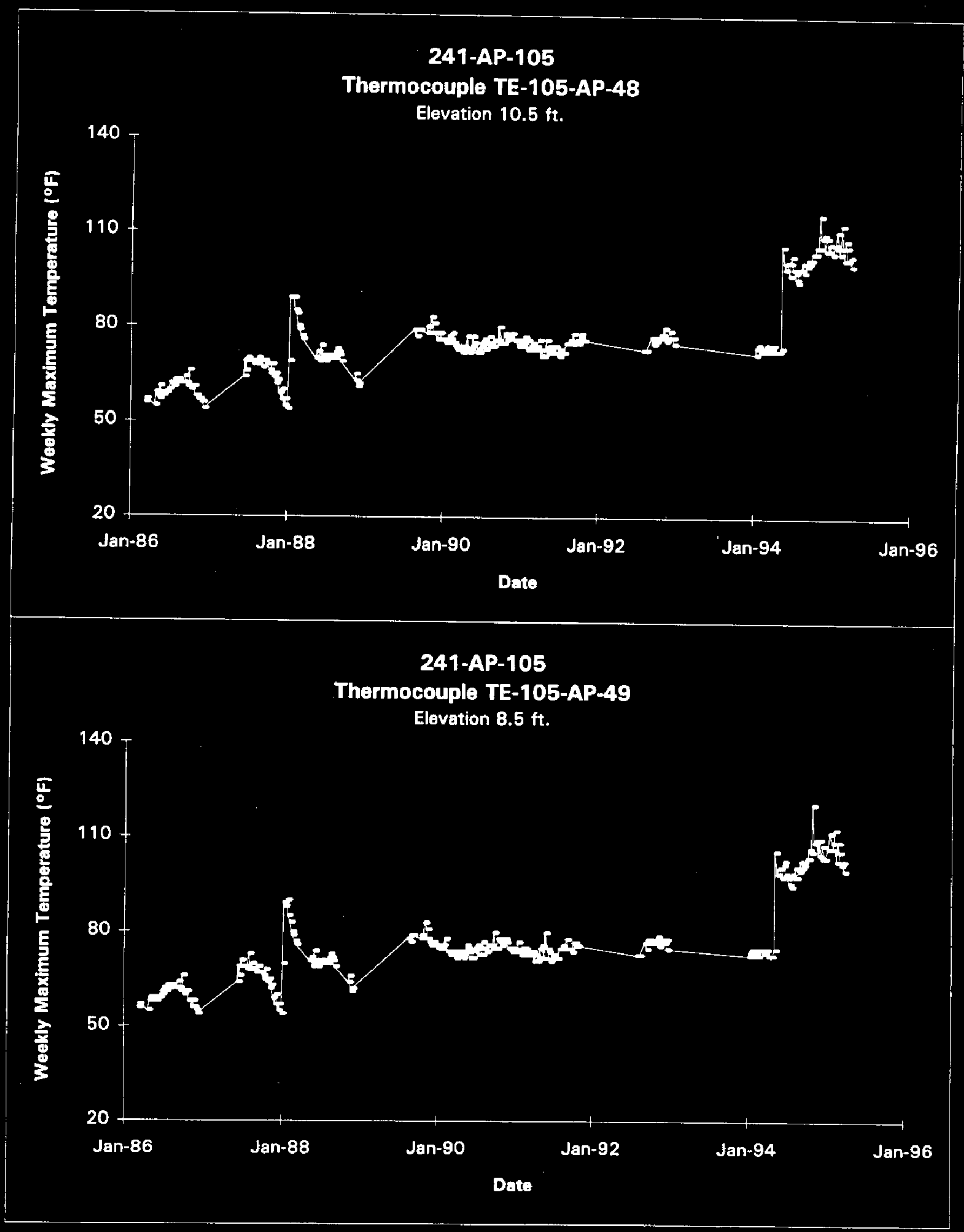




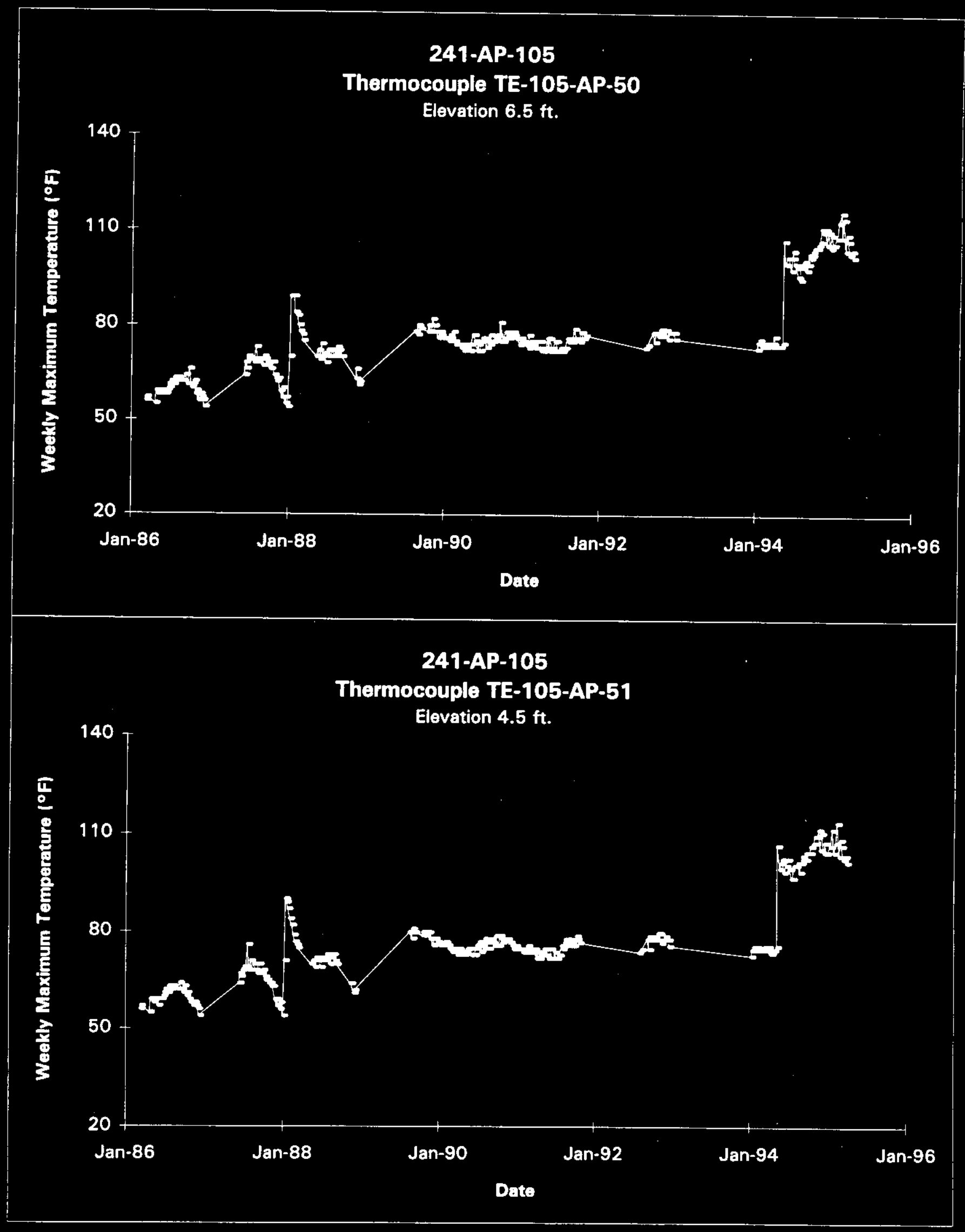

Data obtained from Computer Automated Surveillance Syatem (CASS), April 1995; and Surveillance Analysis Computer System (SACS), June 1995. D-44 


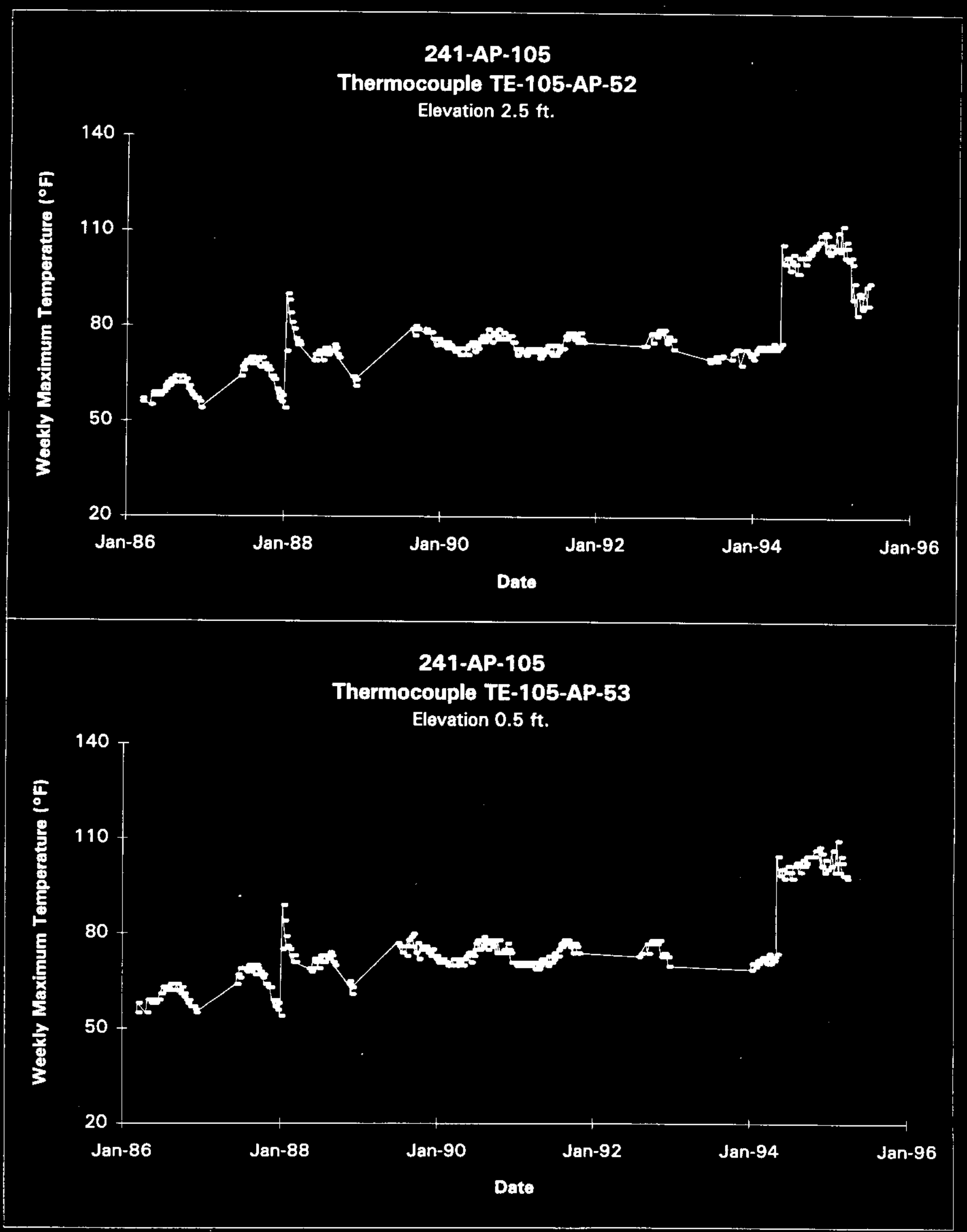




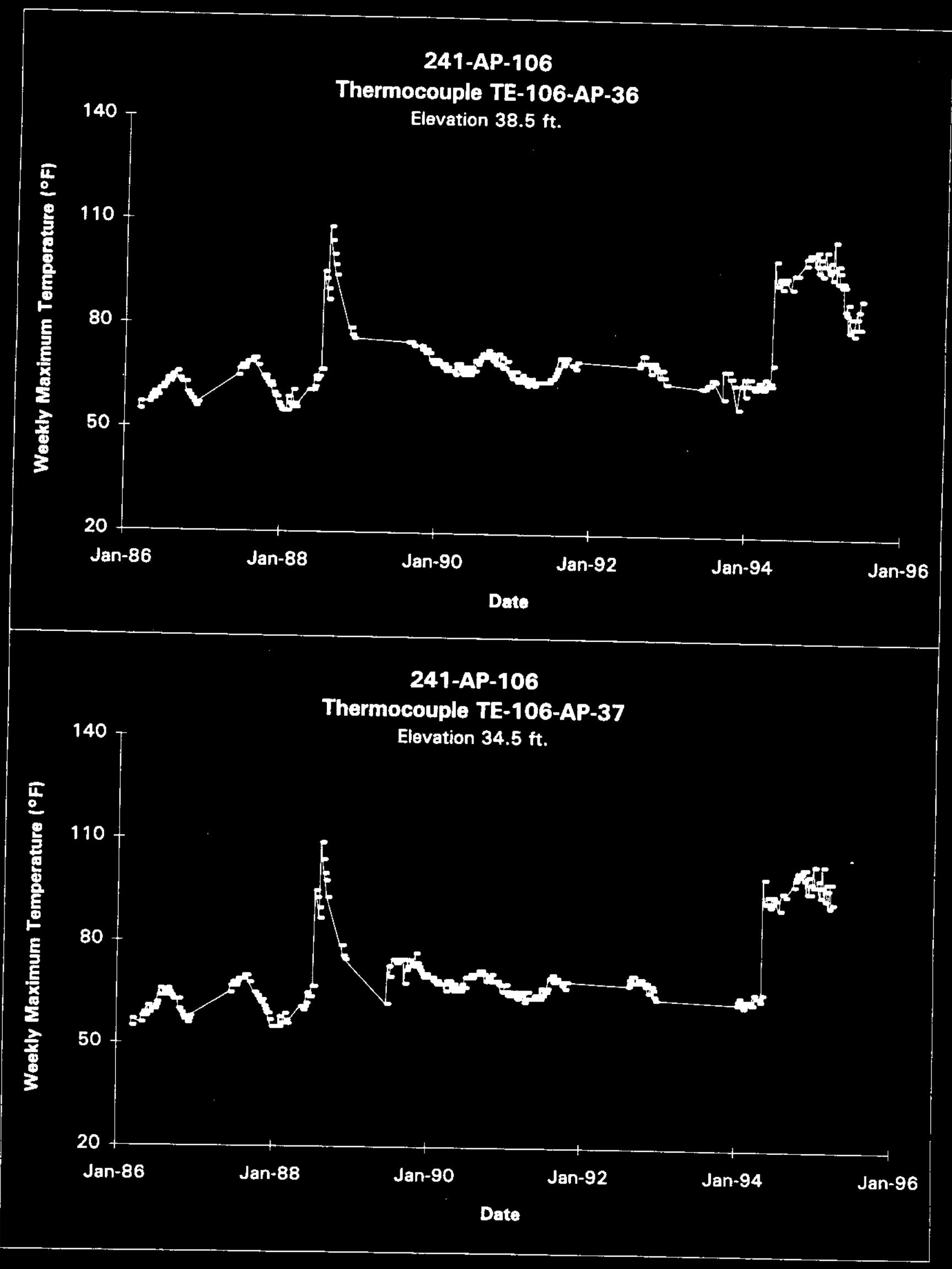

Date obtained from Computer Automated Survaillance System (CASS), April 1995; and Surveillance Analyais Computer System (SACS), June 1995. D-46 


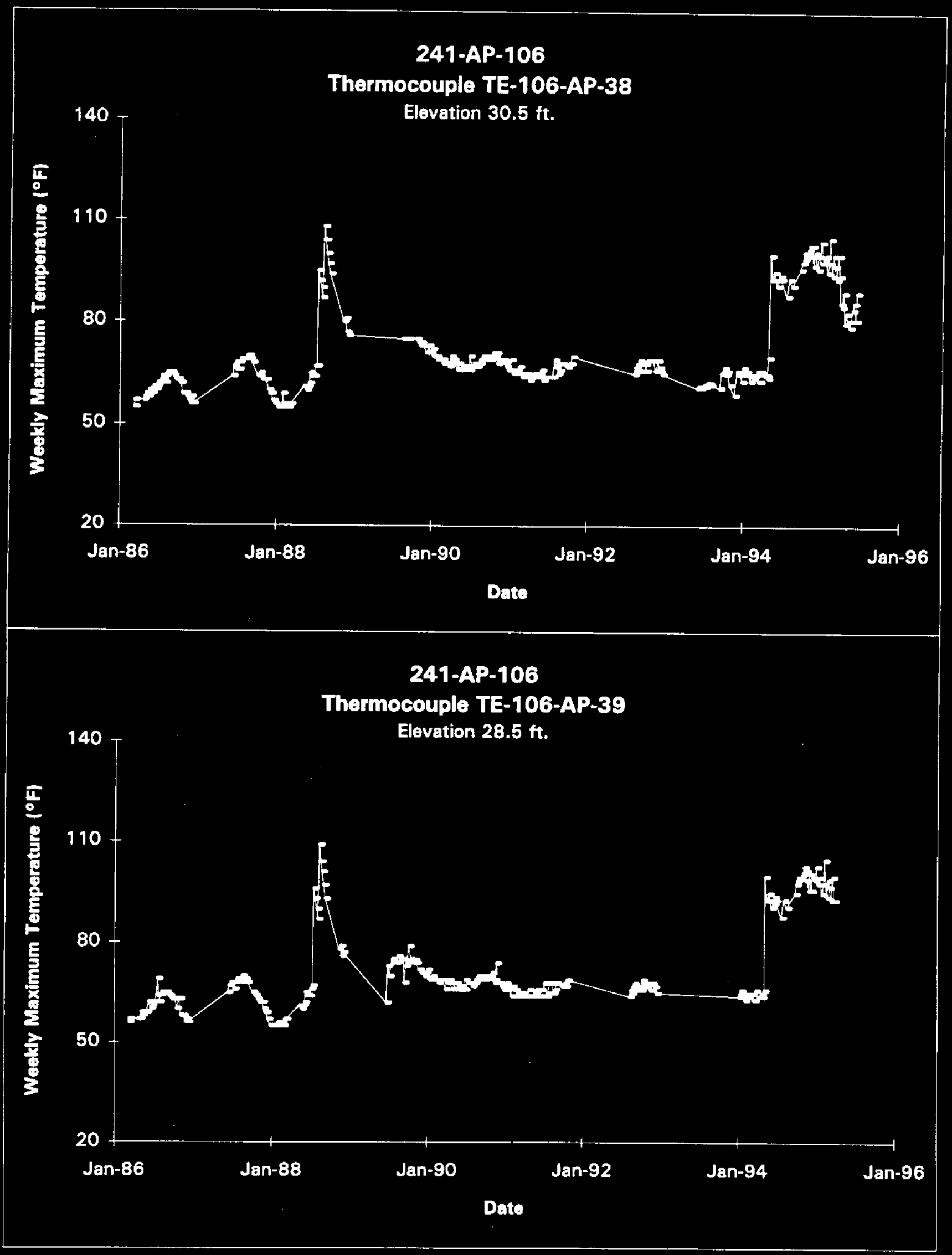

Date obtained from Computer Automated Surveillance Svatem (CASS), April 1995; and Survaillance Analyais Computer Syatem (SACS), June 1995. 


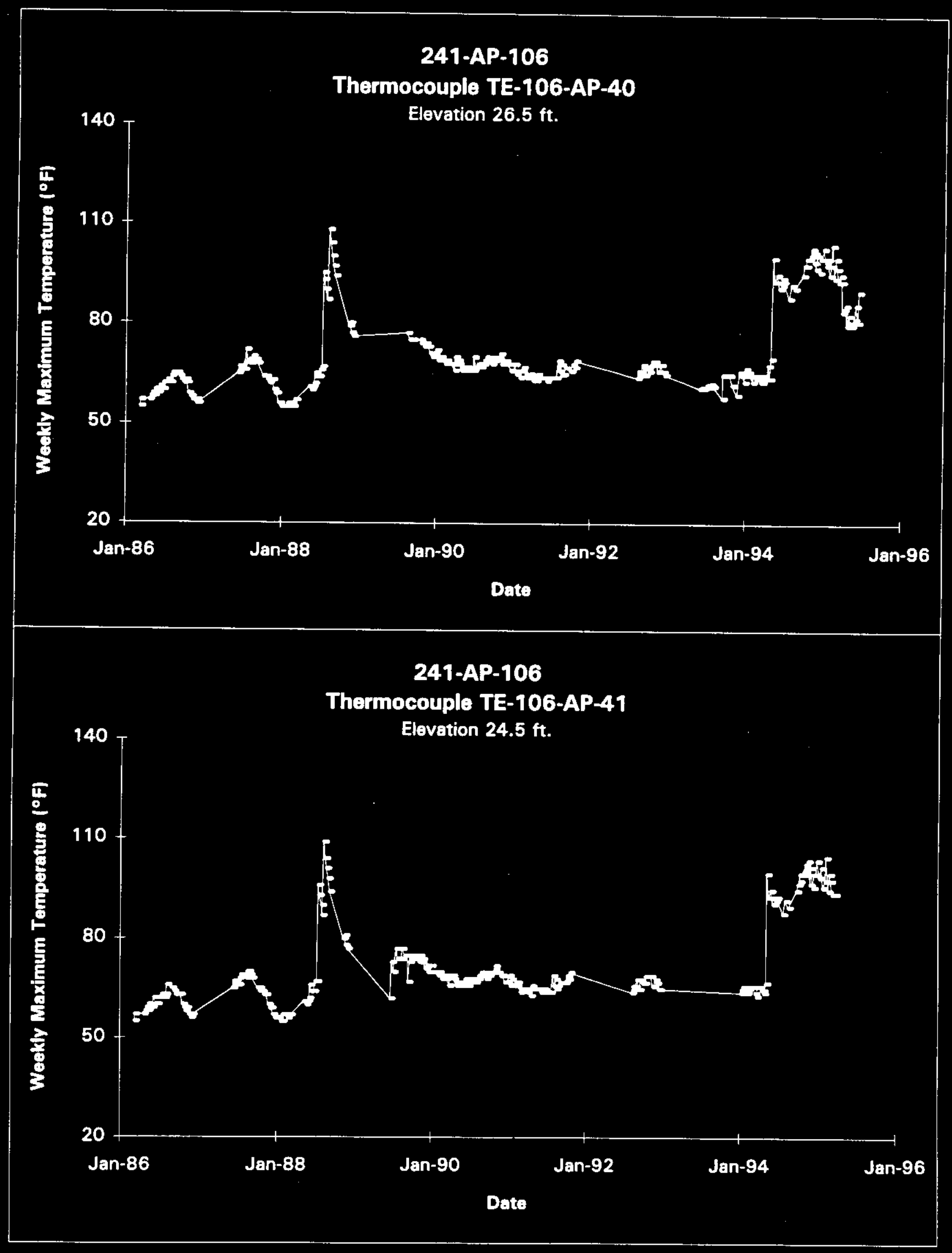

Date obtained from Computer Automated Surveillance Syatem (CASS), April 1995; and Surveillance Analyais Computer Syatem (SACS), June 1995. D-48 


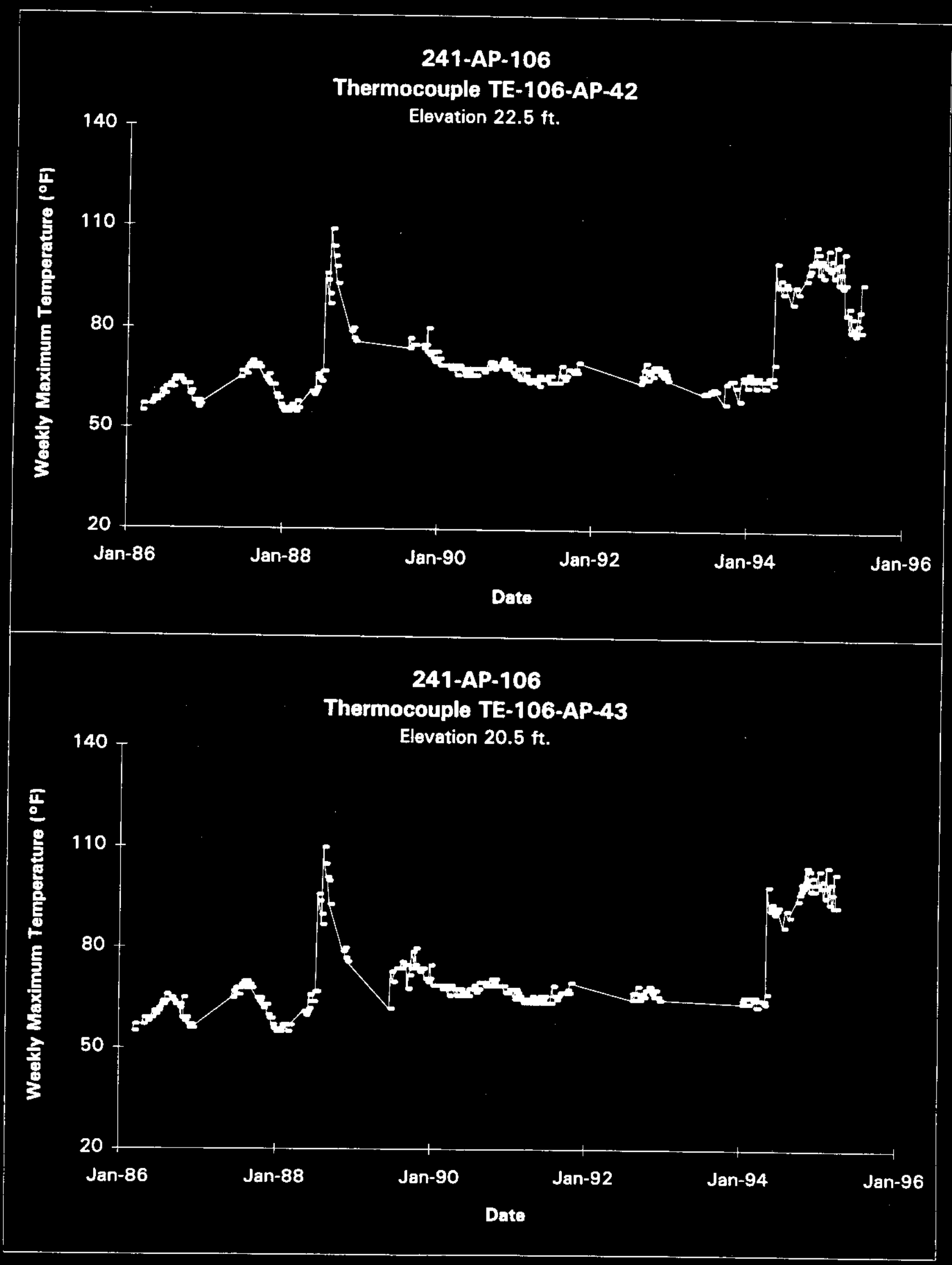

Data obtained from Computer Automuted Surveillance Syatem (CASS), April 1995; und Surveillanee Analysis Computer System (SACS), June 1995. $D-49$ 


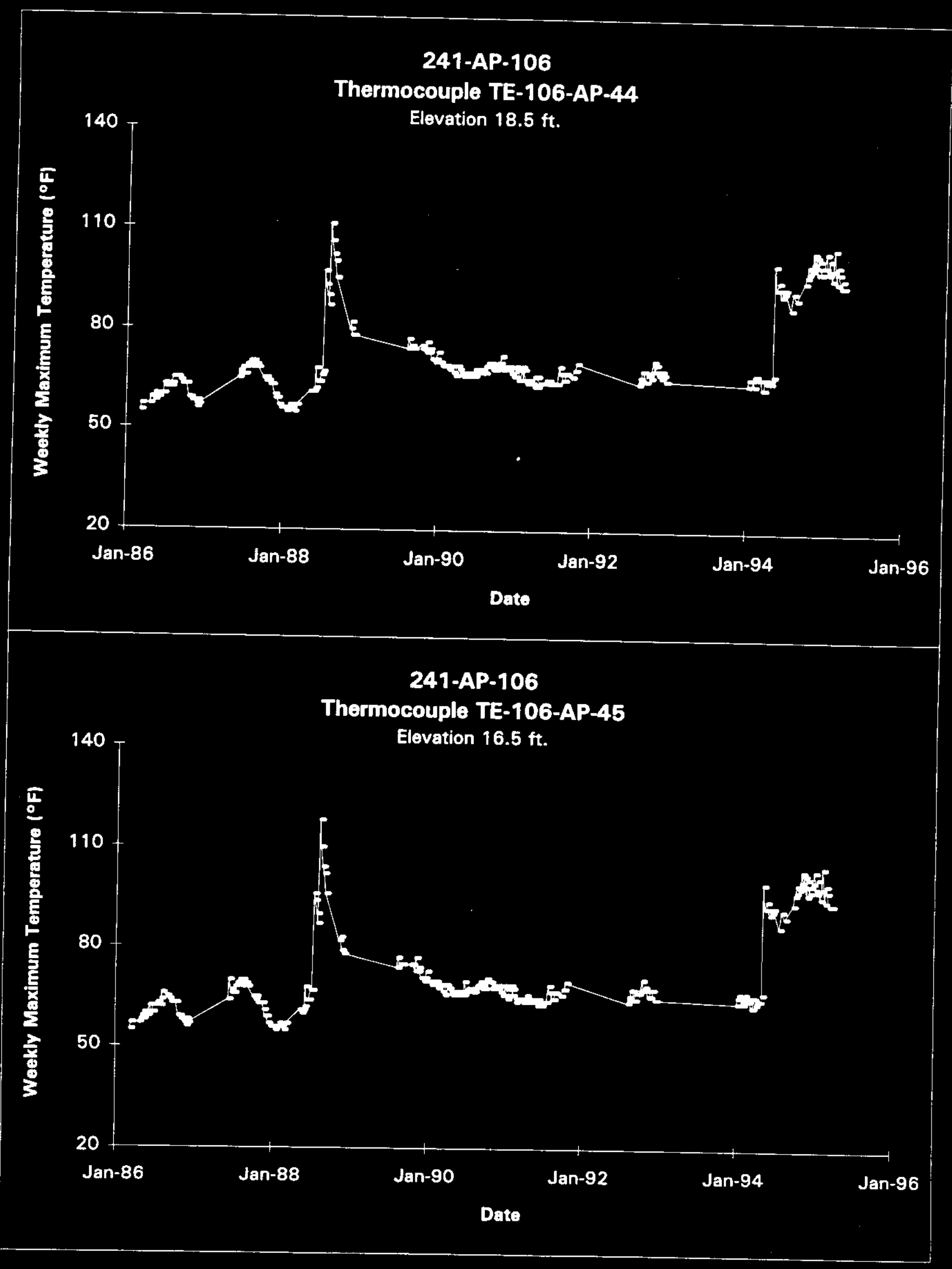

Data obtained from Computer Automated Surveillence System (CASS), April 1995; and Surveillance Analysis Computer System (SACS), June 1995. D-50 


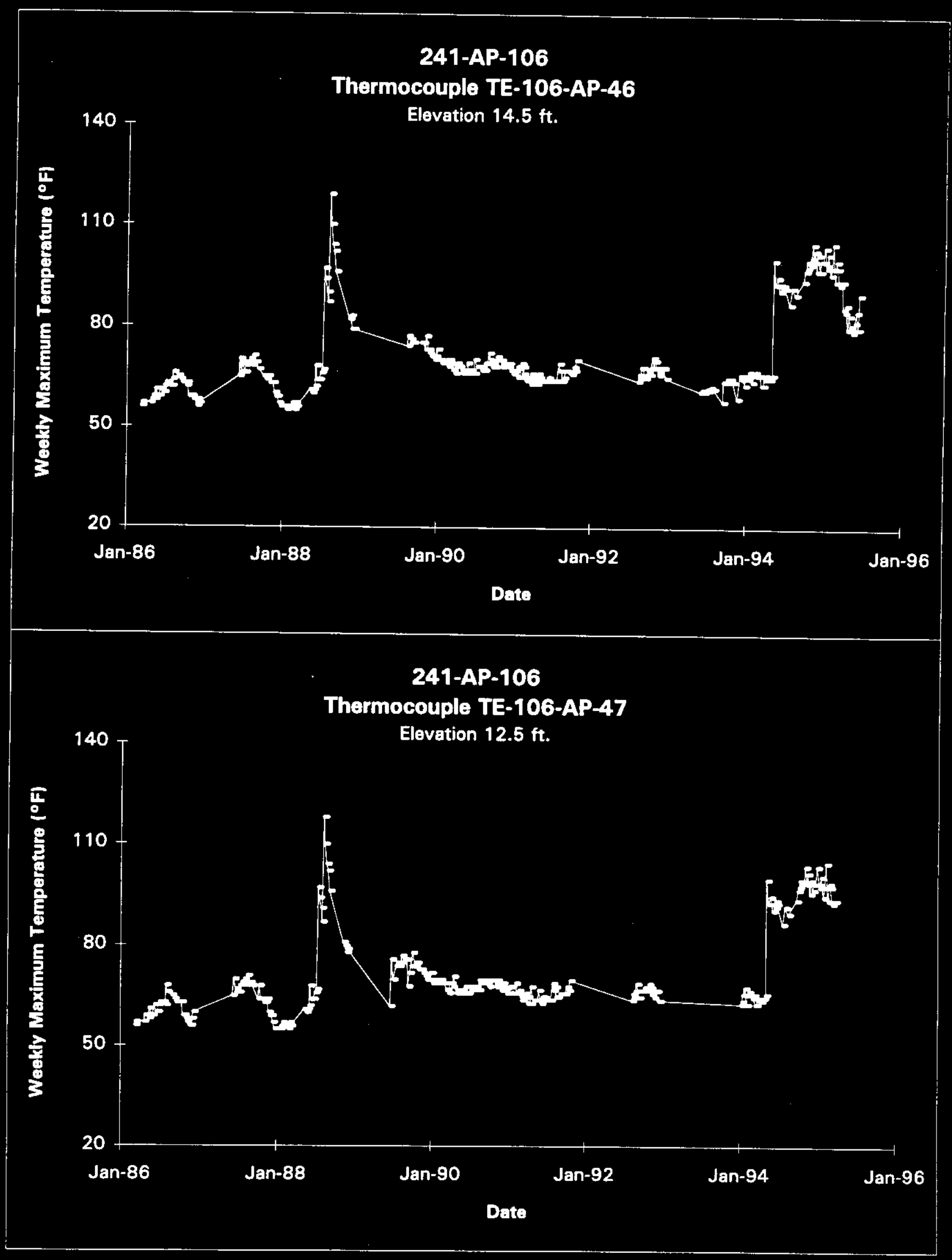




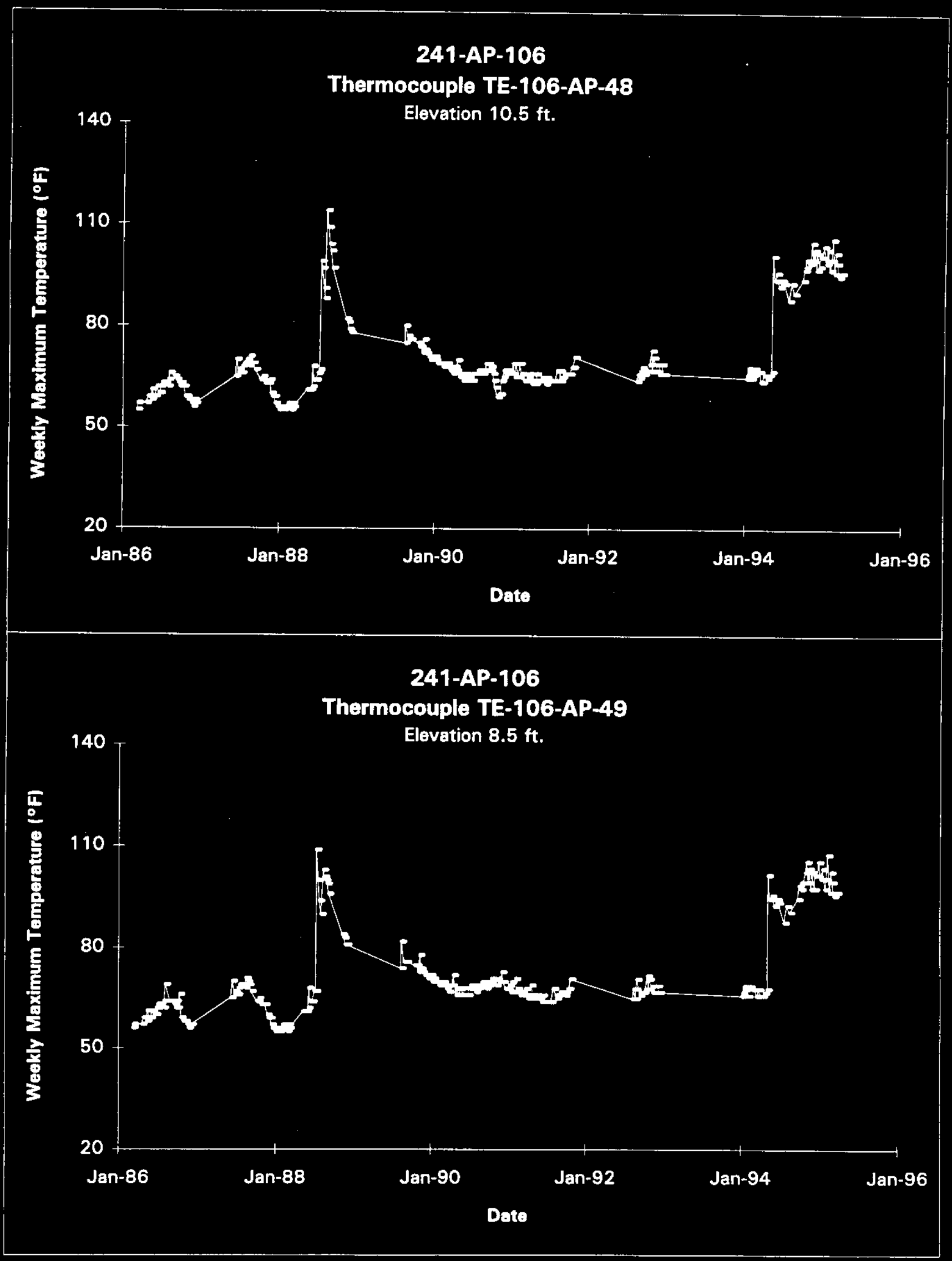

Data obtained from Computer Automated Surveillonee System (CASS), April 1995; and Surveillance Analysis Computer Syatem (SACS), June 1995. D-52 


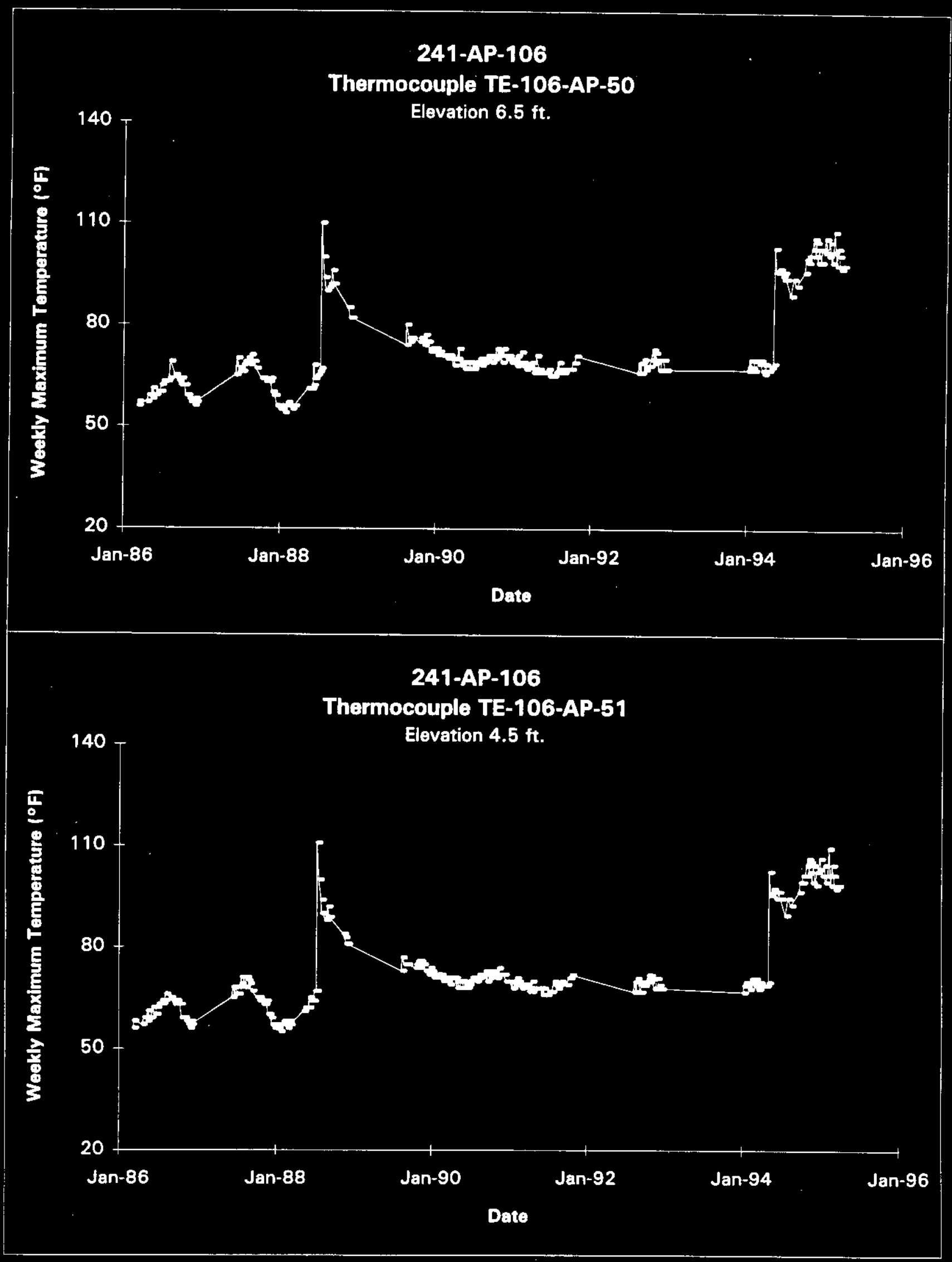

Data obtained from Computer Automated Surveillence Syatem (CASS), April 1995; and Surveillance Analyais Computer Syatem (SACS), June 1995. 


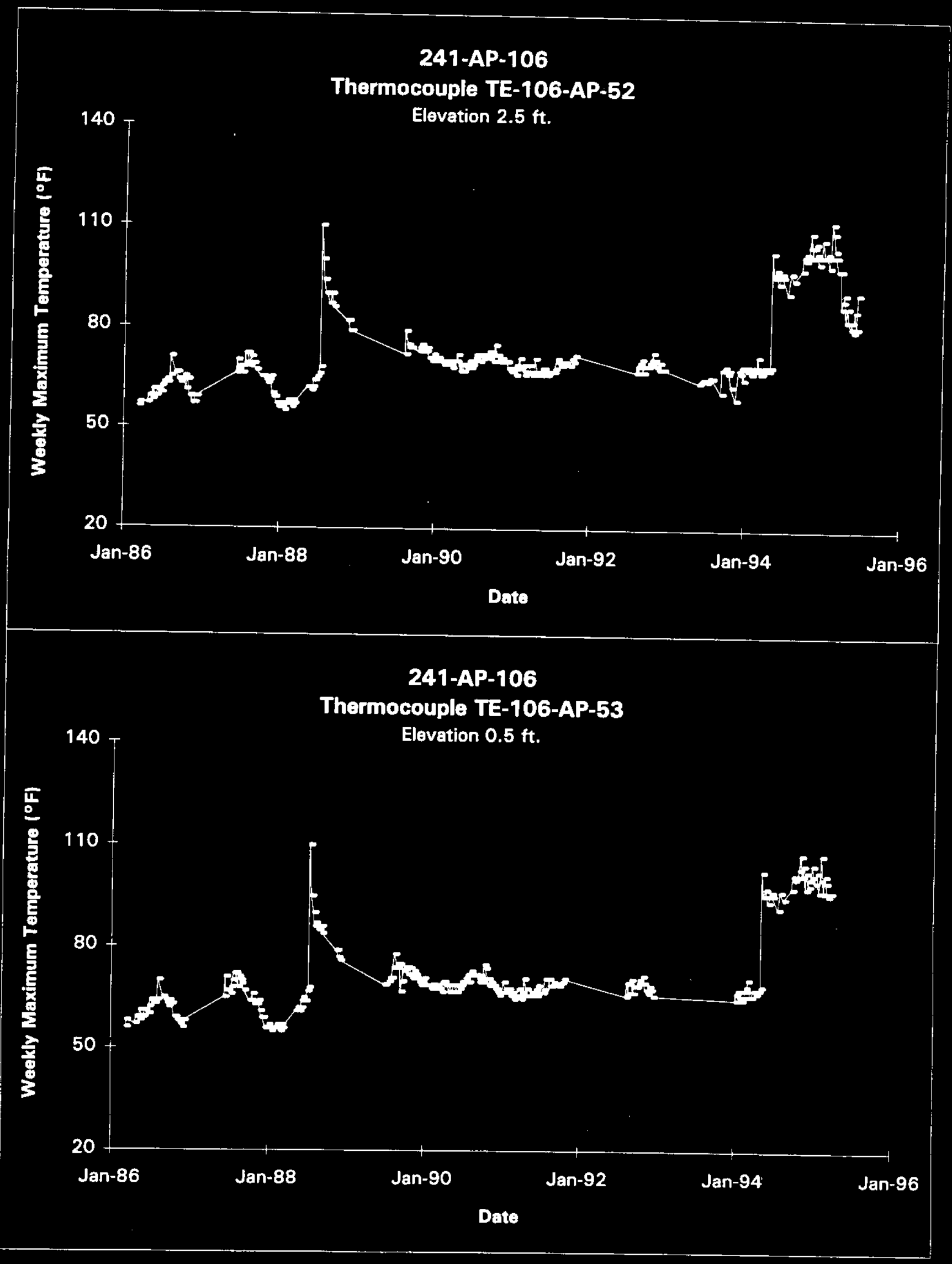

Data obtained from Computer Automated Surveillance System (CASS), April 1995; and Survaillance Analysis Computer System (SACS), June 1995. $D-54$ 


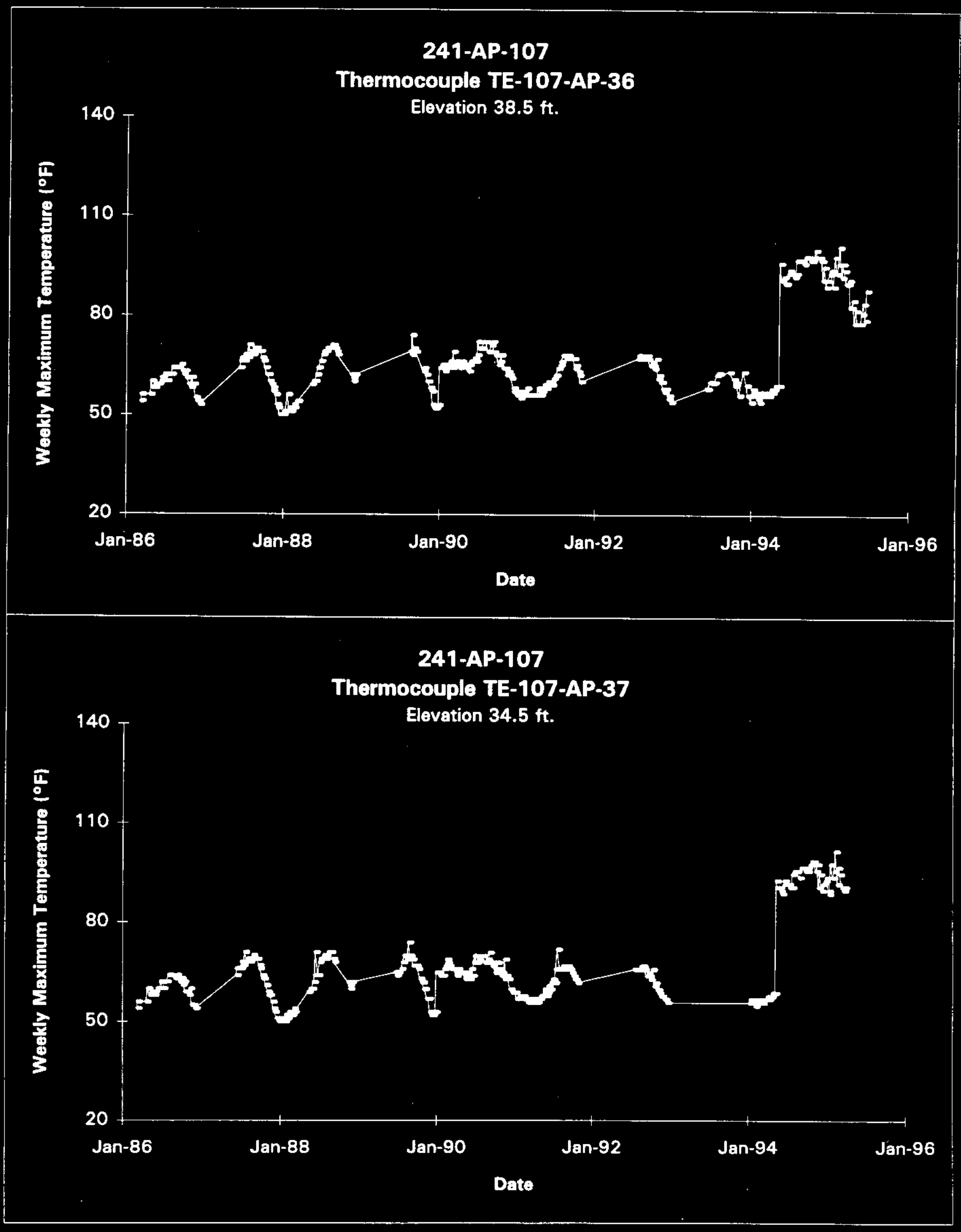

Date obtained from Computer Automated Survaillance System (CASS), April 1995; and Surveillance Analysis Computer Syatem (SACS), June 1995. D-55 


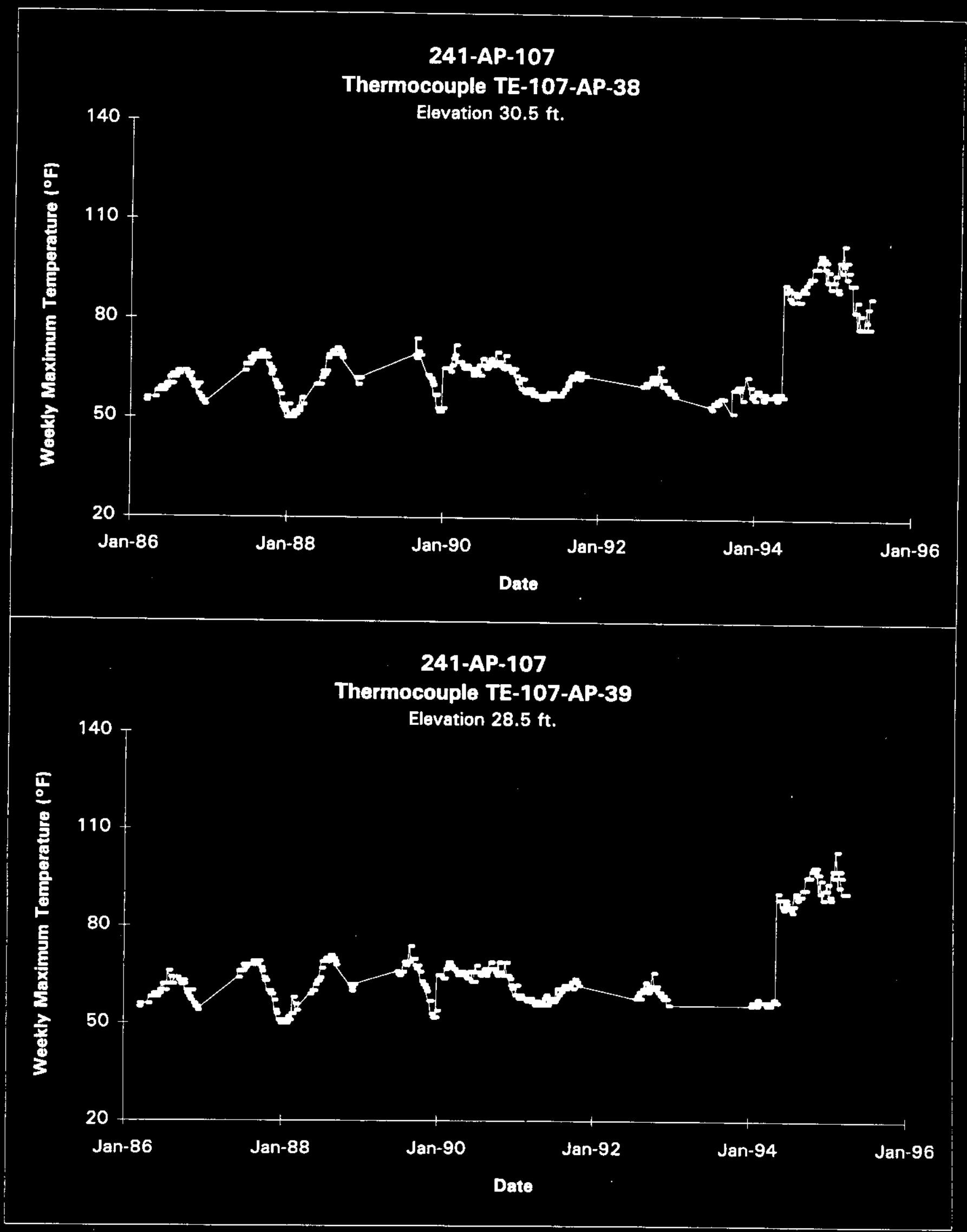




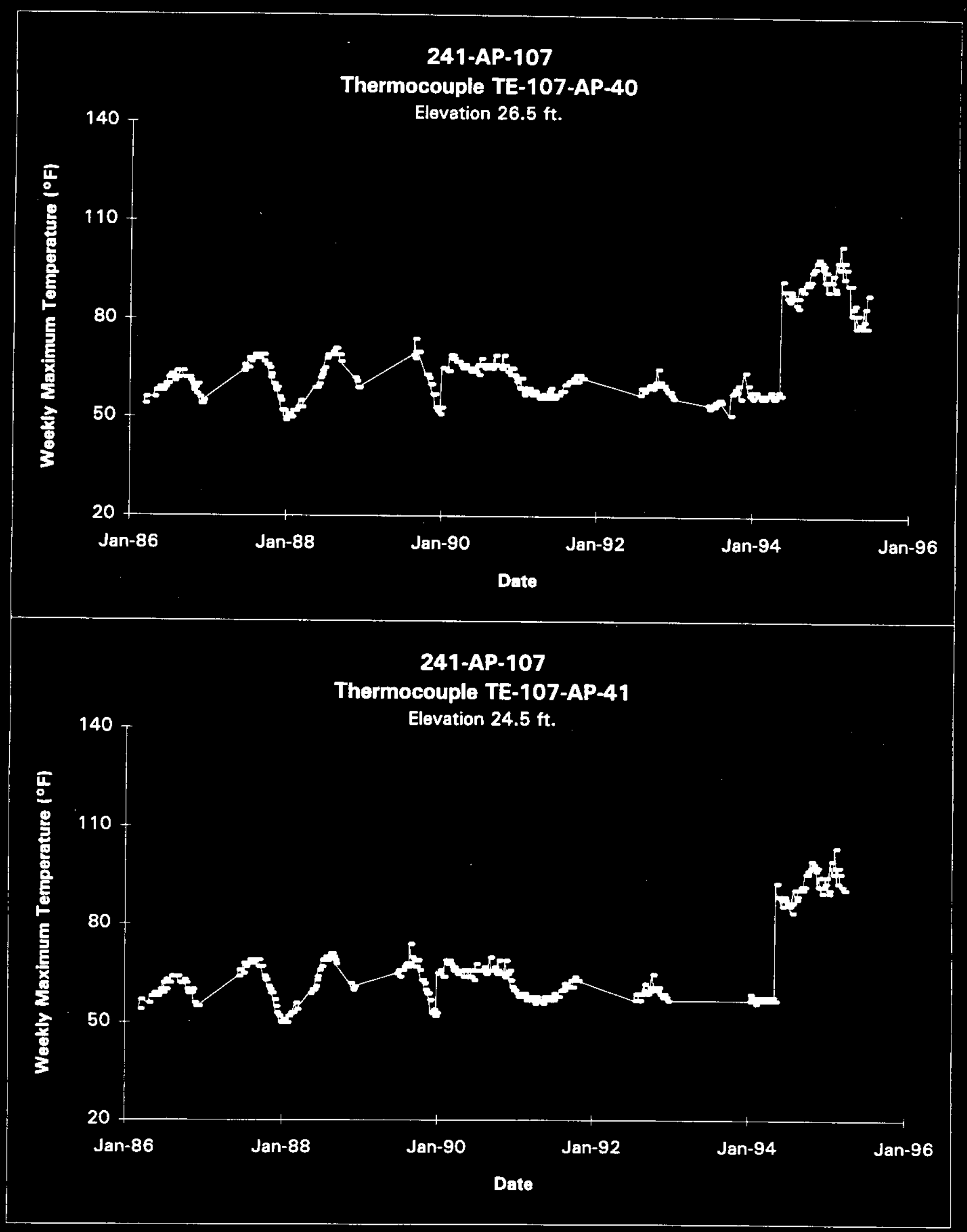

Data obtained from Computer Automated Surveillance Syatem (CASS), April 1995; and Surveillance Anslyais Computer Syatem (SACS), June 1995. 


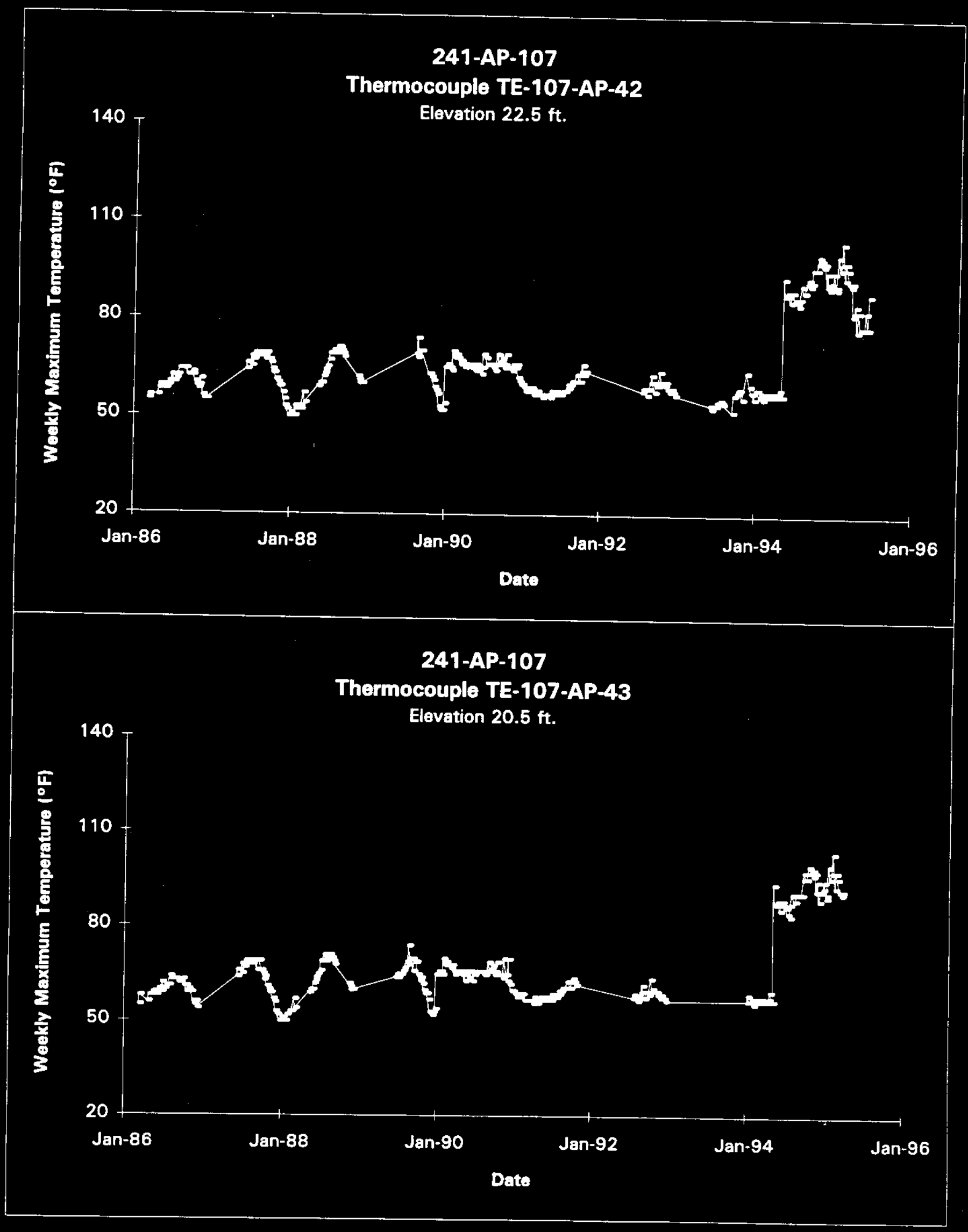




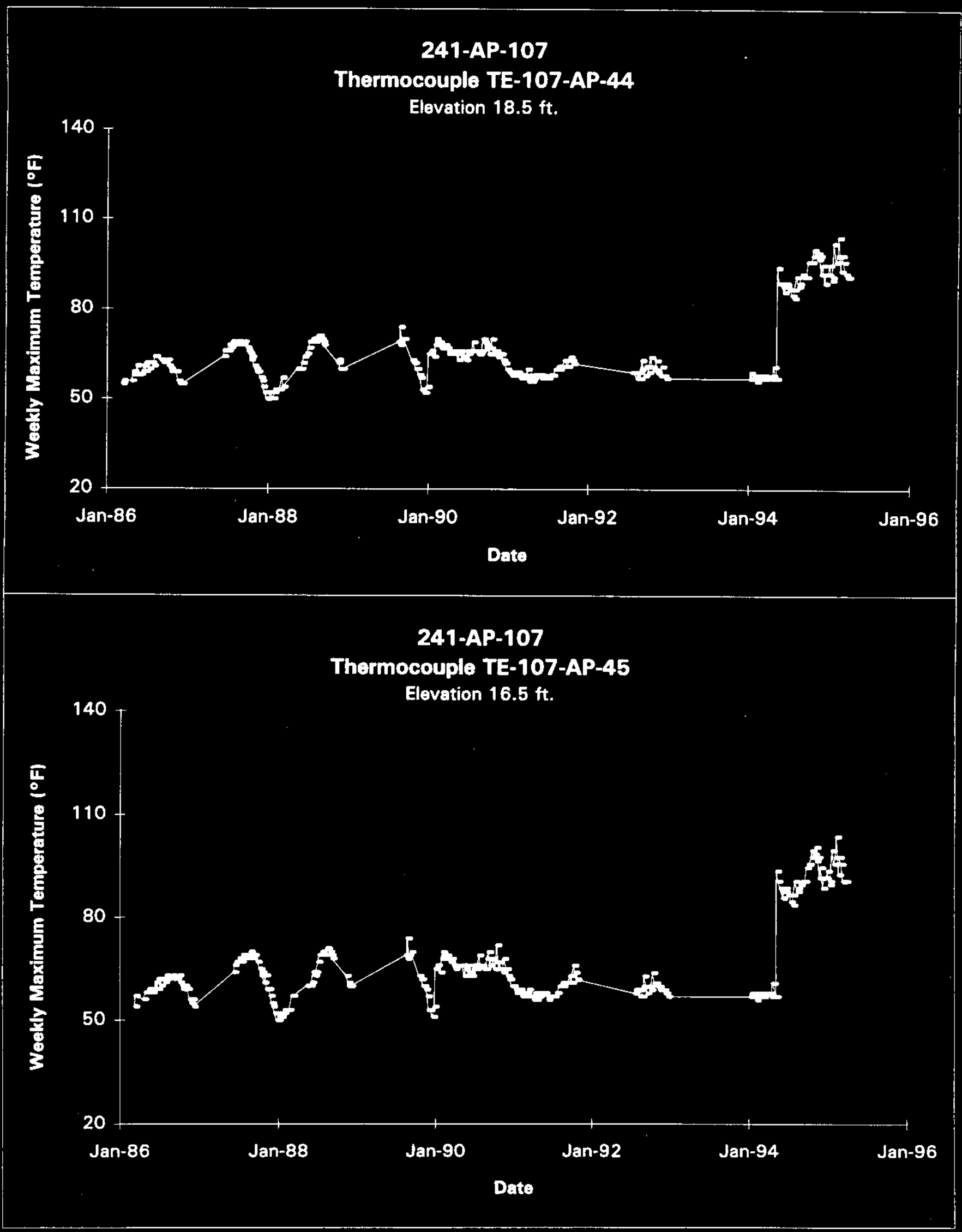

Date obtained from Computer Automated Surveillance System (CASS), April 1995; end Surveillance Analyais Computer System (SACS), June 1995. 


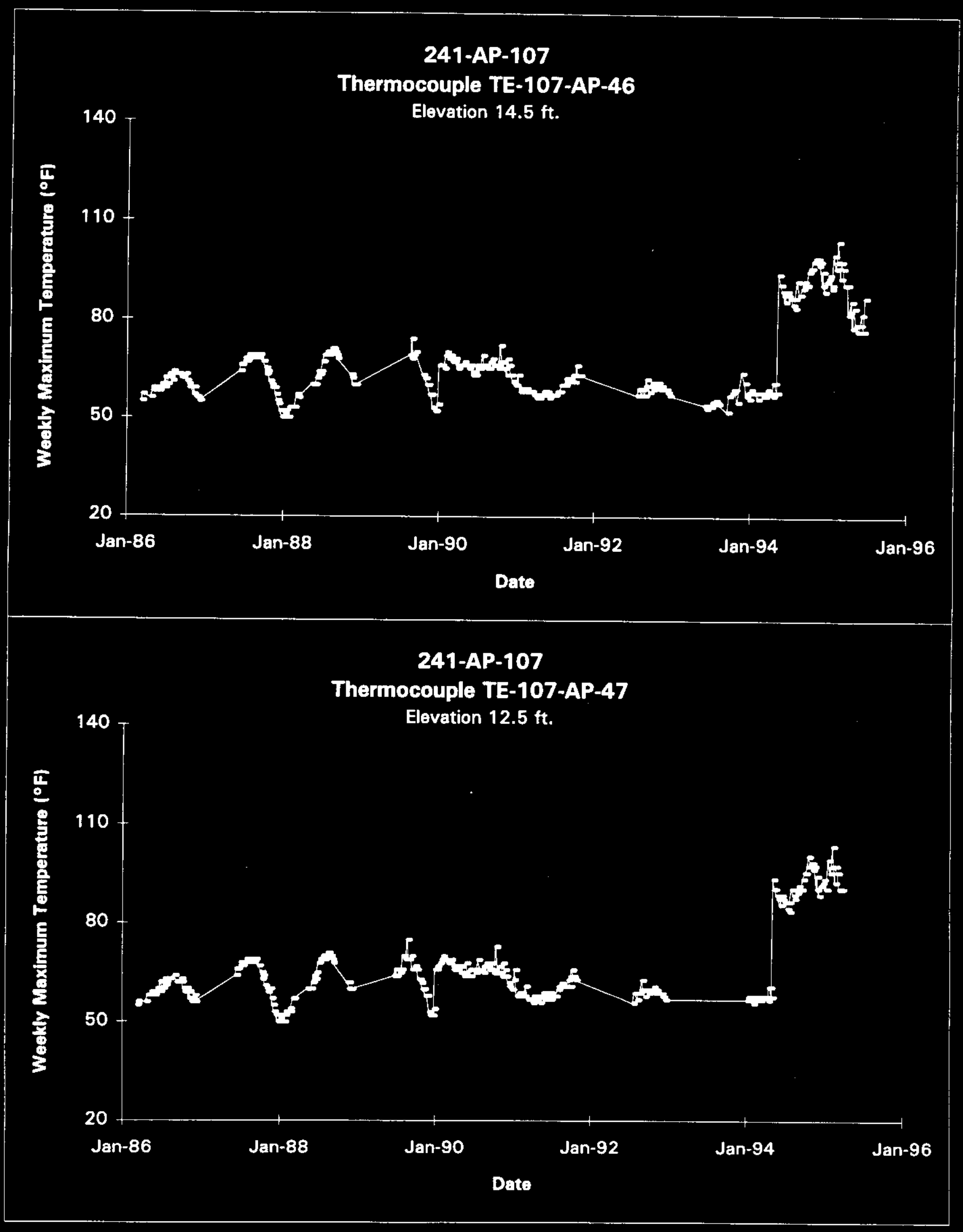




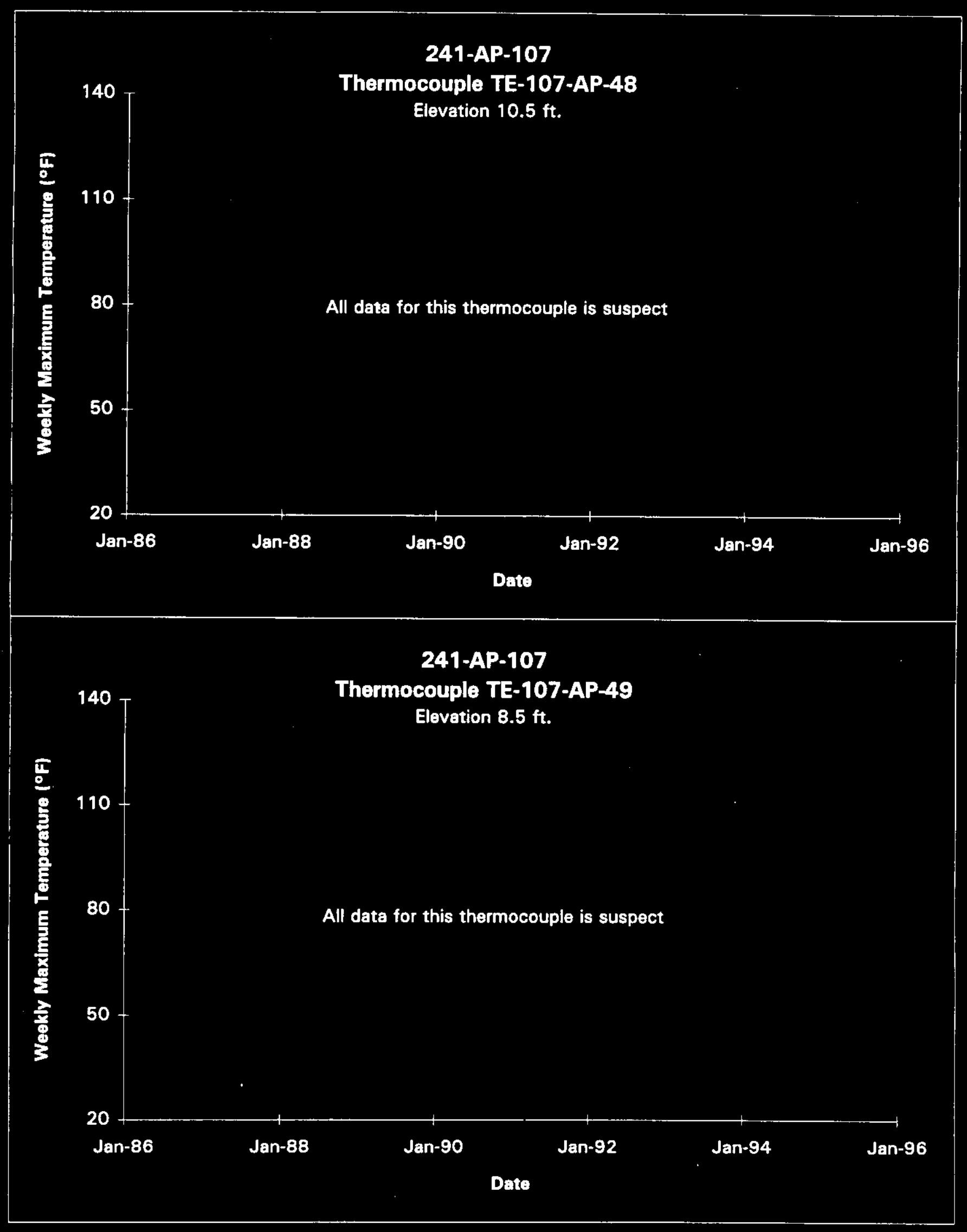




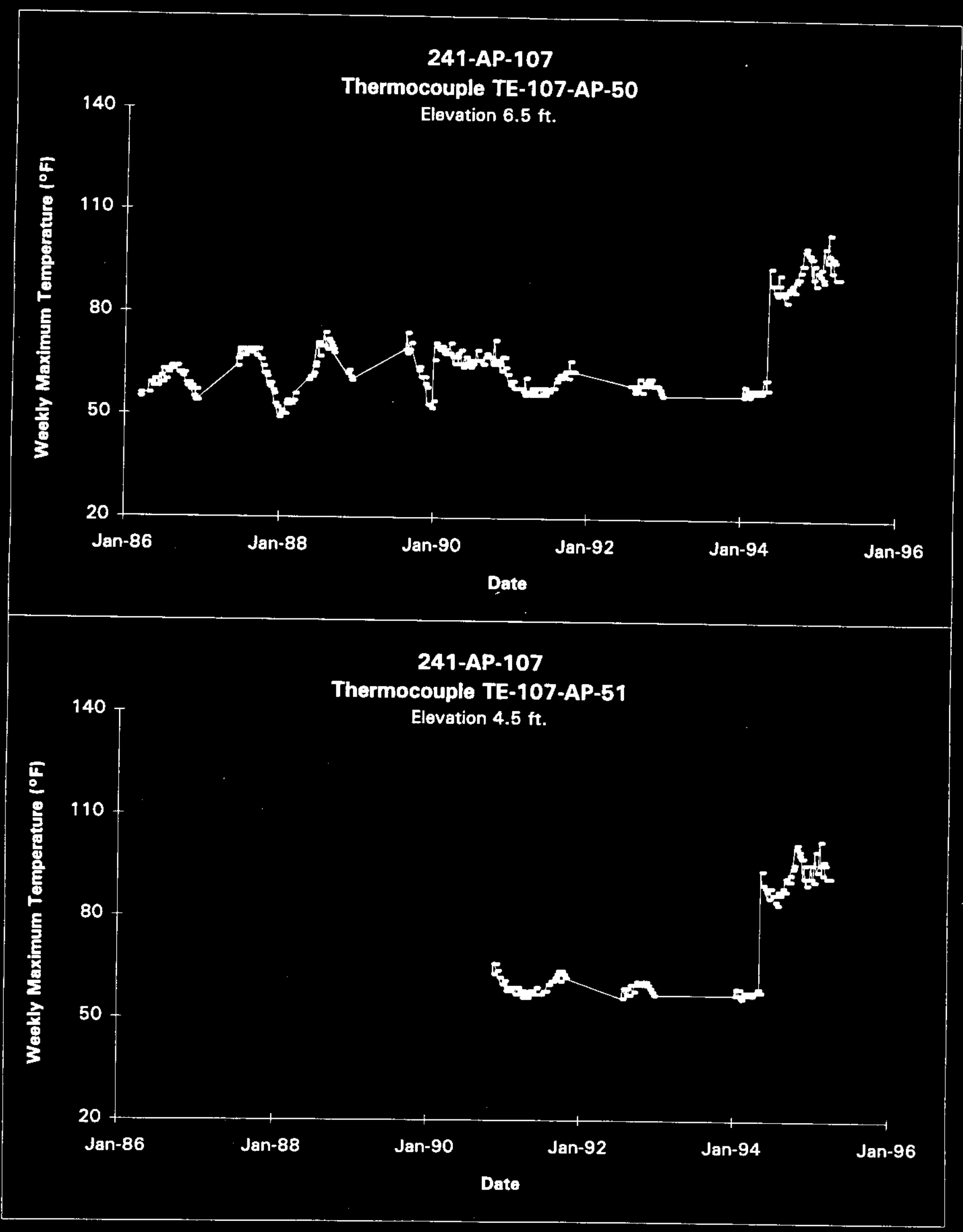




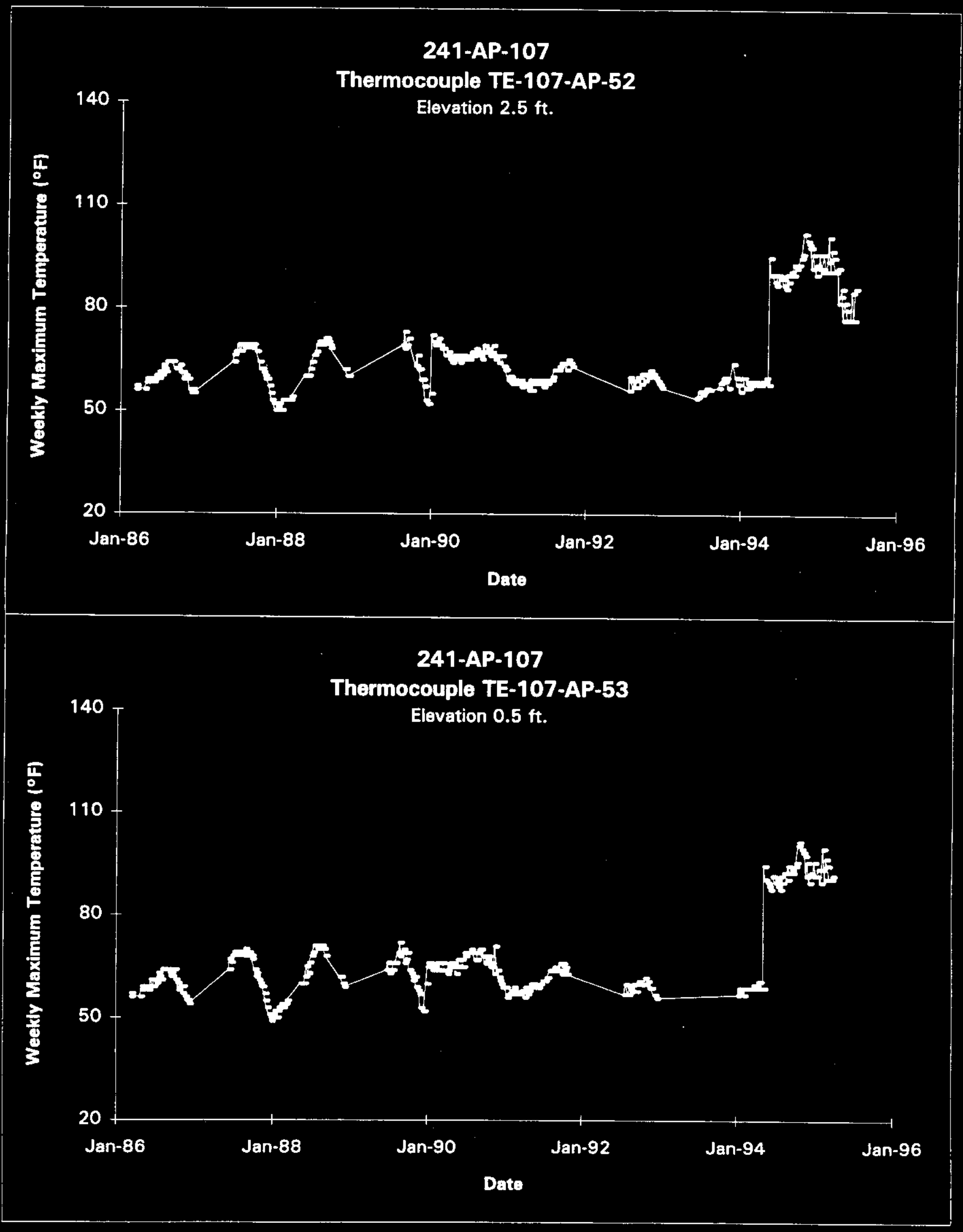

Data obtained from Computer Automuted Surveillance Syatem (CASS), Aprib 1995; and Survoillance Analyais Computer System (SACS), June 1995. 


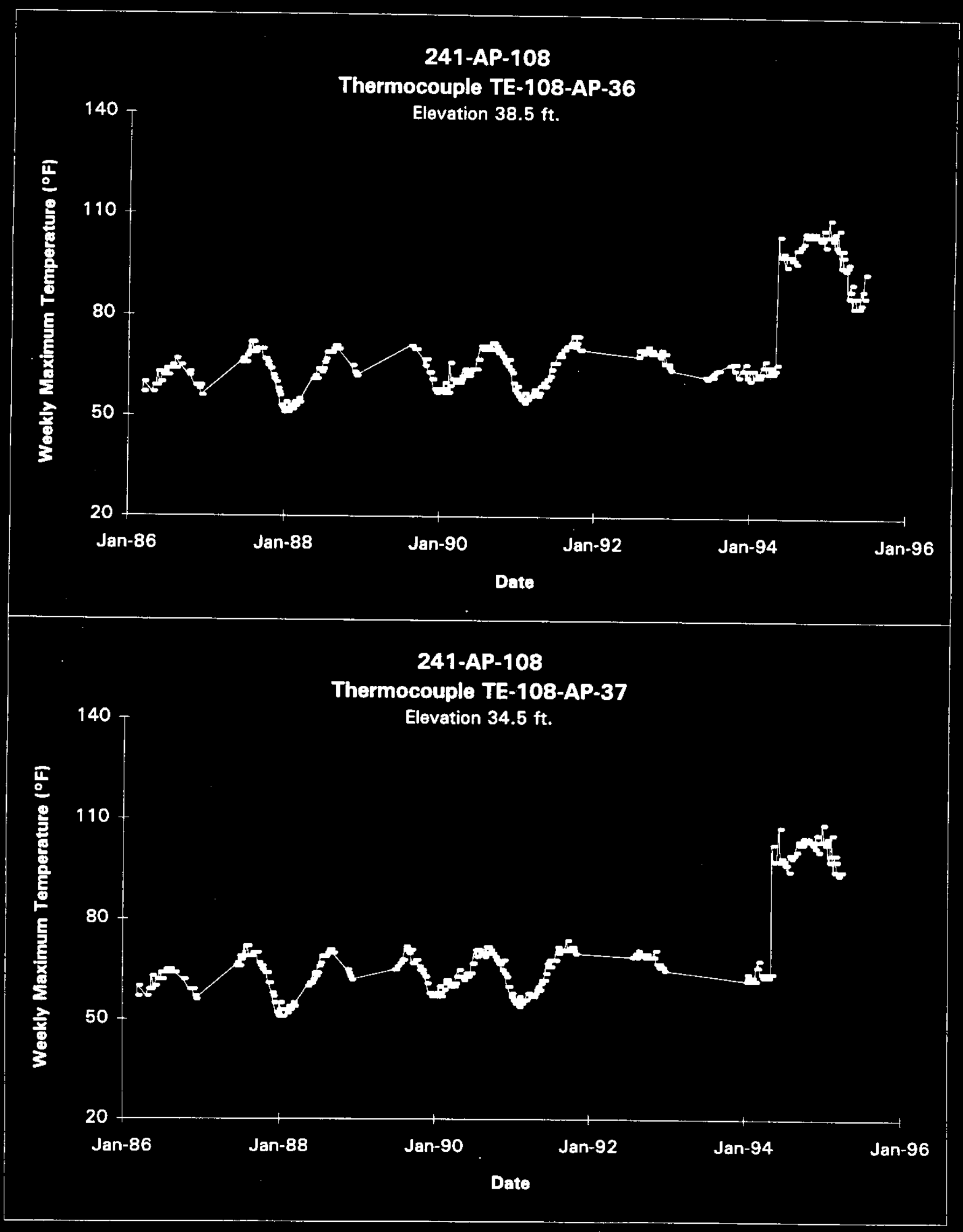

Dats obtained from Computer Automated Surveillance Syatem (CASS), April 1995; and Surveillance Analysis Computer System (SACS), June 1995. D- 64 


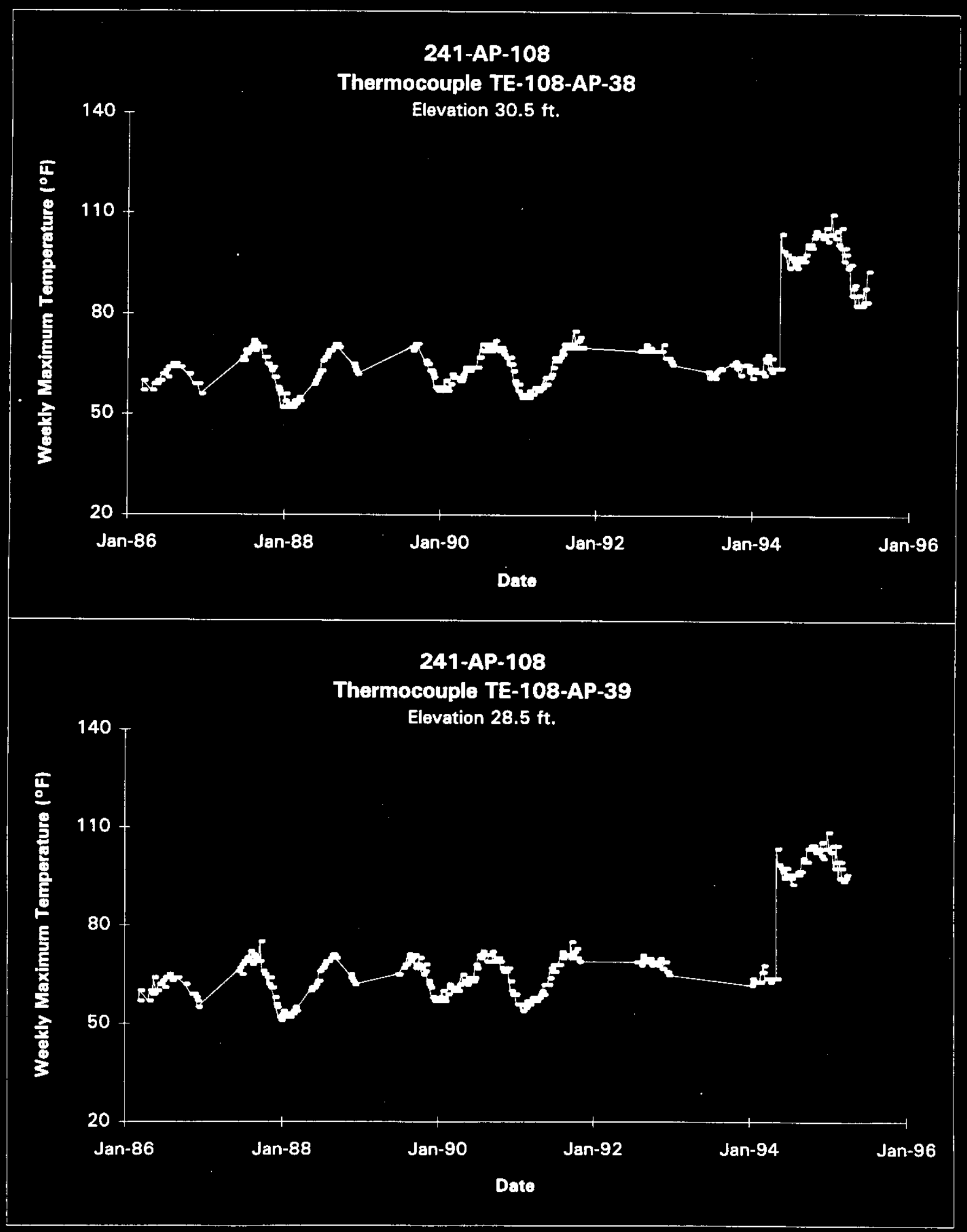




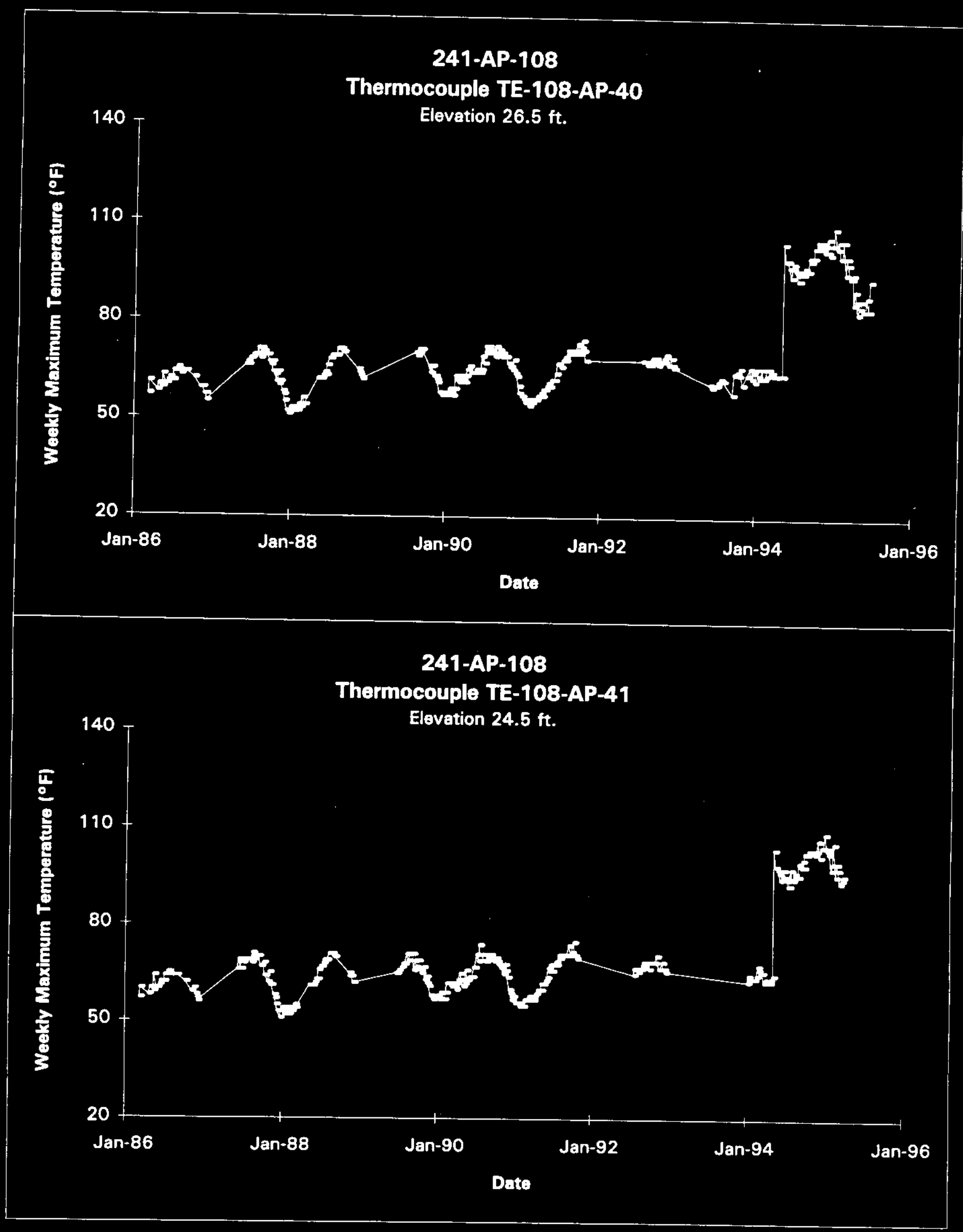

Date obteined from Computer Automated Surveillance System (CASS), April 1995; and Surveillance Analyais Computer System (SACS), June 1995. D- 66 


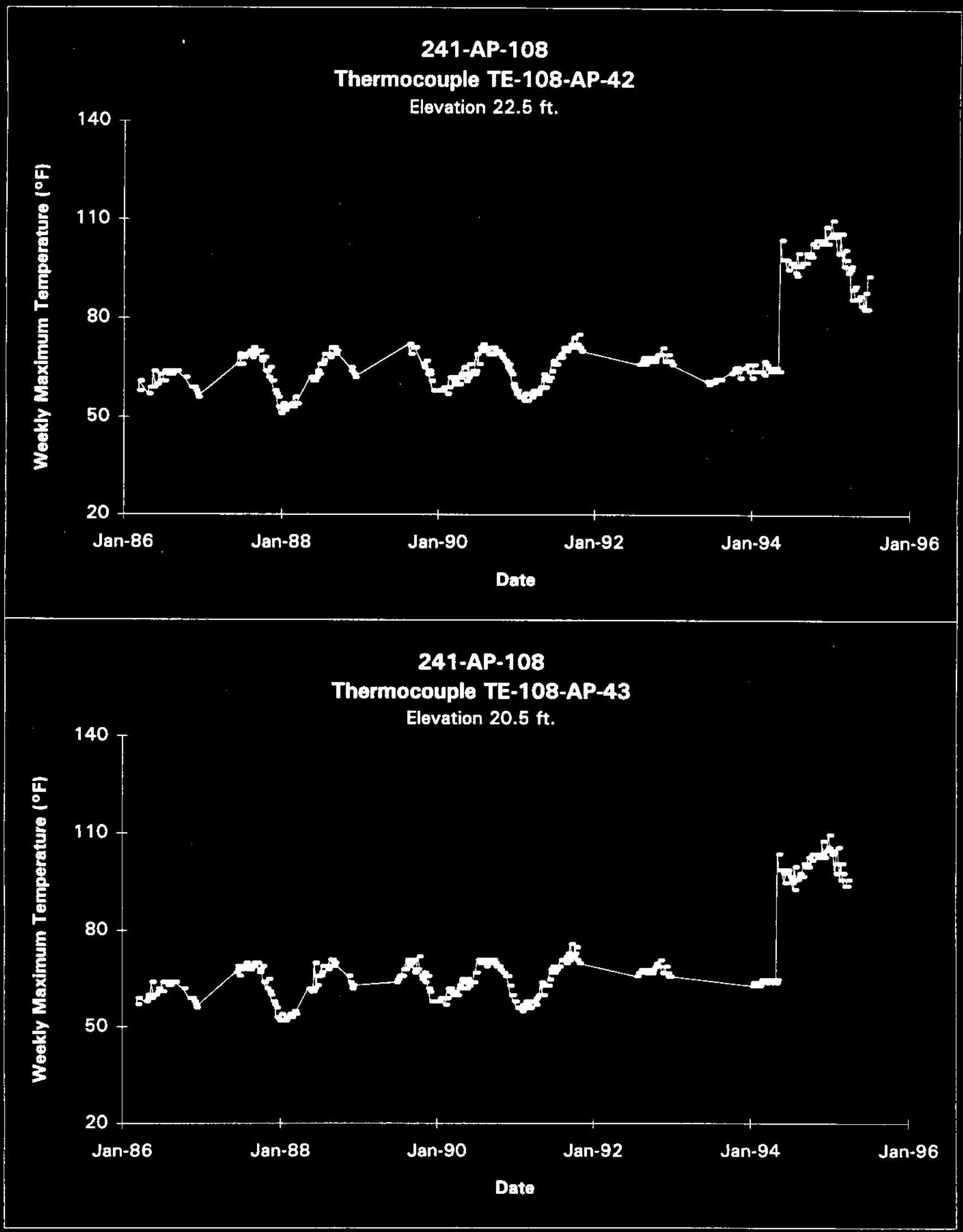




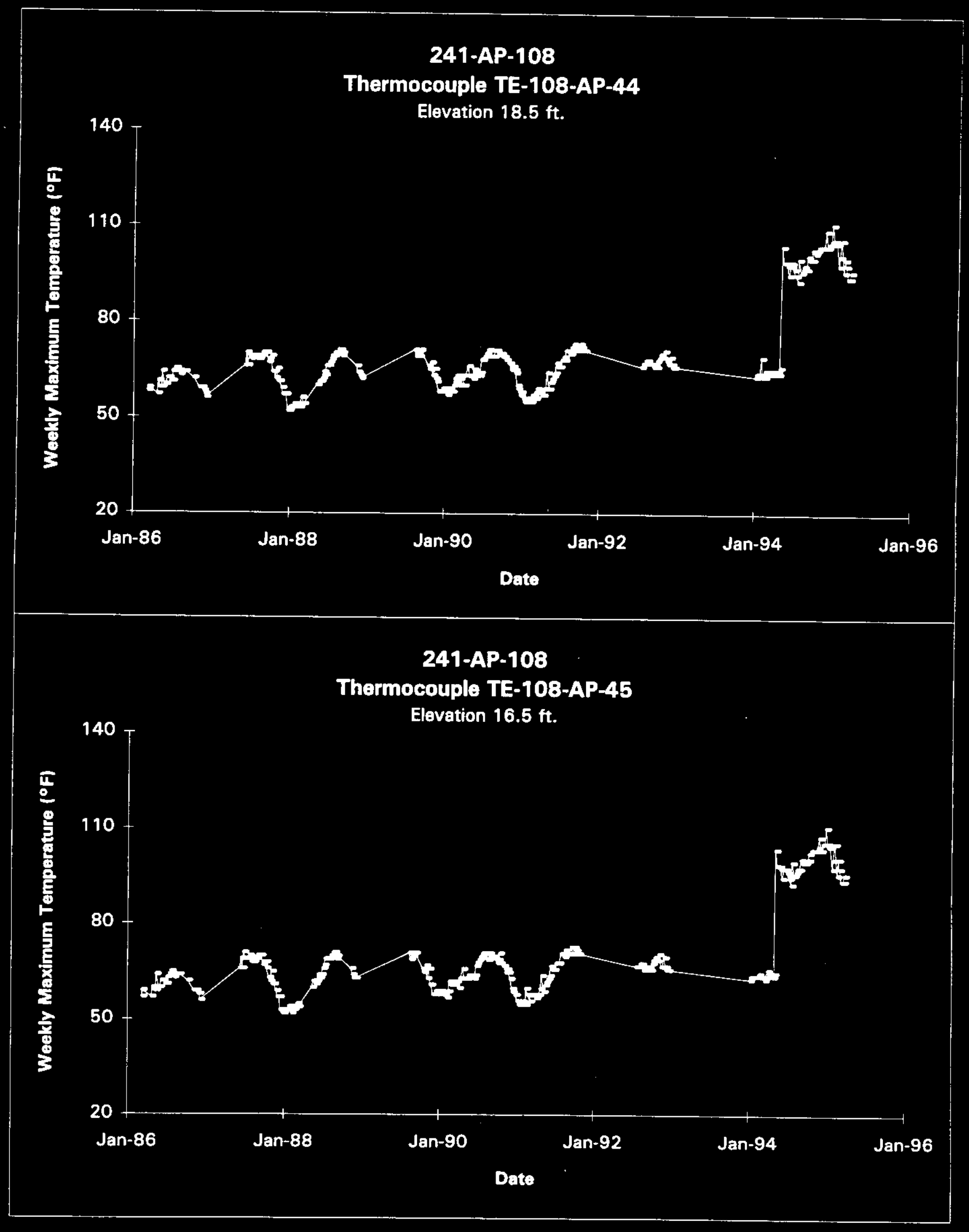

Date obtained from Computer Automated Surveillance System (CASS), April 1995; and Surveillance Analysis Computer System (SACS), June 1995. D- 68 


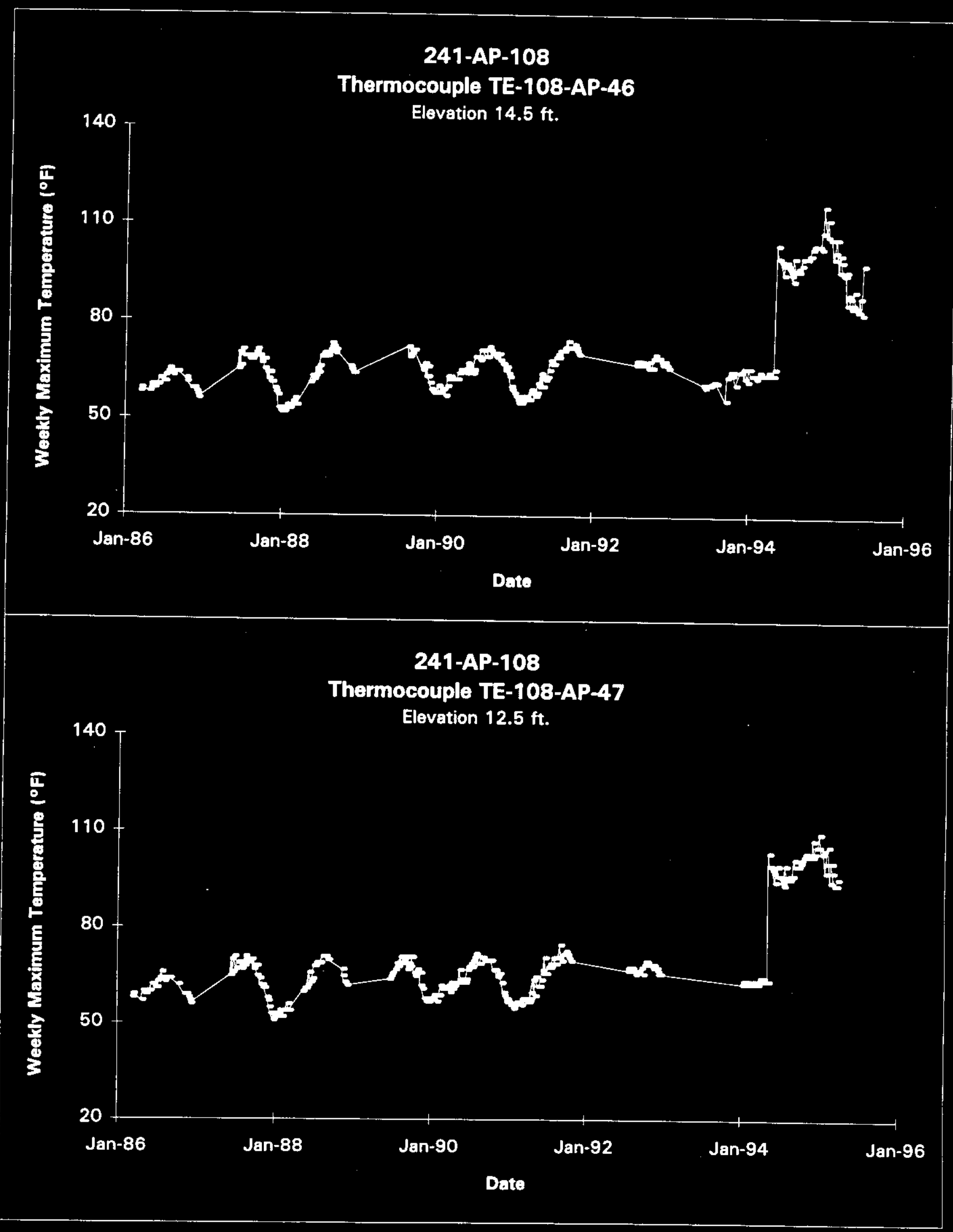

Date obteined from Computer Automated Surveillance System (CASS), April 1995; and Surveillance Analysis Computer Syatem (SACS), June 1995. D- 69 


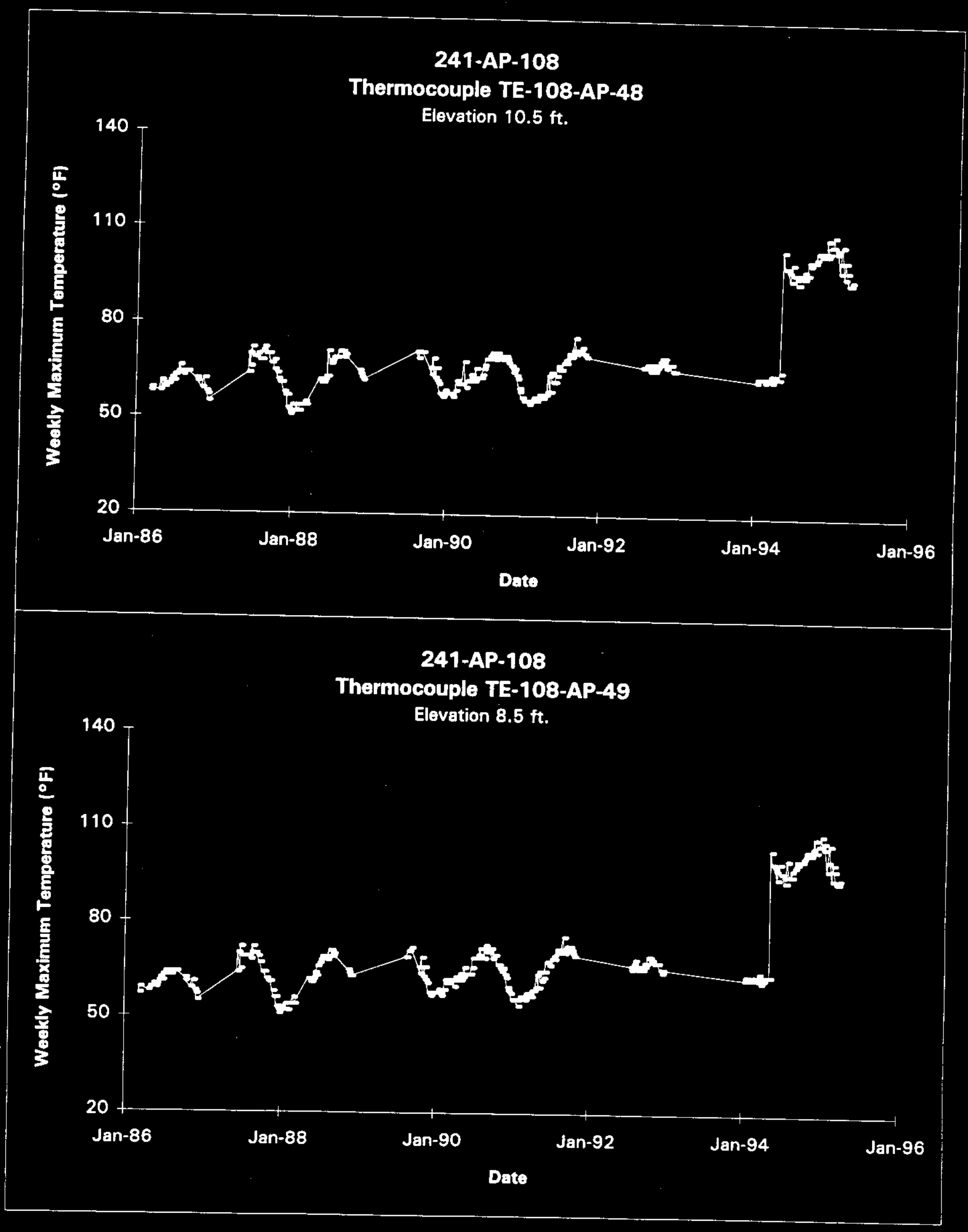

Date obtained from Computer Automated Survaillance Syatem (CASS), April 1995; and Surveillance Analysis Computer Syatem (SACS), June 1995. 


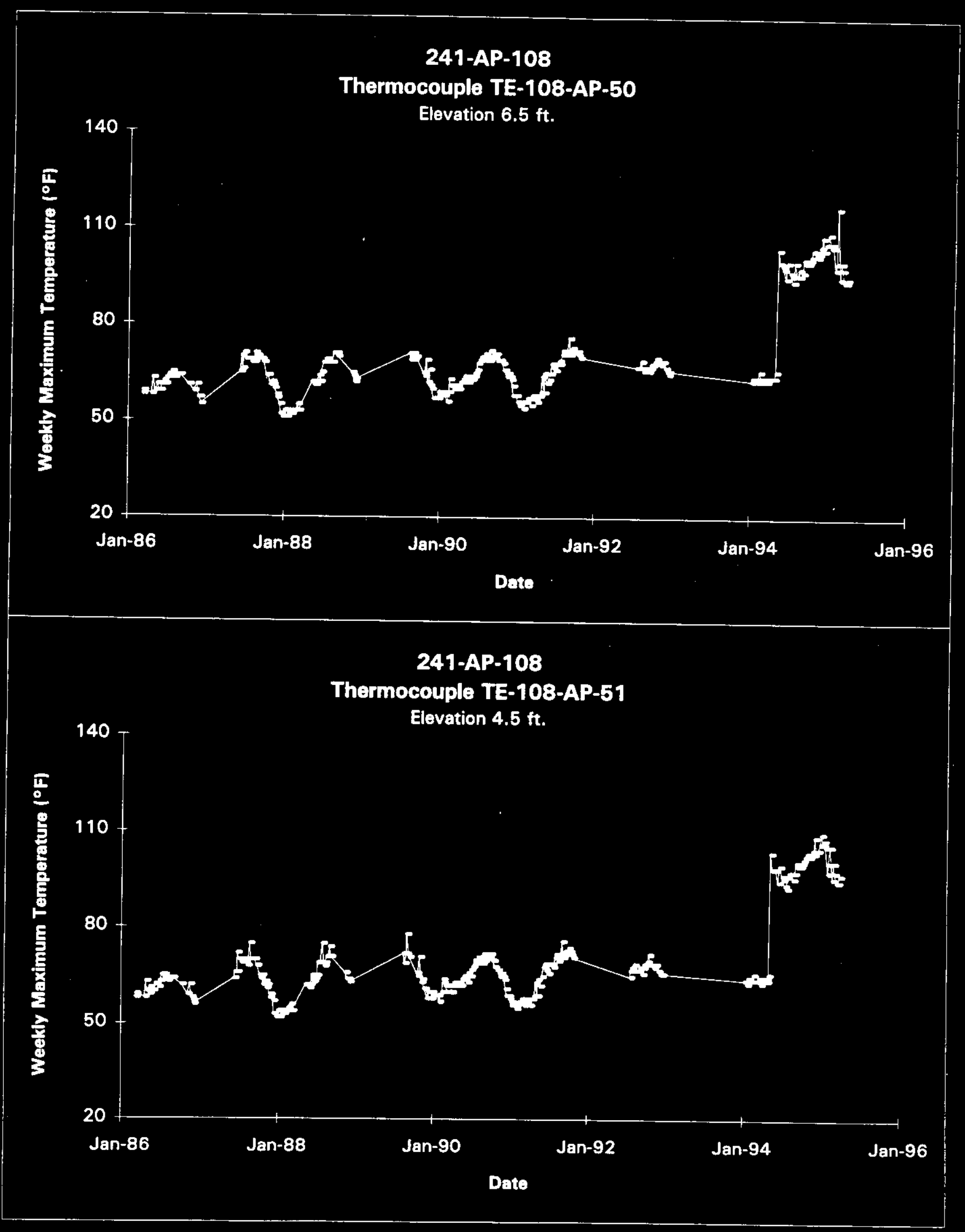

Data obtained from Computer Automated Surveillance System (CASS), April 1995; and Surveillance Analysis Computer System (SACS), June 1995. 


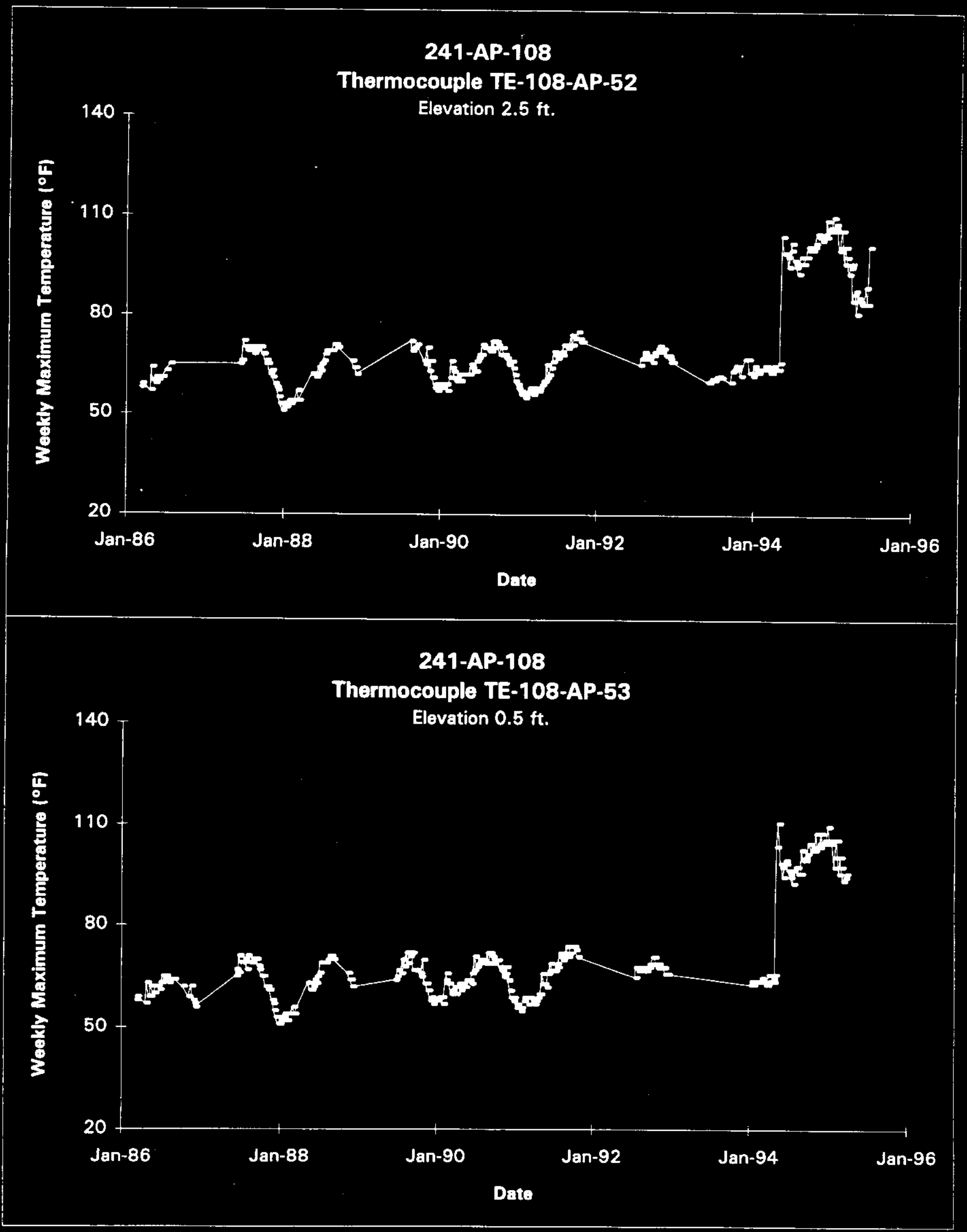

Data obtained from Computer Automated Survellence Syatem (CASS), April 1995; and Surveillance Analysis Computer System (SACS), June 1995. $\mathrm{D}-72$ 


\begin{tabular}{|c|c|c|c|c|c|}
\hline \multicolumn{6}{|c|}{$\begin{array}{l}\text { TANK 241-AP-101 } \\
\text { THIAMOCOUPLE DATA }\end{array}$} \\
\hline & & & & & \\
\hline THEPMOCOUPL * $*$ & DATE & TEMPERATUAE $\left({ }^{\circ} \mathrm{F}\right)$ & DATA SOURCE & QUAUTY & COMMENT \\
\hline TE-101-AP-36 & $4 / 5 / 86$ & 55 & CASS & $\bar{T}$ & \\
\hline TE-101-AP-36 & $4 / 7186$ & $\frac{56}{58}$ & $\begin{array}{l}\text { CASS } \\
\text { CASS }\end{array}$ & $T$ & \\
\hline TE-101-AP-36 & $4 / 13 / 86$ & \begin{tabular}{|l|}
58 \\
94
\end{tabular} & $\frac{\text { CASS }}{\text { CASS }}$ & $\frac{T}{\mathbf{S}}$ & \\
\hline $\begin{array}{l}\text { TE-101-AP-36 } \\
\text { TE-101-AP-36 }\end{array}$ & \begin{tabular}{l|}
$4 / 18 / 36$ \\
$4 / 24 / 86$
\end{tabular} & 56 & $\frac{\text { CASS }}{\text { CASS }}$ & $\frac{S}{T}$ & Data point did not agrae with local trend \\
\hline TE-101-AP-36 & $4 / 29 / 86$ & 55 & CASS & $T$ & \\
\hline TE-101-AP-36 & 5/16/36 & 56 & CASS & $\bar{T}$ & \\
\hline TE-101-AP-36 & $5 / 23 / 86$ & 57 & CASS & $\mathbf{T}$ & \\
\hline TE-101-AP-36 & $5 / 30 / 96$ & 58 & CASS & $T$ & \\
\hline TE-101-AP-36 & $6 / 7 / 86$ & 57 & CASS & $T$ & \\
\hline TE-101-AP-36 & 6/17/86 & 58 & CASS & $\mathbf{T}$ & \\
\hline TE-101-AP-36 & 6/15/86 & 59 & CASS & $T$ & \\
\hline TE-101-AP-36 & $6 / 26 / 86$ & 59 & CASS & $\bar{T}$ & \\
\hline TE-101-AP-36 & $7 / 4 / 86$ & ह9 & CASS & $T$ & \\
\hline TE-101-AP-36 & $7 / 12 / 86$ & 59 & CASS & $\bar{T}$ & \\
\hline TE-101-AP-36 & $7 / 19 / 86$ & 59 & CASS & $T$ & \\
\hline TE-101-AP-36 & $7121 / 86$ & 60 & CASS & $T$ & \\
\hline TE-101-AP-36 & $7 / 29 / 86$ & 60 & CASS & $\bar{T}$ & \\
\hline TE-101-AP-36 & $8 / 5 / 86$ & 61 & CASS & $T$ & \\
\hline TE-101-AP-36 & $8 / 16 / 86$ & 60 & CASS & $\bar{T}$ & \\
\hline TE-107-AP-36 & $8 / 22766$ & 61 & CASS & $T$ & \\
\hline TE-101-AP-36 & $8 / 30 / 86$ & 62 & CASS & $T$ & \\
\hline TE-101-AP-36 & $9 / 6 / 86$ & 63 & CASS & $\mathbf{T}$ & \\
\hline TE-101-AP-36 & 9/9/86 & 64 & CASS & $T$ & \\
\hline TE-101-AP- 36 & 9/19/36 & 64 & CASS & $T$ & \\
\hline TE-101-AP-36 & $9 / 26 / 86$ & 64 & CASS & $T$ & \\
\hline TE-101-AP-36 & $10 / 2 / 86$ & 63 & CASS & $\bar{T}$ & \\
\hline TE-101-AP-36 & $10 / 10 / 86$ & 62 & CASS & $T$ & \\
\hline TE-101-AP-36 & $10 / 15 / 66$ & 62 & CASS & $T$ & \\
\hline TE-101-AP-36 & $10 / 25 / 86$ & 60 & CASS & $\bar{T}$ & \\
\hline TE-101-AP-36 & $10 / 30 / 86$ & 61 & CASS & $T$ & \\
\hline TE-101-AP-36 & $11 / 5 / 86$ & 61 & CASS & $T$ & \\
\hline TE-101-AP-36 & $11 / 15 / 86$ & 58 & CASS & $T$ & \\
\hline TE-101-AP-36 & $11 / 21 / 86$ & 57 & CASS & $T$ & \\
\hline$T E-101-A P=36$ & $11 / 25 / 66$ & 57 & CASS & $T$ & \\
\hline TE-101-AP-36 & $12 / 2 / 66$ & 58 & CASS & $T$ & \\
\hline TE-101-AP-36 & $12 / 13 / 86$ & 54 & CASS & $T$ & \\
\hline TE-10\}-AP-36 & $12 / 20 / 86$ & 53 & CASS & $T$ & \\
\hline TE-101-AP-36 & $12 / 25 / 86$ & 5 & CASS & $T$ & \\
\hline TE-101-AP-36 & $12 / 30 / 86$ & 5 & CASS & $T$ & \\
\hline TE-101-AP-36 & $7 / 4 / 67$ & 64 & CASS & $T$ & \\
\hline TE-101-AP-36 & $7 / 11 / 87$ & 64 & CASS & $T$ & \\
\hline TE-101-AP-36 & $7 / 16 / 87$ & 65 & CASS & $\mathbf{T}$ & \\
\hline TE-101-AP-36 & $7 / 23 / 87$ & 64 & CASS & $T$ & \\
\hline TE-101-AP-36 & 7/29/87 & 66 & CASS & $\mathbf{T}$ & \\
\hline TE-101-AP-36 & 8/8/87 & 65 & CASS & $T$ & \\
\hline TE-101-AP-36 & $8 / 14 / 67$ & 67 & CASS & $T$ & \\
\hline TE-101-AP-36 & B/18/87 & 67 & CASS & $T$ & \\
\hline TE-101-AP-36 & $8 / 27 / 87$ & 67 & CASS & $T$ & \\
\hline TE-101-AP-36 & $9 / 5 / 87$ & 67 & CASS & $T$ & \\
\hline TE-101-AP.36 & $9 / 11 / 87$ & 68 & CASS & $T$ & \\
\hline TE-101-AP-36 & $9 / 13 / 87$ & 69 & CASS & $T$ & \\
\hline TE-101-AP-36 & $9 / 23 / 87$ & 68 & CASS & $\bar{T}$ & \\
\hline TE-101-AP-36 & $10 / 1 / 97$ & 67 & CASS & $T$ & \\
\hline TE-101-AP-36 & $10 / 5 / 87$ & 67 & CASS & $\bar{T}$ & \\
\hline TE-101-AP-36 & $10 / 14 / 87$ & 65 & CASS & $T$ & \\
\hline TE-101-AP-36 & $10 / 24 / 87$ & 64 & CASS & 7 & \\
\hline TE-101-AP-36 & $10 / 30 / 87$ & 63 & CASS & $T$ & \\
\hline TE-101-AP-36 & $11 / 5 / 87$ & 63 & CASS & $T$ & \\
\hline TE-101-AP-36 & $11 / 12 / 87$ & 64 & CASS & $\bar{T}$ & \\
\hline TE-101-AP-36 & $11 / 16 / 87$ & 61! & CASS & $\mathbf{T}$ & \\
\hline TE-101-AP-36 & $11 / 27 / 87$ & 59 & CASS & $T$ & \\
\hline TE-101-AP-36 & $12 / 5 / 87$ & 61 & CASS & $T$ & \\
\hline TE-101-AP-36 & $12 / 9 / 87$ & S9: & CASS & $\bar{T}$ & \\
\hline TE-101-AP-36 & $12 / 18 / 87$ & 60 & CASS & $T$ & \\
\hline TE-101-AP-36 & $12 / 24 / 87$ & 57 & CASS & $T$ & \\
\hline TE-101-AP-36 & $12 / 28 / 87$ & 56 & CASS & $\mathrm{T}$ & \\
\hline TE-101-AP-36 & $1 / 8 / 83$ & 54 & CASS & $T$ & \\
\hline TE-101-AP-36 & $1 / 11 / 88$ & 5 & CASS & $T$ & \\
\hline TE-101-AP-36 & 1/18/88 & 53 & CASS & $T$ & \\
\hline TE-101-AP-36 & $1 / 29 / 88$ & 53 & CASS & $T$ & \\
\hline TE-101-AP-36 & $1 / 31 / 88$ & 52 & CASS & $T$ & \\
\hline TE-101-AP-36 & $2 / 11 / 8 B$ & B2! & CASS & $\mathbf{T}$ & \\
\hline
\end{tabular}




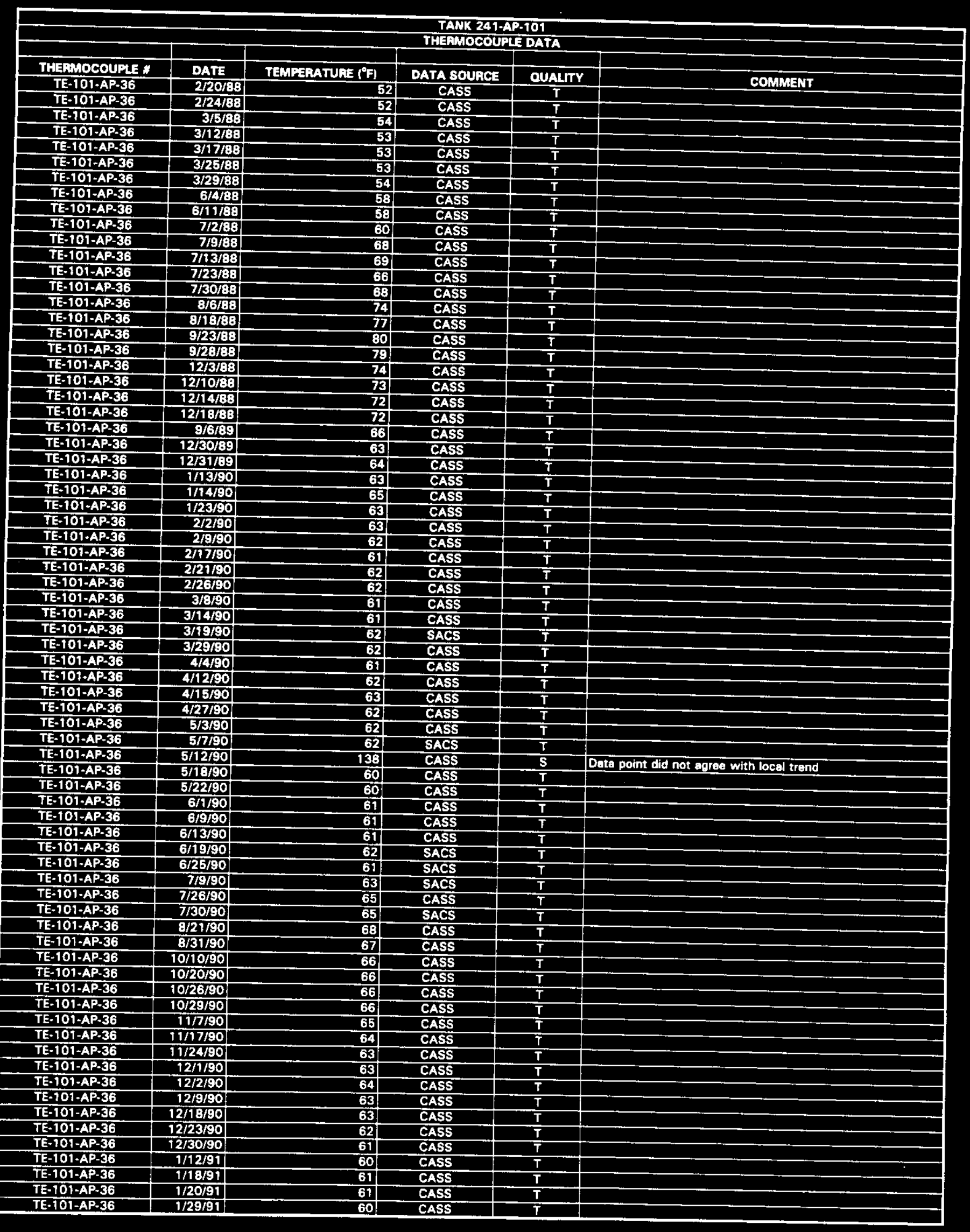




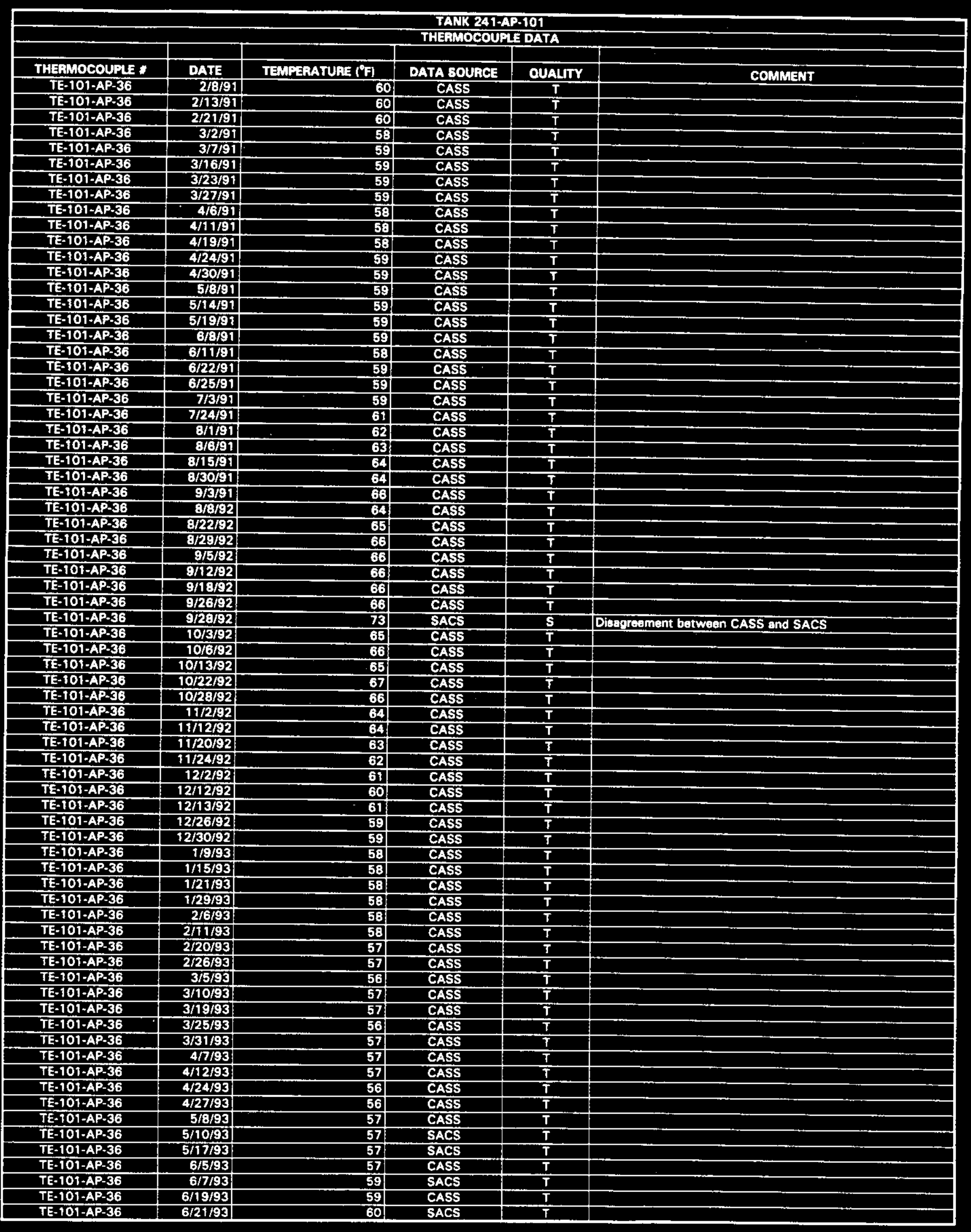

Data obtained from Computer Automated Surveillance System (CASS), April 1995; and Surveillance Analysis Computer System (SACS). June 1995. 


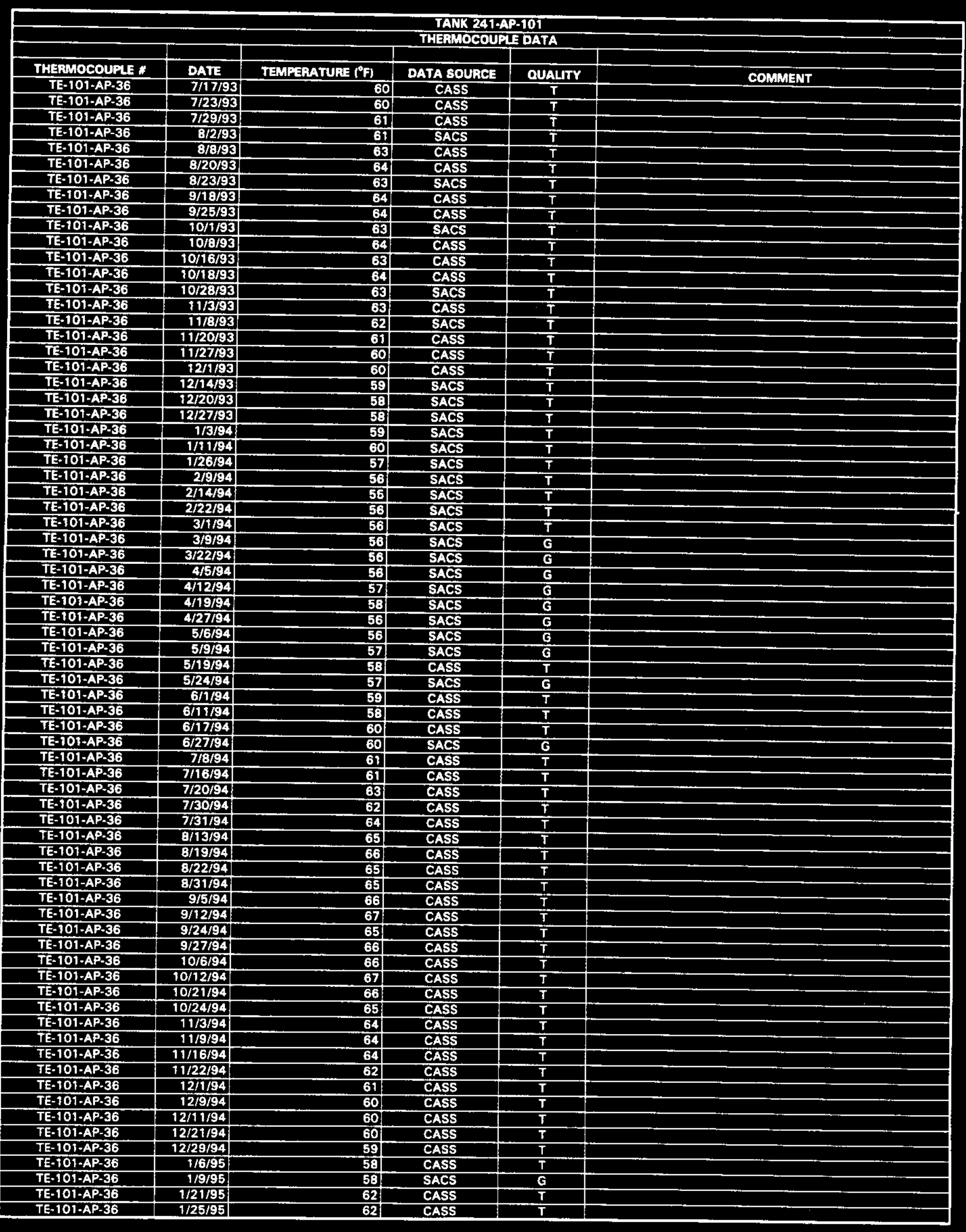

Data obtained from Computer Automated Surveillance System (CASS), April 1995; and Surveillance Analysis Computer System (SACS), June 1995.

D-76 


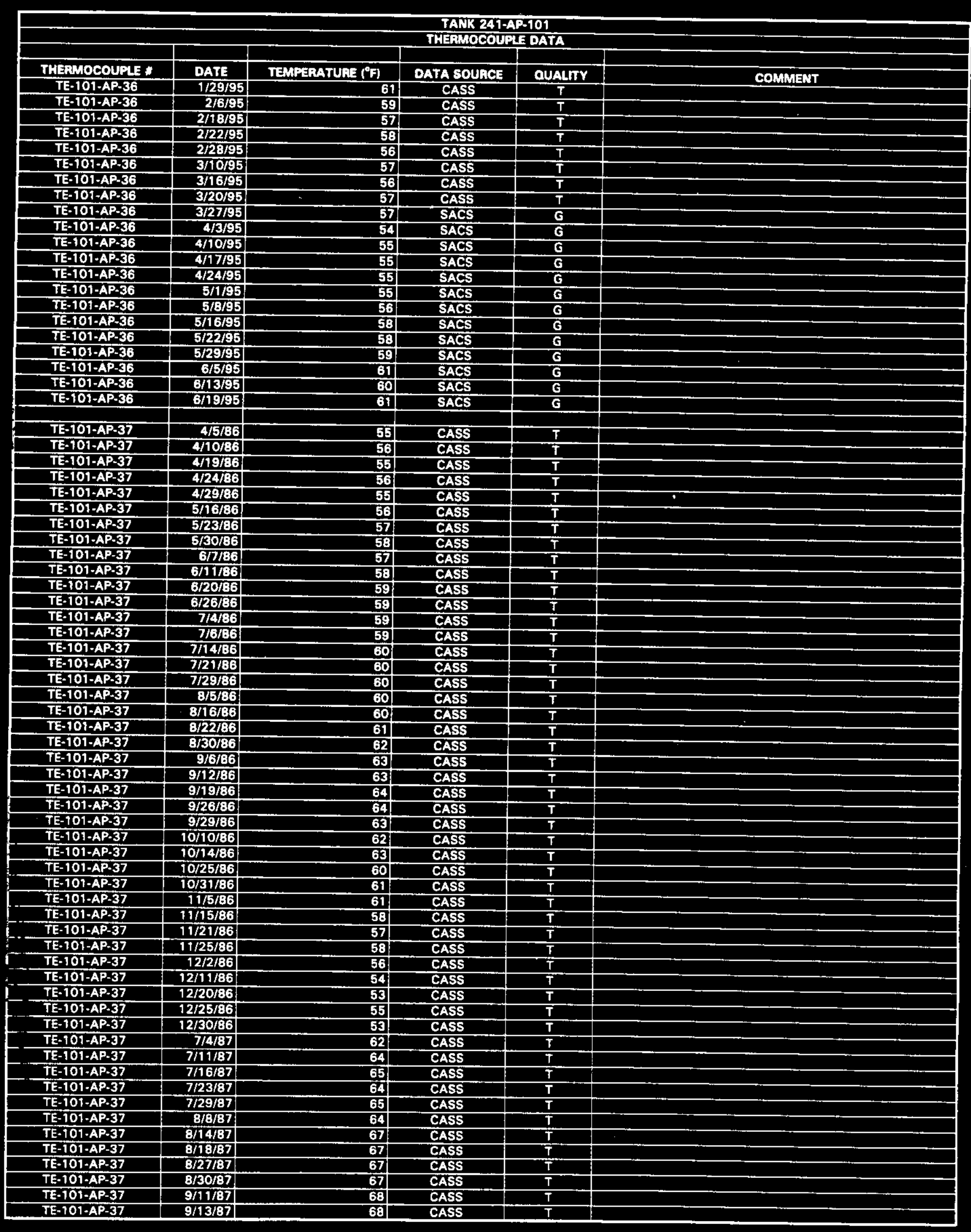

Date obtained from Computer Automated Surveillance System (CASS), April 1995; and Surveillance Analysis Computer System (SACS), June 1995. 


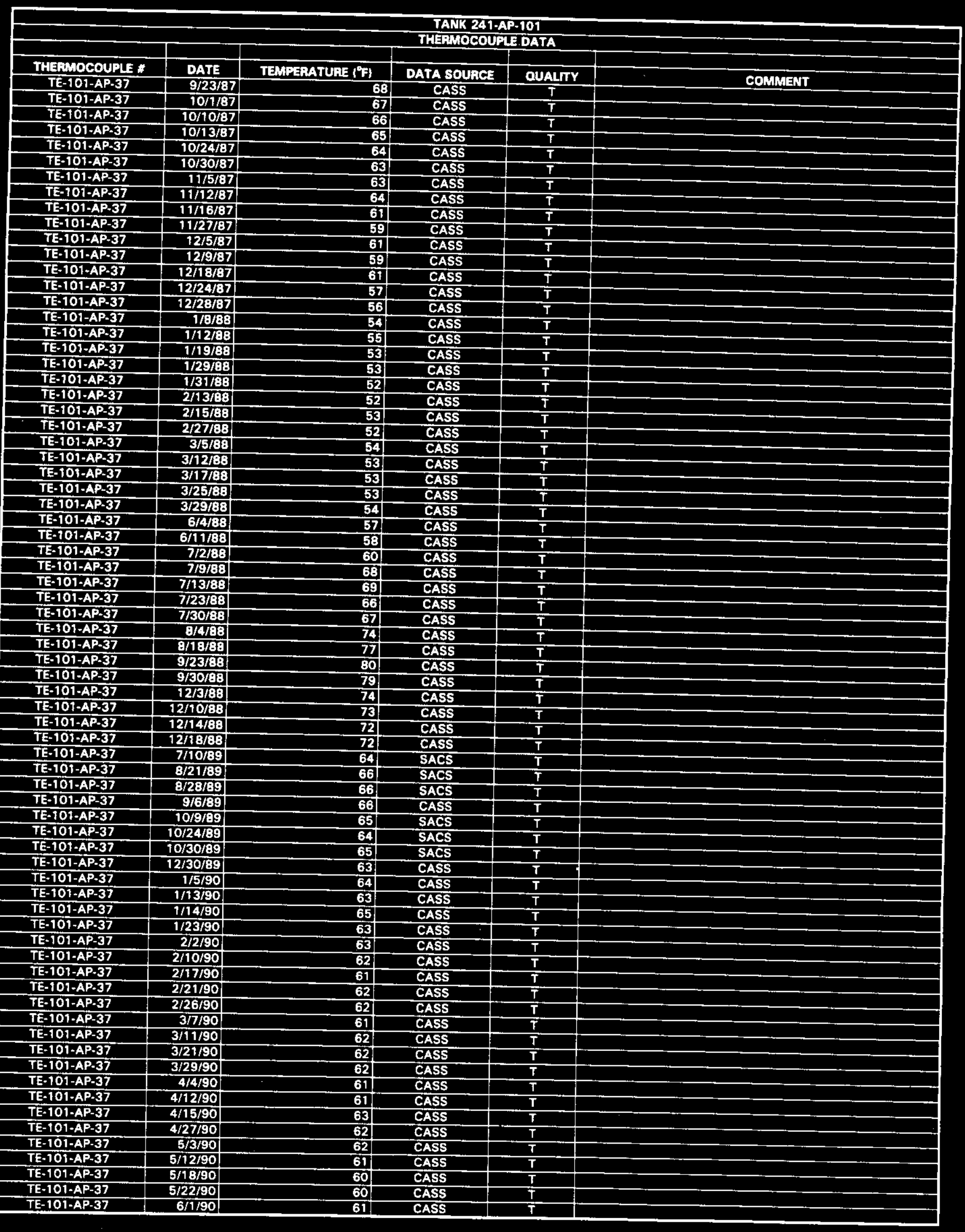




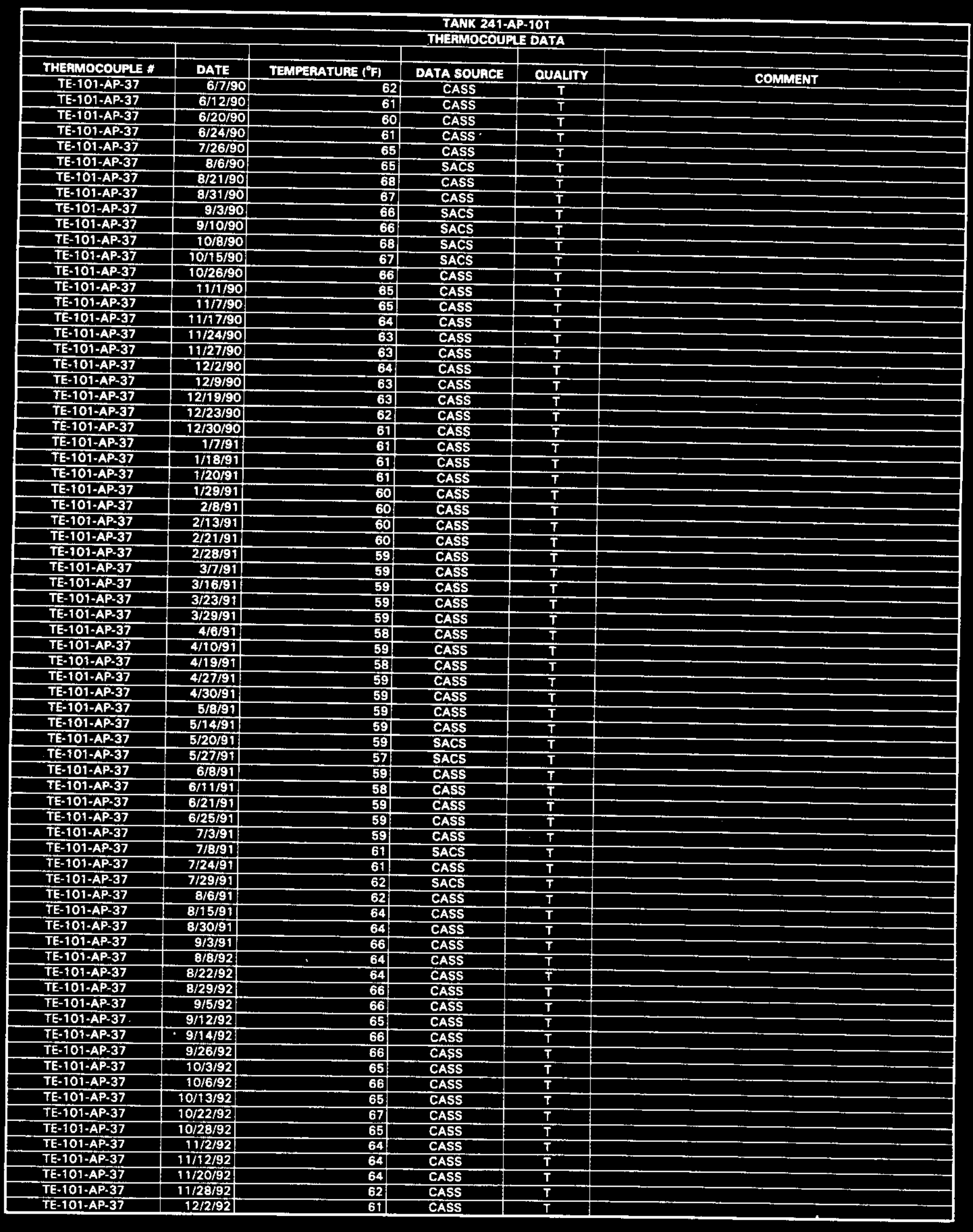




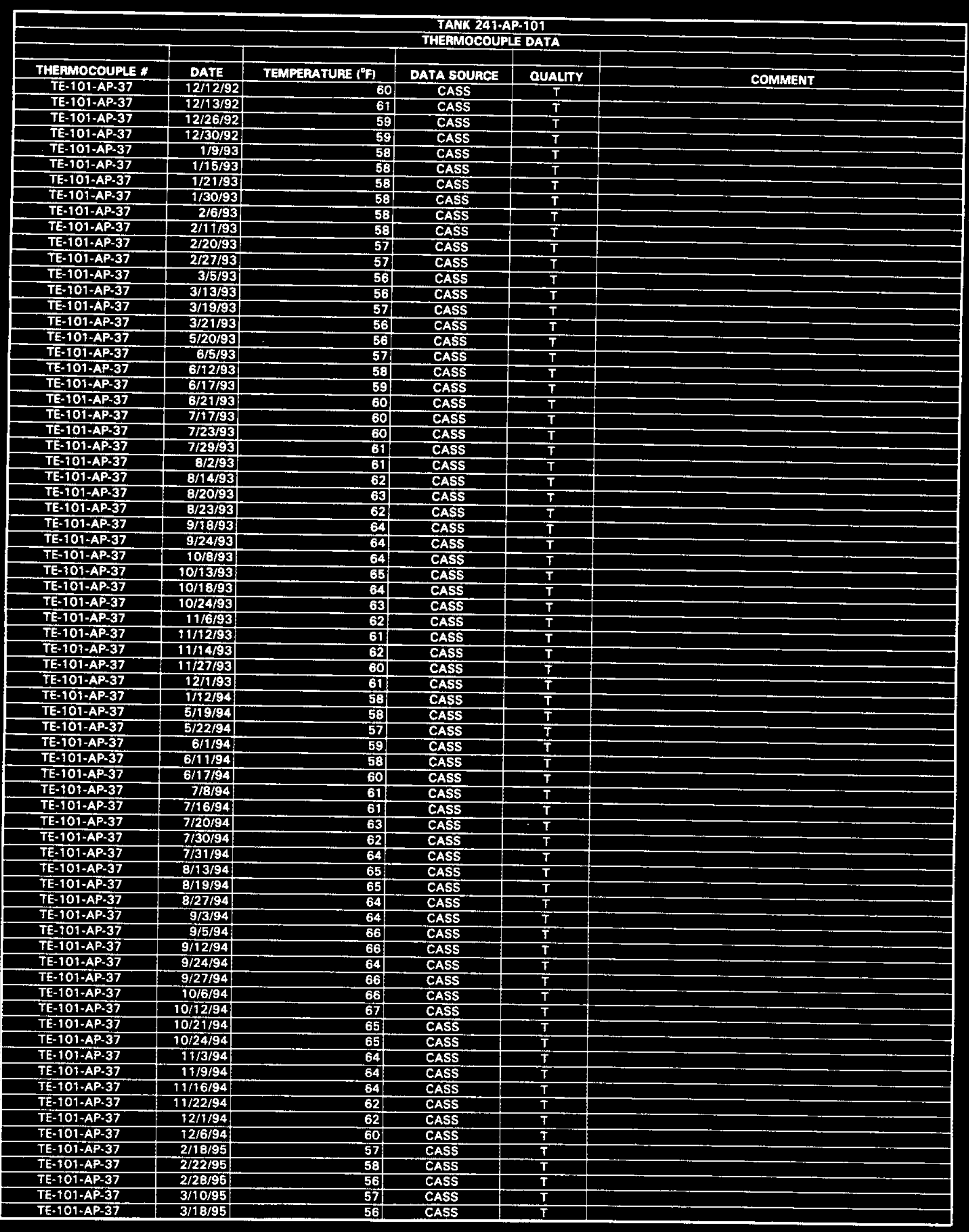




\begin{tabular}{|c|c|c|c|c|c|}
\hline \multicolumn{6}{|c|}{ TANK 241-AP-101 } \\
\hline \multicolumn{6}{|c|}{ THETMOCOUPLE DATA } \\
\hline & & & & & . \\
\hline THERMOCOUPLE * & DATE & TEMPERATUAE $\left.f^{\circ} \mathrm{F}\right\}$ & DATA SOURCE & QUALTY & COMMENT \\
\hline TE-101-AP-37 & $3 / 20 / 95$ & 57 & CASS & $T$ & \\
\hline TE-101-AP-37 & $3 / 26 / 95$ & 57 & CASS & $T$ & \\
\hline & & & & & \\
\hline TE-101-AP-38 & $4 / 5 / 36$ & 55 & CASS & $T$ & \\
\hline TE-101-AP-38 & $4 / 10 / 86$ & 56 & CASS & $T$ & \\
\hline TE-101-AP-38 & $4 / 19 / 36$ & 5 & CASS & $\bar{T}$ & \\
\hline TE-101-AP-38 & $4 / 24 / 86$ & 56 & CASS & $\bar{T}$ & \\
\hline TE-101-AP-38 & $4 / 29 / 86$ & 55 & CASS & $T$ & \\
\hline TE-101-AP-38 & $5 / 16 / 86$ & 56 & CASS & $T$ & \\
\hline TE-101-AP-38 & 5/23/86 & 57 & CASS & $T$ & \\
\hline TE-101.AP-3B & 5/30/86 & 58 & CASS & $\mathrm{T}$ & \\
\hline TE-101-AP-38 & $6 / 7 / 86$ & 57 & CASS & $T$ & \\
\hline TE-101-AP-38 & 6/11/86 & 58 & CASS & $T$ & \\
\hline TE-101.AP-3B & $6 / 20 / 86$ & 59 & CASS & $T$ & \\
\hline TE-101-AP-3B & $6 / 26 / 86$ & 59 & CASS & $\bar{T}$ & \\
\hline TE-101-AP-38 & $7 / 4 / 86$ & 59 & CASS & $T$ & \\
\hline TE-101-AP-38 & 7/6/86 & 59 & CASS & $T$ & \\
\hline TE-103-AP-3B & 7/14/86 & 60 & CASS & $T$ & \\
\hline TE-101-AP-38 & 7/21/86 & 60 & CASS & $T$ & \\
\hline TE-101-AP-38 & $7 / 29 / 86$ & 60 & CASS & $T$ & \\
\hline TE-101-AP-38 & $8 / 5 / 86$ & 60 & CASS & $\mathbf{T}$ & \\
\hline TE-101-AP-38 & $8 / 16 / 36$ & 60 & CASS & $T$ & \\
\hline TE-101-AP-38 & $8 / 22 / 86$ & 61 & CASS & $\bar{T}$ & \\
\hline TE-101-AP-38 & $8 / 30 / 86$ & 61 & CASS & $T$ & \\
\hline TE-101-AP-38 & $9 / 6 / 36$ & 63 & CASS & $T$ & \\
\hline TE-101-AP-38 & 8/9/86 & 63 & CASS & $T$ & \\
\hline TE-101-AP-3B & 9/19/36 & 64 & CASS & $\mathbf{T}$ & \\
\hline TE-101-AP-38 & 9/22/36 & 64 & CASS & $T$ & \\
\hline TE-101-AP-38 & $10 / 2 / 86$ & 62 & CASS & $T$ & \\
\hline TE-101-AP-3B & 10/9/36 & 62 & CASS & $\mathbf{T}$ & \\
\hline TE-101-AP-3B & $10 / 15 / 36$ & 62 & CASS & $\bar{T}$ & \\
\hline TE-101-AP-38 & $10 / 23 / 86$ & 60 & CASS & $T$ & \\
\hline TE-101-AP-38 & $10 / 31 / 86$ & 61. & CASS & $\bar{T}$ & \\
\hline TE-101-AP-38 & $31 / 5 / 86$ & 67 & CASS & $T$ & \\
\hline TE-101-AP-38 & $19 / 15 / 86$ & 58 & CASS & $\mathbf{T}$ & \\
\hline TE-10T-AP-38 & $11 / 21 / 86$ & 57 & CASS & $T$ & \\
\hline TE-101-AP-38 & $11 / 25 / 68$ & 57 & CASS & $T$ & \\
\hline TE-101.AP.38 & $12 / 2 / 86$ & 56 & CASS & $T$ & \\
\hline TE-101-AP-38 & $12 / 11 / 86$ & 54 & CASS & $\mathbf{T}$ & \\
\hline TE-101-AP-38 & $12 / 20 / 66$ & 53 & CASS & $T$ & \\
\hline TE-101-AP-38 & $12 / 27 / 86$ & 54 & CASS & $T$ & \\
\hline TE-101-AP-38 & $12 / 31 / 86$ & 53 & CASS & $\mathbf{T}$ & \\
\hline$T E-101-A P-38$ & $7 / 4 / 87$ & 62 & CASS & $\mathbf{T}$ & \\
\hline TE-101-AP-38 & $7 / 11 / 87$ & 64 & CASS & $\mathbf{T}$ & \\
\hline$T E \cdot 101 \cdot A P \cdot 38$ & $7 / 16 / 87$ & 65 & CASS & $\mathbf{T}$ & \\
\hline TE-101-AP-38 & $7 / 23 / 87$ & 64 & CASS & $T$ & \\
\hline TE-101-AP-38 & 7/29/87 & 65 & CASS & $T$ & \\
\hline TE-101-AP-38 & $8 / 8 / 87$ & 64 & CASS & $\mathbf{T}$ & \\
\hline TE-101-AP-38 & 8/14/37 & 67! & CASS & $T$ & \\
\hline TE-101-AP-38 & 8/18/87 & 67 & CASS & $T$ & \\
\hline TE-101-AP-38 & $8 / 27 / 67$ & 67 & CASS & $\mathbf{T}$ & \\
\hline TE-101-AP-38 & $8 / 30 / 87$ & 67 & CASS & $\bar{T}$ & \\
\hline TE-101-AP-38 & 9/11/87 & 68 & CASS & $T$ & \\
\hline TE-101-AP-38 & $9 / 13 / 87$ & 68 & CASS & $T$ & \\
\hline TE-101-AP-38 & $9 / 23 / 87$ & 68 & CASS & $T$ & \\
\hline TE-101-AP-38 & $10 / 2 / 87$ & 66 & CASS & $\bar{T}$ & \\
\hline TE-101-AP-3B & $10 / 10 / 87$ & 66 & CASS & $T$ & \\
\hline TE-101-AP-38 & $10 / 12 / 87$ & 65 & CASS & $T$ & \\
\hline TE-101-AP-3B & $10 / 22 / 87$ & 64 & CASS & $T$ & \\
\hline TE-101-AP-38 & $10 / 30 / 87$ & 63 & CASS & $T$ & \\
\hline TE-101-AP-38 & $11 / 5 / 87$ & 63 & CASS & $T$ & \\
\hline TE-101-AP-38 & $11 / 12 / 87$ & 64 & CASS & $\mathrm{T}$ & \\
\hline TE-101-AP-38 & $11 / 18 / 87$ & 61 & CASS & $T$ & \\
\hline TE-101-AP-3B & $11 / 27 / 87$ & 59! & CASS & $\mathbf{T}$ & \\
\hline TE-101-AP-38 & $12 / 5 / 87$ & 61 & CASS & $\mathbf{T}$ & \\
\hline TE-101-AP-3B & $12 / 9 / 87$ & 59| & CASS & $\mathbf{T}$ & \\
\hline TE-101-AP-38 & $12 / 18 / 67$ & 60 & CASS & $T$ & \\
\hline TE-101-AP-3B & $12 / 24 / 87$ & 57 & CASS & $T$ & \\
\hline TE-101-AP-38 & $12 / 28 / 87$ & 56 & CASS & $T$ & \\
\hline TE-101-AP-38 & $1 / 8 / 88$ & 54 & CASS & $\mathbf{T}$ & \\
\hline TE-101-AP-38 & $1 / 11 / 88$ & 55! & CASS & $T$ & \\
\hline TE-101-AP-38 & $1 / 18 / 88$ & $53:$ & CASS & $\mathbf{T}$ & \\
\hline TE-101-AP-38 & $1 / 29 / 83$ & 53 & CASS & $\mathbf{T}$ & \\
\hline
\end{tabular}




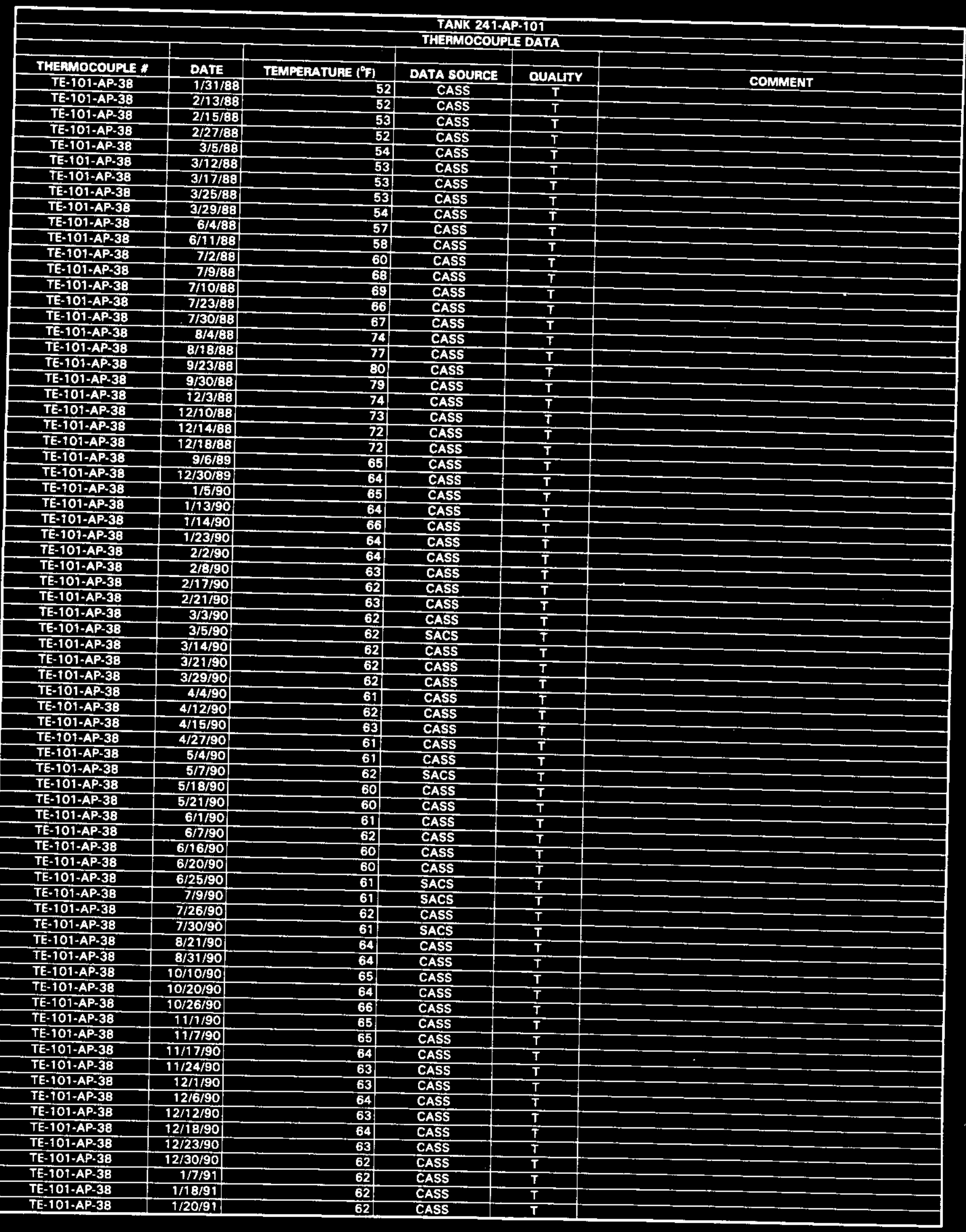

Data obtained from Computer Autometed Surveillance Syatem (CASS), April 1995; and Surveillance Analysis Computer System (SACS), June 1995. 


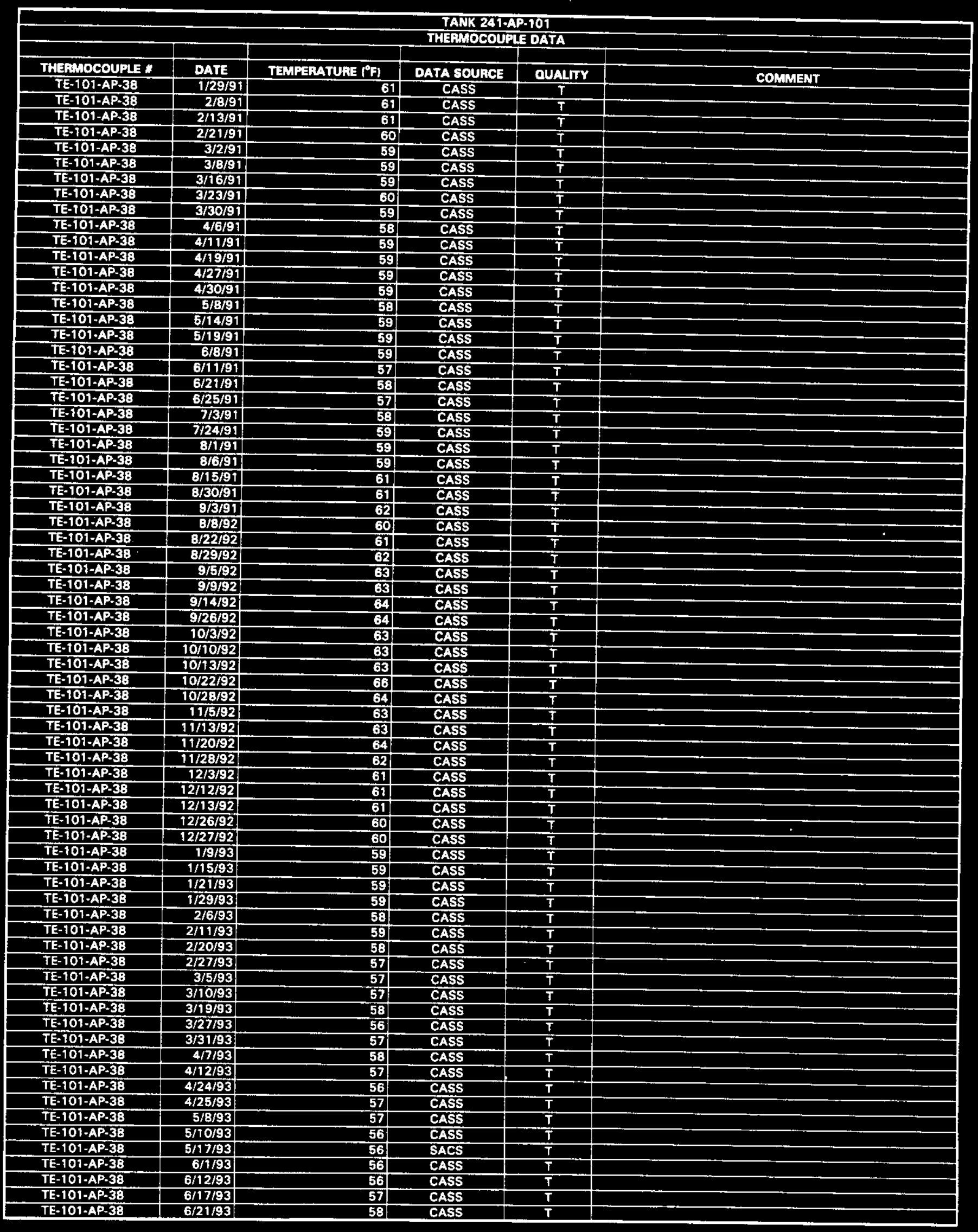




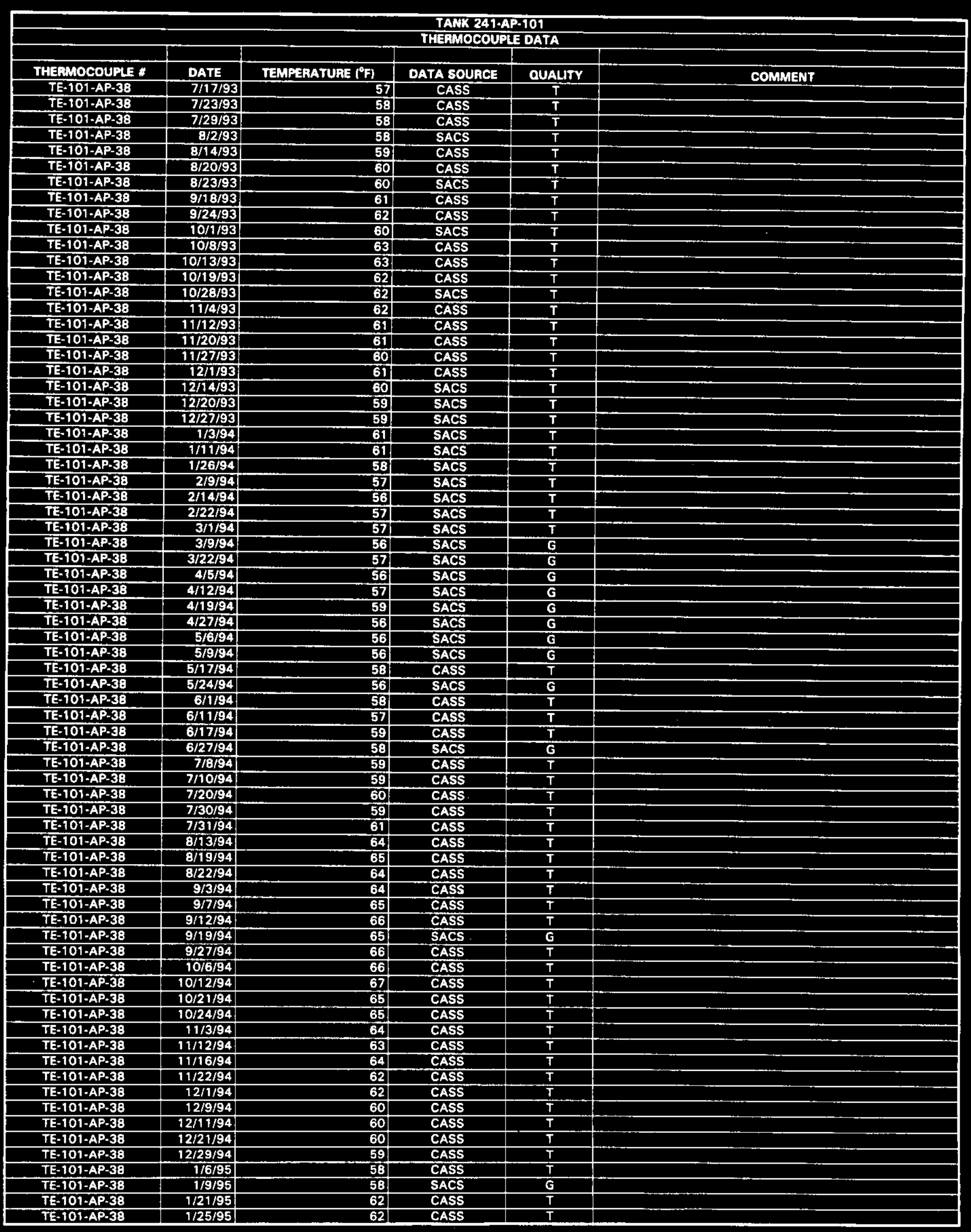

Data obtained from Computer Automated Survellance System (CASS), Aprif 1995; and Surveillance Analysis Computer System (SACS), June 1995. 


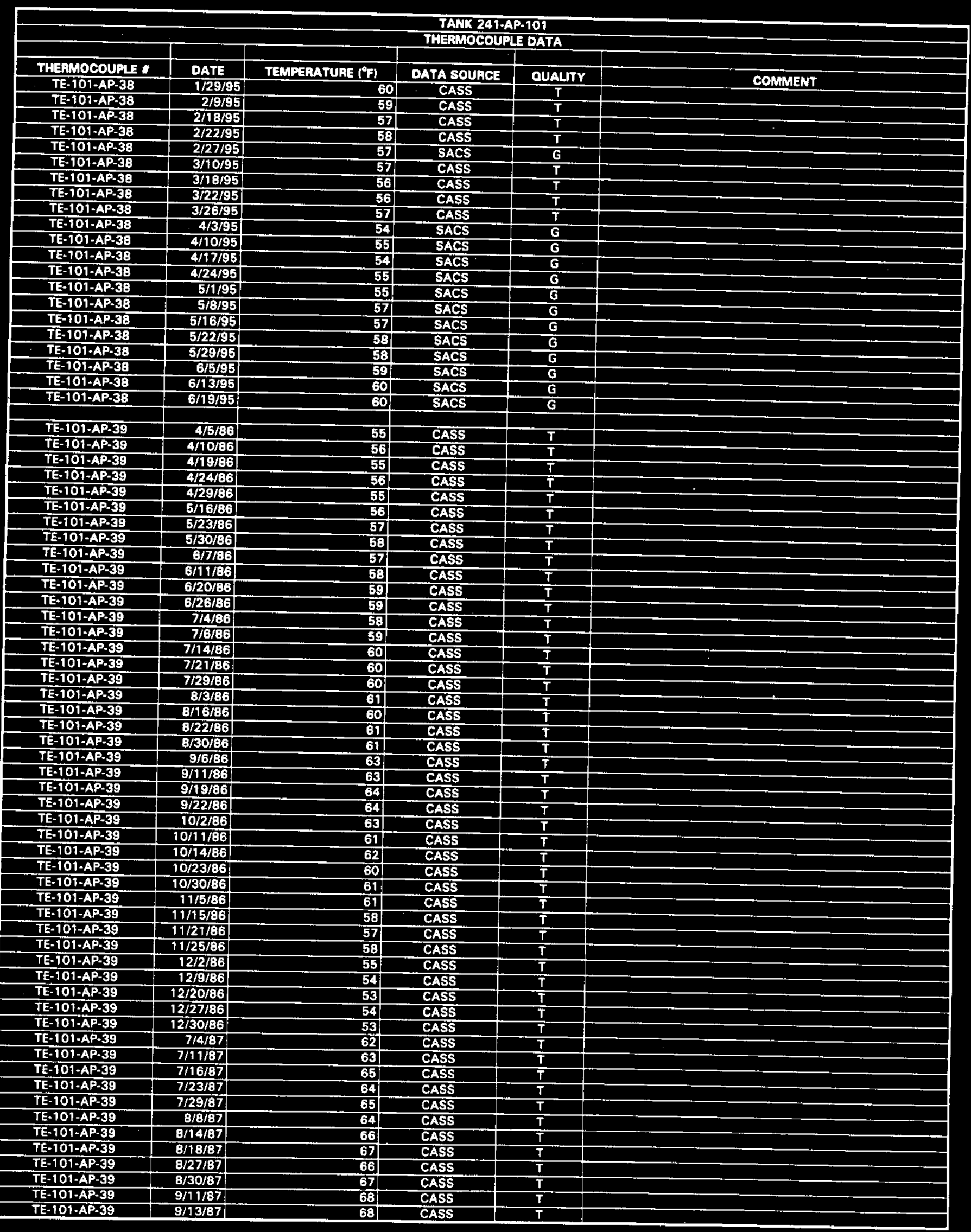




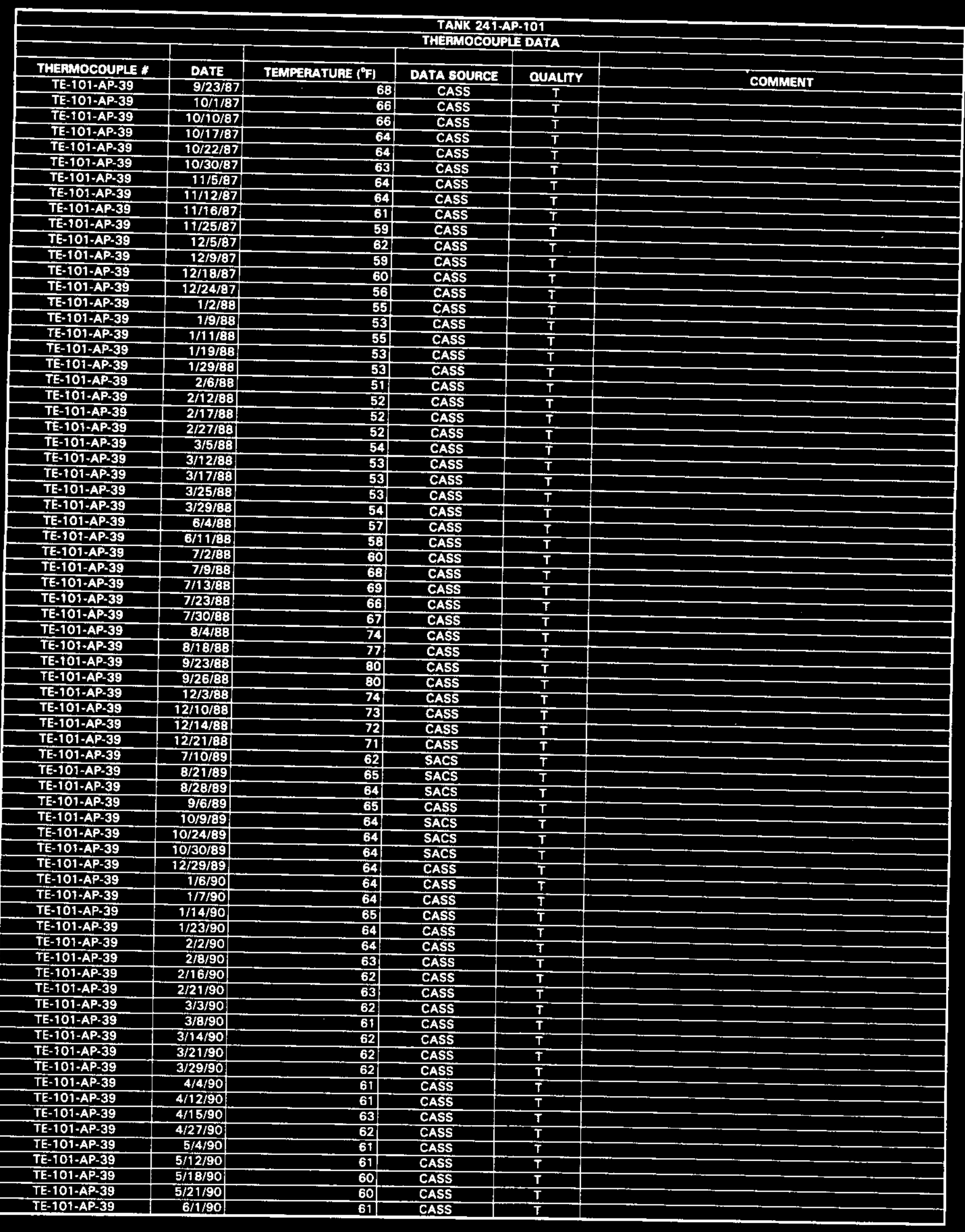

Data obtained from Computer Automated Surveillance System (CASS), April 1995; and Surveillance Analysis Computer System (SACS), June 1995. 


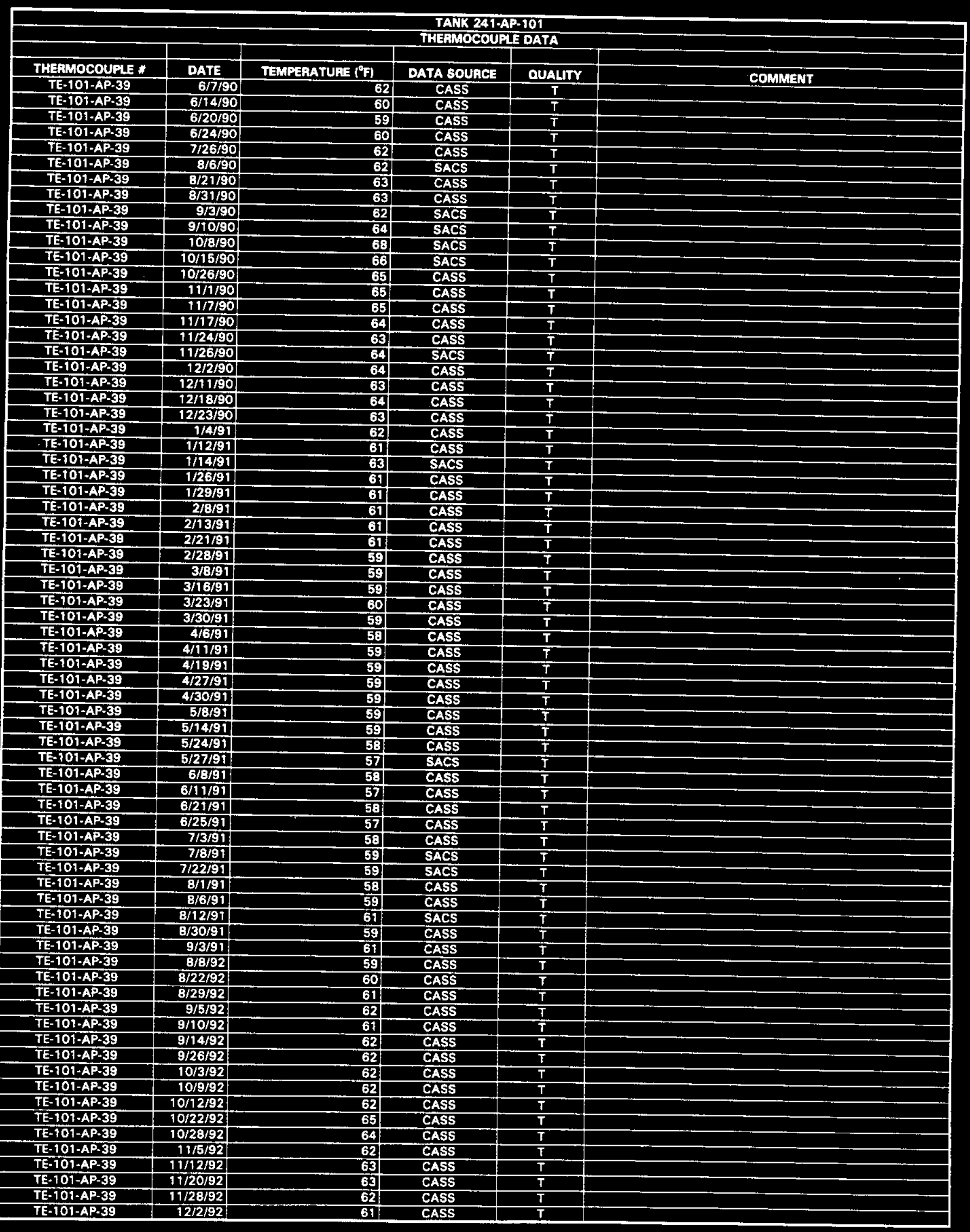




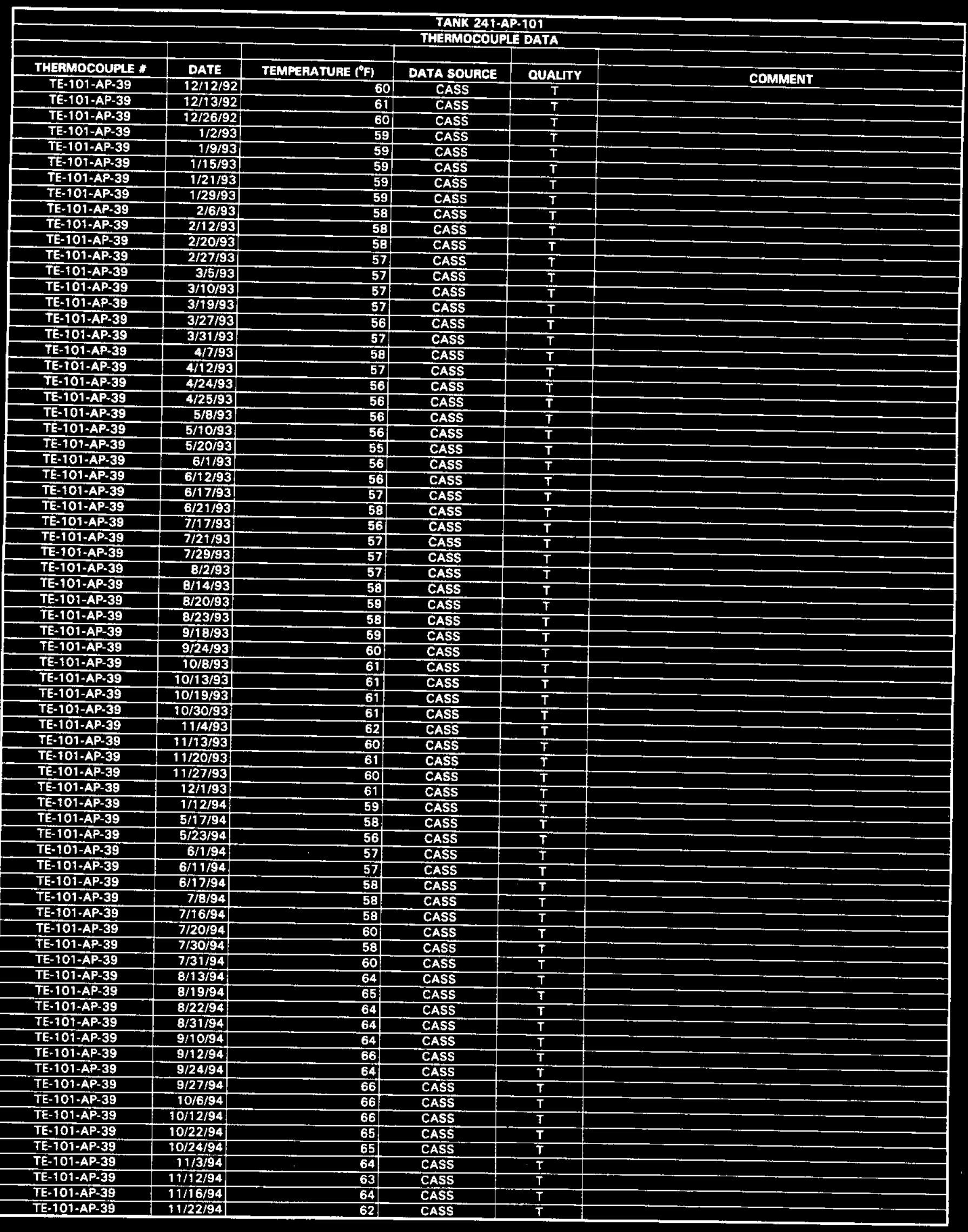




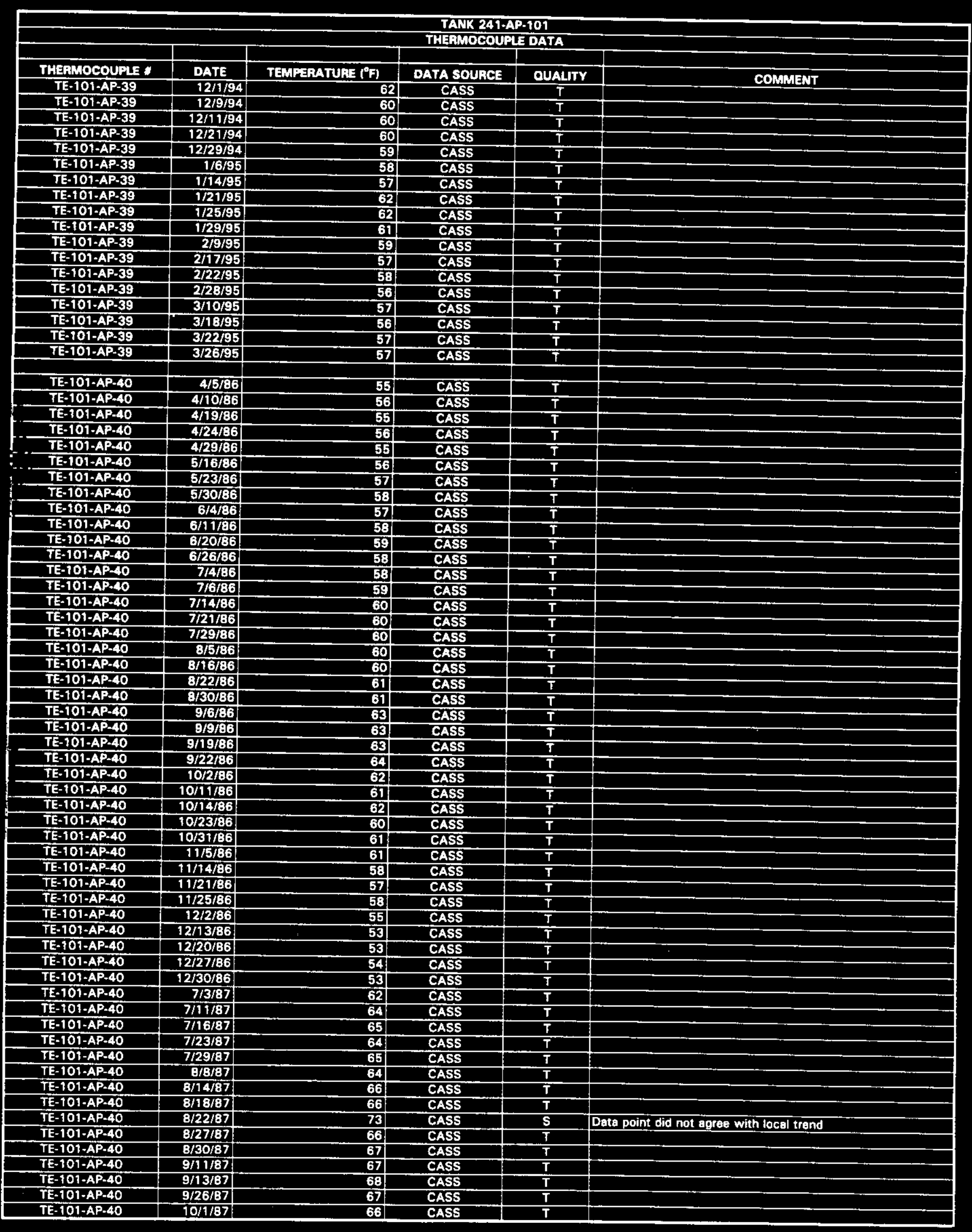

Data obtained from Computer Automated Surveillance Syatem ICASSI, April 1995; and Surveillance Analysis Computer System (SACS), June 1995. 


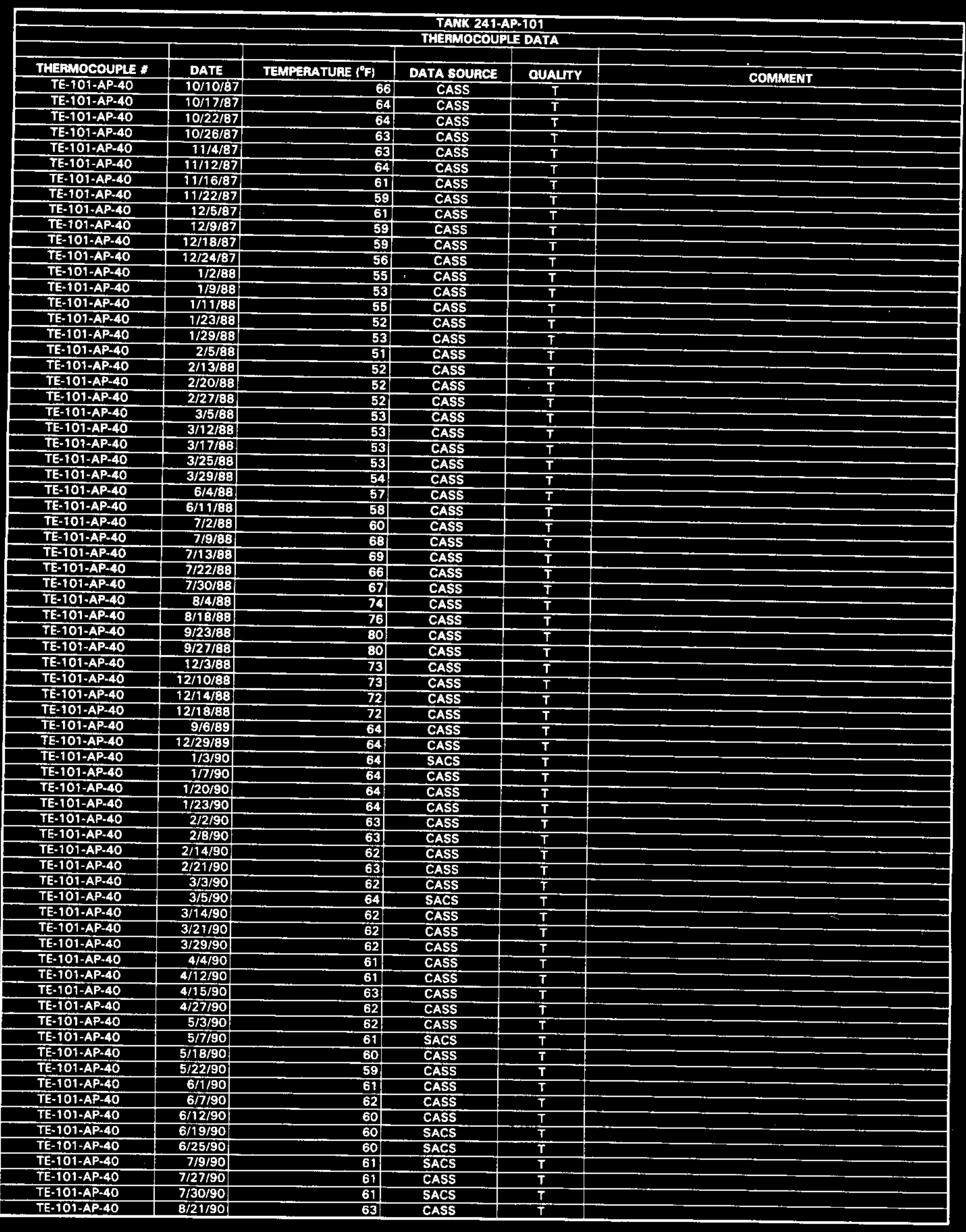

Data obtained from Computer Automated Surveillance System (CASS), April 1995; and Surveillance Analysis Computer System (SACS), June 1995. 


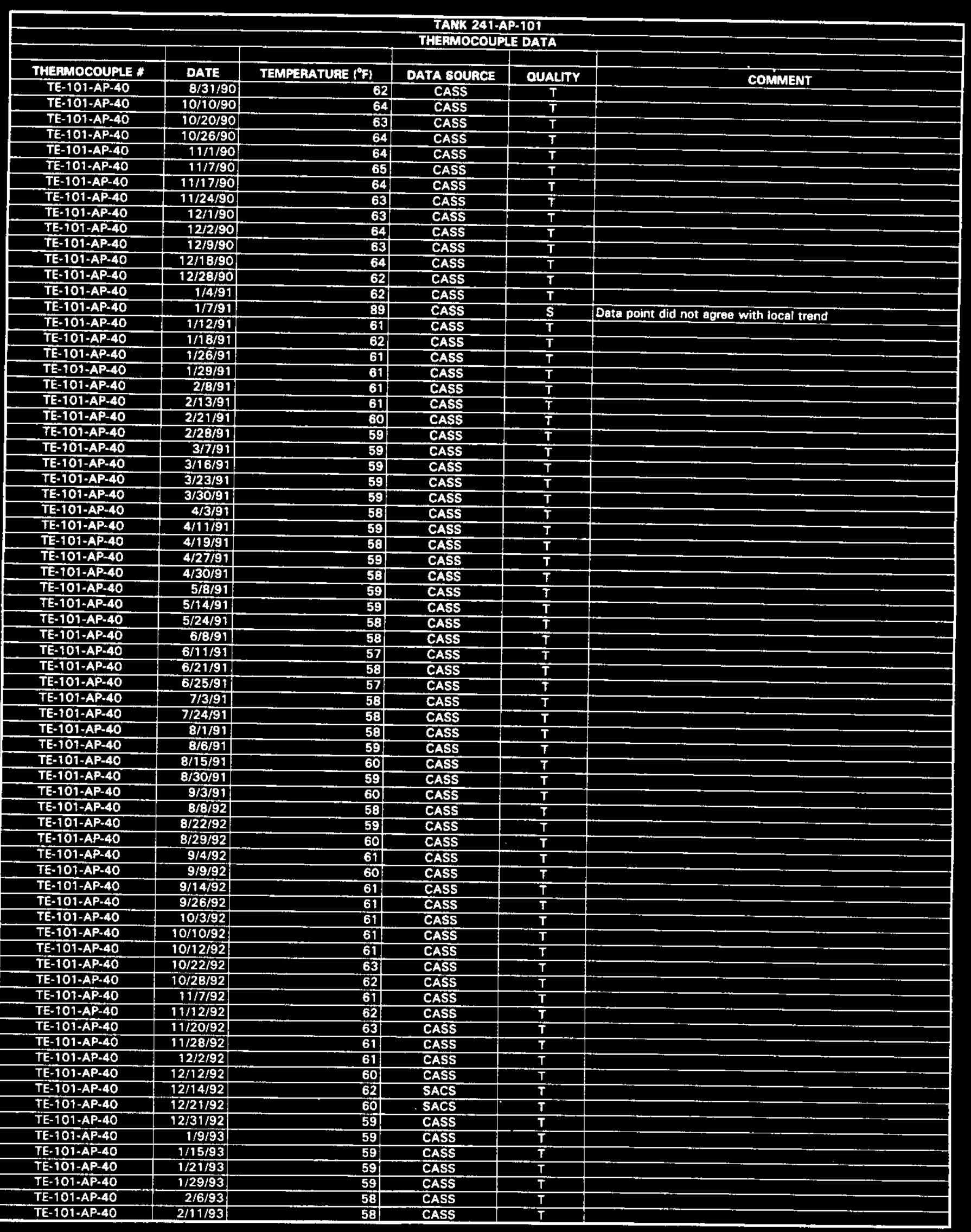




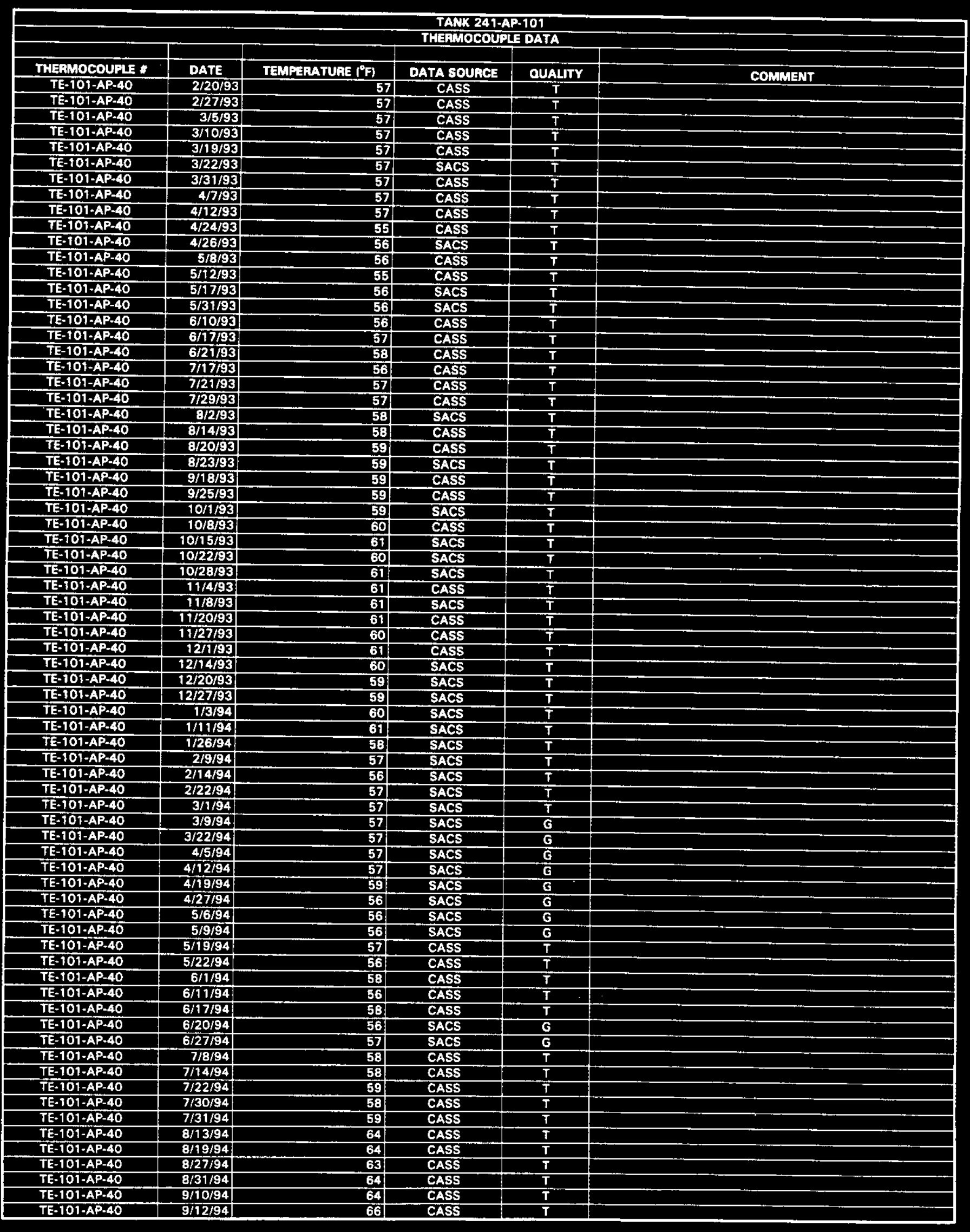




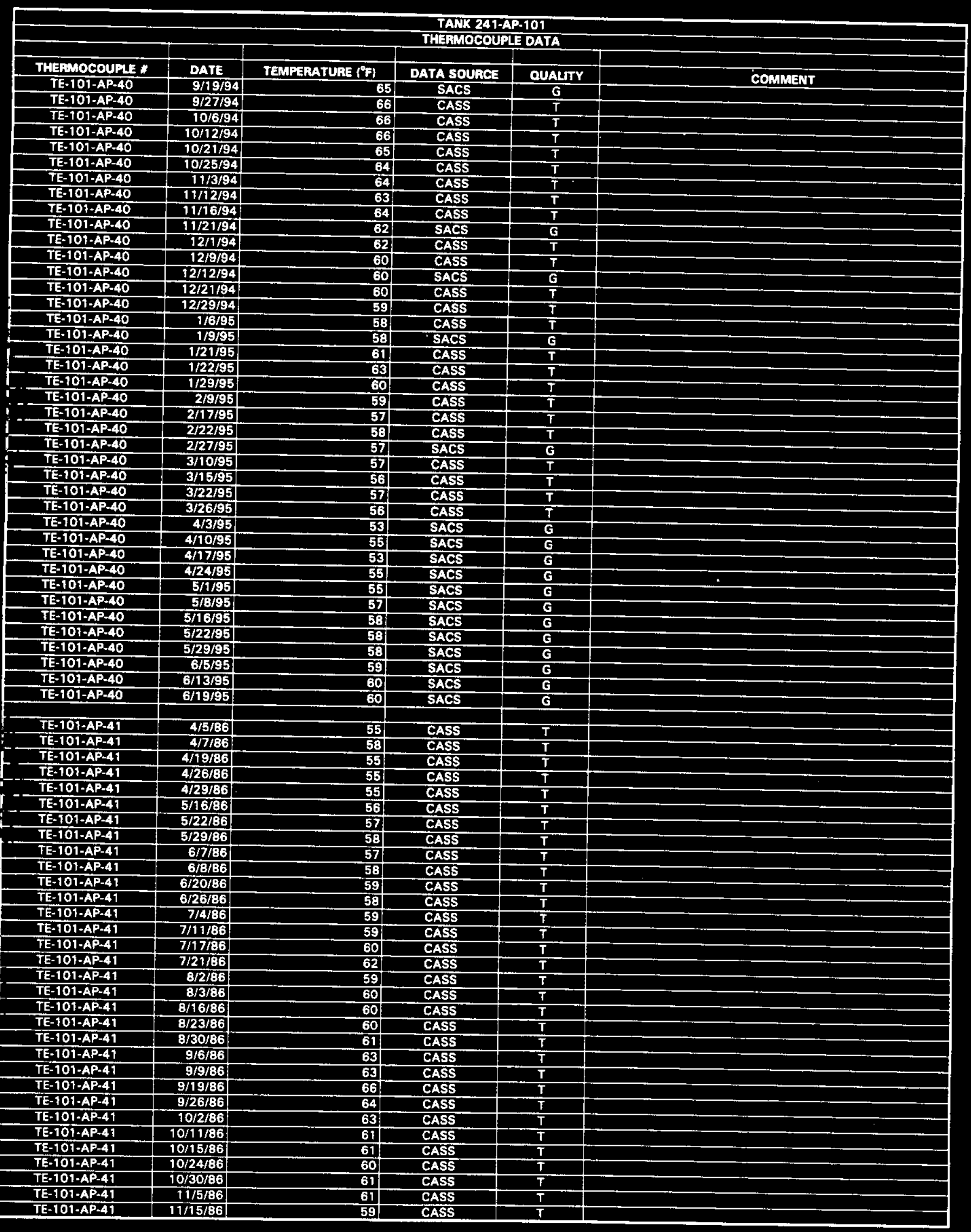




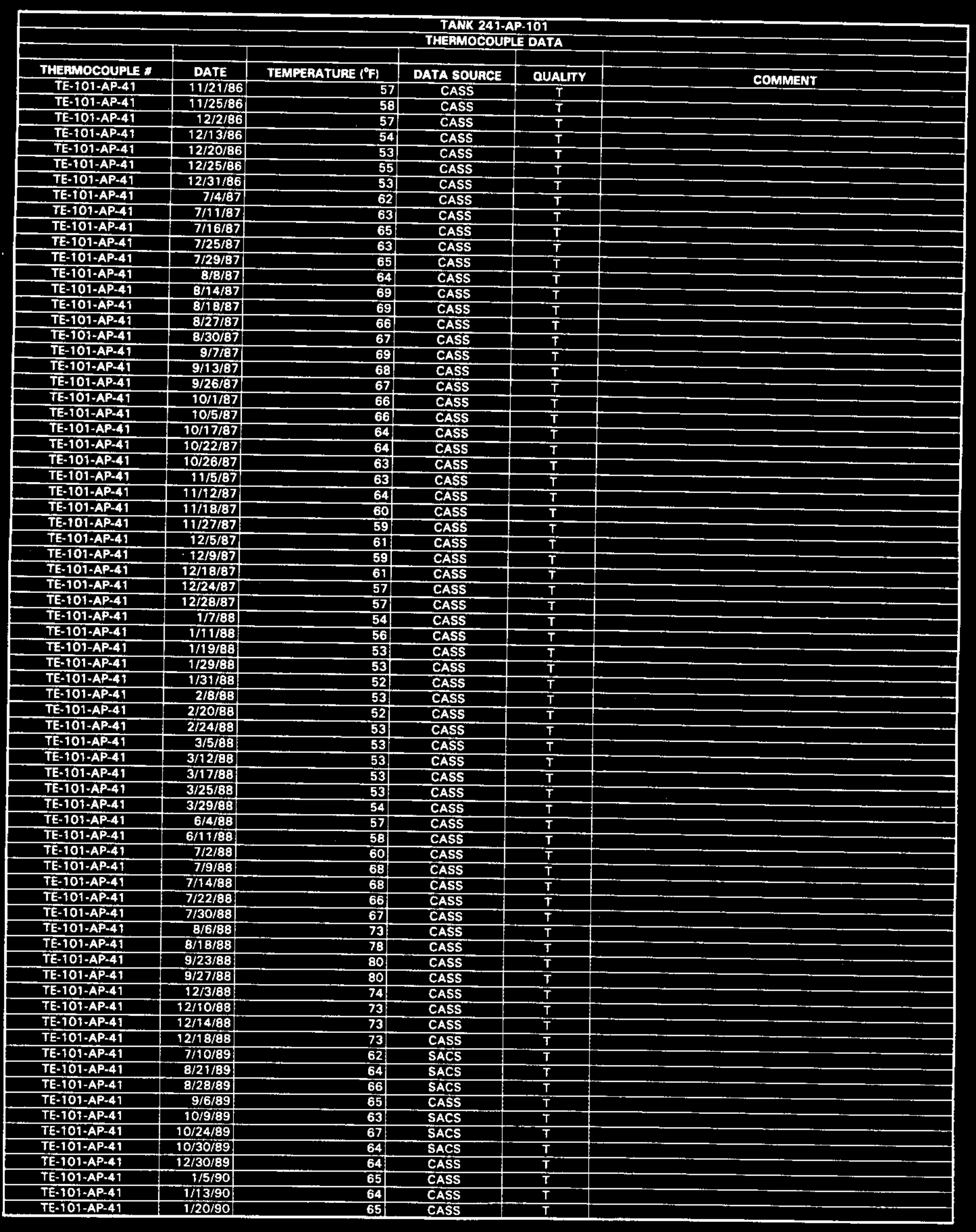




\begin{tabular}{|c|c|c|c|c|c|}
\hline \multicolumn{6}{|c|}{$\begin{array}{l}\text { TANK 241-AP-101 } \\
\text { THERMOCOUFLE DATA }\end{array}$} \\
\hline THERMOCOUPLE \# & DATE & TEMPERATUAE $\left({ }^{\circ} \mathrm{F}\right)$ & DATA sOUACE & ounery & \\
\hline TE-101-AP-41 & $1 / 23 / 90$ & 64 & $\frac{{ }^{2}}{\text { CASS }}$ & $\frac{\text { QUALTYY }}{T}$ & COMMENT \\
\hline TE-101-AP-41 & $2 / 3 / 90$ & 63 & CASS & $T$ & \\
\hline TE-101-AP-41 & $2 / 10 / 90$ & 63 & CASS & $T$ & \\
\hline TE-101-AP-41 & $2 / 17 / 90$ & 62 & CASS & $T$ & \\
\hline TE-101-AP-41 & $2 / 21 / 90$ & 64 & CASS & $\bar{T}$ & \\
\hline TE-101-AP-41 & $3 / 3 / 90$ & 62 & CASS & $T$ & \\
\hline TE-101-AP-41 & $3 / 8 / 90$ & 62 & CASS & $T$ & \\
\hline TE-101-AP-41 & $3 / 14 / 90$ & 63 & CASS & $T$ & \\
\hline TE-101-AP-41 & $3 / 21 / 90$ & 62 & CASS & $T$ & \\
\hline TE-101-AP-41 & $3 / 29 / 90$ & 63 & CASS & $T$ & \\
\hline TE-101-AP-41 & $4 / 4 / 90$ & 62 & CASS & $T$ & \\
\hline TE-101-AP-41 & 4/12/90 & 62 & CASS & $\mathbf{T}$ & \\
\hline TE-101-AP-41 & $4 / 15 / 90$ & 63 & CASS & $T$ & \\
\hline TE-101-AP-41 & $4 / 27 / 90$ & 62 & CASS & $\mathrm{T}$ & \\
\hline TE-101-AP-41 & $4 / 30 / 90$ & 64 & CASS & $\bar{T}$ & \\
\hline TE-101-AP-41 & $5 / 12 / 90$ & 61 & CASS & $\mathbf{T}$ & \\
\hline TE-101-AP-41 & $5 / 18 / 90$ & 60 & CASS & $T$ & \\
\hline TE-101-AP-41 & $5 / 22 / 90$ & 60 & CASS & $\bar{T}$ & \\
\hline TE-101-AP-41 & $6 / 1 / 90$ & 61 & CASS & $T$ & \\
\hline TE-101-AP-41 & $6 / 7 / 90$ & 62 & CASS & $T$ & \\
\hline TE-101-AP-41 & $6 / 16 / 90$ & 60 & CASS & $T$ & \\
\hline TE-101-AP-41 & $6 / 20 / 90$ & 60 & CASS & $\mathbf{T}$ & \\
\hline TE-101-AP-41 & $6 / 23 / 90$ & 107 & CASS & $\mathbf{s}$ & Data point did not agrea with local trend \\
\hline TE-101-AP-41 & 6/24/90 & 59 & CASS & $\bar{T}$ & \\
\hline$T E-101-A P-41$ & $7 / 27 / 90$ & 61 & CASS & $T$ & \\
\hline TE-107-AP-41 & $8 / 6 / 90$ & 61 & SACS & $T$ & \\
\hline TE-101-AP-41 & $8 / 21 / 90$ & 83 & CASS & $\mathbf{T}$ & \\
\hline TE-101-AP-41 & $8 / 31 / 90$ & 63 & CASS & $\mathbf{T}$ & \\
\hline TE-107-AP-41 & $9 / 3 / 90$ & 62 & SACS & $\bar{T}$ & \\
\hline TE-101-AP-41 & $9 / 10 / 90$ & 62 & SACS & $\mathbf{T}$ & \\
\hline$T E-101-A P-41$ & $10 / 8 / 90$ & 68 & SACS & 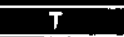 & \\
\hline TE-101-AP-41 & $10 / 20 / 90$ & 64 & CASS & $T$ & \\
\hline TE-101-AP-41 & $10 / 27 / 90$ & 64 & CASS & $T$ & \\
\hline TE-101-AP-41 & $11 / 3 / 90$ & 64 & CASS & $T$ & \\
\hline TE-101-AP-41 & $\pi 1 / 7 / 90$ & 66 & CASS & $\mathbf{T}$ & \\
\hline TE-101-AP-41 & $11 / 17 / 90$ & 64 & CASS & $T$ & \\
\hline TE-101-AP-41 & $11 / 18 / 90$ & 64 & CASS & $T$ & \\
\hline TE-101-AP-41 & $12 / 190$ & 63 & CASS & $T$ & \\
\hline TE-108-AP-41 & $12 / 2 / 90$ & 66 & CASS & $T$ & \\
\hline TE-101-AP-41 & $12 / 14 / 90$ & 63 & CASS & $T$ & \\
\hline TE-101-AP-41 & $12 / 18 / 90$ & 66 & CASS & $T$ & \\
\hline TE-101-AP-41 & $12 / 23 / 90$ & 63 & CASS & $T$ & \\
\hline$T E-101-A P-41$ & $1 / 4 / 91$ & 63 & CASS & $\bar{T}$ & \\
\hline$T E-101-A P-41$ & $1 / 7 / 91$ & 62 & CASS & $T$ & \\
\hline TE-107-AP-41 & $1 / 14 / 91$ & 64 & SACS & $\bar{T}$ & \\
\hline TE-101-AP-41 & $1 / 20 / 91$ & 64 & CASS & $\overline{\mathbf{T}}$ & \\
\hline TE-101-AP-41 & $1 / 29 / 91$ & 61 & CASS & $T$ & \\
\hline TE-101-AP-41 & $2 / 7 / 91$ & 62 & CASS & $T$ & \\
\hline TE-101-AP-41 & $2 / 13 / 91$ & 61 & CASS & $T$ & \\
\hline TE-101-AP-41 & $2 / 21 / 91$ & 61 & CASS & $T$ & \\
\hline TE-101-AP-41 & $3 / 2 / 91$ & 59 & CASS & $T$ & \\
\hline TE-101-AP-41 & $3 / 9 / 91$ & 59 & CASS & $T$ & \\
\hline TE-101-AP-41 & $3 / 16 / 91$ & 59 & CASS & $T$ & \\
\hline TE-101-AP-41 & $3 / 21 / 91$ & 62 & CASS & $T$ & \\
\hline TE-101-AP-41 & $3 / 29 / 91$ & 60 & CASS & $T$ & \\
\hline TE-101-AP-41 & $4 / 6 / 91$ & 58 & CASS & $T$ & \\
\hline TE-101-AP-41 & $4 / 11 / 91$ & 58 & CASS & $T$ & \\
\hline TE-101-AP-41 & $4 / 19 / 91$ & 58 & CASS & $T$ & \\
\hline TE-101-AP-41 & 4/27/91 & 62 & CASS & $T$ & \\
\hline TE-101-AP-41 & $5 / 3 / 91$ & 58 & CASS & $\bar{T}$ & \\
\hline$T E-101-A P-41$ & 5/9/91 & 58 & CASS & $T$ & \\
\hline TE-101-AP-41 & $5 / 14 / 91$ & 59 & CASS & $T$ & \\
\hline TE-101-AP-41 & 5/19/91 & 59 & CASS & $\bar{T}$ & \\
\hline TE-101-AP-41 & $5 / 27 / 91$ & 57 & SACS & $T$ & \\
\hline TE-101-AP-41 & $6 / 8 / 91$ & 58 & CASS & $T$ & \\
\hline TE-101-AP-41 & $6 / 11 / 91$ & 57 & CASS & $T$ & \\
\hline$T E-101-A P-41$ & $6 / 21 / 91$ & 59 & CASS & $T$ & \\
\hline TE-101-AP-41 & $6 / 23 / 91$ & 58 & CASS & $T$ & \\
\hline TE-101-AP-41 & $7 / 3 / 91$ & 58 & CASS & $T$ & \\
\hline TE-101-AP-41 & $7 / 8 / 91$ & 58 & SACS & $T$ & \\
\hline TE-101-AP-41 & $7 / 24 / 91$ & 58 & CASS & $T$ & \\
\hline TE-101-AP-41 & $7 / 29 / 91$ & 59 & SACS & $T$ & \\
\hline TE-101-AP.41 & $8 / 6 / 91$ & 59 & CASS & $\bar{I}$ & \\
\hline
\end{tabular}




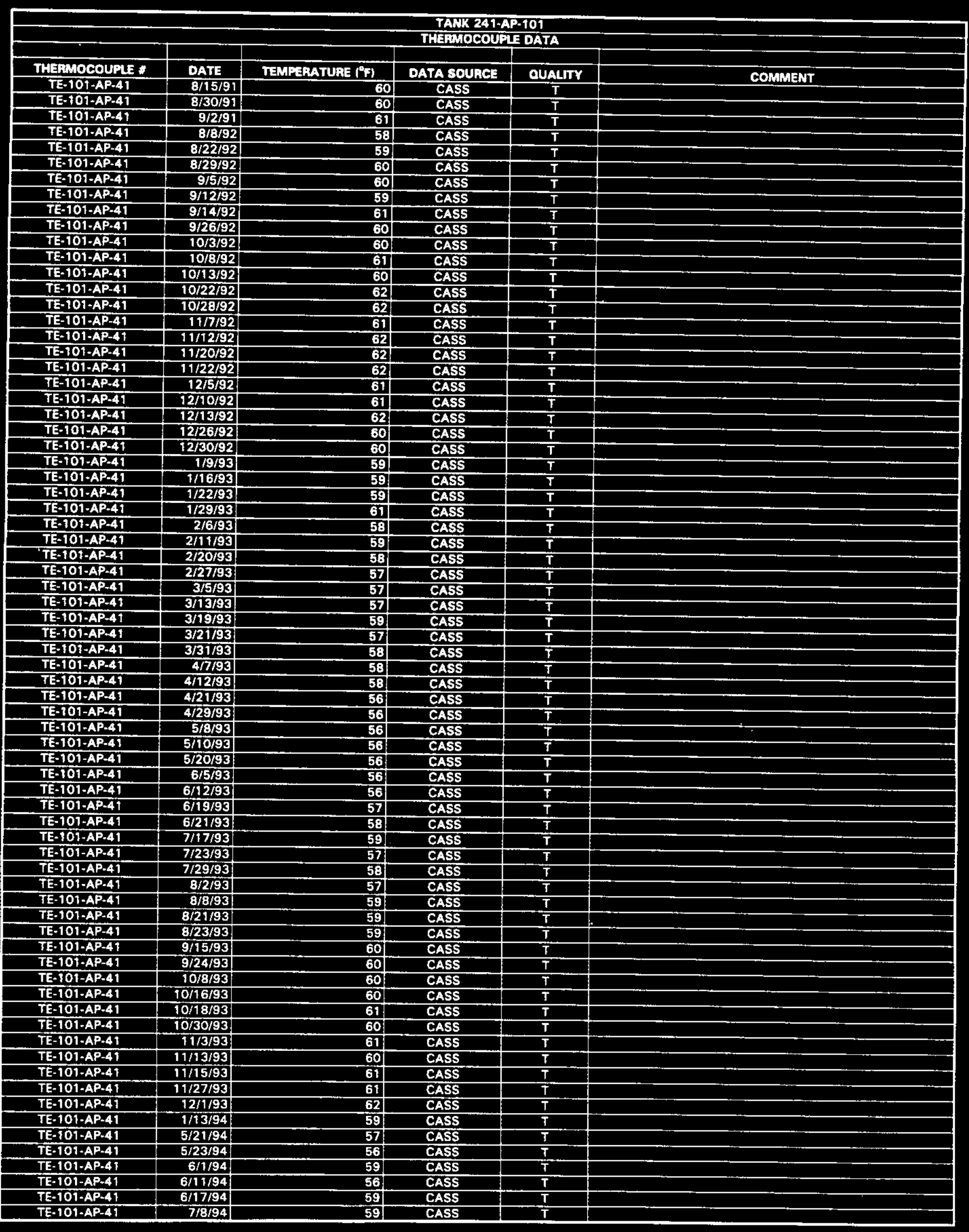




\begin{tabular}{|c|c|c|c|c|c|}
\hline \multicolumn{6}{|c|}{$\begin{array}{l}\text { TAMK 241-AP-101 } \\
\text { THEAMOCOUPLE DATA }\end{array}$} \\
\hline & & & & & \\
\hline THERMOCOUPLE * & DATE & TEMPERATURE $\left({ }^{\circ} \mathrm{F}\right)$ & DATA SOURCE & OUALTY & COMMENT \\
\hline TE-101-AP-41 & $7 / 16 / 94$ & 58 & CASS & $\mathbf{T}$ & \\
\hline TE-101-AP-41 & $7 / 20 / 94$ & 60 & CASS & $T$ & \\
\hline TE-101-AP-41 & $7 / 30 / 94$ & 58 & CASS & $T$ & \\
\hline TE-101-AP-41 & $7 / 31 / 94$ & 61 & CASS & $\bar{T}$ & \\
\hline TE-101-AP-41 & $8 / 13 / 94$ & 65 & CASS & $T$ & \\
\hline TE-101-AP-41 & $8 / 19 / 94$ & 65 & CASS & $T$ & \\
\hline TE-101-AP-41 & $8 / 27 / 94$ & 63 & CASS & $T$ & \\
\hline TE-101-AP-41 & $8 / 37 / 94$ & 64 & CASS & $T$ & \\
\hline TE-101-AP-41 & $9 / 10 / 94$ & 64 & CASS & $T$ & \\
\hline TE-101-AP-41 & $9 / 12 / 94$ & 66 & CASS & $T$ & \\
\hline TE-101-AP-41 & $9 / 24 / 94$ & 64 & CASS & $\bar{T}$ & \\
\hline TE-101-AP-41 & $9 / 27 / 94$ & 68 & CASS & $T$ & \\
\hline TE-101-AP-41 & $10 / 8 / 94$ & 65 & CASS & $T$ & \\
\hline TE-101-AP-41 & $10 / 12 / 94$ & 68 & CASS & $\mathbf{T}$ & \\
\hline TE-101-AP-41 & $10 / 21 / 94$ & 65 & CASS & $T$ & \\
\hline TE-101.AP-41 & $10 / 29 / 94$ & 64 & CASS & $T$ & \\
\hline TE-101-AP-41 & $11 / 3 / 94$ & 64 & CASS & $\mathrm{T}$ & \\
\hline TE-101-AP-41 & $11 / 9 / 94$ & 66 & CASS & $T$ & \\
\hline TE-101-AP-41 & $11 / 19 / 94$ & 65 & CASS & $T$ & \\
\hline TE-101-AP-41 & $11 / 20 / 94$ & 62 & CASS & $T$ & \\
\hline$T E-101 \cdot A P-41$ & $12 / 1 / 94$ & 62 & CASS & $T$ & \\
\hline TE-10T-AP-41 & $12 / 9 / 94$ & 60 & CASS & $T$ & \\
\hline TE-101-AP-4t & $12 / 11 / 94$ & 61) & CASS & $T$ & \\
\hline TE-101-AP-41 & $12 / 21 / 94$ & 62 & CASS & $T$ & \\
\hline TE-101-AP-49 & $12 / 29 / 94$ & 59 & CASS & $T$ & \\
\hline TE-101-AP-41 & $1 / 6 / 95$ & 58 & CASS & $T$ & \\
\hline TE-T01-AP-41 & $1 / 13 / 95$ & 58 & CASS & $T$ & \\
\hline TE-101-AP-41 & $1 / 21 / 95$ & 62 & CASS & $T$ & \\
\hline TE-101-AP-41 & $1 / 22 / 95$ & 64 & CASS & $\bar{T}$ & \\
\hline TE-101-AP-41 & $1 / 29 / 95$ & 63 & CASS & $T$ & \\
\hline TE-101-AP-41 & $2 / 9 / 95$ & 60 & CASS & $\bar{T}$ & \\
\hline TE-101-AP-41 & $2 / 18 / 95$ & 57 & CASS & $\mathbf{T}$ & \\
\hline TE-101-AP-41 & $2 / 22 / 95$ & 59 & CASS & $T$ & \\
\hline TE-101-AP-47 & $2 / 28 / 95$ & 57 & CASS & $T$ & \\
\hline TE-101-AP-41 & 310/85 & 58 & CASS & $\mathbf{T}$ & \\
\hline TE-101-AP-41 & $3 / 18 / 95$ & 56 & CASS & $T$ & \\
\hline TE-101-AP-41 & $3 / 23 / 95$ & 56 & CASS & $\mathbf{T}$ & \\
\hline TE-101-AP-41 & $3 / 26 / 85$ & 57 & CASS & $T$ & \\
\hline & & & & & \\
\hline TE-101-AP-42 & 4/5/86 & 55 & CASS & $T$ & \\
\hline TE-101-AP-42 & $4 / 10 / 86$ & 56 & CASS & $T$ & \\
\hline TE-101-AP-42 & $4 / 19 / 86$ & 55 & CASS & $T$ & \\
\hline TE-101-AP-42 & $4 / 24 / 86$ & 56 & CASS & $T$ & \\
\hline TE-101-AP-42 & $4 / 29 / 86$ & $\mathbf{5 5}$ & CASS & $\bar{T}$ & \\
\hline TE-101-AP-42 & $5 / 16 / 86$ & 56 & CASS & $\mathbf{T}$ & \\
\hline TE-101-AP-42 & $5 / 20 / 86$ & 55 & CASS & $T$ & \\
\hline TE-101-AP-42 & $5 / 23 / 86$ & 63 & CASS & $\mathbf{S}$ & Data point did not agree with local trend \\
\hline TE-101-AP-42 & $5 / 24 / 86$ & 36 & CASS & $\mathbf{5}$ & Data point did not agree with local trend \\
\hline TE-101-AP-42 & $5 / 25 / 86$ & 37 & CASS & $\mathbf{S}$ & Data point did not agree with local trend \\
\hline TE-101-AP-42 & $5 / 26 / 86$ & 36. & CASS & $\mathbf{S}$ & Data point did not agree with local trend \\
\hline TE-101-AP-42 & $5 / 31 / 86$ & 50 & CASS & $T$ & \\
\hline TE-101-AP-42 & $6 / 7 / 26$ & 56 & CASS & $\mathrm{T}$ & \\
\hline TE-101-AP-42 & $6 / 9 / 86$ & 57 & CASS & $T$ & \\
\hline TE-101-AP-42 & $6 / 20 / 86$ & 58 & CASS & $\bar{T}$ & \\
\hline TE-101-AP-42 & $6 / 26 / 86$ & $\mathbf{5 8}$ & CASS & $T$ & \\
\hline TE-101-AP-42 & $7 / 4 / 86$ & 58 & CASS & $\mathbf{T}$ & \\
\hline TE-101-AP-42 & $7 / 6 / 86$ & 59 & CASS & $\mathbf{T}$ & \\
\hline TE-101-AP-42 & $7 / 14 / 86$ & 60 & CASS & $T$ & \\
\hline TE-101-AP-42 & $7 / 21 / 86$ & 60 & CASS & $T$ & \\
\hline TE-101-AP-42 & $7 / 29 / 86$ & 60 & CASS & $\mathbf{T}$ & \\
\hline TE-101-AP-42 & $8 / 5 / 86$ & 60 & CASS & $T$ & \\
\hline TE-101-AP-42 & $8 / 16 / 86$ & 60 & CASS & $T$ & \\
\hline TE-101-AP-42 & $8 / 19 / 86$ & 68 & CASS & $\mathbf{s}$ & Data point did not agree with local trend \\
\hline TE-101-AP-42 & $8 / 22 / 86$ & 61 & CASS & $T$ & \\
\hline TE-101-AP-42 & $8 / 30 / 86$ & 61 & CASS & $T$ & \\
\hline TE-101-AP-42 & $9 / 6 / 86$ & 62 & CASS & $T$ & \\
\hline TE-101-AP-42 & $9 / 9 / 86$ & 63 & CASS & $\bar{T}$ & 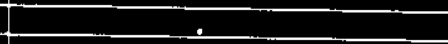 \\
\hline TE-101-AP-42 & $9 / 19 / 86$ & 64 & CASS & $T$ & \\
\hline TE-101-AP-42 & $9 / 22 / 86$ & 64 & CASS & $T$ & \\
\hline TE-101-AP-42 & $10 / 2 / 86$ & 62 & CASS & $T$ & \\
\hline TE-101-AP-42 & $10 / 11 / 86$ & 61 & CASS & $T$ & \\
\hline TE-101-AP-42 & $10 / 14 / 86$ & 62 & CASS & $\mathbf{T}$ & \\
\hline TE-101-AP-42 & $10 / 24 / 86$ & 60 & CASS & $T$ & \\
\hline
\end{tabular}




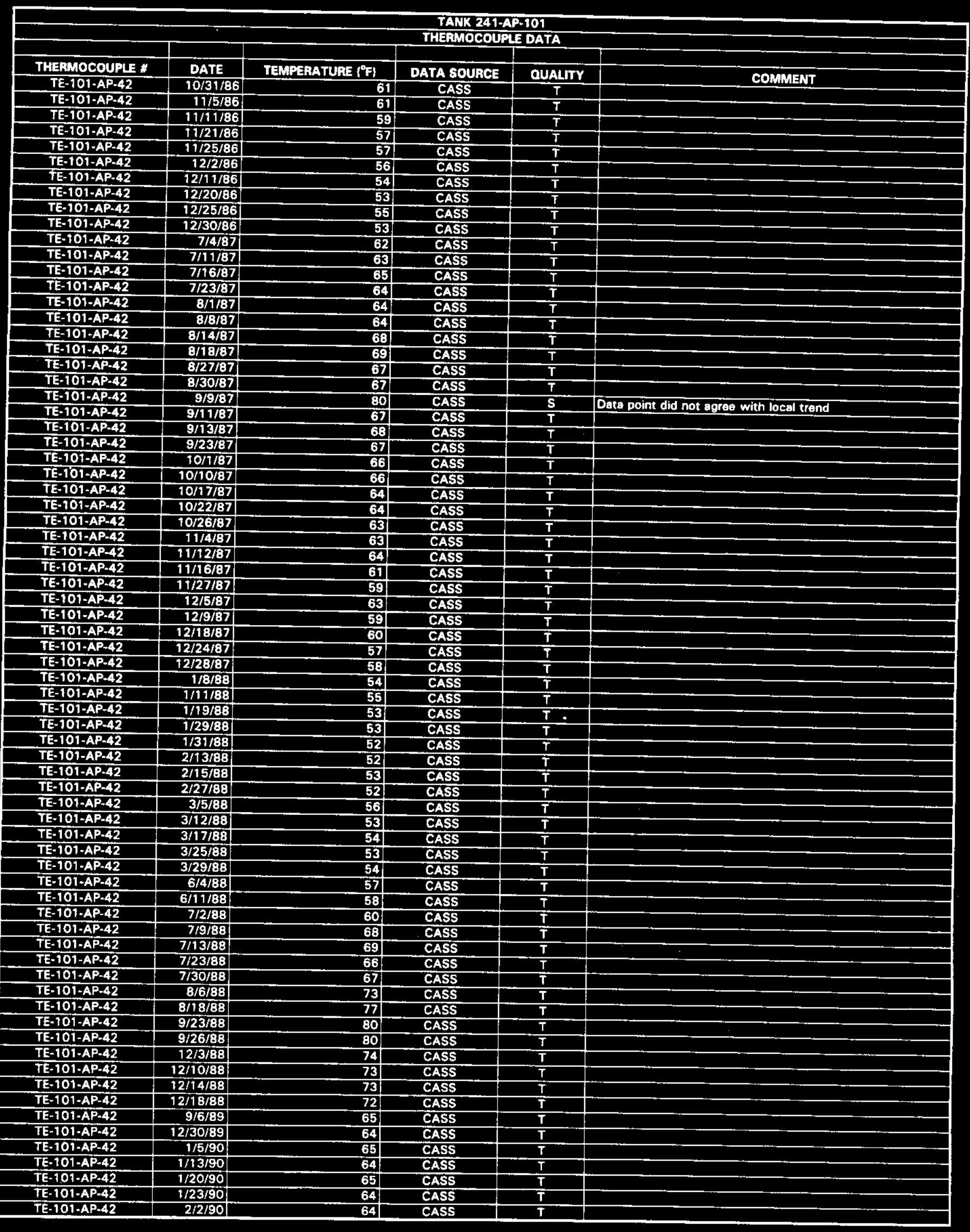

Data obtained from Computer Automated Surveillance System (CASS), April 1995; and Surveillance Analysis Computer System (SACS), June 1995. 


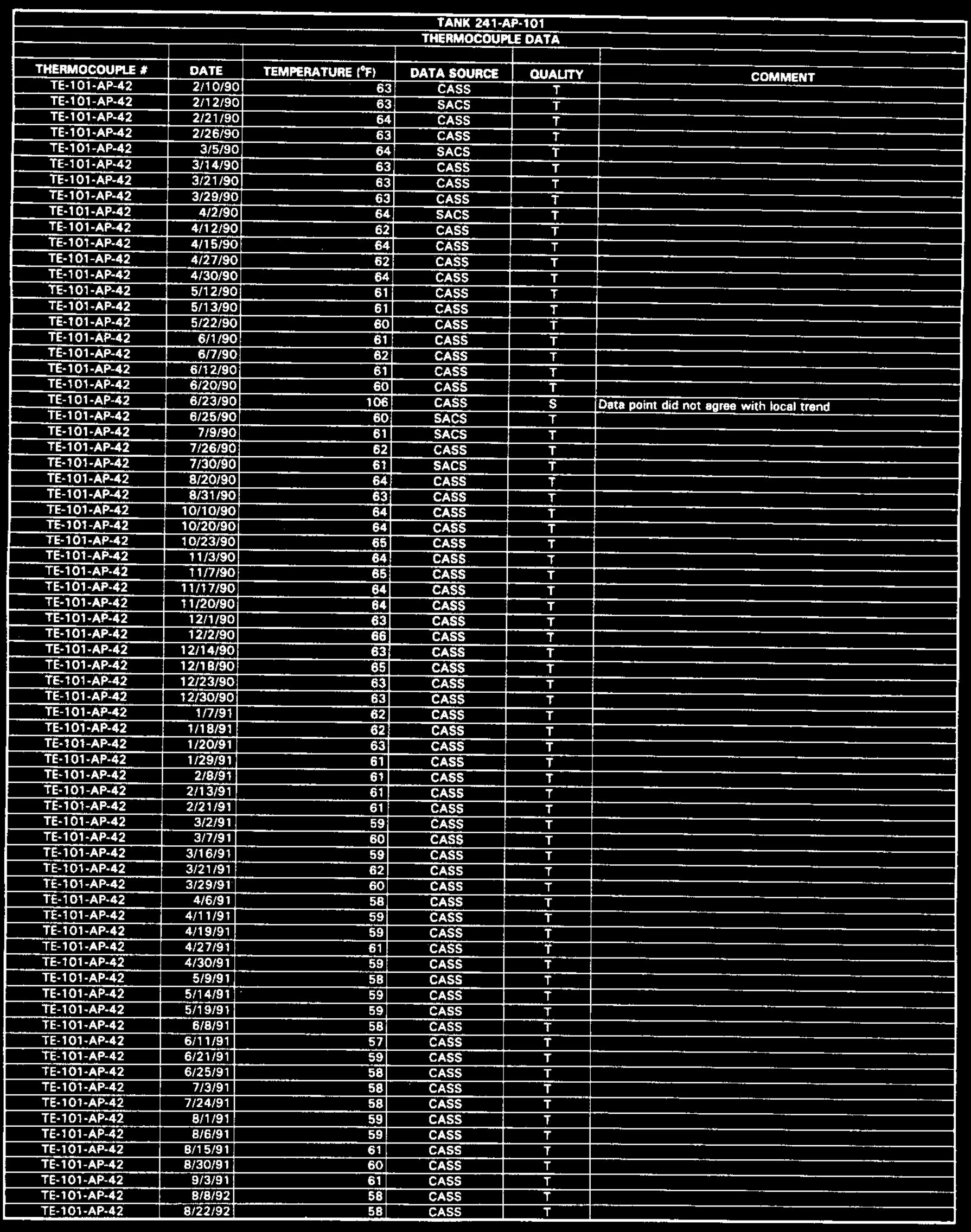


WHC-SD-WM-ER-315, Rev. 0

\begin{tabular}{|c|c|c|c|c|c|}
\hline \multicolumn{6}{|c|}{$\begin{array}{l}\text { TANK 241-AP-101 } \\
\text { THEFMOCOUFLE DATA }\end{array}$} \\
\hline & & & & & \\
\hline THERMOCOUPLE $*$ & DATE & TEMPERATURE ( $\left.{ }^{\circ} \mathrm{F}\right)$ & DATA SOURCE & QUALITY & COMMENT \\
\hline TE-101-AP-42 & $8 / 29 / 92$ & 59 & CASS & $T$ & \\
\hline TE-101-AP-42 & $9 / 5 / 92$ & 60 & CASS & $T$ & \\
\hline TE-101-AP-42 & 9/12/92 & 59 & CASS & $T$ & \\
\hline TE-101-AP-42 & $9 / 18 / 92$ & $\frac{60}{60}$ & $\frac{\text { CASS }}{\text { CASS }}$ & $\frac{T}{T}$ & \\
\hline$\frac{\text { TE-101-AP-42 }}{\text { TE-101-AP-42 }}$ & $\frac{9 / 26 / 92}{10 / 3 / 92}$ & 60 & $\frac{\text { CASS }}{\text { CASS }}$ & $\frac{1}{T}$ & \\
\hline$\frac{T E-101-A P-42}{T E-101-A P=42}$ & $\begin{array}{l}10 / 3 / 92 \\
10 / 6 / 92\end{array}$ & $\frac{60}{61}$ & CASS & $T$ & \\
\hline TE-101-AP-42 & $10 / 12 / 92$ & 60 & CASS & $\mathbf{T}$ & \\
\hline TE-101-AP-42 & $10 / 22 / 92$ & 62 & CASS & $T$ & \\
\hline TE-101-AP-42 & $10 / 28 / 92$ & 62 & CASS & $T$ & \\
\hline TE-101-AP-42 & $11 / 5 / 92$ & 61 & CASS & $\bar{T}$ & \\
\hline$T E-101-A P-42$ & $11 / 12 / 92$ & 61 & CASS & $\mathrm{T}$ & \\
\hline TE-101-AP-42 & $11 / 20 / 92$ & 63 & CASS & $T$ & \\
\hline$T E-101-A P-42$ & $11 / 28 / 92$ & 61 & CASS & $\mathbf{T}$ & \\
\hline TE-101-AP-42 & $12 / 5 / 92$ & 60 & CASS & $T$ & \\
\hline TE-101-AP-42 & $12 / 12 / 92$ & 61 & CASS & $\bar{T}$ & \\
\hline TE-101-AP-42 & $12 / 13 / 92$ & 62 & CASS & $T$ & \\
\hline$T E-101-A P-42$ & $12 / 26 / 92$ & 60 & CASS & $T$ & \\
\hline TE-101-AP-42 & $12 / 30 / 92$ & 60 & CASS & $T$ & \\
\hline TE-101-AP-42 & $1 / 9 / 93$ & 59 & CASS & $T$ & \\
\hline TE-101-AP-42 & 1/15/93 & 59 & CASS & $\bar{T}$ & \\
\hline TE-101-AP-42 & $1 / 22 / 93$ & 59 & CASS & $\mathbf{T}$ & \\
\hline TE-101-AP-42 & $1 / 29 / 93$ & 61 & CASS & $\mathbf{T}$ & \\
\hline TE-101-AP-42 & $2 / 6 / 93$ & 59 & CASS & $\bar{T}$ & \\
\hline TE-101-AP-42 & $2 / 11 / 93$ & 59 & CASS & $T$ & \\
\hline TE-101-AP-42 & $2 / 20 / 93$ & 58 & CASS & $\mathbf{T}$ & \\
\hline TE-101-AP-42 & $2 / 26 / 93$ & 58 & CASS & $T$ & \\
\hline TE-101-AP-42 & $3 / 5 / 93$ & 58 & CASS & $\mathbf{T}$ & \\
\hline TE-101-AP-42 & $3 / 12 / 93$ & 57 & CASS & $\mathbf{T}$ & \\
\hline TE-101-AP-42 & $3 / 19 / 93$ & 58 & CASS & $T$ & \\
\hline TE-101-AP-42 & $3 / 22 / 93$ & 57 & SACS & $T$ & \\
\hline TE-101-AP-42 & 3/31/93 & 58 & CASS & $T$ & \\
\hline TE-101-AP-42 & $4 / 7 / 93$ & 58 & CASS & $\mathbf{T}$ & \\
\hline TE-101-AP-42 & $4 / 12 / 93$ & 58 & CASS & $\mathbf{T}$ & \\
\hline TE-101-AP-42 & $4 / 24 / 93$ & 57 & CASS & $T$ & \\
\hline TE-107.AP-42 & $4 / 26 / 93$ & 57 & SACS & $T$ & \\
\hline TE-101-AP-42 & $5 / 8 / 93$ & 57 & CASS & $\mathbf{T}$ & \\
\hline TE-101-AP-42 & $5 / 10 / 93$ & 56 & SACS & $T$ & \\
\hline TE-101-AP-42 & $5 / 17 / 93$ & 58 & SACS & $T$ & \\
\hline TE-101-AP-42 & 6/5/93 & 56 & $\overline{\text { CASS }}$ & $T$ & \\
\hline TE-101-AP-42 & $6 / 12 / 93$ & 56 & CASS & $\overline{\mathbf{T}}$ & \\
\hline TE-101-AP-42 & 6/19/93 & 57 & CASS & $T$ & \\
\hline$T E-101-A P-42$ & $6 / 21 / 93$ & 58 & CASS & $T$ & \\
\hline TE-101-AP-42 & $7 / 17 / 93$ & 59 & CASS & $T$ & \\
\hline TE-101-AP-42 & $7 / 23 / 93$ & 57 & CASS & $T$ & \\
\hline TE-101-AP-A2 & $7 / 29 / 93$ & 58 & CASS & $T$ & \\
\hline TE-101-AP-42 & $8 / 2 / 93$ & 58 & SACS & $T$ & \\
\hline TE-101-AP-42 & $8 / 8 / 93$ & 59 & CASS & $\mathbf{T}$ & \\
\hline TE-101-AP-42 & $8 / 21 / 93$ & 59 & CASS & $\mathbf{T}$ & \\
\hline TE-101-AP-42 & $8 / 23 / 93$ & 59 & SACS & $\mathbf{T}$ & \\
\hline TE-101-AP-42 & $9 / 15 / 93$ & 60 & CASS & $\mathbf{T}$ & \\
\hline TE-101-AP-42 & $9 / 24 / 93$ & 60 & CASS & $T$ & \\
\hline TE-101-AP-42 & $10 / 1 / 93$ & 59 & SACS & $T$ & \\
\hline TE-101-AP-42 & $10 / 8 / 93$ & 61 & CASS & $T$ & \\
\hline TE-101-AP-42 & $\begin{array}{l}10 / 15 / 93 \\
\end{array}$ & 61 & SACS & $T$ & \\
\hline TE-101-AP-42 & $10 / 18 / 93$ & 61 & CASS & $T$ & \\
\hline TE-101-AP-42 & $10 / 30 / 93$ & 60 & CASS & $T$ & \\
\hline TE-101-AP-42 & $11 / 4 / 93$ & 61 & CASS & $T$ & \\
\hline TE-101-AP-42 & $11 / 8 / 93$ & 62 & SACS & $T$ & \\
\hline TE-101-AP-42 & $11 / 19 / 93$ & 61: & CASS & $\bar{T}$ & \\
\hline TE-101-AP-42 & $11 / 27 / 93$ & 61 & CASS & $T$ & \\
\hline TE-101-AP-42 & $12 / 1 / 93$ & 61 & CASS & $\bar{T}$ & \\
\hline TE-101-AP-42 & $12 / 14 / 93$ & 60 & SACS & $T$ & \\
\hline TE-101-AP-42 & $12 / 20 / 93$ & 59 & SACS & $T$ & \\
\hline TE-101-AP-42 & $12 / 27 / 93$ & 59 & SACS & $T$ & \\
\hline TE-101.AP-42 & $1 / 3 / 94$ & 60 & SACS & $\bar{T}$ & \\
\hline TE-101-AP-42 & $1 / 11 / 94$ & 61: & SACS & $T$ & \\
\hline TE-101-AP-42 & $1 / 26 / 94$ & 58 & SACS & $\bar{T}$ & \\
\hline TE-101-AP-42 & $2 / 9 / 94$ & 57 & SACS & $\mathrm{T}$ & \\
\hline TE-101-AP-42 & $2 / 14 / 94$ & 56 & SACS & $T$ & \\
\hline TE-101-AP-42 & $2 / 22 / 94$ & 57 & SACS & $T$ & \\
\hline TE-101-AP-42 & $3 / 1 / 94$ & 57 & SACS & $T$ & \\
\hline TE-101-AP-42 & $3 / 9 / 94$ & 57 & SACS & $\mathbf{G}$ & \\
\hline
\end{tabular}




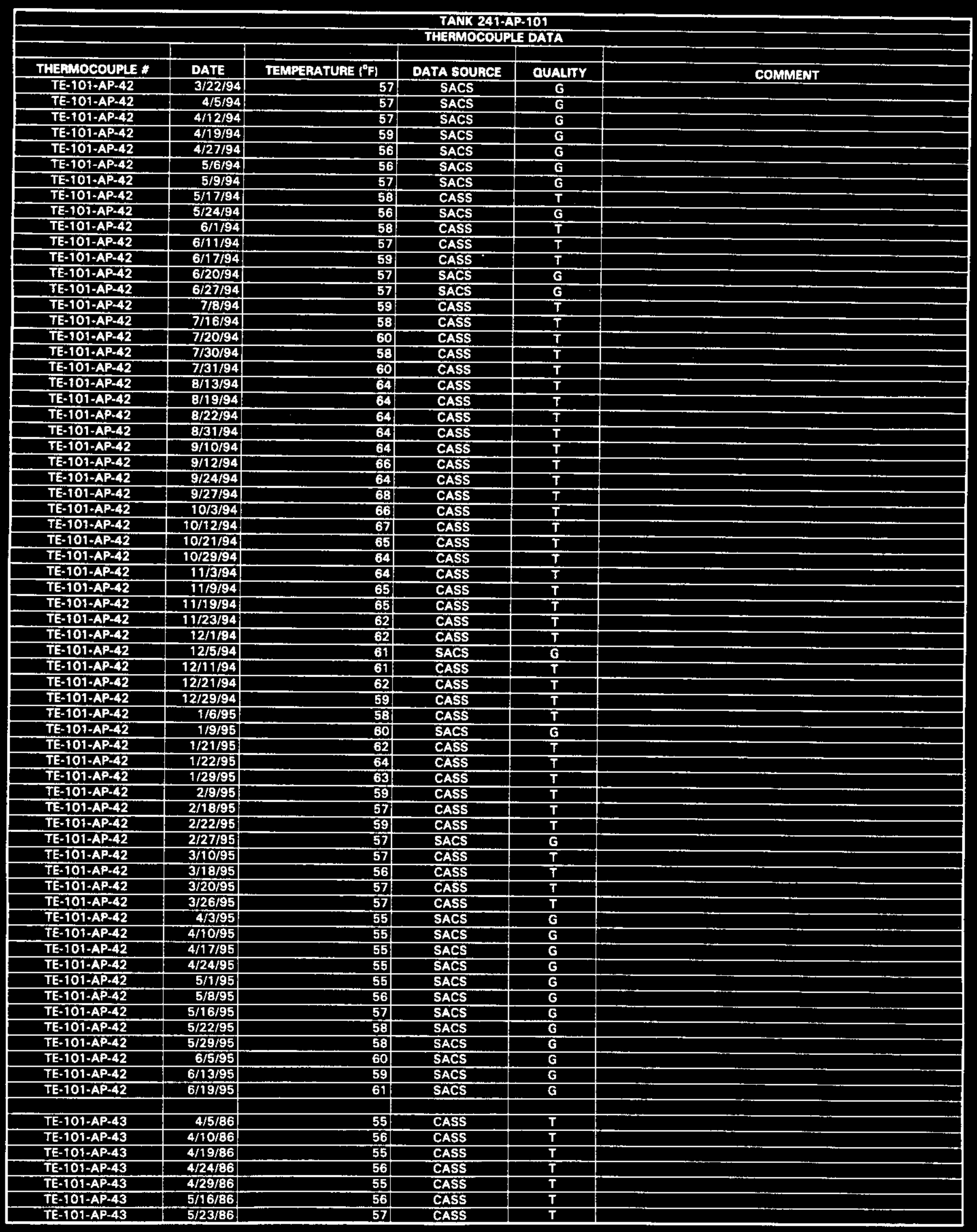




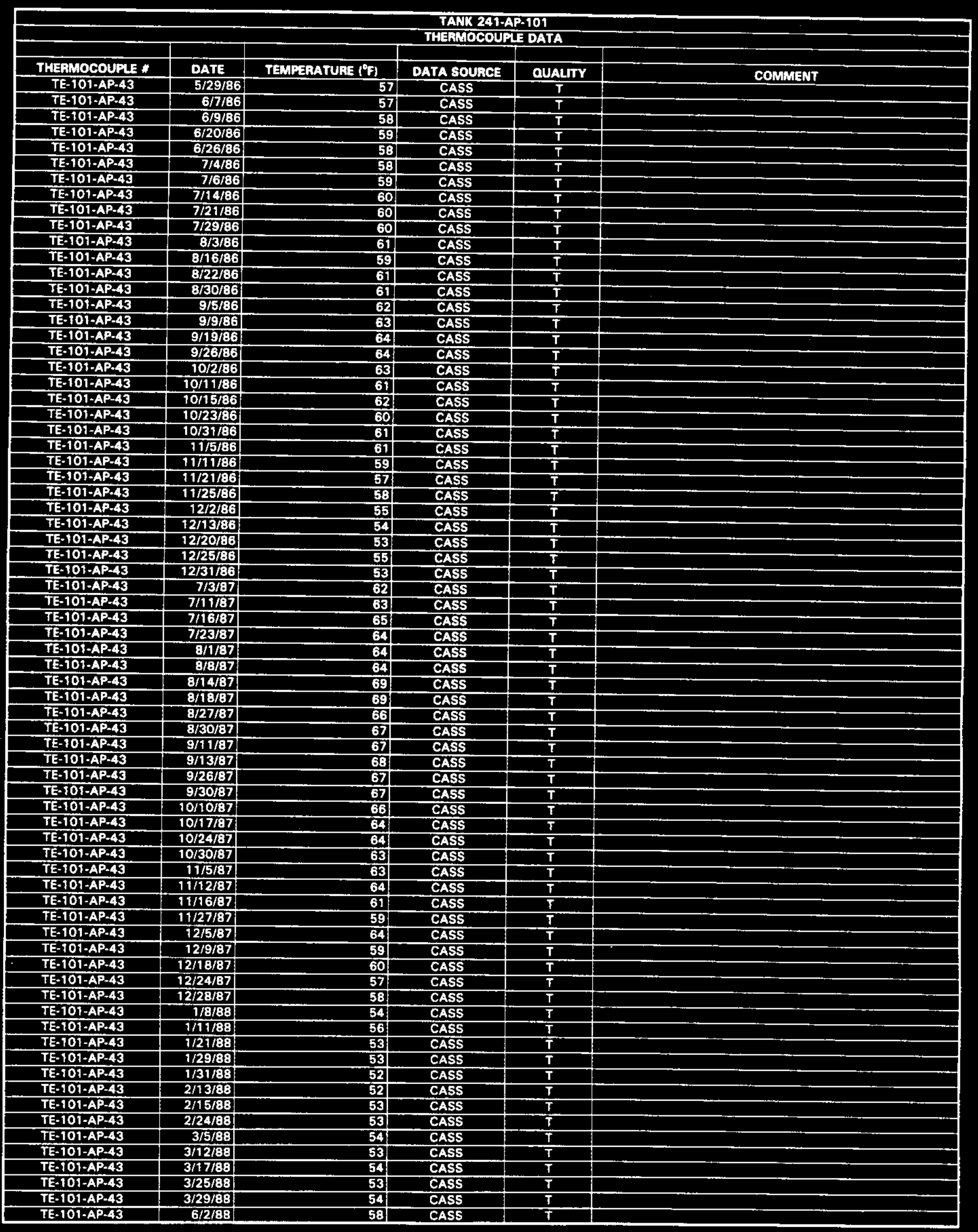




\begin{tabular}{|c|c|c|c|c|c|}
\hline \multicolumn{6}{|c|}{$\begin{array}{l}\text { TANK 241-AP:101 } \\
\text { THEFWOCOUFL DATA }\end{array}$} \\
\hline & & & & & \\
\hline THERMOCOUPLE * & DATE & TEMPERATURE $\left({ }^{\circ} \mathrm{F}\right)$ & DATA SOUACE & QUALITY & COMMENT \\
\hline TE-101-AP-43 & $6 / 11 / 88$ & $\begin{array}{rr}58 \\
\end{array}$ & CASS & $T$ & \\
\hline$\frac{T E-101-A P-43}{T E-101-A P-43}$ & $7 / 2 / 88$ & $\frac{60}{69}$ & $\frac{\text { CASS }}{\text { CASS }}$ & $T$ & \\
\hline $\begin{array}{l}\text { TE-101-AP-43 } \\
\text { TE-101-AP-43 }\end{array}$ & $\begin{array}{r}7 / 9 / 88 \\
7 / 7 / 88\end{array}$ & $\frac{69}{69}$ & $\frac{\text { CASS }}{\text { CASS }}$ & $\frac{T}{T}$ & \\
\hline$\frac{T E-101-A P-43}{\text { TE-101-AP-43 }}$ & $\begin{array}{l}7 / 13 / 88 \\
7 / 22 / 88 \\
\end{array}$ & 69 & $\frac{\text { CASS }}{\text { CASS }}$ & $T$ & \\
\hline TE-101-AP-43 & $7 / 30 / 88$ & 68 & CASS & $T$ & \\
\hline TE-101-AP-43 & $8 / 6 / 88$ & 74 & CASS & $T$ & \\
\hline TE-101-AP-43 & $8 / 18 / 88$ & 77 & CASS & $T$ & \\
\hline TE-101-AP-43 & $9 / 21 / 88$ & 81 & CASS & $\mathrm{T}$ & \\
\hline TE-101-AP-43 & $9 / 30 / 88$ & 80 & CASS & $\mathbf{T}$ & \\
\hline TE-101-AP-43 & $12 / 3 / 88$ & 75 & CASS & $\mathbf{T}$ & \\
\hline TE-101-AP-43 & $12 / 8 / 38$ & 74 & CASS & $T$ & \\
\hline TE-101-AP-43 & $12 / 14 / 88$ & 74 & CASS & $T$ & \\
\hline TE-101-AP-43 & $12 / 18 / 88$ & 73 & CASS & $T$ & \\
\hline TE-101-AP-43 & 7/10/89 & 62 & SACS & $\bar{T}$ & \\
\hline TE-101-AP-43 & $8 / 29 / 89$ & 66 & SACS & $\bar{T}$ & \\
\hline TE-101-AP-43 & $8 / 28 / 89$ & 66 & SACS & $\mathbf{T}$ & \\
\hline TE-101-AP-43 & $9 / 6 / 39$ & 65 & CASS & $T$ & \\
\hline TE-101-AP-43 & $10 / 9 / 89$ & 65 & SACS & $T$ & \\
\hline TE-101-AP-43 & $10 / 24 / 89$ & 69 & SACS & $T$ & \\
\hline TE-101-AP-43 & $10 / 30 / 89$ & 65 & SACS & $T$ & \\
\hline TE-101-AP-43 & $12 / 30 / 89$ & 64 & CASS & $\bar{T}$ & \\
\hline TE-101-AP-43 & $1 / 5 / 90$ & 65 & CASS & $\mathbf{T}$ & \\
\hline TE-101-AP-43 & 1/13/90 & 64 & CASS & $T$ & \\
\hline TE-101-AP-43 & $1 / 20 / 90$ & 65 & CASS & $T$ & \\
\hline TE-101.AP-43 & $1 / 23 / 90$ & 64 & CASS & $T$ & \\
\hline TE-101-AP-43 & $2 / 2190$ & 64! & CASS & $\bar{T}$ & \\
\hline TE-10T-AP-43 & $2 / 10 / 90$ & 63 & CABS & $T$ & \\
\hline TE-101-AP-43 & $2 / 16 / 90$ & 63 & CASS & $T$ & \\
\hline TE-101-AP-43 & $2 / 21 / 90$ & 64 & CASS & $\bar{T}$ & \\
\hline TE-101-AP-43 & $2 / 26 / 90$ & 63 & CASS & $\bar{T}$ & \\
\hline TE-101.AP-43 & $3 / 3 / 90$ & 62 & CASS & $T$ & \\
\hline TE-101-AP-43 & $3 / 14 / 90$ & 63 & CASS & $\mathbf{T}$ & \\
\hline TE-101-AP-43 & $3 / 24 / 90$ & 62 & CASS & $T$ & \\
\hline$T E-101-A P-43$ & $3 / 29 / 90$ & 63 & CASS & $T$ & \\
\hline$T E-101-A P-43$ & $4 / 4 / 90$ & 62 & CASS & $T$ & \\
\hline TE-101-AP-43 & $4 / 12 / 90$ & 62 & CASS & $T$ & \\
\hline TE-101-AP-43 & $4 / 15 / 90$ & 63 & CASS & $T$ & \\
\hline TE-101-AP-43 & $4 / 27 / 90$ & 62 & CASS & $T$ & \\
\hline TE-101-AP-43 & $4 / 30 / 90$ & 64 & CASS & $T$ & \\
\hline$T E-101-A P-43$ & $5 / 12 / 90$ & 61 & CASS & $\mathbf{T}$ & \\
\hline TE-101-AP-43 & $5 / 18 / 90$ & 60 & CASS & $\bar{T}$ & \\
\hline TE-101-AP-A3 & $5 / 21 / 90$ & 60 & CASS & $T$ & \\
\hline TE-101-AP-43 & $6 / 1 / 90$ & 61 & CASS & $T$ & \\
\hline TE-101-AP-43 & $6 / 7 / 90$ & 62 & CASS & $\bar{T}$ & \\
\hline TE-101-AP-43 & $6 / 12 / 90$ & 61 & CASS & $T$ & \\
\hline TE-101-AP-43 & $6 / 20 / 90$ & 60 & CASS & $T$ & \\
\hline TE-101-AP-43 & $6 / 23 / 90$ & 105 & CASS & $\mathbf{s}$ & Data point did not agree with local trend \\
\hline TE-101-AP-43 & $6 / 24 / 90$ & 60 & CASS & $T$ & \\
\hline TE-101-AP-43 & $7 / 26 / 90$ & 62 & CASS & $\mathbf{T}$ & \\
\hline TE-101-AP-43 & $8 / 6 / 90$ & 63 & SACS & $T$ & \\
\hline TE-101-AP-43 & $8 / 21 / 90$ & 63 & CASS & $T$ & \\
\hline TE-101-AP-43 & $8 / 31 / 90$ & 63 & CASS & $T$ & \\
\hline TE-101-AP-43 & $9 / 3 / 90$ & 62 & SACS & $\bar{T}$ & \\
\hline TE-101-AP-43 & $9 / 10 / 90$ & 62 & SACS & $T$ & \\
\hline TE-101-AP-43 & $10 / 8 / 90$ & 69 & SACS & $\mathbf{T}$ & \\
\hline TE-101-AP-43 & $10 / 20 / 90$ & 64 & CASS & $\mathbf{T}$ & \\
\hline TE-101-AP-43 & $10 / 26 / 90$ & 65 & CASS & $\mathbf{T}$ & \\
\hline TE-101-AP-43 & $10 / 29 / 90$ & 65 & CASS & $T$ & \\
\hline TE-103-AP-43 & $11 / 7 / 90$ & 65 & CASS & $T$ & \\
\hline TE-101-AP-43 & $11 / 17 / 90$ & 64 & CASS & $T$ & \\
\hline TE-101-AP-43 & $11 / 20 / 90$ & 64 & CASS & $T$ & \\
\hline TE-101-AP-43 & $12 / 1 / 90$ & 63 & CASS & $T$ & \\
\hline TE-101-AP-43 & $12 / 2 / 90$ & 65 & CASS & $\mathbf{T}$ & \\
\hline TE-101.AP-43 & $12 / 9 / 90$ & 64 & CASS & $\mathbf{T}$ & \\
\hline TE-101-AP-43 & $12 / 18 / 90$ & 65 & CASS & $\bar{T}$ & \\
\hline TE-101-AP-43 & $12 / 23 / 90$ & 63 & CASS & $T$ & \\
\hline TE-101-AP-43 & $1 / 4 / 91$ & 63 & CASS & $\bar{T}$ & \\
\hline TE-101-AP-43 & $1 / 7 / 91$ & 62 & CASS & $T$ & \\
\hline TE-101-AP-43 & $1 / 14 / 91$ & 65 & SACS & $T$ & \\
\hline$T E-101-A P-43$ & $1 / 21 / 91$ & 62 & SACS & $T$ & \\
\hline TE-101-AP-43 & $1 / 29 / 91$ & 61 & CASS & $T$ & \\
\hline TE-101-AP-43 & $2 / 8 / 91$ & 61 & CASS & $\mathbf{T}$ & \\
\hline
\end{tabular}




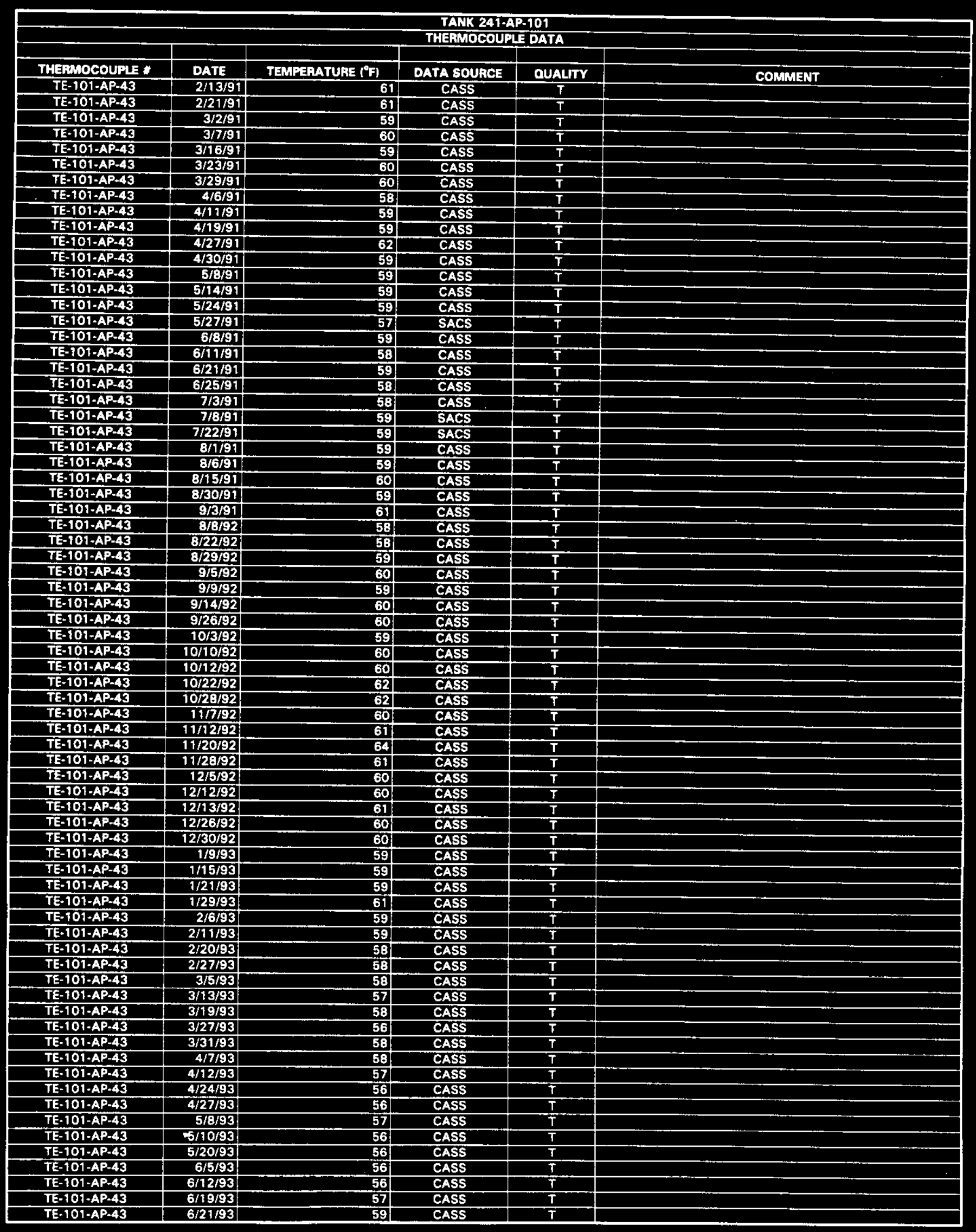




\begin{tabular}{|c|c|c|c|c|c|}
\hline & & & $\begin{array}{r}\text { TANK 241.A } \\
\text { THEAMOCOUA }\end{array}$ & $\frac{101}{\text { DATA }}$ & \\
\hline & & & 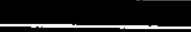 & & \\
\hline THERMOCOUPLE * & DATE & TEMPERATURE $\left({ }^{\circ} \mathrm{F}\right)$ & DATA SOUACE & OUALITY & COMMENT \\
\hline TE-101-AP-43 & $7 / 17 / 93$ & 59 & CASS & $T$ & \\
\hline TE-101-AP-43 & $7 / 23 / 93$ & 57 & $\begin{array}{l}\text { CASS } \\
\text { CASS }\end{array}$ & $\frac{T}{T}$ & \\
\hline TE-101-AP-43 & $7 / 29 / 93$ & 58 & $\frac{\text { CASS }}{\text { CASS }}$ & $\frac{1}{T}$ & \\
\hline$\frac{\text { TE-101-AP-43 }}{\text { TE-101-AP-43 }}$ & $\begin{array}{l}8 / 2 / 93 \\
8 / 8 / 93\end{array}$ & $\frac{58}{59}$ & $\frac{\text { CASS }}{\text { CASS }}$ & $T$ & \\
\hline TE-101-AP-43 & $8 / 21 / 93$ & 59 & CASS & $\mathrm{T}$ & \\
\hline TE-101-AP-43 & $8 / 23 / 93$ & 59 & CASS & $T$ & \\
\hline$T E-101-A P \cdot 43$ & $9 / 16 / 93$ & 60 & CASS & $T$ & \\
\hline$T E-101 \cdot A P-43$ & $9 / 24 / 93$ & 60 & CASS & $T$ & \\
\hline TE-101-AP-43 & $10 / 8 / 93$ & 61 & CASS & $\mathbf{T}$ & \\
\hline TE-101-AP-43 & $10 / 16 / 93$ & 60 & CASS & $\mathbf{T}$ & \\
\hline TE-101-AP-43 & $10 / 23 / 93$ & 61 & CASS & $T$ & \\
\hline TE-101-AP-43 & $10 / 30 / 93$ & 60 & CASS & $\mathbf{T}$ & \\
\hline TE-101-AP-43 & $11 / 6 / 93$ & 61 & CASS & $T$ & \\
\hline TE-101-AP-43 & $11 / 10 / 93$ & 61 & CASS & $T$ & \\
\hline TE-101-AP-43 & $11 / 19 / 93$ & 61 & CASS & $T$ & \\
\hline TE-101-AP-43 & $11 / 27 / 93$ & 61 & CASS & $\mathbf{T}$ & \\
\hline TE-101-AP-43 & 12/1/93 & 62 & CASS & $T$ & \\
\hline TE-101-AP-43 & $1 / 13 / 94$ & 59 & CASS & $T$ & \\
\hline TE-101-AP-43 & $5 / 19 / 94$ & 58 & CASS & $T$ & \\
\hline$T E-101-A P-43$ & $5 / 22 / 94$ & 57 & CASS & $T$ & \\
\hline TE-101-AP-43 & $6 / 1 / 94$ & 59 & CASS & $T$ & \\
\hline TE-101-AP-43 & $6 / 11 / 94$ & 57 & CASS & $\mathbf{T}$ & \\
\hline TE-101-AP-43 & $6 / 17 / 94$ & 59 & CASS & $T$ & \\
\hline TE-101-AP-43 & $7 / 8 / 94$ & 59 & CASS & $T$ & \\
\hline TE-101-AP-43 & $7 / 16 / 94$ & 58 & CASS & $T$ & \\
\hline TE-101-AP-43 & $7 / 20 / 94$ & 60 & CASS & $T$ & \\
\hline TE-101-AP-43 & $7 / 30 / 94$ & 58 & CASS & $\mathbf{T}$ & \\
\hline TE-101-AP-43 & $7 / 31 / 94$ & 60 & CASS & $T$ & \\
\hline TE-101-AP-43 & $B / 13 / 94$ & 61 & CASS & $T$ & \\
\hline TE-101-AP-43 & $8 / 19 / 94$ & 62 & CASS & $T$ & \\
\hline TE-101-AP-43 & $8 / 22 / 94$ & 64 & CASS & $T$ & \\
\hline TE-101-AP-43 & $8 / 31 / 94$ & 64 & CASS & $\bar{T}$ & \\
\hline TE-101-AP.43 & $9 / 10 / 94$ & 64 & CASS & $\bar{T}$ & \\
\hline TE-101-AP-43 & $9 / 12 / 94$ & 66 & CASS & $T$ & \\
\hline TE-101-AP-43 & $9 / 24 / 94$ & 64 & CASS & $T$ & \\
\hline TE-101-AP-43 & $9 / 27 / 94$ & 68 & CASS & $T$ & \\
\hline TE-101-AP-43 & $10 / 3 / 94$ & 66 & CASS & $\mathbf{T}$ & \\
\hline TE-103-AP-43 & $10 / 12 / 94$ & 67 & CASS & $\mathbf{T}$ & \\
\hline TE-101-AP-43 & $10 / 21 / 94$ & 65 & CASS & $T$ & \\
\hline TE-101-AP-43 & $10 / 24 / 94$ & 65 & CASS & $\bar{T}$ & \\
\hline TE-101-AP-43 & $11 / 3 / 94$ & 64 & CASS & $T$ & \\
\hline TE-101-AP-43 & $11 / 9 / 94$ & 66 & CASS & $\mathbf{T}$ & \\
\hline TE-101-AP-43 & $11 / 16 / 94$ & 65 & CASS & $\bar{T}$ & \\
\hline TE-101-AP-43 & $11 / 23 / 94$ & 62 & CASS & $T$ & \\
\hline$T E-101-A P-43$ & $12 / 1 / 94$ & 62 & CASS & $T$ & \\
\hline TE-101-AP-43 & $12 / 9 / 94$ & 60 & CASS & $\bar{T}$ & \\
\hline TE-101-AP-43 & $12 / 11 / 94$ & 61 & CASS & $T$ & \\
\hline TE-101-AP-43 & $12 / 21 / 94$ & 62 & CASS & $T$ & \\
\hline$T E-101 \cdot A P-43$ & $12 / 29 / 94$ & 59 & CASS & $T$ & \\
\hline TE-101-AP-43 & $1 / 6 / 95$ & 59 & CASS & $T$ & \\
\hline TE-101-AP-43 & $1 / 13 / 95$ & 58 & CASS & $T$ & \\
\hline TE-101-AP-43 & $1 / 21 / 95$ & 63 & CASS & $T$ & \\
\hline TE-101-AP-43 & $1 / 23 / 95$ & 64 & CASS & $T$ & \\
\hline TE-101-AP-43 & $1 / 29 / 95$ & 63 & CASS & $\bar{T}$ & \\
\hline TE-101-AP-43 & $2 / 9 / 95$ & 60 & CASS & $T$ & \\
\hline TE-101-AP-43 & $2 / 12 / 95$ & 58 & CASS & $T$ & \\
\hline TE-101-AP-43 & $2 / 22 / 95$ & 59 & CASS & $T$ & \\
\hline TE-101-AP-43 & $2 / 28 / 95$ & 57 & CASS & $T$ & \\
\hline TE-101-AP-43 & $3 / 10 / 95$ & 58 & CASS & $T$ & \\
\hline TE-101-AP-43 & $3 / 18 / 95$ & 56 & CASS & $T$ & \\
\hline TE-101-AP-43 & $3 / 22 / 95$ & 57 & CASS & $T$ & \\
\hline TE-101-AP-43 & $3 / 26 / 95$ & 57. & CASS & $T$ & \\
\hline TE-101-AP-44 & $4 / 5 / 36$ & 5 & CASS & $T$ & \\
\hline TE-101-AP-44 & $\frac{4 / 5 / 86}{4 / 10 / 36}$ & 57 & CASS & $T$ & \\
\hline TE-101-AP-44 & $4 / 19 / 86$ & 55 & CASS & $T$ & \\
\hline$T E-101-A P-44$ & $4 / 24 / 86$ & 56 & CASS & $T$ & \\
\hline TE-101-AP-44 & $4 / 29 / 86$ & 55 & CASS & $T$ & \\
\hline TE-101-AP-44 & $5 / 16 / 86$ & 56 & CASS & $T$ & \\
\hline TE-101-AP-44 & $5 / 23 / 86$ & 57 & CASS & $T$ & \\
\hline TE-101-AP-44 & $5 / 30 / 86$ & 58 & CASS & $\bar{T}$ & \\
\hline TE-101-AP-44 & $677 / 86$ & 57 & CASS & $\mathbf{T}$ & \\
\hline
\end{tabular}




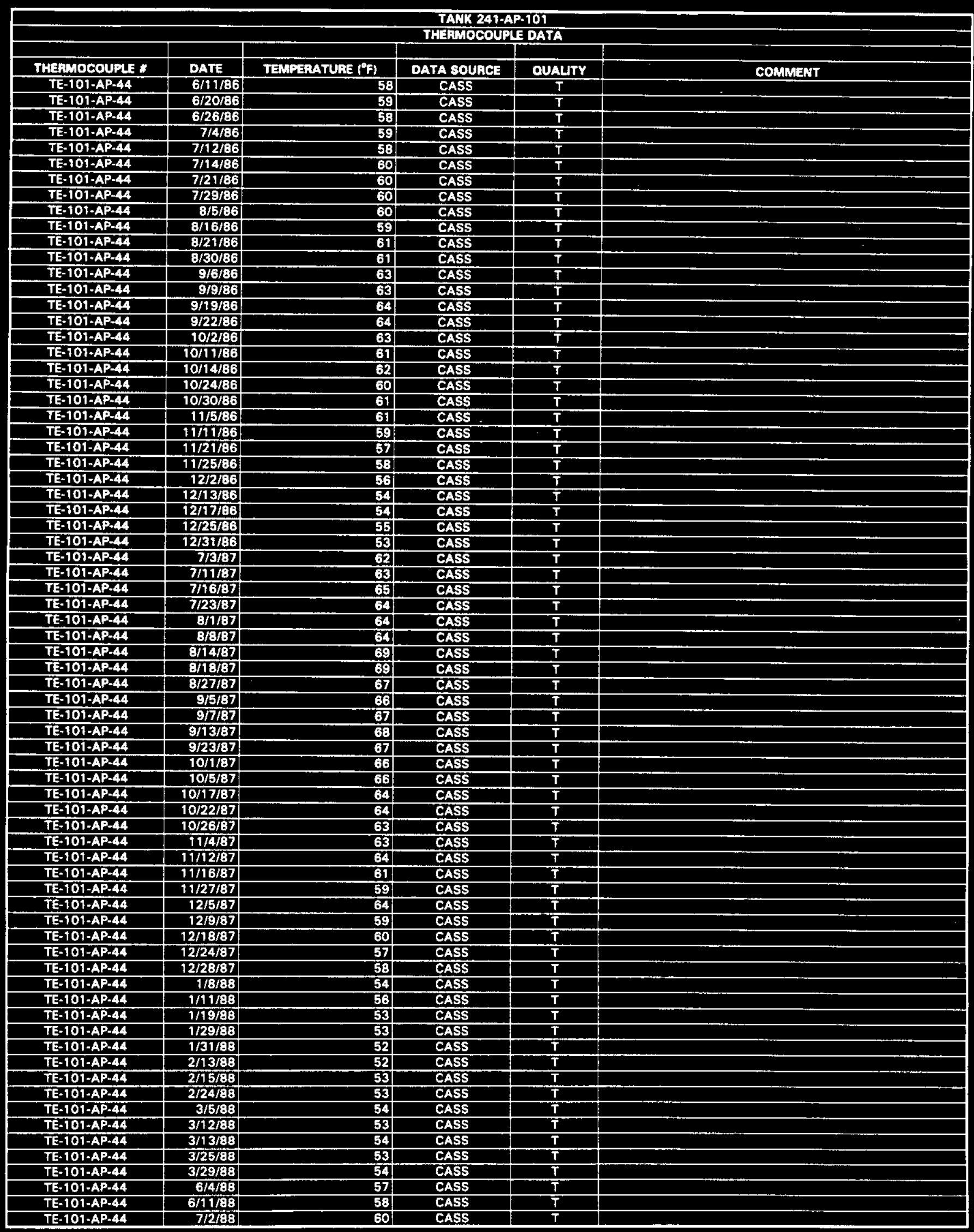




\begin{tabular}{|c|c|c|c|c|c|}
\hline \multicolumn{6}{|c|}{$\begin{array}{l}\text { TANK 241-AP-101 } \\
\text { THEAMOCOUPLE DATA }\end{array}$} \\
\hline & & & & & \\
\hline THEAMOCOUPLE * & DATE & TEMPERATURE $\left({ }^{\circ} \mathrm{F}\right)$ & DATA SOURCE & OUALTY & COMMENT \\
\hline TE-101-AP.44 & $7 / 9 / 8 \mathrm{8}$ & 68 & CASS & $\mathrm{T}$ & \\
\hline TE-101-AP-44 & $7 / 13 / 88$ & 69 & CASS & $T$ & \\
\hline TE-101-AP-44 & $7 / 23 / 88$ & 66 & CASS & $T$ & \\
\hline TE-101-AP-44 & $7 / 30 / 88$ & 67 & CASS & $T$ & \\
\hline TE-101-AP-44 & $8 / 6 / 88$ & 74 & CASS & $T$ & \\
\hline TE-101-AP-44 & $8 / 18 / 88$ & 76 & CASS & $T$ & \\
\hline TE-101-AP-44 & $9 / 23 / 88$ & 80 & CASS & $T$ & \\
\hline TE-101-AP-44 & $9 / 27 / 88$ & 80 & CASS & $T$ & \\
\hline TE-101-AP-44 & $12 / 3 / 88$ & 75 & CASS & $T$ & \\
\hline TE-101-AP-44 & $12 / 6 / 88$ & 74 & CASS & $T$ & \\
\hline$T E-101-A P-44$ & $12 / 14 / 88$ & 74 & CASS & $T$ & \\
\hline TE-101-AP-44 & $12 / 18 / 88$ & 73 & CASS & $T$ & \\
\hline TE-101-AP-44 & $9 / 6 / 89$ & 65 & CASS & $T$ & \\
\hline TE-101-AP-44 & $12 / 30 / 89$ & 64 & CASS & $T$ & \\
\hline TE-101-AP-44 & $1 / 5 / 90$ & 65 & CASS & $\bar{T}$ & \\
\hline TE-101-AP+44 & $1 / 13 / 90$ & 64 & CASS & $T$ & \\
\hline TE-101-AP-44 & $1 / 20 / 90$ & 65 & CASS & $T$ & \\
\hline TE-101-AP=44 & $1 / 23 / 90$ & 64 & CASS & $T$ & \\
\hline TE-101-AP-44 & $2 / 2 / 90$ & 64 & CASS & $T$ & \\
\hline TE-101-AP-44 & $2 / 7 / 90$ & 64 & CASS & $T$ & \\
\hline TE-101-AP-44 & $2 / 12 / 90$ & 63 & CASS & $T$ & \\
\hline TE-101-AP-44 & $2 / 24 / 90$ & 62 & CASS & $T$ & \\
\hline TE-101-AP-44 & $2 / 26 / 90$ & 63 & CASS & $T$ & \\
\hline TE-101-AP-44 & $3 / 6 / 90$ & 62 & CASS & $T$ & \\
\hline TE-101-AP-44 & $3 / 14 / 90$ & 63 & CASS & $\mathbf{T}$ & \\
\hline TE-101-AP-44 & $3 / 21 / 90$ & 63 & CASS & $T$ & $\therefore$ \\
\hline TE-101-AP-44 & $3 / 29 / 90$ & 63 & CASS & $\mathbf{T}$ & \\
\hline TE-101-AP=44 & $4 / 4 / 90$ & 62 & CASS & $\mathbf{T}$ & \\
\hline TE-101-AP-44 & $4 / 12 / 90$ & 62 & CASS & $T$ & \\
\hline TE-101-AP-44 & $4 / 15 / 90$ & 63 & CASS & $T$ & \\
\hline TE-107-AP-44 & $4 / 27 / 90$ & 62 & CASS & $T$ & \\
\hline TE-101-AP-44 & $4 / 30 / 90$ & 64 & CASS & $T$ & \\
\hline TE-101-AP-44 & $5 / 12 / 90$ & 61 & CASS & $T$ & \\
\hline TE-101-AP-44 & $5 / 18 / 90$ & 60 & CASS & $T$ & \\
\hline TE-101-AP-44 & $5 / 22 / 90$ & 60 & CASS & $T$ & \\
\hline TE-101-AP-44 & $6 / 1 / 90$ & 61 & CASS & $T$ & \\
\hline TE-101-AP-44 & $6 / 7 / 90$ & 62 & CASS & $T$ & \\
\hline TE-101-AP-44 & $6 / 14 / 90$ & 60 & CASS & $T$ & \\
\hline TE-101-AP-44 & $6 / 20 / 90$ & 59 & CASS & $T$ & \\
\hline TE-101-AP-44 & $6 / 23 / 90$ & 106 & CASS & $\mathrm{S}$ & Data point did not agree with local trend \\
\hline TE-101-AP-44 & $6 / 24 / 90$ & $\mathbf{5 9}$ & CASS & $T$ & \\
\hline TE-101-AP-44 & $7 / 26 / 90$ & 61 & CASS & $T$ & \\
\hline TE-101-AP-44 & $\mathrm{B} / 23 / 90$ & 59 & CASS & $T$ & \\
\hline TE-101-AP-44 & $8 / 26 / 90$ & 60 & CASS & $T$ & \\
\hline TE-101-AP-44 & $10 / 10 / 90$ & 63 & CASS & $\mathbf{T}$ & \\
\hline TE-101-AP-44 & $10 / 20 / 90$ & 63 & CASS & $T$ & \\
\hline TE-101-AP-44 & $10 / 26 / 90$ & 64 & CASS & $T$ & \\
\hline TE-101-AP-44 & $11 / 1 / 90$ & 64 & CASS & $T$ & \\
\hline TE-101-AP-44 & $11 / 7 / 90$ & 65 & CASS & $\bar{T}$ & \\
\hline TE-101-AP-44 & $11 / 17 / 90$ & 64 & CASS & $T$ & \\
\hline TE-101-AP-44 & $11 / 24 / 90$ & 63 & CASS & $T$ & \\
\hline TE-101-AP-44 & $12 / 1 / 90$ & 63 & CASS & $T$ & \\
\hline TE-101-AP-44 & $12 / 6 / 90$ & 64 & CASS & $T$ & \\
\hline TE-101-AP-44 & $12 / 13 / 90$ & 63 & CASS & $T$ & \\
\hline TE-101-AP-44 & $12 / 21 / 90$ & 63 & CASS & $T$ & \\
\hline TE-101-AP-44 & $12 / 23 / 90$ & 63 & CASS & $\bar{T}$ & \\
\hline TE-101-AP-44 & $12 / 30 / 90$ & 63 & CASS & $T$ & \\
\hline TE-101-AP-44 & $1 / 7 / 91$ & 62 & CASS & $T$ & \\
\hline TE-101-AP-44 & $1 / 19 / 91$ & 62 & CASS & $T$ & \\
\hline TE-101-AP-44 & $1 / 20 / 91$ & 62 & CASS & $T$ & \\
\hline TE-101-AP-44 & $1 / 29 / 91$ & 61 & CASS & $T$ & \\
\hline TE-101-AP+44 & $2 / 8 / 91$ & 61 & CASS & $T$ & \\
\hline TE-101-AP-44 & $2 / 13 / 91$ & 61 & CASS & $T$ & \\
\hline TE-101-AP-44 & $2 / 21 / 91$ & 61 & CASS & $T$ & \\
\hline TE-101-AP-44 & $3 / 2 / 91$ & 59 & CASS & $T$ & \\
\hline TE-101-AP-44 & $3 / 9 / 91$ & 59 & CASS & $T$ & \\
\hline TE-101-AP-44 & $3 / 16 / 91$ & 59 & CASS & $T$ & \\
\hline TE-101-AP-44 & $3 / 23 / 91$ & 60 & CASS & $T$ & \\
\hline TE-101-AP-44 & $3 / 29 / 91$ & 60 & CASS & $T$ & \\
\hline TE-101-AP-44 & $4 / 6 / 91$ & 58 & CASS & $T$ & \\
\hline TE-101-AP-44 & $4 / 11 / 91$ & 59 & CASS & $T$ & \\
\hline TE-101-AP-44 & $4 / 19 / 91$ & 59 & CASS & $T$ & \\
\hline TE-101-AP-44 & $4 / 27 / 91$ & 62. & CASS & $T$ & \\
\hline
\end{tabular}




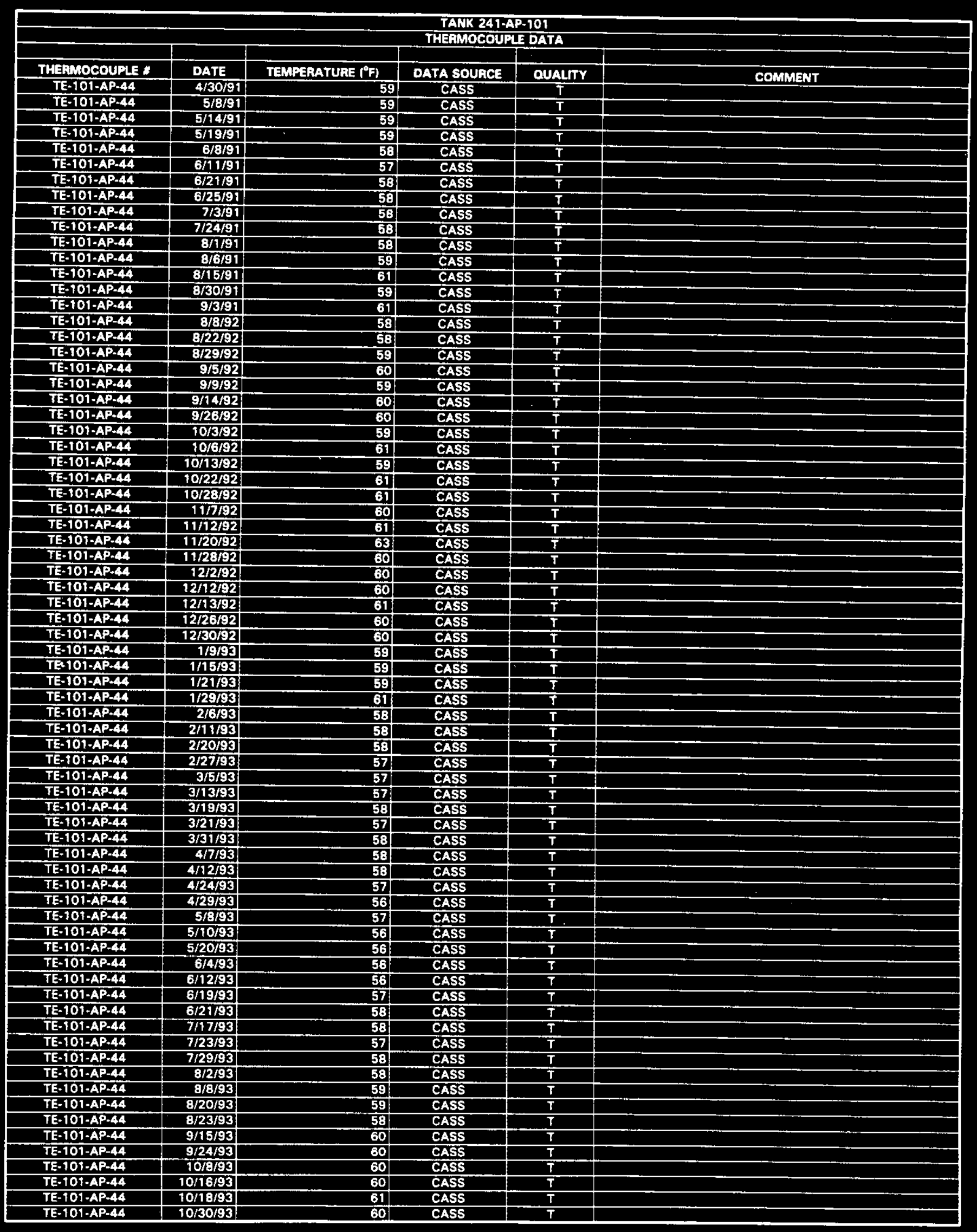




\begin{tabular}{|c|c|c|c|c|c|}
\hline \multicolumn{6}{|c|}{$\begin{array}{l}\text { TANK 241-AP-101 } \\
\text { THERAMOCOUPLE DATA }\end{array}$} \\
\hline & & & & & \\
\hline THEFMOCOUPLE * & DATE & TEMPERATURE $\left({ }^{\circ} \mathrm{F}\right)$ & DATA SOUACE & QUALTY & COMMENT \\
\hline TE-101-AP-44 & $11 / 4 / 93$ & 61 & CASS & $T$ & \\
\hline TE-101-AP-44 & $11 / 13 / 93$ & 60 & CASS & $T$ & \\
\hline TE-101-AP-44 & $11 / 15 / 93$ & 61 & CASS & $\mathbf{T}$ & \\
\hline TE-101-AP.44 & $11 / 27 / 93$ & $\frac{61}{61}$ & CASS & $T$ & \\
\hline$\frac{\text { TE-101-AP-44 }}{\text { TE-101-AP-44 }}$ & $12 / 1 / 93$ & $\frac{61}{59}$ & $\begin{array}{l}\text { CASS } \\
\text { CASS }\end{array}$ & $\frac{T}{T}$ & \\
\hline$\frac{\text { TE-101-AP-44 }}{\text { TE-101-AP-44 }}$ & $\begin{array}{l}1 / 12 / 94 \\
5 / 17 / 94\end{array}$ & 59 & $\begin{array}{l}\text { CASS } \\
\text { CASS }\end{array}$ & $T$ & \\
\hline TE-101-AP-44 & $5 / 22 / 94$ & 57 & CASS & $T$ & \\
\hline TE-101-AP-44 & 6/1/94 & 59 & CASS & $\mathbf{T}$ & \\
\hline TE-101-AP-44 & $6 / 11 / 94$ & 57 & CASS & $T$ & \\
\hline TE-101-AP-44 & $6 / 17 / 194$ & 59 & CASS & $T$ & \\
\hline TE-101-AP-44 & $7 / 8 / 94$ & 60 & CASS & $\mathbf{T}$ & \\
\hline TE-101-AP-44 & $7 / 10 / 94$ & 59 & CASS & $\mathbf{T}$ & \\
\hline TE-101-AP-44 & $7 / 20 / 94$ & 61 & CASS & $\mathrm{T}$ & \\
\hline TE-101-AP-44 & $7 / 30 / 94$ & 59 & CASS & $\bar{T}$ & \\
\hline TE-101-AP-44 & $7 / 31 / 94$ & 61 & CASS & $\bar{T}$ & \\
\hline TE-101-AP-44 & $8 / 13 / 94$ & 62 & CASS & $\mathbf{T}$ & \\
\hline TE-101-AP-44 & $8 / 19 / 94$ & 62 & CASS & $T$ & \\
\hline TE-101-AP-44 & $8 / 27 / 94$ & 64 & CASS & $\bar{T}$ & \\
\hline TE-101-AP-44 & $8 / 31 / 94$ & 65 & CASS & $\bar{T}$ & \\
\hline$T E-101-\overline{A P}-44$ & $9 / 10 / 94$ & 65 & CASS & $\bar{T}$ & \\
\hline TE-101-AP-44 & $9 / 12 / 94$ & 67. & CASS & $T$ & \\
\hline TE-101-AP-44 & $9 / 24 / 94$ & 85 & CASS & $\mathbf{T}$ & \\
\hline TE-101-AP-44 & $9 / 27 / 94$ & 70 & CASS & $T$ & \\
\hline TE-101-AP-44 & $10 / 6 / 94$ & 67! & CASS & $T$ & \\
\hline TE-101-AP-44 & $10 / 12 / 94$ & 68 & CASS & $T$ & \\
\hline TE-101-AP-44 & $10 / 22194$ & 66 & CASS & $\mathbf{T}$ & \\
\hline TE-101-AP-44 & $10 / 27 / 94$ & 66 & CASS & $\mathbf{T}$ & \\
\hline TE-101-AP-44 & $11 / 3 / 94$ & 66 & CASS & $T$ & \\
\hline TE-101-AP-44 & $11 / 12 / 94$ & 65 & CASS & $\bar{T}$ & \\
\hline TE-101-AP-44 & $11 / 19 / 94$ & 66 & CASS & $T$ & \\
\hline TE-101-AP-44 & $11 / 20 / 94$ & 64 & CASS & $T$ & \\
\hline TE-101-AP-44 & $12 / 1 / 34$ & 63. & CASS & $\mathrm{T}$ & \\
\hline TE-101-AP-44 & $12 / 9 / 94$ & 62 & CASS & $T$ & \\
\hline TE-101-AP-44 & $12 / 11 / 94$ & 62 & CASSS & $\mathbf{T}$ & \\
\hline TE-101-AP-44 & $12 / 21 / 94$ & 61 & CASS & $T$ & \\
\hline TE-101-AP-44 & $12 / 29 / 94$ & 60 & CASS & $T$ & \\
\hline TE-101-AP-44 & $1 / 6 / 95$ & 59 & CASS & $\bar{T}$ & \\
\hline TE-101-AP-44 & $1 / 13 / 95$ & 59: & CASS & $\mathbf{T}$ & \\
\hline TE-101-AP-44 & $1 / 21 / 95$ & 64 & CASS & $T$ & \\
\hline TE-101-AP-44 & $1 / 22 / 95$ & 65 & CASS & $\mathbf{I}$ & \\
\hline TE-101-AP-44 & $1 / 29 / 95$ & 64 & CASS & $T$ & \\
\hline TE-101-AP-44 & $2 / 9 / 95$ & 60 & CASS & $T$ & \\
\hline TE-10T-AP-44 & $2 / 16 / 95$ & 58 & CASS & $\bar{T}$ & \\
\hline TE-101-AP-44 & $2 / 22 / 95$ & 59 & CASS & $\mathbf{T}$ & \\
\hline TE-101-AP-44 & $2 / 28 / 95$ & 57 & CASS & $T$ & \\
\hline TE-101-AP-44 & $3 / 10 / 95$ & 58: & CASS & $T$ & \\
\hline TE-101-AP-44 & $3 / 18 / 95$ & 57 & CASS & $T$ & \\
\hline TE-101-AP-44 & $3 / 22 / 95$ & 57 & CASS & $T$ & \\
\hline TE-101-AP-44 & $3 / 26 / 95$ & 58 & CASS & $T$ & \\
\hline & & & & 7 & \\
\hline TE-101-AP-45 & 4/5/86 & 55 & CASS & $T$ & \\
\hline TE-101-AP-45 & $4 / 10 / 86$ & $\begin{array}{l}56 \\
55\end{array}$ & CASS & $T$ & \\
\hline TE-101-AP-45 & $4 / 19 / 86$ & 55 & CASS & $\mathbf{T}$ & \\
\hline TE-101-AP-45 & $4 / 24 / 86$ & 56 & CASS & $\mathbf{T}$ & \\
\hline TE-101-AP-45 & $4 / 29 / 86$ & 55 & CASS & $\mathbf{T}$ & \\
\hline TE-101-AP-A5 & $5 / 16 / 86$ & 56 & CASS & $\mathbf{T}$ & \\
\hline TE-101-AP-45 & $5 / 23 / 86$ & 57 & CASS & $T$ & \\
\hline TE-101-AP-45 & $5 / 30 / 86$ & 58 & CASS & $T$ & \\
\hline TE-101-AP-45 & $6 / 7 / 86$ & 57 & CASS & $T$ & \\
\hline TE-101-AP-45 & $6 / 11 / 86$ & 58 & CASS & $\mathbf{T}$ & \\
\hline TE-101-AP-45 & $6 / 20 / 86$ & 59 & CASS & $\mathbf{T}$ & \\
\hline TE-101-AP-45 & $6 / 26 / 86$ & 58 & CASS & $T$ & \\
\hline TE-101-AP-45 & $7 / 4 / 86$ & 58 & CASS & $T$ & \\
\hline TE-101-AP-45 & $7 / 6 / 86$ & 59 & CASS & $\mathbf{T}$ & \\
\hline TE-101-AP-45 & $7 / 14 / 86$ & 62 & CASS & $\bar{T}$ & \\
\hline TE-101-AP-45 & $7 / 21 / 86$ & 60 & CASS & $T$ & \\
\hline TE-101-AP-45 & $7 / 29 / 86$ & 60 & CASS & $\mathrm{T}$ & \\
\hline TE-101-AP-45 & $8 / 3 / 36$ & 61 & CASS & $T$ & \\
\hline TE-101-AP-45 & B/16/86 & 59 & CASS & $T$ & \\
\hline TE-101-AP-45 & $8 / 21 / 86$ & 61 & CASS & $T$ & \\
\hline TE-101-AP-45 & $8 / 30 / 86$ & 61 & CASS & $T$ & \\
\hline TE-101-AP-45 & $9 / 6 / 86$ & 62 & CASS & $T$ & \\
\hline
\end{tabular}




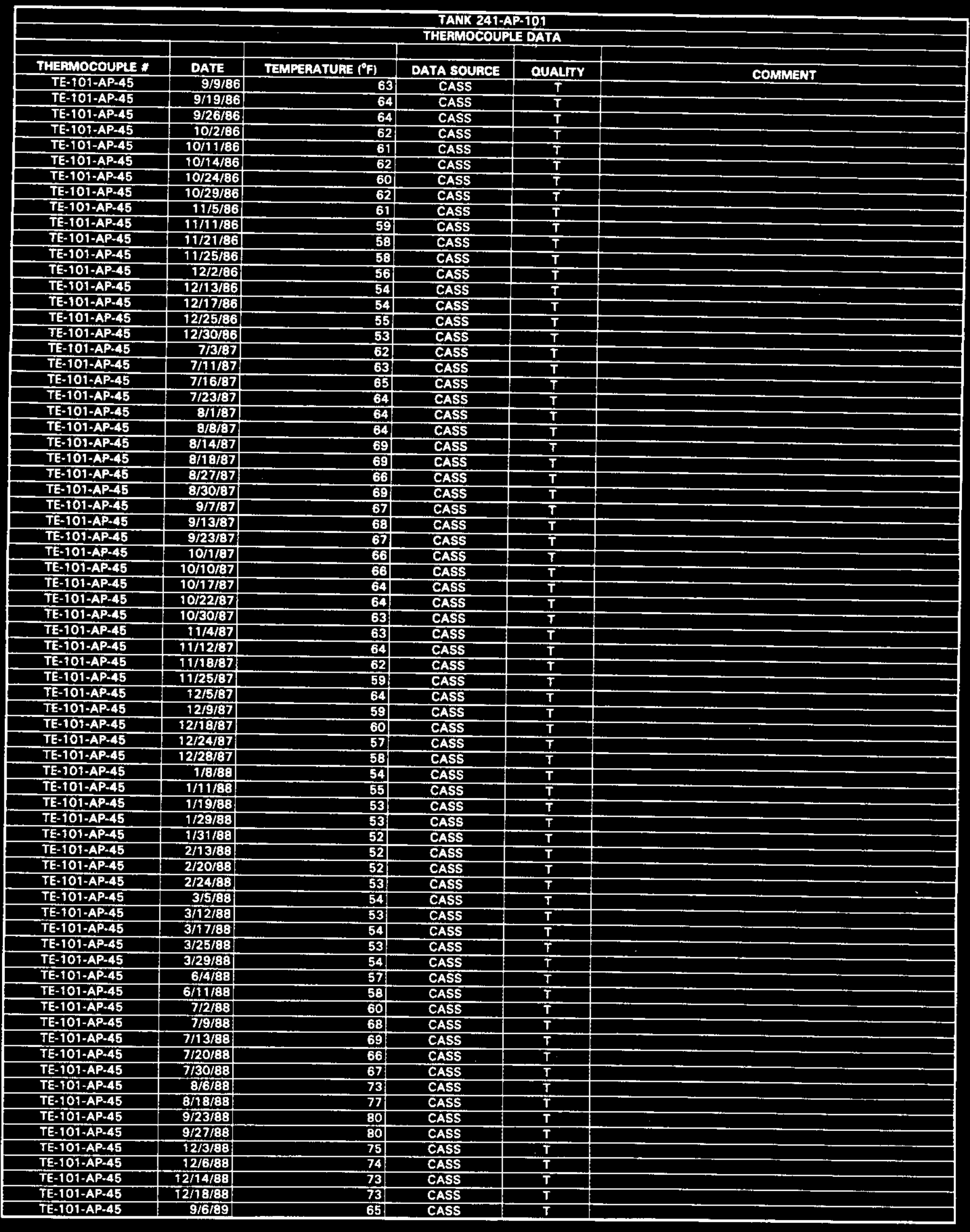




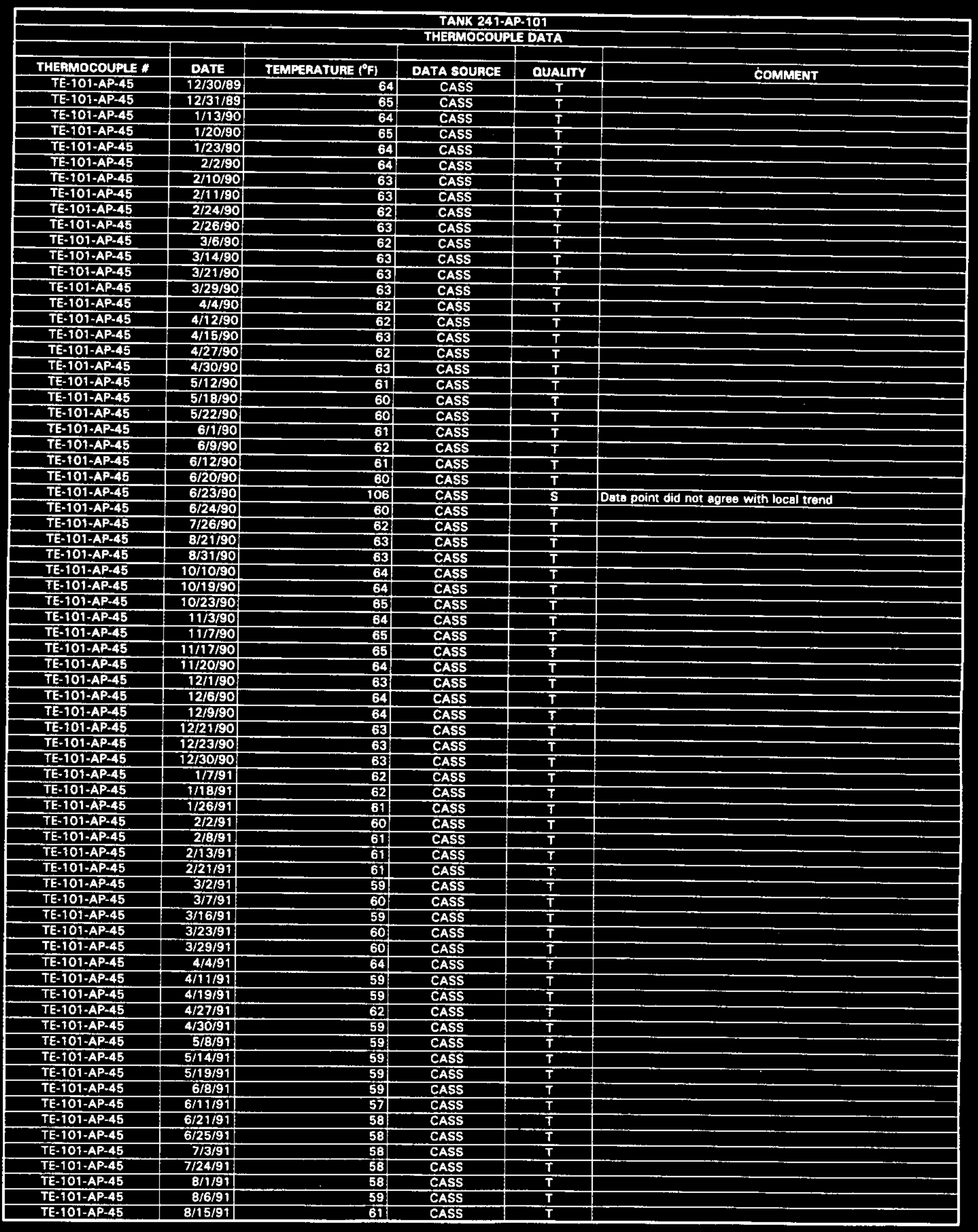




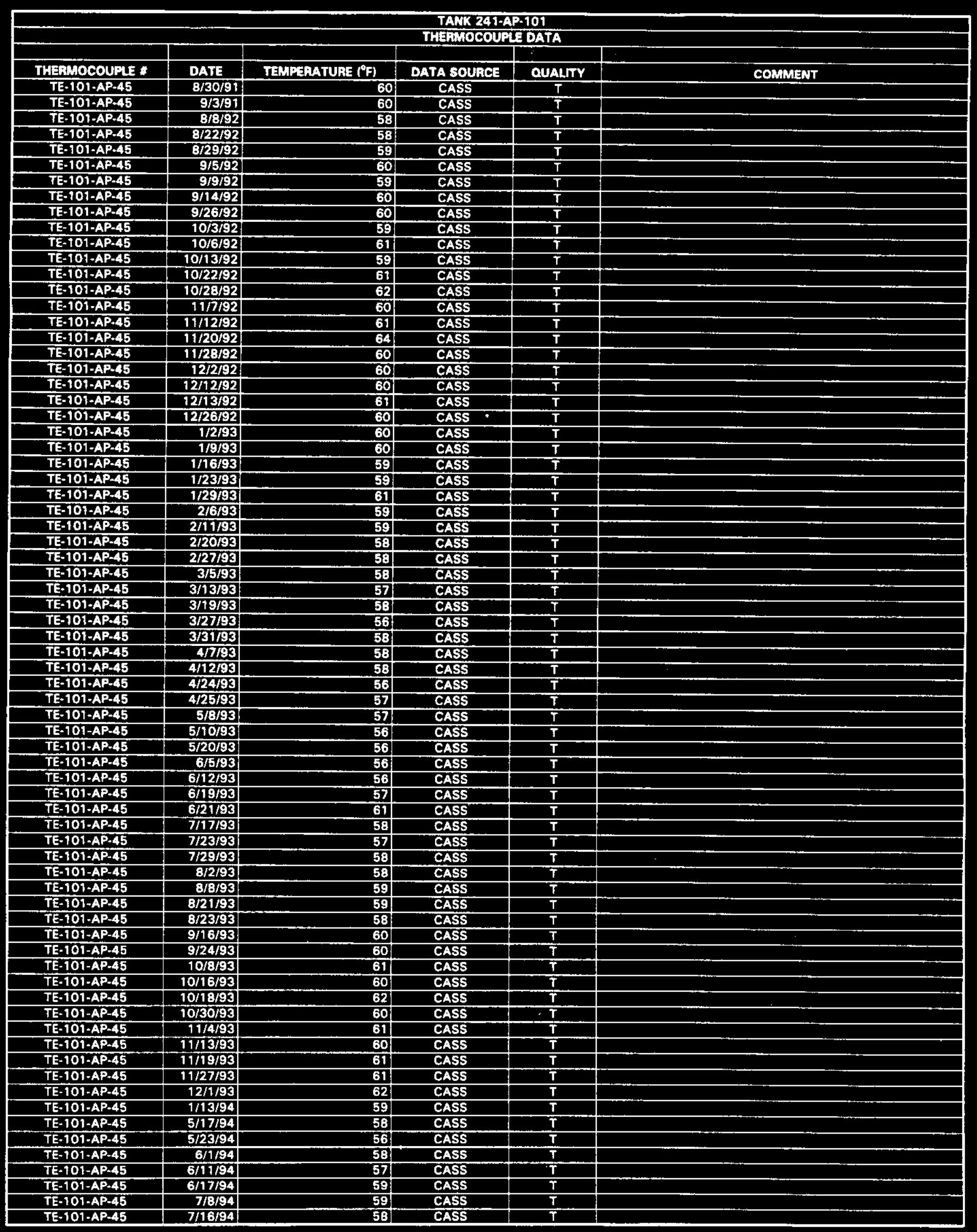




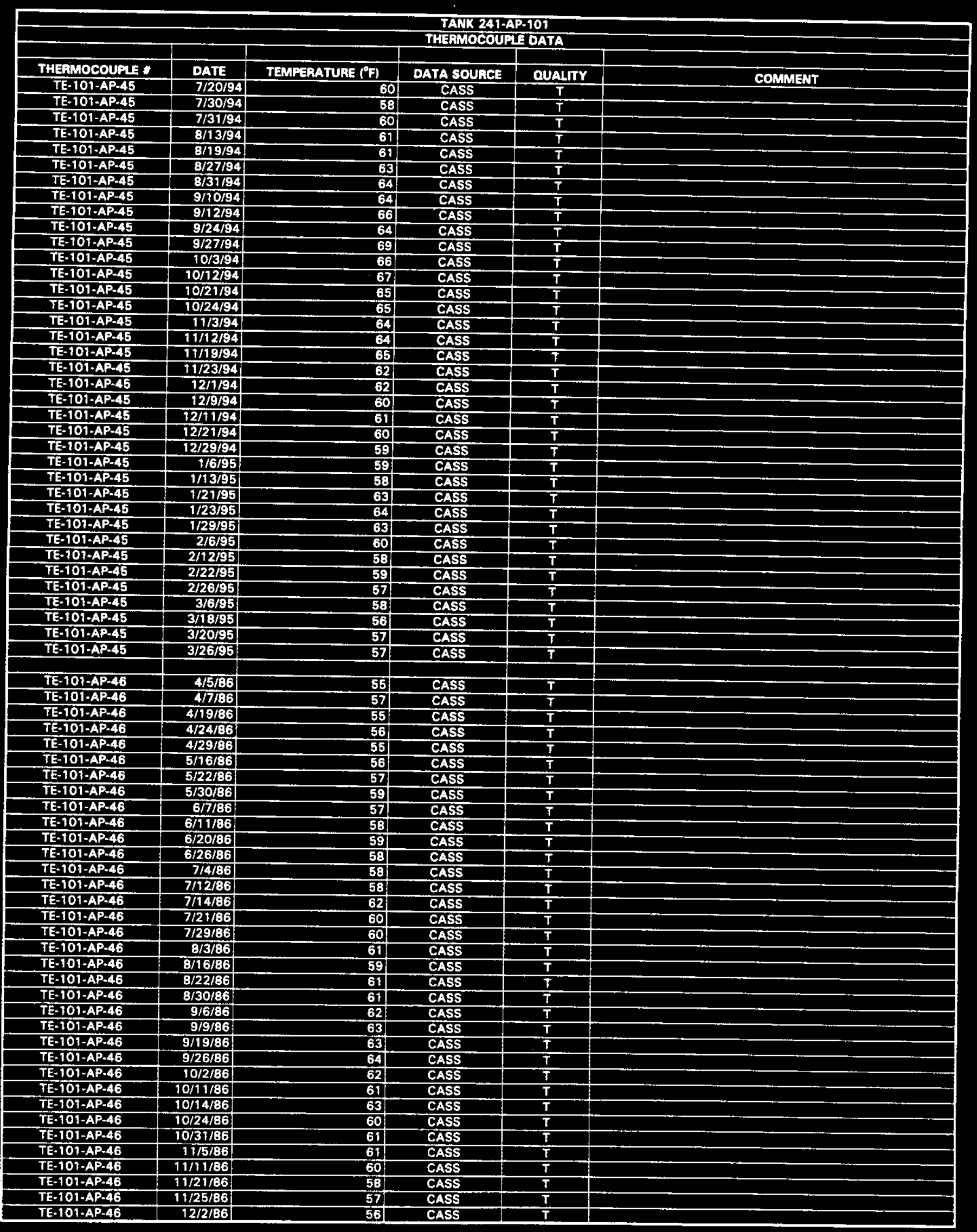




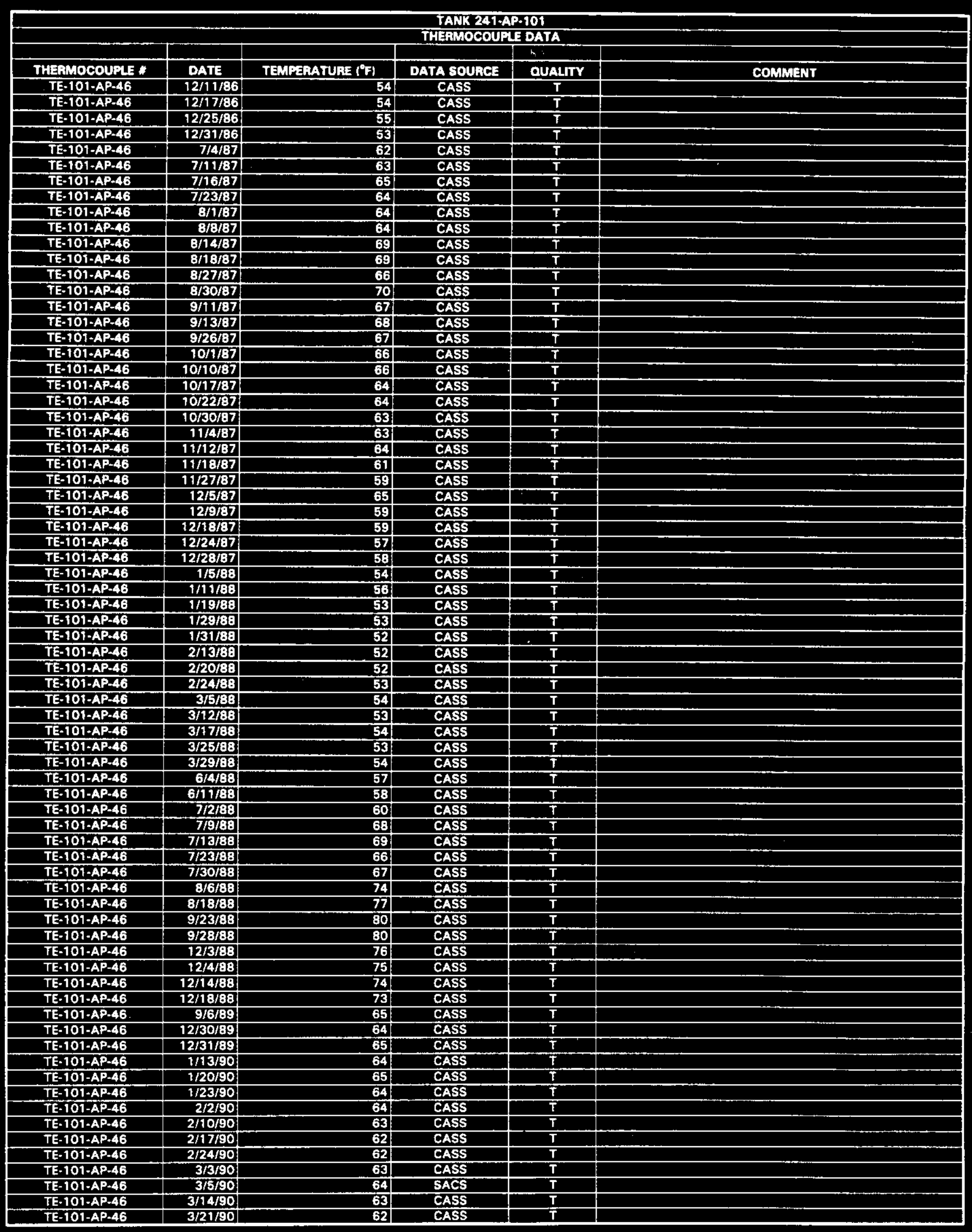




\begin{tabular}{|c|c|c|c|c|c|}
\hline \multicolumn{6}{|c|}{$\begin{array}{l}\text { TAWK 241-AP-101 } \\
\text { THEMMOCOUPE DATA }\end{array}$} \\
\hline & & & & & \\
\hline THERMOCOUPLE * & DATE & TEMPERATUAE $\left({ }^{\circ} \mathrm{F}\right)$ & DATA SOURCE & OUALITY & COMMENT \\
\hline TE-101-AP-46 & $3 / 29 / 90$ & 63 & CASS & $T$ & \\
\hline TE-101-AP-46 & $4 / 4 / 90$ & 62 & CASS & $T$ & \\
\hline TE-101-AP-46 & $4 / 12 / 90$ & 62 & CASS & $T$ & \\
\hline TE-101-AP-46 & $4 / 15 / 90$ & 63 & CASS & $T$ & \\
\hline TE-101-AP-46 & $4 / 27 / 90$ & 62 & CASS & $T$ & \\
\hline TE-101-AP+46 & $5 / 3 / 90$ & 62 & CASS & $\mathbf{T}$ & \\
\hline TE-101-AP-46 & $5 / 7 / 90$ & 62 & SACS & $T$ & \\
\hline TE-101-AP-46 & $5 / 18 / 90$ & 60 & CASS & $T$ & \\
\hline TE-101-AP-46 & $5 / 22 / 90$ & 60 & CASS & $T$ & \\
\hline TE-101-AP-46 & $6 / 1 / 90$ & 61 & CASS & $T=$ & \\
\hline TE-101-AP=46 & $6 / 7 / 90$ & 62 & CASS & $T$ & \\
\hline TE-101-AP.46 & $6 / 16 / 90$ & 60 & CASS & $\mathbf{T}$ & \\
\hline TE-101-AP-46 & $6 / 20 / 90$ & 60 & CASS & $T$ & \\
\hline TE-101-AP-46 & $6 / 23 / 90$ & 105 & CASS & $\mathbf{s}$ & Data point did not agrea with local trend \\
\hline TE-10T-AP-46 & $6 / 25 / 90$ & 62 & SACS & $\bar{T}$ & \\
\hline TE-101-AP-46 & $7 / 9 / 90$ & 61 & SACS & $T$ & \\
\hline TE-101-AP-46 & $7 / 26 / 90$ & 62 & CASS & $T$ & \\
\hline TE-101-AP-46 & $7 / 30 / 90$ & 61 & SACS & $\mathbf{T}$ & \\
\hline TE-101-AP-46 & $8 / 20 / 90$ & 64 & CASS & $T$ & \\
\hline TE-101-AP-46 & $8 / 31 / 90$ & 63 & CASS & $T$ & \\
\hline TE-101-AP+46 & $10 / 10 / 90$ & 64 & CASS & $T$ & \\
\hline TE-101-AP-46 & $10 / 19 / 90$ & 64 & CASS & $T$ & \\
\hline TE-101-AP-46 & $10 / 26 / 90$ & 65 & CASS & $T$ & \\
\hline TE-101-AP+46 & $11 / 2 / 90$ & 64 & CASS & $\bar{T}$ & \\
\hline TE-101-AP-46 & $11 / 7 / 30$ & 65 & CASS & $T$ & \\
\hline TE-101-AP-46 & $11 / 17 / 90$ & 64 & CASS & $T$ & \\
\hline TE-101-AP-46 & $11 / 24 / 90$ & 63 & CASS & $T$ & \\
\hline TE-101-AP-46 & $11 / 25 / 90$ & 64 & CASS & $T$ & \\
\hline TE-101-AP-46 & $12 / 6 / 90$ & 641 & CASS & $T$ & \\
\hline TE-101-AP-46 & $12 / 9 / 90$ & 64 & CASS & $T$ & \\
\hline TE-101-AP-46 & $12 / 21 / 90$ & 63 & CASS & $\bar{T}$ & \\
\hline TE-101-AP-46 & $12 / 23 / 90$ & 63 & CASS & $T$ & \\
\hline TE-101-AP-46 & $12 / 30 / 90$ & 63 & CASS & $\mathbf{T}$ & \\
\hline TE-101-AP-46 & $1 / 7 / 91$ & 62 & CASS & $T$ & \\
\hline TE-101-AP.46 & $1 / 18 / 91$ & 62 & CASS & $T$ & \\
\hline TE-101-AP-46 & $1 / 26 / 91$ & 62 & CASS & $T$ & \\
\hline TE-101-AP-46 & $2 / 2 / 91$ & 60 & CASS & $T$ & \\
\hline TE-101-AP-46 & $2 / 8 / 91$ & 61 & CASS & $T$ & \\
\hline TE-101-AP-46 & $2 / 13 / 91$ & 61 & CASS & $T$ & \\
\hline TE-101-AP-46 & $2 / 21 / 91$ & 61 & CASS & $T$ & \\
\hline TE-101-AP-46 & $3 / 2 / 91$ & 59 & CASS & $T$ & \\
\hline TE-101-AP-46 & $3 / 7 / 91$ & 60 & CASS & $T$ & \\
\hline TE-101-AP-46 & आ/6/9t & 59 & CASS & $\mathbf{T}$ & \\
\hline TE-101-AP-46 & $3 / 23 / 91$ & 60 & CASS & $T$ & \\
\hline TE-101-AP-46 & $3 / 29 / 91$ & 60 & CASS & $T$ & \\
\hline TE-101-AP-46 & $4 / 6 / 97$ & 58 & CASS & $T$ & \\
\hline TE-101-AP-46 & $4 / 11 / 91$ & 59 & CASS & $T$ & \\
\hline TE-101-AP-46 & $4 / 17 / 91$ & 61 & CASS & $T$ & \\
\hline TE-101-AP-46 & $4 / 27 / 91$ & 60 & CASS & $T$ & \\
\hline TE-101-AP-46 & $4 / 30 / 91$ & 59 & CASS & $T$ & \\
\hline TE-101-AP-46 & $5 / 8 / 91$ & 59 & CASS & $\bar{T}$ & \\
\hline TE-101-AP-46 & $5 / 14 / 91$ & 59 & CASS & $T$ & \\
\hline TE-101-AP-46 & $5 / 19 / 91$ & 59 & CASS & $T$ & \\
\hline TE-101-AP-46 & $6 / 8 / 91$ & 58 & CASS & $T$ & \\
\hline TE-101-AP-46 & $6 / 11 / 91$ & 57 & CASS & $\bar{T}$ & \\
\hline TE-101-AP-46 & $6 / 21 / 91$ & 58 & CASS & $T$ & \\
\hline TE-101-AP-46 & $6 / 25 / 91$ & 58 & CASS & $T$ & \\
\hline TE-101-AP-46 & $7 / 3 / 91$ & 58 & CASS & $T$ & \\
\hline TE-101-AP-46 & $7 / 24 / 91$ & 58 & CASS & $T$ & \\
\hline TE-101-AP-46 & $8 / 1 / 91$ & 58 & CASS & $T$ & \\
\hline TE-101-AP-46 & $8 / 6 / 91$ & 59 & CASS & $T$ & \\
\hline TE-101-AP-46 & $8 / 15 / 91$ & 67 & CASS & $\mathbf{T}$ & \\
\hline TE-101-AP-46 & $8 / 30 / 91$ & 59 & CASS & $T$ & \\
\hline TE-101-AP-46 & $9 / 3 / 91$ & 61 & CASS & $T$ & \\
\hline TE-101-AP-46 & $8 / 8 / 92$ & 58 & CASS & $T$ & \\
\hline TE-101-AP-46 & $8 / 22 / 92$ & 58 & CASS & $T$ & \\
\hline TE-101-AP-46 & $\mathrm{B} / 29 / 92$ & 59 & CASS & $T$ & \\
\hline TE-101-AP-46 & $9 / 5 / 92$ & 60 & CASS & $T$ & \\
\hline TE-101-AP-46 & $9 / 9 / 92$ & 59 & CASS & $T$ & \\
\hline TE-101-AP-46 & $9 / 14 / 92$ & 60 & CASS & $T$ & \\
\hline TE-101-AP-46 & $9 / 26 / 92$ & 60 & CASS & $T$ & \\
\hline TE-101-AP-46 & $10 / 3 / 92$ & 59 & CASS & $\bar{T}$ & \\
\hline TE-101-AP-46 & $10 / 6 / 92$ & 61 & CASS & $T$ & \\
\hline
\end{tabular}




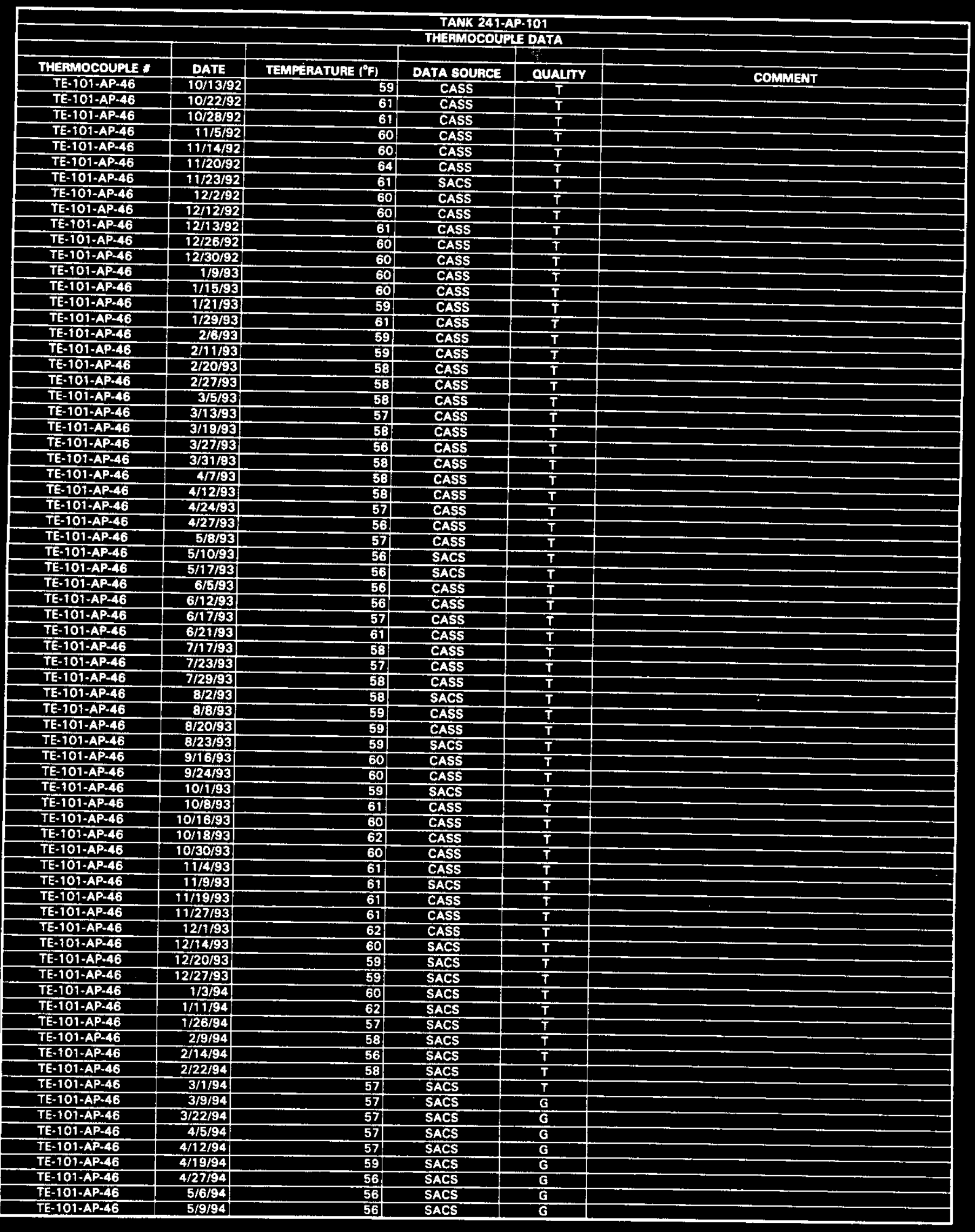




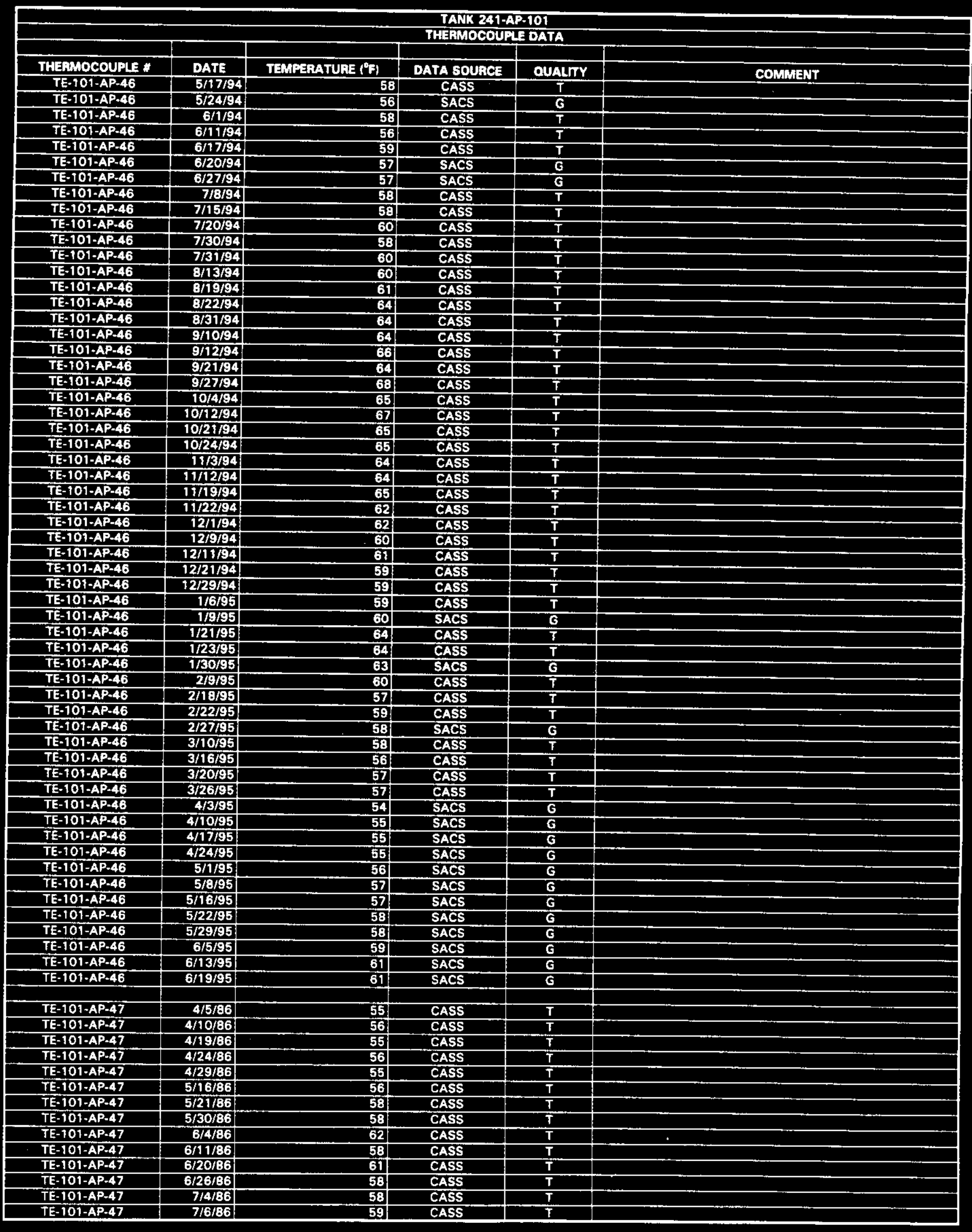




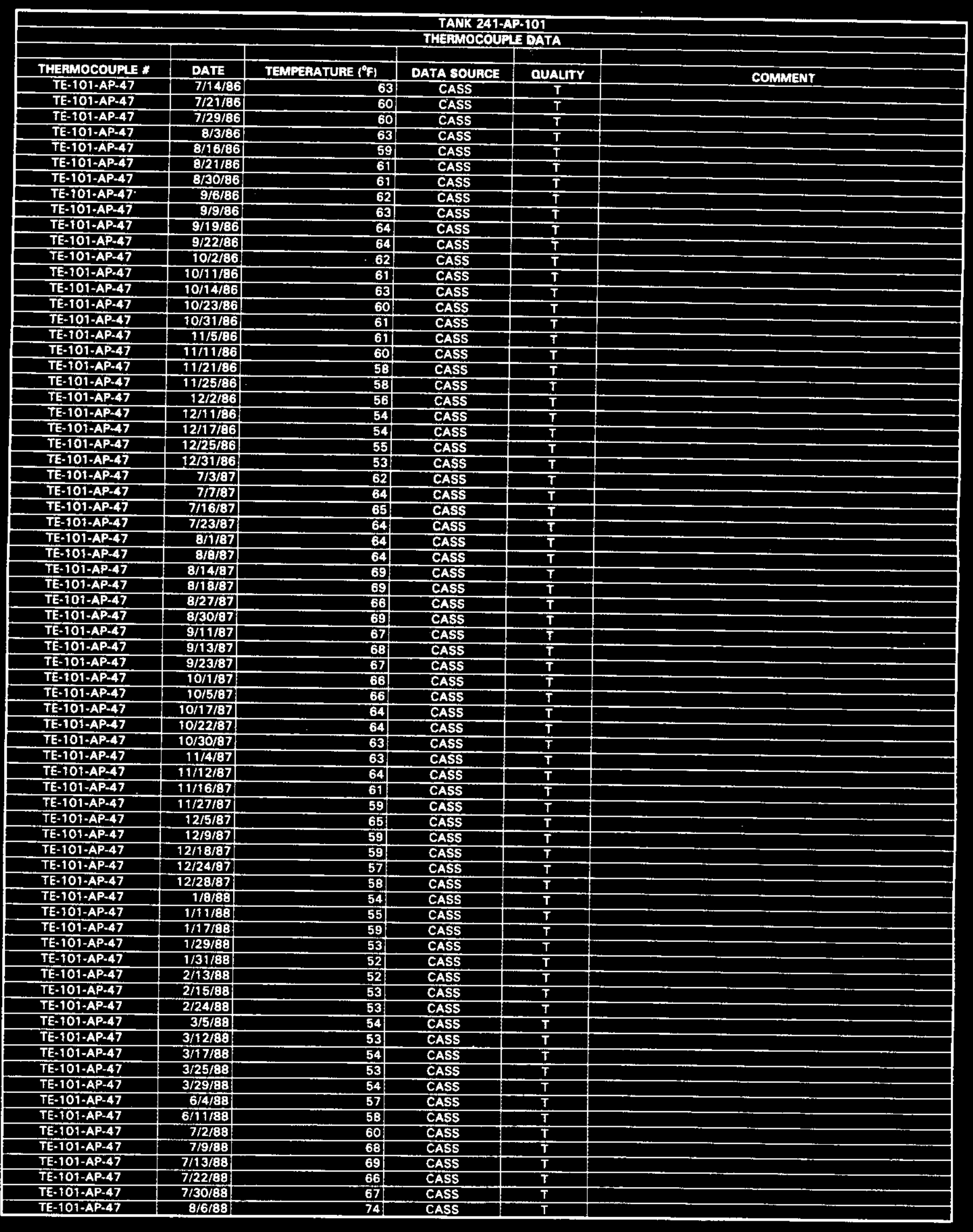

Data obtained from Computer Automated Surveillance System (CASS), April 1995; and Surveillance Analyais Computer System (SACS), June 1995.

D- 118 


\begin{tabular}{|c|c|c|c|c|c|}
\hline & & & & & \\
\hline THEAMOCOUPLE * & DATE & TEMPERATUAE $\left({ }^{\circ} \mathrm{F}\right)$ & DATA SOUACE & QUALTY & COMMENT \\
\hline TE-101-AP-47 & $8 / 18 / 88$ & 77 & CASS & $T$ & \\
\hline TE-101-AP-47 & $9 / 23 / 88$ & 80 & CASS & $\mathbf{T}$ & \\
\hline TE-101-AP-47 & $9 / 28 / 88$ & 80 & CASS & $\bar{T}$ & \\
\hline TE-101-AP-47 & $12 / 3 / 88$ & 76 & CASS & $T$ & \\
\hline TE-101.AP-47 & $12 / 4 / 88$ & 75 & CASS & $T$ & \\
\hline TE-101-AP-47 & $12 / 14 / 8 B$ & 74 & CASS & $\mathbf{T}$ & \\
\hline TE-101-AP-47 & $12 / 18 / 88$ & 73 & CASS & $T$ & \\
\hline TE-101-AP-47 & $7 / 10 / 89$ & 62 & SACS & $T$ & \\
\hline TE-101-AP-47 & $8 / 21 / 89$ & 65 & SACS & $T$ & \\
\hline TE-101-AP-47 & $8 / 28 / 89$ & 65 & SACS & $T$ & \\
\hline TE-101-AP-47 & $9 / 6 / 89$ & 65 & CASS & $T$ & \\
\hline TE-101-AP-47 & $10 / 9 / 89$ & 64 & SACS & $T$ & \\
\hline TE-101-AP-47 & $10 / 30 / 89$ & 65 & SACS & $T$ & \\
\hline TE-101-AP-47 & $12 / 29 / 89$ & 65 & CASS & $T$ & \\
\hline TE-101-AP-47 & $12 / 31 / 89$ & 65 & CASS & $T$ & \\
\hline TE-101-AP-47 & $1 / 13 / 90$ & 64 & CASS & $T$ & \\
\hline TE-101-AP-47 & $1 / 20 / 90$ & 65 & CASS & $T$ & \\
\hline TE-101-AP-47 & $1 / 23 / 90$ & 64 & CASS & $T$ & \\
\hline TE-101-AP-47 & $2 / 2 / 90$ & 64 & CASS & $\bar{T}$ & \\
\hline TE-101-AP-47 & $2 / 7 / 90$ & 64 & CASS & $\bar{T}$ & \\
\hline TE-101-AP-47 & 2/16/90 & 63 & CASS & $T$ & \\
\hline TE-101-AP+47 & $2 / 21 / 90$ & 63 & CASS & $T$ & \\
\hline TE-101-AP-47 & $2 / 26 / 90$ & 63 & CASS & $\bar{T}$ & \\
\hline TE-101-AP-A7 & $3 / 8 / 90$ & 62 & CASS & $\bar{T}$ & \\
\hline TE-101-AP-47 & $3 / 14 / 90$ & 63 & CASS & $\bar{T}$ & \\
\hline TE-101-AP-47 & $3 / 21 / 90$ & 63 & CASS & $T$ & \\
\hline TE-101-AP-47 & $3 / 29 / 90$ & 63 & CASS & $T$ & \\
\hline TE-101-AP-47 & $4 / 4 / 90$ & 62 & CASS & $T$ & \\
\hline TE-101-AP-47 & $4 / 12 / 90$ & 62 & CASS & $T$ & \\
\hline TE-101-AP-47 & $4 / 15 / 90$ & 64 & CASS & $T$ & \\
\hline TE-101-AP-47 & $4 / 27 / 90$ & 62 & CASS & $T$ & \\
\hline TE-101-AP-47 & $5 / 4 / \Omega 0$ & 62 & CASS & $T$ & \\
\hline TE-101-AP-47 & $5 / 12 / 90$ & 62 & CASS & $\mathrm{T}$ & \\
\hline TE-101-AP+47 & $5 / 18 / 90$ & 60 & CASS & $T$ & \\
\hline TE-101-AP-47 & $5 / 22 / 90$ & 60 & CASS & $\bar{T}$ & \\
\hline TE-101-AP-47 & $6 / 1 / 90$ & 61 & CASS & $T$ & \\
\hline TE-101-AP-47 & $6 / 7 / 90$ & 62 & CASS & $T$ & \\
\hline TE-101-AP-47 & $8 / 16 / 90$ & 60 & CASS & $T$ & \\
\hline TE-101-AP-47 & $6 / 20 / 90$ & 60 & CASS & $T$ & \\
\hline TE-101-AP-47 & $6 / 23 / 90$ & 106 & CASS & $\mathbf{S}$ & Data point did not agres with local trend \\
\hline TE-101-AP-47 & $6 / 24 / 90$ & 60 & CASS & $T$ & \\
\hline TE-101-AP-47 & $7 / 26190$ & 62 & CASS & $T$ & \\
\hline TE-101-AP.47 & $8 / 6 / 90$ & 64 & SACS & $T$ & \\
\hline TE-101-AP-47 & $8 / 21 / 90$ & 63 & CASS & $T$ & \\
\hline TE-101-AP-47 & $8 / 31 / 90$ & 63 & CASS & $T$ & \\
\hline TE-101-AP-47 & $9 / 3 / 90$ & 62 & SACS & $T$ & \\
\hline TE-101-AP-47 & $9 / 10 / 90$ & 63 & SACS & $T$ & \\
\hline TE-101-AP-47 & 10/8/90 & 67 & SACS & $T$ & \\
\hline TE-101-AP-47 & $10 / 20 / 90$ & 64 & CASS & $\mathbf{T}$ & \\
\hline TE-101-AP-47 & $10 / 23 / 90$ & 65 & CASS & $T$ & \\
\hline TE-101-AP-47 & $11 / 2 / 90$ & 64 & CASS & $T$ & \\
\hline TE-101-AP-47 & $11 / 7 / 90$ & 65 & CASS & $\bar{T}$ & \\
\hline TE-101-AP-47 & $11 / 17 / 90$ & 64 & CASS & $T$ & \\
\hline TE-101-AP-47 & $11 / 24 / 90$ & 63 & CASS & $T$ & \\
\hline TE-101-AP-47 & $11 / 25 / 90$ & 64 & CASS & $T$ & \\
\hline TE-101-AP-47 & $12 / 2 / 90$ & 65 & CASS & $T$ & \\
\hline TE-101-AP-47 & $12 / 9 / 90$ & 64 & CASS & $T$ & \\
\hline TE-101-AP-47 & $12 / 21 / 90$ & 63 & CASS & $T$ & \\
\hline TE-101-AP-47 & $12 / 23 / 90$ & 63 & CASS & $T$ & \\
\hline TE-101-AP-47 & $1 / 4 / 91$ & 63 & CASS & $\bar{T}$ & \\
\hline TE-101-AP-47 & $1 / 7 / 91$ & 63 & CASS & $T$ & \\
\hline TE-101-AP-47 & $1 / 14 / 91$ & 66 & SACS & $T$ & \\
\hline TE-101-AP-47 & $1 / 26 / 91$ & 62 & CASS & $T$ & \\
\hline TE-101-AP-47 & $2 / 2 / 91$ & 60 & CASS & $T$ & \\
\hline TE-101-AP-47 & $2 / 7 / 91$ & 62 & CASS & $T$ & \\
\hline TE-101-AP-47 & $2 / 13 / 91$ & 61 & CASS & $T$ & \\
\hline TE-101-AP-47 & $2 / 21 / 91$ & 61 & CASS & $T$ & \\
\hline TE-101-AP-47 & $2 / 25 / 91$ & 60 & CASS & $T$ & \\
\hline TE-101-AP-47 & $3 / 6 / 91$ & 60 & CASS & $T$ & \\
\hline TE-101-AP-47 & $3 / 10 / 91$ & 60 & CASS & $T$ & \\
\hline TE-101-AP-47 & $3 / 23 / 91$ & 60 & CASS & $\bar{T}$ & \\
\hline TE-101-AP-47 & $3 / 29 / 91$ & 60 & CASS & $\mathbf{T}$ & \\
\hline TE-101-AP-47 & $4 / 6 / 91$ & 58 & CASS & $T$ & \\
\hline
\end{tabular}




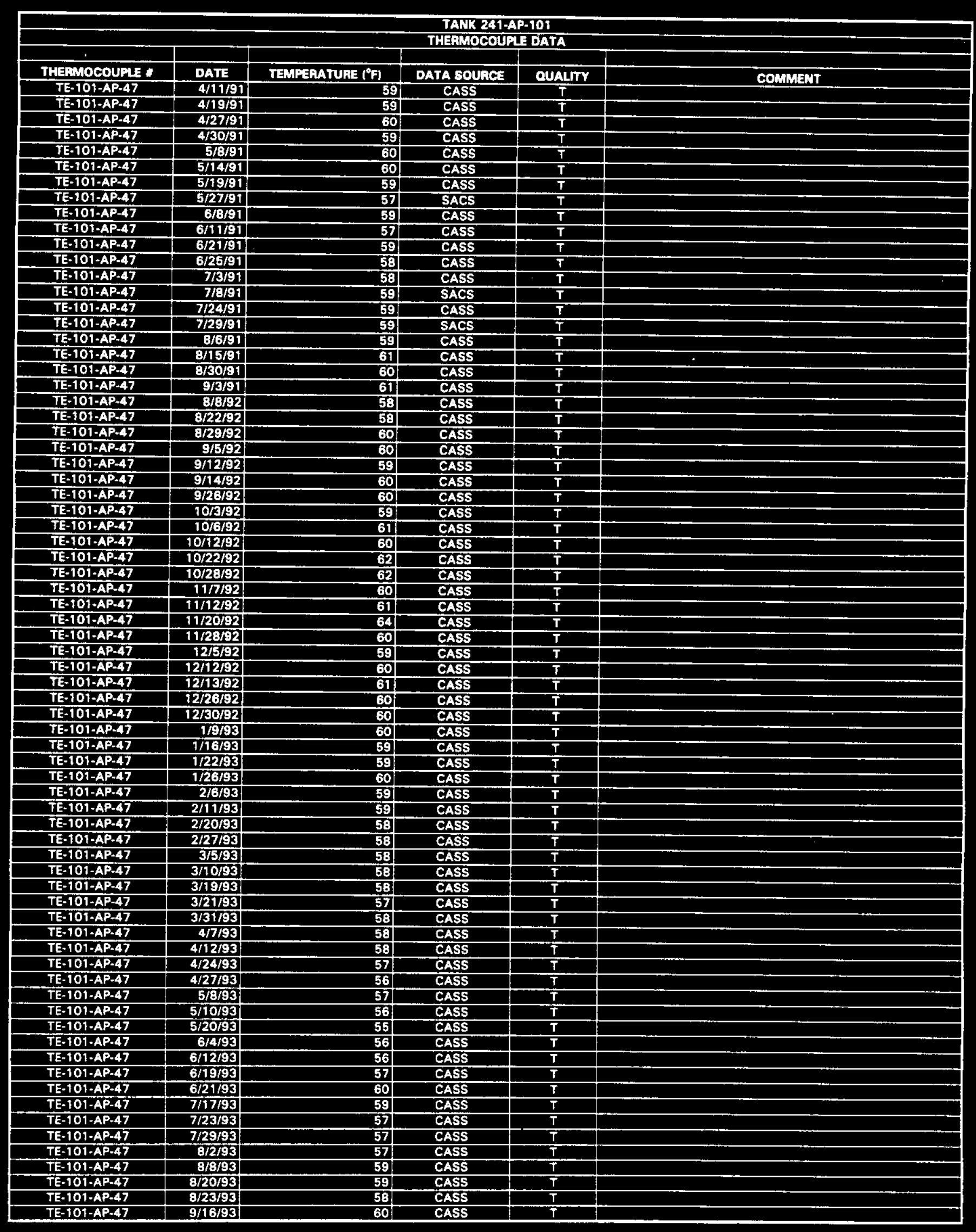




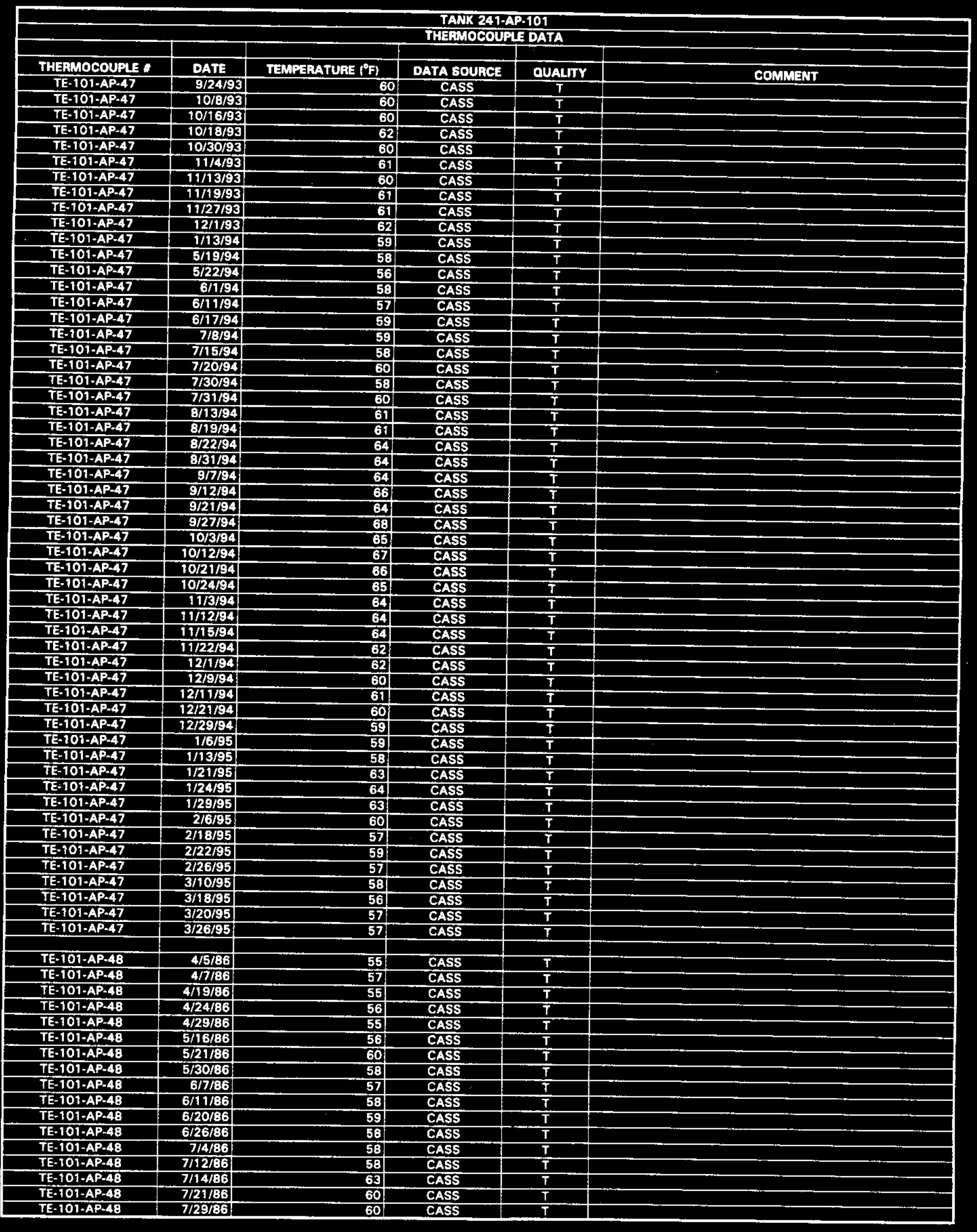

Data obtained from Camputer Automated Surveillance System (CASS). April 1995; and Surveillance Analysis Computer System (SACS), June 1995. 


\begin{tabular}{|c|c|c|c|c|c|}
\hline \multicolumn{6}{|c|}{$\begin{array}{l}\text { TANK 241-AP-101 } \\
\text { THERMOCOUPLE DATA }\end{array}$} \\
\hline & & & & & \\
\hline THERMOCOUPLE * & DATE & TEMPERATURE ( $\left.{ }^{\circ} \mathrm{F}\right)$ & DATA SOURCE & QUALITY & COMMENT \\
\hline TE-101-AP-48 & $8 / 5 / 86$ & 60 & CASS & $T$ & \\
\hline $\mathrm{TE}-104 \cdot \mathrm{AP}-48$ & $8 / 16 / 36$ & 59 & CASS & $T$ & \\
\hline TE-101-AP-48 & $8 / 21 / 86$ & 61 & CASS & $T$ & \\
\hline TE-101-AP-48 & $8 / 30 / 86$ & 61 & CASS & $\mathbf{T}$ & \\
\hline TE-101-AP-48 & $9 / 6 / 86$ & 62 & CASS & $T$ & \\
\hline TE-101-AP-48 & $9 / 9 / 86$ & 63 & CASS & $\mathrm{T}$ & \\
\hline TE-101-AP-4B & $9 / 19 / 86$ & 64 & CASS & $\mathbf{T}$ & \\
\hline TE-101-AP-48 & $9 / 26 / 36$ & 64 & CASS & $T$ & \\
\hline TE-107-AP-4B & $10 / 2 / 86$ & 62 & CASS & $T$ & \\
\hline TE-101-AP-48 & $10 / 11 / 86$ & 61: & CASS & $\mathbf{T}$ & \\
\hline TE-101-AP-48 & $10 / 14 / 86$ & 62 & CASS & $T$ & \\
\hline TE-101-AP-48 & $10 / 23 / 86$ & 60 & CASS & $T$ & \\
\hline TE-101-AP-48 & $10 / 31 / 86$ & 61 & CASS & $\bar{T}$ & \\
\hline TE-101-AP-48 & $11 / 5 / 86$ & 61 & CASS & $T$ & \\
\hline TE-101-AP-48 & $11 / 11 / 86$ & 60 & CASS & $T$ & \\
\hline TE-101-AP-48 & $11 / 21 / 86$ & 58 & CASS & $\mathbf{T}$ & \\
\hline TE-101-AP-48 & $11 / 25 / 86$ & 57 & CASS & $T$ & \\
\hline$T E-101-A P-48$ & $12 / 2 / 86$ & 56 & CASS & $T$ & \\
\hline TE-101-AP-48 & $12 / 11 / 86$ & 54 & CASS & $\bar{T}$ & \\
\hline TE-101-AP-48 & $12 / 17 / 86$ & 54 & CASS & $\mathbf{T}$ & \\
\hline TE-101-AP-48 & $12 / 25 / 86$ & 55 & CASS & $\mathbf{T}$ & \\
\hline TE-101-AP-4B & $12 / 30 / 86$ & 53: & CASS & $\bar{T}$ & \\
\hline TE-101-AP-48 & $7 / 4 / 87$ & 62 & CASS & $\mathbf{T}$ & \\
\hline TE-101-AP-48 & $7 / 11 / 87$ & 64 & CASS & $\mathbf{T}$ & \\
\hline TE-107-AP-48 & 7/16/87 & 65 & CASS & $\bar{T}$ & \\
\hline TE-101-AP-48 & 7/23/87 & 64 & CASS & $\mathbf{T}$ & \\
\hline TE-101-AP-48 & 7/29/87 & 65 & CASS & $T$ & \\
\hline TE-101-AP-48 & $8 / 8 / 87$ & 64 & CASS & $\mathbf{T}$ & \\
\hline TE-101-AP-48 & $8 / 14 / 87$ & 69 & CASS & $T$ & \\
\hline TE-101-AP:48 & $8 / 18 / 87$ & 69 & CASS & $T$ & \\
\hline TE-101-AP-48 & $8 / 27 / 87$ & 66 & CASS & $\mathbf{T}$ & \\
\hline TE-101-AP-48 & $8 / 30 / 87$ & 70 & CASS & $\bar{T}$ & \\
\hline TE-101-AP-48 & $9 / 11 / 37$ & 67 & CASS & $T$ & \\
\hline TE-101-AP-48 & 9/13/37 & 68 & CASS & $T$ & \\
\hline TE-10T-AP-48 & 9/26/87 & 67 & CASS & $T$ & \\
\hline TE-101-AP-48 & $10 / 1 / 87$ & 66 & CASS & $T$ & \\
\hline TE-101-AP-4B & $10 / 10 / 87$ & 66 & CASS & $\mathbf{T}$ & \\
\hline TE-101-AP.4B & 10/17/87 & 64 & CASS & $T$ & \\
\hline TE-101-AP-48 & $10 / 22 / 87$ & 64 & CASS & $\mathbf{T}$ & \\
\hline TE-101-AP-48 & $10 / 30 / 87$ & 63 & CASS & $T$ & \\
\hline TE-101-AP-48 & $11 / 5 / 87$ & 63 & CASS & $T$ & \\
\hline TE-101-AP-48 & $11 / 12 / 87$ & 64 & CASS & $T$ & \\
\hline TE-101-AP-48 & $11 / 16 / 87$ & 61 & CASS & $T$ & \\
\hline TE-101-AP-48 & $11 / 25 / 87$ & 59: & CASS & $T$ & \\
\hline TE-101-AP-48 & $12 / 4 / 87$ & 58 & CASS & $T$ & \\
\hline TE-101-AP-48 & $12 / 5 / 87$ & 67 & CASS & $\mathbf{S}$ & Data point did not agree with local trend \\
\hline TE-101-AP-48 & $12 / 9 / 87$ & 59 & CASS & $\mathbf{T}$ & \\
\hline TE-101-AP-48 & $12 / 18 / 87$ & 59 & CASS & $\mathbf{T}$ & \\
\hline TE-101-AP-48 & $12 / 24 / 87$ & 57 & CASS & $\mathbf{T}$ & \\
\hline TE-101-AP-48 & $12 / 28 / 87$ & 58 & CASS & $T$ & \\
\hline TE-101-AP-48 & $1 / 8 / 88$ & 54 & CASS & $\mathbf{T}$ & \\
\hline TE-101-AP-48 & 1/11/88 & 56 & CASS & $\mathbf{T}$ & \\
\hline TE-101-AP-48 & 1/19/88 & 53 & CASS & $T$ & \\
\hline TE-101-AP-48 & 1/29/88 & 53: & CASS & $T$ & \\
\hline TE-101-AP-4B & $1 / 31 / 88$ & 52 & CASS & $T$ & \\
\hline TE-101-AP-48 & $2 / 13 / 88$ & 52. & CASS & $\bar{T}$ & \\
\hline TE-101-AP-4B & $2 / 20 / 88$ & 52 & CASS & $T$ & \\
\hline TE-101-AP-48 & $2 / 24 / 88$ & 53 & CASS & $\mathbf{T}$ & \\
\hline TE-101-AP-48 & $3 / 5 / 88$ & 54 & CASS & $T$ & \\
\hline TE-101-AP-48 & $3 / 12 / 88$ & 53 & CASS & $T$ & \\
\hline TE-101-AP-48 & 3/17/88 & 53 & CASS & $T$ & \\
\hline TE-101-AP-48 & $3 / 25 / 88$ & 53 & CASS & $\mathbf{T}$ & \\
\hline TE-101-AP-48 & $3 / 29 / 88$ & 54 & CASS & $T$ & \\
\hline TE-101-AP-48 & 6/4/88 & 57 & CASS & $T$ & \\
\hline TE-101-AP-48 & $6 / 11 / 88$ & 58 & CASS & $T$ & \\
\hline TE-101-AP-48 & $7 / 2 / 88$ & 60 & CASS & $T$ & \\
\hline TE-101-AP-48 & 7/9/88 & 68 & CASS & $T$ & \\
\hline TE-101-AP-4B & $7 / 13 / 88$ & 69 & CASS & $\mathrm{T}$ & \\
\hline TE-101-AP-48 & $7 / 23 / 88$ & 66 & CASS & $T$ & \\
\hline TE-101-AP-48 & $7 / 30 / 88$ & 67 & CASS & $T$ & \\
\hline TE-101-AP-48 & $8 / 6 / 88$ & 74 & CASS & $T$ & \\
\hline TE-101-AP-48 & $8 / 18 / 88$ & 78 & CASS & $T$ & \\
\hline$T E-101-A P-48$ & $9 / 23 / 88$ & 80 & CASS & $\mathbf{T}$ & \\
\hline
\end{tabular}




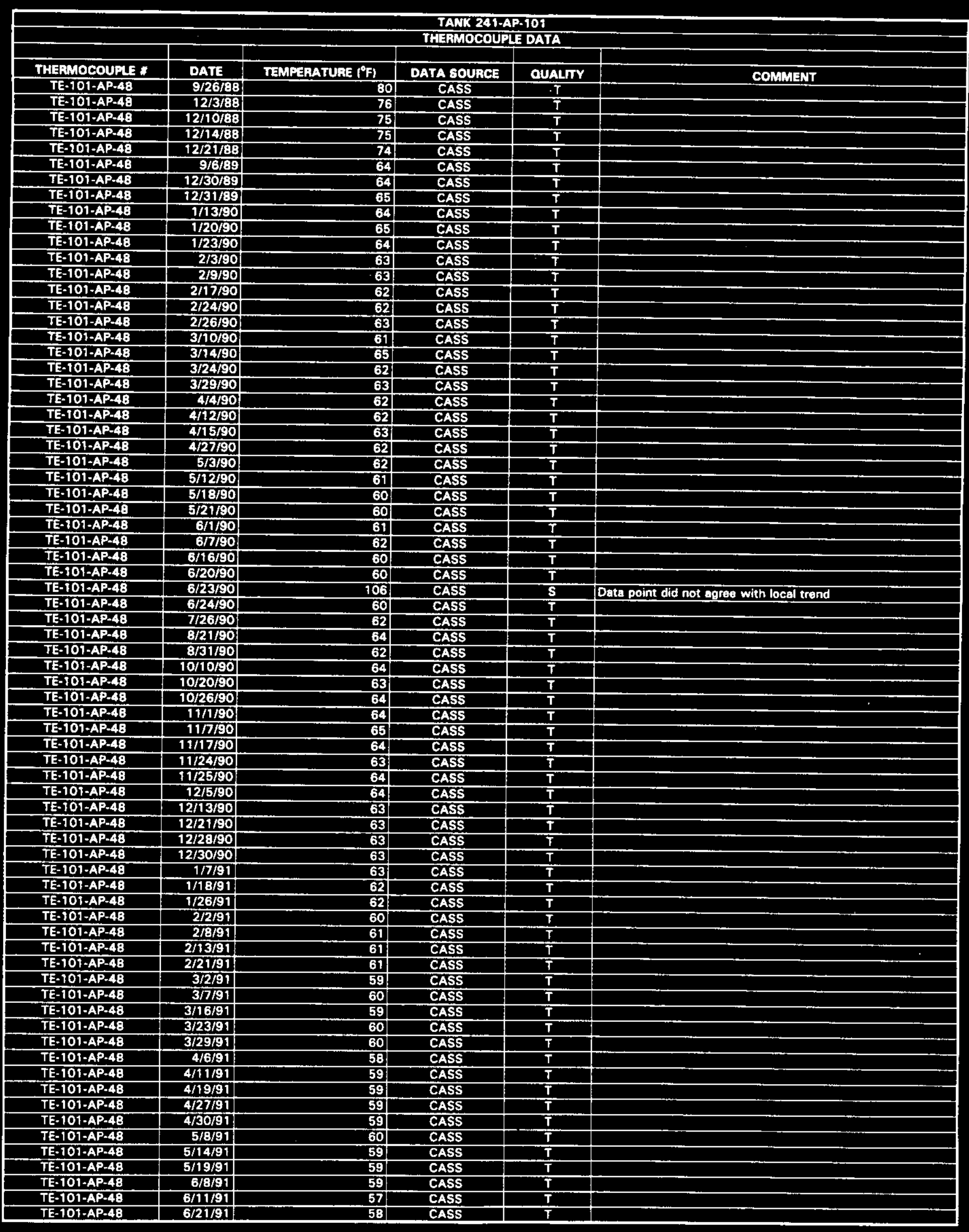




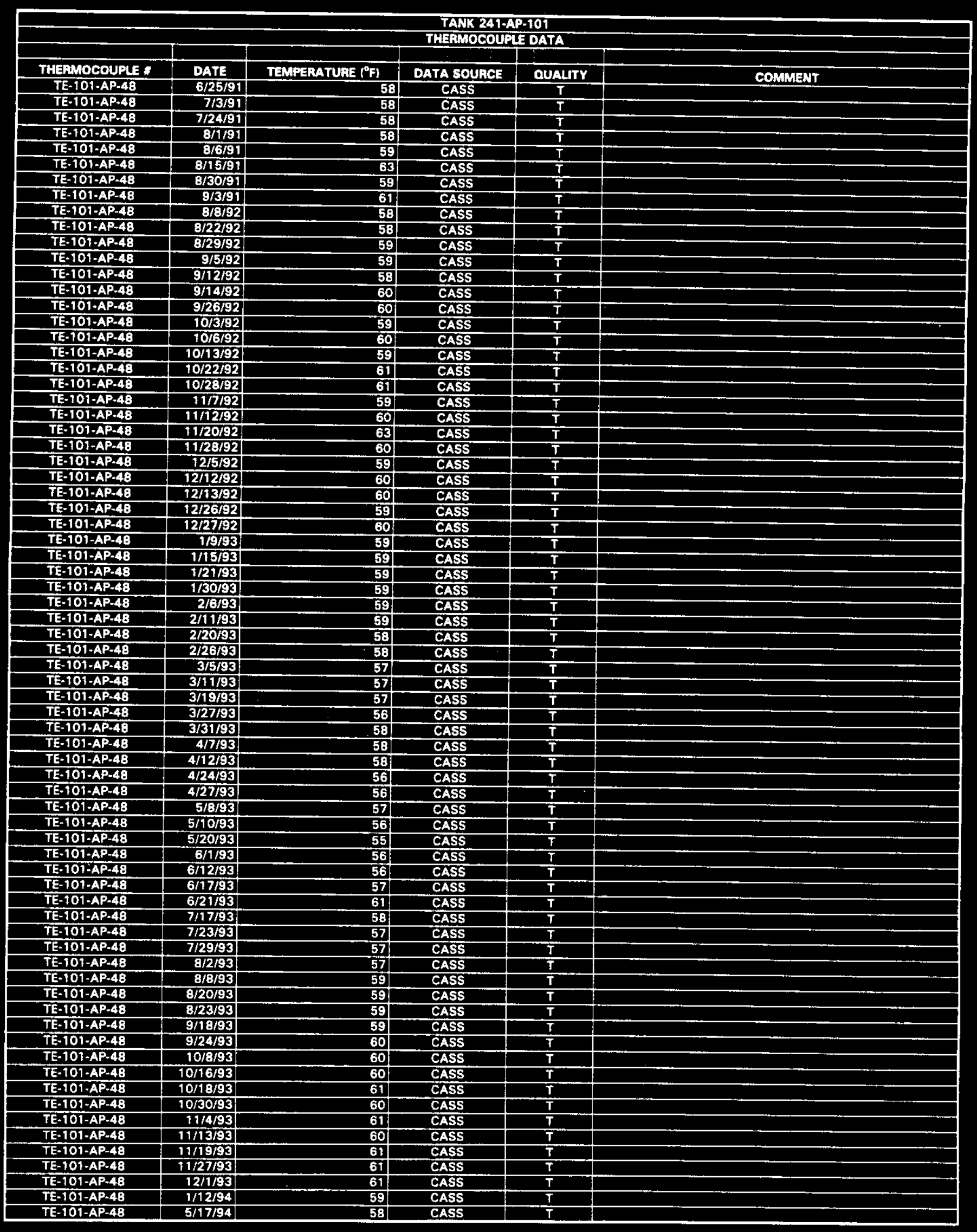




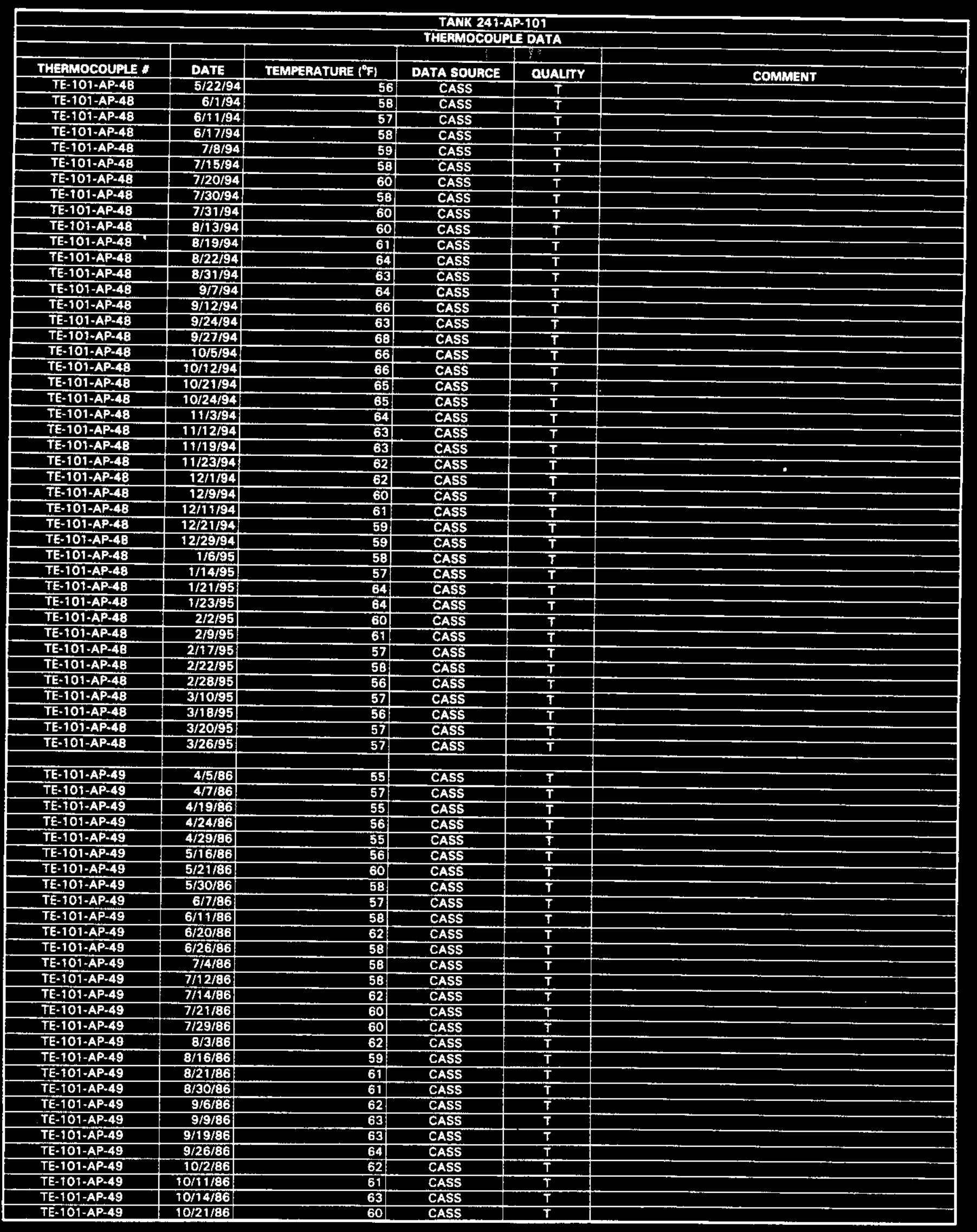




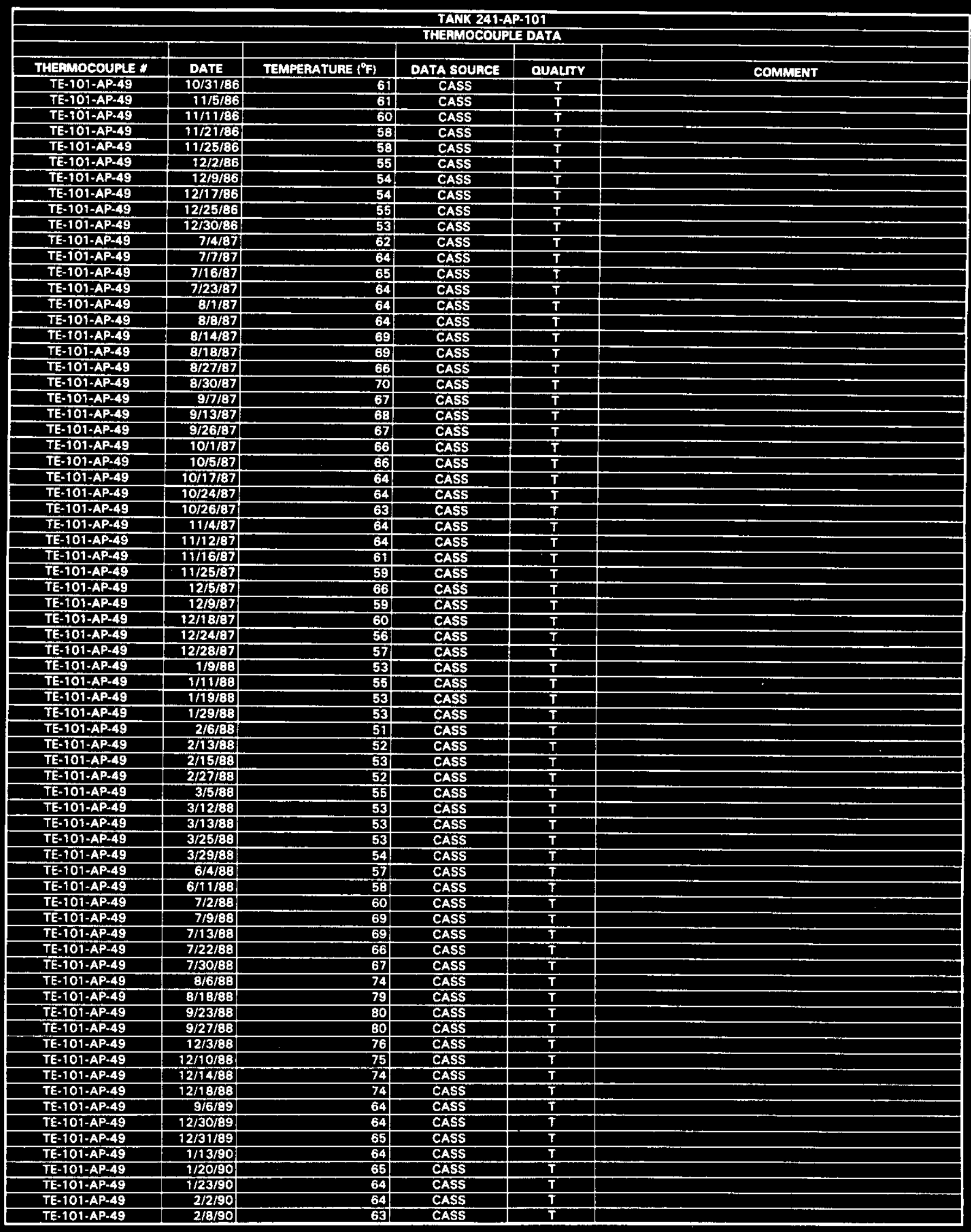




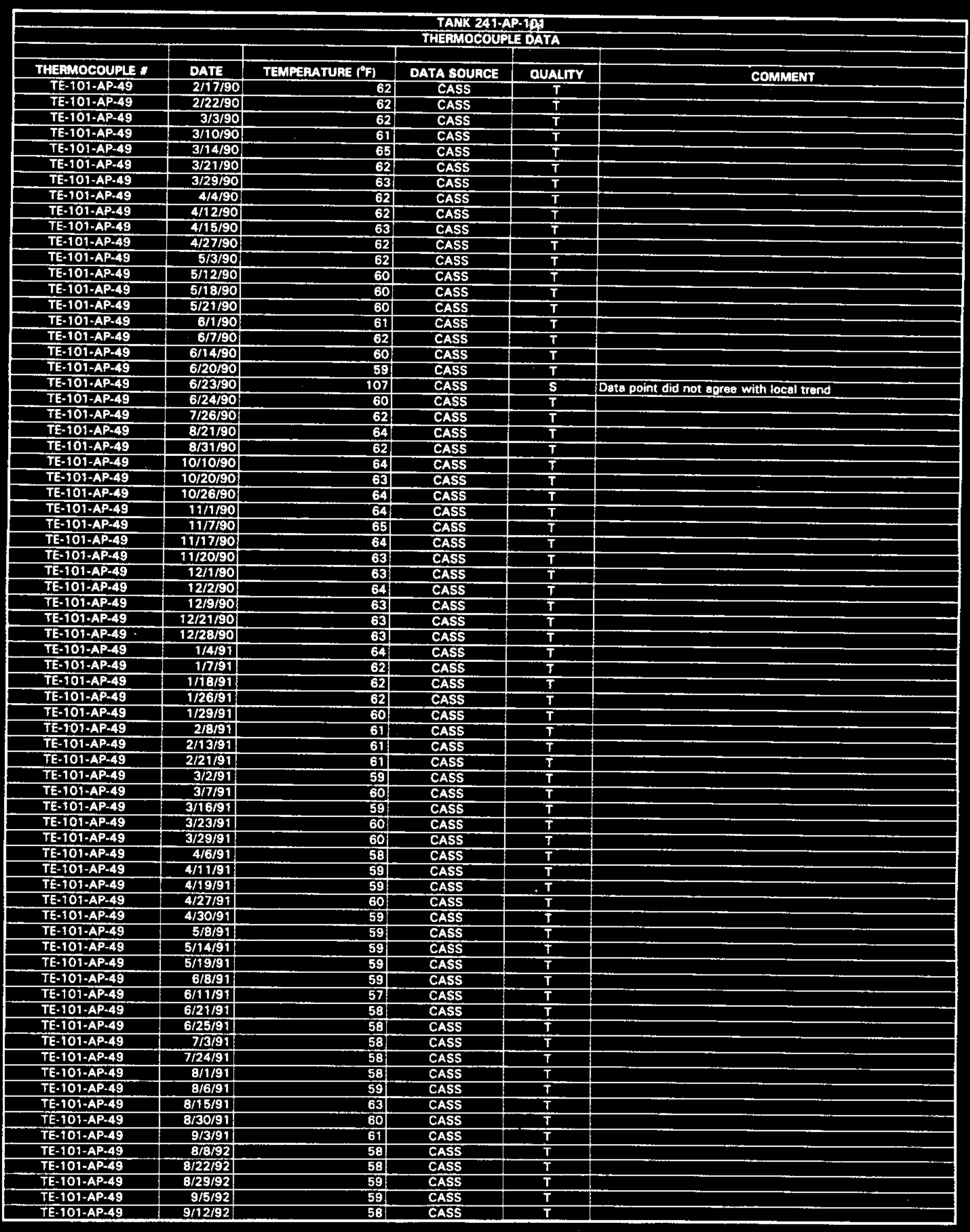




\begin{tabular}{|c|c|c|c|c|c|}
\hline \multicolumn{6}{|c|}{$\begin{array}{l}\text { TANK 241+AP-10T } \\
\text { THEAMOCOUPLE DATA }\end{array}$} \\
\hline THERMOCOUPLE * & DATE & TEMPERATURE $\left({ }^{\circ} \mathrm{F}\right)$ & DATA SOURCE & OUALITY & COMMENT \\
\hline TE-101-AP-49 & $9 / 14 / 92$ & 60 & CASS & $T$ & \\
\hline TE-101-AP-49 & $9 / 26 / 92$ & 60 & CASS & $T$ & \\
\hline TE-101-AP-49 & $10 / 3 / 92$ & 59 & CASS & $\bar{T}$ & \\
\hline TE-101-AP-49 & $10 / 6 / 92$ & 60 & CASS & $T$ & \\
\hline TE-101-AP-49 & $10 / 13 / 92$ & 60 & CASS & $T$ & \\
\hline TE-101-AP-49 & $10 / 22 / 92$ & 62 & CASS & $T$ & \\
\hline TE-101-AP-49 & $10 / 28 / 92$ & 62 & CASS & $\bar{T}$ & \\
\hline TE-10T-AP-49 & $11 / 7 / 92$ & 60 & CASS & $T$ & \\
\hline TE-101-AP-49 & $11 / 12 / 92$ & 61 & CASS & $T$ & \\
\hline TE-101-AP-49 & $11 / 20 / 92$ & 64 & CASS & $\mathbf{T}$ & \\
\hline TE-101-AP-49 & $11 / 28 / 92$ & 60 & CASS & $\bar{T}$ & \\
\hline TE-101-AP-49 & $12 / 5 / 92$ & 59 & CASS & $\bar{T}$ & \\
\hline TE-101-AP-49 & $12 / 12 / 92$ & 59 & CASS & $T$ & \\
\hline TE-101-AP-49 & $12 / 13 / 92$ & 60 & CASS & $\bar{T}$ & \\
\hline TE-101-AP-49 & $12 / 26 / 92$ & 59 & CASS & $T$ & \\
\hline TE-101-AP-49 & $1 / 2 / 93$ & 59 & CASS & $T$ & \\
\hline TE-101-AP-49 & $1 / 9 / 93$ & 59 & CASS & $\bar{T}$ & \\
\hline TE-101-AP-49 & $1 / 15 / 93$ & 59 & CASS & $\bar{T}$ & \\
\hline TE-101-AP-49 & $1 / 21 / 93$ & 59 & CASS & $T$ & \\
\hline TE-101-AP-49 & $1 / 29 / 93$ & 59 & CASS & $T$ & \\
\hline TE-101-AP-49 & $2 / 5 / 93$ & 61 & CASS & $T$ & \\
\hline TE-101-AP-49 & $2 / 11 / 93$ & 59 & CASS & $T$ & \\
\hline TE-101-AP-49 & $2 / 20 / 93$ & 58 & CASS & $T$ & \\
\hline TE-101-AP-49 & $2 / 27 / 93$ & 57 & CASS & $\bar{T}$ & \\
\hline TE-101-AP+49 & $3 / 5 / 93$ & 57 & CASS & $T$ & \\
\hline TE-101-AP-49 & $3 / 10 / 93$ & 57 & CASS & $T$ & \\
\hline TE-101-AP-49 & $3 / 19 / 93$ & 58 & CASS & $T$ & \\
\hline TE-101-AP-49 & $3 / 27 / 93$ & 56 & CASS & $T$ & \\
\hline TE-101-AP-49 & $3 / 31 / 93$ & 58 & CASS & $\mathbf{T}$ & \\
\hline TE-101-AP-49 & $4 / 7 / 93$ & 58 & CA5S & $T$ & \\
\hline TE-101-AP-49 & $4 / 12 / 93$ & 58 & CASS & $T$ & \\
\hline TE-101-AP-49 & $4 / 24 / 93$ & 57 & CASS & $\bar{T}$ & \\
\hline TE-101-AP-49 & $4 / 26 / 93$ & 56 & CASS & $T$ & \\
\hline TE-101-AP-49 & $5 / 7 / 93$ & 57 & CASS & $\mathbf{T}$ & \\
\hline TE-101-AP-49 & $5 / 10 / 93$ & 56 & CASS & $T$ & \\
\hline TE-101-AP-49 & $5 / 20 / 93$ & 55 & CASS & $\mathbf{T}$ & \\
\hline TE-101-AP-49 & $6 / 1 / 93$ & 56 & CASS & $\bar{T}$ & \\
\hline TE-101-AP-49 & $6 / 12 / 93$ & 56 & CASS & $T$ & \\
\hline TE-101-AP-49 & $6 / 17 / 93$ & 57 & CASS & $\mathbf{T}$ & \\
\hline TE-101-AP-49 & $6 / 21 / 93$ & 61 & CASS & $T$ & \\
\hline TE-101-AP-49 & $7 / 17 / 93$ & 58 & CASS & $T$ & \\
\hline TE-101-AP-49 & $7 / 23 / 93$ & 57 & CASS & $T$ & \\
\hline$T E-101-A P-49$ & $7 / 29 / 93$ & 57 & CASS & $T$ & \\
\hline TE-101-AP-49 & $8 / 2 / 93$ & 57 & CASS & $T$ & \\
\hline TE-101-AP- 49 & $8 / 14 / 93$ & 58 & CASS & $T$ & \\
\hline TE-101-AP-49 & $8 / 20 / 93$ & 59 & CASS & $T$ & \\
\hline TE-101-AP-49 & $8 / 23 / 93$ & 58 & CASS & $T$ & \\
\hline TE-101-AP+49 & $9 / 18 / 93$ & 59 & CASS & $T$ & \\
\hline TE-101-AP-49 & $9 / 24 / 93$ & 60 & CASS & $\mathbf{T}$ & \\
\hline TE-101-AP-49 & $10 / \mathrm{a} / \mathrm{s}$ & 60 & CASS & $T$ & \\
\hline TE-101-AP-49 & $10 / 16 / 93$ & 59 & CASS & $T$ & \\
\hline TE-101-AP-49 & $10 / 18 / 93$ & 61 & CASS & $\bar{T}$ & \\
\hline TE-101-AP-49 & $10 / 30 / 93$ & 60 & CASS & $T$ & \\
\hline TE-101-AP-49 & $11 / 4 / 93$ & 61 & CASS & $T$ & \\
\hline TE-101-AP-49 & $11 / 12 / 93$ & 60 & CASS & $T$ & \\
\hline TE-101-AP-49 & $11 / 15 / 93$ & 61 & CASS & $T$ & \\
\hline TE-101-AP-49 & $11 / 27 / 93$ & 60 & CASS & $T$ & \\
\hline TE-101-AP-49 & $12 / 1 / 93$ & 61 & CASS & $T$ & \\
\hline TE-101-AP-49 & $1 / 12 / 94$ & 59 & CASS & $T$ & \\
\hline TE-101-AP-49 & $5 / 17 / 94$ & 58 & CASS & $T$ & \\
\hline TE-101-AP-49 & $5 / 22 / 94$ & 56 & CASS & $T$ & \\
\hline TE-101-AP-49 & $6 / 1 / 94$ & 56 & CASS & $T$ & \\
\hline TE-101-AP-49 & $6 / 11 / 94$ & 56 & CASS & $\bar{T}$ & \\
\hline TE-101-AP-49 & $6 / 17 / 94$ & 58 & CASS & $T$ & \\
\hline TE-101-AP-49 & $7 / 8 / 94$ & 58 & CASS & $T$ & \\
\hline TE-101-AP-49 & $7 / 14 / 94$ & 58 & CASS & $T$ & \\
\hline TE-101-AP-49 & $7 / 20 / 94$ & 60 & CASS & $T$ & \\
\hline TE-101-AP-49 & $7 / 30 / 94$ & 58 & CASS & $T$ & \\
\hline TE-101-AP-49 & $7 / 31 / 94$ & 60 & CASS & $\bar{T}$ & \\
\hline TE-101-AP-49 & $8 / 13 / 94$ & 61 & CASS & $T$ & \\
\hline TE-101-AP-49 & $8 / 19 / 94$ & 60 & CASS & $T$ & \\
\hline TE-101-AP-49 & $8 / 22 / 94$ & 62 & CASS & $T$ & \\
\hline TE-101-AP-49 & $8 / 31 / 94$ & 61 & CASS & $T$ & \\
\hline
\end{tabular}




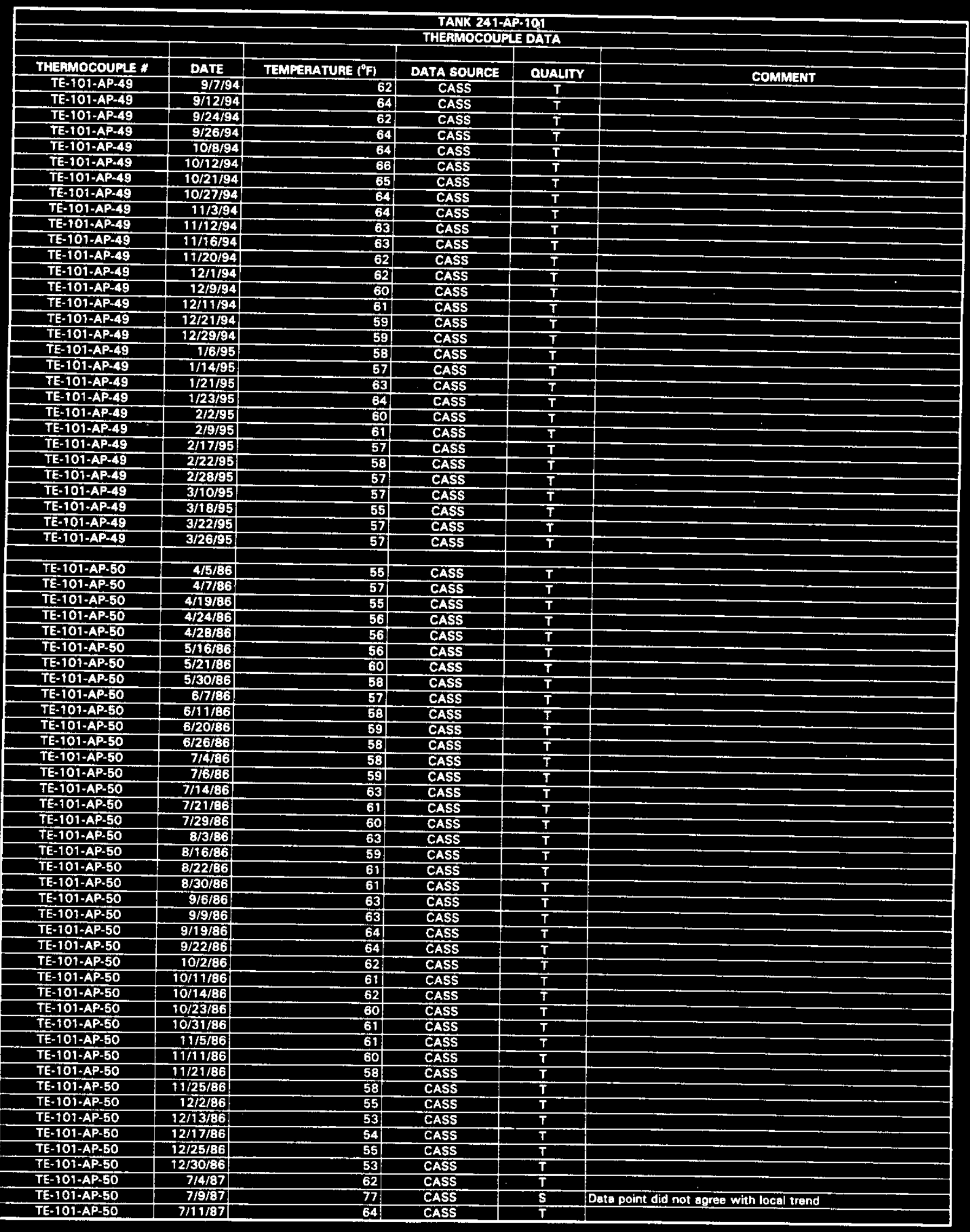

Data obtained from Computer Automated Surveillance Syatem (CASS), April 1995; and Surveillance Analysis Computer Syatem (SACS), June 1995. D-129 


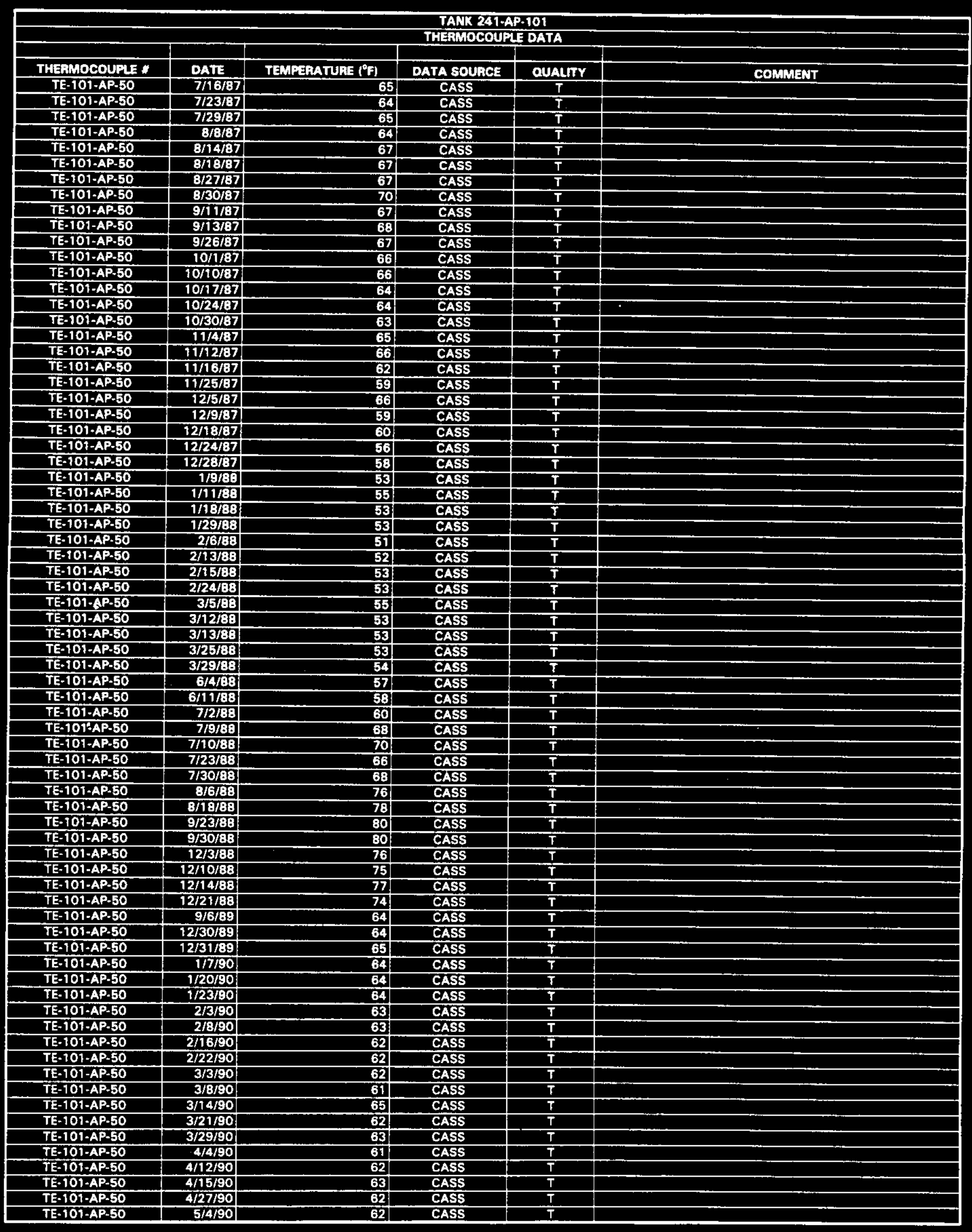




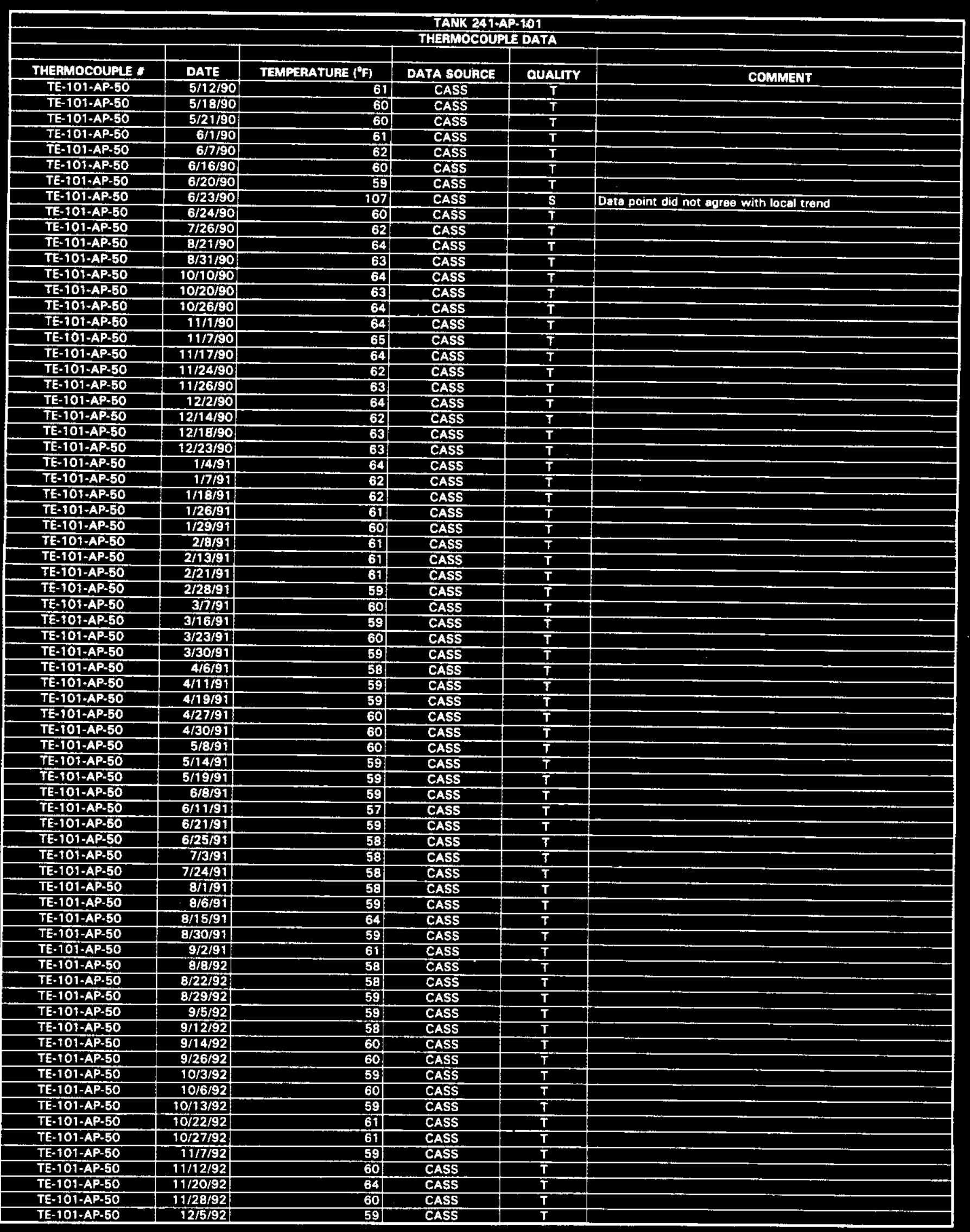




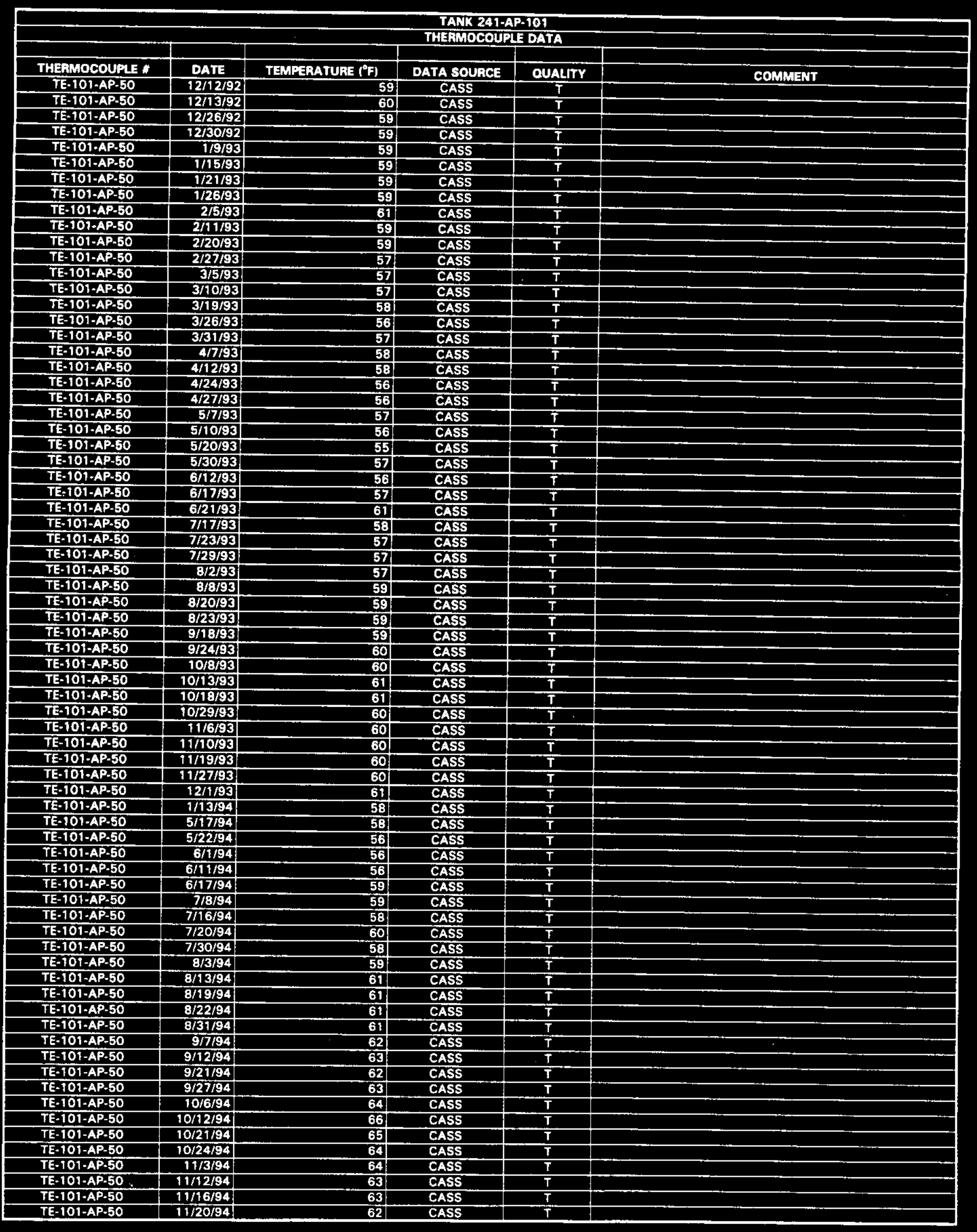




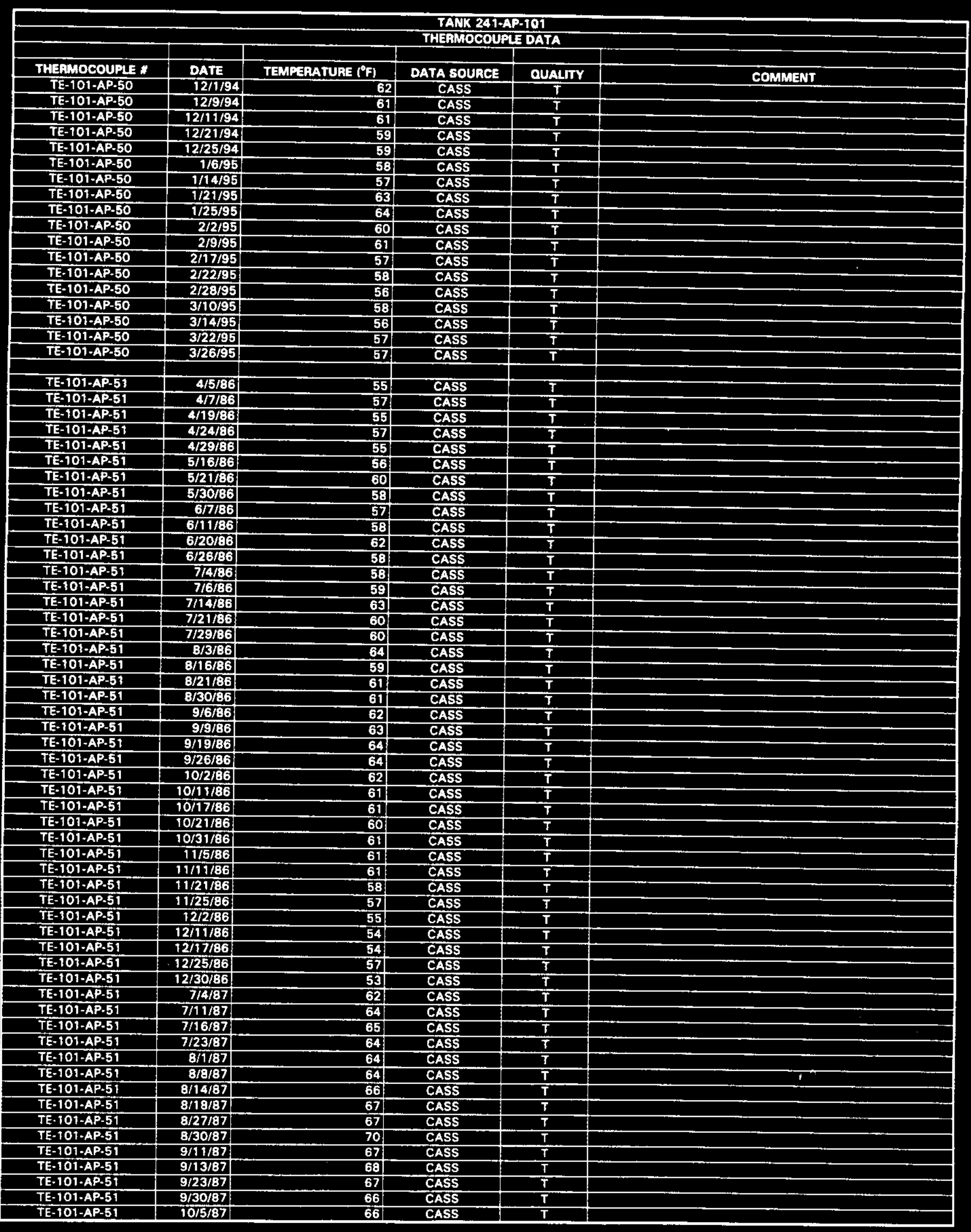




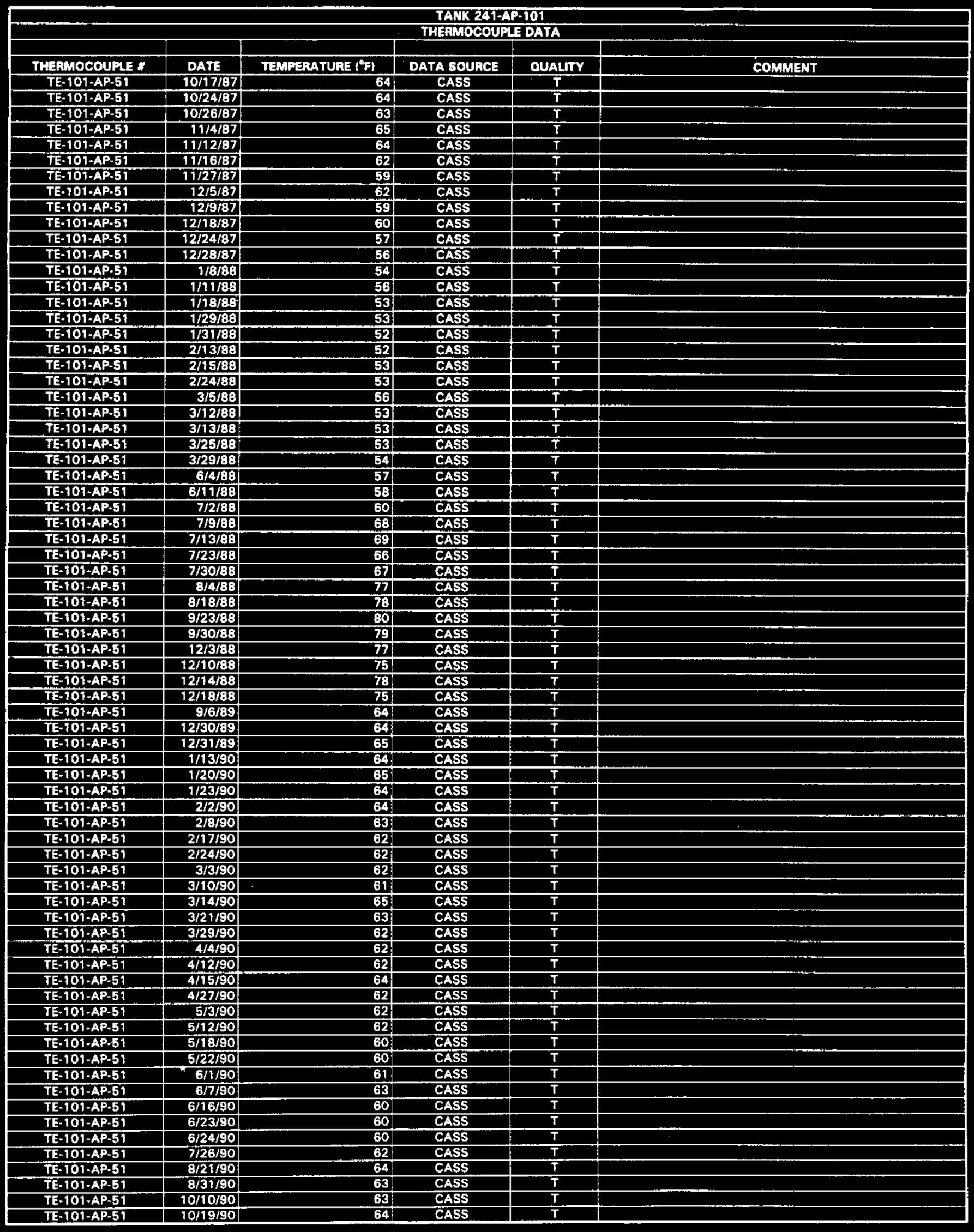




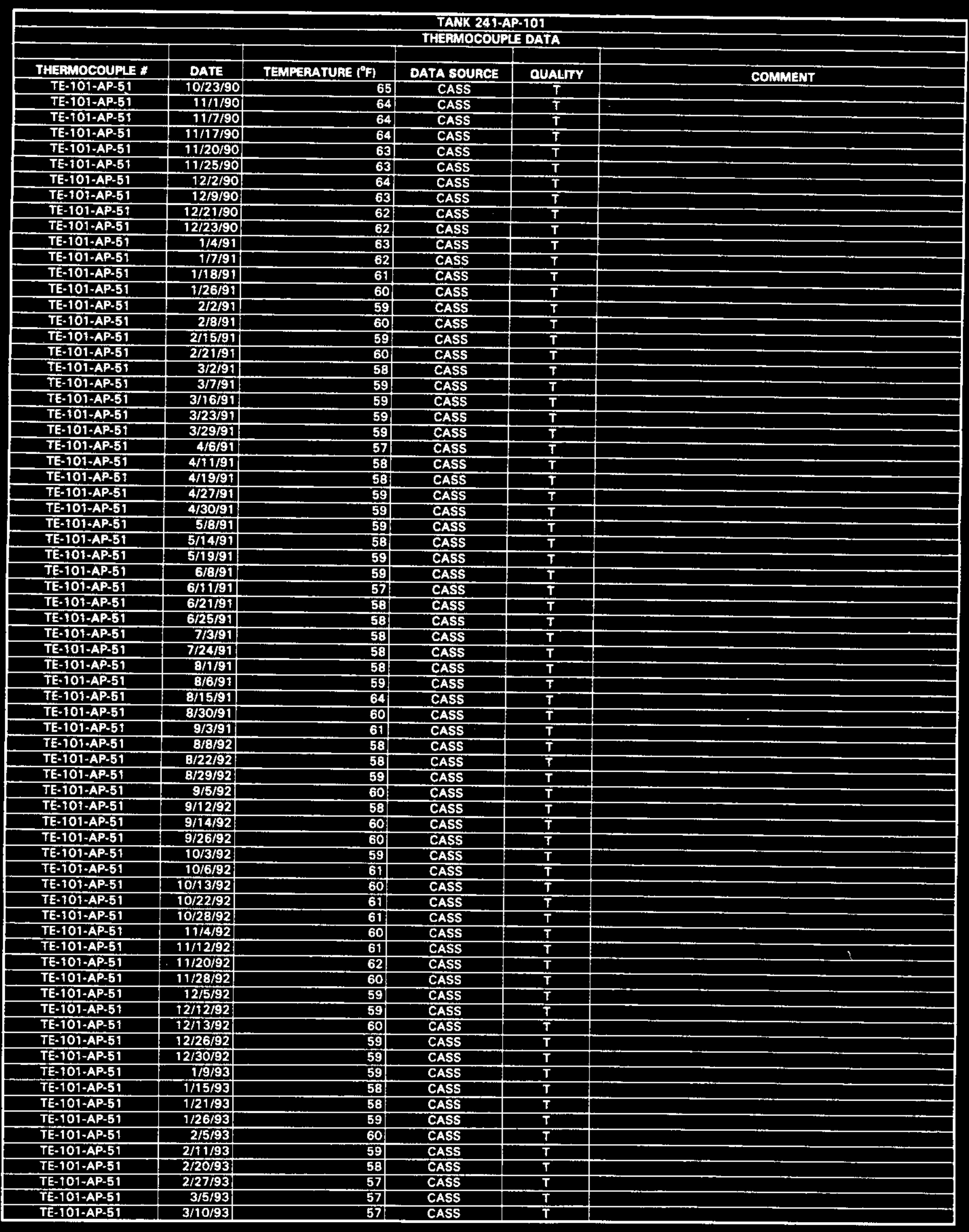




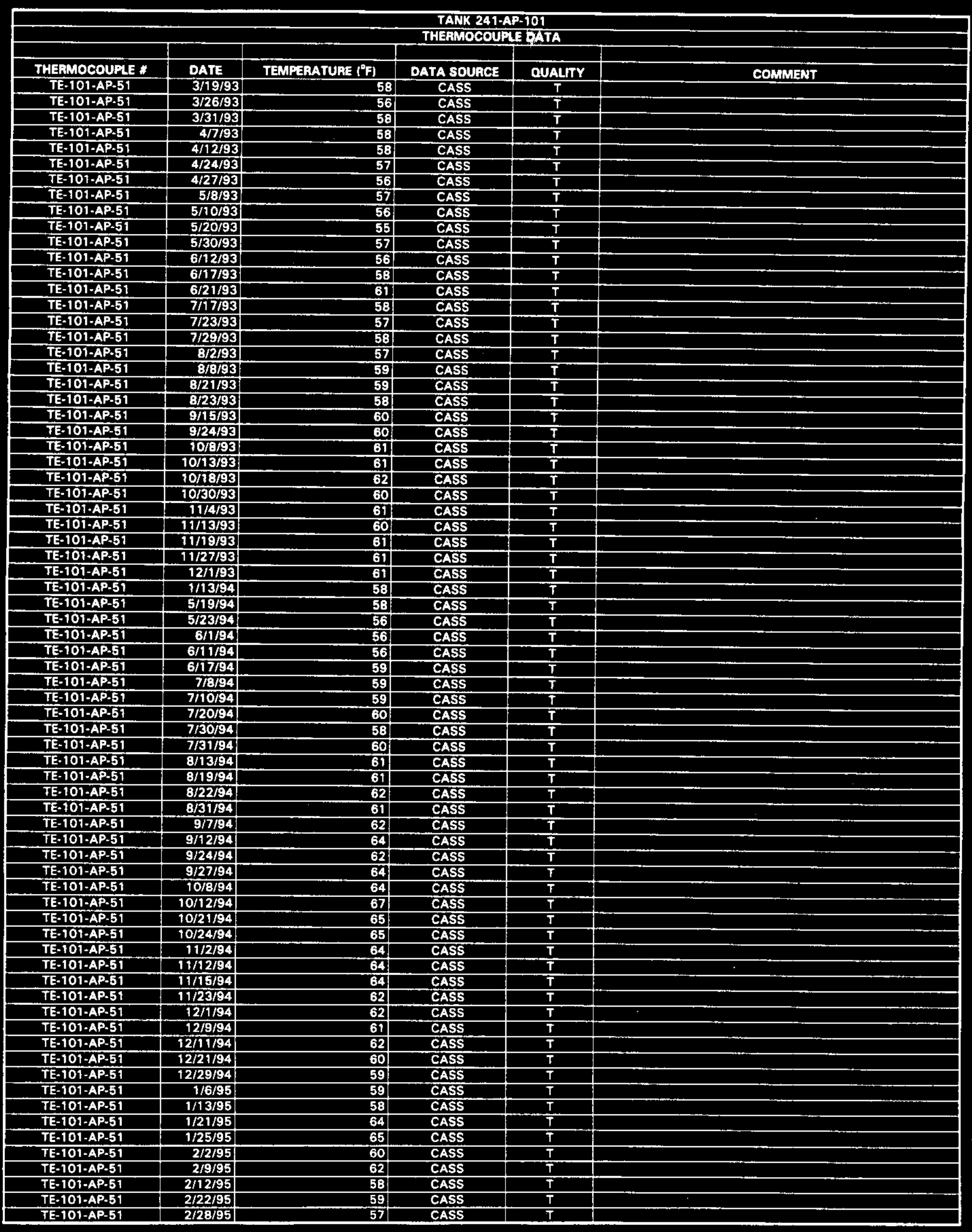




\begin{tabular}{|c|c|c|c|c|c|}
\hline \multicolumn{6}{|c|}{$\begin{array}{l}\text { YANK 241-AP-101 } \\
\text { THEFMOCOUPLE DATA }\end{array}$} \\
\hline & & & & & \\
\hline THEAMOCOUPLE : & DATE & TEMPERATUAE $\left({ }^{\circ} \mathrm{F}\right)$ & DATA SOUACE & OUALTY & COMMENT \\
\hline TE-101-AP-51 & $3 / 10 / 95$ & 58 & CASS & $T$ & \\
\hline TE-101-AP-51 & 3/18/95 & 56 & CASS & $\mathrm{T}$ & \\
\hline TE-101-AP-S1 & $3 / 22 / 95$ & 57 & CASS & $\bar{T}$ & \\
\hline TE-101-AP-51 & $3 / 26 / 95$ & 58 & CASS & $T$ & \\
\hline & $4 / 5 / 86$ & & & & \\
\hline TE-101-AP-52 & $\frac{4 / 5 / 86}{4 / 7 / 36}$ & 55 & CASS & $T$ & \\
\hline$\frac{\text { TE-101-AP-52 }}{\text { TE-101-AP-52 }}$ & $\begin{aligned} 4 / 7 / 36 \\
4 / 19 / 86\end{aligned}$ & 57 & CASS & $T$ & \\
\hline $\begin{array}{l}\text { TE-101-AP-52 } \\
\text { TE-101-AP-52 }\end{array}$ & $\frac{4 / 19 / 86}{4 / 24 / 86}$ & 55 & CASS & $\mathrm{T}$ & \\
\hline TE-101-AP-52 & $\frac{4 / 24 / 86}{4 / 29 / 86}$ & 56 & CASS & $\bar{T}$ & \\
\hline TE-101-AP-52 & $4 / 29 / 86$ & 55 & CASS & $T$ & \\
\hline TE-101-AP-52 & 5/16/86 & 56 & CASS & $T$ & \\
\hline TE-101-AP-52 & $5 / 21 / 86$ & 60 & CASS & $\mathbf{T}$ & \\
\hline TE-101-AP-52 & $5 / 30 / 86$ & 58 & CASS & $\mathbf{T}$ & \\
\hline TE-101-AP-52 & $6 / 7 / 36$ & 57 & CASS & $\bar{T}$ & \\
\hline TE-101-AP-52 & $6 / 11 / 86$ & 58 & CASS & $\bar{T}$ & \\
\hline TE-101-AP-52 & $6 / 20 / 36$ & 63 & CASS & $\mathbf{T}$ & \\
\hline TE-101-AP-52 & $6 / 26 / 86$ & 58: & CASS & $T$ & \\
\hline TE-101-AP-52 & $7 / 4 / 86$ & 58 & CASS & $\mathbf{T}$ & \\
\hline TE-101-AP-52 & $7 / 6 / 86$ & 59 & CASS & $\mathbf{T}$ & \\
\hline TE-101-AP-52 & $7 / 34 / 86$ & 63 & CASS & $\bar{T}$ & \\
\hline TE-10T-AP-52 & $7 / 21 / 86$ & 60 & CASS & $\bar{T}$ & \\
\hline TE-101-AP-52 & $7 / 29 / 86$ & 60 & CASS & $\mathbf{T}$ & \\
\hline TE-101-AP-52 & $8 / 3 / 86$ & 64 & CASS & $T$ & \\
\hline TE-101-AP-52 & $8 / 76 / 86$ & 59 & CASS & $T$ & \\
\hline TE-101-AP.52 & $8 / 22 / 86$ & 61 & CASS & $T$ & \\
\hline TE-101-AP-52 & $8 / 30 / 86$ & 61 & CASS & $\mathbf{T}$ & \\
\hline TE-101-AP-52 & 9/3/36 & 88 & CASS & $\mathbf{S}$ & Date point did not agree with lacal trend \\
\hline TE-101-AP-52 & $9 / 6 / 86$ & 62 & CASS & $\mathbf{T}$ & \\
\hline TE-101-AP-52 & 9/9/86 & 63 & CASS & $\mathbf{T}$ & \\
\hline$\overline{T E-101-A \bar{A}-\overline{52}}$ & $9 / 19 / 86$ & 64 & CASS & $T$ & \\
\hline TE-101-AP-52 & $9 / 26 / 86$ & 64 & CASS & $\mathbf{T}$ & \\
\hline TE-101-AP-52 & $10 / 2 / 86$ & 62 & CASS & $T$ & \\
\hline TE-101-AP-52 & $10 / 11 / 86$ & 61 & CASS & $\mathbf{T}$ & \\
\hline TE-101-AP-52 & $10 / 1486$ & 62 & CASS & $\mathbf{T}$ & \\
\hline IE-101-AP-52 & $10 / 21 / 36$ & 60 & CASS & $\mathbf{T}$ & \\
\hline TE-101-AP-52 & $10 / 31 / 86$ & 61 & CASS & $T$ & \\
\hline TE-101-AP-52 & $11 / 5 / 86$ & 61 & CASS & $T$ & \\
\hline TE-101-AP-52 & $11 / 11 / 86$ & 61 & CASS & $T$ & \\
\hline TE-101-AP-52 & $11 / 21 / 86$ & 58 & CASS & $\mathbf{T}$ & \\
\hline TE-101-AP-52 & $11 / 25 / 36$ & 58 & CASS & $\bar{T}$ & \\
\hline TE-101-AP-52 & $12 / 2 / 86$ & 55 & CASS & $\mathbf{T}$ & \\
\hline TE-101-AP-52 & $12 / 11 / 86$ & 54: & CASS & $\mathbf{I}$ & \\
\hline TE-101-AP-52 & $12 / 17 / 86$ & 54. & CASS & $T$ & \\
\hline TE-101-AP-52 & $12 / 25 / 86$ & 56 & CASS & $\mathrm{T}$ & \\
\hline TE-101-AP-52 & $12 / 31 / 86$ & 53 & CASS & $\mathbf{T}$ & \\
\hline TE-101-AP-52 & 7/4/87 & 62 & CASS & $\mathbf{T}$ & \\
\hline TE-101-AR-52 & $7 / 11 / 87$ & 64 & CASS & $T$ & \\
\hline TE-101-AP-52 & $7 / 16 / 87$ & 65 & CASS & $\mathbf{T}$ & \\
\hline TE-101-AP-52 & $7 / 23 / 67$ & 64 & CASS & $\mathbf{T}$ & \\
\hline TE-101-AP-52 & $8 / 1 / 87$ & 64 & CASS & $T$ & \\
\hline TE-101-AP-52 & $8 / 3 / 87$ & 64 & CASS & $T$ & \\
\hline TE-101-AP.52 & $8 / 14 / 87$ & 67 & CASS & $T$ & \\
\hline TE-101-AP-52 & $8 / 18 / 87$ & 67 & CAŚS & $\bar{T}$ & \\
\hline TE-101-AP-52 & $8 / 27 / 87$ & 67 & CASS & $\mathbf{T}$ & \\
\hline TE-101-AP-52 & $8 / 30 / 87$ & 70 & CASS & $\mathbf{T}$ & \\
\hline TE-101-AP- 52 & 9/7/87 & 68 & CASS & $\mathbf{T}$ & \\
\hline TE-101-AP-52 & $9 / 13 / 87$ & 69 & CASS & $\mathbf{T}$ & \\
\hline TE-101-AP-52 & $9 / 23 / 87$ & 68 & CASS & $T$ & \\
\hline TE-101-AP-52 & 10/1/87 & 66 & CASS & $T$ & \\
\hline TE-101-AP-52 & \begin{tabular}{|l|}
$10 / 10 / 87$ \\
\end{tabular} & 66 & CASS & $T$ & \\
\hline TE-101-AP-52 & $10 / 17 / 87$ & 64 & CASS & $\bar{T}$ & \\
\hline TE-101-AP-52 & $10 / 19 / 87$ & 69 & CASS & $T$ & \\
\hline TE-101-AP-52 & $10 / 30 / 87$ & 63 & CASS & $T$ & \\
\hline TE-101-AP-52 & $11 / 4 / 87$ & 64 & CASS & $\mathbf{T}$ & \\
\hline TE-101.AP-52 & $11 / 12 / 87$ & 65 & CASS & $T$ & \\
\hline TE-101-AP-52 & $11 / 16 / 87$ & 62 & CASS & $T$ & \\
\hline TE-101-AP-52 & 11/25/87 & 59 & CASS & $T$ & \\
\hline TE-101-AP-52 & $11 / 29 / 87$ & 59 & CASS & $T$ & \\
\hline TE-101-AP-52 & 12/5/87 & 67 & CASS & $\mathbf{S}$ & Data point did not agres with local trend \\
\hline TE-101-AP-52 & $12 / 9 / 87$ & 60 & CASS & $\mathbf{T}$ & \\
\hline TE-101-AP-52 & $12 / 18 / 87$ & 60 & CASS & $\mathbf{T}$ & \\
\hline TE-101-AP-52 & $12 / 24 / 87$ & 56 & CASS & $\mathbf{T}$ & \\
\hline TE-101.AP.52 & $1 / 2 / 88$ & 56 & CASS & $\mathbf{T}$ & \\
\hline
\end{tabular}




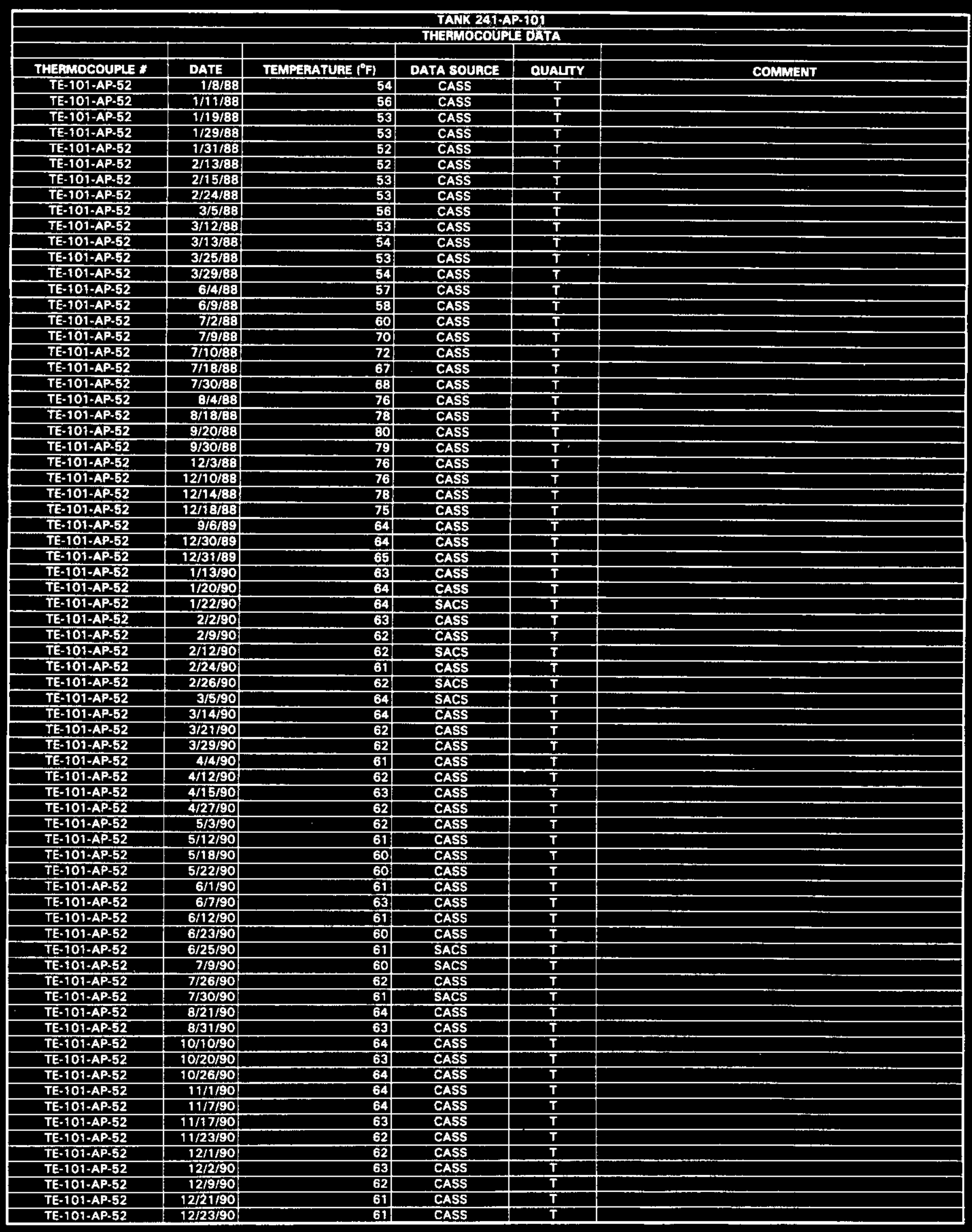

Data obtained from Computer Automated Surveillance System (CASS), April 1995; and Surveillance Analysis Computer System (SACS), June 1995. 


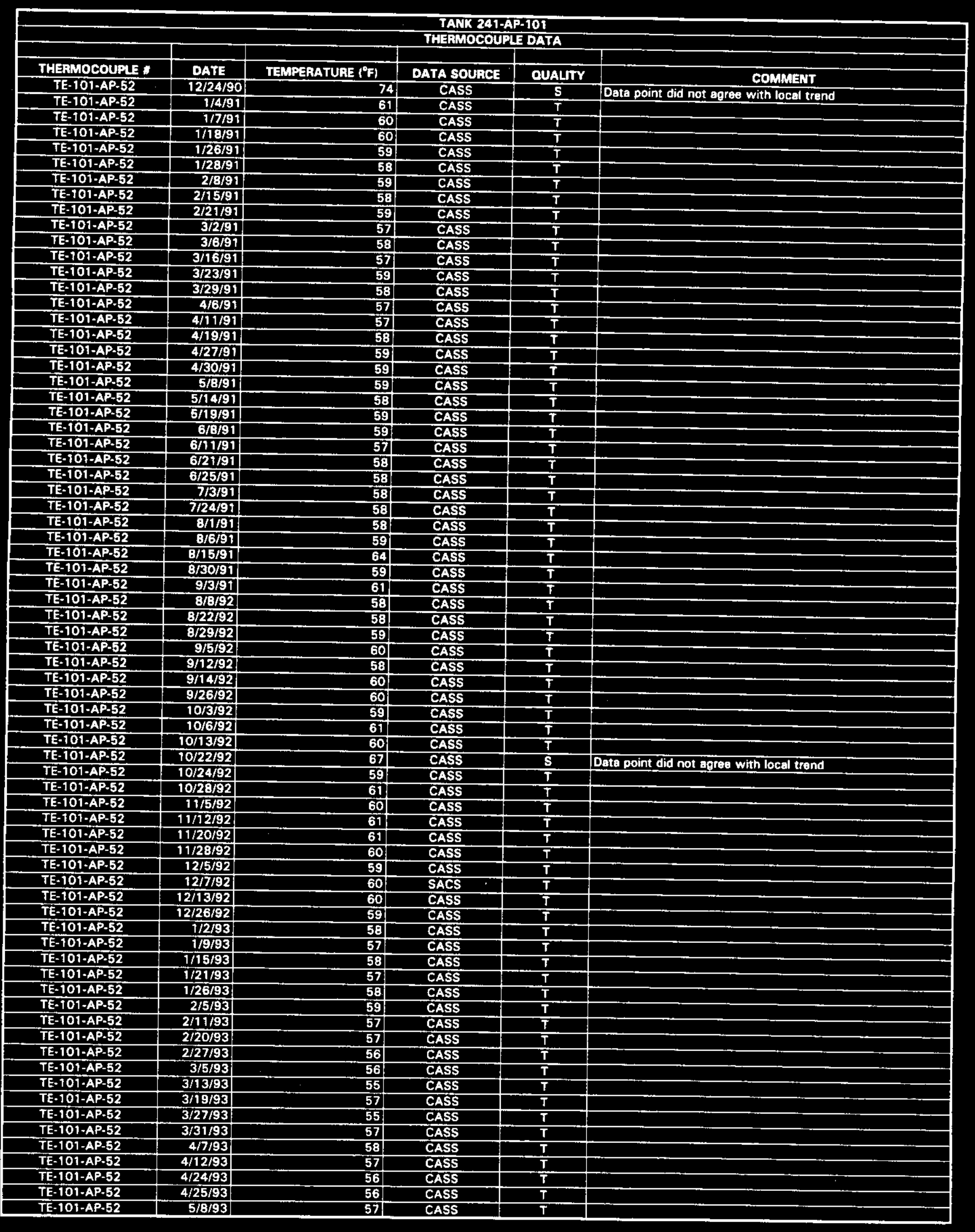

Data obtained from Computer Automated Surveillance System (CASS), April 1995; and Surveillance Analysis Computer System (SACS), June 1995. 


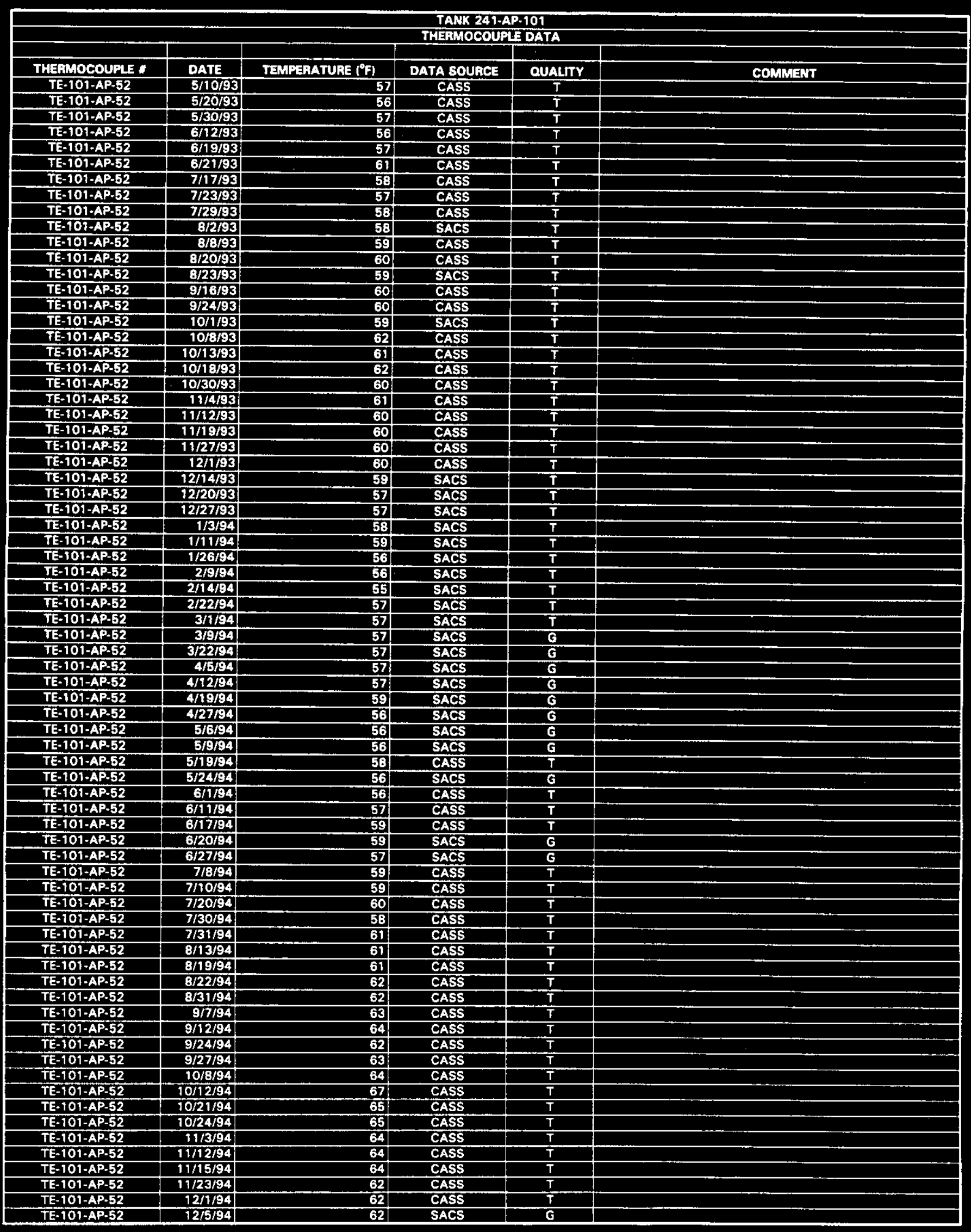




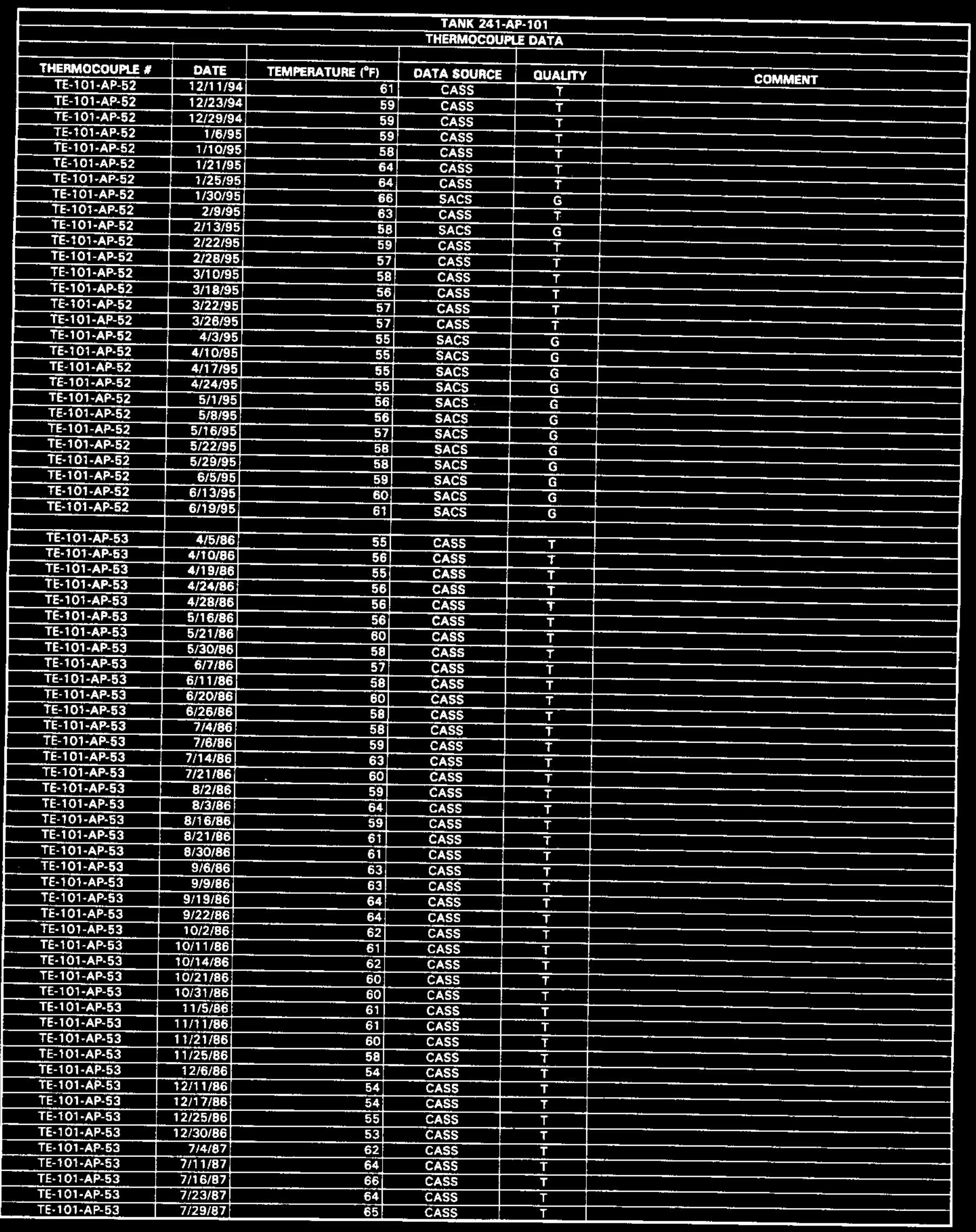




\begin{tabular}{|c|c|c|c|c|c|}
\hline \multicolumn{6}{|c|}{$\begin{array}{l}\text { TANK 241-AP.101 } \\
\text { THEAMOCOUAL DATA }\end{array}$} \\
\hline & & & & & \\
\hline THERMOCOUPLE $*$ & DATE & TEMPERATURE $\left({ }^{\circ} \mathrm{F}\right)$ & DATA SOURCE & GUALTY & COMMENT \\
\hline TE-101.AP.53 & $8 / 8 / 87$ & 64 & CASS & $T$ & \\
\hline TE-101-AP-5 5 & $8 / 14 / 87$ & 67 & CASS & $\mathbf{T}$ & \\
\hline TE-101-AP-53 & B/18/87 & 67 & CASS & $T$ & \\
\hline TE-101-AP-53 & $8 / 27 / 87$ & 67 & CASS & $T$ & \\
\hline TE-101-AP-53 & $8 / 30 / 87$ & 70 & CASS & $T$ & \\
\hline TE-101-AP-53 & $9 / 11 / 87$ & 67 & CASS & $T$ & \\
\hline TE-101.APES3 & $9 / 13 / 37$ & 68 & CASS & $T$ & \\
\hline TE-101-AP-53 & $9 / 26 / 87$ & 67 & CASS & $T$ & \\
\hline TE-101.AP-53 & $10 / 1 / 87$ & 66 & CASS & $T$ & \\
\hline TE-101.AP.53 & $10 / 10 / 87$ & 66 & CASS & $T$ & \\
\hline TE-101-AP-53 & $10 / 17 / 87$ & 64 & CASS & $T$ & \\
\hline TE-101-AP-53 & $10 / 24 / 87$ & 64 & CASS & $T$ & \\
\hline TE-101-AP-53 & $10 / 30 / 87$ & 63 & CASS & $T$ & \\
\hline TE-101-AP-53 & $11 / 4 / 87$ & 65 & CASS & $T$ & \\
\hline TE-101-AP-53 & $11 / 12 / 87$ & 64 & CASS & $T$ & \\
\hline TE-101-AP-53 & $11 / 16 / 87$ & 62 & CASS & $T$ & \\
\hline TE-101+AP-53 & $11 / 25 / 87$ & 59 & CASS & $T$ & \\
\hline TE-107.AP-53 & $11 / 29 / 87$ & 59 & CASS & $T$ & \\
\hline TE-101-AP-53 & $12 / 5 / 87$ & 67 & CASS & $\mathbf{S}$ & Data point did not agree with local trend \\
\hline TE-101-AP-53 & $12 / 8 / 87$ & 59 & CASS & $\bar{T}$ & \\
\hline TE-101-AP-53 & $12 / 18 / 87$ & 61 & CASS & $T$ & \\
\hline TE-101-AP-63 & $12 / 24 / 87$ & 56 & CASS & $T$ & \\
\hline TE-101-AP-53 & $1 / 2 / 83$ & 56 & CASS & $\bar{T}$ & \\
\hline TE-101-AP-53 & $1 / 8 / 88$ & 54 & CASS & $T$ & \\
\hline TE-101-AP-53 & $1 / 11 / 8$ & 56 & CASS & $T$ & \\
\hline TE-101-AP-53 & $1 / 18 / 88$ & 53 & CASS & $T$ & \\
\hline TE-101-AP-53 & $1 / 29 / 88$ & 53 & CASS & $\bar{T}$ & \\
\hline TE-101-AP-53 & $1 / 31 / 88$ & $\mathbf{5 2}$ & CASS & $\bar{T}$ & \\
\hline TE-101-AP-53 & $2 / 13 / 8$ & 52 & CASS & $T$ & \\
\hline TE-101-AP-53 & $2 / 15 / 88$ & 53 & CASS & $T$ & \\
\hline TE-10T-AP-53 & $2 / 24 / 88$ & 533 & CASS & $T$ & \\
\hline TE-101.AP.53 & $3 / 5 / 83$ & 56 & CASS & $T$ & \\
\hline TE-101-AP-53 & $3 / 12 / 88$ & 53 & CASS & $\bar{T}$ & \\
\hline TE-101-AP-53 & $3 / 13 / 88$ & 54 & CASS & $T$ & \\
\hline TE-101-AP-53 & $3 / 25 / 88$ & 53 & CASS & $T$ & \\
\hline TE-101-AP-53 & $3 / 29 / 88$ & 54 & CASS & $T$ & \\
\hline TE-101-AP-53 & $6 / 4 / 63$ & 57 & CASS & $\bar{T}$ & \\
\hline TE-101-AP-53 & $6 / 11 / 88$ & 58 & CASS & $T$ & \\
\hline TE-101.AP.53 & $7 / 2 / 88$ & 60 & CASS & $T$ & \\
\hline TE-101.AP-53 & 7/9/88 & 72 & CASS & $T$ & \\
\hline TE-101-AP-53 & $7 / 10 / 88$ & 73 & CASS & $T$ & \\
\hline TE-101-AP-53 & $7 / 18 / 88$ & 67 & CASS & $T$ & \\
\hline TE-101-AP-53 & $7 / 30 / 8$ & 68 & CASS & $T$ & \\
\hline TE-101-AP-53 & $8 / 6 / 88$ & 70 & CASS & $\bar{T}$ & \\
\hline TE-101-AP-53 & $8 / 18 / 88$ & 74 & CASS & $T$ & \\
\hline TE-101-AP-53 & 9/23/88 & 78 & CASS & $T$ & \\
\hline TE-101-AP-53 & $9 / 30 / 83$ & 76 & CASS & $T$ & \\
\hline TE-101-AP-5B & $12 / 3 / 8 B$ & 74 & CASS & $T$ & \\
\hline TE-101-AP-53 & $12 / 10 / 83$ & 74 & CASS & $\bar{T}$ & \\
\hline TE-101-AP-53 & $12 / 14 / 88$ & 75 & CASS & $T$ & \\
\hline TE-10T-AP-53 & $12 / 18 / 88$ & 72 & CASS & $T$ & \\
\hline TE-101-AP-53 & $7 / 10 / 89$ & 61 & SACS & $\bar{T}$ & \\
\hline TE-101-AP-53 & $8 / 21 / 89$ & 64 & SACS & $T$ & \\
\hline TE-101-AP-53 & $8 / 28 / 89$ & 64 & SACS & $T$ & \\
\hline TE-101-AP-53 & $9 / 6 / 89$ & 64 & CASS & $T$ & \\
\hline TE-101-AP-53 & $10 / 30 / 89$ & 66 & SACS & $\bar{T}$ & \\
\hline TE-101-AP-53 & $12 / 26 / 89$ & 64 & SACS & $\bar{T}$ & \\
\hline TE.101-AP-53 & $12 / 31 / 89$ & 64 & CASS & $T$ & \\
\hline TE-101-AP-53 & $1 / 13 / 90$ & 62 & CASS & $T$ & \\
\hline TE-101-AP-53 & $1 / 20 / 90$ & 63 & CASS & $T$ & \\
\hline TE-101-AP-53 & $1 / 23 / 90$ & 62 & CASS & $T$ & \\
\hline TE-101-AP-53 & $2 / 3 / 90$ & 61 & CASS & $T$ & \\
\hline TE-101-AP.53 & $2 / 8 / 90$ & 61 & CASS & $T$ & \\
\hline TE-101-AP-53 & $2 / 17 / 90$ & 60 & CASS & $\bar{T}$ & \\
\hline TE-101-AP-53 & $2 / 24 / 90$ & 59 & CASS & $T$ & \\
\hline TE-101-AP. 53 & $3 / 3 / 90$ & 60 & CASS & $T$ & \\
\hline TE-101-AP-53 & $3 / 10 / 90$ & 59 & CASS & $T$ & \\
\hline TE-101-AP-53 & $3 / 14 / 90$ & 64 & CASS & $\bar{T}$ & \\
\hline TE-101-AP.53 & $3 / 21 / 90$ & 6ी & CASS & $\bar{T}$ & \\
\hline TE-101-AP.53 & $3 / 29 / 90$ & 61. & CASS & $T$ & \\
\hline TE-101-AP.53 & $4 / 4 / 90$ & 61 & CASS & $\bar{T}$ & \\
\hline TE-101.AP.53 & $4 / 12 / 90$ & 61 & CASS & $T$ & \\
\hline TE-101-AP-53 & $4 / 15 / 90$ & 63 & CASS & $T$ & \\
\hline
\end{tabular}




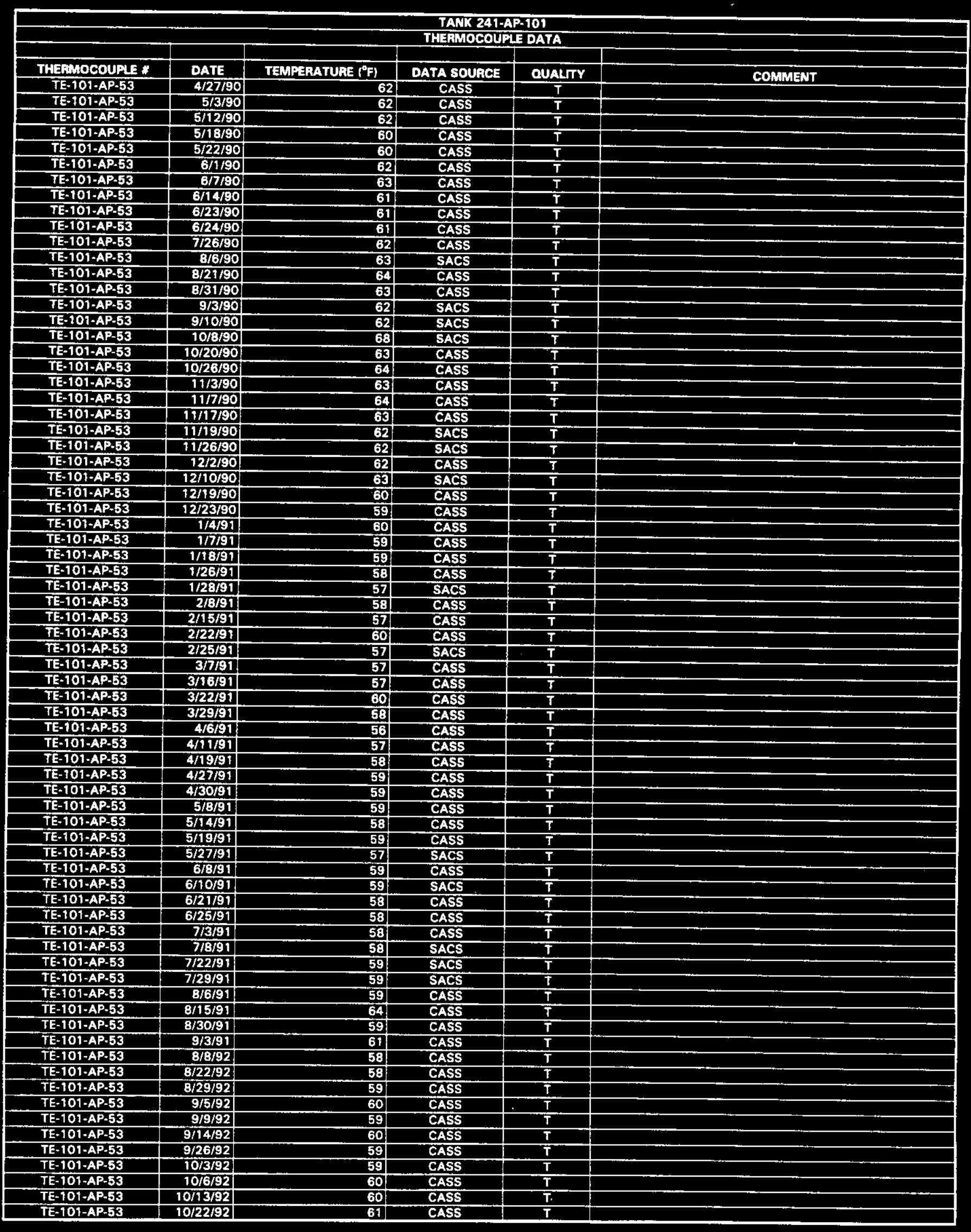




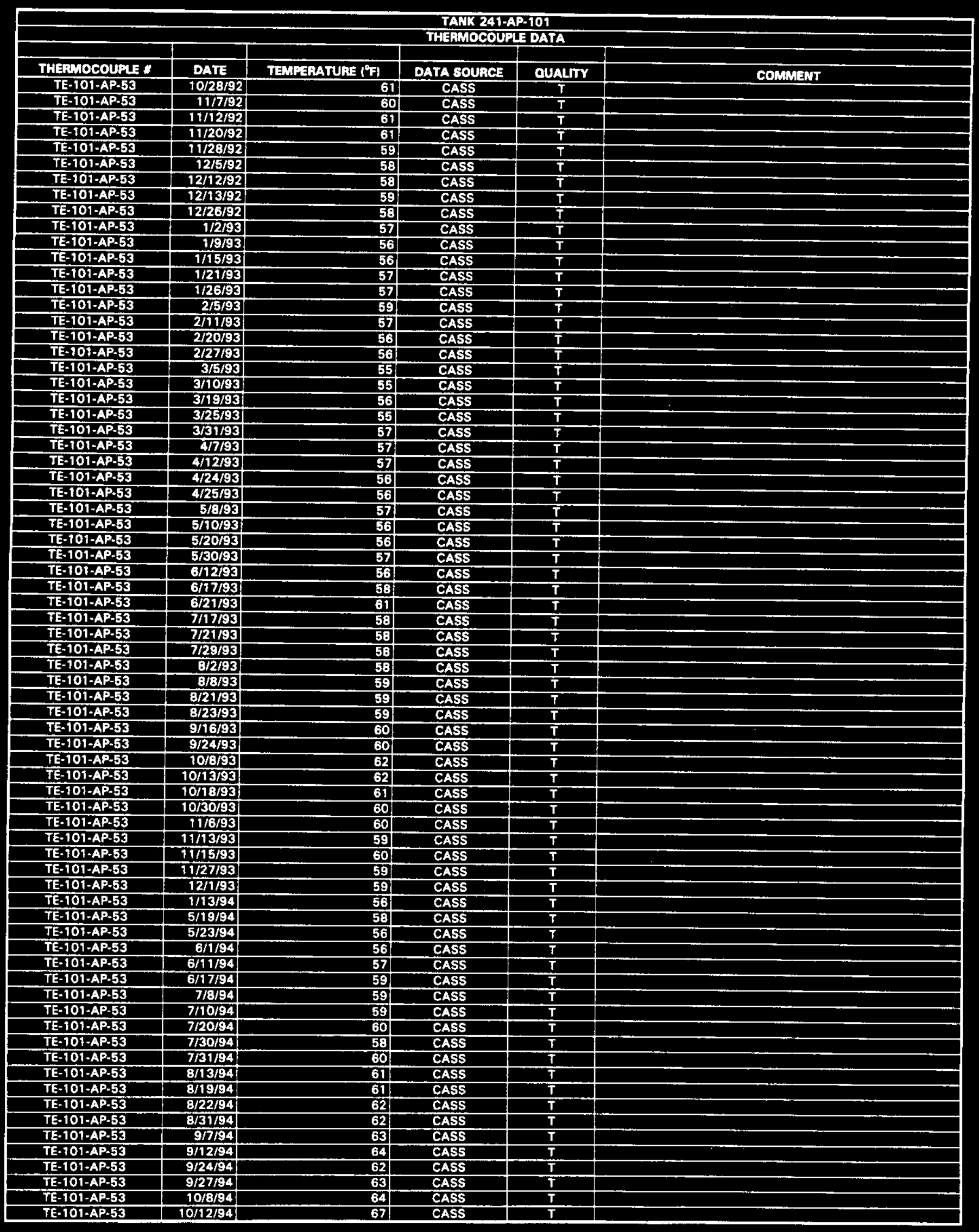




\begin{tabular}{|c|c|c|c|c|c|}
\hline \multicolumn{6}{|c|}{$\begin{array}{l}\text { TANK 241-AP-101 } \\
\text { THEAMOCOUPLE DATA }\end{array}$} \\
\hline & & & & & \\
\hline THEPMOCOUPLE * & DATE & TEMPERATURE ( $\left.{ }^{\circ} \mathrm{F}\right)$ & DATA SOURCE & OUALITY & COMMENT \\
\hline TE-101-AP-53 & $10 / 21 / 94$ & 64 & CASS & $T$ & \\
\hline TE-101-AP-53 & $10 / 24 / 94$ & 64 & CASS & $T$ & \\
\hline TE-101-AP-53 & $11 / 3 / 94$ & 63 & CASS & $\bar{T}$ & \\
\hline TE-101-AP-53 & $11 / 12 / 94$ & 63 & CASS & $\bar{T}$ & \\
\hline TE-101.AP-53 & $11 / 19 / 94$ & 63 & CASS & $T$ & \\
\hline TE-101-AP-53 & $11 / 23 / 94$ & 61 & CASS & $T$ & \\
\hline TE-101-AP-53 & $12 / 1 / 94$ & 62 & CASS & $T$ & \\
\hline TE-101-AP-53 & $12 / 9 / 94$ & 60 & CASS & $T$ & \\
\hline TE-101-AP-53 & $12 / 11 / 94$ & 61 & CASS & $T$ & \\
\hline TE-101-AP-53 & $12 / 23 / 94$ & 59 & CASS & $T$ & \\
\hline TE-101-AP-53 & $12 / 29 / 94$ & 59 & CASS & $\bar{T}$ & \\
\hline TE-101-AP-53 & $1 / 6 / 95$ & 59 & CASS & $T$ & \\
\hline TE-101-AP-53 & $1 / 13 / 95$ & 58 & CASS & $T$ & \\
\hline TE-101-AP-53 & $1 / 21 / 95$ & 64 & CASS & $\mathbf{T}$ & \\
\hline TE-101-AP-53 & $1 / 25 / 95$ & 64. & CASS & $\bar{T}$ & \\
\hline TE-101-AP-53 & $2 / 2 / 95$ & 61 & CASS & $T$ & \\
\hline TE-101-AP-53 & $2 / 9 / 95$ & 62 & CASS & $T$ & \\
\hline TE-103-AP.53 & $2 / 3 / 95$ & 58 & CASS & $T$ & \\
\hline TE-101-AP-53 & $2 / 22 / 95$ & 59 & CASS & $T$ & \\
\hline TE-101-AP-53 & $2 / 28 / 95$ & 57 & CASS & $T$ & \\
\hline TE-101-AP-53 & $3 / 10 / 95$ & 58 & CASS & $T$ & \\
\hline TE-101-AP-53 & $3 / 18 / 95$ & 56 & CASS & $\mathbf{T}$ & \\
\hline TE-101-AP-53 & $3 / 22 / 95$ & 57 & CASS & $T$ & \\
\hline TE-101-AP-53 & $3 / 26 / 95$ & 58 & CASS & $T$ & \\
\hline
\end{tabular}




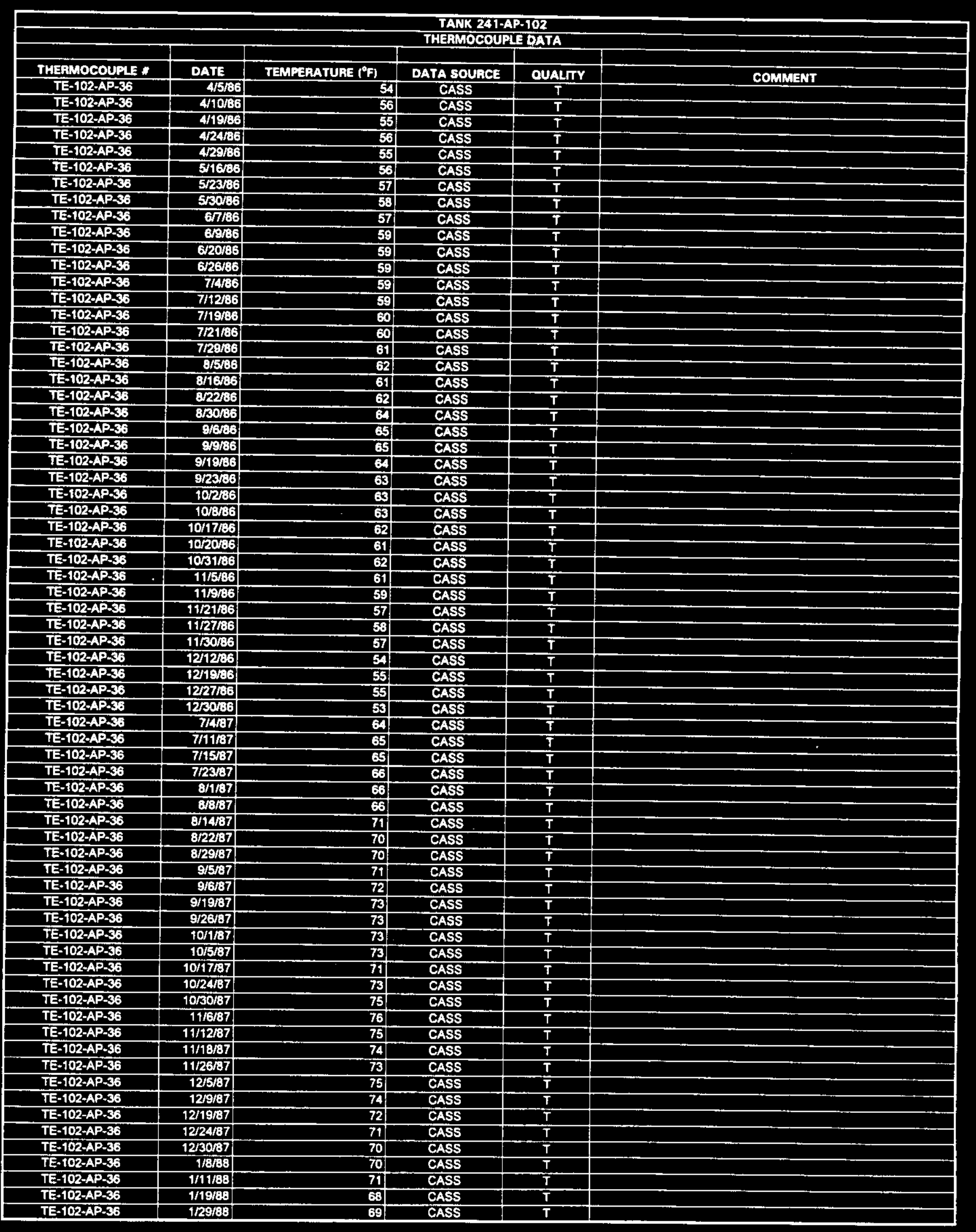




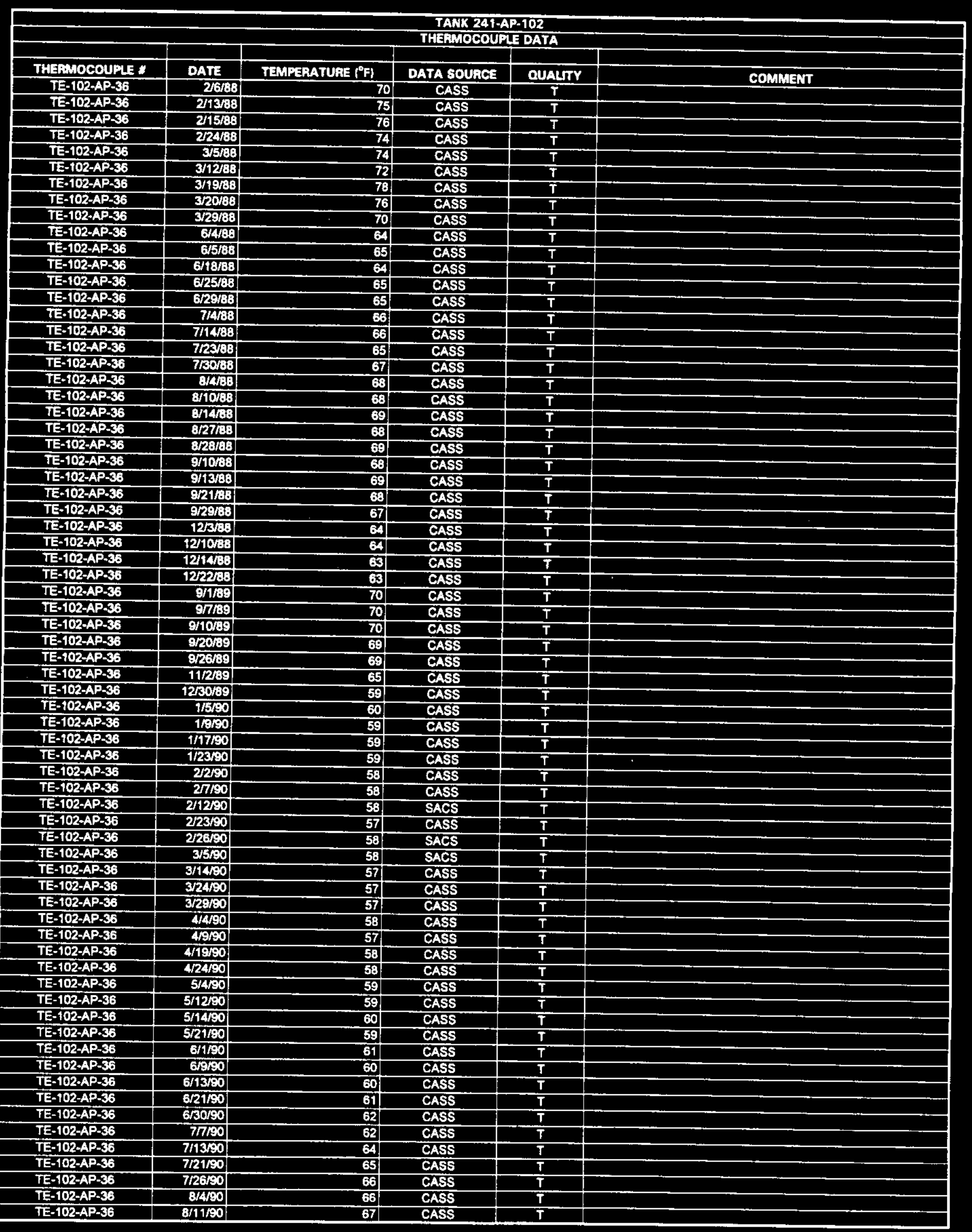




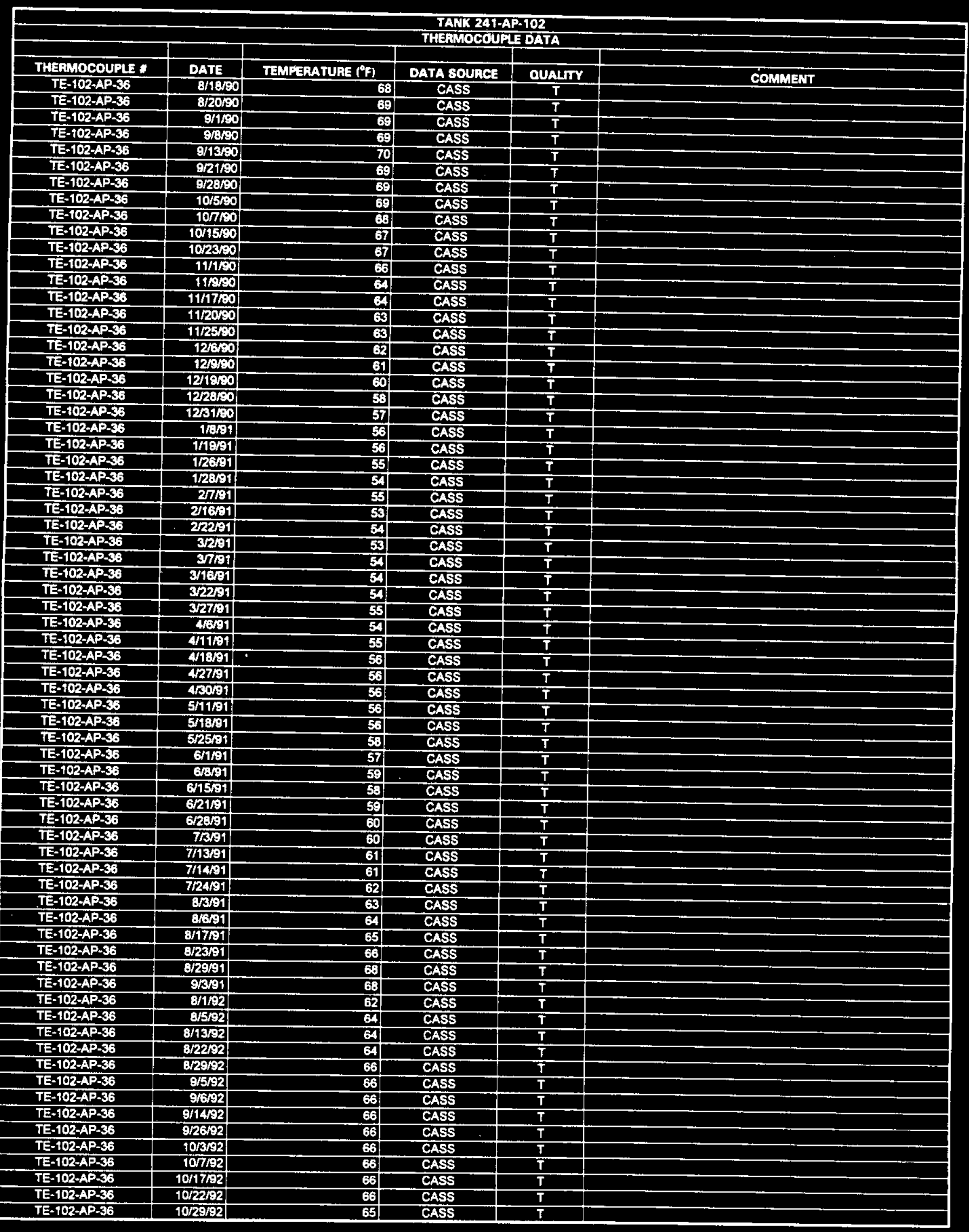




\begin{tabular}{|c|c|c|c|c|c|}
\hline \multicolumn{6}{|c|}{$\begin{array}{l}\text { TANK 241-AP-102 } \\
\text { THERMOCOUPLE DATA }\end{array}$} \\
\hline & & & & & \\
\hline THERMOCOUPLE $*$ & DATE & TEMPGRATUAE $\left(^{\circ} F\right\}$ & DATA SOURCE & QUALITY & COMMENT \\
\hline TE-102-AP-36 & $11 / 7 \% 2$ & 64 & CASS & $T$ & \\
\hline TE-102-AP-36 & $11 / 8 / 92$ & 65 & CASS & $T$ & \\
\hline TE-102-AP-36 & $11 / 21 / 92$ & 65 & CASS & $T$ & \\
\hline TE-102-AP-36 & $11 / 28 / 92$ & 71 & CASS & $\mathbf{T}$ & \\
\hline TE-102-AP-36 & $125 \times 2$ & 70 & CASS & $T$ & \\
\hline TE-102-AP-36 & $12 / 1292$ & 70 & CASS & $T$ & \\
\hline TE-102-AP-33 & $12 / 7 \% 2$ & 73 & CASS & $T$ & \\
\hline TE-102-APS6 & $12 / 21 \% 2$ & 70 & SACS & $T$ & \\
\hline TE-102-AP-36 & $12 / 2392$ & 69: & SACS & $T$ & \\
\hline TE-102-AP-36 & $1 / 0 / 93$ & 67 & CASS & $T$ & \\
\hline TE-102-AP-36 & $1 / 16 / 93$ & 66 & CASS & $T$ & \\
\hline TE-102-AP-36 & $1 / 21 / 93$ & 67 & CASS & $T$ & \\
\hline TE-102-AP-36 & $1 / 30193$ & 60: & CASS & $\bar{T}$ & \\
\hline TE-102-AP-36 & $2 / 493$ & 66 & CASS & $T$ & \\
\hline TE-102-AP-36 & $2 / 9 / 93$ & 64. & CASS & $\bar{T}$ & \\
\hline TE-102-AP-36 & ग1893 & 64 & CASS & $T$ & \\
\hline TE-102-AP-36 & $2 / 2703$ & 62 & CASS & $\mathbf{T}$ & \\
\hline TE-102-AP-36 & 3/5E3 & 66 & CASS & $T$ & \\
\hline TE-102-AP-36 & $3 / 4003$ & 69 & CASS & $\mathbf{T}$ & \\
\hline TE-102-AP-36 & 317\%3 & 71 & SACS & $T$ & \\
\hline TE-102-AP-36 & $3 / 2653$ & 75 & CASS & $T$ & \\
\hline TE-102-AP-36 & $4 \sqrt{303}$ & 77 & CASS & $T$ & \\
\hline TE-102-AP-36 & $4 / 1003$ & 79 & CASS & $\bar{T}$ & \\
\hline TE-102-AP-s6 & $4 / 763$ & 82 & CASS & $T$ & \\
\hline TE-102-AP-36 & $4 \sqrt{2403}$ & 83 & CASS & $\bar{T}$ & \\
\hline TE-102-AP-36 & $4 / 2903$ & 84 & CASS & $T$ & \\
\hline TE-102-AP-36 & 5/8/93 & 84 & CASS & $T$ & \\
\hline TE-102-AP-36 & $5 / 1213$ & 83 & CASS & $T$ & \\
\hline TE-102-AP-36 & $5 / 2263$ & 83. & CASS & $T$ & \\
\hline$T E-102-A P-36$ & 527193 & 83 & CASS & $T$ & \\
\hline TE-102-AP-36 & 5/003 & 83 & CASS & $T$ & \\
\hline TE-102-AP-36 & 6693 & 82 & CASS & $T$ & \\
\hline TE-102-AP-36 & 6/17:3 & 82 & CASS & $T$ & \\
\hline TE-102-AP-33 & 6/24,93 & 82 & CASS & $\mathbf{T}$ & \\
\hline TE-102-AP-35 & $7 / 3 / 93$ & 81 & CASS & $T$ & \\
\hline$T E-102 \cdot A P-36$ & $7 / 483$ & 82 & CASS & $T$ & \\
\hline TE-102-AP-36 & $7 / 17 / 93$ & 80 & CASS & $T$ & \\
\hline TE-102-AP-33 & $7 / 18+3$ & 82 & SACS & $T$ & \\
\hline TE-102-AP-36 & $7 / 3003$ & 82 & CASS & $T$ & \\
\hline TE-102-AP-36 & $8 / 793$ & 81 & CASS & $T$ & \\
\hline TE-102-AP-36 & $8 / 14 / 93$ & 81 & CASS & $T$ & \\
\hline TE-102-AP-36 & 8720193 & 82 & CASS & $T$ & \\
\hline TE-102-AP-36 & $8 / 25 / 93$ & 81 & CASS & $T$ & \\
\hline TE-102-AP-36 & 8/293 & 81 & CASS & $\bar{T}$ & \\
\hline TE-102-AP-36 & $8 / 11 / 03$ & 80 & CASS & $T$ & \\
\hline TE-102-AP-36 & $9 / 13 / 93$ & 81. & CASS & $T$ & \\
\hline TE-102-AP-36 & $9 / 25 / 93$ & 80 & CASS & $\bar{T}$ & \\
\hline TE-102-AP-36 & $10 / 2 / 93$ & 80 & CASS & $T$ & \\
\hline TE-102-AP-36 & $10 / 793$ & 81) & CASS & $T$ & \\
\hline TE-102-AP-36 & $10 / 11 / 93$ & 81 & CASS & $T$ & \\
\hline $\mathrm{TE}-102-\mathrm{AP}-36$ & $10 / 1193$ & 70 & SACS & $\mathbf{s}$ & Disagreement between CASS and SACS \\
\hline TE-102-AP.36 & $10 / 22193$ & 80 & CASS & $T$ & \\
\hline TE-102-AP-36 & $10 / 27 / 93$ & 80 & CASS & $T$ & \\
\hline TE-102-AP-36 & $11 / 493$ & 79 & CASS & $\mathbf{T}$ & \\
\hline TE-102-AP-36 & $11 \overline{18} 93$ & 79 & CASS & $T$ & \\
\hline$T E-102-A P-36$ & $11 / 14 / 93$ & 79 & CASS & $\mathbf{T}$ & \\
\hline TE-102-AP-36 & $11 / 26 / 93$ & 77 & CASS & $T$ & \\
\hline$T E-102-A P-36$ & $12 / 1 / 93$ & 77 & CASS & $T$ & \\
\hline TE-102-AP-36 & 121093 & 80 & SACS & $\bar{T}$ & \\
\hline TE-102-AP-36 & $12 / 14 / 93$ & 76 & SACS & $T$ & \\
\hline TE-102-AP-36 & $12 / 20193$ & 82 & SACS & $T$ & \\
\hline TE-102-AP-36 & $12 / 27 / 93$ & 79 & SACS & $T$ & \\
\hline TE-102-AP-36 & $1 / 3 / 94$ & 72 & SACS & $T$ & \\
\hline TE-102-AP-36 & $1 / 11 / 94$ & 81 & SACS & $T$ & \\
\hline TE-102-AP-36 & $1 / 26 / 94$ & 71 & SACS & $T$ & \\
\hline TE-102-AP-36 & $2 / 9194$ & 70 & SACS & $T$ & \\
\hline TE-102-AP-36 & $2 / 14194$ & 69 & SACS & $\mathbf{T}$ & \\
\hline TE-102-AP-36 & $2 / 22 / 24$ & 71 & SACS & $\bar{T}$ & \\
\hline TE-102-AP-36 & $3 / 1 / 94$ & 73 & SACS & $T$ & \\
\hline TE-102-AP-3S & $30 / 94$ & 70 & SACS & G & \\
\hline
\end{tabular}




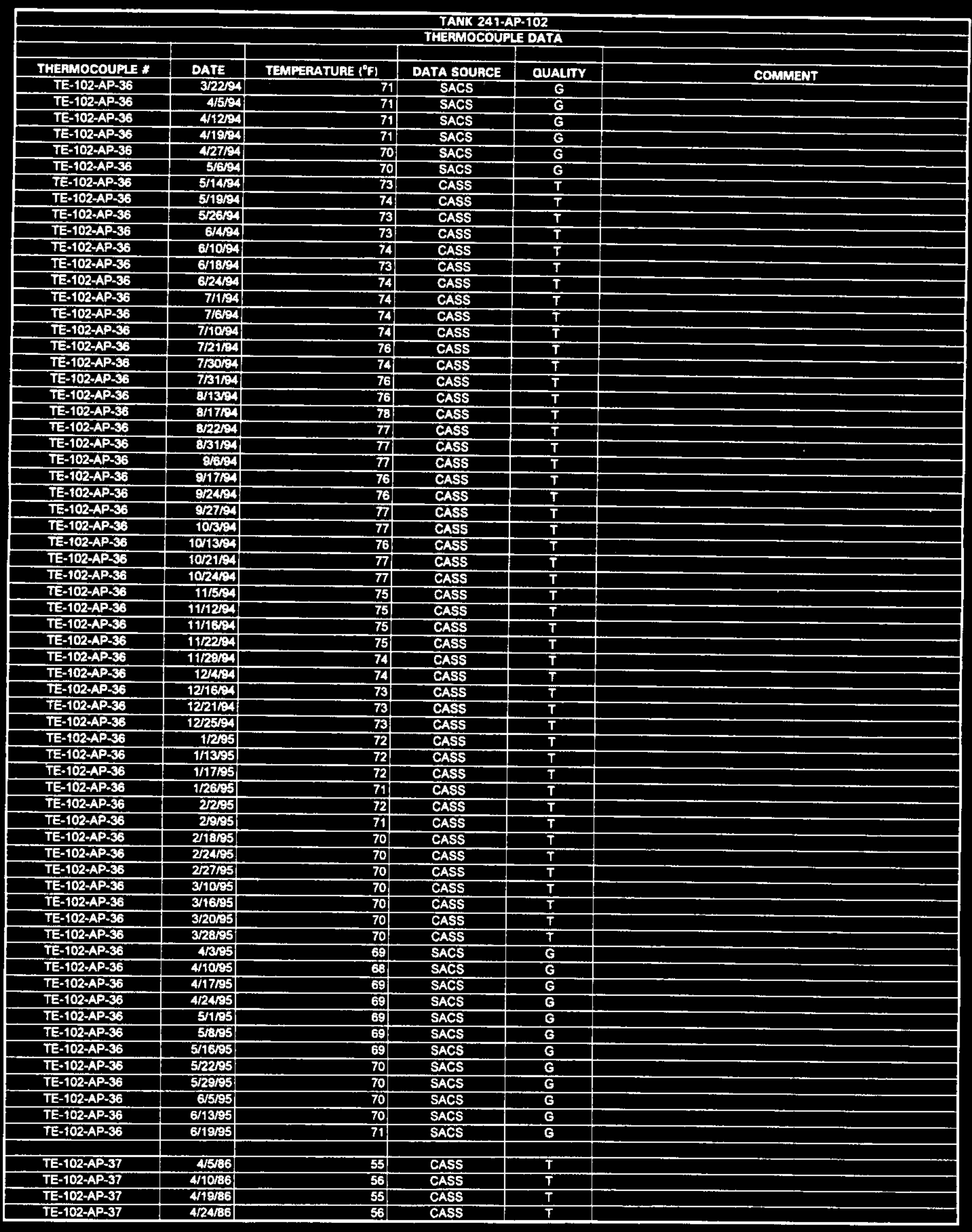




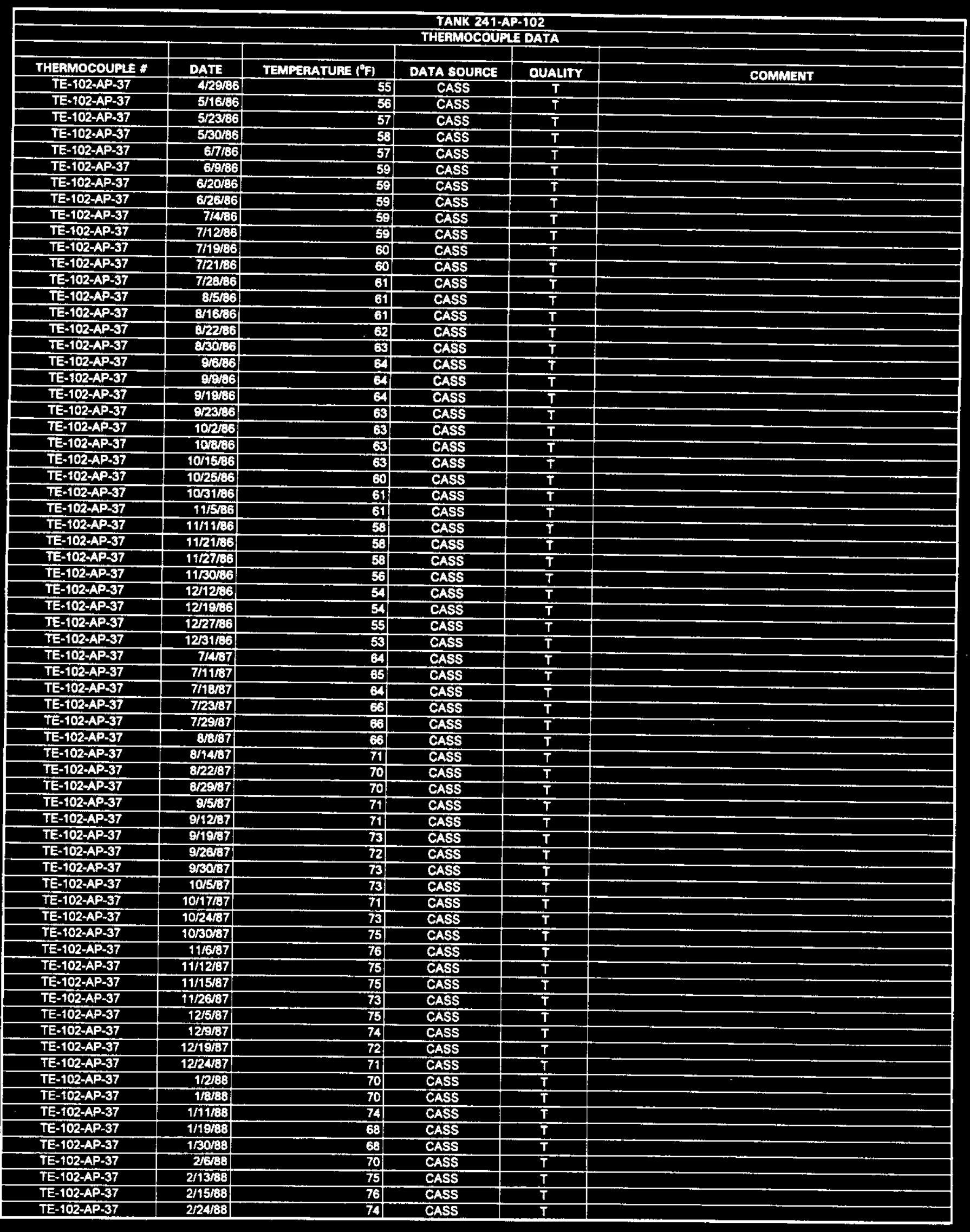

Data obtained from Computer Automated Surveillance Syatem (CASS), April 1995; and Surveillance Analysis Computer System (SACS), June 1995. D-151 
TANK 241-AP-102

THERMOCOUPE DATA

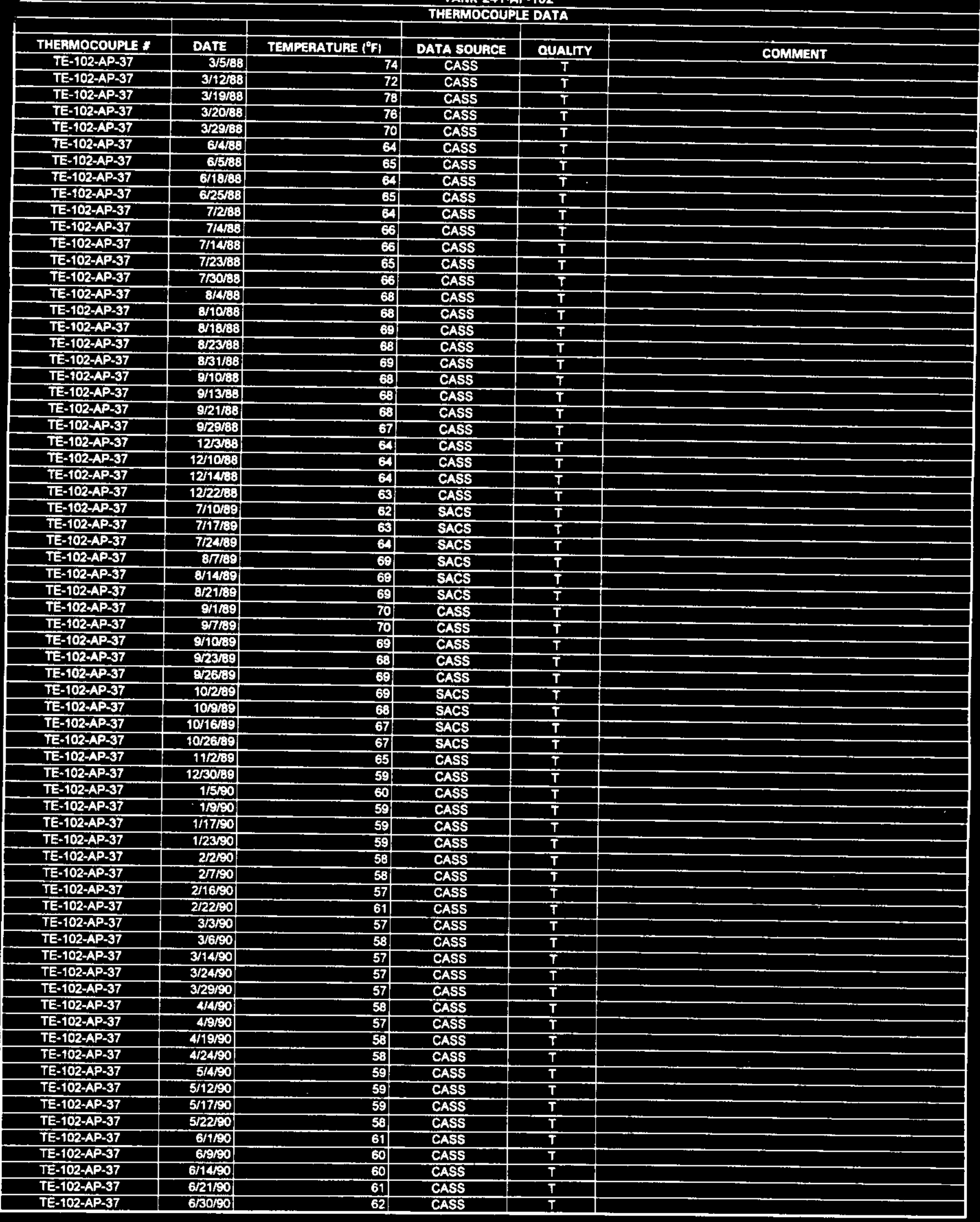




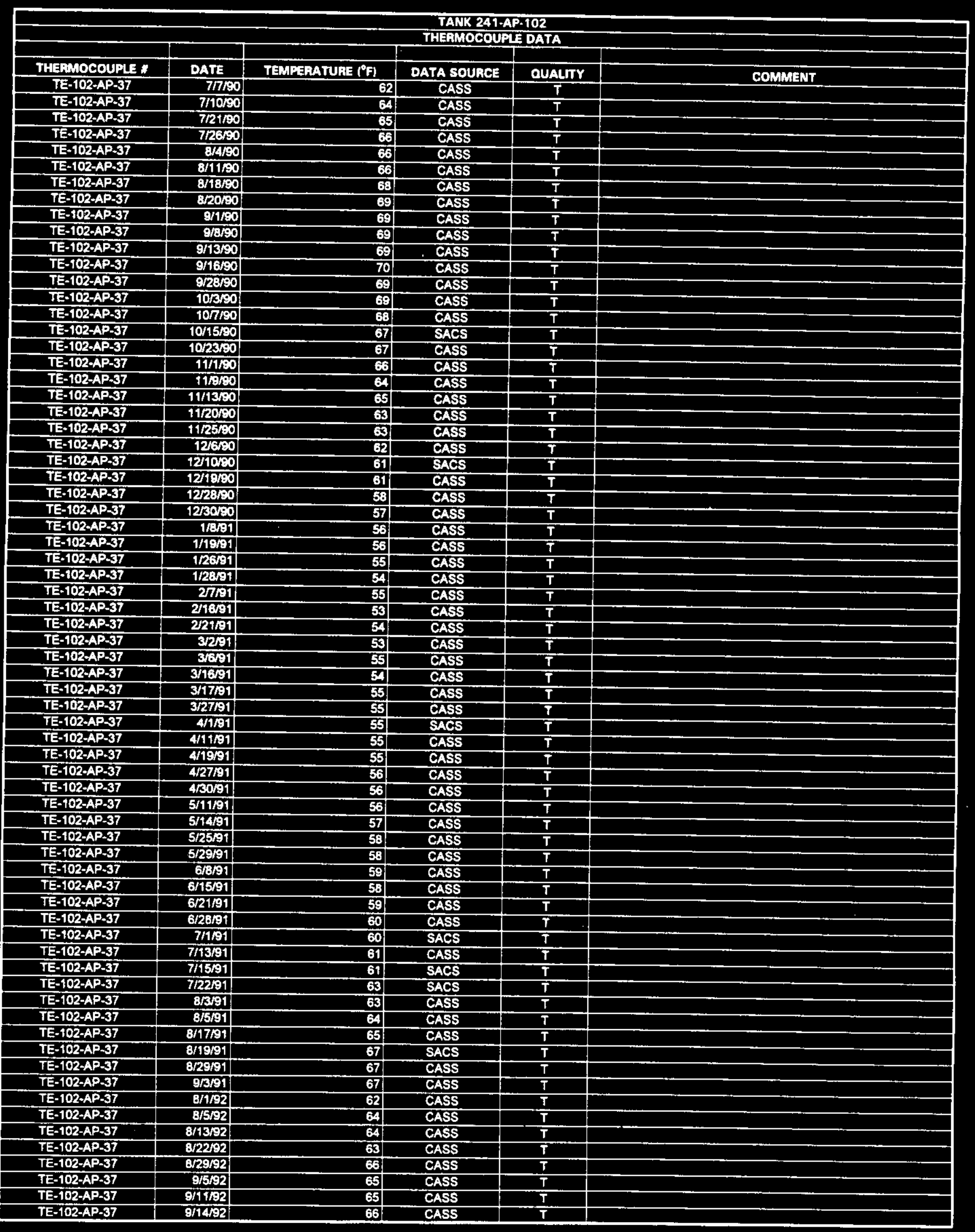

Date obtainad from Computer Automated Surveillance Syatem (CASS), April 1995; and Surveillance Anelysis Computer System (SACS), June 1995. 


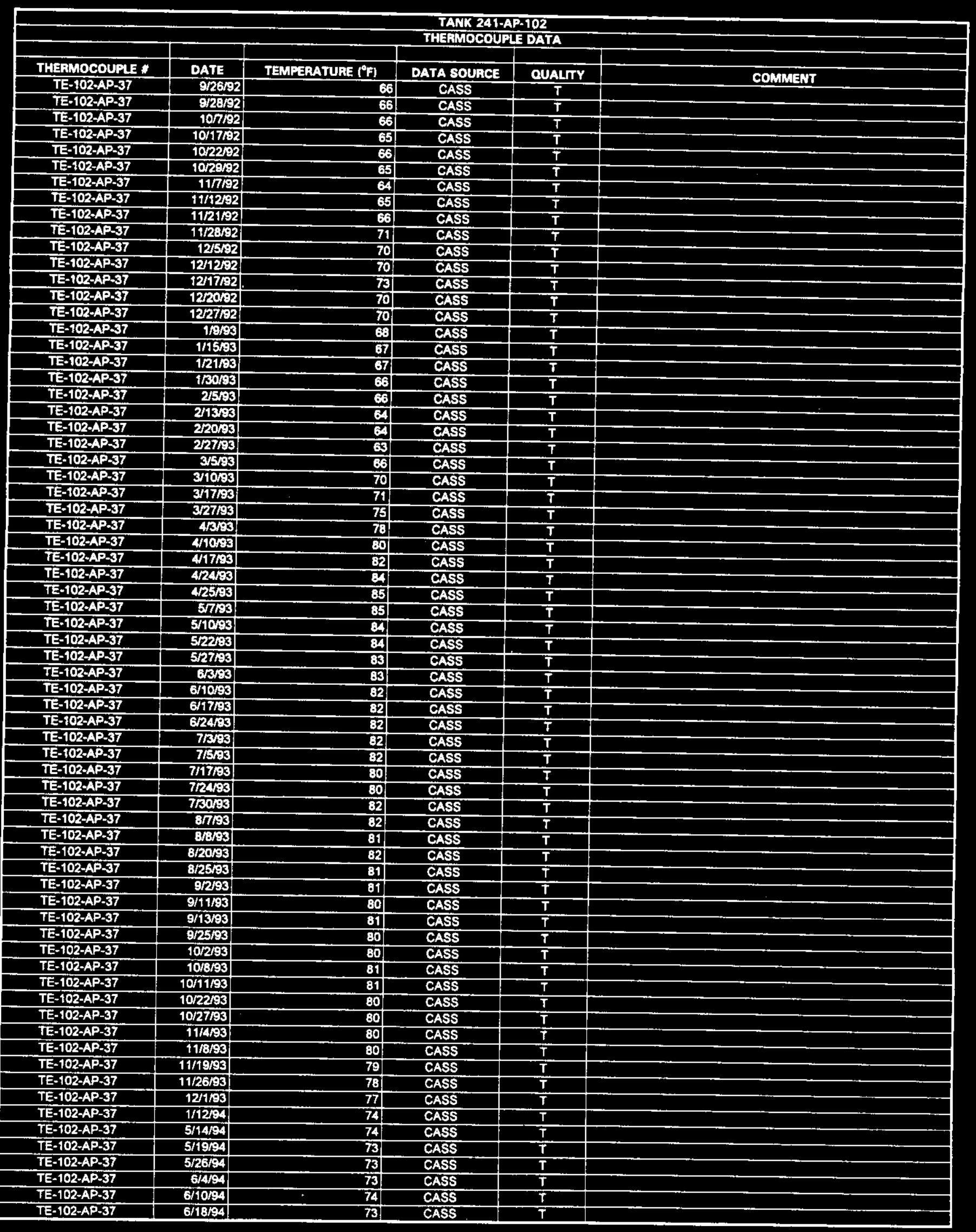




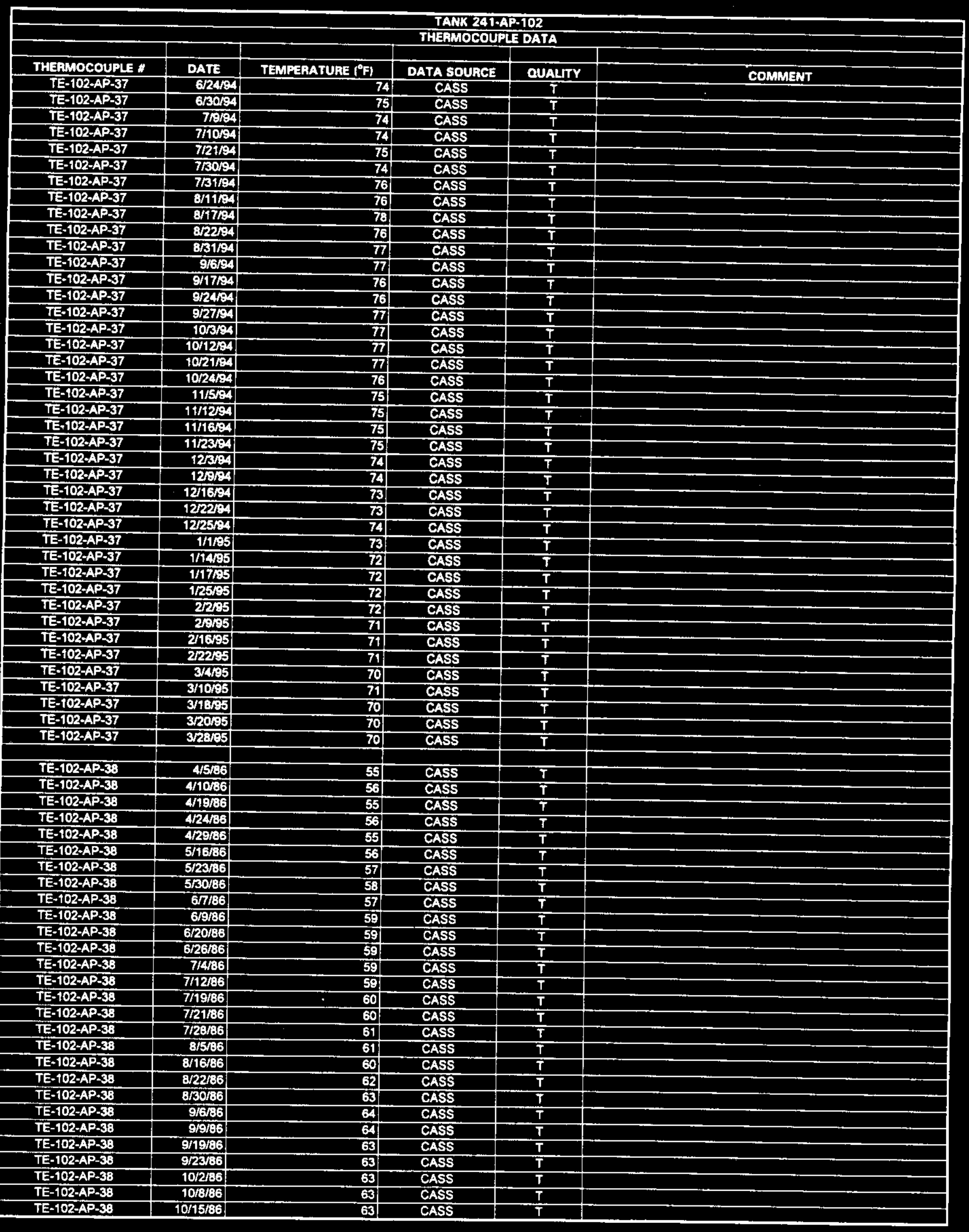

Data obtained from Computer Automated Surveillance System (CASS), April 1995; and Surveillance Analysis Computer Syatem (SACS), June 1995. 


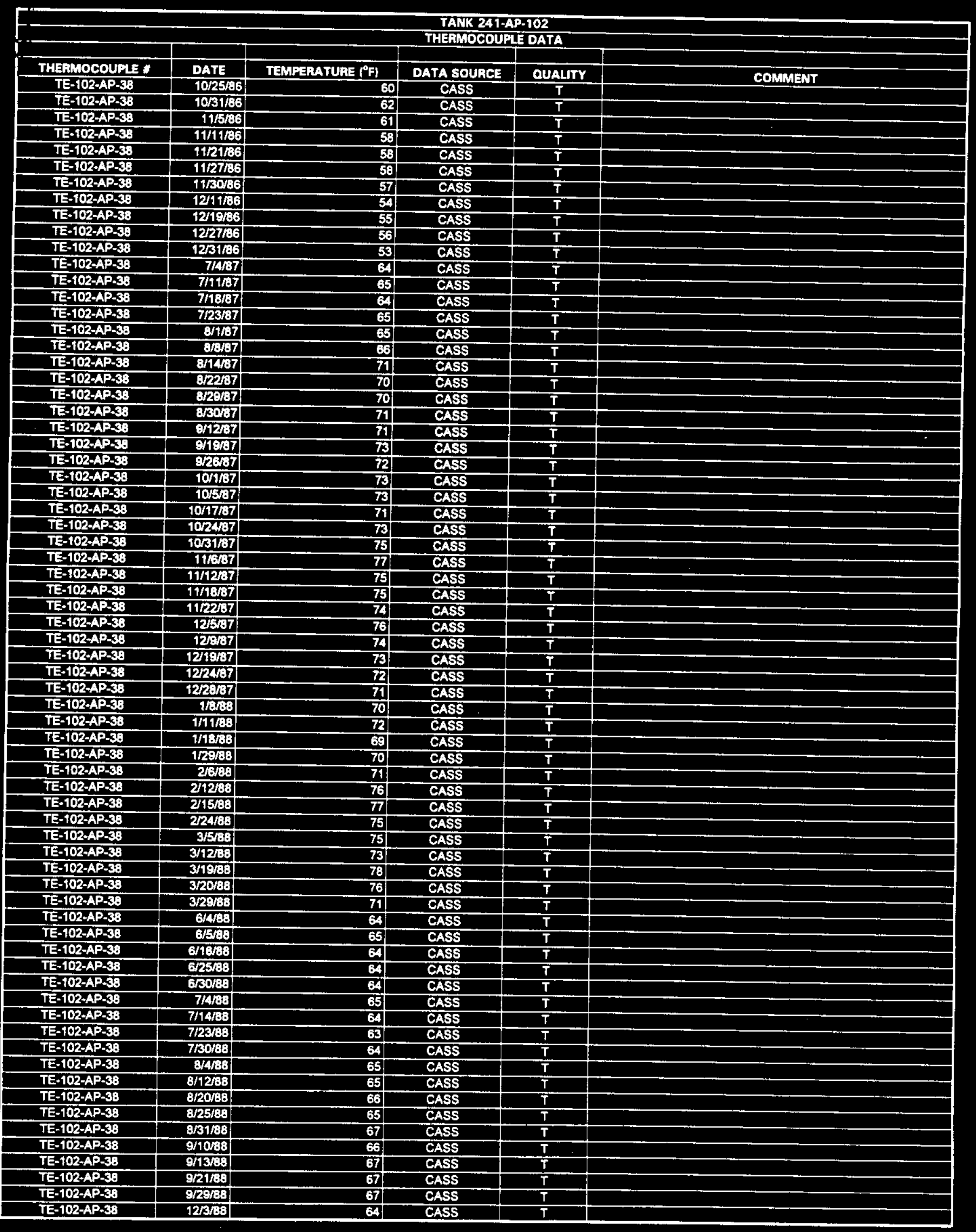




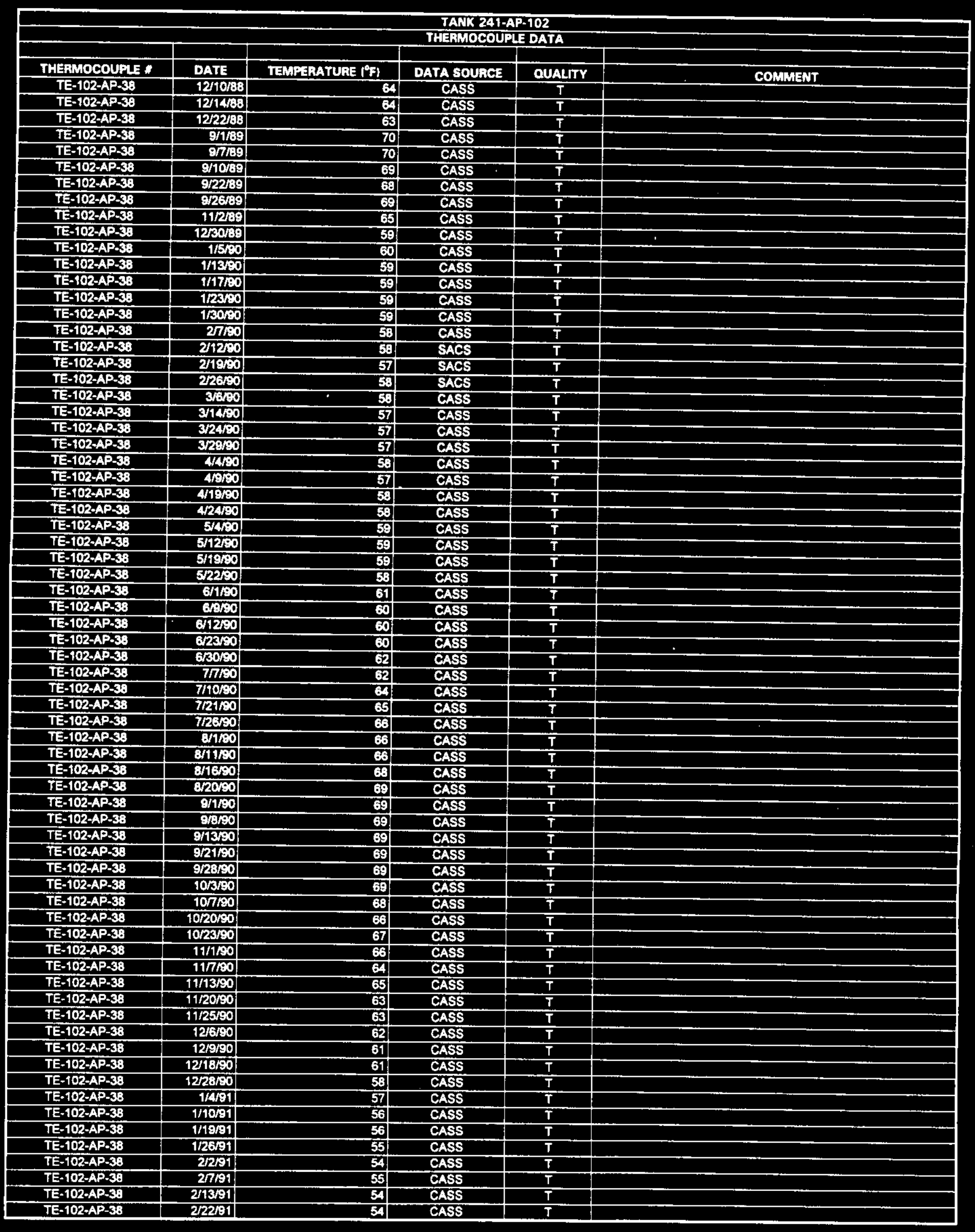




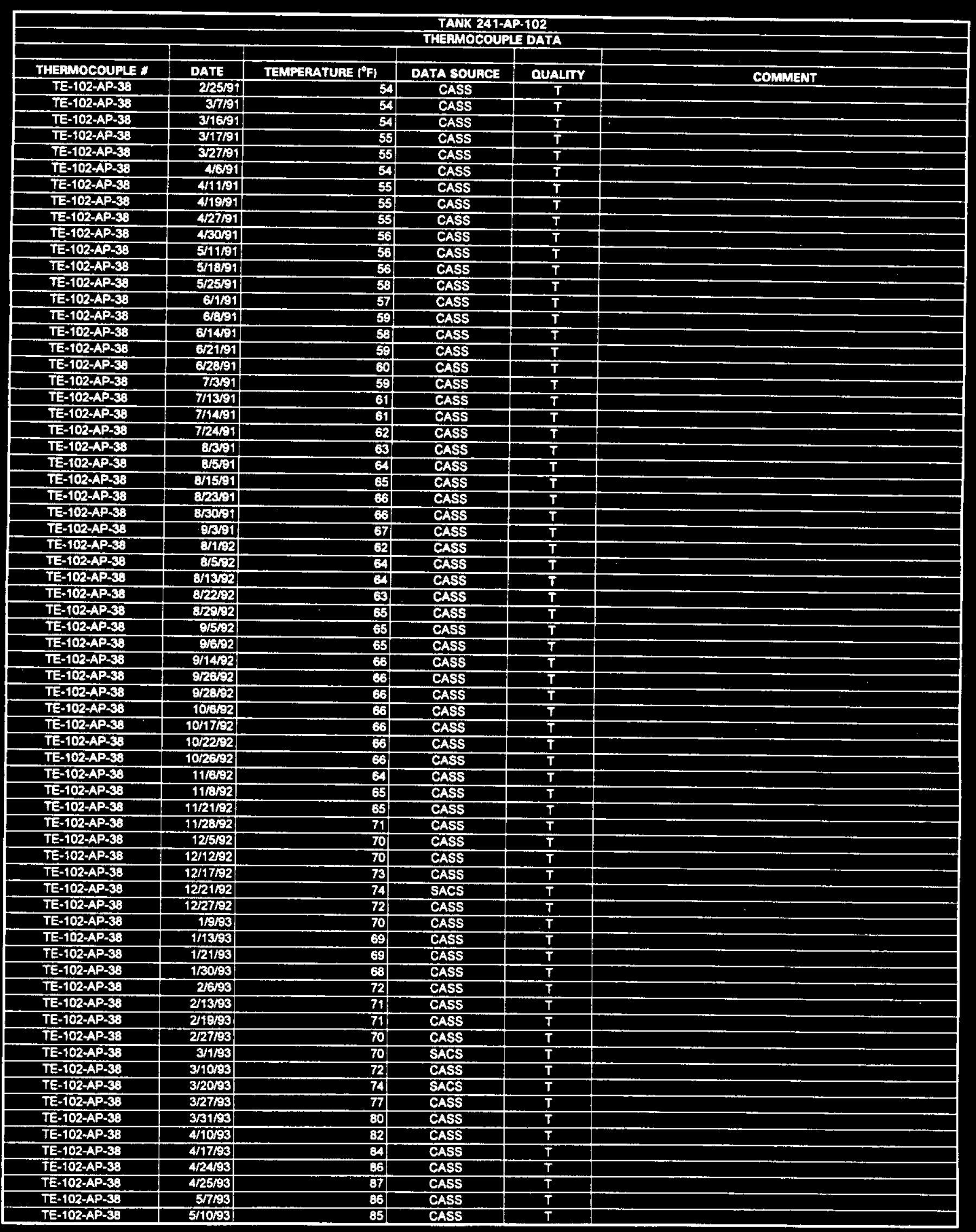




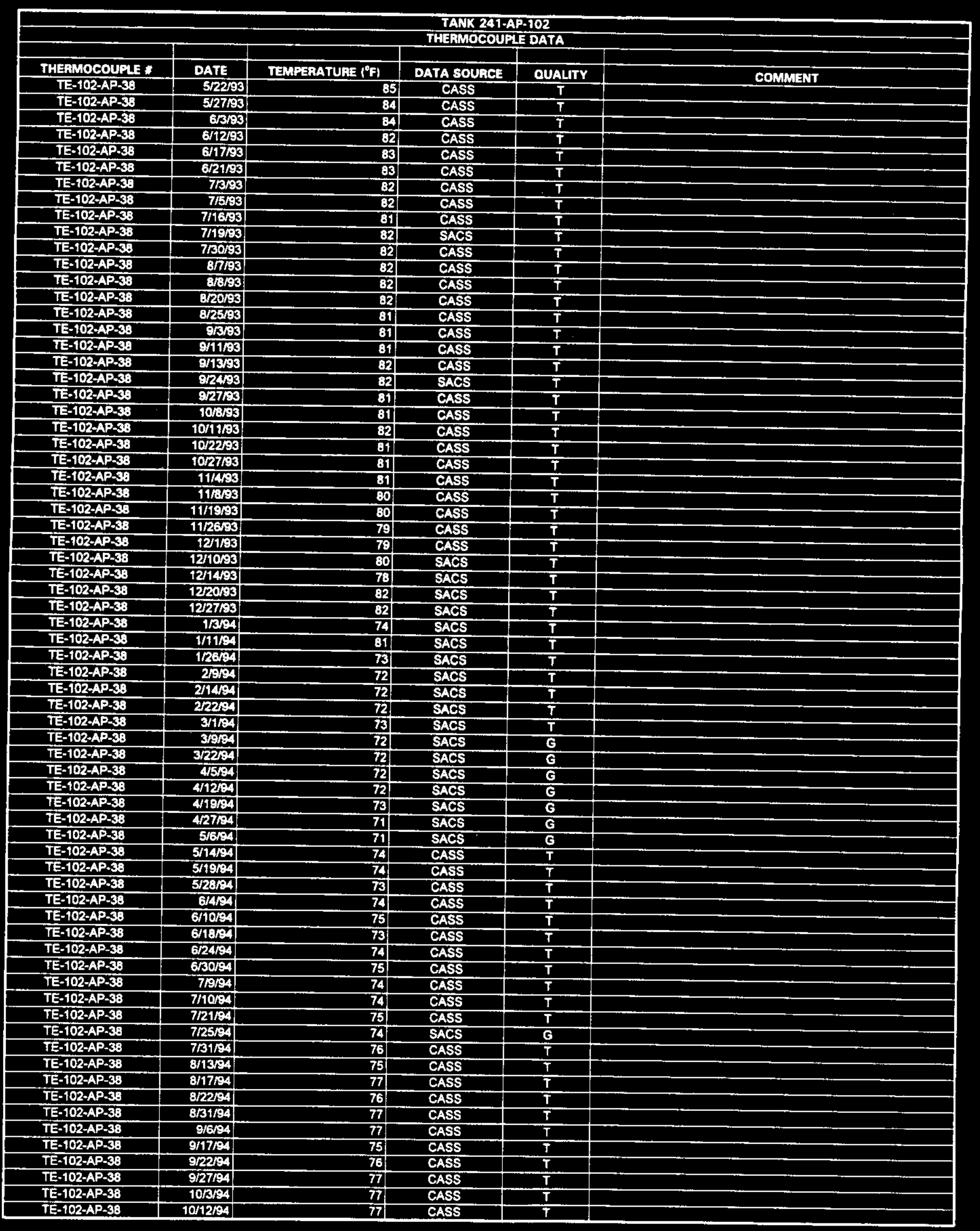




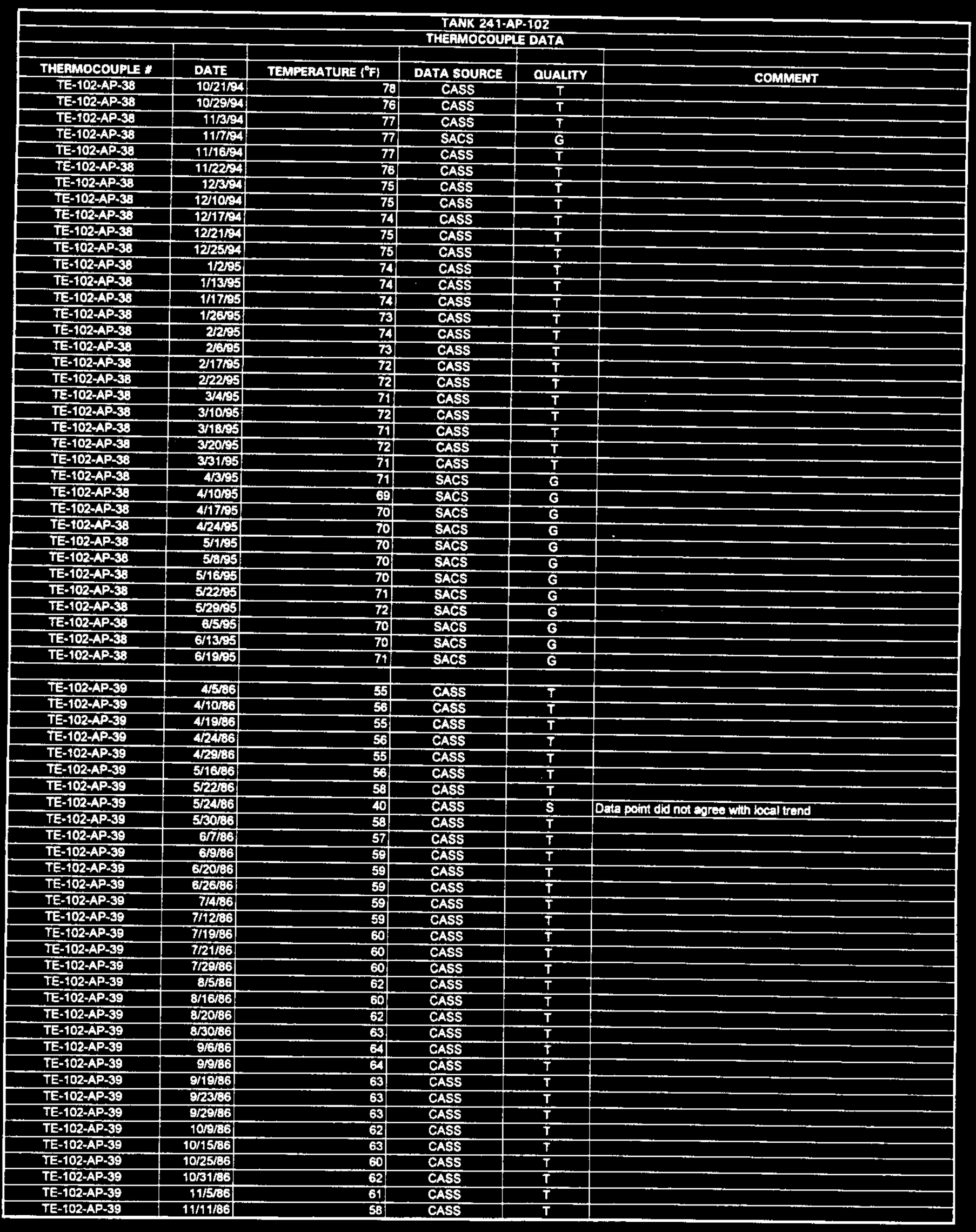




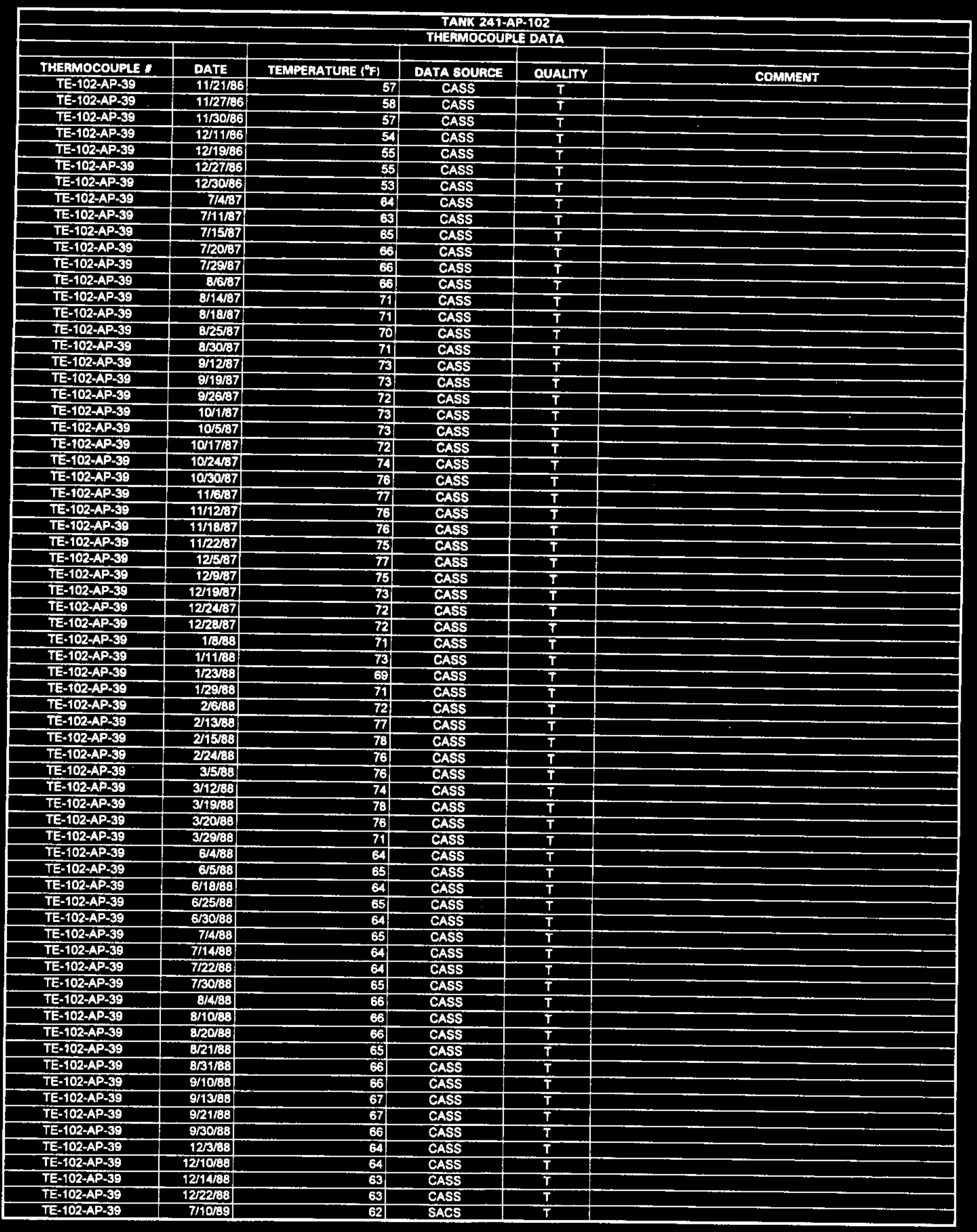




\begin{tabular}{|c|c|c|c|c|c|}
\hline \multicolumn{6}{|c|}{$\begin{array}{l}\text { TANK 241-AP*102 } \\
\text { THERMOCOUPLE DATA }\end{array}$} \\
\hline & & & & & \\
\hline THERMOCOUPLE * & DATE & TEMPERATURE $\left({ }^{\circ} \mathrm{F}\right)$ & DATA SOURCE & QUALITY & COMMENT \\
\hline TE-102-AP-39 & 717789 & 63 & SACS & $T$ & \\
\hline TE-102-AP-39 & $7 / 24 / 89$ & 64 & SACS & $T$ & \\
\hline TE-102-AP-39 & $8 / 7 / 89$ & 69 & SACS & $\mathbf{T}$ & \\
\hline TE-102-AP-39 & $8 / 14 / 89$ & 68 & SACS & $T$ & \\
\hline TE-102-AP-39 & $8 / 21 / 89$ & 68 & SACS & $\bar{T}$ & \\
\hline TE-102-AP-39 & $9 / 1 / 39$ & 70 & CASS & $T$ & \\
\hline TE-102-AP.39 & $8 \pi / 89$ & 70 & CASS & $T$ & \\
\hline TE-102-AP-39 & 9/10/89 & 69 & CASS & $T$ & \\
\hline TE-102-AP-39 & 9/20/89 & 69 & CASS & $\bar{T}$ & \\
\hline TE-102-AP-39 & 9/29/89 & 63 & CASS & $\bar{T}$ & \\
\hline TE-102-AP-39 & $10 / 2 / 89$ & 69 & SACS & $\mathbf{T}$ & \\
\hline TE-102-AP-39 & $10 / 8 / 89$ & 68 & SACS & $\mathbf{T}$ & \\
\hline TE-102-AP-39 & $10 / 1609$ & 66 & SACS & $T$ & \\
\hline TE-102-AP-39 & $10 / 26 / 69$ & 67 & SACS & $T$ & \\
\hline TE-102-AP-39 & $11 / 2 / 69$ & 65 & CASS & $\mathbf{T}$ & \\
\hline TE-102-AP-39 & $12 / 3069$ & 59 & CASS & $T$ & \\
\hline TE-102-AP-39 & $1 / 5 \mathrm{E} \theta 0$ & 60 & CASS & $\bar{T}$ & \\
\hline TE-102-AP-39 & $1 / 10 \%$ & 59 & CASS & $T$ & \\
\hline TE-102-AP-39 & $1 / 17 / 80$ & 59 & CASS & $\overline{\mathbf{T}}$ & \\
\hline TE-102-AP-39 & 1/23/50 & 59 & CASS & $\bar{T}$ & \\
\hline TE-102-AP-39 & $2 / 200$ & 58 & CASS & $T$ & \\
\hline TE-102-AP-39 & $2 \pi / 50$ & 58 & CASS & $T$ & \\
\hline TE-102-AP-38 & $217 \% 0$ & 57 & CASS & $T$ & \\
\hline TE-102-AP-39 & $2 / 23 / 20$ & 57 & CASS & $T$ & \\
\hline TE-102-AP-39 & $3 \sqrt{350}$ & 57 & CASS & $T$ & \\
\hline TE-102-AP-39 & $39 \%$ & 57 & CASS & $T$ & \\
\hline TE-102-AP-39 & $3 / 1450$ & 57 & CASS & $T$ & \\
\hline TE-102-APS39 & $3 / 2450$ & 57 & CASS & $T$ & \\
\hline TE-102-AP-39 & $3 / 2900$ & 57 & CASS & $T$ & \\
\hline TE-102-AP-39 & 4heo & 58 & CASS & $T$ & \\
\hline TE-102-AP-39 & 4800 & 57 & CASS & $T$ & \\
\hline TE-102-AP-39 & M15EO & 58 & CASS & $\mathbf{T}$ & \\
\hline TE-102-AP-39 & $4 / 23 / 50$ & 5 & CASS & $T$ & \\
\hline TE-102-AP-39 & 54190 & 59 & CASS & $\mathbf{T}$ & \\
\hline TE-102-AP-39 & 5/1250 & 59 & CASS & $T$ & \\
\hline TE-102-AP-35 & 511750 & 59 & CASS & $T$ & \\
\hline TE-102-AP-39 & $5 / 2250$ & S3! & CASS & $T$ & \\
\hline TE-102-AP-39 & $6 / 2100$ & 50 & CASS & $\mathbf{T}$ & \\
\hline TE-102-AP-39 & $6 / 9 \%$ & 60 & CASS & $T$ & \\
\hline TE-102.AP-39 & 6/1690 & 59 & CASS & $T$ & \\
\hline TE-102-AP-39 & 6/2150 & 61 & CASS & $T$ & . \\
\hline TE-102-AP-39 & 6/27150 & 63 & CASS & $T$ & \\
\hline TE-102-AP-38 & $7 / 7 / 00$ & 62 & CASS & $T$ & \\
\hline TE-102-AP-39 & 71090 & 64 & CASS & $T$ & \\
\hline TE-102-AP-39 & $7 / 21 / 90$ & 65 & CASS & $\mathbf{T}$ & \\
\hline TE-102-AP-39 & 7/26190 & 66 & CASS & $T$ & \\
\hline TE-102-AP-39 & $8 / 1900$ & 66 & CASS & $T$ & \\
\hline TE-102-AP-38 & $8 / 6 / 50$ & 67 & SACS & $T$ & \\
\hline TE-102-AP-39 & $8 / 16 \% 0$ & 68 & CASS & $\bar{T}$ & \\
\hline TE-102-AP-39 & $8 / 23 / 50$ & 68 & CASS & $T$ & \\
\hline TE-102-AP-39 & $9 / 1 / 90$ & 69. & CASS & $T$ & \\
\hline TE-102-AP-38 & 9/8/90 & 69 & CASS & $\mathbf{T}$ & \\
\hline TE-102-AP-39 & 9/13/80 & 69 & CASS & $T$ & \\
\hline TE-102-AP-39 & 9/16/90 & 70 & CASS & $T$ & \\
\hline TE-102-AP-39 & 9/23800 & 69 & CASS & $T$ & \\
\hline TE-102-AP-39 & $10 / 5 r 00$ & 68 & CASS & $T$ & \\
\hline TE-102-AP-39 & $10 / 7 / 50$ & 68 & CASS & $T$ & \\
\hline TE-102-AP-39 & $10 / 15 / 90$ & 68 & SACS & $T$ & \\
\hline TE-102-AP-39 & $10 / 23 / 90$ & 67 & CASS & $\mathbf{T}$ & \\
\hline TE-102-AP-39 & $10 / 29 / 90$ & 66 & CASS & $\bar{T}$ & \\
\hline TE-102-AP-39 & $11 / 7 / 90$ & 64 & CASS & $T$ & \\
\hline TE-102-AP-39 & $11 / 17 / 90$ & 64 & CASS & $T$ & \\
\hline TE-102-AP-39 & $11 / 19 / 90$ & 64 & SACS & $T$ & \\
\hline TE-102-AP-39 & $11 / 25 \% 90$ & 63 & CASS & $\bar{T}$ & \\
\hline TE-102-AP-39 & $12 / 6 / 90$ & 62 & CASS & $\bar{T}$ & \\
\hline TE-102-AP-39 & $12 / 10 / 90$ & 62 & SACS & $T$ & \\
\hline TE-102-AP.39 & 12/1900 & 61 & CASS & $T$ & \\
\hline TE-102-AP-39 & $12 / 28 / 90$ & 58 & CASS & $T$ & \\
\hline TE-102-AP-39 & $12 / 30 / 90$ & 57 & CASS & $T$ & \\
\hline TE-102-AP-39 & $1 / 12 / 91$ & 56 & CASS & $T$ & \\
\hline
\end{tabular}




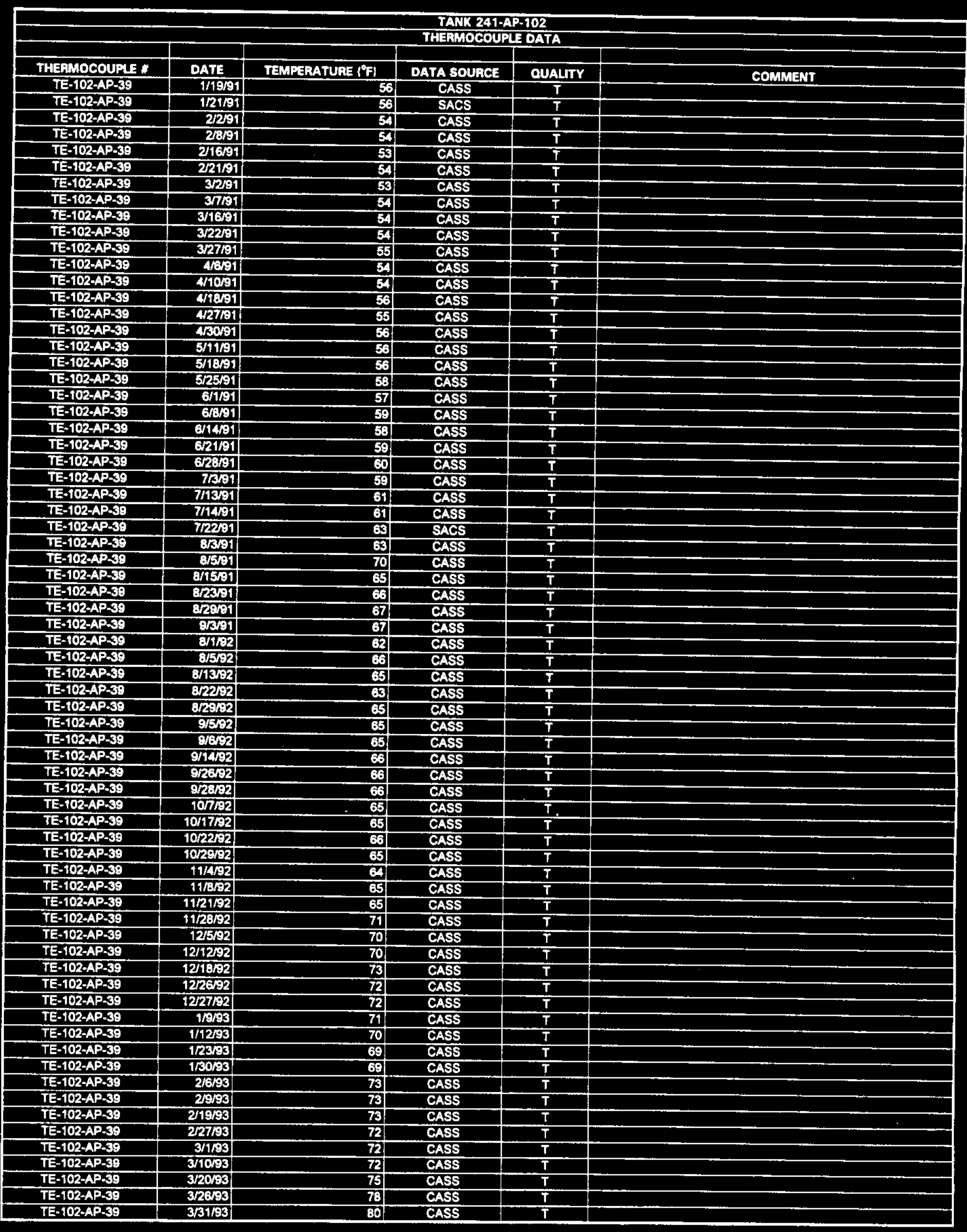

Data obtained from Computer Automated Survaillance System (CASS). April 1995; and Surveillance Analysis Computer System (SACS), June 1995. 


\begin{tabular}{|c|c|c|c|c|c|}
\hline \multicolumn{6}{|c|}{$\begin{array}{l}\text { TANK 241-AP-102 } \\
\text { THETMOCOUFL DATA }\end{array}$} \\
\hline & & & & & \\
\hline THERMOCOUPLE $*$ & Date & TEMPERATUAE $\left({ }^{\circ} \mathrm{F}\right)$ & DATA SOURCE & QUALITY & COMMENT \\
\hline TE.102-AP.39 & $4 / 6 / 93$ & 82 & CASS & $T$ & \\
\hline TE-102-AP-39 & $4 / 17 / 93$ & 84 & CASS & $\bar{T}$ & \\
\hline TE-102-AP-39 & $4 / 24 / 93$ & 86 & CASS & $T$ & \\
\hline TE-102-AP-39 & $4 / 2 5 \longdiv { 1 9 3 }$ & 87 & CASS & $T$ & \\
\hline TE-102-AP-39 & $5 / 7 / 93$ & 86 & CASS & $T$ & \\
\hline TE-102-AP-39 & $5 / 10 / 93$ & 85 & CASS & $\bar{T}$ & \\
\hline TE-102-AP-39 & $5 / 22 / 93$ & 85 & CASS & $T$ & \\
\hline TE-102-AP-39 & $5 / 27 / 93$ & 88 & CASS & $T$ & \\
\hline TE-102-AP-39 & $6 / 3993$ & 84 & CASS & $T$ & \\
\hline TE-102-AP-39 & $6 / 12 / 3$ & 82 & CASS & $T$ & \\
\hline TE-102-AP-39 & $6 1 5 \longdiv { 9 3 }$ & 85 & CASS & $T$ & \\
\hline TE-102-AP-39 & $6 / 21 / 93$ & 141 & CASS & $\mathbf{s}$ & Data point did not garee with local trend \\
\hline TE-102-AP-39 & $6 / 2403$ & 82 & CASS & $\bar{T}$ & \\
\hline TE-102-AP-39 & $7 / 3 / 53$ & 83 & CASS & $\mathbf{T}$ & \\
\hline TE-102-AP-39 & $7 / 5 / 93$ & 83 & CASS & $\mathbf{T}$ & \\
\hline TE-102-AP-39 & $716 \times 3$ & 81 & CASS & $T$ & \\
\hline TE-102-AP-39 & $7 / 22 / 93$ & 81 & CASS & $T$ & \\
\hline TE-102-AP-39 & $7 / 30 / 93$ & 82 & CASS & $T$ & \\
\hline TE-102-AP-39 & $8 / 403$ & 81 & CASS & $T$ & \\
\hline TE-102-AP-39 & $8 / 8 / 93$ & 82 & CASS & $T$ & \\
\hline TE-102-AP-39 & $8 / 48 / 93$ & 84 & CASS & $T$ & \\
\hline TE-102-AP-39 & $8 / 24 / 93$ & 83 & CASS & $T$ & \\
\hline TE-102-AP-39 & 8/253 & 97 & CASS & $\mathbf{S}$ & Drta point did not eqroes with local trend \\
\hline TE-102-AP-39 & Q/403 & 80 & CASS & $T$ & \\
\hline TE-102-AP-39 & $8 / 11 \ldots 3$ & 80 & CASS & $T$ & \\
\hline TE-102-AP-39 & 9/3/s3 & 85 & CASS & $T$ & \\
\hline TE-102-AP-39 & $9 / 25 \times 3$ & 80 & CASS & $T$ & \\
\hline TE-102-AP-39 & 9/27/:3 & 81 & CASS & $T$ & \\
\hline TE-102AP-39 & 10/ars & 82 & CASS & $\bar{T}$ & \\
\hline TE-102-AP-39 & $10 / 11 / 93$ & 82 & CASS & $T$ & \\
\hline TE-102-AP-39 & $10 / 2293$ & 81 & CASS & $T$ & \\
\hline TE-102-AP-39 & $10 / 27 / 93$ & 81 & CASS & $T$ & \\
\hline TE-102-AP-39 & $11 / 4 \sqrt{3}$ & 81 & CASS & $T$ & \\
\hline TE-102-AP-39 & $11 / \mathrm{Br} 3$ & 80 & CASS & $T$ & \\
\hline TE-102-AP-39 & 11/19/93 & 80 & CASS & $T$ & \\
\hline TE-102-AP-39 & $11 / 2693$ & 79 & CASS & $\bar{T}$ & \\
\hline TE-102-AP-39 & $12 / 3 / 93$ & 78 & CASS & $T$ & \\
\hline TE-102-AP-39 & 111254 & 76 & CASS & $T$ & \\
\hline TE-102-AP-39 & 5/12/ & 87 & CASS & $\mathbf{S}$ & Data point did not agree with local trend \\
\hline TE-102-AP-39 & 5/14/84 & 73 & CASS & $\bar{T}$ & \\
\hline TE-102-AP-39 & 5/19/94 & 74 & CASS & $T$ & \\
\hline TE-102-AP+39 & $5 / 26 \times 4$ & 73 & CASS & $\mathbf{T}$ & \\
\hline TE-102-AP-39 & $6 / 4 / 94$ & 74 & CASS & $\bar{T}$ & \\
\hline TE-102-AP-39 & 610/94 & 75 & CASS & $T$ & \\
\hline TE-102-AP-39 & $6 / 2 / 94$ & 74 & CASS & $T$ & \\
\hline TE-102-AP-39 & 6/2494 & 74 & CASS & $T$ & \\
\hline TE-102-AP-39 & 6/2654 & 87 & CASS & $\mathbf{s}$ & Data point did not sgree with local frend \\
\hline TE-102-AP-39 & $6 / 30 / 94$ & 75 & CASS & $T$ & (2) \\
\hline TE-102-AP-39 & $74 / 94$ & 82 & CASS & $T$ & \\
\hline TE-102-AP-39 & $7 / 10 / 94$ & 74 & CASS & $T$ & \\
\hline TE-102-AP-39 & 7/20194 & 75 & CASS & $\mathbf{T}$ & \\
\hline TE-102-AP-39 & $7 / 31 / 94$ & 76 & CASS & $T$ & \\
\hline TE-102-AP-39 & $8 / 11 / 94$ & 87 & CASS & $\mathbf{S}$ & Data point did not agree with local trend \\
\hline TE-102-AP-39 & $8 / 13194$ & 75 & CASS & $T$ & \\
\hline TE-102-AP-39 & $8 / 17 / 94$ & 77 & CASS & $\mathbf{T}$ & \\
\hline TE-102-AP-39 & $8 / 2294$ & 77 & CASS & $\mathbf{T}$ & \\
\hline TE-102-AP-39 & $8 / 28 / 94$ & 86 & CASS & $\mathbf{S}$ & Data point did not agree with local trend \\
\hline TE+102-AP-39 & 8/31/94 & 82 & CASS & $\mathbf{T}$ & \\
\hline TE-102-AP-39 & $9 / 6 / 94$ & 77 & CASS & $\mathbf{T}$ & \\
\hline TE-102-AP-39 & $9 / 17 / 94$ & 76 & CASS & $T$ & \\
\hline TE-102-AP-39 & $9 / 23194$ & 76 & CASS & $T$ & \\
\hline TE-102-AP-39 & $9 / 27 / 94$ & 77 & CASS & $T$ & \\
\hline TE-102-AP-39 & $10 / 3 / 94$ & 77 & CASS & $T$ & \\
\hline TE-102-AP-39 & 10/1294 & 77 & CASS & $T$ & \\
\hline TE-102-AP-39 & $10 / 21 / 94$ & 77 & CASS & $\bar{T}$ & \\
\hline TE-102-AP-39 & $10 / 24 / 94$ & 77 & CASS & $\bar{T}$ & \\
\hline TE-102-AP-39 & $11 / 3 / 94$ & 77 & CASS & $T$ & \\
\hline TE-102-AP-39 & $11 / 12 / 94$ & 76 & CASS & $\mathbf{T}$ & \\
\hline TE-102-AP-39 & $11 / 16 / 94$ & 77 & CASS & $T$ & \\
\hline TE-102-AP-39 & $11 / 20 / 94$ & 77 & CASS & $\bar{T}$ & \\
\hline
\end{tabular}




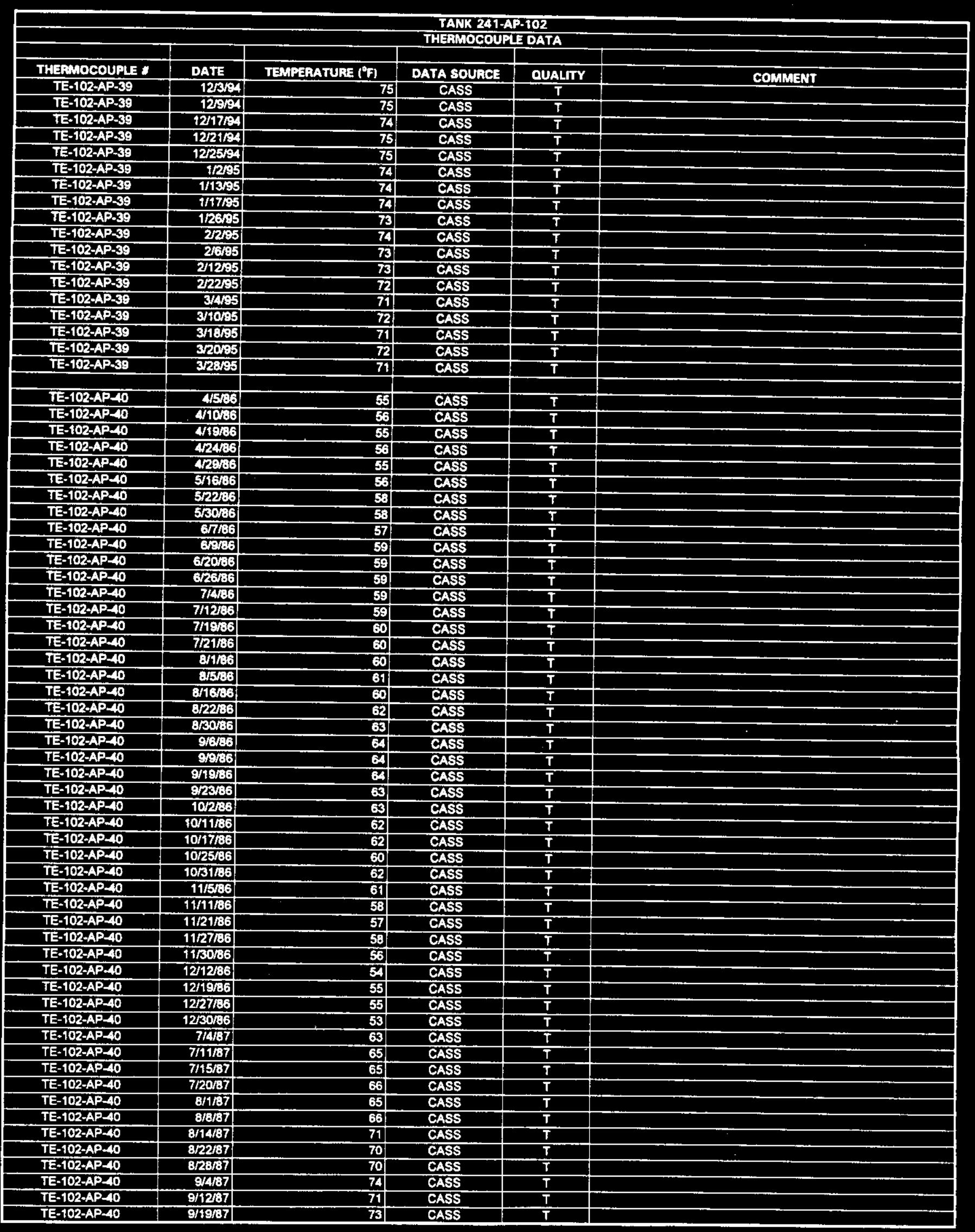




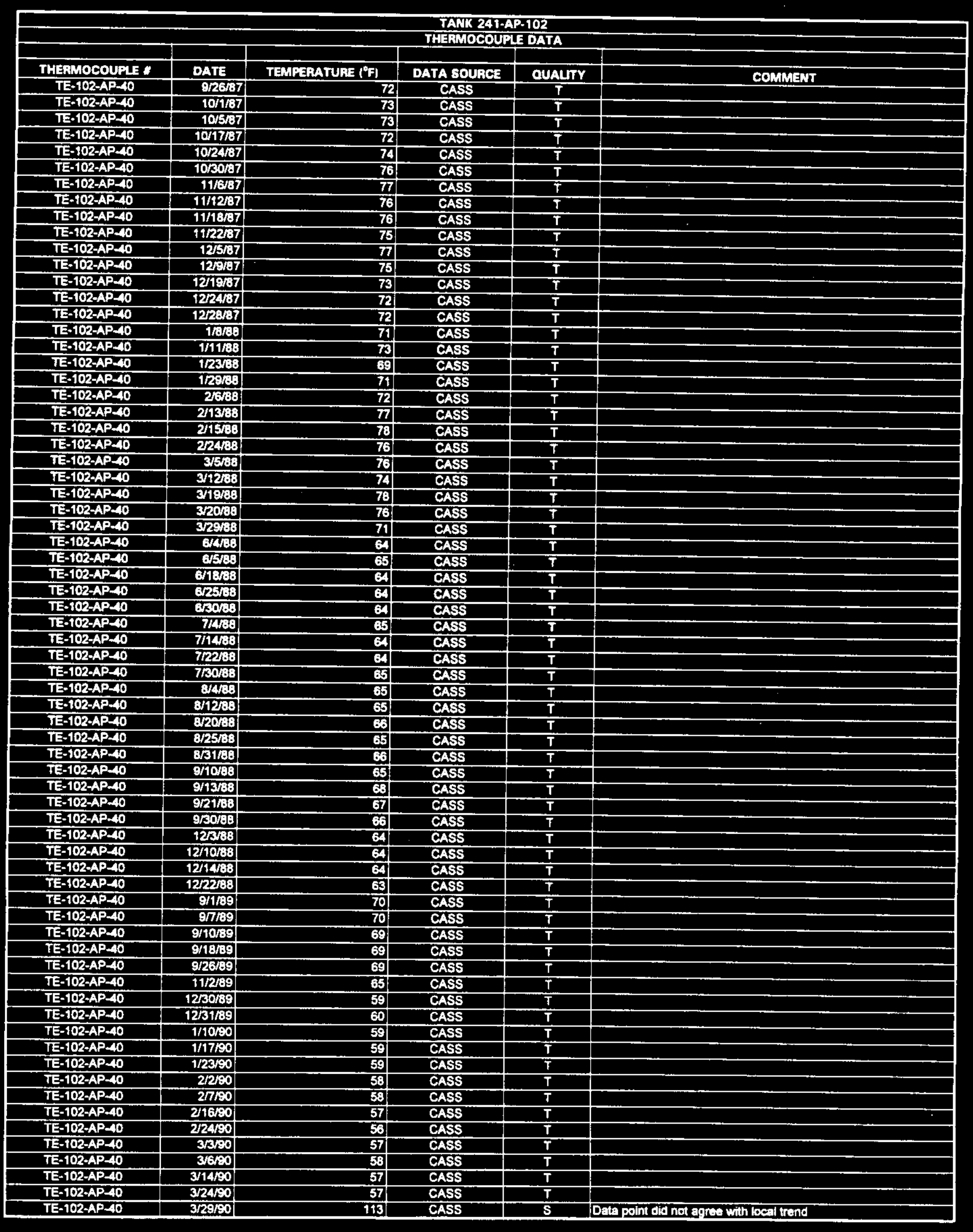




\begin{tabular}{|c|c|c|c|c|c|}
\hline \multicolumn{6}{|c|}{$\begin{array}{c}\text { TAMK 241-AP-102 } \\
\text { THERMOCOUPLE DATA }\end{array}$} \\
\hline THEAMOCOUPLE * & DATE & TEMPEAATUAE ( $\left.{ }^{\circ} \mathrm{F}\right)$ & DATA SOURCE & QUALITY & COMMENT \\
\hline TE-102-AP-40 & $3 / 31 / 90$ & 56 & CASS & $T$ & \\
\hline TE-102-AP-40 & $4 / 4 / 90$ & 58 & CASS & $T$ & \\
\hline TE-102-AP-40 & 4/990 & 57 & CASS & $T$ & \\
\hline TE-102-AP-40 & 415150 & 58 & CASS & $T$ & \\
\hline$T E-102-A P-40$ & $4 / 27 / 100$ & 63 & CASS & $T$ & \\
\hline$T E-102-A P-40$ & 5400 & 39. & CASS & $T$ & \\
\hline TE-102-AP-40 & $5 / 1250$ & 59 & CASS & $T$ & \\
\hline$T E-102-A P-40$ & 517/s0 & 59 & CASS & $T$ & \\
\hline$T E-102-A P-40$ & $5 / 22190$ & 58 & CASS & $T$ & \\
\hline$T E-102-A P-40$ & $6 / 1 / 90$ & 80 & CASS & $T$ & \\
\hline TE-102-AP-40 & G/9/90 & 60 & CASS & $T$ & \\
\hline$T E-102-A P-40$ & 616/90 & 59 & CASS & $\mathbf{T}$ & \\
\hline TE-102-AP-40 & $6 \sqrt{2350}$ & 60 & CASS & $T$ & \\
\hline TE-102-AP-40 & $6 / 25: 00$ & 63 & CASS & $T$ & \\
\hline$T E-102-A P-40$ & $7 / 6 / 00$ & 62 & CASS & $\mathbf{T}$ & \\
\hline TE-102-AP-40 & $7 / 10=0$ & 64 & CASS & $\mathbf{T}$ & \\
\hline TE-102-AP-40 & $7 / 21 / 00$ & 65 & CASS & $T$ & \\
\hline TE-102-AP-40 & $7 / 26 / 90$ & 66 & CASS & $T$ & \\
\hline$T E-102-A P-40$ & $8 / 100$ & 66 & CASS & $T$ & \\
\hline TE-102-AP-40 & $8 / 1190$ & 66 & CASS & $T$ & \\
\hline TE-102-AP-40 & 8/1690 & 68 & CASS & $\bar{T}$ & \\
\hline TE-102-AP-40 & $8 / 2000$ & 69 & CASS & $T$ & \\
\hline TE-102-AP-10 & 91100 & 69 & CASS & $T$ & \\
\hline TE-102-AP-40 & 9860 & 68 & CASS & $T$ & \\
\hline TE-102-AP-40 & 9/13/90 & 69 & CASS & $T$ & \\
\hline$T E-102-A P=40$ & $9 / 21 / 00$ & 69 & CASS & $T$ & \\
\hline TE-102-AP-40 & $9 / 25100$ & 69 & CASS & $T$ & \\
\hline TE-102-AP-40 & $105 \times 20$ & 68 & CASS & $T$ & \\
\hline TE-102-AP-40 & $107 \times 0$ & 83 & CASS & $\bar{T}$ & \\
\hline TE-102-AP-40 & $10 / 19 / 00$ & 66: & CASS & $T$ & \\
\hline$T E-102-A P-40$ & tor2350 & 67 & CASS & $T$ & \\
\hline TE-102-AP-40 & $10 / 29190$ & 66 & CASS & $T$ & \\
\hline$T E-102-A P-40$ & $11 / \pi \infty 0$ & 64 & CASS & $T$ & \\
\hline TE-102-AP-40 & $11 / 17 / 00$ & 64 & CASS & $T$ & \\
\hline TE-102-AP-40 & $11 / 2480$ & 62 & CASS & $T$ & \\
\hline TE-102-AP-40 & $11 / 25 / 80$ & 63 & CASS & $T$ & \\
\hline TE-102-AP-10 & $12 / 6 / 80$ & 62 & CASS & $T$ & \\
\hline$T E-102-4 P-40$ & $12 \mathrm{NSOO}$ & 61 & CASS & $\mathbf{T}$ & \\
\hline TE-102-AP-40 & $12 / 18 / 50$ & 61 & CASS & $T$ & \\
\hline TE-102-AP-40 & $12 / 2890$ & 5 & CASS & $T$ & \\
\hline TE-102-AP-40 & $12 / 30 \% 0$ & 57 & CASS & $T$ & \\
\hline TE-102-AP-40 & $1 \pi m$ & 56 & CASS & $T$ & \\
\hline TE-102-AP=40 & 1/19/91 & 56 & CASS & $T$ & \\
\hline TE-102-AP-40 & $1 / 26 / 91$ & 55 & CASS & $T$ & \\
\hline$T E \cdot 102-A P-40$ & I/28/91 & 54 & CASS & $T$ & \\
\hline$T E-102-A P-40$ & $2 \pi / 91$ & 54 & CASS & $T$ & \\
\hline$T E-102-A P-40$ & $2 / 16 / 91$ & 53 & CASS & $T$ & \\
\hline$T E-102-A P-40$ & $2 / 21 / 91$ & 54 & CASS & $\mathbf{T}$ & \\
\hline TE-102-AP-40 & $3 / 2 / 91$ & 53 & CASS & $T$ & \\
\hline TE-102-AP-40 & $3 / 6 / 91$ & 54 & CASS & $T$ & \\
\hline$T E-102+A P-40$ & $3 / 16 / 91$ & 54 & CASS & $T$ & \\
\hline TE-102-AP-40 & $3 / 21 / 91$ & 54 & CASS & $T$ & \\
\hline TE-102-AP-40 & 3/30191 & 54 & CASS & $T$ & \\
\hline$T E-102+A P-10$ & 4/6/91 & 53 & CASS & $T$ & \\
\hline TE-102-AP-40 & $4 / 10 / 91$ & 54 & CASS & $\mathbf{T}$ & \\
\hline$T E-102-A P-40$ & 4/19/91 & 55 & CASS & $T$ & \\
\hline IE-102-AP-40 & $4 / 27 / 91$ & 55 & CASS & $T$ & \\
\hline TE-102-AP-40 & 4/30/91 & 56 & CASS & $T$ & \\
\hline$T E-102-A P-40$ & $5 / 11 / 91$ & 56 & CASS & $T$ & \\
\hline$T E-102-A P-40$ & $5 / 15 \times 91$ & 56 & CASS & $\mathbf{T}$ & \\
\hline TE-102-AP=40 & $5 / 25 / 91$ & 57 & CASS & $\mathbf{T}$ & \\
\hline TE-102-AP-40 & 6/1/91 & 57 & CASS & $T$ & \\
\hline TE-102-AP-40 & 6/8/91 & 59 & CASS & $T$ & \\
\hline TE-102-AP-40 & $6 / 14 / 91$ & 58 & CASS & $T$ & \\
\hline TE-102-AP-40 & $6 / 21 / 91$ & 59 & CASS & $T$ & \\
\hline$T E-102-A P-40$ & $6 / 28 / 91$ & 60 & CASS & $T$ & \\
\hline TE-102-AP-40 & 7/3/91 & 59 & CASS & $T$ & \\
\hline$T E-102-A P-40$ & $7 / 13 / 91$ & 61 & CASS & $T$ & \\
\hline TE-102-AP-40 & $7 / 14191$ & 61 & CASS & $T$ & \\
\hline TE-102-AP-40 & $7 / 24 / 91$ & 62 & CASS & $T$ & \\
\hline
\end{tabular}




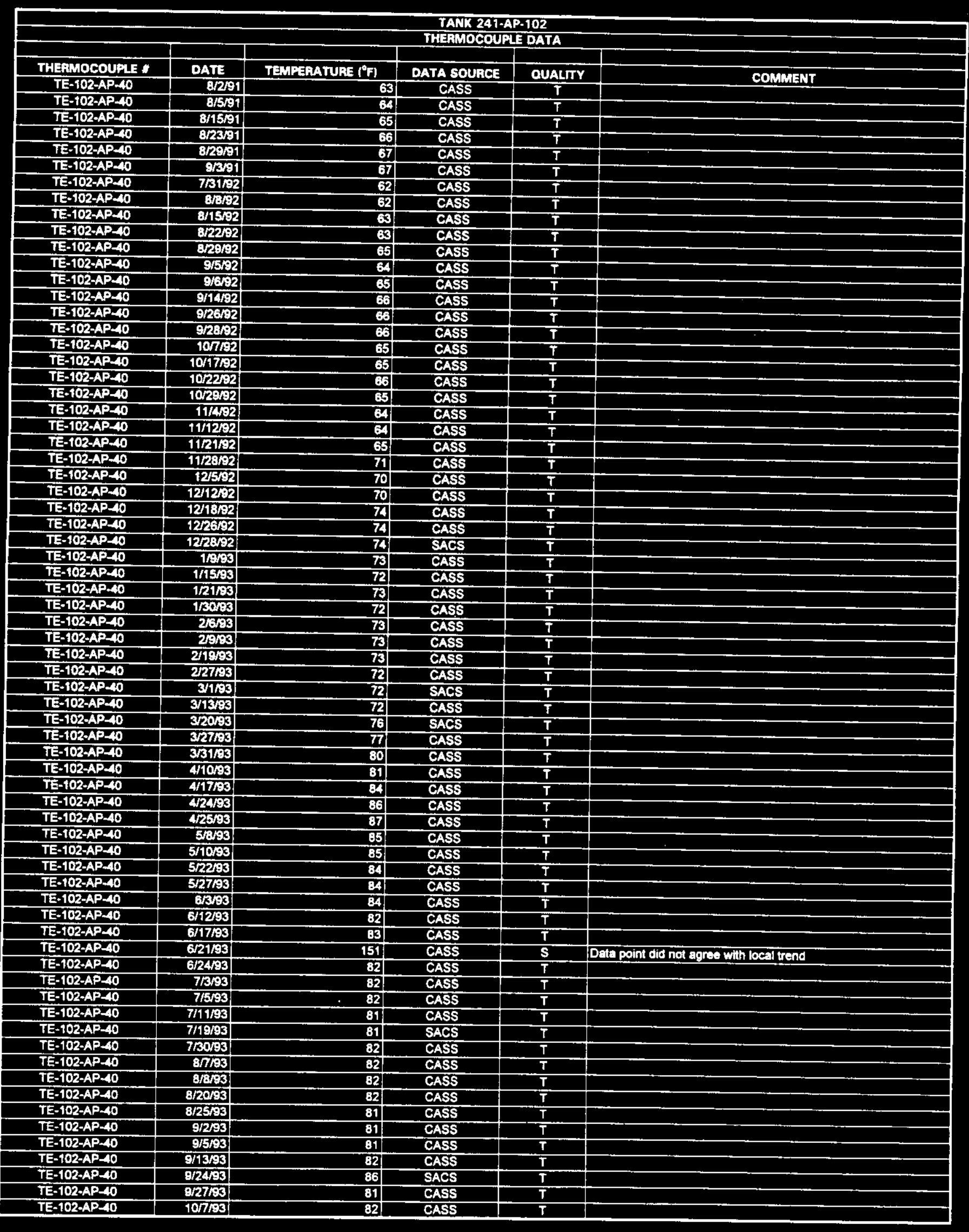

Data obtained trom Computer Automated Surveillance Syatem (CASS), April 1995; and Surveillance Analysis Compurer System (SACS), Juna 1995. D-168 


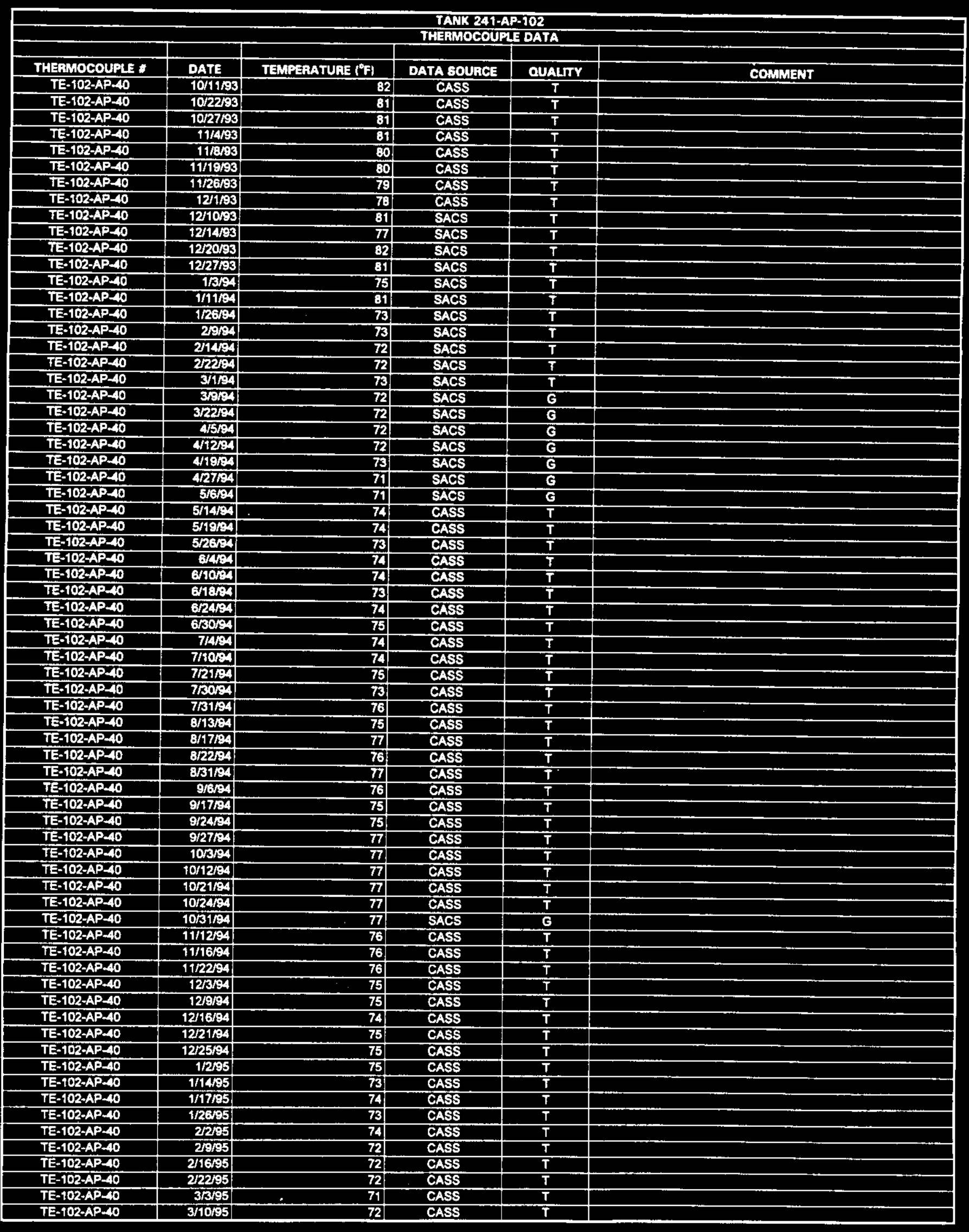




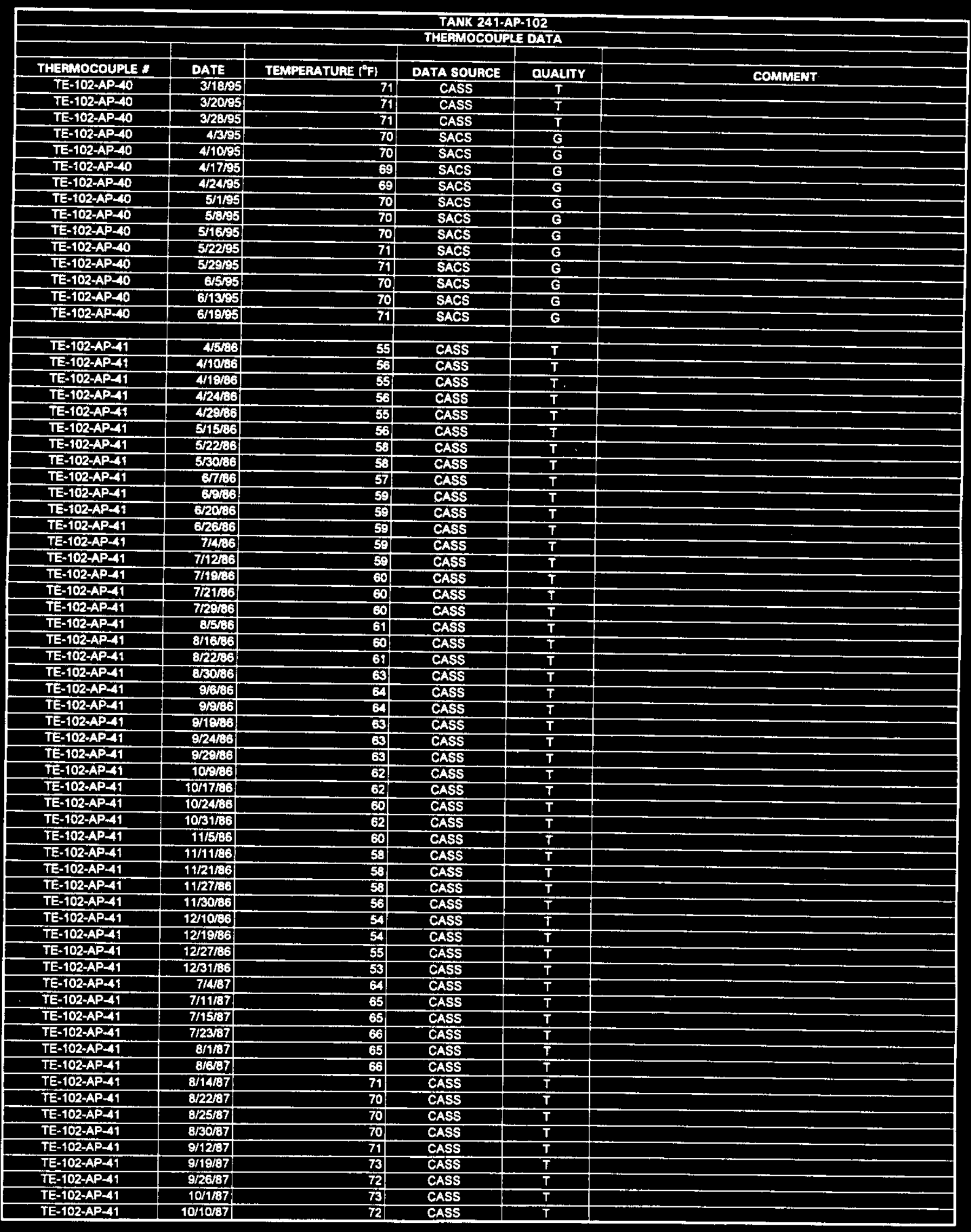




\begin{tabular}{|c|c|c|c|c|c|}
\hline \multicolumn{6}{|c|}{$\begin{array}{c}\text { TANK 241.AP-102 } \\
\text { THJFIMOCOUPLE DATA }\end{array}$} \\
\hline & & & & & \\
\hline THEPMOCOUPL * & DATE & TEMPERATURE $\left({ }^{\circ} F\right)$ & DATA SOUACE & OUALITY & COMMENT \\
\hline TE-102-AP-41 & $10 / 17 / 87$ & 72 & CASS & $T$ & \\
\hline TE-102-AP-41 & $10 / 24 / 87$ & 74 & CASS & $T$ & \\
\hline TE-102-AP-41 & 10/30/87 & 77 & CASS & $\bar{T}$ & \\
\hline TE-102-AP-A1 & $11 / 5 / 87$ & 77 & CASS & $T$ & \\
\hline TE-102-AP-41 & $11 / 12 / 87$ & 76 & CASS & $T$ & \\
\hline TE-102-AP-41 & $11 / 18 / 67$ & 76 & CASS & $T$ & \\
\hline TE-102-AP-41 & $14 / 22 / 37$ & 75 & CASS & $T$ & \\
\hline$T E-102-A P-41$ & $12 / 5 / 87$ & 77 & CASS & $T$ & \\
\hline TE-102-AP-41 & $125 / 67$ & 75 & CASS & $T$ & \\
\hline TE-102-AP-41 & $12 / 1987$ & 74 & CASS & $T$ & \\
\hline TE-102-AP-41 & $12 / 24 / 87$ & 72 & CASS & $T$ & \\
\hline TE-102-AP-41 & $12 / 29 / 87$ & 72 & CASS & $T$ & \\
\hline$T E-102-A P-41$ & $1 / 3 / 38$ & 71 & CASS & $T$ & \\
\hline TE-102-AP-41 & $1 / 12 / 88$ & 71 & CASS & $T$ & \\
\hline TE-102-AP-41 & 1/18/68 & 70 & CASS & $\mathbf{T}$ & \\
\hline$T E-102-A P-41$ & $1 / 29138$ & 70 & CASS & $T$ & \\
\hline$T E-102-A P-41$ & $26 / 8$ & 73 & CASS & $T$ & \\
\hline TE-102-AP-41 & $2 / 138$ & 77 & CASS & $T$ & \\
\hline$T E-102-A P-41$ & $2 / 1538$ & 77 & CASS & $T$ & \\
\hline TE-102-AP-41 & $2 / 24 / 38$ & 76 & CASS & $T$ & \\
\hline $\mathrm{TE}-102-\mathrm{AP}=11$ & 3/S/B8 & 76 & CASS & $T$ & \\
\hline TE-102-AP-41 & 3/2/38 & 74 & CASS & $T$ & \\
\hline$T E-102-A P-41$ & 3/968 & 78 & CASS & $\mathbf{T}$ & \\
\hline TE-102-AP-41 & $3 / 20 / 88$ & 76 & CASS & $\mathbf{T}$ & \\
\hline TE-102-AP-41 & $3 / 2988$ & 70 & CASS & $T$ & \\
\hline TE-102-AP-41 & 6/388 & 64 & CASS & $T$ & \\
\hline$T E-102-A P-41$ & 658B & 65 & CASS & $T$ & \\
\hline TE-102-AP-A1 & Grabs & 64 & CASS & $T$ & \\
\hline TE-102-AP-41 & 62563 & 64 & CASS & $T$ & \\
\hline IE-102-AP-41 & erares & 64 & CASS & $T$ & \\
\hline$T E-102-A P-41$ & 74488 & 65 & CASS & $T$ & \\
\hline$T E-102-A P-41$ & $7 / 10 / 88$ & 65 & CASS & $T$ & \\
\hline TE-102-AP-41 & $7 / 23 / 88$ & 63 & CASS & $T$ & \\
\hline TE-102-AP-41 & $7 / 00 / 88$ & 64 & CASS & $T$ & \\
\hline TE-102-AP-41 & $8 / 48 B$ & 65 & CASS & $T$ & \\
\hline TE-102-AP-41 & $8 / 12 / 88$ & 65 & CASS & $T$ & \\
\hline TE-102-AP-41 & 8/2008 & 66 & CASS & $T$ & \\
\hline TE-102-AP-41 & 8/21/89 & 65 & CASS & $T$ & \\
\hline$T E-102-A P-41$ & Bzars & 112 & CASS & $\mathrm{s}$ & Dota point did not agree with local trend \\
\hline TE-102-AP-41 & $8 / 31 / 88$ & 66 & CASS & $T$ & \\
\hline TE-102-AP-41 & 9/10/36 & 65 & CASS & $T$ & \\
\hline TE-102-AP-41 & 9/1308 & 67 & CASS & $T$ & \\
\hline TE-102-AP-41 & $9 / 2488$ & 65 & CASS & $T$ & \\
\hline$T E-102-A P-41$ & $9 / 30 / 38$ & 66 & CASS & $T$ & \\
\hline TE-102-AP-41 & 12Kusa & 64 & CASS & $T$ & \\
\hline TE-102-AP-41 & $12 / 10 / 88$ & 65 & CASS & $T$ & \\
\hline TE-102-AP-41 & $12 / 14 / 88$ & 64 & CASS & $T$ & \\
\hline TE-102-AP-41 & $12 / 18 / 38$ & 63 & CASS & $T$ & \\
\hline TE-102-AP-41 & $710 / 39$ & 62 & SACS & $\mathrm{T}$ & \\
\hline TE-102.AP-41 & 711789 & 63! & SACS & $T$ & \\
\hline$T E-102-A P-41$ & $7 / 24189$ & 63 & SACS & $T$ & \\
\hline$T E-102-A P-41$ & $87 / 89$ & 69 & SACS & $T$ & \\
\hline TE-102-AP-41 & $8 / 14 / 89$ & 68 & SACS & $T$ & \\
\hline TE-102-AP-41 & $8 / 21 / 89$ & 68 & SACS & $T$ & \\
\hline TE-102-AP-41 & $9 / 1 / 39$ & 69 & CASS & $\mathbf{T}$ & \\
\hline TE-102-AP-41 & \%/4/89 & 70 & CASS & $T$ & \\
\hline TE-102-AP-41 & $9 / 10 / 89$ & 69 & CASS & $T$ & \\
\hline TE-102-AP-41 & 9/20/39 & 68 & CASS & $T$ & \\
\hline$T E-102-A P-41$ & $9 / 29 / 89$ & 68 & CASS & $T$ & \\
\hline TE-102-AP-41 & $10 / 2 / 89$ & 68 & SACS & $T$ & \\
\hline TE-102-AP-41 & $10 / 9 / 89$ & 66 & SACS & $T$ & \\
\hline$T E-102-A P-41$ & $10 / 16 / 89$ & 66 & SACS & $T$ & \\
\hline TE-102-AP-41 & $10 / 26 / 89$ & 66 & SACS & $T$ & \\
\hline TE-102-AP-41 & $11 / 2 / 89$ & 64 & CASS & $T$ & \\
\hline TE-102-AP-41 & $12 / 30 / 89$ & 59 & CASS & $T$ & \\
\hline TE-102-AP-41 & $1 / 5 / 90$ & 60 & CASS & $T$ & \\
\hline TE-102-AP-41 & $1 / 13 / 90$ & 59 & CASS & $T$ & \\
\hline TE-102-AP-41 & $1 / 20190$ & 59 & CASS & $T$ & \\
\hline TE-102-AP-41 & $1 / 2490$ & 59 & CASS & $T$ & \\
\hline$T E-102-A P-41$ & $2 / 2190$ & 58 & CASS & $T$ & \\
\hline
\end{tabular}




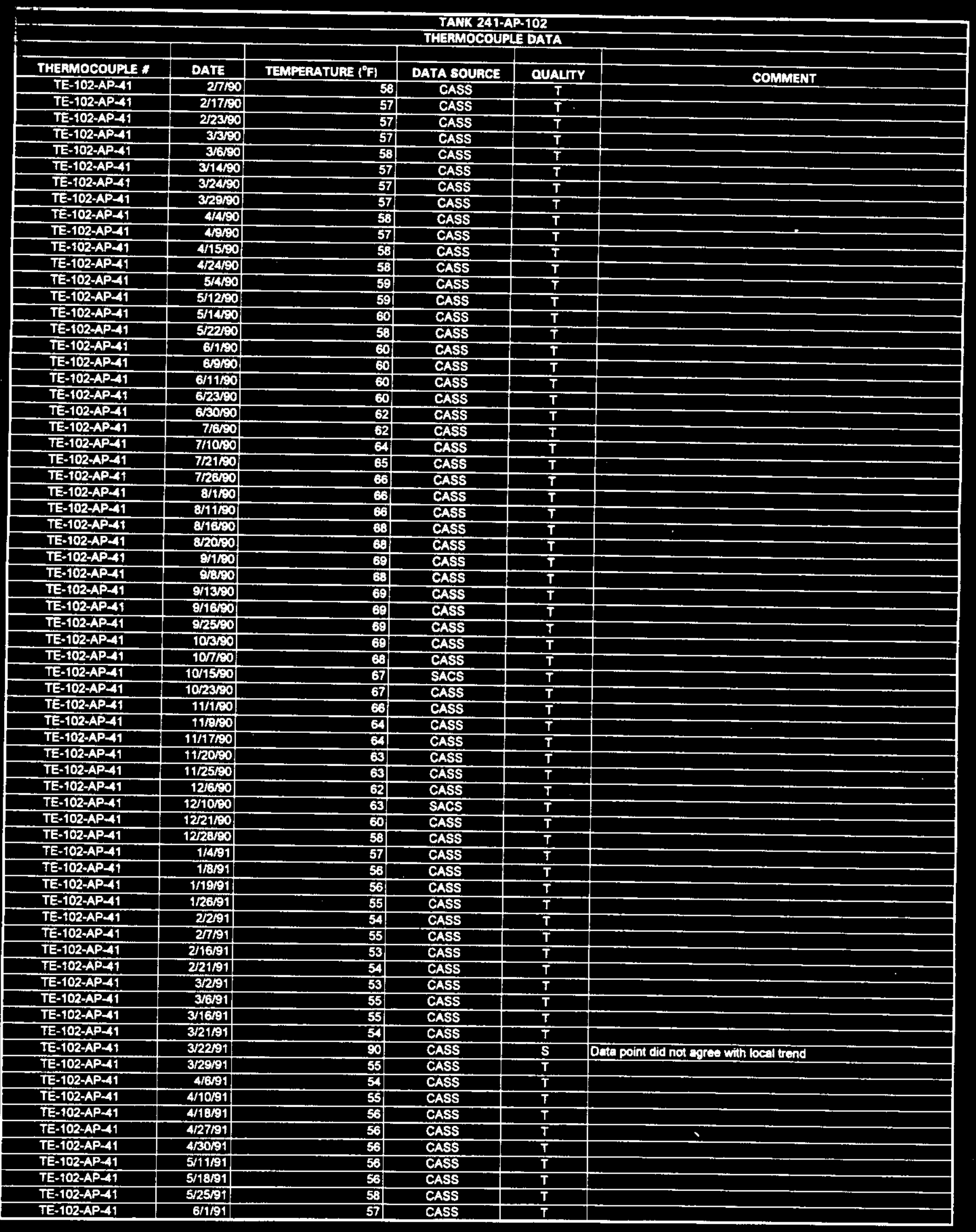




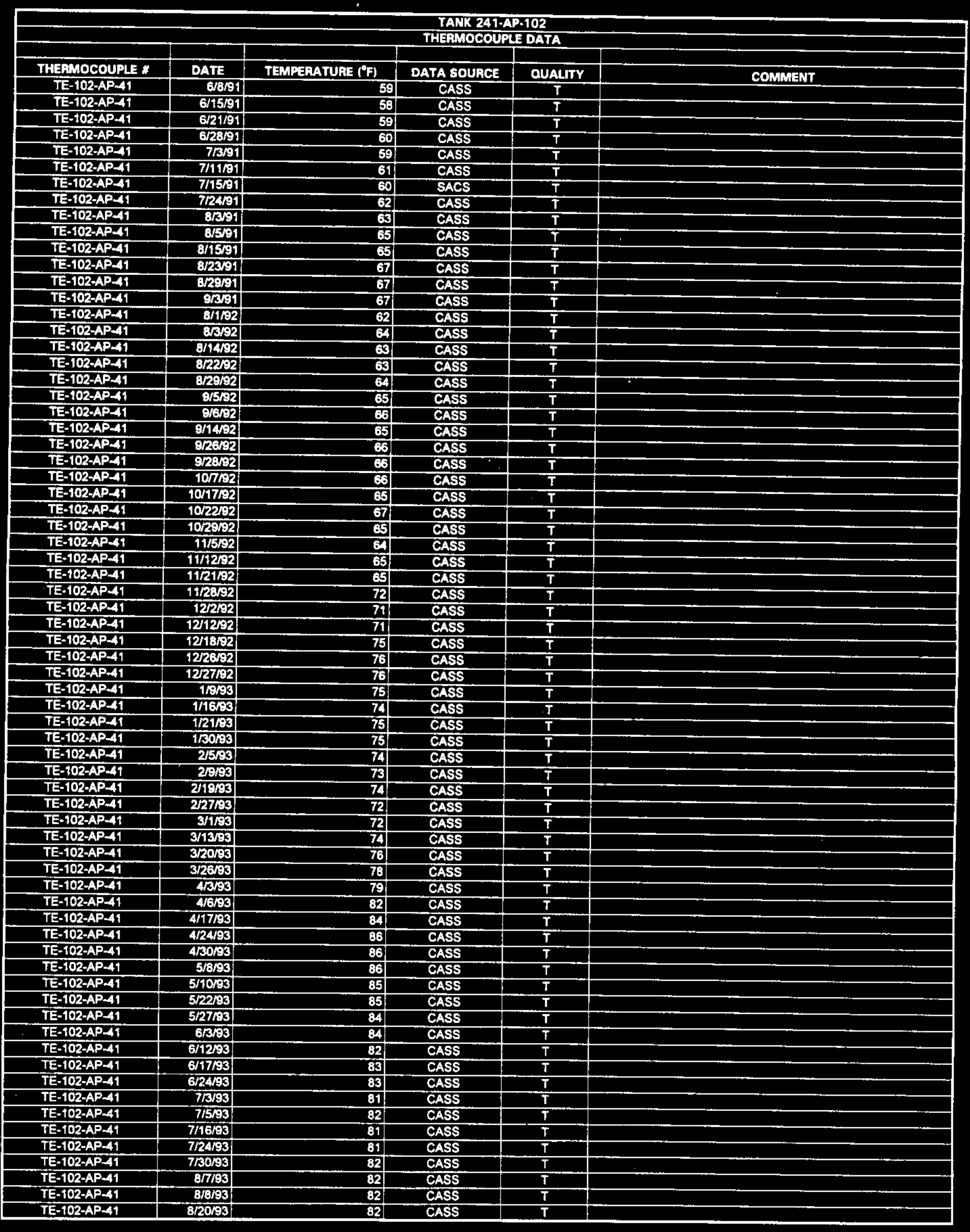

Data obteined from Computer Automated Surveillance System (CASS), April 1995; and Surveillance Analysis Computer Syatem (SACS), June 1995. 


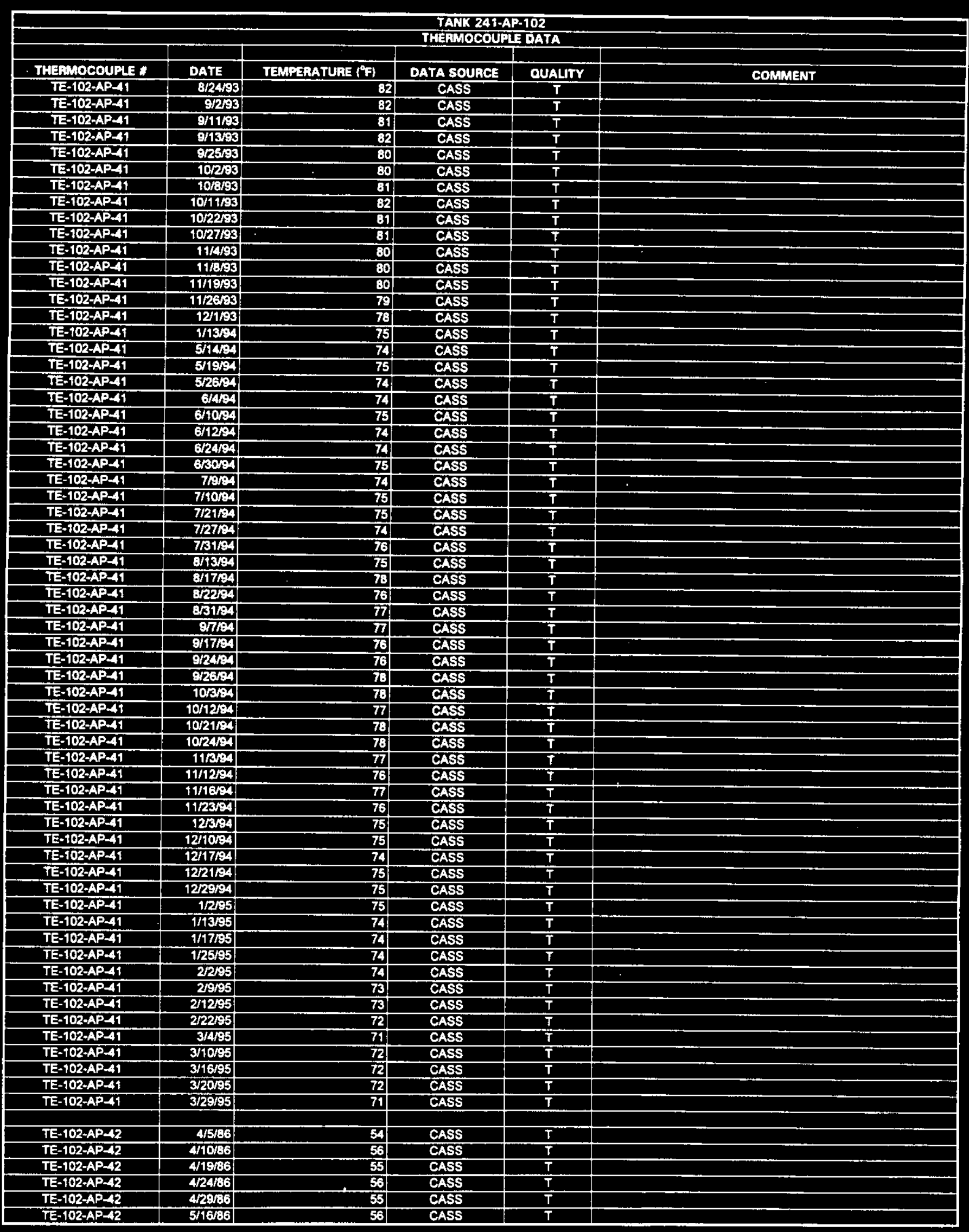




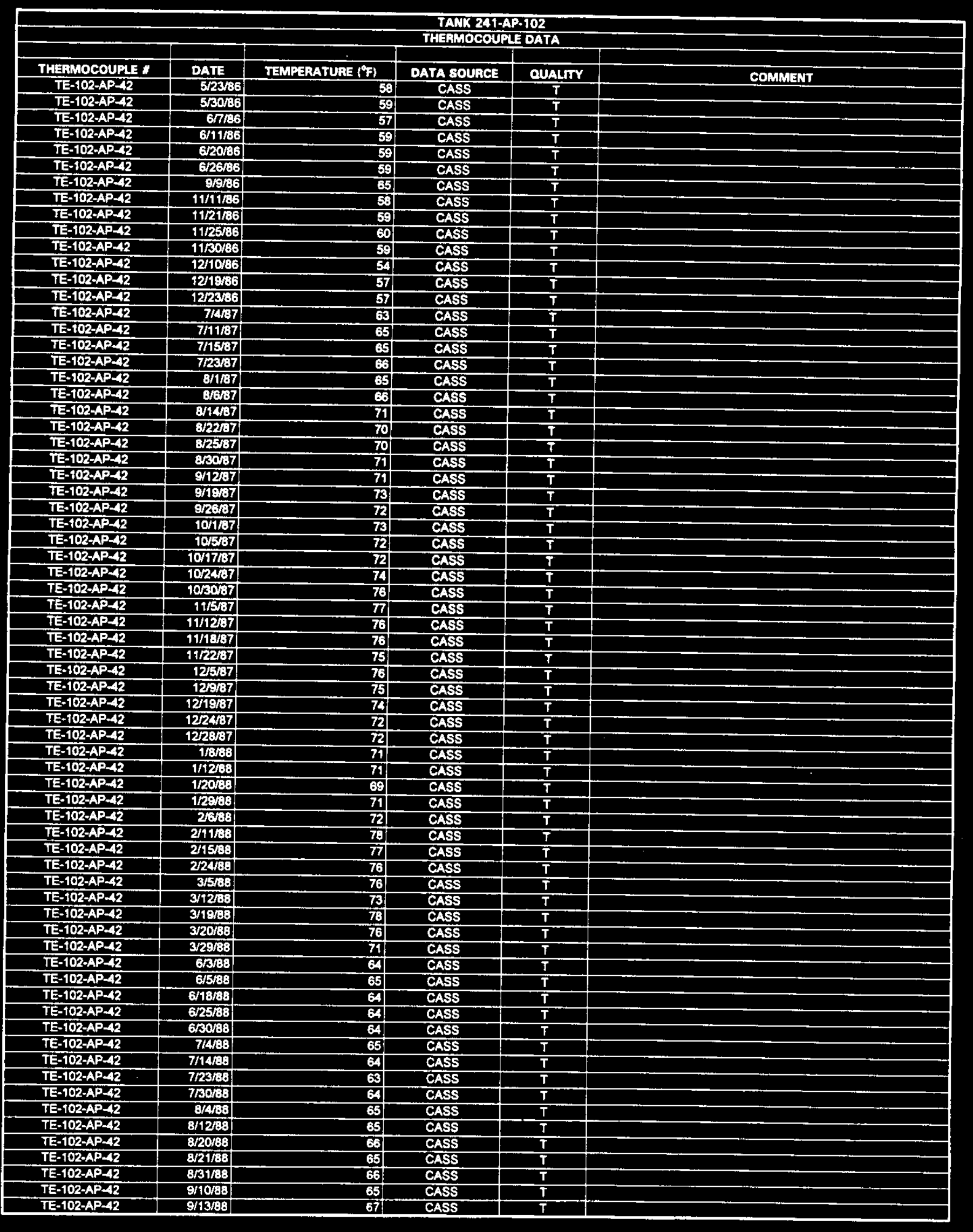




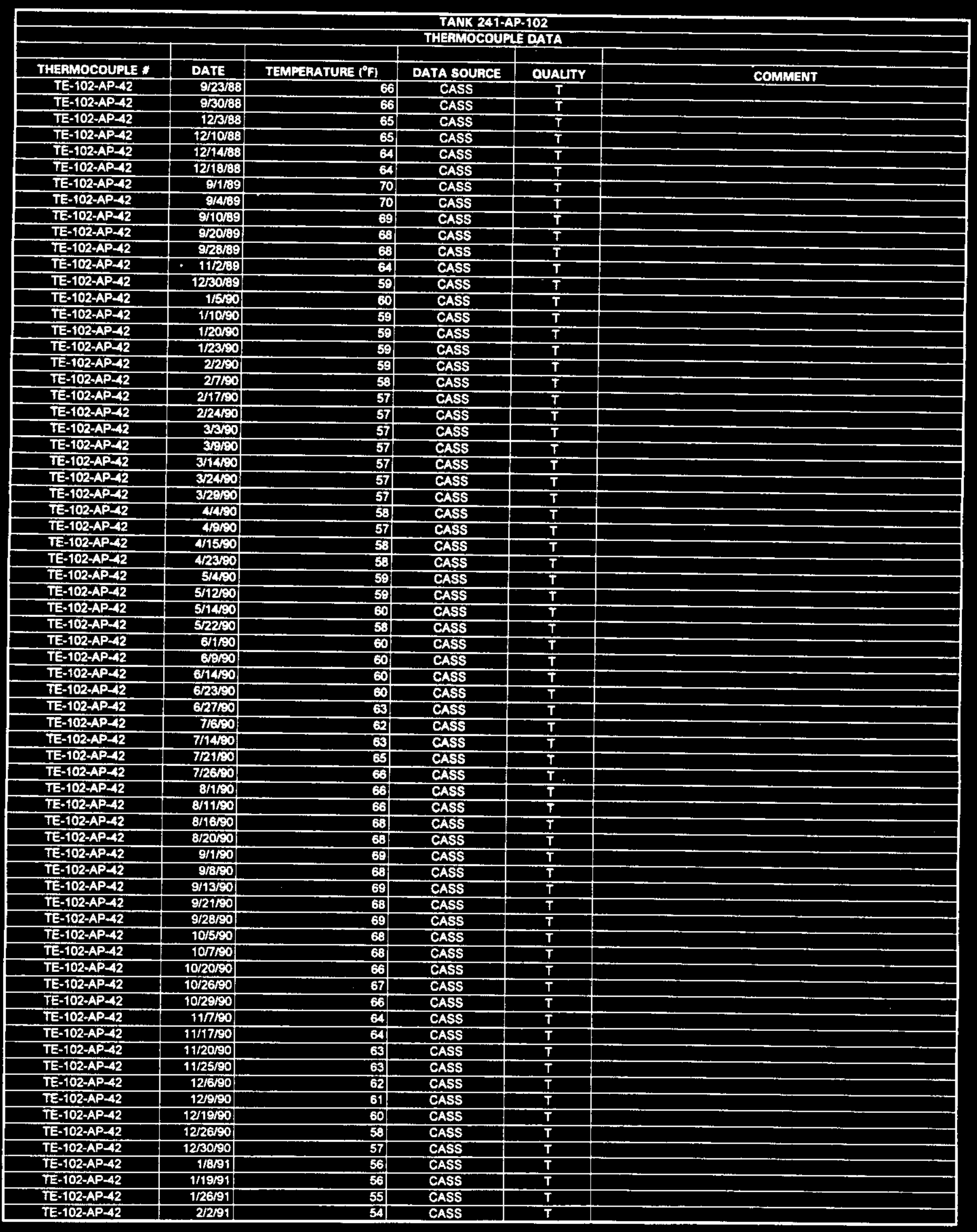




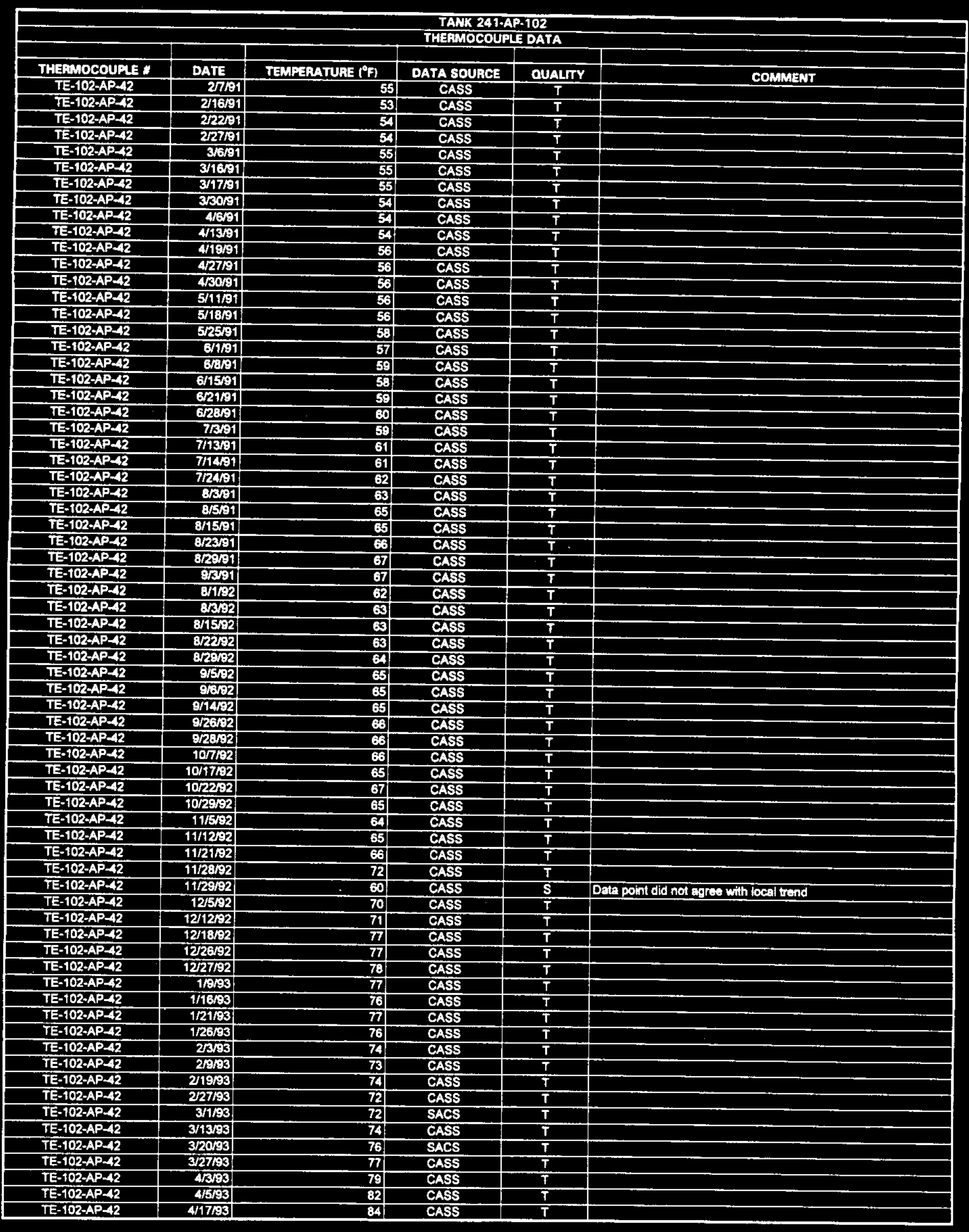

Data obtained from Computer Automated Surveillance Syatem (CASS), April 1995; and Survaillance Analysis Computer System (SACS), June 1995. 


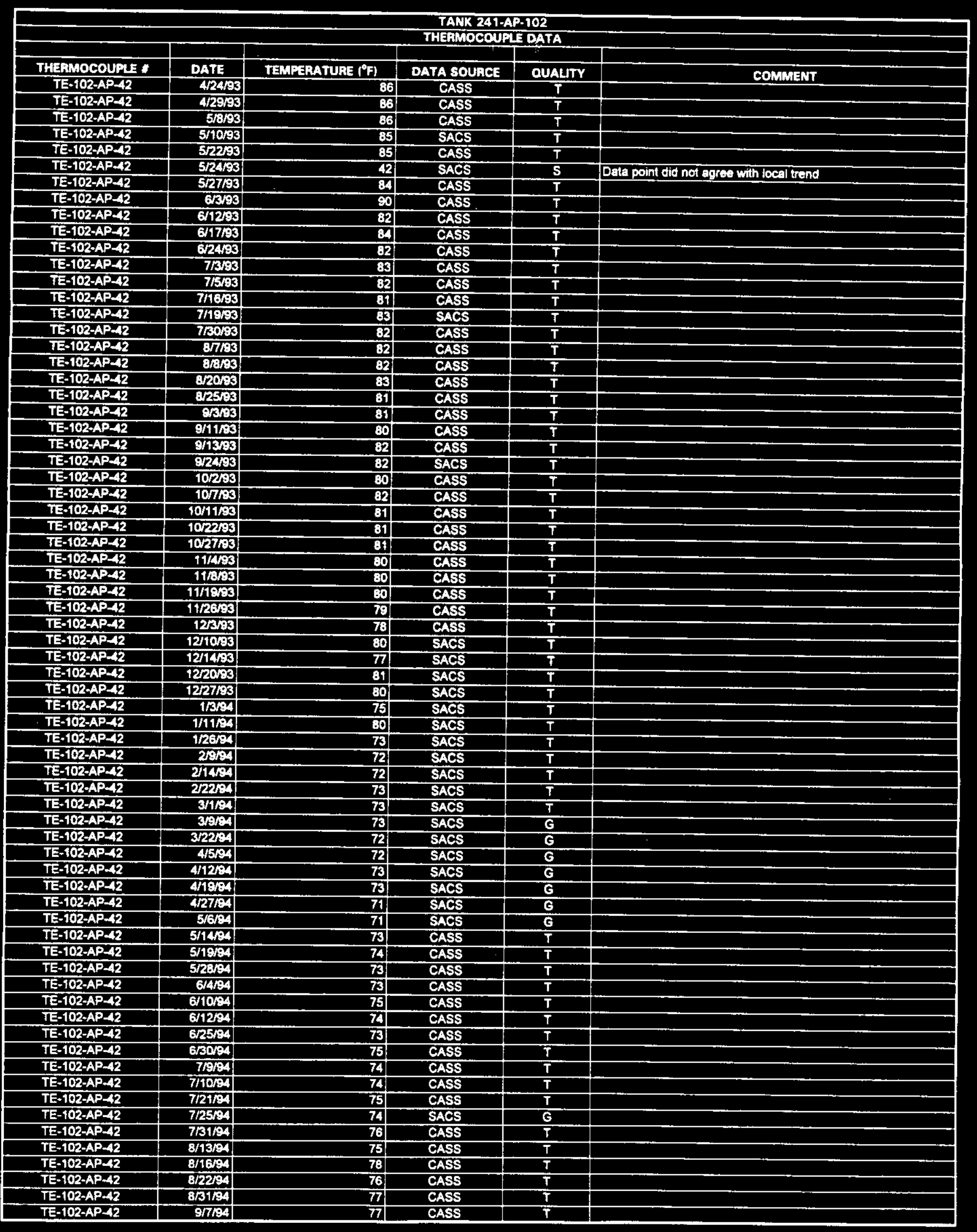




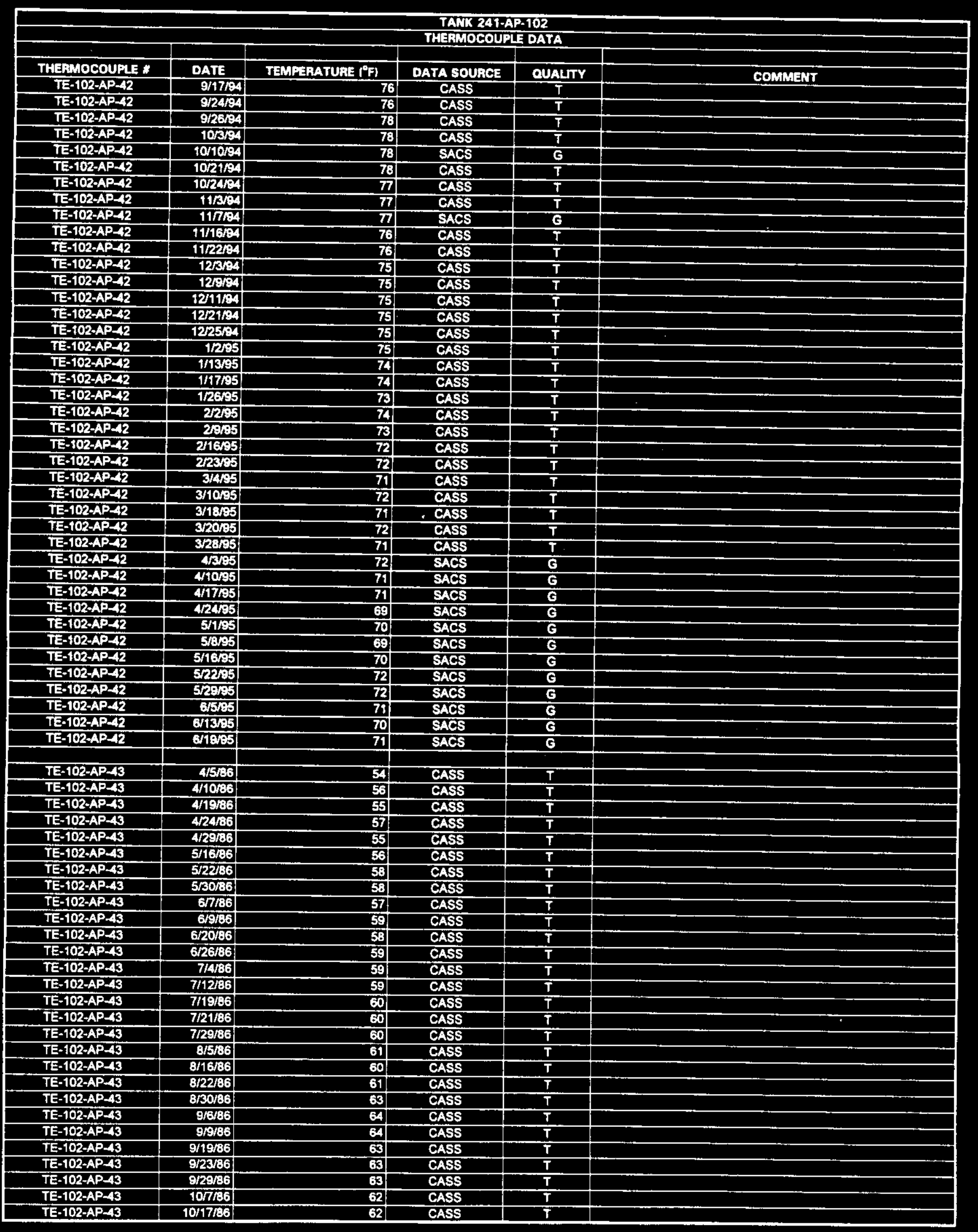




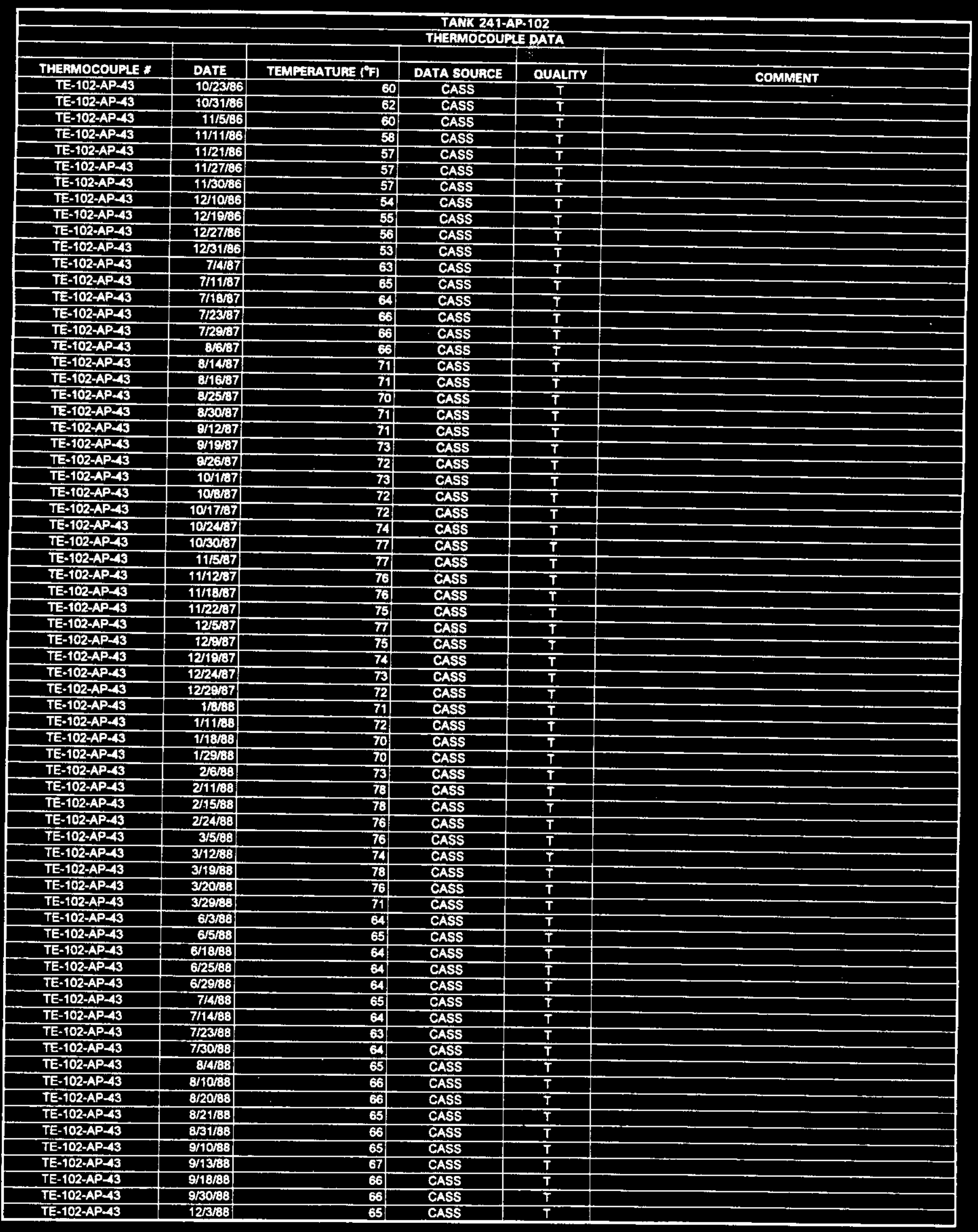




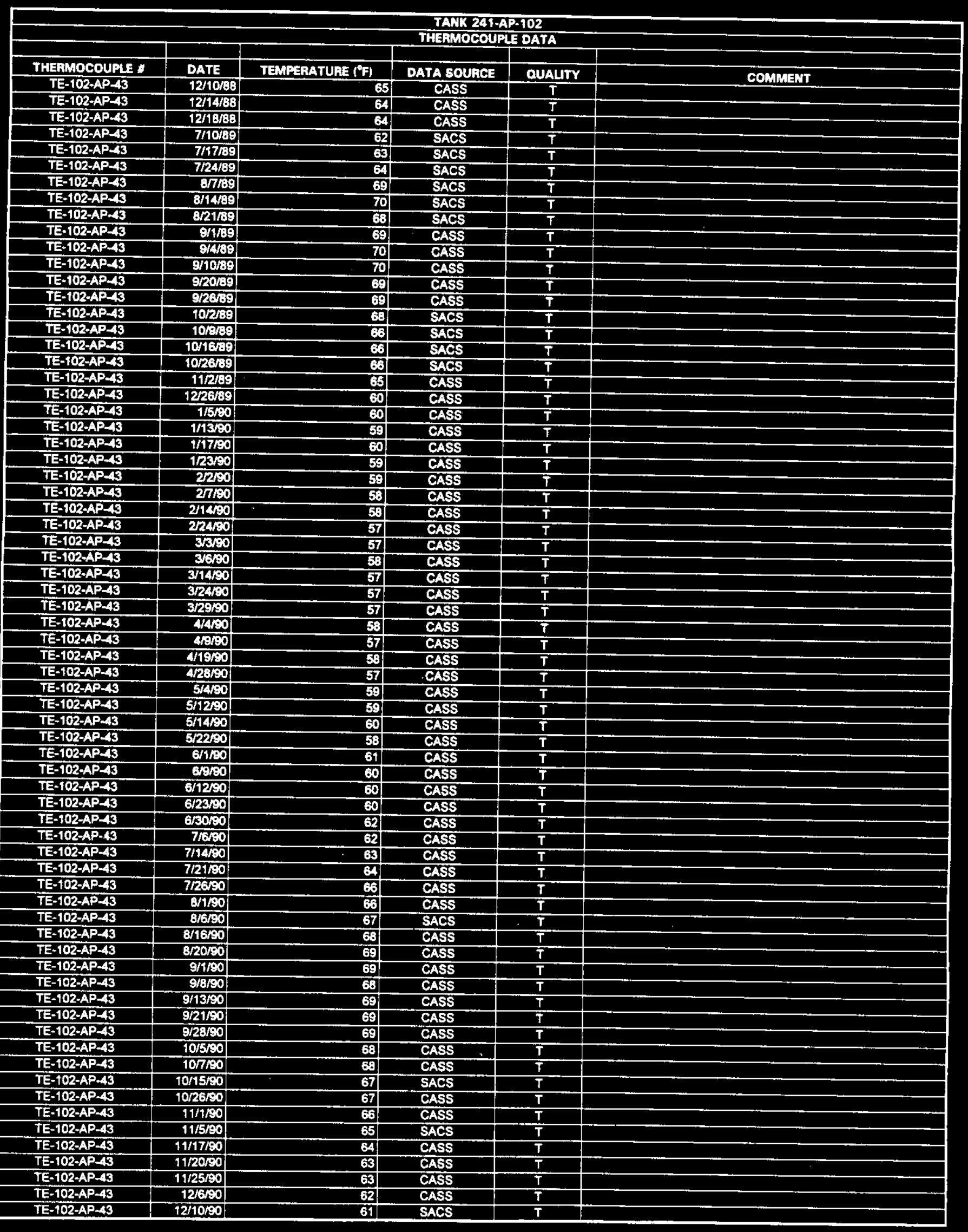

Data obtainad from Computer Automated Surveillance System (CASS), April 1995; and Surveillance Analysis Computer System (SACS), Juna 1995. D-181 


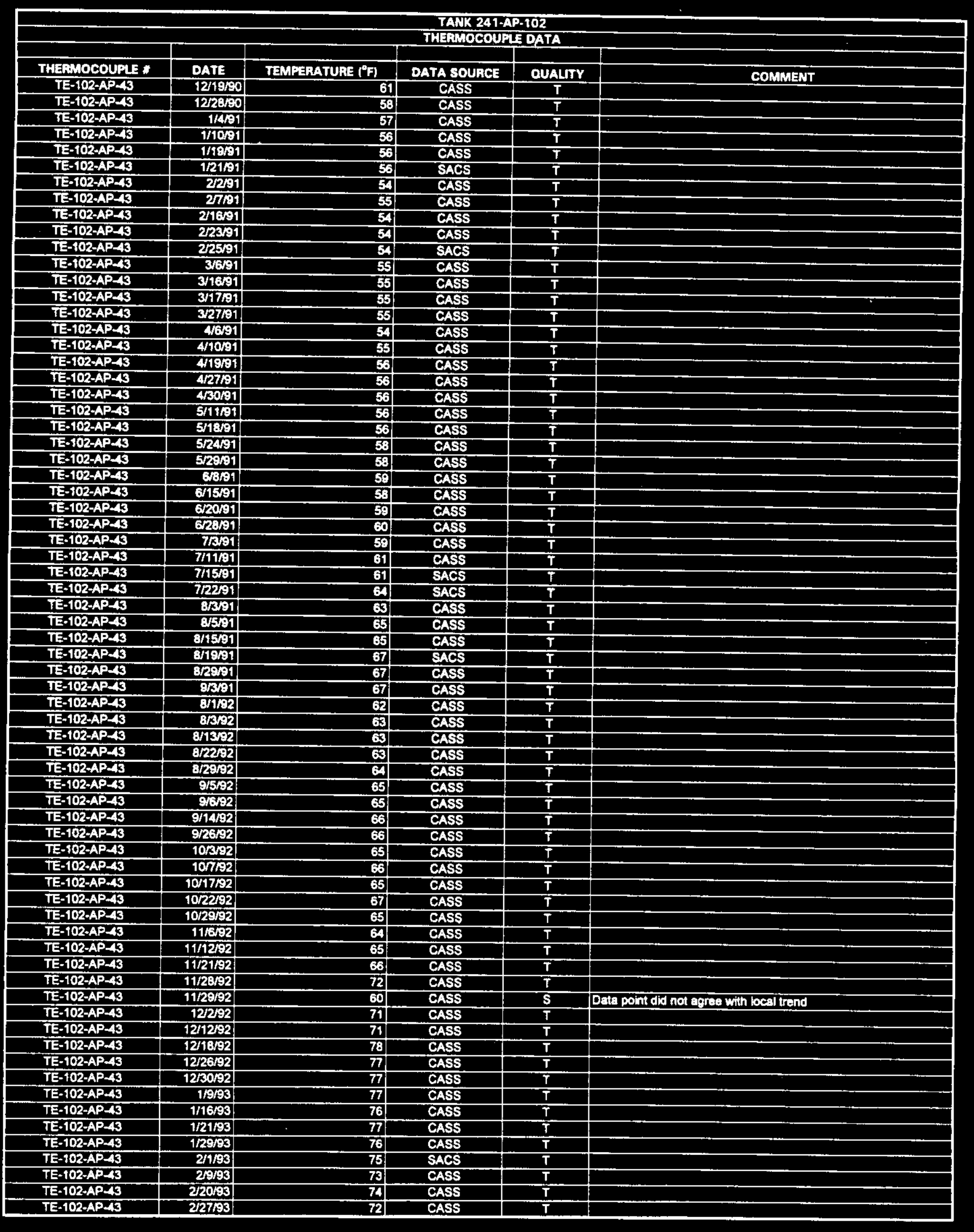




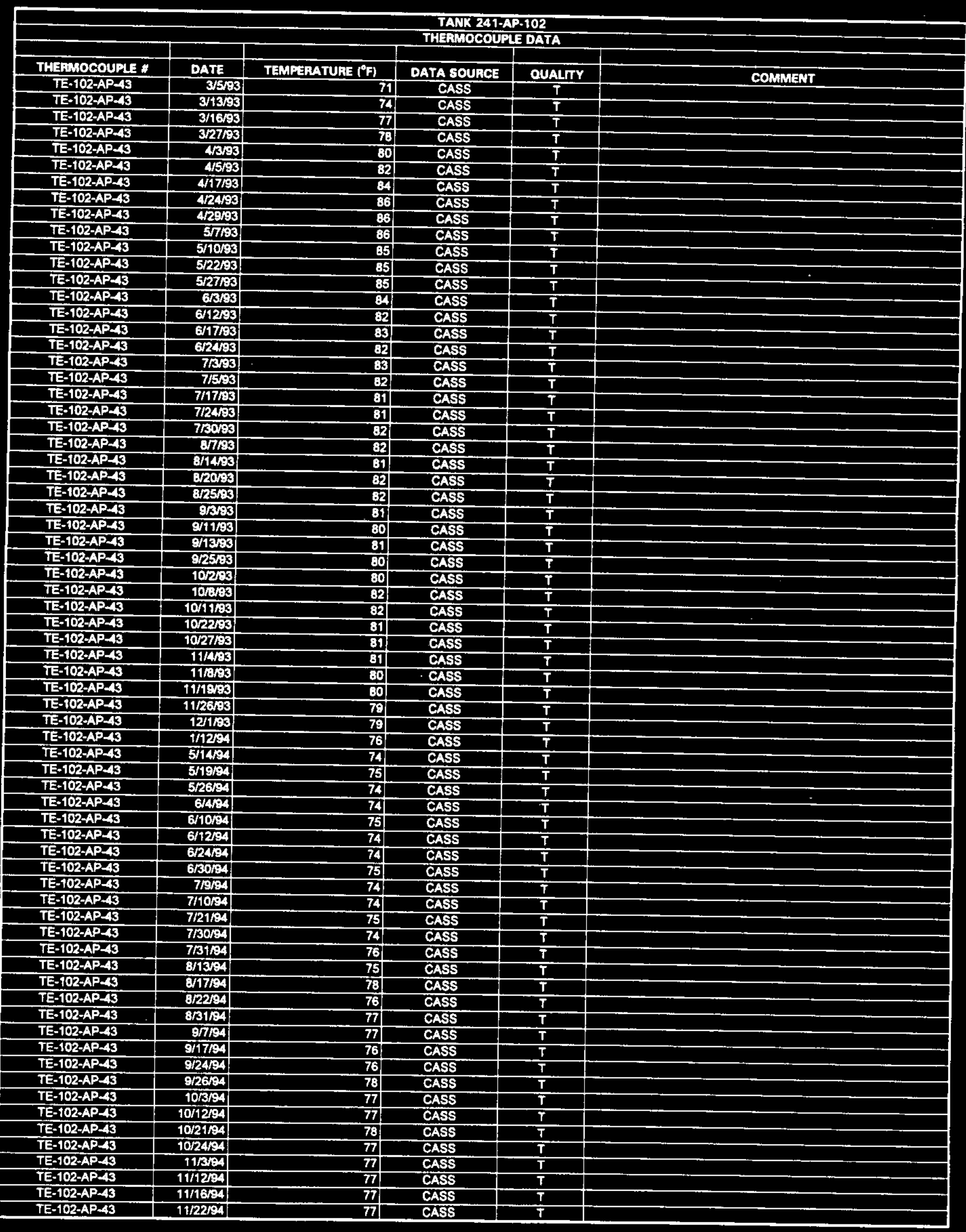

Data obtained from Computer Automated Surveillance System (CASS). April 1995; and Surveillance Analysis Computer System (SACS), June 1995. D-183 


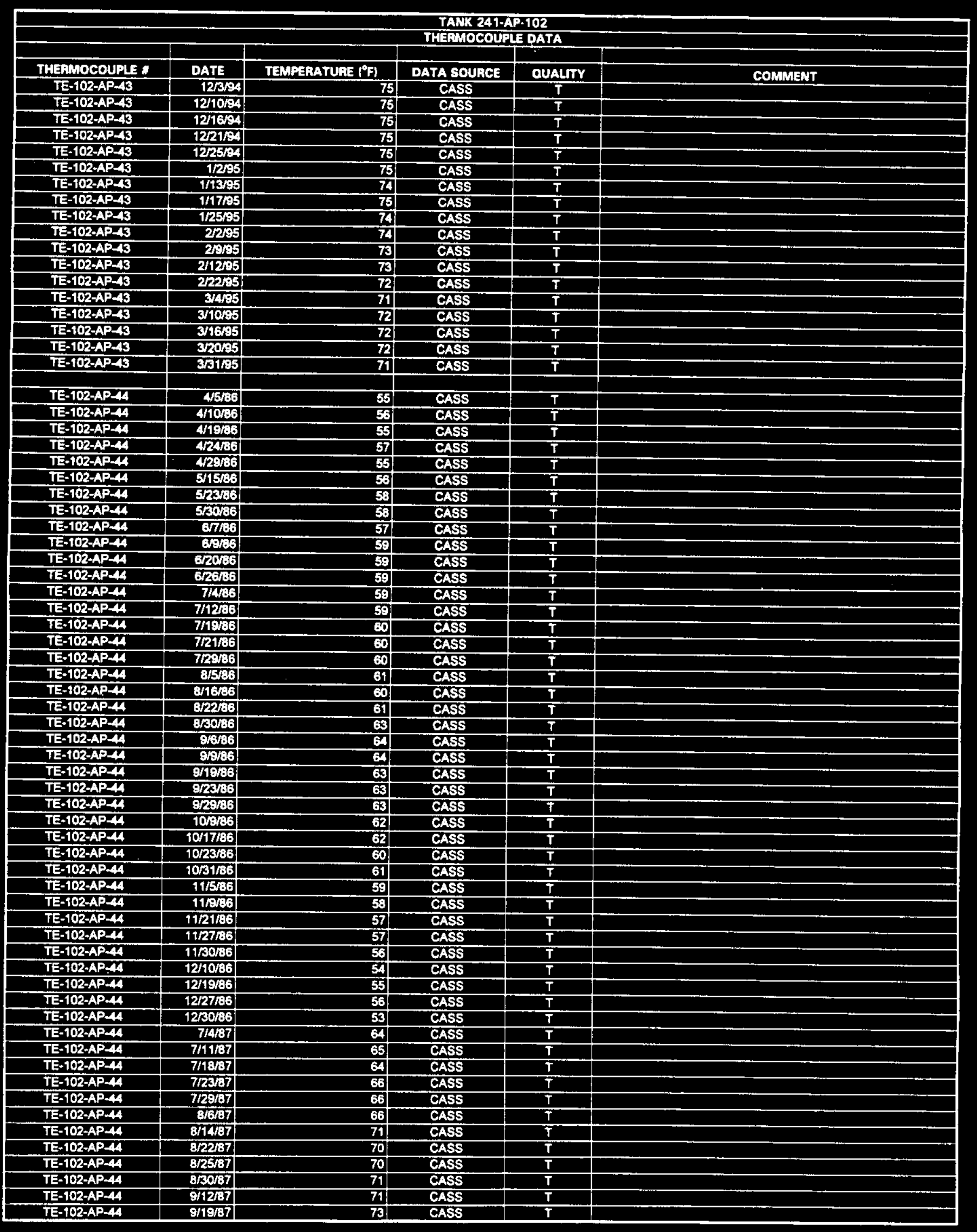




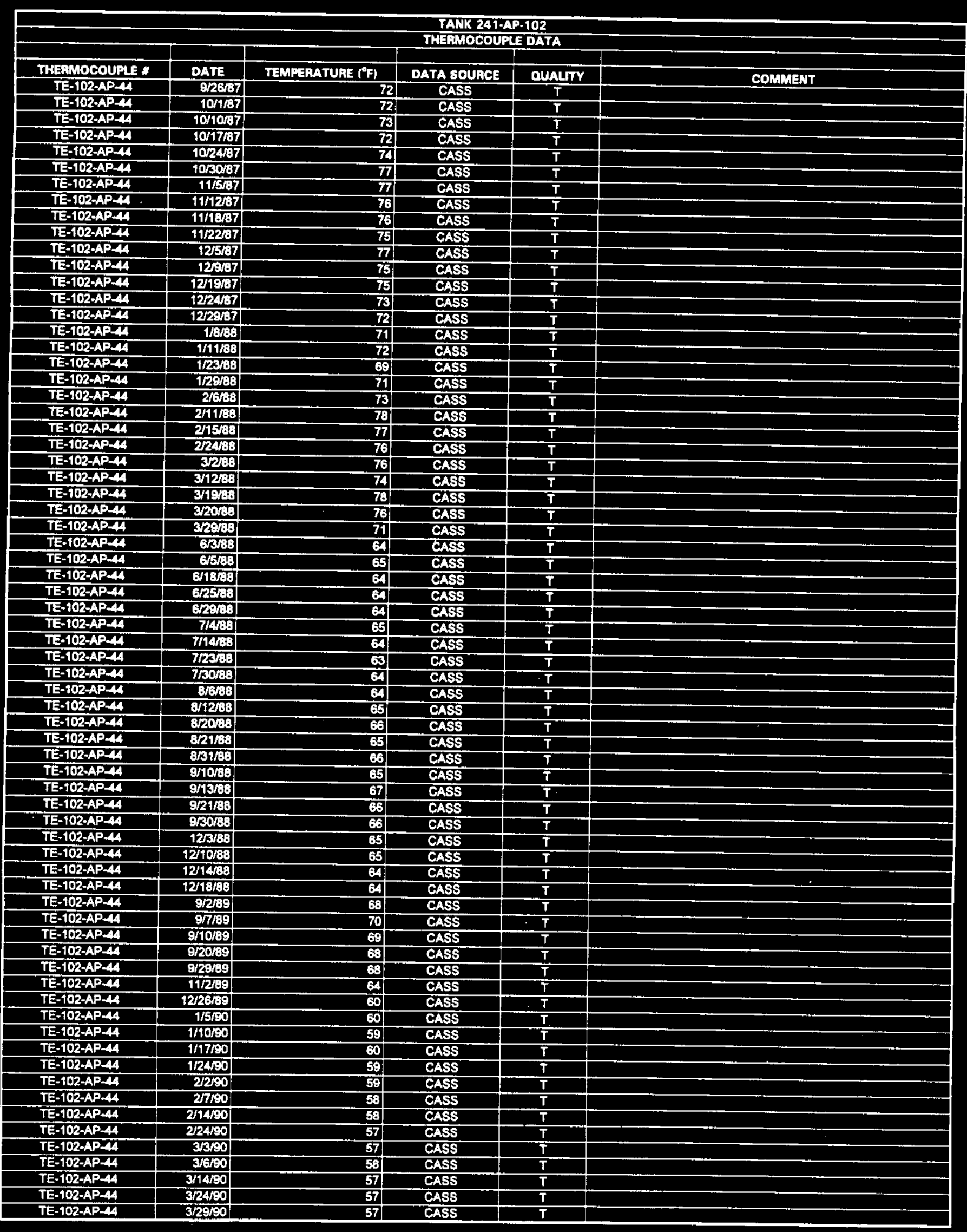




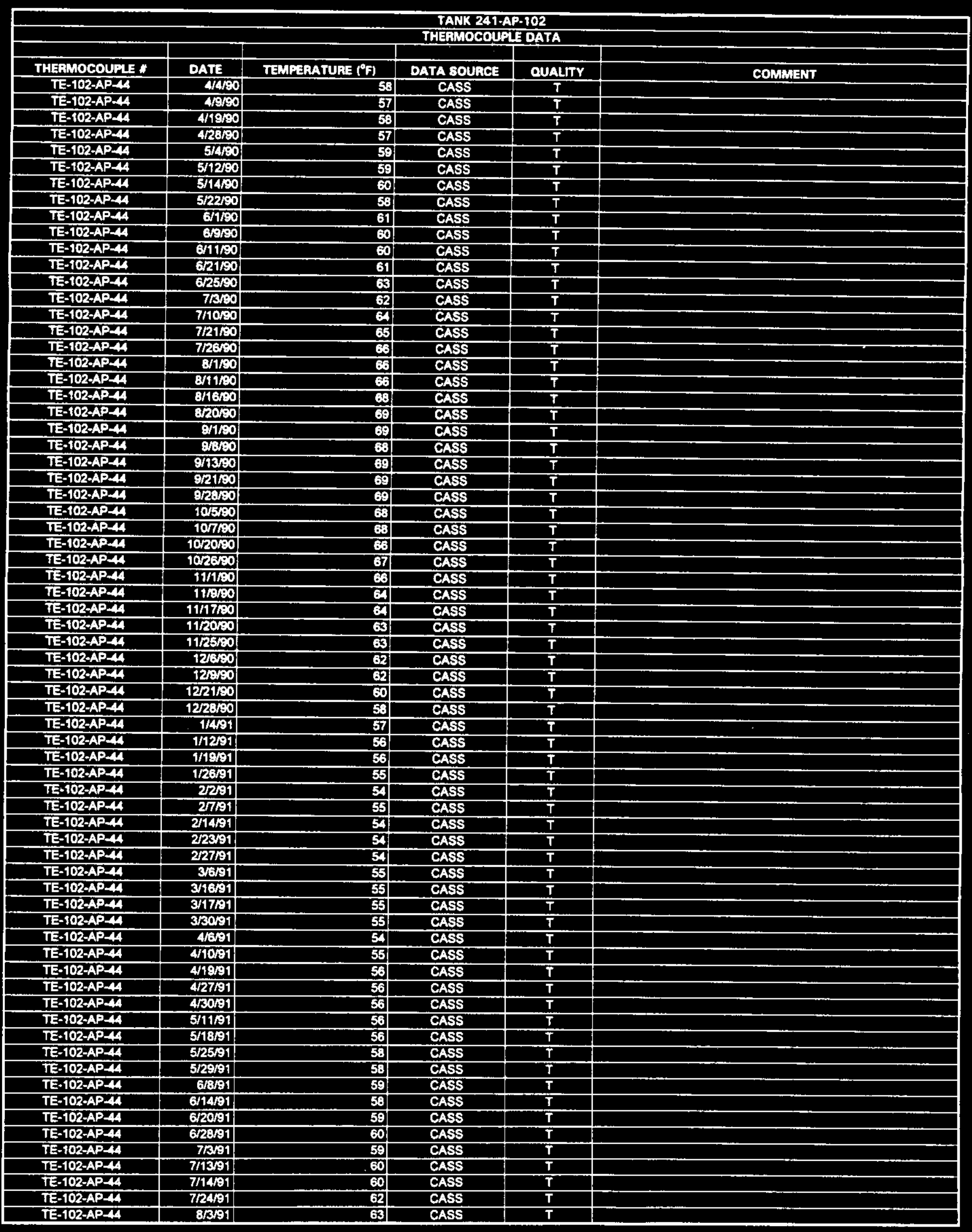




\begin{tabular}{|c|c|c|c|c|c|}
\hline \multicolumn{6}{|c|}{$\begin{array}{l}\text { TACK 241.AP-102 } \\
\text { THERMOCOUPLE DATA }\end{array}$} \\
\hline THEFMOCOUPLE : & DATE & TEMPERATUAE $1^{\circ} \mathrm{F}$ ) & DATA SOUACE & OUAITY & \\
\hline TE-102-AP-A4 & $8 / 5 / 91$ & 65 & CASS & $\frac{T}{T}$ & COMMENT \\
\hline TE-102-AP-14 & $8 / 15 / 91$ & 65: & CASS & $T$ & \\
\hline TE-102-AP-44 & $8 / 23 / 91$ & 86 & CASS & $T$ & \\
\hline TE-102-AP-4A & $8 / 29 / 91$ & 66 & CASS & $T$ & \\
\hline TE-102-AP-44 & 93191 & 67 & CASS & $\bar{T}$ & \\
\hline TE-102-AP-44 & $8 / 1 / 92$ & 62 & CASS & $T$ & \\
\hline TE-102-AP-44 & 8/3/92 & 63 & CASS & $T$ & \\
\hline$T E-102-A P-44$ & $8 / 15 / 92$ & 63 & CASS & $T$ & \\
\hline TE-102-AP-4A & 8/22/82 & 63 & CASS & $T$ & \\
\hline$T E-102-A P-4$ & B/29/92 & 64 & CASS & $T$ & \\
\hline TE-102-AP-4A & 95:32 & 65 & CASS & $T$ & \\
\hline$T E-102 \cdot A P-44$ & 9/6/92 & 65 & CASS & $T$ & \\
\hline TE-102-AP-4A & 91492 & 65 & CASS & $\bar{T}$ & \\
\hline TE-102-AP-4A & $8 / 26 / 92$ & 60 & CASS & $\mathbf{T}$ & \\
\hline TE-102-AP-44 & $10 / 3 / 92$ & 65 & CASS & $\bar{T}$ & \\
\hline TE-102-AP-44 & $107 \% 2$ & 66 & CASS & $T$ & \\
\hline TE-102-AP-44 & $10 / 17 \% 2$ & 66 & CASS & $T$ & \\
\hline TE-102-AP-44 & $10 / 22292$ & 67 & CASS & $T$ & \\
\hline TE-102-AP-44 & $10 / 29 / 92$ & 66 & CASS & $T$ & \\
\hline$T E-102-A P-44$ & 11/562: & EA & CASS & $\mathbf{T}$ & \\
\hline TE-102-AP-A4 & $11 / 12292$ & 65 & CASS & $\mathbf{T}$ & \\
\hline TE-102-AP-44 & $11 / 21 / 02$ & 66 & CASS & $\mathbf{T}$ & \\
\hline TE-102-AP-44 & $11 / 28 / 92$ & 72 & CASS & $T$ & \\
\hline TE-102-AP-A4 & $11 / 29 / 92$ & 60 & CASS & $\mathbf{5}$ & Data point did not agree with local trend \\
\hline TE-102-AP-4A & $12 / 2192$ & 71 & CASS & $\bar{T}$ & \\
\hline TE-102-AP-4A & $12 / 12 / 2$ & 71 & CASS & $\bar{T}$ & \\
\hline TE-102-AP-A4 & $12 / 18 / 92$ & 76 & CASS & $T$ & \\
\hline TE-102-AP-A & $12 / 26 \%$ & 75 & CASS & $\mathbf{T}$ & \\
\hline TE-102-AP-A4 & $1 / 2 / 93$ & 75 & CASS & $\mathbf{T}$ & \\
\hline TE-102-AP-A4 & $1 / 5 / 8$ & 76 & CASS & $\mathbf{T}$ & \\
\hline TE-102-AP-44 & 1/1693 & 75 & CASS & $T$ & \\
\hline TE-102-AP-44 & 1/21/93 & 76 & CASS & $\mathbf{T}$ & \\
\hline TE-102-AP-44 & 1/29/93 & 75 & CASS & $\mathbf{T}$ & \\
\hline TE-102-AP-44 & $2 / 693$ & 73 & CASS & $\mathbf{T}$ & \\
\hline TE-102-AP-44 & $2 / 9 / 93$ & 73 & CASS & $T$ & \\
\hline TE-102-AP-AA & $2 / 1993$ & 74 & CASS & $\mathbf{T}$ & \\
\hline TE-102-AP-44 & $2 / 27 / 93$ & 72 & CASS & $\bar{T}$ & \\
\hline TE-102-AP-4A & 3/193 & 72 & CASS & $\mathbf{T}$ & \\
\hline TE-102-AP-AA & $3 / 13 / 93$ & 74 & CASS & $T$ & \\
\hline TE-102-AP-4A & 3/1698 & 77 & CASS & $\bar{T}$ & \\
\hline$T E-102 \cdot A P-A$ & $3 \sqrt{2693}$ & 78 & CASS & $T$ & \\
\hline TE-102-AP-44 & 43593 & 80 & CASS & $\mathbf{T}$ & \\
\hline TE-102-AP-4A & $4 / 5 / 93$ & 82 & CASS & $T$ & \\
\hline TE-102-AP-4A & $4 / 17 / 33$ & 84 & CASS & $\bar{T}$ & \\
\hline TE-102-AP-44 & $4 / 24 / 93$ & 86 & CASS & $\bar{T}$ & \\
\hline TE-102-AP-4A & $4 / 29 / 93$ & 87 & CASS & $T$ & \\
\hline TE-102-AP-A & 5/8193 & 86 & CASS & $T$ & \\
\hline TE-102-AP-A4 & $5 / 10 / 63$ & 86 & CASS & $T$ & \\
\hline TE-102-AP-44 & $5 / 22 / 93$ & 85 & CASS & $T$ & \\
\hline TE-102-AP-44 & $5 / 27 / 93$ & 84 & CASS & $T$ & \\
\hline TE-102-AP-A4 & $6 / 3 / 93$ & 84 & CASS & $\mathbf{T}$ & \\
\hline TE-102-AP-44 & $6 / 1263$ & 82 & CASS & $\mathbf{T}$ & \\
\hline TE-102-AP-44 & 6/1793 & 83 & CASS & $\bar{T}$ & \\
\hline TE-102-AP-44 & $6 / 24 / 93$ & 83 & CASS & $T$ & \\
\hline TE-102-AP-44 & $7 / 3 / 93$ & 83 & CASS & $T$ & \\
\hline TE-102-AP-44 & $7 / 5 / 93$ & 82 & CASS & $T$ & \\
\hline TE-102-AP-44 & 7/16/93 & 81 & CASS & $T$ & \\
\hline TE-102-AP-44 & $7 / 24 / 93$ & 81 & CASS & $T$ & \\
\hline TE-102-AP-4A & $7 / 30 / 93$ & 82 & CASS & $T$ & \\
\hline TE-102-AP-44 & $87 / 93$ & 82 & CASS & $T$ & \\
\hline TE-102-AP-44 & $8 / 8 / 93$ & 82 & CASS & $T$ & \\
\hline TE-102-AP-44 & $8 / 20 / 93$ & 82 & CASS & $T$ & \\
\hline TE-102-AP-44 & $8 / 25 / 93$ & 82 & CASS & $T$ & \\
\hline$T E-102-A P-44$ & $9 / 293$ & 82 & CASS & $T$ & \\
\hline TE-102-AP-4A & 9110/93 & B1 & CASS & $T$ & \\
\hline TE-102-AP-44 & $9 / 13 / 93$ & 82 & CASS & $T$ & \\
\hline TE-102-AP-44 & 9/25/93 & 80 & CASS & $T$ & \\
\hline$T E-102-A P-44$ & $10 / 2193$ & 80 & CASS & $T$ & \\
\hline TE-102-AP-44 & 10/193 & 82 & CASS & $\mathbf{T}$ & \\
\hline$T E-102-A P-44$ & $10 / 11 / 93$ & 82 & CASS & $T$ & \\
\hline
\end{tabular}




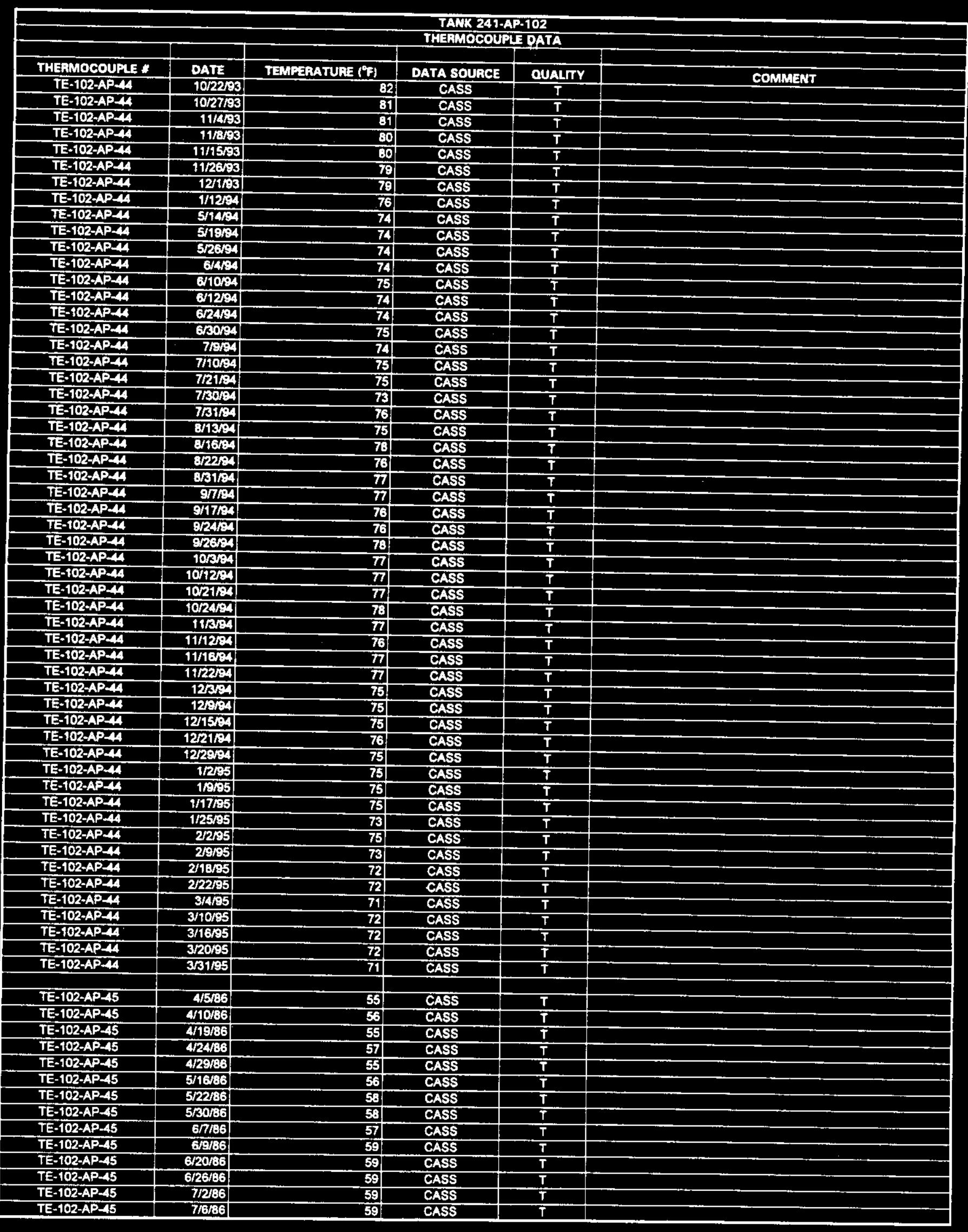

Date obtained from Computer Automated Surveillance System (CASS), April 1995; and Survellance Analyais Computer Systam (SACS), June 1995. 


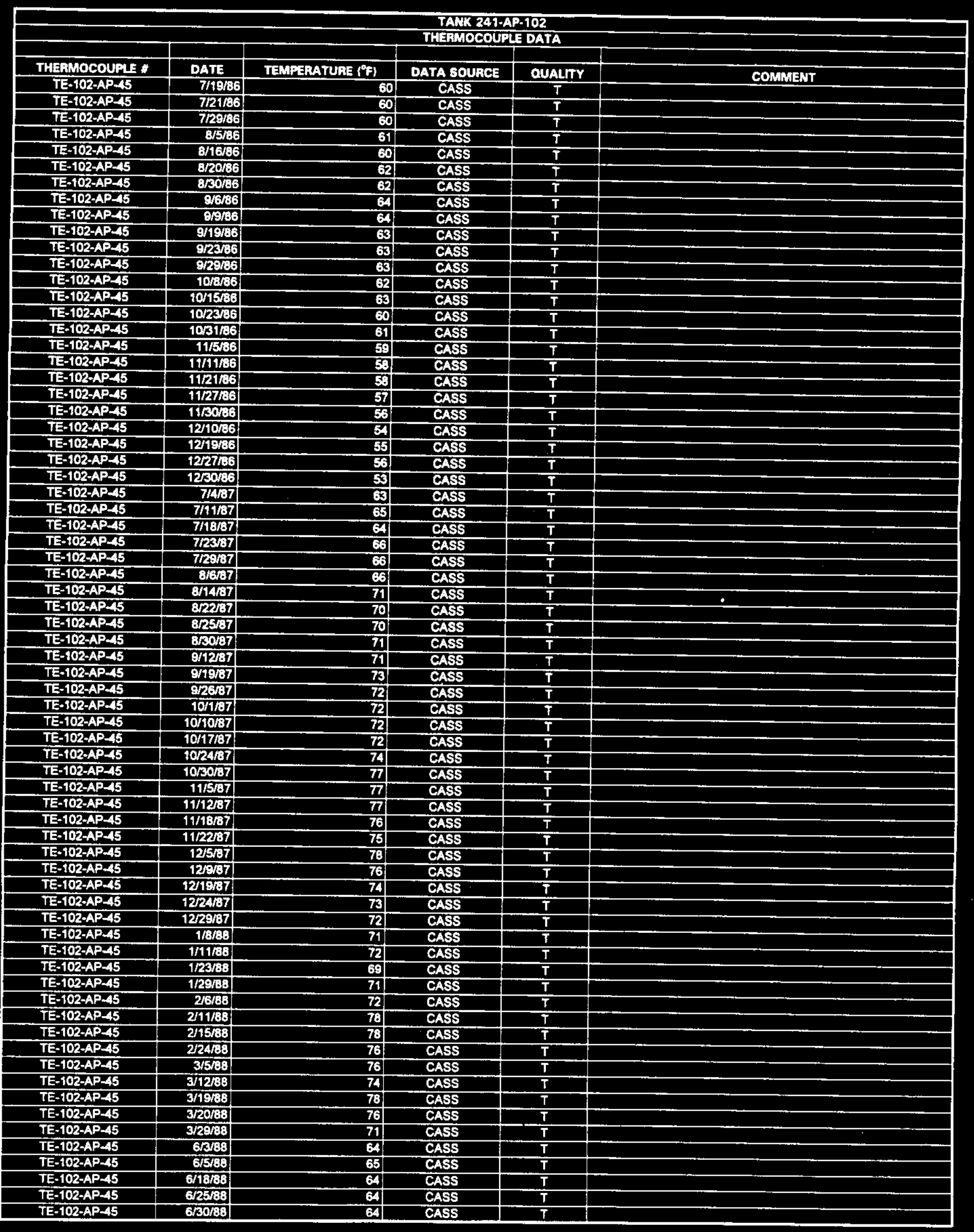

Date obtained from Computer Automated Surveillance System (CASS), April 1995; and Survellance Analysis Computer Syatem (SACS), June 1995. 


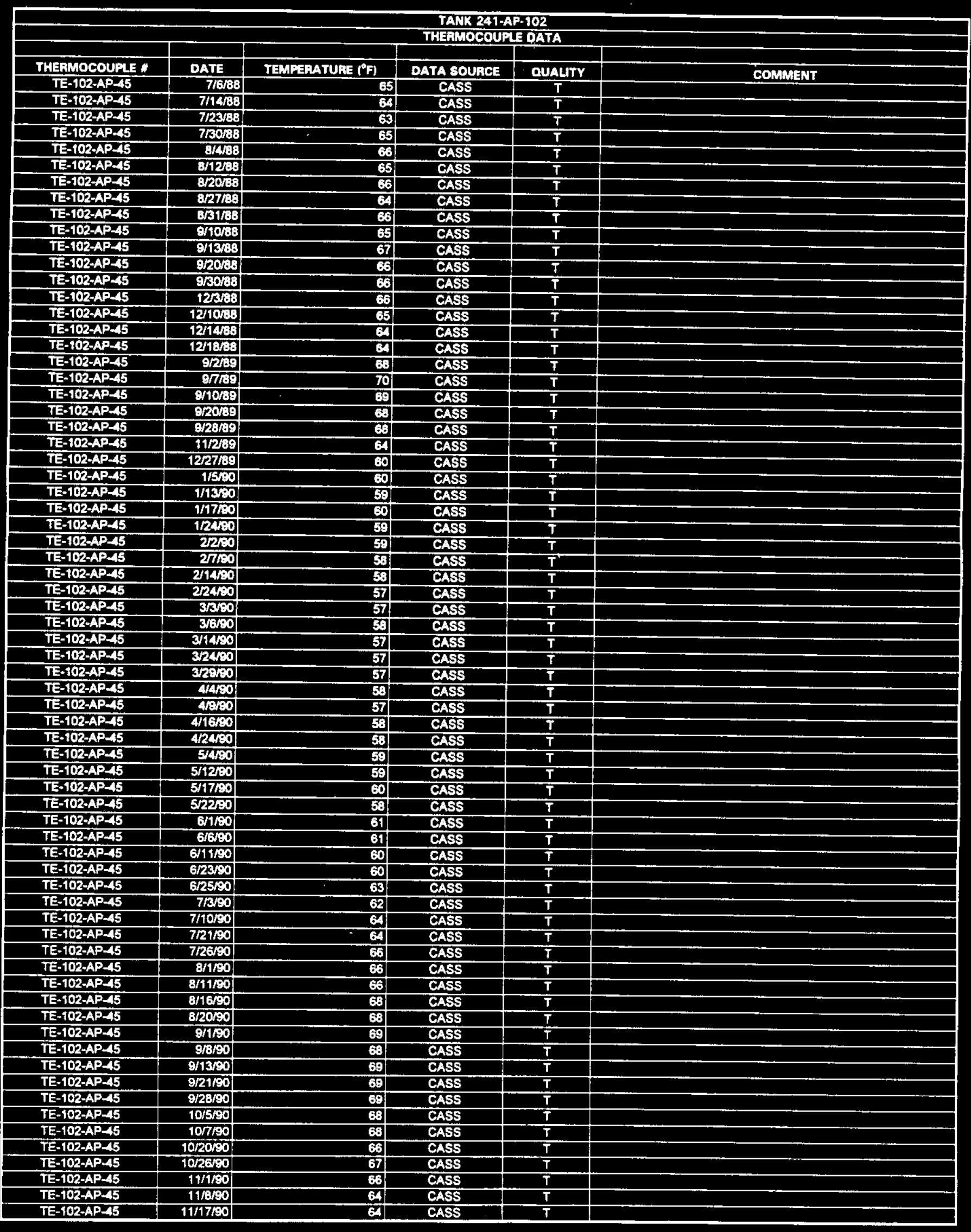




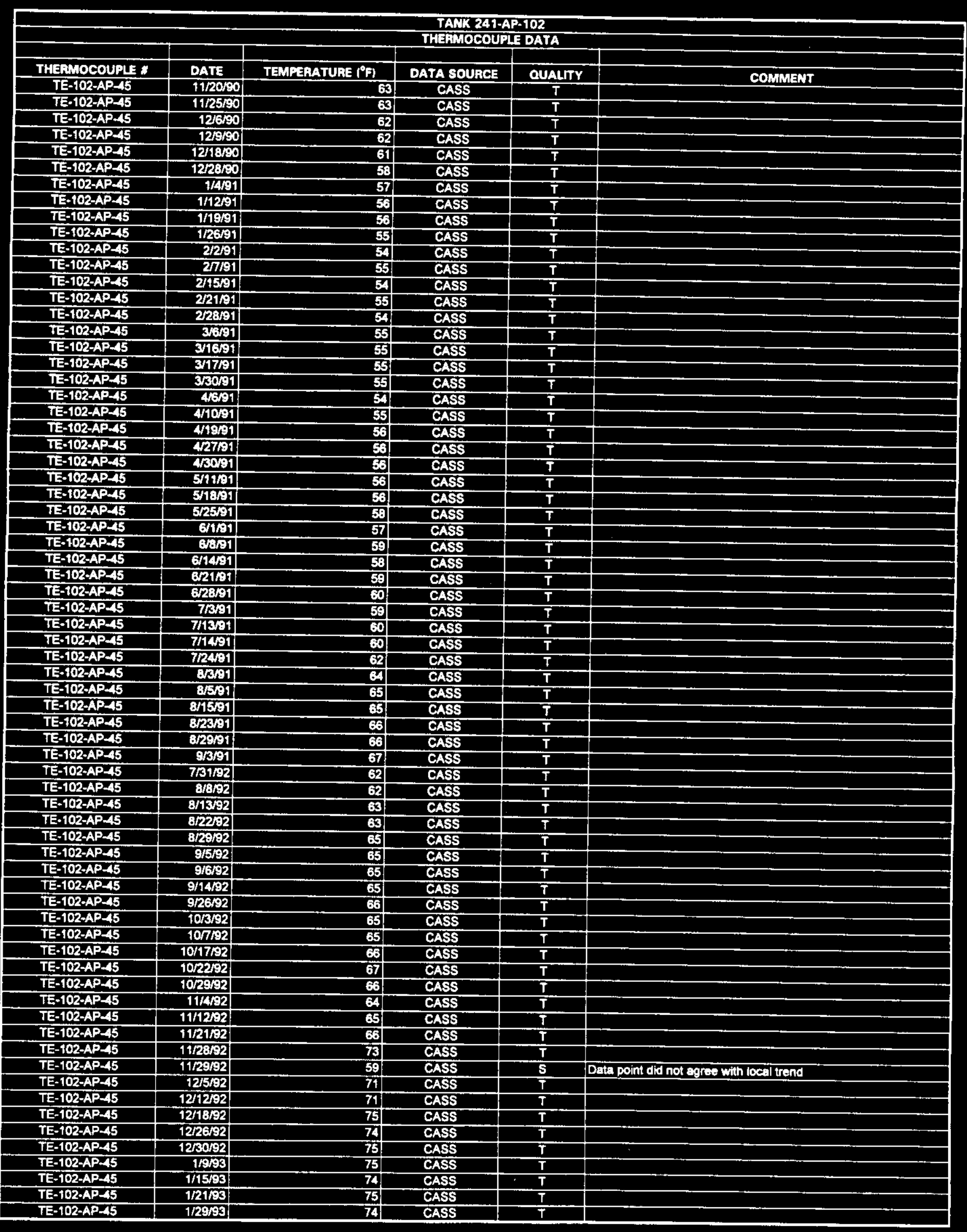

Data obtained from Computer Automated Surveillance System (CASS), Apri 1995; and Surveillance Anelysis Computer System (SACS), June 1995. D-191 


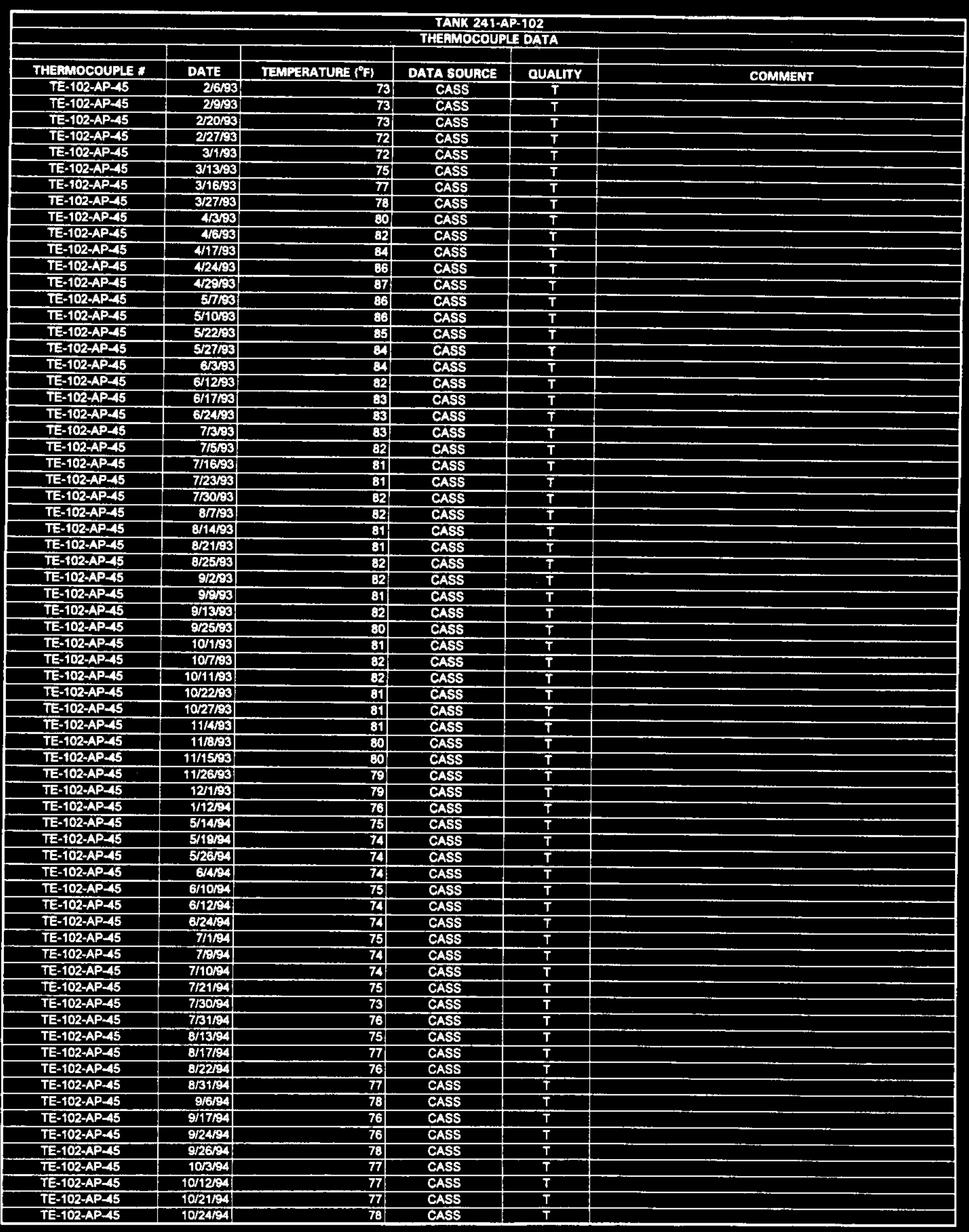




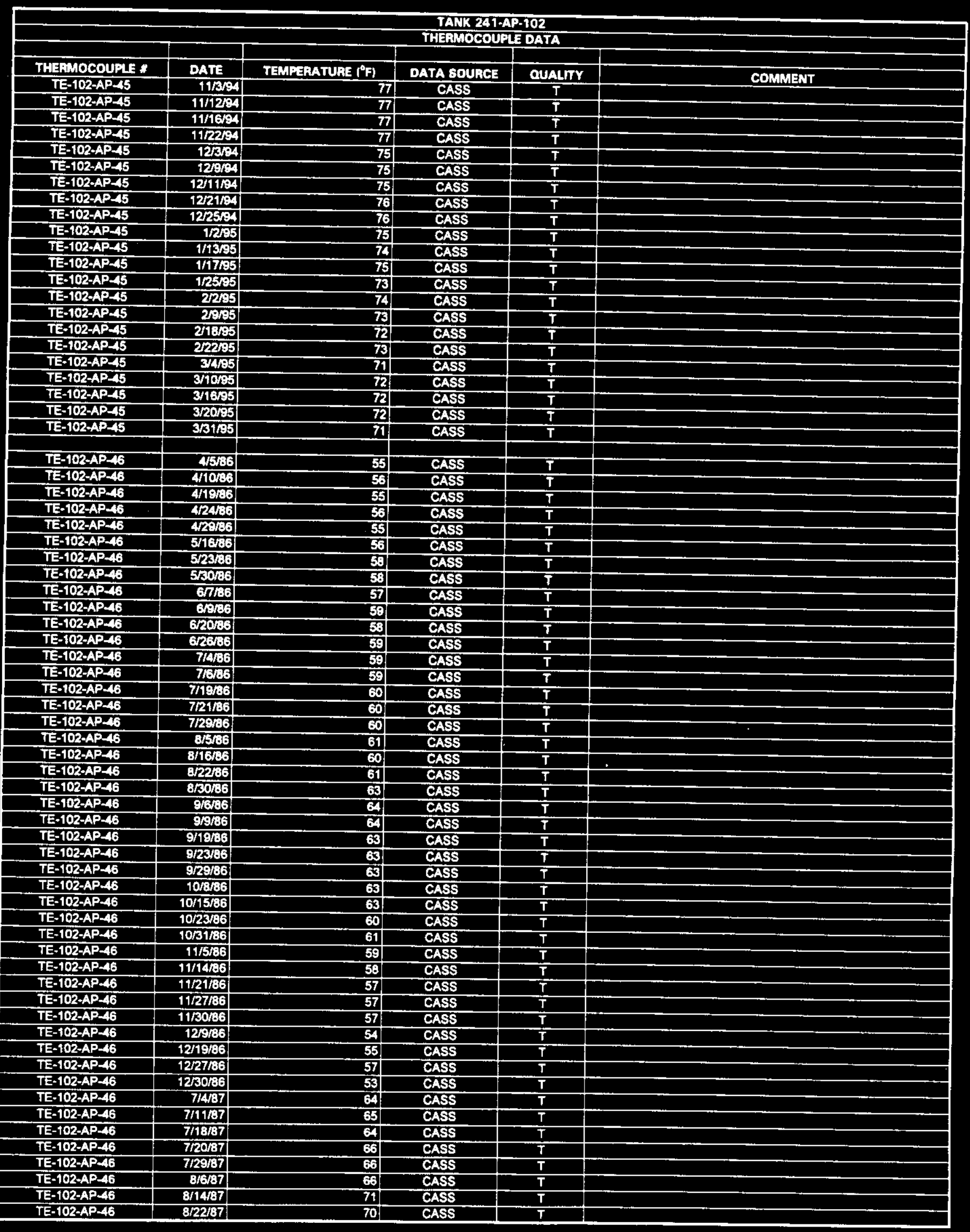




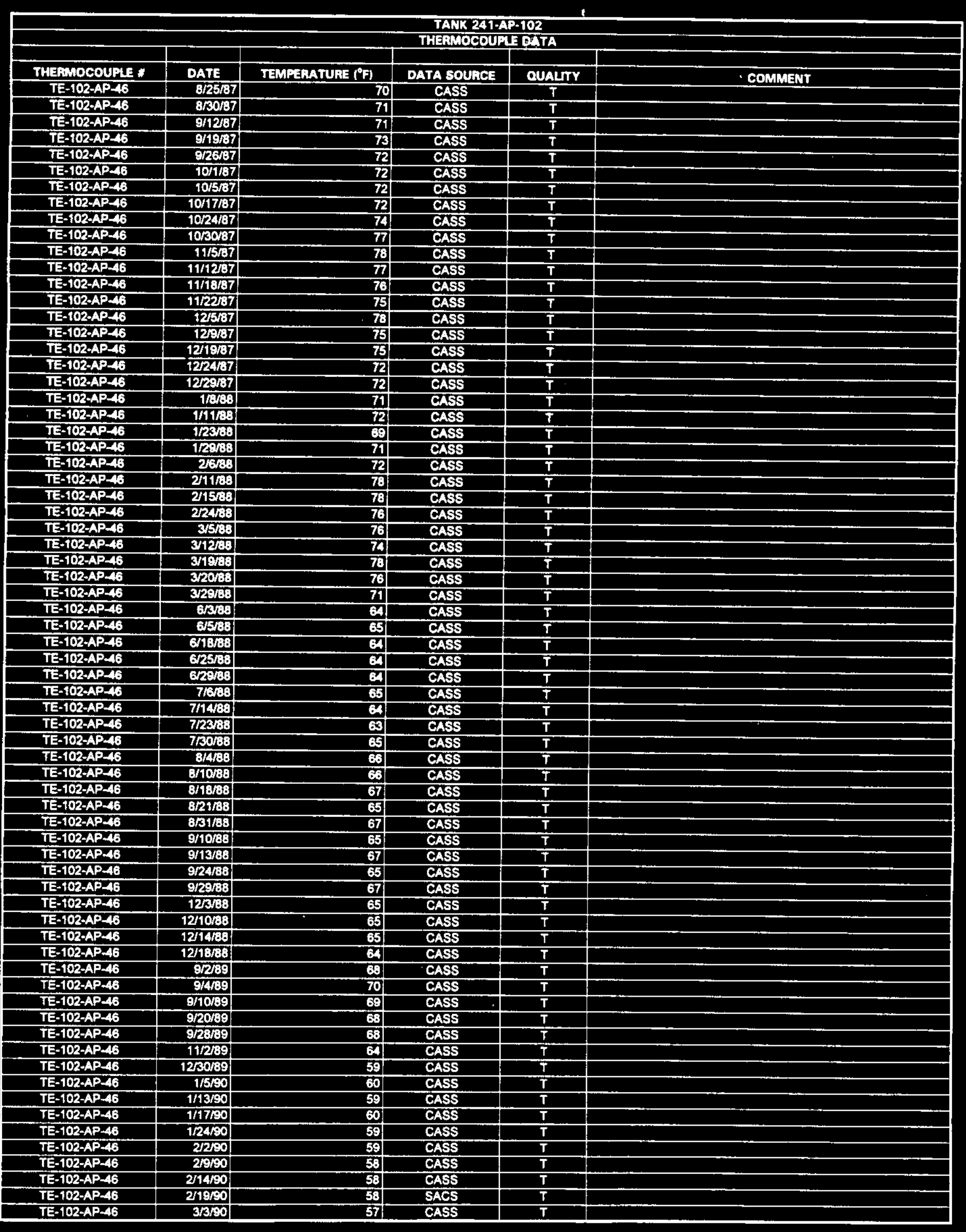

Date obteined from Computer Automatod Surveillance System (CASS), April 1995; and Surveillance Analysis Computer System (SACS), June 1995. 


\begin{tabular}{|c|c|c|c|c|c|}
\hline \multicolumn{6}{|c|}{$\begin{array}{l}\text { TAMK 241-AP-102 } \\
\text { THERMOCOUPLE DATA }\end{array}$} \\
\hline & & & & & \\
\hline THERMOCOUPLE & DATE & TEMPERATUAE ( $\left.{ }^{\circ} F\right)$ & DATA SOURCE & QUALITY & COMMENT \\
\hline TE-102-AP-46 & 3/600 & 58 & CASS & $T$ & \\
\hline TE-102-AP-46 & $3 / 14 \% 0$ & 57 & CASS & $T$ & \\
\hline TE-102-AP-46 & $3 / 24 / 50$ & 57 & CASS & $T$ & \\
\hline$T E-102-A P-46$ & $3 / 29 / 50$ & 57 & CASS & $\mathbf{T}$ & \\
\hline TE-102-AP-46 & $4 / 490$ & 58 & CASS & $T$ & \\
\hline TE-102-AP-46 & 4ASO & 58 & CASS & $T$ & \\
\hline TE-102-AP-46 & $4 / 19 / 60$ & 58 & CASS & $\mathbf{T}$ & \\
\hline TE-102-AP-46 & $4 / 2390$ & 58 & CASS & $T$ & \\
\hline TE-102-AP-46 & 5IASO & 59 & CASS & $T$ & \\
\hline TE-102AP-46 & 5/12/50 & 59 & CASS & $T$ & \\
\hline TE-102-AP 46 & 5/14No & 60 & CASS & $T$ & \\
\hline TE-102-AP-46 & $5 / 2250$ & 38 & CASS & $T$ & \\
\hline TE-102-AP-46 & 6/1:0 & 61 & CASS & $T$ & \\
\hline$T Z-102-A P=46$ & 6850 & 67 & CASS & $\mathbf{T}$ & \\
\hline TE-102-AP-46 & 61680 & 59 & CASS & $T$ & \\
\hline TE-102AP-46 & $6 \sqrt{2300}$ & 60 & CASS & $T$ & \\
\hline$T Z-102-A P-46$ & $6301: 0$ & 62 & CASS & $T$ & \\
\hline TE-102-AP-46 & $7 / 3 / 50$ & 62 & CASS & $T$ & \\
\hline$T E-102-A P-16$ & 7/10s0 & 64 & CASS & $T$ & \\
\hline TE-102-AP-46 & $7 / 2150$ & 64 & CASS & $T$ & \\
\hline TE-102-AP-46 & $7 / 2600$ & 66 & CASS & $T$ & \\
\hline$T E-102-A P-46$ & $8 / 180$ & 63 & CASS & $T$ & \\
\hline TE-102-AP-46 & $6 / 11 \% 0$ & 66 & CASS & $T$ & \\
\hline TE-102-AP-46 & $8 / 16: 0$ & 68 & CASS & $T$ & \\
\hline$T Z-102-A P-46$ & $8 / 2000$ & 68 & CASS & $T$ & \\
\hline$T E-102-A P-46$ & $9 / 1 / 90$ & 69 & CASS & $\bar{T}$ & \\
\hline TE-102-APL66 & groso & 68 & CASS & $\bar{T}$ & \\
\hline TE-102-AP-46 & 913:00 & 69 & CASS & $T$ & \\
\hline TE-102-AP-46 & 9/21/s0 & 69 & CASS & $T$ & \\
\hline$T E-102-A P=46$ & 9r2a/so & 69 & CASS & $T$ & \\
\hline$T E-102-A P=46$ & 105300 & 69 & CASS & $T$ & \\
\hline TE-102-AP-46 & $10 h r=0$ & 68 & CASS & $T$ & \\
\hline$T E-102-A P=46$ & $10 / 20 / 0$ & 63 & CASS & $T$ & \\
\hline TE-102-AP-46 & 102680 & 67 & CASS & $T$ & \\
\hline TE-102-AP-46 & $11 / 1 / 80$ & 63 & CASS & $T$ & \\
\hline$T E-102-A P-16$ & $11 \pi r=0$ & 64 & CASS & $T$ & \\
\hline TE-102-AP-46 & $11 / 17 \% 0$ & 64 & CASS & $T$ & \\
\hline TE-102-AP 46 & $11 / 20 \% 0$ & 63 & CASS & $T$ & \\
\hline TE-102-AP-46 & $11 / 25 / 00$ & 64 & CASS & $T$ & \\
\hline TE-102-AP-46 & $12 / \mathrm{B} \times 0$ & 62 & CASS & $T$ & \\
\hline TE-102-AP-46 & $129 \% 0$ & 62 & CASS & $T$ & \\
\hline TE-102A $A-46$ & $12 / 21 / 00$ & 60 & CASS & $T$ & \\
\hline$T E-102 A A P-46$ & $12 / 2890$ & 58 & CASS & $T$ & \\
\hline TE-102-AP-46 & $1 / 491$ & 57 & CASS & $T$ & \\
\hline$T E-102-A P-46$ & $1 / 12 \times 1$ & 56 & CASS & $T$ & \\
\hline TE-102-AP-46 & $1 / 1901$ & 56 & CASS & $T$ & \\
\hline TE-102-AP-46 & $1 / 26 / 91$ & 55 & CASS & $T$ & \\
\hline$T E-102-A P-46$ & $1 / 30 / 91$ & 54 & CASS & $T$ & \\
\hline TE-102-AP-46 & $2 \pi / 91$ & 55 & CASS & $T$ & \\
\hline TE-102-AP-46 & $2 / 1391$ & 54 & CASS & $T$ & \\
\hline TE-102-AP-46 & $2 / 21 / 91$ & 55 & CASS & $T$ & \\
\hline TE-102-AP-46 & $2 / 28 / 91$ & 54 & CASS & $T$ & \\
\hline TE-102-AP-46 & 3691 & 55 & CASS & $T$ & \\
\hline$T E=102-A P-46$ & $3 / 1691$ & 55 & CASS & $T$ & \\
\hline TE-102-AP-46 & $3 / 22 / 91$ & 54 & CASS & $T$ & \\
\hline TE-102-AP-46 & $3 / 27 / 91$ & 55 & CASS & $T$ & \\
\hline$T E-102-A P-46$ & $4 / 3 / 91$ & 54 & CASS & $T$ & \\
\hline TE-102-AP-46 & $4 / 10 / 91$ & 55 & CASS & $T$ & \\
\hline TE-102-AP-46 & 4/9/91 & 56 & CASS & $T$ & \\
\hline$T E-102-A P-46$ & $4 / 27 / 91$ & 56 & CASS & $T$ & \\
\hline$T E-102-A P-46$ & $4 / 30 / 91$ & 56 & CASS & $T$ & \\
\hline TE-102-AP 46 & $5 / 11 / 91$ & 56: & CASS & $T$ & \\
\hline TE-102-AP-46 & S/1BRI & 56 & CASS & $\mathbf{T}$ & \\
\hline $\mathrm{TE}-102-A P-46$ & $5 / 25 / 91$ & 58 & CASS & $T$ & \\
\hline$T E-102-A P-46$ & $6 / 1 / 91$ & \begin{tabular}{l|l}
57 \\
\end{tabular} & CASS & $T$ & \\
\hline TE-102-AP-46 & $6 / 8 / 91$ & 59 & CASS & $T$ & \\
\hline TE-102-AP-46 & $6 / 14 / 91$ & 58 & CASS & $T$ & \\
\hline TE-102-AP-46 & $6 / 21 / 91$ & 59 & CASS & $T$ & \\
\hline$T E-102-A P-46$ & $6 / 28 / 91$ & 60 & CASS & $T$ & \\
\hline$T E-102-A P-46$ & $7 / 3 / 91$ & 59 & CASS & $T$ & \\
\hline
\end{tabular}




\begin{tabular}{|c|c|c|c|c|c|}
\hline \multicolumn{6}{|c|}{$\begin{array}{l}\text { TANK 241-AP-102 } \\
\text { THERMOCOUPL DATA }\end{array}$} \\
\hline & & & & & \\
\hline THERMOCOUPLE * & DATE & TEMPERATURE ( $\left.{ }^{\circ} \mathrm{F}\right)$ & DATA SOURCE & OUALTY & COMMENT \\
\hline TE-102-AP-46 & 711391 & 60 & CASS & $T$ & \\
\hline TE-102-AP-46 & $7 / 14 / 91$ & 60 & CASS & $T$ & \\
\hline TE-102-AP-46 & $7 / 24 / 91$ & 62 & CASS & $T$ & \\
\hline TE-102-AP-46 & 8/3/91 & 63 & CASS & $T$ & \\
\hline$T E-102-A P-16$ & $8 / 591$ & 65 & CASS & $T$ & \\
\hline$T E-102-A P-46$ & 8/15/31 & 65 & CASS & $T$ & \\
\hline TE-102-AP-46 & B/23/91 & 66 & CASS & $T$ & \\
\hline$T E-102-A P-46$ & 8/29/91 & 66 & CASS & $T$ & \\
\hline TE-102-AP-46 & 93194 & 67 & CASS & $T$ & \\
\hline TE-102-AP-46 & $7 / 31 / 21$ & 62 & CASS & $T$ & \\
\hline$T E-102-A P-46$ & $8 / 82$ & 62 & CASS & $T$ & \\
\hline TE-102-AP-46 & $8 / 13 / 92$ & 63 & CASS & $T$ & \\
\hline TE-102-AP-46 & $8 / 22 / 92$ & 63 & CASS & $T$ & \\
\hline$T E-102-A P-46$ & $8 / 29 / 92$ & 65 & CASS & $T$ & \\
\hline$T E-102-A P-46$ & 98/592 & 65 & CASS & $\bar{T}$ & \\
\hline TE-TO2AAP-A6 & QR/392 & 65 & CASS & $\bar{T}$ & \\
\hline TE-102-AP-46 & $9 / 14 / 92$ & 66 & CASS & $T$ & \\
\hline TE-102-AP-46 & 9/26/92: & 66 & CASS & $T$ & \\
\hline TE-102-AP-46 & $10 / 1 / 92$ & 65 & CASS & $T$ & \\
\hline TE-102-AP-46 & $10 \% 92$ & 66 & CASS & $T$ & \\
\hline TE-102-AP-46 & $10 / 17 / 02$ & 66 & CASS & $T$ & \\
\hline TE-102-AP-46 & $10 / 2202$ & 67 & CASS & $T$ & \\
\hline TE-102-AP-46 & $10 / 2902$ & 65 & CASS & $T$ & \\
\hline TE-102-AP-46 & $115 \% 2$ & 64 & CASS & $\bar{T}$ & \\
\hline TE-102-AP-46 & $11 / 12 / 2$ & 65 & CASS & $T$ & \\
\hline TE-102-AP-46 & $11 / 21 / 92$ & 66 & CASS & $T$ & \\
\hline TE-102-AP-AB & $11 / 26 / 92$ & 76 & CASS & $T$ & \\
\hline$T E-102-A P-46$ & $11 / 2902$ & 59 & CASS & s & Deta point did net wiroe with locel trend \\
\hline TE-102-AP-46 & $11 / 30192$ & 74 & CASS & $T$ & \\
\hline TE-102-APA 46 & $32 / 2 / 2$ & 73 & CASS & $T$ & \\
\hline TE-102-AP-46 & 12/18/92 & 75 & CASS & $T$ & \\
\hline TE-102AP-46 & $12 / 23,92$ & 74 & CASS & $T$ & \\
\hline TE-102-AP-46 & $12 / 27 / 92$ & 75 & CASS & $T$ & \\
\hline TE-102-AP-46 & $1 / \mathrm{has}$ & 74 & CASS & $T$ & \\
\hline TE-102.AP-46 & $1 / 16 / 93$ & 73 & CASS & $T$ & \\
\hline TE-102-AP-46 & $1 / 21 / 8$ & 74 & CASS & $T$ & \\
\hline TE-102-AP-16 & $1 / 29 / 93$ & 74 & CASS & $T$ & \\
\hline TE-102-AP-46 & $2 / 5 \% 3$ & 74 & CASS & $\bar{T}$ & \\
\hline TE-102AP-46 & $2 \pi 93$ & 73 & CASS & $T$ & \\
\hline TE-102-AP-46 & $2 / 15 / 93$ & 85 & CASS & $\mathbf{S}$ & Data point did not agree with local trand \\
\hline TE-102-AP-46 & 220093 & 73 & CASS & $\bar{T}$ & \\
\hline TE-102-AP-16 & $2 / 27193$ & 72 & CASS & $\bar{T}$ & \\
\hline$T E-102-A P-46$ & आ193 & 73 & SACS & $T$ & \\
\hline TE-102-AP-46 & $3 / 13 / 93$ & 75 & CASS & $T$ & \\
\hline TE-102-AP-46 & $3 / 20 / 93$ & 77. & SACS & $\mathbf{T}$ & \\
\hline TE-102-AP-46 & $3 / 27 / 93$ & 78 & CASS & $T$ & \\
\hline TE-102-AP-46 & $4 / 3 / 93$ & 80 & CASS & $T$ & \\
\hline TE-102-AP-46 & $46 \sqrt{93}$ & 82 & SACS & $T$ & \\
\hline TE-102-AP-46 & $417 / 93$ & 84 & CASS & $T$ & \\
\hline TE-102-APA6 & $4 / 2493$ & 86 & CASS & $T$ & \\
\hline TE-102-AP-46 & $4 / 29 \% 3$ & 87 & CASS & $T$ & \\
\hline TE-102-AP-46 & $5 / 7 / 93$ & 86 & CASS & $T$ & \\
\hline$T E-102-A P-46$ & 5/10/93 & 85 & SACS & $T$ & \\
\hline TE-102-AP-46 & $5 / 22193$ & 85 & CASS & $T$ & \\
\hline TE-102-AP-46 & $5 / 24 / 93$ & 74 & SACS & $\mathbf{S}$ & Disagreement between CASS and SACS \\
\hline TE-102-AP-46 & $\begin{array}{ll}5 / 27 / 93 \\
\end{array}$ & 85 & CASS & $T$ & \\
\hline TE-102-AP-A6 & $6 / 3 / 93$ & 84 & CASS & $T$ & \\
\hline TE-102-AP-A6 & $6 / 12 \times 3$ & 82 & CASS & $T$ & \\
\hline TE-102-AP-A6 & $6 / 17193$ & 8 & CASS & $\mathrm{T}$ & \\
\hline TE-102-AP-16 & $6 / 24 / 93$ & 82 & CASS & $\bar{T}$ & \\
\hline TE-102-AP-46 & $73 \times 3$ & 83 & CASS & $T$ & \\
\hline$T E-102 \cdot A P=46$ & 71593 & 82 & SACS & $T$ & \\
\hline TE-102-AP-46 & 7/16r93 & B1 & CASS & $T$ & \\
\hline TE-102-AP-46 & $7 / 23 / 93$ & 84 & CASS & $T$ & \\
\hline TE-102-AP-46 & $7 / 30 / 93$ & 82 & CASS & $T$ & \\
\hline TE-102-AP-46 & 8n/63 & 82 & CASS & $T$ & \\
\hline TE-102-AP-46 & $8 / 4493$ & 81 & CASS & $\bar{T}$ & \\
\hline TE-102-AP-46 & $8 / 20 / 93$ & 82 & CASS & $T$ & \\
\hline TE-102-AP= 46 & $8 / 25 / 93$ & 82 & CASS & $\mathrm{T}$ & \\
\hline TE-102-AP-46 & $9 / 2 / 93$ & 82 & CASS & $\bar{T}$ & \\
\hline
\end{tabular}




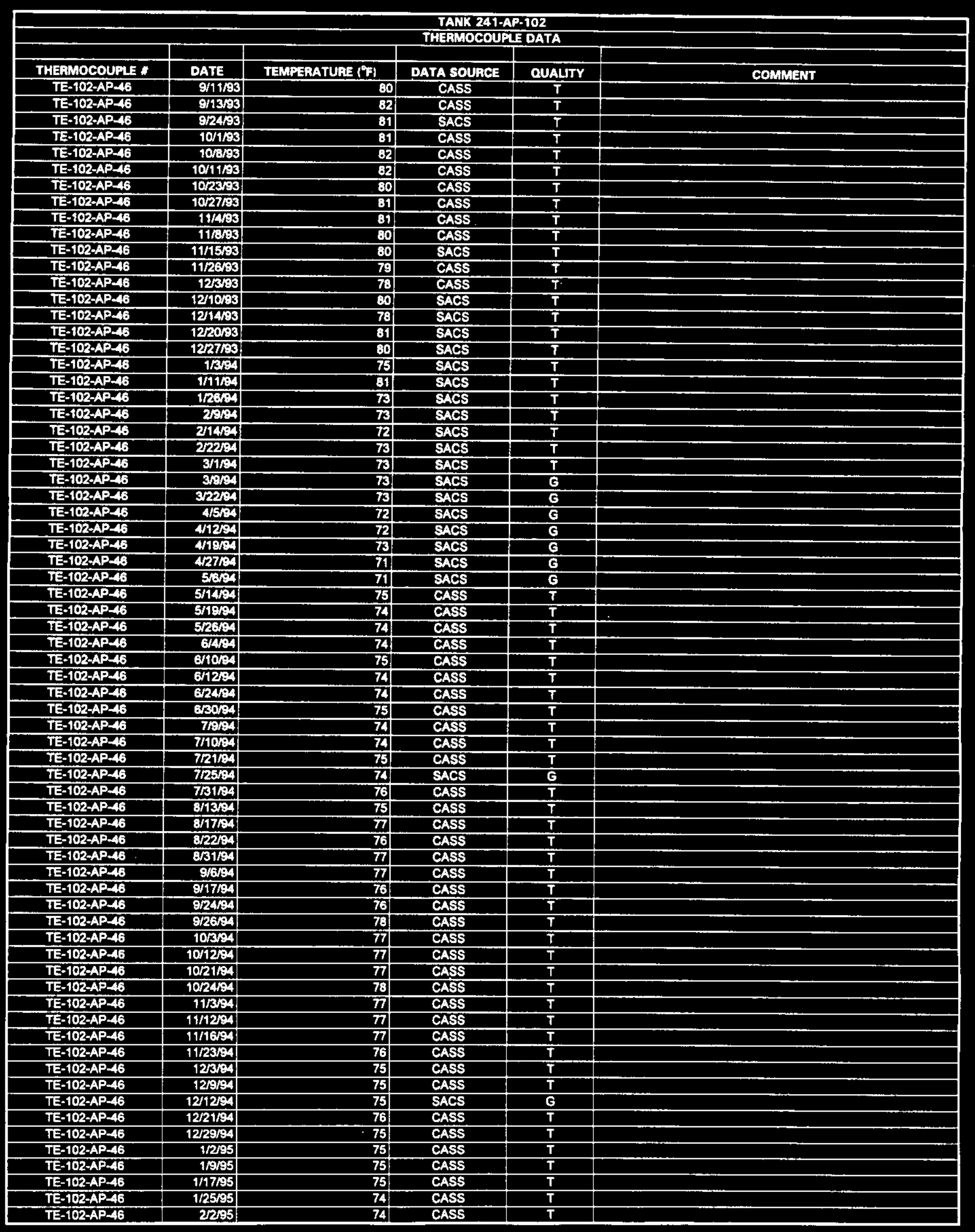




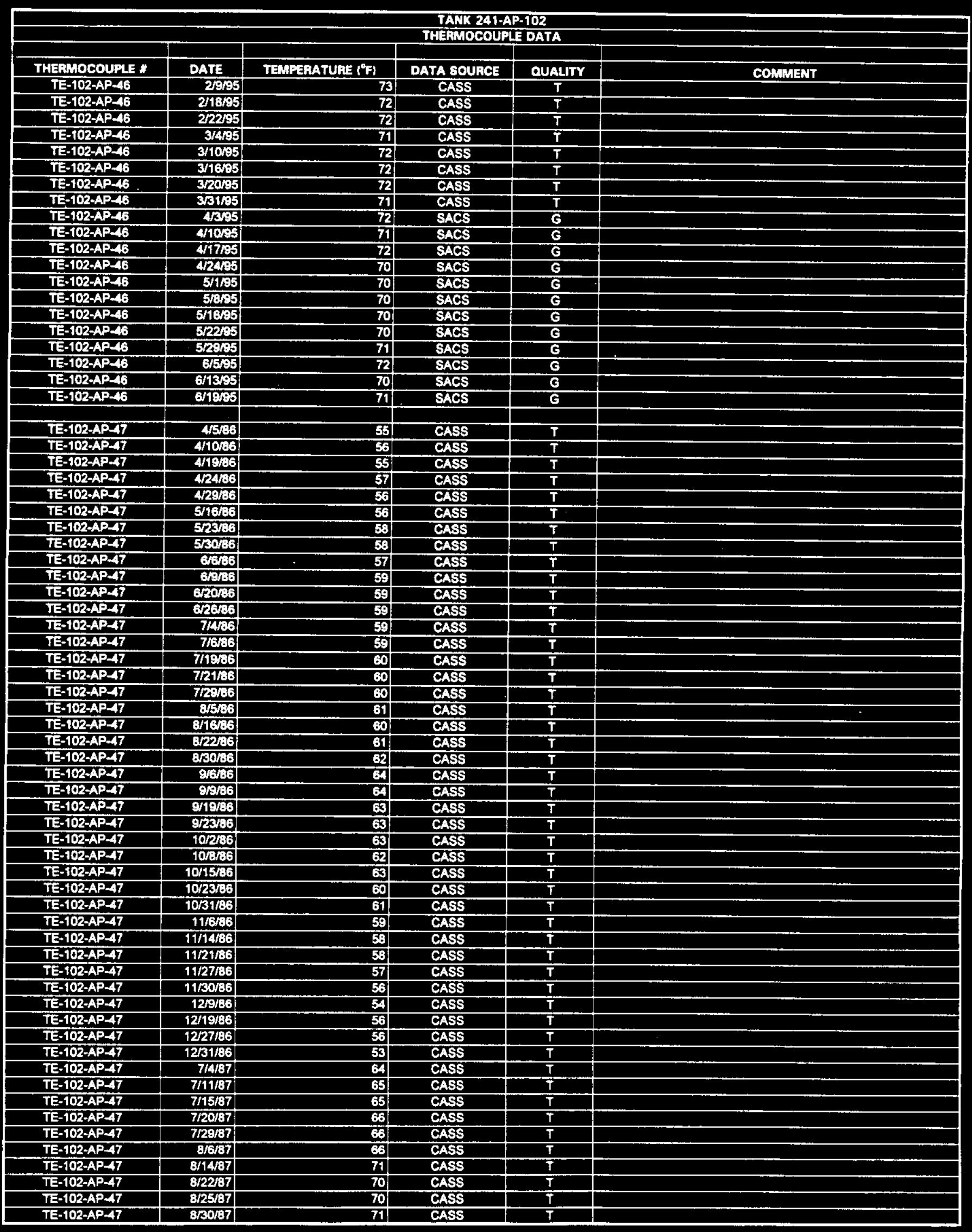

Data obtained from Computer Automated Surveillance System (CASS), April 1995; and Surveillance Analysis Computer System iSACS), June 1995.

$$
\text { D-198 }
$$




\begin{tabular}{|c|c|c|c|c|c|}
\hline \multicolumn{6}{|c|}{$\begin{array}{l}\text { TANK 241-AP-102 } \\
\text { THIROCOURE DATA }\end{array}$} \\
\hline & & & & & \\
\hline THEPMOCOUPLE * & DATE & TEMPGRATURE ( $\left.{ }^{\circ} \mathrm{F}\right)$ & DATA sounce & QUALTY & COMMENT \\
\hline TE-102-APAC & $9 / 11 / 87$ & 72 & CASS & $T$ & \\
\hline TE-102-AP-47 & $9 / 19 / 87$ & 73 & CASS & $T$ & \\
\hline TE-102-AP-47 & $9 / 26 / 67$ & 72 & CASS & $T$ & \\
\hline TE-102-AP-47 & $10 / 1 / 87$ & 72 & CASS & $T$ & \\
\hline$T E-102-A P-47$ & 10\%10/37 & 72 & CASS & $T$ & \\
\hline$T E-102-A P-47$ & $10 / 17 / 37$ & 72 & CASS & $T$ & \\
\hline$T E-102-A P-47$ & $10 / 24 / 37$ & 74 & CASS & $T$ & \\
\hline TE-102-AP-47 & $10 / 30187$ & 77 & CASS & $T$ & \\
\hline TE-102-AP-47 & $11 / 537$ & 78 & CASS & $\mathbf{T}$ & \\
\hline$T E-102+A P-47$ & $11 / 12 / 87$ & 77 & CASS & $\bar{T}$ & \\
\hline TE-102-AP-47 & $11198 \mathrm{~B} 7$ & 76 & CASS & $T$ & \\
\hline $7 E-102-A P-47$ & $11 / 22287$ & 75 & CASS & $T$ & \\
\hline TE-102-AP47 & 12587 & 78 & CASS & $T$ & \\
\hline TE-102-AP-47 & $12 / 87$ & 78 & CASS & $T$ & \\
\hline TE-102-AP-47 & 121987 & 75 & CASS & $T$ & \\
\hline TE-102-AP-47 & $12 / 2487$ & 72 & CASS & $T$ & \\
\hline TE-102-AP-47 & $12 / 29 / 87$ & 72 & CASS & $T$ & \\
\hline$T E-102-A P-47$ & $1 / \mathrm{a} / \mathrm{es}$ & 71 & CASS & $T$ & \\
\hline$T E-102-A P-47$ & $1 / 11 / 88$ & 72 & CASS & $T$ & \\
\hline$T E-102-A P-47$ & $1 / 23 / 88$ & 69 & CASS & $T$ & \\
\hline TE-102-AP-47 & $1 / 29 / 38$ & 71 & CASS & $T$ & \\
\hline$T E-102-A P=47$ & $2 / 6 / 38$ & 73 & CASS & $T$ & \\
\hline TE-102-AP-47 & $2 / 1388$ & 7 & CASS & $T$ & \\
\hline TE-102-AP-47 & 2/15/38 & 78 & CASS & $T$ & \\
\hline TE-102-AP-47 & 2/24/88 & 76 & CASS & $T$ & \\
\hline TE-102-AP-47 & 3508 & 78 & CASS & $T$ & \\
\hline TE-102-AP-47 & 3/2/8 & 74 & CASS & $\bar{T}$ & \\
\hline$T E-102-A P-47$ & $3 / 18 / 68$ & 78 & CASS & $T$ & \\
\hline TE-102-AP-47 & 3/20/68 & 76 & CASS & $T$ & \\
\hline TE-102-AP-47 & 3/2988 & 71 & CASS & $T$ & \\
\hline$T E-102-A P-47$ & 6/3/3a & 6 & CASS & $T$ & \\
\hline TE-102-AP-47 & 81538 & 65 & CASS & $T$ & \\
\hline TE-102-AP-A7 & 8/18/88 & 64 & CASS & $T$ & \\
\hline TE-102-AP-47 & 625183 & 64 & CASS & $T$ & \\
\hline TE-102-AP-47 & 6/29/38 & 64 & CASS & $T$ & \\
\hline TE-102-AP-47 & $7 / 6 / B a$ & 66 & CASS & $T$ & \\
\hline$T E-102-A P-47$ & $7 / 13 / 88$ & 65 & CASS & $T$ & \\
\hline TE-102-AP-47 & $7 / 22 / 38$ & 64 & CASS & $T$ & \\
\hline TE-102-AP-47 & 713008 & 65 & CASS & $T$ & \\
\hline TE-102-AP-47 & $8 / 488$ & 66 & CASS & $\bar{T}$ & \\
\hline$T E-102 \cdot A P-47$ & $8 / 10 / 0$ & 66 & CASS & $T$ & \\
\hline$T E-102-A P-47$ & $8 / 2083$ & E6 & CASS & $\mathrm{T}$ & \\
\hline TE-102-AP-47 & 8/21/88 & 65 & CASS & $\bar{T}$ & \\
\hline TE-102-AP-47 & $8 / 3183$ & 66 & CASS & $T$ & \\
\hline$T E-102-A P-47$ & 9/10/88 & 65 & CASS & $T$ & \\
\hline TE-102-AP-A7 & 9/13/83 & 67 & CASS & $T$ & \\
\hline TE-102-AP-47 & 9/22/88 & 66 & CASS & $T$ & \\
\hline E-102-AP-47 & $9 / 29 / 88$ & 67 & CASS & $T$ & \\
\hline TE-102-AP-47 & $12 / 3 / 88$ & 65 & CASS & $T$ & \\
\hline TE-102-AP-47 & 12/10/88 & 65 & CASS & $T$ & \\
\hline$T E-102 \cdot A P-47$ & $12 / 14 / 88$ & 64 & CASS & $T$ & \\
\hline$T E-102-A P-47$ & $12 / 18 / 88$ & 64 & CASS & $\mathbf{T}$ & \\
\hline$T E-102-A P-47$ & $7 / 10 / 89$ & 62 & SACS & $T$ & \\
\hline TE-102-AP-47 & $7 / 17 / 89$ & 63 & SACS & $T$ & \\
\hline TE-102-AP-47 & 7/24/89 & 64 & SACS & $T$ & \\
\hline TE-102-AP-47 & $8 / 7 \mathrm{se}$ & 69 & SACS & $T$ & \\
\hline TE-102-AP-47 & $8 / 1489$ & 69 & SACS & $T$ & \\
\hline TE-102-AP-47 & $8 / 21 / 89$ & 69 & SACS & $T$ & \\
\hline TE-102-APA7 & $9 / 2139$ & 68 & CASS & $T$ & \\
\hline$T E-102-A P-47$ & $9 \pi / 39$ & 70 & CASS & $T$ & \\
\hline TE-102-AP-47 & 9/10/80 & 69 & CASS & $T$ & \\
\hline$T E-102-A P-47$ & 9/20/89: & 68 & CASS & $T$ & \\
\hline TE-102-AP-47 & $9 / 28 / 89$ & 68 & CASS & $T$ & \\
\hline TE-102-AP-47 & $10 / 289$ & 68 & SACS & $T$ & \\
\hline TE-102-AP-47 & $10 / 9 / 89$ & 67 & SACS & $T$ & \\
\hline TE-102-AP-47 & $10 / 16 / 8$ & 66 & SACS & $T$ & \\
\hline$T E-102-A P-47$ & 10/26/99 & 66 & SACS & $T$ & \\
\hline TE-102-AP-47 & $11 / 2 / 89$ & 65 & CASS & $T$ & \\
\hline TE-102-AP-47 & 12/30/89 & 59 & CASS & $T$ & \\
\hline TE-102-AP-47 & $1 / 5 / 90$ & 60 & CASS & $T$ & \\
\hline
\end{tabular}




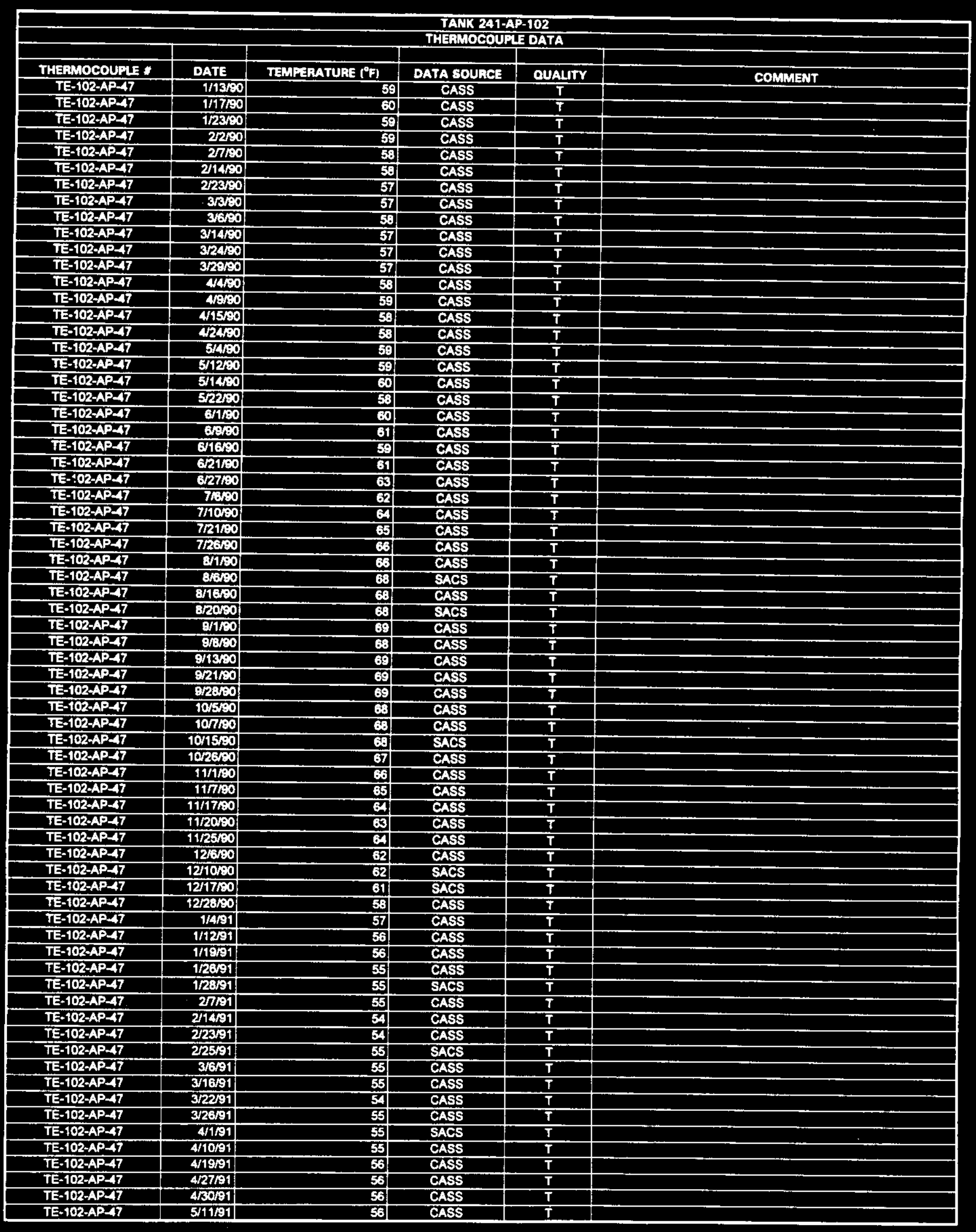




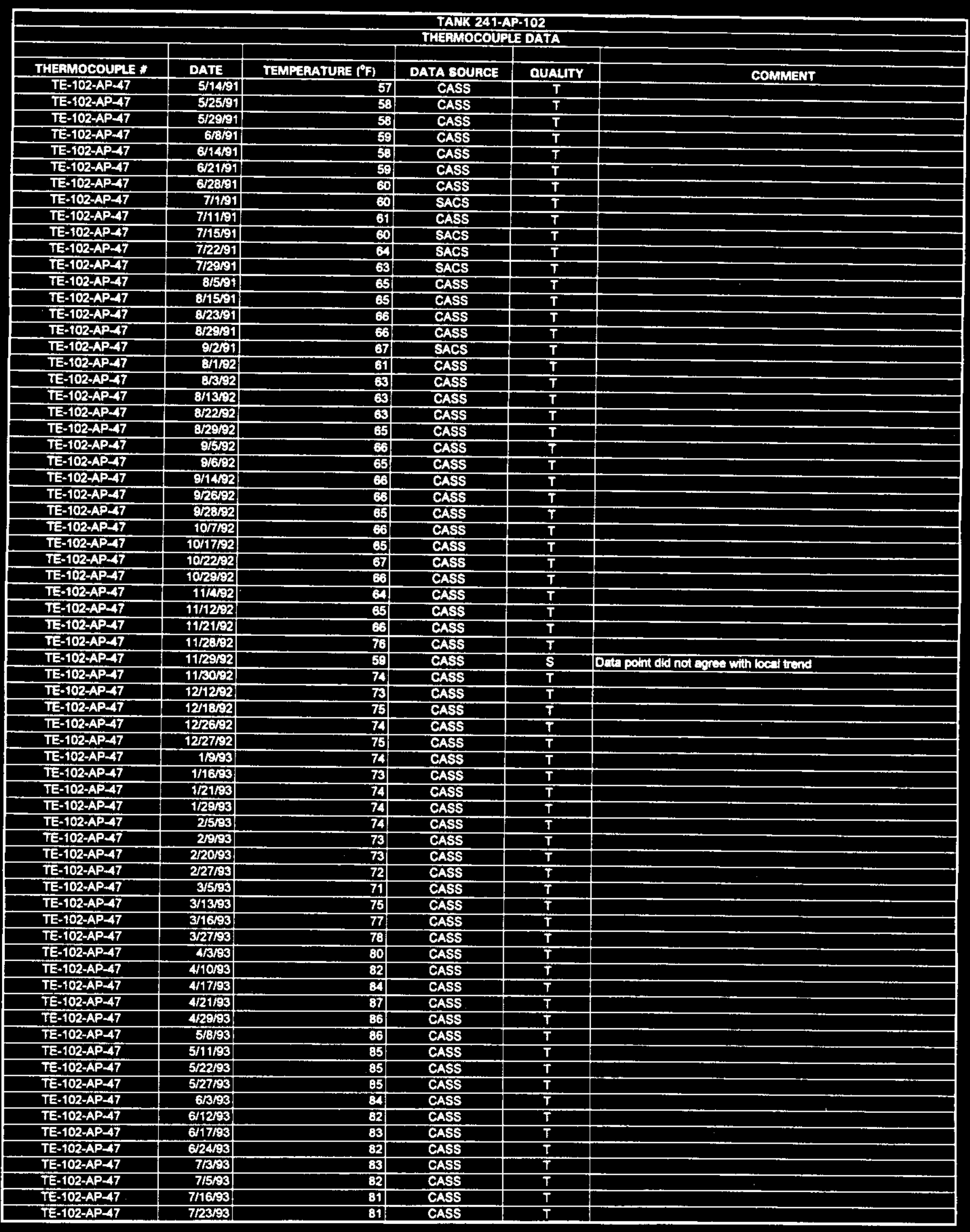




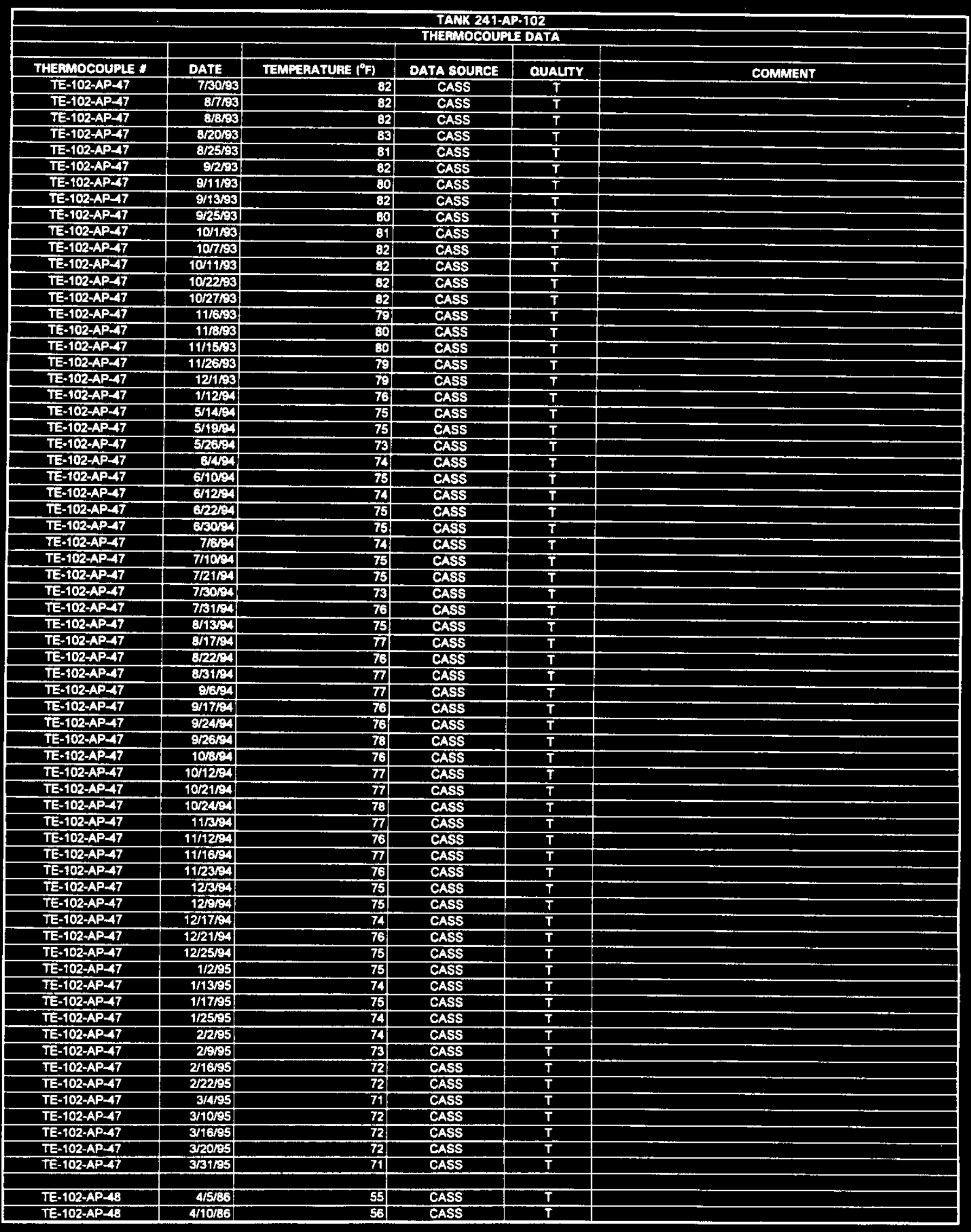

Data obtained from Computer Automated Surveillance Syatem (CASS), April 1995; and Surveillance Analysis Computer System (SACS), June 1995.

$$
\text { D-202 }
$$




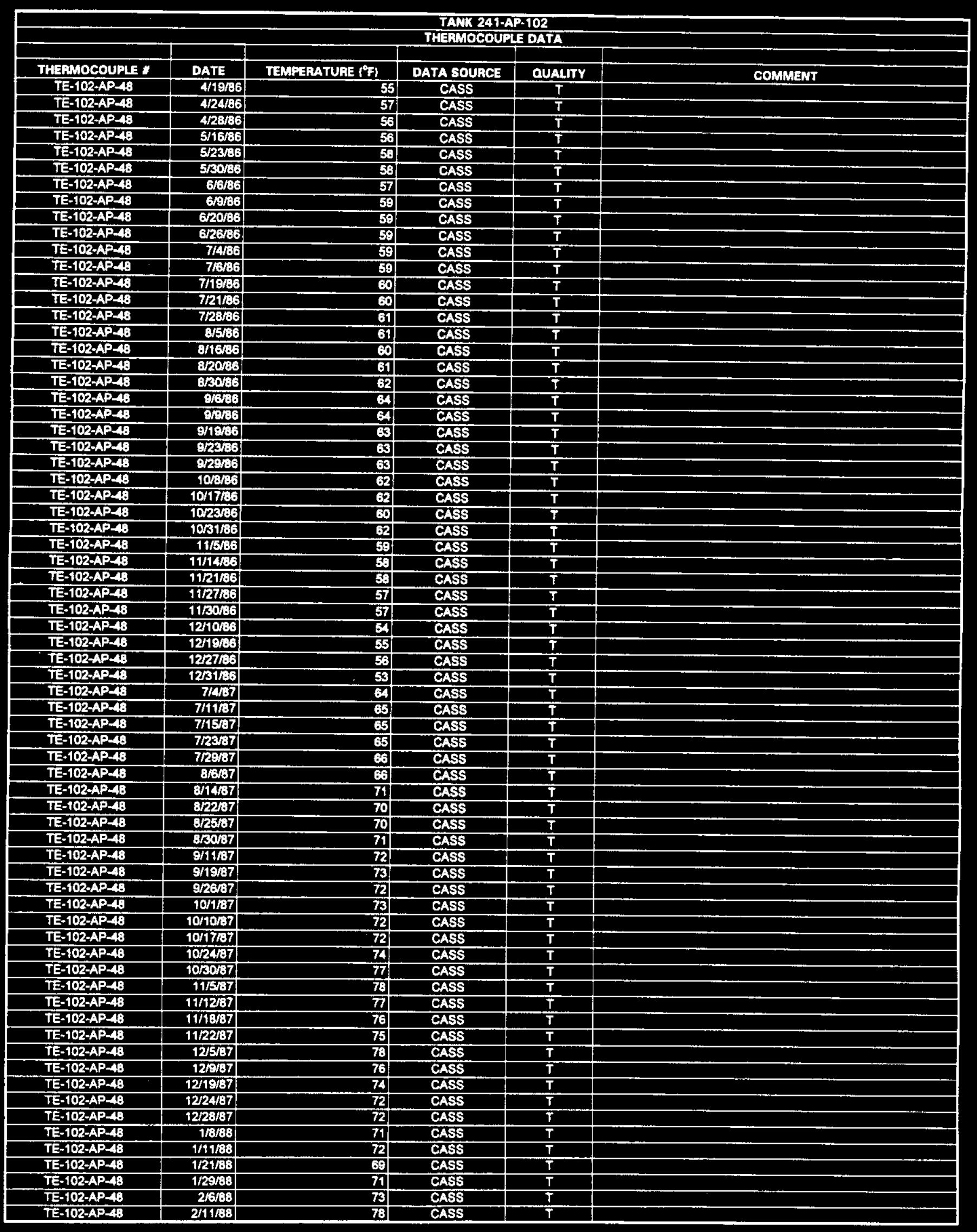




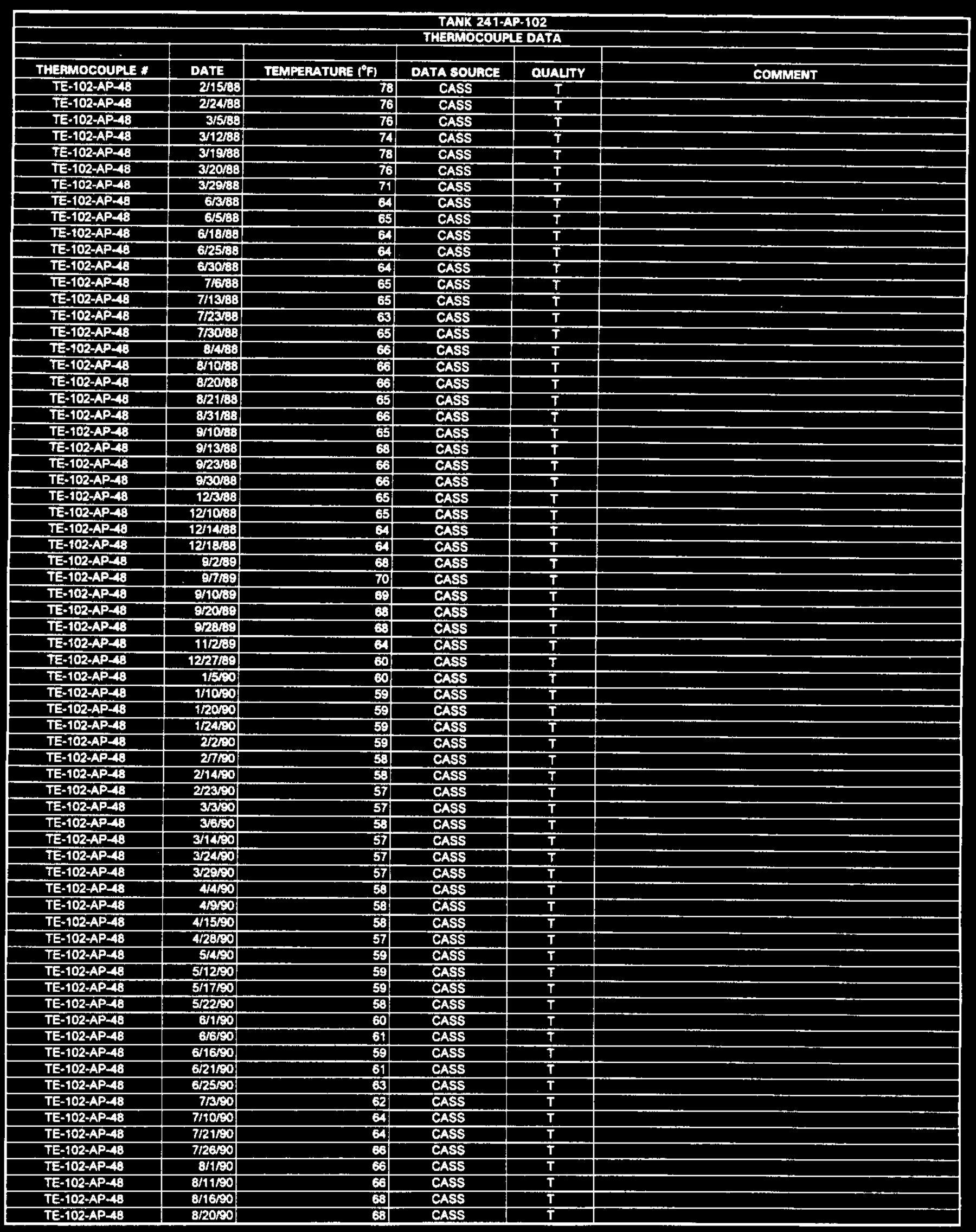




\begin{tabular}{|c|c|c|c|c|c|}
\hline \multicolumn{6}{|c|}{$\begin{array}{l}\text { TANK 241-AP.102 } \\
\text { THERMOCOUPLE DATA }\end{array}$} \\
\hline & & & & & \\
\hline THERMOCOUPLE: & DATE & TEMPERATUAE ( ${ }^{\circ} \mathrm{F}$ ) & DATA SOUACE & QUALITY & COMMENT \\
\hline$T E-102-A P-48$ & 9/1/50 & 69 & CASS & $T$ & \\
\hline TE-102-AP-48 & 98BOO & 68 & CASS & $T$ & \\
\hline$T E-102-A P-48$ & 9/13/90 & 69 & CASS & $\mathbf{T}$ & \\
\hline$T E-102-A P-48$ & $9 / 16 / 0$ & 69 & CASS & $T$ & \\
\hline TE-102-AP-48 & $9 / 28 / 90$ & 69 & CASS & $\bar{T}$ & \\
\hline TE-102-AP-48 & 10 sweo & 69. & CASS & $T$ & \\
\hline TE-102-AP-48 & $10 / 7 \% 0$ & 68 & CASS & $\mathbf{T}$ & \\
\hline TE-102-AP-48 & $10 / 2090$ & 66 & CASS & $T$ & \\
\hline$T E-102-A P-48$ & 102390 & 67 & CASS & $T$ & \\
\hline TE-102-AP-48 & $11 / 1 / 0$ & 66 & CASS & $\bar{T}$ & \\
\hline TE-102-AP-48 & $11 / 7 \% 0$ & 64 & CASS & $\mathbf{T}$ & \\
\hline TE-102-AP-48 & $11 / 1690$ & 64 & CASS & $\mathbf{T}$ & \\
\hline TE-102-AP-48 & $11 / 24 / 90$ & 62 & CASS & $T$ & \\
\hline TE-102-AP-4B & $11 / 2500$ & 64 & CASS & $T$ & \\
\hline TE-102-AP-48 & $12 \mathrm{Br} 00$ & 62 & CASS & $\bar{T}$ & \\
\hline$T E-102-A P-48$ & $12 / 010$ & 62 & CASS & $T$ & \\
\hline$T E-102-A P-48$ & 12/19se & 60 & CASS & $T$ & \\
\hline TE-102-AP-48 & $12 / 2890$ & 53 & CASS & $T$ & \\
\hline TE-102-AP-48 & $1 / 4 / 91$ & 57 & CASS & $T$ & \\
\hline TE-102-AP-4B & $18 \% 1$ & 56 & CASS & $T$ & \\
\hline TE-102-AP-48 & 1/1901 & 55 & CASS & $T$ & \\
\hline TE-102-AP-48 & 1/2601 & 55 & CASS & $T$ & \\
\hline TE-102-AP-48 & $2 / 291$ & 54 & CASS & $T$ & \\
\hline TE-102-AP-4B & $27 \% 1$ & 55. & CASS & $T$ & \\
\hline TE-102-AP-4B & 2/1691 & 53 & CASS & $T$ & \\
\hline JE-102-AP-43 & 272231 & 54 & CASS & $T$ & \\
\hline$T E-102-A P-48$ & 3201: & 53 & CASS & $T$ & \\
\hline TE-102-AP 48 & 3681 & 5 & CASS & $T$ & \\
\hline TE-102-AP-40 & 31091 & 55 & CASS & $T$ & \\
\hline$T E-102-A P-48$ & $322 \times 1$ & 54 & CASS & $T$ & \\
\hline TE-102-AP-48 & $3 \times 091$ & E4 & CASS & $T$ & \\
\hline TE-102-AP-4B & 4601 & 54 & CASS & $T$ & \\
\hline$T E-102-A P-48$ & 41291 & 54 & CASS & $T$ & \\
\hline TE-102-AP-48 & 4/PA1 & 56 & CASS & $T$ & \\
\hline TE-102-AP-4B & 427191 & 56 & CASS & $T$ & \\
\hline TE-102-AP-48 & 430A1 & 56 & CASS & $T$ & \\
\hline TE-102-AP448 & EI11A1 & 56) & CASS & $\mathbf{T}$ & \\
\hline TE-102-AP-48 & $5 / 1494$ & 57 & CASS & $T$ & \\
\hline TE-102-AP-48 & $5 / 2501$ & 58 & CASS & $T$ & \\
\hline TE-102-AP-46 & 6/1/91 & 57 & CASS & $T$ & \\
\hline TE-102-AP-48 & 6/B/91 & 59 & CASS & $T$ & \\
\hline TE-102-AP-43 & 61491 & 58 & CASS & $T$ & \\
\hline TE-102-AP-48 & 62001 & 59 & CASS & $T$ & \\
\hline$T E-102-A P-48$ & 6/20191 & 60 & CASS & $T$ & \\
\hline$T E-102-A P-48$ & $7 / 3191$ & 59 & CASS & $T$ & \\
\hline$T E-102-A P-40$ & 71191 & 61 & CASS & $T$ & \\
\hline TE-102-AP-48 & 71491 & 60 & CASS & $I$ & \\
\hline TE-102-AP-48 & $7 / 2491$ & 61 & CASS & $T$ & \\
\hline TE-102-AP-48 & $8 / 3 \times 1$ & 62 & CASS & $T$ & \\
\hline$T E-102-A P-48$ & 8/5/91 & 65 & CASS & $T$ & \\
\hline TE-102-AP-48 & $8 / 15 \wedge 1$ & 65 & CASS & $T$ & \\
\hline$T E-102-A P-48$ & 8/23491 & 66 & CASS & $T$ & \\
\hline TE-102-AP-48 & $8 / 29 / 91$ & 67 & CASS & $\mathbf{T}$ & \\
\hline TE-102-AP-16 & 9/31. & 67 & CASS & $T$ & \\
\hline TE-102AP-48 & $8 / 1 / 92$ & 61 & CASS & $T$ & \\
\hline TE-102-AP-48 & $8 / 8 / 92$ & 62 & CASS & $\bar{T}$ & \\
\hline TE-102-AP-48 & $8 / 13 / 92$ & 63 & CASS & $T$ & \\
\hline$T E-102-A P-48$ & $8 / 22192$ & 63 & CASS & $T$ & \\
\hline$T E-102-A P-48$ & $8 / 29 / 92$ & 64 & CASS & $T$ & \\
\hline TE-102-AP-48 & 9HS92 & 65 & CASS & $T$ & \\
\hline$T E-102-A P-48$ & $911 / 92$ & 64 & CASS & $T$ & \\
\hline TE-102-AP-48 & $9 / 14 / 22$ & 66 & CASS & $T$ & \\
\hline TE-102-AP-48 & $9 / 26 / 92$ & 66 & CASS & $T$ & \\
\hline TE-102-AP-48 & $10 / 1 / 92$ & 65 & CASS & $\mathbf{T}$ & \\
\hline TE-102-AP-48 & $107 / 92$ & 66 & CASS & $\mathbf{T}$ & \\
\hline TE-102-AP-48 & $10 / 17 / 92$ & 65. & CASS & $\mathbf{T}$ & \\
\hline TE-102-AP-48 & $10 / 22 / 92$ & 67 & CASS & $T$ & \\
\hline TE-102-AP-48 & $10 / 29 / 92$ & 65 & CASS & $T$ & \\
\hline TE-102-AP-48 & $11 / 492$ & 64 & CASS & $\bar{T}$ & \\
\hline TE-102-AP-48 & $11 / 12 / 92$ & 65 & CASS & $T$ & \\
\hline
\end{tabular}




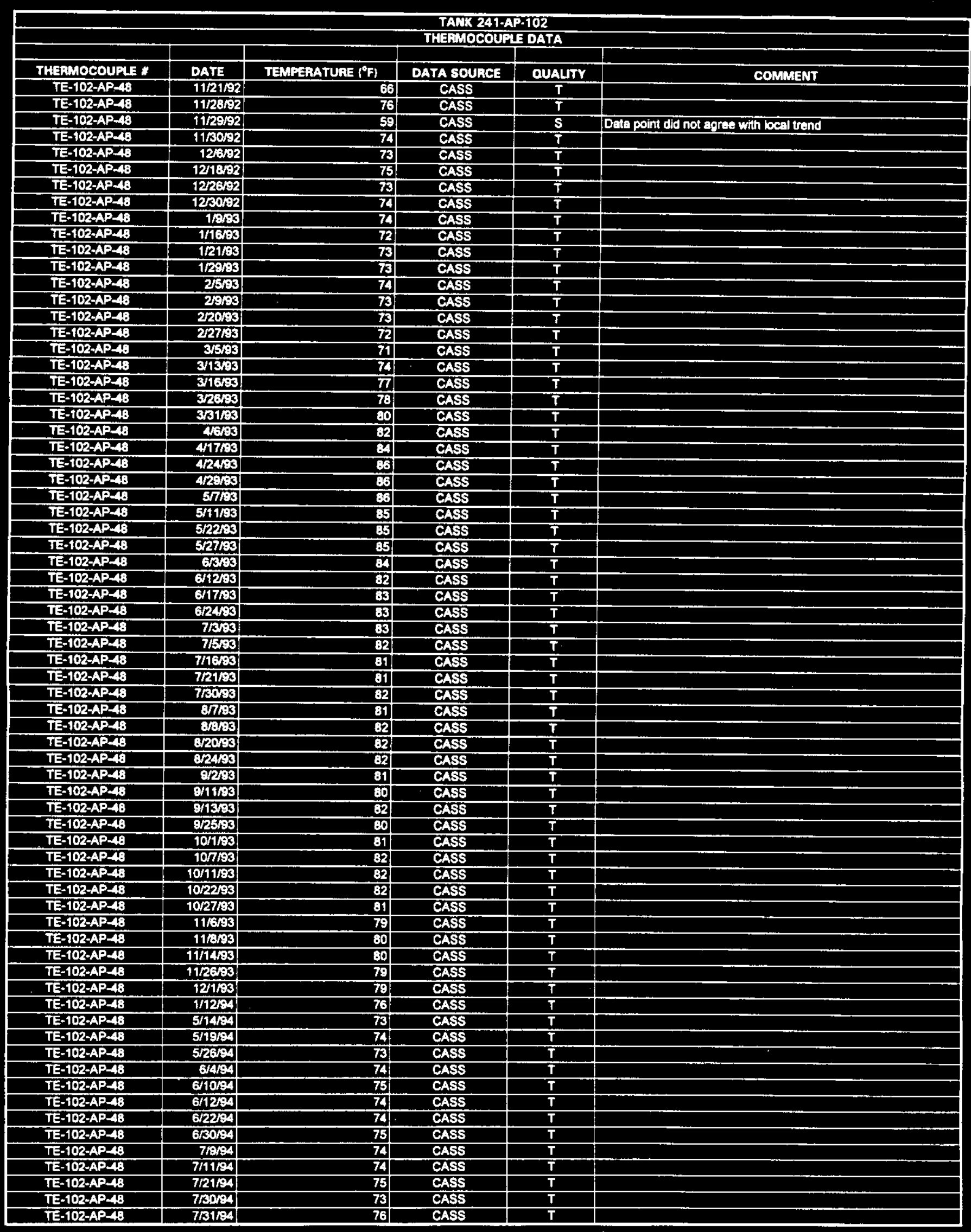




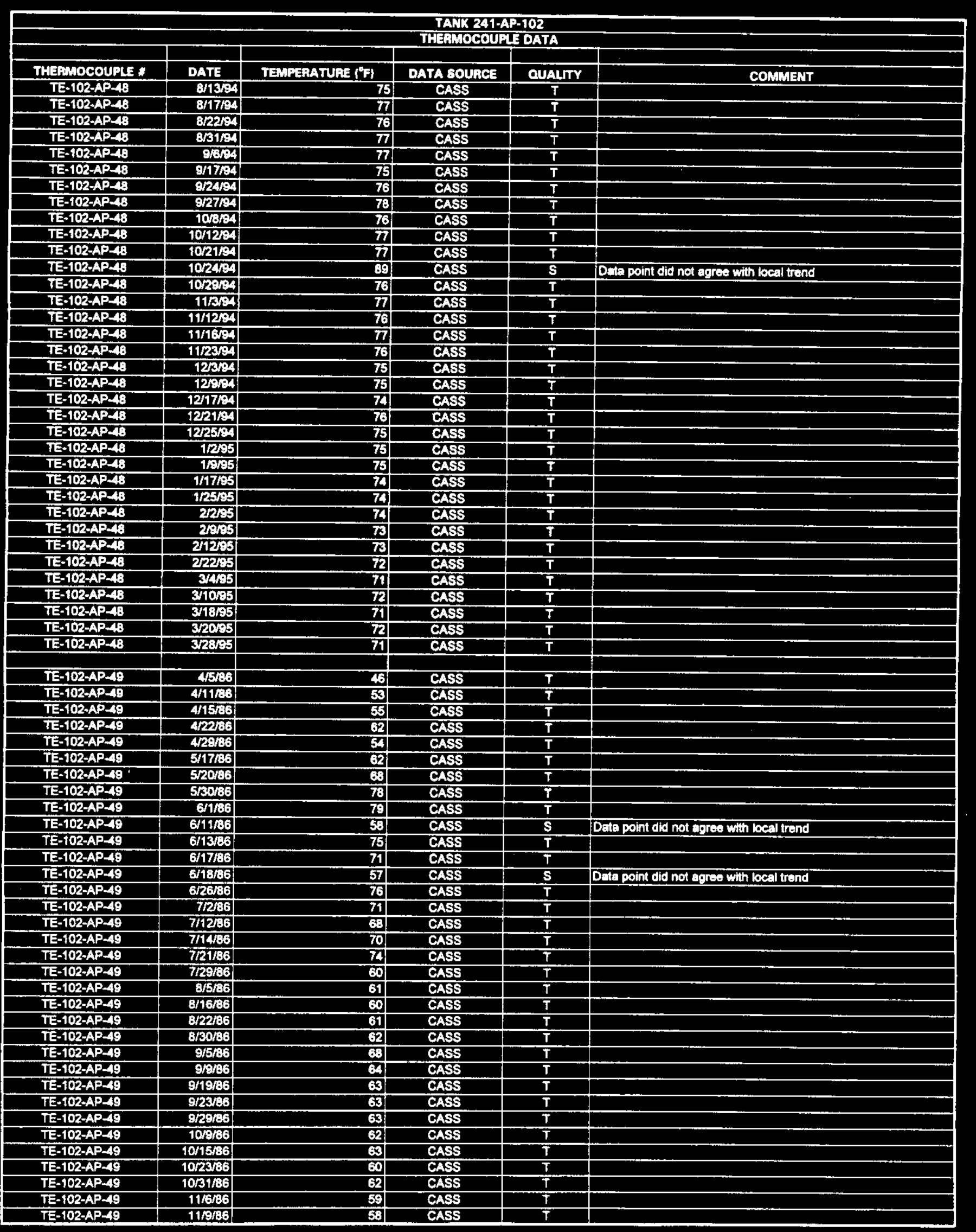




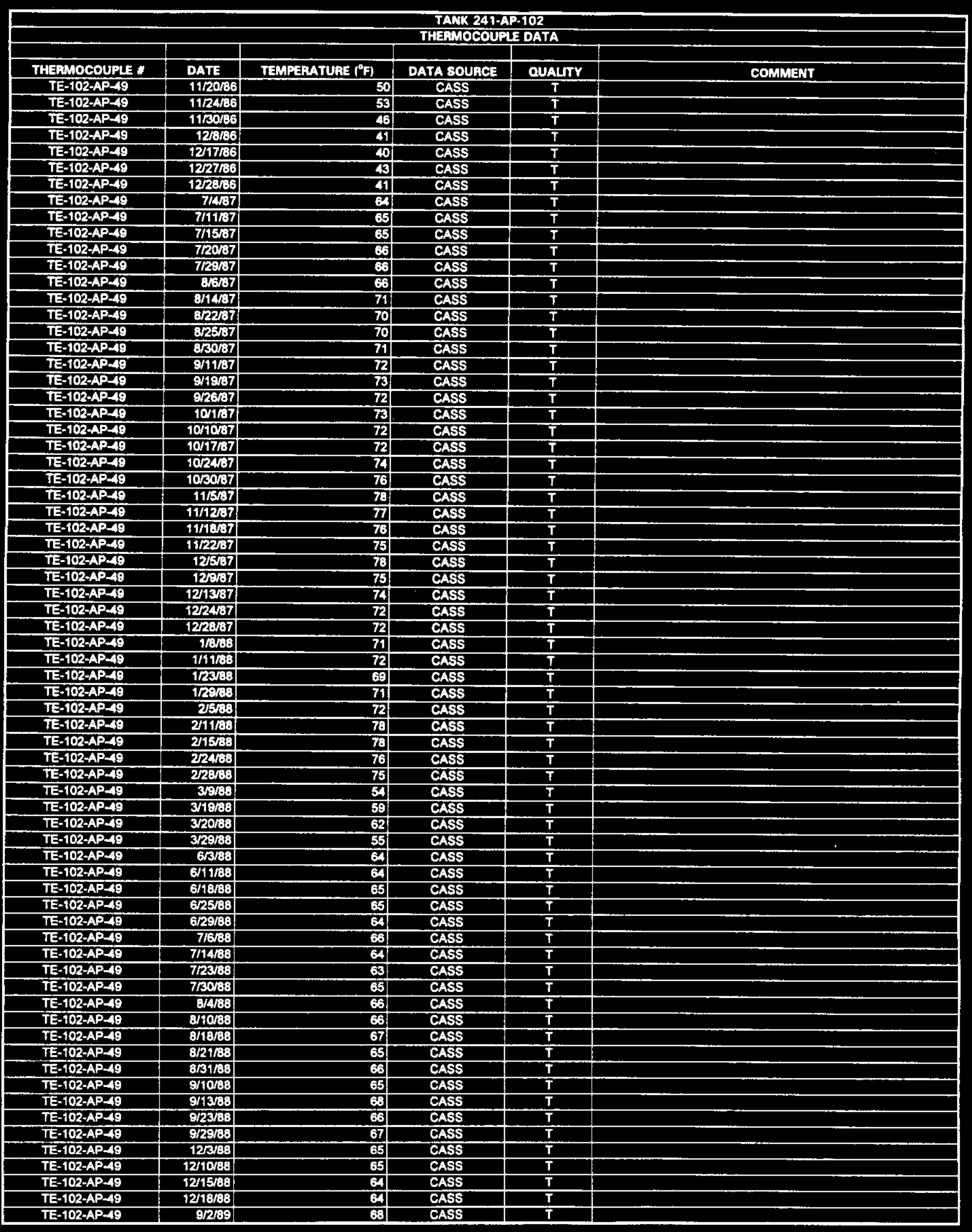

Data obtained from Computer Automated Surveillence System (CASS), April 1995; and Surveillance Analysis Computer System (SACS), June 1995.

$$
\text { D-208 }
$$




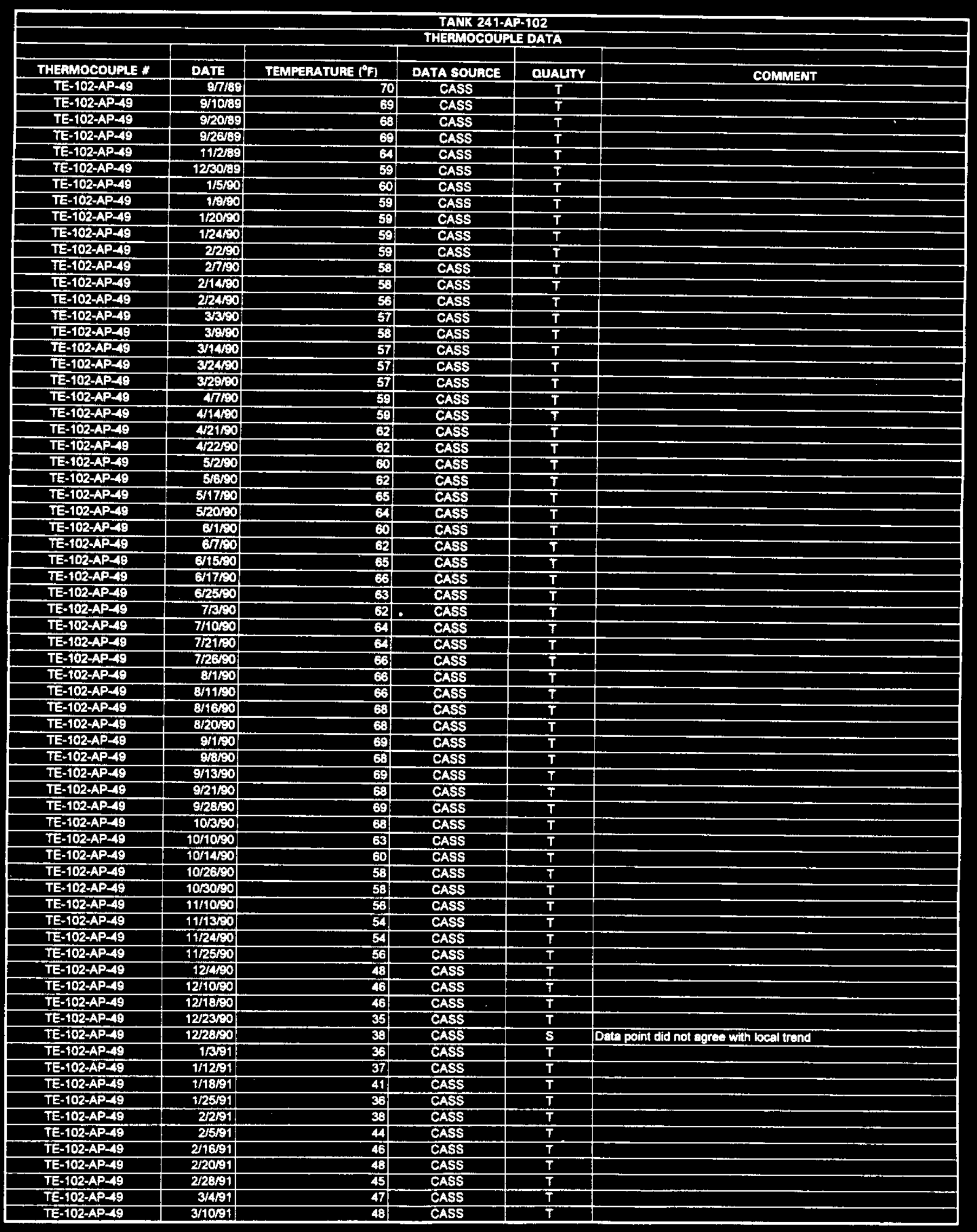




\begin{tabular}{|c|c|c|c|c|c|}
\hline \multicolumn{6}{|c|}{$\begin{array}{l}\text { TATK 241-AP-102 } \\
\text { THEFIMOCOUPLEDATA }\end{array}$} \\
\hline THERMOCOUPLE * & DATE & TEMPERATUAE $1^{\circ} \mathrm{F}$ ) & DATA sougce & gualty & \\
\hline TE-102-AP-49 & $3 / 22291$ & 50 & CASS & $\frac{\text { QUALIY }}{T}$ & COMMENT \\
\hline$T E-102-A P-49$ & 3/30/91 & 49 & CASS & $\bar{T}$ & \\
\hline TE-102-AP-19 & 45/91 & 55 & CASS & $T$ & \\
\hline TE-102-AP-49 & $4 / 13 \times 91$ & 5 & CASS & $T$ & \\
\hline TE-102-AP 49 & 420191 & 56 & CASS & $T$ & \\
\hline TE-102-AP-49 & $4 / 23 / 91$ & 59 & CASS & $T$ & \\
\hline TE-102-AP-49 & $5 / 491$ & 59 & CASS & $T$ & \\
\hline TE-102-AP- 49 & 5/591 & 60 & CASS & $T$ & \\
\hline TE-102-AP-A9 & 5/1691 & $\mathbf{6 0}$ & CASS & $T$ & \\
\hline TE-102-AP-49 & $5 / 22 / 91$ & 61 & CASS & $T$ & \\
\hline TE-102-AP- $4 \theta$ & $5 / 28 / 91$ & 611 & CASS & $T$ & \\
\hline TE-102-AP-A9 & $6 / 3 / 91$ & 59 & CASS & $T$ & \\
\hline TE-102-AP- 49 & 6/401 & 53 & CASS & $\mathbf{T}$ & \\
\hline TE-102-AP-49 & G/2001 & $59 !$ & CASS & $T$ & \\
\hline TE-102-AP-19 & 6/28\%1 & EO & CASS & $\bar{T}$ & \\
\hline TE-102-AP-18 & $7 / 3 / 91$ & 59 & CASS & $T$ & \\
\hline TE-102-AP- 19 & 7111/91 & 61 & CASS & $\mathbf{T}$ & \\
\hline TE-102-AP-49 & 771401 & 60 & CASS & $T$ & \\
\hline TE-102-AP-49 & 72491 & 61 & CASS & $T$ & \\
\hline TE-102-AP-49 & 6/3/91 & 62 & CASS & $\mathbf{T}$ & \\
\hline$T E-102-A P-19$ & B/5/91 & 65 & CASS & $\mathbf{T}$ & \\
\hline$T E=102-A P=-19$ & 8/15: & 85 & CASS & $T$ & \\
\hline TE-102:AP-49 & $8 / 2 \times 191$ & 66 & CASS & $T$ & \\
\hline TE-102-AP-49 & 8/2891 & 67 & CASS & $T$ & \\
\hline$T E-102 A P-49$ & 8/301 & 67 & CASS & $\bar{T}$ & \\
\hline$T E-102-A P-49$ & $8 / 192$ & 61 & CASS & $T$ & \\
\hline TE-102-AP- 49 & 8362 & 63 & CASS & $\mathbf{T}$ & \\
\hline TE-102-AP-A9 & 8/13:02 & 63 & CASS & $T$ & \\
\hline TE-102-AP-A9 & $8 / 2292$ & 63 & CASS & $T$ & \\
\hline$T E-102 \cdot A P-\triangle B$ & $8 / 2932$ & 85 & CASS & $\mathrm{T}$ & \\
\hline TE-102-AP-49 & 8/502 & 84 & CASS & $\bar{T}$ & \\
\hline TE-102-AP-49 & $9 / 62$ & 65 & CASS & $\mathbf{T}$ & \\
\hline$T E-102-A P-19$ & 9/1402 & 68 & CASS & $T$ & \\
\hline TE-102-AP-49 & 8/26/92 & G6 & CASS & $T$ & \\
\hline TE-102-AP- 48 & 10/192 & 65 & CASS & $T$ & \\
\hline TE-102-AP-49 & $10 \pi / 92$ & 6 & CASS & $\bar{T}$ & \\
\hline TE-102-AP-49 & $10 / 1762$ & 65) & CAss & $T$ & \\
\hline$T E-102-A P-49$ & $10 / 22192$ & 67 & CASS & $T$ & \\
\hline TE-102-AP-49 & $10 / 29182$ & 65 & CASS & $T$ & \\
\hline$T E-102-A P-49$ & 11/492 & EA & CASS & $T$ & \\
\hline TE-102-AP-A8 & $11 / \mathrm{a} / 2$ & 65 & CASS & $T$ & \\
\hline TE-102-AP-49 & $11 / 21 / 92$ & 66 & CASS & $T$ & \\
\hline TE-102-AP-49 & $11 / 28 / 92$ & 76 & CASS & $T$ & \\
\hline TE-102-AP-49 & $11 / 2902$ & 59 & CASS & $\mathrm{s}$ & De ta point did not coree with locel trend \\
\hline TE-TO2AP-A9 & $11 / 30 / 22$ & 74 & CASS & $\bar{T}$ & \\
\hline TE-102_AP-A9 & $12 / 6 / 92$ & 73 & CASS & $T$ & \\
\hline TE-102.AP- 49 & $12 / 18 / 92$ & 75 & CASS & $T$ & \\
\hline TE-102.AP- 49 & $12 / 26 / 92$ & 73 & CASS & $T$ & \\
\hline TE-102-AP-49 & 1213002 & 74 & CASS & $T$ & \\
\hline$T E-102-A P-49$ & $1 / 9 / 9$ & 73 & CASS & $T$ & \\
\hline TE-102-AP-49 & 1/16/93 & 72 & CASS & $\bar{T}$ & \\
\hline TE-102-AP-A9 & $1 / 21 / 93$ & 73 & CASS & $T$ & \\
\hline$T E-102-A P-49$ & 1/30/93 & 72 & CASS & $T$ & \\
\hline TE-102-AP-49 & $215 \% 3$ & 74 & CASS & $T$ & \\
\hline TE-102-AP-49 & $2 / 9193$ & 73 & CASS & $T$ & \\
\hline TE-102-AP-A9 & $2 / 19 / 93$ & 73 & CASS & $T$ & \\
\hline TE-102-AP-A9 & 2/27/93 & 72 & CASS & $\bar{T}$ & \\
\hline$T E-102-A P-49$ & 3/5/93 & 71 & CASS & $T$ & \\
\hline TE-102-AP-49 & 3/13/93 & 74 & CASS & $\mathbf{T}$ & \\
\hline TE-102-AP-49 & $3 / 16 / 93$ & 77 & CASS & $T$ & \\
\hline$T E-102-A P=49$ & $3 / 27 / 93$ & 77 & CASS & $T$ & \\
\hline TE-102-AP-A9 & $3 / 31 / 43$ & 80 & CASS & $T$ & \\
\hline TE-102-AP- 19 & $4 / 6 / 93$ & 82 & CASS & $T$ & \\
\hline TE-102-AP-49 & $4 / 17193$ & 84 & CASS & $T$ & \\
\hline TE-102-AP-49 & $4 / 24 / 93$ & 86 & CASS & $T$ & \\
\hline TE-102-AP-49 & 4/29/93 & 86 & CASS & $T$ & \\
\hline TE-T02-AP-49 & $5 / 8 / 93$ & 86 & CASS & $\bar{T}$ & \\
\hline$T E-102-A P-19$ & 5/11/93 & 85 & CASS & $T$ & \\
\hline TE-102-AP-A9 & $5 / 22 / 93$ & 85 & CASS & $T$ & \\
\hline$T E-102-A P-49$ & 5/27/93 & 85 & CASS & $T$ & \\
\hline
\end{tabular}




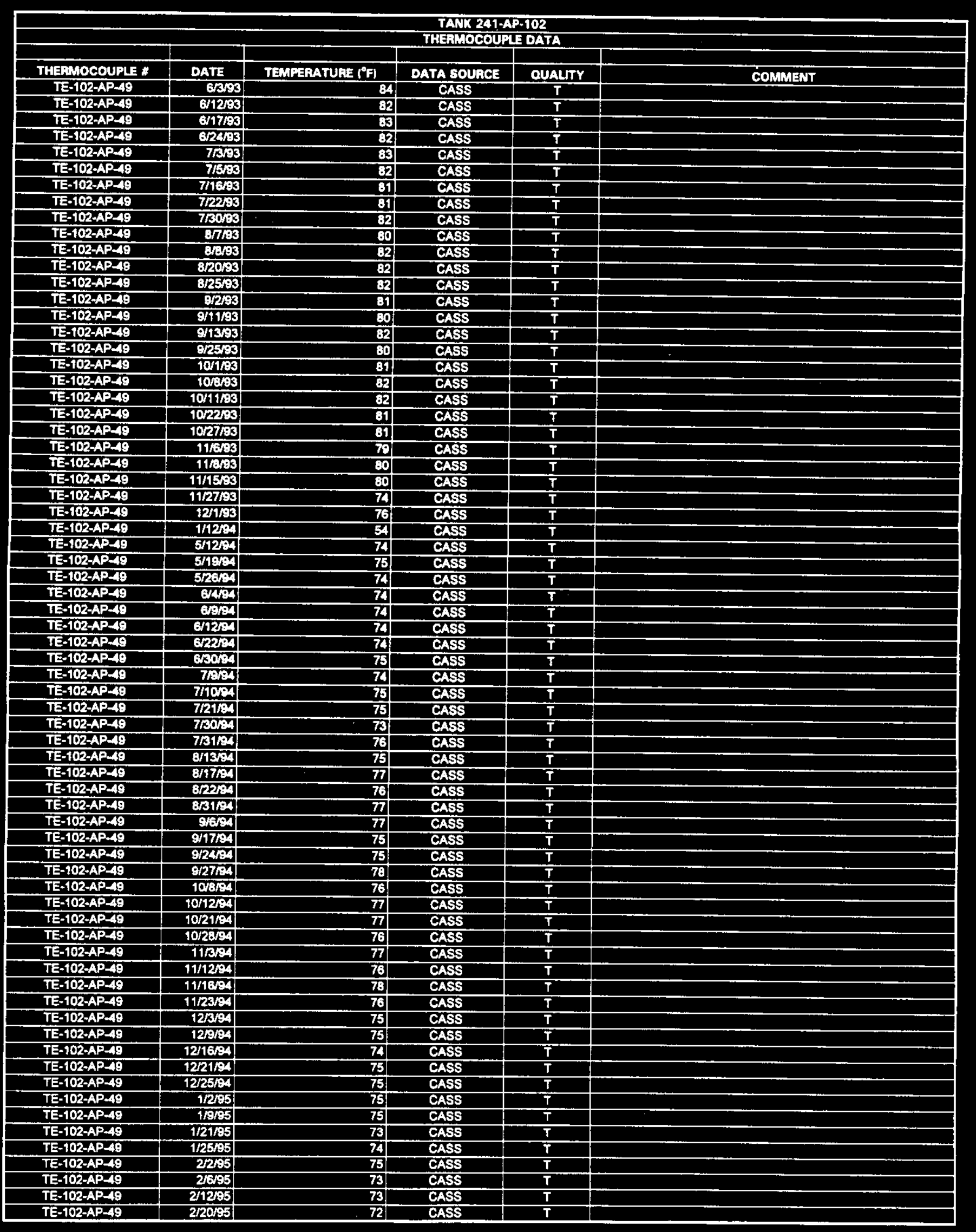




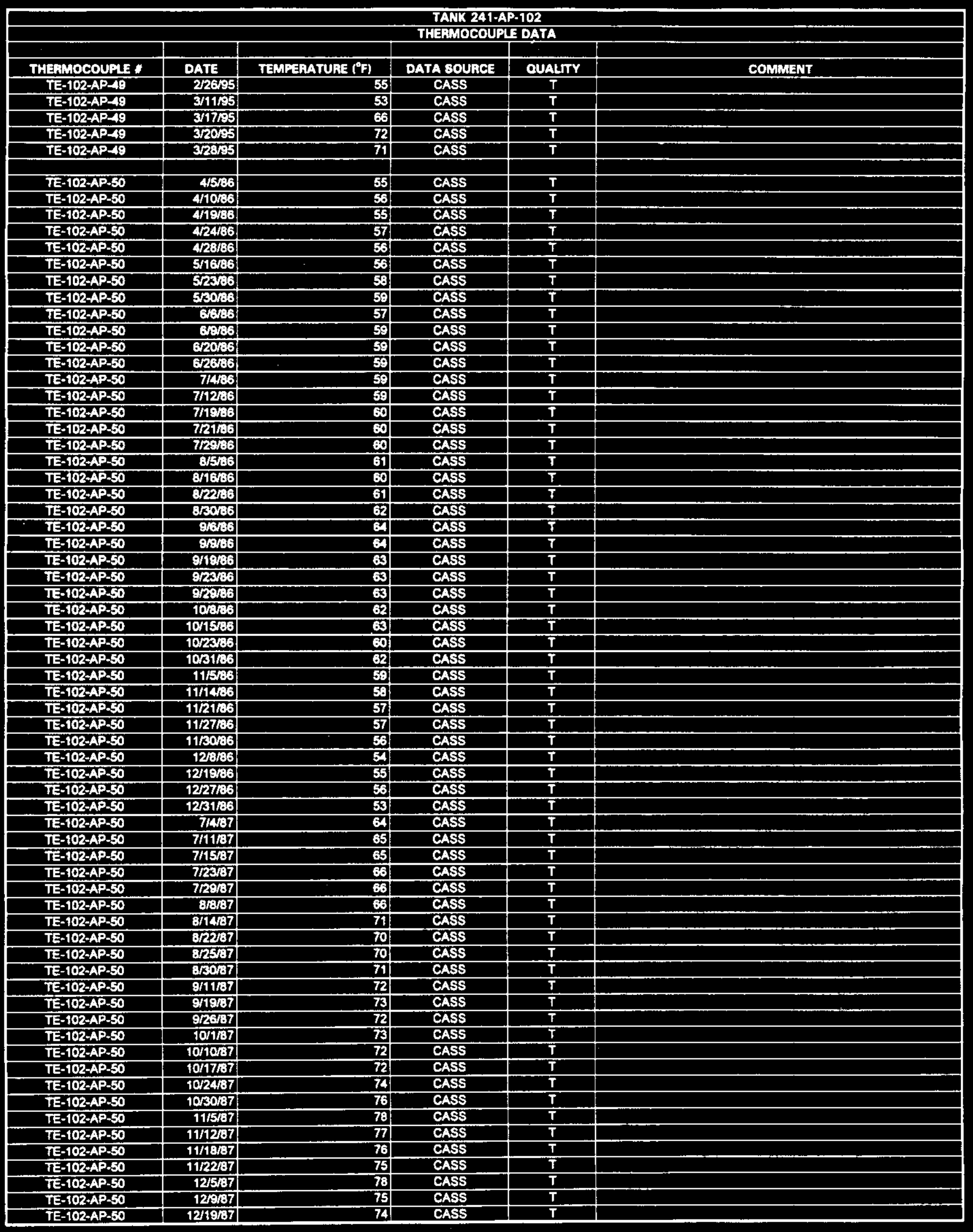

Data obtained from Computer Automated Surveillance System (CASS), April 1995; and Surveillance Analysis Computer System (SACS), June 1995. 


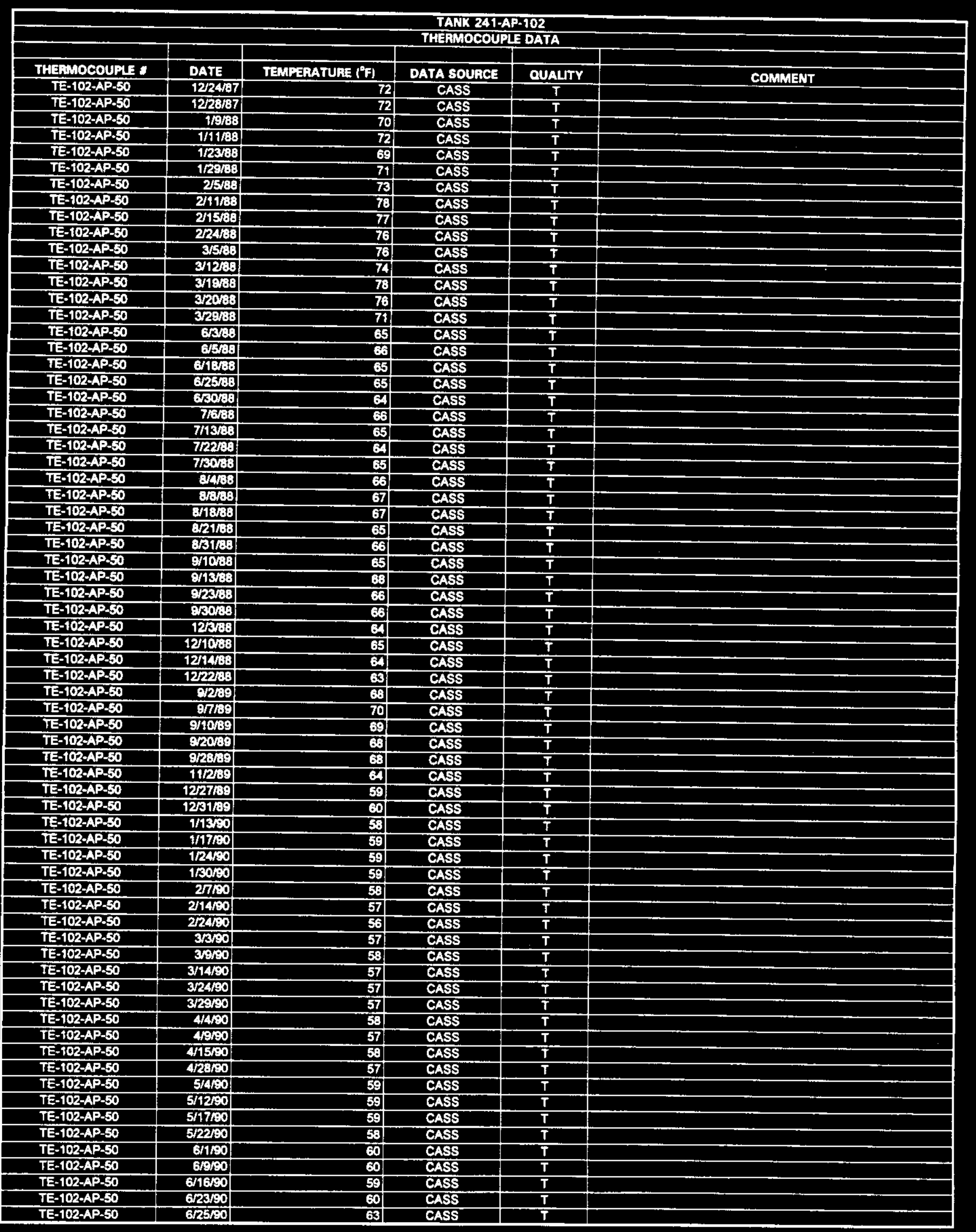




\begin{tabular}{|c|c|c|c|c|c|}
\hline \multicolumn{6}{|c|}{$\begin{array}{l}\text { TANK 241.AP-102 } \\
\text { IHEAMOCOUPLE DATA }\end{array}$} \\
\hline & & & & & \\
\hline THEPMOCOUPLE * & DATE & TEMPERATUAE $\left({ }^{\circ} \mathrm{F}\right)$ & DATA SOUACE & QUALITY & COMMENT \\
\hline TE-102.AP-50 & 73150 & 62 & CASS & $T$ & \\
\hline TE-102-AP-50 & $7 / 10 / 00$ & 64 & CASS & $\bar{T}$ & \\
\hline TE-102-AP-50 & $7 / 2160$ & 64 & CASS & $T$ & \\
\hline TE-102-AP-50 & 72650 & 66 & CASS & $T$ & \\
\hline TE-102-AP-50 & B/150 & 63 & CASS & $T$ & \\
\hline TE-102-AP-S0 & 8/11/50 & 66 & CASS & $T$ & \\
\hline TE-102-AP-50 & $8 / 16 \% 0$ & 68 & CASS & $T$ & \\
\hline$T E=102-A P-50$ & $8 / 2060$ & 68 & CASS & $T$ & \\
\hline TE-102-AP-50 & 8/1/00 & 69 & CASS & $T$ & \\
\hline TE-102-AP-50 & gaso & 68 & CASS & $T$ & \\
\hline TE-102-AP-50 & 9/1350 & 69 & CASS & $T$ & \\
\hline TE-102-AP-50 & 9/19/80 & 69 & CASS & $T$ & \\
\hline TE-102-AP-50 & 8/2390 & 69 & CASS & $T$ & \\
\hline TE-102-AP-S0 & 10/560! & 63 & CASS & $\bar{T}$ & \\
\hline TE-102-AP-50 & $10 / 10 \% 0$ & 67 & CASS & $T$ & \\
\hline TE-102-AP-50 & $10 / 1500$ & 66 & CASS & $T$ & \\
\hline TE-102-AP-50 & $10 / 26 / 50$ & 67 & CASS & $T$ & \\
\hline TE-102-AP-S0 & 102000 & 66 & CASS & $T$ & \\
\hline TE-102-AP-50 & $11 \pi / 50$ & 64 & CASS & $T$ & \\
\hline TE-102-AP-50 & $11 / 1350$ & EA & CASS & $T$ & \\
\hline TE-102-AP-50 & $11 / 24 / 80$ & 62 & CASS & $T$ & \\
\hline TE-102-AP-S0 & $11 / 2500$ & 63 & CASS & $T$ & \\
\hline TE-102-AP. 50 & 1213:0 & 62 & CASS & $T$ & \\
\hline TE-102.AP-BO & $12 / 13 \mathrm{SO}$ & 60 & CASS & $T$ & \\
\hline TE-102-AP-50 & $12 \pi 1600$ & 61 & CASS & $T$ & \\
\hline TE-102-AP-SO & 12128300 & 59 & CASS & $T$ & \\
\hline TE-102-AP-S0 & $12 / 3000$ & 57 & CASS & $T$ & \\
\hline TE-102.AP-SO & $1 / 7 \% 1$ & $\mathbf{5 6}$ & CASS & $\bar{T}$ & \\
\hline TE-102-AP-50 & $1 / 1 \% 91$ & S3 & CASS & $T$ & \\
\hline TE-102-AP-50 & $1 / 2691$ & 3 & CASS & $T$ & \\
\hline TE-102-AP-S0 & 1/2008 & 51 & CASS & $\bar{T}$ & \\
\hline TE-102-AP-50 & 21301 & S4 & CASS & $T$ & \\
\hline TE-102-AP-50 & 21691 & 53 & CASS & $T$ & \\
\hline TE-102-AP-SO & $2 / 21 / 31$ & 5. & CASS & $T$ & \\
\hline TE-102-AP-50 & 32291 & $\mathbf{5 3}$ & CASS & $T$ & \\
\hline TE-102-AP.50 & 36601 & 54 & CASS & $T$ & \\
\hline TE-102AP.50 & 31091 & 55 & CASS & $T$ & \\
\hline TE-102-APSDO & $3 / 2251$ & 54 & CASS & $T$ & \\
\hline TE-102-AP.50 & $3 / 26 \% 1$ & 55 & CASS & $T$ & \\
\hline TE-102-AP-50 & 4691 & $\mathbf{5 3}$ & CASS & $T$ & \\
\hline TE-102-AP-50 & $4 / 1091$ & 54 & CASS & $T$ & \\
\hline TE-102-AP-S0 & $4 / 1901$ & 55 & CASS & $\bar{T}$ & \\
\hline TE-102-AP-50 & 4/2791 & S6 & CASS & $T$ & \\
\hline TE-102-AP-50 & 43001 & S6 & CASS & $T$ & \\
\hline TE-102-AP-SO & 5/11/91 & 56 & CASS & $T$ & \\
\hline TE-102-AP-50 & 5/14/91 & 57 & CASS & $T$ & \\
\hline TE-102-AP-50 & 5/2561 & 57 & CASS & $T$ & \\
\hline TE-102-AP-50 & $6 / 1 / 91$ & 57 & CASS & $T$ & \\
\hline TE-102-AP-50 & 6/3/91 & 59 & CASS & $T$ & \\
\hline TE-102-AP-50 & 6/14/91 & 58 & CASS & $T$ & \\
\hline TE-102-AP-50 & 6/20/91 & 59 & CASS & $T$ & \\
\hline TE-102-AP-SO & $6 / 28 / 91$ & 60 & CASS & $\bar{T}$ & \\
\hline TE-102-AP.SO & $73 / 91$ & 59 & CASS & $T$ & \\
\hline TE-102-AP-50 & 7/13/91 & 61 & CASS & $T$ & \\
\hline TE-102-AP-50 & 7/14/91 & 61 & CASS & $\bar{T}$ & \\
\hline TE-102-AP-50 & $7 / 24 / 91$ & 62 & CASS & $T$ & \\
\hline TE-102-AP-50 & $8 / 3 / 91$ & 62 & CASS & $T$ & \\
\hline TE-102-AP-50 & $8 / 5 / 91$ & 65 & CASS & $\bar{T}$ & \\
\hline TE-102-AP-50 & $8 / 15 \% 1$ & 65 & CASS & $T$ & \\
\hline TE-102-AP-50 & $8 / 23191$ & 66 & CASS & $T$ & \\
\hline TE-102-AP-S0 & $8 / 2901$ & 67 & CASS & $T$ & \\
\hline TE-102-AP-SO & $9 / 3 / 91$ & 66 & CASS & $T$ & \\
\hline TE-102-AP-50 & $8 / 1 / 12$ & 61 & CASS & $T$ & \\
\hline TE-102-AP-50 & $8 / 6192$ & 62 & CASS & $\bar{T}$ & \\
\hline TE-102-AP-50 & $8 / 13 / 92$ & 63 & CASS & $T$ & \\
\hline TE-102-AP-SO & $8 / 22 / 92$ & 63 & CASS & $T$ & \\
\hline TE-102-AP-50 & $8 / 29 / 92$ & 63 & CASS & $T$ & \\
\hline TE-102-AP-SO & 9/B/2 & 64 & CASS & $T$ & \\
\hline TE-102-AP-50 & 9602 & 65 & CASS & $T$ & \\
\hline TE-102-AP-50 & $9 / 1492$ & 65 & CASS & $T$ & \\
\hline
\end{tabular}




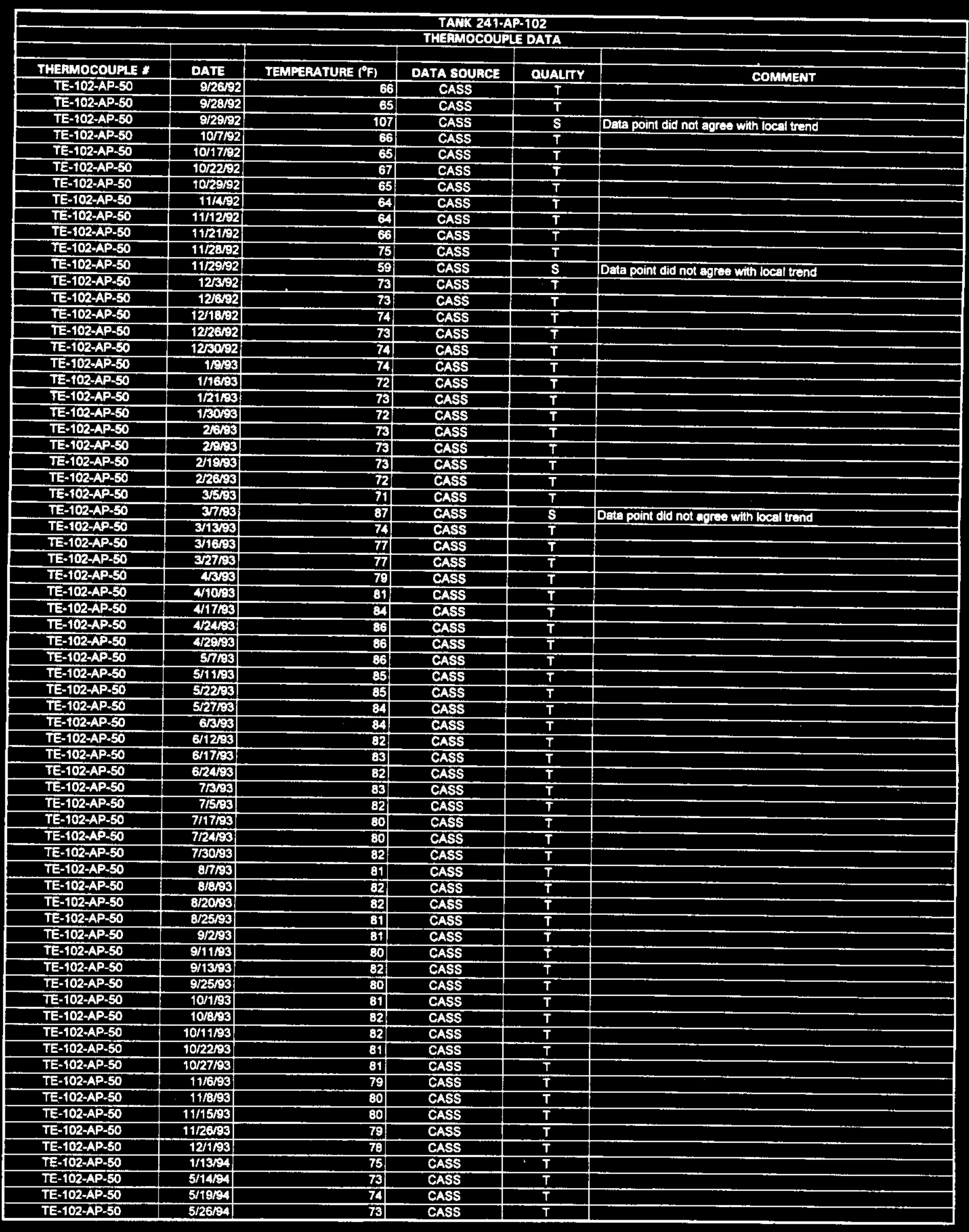




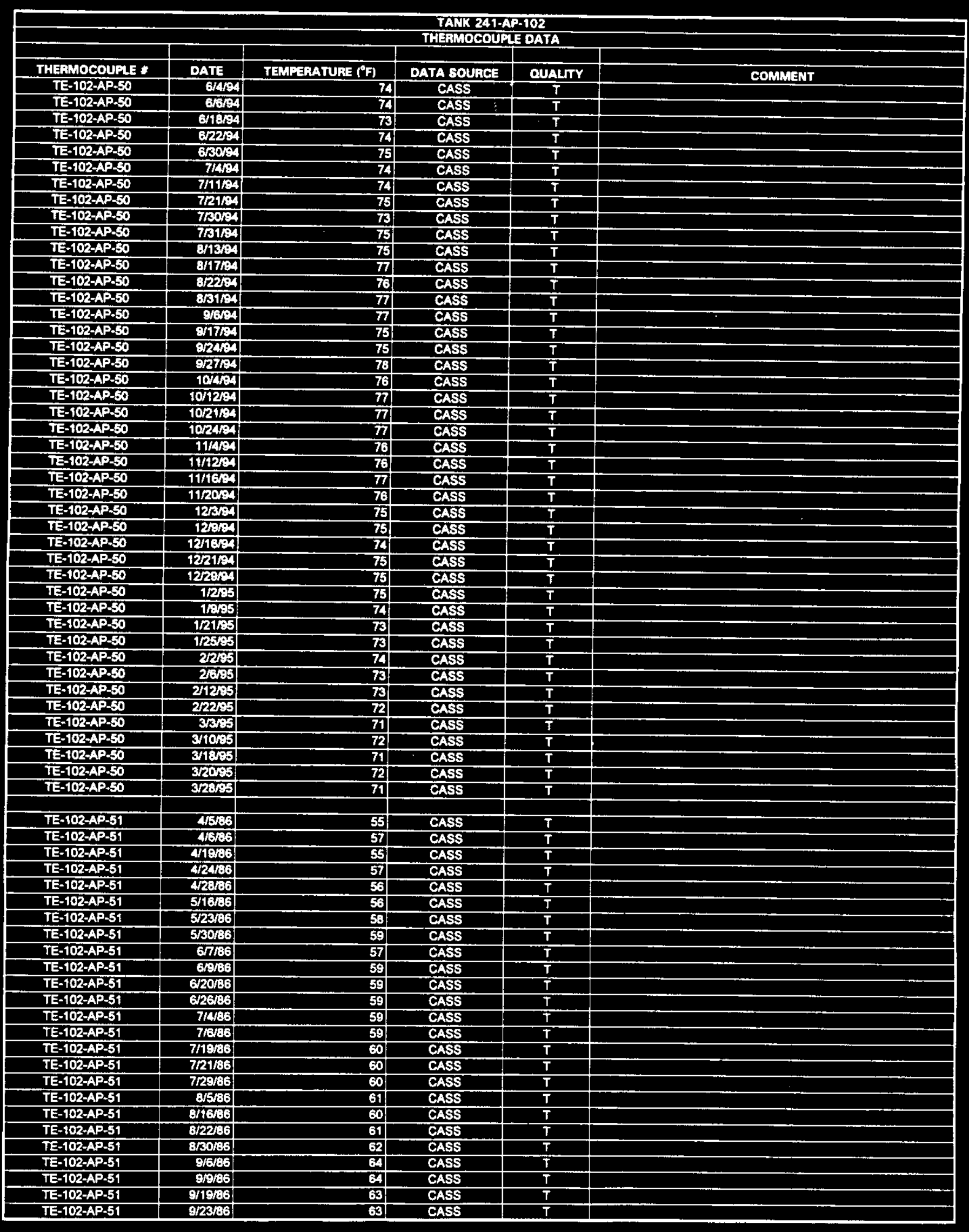

Data obtained from Computer Automated Surveillance Syatem (CASS), April 1995; and Surveillance Analysis Computer Syatem (SACS), June 1995. 


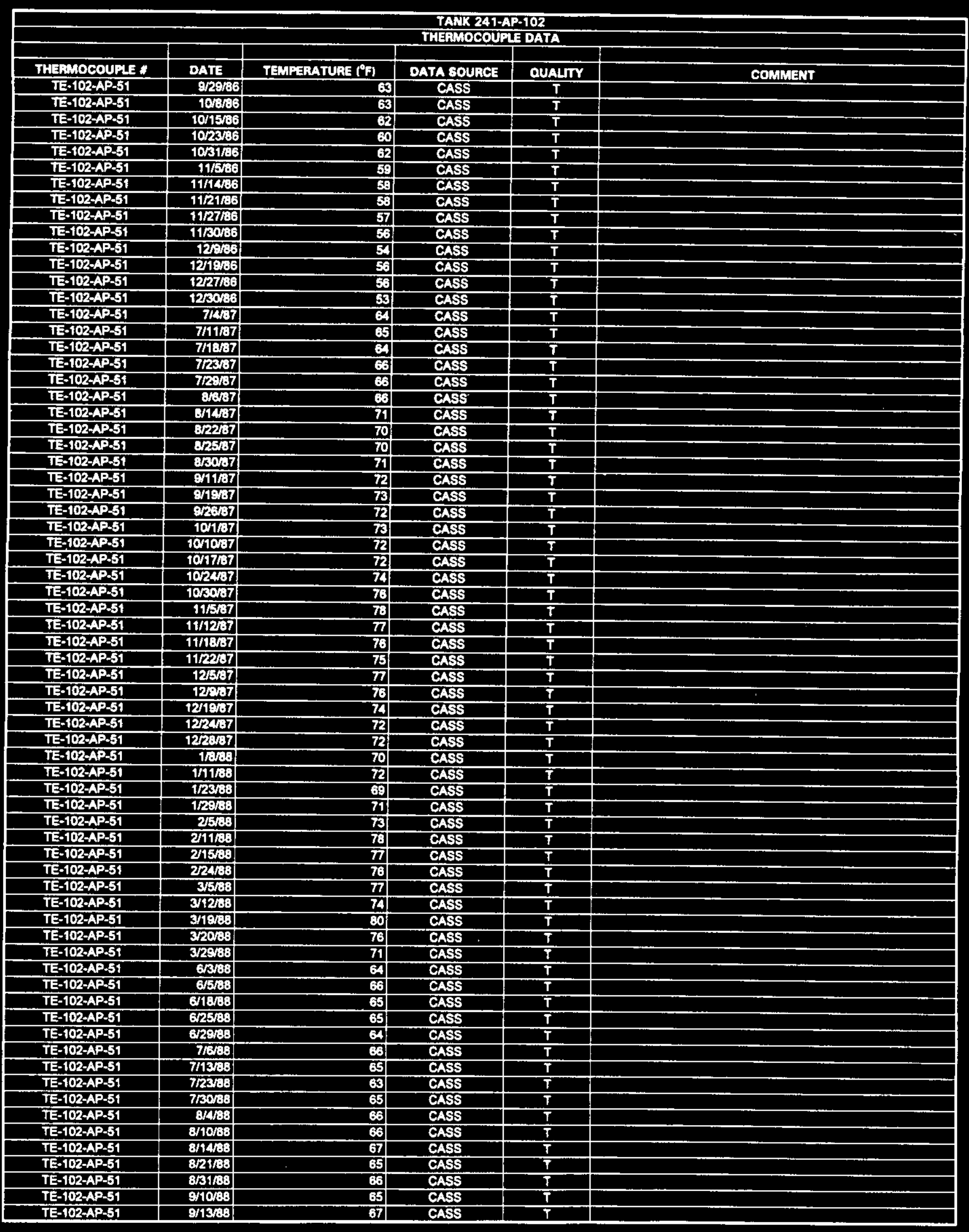




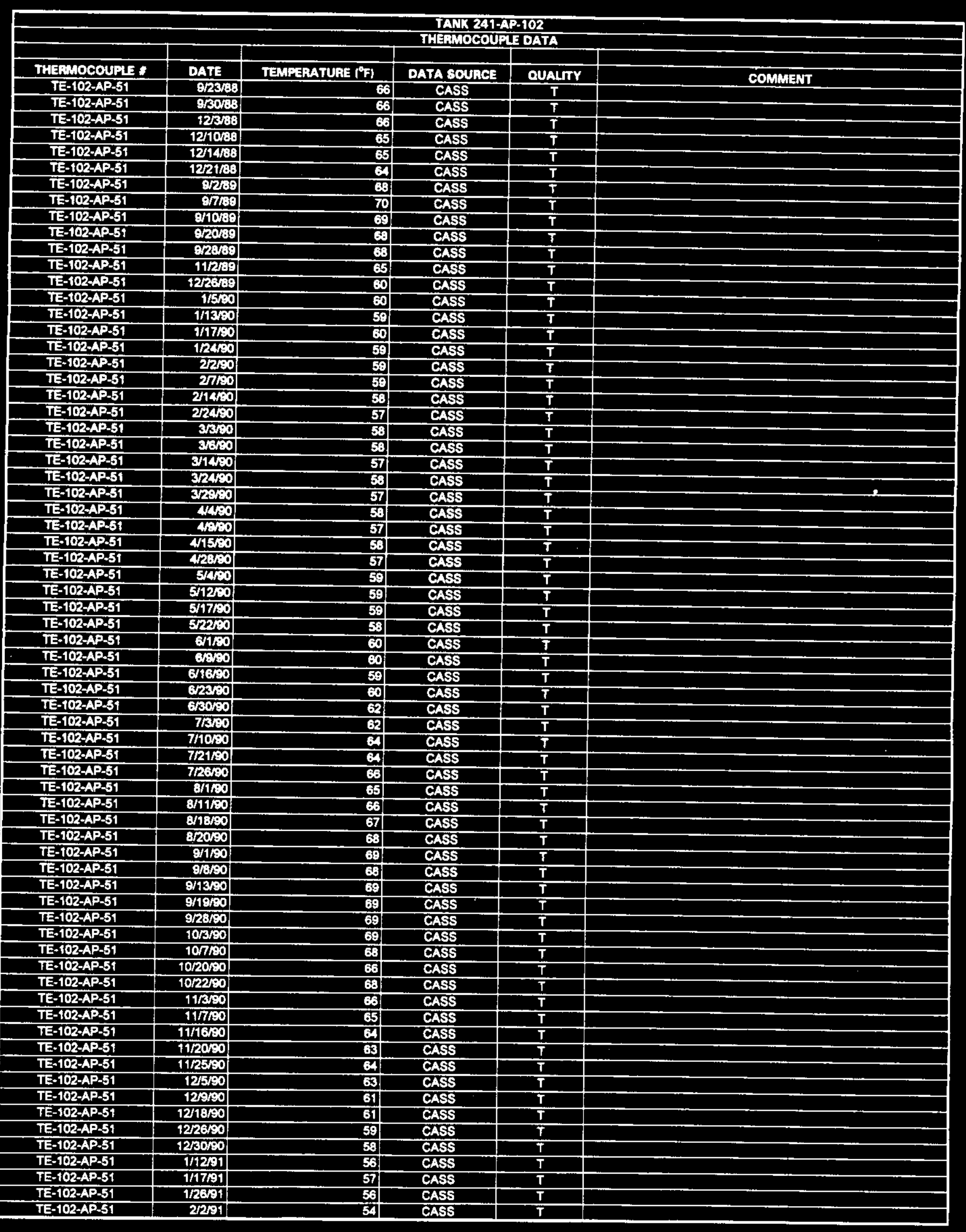




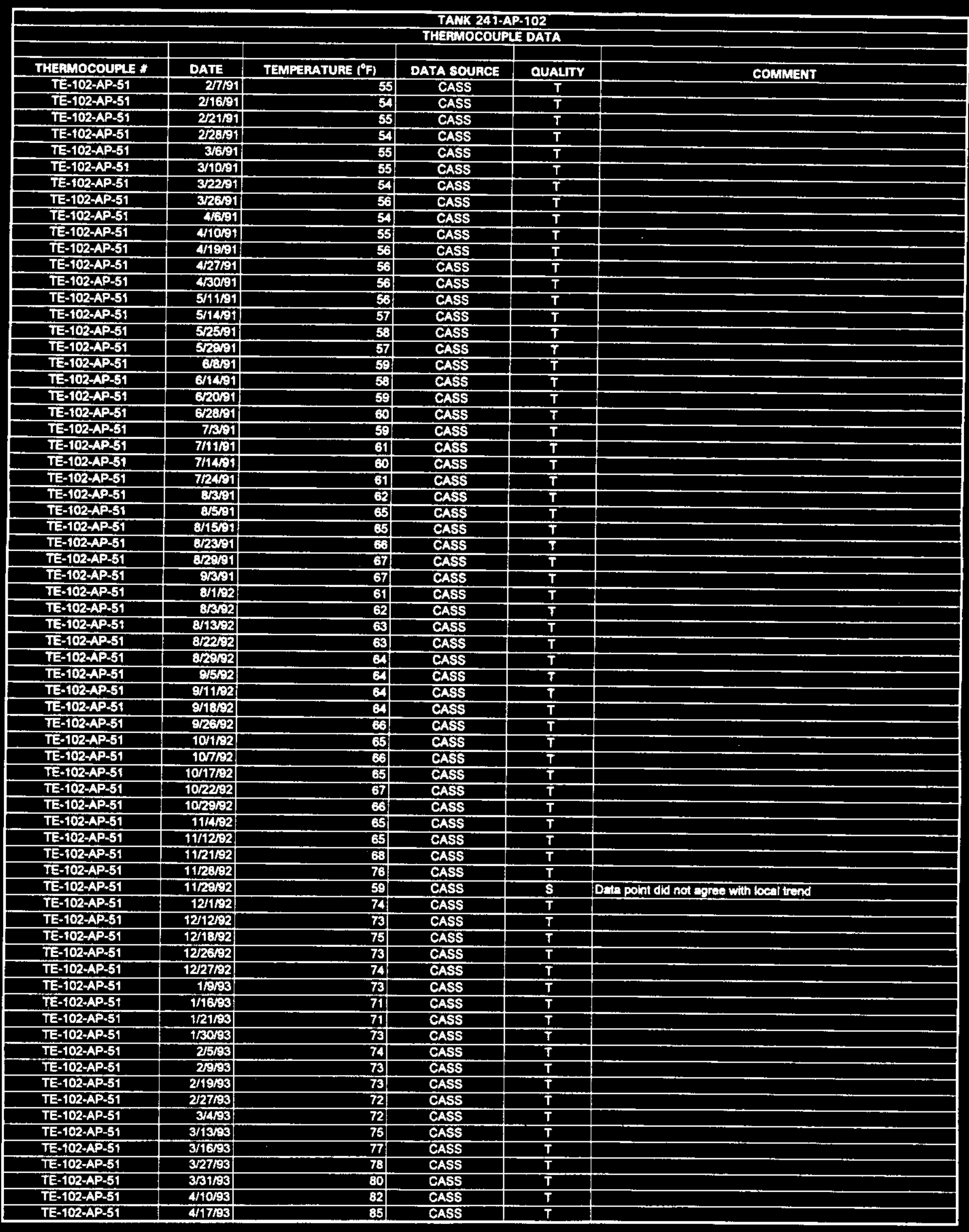




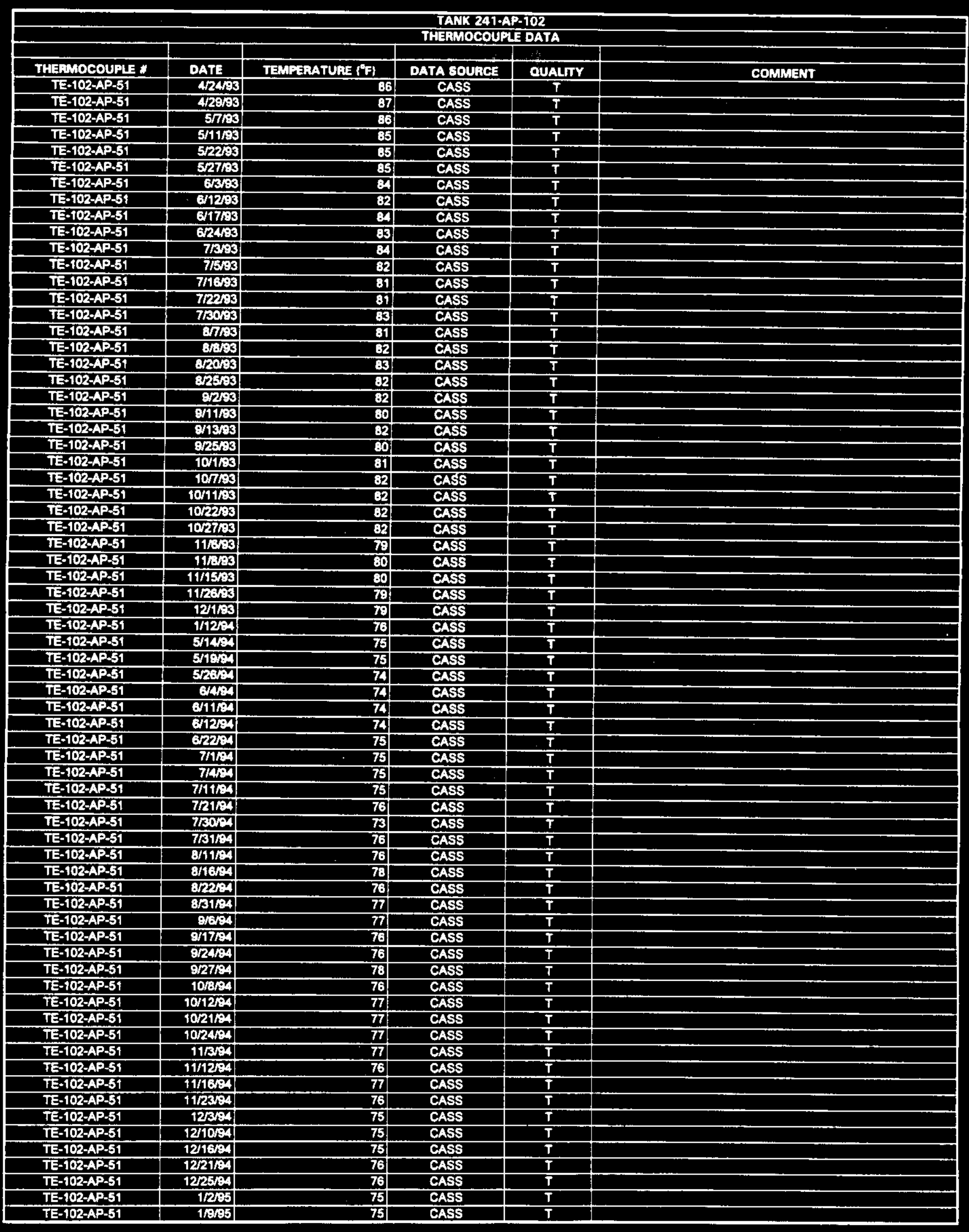




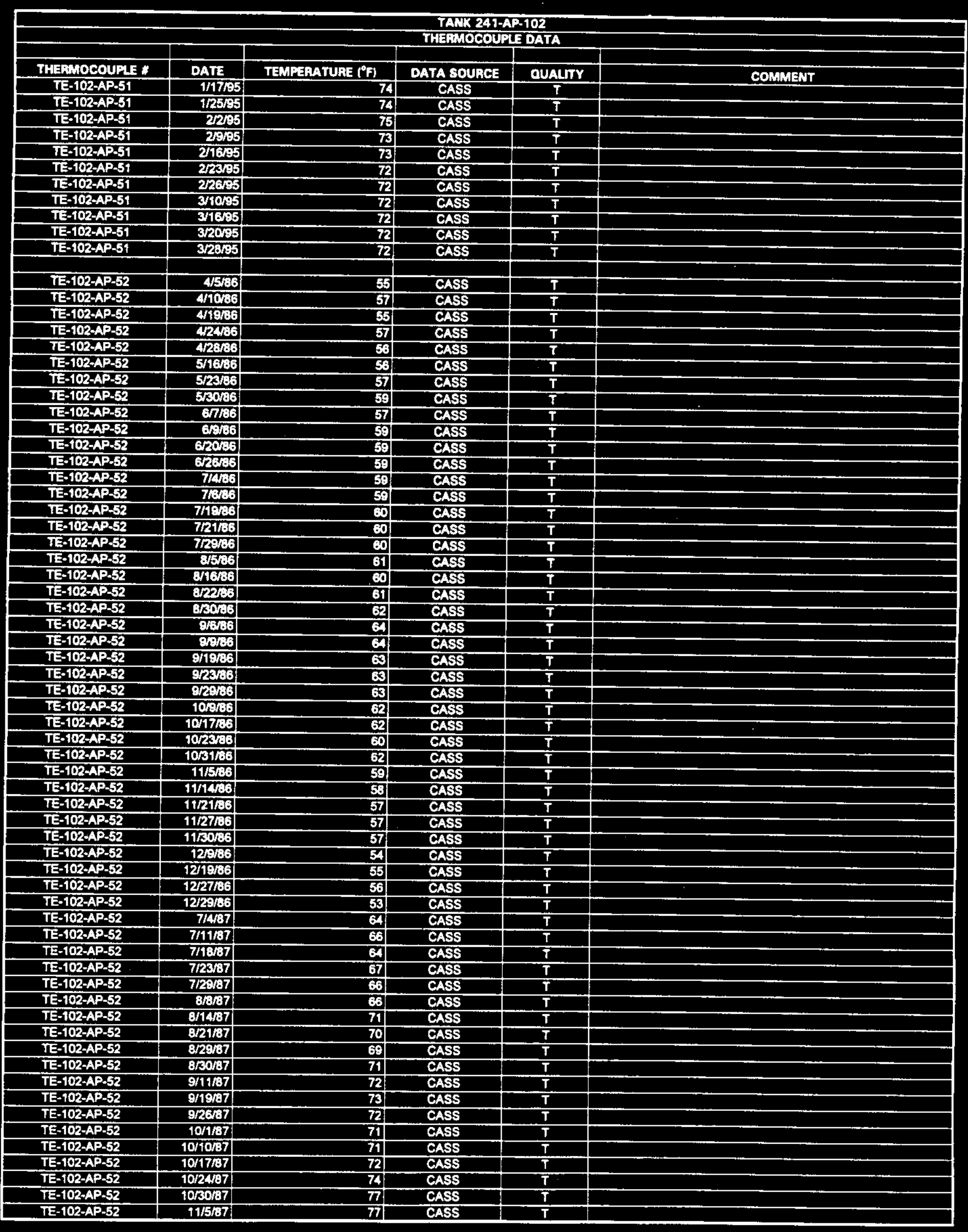




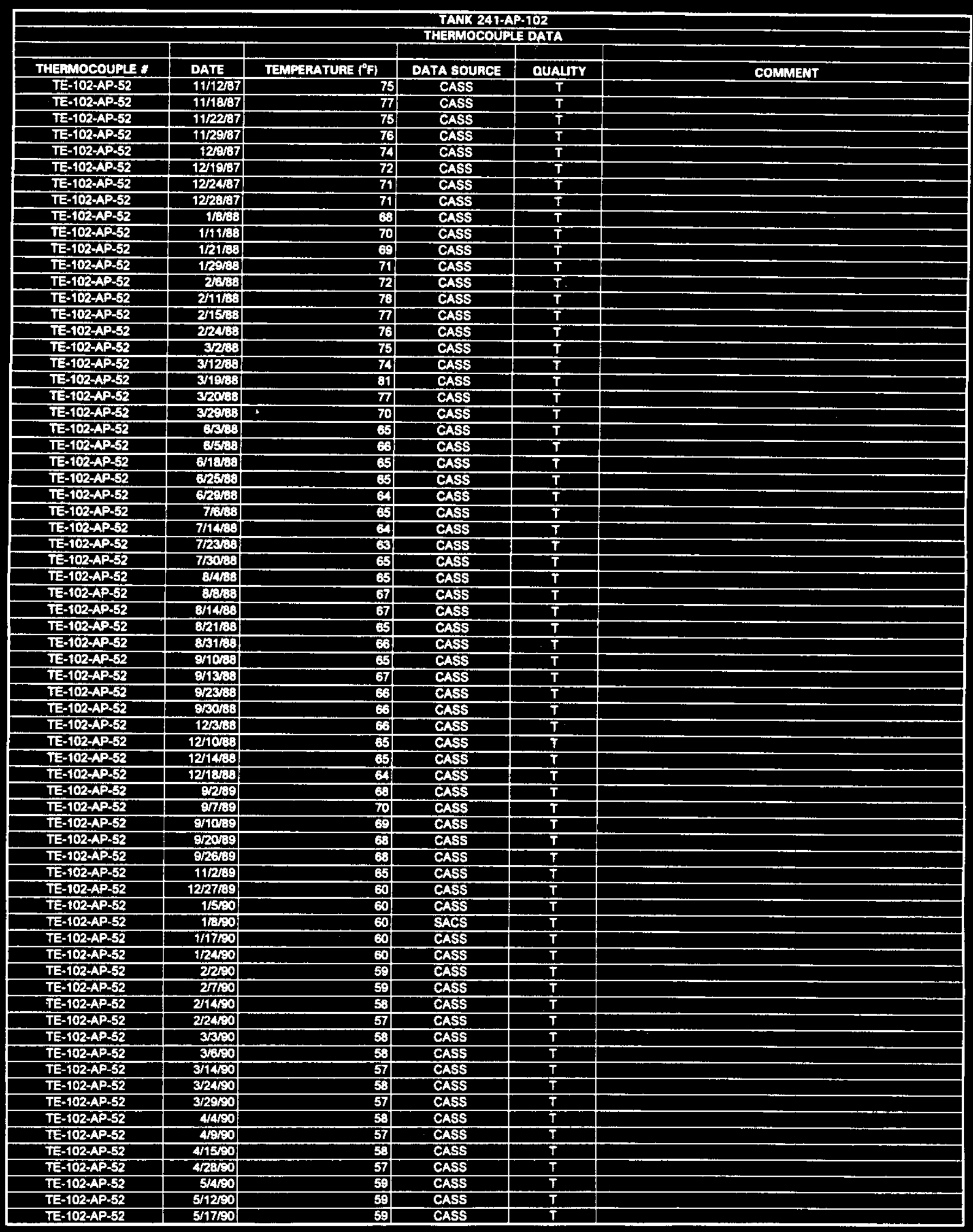




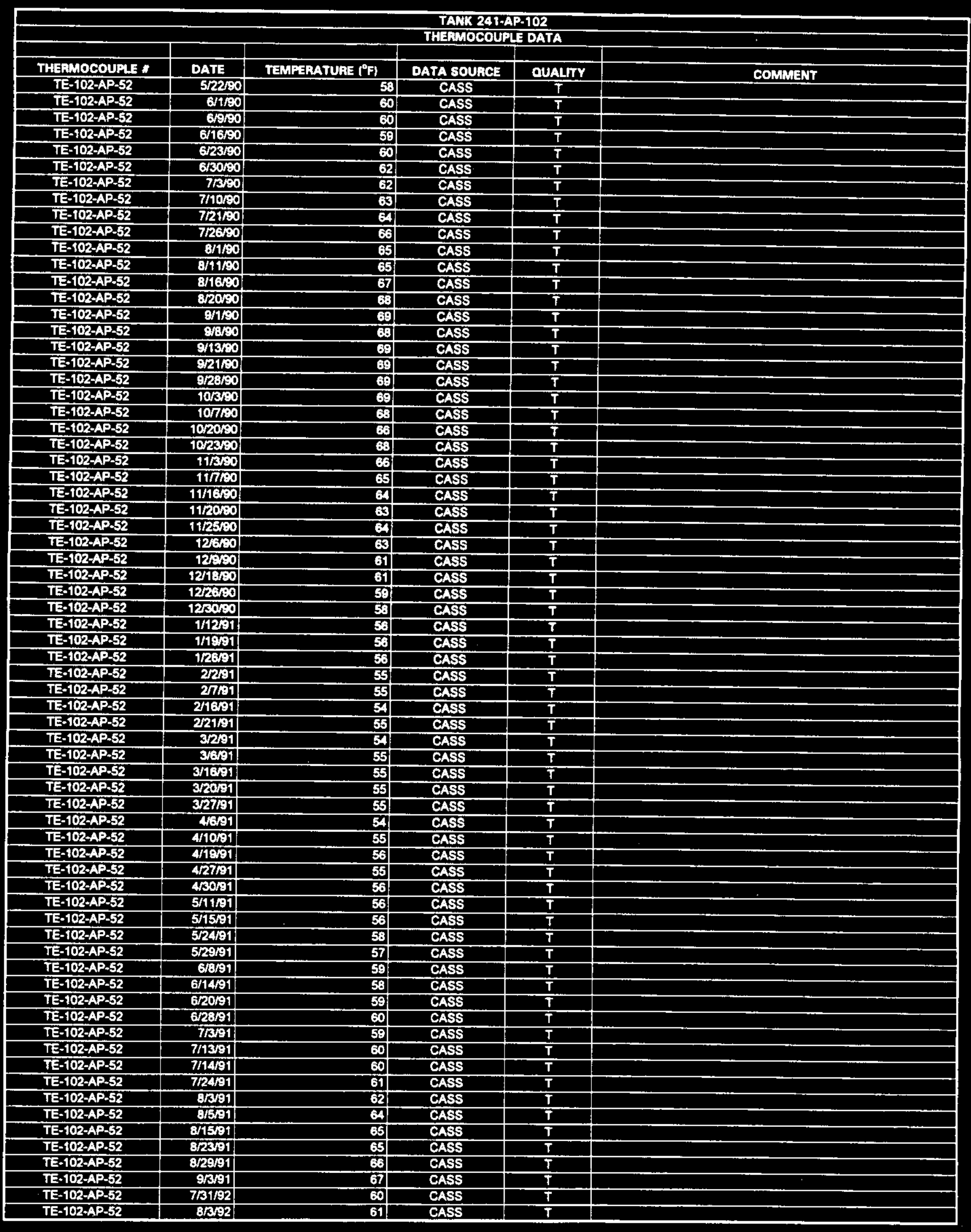




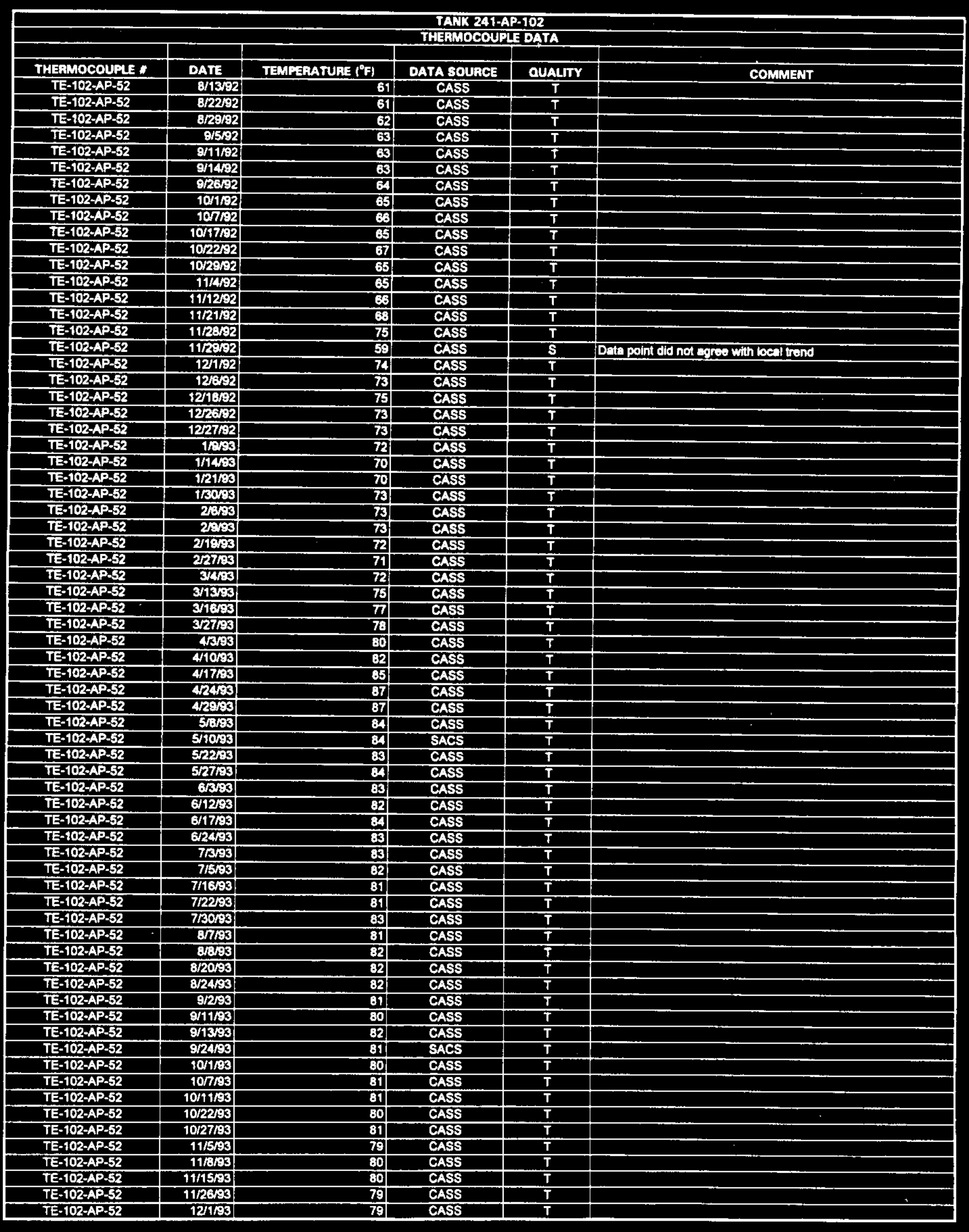

Data obtained from Computer Automated Surveillanca System (CASS), April 1995; and Surveillonce Analysis Computer System (SACS), June 1995.

D-224 


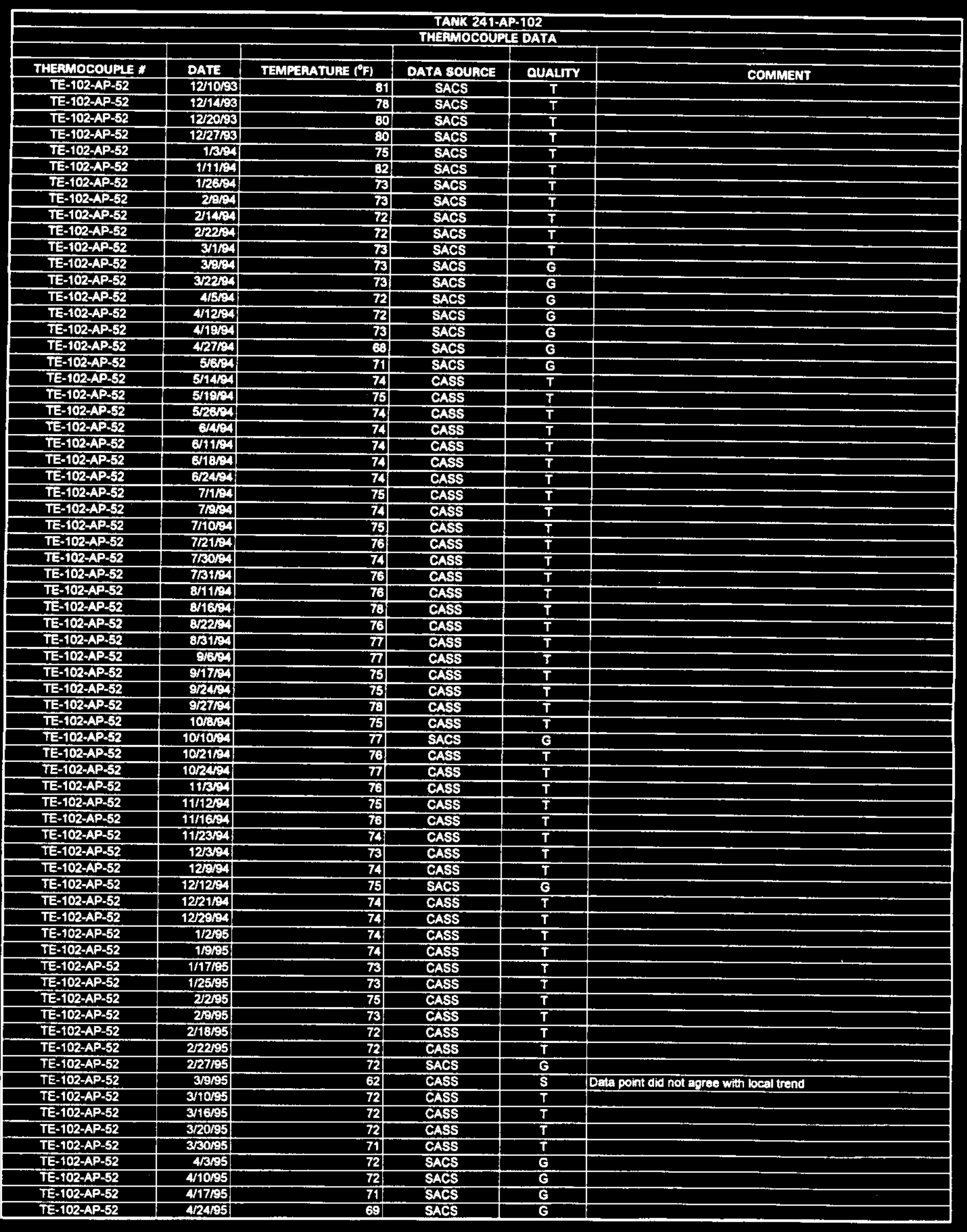




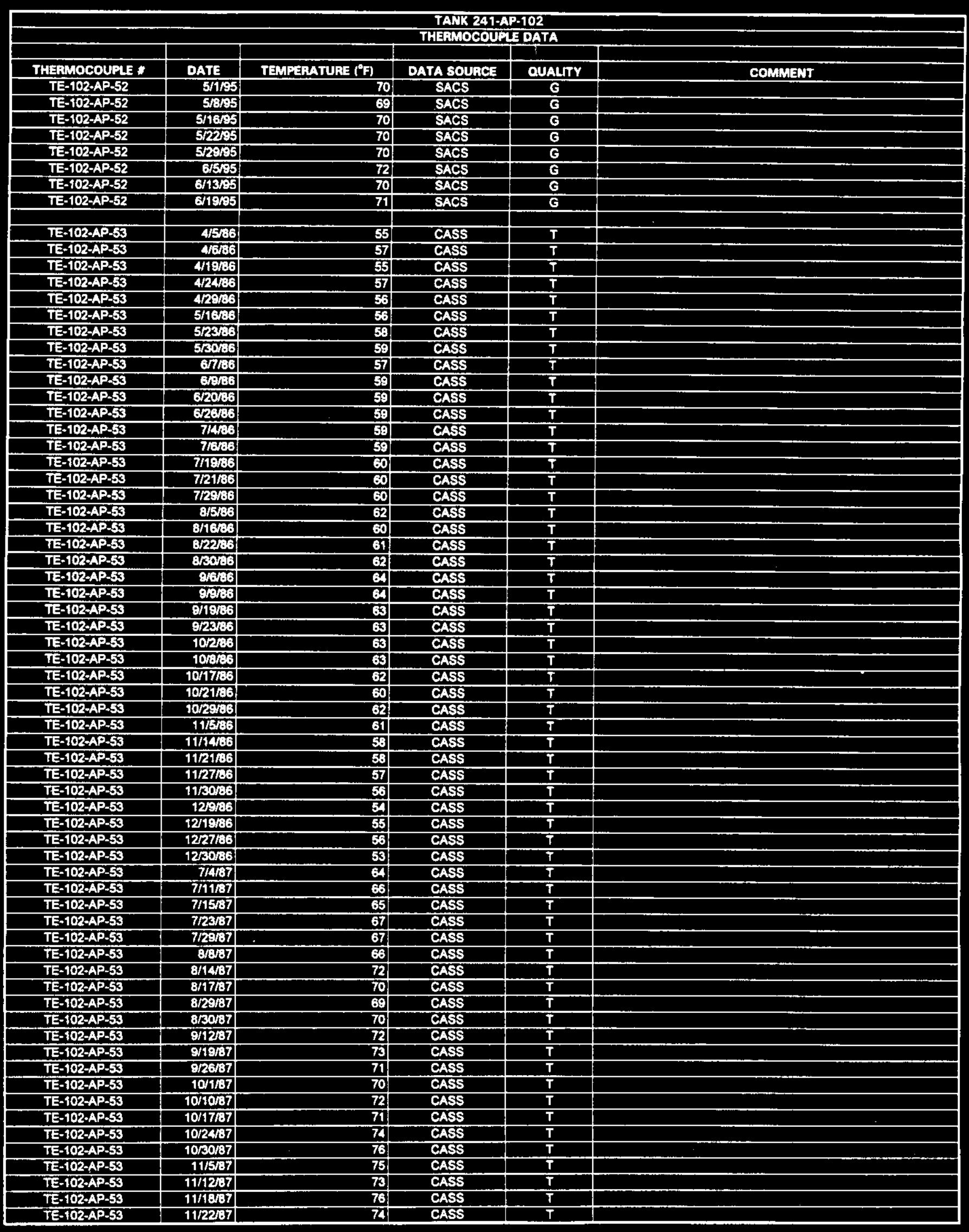




\begin{tabular}{|c|c|c|c|c|c|}
\hline \multicolumn{6}{|c|}{$\begin{array}{l}\text { TANK 241-AP-102 } \\
\text { THEAMOCOUFLE DATA }\end{array}$} \\
\hline & & & & & \\
\hline THERMOCOUPLE : & DATE & TEMPERATURE $\left({ }^{\circ} \mathrm{F}\right)$ & DATA SOURCE & OUAUTY & COMMENT \\
\hline$T E-102-A P-53$ & 12/5/87 & 74 & CASS & $T$ & \\
\hline TE-102-AP-53 & $1219 / 67$ & 72 & CASS & $\mathbf{T}$ & \\
\hline TE-102-AP-53 & $12 / 1987$ & 70 & CASS & $T$ & \\
\hline TE-102-AP-53 & $12 / 24 / 87$ & 68 & CASS & $T$ & \\
\hline$\frac{\text { TE-102-AP-53 }}{\text { TE-102-AP-53 }}$ & \begin{tabular}{r|}
$12 / 28 / 87$ \\
$1 / 3 / 88$
\end{tabular} & $\frac{68}{66}$ & CASS & $T$ & \\
\hline$\frac{T E-102-A P-53}{T E-102-A P \cdot 53}$ & $\frac{1 / 3 / 88}{9 / 41 / 88}$ & $\frac{66}{68}$ & $\begin{array}{l}\text { CASS } \\
\text { CASS }\end{array}$ & $\frac{T}{T}$ & \\
\hline TE-102-AP-53 & 1/23/38 & 68 & CASS & $T$ & \\
\hline TE-102-AP-53 & 1/2983 & 71 & CASS & $T$ & \\
\hline TE-102-AP-53 & $2 / 5 / 88$ & 73 & CASS & $T$ & \\
\hline TE-102-AP-53 & 2/12/88 & 77 & CASS & $T$ & \\
\hline TE-102-AP-53 & $2 / 18 / 88$ & 75 & CASS & $\bar{T}$ & \\
\hline TE-102-AP-53 & 22488 & 73 & CASS & $\bar{T}$ & \\
\hline TE-102-AP.53 & 3588 & 73 & CASS & $\bar{T}$ & \\
\hline TE-102-AP-53 & 3/1268 & 74 & CASS & $\mathbf{T}$ & \\
\hline TE-102-AP-53 & $3 / 1838$ & 81 & CASS & $\bar{T}$ & \\
\hline TE-102.AP-53 & $3 / 2008$ & 78 & CASS & $T$ & \\
\hline TE-102.AP-53 & $3 / 2908$ & 69 & CASS & $T$ & \\
\hline TE-102-AP-53 & $6 / 3 / 38$ & 65 & CASS & $\overline{\mathbf{T}}$ & \\
\hline TE-102-AP-53 & $6 / 5 / 38$ & 63) & CASS & $\mathbf{T}$ & \\
\hline TE-102-AP.53 & 6/14/83 & 60 & CASS & $T$ & \\
\hline TE-102-AP-63 & $6 / 2650$ & 65 & CASS & $T$ & \\
\hline TE-102-AP-53 & 6/3038 & EA & CASS & $\mathbf{T}$ & \\
\hline TE-102-AP-53 & 76638 & 63 & CASS & $\mathbf{T}$ & \\
\hline$T E-102-A P-53$ & $7113 / 68$ & 65 & CASS & $T$ & \\
\hline TE-102-AP-63 & $723 / 38$ & 63 & CASS & $T$ & \\
\hline$T E-102-A P-53$ & 7 73000 & 65 & CASS & $\bar{T}$ & \\
\hline TE-102.AP.53 & 8408 & 65 & CASS & $T$ & \\
\hline TE-102-AP. 53 & 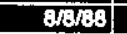 & 67 & CASS & $T$ & \\
\hline TE-102-AP-53 & $8 / 1488$ & 67 & CASS & $\bar{T}$ & \\
\hline TE-102-AP-53 & 8/21/38 & 65 & CASS & $T$ & \\
\hline TE-102-AP-53 & $8 / 31 / 38$ & 68 & CASS & $\mathbf{T}$ & \\
\hline TE-102-AP-53 & 9/10/88 & 65 & CASS & $T$ & \\
\hline TE-102-AP-53 & 9/13/38 & 67 & CASS & $T$ & \\
\hline TE-102-AP-53 & 9/23/38 & 66 & CASS & $T$ & \\
\hline TE-102-AP-53 & $9 / 30 / 88$ & 66 & CASS & $T$ & \\
\hline TE-102.AP-S3 & $12 / 3 / 38$ & 66 & CASS & $\mathbf{T}$ & \\
\hline TE-102-AP-53 & $12 / 0 / 38$ & 64 & CASS & $\bar{T}$ & \\
\hline TE-102-AP-53 & $12 / 14 / 38$ & 64 & CASS & $\bar{T}$ & \\
\hline TE-102-AP-53 & 12/21/30 & 63 & CASS & $T$ & \\
\hline TE-102-AP-53 & $710 / 89$ & 61 & SACS & $T$ & \\
\hline TE-102-AP-53 & $717 / 39$ & 63 & SACS & $\bar{T}$ & \\
\hline TE-102-AP-53 & $7 / 24 / 88$ & 64 & SACS & $\bar{T}$ & \\
\hline TE-102-AP-53 & $87 / 73$ & 70 & SACS & $T$ & \\
\hline TE-102-AP-53 & $8 / 14 / 89$ & 70 & SACS & $\bar{T}$ & \\
\hline TE-102-AP.53 & 8/21/89 & 70 & SACS & $\bar{T}$ & \\
\hline TE-102-AP-53 & 9/2/89 & 69 & CASS & $T$ & \\
\hline TE-102-AP-53 & 94469 & 71 & CASS & $T$ & \\
\hline TE-102-AP-53 & //10/39 & 70 & CASS & $T$ & \\
\hline TE-102-AP-53 & $9 / 20 / 89$ & 69 & CASS & $T$ & \\
\hline TE-102-AP-53 & 9/26/69 & 69 & CASS & $T$ & \\
\hline TE-102-AP-53 & 10/2/39 & 68 & SACS & $\bar{T}$ & \\
\hline TE-102-AP-53 & 10/9/89 & 66 & SACS & $T$ & \\
\hline TE-102-AP-53 & $10 / 16 / 89$ & 68 & SACS & $T$ & \\
\hline TE-102-AP-53 & $10 / 26 / 39$ & 66 & SACS & $T$ & \\
\hline TE-102-AP-53 & 11/2/89 & 65 & CASS & $T$ & \\
\hline TE-102-AP-53 & $12 / 26 / 89$ & 62 & SACS & $T$ & \\
\hline TE-102-AP-53 & $12 / 31 / 89$ & 61 & CASS & $T$ & \\
\hline TE-102-AP-53 & $1 / 13 / 90$ & 59 & CASS & $\bar{T}$ & \\
\hline TE-102-AP-53 & 1/17\%o & 61 & CASS & $T$ & \\
\hline TE-102-AP-53 & 1/2490 & 60 & CASS & $T$ & \\
\hline TE-102-AP-53 & 212100 & 60 & CASS & $T$ & \\
\hline TE-102-AP-53 & $2 \pi 100$ & 59 & CASS & $T$ & \\
\hline TE-102-AP-53 & $2 / 14 \% 0$ & 58 & CASS & $T$ & \\
\hline TE-102-AP-53 & $2 / 2490$ & 57 & CASS & $T$ & \\
\hline TE-102-AP-53 & $3 / 3 / 90$ & 58 & CASS & $T$ & \\
\hline TE-102-AP-53 & $3 / 6 / 90$ & 59 & CASS & $T$ & \\
\hline TE-102-AP-53 & 3/4/90 & 57 & CASS & $T$ & \\
\hline TE-102-AP-53 & $3 / 24 / 90$ & 56 & CASS & $T$ & \\
\hline TE-102-AP-53 & 3/29/00 & 57 & CASS & $T$ & \\
\hline
\end{tabular}




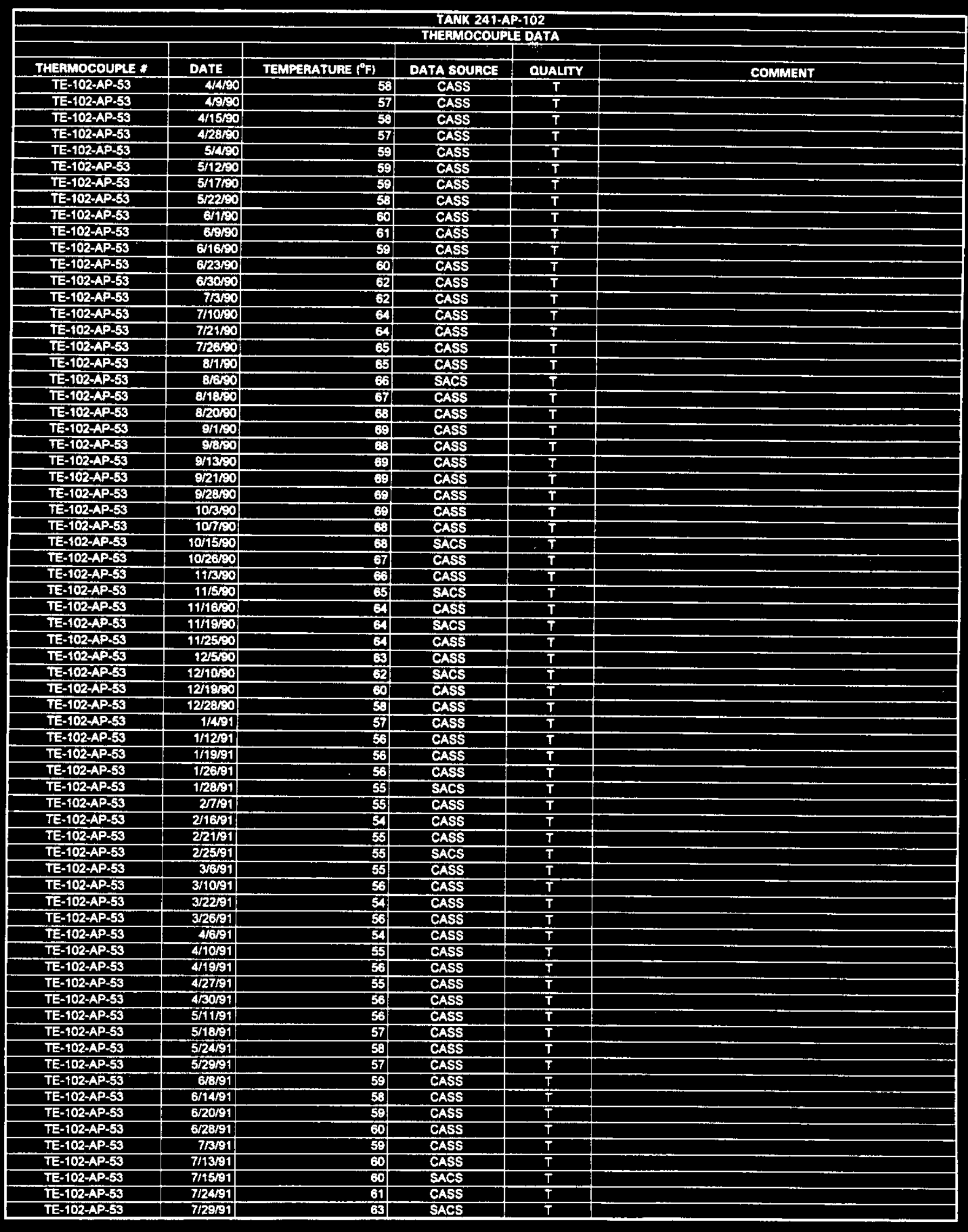




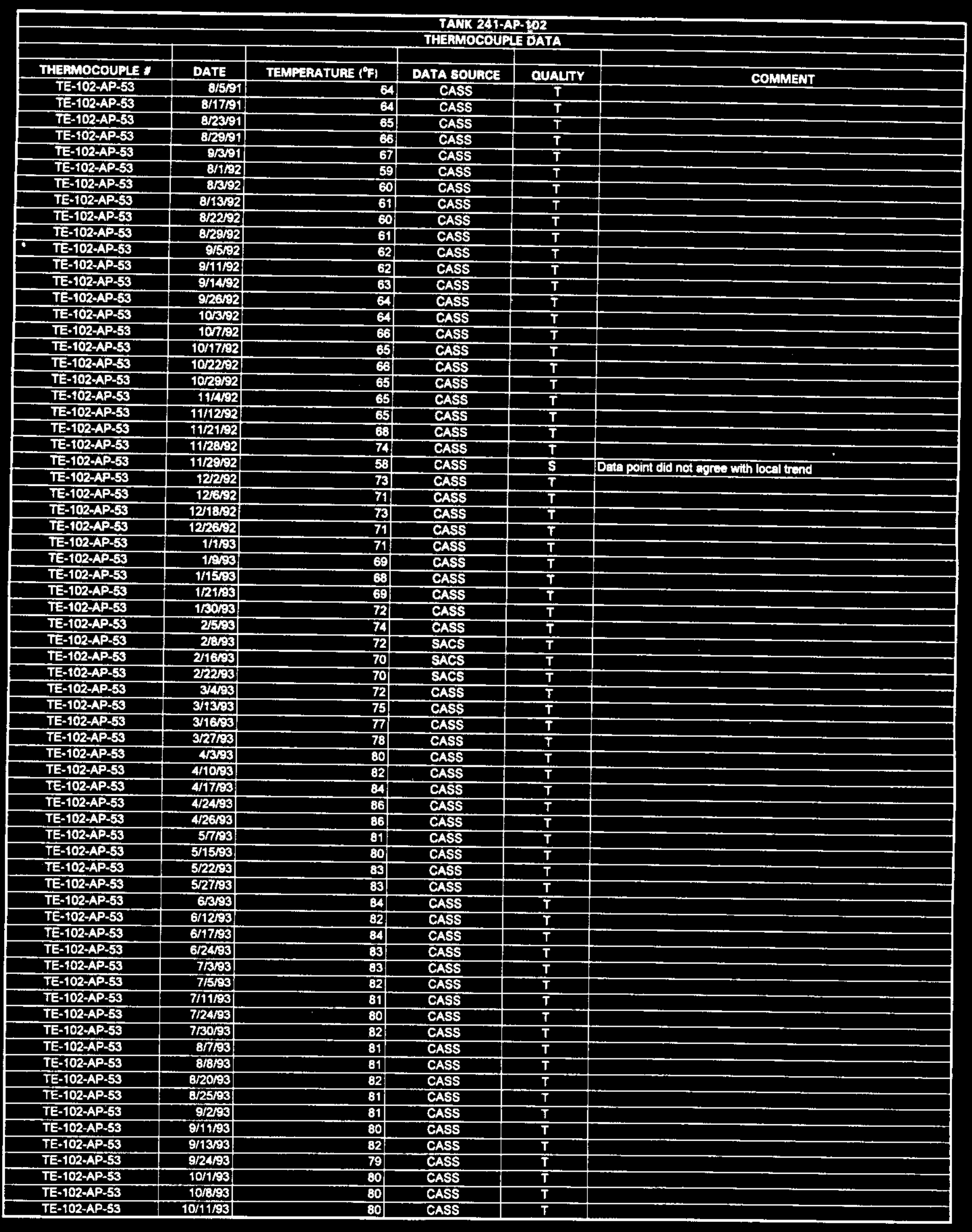




\begin{tabular}{|c|c|c|c|c|c|}
\hline \multicolumn{6}{|c|}{$\begin{array}{l}\text { TANK 247-AP-102 } \\
\text { THETMOCOUPE DATA }\end{array}$} \\
\hline & & & & . & \\
\hline THEPMOCOUPLE * & DATE & TEMPERATUAE $\left({ }^{\circ} \mathrm{F}\right)$ & DATA SOURCE & QUALTY & COMMENT \\
\hline TE-102-AP-53 & $10 / 22 / 93$ & 80 & CASS & $T$ & \\
\hline TE-102-AP-53 & $10 / 2793$ & 80 & CASS & $\bar{T}$ & \\
\hline TE-102-AP-53 & $11 / 6 r 93$ & 77 & CASS & $T$ & \\
\hline TE-102-AP-53 & 11 kas & 78 & CASS & $T$ & \\
\hline TE-102-AP-53 & 11/15/93 & 79 & CASS & $T$ & \\
\hline TE-102-APS3 & $11 / 26 / 53$ & 78 & CASS & $T$ & \\
\hline TE-102-AP-53 & $121 / 93$ & 77 & CASS & $T$ & \\
\hline TE-102-AP-63 & $1 / 1394$ & 75 & CASS & $T$ & \\
\hline TE-102-AP-53 & 5/494 & 74 & CASS & $T$ & \\
\hline$T E-102-A P-53$ & $5 / 1204$ & 75 & CASS & $\mathbf{T}$ & \\
\hline TE-102-AP-53 & 5rat & 74 & CASS & $T$ & \\
\hline TE-102-AP-53 & 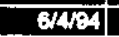 & 74 & CASS & $T$ & \\
\hline TE-102-AP-53 & 6\%9 & 74 & CASS & $\bar{T}$ & \\
\hline TE-102-AP-53 & 61254 & 75 & CASS & $T$ & \\
\hline TE-102-AP-53 & E2454 & 74 & CASS & $\mathbf{T}$ & \\
\hline TE-102-AP-53 & 7194 & 74 & CASS & $T$ & \\
\hline TE-102-AP-53 & Tres & 74 & CASS & $T$ & \\
\hline TE-102-AP-53 & 71094 & 75 & CASS & $T$ & \\
\hline JE-102-AP-53 & $7 / 21 / 94$ & 76 & CASS & $T$ & \\
\hline $\mathrm{TE}-102-A P-53$ & $7130 \mathrm{~s} 4$ & 74 & CASS & $T$ & \\
\hline$T E-102-A P-53$ & $7 / 31 / 94$ & 76 & CASS & $T$ & \\
\hline $\mathrm{TE}-102,4 \mathrm{P}-53$ & 6/13:4 & 75 & CASS & $T$ & \\
\hline TE-102-AP-53 & 817\% & 78 & CASS & $T$ & \\
\hline TE-102-AP-S3 & 82254 & 78 & CASS & $\mathbf{T}$ & \\
\hline TE-102-AP-53 & $8 / 314$ & 7 & CASS & $T$ & \\
\hline TE-102-AP-53 & otan & 7 & CASS & $T$ & \\
\hline TE-102-APS3 & $8 / 17 / 4$ & 75 & CASS & $T$ & \\
\hline TE-102.AP.53 & S/24:4 & 76 & CASS & $T$ & \\
\hline TE-102-AP-63 & 927א: & 77 & CASS & $T$ & \\
\hline TE-102-AP-53 & $10 \times 3$ & 75 & CASS & $T$ & \\
\hline TE-102-AP-53 & $10 / 2234$ & 75 & CASS & $T$ & \\
\hline TE-102AP-53 & $10 / 21 / 34$ & 75 & CASS & $\mathbf{T}$ & \\
\hline TE-102-AP-53 & $10 / 24 \times 4$ & 75 & CASS & $T$ & \\
\hline TE-102-AP-S3 & $11 / \mathrm{K} / \mathrm{M}$ & 74 & CASS & $T$ & \\
\hline TE-102-AP-53 & 11/1254 & 73 & CASS & $T$ & \\
\hline IE-102AP-53 & $11 / 162$ & 75 & CASS & $T$ & \\
\hline TE-102-AP-S3 & $11 / 2494$ & 72 & CASS & $T$ & \\
\hline TE-102-AP-53 & $12 / 3 \mathrm{se}$ & 71 & CASS & $T$ & \\
\hline TE-102-AP-5X & 12994 & 72 & CASS & $T$ & \\
\hline TE-102-AP-53 & $12 / 1704$ & 71 & CASS & $T$ & \\
\hline$T E-102-A P-53$ & $12 / 21 \% 4$ & 73 & CASS & $T$ & \\
\hline TE-102AP-53 & 122589 & 73 & CASS & $T$ & \\
\hline TE-102-AP-53 & 1/2205 & 72 & CASS & $T$ & \\
\hline TE-102-AP-S3 & 1205 & 72 & CASS & $T$ & \\
\hline TE-102-AP-53 & $1 / 1985$ & 71 & CASS & $T$ & \\
\hline$T E-102-A P-53$ & 1/25SS & 71 & CASS & $T$ & \\
\hline$T E-102 \cdot A P \cdot 53$ & 2/2S5 & 72 & CASS & $T$ & \\
\hline$T E-102-A P-53$ & $20 / 95$ & 71 & CASS & $T$ & \\
\hline TE-102-AP-53 & $2 / 6 \mathrm{sS}$ & 71 & CASS & $T$ & \\
\hline TE-102-AP-53 & $2 / 22255$ & 71 & CASS & $T$ & \\
\hline TE-102-AP-53 & $2 / 27 / 95$ & 70 & CASS & $\bar{T}$ & \\
\hline $\mathrm{TE}-102-\mathrm{AP}-53$ & 3ars & 74 & CASS & $T$ & \\
\hline TE-102-AP,53 & अ1605 & 70 & CASS & $T$ & \\
\hline TE-102-AP-53 & $3 / 20195$ & 71 & CASS & $T$ & \\
\hline TE-102-AP-53 & 3/28/:5 & 70 & CASS & $\mathbf{T}$ & \\
\hline
\end{tabular}




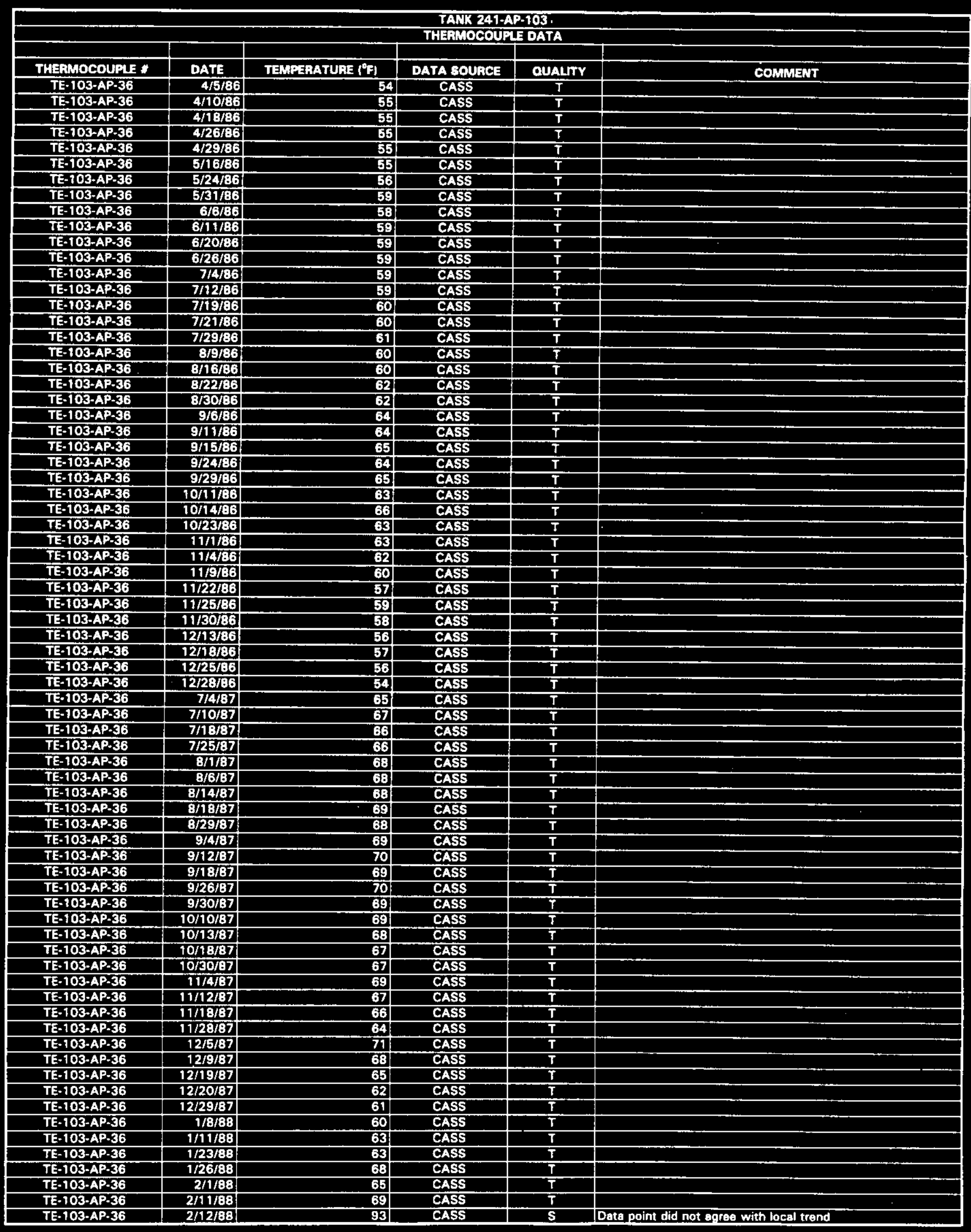




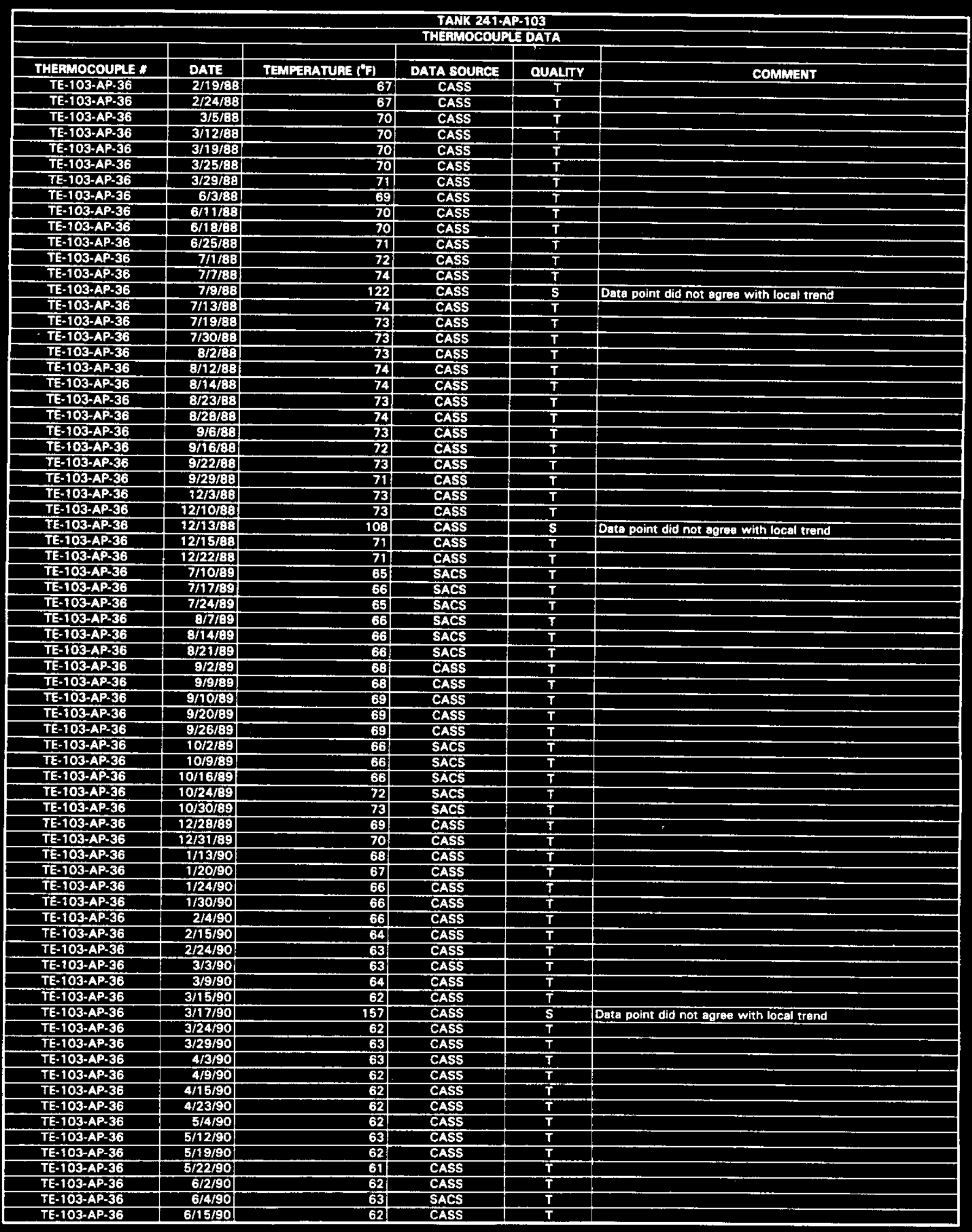

Date obtained from Computer Automated Survelllance System (CASS). April 1995; and Surveillance Anelysis Computer System (SACS), JUne 1995.

D-232 


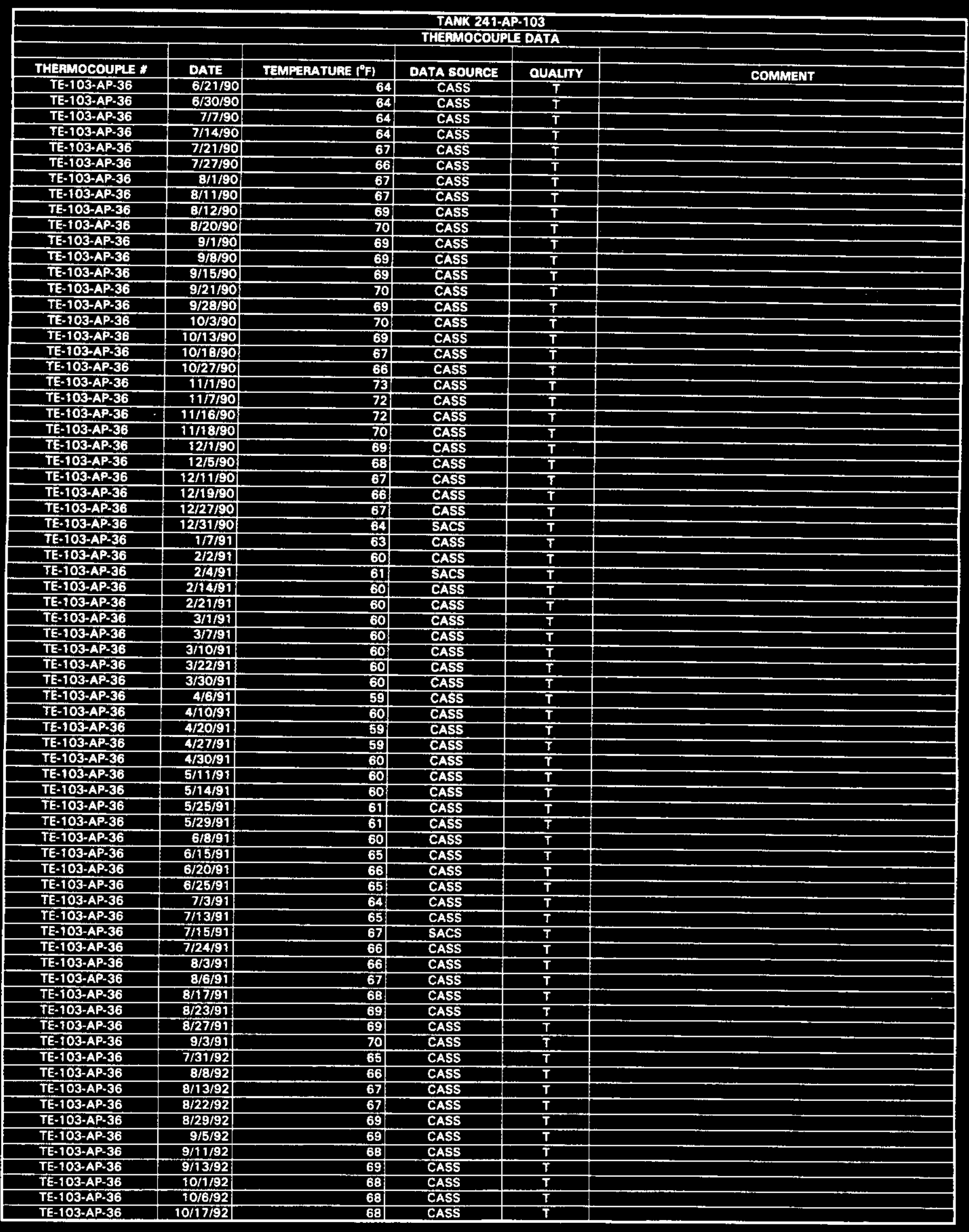




\begin{tabular}{|c|c|c|c|c|c|}
\hline \multicolumn{6}{|c|}{$\begin{array}{l}\text { TANK 241-AP-103 } \\
\text { THEAMOCOUPLE DATA }\end{array}$} \\
\hline & & & & & \\
\hline THEAMOCOUPL : & DATE & TEMPERATURE ("F) & DATA SOUACE & OUALITY & COMMENT \\
\hline TE-103-AP-36 & $10 / 22 / 92$ & 68 & CASS & $T$ & \\
\hline TE-103.AP-36 & $10 / 27 / 92$ & 68 & CASS & $\mathbf{T}$ & \\
\hline TE-103-AP-36 & $11 / 4 / 92$ & 66 & CASS & $T$ & \\
\hline TE-103-AP-36 & $11 / 10 / 92$ & 65 & CASS & $T$ & \\
\hline TE-103-AP-36 & $11 / 17 / 92$ & 66 & CASS & $T$ & \\
\hline TE-103-AP-36 & $11 / 24 / 92$ & 64 & CASS & $T$ & \\
\hline TE-103-AP-36 & $11 / 29 / 92$ & 70 & CASS & $\mathbf{S}$ & Data point did not agree with local trend \\
\hline TE-103-AP-36 & $12 / 5 / 92$ & 62 & CASS & $\mathbf{T}$ & \\
\hline TE-103-AP-36 & $12 / 11 / 92$ & 62 & CASS & $\mathbf{T}$ & \\
\hline TE-103-AP-36 & $12 / 18 / 92$ & 61 & CASS & $T$ & \\
\hline TE-108-AP-36 & $12 / 26 / 92$ & 61 & CASS & $\bar{T}$ & \\
\hline TE-103-AP-36 & $1 / 2 / 93$ & 60 & CASS & $\bar{T}$ & \\
\hline TE-103-AP-36 & $1 / 9 / 93$ & 59 & CASS & $T$ & \\
\hline TE-103-AP-36 & $1 / 15 / 93$ & 58 & CASS & $T$ & \\
\hline TE-103-AP-36 & $1 / 23 / 93$ & $\mathbf{5 7}$ & CASS & $T$ & \\
\hline TE-103-AP-36 & $1 / 26 / 93$ & 59 & CASS & $\bar{T}$ & \\
\hline TE-103-AP-36 & $2 / 6 / 93$ & 57 & CASS & $T$ & \\
\hline$T E-103-A P-36$ & $2 / 12 / 93$ & 57 & CASS & $T$ & \\
\hline TE-103-AP-36 & $2120 / 93$ & 57 & CASS & $T$ & \\
\hline TE-103-AP-36 & $2 / 26 / 93$ & 57 & CASS & $T$ & \\
\hline TE-103-AP-36 & $3 / 4 / 93$ & 56 & CASS & $T$ & \\
\hline TE-108-AP-36 & 3/10/93 & 56 & CASS & $T$ & \\
\hline TE-10\}.AP-36 & $3 / 19 / 93$ & 56 & CASS & $T$ & \\
\hline TE-103-AP-36 & $3 / 27 / 93$ & 56 & CASS & $\bar{T}$ & \\
\hline TE-103-AP-36 & $3 / 31 / 93$ & 56 & CASS & $T$ & \\
\hline TE-103-AP-36 & $4 / 6 / 93$ & 56 & CASS & $T$ & \\
\hline TE-103-AP-36 & $4 / 12 / 93$ & 56 & SACS & $T$ & \\
\hline TE-103-AP-36 & $4 / 24 / 93$ & 57 & CASS & $T$ & \\
\hline TE-103-AP-36 & $4 / 29 / 93$ & 56 & CASS & $T$ & \\
\hline TE-103-AP-36 & $5 / 3 / 93$ & 57 & CASS & $T$ & \\
\hline TE-103-AP-36 & 5/10/93 & 57 & CASS & $T$ & \\
\hline TE-103-AP-36 & $5 / 22 / 93$ & 58 & CASS & $T$ & \\
\hline TE-103-AP-36 & $5 / 29 / 93$ & 59 & CASS & $T$ & \\
\hline TE-103-AP-36 & $6 / 5 / 93$ & 59 & CASS & $\mathrm{T}$ & \\
\hline TE-103-AP-36 & 6/7/93 & 60 & SACS & $T$ & \\
\hline TE-103-AP-36 & 6/19/93 & 60 & CASS & $T$ & \\
\hline TE-103-AP-36 & $6 / 24 / 93$ & 61 & CASS & $\bar{I}$ & \\
\hline TE-103-AP-36 & $6 / 30 / 93$ & 80 & CASS & $\mathbf{s}$ & Dote point did not agree with local trend \\
\hline TE-103-AP-36 & $7 / 3 / 93$ & 61 & CASS & $\bar{T}$ & \\
\hline TE-103-AP-36 & 7/8/93 & 63 & CASS & $T$ & \\
\hline TE-103-AP-36 & $7 / 17 / 93$ & 62 & CASS & $T$ & \\
\hline TE-103-AP-36 & $7 / 21 / 93$ & 63 & CASS & $\mathbf{T}$ & \\
\hline TE-103-AP-36 & $7 / 31 / 93$ & 63 & CASS & $T$ & \\
\hline TE-103-AP-36 & $8 / 7 / 93$ & 64 & CASS & $\mathbf{T}$ & \\
\hline TE-103-AP-36 & $8 / 14 / 93$ & 64 & CASS & $\bar{T}$ & \\
\hline TE-103-AP-36 & $8 / 20 / 93$ & 66 & CASS & $T$ & \\
\hline TE-103-AP.36 & $8 / 28 / 93$ & 66) & CASS & $T$ & \\
\hline TE-103-AP-36 & $9 / 2 / 93$ & 66 & CASS & $T$ & \\
\hline TE-103-AP-36 & 9/9/93 & 67 & CASS & $T$ & \\
\hline TE-103-AP-36 & $9 / 13 / 93$ & 67 & CASS & $\mathbf{T}$ & \\
\hline TE-103-AP-36 & 9/19/93 & 65 & CASS & $T$ & \\
\hline TE-103-AP-36 & $9 / 25 / 93$ & 115 & CASS & $\mathbf{S}$ & Data point did nof agree with local trend \\
\hline$T E-103 \cdot A P \cdot 36$ & $10 / 1 / 93$ & 65 & CASS & $\bar{T}$ & \\
\hline TE-103-AP-36 & $10 / 8 / 93$ & 66 & CASS & $T$ & \\
\hline TE-103-AP-36 & $10 / 11 / 93$ & 65 & CASS & $T$ & \\
\hline TE-103-AP-36 & $10 / 18 / 93$ & 64 & CASS & $\bar{T}$ & \\
\hline TE-103.AP-36 & $10 / 29 / 93$ & 62. & CASS & $T$ & \\
\hline TE-103-AP-36 & $11 / 6 / 93$ & 61 & CASS & $T$ & \\
\hline TE-103-AP-36 & 11/8/93 & 62 & SACS & $T$ & \\
\hline TE-103-AP-36 & $11 / 19 / 93$ & 60 & CASS & $T$ & \\
\hline TE-103-AP-36 & $11 / 23 / 93$ & 59 & CASS & $\bar{T}$ & \\
\hline TE-103-AP-36 & $12 / 1 / 93$ & 59 & CASS & $\mathbf{T}$ & \\
\hline TE-103-AP-36 & $12 / 10 / 93$ & 58 & SACS & $\bar{T}$ & \\
\hline TE-103-AP-36 & $12 / 14 / 93$ & 59 & SACS & $T$ & \\
\hline TE-103-AP-36 & $12 / 20 / 93$ & 56 & SACS & $T$ & \\
\hline TE-103-AP-36 & $12 / 27 / 93$ & 55 & SACS & $\bar{T}$ & \\
\hline TE-103-AP-36 & $1 / 3 / 94$ & 56 & SACS & $T$ & \\
\hline TE-103-AP-36 & $1 / 11 / 94$ & 58 & SACS & $T$ & \\
\hline TE-103-AP-36 & $1 / 26 / 94$ & 5 & SACS & $\bar{T}$ & \\
\hline$T E-103-A P-36$ & $2 / 9 / 94$ & 53 & SACS & $T$ & \\
\hline TE-103-AP-36 & $2 / 14 / 94$ & 52 & SACS & $T$ & \\
\hline TE-103-AP-36 & $2 / 22 / 94$ & 53 & SACS & $T$ & \\
\hline TE-103-AP-36 & $3 / 1 / 94$ & 54 & SACS & $\mathbf{T}$ & \\
\hline
\end{tabular}




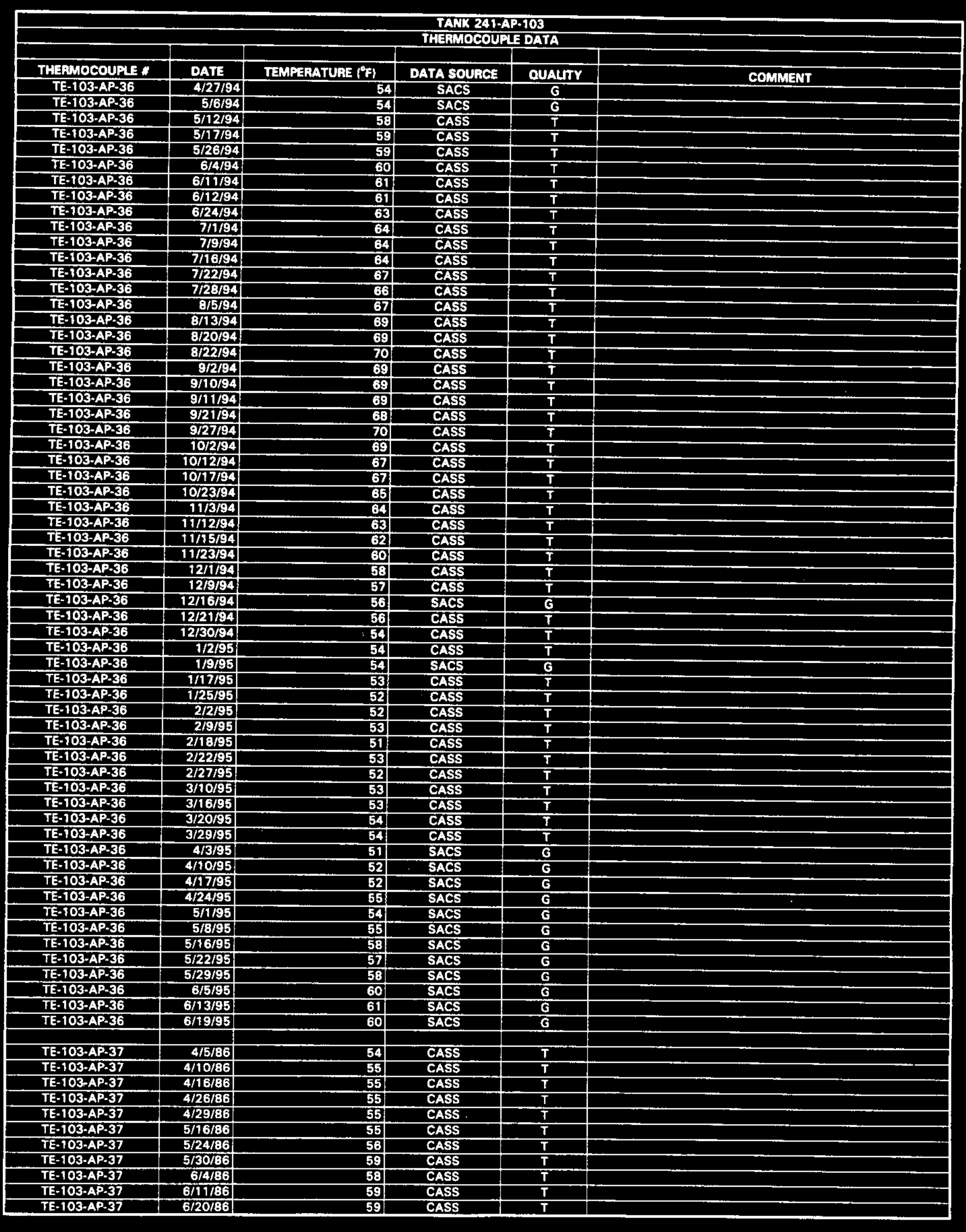




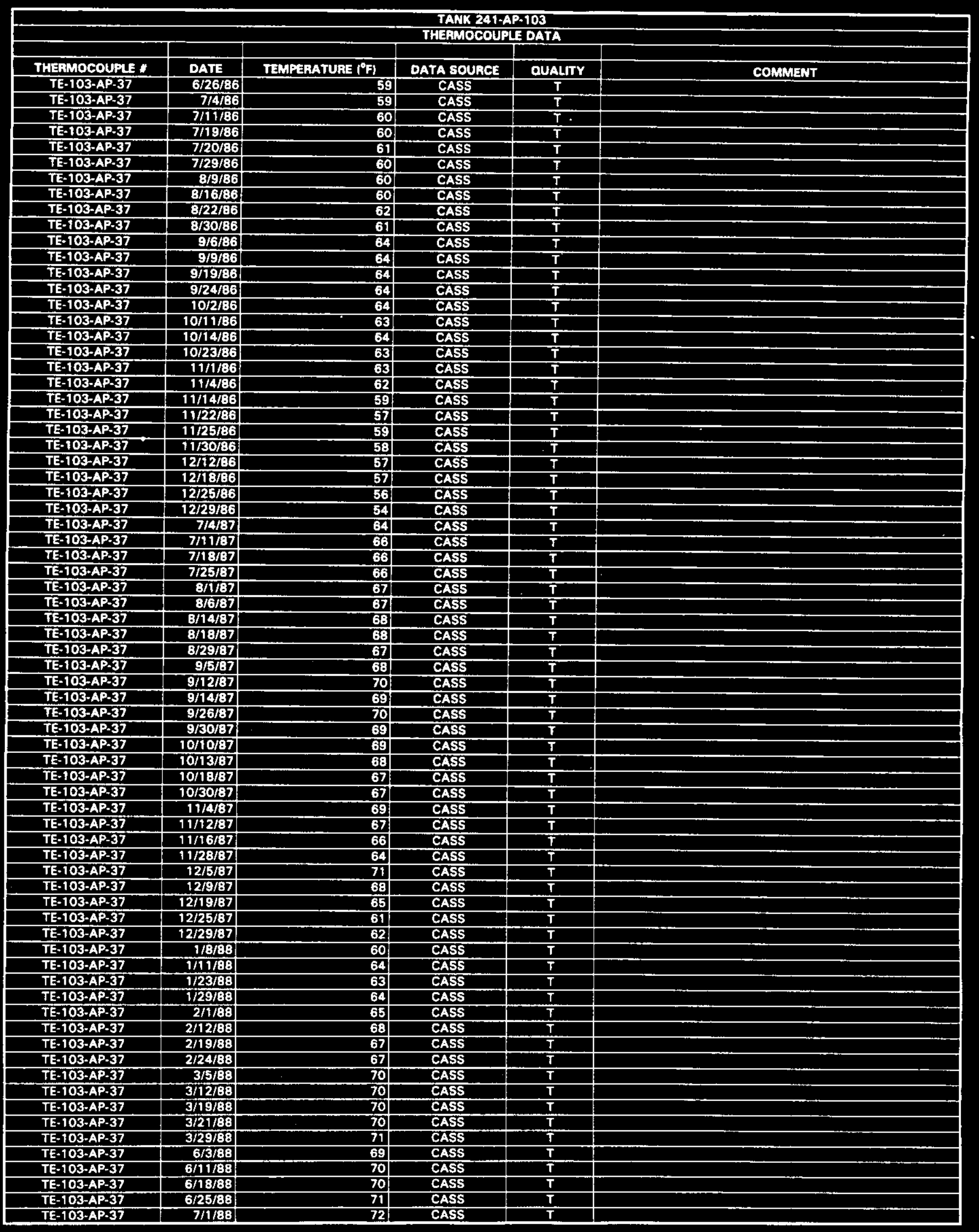




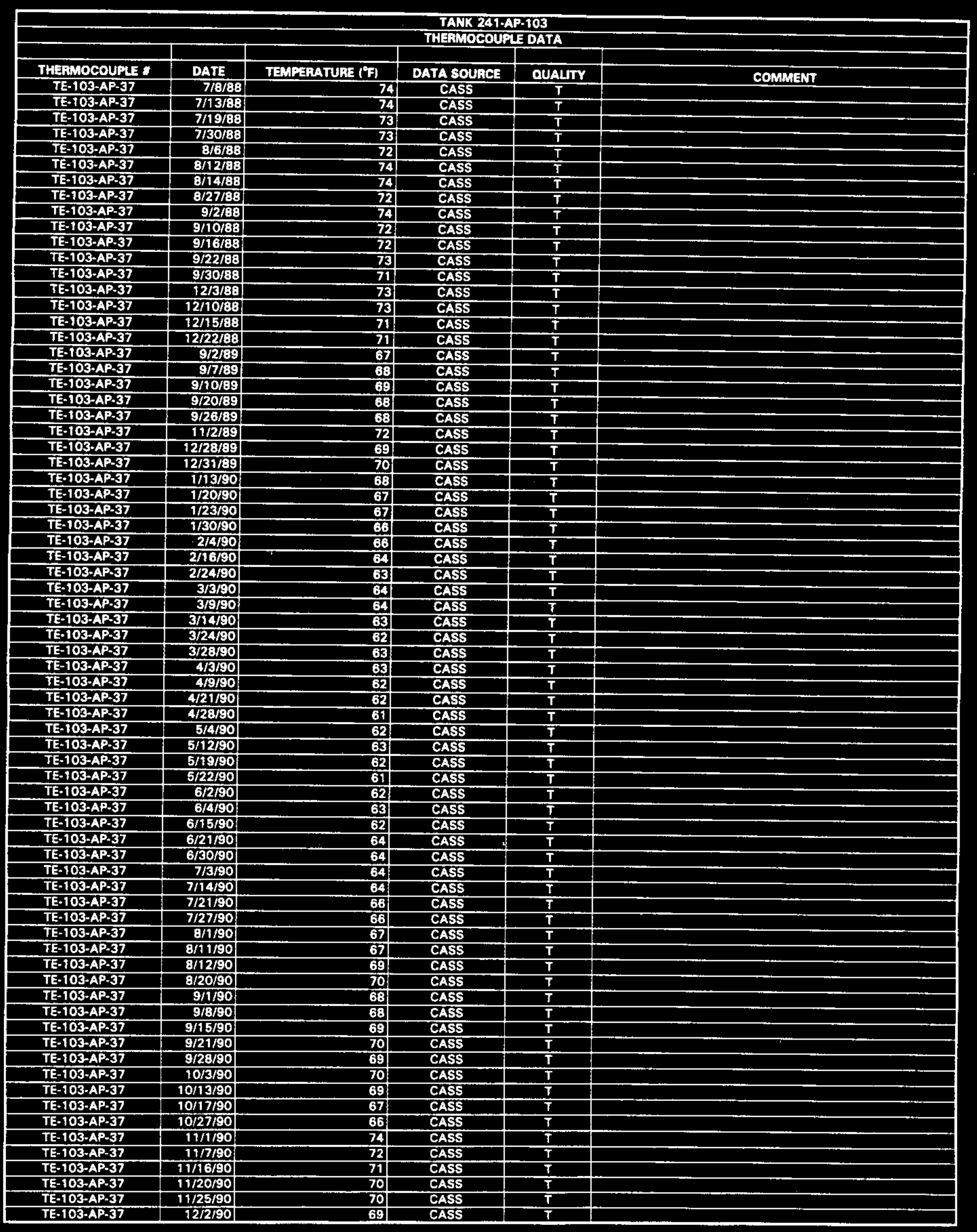




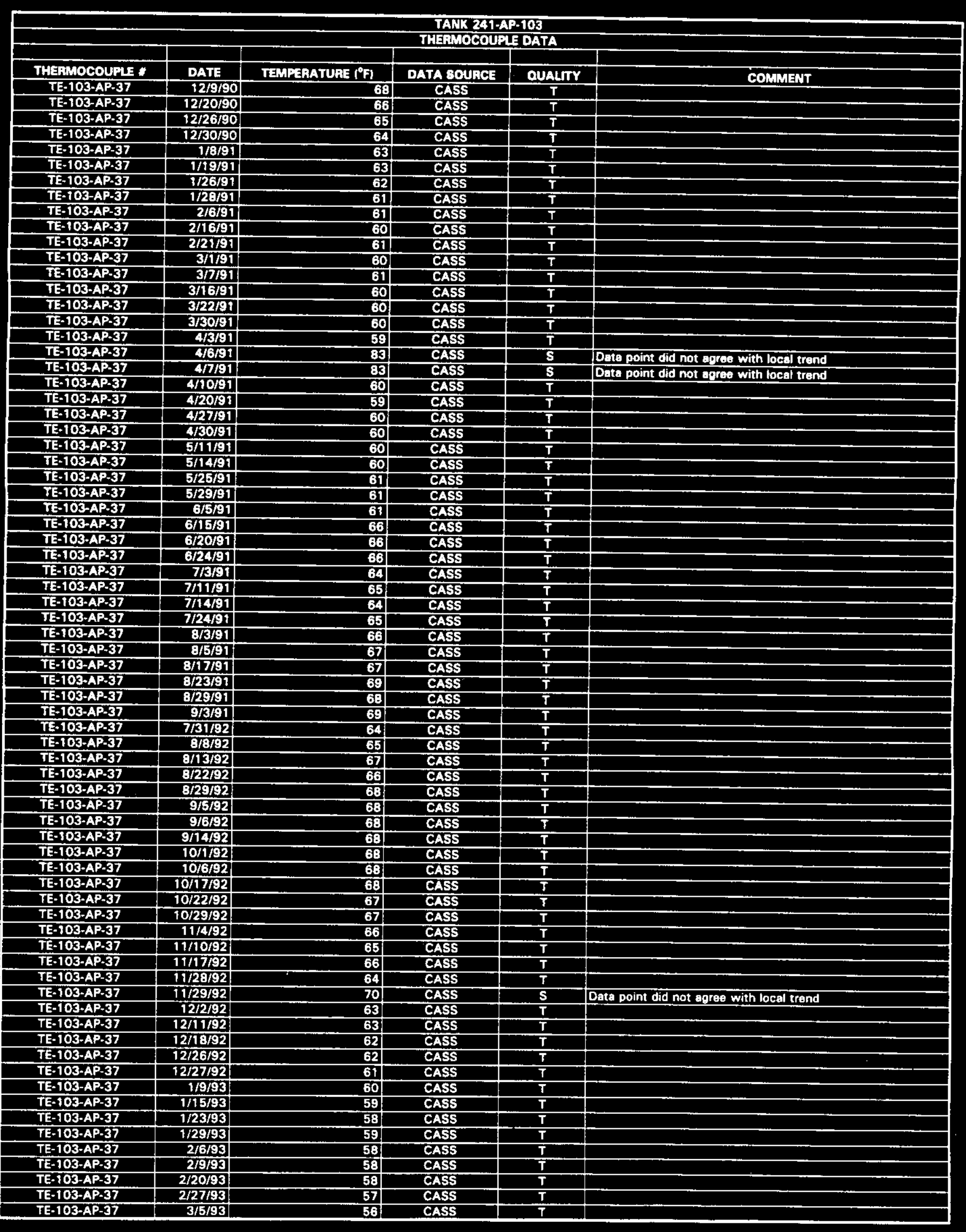




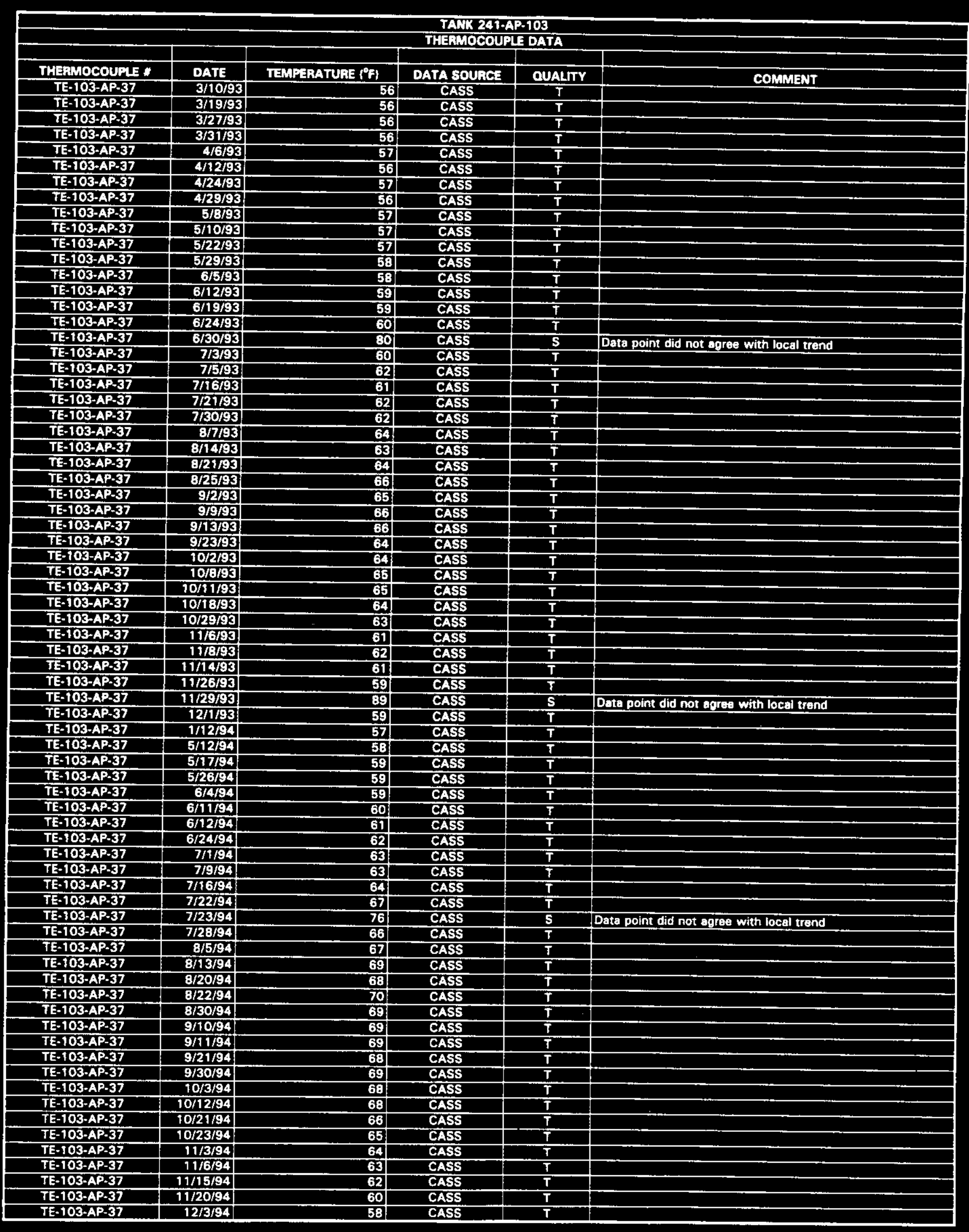

Data obtained from Computer Automated Surveillance System (CASS), April 1995; and Surveillance Analysis Computer Syatem (SACS), June 1995.

$D-239$ 


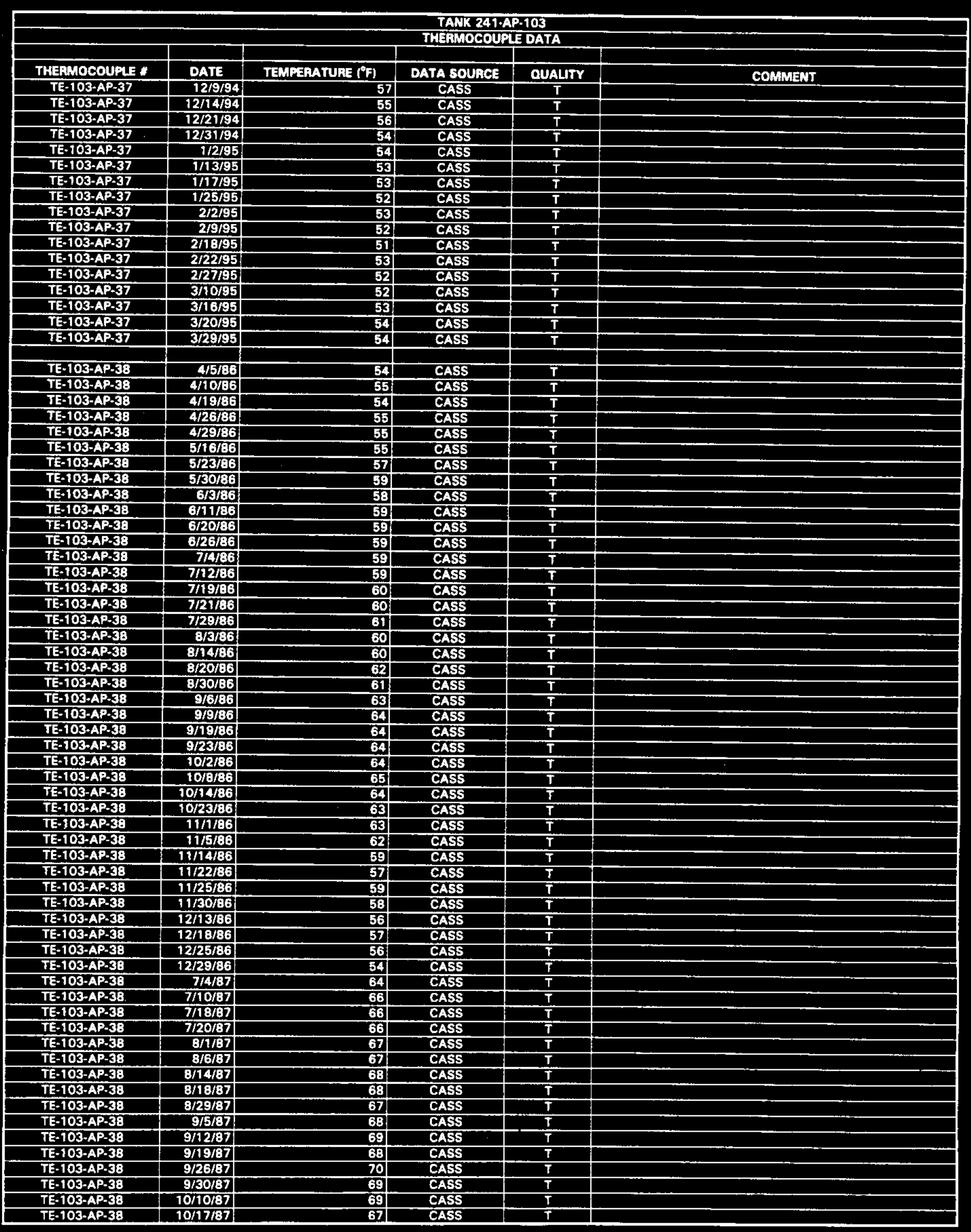




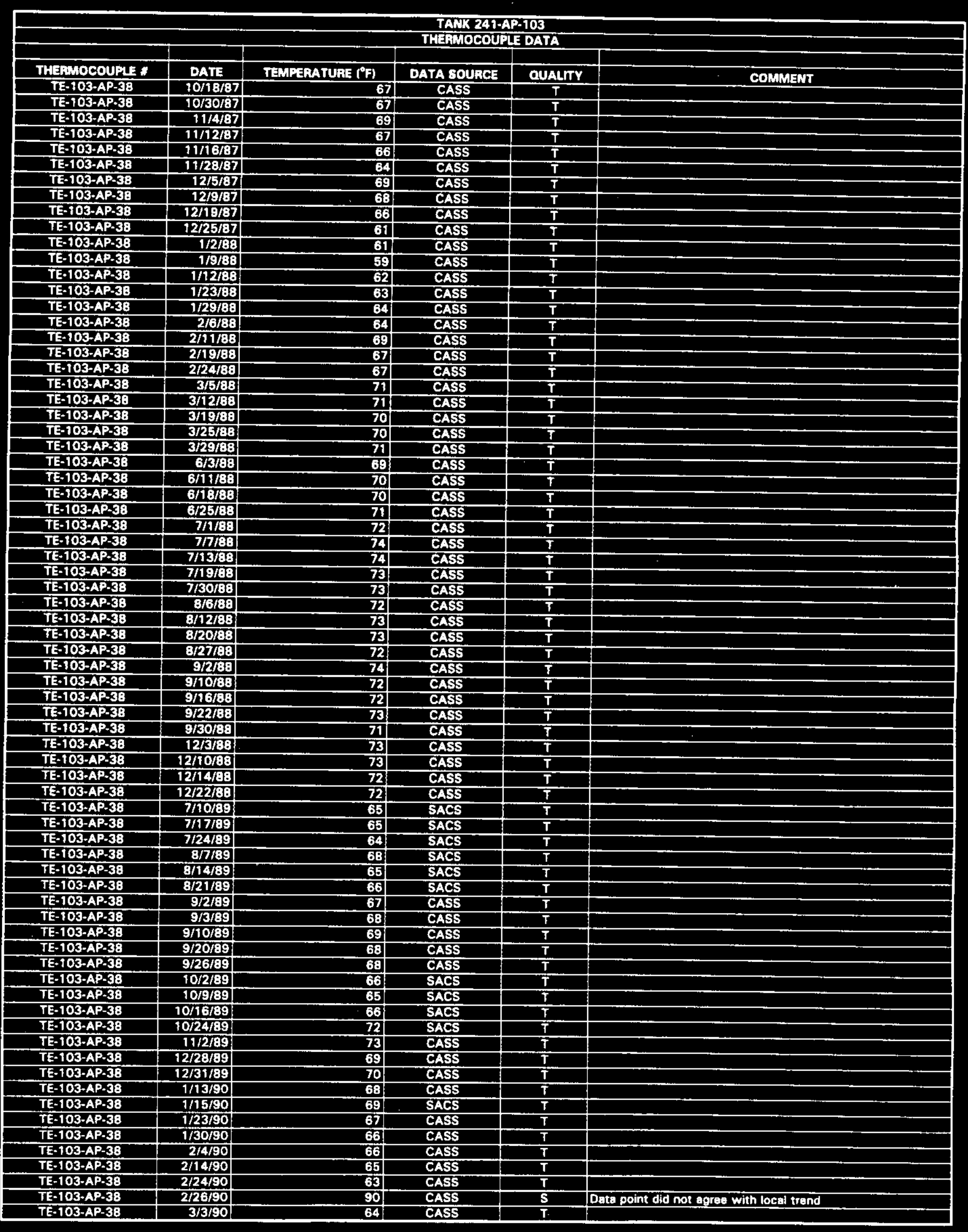




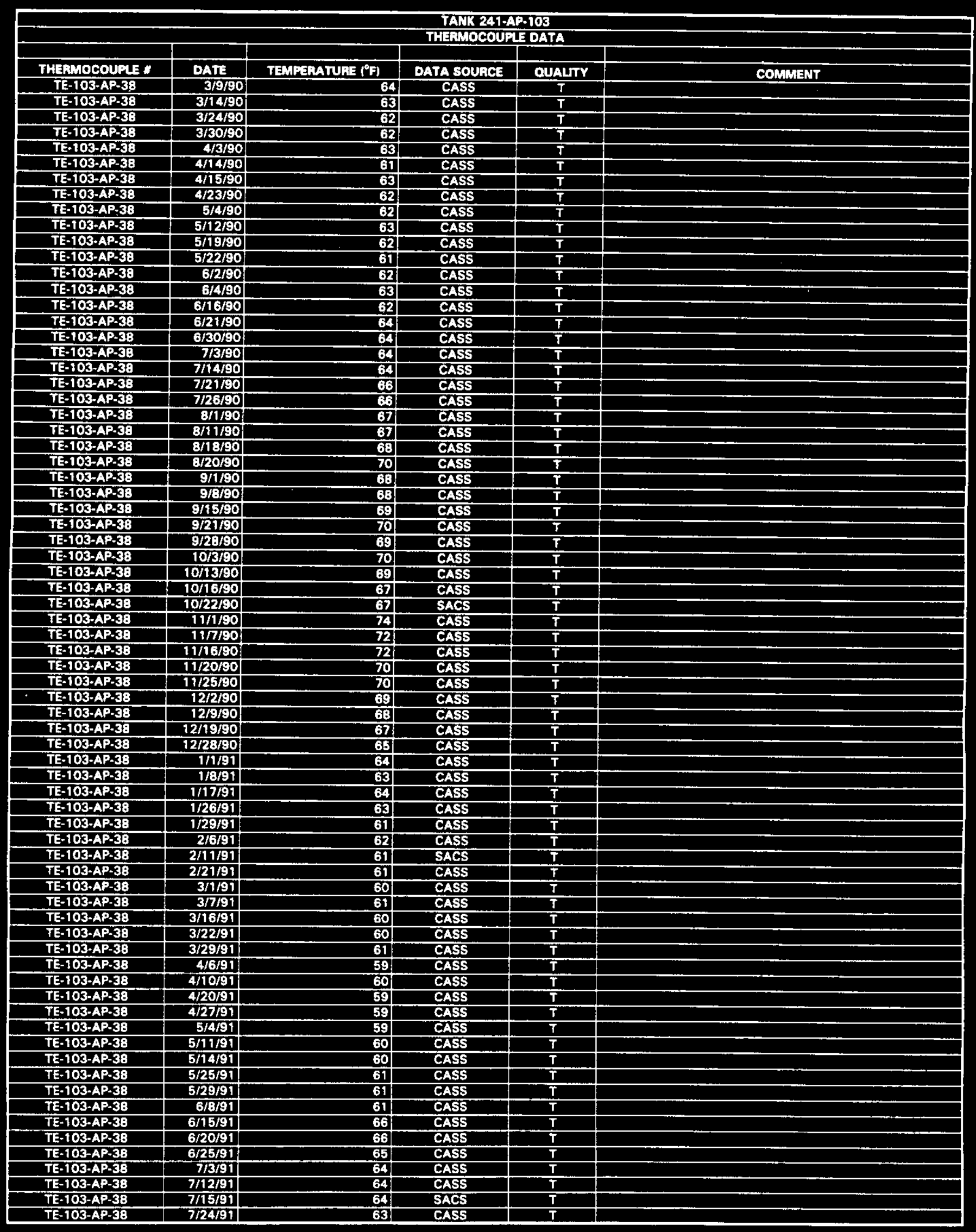




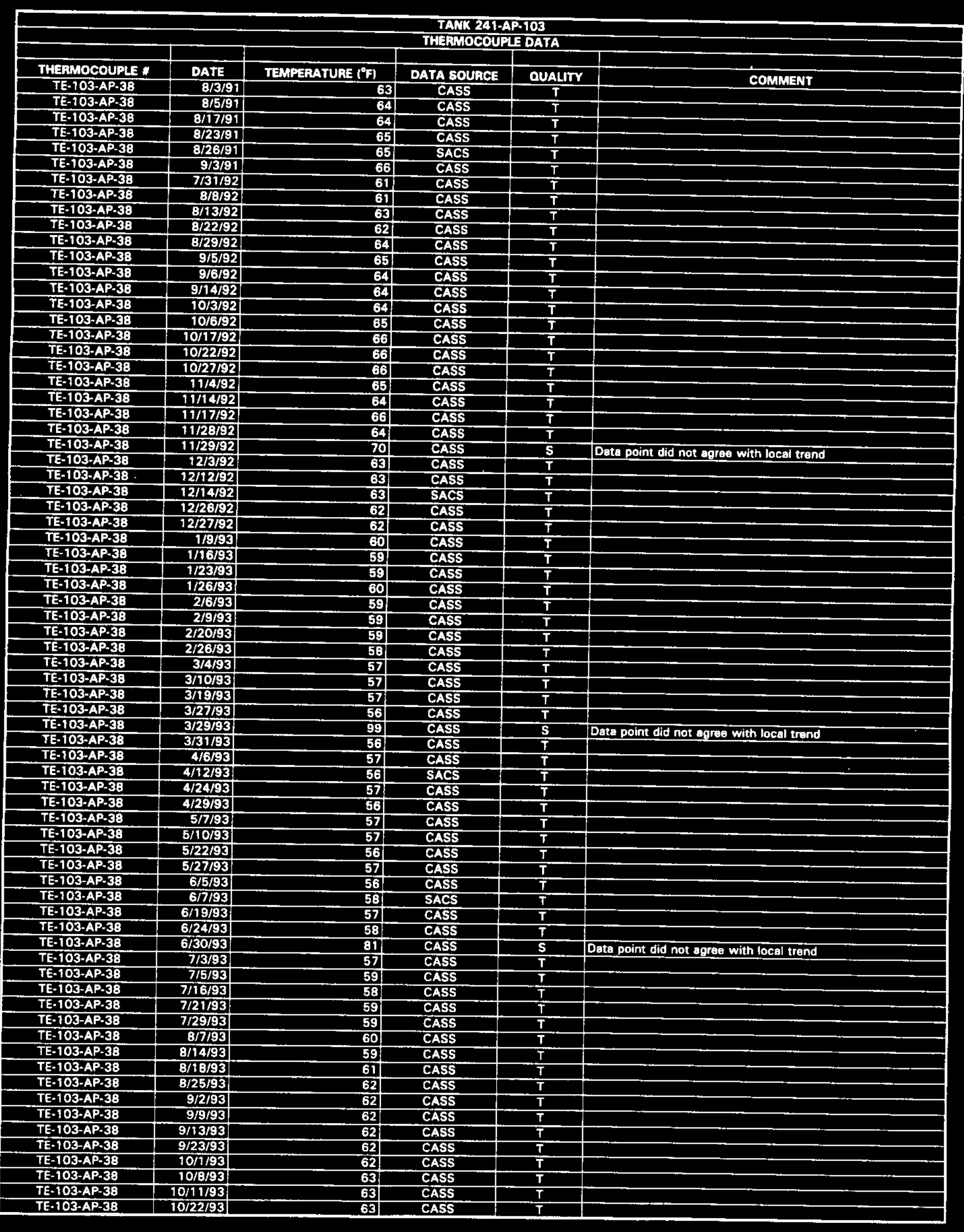




\begin{tabular}{|c|c|c|c|c|c|}
\hline \multicolumn{6}{|c|}{$\begin{array}{l}\text { TANK 241-AP-103 } \\
\text { THJAMOCOUPLE DATA }\end{array}$} \\
\hline THERMOCOUPLE * & DATE & TEMPERATUAE ( $\left.^{\circ} \mathrm{F}\right)$ & DATA SOURCE & OUALITY & COMMENT \\
\hline TE-103-AP-3B & $10 / 27 / 93$ & 63 & CASS & $\mathbf{T}$ & \\
\hline TE-103-AP-3B & $11 / 6 / 93$ & 61 & CASS & $T$ & \\
\hline TE-103-AP-38 & $11 / 8 / 93$ & 63 & CASS & $T$ & \\
\hline TE-103.AP-38 & $71 / 19 / 93$ & 61 & CASS & $T$ & \\
\hline TE-103-AP-3B & $11 / 27 / 93$ & 59 & CASS & $\bar{T}$ & \\
\hline TE-103-AP-38 & $12 / 1 / 93$ & 60 & CASS & $\mathbf{T}$ & \\
\hline TE-103-AP-38 & $12 / 10 / 93$ & 60 & SACS & $T$ & \\
\hline TE-103-AP-38 & $12 / 14 / 93$ & 60 & SACS & $\bar{T}$ & \\
\hline TE-103-AP-38 & $12 / 20 / 93$ & 57 & SACS & $\mathbf{T}$ & \\
\hline TE-TOS-AP-3B & $12 / 27 / 93$ & 57 & SACS & $\mathbf{T}$ & \\
\hline TE-103-AP-38 & $1 / 3 / 94$ & 57 & SACS & $\mathbf{T}$ & \\
\hline TE-103-AP-3B & $1 / 11 / 94$ & 60 & SACS & $\mathbf{T}$ & \\
\hline TE-103-AP-38 & $1 / 26 / 94$ & 55 & SACS & $T$ & \\
\hline TE-103-AP-38 & $2 / 9 / 94$ & 54 & SACS & $T$ & \\
\hline TE-103-AP-38 & $2 / 14 / 94$ & 53 & SACS & $\bar{T}$ & \\
\hline TE-103-AP-38 & $2 / 22 / 94$ & 54 & SACS & $\bar{T}$ & \\
\hline TE-103-AP-38 & $3 / 1 / 94$ & 55 & SACS & $T$ & \\
\hline TE-103-AP-3B & $4 / 27 / 94$ & 54 & SACS & $\overline{\mathbf{G}}$ & \\
\hline TE-103-AP-3B & $5 / 6 / 94$ & 54 & SACS & $\mathbf{G}$ & \\
\hline TE-103-AP-38 & $5 / 12 / 94$ & 57 & CASS & $T$ & \\
\hline TE-103-AP-38 & $5 / 17 / 94$ & 59 & CASS & $T$ & \\
\hline TE-103-AP-38 & $5 / 26 / 94$ & 59 & CASS & $T$ & \\
\hline TE-103-AP-38 & $6 / 4 / 94$ & 60 & CASS & $T$ & \\
\hline TE-103-AP-38 & $6 / 9 / 94$ & 61 & CASS & $T$ & \\
\hline TE-103-AP-38 & $6 / 18 / 94$ & 60 & CASS & $T$ & \\
\hline TE-103-AP-38 & $6 / 24 / 94$ & 62 & CASS & $T$ & \\
\hline TE-103-AP-38 & $7 / 1 / 94$ & 63 & CASS & $T$ & \\
\hline TE-103-AP-38 & $7 / 9 / 94$ & 64 & CASS & $T$ & \\
\hline TE-103-AP-36 & $7 / 16 / 94$ & 64 & CASS & $T$ & \\
\hline TE-103-AP-38 & $7 / 22 / 94$ & 66 & CASS & $T$ & \\
\hline TE-103-AP-3B & $7 / 28 / 94$ & 66 & CASS & $T$ & \\
\hline TE-103-AP-38 & $8 / 5 / 94$ & 66 & CASS & $T$ & \\
\hline TE-103-AP-38 & $8 / 11 / 94$ & 69 & CASS & $T$ & \\
\hline TE-103-AP-38 & $8 / 20 / 94$ & 68 & CASS & $T$ & \\
\hline TE-103-AP-3B & $8 / 22 / 94$ & 89 & CASS & $T$ & \\
\hline TE-103-AP-3B & $9 / 2 / 94$ & 88 & CASS & $T$ & \\
\hline TE-108-AP-38 & $9 / 10 / 94$ & 68 & CASS & $\bar{T}$ & \\
\hline TE-103-AP-38 & $9 / 11 / 94$ & 69 & CASS & $T$ & \\
\hline TE-103-AP-38 & $9 / 17 / 94$ & 120 & CASS & $\mathbf{S}$ & Data point did not agree with local trend \\
\hline TE-103-AP-3B & $9 / 21 / 94$ & 68 & CASS & $T$ & \\
\hline TE-103-AP-38 & $9 / 27 / 94$ & 69 & CAS5 & $\bar{T}$ & \\
\hline TE-103-AP-3B & $10 / 3 / 94$ & 68: & CASS & $\mathbf{T}$ & \\
\hline TE-103-AP-3B & $10 / 12 / 94$ & 67 & CASS & $\bar{T}$ & \\
\hline TE-103-AP-38 & $10 / 17 / 94$ & 82 & CASS & $\mathbf{S}$ & Data point did not agree with local trend \\
\hline TE-103-AP-38 & $10 / 21 / 94$ & 66 & CASS & $\bar{T}$ & \\
\hline TE-103-AP-38 & $10 / 29 / 94$ & 64 & CASS & $T$ & \\
\hline TE-103-AP-38 & $11 / 3 / 94$ & 64 & CASS & $T$ & \\
\hline TE-103-AP-38 & $11 / 12 / 94$ & 63 & CASS & $T$ & \\
\hline TE-103-AP-38 & $11 / 15 / 94$ & 62 & CASS & $\bar{T}$ & \\
\hline TE-103-AP-38 & $11 / 20 / 94$ & 60 & CASS & $T$ & \\
\hline TE-103-AP-38 & $12 / 1 / 94$ & 58 & CASS & $T$ & \\
\hline TE-103-AP-38 & $12 / 9 / 94$ & 57 & CASS & $T$ & \\
\hline TE-103-AP-38 & $12 / 16 / 94$ & 55 & SACS & G & \\
\hline TE-103-AP-38 & $12 / 21 / 94$ & 56 & CASS & $\mathbf{T}$ & \\
\hline TE-103-AP-36 & $12 / 31 / 94$ & 54 & CASS & $T$ & \\
\hline TE-103-AP-38 & $1 / 2 / 95$ & 54 & CASS & $T$ & \\
\hline TE-103-AP-38 & $1 / 9 / 95$ & 54 & SACS & $\mathbf{G}$ & \\
\hline TE-103-AP-38 & $1 / 17 / 95$ & 53 & CASS & $T$ & \\
\hline TE-103-AP-38 & $1 / 25 / 95$ & 52 & CASS & $T$ & \\
\hline TE-103-AP-38 & $2 / 2 / 95$ & 52 & CASS & $T$ & \\
\hline TE-103-AP-38 & $2 / 9 / 95$ & 52 & CASS & $T$ & \\
\hline TE-103-AP.38 & $2 / 18 / 95$ & 51 & CASS & $T$ & \\
\hline TE-103-AP-38 & $2 / 22 / 95$ & 53 & CASS & $T$ & \\
\hline TE-103-AP-38 & $2 / 27 / 95$ & 52 & CASS & $T$ & \\
\hline TE-103-AP-38 & $3 / 10 / 95$ & 52 & CASS & $T$ & \\
\hline TE-103-AP-38 & $3 / 16 / 95$ & 53 & CASS & $T$ & \\
\hline TE-103-AP-3B & $3 / 20 / 95$ & 54 & CASS & $T$ & \\
\hline TE-103-AP-38 & $3 / 29 / 95$ & 54 & CASS & $T$ & \\
\hline TE-103-AP-38 & $4 / 3 / 95$ & 51 & SACS & $\mathbf{G}$ & \\
\hline TE-103-AP-38 & 4/10/95 & 52 & SACS & $\mathbf{G}$ & \\
\hline TE-103-AP-38 & $4 / 17 / 95$ & 52 & SACS & $\mathbf{G}$ & \\
\hline TE-103-AP-3B & $4 / 24 / 95$ & 55 & SACS & $\mathbf{G}$ & \\
\hline TE-103-AP-3B & $5 / 1 / 95$ & 54 & SACS & $\mathbf{G}$ & \\
\hline
\end{tabular}




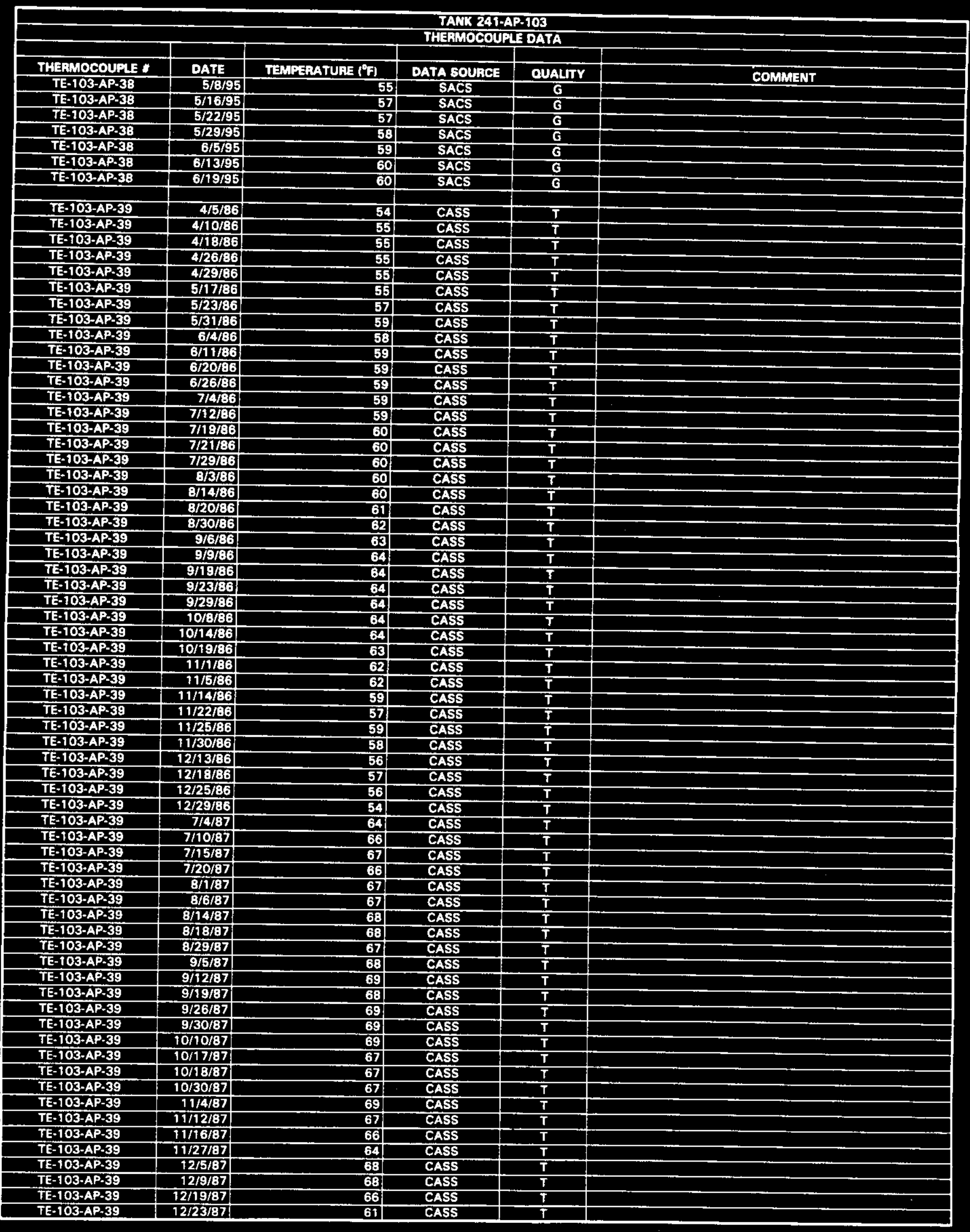

Data obtained from Computer Automated Surveillance System (CASS), April 1995; and Surveillance Analysis Computer System (SACS), June 1995. 


\begin{tabular}{|c|c|c|c|c|c|}
\hline \multicolumn{6}{|c|}{$\begin{array}{l}\text { TANK 241-AP-103 } \\
\text { THEFIMOCOUPLE DATA }\end{array}$} \\
\hline THEPMOCOUPLE * & DATE & TEMPERATURE $\left({ }^{\circ} \mathrm{F}\right)$ & DATA SOUACE & OUALITY & COMMENT \\
\hline TE-103-AP-39 & $12 / 29 / 87$ & 61 & CASS & $\mathbf{T}$ & \\
\hline TE-103-AP-39 & $1 / 9 / 88$ & 59 & CASS & $\mathbf{T}$ & \\
\hline TE-103-AP-39 & $1 / 12 / 88$ & 62 & CASS & $T$ & \\
\hline TE-103-AP-39 & $1 / 23 / 88$ & 63 & CASS & $T$ & \\
\hline TE-103-AP-39 & $1 / 29 / 88$ & 64 & CASS & $T$ & \\
\hline TE-103-AP-39 & $2 / 6 / 88$ & 64 & CASS & $T$ & \\
\hline TE-103-AP-39 & $2 / 91 / 38$ & 69 & CASS & $\mathbf{T}$ & \\
\hline TE-103-AP-39 & $2 / 19 / 88$ & 67 & CASS & $T$ & \\
\hline TE-103-AP-39 & $2 / 24 / 88$ & 67 & CASS & $T$ & \\
\hline TE-103.AP-39 & $3 / 5 / 88$ & 71 & CASS & $\mathbf{T}$ & \\
\hline TE-103-AP-39 & $3 / 12 / 88$ & 70 & CASS & $\bar{T}$ & \\
\hline TE-103-AP-39 & $3 / 19 / 88$ & 70 & CASS & $\mathbf{T}$ & \\
\hline TE-103-AP-39 & $3 / 25 / 68$ & 70 & CASS & $T$ & \\
\hline TE-103-AP-39 & $3 / 29 / 38$ & 71 & CASS & $\mathbf{T}$ & \\
\hline TE-103-AP-39 & $6 / 3 / 38$ & 69 & CASS & $\mathbf{T}$ & \\
\hline TE-103-AP-39 & $6 / 11 / 88$ & 70 & CASS & $T$ & \\
\hline TE-103-AP-39 & 6/18/38 & 70 & CASS & $T$ & \\
\hline TE-103-AP-39 & $6 / 25 / 86$ & 71 & CASS & $\mathbf{T}$ & \\
\hline TE-103-AP-39 & $711 / 83$ & 72 & CASS & $\mathbf{T}$ & \\
\hline TE-103-AP-39 & $7 / 7 / 38$ & 74 & CASS & $T$ & \\
\hline TE-103-AP-39 & $7 / 13 / 68$ & 74 & CASS & $T$ & \\
\hline TE-108-AP-39 & 7/19/88 & 73 & CASS & $\bar{T}$ & \\
\hline TE-103-AP-39 & $7 / 30 / 88$ & 73 & CASS & $T$ & \\
\hline TE-108.AP-39 & $8 / 6 / 88$ & 72 & CASS & $T$ & \\
\hline TE-103-AP-39 & $8 / 9 / 88$ & 124 & CASS & $\mathbf{S}$ & Data point did not agree with local trend \\
\hline TE-103-AP-39 & $8 / 12 / 38$ & 73 & CASS & $T$ & \\
\hline TE-103-AP-39 & $8 / 20 / 88$ & 73 & CASS & $\mathbf{T}$ & \\
\hline TE-103-AP-39 & $8 / 25 / 38$ & 73 & CASS & $\mathbf{T}$ & \\
\hline TE-103-AP-39 & 9/2/8B & 74 & CASS & $\mathbf{T}$ & \\
\hline TE-103-AP-39 & 9/10/88 & 72 & CASS & $T$ & \\
\hline TE-103-AP-39 & $9 / 14 / 88$ & 72 & CASS & $T$ & \\
\hline TE-103-AP-39 & $9 / 22 / 88$ & 73 & CASS & $\mathbf{T}$ & \\
\hline TE-103-AP-39 & 9/29/88 & 71 & CASS & $\mathbf{T}$ & \\
\hline TE-103-AP-39 & $12 / 3 / 88$ & 73 & CABS & $T$ & \\
\hline TE-103-AP-39 & $12 / 10 / 88$ & 73 & CASS & $\mathbf{T}$ & \\
\hline TE-103-AP-39 & 12/15/88 & 71 & CASS & $\mathbf{T}$ & \\
\hline IE-103-AP-39 & $12 / 22 / 88$ & 71 & CASS & $T$ & \\
\hline TE-103-AP-39 & $9 / 2 / 69$ & 67 & CASS & $\bar{T}$ & \\
\hline TE-103-AP-39 & $9 / 3 / 69$ & 68 & CASS & $\bar{T}$ & \\
\hline TE-103-AP-39 & $9 / 10 / 89$ & 69 & CASS & $\mathbf{T}$ & \\
\hline TE-103-AP-39 & 9/20/89 & 68 & CASS & $\mathbf{T}$ & \\
\hline TE-103-AP-39 & 9/26/89 & 68. & CASS & $\mathbf{T}$ & \\
\hline TE-103-AP-39 & $111 / 2 / 39$ & 72 & CASS & $\mathbf{T}$ & \\
\hline TE-103-AP-39 & $12128 / 89$ & 69 & CASS & $T$ & \\
\hline TE-103-AP-39 & $12 / 31 / 89$ & 70 & CASS & $\bar{T}$ & \\
\hline TE-103-AP-39 & $1 / 7 / 180$ & 68 & CASS & $\mathbf{T}$ & \\
\hline TE-103-AP-39 & 1/17/90 & 68 & CASS & $T$ & \\
\hline TE-103-AP-39 & $1 / 23 / 90$ & 67 & CASS & $\mathbf{T}$ & \\
\hline TE-103-AP-39 & $1 / 30 / 90$ & 86 & CASS & $T$ & \\
\hline TE-103-AP-39 & $2 / 4 / 90$ & 66 & CASS & $\bar{T}$ & \\
\hline TE-103-AP-39 & $2 / 14 / 90$ & 65 & CASS & $\mathbf{T}$ & \\
\hline TE-103-AP-39 & $2120 / 90$ & 64 & CASS & $T$ & \\
\hline TE-103-AP-39 & $3 / 3 / 90$ & 64 & CASS & $T$ & \\
\hline TE-103-AP-39 & $3 / 9 / 90$ & 64 & CASS & $\mathbf{T}$ & \\
\hline TE-103-AP-39 & $3 / 14 / 90$ & 63 & CASS & $\mathbf{T}$ & \\
\hline TE-103-AP-39 & $3 / 24 / 90$ & 62 & CASS & $\mathbf{T}$ & \\
\hline TE-103-AP-39 & $3 / 28 / 90$ & 63 & CASS & $T$ & \\
\hline TE-103-AP-39 & $4 / 3 / 90$ & 63 & CASS & $T$ & \\
\hline TE-103-AP-39 & $4 / 14 / 90$ & 61 & CASS & $T$ & \\
\hline TE-103-AP-39 & 4/15/90 & 63. & CASS & $\mathbf{T}$ & \\
\hline TE-103-AP-39 & 4/28/90 & 61 & CASS & $T$ & \\
\hline TE-103-AP-39 & $5 / 4 / 90$ & 62 & CASS & $T$ & \\
\hline TE-108-AP-39 & $5 / 12 / 90$ & 63 & CASS & $T$ & \\
\hline TE-103-AP-39 & $5 / 14 / 90$ & 62 & CASS & $T$ & \\
\hline TE-103-AP-39 & $5 / 22 / 90$ & 61 & CASS & $T$ & \\
\hline TE-103-AP-39 & $6 / 1 / 90$ & 62 & CASS & $\mathbf{T}$ & \\
\hline TE-103-AP-39 & $6 / 4 / 90$ & 63 & CASS & $\mathbf{T}$ & \\
\hline TE-103-AP-39 & 6/15/90 & 62 & CASS & $\bar{T}$ & \\
\hline TE-103-AP-39 & $6 / 21 / 90$ & 64 & CASS & $T$ & \\
\hline TE-103-AP-39 & $6 / 30 / 90$ & 64 & CASS & $T$ & \\
\hline TE-103-AP-39 & $7 / 3 / 90$ & 64 & CASS & $T$ & \\
\hline TE-103-AP-39 & $7 / 7 / 4 / 90$ & 64 & CASS & $T$ & \\
\hline TE-103-AP-39 & $7 / 21 / 90$ & 67 & CASS & $\mathbf{T}$ & \\
\hline
\end{tabular}




\begin{tabular}{|c|c|c|c|c|c|}
\hline \multicolumn{6}{|c|}{$\begin{array}{l}\text { TANK 241-AP-103 } \\
\text { THEAMOCOUPLE DATA }\end{array}$} \\
\hline & & & & & \\
\hline THERMOCOUPLE * & DATE & TEMPGPATURE $\left({ }^{\circ} \mathrm{F}\right)$ & DATA SOUACE & OUALTY & COMMENT \\
\hline TE-103-AP-39 & $7 / 26 / 90$ & 66 & CASS & $T$ & \\
\hline TE-103-AP-39 & $8 / 1 / 90$ & 67 & CASS & $\bar{T}$ & \\
\hline TE-103-AP-39 & $8 / 11 / 90$ & 67 & CASS & $T$ & \\
\hline TE-103-AP-39 & $8 / 12 / 90$ & 69 & CASS & $T$ & \\
\hline TE-103-AP-39 & $8 / 20 / 90$ & 69 & CASS & $\mathbf{T}$ & \\
\hline TE-103-AP-39 & $\overline{9} / 1 / 90$ & 68 & CASS & $\mathbf{T}$ & \\
\hline TE-103-AP-39 & $9 / 8 / 90$ & 68 & CASS & $\bar{T}$ & \\
\hline TE-103-AP-39 & $9 / 15 / 90$ & 69 & CASS & $\mathbf{T}$ & \\
\hline TE-103-AP-39 & $9 / 21 / 90$ & 70 & CASS & $\bar{T}$ & \\
\hline TE-103-AP-39 & $9 / 28 / 90$ & 69 & CASS & $T$ & \\
\hline TE-103-AP-39 & $10 / 5 / 90$ & 69 & CASS & $T$ & \\
\hline TE-103-AP-39 & $10 / 13 / 90$ & 68 & CASS & $\mathbf{T}$ & \\
\hline TE-103-AP-39 & $10 / 16 / 90$ & 67 & CASS & $T$ & \\
\hline TE-\$03-AP-39 & $10 / 22 / 90$ & 99 & CASS & $\mathbf{S}$ & Data point did not agree with local trend \\
\hline TE-103-AP-39 & $10 / 23 / 90$ & 67 & CASS & $T$ & \\
\hline TE-103-AP-39 & $11 / 1 / 90$ & 75 & CASS & $T$ & \\
\hline TE-103-AP-39 & $11 / 7 / 90$ & 73 & CASS & $T$ & \\
\hline TE-103-AP-39 & $11 / 16 / 90$ & 72 & CASS & $T$ & \\
\hline TE-103-AP-39 & $11 / 18 / 80$ & 71 & CASS & $T$ & \\
\hline TE-103-AP-39 & $12 / 1 / 90$ & 70 & CASS & $T$ & \\
\hline TE-103-AP-39 & $12 / 4 / 90$ & 69 & CASS & $T$ & \\
\hline TE-103-AP-39 & $12 / 9 / 90$ & 69 & CASS & $T$ & \\
\hline TE-103-AP-39 & $12 / 19 / 90$ & 68 & CASS & $T$ & \\
\hline TE-103-AP-39 & $12 / 29 / 90$ & 66 & CASS & $T$ & \\
\hline TE-103-AP-39 & $12 / 30 / 90$ & 66 & CASS & $T$ & \\
\hline TE-103-AP-39 & $1 / 12 / 91$ & 64 & CASS & $T$ & \\
\hline TE-103-AP-39 & $1 / 17 / 91$ & 65 & CAS5 & $T$ & \\
\hline TE-103-AP-3Q & $1 / 26 / 91$ & 64 & CASS & $T$ & \\
\hline TE-103-AP-39 & $1 / 30 / 91$ & 62 & CASS & 7 & \\
\hline TE-103-AP-39 & $2 / 6 / 91$ & 63 & CASS & $\bar{T}$ & \\
\hline TE-103-AP-39 & $2 / 15 / 91$ & 61 & CASS & $T$ & \\
\hline TE-103-AP-39 & $2 / 21 / 91$ & 61 & CASS & $T$ & \\
\hline TE-103-AP-39 & $3 / 1 / 91$ & 61 & CASS & $T$ & \\
\hline TE-103-AP-39 & $3 / 7 / 91$ & 61 & CASS & $T$ & \\
\hline TE-103-AP-39 & $3 / 10 / 91$ & 61 & CASS & $T$ & \\
\hline TE-103-AP-39 & $3 / 22 / 91$ & 61 & CASS & $\bar{T}$ & \\
\hline TE-103-AP-39 & $3 / 30 / 91$ & 61 & CASS & $T$ & \\
\hline TE-103-AP-39 & $4 / 6 / 91$ & 59 & CASS & $T$ & \\
\hline TE-103-AP-39 & $4 / 10 / 91$ & 61 & CASS & $T$ & \\
\hline TE-103.AP-39 & $4 / 20 / 91$ & 59 & CASS & $T$ & \\
\hline TE-103-AP-39 & $4 / 23 / 91$ & 60 & CASS & $\mathbf{T}$ & \\
\hline TE-103-AP-39 & $4 / 30 / 91$ & 61 & CASS & $T$ & \\
\hline TE-103-AP-39 & $5 / 11 / 91$ & 601 & CASS & $T$ & \\
\hline TE-103-AP-39 & $5 / 14 / 91$ & 60 & CASS & $T$ & \\
\hline TE-103-AP-39 & $5 / 25 / 91$ & 61 & CASS & $T$ & \\
\hline TE-103-AP-39 & $5 / 29 / 91$ & 61 & CASS & $T$ & \\
\hline TE-103-AP-39 & 6/8/91 & 61 & CASS & $T$ & \\
\hline TE-103-AP-39 & $6 / 15 / 91$ & 66 & CASS & $T$ & \\
\hline TE-103-AP-39 & $6 / 20 / 91$ & 66 & CASS & $T$ & \\
\hline TE-103-AP-39 & $6 / 24 / 91$ & 65 & CASS & $\mathbf{T}$ & \\
\hline TE-103-AP-39 & $7 / 3 / 91$ & 63 & CASS & $\mathbf{T}$ & \\
\hline TE-103-AP-39 & $7 / 11 / 91$ & 64 & CASS & $T$ & \\
\hline TE-103-AP-39 & $7 / 14 / 91$ & 63 & CASS & $T$ & \\
\hline TE-103-AP-39 & $7 / 24 / 91$ & 63 & CASS & $T$ & \\
\hline TE-103-AP-39 & $8 / 2 / 91$ & 63 & CASS & $T$ & \\
\hline TE-103-AP-39 & $8 / 5 / 91$ & 64 & CASS & $T$ & \\
\hline TE-103-AP-39 & $8 / 17 / 91$ & 63 & CASS & $T$ & \\
\hline TE-103-AP-39 & $8 / 23 / 91$ & 64 & CASS & $T$ & \\
\hline TE-103-AP-39 & $8 / 27 / 91$ & 64 & CASS & $T$ & \\
\hline TE-103-AP-39 & $9 / 3 / 91$ & 65 & CASS & $T$ & \\
\hline TE-103-AP-39 & $7 / 31 / 92$ & 60 & CASS & $T$ & \\
\hline TE-103-AP-39 & $8 / 8 / 92$ & 61 & CASS & $T$ & \\
\hline TE-103-AP-39 & $8 / 13 / 92$ & 63 & CASS & $T$ & \\
\hline TE-103-AP-39 & $8 / 22 / 92$ & 61 & CASS & $T$ & \\
\hline TE-103-AP-39 & $8 / 29 / 92$ & 63 & CASS & $\cdot \mathbf{T}$ & \\
\hline TE-103-AP-39 & $9 / 5 / 92$ & 64 & CASS & $T$ & \\
\hline TE-103-AP-39 & $9 / 11 / 92$ & 63 & CASS & $T$ & \\
\hline TE-103-AP-39 & $9 / 14 / 92$ & 63 & CASS & $T$ & \\
\hline TE-103-AP-39 & $10 / 3 / 92$ & 63 & CASS & $T$ & \\
\hline TE-103-AP-39 & $10 / 6 / 92$ & 64 & CASS & $T$ & \\
\hline TE-103-AP-39 & $10 / 17 / 92$ & 65 & CASS & $T$ & \\
\hline TE-103-AP-39 & $10 / 22 / 92$ & 65 & CASS & $T$ & \\
\hline TE-103-AP-39 & $10 / 28 / 92$ & 65 & CASS & $T$ & \\
\hline
\end{tabular}




\begin{tabular}{|c|c|c|c|c|c|}
\hline \multicolumn{6}{|c|}{$\begin{array}{l}\text { TAKK 241-AP-103 } \\
\text { THSAMOCOUPIE DATA }\end{array}$} \\
\hline & & & & & \\
\hline THERMOCOUPLE * & DATE & TEMPERATURE ( $\left.{ }^{\circ} F\right)$ & DATA SOUACE & QUALTY & COMMENT \\
\hline TE-103-AP-39 & $11 / 5 / 92$ & 64 & CASS & $T$ & \\
\hline TE-103-AP-39 & $11 / 9 / 92$ & 64 & CASS & $\mathbf{T}$ & \\
\hline TE-103-AP-39 & $11 / 17 / 92$ & 65 & CASS & $T$ & \\
\hline TE-103-AP-39 & $11 / 28 / 92$ & 64 & CASS & $T$ & \\
\hline TE-103-AP-39 & $\$ 1 / 29 / 92$ & 70 & CASS & $\mathbf{S}$ & Data point did not egree with local trend \\
\hline TE-103-AP-39 & $12 / 2 / 92$ & 63 & CASS & $T$ & \\
\hline TE-103-AP-39 & $12 / 12 / 92$ & 63 & CASS & $\bar{T}$ & \\
\hline TE-103-AP-39 & $12 / 13 / 92$ & 63 & CASS & $\mathbf{T}$ & \\
\hline TE-108-AP-39 & $12 / 26 / 92$ & 62 & CASS & $T$ & \\
\hline TE-103-AP-39 & $1 / 2 / 93$ & 61 & CASS & $T$ & \\
\hline TE-103-AP-39 & $1 / 9 / 93$ & 60 & CASS & $T$ & \\
\hline TE-103-AP-39 & $1 / 14 / 93$ & 60 & CASS & $T$ & \\
\hline TE-103-AP-39 & $1 / 23 / 93$ & 59 & CASS & $T$ & \\
\hline TE-103-AP-39 & $1 / 26 / 93$ & 80 & CASS & $T$ & \\
\hline TE-103-AP-39 & $2 / 6 / 93$ & 59 & CASS & $\mathbf{T}$ & \\
\hline TE-103-AP-39 & $2 / 9 / 93$ & 59 & CASS & $T$ & \\
\hline TE-103-AP-39 & $2 / 19 / 93$ & 58 & CASS & $T$ & \\
\hline TE-103-AP-39 & $2 / 27 / 93$ & 58 & CASS & $\bar{T}$ & \\
\hline TE-103-AP-39 & $3 / 4 / 93$ & 57 & CASS & $\mathbf{T}$ & \\
\hline TE-103-AP-39 & $3 / 10 / 93$ & 57. & CASS & $T$ & \\
\hline TE-103-AP-39 & 3/19/93 & 56 & CASS & $T$ & \\
\hline TE-103-AP-39 & $3 / 27 / 93$ & 56 & CASS & $T$ & \\
\hline TE-103-AP-39 & $3 / 31 / 93$ & 56 & CASS & $T$ & \\
\hline TE-103-AP-39 & $4 / 6 / 93$ & 57 & CASS & $T$ & \\
\hline TE-103-AP-39 & $4 / 12 / 93$ & 56 & CASS & $T$ & \\
\hline TE-103-AP-39 & $4 / 24 / 93$ & 57 & CASS & $T$ & \\
\hline TE-103-AP-39 & $4 / 29 / 93$ & 56 & CASS & $T$ & \\
\hline TE-103-AP-39 & $5 / 7 / 93$ & 57 & CASS & $T$ & \\
\hline TE-103-AP-39 & $5 / 11 / 93$ & 56 & CASS & $T$ & \\
\hline TE-103-AP-39 & $5 / 22 / 93$ & 56 & CASS & $T$ & \\
\hline TE-103-AP-39 & $5 / 27 / 93$ & 57 & CASS & $T$ & \\
\hline TE-103-AP-39 & $6 / 5 / 93$ & 58 & CASS & $T$ & \\
\hline TE-103.AP-39 & $6 / 12 / 93$ & 56 & CASS & $T$ & \\
\hline TE-103-AP-39 & $6 / 15 / 93$ & 121 & CASS & $\mathbf{S}$ & Date point did not agree with local trend \\
\hline TE-103-AP-39 & $6 / 16 / 93$ & 57 & CASS & $T$ & \\
\hline TE-103-AP-39 & $6 / 24 / 83$ & 57 & CASS & $\bar{T}$ & \\
\hline TE-103-AP-39 & 6/30/93 & 81 & CASS & $\mathbf{S}$ & Data point did not agree with locel trend \\
\hline TE-103-AP-39 & $7 / 3 / 93$ & 57 & CASS & $T$ & \\
\hline TE-103-AP-39 & $7 / 5 / 93$ & 59 & CASS & $\mathbf{T}$ & \\
\hline TE-103-AP-39 & $7117 / 93$ & 57 & CASS & $\mathbf{T}$ & \\
\hline TE-103-AP-39 & $7 / 21 / 93$ & 59 & CASS & $T$ & \\
\hline TE-103+AP-39 & $7 / 31 / 93$ & 58 & CASS & $\bar{T}$ & \\
\hline TE-103-AP-39 & 6/7/93 & 60 & CASS & $T$ & \\
\hline TE-103-AP-39 & $8 / 14 / 93$ & 59 & CASS & $T$ & \\
\hline TE-103-AP-39 & $8 / 20 / 93$ & 60 & CASS & $T$ & \\
\hline TE-103-AP-39 & $8 / 28 / 93$ & 61 & CASS & $T$ & \\
\hline TE-103-AP-39 & $9 / 2 / 93$ & 61 & CASS & $T$ & \\
\hline TE-103-AP-39 & $9 / 9 / 93$ & 61 & CASS & $\mathbf{T}$ & \\
\hline TE-103-AP-39 & $9 / 13 / 93$ & 81! & CASS & $\mathbf{T}$ & \\
\hline TE-103-AP-39 & $9 / 23 / 93$ & 61 & CASS & $T$ & \\
\hline TE-103-AP-39 & $10 / 1 / 93$ & 61 & CASS & $T$ & \\
\hline TE-103-AP-39 & $10 / 8 / 93$ & 62 & CASS & $\mathbf{T}$ & \\
\hline TE-103-AP-39 & $10 / 11 / 93$ & 63 & CASS & $T$ & \\
\hline TE-103-AP-39 & $10 / 22 / 93$ & 62 & CASS & $T$ & \\
\hline TE-103-AP-39 & $10 / 29 / 93$ & 62 & CASS & $T$ & \\
\hline TE-103-AP-39 & $11 / 6 / 93$ & 61 & CASS & $T$ & \\
\hline TE-103-AP-39 & $11 / 8 / 93$ & 62 & CASS & $T$ & \\
\hline TE-103-AP-39 & $11 / 19 / 93$ & 61 & CASS & $T$ & \\
\hline TE-103-AP-39 & $11 / 23 / 93$ & 60 & CASS & $T$ & \\
\hline TE-103-AP-39 & $12 / 1 / 93$ & 60 & CASS & $T$ & \\
\hline TE-103-AP-39 & $1 / 12 / 94$ & 57 & CASS & $T$ & \\
\hline TE-103-AP.39 & $5 / 12 / 94$ & 58 & CASS & $T$ & \\
\hline TE-103-AP-39 & $5 / 16 / 94$ & 104 & CASS & $\mathbf{S}$ & Date point did not egree with local trend \\
\hline TE-103-AP-39 & $5 / 17 / 94$ & 59 & CASS & $T$ & \\
\hline TE-103-AP-39 & $5 / 26 / 94$ & 59 & CASS & $T$ & \\
\hline TE-103-AP-39 & $6 / 4 / 94$ & 59 & CASS & $\mathbf{T}$ & \\
\hline TE-103-AP-39 & $6 / 11 / 94$ & 60 & CASS & $T$ & \\
\hline TE-103-AP-39 & $6 / 12 / 94$ & 61 & CASS & $\mathbf{T}$ & \\
\hline TE-103-AP-39 & $6 / 24 / 94$ & 62 & CASS & $T$ & \\
\hline TE-103-AP-39 & $7 / 1 / 94$ & 63 & CASS & $T$ & \\
\hline TE-103-AP-39 & $7 / 9 / 94$ & 64 & CASS & $T$ & \\
\hline TE-103-AP-39 & $7 / 16 / 94$ & 64 & CASS & $T$ & \\
\hline TE-103-AP-39 & $7 / 22 / 94$ & 66 & CASS & $T$ & \\
\hline
\end{tabular}




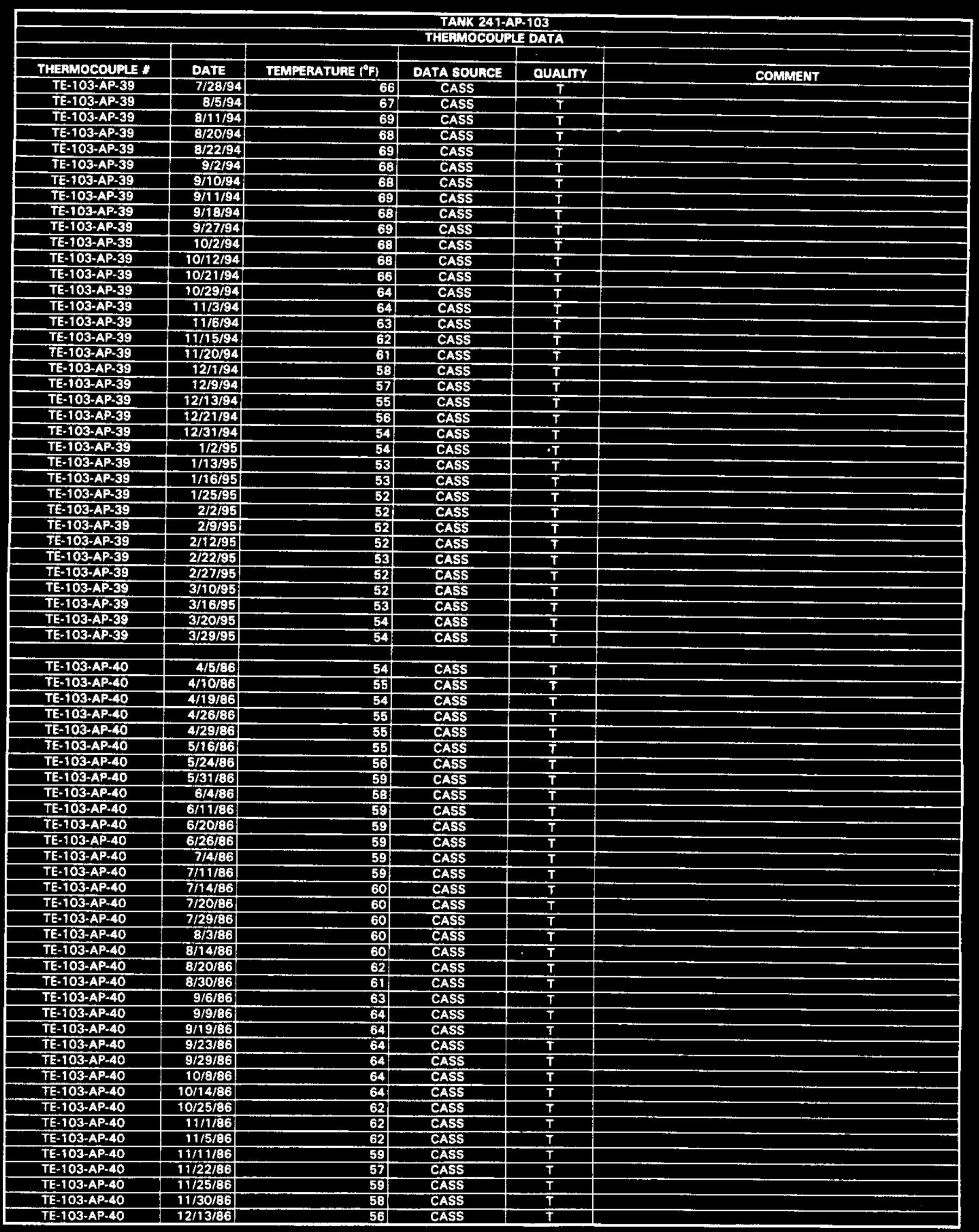




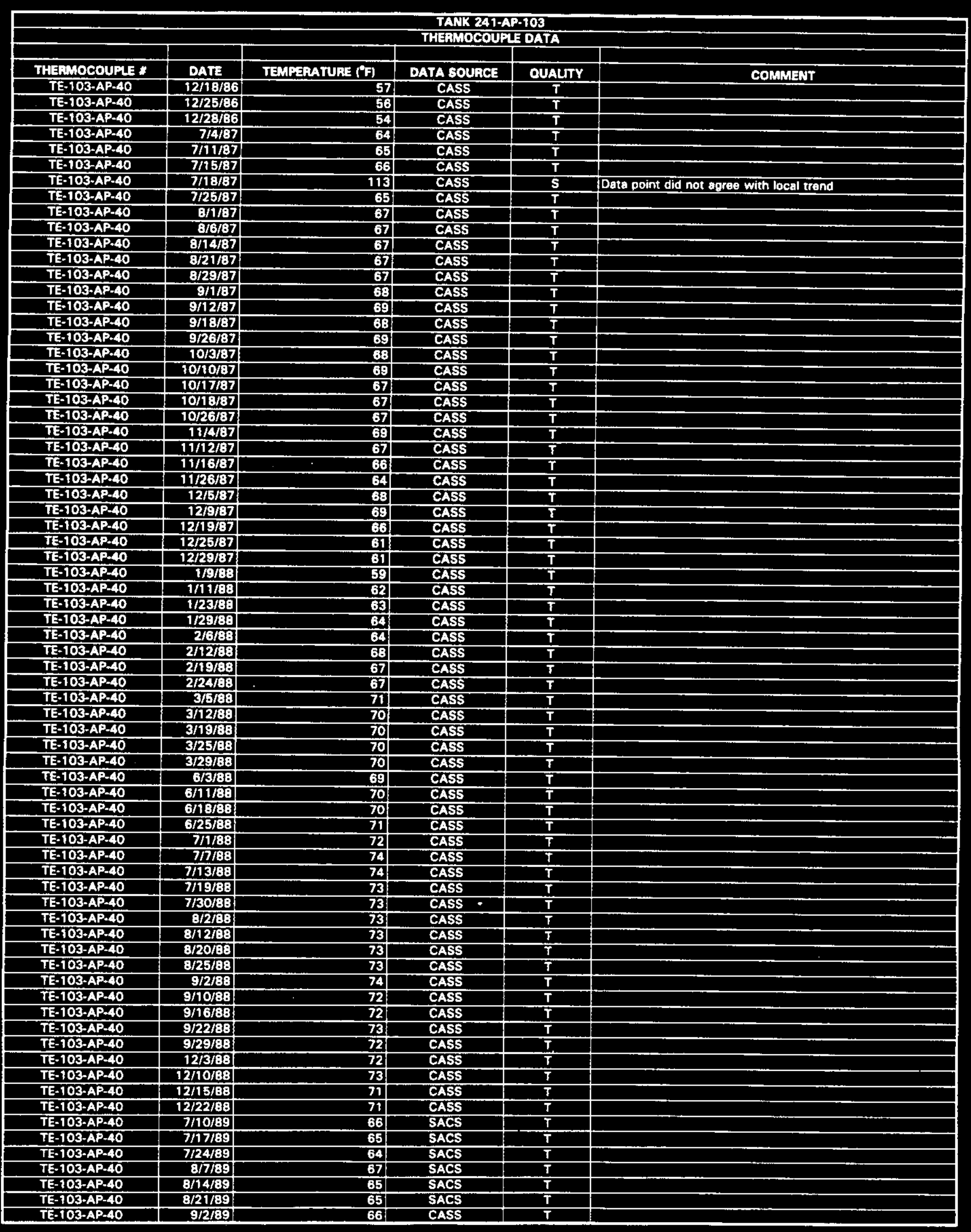




\begin{tabular}{|c|c|c|c|c|c|}
\hline \multicolumn{6}{|c|}{$\begin{array}{l}\text { TANK 241-AP-103 } \\
\text { THEFMOCOUPLE DATA }\end{array}$} \\
\hline THEPMOCOUPE * & DATE & TEMPYRATUAE $0^{\circ} \mathrm{F}$ & DATA SOUACE & & \\
\hline TE-103-AP-40 & $9 / 7 / 89$ & 67 & $\frac{\text { DAMA BOUREE }}{\text { CASS }}$ & $\frac{\text { OUALTYY }}{T}$ & COMMENT \\
\hline TE-103-AP-40 & $9 / 10 / 89$ & 68 & CASS & $T$ & \\
\hline TE-103-AP-40 & 9/20/89 & 67: & CASS & $T$ & \\
\hline TE-103-AP-40 & $8 / 23 / 69$ & 116 & CASS & $\mathbf{S}$ & Data point did not agree with local trend \\
\hline$T E-103-A P-40$ & 9/26/89 & 67 & CASS & $\bar{T}$ & \\
\hline TE-103-AP-40 & 10/2/89 & 66 & SACS & $\bar{T}$ & \\
\hline TE-103-AP-40 & 10/9/89 & 64 & SACS & $T$ & \\
\hline TE-103-AP-40 & $10 / 16 / 89$ & 86 & SACS & $T$ & \\
\hline TE-103-AP-40 & $10 / 24 / 69$ & 72 & SACS & $T$ & \\
\hline TE-103-AP-40 & $31 / 2 / 69$ & 72 & CASS & $T$ & \\
\hline TE-103-AP-40 & $12 / 28 / 69$ & 69 & CASS & $T$ & \\
\hline TE-103-AP-40 & 12/31/89 & 70 & CASS & $T$ & \\
\hline TE-103-AP-40 & $1 / 7190$ & 68 & CASS & $T$ & \\
\hline TE-103-AP-40 & $1 / 15 / 90$ & 69 & SACS & $T$ & \\
\hline TE-103-AP-40 & $1 / 23 / 90$ & 67 & CASS & $T$ & \\
\hline TE-103-AP-40 & $1 / 30 / 90$ & 66 & CASS & $T$ & \\
\hline TE-103-AP-40 & $2 / 4 / 90$ & 86 & CASS & $\mathbf{T}$ & \\
\hline TE-103-AP-40 & $2 / 14 / 90$ & 86 & CASS & $\bar{T}$ & \\
\hline TE-103-AP-40 & $2 / 23 / 90$ & 83 & CASS & $T$ & \\
\hline TE-103-AP-40 & $3 / 3 / 90$ & 64 & CASS & $\bar{T}$ & \\
\hline TE-103-AP-AO & $3 / 9 / 90$ & 64 & CASS & $\mathbf{T}$ & \\
\hline TE-103-AP-40 & $3 / 17 / 90$ & 62 & CASS & $T$ & \\
\hline$T E-103-A P-40$ & $3 / 24 / 90$ & 62 & CASS & $\bar{T}$ & \\
\hline TE-103.AP-40 & $3 / 29 / 90$ & 62 & CASS & $\bar{T}$ & \\
\hline TE-103-AP-40 & $4 / 3 / 90$ & 62 & CASS & $T$ & \\
\hline TE-103-AP-40 & $4 / 9 / 90$ & 62 & CASS & $T$ & \\
\hline TE-103-AP-40 & $4 / 16 / 90$ & 62 & SACS & $T$ & \\
\hline TE-103-AP-40 & $4 / 28 / 80$ & 61 & CASS & $\bar{T}$ & \\
\hline$T E-103-A P-40$ & $5 / 4 / 90$ & 62 & CASS & $\bar{T}$ & \\
\hline TE-103-AP-40 & $5 / 12 / 90$ & 63 & CASS & $T$ & \\
\hline TE-103-AP-40 & $5 / 14 / 80$ & 62 & CASS & $T$ & \\
\hline TE-103-AP-40 & $5 / 22 / 90$ & 631 & CASS & $T$ & \\
\hline TE-103-AP-40 & $5 / 28 / 90$ & 63 & SACS & $\bar{T}$ & \\
\hline TE-103-AP-40 & 6/9/90 & 62 & CASS & $T$ & \\
\hline TE-103-AP-40 & 6/14/80 & 62 & CASS & $T$ & \\
\hline TE-103-AP-40 & 6/19/90 & 63 & SACS & $\bar{T}$ & \\
\hline TE-103-AP.40 & $6 / 30 / 90$ & 84 & CASS & $\mathbf{T}$ & \\
\hline$T E-103-A P-40$ & 717/80 & 63 & CASS & $T$ & \\
\hline TE-103-AP-40 & $7 / 74 / 90$ & 64 & CASS & $T$ & \\
\hline TE-103-AP-40 & $7 / 21 / 90$ & 661 & CASS & $T$ & \\
\hline TE-103-AP-40 & $7 / 26 / 90$ & 68 & CASS & $\bar{T}$ & \\
\hline TE-103-AP-40 & $8 / 1 / 90$ & 67 & CASS & $\mathrm{T}$ & \\
\hline TE-103-AP-40 & $8 / 5 / 90$ & 67 & CASS & $T$ & \\
\hline TE-103-AP-40 & $8 / 16 / 90$ & 68 & CASS & 7 & \\
\hline TE-103-AP-40 & 8/20/90 & 69 & CASS & $T$ & \\
\hline TE-103-AP-40 & $9 / 1 / 90$ & 68 & CASS & $T^{*}$ & \\
\hline TE-103-AP-40 & $978 / 90$ & 69 & CASS & $T$ & \\
\hline TE-103-AP-40 & $9 / 15 / 90$ & 69 & CASS & $T$ & \\
\hline TE-103-AP-40 & $9 / 16 / 90$ & 69 & CASS & $T$ & \\
\hline TE-103-AP-40 & 9/25/90 & 70 & CASS & $T$ & \\
\hline TE-103-AP-40 & $10 / 5 / 90$ & 69 & CASS & $T$ & \\
\hline TE-103-AP-40 & $10 / 13 / 90$ & 69 & CASS & $T$ & \\
\hline TE-103-AP-40 & $10 / 18 / 90$ & 104 & CASS & s & Data point did not agree with local trend \\
\hline TE-103-AP-40 & $10 / 20 / 90$ & 66 & CASS & $T$ & \\
\hline TE-103-AP-40 & $10 / 23 / 90$ & 67 & CASS & $T$ & \\
\hline TE-103-AP-40 & $11 / 1 / 90$ & 75 & CASS & $T$ & \\
\hline TE-103-AP-40 & $11 / 5 / 30$ & 73 & CASS & $\mathbf{T}$ & \\
\hline TE-103-AP-40 & $11 / 16 / 90$ & 72 & CASS & T & \\
\hline TE-103-AP-40 & $11 / 18 / 90$ & 71 & CASS & $T$ & \\
\hline TE-103-AP-40 & $12 / 1 / 90$ & 70 & CASS & $T$ & \\
\hline TE-103-AP-40 & $12 / 4 / 90$ & 69 & CASS & $T$ & \\
\hline TE-103-AP-40 & $12 / 9 / 90$ & 69 & CASS & $T$ & \\
\hline TE-103-AP-40 & $12 / 19 / 90$ & 68 & CASS & $T$ & \\
\hline TE-103-AP-40 & $12 / 28 / 90$ & 66 & CASS & $\mathbf{T}$ & \\
\hline TE-103-AP-40 & $1 / 1 / 91$ & 65 & CASS & $\bar{T}$ & \\
\hline$T E-103-A P-40$ & 1/9/91 & 64 & CASS & $\bar{T}$ & \\
\hline$T E-103-A P-40$ & $1 / 19 / 91$ & 64 & CASS & $T$ & \\
\hline TE-108-AP-40 & $1 / 26 / 91$ & 64 & CASS & $T$ & \\
\hline$T E-103-A P-40$ & $1 / 29 / 91$ & 62 & CASS & $T$ & \\
\hline TE-103-AP-40 & $2 / 4 / 91$ & 62 & SACS & $T$ & \\
\hline TE-103-AP-40 & $2 / 11 / 91$ & 62 & SACS & $T$ & \\
\hline$T E-103-A P-40$ & $2 / 21 / 91$ & 61 & CASS & $T$ & \\
\hline TE-103-AP-40 & 3/1/91 & 61 & CASS & $T$ & \\
\hline
\end{tabular}




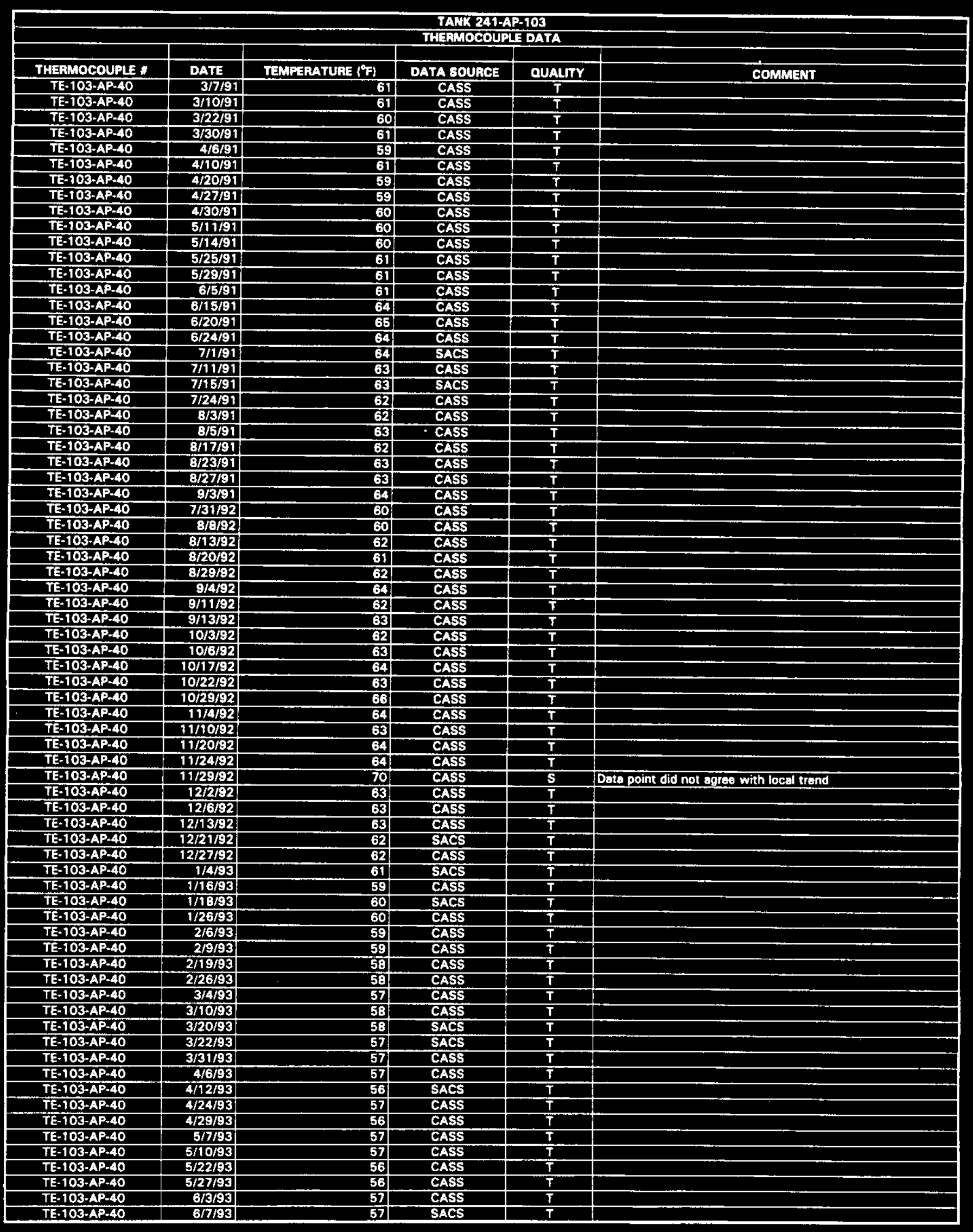

Data obtained from Computer Automated Surveillance System (CASS), April 1995; and Surveillance Analyais Computer Syatem (SACS), June 1995.

D-252 


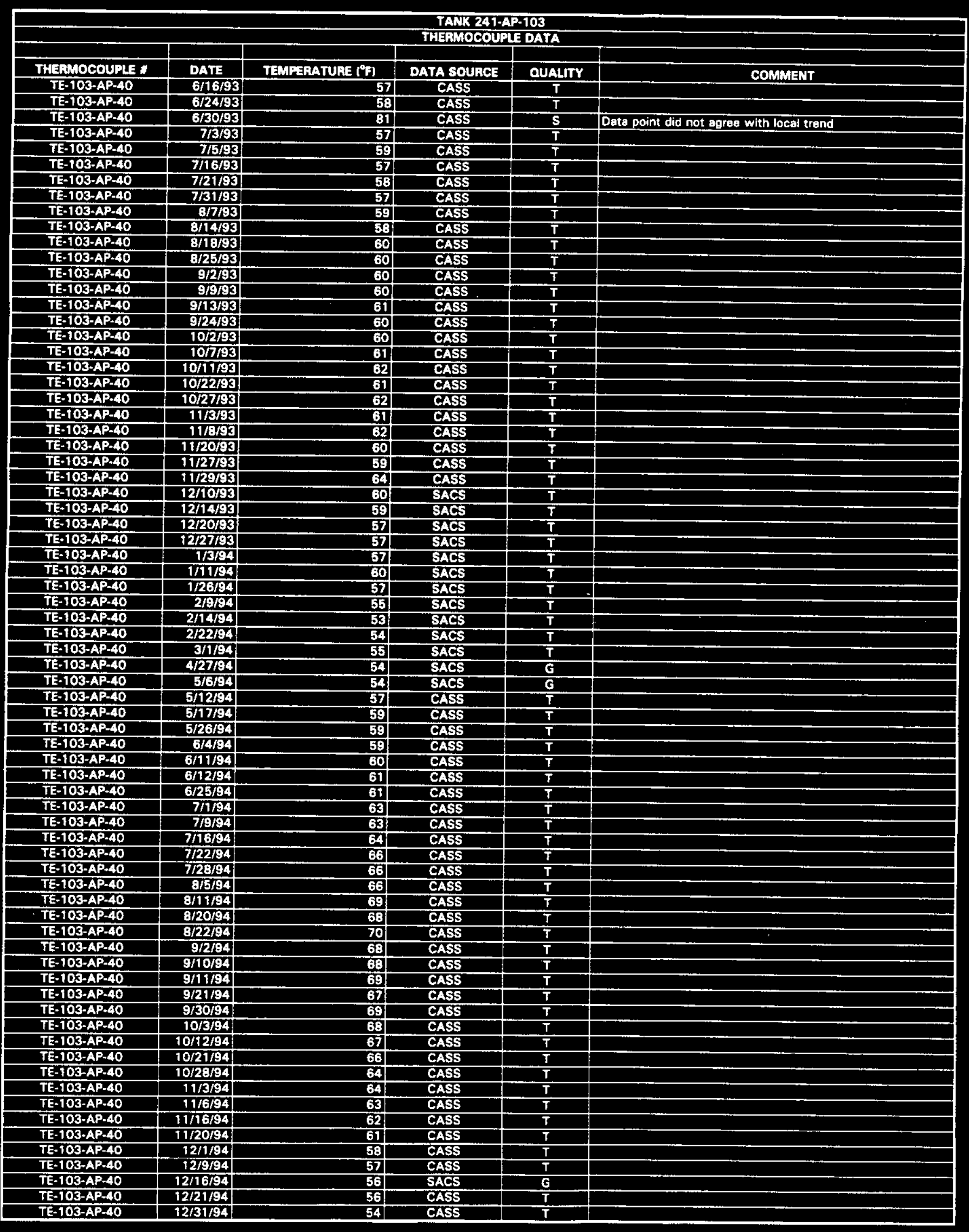




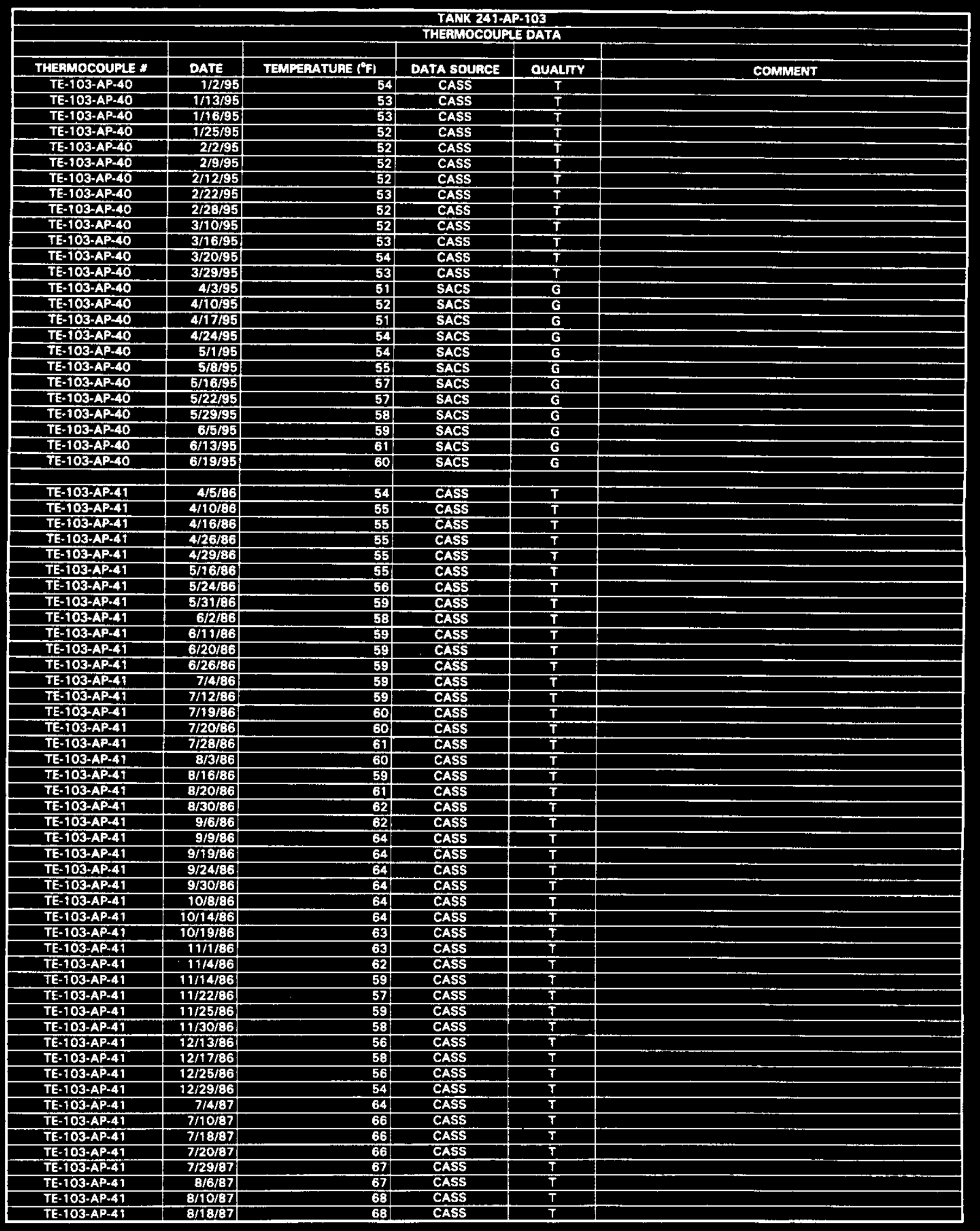




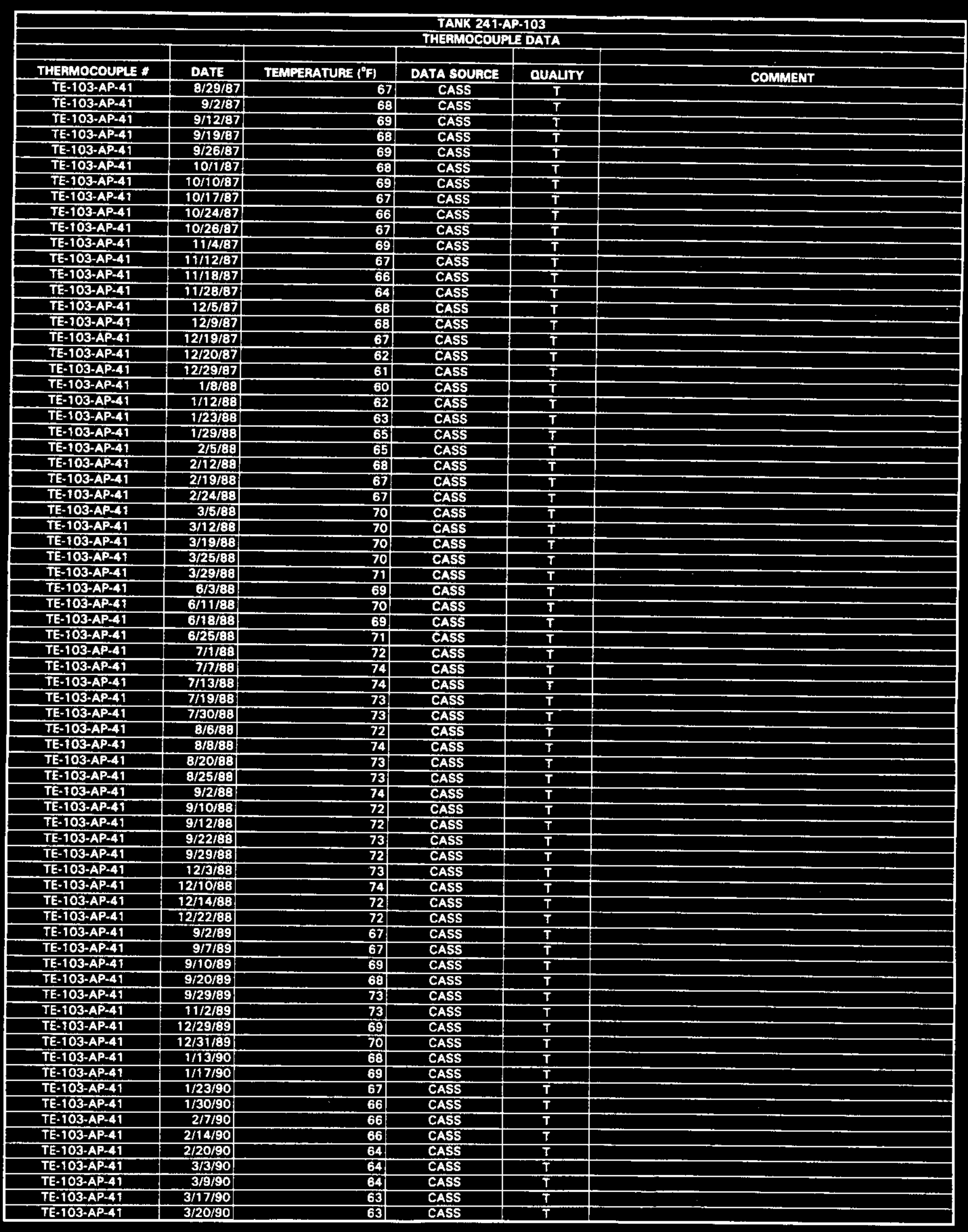

Data obtained from Computer Automated Surveillance Syatem (CASS), April 1995; and Surveillance Analysis Computer Syatem (SACS), June 1995.

D-255 


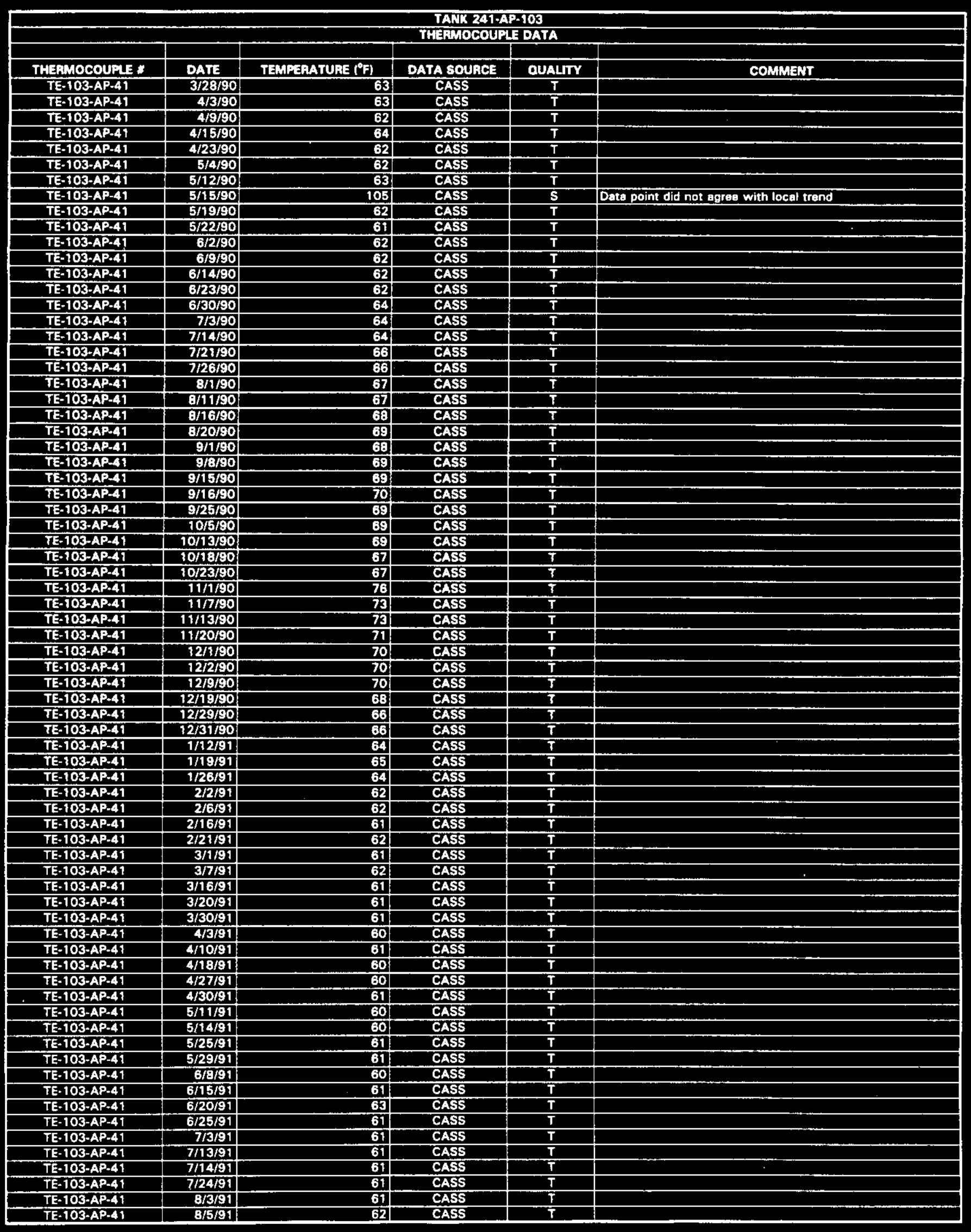

Data obtained from Computer Automated Surveillance System (CASS), April 1995; and Surveillance Anslysis Computer System (SACS), June 1995. 


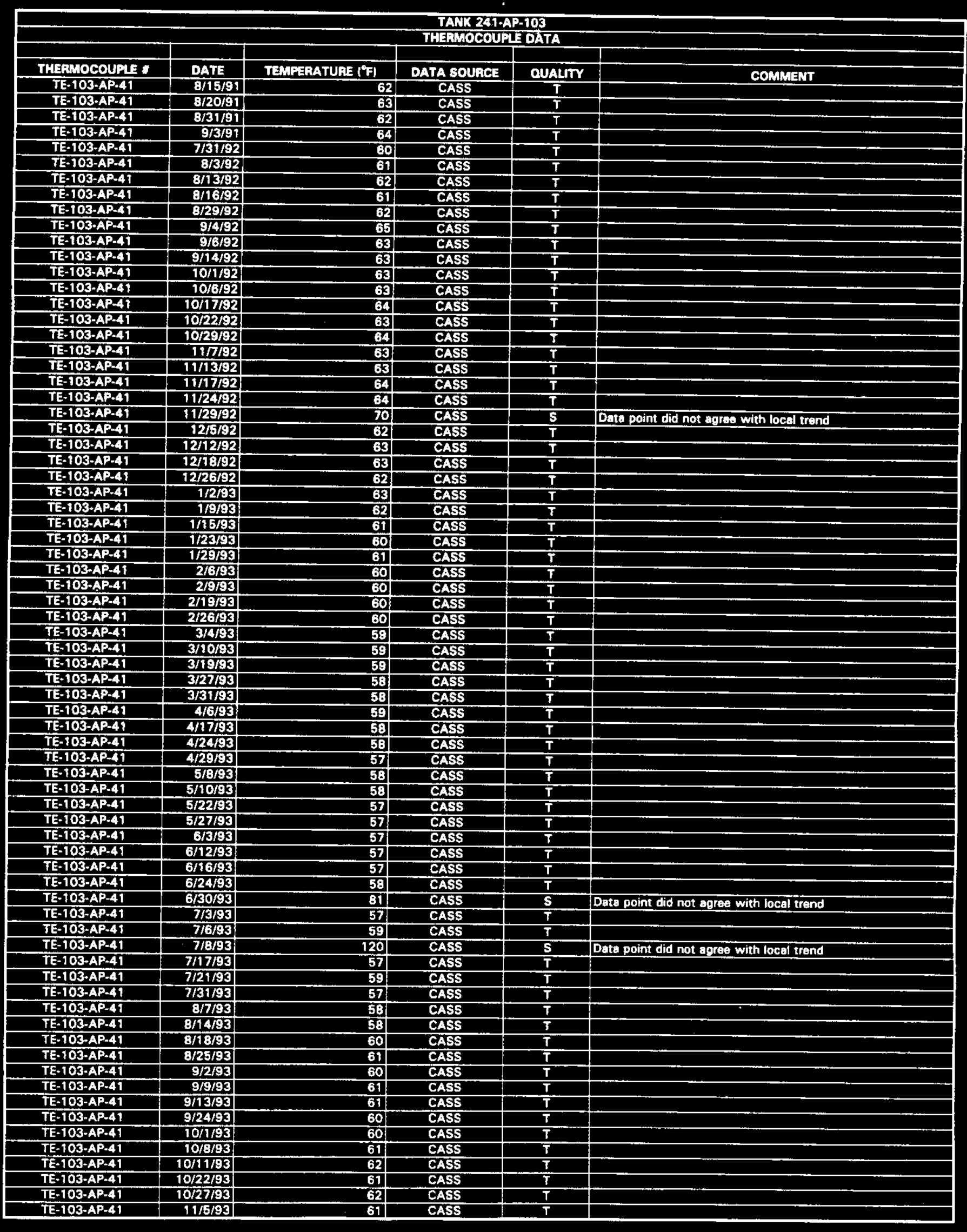




\begin{tabular}{|c|c|c|c|c|c|}
\hline \multicolumn{6}{|c|}{$\begin{array}{l}\text { TANK 241-AP-103 } \\
\text { THEAIMOCOUAL DATA }\end{array}$} \\
\hline & & & & & \\
\hline THERMOCOUPLE & DATE & TEMPERATURE $\left\{{ }^{\circ} \mathrm{F}\right\}$ & DATA SOUACE & OUALIY & COMMENT \\
\hline TE-103-AP-41 & $11 / 8 / 93$ & 62 & CASS & $T$ & \\
\hline TE-103-AP-41 & $11 / 19 / 93$ & 61 & CASS & $\bar{T}$ & \\
\hline TE-103-AP-41 & $11 / 27 / 93$ & 60 & CASS & $T$ & \\
\hline TE-103-AP.41 & 12/1/83 & 61 & CASS & $\overline{\mathbf{T}}$ & \\
\hline TE-103-AP+41 & $1212 / 93$ & 97 & CASS & $\mathbf{S}$ & Data point did not agree with locel trand \\
\hline TE-103-AP-41 & $1 / 12 / 94$ & 58 & CASS & $T$ & \\
\hline TE-103-AP-41 & 5/12/94 & 57 & CASS & $\bar{T}$ & \\
\hline TE-103-AP-41 & 5/17/94 & 69 & CASS & $\mathbf{T}$ & \\
\hline TE-103-AP-41 & $5 / 26 / 94$ & 59 & CASS & $\mathbf{T}$ & \\
\hline TE-103-AP-4T & $6 / 4 / 94$ & 60 & CASS & $T$ & \\
\hline TE-103-AP-41 & 6/11/94 & 60 & CASS & $\mathbf{T}$ & \\
\hline TE-103-AP-41 & 6/12/94 & 61 & CASS & $\mathbf{T}$ & \\
\hline IE-103-AP-41 & $6 / 25 / 94$ & 61 & CASS & $\mathbf{T}$ & \\
\hline TE-103-AP-41 & $7 / 1 / 94$ & 64 & CASS & $T$ & \\
\hline TE-103-AP-41 & $7 / 9 / 94$ & 64 & CASS & $T$ & \\
\hline TE-103-AP. 41 & $7 / 16 / 94$ & 84 & CASS & $\mathbf{T}$ & \\
\hline TE-103-AP-41 & $7 / 22 / 34$ & 68 & CASS & $T$ & \\
\hline TE-103-AP-41 & $7 / 28 / 94$ & 66 & CASS & $T$ & \\
\hline TE-103-AP-41 & $8 / 5 / 94$ & 67 & CASS & $T$ & \\
\hline TE-103-AP-41 & $8 / 13 / 94$ & 68 & CASS & $T$ & \\
\hline TE-103.AP-41 & $8 / 20 / 94$ & 68 & CASS & $T$ & \\
\hline TE-103-AP-41 & B/22/94 & 69 & CASS & $\bar{T}$ & \\
\hline TE-103-AP-41 & 9/3/34 & 68 & CASS & $T$ & \\
\hline TE-103.AP-A1 & $9 / 10 / 94$ & 68 & CASS & $T$ & \\
\hline TE-103-AP-41 & $9 / 11 / 94$ & 69 & CASS & $T$ & \\
\hline TE-103-AP-41 & $9 / 18 / 34$ & 68 & CASS & $T$ & \\
\hline TE-103-AP=41 & 9/30/94 & 69 & CASS & $\mathbf{T}$ & \\
\hline TE-103-AP-41 & $10 / 2 / 94$ & 68 & CASS & $T$ & \\
\hline TE-103-AP-41 & $10 / 12 / 94$ & 68 & CASS & $\mathbf{T}$ & \\
\hline TE-103-AP-41 & $10 / 21 / 94$ & 66 & CASS & $T$ & \\
\hline TE-103-AP-41 & $10 / 28 / 94$ & 84 & CASS & $T$ & \\
\hline TE-103-AP-41 & $11 / 3 / 94$ & 64 & CASS & $\mathbf{T}$ & \\
\hline TE-103-AP-41 & $11 / 12 / 34$ & 63 & CASS & $T$ & \\
\hline TE-103-AP-41 & $11 / 15 / 94$ & 61 & CASS & $\mathbf{T}$ & \\
\hline TE-103-AP-A1 & $11 / 20 / 94$ & 61 & CASS & $T$ & \\
\hline TE-103-AP-41 & 12/2/94 & 58. & CASS & $T$ & \\
\hline TE-103-AP-A1 & $1214 / 94$ & 58 & CASS & $\mathbf{T}$ & \\
\hline TE-103-AP-AT & $12 / 8 / 94$ & 80 & CASS & $\mathbf{S}$ & Data point did not apree with local trend \\
\hline TE-103-AP-4I & $12 / 14 / 94$ & 55 & CASS & $\mathbf{T}$ & \\
\hline TE-103.AP-41 & $12 / 21 / 94$ & 56 & CASS & $\mathbf{T}$ & \\
\hline$T E-103-A P-41$ & $72 / 30 / 94$ & 54 & CASS & $\mathbf{T}$ & \\
\hline TE-103-AP-41 & $1 / 2 / 95$ & 54 & CASS & $\mathbf{T}$ & \\
\hline TE-103-AP-41 & 1/13/95 & 53 & CASS & $\mathbf{T}$ & \\
\hline TE-103-AP-41 & W16/95 & $\mathbf{5 3}$ & CASS & $\mathbf{T}$ & \\
\hline TE-103-AP-41 & 1/25/95 & 53 & CASS & $\mathbf{T}$ & \\
\hline TE-103-AP-41 & $2 / 2 / 95$ & 53. & CASS & $\mathbf{T}$ & \\
\hline TE-103-AP-41 & $2 / 9 / 95$ & 53 & CASS & $\mathbf{T}$ & \\
\hline TE-103-AP-41 & $2 / 18 / 95$ & 52 & CASS & $\mathbf{T}$ & \\
\hline TE-103-AP-41 & $2 / 25 / 95$ & 53 & CASS & $\mathbf{T}$ & \\
\hline TE-103-AP-41 & $2 / 27 / 95$ & 52 & CASS & $T$ & \\
\hline TE-103-AP-41 & 3/10/95 & 52 & CASS & $\bar{T}$ & \\
\hline TE-103-AP-41 & 3/16/95 & 53 & CASS & $\mathbf{T}$ & \\
\hline TE-103-AP-41 & $3 / 20 / 95$ & 54 & CASS & $\mathbf{T}$ & \\
\hline TE-103-AP-41 & 3/29/95 & 54 & CASS & $T$ & \\
\hline TE-103-AP-42 & $4 / 5 / 86$ & 54 & CASS & $\bar{T}$ & \\
\hline TE-103+AP-A2 & $4 / 6 / 86$ & 56 & CASS & $T$ & \\
\hline TE-103-AP-42 & 4/19/86 & 57 & CASS & $\bar{T}$ & \\
\hline TE-103-AP-42 & $4 / 26 / 86$ & 55 & CASS & $\mathbf{T}$ & \\
\hline TE-103-AP-42 & 4/29/86 & 55 & CASS & $\mathbf{T}$ & \\
\hline TE-103-AP-42 & $5 / 16 / 86$ & 55 & CASS & $T$ & \\
\hline TE-103-AP-42 & $5 / 24 / 86$ & 56 & CASS & $T$ & \\
\hline TE-103-AP-A2 & $5 / 30 / 86$ & 60 & CASS & $\mathbf{T}$ & \\
\hline TE-103-AP-42 & $6 / 3 / 86$ & 58 & CASS & $T$ & \\
\hline TE-103-AP-42 & $6 / 11 / 86$ & 59 & CASS & $\mathbf{T}$ & \\
\hline TE-103-AP-42 & 6/20/86 & 59 & CASS & $\mathbf{T}$ & \\
\hline TE-103-AP-42 & $6 / 26 / 86$ & 59 & CASS & $\mathbf{T}$ & \\
\hline TE-103-AP-42 & $7 / 4 / 86$ & 59 & CASS & $\mathbf{T}$ & \\
\hline TE-103-AP-42 & $7 / 12 / 86$ & 59! & CASS & $\mathbf{T}$ & \\
\hline TE-103-AP-42 & 7/19/86 & 59 & CASS & $\mathbf{T}$ & \\
\hline TE-103-AP-42 & $7120 / 86$ & 60 & CASS & $T$ & \\
\hline TE-103-AP-42 & $7128 / 86$ & 61 & CASS & $T$ & \\
\hline TE-103-AP-42 & $8 / 3 / 3 / 66$ & 60 & CASS & $T$ & \\
\hline
\end{tabular}




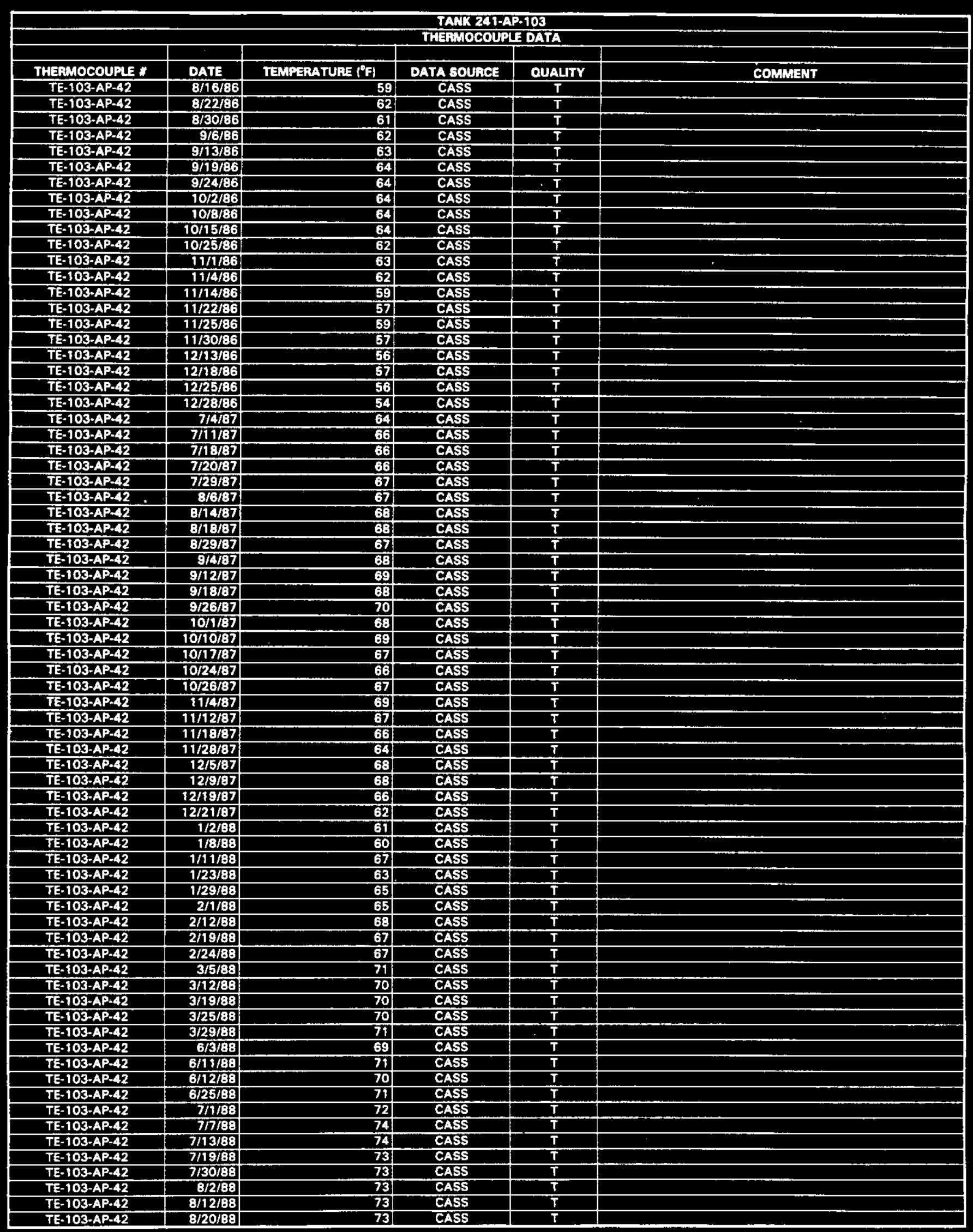




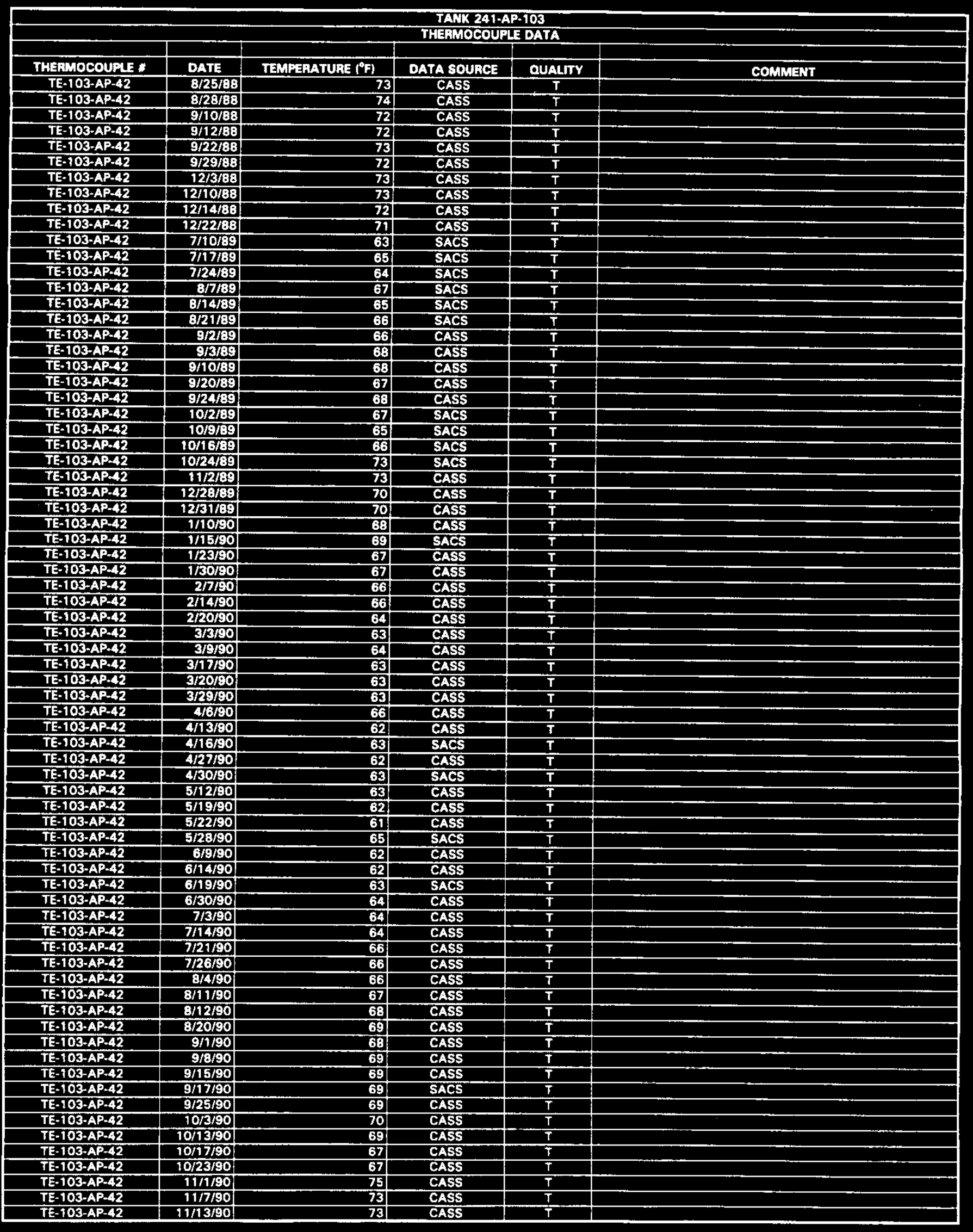




\begin{tabular}{|c|c|c|c|c|c|}
\hline \multicolumn{6}{|c|}{$\begin{array}{l}\text { TAWK 241-AP.103 } \\
\text { THIFRMOCOUPLE DATA }\end{array}$} \\
\hline & & & & & \\
\hline THEAMOCOUPLE $\boldsymbol{x}$ & DATE & TEMPERATUAE ( $\left.{ }^{\circ} F\right)$ & DATA SOURCE & OUALTY & COMMENT \\
\hline TE-103-AP-42 & $11 / 20 / 90$ & 71 & CASS & $T$ & \\
\hline TE-102-AP-42 & $11 / 25 / 90$ & 71 & CASS & $\mathrm{T}$ & \\
\hline TE-108-AP-42 & $12 / 2 / 90$ & 71 & CASS & $T$ & \\
\hline$\frac{T E-103-A P-42}{T E-103-A P-42}$ & $12 / 9 / 90$ & 69 & $\begin{array}{ll}\text { CASS } \\
\text { CASG }\end{array}$ & $\frac{T}{T}$ & \\
\hline$\frac{T E-103-A P-42}{T E-103-A P-42}$ & $\frac{12 / 19 / 90}{12 / 21 / 90}$ & $\begin{array}{l}68 \\
57\end{array}$ & $\frac{\text { CASS }}{\text { CASS }}$ & $\frac{T}{S}$ & Data point did not agree with local trend \\
\hline$T E-103-A P-42$ & $12 / 29 / 90$ & 66 & CASS & $\mathbf{T}$ & \\
\hline TE-103-AP-42 & $12 / 31 / 90$ & 66 & SACS & $T$ & \\
\hline TE-103-AP-42 & $1 / 12 / 98$ & 64. & CASS & $T$ & \\
\hline TE-103-AP-42 & $1 / 19 / 91$ & 65 & CASS & $\mathbf{T}$ & \\
\hline TE-103-AP-42 & $1 / 26 / 91$ & 64 & CASS & $\mathbf{T}$ & \\
\hline TE-103-AP-42 & $2 / 219$ & 62 & CASS & $\bar{T}$ & \\
\hline TE-103-AP-42 & $2 / 8 / 91$ & 62: & CASS & $\mathbf{T}$ & \\
\hline$T E-103-A P-42$ & $2 / 16 / 91$ & 61 & CASS & $\mathbf{T}$ & \\
\hline TE-103-AP-42 & $2 / 21 / 91$ & 62 & CASS & $\mathbf{T}$ & \\
\hline TE-103-AP.42 & 3/1/21 & 61 & CASS & $T$ & \\
\hline TE-108-AP-42 & 3/7/91 & 62 & CASS & $\bar{T}$ & \\
\hline TE-103-AP-42 & 3/16/91 & 61 & CASS & $T$ & \\
\hline$T E-103-A P-42$ & $3 / 22 / 81$ & 60 & CASS & $T$ & \\
\hline TE-103-AP.42 & $3 / 30 / 91$ & 61 & CASS & $T$ & \\
\hline TE-103-AP-42 & $4 / 6 / 91$ & 59 & CASS & $\bar{T}$ & \\
\hline TE-103-AP-42 & $4 / 10 / 31$ & 61 & CASS & $\mathrm{T}$ & \\
\hline TE-103-AP-42 & 4/18/91 & 60 & CASS & $T$ & \\
\hline TE-103-AP-42 & $4 / 27 / 91$ & 60 & CASS & $\mathbf{T}$ & \\
\hline TE-103-AP- 42 & $4 / 30 / 91$ & 61 & CASS & $T$ & \\
\hline TE-103-AP-42 & 5/11/9? & 60 & CASS & $T$ & \\
\hline TE-103-AP-42 & $5 / 14 / 91$ & 60 & CASS & $T$ & \\
\hline TE-103.AP-42 & $5 / 25 / 91$ & 61 & CASS & $T$ & \\
\hline TE-103.AP-42 & $5 / 29 / 91$ & 61 & CASS & $\mathbf{T}$ & \\
\hline TE-103-AP-42 & $6 / 7 / 31$ & 60 & CASS & $\bar{T}$ & \\
\hline$T E=103-A P-42$ & $6 / 13 / 91$ & 60 & CASS & $\bar{T}$ & \\
\hline TE-103-AP-42 & $6 / 20 / 91$ & 61 & CASS & $\bar{T}$ & \\
\hline TE-103-AP-42 & $6 / 25 / 91$ & 60 & CASS & 7 & \\
\hline TE-103-AP-42 & $7 / 3 / 91$ & 61 & CASS & $T$ & \\
\hline TE-103-AP-42 & 7/11/91 & 61 & CASS & $T$ & \\
\hline TE-103-AP-A2 & 7/15/94 & 60 & SACS & $T$ & \\
\hline TE-103-AP-42 & $7 / 24 / 91$ & 61 & CASS & $T$ & \\
\hline TE-103-AP-42 & $8 / 3 / 91$ & 61 & CASS & $T$ & \\
\hline TE-103-AP-42 & $8 / 5 / 91$ & 62 & CASS & $T$ & \\
\hline TE-103-AP-42 & $8 / 17 / 91$ & 61 & CASS & $\mathbf{T}$ & \\
\hline TE-103-AP-42 & $8 / 19 / 91$ & 63 & CASS & $T$ & \\
\hline TE-103-AP-42 & $8 / 31 / 91$ & 62 & CASS & $T$ & \\
\hline TE-103-AP-A2 & $9 / 3 / 91$ & 64 & CASS & $T$ & \\
\hline TE-103-AP-42 & $7 / 31 / 92$ & 60 & CASS & $T$ & \\
\hline TE-103-AP-42 & $8 / 8 / 92$ & 61 & CASS & $T$ & \\
\hline TE-103-AP-42 & $8 / 13 / 92$ & 62 & CASS & $T$ & \\
\hline TE-108-AP-42 & $8 / 22 / 92$ & 61. & CASS & $\mathbf{T}$ & \\
\hline TE-103-AP-42 & $8 / 29 / 92$ & 63 & CASS & $T$ & \\
\hline TE-103-AP-42 & 9/4/92 & 64 & CASS & $\mathbf{T}$ & \\
\hline TE-103-AP-42 & $9 / 6 / 92$ & 63 & CASS & $T$ & \\
\hline TE-103-AP-42 & $9 / 13 / 92$ & 63 & CASS & $T$ & \\
\hline TE-103-AP-42 & $10 / 1 / 92$ & 63 & CASS & $T$ & \\
\hline TE-103-AP-42 & 10/6/92 & 63 & CASS & $T$ & \\
\hline TE-103-AP-42 & $10 / 17 / 92$ & 62 & CASS & $T$ & \\
\hline TE-103-AP-42 & $10 / 22 / 92$ & 63 & CASS & $T$ & \\
\hline TE-103-AP-42 & $10 / 29 / 92$ & 64 & CASS & $T$ & \\
\hline$T E-103-A P-42$ & $11 / 4 / 92$ & 63 & CASS & $T$ & \\
\hline TE-103-AP-42 & $11 / 14 / 92$ & 62 & CASS & $T$ & \\
\hline TE-103-AP-42 & $11 / 17 / 92$ & 64 & CASS & $T$ & \\
\hline TE-103-AP-42 & $11 / 24 / 92$ & 64 & CASS & $T$ & \\
\hline TE-10X-AP-42 & $11 / 29 / 92$ & 70 & CASS & $\mathbf{S}$ & Data point did not agree whth local trend \\
\hline TE-103-AP-42 & $12 / 5 / 92$ & 62 & CASS & $T$ & \\
\hline TE-103-AP-42 & $12 / 12 / 92$ & 63 & CASS & $\mathbf{T}$ & \\
\hline$T E-103-A P-42$ & $12 / 18 / 92$ & 63. & CASS & $\mathbf{T}$ & \\
\hline TE-103-AP-42 & $12 / 26 / 92$ & 63 & CASS & $T$ & \\
\hline$T E-103-A P-42$ & $12 / 27 / 92$ & 63 & CASS & $T$ & \\
\hline TE-103-AP-42 & $1 / 9 / 93$ & 62 & CASS & $T$ & \\
\hline TE-103-AP-42 & $1 / 14 / 93$ & 62 & CASS & $T$ & \\
\hline TE-103-AP-42 & $1 / 21 / 93$ & 61 & CASS & $T$ & \\
\hline TE-103-AP-42 & $1 / 26 / 93$ & 62 & CASS & $T$ & \\
\hline$T E-103-A P-42$ & $2 / 6 / 93$ & 61 & CASS & $T$ & \\
\hline TE-103-AP-42 & $2 / 13 / 93$ & 60 & CASS & $T$ & \\
\hline TE-103-AP-42 & $2 / 19 / 93$ & 60 & CASS & $\bar{T}$ & \\
\hline
\end{tabular}




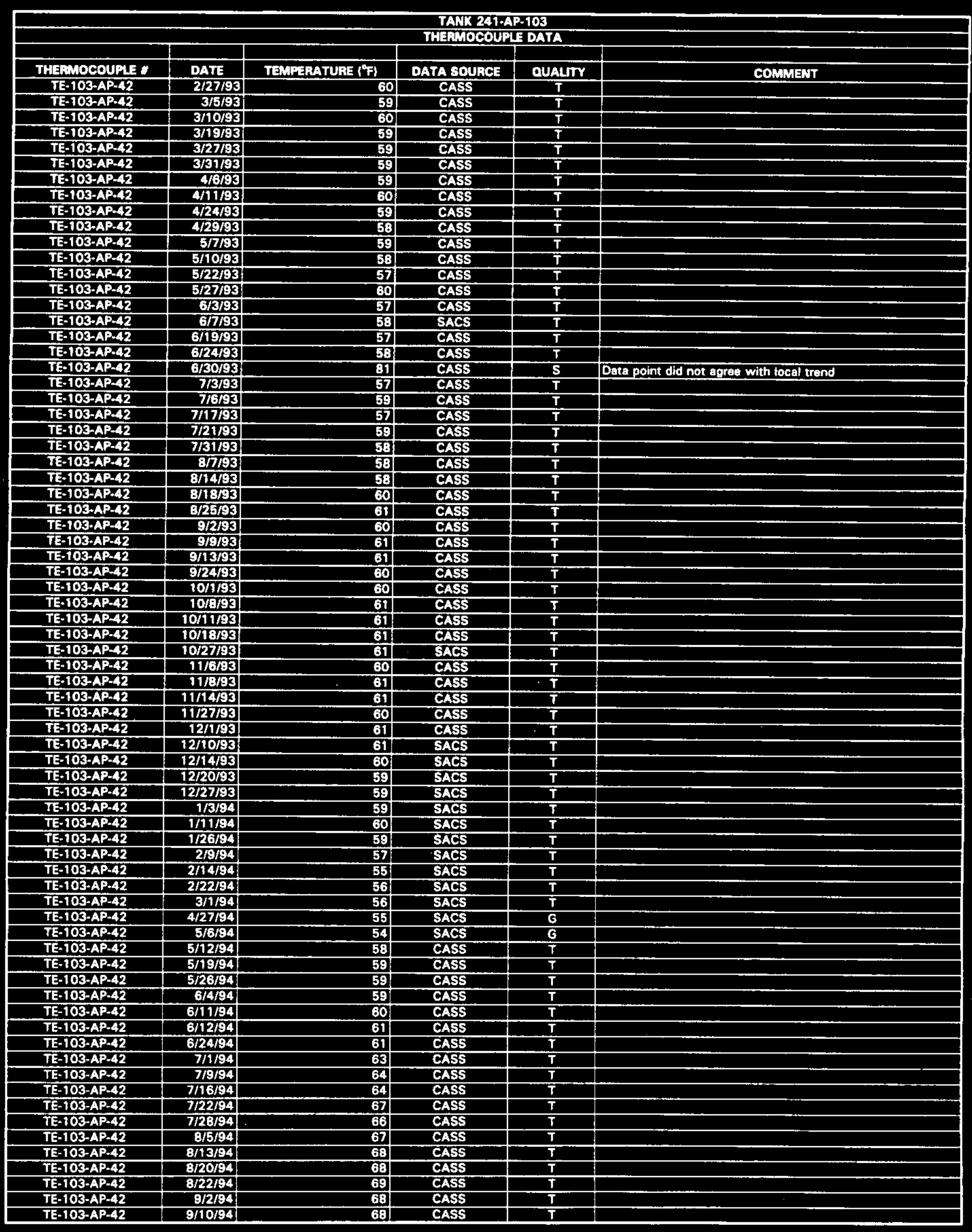

Date obteined from Computer Autometed Surveillence System (CASS), April 1995; and Surveillance Aralyais Computer System (SACS), June 1995.

$$
\text { D-262 }
$$




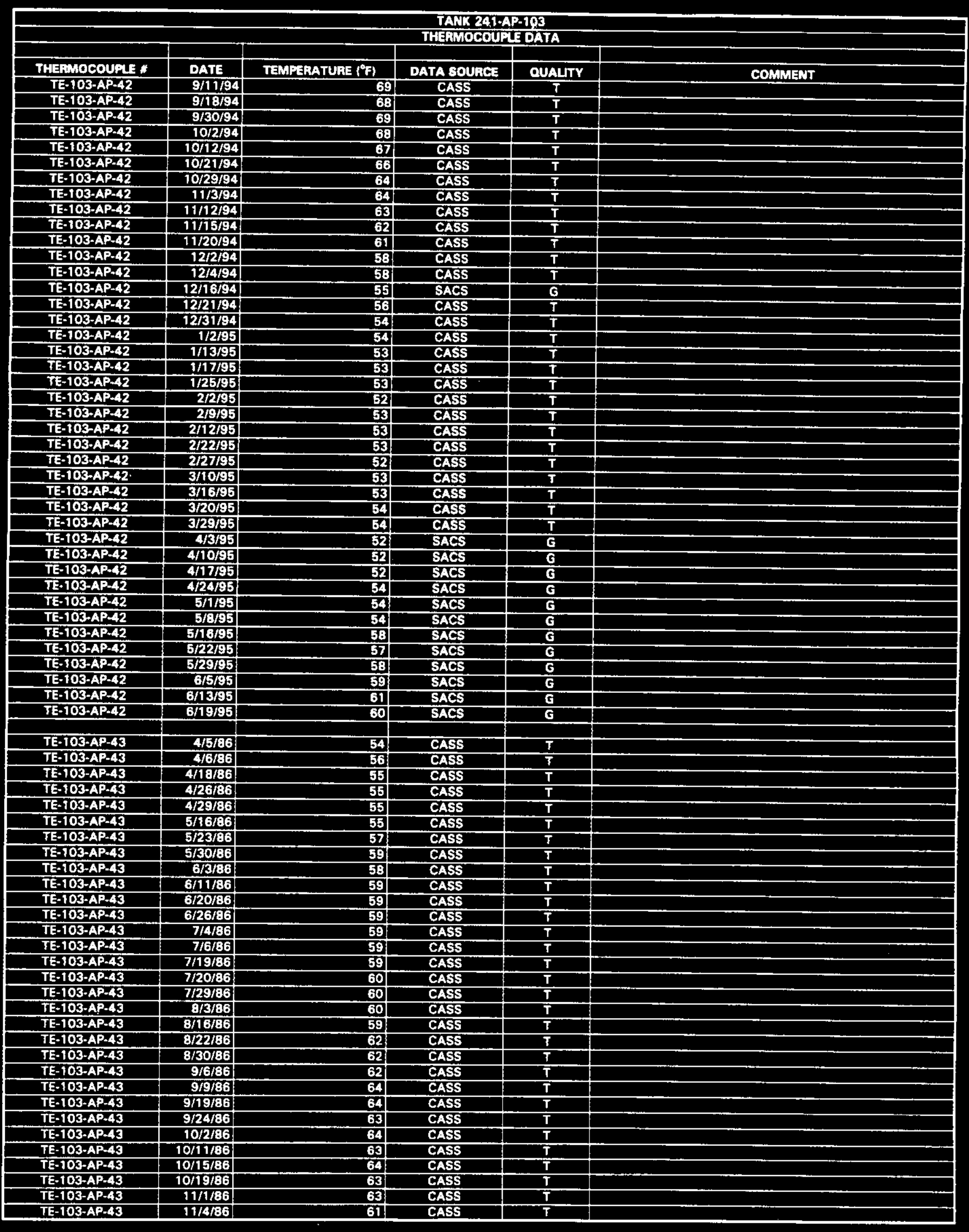




\begin{tabular}{|c|c|c|c|c|c|}
\hline \multicolumn{6}{|c|}{$\begin{array}{l}\text { TANK 24T-AP=103 } \\
\text { THEAMOCOOPLE DATA }\end{array}$} \\
\hline THERMOCOUPLE * & & & & & \\
\hline TE-103-AP-43 & $11 / 14 / 86$ & & DATA SOURCE & OUALIY & COMMENT \\
\hline TE-103-AP-43 & $11 / 22 / 66$ & $\begin{array}{l}59 \\
57\end{array}$ & $\frac{\text { CASS }}{\text { CASS }}$ & $\frac{T}{T}$ & \\
\hline TE-103-AP-43 & $11 / 25 / 86$ & 59. & $\begin{array}{l}\text { CASS } \\
\text { CASS }\end{array}$ & $\frac{T}{T}$ & \\
\hline TE-103-AP-43 & $11 / 30 / 86$ & 57 & CASS & $\bar{T}$ & \\
\hline TE-103-AP-43 & $12 / 12 / 86$ & 57| & CASS & $\mathbf{T}$ & \\
\hline TE-103-AP-43 & $12 / 18 / 86$ & 57 & CASS & $\mathbf{T}$ & \\
\hline TE-103-AP-43 & $12 / 25 / 86$ & 56 & CASS & $T$ & \\
\hline TE-103-AP-43 & $12 / 28 / 86$ & 54 & CASS & $T$ & \\
\hline TE-103-AP-43 & 7/4/87 & 64 & CASS & $\mathbf{T}$ & \\
\hline TE-103-AP-43 & 7711/87 & 66 & CASS & $\mathbf{T}$ & \\
\hline TE-103-AP-43 & $7 / 15 / 87$ & 66 & CASS & $\bar{T}$ & \\
\hline TE-103-AP-43 & 7/18/87 & 112 & CASS & $\mathbf{S}$ & Data point did not apree with locsl trend \\
\hline TE-103-AP-43 & 7/20/87 & 66 & CASS & $\mathbf{T}$ & \\
\hline TE-103-AP-43 & 7/29/87 & 67 & CASS & $\mathbf{T}$ & \\
\hline TE-103-AP-43 & $8 / 6 / 87$ & 67 & CASS & $\mathbf{T}$ & \\
\hline TE-103-AP-43 & $8 / 10 / 87$ & 68 & CASS & $\mathbf{T}$ & \\
\hline TE-108-AP-43 & 6/18/87 & 68 & CASS & $\bar{T}$ & \\
\hline TE-103-AP-43 & $8 / 29 / 87$ & 67 & CASS & $T$ & \\
\hline TE-103-AP-43 & 8/2/87 & 68 & CASS & $\bar{T}$ & \\
\hline TE-103-AP-43 & $9 / 12 / 87$ & 69 & CASS & $\mathbf{T}$ & \\
\hline TE-103-AP-43 & 9/18/87 & 68 & CASS & $\mathrm{T}$. & \\
\hline TE-103-AP-43 & $9 / 26 / 87$ & 69 & CASS & $T$ & \\
\hline$T E-103-A P-43$ & $9 / 30 / 87$ & 68 & CASS & $T$ & \\
\hline TE-103-AP-43 & $10 / 10 / 87$ & 69 & CASS & $\mathbf{T}$ & \\
\hline TE-103-AP-43 & $10 / 17 / 87$ & 67 & CASS & $\mathbf{T}$ & \\
\hline TE-103-AP-43 & $10 / 24 / 87$ & 66 & CASS & $\mathbf{T}$ & \\
\hline TE-103-AP-43 & $10 / 30 / 87$ & 67 & CASS & $T$ & \\
\hline TE-103-AP.43 & $11 / 4 / 87$ & 69 & CASS & $T$ & \\
\hline$T E-103-A P-43$ & $11 / 12 / 87$ & 67 & CASS & $T$ & \\
\hline TE-103-AP-A3 & $\$ 11 / 18 / 87$ & 66 & CASS & $\mathbf{T}$ & \\
\hline TE-103-AP-43 & $11 / 28 / 87$ & 64) & CASS & $\mathbf{T}$. & \\
\hline TE-103-AP-43 & $12 / 5 / 87$ & 68 & CASS & $\mathbf{T}$ & \\
\hline$T E-103-A P-43$ & $12 / 9 / 87$ & 69 & CASS & $T$ & \\
\hline TE-103-AP-43 & $12 / 19 / 87$ & 67 & CASS & $T$ & \\
\hline TE-103-AP-43 & $12 / 26 / 87$ & 61 & CASS & $T$ & \\
\hline$T E-103-A P-43$ & $12 / 29 / 87$ & 62 & CASS & $\bar{T}$ & \\
\hline TE-103-AP-43 & $1 / 8 / 88$ & 61 & CASS & $\mathbf{T}$ & \\
\hline TE-103-AP-43 & 1/11/88 & 64 & CASS & $\mathbf{T}$ & \\
\hline TE-103-AP-43 & $1 / 23 / 88$ & 63 & CASS & $T$ & \\
\hline TE-103-AP-43 & $1 / 29 / 88$ & 65 & CASS & $T$ & \\
\hline TE-103-AP-43 & $2 / 5 / 88$ & 65 & CASS & $\mathrm{T}$ & \\
\hline TE-103-AP-43 & $2 / 11 / 83$ & 69 & CASS & $\mathbf{T}$ & \\
\hline TE-103-AP-43 & $2 / 19 / 8 \mathrm{~B}$ & 67 & CASS & $\mathbf{T}$ & \\
\hline TE-103.AP-43 & $2 / 27 / 88$ & 67 & CASS & $\mathbf{T}$ & \\
\hline TE-103-AP-43 & $3 / 5 / 88$ & 70 & CASS & $\mathbf{T}$ & \\
\hline$T E-103 \cdot A P-43$ & 3/12/88 & 71 & CASS & $T$ & \\
\hline TE-103-AP-43 & 3/19/89 & 70 & CASS & $T$ & \\
\hline TE-103-AP-43 & $3 / 21 / 83$ & 71 & CASS & $\mathbf{T}$ & \\
\hline TE-103-AP-43 & $3 / 29 / 88$ & 71 & CASS & $T$ & \\
\hline TE-103-AP-43 & $6 / 3 / 88$ & 69 & CASS & $T$ & \\
\hline TE-103-AP-43 & 6/11/88 & 71 & CASS & $\mathbf{T}$ & \\
\hline TE-103-AP-43 & $6 / 18 / 88$ & 70 & CASS & $T$ & \\
\hline TE-103-AP-43 & $6 / 25 / 88$ & 72 & CASS & $\mathbf{T}$ & \\
\hline TE-103-AP-43 & $7 / 1 / 88$ & 72 & CASS & $\mathbf{T}$ & \\
\hline TE-103-AP-43 & 717188 & 74 & CASS & $\mathbf{T}$ & \\
\hline TE-103-AP-43 & $7 / 13 / 88$ & 74 & CASS & $T$ & \\
\hline TE-103-AP-43 & $7 / 19 / 88$ & 73 & CASS & $T$ & \\
\hline TE-103-AP-43 & $7 / 30 / 88$ & 73 & CASS & $T$ & \\
\hline TE-103-AP-43 & $8 / 2 / 88$ & 73 & CASS & $T$ & \\
\hline TE-103-AP-43 & $8 / 12 / 88$ & 73 & CASS & $T$ & \\
\hline TE-103-AP-43 & $8 / 20 / 88$ & 73 & CASS & $T$ & \\
\hline TE-103-AP-43 & $8 / 25 / 88$ & 73 & CASS & $\mathbf{T}$ & \\
\hline TE-102-AP-43 & $9 / 2 / 88$ & 74 & CASS & $T$ & \\
\hline TE-103-AP. 43 & $9 / 10 / 8$ & 72 & CASS & $T$ & \\
\hline TE-103-AP-43 & 9/12/88 & 72 & CASS & $T$ & \\
\hline TE-103-AP-A3 & $9 / 22 / 88$ & 73 & CASS & $\mathbf{T}$ & \\
\hline TE-103-AP-43 & $9 / 29 / 88$ & 72 & CASS & $T$ & \\
\hline TE-103-AP-43 & $12 / 3 / 88$ & 73 & CASS & $T$ & \\
\hline TE-103-AP-43 & $12 / 10 / 88$ & 74 & CASS & $\mathbf{T}$ & \\
\hline TE-103-AP-43 & $12 / 14 / 88$ & 72 & CASS & $T$ & \\
\hline TE-103-AP-43 & $12 / 22 / 88$ & 74 & CASS & $T$ & \\
\hline TE-103-AP-43 & 9/2/89 & 66 & CASS & $T$ & \\
\hline TE-103-AP-43 & $9 / 3 / 39$ & 68 & CASS & $\mathbf{T}$ & \\
\hline
\end{tabular}




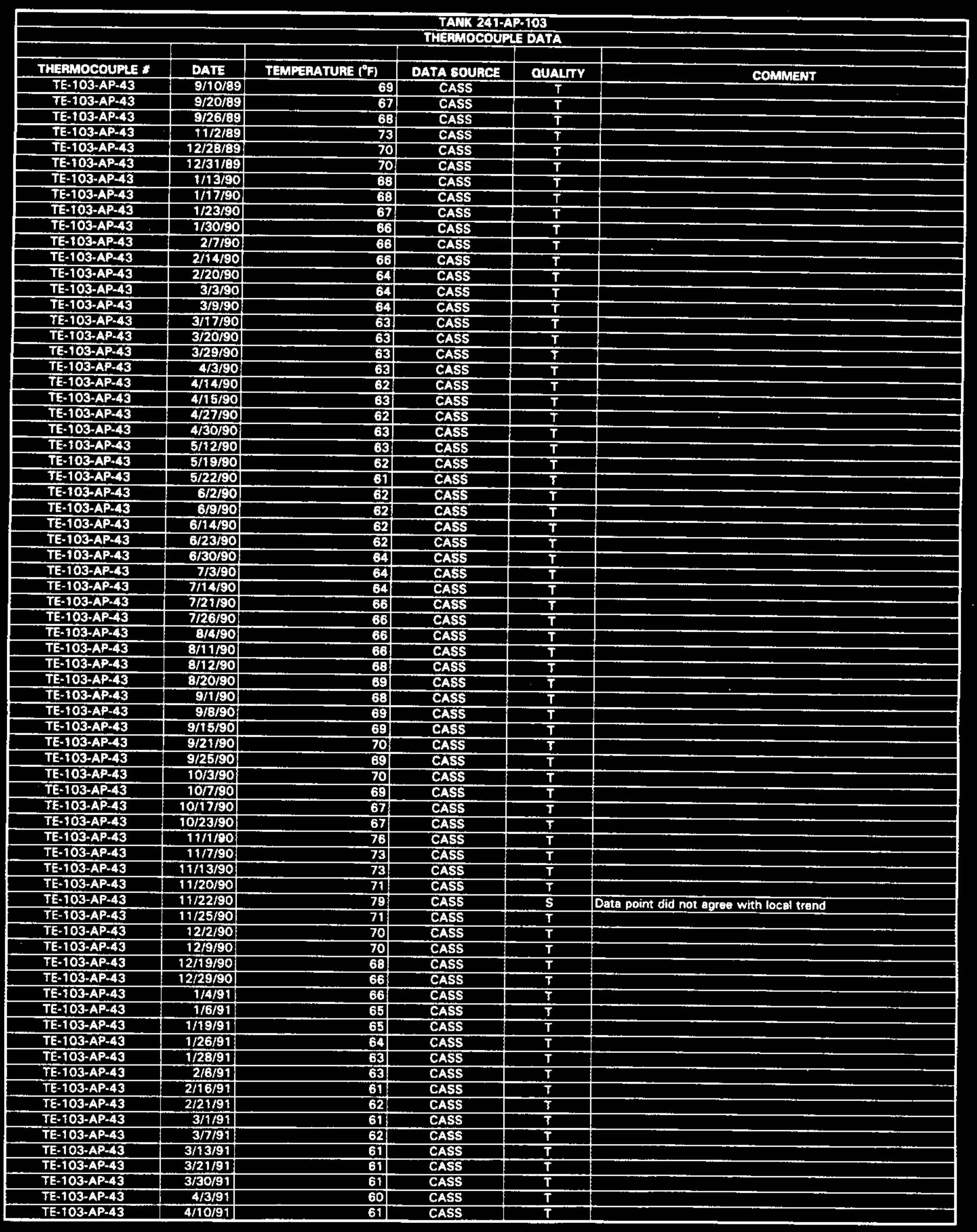




\begin{tabular}{|c|c|c|c|c|c|}
\hline \multicolumn{6}{|c|}{$\begin{array}{l}\text { TANK 241-AP-103 } \\
\text { THERMOCOUPIE DATA }\end{array}$} \\
\hline THEAMOCOUPLE * & DATE & TEMPERATURE ( $\left.{ }^{\circ} \mathrm{F}\right)$ & DATA SOUACE & OUAUTY & COMMENT \\
\hline TE-103-AP-43 & 4/18/91 & 60 & CASS & $T$ & \\
\hline TE-103-AP-43 & 4/27/91 & 60 & CASS & $T$ & \\
\hline TE-103-AP-43 & $5 / 4 / 91$ & 59 & CASS & $T$ & \\
\hline TE-103-AP-43 & $5 / 11 / 91$ & 60 & CASS & $T$ & \\
\hline TE-108-AP-A3 & 5/14/91 & 60 & CASS & $T$ & \\
\hline TE-103-AP-43 & 5/25/91 & 61 & CASS & $\mathbf{T}$ & \\
\hline TE-103-AP-43 & 5/29/91 & 61 & CASS & $\mathbf{T}$ & \\
\hline TE-103-AP-43 & $6 / 7 / 91$ & 60 & CASS & $\mathbf{T}$ & \\
\hline TE-103-AP-43 & 6/15/91 & 59 & CASS & $\mathbf{T}$ & \\
\hline TE-103-AP-43 & 6/20/91 & 61 & CASS & $\mathbf{T}$ & \\
\hline TE-103-AP-43 & $6 / 25 / 91$ & 59 & CASS & $\bar{T}$ & \\
\hline TE-103-AP-43 & $7 / 3 / 91$ & 59 & CASS & $\bar{T}$ & \\
\hline TE-103-AP-43 & $7 / 13 / 91$ & 60 & CASS & $T$ & \\
\hline TE-103-AP-43 & $7 / 14 / 91$ & 60 & CASS & $\mathbf{T}$ & \\
\hline TE-103-AP-43 & $7 / 24 / 91$ & 60 & CASS & $T$ & \\
\hline TE-103-AP-43 & $8 / 3 / 94$ & 60 & CASS & $T$ & \\
\hline TE-103-AP-43 & $8 / 5 / 91$ & 61 & CASS & $T$ & \\
\hline TE-103.AP-43 & 8/17/91 & 61 & CASS & $T$ & \\
\hline TE-108-AP-43 & $8 / 19 / 91$ & 63 & CASS & $\bar{T}$ & \\
\hline TE-103-AP-43 & $8 / 27 / 91$ & 62 & CASS & $T$ & \\
\hline TE-103-AP-43 & 9/2/91 & 64 & CASS & $\mathbf{T}$ & \\
\hline TE-103-AP-43 & $7 / 31 / 92$ & 60 & CASS & $\mathbf{T}$ & \\
\hline TE-103-AP-43 & $8 / 3 / 92$ & 61 & CASS & $\mathbf{T}$ & \\
\hline TE-103-AP-43 & $8 / 13 / 92$ & 62 & CASS & $\mathbf{T}$ & \\
\hline TE-108-AP-43 & 8/16/92 & 61 & CASS & $T$ & \\
\hline$T E-103-A P-43$ & $8 / 29 / 92$ & 62 & CASS & $\mathbf{T}$ & \\
\hline TE-103-AP-43 & $9 / 4 / 92$ & 64 & CASS & $T$ & \\
\hline TE-103-AP-43 & 9/11/92 & 62 & CASS & $\mathbf{T}$ & \\
\hline TE-103.AP-43 & $9 / 13 / 92$ & 63 & CASS & $\bar{T}$ & \\
\hline TE-103-AP-43 & $10 / 3 / 92$ & 62 & CASS & $\bar{T}$ & \\
\hline TE-103-AP.43 & $10 / 10 / 92$ & 62 & CASS & $T$ & \\
\hline TE-103-AP-43 & $10 / 17 / 92$ & 62 & CASS & $\mathbf{T}$ & \\
\hline TE-103.AP-A3 & $10 / 22 / 92$ & 63 & CASS & $T$ & \\
\hline TE-103-AP-43 & $10 / 26 / 92$ & 84 & CASS & $\mathbf{T}$ & \\
\hline IE-103-AP-43 & $11 / 4 / 92$ & 63 & CASS & $\mathbf{T}$ & \\
\hline$T E-103-A P-43$ & $11 / 14 / 92$ & 62 & CASS & $\bar{T}$ & \\
\hline$T E-103-A P-43$ & $11 / 17 / 92$ & 64 & CASS & $\mathbf{T}$ & \\
\hline TE-103-AP-A3 & $11 / 24 / 92$ & 64 & CASS & $T$ & \\
\hline TE-103-AP-43 & $11 / 29 / 92$ & 71 & CASS & $\mathbf{s}$ & Dote point did not agree with local trend \\
\hline TE-103-AP-A3 & $12 / 5 / 92$ & 62 & CASS & $T$ & \\
\hline TE-103-AP-43 & $12 / 11 / 92$ & 63 & CASS & $\mathbf{T}$ & \\
\hline TE-103-AP-43 & $12 / 18 / 92$ & 63 & CASS & $T$ & \\
\hline TE-103-AP-43 & $12 / 26 / 92$ & 63 & CASS & $\bar{T}$ & \\
\hline TE-103-AP-43 & $1 / 2 / 93$ & 63 & CASS & $T$ & \\
\hline TE-103-AP-43 & $1 / 9 / 93$ & 62 & CASS & $\bar{T}$ & \\
\hline TE-103-AP-43 & 1/14/93 & 62 & CASS & $T$ & \\
\hline TE-103-AP-43 & 1/23/93 & 61 & CASS & $\bar{T}$ & \\
\hline TE-103-AP-43 & $1 / 26 / 93$ & 62 & CASS & $T$ & \\
\hline TE-103-AP-43 & $2 / 3 / 93$ & 63: & CASS & $\bar{T}$ & \\
\hline TE-103-AP-43 & $2 / 9 / 93$ & $61\}$ & CASS & $\mathbf{T}$ & \\
\hline TE-103-AP-43 & $2 / 19 / 93$ & 61 & CASS & $\mathbf{T}$ & \\
\hline TE-103-AP-43 & $2 / 27 / 93$ & 61 & CASS & $T$ & \\
\hline TE-103-AP-43 & 3/4/33 & 60 & CASS & $\bar{T}$ & \\
\hline TE-103-AP-43 & $3 / 10 / 93$ & 60 & CASS & $T$ & \\
\hline TE-103-AP-43 & $3 / 20 / 93$ & 59 & CASS & $T$ & \\
\hline TE-103-AP-43 & $3 / 27 / 93$ & 60 & CASS & $T$ & \\
\hline TE-103-AP-43 & $3 / 31 / 93$ & 59 & CASS & 7 & \\
\hline TE-103-AP-43 & $4 / 6 / 93$ & 60 & CASS & $T$ & \\
\hline$T E-103-A P-43$ & 4/17/93 & 59 & CASS & $\bar{T}$ & \\
\hline TE-103-AP-43 & 4/24/98 & 59 & CASS & $T$ & \\
\hline TE-103-AP-43 & $5 / 1 / 93$ & 58 & CASS & $T$ & \\
\hline TE-103-AP-43 & $5 / 7 / 93$ & 59 & CASS & $T$ & \\
\hline TE-103-AP-43 & $5 / 10 / 93$ & 59 & CASS & $T$ & \\
\hline TE-103-AP-43 & $5 / 22 / 93$ & 58: & CASS & $\mathbf{T}$ & \\
\hline TE-103-AP-43 & $5 / 29 / 93$ & 57! & CASS & $\mathbf{T}$ & \\
\hline TE-103-AP-43 & 6/3/93: & 58) & CASS & $\mathbf{T}$ & \\
\hline TE-103-AP-43 & $6 / 12 / 93$ & 57 & CASS & $\mathbf{T}$ & \\
\hline TE-103-AP-43 & 6/16/93 & 58 & CASS & $\mathbf{T}$ & \\
\hline TE-103-AP-43 & $6 / 24 / 93$ & 58 & CASS & $\mathbf{T}$ & \\
\hline TE-103-AP-43 & 6/30/93 & 811 & CASS & $\mathbf{S}$ & Date point did not agree with local trend \\
\hline TE-103-AP-43 & $7 / 3 / 93$ & 57 & CASS & $\mathbf{T}$ & \\
\hline TE-103-AP-43 & $7 / 6 / 93$ & 59 & CASS & $\mathbf{T}$ & \\
\hline TE-103-AP-43 & 7/17/93 & 57 & CASS & $\bar{T}$ & \\
\hline
\end{tabular}




\begin{tabular}{|c|c|c|c|c|c|}
\hline \multicolumn{6}{|c|}{$\begin{array}{l}\text { TANKK 241-AP.103 } \\
\text { THERIMOCOUPLE DATA }\end{array}$} \\
\hline & & & & & \\
\hline THERMOCOUPLE * & DATE & TEMFERATURE ( & DATA SOUACE & QUALITY & COMMENT \\
\hline TE-103-AP-43 & $7 / 21 / 93$ & 59 & CASS & $T$ & \\
\hline TE-103-AP-43 & $7 / 27 / 93$ & 58 & CASS & $\mathbf{T}$ & \\
\hline TE-103-AP-43 & $8 / 7 / 93$ & 59 & CASS & $T$ & \\
\hline TE-103-AP-43 & $8 / 14 / 93$ & 58 & CASS & $T$ & \\
\hline TE-103-AP-43 & $8 / 20 / 93$ & 59 & CASS & $T$ & \\
\hline TE-103-AP-43 & $8 / 25 / 93$ & 61 & CASS & $\mathbf{T}$ & \\
\hline TE-103-AP-43 & $8 / 29 / 93$ & 60 & CASS & $T$ & \\
\hline TE-103-AP-43 & 9/9/93 & 60 & CASS & $\mathbf{T}$ & \\
\hline TE-103-AP-43 & $9 / 13 / 93$ & 60 & CASS & $\mathbf{T}$ & \\
\hline TE-103-AP-43 & $9 / 25 / 93$ & 59 & CASS & $T$ & \\
\hline TE-103-AP-43 & $10 / 1 / 93$ & 60 & CASS & $T$ & \\
\hline TE-103-AP-43 & 10/8/93 & 61 & CASS & $\mathbf{T}$ & \\
\hline TE-103-AP-43 & 10/11/93 & 62 & CASS & $\mathbf{T}$ & \\
\hline TE-103-AP-43 & $10 / 18 / 93$ & 61 & CASS & $\mathbf{T}$ & \\
\hline TE-103-AP-43 & $10 / 27 / 93$ & 61 & CASS & $\mathbf{T}$ & \\
\hline TE-103-AP-43 & $31 / 6 / 93$ & 60 & CASS & $T$ & \\
\hline TE-103-AP-43 & $11 / 8 / 93$ & 61 & CASS & $T$ & \\
\hline TE-103-AP-43 & $11 / 19 / 93$ & 61 & CASS & $T$ & \\
\hline TE-103-AP-43 & $11 / 27 / 93$ & 60 & CASS & $\mathbf{T}$ & \\
\hline IE-103-AP-43 & $12 / 1 / 93$ & 61 & CASS & $\mathbf{T}$ & \\
\hline TE-103-AP-43 & $1 / 12 / 94$ & 60 & CASS & $\bar{T}$ & \\
\hline$T E-103 \cdot A P-43$ & 5/12/94 & 57 & CASS & $T$ & \\
\hline TE-103-AP-43 & $5 / 17 / 94$ & 59 & CASS & $T$ & \\
\hline TE-103-AP-43 & 5/26/94 & 59 & CASS & $T$ & \\
\hline TE-103-AP-43 & $6 / 4 / 94$ & 59 & CASS & $\mathbf{T}$ & \\
\hline TE-103-AP-43 & $6 / 11 / 94$ & 80 & CASS & $T$ & \\
\hline TE-102-AP-43 & 6/12/94 & 61 & CASS & $\mathbf{T}$ & \\
\hline TE-103-AP-43 & $6 / 24 / 94$ & 62 & CASS & $T$ & \\
\hline TE-103-AP-43 & $711 / 94$ & 64 & CASS & $T$ & \\
\hline$T E-103-A P-43$ & $7 / 9 / 94$ & 64 & CASS & $T$ & \\
\hline TE-103-AP=43 & 7/16/94 & 64 & CASS & $\mathbf{T}$ & \\
\hline TE-103-AP-43 & $7 / 22 / 94$ & 67 & CASS & $T$ & \\
\hline TE-103-AP-43 & $7 / 28 / 94$ & 66 & CASS & $T$ & \\
\hline TE-103-AP-43 & $8 / 5 / 94$ & 66 & CASS & $\mathbf{T}$ & \\
\hline TE-103-AP-43 & $8 / 13 / 94$ & 68 & CASS & $\mathbf{T}$ & \\
\hline$T E-103-A P-43$ & $8 / 20 / 94$ & 68 & CASS & $\mathbf{T}$ & \\
\hline TE-103-AP-43 & $8 / 22 / 94$ & 69 & CASS & $\mathbf{T}$ & \\
\hline$T E-103-A P-43$ & $9 / 2 / 94$ & 68 & CASS & $T$ & \\
\hline TE-108-AP-43 & 9/10/94 & 68 & CASS & $\bar{T}$ & \\
\hline TE-103-AP-43 & $9 / 11 / 94$ & 69 & CASS & $T$ & \\
\hline TE-103-AP-43 & 9/18/94 & 68 & CASS & $\mathbf{T}$ & \\
\hline TE-103-AP-43 & 9/20/94 & 122 & CASS & $\mathbf{s}$ & Data point did not agroe with local trend \\
\hline$T E-103-A P-43$ & 9/30/94 & 69 & CASS & $\mathbf{T}$ & \\
\hline TE-103-AP-43 & 10/2/94 & 68 & CASS & $\mathbf{T}$ & \\
\hline TE-108-AP-43 & $10 / 12 / 94$ & 67. & CASS & $\mathbf{T}$ & \\
\hline TE-103-AP-43 & $10 / 21 / 94$ & 67) & CASS & $\mathbf{T}$ & \\
\hline TE-103-AP-43 & $10 / 29 / 94$ & 64) & CASS & $T$ & \\
\hline TE-103-AP-A3 & $11 / 3 / 94$ & 64 & CASS & $T$ & \\
\hline TE-103-AP-43 & $11 / 12 / 94$ & 63 & CASS & $\bar{T}$ & \\
\hline TE-103-AP-43 & $11 / 15 / 94$ & 62 & CASS & $T$ & \\
\hline TE-103-AP-43 & $11 / 20 / 94$ & 61 & CASS & $\mathbf{T}$ & \\
\hline TE-103-AP-43 & $12 / 3 / 94$ & 58 & CASS & $\mathbf{T}$ & \\
\hline TE-103-AP-43 & $12 / 4 / 94$ & 58 & CASS & $\mathbf{T}$ & \\
\hline TE-103-AP-43 & $12 / 15 / 94$ & 55 & CASS & $T$ & \\
\hline TE-103-AP-43 & $12 / 21 / 94$ & 56 & CASS & $T$ & \\
\hline TE-103-AP-43 & $12 / 31 / 94$ & 54 & CASS & $T$ & \\
\hline TE-103-AP-43 & $1 / 2 / 95$ & 54 & CASS & $\mathbf{T}$ & \\
\hline TE-103-AP-43 & $1 / 13 / 95$ & 53 & CASS & $\mathbf{T}$ & \\
\hline TE-103-AP-43 & $1 / 17 / 95$ & 53 & CASS & $\mathbf{T}$ & \\
\hline$T E-103-A P-43$ & $1 / 25 / 95$ & 52 & CASS & $T$ & \\
\hline TE-103-AP-43 & $2 / 2 / 95$ & 53 & CASS & $T$ & \\
\hline TE-103-AP-43 & $2 / 9 / 95$ & 53 & CASS & $T$ & \\
\hline TE-103-AP-43 & $2 / 12 / 95$ & 53 & CASS & $T$ & \\
\hline TE-103-AP-43 & 2/22/95 & 53 & CASS & $T$ & \\
\hline TE-103-AP-43 & 2/28/95 & 52 & CASS & $T$ & \\
\hline TE-103-AP-43 & $3 / 10 / 95$ & 53 & CASS & $\mathrm{T}$ & \\
\hline$T E-103-A P-43$ & 3/16/95 & 53 & CASS & $T$ & \\
\hline TE-103-AP-43 & 3/20/95 & 54 & CASS & $T$ & \\
\hline TE-103-AP-43 & $3 / 29 / 95$ & 54 & CASS & $T$ & \\
\hline & & & & & \\
\hline TE-103-AP-44 & $4 / 5 / 86$ & 54 & CASS & $T$ & \\
\hline TE-103-AP-A4 & $4 / 10 / 86$ & 56 & CASS & $T$ & \\
\hline TE-103-AP-44 & $4 / 18 / 86$ & 55 & CASS & $\mathbf{T}$ & \\
\hline
\end{tabular}




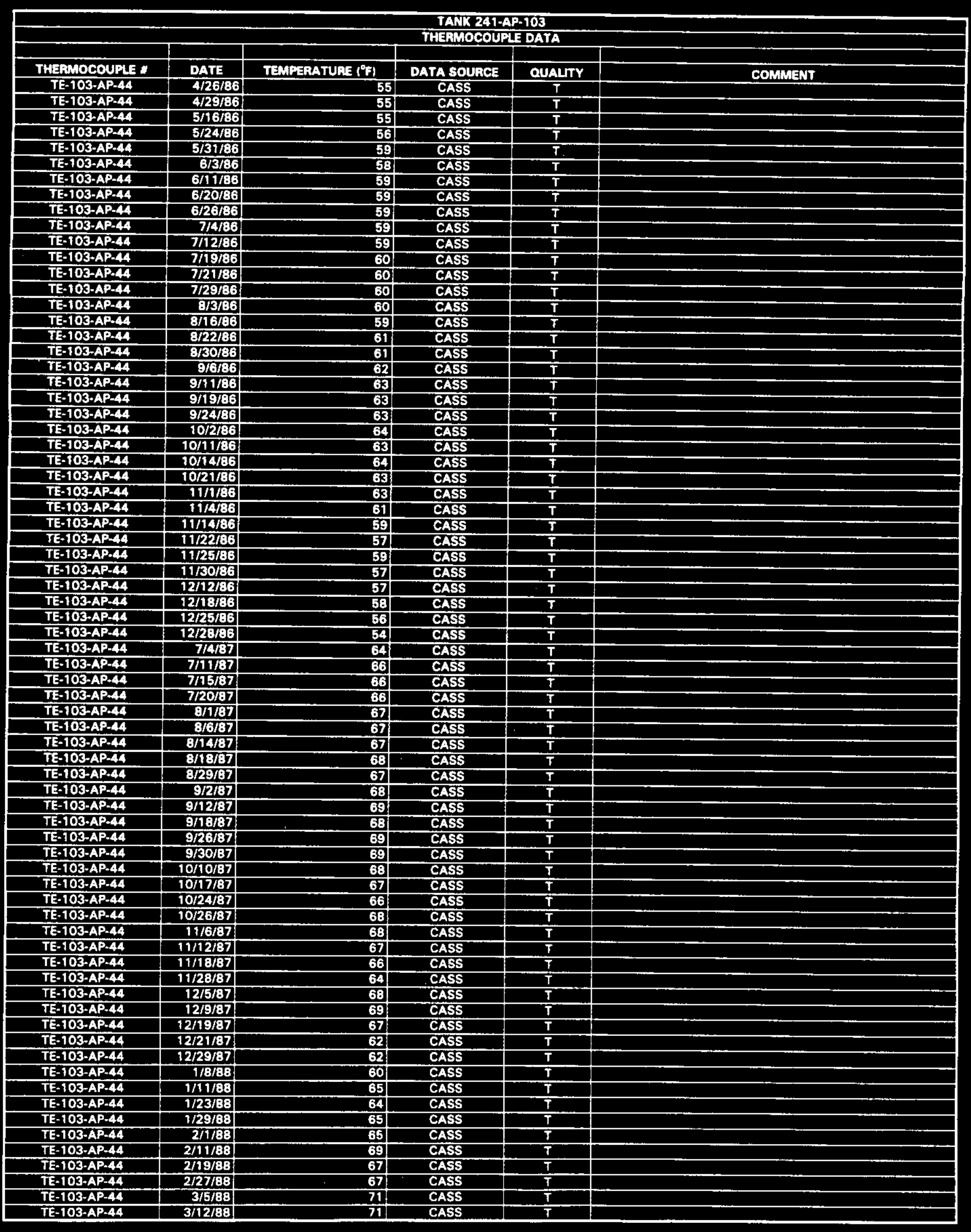




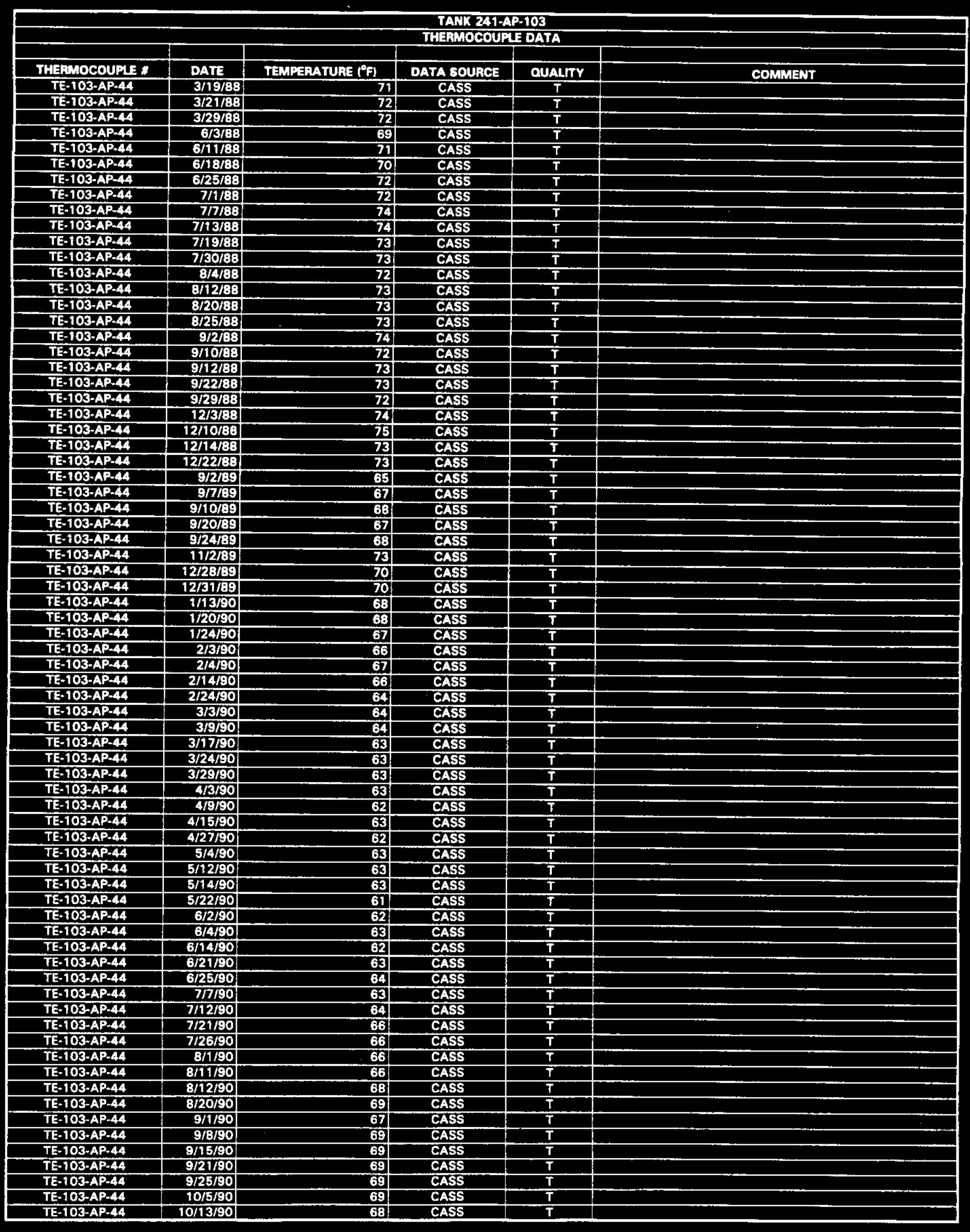




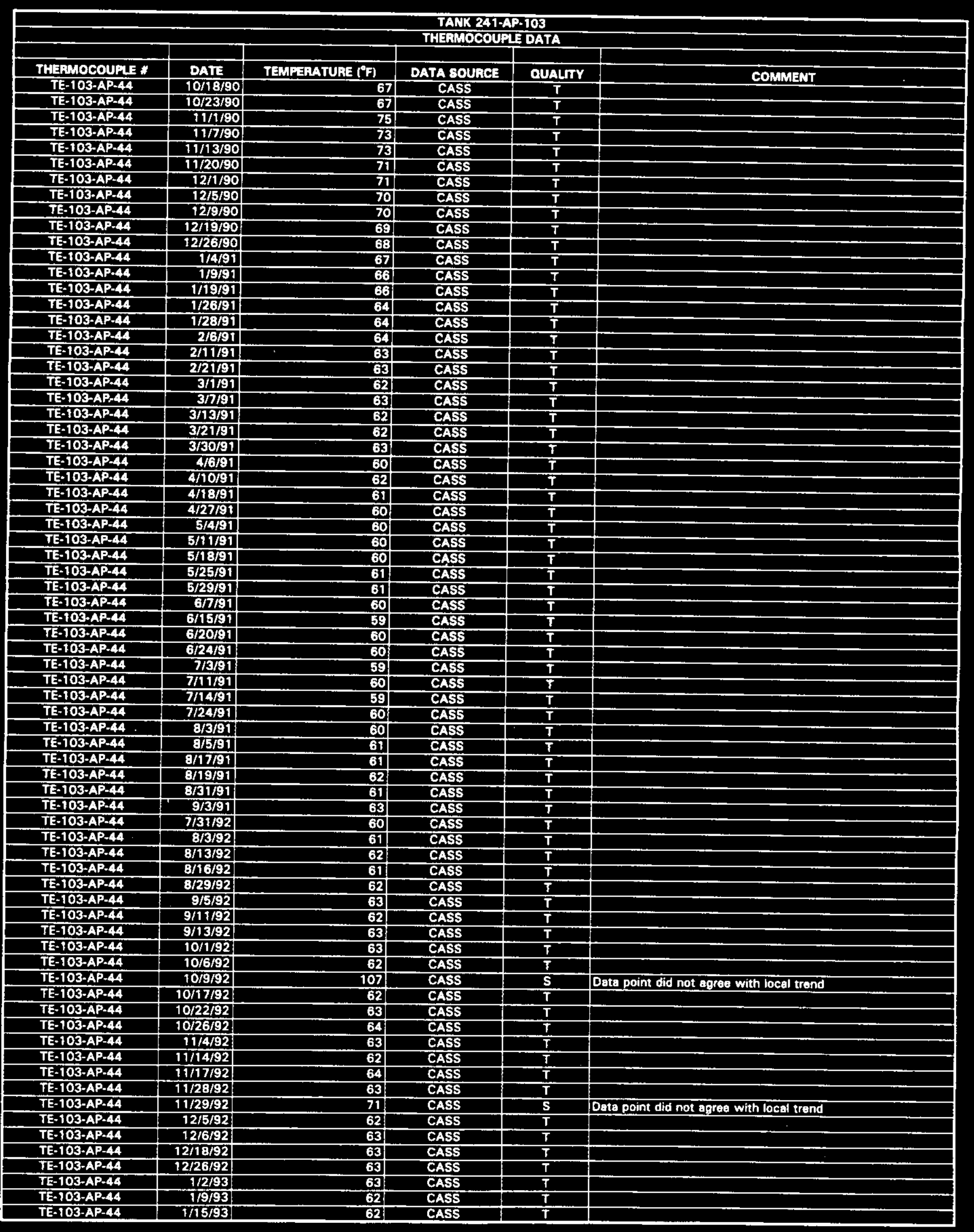




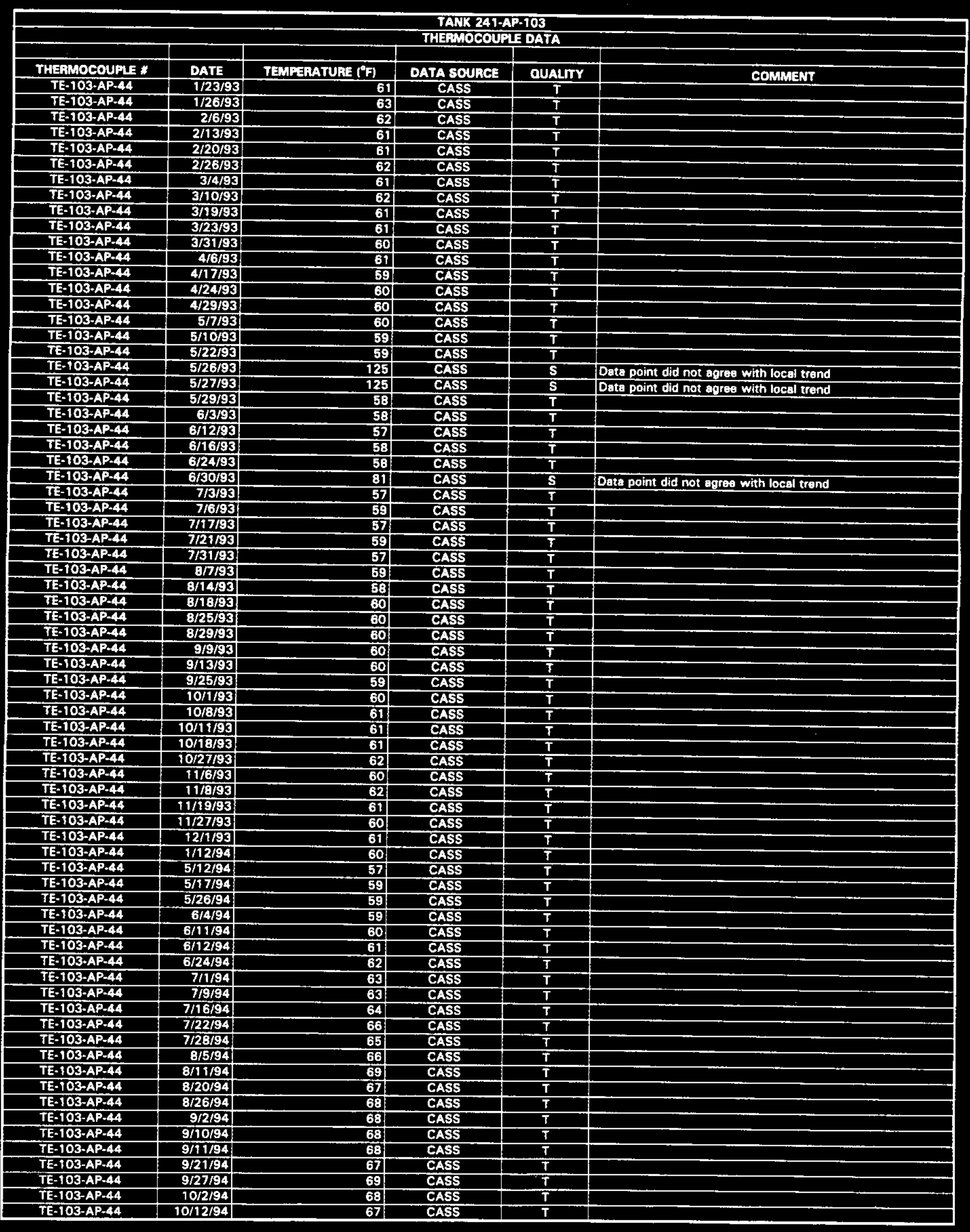

Data obtained from Computer Automated Surveillance Syatem (CASS), April 1995; and Surveillance Analysis Computer System (SACS), June 1995. 


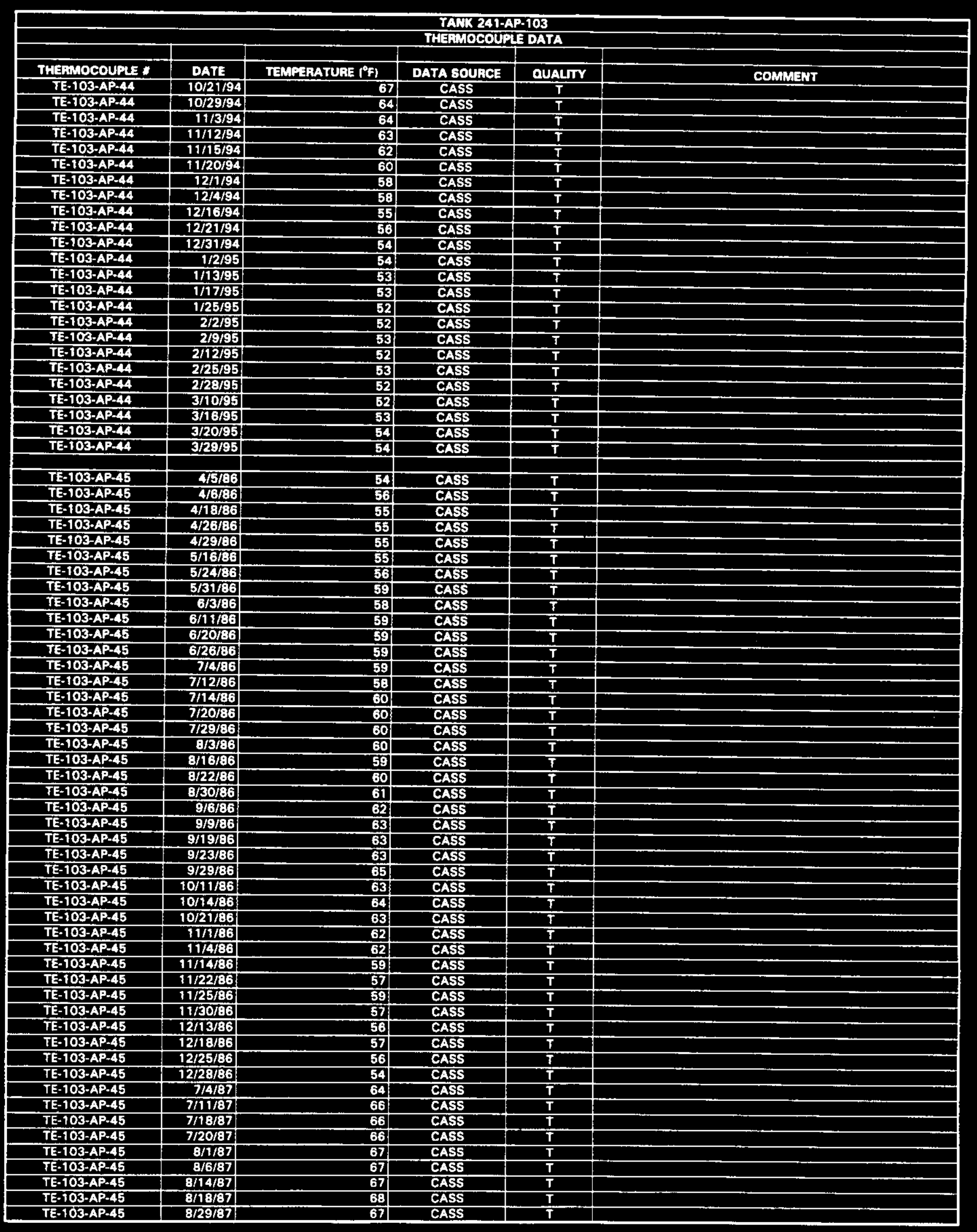




\begin{tabular}{|c|c|c|c|c|c|}
\hline \multicolumn{6}{|c|}{$\begin{array}{l}\text { TANK 241-AP-103 } \\
\text { THLRMOCOUFLE DATA }\end{array}$} \\
\hline & & & & & \\
\hline THERMOCOUPIE * & DATE & TEMPERATURE ( $\left.{ }^{\circ} \mathrm{F}\right)$ & DATA SOURCE & QUALTY & COMMENT \\
\hline TE-103-AP-45 & $9 / 2 / 87$ & 68 & CASS & $T$ & \\
\hline TE-103-AP-45 & $9 / 12 / 87$ & 69 & CASS & $\mathbf{T}$ & \\
\hline TE-103-AP-45 & $9 / 16 / 87$ & 69 & CASS & $T$ & \\
\hline TE-103-AP-45 & 9/26/87 & 69 & CASS & $T$ & \\
\hline TE-103-AP-45 & $9 / 30 / 87$ & 69 & CASS & $T$ & \\
\hline TE-103-AP-45 & $10 / 10 / 87$ & 68 & CASS & $T$ & \\
\hline$T E-103-A P-45$ & $10 / 17 / 87$ & 67 & CASS & $T$ & \\
\hline TE-103-AP-45 & $10 / 24 / 87$ & 66 & CASS & $T$ & \\
\hline$T E-103-A P-45$ & $10 / 30 / 87$ & 67 & CASS & $T$ & \\
\hline$T E=103-A P-45$ & $11 / 6 / 87$ & 68 & CASS & $T$ & \\
\hline TE-103-AP-45 & $11 / 12 / 87$ & 67 & CASS & $T$ & \\
\hline TE-103-AP-45 & $11 / 16 / 87$ & 67 & CASS & $T$ & \\
\hline TE-103-AP-45 & $11 / 28 / 87$ & 64 & CASS & $T$ & \\
\hline TE-108-AP-45 & $12 / 4 / 87$ & 73 & CASS & $T$ & \\
\hline TE-103-AP-45 & $12 / 9 / 87$ & 69 & CASS & $T$ & \\
\hline TE-103-AP-45 & $12 / 19 / 87$ & 67 & CASS & $\bar{T}$ & \\
\hline TE-103-AP-45 & $12 / 21 / 87$ & 62 & CASS & $T$ & \\
\hline TE-103-AP-45 & $12 / 29 / 97$ & 62: & CASS & $T$ & \\
\hline TE-103-AP-45 & //B/8B & 61 & CASS & $\mathbf{T}$ & \\
\hline TE-103-AP-45 & $1 / 17 / 88$ & 64 & CASS & $T$ & \\
\hline TE-103-AP-45 & $1 / 18 / 88$ & 63 & CASS & $T$ & \\
\hline TE-103-AP-45 & 1/29/8 & 64 & CASS & $T$ & \\
\hline TE-103-AP-45 & $2 / 1 / 88$ & 65 & CASS & $T$ & \\
\hline$T E=103-A P-45$ & $2 / 5 / 80$ & 80 & CASS & $\mathbf{S}$ & Dats point did not agree with local trand \\
\hline TE-103-AP-45 & $2 / 11 / 88$ & 69 & CASS & $T$ & \\
\hline$T E-108-A P-45$ & $2 / 19 / 88$ & 67 & CASS & $\bar{T}$ & \\
\hline TE-103-AP-45 & $2 / 24 / 88$ & 68 & CASS & $T$ & \\
\hline TE-103-AP-45 & 3/5/38 & $7 \pi$ & CASS & $T$ & \\
\hline TE-103-AP-45 & $3 / 12 / 8$ & 72 & CASS & $T$ & \\
\hline$T E-103-A P-45$ & 3/19/88 & 72 & CASS & $T$ & \\
\hline TE-103-AP-45 & $3 / 21 / 88$ & 73 & CASS & $T$ & \\
\hline$T E-103-A P-45$ & $3 / 29 / 88$ & 72 & CASS & $T$ & \\
\hline TE-103-AP-A5 & $6 / 3 / 88$ & 69 & CASS & $T$ & \\
\hline TE-103-AP-45 & $6 / 11 / 88$ & 71 & CASS & $T$ & \\
\hline TE-103-AP-45 & 6/18/88 & 70 & CASS & $\mathbf{T}$ & \\
\hline TE-103-AP-45 & 6/25/8 & 71 & CASS & $T$ & \\
\hline TE-103-AP-45 & $7 / 1 / 8 B$ & 72 & CASS & $T$ & \\
\hline TE-103-AP-45 & $7 / 7 / 88$ & 74 & CASS & $T$ & \\
\hline TE-103-AP-45 & $7 / 13 / 88$ & 74 & CASS & $T$ & \\
\hline TE-103-AP-45 & $7 / 19 / 88$ & 73 & CASS & $T$ & \\
\hline TE-103-AP-45 & $7 / 30 / 88$ & 73 & CASS & $T$ & \\
\hline TE-103-AP-45 & $8 / 4 / 88$ & 72 & CASS & $T$ & \\
\hline TE-103-AP-45 & $8 / 12 / 89$ & 73 & CASS & $T$ & \\
\hline TE-103-AP-45 & $8 / 20 / 80$ & 73 & CASS & $T$ & \\
\hline TE-103-AP-45 & $8 / 25 / 88$ & 73 & CASS & 3 & \\
\hline TE-103-AP-45 & $8 / 28 / 88$ & 73 & CASS & $\mathbf{T}$ & \\
\hline TE-103-AP-45 & $9 / 8 / 88$ & 72 & CASS & $T$ & \\
\hline TE-103-AP-45 & 9/12/88 & $74 !$ & CASS & $T$ & \\
\hline TE-103-AP-45 & 9/22/89 & 73 & CASS & $T$ & \\
\hline TE-103-AP-45 & $9 / 29 / 80$ & 71 & CASS & $T$ & \\
\hline TE-103-AP-45 & $12 / 3 / 88$ & 74 & CASS & $T$ & \\
\hline TE-103-AP-45 & $12 / 10 / 88$ & 75 & CASS & $\bar{T}$ & \\
\hline TE-103-AP-45 & $12 / 74 / 88$ & 72 & CASS & $T$ & \\
\hline TE-103-AP-45 & $12 / 22 / 88$ & 74 & CASS & $\bar{T}$ & \\
\hline TE-103-AP-45 & $9 / 2 / 89$ & 65 & CASS & $\mathbf{T}$ & \\
\hline TE-103-AP-45 & $9 / 3 / 89$ & 67. & CASS & $T$ & \\
\hline TE-103-AP-45 & $9 / 10 / 89$ & 68 & CASS & $T$ & \\
\hline TE-103-AP-45 & $9 / 20 / 89$ & 67 & CASS & $T$ & \\
\hline TE-103-AP-45 & 9/26/89 & 68 & CASS & $T$ & \\
\hline TE-103-AP-45 & 11/2/89 & 74 & CASS & $T$ & \\
\hline TE-103-AP-45 & $12 / 28 / 89$ & 71 & CASS & $T$ & \\
\hline TE-103-AP-45 & $12 / 31 / 89$ & 71 & CASS & $T$ & \\
\hline TE-103-AP-45 & $1 / 13 / 90$ & 69 & CASS & $\mathrm{T}$ & \\
\hline TE-103-AP-45 & $1 / 20 / 90$ & 69 & CASS & $T$ & \\
\hline TE-103-AP-45 & $1 / 23 / 90$ & 68 & CASS & $T$ & \\
\hline TE-103-AP-45 & $1 / 30 / 90$ & 67 & CASS & $T$ & \\
\hline TE-103-AP-45 & $2 / 4 / 90$ & 67 & CASS & $T$ & \\
\hline TE-103-AP-45 & $2 / 14 / 90$ & 67 & CASS & $T$ & \\
\hline TE-103-AP-45 & $2 / 18 / 90$ & 65 & CASS & $T$ & \\
\hline TE-103-AP-45 & $3 / 3 / 90$ & 65 & CASS & $T$ & \\
\hline TE-103-AP-45 & $3 / 9 / 90$ & 65 & CASS & $T$ & \\
\hline TE-103-AP-45 & $3 / 17 / 90$ & 64 & CASS & $\bar{T}$ & \\
\hline TE-103-AP-45 & $3 / 20 / 90$ & 64 & CASS & $\mathbf{T}$ & \\
\hline
\end{tabular}




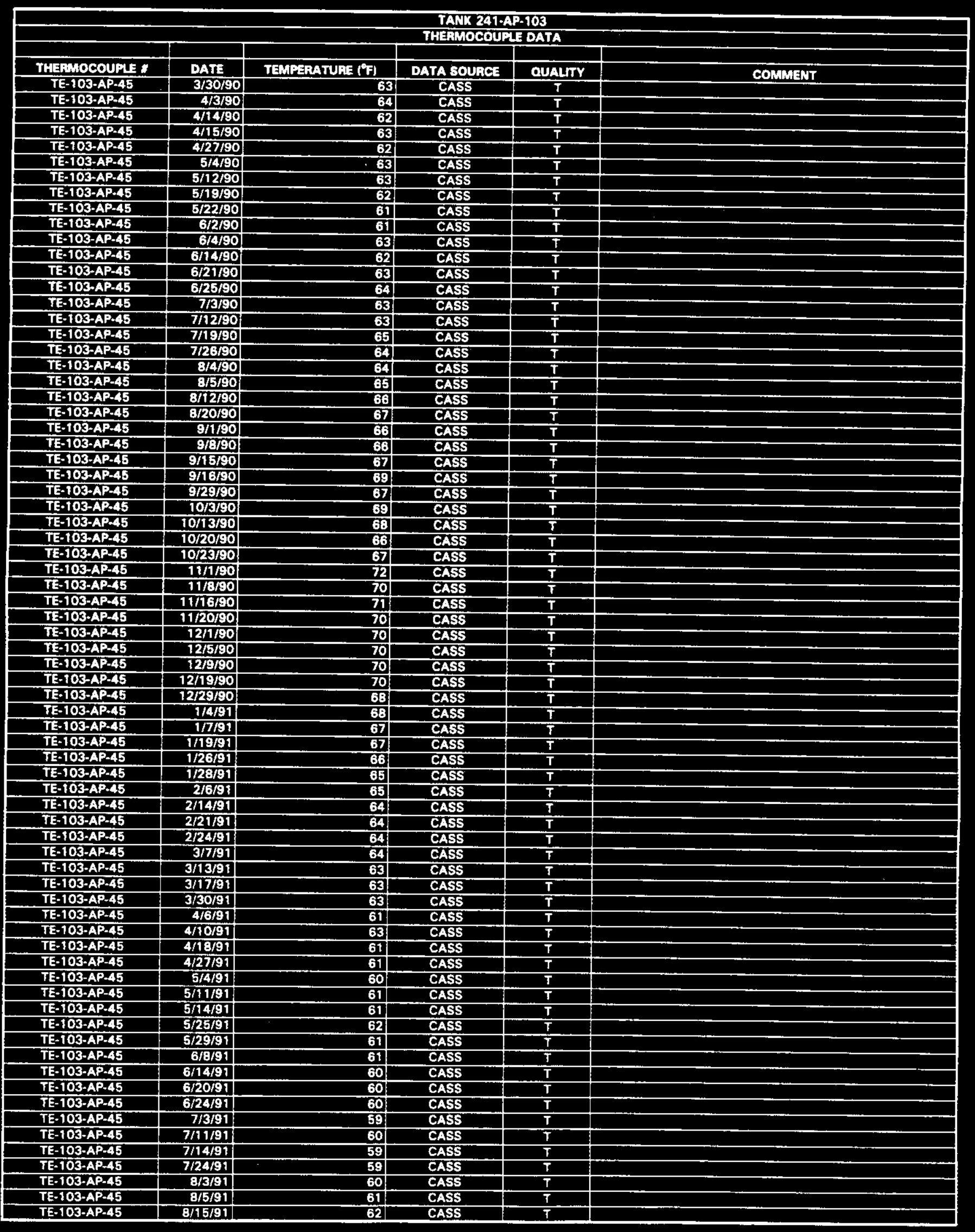




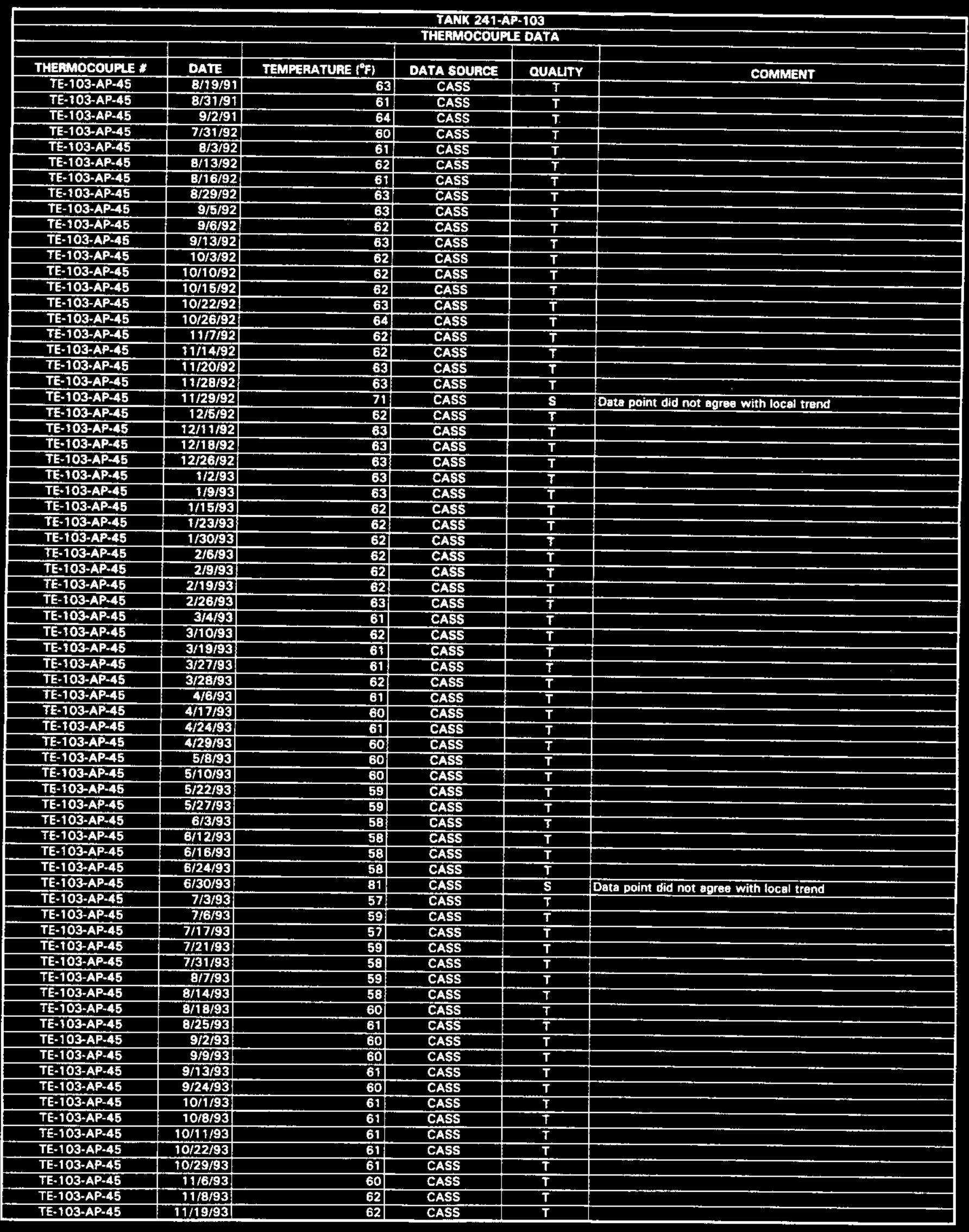




\begin{tabular}{|c|c|c|c|c|c|}
\hline \multicolumn{6}{|c|}{$\begin{array}{l}\text { TATK 241-AP-103 } \\
\text { THEFMOCOUPLE DATA }\end{array}$} \\
\hline & & & & & \\
\hline THERMOCOUPLE * & DATE & TEMPERATUAE $\left({ }^{\circ} \mathrm{F}\right)$ & DATA SOURCE & QUALITY & COMMENT \\
\hline TE-103-AP-45 & $11 / 27 / 93$ & 60 & CASS & $T$ & \\
\hline TE-103-AP-45 & $12 / 3 / 93$ & 61 & CASS & $T$ & \\
\hline TE-103-AP-45 & $1 / 13 / 94$ & 60 & CASS & $T$ & \\
\hline TE-103-AP.45 & 5/12/94 & 57 & CASS & $T$ & \\
\hline TE-103-AP.45 & $5 / 17 / 94$ & 58 & CASS & $T$ & \\
\hline$T E-103-A P-45$ & $5 / 26 / 94$ & 59 & CASS & $T$ & \\
\hline TE-103-AP-45 & $6 / 4 / 94$ & 59 & CASS & $T$ & \\
\hline TE-103-AP-45 & $6 / 11 / 94$ & 60 & CASS & $\mathbf{T}$ & \\
\hline TE-103-AP-45 & $6 / 12 / 94$ & 61. & CASS & $\bar{T}$ & \\
\hline TE-103-AP-45 & 6/24/94 & 63 & CASS & $T$ & \\
\hline TE-103-AP-45 & $7 / 1 / 94$ & 63 & CASS & $T$ & \\
\hline TE-103-AP-45 & $7 / 9 / 34$ & 63 & CASS & $\bar{T}$ & \\
\hline TE-103-AP-45 & $711 / 34$ & 65 & CASS & $T$ & \\
\hline TE-103-AP-45 & $7 / 22 / 94$ & 67 & CASS & $T$ & \\
\hline TE-108-AP-45 & $7 / 28 / 94$ & 65 & CASS & $T$ & \\
\hline TE-103-AP-45 & $8 / 5 / 94$ & 66 & CASS & $\mathbf{T}$ & \\
\hline TE-103-AP-45 & $8 / 13 / 94$ & 68 & CASS & $T$ & \\
\hline TE-103-AP-45 & $8 / 20 / 94$ & 67 & CASS & $T$ & \\
\hline TE-103-AP-45 & $8 / 22 / 94$ & 69 & CASS & $T$ & \\
\hline TE-103-AP-45 & $9 / 2 / 94$ & 68 & CASS & $\mathrm{T}$ & \\
\hline TE-103-AP-45 & $9 / 10 / 94$ & 68 & CASS & $\mathbf{T}$ & \\
\hline TE-103-AP- 45 & $9 / 11 / 94$ & 69 & CASS & $T$ & \\
\hline TE-103-AP-45 & 8/21/94 & 67 & CASS & $T$ & \\
\hline TE-103-AP-45 & 9/27/94 & 69 & CASS & $T$ & \\
\hline TE-103-AP-45 & $10 / 3 / 94$ & 68 & CASS & $\mathbf{T}$ & \\
\hline TE-103-AP-45 & $10 / 15 / 94$ & 66 & CASS & $\mathbf{T}$ & \\
\hline TE-103-AP-45 & $10 / 21 / 94$ & 67 & CASS & $T$ & \\
\hline TE-103-AP-45 & $10 / 29 / 94$ & 64 & CASS & $\mathbf{T}$ & \\
\hline TE-103-AP-45 & $11 / 3 / 94$ & 64 & CASS & $T$ & \\
\hline TE-103-AP-45 & $11 / 12 / 94$ & 63 & CASS & $T$ & \\
\hline TE-103-AP-45 & $11 / 15 / 94$ & 62 & CASS & $\mathbf{T}$ & \\
\hline TE-103-AP-45 & $11 / 20 / 94$ & 60 & CASS & $T$ & \\
\hline TE-103-AP-45 & $12 / 1 / 94$ & 58 & CASS & $T$ & \\
\hline TE-103-AP-45 & $12 / 9 / 94$ & 57 & CASS & $T$ & \\
\hline TE-103.AP-45 & $12 / 15 / 94$ & 55 & CASS & $T$ & \\
\hline TE-103-AP-45 & $12 / 21 / 94$ & 56 & CASS & $T$ & \\
\hline TE-103-AP-45 & $12 / 31 / 94$ & 54 & CASS & $T$ & \\
\hline TE-103-AP-45 & i/2/95 & 54. & CASS & $\bar{T}$ & \\
\hline TE-103-AP-45 & $1 / 13 / 95$ & 54 & CASS & $\mathbf{T}$ & \\
\hline TE-103-AP-45 & $1 / 17 / 95$ & 53 & CASS & $\mathbf{T}$ & \\
\hline TE-103-AP-45 & $1 / 25 / 95$ & 52 & CASS & $T$ & \\
\hline TE-103-AP-45 & $1 / 27 / 95$ & 93 & CASS & $\mathrm{s}$ & Data point did not agree with local trend \\
\hline TE-103-AP-45 & $2 / 2 / 95$ & 53 & CASS & $T$ & \\
\hline TE-103-AP-45 & $2 / 9 / 95$ & 52 & CASS & $T$ & \\
\hline TE-103-AP-45 & $2 / 18 / 85$ & 52 & CASS & $\mathbf{T}$ & \\
\hline TE-103-AP-45 & $2 / 22 / 95$ & 53 & CASS & $T$ & \\
\hline TE-103-AP-45 & $2 / 28 / 95$ & 52 & CASS & $T$ & \\
\hline TE-103-AP-45 & $3 / 10 / 95$ & 52 & CASS & $T$ & \\
\hline TE-103-AP-45 & $3 / 16 / 95$ & 53 & CASS & $T$ & \\
\hline TE-103-AP-45 & $3 / 20 / 95$ & 54 & CASS & $\mathbf{T}$ & \\
\hline TE-103-AP-45 & $3 / 29 / 95$ & 54 & CASS & $T$ & \\
\hline & & & & & \\
\hline TE-103-AP-46 & $4 / 5 / 86$ & 54 & CASS & $\mathbf{T}$ & \\
\hline TE-103-AP-46 & $4 / 6 / 86$ & 56 & CASS & $T$ & \\
\hline TE-103-AP-46 & $4 / 18 / 86$ & 55 & CASS & $T$ & \\
\hline TE-103-AP-46 & 4/26/86 & 55 & CASS & $\mathrm{T}$ & \\
\hline TE-103-AP-46 & $4 / 29 / 36$ & 55 & CASS & $\bar{T}$ & \\
\hline TE-103-AP-46 & $5 / 16 / 86$ & 55 & CASS & $T$ & \\
\hline TE-103-AP-46 & $5 / 23 / 86$ & 57 & CASS & $T$ & \\
\hline TE-103-AP-46 & $5 / 31 / 86$ & 59 & CASS & $T$ & \\
\hline TE-103-AP-46 & $6 / 3 / 86$ & 58. & CASS & $T$ & \\
\hline TE-103-AP-46 & $6 / 11 / 86$ & 59 & CASS & $\mathbf{T}$ & \\
\hline TE-103-AP-46 & $6 / 20 / 66$ & 59 & CASS & $\mathbf{T}$ & \\
\hline TE-103-AP-46 & $6 / 26 / 36$ & 59 & CASS & $T$ & \\
\hline TE-103-AP-46 & $7 / 4 / 66$ & 59 & CASS & $T$ & \\
\hline TE-103-AP-46 & $7 / 11 / 86$ & 59 & CASS & $T$ & \\
\hline TE-103-AP-46 & $7 / 19 / 66$ & 59 & CASS & $\mathbf{T}$ & \\
\hline TE-103-AP-46 & $7 / 21 / 86$ & 60 & CASS & $T$ & \\
\hline TE-103.AP-46 & $7 / 28 / 86$ & 61 & CASS & $T$ & \\
\hline TE-103-AP-46 & $8 / 3 / 86$ & 60 & CASS & $\bar{T}$ & \\
\hline TE-103-AP-46 & $8 / 16 / 86$ & 59 & CASS & $T$ & \\
\hline TE-103-AP-46 & $8 / 22 / 86$ & 60 & CASS & $T$ & \\
\hline TE-103-AP-46 & $8 / 30 / 86$ & 61 & CASS & $\bar{T}$ & \\
\hline
\end{tabular}




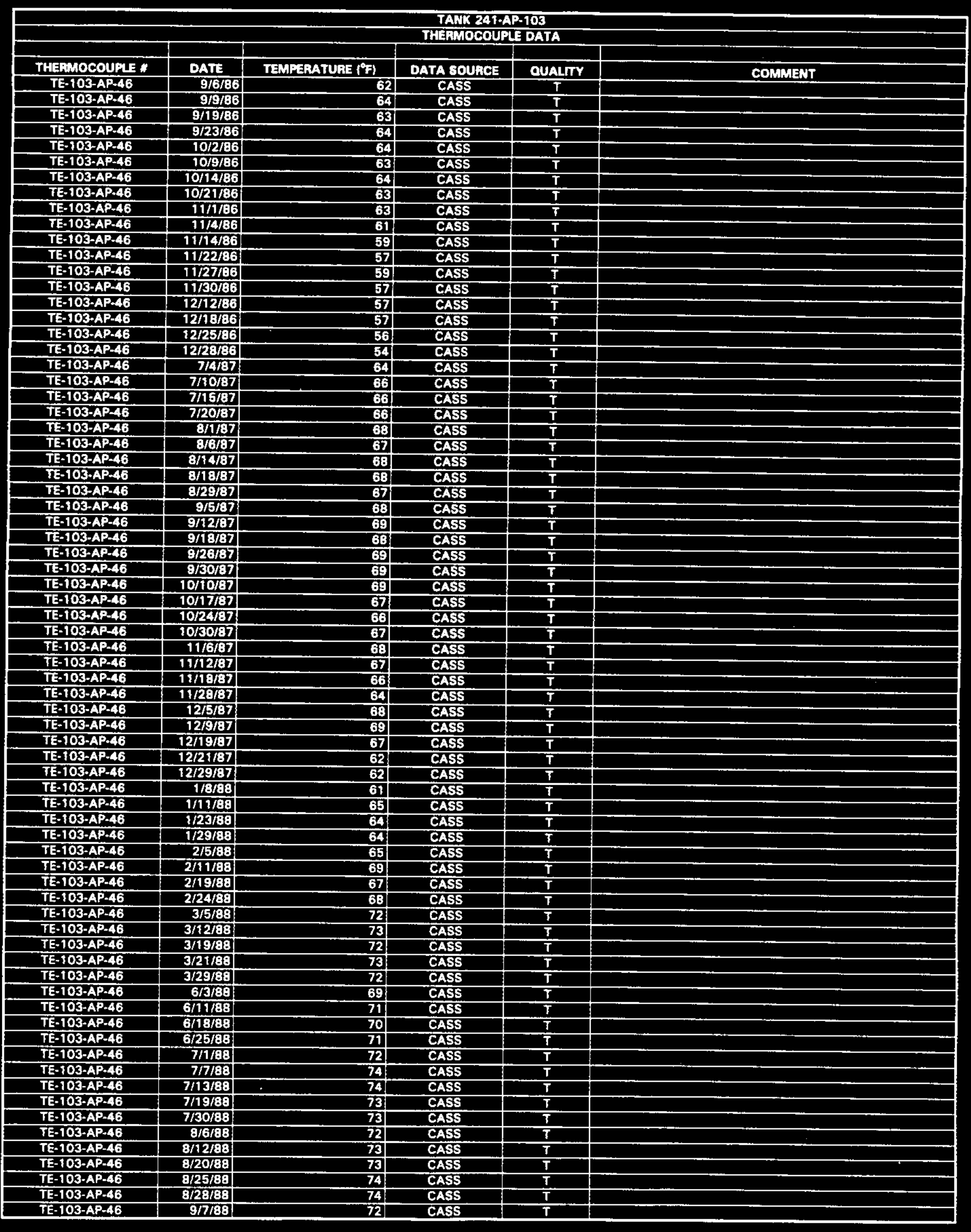

Data obtained from Computer Automated Survaillance System (CASS). April 1995; and Surveillance Analyais Computer System (SACS). June 1995. 


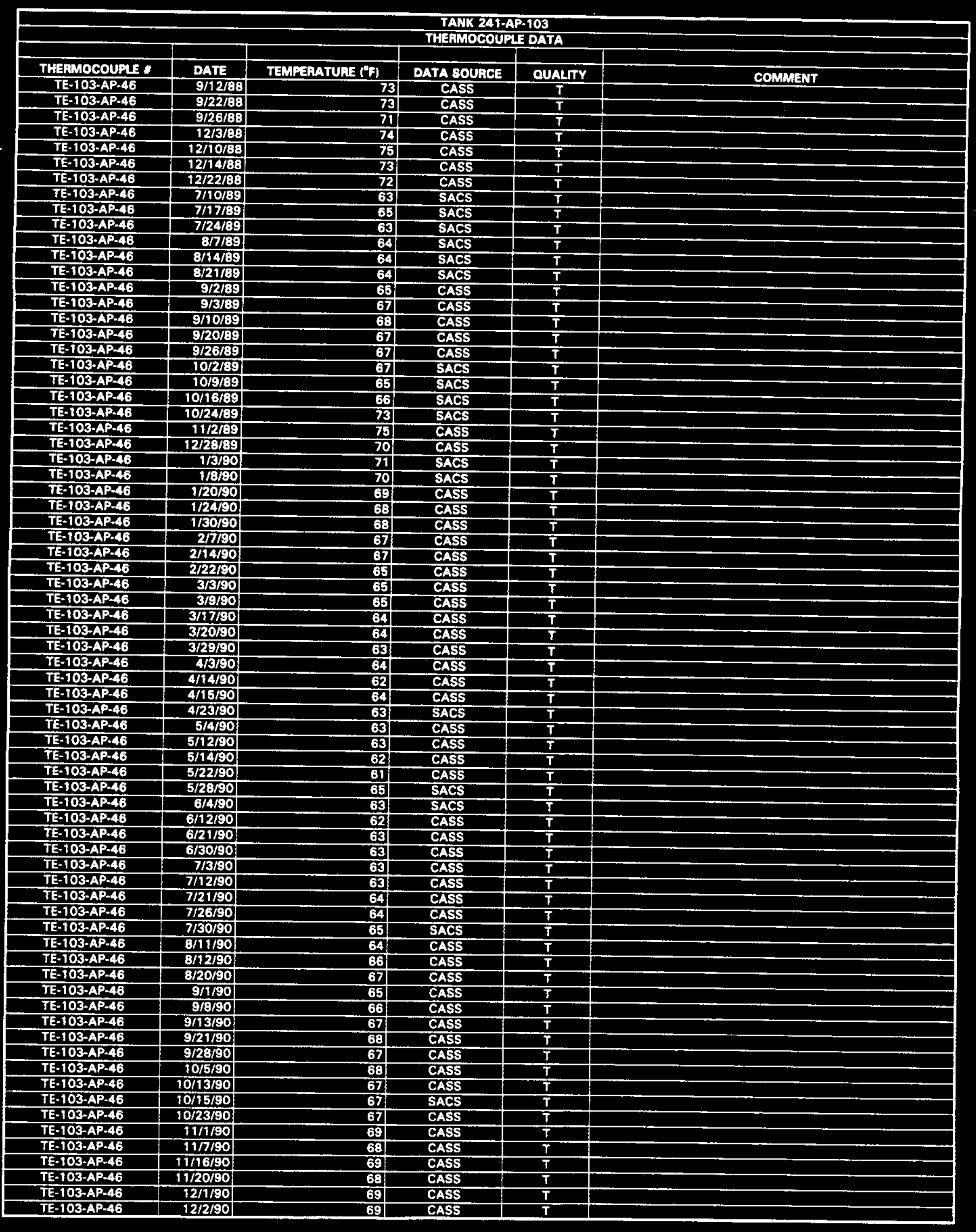

Data obtained from Computer Automated Survaillance System (CASS), April 1995; and Surveillance Analysis Computer System (SACS), June 1995. 


\begin{tabular}{|c|c|c|c|c|c|}
\hline \multicolumn{6}{|c|}{$\begin{array}{l}\text { TAKK 241-AP-103 } \\
\text { THERMOCOUPLE DATA }\end{array}$} \\
\hline & & & & & \\
\hline$\frac{\text { THE:MOCOUPLE * }}{\text { TE-TO3-AP-46 }}$ & $\frac{\text { DATE }}{12 / 9 / 90}$ & $\frac{\text { TEMPERATURE }\left({ }^{\circ} \mathrm{F}\right)}{69}$ & DATA SOUACE & QUALITY & COMMENT \\
\hline$\frac{T E-103-A P-46}{\text { TE-103-AP-46 }}$ & \begin{tabular}{r|}
$12 / 9 / 90$ \\
$12 / 19 / 90$
\end{tabular} & \begin{tabular}{l|}
69 \\
69
\end{tabular} & CASS & $\mathrm{T}$ & \\
\hline TE-103-AP-46 & $12 / 28 / 90$ & $\frac{69}{67}$ & $\frac{\text { CASS }}{\text { CASS }}$ & $\frac{T}{T}$ & \\
\hline TE-103-AP-46 & $12 / 31 / 90$ & 68 & SACS & $T$ & \\
\hline TE-103-AP-46 & $1 / 12 / 91$ & 66 & CASS & $T$ & \\
\hline TE-103-AP-46 & 1/19/91 & 67 & CASS & $\bar{T}$ & \\
\hline TE-103-AP-46 & $1 / 26 / 91$ & 66 & CASS & $T$ & \\
\hline TE-103-AP-46 & 1/28/91 & 65 & CASS & $T$ & \\
\hline TE-103-AP-46 & $2 / 6 / 91$ & 66 & CASS & $T$ & \\
\hline$T E-103-A P-46$ & $2 / 13 / 91$ & 64 & CASS & $T$ & \\
\hline TE-103-AP-46 & $2 / 21 / 91$ & 64 & CASS & $\bar{T}$ & \\
\hline TE-103-AP-46 & $2 / 24 / 91$ & 64 & CASS & $T$ & \\
\hline TE-103-AP-46 & $3 / 7 / 91$ & 64 & CASS & $T$ & \\
\hline TE-103-AP-46 & आ16/91 & 63 & CASS & $\bar{T}$ & \\
\hline TE-103-AP-46 & $3 / 21 / 91$ & 63 & CASS & $\bar{T}$ & \\
\hline TE-103-AP-46 & 3/27/91 & 64. & CASS & $T$ & \\
\hline TE-103-AP-46 & $4 / 6 / 91$ & 61 & CASS & $T$ & \\
\hline TE-103-AP-46 & $4 / 10 / 91$ & 63 & CASS & $T$ & \\
\hline TE-103-AP-46 & $4 / 19 / 91$ & 61 & CASS & $T$ & \\
\hline TE-103-AP-46 & $4 / 23 / 91$ & 62 & CASS & $T$ & \\
\hline TE-103-AP-46 & $5 / 4 / 91$ & 60 & CASS & $T$ & \\
\hline TE-103-AP-46 & 5/11/91 & 61 & CASS & $T$ & \\
\hline TE-103-AP+46 & 5/14/91 & 61 & CASS & $\mathbf{T}$ & \\
\hline TE-103-AP-A6 & $5 / 25 / 91$ & 62 & CASS & $T$ & \\
\hline$T E \cdot 103-A P-46$ & 5/29/91 & 62 & CASS & $T$ & \\
\hline$T E-103-A P-46$ & 6/8/91 & 61 & CASS & $T$ & \\
\hline TE-103-AP-46 & 6/15/97 & 60 & CASS & $T$ & \\
\hline TE-103-AP-46 & $6 / 20 / 91$ & 61 & CASS & $T$ & \\
\hline TE-103-AP-46 & $6 / 25 / 91$ & 60 & CASS & $\bar{T}$ & \\
\hline TE-103-AP-46 & $7 / 1 / 91$ & 60 & SACS & $\bar{T}$ & \\
\hline$T E-103-A P-46$ & 7/11/91 & 61 & CASS & $T$ & \\
\hline TE-103-AP-46 & $7 / 15 / 91$ & 60 & SACS & $T$ & \\
\hline TE-103-AP-46 & $7 / 22 / 91$ & 62 & SACS & $T$ & \\
\hline TE-103-AP-46 & $8 / 191$ & 61 & CASS & $\bar{T}$ & \\
\hline TE-103-AP-46 & $8 / 5 / 91$ & 82 & CASS & $T$ & \\
\hline TE-103-AP-46 & $8 / 15 / 91$ & 62 & CASS & $T$ & \\
\hline TE-103-AP-46 & $8 / 23 / 91$ & 63. & CASS & $\bar{T}$ & \\
\hline$T E-103-A P-46$ & $8 / 26 / 91$ & 63 & SACS & $T$ & \\
\hline TE-103-AP-46 & $8 / 3 / 99$ & 64 & CASS & $T$ & \\
\hline TE-103-AP-46 & $7 / 31 / 92$ & 61 & CASS & $T$ & \\
\hline TE-103-AP-46 & $8 / 8 / 92$ & 61 & CASS & $T$ & \\
\hline TE-103-AP-46 & $8 / 13 / 92$ & 62 & CASS & $\bar{T}$ & \\
\hline TE-103-AP-46 & $8 / 20 / 92$ & $61\}$ & CASS & $T$ & \\
\hline TE-103-AP-46 & 8/29/92 & 82 & CASS & $T$ & \\
\hline$T E-103-A P-46$ & $9 / 5 / 92$ & 63 & CASS & $T$ & \\
\hline TE-103-AP-46 & $9 / 11 / 92$ & 62 & CASS & $T$ & \\
\hline TE-103-AP-46 & $9 / 14 / 92$ & 63 & CASS & 7 & \\
\hline TE-103-AP-46 & 10/3/92 & 62 & CASS & $\bar{T}$ & \\
\hline TE-103-AP-46 & $10 / 8 / 92$ & 62 & CASS & $T$ & \\
\hline TE-103-AP-46 & $10 / 17 / 92$ & 62 & CASS & $T$ & \\
\hline TE-103-AP-46 & $10 / 22 / 92$ & 64 & CASS & $T$ & \\
\hline TE-103-AP.46 & $10 / 29 / 92$ & 63 & CASS & $T$ & \\
\hline TE-103-AP-46 & $11 / 4 / 92$ & 63 & CASS & $T$ & \\
\hline TE-103-AP-46 & $11 / 14 / 92$ & 62 & CASS & $T$ & \\
\hline TE-103-AP-46 & $11 / 17 / 92$ & 64 & CASS & $T$ & \\
\hline TE-103-AP-A6 & $11 / 28 / 92$ & 62 & CASS & $T$ & \\
\hline TE-103-AP-46 & $11 / 29 / 92$ & 74 & CASS & $\mathbf{s}$ & Data point did not agree with local trend \\
\hline TE-103-AP-46 & $12 / 2 / 92$ & 62 & CASS & $T$ & \\
\hline TE-103-AP-46 & $12 / 11 / 92$ & 63 & CASS & $T$ & \\
\hline TE-103-AP-46 & $12 / 18 / 92$ & 63 & CASS & $T$ & \\
\hline TE-103-AP-46 & $12 / 26 / 92$ & 63 & CASS & $T$ & \\
\hline TE-103-AP-46 & $12 / 30 / 92$ & 62 & CASS & $T$ & \\
\hline TE-108-AP-46 & $1 / 9 / 93$ & 62 & CASS & $T$ & \\
\hline TE-103-AP-46 & 1/16/93 & 61 & CASS & $T$ & \\
\hline TE-103-AP-46 & $1 / 23 / 93$ & 61 & CASS & $T$ & \\
\hline TE-103-AP-46 & $1 / 30 / 93$ & 63 & CASS & $T$ & \\
\hline TE-103-AP-46 & $2 / 6 / 93$ & 62 & CASS & $\bar{T}$ & \\
\hline TE-103-AP-46 & 2/9/93 & 62 & CASS & $\bar{T}$ & \\
\hline TE-103-AP-46 & $2 / 18 / 93$ & 61 & CASS & $T$ & \\
\hline TE-103-AP-46 & $2 / 27 / 93$ & 61 & CASS & $T$ & \\
\hline TE-103-AP-46 & $3 / 4 / 93$ & 61: & CASS & $\bar{T}$ & \\
\hline$T E-103-A P-46$ & $3 / 10 / 93$ & 61 & CASS & $T$ & \\
\hline TE-103-AP-46 & 3/19/93 & 61 & CASS & $\mathbf{T}$ & \\
\hline
\end{tabular}




\begin{tabular}{|c|c|c|c|c|c|}
\hline & & & $\begin{array}{l}\text { TAMK 24\%-A } \\
\text { THEPHOCOUP }\end{array}$ & $\frac{103}{\text { DATA }}$ & \\
\hline & & & & & \\
\hline THERMOCOUPLE * & DATE & TEMPERATUAE ( $\left.{ }^{\circ} \mathrm{F}\right)$ & DATA SOUACE & OUALITY & COMMENT \\
\hline TE-103-AP-46 & $3 / 27 / 93$ & 61 & CASS & $T$ & \\
\hline TE-103-AP-46 & $3 / 28 / 93$ & 61 & CASS & $T$ & \\
\hline TE-103-AP-46 & $4 / 6 / 93$ & 61 & CASS & $T$ & \\
\hline TE-103-AP-46 & $4 / 17 / 93$ & 60 & CASS & $\bar{T}$ & \\
\hline TE-103-AP-46 & $4 / 24 / 93$ & 60 & CASS & $T$ & \\
\hline TE-103-AP-46 & $4 / 29 / 93$ & 60 & CASS & $T$ & \\
\hline TE-103-AP-46 & $5 / 7 / 93$ & 60 & CASS & $T$ & \\
\hline TE-103-AP-46 & $5 / 70 / 93$ & 60 & CASS & $\mathbf{T}$ & \\
\hline TE-103-AP-46 & $5 / 22 / 93$ & 59 & CASS & $T$ & \\
\hline TE-103-AP-46 & $5 / 24 / 93$ & 60 & SACS & $T$ & \\
\hline TE-103-AP-46 & $6 / 3 / 93$ & 58 & CASS & $\mathbf{T}$ & \\
\hline TE-103-AP-46 & $6 / 7 / 93$ & 59 & SACS & $\mathbf{T}$ & \\
\hline TE-103-AP-46 & 6/19/93 & 58 & CASS & $T$ & . \\
\hline TE-103-AP-46 & $6 / 24 / 93$ & 59 & CASS & $T$ & \\
\hline TE-103-AP-46 & $6 / 30 / 93$ & 81 & CASS & $\mathbf{s}$ & Data point did not agree with local trend \\
\hline TE-103-AP-46 & $\therefore 7 / 1 / 93$ & 58 & CASS & $\bar{T}$ & \\
\hline TE-103-AP-46 & $7 / 6 / 93$ & 59 & CASS & $T$ & \\
\hline TE-103-AP-46 & $7 / 16 / 93$ & 58 & CASS & $\mathbf{T}$ & \\
\hline TE.103-AP.46 & $7 / 21 / 93$ & 59 & CASS & $T$ & \\
\hline TE-103-AP-46 & $7 / 31 / 93$ & 58 & CASS & $T$ & \\
\hline TE-103-AP-A6 & $8 / 7 / 93$ & 60 & CASS & $\mathbf{T}$ & \\
\hline TE-103-AP-46 & $8 / 14 / 93$ & 59 & CASS & $T$ & \\
\hline TE-103-AP-46 & 8/18/93 & 61 & CASS & $T$ & \\
\hline TE-103-AP-46 & $8 / 25 / 93$ & 62 & CASS & $T$ & \\
\hline TE-108-AP-46 & 8/29/93 & 61 & CASS & $T$ & \\
\hline TE-103-AP-46 & $9 / 9 / 93$ & 62 & CASS & $T$ & \\
\hline TE-103-AP46 & 9/13/93 & 61 & CASS & $T$ & \\
\hline$T E-103-A P-46$ & $9 / 23 / 93$ & 61 & CASS & $T$ & \\
\hline TE-103-AP-46 & $10 / 1 / 93$ & 61 & CASS & $\mathbf{T}$ & \\
\hline TE-103-AP-46 & $10 / 8 / 93$ & 63 & CASS & $\bar{T}$ & \\
\hline TE-103-AP-46 & $10 / 1 / / 93$ & 62 & CASS & $T$ & \\
\hline TE-103-AP-46 & $10 / 18 / 93$ & 62 & CASS & $\bar{T}$ & \\
\hline TE-103-AP.46 & $10 / 29 / 93$ & 62 & CASS & $T$ & \\
\hline TE-103-AP-46 & $11 / 6 / 93$ & 61 & CASS & $T$ & \\
\hline TE-103-AP-46 & 11/8/93 & 63 & CASS & $T$ & \\
\hline TE-103-AP-46 & $11 / 18 / 93$ & 62 & CASS & $T$ & \\
\hline$T E-103-A P-46$ & $11 / 27 / 83$ & 60 & CASS & $T$ & \\
\hline TE-103-AP-46 & $12 / 1 / 93$ & 61 & CASS & $T$ & \\
\hline TE-103-AP-46 & $12 / 10 / 93$ & 62 & SACS & $\mathbf{T}$ & \\
\hline TE-103-AP-46 & $12 / 14 / 93$ & 61 & SACS & $T$ & \\
\hline TE-103-AP-46 & $12 / 20 / 83$ & 60 & SACS & $T$ & \\
\hline TE-103-AP-46 & $12 / 27 / 93$ & 60 & SACS & $T$ & \\
\hline TE-103-AP-46 & $1 / 3 / 94$ & 60 & SACS & $T$ & \\
\hline TE-103-AP-46 & $1 / 11 / 94$ & 64 & SACS & $\mathbf{T}$ & \\
\hline$T E-103-A P-46$ & $1 / 26 / 94$ & 56 & SACS & $T$ & \\
\hline TE-103-AP-46 & $2 / 9 / 94$ & 59 & SACS & $T$ & \\
\hline TE-103-AP.46 & $2 / 14 / 84$ & 58 & SACS & $\mathbf{T}$ & \\
\hline TE-103-AP+46 & $2 / 22 / 94$ & 59 & SACS & $T$ & \\
\hline$T E-103-A P-46$ & $3 / 1 / 94$ & 59 & SACS & $T$ & \\
\hline$T E-103-A P-46$ & $4 / 27 / 94$ & 57 & SACS & $\mathbf{G}$ & \\
\hline TE-103-AP-46 & $5 / 6 / 94$ & 55 & SACS & $\mathbf{G}$ & \\
\hline TE-103-AP-46 & $5 / 12 / 94$ & 56 & CASS & $\mathbf{T}$ & \\
\hline TE-103-AP-46 & $5 / 17 / 94$ & 58 & CASS & $\mathbf{T}$ & \\
\hline TE-103-AP-46 & $5 / 26 / 94$ & 59 & CASS & $T$ & \\
\hline TE-103-AP-46 & $6 / 1 / 94$ & 60 & CASS & $T$ & \\
\hline TE-103-AP-46 & $6 / 11 / 94$ & 60 & CASS & $T$ & \\
\hline TE-103-AP-46 & $6 / 12194$ & 61 & CASS & $T$ & \\
\hline$T E-103-A P=46$ & $6 / 24 / 94$ & 62 & CASS & $\bar{T}$ & \\
\hline TE-103-AP-46 & $7 / 1 / 94$ & 63 & CASS & $\mathbf{T}$ & . \\
\hline TE-103-AP-46 & $7 / 9 / 94$ & 63 & CASS & $T$ & \\
\hline TE-103-AP-46 & $7 / 16 / 94$ & 64 & CASS & $T$ & \\
\hline TE-103-AP-46 & $7 / 22 / 94$ & 66 & CASS & $T$ & \\
\hline TE-103-AP-46 & $7 / 28 / 94$ & 66 & CASS & $T$ & \\
\hline TE-103-AP-46 & $8 / 5 / 94$ & 66 & CASS & $T$ & \\
\hline TE-103-AP-46 & $8 / 13 / 94$ & 69 & CASS & $T$ & \\
\hline TE-103-AP-46 & $8 / 15 / 94$ & 68 & SACS & G & \\
\hline TE-103-AP-46 & $8 / 22 / 94$ & 69 & CASS & $\bar{T}$ & \\
\hline TE-103-AP-46 & $9 / 2 / 94$ & 68 & CASS & $T$ & \\
\hline TE-103-AP-46 & $9 / 10 / 94$ & 68 & CASS & $T$ & \\
\hline TE-103-AP-46 & $9 / 11 / 94$ & 69 & CASS & $T$ & \\
\hline TE-103-AP-46 & $9 / 21 / 94$ & 67 & CASS & $T$ & \\
\hline TE-103-AP-46 & $9 / 27 / 94$ & 69 & CASS & $T$ & \\
\hline TE-103-AP-46 & $10 / 2 / 94$ & 68 & CASS & $T$ & \\
\hline
\end{tabular}




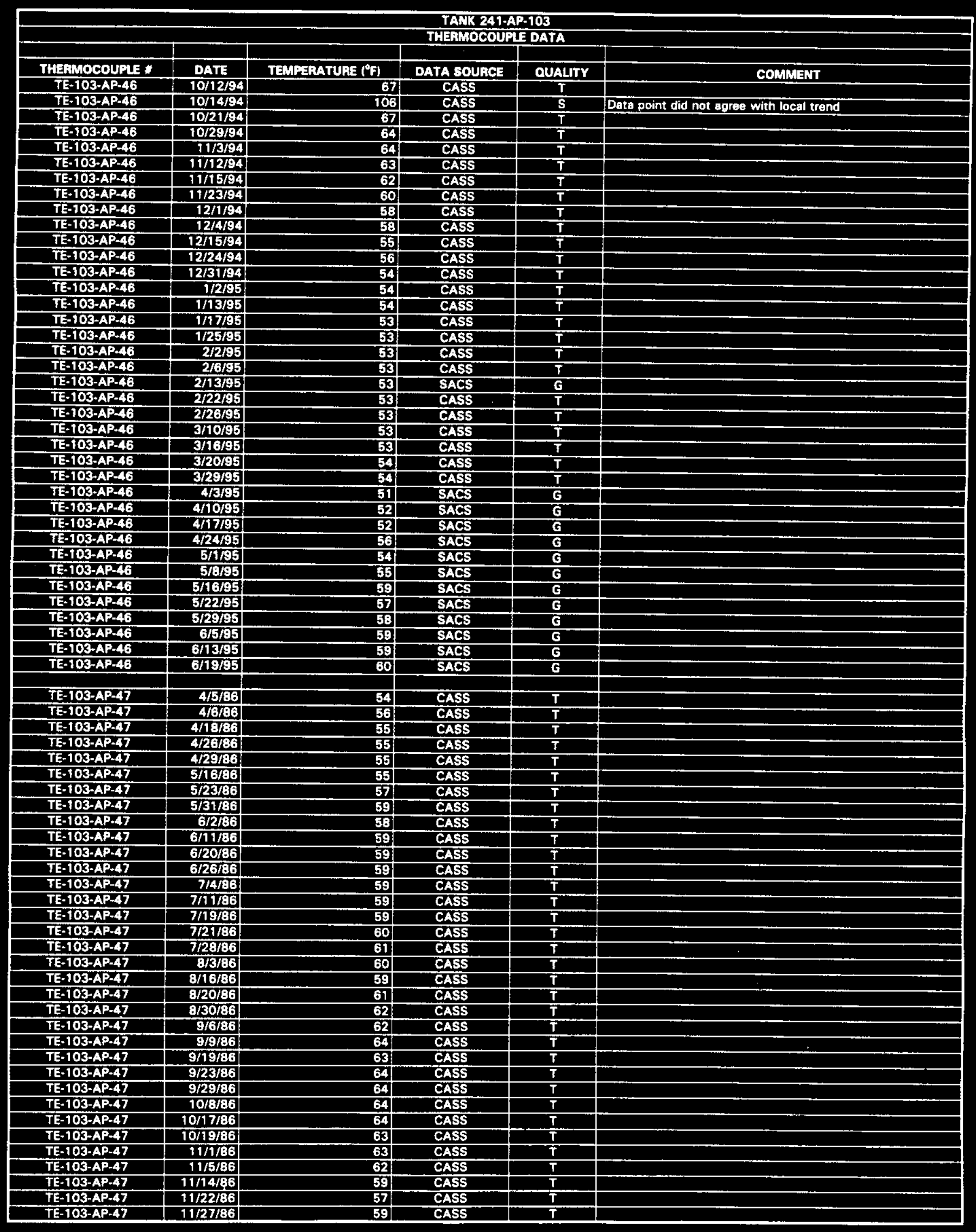




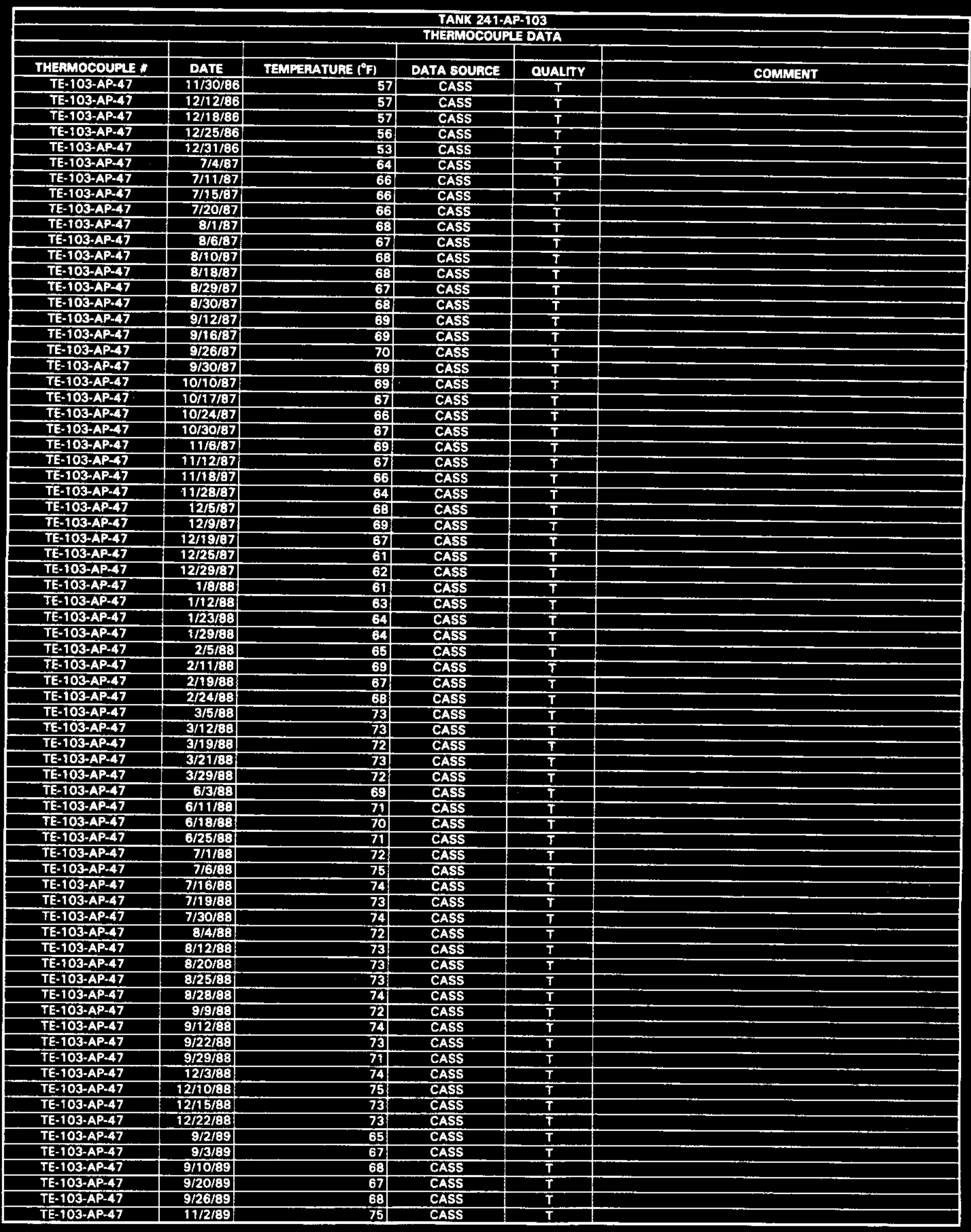




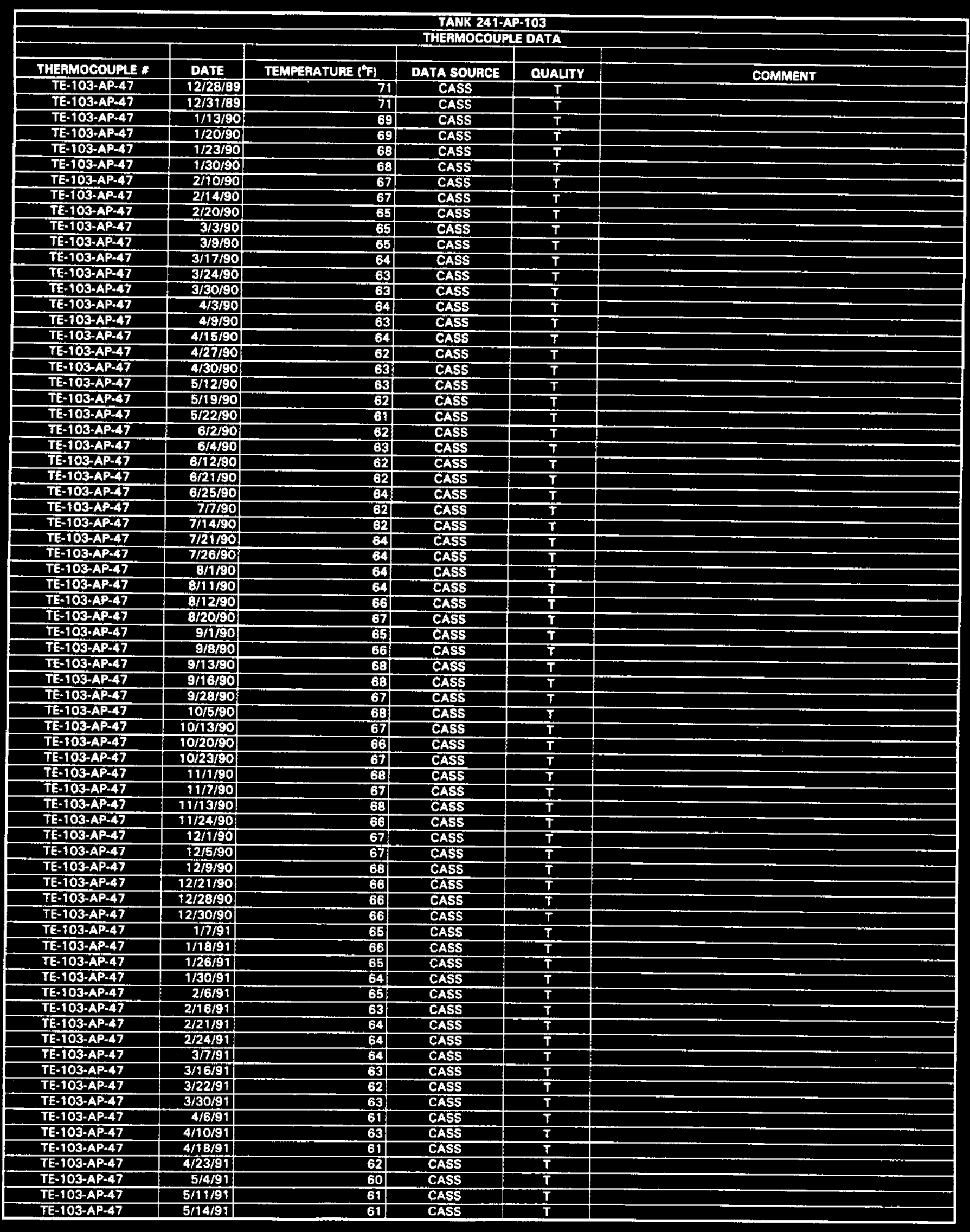

Data obtained from Computer Automated Surveillance Syatem (CASS), April 1995; and Surveillance Analysis Computer Syatem (SACS), June 1995. 


\begin{tabular}{|c|c|c|c|c|c|}
\hline \multicolumn{6}{|c|}{$\begin{array}{l}\text { TANK 241-AP.103 } \\
\text { THERMOCOUPLE DATA }\end{array}$} \\
\hline & DATE & & & & \\
\hline$\frac{\text { THEFMOCOUPE : }}{\text { TE-103-AP-47 }}$ & $\frac{\text { DAIE }}{5 / 25 / 91}$ & $\frac{\text { TEMPERATURE }\left({ }^{\circ} \mathrm{F}\right)}{62}$ & DATA SOUACE & QUALITY & COMMENT \\
\hline TE-103-AP-47 & $5 / 29 / 91$ & \begin{tabular}{r|}
62 \\
62
\end{tabular} & $\frac{\text { CASS }}{\text { CASS }}$ & $\mathrm{T}$ & \\
\hline TE-103-AP-47 & $6 / 5 / 91$ & 81 & $\begin{array}{l}\text { CASS } \\
\text { CASS }\end{array}$ & $\frac{T}{T}$ & \\
\hline TE-103-AP-47 & $6 / 15 / 91$ & 60 & CASSS & $\frac{T}{T}$ & \\
\hline TE-103-AP-47 & $6 / 20 / 91$ & 61 & CASS & $T$ & \\
\hline TE-103-AP-47 & $6 / 25 / 91$ & 60 & CASS & $T$ & \\
\hline TE-103-AP-47 & 7/3/9i & 59 & CASS & $T$ & \\
\hline TE-103-AP-47 & $7 / 11 / 91$ & 61 & CASS & $T$ & \\
\hline TE-103-AP-47 & $7 / 74 / 91$ & 60 & CASS & $T$ & \\
\hline TE-103-AP-47 & $7 / 24 / 91$ & 60 & CASS & $T$ & \\
\hline TE-103-AP-47 & $8 / 3 / 91$ & 61 & CASS & $T$ & \\
\hline$T E-103-A P-47$ & $8 / 5 / 91$ & 62 & CASS & $\frac{T}{T}$ & \\
\hline TE-103-AP-47 & $8 / 15 / 91$ & 62 & CASS & $T$ & \\
\hline TE $=103-A P-47$ & $8 / 23 / 91$ & 63 & CASS & $T$ & \\
\hline TE-103-AP-47 & $8 / 27 / 91$ & 63 & CASS & $T$ & \\
\hline TE-103-AP-47 & $9 / 2 / 91$ & 65 & CASS & $T$ & \\
\hline TE-103-AP-47 & $7 / 31 / 92$ & 61 & CASS & $T$ & \\
\hline TE-103-AP-47 & $8 / 8 / 92$ & 61 & CASS & $T$ & \\
\hline$T E-103-A P-47$ & $8 / 73 / 92$ & 63 & CASS & $T$ & \\
\hline TE-103-AP-47 & $8 / 22 / 92$ & 61 & CASS & $T$ & \\
\hline TE-108-AP-47 & $8 / 29 / 92$ & 63 & CASS & $\bar{T}$ & \\
\hline TE-103-AP-47 & 9/5/92 & 63 & CASS & $T$ & \\
\hline TE-103-AP-47 & 9/11/A2 & 62 & CASS & $T$ & \\
\hline TE-103-AP-47 & 9/13/92 & 63 & CASS & $T$ & \\
\hline TE-103.AP-47 & $10 / 3 / 92$ & 62 & CASS & $T$ & \\
\hline TE-103-AP-47 & $10 / 10 / 92$ & 62 & CASS & $T$ & \\
\hline TE-103-AP-47 & $10 / 17 / 92$ & 62 & CASS & $T$ & \\
\hline TE-103-AP-47 & $10 / 22 / 92$ & 63 & CASS & $\bar{T}$ & \\
\hline TE-103-AP-47 & $10 / 29 / 92$ & 63 & CASS & $T$ & \\
\hline TE-103-AP-47 & $11 / 4 / 92$ & 63 & CASS & $T$ & \\
\hline TE-103-AP-47 & $11 / 13 / 92$ & 62 & CASS & $T$ & \\
\hline TE-103-AP-47 & $11 / 17 / 92$ & 64 & CASS & $T$ & \\
\hline TE-103-AP=47 & $11 / 24 / 92$ & 63 & CASS & $T$ & \\
\hline TE-103-AP-47 & $11 / 29 / 92$ & 74 & CASS & $\mathrm{s}$ & Data point did not agree with local trend \\
\hline TE-103-AP-47 & $12 / 2 / 92$ & 62 & CASS & $T$ & \\
\hline TE-103-AP-47 & $12 / 12 / 92$ & 63 & CASS & $T$ & \\
\hline TE-103-AP-47 & $12 / 18 / 92$ & 62 & CASS & $T$ & \\
\hline TE-103-AP.47 & $12 / 26 / 92$ & 63| & CASS & $T$ & \\
\hline TE-103-AP-47 & $12 / 30 / 92$ & 62 & CASS & $T$ & \\
\hline TE-103-AP-47 & $1 / 9 / 93$ & 6i & CASS & $T$ & \\
\hline$T E-103-A P-47$ & 1/14/93 & 61 & CASS & $T$ & \\
\hline TE-103-AP-47 & $1 / 23 / 93$ & 60) & CASS & $\bar{T}$ & \\
\hline TE-103-AP-47 & $1 / 30 / 93$ & 62 & CASS & $T$ & \\
\hline TE-103-AP-47 & $2 / 6 / 93$ & 61 & CASS & $T$ & \\
\hline TE-103-AP-47 & $2 / 9 / 93$ & 61 & CASS & $T$ & \\
\hline TE-103-AP-47 & $2 \sqrt{20 / 93}$ & 60 & CASS & $T$ & \\
\hline TE-103-AP-47 & $2 / 26 / 93$ & 61 & CASS & $\bar{T}$ & \\
\hline TE-103-AP-47 & $3 / 5 / 93$ & 59 & CASS & $T$ & \\
\hline TE-103-AP-47 & $3 / 10 / 93$ & 60 & CASS & $T$ & \\
\hline TE-103-AP-47 & $3 / 19 / 93$ & 59 & CASS & $T$ & \\
\hline TE-108-AP-47 & $3 / 27 / 93$ & 59 & CASS & $T$ & \\
\hline TE-103-AP-47 & $3 / 28 / 93$ & 60 & CASS & $T$ & \\
\hline TE-103-AP-47 & $4 / 6 / 93$ & 60 & CASS & $T$ & \\
\hline TE-103-AP-47 & 4/17/93 & 59 & CASS & $T$ & \\
\hline TE-103-AP-47 & $4 / 24 / 93$ & 60 & CASS & $T$ & \\
\hline TE-103-AP-47 & $4 / 29 / 93$ & 59 & CASS & $\bar{T}$ & \\
\hline TE-103-AP-47 & $5 / 8 / 93$ & 59 & CASS & $T$ & \\
\hline TE-103-AP-47 & $5 / 11 / 93$ & 59 & CASS & $T$ & \\
\hline TE-103-AP-47 & $5 / 22 / 93$ & 58 & CASS & $T$ & \\
\hline TE-103-AP-47 & $5 / 27 / 93$ & 59 & CASS & $T$ & \\
\hline TE-103-AP-47 & $6 / 2 / 93$ & 112 & CASS & $\mathbf{s}$ & Data point did not agres with local trend \\
\hline TE-103-AP-47 & $6 / 5 / 93$ & 57 & CASS & $\bar{T}$ & \\
\hline TE-108-AP-47 & $6 / 12 / 93$ & 58 & CASS & $\bar{T}$ & \\
\hline TE-103-AP-47 & 6/17/93 & 58) & CASS & $T$ & \\
\hline TE-103-AP-47 & $6 / 24 / 93$ & 59 & CASS & $T$ & \\
\hline TE-103-AP-47 & $6 / 30 / 93$ & 81 & CASS & $\mathbf{s}$ & Data point did not agres with local trend \\
\hline TE-103-AP-47 & $7 / 3 / 93$ & 58 & CASS & $T$ & \\
\hline TE-103-AP-47 & $7 / 5 / 93$ & 60 & CASS & $T$ & \\
\hline TE-103-AP-47 & $7 / 17 / 93$ & 58 & CASS & $T$ & \\
\hline TE-108-AP-47 & $7 / 21 / 93$ & 60 & CASS & $T$ & \\
\hline TE-103-AP-47 & $7 / 31 / 93$ & 59 & CASS & $T$ & \\
\hline TE-103-AP-47 & $8 / 7 / 93$ & 60 & CASS & $T$ & \\
\hline$T E-103-A P-47$ & $8 / 14 / 93$ & 59 & CASS & $T$ & \\
\hline
\end{tabular}




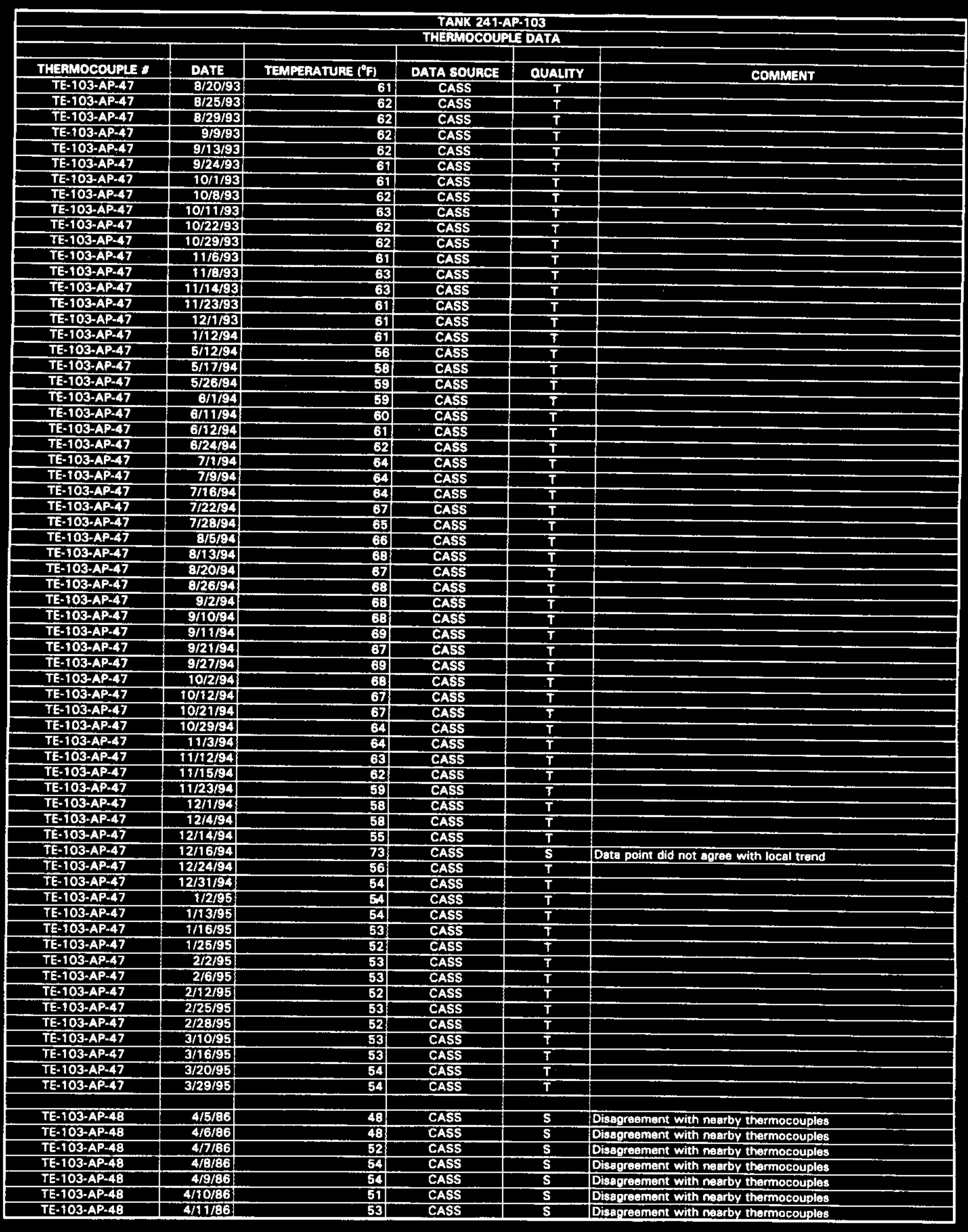

Data obtainad from Computer Automated Surveillance System (CASS), Aprił 1995; and Surveillance Anslyais Computer Syatem (SACS), June 1995. 


\begin{tabular}{|c|c|c|c|c|c|}
\hline \multicolumn{6}{|c|}{$\begin{array}{l}\text { TANK 241-AP-103 } \\
\text { THEFOMOCOUPLE DATA }\end{array}$} \\
\hline & & & & & \\
\hline THERMOCOUPLE * & DATE & TEMPERATURE $\left({ }^{\circ} \mathrm{F}\right)$ & DATA SOURCE & QUALTY & COMMENT \\
\hline TE-103-AP-48 & $4 / 12 / 86$ & 53 & CASS & $\mathbf{s}$ & Disagreement with nearby thermocouplas \\
\hline TE-103-AP.48 & $4 / 13 / 86$ & 52 & CASS & $\mathbf{S}$ & Disegreement with nearby thermocouples \\
\hline TE-103-AP-48 & $4 / 14 / 86$ & 53 & CASS & $\mathbf{s}$ & Disegreement with nearby thermocouples \\
\hline TE-103-AP-48 & $4 / 15 / 86$ & 57 & CASS & $\mathbf{s}$ & Disagreement with nearby thermocouples \\
\hline TE-103-AP-48 & 4/16/86 & 53 & CASS & $\mathbf{s}$ & Disagreement with nearby thermocouples \\
\hline$T E-103-A P-48$ & $4 / 17 / 86$ & 53: & CASS & $\mathbf{S}$ & Disagreament with nearby thermocouples \\
\hline TE-103-AP-48 & 4/18/86 & 52 & CASS & $\mathbf{S}$ & Disdgreement with nearby thermocouples \\
\hline TE-103-AP-48 & 4/19/86 & 55 & CASS & $\overline{\mathbf{S}}$ & Disagreament with neaby thermocouples \\
\hline TE-103-AP-AB & $4 / 20 / 86$ & 57! & CASS & $\mathbf{s}$ & Disagreement with nearby thormocouples \\
\hline TE-103-AP-48 & $4 / 21 / 36$ & 59 & CASS & $\mathbf{S}$ & Digacreement with nearby thermocouples \\
\hline TE-103-AP-4B & 4/22/86 & 61 & CASS & $\mathbf{s}$ & Disagreement with nearby thermocouples \\
\hline TE-103-AP-48 & 4/24/86 & 52 & CASS & $\mathbf{S}$ & Disagreement with nearby thermocouplas \\
\hline TE-103-AP-48 & $4 / 25 / 66$ & 54! & CASS & $\mathbf{S}$ & Disogreement with nearby thermocouplas \\
\hline TE-103-AP-48 & 4/26/36 & 53 & CASS & $\mathbf{S}$ & Disegreement with nearby thermocouples \\
\hline TE-103-AP-4B & $4 / 27 / 86$ & 53 & CASS & $\mathbf{s}$ & Disagresment with nearby them mocouples \\
\hline$T E-103-A P-48$ & $4 / 28 / 86$ & 54 & CASS & $\mathbf{S}$ & Disegreement with nearby thermocouples \\
\hline TE-103-AP-48 & $4 / 29 / 86$ & 54 & CÁSS & $\mathbf{5}$ & Oisagreement with nearby thermocouples \\
\hline TE-103-AP-48 & $5 / 15 / 86$ & 57 & CASS & $\mathbf{s}$ & Disaproement with nearby thermocouples \\
\hline$T E-103 \cdot A P-48$ & $5 / 16 / 96$ & 59 & CASS & S & Disapreement with noarby thermocouples \\
\hline TE-103-AP-48 & 5/17/86 & 62 & CASS & $\mathbf{5}$ & Disagreament with nearby thermocouplos \\
\hline TE-103-AP-48 & 5/18/86 & 68: & CASS & $\mathbf{S}$ & Disegreament with nearby thermocouples \\
\hline TE-103-AP-48 & $5 / 20 / 86$ & 66 & CASS & $\mathbf{S}$ & Disagreement with nearby thermocouples \\
\hline TE-103.AP-4B & $5 / 21 / 86$ & 63 & CASS & $\mathbf{s}$ & Disagreement with nearby thermocouplas \\
\hline TE-TO3-AP-4B & $5 / 22 / 36$ & 58 & CASS & $\mathbf{S}$ & Disagreament with naarby thermocouples \\
\hline$T E-103-A P-48$ & $5 / 23 / 86$ & 60 & CASS & $\mathbf{S}$ & Disagreement with noarty thermocouples \\
\hline TE-103-AP-48 & $5 / 24 / 86$ & 63 & CASS & $\mathbf{s}$ & Disegreement with nearby thermocouples \\
\hline TE-103-AP-48 & $5 / 25 / 86$ & 68 & CASS & $\mathbf{s}$ & Diggaresment with neachy thermocouples \\
\hline TE-103-AP-48 & $5 / 26 / 86$ & 72 & CASS & $\mathbf{S}$ & Disaqreament with nearby thermocouples \\
\hline$T E-103-A P-4 B$ & $5 / 28 / 86$ & 58 & CASS & $\mathbf{S}$ & Diengreement with nearby thermocouplas \\
\hline TE-103-AP-48 & $5 / 29 / 86$ & 59 & CASS & $\mathbf{s}$ & Disagresment with nearby thermocouples \\
\hline TE-103-AP-48 & $5 / 30 / 86$ & 59 & CASS & $\mathbf{S}$ & Disusreement with nearby thermocouples \\
\hline TE-103-AP-48 & $5 / 31 /: 6$ & 59 & CASS & $\mathbf{s}$ & Diseareament with noarby thermocouples \\
\hline TE-103-AP-48 & $6 / 1 / 86$ & 58 & CASS & $\mathbf{s}$ & Disagreament with noarby thermocouples \\
\hline TE-103-AP-4B & 6/2/86 & 58 & CASS & $\mathbf{S}$ & Diasdreament with noarby thempocouples \\
\hline TE-103-AP-48 & $6 / 3 / 86$ & 58) & CASS & $\mathbf{5}$ & Disegreement with nearby thaimocouples \\
\hline TE-103-AP-48 & $6 / 4 / 86$ & 57 & CASS & $\mathbf{S}$ & Ditsagreament with nearby thermocouplos \\
\hline TE-103-AP-48 & $6 / 5 / 36$ & 57 & CASS & $\mathbf{S}$ & Diagreement with noarby thermocouplas \\
\hline TE-103-AP-48 & 6/3/86 & 57 & CASS & $\mathbf{s}$ & Diagareement with neaby thermocouples \\
\hline TE-103.AP-48 & $6 / 7 / 86$ & 57 & CASS & $\mathbf{S}$ & Disugreament with nearby thermocouples \\
\hline TE-103-AP-4B & 6/8/86 & 57) & CASS & $\mathbf{S}$ & Diagreament with nearby thermocouplos \\
\hline TE-103.AP-48 & 6/9/86 & 58 & CASS & $\mathbf{s}$ & Disagreement with nearby thermocouplas \\
\hline TE-108-AP-48 & 6/10/B6 & 59 & CASS & $\mathbf{s}$ & Disedreament with nearby theinocouplas \\
\hline TE-103-AP-48 & $6 / 11 / 80$ & 59 & CASS & $\mathbf{S}$ & Diecoreement with nearby thormocouples \\
\hline TE-103-AP-48 & 6/12/86 & 57. & CASS & 5 & Disegreemant with neasby thermocouples \\
\hline TE-103-AP-48 & 6/13/36 & 57 & CASS & S & Disagreement with nearby tharmocouples \\
\hline TE-T03-AP-48 & $6 / 14 / 86$ & 57 & CASS & $\mathbf{s}$ & Diagoreament with nearby thermocouplos \\
\hline TE-103-AP-48 & $6 / 15 / 86$ & 57 & CASS & $\mathbf{S}$ & Disagreement with noarby thermocouples \\
\hline TE-103-AP-48 & $6 / 16 / 86$ & 57 & CASS & $\mathbf{s}$ & Disagreernent with nearby thermocouplas \\
\hline TE-103-AP-48 & $6 / 17 / 86$ & 57 & CASS & $\mathbf{s}$ & Oisagreement with nearby tharmocouples \\
\hline TE-103-AP-48 & $6 / 18 / 86$ & 57! & CASS & $\mathbf{s}$ & Disagreament with nearby thermocouples \\
\hline TE-103-AP-48 & $6 / 20 / 86$ & 59 & CASS & S & Disagreement with nearby thermocouples \\
\hline TE-103-AP-48 & $6 / 22 / 36$ & $5 B$ & CASS & $\mathbf{s}$ & Disagreemant with nearby thermocouplas \\
\hline TE-103-AP-48 & $6 / 23 / 96$ & 57 & CASS & $\mathbf{s}$ & Disagreement with nearby thermocouples \\
\hline TE-103-AP-48 & $6 / 24 / 86$ & 57 & CASS & S & Disagreement with nearby thermocouplas \\
\hline TE-103-AP-48 & $6 / 25 / 86$ & 57 & CASS & $\overline{\mathbf{s}}$ & Disagreement with nearby thermocouples \\
\hline TE-103-AP-48 & $6 / 26 / 86$ & 59 & CASS & $\mathbf{S}$ & Disagresment with nearby thermocouplos \\
\hline TE-103-AP-4B & $7 / 2 / 96$ & 59 & CASS & S & Oisagresment with nearby thermocouples \\
\hline TE-103-AP-48 & $7 / 3 / 86$ & 59 & CASS & $\mathbf{S}$ & Disagreement with nearby thermocouples \\
\hline TE-103-AP-48 & $7 / 4 / 86$ & 63 & CASS & $\mathbf{s}$ & Disagreement with nearby tharmocouples \\
\hline TE-103-AP-48 & $\begin{array}{l}7 / 6 / 86 \\
\end{array}$ & 67 & CASS & $\mathbf{S}$ & Disagreement with nearby thermocouples \\
\hline TE-103-AP-48 & 7/7/86 & 69 & CASS & $\mathbf{s}$ & Disagreament with nearby thermocouples \\
\hline TE-103-AP-48 & $7 / 11 / 86$ & 61 & CASS & $\mathbf{S}$ & Disegreement with nearby thermocouples \\
\hline TE-103-AP-48 & $7 / 12 / 86$ & 60 & CASS & 5 & Disagreement with nearby tharmocouples \\
\hline TE-103-AP-48 & $7 / 13 / 86$ & 59 & CASS & $\mathbf{s}$ & Dissgreament with nearby tharmocouples \\
\hline TE-103-AP-48 & $7 / 14 / 86$ & 59 & CASS & $\mathbf{S}$ & Disagreement with nearby tharmocouples \\
\hline TE-103-AP-48 & $7 / 16 / 36$ & 59 & CASS & $\mathbf{s}$ & Diseqreememt with nearby thermocouples \\
\hline TE-103-AP-48 & 717166 & 59 & CASS & $\mathbf{S}$ & Disagreament with nearby thermocouples \\
\hline TE-103-AP-48 & $7 / 18 / 86$ & 58 & CASS & $\mathbf{s}$ & Disagraement with nearby thermocouples \\
\hline TE-103-AP-48 & 7/19/86 & 59 & CASS & $\mathbf{s}$ & Disugreement with nearby thermocouples \\
\hline TE-103-AP-48 & $7120 / 86$ & 60 & CASS & $\mathbf{S}$ & Disegreement with nearby thermocouplas \\
\hline TE-103-AP-48 & $7 / 21 / 86$ & 60 & CASS & $\mathbf{S}$ & Disagreenent with nearby tharmocouples \\
\hline TE-103-AP-4B & $7 / 22 / 86$ & 58 & CASS & $\mathbf{s}$ & Disegreement with nearby thermocouples \\
\hline TE-103-AP-4B & $7123 / 86$ & 59 & CASS & $\mathbf{S}$ & Dissoreement with nearby thermocouplas \\
\hline
\end{tabular}




\begin{tabular}{|c|c|c|c|c|c|}
\hline \multicolumn{6}{|c|}{$\begin{array}{l}\text { TANK 241-AP-103 } \\
\text { THERMOCOUFLE DATA }\end{array}$} \\
\hline & & TEMPERATURE ( $(\mathrm{F})$ & DATA SOUACE & & \\
\hline$\frac{\text { THEAMOCOUPLE: }}{\text { TE-103-APAB }}$ & $\begin{array}{l}\text { DATE } \\
7 / 24 / 86\end{array}$ & 59 & $\frac{\text { DATA SOUACE }}{\text { CASS }}$ & QUALTY & COMMENT \\
\hline TE-103-AP-48 & $7 / 25 / 86$ & $\mathbf{5 8}$ & $\begin{array}{l}\text { CASS } \\
\text { CASS }\end{array}$ & $\mathbf{s}$ & Disagreement with nearby tharmocouples \\
\hline TE-103-AP-48 & $7 / 26 / 86$ & 58 & $\frac{\text { CASS }}{\text { CASS }}$ & $\mathbf{S}$ & Disagreament with nearby thermocouples \\
\hline TE-103-AP-48 & $7 / 27 / 86$ & 59. & CASS & S & Disagreement with nearby thermocouplas \\
\hline TE-103-AP-48 & $7 / 28 / 86$ & 61 & CASS & $\frac{\mathbf{s}}{\mathbf{S}}$ & $\begin{array}{l}\text { Oisagreement with nearby thermocouples } \\
\text { Disarrement with negry there }\end{array}$ \\
\hline TE-103-AP-48 & 7/29/86 & 59: & CASS & $\mathbf{S}$ & $\begin{array}{l}\text { Disagreement with neaby thermocouples } \\
\text { Disagreement with nearby thermocouples }\end{array}$ \\
\hline TE-103-AP-48 & $7 / 30 / 86$ & 59 & CASS & $\mathbf{s}$ & Disagreoment with nearby thermocouples \\
\hline TE-103-AP-48 & $7731 / 86$ & 59 & CASS & $\mathbf{S}$ & Disagreament whth nearby thermocouplos \\
\hline TE-103-AP-48 & $8 / 1 / 86$ & 59 & CASS & $\mathbf{s}$ & Disagraement whe nearby thermocouples \\
\hline TE-103-AP-48 & $8 / 2 / 86$ & 59 & CASS & $\mathbf{s}$ & Disapreament with nearby thermocouples \\
\hline TE-103-AP-48 & $8 / 3 / 86$ & 60 & CASS & $\mathbf{s}$ & Disagreement with nearby thermocouples \\
\hline TE-103-AP-48 & $8 / 4 / 86$ & 58 & CASS & $\mathbf{5}$ & Disagreement with nearby thermocouples \\
\hline TE-103-AP-48 & $8 / 5 / 36$ & 59 & CASS & $\mathbf{s}$ & Disagreament with nearby tharmocouples \\
\hline TE-103-AP-48 & $8 / 6 / 86$ & 59 & CASS & $\mathbf{S}$ & Disagreement with nearby thermocouples \\
\hline TE-103-AP-48 & $8 / 7 / 86$ & 59! & CASS & $\mathbf{S}$ & Disagreement with noarby thermocouples \\
\hline TE-103-AP-48 & $8 / 8 / 36$ & 59 & CASS & $\mathbf{s}$ & Disagreement with nearby thermocouples \\
\hline TE-103-AP-48 & $8 / 9 / 86$ & 59 & CASS & $\mathbf{S}$ & Disagresment with noarby thermocouples \\
\hline TE-108-AP-48 & $8 / 10 / 86$ & 59 & CASS & $\mathbf{s}$ & Disagreement with nearby thermocouples \\
\hline TE-103-AP-48 & $8 / 11 / 86$ & 59 & CASS & $\mathbf{S}$ & Disegreement with nearby thermocouples \\
\hline TE-108-AP-48 & 8/12/86 & 59. & CASS & $\mathbf{S}$ & Disapreament with nearby thermocouples \\
\hline TE-103.AP-48 & $8 / 13 / 36$ & 69t & CASS & $\mathbf{5}$ & Diagrearnent with nearby thermocouples \\
\hline TE-108-AP-48 & $8 / 14 / 86$ & 59 & CASS & $\mathbf{S}$ & Disagroentent with notby thermocouples \\
\hline TE-108-AP- 48 & $8 / 15 / 86$ & 59 & CASS & $\mathbf{s}$ & Disagreement with nearby tharmocouples \\
\hline TE-103-AP-4B & 8/16/86 & 59 & CASS & $\mathbf{S}$ & Disspresment with nearby tharmocouples \\
\hline TE-103.AP-48 & $8 / 1786$ & 59 & CASS & $\mathbf{S}$ & Disagreament with nearby thermocouples \\
\hline TE-103-AP-48 & $8 / 18 / 86$ & 61 & CASS & $\mathbf{S}$ & Disapreament with nearby themocouples \\
\hline TE-103-AP-48 & $8 / 19 / 36$ & 61 & CASS & $\mathbf{S}$ & Disopreemem with nearby tharmocouples \\
\hline TE-103-AP-48 & $8 / 20 / 86$ & 62 & CASS & $\mathbf{S}$ & Disepreement with nearby thermocouples \\
\hline TE-103-AP-48 & $6 / 21 / 86$ & 60 & CASS & $\mathbf{S}$ & Disapreement with nearby thermocouplas \\
\hline TE-103-AP-48 & $8 / 22 / 86$ & 61 & CASS & $\mathbf{s}$ & Disagreement with nearby thermocouples \\
\hline TE-103-AP-48 & $8 / 23 / 86$ & 59 & CASS & $\mathbf{s}$ & Disegreement with nearby thermocouples \\
\hline TE-103-AP-48 & $8 / 24 / 86$ & 59 & CASS & $\mathbf{S}$ & Disegreement with nearby thermocouples \\
\hline TE-103-AP-48 & $8 / 25 / 86$ & 60: & CASS & $\mathbf{S}$ & Disergreement with nestby thermocouples \\
\hline$T E-103-A P-48$ & $8 / 27 / 86$ & 60 & CASS & $\mathbf{s}$ & Disegresment with nearby tharmocouples \\
\hline TE-103-AP-48 & 8/28/88 & 60 & CASS & $\mathbf{S}$ & Disegreament with nearby thermocouples \\
\hline TE-103-AP-46 & $8 / 29 / 86$ & 60 & CASS & $\mathbf{s}$ & Diadereament with nosiby thermocouples \\
\hline TE-103-AP-48 & $8 / 30 / 86$ & 73 & CASS & $\mathbf{s}$ & Disapreament with nearby thermocouples \\
\hline TE-103-AP-48 & $8 / 31 / 36$ & 62 & CASS & $\mathbf{s}$ & Disegroement with nesrby tharmocouples \\
\hline TE-103-AP-48 & $9 / 1 / 86$ & 61 & CASS & $\bar{s}$ & Diegoreement with neaby thermocouples \\
\hline TE-103-AP-48 & $9 / 2 / 86$ & 61! & CASS & $\mathbf{S}$ & Diserpresment with respy thermocouplas \\
\hline TE-103-AP-48 & $9 / 3 / 86$ & 61 & CASS & $\mathbf{s}$ & Disereament with nearby thermocouples \\
\hline$T E-103-A P-48$ & 9/4/86 & 61 & CASS & $\mathrm{s}$ & Disagrearnent with nearby thermocouples \\
\hline TE-103-AP-48 & $9 / 5 / 36$ & 61 & CASS & $\mathbf{S}$ & Disagreernent with nearby thermocouples \\
\hline TE-103-AP-48 & $9 / 6 / 66$ & 62 & CASS & $\mathbf{s}$ & Disagreement with nearby thermocouples \\
\hline TE-103-AP-48 & $9 / 7 / 86$ & 61 & CASS & $\mathbf{s}$ & Disepreement with nearby thermocouples \\
\hline TE-103-AP-48 & $9 / 6 / 36$ & 61 & CASS & $\mathrm{s}$ & Disspreement with nearby thermocouples \\
\hline TE-103-AP-48 & $9 / 9 / 86$ & 64 & CASS & $\mathbf{S}$ & Disagreament with nouby thermocouples \\
\hline TE-103-AP-48 & $9 / 10 / 86$ & 64 & CASS & $\mathbf{S}$ & Disegreement with neaby thermocouplas \\
\hline TE-103-AP-AB & $9 / 71 / 86$ & 64 & CASS & $\mathbf{s}$ & Discgreement with nearby thermocouples \\
\hline TE-103-AP-AB & $9 / 12 / 86$ & 66 & CASS & $\mathbf{S}$ & Disegreement with nearby tharmocouples \\
\hline TE-103-AP-48 & $9 / 13 / 86$ & 64 & CASS & $\mathbf{s}$ & Disagreament with nearby thermocouples \\
\hline TE-103-AP-48 & $9 / 14 / 86$ & 62 & CASS & $\mathbf{S}$ & Disagreement with nearby thermocouples \\
\hline TE-103-AP-48 & $9 / 15 / 86$ & 62 & CASS & $\mathbf{s}$ & Disagreement with nearby thermocouples \\
\hline TE-103-AP-48 & $9 / 16 / 86$ & 63 & CASS & $\mathbf{S}$ & Disagreement with nearby thermocouples \\
\hline TE-103-AP-48 & $8 / 17 / 86$ & 64 & CASS & $\mathbf{S}$ & Disagreement with nearby thermocouples \\
\hline TE-103-AP-48 & $9 / 18 / 86$ & 62 & CASS & $\mathbf{s}$ & Diasgreament with nearby tharmocouples \\
\hline TE-103-AP-4B & $9 / 19 / 86$ & 63 & CASS & $\mathbf{S}$ & Disagreement with nearby thormocouples \\
\hline TE-103-AP-48 & $9 / 21 / 86$ & 63 & CASS & $s$ & Disagreement with nearby thermocouples \\
\hline TE-103-AP-48 & $9 / 22 / 86$ & 62 & CASS & $\mathbf{s}$ & Disegraenent with nearby thermocouples \\
\hline TE-103-AP-48 & $9 / 23 / 86$ & 63 & CASS & $\mathbf{S}$ & Disagraement with nearby thermocouplas \\
\hline$T E-103-A P-48$ & $9 / 24 / 86$ & 59 & CASS & $\mathbf{s}$ & Diasgreement with nearby tharmocouples \\
\hline TE-103-AP-48 & 9/26/86 & 59: & CASS & $\mathbf{S}$ & Disegreement with nearby thermocouples \\
\hline TE-103-AP-48 & $9 / 27 / 86$ & 59 & CASS & $\mathbf{S}$ & Disagreement with noarby thermocouples \\
\hline$T E-103-A P-48$ & 9/29/86 & 63 & CASS & $\mathbf{s}$ & Disagreement with noarby thermocouples \\
\hline TE-103-AP-48 & $9 / 30 / 86$ & 59 & CASS & $\mathbf{S}$ & Disagreament with nearby thermocouples \\
\hline TE-103-AQ-48 & $10 / 1 / 86$ & 59 & CASS & $\mathbf{S}$ & Dissgreament with nearby thermocouples \\
\hline TE-103-AP-48 & $10 / 2 / 86$ & 59 & CASS & $\mathbf{S}$ & Dissgreement with nearby thermocouples \\
\hline TE-103-AP-4B & $10 / 3 / 86$ & 59 & CASS & $\mathbf{s}$ & Disagreement with nearby thermocouples \\
\hline TE-103-AP-4B & $10 / 4 / 96$ & 60 & CASS & $\mathbf{s}$ & Disagraement with nearby thermocouples \\
\hline TE-103-AP-48 & $10 / 5 / 86$ & 61 & CASS & $\mathbf{S}$ & Disagreament with nearby thermocouples \\
\hline TE-103-AP-48 & $10 / 6 / 86$ & 62 & CASS & $\mathbf{S}$ & Disegreement with nearby thermocouples \\
\hline TE-103-AP-48 & $10 / 7 / 86$ & 61 & CASS & $\mathbf{s}$ & Disagreement with narby thermocouples \\
\hline TE-103-AP-48 & $10 / 8 / 86$ & 64 & CASS & $\mathbf{s}$ & Disegreament with noarby thermocougles \\
\hline
\end{tabular}




\begin{tabular}{|c|c|c|c|c|c|}
\hline \multicolumn{6}{|c|}{$\begin{array}{l}\text { TAKK 241-AP\&103 } \\
\text { THEFWOCOUPLE DATA }\end{array}$} \\
\hline & & & & & \\
\hline THERMOCOUPLE & DATE & TEMPERATUAE $\left(^{\circ} \mathrm{k}\right)$ & DATA SOURCE & QUALITY & COMMENT \\
\hline TE-103-AP-48 & 10/9/86 & 62 & CASS & $\mathbf{s}$ & Disagreement with nearby thermocouples \\
\hline TE-103-AP-48 & $10 / 10 / 86$ & 63. & CASS & $\mathbf{s}$ & Disagreement with nearby thermocouples \\
\hline TE-103-AP-48 & $10 / 11 / 86$ & 59 & CASS & $\mathbf{S}$ & Disagreement with nearby tharmocouples \\
\hline TE-103-AP-48 & $10 / 12 / 86$ & 58 & CASS & $\mathbf{s}$ & Disagreament with nearby thermocouples \\
\hline TE-103-AP-48 & $10 / 13 / 86$ & 61 & CASS & $\mathbf{s}$ & Disegreement with nearby thermocouples \\
\hline TE-103-AP-48 & $10 / 14 / 86$ & 63 & CASS & $\mathbf{s}$ & Disegreement with nearby tharmocouples \\
\hline$T E-103-A P-48$ & $10 / 15 / 86$ & 61 & CASS & $\mathbf{S}$ & Disagreement with nearby thermocouplas \\
\hline TE-103-AP-4B & $10 / 16 / 86$ & 61 & CASS & $\mathbf{s}$ & Disegreement with nearby thermocouplas \\
\hline TE-103-AP-48 & $10 / 17 / 86$ & 62 & CASS & $\mathbf{S}$ & Disapreement with nadby thermocouples \\
\hline TE-103-AP-48 & $10 / 18 / 86$ & 61 & CASS & $\mathbf{S}$ & Disegreement with nearby thermocouples \\
\hline TE-103-AP-4B & $10 / 19 / 86$ & 61 & CASS & $\mathbf{s}$ & Diagereament with nearby thermocouples \\
\hline TE-103-AP-48 & $10 / 20 / 86$ & 61 & CASS & $\mathbf{S}$ & Disagrearnent with nearby thermocouples \\
\hline TE-108-AP-48 & $10 / 21 / 86$ & 61 & CASS & $\mathbf{S}$ & Disegreement with nearby thermocouples \\
\hline TE-103-AP-48 & $10 / 22 / 96$ & 61 & CASS & $\mathbf{S}$ & Disagreement with nearby thermocouples \\
\hline TE-108-AP.48 & $10 / 23 / 86$ & 61 & CASS & $\mathbf{S}$ & Dieagreement with naarby thermocouples \\
\hline TE-103-AP-48 & 10/24/86 & 61 & CASS & $\mathbf{S}$ & Diesegreament with nearby thermocouples \\
\hline TE-103-AP-48 & $10 / 25 / 86$ & 61 & CASS & $\mathbf{S}$ & Diagareament with nearby tharmocouples \\
\hline TE-103-AP-48 & 10/26/86 & 61 & CASS & $\mathbf{s}$ & Disapreoment with nasby thormocouplas \\
\hline TE-103-AP-48 & $10 / 27 / 86$ & 61 & CASS & $\mathbf{S}$ & Disegroement with nearby tharmocouplas \\
\hline TE-103-AP-48 & $10 / 28 / 86$ & 60 & CASS & $\mathbf{S}$ & Disagreement with nearby thornocouples \\
\hline TE-103-AP-48 & $10 / 29 / 86$ & 60 & CASS & $\mathbf{S}$ & Disagreement with nearby tharmocouples \\
\hline TE-103-AP-48 & $10 / 30 / 86$ & 60 & CASS & $\mathbf{S}$ & Disegroement with nestby tharmocoupies \\
\hline TE-103-AP-48 & $10 / 31 / 86$ & 60 & CASS & $\mathbf{S}$ & Disagreament with nearby thermocouples \\
\hline TE-103-AP-48 & $11 / 1 / 86$ & 61 & CASS & $\mathbf{S}$ & Diasgreement with nearby thermocouples \\
\hline TE-103-AP-48 & $91 / 2 / 86$ & 59 & CASS & $\mathbf{s}$ & Disagreement with nearby thermocouples \\
\hline TE-103-AP-48 & 11/3/86 & 59. & CASS & $\mathbf{S}$ & Disegreement with nearby thermocouplas \\
\hline TE-103-AP-48 & $11 / 4 / 86$ & 61 & CASS & $\mathbf{S}$ & Dissoreement with nearby thermocouples \\
\hline TE-103-AP-48 & $11 / 5 / 36$ & 61 & CASS & $\overline{\mathbf{S}}$ & Disegreament with nodrby thermocouples \\
\hline TE-103-AP.48 & $11 / 6 / 86$ & 59 & CASS & $\mathbf{S}$ & Disegreement with nearby thermocouples \\
\hline TE-103-AP-48 & $11 / 7 / 86$ & 58 & CASS & $\mathbf{S}$ & Oisegreement with nearby thermocouples \\
\hline TE-103-AP-48 & $11 / 8 / 86$ & 58: & CASS & $\mathbf{S}$ & Disepreament with nearby thermocouples \\
\hline TE-103-AP-48 & $11 / 9 / 86$ & 57 & CASS & $\mathbf{S}$ & Disagrearnent with nasitoy thermocouples \\
\hline TE-103-AP-49 & $11 / 10 / 86$ & 54 & CASS & $\mathbf{S}$ & Diacoreement wich nasiby thermocouples \\
\hline TE-103-AP-48 & $11 / 11 / 86$ & 56 & CASS & $\mathbf{S}$ & Disegreament with nearty thermosouples \\
\hline TE-103-AP-48 & $11 / 12 / 86$ & 55 & CASS & $\mathbf{S}$ & Disepreament with nearby themocouples \\
\hline TE-103-AP.48 & $11 / 13 / 86$ & 55 & CASS & $\mathbf{S}$ & Disapresment whth nestby thempcouplas \\
\hline TE-103-AP-48 & $11 / 14 / 86$ & 57 & CASS & $\mathbf{S}$ & Diagrreement with nearby thermocouples \\
\hline TE-103-AP-48 & $11 / 15 / 86$ & 55 & CASS & s & Disagreernent with nearby thermocouples \\
\hline TE-103-AP-4B & $11 / 16 / 68$ & S5 & CASS & $\mathbf{5}$ & Diagpreament with nearby themocouples \\
\hline TE-103.AP-48 & $11 / 17 / 86$ & S5! & CASS & $\mathbf{S}$ & Diagr reement with nearby tharmocouplas \\
\hline TE-108.AP-48 & $11 / 18 / 86$ & S5) & CASS & $\mathbf{s}$ & Disagreemant with neadby thermocouples \\
\hline TE-103-AP-48 & $11 / 19 / 86$ & 55 & CASS & $\mathbf{S}$ & Disepreement with neacby thermocouplos \\
\hline TE-103-AP-48 & $11 / 20 / 36$ & 55 & CASS & $\mathbf{S}$ & Disagreement with naarby tharmocouples \\
\hline TE-103-AP-48 & $11 / 22 / 86$ & 56 & CASS & $\mathbf{S}$ & Dideqreement with nearby thermocouples \\
\hline TE-T08-AP-48 & $11 / 23 / 86$ & 5 & CASS & $\mathbf{s}$ & Disagraement with nearby thermocouplas \\
\hline TE-103-AP-48 & $11 / 24 / 86$ & 56 & CASS & $\mathbf{s}$ & Disegresment with nsarby thermocouples \\
\hline TE-103-AP-4B & $11 / 25 / 86$ & 57 & CASS & $\mathbf{S}$ & Disapreement with nearby thermocouples \\
\hline TE-103-AP-A8 & $11 / 26 / 86$ & 55 & CASS & $\mathbf{s}$ & Disagreement with nearby thermocouplas \\
\hline TE-103-AP-48 & $11 / 27 / 86$ & 58 & CASS & $\mathbf{S}$ & Disagreernent with neartby thermocouplas \\
\hline TE-103-AP-48 & $11 / 2 \mathrm{~g} / 86$ & 55 & CASS & s & Disagreament with nearby thermocouples \\
\hline TE-103-AP-48 & $11 / 30 / 86$ & 55 & CASS & $\mathbf{S}$ & Disagroement with nearby thermocouplas \\
\hline TE-103-AP-48 & $12 / 1 / 86$ & 53 & CASS & $\mathbf{s}$ & Diaapreement with nearby tharmocouplas \\
\hline$T E-103-A P-48$ & $12 / 3 / 86$ & 52 & CASS & $\mathbf{s}$ & Disapreement with nearby thermocouples \\
\hline TE-103-AP-4B & $12 / 4 / 66$ & 53 & CASS & $\mathbf{S}$ & Disagreement with nearby thermocouples \\
\hline TE-103-AP-AB & $12 / 5 / 86$ & 53 & CASS & $\mathbf{S}$ & Disagreement with nearby thormocouples \\
\hline TE-103-AP-48 & $12 / 6 / 26$ & 53 & CASS & $\mathbf{s}$ & Diseqreement with nearby thermocouples \\
\hline TE-103-AP-48 & $12 / 786$ & 53 & CASS & $\mathbf{S}$ & Digagraement with nearby thermocouples \\
\hline TE-103-AP-48 & $12 / 9 / 86$ & 53 & CASS & $\mathbf{s}$ & Disagraement with nearby thermocouples \\
\hline TE-103-AP-48 & $12 / 10 / 86$ & 52 & CASS & $\mathbf{s}$ & Disagreement with nearby tharmocouples \\
\hline TE-103-AP-48 & $12 / 11 / 86$ & 53 & CASS & $\mathrm{S}$ & Disagreament with nearby thermocouples \\
\hline TE-103-AP-48 & $12 / 12 / 86$ & 53 & CASS & $\mathbf{S}$ & Disagraement with nearby thermocouples \\
\hline TE-103-AP-48 & $12 / 13 / 86$ & 53 & CASS & $\mathbf{s}$ & Disagreement with nearby tharmocouples \\
\hline TE-103-AP-48 & $12 / 14 / 86$ & 53 & CASS & $\mathbf{S}$ & Disagreament with nearby thermocouples \\
\hline TE-TO3-AP-48 & $12 / 15 / 86$ & 53 & CASS & $\mathbf{S}$ & Disagreement with nearby thermacouples \\
\hline TE-103-AP-4B & $12 / 16 / 86$ & 53 & CASS & $\mathbf{S}$ & Disapreoment with nearby thermocouplas \\
\hline TE-103-AP-48 & $12 / 17 / 86$ & 55 & CASS & $\mathbf{S}$ & Disapreement with noarby thermocouples \\
\hline TE-103-AP-48 & $12 / 18 / 86$ & 55 & CASS & $\mathbf{S}$ & Disagreement with nearby thermocouples \\
\hline$T E-103-A P-48$ & $12 / 19 / 86$ & 53. & CASS & $\mathbf{s}$ & Disscreament with nearby thermocouplas \\
\hline TE-103-AP-48 & $12 / 20 / 86$ & 53 & CASS & $\mathbf{s}$ & Disarreement with naarby thermocouples \\
\hline TE-T03-AP-48 & $12 / 21 / 86$ & 53 & CASS & $\mathbf{S}$ & Disagrement with nesrby thermocouples \\
\hline TE-103-AP-48 & $12 / 22 / 86$ & 53 & CASS & $\mathbf{s}$ & Disagreement with nearby thermocouples \\
\hline TE-103-AP-48 & $12 / 23 / 86$ & 52 & CASS & $\mathbf{S}$ & Disagreement with nearby thermocouples \\
\hline TE-103-AP-48 & $12 / 24 / 86$ & 54 & CASS & $\mathbf{s}$ & Diserresment with nearby thermocouplas \\
\hline
\end{tabular}




\begin{tabular}{|c|c|c|c|c|c|}
\hline \multicolumn{6}{|c|}{$\begin{array}{l}\text { TALK 241-AP.103 } \\
\text { THEFMOCOUPLE DATA }\end{array}$} \\
\hline & & & & & \\
\hline THEPMOCOUPLE $*$ & DATE & TEMPERATURE P $\left.^{\circ} \mathrm{F}\right\}$ & DATA SOURCE & QUALTYY & COMHENT \\
\hline TE-108-AP-48 & $12 / 25 / 86$ & 54 & CASS & $\mathbf{S}$ & Disagreement with nearby thermocouples \\
\hline TE-103-AP-48 & $12 / 26 / 86$ & 52 & CASS & $\mathbf{S}$ & Disagreement with nearby thermocouples \\
\hline TE-103-AP-48 & $12 / 27 / 86$ & 53 & CASS & $\mathbf{S}$ & Disagreement with nearby tharmocouples \\
\hline TE-103-AP-48 & $12 / 28 / 86$ & 51 & CASS & $\mathbf{S}$ & Disegreament with nearby tharmocouples \\
\hline TE-103-AP-48 & $12 / 29 / 86$ & 51 & CASS & $\mathbf{S}$ & Disagreement with nearby thermocouplas \\
\hline TE-103-AP-48 & $12 / 30 / 86$ & 51 & CASS & $\mathbf{s}$ & Disagreament with nearby thermocouples \\
\hline TE-103-AP-48 & $12 / 31 / 86$ & 50: & CASS & $\mathbf{s}$ & Disacreement with nearby thermocouples \\
\hline TE-103-AP-48 & $7 / 5 / 87$ & 64 & CASS & $\mathbf{s}$ & Disegreement with nearby thermocouples \\
\hline TE-103-AP-48 & $7 / 2 / 87$ & 64 & CASS & $\mathbf{S}$ & Disugreernent with nesiby thermocouples \\
\hline TE-103-AP-48 & $7 / 3 / 87$ & 64 & CASS & $\mathbf{S}$ & Disagreement with nearby tharmocouplas \\
\hline TE-103-AP-48 & 7/4/87 & 64 & CASS & $\mathbf{S}$ & Diesgreamant with nearby thermocouples \\
\hline TE-103-AP-48 & $7 / 5 / 87$ & 65 & CASS & $\mathbf{S}$ & Disegreament with nearby thermocouples \\
\hline TE-103-AP-48 & 7/6/87 & 64 & CASS & $\mathbf{S}$ & Disagreement with neaby thermocouples \\
\hline TE-103-AP-4B & $7 / 7 / 87$ & 64 & CASS & $\mathbf{s}$ & Disagreement with neartoy thermocouples \\
\hline TE-103-AP-48 & $7 / 3 / 87$ & 64 & CASS & $\underline{S}$ & Disegreernent with nearby thermocouplas \\
\hline TE-103-AP-4B & $719 / 87$ & 64 & CASS & $\mathbf{s}$ & Disepreement with nearby thermocouplas \\
\hline TE-103-AP-4B & 7/10/87 & 66 & CASS & $\mathbf{s}$ & Disagreement with nearby thermocouples \\
\hline TE-108-AP-4B & $7 / 31 / 87$ & 66 & CASS & $\mathbf{S}$ & Diasareament with nearby thermocouples \\
\hline TE-103-AP-48 & $7 / 13 / 87$ & 64 & CASS & $\mathbf{s}$ & Disagreement with nearby thermocouples \\
\hline TE-103-AP-48 & 7/15/37 & 66 & CASS & $\mathbf{S}$ & Disegreement with nearby thermocouples \\
\hline TE-103-AP-48 & $796 / 87$ & 65 & CASS & $\mathbf{S}$ & Disagreement with nearby thermocouples \\
\hline TE-103-AP-48 & 7/17/87 & 64 & CASS & $\mathbf{S}$ & Disagreament with nearby thermocouples \\
\hline TE-103-AP-4B & $7 / 18 / 87$ & 65 & CASS & $\mathbf{S}$ & Disegreement with nearby thermocouples \\
\hline TE-103-AP-A8 & $7 / 99 / 87$ & 69 & CASS & $\mathbf{s}$ & Disegreement with nesiby thermocouples \\
\hline TE-103-AP-48 & $7 / 20 / 87$ & 68 & CASS & $\mathbf{S}$ & Disegreament with neaby thermocouples \\
\hline TE-103-AP-48 & $7 / 21 / 87$ & 66 & CASS & $\mathbf{s}$ & Oiseproement with nearby tharmocouplas \\
\hline TE-103-AP-48 & $7 / 22 / 87$ & 85 & CASS & $\mathbf{s}$ & Diseproement with noerby thermocouples \\
\hline TE-103-AP-48 & $7 / 23 / 87$ & 64 & CASS & $\overline{\mathbf{S}}$ & Disugreament with nadby thermocouples \\
\hline TE-103-AP-48 & 7/24/87! & 65 & CASS & $\mathbf{S}$ & Ditegreement with nearby thermocouples \\
\hline TE-103-AP-48 & 7/25/87 & 65 & CASS & $\mathbf{s}$ & Disagreernem with nearby thermocouples \\
\hline TE-103-AP-48 & $7 / 26 / 37$ & 65 & CASS & $\mathbf{s}$ & Disedreomant with nearby tharmosouples \\
\hline TE-103-AP-48 & $7 / 27 / 87$ & 66 & CASS & $\mathbf{S}$ & Disegreoment with neaphy thermocouplos \\
\hline TE-103-AP-48 & $7 / 28 / 87$ & 66 & CASS & $\mathbf{s}$ & Dinepreement with nearby thermocouples \\
\hline TE-103-AP-48 & $7 / 29 / 87$ & 67 & CASS & $\mathbf{S}$ & Dienpreament with madby thempocouples \\
\hline TE-103-AP-48 & $7 / 30 / 87$ & 66 & CASS & $\mathbf{s}$ & Disagreament whe nearby thermocouples \\
\hline TE-103-AP-48 & 7/31/87 & 66 & CASS & $\mathbf{s}$ & Diagereement with nearby thermocouples \\
\hline TE-103-AP-48 & $8 / 1 / 87$ & 66 & CASS & $\mathbf{s}$ & Disagreement with nearty thermocouples \\
\hline TE-103-AP-48 & $8 / 2 / 87$ & 67 & CASS & $\mathbf{s}$ & Oigurgeomont with nashy thermocouples \\
\hline TE-103-AP-48 & $8 / 3 / 37$ & 86 & CASS & $\overline{\mathbf{S}}$ & Disspreement with noarby thermocouplas \\
\hline TE-103-AP-48 & $8 / 4 / 87$ & 65 & CASS & $\mathbf{S}$ & Digerreament with nearby thermocouples \\
\hline TE-103-AP-48 & $8 / 5 / 87$ & 66 & CASS & $\mathbf{S}$ & Disagreament with naarby thermocouplas \\
\hline TE-103-AP-48 & $8 / 6 / 87$ & 67 & CASS & $\mathbf{S}$ & Distrgrearnent with nearby thormocouples \\
\hline TE-103-AP-4B & $8 / 7 / 87$ & 66 & CASS & $\mathbf{s}$ & Disargeement with nearby thermocouples \\
\hline TE-103-AP-48 & $8 / 3 / 87$ & 86 & CASS & $\mathbf{s}$ & Disapreament with nearby thermocouples \\
\hline TE-103-AP-48 & B/9/a7 & 66 & CASS & $\mathbf{s}$ & Disagreament with nearby thermocouplos \\
\hline TE-103-AP-48 & $8 / 10 / 37$ & 67. & CASS & $\mathbf{S}$ & Disegreoment with nearby thermocouples \\
\hline TE-103-AP-48 & $8 / 12 / 87$ & 66 & CASS & $\mathbf{s}$ & Disagreement with nearby thermocouples \\
\hline TE-103-AP-48 & $8 / 13 / 87$ & 66 & CASS & $\mathbf{s}$ & Dissoreament with nathy thermocouples \\
\hline$T E-103-A P-48$ & $8 / 14 / 87$ & 73 & CASS & $\mathbf{S}$ & Disegreament with nearby therimocouplos \\
\hline TE-103-AP-48 & $8 / 15 / 87$ & 72 & CASS & $\mathbf{S}$ & Disagreement with nearby tharmocouples \\
\hline TE-108-AP-48 & $8 / 16 / 87$ & 70 & CASS & $\overline{\mathbf{S}}$ & Disagreament with nearby thermocouples \\
\hline TE-103-AP-48 & $8 / 17 / 87$ & 67 & CASS & $\mathbf{s}$ & Disagreament with nearby thermocouples \\
\hline TE-103-AP-48 & $8 / 18 / 87$ & 68 & CASS & $\mathbf{S}$ & Disagraement with nearby thermocouples \\
\hline TE-103-AP-48 & $8 / 19 / 87$ & 66 & CASS & $\mathbf{s}$ & Disagreement with nearby thermocouples \\
\hline TE-103-AP-48 & $8 / 20 / 87$ & 66 & CASS & $\mathbf{S}$ & Disagreement with nearby thermocouples \\
\hline TE-103-AP-48 & $8 / 21 / 87$ & 67) & CASS & $\mathbf{S}$ & Disagreement with nearby thermocouples \\
\hline TE-103-AP-4B & $8 / 22 / 87$ & 68: & CASS & $\mathbf{s}$ & Disegreement with nearby thermocouples \\
\hline TE-103-AP-48 & $8 / 23 / 87$ & 66 & CASS & $\mathbf{s}$ & Disagreement with nearby thermocouples \\
\hline TE-103-AP-48 & $8 / 24 / 87$ & 66 & CASS & $\mathbf{S}$ & Disagreament with nearby thermocouples \\
\hline TE-103-AP-48 & $8 / 25 / 87$ & 72 & CASS & $\mathbf{S}$ & Disagreement with nearby tharmocouples \\
\hline TE-103-AP-48 & $8 / 27 / 87$ & 72 & CASS & $\mathbf{S}$ & Disagreament with nearby thermocouples \\
\hline TE-103-AP-48 & $8 / 28 / 87$ & 67 & CASS & $\mathbf{s}$ & Disagreatnent with nearby thermocouples \\
\hline TE-103-AP-48 & $8 / 29 / 37$ & 67 & CASS & $\mathbf{S}$ & Disarreament with nearby thermocouples \\
\hline TE-103-AP-48 & $8 / 30 / 87$ & 68 & CASS & $\mathbf{s}$ & Disegreemont with nearby thermocouples \\
\hline TE-103-AP-48 & $8 / 31 / 87$ & 67 & CASS & $\mathbf{s}$ & Disagreoment with nearby thermocouplas \\
\hline TE-103-AP-48 & $9 / 1 / 87$ & 67 & CASS & S & Disagreement with nearby thermocouples \\
\hline TE-103-AP-48 & $9 / 2 / 87$ & 67 & CASS & $\mathbf{s}$ & Disagreament with nearby thermocouples \\
\hline TE-103-AP-48 & $9 / 4 / 87$ & 67 & CASS & $\mathbf{s}$ & Disapreement with nearby thermocouples \\
\hline TE-103-AP-48 & $9 / 5 / 87$ & 67 & CASS & $\mathbf{s}$ & Disagreement with nearby thermocouplas \\
\hline TE-103-AP-48 & $9 / 6 / 97$ & 69 & CASS & $\mathbf{s}$ & Disegreement with nearby thermocouples \\
\hline TE-103-AP-48 & $9 / 7 / 37$ & 68 & CASS & .5 & Disagreement with nearby thermocouples \\
\hline TE-103-AP-48 & $9 / 8 / 37$ & 67 & CASS & $\mathbf{s}$ & Disagresment with nearby thermocouples \\
\hline TE-103-AP-48 & $9 / 9 / 87$ & 68 & CASS & $\overline{\mathbf{S}}$ & Disagreement with nearby thermocouples \\
\hline
\end{tabular}




\begin{tabular}{|c|c|c|c|c|c|}
\hline \multicolumn{6}{|c|}{$\begin{array}{l}\text { TAKK 241-AP-103 } \\
\text { THEAMOCOUFIE DATA }\end{array}$} \\
\hline & & & & & \\
\hline THERMOCOUPE * & DATE & TEMPERATURE ('F) & DATA SOUACE & QUALTYY & COMMENT \\
\hline TE-103-AP-48 & 9/10/87 & 69 & CASS & $\mathbf{s}$ & Disagreement with nearby thermocouples \\
\hline TE-103-AP-4B & $9 / 11 / 87$ & 67 & CASS & $\mathbf{S}$ & Disagreement with nearby thermocouples \\
\hline TE-103-AP-48 & 9/12/37 & 69 & CASS & $\mathbf{S}$ & Disagreernent with nearby thermocouples \\
\hline TE-103-AP-48 & $9 / 13 / 87$ & 68 & CASS & $\mathbf{S}$ & Disegreament with resiby thermocouples \\
\hline TE-103-AP-48 & $9 / 14 / 87$ & 68 & CASS & $\mathbf{s}$ & Diadgreement with nearby thermocouples \\
\hline TE-103-AP-48 & $9 / 15 / 87$ & 68 & CASS & $\mathbf{S}$ & Disegreement with nearby thermocouples \\
\hline TE-103-AP-48 & 9/16/87 & 69 & CASS & $\mathbf{S}$ & Disagreament with nearby thermocouples \\
\hline TE-T03-AP-48 & $9 / 17 / 87$ & 67 & CASS & $\mathbf{S}$ & Disegroement with noarby thermocouples \\
\hline TE-103-AP-48 & $9 / 18 / 87$ & 68 & CASS & $\mathbf{s}$ & Disaproement whth neartoy thermocouples \\
\hline TE-103-AP-48 & $9 / 19 / 87$ & 69 & CASS & $\mathbf{s}$ & Disagreement with nearby themrocouples \\
\hline TE-103-AP-48 & $9 / 20 / 87$ & 69 & CASS & 5 & Disagreament with nearby thermocouplas \\
\hline TE-103-AP-48 & $9 / 21 / 87$ & 70 & CASS & $\mathbf{s}$ & Disargeament with nearby thermocouples \\
\hline TE-103-AP-48 & $9 / 22 / 87$ & 71 & CASS & $\mathbf{s}$ & Disegreament with nearby thermocouples \\
\hline TE-103-AP-48 & 9/23/87 & 71 & CASS & $\mathbf{s}$ & Diagorement with neaby tharmocouplas \\
\hline$T E-103-A P-48$ & $8 / 24 / 67$ & 70 & CASS & $\mathbf{s}$ & Diagrgeament with nearby thormocouples \\
\hline TE-103-AP-48 & $9 / 25 / 87$ & 69 & CASS & $\mathbf{s}$ & Diaergeament with noerby thermocouples \\
\hline TE-103-AP-48 & $9 / 26 / 87$ & 70 & CASS & $\mathbf{s}$ & Diagpreament with nearty thermocouples \\
\hline TE-103-AP-48 & $9 / 27 / 87$ & 65 & CASS & $\mathbf{s}$ & Disagreament with nearby thermocouples \\
\hline$\overline{T E}=103-A P-48$ & 9/28/87 & 65 & CASS & $\mathbf{S}$ & Disepresment with noarby thermocouples \\
\hline$\overline{T E-103-A P-48}$ & 9/29/67 & 66 & CASS & $\mathbf{5}$ & Diagrreement with nagrby thermocouples \\
\hline TE-103-AP-48 & $9 / 30 / 67$ & 68 & CASS & $\mathbf{s}$ & Digarreement with nearby thermocouples \\
\hline$T E-103-A P-48$ & $10 / 1 / 87$ & 67 & CASS & $\mathbf{5}$ & Diesprosment with nearby thermocouples \\
\hline TE-103-AP-48 & $10 / 2 / 87$ & 67 & CASS & $\overline{\mathbf{S}}$ & Ditaprivement with nearby thermocouplas \\
\hline TE-103-AP-48 & 10/3/67 & 68 & CASS & $\mathbf{S}$ & Diseroement with nastby thermocouples \\
\hline TE-103-AP.48 & $10 / 4 / 87$ & 68 & CASS & $\mathbf{S}$ & Ditergreement with nearty thermocouples \\
\hline$T E-103-A P-48$ & $10 / 5 / 87$ & 67 & CASS & $\mathbf{5}$ & Diagoreement with nearty thermocouples \\
\hline$T E-103-A P \cdot 48$ & 10/6/87 & 86 & CASS & $\mathbf{s}$ & Diagreanent with nedroy thermocoupless \\
\hline TE-103-AP-48 & $10 / 7 / 87$ & 67 & CASS & s & Disegreanent with naarby theimocouples \\
\hline TE-103-AP-48 & 10/8/87 & 68 & CASS & $\mathbf{S}$ & Disegreement with nearty thermocouples \\
\hline TE-108-AP-48 & 10/9/37 & 66: & CASS & $\mathbf{S}$ & Disepreament with noarby thermocouples \\
\hline YE-103-AP-48 & $10 / 10 / 87$ & 64 & CASS & $\mathbf{s}$ & Disaproement with nearby thermocouples \\
\hline TE-103-AP-4B & $10 / 21 / 87$ & 62 & CASS & $\mathbf{s}$ & Diragreoment with noarby thermocouples \\
\hline TE-103-AP-48 & $10 / 12 / 87$ & 61 & CASS & $\mathbf{5}$ & Dicegreamont with noaby themrocouples \\
\hline TE-103-AP-48 & $10 / 13 / 87$ & 60 & CASS & $\mathbf{S}$ & Disepreement with neaby thermocouples \\
\hline$T E-103-A P-48$ & $10 / 14 / 87$ & 59 & CASS & $\mathbf{s}$ & Didepresment with nearby thormocouples \\
\hline TE-103-AP-48 & $10 / 15 / 87$ & Bg. & CASS & $\mathbf{S}$ & Diagreement with neaby thermocouples \\
\hline TE-103-AP-48 & $10 / 16 / 87$ & 57| & CASS & $\mathbf{s}$ & Diagroemont whth nosiby tharmocouplas \\
\hline TE-103.AP-48 & $10 / 17 / 87$ & 58 & CASS & $\mathbf{s}$ & Diagreement with nesiby thermocouples \\
\hline TE-103-AP-48 & $10 / 18 / 87$ & 58 & CASS & S & Diacorreament with nearby thamocouples \\
\hline TE-103-AP-48 & 10/19/87 & 57 & CASS & $\mathbf{S}$ & Disagreement with nasby thermocouples \\
\hline TE-103-AP-48 & $10 / 20 / 87$ & 56: & CASS & $\mathbf{S}$ & Diagereament with naaby thermocouples \\
\hline TE-103.AP-48 & $10 / 21 / 87$ & S5 & CASS & $\mathbf{s}$ & Disegroement with neaby themocouplas \\
\hline TE-103-AP-48 & $10 / 22 / 87$ & $\mathbf{5 5}$ & CASS & $\overline{\mathbf{S}}$ & Disupreament with noaby thermocouples \\
\hline TE-103-AP-48 & $10 / 23 / 87$ & 56 & CASS & $\mathbf{S}$ & Disepreement with neasby thermocouples \\
\hline$T E-103 \cdot A P-48$ & $10 / 24 / 87$ & 56 & CASS & $\mathbf{s}$ & Disepreement with noniby thormocouples \\
\hline TE-103-AP-48 & $10 / 25 / 87$ & 57 & CASS & $\mathbf{S}$ & Disagreament with nearby thermocouples \\
\hline TE-103-AP-48 & $10 / 26 / 37$ & 59 & CASS & $\mathbf{s}$ & Disegreament with nearby thermocouples \\
\hline TE-103-AP-48 & $10 / 27 / 87$ & 56 & CASS & $\mathbf{5}$ & Digagreoment with neaby thermocouples \\
\hline TE-103-AP-48 & $10 / 28 / 87$ & 5. & CASS & $\mathbf{S}$ & Oiseqreement with nearby thermocouples \\
\hline TE-103.AP-48 & $10 / 29 / 87$ & 55 & CASS & $\mathbf{s}$ & Diasegreament with natby thermocouples \\
\hline TE-108-AP-48 & $10 / 30 / 87$ & 58 & CASS & $\mathbf{s}$ & Diagreement with nearby thermocouples \\
\hline TE-108-AP-48 & $10 / 31 / 87$ & 58 & CASS & $\mathbf{s}$ & Disagreement with narby thermocouples \\
\hline TE-103-AP-48 & $11 / 1 / 87$ & 58 & CASS & $\mathbf{s}$ & Disegreament with noarby thermocouples \\
\hline TE-103-AP-48 & $11 / 2 / 87$ & 59 & CASS & $\mathbf{s}$ & Disaproement with noarby thermocouplas \\
\hline TE-103-AP-48 & $11 / 3 / 87$ & 59 & CASS & $\mathbf{S}$ & Disagreement with neaby tharmocouples \\
\hline TE-103-AP-48 & $11 / 4 / 87$ & 61 & CASS & $\mathbf{s}$ & Disagreement with nearby tharmocouples \\
\hline TE-103-AP-48 & $11 / 5 / 87$ & 60 & CASS & $\mathbf{S}$ & Disegroement with nearby thermocouples \\
\hline TE-103-AP-48 & $11 / 6 / 87$ & 61 & CASS & $\mathbf{s}$ & Diasegreement with nearby thermocouples \\
\hline TE-103-AP-48 & $11 / 7 / 87$ & 59 & CASS & $\mathbf{s}$ & Disagreement with nearby thermocouples \\
\hline TE-103-AP-4B & $11 / 8 / 87$ & 58 & CASS & s & Disagreement with nearby thermocouples \\
\hline TE-103-AP-48 & $11 / 3 / 87$ & 59 & CASS & $\mathbf{S}$ & Disatraement with rearby thermocouplos \\
\hline TE-103-AP-48 & $11 / 10 / 87$ & 59! & CASS & $\mathbf{s}$ & Disagreement whth nearty thermocouples \\
\hline TE-103-AP-48 & $11 / 11 / 87$ & 60 & CASS & $\mathrm{s}$ & Disagreement with nearby thermocouples \\
\hline TE-103-AP-48 & $11 / 12 / 87$ & 62 & CASS & $\mathrm{s}$ & Disagreement with nearby thermocouples \\
\hline TE-103-AP-48 & $11 / 14 / 87$ & 58 & CASS & $\mathbf{S}$ & Disagreement with nearby thermocouples \\
\hline TE-103-AP-4B & $11 / 15 / 87$ & 58 & CASS & $\mathbf{S}$ & Disepreement with nearby thermocouples \\
\hline TE-T03-AP-4B & $11 / 16 / 87$ & 59 & CASS & $\mathbf{s}$ & Disagreement with nearby thermocouples \\
\hline TE-103-AP-48 & 11/17/87 & 55 & CASS & $\mathbf{s}$ & Disagreement with nearby thermocouples \\
\hline$T E-103-A P-48$ & $11 / 18 / 87$ & 57 & CASS & $\mathbf{s}$ & Disegreement with nearby thermocouples \\
\hline TE-103-AP-48 & $11 / 19 / 87$ & 57 & CASS & $\mathbf{s}$ & Disagreement with nearby thermocouplos \\
\hline TE-103-AP-48 & $11 / 20 / 87$ & 57 & CASS & $\mathbf{s}$ & Disagreement with nearby thermocouples \\
\hline TE-103-AP-48 & $11 / 21 / 87$ & 57 & CASS & $\mathbf{S}$ & Disapreement with nearby thermocouples \\
\hline TE-103-AP-48 & $11 / 22 / 87$ & 57 & CASS & $\mathbf{s}$ & Diserreement with nearby thermocouples \\
\hline
\end{tabular}




\begin{tabular}{|c|c|c|c|c|c|}
\hline \multicolumn{6}{|c|}{$\begin{array}{l}\text { TAWK 241-AP-103 } \\
\text { THEFIOOCOUPE DATA }\end{array}$} \\
\hline & & & & & \\
\hline THERMOCOUPLE \# & DATE & TEMPERATURE ( $\left.{ }^{\circ} \mathrm{F}\right)$ & DATA SOURCE & QUALITY & COMMENT \\
\hline TE-103-AP-48 & $11 / 25 / 87$ & 57 & CASS & $\mathbf{s}$ & Disagreement with nearby thermocouples \\
\hline TE-103-AP.4B & $11 / 26 / 87$ & 54 & CASS & 5 & Diasgreement with nearby thermocouples \\
\hline TE-103-AP-4B & $11 / 27 / 87$ & 55 & CASS & s & Disagreement with nearby thermocouples \\
\hline TE-103-AP-48 & $11 / 28 / 87$ & 55 & CASS & $\mathbf{S}$ & Disagreament with nearby thermocouples \\
\hline TE-103-AP-4B & $11 / 29 / 37$ & 56 & CASS & $\mathbf{S}$ & Disagreement with nearby thermocouples \\
\hline TE-103-AP-4B & $11 / 30 / 87$ & 55! & CASS & $\mathbf{S}$ & Disagreement with nearby thermocouples \\
\hline TE-103-AP-48 & 12/1/87 & 56 & CASS & $\mathbf{S}$ & Disegroement with nearby thermocouples \\
\hline TE-103-AP-48 & $12 / 2 / 37$ & 57 & CASS & $\mathbf{S}$ & Disagreement with naarby thermocouples \\
\hline TE-103-AP-48 & 12/3/37 & 59 & CASS & $\mathbf{s}$ & Disagreament with nesiby thermocouples \\
\hline TE-103-AP-48 & 12/4/87 & 62 & CASS & $\mathbf{s}$ & Disugroement with nastby thermocouples \\
\hline TE-103.AP-48 & $12 / 5 / 37$ & 62 & CASS & $\mathbf{s}$ & Diagreement with nearby thermocouples \\
\hline TE-103-AP-48 & $12 / 6 / 67$ & 5 & CASS & $\mathbf{S}$ & Disapreement with nearby thermocouples \\
\hline TE-103-AP-48 & I2/7/87 & 58 & CASS & $\mathbf{s}$ & Disegreament with nearby thermocouples \\
\hline TE-103-AP.4B & 12/3/87 & 56 & CASS & $\mathbf{S}$ & Disapreement with nestby thermocouples \\
\hline TE-103-AP-48 & $12 / 9 / 87$ & 62 & CASS & $\mathbf{s}$ & Disegreement with neartey thermocouples \\
\hline TE-103-AP-48 & $12 / 10 / 87$ & 58 & CASS & $\mathbf{S}$ & Disegreement with nearby tharmocouples \\
\hline TE-103-AP-48 & $12 / 11 / 87$ & 57 & CASS & $\mathbf{s}$ & Disagreement with nearby thormocouples \\
\hline TE-103-AP-48 & $12 / 12 / 87$ & 56| & CASS & $\mathbf{S}$ & Diegoreement with nearby thermocouples \\
\hline TE-108-AP-48 & $12 / 13 / 37$ & $\mathbf{5 6}$ & CASS & $\mathbf{S}$ & Ditagreament with nosiby thermocouples \\
\hline TE-103-AP-48 & $12 / 14 / 87$ & 55 & CASS & $\mathbf{s}$ & Disagreement with nearby thermocouples \\
\hline TE-103-AP-48 & $12 / 15 / 87$ & 54 & CASS & $\mathbf{S}$ & Diagoreement with nasby thermocouples \\
\hline TE-103-AP-48 & $12 / 16 / 87$ & 54 & CASS & $\mathbf{s}$ & Diespreament with nearby thermocouples \\
\hline TE-103-AP-48 & $12 / 17 / 87$ & 64! & CASS & $\mathbf{S}$ & Disagreement with noaby thermocouples \\
\hline TE-103-AP-48 & $12 / 18 / 87$ & 55 & CASS & $\mathbf{s}$ & Dieagreament with nearby thermocouplas \\
\hline$\overline{T E-103-A P-48}$ & 12/19/97 & 56: & CASS & $\mathbf{S}$ & Disagreement with nearby thermocouples \\
\hline TE-103-AP-4B & $12 / 20 / 87$ & 53 & CASS & $\mathbf{S}$ & Diagagreanent with nearby thermocouples \\
\hline TE-103-AP-48 & $12 / 21 / 87$ & 53 & CASS & $\mathbf{S}$ & Disarreement with nearby thermocouples \\
\hline TE-103-AP-4B & $12 / 22 / 37$ & 53 & CASS & S & Disagreement with nearby thermocouples \\
\hline TE-103-AP-48 & $12 / 23 / 87$ & 53 & CASS & $\mathbf{s}$ & Disagreement with nearby thermocouples \\
\hline TE-T03-AP-48 & $12 / 25 / 87$ & 53 & CASS & $\mathbf{s}$ & Disagreement with nearby thermocouples \\
\hline TE-103-AP-48 & 12/26/87 & 53 & CASS & $\overline{\mathbf{5}}$ & Disepreament with nearty thermocouples \\
\hline TE-103-AP.4B & $12 / 27 / 87$ & 52 & CASS & $\mathbf{S}$ & Disagreament with nearby thermocouples \\
\hline TE-103-AP-AB & $12 / 28 / 87$ & 53 & CASS & $\mathbf{S}$ & Dieagreament with nearby thermocouples \\
\hline TE-103-AP-48 & $12 / 29 / 87$ & 54 & CASS & $\mathbf{s}$ & Disergreement with nearby thermocouples \\
\hline$T E-103-A P-48$ & $12 / 30 / 87$ & 53 & CASS & $\mathbf{S}$ & Disagreement with nearby thermocouples \\
\hline TE-103-AP-48 & $12 / 31 / 87$ & 53 & CASS & $\mathbf{s}$ & Dladreament with neaby thermocouples \\
\hline TE-103-AP-48 & $1 / 1 / 88$ & 53 & CASS & $\mathbf{S}$ & Ditagrearnent with naeby thermocouples \\
\hline TE-103-AP-4B & $1 / 2 / 88$ & 54 & CASS & $\mathbf{S}$ & Disegrearnent with nearty tharmocouples \\
\hline TE-103-AP-4B & $1 / 3 / 68$ & 53 & CASS & $\mathbf{S}$ & Disegreomant with nearby thormocouples \\
\hline TE-103-AP-48 & T/4/88 & 51 & CASS & $\mathbf{s}$ & Disegreement with nearby thermocouples \\
\hline$T E-103 \cdot A P-48$ & $1 / 5 / 88$ & 50 & CASS & $\overline{\mathbf{S}}$ & Disepreement with naerby thermocouples \\
\hline TE-103-AP-48 & $1 / 6 / 88$ & 50 & CASS & $\mathbf{s}$ & Divegreement with nearby thermocouplas \\
\hline TE-103.AP-48 & 1/7/88 & 51 & CASS & $\mathbf{S}$ & Disegreament with nearby thermocouples \\
\hline TE-103-AP-48 & $1 / 6 / 8$ & 53 & CASS & $\mathbf{s}$ & Diseqreement with nearby theimocouples \\
\hline$T E-103-A P-48$ & $1 / 8 / 88$ & 51 & CASS & $\mathbf{S}$ & Diasgreament with nearby thermocouples \\
\hline TE-103-AP-48 & $1 / 10 / 88$ & 52 & CASS & $\mathbf{S}$ & Disagreement with nearby thermocouplas \\
\hline TE-103-AP-48 & $1 / 11 / 88$ & 54 & CASS & $\mathbf{S}$ & Disagreement with nearby thermocouples \\
\hline TE-103-AP-48 & $1 / 12 / 38$ & 52 & CASS & $\mathbf{s}$ & Disagreement with nearby thermocouplas \\
\hline TE-103-AP-48 & $1 / 13 / 88$ & 51 & CASS & $\mathbf{s}$ & Dissoreement with nearby thermocouples \\
\hline TE-103-AP-48 & $1 / 14 / 38$ & 51 & CASS & $\mathbf{s}$ & Disagreament with nasby thermocouples \\
\hline TE-103-AP-48 & $1 / 15 / 89$ & 53 & CASS & $\overline{\mathbf{s}}$ & Disagreement with nearby thermocouples \\
\hline TE-103-AP-48 & $1 / 16 / 88$ & 56 & CASS & $\mathbf{S}$ & Disagresment with naaby thermocouples \\
\hline TE-103-AP-48 & $1 / 17 / 88$ & 54 & CASS & $\overline{\mathbf{S}}$ & Disagreement with nearby thermocouples \\
\hline TE-103-AP-48 & $1 / 18 / 88$ & 55 & CASS & $\mathbf{S}$ & Disagraement with nearby thermocouples \\
\hline TE-103-AP-48 & $1 / 19 / 88$ & 54 & CASS & $\mathbf{s}$ & Disagreamem with nearby thermocouples \\
\hline TE-103-AP-48 & $1 / 20 / 88$ & 55 & CASS & $\mathbf{S}$ & Disagreament with nearby thermocouples \\
\hline TE-103-AP-48 & $1 / 21 / 88$ & 54 & CASS & $\mathbf{s}$ & Disagreament with nearby tharmocouples \\
\hline TE-103-AP-48 & $1 / 22 / 88$ & 54 & CASS & $\overline{\mathbf{S}}$ & Disapreamem with nearby thermocouples \\
\hline TE-103-AP-48 & $1 / 23 / 38$ & 57 & CASS & $\mathbf{S}$ & Disagreement with nearby thermocouples \\
\hline TE-103-AP-48 & $1 / 24 / 38$ & 56 & CASS & $\mathbf{s}$ & Disegreement with nasiby thermocouples \\
\hline TE-103-AP-48 & $1 / 25 / 88$ & 55! & CASS & $\mathbf{s}$ & Disagroement with nearby thermocouples \\
\hline TE-103-AP-48 & $1 / 26 / 88$ & 54 & CASS & $\mathbf{S}$ & Disagreement with nearby thermocouples \\
\hline TE-103-AP-48 & $1 / 27 / 88$ & 55 & CASS & $\mathbf{S}$ & Disagreement with narby thermocouples \\
\hline TE-103-AP-48 & $1 / 28 / 88$ & 55: & CASS & $\overline{\mathbf{s}}$ & Disagreement with narby thermocouples \\
\hline TE-103-AP-48 & $1 / 29 / 88$ & 59 & CASS & $\overline{\mathbf{s}}$ & Disagreement with nearby thermocouples \\
\hline TE-103-AP-48 & $1 / 30 / 88$ & 57 & CASS & $\mathbf{s}$ & Disagreement with nearby thermocouples \\
\hline TE-103-AP-48 & $1 / 31 / 88$ & 57 & CASS & $\mathbf{S}$ & Disagreement with nearby thermocouples \\
\hline TE-103-AP-48 & $2 / 1 / 88$ & 55 & CASS & $\mathbf{S}$ & Disagreement with nesby thermocouples \\
\hline TE-103-AP-4B & 212188 & 54 & CASS & $\overline{\mathbf{S}}$ & Disagreement with nasby thermocouplas \\
\hline TE-103-AP-4B & $2 / 3 / 88$ & 53 & CASS & $\mathbf{S}$ & Discoreament with nearby thermocouples \\
\hline TE-103-AP-4B & $2 / 4 / 88$ & 54 & CASS & $\overline{\mathbf{S}}$ & Disagreement with nearby thermocouplas \\
\hline TE-103-AP.4B & $2 / 5 / 88$ & 56 & CASS & $\mathbf{S}$ & Disagreement with pearby thermocouples \\
\hline TE-103-AP-48 & $2 / 6 / 88$ & 56 & CASS & $\mathbf{s}$ & Disagreement with nearby thermocouples \\
\hline
\end{tabular}




\begin{tabular}{|c|c|c|c|c|c|}
\hline \multicolumn{6}{|c|}{$\begin{array}{l}\text { TANK 241-AP-103 } \\
\text { THERMOCOWAE DATA }\end{array}$} \\
\hline & & & & & \\
\hline THERMOCOUPLE * & DATE & TEMPERATURE ("F) & DATA BOURCE & OUALITY & COMMENT \\
\hline TE-103-AP-48 & $2 / 7 / 88$ & 56 & CASS & $\mathbf{s}$ & Disagreement with nearby thermocouples \\
\hline$T E=103-A P-48$ & $2 / 8 / 88$ & 58 & CASS & $\mathbf{S}$ & Disagreement with nearby thermocouples \\
\hline TE-103-AP-48 & $2 / 9 / 88$ & 56 & CASS & $\mathrm{s}$ & Disagreament with nearby thermocouples \\
\hline TE-103-AP-48 & $2 / 10 / 88$ & 59 & CASS & $\overline{\mathbf{S}}$ & Disagreament with nearby thermocouples \\
\hline TE-103-AP-48 & $2 / 11 / 88$ & 62 & CASS & $\mathbf{S}$ & Disagreement with nearby thermocouples \\
\hline TE-103-AP-48 & $2 / 12 / 88$ & 63 & CASS & $\mathbf{s}$ & Disagreement with nearby thermocouples \\
\hline TE-103-AP-48 & $2 / 13 / 88$ & 62 & CASS & $\mathrm{s}$ & Disegreament with nearby thermocouples \\
\hline TE-103-AP-48 & $2 / 4 / 88$ & 53 & CASS & $\mathbf{s}$ & Disagreement with nearby tharmocouples \\
\hline TE-103-AP-48 & $2 / 15 / 80$ & 63 & CASS & $\mathbf{S}$ & Disacreament with nearby themocouples \\
\hline$T E-103-A P-48$ & $2 / 16 / 38$ & 62 & CASS & $\mathbf{S}$ & Disagreement with nearby thermocouplas \\
\hline TE-103.AP-48 & $2 / 17 / 88$ & 57 & CASS & $\mathbf{S}$ & Disapreement with nearby thermocouples \\
\hline TE-103-AP-48 & $2 / 18 / 88$ & 56 & CASS & $\mathbf{S}$ & Disapreement with nearby thermocouples \\
\hline TE-103-AP-48 & $2 / 19 / 83$ & 57 & CASS & $\mathbf{s}$ & Disagreement with nearby thermocouples \\
\hline TE-103-AP-48 & $2 / 20 / 88$ & 55 & CASS & $\mathbf{s}$ & Disegreement with neatby thermocouples \\
\hline TE-103-AP-48 & $2 / 21 / 88$ & 54 & CASS & 5 & Disagreament with nearby thermocouples \\
\hline TE-103-AP-48 & $2 / 22 / 88$ & 56 & CASS & $\mathbf{S}$ & Disagreement with nearby thermocouples \\
\hline TE-103-AP-48 & $2 / 23 / 88$ & 53 & CASS & $\mathbf{s}$ & Disagreement with nearty thermocouplas \\
\hline TE-103-AP-4B & $2 / 24 / 83$ & 54 & CASS & $\mathbf{S}$ & Disagreement with nearby thermocouples \\
\hline TE-103-AP-48 & $2 / 25 / 88$ & 58 & CASS & s & Disegreement with naarby thermocouples \\
\hline TE-103-AP-48 & $2 / 26 / 88$ & 57: & CASS & $\mathbf{S}$ & Disagreament with neartoy therimocouples \\
\hline$T E-103 \cdot A P=48$ & $2 / 27 / 88$ & 561 & CASS & $\mathbf{S}$ & Disagreement with nearby thermocouples \\
\hline$T E-103-A P-48$ & $2 / 28 / 88$ & 57 & CASS & $\mathbf{s}$ & Disagreement with nearby thermocouples \\
\hline TE-103-AP.48 & $2 / 29 / 88$ & 56 & CASS & $\mathbf{S}$ & Disegreement with neartey thermocouples \\
\hline TE-103-AP-48 & $3 / 1 / 88$ & 55) & CASS & $\mathbf{s}$ & Disegreament with nearby thermocouples \\
\hline TE-103-AP-48 & $3 / 2 / 8$ & 55 & CASS & s & Disegreement with nearby themocouples \\
\hline TE-103-AP-48 & $3 / 3 / 88$ & 55 & CASS & $\mathbf{S}$ & Disagreerment with nearby thermocouples \\
\hline TE-103.AP-48 & $3 / 4 / 38$ & 53 & CASS & $\mathbf{s}$ & Disegreement with nesphy thermocouples \\
\hline TE-103-AP-4B & $3 / 5 / 68$ & 51 & CASS & $\mathbf{s}$ & Diasagreement with noarby thermocouplas \\
\hline TE-103-AP-48 & $3 / 6 / 80$ & 51 & CASS & $\mathbf{s}$ & Dieagreament with nearby thermocouplas \\
\hline TE-103-AP-4B & 37778 & 53 & CASS & $\overline{\mathbf{S}}$ & Disegreament with noaby thermocouples \\
\hline TE-103-AP-48 & 3/8/88 & 53: & CASS & $\mathbf{s}$ & Dissproement with nearby thermocouplas \\
\hline TE-103-AP-48 & 3/9/88 & 52! & CASS & $\mathbf{5}$ & Disagreament with noarby tharmocousples \\
\hline TE-103-AP-48 & $3 / 10 / 88$ & S0| & CASS & $\mathbf{s}$ & Disegreament with nearby themocouplas \\
\hline TE-103-AP-4B & $3 / 11 / 83$ & 50 & CASS & $\mathbf{s}$ & Oisagreemem with nearby thermocouples \\
\hline TE-103-AP-48 & 3/12/88 & 51 & CASS & $\overline{\mathbf{S}}$ & Disaproament with naarby thermocouples \\
\hline TE-103-AP-48 & $3 / 13 / 68$ & 51 & CASS & $\mathbf{S}$ & Disaprearnent with nearty thermocouples \\
\hline TE-103-AP-48 & आ4/88 & 53: & CASS & $\mathbf{s}$ & Disagreernent with nearby thermocouples \\
\hline TE-103-AP-48 & $3 / 15 / 88$ & 51 & CASS & $\mathbf{s}$ & Disegreement with nearby thermocouples \\
\hline$T E-103-A P-4 B$ & $3 / 16 / 88$ & $5 \mathbf{5 i}$ & CASS & $\mathbf{s}$ & Diagareement with nearby thermocouples \\
\hline TE-103-AP-4B & $3 / 17 / 88$ & 51 & CASS & 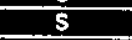 & Disagreament with nearby thermocouples \\
\hline TE-103-AP-48 & $3 / 18 / 88$ & 52 & CASS & S & Disapraement with nearby thermocouples \\
\hline TE-303-AP-48 & $3 / 19 / 88$ & 54 & CASS & $\mathbf{s}$ & Disagreement with noarby thermocouples \\
\hline TE-103-AP-4B & $3 / 20 / 38$ & 58 & CASS & $\mathbf{S}$ & Disagreament with nearby thermocouples \\
\hline$T E-103-A P-48$ & $3 / 21 / 80$ & 58 & CASS & 5 & Diagareement with nearby themocouples \\
\hline TE-103-AP-4B & $3 / 22 / 88$ & 53) & CASS & s & Diasagreement with nearby thermocouples \\
\hline TE-108-AP-4B & $3 / 23 / 88$ & 54 & CASS & $\mathbf{s}$ & Disegreement with nearby thermocouples \\
\hline TE-103-AP-4B & $3 / 24 / 88$ & 52 & CASS & $\mathbf{s}$ & Disapreament with nearby thermocouples \\
\hline TE-103-AP-48 & $3 / 25 / 88$ & 52 & CASS & $\mathbf{S}$ & Diagareament with nearby thermocouples \\
\hline TE-103-AP-48 & $3 / 26 / 88$ & 56 & CASS & s & Disaprearnent with nearby thermocouples \\
\hline TE-103-AP-48 & $3 / 27 / 88$ & 51 & CASS & $\mathbf{s}$ & Disepreement with nearby thermocouples \\
\hline TE-108-AP-4B & $3 / 28 / 88$ & 51 & CASS & S & Disagreement with nearby thermocouples \\
\hline$T E-103-A P-4 B$ & $3 / 29 / 88$ & 54 & CASS & $\mathbf{s}$ & Disagreement with nearby thermocouplas \\
\hline TE-103-AP-48 & $3 / 30 / 88$ & 53 & CASS & s & Disagraement with nearby thermocouples \\
\hline TE-103-AP-48 & $3 / 31 / 88$ & 52 & CASS & $\mathbf{s}$ & Disagreament with nearby thermocouples \\
\hline TE-103-AP-48 & $6 / 1 / 88$ & 66 & CASS & $\mathbf{S}$ & Disagreement with nearby thermocouples \\
\hline TE-103-AP-48 & $6 / 2 / 88$ & 67 & CASS & $\mathbf{s}$ & Disagreement with nearby thermocouples \\
\hline TE-103-AP-48 & 6/3/88 & 67! & CASS & $\mathbf{S}$ & Disapreament with nearby thermocouples \\
\hline TE-103-AP-48 & 6/4/88 & 68 & CASS & $\mathbf{S}$ & Disagreament with nearby thermocouples \\
\hline TE-103-AP-48 & 6/5/88 & 69: & CASS & $\mathbf{S}$ & Disagreament with nearby thermocouples \\
\hline TE-103-AP-48 & 6/6/8B & 71 & CASS & 5 & Disagreement with nearby thermocouples \\
\hline TE-103-AP-48 & $6 / 7 / 68$ & 69 & CASS & $\mathbf{S}$ & Disegreement with nearby thermocouples \\
\hline TE-103-AP-48 & $6 / 8 / 88$ & 64 & CASS & $\mathbf{s}$ & Disagreement with nearby thermocouples \\
\hline TE-103-AP-48 & $6 / 9 / 80$ & 65 & CASS & $\mathbf{s}$ & Disagreement with nearby thermocouples \\
\hline TE-103-AP-48 & $6 / 11 / 88$ & 66 & CASS & $\mathbf{S}$ & Disagreement with nearby thermocouples \\
\hline TE-103-AP-48 & $6 / 12 / 88$ & 69: & CASS & $\mathbf{S}$ & Dissqreement with nearby thermocouples \\
\hline TE-103-AP-48 & $6 / 13 / 88$ & 69 & CASS & s & Disagreement with nearby thermocouples \\
\hline TE-103-AP-48 & $6 / 14 / 88$ & 69 & CASS & $\mathbf{s}$ & Disagreament with nearby tharmocouples \\
\hline TE-103-AP-48 & $6 / 15 / 8$ & 69 & CASS & $\mathbf{s}$ & Disagreement with nearby thermocouples \\
\hline$T E-103-A P-48$ & $6 / 16 / 88$ & 69 & CASS & $\mathbf{s}$ & Disagraement with nearby thermocouples \\
\hline TE-103-AP-48 & $6 / 17 / 88$ & 69 & CASS & S & Disegreement with nearby thermocouples \\
\hline TE-103-AP-48 & 6/18/88: & 69 & CASS & $\mathbf{s}$ & Disagregment with nearby thermocouples \\
\hline TE-103-AP-4B & $6 / 19 / 88$ & 69 & CASS & $\mathbf{s}$ & Disagreement with nearby thermocouples \\
\hline TE-103-AP-48 & $6 / 20 / 88$ & 69 & CASS & $\mathbf{S}$ & Disa?reament with nasrby tharmacouples \\
\hline
\end{tabular}




\begin{tabular}{|c|c|c|c|c|c|}
\hline \multicolumn{6}{|c|}{ TANK 241-AP-103 } \\
\hline & & & & & \\
\hline THERMOCOUPLE * & DATE & TEMPEAATURE ( $\left.{ }^{\circ} \mathrm{F}\right)$ & DATA SOUACE & QUALTY & COMMENT \\
\hline TE-103-AP-48 & $6 / 21 / 88$ & 69 & CASS & $\mathbf{s}$ & Disagreament with nearby thermocouples \\
\hline TE-103-AP-4B & $6 / 22 / 88$ & 70 & CASS & $\mathbf{S}$ & Disagreement with nearby thermocouples \\
\hline TE-103-AP-4B & $6 / 23 / 88$ & 70 & CASS & $\mathbf{s}$ & Disapreament with nearby thermocouples \\
\hline TE-103-AP-48 & $6 / 24 / 88$ & 71 & CASS & $\mathbf{s}$ & Disagreement with nearby thermocouples \\
\hline TE-103-AP-48 & $6 / 25 / 88$ & 71 & CASS & $\mathbf{S}$ & Disagreement with nearby tharmocouples \\
\hline TE-103-AP-48 & $6 / 26 / 88$ & 71 & CASS & $\mathbf{5}$ & Disagreement with nearty thermocouples \\
\hline TE-103-AP-48 & $6 / 27 / 88$ & 71 & CASS & $\mathbf{s}$ & Oisegreament with nearby thermocouples \\
\hline TE-103-AP-48 & $6 / 28 / 88$ & 71 & CASS & $\mathbf{s}$ & Disegreement with nearby thermocouples \\
\hline TE-103-AP-48 & $6 / 29 / 8 B$ & 72 & CASS & $\mathbf{s}$ & Disagreement with nearby thermocouplas \\
\hline TE-103-AP-4B & $6 / 30 / 88$ & 72 & CASS & $\mathbf{S}$ & Disagreament with neacby thermocouples \\
\hline TE-103-AP-48 & 7/1/83 & 72 & CASS & $\mathbf{S}$ & Disedreement with nearby thermocouples \\
\hline TE-103-AP-48 & $7 / 3 / 88$ & 73 & CASS & $\mathbf{S}$ & Disegreament with nearby thermocouples \\
\hline TE-103-AP-48 & 7/4/88 & 74 & CASS & $\mathbf{S}$ & Disegreament with nearby thermocouples \\
\hline TE-103-AP-48 & $7 / 5 / 80$ & 73 & CASS & $\mathbf{s}$ & Disagreament with nearby thermocouples \\
\hline TE-103-AP-4B & $7 / 6 / 88$ & 75 & CASS & $\mathbf{S}$ & Disagreament with naarby thermocouplas \\
\hline TE-103-AP-48 & 7/7/88 & 74 & CASS & $\mathbf{S}$ & Disagreement with nearby thermocouples \\
\hline TE-103-AP-A8 & 7788 & 73 & CASS & $\mathbf{S}$ & Disagreement with nearby thermocouples \\
\hline TE-103-AP-48 & 7/9/38 & 72 & CASS & $\mathbf{S}$ & Disspredment with nearby thermocouples \\
\hline TE-103-AP-4B & $7 / 10 / 88$ & 74 & CASS & $\mathbf{s}$ & Disagreemant with nearby thermocouples \\
\hline TE-103-AP-4B & $7 / 11 / 88$ & 72 & CASS & $\mathbf{S}$ & Disagreement with nearby thermocouples \\
\hline TE-103-AP-48 & $7 / 12 / 88$ & 72 & CASS & $\mathbf{s}$ & Disagreament with nearby thermocouplas \\
\hline TE-108-AP-48 & $7 / 13 / 68$ & 74 & CASS & $\mathbf{S}$ & Disegreement with nearby thormocouples \\
\hline TE-103-AP-48 & $7 / 14 / 88$ & 72 & CASS & $\mathbf{s}$ & Disagreament with nearby thermocouples \\
\hline TE-103-AP-48 & $7 / 15 / 68$ & 72 & CASS & $\mathbf{S}$ & Disegraement with raerby thermocouples \\
\hline TE-103-AP-48 & $7 / 16 / 8$ & 74 & CASS & S & Disegroemam with nearby tharmocouples \\
\hline TE-103-AP-48 & $7 / 17 / 88$ & 72 & CASS & $\mathbf{S}$ & Disagreement with nearby thermocouples \\
\hline TE-108-AP-48 & $7 / 78 / B^{-1}$ & 72 & CASS & $\mathbf{S}$ & Diagreament with nearby thermocouples \\
\hline TE-103.AP-48 & $7 / 19 / 8 \mathrm{~B}$ & 73 & CASS & $\mathbf{s}$ & Disagreernent with nearby thermacouplas \\
\hline TE-103-AP-AB & $7 / 20 / 88$ & 71 & CASS & $\mathbf{s}$ & Disapraement with nearby thermocouples \\
\hline TE-103.AP-48 & $7 / 21 / 88$ & 71 & CASS & $\mathbf{s}$ & Disagreement with nearby thermocouples \\
\hline TE-103-AP-48 & $7 / 22 / 88$ & 71 & CASS & $\mathbf{S}$ & Disaproement with nearby thermocouples \\
\hline TE-103-AP-48 & $7 / 23 / 88$ & 72 & CASS & $\mathbf{s}$ & Distapreement with naarby thermocouplos \\
\hline TE-103-AP-48 & $7 / 24 / 88$ & 71 & CASS & $\mathbf{S}$ & Disagreement with naarby thermocouples \\
\hline TE-103-AP-48 & $7 / 25 / 88$ & 71 & CASS & s & Dieagreament with nearby thermocouples \\
\hline TE-103-AP-48 & $7 / 26 / 88$ & 72 & CASS & $\mathbf{S}$ & Disegreement with neaby tharmocouples \\
\hline TE-103-AP-48 & $7 / 27 / 88$ & 71 & CASS & $\mathbf{S}$ & Oisegreement with neaby thermocouples \\
\hline TE-103-AP-48 & $7 / 28 / 88$ & 72 & CASS & $\mathbf{S}$ & Dirapreament with nearby thermocouples \\
\hline TE-103-AP-48 & $7 / 29 / 88$ & 72 & CASS & $\mathbf{5}$ & Disagreament with nearby thermocouples \\
\hline TE-103-AP-48 & $7 / 30 / 38$ & 73 & CASS & $\mathbf{s}$ & Disagreement with nearby thermocouples \\
\hline TE-103-AP-48 & $7 / 31 / 88$ & 72 & CASS & $\mathbf{5}$ & Ditagraemont with nearby thermocouples \\
\hline TE-103-AP-48 & $8 / 1 / 88$ & 72 & CASS & $\mathbf{S}$ & Disegraement with neaby thormocouples \\
\hline TE-103-AP-48 & $8 / 2 / 38$ & 72 & CASS & $\mathbf{s}$ & Giaspreement with nearby thermocouples \\
\hline$T E-103-A P-48$ & $8 / 4 / 88$ & 72 & CASS & $\mathbf{S}$ & Oizapreament with naaby thermocouplas \\
\hline TE-103-AP-48 & B/5/B8 & 71 & CASS & $\mathbf{s}$ & Diasogeennent with nearby tharmocouples \\
\hline TE-103-AP-48 & 8/6/88 & 72 & CASS & $\mathbf{5}$ & Disapreement with nearby thermocouples \\
\hline TE-103-AP-48 & $8 / 8 / 88$ & 73 & CASS & $\mathbf{5}$ & Disagraement with nearby thermocouples \\
\hline TE-103-AP-48 & $8 / 9 / 88$ & 71 & CASS & $\overline{\mathbf{S}}$ & Disagreement with nearby thermocouples \\
\hline TE-103-AP-48 & $8 / 10 / 88$ & 71 & CASS & $\mathbf{S}$ & Diseoroement with nearby thermocouples \\
\hline TE-103-AP-48 & $8 / 11 / 88$ & 72 & CASS & s & Disapreement with nearby thermocouples \\
\hline TE-103-AP-48 & $8 / 12 / 88$ & 73 & CASS & $\mathbf{S}$ & Disagreement with nearby thermocouples \\
\hline TE-103-AP-48 & $8 / 13 / 88$ & 72 & CASS & $\mathbf{S}$ & Disagreement with nearby thermocouples \\
\hline TE-103-AP-48 & $8 / 14 / 88$ & 73 & CASS & $\mathbf{s}$ & Disagreement with nearby thermocouples \\
\hline TE-103-AP-4B & $8 / 15 / 88$ & 72 & CASS & $\mathrm{s}$ & Disagreement with nearby tharmocouples \\
\hline TE-103-AP-4B & $8 / 16 / 88$ & 72 & CASS & $\mathbf{S}$ & Disagreement with naarby thermocouples \\
\hline TE-103-AP-48 & $8 / 17 / 8$ & 71. & CASS & $\mathbf{s}$ & Disagreement with nearby thermocouples \\
\hline TE-103-AP-48 & $8 / 19 / 88$ & 72 & CASS & $\mathbf{s}$ & Dissgreement with nearby thermocouples \\
\hline TE-103-AP-48 & $8 / 20 / 88$ & 73 & CASS & $\mathbf{S}$ & Disagreement with nearby thermocouples \\
\hline TE-103-AP-48 & $8 / 21 / 88$ & 72 & CASS & $\mathbf{S}$ & Disagreament with nearby thermocouples \\
\hline TE-103-AP-48 & $8 / 23 / 88$ & 72 & CASS & $\mathbf{s}$ & Disapreament with nearby thermocouples \\
\hline TE-103-AP-48 & $8 / 24 / 88$ & 71 & CASS & $\mathbf{S}$ & Disagreament with nearby thermocouples \\
\hline TE-103-AP-48 & $8 / 25 / 38$ & 73 & CASS & $\mathbf{S}$ & Disagreement with nearby thermocouples \\
\hline TE-10B-AP-4B & $8 / 27 / 88$ & 72 & CASS & $\mathbf{S}$ & Disegreement with nearby thermocouples \\
\hline TE-103-AP-48 & $8 / 28 / 88$ & 74 & CASS & $\mathbf{s}$ & Disagreement with nearby thermocouples \\
\hline TE-108-AP-48 & $8 / 29 / 38$ & 71 & CASS & $\mathbf{S}$ & Disopreament with nearby thermocouples \\
\hline TE-103-AP-48 & $8 / 30 / 88$ & 72 & CASS & $\mathbf{S}$ & Disagreement with nearby thermocouples \\
\hline TE-103-AP-48 & $8 / 31 / 88$ & 72 & CASS & $\mathbf{S}$ & Disagreament with nearby thermocouples \\
\hline TE-103-AP-48 & $9 / 1 / 88$ & 72 & CASS & $\mathbf{s}$ & Disepreement with nearby thermocouples \\
\hline TE-103-AP-48 & $9 / 2 / 38$ & 72 & CASS & $\mathbf{s}$ & Disegreement with nearby thermocouplos \\
\hline TE-103-AP-48 & $9 / 3 / 38$ & 71 & CASS & $\mathbf{s}$ & Disaproement with nearby thermocouples \\
\hline TE-103-AP-48 & 9/4/8B & 72 & CASS & $\mathbf{S}$ & Disagreament with nearby thermocouples \\
\hline TE-103-AP-48 & $9 / 5 / 88$ & 71 & CASS & $\mathbf{S}$ & Disegreement with nearby thermocouples \\
\hline TE-103-AP-48 & $9 / 6 / 88$ & 72 & CASS & $\mathbf{S}$ & Disegreement with nearby thermocouples \\
\hline TE-103-AP-48 & 9/7/88 & 72 & CASS & $\mathbf{s}$ & Disegreement with naarby thermocouples \\
\hline
\end{tabular}




\begin{tabular}{|c|c|c|c|c|c|}
\hline \multicolumn{6}{|c|}{$\begin{array}{l}\text { TANK 241-AP-10S } \\
\text { THEFIMOCOUFLE DATA }\end{array}$} \\
\hline & & & & & \\
\hline THERMOCOUPL : & DATE & TEMPEAATURE ("F) & DATA SOUACE & QUAUTYY & COMMENT \\
\hline TE-103-AP-48 & $8 / 8 / 88$ & 71 & CASS & $\overline{\mathbf{S}}$ & Disepreement with nearby thermocouples \\
\hline TE-103-AP-48 & $9 / 9 / 88$ & 72 & CASS & $\mathbf{S}$ & Disagreement with nearby thermocouples \\
\hline TE-103-AP-4B & 9/10/88 & 71 & CASS & $\mathbf{S}$ & Disarreement with nearby thermocouples \\
\hline TE-103-AP-48 & $9 / 11 / 88$ & 71 & CASS & $\mathbf{S}$ & Disepreament whi nearby thermocouplos \\
\hline TE-TO2-AP-48 & $9 / 12 / 83$ & 73 & CASS & $\mathbf{s}$ & Disegroement with neaby thermocouples \\
\hline TE-103.AP-48 & $9 / 13 / 88$ & 71 & CASS & $\mathbf{s}$ & Disagreament with noarby tharmocouplos \\
\hline TE-103-AP-48 & 9/14/88 & 71 & CABSS & $\mathbf{S}$ & Disegreement with noarby thermocouples \\
\hline TE-103-AP-48 & 9/15/88 & 71 & CASS & $\mathbf{S}$ & Disegreement with nearby thermoccuples \\
\hline TE-103-AP-48 & $9 / 16 / 88$ & 71 & CASS & $\mathbf{S}$ & Disapreement with nearby thermocouples \\
\hline$T E-103-A P-4 B$ & $8 / 17 / 88$ & 71 & CASS & $\mathbf{S}$ & Disapreament with nearby tharmocouples \\
\hline TE-103-AP-48 & 9/18/88 & 71 & CASS & $\mathbf{S}$ & Oisagreement with nearby thermocouples \\
\hline TE-103.AP-4B & $9 / 19 / 88$ & 71 & CASS & $\mathbf{s}$ & Disapreement with nearby thermocouples \\
\hline TE-103-AP-48 & 9/20/88 & 71 & CASS & $\mathbf{S}$ & Disagreement with nearby thermocouplas \\
\hline TE-103-AP-48 & $9 / 21 / 88$ & 65 & CASS & $\mathbf{S}$ & Disarroement with naarby thermocouples \\
\hline TE-103-AP-48 & $9 / 22 / 88$ & 70 & CASS & $\mathbf{S}$ & Digergeernemt whth nearby thermocouples \\
\hline TE-103-AP-48 & $9 / 23 / 83$ & 66 & CASS & $\mathbf{S}$ & Disary reement with noarby thermocouples \\
\hline TE-103-AP-4B & $9 / 24 / 68$ & 65 & CASS & s & Diagreement with noarby tharmocouples \\
\hline TE-103-AP-4B & $9 / 25 / 88$ & 70 & CASS & $\mathbf{5}$ & Disagreernent with neaby thermocouples \\
\hline TE-103-AP.4B & $9 / 26 / 88$ & 67 & CASS & $\mathbf{S}$ & Disagreement with nearty thermacouples \\
\hline$T E-103-A P-48$ & $9 / 27 / 88$ & 86 & CASS & $\mathbf{S}$ & Disagreement with nearby thermocouples \\
\hline TE-103-AP-48 & $9 / 29 / 83$ & 69 & CASS & $\mathbf{s}$ & Digergeament with nearby thermocouples \\
\hline TE-103-AP-48 & 9/30/38 & 68 & CASS & $\mathbf{S}$ & Diaegresment with nearby thermocouples \\
\hline TE-103-AP-48 & $\sqrt{2 \pi / 88}$ & 50. & CASS & $\mathbf{S}$ & Oiasogreament whth neshy thermocouples \\
\hline TE-103-AP-48 & 12/2/38 & $\mathbf{5 2}$ & CASS & $\mathbf{S}$ & Disegreament with nearby themocouples \\
\hline$T E \cdot 103-A P-48$ & $1213 / 88$ & Bo & CASS & $\mathbf{s}$ & Diagoreament with noarby thermocouples \\
\hline TE-103-AP-48 & 12/4/88 & E0 & CASS & $\mathbf{s}$ & Diaspreament with nosiby thermocouples \\
\hline TE-103-AP-4B & $12 / 5 / 88$ & 49 & CASS & $\mathbf{s}$ & Dieseproement with nearby thermocouples \\
\hline TE-103-AP-48 & $12 / 6 / 88$ & 49 & CASS & $\mathbf{s}$ & Disagreoment with naarby thermocouples \\
\hline TE-103-AP-4B & $12 / 7 / 88$ & 501 & CASS & $\mathbf{S}$ & Diagroement with nearby thermocouples \\
\hline TE-103-AP-4B & 12/8/88 & 51 & CASS & $\mathbf{s}$ & Disegreement with nearby thermocouples \\
\hline$T E-103-A P-48$ & 12/9/98 & 53: & CASS & $\mathbf{s}$ & Diasereement with neaby thermocouples \\
\hline TE-103-AP-48 & $12 / 10 / 8$ & 54. & CASS & $\mathbf{S}$ & Diepreament with mearby thormocouples \\
\hline TE-103.AP.AB & $12 / 11 / 88$ & 52 & CASS & $\mathbf{s}$ & Diedresment with neaby thermocouples \\
\hline TE-103-AP-48 & $12 / 12 / 88$ & 31 & CASS & $\mathbf{s}$ & Diagereement with nearby thesmocouples \\
\hline TE-108-AP-48 & 12/13/88 & 53 & CASS & $\mathbf{s}$ & Disepreament with nearby tharmocouplos \\
\hline TE-103-AP-48 & $12 / 14 / 88$ & 52 & CASS & $\mathbf{s}$ & Diangreament with nearby thermoccuples \\
\hline TE-103-AP-48 & $12 / 15 / 88$ & 49 & CASS & $\mathbf{s}$ & Diaspreement with nestiby thermocouples \\
\hline TE-103-AP-48 & $12 / 16 / 83$ & 48 & CASS & $\mathbf{s}$ & Diagreernent with nearby thermocouplas \\
\hline TE-103.AP-48 & $12 / 17 / 88$ & 46 & CASS & $\mathbf{s}$ & Diapgreement with nearby thamocouples \\
\hline TE-103-AP-48 & $12 / 18 / 8$ & 47 & CASS & $\mathbf{S}$ & Diesproement with nearby thormoccuples \\
\hline TE-108-AP-48 & $12 / 19 / 88$ & 46 & CASS & $\mathbf{s}$ & Disgareement with nearby theimocouples \\
\hline TE-103-AP-48 & $12120 / 89$ & 45 & CASS & $\mathbf{s}$ & Ditagreamem with nearby thermocouplas \\
\hline TE-103-AP-A8 & $12 / 21 / 88$ & 45 & CASS & $\mathbf{s}$ & Dieagreament with neaby thermocouples \\
\hline TE-103-AP-48 & $12 / 22 / 88$ & 46 & CASS & $\mathbf{s}$ & Disegreement with nearby thormocouples \\
\hline TE-103-AP-48 & $9 / 1 / 39$ & 64 & CASS & $\mathbf{s}$ & Disapreament with nearby thormocouples \\
\hline$T E-103-A P-48$ & 9/2/89 & 65 & CASS & $\mathbf{s}$ & Diasgreament with narby tharmocouples \\
\hline IE-103-AP-48 & $9 / 3 / 89$ & 67| & CASS & $\mathbf{s}$ & Disugreament with nearby thermocouplas \\
\hline TE-103-AP-48 & $9 / 4 / 89$ & 85 & CASS & $\overline{\mathbf{S}}$ & Disagroement with nearby thermocouplas \\
\hline TE-103-AP-48 & 9/5/89 & 65 & CASS & $\mathbf{S}$ & Disepreement with nearby thermocouples \\
\hline TE-103-AP-48 & 9/6/89 & 65 & CASS & $\mathbf{s}$ & Disagreament with nearby thermocouples \\
\hline TE-103-AP-48 & $9 / 7 / 89$ & 66 & CASS & $\mathbf{s}$ & Disagreament with nasiby thermocouplas \\
\hline TE-103-AP-48 & $9 / 8 / 89$ & 65 & CASS & $\mathbf{s}$ & Disagreement with nearby thormocouples \\
\hline TE-103-AP-48 & $9 / 9 / 89$ & 66 & CASS & $\mathbf{s}$ & Disapreement with nearby thermocouples \\
\hline$\overline{T E}-103-A P-48$ & 9/10/89 & 68. & CASS & $\mathbf{S}$ & Disagreement with nesby thermocouples \\
\hline IE-103-AP-4B & 9/11/89 & 65 & CASS & $\mathbf{S}$ & Disaproement with naarby tharmocouples \\
\hline TE-108-AP-48 & $9 / 13 / 69$ & 65 & CASS & $\mathbf{S}$ & Disepreament with nearby thermocouples \\
\hline TE-103-AP-48 & $9 / 14 / 89$ & 66 & CASS & $\mathbf{s}$ & Disapreement with nearby thermocouples \\
\hline TE-103-AP-49 & $9 / 15 / 89$ & 65 & CASS & $\mathbf{S}$ & Disegreament with naarby thermocouples \\
\hline TE-103-AP-48 & $9 / 16 / 89$ & 65: & CASS & $\mathbf{S}$ & Diasogreement with neartoy tharmocouplas \\
\hline TE-103-AP-48 & $9 / 17 / 89$ & 65 & CASS & $\mathbf{s}$ & Disagreement with nearby tharmocouples \\
\hline TE-103-AP-48 & $9 / 18 / 89$ & 65 & CASS & $\mathbf{S}$ & Disepreement with nearby thermocouples \\
\hline TE-103-AP-48 & 9/19/39 & 66 & CASS & S & Disapreament with naarby thermocouples \\
\hline TE-103-AP-48 & $8 / 20 / 89$ & 67 & CASS & $\mathbf{S}$ & Disagreament with nearby thermocouples \\
\hline TE-103-AP-48 & $9 / 22 / 89$ & 65 & CASS & $\overline{\mathbf{S}}$ & Disacreement with nearby thermocouples \\
\hline TE-103-AP-48 & $8 / 23 / 89$ & 65 & CASS & $\mathbf{S}$ & Disagreament with nearby thermocouplas \\
\hline TE-103-AP-48 & $9 / 24 / 89$ & 66 & CASS & $\mathbf{S}$ & Disegreement with nearby tharmocouples \\
\hline TE-103-AP-4B & 9/25/89 & 65 & CASS & $\mathbf{S}$ & Disegreement with nearty tharmocouples \\
\hline TE-TOS-AP.48 & $9 / 26 / 89$ & 66 & CASS & $\mathbf{s}$ & Disegreament with nearty tharmocouplas \\
\hline TE-103-AP-48 & 9/27/89 & 66 & CASS & $\mathbf{S}$ & Disagreament with nesiby thermocouples \\
\hline TE-103-AP-48 & $9 / 28 / 89$ & 66 & CASS & $\mathbf{s}$ & Diseqreament with nearby thermocouples \\
\hline TE-103-AP-48 & $9 / 29 / 89$ & 65 & CASS & $\mathbf{s}$ & Disagreement with nearby thermocouples \\
\hline TE-103-AP-48 & $9 / 30 / 89$ & 65 & CASS & $\mathbf{S}$ & Disegreament with nearby thermocouples \\
\hline TE-103-AP-48 & $11 / 2 / 89$ & 72 & CASS & $\mathbf{s}$ & Disepreament with nearby thermocouples \\
\hline
\end{tabular}


*als View/Print Document Cover Sheet tok

This document was retrieved from the Boeing ISEARCH System.

Accession \#: D196051032

Document \#: SD-WM-ER-315

Title/Desc:

SUPPORTING DOCUMENT FOR SOUTHEAST QUADRANT HISTORICAL TANK CONTENT ESTIMATE REPORT FOR AP

TANK FARM [VOL I SEC 2 OF 2 PAGE D-295 TO D-720] 
THIS DOCUMENT WAS TOO LARGE TO SCAN AS A SINGLE DOCUMENT. IT REQUIRED BREAKING INTO SMALLER SECTIONS.

DOCUMENT NUMBER: $S D-\omega m-E R-315(0) I)$

SECTION: 2 OF 2

TTLE: Southeast Quadront +fistoridal Yoah Oonlent Estimate Reporl for AP Jank

AUTHOR: $\angle A$ Taddes $/$ SD Conert RECIPIENT:

DATE:

$91 / 3 / 95$

KEYWORDS:

andis

REFERENCES: 
THEIS PAGT INYFNTONAUY

IFTH BIANK

- 


\begin{tabular}{|c|c|c|c|c|c|}
\hline \multicolumn{6}{|c|}{$\begin{array}{l}\text { TANK 241-AP-103 } \\
\text { THERMOCOUPE DATA }\end{array}$} \\
\hline THERMOCOUPLE * & DATE & TEMPERATUAE $\left({ }^{\circ} \mathrm{F}\right)$ & DATA SOURCE & QUALITY & COММЕМT \\
\hline TE-103-AP-48 & $12 / 26 / 89$ & 47 & CASS & $\mathrm{s}$ & $\begin{array}{l}\text { ComimENT } \\
\text { Disagreement with nearby thermocouples }\end{array}$ \\
\hline TE-103-AP-48 & $12 / 27 / 89$ & 47 & CASS & $\mathbf{S}$ & Disagreament with nearby thermocouples \\
\hline TE-103-AP-48 & $12 / 28 / 89$ & 46 & CASS & $\mathbf{s}$ & Disegreement with nearby thermocouplas \\
\hline TE-103-AP-48 & $12 / 29 / 89$ & 46 & CASS & $\mathbf{S}$ & Disagreement with nearby thermocouples \\
\hline TE-103-AP-48 & $12 / 30 / 89$ & 46 & CASS & $\mathbf{S}$ & Dissgreement with nearby thermocouples \\
\hline TE-103-AP-48 & $12 / 31 / 89$ & 48 & CASS & $\mathbf{S}$ & Dissgreement with nearby thermocouples \\
\hline TE-103-AP-48 & $1 / 1 / 90$ & 46 & CASS & $\mathbf{S}$ & Disagreement with nearby thermocouplas \\
\hline TE-103-AP-48 & $1 / 2 / 90$ & 45 & CASS & $\mathbf{s}$ & Disagreement with nearby thermocouples \\
\hline TE-103-AP-48 & $1 / 3 / 80$ & 45 & CASS & $\mathbf{S}$ & Disagreement with nearby thermocouples \\
\hline TE-103-AP-48 & $1 / 4 / 90$ & 47 & CASS & $\mathbf{s}$ & Disagreement with nearby thermocouples \\
\hline TE-103-AP-48 & $1 / 5 / 90$ & 45 & CASS & $\underline{\mathbf{S}}$ & Disagreement with nearby thermocouples \\
\hline TE-108-AP-48 & $1 / 6 / 90$ & 50 & CASS & $\mathbf{S}$ & Disagreament with nearby thermocouples \\
\hline TE-YOB-AP-4B & $1 / 7 / 90$ & 53 & CASS & $\mathbf{S}$ & Disagreement with nearby thermocouples \\
\hline TE-103-AP-48 & 1/9/80 & 53 & CASS & $\mathbf{s}$ & Disagreament with nearby thermocouples \\
\hline TE-108-AP-48 & 1/10/90 & 63 & CASS & $\mathbf{S}$ & Disagreament with nearby thermocouples \\
\hline TE-103-AP-48 & $1 / 11 / 90$ & 59 & CASS & $\mathbf{s}$ & Disegreement with nearby thermocouples \\
\hline TE-103-AP-48 & //12/90 & 58 & CASS & $\mathbf{S}$ & Disepraement with nearby thermocouples \\
\hline TE-103-AP-48 & //13/90 & 59 & CASS & $\mathbf{S}$ & Disagreement with nearby thermocouples \\
\hline IE-103-AP-48 & 1/14/80 & 57 & CASS & $\mathbf{5}$ & Dieagreement with neaby thermocouples \\
\hline TE-103-AP-4B & $1 / 125 / 90$ & 54 & CASS & $\mathbf{s}$ & Oisegreemem with nearby thermocouples \\
\hline TE-103-AP-48 & $1 / 16 / 90$ & 55 & CASS & $\bar{s}$ & Disagreement with naarby tharmocouplas \\
\hline TE-103-AP-48 & $1 / 17 / 90$ & 54 & CASS & $\mathbf{s}$ & Disegreement with noaby thermocouplas \\
\hline TE-103-AP-46 & 1/18/90 & 46 & CASS & $\mathbf{S}$ & Disapreement with nasby thermocouples \\
\hline TE-103.AP-48 & $1 / 19 / 90$ & 48 & CASS & $\mathbf{S}$ & Disepresmant with nearby thermocouples \\
\hline TE-103-AP-48 & $1 / 20 / 90$ & 49 & CASS & $\mathbf{s}$ & Disapreement with nearby thermocouples \\
\hline TE-103-AP-48 & $1 / 21 / 90$ & 48 & CASS & $\overline{\mathrm{S}}$ & Disegreement with nearby thermocouples \\
\hline TE-103-AP-48 & $1 / 22 / 90$ & 48 & CASS & $\mathbf{s}$ & Disagreement with nearby thermocouples \\
\hline TE-103-AP-4B & $1 / 23 / 90$ & 49 & CASS & $\mathbf{S}$ & Diseqreement with nestby thormocouplas \\
\hline TE-103-AP-48 & $1 / 24 / 80$ & 47 & CASS & $\mathbf{S}$ & Disegreement with nearby thermocouples \\
\hline TE-103.AP-48 & $1 / 25 / 90$ & 48 & CASS & $\mathbf{S}$ & Disagreement with nearty thermocouples \\
\hline TE-103-AP-4B, & $1 / 26 / 90$ & 48 & CASS & $\mathbf{S}$ & Disagreament with nearby thermocouples \\
\hline TE-103-AP-48 & $1 / 27 / 90$ & 46 & CASS & $\mathbf{s}$ & Disagrearnent with neaby thermocouples \\
\hline TE-103-AP-4B & $1 / 23 / 90$ & 48 & CASS & $\mathbf{s}$ & Disargreement with nearby thermocouples \\
\hline TE-103-AP-48 & 1/29/80 & 48: & CASS & $\mathbf{s}$ & Disapresment with nearby thermocouples \\
\hline TE-103-AP-48 & $1 / 30 / 90$ & 48 & CASS & $\mathbf{s}$ & Diagreament with nastby thormocouplas \\
\hline TE-103.AP-48 & $1 / 31 / 90$ & 46 & CASS & $\mathbf{S}$ & Disagrearnent with nearby thermocouples \\
\hline TE-103-AP-48 & $2 / 1 / 90$ & 45 & CASS & $\mathbf{S}$ & Disagreement with nearby thormocouples \\
\hline TE-103-AP-4B & $2 / 2 / 90$ & 46 & CASS & $\mathbf{s}$ & Diadreement with naarby thermocouples \\
\hline TE-103-AP-48 & $2 / 3 / 90$ & 49 & CASS & $\mathbf{S}$ & Disagreament with nearby thermocouples \\
\hline TE-103-AP-48 & $2 / 4 / 90$ & 52 & CASS & $\mathbf{S}$ & Disagreament with nearby tharmocouples \\
\hline TE-103-AP-48 & $2 / 5 / 90$ & 54 & CASS & $\mathbf{s}$ & Disagreemant with nearby thormocouples \\
\hline TE-103-AP-48 & $2 / 6190$ & 47 & CASS & $\mathbf{5}$ & Disagreement with nearby thermocouples \\
\hline TE-103-AP-48 & $2 / 7 / 90$ & 46 & CASS & $\mathbf{s}$ & Disegreement with nearby thermocouples \\
\hline TE-103-AP-48 & $2 / 3 / 90$ & 5i & CASS & s & Disegreement with nearby thermocouples \\
\hline TE-103-AP-48 & $2 / 9 / 90$ & 51 & CASS & $\mathbf{S}$ & Disagreement with nearby thermocouples \\
\hline TE-103-AP-48 & $2 / 10 / 90$ & 53 & CASS & $\mathbf{s}$ & Diaagreement with nearby thermocouples \\
\hline TE-103-AP-48 & $2 / 11 / 90$ & 53 & CASS & $\mathbf{s}$ & Disagreament whth nearby thermocouples \\
\hline TE-103-AP-48 & $2 / 12 / 90$ & 45 & CASS & $\mathbf{S}$ & Disagraement with nearby tharmocouples \\
\hline TE-103-AP-4B & $2 / 13 / 90$ & 47 & CASS & $\mathbf{S}$ & Disagreement with nearby thermocouples \\
\hline TE-103-AP.48 & $2 / 14 / 90$ & 46 & CASS & $\mathbf{S}$ & Disegreement with nearby thermocouples \\
\hline TE-103-AP-48 & $2 / 15 / 90$ & 44 & CASS & S & Disegreement with nearby thermocouples \\
\hline TE-103-AP-48 & $2216 / 30$ & 44 & CASS & $\mathbf{S}$ & Disagreement with nearby thermocouples \\
\hline TE-103-AP-48 & $2 / 17 / 90$ & 44 & CASS & $\mathbf{S}$ & Disapreament with nearby thermocouples \\
\hline TE-103-AP-48 & $2 / 18 / 90$ & 40 & CASS & $\mathbf{S}$ & Disagresment with nearby thermocouples \\
\hline TE-103-AP-48 & $2 / 19 / 90$ & 40 & CASS & $\mathbf{S}$ & Disagreemant with nearby thermocouples \\
\hline TE-103-AP-48 & $2 / 20 / 90$ & 43 & CASS & $\mathbf{S}$ & Disagreement with nearby thermocouples \\
\hline TE-103-AP-48 & $2 / 21 / 90$ & 44 & CASS & $\mathbf{S}$ & Disagraement with nearby thermocouples \\
\hline TE-103-AP-48 & $2 / 22 / 90$ & 48 & CASS & $\mathbf{S}$ & Disagraement with naarby thermocouples \\
\hline TE-103-AP-48 & $2 / 23 / 90$ & 46 & CASS & $\mathbf{5}$ & Disagreement with nearby thermocouples \\
\hline TE-103-AP-48 & $2 / 24 / 90$ & 49 & CASS & $\mathbf{s}$ & Disagreement with nearby thermocouples \\
\hline TE-103-AP-48 & $2 / 25 / 90$ & 47 & CASS & $\mathbf{s}$ & Disagreament with nearby thermocouples \\
\hline TE-103-AP-48 & $2 / 26 / 90$ & 46 & CASS & $\mathbf{s}$ & Disagreement with nearby thermocouples \\
\hline TE-103-AP-48 & $2 / 28 / 90$ & 50 & CASS & $\mathbf{S}$ & Disagraement with nearby thermocouples \\
\hline TE-103-AP-4B & $3 / 2 / 90$ & 49 & CASS & $\mathbf{S}$ & Disagreement with nearby thermocouples \\
\hline TE-103-AP-48 & $3 / 3 / 90$ & 51 & CASS & $\mathbf{s}$ & Disagreement with nearby thermocouples \\
\hline TE-103-AP-4B & $3 / 4 / 90$ & 50 & CASS & $\mathbf{S}$ & Disegreement with nearby thermocouples \\
\hline TE-103-AP-48 & $3 / 5 / 90$ & 50 & CASS & $\mathbf{S}$ & Disegreement with nearby thermocouplas \\
\hline TE-103-AP-48 & $3 / 6 / 90$ & 49 & CASS & $\mathbf{S}$ & Disegreement with nearby thermocouples \\
\hline TE-103-AP-48 & $3 / 7 / 90$ & 55 & CASS & $\mathbf{S}$ & Disagreement with nearby thermocouples \\
\hline$T E=103-A P-48$ & $3 / 8 / 90$ & 62 & CASS & $\mathbf{S}$ & Disegreement with nearby tharmocouples \\
\hline TE-103-AP-48 & $3 / 9 / 90$ & 52 & CASS & $\mathbf{S}$ & Disagreement with nearby thermocouples \\
\hline TE-103-AP-48 & $3 / 10 / 90$ & 57 & CASS & $\mathbf{s}$ & Disagreement with nearby thermocouples \\
\hline IE-103-AP-48 & $3 / 11 / 90$ & 49 & CASS & $\mathbf{S}$ & Disagreement with nearby thermocouplas \\
\hline
\end{tabular}


ANTK 241-AP-103

\begin{tabular}{|c|c|c|c|c|c|}
\hline \multicolumn{6}{|c|}{$\begin{array}{l}\text { TANK 241-AP-103 } \\
\text { THERHOCOUPLE DATA }\end{array}$} \\
\hline & & & & & \\
\hline THERMOCOUPLE * & DATE & TEMPERATUAE $\left({ }^{\circ} \mathrm{F}\right)$ & DATA SOURCE & GUALITY & COMMENT \\
\hline TE-103-AP-48 & $3 / 12 / 90$ & 49 & CASS & $\mathbf{S}$ & Disagreament with nearby thermocouples \\
\hline TE-103-AP-48 & $3 / 13 / 90$ & 48 & CASS & $\mathbf{S}$ & Disagreement with nearby thermocouples \\
\hline TE-103-AP-48 & $3 / 14 / 90$ & 53 & CASS & $\mathbf{S}$ & Disagraement with nearby thermocouples \\
\hline TE-103-AP-4B & 3/15/90 & 56 & CASS & $\mathbf{s}$ & Disagreament with nearby thermocouples \\
\hline TE-108-AP-48 & 3/16/90/ & 58 & CASS & $\mathbf{S}$ & Disagreement with nearby thermocouples \\
\hline TE-103-AP-48 & $3 / 17 / 90$ & 62 & CASS & $\mathbf{S}$ & Disagreement with nearby thermocouples \\
\hline TE-103-AP-48 & $3 / 18 / 90$ & 62: & CASS & $\mathbf{S}$ & Disagreement with nearby thermocouples \\
\hline TE-103-AP-48 & $3 / 19 / 90$ & 62 & CASS & $\mathbf{S}$ & Disagreement with nearby thermocouples \\
\hline TE-103-AP-48 & $3 / 20 / 90$ & 63 & CASS & $\underline{s}$ & Disagreement with nearby thermocouples \\
\hline TE-103-AP-48 & $3 / 21 / 20$ & 62 & CASS & $\mathbf{s}$ & Disagreement with nearby tharmocouples \\
\hline TE-103-AP-48 & $3 / 22 / 90$ & 62 & CASS & $\mathbf{S}$ & Disagreement with nearby thermocouples \\
\hline TE-103-AP-4B & $3 / 23 / 90$ & 62 & CASS & $\mathbf{S}$ & Disagreement with nearby thermocouples \\
\hline TE-103-AP-48 & $3 / 24 / 90$ & 63. & CASS & $\mathbf{S}$ & Disagreament with nearby thormocouples \\
\hline TE-103-AP-48 & $3 / 26 / 90$ & 62 & CASS & $\mathbf{s}$ & Disagreement with nearty themocouples \\
\hline TE-103-AP-48 & $3 / 27 / 90$ & 62 & CASS & 5 & Disagreament with nearby tharmocouples \\
\hline TE-103-AP-48 & $3 / 28 / 90$ & 62 & CASS & $\mathbf{S}$ & Disagreament whth nearby thermocouples \\
\hline TE-103-AP-48 & $3 / 29 / 90$ & 64 & CASS & $\mathbf{s}$ & Disagreament with nearby thermocouples \\
\hline TE-103-AP-48 & $3 / 30 / 90$ & 62 & CASS & $\mathbf{S}$ & Disagreament with naarby thermocouples \\
\hline TE-103-AP-48 & $3 / 31 / 90$ & 62 & CASS & $\mathbf{S}$ & Disagreement with nearby thermocouples \\
\hline TE-103-AP-48 & $4 / 1 / 90$ & 62 & CASS & $\mathbf{S}$ & Disagreement with nearby thermocouples \\
\hline TE-103-AP-AB & $4 / 2 / 90$ & 62 & CASS & $\mathbf{S}$ & Disagreament with nearby thermocouples \\
\hline TE-103-AP-4B & $4 / 3 / 90$ & 64 & CASS & $\mathbf{S}$ & Disagreement with nearby thermocouples \\
\hline TE-103-AP-48 & 4/4/50 & 62. & CASS & $\mathbf{S}$ & Disagreement with nestby thermocouples \\
\hline TE-103-AP-4B & $4 / 5 / 90$ & 62 & CASS & $\mathbf{s}$ & Disagraement with neaby thermocouples \\
\hline TE-103-AP-48 & $4 / 6 / 90$ & 62 & CASS & $\mathbf{S}$ & Disagreament with noarby thermocouples \\
\hline TE-103-AP-4B & $4 / 7 / 90$ & 62 & CASS & $\mathbf{S}$ & Disagresmont with nearby thermocouples \\
\hline TE-103-AP-4B & $4 / 8 / 90$ & 62 & CASS & $\mathbf{s}$ & Disagreement with neaby thermocouples \\
\hline TE-103-AP-48 & $4 / 9 / 90$ & 62 & CASS & $\mathbf{S}$ & Disagreament with nearby thermocouples \\
\hline TE-103-AP-48 & $4 / 10 / 90$ & 61 & CASS & $\mathbf{s}$ & Disugreement with nearby tharmocouples \\
\hline TE-103-AP-48 & $4 / 11 / 90$ & 61) & CASS & $\mathbf{s}$ & Disagreament with nearby thermocouples \\
\hline TE-103-AP-48 & $4 / 2 / 90$ & 61 & CASS & $\mathbf{s}$ & Disagreement with noarby thermocouples. \\
\hline TE-103-AP-48 & $4 / 13 / 90$ & 62 & CASS & $\mathbf{S}$ & Disagraement with neartoy thermocouples \\
\hline TE-103-AP-48 & 4/14/90 & 61 & CASS & $\mathbf{S}$ & Disagreement with nearby thermocouples \\
\hline TE-103-AP-48 & $4 / 15 / 90$ & 64) & CASS & $\mathbf{s}$ & Disagreement with nearby thermocouples \\
\hline TE-103-AP-48 & $4 / 16 / 90$ & 61 & CASS & $\mathbf{s}$ & Disagreemant with nearby thermocouples \\
\hline TE-103-AP-4B & $4 / 17 / 30$ & 61 & CASS & $\mathbf{S}$ & Disagreement with nearby thermocouples \\
\hline TE-103-AP-4B & $4 / 18 / 90$ & 61 & CASS & $\mathbf{s}$ & Disagreement with narby thermocouples \\
\hline TE-103-AP-48 & $4 / 19 / 90$ & 62 & CASS & $\mathbf{s}$ & Disagreament with nearby thermocouples \\
\hline TE-103-AP-48 & $4 / 20 / 90$ & 60 & CASS & $\mathbf{s}$ & Dissoreement with nearby thermocouples \\
\hline TE-103-AP-AB & $4 / 21 / 90$ & 61 & CASS & $\mathbf{s}$ & Distagreement with nearby thermocouples \\
\hline TE-103-AP-48 & $4 / 22 / 90$ & 61 & CASS & $\mathbf{s}$ & Disagreament with nearby thermocouplas \\
\hline TE-103-AP-48 & $4 / 23 / 90$ & 62 & CASS & $\mathbf{5}$ & Disagraement with nearby tharmocouples \\
\hline TE-103-AP-48 & $4 / 24 / 90$ & 61 & CASS & $\mathbf{S}$ & Disagroement with naarby tharmocouplas \\
\hline TE-103-AP-48 & $4 / 25 / 90$ & 61 & CASS & $\mathbf{S}$ & Disagreament with nearby thermocouplas \\
\hline TE-103-AP-48 & $4 / 26 / 90$ & 61) & CASS & $\mathbf{S}$ & Disegreement with neartoy thermocouplas \\
\hline$T E \cdot 103-A P-48$ & $4 / 27 / 90$ & 61 & CASS & $\mathbf{s}$ & Disegreament with nearby thermocouples \\
\hline TE-103-AP-48 & $4 / 28 / 90$ & 61 & CASS & $\mathbf{S}$ & Disegreament with nearby themocouples \\
\hline TE-103-AP-48 & $4 / 29 / 90$ & 62 & CASS & $\mathbf{S}$ & Disagreement with nearby thermacouples \\
\hline TE-103-AP-48 & $4 / 30 / 80$ & 63 & CASS & $\mathbf{s}$ & Disagreement with nearby thermocouples \\
\hline TE-103-AP-48 & $5 / 1 / 90$ & 61 & CASS & $\mathbf{S}$ & Disagreemant with naarby thermocouples \\
\hline TE-103-AP+48 & $5 / 2 / 90$ & 61 & CASS & $\mathbf{s}$ & Disagreement with nearby thermocouples \\
\hline TE-103-AP-4B & $5 / 3 / 90$ & 61 & CASS & $\mathbf{s}$ & Disagreament with nearby thermocouples \\
\hline TE-103-AP-4B & $5 / 4 / 90$ & 62 & CASS & $\mathbf{s}$ & Disagreament with nearby thermocouplas \\
\hline$T E-103-A P-48$ & 5/5/90 & 60 & CASS & $\mathbf{S}$ & Disagreement with nearby thermocouples \\
\hline TE-103-AP-48 & $5 / 6 / 90$ & 61. & CASS & $\mathbf{s}$ & Disagreement with nearby thermocouples \\
\hline TE-103-AP-48 & $5 / 7 / 50$ & 61 & CASS & $\mathbf{s}$ & Disagreement with nearby thormocouples \\
\hline TE-103-AP-48 & 5/8/90 & 62 & CASS & $\mathbf{s}$ & Disagreement with nearby thermocouples \\
\hline TE-103-AP-48 & $5 / 9 / 90$ & 61: & CASS & $\mathbf{S}$ & Disagreement with naarby thermocouples \\
\hline TE-103-AP-48 & $5 / 10 / 90$ & 61: & CASS & $\mathbf{S}$ & Disagreement with nearby thermocouples \\
\hline TE-103-AP-4B & $5 / 11 / 90$ & 61 & CASS & $\mathbf{S}$ & Disagreement with nearby thermocouples \\
\hline TE-103-AP-48 & $5 / 12 / 90$ & 63 & CASS & $\mathbf{S}$ & Disagreement with nearby thermocouples \\
\hline TE-103-AP-48 & $5 / 13 / 90$ & 61 & CASS & $\mathbf{S}$ & Disagreement with nearby thermocouples \\
\hline TE-103-AP-48 & $5 / 14 / 90$ & 62 & CASS & $\mathbf{s}$ & Disagreement with nearby thermocouples \\
\hline TE-103-AP-48 & $5 / 15 / 90$ & 61. & CASS & $\mathbf{S}$ & Disagreament with nearby thermocouples \\
\hline TE-103-AP-48 & $5 / 16 / 90$ & 61: & CASS & $\mathbf{5}$ & Disagreament with naarby thermocouples \\
\hline TE-103-AP-48 & $5 / 17 / 90$ & 61 & CASS & $\mathbf{s}$ & Disagreement with nearby thermocouples \\
\hline TE-103-AP-48 & $5 / 18 / 90$ & 61) & CASS & $\mathbf{S}$ & Disagreement with nearby thermocouples \\
\hline TE-103-AP-48 & $5 / 19 / 90$ & 62 & CASS & $\mathbf{S}$ & Disagreement with nearby thermocouples \\
\hline TE-103-AP-48 & $5 / 20 / 90$ & 61 & CASS & $\mathbf{S}$ & Disagreament with nearby thermocouples \\
\hline TE-103-AP-48 & $5 / 21 / 90$ & 61 & CASS & S. & Disagreement with nearby thermocouples \\
\hline TE-103-AP-48 & $5 / 22190$ & 61 & CASS & $\mathbf{S}$ & Disagreement with naarby thermocouples \\
\hline TE-103-AP-48 & $6 / 1 / 90$ & 61. & CASS & $\mathbf{S}$ & Disagreament with nearby thermocouplos \\
\hline TE-103-AP-48 & 6/2/90 & 63 & CASS & $\mathbf{S}$ & Disagreement with nearby thermocouples \\
\hline
\end{tabular}




\begin{tabular}{|c|c|c|c|c|c|}
\hline \multicolumn{6}{|c|}{$\begin{array}{l}\text { TANK 241-AP:108 } \\
\text { THERMOCOUPLE DATA }\end{array}$} \\
\hline THEAMOCOUPLE * & DATE & TEMPERATUAE $\left({ }^{\circ} \mathrm{F}\right\}$ & DATA SOURCE & OUALTY & COMMENT \\
\hline TE-103-AP-48 & 6/3/90 & $\begin{array}{r}62 \\
\end{array}$ & CASS & $\mathbf{s}$ & Disagreement with nearby thermocouples \\
\hline TE-103-AP-48 & 6/4/90 & 62 & CASS & $\mathbf{S}$ & Disagreament with nearby thermocouples \\
\hline TE-103-AP-4B & 6/5/90 & 61 & CASS & $\mathbf{S}$ & Disagreement with naarby thermocouplas \\
\hline TE-103-AP-4B & 6/6/90 & 61 & CASS & $\mathbf{S}$ & Disagreement with nearby thermocouples \\
\hline TE-108-AP-48 & $6 / 7 / 90$ & 61 & CASS & $\mathbf{S}$ & Disagreement with nearty thermocouples \\
\hline TE-103-AP-48 & 6/8/90 & 60 & CASS & $\mathbf{S}$ & Disagreement with nearby thermocouples \\
\hline TE-103-AP-4B & 6/9/90 & 62 & CASS & $\mathbf{S}$ & Disagreement with nearby thermocouples \\
\hline TE-103-AP-48 & 6/10/90/ & 61 & CASS & $\mathbf{S}$ & Disagreement with nearty thermocouples \\
\hline TE-103-AP-4B & 6/11/90 & 61 & CASS & $\mathbf{s}$ & Disegreement with nearby thermocouples \\
\hline TE-TOS-AP-48 & 6/12/80 & 62 & CASS & $\mathbf{s}$ & Diaegreement with nearby thermocouples \\
\hline TE-103-AP-48 & 6/13/90 & 61 & CASS & $\mathbf{S}$ & Disagreement with nearby thermocouples \\
\hline TE-103-AP-48 & $6 / 14 / 90$ & 62 & CASS & $\mathbf{s}$ & Disagreement with nearby thermocouples \\
\hline TE-103-AP-48 & $6 / 15 / 90$ & 81 & CASS & $\mathbf{S}$ & Disagreement with nearby thermocouples \\
\hline TE-103-AP-48 & $6 / 16 / 90$ & 61 & CASS & $\mathbf{S}$ & Disagreement with noarby thermocouples \\
\hline TE-103-AP-48 & $6 / 17 / 20$ & 62 & CASS & $\mathbf{S}$ & Disagreement with nearby thermocouples \\
\hline TE-103-AP-48 & $6 / 18 / 90$ & 61. & CASS & $\mathbf{s}$ & Disagreement with nearby thermocouples \\
\hline TE-103-AP-48 & 6/19/90 & 61 & CASS & $\mathbf{S}$ & Diagrreement with nearby thermocouples \\
\hline TE-103-AP-4B & $6 / 20 / 90$ & 61 & CASS & $\mathbf{S}$ & Disegreament with nearby thermocouples \\
\hline TE-103-AP-48 & $6 / 21 / 180$ & 62 & CASS & $\mathbf{S}$ & Disagreement with nearby tharmocouples \\
\hline TE-103-AP-48 & $6 / 22 / 90$ & 61 & CASS & $\mathbf{S}$ & Disegrresment with nearby thermocouples \\
\hline TE-103-AP-48 & $6 / 23 / 90$ & 61 & CASS & $\mathbf{S}$ & Dieagreament with naasty thermocouples \\
\hline TE-103-AP-48 & 6/24/90 & 61 & CASS & $\mathbf{S}$ & Dinagreament with nearby thermocouples \\
\hline TE-103-AP-48 & 6/25/90 & 64 & CASS & $\mathbf{S}$ & Disepreament with nodby thermocouplas \\
\hline TE-103-AP-48 & $6 / 26 / 90$ & 61 & CASS & $\mathbf{S}$ & Disagreement with nearby tharmocouples \\
\hline TE-103-AP-48 & $6 / 27 / 90$ & 63 & CASS & $\mathbf{S}$ & Disegreement with nearby thermocouples \\
\hline TE-103-AP-48 & $6 / 28 / 90$ & 61 & CASS & $\mathbf{s}$ & Diasagreement with nearby thermocouplas \\
\hline TE-103-AP-48 & $6 / 29 / 90$ & 61 & CASS & $\mathbf{5}$ & Disegreament with nearby thermocouplas \\
\hline TE-103-AP.4B & $6 / 30 / 90$ & 63 & CASS & $\mathbf{S}$ & Disagreernent with nearby thermocouples \\
\hline TE-108-AP-48 & 7 miso & 62 & CASS & $\mathbf{S}$ & Diadroement with nearby thermocouples \\
\hline TE-103-AP-48 & $7 / 2190$ & 61 & CASS & $\mathbf{S}$ & Oisepreament with nearby thermocouples \\
\hline TE-103-AP-48 & $7 / 3 / 90$ & 62 & CASS & $\mathbf{S}$ & Diaspreament with nearby thermocouples \\
\hline TE-103.AP-48 & $7 / 4 / 80$ & 62 & CASS & $\mathbf{s}$ & Disagreament with neaby therimoccuples \\
\hline TE-103-AP-48 & $7 / 5 / 90$ & 61 & CASS & $\mathbf{s}$ & Disagreament with nearby thermocouples \\
\hline TE-103-AP-48 & $7 / 6150$ & 62 & CASS & $\mathbf{S}$ & Disegreement with nearby thormocouples \\
\hline TE-103-AP-48 & $7 / 7 / 90$ & 62 & CASS & $\mathbf{s}$ & Disegreament with nearby tharmocouples \\
\hline TE-103-AP-48 & $7 / 8 / 90$ & 62 & CASS & $\mathbf{S}$ & Disegreement with nearby thermocouplas \\
\hline TE-103-AP-48 & $7 / 9 / 90$ & 62 & CASS & $\mathbf{S}$ & Disegreement with nearby thermocouplas \\
\hline TE-103-AP-48 & $7 / 10 / 80$ & 62 & CASS & $\mathbf{S}$ & Disagreament with nearby theimocouples \\
\hline TE-103-AP-AB & $7 / 12 / 90$ & 62 & CASS & $\mathbf{S}$ & Disagreement with nearby thermocouples \\
\hline TE-103-AP-48 & $7 / 13 / 90$ & 62 & CASS & $\mathbf{s}$ & Dieagreement with nearby thermocouples \\
\hline TE-103-AP-48 & $7 / 14 / 90$ & 62 & CASS & $\mathbf{S}$ & Disapreement with neasby thermocouples \\
\hline TE-103-AP-48 & $7 / 15 / 90$ & 62 & CASS & $\mathbf{S}$ & Disagreement with nearby thormocouples \\
\hline TE-103-AP-4a & $7 / 16 / 90$ & 62 & CASS & $\mathbf{S}$ & Disagreament with nearby thormocouples \\
\hline TE-103-AP-4B & $7 / 18 / 90$ & 62 & CASS & $\mathbf{s}$ & Disapreement with nearby thermocouples \\
\hline TE-108-AP-48 & $719 / 90$ & 65 & CASS & $\mathbf{S}$ & Disagreament with nearby thermocouples \\
\hline TE-108-AP-48 & $7 / 20 / 90$ & 62 & CASS & $\mathbf{S}$ & Disegreement with nearby thermocouples \\
\hline TE-103-AP-48 & $7 / 21 / 90$ & 64 & CASS & $\mathbf{s}$ & Disegreement with nearby thermocouples \\
\hline TE-103-AP-48 & $7122 / 90$ & 63 & CASS & $\mathbf{s}$ & Disegreement with nearby thermocouples \\
\hline TE-103-AP-48 & $7 / 24 / 90$ & 63 & CASS & $\mathbf{S}$ & Disegreement with nearby thermocouples \\
\hline TE-103-AP-48 & 7/25/90 & 63 & CASS & $\mathbf{S}$ & Disagreement with nearby thermocouples \\
\hline TE-103-AP-48 & $7 / 26 / 90$ & 64 & CASS & $\mathbf{S}$ & Disagreament with nearby thermocouplas \\
\hline TE-103-AP-48 & $7 / 27190$ & 63 & CASS & $\mathbf{S}$ & Disagreement with nearby thermocouples \\
\hline TE-103-AP-48 & $7129 / 90$ & 63 & CASS & $\mathbf{S}$ & Disagreement with nearby thermocouples \\
\hline TE-103-AP-4B & $7 / 30 / 90$ & 63 & CASS & $\mathbf{s}$ & Disagraement with nearby thermocouples \\
\hline TE-103-AP-48 & $7 / 31 / 90$ & 63 & CASS & $\mathbf{5}$ & Disagraement with nearby tharmocouples \\
\hline TE-103-AP-48 & $8 / 1 / 90$ & 64 & CASS & $\mathbf{5}$ & Disegreement with nearby thermocouples \\
\hline TE-103-AP-48 & $8 / 2 / 90$ & 63 & CASS & $\mathbf{S}$ & Disegreement with nearby thermocouplas \\
\hline TE-103-AP-48 & $8 / 3 / 90$ & 63) & CASS & $\mathbf{s}$ & Disagreement with nearby thermocouples \\
\hline TE-103-AP-48 & $8 / 4 / 90$ & 63 & CASS & $\mathbf{S}$ & Disagreement with nearby thermocouples \\
\hline TE-103-AP-48 & $8 / 5 / 90$ & 64 & CASS & $\mathbf{S}$ & Disagreement with nearby thermocouples \\
\hline TE-103-AP-48 & $8 / 6 / 90$ & 63 & CASS & $\mathbf{S}$ & Disugraement with nearby thermocouples \\
\hline TE-103-AP-48 & 8/7/90 & 64. & CASS & $\overline{\mathbf{S}}$ & Disagreament with nearby thermocouples \\
\hline TE-103-AP-48 & $8 / 8 / 90$ & 64| & CASS & $\overline{\mathbf{S}}$ & Disegreement with nearby thermocouplas \\
\hline TE-103-AP-48 & $8 / 9 / 90$ & 64 & CASS & $\mathbf{S}$ & Disagreament with nesby thermocouples \\
\hline TE-103-AP-48 & $8 / 10 / 90$ & 64 & CASS & $\mathbf{S}$ & Disagreament with nearby tharmocouples \\
\hline TE-103-AP-48 & $8 / 11 / 90$ & 64 & CASS & $\mathbf{S}$ & Disagreement with nearby thermocouples \\
\hline TE-103-AP-48 & $8 / 12 / 90$ & 66 & CASS & $\mathbf{S}$ & Disagreement with nearby thermocouples \\
\hline TE-103-AP-48 & 8/13/90 & 64 & CASS & $\mathbf{S}$ & Disagreement with nearby thermocouples \\
\hline TE-103-AP-48 & $8 / 14 / 90$ & 64 & CASS & $\mathbf{s}$ & Disagreement with nearby thermocouples \\
\hline TE-103-AP-48 & $8 / 15 / 90$ & 64. & CASS & $\mathbf{S}$ & Disagreament with nearby thermocouples \\
\hline TE-103-AP-48 & $8 / 16 / 90$ & 65 & CASS & $\mathbf{S}$ & Disegreement with nearby thermocouples \\
\hline TE-103-AP-4B & $8 / 17 / 90$ & 64 & CASS & $\mathbf{s}$ & Disagreement with nearby thermocouples \\
\hline TE-103-AP-4B & $8 / 18 / 90$ & 64 & CASS & $\overline{\mathbf{s}}$ & Disagreement with nearby thermocouples \\
\hline
\end{tabular}




\begin{tabular}{|c|c|c|c|c|c|}
\hline \multicolumn{6}{|c|}{$\begin{array}{l}\text { TANKK 241-AP-103 } \\
\text { THEAMOCOUPLE DATA }\end{array}$} \\
\hline & & & & & \\
\hline THERMOCOUPE $*$ & DATE & TEMPERATURE ( $\left.{ }^{\circ} \mathrm{F}\right)$ & DATA SOUACE & QUALTY & COMMENT \\
\hline TE-103-AP-48 & $8 / 19 / 90$ & 64 & CASS & $\mathbf{s}$ & Disagreement with nearby thermocouples \\
\hline TE-103-AP-48 & 8/20/90 & 67 & CASS & 5 & Disagreament with noarby thermocouples \\
\hline TE-103-AP-4B & $8 / 21 / 90$ & 64 & CASS & S & Dissgreement with nearby thermocouples \\
\hline TE-103-AP-48 & $8 / 22 / 90$ & 64 & CASS & $\mathbf{S}$ & Disagreement with nearby thermocouples \\
\hline TE-103-AP-4B & B/23/90 & 65 & CASS & 5 & Disegreement with nearby thermocouples \\
\hline TE-103-AP-48 & $8 / 24 / 90$ & 65 & CASS & $\mathbf{S}$ & Disagreement with nearty thermocouples \\
\hline TE-103-AP-4B & $8 / 25 / 90$ & 64 & CASS & S & Disagreament with nearby thermocouplas \\
\hline$T E-103-A P-4 B$ & $8 / 26 / 90$ & 65 & CASS & $\mathbf{s}$ & Disagreement with neerby thermocouples \\
\hline TE-103-AP-48 & $8 / 27 / 190$ & 65 & CASS & $\mathbf{S}$ & Disagreament with nearby thermocouples \\
\hline TE-103-AP-48 & $8 / 28 / 90$ & 64 & CASS & $\mathbf{s}$ & Disagreement with nearby thermocouples \\
\hline TE-103-AP-48 & $8 / 29 / 90$ & 64 & CASS & $\mathbf{S}$ & Disagreement with naarby thermocouples \\
\hline TE-103-AP-48 & $8 / 30 / 90$ & 65 & CASS & s & Disagreement with nearby thermocouples \\
\hline TE-103-AP-48 & B/31/80 & 65 & CASS & $\mathbf{S}$ & Oisegreament with nearby thermocouples \\
\hline TE-103-AP-48 & $9 / 1 / 30$ & 65 & CASS & $\mathbf{s}$ & Disagreement with nearby thermocouples \\
\hline TE-103-AP-48 & 9/2/30 & 65 & CASS & $\overrightarrow{\mathbf{S}}$ & Disegreement with nearby thermocouples \\
\hline TE-103-AP-48 & 9/3/90 & 65 & CASS & $\underline{\mathbf{s}}$ & Disagreement with neaby thermocouples \\
\hline TE-103-AP-48 & $9 / 4 / 90$ & 64 & CASS & $\mathbf{S}$ & Disagreement with nearby thermocouples \\
\hline TE-103-AP-48 & $9 / 8 / 80$ & 66 & CASS & $\mathbf{s}$ & Disagreement with nasiby thermocouples \\
\hline TE-103-AP-48 & $8 / 9 / 90$ & 65 & CASS & $\mathbf{s}$ & Disagreement with nearby thermocouplas \\
\hline TE-103-AP-4B & $9 / 10 / 90$ & 65 & CASS & s & Diasgreement with nearby tharmocouples \\
\hline TE-103-AP-48 & $9 / 11 / 80$ & 65 & CASS & $\mathbf{S}$ & Disegroement with neaby thermocouples \\
\hline TE-103-AP-48 & $8 / 12 / 90$ & 65 & CASS & 5 & Disagreament with nearby tharmocouples \\
\hline TE-103-AP-48 & 9/13/ब0 & 67 & CASS & $\mathbf{s}$ & Disagroement with naarby thermacouplas \\
\hline TE-108-AP-48 & $9 / 14 / 90$ & 65 & CASS & $\mathbf{s}$ & Disagresment with neaby thermocouples \\
\hline TE-103-AP-48 & $9 / 15 / 90$ & 67 & CASS & $\mathbf{s}$ & Disegreement with neaby thermocouples \\
\hline TE-108-AP-48 & 9/16/90 & 69 & CASS & $\mathbf{S}$ & Disapreensent with nearby thermocouples \\
\hline TE-103-AP-48 & $9 / 17 / 90$ & 66 & CASS & $\mathbf{s}$ & Disagreament with nearby thermocouples \\
\hline TE-103-AP-48 & $9 / 18 / 90$ & 86 & CASS & $\mathbf{s}$ & Disagreament with nearby thermocouples \\
\hline TE-103-AP-48 & 9/19/90 & 65 & CASS & $\mathbf{s}$ & Disagreament whith nearby thermacouplas \\
\hline TE-103-AP-48 & $9 / 20 / 90$ & 65: & CASS & $\mathbf{s}$ & Disagreement with nearby thermocouples \\
\hline $\mathrm{TE} \cdot 103-A \mathrm{P}-48$ & 9/21/30 & 66 & CASS & $\mathbf{s}$ & Disagreement with naarby thermocouples \\
\hline TE-103-AP-48 & $9 / 22 / 90$ & 65 & CASS & $\mathbf{s}$ & Disagraement with nearby thermocouples \\
\hline TE-103-AP-48 & 9/23/90 & 65 & CASS & $\mathbf{s}$ & Disegreement with nearby thermocouples \\
\hline TE-103-AP:48 & $9 / 24 / 30$ & 66 & CASS & $\mathbf{s}$ & Disagreament with nearby thermocouples \\
\hline TE-103-AP-48 & $9 / 25 / 90$ & 67 & CASS & $\overline{\mathbf{s}}$ & Disegreement with nestby thermocougles \\
\hline TE-103-AP-48 & $9 / 26 / 90$ & 65 & CASS & s & Disegreament with nearby thermocouples \\
\hline TE-103-AP-4B & $9 / 27 / 90$ & 65 & CASS & $\mathbf{S}$ & Disarreament with nearby thermocouples \\
\hline TE-103-AP-48 & $9 / 28 / 90$ & 67 & CASS & $\mathbf{S}$ & Disegreement with nearby thermocouples \\
\hline TE-103-AP-48 & 9/29/30 & 66 & CASS & $\mathbf{S}$ & Disagreement with nearby thermocouples \\
\hline$T E-103-A P-48$ & $9 / 30 / 90$ & 66 & CASS & $\mathbf{S}$ & Disagreement with nearby thermocouplas \\
\hline TE-103-AP-48 & $10 / 1 / 100$ & 66 & CASS & $\mathbf{s}$ & Disagroement with nearby thermocouples \\
\hline TE-T03-AP-4B & $10 / 2 / 90$ & 66 & CASS & $\mathbf{s}$ & Disagreament with nearby thermocouples \\
\hline TE-103-AP-48 & $10 / 3 / 90$ & 68) & CASS & $\mathbf{s}$ & Disegreament with nearby thermocouples \\
\hline TE-103-AP-48 & $10 / 4 / 90$ & 65 & CASS & $\mathbf{s}$ & Disagreement with nearby thermocouples \\
\hline TE-103-AP-48 & $10 / 5 / 90$ & 66 & CASS & $\mathbf{s}$ & Disapreement with neartby thermocouples \\
\hline$T E-103-A P-48$ & $10 / 6 / 90$ & 66 & CASS & S & Disegreement with nearby thermocouples \\
\hline TE-103-AP-48 & $10 / 7 / 60$ & 67 & CASS & $\mathrm{s}$ & Diaggreament with nearby thermocouples \\
\hline TE-103-AP-48 & $10 / 8 / 90$ & 66 & CASS & $\mathbf{S}$ & Disegreement with neaby thermocouplas \\
\hline TE-103-AP-48 & $10 / 9 / 90$ & 66 & CASS & $\mathbf{S}$ & Disagreement with nearby thermocouplas \\
\hline TE-103.AP-48 & $10 / 10 / 90$ & 66- & CASS & $\mathrm{s}$ & Disapreament with nearby tharmocouplas \\
\hline TE-103-AP-4B & $10 / 71 / 90$ & 66 & CASS & $\mathbf{S}$ & Diagareament with nearby thermocouples \\
\hline TE-103-AP-48 & $10 / 12 / 90$ & 66 & CASS & $\mathbf{s}$ & Disagroement with nearby thermocouples \\
\hline TE-103-AP-48 & $10 / 13 / 90$ & 67 & CASS & $\mathbf{s}$ & Disapreement with nasby thermocouples \\
\hline TE-103-AP-48 & $10 / 14 / 90$ & 66 & CASS & $\mathbf{S}$ & Disagreement with neapy thermocouples \\
\hline TE-103-AP.48 & $10 / 15 / 90$ & 66 & CASS & $\overline{\mathbf{S}}$ & Disagreement with nearby thermocouples \\
\hline TE-103-AP-48 & $10 / 16 / 90$ & 66 & CASS & $\mathbf{s}$ & Disagreement with nearby thermocouples \\
\hline TE-103-AP-48 & $10 / 17 / 90$ & 66 & CASS & $\mathbf{S}$ & Disagreament with nearby thermocouples \\
\hline TE-103-AP-48 & $10 / 18 / 90$ & 66 & CASS & $\mathbf{s}$ & Disagreement with nearby thermocouples \\
\hline TE-103-AP-48 & $10 / 19 / 90$ & 66 & CASS & $\mathbf{S}$ & Disagreement with naerby thermocouples \\
\hline TE-103-AP-48 & $10 / 20 / 90$ & 55 & CASS & $\mathbf{s}$ & Disagreement with nearby thermocouples \\
\hline TE-103-AP-48 & $10 / 21 / 80$ & 66 & CASS & $\mathbf{S}$ & Disagreament with nearby tharmocouples \\
\hline$T E-103-A P-48$ & $10 / 22 / 90$ & 66 & CASS & $\mathbf{S}$ & Disagreement with nearby thermocouples \\
\hline TE-108-AP-48 & $10 / 23 / 90$ & 66 & CASS & $\mathbf{s}$ & Disagreament with nearby thermocouples \\
\hline TE-103-AP-48 & $10 / 24 / 90$ & 61 & CASS & $\mathbf{s}$ & Disagreament with nearby thermocouples \\
\hline TE-103-AP-48 & $10 / 25 / 90$ & 60 & CASS & $\mathbf{s}$ & Disagreament with nearby thermocouples \\
\hline TE-103-AP-4B & $10 / 26 / 90$ & 66 & CASS & s & Disegreament with nearby thermocouples \\
\hline$T E-103-A P-48$ & $10 / 27 / 90$ & 65 & CASS & s & Disagreament with nearby thermocouples \\
\hline TE-103-AP-48 & $10 / 28 / 90$ & 66 & CASS & s & Diaagreement with nearby thermocouples \\
\hline TE-103-AP-48 & $10 / 29 / 90$ & 66. & CASS & $\mathbf{s}$ & Disapreement with nasiby thermocouples \\
\hline TE-103-AP-48 & $10 / 30 / 90$ & 66 & CASS & $\mathbf{s}$ & Disagreement with nearby thermocouples \\
\hline TE-108-AP-48 & $10 / 31 / 90$ & 66 & CASS & $\mathbf{5}$ & Disagreement with nearby thermocouples \\
\hline TE-103-AP-48 & $11 / 1 / 90$ & 67 & CASS & 5 & Disagreement with nearby thermocouples \\
\hline TE-103-AP-48 & $11 / 2 / 90$ & 66 & CASS & $\mathbf{s}$ & Disepreement with nearby thermocauples \\
\hline
\end{tabular}




\begin{tabular}{|c|c|c|c|c|c|}
\hline \multicolumn{6}{|c|}{$\begin{array}{l}\text { TAKK 241-AP-103 } \\
\text { THEFMOCOUPIF DATA }\end{array}$} \\
\hline THEAMOCOUPLE * & DATE & TEMPERATURE $\left(^{\circ} \mathrm{F}\right)$ & DATA SOUACE & OUALTY & COMMENT \\
\hline$T E-103-A P-48$ & $11 / 3 / 90$ & 54 & CASS & $\mathbf{S}$ & Disagreement with naarby thermocouplas \\
\hline TE-103-AP-48 & $11 / 4 / 90$ & 61 & CASS & $\mathbf{s}$ & Disagreement with nearby thermocouples \\
\hline TE-103-AP-48 & $11 / 5 / 90$ & 64 & CASS & $\mathbf{s}$ & Disagreement with nearby thermocouples \\
\hline TE-103-AP-48 & $11 / 6 / 90$ & 62. & CASS & $\mathbf{s}$ & Disagreement with nearby thermocouples \\
\hline TE-103-AP-4B & $11 / 7 / 90$ & 61 & CASS & $\mathbf{s}$ & Disagreement with nearby thermocouples \\
\hline TE-103-AP-48 & $11 / 8 / 30$ & 66 & CASS & $\mathbf{S}$ & Disapreament with nearby thermocouples \\
\hline TE-T03-AP-48 & $11 / 9 / 90$ & 65 & CASS & $\mathbf{S}$ & Disagreement with nearby thermocouples \\
\hline TE-103-AP-48 & $11 / 10 / 90$ & 85 & CASS & $\mathbf{S}$ & Disagreament with nostby thermocouples \\
\hline TE-103-AP-4B & $11 / 11 / 90$ & 66 & CASS & $\mathbf{S}$ & Disapreament with nearby thermocouples \\
\hline TE-103-AP-48 & $11 / 12 / 90$ & 66 & CASS & $\mathbf{s}$ & Disagreement with nearby thermocouples \\
\hline TE-103-AP-48 & $11 / 13 / 90$ & 67 & CASS & $\mathbf{s}$ & Disegreement with nearby thormocouples \\
\hline TE-103-AP-48 & $11 / 14 / 90$ & 86 & CASS & $\mathbf{S}$ & Disagreement with nearby thermocouples \\
\hline TE-103-AP-48 & $11 / 15 / 90$ & 65 & CASS & $\mathbf{s}$ & Disagreament with nearby thermocouples \\
\hline TE-103-AP-48 & $11 / 16 / 90$ & 66 & CASS & $\mathbf{S}$ & Disegreament with nearby thermocouples \\
\hline TE-103-AP-48 & $11 / 17 / 90$ & 66 & CASS & $\mathbf{s}$ & Disagreement with nearby thermocouples \\
\hline TE-103-AP-48 & $11 / 18 / 90$ & 66 & CASS & $\mathbf{s}$ & Disagreenent with nearby thermocouples \\
\hline TE-108-AP-48 & $11 / 19 / 90$ & 66 & CASS & $\mathbf{S}$ & Disegraement with nearby thermocouples \\
\hline TE-103-AP-48 & $11 / 20 / 90$ & 66 & CASS & $\mathbf{S}$ & Disagreennent with neaby thermocouples \\
\hline TE-103-AP-48 & $11 / 21 / 90$ & 65 & CASS & $\mathbf{S}$ & Disagreament with noarby thermocouples \\
\hline TE-103-AP-48 & $11 / 22 / 90$ & 65. & CASS & $\mathbf{s}$ & Disagreement with naarby thermocouplas \\
\hline TE-103-AP-48 & $11 / 23 / 90$ & 65 & CASS & $\overline{\mathbf{S}}$ & Disagreament with nearby thermocouples \\
\hline TE-103-AP-48 & $11 / 24 / 90$ & 65 & CASS & $\mathbf{s}$ & Disegreement with nearby thermocouples \\
\hline TE-103-AP-48 & $11 / 25 / 90$ & 66 & CASS & $\mathbf{S}$ & Disagreement with nearby thermocouples \\
\hline TE-103-AP-48 & $11 / 26 / 90$ & 66 & CASS & $\mathbf{s}$ & Disegreement with noarby thermocouples \\
\hline TE-103-AP-4B & $11 / 27 / 90$ & 66 & CASS & $\mathbf{s}$ & Disegreement with nesiby thermocouples \\
\hline TE-108-AP-48 & $11 / 28 / 90$ & 65 & CASS & $\mathbf{S}$ & Disagreement with neaby thermocouples \\
\hline TE-103-AP-48 & $11 / 29 / 90$ & 65 & CASS & $\mathbf{S}$ & Disenreement with neaby thermocouples \\
\hline TE-103-AP-48 & $11 / 30 / 90$ & 65 & CASS & $\mathbf{s}$ & Diasgreament with nearby thermocouples \\
\hline TE-103-AP-48 & $12 / 1 / 30$ & 68] & CASS & $\mathbf{s}$ & Disegreement with nearby thermocouples \\
\hline TE-103-AP-4B & $12 / 2190$ & 66 & CASS & $\mathbf{s}$ & Disagreement with nearby theimocouples \\
\hline TE-108-AP-48 & 12/3/30 & 65 & CASS & $\mathbf{5}$ & Disagreement with nearby thermocouples \\
\hline TE-103-AP-4B & $12 / 4 / 90$ & 65 & CASS & $\mathbf{s}$ & Disupreement with nearby thermocouplas \\
\hline TE-103-AP-48 & $12 / 5 / 90$ & 65 & CASS & $\mathbf{S}$ & Distpreament with maarby thermocouples \\
\hline TE-103-AP-48 & $12 / 6 / 90$ & 65 & CASS & $\mathbf{S}$ & Diserprement with noaby tharmocouples \\
\hline TE-103-AP-4B & $12 / 7 / 90$ & 65 & CASS & $\mathbf{S}$ & Diasercement with nearby tharmocouples \\
\hline TE-103-AP-48 & $12 / 8 / 90$ & 65 & CASS & $\mathbf{S}$ & Disagreement with nearby thermocouples \\
\hline TE-103-AP-48 & $1219 / 90$ & 67 & CASS & $\mathbf{S}$ & Diagroement with nearby thermocouples \\
\hline TE-103-AP-48 & $12 / 10 / 90$ & 65 & CASS & $\mathbf{S}$ & Digangerment with nearby thermocouplas \\
\hline TE-103-AP-48 & $12 / 11 / 90$ & 66 & CASS & $\mathbf{S}$ & Disagreament with nearby thermocouples \\
\hline TE-103-AP-48 & $12 / 12 / 30$ & 65 & CASS & $\mathbf{S}$ & Diaspreement with nearby thermocouples \\
\hline TE-103-AP-48 & $12 / 13 / 90$ & 66 & CASS & $\mathbf{S}$ & Oisapreement with nearby thermocouples \\
\hline TE-103-AP-48 & $12 / 14 / 90$ & 65: & CASS & $\mathbf{s}$ & Disegreement with naarby tharmocouples \\
\hline TE-103-AP-4B & $12 / 17 / 90$ & 65 & CASS & $\mathbf{S}$ & Disegreement with nearby thermocouples \\
\hline TE-103-AP-48 & $12 / 18 / 90$ & 65 & CASS & $\mathbf{S}$ & Disegreement with nearby thermocouples \\
\hline TE-108-AP-48 & $12 / 19 / 90$ & 68 & CASS & $\mathbf{S}$ & Diaggreement with nearby thermocouples \\
\hline TE-103-AP-48 & $12 / 20 / 90$ & 65 & CASS & $\mathbf{S}$ & Diagreement with nearby thermocouples \\
\hline TE-103-AP-48 & $12 / 21 / 90$ & 38 & CASS & $\mathbf{S}$ & Disagreement whth nearby thormocouples \\
\hline TE-103-AP-48 & $12 / 22 / 90$ & 34 & CASS & $\mathbf{S}$ & Disegreement with nearby thermocouples \\
\hline TE-103-AP-48 & $12 / 23 / 90$ & 36 & CASS & $\mathbf{S}$ & Disagreament with nearby thermocouplas \\
\hline TE-103-AP-48 & $12 / 24 / 90$ & 37 & CASS & $\mathbf{S}$ & Disegreement with nearby thermocouples \\
\hline TE-103-AP-48 & $12 / 25 / 90$ & 37 & CASS & $\mathbf{S}$ & Disagraement with nearby thermocouplas \\
\hline TE-103-AP-48 & $12 / 26 / 90$ & 39 & CASS & $\mathbf{s}$ & Disagraement with nearby thermocouplas \\
\hline TE-103-AP-48 & $12 / 27 / 90$ & 40 & CASS & $\mathbf{S}$ & Digagreement with nearby thermocouples \\
\hline TE-103-AP-48 & $12 / 28 / 90$ & 43 & CASS & $\mathbf{S}$ & Disagreement with nearby thermocouples \\
\hline TE-103-AP-48 & $12 / 29 / 90$ & 38 & CASS & $\mathbf{S}$ & Disagreement with nearby thermocouples \\
\hline TE-103-AP-48 & $12 / 30 / 90$ & 38 & CASS & $\mathbf{S}$ & Disagreement with nearby thermocouples \\
\hline TE-103-AP-AB & $12 / 31 / 90$ & 40 & CASS & $\mathbf{s}$ & Disagreement with nearby thermocouples \\
\hline TE-103-AP-48 & $1 / 1 / 91$ & 41 & CASS & $\mathbf{s}$ & Disagreament with nearby thermocouples \\
\hline TE-103-AP-48 & $1 / 2 / 91$ & 41. & CASS & $\mathbf{S}$ & Disagreement with nearby tharmocouples \\
\hline TE-103-AP-4B & 1/3/91 & 45 & CASS & $\mathbf{S}$ & Disagraement with nearby thermocouples \\
\hline TE-103-AP-48 & $1 / 4 / 91$ & 40 & CASS & $\mathbf{S}$ & Disagreement with nearby thermocouples \\
\hline TE-103-AP-48 & $1 / 5 / 91$ & 40 & CASS & $\mathbf{S}$ & Diaspreament with nearby thermocouples \\
\hline TE-103-AP-48 & $1 / 6 / 91$ & 411 & CASS & $\mathbf{S}$ & Disagreement with nearby thermocouplas \\
\hline TE-103-AP-48 & $1 / 7 / 91$ & 41 & CASS & $\mathbf{s}$ & Disegreement with nearby thermocouples \\
\hline TE-103-AP-48 & $1 / 8 / 91$ & 42 & CASS & $\mathbf{S}$ & Disagraement with nearby thermocouples \\
\hline TE-103-AP-4B & $1 / 9 / 91$ & 42 & CASS & $\mathbf{S}$ & Disagreement with nearby thermocouples \\
\hline$T E-103-A P-48$ & 1/10/91 & 42 & CASS & $\mathbf{S}$ & Disagreement with nearby thermocouplas \\
\hline TE-103-AP-48 & 1/11/91 & 42 & CASS & $\mathbf{S}$ & Disagraement with nearby thermocouples \\
\hline TE-103-AP-48 & 1/12/91 & 42 & CASS & $\mathbf{s}$ & Disagreament with nearby thermocouples \\
\hline$T E-103 \cdot A P-4 B$ & 1/13/91 & 44 & CASS & $\mathbf{S}$ & Disagreement with nearby thermocouples \\
\hline TE-103-AP-48 & $1 / 14 / 91$ & 63 & CASS & $\mathbf{s}$ & Disegreement with nearby thermocouples \\
\hline TE-103-AP-48 & $1 / 15 / 91$ & 64 & CASS & $\mathbf{S}$ & Disegreement with nearby thermocauples \\
\hline TE-103-AP-48 & $1 / 16 / 91$ & 64 & CASS & $\mathbf{S}$ & Diss greament with nearby thermocouples \\
\hline
\end{tabular}




\begin{tabular}{|c|c|c|c|c|c|}
\hline \multicolumn{6}{|c|}{$\begin{array}{l}\text { TANK 241-AP-103 } \\
\text { THEFMOCOUPLE DATA }\end{array}$} \\
\hline & & & & & \\
\hline THEFHOCOUPL * & DATE & TEMPERATUAE $\left({ }^{\circ} \mathrm{F}\right)$ & DATA BOURCE & OUALTY & COMMENT \\
\hline TE-103-AP-48 & $1 / 17 / 91$ & 64 & CASS & $\mathbf{s}$ & Disagreement with nearby thermocouples \\
\hline TE-103-AP-48 & $1 / 18 / 91$ & 64 & CASS & $\mathbf{s}$ & Disagreement with nearby thermocouples \\
\hline TE-103.AP-48 & $1 / 19 / 91$ & 64 & CASS & $\mathbf{S}$ & Disagreement with nearby thermocouples \\
\hline TE-103-AP-48 & $1 / 20 / 91$ & 63 & CASS & $\mathbf{S}$ & Disegraement with nestby thermocouples \\
\hline TE-103-AP-4B & $1 / 21 / 91$ & 60 & CASS & $\mathbf{s}$ & Disagreement with nearby thermocouples \\
\hline TE-103-AP-48 & $1 / 22 / 91$ & 61 & CASS & $\mathbf{s}$ & Disapreement with nearby thermocouples \\
\hline TE-103-AP-48 & $1 / 23 / 91$ & 42 & CASS & $\mathbf{S}$ & Disegraement with noarby thermocouples \\
\hline TE-108-AP-48 & $1 / 24 / 91$ & 41 & CASS & S & Disegreement with nearby tharmocouples \\
\hline TE-103-AP-48 & $1 / 26 / 91$ & 41 & CASS & $\mathbf{s}$ & Disagreament with nasiby thermocouples \\
\hline TE-103-AP-48 & $1 / 26 / 97$ & 40 & CASS & $\mathbf{s}$ & Disegreement with nearby thermocouples \\
\hline TE-103-AP-48 & $1 / 27 / 91$ & 38 & CASS & $\mathbf{s}$ & Diseqreement with noarby thermocouples \\
\hline TE-103-AP-4B & $1 / 28 / 91$ & 40 & CASS & $\mathbf{s}$ & Disagreement with neartby thermocouples \\
\hline TE-103-AP-4B & $1 / 29 / 91$ & 38 & CASS & $\mathbf{s}$ & Disegreement with nearby thermocouples \\
\hline TE-103-AP-4B & $1 / 30 / 91$ & 40 & CASS & $\mathbf{s}$ & Disagreement with nearby thermocouples \\
\hline TE-103-AP-48 & $2 / 2 / 91$ & 43 & CASS & $\mathbf{s}$ & Disagreement with naarby thermocouples \\
\hline TE-108-AP-48 & $2 / 4 / 91$ & 46 & CASS & $\mathbf{s}$ & Disagreemant with nearby tharmocouples \\
\hline TE-103-AP-48 & $2 / 5 / 91$ & 48 & CASS & $\mathbf{s}$ & Disapreement with neaby thermocouples \\
\hline TE-103-AP-48 & $2 / 6 / 91$ & 46 & CASS & $\mathbf{S}$ & Disagreement with nearby thermocouplos \\
\hline TE-103-AP-48 & $2 / 3 / 91$ & 48 & CASS & s & Disegreemant with nearby tharmocouples \\
\hline TE-103-AP-48 & $2 / 9 / 91$ & 49 & CASS & $\mathbf{s}$ & Disagreement with nearby thermocouples \\
\hline TE-103-AP-4B & $2 / 11 / 91$ & 50 & CASS & S & Disegreement with neably thermocouples \\
\hline TE-103-AP-4B & $2 / 12 / 91$ & 45 & CASS & $\mathbf{s}$ & Disagreament with nearby tharmocouples \\
\hline TE-103-AP-4B & $2 / 13 / 91$ & 48 & CASS & $s$ & Disaqreement with neaby thermocouples \\
\hline TE-108-AP-48 & $2 / 14 / 91$ & 55 & CASS & $\mathbf{S}$ & Disedreement with nearby thasmocouples \\
\hline TE-103-AP-48 & $2 / 15 / 91$ & 62 & CASS & $\frac{5}{S}$ & Disagreament with noarty themocouples \\
\hline TE-103-AP-4B & $2 / 16 / 91$ & 62 & CASS & s & Diagrreament with naarby thermocouples \\
\hline TE-108-AP-48 & $2 / 17 / 91$ & 62 & CASS & $\mathbf{S}$ & Diaspreement with nearby themocouples \\
\hline TE-103-AP-48 & $2 / 18 / 91$ & 47 & CASS & $\mathbf{S}$ & Disergeement with neasby thermocouples \\
\hline TE-10B-AP-4B & $2 / 19 / 91$ & 48 & CASS & $\mathbf{s}$ & Diacoreement with nearby thermocouples \\
\hline$T E-103-A P-48$ & $2 / 20 / 91$ & 62 & CASS & $\mathbf{S}$ & Dicagresment whe nearby theimocouples \\
\hline TE-103-AP-48 & $2 / 21 / 31$ & 63 & CASS & $\mathbf{S}$ & OFaagreernont with neaby thermocouples \\
\hline$T E-103 \cdot A P-48$ & $2 / 22 / 91$ & 62 & CASS & s & Dissagreement with nearby thermocouples \\
\hline TE-103-AP-4B & $2 / 23 / 91$ & 53 & CASS & $\mathbf{S}$ & Disagreement with nearby thermocouplas \\
\hline TE- T0B-AP-4B & $2 / 24 / 91$ & 63 & CASS & $\mathbf{s}$ & Disagroement with nearby thermocouples \\
\hline TE-103-AP-4B & $2 / 25 / 91$ & 62 & CASS & $\mathbf{S}$ & Disegreement with nearby thermocouples \\
\hline$T E-103-A P-4 B$ & $2 / 26 / 91$ & 61 & CASS & $\mathbf{s}$ & Disagreement with naarby thermocouples \\
\hline$T E-103-A P \cdot 48$ & $2 / 27 / 91$ & 62 & CASS & $\mathbf{s}$ & Disagresenent with nastby themocouples \\
\hline TE-103-AP-48 & $2 / 28 / 91$ & 62! & CASS & $\mathbf{s}$ & Disergerment whth neaby tharmocouplos \\
\hline TE-103-AP-48 & 3/1/81 & 62 & CASS & $\mathbf{S}$ & Diasrreement with neaby thermocouples \\
\hline$T E-103-A P-48$ & 3/2/91 & 61 & CASS & s & Dingrreament with nearby thermocouples \\
\hline TE-103-AP- 48 & $3 / 3 / 98$ & 63 & CASS & $\mathbf{s}$ & Disegreement with nearby themocouples \\
\hline TE-103-AP-48 & $3 / 4 / 91$ & 61 & CASS & $\mathbf{S}$ & Distagreamem with nearby thermocouples \\
\hline TE-103-AP-48 & 3/5/91\} & 62 & CASS & $\mathbf{S}$ & Disepreemont with nearby thermocouples \\
\hline TE-103-AP-48 & 3/6/91 & 62 & CASS & $\mathbf{s}$ & Disegreement with nearby thermocouples \\
\hline$T E-103-A P-48$ & 3/191 & 63 & CASS & s & Disagreement with coarby thermocouples \\
\hline TE-103-AP-48 & $3 / 8 / 91$ & 61 & CASS & s & Disagreement with nearby thermocouples \\
\hline TE-103-AP-48 & $3 / 9 / 91$ & 61 & CASS & $\mathbf{S}$ & Disagreement with nearby thermocouples \\
\hline$T E-103-A P-4 B$ & 3/10/91 & 62 & CASS & $\mathbf{s}$ & Disugreament with nearby thormocouples \\
\hline TE-103-AP-48 & $3 / 11 / 91$ & 61 & CASS & $\mathbf{S}$ & Disagreament with naarby thermocouples \\
\hline TE-103-AP-48 & $3 / 12 / 97$ & 61 & CASS & $\mathbf{S}$ & Disapreament with nearby thermocouples \\
\hline TE-103-AP-48 & 3/13/91 & 63 & CASS & $\mathbf{s}$ & Disagrcement with nearby thermocouples \\
\hline TE-103-AP-48 & $3 / 15 / 91$ & 61 & CASS & $\bar{s}$ & Disagreement with nearby thermocouples \\
\hline TE-103-AP-4B & $3 / 16 / 91$ & 63 & CASS & S & Disagreement with naarby thermocouples \\
\hline TE-108-AP-48 & $3 / 17 / 91$ & 61 & CASS & $\mathbf{s}$ & Disagreement with nearby thermocouples \\
\hline$T E-103-A P+48$ & $3 / 18 / 91$ & 61 & CASS & $\mathbf{s}$ & Disagreement with nearby thermocouples \\
\hline TE-103-AP-48 & $3 / 19 / 91$ & 6i & CASS & $\mathbf{S}$ & Disagreament with nearby thermocouples \\
\hline TE-103-AP-48 & $3 / 20 / 91$ & 62 & CASS & S & Disagreament with nearby thermocouples \\
\hline TE-103-AP-48 & $3 / 21 / 91$ & 62 & CASS & $\frac{1}{s}$ & Disagreamant with nearby tharmocouples \\
\hline TE-103-AP-4B & $3 / 22 / 91$ & 62 & CASS & $\mathbf{s}$ & Disagreement with nearby thermocouples \\
\hline$T E-103-A P-48$ & $3 / 23 / 91$ & 61 & CASS & $\mathbf{S}$ & Disapreament with nearby thermocouples \\
\hline$T E-103 \cdot A P-48$ & $3 / 24 / 91$ & 61 & CASS & S & Disepraement with nasiby thermocouplas \\
\hline TE-103-AP-48 & $3 / 25 / 91$ & 61 & CASS & s & Disegreement with nearby thermocouples \\
\hline TE-103.AP-48 & $3 / 26 / 91$ & 56: & CASS & $\mathbf{S}$ & Disepreement with nearby thermocouples \\
\hline TE-10B-AP-4B & $3 / 27 / 91$ & 63 & CASS & $\mathbf{s}$ & Disegreement with nearby thermocouples \\
\hline TE-103-AP-48 & $3 / 28 / 91$ & 61 & CASS & $\mathbf{S}$ & Disapreement with nearby thermocouples \\
\hline TE-103-AP-48 & $3 / 29 / 91$ & 62 & CASS & $\mathbf{S}$ & Disugreement with nearby thermocouples \\
\hline TE-103-AP-48 & $3 / 30 / 91$ & 62 & CASS & $\mathbf{S}$ & Disagreement with nearby thermocouples \\
\hline TE-103-AP-48 & $3 / 31 / 91$ & 60 & CASS & $\mathbf{S}$ & Disapresment with nearby thermocouples \\
\hline$T E-103-A P-48$ & $4 / 1 / 91$ & 60 & CASS & $\mathbf{s}$ & Disagreement with nearby thermocouples \\
\hline TE-103-AP-48 & $4 / 2 / 91$ & 60 & CASS & $\mathbf{s}$ & Disagreement with nearby thermocouples \\
\hline TE-103-AP-48 & $4 / 3 / 91$ & 61 & CASS & $\mathbf{s}$ & Disagreament with nearby thermocouples \\
\hline$T E=103-A P+48$ & 4/4/91 & 60 & CASS & $\bar{s}$ & Disegreement with nastby tharmocouplas \\
\hline TE-103-AP-48 & $4 / 5 / 91$ & 60 & CASS & $\mathbf{S}$ & Diaggreament with naarby thermocouples \\
\hline
\end{tabular}




\begin{tabular}{|c|c|c|c|c|c|}
\hline \multicolumn{6}{|c|}{$\begin{array}{l}\text { TANK 241-AP-103 } \\
\text { THERMOCOUPLE DATA }\end{array}$} \\
\hline & & & & & \\
\hline THËMOCOUPLE \# & DATE & TEMPERATUAE ( $\left.{ }^{\circ} \mathrm{F}\right)$ & DATA SOURCE & OUALTYY & COMMENT \\
\hline TE-103-AP-48 & 4/6/91 & 61 & CASS & $\mathbf{S}$ & Disagreament with nearby thermocouples \\
\hline TE-103-AP-48 & 4/7/91 & 61 & CASS & $\mathbf{S}$ & Disagreament with nearby thermocouples \\
\hline TE-103-AP-48 & $4 / 8 / 91$ & 60 & CASS & $\mathbf{S}$ & Disagreament with nearby thermocouples \\
\hline TE-103-AP-48 & 4/9/91 & 60 & CASS & $\mathbf{s}$ & Disegraement with nearby thermocouples \\
\hline TE-103-AP-48 & 4/10/91 & 62 & CASS & $\mathbf{S}$ & Disagreement with nearby thermocouples \\
\hline TE-103-AP-4B & 4/11/91 & 60 & CASS & $\mathbf{S}$ & Disagreament with nearby thermocouples \\
\hline TE-103-AP-48 & 4/12/91 & 60 & CASS & $\mathbf{S}$ & Disagreement with nearby thermocouples \\
\hline TE-103-AP-48 & $4 / 13 / 91$ & 60) & CASS & $\mathbf{s}$ & Disegreement with nadrby thermocouples \\
\hline TE-103-AP-48 & 4/14/91 & 60 & CASS & $\mathbf{S}$ & Disapreement with nearby thermocouples \\
\hline TE-103-AP-48 & 4/15/91 & 60 & CASS & $\mathbf{s}$ & Disagreament with nearby thermocouples \\
\hline TE-103-AP:48 & $4 / 16 / 91$ & 60 & CASS & $\mathbf{S}$ & Disegreement with nearby thermocouples \\
\hline TE-108-AP-48 & 4/17/91 & 60 & CASS & $\mathbf{S}$ & Disagreament with nearby thermocouples \\
\hline TE-103-AP-4B & 4/18/9: & 60: & CASS & $\overline{\mathbf{s}}$ & Disagreement with nearby thermocouples \\
\hline TE-103-AP-48 & $4 / 19 / 91$ & 60 & CASS & $\mathbf{S}$ & Disagreement with nearby thermocouples \\
\hline TE-103-AP.48 & 4/20/91 & 60 & CASS & $\mathbf{s}$ & Disagreement with nearby thermocouples \\
\hline TE-108-AP-48 & 4/21/91 & 59 & CASS & $\mathbf{S}$ & Diseproement with nearby thermocouples \\
\hline TE-103-AP-4B & $4 / 22 / 91$ & 60 & CASS & $\mathbf{S}$ & Disegreement with nesiby thermocouples \\
\hline TE-108-AP-48 & $4 / 23 / 97$ & 61. & CASS & $\mathbf{s}$ & Diesegreement with nearby thermocouples \\
\hline TE-103-AP-48 & $4 / 24 / 91$ & 60 & CASS & $\mathbf{S}$ & Disagreement with nearby tharmocouples \\
\hline TE-103-AP-48 & $4 / 25 / 91$ & 60 & CASS & $\mathbf{s}$ & Disagreement with nearby thermocouples \\
\hline TE-103-AP-48 & $4 / 26 / 91$ & 60 & CASS & $\mathbf{S}$ & Disagroement wikh noarby thermocouples \\
\hline TE-103-AP-48 & $4 / 27 / 93$ & 61 & CASS & $\mathbf{S}$ & Dicaproement with nearby thermocouples \\
\hline IE-103-AP-48 & $4 / 28 / 91$ & 60 & CASS & $\mathbf{S}$ & Disagresment with nearby thermocouples \\
\hline TE-103-AP-48 & $4 / 29 / 91$ & 60 & CASS & $\mathbf{5}$ & Disegreement with nearby thermocouples \\
\hline$T E-103 \cdot A P-48$ & $4 / 30 / 91$ & 60 & CASS & $\mathbf{S}$ & Disagreement with nearby thermocouples \\
\hline TE-103-AP+48 & $5 / 1 / 91$ & 60 & CASS & $\mathbf{s}$ & Oisagreement with neaby thermocouples \\
\hline TE-103-AP-48 & 5/2/93 & 60 & CASS & $\mathbf{S}$ & Disapreament with nearby thermocouples \\
\hline TE-103-AP-48 & E/3/91 & 60 & CASS & $\mathbf{S}$ & Disagreament with nearby thermocouples \\
\hline TE-103-AP-48 & 5/4/91 & 60 & CASS & $\mathbf{S}$ & Disapreement with nasby thermocouples \\
\hline TE-103-AP-48 & 5/5/91 & 61 & CASS & $\mathbf{S}$ & Disegresmem with nearby thermocouples \\
\hline TE-103-AP-46 & 5/6/91 & 59 & CASS & $\mathbf{s}$ & Disegreement with neaby thermocouples \\
\hline TE-103-AP-48 & 5/7/91 & 60 & CASS & $\mathbf{s}$ & Disepreement with nearby thermocouples \\
\hline TE-103-AP-48 & 5/891 & 61) & CASS & $\mathbf{S}$ & Diagproement with naarby thermocouples \\
\hline TE-103-AP-48 & 5/9/91 & 60 & CASS & $\mathbf{s}$ & Disagreament with naerby tharmocouples \\
\hline TE-10B-AP-48 & $5 / 10 / 91$ & 59 & CASS & $\mathbf{8}$ & Disarreanent with nosiby thermocouples \\
\hline TE-103-AP-4B & $5 / 11 / 91$ & 61 & CASS & $\mathbf{S}$ & Oisegreement with nearby thermocouplas \\
\hline TE-108-AP-48 & $5 / 12 / 91$ & 60 & CASS & $\mathbf{S}$ & Disepreament with nearby thermocouples \\
\hline TE-103-AP-48 & $5 / 13 / 91$ & 60 & CASS & 5 & Dibagroement with nearby thermocouples \\
\hline TE-103-AP-48 & 5/14/91 & 61 & CASS & $\mathbf{S}$ & Diagoreament with noerby tharmocouplas \\
\hline TE-103-AP-48 & $5 / 15 / 91$ & 60 & CASS & $\mathbf{s}$ & Disapreament with nearby theimocouples \\
\hline TE-103-AP-48 & $5 / 16 / 91$ & 59) & CASS & $\mathbf{s}$ & Disagreament with nearby thermocouples \\
\hline TE-103-AP-4B & 5/77/91 & 60 & CASS & $\mathbf{s}$ & Disepreament with nearby thermocouples \\
\hline TE-103-AP-4B & 5/18/91 & 60 & CASS & $\mathbf{s}$ & Disagreament with nearby thermocouples \\
\hline TE-103-AP-48 & $5 / 19 / 91$ & 61 & CASS & $\mathbf{s}$ & Disagreament with nearby thermocouples \\
\hline$T E-103-A P-4 B$ & $5 / 20 / 91$ & 60 & CASS & $\mathbf{S}$ & Diagsreament with nearby thermocouples \\
\hline TE-103-AP-48 & $5 / 21 / 91$ & 59. & CASS & $\mathbf{s}$ & Disagreament with nourby thermocouples \\
\hline TE-103-AP-48 & $5 / 22 / 91$ & 60 & CASS & $\mathbf{S}$ & Dieagreament with nearby thermocouples \\
\hline TE-TOB-AP-4B & $5 / 23 / 91$ & 60 & CASS & $\mathbf{s}$ & Disagreament with nearby thermocouples \\
\hline TE-103.AP-48 & $5 / 24 / 91$ & 59 & CASS & $\mathbf{s}$ & Disupreament with naarby thermocouples \\
\hline TE-103-AP-48 & $5 / 25 / 91$ & 61 & CASS & $\mathbf{S}$ & Diadgreament with nearby thermocouplas \\
\hline TE-103-AP-48 & $5 / 26 / 91$ & 59 & CASS & $\mathbf{S}$ & Disegreement with nearby thermocouples \\
\hline TE-103-AP-48 & $5 / 27 / 91$ & 60 & CASS & $\mathbf{S}$ & Disepreement with nearby thermocouples \\
\hline TE-103-AP-48 & $5 / 28 / 91$ & 60: & CASS & $\mathbf{s}$ & Disegroement with neaby thermocouples \\
\hline TE-103-AP-48 & $5 / 29 / 91$ & 62 & CASS & $\mathbf{s}$ & Disagreament with nearby thermocouples \\
\hline TE-103-AP-48 & $5 / 30 / 91$ & 60 & CASS & $\mathbf{S}$ & Disagreement with nearby thermocouples \\
\hline TE-103-AP-48 & 6/1/91 & 59 & CASS & $\mathbf{S}$ & Disagreement with nearby thermocouples \\
\hline TE-103-AP-48 & 6/2/91 & 59 & CASS & $\mathbf{S}$ & Disegreement with nearby thamocouplas \\
\hline TE-103-AP-48 & $6 / 3 / 91$ & 60 & CASS & $\mathbf{S}$ & Disagreement with nearby themocouplas \\
\hline TE-103-AP-48 & $6 / 5 / 91$ & 61 & CASS & $\mathbf{S}$ & Disagreement with nearby thermocouples \\
\hline$T E-103-A P-4 B$ & 6/6/91 & 59 & CASS & $\mathbf{s}$ & Disapreement with nearby thermocouples \\
\hline TE-103-AP-48 & 6/7/91 & 60 & CASS & $\mathbf{s}$ & Disagreament with nearby thermocouples \\
\hline TE-103-AP-48 & 6/3/91 & 60 & CASS & $\mathbf{S}$ & Disegreement with nearby thermocouples \\
\hline TE-103-AP-48 & 6/9/91 & 59 & CASS & $\mathbf{S}$ & Disagreement with nearby thermocouples \\
\hline TE-103-AP-AB & $6 / 10 / 91$ & $\mathbf{5 9}$ & CASS & $\mathbf{S}$ & Disagreement with nearby thermocouples \\
\hline TE-103-AP-48 & $6 / 11 / 91$ & 59 & CASS & $\mathbf{S}$ & Disagreement with nearby thermocouples \\
\hline TE-103-AP-4B & $6 / 12 / 91$ & 60 & CASS & $\mathbf{S}$ & Disagreement with nearby thermocouples \\
\hline TE-103-AP-48 & $6 / 13 / 91$ & 60 & CASS & $\mathbf{S}$ & Diagaraement with nearby themnocouples \\
\hline TE-103-AP-48 & $6 / 14 / 91$ & 60 & CASS & $\mathbf{S}$ & Disagreement with nearby thermocouplas \\
\hline TE-103-AP-4g & $6 / 15 / 91$ & 60 & CASS & s & Disagreement with nearby thermocouples \\
\hline TE-103-AP-48 & $6 / 18 / 91$ & 60 & CASS & $\mathbf{S}$ & Disagreement with nearby thermocouples \\
\hline TE-103-AP-48 & 6/19/91 & 59 & CASS & $\mathbf{s}$ & Disagreement with nearby thermocouples \\
\hline TE-103-AP-48 & $6 / 20 / 91$ & 61: & CASS & $\mathbf{s}$ & Disagreement with nearby thermocouples \\
\hline TE-103-AP-48 & $6 / 21 / 91$ & 601 & CASS & $\mathbf{S}$ & Dissegeement with nearby thermocouples \\
\hline
\end{tabular}




\begin{tabular}{|c|c|c|c|c|c|}
\hline \multicolumn{6}{|c|}{$\begin{array}{l}\text { TANK 248-AP-103 } \\
\text { THEAMOCOUНLE DATA }\end{array}$} \\
\hline & & & & & \\
\hline THERMOCOUPLE : & DATE & TEMPERATUAE ( $^{\circ} \mathrm{Fl}$ & DATA SOURCE & QUALITY & COMMENT \\
\hline TE-103-AP-48 & $6 / 22 / 91$ & 69 & CASS & $\mathbf{5}$ & Disagreement with nearby thermocouples \\
\hline TE-103-AP-48 & $6 / 23 / 91$ & 59 & CASS & $\mathbf{S}$ & Disagreement with nearby thermocouples \\
\hline TE-103-AP-48 & $6 / 24 / 91$ & 59 & CASS & $\mathbf{S}$ & Disagreement with nearby thermocouples \\
\hline TE-103-AP-48 & $6 / 25 / 91$ & 60 & CASS & $\mathbf{s}$ & Disagreement with nearby tharmocouples \\
\hline TE-103-AP-48 & $7 / 2 / 91$ & 59 & CASS & $\mathbf{S}$ & Disagreament with nearby thermocouples \\
\hline TE-103-AP-48 & $7 / 3 / 91$ & 59 & CASS & $\mathbf{S}$ & Disagresmont with nearby thermacouples \\
\hline TE-103-AP-48 & $7 / 11 / 91$ & 61 & CASS & 5 & Disagreement with nearby tharmocouples \\
\hline TE-103-AP-48 & $7 / 2 / 91$ & 60 & CASS & $\mathbf{S}$ & Disagreement with nearby tharmocouples \\
\hline TE-103-AP-48 & $7 / 13 / 91$ & 60 & CASS & $\mathbf{S}$ & Disagreement with nearby thermocouples \\
\hline TE-103-AP-48 & $7 / 74 / 91$ & 60 & CASS & $\mathbf{5}$ & Disagreement with nearby tharmocouples \\
\hline TE-103-AP-48 & $7 / 24 / 91$ & 60 & CASS & $\mathbf{S}$ & Disagreament with noarby tharmocouples \\
\hline TE-103-AP-48 & $7 / 30 / 91$ & 60 & CASS & $\overline{\mathbf{S}}$ & Disagreement with nearby tharmocouples \\
\hline TE-103-AP-48 & $7 / 31 / 91$ & 61 & CASS & $\mathbf{S}$ & Disagreament with naarby themocouples \\
\hline TE-103-AP-48 & $8 / 1 / 91$ & 61 & CASS & $\mathbf{s}$ & Disagreement with nearby thermocouples \\
\hline TE-103-AP-4B & $8 / 2 / 91$ & 61 & CASS & $\mathbf{S}$ & Disagreement with nearby thermocouples \\
\hline TE-103-AP-48 & $8 / 3 / 91$ & 61 & CASS & 5 & Disagreemant with nearby thermocouples \\
\hline TE-103-AP-48 & $8 / 4 / 91$ & 61 & CASS & $\mathbf{s}$ & Disagreament with nearby thermocouples \\
\hline TE-103-AP-48 & $8 / 5 / 91$ & 62 & CASS & $\overline{\mathbf{S}}$ & Disagreement with neartoy thermoccuples \\
\hline TE-103-AP-48 & $8 / 6 / 91$ & 61 & CASS & $\mathbf{S}$ & Diagreament with noarby tharmocouples \\
\hline TE-103-AP-4B & $8 / 12 / 91$ & 61 & CASS & $\mathbf{s}$ & Oisagreement with naarby thermocouples \\
\hline TE-103-AP-4B & $8 / 13 / 91$ & 61 & CASS & $\mathbf{s}$ & Disagresment with nearby thermocouples \\
\hline TE-103-AP-48 & $8 / 14 / 91$ & 61 & CASS & $\mathbf{s}$ & Disagreament with nearby thermocouples \\
\hline TE-103-AP-AB & $8 / 15 / 91$ & 62 & CASS & $\mathbf{S}$ & Disagreement with nearby thermocouples \\
\hline TE-103-AP-48 & $8 / 16 / 91$ & 61 & CASS & $\mathbf{S}$ & Disagreement with nearby themnocouples \\
\hline TE-103-AP-48 & $8 / 17 / 91$ & 62 & CASS & $\mathbf{s}$ & Disagreament with nearby thormocouples \\
\hline TE-103-AP-48 & 8/18/91 & 61 & CASS & $\mathbf{S}$ & Disagreement with nearby thermocouples \\
\hline TE-103.AP-48 & $8 / 19 / 91$ & 62 & CASS & $\mathbf{S}$ & Disegreament with nearby tharmocouplas \\
\hline TE-103-AP-48 & $8 / 20 / 91$ & 63 & CASS & $\mathbf{S}$ & Disagreament with nearby thermocouples \\
\hline TE-103-AP-48 & $8 / 22191$ & 61 & CASS & $\mathbf{S}$ & Disagraement with noarby thermocouples \\
\hline TE-103-AP-48 & $8 / 23 / 91$ & 63) & CASS & $\mathbf{S}$ & Disagreement with nearby tharmocouples \\
\hline TE-103-AP-48 & $8 / 24 / 91$ & 61 & CASS & $\mathbf{s}$ & Disagreement with nearby thormocouples \\
\hline TE-103-AP-48 & 8/25/9? & 62 & CASS & $\mathbf{s}$ & Disarreament with nearby thomocouples \\
\hline TE-T03-AP-48 & $8 / 26 / 91$ & 62 & CASS & $\mathbf{S}$ & Disagreament with nearby thermocouples \\
\hline TE-103-AP-48 & $8 / 27 / 91$ & 62 & CASS & $\mathbf{s}$ & Diagrreement with nearby themocouples \\
\hline$T E+103-A P=48$ & $8 / 28 / 91$ & 62 & CASS & S & Disagreament with nearby tharnocouples \\
\hline TE-103-AP-AB & $8 / 29 / 91$ & 62 & CASS & $\mathbf{s}$ & Disagreement with noarby thermocouples \\
\hline TE-103-AP-4B & $8 / 30 / 91$ & 62 & CASS & $\mathbf{S}$ & Disepreament with nearty themocouples \\
\hline TE-103-AP+48 & $8 / 31 / 91$ & 621 & CASS & $\mathbf{S}$ & Disagreement with nearby tharmocouples \\
\hline TE-103-AP-48 & g/1/91 & 62 & CASS & 5 & Disagreement with naarby thermocouples \\
\hline TE-103-AP-48 & $9 / 2 / 91$ & 64 & CASS & $\mathbf{s}$ & Disagreement with nasiby themocouplas \\
\hline TE-103-AP-48 & $9 / 3 / 91$ & 65 & CASS & $\mathbf{S}$ & Disagreement with nearby thermocouples \\
\hline TE-103.AP-48 & $7 / 31 / 92$ & 60 & CASS & $T$ & \\
\hline TE-103-AP-48 & $8 / 4 / 92$ & 61 & CASS & $T$ & \\
\hline TE-103-AP-48 & $8 / 13 / 92$ & 62 & CASS & $T$ & \\
\hline TE-103-AP-48 & $8 / 20 / 92$ & 61 & CASS & $T$ & \\
\hline$T E=103-A P-48$ & $8 / 29 / 92$ & 63 & CASS & $T$ & \\
\hline TE-103-AP.48 & $9 / 5 / 92$ & 63 & CASS & $T$ & \\
\hline TE-103-AP-48 & $9 / 11 / 92$ & 62 & CASS & $\bar{T}$ & \\
\hline$T E-103-A P-48$ & $9 / 14 / 92$ & 63 & CASS & $T$ & \\
\hline$T E-103-A P-48$ & $10 / 3 / 92$ & 62 & CASS & $T$ & \\
\hline$T E-103-A P-48$ & $10 / 6 / 92$ & 62 & CASS & $T$ & \\
\hline TE-103-AP-48 & $10 / 17 / 92$ & 62 & CASS & $T$ & \\
\hline TE-103-AP-48 & $10 / 22 / 92$ & 63 & CASS & $T$ & \\
\hline TE-103-AP-40 & $10 / 29 / 92$ & 63 & CASS & $T$ & \\
\hline TE-103-AP-4B & $11 / 4 / 92$ & 63 & CASS & $T$ & \\
\hline TE-103-AP-48 & $11 / 9 / 92$ & 62 & CASS & $T$ & \\
\hline TE-103-AP-48 & $11 / 17 / 92$ & 64 & CASS & $\mathbf{T}$ & \\
\hline TE-103-AP-48 & $11 / 28 / 92$ & 63 & CASS & $T$ & \\
\hline$T E-103-A P=48$ & $11 / 29 / 92$ & 74 & CASS & $\mathbf{s}$ & Data point did not agres with local trend \\
\hline TE-103-AP-48 & $12 / 2 / 92$ & 62 & CASS & $T$ & \\
\hline TE-103-AP-48 & $12 / 12 / 92$ & 63 & CASS & $T$ & \\
\hline$T E-103-A P=48$ & $12 / 18 / 92$ & 62 & CASS & $T$ & \\
\hline TE-103-AP-48 & $12 / 26 / 92$ & 62 & CASS & $T$ & \\
\hline TE-103-AP-48 & $12 / 27 / 92$ & 62 & CASS & $T$ & \\
\hline TE-103-AP-48 & $1 / 9 / 93$ & 60 & CASS & $T$ & \\
\hline TE-103-AP-48 & $1 / 16 / 93$ & 60 & CASS & $T$ & \\
\hline TE-103-AP-48 & $1 / 23 / 93$ & 60 & CASS & $T$ & \\
\hline TE-103-AP-48 & $1 / 30 / 93$ & 61 & CASS & $T$ & \\
\hline TE-103-AP-48 & $2 / 4 / 93$ & 61 & CASS & $T$ & \\
\hline TE-103-AP+48 & $2 / 9 / 93$ & 60 & CASS & $T$ & \\
\hline TE-103-AP-48 & $2 / 17 / 93$ & 51 & CASS & S & Dota point did not egree with local trend \\
\hline TE-103-AP-48 & $2 / 20 / 93$ & 59 & CASS & $T$ & \\
\hline TE-103-AP-48 & $2 / 26 / 93$ & 60 & CASS & $T$ & \\
\hline
\end{tabular}




\begin{tabular}{|c|c|c|c|c|c|}
\hline & & & $\begin{array}{l}\text { TANK 241\% } \\
\text { THERMOCOUP }\end{array}$ & $\frac{103}{\text { DATA }}$ & \\
\hline THERMOCOUPLE * & DATE & TEMPERATUAE ( $\left.{ }^{\circ} \mathrm{F}\right)$ & DATA SOURCE & QUALITY & COMMENT \\
\hline TE-103-AP.48 & $3 / 4 / 93$ & 59 & CASS & $\mathbf{T}$ & \\
\hline TE-103-AP-48 & $3 / 10 / 93$ & 59 & CASS & $T$ & \\
\hline TE-103-AP-48 & $3 / 19 / 93$ & 59 & CASS & $T$ & \\
\hline TE-103-AP-48 & $3 / 27 / 93$ & 59 & CASS & $T$ & \\
\hline TE-103-AP-4B & $3 / 31 / 93$ & 59 & CASS & $\mathbf{T}$ & \\
\hline TE-103-AP-48 & $4 / 6 / 93$ & 59 & CASS & $T$ & \\
\hline TE-103-AP-48 & $4 / 17 / 93$ & 58 & CASS & $\mathbf{T}$ & \\
\hline TE-103-AP-48 & $4 / 24 / 93$ & 59 & CASS & $T$ & \\
\hline TE-103-AP-4B & $4 / 29 / 93$ & 58 & CASS & $T$ & \\
\hline TE-103-AP-48 & $5 / 7 / 93$ & 59 & CASS & $T$ & \\
\hline$T E+103-A P-48$ & $5 / 10 / 93$ & 59 & CASS & $T$ & \\
\hline TE-103-AP-48 & $5 / 22 / 93$ & 58 & CASS & $\mathbf{T}$ & \\
\hline TE-103-AP-48 & $5 / 27 / 93$ & $\mathbf{5 8}$ & CASS & $T$ & \\
\hline TE-103-AP-48 & $6 / 3 / 93$ & 58 & CASS & $T$ & \\
\hline TE-103-AP.48 & $6 / 12 / 93$ & 57 & CASS & $\bar{T}$ & \\
\hline TE-103-AP-48 & $6 / 16 / 93$ & 58 & CASS & $T$ & \\
\hline TE-103-AP-48 & $6 / 24 / 93$ & 59 & CASS & $T$ & \\
\hline TE-103-AP-48 & 6/30/93 & 81 & CASS & $\mathrm{s}$ & Data point did not agree with local trend \\
\hline TE-103-AP-4B & $7 / 3 / 93$ & 58 & CASS & $\bar{T}$ & \\
\hline TE-103-AP-48 & $7 / 6 / 93$ & 60 & CASS & $T$ & \\
\hline TE-103-AP-48 & 71793 & 58 & CASS & $T$ & \\
\hline TE-103-AP-48 & $7 / 21 / 93$ & 60 & CASS & $T$ & \\
\hline TE-103-AP-48 & $7 / 31 / 93$ & 59 & CASS & $\bar{T}$ & \\
\hline TE-103-AP-48 & $8 / 7 / 93$ & 59 & CASS & $\bar{T}$ & \\
\hline TE-103-AP-48 & $8 / 74 / 93$ & 59 & CASS & $T$ & \\
\hline$T E-103-A P-48$ & $8 / 18 / 93$ & 61 & CASS & $T$ & \\
\hline TE-103-AP-4B & $8 / 25 / 93$ & 63 & CASS & $\bar{T}$ & \\
\hline TE-103-AP-48 & $9 / 2 / 93$ & 61 & CASS & $\mathbf{T}$ & \\
\hline TE-103-AP-48 & $9 / 9 / 93$ & 62 & CASS & $\mathbf{T}$ & \\
\hline$T E-103 \cdot A P-48$ & 9/13/93 & 62 & CASS & $T$ & \\
\hline$T E-103-A P-49$ & $9 / 24 / 93$ & 61 & CASS & $T$ & \\
\hline TE-103-AP-48 & $10 / 1 / 93$ & 62 & CASS & $T$ & \\
\hline TE-103-AP-48 & $10 / 9 / 93$ & 61 & CASS & $T$ & \\
\hline TE-103-AP-48 & $10 / 11 / 93$ & 62 & CASS & $T$ & \\
\hline TE-103-AP-48 & $10 / 22 / 93$ & 62 & CASS & $T$ & \\
\hline TE-103-AP-48 & $10 / 29 / 93$ & 62 & CASS & $T$ & \\
\hline TE-103-AP-4B & $11 / 6 / 93$ & 61 & CASS & $T$ & \\
\hline TE-103-AP-48 & $11 / \mathrm{B} / \mathrm{A3}$ & 63 & CASS & $T$ & \\
\hline TE-103-AP-48 & $11 / 20 / 93$ & 61 & CASS & $\mathbf{T}$ & \\
\hline$T E-103-A P-48$ & $11 / 27 / 93$ & 60 & CASS & $T$ & \\
\hline TE-103-AP-4B & $12 / 1 / 93$ & 61 & CASS & $T$ & \\
\hline TE-103-AP-48 & $1 / 13 / 94$ & .59 & CASS & $T$ & \\
\hline TE-103-AP-48 & $5 / 12 / 94$ & 56 & CASS & $T$ & \\
\hline TE-103-AP-48 & $5 / 17 / 94$ & 58 & CASS & $T$ & \\
\hline TE-103-AP-48 & $5 / 26 / 94$ & 58 & CASS & $T$ & \\
\hline TE-103-AP-48 & $6 / 4 / 94$ & 59 & CASS & $\mathbf{T}$ & \\
\hline TE-103-AP-48 & $6 / 11 / 94$ & 60 & CASS & $T$ & \\
\hline TE-103-AP-4B & $6 / 12 / 94$ & 61 & CASS & $T$ & \\
\hline TE-103-AP-4S & $6 / 24 / 94$ & 61 & CASS & $T$ & \\
\hline TE-103-AP-48 & $7 / 1 / 94$ & 63 & CASS & $T$ & \\
\hline TE-103-AP-48 & $7 / 9 / 94$ & 63 & CASS & $T$ & \\
\hline TE-103-AP-4B & $7 / 11 / 94$ & 64 & CASS & $T$ & \\
\hline TE-103-AP-48 & $7 / 22 / 94$ & 66 & CASS & $T$ & \\
\hline TE-103-AP-48 & $7 / 28 / 94$ & 65 & CASS & $T$ & \\
\hline TE-103-AP-48 & $8 / 5 / 94$ & 66. & CASS & $T$ & \\
\hline TE-103-AP-48 & $8 / 13 / 94$ & 68 & CASS & $T$ & \\
\hline TE-103-AP-48 & $8 / 20 / 94$ & 67 & CASS & $T$ & \\
\hline TE-103-AP-48 & $8 / 26 / 94$ & 68 & CASS & $T$ & \\
\hline TE-103-AP-4Q & $9 / 2 / 94$ & 88 & CASS & $T$ & \\
\hline TE-103-AP-48 & $9 / 10 / 94$ & 68 & CASS & $T$ & \\
\hline TE-103-AP-48 & $9 / 11 / 94$ & 69 & CASS & $T$ & \\
\hline TE-103-AP-48 & $9 / 21 / 94$ & 67 & CASS & $T$ & \\
\hline TE-103-AP-48 & $9 / 30 / 94$ & 69 & CASS & $T$ & \\
\hline TE-103-AP-48 & $10 / 2 / 94$ & 68 & CASS & $T$ & \\
\hline TE-103-AP-49 & $10 / 12 / 94$ & 67 & CASS & $\bar{T}$ & \\
\hline TE-103-AP-48 & $10 / 21 / 94$ & 67 & CASS & $\mathbf{T}$ & \\
\hline TE-103-AP-48 & $10 / 28 / 94$ & 64 & CASS & $T$ & \\
\hline TE-103-AP-48 & $11 / 3 / 94$ & 64 & CASS & $T$ & \\
\hline TE-103-AP-48 & $11 / 12 / 94$ & 63 & CASS & $T$ & \\
\hline TE-103-AP-4B & $11 / 15 / 94$ & 62 & CASS & $T$ & \\
\hline TE-103-AP-43 & $11 / 20 / 94$ & 61 & CASS & $T$ & \\
\hline TE-103-AP-48 & $12 / 1 / 94$ & 58 & CASS & $T$ & \\
\hline TE-103-AP-48 & $12 / 9 / 94$ & 57 & CASS & $T$ & \\
\hline
\end{tabular}




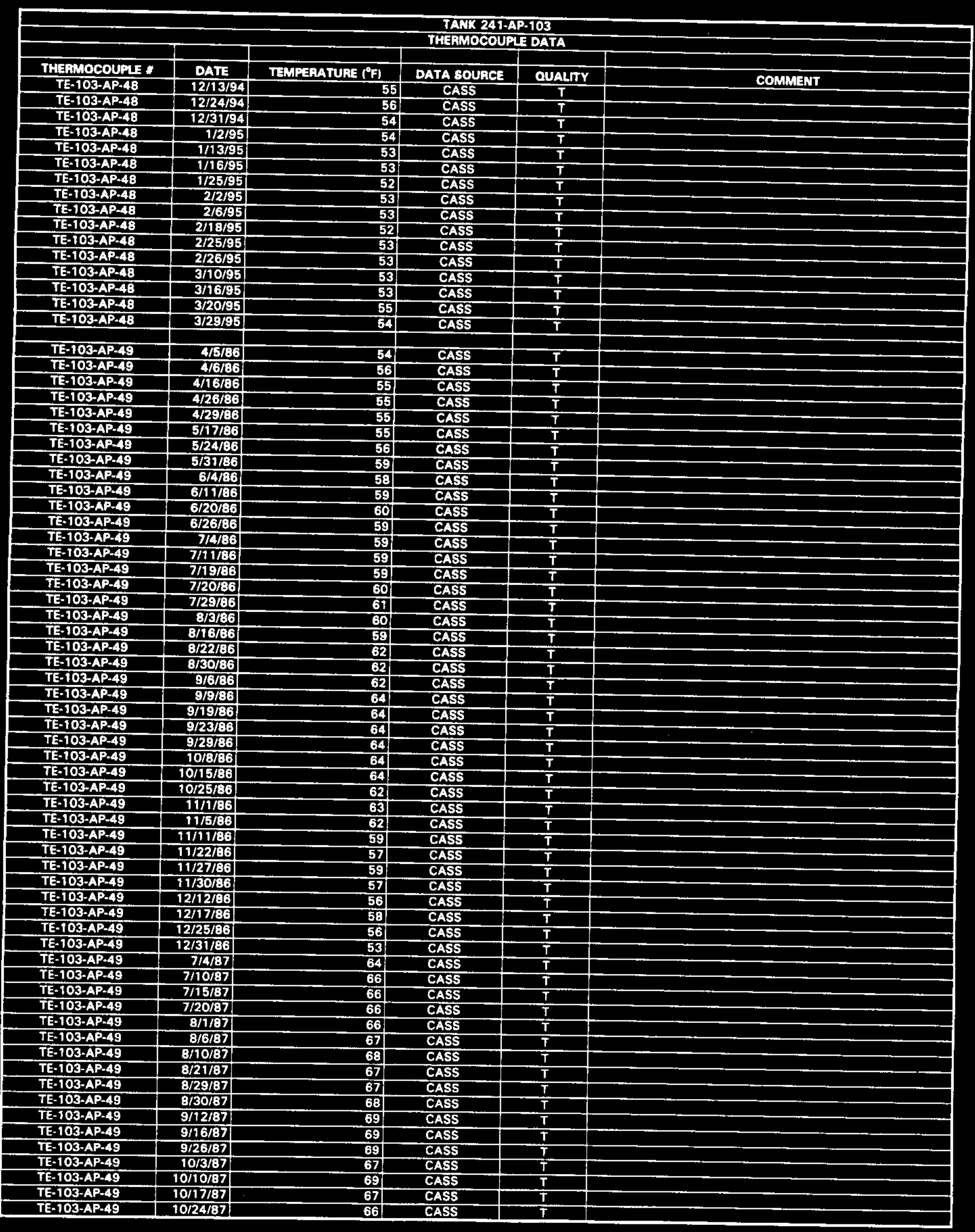

Data obtained from Computer Automated Surveillance System (CASS), April 1995; and Survellence Analysis Computer System (SACS). June 1995. 


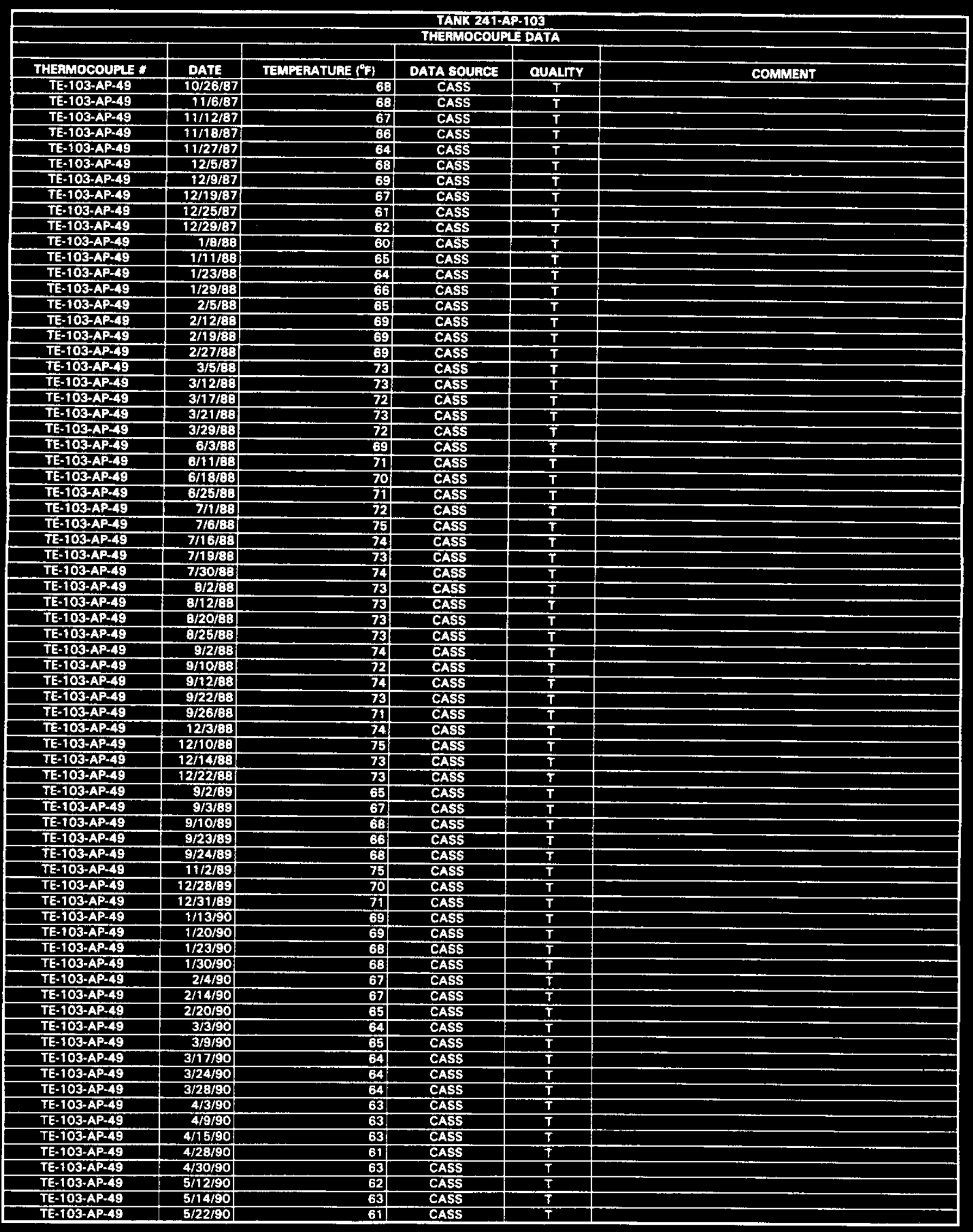

Data obtainad from Computer Automated Surveillance System (CASS), April 1995; and Survaillance Analysis Computer Syatem (SACS), June 1995. D-305 


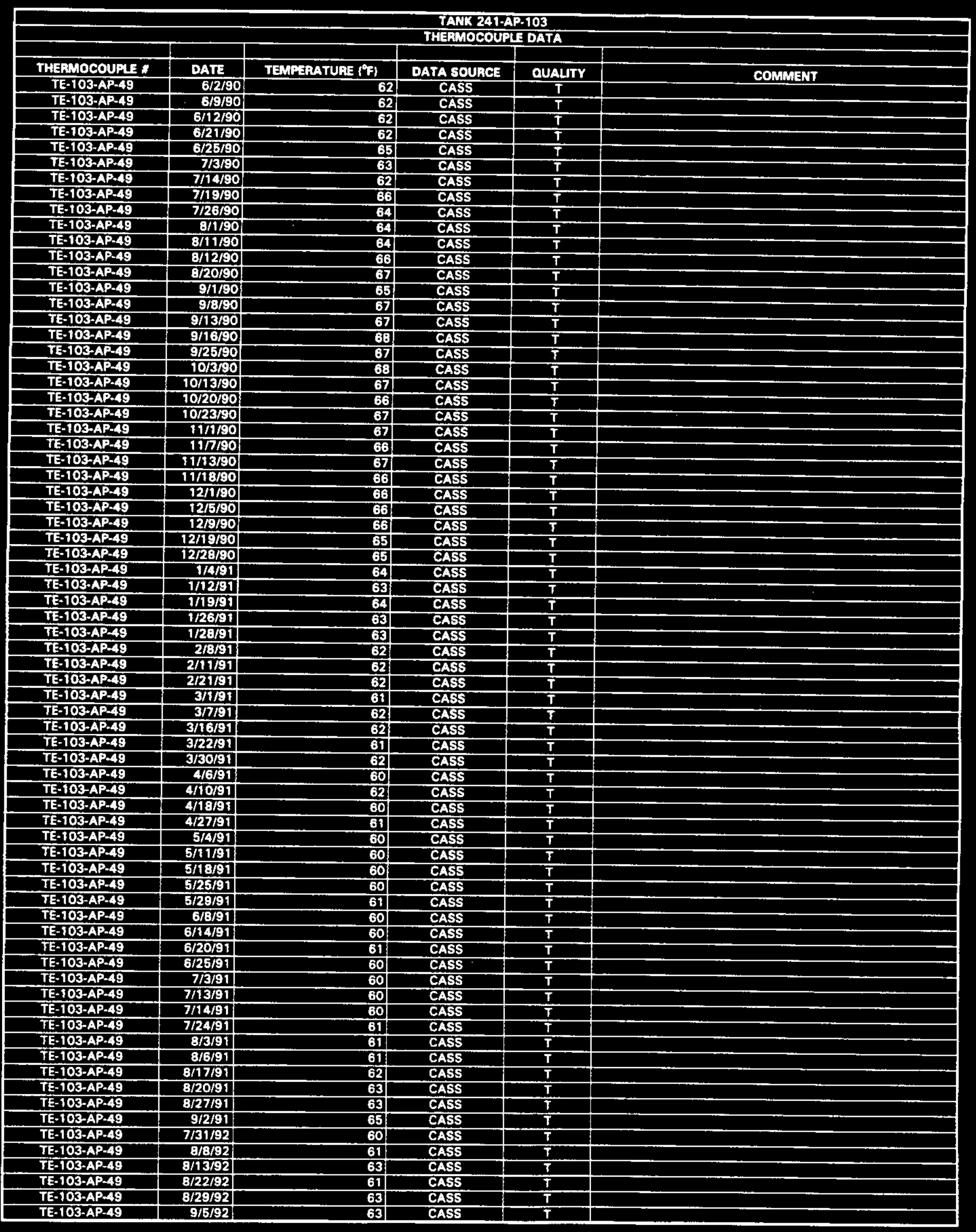




\begin{tabular}{|c|c|c|c|c|c|}
\hline \multicolumn{6}{|c|}{$\begin{array}{l}\text { TANK 241-AP-103 } \\
\text { THEFMOCOUPLE DATA }\end{array}$} \\
\hline & & & & & \\
\hline THERMOCOUPLE $*$ & DATE & TEMPEATUUAE ( $\left.{ }^{\circ} \mathrm{F}\right)$ & DATA SOUACE & OUALITY & COMMENT \\
\hline$T E-103-A P-49$ & $9 / 8 / 92$ & 62 & CASS & $T$ & \\
\hline TE-103-AP-49 & 9/13/92 & 63 & CASS & $T$ & \\
\hline TE-108-AP.49 & $10 / 1 / 92$ & 62 & CASS & $T$ & \\
\hline TE-103-AP-49 & $10 / 6 / 92$ & 62 & CASS & $T$ & \\
\hline TE-103-AP-49 & $10 / 15 / 92$ & 62 & CASS & $T$ & \\
\hline TE-103-AP-49 & $10 / 22 / 92$ & 63 & CÁSS & $T$ & \\
\hline TE-103-AP-49 & $10 / 29 / 92$ & 63 & CASS & $\bar{T}$ & \\
\hline TE-103-AP-49 & $11 / 4 / 92$ & 63 & CASS & $T$ & \\
\hline TE-103-AP-49 & $11 / 9 / 92$ & 62 & CASS & $\bar{T}$ & \\
\hline TE-103-AP-49 & $11 / 17 / 92$ & 64 & CASS & $T$ & \\
\hline TE-103-AP-49 & $11 / 24 / 92$ & 63 & CASS & $T$ & \\
\hline TE-103-AP-49 & $11 / 29 / 92$ & 74 & CASS & S & Date point did not agree with locsl trend \\
\hline TE-103-AP-49 & $12 / 5 / 92$ & 61 & CASS & $T$ & \\
\hline TE-103-AP-49 & $12 / 12 / 92$ & 62 & CASS & $T$ & \\
\hline TE-103-AP-49 & $12 / 18 / 92$ & 62 & CASS & $\bar{T}$ & \\
\hline TE-103-AP-49 & $12 / 26 / 92$ & 61 & CASS & $T$ & \\
\hline TE-103-AP-49 & $12 / 27 / 92$ & 61 & CASS & $T$ & \\
\hline TE-103-AP-49 & 7/9/93 & 60 & CASS & $T$ & \\
\hline TE-103-AP-49 & $1 / 15 / 93$ & 60 & CASS & $T$ & \\
\hline TE-103-AP-49 & $1 / 23 / 93$ & 59 & CASS & $T$ & \\
\hline TE-103-AP-A9 & $1 / 30 / 93$ & 61 & CASS & $T$ & \\
\hline TE-103-AP-49 & $2 / 5 / 93$ & 60 & CASS & $\bar{T}$ & \\
\hline$T E-103-A P-49$ & $2 / 9 / 93$ & 60 & CASS & $T$ & \\
\hline TE-103-AP-49 & $2 / 19 / 93$ & 59 & CASS & $T$ & \\
\hline TE-103-AP-49 & $2 / 26 / 93$ & 59 & CASS & $T$ & \\
\hline TE-103-AP-49 & $3 / 4 / 83$ & 58 & CASS & $T$ & \\
\hline$T E-103-A P-49$ & $3 / 10 / 93$ & 69 & CASS & $T$ & \\
\hline TE-103-AP-49 & 3/19/93 & 58 & CASS & $T$ & \\
\hline TE-103-AP-49 & $3 / 27 / 93$ & 58 & CASS & $T$ & \\
\hline TE-103-AP-49 & $3 / 31 / 93$ & 58 & CASS & $T$ & \\
\hline TE-103-AP-49 & 4/8/93 & 59 & CASS & $T$ & \\
\hline TE-103-AP-49 & $4 / 12 / 93$ & 58 & CASS & $T$ & \\
\hline TE-103-AP.49 & $4 / 24 / 93$ & 58 & CASS & $T$ & \\
\hline TE-103-AP-49 & $4 / 29 / 93$ & 57 & CASS & $T$ & \\
\hline TE-103-AP-49 & $5 / 8 / 93$ & 58 & CASS & $T$ & \\
\hline TE-103-AP-49 & $5 / 10 / 93$ & 58 & CASS & $\bar{T}$ & \\
\hline TE-103-AP-49 & $5 / 22 / 93$ & 57 & CASS & $T$ & \\
\hline TE-103-AP-49 & $5 / 29 / 93$ & 57 & CASS & $T$ & \\
\hline TE-103-AP-49 & $6 / 3 / 93$ & 5 & CASS & $\bar{T}$ & \\
\hline TE-103-AP-49 & $6 / 12 / 93$ & 57) & CASS & $T$ & \\
\hline TE-103-AP-49 & 6/19/93 & 58 & CASS & $T$ & \\
\hline TE-103-AP-49 & $6 / 24 / 93$ & 59 & CASS & $T$ & \\
\hline TE-103-AP-AS & 6/30/93 & 81 & CASS & $\dot{s}$ & Data point did not agree with local trend \\
\hline TE-103-AP-49 & 7/3/93 & 58 & CASS & $T$ & \\
\hline TE-103-AP-49 & $7 / 6 / 93$ & 60 & CASS & $T$ & \\
\hline TE-103-AP-A9 & $7 / 17 / 93$ & 58 & CASS & $T$ & \\
\hline TE-103-AP-49 & $7 / 21 / 83$ & 60 & CASS & $T$ & \\
\hline$T E-103-A P-49$ & $7 / 31 / 93$ & 59 & CASS & $T$ & \\
\hline TE-103-AP-49 & $8 / 7 / 33$ & 60 & CASS & $T$ & \\
\hline TE-103-AP-49 & $8 / 74 / 93$ & 59 & CASS & $\mathbf{T}$ & \\
\hline TE-103-AP-49 & $8 / 20 / 93$ & 61 & CASS & $T$ & \\
\hline TE-103-AP-49 & $8 / 25 / 93$ & 62 & CASS & $T$ & \\
\hline TE-103-AP-49 & $8 / 29 / 93$ & 62 & CASS & $T$ & \\
\hline TE-103-AP-49 & $9 / 9 / 93$ & 62 & CASS & $\mathbf{T}$ & \\
\hline TE-103-AP-49 & 9/13/93 & 62 & CASS & $T$ & \\
\hline TE-103-AP-49 & 9/24/93 & 61 & CASS & $T$ & \\
\hline TE-103-AP-49 & $10 / 1 / 93$ & 61 & CASS & $T$ & \\
\hline TE-103-AP-49 & $10 / 8 / 93$ & 62 & CASS & $T$ & \\
\hline TE-103-AP-49 & 10/11/93 & 62 & CASS & $T$ & \\
\hline TE-103-AP-49 & $10 / 22 / 93$ & 62 & CASS & $T$ & \\
\hline TE-103-AP-49 & $10 / 29 / 93$ & 62 & CASS & $T$ & \\
\hline TE-103-AP-49 & $11 / 6 / 93$ & 61 & CASS & $T$ & \\
\hline TE-103-AP-49 & $11 / 8 / 93$ & 62 & CASS & $T$ & \\
\hline TE-103-AP-49 & $11 / 19193$ & 61 & CASS & $T$ & \\
\hline TE-103-AP-49 & $11 / 27 / 93$ & 60 & CASS & $T$ & \\
\hline TE-103-AP-49 & $12 / 1 / 93$ & 64 & CASS & $T$ & \\
\hline TE-103-AP-49 & $1 / 13 / 94$ & 58 & CASS & $T$ & \\
\hline$T E-103-A P-49$ & $5 / 12194$ & 57 & CASS & $T$ & \\
\hline TE-103-AP-49 & $5 / 17 / 94$ & 58 & CASS & $T$ & \\
\hline TE-103-AP-49 & $5 / 26 / 94$ & 59 & CASS & $T$ & \\
\hline TE-103-AP-49 & $6 / 4 / 94$ & 59 & CASS & $T$ & \\
\hline TE-103-AP-49 & $6 / 11 / 94$ & 60 & CASS & $\mathbf{T}$ & \\
\hline TE-103-AP-49 & $6 / 12 / 94$ & 61 & CASS & $\mathbf{T}$ & \\
\hline
\end{tabular}




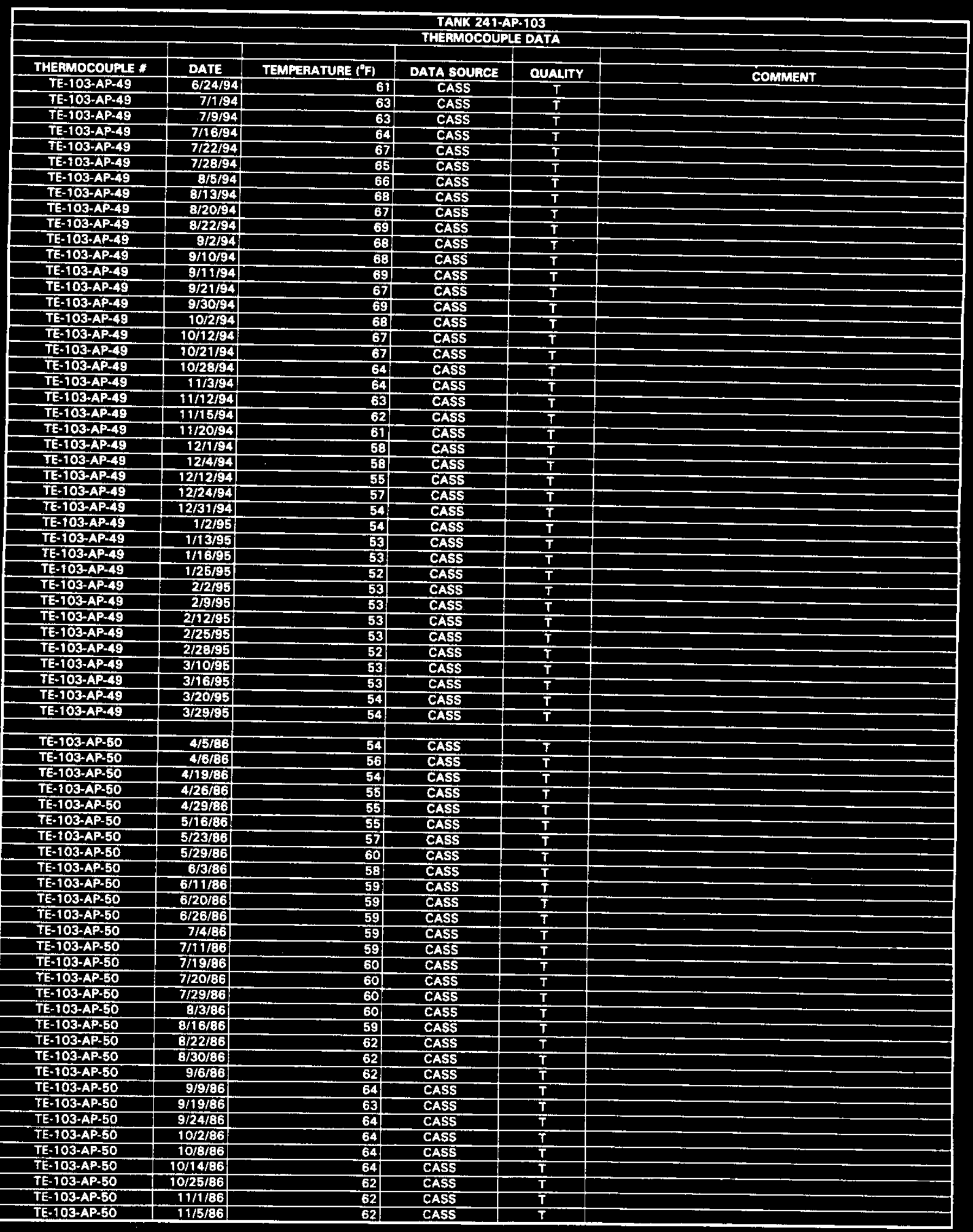

Data obtained from Computer Automated Surveillance System (CASS), April 1995; and Surveillance Analyais Computer Syatem (SACS), June 1995. D-308 


\begin{tabular}{|c|c|c|c|c|c|}
\hline \multicolumn{6}{|c|}{$\begin{array}{l}\text { TANK 241-AP-103 } \\
\text { THEAMOCOUPLE DATA }\end{array}$} \\
\hline THEHMOCOUPLE * & DATE & TEMPERATUAE $\left({ }^{\circ} \mathrm{F}\right)$ & DATA SOUACE & & \\
\hline TE-103-AP-50 & $11 / 11 / 86$ & 59 & CASS & $\frac{\text { DUALIIV }}{T}$ & COMRIENT \\
\hline TE-103-AP-50 & $11 / 22 / 86$ & 57 & CASS & $\frac{T}{T}$ & \\
\hline TE-103-AP-50 & $11 / 27 / 86$ & 59: & CASS & $T$ & \\
\hline TE-103-AP-50 & 12/1/36 & 56 & CASS & $\bar{T}$ & \\
\hline TE-103-AP-50 & $12 / 11 / 86$ & 66 & CASS & $\mathbf{s}$ & Date point did not agree with local trend \\
\hline TE-103-AP-50 & $12 / 13 / 86$ & 55 & CASS & $T$ & \\
\hline TE-103-AP-BO & $12 / 17 / 86$ & 84 & CASS & $\mathbf{S}$ & Data paint did not agree with local trend \\
\hline TE-103-AP-50 & $12 / 18 / 86$ & $\mathbf{5 7}$ & CASS & 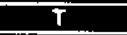 & \\
\hline TE-10S-AP-50 & $12 / 25 / 86$ & 56 & CASS & $\mathbf{T}$ & \\
\hline TE-103-AP-50 & $12 / 31 / 86$ & 53 & CASS & $\mathbf{T}$ & \\
\hline TE-103-AP-50 & $7 / 4 / 67$ & 64 & CASS & $\mathbf{T}$ & \\
\hline TE.103-AP-50 & $7 / 11 / 87$ & 65 & CASS & $\mathbf{T}$ & \\
\hline TE-103-AP-50 & 7/15/87 & 66 & CASS & $\mathbf{T}$ & \\
\hline TE-103-AP-50 & $7 / 25 / 87$ & 65 & CASS & $T$ & \\
\hline TE-103-AP-S0 & $8 / 1 / 87$ & 66 & CASS & $\bar{T}$ & \\
\hline TE-103-AP-50 & $8 / 6 / 97$ & 67 & CASS & $T$ & \\
\hline TE-103-AP-EO & $8 / 14 / 87$ & 67 & CASS & $\mathbf{T}$ & \\
\hline TE-103-APSO & $8 / 18 / 37$ & 67 & CASS & $T \quad 1$ & \\
\hline TE-108-AP-SO & $8 / 28187$ & 67 & CASS & $T$ & \\
\hline TE-103-AP-50 & $8 / 30 / 87$ & 68 & CASS & $T$ & \\
\hline TE-103-AP-50 & $9 / 12 / 87$ & 69 & CASS & $T$ & \\
\hline TE-103-AP-50 & $9 / 16 / 87$ & 69 & CASS & $T$ & \\
\hline TE-103-AP-50 & $9 / 26 / 87$ & 69 & CASS & $T$ & \\
\hline TE-103-AP-50 & $10 / 3 / 87$ & 67 & CASS & $T$ & \\
\hline TE-103-AP-50 & $10 / 10 / 87$ & 69 & CASS & $\mathrm{T}$ & \\
\hline TE-103.AP-50 & $10 / 27 / 87$ & 67 & CASS & $T$ & \\
\hline TE-103-AP-50 & $10 / 24 / 87$ & 66 & CASS & $T$ & \\
\hline TE-103-AP-60 & $10 / 26 / 87$ & 68 & CASS & $T$ & \\
\hline TE-103-AP-50 & $11 / 6 / 87$ & 69 & CASS & $T$ & \\
\hline TE-108-AP-50 & $11 / 12 / 87$ & 67 & CASS & $\bar{T}$ & \\
\hline TE-103-AP-50 & $11 / 18 / 87$ & 66 & CASS & $T$ & \\
\hline TE-103-AP-50 & $11 / 27 / 87$ & 64 & CASS & $T$ & \\
\hline TE-103-AP-50 & 12/5/87 & 68 & CASS & $\mathbf{T}$ & \\
\hline TE-103-AP-SO & $12 / 9 / 67$ & 69 & CASS & $T$ & \\
\hline TE-10S-AP-50 & $12 / 19 / 87$ & 67 & CASS & $\mathbf{T}$ & \\
\hline TE-103.AP.50 & $12123 / 87$ & 61 & CASS & $T$ & \\
\hline TE-103-AP-50 & $12 / 28 / 87$ & 61 & CASS & $T$ & \\
\hline TE-103-AP-50 & $1 / 8 / 38$ & 60 & CASS & $T$ & \\
\hline TE-108-AP-50 & 1/11/88 & 66! & CASS & $T$ & \\
\hline TE-103-AP-50 & $1 / 23 / 88$ & 64 & CASS & $T$ & \\
\hline TE-103-AP-50 & 1/29/88 & 65 & CASS & $\mathbf{T}$ & \\
\hline TE-108-AP-50 & $2 / 8 / 88$ & 65 & CASS & $T$ & \\
\hline TE-103-AP-50 & $2 / 13 / 88$ & 69 & CASS & $T$ & \\
\hline TE-103-AP-50 & $2 / 19 / 88$ & 69 & CASS & $T$ & \\
\hline TE-103-AP-50 & $2 / 27 / 38$ & 69 & CASS & $T$ & \\
\hline TE-103-AP-50 & $3 / 5 / 88$ & 73 & CASS & $\bar{T}$ & \\
\hline TE-103-AP-50 & 3/12/88 & 72 & CASS & $T$ & \\
\hline TE-103-AP-50 & $3 / 19 / 88$ & 71 & CASS & $T$ & \\
\hline TE-103-AP-50 & $3 / 21 / 88$ & 73 & CASS & $T$ & \\
\hline TE-103-AP-50 & 3/29/88 & 72 & CASS & $\mathbf{T}$ & \\
\hline TE-103-AP-50 & $6 / 3 / 88$ & 69 & CASS & $\bar{T}$ & \\
\hline TE-103-AP-50 & 6/11/88 & 71 & CASS & $\mathbf{T}$ & \\
\hline TE-103-AP-50 & 6/18/88 & 69 & CASS & $T$ & \\
\hline TE-108-AP-50 & $6 / 25 / 68$ & 72 & CASS & $T$ & \\
\hline TE-103-AP-50 & $7 / 1 / 88$ & 72 & CASS & $T$ & \\
\hline TE-103-AP-50 & $7 / 6 / 88$ & 75 & CASS & $\mathbf{T}$ & \\
\hline TE-103-AP-50 & 7/13/88 & 74 & CASS & $T$ & \\
\hline TE-103-AP-50 & 7/19/88 & 73 & CASS & $\mathbf{T}$ & \\
\hline TE-103-AP-50 & $7 / 30 / 88$ & 74 & CASS & $\mathbf{T}$ & \\
\hline IE-103-AP-50 & $8 / 2 / 88$ & 73 & CASS & $\mathbf{T}$ & \\
\hline TE-108-AP-50 & $8 / 12 / 80$ & 73 & CASS & $\mathbf{T}$ & \\
\hline TE-103-AP-50 & $8 / 20 / 8$ & 73 & CASS & $\mathbf{T}$ & \\
\hline TE-103-AP-50 & $8 / 25 / 82$ & 73 & CASS & $\mathbf{T}$ & \\
\hline TE-103-AP-50 & 9/2/88 & 74 & CASS & $T$ & \\
\hline TE-103-AP-50 & $9 / 10 / 88$ & 71 & CASS & $T$ & \\
\hline TE-103-AP-50 & $9 / 12 / 88$ & 73 & CASS & $T$ & \\
\hline TE-103-AP-50 & $9 / 22 / 88$ & 73 & CASS & $\mathrm{T}$ & \\
\hline TE-103-AP-50 & $9 / 26 / 83$ & 71 & CASS & $\mathbf{T}$ & \\
\hline TE-103-AP-SO & $12 / 3 / 88$ & 74 & CASS & $T$ & \\
\hline TE-103-AP-50 & $12 / 10 / 88$ & 75 & CASS & $T$ & \\
\hline TE-103-AP-50 & $12 / 14 / 88$ & 73 & CASS & $T$ & \\
\hline TE-103-AP-50 & 12122188 & 72 & CASS & $T$ & \\
\hline TE-103-AP.50 & $9 / 2 / 89$ & 65 & CASS & $T$ & \\
\hline
\end{tabular}




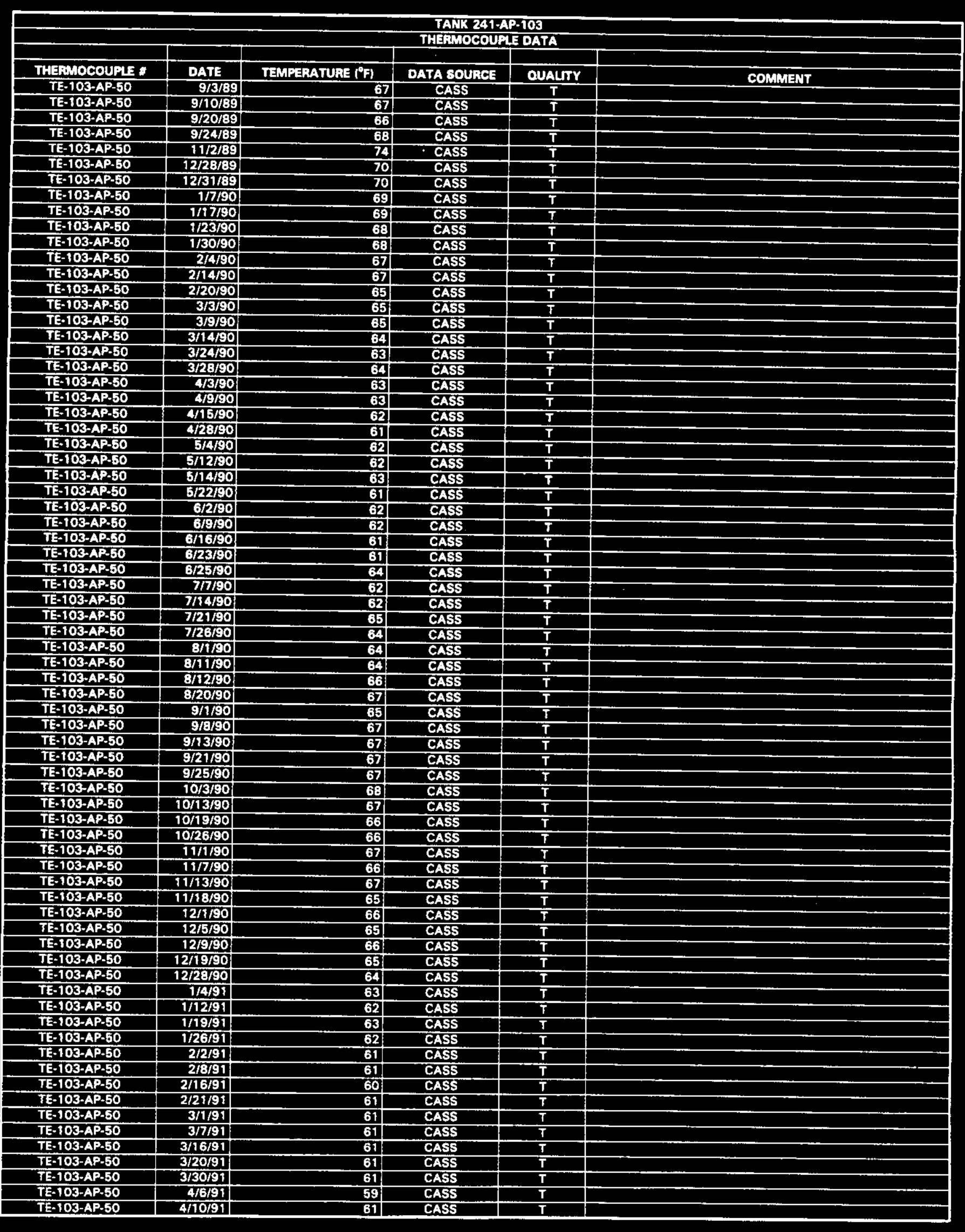




\begin{tabular}{|c|c|c|c|c|c|}
\hline \multicolumn{6}{|c|}{$\begin{array}{l}\text { TAKK 241-AP-103 } \\
\text { THERMOCOUPLE DATA }\end{array}$} \\
\hline & & & & & \\
\hline THERMOCOUPLE & DATE & TEMPERATURE $\left({ }^{\circ} \mathrm{F}\right)$ & DATA SOURCE & OUALTY & COMMENT \\
\hline TE-103-AP-50 & $4 / 18 / 91$ & 60 & CASS & $T$ & \\
\hline TE-103-AP-50 & $4 / 27 / 91$ & 60 & CASS & $\mathrm{T}$ & \\
\hline TE-103-AP-60 & $5 / 4 / 91$ & 60 & CASS & $T$ & \\
\hline TE-103-AP-50 & 5/11/91 & 60 & CASS & $T$ & \\
\hline$\frac{\text { TE-103-AP-50 }}{\text { TE-103-AP-50 }}$ & $5 / 14 / 91$ & 60 & CASS & $T$ & \\
\hline$\frac{T E-103-A P-50}{\text { TE-103-AP-50 }}$ & $5 / 25 / 91$ & 60 & CASS & $\frac{T}{T}$ & \\
\hline$\frac{\text { TE-103-AP-50 }}{\text { TE-103-AP-50 }}$ & $\frac{5 / 29 / 91}{6 / 8 / 91}$ & $\frac{62}{61}$ & $\frac{\text { CASS }}{\text { CASS }}$ & $\frac{T}{T}$ & \\
\hline TE-103-AP-50 & $6 / 15 / 91$ & 60 & CASS & $T$ & \\
\hline TE-103-AP-50 & $6 / 20 / 91$ & 61 & CASS & $T$ & \\
\hline TE-103-AP-50 & $6 / 25 / 91$ & 60 & CASS & $\mathbf{T}$ & \\
\hline TE-103-AP-50 & $7 / 3 / 91$ & 59 & CASS & $T$ & \\
\hline TE-103-AP-50 & $7 / 11 / 91$ & 61 & CASS & $T$ & \\
\hline TE-103-AP-50 & $7 / 14 / 81$ & 60 & CASS & $T$ & \\
\hline TE-103-AP-50 & $7 / 24 / 91$ & 60 & CASS & $\bar{T}$ & \\
\hline TE-103-AP-50 & $8 / 3 / 91$ & 61 & CASS & $T$ & \\
\hline TE-103-AP-50 & $8 / 5 / 91$ & 62 & CASS & $T$ & \\
\hline TE-103-AP-50 & $8 / 15 / 91$ & 62. & CASS & $T$ & \\
\hline TE-103-AP-50 & $8 / 19 / 91$ & 64 & CASS & $T$ & \\
\hline TE-103-AP-SO & $8 / 26 / 91$ & 63 & CASS & $T$ & \\
\hline TE-103-AP-50 & 9/3/91 & 64 & CASS & $T$ & \\
\hline TE-103-AP-50 & $7 / 31 / 92$ & 60 & CASS & $T$ & \\
\hline TE-103-AP-50 & $8 / 3 / 92$ & 61 & CASS & $T$ & \\
\hline TE-103-AP.50 & $8 / 13 / 92$ & 62 & CASS & $T$ & \\
\hline TE-10S-AP-50 & $8 / 18 / 92$ & 61 & CASS & $T$ & \\
\hline TE-103-AP-50 & 8/29/92 & 62 & CASS & $T$ & \\
\hline TE-103-AP-50 & $8 / 4 / 92$ & 63 & CASS & $T$ & \\
\hline TE-103.AP-50 & 9/6/92 & 62 & CASS & $\bar{T}$ & \\
\hline TE-103-AP-50 & $9 / 13 / 92$ & 63 & CASS & $T$ & \\
\hline TE-103-AP-50 & $10 / 1 / 92$ & 63 & CASS & $T$ & \\
\hline TE-103-AP-50 & $10 / 6 / 92$ & 62 & CASS & $T$ & \\
\hline TE-103-AP-50 & $10 / 14 / 92$ & 62 & CASS & $T$ & \\
\hline TE-103-AP-50 & $10 / 22 / 92$ & 63 & CASS & $T$ & \\
\hline TE-103-AP-50 & $10 / 29 / 92$ & 63 & CASS & $T$ & \\
\hline TE-103-AP-5O & $11 / 4 / 92$ & 62 & CASS & $T$ & \\
\hline TE-103-AP-50 & $11 / 9 / 92$ & 62 & CASS & $T$ & \\
\hline TE-103-AP-50 & $11 / 20 / 92$ & 63 & CASS & $\mathbf{T}$ & \\
\hline TE-103-AP-50 & $11 / 24 / 92$ & 63 & CASS & $T$ & \\
\hline TE-103-AP-50 & $11 / 29 / 92$ & 74 & CASS & s & Data point did not agree with local trend \\
\hline TE-103-AP-50 & $12 / 3 / 92$ & 61 & CASS & $T$ & \\
\hline TE-103-AP-50 & 12/2/92 & 62 & CASS & $\mathbf{T}$ & \\
\hline TE-103-AP-50 & $12 / 18 / 92$ & 61 & CASS & $T$ & \\
\hline TE-103-AP-50 & $12 / 26 / 92$ & 60 & CASS & $\bar{T}$ & \\
\hline TE-103-AP-50 & $12 / 27 / 92$ & 61) & CASS & $\mathbf{T}$ & \\
\hline$T E \cdot 103-A P-50$ & $1 / 9 / 93$ & 60 & CASS & $T$ & \\
\hline TE-103-AP-50 & $1 / 36 / 93$ & 59 & CASS & $\mathbf{T}$ & \\
\hline TE-103-AP-50 & $1 / 23 / 93$ & 59 & CASS & $\mathbf{T}$ & \\
\hline TE-103-AP-50 & $1 / 30 / 93$ & 60. & CASS & $T$ & \\
\hline TE-103-AP-50 & $2 / 6 / 93$ & 59 & CASS & $T$ & \\
\hline TE-103-AP-50 & $2 / 12 / 93$ & 59 & CASS & $\frac{T}{T}$ & \\
\hline TE-103-AP-50 & $2 / 20 / 93$ & 5 & $\frac{\text { CASS }}{\text { CASS }}$ & $\frac{T}{T}$ & \\
\hline TE-103-AP-50 & $2 / 26 / 93$ & \begin{tabular}{l|l}
58 \\
58
\end{tabular} & $\frac{\text { CASS }}{\text { CASS }}$ & $T$ & \\
\hline TE-103-AP-50 & $3 / 4 / 93$ & $\frac{58}{58}$ & $\begin{array}{l}\text { CASS } \\
\text { CASS }\end{array}$ & $T$ & \\
\hline TE-108-AP-50 & $3 / 10 / 93$ & $\frac{58}{57}$ & CASS & $T$ & \\
\hline TE-103+AP-50 & $3 / 19 / 93$ & \begin{tabular}{l|}
57 \\
57
\end{tabular} & $\frac{\text { CASS }}{\text { CASS }}$ & $\mathbf{T}$ & \\
\hline TE-103-AP-50 & $\begin{array}{l}3 / 27 / 93 \\
3 / 37 / 93\end{array}$ & 57| & $\frac{\text { CASS }}{\text { CASS }}$ & $\bar{T}$ & \\
\hline TE-103-AP-50 & $\frac{3 / 31 / 93}{4 / 6 / 93}$ & \begin{tabular}{l|}
58 \\
58
\end{tabular} & $\frac{\text { CASS }}{\text { CASS }}$ & $\bar{T}$ & \\
\hline TE-103-AP-50 & \begin{tabular}{|r|}
$4 / 6 / 93$ \\
$4 / 12 / 93$
\end{tabular} & 57 & $\frac{\text { CASS }}{\text { CASS }}$ & $\bar{T}$ & \\
\hline TE-108-AP-50 & \begin{tabular}{|l|}
$4 / 12 / 93$ \\
$4 / 24 / 93$
\end{tabular} & 58 & $\frac{\text { CASS }}{\text { CASS }}$ & $\bar{T}$ & \\
\hline TE-103-AP-50 & $\begin{array}{l}4 / 24 / 93 \\
4 / 29 / 33\end{array}$ & $56 !$ & CASS & $T$ & \\
\hline TE-103-AP-50 & $\frac{4 / 29 / 93}{5 / 8 / 93}$ & 57 & CASS & $T$ & \\
\hline TE-103-AP-50 & \begin{tabular}{r|}
$5 / 8 / 93$ \\
$5 / 10 / 93$
\end{tabular} & 58 & CASS & $T$ & \\
\hline TE-103-AP-50 & $\frac{5 / 10 / 93}{5 / 22 / 93}$ & 57 & CASS & $T$ & \\
\hline TE-103-AP-50 & \begin{tabular}{l|}
$5 / 22 / 93$ \\
$5 / 29 / 93$
\end{tabular} & 57 & CASS & $T$ & \\
\hline $\begin{array}{l}\text { TE-103-AP-50 } \\
\text { TE-103-AP-50 }\end{array}$ & \begin{tabular}{r|}
$5 / 29 / 93$ \\
$6 / 3 / 93$
\end{tabular} & 58 & CASS & $\mathbf{T}$ & \\
\hline$\frac{\text { TE-103-AP-50 }}{\text { TE-103-AP-50 }}$ & $\begin{array}{r}6 / 3 / 93 \\
6 / 12 / 93 \\
\end{array}$ & 57 & CASS & $T$ & \\
\hline$\frac{\text { TE-103-AP-50 }}{\text { TE-103-AP-50 }}$ & $\begin{array}{l}6 / 12 / 93 \\
6 / 16 / 93\end{array}$ & 58 & CASS & $T$ & \\
\hline$\frac{\text { TE-103-AP-50 }}{\text { TE-103-AP-50 }}$ & $\begin{array}{l}6 / 16 / 93 \\
6 / 24 / 93\end{array}$ & 59 & CASS & $\mathbf{T}$ & \\
\hline$\frac{\text { TE-103-AP-50 }}{\text { TE-103-AP-50 }}$ & $\begin{array}{l}6 / 24 / 93 \\
6 / 30 / 93\end{array}$ & 80 & CASS & s & Data point did not agree with local trand \\
\hline $\begin{array}{l}\text { TE-103-AP-50 } \\
\text { TE-103-AP-50 }\end{array}$ & \begin{tabular}{r|}
$6 / 30 / 93$ \\
$7 / 3 / 93$
\end{tabular} & 58 & CASS & $T$ & \\
\hline $\begin{array}{l}\text { TE-103-AP-50 } \\
\text { TE-103-AP-50 }\end{array}$ & $\begin{array}{l}7 / 3 / 93 \\
7 / 6 / 93\end{array}$ & 60 & CASS & $\mathrm{T}$ & \\
\hline $\begin{array}{l}\text { TE-103-AP-50 } \\
\text { TE-103-AP-50 }\end{array}$ & \begin{tabular}{r|}
$7 / 6 / 93$ \\
$7 / 16 / 93$
\end{tabular} & 59 & CASS & $T$ & \\
\hline
\end{tabular}




\begin{tabular}{|c|c|c|c|c|c|}
\hline \multicolumn{6}{|c|}{$\begin{array}{l}\text { TANK 241-AP-10S } \\
\text { THEFMOCOUPLE DATA }\end{array}$} \\
\hline THEPMOCOUPLE * & DATE & TEMPERATURE ( $\left.{ }^{\circ} \mathrm{F}\right)$ & DATA SOUACE & oustry & \\
\hline TE-103-AP-50 & $7 / 23 / 93$ & 59 & CASS & $T$ & COMMENT \\
\hline TE-103-AP-50 & $7 / 31 / 93$ & 59 & CASS & $T$ & \\
\hline TE-103-AP-50 & $8 / 7 / 93$ & 60 & CASS & $T$ & \\
\hline TE-103-AP-50 & $8 / 14 / 93$ & 59 & CASS & $T$ & \\
\hline TE-103-AP-50 & $8 / 20 / 93$ & 61 & CASS & $T$ & \\
\hline TE-103-AP-50 & $8 / 25 / 93$ & 62 & CASS & $T$ & \\
\hline TE-103-AP-50 & $8 / 29 / 93$ & 62 & CASS & $\mathbf{T}$ & \\
\hline TE-103-AP-50 & 9/9/93 & 62 & CASS & $\bar{T}$ & \\
\hline TE-103-AP-50 & 9/16/93 & 61 & CASS & $T$ & \\
\hline TE-103-AP-50 & $9 / 23 / 93$ & 61 & CASS & $T$ & \\
\hline TE-103-AP-50 & $10 / 1 / 93$ & 61 & CASS & $T$ & \\
\hline TE-103-AP-50 & $10 / 7 / 93$ & 62 & CASS & $T$ & \\
\hline TE-103-AP-50 & $10 / 11 / 93$ & 62 & CASS & $T$ & \\
\hline TE-108-AP-50 & $10 / 18 / 93$ & 62 & CASS & $T$ & \\
\hline TE-103-AP-50 & $10 / 29 / 93$ & 61 & CASS & $T$ & \\
\hline TE-108-AP-50 & $11 / 5 / 93$ & 61 & CASS & $T$ & \\
\hline TE-103-AP-50 & 11/8/93 & 63 & CASS & $T$ & \\
\hline TE-103-AP-SO & $11 / 14 / 93$ & 62 & CASS & $T$ & \\
\hline TE-103-AP-50 & $11 / 26 / 93$ & 60 & CASS & $T$ & \\
\hline TE-103-AP-50 & $12 / 1 / 93$ & 61 & CASS & $\mathbf{T}$ & \\
\hline TE-103-AP-50 & $1 / 13 / 94$ & 58 & CASS & $T$ & \\
\hline TE-103-AP-SO & 5/12/94 & 57 & CASS & $T$ & \\
\hline TE-103-APASO & $5 / 17 / 94$ & 59 & CASS & $T$ & \\
\hline TE-10Q-AP-SO & 5/26/94 & 58 & CASS & $T$ & \\
\hline TE-103-AP=50 & $6 / 4 / 94$ & 59 & CASS & $T$ & \\
\hline TE-103-AP-BO & 6/11/94 & 60 & CASS & $T$ & \\
\hline TE-103-AP-50 & 6/12/94 & 611 & CASS & $\mathbf{T}$ & \\
\hline TE-103-AP-50 & $6 / 24 / 94$ & 61 & CASS & $T$ & \\
\hline TE-103-AP-50 & $7 / 1 / 94$ & 63 & CASS & $\mathbf{T}$ & \\
\hline TE-108-AP-50 & 7/9/94 & 63 & CASS & $\bar{T}$ & \\
\hline TE-103-AP-50 & $7 / 16 / 94$ & 63 & CABS & $\bar{T}$ & \\
\hline TE-103.AP-50 & $7 / 22 / 94$ & 66 & CASS & $\bar{T}$ & \\
\hline TE-103-AP-50 & $7 / 28 / 94$ & 85 & CASS & $T$ & \\
\hline TE-103-AP-60 & $8 / 5 / 94$ & 66 & CASS & $T$ & \\
\hline TE-103-AP-50 & $8 / 13 / 94$ & 68 & CASS & $\bar{T}$ & \\
\hline TE-103-AP-50 & $8 / 20 / 94$ & 67. & CASS & $T$ & \\
\hline TE-103-AP-50 & $0 / 22 / 94$ & 69 & CASS & $\bar{T}$ & \\
\hline TE-103-AP-50 & $8 / 28 / 94$ & 68 & CASS & $T$ & \\
\hline TE-103.AP-50 & $9 / 5 / 94$ & 68 & CASS & $T$ & \\
\hline TE-103-AP-50 & $9 / 11 / 94$ & 69 & CASS & $T$ & \\
\hline TE-103-AP-50 & $9 / 21 / 94$ & 67! & CASS & $T$ & \\
\hline TE-103-APS50 & $9 / 30 / 94$ & 69 & CASS & $T$ & \\
\hline TE-103-AP-50 & $10 / 2 / 94$ & 68 & CASS & $T$ & \\
\hline TE-108-AP-50 & $10 / 14 / 94$ & 66 & CASS & $T$ & \\
\hline TE-103-AP-50 & $10 / 21 / 94$ & 67 & CASS & $\bar{T}$ & \\
\hline TE-103-AP-50 & $10 / 24 / 94$ & 64 & CASS & $\bar{T}$ & \\
\hline TE-103-AP-50 & $11 / 3 / 94$ & 64 & CASS & $T$ & \\
\hline TE-103-AP-50 & $11 / 12 / 94$ & 62 & CASS & $T$ & \\
\hline TE-103-AP-50 & $11 / 15 / 94$ & 62 & CASS & $T$ & \\
\hline TE-103-AP-50 & $11 / 20 / 94$ & 61 & CASS & $T$ & \\
\hline TE-103-AP-50 & $12 / 1 / 94$ & 58 & CASS & $T$ & \\
\hline TE-103-AP-50 & $12 / 5 / 94$ & 57 & CASS & $\mathbf{T}$ & \\
\hline TE-103-AP-50 & $12 / 12 / 94$ & 55 & CASS & $T$ & \\
\hline TE-103-AP-50 & $12 / 21 / 94$ & 56 & CASS & $T$ & \\
\hline TE-103-AP-50 & $12 / 30 / 94$ & 54 & CASS & $T$ & \\
\hline TE-103-AP-50 & $1 / 2 / 95$ & 54 & CASS & $T$ & \\
\hline TE-103-AP-50 & $1 / 13 / 95$ & 53 & CASS & $T$ & \\
\hline TE-103-AP-50 & $1 / 17 / 95$ & 53 & CASS & $T$ & \\
\hline TE-103-AP-50 & $1 / 25 / 95$ & 52 & CASS & $T$ & \\
\hline TE-103-AP-50 & $1 / 29 / 95$ & 52 & CASS & $T$ & \\
\hline TE-103-AP-50 & $2 / 9 / 95$ & 52 & CASS & $\mathrm{T}$ & \\
\hline TE-103.APAO & $2 / 12 / 95$ & 53 & CASS & $T$ & \\
\hline TE-103-AP-50 & $2 / 22 / 95$ & 53 & CASS & $T$ & \\
\hline TE-103-AP-50 & $2 / 28 / 95$ & 52 & CASS & $T$ & \\
\hline TE-103-AP-50 & $3 / 10 / 95$ & 53 & CASS & $T$ & \\
\hline TE-103.AP-50 & 3/16/95 & 53 & CASS & $T$ & \\
\hline TE-103-AP-50 & $3 / 20 / 95$ & 55 & CASS & $T$ & \\
\hline TE-103-AP-50 & $3 / 29 / 95$ & 54 & CASS & $\mathbf{T}$ & \\
\hline TE-103-AP-51 & $4 / 5 / 86$ & 54 & CASS & $\bar{T}$ & \\
\hline TE-103-AP-51 & $4 / 6 / 86$ & 56 & CASS & $\bar{T}$ & \\
\hline TE-103-AP-51 & $4 / 18 / 86$ & 55 & CASS & $T$ & \\
\hline TE-103-AP-51 & $4 / 26 / 86$ & 55 & CASS & $\mathbf{I}$ & \\
\hline
\end{tabular}




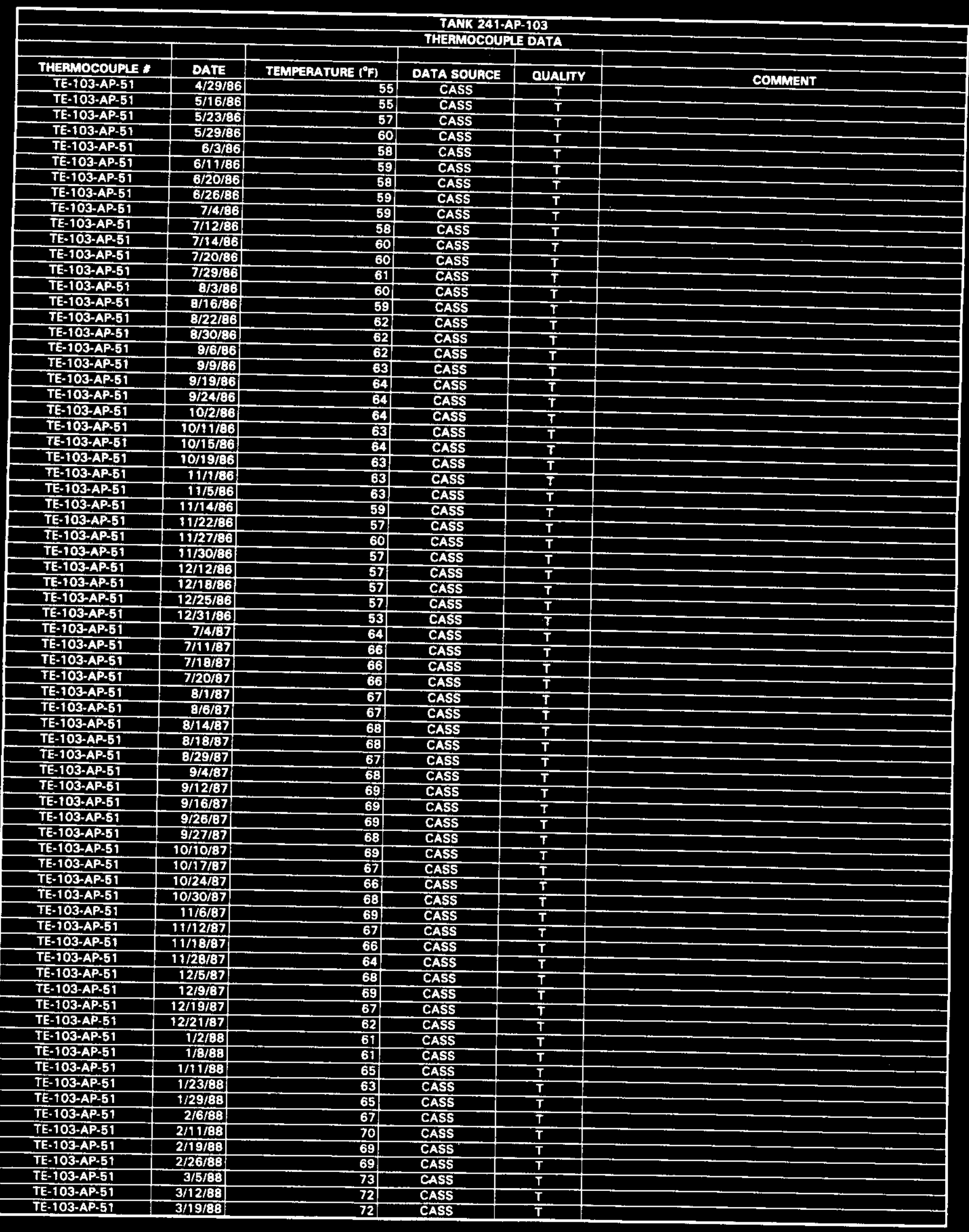

Date obtained from Computer Automated Surveillance System (CASS), April 1995; and Surveillance Analysis Computer System (SACS), June 1995. 


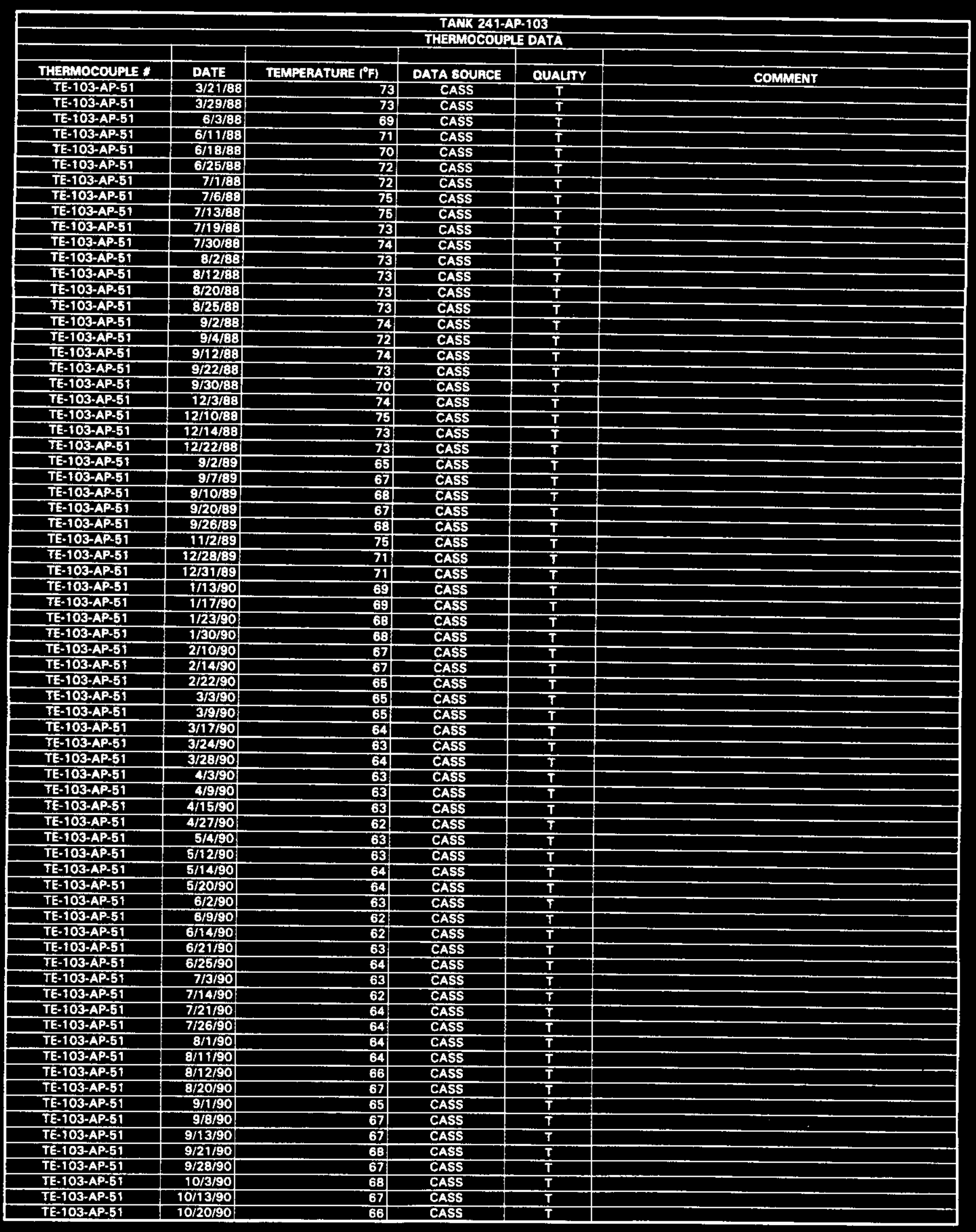




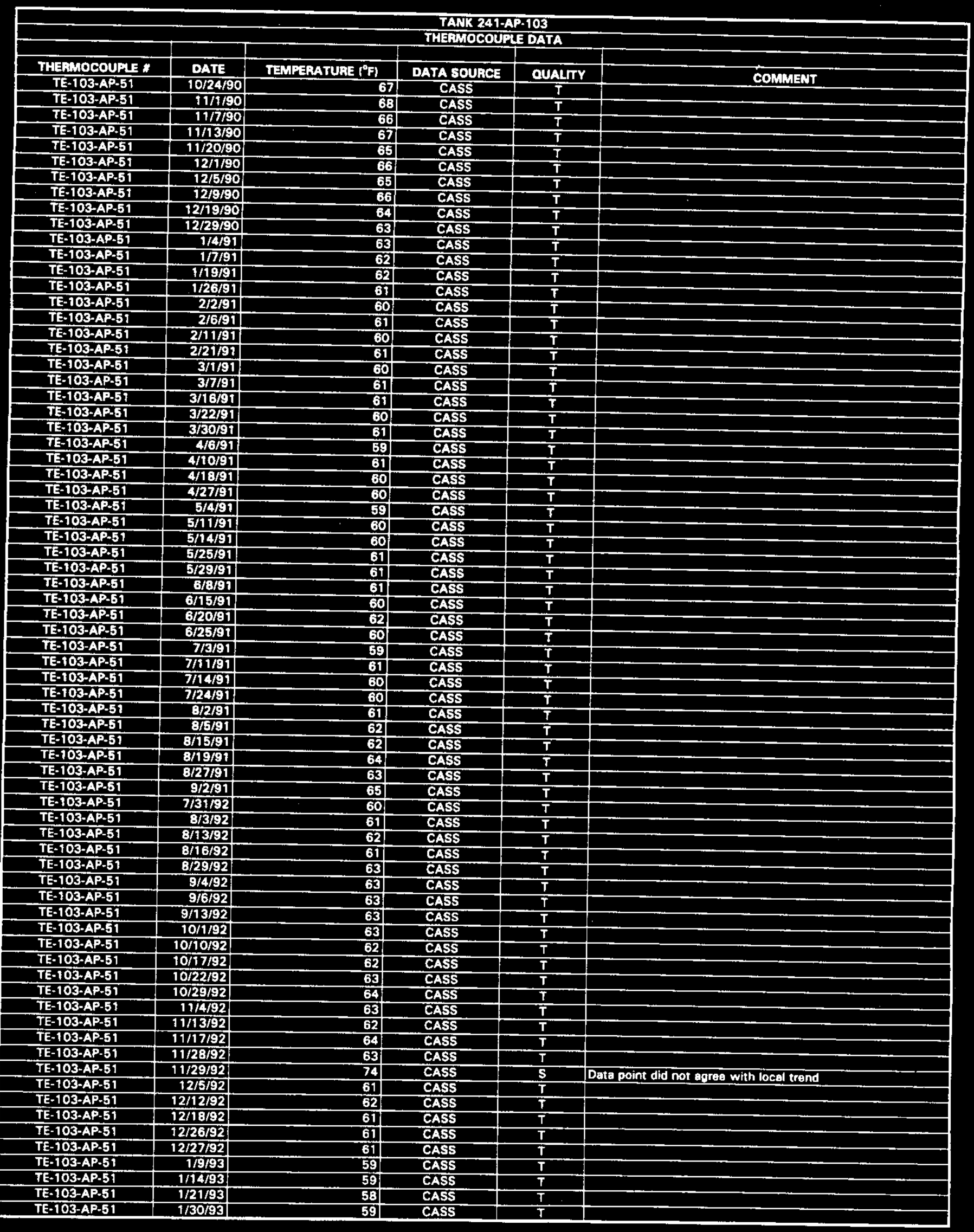

Data obtained from Computer Automated Surveillence Syatem (CASS), April 1995; and Surveillance Anelysis Computer System (SACS), June 1995. D-315 


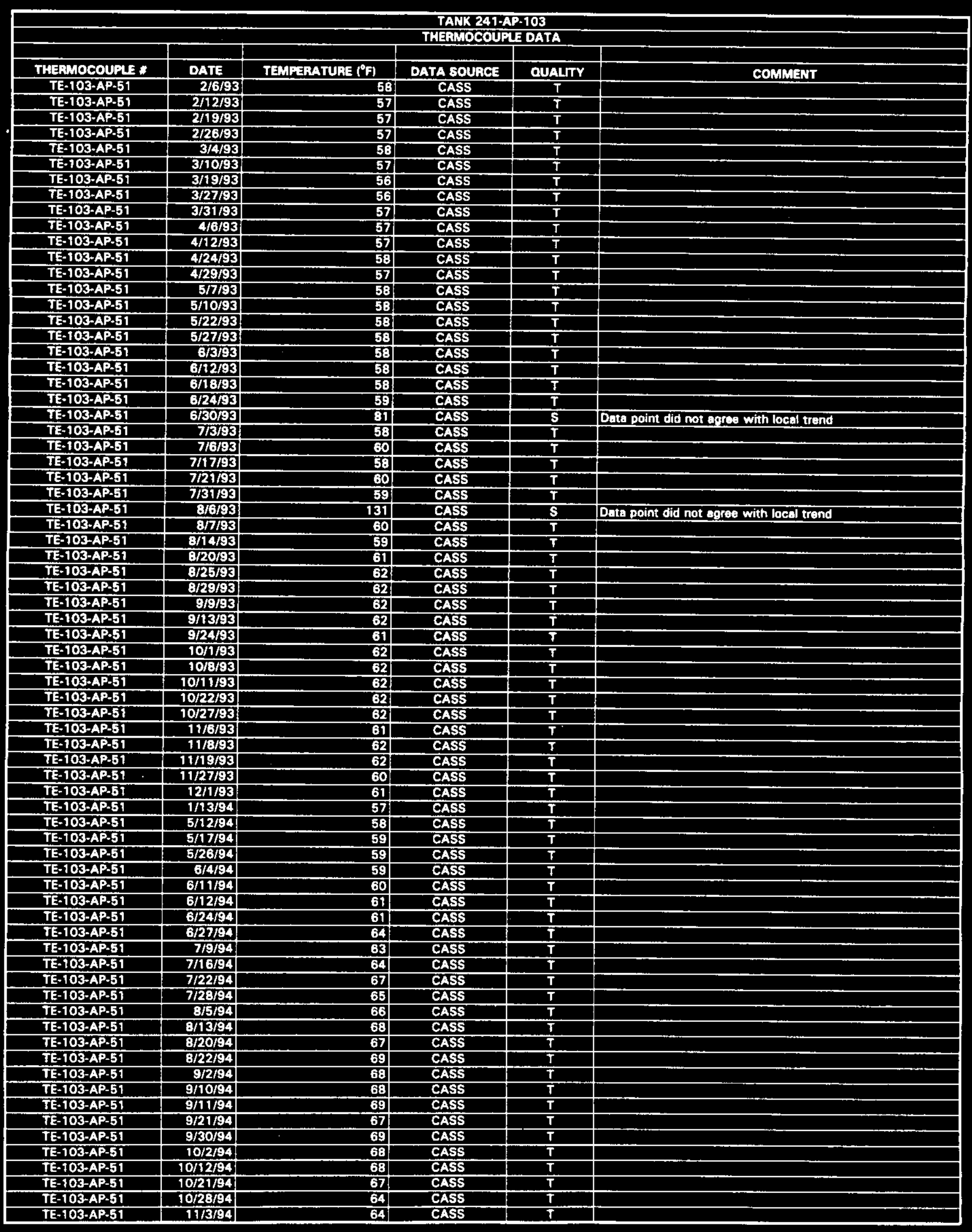




\begin{tabular}{|c|c|c|c|c|c|}
\hline \multicolumn{6}{|c|}{$\begin{array}{l}\text { TANK 241-AP-TOB } \\
\text { THERMOCOUFLE DATA }\end{array}$} \\
\hline & DATE & & & & \\
\hline TE-103-AP-51 & \begin{tabular}{l|l} 
DA/E \\
$11 / 12 / 94$ \\
\end{tabular} & $\begin{array}{r}\text { TEMPEAATUAE }\left({ }^{\circ} \mathrm{F}\right) \\
63\end{array}$ & DATA SOURCE & QUALTYY & COMMENT \\
\hline TE-103-AP-51 & $11 / 15 / 94$ & $\begin{array}{l}63 \\
62\end{array}$ & $\frac{\text { CASS }}{\text { CASS }}$ & $T$ & \\
\hline TE-103-AP-51 & $11 / 20 / 94$ & 60 & CASS & $\frac{T}{T}$ & \\
\hline TE-103-AP-51 & $11 / 25 / 94$ & 93 & CASS & 5 & \\
\hline TE-103-AP-51 & $12 / 2 / 94$ & 58 & CASS & $T$ & Data point did not agree with local trend \\
\hline TE-103-AP-51 & $12 / 4 / 94$ & 58 & CASS & $\mathrm{T}$ & \\
\hline TE-103-AP-51 & $12 / 11 / 94$ & 55 & CASS & $T$ & \\
\hline TE-103-AP-51 & $12 / 14 / 94$ & 90 & CASS & $\mathbf{s}$ & Data point did not agree with locel trend \\
\hline TE-103-AP-61 & $12 / 21 / 94$ & 56 & CASS & $\mathbf{T}$ & \\
\hline TE-103-AP-51 & $12 / 30 / 34$ & 54 & CASS & $T$ & \\
\hline TE-103-AP-51 & $1 / 2 / 95$ & 54 & CASS & $\mathbf{T}$ & \\
\hline TE-103-AP-51 & $1 / 13 / 95$ & 53 & CASS & $T$ & \\
\hline TE-103-AP-51 & $1 / 17 / 95$ & 53 & CASS & $T$ & \\
\hline TE-103-AP-51 & 1/25/95 & 53 & CASS & $T$ & \\
\hline TE-103-AP-51 & $2 / 2 / 95$ & 52 & CASS & $T$ & \\
\hline TE-103-AP-51 & $2 / 9 / 95$ & 53 & CASS & $T$ & \\
\hline TE-103-AP-51 & $2 / 18 / 96$ & 52 & CASS & $T$ & \\
\hline TE-103-AP-51 & 2/25/95 & 53 & CASS & $T$ & \\
\hline TE-103-AP-51 & $2 / 28 / 95$ & 52 & CASS & $\mathbf{T}$ & \\
\hline TE-103-AP-51 & $3 / 10 / 95$ & 53 & CASS & $T$ & \\
\hline TE-103-AP-51 & $3 / 16 / 95$ & 53 & CASS & $T$ & \\
\hline TE-103-AP-51 & $3 / 22 / 95$ & 54 & CASS & $\mathbf{T}$ & \\
\hline TE-103-AP.51 & $3 / 29 / 95$ & 54 & CASS & $T$ & \\
\hline & & & & & \\
\hline TE-103-AP-52 & $4 / 5 / 36$ & 54 & CASS & $T$ & \\
\hline TE-103-AP-52 & 4/6/36 & 56 & $\frac{\text { CASS }}{\text { CASS }}$ & $T$ & \\
\hline$\frac{T \text { TE-103-AP-52 }}{\text { TE-103-AP-52 }}$ & \begin{tabular}{l|}
$4 / 19 / 86$ \\
$4 / 26 / 86$
\end{tabular} & \begin{tabular}{l|l}
555 \\
55
\end{tabular} & $\frac{\text { CASS }}{\text { CASS }}$ & $T$ & \\
\hline $\begin{array}{l}\text { TE-103-AP-52 } \\
\text { TE-103-AP-52 }\end{array}$ & $4 / 26 / 86$ & 55 & $\frac{\text { CASS }}{\text { CASS }}$ & $\frac{T}{T}$ & \\
\hline$\frac{T E-103 \cdot A P \cdot 52}{T E-103 \cdot A P-52}$ & $5 / 16 / 86$ & 55 & CASS & $T$ & \\
\hline TE-103-AP-52 & $5 / 23 / 86$ & 57 & CASS & $T$ & \\
\hline TE-103-AP-52 & $5 / 30 / 86$ & 60 & CASS & $\mathbf{T}$ & \\
\hline TE-103-AP-52 & $6 / 1 / 86$ & 59! & CASS & $T$ & \\
\hline TE-103-AP-52 & $6 / 11 / 86$ & 59 & CASS & $T$ & \\
\hline TE-103-AP-52 & $6 / 20 / 86$ & 59 & CASS & $T$ & \\
\hline TE-103-AP-62 & $6 / 26 / 16$ & 59 & CASS & $T$ & \\
\hline TE-103-AP-52 & $7 / 4 / 86$ & 59 & CASS & $\mathbf{T}$ & \\
\hline TE-103-AP-52 & $7 / 12 / 86$ & 58 & CASS & $\bar{T}$ & \\
\hline TE-103-AP-52 & 7/19/86 & 59 & CASS & $\mathbf{T}$ & \\
\hline TE-103-AP-52 & $7 / 21 / 86$ & 59 & CASS & $\bar{T}$ & \\
\hline TE-103-AP-52 & $7 / 29 / 86$ & 60 & CASS & $\mathbf{T}$ & \\
\hline TE-103.AP-52 & $8 / 3 / 36$ & 60 & CASS & $T$ & \\
\hline TE-103-AP-52 & $8 / 16 / 86$ & 59 & CASS & $T$ & \\
\hline TE-103.AP-52 & $8 / 22 / 86$ & 62 & CASS & $T$ & \\
\hline TE-103-AP-52 & $8 / 30 / 86$ & 62 & CASS & $T$ & \\
\hline TE-103-AP-52 & $9 / 6 / 86$ & 62 & CASS & $T$ & \\
\hline TE-103-AP-52 & 9/9/86 & 63 & CASS & $T$ & \\
\hline TE-103-AP-52 & $9 / 29 / 86$ & 64 & CASS & $\mathbf{T}$ & \\
\hline TE-103-AP-52 & $9 / 24 / 86$ & 64: & CASS & $T$ & \\
\hline TE-103-AP-52 & $10 / 2 / 86$ & 64 & CASS & $T$ & \\
\hline TE-103-AP-52 & $10 / 11 / 86$ & 62 & CASS & $T$ & \\
\hline TE-103-AP-52 & $10 / 17 / 86$ & 64 & CASS & $T$ & \\
\hline TE-103-AP-52 & $10 / 19 / 86$ & 63 & CASS & $\mathbf{T}$ & \\
\hline TE-103-AP-52 & $11 / 1 / 86$ & 63 & CASS & $T$ & \\
\hline TE-103-AP-52 & $11 / 5 / 86$ & 62 & CASS & $T$ & \\
\hline TE-103-AP-52 & $11 / 114 / 86$ & 59 & CASS & $T$ & \\
\hline TE-103-AP-52 & $11 / 22 / 86$ & 57 & CASS & $\mathrm{T}$ & \\
\hline TE-103-AP-52 & $11 / 25 / 86$ & 60 & CASS & $T$ & \\
\hline TE-103-AP-52 & $11 / 30 / 86$ & 57 & CASS & $\mathbf{T}$ & \\
\hline TE-103-AP.52 & $12 / 12 / 36$ & 57 & CASS & $T$ & \\
\hline TE-103-AP-52 & $12 / 17 / 86$ & 58 & CASS & $T$ & \\
\hline TE-103-AP-52 & $12 / 25 / 86$ & 56 & CASS & $T$ & \\
\hline TE-103-AP-52 & $12 / 28 / 86$ & 54 & CASS & $\bar{T}$ & \\
\hline TE-103-AP-52 & $7 / 4 / 87$ & 64 & CASS & $\mathbf{T}$ & \\
\hline TE-103-AP-52 & 7/10/87 & 69 & CASS & $\mathbf{T}$ & \\
\hline TE-103-AP-52 & 7/18/87 & 66 & CASS & $T$ & \\
\hline TE-103-AP-52 & 7/20/87 & 66 & CASS & $T$ & \\
\hline TE-103-AP-52 & $8 / 1 / 87$ & 67 & CASS & $\bar{T}$ & \\
\hline TE-103-AP-52 & $8 / 6 / 87$ & 67 & CASS & $T$ & \\
\hline TE-103-AP-52 & $8 / 14 / 87$ & 68 & CASS & $\bar{T}$ & \\
\hline TE-103-AP-52 & $8 / 18 / 87$ & 68 & CASS & $\mathbf{T}$ & \\
\hline TE-103-AP-52 & $8 / 29 / 87$ & 67 & CASS & $\mathbf{T}$ & \\
\hline TE-103.AP-52 & $9 / 2 / 87$ & 68 & CASS & $\mathbf{T}$ & \\
\hline
\end{tabular}




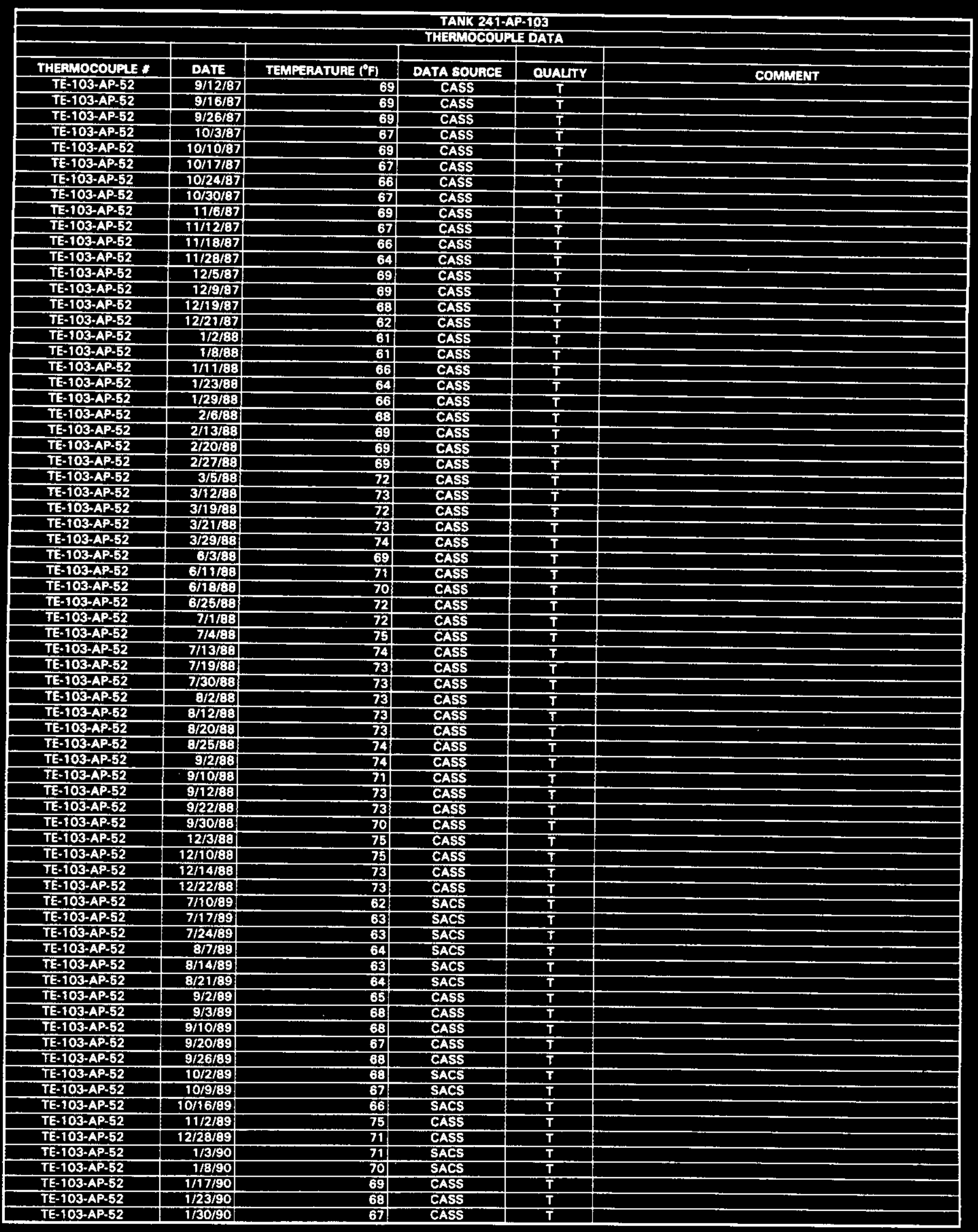




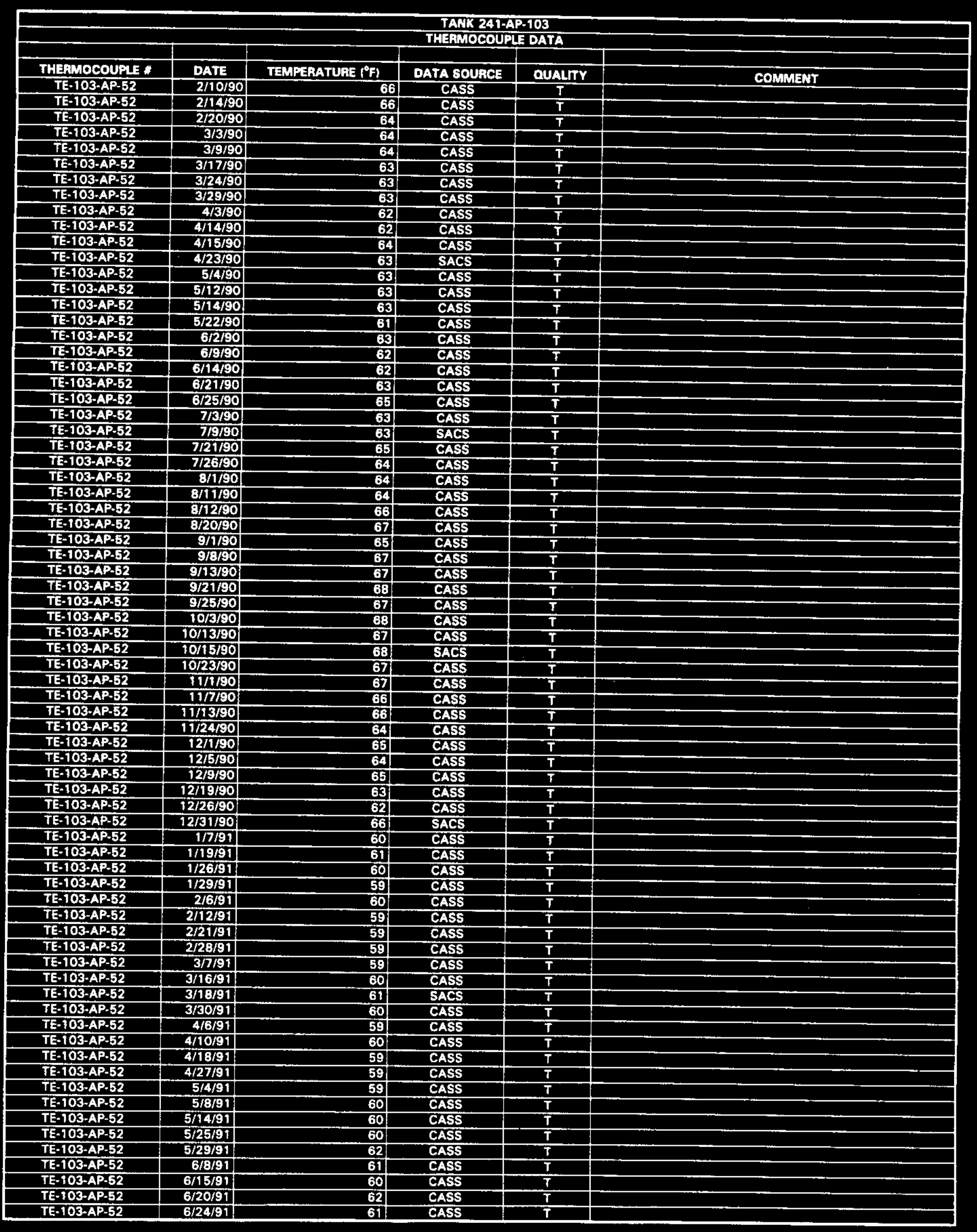




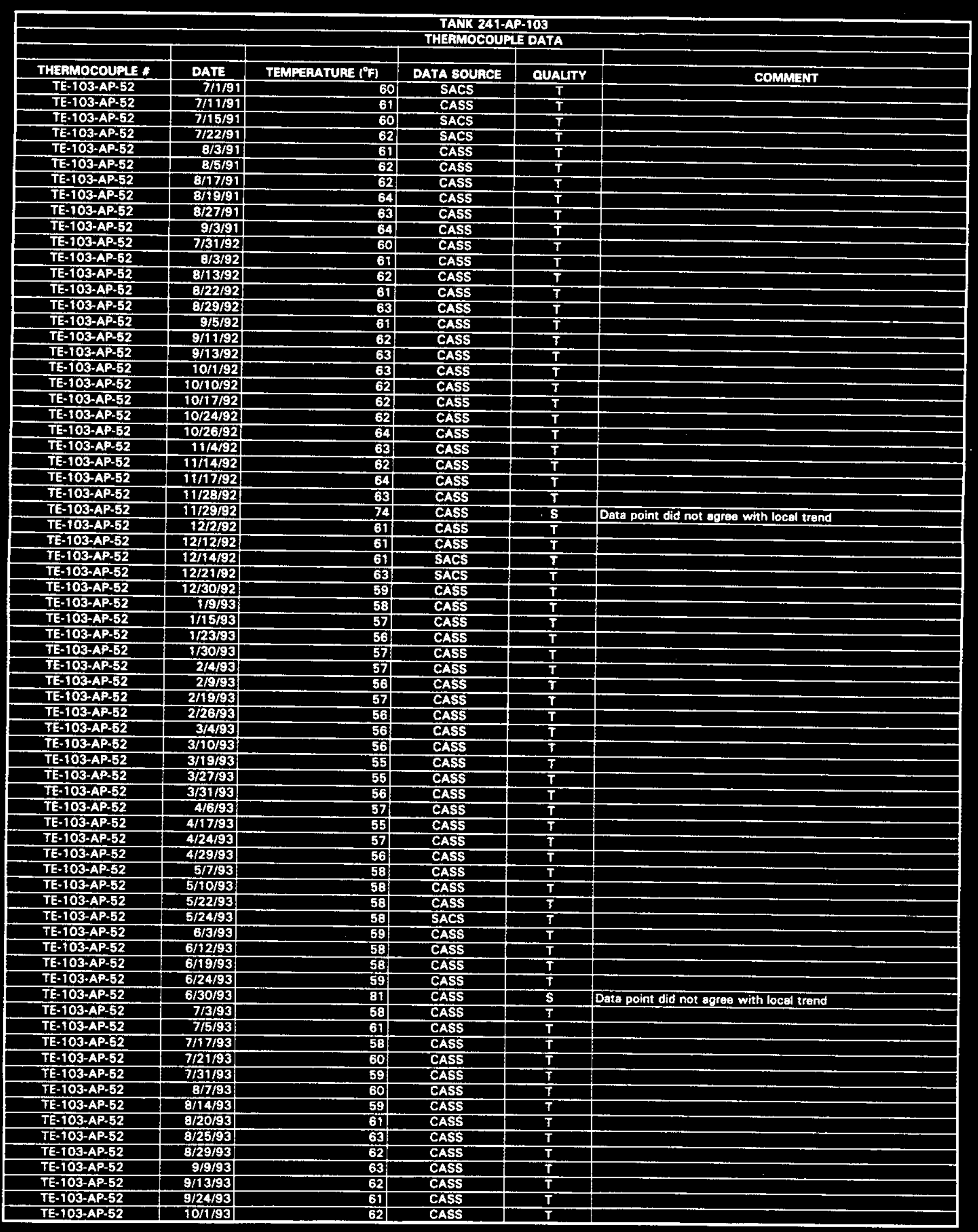

Data obtained from Computer Automated Surveiliance System (CASS), April 1995; and Surveillance Analyais Computer System (SACS), June 1995.

D-320 


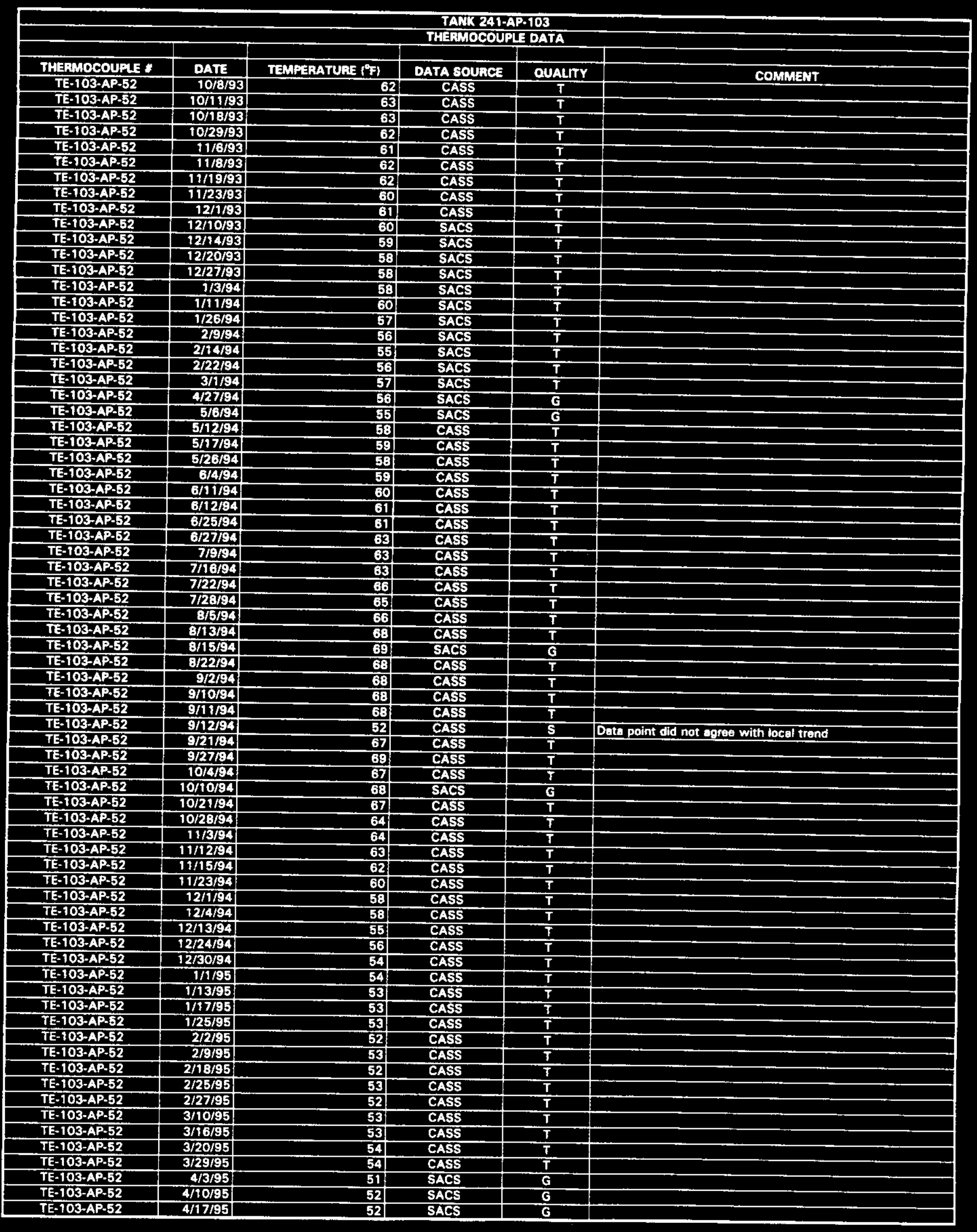

Date obtained from Computer Automated Surveillance Syatem (CASS), April 1995; and Surveillance Analysis Computer Syatem (SACS), June 1995. $D-321$ 


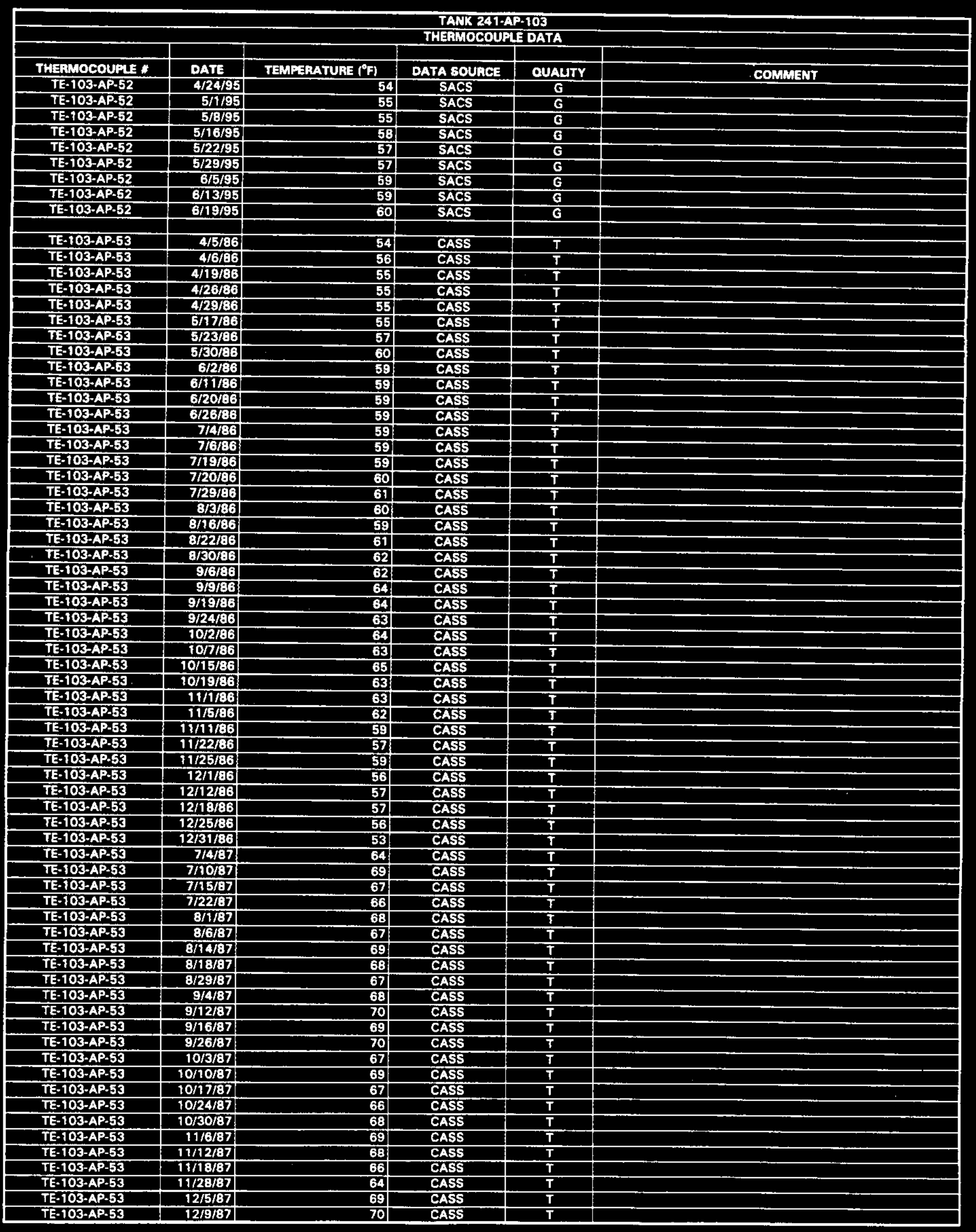




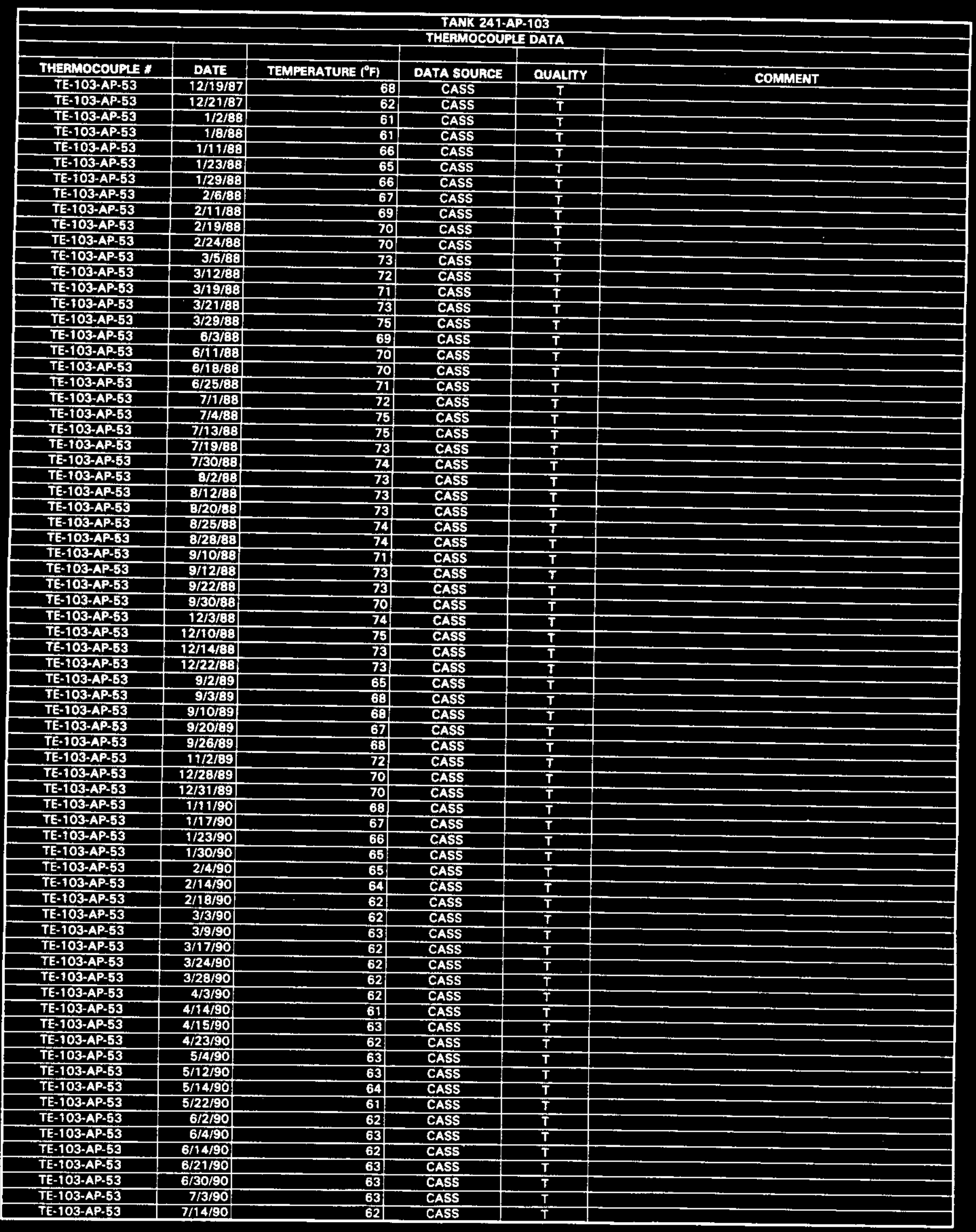

Date obtained from Computer Automated Surveillance System (CASS), April 1995; and Surveillance Analysis Computer Syatem (SACS), June 1995. 


\begin{tabular}{|c|c|c|c|c|c|}
\hline \multicolumn{6}{|c|}{$\begin{array}{l}\text { TANK 241-AP+103 } \\
\text { THERMOCOUPLE DATA }\end{array}$} \\
\hline & & & & & \\
\hline THERMOCOUPLE $\boldsymbol{*}$ & DATE & TEMPERATURE $\left({ }^{\circ} \mathrm{F}\right)$ & DATA SOURCE & QUALITY & COMMENT \\
\hline TE-103-AP-53 & $7 / 21 / 90$ & 65 & CASS & $T$ & \\
\hline TE-103-AP-53 & $7 / 26 / 90$ & 64 & CASS & $\mathbf{T}$ & \\
\hline TE-103-AP-53 & $8 / 1 / 90$ & 64 & CASS & $\mathbf{T}$ & \\
\hline TE-103-AP-53 & $8 / 5 / 90$ & 65 & CASS & $T$ & \\
\hline TE-103-AP-53 & $8 / 12 / 90$ & 66 & CASS & $\bar{T}$ & \\
\hline TE-103-AP-53 & $8 / 20 / 90$ & 67 & CASS & $T$ & \\
\hline TE-103.AP-53 & $9 / 1 / 90$ & 65 & CASS & $T$ & \\
\hline TE-103-AP-53 & 9/3/90 & 67 & CASS & $T$ & \\
\hline TE-103-AP-53 & 9/13/90 & 67 & CASS & $T$ & \\
\hline TE-103-AP-53 & $9 / 21 / 90$ & 68 & CASS & $T$ & \\
\hline TE-103-AP-53 & $9 / 28 / 90$ & 67 & CASS & $\bar{T}$ & \\
\hline TE-103-AP-53 & $10 / 3 / 90$ & 69 & CASS & $\bar{T}$ & \\
\hline TE-103-AP-53 & $10 / 7 / 90$ & 68 & CASS & $\bar{T}$ & \\
\hline TE-103-AP-53 & $10 / 20 / 90$ & 65 & CASS & $T$ & \\
\hline TE-103-AP-53 & $10 / 24 / 90$ & 66 & CASS & $T$ & \\
\hline TE-103-AP-53 & $11 / 1 / 90$ & 66 & CASS & $T$ & \\
\hline TE-103-AP-53 & $11 / 7 / 90$ & 65 & CASS & $\mathbf{T}$ & \\
\hline TE-103-AP-53 & $11 / 13 / 90$ & 66 & CASS & $T$ & \\
\hline TE-103-AP-53 & $11 / 20 / 90$ & 64 & CASS & $T$ & \\
\hline TE-103-AP-53 & $12 / 1 / 90$ & 64 & CASS & $T$ & \\
\hline TE-103-AP-53 & $12 / 5 / 90$ & 64 & CASS & $T$ & \\
\hline TE-103-AP-53 & $1219 / 90$ & 63 & CASS & $T$ & \\
\hline TE-103.AP-53 & $12 / 19 / 90$ & 61 & CASS & $T$ & \\
\hline TE-103-AP-53 & $12 / 26 / 90$ & 59 & CASS & $T$ & \\
\hline TE-103.AP-53 & $12 / 30 / 80$ & 59 & CASS & $T$ & \\
\hline TE-103-AP-53 & $1 / 12 / 91$ & 57 & CASS & $T$ & \\
\hline TE-103-AP-53 & I/19/91 & 59 & CASS & $T$ & \\
\hline TE-103-AP-53 & $1 / 26 / 91$ & 58 & CASS & $\mathbf{T}$ & \\
\hline TE-103-AP.53 & $2 / 2 / 91$ & 57 & CASS & $T$ & \\
\hline TE-103-AP-53 & $2 / 6 / 91$ & 59 & CASS & $T$ & \\
\hline TE-103-AP-53 & $2 / 16 / 91$ & 57 & CASS & $\bar{T}$ & \\
\hline TE-103-AP-53 & $2 / 21 / 91$ & 59 & CASS & $\bar{T}$ & \\
\hline TE-103-AP-53 & $2 / 24 / 91$ & 59 & CASS & $T$ & \\
\hline TE-103-AP-53 & $3 / 7 / 91$ & 59 & CASS & $T$ & \\
\hline TE-103-AP-63 & आ/16/91 & 59 & CASS & $T$ & \\
\hline TE-10S-AP-53 & $3 / 21 / 91$ & 59 & CASS & $T$ & \\
\hline TE-103-AP-53 & $3 / 30 / 91$ & 60 & CASS & $T$ & \\
\hline TE-103-AP-53 & $4 / 6 / 91$ & 58 & CASS & $T$ & \\
\hline TE-103-AP-53 & $4 / 10 / 91$ & 60 & CASS & $T$ & \\
\hline TE-103-AP-53 & $4 / 20 / 91$ & 58 & CASS & $T$ & \\
\hline TE-103.AP-53 & $4 / 23 / 91$ & 60 & CASS & $T$ & \\
\hline TE-103-AP-53 & $5 / 4 / 91$ & 59 & CASS & $\bar{T}$ & \\
\hline TE-103-AP-53 & $5 / 11 / 91$ & 60 & CASS & $T$ & \\
\hline TE-103-AP-53 & $5 / 14 / 91$ & 61 & CASS & $T$ & \\
\hline TE-103-AP-53 & $5 / 25 / 91$ & 61 & CASS & $T$ & \\
\hline TE-103-AP-53 & $5 / 28 / 91$ & 61 & CASS & $T$ & \\
\hline TE-103-AP-53 & $6 / 8 / 97$ & 61 & CASS & $T$ & \\
\hline TE-103-AP-53 & $6 / 15 / 91$ & 60 & CASS & $T$. & \\
\hline TE-103-AP-53 & $6 / 19 / 91$ & 116 & CASS & 5 & Data point did not agree with focal trend \\
\hline TE-103-AP-53 & $6 / 20 / 91$ & 61 & CASS & $T$ & \\
\hline TE-103-AP-53 & $6 / 24 / 91$ & 61 & CASS & $T$ & \\
\hline TE-103-AP-53 & $7 / 2 / 91$ & 60 & CASS & $T$ & \\
\hline TE-103-AP-53 & $7 / 11 / 91$ & 61 & CASS & $T$ & \\
\hline TE-103-AP-53 & $7 / 14 / 91$ & 60 & CASS & $T$ & \\
\hline TE-103-AP-53 & $7 / 24 / 91$ & 60 & CASS & $T$ & \\
\hline TE-103-AP-53 & 8/3/91 & 61 & CASS & $T$ & \\
\hline TE-103-AP-53 & $8 / 5 / 91$ & 62 & CASS & $T$ & \\
\hline TE-103-AP-53 & $8 / 15 / 91$ & 63 & CASS & $\bar{T}$ & \\
\hline TE-103-AP-53 & $8 / 23 / 91$ & 63 & CASS & $T$ & \\
\hline TE-103-AP-53 & $8 / 27 / 91$ & 63 & CASS & $T$ & \\
\hline TE-103-AP-53 & $9 / 2 / 91$ & 65 & CASS & $T$ & \\
\hline TE-103-AP-53 & $7 / 31 / 92$ & 60) & CASS & $T$ & \\
\hline TE-103-AP-53 & $8 / 3 / 92$ & 61 & CASS & $T$ & \\
\hline TE-103-AP-53 & $8 / 13 / 92$ & 63 & CASS & $T$ & \\
\hline TE-103-AP-53 & $8 / 18 / 92$ & 61 & CASS & $T$ & \\
\hline TE-103.AP.53 & $8 / 29 / 92$ & 63 & CASS & $T$ & \\
\hline TE-103-AP-53 & $9 / 5 / 92$ & 61 & CASS & $T$ & \\
\hline TE-103-AP-53 & $9 / 11 / 92$ & 62 & CASS & $T$ & \\
\hline TE-103-AP-53 & $9 / 13 / 92$ & 63 & CASS & $T$ & \\
\hline TE-103-AP-53 & $10 / 1 / 92$ & 63 & CASS & $T$ & \\
\hline TE-103-AP-53 & $10 / 10 / 92$ & 62 & CASS & $T$ & \\
\hline TE-103-AP-53 & $10 / 16 / 92$ & 62 & CASS & $T$ & \\
\hline TE-103-AP-53 & $10 / 22 / 92$ & 63 & CASS & $T$ & \\
\hline
\end{tabular}




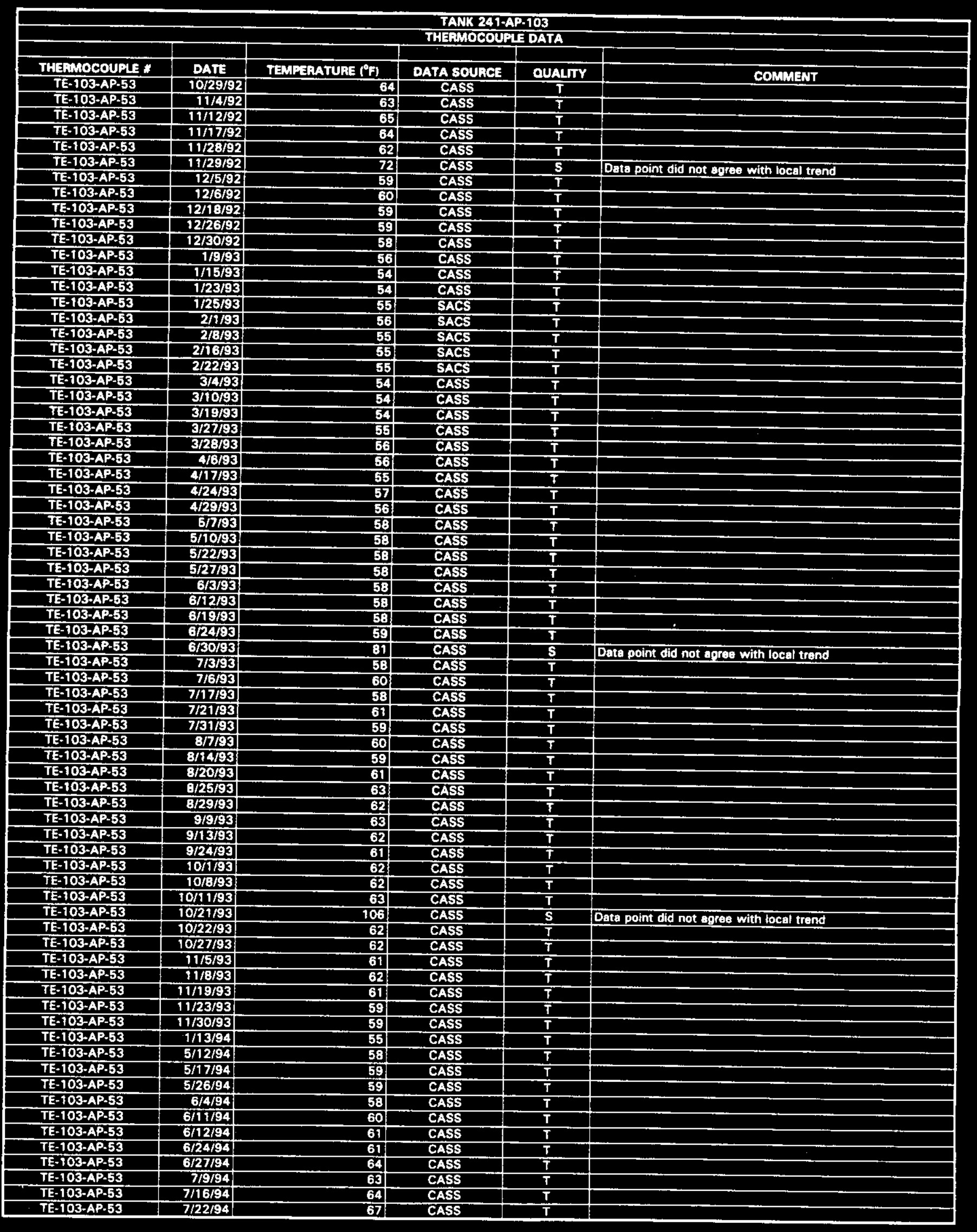

Data obtained from Computer Automated Surveillance Syatem (CASS). April 1995; and Surveillance Analyais Camputer Syatem (SACS), Jung 1995. 


\begin{tabular}{|c|c|c|c|c|c|}
\hline \multicolumn{6}{|c|}{$\begin{array}{l}\text { TANK 241-AP-103 } \\
\text { THERMOCOUFL DATA }\end{array}$} \\
\hline & & & & & \\
\hline THEPMOCOUPL & DATE & TEMPEAATUAE $\left.f^{\circ} \mathrm{F}\right\}$ & DATA SOURCE & OUALITY & COMMENT \\
\hline TE-103-AP-53 & $7 / 28 / 94$ & 66 & CASS & $T$ & \\
\hline TE-103-AP-53 & $8 / 5 / 94$ & 66 & CASS & $T$ & \\
\hline TE-103-AP-53 & $8 / 13 / 94$ & 68 & CASS & $T$ & \\
\hline TE-103-AP-53 & $8 / 20 / 94$ & 67 & CASS & $\mathbf{T}$ & \\
\hline TE-103-AP-53 & $8 / 22 / 94$ & 69 & CASS & $T$ & \\
\hline TE-103-AP-SB & $8 / 28 / 94$ & 68 & CASS & $\mathbf{T}$ & \\
\hline TE-103-AP-53 & $9 / 10 / 94$ & 68 & CASS & $\mathbf{T}$ & \\
\hline TE-103-AP-53 & $9 / 11 / 94$ & 68 & CASS & $\bar{T}$ & \\
\hline TE-103-AP-53 & $9 / 12 / 94$ & 52 & CASS & $\mathbf{s}$ & Data point did not agree with local trend \\
\hline TE+103-AP+53 & $9 / 21 / 94$ & 67 & CASS & $\bar{T}$ & \\
\hline TE-103-AP-53 & $9 / 27 / 94$ & 69 & CASS & $\mathbf{T}$ & \\
\hline TE-103-AP-53 & $10 / 4 / 94$ & 67 & CASS & $T$ & \\
\hline TE-103.AP-53 & 10/12/94 & 67 & CASS & $\mathrm{T}$ & \\
\hline TE-103-AP-S3 & $10 / 21 / 94$ & 67 & CASS & $\bar{T}$ & \\
\hline TE-103-AP-53 & $10 / 27 / 94$ & 64 & CASS & $T$ & \\
\hline TE-103-AP-53 & $11 / 3 / 94$ & 64 & CASS & $\bar{T}$ & \\
\hline TE-103-AP-53 & $11 / 12 / 94$ & 63 & CASS & $T$ & \\
\hline TE-103-AP-53 & $11 / 16 / 94$ & 61 & CASS & $\bar{T}$ & \\
\hline TE-103-AP-53 & $11 / 23 / 94$ & 59 & CASS & $T$ & \\
\hline TE-103-AP-53 & $12 / 3 / 94$ & 57 & CASS & $T$ & \\
\hline TE-103-AP-53 & $12 / 9 / 94$ & 57 & CASS & $T$ & \\
\hline TE-103-AP-53 & $12117 / 94$ & 54 & CASS & $T$ & \\
\hline TE-103-AP-53 & $12 / 24 / 94$ & 56 & CASS & $T$ & \\
\hline TE-10B-AP.53 & $12 / 26 / 94$ & 54 & CASS & $T$ & \\
\hline TE-103-AP-53 & $1 / 1 / 96$ & 54 & CASS & $T$ & \\
\hline TE-103-AP-SB & $1 / 13 / 95$ & 53 & CASS & $\bar{T}$ & \\
\hline TE-103-AP-S3 & $1 / 17 / 95$ & 53 & CASS & $T$ & \\
\hline TE-103.AP-53 & $1 / 25 / 95$ & 52 & CASS & $T$ & \\
\hline TE-103-AP-53 & $2 / 2 / 95$ & 53 & CASS & $T$ & \\
\hline TE-103-AP-53 & $2 / 9 / 95$ & 53 & CASS & $T$ & \\
\hline TE-103-AP-53 & $2 / 18 / 95$ & 51 & CASS & $T$ & \\
\hline TE-103-AP-53 & 2122195 & 53 & CASS & $T$ & \\
\hline$T E-103-A P-53$ & $2 / 27 / 95$ & 52 & CASS & $T$ & \\
\hline TE-103-AP-53 & $3 / 10 / 95$ & 52 & CASS & $T$ & \\
\hline TE-103-AP-53 & आ16/95 & 53 & CASS & $T$ & \\
\hline TE-103-AP-53 & $3 / 20 / 85$ & 54 & CASS & $T$ & \\
\hline TE-103-AP-53 & 3/29/95 & 54 & CASS & $T$ & \\
\hline
\end{tabular}




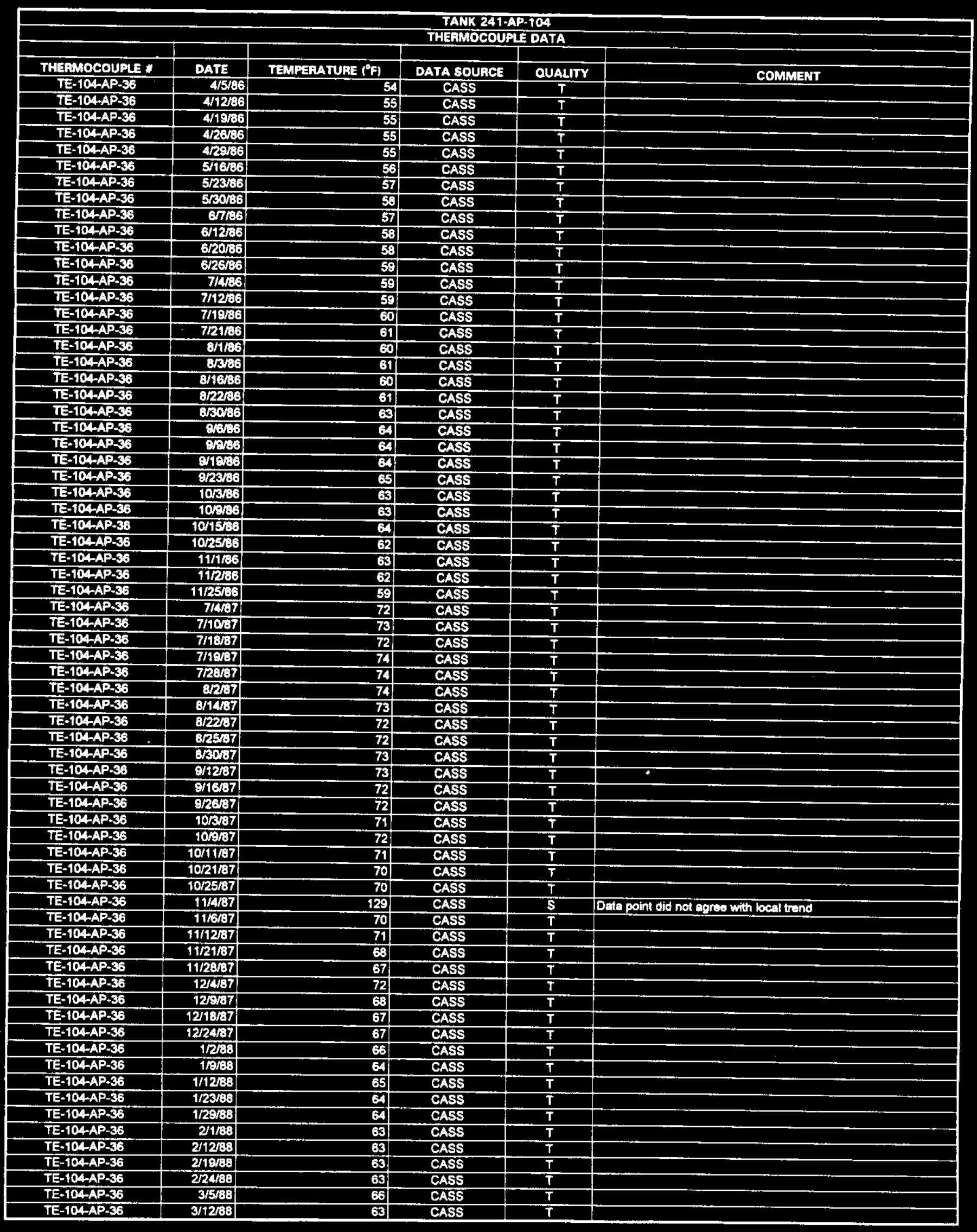

Data obtained from Computer Automated Surveillance System (CASS), April 1995; and Surveillance Analysis Computer System (SACS), June 1995.

D-327 


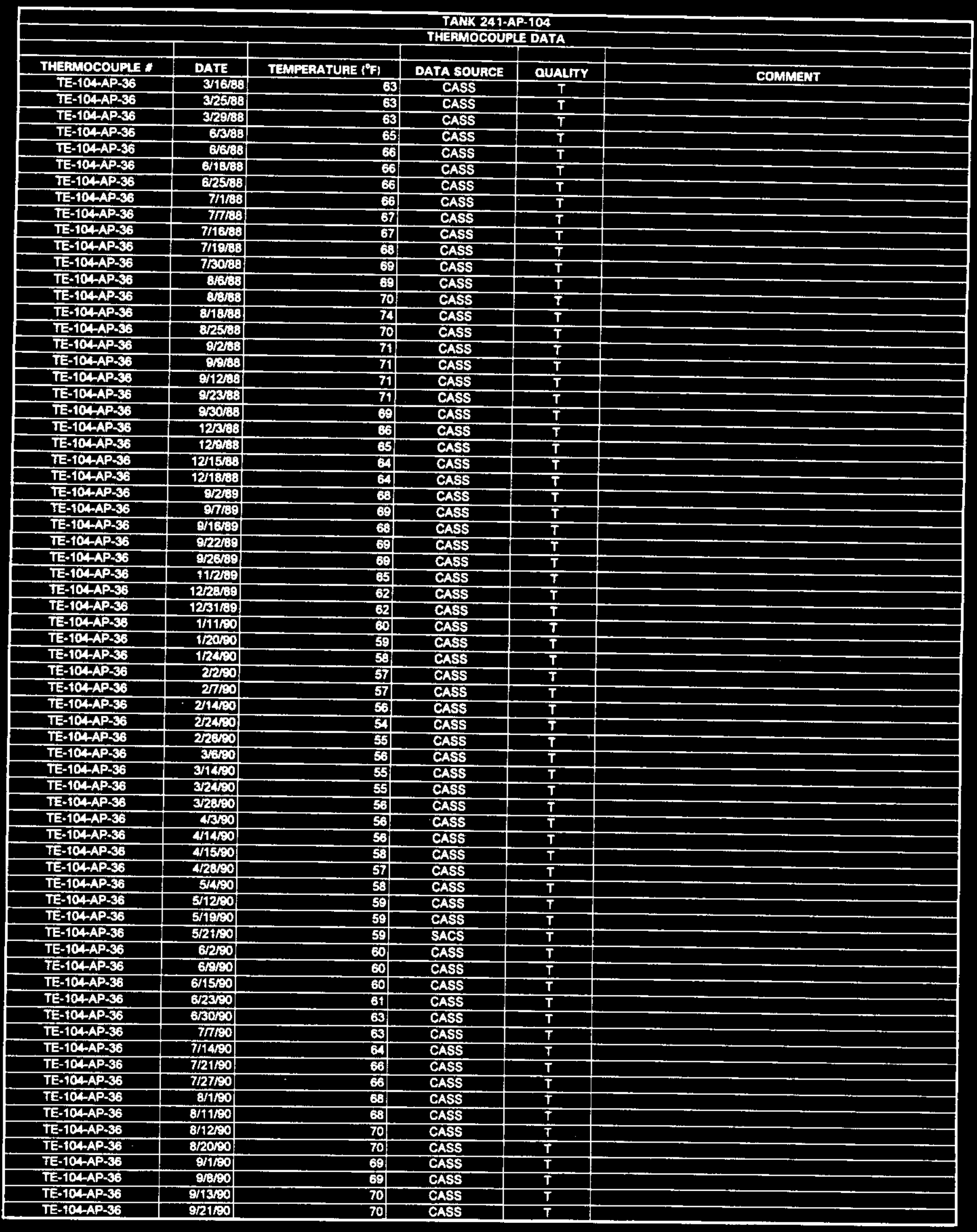




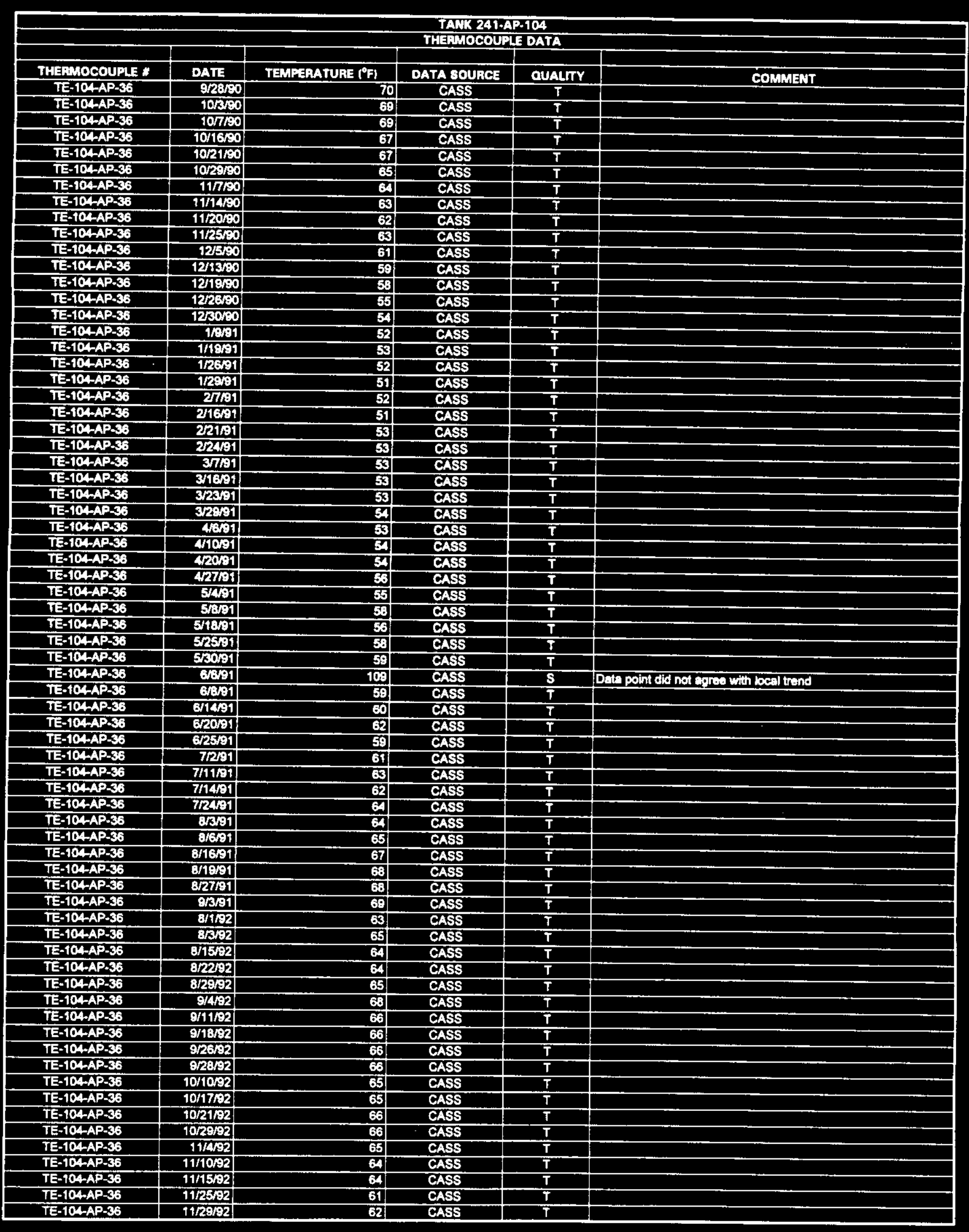




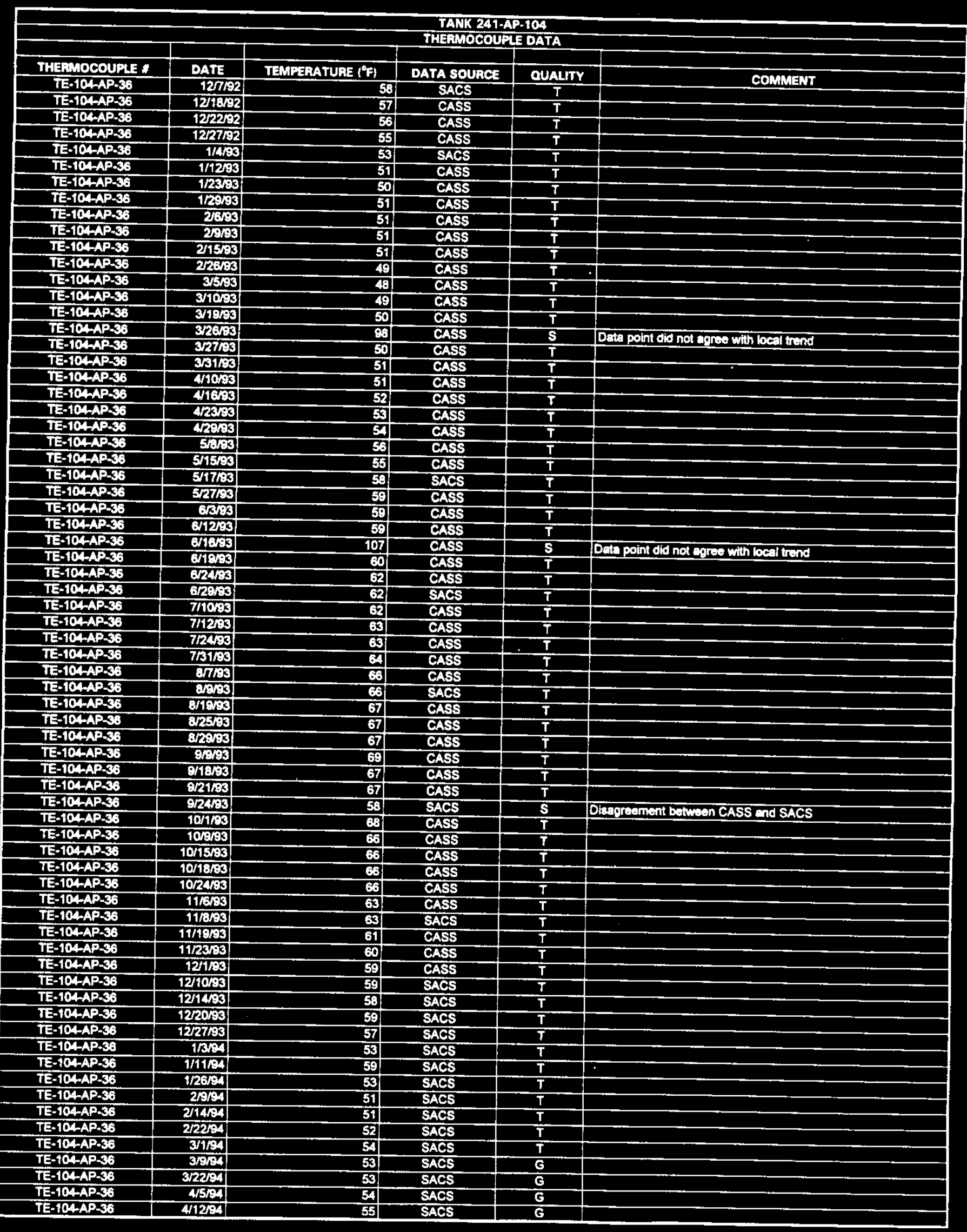




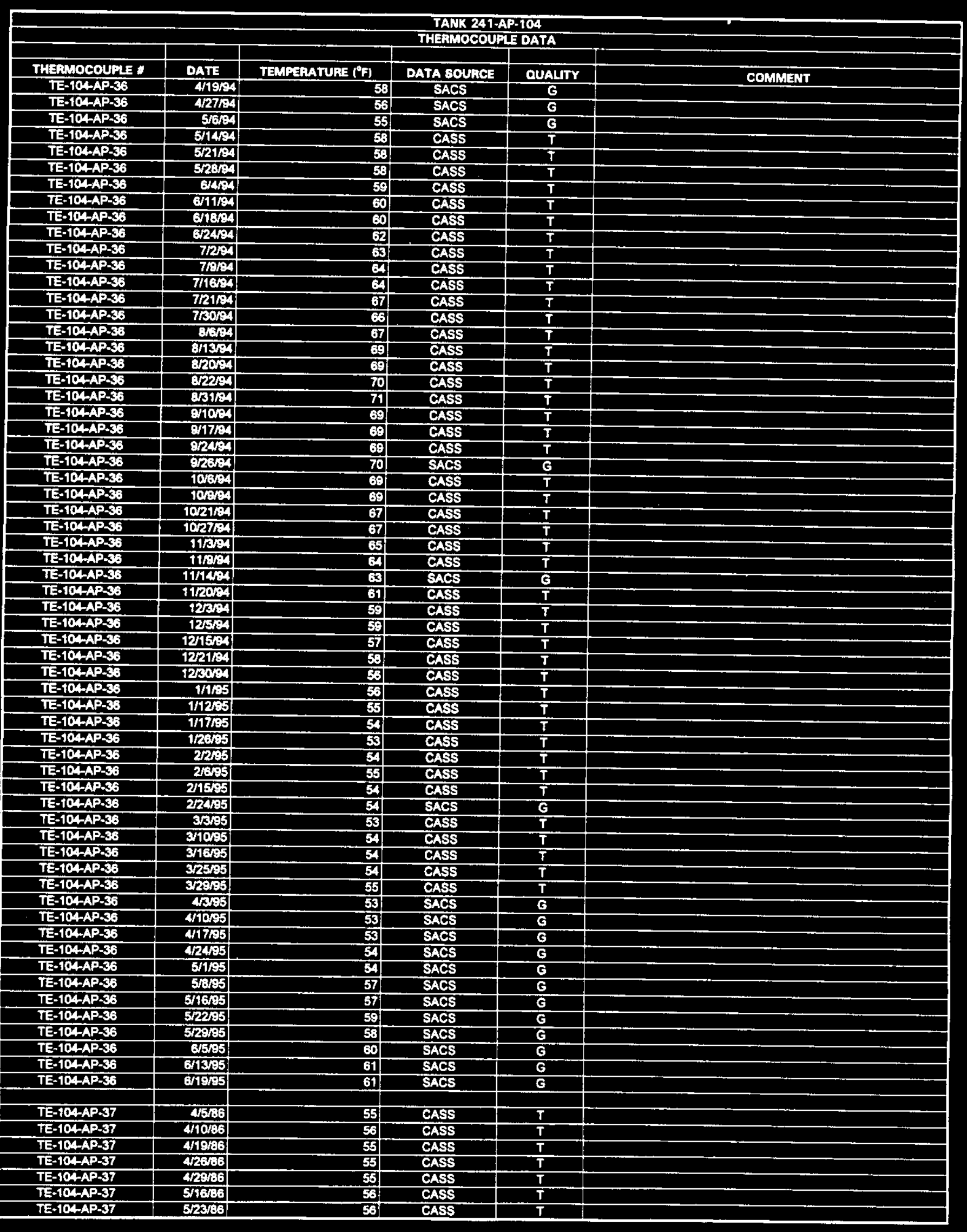




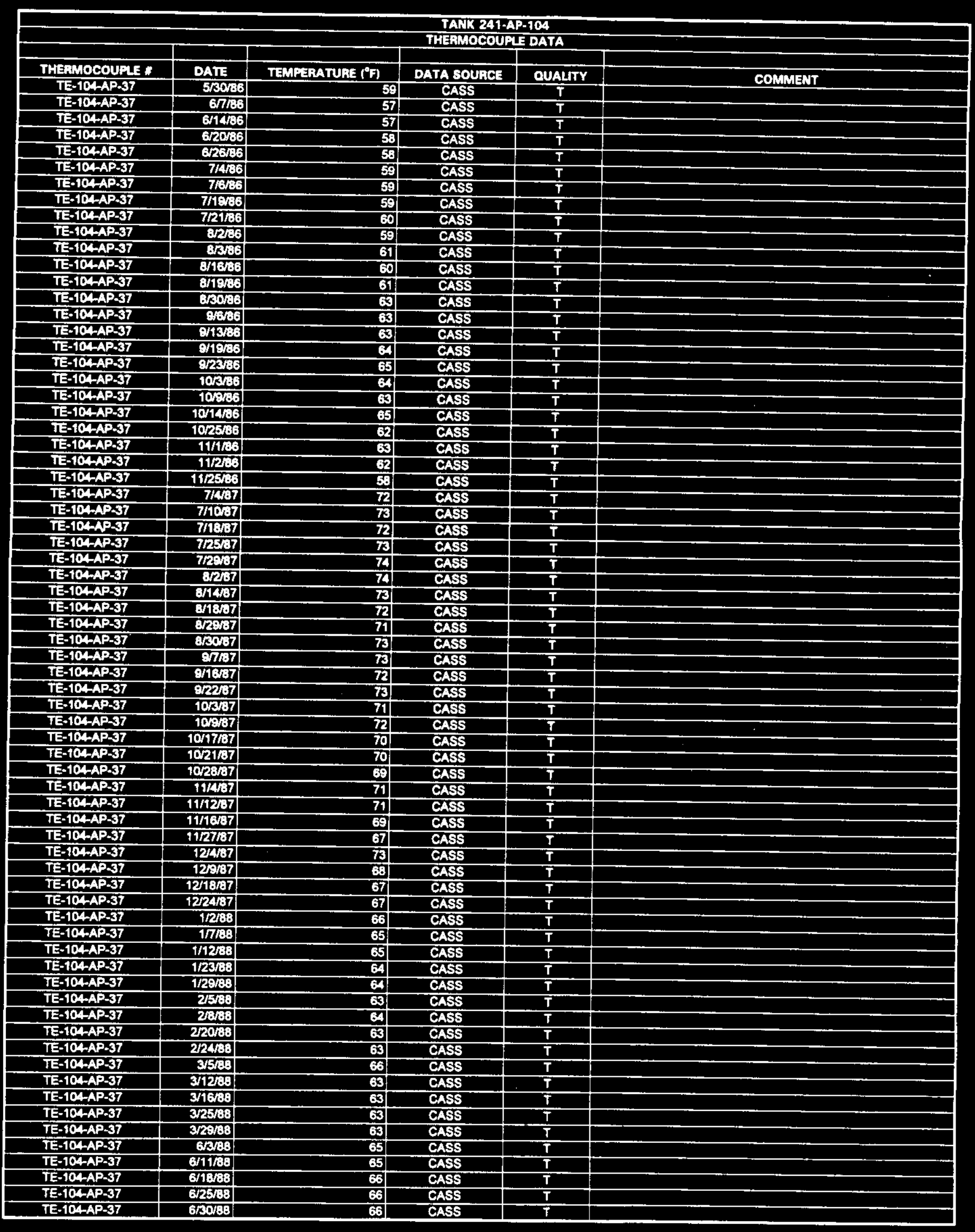

Data obtained from Computer Automated Survaillence System (CASS), April 1995; and Surveillance Anelysis Computer System (SACS). Juna 1995.

D-332 


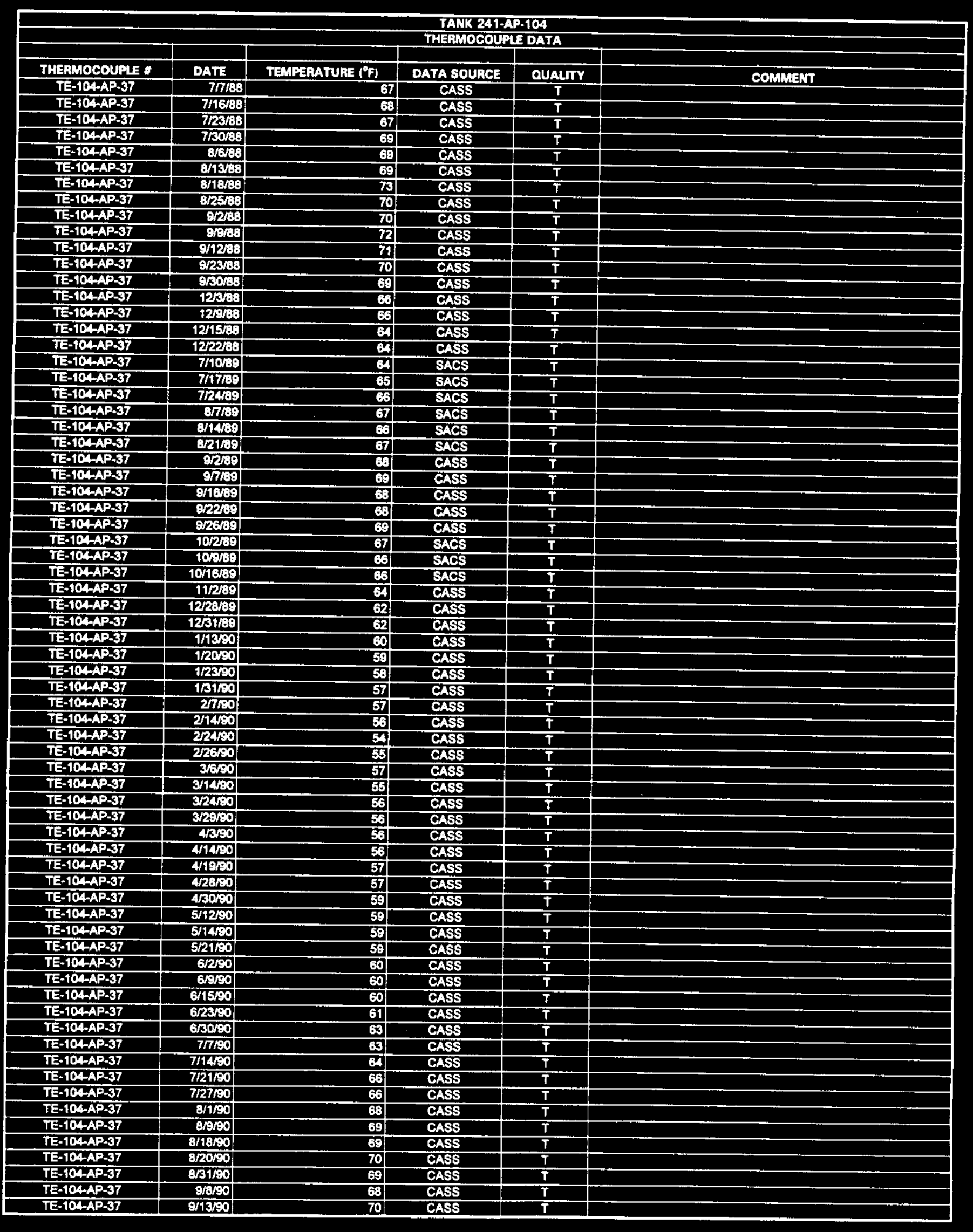




\begin{tabular}{|c|c|c|c|c|c|}
\hline \multicolumn{6}{|c|}{$\begin{array}{l}\text { TANK 241.AP-104 } \\
\text { THERMOCOUALE DATA }\end{array}$} \\
\hline & & & & & \\
\hline THERMOCOUPE * & DATE & TEMPERATUAE $\left({ }^{\circ} \mathrm{F}\right)$ & DATA SOURCE & QUALTY & COMMENT \\
\hline TE.104AP.37 & $9 / 21 / 90$ & 70 & CASS & $T$ & \\
\hline TE-104AP-37 & $9 / 2500$ & 69 & CASS & $T$ & \\
\hline TE-104-AP-37 & $10 \mathrm{~s} / 90$ & 69 & CASS & $T$ & \\
\hline$\frac{T E-104 A P-37}{T E-104-A P-37}$ & $107 / 30$ & 69 & $\frac{\text { CASS }}{\text { CASS }}$ & $\frac{T}{T}$ & \\
\hline $\begin{array}{l}\text { TE-104AP-37 } \\
\text { TE-104AP-37 }\end{array}$ & $\frac{10 / 19 / 50}{10 / 2350}$ & 66 & $\frac{\text { CASS }}{\text { CASS }}$ & $T$ & \\
\hline$\frac{\text { TE-104-AP-37 }}{\text { TE-104-AP-37 }}$ & $\frac{10 / 2390}{10 / 28 / 90}$ & 67 & $\begin{array}{l}\text { CASS } \\
\text { CASS }\end{array}$ & $T$ & \\
\hline TE-104-AP-37 & $11 \pi / 00$ & 64: & CASS & $T$ & \\
\hline TE-104AP-37 & $19 / 16 / 0$ & 63 & CASS & $T$ & \\
\hline TE-104AP-37 & 11/20\% & 62 & CASS & $T$ & \\
\hline TE-104-AP-37 & $91 / 25500$ & 63 & CASS & $T$ & \\
\hline TE-104AP-37 & 121590 & 61 & CASS & $T$ & \\
\hline TE-104AP-37 & $120 \%$ & 60 & CASS & $T$ & \\
\hline TE-104AP-37 & $1219 \%$ & 58 & CASS & $T$ & \\
\hline TE-104-AP-37 & $12 / 26 \% 0$ & 55 & CASS & $T$ & \\
\hline TE-104AP-37 & $12130 \% 0$ & 59 & CASS & $T$ & \\
\hline TE-104AP-37 & $1 / 3 / 31$ & 52 & CASS & $T$ & \\
\hline TE-104-AP-37 & 1/1891 & 53 & CASS & $T$ & \\
\hline TE-104-AP-37 & $1 / 2001$ & $\mathbf{5 3}$ & CASS & $T$ & \\
\hline TE-104-AP-37 & 22291 & 51 & CASS & $\bar{T}$ & \\
\hline TE-104AP-37 & $2 \pi 181$ & 52 & CASS & $\bar{T}$ & \\
\hline TE-104AP-37 & $211 \% 1$ & 57. & SACS & $\bar{T}$ & \\
\hline TE-104-AP-37 & $221 / 91$ & 5 & CASS & $\bar{T}$ & \\
\hline TE-104AP-37 & 2/2301 & 52 & CASS & $T$ & \\
\hline TE-104AP-37 & 3rwot & 53 & CASS & $T$ & \\
\hline TE-104AP-37 & 3/6:1 & 5 & CASS & $T$ & \\
\hline TE-104-AP-37 & $3 / 2101$ & 54 & CASS & $\mathbf{T}$ & \\
\hline TE-104-AP-37 & 32901 & 5-1 & CASS & $T$ & \\
\hline TE-104AP-37 & D6R日 & $5 \mathbf{5 3}$ & CASS & $T$ & \\
\hline TE-104-AP-37 & 4ros1 & 54 & CASS & $\mathbf{T}$ & \\
\hline TE-104AP-37 & 4/1798 & 55) & CASS & $T$ & \\
\hline TE-104AP-37 & $4 / 27091$ & 56 & CASS & $\bar{T}$ & \\
\hline TE-104-AP-37 & 5मQब1 & 55 & CASS & $T$ & \\
\hline TE-104AP-37 & StaI & 50 & CASS & $\mathbf{T}$ & \\
\hline TE-104AP-37 & 5/1861 & 5 & CASS & $T$ & \\
\hline TE-104-APA7 & 5/2531 & 58 & CASS & $T$ & \\
\hline TE-104-AP-37 & 523901 & 58 & CASS & $T$ & \\
\hline TE-104AP-37 & 6/8/91 & 60 & CASS & $T$ & \\
\hline TE-104-AP-37 & 6/14/01 & 60 & CASS & $T$ & \\
\hline TE-104-AP-37 & 6/2001 & 61 & CASS & $T$ & \\
\hline TE-104AP-37 & 6/2501 & 59 & CASS & $T$ & \\
\hline TE-104AP-37 & 7/161 & 62 & SACS & $T$ & \\
\hline$T E-104-A P-37$ & 7/1101 & 63 & CASS & $T$ & \\
\hline TE-104-AP-37 & $7 / 5$ 1591 & 63 & SACS & $T$ & \\
\hline TE-104-AP-37 & $7 / 22191$ & EA & SACS & $\bar{T}$ & \\
\hline TE-104AP-37 & $7 / 2901$ & 65 & SACS & $T$ & \\
\hline TE-104AP-37 & $8 / 301$ & 65. & CASS & $T$ & \\
\hline TE-104-AP-37 & 8/17/91 & 66 & CASS & $T$ & \\
\hline TE-104AP-37 & $8 / 23 / 91$ & 67 & CASS & $T$ & \\
\hline TE-104-AP-37 & $8 / 31 / 31$ & 67 & CASS & $T$ & \\
\hline TE-104-AP-37 & 9/3ra1 & 68 & CASS & $T$ & \\
\hline TE-104-AP-37 & $8 / 1 / 92$ & 63 & CASS & $\bar{T}$ & \\
\hline TE-104-AP-37 & 86192 & 63 & CASS & $T$ & \\
\hline TE-104AP-37 & $8 / 13 / 92$ & 64 & CASS & $T$ & \\
\hline TE-104-AP-37 & $8 / 22192$ & 64 & CASS & $T$ & \\
\hline TE-104AP-37 & $8 / 29 / 92$ & 65 & CASS & $T$ & \\
\hline TE-104.AP-37 & $8 / 4,92$ & 67 & CASS & $T$ & \\
\hline TE-104-AP-37 & 9/11/02 & 66 & CASS & $T$ & \\
\hline TE-104-AP-37 & $9 / 14 / 92$ & 66 & CASS & $\bar{T}$ & \\
\hline TE-104AP-37 & $9 / 26 / 92$ & 66 & CASS & $\mathbf{T}$ & \\
\hline TE-104-AP-37 & 9/23/92 & 66 & CASS & $T$ & \\
\hline TE-104AP-37 & $10 / 392$ & 66 & CASS & $T$ & \\
\hline TE-104AP-37 & $10 / 15 / 92$ & 65 & CASS & $T$ & \\
\hline 104AP-37 & $10 / 21 / 92$ & 65 & CASS & $T$ & \\
\hline TS-104-AP-37 & $10 / 29 / 92$ & 66 & CASS & $T$ & \\
\hline TE-104AP-37 & $11 / 462$ & 65 & CASS & $T$ & \\
\hline TE-104-AP-37 & $11 / 10 / 92$ & 69. & CASS & $T$ & \\
\hline TE-104AP-37 & $11 / 15 / 22$ & 64 & CASS & $T$ & \\
\hline TE-104-AP-37 & $11 / 25 / 92$ & 61 & CASS & $T$ & \\
\hline TE-104-AP-37 & $11 / 29 / 92$ & 63 & CASS & $T$ & \\
\hline
\end{tabular}




\begin{tabular}{|c|c|c|c|c|c|}
\hline \multicolumn{6}{|c|}{$\begin{array}{l}\text { TAMK 241-AP-104 } \\
\text { THERMOCOUPL DATA }\end{array}$} \\
\hline & & & & & \\
\hline THERMOCOUPLE * & DATE & TEMPERATUAE $\left.f^{\circ} \mathrm{F}\right\}$ & DATA BOURCE & QUAUTY & COMMENT \\
\hline TE-104-AP-37 & $126 \% 92$ & 59 & CASS & $T$ & \\
\hline TE-104-AP-37 & $12 / 1892$ & 57 & CASS & $T$ & \\
\hline TE-104AP-37 & $12 / 2292$ & 56 & CASS & $T$ & \\
\hline TE-10.AP-37 & $12 / 27 \% 2$ & 55 & CASS & $T$ & \\
\hline TE-104-AP-37 & $1 / 4 / 93$ & 53 & CASS & $T$ & \\
\hline TE-104-AP-37 & $1 / 1293$ & 51 & CASS & $\mathbf{T}$ & \\
\hline TE-104-AP-37 & $1 / 23 / 93$ & 50 & CASS & $T$ & \\
\hline TE-104-AP-37 & T/29:S3 & 51 & CASS & $\bar{T}$ & \\
\hline TE-104-AP-37 & 26,93 & 51 & CASS & $T$ & \\
\hline TE-104AP-37 & $2 / 903$ & 57 & CASS & $T$ & \\
\hline TE-104-AP-37 & $2 / 1593$ & 51 & CASS & $\bar{T}$ & \\
\hline TE-104AP-37 & $2 / 1893$ & 92 & CASS & $\underline{\mathrm{s}}$ & Data point did not agree with local trend \\
\hline TE-104-AP-37 & $2 / 2693$ & 49 & CASS & $T$ & \\
\hline TE-104AP-37 & 3593 & 48 & CASS & $T$ & \\
\hline TE-104-AP-37 & $3 / 1293$ & 49 & CASS & $T$ & \\
\hline TE-104-AP-37 & $3 / 4 / 93$ & 89 & CASS & 8 & Data point did not eqree with locel trend \\
\hline TE-104-AP-37 & 31993 & 50 & CASS & $T$ & \\
\hline TE-104-AP-37 & 3/27/53 & 51 & CASS & $\mathbf{T}$ & \\
\hline TE-104AP-37 & $3 / 31 / 93$ & 51 & CASS & $\mathbf{T}$ & \\
\hline TE-104-AP-37 & 46283 & 52 & CASS & $\mathbf{T}$ & \\
\hline TE-104-AP-37 & 41203 & 53 & CASS & $\mathbf{T}$ & \\
\hline TE-104-AP-37 & $4 / 23 / 93$ & 53 & CASS & $\mathbf{T}$ & \\
\hline TE-104-AP.37 & 4 rav3 & 54 & CASS & $T$ & \\
\hline TE-104-AP-37 & 5 Saras & 56) & CASS & $\mathbf{T}$ & \\
\hline TE-104-AP-37 & $5 / 15 \times 3$ & $\mathbf{5 5}$ & CASS & $T$ & \\
\hline TE-104-AP-37 & $5,22 \times 3$ & 57 & CASS & $T$ & \\
\hline$T E-104-A P-37$ & $5 \sqrt{27 / 03}$ & 59 & CASS & $T$ & \\
\hline TE-194-AP-37 & $5 \times 3193$ & 116 & CASS & 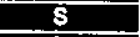 & Drta point did not edrea with local trend \\
\hline TE-104-AP-37 & 6r53 & 5 & CASS & $\mathbf{T}$ & \\
\hline TE-104-AP-37 & $6 / 12103$ & S9: & CASS & $T$ & \\
\hline TE-104-AP-37 & 6/19:3 & 80 & CASS & $T$ & \\
\hline TE-104AP-37 & QR24:33 & 62 & CASS & $T$ & \\
\hline$T E-104-A P-37$ & $7 / \sqrt[3]{3}$ & 61 & CASS & $T$ & \\
\hline TE-104AP-37 & 77tors & 62 & CASS & $T$ & \\
\hline TE-104-AP-37 & $7 / 1293$ & 63 & CASS & $T$ & \\
\hline$T E-104 A P-37$ & $7 / 21 / 3$ & 63 & CASS & $T$ & \\
\hline TE-1Q4-AP-37 & $7 / 30193$ & 64 & CASS & $T$ & \\
\hline TE-104-AP-37 & $8 n+93$ & 86 & CASS & $\mathbf{T}$ & \\
\hline TE-104लAP-37 & $8 / 4 / 93$ & 65 & CASS & $T$ & \\
\hline TE-104-AP-37 & 8/1993 & 67 & CASS & $T$ & \\
\hline TE-104-AP-37 & $8 / 25.93$ & 67 & CASS & $T$ & \\
\hline TE-104-AP-37 & $8 / 203$ & 67 & CASS & $T$ & \\
\hline TE-104-AP-37 & 9/903 & 68 & CASS & $T$ & \\
\hline TE-104-AP-37 & $815 \times 3$ & 67 & CASS & $T$ & \\
\hline TE-104AP-37 & $8 / 2503$ & 66 & CASS & $T$ & \\
\hline $\mathrm{FE}-104-\mathrm{AP}-37$ & $10 / 1 / 23$ & 68 & CASS & $T$ & \\
\hline TE-104-AP-37 & 10/9res & 66 & CASS & $T$ & \\
\hline TE-104-AP-37 & $10 / 13 / 93$ & 66 & CASS & $\bar{T}$ & \\
\hline TE-104-AP-37 & $10 / 2293$ & 63 & CASS & $T$ & \\
\hline TE-104-AP-37 & $10 \mathrm{rop} 93$ & 64 & CASS & $\mathrm{T}$ & \\
\hline TE-104-AP-37 & $11 / 6 / 93$ & 63 & CASS & $T$ & \\
\hline TE-104-AP-37 & $11 / \mathrm{Br} \theta 3$ & 63 & CASS & $T$ & \\
\hline TE-104-AP-37 & $11 / 14 / 93$ & 62 & CASS & $T$ & \\
\hline TE-104-AP-37 & $11 / 22 / 93$ & EO & CASS & $T$ & . \\
\hline TE-104-AP-37 & $12 / 1 / 93$ & 59 & CASS & $T$ & \\
\hline TE-104AP-37 & $1 / 13 / 94$ & 53 & CASS & $T$ & \\
\hline TE-104-AP-37 & $5 / 14 / 94$ & 57 & CASS & $T$ & \\
\hline TE-104-AP-37 & 51984 & 58 & CASS & $T$ & \\
\hline$T E-104-A P-37$ & $5 n 2894$ & 58 & CASS & $T$ & \\
\hline TE-104-AP-37 & 64Ma4 & 59 & CASS & $T$ & \\
\hline TE-104AP-37 & $6 / 11 / 9$ & 60 & CASS & $T$ & \\
\hline TE-104-AP-37 & $6 / 18 / 94$ & 60 & CASS & $T$ & \\
\hline TE-104-AP-37 & $6 / 22194$ & 62 . & CASS & $T$ & \\
\hline TE-104-AP-37 & $7 / 2 / 4$ & 63 & CASS & $T$ & \\
\hline TE-104-AP-37 & $7 / 994$ & 64 & CASS & $T$ & \\
\hline TE-104-AP-37 & $7 / 15 / 94$ & 64 & CASS & $T$ & \\
\hline TE-104AP-37 & $7116 / 94$ & 131 & CASS & $\mathbf{s}$ & Data point did not eqree with local trend \\
\hline TE-104-AP-37 & $7 / 21 / 94$ & 67 & CASS & $T$ & \\
\hline TE-104-AP-37 & $7 / 30 / 94$ & 66 & CASS & $T$ & \\
\hline TE-104-AP-37 & 8/6/94 & 67 & CASS & $\bar{T}$ & \\
\hline
\end{tabular}




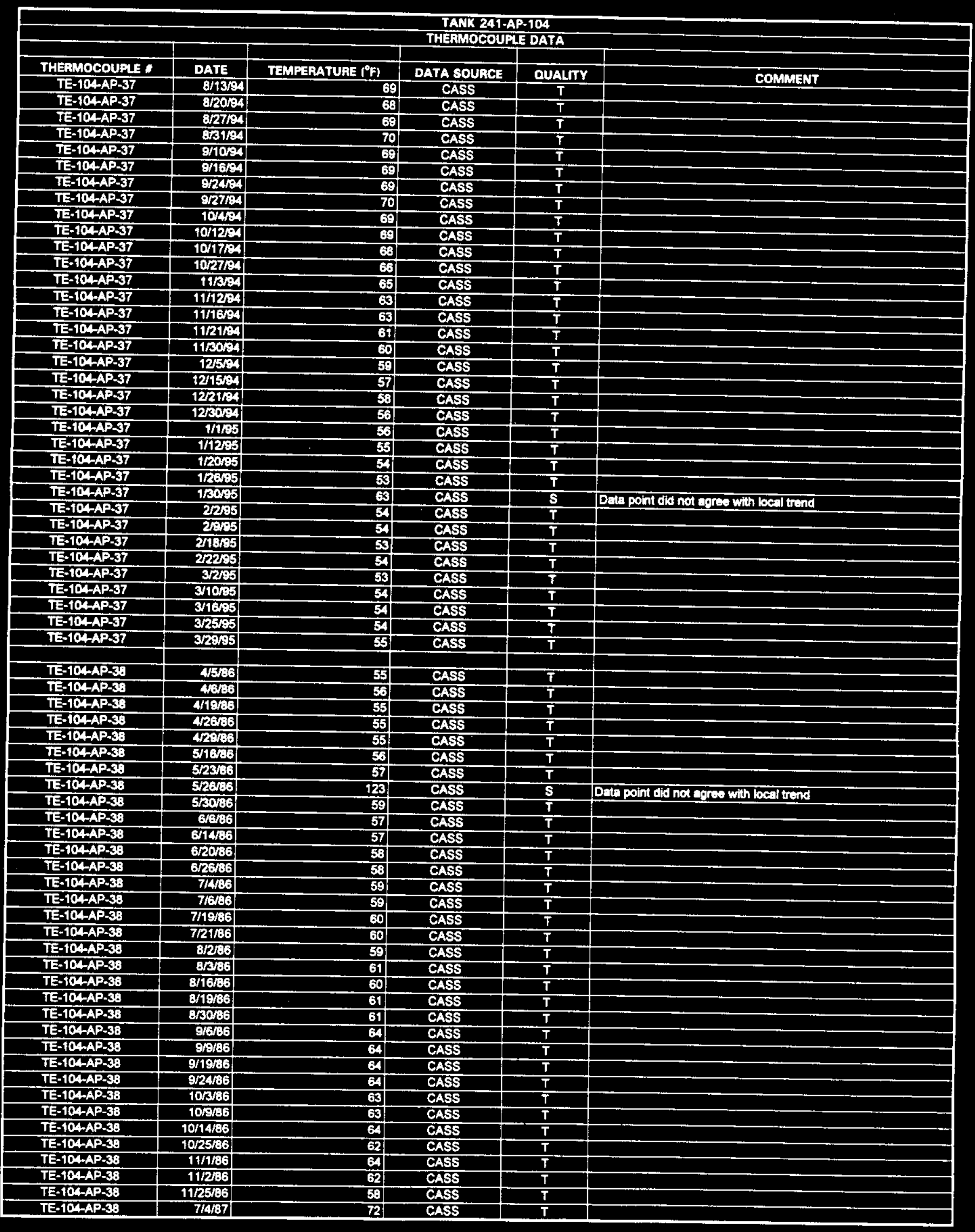

Data obtained from Computer Automated Surveillance Syatem (CASS), April 1995; and Surveillance Analyais Computer System (SACS\}, June 1995. 


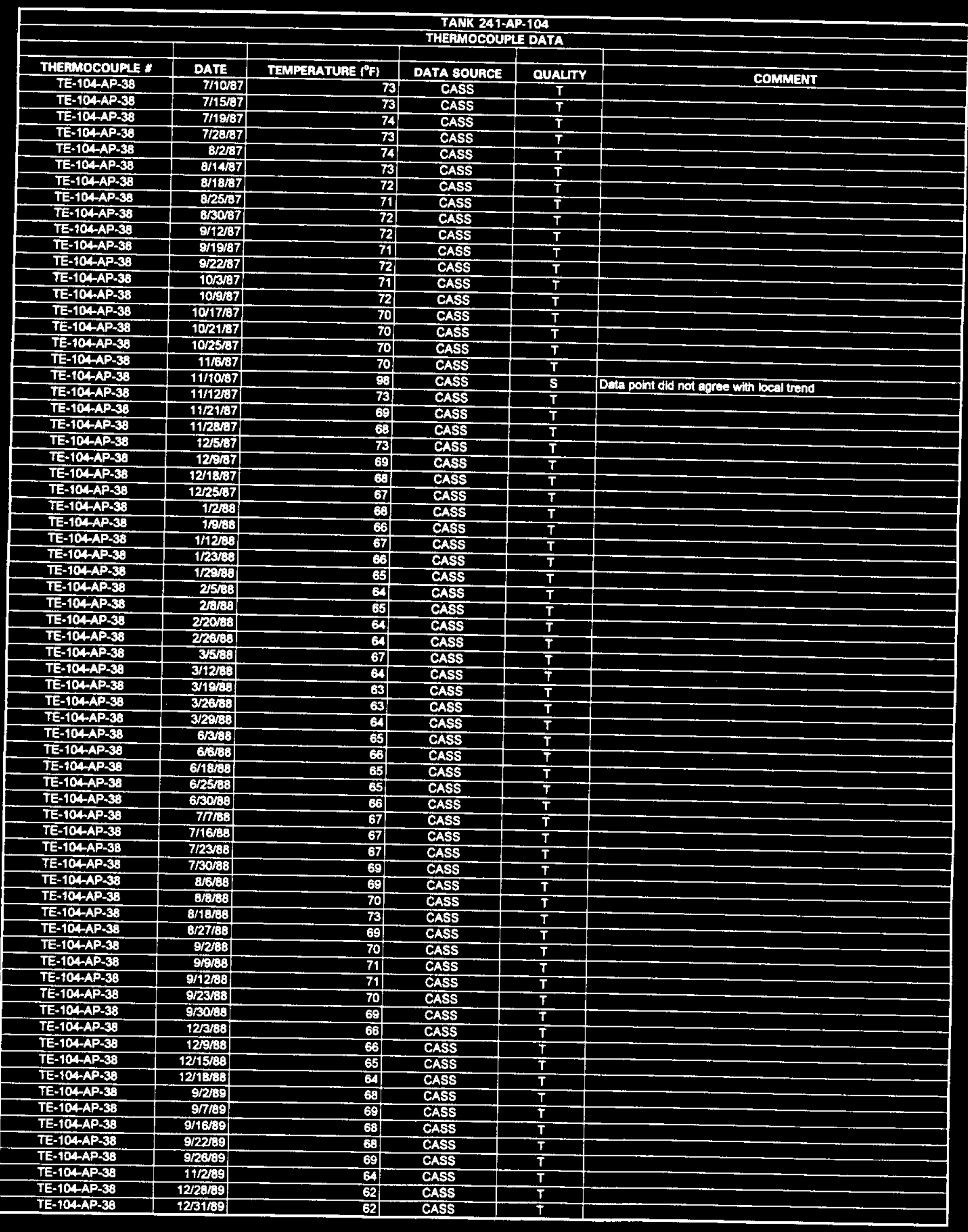

Data obtained from Computer Automated Survaillance Syatem (CASS). April 1995; and Surveillance Analysis Computer Syatem (SACS), June 1995. 


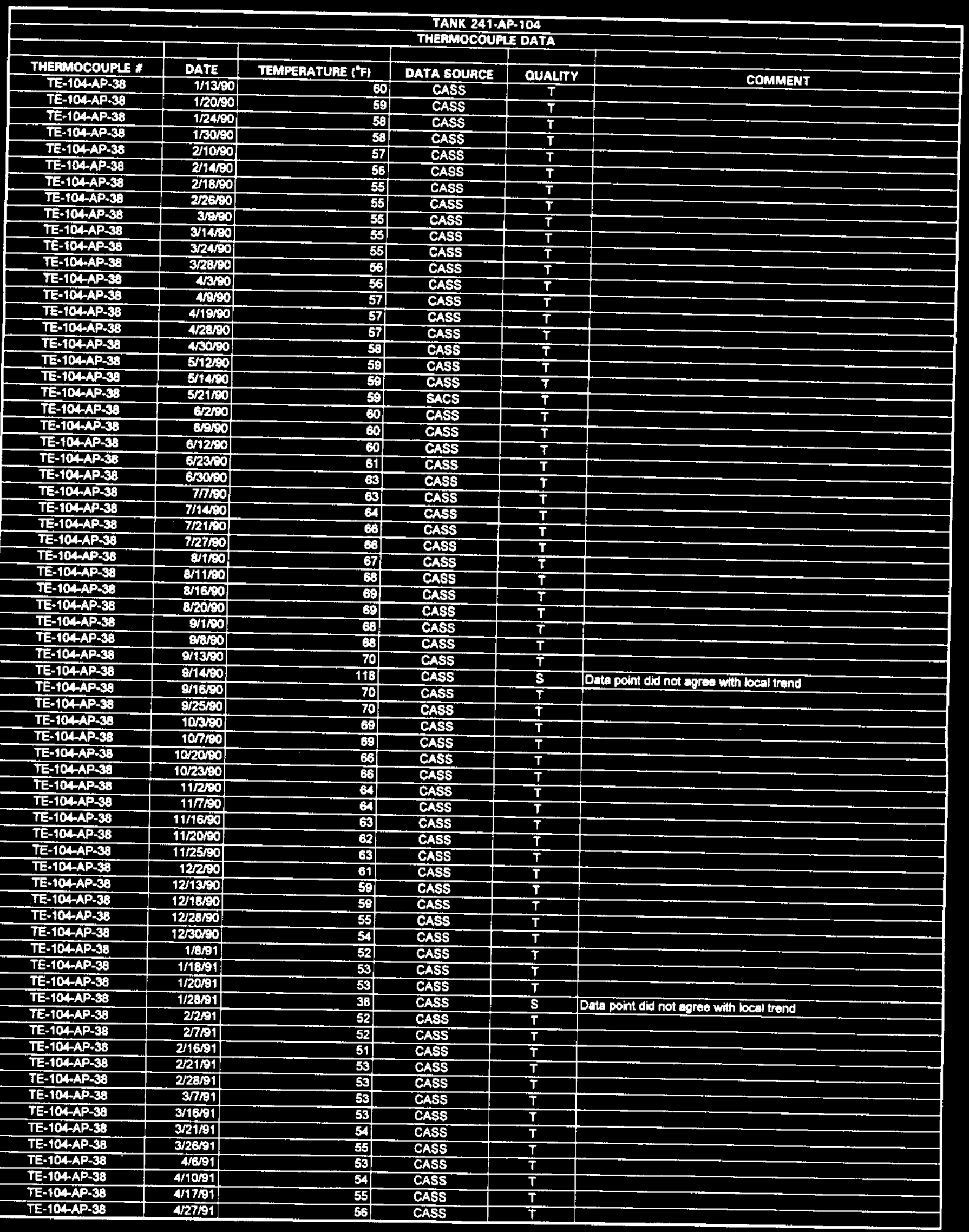

Data obtained from Computer Automated Survaillance System (CASS), April 1995; and Surveillance Anelysis Computer System (SACS), June 1995. 


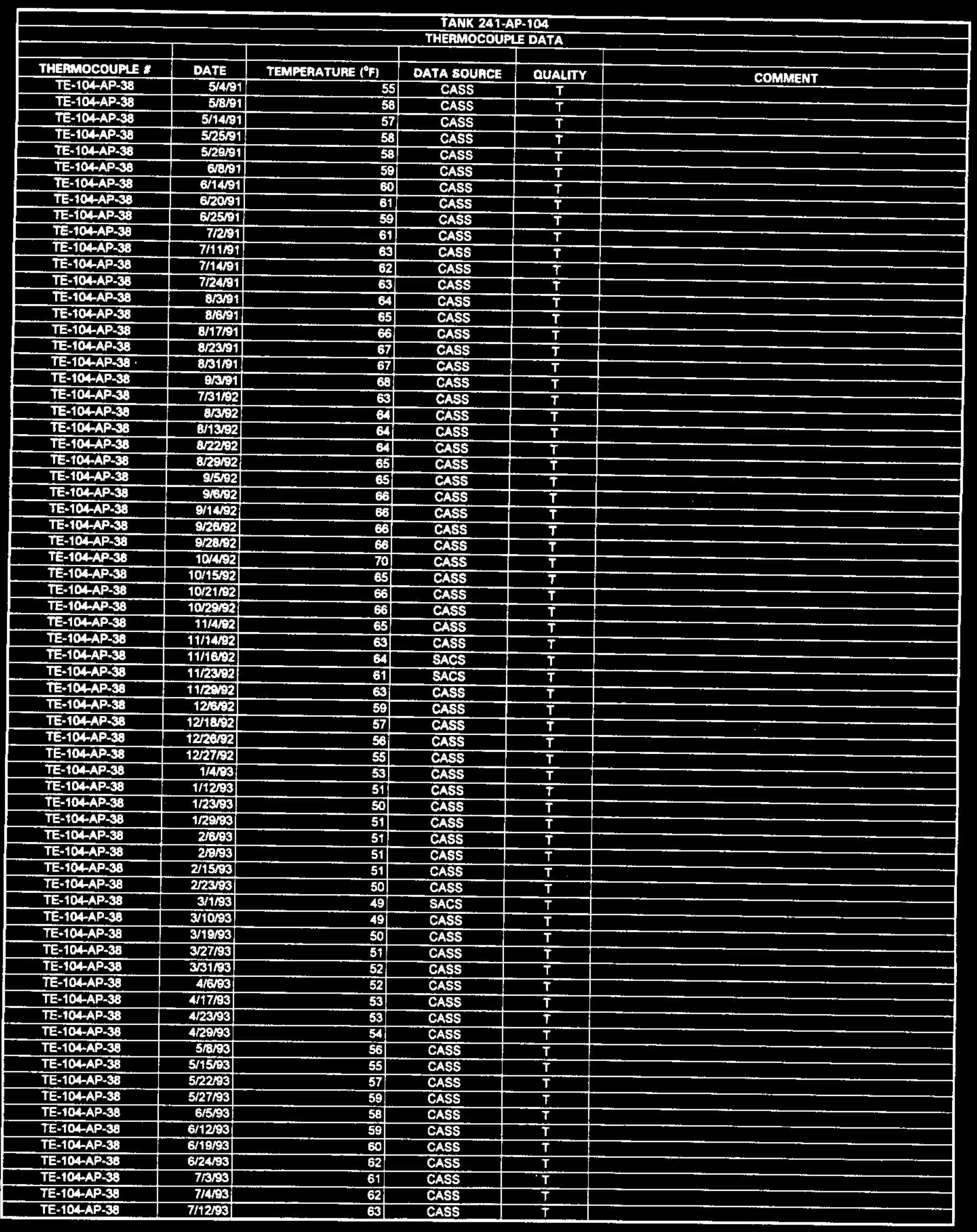

Data obtained from Computer Automated Surveillance Syatem \{CASS), April 1995; and Surveillance Analysis Computer Syotem (SACS), June 1995.

D-339 


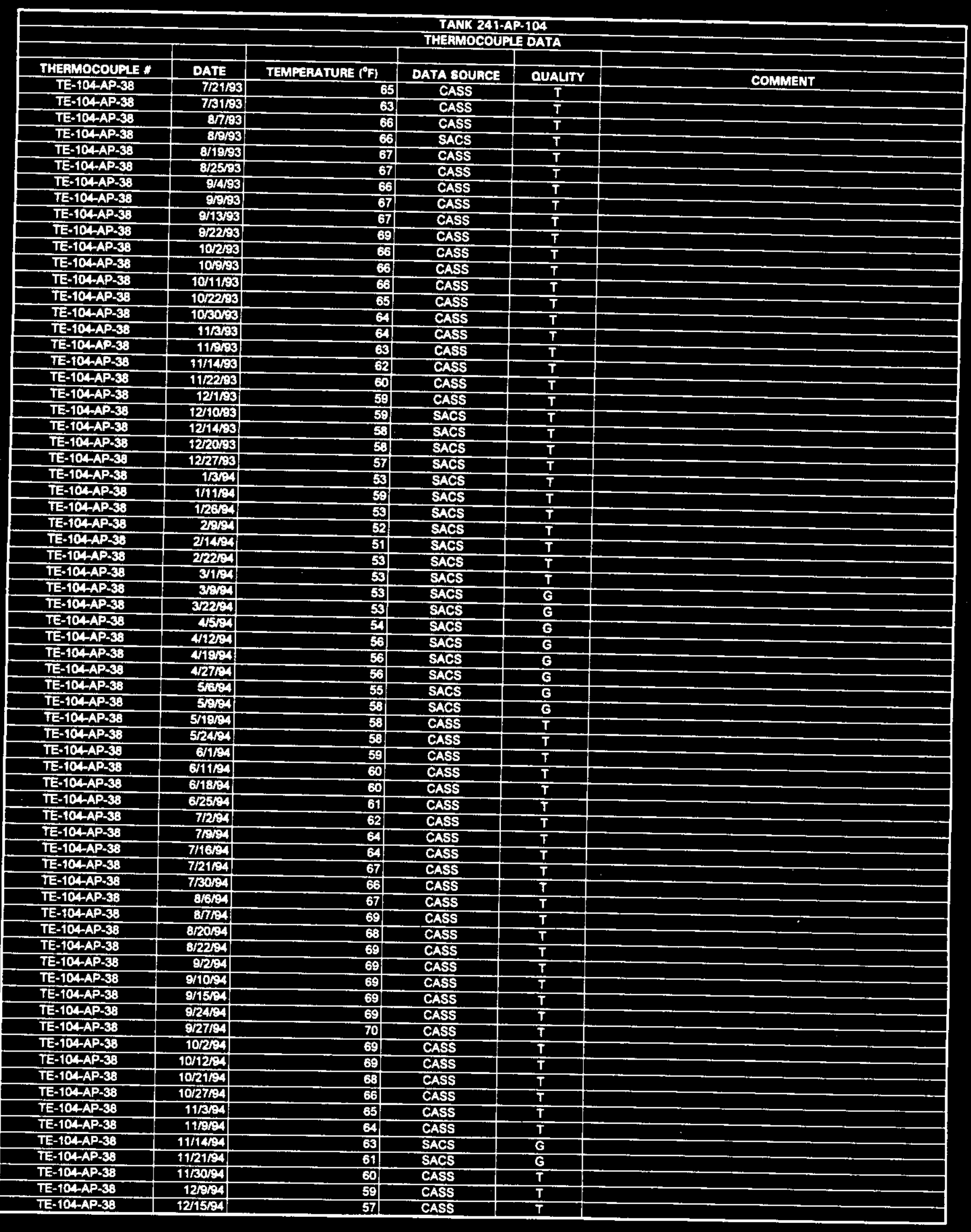

Date obtained from Computer Automated Surveillance System (CASS). April 1995; and Surveillance Analysis Computer Syatem (SACS). June 1995. $\mathrm{D}-340$ 


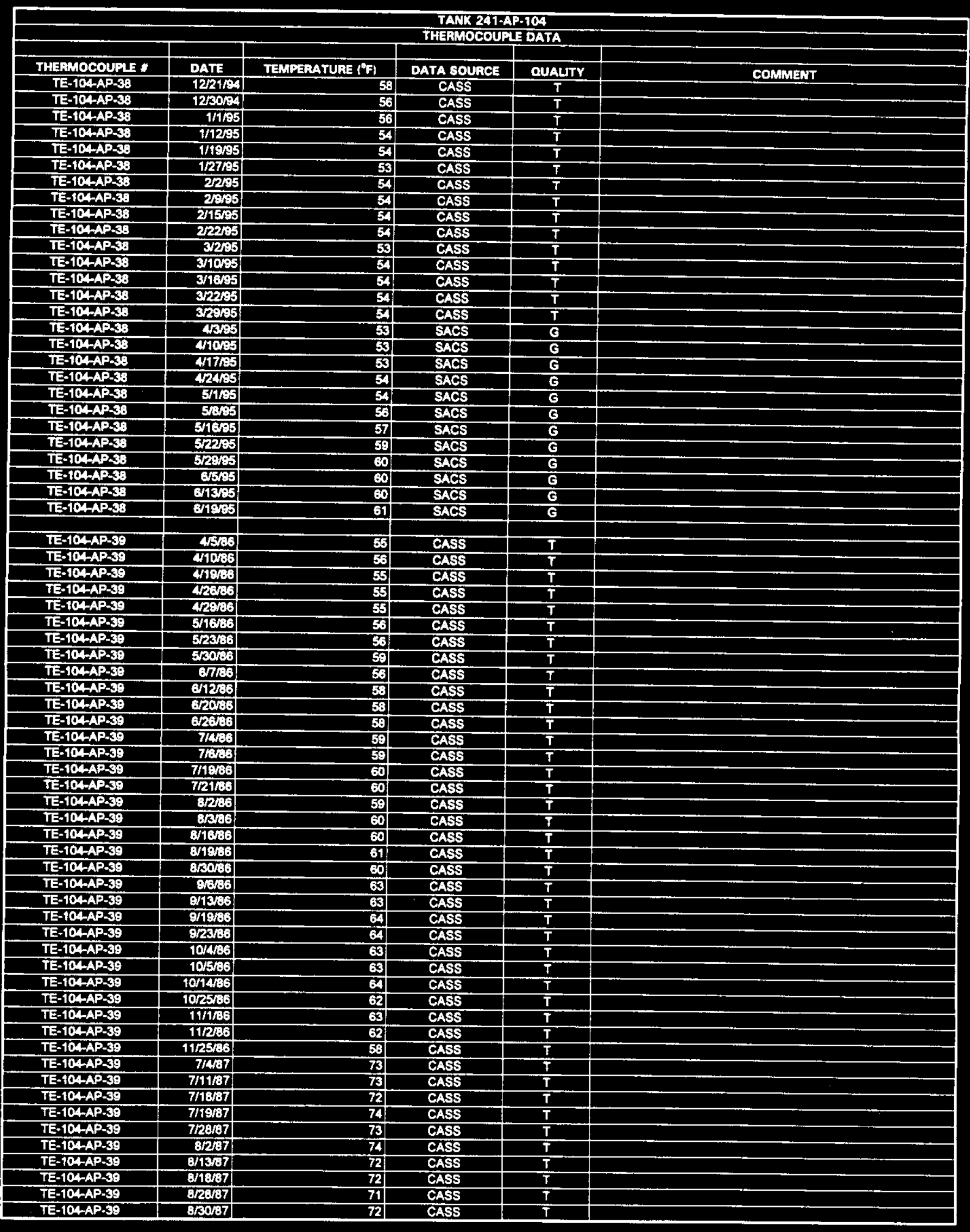

Data obtainad from Computer Automated Surveillance System (CASS), April 1995; and Surveillance Analysis Computer System (SACS), June 1995. 


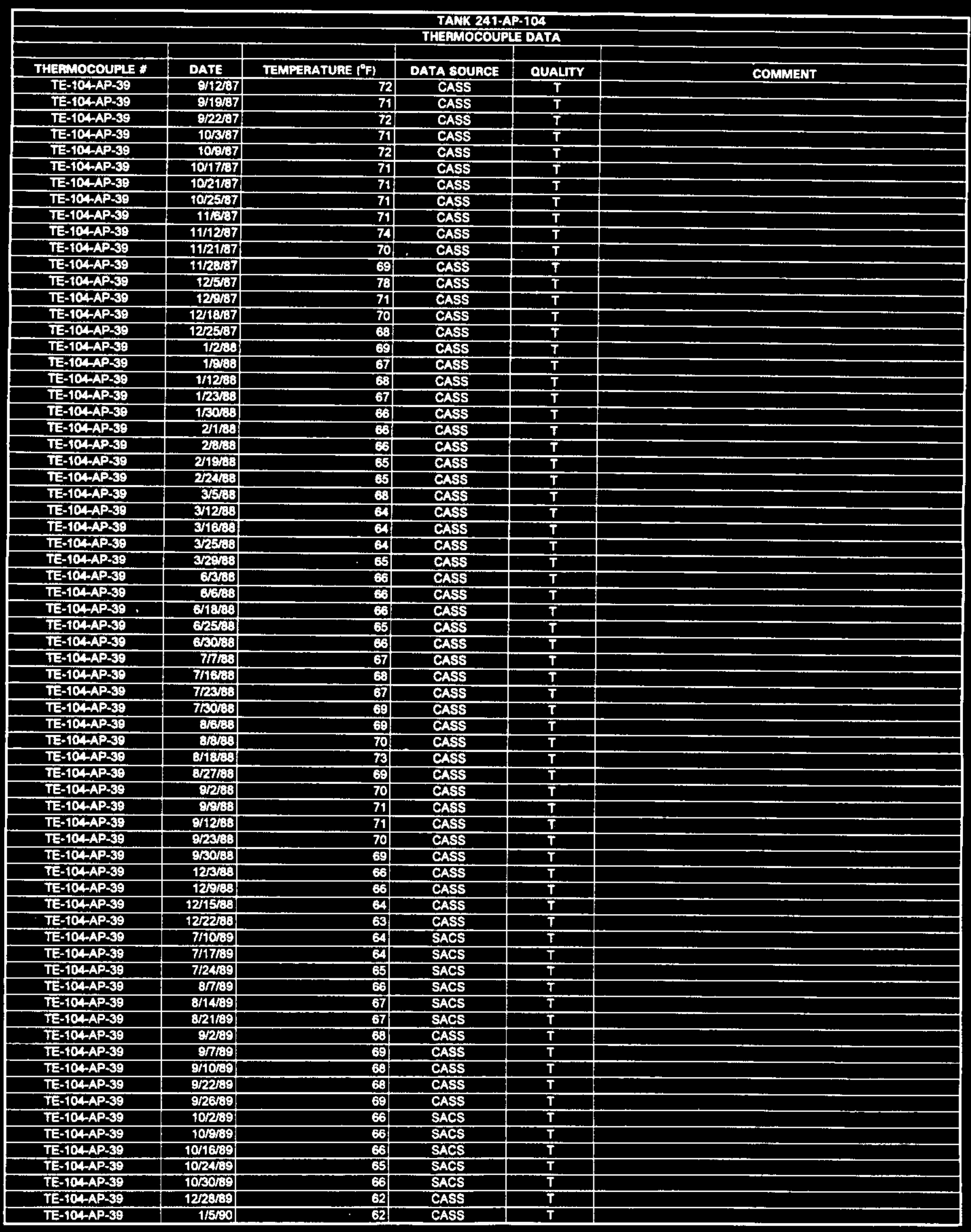




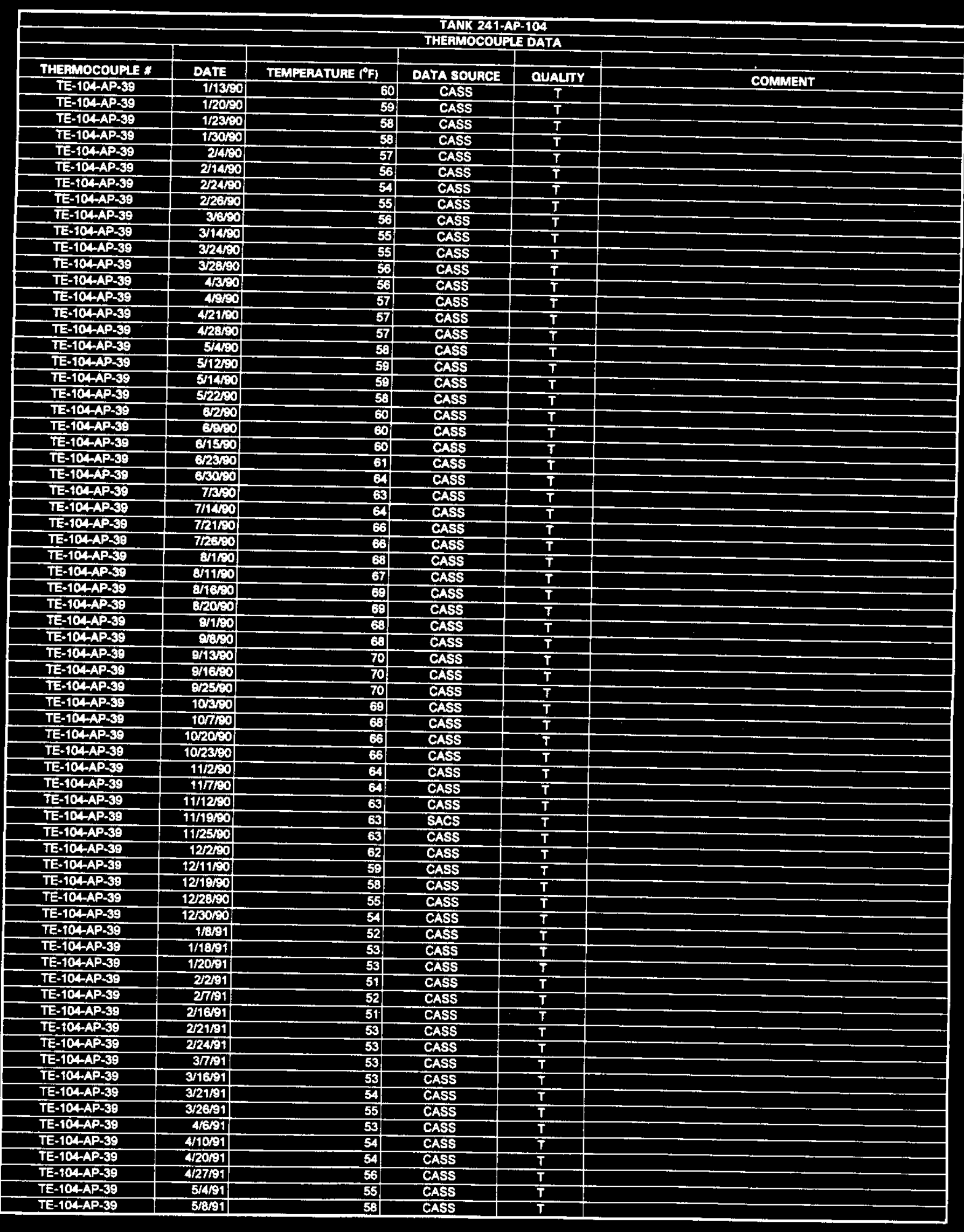

Data obtained from Computer Automated Surveillance Syatem (CASS), April 1995; and Surveillance Analysis Computer System (SACS), June 1995.

D-343 


\begin{tabular}{|c|c|c|c|c|c|}
\hline \multicolumn{6}{|c|}{$\begin{array}{l}\text { TANK 241-AP-104 } \\
\text { THERMOCOUPIE DATA }\end{array}$} \\
\hline & & & & & \\
\hline THERMOCOUPLE * & DATE & TEMPERATUAE $\left({ }^{\circ} \mathrm{F}\right)$ & DATA sOURCE & QUALITY & COMMENT \\
\hline TE-104-AP-39 & 5/1491 & 57 & CASS & $\bar{T}$ & \\
\hline TE-104AP-39 & 5/25\%91 & 58 & CASS & $T$ & \\
\hline TE-104AP-39 & $5 / 30 / 91$ & 58 & CASS & $T$ & \\
\hline TE-104-AP-39 & $6 / 3 / 91$ & 59 & SACS & $T$ & \\
\hline TE-104-AP-39 & 8/1461 & 60 & CASS & $\bar{T}$ & \\
\hline TE-104AP-39 & $6 / 2001$ & 62 & CASS & $T$ & \\
\hline TE-104AP-30 & 6/2551 & 59 & CASS & $T$ & \\
\hline TE-104AP-39 & 7201 & 62 & CASS & $\bar{T}$ & \\
\hline TE-104AP:38 & 7/11/91 & 63 & CASS & $T$ & \\
\hline TE-104-AP-39 & 7/1581 & 63 & SACS & $\bar{T}$ & \\
\hline TE-104-AP-39 & 722391 & 64 & SACS & $T$ & \\
\hline TE-104AP-30 & 8rav1 & 64 & CASS & $T$ & \\
\hline TE-104AP-S9 & 8/Ga1 & 65 & CASS & $T$ & \\
\hline TE-104AP-39 & $8 / 17 \%$ & 66 & CASS & $T$ & \\
\hline TE-104AP-39 & $8 / 2391$ & 68 & CASS & $T$ & \\
\hline TE-104-AP-38 & $831 \% 1$ & 67 & CASS & $T$ & \\
\hline TE-104-AP-S9 & 9/3/91 & 68 & CASS & $T$ & \\
\hline TE-104AP-S0 & 8/1/32 & 62 & CASS & $T$ & \\
\hline$T E-104-A P-39$ & $8 / 392$ & 64 & CASS & $T$ & \\
\hline TE-104-AP-39 & $8 / 1362$ & 6a & CASS & $T$ & \\
\hline TE-104-AP-39 & $8 / 1892$ & 6 & CASS & $\bar{T}$ & \\
\hline TE-104AP-39 & 6/29/92 & 65 & CASS & $\bar{T}$ & \\
\hline TE-104AP-38 & 9/5252 & 64 & CASS & $T$ & \\
\hline TE-104AP-38 & $8 / 6 / 2$ & 66 & CASS & $\bar{T}$ & \\
\hline TE-104-AP-39 & G/13/252 & 66 & CASS & $T$ & \\
\hline TE-104AP-39 & 8R6\%2 & 65 & CASS & $T$ & \\
\hline$T E-1 D A-A P-30$ & graro2 & 66 & CASS & $\bar{T}$ & \\
\hline TE-104AP-39 & 10062 & 66 & CASS & $T$ & \\
\hline TE-104AP-39 & 1017792 & 64 & CASS & $T$ & \\
\hline TE-104AP-39 & $10 / 21 / 22$ & 65 & CASS & $\bar{T}$ & \\
\hline TE-104AP-38 & 10rage2 & 60 & CASS & $\bar{T}$ & \\
\hline TE-104-AP-39 & 114492 & 65 & CASS & $T$ & \\
\hline TE-104AP-39 & $11 / 9 / 92$ & 6-1 & CASS & $T$ & \\
\hline TE-104-AP-38 & $11 / 2002$ & 63 & CASS & $T$ & \\
\hline TE-104AP-38 & $11 / 2392$ & 61 & CAsS & $\bar{T}$ & \\
\hline TE-104AP-38 & $11 / 29 / 2$ & 63 & CASS & $\bar{T}$ & \\
\hline TE-104AP-39 & 12602 & 59 & CASS & $T$ & \\
\hline TE-104-AP-39 & $12 / 13 / 92$ & 58 & CASS & $\bar{T}$ & \\
\hline TE-104-AP-39 & $12 / 26 / 92$ & 55 & CASS & $T$ & \\
\hline TE-104AP-39 & 1227792 & S⿹ & CASS & $T$ & \\
\hline TE-104AP.39 & $1 / 4 \mathrm{as}$ & 53 & CASS & $T$ & \\
\hline TE-104AP-38 & $1 / 12 \times 3$ & 5t & CASS & $T$ & \\
\hline TE-104AP-39 & $1 / 23 / 03$ & So & CASS & $\bar{T}$ & \\
\hline TE-104-AP-39 & $1 / 2903$ & S1 & CASS & $\mathbf{T}$ & \\
\hline TE-104-AP-39 & $20 / 93$ & 51 & CASS & $T$ & \\
\hline$T E-104-A P-39$ & $20 / 93$ & 51 & CASS & $\bar{T}$ & \\
\hline TE-104-AP-39 & 2/1593 & 51 & CASS & $T$ & \\
\hline TE-104AP-39 & $2 / 2363$ & 50 & CASS & $T$ & \\
\hline TE-104-AP-39 & $3 / 5 / 93$ & 48 & CASS & $T$ & \\
\hline TE-104AP-39 & 3/10/93 & 49 & CASS & $T$ & \\
\hline TE-104AP-38 & 3/19/93 & $\mathbf{5 0}$ & CASS & $\mathbf{T}$ & \\
\hline TE-104AP-39 & $3 / 27 / 93$ & $\mathbf{5 1}$ & CASS & $\mathbf{T}$ & \\
\hline TE-104AP-39 & $3 / 31 / 93$ & 52 & CASS & $T$ & \\
\hline TE-104-AP-39 & 46/93 & 52 & CASS & $T$ & \\
\hline TE-104-AP-39 & $4 / 17 / 93$ & $\mathbf{5 3}$ & CASS & $T$ & \\
\hline TE-104AP-39 & $4 \sqrt{23 / 93}$ & 53 & CASS & $\bar{T}$ & \\
\hline TE-104-AP-39 & $4 / 27193$ & 9 & CASS & $\mathbf{s}$ & Data point did not agroe with local trend \\
\hline TE-104AP-39 & 4/2963 & 54 & CASS & $\bar{T}$ & \\
\hline TE-104-AP-39 & $5 / 563$ & 55 & CASS & $T$ & \\
\hline TE-104-AP-39 & 5/15/A3 & 35 & CASS & $T$ & \\
\hline TE-104-AP-39 & $5 / 22193$ & 57 & CASS & $T$ & \\
\hline TE-104AP-39 & $5 / 27 / 93$ & 60 & CASS & $T$ & \\
\hline TE-104-AP-39 & 6/5/93 & 58 & CASS & $T$ & \\
\hline TE-104-AP-39 & $6 / 12193$ & 59 & CASS & $T$ & \\
\hline TE-104-AP-39 & $6 / 16 \times 3$ & 61 & CASS & $T$ & \\
\hline TE-104-AP-39 & $6 / 24 / 93$ & 62 & CASS & $\bar{T}$ & \\
\hline TE-104-AP-39 & $7 / / 3 / 93$ & 61 & CASS & $T$ & \\
\hline TE-104AP-38 & $7 / 6 / 93$ & 62 & CASS & $T$ & \\
\hline TE-104-AP-39 & $7 / 2 / 93$ & 63 & CASS & $\bar{T}$ & \\
\hline TE-104AP-39 & $7 / 21 / 93$ & 63 & CASS & $T$ & \\
\hline
\end{tabular}




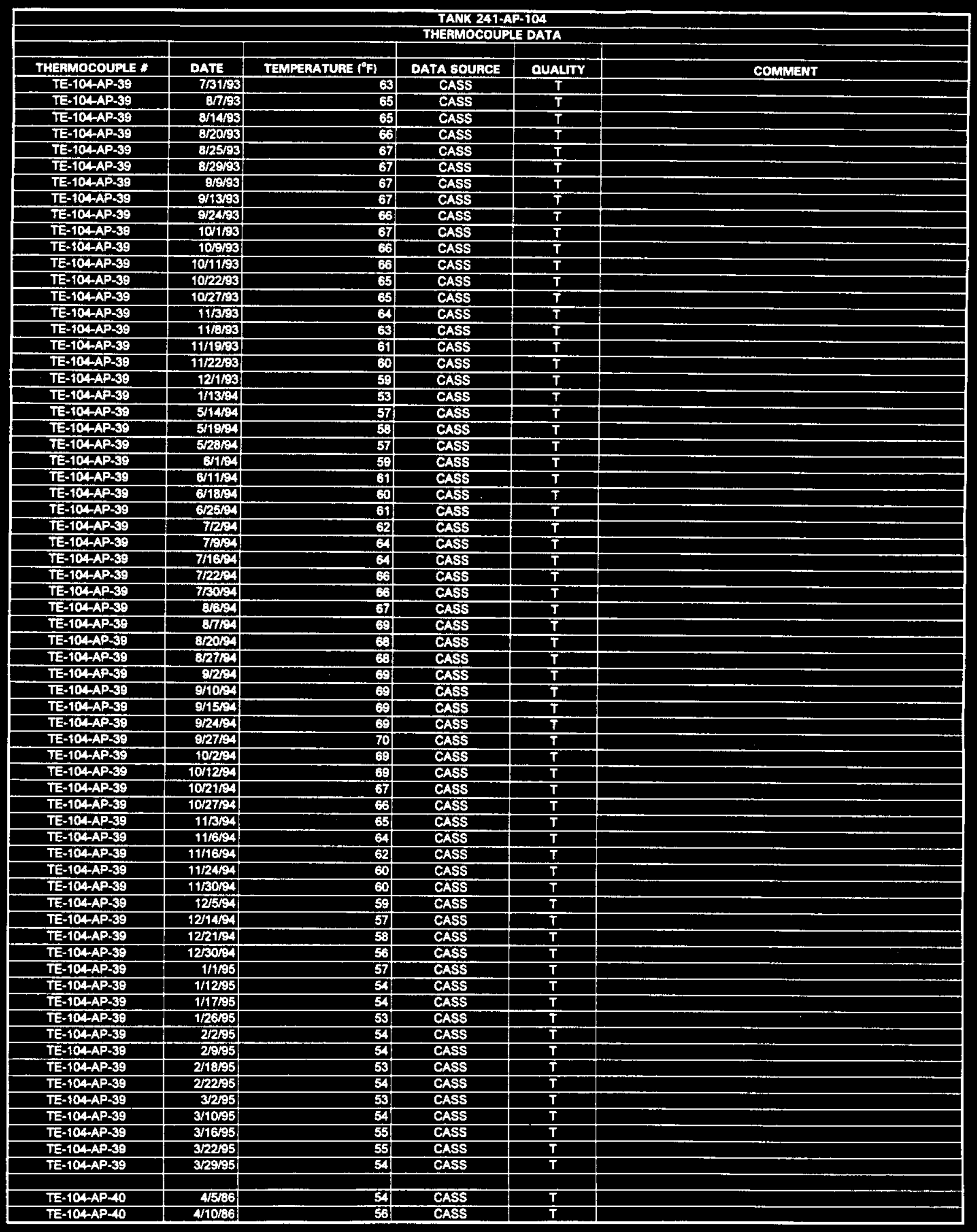




\begin{tabular}{|c|c|c|c|c|c|}
\hline \multicolumn{6}{|c|}{$\begin{array}{l}\text { TANK 241-AP-104 } \\
\text { THEAMOCOUAIE DATA }\end{array}$} \\
\hline THEAMOCOUPL * & DATE & TEMPEAATURE $\left({ }^{\circ} \mathrm{F}\right)$ & DATA SOURCE & OUALTY & COMMENT \\
\hline TE-104-AP 40 & $4 / 19 / 86$ & 55 & CASS & $T$ & 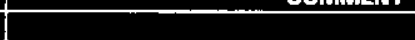 \\
\hline TE-104AP-40 & $4 / 24 / 86$ & 56 & CASS & $\bar{T}$ & \\
\hline TE-104AP-40 & $4 / 29 / 86$ & 55 & CASS & $T$ & \\
\hline TE-104-AP-40 & 5/16/36 & 56 & CASS & $T$ & \\
\hline TE-104-AP-40 & $5 / 23 / 86$ & 57 & CASS & $T$ & \\
\hline TE-104AP-10 & $5 / 30 / 86$ & 59 & CASS & $T$ & \\
\hline$T E-104-A P-40$ & 67186 & 57 & CASS & $T$ & \\
\hline TE-104-AP-40 & $6 / 12 / 86$ & 58 & CASS & $T$ & \\
\hline $\mathrm{TE}-104-A P-40$ & 6/20/86 & 58 & CASS & $T$ & \\
\hline$T E-104-A P=40$ & 6/26/86 & 58 & CASS & $T$ & \\
\hline TE-104AP-10 & $7 / 4 / 66$ & 58 & CASS & $T$ & \\
\hline TE-104-AP-10 & 76 & 59 & CASS & $T$ & \\
\hline TE-104AP-10 & 71986 & 60 & CASS & $\bar{T}$ & \\
\hline TE-10AAP $=40$ & $7 / 21 / 86$ & 6 & CASS & $T$ & \\
\hline$T E=104-A P-40$ & $8 / 2186$ & 58 & CASS & $T$ & \\
\hline TE-104-AP- 40 & $8 / 318$ & 60 & CASS & $T$ & \\
\hline TE-TOA-AP-10 & B/16\%6 & 60 & CASS & $\bar{T}$ & \\
\hline TE-104AP-10 & $8 / 1986$ & 61 & CASS & $T$ & \\
\hline TE-104AP-A0 & 8/3006 & 60 & CASS & $\bar{T}$ & \\
\hline TE-104AP-10 & $8 / 686$ & 63 & CASS & $T$ & \\
\hline TE-104AP- 40 & 90/86 & GA & CASS & $T$ & \\
\hline TE-104AP-A0 & 91986 & E4 & CASS & $T$ & \\
\hline TE-104-AP-40 & $8 / 23 / 66$ & 64 & CASS & $T$ & \\
\hline TE-104-AP-40 & 10486 & 63 & CASS & $T$ & \\
\hline TE-104AP-40 & 10/5/36 & 63 & CASS & $T$ & \\
\hline TE-104AP-40 & 10/4486 & E4 & CASS & $\bar{T}$ & \\
\hline TE-104AP-40 & $10 / 2538$ & 62 & CASS & $T$ & \\
\hline TE-TOA-AP-10 & 11/1/36 & 64 & CASS & $T$ & \\
\hline TE-104AP-40 & $11 / 2 / 86$ & 62 & CASS & $T$ & \\
\hline TE-104AP- 10 & $11 / 2566$ & 58 & CASS & $T$ & \\
\hline TE-104AP-10 & 71407 & 73 & CASS & $T$ & \\
\hline TE-104AP- 10 & $7 / 11 / 37$ & 73 & CASS & $T$ & \\
\hline TE-104AP-40 & $7 / 18 / 87$ & 72 & CASS & $T$ & \\
\hline TE-104AP- 10 & 71887 & 73 & CASS & $\bar{T}$ & \\
\hline TE-TOA-AP-10 & $7 / 28 / 67$ & 72 & CASS & $T$ & \\
\hline TE-104AP-40 & $8 / 2 / 87$ & 73 & CASS & $T$ & \\
\hline TE-104AP=40 & $8 / 13 / 67$ & 72 & CASS & $\bar{T}$ & \\
\hline TE-104-AP-40 & 8/1887 & 72 & CASS & $T$ & \\
\hline TE-104AP-40 & 8/25537 & 71 & CASS & $T$ & \\
\hline TE-104-AP-40 & B/30/87 & 72 & CASS & $\bar{T}$ & \\
\hline TE-104-AP- 10 & $8 / 12 / 37$ & 71 & CASS & $T$ & \\
\hline TE-104AP-40 & 9/18/87 & 71 & CASS & $T$ & \\
\hline TE-104AP-40 & $9 / 22 / 87$ & 72 & CASS & $T$ & \\
\hline TE-104AP-40 & 8/28/87 & 57 & CASS & $\mathbf{S}$ & Data point did not erree with locsal trend \\
\hline TE-104-AP-40 & 30/3/37 & 71 & CASS & $T$ & \\
\hline TE-104AP-40 & 10/9/87 & 72 & CASS & $\mathbf{T}$ & \\
\hline TE-104-AP-40 & $10 / 17 / 37$ & 71 & CASS & $T$ & \\
\hline TE-104-AP-40 & $10 / 21 / 87$ & 71 & CASS & $\bar{T}$ & \\
\hline$T E-104 A P=40$ & 10/28/37 & 70. & CASS & $\bar{T}$ & \\
\hline TE-104AP- 10 & 11/6/87 & 71 & CASS & $\mathbf{T}$ & \\
\hline TE-104AP-40 & 11/12/67 & 73 & CASS & $\bar{T}$ & \\
\hline TE-104AP-40 & $11 / 16 / 87$ & 71 & CASS & $T$ & \\
\hline$T E-104 A-40$ & $11 / 28 / 87$ & 69 & CASS & $\bar{T}$ & \\
\hline TE-104AP-10 & 12/587 & 76 & CASS & $\mathbf{T}$ & \\
\hline TE-104-AP-40 & 129687 & 70 & CASS & $\bar{T}$ & \\
\hline TE-104AP-40 & $12 / 18 / 87$ & 69 & CASS & $\mathbf{T}$ & \\
\hline TE-104AP-40 & $12125 / 87$ & 68 & CASS & $\mathbf{T}$ & \\
\hline TE-104AP-10 & $1 / 218$ & 68 & CASS & $T$ & \\
\hline TE-104AP-40 & $1 / 7 / 8$ & 67 & CASS & $T$ & \\
\hline TE-104AP-40 & $1 / 12 / 88$ & 67 & CASS & $\bar{T}$ & \\
\hline TE-104AP-40 & 1/23/38 & 69 & CASS & $\mathbf{T}$ & \\
\hline TE-104-AP-10 & 1/29/88 & 66 & CASS & $T$ & \\
\hline TE-104AP-40 & $2 / 5 / 88$ & 65 & CASS & $T$ & \\
\hline TE-104AP-40 & $2 / 12 / 68$ & 65 & CASS & $T$ & \\
\hline TE-104AP=40 & $2 / 19 / 68$ & 65 & CASS & $T$ & \\
\hline TE-104-AP-40 & $2 / 23 / 68$ & 65 & CASS & $T$ & \\
\hline TE-104AP $=40$ & $3 / 5 / 86$ & 67 & CASS & $T$ & \\
\hline TE-104-AP=10 & $3 / 10 / 88$ & 64 & CASS & $T$ & \\
\hline TE-104-AP-40 & 3/1688 & 64 & CASS & $T$ & \\
\hline TE-104AP-40 & $3 / 25 / 38$ & 64 & CASS & $T$ & \\
\hline
\end{tabular}




\begin{tabular}{|c|c|c|c|c|c|}
\hline \multicolumn{6}{|c|}{$\begin{array}{l}\text { TATK 241-AP-104 } \\
\text { TH:FMOCOUPLE DATA }\end{array}$} \\
\hline THERMOCOUPLE * & DATE & TEMPERATURE ( $\left.{ }^{\circ} \mathrm{F}\right)$ & DATA sOURCE & QUALTY & COMMENT \\
\hline TE-104AP-40 & $3 / 29 / 38$ & 64 & CASS & $T$ & \\
\hline TE-104AP 40 & $6 / 3 / 38$ & 66 & CASS & $T$ & \\
\hline TE-104-AP-40 & $6 / 3 / 38$ & 104 & CASS & $\mathbf{s}$ & Data point did not agree with locsl trend \\
\hline TE-104AP-40 & $6 / 11 / 68$ & 65 & CASS & $\mathbf{T}$ & (2) \\
\hline TE-104-AP-40 & ETBas & 65 & CASS & $T$ & \\
\hline TE-104-AP-40 & 6/2508 & 65 & CASS & $\mathbf{T}$ & \\
\hline TE-104-AP-40 & $6 / 29 / 88$ & 66 & CASS & $T$ & \\
\hline TE-104AP-40 & $7 / 7 / 88$ & 66 & CASS & $T$ & \\
\hline TE-104AP-40 & $716 \mathrm{~B}$ & 67 & CASS & $T$ & \\
\hline TE-104AP-40 & $771 \mathrm{SBO}$ & 68 & CASS & $\bar{T}$ & \\
\hline TE-104AP-10 & $7 / 30 / 88$ & 69 & CASS & $T$ & \\
\hline TE-104AP-40 & 8/68: & 69 & CASS & $T$ & \\
\hline TE-104-AP-40 & $83 / 3$ & 70 & CASS & $T$ & \\
\hline TE-104AP-40 & $8 / 18 / 38$ & 73 & CASS & $T$ & \\
\hline TE-104-AP-40 & 872588 & 71 & CASS & $T$ & \\
\hline$T E-104-A P-40$ & 9/2/88 & 70 & CASS & $T$ & \\
\hline TE-104-AP-40 & 8mes & 71 & CASS & $T$ & \\
\hline TE-104AP-40 & 91288 & 71 & CASS & $T$ & \\
\hline TE-104-AP-10 & 9/23/88 & 70 & CASS & $T$ & \\
\hline TE-104-AP-40 & 930/88 & 69 & CASS & $\bar{T}$ & \\
\hline TE-104AP 40 & $12 \pi 309$ & 86 & CASS & $T$ & \\
\hline TE-104-AP- 10 & 12 sed & 66 & CASS & $\mathbf{T}$ & \\
\hline$T E-104 A P=40$ & $12 / 1438$ & 65 & CASS & $T$ & \\
\hline$T E-104-A P=40$ & 1222238 & 63 & CASS & $T$ & \\
\hline TE-104AP-40 & 8/2/89 & 68 & CASS & $T$ & \\
\hline TE-104AP-40 & 9r/69 & 69 & CASS & $T$ & \\
\hline TE-104AP-40 & 916/39 & 67 & CASS & $T$ & \\
\hline TE-104AP-40 & $9 / 2219$ & 68 & CASS & $T$ & \\
\hline TE-104-AP-10 & 8/26309 & 69 & CASS & $\mathbf{T}$ & \\
\hline TE-104AP-AO & $11 / 280$ & 64 & CASS & $T$ & \\
\hline$T E-104 A P-10$ & $12 / 28 / 89$ & 62 & CASS & $T$ & \\
\hline TE-104AP-40 & $12 / 31 / 89$ & 62 & CASS & $T$ & \\
\hline TE-104AP-40 & $1 / 1080$ & 60 & CASS & $T$ & \\
\hline TE-104AP-40 & 1 Rores & 59 & CASS & $T$ & \\
\hline$T E-104 A P-40$ & $1 / 23 / 80$ & 38 & CASS & $\bar{T}$ & \\
\hline TE-104-AP-40 & $1 / 3050$ & 58 & CASS & $T$ & \\
\hline TE-104AP-40 & $2 / 4: 0$ & 57 & CASS & $T$ & \\
\hline TE-104AP-40 & $2 / 1450$ & 56 & CASS & $T$ & \\
\hline$T E+104 A P=40$ & $2 / 2390$ & 54 & CAss & $T$ & \\
\hline TE-104AP 10 & $2 / 2650$ & 55 & CASS & $T$ & \\
\hline TE-104AP-40 & 36s0 & 55 & CASS & $T$ & \\
\hline TE-104-AP-40 & 3/490 & 55 & CASS & $T$ & \\
\hline TE-104AP-40 & $3 / 2400$ & 55 & CASS & $T$ & \\
\hline$T E-104 A P-40$ & $330 / 80$ & 56 & CASS & $T$ & \\
\hline$T E-104-A P-40$ & $4 \pi / 50$ & 55 & CASS & $\bar{T}$ & \\
\hline TE-104-AP-10 & ASEO & 57 & CASS & $T$ & \\
\hline$T E-104-A P-10$ & 4/5EO & 58 & CASS & $T$ & \\
\hline TE-104-AP-40 & 4/28/90 & 57 & CASS & $T$ & \\
\hline TE-104AP-40 & $4 / 30100$ & 58 & CASS & $T$ & \\
\hline TE-104AP+40 & 51280 & 59 & CASS & $T$ & \\
\hline TE-104-AP-40 & 51490 & 59 & CASS & 7 & \\
\hline TE-104-AP-40 & $5 / 21 / 00$ & 59 & SACS & $T$ & \\
\hline TE-104AP-40 & $6 / 1 / 00$ & 60 & CASS & $T$ & \\
\hline TE-104-AP-40 & Geso & 60 & CASS & $T$ & \\
\hline TE-104-AP-10 & $6 / 2 / 50$ & 60 & CASS & $T$ & \\
\hline TE-104AP-40 & $6 / 18 \times 0$ & 114 & CASS & $\mathrm{s}$ & Data point did not agree with local trend \\
\hline TE-104AP-40 & 6ras:0: & 61 & CASS & $\bar{T}$ & \\
\hline TE-104-AP-10 & 6/30190 & 63 & CASS & $\mathbf{T}$ & \\
\hline TE-104-AP-AO & 7390 & 63 & CASS & $T$ & \\
\hline TE-104-AP-40 & $714 \% 0$ & 64 & CASS & $T$ & \\
\hline TE-104-AP-40 & $7 / 21 / 90$ & 67 & CASS & $T$ & \\
\hline TE-104-AP-40 & $7 / 26 / 90$ & 66 & CASS & $T$ & \\
\hline TE-104-AP-40 & $8 / 150$ & 67 & CASS & $\mathbf{T}$ & \\
\hline TE-104AP-40 & $8 / 11 / 00$ & 68 & CASS & $\mathbf{T}$ & \\
\hline TE-104-AP-40 & $8 / 16 / 90$ & 69 & CASS & $\mathbf{T}$ & \\
\hline TE-104-AP-40 & 6/20/90 & 69 & CASS & $T$ & \\
\hline TE-104-AP-40 & $9 / 1 / 30$ & 68 & CASS & $T$ & \\
\hline TE-104-AP-40 & 9/8/s0 & 68 & CASS & $\bar{T}$ & \\
\hline TE-104-AP-40 & $9 / 15 / 00$ & 69 & CASS & $T$ & \\
\hline TE-104-AP-40 & 9/16/so & 70 & CASS & $T$ & \\
\hline
\end{tabular}




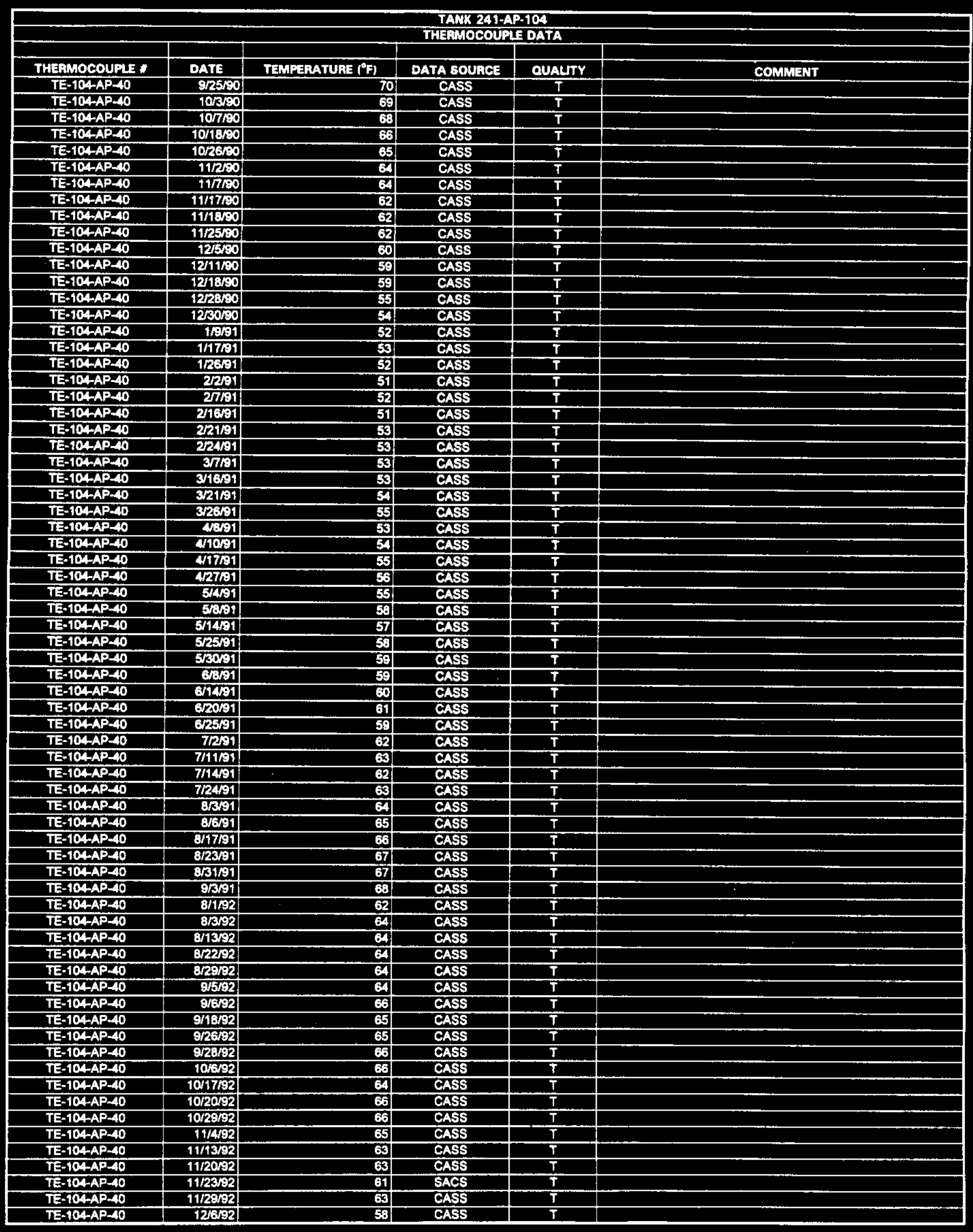




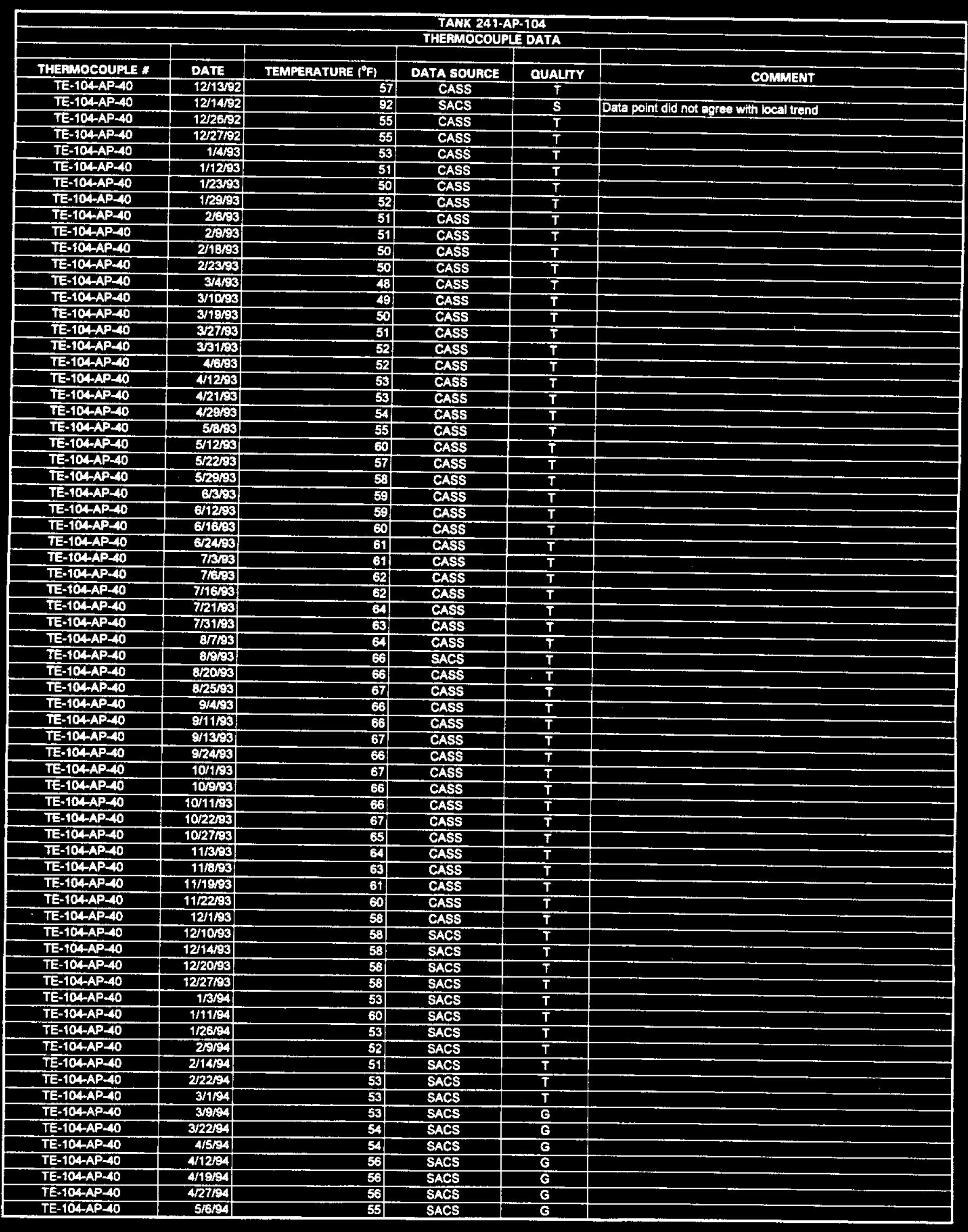




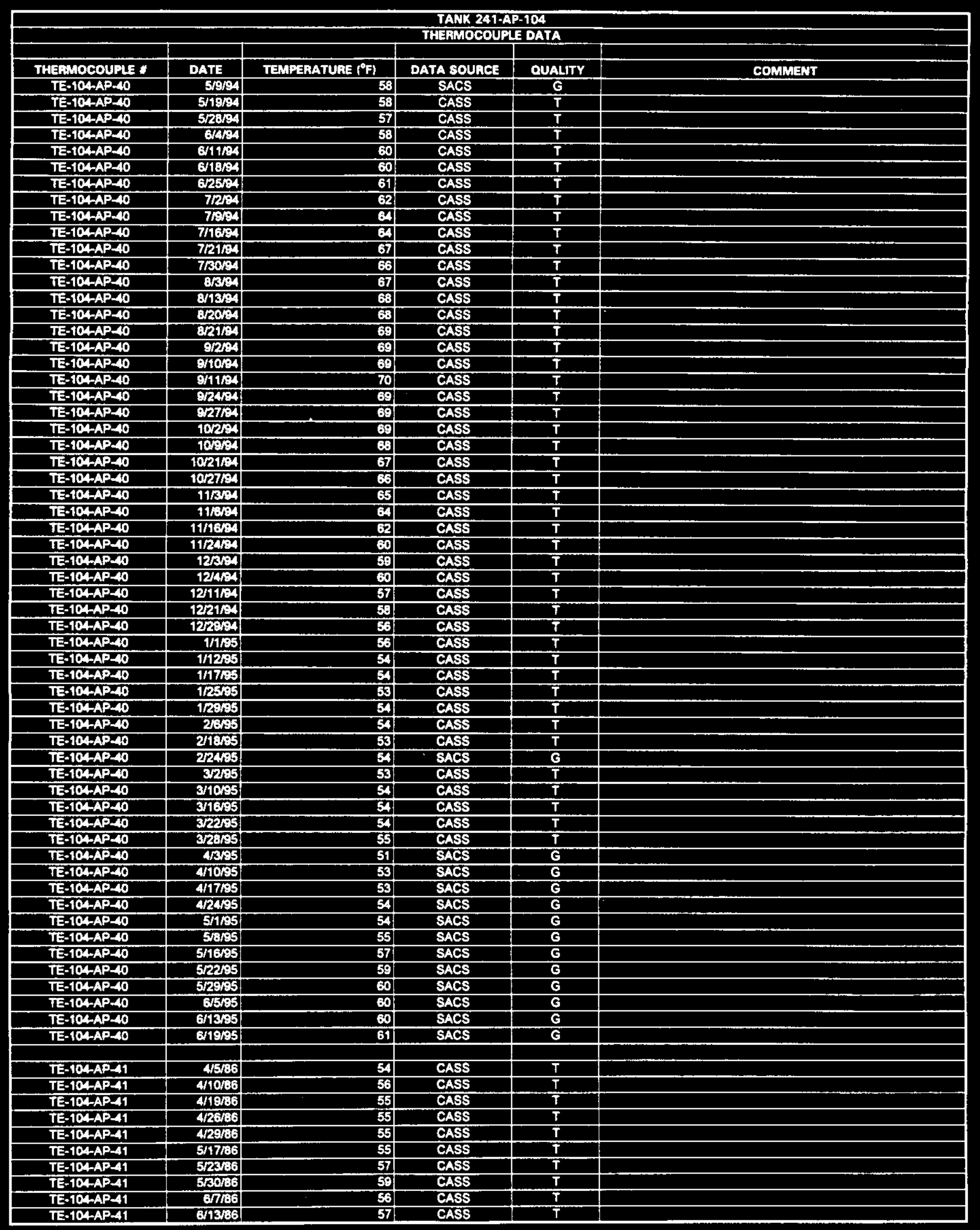




\begin{tabular}{|c|c|c|c|c|c|}
\hline \multicolumn{6}{|c|}{$\begin{array}{l}\text { TANK 241-AP-104 } \\
\text { THEMMOCOUPLE DATA }\end{array}$} \\
\hline & & & & & \\
\hline THERMOCOUPLE * & DATE & TEMPERATUAE $\left({ }^{\circ} \mathrm{F}\right)$ & DATA SOURCE & OUALTY & COMMENT \\
\hline TE-104AP-41 & 6/20/86 & 58 & CASS & $T$ & \\
\hline TE-104AP-41 & 6/26/36 & 58 & CÁSS & $\mathbf{T}$ & \\
\hline TE-104-AP-41 & $7 / 4 / 36$ & 58 & CASS & $T$ & \\
\hline IE-104-AP-41 & $7 / 1266$ & 58 & CASS & $T$ & \\
\hline TE-104-AP-49 & $719 / 66$ & 60 & CASS & $\mathrm{T}$ & \\
\hline TE-104-AP-A1 & $7 / 21 / 36$ & 59 & CASS & $T$ & \\
\hline TE-104-AP-41 & $8 / 286$ & 59 & CASS & $T$ & \\
\hline TE-104-AP-41 & $8 / 3 / 86$ & E0 & CASS & $\mathbf{T}$ & \\
\hline TE-104AP-A1 & 8/16/36 & 60 & CASS & $\mathbf{T}$ & \\
\hline TE-104-AP-41 & $8 / 22 / 86$ & $\overline{60}$ & CASS & $\mathbf{T}$ & \\
\hline TE-104-AP-41 & $8 / 23 / 66$ & 74 & CASS & $\mathbf{S}$ & Data point did not agree with local trend \\
\hline IE-104-AP-41 & $8 / 29 / 86$ & 60 & CASS & $\mathbf{T}$ & \\
\hline TE-104-AP-A1 & $8 / 30 / 86$ & 85 & CASS & $\mathbf{s}$ & Data point did not agree with local trend \\
\hline$T E-104-A P-41$ & 9/6/83 & 63 & CASS & $T$ & \\
\hline TE-104AP-41 & 9/86 & 64 & CASS & $T$ & \\
\hline TE-104AP-11 & Q/19/66 & E4 & CASS & $T$ & \\
\hline TE-104AP-A1 & $8 / 23 / 66$ & 63 & CASS & $T$ & \\
\hline TE-104AP-41 & 10486 & 63 & CASS & $T$ & \\
\hline TE-104APA1 & $10 / 2 / 86$ & 62 & CASS & $T$ & \\
\hline TE-104-AP-A1 & $10 / 14 / 86$ & 64 & CASS & $\mathbf{T}$ & \\
\hline TE-10A-AP-A1 & $10 / 2486$ & 64 & CASS & $T$ & \\
\hline TE-104AP-41 & 11/186 & 63 & CASS & $\bar{T}$ & \\
\hline TE-TOA-AP-A1 & $11 / 2 / 66$ & 62 & CASS & $T$ & \\
\hline IE-104-AP-A1 & $11 / 25 / 36$ & 59 & CASS & $T$ & \\
\hline TE-104-AP-A1 & $7 / 487$ & 73 & CASS & $\bar{T}$ & \\
\hline TE-1CAAP-A1 & 710087 & 74 & CASS & $\mathbf{T}$ & \\
\hline TE-104-AP-A1 & $718 / 87$ & 72 & CASS & $T$ & \\
\hline TE-104-AP-A1 & $7 / 1967$ & 75 & CASS & $T$ & \\
\hline TE-104AP-A1 & $7 / 28 / 67$ & 72 & CASS & $T$ & \\
\hline TE-104AP-AI & $8 / 267$ & 73 & CASS & $T$ & \\
\hline IE-104-AP-A1 & $8 / 14 / 87$ & 72 & CASS & $T$ & \\
\hline TE-104AP $=41$ & $8 / 18 / 87$ & 72 & CASS & $T$ & \\
\hline TE-104-AP-AT & 8/29:87 & 71 & CASS & $T$ & \\
\hline$T E-104-A P=1$ & 83067 & 72 & CASS & $T$ & \\
\hline TE-104AP-41 & 9607 & 73 & CASS & $T$ & \\
\hline TE-104-AP-11 & 91687 & 72 & CASS & $T$ & \\
\hline TE-104APDA1 & $9 / 2227$ & 72 & CASS & $T$ & \\
\hline TE-10-AP-A1 & $10 \times 3 / 87$ & 71 & CASS & $\mathbf{T}$ & \\
\hline TE-104-AP-11 & 100/7 & 72 & CASS & $T$ & \\
\hline TE-104-AP-AI & $10 / 17 / 87$ & 71 & CASS & $T$ & \\
\hline TE-104-AP-A1 & $10 / 21 / 37$ & 71 & CASS & $T$ & \\
\hline TE-104-AP-A1 & $10 / 25 / 87$ & 71 & CASS & $\bar{T}$ & \\
\hline TE-104-AP-41 & $11 / 4 / 67$ & 72 & CASS & $\mathbf{T}$ & \\
\hline TE-104-AP-49 & 11/12/87 & 74 & CASS & $\mathbf{T}$ & \\
\hline TE-104-APA1 & $11 / 18 / 87$ & 71 & CASS & $T$ & \\
\hline TE-104AP-A1 & 11/22/87 & 70 & CASS & $\bar{T}$ & \\
\hline TE-104AP-A1 & 1215/87 & 77 & CASS & $T$ & \\
\hline TE-104AP-41 & $12 / 9 / 87$ & 70 & CASS & $T$ & \\
\hline$T E-104 A P-41$ & $12 / 18 / 87$ & 70 & CASS & $T$ & \\
\hline$T E=104-A P-41$ & 1222487 & 70 & CASS & $T$ & \\
\hline TE-104-AP-A1 & 1/2/38 & 69 & CASS & $T$ & \\
\hline TE-104-AP-A1 & 1/0/38 & 67 & CASS & $T$ & \\
\hline TE-104-AP-A1 & 1/12/80 & 68 & CASS & $T$ & \\
\hline TE-104-AP-A1 & $1 / 2008$ & 63 & CASS & $T$ & \\
\hline TE-104-AP-41 & $1 / 2968$ & 67 & CASS & $T$ & \\
\hline TE-104AP-41 & $2 / 1 / 88$ & 66 & CASS & $T$ & \\
\hline TE-104AP-41 & $2 / 11 / 38$ & 66 & CASS & $T$ & \\
\hline TE-104-AP-41 & $2 / 14 / 88$ & 66 & CASS & $T$ & \\
\hline TE-104-AP-41 & 2/24/38 & 65 & CASS & $T$ & \\
\hline TE-104AP-A1 & 3/5/88 & 68 & CASS & $T$ & \\
\hline$T E-104-A P-41$ & 38/88 & 65 & CASS & $T$ & \\
\hline TE-104-AP-41 & 3/19/88 & 64 & CASS & $T$ & \\
\hline TE-104-AP-41 & $3 / 20 / 88$ & 65 & CASS & $\mathrm{T}$ & \\
\hline TE-104AP-41 & $3 / 29 / 88$ & 65 & CASS & $T$ & \\
\hline TE-104-AP-41 & 6/3/88 & 66 & CASS & $T$ & \\
\hline TE-104-AP-41 & 6/6/88 & 66 & CASS & $T$ & \\
\hline TE-104-AP-41 & 6/13/68 & 64 & CASS & $T$ & \\
\hline TE-104-AP-41 & 6/25/88 & 65 & CASS & $\bar{T}$ & \\
\hline TE-104-AP-41 & 6/29/88 & 66 & CASS & $T$ & \\
\hline TE-104-AP-41 & $7 \pi / 88$ & 67 & CASS & $T$ & \\
\hline
\end{tabular}




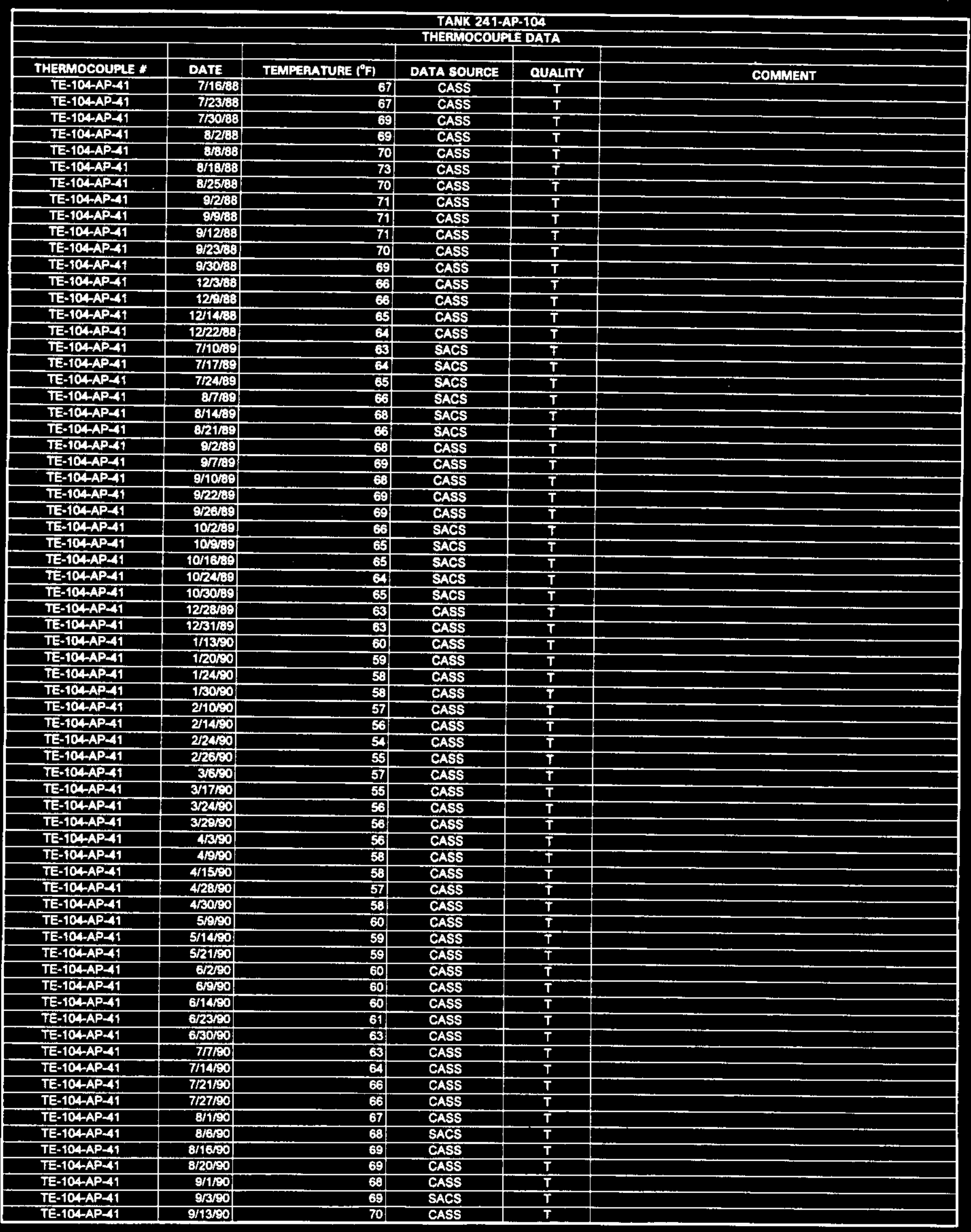

Data obteined from Computer Autometed Surveillance System (CASS), April 1995; and Surveillance Analysis Computer System (SACS), June 1995.

D-352 


\begin{tabular}{|c|c|c|c|c|c|}
\hline \multicolumn{6}{|c|}{$\begin{array}{l}\text { TANK 241-AP-104 } \\
\text { THERMOCOUPLE DATA }\end{array}$} \\
\hline & & & & & \\
\hline THERMOCOUPLE * & DATE & TEMPERATURE $\left({ }^{\circ} \mathrm{F}\right)$ & DATA SOURCE & GUATY & COMMENT \\
\hline TE-104-AP-41 & $9 / 16 / s 0$ & 70 & CASS & $T$ & \\
\hline TE-104AP-41 & $9 / 25190$ & 70 & CASS & $T$ & \\
\hline TE-104APC1 & $10 / 3 / 80$ & 69 & CASS & $T$ & \\
\hline TE-104-AP-A1 & $107 \%$ & 68 & CASS & $\mathbf{T}$ & \\
\hline TE-104-APL1 & 102050 & 66 & CASS & $T$ & \\
\hline TE-104AP-41 & $10 / 23 / 50$ & 86 & CASS & $T$ & \\
\hline TE-104-AP-41 & $11 / 2 / 90$ & 64 & CASS & $\mathbf{T}$ & \\
\hline TE-TO4-AP-41 & $11 / \pi \% 0$ & 64 & CASS & $\mathbf{T}$ & \\
\hline TE-10,A-AP-A1 & $11 / 14 / 80$ & 63 & CASS & $T$ & \\
\hline TE-104AP-A1 & $11 / 20 \% 0$ & 62. & CASS & $T$ & \\
\hline TE-104AP-41 & 11/25:00 & 62 & CASS & $T$ & \\
\hline TE-104AP-41 & 121500 & 61 & CASS & $T$ & \\
\hline TE-104-APA1 & 12NS00 & 80 & CASS & $T$ & \\
\hline TE-104AP-41 & $12 / 1950$ & 58 & CASS & $T$ & \\
\hline TE-104-AP-41 & 12 2650 & 55 & CASS & $\bar{T}$ & \\
\hline TE-104-AP-41 & $12 \times 0 \% 80$ & $\mathbf{5 1}$ & CASS & $T$ & \\
\hline TE-104AP-41 & 1901 & 52 & CASS & $T$ & \\
\hline TE-1D4-AP-41 & $1 / 19 / 91$ & 53 & CASS & $T$ & \\
\hline TE-104-AP-49 & $1 / 2001$ & 53 & CASS & $T$ & \\
\hline TE-104AP-A1 & $2 / 2 \times 1$ & 51 & CASS & $\bar{T}$ & \\
\hline TE-104-AP-41 & 2का & 52 & CASS & $T$ & \\
\hline TE-104AP-A1 & 21691 & 51 & CASS & $T$ & \\
\hline$T E-104 A P=41$ & $221 / 91$ & 53 & CASS & $T$ & \\
\hline TE-104-AP-41 & 2/2691 & $\mathbf{5 3}$ & CASS & $\mathbf{T}$ & \\
\hline$T E-104 \times A P-41$ & आnकt & 53 & CASS & $T$ & \\
\hline TE-104AP-41 & आ1691 & 54 & CASS & $T$ & \\
\hline TE-104AP-41 & $3 / 21 / 91$ & 54 & CASS & $T$ & \\
\hline TE-104-AP-A1 & 3ra91 & 54 & CASS & $T$ & \\
\hline TE-104-AP-41 & 4391 & 5 & CASS & $T$ & \\
\hline$T E-104 A P-41$ & 41001 & 54 & CASS & $T$ & \\
\hline TE-104-AP-A1 & 42091 & 54 & CASS & $T$ & \\
\hline TE-104-AP-41 & 42761 & 53 & CASS & $T$ & \\
\hline TE-104APA1 & skast & 55) & CASS & $T$ & \\
\hline TE-104AP-41 & Erast & 57 & CASS & $T$ & \\
\hline TE-104-AP=4 & 51761 & 53 & CASS & $T$ & \\
\hline TE-104AP-41 & $5 / 10 / 01$ & 58 & CASS & $T$ & \\
\hline TE-104-AP-41 & 5roos1 & 59 & CASS & $T$ & \\
\hline IE-104-AP-41 & 6801 & 58 & CASS & $T$ & \\
\hline$T E-104 A P-41$ & 6/1491 & 60 & CASS & 7 & \\
\hline TE-104-AP-41 & E/2001 & 61 & CASS & $T$ & \\
\hline TE-104-AP-41 & 6/2591 & 59 & CASS & $T$ & \\
\hline TE-104-AP-A1 & 7291 & 62 & CASS & $T$ & \\
\hline TE-104-AP-11 & $7 / 11 / 91$ & 63 & CASS & $T$ & \\
\hline TE-104AP-11 & $7 / 1591$ & 64 & SACS & $T$ & \\
\hline TE-104-AP-41 & $7 / 2291$ & 64 & SACS & $T$ & \\
\hline TE-104-AP-41 & 7/29/91 & 6 & SACS & $T$ & \\
\hline TE-104AP-41 & 8\%91 & 64 & CASS & $T$ & \\
\hline$T E-104-A P-41$ & $8 / 1791$ & 66 & CASS & $T$ & \\
\hline$T E-104-A P-41$ & $8 / 20191$ & 67 & CASS & $T$ & \\
\hline TE-104-AP-41 & $8 / 2969$ & 67 & CASS & $T$ & \\
\hline TE-104-AP-41 & $9 / 3191$ & 68 & CASS & $T$ & \\
\hline TE-104+AP-41 & $8 / 1 / 92$ & 62 & CASS & $\bar{T}$ & \\
\hline TE-104-AP-41 & $8 / 3 / 92$ & 64 & CASS & $T$ & \\
\hline TE-104-AP-41 & $8 / 1392$ & 64 & CASS & $T$ & \\
\hline$T B-104-A P-41$ & $8 / 2202$ & 63 & CASS & $T$ & \\
\hline TE-104AP-41 & $8 / 29192$ & 65 & CASS & $T$ & \\
\hline TE-104AP-41 & 9r502 & 64 & CASS & $T$ & \\
\hline TE-104-AP-AT & 962 & 66 & CASS & $T$ & \\
\hline TE-104-AP-41 & 9/1492 & 66 & CASS & $T$ & \\
\hline TE-104-AP-41 & 9/2692 & 66 & CASS & $T$ & \\
\hline TE-104-AP-41 & 9/2892 & 66 & CASS & $\bar{T}$ & \\
\hline TE-104AP 41 & $10 / 6 / 92$ & 66 & CASS & $T$ & \\
\hline$T E+104-A P-41$ & $10 / 1592$ & 65 & CASS & $T$ & \\
\hline TE-104AP-41 & $10 / 21 / 92$ & 65 & CASS & $T$ & \\
\hline$T E-104-A P-41$ & 102902 & 68 & CASS & $T$ & \\
\hline TE-104-AP-A1 & $11 / 492$ & 65 & CASS & $T$ & \\
\hline TE-104-AP-41 & $11 / 1392$ & 63 & CASS & $T$ & \\
\hline$T E-104-A P-41$ & $11 / 20 / 92$ & 63 & CASS & $T$ & \\
\hline TE-104AP-11 & $11 / 23 / 92$ & 61 & CASS & $T$ & \\
\hline TE-104-AP-41 & 11/29/92 & 62 & CASS & $T$ & \\
\hline
\end{tabular}




\begin{tabular}{|c|c|c|c|c|c|}
\hline \multicolumn{6}{|c|}{$\begin{array}{l}\text { TANK 241-AP-104 } \\
\text { THEPMOCOUPLE DATA }\end{array}$} \\
\hline & & & & & \\
\hline THERAMOCOUPLE * & DATE & TEMPERATURE $\left({ }^{\circ} \mathrm{F}\right)$ & DATA SOUACE & QUALITY & COMHENT \\
\hline$T E-104-A P-41$ & 126392 & 59 & CASS & $T$ & \\
\hline TE-104-AP-41 & $12 / 13 / 92$ & 58 & CASS & $T$ & \\
\hline TE-104-AP-41 & $12 / 2292$ & 56 & CASS & $T$ & \\
\hline TE-10A-AP-A1 & 123002 & 55 & CASS & $T$ & \\
\hline TE-104-AP-A1 & 1 19/93 & 53 & CASS & $\bar{T}$ & \\
\hline TE-104-AP-A1 & $1 / 1233$ & 51 & CASS & $T$ & \\
\hline TE-104-AP-41 & $1 / 235$ & so & CASS & $T$ & \\
\hline TE-104-AP-41 & 1/29/93 & 52 & CASS & $T$ & \\
\hline TE-104-AP-41 & 2659 & 52 & CASS & $\mathbf{T}$ & \\
\hline TE-104-AP-41 & 2993 & 52 & CASS & $\mathbf{T}$ & \\
\hline TE-104-AP-41 & $2 / 8 / 93$ & 51 & CASS & $\bar{T}$ & \\
\hline TE-TOMAP-41 & $2 / 23 \times 93$ & 50 & CASS & $T$ & \\
\hline$T E-104-A P-41$ & अ/293 & 49 & CASS & $T$ & \\
\hline TE-104-AP-41 & 310/3 & 50 & CASS & $T$ & \\
\hline TE-104-AP-41 & 3/1293 & 50 & CASS & $T$ & \\
\hline TE-104-APA1 & $3 / 27 \% 3$ & 51 & CASS & $T$ & \\
\hline TE-TO4AP-A1 & $3 / 31 / 83$ & 52 & CASS & $\bar{T}$ & \\
\hline TE-104-APA1 & $40 \% 3$ & 52 & CASS & $T$ & \\
\hline TE-104AP-41 & 417133 & 53 & CASS & $\bar{T}$ & \\
\hline TE-104AP-41 & 41203 & 88 & CASS & 8 & Oata point did not agree with local trend \\
\hline TE-104-AP-A1 & $4 / 23193$ & 5 & CASS & $T$ & \\
\hline TE-104-AP-A1 & 420103 & 54 & CASS & $\mathbf{T}$ & \\
\hline TE-104-AP-41 & $5 \mathrm{ags}$ & 56: & CASS & $T$ & \\
\hline TE-104-AP-41 & S15:3 & 55 & CASS & $T$ & \\
\hline TE-104APAT & 572203 & $\mathbf{3 3}$ & CASS & $\bar{T}$ & \\
\hline TE-104-AP-4 & $5 / 29 / 33$ & 58 & CASS & $T$ & \\
\hline TE-104-AP-A1 & 6ra93 & 59 & CASS & $\bar{T}$ & \\
\hline TE-104AP-41 & $6 / 12 \times 3$ & 59 & CASS & $T$ & \\
\hline TE-104-AP-41 & 6/903 & 60 & CASS & $T$ & \\
\hline$T E-104-A P-41$ & 6r24/03 & 62 & CASS & $T$ & \\
\hline TB-104AP-41 & $7 / 363$ & 61 & CASS & $T$ & \\
\hline TE-104-AP-41 & $7 / 10 \% 3$ & 62 & CASS & $T$ & \\
\hline TE-T04AP-A1 & $7 / 1293$ & 6 & CAss & $T$ & \\
\hline TE-104-AP-41 & $7 / 21 / 93$ & 63 & CASS & $T$ & \\
\hline TE-104-AP-41 & $7 / 3003$ & 64 & CASS & $T$ & \\
\hline TE-104-AP-41 & $8 \pi / 93$ & 65 & CASS & $T$ & \\
\hline TE-104-AP-41 & $8 / 14 / 93$ & 65 & CASS & $T$ & \\
\hline TE-104-AP-41 & 8/20153 & 63) & CASS & $T$ & \\
\hline TE-104-AP-A1 & $8 / 25 \times 3$ & 67 & CASS & $T$ & \\
\hline TE-104-AP-41 & $8 / 29 / 83$ & 67 & CASS & $T$ & \\
\hline TE-104AP-41 & 9093 & 67 & CASS & $T$ & \\
\hline$T E-104-A P-41$ & $913 \times 3$ & 67 & CASS & $T$ & \\
\hline TE-104-AP-41 & $8 / 2403$ & 66 & CASS & $T$ & \\
\hline$T E-104-A P-41$ & $10 / 1 / 83$ & 68 & CASS & $T$ & \\
\hline TE-104-AP-41 & $10 / 763$ & 67 & CASS & $T$ & 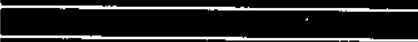 \\
\hline TE-104-AP-41 & 10/1193 & 66 & CASS & $T$ & \\
\hline TE-104-AP-41 & $10 / 22193$ & 66 & CASS & $T$ & \\
\hline TE-104-AP-41 & 10/27/93: & 66 & CASS & $T$ & \\
\hline TE-104AP-41 & $11 / 3193$ & 64 & CASS & $T$ & \\
\hline TE-104-AP-41 & $11 / 8 / 93$ & 63 & CASS & $T$ & \\
\hline TE-104-AP-41 & $11 / 14 / 93$ & 62 & CASS & $T$ & \\
\hline TE-104-AP-41 & $11 / 22 / 93$ & 60 & CASS & $T$ & \\
\hline TE-104-AP-41 & 121193 & 53 & CASS & $T$ & \\
\hline TE-104-AP-A1 & $1 / 1364$ & 53 & CASS & $T$ & \\
\hline TE-104-AP-44 & 514194 & 58 & CASS & $T$ & \\
\hline$T E-104-A P-41$ & $5 / 21 \% 4$ & 58! & CASS & $T$ & \\
\hline TE-104-AP-41 & $5 / 2494$ & 59 & CASS & $T$ & \\
\hline TE-104-AP-41 & 64a4 & 59 & CASS & $T$ & \\
\hline TE-104-AP-41 & 61164 & 60 & CASS & $T$ & \\
\hline TE-104-AP-41 & 6/18kn & 60 & CASS & $T$ & \\
\hline TE-104-AP-41 & 625194 & 61 & CASS & $T$ & \\
\hline$T E-104-A P-41$ & 6/30/94 & 63 & CASS & $T$ & \\
\hline TE-104-AP-41 & 7 Fis4 & 64 & CASS & $T$ & \\
\hline TE-104-AP-41 & $7 / 16194$ & 64 & CASS & $T$ & \\
\hline TE-104-AP-41 & $7 / 21 / 94$ & 66 & CASS & $\bar{T}$ & \\
\hline TE-104AP-A1 & $7 / 3009$ & 68) & CASS & $T$ & \\
\hline TE-104AP-A1 & BAS4 & 67 & CASS & $T$ & \\
\hline TE-104-AP-41 & $8 / 13 / 94$ & 69 & CASS & $T$ & \\
\hline TE-104-AP-A1 & 8/20194 & 68 & CASS & $T$ & \\
\hline TE-104AP-A1 & $8 / 22 / 94$ & 69 & CASS & $T$ & \\
\hline
\end{tabular}




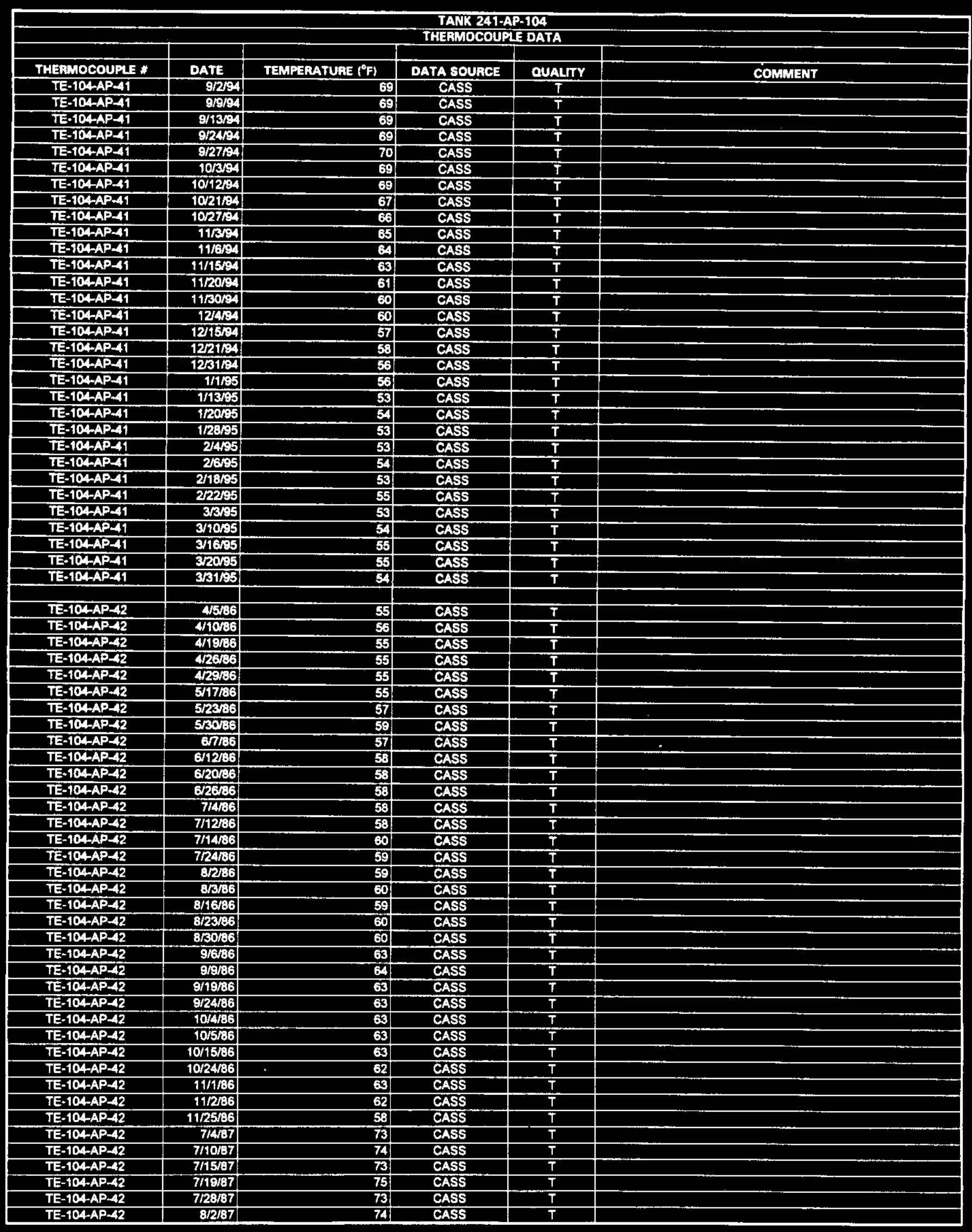




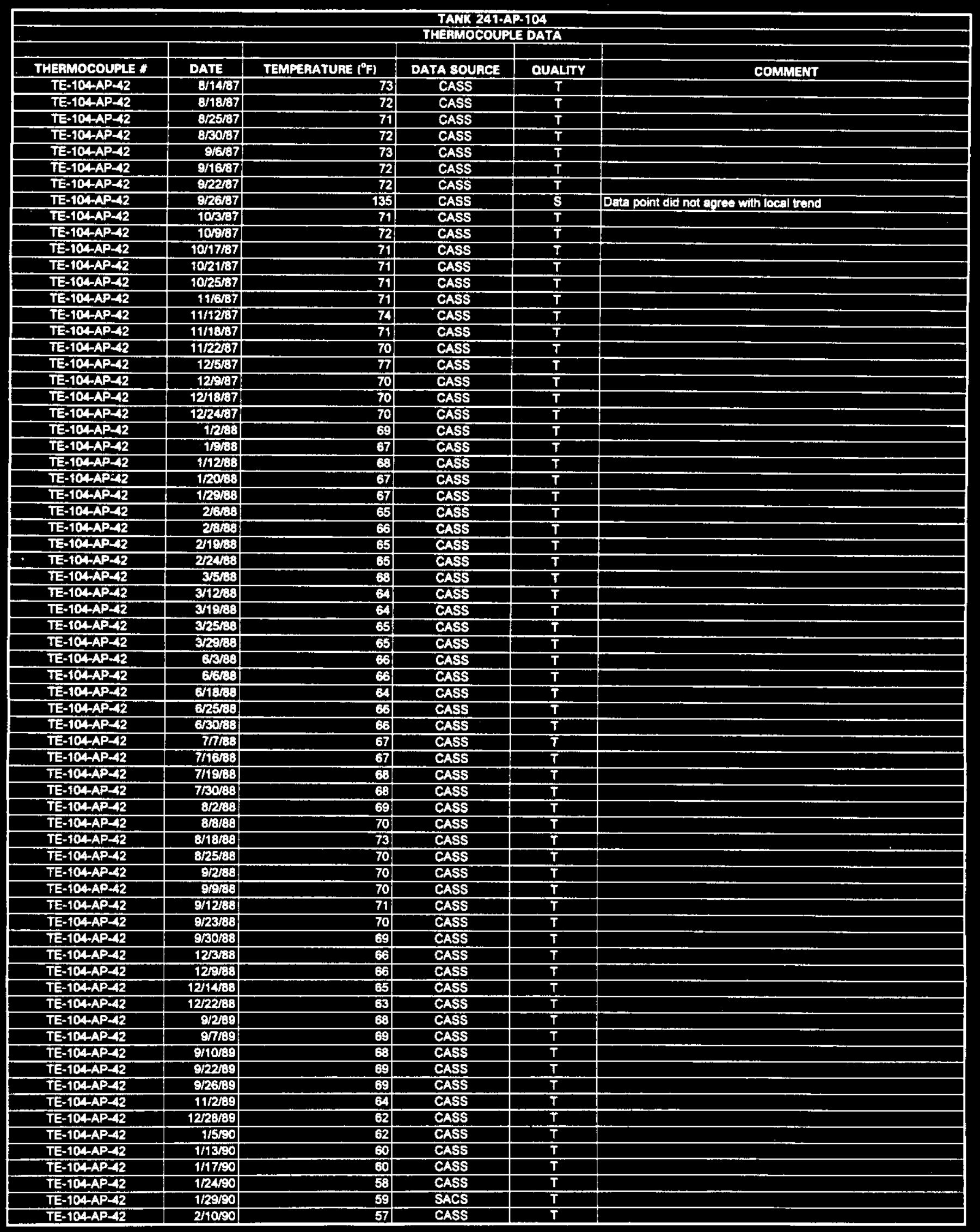




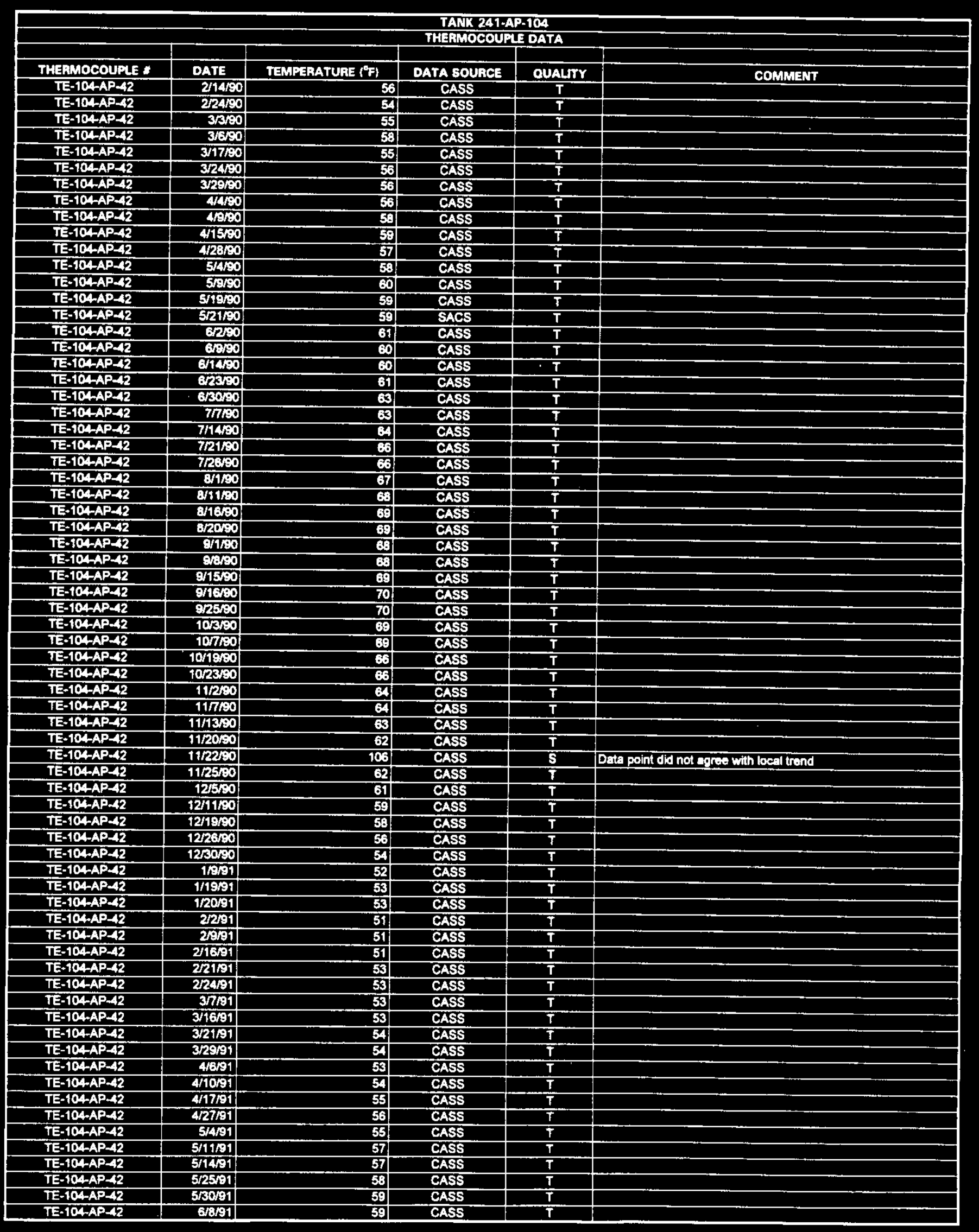

Data obtained from Computer Automated Surveiliance System \{CASS), April 1995; and Surveillance Analyais Computer System (SACS), June 1995, D-357 


\begin{tabular}{|c|c|c|c|c|c|}
\hline \multicolumn{6}{|c|}{$\begin{array}{l}\text { TACKK 241-AP-104 } \\
\text { THEGHOCOUPLE DATA }\end{array}$} \\
\hline & & & & & \\
\hline THERMOCOUPLE * & DATE & TEMPERATURE $\left({ }^{\circ} \mathrm{F}\right)$ & DATA SOURCE & QUALTY & COMMENT \\
\hline TE-104.AP $=-12$ & 6/14/91 & 60 & CASS & $T$ & \\
\hline TE-104-AP-A2 & 6/20/91 & 61 & CASS & $T$ & \\
\hline TE-104-AP-42 & 6/25391 & 59 & CASS & $T$ & \\
\hline TE-104AP-42 & $7 / 291$ & 62 & CASS & $T$ & \\
\hline TE-104AP-42 & $7 / 11 / 91$ & 63 & CASS & $\mathbf{T}$ & \\
\hline TE-104AP-A2 & $7 / 14 / 81$ & 62 & CASS & $T$ & \\
\hline TE-104-AP-A2 & $7 / 2461$ & 63 & CASS & $\bar{T}$ & \\
\hline TE-104-AP-12 & $8 / 3191$ & G & CASS & $T$ & \\
\hline TE-104-AP-12 & $8 / 6 / 91$ & G4 & CASS & $T$ & \\
\hline TE-104-AP-A2 & $8 / 37 / 31$ & 66 & CASS & $\mathbf{T}$ & \\
\hline TE-104AP-12 & 8/20/91 & 68 & CASS & $\mathbf{T}$ & \\
\hline TE-104AP-A2 & 8/3151 & 67? & CASS & $T$ & \\
\hline TE-104AP-42 & $8 / 3 / 91$ & 6 & CASS & $T$ & \\
\hline$T E-104-A P-42$ & $7 / 31 / 62$ & 63 & CASS & $T$ & \\
\hline TE-TOA-AP-42 & $8 / 3192$ & 64 & CASS & $\mathbf{T}$ & \\
\hline TE-104-AP-12 & $8 / 1392$ & 64 & CASS & $\bar{T}$ & \\
\hline TE-104AP-A2 & $8 / 18,92$ & 64 & CASS & $\bar{T}$ & \\
\hline TE-104AP-12 & $8 / 29 / 92$ & 65 & CASS & $T$ & \\
\hline TE-104AP-A2 & 8/562 & EA & CASS & $T$ & \\
\hline$T E-104 A P-42$ & 9/1102 & 66 & CASS & $T$ & \\
\hline TE-104AP-42 & g1402 & 66 & CASS & $\bar{T}$ & \\
\hline TE-104AP-A2 & 9/2612 & 67 & CASS & $T$ & \\
\hline TE-104AP-A2 & 9/28/92 & 65 & CASS & $\mathbf{T}$ & \\
\hline TE-104.AP-A2 & 106/2 & 66 & CASS & $\mathbf{T}$ & \\
\hline$T E=104-A P-42$ & $10 / 17 / 82$ & EA & CASS & $\mathbf{T}$ & \\
\hline TE-104-AP-42 & $10 / 21 / 92$ & 65 & CASS & $\mathbf{T}$ & \\
\hline TE-104-AP-12 & 10/2902 & 65 & CASS & $T$ & \\
\hline$T E-104-A P-A 2$ & $11 / 492$ & 65 & CASS & $\mathbf{T}$ & \\
\hline TE-104-AP-A2 & $11 / 13 / 92$ & 63 & CASS & $\mathbf{T}$ & \\
\hline TE-104-AP-22 & $11 / 20092$ & 63 & CASS & $\mathbf{T}$ & \\
\hline TE-104-AP-A2 & $11 / 23402$ & 61 & SACS & $T$ & \\
\hline TE-104AP-42 & $11 / 2602$ & 84 & CASS & $\mathbf{s}$ & Data point did not eqree with local trend \\
\hline TE-104AP-A2 & $11 / 29 \%$ & 62 & CASS & $T$ & \\
\hline TE-104AP-42 & $127 \% 2$ & Sa: & SAcs & $T$ & \\
\hline TE-104AP-12 & $1213 / 22$ & $\mathbf{S 8}$ & CAsS & $\mathbf{T}$ & \\
\hline TE-104AP-42 & $12 / 22 / 92$ & 56 & CASS & $\mathbf{T}$ & \\
\hline TE-104AP-A2 & $12 / 26 / 92$ & 55 & SACS & $T$ & \\
\hline TE-104AP-42 & $1 / 403$ & 53 & CASS & $T$ & \\
\hline TE-104-AP-42 & $1 / 11 / 3$ & 53 & SACS & $T$ & \\
\hline TE-104AP-A2 & $1 / 23 / 93$ & 50 & CASS & $T$ & - \\
\hline TE-104AP-A2 & $1 / 29 \% 3$ & 51) & CASS & $T$ & \\
\hline TE-104-AP-12 & $26 \sqrt{3}$ & 51 & CASS & $\mathbf{T}$ & \\
\hline TE-104AP-A2 & $2 / 129$ & 51 & CASS & $\mathbf{T}$ & \\
\hline TE-104AP-42 & $2 / 18 / 93$ & 51 & CASS & $\mathbf{T}$ & \\
\hline TE-104AP-A2 & $2 / 2693$ & 50 & CASS & $T$ & \\
\hline TE-104AP-42 & $3 / 653$ & 48 & CASS & $T$ & \\
\hline TE-104AP-12 & 3/13/93 & 4 & CASS & $\mathbf{T}$ & \\
\hline TE-104AP-A2 & $3 / 19 / 93$ & 50 & CASS & $T$ & \\
\hline TE-104-AP-A2 & $3 / 2723$ & 51 & CASS & $\bar{T}$ & \\
\hline TE-104AP-42 & $3 \sqrt{31 / 93}$ & 52 & CASS & $T$ & \\
\hline TE-104AP-42 & $4 / 693$ & 52 & CASS & $T$ & \\
\hline TE-104APA2 & $4 / 1793$ & 53 & CASS & $T$ & \\
\hline TE-104AP-A2 & 4/23/93 & 53 & CASS & $T$ & \\
\hline TE-104-AP-A2 & 4/29/93 & 54 & CASS & $T$ & \\
\hline TE-104AP-42 & $5 / 8 / 93$ & 56 & CASS & $T$ & \\
\hline TE-104-AP-4Z & 5/15/93 & 55 & CASS & $T$ & \\
\hline TE-104AP-42 & 5/17/93. & 58 & SACS & $T$ & \\
\hline TE-104-AP-42 & $572 \% 93$ & 57 & CASS & $T$ & \\
\hline TE-104AP-42 & 6/3/93 & 59 & CASS & $\mathbf{T}$ & \\
\hline TE-104AP-42 & 6/12/93 & 59. & CASS & $T$ & \\
\hline TE-104-AP-12 & 6/16/93 & 61 & CASS & $\mathbf{T}$ & \\
\hline TE-104AP-42 & $6 / 24 / 93$ & 61 & CASS & $\mathbf{T}$ & \\
\hline TE-104-AP-42 & 6/29/93 & 62 & SACS & $\mathbf{T}$ & \\
\hline TE-104-AP-42 & 7/10/93 & 62 & CASS & $\mathbf{T}$ & \\
\hline TE-104AP-A2 & $7 / 16 / 93$ & 62 & CASS & $\mathbf{T}$ & \\
\hline TE-104AP-42 & $7 / 21 / 93$ & 63 & CASS & $T$ & \\
\hline TE-104-AP-42 & $7 / 30 / 93$ & 64 & CASS & $T$ & \\
\hline TE-104AP-42 & $8 / 793$ & 66 & CASS & $\mathbf{T}$ & \\
\hline$T E-104-A P-12$ & $89 / 93$ & 66 & SACS & $T$ & \\
\hline TE-104AP-A2 & $8 / 20 \% 3$ & 66 & CASS & $\bar{T}$ & \\
\hline
\end{tabular}




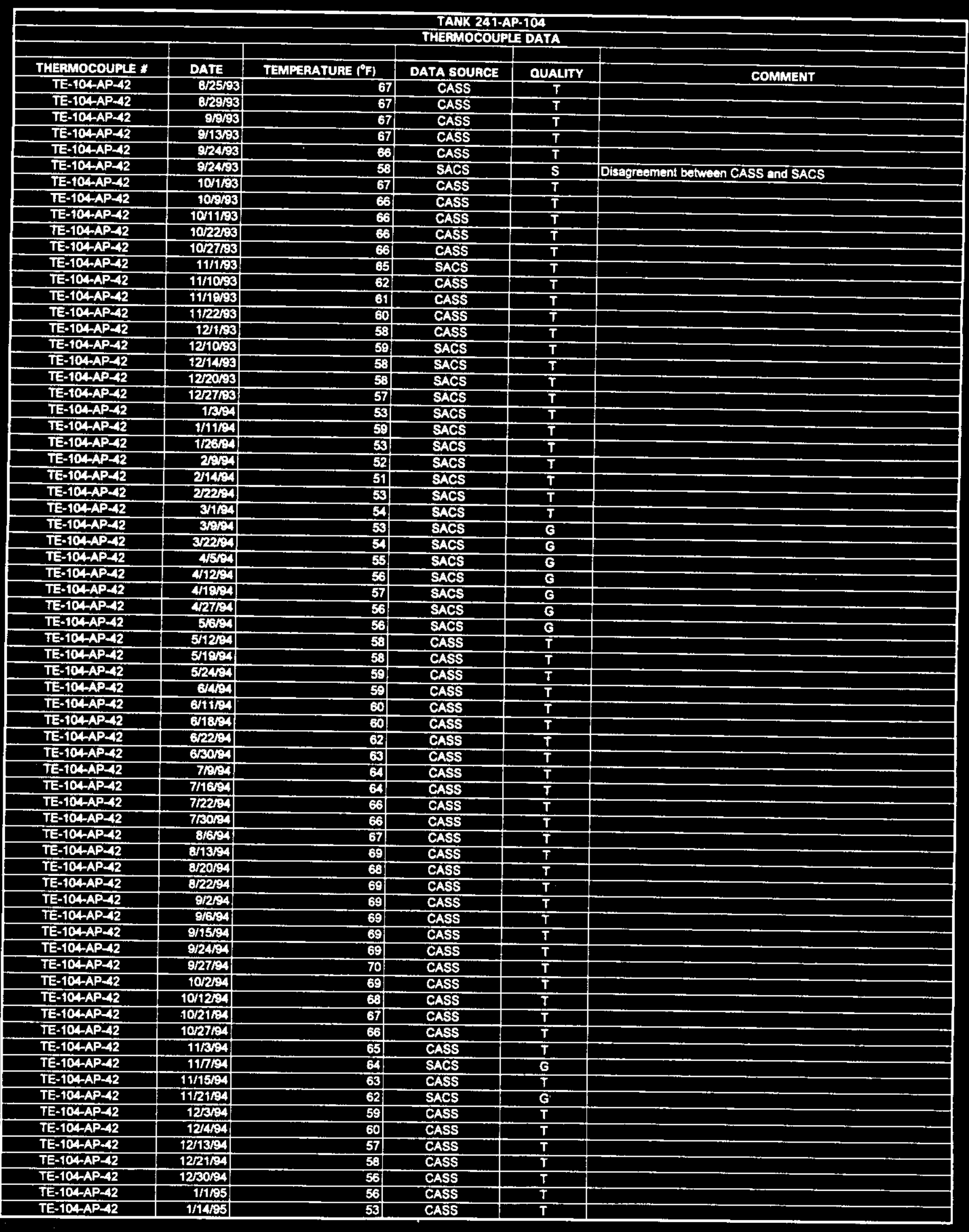

Data obtained from Computer Automated Surveillance System (CASS), April 1995; and Surveillance Analyais Computer System (SACS), June 1995. 


\begin{tabular}{|c|c|c|c|c|c|}
\hline \multicolumn{6}{|c|}{$\begin{array}{l}\text { TAWK 241-AP-104 } \\
\text { THERMOCOUPLE DATA }\end{array}$} \\
\hline & & & & & \\
\hline THERMOCOUPLE & DATE & TEMPERATURE $\left({ }^{\circ} \mathrm{F}\right)$ & DATA SOUACE & OUALITY & COMMENT \\
\hline$T E-104-A P-42$ & $1 / 1765$ & 54 & CASS & $T$ & \\
\hline$T E-104-A P-42$ & $1 / 25 / 95$ & 53 & CASS & $T$ & \\
\hline TE-104AP-42 & $2 / 4 / 95$ & 53 & CASS & $\bar{T}$ & \\
\hline$T E-104 A P-42$ & 21695 & 54 & CASS & $T$ & \\
\hline TE-104-AP-42 & 2/1395 & 54 & SACS & $\bar{G}$ & \\
\hline TE-104-AP-42 & 212205 & 55. & CASS & $T$ & \\
\hline TE-104-AP-42 & 3/3/95 & 53 & CASS & $T$ & \\
\hline TE-10-AP- 42 & 3/1005 & 54 & CASS & $T$ & \\
\hline TE-104-AP-42 & 3/16/95 & 54 & CASS & $T$ & \\
\hline TE-104-AP-42 & $3 / 25 r 95$ & 54 & CASS & $T$ & \\
\hline TE-104AP-A2 & $3 / 29 / 95$ & 54 & CASS & $T$ & \\
\hline TE-104AP-A2 & $4 / 3 / 95$ & 53 & SACS & G & \\
\hline TE-104AP-A2 & $4 / 10 / 95$ & 53 & SACS & G & \\
\hline TE-104AP-A2 & $417 / 5$ & 54 & SACS & $G$ & \\
\hline TE-104-AP-A2 & 42495 & 54 & SACS & G & \\
\hline TE-104-AP-A2 & S1105 & 55 & SACS & G & \\
\hline TE-TQA-AP- 12 & Sors & 5 & SACS & $\bar{G}$ & \\
\hline TE-104AP C2 & 516.5 & 57 & SACS & G & \\
\hline$\pi E-104-A P-\sqrt{2}$ & 5/22195 & 59 & SACS & 6 & \\
\hline$T E-104-A P-42$ & 5/2903 & 60 & SACS & G & \\
\hline TE-104AP-A2 & 65B53 & 60 & SACS & G & \\
\hline TE-104-AP-42 & E/355 & 60 & SACS & G & \\
\hline TE-104AP-A2 & 61985: & 60 & SACS & $\bar{G}$ & \\
\hline TE-104AP-43 & 4586 & & & & \\
\hline$\frac{T E-104-A P-3}{T E-104-A P-43}$ & $4 / 0 / 86$ & $\frac{55}{56}$ & $\frac{\text { CASS }}{\text { CASS }}$ & $T$ & \\
\hline TE-TDA-AP- 13 & $419 / 86$ & 55 & $\frac{\text { CASS }}{\text { CASS }}$ & $T$ & \\
\hline TE-1OA-AP- 43 & 42686 & 55 & $\frac{\text { CASS }}{\text { CASS }}$ & $\frac{T}{T}$ & \\
\hline TE-104AP-A3 & 4/2966 & 5 & CASS & $\frac{T}{T}$ & \\
\hline TE-104-APA-A3 & $5 / 36 / 6$ & E6 & CASS & $T$ & \\
\hline TE-104-AP-13 & $5 / 23 / 66$ & 56 & CASS & $T$ & \\
\hline TE-TOA-AP- 13 & $5 / 30 / 86$ & 59 & CASS & $\bar{T}$ & \\
\hline TE-104-AP-13 & 8776 & E6 & CASS & $T$ & \\
\hline TE-104AP-43 & $6 / 1236$ & $\mathbf{5 3}$ & CASS & $\bar{T}$ & \\
\hline TE-10-AP-43 & $6 / 15 / 36$ & 58 & CASS & $T$ & \\
\hline TE-104-AP-43 & 6/26/36 & 58 & CASS & $\bar{T}$ & \\
\hline TE-104-AP-43 & $7 / 4 / B 6$ & 58 & CASS & $T$ & \\
\hline TE-104AP-43 & $7 / 12 / 86$ & 58 & CASS & $T$ & \\
\hline TE-104AP-43 & 711986 & 60 & CASS & $T$ & \\
\hline TE-104AP-A3 & 712366 & 59 & CASS & $T$ & \\
\hline TE-104AP-A3 & $8 / 2 / 36$ & 59 & CASS & $\bar{T}$ & \\
\hline TE-104-AP-43 & $8 / 3 / 56$ & 60 & CASS & $T$ & \\
\hline TE-104-AP-43 & $8 / 16 / 66$ & 59 & CASS & $T$ & \\
\hline TE-104-AP-43 & $8 / 22166$ & 60 & CASS & $\mathbf{T}$ & \\
\hline TE-104AP- 43 & $8 / 30 / 66$ & 60 & CASS & $\mathbf{T}$ & \\
\hline$T E-104 A P-43$ & 8/686 & 62 & CASS & $T$ & \\
\hline$T E-104-A P-43$ & 9/12/36 & 63 & CASS & $T$ & \\
\hline$T E-104-A P-43$ & $9 / 18 / 86$ & 63 & CASS & $T$ & \\
\hline TE-104AP-13 & $9 / 24 / 36$ & 63 & CASS & $T$ & \\
\hline TE-104AP-43 & 10/3/86 & 64 & CASS & $T$ & \\
\hline TE-104-APAA3 & 10/9/36 & 63 & CASS & $T$ & \\
\hline TE-104AP-A3 & 10/15/86 & 63 & CASS & $T$ & \\
\hline TE-10A-AP-A3 & $10 / 24 / 86$ & 62 & CASS & $T$ & \\
\hline$T E-104-A P-43$ & $11 / 1 / 86$ & 64 & CASS & $T$ & \\
\hline TE-104AP-43 & 19/2/66 & 62 & CASS & $T$ & \\
\hline TE-104-AP-43 & $11 / 25 / 86$ & 59 & CASS & $T$ & \\
\hline TE-104AP-43 & $\begin{array}{l}7 / 87 \\
\end{array}$ & 73 & CASS & $T$ & \\
\hline TE-104AP-13 & $7710 / 87$ & 74 & CASS & $T$ & \\
\hline TE-104AP-43 & $7 / 18 / 87$ & 73 & CASS & $T$ & \\
\hline TE-104-AP-43 & 7/19/87! & 75 & CASS & $\mathbf{T}$ & \\
\hline TE-104-AP-43 & $7 / 28 / 87$ & 73 & CASS & $T$ & \\
\hline TE-104AP-43 & $8 / 6 / 87$ & 73 & CASS & $T$ & \\
\hline TE-104-AP-43 & $8 / 14 / 87$ & 73 & CASS & $T$ & \\
\hline TE-104AP-43 & $8 / 18 / 87$ & 72 & CASS & $T$ & \\
\hline TE-104-AP-43 & $8 / 28 / 87$ & 71 & CASS & $T$ & \\
\hline TE-104AP-43 & $8 / 30 / 87$ & 72 & CASS & $T$ & \\
\hline$T E=104 A P-43$ & $9 / 12 / 87$ & 72 & CASS & $T$ & \\
\hline TE-104AP-13 & 9/19/87 & 71 & CASS & $T$ & \\
\hline TE-104APA43 & $9 / 22 / 87$ & 72 & CASS & $T$ & \\
\hline TE-104-AP-43 & 10/3/67 & 711 & CASS & $\bar{T}$ & \\
\hline
\end{tabular}




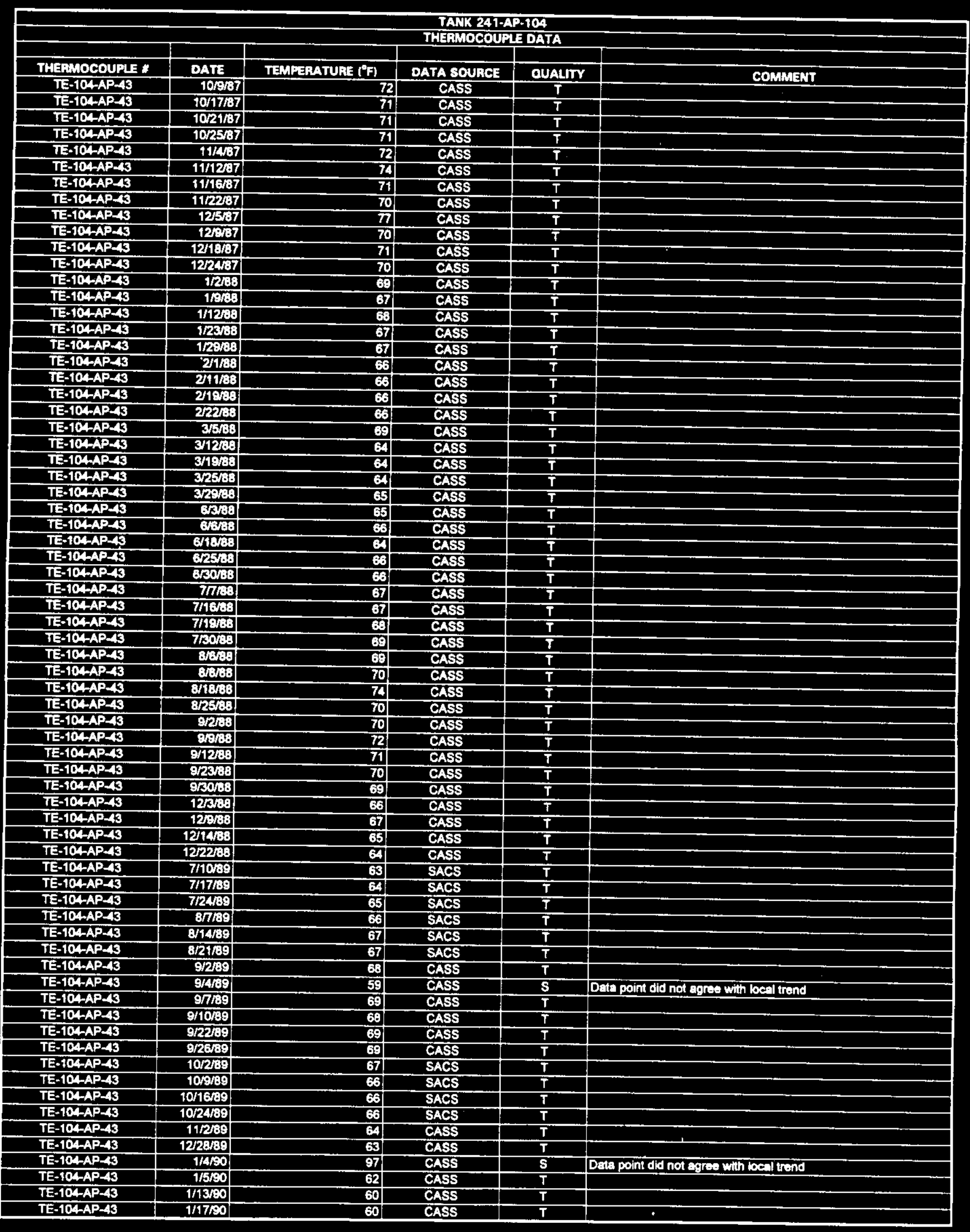




\begin{tabular}{|c|c|c|c|c|c|}
\hline \multicolumn{6}{|c|}{$\begin{array}{l}\text { TANK 241-AP. } 104 \\
\text { THEFMOCOUPLE DATA }\end{array}$} \\
\hline TYERMocoupi = & MATE & & & & \\
\hline TE-104-ADC & & 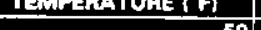 & DATA SOURCE & QUALITY & COMMENT \\
\hline TE-104-AP- 3 & 21/25rou & $\frac{99}{57}$ & CASS & $T$ & \\
\hline IE-104AP-43 & $2 / 10 / 50$ & $\frac{37}{57}$ & CAss & $T$ & \\
\hline TE-104-AP-43 & $2 / 4 / 90$ & 56 & $\frac{\text { CASS }}{\text { CASS }}$ & $T$ & \\
\hline TE-104-AP-43 & $2 / 2480$ & 54 & $\frac{\text { CASS }}{\text { CASS }}$ & $T$ & \\
\hline TE-104AP-A3 & 3390 & 55 & $\frac{\text { CASS }}{\text { CASS }}$ & $\frac{T}{T}$ & \\
\hline TE-104-AP-43 & 3/690 & 58 & $\frac{\text { CASS }}{\text { CASS }}$ & $\frac{T}{T}$ & \\
\hline TE-104-AP-43 & $3 / 7 / 90$ & 55 & CASS & $T$ & \\
\hline TE-104-AP-A3 & 3rars: & 56 & CASS & $T$ & \\
\hline TE-104-AP-43 & $3 / 2850$ & 56 & CASS & $T$ & \\
\hline TE-104-AP-43 & $44 \times 0$ & 56 & CASS & $T$ & \\
\hline$T E-104-A P=43$ & 4aso & 57 & CASS & $T$ & \\
\hline$T E-104-A P-43$ & $415: 0$ & 58 & CASS & $T$ & \\
\hline$T E-104-A P-43$ & $4 / 27 / 90$ & S8 & CASS & $T$ & \\
\hline TE-104-APCA3 & 4k01:0 & 59 & CASS & $\mathbf{T}$ & \\
\hline TE-104AP-43 & 51220 & 59 & CASS & $T$ & \\
\hline TE-104-AP-A3 & $5 / 14 / 00$ & 59 & CASS & $T$ & \\
\hline$T E-104-A P=43$ & 521100 & 59 & CASS & $T$ & \\
\hline TE-104-AP-A3 & G/1so & 61 & CASS & $T$ & \\
\hline TE-104-AP-A3 & 6900 & 80 & CASS & $T$ & \\
\hline TE-104-AP-43 & 614:00 & 60 & CASS & $T$ & \\
\hline$T E-104-A P=43$ & erzaso: & 61 & CASS & $\mathbf{T}$ & \\
\hline TE-104AP-43 & 6roxso & 63 & CASS & $T$ & \\
\hline$T E-104 A P=43$ & $7 \pi / 50$ & 63 & CASS & $T$ & \\
\hline$T E-104-A P-43$ & $7 / 14: 0$ & 64 & CASS & $T$ & \\
\hline TE-104-AP-43 & $7 / 2+100$ & 65 & CASS & $T$ & \\
\hline TE-104AP-43 & 7/265:0 & 66 & CASS & $T$ & \\
\hline TE-TQ4-AP-43 & 6/160 & 67 & CASS & $T$ & \\
\hline TE-104-AP-43 & Broso & 68 & CASS & $T$ & \\
\hline TE-TO4-AP-43 & 8/1600 & 68 & CASS & $T$ & \\
\hline$T E-104-A P-43$ & $8 / 20100$ & 69 & CASS & $T$ & \\
\hline TE-104-AP- 43 & $8 \sqrt{2760}$ & 69 & CASS & $T$ & \\
\hline$\overline{T E}-104-A P-43$ & Eres & 63 & CASS & $T$ & \\
\hline TE-104-AP-43 & 9T5E0 & 69 & CASS & $T$ & \\
\hline TE-104-AP-43 & $9 / 16 \times 0$ & 70 & CASS & $T$ & \\
\hline TE-104-AP-A3 & grzasio & 70 & CASS & $T$ & \\
\hline TE-104AP-43 & 10axes & 69 & CASS & $T$ & \\
\hline TE-TO4-AP-43 & $10 \pi k 0$ & 69 & CASS & $T$ & \\
\hline TE-104-AP-43 & $10 / 1890$ & 66 & CASS & $T$ & \\
\hline TE-104-AP-43 & 1023500 & 66 & CASS & $T$ & \\
\hline TE-104-AP-43 & $11 / 350$ & 64 & CASS & $T$ & \\
\hline TE-104AP-43 & $11 / 7 / 90$ & 64) & CASS & $T$ & \\
\hline TE-104-AP-43 & $11 / 1490$ & 63 & CASS & $T$ & \\
\hline TE-104-AP-43 & $11 / 2000$ & 62 & CASS & $T$ & \\
\hline TE-104-AP-43 & $11 / 2500$ & 62 & CASS & $T$ & \\
\hline TE-104-AP-13 & $12 / 290$ & 62 & CASS & $T$ & \\
\hline TE-10M-AP-43 & $12 \operatorname{sareO}$ & 60 & CASS & $\mathbf{T}$ & \\
\hline TE-104-AP-43 & $12 / 19800$ & 58 & CASS & $\bar{T}$ & \\
\hline TE-104-AP-A3 & $12 / 2490$ & 56 & SACS & $T$ & \\
\hline$T E+104-A P-43$ & 123090 & 55 & CASS & $T$ & \\
\hline TE-104-AP-43 & $1 / 991$ & 52 & CASS & $T$ & \\
\hline TE-104-AP-43 & $1 / 17 / 91$ & 54 & CASS & $T$ & \\
\hline TE-104-AP-43 & $1 / 2641$ & 53 & CASS & $T$ & \\
\hline TE-104AP-43 & $2 / 2 / 91$ & 51. & CASS & $T$ & \\
\hline $\mathrm{TE}-104-A P-43$ & 2501 & 51 & CASS & $T$ & \\
\hline TE-104-AP-43 & 21301 & 52 & CASS & $T$ & \\
\hline TE-104-AP-43 & $2 / 21 / 91$ & 53 & CASS & $T$ & \\
\hline TE-104-AP-43 & $2 / 2461$ & $\mathbf{5 3}$ & CASS & $T$ & \\
\hline TE-104AP-43 & 37\%1 & 53 & CASS & $\bar{T}$ & \\
\hline TE-104-AP-43 & 3/1691 & 5 & CASS & $T$ & \\
\hline$T E-104-A P-43$ & $3 / 20 / 91$ & 55 & CASS & $T$ & \\
\hline TE-104-AP-A3 & 3/29/91 & 54 & CASS & $T$ & \\
\hline TE-104-AP-43 & $4 / 601$ & 53 & CASS & $T$ & \\
\hline TE-104AP-43 & 4/1091 & 54. & CASS & $T$ & \\
\hline TE-104AP-43 & $4 / 17 / 91$ & 55 & CASS & $T$ & \\
\hline TE-104-AP-43 & $4 / 27 / 91$ & 57 & CASS & $T$ & \\
\hline TE-104-AP-43 & S/4/O1 & 55 & CASS & $T$ & \\
\hline TE-104-AP-43 & $5 / 11 / 91$ & 57 & CASS & $T$ & \\
\hline TE-104-AP-A3 & $5 / 1491$ & 57 & CASS & $T$ & \\
\hline TE-104-AP-43 & $5 / 2361$ & 59 & CASS & $T$ & \\
\hline
\end{tabular}




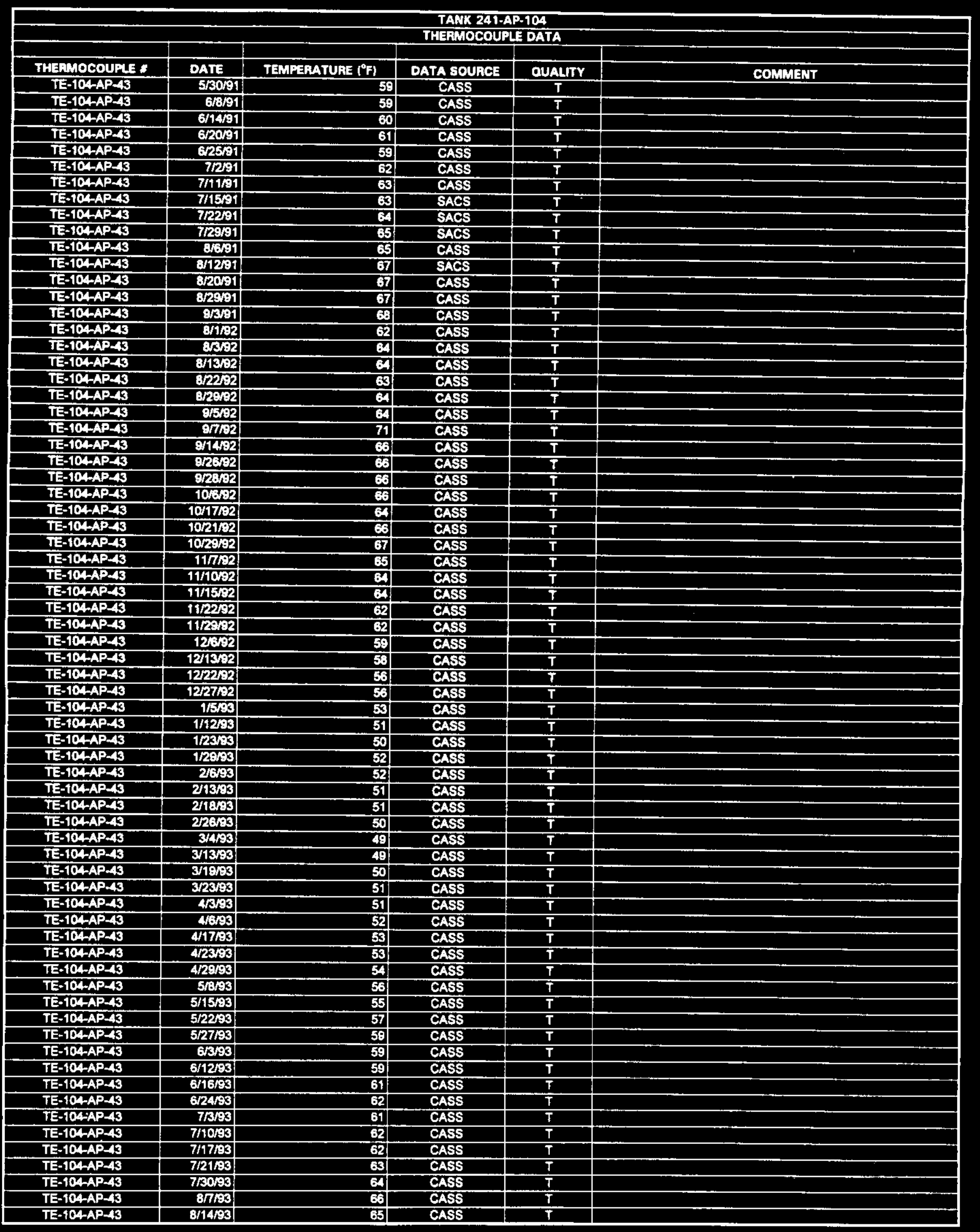




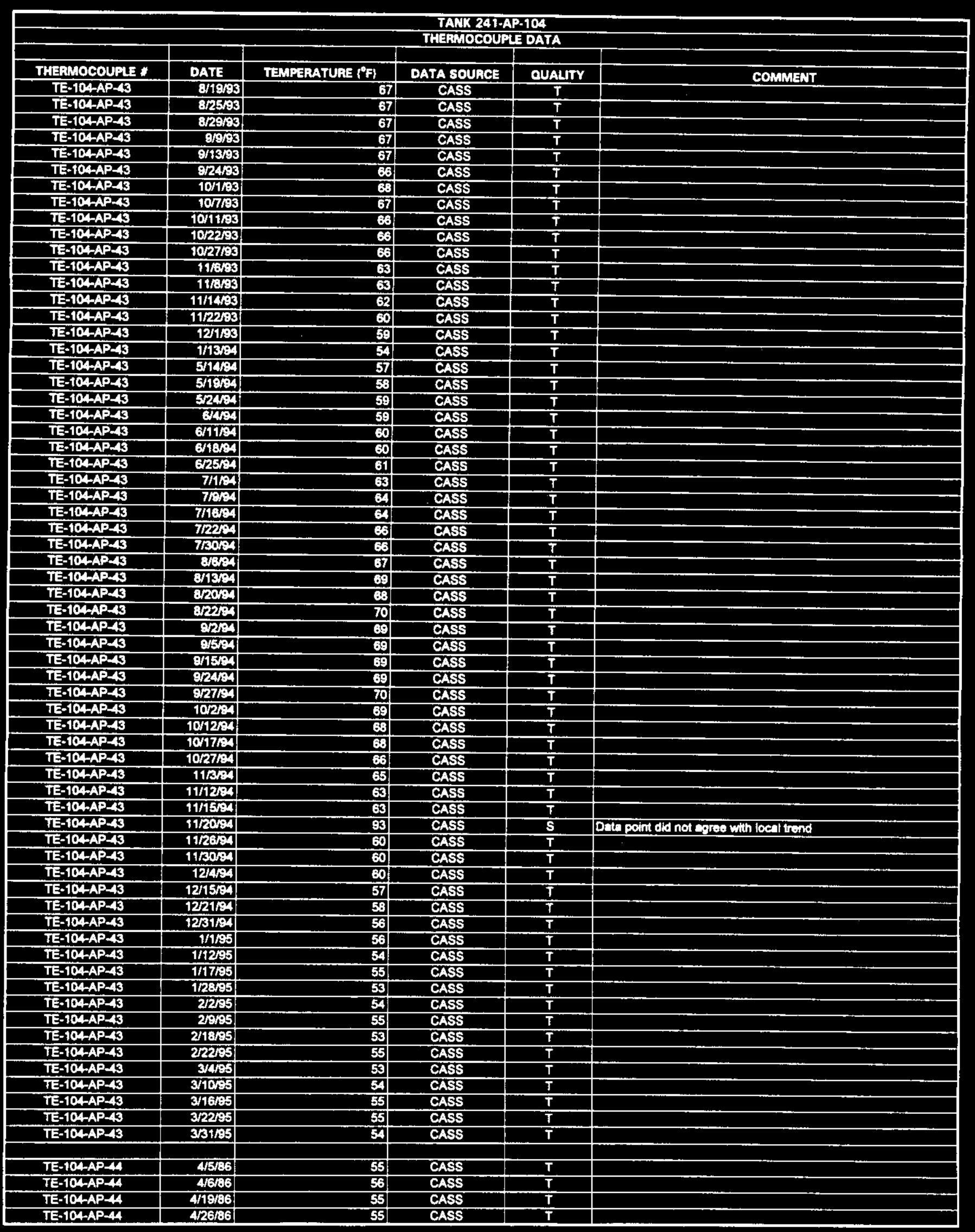




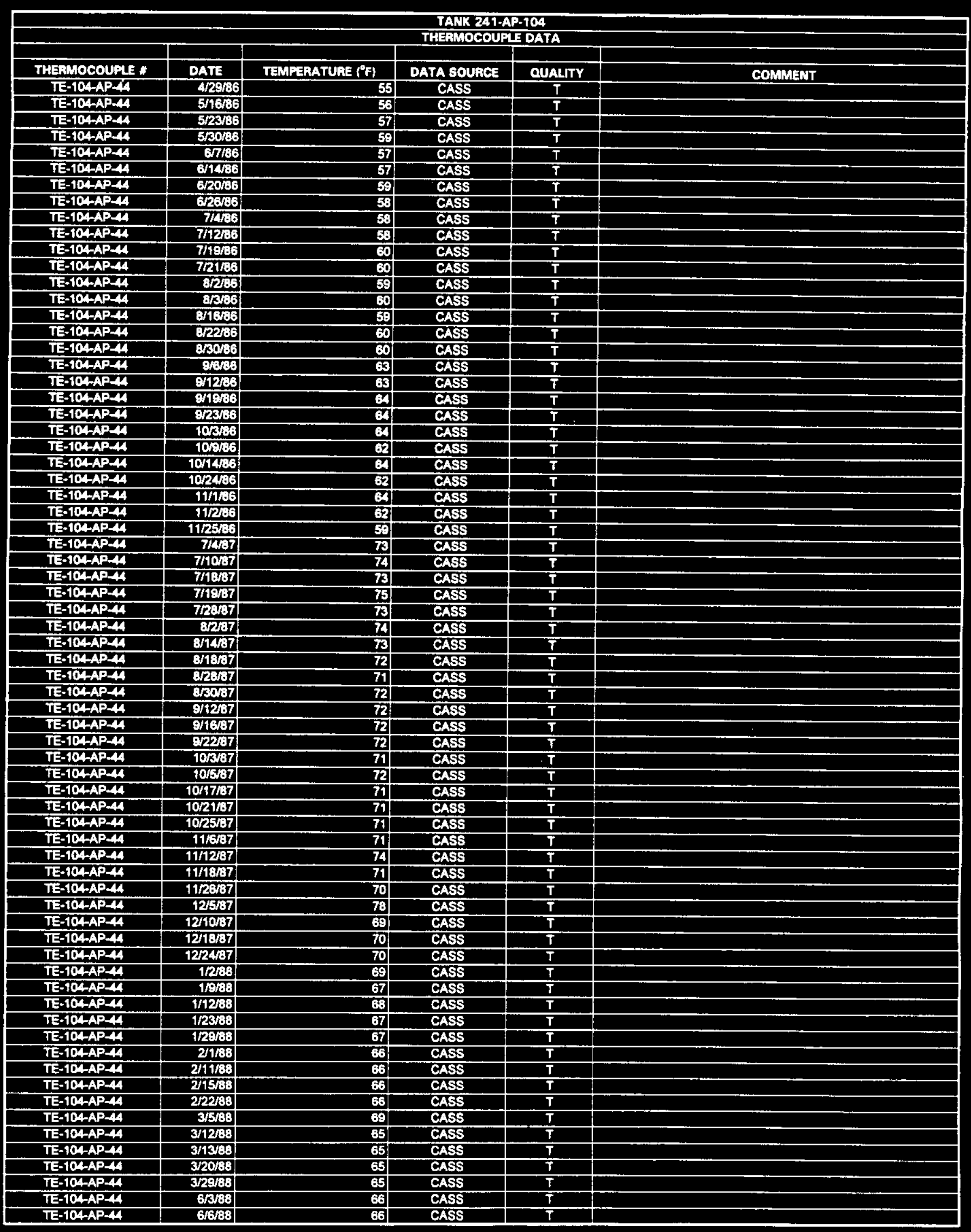




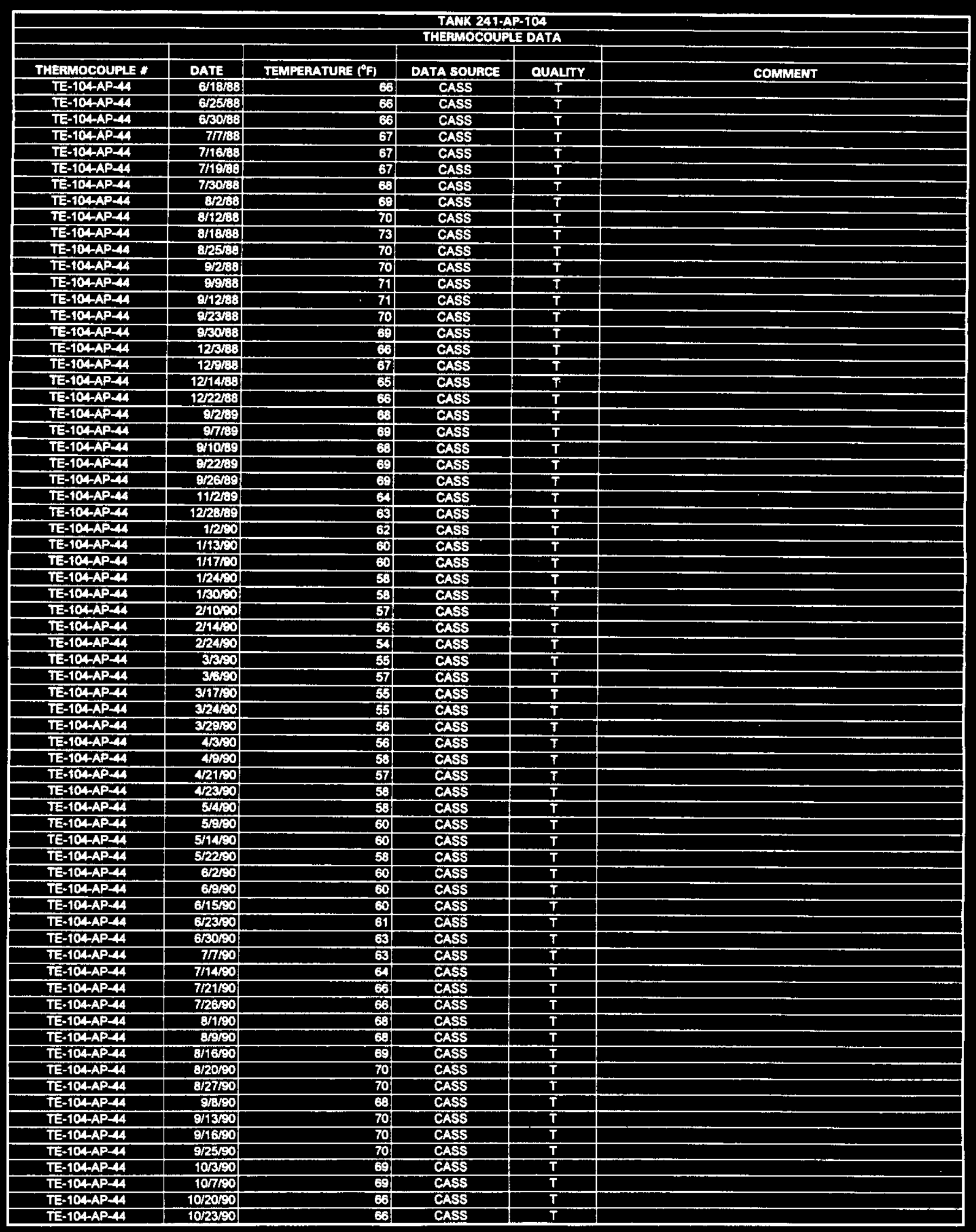




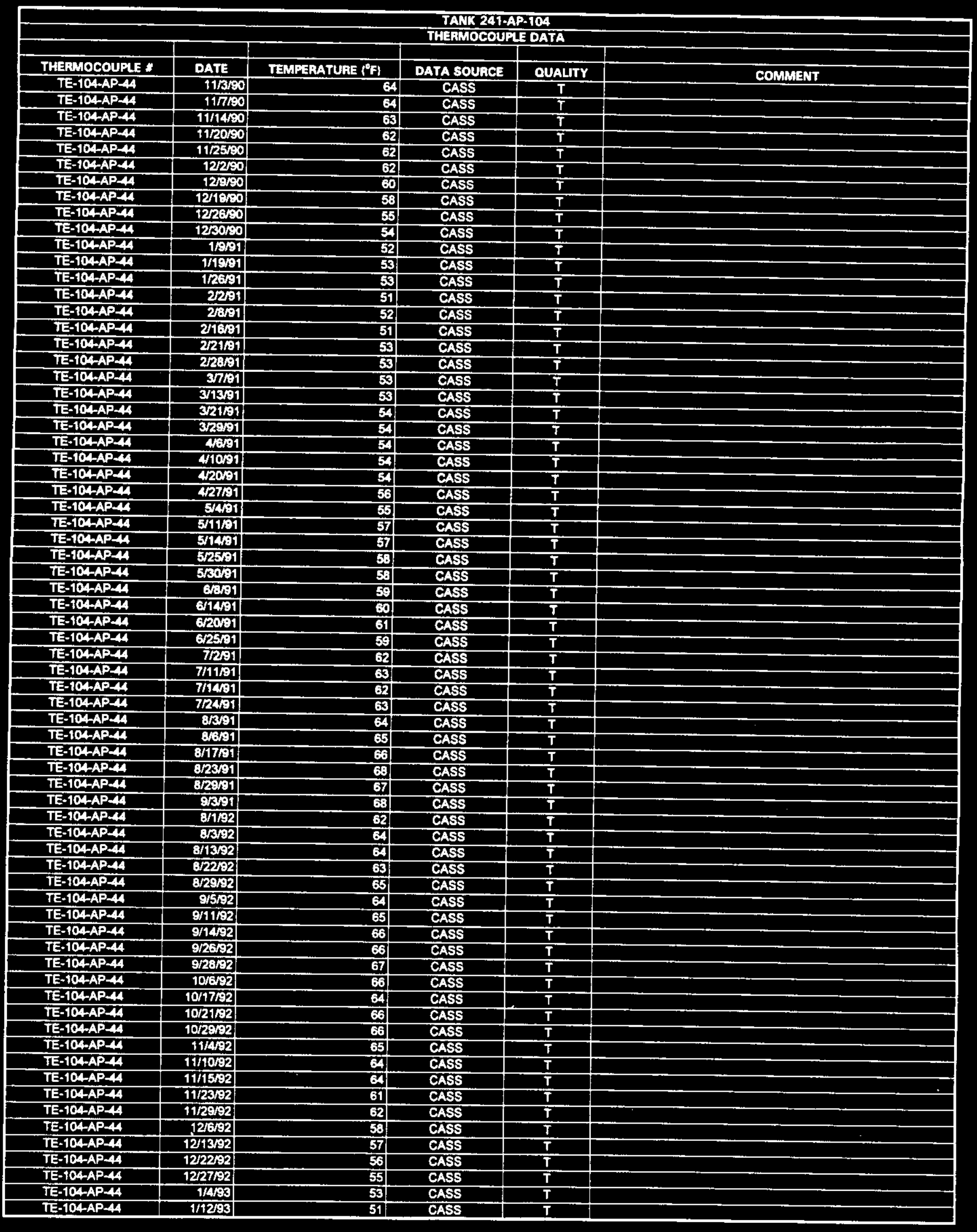

Data obtained from Computer Automated Surveillance Syatem ICASS), April 1995; and Surveillance Analysis Computer System (SACS), June 1995.

D-367 


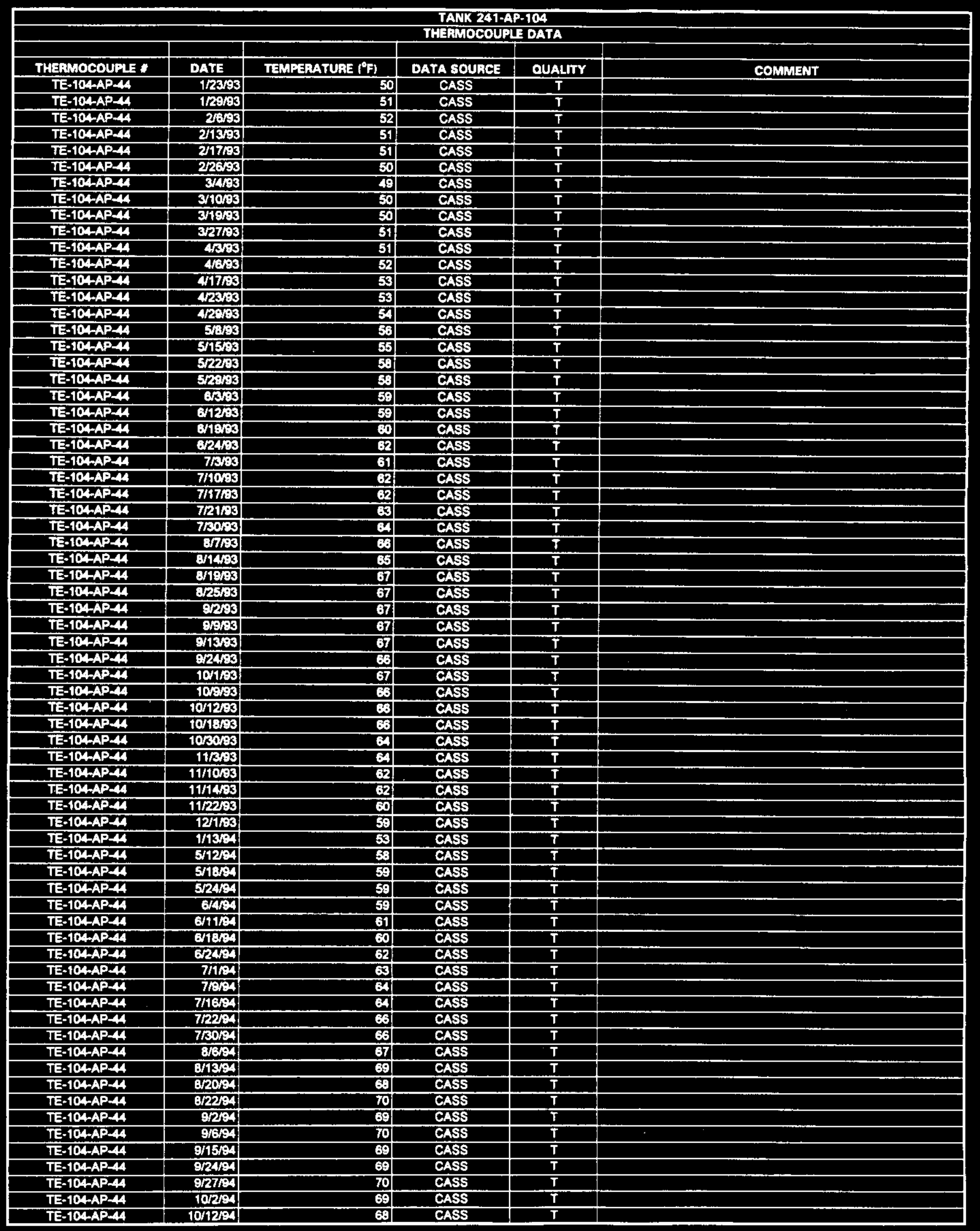




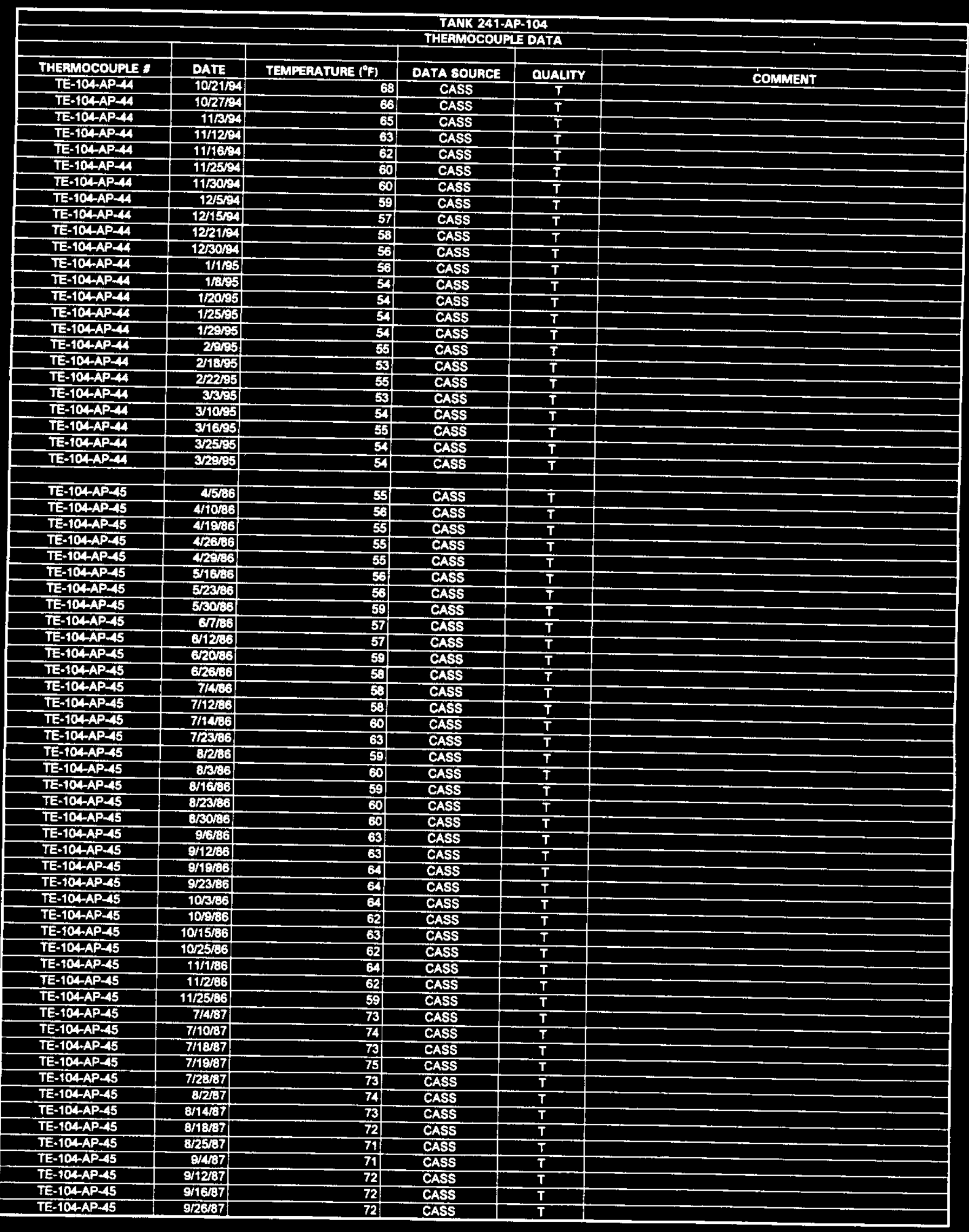

Date obtained from Computer Automated Surveillance System (CASS), April 1995; and Surveillance Analysis Computer Syatem (SACS), June 1995. 


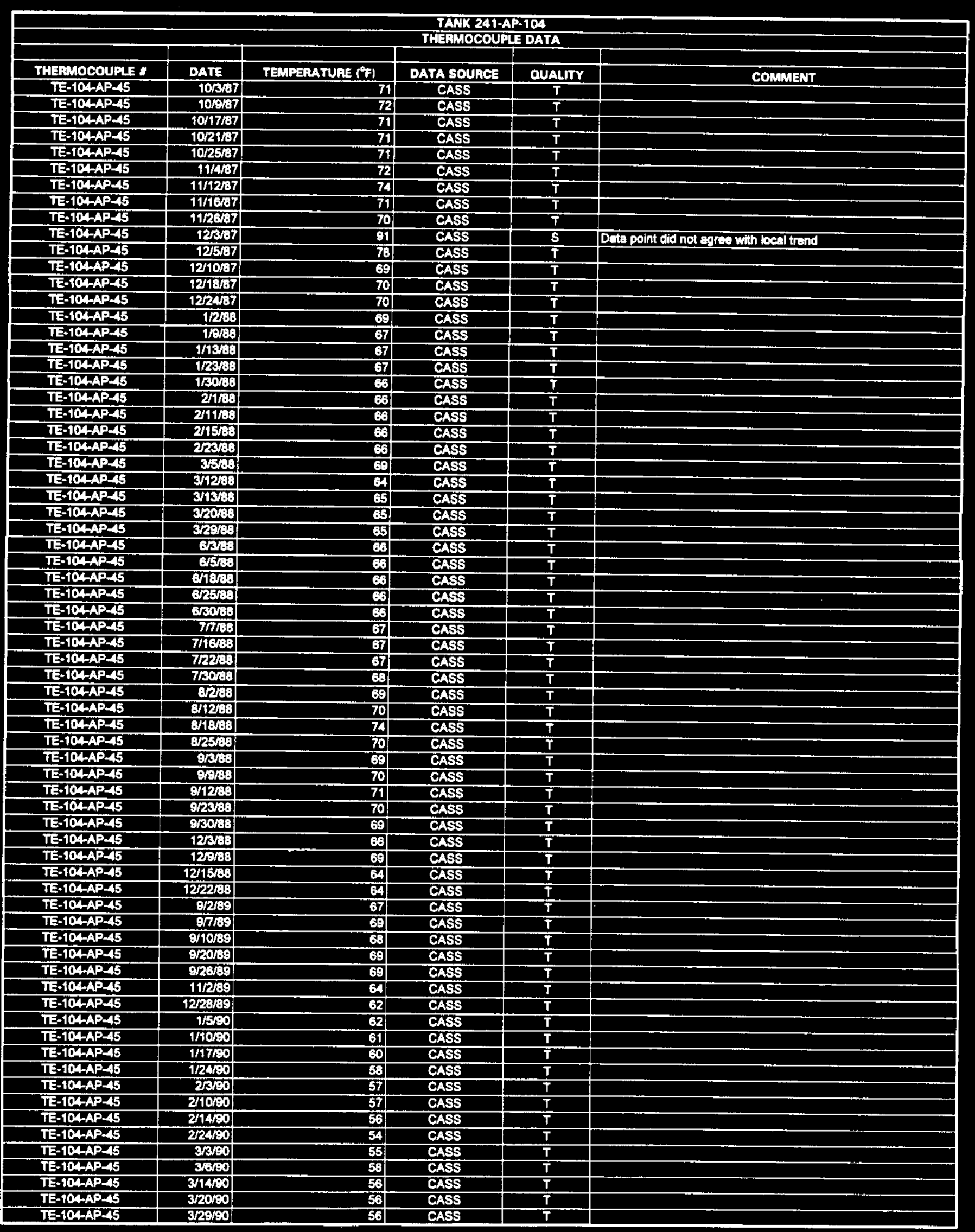




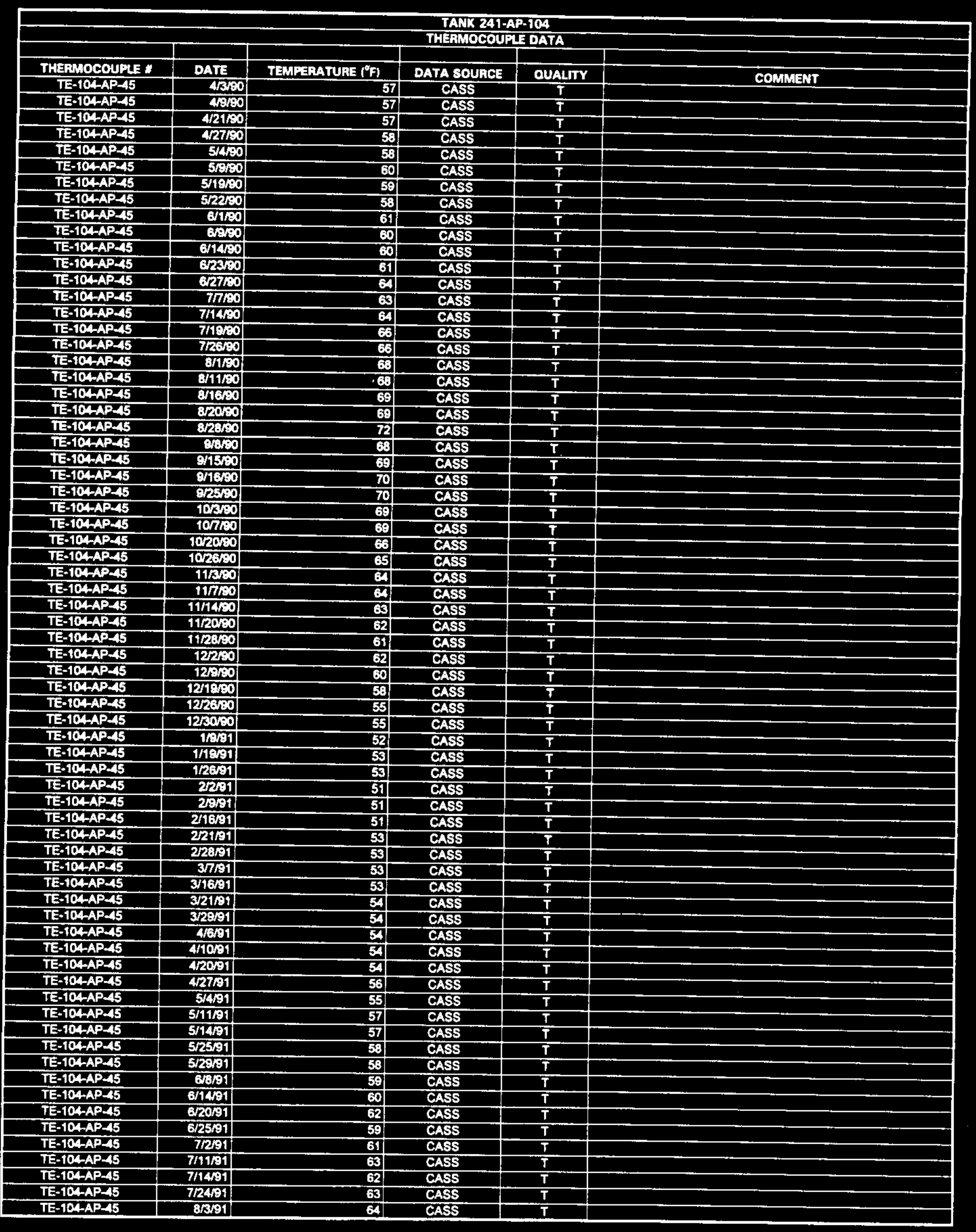




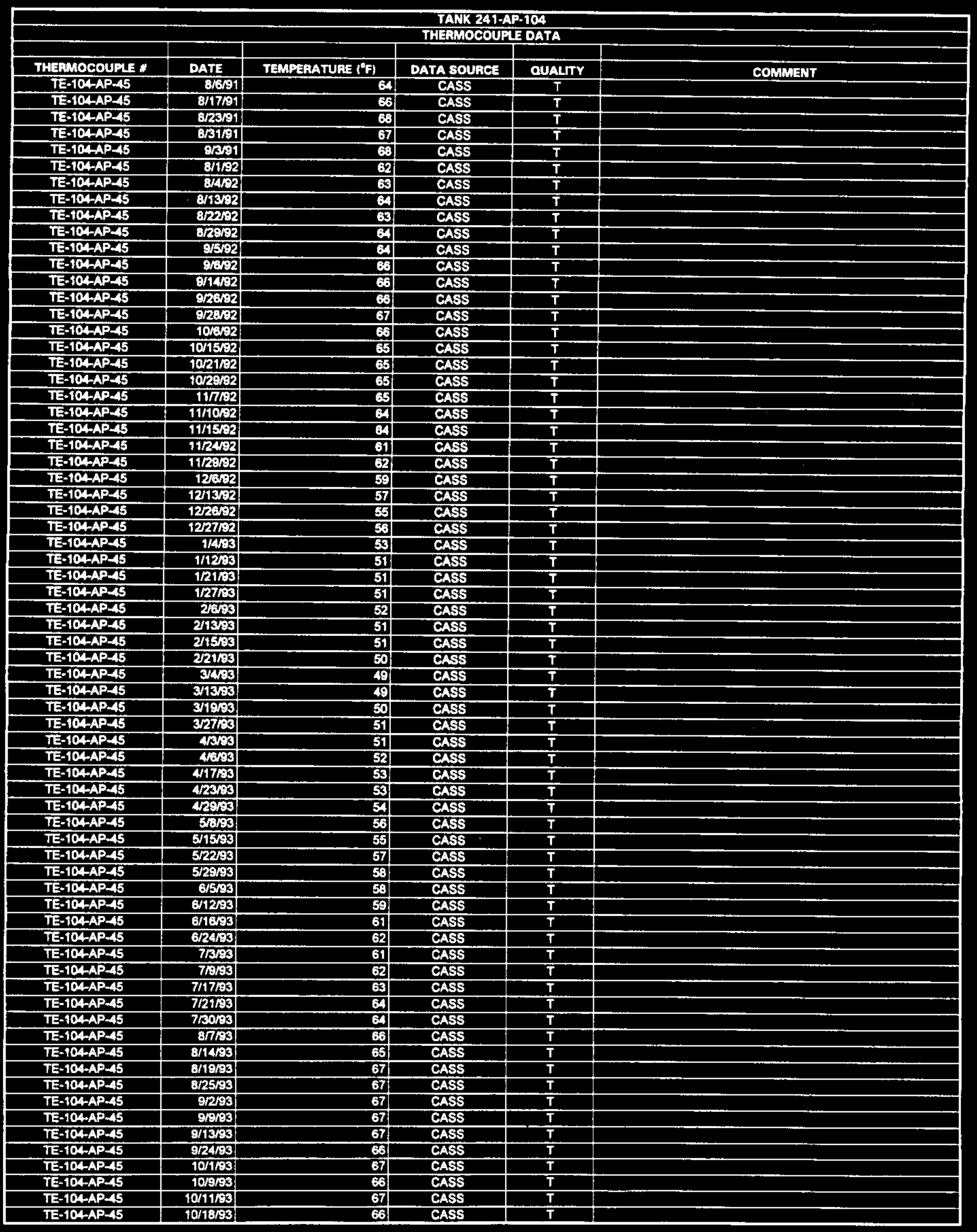




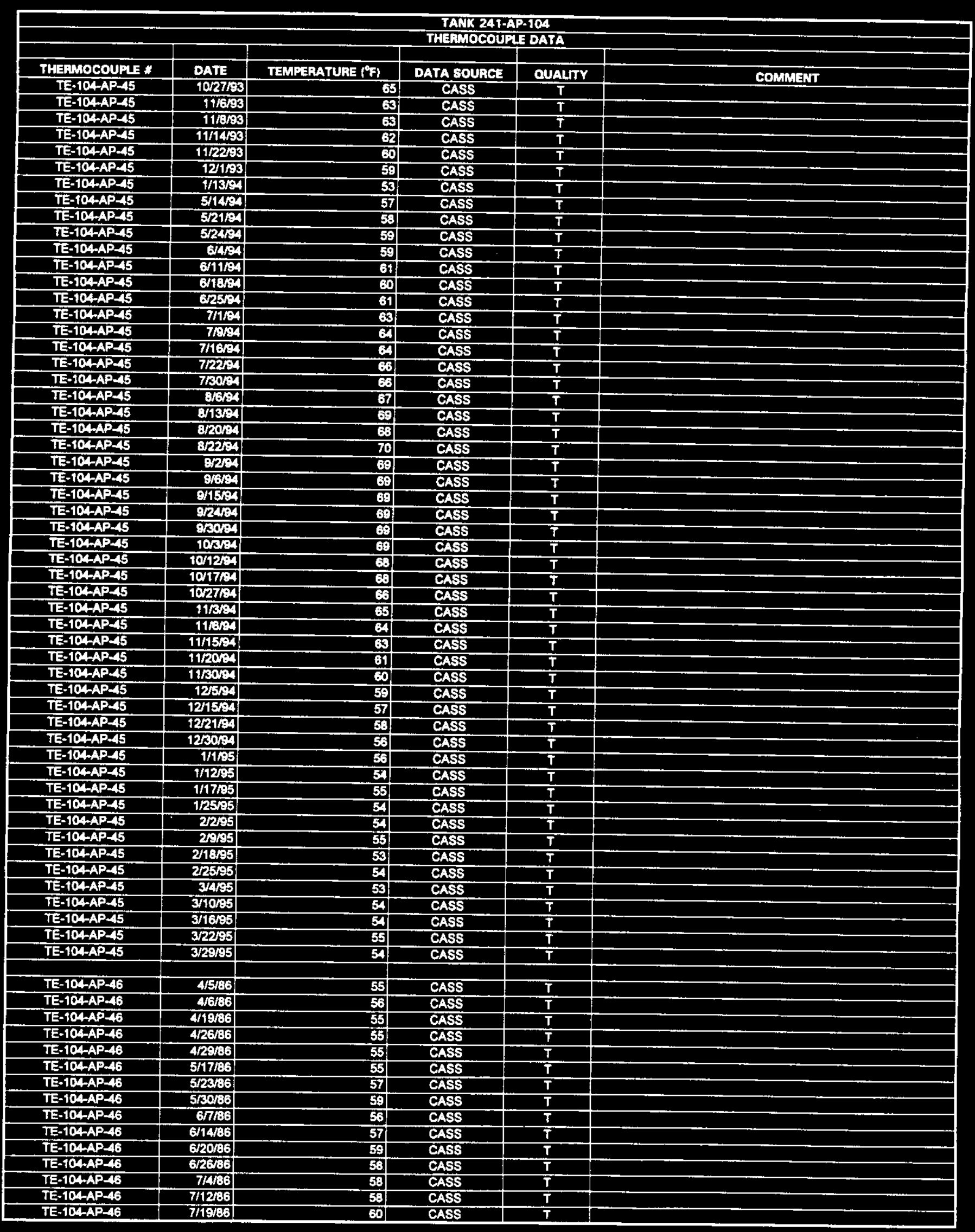




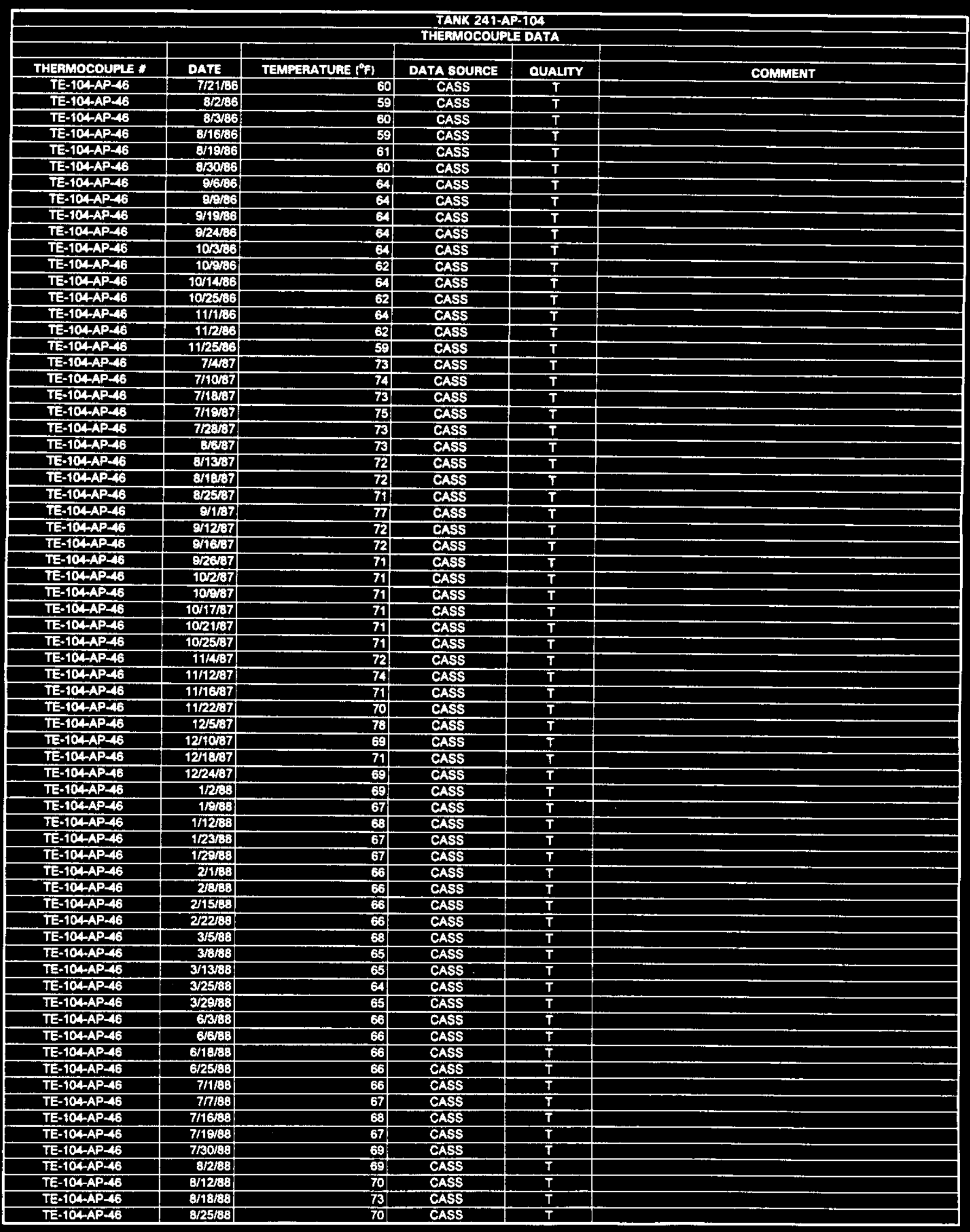




\begin{tabular}{|c|c|c|c|c|c|}
\hline \multicolumn{6}{|c|}{$\begin{array}{l}\text { TANK 241-AP-104 } \\
\text { THERMOCOUPLE DATA }\end{array}$} \\
\hline & & & & & \\
\hline THEPMOCOUPLE : & DATE & TEMPERATURE $\left({ }^{\circ} \mathrm{F}\right)$ & DATA SOURCE & QUALITY & COMMENT \\
\hline TE-104-AP-46 & $8 / 3 / 88$ & r & CASS & $T$ & \\
\hline TE-104AP-46 & 9/8/B8 & 71 & CASS & $T$ & \\
\hline TE-104-AP-46 & $9 / 12 / 38$ & 71 & CASS & $\mathbf{T}$ & \\
\hline TE-104AP-46 & 9/23/38 & 70 & CASS & $T$ & \\
\hline TE-104AP-46 & $8 / 30 / 68$ & 69 & CASS & $\bar{T}$ & \\
\hline TE-10A-AP-46 & $12 / 368$ & 66 & CASS & $T$ & \\
\hline TE-104-AP-16 & $129 / 88$ & 67 & CASS & $T$ & \\
\hline TE-104AP-46 & $12 / 15 / 3$ & GA & CASS & $T$ & \\
\hline TE-104AP-46 & 1212238 & 64 & CASS & $\mathbf{T}$ & \\
\hline TE-104-AP=46 & $8 / 2 / 39$ & 68 & CASS & $T$ & \\
\hline TE-104AP-A6 & 97/39 & 69 & CASS & $T$ & \\
\hline TE-104AP-46 & $9 / 10 / 3$ & 63 & CASS & $\mathbf{T}$ & \\
\hline TE-104-AP-46 & 9r20/00 & 69 & CASS & $T$ & \\
\hline TE-10A-AP-46 & $9 / 26 / 69$ & 69 & CASS & $\mathbf{T}$ & \\
\hline TE-104AP-46 & $11 / 2189$ & 84 & CASS & $\bar{T}$ & \\
\hline TE-104-AP-46 & 12/28/89 & 62 & CASS & $\mathbf{T}$ & \\
\hline TE-104AP-46 & T/BSO & 62 & CASS & $T$ & \\
\hline TE-104AP- 66 & traso & 81 & SACS & $T$ & \\
\hline TE-104-AP-46 & $1 / 17 \%$ & 80 & CASS & $T$ & \\
\hline TE-104AP-46 & $1 / 18 \% 0$ & B5 & CASS & $\mathbf{s}$ & Data point did not egree with local trend \\
\hline TE-104AP-46 & 1/24100 & $\mathbf{5 8}$ & CASS & $T$ & \\
\hline TE-104AP-46 & $1 / 2900$ & 58 & SACS & $T$ & \\
\hline TE-104-AP-46 & $2 / 1000$ & 57 & CASS & $T$ & \\
\hline TE-104AP-46 & 21480 & 56 & CASS & $T$ & \\
\hline TE-104-AP-16 & 22490 & 54 & CASS & $T$ & \\
\hline TE-104-AP-46 & $3 / \sqrt{100}$ & 56 & CASS & $T$ & \\
\hline TE-10,AP-46 & 3860 & 56 & CASS & $\mathbf{T}$ & \\
\hline TE-104AP-46 & 3/1400 & 56 & CASS & $\mathbf{T}$ & \\
\hline$T E-104 A P-16$ & $3 / 2400$ & 56 & CASS & $T$ & \\
\hline TE-104-AP= 66 & $3 \sqrt{28900}$ & 56 & CASS & $T$ & \\
\hline TE-104AP-16 & 46100 & 56 & CASS & $T$ & \\
\hline TE-104AP-16 & 4200 & 58 & CASS & $T$ & \\
\hline TE-10MAP- 16 & A/21k:0 & 57 & CASS & $\mathbf{T}$ & \\
\hline TE-104AP-16 & 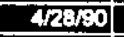 & 57 & CASS & $T$ & \\
\hline TE-104AP-46 & 5itio & 53 & CASS & $T$ & \\
\hline TE-TOA-AP-A6 & 5NOSO & 60 & CASS & $\bar{T}$ & \\
\hline TE-104APA 46 & 5/1400 & 58 & CASS & $T$ & \\
\hline TE-104-AP-46 & $5 / 2160$ & 59 & CASS & $\bar{T}$ & \\
\hline$T E-104-A P-46$ & $6 / 250$ & 61 & CASS & $T$ & \\
\hline TE-104AP-46 & 6onol & 60 & CASS & $\bar{T}$ & \\
\hline TE-104AP-46 & 6/1480 & 60 & CASS & $\bar{T}$ & \\
\hline TE-104-AP-46 & $6 / 23100$ & 61 & CASS & $T$ & \\
\hline TE-104AP-46 & $6 / 3000$ & 64 & CASS & $T$ & \\
\hline TE-104AP-46 & $7 / 3 / 90$ & 63 & CASS & $\bar{T}$ & \\
\hline TE-1BA-AP-46 & 71460 & 64 & CASS & $T$ & \\
\hline TE-104-AP-46 & 71900 & 66 & CASS & $T$ & \\
\hline TE-104-AP-46 & $7 / 26100$ & 68 & CASS & $T$ & \\
\hline E-104AP-16 & $8 / 1 / 50$ & 68 & CASS & $T$ & \\
\hline TE-104AP-46 & $8 / 11 / 90$ & 67. & CASS & $T$ & \\
\hline TE-104-AP-46 & $8 / 600$ & 69 & CASS & $T$ & \\
\hline TE-104-AP-46 & 8/20ral & $6 \theta$ & CASS & $T$ & \\
\hline TE-104-AP-46 & B/27/50 & 70 & CASS & $T$ & \\
\hline TE-104-AP-AB & arso & 68 & CASS & $T$ & \\
\hline TE-104AP-46 & $9 / 13 / 30$ & 69 & CASS & $\mathbf{T}$ & \\
\hline TE-104AP-46 & 9/16/00 & 70 & CASS & $T$ & \\
\hline TE-104AP-46 & 8/25500 & 70 & CASS & $T$ & \\
\hline TE-104AP-46 & $10 \mathrm{r} s=0$ & 69 & CASS & $T$ & \\
\hline TE-104AP-46 & 107roo & 69 & CASS & $T$ & \\
\hline TE-104-AP-46 & $10 / 1800$ & 66 & CASS & $T$ & \\
\hline TE-104-AP-46 & $10 / 2680$ & 65 & CASS & $T$ & \\
\hline TE-104AP-46 & $11 / 200$ & 64 & CASS & $T$ & \\
\hline TE-104-AP-A6 & $11 \overline{7150}$ & 64 & CASS & $T$ & \\
\hline TE-104-AP-46 & $11 / 13 / 90$ & 64 & CASS & $T$ & \\
\hline TE-104-AP-46 & $11 / 20 / 80$ & 62 & CASS & $\bar{T}$ & \\
\hline TE-104-AP-46 & $11 / 25 / 90$ & 62 & CASS & $T$ & \\
\hline TE-104AP-46 & 12/2150 & 62 & CASS & $T$ & \\
\hline TE-104AP-46 & $12 / 9100$ & 60 & CASS & $\bar{T}$ & \\
\hline TE-104-AP-46 & 12/990 & 58 & CASS & $T$ & \\
\hline TE-104AP- 16 & 1212600 & 55 & CASS & $T$ & \\
\hline TE-104.AP-46 & $12 \pi 30 / 90$ & 54 & CASS & $T$ & \\
\hline
\end{tabular}




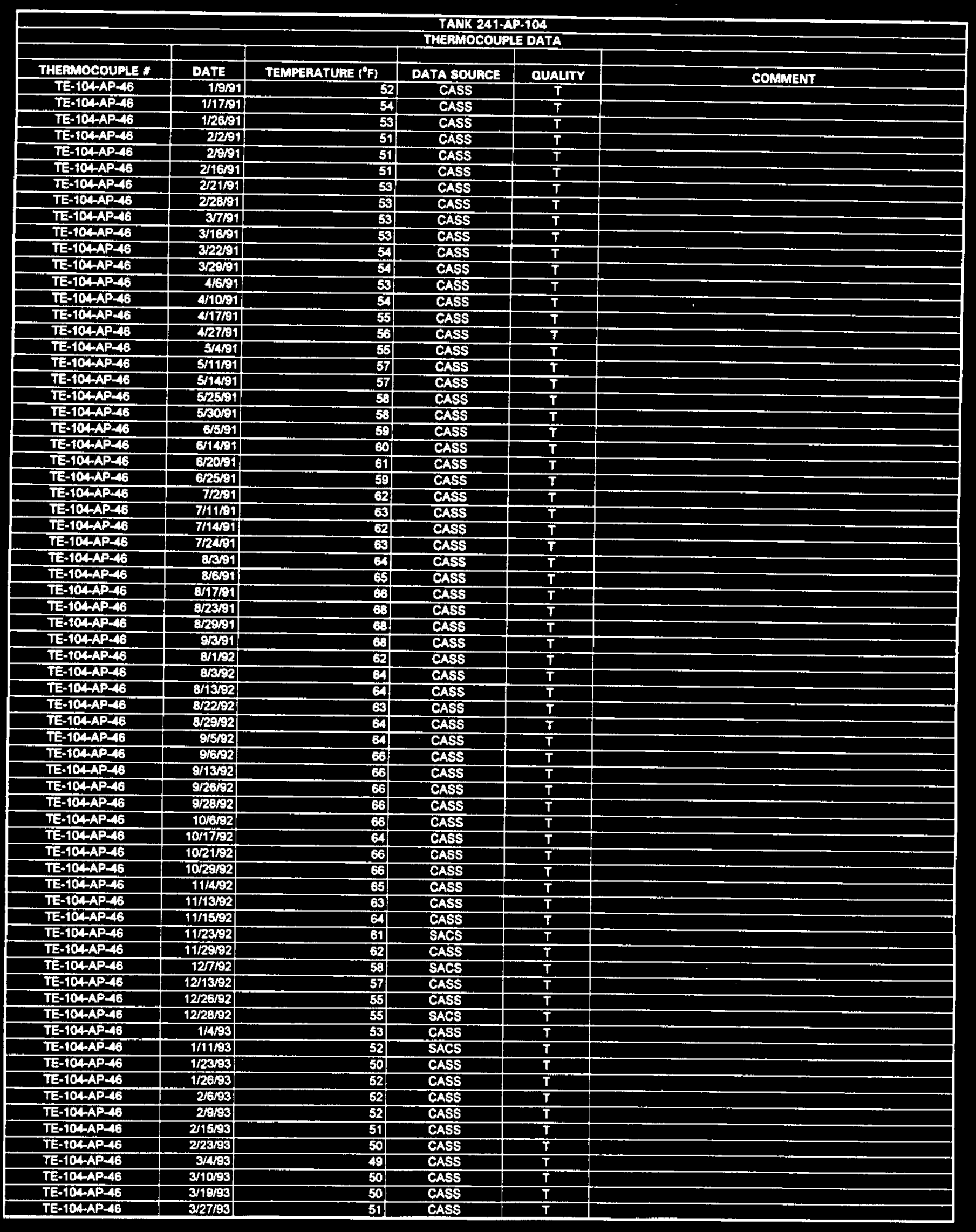




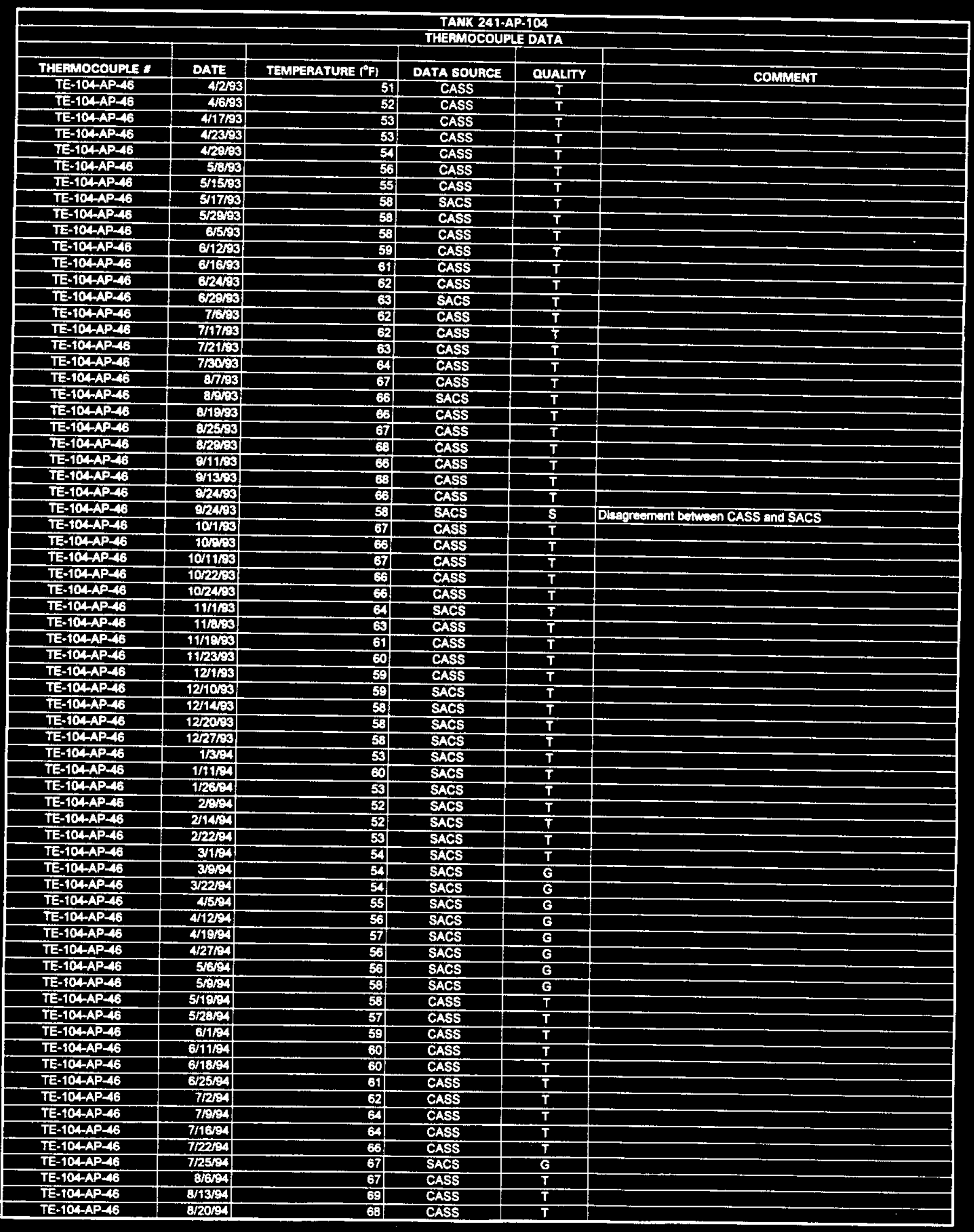

Data obtained from Computer ALtomated Surveillance System (CASS), April 1995; and Surveillance Analysis Computer System (SACS), June 1995. D-377 


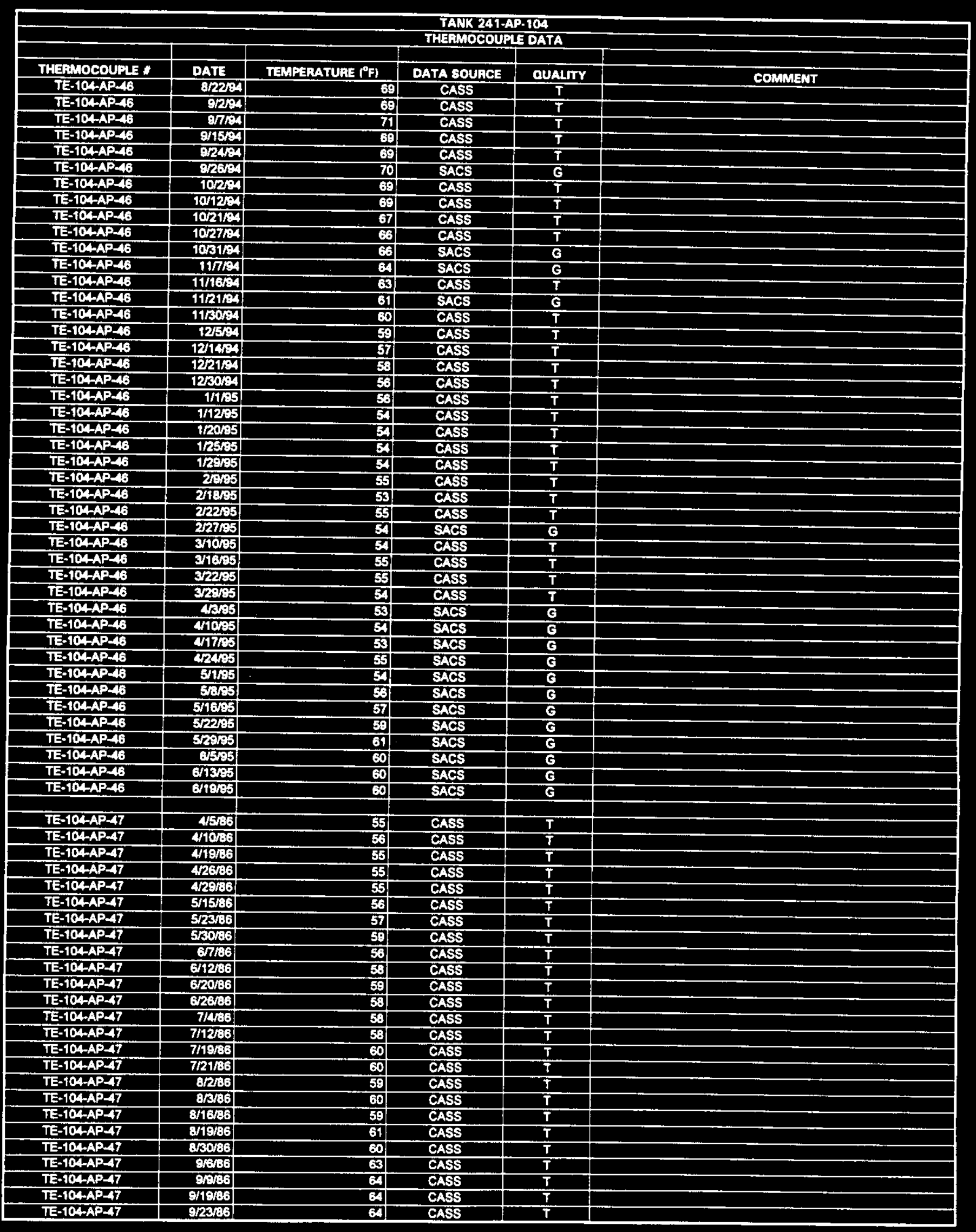




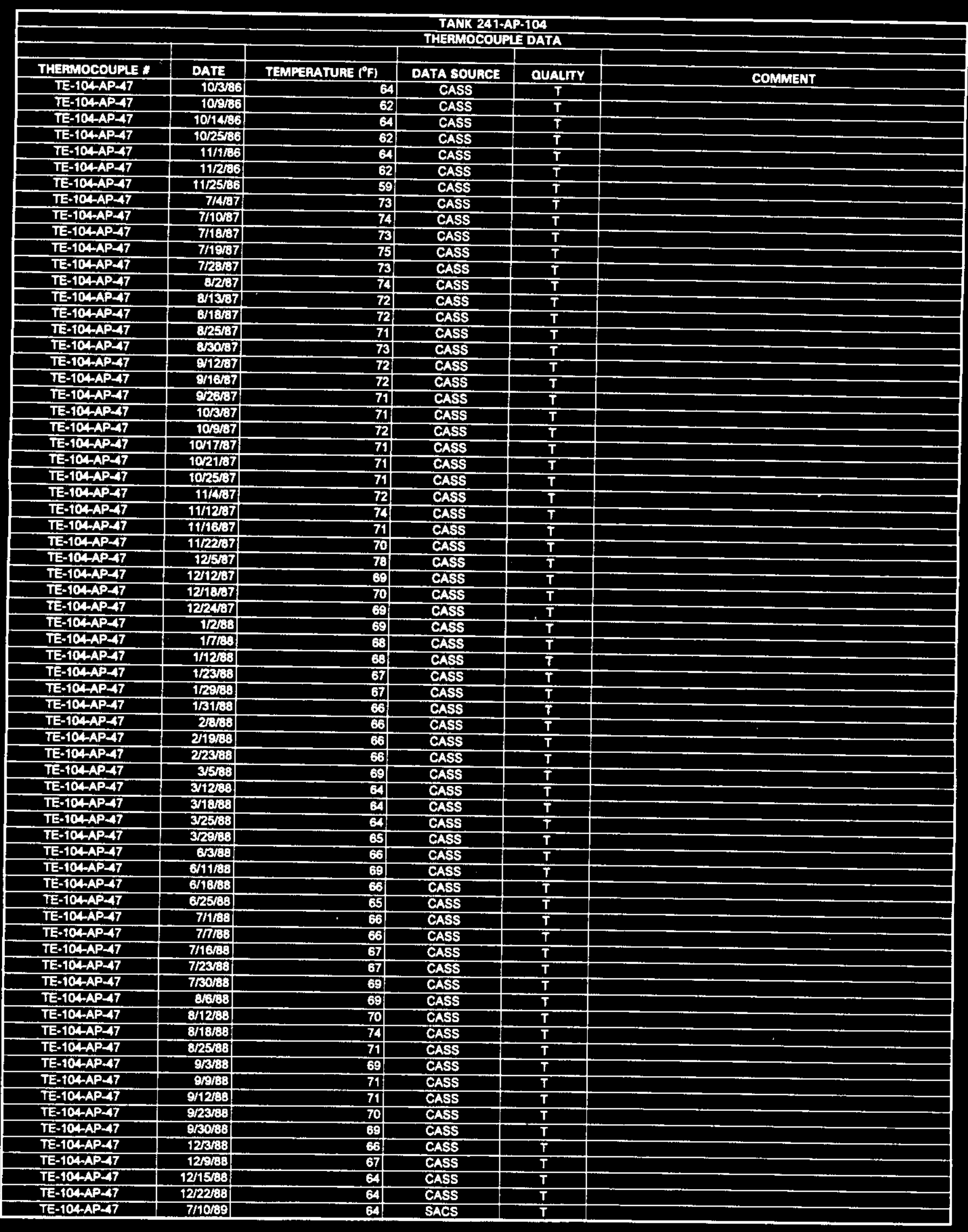

Data obtained from Computer Automated Surveillance System (CASS), April 1995; and Surveillance Analysis Computer System (SACS), June 1995. 


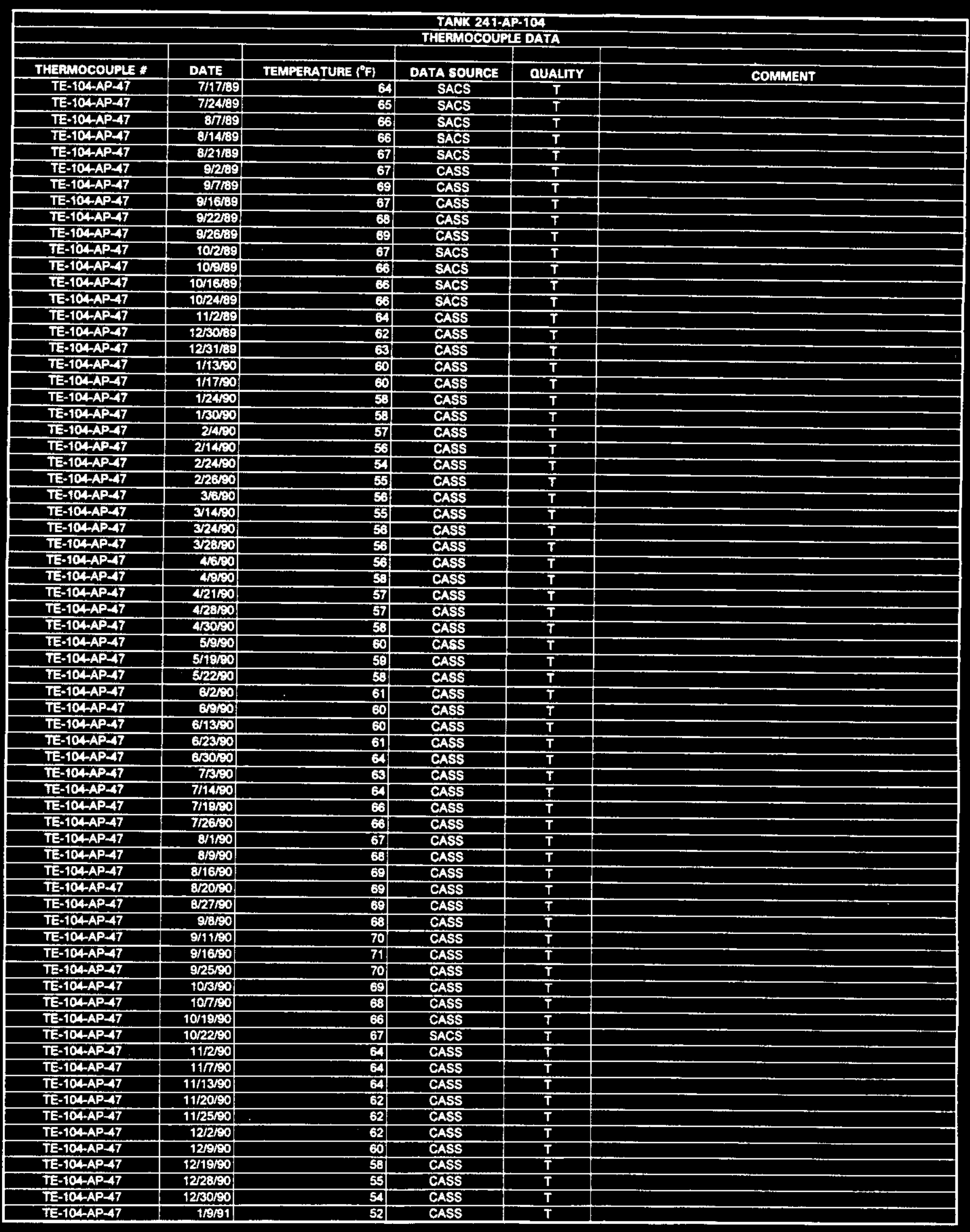

Data obtained from Computer Automoted Surveillance Syatem (CASS), April 1995; and Surveillonce Analysis Computer System (SACS), June 1995.

D-380 


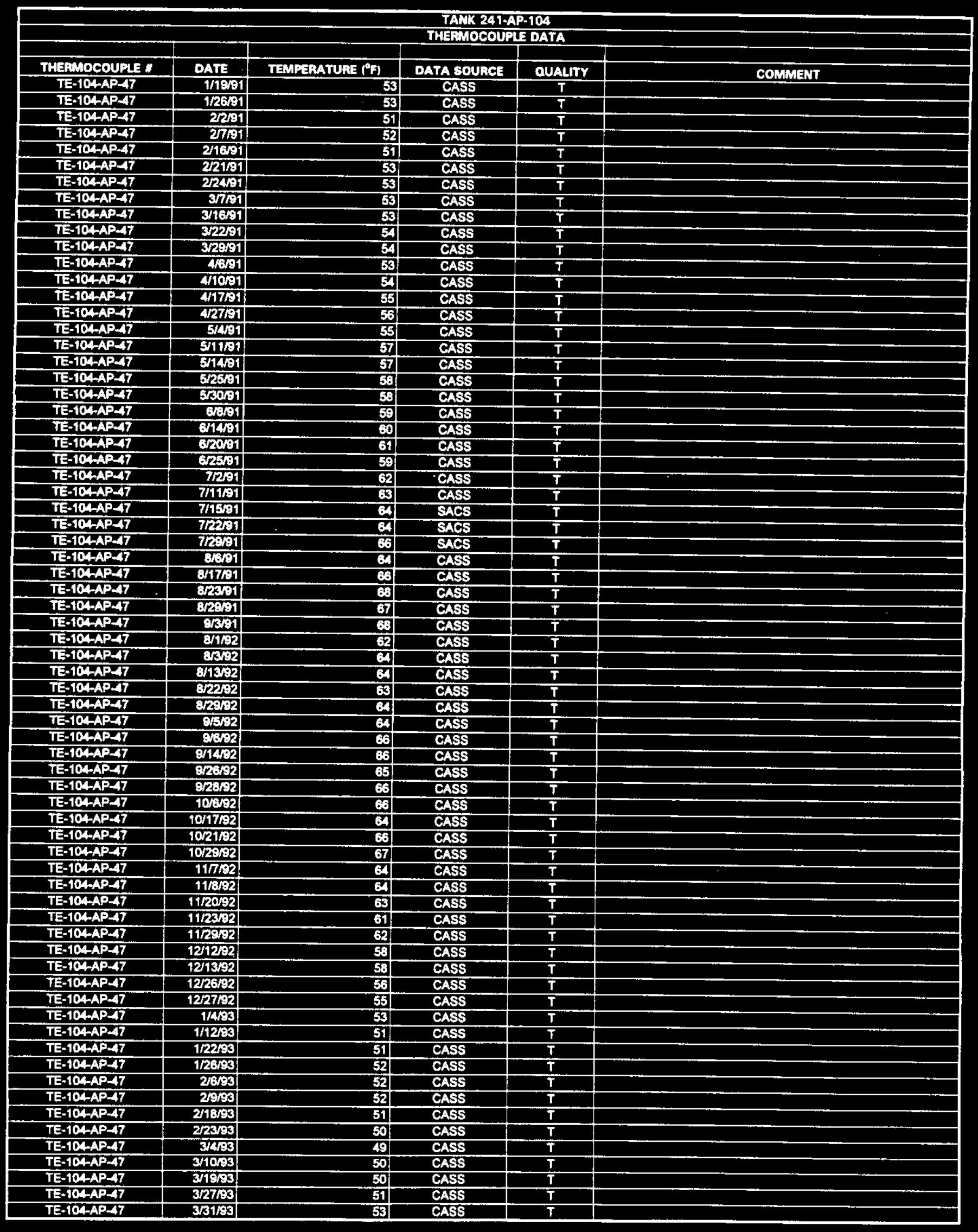

Data obtainad from Computer Automated Surveillance System (CASS), April 1995; and Surveillance Analysis Computer System (SACS), June 1995. 


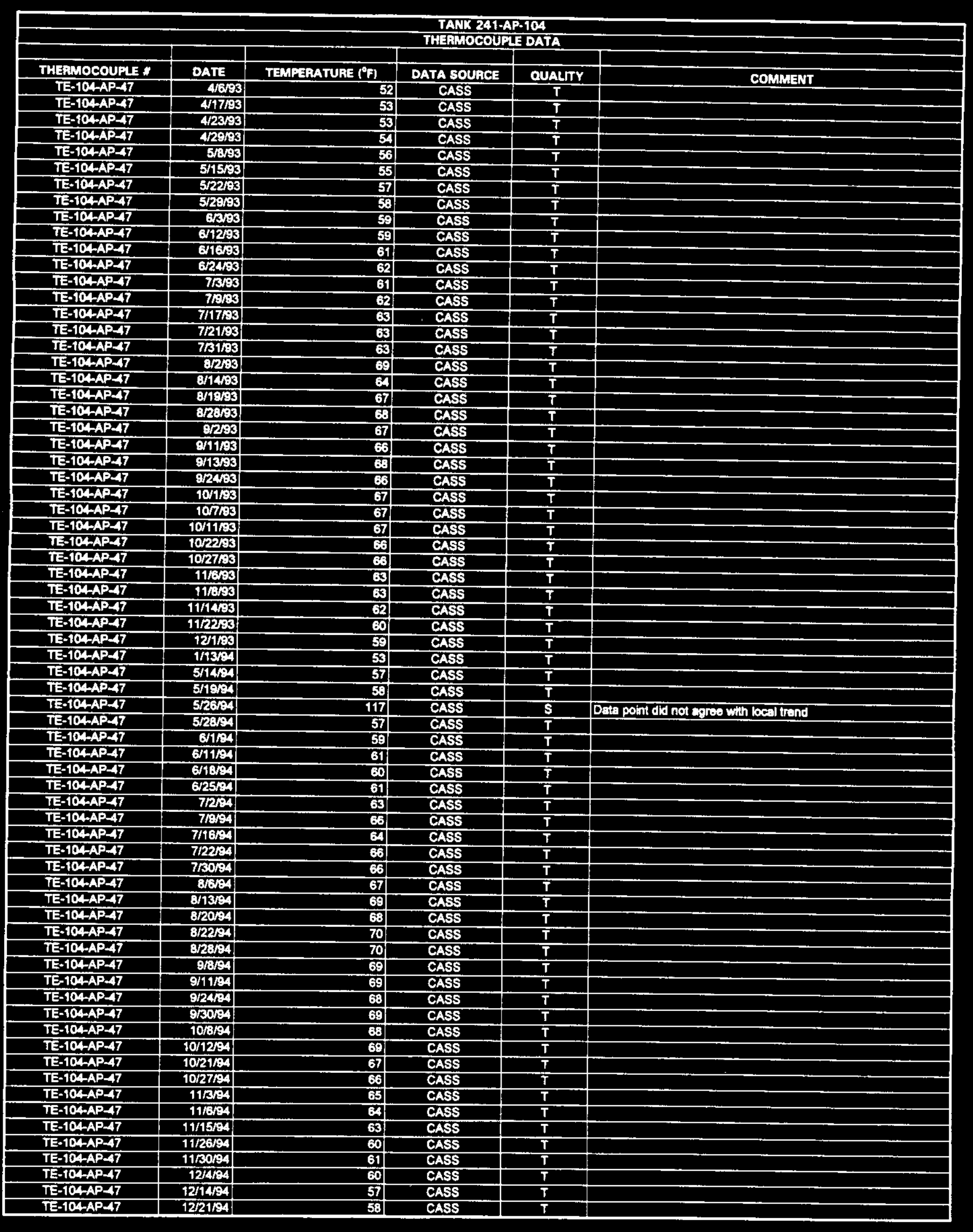




\begin{tabular}{|c|c|c|c|c|c|}
\hline \multicolumn{6}{|c|}{$\begin{array}{l}\text { TATK 241-AP-104 } \\
\text { THERMOCOURL DATA }\end{array}$} \\
\hline THEAMOCOUPLE & DATE & TEMPERATUAE $\left({ }^{\circ} \mathrm{F}\right)$ & DATA SOUACE & & \\
\hline TE-104AP-A7 & $12 / 30 / 94$ & 56 & CASS & $\frac{\text { QUALITY }}{T}$ & COMMENT \\
\hline TE-104AP-47 & $1 / 1 / 95$ & 56 & CASS & $\frac{T}{T}$ & \\
\hline TE-104-AP-A7 & $1 / 12 / 95$ & 54 & CASS & $\frac{T}{T}$ & \\
\hline TE-104-AP-17 & $1 / 17 / 65$ & 54 & CASS & $T$ & \\
\hline TE-104-AP-47 & 1/25/95 & 54 & CASS & $T$ & \\
\hline TE-104-AP-47 & $2 / 2155$ & 54 & CASS & $T$ & \\
\hline IE-104-AP-47 & $29 / 95$ & 54 & CASS & $T$ & \\
\hline TE-104AP-47 & $2 / 18 / 95$ & 54 & CASS & $\bar{T}$ & \\
\hline TE-104AP-A7 & $2 / 2253$ & S1 & CASS & $T$ & \\
\hline TE-104-AP-A7 & 3365 & $\overline{53}$ & CASS & $T$ & \\
\hline$T E-104 A P-47$ & 3/10/95 & SA & CASS & $T$ & \\
\hline TE-104-AP-47 & 3/1695 & 55 & CASS & $T$ & \\
\hline TE-104AP-A7 & $3 / 2295$ & 55 & CASS & $T$ & \\
\hline TE-104AP-A7 & 3/2905 & 54 & CASS & $T$ & \\
\hline & & & & & \\
\hline TE-104-AP-48 & $4 / 5 / 36$ & 55 & CASS & $T$ & \\
\hline TE-104AP-48 & $410 / 86$ & 57 & CASS & $T$ & \\
\hline TE-104AP-48 & 4/9/86 & 55 & CASS & $T$ & \\
\hline TE-104AP-A3 & $426 / 66$ & 55 & CASS & $T$ & \\
\hline TE-104AP- 49 & $4 / 2986$ & B5 & CASS & $T$ & \\
\hline TE-104-AP-43 & 5/16/66 & 56 & CASS & $T$ & \\
\hline TE-10A-AP-48 & $5 \sqrt{23 / 66}$ & 56 & CASS & $\bar{T}$ & \\
\hline TE-104AP-AB & 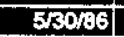 & 59 & CASS & $\bar{T}$ & \\
\hline$T E-104-A P-48$ & 6786 & 56 & CASS & $T$ & \\
\hline TE-104AP- 48 & 6/2B6 & 53 & CASS & $T$ & \\
\hline TE-104AP-48 & 6/20186 & 59 & CASS & $T$ & \\
\hline TE-104-AP-48 & 6/2686 & $53 !$ & CASS & $\mathrm{T}$ & \\
\hline TE-104AP- 48 & $7 / 486$ & 53 & CASS & $T$ & \\
\hline TE-104-AP-4B & $772 B 6$ & 53 & CASS & $T$ & \\
\hline TE-104AP-18 & 719 & 60 & CASS & $T$ & \\
\hline TE-104-AP- $\overline{48}$ & $7 / 21 / 36$ & 60 & CASS & $T$ & \\
\hline TE-104-APAS & $8 / 286$ & 59 & CASS & $T$ & \\
\hline TE-104-AP-4B & $8 / 3 / 66$ & 60 & CASS & $\bar{T}$ & \\
\hline TE-104-AP-48 & $8 / 18 / 6$ & 59 & CASS & $T$ & \\
\hline TE-104-AP-4B & $8 / 19 / 66$ & 61 & CASS & $T$ & \\
\hline TE-104AP-48 & B/30/36 & 60 & CASS & $T$ & \\
\hline TE-104-AP-48 & 8666 & 63 & CASS & $T$ & \\
\hline TE-104AP-AB & \%o6 & GA & CASS & $\bar{T}$ & \\
\hline TE-104-AP-48 & 919/8 & 63 & CASS & $T$ & \\
\hline TE-104AP- 48 & $8 / 23106$ & 64 & CASS & $T$ & \\
\hline TE-104AP-4B & $10 / 3166$ & 64 & CASS & $T$ & \\
\hline TE-104AP-48 & 10/9/86 & 62 & CASS & $T$ & \\
\hline TE-104AP-48 & $10 / 14 / 86$ & 64 & CASS & $T$ & \\
\hline TE-104-AP-48 & $10 / 2306$ & 62 & CASS & $T$ & \\
\hline TE-104AP-AB & $10 / 24 / 86$ & 102 & CASS & $\mathbf{s}$ & Data point did not agree with local trend \\
\hline TE-104-AP-48 & $11 / 1 / 186$ & 64 & CASS & $\bar{T}$ & \\
\hline TE-104AP-48 & $11 / 2 / 66$ & 62 & CASS & $\mathbf{T}$ & \\
\hline TE-104AP-48 & $11 / 25 / 86$ & 58 & CASS & $T$ & \\
\hline TE-104AP-48 & $7 / 4 / 87$ & 73 & CASS & $\mathbf{T}$ & \\
\hline TE-104-AP-48 & $7 / 10 / 87$ & 74 & CASS & $T$ & \\
\hline TE-104-AP-48 & $7 / 18 / 87$ & 72 & CASS & $T$ & \\
\hline TE-104-AP-48 & $7 / 19 / 87$ & 75 & CASS & $T$ & \\
\hline$T E-104 A P-48$ & $7 / 28 / 27$ & 73 & CASS & $T$ & \\
\hline TE-104-AP=48 & $8 / 6187$ & 73 & CASS & $T$ & \\
\hline TE-104-AP-48 & $8 / 13 / 67$ & 72 & CASS & $\mathbf{T}$ & \\
\hline TE-104-AP-48 & $8 / 18 / 87$ & 72 & CASS & $T$ & \\
\hline TE-104AP-48 & $8 / 25 / 87$ & 71 & CASS & $T$ & \\
\hline TE-104-AP-48 & $8 / 30 / 87$ & 73 & CASS & $T$ & \\
\hline TE-104AP-48 & $9 / 12 / 67$ & 72 & CASS & $T$ & \\
\hline TE-104AP-48 & 9/16/87 & 72 & CASS & $T$ & \\
\hline TE-104-AP-48 & $9 / 26 / 67$ & 71 & CASS & $T$ & \\
\hline TE-104-AP-AB & $10 / 3 / 67$ & 71 & CASS & $\bar{T}$ & \\
\hline TE-104-AP-4B & 10/9/87 & 71 & CASS & $T$ & \\
\hline TE-104-AP-48 & 10/17/87 & 71 & CASS & $T$ & \\
\hline$T E-104 A P-4 B$ & 10/21/87 & 71 & CASS & $T$ & \\
\hline TE-104APAB & $10 / 25 / 87$ & 71 & CASS & $T$ & \\
\hline TE-104-AP-48 & 11/4/87 & 74 & CASS & $T$ & \\
\hline TE-104-AP-48 & $11 / 12 / 87$ & 74 & CASS & $T$ & \\
\hline TE-104-AP-48 & $11 / 16 / 87$ & 71 & CASS & $T$ & \\
\hline TE-104AP-48 & $11 / 22187$ & 70 & CASS & $\mathrm{T}$ & \\
\hline
\end{tabular}




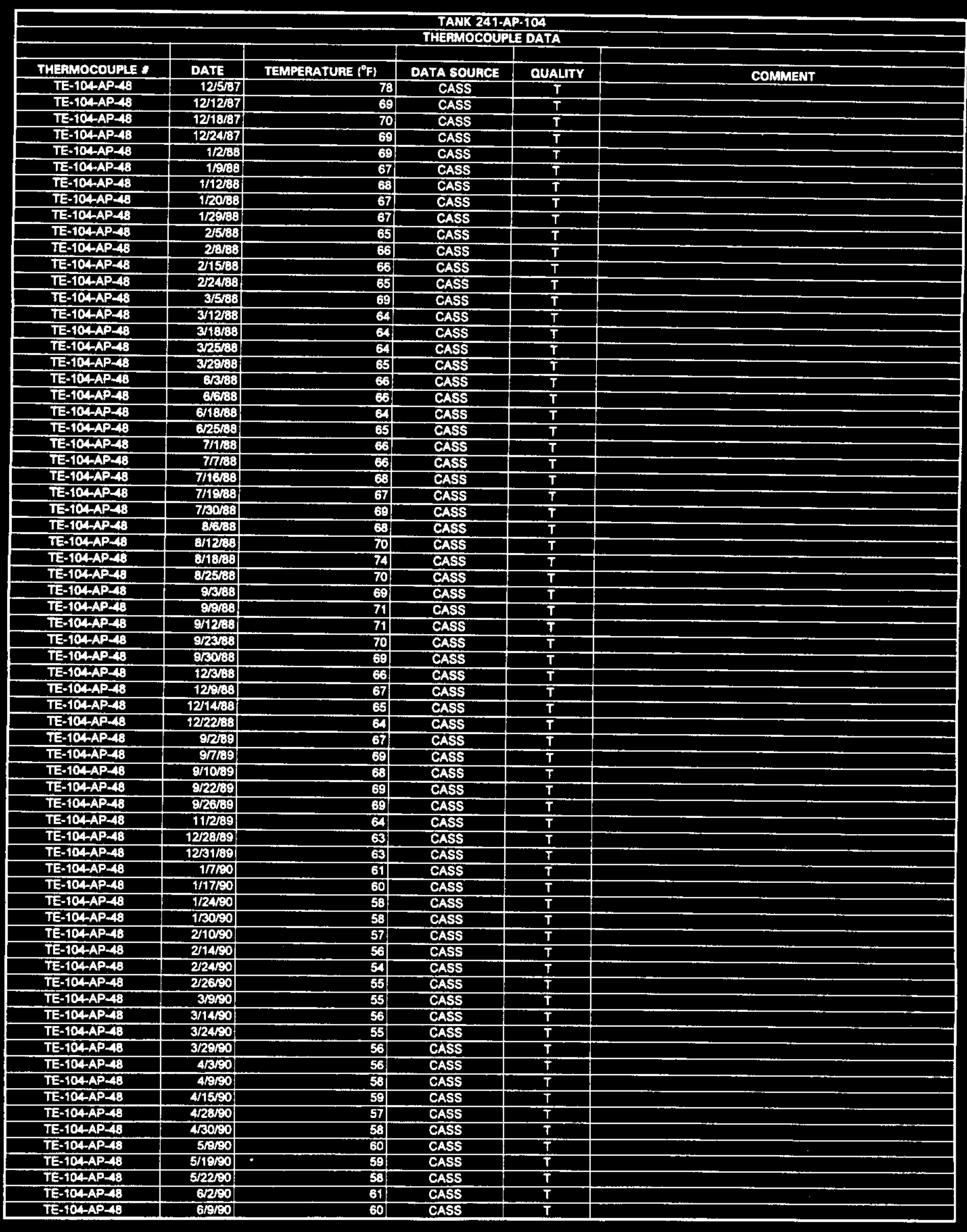

Data obtained from Computer Automated Surveillance System (CASS), April 1995; and Surveillance Analysis Computer System (SACS), June 1995.

D-384 


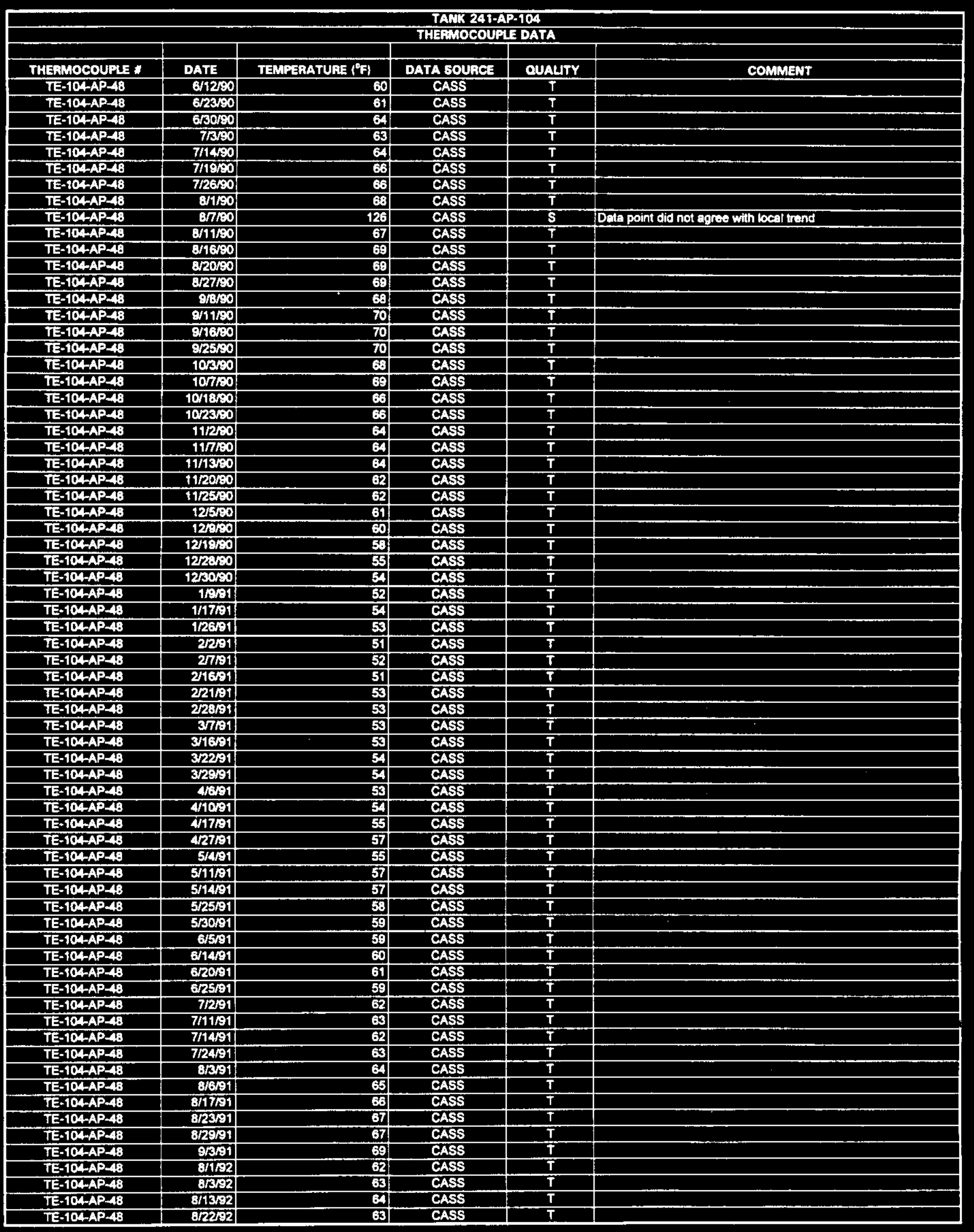




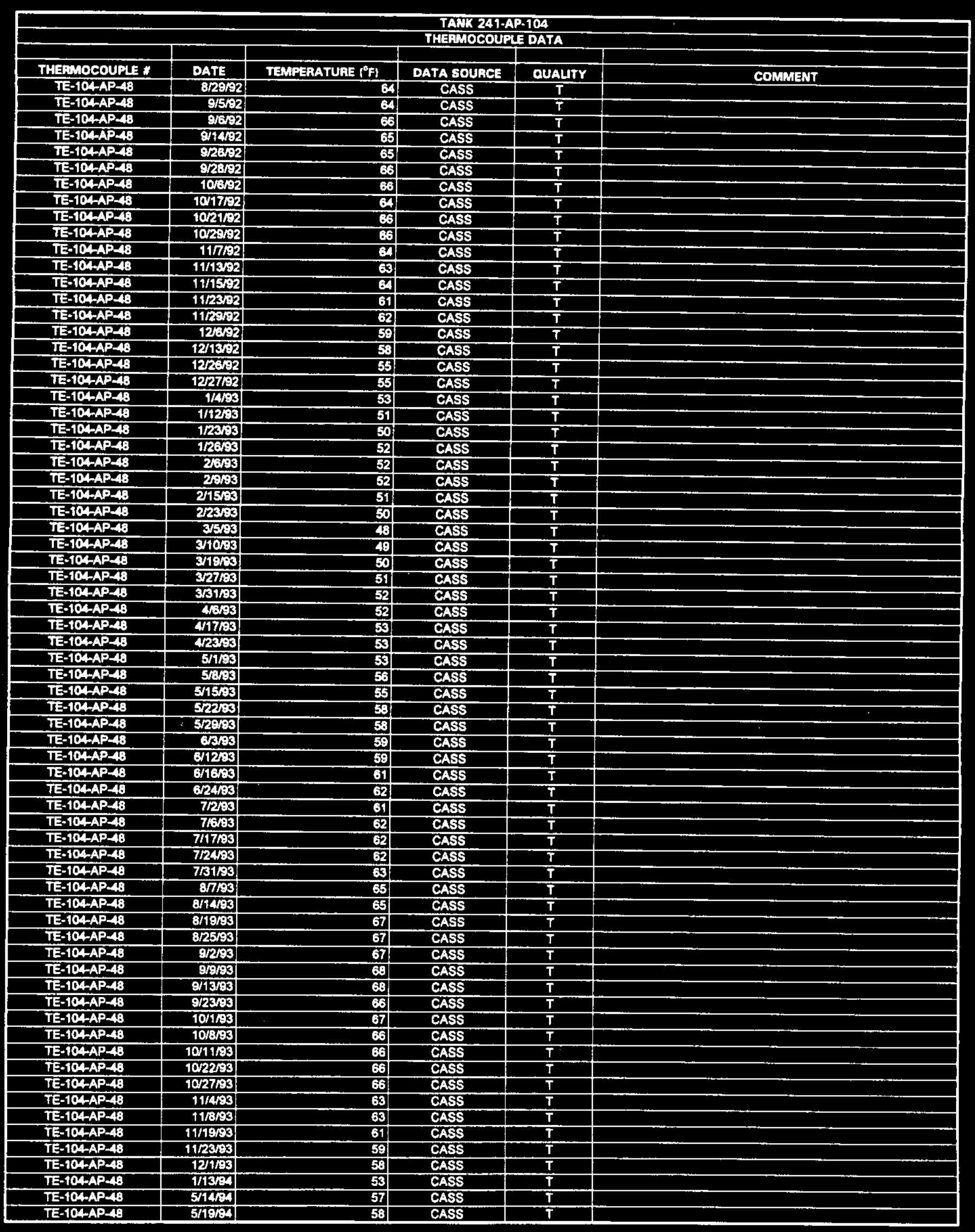

Dote obteined from Computer Automated Surveillance System (CASS), April 1995; and Surveillance Analysis Computer System (SACS), June 1995.

$$
\text { D-386 }
$$




\begin{tabular}{|c|c|c|c|c|c|}
\hline \multicolumn{6}{|c|}{$\begin{array}{l}\text { TANK 241-AP-104 } \\
\text { THERMOCOUPLE DATA }\end{array}$} \\
\hline THEAMOCOUPLE & & & & & \\
\hline & DAIE & TEMPIAAUUAE $(F)$ & DATA SOURCE & QUALTY & COMMENT \\
\hline & $\frac{5 / 2494}{6 / 404}$ & 59 & CASS & $T$ & \\
\hline TE-104-AP-4B & $\frac{6 / 4 / 94}{6 / 11 / 94}$ & 59 & CASS & $T$ & \\
\hline$T E-104-A P-48$ & $\begin{array}{l}6 / 11 / 94 \\
6 / 18 / 94\end{array}$ & 61 & CASS & $\mathbf{T}$ & \\
\hline TE-104-AP-48 & $\frac{6 / 18 / 94}{6 / 25 / 94}$ & $\frac{60}{61}$ & CASS & $T$ & \\
\hline TE-104-AP-48 & $6 r 30 / 94$ & 63 & $\begin{array}{l}\text { CASS } \\
\text { CASS }\end{array}$ & $\frac{T}{T}$ & \\
\hline$T E-104-A P-48$ & $7 / 4 / 94$ & 65 & CASS & $T$ & \\
\hline TE-104-AP-48 & $7710 \mathrm{~A}$ & 65 & CASS & $T$ & \\
\hline TE-104AP-48 & $7 / 22294$ & 66 & CASS & $T$ & \\
\hline TE-104-AP- 48 & $7 / 30 / 94$ & 66 & CASS & $T$ & \\
\hline TE-104-AP-48 & $815 \% 4$ & 67 & CASS & $T$ & \\
\hline TE-104AP-48 & $8 / 13 / 94$ & 69 & CASS & $T$ & \\
\hline IE-104AP-48 & $8 / 20194$ & 68 & CASS & $T$ & \\
\hline TE-104-AP-48 & $8 / 22194$ & 70 & CASS & $T$ & \\
\hline TE-104-AP-48 & $823 \% 4$ & 70 & CASS & $T$ & \\
\hline TE-104-AP-48 & 8/84 & 69 & CASS & $T$ & \\
\hline TE-104AP-48 & g11\% & 69 & CASS & $\mathbf{T}$ & \\
\hline TE-104AP-AB & $8 / 2494$ & 68 & CASS & $T$ & \\
\hline JE-104-AP 48 & SKOKA & 69 & CASS & $T$ & \\
\hline TE-10A-AP-AB & $10 / 294$ & 69. & CASS & $T$ & \\
\hline TE-104-AP-AB & $10 / 12 \%$ & 69 & CASS & $T$ & \\
\hline$T E-104-A P-4 B$ & 10/21/39 & 67 & CASS & $T$ & \\
\hline$T E-104-A P-48$ & $10 / 27 / 94$ & 66 & CASS & $T$ & \\
\hline$T E-104-A P-48$ & TISTH & 65 & CASS & $T$ & \\
\hline TE-104AP-4B & 11/BA & 64 & CASS & $T$ & \\
\hline TE-104-AP-48 & $11 / 16 \mathrm{ax}$ & 62 & CASS & $T$ & \\
\hline TE-104-AP-48 & $11 / 249$ & 60. & CASS & $T$ & \\
\hline TE-104-AP-48 & $11 / 30 \mathrm{OA}$ & 6 & CASS & $T$ & \\
\hline$T E-104-A P-48$ & 12404 & 60 & CASS & $T$ & \\
\hline TE-104-AP-48 & 121409 & 57 & CASS & $\mathbf{T}$ & \\
\hline TE-104AP-48 & $12 / 21 \%$ & 53 & CASS & $T$ & \\
\hline TE-104AP-48 & $1228 \%$ & 56 & CASS & $T$ & \\
\hline TE-104AP-48 & $1 / 1 / 55$ & 56. & CASS & $T$ & \\
\hline TE-104AP-18 & $1 / 14 / 8$ & 53 & CASS & 7 & \\
\hline TE-104AP- 48 & 1 120/05 & 54 & CASS & $\mathbf{T}$ & \\
\hline TE-104AP-AB & $1 / 2565$ & 53 & CASS & $T$ & \\
\hline TE-104-AP-43 & 2205 & 54 & CASS & $T$ & \\
\hline TE-104-AP-48 & 2605 & 54 & CASS & $\mathbf{T}$ & \\
\hline TE-104-AP-48 & $2 / 1845$ & 53 & CASS & $T$ & \\
\hline$T E-104-A P-48$ & $2 / 2225$ & 55 & CASS & $T$ & \\
\hline TE-10A-AP-48 & 3/35 & 53 & CASS & $\bar{T}$ & \\
\hline TE-104AP-AB & $3 / 10 / 5$ & 54 & CASS & $T$ & \\
\hline$T E=104-A P-40$ & 3/16/95 & 55 & CASS & $T$ & \\
\hline TE-104-AP-48 & $3 / 22295$ & 55 & CASS & $T$ & \\
\hline TE-104-AP-48 & $3 / 29195$ & 54 & CASS & $\bar{T}$ & \\
\hline TE-104-AP-49 & 45363 & 55 & CASS & & \\
\hline TE-104-AP-49 & $4 / 10 / 66$ & 56 & $\frac{\text { CASS }}{\text { CASS }}$ & $\frac{T}{T}$ & \\
\hline TE-104-AP- 49 & 4/19/66 & 55 & CASS & $T$ & \\
\hline TE-104-AP-19 & 4/26/86 & 55 & CASS & $T$ & \\
\hline TE-104-AP-49 & $4 / 29 / 86$ & 55 & CASS & $T$ & \\
\hline TE-104-AP- 19 & $5 / 16 / 66$ & 56! & CASS & $T$ & \\
\hline TE-104-AP- 49 & $5 / 23 / 86$ & 57 & CASS & $\bar{T}$ & \\
\hline TE-104AP-19 & $5 / 30 / 86$ & 59 & CASS & $T$ & \\
\hline TE-104-AP-19 & 6/486 & 57 & CASS & $T$ & \\
\hline$T E-104-A P-19$ & $6 / 12266$ & 5 & CASS & $T$ & \\
\hline TE-104AP- 49 & $6 / 2008$ & 59 & CASS & $T$ & \\
\hline TE-104-AP- 49 & 6/26186 & 58 & CASS & $T$ & \\
\hline TE-104-AP-49 & 71486 & 53 & CASS & $T$ & \\
\hline TE-104AP-49 & $7 / 11 / 86$ & 114 & CASS & s & Data point did not agree with local trend \\
\hline TE-104-AP-49 & 71/2236 & 58 & CASS & $T$ & \\
\hline TE-104.AP 49 & 714486 & 60 & CASS & $\mathbf{T}$ & \\
\hline TE-104.AP-49 & 7/20/86 & 61 & CASS & $\mathbf{T}$ & \\
\hline TE-104AP-49 & $8 / 2 / 86$ & 59 & CASS & $\mathrm{T}$ & \\
\hline TE-104AP- 49 & $8 / 3 / 66$ & 60 & CASS & $\mathbf{T}$ & \\
\hline TE-104-AP-19 & $8 / 16 / 86$ & 59 & CASS & $T$ & \\
\hline TE-104-AP-49 & $8 / 19 / 86$ & 61 & CASS & $\mathrm{T}$ & \\
\hline TE-104APA9 & $8 / 30 / 86$ & 60 & CASS & $T$ & \\
\hline TE-104AP-49 & 9660 & 64 & CASS & $T$ & \\
\hline TE-104-AP-49 & 99/86 & 64 & CASS & $\mathbf{T}$ & \\
\hline
\end{tabular}




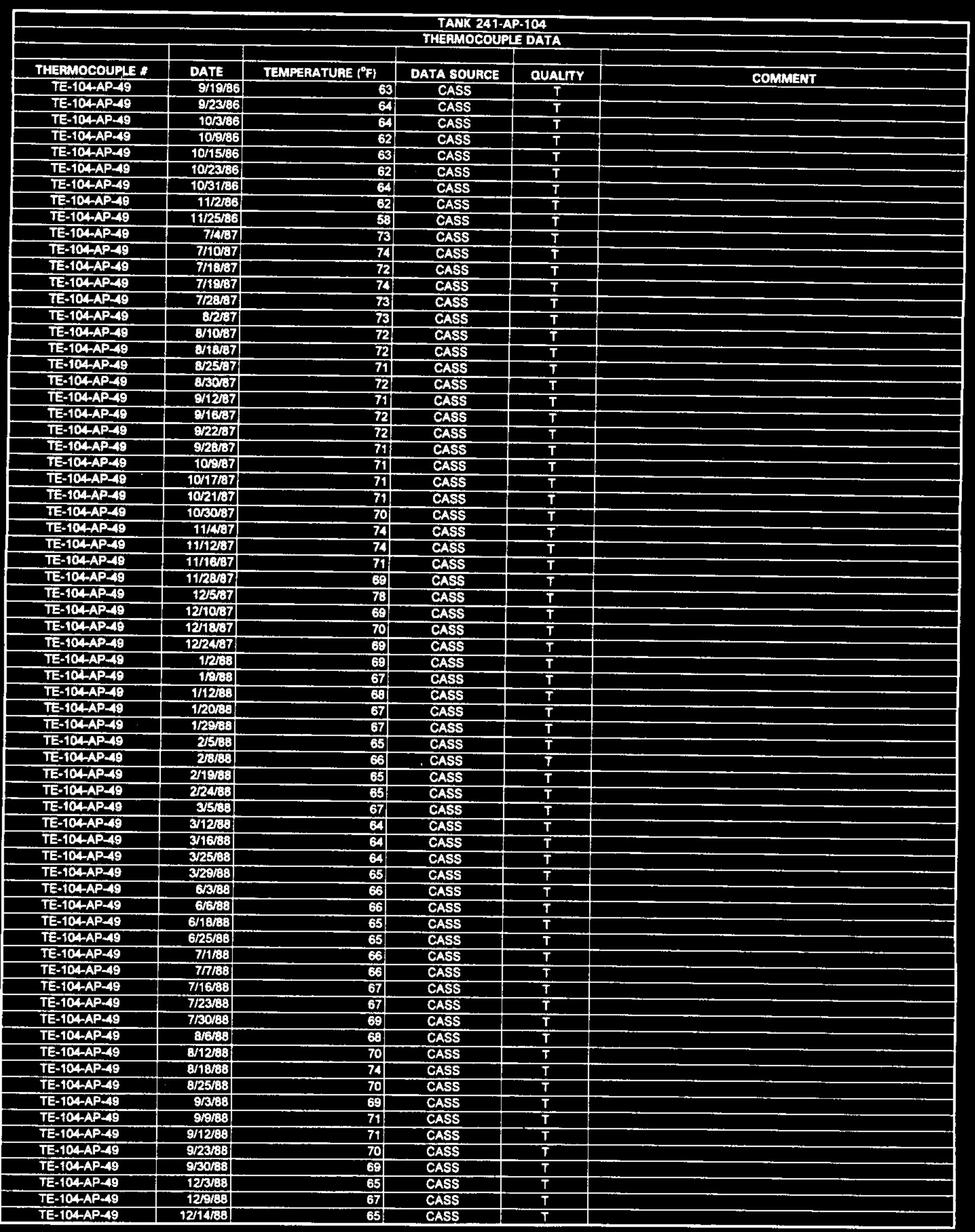




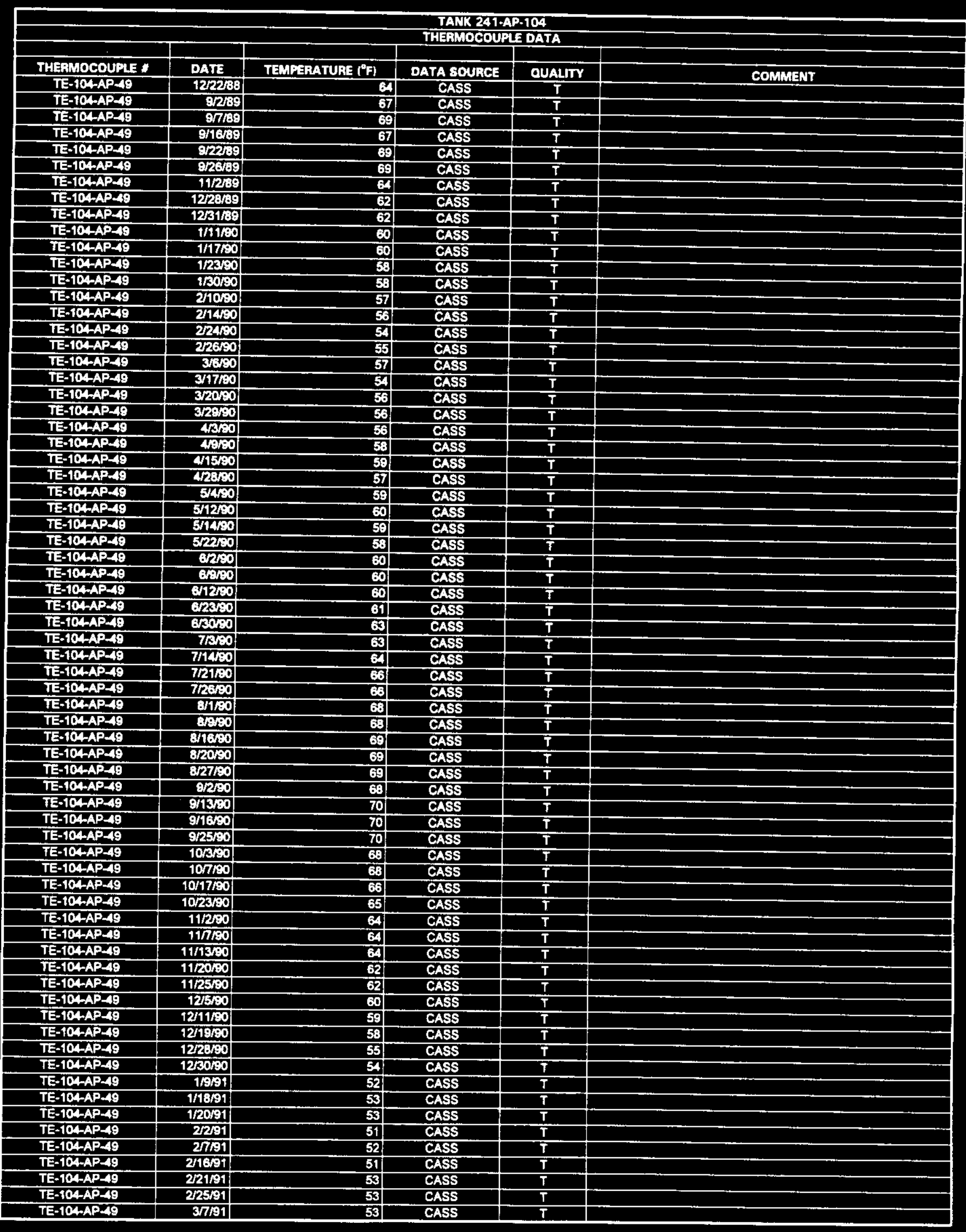

Data obtained from Computer Automated Surveillance System (CASS), April 1995; and Surveillance Analyais Computer Syatem (SACS), June 1995. $D-389$ 


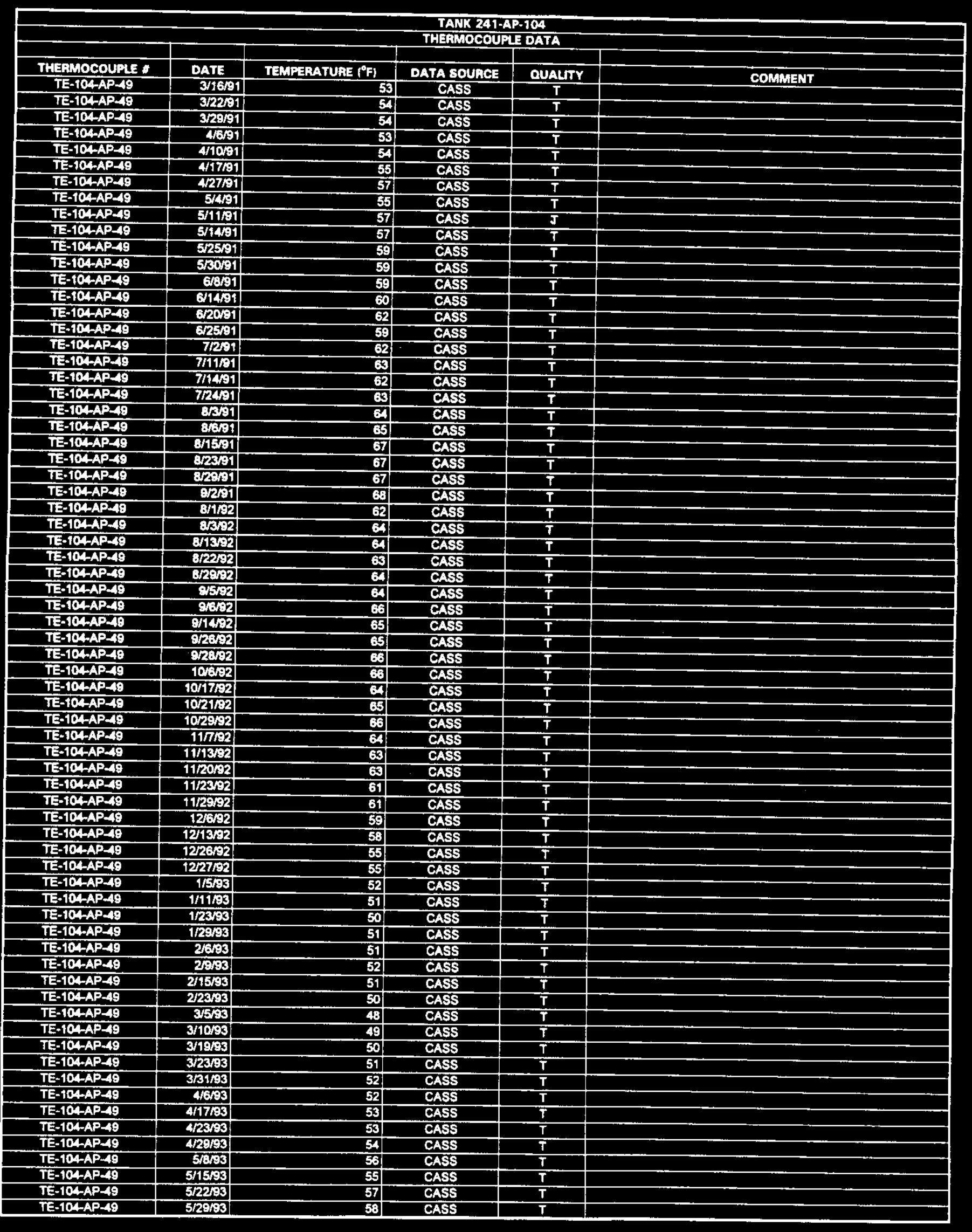




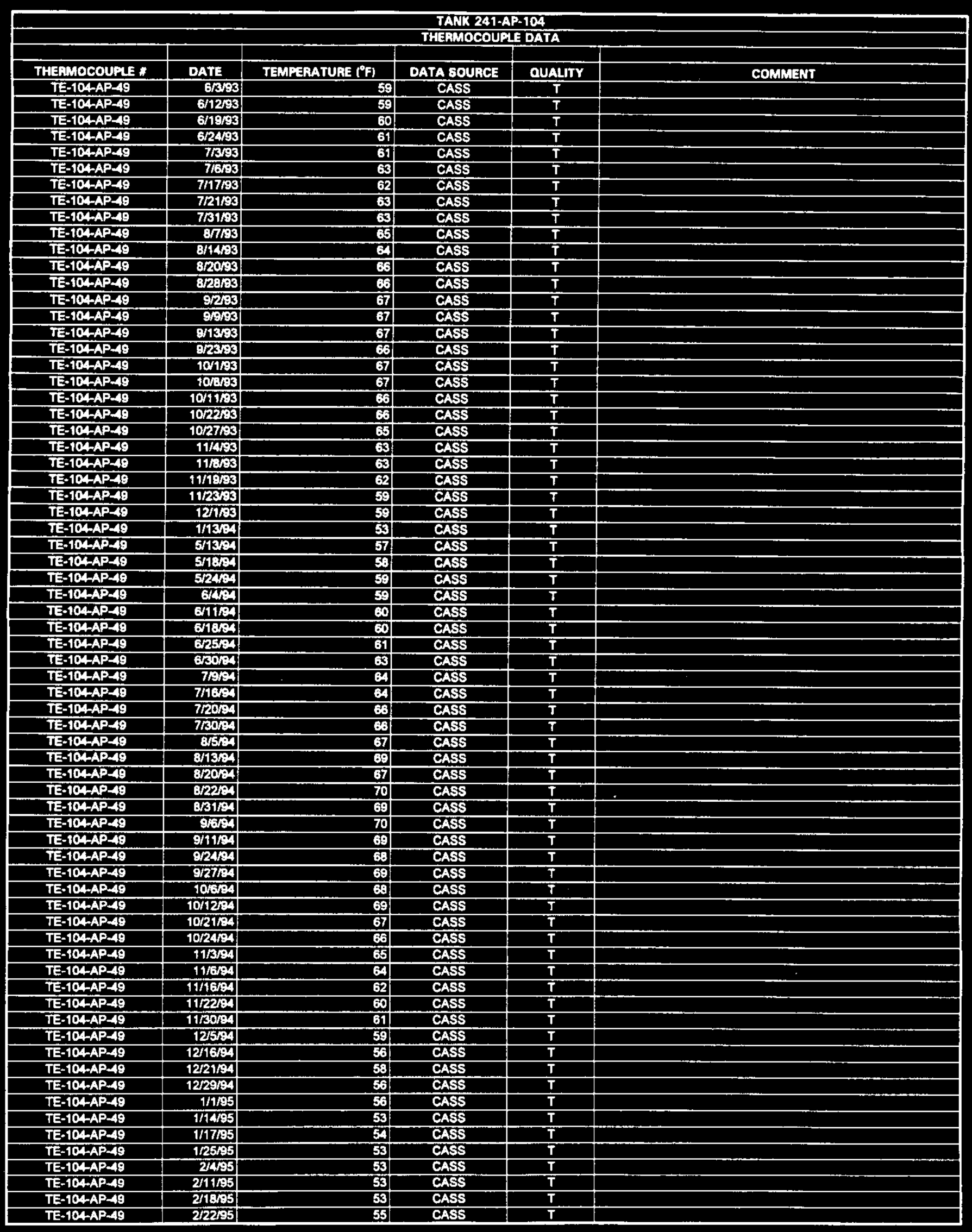

Data obtained from Computer Automated Survaillance System \{CASS), Aprił 1995; and Surveillance Analysis Computer System (SACS), June 1995.

$$
\text { D-391 }
$$




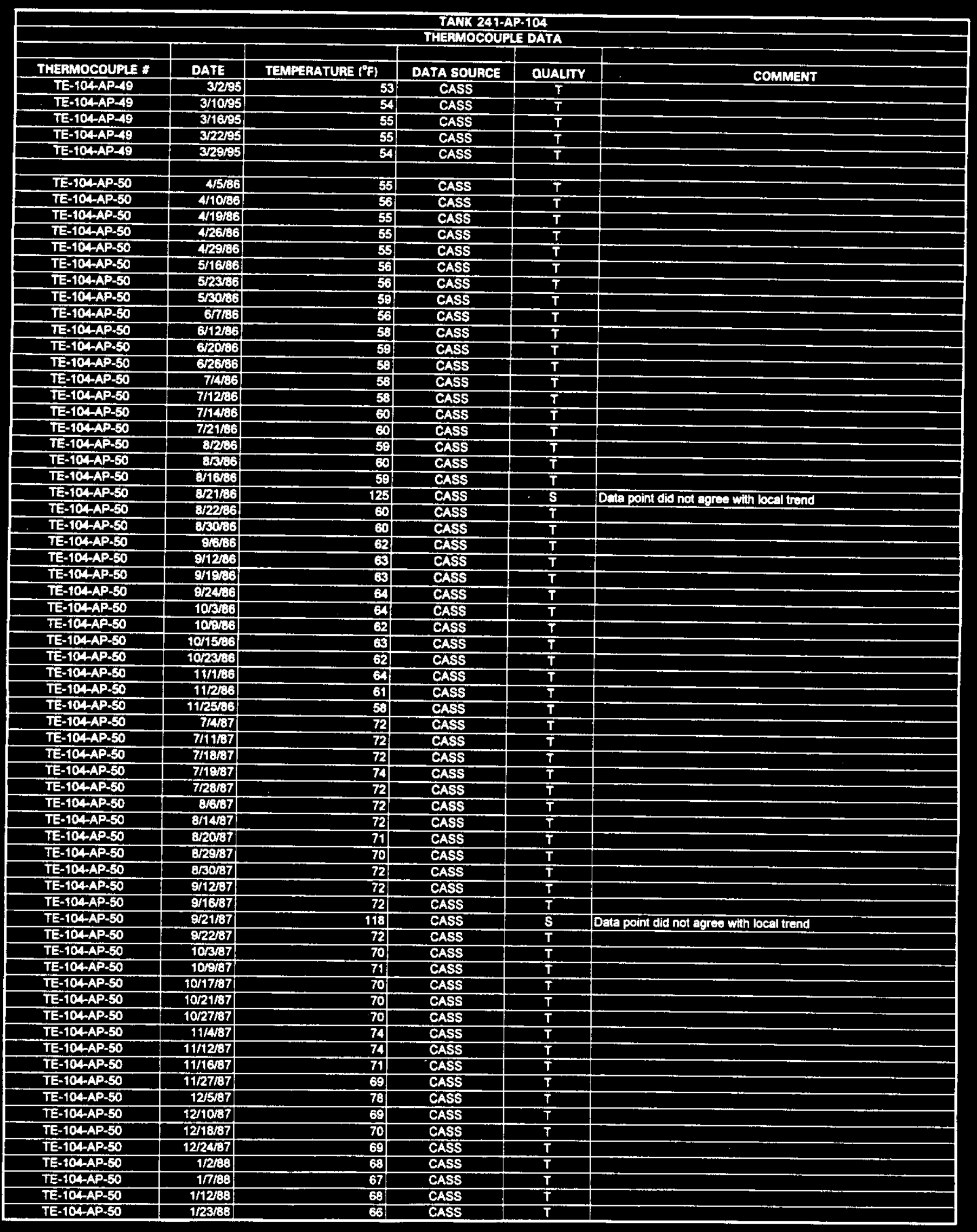

Data obteined from Computer Automated Surveillance Syatem (CASS), April 1995; and Surveillance Analysis Computer System (SACS), June 1995. D-392 


\begin{tabular}{|c|c|c|c|c|c|}
\hline \multicolumn{6}{|c|}{$\begin{array}{l}\text { TANK 241-AP-1O4 } \\
\text { THERMOCOUPLE DATA }\end{array}$} \\
\hline THERMOCOUPLE * & DATE & TEMPERATUAE $\left({ }^{\circ} \mathrm{F}\right)$ & Data souace & & \\
\hline$\frac{\text { THERMOCOUPL }}{\text { TE-104AP-SO }}$ & D/ALE & & & QUALTY & COMMENT \\
\hline$\frac{T E-104 A P-50}{T E-104 A P-50}$ & $2 / 1 / 88$ & $\begin{array}{l}66 \\
65\end{array}$ & CASS & $T$ & \\
\hline$\frac{T E-104 A A P-50}{\text { TE-104AP-50 }}$ & $2 / 2 / 88$ & 65 & CASS & $T$ & \\
\hline TE-104-AP-SO & 2/49/88 & 65 & $\frac{\text { CASS }}{\text { CASS }}$ & $\mathbf{T}$ & \\
\hline TE-104-AP.50 & 2/23/38 & 6 & $\begin{array}{l}\text { CASS } \\
\text { CASS }\end{array}$ & $T$ & \\
\hline TE-104AP-50 & $3 / 5 / 88$ & 66 & $\begin{array}{l}\text { CASS } \\
\text { CASS }\end{array}$ & $\frac{T}{T}$ & \\
\hline TE-104-AP-50 & $3 / 10 / 38$ & 64 & CASS & $T$ & \\
\hline TE-104AP-S0 & 3/16/38 & 64 & CASS & $\bar{T}$ & \\
\hline TE-104-AP-50 & 3/25/38 & 84 & CASS & $\mathbf{T}$ & \\
\hline TE-104-AP-50 & $3 / 29 / 88$ & 65 & CASS & $T$ & \\
\hline TE-104AP-50 & $6 / 3 / 38$ & 66 & CASS & $T$ & \\
\hline TE-104-AP-50 & $6 / 6 / 68$ & 65 & CASS & $T$ & \\
\hline TE-104-AP-50 & 6/98/88 & 66 & CASS & $\bar{T}$ & \\
\hline TE-104-AP-50 & 6/25/38 & 66 & CASS & $T$ & \\
\hline TE-104-AP-50 & $7 / 238$ & 65 & CASS & $T$ & \\
\hline TE-104-AP-50 & $7 \pi / 38$ & 66 & CASS & $\mathbf{T}$ & \\
\hline TE-104AP-50 & $7111 / 88$ & 124 & CASS & $\mathbf{S}$ & Data point did not agree with local trend \\
\hline TE-104AP-50 & 7/13/68 & 67 & CASS & $\mathbf{T}$ & \\
\hline TE-104AP-50 & 7 723/38 & 67 & CASS & $T$ & \\
\hline TE-104-AP-50 & $7 / 30 / 68$ & 60 & CASS & $T$ & \\
\hline TE-104AP-50 & $8 / 233$ & 69 & CASS & $T$ & \\
\hline TE-104AP-50 & $8 / 12 / 38$ & 70 & CASS & $T$ & \\
\hline TE-104AP-50 & A/18/88 & 74 & CASS & $T$ & \\
\hline TE-104AP-50 & $8 / 25368$ & 71 & CASS & $T$ & \\
\hline TE-104AP-50 & $9 / 3 / 8$ & 69 & CASS & $T$ & \\
\hline TE-104-AP-50 & SEB & 70 & CASS & $T$ & \\
\hline TE-104-AP-50 & $9 / 12 / 36$ & 71 & CASS & $T$ & \\
\hline TE-104-AP-50 & $9 / 23 / 68$ & 70 & CASS & $\mathbf{T}$ & \\
\hline TE-104-AP-50 & 9/28/38 & 69 & CASS & $T$ & \\
\hline TE-104-AP-S0 & 93063 & 111 & CASS & $\mathbf{s}$ & Data point did not agree with local trend \\
\hline TE:104AP-50 & 123300 & 85 & CASS & $T$ & \\
\hline TE-1OYAP-50 & $120 / 8$ & 66 & CASS & $T$ & \\
\hline TE-104-AP-50 & 121438 & 65 & CASS & $\bar{T}$ & \\
\hline TE-104AP-50 & $12 / 2230$ & 64 & CASS & $T$ & \\
\hline TE-104AP-50 & $8 / 269$ & 67 & CASS & $T$ & \\
\hline TE-104-AP.SO & $8 / 739$ & eी & CASS & $T$ & \\
\hline TE-104AP-50 & 8/16/89 & 67 & CASS & $T$ & \\
\hline TE-104AP-50 & 8/22/39 & 69 & CASS & $\mathbf{T}$ & \\
\hline TE-104AP-50 & $9 / 2669$ & 69 & CASS & $T$ & \\
\hline TE-104AP-50 & $11 / 2 / 39$ & 64 & CASS & $\bar{T}$ & \\
\hline TE-104-AP-SO & 1212369 & 62 & CASS & $T$ & \\
\hline TE-104-AP-SO & 12/31/39 & 62 & CASS & $T$ & \\
\hline TE-104-AP-50 & $1 / 10 \% 0$ & 60 & CASS & $\bar{T}$ & \\
\hline TE-104-AP-50 & 1/20\%0 & 59 & CASS & $T$ & \\
\hline TE-104-AP-50 & $1 / 23100$ & 58 & CASS & $T$ & \\
\hline TE-104-AP-50 & $1 / 30 / 90$ & 58 & CASS & $\mathbf{T}$ & \\
\hline TE-104-AP-50 & 27100 & 57 & CASS & $\mathbf{T}$ & \\
\hline TE-104-AP-BO & 2/4190 & 56 & CASS & $T$ & \\
\hline TE-104AP-50 & $2 / 23 / 90$ & 54 & CASS & $T$ & \\
\hline TE-104AP-SD & 2/26\%00 & 55 & CASS & $T$ & \\
\hline TE-104-AP-50 & $3 / 690$ & 57 & CASS & $\mathbf{T}$ & \\
\hline TE-104AP-50 & 3/1750 & 54 & CASS & $\mathbf{T}$ & \\
\hline TE-104-AP-50 & $3 / 24 / 90$ & 55) & CASS & $\bar{T}$ & \\
\hline TE-104-AP-50 & $3 / 28 / 90$ & 56 & CASS & $\mathbf{T}$ & \\
\hline TE-104-AP-50 & 47180 & 55 & CASS & $T$. & \\
\hline TE-104-AP-50 & $4 / 1250$ & 56 & CASS & $T$ & \\
\hline TE-104-AP-50 & $4 / 15 / 20$ & 59. & CASS & $T$ & \\
\hline TE-10A-AP-50 & $4 / 28 / 00$ & 57 & CASS & $T$ & \\
\hline TE-104AP.50 & 51400 & 58 & CASS & $T$ & \\
\hline TE-104-AP-50 & 5/12/90 & 60 & CASS & $T$ & \\
\hline TE-104AP-50 & 5/14/00 & 59 & CASS & $T$ & \\
\hline TE-104-AP-50 & 5/22/00 & 53 & CASS & $T$ & \\
\hline TE-104AP-50 & 6/2/50 & 60 & CASS & $T$ & \\
\hline TE-104-AP-50 & 6raso & का & CASS & $T$ & \\
\hline TE-104AP-50 & 6/16/90 & 59 & CASS & $\bar{T}$ & \\
\hline TE-104-AP-50 & 6/23/90 & 61 & CASS & $\mathbf{T}$ & \\
\hline TE-104-AP-50 & 6/30/00 & 63 & CASS & $T$ & \\
\hline TE-104AP-50 & $77 \% 90$ & 62 & CASS & $T$ & \\
\hline $\mathrm{TE}-104$ AP- 50 & 714600 & 64 & CASS & $T$ & \\
\hline TE-104-AP-50 & 7121100 & 66 & CASS & $\bar{T}$ & \\
\hline
\end{tabular}




\begin{tabular}{|c|c|c|c|c|c|}
\hline \multicolumn{6}{|c|}{$\begin{array}{l}\text { TANK 241-AP-104 } \\
\text { THERMOCOUFLE DATA }\end{array}$} \\
\hline & & & & & \\
\hline THEAMOCOUPL * & DATE & TEMFERATURE ( $\left.{ }^{\circ} \mathrm{F}\right)$ & DATA SOUACE & QUALTY & COMMENT \\
\hline TE-104-AP-50 & $7 / 26 \%$ & 66 & CASS & $T$ & \\
\hline TE-104-AP-50 & $8 / 1 / 50$ & 68 & CASS & $T$ & \\
\hline TE-104AP-50 & B/O/SO & 69 & CASS & $\mathbf{T}$ & \\
\hline TE-104AP-50 & $8 / 16 / 90$ & 69 & CASS & $\mathbf{T}$ & \\
\hline TE-104-AP-50 & $8 / 20 / 00$ & 69 & CASS & $T$ & \\
\hline TE-104-AP-50 & $8 / 27100$ & 68 & CASS & $T$ & \\
\hline TE-104-AP.50 & 92/50 & 68 & CASS & $\mathbf{T}$ & \\
\hline TE-104AP-S0 & 9/1\%0 & 70 & CASS & $T$ & \\
\hline TE-104AP-S0 & 916\%0 & 70 & CASS & $T$ & \\
\hline TE-104-AP-50 & 9/25rs0 & 70 & CASS & $T$ & \\
\hline TE-104-AP-SO & 10/3/50 & 68 & CASS & $T$ & \\
\hline TE-104-AP-50 & $107 / 50$ & 68 & CASS & $T$ & \\
\hline TE-104-AP-50 & $10 / 1600$ & ES & CASS & $T$ & \\
\hline TE-104AP-50 & $10 / 23100$ & 65 & CASS & $T$ & \\
\hline TE-104AP-50 & $10 / 3000$ & (2) & CASS & $T$ & \\
\hline TE-104AP-SO & $11 \pi / 20$ & 64 & CASS & $T$ & \\
\hline TE-104APS.50 & $11 / 1350$ & 81 & CASS & $T$ & \\
\hline TE-104AP. 50 & $11 / 1850$ & 62 & CASS & $T$ & \\
\hline TE-104AP-50 & $11 / 2550$ & 62 & CASS & $\mathbf{T}$ & \\
\hline TE-104AP-50 & 121580 & 60 & CASS & $\mathbf{T}$ & \\
\hline TE-104AP-60 & $12 / 1150$ & 59 & CASS & $T$ & \\
\hline TE-104-APSEO & $12 \pi 1900$ & 53 & CASS & $\mathbf{T}$ & \\
\hline TE-104AP-EO & $12 / 2200$ & 83. & CASS & $\mathbf{s}$ & Data point did not agrese with local frend \\
\hline TE-104AP.SO & 12/28/so & 55 & CASS & $\bar{T}$ & \\
\hline TE-104APSB & 123000 & BA & CASS & $T$ & \\
\hline TE-TOAAPSE & 1FA1 & 52 & CASS & $T$ & \\
\hline TE-104-AP-50 & 1/10\%1 & 53 & CASS & $T$ & \\
\hline TE-104-AP-50 & $1 / 2301$ & 52: & CASS & $T$ & \\
\hline TE-104-AP-50 & $2 / 2 \omega 1$ & 51 & CASS & $T$ & \\
\hline TE-104AP-SO & 27191 & 52 & CASS & $T$ & \\
\hline TE-104-AP-SO & $2716 / 21$ & 51 & CASS & $\mathbf{T}$ & \\
\hline TE-104AP-50 & $2 / 2101$ & 53 & CASS & $\bar{T}$ & \\
\hline TE-104-AP.50 & $2 / 25191$ & 53 & CASS & $T$ & \\
\hline TE-104AP-50 & 3761 & 5 & CASS & $\mathbf{T}$ & \\
\hline TE-104-AP-50 & 3/1609 & 5 & CASS & $T$ & \\
\hline TE-10.AP-60 & 3/21/31 & SA & CASS & $T$ & \\
\hline$T E-104 A P-50$ & 3/2981 & SA & CASS & $T$ & \\
\hline TE-104-AP-50 & 4391 & $5 \mathbf{5 3}$ & CASS & $\mathbf{T}$ & \\
\hline TE-104-AP-50 & 4/1091 & SA & CASS & $\mathbf{T}$ & \\
\hline TE-104AP-50 & 4/7631 & 55 & CASS & $T$ & \\
\hline TE-104AP.50 & $4 / 27 / 61$ & 57. & CASS & $\mathbf{T}$ & \\
\hline TE-10A-AP-50 & 54at: & 55 & CASS & $\mathbf{T}$ & \\
\hline TE-104AP-50 & 511\%1 & 57 & CASS & $T$ & \\
\hline TE-104-AP-50 & 5/1691 & 57 & CASS & $\mathbf{T}$ & \\
\hline TE-104-APS50 & $5 / 25 / 91$ & 59 & CASS & $T$ & \\
\hline TE-104AP.50 & $5 / 3001$ & 59 & CASS & $\mathbf{T}$ & \\
\hline TE-104AP-SO & G/91 & 59 & CASS & $T$ & \\
\hline TE-104AP-50 & 6/14/91 & 61 & CASS & $\mathbf{T}$ & \\
\hline TE-104-AP-50 & $6 / 20 \% 1$ & 62 & CASS & $\mathbf{T}$ & \\
\hline TE-104-AP-50 & $6 / 2591$ & 59 & CASS & $T$ & \\
\hline TE-104AP-50 & $7 / 291$ & 62 & CASS & $\mathbf{T}$ & \\
\hline TE-104AP-50 & 7/11/91 & 64 & CASS & $\mathbf{T}$ & \\
\hline TE-104AP-50 & 77491 & 62 & CASS & $T$ & \\
\hline TE-104-AP-50 & 712491 & 63 & CASS & $T$ & \\
\hline TE-104-AP-SO & 8/391 & 64 & CASS & $T$ & \\
\hline TE-104-AP-50 & $8 / 6 / 91$ & 64) & CASS & $T$ & \\
\hline TE-104-AP-50 & $8 / 15 / 91$ & 67 & CASS & $T$ & \\
\hline TE-104-AP-50 & $8 / 20 / 91$ & 68 & CASS & $T$ & \\
\hline TE-104-AP-50 & 8/29/91 & 67 & CASS & $T$ & \\
\hline TE-104APS50 & $9 / 3 / 91$ & 69 & CASS & $\bar{T}$ & \\
\hline TE-104-AP-50 & $8 / 1 / 92$ & 62 & CASS & $T$ & \\
\hline TE-104AP-50 & B/262 & 127 & CASS & $\mathbf{s}$ & Data point did not agree with locel trend \\
\hline TE-104AP-50 & $8 / 3 / 92$ & EA & CASS & $T$ & \\
\hline TE-104AP-50 & $8 / 43 / 92$ & 64) & CASS & $\mathbf{T}$ & \\
\hline TE-104AP-50 & $8 / 22 / 92$ & 63. & CASS & $T$ & \\
\hline TE-104-AP-50 & $8 / 29 / 92$ & 64 & CASS & $T$ & \\
\hline TE-104AP.50 & 9/5/2 & 63 & CASS & $T$ & \\
\hline TE-104-AP-50 & 9/11/92 & 65 & CASS & $T$ & \\
\hline TE-TOA-AP-50 & 9/14/22 & 65 & CASS & $T$ & \\
\hline TE-104AP-SO & $9 / 26 / 92$ & 65 & CASS & $T$ & \\
\hline
\end{tabular}




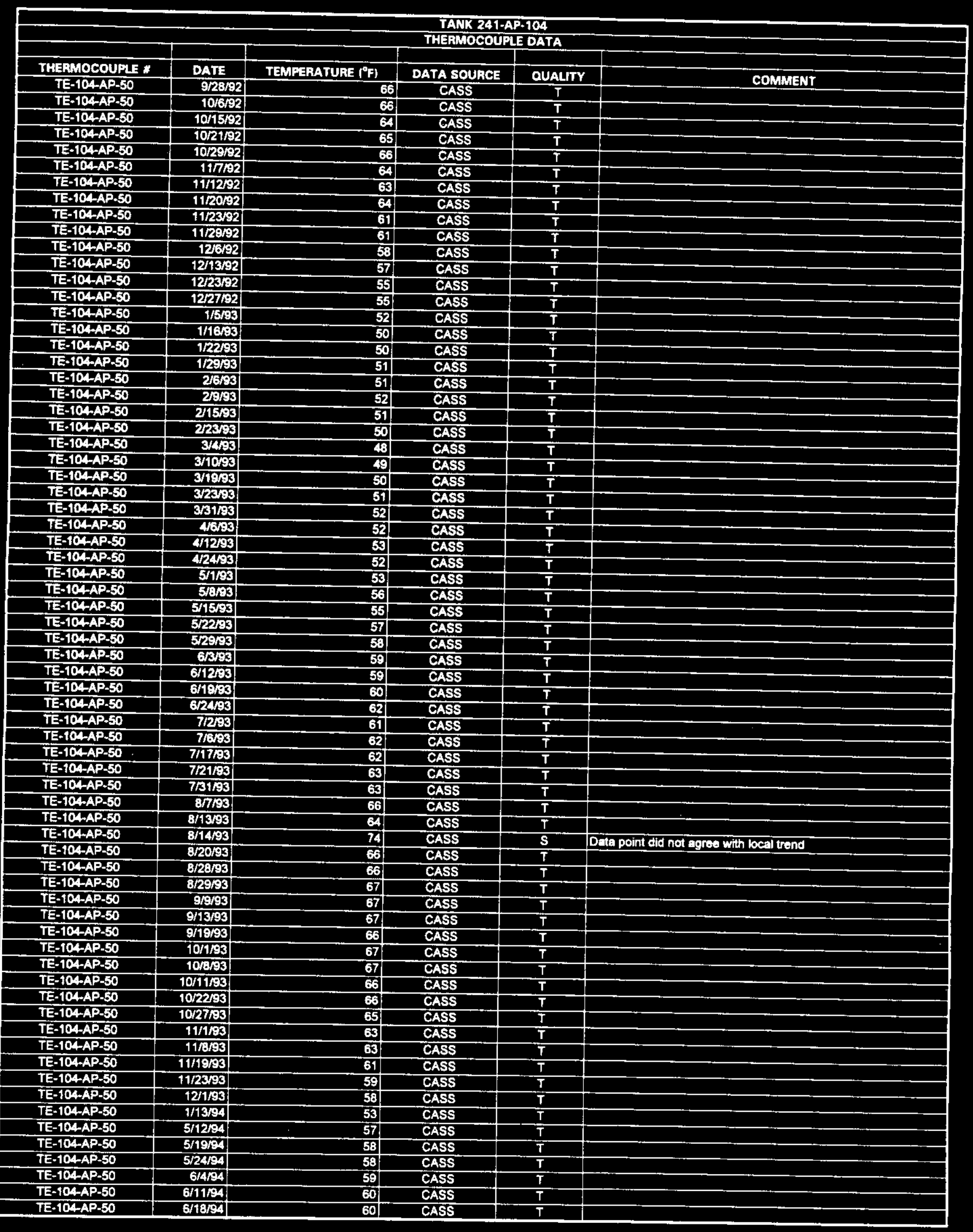

Data obtained from Computer Automated Surveillance System (CASS). April 1995; and Surveillance Analysis Computer System (SACS), June 1995. 


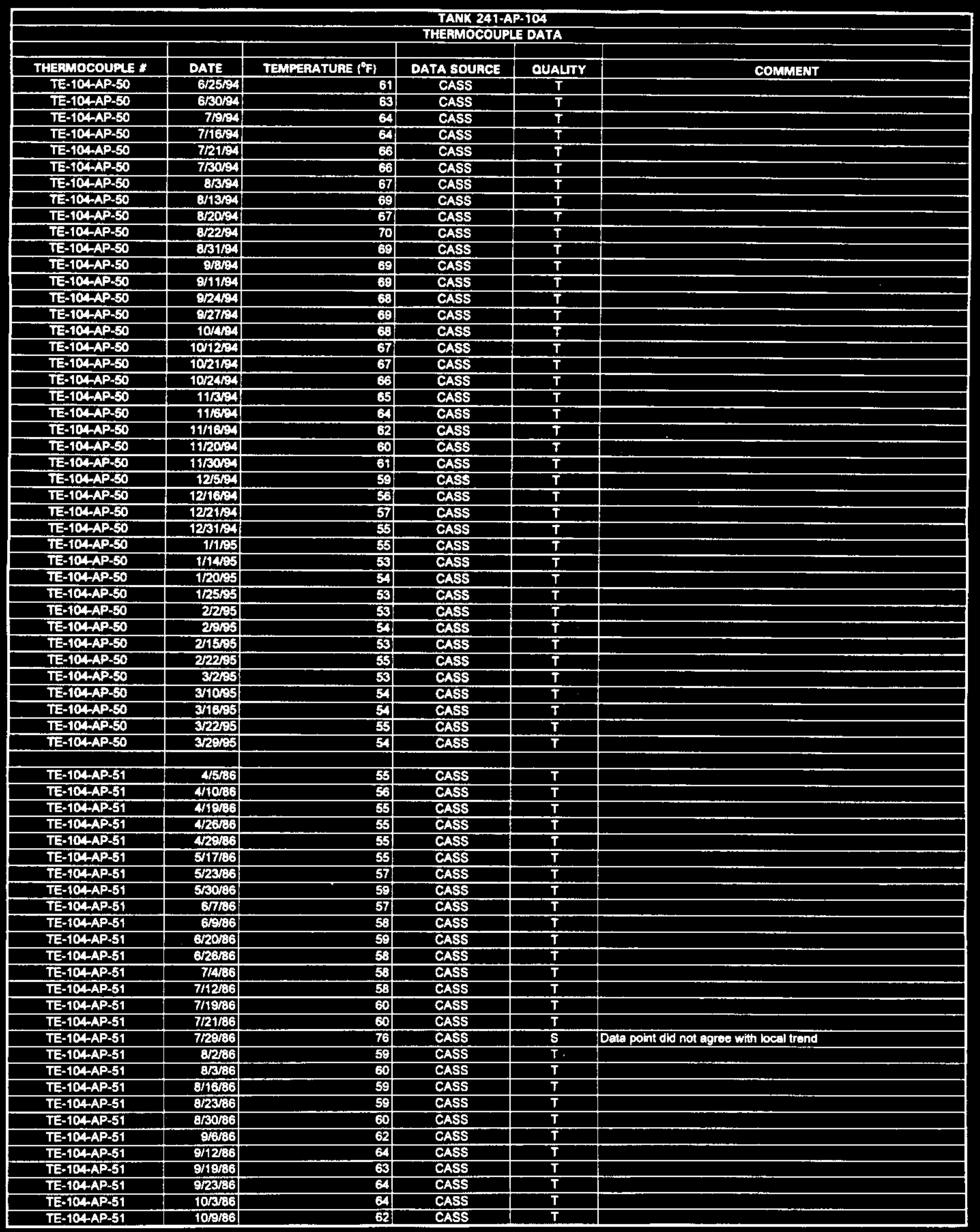




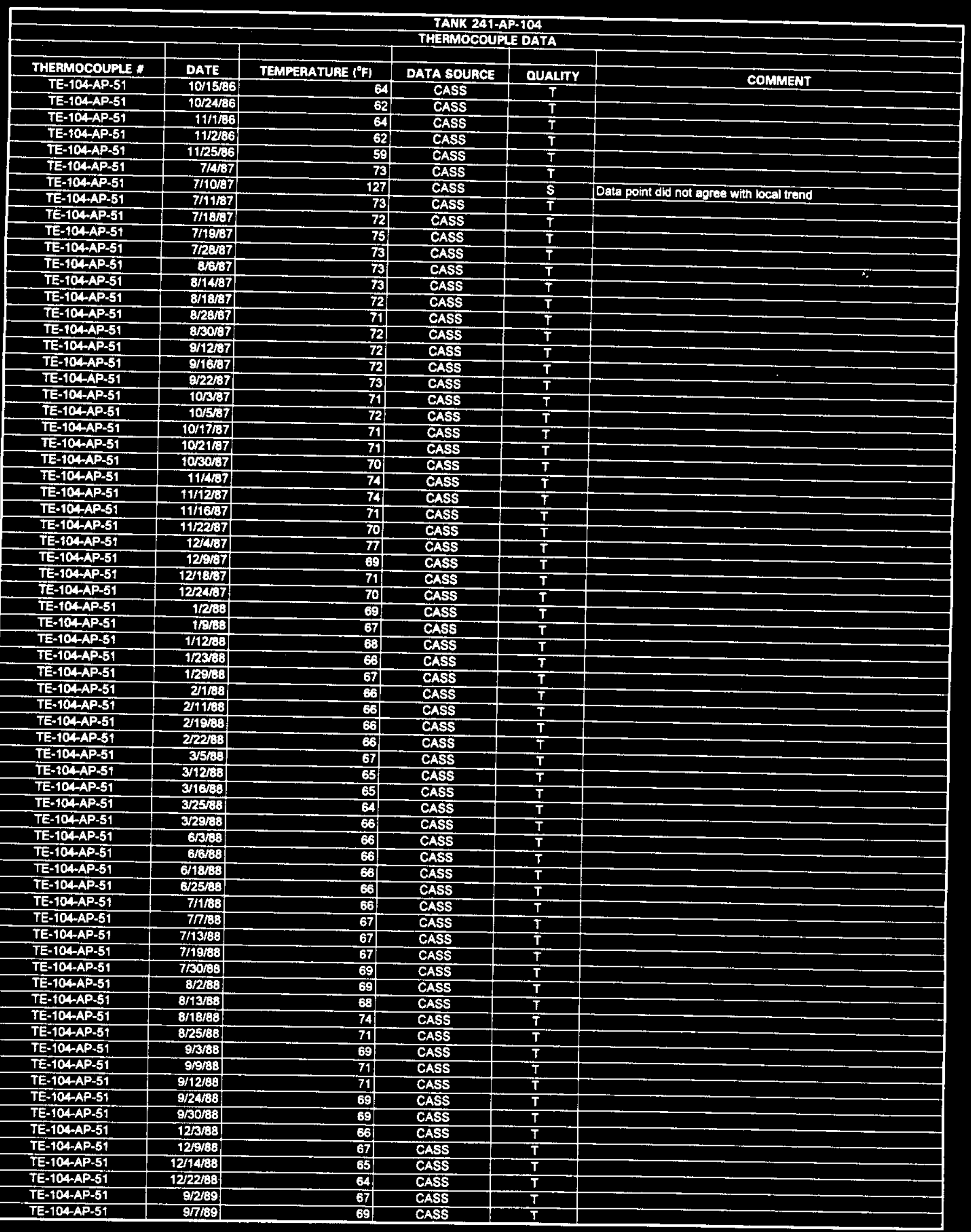

Data obtained from Computer Automated Surveillance System (CASS), April 1995; and Surveillance Analysis Computer System (SACS), June 1995. D-397 


\begin{tabular}{|c|c|c|c|c|c|}
\hline \multicolumn{6}{|c|}{$\begin{array}{l}\text { TANK 291.AP-104 } \\
\text { THERMOCOUPLE DATA }\end{array}$} \\
\hline & & & & & \\
\hline THERMOCOUPLE * & DATE & TEMPERATURE $\left({ }^{\circ} \mathrm{F}\right)$ & DATA SOURCE & OUALITY & COMMENT \\
\hline TE-104-AP-51 & $9 / 10 / 39$ & 63 & CASS & $T$ & \\
\hline TE-104-AP-51 & 8/22189 & 69 & CASS & $\bar{T}$ & \\
\hline TE-104-AP-51 & 9/26/89 & 69 & CASS & $T$ & \\
\hline TE-104AP-51 & $11 / 2369$ & 64 & CASS & $\bar{T}$ & \\
\hline TE-104AP-51 & 12/2B/69 & 63 & CASS & $T$ & \\
\hline TE-104AP-51 & $1 / 5 \% 0$ & 62 & CASS & $T$ & \\
\hline TE-104-AP-51 & 1/13/50 & 60 & CASS & $\bar{T}$ & \\
\hline TE-104-AP-51 & $1 / 2000$ & 59 & CASS & $\bar{T}$ & \\
\hline TE-104-AP-51 & $1 / 2400$ & 58 & CASS & $\bar{T}$ & \\
\hline TE-104-AP-51 & $1 / 3000$ & 38 & CASS & $T$ & \\
\hline TE-104AP-51 & 24100 & 58 & CASS & $T$ & \\
\hline TE-104AP-51 & $2 / 1450$ & 57: & CASS & $T$ & \\
\hline TE-104AP-51 & $2 / 2490$ & SA: & CASS & $T$ & \\
\hline TE-104AP-51 & $3 \sqrt{360}$ & 55 & CASS & $T$ & \\
\hline IE-104AP-51 & 3/600 & 57 & CASS & $T$ & \\
\hline TE-104AP-51 & 3/17:0 & 55 & CASS & $T$ & \\
\hline TE-104AP-51 & 32480 & 5 & CASS & $T$ & \\
\hline IE-104AP-51 & $3 / 2900$ & 56 & CASS & $T$ & \\
\hline TE-104AP-51 & 4600 & 56 & CASS & $\bar{T}$ & \\
\hline$T E-104-A P-51$ & 400 & 58 & CASS & $T$ & \\
\hline TE-104AP-51 & 4/1550 & 59 & CASS & $T$ & \\
\hline TE-104AP-51 & Traso & 37 & CASS & $T$ & \\
\hline TE-104AP-51 & 5WEO & 69 & CASS & $T$ & \\
\hline TE-104AP-51 & 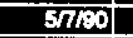 & 100 & CASS & $\overline{\mathbf{S}}$ & Dota point did not apree with local trend \\
\hline TE-104-AP-ST & $5 / 200$ & 60 & CASS & $T$ & \\
\hline TE-104AP-51 & 51400 & 50 & CASS & $\bar{T}$ & \\
\hline IE-1C4AP-51 & $5 / 2100$ & 50 & CASS & $\mathbf{T}$ & \\
\hline TE-104AP-51 & $6 / 2100$ & 61 & CASS & $T$ & \\
\hline TE-104AP-51 & BEOO & 60 & CASS & $\mathbf{T}$ & \\
\hline TE-104AP-51 & 61400 & 60 & CASS & $\mathbf{T}$ & \\
\hline TE.104AP-51 & 6/23/90 & 61 & CASS & $\mathbf{T}$ & \\
\hline TE-104AP-51 & ERerso & 6A & CASS & $T$ & \\
\hline TE-104APST & 7 TSOOL & 63 & CASS & $\mathbf{T}$ & \\
\hline TE-104AP-51 & 71480 & 64 & CASS & $\mathbf{T}$ & \\
\hline TE-104AP-51 & 77800 & 67 & CASS & $\bar{T}$ & \\
\hline TE-104AP-51 & 772630| & 66 & CASS & $T$ & \\
\hline TE-104AP-51 & $8 / 100$ & 68) & CASS & $T$ & \\
\hline TE-104AP-51 & $8 / 11 \%$ & 67 & CASS & $\mathbf{T}$ & \\
\hline TE-104AP-51 & 8/18:0 & 70 & CASS & $\mathbf{T}$ & \\
\hline TE TOLAP-51 & $8 \pi$ 2000 & 68 & CASS & $\bar{T}$ & \\
\hline 7.104 AP-51 & $8 / 27 / 00$ & 69 & CASS & $\bar{T}$ & \\
\hline$=104 . A P-51$ & 8\%O0 & 68) & CASS & $\mathbf{T}$ & \\
\hline TE. $104-A P-51$ & 9/13/90 & 70 & CASS & $T$ & \\
\hline TE-104-AP-51 & $9 / 1600$ & 70 & CASS & $T$ & \\
\hline TE=104AP-51 & 9/25/30 & 70 & CASS & $\bar{T}$ & \\
\hline TE-104AP-51 & $10 / 350$ & 69 & CASS & $\mathbf{T}$ & \\
\hline TE-104-AP-51 & $10 / 750$ & 69 & CASS & $T$ & \\
\hline TE-104-AP-51 & 10/19/90 & 66 & CASS & $T$ & \\
\hline TE-104-AP-51 & $10 / 233100$ & 66 & CASS & $T$ & \\
\hline TE-104-AP-51 & $11 / 3 / 300$ & 64) & CASS & $T$ & \\
\hline TE-104-AP-51 & 11/7r: & 65 & CASS & $T$ & \\
\hline TE-104AP-51 & $11 / 13 \%$ & 64 & CASS & $T$ & \\
\hline TE-104AP-51 & $11 / 2000$ & 62 & CASS & $T$ & \\
\hline TE-104-AP-51 & $11 / 2500$ & 62 & CASS & $T$ & \\
\hline TE-104-AP-51 & $12 \pi 250$ & 62 & CASS & $T$ & \\
\hline TE-104AP-51 & 12290 & 60 & CASS & $T$ & \\
\hline TE-104-AP-51 & 12/1980 & 50 & CASS & $T$ & \\
\hline TE-104AP-51 & 12 2668 & s5 & CASS & $T$ & \\
\hline TE-104-AP-51 & 123000 & 54 & CASS & $T$ & \\
\hline TE-104-AP-51 & $10 \times 1$ & 52 & CASS & $T$ & \\
\hline TE-104AP-S1 & 1/17/81 & 54 & CASS & $T$ & \\
\hline TE-104AP-51 & $1 / 2601$ & 53) & CASS & $T$ & \\
\hline TE-104-AP-51 & $2 / 2191$ & 51 & CASS & $T$ & \\
\hline TE-104AP-51 & 27191 & 53 & CASS & $T$ & \\
\hline TE-104-AP-51 & $2 / 16 / 91$ & 51 & CASS & $T$ & \\
\hline TE-104-AP-51 & $2 / 21 / 91$ & 53 & CASS & $T$ & \\
\hline TE-104AP-51 & 2/2491 & 53 & CASS & $T$ & \\
\hline TE-104-AP-51 & $3 / 691$ & 54 & CASS & $T$ & \\
\hline TE-104-AP-51 & 3/1691 & 53 & CASS & $T$ & \\
\hline TE-104-AP-51 & 3/20/91 & 55 & CASS & $T$ & \\
\hline
\end{tabular}




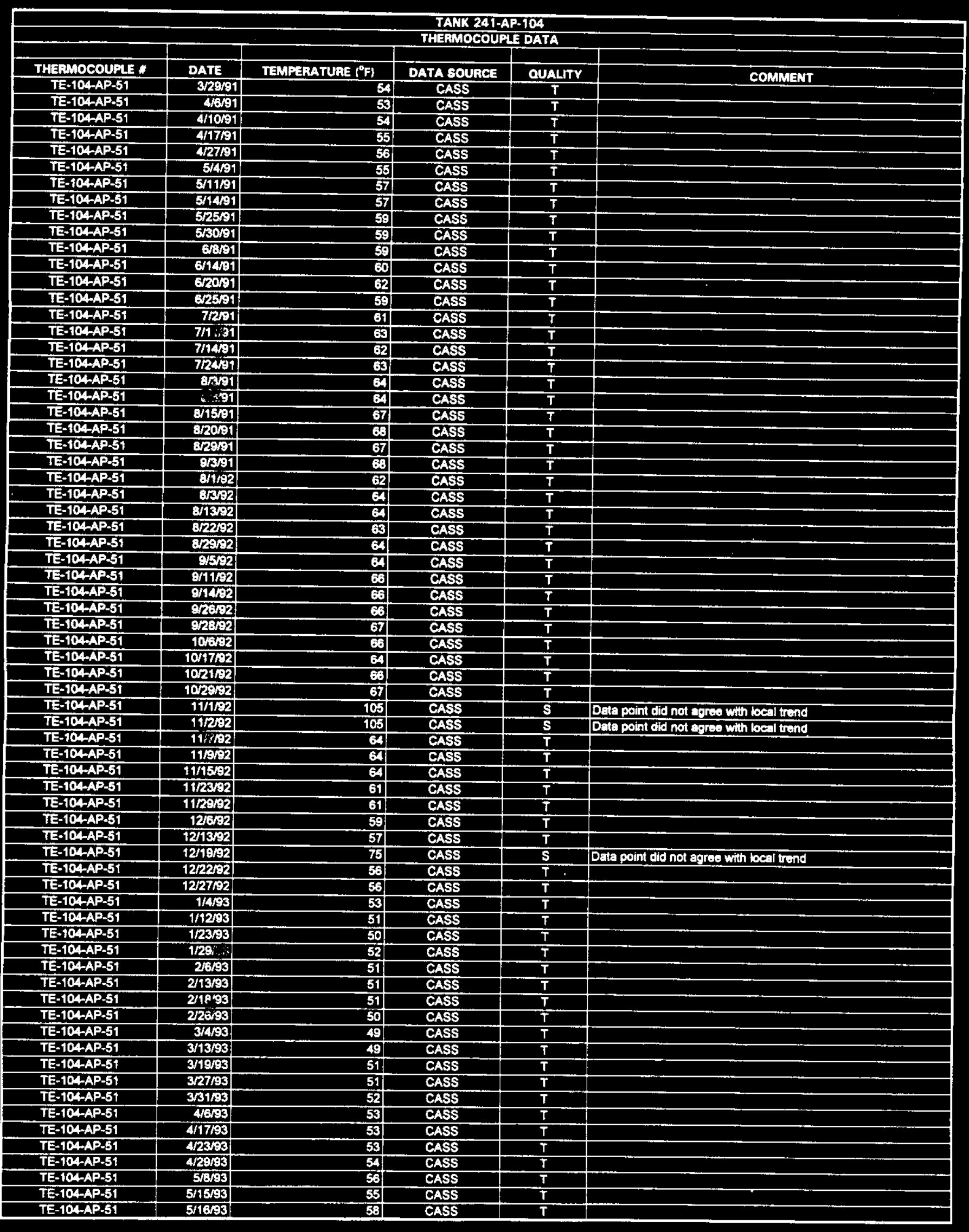

Date obtained from Computer Automated Surveillance Syatem (CASS), April 1995; and Surveillance Analysis Computer System (SACS), June 1995. D-399 


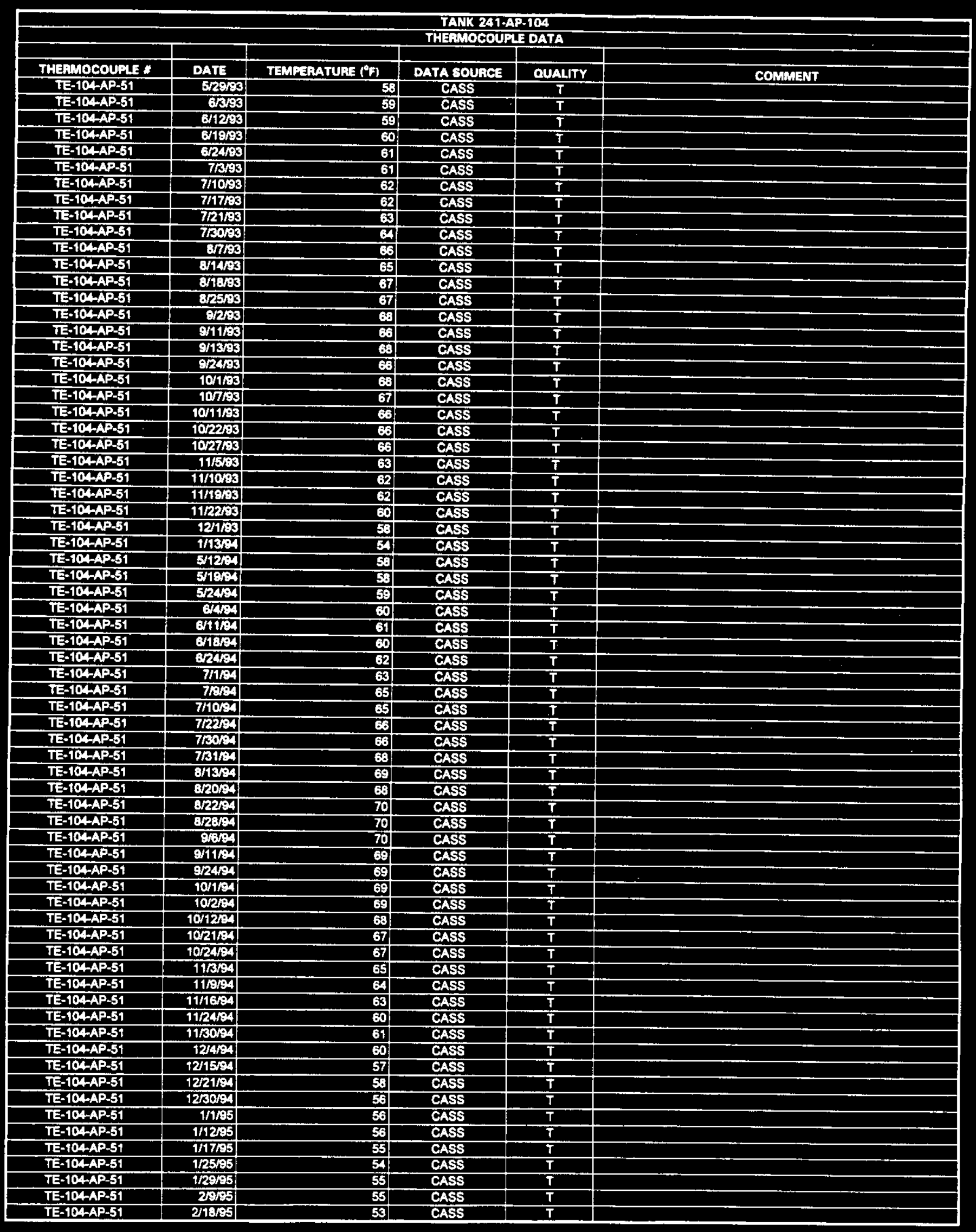




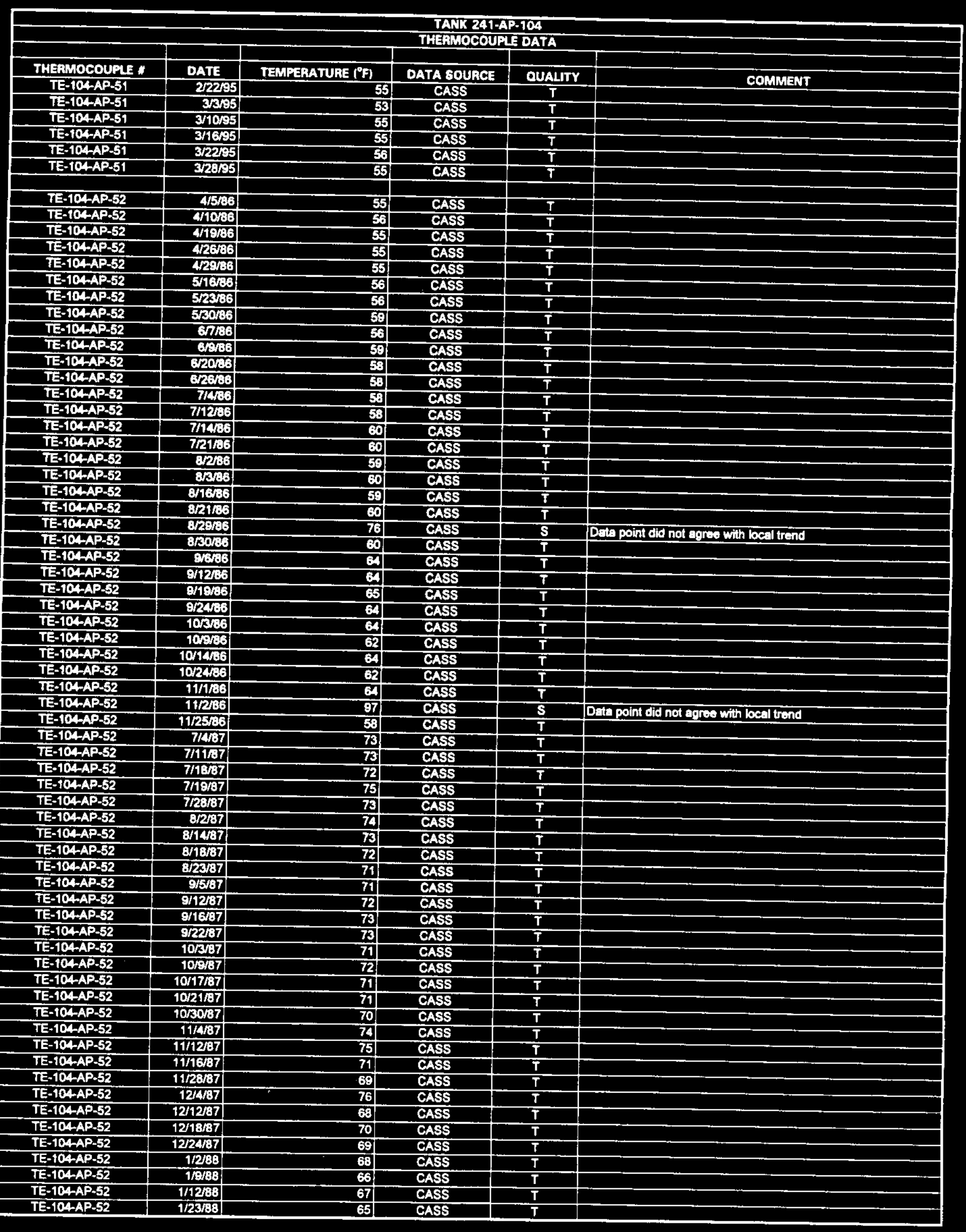

Data obtained from Computer Automated Surveillance System (CASS), April 1995; and Surveillance Analysis Computer System (SACS), June 1995.

D- 401 


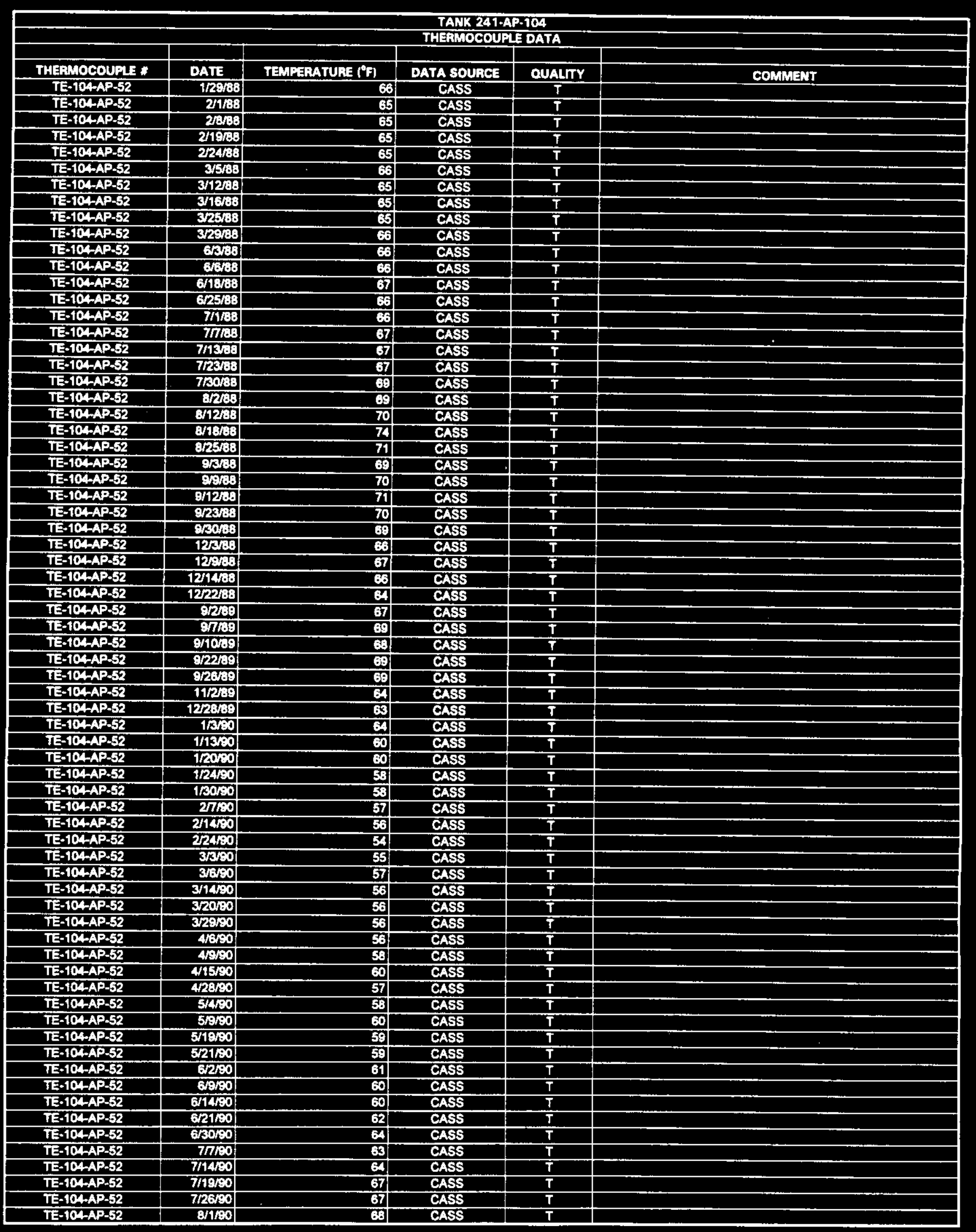




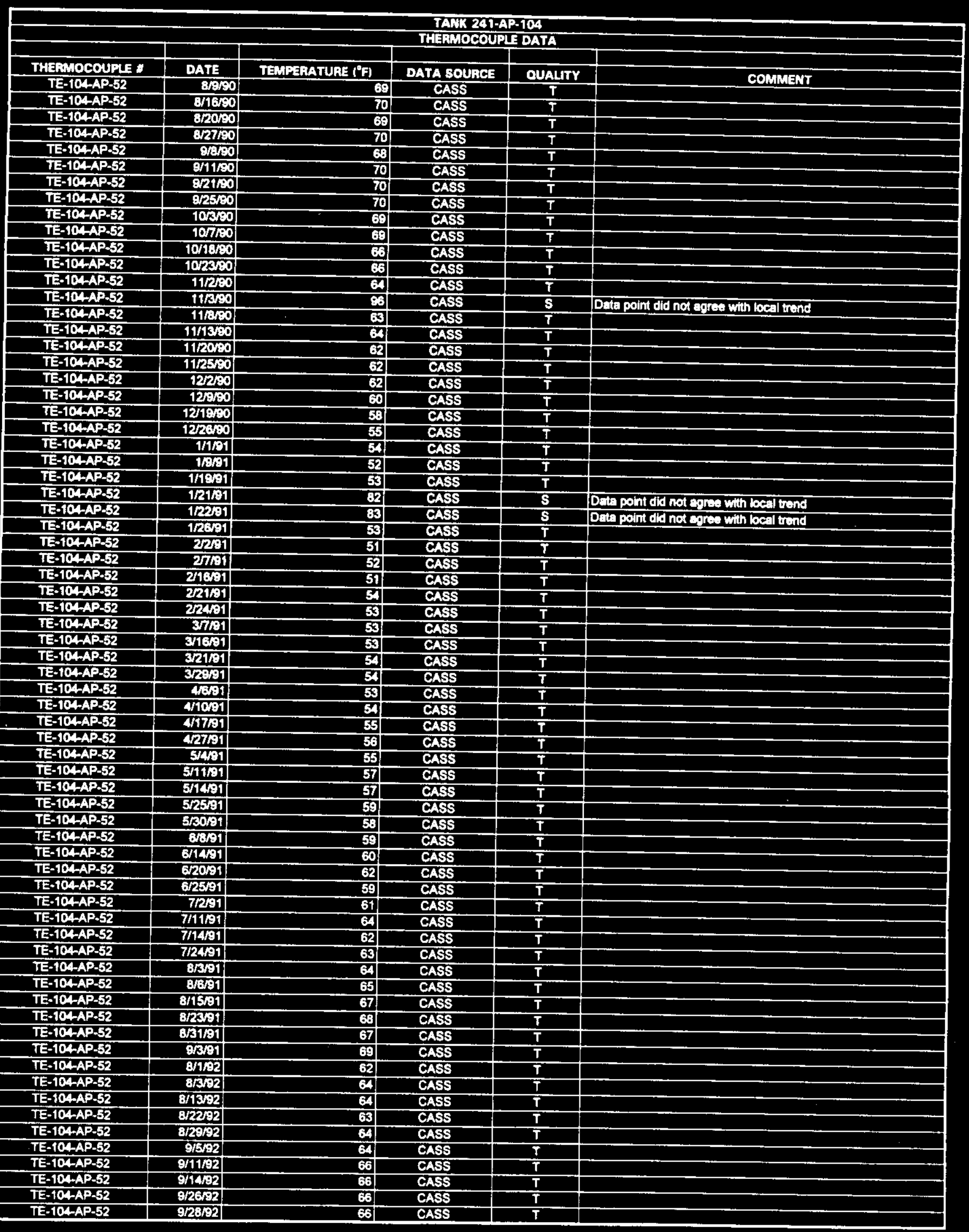

Data obtained from Computer Auromated Surveillence System (CASS), April 1995; and Surveillance Analysis Computer System (SACS), June 1995. 


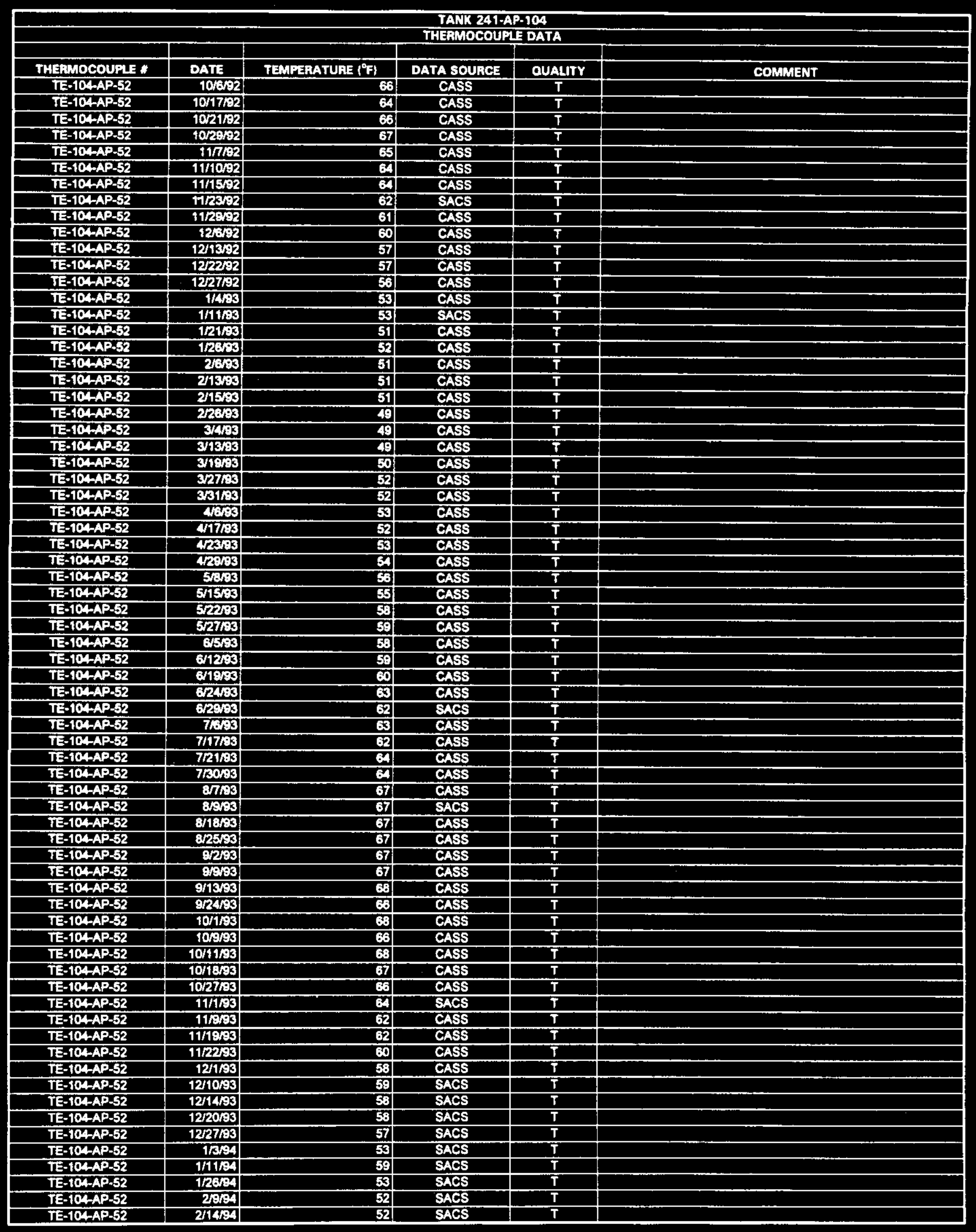

Data obtained from Computer Automated Surveillance Syatem (CASS), April 1995; ond Surveillance Analyais Computer Syatem (SACS), June 1995. 


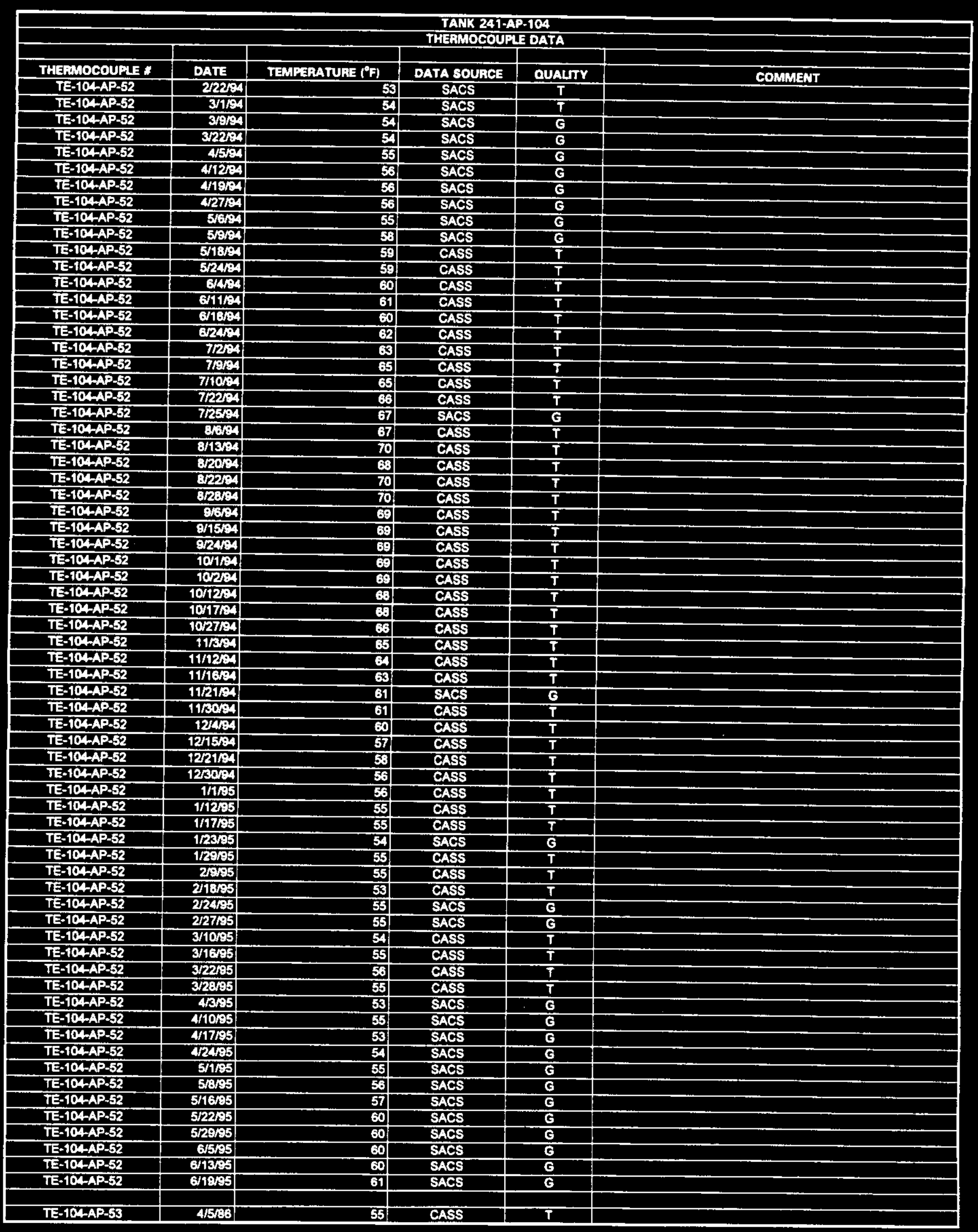




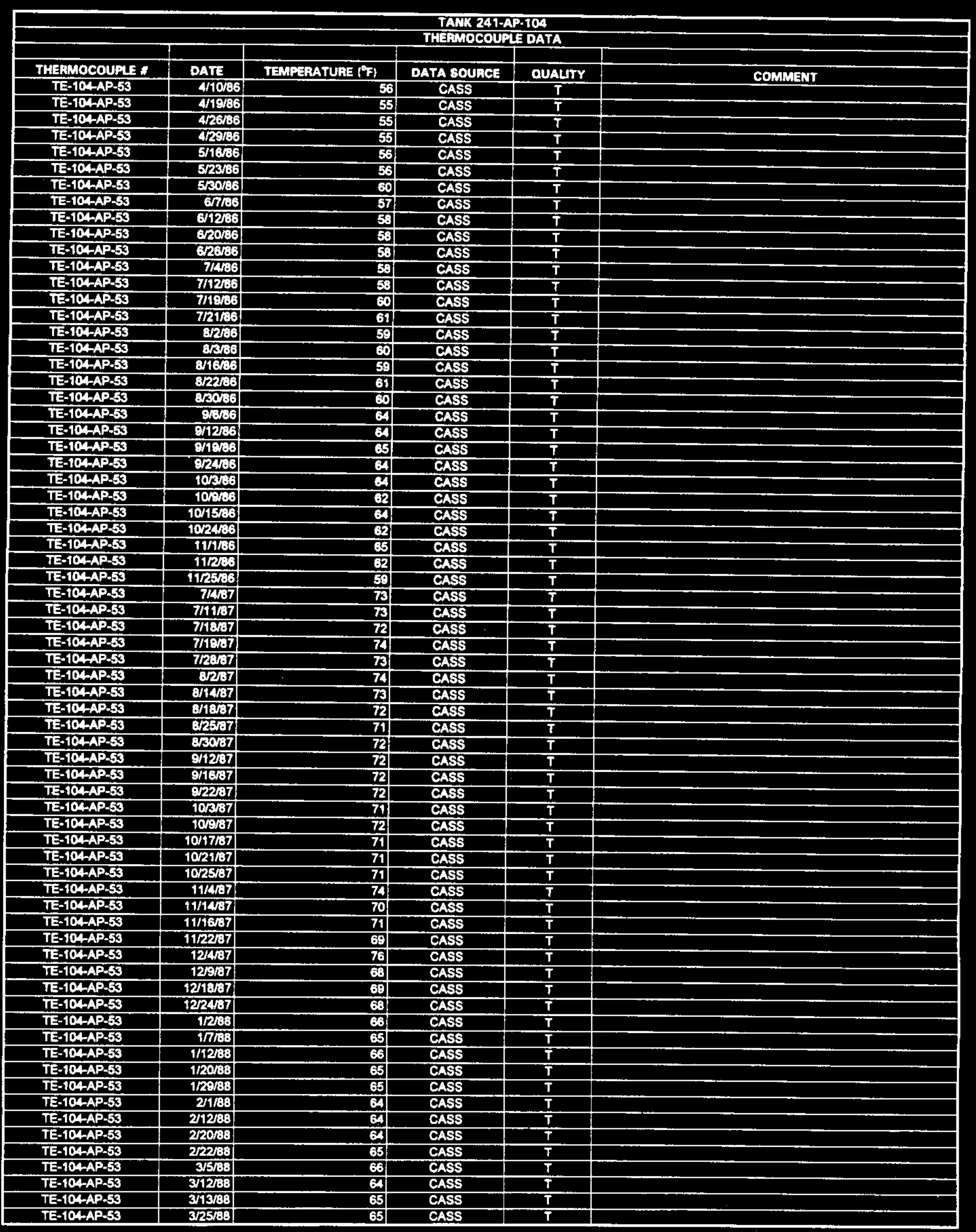




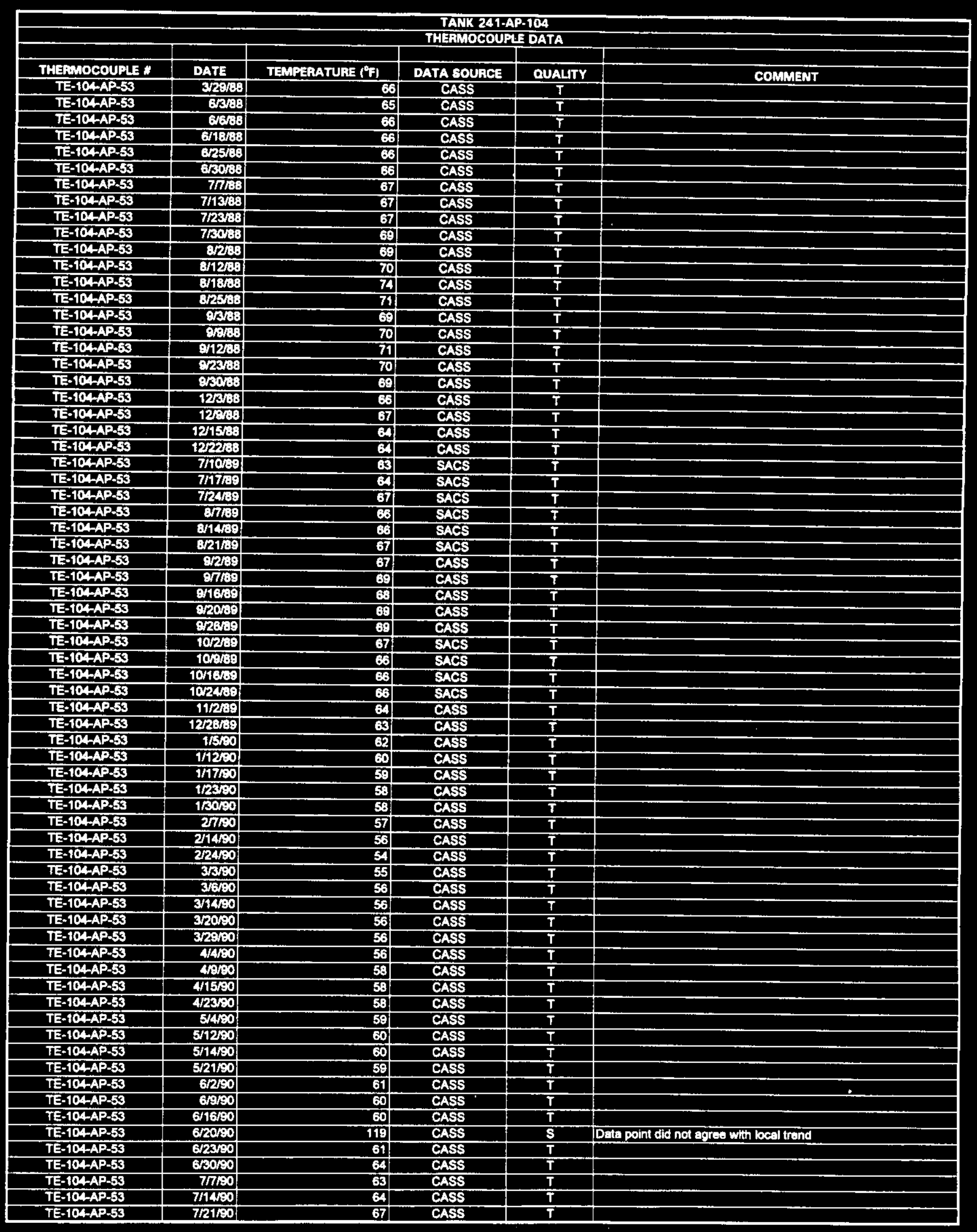




\begin{tabular}{|c|c|c|c|c|c|}
\hline \multicolumn{6}{|c|}{$\begin{array}{l}\text { TANK 241-AP-104 } \\
\text { THERMOCOUPLIF DATA }\end{array}$} \\
\hline & & & & & \\
\hline THERMOCOUPLE & DATE & TEMPERATUAE $\left({ }^{\circ} F\right)$ & DATA SOURCE & QUALTYY & COMMENT \\
\hline TE-104-AP.53 & $7 / 2690$ & $\begin{array}{r}67 \\
\end{array}$ & CASS & $T$ & \\
\hline TE-104AP-53 & $8 / 190$ & 68 & CASS & $T$ & \\
\hline TE-104-AP-53 & 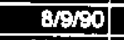 & 69 & CASS & $\mathbf{T}$ & \\
\hline TE-104-AP-53 & $8 / 1600$ & 70 & CASS & $T$ & \\
\hline TE-104-AP-53 & $8 / 20 \%$ & 70 & CASS & $T$ & \\
\hline TE-104-AP-53 & $8 / 27 / 50$ & 70 & $\begin{array}{l}\text { CASS } \\
\text { CASS }\end{array}$ & $\frac{T}{T}$ & \\
\hline $\begin{array}{l}\text { TE-104AP-53 } \\
\text { TE-104AP-53 }\end{array}$ & $9 / 8 / 90$ & $\frac{68}{70}$ & $\frac{\text { CASS }}{\text { CASS }}$ & $T$. & \\
\hline $\begin{array}{l}\text { TE-104AP-53 } \\
\text { TE-104-AP-53 }\end{array}$ & $\begin{array}{l}9 / 11 / 50 \\
9 / 21 / 50\end{array}$ & $\begin{array}{l}70 \\
70\end{array}$ & CASS & $T$ & \\
\hline TE-104AP.53 & $9 / 27 / 90$ & 71 & CASS & $\mathbf{T}$ & \\
\hline TE-104AP-63 & $10 / 360$ & 69 & CASS & $\bar{T}$ & \\
\hline TE-104-AP-53 & $107 / 50$ & 69 & CASS & $\bar{T}$ & \\
\hline TE-104AP-53 & $10 / 18 / 90$ & 66 & CASS & $T$ & \\
\hline TE-104AP-53 & $10 / 2350$ & 66 & CASS & $\mathbf{T}$ & \\
\hline TE-104AP-53 & $11 / 200$ & 64 & CASS & $T$ & \\
\hline TE-104AP-53 & $11 \pi / 100$ & 64 & CASS & $T$ & \\
\hline TE-104AP-53 & $11 / 13 / 90$ & 64 & CASS & $T$ & \\
\hline TE-104-AP-53 & $11 / 1980$ & 63 & SACS & $T$ & \\
\hline TE-104-AP-53 & $11 / 25100$ & 62 & CASS & $T$ & \\
\hline TE-104-AP-53 & 12200 & 62 & CASS & $\mathbf{T}$ & \\
\hline TE-104AP-53 & $120 \% 0$ & 59 & CASS & $\mathbf{T}$ & \\
\hline TE-104AP-53 & $12 / 13 / 00$ & 46 & CASS & $\mathbf{S}$ & Deta point did not agree with locil trend \\
\hline TE-104AP-53 & 121980 & 58 & CASS & $T$ & \\
\hline TE-104-AP-53 & $12 / 2400$ & S9) & SACS & $\bar{T}$ & \\
\hline TE-104AP-53 & $1230 / 00$ & 54 & CASS & $T$ & \\
\hline TE-104AP-53 & 1091 & 52 & CASS & $T$ & \\
\hline TE-104-AP-53 & 1/18/91 & 53 & CASS & $T$ & \\
\hline TE:104AP-53 & $1 / 20 / 91$ & $5 \mathbf{5}$ & CASS & $\bar{T}$ & \\
\hline TE-104AP.53 & $2 / 2 / 31$ & 51 & CASS & $T$ & \\
\hline TE-104-AP.53 & $27 / 21$ & 52. & CASS & $T$ & \\
\hline TE-104APS53 & 2/161 & 51 & CASS & $\bar{T}$ & \\
\hline TE-104AP-53 & $221 / 91$ & 5. & CASS & $T$ & \\
\hline TE-104AP-53 & 22491 & 53 & CASs & $\bar{T}$ & \\
\hline TE-104AP-63 & अन191 & 53 & CASS & $\mathbf{T}$ & \\
\hline TE-1OAAP.53 & 3/1691 & 53 & CASS & $\bar{T}$ & \\
\hline TE-104AP-53 & 3/2201 & 5a & CASS & $T$ & \\
\hline TE-104AP-53 & $3 / 2891$ & 5A & CASS & $T$ & \\
\hline TE-104AP-53 & 4651 & (53) & CASS & $\mathbf{T}$ & \\
\hline TE-104-AP-53 & 4/1001 & 54 & CASS & $T$ & \\
\hline TE-104-AP-53 & $4 / 2001$ & En & CASS & $T$ & \\
\hline TE-104-AP-53 & ब12781 & 56 & CASS & $T$ & \\
\hline TE-104AP-53 & S261 & 55. & CASS & $T$ & \\
\hline TE-104AP-53 & 51161 & 57 & CASS & $T$ & \\
\hline TE-104-AP-63 & 5/14/91 & 57 & CASS & $T$ & \\
\hline TE-104-AP-53 & 5/25/31 & 59 & CASS & $\bar{T}$ & \\
\hline TE-104-AP-53 & $5 / 30 / 91$ & 50 & CASS & $T$ & \\
\hline TE-104AP-53 & 6/8/91 & 59 & CASS & $T$ & \\
\hline TE-104-AP-53 & 6/14/91 & 61 & CASS & $T$ & \\
\hline TE-104-AP-53 & 6/20/91 & 62 & CASS & $T$ & \\
\hline TE-104-AP-53 & 6/25/91 & 59: & CASS & $\mathbf{T}$ & \\
\hline TE-104AP-53 & $7 / 2191$ & 62 & CASS & $T$ & \\
\hline TE-104-AP-53 & $7711 / 91$ & EA & CASS & $T$ & \\
\hline TE-104AP-53 & 7/15/91 & 63 & SACS & $T$ & \\
\hline TE-104AP-53 & $7 / 2231$ & 64 & SACS & $T$ & \\
\hline TE-104AP-53 & 7129191 & 65 & SACS & $\mathbf{T}$ & \\
\hline TE-104-AP-53 & $8 / 6 / 91$ & 65 & CASS & $T$ & \\
\hline TE-104AP-53 & $8 / 16 / 91$ & 67 & CASS & $T$ & \\
\hline TE-104AP-53 & $8 / 23 / 91$ & 68 & CASS & $T$ & \\
\hline TE-104-AP-53 & $8 / 31 / 91$ & 67 & CASS & $T$ & \\
\hline TE-104-AP-53 & 9/3/91 & 69 & CASS & $\bar{T}$ & \\
\hline TE-104-AP-53 & $8 / 1192$ & 62 & CASS & $T$ & \\
\hline TE-104-AP-53 & $8 / 3 / 92$ & 63 & CASS & $T$ & \\
\hline TE-104-AP-53 & $8 / 13 / 92$ & 64 & CASS & $T$ & \\
\hline TE-104-AP-53 & $8 / 22 / 92$ & 63 & CASS & $\bar{T}$ & \\
\hline TE-104-AP-53 & 8/28/2: & 64 & CASS & $\bar{T}$ & \\
\hline$T E=104-A P-53$ & 9/5/92 & 64 & CASS & $T$ & \\
\hline TE-104-AP-53 & 9/6/2 & 66 & CASS & $T$ & \\
\hline TE-104AP-53 & 9/1402 & 66 & CASS & $\mathbf{T}$ & \\
\hline TE-104-AP-53 & $8 / 2692$ & 69 & CASS & $\bar{T}$ & \\
\hline TE-104AP-53 & $9 / 23 / 92$ & 68 & CASS & $T$ & \\
\hline
\end{tabular}




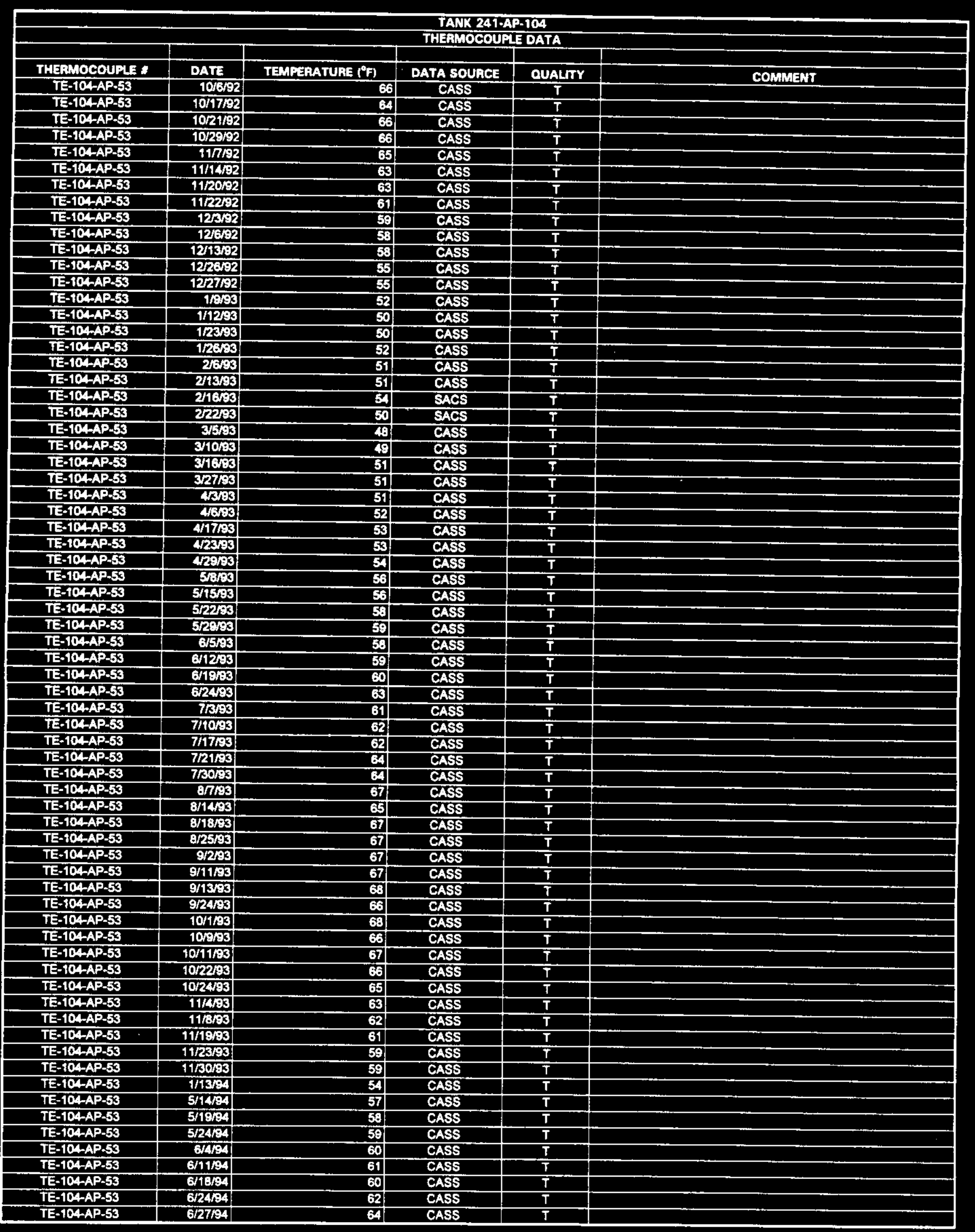




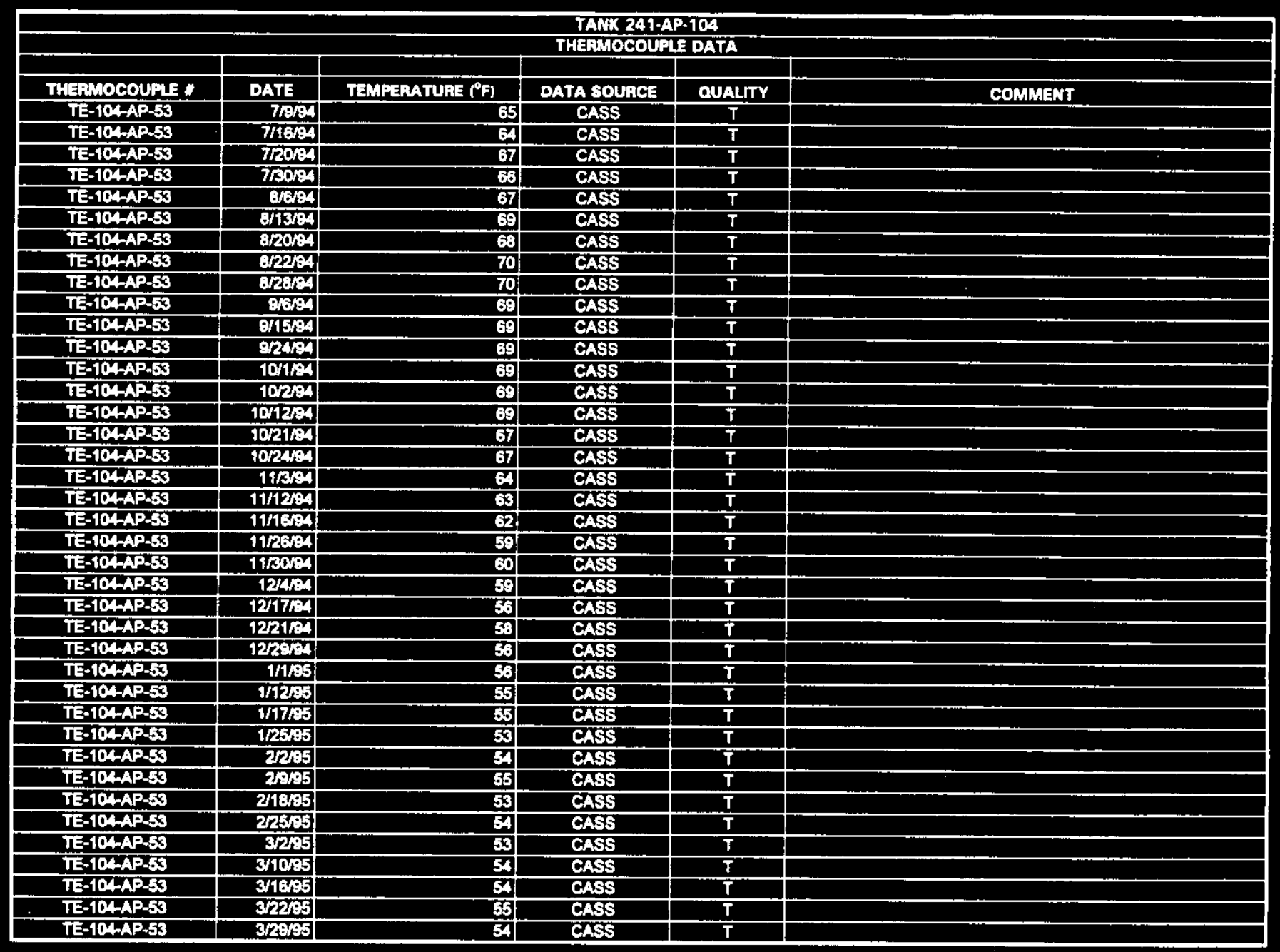




\begin{tabular}{|c|c|c|c|c|c|}
\hline \multicolumn{6}{|c|}{$\begin{array}{l}\text { TANK 241-AP-105 } \\
\text { THERMOCOUPLE DATA }\end{array}$} \\
\hline & & & DATA SOUACE & & \\
\hline$\frac{\text { THERMOCOUPLE: }}{\text { TE-105-AP-36 }}$ & $\frac{\text { DATE }}{4 / 5 / 86}$ & TEMPEAATURE $\left({ }^{\circ} \mathrm{F}\right)$ & $\frac{\text { DATA SOUACE }}{\text { CASS }}$ & $\frac{\text { QUALTTY }}{T}$ & COMMENT \\
\hline$T E-105-A P-36$ & $4 / 7 / 86$ & 57 & CASS & $T$ & \\
\hline TE-105-AP-36 & $5 / 17 / 86$ & 55 & CASS & $\mathbf{T}$ & \\
\hline TE-105-AP-36 & $5 / 22 / 86$ & 57 & CASS & $T$ & \\
\hline TE-105-AP-36 & $5 / 26 / 86$ & 60 & CASS & $T$ & \\
\hline TE-105-AP-36 & $6 / 6 / 86$ & 58 & CASS & $\bar{T}$ & \\
\hline TE-105-AP-36 & $6 / 9 / 86$ & 59 & CASS & $T$ & \\
\hline TE-105-AP-36 & $6 / 17 / 86$ & 59 & CASS & $\bar{T}$ & \\
\hline TE-105-AP-36 & $6 / 25 / 66$ & 58 & CASS & $T$ & \\
\hline TE-105-AP-36 & $7 / 5 / 86$ & 59 & CASS & $T$ & \\
\hline TE-105-AP-36 & $7 / 12 / 36$ & 59 & CASS & $T$ & \\
\hline TE-105-AP-36 & $7 / 19 / 86$ & 61 & CASS & $T$ & \\
\hline TE-105-AP-36 & $7 / 22 / 86$ & 61 & CASS & $T$ & \\
\hline TE-105-AP-36 & $7 / 29 / 86$ & 63 & CASS & $T$ & \\
\hline TE-105-AP-36 & 8/9/36 & 62 & CASS & $T$ & \\
\hline TE-105-AP-36 & $8 / 13 / 36$ & 62 & CASS & $T$ & \\
\hline TE-105-AP-36 & $8 / 17 / 86$ & 63 & CASS & $T$ & \\
\hline TE-105-AP-36 & $8 / 27 / 186$ & 63 & CASS & $\bar{T}$ & \\
\hline TE-105.AP-36 & $9 / 1 / 86$ & 65 & CASS & $T$ & \\
\hline TE-105-AP-36 & $9 / 25 / 86$ & 63 & CASS & $T$ & \\
\hline TE-105-AP-36 & $10 / 3 / 86$ & 63 & CASS & $T$ & \\
\hline TE-105-AP-36 & $10 / 11 / 86$ & 62 & CASS & $\mathbf{T}$ & \\
\hline TE-105-AP-36 & $10 / 12 / 86$ & 62 & CASS & $T$ & \\
\hline TE-105-AP-36 & $10 / 22 / 86$ & 62 & CASS & $\mathbf{T}$ & \\
\hline TE-105-AP-36 & $11 / 1 / 86$ & 60 & CASS & $T$ & \\
\hline TE-105-AP-36 & 11/2/86 & 70 & CASS & $\mathbf{s}$ & Date point did not agree with local trend \\
\hline TE-105-AP-36 & 11/6/86 & 60 & CASS & $T$ & \\
\hline TE-105-AP-36 & 12/9/36 & 59 & CASS & $\mathbf{T}$ & \\
\hline TE-105-AP-36 & $11 / 19 / 86$ & 57 & CASS & $\mathbf{T}$ & \\
\hline TE-105-AP-36 & $19 / 26 / 86$ & 57 & CASS & $\mathbf{T}$ & \\
\hline TE-105-AP-36 & $12 / 5 / 86$ & 56 & CASS & $T$ & \\
\hline TE-105-AP-36 & $12 / 11 / 86$ & 58 & CASS & $T$ & \\
\hline TE-105-AP-36 & $12 / 14 / 86$ & 65 & CASS & $\mathbf{S}$ & Data point did not apres with local trend \\
\hline TE-105-AP-36 & $12 / 17 / 86$ & 56 & CASS & $T$ & \\
\hline TE-105+AP-36 & $12 / 24 / 86$ & 56. & CASS & $T$ & \\
\hline TE-105-AP-36 & $12 / 31 / 36$ & 55 & CASS & $\mathbf{T}$ & \\
\hline IE-105-AP-36 & $7 / 4 / 87$ & 65 & CASS & $T$ & \\
\hline TE-105-AP-36 & 7/9/37 & 69 & CASS & $T$ & \\
\hline TE-105-AP-36 & 7/18/87 & 86 & CASS & $T$ & \\
\hline TE-105-AP-36 & $7 / 19 / 87$ & 68 & CASS & $T$ & \\
\hline TE-105-AP-36 & $7 / 27 / 87$ & 70 & CASS & $\mathbf{T}$ & \\
\hline TE-105-AP-36 & $8 / 5 / 37$ & 69 & CASS & $T$ & \\
\hline TE-105-AP-36 & $8 / 14 / 87$ & 71 & CASS & $\mathbf{T}$ & \\
\hline TE-105-AP-36 & $8 / 17 / 87$ & 72 & CASS & $\mathbf{T}$ & \\
\hline TE-105-AP-36 & B/26/87 & 69 & CASS & $\mathbf{T}$ & \\
\hline IE-105-AP-36 & $9 / 4 / 87$ & 69 & CASS & $\mathbf{t}$ & \\
\hline TE-105-AP-36 & $9 / 9 / 87$ & 71 & CASS & $T$ & \\
\hline TE-105-AP-36 & 9/17/87 & 71 & CASS & $\mathbf{T}$ & \\
\hline TE-105-AP-36 & 9/26/87 & 68 & CASS & $\mathbf{T}$ & \\
\hline TE-105-AP-36 & 10/2/97 & 70 & CASS & $\mathbf{T}$ & \\
\hline TE-105-AP-36 & $10 / 5 / 67$ & 70 & CASS & $\mathbf{T}$ & \\
\hline TE-105-AP-36 & $10 / 17 / 87$ & 67 & CASS & $\mathbf{T}$ & \\
\hline TE-105-AP-36 & $10 / 20 / 87$ & 69 & CASS & $\mathbf{T}$ & \\
\hline TE-105-AP-36 & $10 / 23 / 87$ & 97 & CASS & $\mathbf{S}$ & Data point did not agree with local trend \\
\hline TE-105.AP-36 & $10 / 30 / 87$ & 67 & CASS & $\mathbf{T}$ & \\
\hline TE-105-AP-36 & $11 / 3 / 87$ & 66 & CASS & $T$ & \\
\hline TE-105-AP-36 & $11 / 11 / 87$ & 65 & CASS & $T$ & \\
\hline TE-105-AP-36 & $11 / 18 / 87$ & 65 & CASS & $\bar{T}$ & \\
\hline TE-105-AP-36 & $11 / 26 / 87$ & 64 & CASS & $\bar{T}$ & \\
\hline TE-105-AP-36 & $11 / 27 / 87$ & 72 & CASS & $\mathbf{S}$ & Data point did not agres with local trend \\
\hline TE-105-AP-36 & $12 / 4 / 87$ & 62 & CASS & $\bar{T}$ & \\
\hline TE-105-AP-36 & 12/6/87 & 63 & CASS & $T$ & \\
\hline TE-105-AP-36 & 12/17/87 & 59 & CASS & $\mathbf{T}$ & \\
\hline TE-105-AP-36 & $12 / 21 / 87$ & 58 & CASS & $T$ & \\
\hline TE-105-AP-36 & $12 / 28 / 87$ & 59 & CASS & $\mathbf{T}$ & \\
\hline TE-105-AP-36 & $1 / 7 / 88$ & 56 & CASS & $\mathbf{T}$ & \\
\hline TE-105-AP-36 & $1 / 15 / 88$ & 57 & CASS & $T$ & \\
\hline TE-105-AP-36 & $1 / 23 / 8$ & 54 & CASS & $\mathbf{T}$ & \\
\hline TE-105-AP-36 & $1 / 30 / 88$ & 68 & CASS & $\mathbf{T}$ & \\
\hline TE-105-AP-36 & $2 / 3 / 38$ & 84 & CASS & $T$ & \\
\hline TE-105-AP-36 & $2 / 7 / 88$ & 79 & CASS & $\mathbf{T}$ & \\
\hline TE-105-AP-36 & $2 / 14 / 88$ & 77 & CASS & $T$ & \\
\hline TE-105-AP-36 & 2/25/83 & 75 & CASS & $T$ & \\
\hline
\end{tabular}




\begin{tabular}{|c|c|c|c|c|c|}
\hline \multicolumn{6}{|c|}{$\begin{array}{l}\text { TANK 241-AP-105 } \\
\text { THERMOCOUPLE DATA }\end{array}$} \\
\hline & & & & & \\
\hline THERMOCOUPE * & DATE & TEMPEAATURE $\left.t^{\circ} \mathrm{F}\right\}$ & DATA SOUACE & QUALTY & COMMENT \\
\hline TE-105-AP-36 & $3 / 3 / 88$ & 75 & CASS & $T$ & \\
\hline TE-105-AP.36 & $3 / 11 / 88$ & 73 & CASS & $T$ & \\
\hline TE-105-AP-36 & 3/19/88 & 71 & CASS & $\mathbf{T}$ & \\
\hline TE-105-AP-36 & $3 / 25 / 88$ & 72 & CASS & $\mathbf{T}$ & \\
\hline TE-105-AP-36 & $3 / 31 / 88$ & 71 & CASS & $T$ & \\
\hline TE-105-AP-36 & $6 / 1 / 88$ & 71 & CASS & $T$ & \\
\hline TE-105-AP-36 & $6 / 10 / 88$ & 70 & CASS & $\bar{T}$ & \\
\hline TE-105-AP-36 & $6 / 18 / 88$ & 68 & CASS & $\mathbf{T}$ & \\
\hline TE-105.AP-36 & $6 / 25 / 88$ & 70. & CASS & $\bar{T}$ & \\
\hline TE-105-AP-36 & 6/29/88 & 71 & CASS & $T$ & \\
\hline TE-105-AP-36 & $7 / 8 / 88$ & 71 & CASS & $T$ & \\
\hline TE-105-AP-36 & $7 / 16 / 8 a$ & 69 & CASS & $T$ & \\
\hline TE-105-AP-36 & $7 / 23 / 88$ & 72 & CASS & $T$ & \\
\hline TE-105-AP-36 & $7 / 27 / 8$ & 71 & CASS & $T$ & \\
\hline TE-105-AP-36 & $8 / 3 / 88$ & 72 & CASS & $T$ & \\
\hline TE-105-AP-36 & 8/13/88 & 71 & CASS & $T$ & \\
\hline TE-105-AP-36 & 8/18/88 & 73 & CASS & $T$ & \\
\hline TE-105-AP-36 & $8 / 27 / 88$ & 71 & CASS & $T$ & \\
\hline TE-105-AP-36 & 9/2/Ba & 73 & CASS & $T$ & \\
\hline TE-105-AP-36 & $9 / 6 / 8 \mathrm{~B}$ & 73 & CASS & $T$ & \\
\hline IE-105-AP-36 & 9/13/8B & 73 & CASS & $T$ & \\
\hline TE-105.AP-36 & 9/19/8B & 71 & CASS & $T$ & \\
\hline TE-105-AP-36 & $9 / 27 / 68$ & 70 & CASS & $T$ & \\
\hline TE-10S-AP-36 & $12 / 1 / 8$ & 64 & CASS & $\bar{T}$ & \\
\hline TE-105-AP-36 & $12 / 8 / 8 \mathrm{~B}$ & 70 & CASS & $T$ & \\
\hline TE-105-AP-36 & $12 / 14 / 88$ & 61 & CASS & $T$ & $\cdot$ \\
\hline TE-105-AP-36 & $12 / 18 / 88$ & 61 & CASS & $T$ & \\
\hline TE-105+AP-36 & $7 / 17 / 89$ & 60 & SACS & $\mathbf{5}$ & Disagreement botwaen CASS and SACS \\
\hline TE-105-AP-36 & $7 / 24 / 89$ & 61 & SACS & $\mathbf{S}$ & Diagreament batwean CASS and SACS \\
\hline TE-105-AP-36 & $8 / 7 / 89$ & 61 & SACS & $\mathbf{S}$ & Disagreament betwean CASS and SACS \\
\hline TE-105-AP-36 & 8/74/89 & 62 & SACS & $\mathbf{S}$ & Disagreament betwean CASS and SACS \\
\hline TE-105-AP-36 & $8 / 21 / 89$ & 81 & SACS & $\mathbf{S}$ & Disapreement botwoen CASS and SACS \\
\hline TE+105-AP+36 & $8 / 28 / 09$ & 61 & SACS & $\mathbf{s}$ & Disagreament botween CASS and SACS \\
\hline TE-105-AP-36 & $9 / 2 / 68$ & 77 & CASS & $T$ & \\
\hline TE-105-AP-36 & $9 / 4 / 89$ & 77 & CASS & $T$ & \\
\hline TE-105-AP-36 & $9 / 4 / 89$ & 62 & SACS & $\mathbf{S}$ & Disagreament between CASS and SACS \\
\hline TE-105+AP-36 & $9 / 11 / 89$ & 6i & SACS & $\mathbf{s}$ & Disagreement botween CASS end SACS \\
\hline TE-105-AP-36 & 9/14/89 & 75 & CASS & $T$ & \\
\hline $\mathrm{TE}+105-\mathrm{AP}-36$ & $9 / 18 / 89$ & 76 & CASS & $\mathbf{T}$ & \\
\hline TE-105-AP-36 & $9 / 18 / 89$ & 62 & SACS & $\mathbf{5}$ & Disagreament between CASS and SACS \\
\hline TE-105-AP-36 & 9/26/89 & 61 & SACS & 5 & Disagreament between CASS and SACS \\
\hline TE-105-AP-36 & $9 / 28 / 89$ & 75 & CASS & $T$ & \\
\hline TE-105-AP-36 & $10 / 16 / 89$ & 62 & SACS & $\mathbf{S}$ & Disagreement between CASS and SACS \\
\hline TE-105-AP-36 & $10 / 24 / 89$ & 61 & SACS & $\mathbf{S}$ & Disagreement between CASS and SACS \\
\hline TE-105-AP-36 & 10/30/A9 & 61 & SACS & $\mathbf{S}$ & Disagreement between CASS and SACS \\
\hline TE-105-AP-36 & $11 / 4 / 89$ & 72 & CASS & $T$ & \\
\hline TE-105-AP-36 & $11 / 5 / 89$ & 74 & CASS & $T$ & \\
\hline TE-105-AP-36 & $11 / 7 / 89$ & 60 & SACS & $\mathbf{S}$ & Disagreement between CASS and SACS \\
\hline TE-105-AP-36 & $11 / 13 / 89$ & 62. & SACS & $\mathbf{s}$ & Disagreement between CASS and SACS \\
\hline TE-105-AP-36 & $11 / 18 / 89$ & 73 & CASS & $T$ & \\
\hline TE-105-AP-36 & $11 / 20 / 89$ & 62 & SACS & $\mathbf{S}$ & Disagreament between CASS and SACS \\
\hline TE-105-AP-36 & $11 / 23 / 89$ & 72 & CASS & $T$ & \\
\hline TE-105-AP-36 & $11 / 27 / 89$ & 72 & CASS & $T$ & \\
\hline TE-105-AP-36 & $11 / 27 / 89$ & 60 & SACS & $\mathrm{S}$ & Disagreement between CASS and SACS \\
\hline TE-105-AP-36 & $12 / 4 / 89$ & 63 & SACS & $\mathbf{s}$ & Disagreement between CASS and SACS \\
\hline TE-105-AP-36 & $12 / 6 / 89$ & 75 & CASS & $\mathbf{T}$ & \\
\hline TE-105-AP-36 & $12 / 11 / 89$ & 60 & SACS & $\mathbf{s}$ & Disagreament batween CASS and SACS \\
\hline TE-105-AP-36 & $12 / 16 / 89$ & 69 & CASS & $\mathbf{T}$ & \\
\hline TE-105-AP-36 & $12 / 18 / 89$ & 63 & SACS & $\mathbf{S}$ & Disagreement between CASS and SACS \\
\hline TE-105-AP-36 & $12 / 19 / 89$ & 69 & CASS & $\mathbf{T}$ & \\
\hline TE-105-AP-36 & $12 / 26 / 89$ & 61 & SACS & $\mathbf{s}$ & Disegreement batween CASS and SACS \\
\hline TE-105-AP-36 & $12 / 30 / 89$ & 69 & CASS & 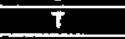 & \\
\hline TE-105-AP-36 & $1 / 2 / 90$ & 70 & CASS & $T$ & \\
\hline TE-105-AP-36 & $1 / 3 / 90$ & 68 & SACS & $\mathbf{S}$ & Disagreament between CASS and SACS \\
\hline TE-105-AP-36 & $1 / 8 / 90$ & 68 & SACS & $\mathbf{s}$ & Disagreament between CASS and SACS \\
\hline TE-105-AP-36 & $1 / 13 / 90$ & 67 & CASS & $\mathbf{T}$ & \\
\hline TE-105-AP-36 & $1 / 15 / 90$ & 67 & SACS & $\mathbf{S}$ & Disagreement between CASS and SACS \\
\hline TE-105-AP-36 & $1 / 20 / 90$ & 68 & CASS & $\mathbf{T}$ & \\
\hline TE-105-AP-36 & $1 / 22 / 90$ & 65 & SACS & $\mathbf{S}$ & Disagreament between CASS and SACS \\
\hline TE-105-AP-36 & $1 / 27 / 90$ & 66 & CASS & $\mathbf{T}$ & \\
\hline TE-105.AP-36 & $1 / 29 / 90$ & 66 & SACS & $\mathbf{S}$ & Disagreement between CASS and SACS \\
\hline TE-105-AP-36 & $2 / 1 / 90$ & 72 & CASS & $T$ & \\
\hline TE-105-AP-36 & $2 / 5 / 90$ & 65 & SACS & $\mathbf{s}$ & Disagreament between CASS and SACS \\
\hline
\end{tabular}




\begin{tabular}{|c|c|c|c|c|c|}
\hline \multicolumn{6}{|c|}{$\begin{array}{l}\text { TANK 241-AP-105 } \\
\text { THERMOCOUPLE DATA }\end{array}$} \\
\hline & & & & & \\
\hline THERMOCOUPLE: & DATE & TEMPERATUAE ( $\mathrm{F}$ ) & DATA SOUACE & QUALTY & COMMENT \\
\hline TE-105-AP-36 & $2 / 10 / 90$ & 67 & CASS & $T$ & \\
\hline TE-105-AP-36 & $2 / 12 / 90$ & 65 & SACS & $\mathbf{s}$ & Disagreement between CASS and SACS \\
\hline TE-105-AP-36 & $2 / 13 / 90$ & 68 & CASS & $\mathbf{T}$ & \\
\hline TE-105-AP-36 & $2 / 19 / 90$ & 64 & SACS & $\mathbf{S}$ & Disagreament between CASS and SACS \\
\hline TE-105-AP-36 & $2 / 21 / 90$ & 68 & CASS & $T$ & \\
\hline TE-105-AP-36 & $3 / 3 / 90$ & 67 & CASS & $T$ & \\
\hline TE-105-AP-36 & $3 / 5 / 90$ & 71 & SACS & S & Disagreement between CASS and SACS \\
\hline TE-105-AP-36 & $3 / 8 / 90$ & 66 & CASS & $T$ & \\
\hline TE-105-AP-36 & $3 / 12 / 90$ & 66 & CASS & $T$ & \\
\hline TE-105-AP-36 & $3 / 12 / 90$ & 63 & SACS & $\mathbf{S}$ & Disagreement between CASS and SACS \\
\hline TE-105-AP-36 & $3 / 19 / 90$ & 65 & SACS & $\mathbf{S}$ & Disagreement between CASS and SACS \\
\hline TE-105-AP-36 & $3 / 20 / 90$ & 67 & CASS & $\mathbf{T}$ & \\
\hline TE-105-AP-36 & $3 / 25 / 90$ & 67 & CASS & $\mathbf{T}$ & \\
\hline TE-105-AP-36 & $3 / 26 / 90$ & 65 & SACS & $\mathbf{S}$ & Disargreement batween CASS and SACS \\
\hline TE.105-AP-X6 & $4 / 2 / 90$ & 65 & SACS & $\mathbf{S}$ & Disagreement between CASS and SACS \\
\hline TE-105-AP-36 & $4 / 3 / 90$ & 67 & CASS & $T$ & \\
\hline$T E-105-A P-36$ & 4/9/90 & 65 & SACS & $\mathbf{S}$ & Disacreement between CASS and SACS \\
\hline TE-105-AP-36 & $4 / 14 / 90$ & 66 & CASS & $T$ & \\
\hline TE-105-AP-36 & $4 / 16 / 90$ & 88 & SACS & $\mathbf{S}$ & Disagreement between CASS and SACS \\
\hline TE-105-AP-36 & $4 / 20 / 20$ & 69 & CASS & $T$ & \\
\hline TE-105+AP-36 & $4 / 23 / 90$ & 65 & SACS & $\mathbf{S}$ & Disagreement between CASS and SACS \\
\hline TE-105.AP-36 & $4 / 24 / 90$ & 68 & CASS & $T$ & \\
\hline TE-105-AP-36 & $4 / 30 / 90$ & 65 & SACS & $\mathbf{S}$ & Disagreement between CASS and SACS \\
\hline TE-105-AP-36 & $5 / 5 / 90$ & 67 & CASS & $\bar{T}$ & \\
\hline TE-105-AP-36 & $5 / 6 / 90$ & 71 & CASS & $T$ & \\
\hline TE-105-AP-36 & $5 / 7 / 90$ & 67 & SACS & $\mathbf{s}$ & Disagreement betwean CASS and SACS \\
\hline TE-105-AP-36 & $5 / 14 / 90$ & 67) & SACS & $\mathbf{S}$ & Disagreement betwean CASS and SACS \\
\hline TE-105-AP-36 & 5/19/90 & 68 & CASS & $\mathbf{T}$ & \\
\hline TE-105-AP-36 & $5 / 21 / 90$ & 69 & SACS & $\mathbf{S}$ & Disagreement between CASS and SACS \\
\hline TE-105-AP-36 & $5 / 22 / 90$ & 69 & CASS & $T$ & \\
\hline TE-105-AP-36 & $5 / 28 / 90$ & 68 & SACS & $\mathbf{s}$ & Disagreement between CASS and SACS \\
\hline TE-105-AP-36 & $6 / 2 / 90$ & 69 & CASS & $T$ & \\
\hline TE-105-AP-36 & $6 / 4 / 90$ & 67 & SACS & $\mathbf{s}$ & Disagreemant between CASS and SACS \\
\hline TE-105-AP-36 & $6 / 8 / 90$ & 70 & CASS & $\bar{T}$ & \\
\hline TE-105-AP+36 & $6 / 11 / 90$ & 68 & SACS & $\mathbf{S}$ & Disagreement between CASS and SACS \\
\hline TE-105.AP-36 & $6 / 12 / 90$ & 71 & CASS & $T$ & \\
\hline TE-105-AP-36 & $6 / 19 / 90$ & 69 & SACS & 5 & Disagreement between CASS and SACS \\
\hline TE-105-AP-36 & $6 / 23 / 90$ & 69 & CASS & $\mathbf{T}$ & \\
\hline TE-105-AP-36 & $6 / 25 / 90$ & 69 & SACS & 5 & Disagreement betwean CASS and SACS \\
\hline TE-105-AP-36 & $6 / 27 / 90$ & 72 & CASS & $T$ & \\
\hline TE-105-AP-36 & $7 / 2 / 90$ & 72 & SACS & $\mathbf{S}$ & Disagreement batween CASS and SACS \\
\hline TE-105-AP-36 & $7 / 6 / 90$ & 70 & CASS & $T$ & \\
\hline TE-105-AP-36 & $712 / 90$ & 73 & CASS & $\mathbf{T}$ & \\
\hline TE-105-AP-36 & $716 / 90$ & 73 & CASS & $\mathbf{T}$ & \\
\hline TE-105-AP-36 & $7 / 23 / 90$ & 75 & CASS & $\mathbf{T}$ & \\
\hline TE-105-AP-36 & $7 / 23 / 90$ & 72 & SACS & 5 & Disagreament between CASS and SACS \\
\hline TE-105-AP-36 & $7 / 30 / 90$ & 72 & SACS & $\mathbf{s}$ & Disagreement between CASS und SACS \\
\hline TE-105-AP-36 & $8 / 3 / 90$ & 73 & CASS & $T$ & \\
\hline TE-105-AP-36 & $8 / 6 / 90$ & 64 & SACS & $\mathbf{S}$ & Disapreement between CASS and SACS \\
\hline TE-105-AP-36 & $8 / 11 / 90$ & 75 & CASS & $T$ & \\
\hline TE-105-AP-36 & $8 / 13 / 90$ & 63 & SACS & $\mathbf{S}$ & Disagreement between CASS and SACS \\
\hline TE-105-AP-36 & $8 / 18 / 90$ & 74 & CASS & $T$ & \\
\hline TE-105-AP-36 & $\mathrm{B} / 20 / 90$ & 74 & SACS & $\mathbf{S}$ & Disagreement between CASS and SACS \\
\hline TE-105-AP-36 & $8 / 21 / 90$ & 83 & CASS & $\mathbf{T}$ & \\
\hline TE-105-AP-36 & $8 / 29 / 90$ & 75 & CASS & $\mathbf{T}$ & \\
\hline TE-105.AP-36 & $9 / 3 / 90$ & 62 & SACS & $\$$ & Disagreement betwaen CASS and SACS \\
\hline TE-105-AP-36 & 9/8/SO & 74 & CASS & $\mathbf{T}$ & \\
\hline TE-105-AP-36 & $8 / 10 / 90$ & 74 & SACS & $\mathbf{S}$ & Disagreement between CASS and SACS \\
\hline TE-105-AP-36 & $9 / 15 / 90$ & 76 & CASS & $T$ & \\
\hline TE-105-AP+36 & $9 / 17 / 90$ & 75 & SACS & $\mathbf{S}$ & Disagreement betwaen CASS and SACS \\
\hline TE-105-AP-36 & $9 / 22 / 90$ & 75 & CASS & $\mathbf{T}$ & \\
\hline TE-105-AP-36 & $9 / 28 / 90$ & 75 & CASS & $\mathbf{T}$ & \\
\hline TE-105-AP-36 & $10 / 1 / 90$ & 63 & SACS & $\mathbf{S}$ & Disagreenent between CASS and SACS \\
\hline TE-105-AP-36 & $10 / 6 / 90$ & 73 & CASS & $\mathbf{T}$ & \\
\hline TE-105-AP-36 & $10 / 7 / 90$ & 75 & CASS & $\mathbf{T}$ & \\
\hline TE-105-AP-36 & $10 / 8 / 90$ & 63 & SACS & $\mathbf{S}$ & Disagreament between CASS and SACS \\
\hline TE-105-AP-36 & $10 / 15 / 90$ & 73 & CASS & $\mathbf{T}$ & \\
\hline TE-105+AP-36 & $10 / 15 / 90$ & 62 & SACS & $\mathbf{S}$ & Disagreement betwaen CASS and SACS \\
\hline TE-105-AP-36 & $10 / 22 / 90$ & 61 & SACS & 5 & Disagreement between CASS and SACS \\
\hline TE-105+AP-36 & $10 / 26 / 90$ & 72 & CASS & $\mathbf{T}$ & \\
\hline TE-105-AP-36 & $10 / 29 / 90$ & 82) & CASS & $\mathbf{T}$ & \\
\hline TE-105-AP-36 & $10 / 29 / 90$ & 63 & SACS & $\mathbf{s}$ & Disagraement between CASS and SACS \\
\hline TE-105-AP-36 & $11 / 5 / 90$ & 62 & SACS & $\mathbf{s}$ & Disagreement between CASS and SACS \\
\hline
\end{tabular}




\begin{tabular}{|c|c|c|c|c|c|}
\hline \multirow{2}{*}{\multicolumn{6}{|c|}{$\begin{array}{l}\text { TANK 241-AP:105 } \\
\text { THERMOCOUPLE DATA }\end{array}$}} \\
\hline & & & & & \\
\hline THERMOCOUPLE : & DATE & TEMPERATURE $\left({ }^{\circ} \mathrm{F}\right)$ & DATA SOURCE & QUALTYY & COMMENT \\
\hline TE-105-AP-36 & $11 / 10 / 90$ & 71 & CASS & $T$ & \\
\hline TE-105-AP-36 & $11 / 12 / 90$ & 62 & SACS & $\mathbf{S}$ & Disagreement betwoen CASS and SACS \\
\hline TE-105-AP-36 & $11 / 17 / 90$ & 80 & CASS & $\mathrm{T}$ & \\
\hline TE-105-AP-36 & $11 / 19 / 90$ & 63 & SACS & $\mathbf{S}$ & Disagreement between CASS and SACS \\
\hline TE-105.AP-36 & $11 / 23 / 90$ & 71 & CASS & $T$ & \\
\hline TE-105-AP-36 & $11 / 26 / 90$ & 61 & SACS & $\overline{\mathbf{S}}$ & Disegreement between CASS and SACS \\
\hline TE-105-AP-36 & $11 / 29 / 90$ & 72 & CASS & $\mathbf{T}$ & \\
\hline TE-105-AP-36 & $12 / 3 / 90$ & 62 & SACS & $\mathbf{s}$ & Disapreament between CASS and SACS \\
\hline TE-105-AP-36 & $12 / 6 / 90$ & 72 & CASS & $\mathbf{T}$ & \\
\hline TE-105.AP-36 & $12 / 10 / 90$ & 63 & SACS & $\mathbf{S}$ & Disegreement between CASS and SACS \\
\hline TE-105-AP-36 & $12 / 11 / 90$ & 70 & CASS & $\mathbf{T}$ & \\
\hline TE-105-AP-36 & $12 / 17 / 90$ & 63 & SACS & $\mathbf{s}$ & Oisagreement between CASS and SACS \\
\hline TE-105-AP-36 & $12 / 20 / 90$ & 69 & CASS & $\mathbf{T}$ & \\
\hline TE-105-AP-36 & $12 / 24 / 90$ & 62 & SACS & $\mathbf{s}$ & Disagreament between CASS and SACS \\
\hline TE-105-AP-36 & $12 / 27 / 90$ & 68 & CASS & $T$ & \\
\hline TE-105-AP-36 & $12 / 31 / 90$ & 62 & SACS & $\mathbf{5}$ & Disagreement berween CASS and SACS \\
\hline TE-105-AP-36 & $1 / 4 / 91$ & 66 & CASS & $T$ & \\
\hline TE-105-AP-36 & $1 / 12 / 91$ & 66 & CASS & $\mathbf{T}$ & \\
\hline TE-105-AP-36 & $1 / 14 / 91$ & 67 & CASS & $\mathbf{T}$ & \\
\hline TE-105.AP-36 & I/14/91 & 62 & SACS & $\mathbf{s}$ & Disagreement betwean CASS and SACS \\
\hline TE-105-AP-36 & $1 / 21 / 91$ & 62 & SACS & $\mathbf{S}$ & Disagreement between CASS and SACS \\
\hline TE-105-AP-36 & $1 / 24 / 91$ & 66 & CASS & $\mathbf{T}$ & \\
\hline TE-105.AP-36 & $1 / 27 / 91$ & 66 & CASS & $\mathbf{T}$ & \\
\hline TE-105-AP-36 & $1 / 28 / 91$ & 62 & SACS & $\mathbf{S}$ & Disaqreement betwoen CASS and SACS \\
\hline TE-105-AP-36 & $2 / 4 / 91$ & 62 & SACS & $\bar{s}$ & Disegreement between CASS and SACS \\
\hline TE-105-AP-36 & $2 / 6 / 91$ & 68 & CASS & $T$ & \\
\hline TE-105-AP-36 & $2 / 11 / 91$ & 63 & SACS & $\mathbf{s}$ & Disagreement betwoen CASS and SACS \\
\hline TE-105-AP-36 & $2 / 15 / 91$ & 64 & CASS & $\overline{\mathbf{T}}$ & \\
\hline TE-10S-AP-36 & $2 / 18 / 91$ & 64 & SACS & $\mathbf{s}$ & Disepreement batween CASS and SACS \\
\hline TE-105-AP-36 & $2 / 22 / 91$ & 65 & CASS & $T$ & \\
\hline TE-105-AP-36 & $2 / 25 / 91$ & 61: & SACS & $\mathbf{S}$ & Disagreament between CASS and SACS \\
\hline TE-105-AP-36 & 3/1/91 & 65 & CASS & $\mathbf{T}$ & \\
\hline$T E-105-A P-36$ & $3 / 3 / 97$ & 72 & CASS & $\mathbf{T}$ & \\
\hline TE-105-AP-36 & $3 / 4 / 91$ & 63. & SACS & $\mathbf{s}$ & Oisagreament betwaen CASS and SACS \\
\hline TE-105-AP-36 & $3 / \pi 1 / 31$ & 66 & CASS & $\mathbf{T}$ & \\
\hline TE-105-AP-36 & $3 / 1 / 91$ & 62 & SACS & $\mathbf{S}$ & Disagreement between CASS and SACS \\
\hline TE-105-AP-36 & $3 / 18 / 91$ & 65 & CASS & $\mathbf{T}$ & \\
\hline TE-105-AP-36 & 3/18/91 & 63 & SACS & $\mathbf{S}$ & Disepreement between CASS and SACS \\
\hline TE-105-AP-36 & 3/27/91 & 65 & CASS & $\mathbf{T}$ & \\
\hline TE-106-AP-36 & $4 / 101$ & 66 & CASS & $\bar{T}$ & \\
\hline TE-105-AP-36 & 4/1/91 & 62 & SACS & $\mathbf{S}$ & Disagreement between CASS and SACS \\
\hline TE-105-AP-36 & $4 / 8 / 91$ & 62 & SACS & $\mathbf{5}$ & Disagreement between CASS and SACS \\
\hline TE-105-AP-36 & $4 / 12 / 91$ & 67 & CASS & $T$ & \\
\hline TE-105-AP-36 & $4 / 15 / 91$ & 62 & SACS & $\mathbf{S}$ & Disagreament betwaen CASS and SACS \\
\hline TE-105-AP-36 & $4 / 20 / 91$ & 66 & CASS & $T$ & \\
\hline TE-105-AP-36 & $4 / 22191$ & 64 & SACS & $\mathbf{S}$ & Disagreament between CASS and SACS \\
\hline TE-105-AP-36 & $4 / 24 / 91$ & 66 & CASS & $\mathbf{T}$ & \\
\hline TE-105-AP-36 & $4 / 29 / 91$ & 62 & SACS & $\mathbf{S}$ & Disegreament between CASS and SACS \\
\hline TE-105.AP-36 & $5 / 4 / 91$ & 66 & CASS & $T$ & \\
\hline TE-105-AP-36 & 5/6/91 & 62 & SACS & $\mathbf{S}$ & Disagreement between CASS and SACS \\
\hline TE-105-AP-36 & $5 / 10 / 91$ & 67 & CASS & $\bar{T}$ & \\
\hline TE-105-AP-36 & $5 / 13 / 91$ & 62 & SACS & $\mathbf{s}$ & Disagreement between CASS and SACS \\
\hline TE-105-AP-36 & $5 / 16 / 91$ & 68 & CASS & $T$ & \\
\hline TE-105-AP-36 & $5 / 20 / 91$ & 63 & SACS & $\mathbf{s}$ & Disagreement between CASS and SACS \\
\hline TE-105-AP-36 & $5 / 25 / 91$ & 67: & CASS & $T$ & \\
\hline TE-105-AP-36 & $5 / 26 / 91$ & 68 & CASS & $\mathbf{T}$ & \\
\hline TE-105-AP-36 & 5/27/91 & 63 & SACS & $\mathbf{S}$ & Disapreement between CASS and SACS \\
\hline TE-105-AP-36 & $6 / 3 / 91$ & 62 & SACS & $\mathbf{s}$ & Disagreement botween CASS and SACS \\
\hline TE-105-AP-36 & 6/5/91 & 68: & CASS & $\mathbf{T}$ & \\
\hline TE-105-AP-36 & 6/10/91 & 63 & SACS & $\mathbf{s}$ & Disagreament between CASS and SACS \\
\hline TE-105-AP-36 & $6 / 12 / 91$ & 68 & CASS & $\bar{T}$ & \\
\hline TE-105-AP-36 & 6/17/91 & 63 & SACS & $\mathbf{S}$ & Disagreement between CASS and SACS \\
\hline TE-105-AP-36 & 6/19/91 & 69 & CASS & $T$ & \\
\hline TE-105-AP-36 & $6 / 24 / 91$ & 63 & SACS & $\mathbf{s}$ & Disagreement between CASS and SACS \\
\hline TE-105-AP-36 & $6 / 25 / 91$ & 70 & CASS & $\bar{T}$ & \\
\hline TE-105-AP-36 & $7 / 1 / 91$ & 65 & SACS & $\mathrm{s}$ & Disagreement between CASS and SACS \\
\hline TE-105-AP-36 & $7 / 3 / 91$ & 69 & CASS & $\overline{\mathbf{T}}$ & \\
\hline TE-105-AP-36 & $7 / 8 / 91$ & 72 & SACS & $\mathbf{S}$ & Disagreement between CASS and SACS \\
\hline TE-105-AP-36 & $7 / 13 / 91$ & 70 & CASS & $T$ & \\
\hline TE-105-AP-36 & $7 / 15 / 91$ & 70 & CASS & $T$ & \\
\hline TE-105-AP-36 & $7 / 16 / 91$ & 71 & SACS & $\mathbf{S}$ & Disagreement between CASS and SACS \\
\hline TE-105-AP-36 & 712,2191 & 63 & SACS & $\mathbf{S}$ & Disagraement between CASS and SACS \\
\hline TE-105-AP-36 & $7 / 24 / 91$ & 71 & CASS & $\mathbf{T}$ & \\
\hline
\end{tabular}




\begin{tabular}{|c|c|c|c|c|c|}
\hline \multicolumn{6}{|c|}{$\begin{array}{l}\text { TANK 241-AP-105 } \\
\text { THEAMOCOUPLE DATA }\end{array}$} \\
\hline THEAMOCOUPLE * & DATE & TEMPERATUAE $\mathrm{f}^{\circ} \mathrm{Fr}$ & & & \\
\hline TE-105-AP-36 & $7 / 29 / 91$ & 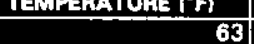 & $\frac{\text { DATA SOULiez }}{\text { SACS }}$ & QUALIY & COMMENT \\
\hline TE-105-AP-36 & $8 / 3 / 91$ & 72 & $\frac{\text { DALS }}{\text { CASS }}$ & $\frac{5}{T}$ & Disagreement batween CASS and SACS \\
\hline TE-105-AP-36 & $8 / 4 / 91$ & 64 & SACS & $\frac{T}{S}$ & \\
\hline TE-105-AP-36 & $8 / 6 / 91$ & 72 & CASS & $\mathbf{T}$ & Disagreement between CASS and SACS \\
\hline TE-105-AP+36 & $8 / 12 / 91$ & 64 & SACS & $\mathbf{S}$ & Disagreement between CASS and SACS \\
\hline TE-105-AP-36 & $8 / 16 / 91$ & 75 & CASS & $T$ & \\
\hline TE-105-AP-36 & $8 / 19 / 91$ & 63 & SACS & $\mathbf{S}$ & Disagreament between CASS and SACS \\
\hline TE-105-AP-36 & $8 / 20 / 91$ & 74 & CASS & $\bar{T}$ & \\
\hline TE-105-AP-36 & $8 / 30 / 91$ & 75 & CASS & $\mathbf{T}$ & \\
\hline TE-105-AP-36 & 9/2/91 & 75 & CASS & $\mathbf{T}$ & \\
\hline TE-105-AP-36 & $9 / 2 / 91$ & 62 & SACS & $\mathbf{s}$ & Disagreement between CASS and SACS \\
\hline TE-105-AP-36 & $9 / 8 / 91$ & 75. & CASS & $\mathbf{T}$ & \\
\hline TE-105-AP-36 & $9 / 16 / 91$ & 64 & SACS & $\mathbf{s}$ & Disagreement between CASS and SACS \\
\hline TE-105-AP-36 & $9 / 18 / 91$ & 75 & CASS & $\mathbf{T}$ & \\
\hline TE-105-AP-36 & $9 / 23 / 91$ & 62 & SACS & $\mathbf{s}$ & Disagreement between CASS and SACS \\
\hline TE-105-AP-36 & $9 / 27 / 91$ & 74 & CASS & $T$ & \\
\hline TE-105-AP-36 & $9 / 30 / 91$ & 75 & CASS & $\bar{T}$ & \\
\hline TE-105-AP-36 & 9/30/91 & 62 & SACS & $\mathbf{s}$ & Disagreament between CASS and SACS \\
\hline TE-105-AP-36 & $10 / 7 / 91$ & 63 & SACS & $\mathbf{s}$ & Disagreement between CASS and SACS \\
\hline TE-105-AP-36 & $10 / 11 / 91$ & 73 & CASS & $\mathbf{T}$ & \\
\hline TE-105-AP-36 & $10 / 14 / 91$ & 62 & SACS & $\mathbf{S}$ & Disagreement between CASS and SACS \\
\hline TE-10S-AP-36 & $10 / 16 / 91$ & 75 & CASS & $T$ & \\
\hline TE-TO5-AP-36 & $10 / 20 / 21$ & 75 & CASS & $\mathbf{T}$ & \\
\hline TE-105-AP-36 & $10 / 21 / 91$ & 62 & SACS & $\mathbf{5}$ & Disagreement between CASS and SACS \\
\hline TE-105-AP-36 & $10 / 28 / 91$ & 62 & SACS & $\mathbf{s}$ & Disagreamant between CASS and SACS \\
\hline TE-105-AP+36 & $10 / 29 / 91$ & 71 & CASS & $T$ & \\
\hline TE+105-AP-36 & $11 / 6 / 91$ & 71 & CASS & $\mathrm{T}$ & \\
\hline TE-105-AP-36 & $8 / 11 / 92$ & 72 & CASS & $T$ & \\
\hline TE-IOS-AP-36 & $8 / 24 / 92$ & 73 & CASS & $T$ & \\
\hline TE-105-AP-36 & $9 / 14 / 92$ & 63 & SACS & $\mathbf{s}$ & Disagreement between CASS and SACS \\
\hline TE-105-AP-36 & $9 / 19 / 92$ & 75 & CASS & $T$ & \\
\hline TE-105-AP-36 & $9 / 21 / 92$ & 63 & SACS & $\mathbf{S}$ & Disagreement batween CASS and SACS \\
\hline TE-105-AP-36 & $9 / 26 / 92$ & 73 & CASS & $T$ & \\
\hline TE-105-AP-36 & $9 / 28 / 92$ & 73 & SACS & $\mathrm{S}$ & Disagreement betwean CASS and SACS \\
\hline TE-105-AP-36 & $10 / 3 / 92$ & 77 & CASS & $T$ & \\
\hline TE-105-AP+36 & $10 / 5 / 92$ & 73 & SACS & $\mathbf{s}$ & Disagreement between CASS and SACS \\
\hline TE-105-AP-36 & $10 / 9 / 92$ & 74. & CASS & $T$ & \\
\hline IE-105-AP-36 & $10 / 12 / 92$ & 73 & SACS & $\mathbf{s}$ & Disagreement between CASS and SACS \\
\hline TE-105-AP-36 & $10 / 13 / 92$ & 74 & CASS & $T$ & \\
\hline TE-105-AP-36 & $10 / 19 / 92$ & 73 & SACS & $\mathbf{S}$ & Disagreement between CASS and SACS \\
\hline TE-105-AP-36 & $10 / 22 / 92$ & 74 & CASS & $\mathbf{T}$ & \\
\hline TE-105-AP-36 & $10 / 27 / 92$ & 73 & SACS & 5 & Disagreement between CASS and SACS \\
\hline TE-105-AP-36 & $10 / 31 / 92$ & 74 & CASS & $\mathbf{T}$ & \\
\hline TE-105-AP-36 & $11 / 2 / 92$ & 72 & SACS & $\mathbf{S}$ & Disegreement between CASS and SACS \\
\hline TE-105-AP-36 & $11 / 6 / 92$ & 74 & CASS & $\bar{T}$ & \\
\hline TE-105-AP-36 & $11 / 9 / 92$ & 73 & CASS & $T$ & \\
\hline TE-105-AP-36 & $11 / 9 / 92$ & $\mathbf{7 2}$ & SACS & $\mathbf{s}$ & Disagreement between CASS and SACS \\
\hline TE-105-AP-36 & $11 / 16 / 92$ & 63 & SACS & $\mathbf{S}$ & Disagreement between CASS and SACS \\
\hline TE-105-AP-36 & $11 / 17 / 92$ & 74 & CASS & $\bar{T}$ & \\
\hline TE-105-AP-36 & $11 / 22 / 92$ & 71 & CASS & $\mathbf{T}$ & \\
\hline TE-105-AP-36 & $11 / 23 / 92$ & 63 & SACS & $\mathbf{s}$ & Disagreement between CASS and SACS \\
\hline TE-105-AP-36 & $11 / 30 / 92$ & 69 & SACS & $\mathbf{S}$ & Disagreement between CASS and SACS \\
\hline TE-105-AP-36 & $12 / 1 / 92$ & 72 & CASS & $T$ & \\
\hline TE-105-AP-36 & $12 / 6 / 92$ & 72 & CASS & $\bar{T}$ & \\
\hline TE-105-AP-36 & $12 / 7 / 92$ & 68 & SACS & $\mathbf{S}$ & Disagteament between CASS and SACS \\
\hline TE-105-AP-36 & $12 / 14 / 92$ & 68 & SACS & $\mathbf{S}$ & Disagreament between CASS and SACS \\
\hline TE-105-AP-36 & $12 / 19 / 92$ & 69 & CASS & $\mathbf{T}$ & \\
\hline TE-105-AP-36 & $12 / 21 / 92$ & 68 & SACS & $\mathbf{S}$ & Disagreement between CASS and SACS \\
\hline TE-105-AP-36 & $12 / 24 / 92$ & 77 & CASS & $\bar{T}$ & \\
\hline TE-105-AP-36 & $12 / 28 / 92$ & 67 & SACS & $\mathbf{S}$ & Disagreement between CASS and SACS \\
\hline TE-105-AP-36 & $12 / 30 / 92$ & 67 & CASS & $T$ & \\
\hline TE-105-AP-36 & $6 / 11 / 93$ & 67 & SACS & $\mathbf{S}$ & Disegreement between CASS and SACS \\
\hline TE-105-AP-36 & $6 / 18 / 93$ & 65 & SACS & $\mathbf{s}$ & Disagraement between CASS and SACS \\
\hline TE-105-AP-36 & $6 / 28 / 93$ & 67 & SACS & $\mathbf{S}$ & Dissgreement between CASS and SACS \\
\hline TE-105-AP-36 & $7 / 9 / 93$ & 68 & SACS & $\mathbf{S}$ & Disagreement between CASS and SACS \\
\hline TE-105-AP-36 & $7 / 15 / 93$ & 68 & SACS & $\mathbf{S}$ & Disagreament betwean CASS and SACS \\
\hline TE-105-AP-36 & $7 / 19 / 93$ & 67 & SACS & $\mathbf{S}$ & Disagreement between CASS and SACS \\
\hline TE-105-AP-36 & $7 / 30 / 93$ & 70 & SACS & $\mathbf{s}$ & Disagreement between CASS and SACS \\
\hline TE-105-AP-36 & $8 / 6 / 93$ & 69 & SACS & $\mathbf{S}$ & Disagreement between CASS end SACS \\
\hline TE-105-AP-36 & $8 / 12 / 93$ & 62 & SACS & $\mathbf{S}$ & Disagreement betwoen CASS and SACS \\
\hline TE-105-AP-36 & $9 / 24 / 93$ & 67 & SACS & $\mathbf{S}$ & Disagraement between CASS and SACS \\
\hline TE-105-AP-36 & $9 / 26 / 93$ & 70 & SACS & $\mathbf{S}$ & Disagraement between CASS and SACS \\
\hline TE-105-AP-36 & $10 / 1 / 93$ & 71 & SACS & $\mathbf{S}$ & Disagreament between CASS and SACS \\
\hline
\end{tabular}




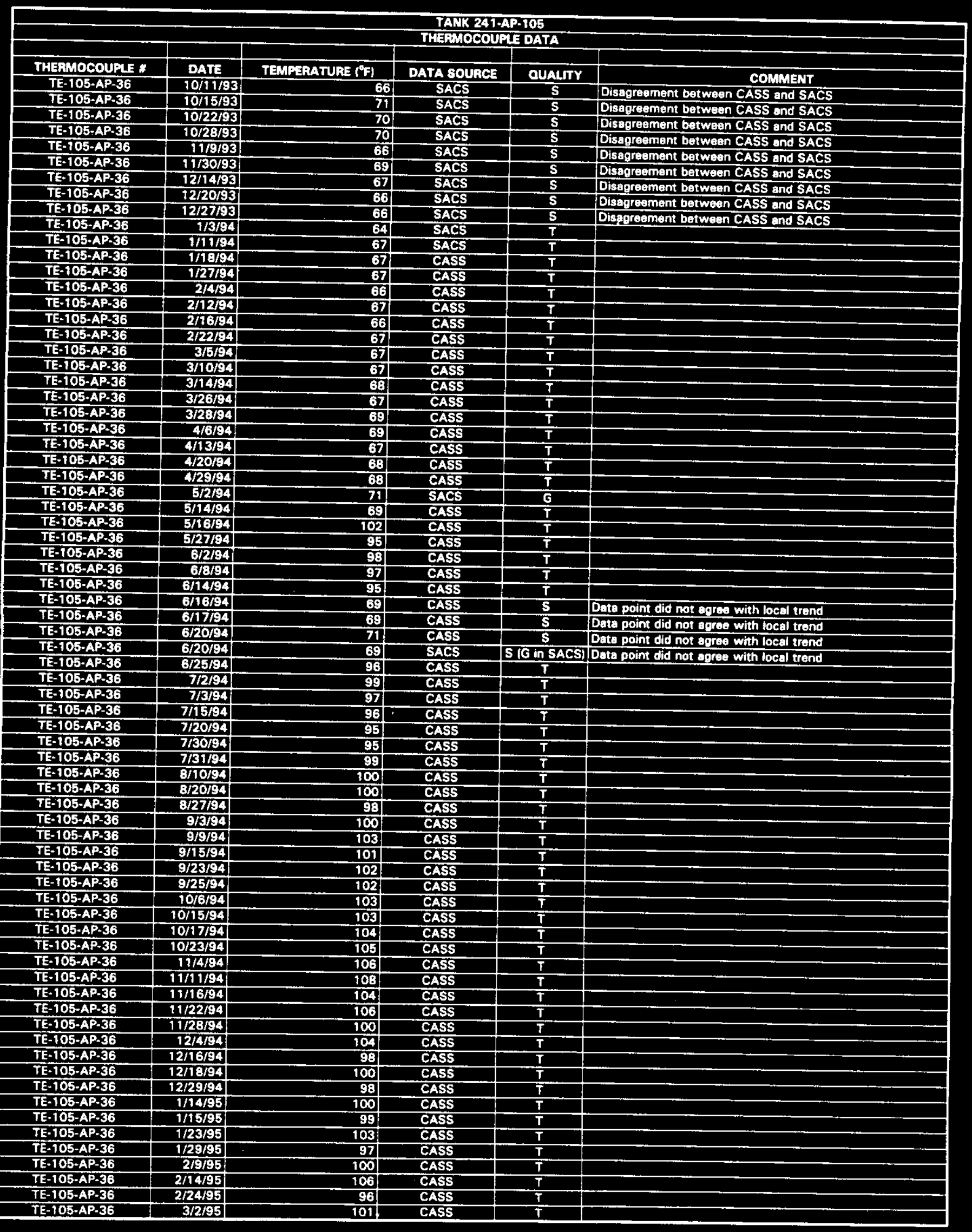




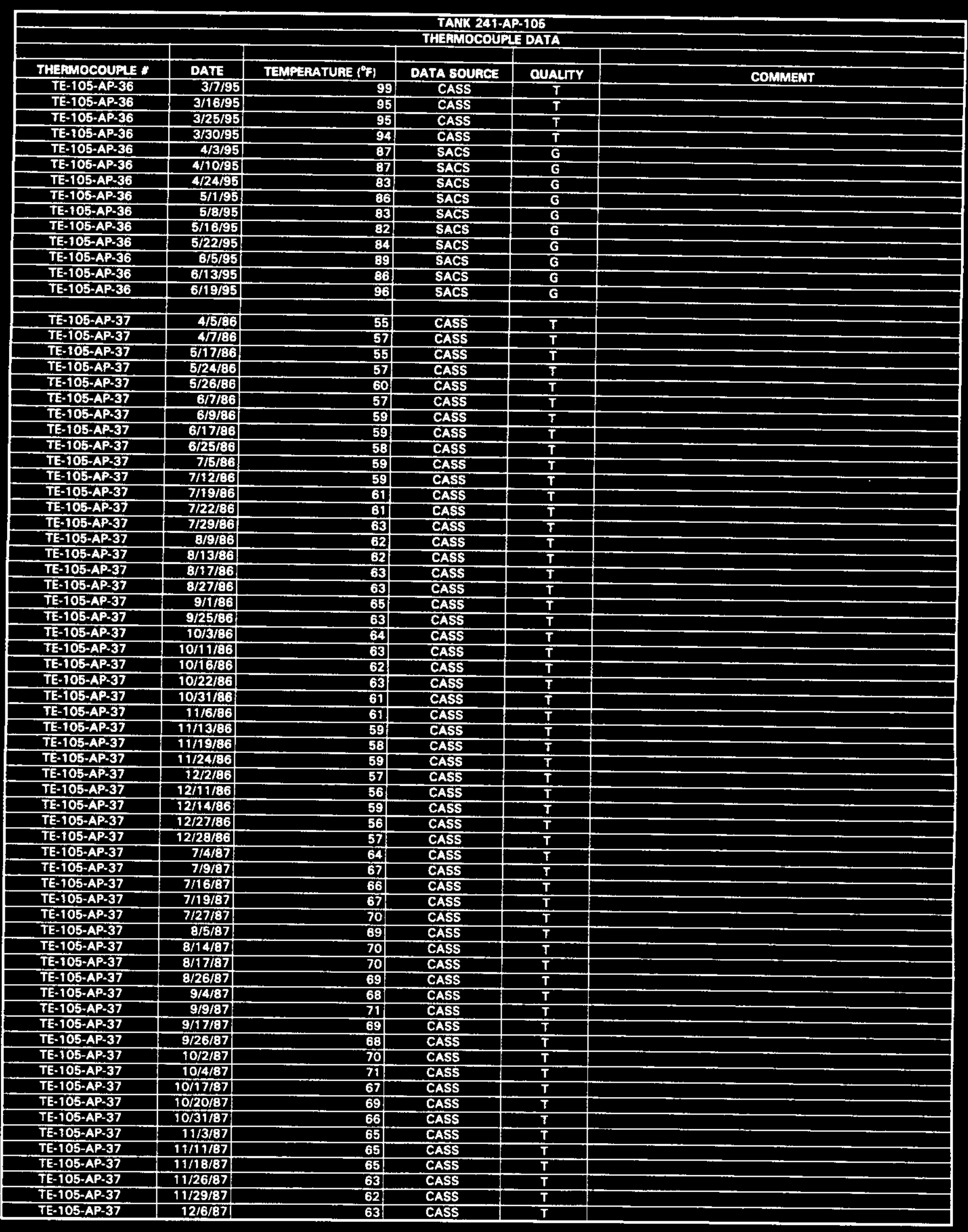

Data obtained from Computer Automated Surveillance System (CASS) in Aprll 1995, and Surviellance Analysis Computer System (SACS) in June 1995. D- 417 


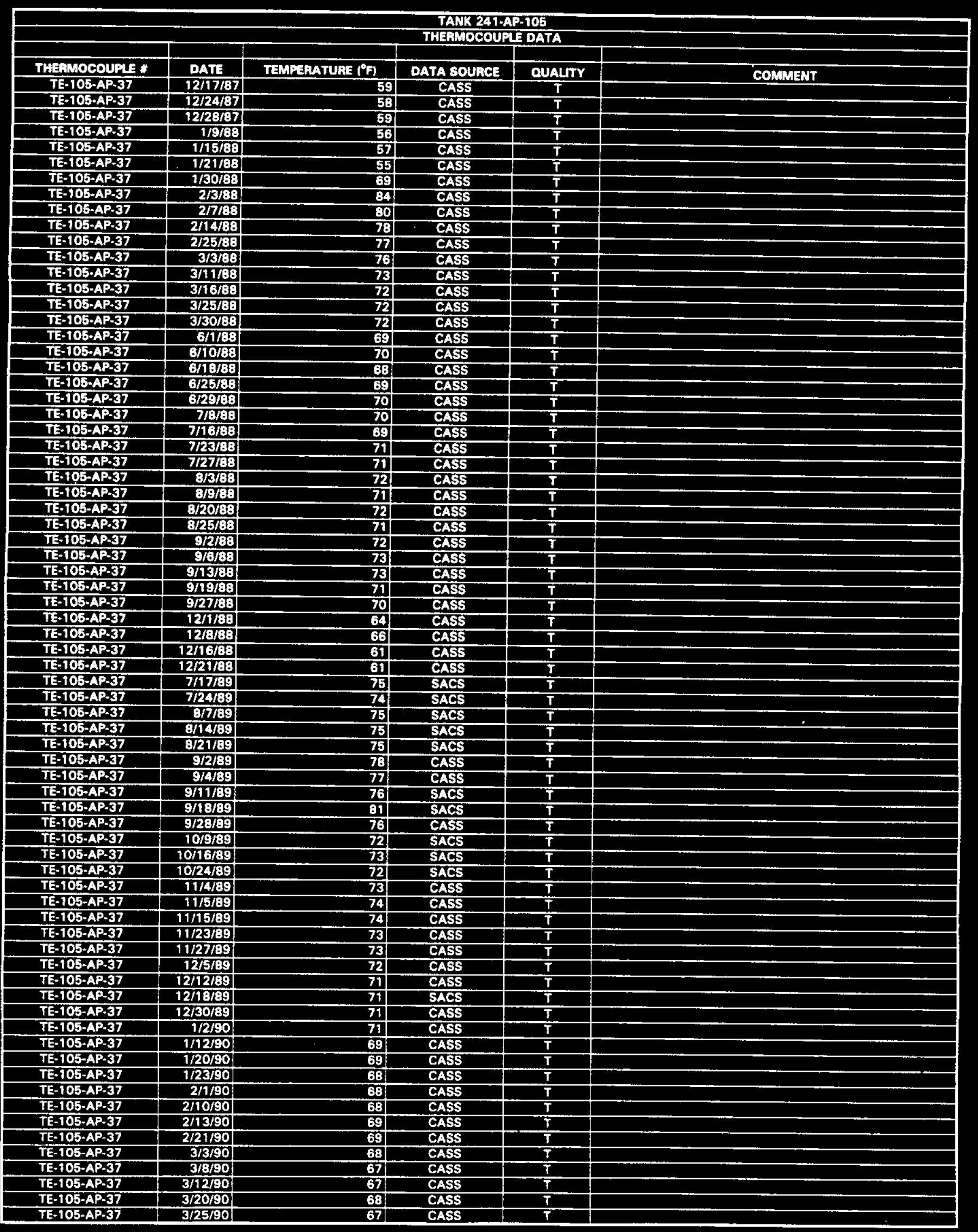




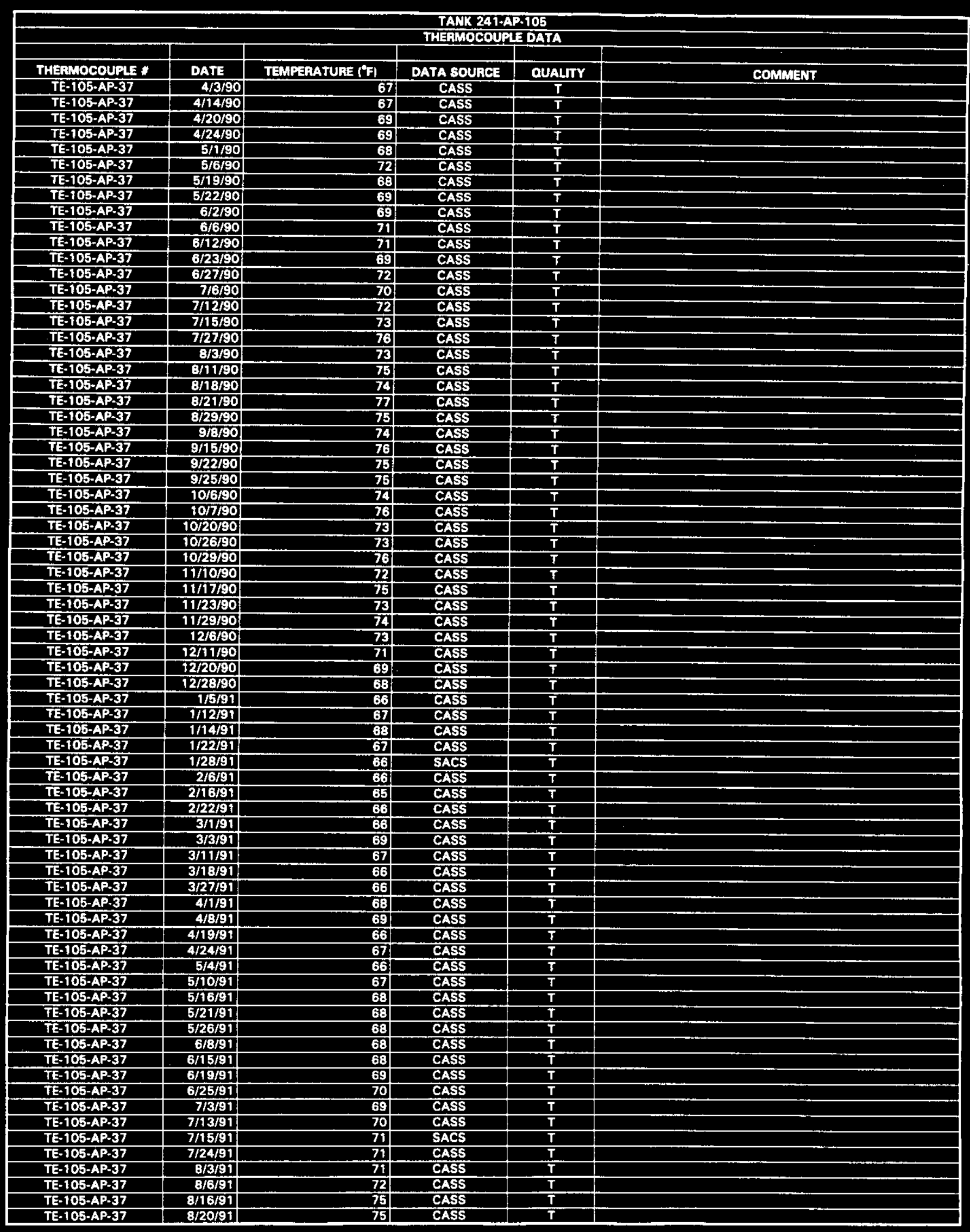

Data obtained from Computer Automated Surveillance System (CASS) in April 1995, and Surviellance Analysis Computer System (SACS) in June 1995. 


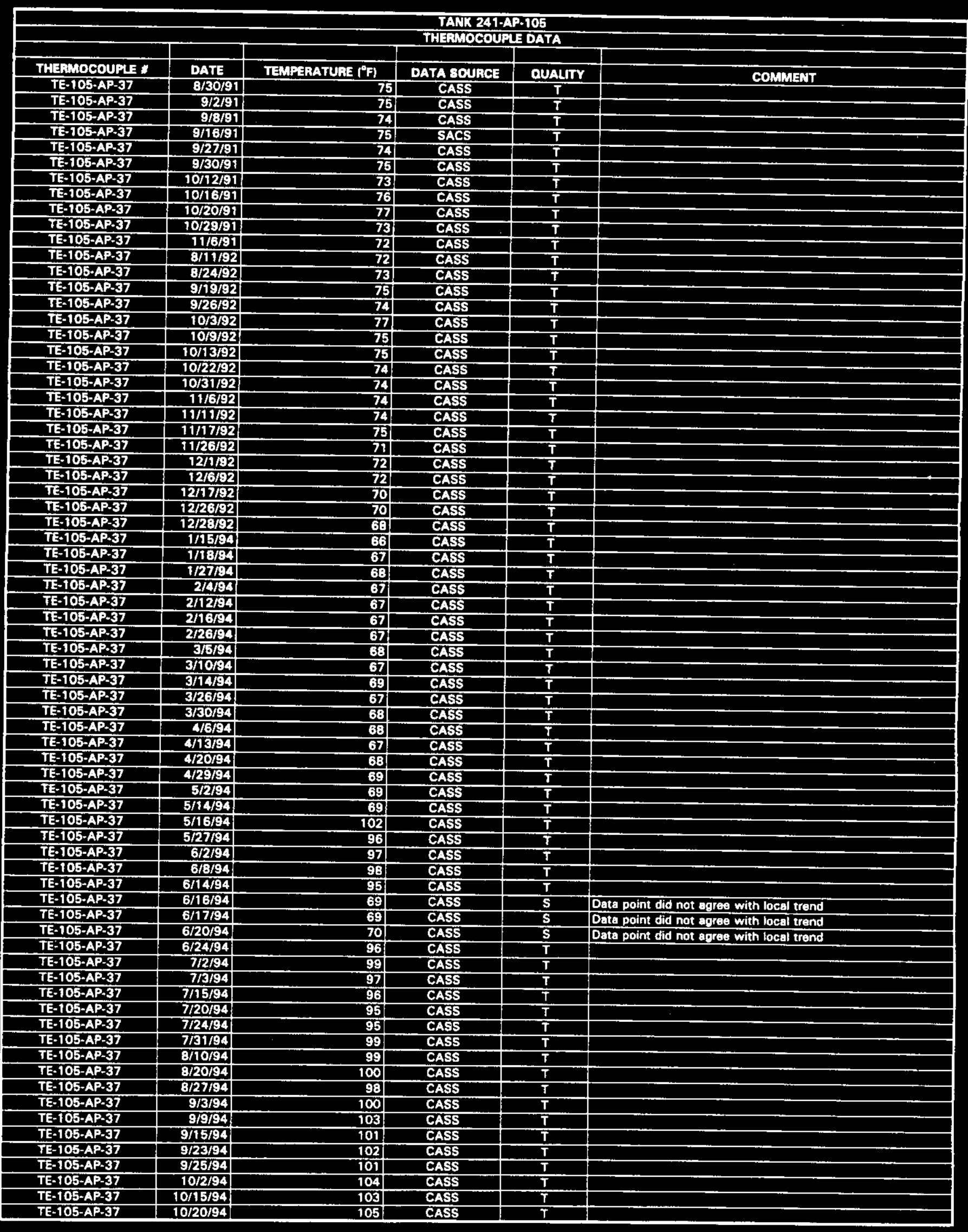

Data obtained from Computer Automated Surveillance System (CASS) in April 1995, and Surviellance Analysis Computer System (SACS) in June 1995. 


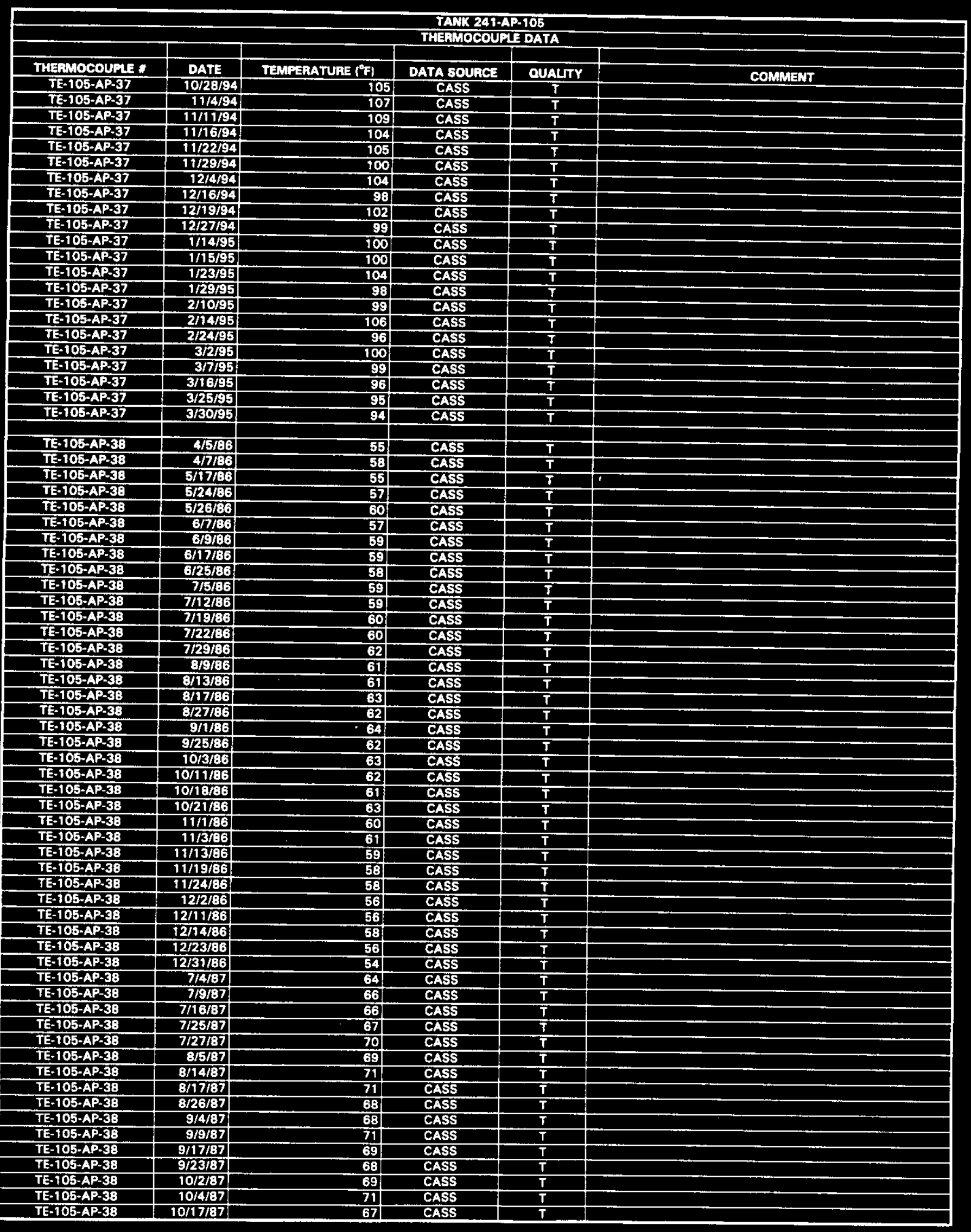

Data obtained from Computer Automated Surveillence System (CASS) in April 1995, and Surviellance Analysis Computer System (SACSI in June 1995. $D-421$ 


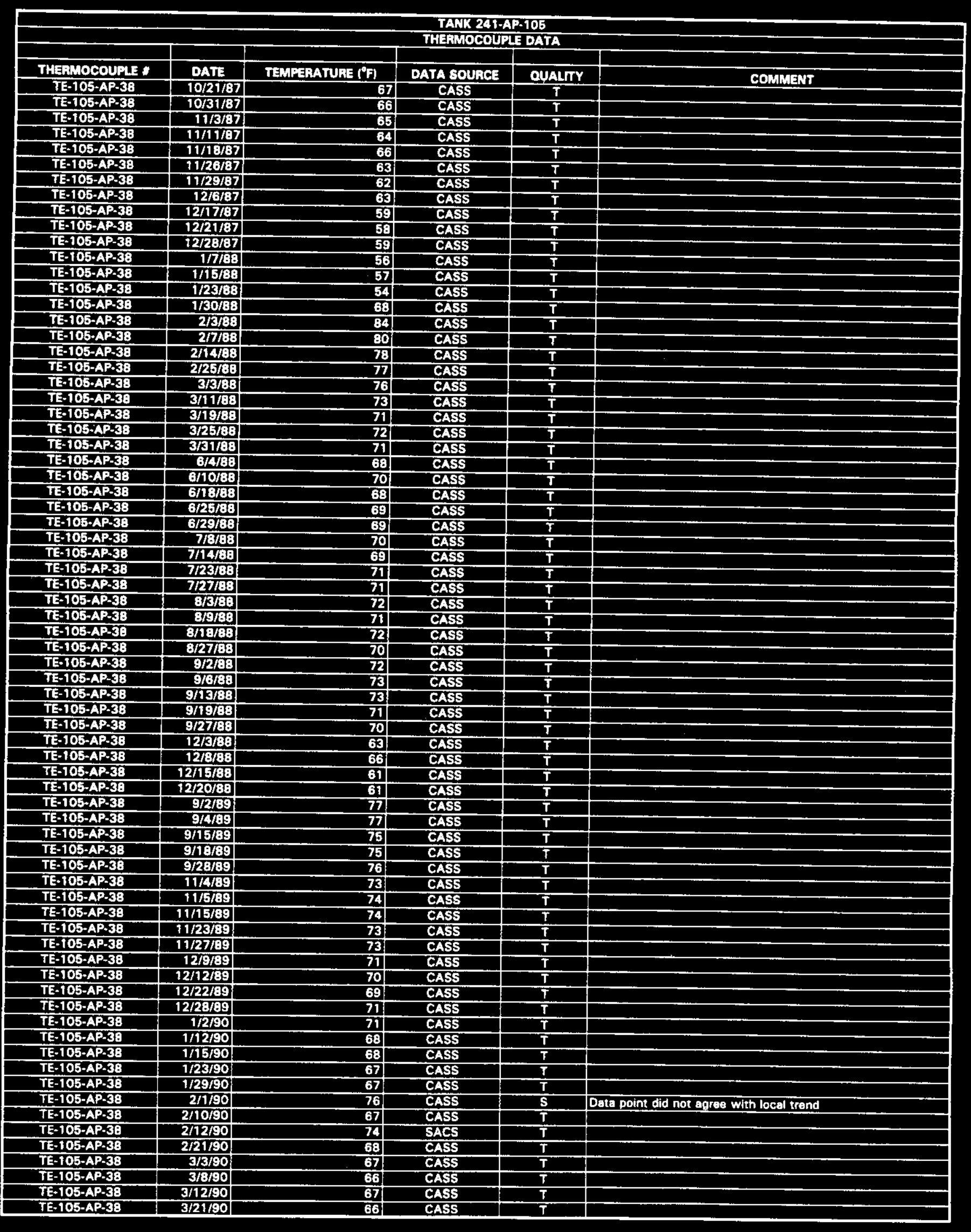

Dato obtained trom Computer Automated Surveillance Syatem (CASS) in April 1995, and Surviellance Analysis Computer System (SACS) in June 1995.

$D-422$ 


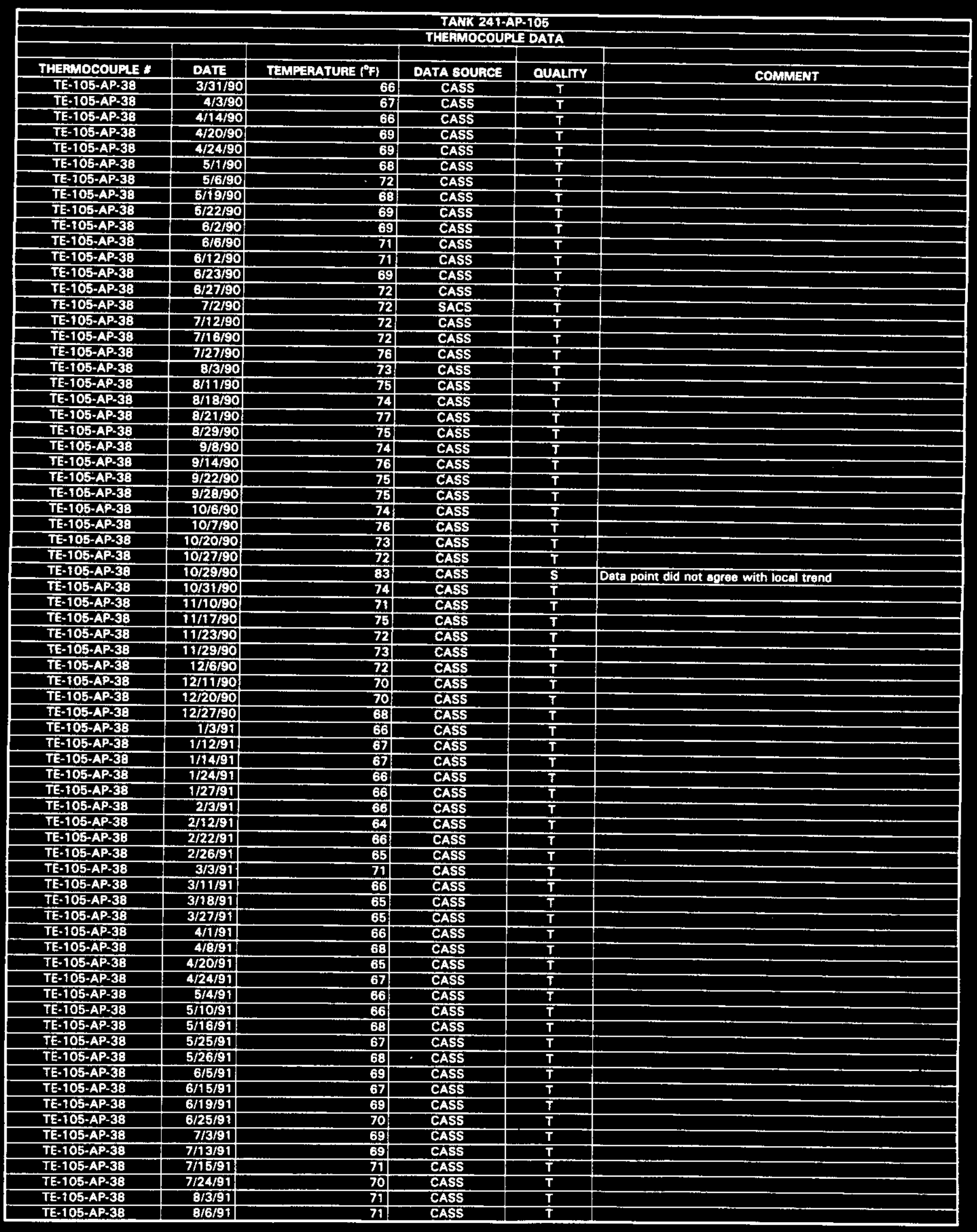

Date obtained from Computer Automated Surveillance Syatem (CASS) in April 1995, and Surviellance Analysis Computer System (SACS) in June 1995. 


\begin{tabular}{|c|c|c|c|c|c|}
\hline & & & $\begin{array}{l}\text { TAMK 241-A } \\
\text { THERMOCOUP }\end{array}$ & DATA & \\
\hline & & & & & \\
\hline THERHOCOUPLE $*$ & DATE & TEMPERATUAE $\left.f^{\circ} \mathrm{F}\right\}$ & DATA SOUACE & OUALITY & COMMENT \\
\hline TE-105-AP-38 & $8 / 16 / 91$ & 75 & CASS & $T$ & \\
\hline TE-105-AP-38 & $8 / 20 / 91$ & 74 & CASS & $\mathbf{T}$ & \\
\hline TE-105-AP-38 & $8 / 30 / 91$ & 75 & CASS & $T$ & \\
\hline TE-105-AP-38 & $9 / 2 / 91$ & 75 & CASS & $T$ & \\
\hline TE-105-AP-38 & 9/8/91 & 74 & CASS & $T$ & \\
\hline TE-105-AP-38 & 9/18/91 & 74 & CASS & $T$ & \\
\hline TE-105-AP-3B & 9/27/91 & 74 & CASS & $T$ & \\
\hline TE-105-AP-38 & $9 / 30 / 91$ & 75 & CASS & $\bar{T}$ & \\
\hline TE-105-AP-38 & $10 / 11 / 91$ & 73 & CASS & $\mathbf{T}$ & \\
\hline TE-105-AP-38 & 10/16/91 & 76 & CASS & $T$ & \\
\hline TE-105-AP-3A & 10/20/91 & 77 & CASS & $\mathbf{T}$ & \\
\hline TE-105-AP-38 & 10/29/91 & 73 & CASS & $T$ & \\
\hline TE-105-AP-38 & $11 / 6 / 91$ & 72 & CASS & $\mathbf{T}$ & \\
\hline TE-105-AP-38 & $8 / 11 / 92$ & 72 & CASS & $\bar{T}$ & \\
\hline TE-105-AP-38 & $8 / 24 / 92$ & 73 & CASS & $\bar{T}$ & \\
\hline TE-105-AP-38 & 9/19/92 & 75 & CASS & $T$ & \\
\hline TE-105-AP-38 & $9 / 26 / 92$ & 73 & CASS & $T$ & \\
\hline TE-105-AP-3B & $10 / 3 / 92$ & 76 & CASS & $T$ & \\
\hline TE-105-AP-38 & 10/9/92 & 74 & CASS & $\bar{T}$ & \\
\hline TE-105.AP-38 & $10 / 13 / 92$ & 74 & CASS & $T$ & \\
\hline TE-105-AP-38 & 10/22/92 & 74 & CASS & $T$ & \\
\hline TE-105-AP-3B & $10 / 31 / 92$ & 74 & CASS & $\bar{T}$ & \\
\hline TE-10S-AP-3B & $11 / 6 / 92$ & 73 & CASS & $\bar{T}$ & \\
\hline TE-105-AP-38 & $11 / 11 / 92$ & 73 & CASS & $\mathbf{T}$ & \\
\hline TE-105-AP-38 & $11 / 17 / 22$ & 74 & CASS & $T$ & \\
\hline TE-105-AP-3B & $11 / 22 / 92$ & 71 & CASS & $T$ & \\
\hline TE-105-AP_38 & $12 \pi / 92$ & 72 & CASS & $T$ & \\
\hline TE-105-AP-38 & $12 / 6 / 92$ & 72 & CASS & $T$ & \\
\hline TE-105-AP-3B & $12 / 17 / 92$ & 69 & CASS & $T$ & \\
\hline TE-105-AP-38 & $12 / 26 / 92$ & 70 & CASS & $T$ & \\
\hline TE-105-AP-38 & $12 / 30 / 92$ & 67 & CASS & $T$ & \\
\hline TE-105-AP-38 & $6 / 11 / 93$ & 67 & SACS & $T$ & \\
\hline TE-105-AP-38 & 6/18/93 & 68 & SACS & $\bar{T}$ & \\
\hline TE-105-AP-3B & 6/28/93 & 67 & SACS & $T$ & \\
\hline TE-105-AP-38 & $7 / 2 /$ & 67 & SACS & $T$ & \\
\hline TE-105-AP-38 & $7 / 15 / 93$ & 68 & SACS & $T$ & \\
\hline TE-10S-AP-38 & $7 / 19 / 93$ & 67 & SACS & $\mathbf{T}$ & \\
\hline TE-105-AP-38 & $7 / 30 / 23$ & 70 & SACS & $T$ & \\
\hline TE-105-AP-38 & 6/6/93 & 69 & SACS & $T$ & \\
\hline TE-105-AP-3B & $8 / 12 / 93$ & 69 & SACS & $T$ & \\
\hline TE-105-AP-3B & $9 / 24 / 93$ & 68 & SACS & $I$ & \\
\hline TE-105-AP-3B & $10 / 1 / 93$ & 70 & SACS & $T$ & \\
\hline TE-105-AP-38 & $10 / 15 / 93$ & 71 & SACS & $T$ & \\
\hline TE-105-AP-38 & $10 / 22 / 93$ & 70 & SACS & $T$ & \\
\hline TE-105-AP-38 & $10 / 28 / 83$ & 70 & SACS & $T$ & \\
\hline TE-105-AP-3B & $11 / 93$ & 65 & SACS & $T$ & \\
\hline TE-105-AP-38 & $11 / 30 / 93$ & 69 & SACS & $T$ & \\
\hline TE-105-AP-38 & $12 / 14 / 93$ & 67 & SACS & $T$ & \\
\hline TE-105-AP-38 & $12 / 20 / 93$ & 661 & SACS & $\bar{T}$ & \\
\hline TE-105-AP-38 & $12 / 27 / 193$ & 66 & SACS & $T$ & \\
\hline TE-105-AP-38 & $1 / 3 / 94$ & 64 & SACS & $T$ & \\
\hline TE-105-AP-3B & $1 / 11 / 94$ & 67 & SACS & $\bar{T}$ & \\
\hline TE-105-AP-38 & $1 / 18 / 94$ & 67 & CASS & $T$ & \\
\hline TE-105.AP-3B & $1 / 27 / 94$ & 68 & CASS & $T$ & \\
\hline TE-105-AP-3B & $2 / 4 / 94$ & 66 & CASS & $\bar{T}$ & \\
\hline TE-105-AP-38 & $2 / 12 / 94$ & 67 & CASS & $T$ & \\
\hline TE-105-AP-38 & $2 / 16 / 94$ & 66 & CASS & $T$ & \\
\hline TE-105-AP-3B & $2 / 26 / 94$ & 66 & CASS & $T$ & \\
\hline TE-105-AP-3B & $3 / 5 / 94$ & 67 & CASS & $T$ & \\
\hline TE-105-AP-3B & $3 / 10 / 94$ & 67 & CASS & $T$ & \\
\hline TE-105-AP-38 & $3 / 14 / 94$ & 69 & CASS & $T$ & \\
\hline TE-105-AP-3B & $3 / 26 / 94$ & 67 & CASS & $\mathbf{T}$ & \\
\hline TE-105-AP-38 & $3 / 30 / 94$ & 67 & CASS & $T$ & \\
\hline TE-105-AP-38 & $4 / 6 / 94$ & 68 & CASS & $T$ & \\
\hline TE-105-AP-38 & $4 / 13 / 94$ & 67 & CASS & $\mathbf{T}$ & \\
\hline TE-105.AP-38 & $4 / 20 / 94$ & 68 & CASS & $T$ & \\
\hline TE-105-AP-38 & $4 / 29 / 94$ & 68 & CASS & $T$ & \\
\hline TE-105-AP-38 & $5 / 2 / 94$ & 68 & CASS & $T$ & \\
\hline TE-105-AP-38 & $5 / 14 / 94$ & 69 & CASS & $T$ & \\
\hline TE-105-AP-38 & $5 / 21 / 94$ & 108 & CASS & $\mathrm{T}$ & \\
\hline TE-105-AP-38 & $5 / 27 / 94$ & 95 & CASS & $\bar{T}$ & \\
\hline TE-105-AP-38 & $6 / 2 / 94$ & 97 & CASS & $T$ & \\
\hline TE-105-AP-3B & 6/8/94 & 97 & CASS & $T$ & \\
\hline
\end{tabular}




\begin{tabular}{|c|c|c|c|c|c|}
\hline \multicolumn{6}{|c|}{$\begin{array}{l}\text { TAKK 241-AP-105 } \\
\text { THERIMOCOUFLE DATA }\end{array}$} \\
\hline & & & & & \\
\hline THERMOCOUPLE * & DATE & TEMPERATURE $\left({ }^{\circ} F\right)$ & DATA SOUACE & OUALTTY & COMMENT \\
\hline TE-105-AP-38 & $6 / 14 / 94$ & 95 & CASS & $T$ & \\
\hline TE-105-AP-38 & 6/16/94 & 69 & CASS & $\overline{\mathbf{S}}$ & Data point did not agree with local trend \\
\hline TE-105-AP-3B & $6 / 17 / 94$ & 69: & CASS & 5 & Data point did not agree with local trend \\
\hline TE-105-AP-3B & $6 / 20 / 94$ & 70 & CASS & s & Data point did not agree with local trend \\
\hline TE-105-AP-38 & $6 / 20 / 94$ & 68 & SACS & $S$ (G in SACS) & Data point did not agree with local trend \\
\hline TE-105-AP-38 & $6 / 24 / 94$ & 96 & CASS & $T$ & \\
\hline TE-105-AP-38 & $7 / 2 / 94$ & 98 & CASS & $T$ & \\
\hline TE-105-AP-3B & $7 / 3 / 94$ & 97 & CASS & $T$ & \\
\hline TE-105.AP-3B & $7 / 15 / 94$ & 96 & CASS & $T$ & \\
\hline TE-105-AP-38 & $7 / 19 / 94$ & 95 & CASS & $\bar{T}$ & \\
\hline TE-105-AP-38 & $7 / 30 / 94$ & 94 & CASS & $\bar{T}$ & \\
\hline TE-105-AP-3B & $7 / 31 / 94$ & 99 & CASS & $\mathbf{T}$ & \\
\hline TE-105-AP-38 & $8 / 10 / 94$ & 98 & CASS & $T$ & \\
\hline TE-105-AP-38 & $8 / 20 / 94$ & 100 & CASS & $T$ & \\
\hline IE-105-AP-38 & $8 / 22 / 94$ & 98 & CASS & $\bar{T}$ & \\
\hline TE-105-AP-3B & $9 / 3 / 94$ & 99 & CASS & $T$ & \\
\hline TE-T05-AP-3B & 9/9/94 & 103 & CASS & $T$ & \\
\hline TE-105-AP-3B & $9 / 15 / 94$ & 101 & CASS & $T$ & \\
\hline TE-105-AP-3B & $9 / 23 / 94$ & 102 & CASS & $T$ & \\
\hline TE-105-AP-38 & $9 / 25 / 94$ & 101 & CASS & $T$ & \\
\hline TE-105-AP-38 & $10 / 6 / 94$ & 103 & CASS & $T$ & \\
\hline TE-105-AP-38 & $10 / 15 / 94$ & 102 & CASS & $\overline{\mathrm{T}}$ & \\
\hline TE-105-AP-38 & $70 / 17 / 94$ & 104 & CASS & $T$ & \\
\hline TE-105-AP-3B & $10 / 28 / 94$ & 105 & CASS & $\bar{T}$ & \\
\hline TE-105-AP-3B & $111 / 4 / 94$ & 106 & CASS & $T$ & \\
\hline TE-105-AP-38 & $11 / 11 / 94$ & 108 & CASS & $T$ & \\
\hline$T E-105-A P=38$ & $11 / 16 / 94$ & 105 & CASS & $T$ & \\
\hline TE-105-AP-3B & $11 / 22 / 94$ & 105 & CASS & $T$ & \\
\hline TE-105-AP-38 & $12 / 3 / 94$ & 99 & CASS & $T$ & \\
\hline TE-105-AP-38 & $12 / 4 / 94$ & 104 & CASS & $T$ & \\
\hline TE-105-AP-38 & $12 / 18 / 94$ & 98 & CASS & $T$ & \\
\hline TE-105-AP-38 & $12 / 19 / 94$ & 101 & CASS & $T$ & \\
\hline TE-105-AP-38 & $12 / 29 / 94$ & 98 & CASS & $T$ & \\
\hline TE-105-AP-38 & $1 / 14 / 95$ & 100 & CASS & $T$ & \\
\hline TE-105-AP-3B & 1/15/95 & 99 & CASS & $T$ & \\
\hline TE-105-AP-3B & $1 / 23 / 95$ & 103 & CASS & $T$ & \\
\hline TE-105-AP-38 & $1 / 30 / 95$ & 98 & SACS & G & \\
\hline TE-T05-AP-38 & 2/9/95 & 100 & CASS & $\bar{T}$ & \\
\hline TE-105-AP-3B & $2 / 14 / 95$ & 106 & CASS & $T$ & \\
\hline TE-105-AP-3B & $2 / 24 / 95$ & 96 & CASS & $T$ & \\
\hline TE-105-AP-38 & 3/2/95 & 100 & CASS & $T$ & \\
\hline TE-105-AP-38 & 3/7/25 & 99 & CASS & $T$ & \\
\hline TE-105-AP-38 & $3 / 12 / 95$ & 96 & CASS & $T$ & \\
\hline TE-10S-AP-3B & $3 / 25 / 95$ & 94 & CASS & $\bar{T}$ & \\
\hline TE-TO5-AP-38 & $3 / 30 / 95$ & 94 & CASS & $T$ & \\
\hline TE-105-AP-38 & 4/3/95 & 86 & SACS & G & \\
\hline TE-105-AP-3B & $4 / 10 / 95$ & 88 & SACS & G & \\
\hline TE-105-AP-3B & $4 / 24 / 95$ & B3! & SACS & G & \\
\hline TE-105-AP-38 & 5/1/95 & 86 & SACS & G & \\
\hline TE-105-AP-38 & $5 / 8 / 95$ & 84 & SACS & $\mathbf{G}$ & \\
\hline$T E-105-A P-38$ & $5 / 16 / 95$ & 84 & SACS & G & \\
\hline TE-105-AP-38 & $5 / 22 / 95$ & 84 & SACS & G & \\
\hline TE-105-AP-38 & 6/5/95 & 88 & SACS & G & \\
\hline TE-105-AP-38 & $6 / 13 / 95$ & 85 & SACS & G & \\
\hline TE-105-AP-38 & 6/19/95 & 98 & SACS & G & \\
\hline & & & & & \\
\hline TE-105-AP-39 & 4/5/86 & 56 & CASS & $T$ & \\
\hline TE-105-AP-39 & $4 / 7 / 86$ & 58 & CASS & $T$ & \\
\hline TE-105-AP-39 & $5 / 17 / 86$ & 55 & CASS & $T$ & \\
\hline TE-105-AP-39 & $5 / 24 / 86$ & 57 & CASS & $\mathbf{T}$ & \\
\hline TE-105-AP-39 & $5 / 26 / 86$ & 60 & CASS & $T$ & \\
\hline TE-105-AP.39 & $6 / 4 / 86$ & 58 & CASS & $T$ & \\
\hline TE-105-AP-39 & $6 / 9 / 86$ & 61 & CASS & $T$ & \\
\hline TE-105-AP-39 & $6 / 17 / 86$ & 59 & CASS & $T$ & \\
\hline TE-105-AP-39 & $6 / 25 / 86$ & 58 & CASS & $T$ & \\
\hline TE-105-AP-39 & $7 / 5 / 86$ & 59 ! & CASS & $T$ & \\
\hline TE-105-AP-39 & $7 / 12 / 86$ & 59 & CASS & $T$ & \\
\hline TE-105-AP-39 & $7 / 19 / 86$ & 60 & CASS & $T$ & \\
\hline TE-105-AP-39 & $7 / 22 / 86$ & 60 & CASS & $T$ & \\
\hline TE-105-AP-39 & $7 / 29 / 86$ & 62 & CASS & $\mathbf{T}$ & \\
\hline TE-105-AP-39 & $8 / 9 / 86$ & 61: & CASS & $T$ & \\
\hline TE-105-AP-39 & $8 / 13 / 86$ & 62 & CASS & $T$ & \\
\hline TE-105-AP-39 & $8 / 17 / 86$ & 63 & CASS & $T$ & \\
\hline
\end{tabular}




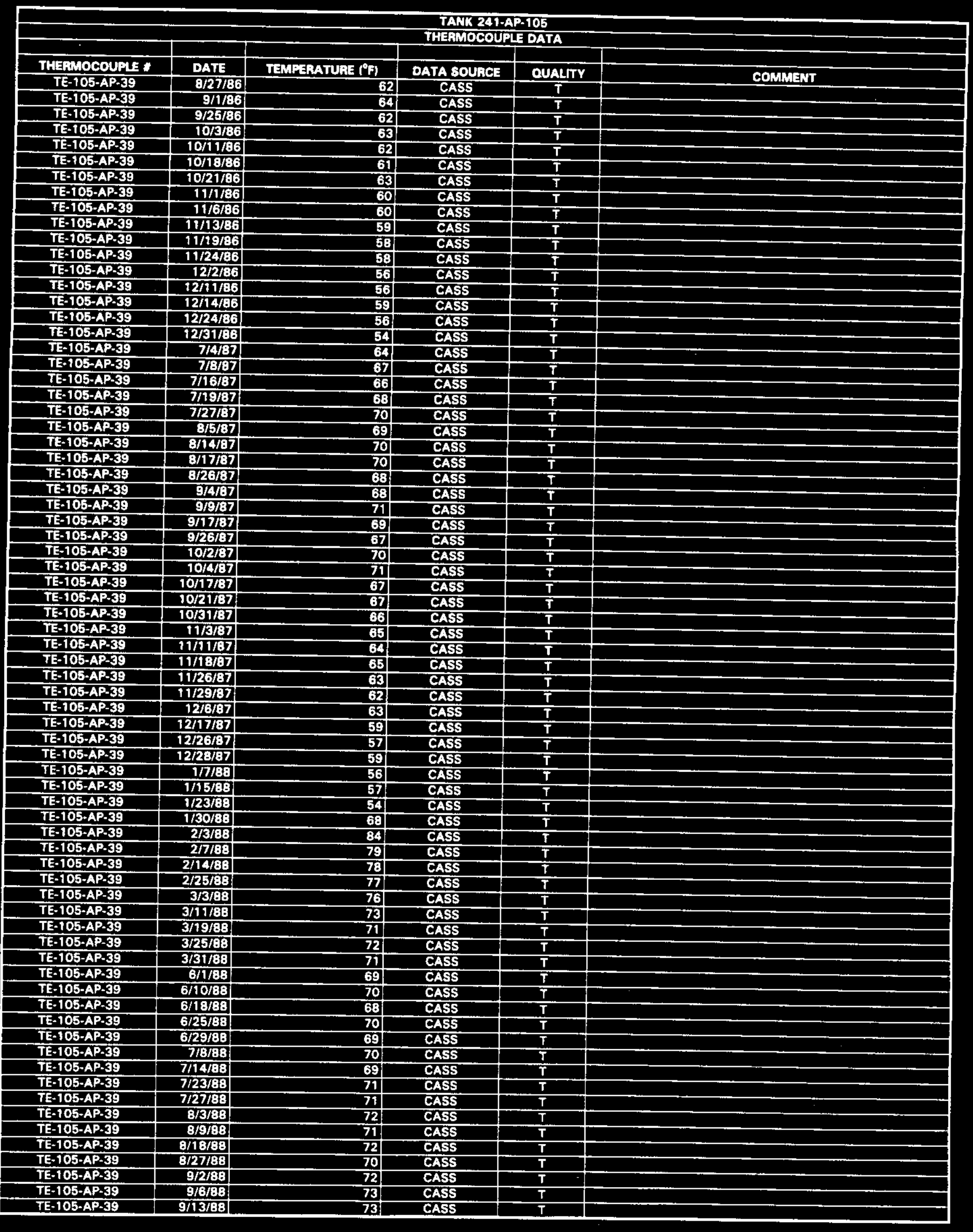

Data obtained from Computer Automated Surveillance Syatem (CASS) in April 1995, and Surviellarce Analyais Computer System (SACS) in June 1995. 


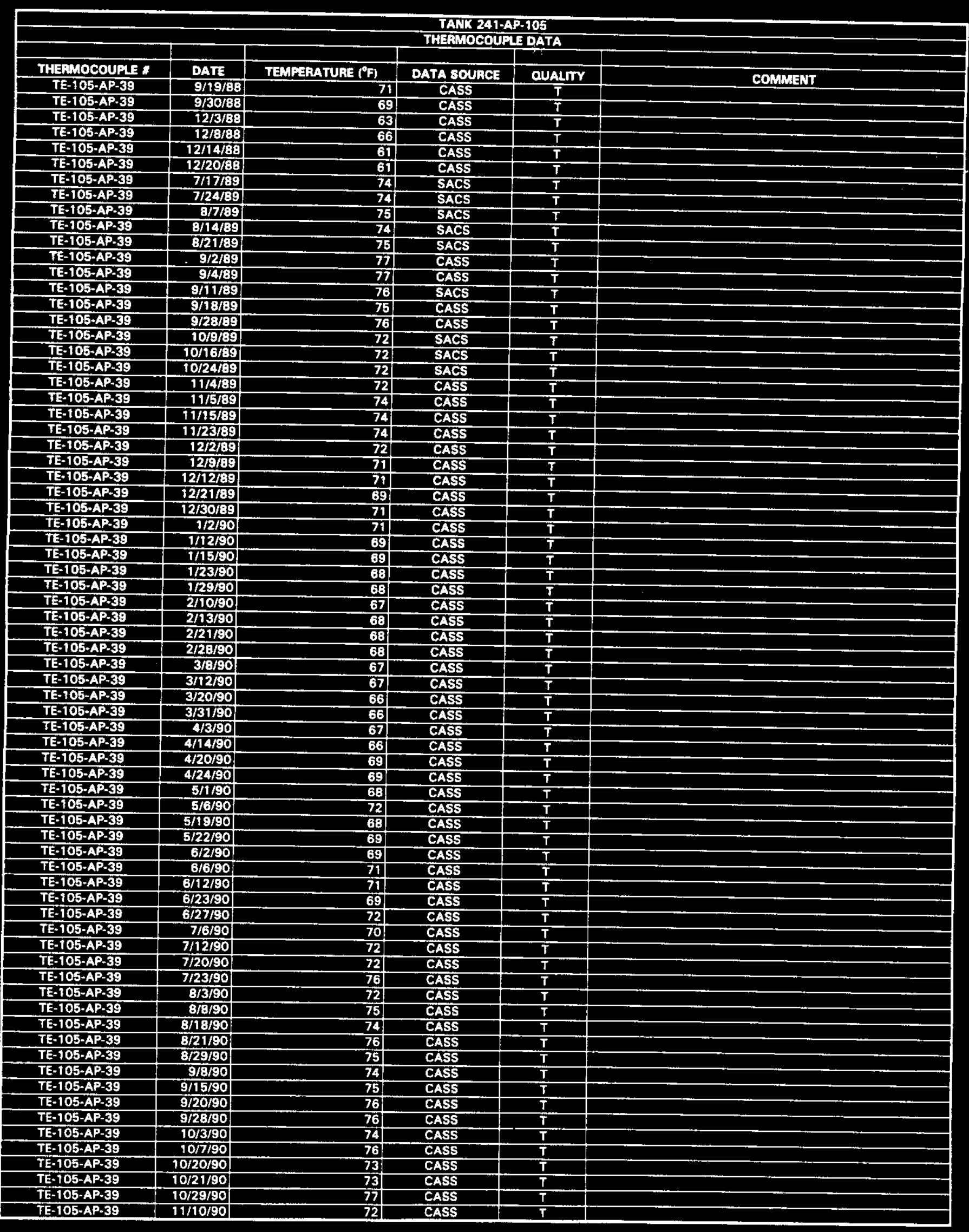

Data obtained from Computer Automated Surveillance System (CASS) in April 1995, and Surviellance Analysis Computer Syatem (SACS) in June 1995. 


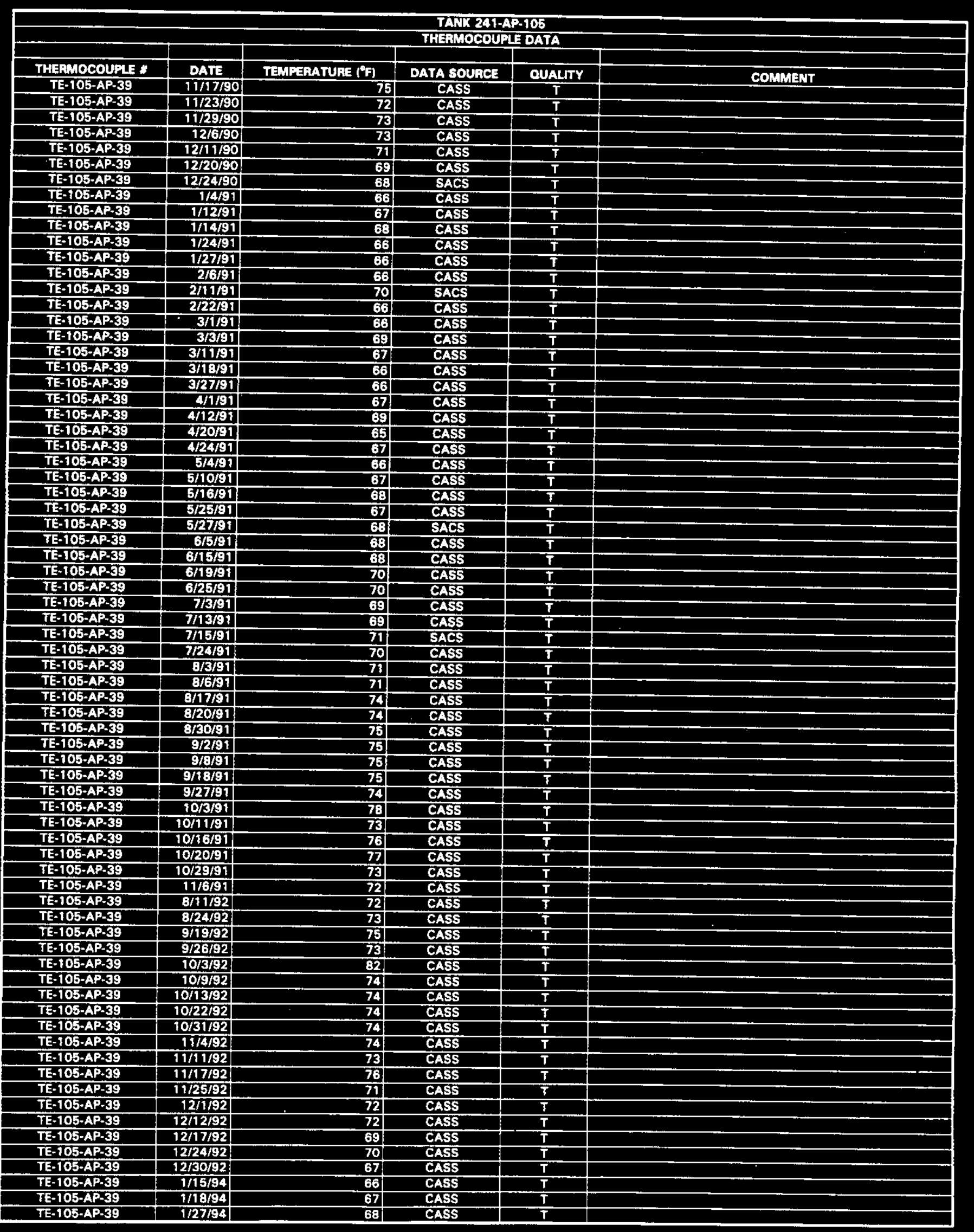

Data obtained from Computer Automated Surveillance System (CASS) in April 1995, and Surviellance Analysis Computer System (SACS) in June 1995. 


\begin{tabular}{|c|c|c|c|c|c|}
\hline \multicolumn{6}{|c|}{$\begin{array}{l}\text { TANK 241-AP-105 } \\
\text { THEZMOCOUPLE DATA }\end{array}$} \\
\hline & & & & & \\
\hline THERMOCOUPL $"$ & DATE & TEMPERATURE $\left({ }^{\circ} \mathrm{F}\right)$ & DATA SOURCE & QUALITY & COMMENT \\
\hline TE-105-AP-39 & $2 / 4 / 94$ & 66 & CASS & $T$ & \\
\hline TE-105-AP-39 & $2 / 12 / 94$ & 67 & CASS & $\mathbf{T}$ & \\
\hline TE-105-AP-39 & $2 / 16 / 94$ & 86 & CASS & $T$ & \\
\hline TE-105-AP-39 & $2 / 22 / 94$ & 67 & CASS & $T$ & \\
\hline TE-105-AP-39 & $3 / 5 / 94$ & 68 & CASS & $T$ & \\
\hline TE-105-AP-39 & $3 / 10 / 94$ & $\frac{67}{69}$ & CASS & $T$ & \\
\hline TE-105-AP-39 & $3 / 14 / 94$ & 69 & $\begin{array}{l}\text { CASS } \\
\text { CASS }\end{array}$ & $T$ & \\
\hline $\begin{array}{l}\text { TE-105-AP-39 } \\
\text { TE-105-AP-39 }\end{array}$ & $\begin{array}{l}3 / 26 / 94 \\
3 / 30 / 94\end{array}$ & 67 & $\frac{\text { CASS }}{\text { CASS }}$ & $T$ & \\
\hline$\frac{T E-105-A P-39}{\text { TE-105-AP-39 }}$ & \begin{tabular}{r|}
$3 / 30 / 94$ \\
$4 / 6 / 94$
\end{tabular} & 69 & $\frac{\text { CASS }}{\text { CASS }}$ & $T$ & \\
\hline$\frac{11-105-A P-39}{T E-105-A P-39}$ & $4 / 13 / 94$ & 67 & CASS & $\frac{T}{T}$ & \\
\hline TE-105-APA39 & $4 / 20 / 94$ & 68 & CASS & $T$ & \\
\hline TE-105-AP-39 & $4 / 29 / 94$ & 68 & CASS & $T$ & \\
\hline TE-105-AP-39 & $5 / 2 / 94$ & 68 & CASS & $T$ & \\
\hline TE-105-AP-39 & $5 / 14 / 94$ & 69 & CASS & $\bar{T}$ & \\
\hline TE-105.AP-39 & 5/16/94 & 102 & CASS & $\bar{T}$ & \\
\hline TE-105-AP-39 & $5 / 27 / 94$ & 95 & CASS & $T$ & \\
\hline TE-105-AP-39 & $6 / 2 / 94$ & 98 & CASS & $T$ & \\
\hline TE-105-AP-39 & $6 / 8 / 94$ & 97 & CASS & $T$ & \\
\hline TE-105-AP-39 & $6 / 14 / 94$ & 95 & CASS & $T$ & \\
\hline TE-105-AP-39 & $6 / 16 / 94$ & 69 & CASS & $\mathbf{s}$ & Data point did not agres with local trend \\
\hline TE-105.AP-39 & $6 / 17 / 94$ & 69 & CASS & s & Data point did not agree with local trend \\
\hline TE-105-AP-39 & $6 / 20 / 94$ & 70 & CASS & $\mathbf{s}$ & Data point did not apres with local trend \\
\hline TE-105-AP-39 & $6 / 25 / 94$ & 96 & CASS & $\mathrm{T}$ & \\
\hline TE-105-AP-39 & $7 / 2 / 94$ & 99 & CASS & $\bar{T}$ & \\
\hline TE-105-AP-39 & $7 / 7 / 94$ & 96 & CASS & $T$ & \\
\hline TE-105-AP-39 & $7 / 11 / 94$ & 148 & CASS & 5 & Data point did not agree with local trend \\
\hline TE-105-AP-39 & $7 / 13 / 94$ & 95 & CASS. & $\bar{T}$ & \\
\hline TE-105-AP-39 & $7 / 20 / 94$ & 94 & CASS & $T$ & \\
\hline TE-105-AP-39 & $7 / 30 / 94$ & 94 & CASS & $T$ & \\
\hline TE-105-AP-39 & $7 / 31 / 94$ & 98 & CASS & $\mathbf{T}$ & \\
\hline TE-105-AP-39 & $8 / 10 / 94$ & 98 & CASS & $T$ & \\
\hline TE-105-AP-39 & $8 / 20 / 94$ & 99 & CASS & $\mathbf{T}$ & \\
\hline TE-105-AP-39 & $8 / 22 / 94$ & 98 & CASS & $\mathbf{T}$ & \\
\hline TE-105-AP-39 & $9 / 3 / 94$ & 99 & CASS & $\mathbf{T}$ & \\
\hline TE-105-AP-39 & 9/9/94 & 102 & CASS & $\bar{T}$ & \\
\hline TE-105-AP-39 & $9 / 15 / 94$ & 101 & CASS & $\bar{T}$ & \\
\hline TE-105-AP.39 & $9 / 23 / 94$ & 102 & CASS & $T$ & \\
\hline TE-105-AP-39 & $9 / 25 / 94$ & 101 & CASS & $\bar{T}$ & \\
\hline TE-105-AP-39 & $10 / 2 / 94$ & 104 & CASS & $T$ & \\
\hline TE-105-AP-39 & $10 / 15 / 94$ & 103 & CASS & $\bar{T}$ & \\
\hline TE-105-AP-39 & $10 / 17 / 94$ & 104 & CASS & $T$ & \\
\hline TE-105-AP-39 & $10 / 28 / 94$ & 105 & CASS & $T$ & \\
\hline TE-105-AP-39 & $11 / 4 / 94$ & 106 & CASS & $\bar{T}$ & \\
\hline TE-105-AP-39 & $11 / 11 / 94$ & 105 & CASS & $T$ & \\
\hline TE-105-AP-39 & $11 / 16 / 94$ & 105 & CASS & $T$ & \\
\hline TE-105-AP-39 & $11 / 22 / 94$ & 106 & CASS & $T$ & \\
\hline TE-105-AP-39 & $11 / 28 / 94$ & 100 & CASS & $T$ & \\
\hline TE-105-AP-39 & $12 / 4 / 94$ & 104 & CASS & $T$ & \\
\hline TE-105-AP-39 & $12 / 16 / 94$ & 98 & CASS & $T$ & \\
\hline TE-105-AP-39 & $12 / 19 / 94$ & 101 & CASS & $T$ & \\
\hline TE-105-AP-39 & $12 / 29 / 94$ & 98 & CASS & $T$ & \\
\hline TE-105-AP-39 & $1 / 14 / 95$ & 100 & CASS & $T$ & \\
\hline TE-105-AP-39 & $1 / 15 / 95$ & 100 & CASS & $T$ & \\
\hline TE-105-AP-39 & $1 / 23 / 95$ & 104 & CASS & $\bar{T}$ & \\
\hline TE-105-AP-39 & $2 / 2 / 95$ & 97 & CASS & $T$ & \\
\hline TE-105-AP-39 & $2 / 9 / 95$ & 100 & CASS & $\bar{T}$ & \\
\hline TE-105-AP-39 & $2 / 14 / 95$ & 106 & CASS & $T$ & \\
\hline TE-105-AP-39 & $2 / 24 / 95$ & 96 & CASS & $T$ & \\
\hline TE-105-AP-39 & $3 / 2 / 95$ & 101 & CASS & $T$ & \\
\hline TE-105-AP-39 & $3 / 7 / 95$ & 99 & CASS & $T$ & \\
\hline TE-105-AP-39 & 3/12/95 & 96 & CASS & $T$ & \\
\hline TE-105-AP-39 & $3 / 25 / 95$ & 95 & CASS & $T$ & \\
\hline TE-105.AP-39 & $3 / 30 / 95$ & 94 & CASS & $\bar{T}$ & \\
\hline 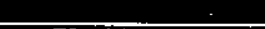 & & & & & \\
\hline TE-105-AP-40 & $4 / 5 / 86$ & 55 & CASS & $\mathbf{T}$ & \\
\hline TE-105-AP-40 & $4 / 7 / 86$ & 58 & CASS & $T$ & \\
\hline TE-105-AP-40 & $5 / 17 / 86$ & 55 & CASS & $T$ & \\
\hline TE-105-AP-40 & $5 / 22 / 86$ & 57 & CASS & $T$ & \\
\hline TE-105-AP-40 & $5 / 26 / 86$ & 60 & CASS & $T$ & \\
\hline$T E-105-A P-40$ & $6 / 7 / 86$ & 57 & CASS & $T$ & \\
\hline TE-105-AP-40 & 6/9/96 & 61 & CASS & $T$ & \\
\hline TE-105-AP-40 & 6/17/86 & 59 & CASS & $\bar{T}$ & \\
\hline
\end{tabular}




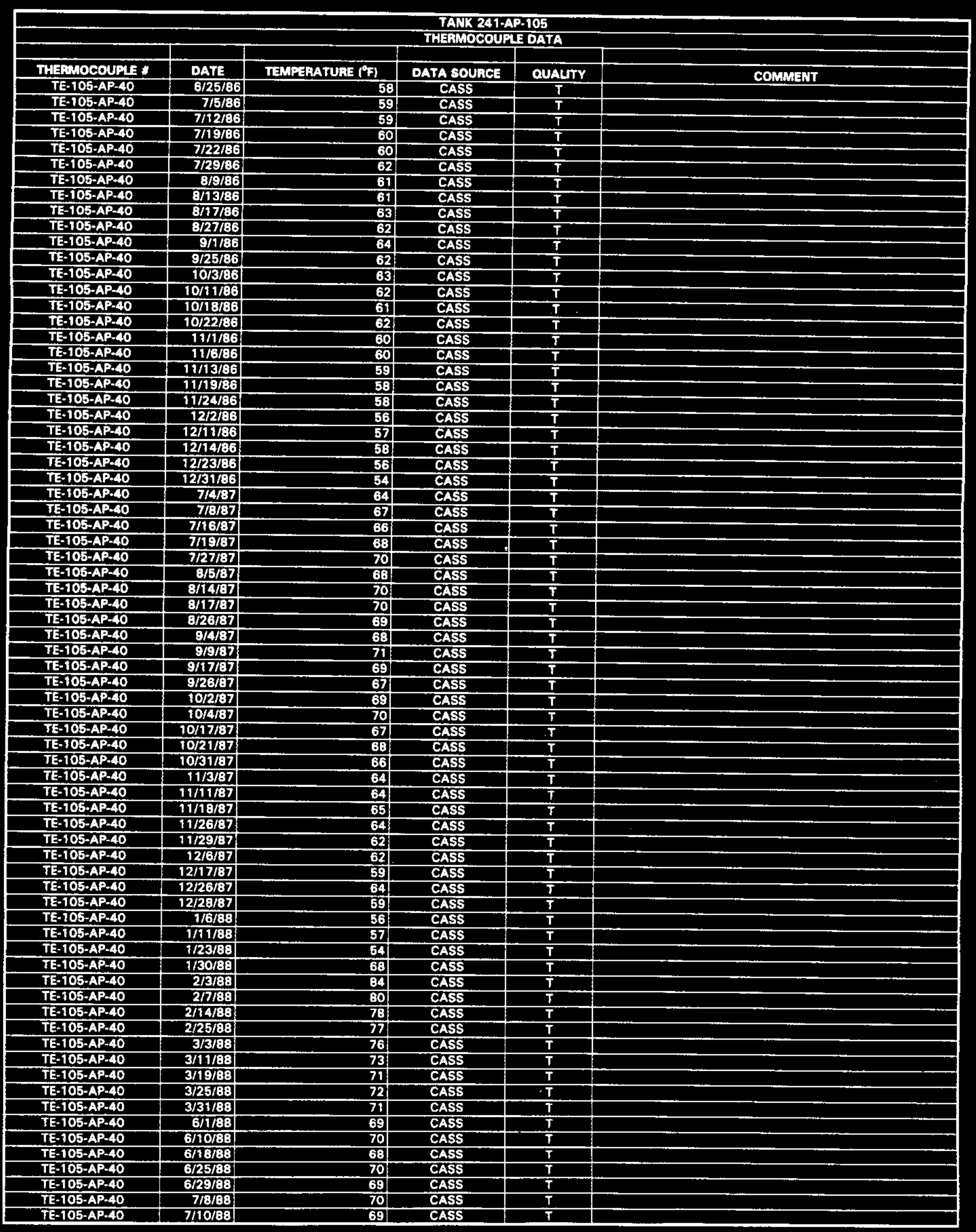




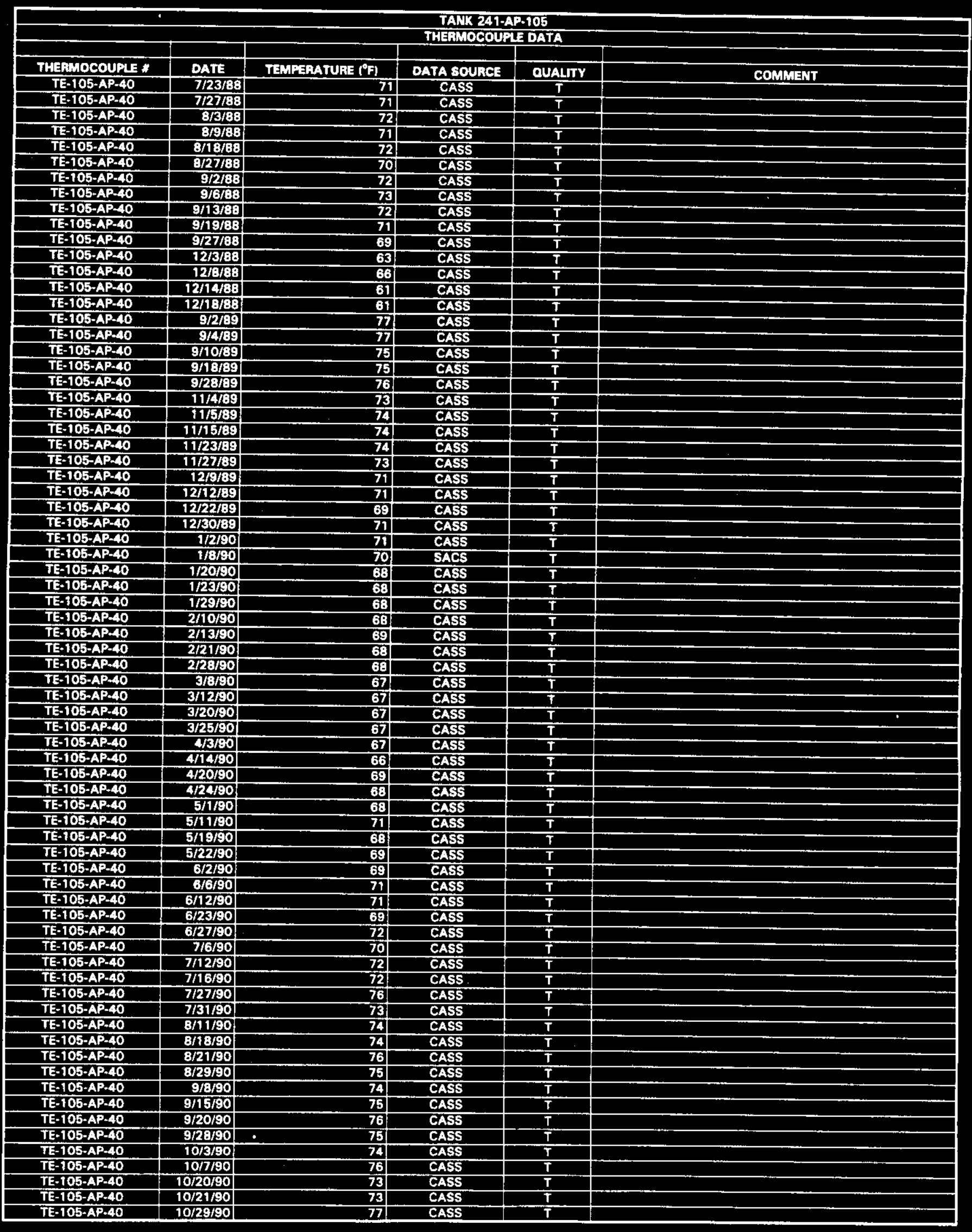

Date obtainad from Computer Automated Surveillance System (CASS) in April 1995, and Surviellance Analysis Computer System (SACS) in June 1995. D-431 


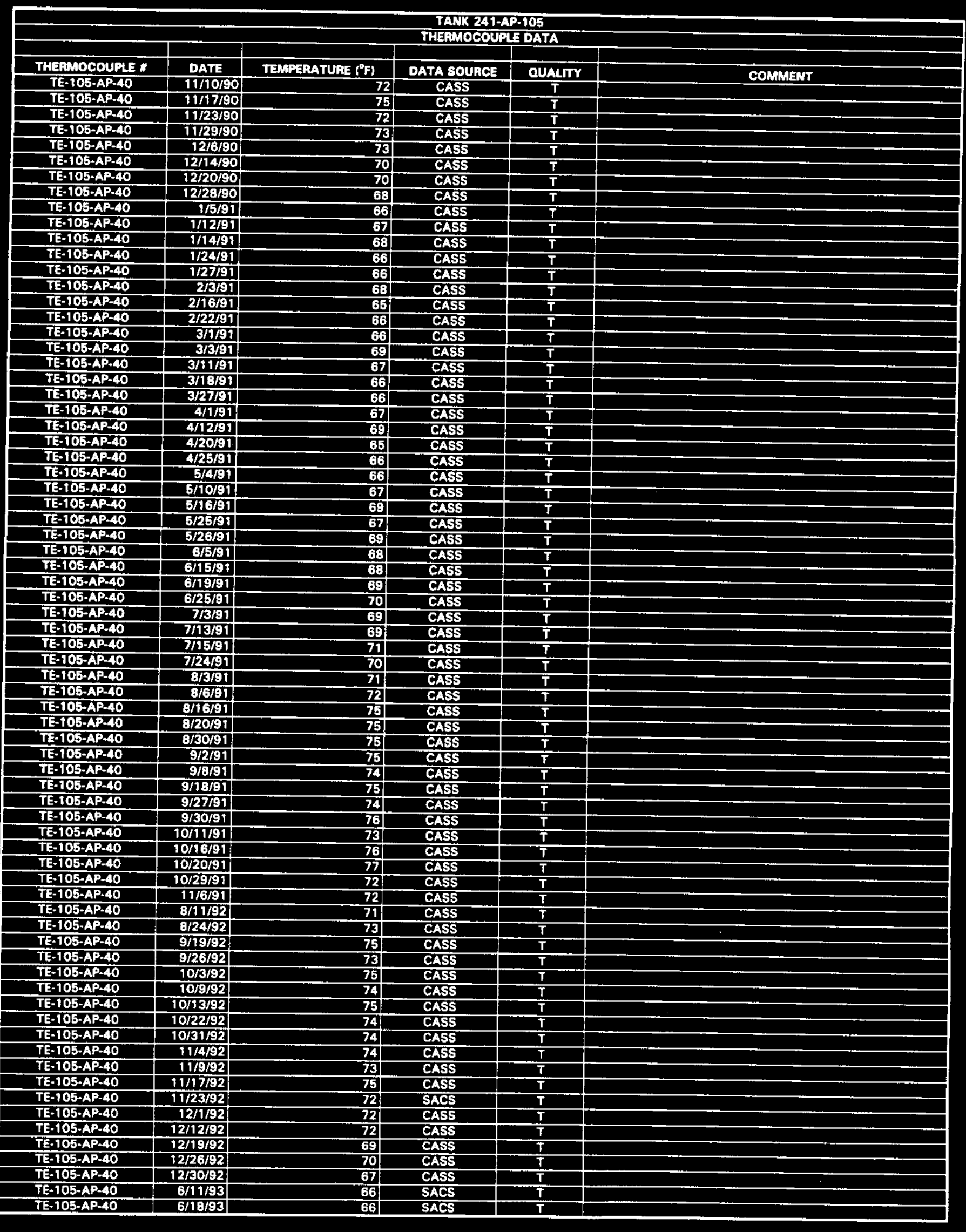

Data obtained from Computer Automated Surveillance System (CASS) in April 1995, and Surviellance Analysis Computer System (SACS) in June 1995.

D-432 


\begin{tabular}{|c|c|c|c|c|c|}
\hline \multicolumn{6}{|c|}{$\begin{array}{l}\text { TANK 241-AP-105 } \\
\text { THEZMMOCOUFLE DATA }\end{array}$} \\
\hline & & & & & \\
\hline THERHOCOUPE * & DATE & TEMPERATURE $\left({ }^{\circ} \mathrm{F}\right)$ & DATA SOUACE & OUALITY & COMMENT \\
\hline TE-105-AP-40 & $6 / 28 / 93$ & 67 & SACS & $T$ & \\
\hline TE-105-AP-40 & 7/9/93 & $\frac{68}{69}$ & $\frac{\text { SACS }}{\text { SACS }}$ & $-T$ & \\
\hline$\frac{T E-105-A P-40}{T E-105-A P-40}$ & $7 / 15 / 93$ & $\frac{69}{67}$ & $\frac{\text { SACS }}{\text { SACS }}$ & $\frac{T}{T}$ & \\
\hline$\frac{T E-105-A P-40}{T E-105-A P-40}$ & $\begin{array}{l}7 / 19 / 93 \\
7 / 30 / 93\end{array}$ & $\frac{67}{70}$ & $\begin{array}{l}\text { SACS } \\
\text { SACS }\end{array}$ & $\frac{T}{T}$ & \\
\hline TE-105-AP-40 & $8 / 6 / 93$ & 69 & SACS & $\mathbf{T}$ & \\
\hline TE-10S-AP-40 & $8 / 12 / 93$ & 69 & SACS & $T$ & \\
\hline TE-105-AP-40 & 9/24/93 & 88 & SACS & $T$ & \\
\hline TE-105-AP-40 & 10/1/93 & 70 & SACS & $T$ & \\
\hline TE-105-AP-40 & $10 / 15 / 93$ & 71 & SACS & $T$ & \\
\hline TE-105-AP-40 & $10 / 22 / 93$ & 70 & SACS & $T$ & \\
\hline TE-105-AP-40 & 10/28/93 & 70 & SACS & $T$ & \\
\hline IE-105-AP-40 & $11 / 9 / 93$ & 67. & SACS & $T$ & \\
\hline TE-105-AP-40 & $11 / 30 / 93$ & 69 & SACS & $T$ & \\
\hline TE-105-AP-40 & $12 / 14 / 93$ & 67 & SACS & $\bar{T}$ & \\
\hline$T E-105-A P=40$ & $12 / 20 / 93$ & 68 & SACS & $\bar{T}$ & \\
\hline TE-10K-AP-40 & $12 / 27 / 93$ & 66 & SACS & $T$ & \\
\hline$T E-105-A P-40$ & $1 / 3 / 94$ & 65 & SACS & $T$ & \\
\hline TE-105-AP-40 & $1 / 11 / 94$ & 67 & SACS & $T$ & \\
\hline IE-105-AP-40 & 1/18/94 & 67 & CASS & $\bar{T}$ & \\
\hline TE-10S-AP-AO & $1 / 24 / 94$ & 69 & SACS & $T$ & \\
\hline TE-105-AP-AO & $2 / 4 / 94$ & 66 & CASS & $\bar{T}$ & \\
\hline TE-105-AP.40 & $2 / 12 / 94$ & 67! & CASS & $T$ & \\
\hline TE-106-AP-AO & $2 / 15 / 94$ & 67 & CASS & $\mathbf{T}$ & \\
\hline TE-105-AP-40 & 2122194 & 67 & CASS & $\mathbf{T}$ & \\
\hline TE-106-AP-AO & $3 / 5 / 94$ & 68 & CASS & $\mathbf{T}$ & \\
\hline TE-105-AP-10 & $3 / 8 / 94$ & 67 & CASS & $\mathbf{T}$ & \\
\hline TE-105-AP-40 & $3 / 14 / 94$ & 69 & CASS & $T$ & \\
\hline TE-105-AP-40 & $3 / 26 / 94$ & 66 & CASS & $\mathbf{T}$ & • \\
\hline TE-105-AP-40 & $3 / 30 / 94$ & 68 & CASS & $T$ & \\
\hline TE-105-AP-40 & $4 / 6 / 94$ & 69 & CASS & $\mathbf{T}$ & \\
\hline TE-105-AP-40 & 4/13/94 & 67 & CASS & $\mathbf{T}$ & \\
\hline TE-105-AP-40 & $4 / 23 / 94$ & 67 & CASS & $\mathbf{T}$ & \\
\hline TE-105-AP-40 & $4 / 29 / 94$ & 68 & CASS & $T$ & \\
\hline TE-105-AP-40 & $5 / 2 / 94$ & 68 & CASS & $\mathbf{T}$ & \\
\hline TE-105-AP-40 & $5 / 14 / 94$ & 68 & CASS & $\mathbf{T}$ & \\
\hline TE-TOS-AP-40 & $5 / 16 / 94$ & 102 & CASS & $T$ & \\
\hline TE-105-AP-40 & $5 / 27 / 94$ & 95 & CASS & $T$ & \\
\hline TE-105-AP-40 & 8/2/94 & 98 & CASS & $\mathbf{T}$ & \\
\hline$T=105-A P-40$ & 6/3/94 & 97 & CASS & $\mathbf{T}$ & \\
\hline TE-105-AP-40 & 6/16/94 & 69 & CASS & $\mathbf{s}$ & Data point did not agres with local trend \\
\hline TE-105-AP-40 & 8/17/94 & 691 & CASS & $\mathbf{S}$ & Data point did not agree with local trend \\
\hline TE-105-AP-40 & 6/19/94 & 94 & CASS & $T$ & \\
\hline TE $=105-A P-40$ & $6 / 20 / 94$ & 70 & CASS & $\mathbf{s}$ & Date point did not egree with locel trend \\
\hline$T E-105-A P-40$ & $6 / 20 / 94$ & 69 & SACS & S $(G$ in SACS & Date point did not egree with local trand \\
\hline TE-105-AP-40 & $6 / 25 / 94$ & 96 & CASS & $T$ & \\
\hline TE-105-AP-40 & 71/2/94 & 98 & CASS & $\mathbf{T}$ & \\
\hline TE-105-AP-40 & $7 / 7 / 94$ & 96 & CASS & $\mathbf{T}$ & \\
\hline TE-105-AP-40 & $7 / 15 / 94$ & 95 & CASS & $T$ & \\
\hline TE-105-AP-40 & $7 / 20 / 94$ & 94 & CASS & $T$ & \\
\hline TE-105-AP-40 & $7 / 29 / 94$ & 95 & CASS & $T$ & \\
\hline TE-105-AP-40 & $8 / 6 / 94$ & 96 & CASS & $T$ & \\
\hline TE-105-AP-40 & $8 / 10 / 94$ & 99 & CASS & $\bar{T}$ & \\
\hline TE-105-AP-AO & $8 / 20 / 94$ & 100 & CASS & $T$ & \\
\hline TE-105-AP-40 & $8 / 22 / 94$ & 98 & CASS & $T$ & \\
\hline TE-105-AP-40 & 9/3/94 & 99 & CASS & $\bar{T}$ & \\
\hline TE-105-AP-40 & 9/9/94 & 103 & CASS & $\bar{T}$ & \\
\hline TE-105-AP-40 & $9 / 15 / 94$ & 101 & CASS & $\mathbf{T}$ & \\
\hline TE-105-AP-40 & $9 / 23 / 94$ & 101 & CASS & $T$ & \\
\hline TE-105-AP-40 & 9/25/94 & 101 & CASS & $T$ & \\
\hline TE-105-AP-40 & $10 / 2 / 94$ & 104 & CASS & $T$ & \\
\hline TE-105-AP-40 & $10 / 15 / 94$ & 102 & CASS & $\mathbf{T}$ & \\
\hline TE-105-AP-40 & $10 / 17 / 94$ & 104 & CASS & $T$ & \\
\hline TE-105-AP-40 & $10 / 28 / 94$ & 105 & CASS & $T$ & \\
\hline TE-105-AP-40 & $11 / 4 / 94$ & 111 & CASS & $T$ & \\
\hline TE-105-AP-A0 & $11 / 11 / 94$ & 105 & CASS & $\mathbf{T}$ & \\
\hline TE-105-AP-AO & $11 / 16 / 94$ & 104 & CASS & $\mathbf{T}$ & \\
\hline TE-105-AP-4O & $11 / 22 / 94$ & 106 & CASS & $T$ & \\
\hline TE-105-AP-40 & $11 / 29 / 94$ & 100 & CASS & $T$ & \\
\hline TE-105-AP-40 & $12 / 4 / 94$ & 104 & CASS & 5 & \\
\hline TE-105-AP-40 & $12 / 16 / 94$ & 99 & CASS & $T$ & \\
\hline TE-105-AP-40 & $12 / 19 / 94$ & 102 & CASS & $T$ & \\
\hline TE-105-AP-40 & $12 / 29 / 94$ & 98 & CASS & $T$ & \\
\hline
\end{tabular}




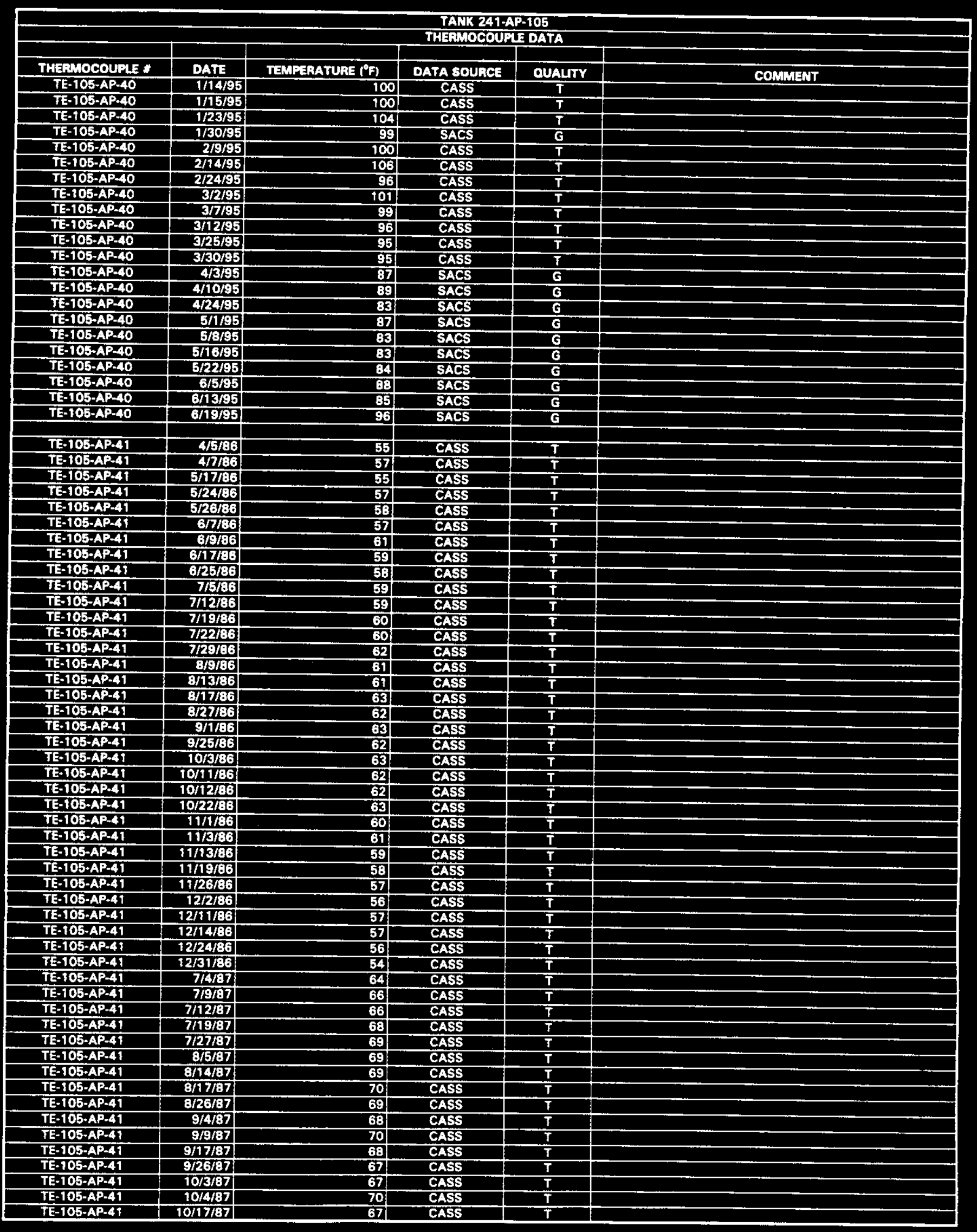




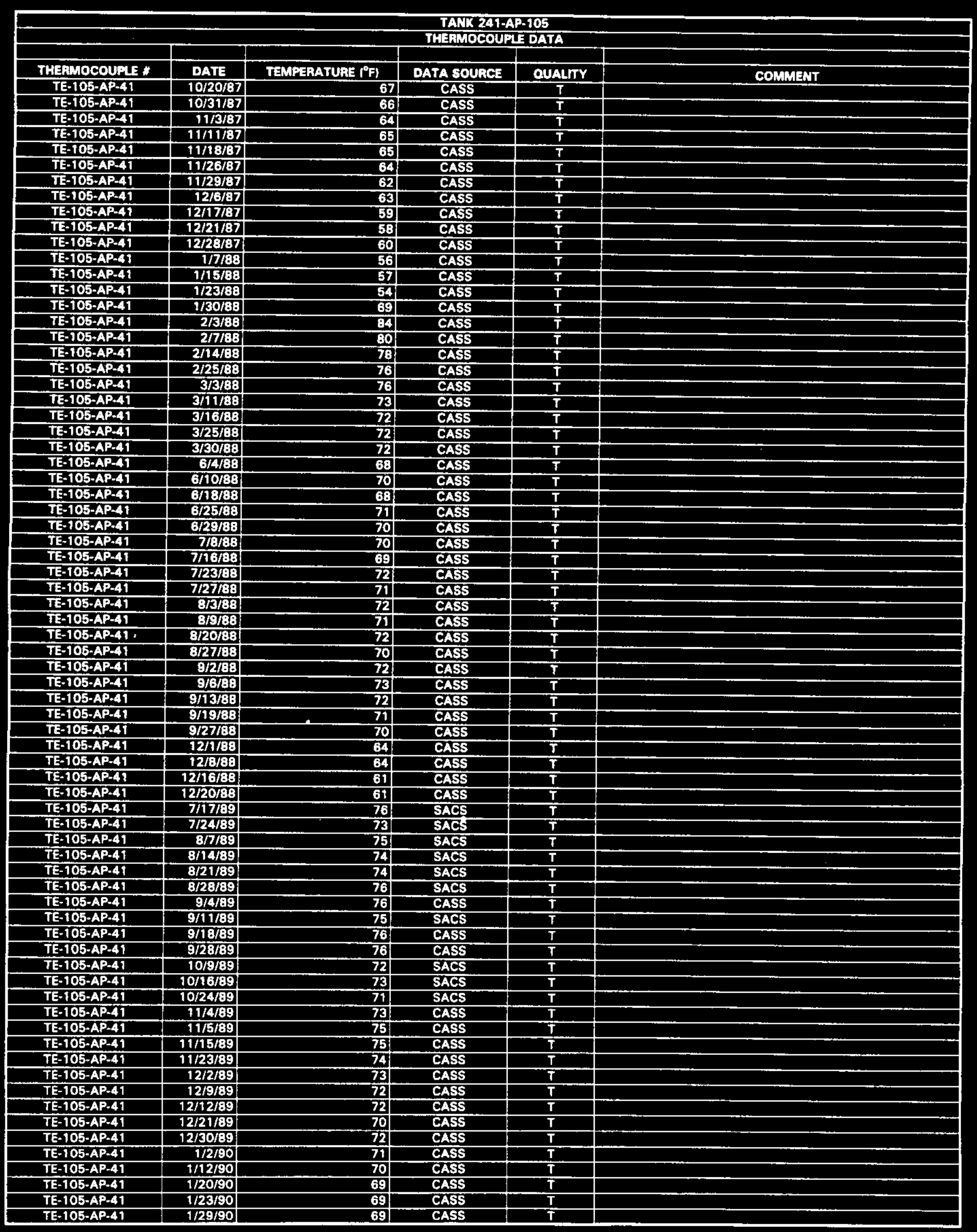




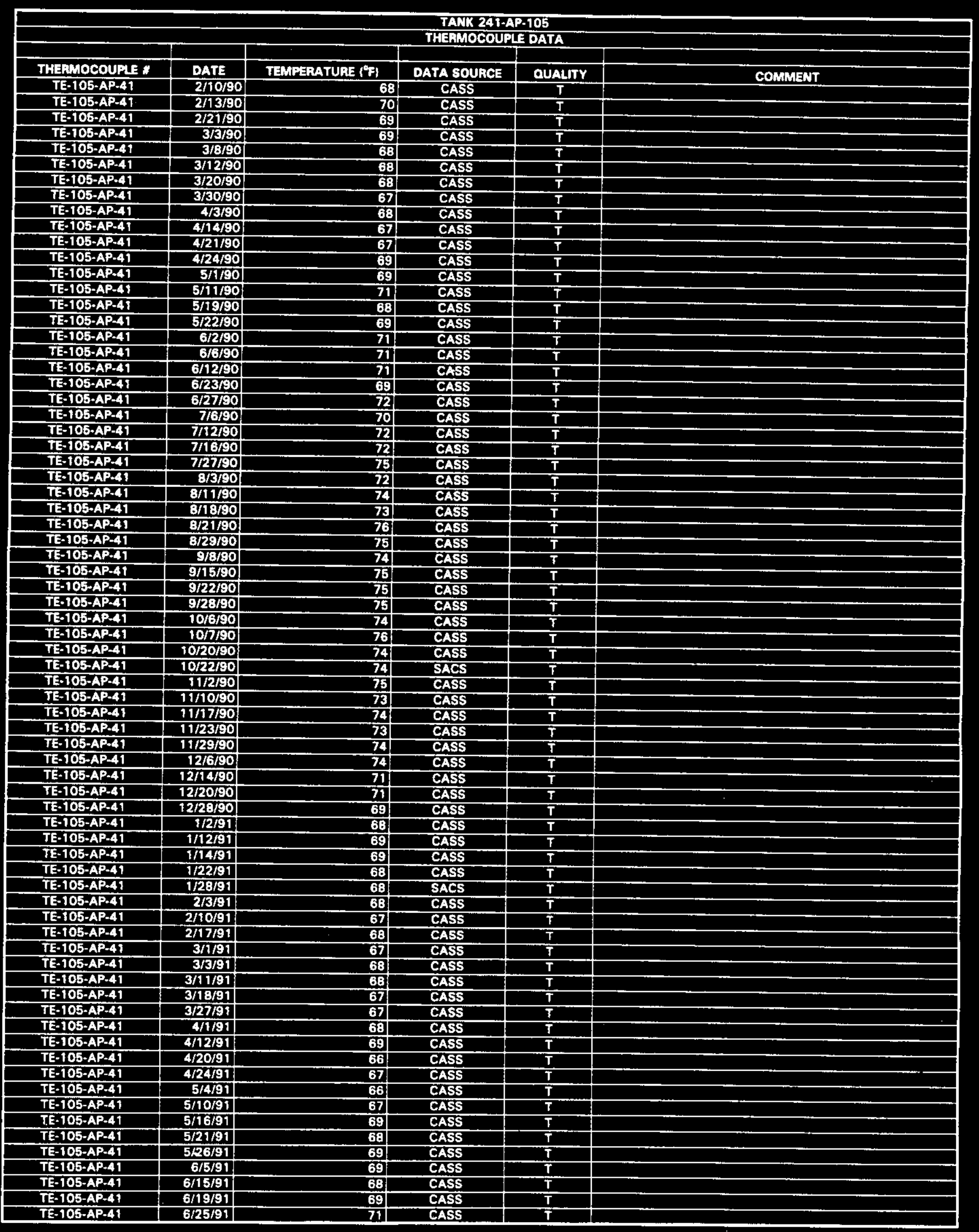




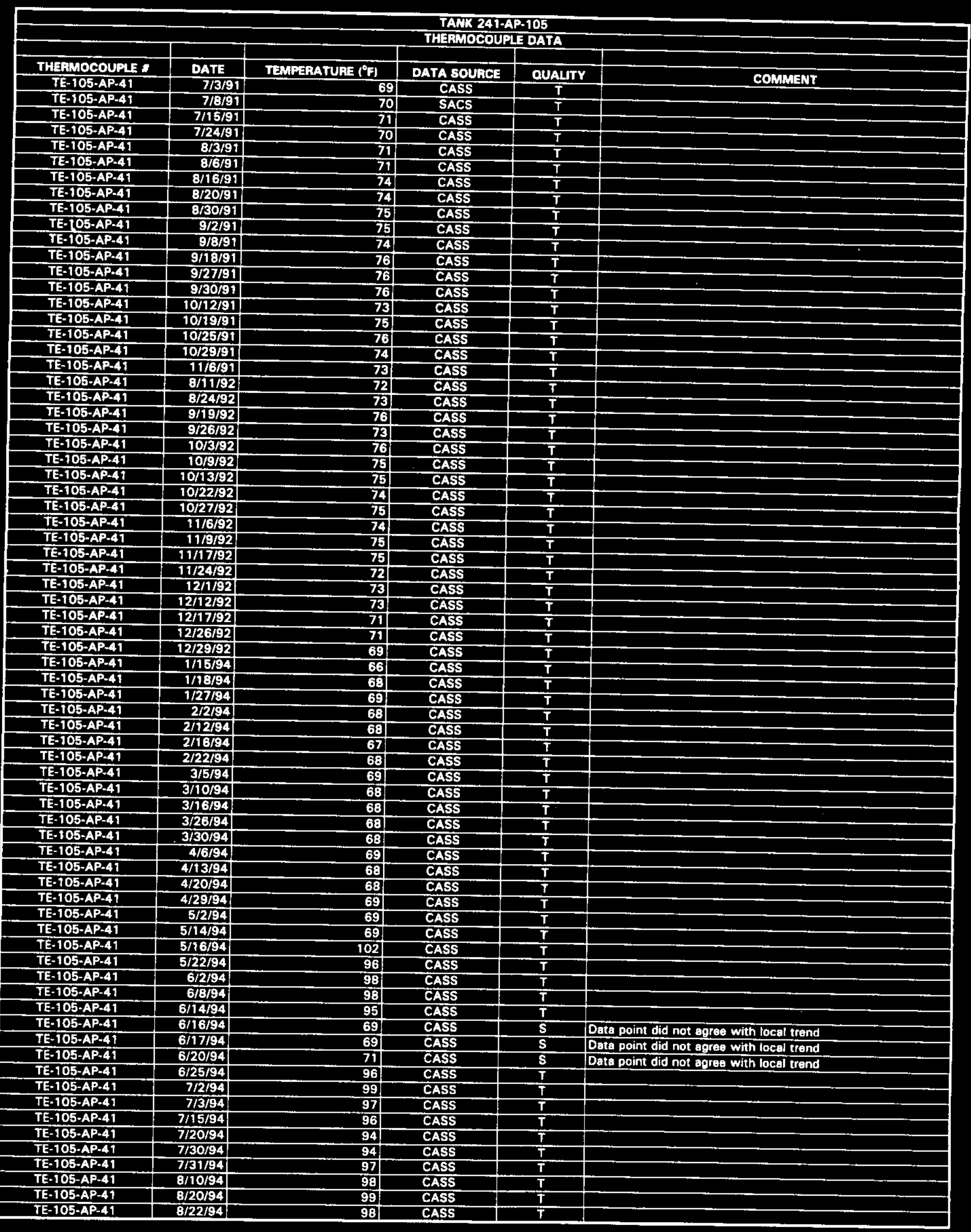

Data obtained from Computer Automated Surveillance System (CASS) in April 1995, ond Surviallance Analysis Computer System (SACS) in June 1995. 


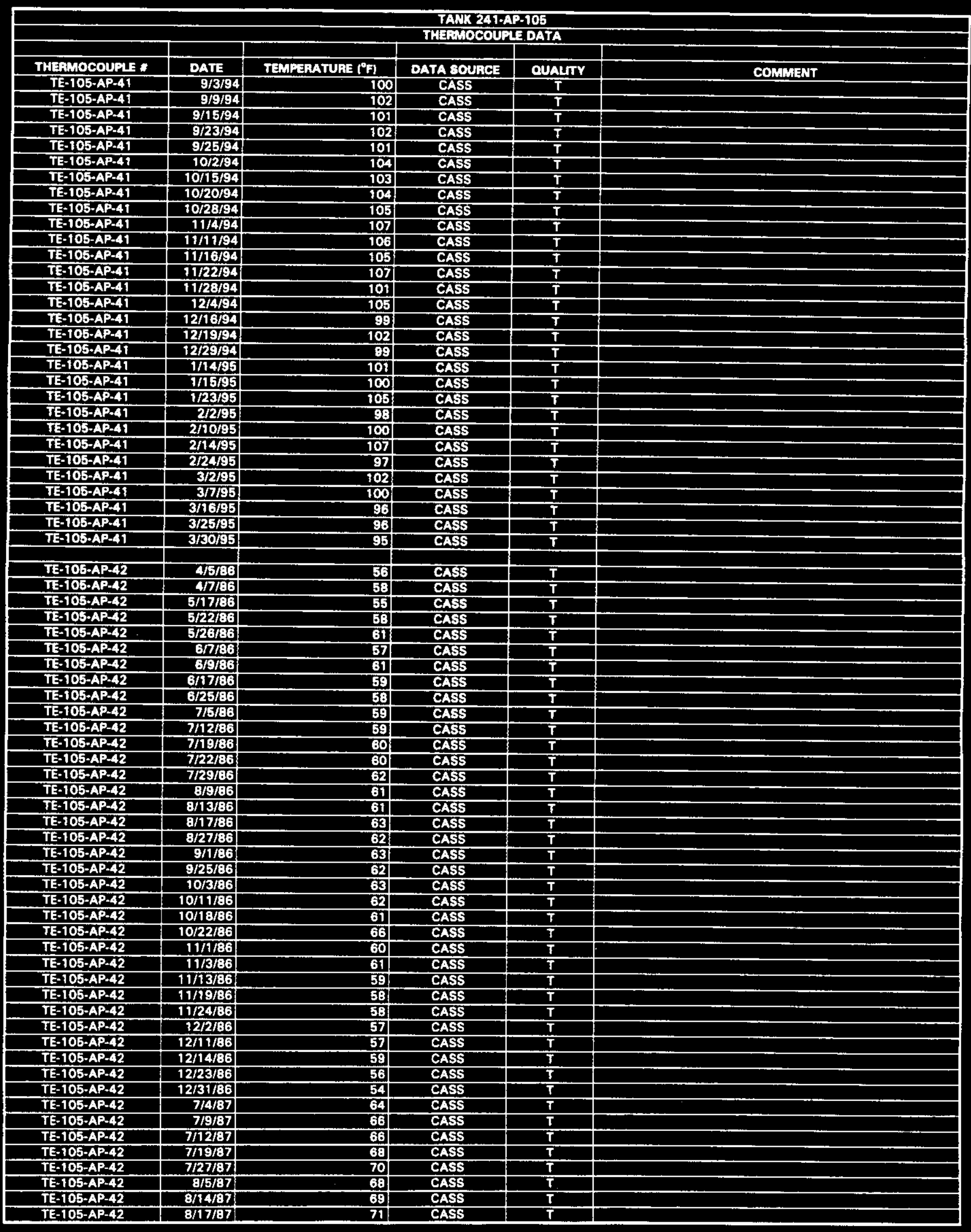




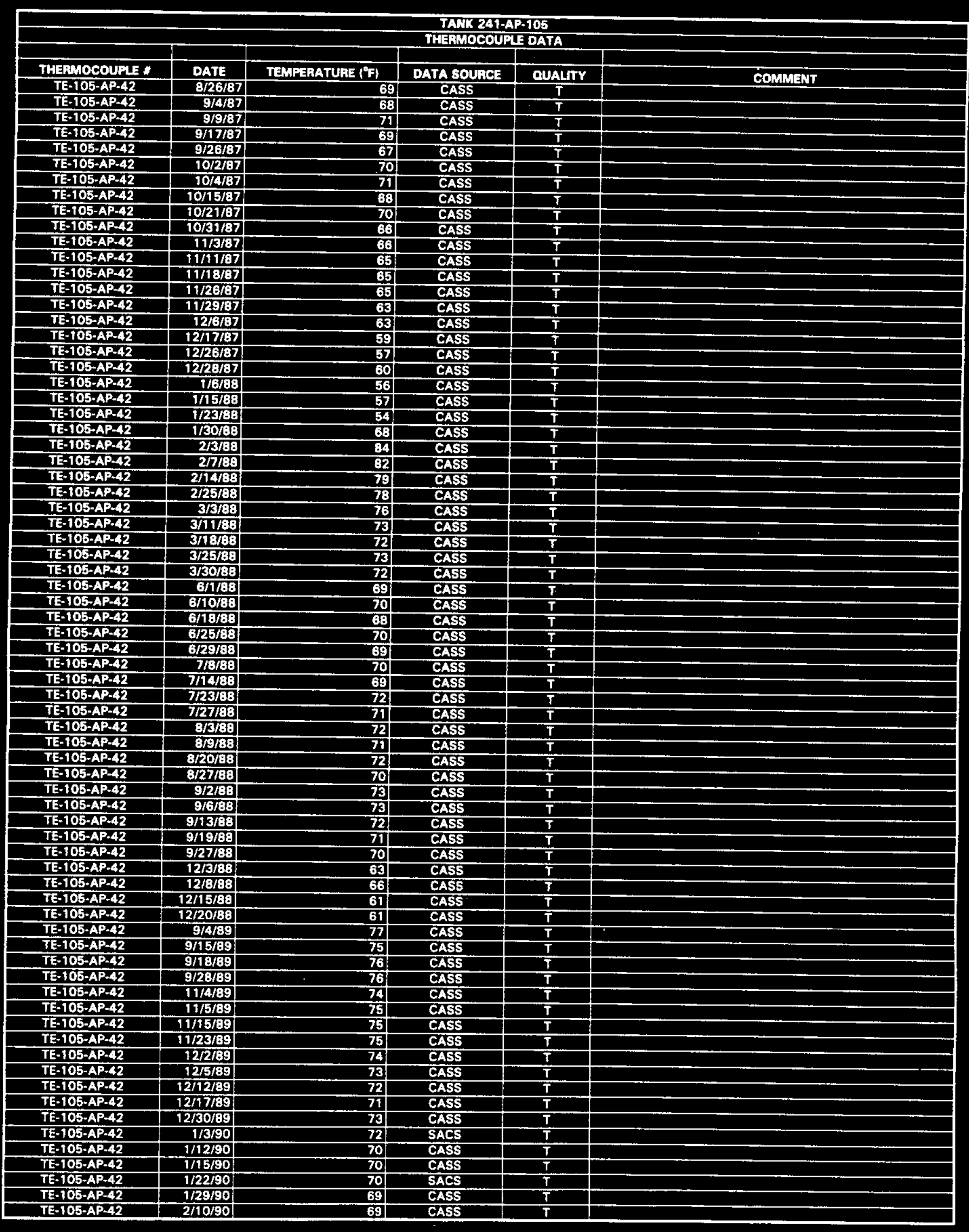

Data obtained from Computer Automated Surveillance Syatem (CASS) in April 1995, and Surviellance Anslysis Computer System (SACS) in June 1995. 


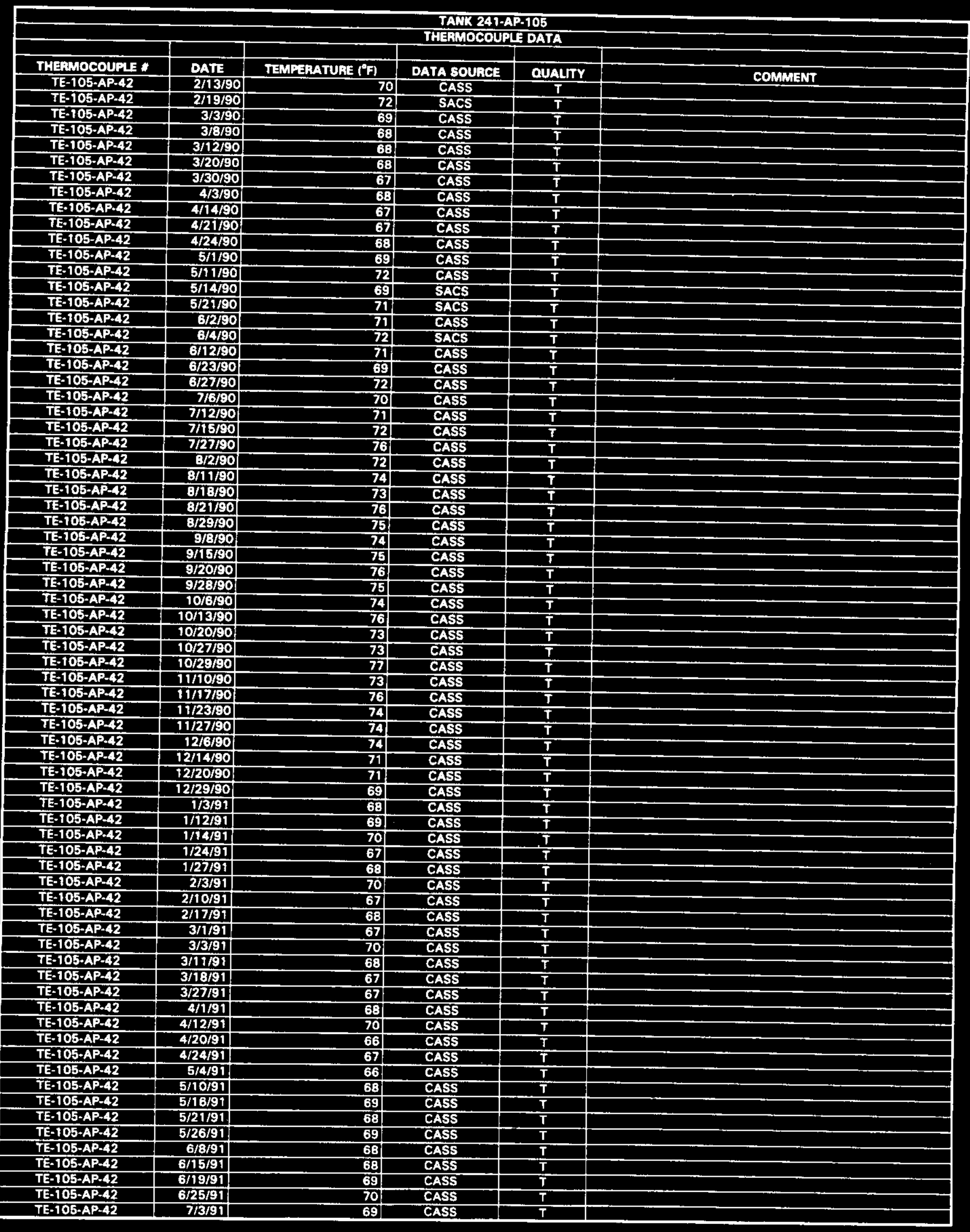




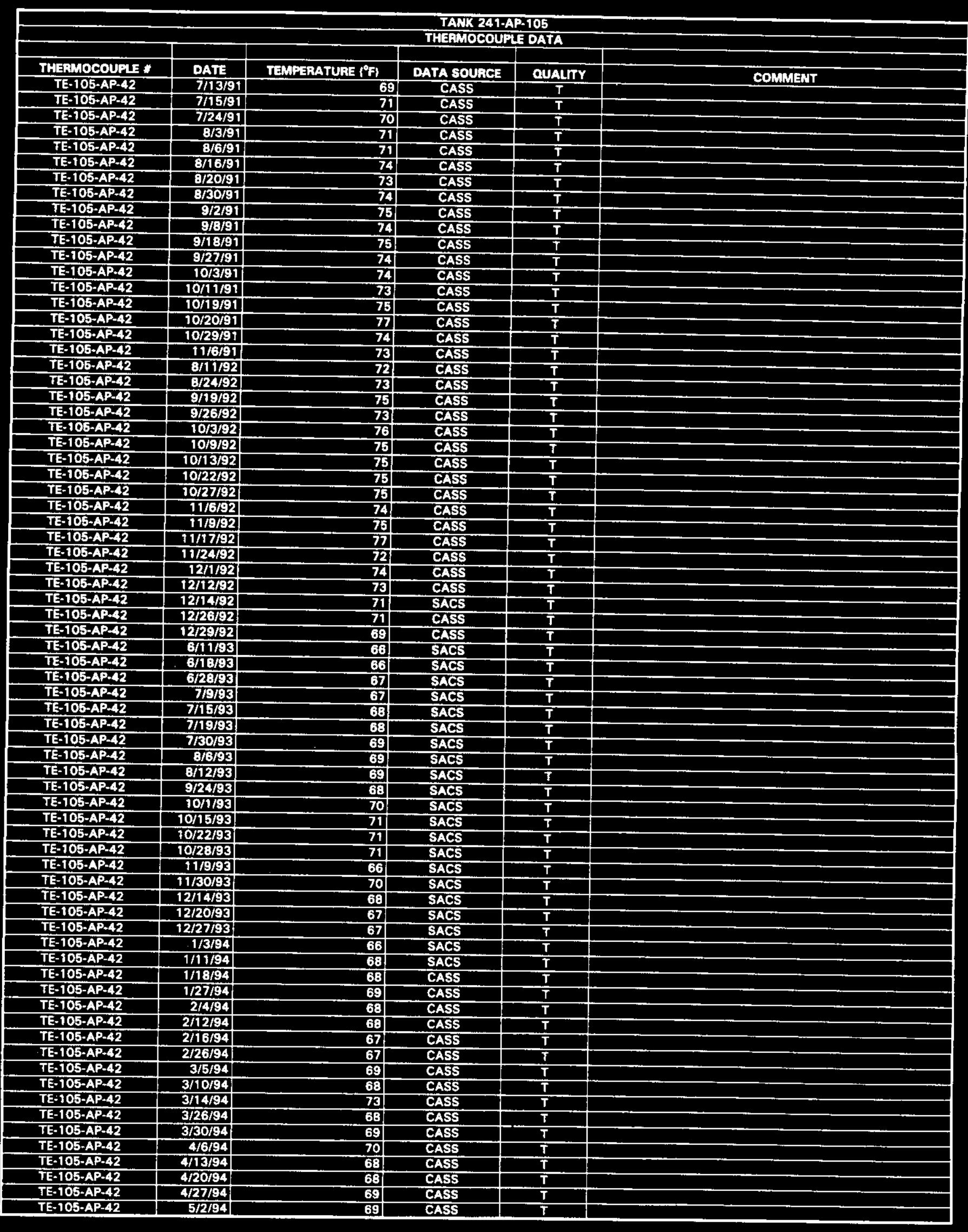

Date obtained from Computer Automated Surveillance Syatem (CASS) in April 1995, and Surviellance Analysis Computer System (SACS) in June 1995. 


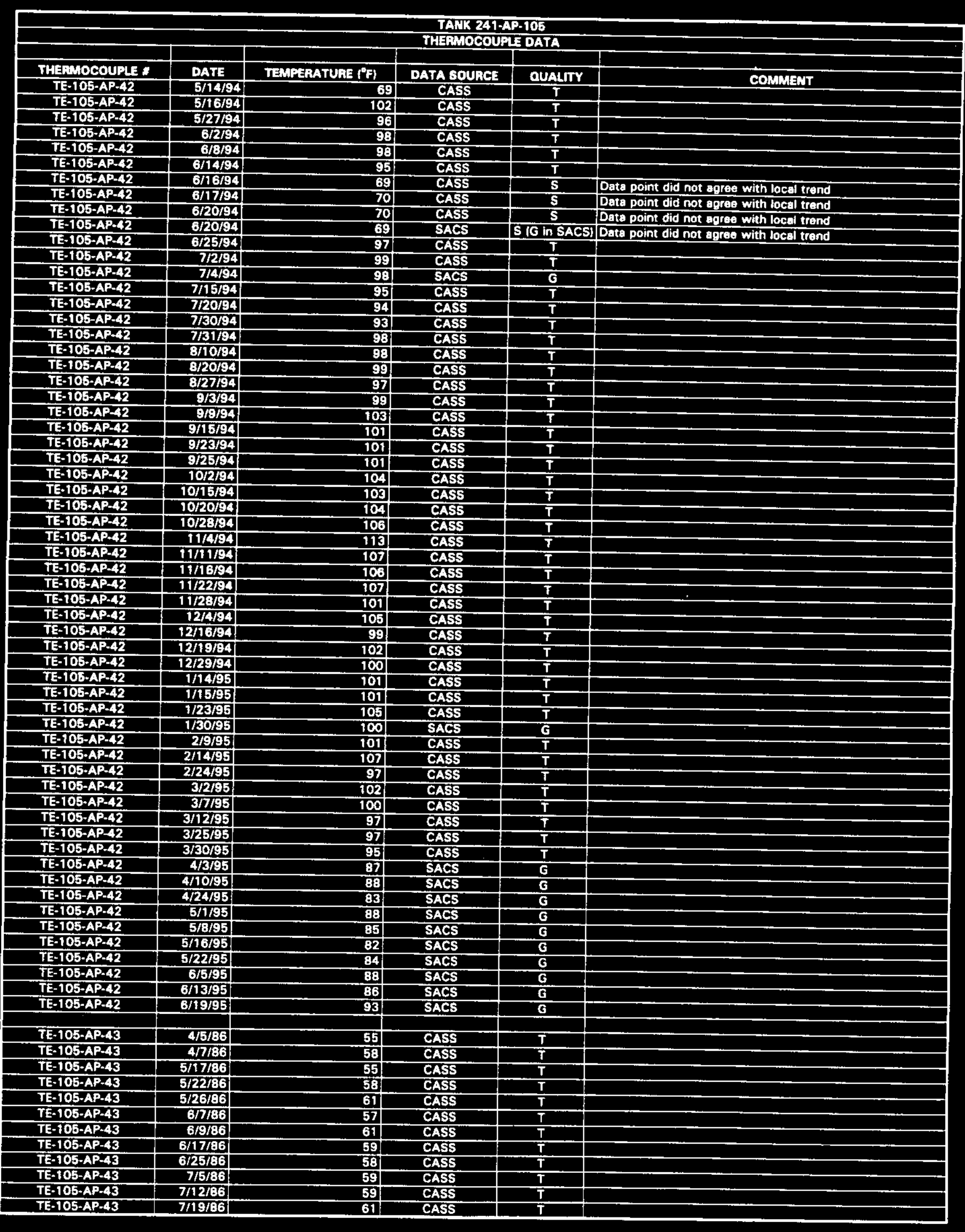

Date obtained from Computer Automoted Surveillance System (CASS) in April 1995, and Surviellance Analysis Computer System (SACS) in June 1995. 


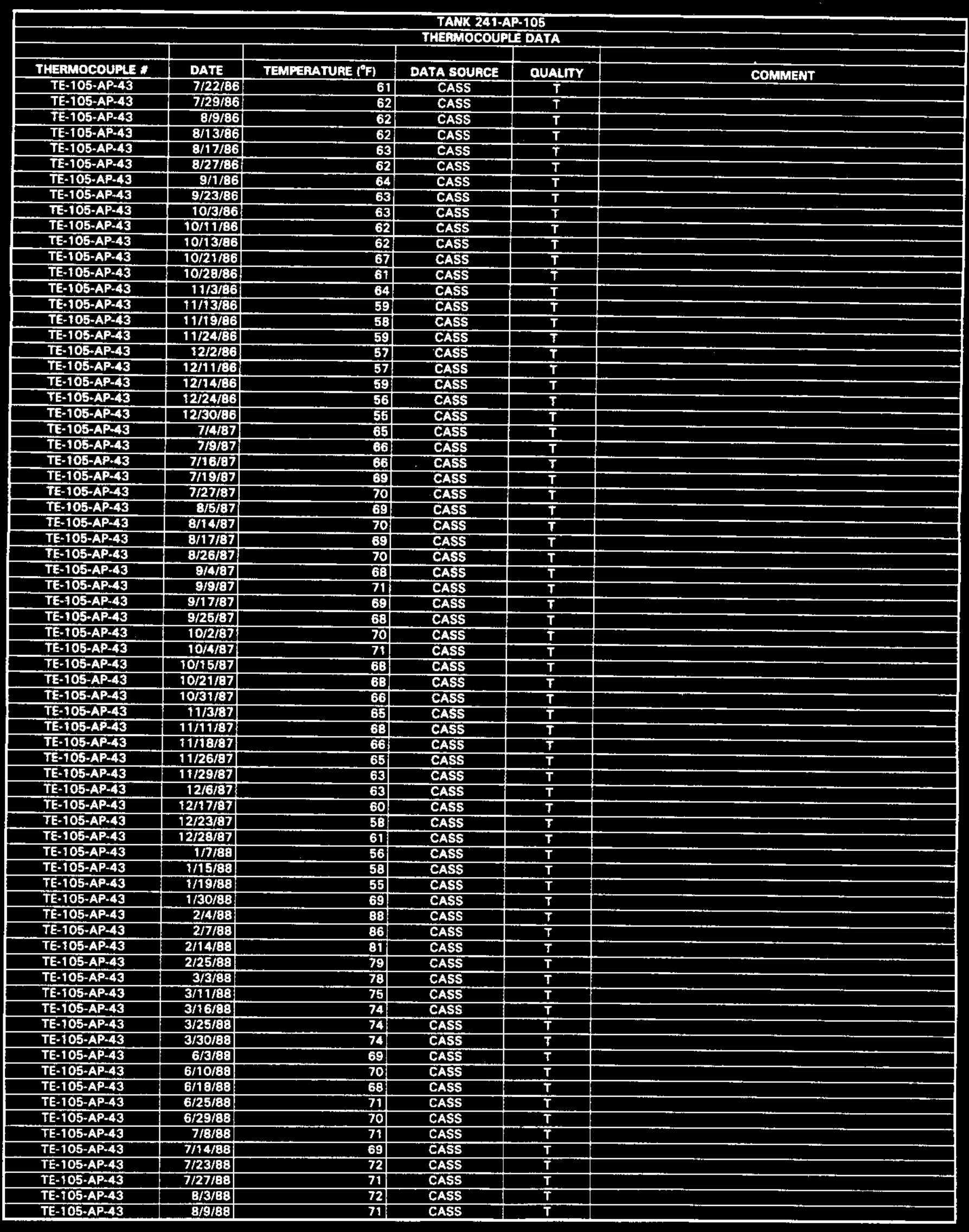




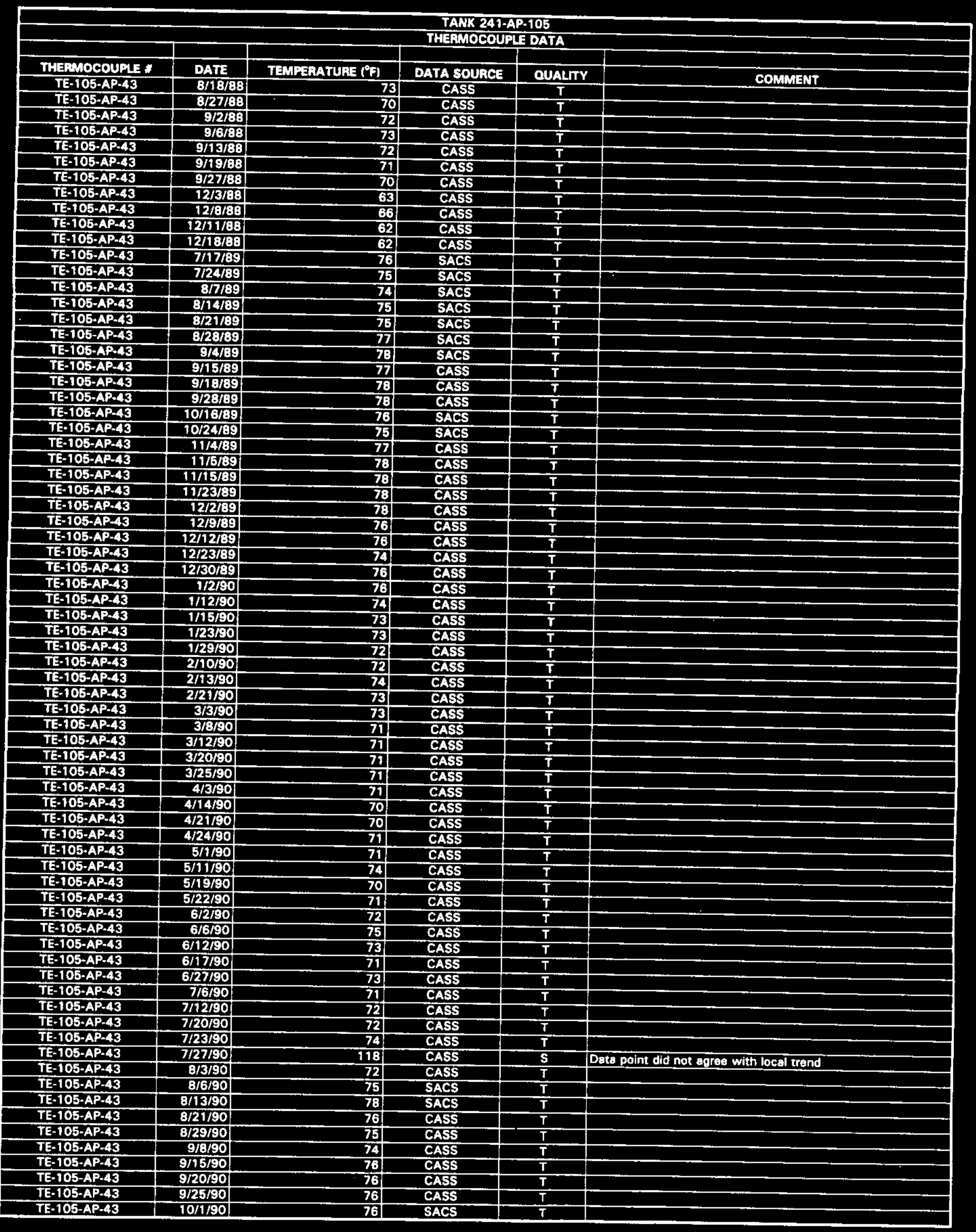

Data obtained from Camputer Automated Surveillance Syatem (CASS) in April 1995, and Surviellance Analysis Computer System (SACS) in June 1995. 


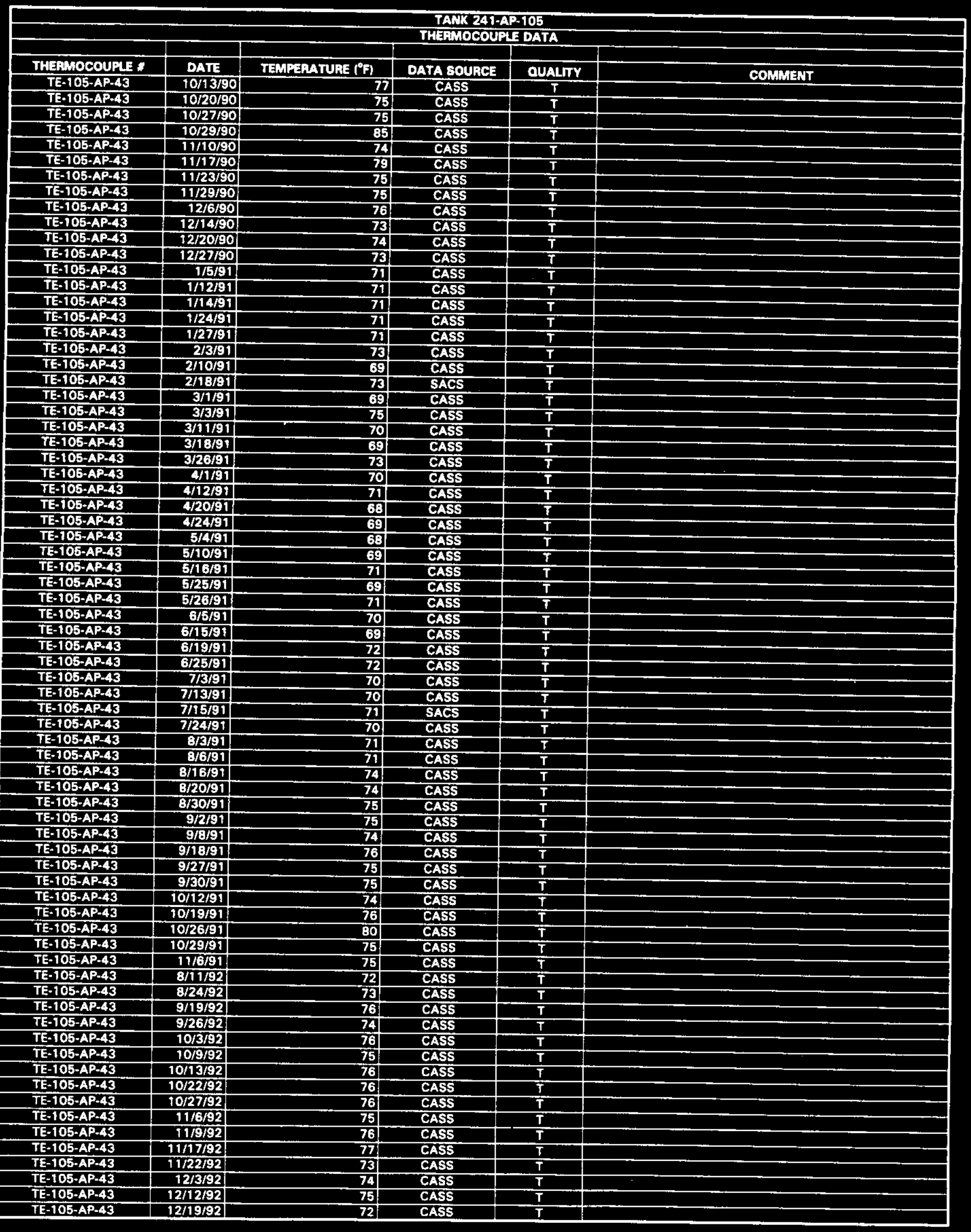

Data obteined from Computer Autometed Surveillance System (CASS) in Aprll 1995, and Surviellance Analysis Computer System (SACS) in June 1995. 


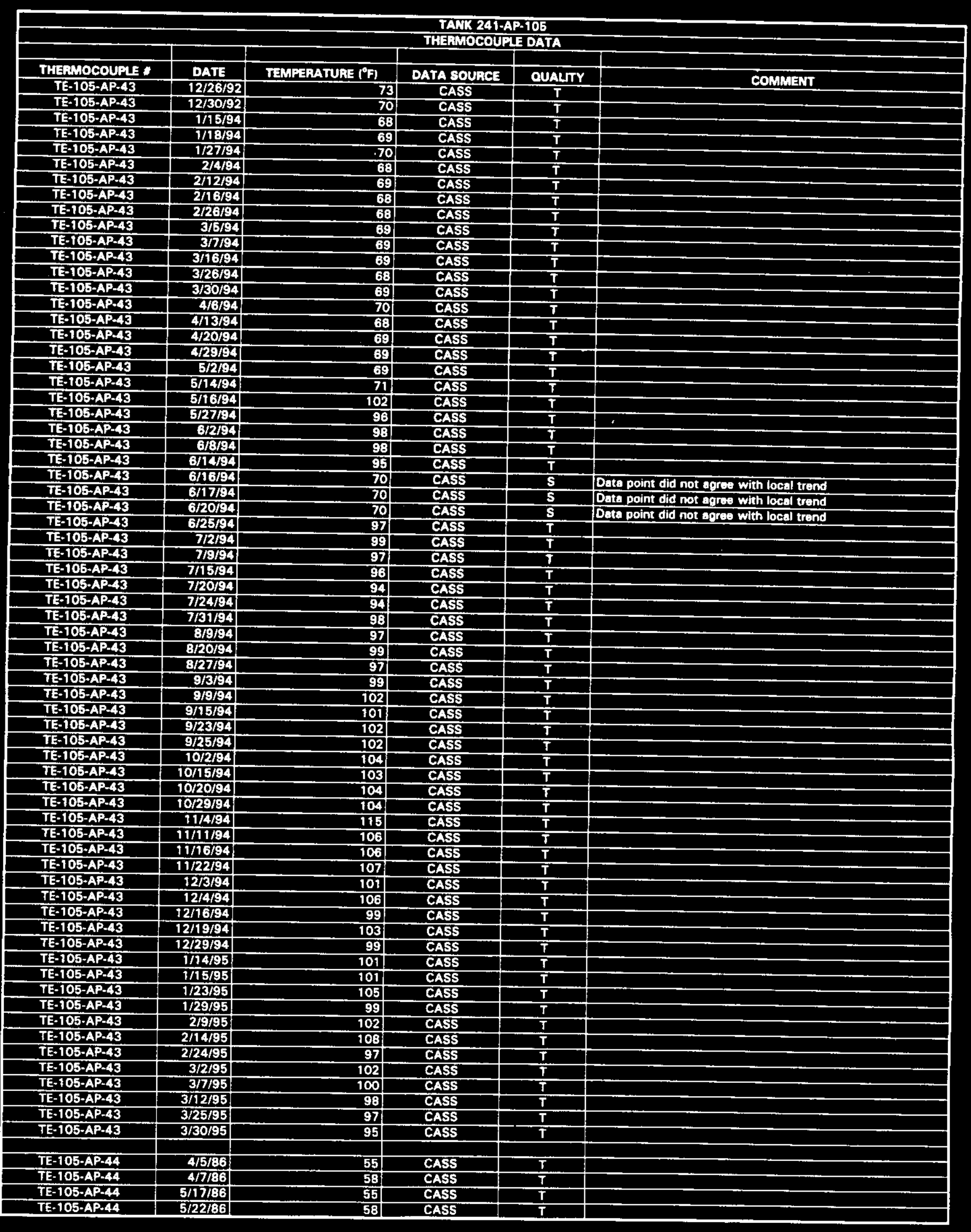

Data obtained from Computer Automated Surveillance System (CASS) in April 1995, and Surviellance Analysis Computer System (SACS) in June 1995. 


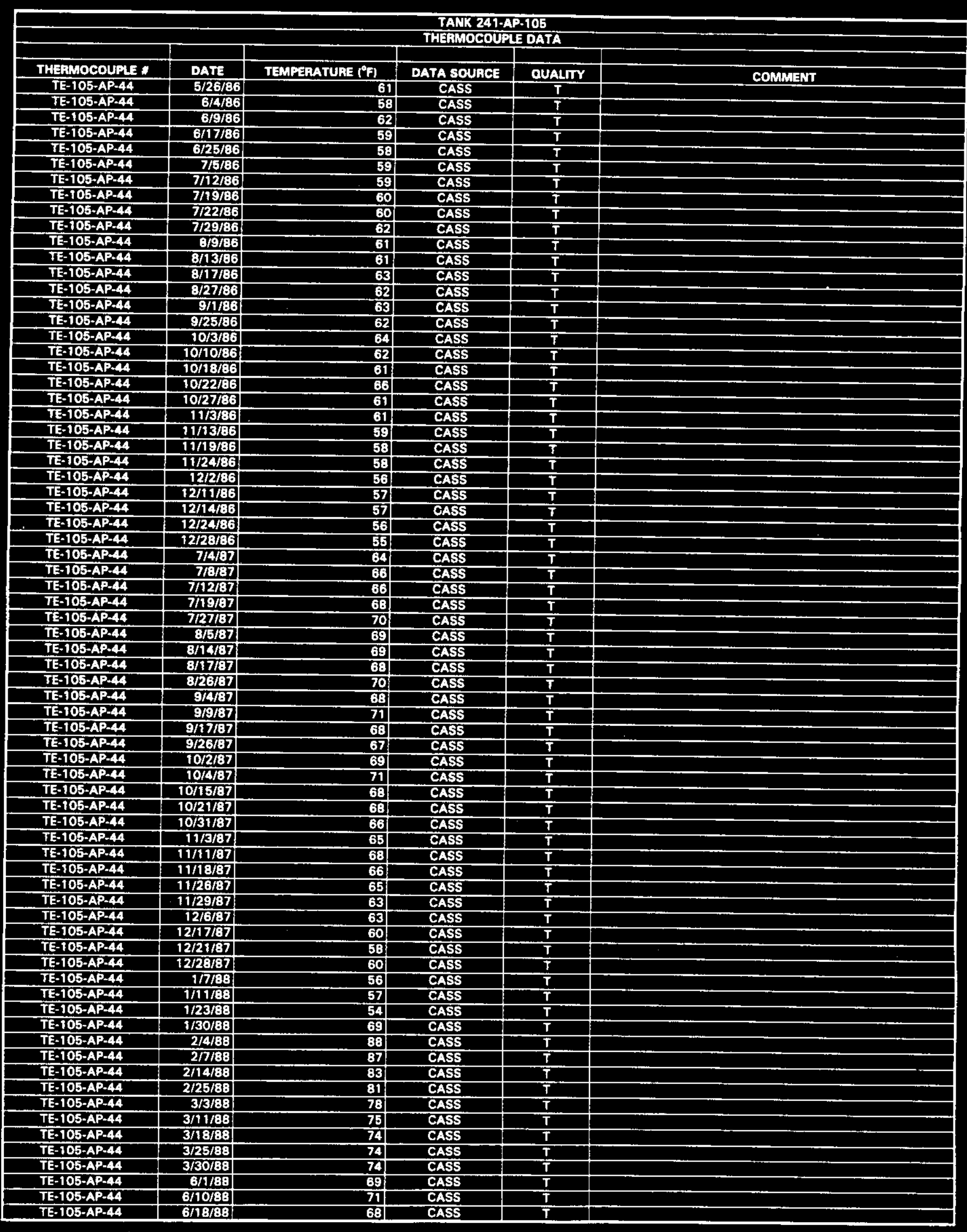

Data obtained from Computer Automated Surveillance Syatem (CASS) in April 1995, and Surviellance Analyais Computer Syatem (SACS) in June 1995. D- 447 


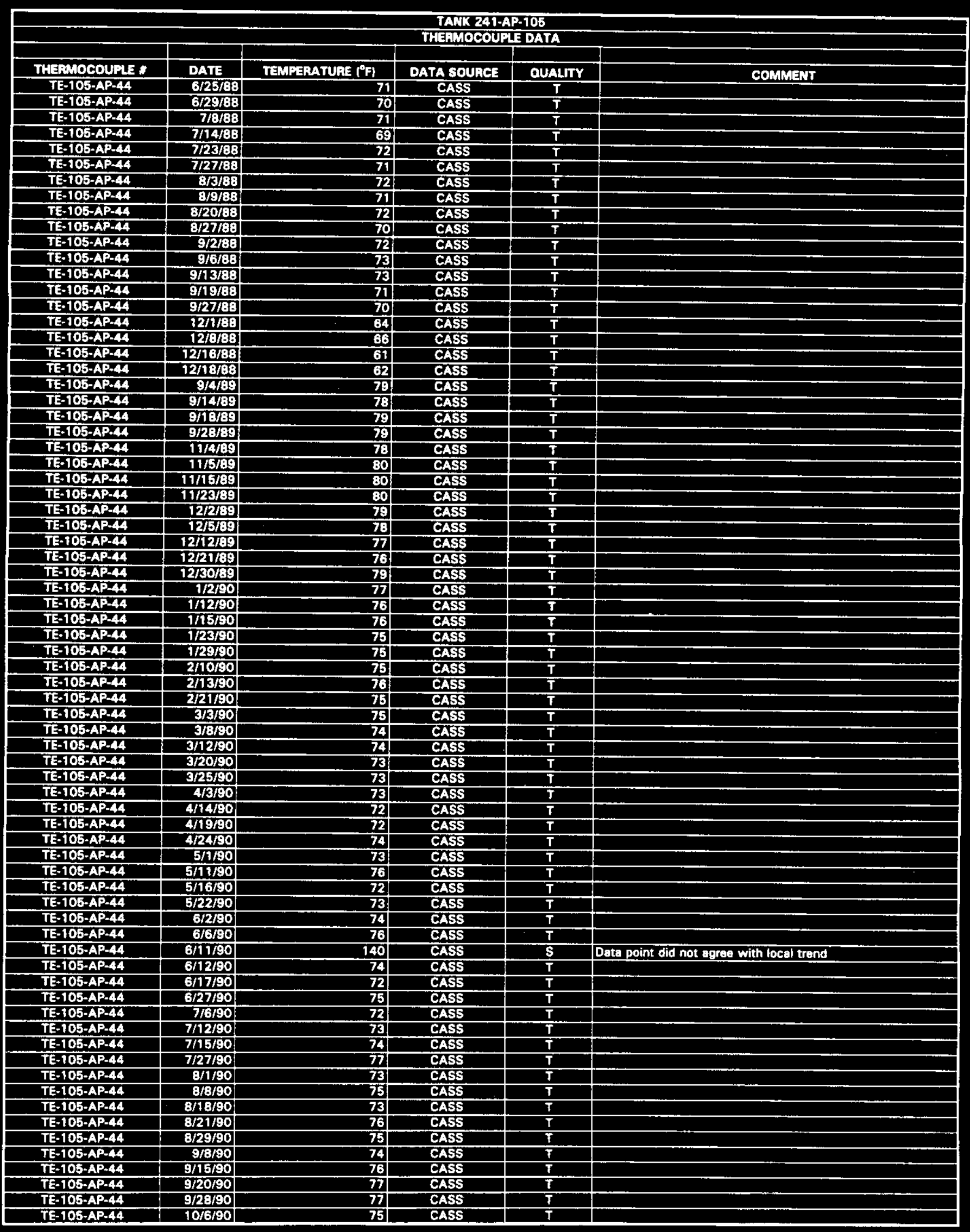




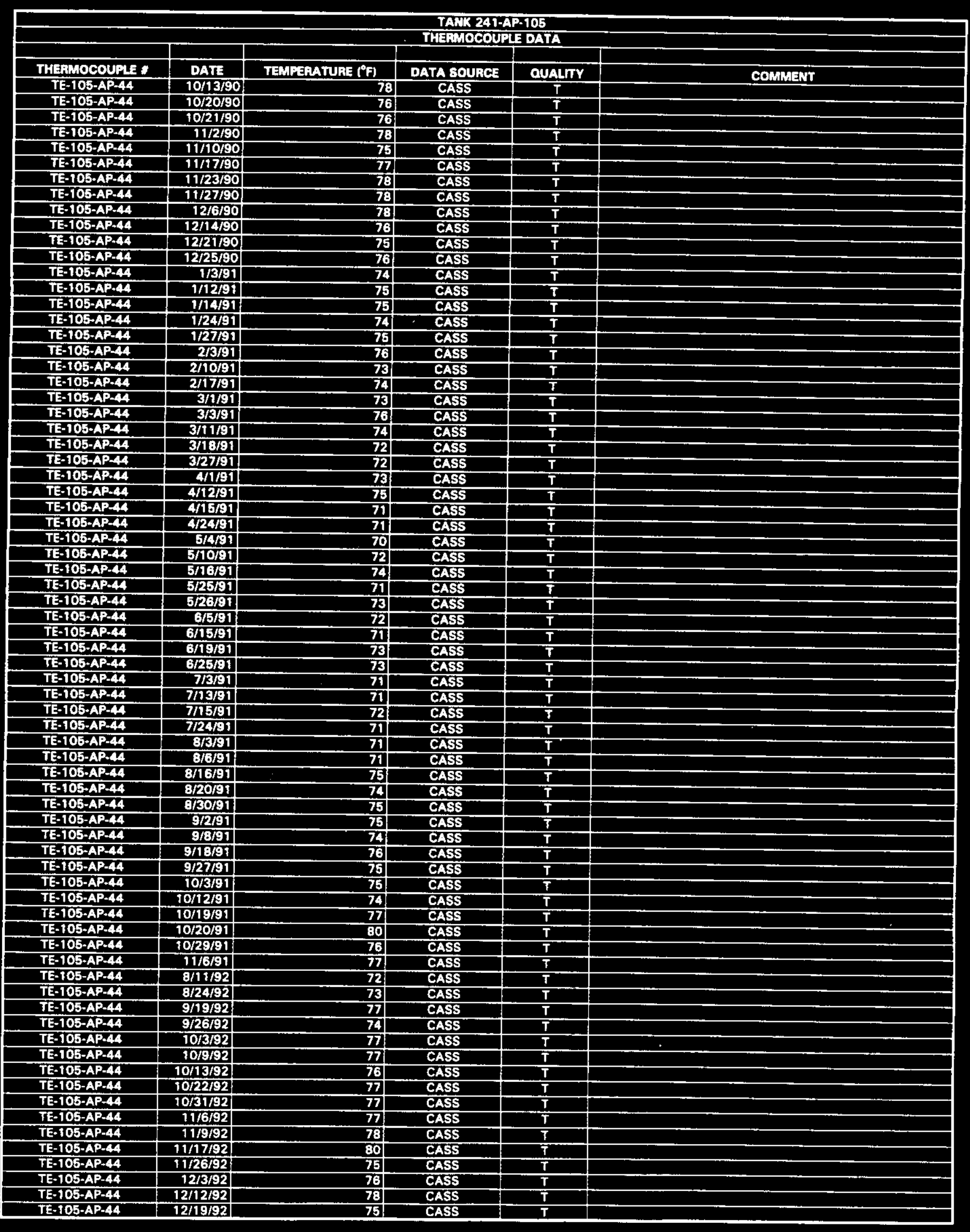

Data obtained from Computer Automated Surveillance System (CASS) in April 1995, and Surviellance Analysis Computer Syatem (SACS) in June 1995. 


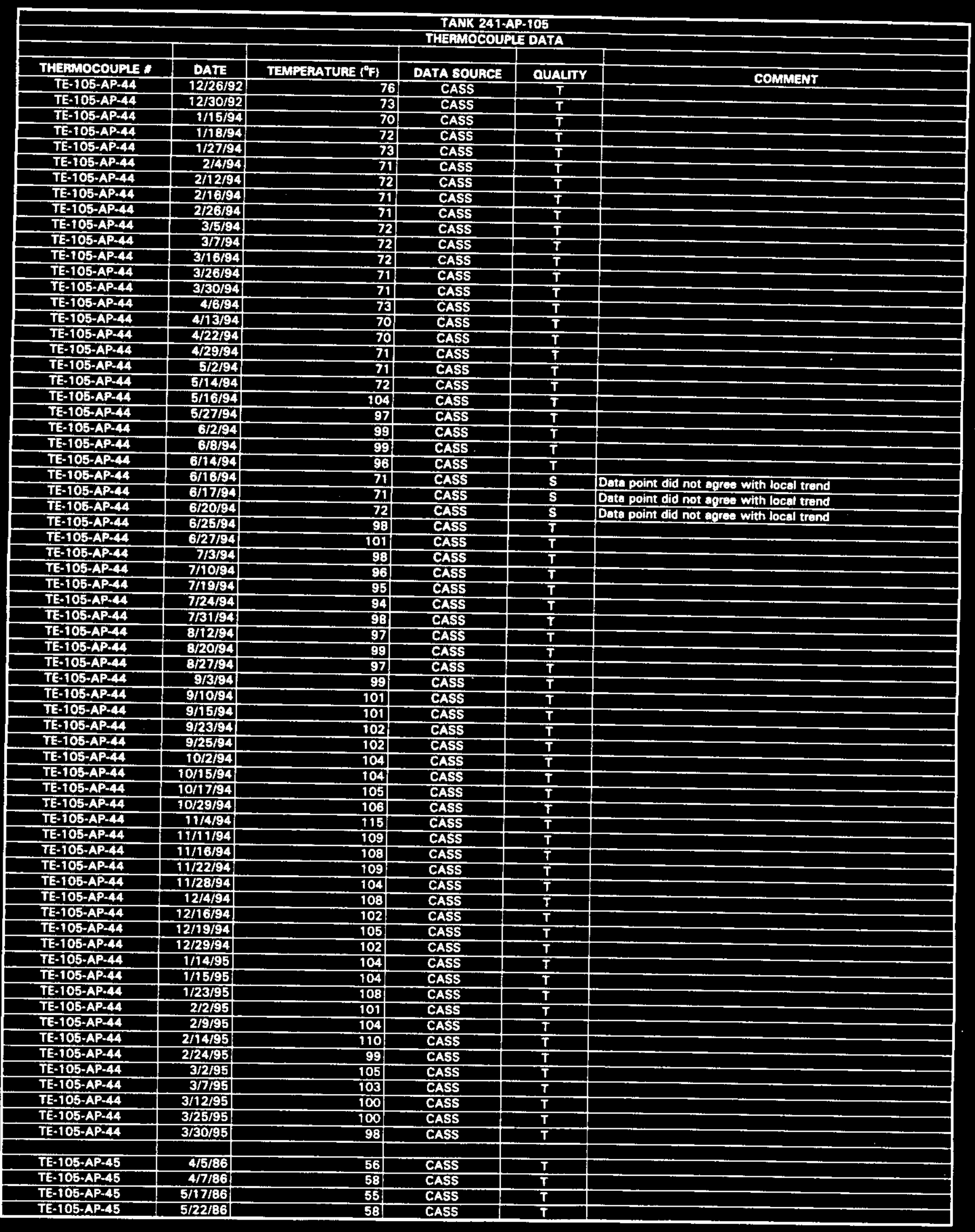

Date obtained from Computer Automated Survaillance Syatem (CASS) in April 1995, and Surviellance Analyais Computer System (SACS) in June 1995.

D-450 


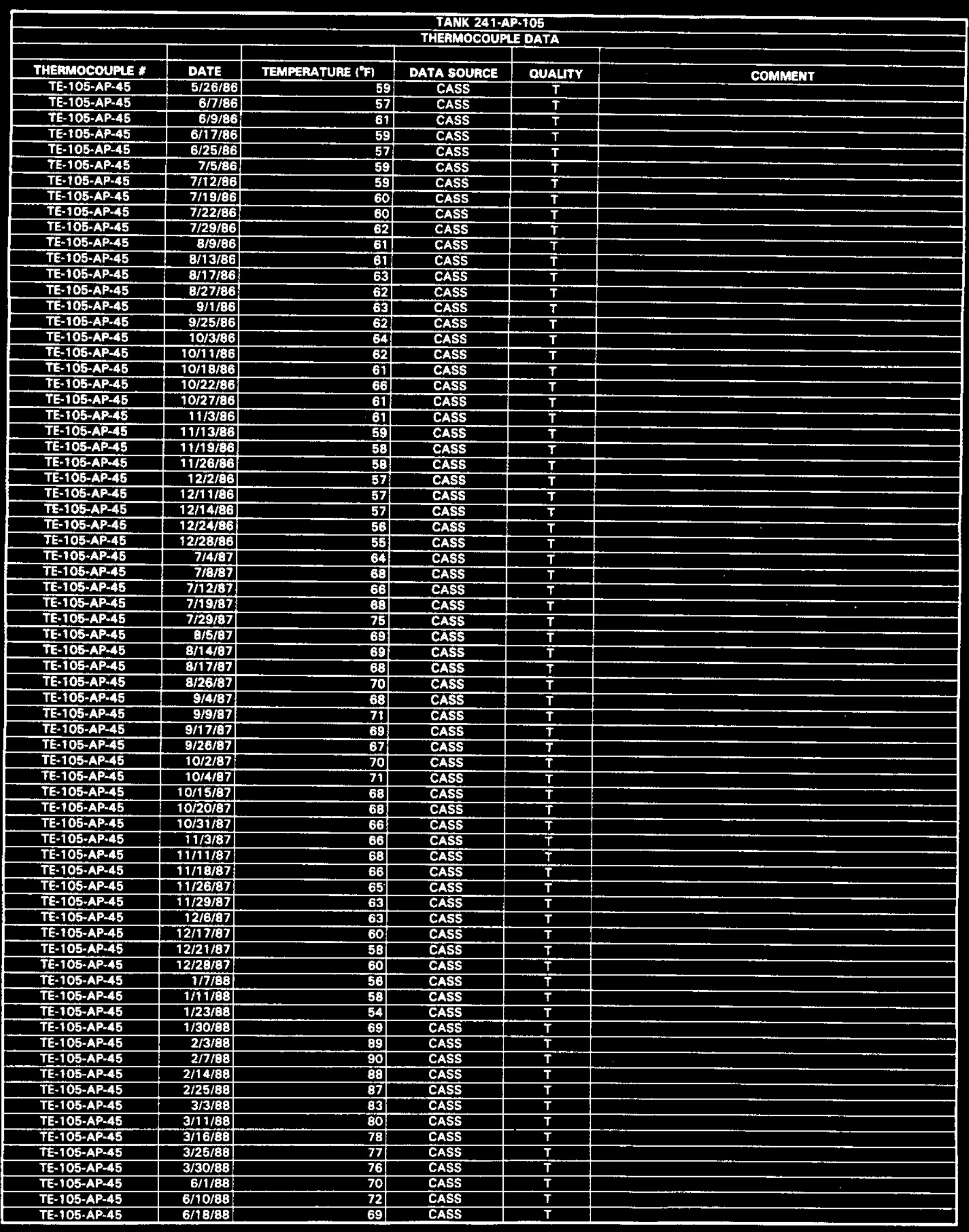

Data obtained from Computer Automated Surveillance System (CASS) in April 1995, and Surviellance Analysis Computer Syatem (SACS) in Jure 1995. 


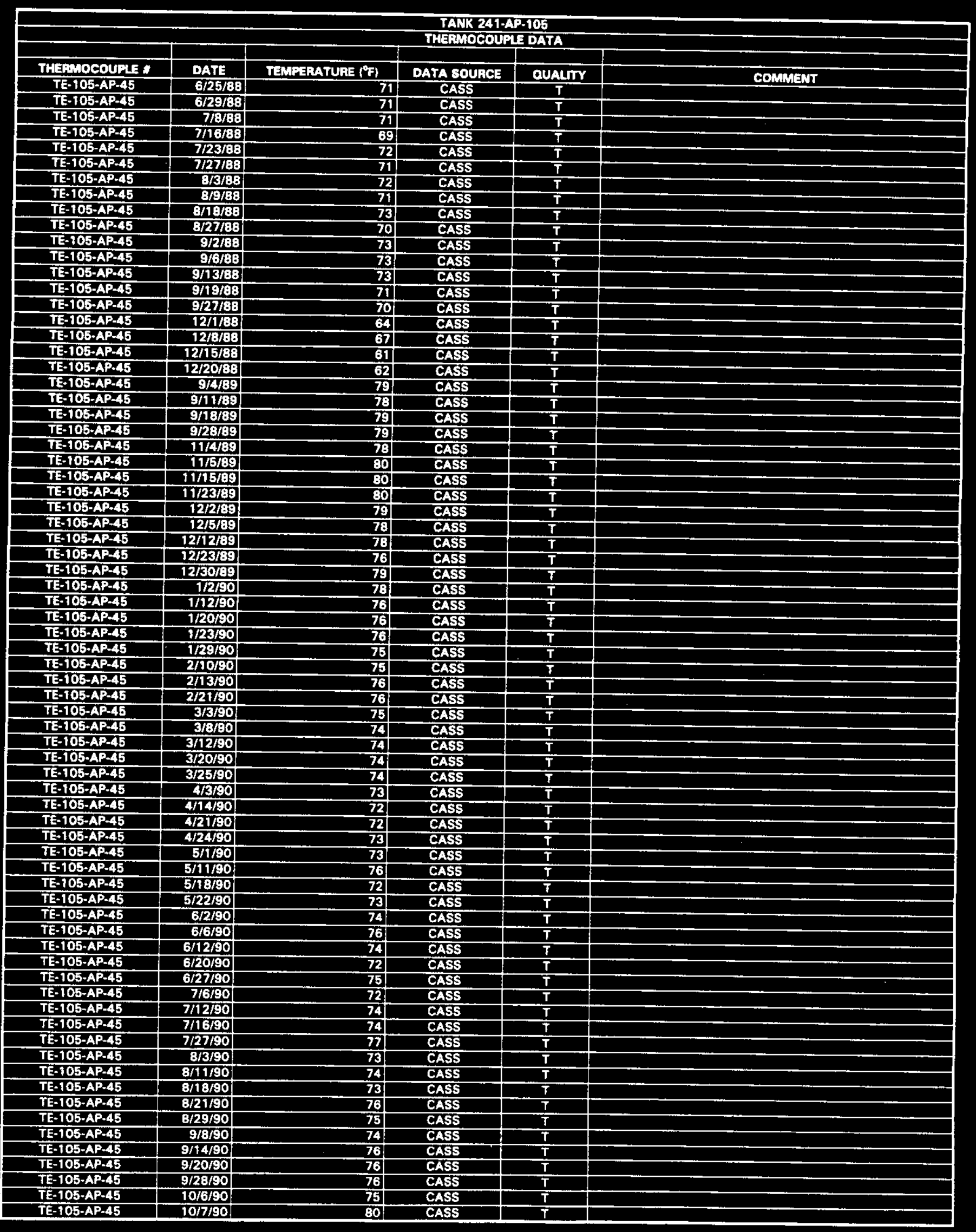

Data obtained from Computer Automated Surveillance Syatem (CASS) in April 1995, and Surviellance Analysis Computer System (SACS) in June 1995.

D-452 


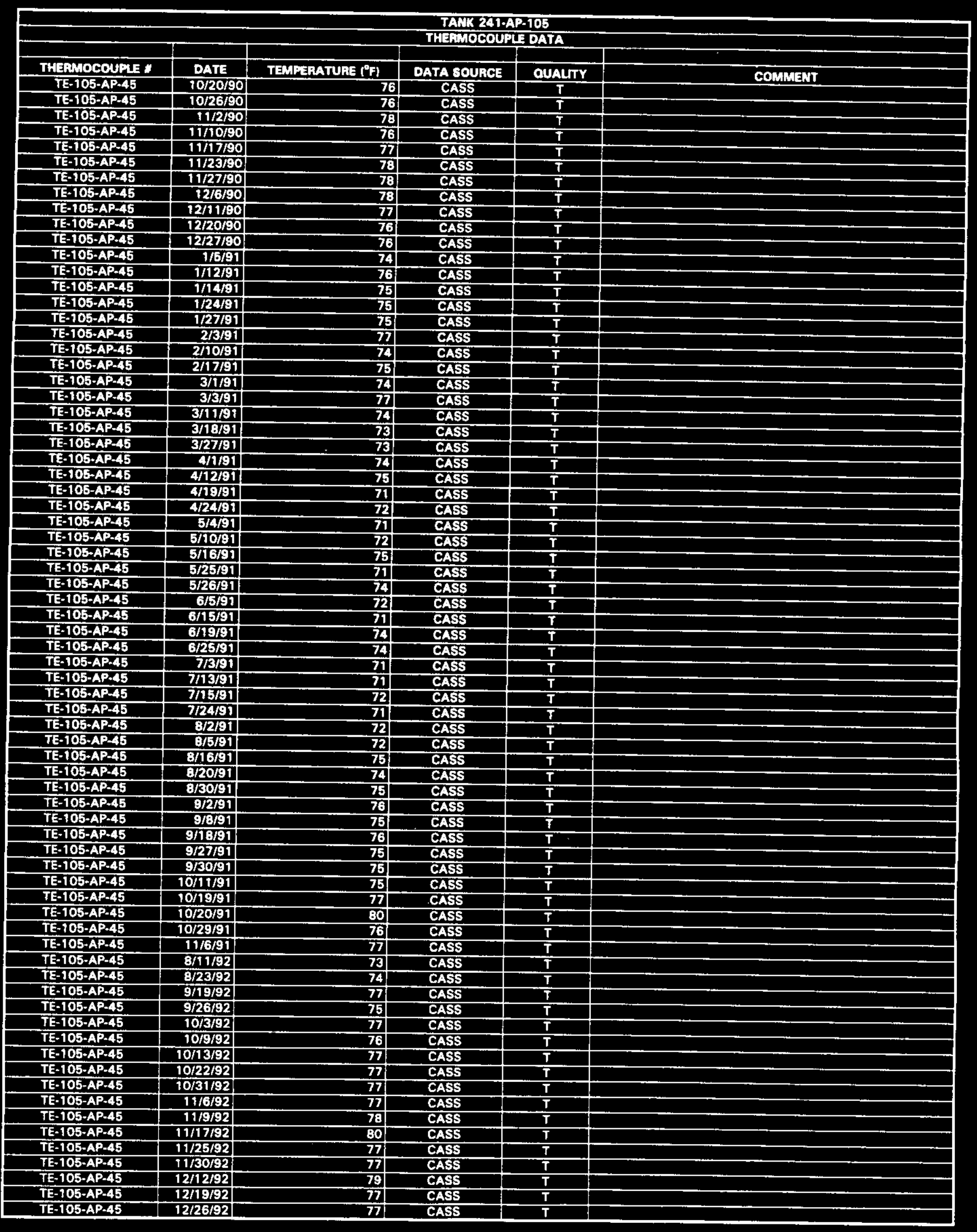

Data obtained from Computer Automated Surveillance Svatem (CASS) in April 1995, and Surviellance Analysis Compurer Syatem (SACS) in June 1995. $D-453$ 


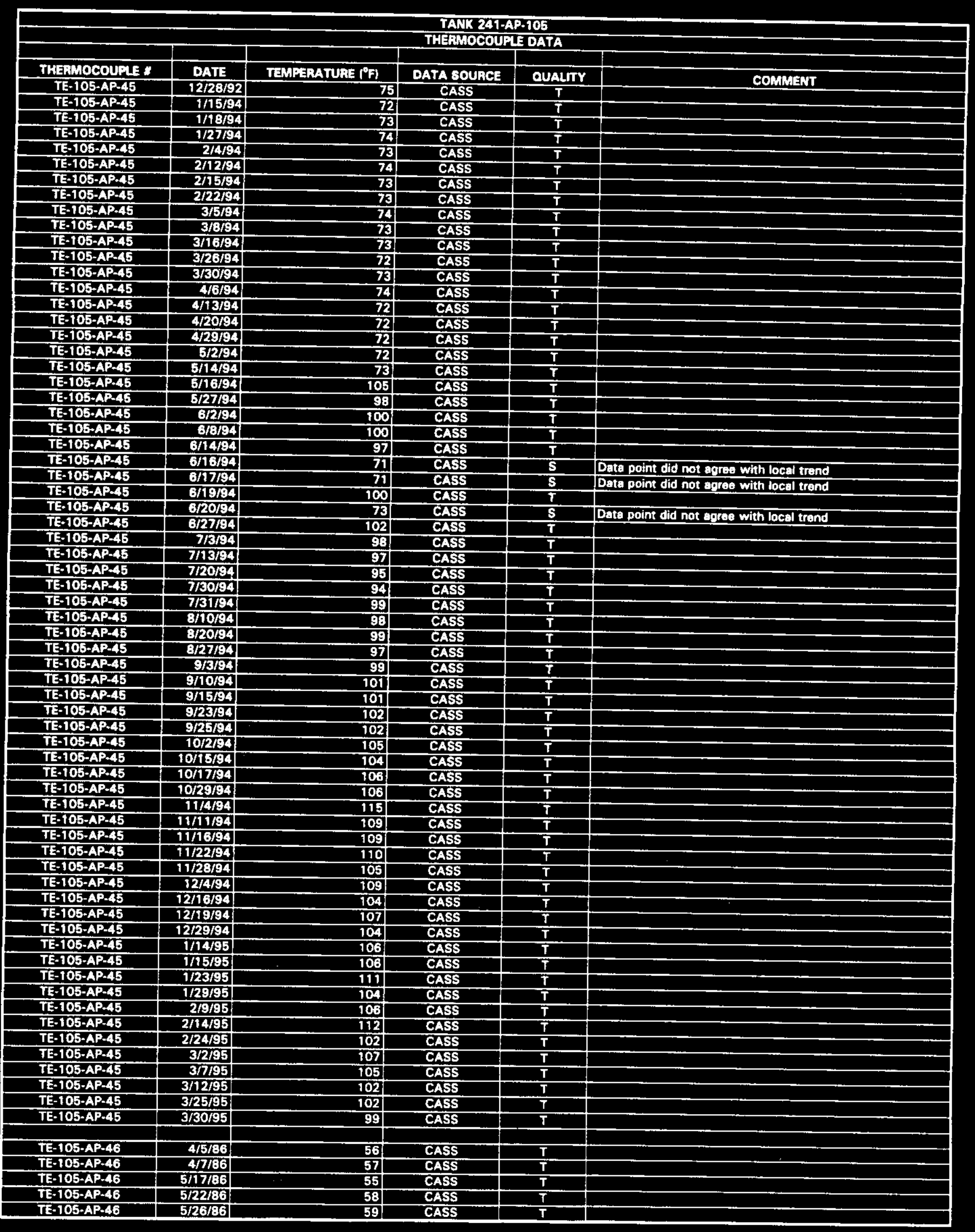

Date obtained from Computer Automated Survaillance System (CASS) in April 1995, and Surviellance Anelyais Computer Syatem (SACS) in June 1995. 


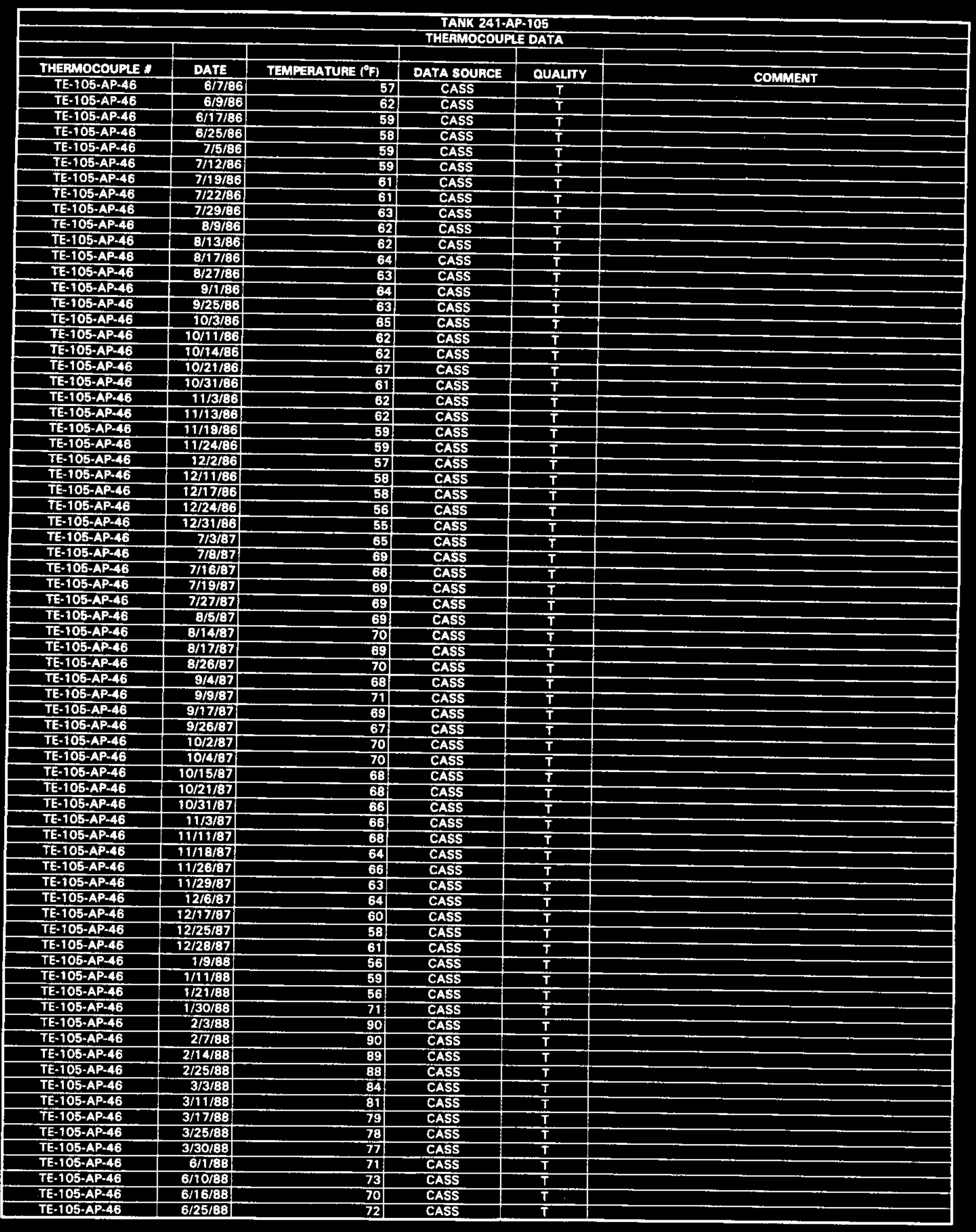

Data obtained from Computer Automated Surveillance System (CASS) in April 1995, and Surviellance Analysis Computer System (SACS) in June 1995. 


\begin{tabular}{|c|c|c|c|c|c|}
\hline \multicolumn{6}{|c|}{$\begin{array}{l}\text { TANK 241-AP-TO5 } \\
\text { THBAMOCOUPLE DATA }\end{array}$} \\
\hline & & & & & \\
\hline THEAMOCOUPLE * & DATE & TEMPERATURE (F) & DATA SOUACE & OUALTY & COMMENT \\
\hline TE-105-AP-46 & $6 / 29 / 88$ & 70 & CASS & $T$ & \\
\hline TE-105-AP-46 & $7 / 8 / 88$ & 71 & CASS & $T$ & \\
\hline TE-105-AP-46 & $7 / 16 / 88$ & 69 & CASS & $T$ & \\
\hline TE-105-AP.46 & $7 / 23 / 8 B$ & 71 & CASS & $\bar{T}$ & \\
\hline TE-105-AP-46 & $7 / 27 / 8 \mathrm{~B}$ & 70 & CASS & $T$ & \\
\hline TE- $105-A P=46$ & $8 / 3 / 88$ & 72 & CASS & $T$ & \\
\hline TE-T05-AP-46 & $8 / 9 / 88$ & 70 & CASS & $T$ & \\
\hline TE-105-AP-46 & $8 / 14 / 88$ & 72 & CASS & $T$ & \\
\hline TE-105-AP-46 & $8 / 27 / 8 \mathrm{a}$ & 70 & CASS & $T$ & \\
\hline TE-105-AP-46 & $9 / 2 / 88$ & 72 & CASS & $\bar{T}$ & \\
\hline TE-105-AP-46 & 9/6/88 & 73 & CASS & $T$ & \\
\hline TE-105-AP.46 & $9 / 13 / 8 \mathrm{~B}$ & 73 & CASS & $\bar{T}$ & \\
\hline TE-105-AP-46 & $9 / 19 / 8 \mathrm{~B}$ & 71 & CASS & $T$ & \\
\hline$T E-105 \cdot A P-46$ & $9 / 27 / 88$ & 70 & CASS & $T$ & \\
\hline$T E=105-A P-46$ & $12 / 1 / 88$ & 64 & CASS & $\mathbf{T}$ & \\
\hline TE-105-AP-46 & $12 / 5 / \mathrm{aB}$ & 66 & CASS & $T$ & \\
\hline TE-105-AP-46 & $12 / 15 / 88$ & 61 & CASS & $\bar{T}$ & \\
\hline TE-105-AP-46 & $12 / 20 / 88$ & 62 & CASS & $T$ & \\
\hline TE-105-AP-46 & $9 / 4 / 69$ & 79 & CASS & $T$ & \\
\hline TE-105-AP-46 & $9 / 14 / 89$ & 78 & CASS & $T$ & \\
\hline TE-105-AP-46 & $9 / 18 / 89$ & 79 & CASS & $T$ & \\
\hline TE-105-AP-46 & $9 / 28 / 89$ & 79 & CASS & $T$ & \\
\hline TE-105-AP-A6 & $11 / 4 / 89$ & 78 & CASS & $T$ & \\
\hline TE-IOS-AP-46 & $11 / 5 / 89$ & Bo & CAES & $T$ & \\
\hline$T E-105-A P-46$ & $11 / 18 / 89$ & 79 & CASS & $\bar{T}$ & \\
\hline$T E-105-A P-46$ & $11 / 23 / 89$ & 80 & CASS & $T$ & \\
\hline TE-105.AP-46 & $12 / 2 / 89$ & 81 & CASS & $T$ & \\
\hline TE-105-AP-46 & $12 / 9 / 89$ & 78 & CASS & $T$ & \\
\hline TE-T0S-AP.46 & $12 / 12 / 89$ & 78 & CASS & $T$ & \\
\hline TE-105-AP-46 & $12 / 23 / 89$ & 76 & CASS & $T$ & \\
\hline TE-105-AP-46 & $12 / 28 / 89$ & 79 & CASS & $T$ & \\
\hline TE-105-AP-46 & $1 / 2 / 90$ & 78 & CASS & $T$ & \\
\hline TE-105-AP-46 & $1 / 12 / 90$ & 77 & CASS & $T$ & \\
\hline TE-105-AP-46 & $1 / 20 / 90$ & 76 & CASS & $T$ & \\
\hline TE-105-AP-46 & $1 / 23 / 90$ & 76 & CASS & $T$ & \\
\hline TE-105-AP-46 & $1 / 29 / 90$ & 75 & CASS & $\bar{T}$ & \\
\hline TE-105-AP-46 & $2 / 10 / 90$ & 75 & CASS & $T$ & \\
\hline TE-105-AP-46 & $2 / 13 / 90$ & 77 & CASS & $T$ & \\
\hline TE-105-AP-46 & $2 / 21 / 90$ & 76 & CASS & $T$ & \\
\hline TE-105-AP-46 & $2 / 28 / 90$ & 76 & CASS & $T$ & \\
\hline TE-105-AP-46 & $3 / 8 / 80$ & 75 & CASS & $T$ & \\
\hline TE-105-AP-46 & $3 / 12 / 90$ & 74 & CASS & $\mathbf{T}$ & \\
\hline TE-105-AP-46 & $3 / 20 / 90$ & 74 & CASS & $T$ & \\
\hline TE-105-AP-46 & $3 / 25 / 90$ & 74 & CASS & $T$ & \\
\hline TE-105-AP-46 & $4 / 3 / 90$ & 74 & CASS & $T$ & \\
\hline TE-105-AP-46 & $4 / 9 / 90$ & 73 & SACS & $T$ & \\
\hline TE-105-AP-46 & $4 / 21 / 90$ & 72 & CASS & $T$ & \\
\hline TE-10S-AP-46 & $4 / 24 / 90$ & 74 & CASS & $T$ & \\
\hline TE-105-AP-46 & $5 / 1 / 90$ & 73 & CASS & $T$ & \\
\hline TE-105-AP-46 & $5 / 11 / 90$ & 76 & CASS & $T$ & \\
\hline$T E-105-A P-46$ & $5 / 19 / 90$ & 72 & CASS & $T$ & \\
\hline TE-105-AP-46 & $5 / 21 / 90$ & 74 & SACS & $T$ & \\
\hline TE-105-AP-46 & $6 / 2 / 90$ & 74 & CASS & $T$ & \\
\hline TE-105-AP-46 & $6 / 6 / 90$ & 76 & CASS & $T$ & \\
\hline TE-105-AP-46 & $6 / 12 / 90$ & 75 & CASS & $T$ & \\
\hline TE-105-AP-46 & $6 / 20 / 90$ & 72 & CASS & $T$ & \\
\hline TE-105-AP-46 & $6 / 27 / 90$ & 75 & CASS & $T$ & \\
\hline TE-105-AP-46 & $7 / 6 / 90$ & 72 & CASS & $T$ & \\
\hline TE-105-AP-46 & $7 / 12 / 90$ & 73 & CASS & $T$ & \\
\hline TE-105-AP-46 & $7 / 16 / 90$ & 74 & CASS & $T$ & \\
\hline TE-105-AP-46 & $7 / 27 / 90$ & 77 & CASS & $T$ & \\
\hline TE-105-AP-46 & $8 / 3 / 90$ & 73 & CASS & $T$ & \\
\hline TE-105-AP-46 & $8 / 8 / 90$ & 75 & CASS & $T$ & \\
\hline TE-105-AP-46 & $8 / 18 / 90$ & 73 & CASS & $\bar{T}$ & \\
\hline TE-105-AP-46 & $8 / 21 / 90$ & 76 & CASS & $T$ & \\
\hline TE-105-AP-46 & $\mathrm{B} / 29 / 90$ & 75 & CASS & $T$ & \\
\hline TE-105-AP-46 & $9 / 8 / 90$ & 74 & CASS & $\bar{T}$ & \\
\hline TE-105-AP-46 & $9 / 75 / 90$ & 76 & CASS & $\bar{T}$ & \\
\hline TE-105-AP-46 & $9 / 20 / 90$ & 76 & CASS & $\mathbf{T}$ & \\
\hline TE-105-AP-46 & $9 / 28 / 90$ & 76 & CASS & $T$ & \\
\hline TE-105-AP-46 & $10 / 6 / 90$ & 75 & CASS & $T$ & \\
\hline TE-105-AP-46 & $10 / 7 / 20$ & 80 & CASS & $T$ & \\
\hline TE-105-AP-46 & $10 / 20 / 90$ & 75 & CASS & $T$ & \\
\hline
\end{tabular}




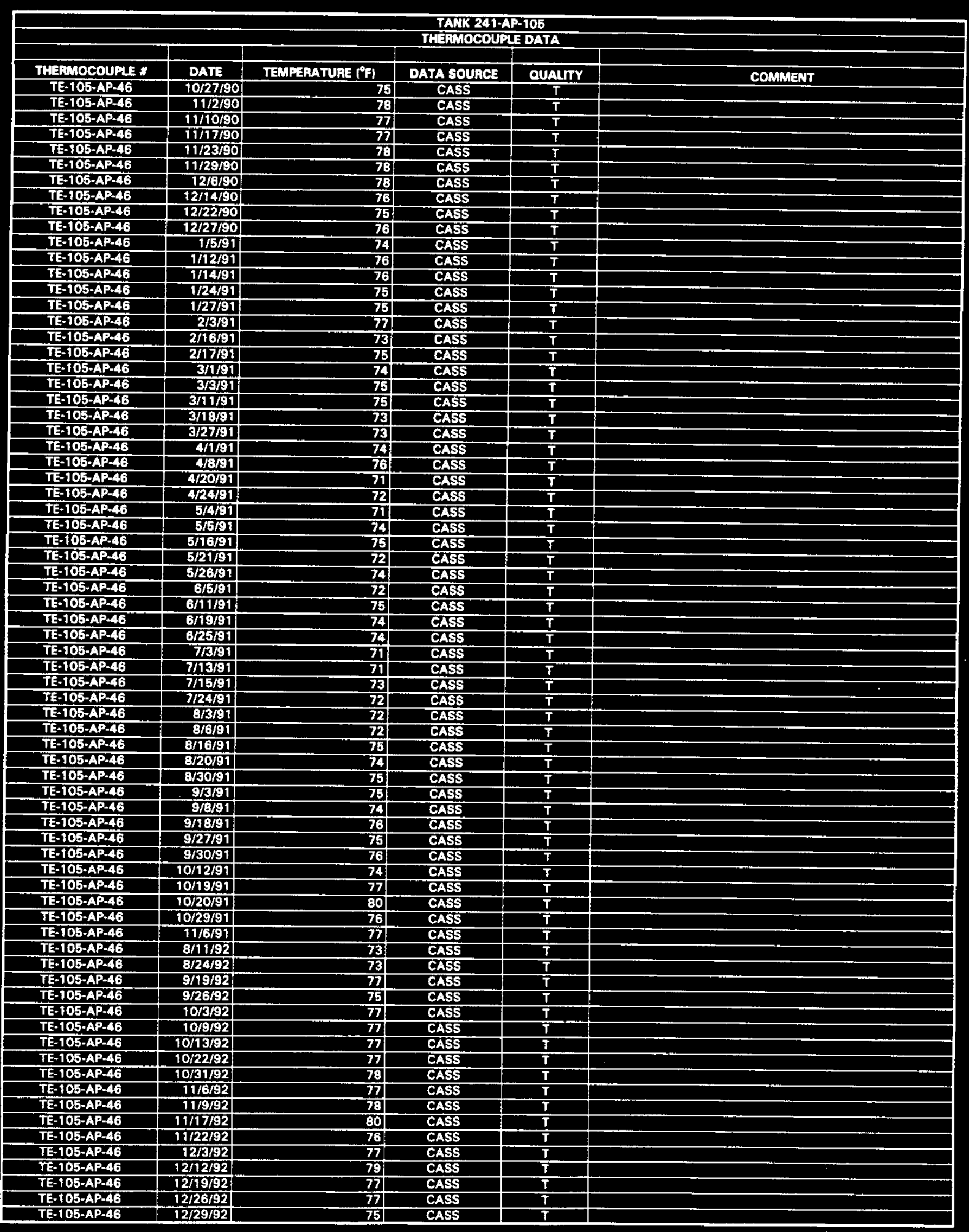

Data obtained from Computer Automated Surveillance Syatem (CASS) in April 1995, and Surviellance Analysis Computer Syatem (SACS) in June 1995. D-457 


\begin{tabular}{|c|c|c|c|c|c|}
\hline \multicolumn{6}{|c|}{$\begin{array}{l}\text { TAKK 241-AP.105 } \\
\text { THERiMOCOUPLE DATA }\end{array}$} \\
\hline & & & & & \\
\hline THERMOCOUPL * & DATE & TEMPERATURE $\left({ }^{\circ} \mathrm{F}\right)$ & DATA SOURCE & OUALTY & COMMENT \\
\hline TE-105-AP-46 & 6/11/93 & 69 & SACS & $T$ & \\
\hline TE-105-AP-46 & 6/18/93 & 68 & SACS & $T$ & \\
\hline TE-105-AP-46 & $6 / 28 / 93$ & 69 & SACS & $T$ & \\
\hline TE-105-AP-46 & $7 / 9 / 93$ & 69 & SACS & $T$ & \\
\hline TE-105-AP-46 & 7/15/93 & 70 & SACS & $\bar{T}$ & \\
\hline TE-105-AP-46 & 7/19/93 & 69 & SACS & $T$ & \\
\hline TE-105-AP-46 & $7 / 30 / 93$ & 70 & SACS & $T$ & \\
\hline TE-105-AP-46 & $8 / 6 / 93$ & 69 & SACS & $T$ & \\
\hline TE-105-AP-46 & $8 / 12 / 93$ & 69 & SACS & $T$ & \\
\hline TE-105-AP+46 & $9 / 24 / 93$ & 69 & SACS & $T$ & \\
\hline TE-105-AP-46 & $10 / 1 / 93$ & 72 & SACS & $T$ & \\
\hline TE-105-AP-46 & $10 / 15 / 93$ & 72 & SACS & $T$ & \\
\hline TE-105-AP-46 & $10 / 22 / 93$ & 72 & SACS & $T$ & \\
\hline TE-105-AP-46 & $10 / 20 / 93$ & 73 & SACS & $T$ & \\
\hline TE-105-AP-46 & 11/9/93 & 68 & SACS & $T$ & \\
\hline TE-105-AP-46 & $11 / 30 / 93$ & 75 & SACS & $T$ & \\
\hline TE-105-AP-46 & $12 / 14 / 93$ & 74 & SACS & $T$ & \\
\hline TE-105-AP-46 & $12 / 20 / 93$ & 72 & SACS & $T$ & \\
\hline$T E-105-A P-46$ & $12 / 27 / 83$ & 73 & SACS & $T$ & \\
\hline TE-105-AP-46 & $1 / 3 / 34$ & 71 & SACS & $T$ & \\
\hline TE-105-AP-46 & $1 / 11 / 94$ & 74 & SACS & $T$ & \\
\hline TE-105-AP-46 & $1 / 18 / 94$ & 74 & CASS & $T$ & \\
\hline TE-105-AP.46 & 1/26/94 & 75 & SACS & $T$ & \\
\hline TE-105-AP-46 & $2 / 4 / 94$ & 73 & CASS & $T$ & \\
\hline TE.105-AP-46 & $2 / 12 / 94$ & 74 & CASS & $\bar{T}$ & \\
\hline TE-105-AP-46 & $2 / 16 / 94$ & 73 & CASS & $T$ & \\
\hline TE-105-AP-46 & $2 / 22 / 94$ & 73 & CASS & $T$ & \\
\hline TE-105-AP-46 & $3 / 5 / 94$ & 74 & CASS & $T$ & \\
\hline TE-105-AP-46 & $3 / 7 / 94$ & 74 & CASS & $T$ & \\
\hline TE-105-AP-46 & $3 / 16 / 94$ & 74 & CASS & $T$ & \\
\hline TE-105-AP-A6 & $3 / 26 / 94$ & 75 & CASS & $\bar{T}$ & \\
\hline$T E-105-A P-46$ & $3 / 30 / 94$ & 73 & CASS & $T$ & \\
\hline TE-105-AP-46 & $4 / 6 / 94$ & 74 & CASS & $T$ & \\
\hline TE-105-AP-46 & $4 / 13 / 94$ & 72 & CASS & $T$ & \\
\hline TE-105-AP-46 & $4 / 20 / 94$ & 73 & CASS & $T$ & \\
\hline TE-105-AP-46 & $4 / 27 / 84$ & 73 & CASS & $T$ & \\
\hline TE-105-AP-46 & $5 / 2 / 94$ & 73 & CASS & $T$ & \\
\hline TE-105-AP-46 & $5 / 14 / 94$ & 74 & CASS & $T$ & \\
\hline TE-105-AP-46 & $5 / 16 / 94$ & 105 & CASS & $T$ & \\
\hline TE-105-AP-46 & $5 \longdiv { 2 7 / 9 4 }$ & 98 & CASS & $T$ & \\
\hline TE-105-AP-46 & $6 / 2 / 94$ & 100 & CASS & $T$ & \\
\hline TE-105-AP-46 & $6 / 2894$ & 100 & CASS & $T$ & \\
\hline TE-105-AP-46 & $6 / 13 / 94$ & 100 & SACS & $\bar{G}$ & \\
\hline TE-105-AP-46 & $6 / 16 / 94$ & 71 & CASS & $\mathbf{s}$ & Data point did not agree with local trend \\
\hline TE-105-AP-46 & $6 / 17 / 94$ & 71 & CASS & $\mathbf{S}$ & Data point did not agree with local trend \\
\hline TE-105-AP-46 & 6/19/94 & 100 & CASS & $T$ & \\
\hline TE-105-AP-46 & $6 / 20 / 94$ & 73 & CASS & 5 & Data point did not agree with locel trend \\
\hline TE-105-AP-46 & $6 / 20 / 94$ & 71 & SACS & $S$ (G in SACS) & Data point did not agree with local trend \\
\hline TE-105-AP-A6 & $6 / 27 / 94$ & 102 & CASS & $T$ & \\
\hline TE-105-AP-46 & $7 / 7 / 94$ & 98 & CASS & $T$ & \\
\hline TE-105-AP-46 & $7 / 13 / 94$ & 9B & CASS & $T$ & \\
\hline TE-105-AP-46 & 7/20/84 & 95 & CASS & $\bar{T}$ & \\
\hline TE-105-AP-46 & $7 / 24 / 94$ & 95 & CASS & $T$ & \\
\hline TE-105-AP-46 & $7 / 31 / 94$ & 99 & CASS & $\bar{T}$ & \\
\hline TE-105-AP-46 & $8 / 10 / 94$ & 98 & CASS & $\bar{T}$ & \\
\hline$T E-105-A P-46$ & 8/20/94 & 99 & CASS & $T$ & \\
\hline TE-105-AP-46 & $8 / 27 / 94$ & 97 & CASS & $T$ & \\
\hline TE-105-AP-46 & $8 / 3 / 94$ & 99 & CASS & $\bar{T}$ & \\
\hline TE-105-AP-46 & 9/9/94 & 102 & CASS & $T$ & \\
\hline TE-105-AP-46 & 9/15/94 & 101 & CASS & $T$ & \\
\hline TE-105-AP-46 & $9 / 23 / 94$ & 102 & CASS & $T$ & \\
\hline$T E-105-A P-46$ & 9/25/94 & 102 & CASS & $T$ & \\
\hline TE-105-AP-46 & $10 / 2 / 94$ & 105 & CASS & $T$ & \\
\hline TE-105-AP-46 & $10 / 15 / 94$ & 104 & CASS & $T$ & \\
\hline TE-105-AP-46 & $10 / 22 / 94$ & 105 & CASS & $T$ & \\
\hline TE-105-AP-46 & $10 / 29 / 94$ & 106 & CASS & $\bar{T}$ & \\
\hline TE-105-AP-46 & $11 / 4 / 94$ & 118 & CASS & $T$ & \\
\hline TE-105-AP-46 & $11 / 11 / 94$ & 109 & CASS & $T$ & \\
\hline TE-105-AP-46 & $11 / 16 / 94$ & 109 & CASS & $\mathbf{T}$ & \\
\hline TE-105-AP-46 & $11 / 22 / 94$ & 110 & CASS & $\mathbf{T}$ & \\
\hline TE-105-AP-46 & $11 / 28 / 94$ & 105 & CASS & $T$ & \\
\hline TE-105-AP-46 & $12 / 4 / 94$ & 109 & CASS & $T$ & \\
\hline TE-105-AP-46 & $12 / 16 / 94$ & 104 & CASS & $T$ & \\
\hline
\end{tabular}




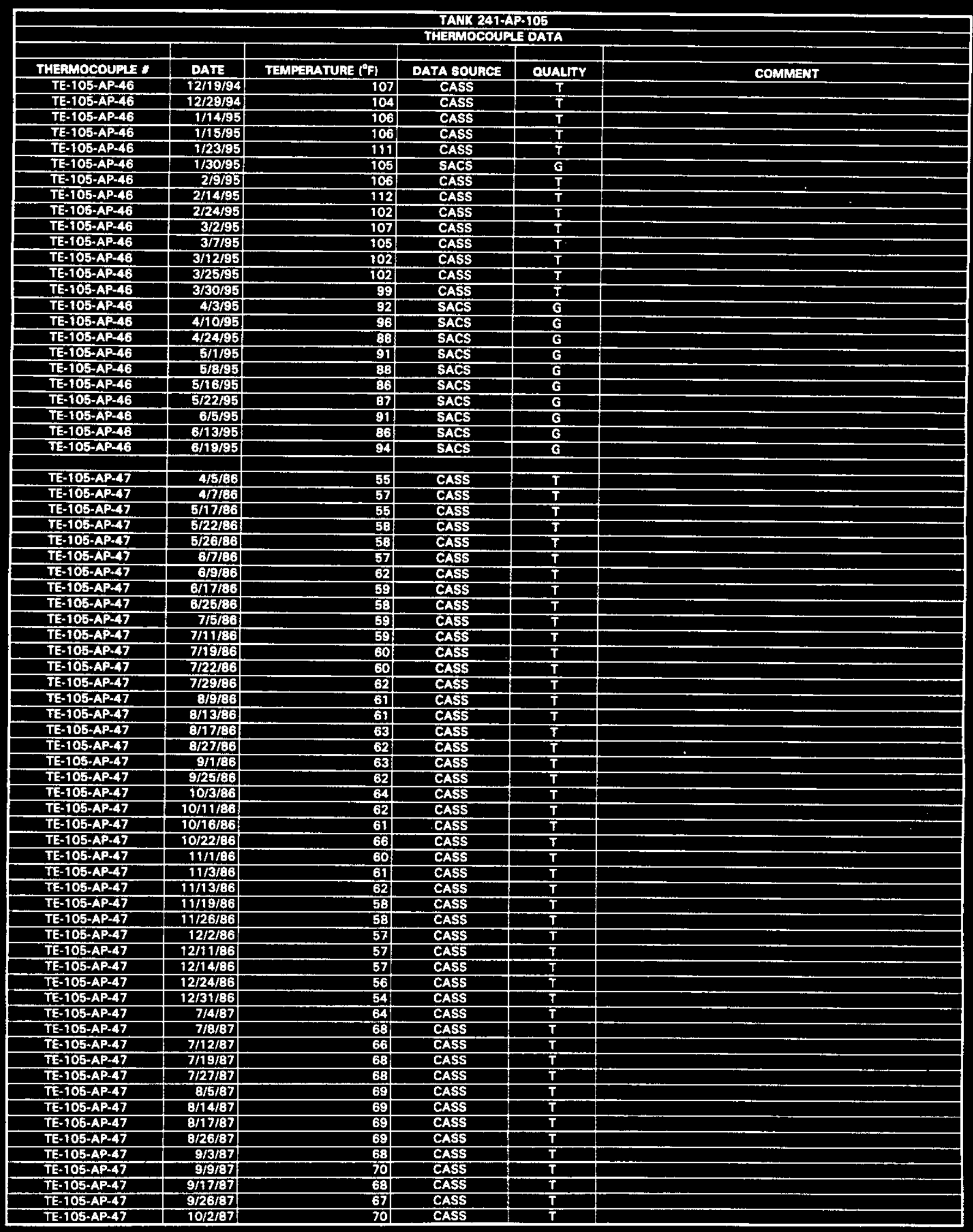




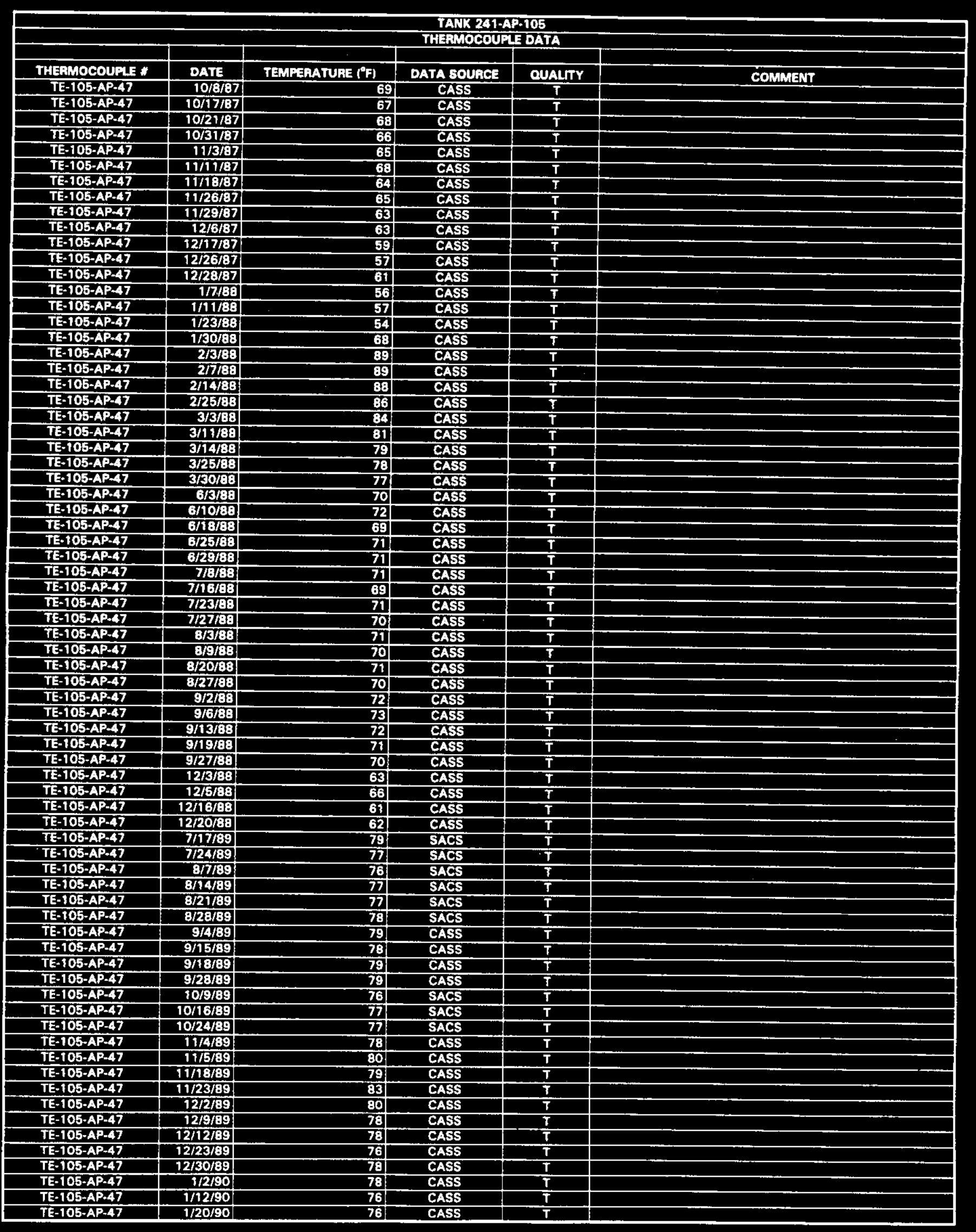




\begin{tabular}{|c|c|c|c|c|c|}
\hline \multicolumn{6}{|c|}{$\begin{array}{l}\text { TANK 241-AP-105 } \\
\text { THEFTOCOUPLE DATA }\end{array}$} \\
\hline & & & & & \\
\hline THERMOCOUPL * & DATE & TEMPERATUAE $\left\{^{\circ} \mathrm{F}\right\}$ & DATA SOURCE & QUALTY & COMMENT \\
\hline TE-105-AP-47 & $1 / 23 / 90$ & -76 & CASS & $T$ & \\
\hline TE-105-AP-47 & $1 / 29 / 90$ & 75 & CASS & $\mathbf{T}$ & \\
\hline$T E-105-A P-47$ & $2 / 10 / 90$ & 75 & CASS & $\bar{T}$ & \\
\hline TE-105-AP-47 & $2 / 13 / 90$ & 76 & CASS & $\mathrm{T}$ & \\
\hline TE-105-AP-47 & $2 / 18 / 90$ & 76 & CASS & $\mathbf{I}$ & \\
\hline TE-105-AP-47 & 2/28/90 & 78 & CASS & $\bar{T}$ & \\
\hline TE-105-AP-47 & 3/3/90 & 74 & CASS & $T$ & \\
\hline TE-105-AP-47 & $3 / 12 / 90$ & 74 & CASS & $T$ & \\
\hline TE-105-AP-47 & $3 / 20 / 90$ & 74 & CASS & $\bar{T}$ & \\
\hline TE-105-AP-47 & $3 / 25 / 90$ & 74 & CASS & $T$ & \\
\hline TE-105-AP-47 & $4 / 3 / 90$ & 74 & CASS & $T$ & \\
\hline TE-105.AP-47 & $4 / 14 / 90$ & 72 & CASS & $\mathbf{T}$ & \\
\hline TE-105-AP-47 & $4 / 21 / 90$ & 72 & CASS & $\bar{T}$ & \\
\hline TE-105-AP-47 & $4 / 24 / 90$ & 74 & CASS & $\bar{T}$ & \\
\hline TE-105-AP-47 & $5 / 1 / 90$ & 73 & CASS & $\bar{T}$ & \\
\hline TE-105-AP-47 & $5 / 11 / 90$ & 77 & CASS & $\mathbf{T}$ & \\
\hline TE-10b-AP-47 & 5/19/90 & 72 & CASS & $T$ & \\
\hline TE-105-AP-47 & $5 / 22 / 80$ & 73 & CASS & $T$ & \\
\hline TE-105-AP-47 & $6 / 2 / 90$ & 74 & CASS & $T$ & \\
\hline TE-105-AP-47 & $6 / 6 / 90$ & 77 & CASS & $T$ & \\
\hline TE-105-AP-47 & 6/12/90 & 75 & CASS & $T$ & \\
\hline TE-105-AP-47 & 6/22/90 & 72 & CASS & $T$ & \\
\hline TE-106-AP-47 & $6 / 26 / 90$ & 74 & CASS & $T$ & \\
\hline TE-105-AP-A7 & $7 / 6 / 90$ & 72 & CASS & $T$ & \\
\hline TE-105-AP-47 & $7 / 32 / 90$ & 74 & CASS & $\bar{T}$ & \\
\hline TE-105-AP-47 & $7 / 16 / 90$ & 75 & CASS & $T$ & \\
\hline TE-105-AP-47 & $7 / 27 / 90$ & 75 & CASS & $\mathbf{T}$ & \\
\hline TE-105-AP-47 & $8 / 3 / 90$ & 73 & CASS & $\mathbf{T}$ & \\
\hline TE-105-AP-47 & $8 / 8 / 90$ & 75 & CASS & $T$ & \\
\hline TE-105-AP-47 & $8 / 18 / 90$ & 73 & CASS & $T$ & \\
\hline TE-105-AP-47 & $8 / 21 / 90$ & 77 & CASS & $\bar{T}$ & . \\
\hline$T E-105-A P-47$ & $8 / 29 / 90$ & 75 & CASS & $\bar{T}$ & \\
\hline TE-105-AP-47 & $9 / 8 / 90$ & 74 & CASS & $\mathbf{T}$ & \\
\hline TE-105-AP-47 & 9/15/90 & 76 & CASS & $\bar{T}$ & \\
\hline TE-105-AP-47 & $9 / 20 / 90$ & 77 & CASS & $T$ & \\
\hline TE-105-AP-47 & $9 / 25190$ & 144 & CASS & $\mathbf{S}$ & Date point did not agree with locel trend \\
\hline TE-105-AP-47 & $9 / 28 / 90$ & 76 & CASS & $T$ & \\
\hline TE-105-AP-47 & $10 / 6 / 90$ & 75 & CASS & $T$ & \\
\hline TE-105-AP-47 & $10 / 13 / 90$ & 80 & CASS & $\mathbf{T}$ & \\
\hline TE-105-AP-47 & $10 / 15 / 90$ & 76 & SACS & $\mathbf{T}$ & \\
\hline TE-105-AP-47 & $10 / 21 / 80$ & 76 & CASS & $\mathbf{T}$ & \\
\hline TE-TOS-AP-47 & $11 / 2190$ & 78 & CASS & $\mathbf{T}$ & \\
\hline TE-105-AP-47 & $31 / 10 / 90$ & 77 & CASS & $T$ & \\
\hline TE-106-AP-47 & $11 / 27 / 90$ & 77 & CASS & $T$ & \\
\hline TE-105-AP-47 & $11 / 23 / 90$ & 7 & CASS & $T$ & \\
\hline TE-105-AP-47 & $11 / 27 / 80$ & 78 & CASS & $T$ & \\
\hline TE-105-AP-47 & $12 / 6 / 80$ & 78 & CASS & $T$ & \\
\hline TE-105-AP-47 & $12 / 11 / 90$ & 77 & CASS & $T$ & \\
\hline TE-105-AP-47 & $12 / 20 / 90$ & 76 & CASS & $T$ & \\
\hline TE-105-AP-47 & $12 / 27 / 90$ & 76 & CASS & $T$ & \\
\hline TE-105-AP-47 & $1 / 2 / 91$ & 75 & CASS & $T$ & \\
\hline TE-105-AP-47 & W/12/91 & 76 & CASS & $T$ & \\
\hline TE-105-AP-47 & 1/14/91 & 76 & CASS & $\bar{T}$ & \\
\hline TE-105-AP,47 & //24/97 & 75 & CASS & $\bar{T}$ & \\
\hline TE-105-AP-47 & $1 / 28 / 91$ & 77 & SACS & $\mathbf{T}$ & \\
\hline TE-105-AP-47 & $2 / 3 / 91$ & 78 & CASS & $\mathbf{T}$ & \\
\hline TE-105-AP-47 & $2 / 16 / 91$ & 73 & CASS & $\bar{T}$ & \\
\hline TE-105-AP-47 & $2 / 17 / 91$ & 76 & CASS & $T$ & \\
\hline TE-105-AP-47 & $3 / 1 / 191$ & 74 & CASS & $T$ & \\
\hline TE-105-AP-47 & $3 / 9 / 91$ & 74 & CASS & $T$ & \\
\hline TE-105-AP-47 & 3/11/91 & 75 & CASS & $T$ & \\
\hline TE-105-AP-47 & 3/18/91 & 74 & SACS & $T$ & \\
\hline TE-105-AP-47 & 3/27/91 & 73 & CASS & $T$ & \\
\hline TE-105-AP-47 & $4 / 1 / 91$ & 74 & CASS & $T$ & \\
\hline TE-105-AP-47 & $4 / 12 / 91$ & 76 & CASS & $T$ & \\
\hline TE-105-AP-47 & $4 / 20 / 91$ & 71 & CASS & $T$ & \\
\hline TE-105-AP-47 & 4/24/91 & 73 & CASS & $\mathbf{T}$ & \\
\hline TE-105-AP-47 & $5 / 4 / 91$ & 71 & CASS & $T$ & \\
\hline TE-105-AP-47 & $5 / 5 / 91$ & 73 & CASS & $T$ & \\
\hline TE-105-AP-47 & $5 / 16 / 91$ & 76 & CASS & $T$ & \\
\hline TE-105-AP-47 & $5 / 21 / 91$ & 72 & CASS & $\mathrm{T}$ & \\
\hline TE-105-AP-47 & $5 / 26 / 91$ & 74 & CASS & $T$ & \\
\hline TE-105-AP-47 & $6 / 5 / 91$ & 72 & CASS & $\mathbf{T}$ & \\
\hline
\end{tabular}




\begin{tabular}{|c|c|c|c|c|c|}
\hline \multicolumn{6}{|c|}{$\begin{array}{l}\text { TACK 241-AP-105 } \\
\text { THIEAMOCOUPLE DAYA }\end{array}$} \\
\hline & & & & & \\
\hline THERMOCOUPLE & DATE & TEMPERATURE ( $\left.^{\circ} \mathrm{F}\right)$ & DATA SOURCE & OUALITY & COMMENT \\
\hline TE-105-AP-47 & 6/11/91 & 72 & CASS & $T$ & \\
\hline TE-105-AP-47 & 6/19/91 & 74 & CASS & $\mathbf{T}$ & \\
\hline TE-105-AP-47 & 6/25/91 & 74 & CASS & $T$ & \\
\hline TE-105-AP-47 & $7 / 3 / 91$ & 72 & CASS & $T$ & \\
\hline TE-105-AP-47 & 7/13/91 & 72 & CASS & $T$ & \\
\hline TE-105-AP-47 & 7/15/91 & 73 & CASS & $T$ & \\
\hline TE-105-AP-47 & $7 / 24 / 91$ & 72 & CASS & $\mathbf{T}$ & \\
\hline TE-105-AP-47 & $8 / 3 / 91$ & 72 & CASS & $T$ & \\
\hline TE-105-AP-47 & $8 / 6 / 91$ & 72 & CASS & $T$ & \\
\hline TE-105-AP-47 & $8 / 17 / 91$ & 75 & CASS & $T$ & \\
\hline TE-105-AP-47 & 8/20/91 & 75 & CASS & $\bar{T}$ & \\
\hline IE-105-AP-47 & $8 / 30 / 91$ & 76 & CASS & $T$ & \\
\hline TE-105-AP-47 & 9/2/91 & 76 & CASS & $T$ & \\
\hline TE-105-AP-47 & 9/8/91 & 75 & CASS & $T$ & \\
\hline TE-105-AP-47 & 9/18/91 & 77 & CASS & $\mathbf{T}$ & \\
\hline TE-105-AP-47 & 8/27/91 & 74 & CASS & $T$ & \\
\hline TE-105-AP:A7 & $10 / 3 / 91$ & 76 & CASS & $\mathbf{T}$ & \\
\hline TE-105-AP-47 & 10/12/91 & 75 & CASS & $\mathbf{T}$ & \\
\hline$T E-105-A P-47$ & $10 / 13 / 91$ & 80 & CASS & $T$ & \\
\hline TE-105-AP-47 & $10 / 20 / 91$ & 78 & CÁSS & $T$ & \\
\hline TE-105-AP-47 & $10 / 29 / 91$ & 77 & CASS & $\mathbf{T}$ & \\
\hline TE-105-AP-47 & $11 / 6 / 91$ & 77 & CASS & $T$ & \\
\hline TE-105-AP-47 & $8 / 11 / 92$ & 73 & CASS & $\mathbf{T}$ & \\
\hline TE-105-AP-47 & $8 / 23 / 92$ & 74 & CASS & $T$ & \\
\hline TE-105-AP-47 & 9/19/92 & 77 & CASS & $\bar{T}$ & \\
\hline TE-105-AP-47 & $9 / 26 / 92$ & 75 & CASS & $\bar{T}$ & \\
\hline TE-105-AP-47 & 10/3/92 & 78 & CASS & $T$ & \\
\hline TE-105-AP-47 & $10 / 9 / 92$ & 77 & CASS & $T$ & \\
\hline TE-105-AP.A7 & $10 / 13 / 92$ & 77 & CASS & $T$ & \\
\hline TE-105-AP-47 & $10 / 22 / 92$ & 77 & CASS & $T$ & \\
\hline TE-105-AP-47 & $10 / 31 / 92$ & 77 & CASS & $T$ & \\
\hline TE-105-AP-47 & $11 / 6 / 92$ & 77 & CASS & $T$ & \\
\hline TE-105-AP-47 & $11 / 11 / 192$ & 78 & CASS & $T$ & \\
\hline TE-105-AP-A7 & $11 / 17 / 92$ & 81 & CASS & $T$ & \\
\hline TE-105-AP-47 & $11 / 24 / 92$ & 76 & CASS & $\mathrm{T}$ & \\
\hline TE-105-AP-47 & 12/3/92 & 77 & CASS & $T$ & \\
\hline TE-105-AP-47 & $12 / 12 / 92$ & 79 & CASS & $\bar{T}$ & \\
\hline TE-105-AP-47 & $12 / 19 / 92$ & 77 & CASS & $T$ & \\
\hline TE-105-AP-47 & $12 / 26 / 92$ & 78 & CASS & $T$ & \\
\hline TE-105-AP-47 & $12 / 29 / 92$ & 75 & CASS & $\mathbf{T}$ & \\
\hline TE-105-AP-47 & $1 / 15 / 94$ & 72 & CASS & $T$ & \\
\hline$T E-105-A P-47$ & $1 / 18 / 94$ & 74 & CASS & $T$ & \\
\hline TE-105-AP-47 & $1 / 27 / 94$ & 76 & CASS & $\mathbf{T}$ & \\
\hline TE-105-AP-47 & $2 / 4 / 94$ & 73 & CASS & $T$ & \\
\hline TE-105-AP-47 & $2 / 12 / 94$ & 74 & CASS & $T$ & \\
\hline TE-105-AP-47 & $2 / 16 / 94$ & 73 & CASS & $T$ & \\
\hline TE-105-AP-47 & $2 / 26 / 94$ & 73 & CASS & $T$ & \\
\hline TE-105-AP-47 & $3 / 5 / 94$ & 75 & CASS & $T$ & \\
\hline TE-105*AP-47 & $3 / 8 / 94$ & 74 & CASS & $T$ & \\
\hline TE-105-AP-47 & $3 / 16 / 94$ & 74 & CASS & $T$ & \\
\hline TE-105-AP-47 & $3 / 25 / 94$ & 74 & CASS & $T$ & \\
\hline TE-105-AP-47 & $3 / 30 / 94$ & 74 & CASS & $T$ & \\
\hline TE-105-AP-47 & $4 / 6 / 94$ & 75 & CASS & $\mathbf{T}$ & \\
\hline TE-105-AP-47 & $4 / 13 / 94$ & 73 & CASS & $T$ & \\
\hline TE-105-AP-47 & $4 / 20 / 94$ & 73 & CASS & $\mathbf{T}$ & \\
\hline$T E-105-A P-47$ & $4 / 29 / 94$ & 73 & CASS & $T$ & \\
\hline TE-105-AP-47 & $5 / 2 / 94$ & 73 & CASS & $T$ & \\
\hline TE-105-AP-47 & $5 / 14 / 94$ & 74 & CASS & $\bar{T}$ & \\
\hline TE-105-AP-47 & $5 / 16 / 94$ & 106 & CASS & $T$ & \\
\hline TE-105-AP-47 & $5 / 27 / 94$ & 99 & CASS & $T$ & \\
\hline TE-105-AP-47 & $6 / 2 / 94$ & 100 & CASS & $T$ & \\
\hline TE-105-AP-47 & $6 / 8 / 94$ & 101 & CASS & $T$ & \\
\hline TE-105-AP-47 & $6 / 16 / 94$ & 72 & CASS & $\mathbf{S}$ & Data point did not egree with local trend \\
\hline TE-105-AP-47 & $6 / 17 / 94$ & 72 & CASS & $\mathbf{s}$ & Data point did not agree with local trend \\
\hline TE-105-AP-47 & 6/18/94 & 97 & CASS & $T$ & \\
\hline TE-105-AP-47 & $6 / 19 / 94$ & 100 & CASS & $\mathbf{T}$ & \\
\hline TE-105-AP-47 & $6 / 20 / 94$ & 73 & CASS & $\mathbf{S}$ & Data point did not sgres with locel trend \\
\hline TE-105-AP-47 & $7 / 2 / 94$ & 102 & CASS & $\mathbf{T}$ & \\
\hline TE-105-AP-47 & $7 / 3 / 94$ & 99 & CASS & $\mathbf{T}$ & \\
\hline TE-105-AP.47 & $7 / 13 / 94$ & 98 & CASS & $T$ & \\
\hline$T E-105-A P=47$ & $7 / 19 / 94$ & 96 & CASS & $T$ & \\
\hline TE-105-AP-47 & $7 / 24 / 94$ & 96 & CASS & $T$ & \\
\hline TE-105-AP-47 & $7 / 31 / 94$ & 99 & CASS & $\mathbf{T}$ & \\
\hline
\end{tabular}




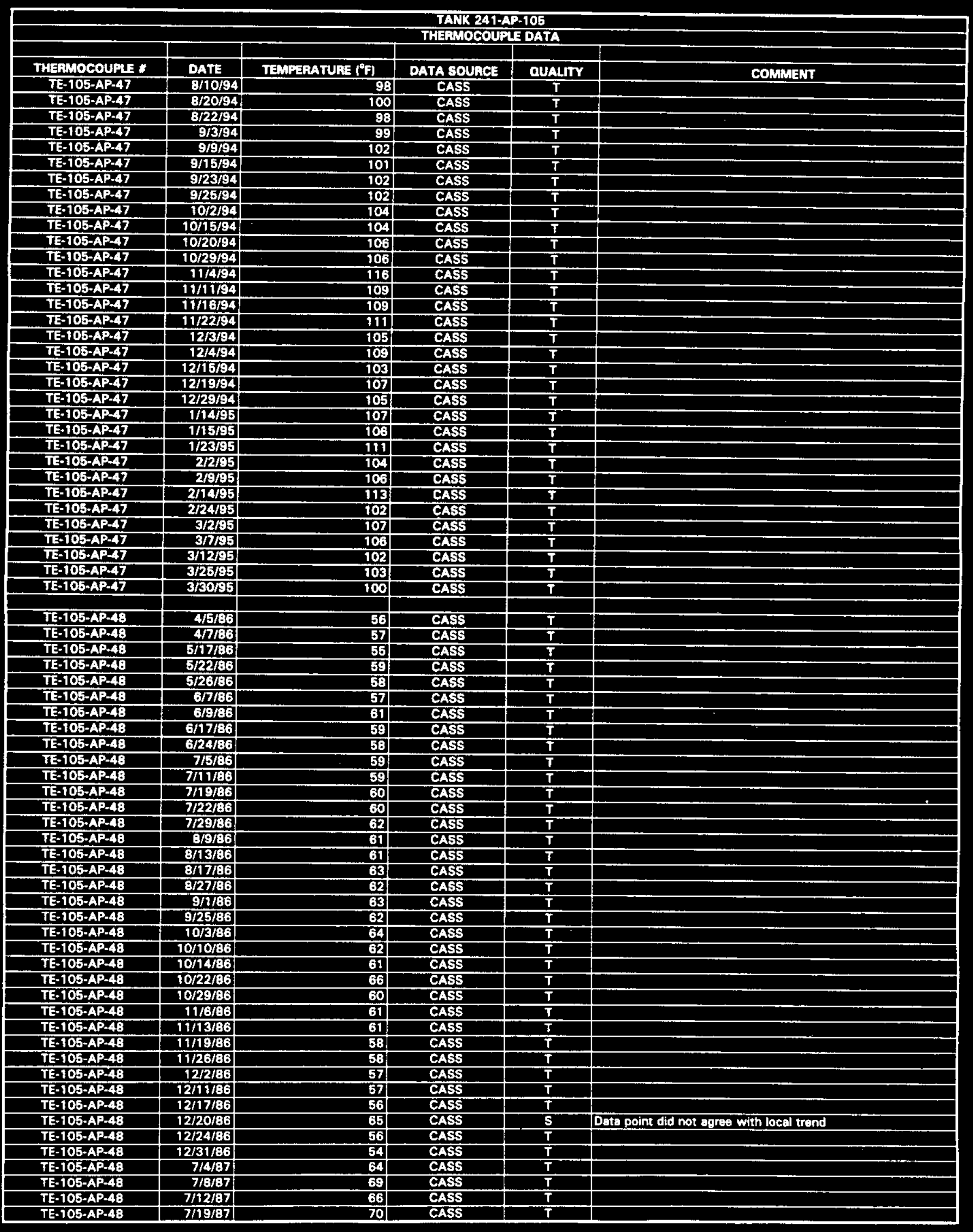

Data obtained from Computer Automated Surveillance System (CASS) in April 1995, and Surviellance Analysis Computer System (SACS) in June 1995. D-463 


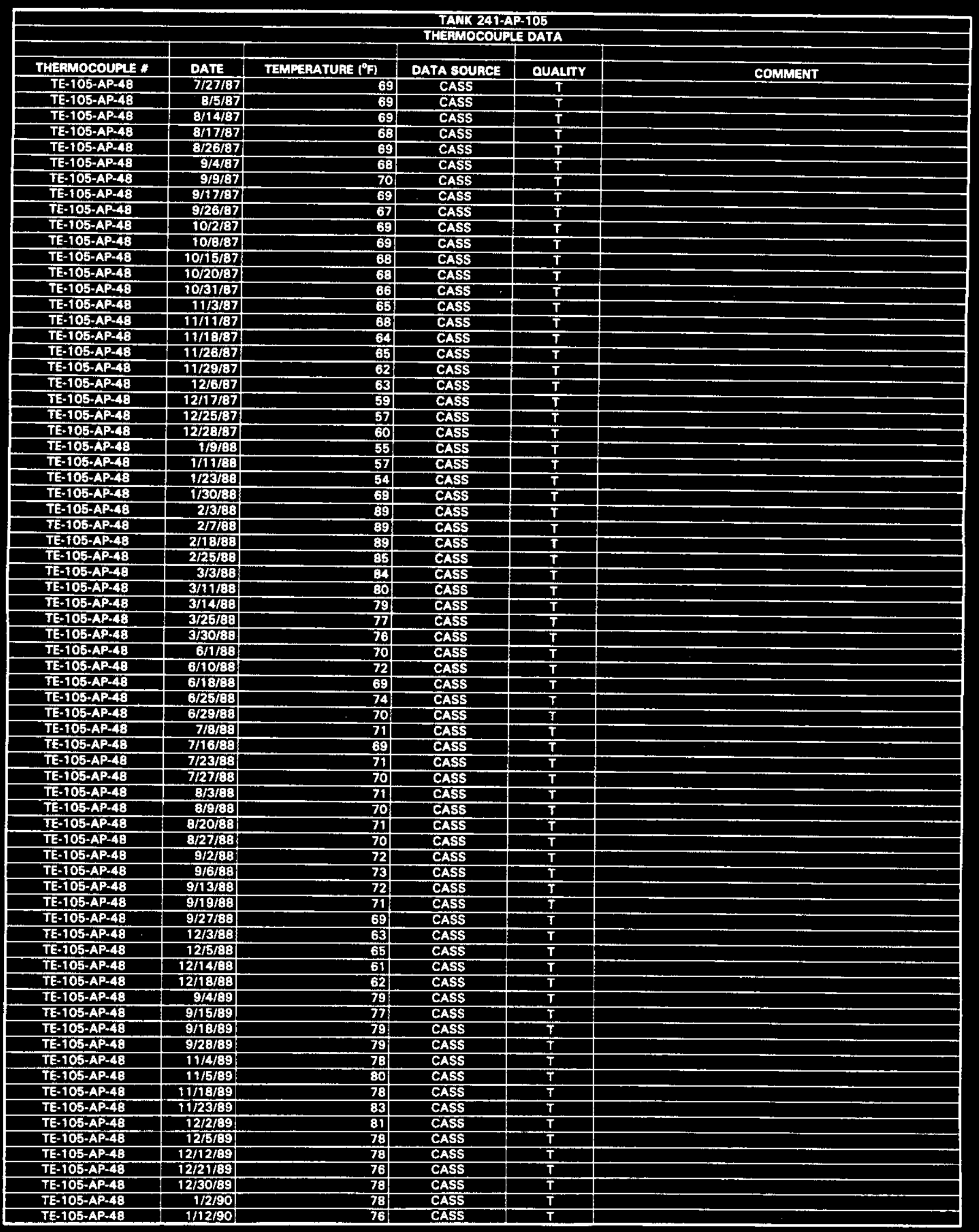




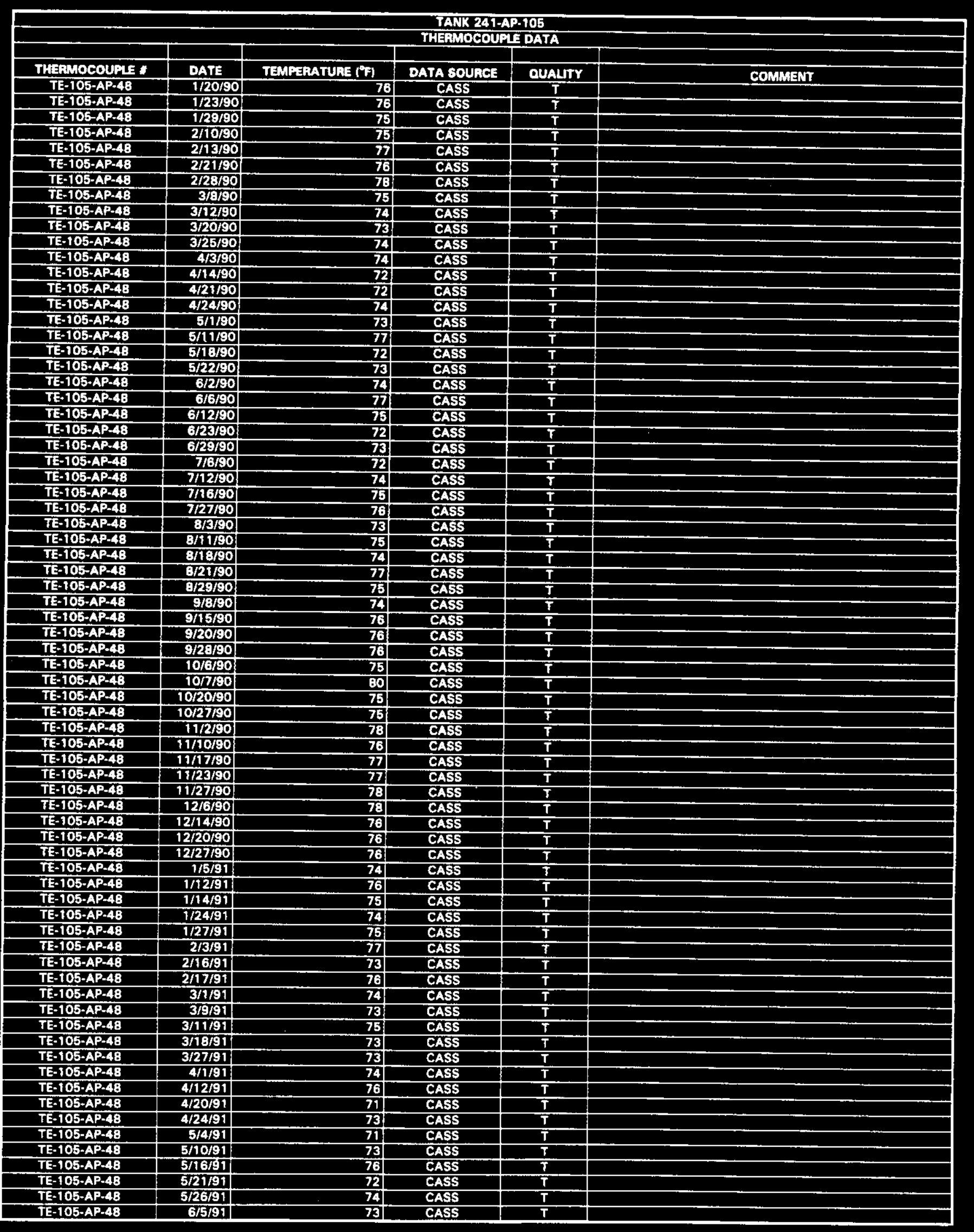

Data obtained from Computer Automated Surveillance System (CASS) in April 1995, and Surviellance Analysis Computer System (SACS) in June 1995.

$D-465$ 


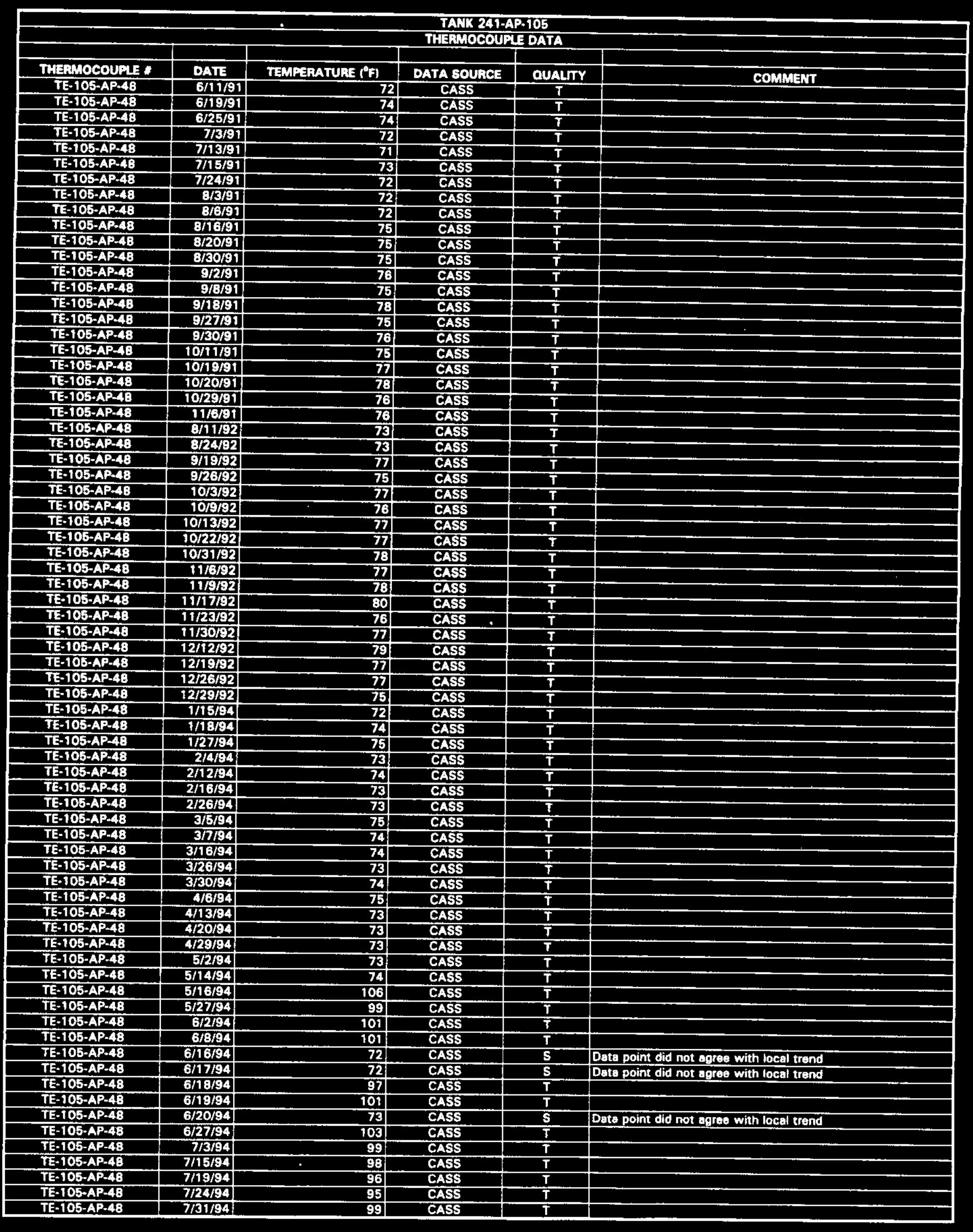

Data obtained from Computer Automated Survoillance System (CASS) in April 1995, and Surviellance Analysis Computer Syatem (SACS) in June 1995.

$$
\text { D-466 }
$$




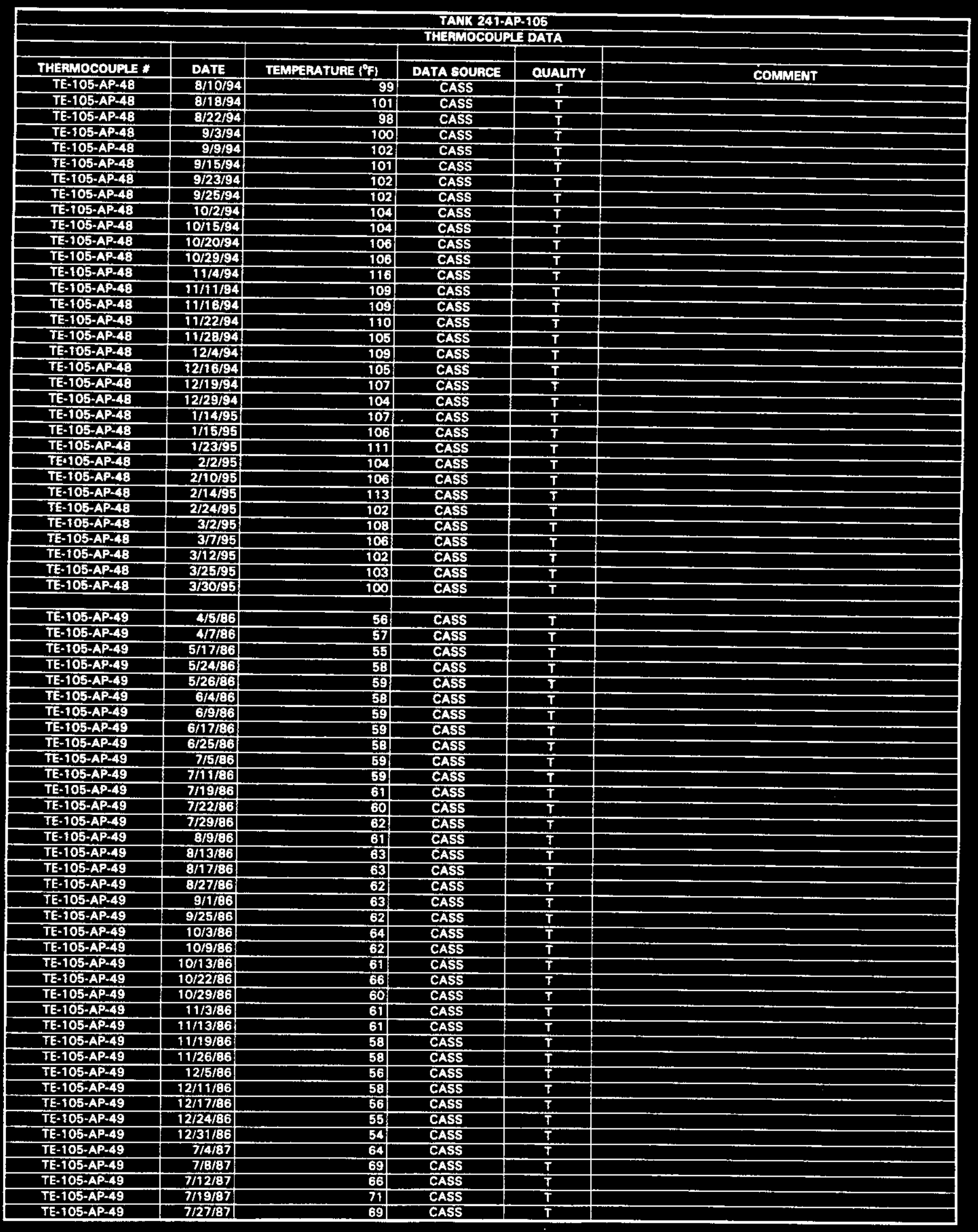

Date obtained from Computer Automated Surveillance Syatem (CASS) in April 1995, and Surviellance Analysis Computer System (SACS) in June 1995.

$D-467$ 


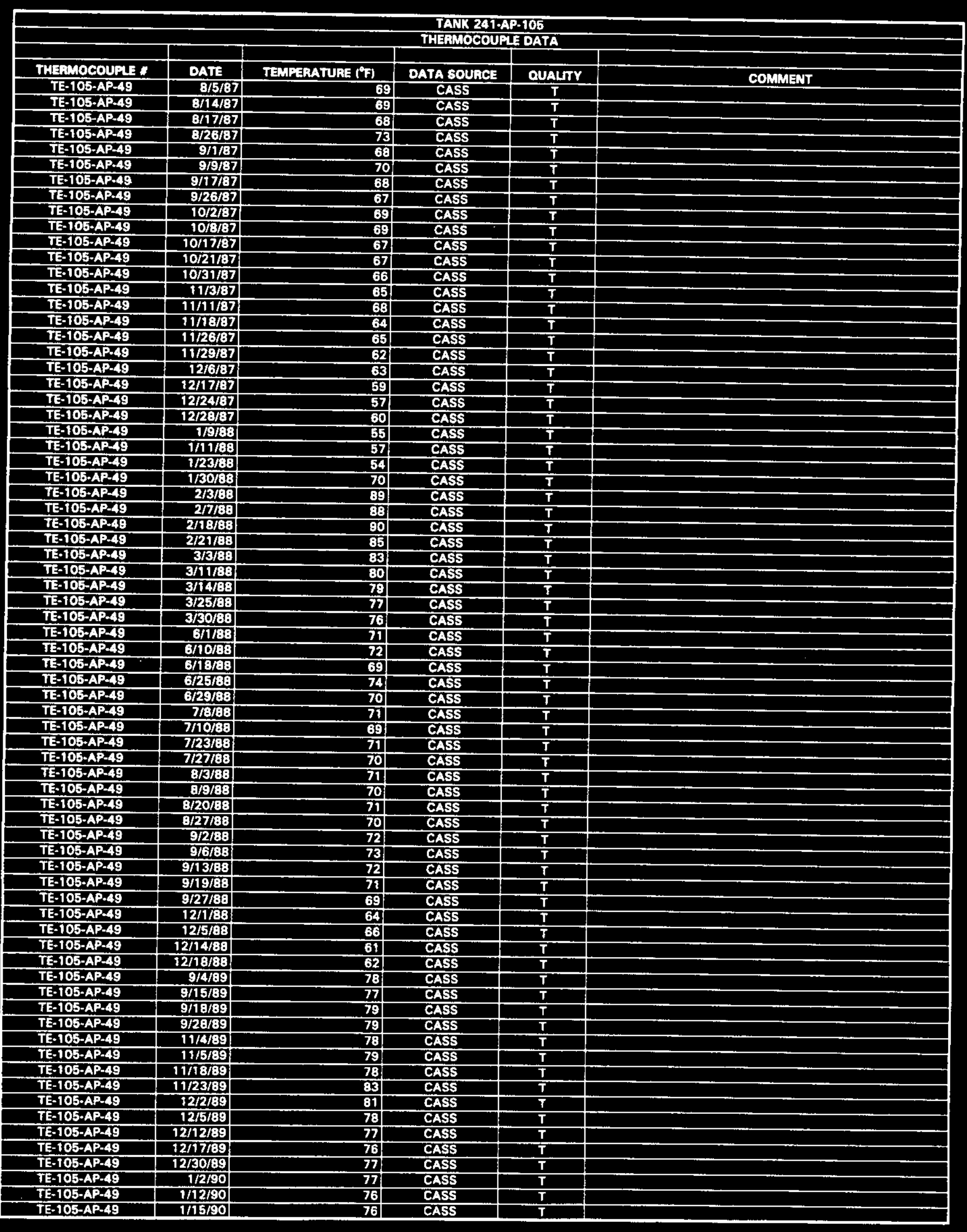




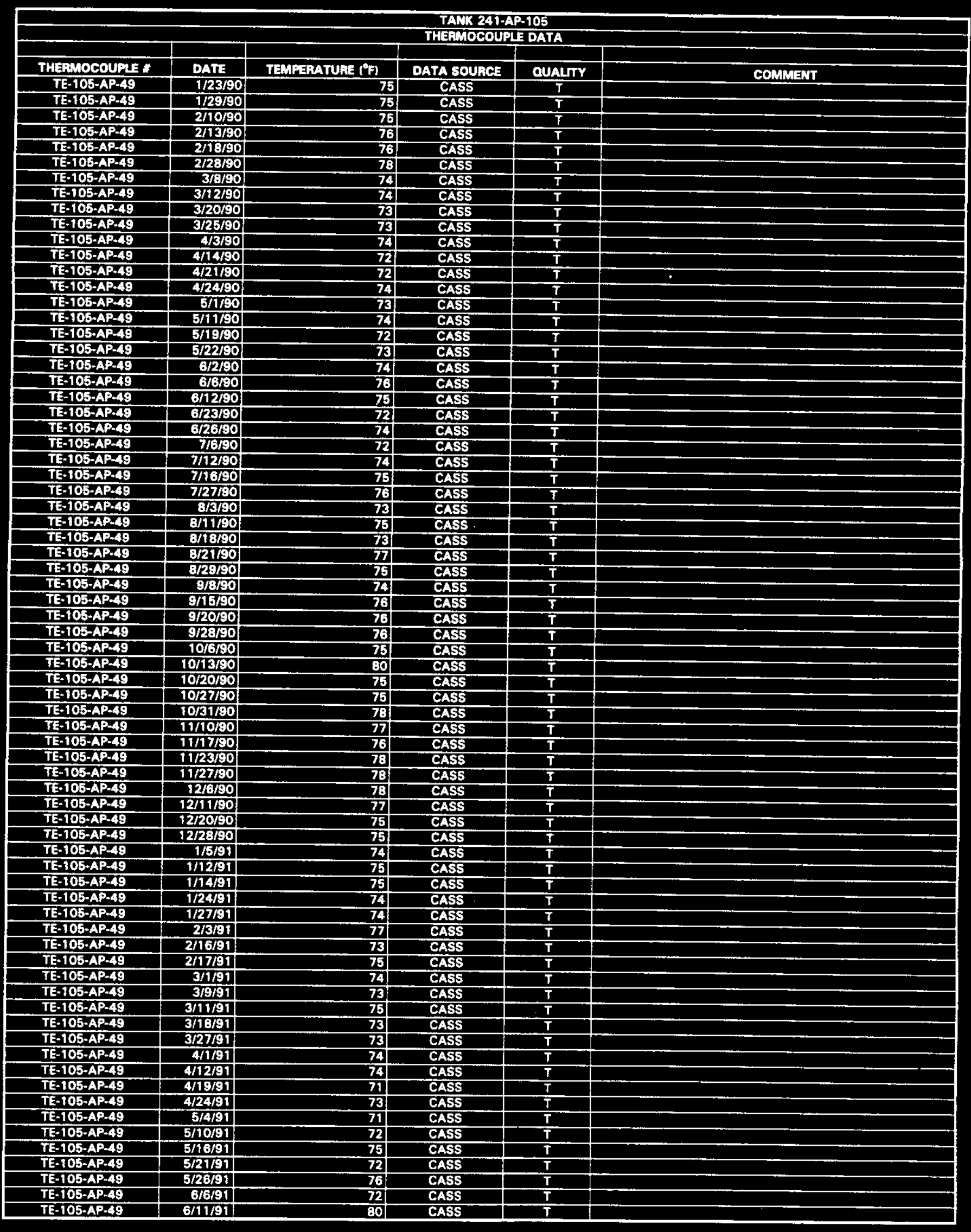

Data obtained from Computer Automated Surveillanes System (CASS) in April 1995, and Surviellence Analysis Computer Syatem (SACS) in June 1995. 


\begin{tabular}{|c|c|c|c|c|c|}
\hline \multicolumn{6}{|c|}{$\begin{array}{l}\text { TANK 241-AP-105 } \\
\text { THEAMOCOUV̈E DATA }\end{array}$} \\
\hline & & & & & \\
\hline THEFMOCOUPLE * & DATE & TEMPERATURE ( $\left.{ }^{\circ} \mathrm{F}\right)$ & DATA BOURCE & OUALTY & COMMENT \\
\hline TE-106-AP-49 & $6 / 19 / 91$ & 75 & CASS & $T$ & \\
\hline TE-105-AP-49 & $6 / 25 / 91$ & 74 & CASS & $T$ & \\
\hline TE-105-AP-49 & $7 / 3 / 91$ & 71 & CASS & $T$ & \\
\hline TE-TOS-AP-49 & $712 / 91$ & 72 & CASS & $T$ & \\
\hline TE-105-AP-49 & $7 / 15 / 91$ & 73 & CASS & $T$ & \\
\hline TE-105-AP-49 & $7 / 24 / 91$ & 72 & CASS & $T$ & \\
\hline TE-105-AP-49 & $8 / 3 / 91$ & 72 & CASS & $T$ & \\
\hline TE-105-AP-49 & 8/6/91 & 72 & CASS & $T$ & \\
\hline TE-105-AP-49 & 8/16/97 & 75 & CASS & $T$ & \\
\hline TE-105-AP-49 & $8 / 20 / 91$ & 75 & CASS & $T$ & \\
\hline TE-105-AP-49 & $8 / 30 / 91$ & 76 & CASS & $\bar{T}$ & \\
\hline TE-105-AP-49 & $9 / 3 / 91$ & 75 & CASS & $T$ & \\
\hline TE-10S-AP-49 & 8/8/91 & 75 & CASS & $\mathbf{T}$ & \\
\hline TE-105-AP-49 & 9/18/91 & 79 & CASS & $\bar{T}$ & \\
\hline TE-105-AP-49 & $9 / 27 / 91$ & 75 & CASS & $T$ & \\
\hline TE-106-AP-49 & $10 / 3 / 91$ & 76 & CASS & $T$ & \\
\hline TE-105-AP-49 & $10 / 12 / 91$ & 74 & CASS & $T$ & \\
\hline TE-105-AP-49 & $10 / 19 / 91$ & 77 & CASS & $T$ & \\
\hline TE-105-AP-49 & $10 / 25 / 91$ & 77 & CASS & $T$ & \\
\hline TE-105-AP- 49 & $10 / 29 / 91$ & 76 & CASS & $T$ & \\
\hline TE-105-AP-49 & $11 / 6 / 91$ & 76 & CASS & $T$ & \\
\hline TE-105-AP-49 & $8 / 11 / A_{2}$ & 73 & CASS & $\mathbf{T}$ & \\
\hline TE-105-AP-49 & $8 / 24 / 92$ & 73 & CASS & $T$ & \\
\hline TE-105-AP-49 & 9/19/22 & 77 & CASS & $T$ & \\
\hline TE-TOS-AP-49 & $9 / 26 / 92$ & 75 & CASS & $T$ & \\
\hline$T E-105-A P-49$ & $9 / 29 / 92$ & 78 & CASS & $T$ & \\
\hline TE-105-AP- 49 & $10 / 9 / 92$ & 78 & CASS & $T$ & \\
\hline TE-105.AP-49 & $10 / 13 / 92$ & 77 & CASS & $T$ & \\
\hline TE-105-AP-49 & $10 / 22 / 92$ & 77 & CASS & $\bar{T}$ & \\
\hline TE-105-AP-49 & $10 / 31 / 92$ & 77 & CASS & $T$ & \\
\hline TE-105-AP-49 & $11 / 6 / 82$ & 77 & CASS & $T$ & \\
\hline TE-105-AP-49 & $11 / 9 / 2$ & 78 & CASS & $T$ & \\
\hline TE-105-AP-49 & $11 / 1792$ & 79 & CASS & $T$ & \\
\hline TE-105-AP- 49 & $11 / 23 / 92$ & 76 & CASS & $T$ & \\
\hline TE-105-AP-49 & $12 / 3 / 92$ & 77 & CASS & $T$ & \\
\hline TE-105-AP- 49 & $12 / 12 / 22$ & 78 & CASS & $\mathbf{T}$ & \\
\hline TE-10S-AP-49 & $12 / 19 / 92$ & 77 & CASS & $T$ & \\
\hline TE-105-AP-49 & $12 / 26 / 92$ & 78 & CASS & $\bar{T}$ & \\
\hline TE-105-AP-49 & $12 / 29 / 92$ & 75 & CASS & $\bar{T}$ & \\
\hline TE-105-AP-49 & $1 / 15 / 94$ & 73 & CASS & $\mathbf{T}$ & \\
\hline TE-105-AP-49 & $1 / 18 / 94$ & 74 & CASS & $T$ & \\
\hline IE-105-AP-49 & $1 / 27 / 24$ & 75 & CASS & $T$ & \\
\hline$T E-105-A P-49$ & $2 / 4 / 94$ & 73 & CASS & $T$ & \\
\hline TE-105-AP-49 & $2 / 12 / 94$ & 75 & CASS & $T$ & \\
\hline TE-105-AP-49 & 216/94 & 74 & CASS & $\bar{T}$ & \\
\hline TE-105-AP-49 & $2 / 26194$ & 73 & CASS & $\bar{T}$ & \\
\hline TE-10S-AP-49 & $3 / 5 / 94$ & 75 & CASS & $T$ & \\
\hline TE-105-AP-49 & $3 / 7 / 94$ & 75 & CASS & $T$ & \\
\hline TE-105-AP-49 & $3 / 16 / 94$ & 75 & CASS & $T$ & \\
\hline TE-105-AP-49 & $3 / 25 / 94$ & 74 & CASS & $T$ & \\
\hline TE-105-AP-49 & $3 / 30 / 94$ & 74 & CASS & $\bar{T}$ & \\
\hline TE-105-AP-49 & $4 / 6 / 94$ & 75 & CASS & $T$ & \\
\hline TE-105-AP-49 & $4 / 13 / 94$ & 73 & CASS & $T$ & \\
\hline TE-105-AP-49 & $4 / 20 / 94$ & 73 & CASS & $T$ & \\
\hline TE-105-AP-49 & $4 / 29 / 94$ & 73 & CASS & $T$ & \\
\hline TE-105-AP-49 & $5 / 2 / 94$ & 73 & CASS & $T$ & \\
\hline TE-105+AP-49 & $5 / 14 / 94$ & 75 & CASS & $T$ & \\
\hline TE-10S-AP-49 & $5 / 16 / 94$ & 106 & CASS & $T$ & \\
\hline TE-105-AP-49 & $5 / 27 / 94$ & 99 & CASS & $T$ & \\
\hline TE-105-AP-49 & $6 / 2 / 94$ & 101 & CASS & $\mathbf{T}$ & \\
\hline TE-105-AP-49 & $6 / 8 / 94$ & 101 & CASS & $\bar{T}$ & \\
\hline TE-105-AP-49 & $6 / 14 / 94$ & 98 & CASS & $T$ & \\
\hline TE-105-AP-49 & $6 / 16 / 94$ & 72 & CASS & $\mathbf{S}$ & Data point did not agree with local trend \\
\hline TE-105-AP-49 & $6 / 17 / 94$ & 72 & CASS & $\mathbf{S}$ & Data point did not agree with local trend \\
\hline TE-105-AP-49 & $6 / 20 / 94$ & 73 & CASS & $\mathbf{S}$ & Data point did not egree with local trend \\
\hline TE-105-AP-49 & $6 / 25 / 94$ & 102 & CASS & $T$ & \\
\hline TE-105-AP-49 & $6 / 27 / 94$ & 103 & CASS & $T$ & \\
\hline TE-105-AP-49 & $7 / 3 / 94$ & 99 & CASS & $\mathbf{T}$ & \\
\hline TE-105-AP-49 & $7 / 13 / 94$ & 98 & CASS & $\mathbf{T}$ & \\
\hline$T E-105-A P-49$ & $7 / 20 / 94$ & 96 & CASS & $T$ & \\
\hline$T E-105-A P-49$ & $7 / 28 / 94$ & 95 & CASS & $\mathbf{T}$ & \\
\hline TE-105-AP-49 & $7 / 31 / 94$ & 99 & CASS & $\mathbf{T}$ & \\
\hline TE-105-AP-49 & $8 / 12 / 94$ & 98 & CASS & $T$ & \\
\hline
\end{tabular}




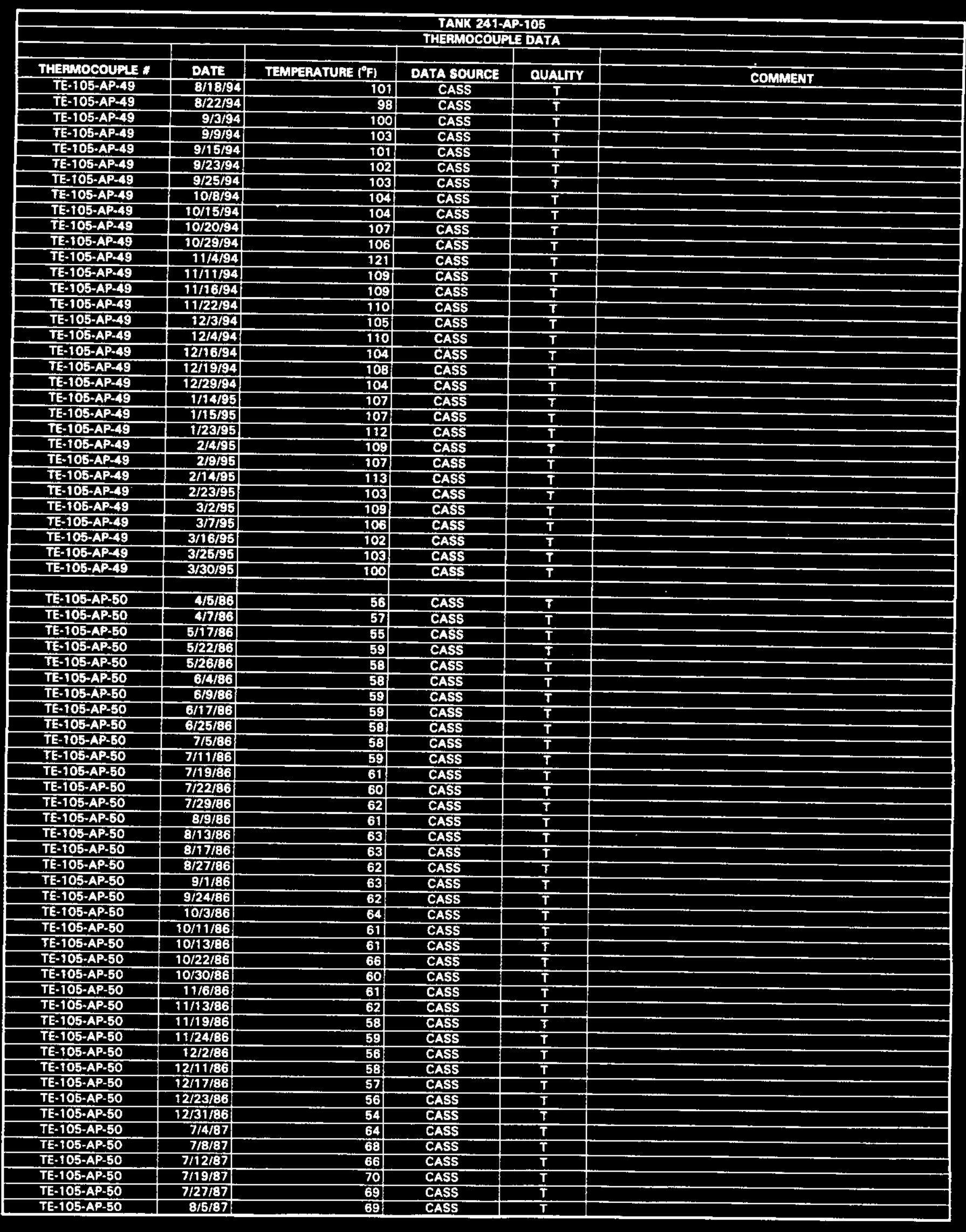




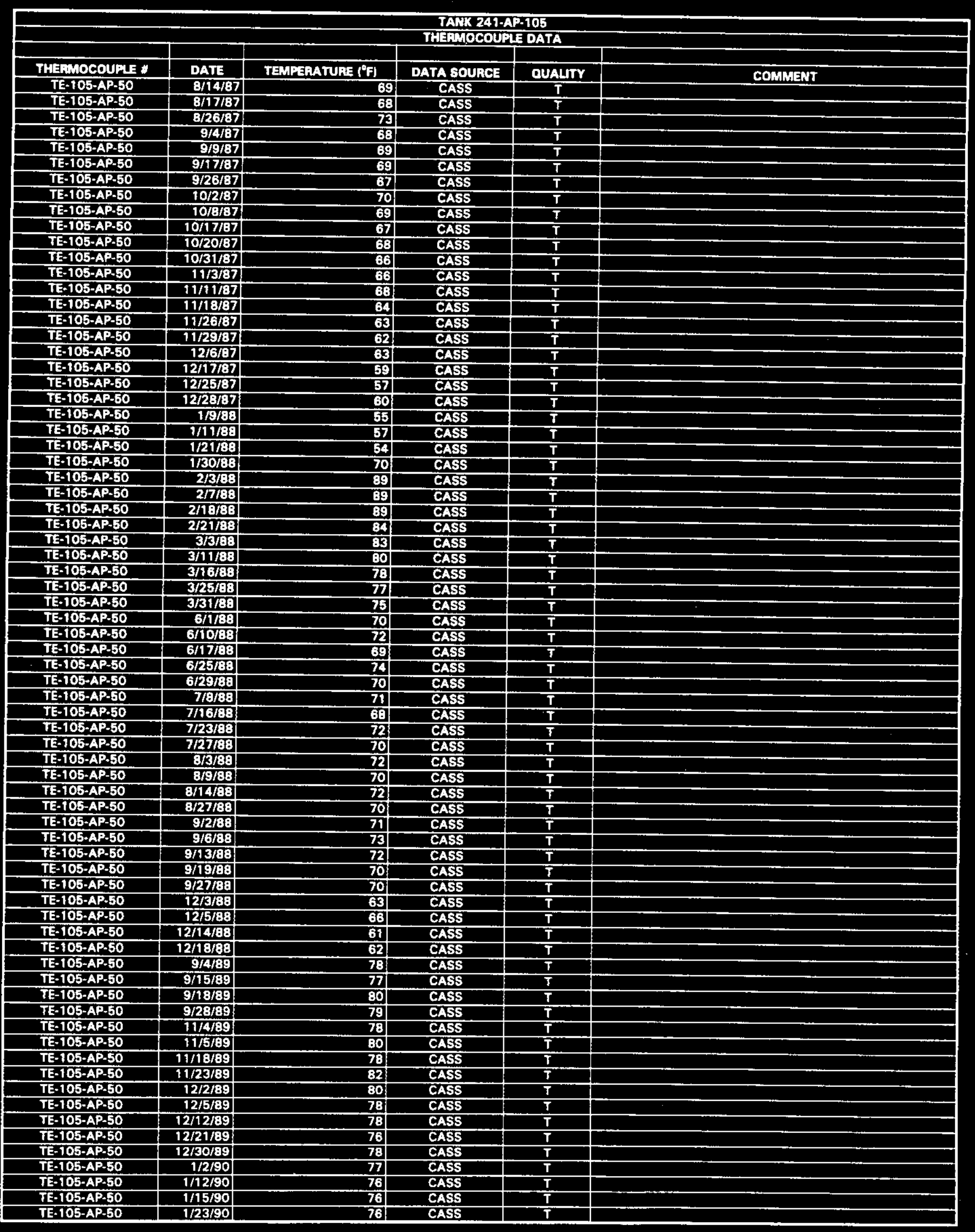

Data obtainad from Computer Automated Surveillance System (CASS) in April 1995, and Surviellance Analysis Computer System (SACS) in June 1995. 


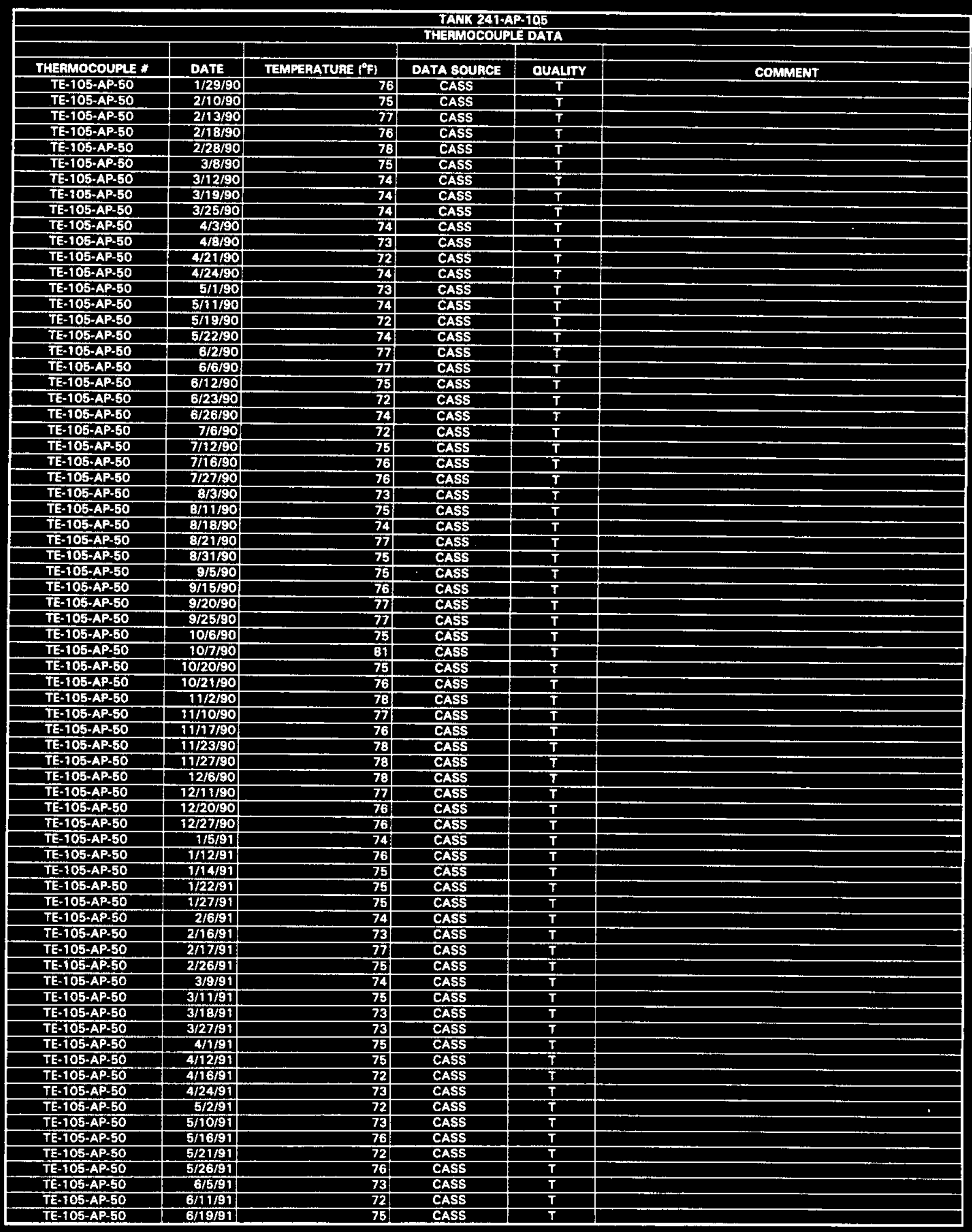




\begin{tabular}{|c|c|c|c|c|c|}
\hline \multicolumn{6}{|c|}{$\begin{array}{l}\text { TANK 241-AP-105 } \\
\text { THEFMOCOUFLE DATA }\end{array}$} \\
\hline THERMOCOUPIE * & DATE & TEMPERATUAE $\left({ }^{\circ} \mathrm{F}\right)$ & DATA SOURCE & OUALTY & \\
\hline TE-105.AP-50 & $6 / 25 / 91$ & 75 & CASS & $\frac{T}{T}$ & COMMENT \\
\hline IE-105-AP-S0 & $7 / 3 / 91$ & 72 & CASS & $T$ & \\
\hline TE-105-AP-50 & $7 / 13 / 91$ & 72 & CASS & $\mathbf{T}$ & \\
\hline TE-105-AP-50 & $7 / 15 / 91$ & 73 & CASS & $\bar{T}$ & \\
\hline TE-105-AP-50 & 7/24/91 & 72 & CASS & $T$ & \\
\hline TE-105-AP-50 & $8 / 3 / 91$ & 73 & CASS & $\mathbf{T}$ & \\
\hline TE-105-AP-SO & $8 / 6 / 91$ & 73 & CASS & $\mathbf{T}$ & \\
\hline TE-105-AP-50 & 8/16/97 & 76 & CASS & $T$ & \\
\hline TE-105.AP-50 & $8 / 20 / 91$ & 75 & CASS & $\mathbf{T}$ & \\
\hline TE-105-AP-50 & $8 / 30 / 91$ & 76 & CASS & $\mathbf{T}$ & \\
\hline TE-105-AP-50 & $9 / 2 / 91$ & 76 & CASS & $\mathbf{T}$ & \\
\hline TE-105-AP-50 & 9/8/91 & 75 & CASS & $T$ & \\
\hline TE-105-AP-50 & 9/18/91 & 79 & CASS & $T$ & \\
\hline TE-105-AP-50 & 8/27/91 & 76 & CASS & $T$ & \\
\hline TE-105-AP-50 & $10 / 3 / 91$ & 76 & CASS & $\mathbf{T}$ & \\
\hline TE-105-AP-50 & $10 / 12 / 91$ & 75 & CASS & $T$ & \\
\hline TE-105-AP-50 & $10 / 16 / 91$ & 78 & CASS & $\mathbf{T}$ & \\
\hline TE-105-AP-50 & $10 / 20 / 91$ & 78 & CASS & $\mathbf{T}$ & \\
\hline TE-105-AP-50 & $10 / 29 / 91$ & 76 & CASS & 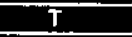 & \\
\hline TE-105-AP-50 & $11 / 6 / 91$ & 77 & CASS & $T$ & \\
\hline TE-105-AP-50 & $8 / 11 / 92$ & 73 & CASS & $T$ & \\
\hline IE-105-AP-50 & $8 / 24 / 92$ & 74 & CASS & $T$ & \\
\hline TE-105-AP-50 & 9/19/22 & 78 & CASS & $\mathbf{T}$ & \\
\hline TE-105-AP-50 & 9/26/92 & 75 & CASS & $T$ & \\
\hline TE-105-AP-50 & $10 / 3 / 92$ & 78 & CASS & $T$ & \\
\hline TE-105-AP-50 & $10 / 9 / 92$ & 78 & CASS & $T$ & \\
\hline TE-105-AP-50 & $10 / 13 / 92$ & 77 & CASS & $T$ & \\
\hline TE-105-AP-50 & $10 / 22 / 92$ & 79 & CASS & $\overline{\mathbf{T}}$ & \\
\hline TE-105-AP-50 & $10 / 27 / 92$ & 78 & CASS & $T$ & \\
\hline TE-105-AP-60 & $11 / 6 / 92$ & 77 & CAS5 & $T$ & \\
\hline TE-106-AP-50 & $11 / 9 / 92$ & 79 & CASS & $\bar{T}$ & \\
\hline TE-105-AP-50 & $11 / 17 / 92$ & 79 & CASS & $T$ & \\
\hline TE-105-AP-50 & 11/26/92 & 76 & CASS & $\bar{T}$ & \\
\hline TE-10S-AP-60 & $12 / 3 / 92$ & 78 & CASS & $T$ & \\
\hline TE-105-AP-GO & $12 / 12 / 92$ & 78 & CASS & $T$ & \\
\hline TE-105-AP-BO & 12/19/92 & 78 & CASS & $T$ & \\
\hline TE-105-AP.5O & $12 / 26 / 92$ & 78 & CASS & $T$ & \\
\hline TE-105-AP-50 & $12 / 29 / 92$ & 76 & CASS & $T$ & \\
\hline$T E-105-A P-50$ & $1 / 15 / 94$ & 73 & CASS & $T$ & \\
\hline TE-105-AP-50 & //18/94 & 75 & CASS & $T$ & \\
\hline TE-105-AP-50 & $1 / 27 / 94$ & 76 & CASS & $T$ & \\
\hline TE-105-AP-50 & $2 / 4 / 94$ & 74 & CASS & $T$ & \\
\hline TE-105-AP-50 & $2 / 12 / 94$ & 75 & CASS & $T$ & \\
\hline TE-105-AP-50 & $2 / 16 / 94$ & 74 & CASS & $\mathbf{T}$ & \\
\hline TE-105-AP:50 & $2 / 26 / 94$ & 74 & CASS & $\bar{T}$ & \\
\hline TE-105-AP-50 & $3 / 5 / 94$ & 75 & CASS & $T$ & \\
\hline TE-105-AP-50 & $3 / 8 / 94$ & 75 & CASS & $\mathbf{T}$ & \\
\hline TE-105-AP-50 & $3 / 16 / 94$ & 75 & CASS & $\mathbf{T}$ & \\
\hline TE-105-AP-50 & $3 / 26 / 94$ & 74 & CASS & $T$ & \\
\hline TE-105-AP-50 & $3 / 30 / 94$ & 75 & CASS & $\mathbf{T}$ & \\
\hline TE-105-AP-50 & $4 / 6 / 94$ & 77 & CASS & $\mathrm{T}$ & \\
\hline TE-105-AP-50 & $4 / 13 / 94$ & 74 & CASS & $T$ & \\
\hline TE-105-AP-50 & $4 / 20 / 94$ & 74 & CASS & $T$ & \\
\hline TE-105-AP-50 & 4/29/94 & 74 & CASS & $T$ & \\
\hline TE-105-AP-50 & $5 / 2 / 94$ & 74 & CASS & $T$ & \\
\hline TE-105-AP-50 & 5/34/94 & 75 & CASS & $T$ & \\
\hline TE-105-AP-50 & $5 / 16 / 94$ & 107 & CASS & $T$ & \\
\hline TE-105-AP-50 & $5 / 22 / 94$ & 100 & CASS & $T$ & \\
\hline TE-105-AP-50 & $6 / 2 / 94$ & 102 & CASS & $\mathbf{T}$ & \\
\hline TE-105-AP-50 & $6 / 8 / 94$ & 102 & CASS & $T$ & \\
\hline TE-105-AP-50 & $6 / 16 / 94$ & 73 & CASS & $\mathrm{s}$ & Data point did not apres with local trend \\
\hline TE-105-AP-50 & $6 / 17 / 94$ & 73 & CASS & $\mathbf{S}$ & Dats point did mot agree with local trend \\
\hline TE-105-AP-50 & $6 / 18 / 94$ & 98 & CASS & $\mathbf{T}$ & \\
\hline TE-105-AP-50 & $6 / 20 / 94$ & 74 & CASS & $\mathbf{S}$ & Date point did not agree with local trend \\
\hline TE-105-AP-50 & $6 / 25 / 94$ & 102 & CASS & $\mathbf{T}$ & \\
\hline TE-105-AP-50 & $6 / 27 / 94$ & 104 & CASS & $\mathbf{T}$ & \\
\hline TE-105-AP-50 & $7 / 3 / 94$ & 100 & CASS & $\mathbf{T}$ & \\
\hline TE-105-AP-50 & $7 / 13 / 94$ & 99 & CASS & $\mathbf{T}$ & \\
\hline TE-105-AP-50 & $7 / 20 / 94$ & 96 & CASS & $\mathbf{T}$ & \\
\hline TE-105-AP-50 & $7 / 30 / 94$ & 95 & CASS & $\mathbf{T}$ & \\
\hline TE-105-AP-50 & $7 / 31 / 94$ & 100 & CASS & $\mathbf{T}$ & \\
\hline TE-105-AP-50 & $8 / 10 / 94$ & 99 & CASS & $\mathbf{T}$ & \\
\hline TE-105.AP-5O & $8 / 18 / 94$ & 101 & CASS & $\mathbf{T}$ & \\
\hline
\end{tabular}




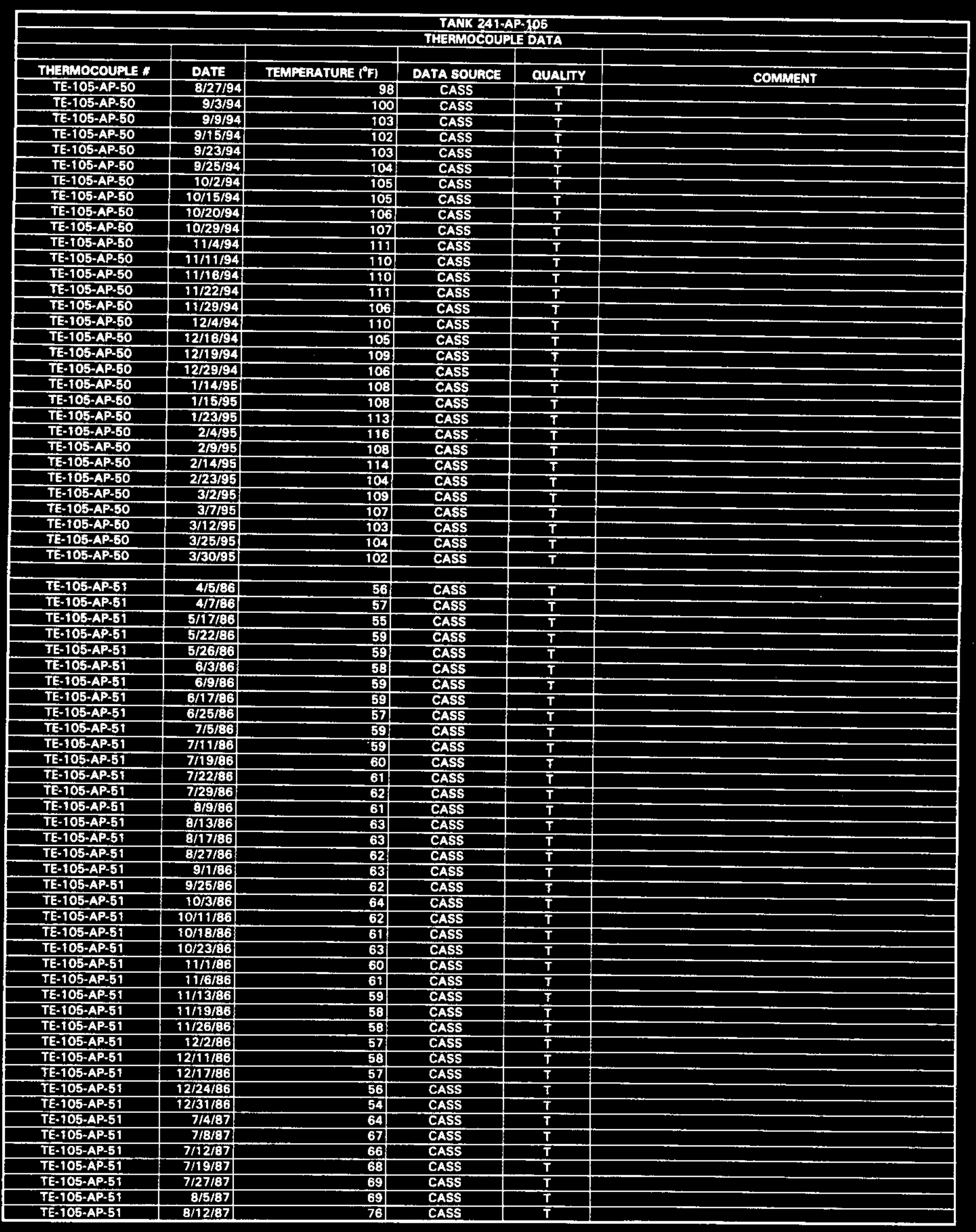




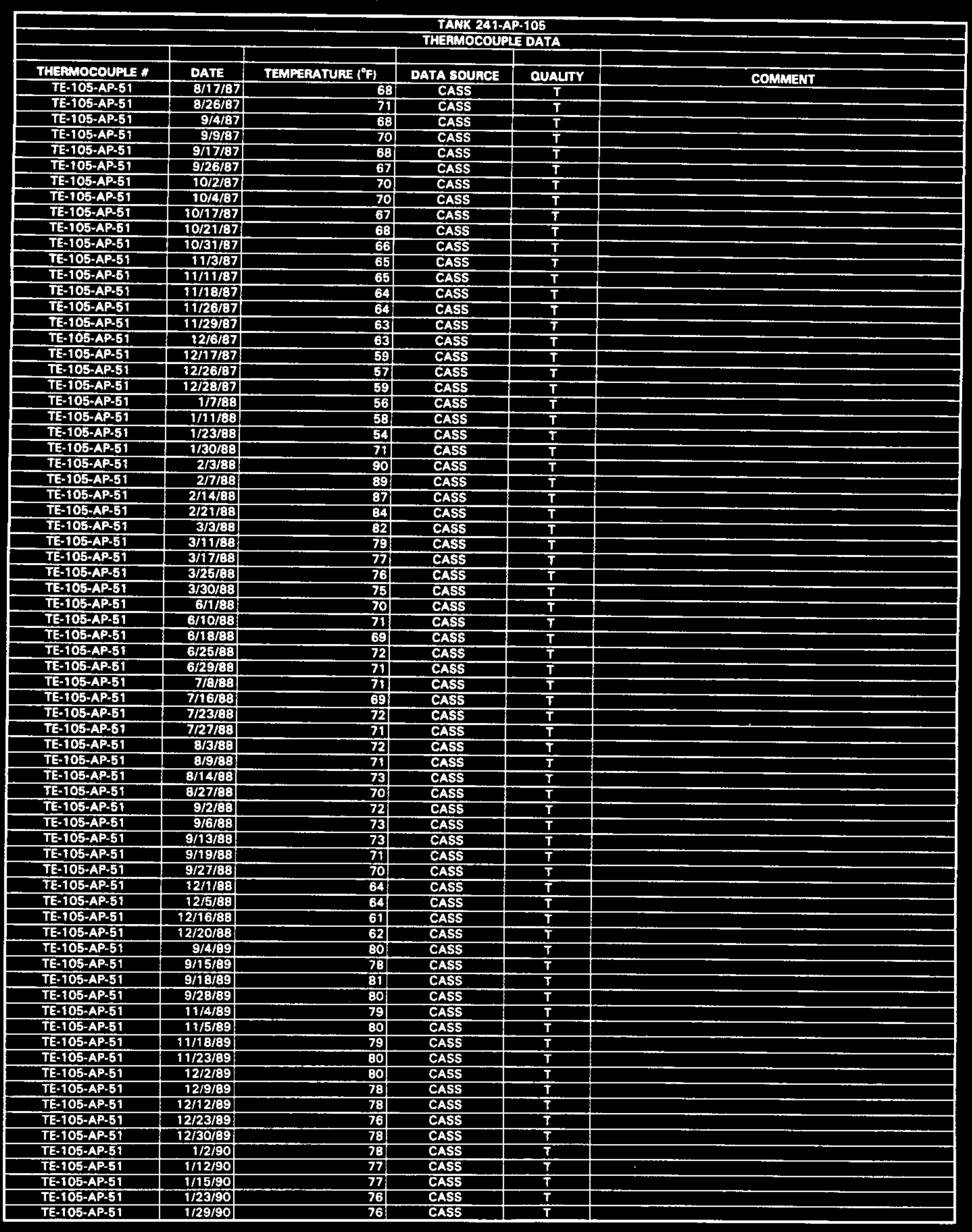

Data obtained from Computer Automated Surveillance System (CASS) in April 1995, and Surviellance Analysis Computer Syatem (SACS) in June 1995. 


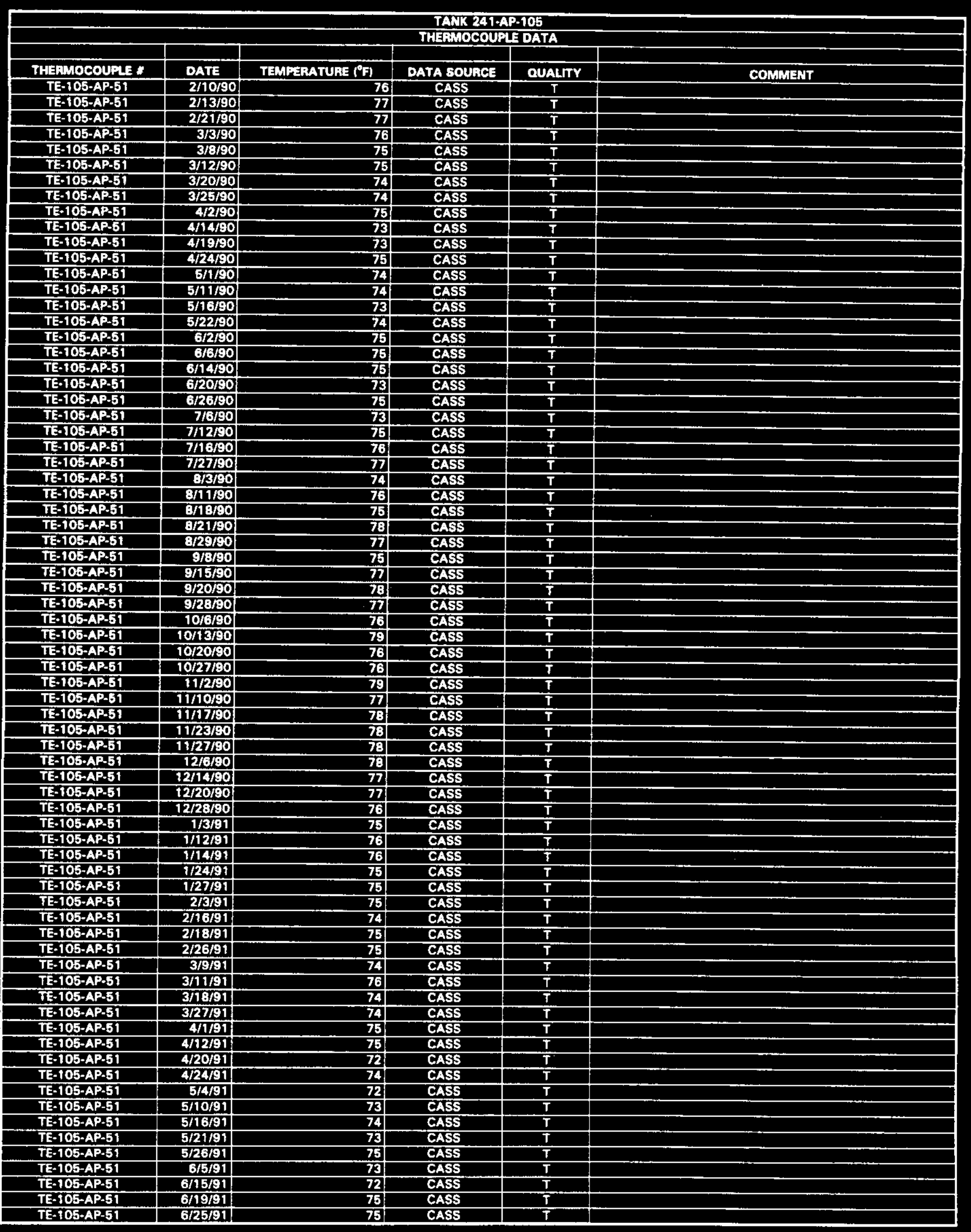




\begin{tabular}{|c|c|c|c|c|c|}
\hline \multirow{2}{*}{\multicolumn{6}{|c|}{$\begin{array}{l}\text { TAKK 241-AP-105 } \\
\text { THIAMOCOUPLE DATA }\end{array}$}} \\
\hline & & & & & \\
\hline THEAMOCOUPL * & DATE & TEMPERATUPE $\left(^{\circ} \mathrm{F}\right)$ & DATA SOUACE & OUALITY & COMMENT \\
\hline TE-105-AP-51 & $7 / 3 / 91$ & 72 & CASS & $T$ & \\
\hline TE-105-AP-51 & $7 / 3 / 91$ & 72 & CASS & $T$ & \\
\hline TE-105-AP-51 & $7 / 15 / 91$ & 74 & CASS & $\bar{T}$ & \\
\hline TE-105-AP-51 & $7 / 24 / 91$ & 72 & CASS & $T$ & \\
\hline TE-105-AP-51 & $8 / 3 / 91$ & 73 & CASS & $T$ & \\
\hline TE-105-AP-51 & $8 / 6 / 91$ & 73 & CASS & $\bar{T}$ & \\
\hline TE-106-AP-51 & $8 / 26 / 91$ & 76 & CASS & $\bar{T}$ & \\
\hline TE-105-AP-51 & $8 / 20 / 91$ & 75 & CASS & $\bar{T}$ & \\
\hline TE-105.AP-51 & $8 / 30 / 91$ & 77 & CASS & $T$ & \\
\hline TE-105-AP-51 & $9 / 3 / 91$ & 78 & CASS & $T$ & \\
\hline TE-105-AP-51 & $9 / 8 / 91$ & 76 & CASS & $T$ & \\
\hline TE-105-AP-51 & $9 / 18 / 91$ & 78 & CASS & $T$ & \\
\hline TE-105-AP-51 & $9 / 27 / 91$ & 76 & CASS & $T$ & \\
\hline TE-105-AP-51 & $10 / 3 / 91$ & 77 & CASS & $T$ & \\
\hline TE-105-AP-51 & $10 / 32 / 91$ & 76 & CASS & $T$ & \\
\hline TE-105-AP-51 & $10 / 19 / 91$ & 78 & CASS & $T$ & \\
\hline TE-105-AP-51 & $10 / 25 / 91$ & 79 & CASS & $T$ & \\
\hline TE-105.AP-51 & $10 / 29 / 91$ & 78 & CASS & $T$ & \\
\hline TE-105-AP-51 & $11 / \mathrm{B} / \mathrm{s}$ & 77 & CASS & $T$ & \\
\hline TE-105-AP-51 & $8 / 11 / 92$ & 74 & CASS & $T$ & \\
\hline TE-105-AP-51 & $8 / 24 / 92$ & 75 & CASS & $T$ & \\
\hline TE-105-AP-5T & $9 / 19 / 92$ & 78) & CASS & $T$ & \\
\hline TE-105-AP-51 & $9 / 26 / 92$ & 75 & CASS & $T$ & \\
\hline TE-105-AP-51 & $9 / 29 / 92$ & 79 & CASS & $T$ & \\
\hline TE-105-AP-51 & $10 / 9 / 92$ & 79 & CASS & $T$ & \\
\hline TE-105-APEST & 10/13/92 & 78 & CASS & $T$ & \\
\hline TE-105-AP-S1 & $10 / 22 / 92$ & 79 & CASS & $T$ & \\
\hline TE-105-AP-51 & $10 / 31 / 92$ & 78 & CASS & $T$ & \\
\hline TE-105-AP-51 & $11 / 6 / 92$ & 78 & CASS & $\mathbf{T}$ & \\
\hline IE-105-AP-51 & $11 / 9 / 92$ & 80 & CASS & $\mathbf{T}$ & \\
\hline TE-106-AP-51 & $11 / 17 / 92$ & 80 & CASS & $T$ & \\
\hline TE-106-AP-51 & $11 / 23 / 92$ & 77 & CASS & $T$ & \\
\hline TE-105-AP-51 & $12 / 3 / 92$ & 78 & CASS & $T$ & \\
\hline TE-105-AP-51 & $12 / 12 / 92$ & 79 & CASS & $\bar{T}$ & \\
\hline TE-105-AP-51 & $12 / 19 / 92$ & 78 & CASS & $T$ & \\
\hline TE-105-AP-51 & $12 / 26 / 92$ & 78 & CASS & $T$ & \\
\hline TE-105-AP-51 & $12 / 29 / 92$ & 76 & CASS & $\bar{T}$ & \\
\hline TE-105-AP-51 & $1 / 15 / 94$ & 73 & CASS & $\bar{T}$ & \\
\hline TE-105-AP-S1 & $1 / 18 / 94$ & 75 & CASS & $T$ & \\
\hline TE-105-AP-51 & $1 / 27 / 94$ & 76 & CASS & $T$ & \\
\hline TE-105-AP-51 & $2 / 4 / 94$ & 75 & CASS & $T$ & \\
\hline TE-105-AP-51 & $2 / 12 / 94$ & 76 & CASS & $T$ & \\
\hline TE-105-AP-51 & $2 / 16 / 94$ & 75 & CASS & $T$ & \\
\hline TE-T05-AP-51 & $2 / 26 / 94$ & 75 & CASS & $T$ & \\
\hline TE-105-AP-51 & $3 / 5 / 94$ & 75 & CASS & $T$ & \\
\hline TE-105-AP-51 & $3 / 10 / 94$ & 75 & CASS & $\mathbf{T}$ & \\
\hline TE-105-AP-51 & $3 / 16 / 94$ & 76 & CASS & $T$ & \\
\hline TE-105-AP-51 & $3 / 26 / 94$ & 75 & CASS & $T$ & \\
\hline TE-105-AP-51 & $3 / 30 / 94$ & 76 & CASS & $T$ & \\
\hline TE-105-AP-51 & $4 / 6 / 94$ & 76 & CASS & $T$ & \\
\hline TE-105-AP-51 & $4 / 13 / 94$ & 74 & CASS & $T$ & \\
\hline TE-I05-AP-51 & $4 / 20 / 94$ & 74 & CASS & $T$ & \\
\hline TE-105-APS1 & $4 / 27 / 94$ & 75 & CASS & $T$ & \\
\hline TE-105-AP-51 & $5 / 2 / 94$ & 75 & CASS & $T$ & \\
\hline TE-105-AP-51 & $5 / 14 / 94$ & 76 & CASS & $T$ & \\
\hline TE-105-AP-51 & $5 / 18 / 94$ & 107 & CASS & $T$ & \\
\hline TE-105-AP-51 & $5 / 27 / 94$ & 100 & CASS & $T$ & \\
\hline TE-105-AP-51 & $6 / 2 / 94$ & 102 & CASS & $T$ & \\
\hline TE-105-AP-51 & $6 / 8 / 94$ & 103 & CASS & $T$ & \\
\hline TE-105-AP-51 & $6 / 14 / 94$ & 99 & CASS & $T$ & \\
\hline TE-105-AP-51 & $6 / 16 / 94$ & 74 & CASS & 5 & Date point did not agree with local trend \\
\hline TE-105-AP-51 & $6 / 17 / 94$ & 73 & CASS & $\mathbf{s}$ & Data point did not agrea with local trend \\
\hline TE-105-AP-51 & $6 / 20 / 94$ & 75 & CASS & $\mathbf{s}$ & Data point did not agree with local trend \\
\hline TE-105-AP-51 & $6 / 25 / 94$ & 101 & CASS & $T$ & \\
\hline TE-105-AP-51 & $7 / 2 / 94$ & 103 & CASS & $\mathbf{T}$ & \\
\hline TE-105-AP-51 & $7 / 7 / 94$ & 100 & CASS & $T$ & \\
\hline TE-105-AP-51 & $7 / 13 / 94$ & 100 & CASS & $T$ & \\
\hline TE-105-AP-51 & $7 / 20 / 94$ & 97 & CASS & $T$ & \\
\hline TE-105-AP-51 & $7 / 24 / 94$ & 97 & CASS & $\mathbf{T}$ & \\
\hline TE-105-AP-51 & $7 / 31 / 94$ & 101. & CASS & $T$ & \\
\hline TE-105-AP-51 & $8 / 10 / 94$ & 101 & CASS & $T$ & \\
\hline TE-105-AP-51 & $8 / 20 / 94$ & 102 & CASS & $T$ & \\
\hline TE-105-AP-51 & $8 / 27 / 94$ & 99 & CASS & $T$ & \\
\hline
\end{tabular}




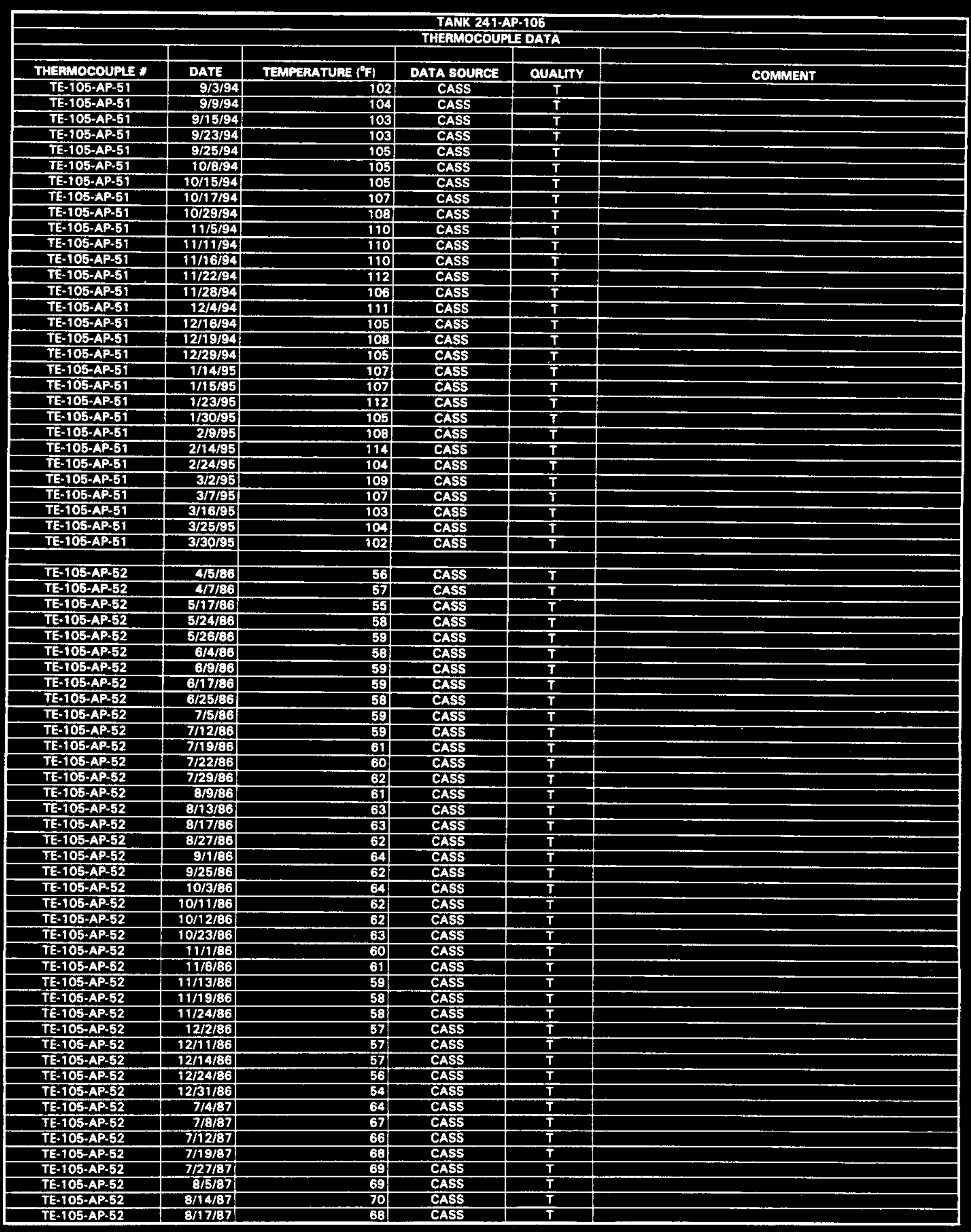




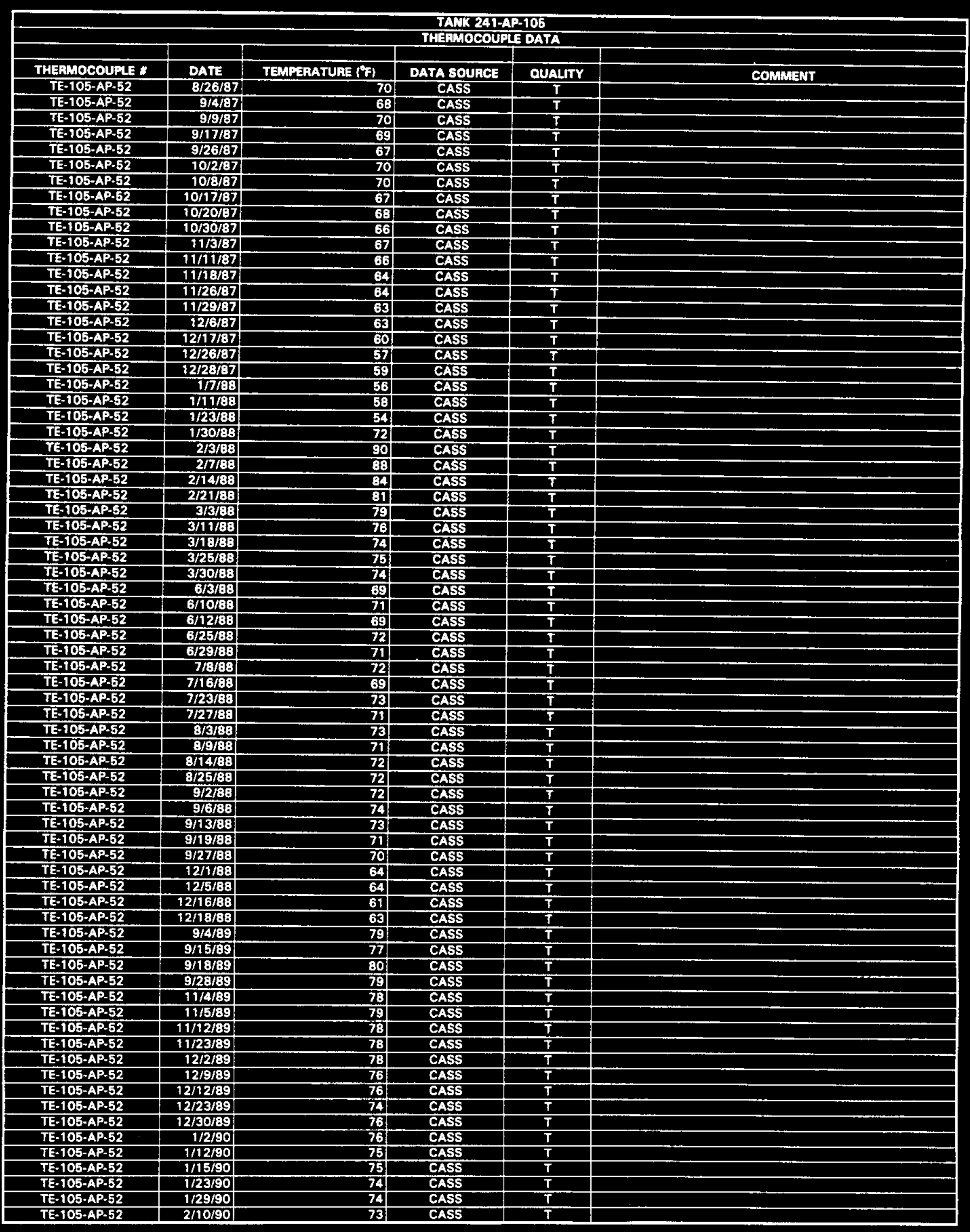




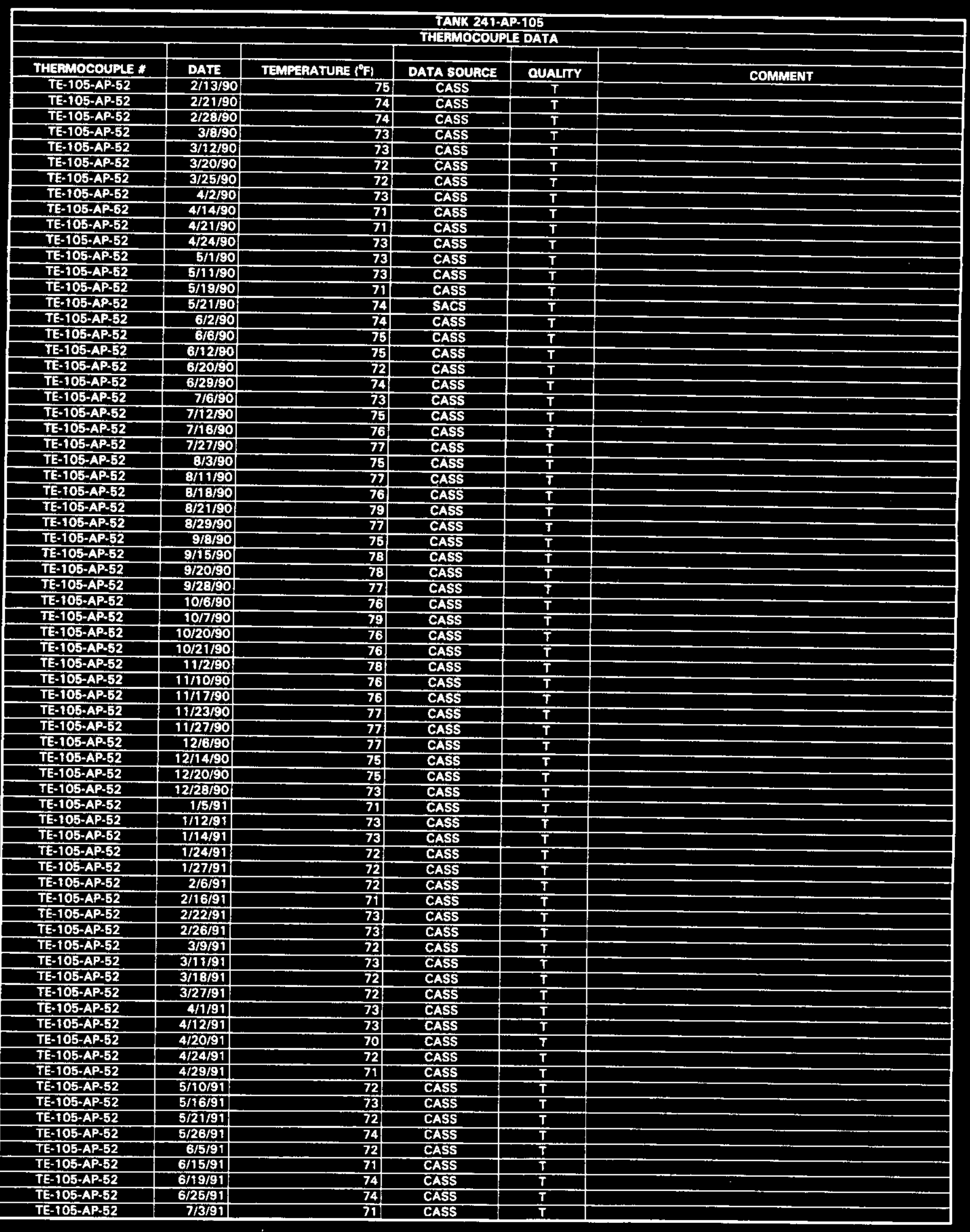

Data obtainad from Computer Automated Survaillance Syatem (CASS) in April 1995, and Surviellence Analyais Computer Syatem (SACS) in June 1995. 


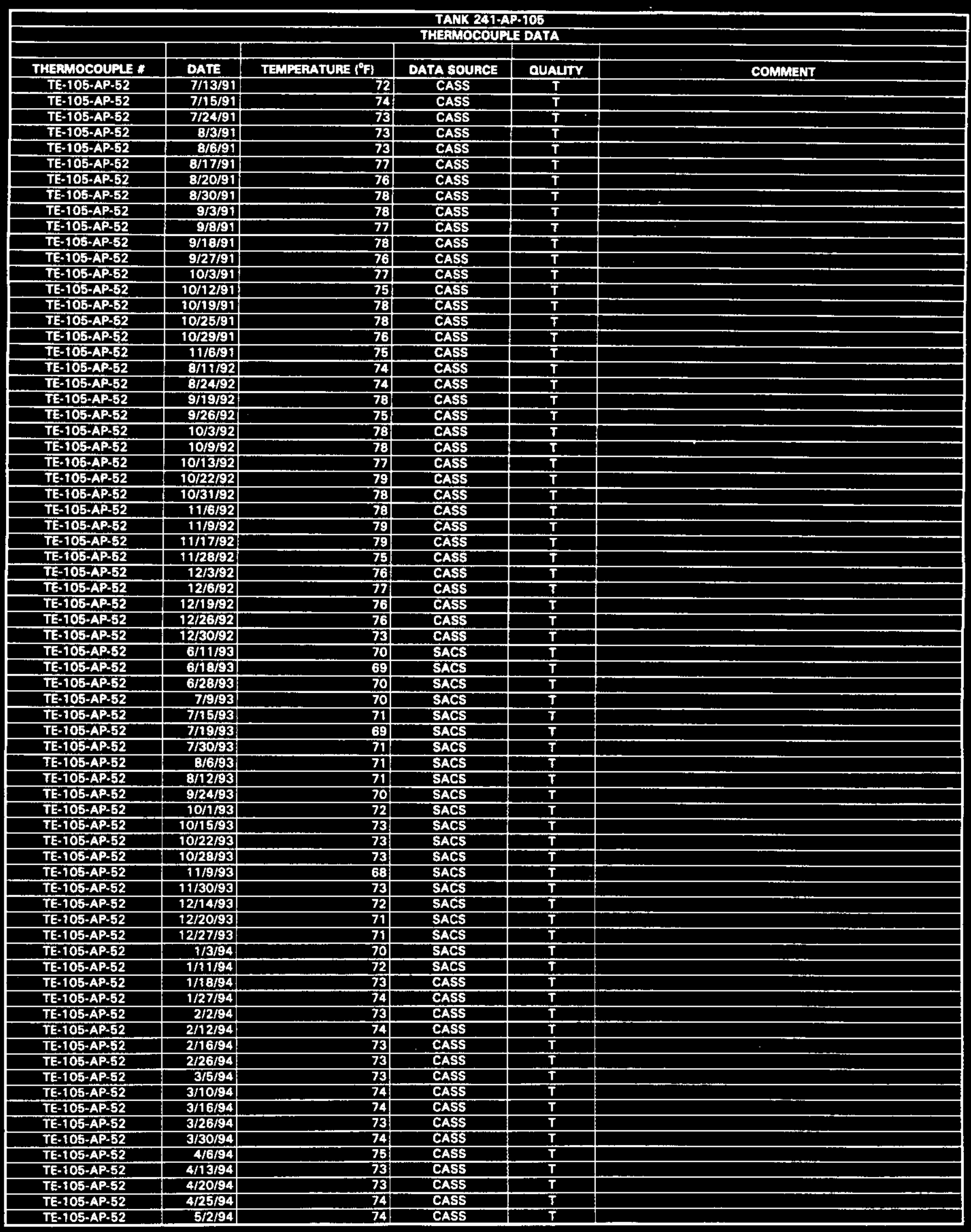

Date obtained from Computer Automated Surveillance System (CASS) in April 1995, and Surviellance Analysis Computer Syatem (SACS) in June 1995. 


\begin{tabular}{|c|c|c|c|c|c|}
\hline \multicolumn{6}{|c|}{$\begin{array}{l}\text { TANK 241-AP-105 } \\
\text { THEMMOCOUPL DATA }\end{array}$} \\
\hline & & & & & \\
\hline THERMOCOUPL * & DATE & TEMPERATUAE $\left({ }^{\circ} \mathrm{F}\right)$ & DATA SOURCE & QUALITY & COMMENT \\
\hline TE-105-AP-52 & $5 / 14 / 94$ & 75 & CASS & $T$ & \\
\hline TE-105+AP-52 & $5 / 16 / 94$ & 106 & CASS & $\mathbf{T}$ & \\
\hline TE-105-AP-52 & $5 / 22 / 94$ & 100 & CASS & $\bar{T}$ & \\
\hline TE-105-AP-52 & $6 / 2 / 94$ & 102 & CASS & $\bar{T}$ & \\
\hline TE-105-AP-52 & $6 / 8 / 94$ & 102 & CASS & $\mathbf{T}$ & \\
\hline TE-105-AP-52 & $6 / 16 / 94$ & 73 & CASS & $\mathbf{S}$ & Data point did not agree with tocal trend \\
\hline TE-105-AP-52 & $6 / 17 / 94$ & 73 & CASS & $\mathbf{S}$ & Date paint did not agres with local trend \\
\hline TE-105-AP-52 & $6 / 18 / 94$ & 98 & CASS & $T$ & \\
\hline TE-105-AP-52 & $6 / 20 / 94$ & 74 & CASS & $\mathbf{S}$ & Data point did not agree with tocal trend \\
\hline TE-105-AP-52 & $6 / 20 / 94$ & 71 & SACS & S $[\mathbf{G}$ in SACS) & Data point did not agree with local trend \\
\hline TE-105-AP-52 & $6 / 25 / 94$ & 101 & CASS & $T$ & \\
\hline TE-105-AP-52 & $7 / 2 / 94$ & 103 & CASS & $\bar{T}$ & \\
\hline TE-105-AP-52 & $7 / 3 / 94$ & 100 & CASS & $T$ & \\
\hline TE-105-AP-52 & $7 / 13 / 94$ & 100 & CASS & $T$ & \\
\hline TE-105-AP-52 & $7 / 20 / 94$ & 97 & CASS & $\mathbf{T}$ & \\
\hline TE-105-AP-52 & $7 / 20 / 94$ & 97 & CASS & $T$ & \\
\hline TE-105-AP-52 & $7 / 31 / 94$ & 102 & CASS & $T$ & \\
\hline TE-105-AP-52 & $8 / 10 / 94$ & 102 & CASS & $\mathbf{T}$ & \\
\hline TE-105-AP-52 & $8 / 20 / 94$ & 102 & CASS & $T$ & \\
\hline TE-105-AP-52 & $8 / 27 / 94$ & 100 & CASS & $\bar{T}$ & \\
\hline TE-105-AP-52 & $9 / 3 / 94$ & 102 & CASS & $T$ & \\
\hline TE-105-AP-52 & $9 / 9 / 94$ & 104 & CASS & $T$ & \\
\hline TE-105-AP-52 & $9 / 17 / 94$ & 103 & CASS & $\cdot T$ & \\
\hline TE-105-AP.52 & $9 / 23 / 94$ & 104 & CASS & $T$ & \\
\hline TE-105-AP-S2 & $9 / 25 / 94$ & 105 & CASS & $T$ & \\
\hline TE-105-AP-52 & $10 / 8 / 94$ & 105 & CASS & $T$ & \\
\hline TE-105-AP-52 & $10 / 9 / 94$ & 106 & CASS & $T$ & \\
\hline TE-105-AP-52 & $10 / 17 / 94$ & 106 & CASS & $T$ & \\
\hline TE-105-AP-52 & $10 / 29 / 94$ & 107 & CASS & $T$ & \\
\hline TE-105-AP-52 & $11 / 4 / 94$ & 109 & CASS & $\bar{T}$ & \\
\hline TE-10S-AP-52 & $11 / 11 / 94$ & 109 & CASS & $T$ & \\
\hline TE-105-AP-52 & $11 / 16 / 94$ & 109 & CASS & $T$ & \\
\hline TE-105-AP-52 & $11 / 22 / 94$ & 110 & CASS & $T$ & \\
\hline TE-105-AP-52 & $12 / 3 / 94$ & 104 & CASS & $T$ & \\
\hline TE-105-AP-52 & $12 / 4 / 94$ & 109 & CASS & $T$ & \\
\hline TE-105-AP-52 & $12 / 16 / 94$ & 103 & CASS & $T$ & \\
\hline TE-105-AP-52 & $12 / 19 / 94$ & 106 & CASS & $T$ & \\
\hline TE-105-AP-52 & $12 / 29 / 94$ & 104 & CASS & $T$ & \\
\hline TE-105-AP-52 & $1 / 14 / 95$ & 105 & CASS & $\mathbf{T}$ & \\
\hline TE.105-AP.52 & $7 / 15 / 95$ & 105 & CASS & $T$ & \\
\hline TE-105-AP-52 & $1 / 23 / 95$ & 110 & CASS & $T$ & \\
\hline TE-105-AP-52 & $1 / 30 / 95$ & 104 & SACS & $\mathbf{G}$ & \\
\hline TE-105-AP-S2 & $2 / 10 / 95$ & 105 & CASS & $T$ & \\
\hline TE-105-AP-52 & $2 / 14 / 95$ & 112 & CASS & $T$ & \\
\hline TE-105-AP-52 & $2 / 24 / 95$ & 102 & CASS & $T$ & \\
\hline TE-105-AP-52 & $3 / 2 / 95$ & 107 & CASS & $\mathbf{T}$ & \\
\hline TE-105-AP-52 & $3 / 7 / 95$ & 105 & CASS & 7 & \\
\hline TE-105-AP-52 & $3 / 12 / 95$ & 101 & CASS & $T$ & \\
\hline TE-105-AP-52 & $3 / 25 / 95$ & 102 & CASS & $T$ & \\
\hline TE-105-AP-52 & $3 / 30 / 95$ & 100 & CASS & $T$ & \\
\hline TE-105-AP-52 & $4 / 3 / 95$ & 89 & SACS & $G$ & \\
\hline TE-105.AP-52 & $4 / 10 / 95$ & 94 & SACS & $G$ & \\
\hline TE-105-AP-52 & $4 / 24 / 95$ & 84 & SACS & G & \\
\hline TE-105-AP-52 & $5 / 1 / 95$ & 91 & SACS & $G$ & \\
\hline TE-105-AP-52 & $5 / 8 / 95$ & 90 & SACS & $\mathbf{G}$ & \\
\hline TE-105-AP-52 & $5 / 16 / 95$ & 86 & SACS & G & \\
\hline TE-105-AP-52 & $5 / 22 / 25$ & 87 & SACS & $\mathbf{G}$ & \\
\hline TE-105-AP-52 & $6 / 5 / 95$ & 93 & SACS & G & \\
\hline TE-105-AP-52 & $6 / 13 / 95$ & 87 & SACS & 6 & \\
\hline TE-105-AP-52 & $6 / 19 / 95$ & 94 & SACS & $\mathbf{G}$ & \\
\hline & & & & & \\
\hline TE-105-AP-53 & $4 / 5 / 86$ & \begin{tabular}{l|l}
$\mathbf{5 5}$ \\
\end{tabular} & CASS & $\mathbf{T}$ & \\
\hline TE-105-AP-53 & $4 / 7 / 86$ & 58 & CASS & $T$ & \\
\hline TE-105-AP-53 & $5 / 17 / 86$ & 55 & CASS & $T$ & \\
\hline TE-105-AP-53 & $5 / 22 / 86$ & 59 & CASS & $T$ & \\
\hline TE-105-AP-53 & $5 / 26 / 86$ & 59 & CASS & $T$ & \\
\hline TE-105-AP-53 & $6 / 6 / 86$ & 58 & CASS & $\mathbf{T}$ & \\
\hline TE-105-AP-53 & $6 / 9 / 86$ & 59 & CASS & $T$ & \\
\hline TE-105-AP-53 & $6 / 17 / 86$ & 59 & CASS & $T$ & \\
\hline TE-105-AP-53 & $6 / 25 / 86$ & 58 & CASS & $\mathbf{T}$ & \\
\hline TE-105-AP-53 & $7 / 5 / 86$ & 59 & CASS & $T$ & \\
\hline TE-105-AP-53 & $7 / 11 / 86$ & 59 & CASS & $T$ & \\
\hline TE-105-AP.53 & $7 / 19 / 86$ & 61 & CASS & $T$ & \\
\hline
\end{tabular}




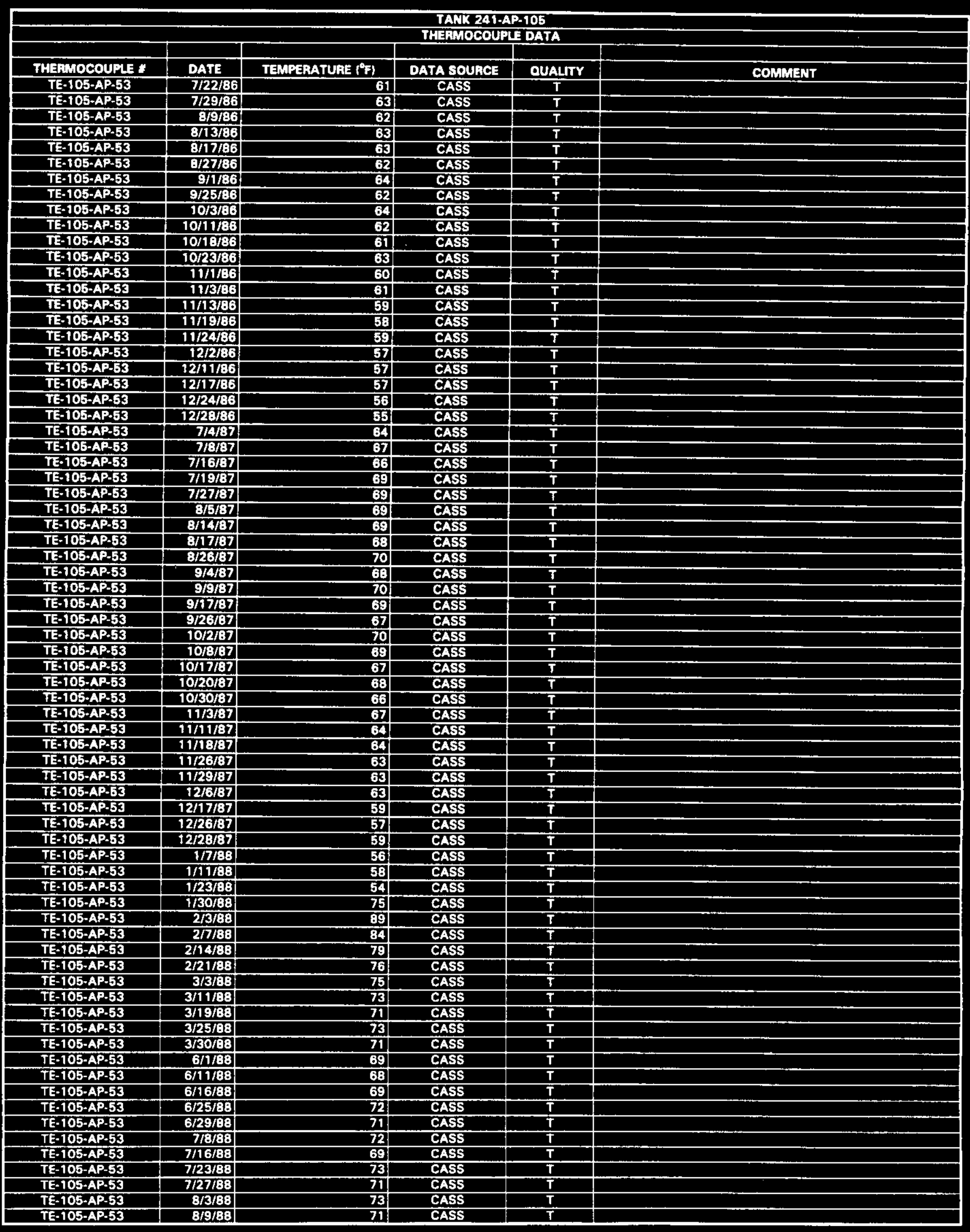




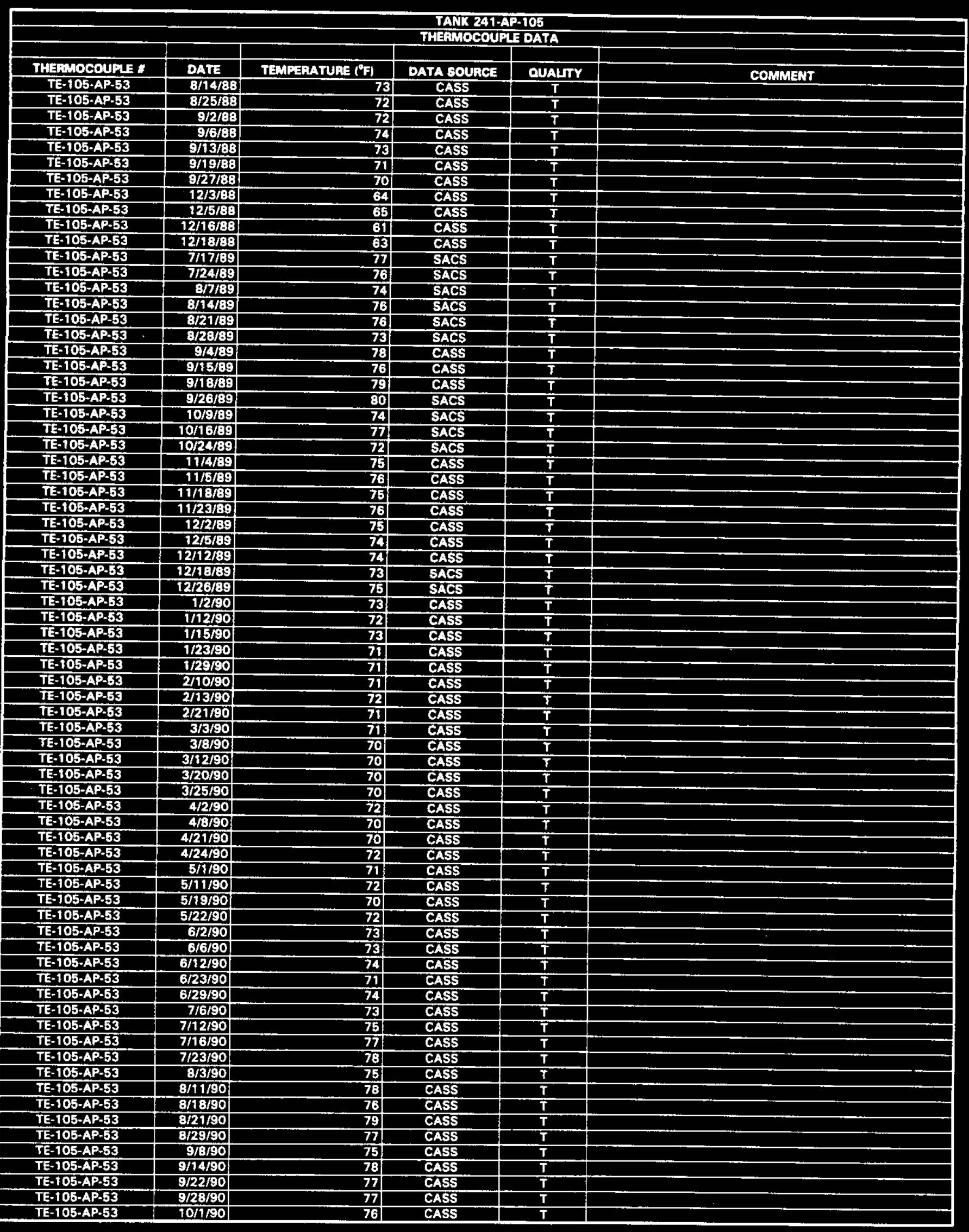

Data obteinad from Computer Automated Surveillance System (CASS) in April 1995, and Surviellance Analyais Computer System (SACS) in June 1995. 


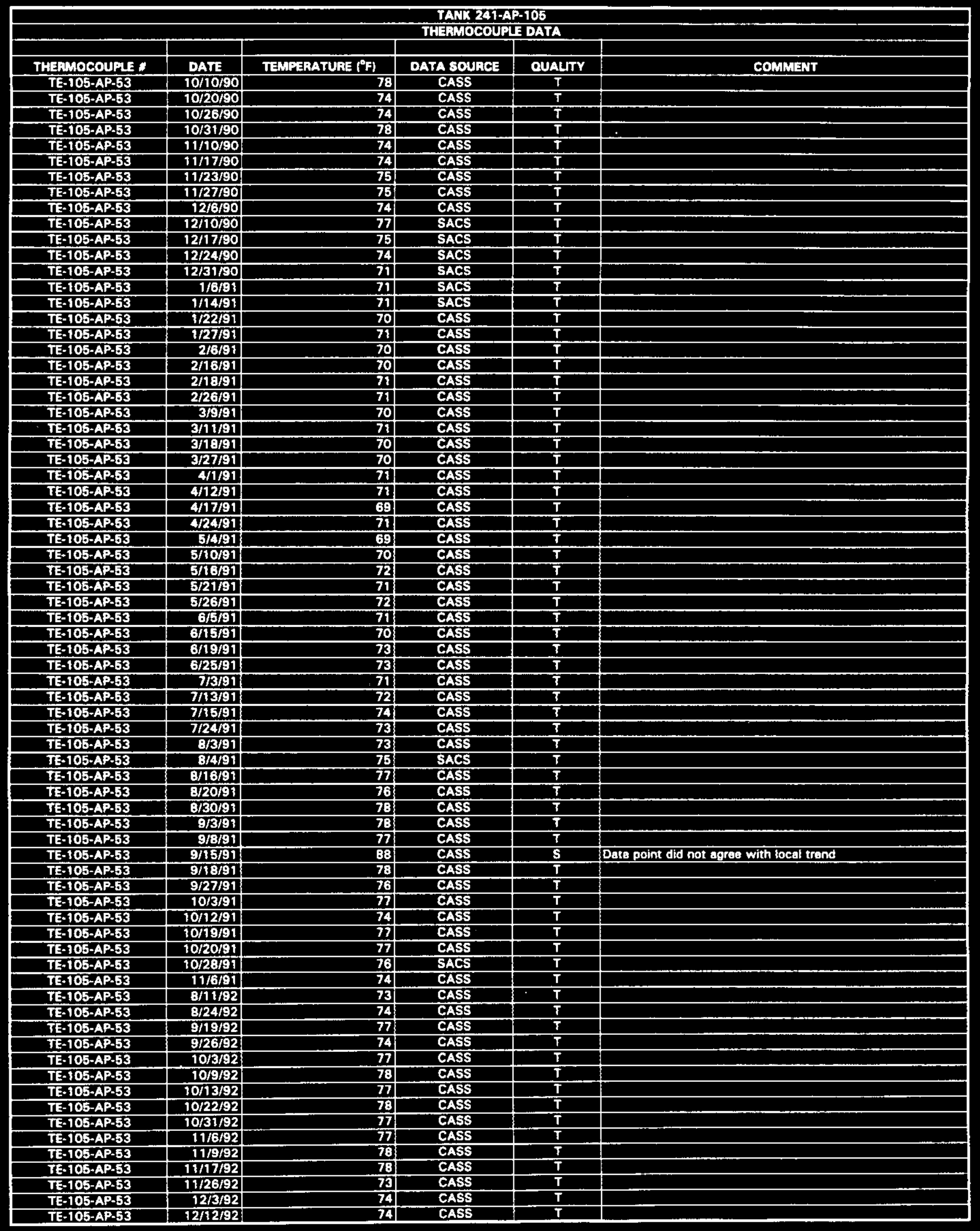

Date obtainad from Computer Automated Surveillance System (CASS) in April 1995, and Surviellance Analysis Computer System (SACS) in June 1995.

$$
\text { D-486 }
$$




\begin{tabular}{|c|c|c|c|c|c|}
\hline \multicolumn{6}{|c|}{$\begin{array}{l}\text { TANK 241-AP-105 } \\
\text { THEFMOCOUPLE DATA }\end{array}$} \\
\hline & DATE & & & & \\
\hline $\begin{array}{c}\text { THERMOCOUPLE } * \\
\text { TE-105.AP-53 }\end{array}$ & $12 / 19 / 92$ & TEMPERATURE $\left({ }^{\circ} \mathrm{F}\right)$ & DATA SOUACE & QUALTY & COMMENT \\
\hline$\frac{T E-105-A P-53}{T E-105-A P-53}$ & $12 / 26 / 92$ & $\begin{array}{ll}73 \\
73\end{array}$ & CASS & $\bar{T}$ & \\
\hline $\begin{array}{l}\text { TE-105-AP-53 } \\
\text { TE-105-AP-53 }\end{array}$ & $12 / 29 / 92$ & $\frac{73}{70}$ & CASS & $T$ & \\
\hline$\frac{\text { TE-105-AP-53 }}{\text { TE-105-AP-53 }}$ & $1 / 15 / 94$ & 70 & CASS & $T$ & \\
\hline TE-105-AP-53 & 1/18/94 & 71 & $\begin{array}{l}\text { CASS } \\
\text { CASS }\end{array}$ & $\frac{T}{T}$ & \\
\hline TE-105-AP-53 & $1 / 27 / 94$ & 71 & CASS & $T$ & \\
\hline TE-105-AP-53 & $2 / 4 / 94$ & 70 & CASS & $T$ & \\
\hline TE-105-AP-53 & $2 / 12 / 94$ & 72 & CASS & $T$ & \\
\hline TE-105-AP-53 & $2 / 16 / 94$ & 71 & CASS & $T$ & \\
\hline TE-105-AP-B3 & $2 / 26 / 94$ & 72 & CASS & $T$ & \\
\hline TE-105-AP-53 & $3 / 5 / 34$ & 72 & CASS & $T$ & \\
\hline TE-105-AP-53 & $3 / 7 / 34$ & 73 & CASS & $T$ & \\
\hline TE-105-AP-53 & 3/16/94 & 72 & CASS & $\bar{I}$ & \\
\hline TE-105-AP-53 & $3 / 26 / 94$ & 71 & CASS & $\bar{T}$ & \\
\hline TE-105-AP-53 & 3/30/94 & 73 & CASS & $T$ & \\
\hline TE-105-AP-53 & $4 / 6 / 94$ & 74 & CASS & $T$ & \\
\hline TE-105.AP-53 & $4 / 13 / 94$ & 71 & CASS & $T$ & \\
\hline TE-105.AP-53 & $4 / 20 / 94$ & 72 & CASS & $T$ & \\
\hline TE-105-AP-53 & $4 / 29 / 94$ & 72 & CASS & $\mathbf{T}$ & \\
\hline TE-105-AP-53 & $5 / 2 / 94$ & 73 & CASS & $\bar{T}$ & \\
\hline TE-105-AP-53 & $5 / 14 / 94$ & 74 & CASS & $T$ & \\
\hline TE-105-AP-63 & $5 / 16 / 94$ & 105 & CASS & $\bar{T}$ & \\
\hline TE-105-AP-53 & $5 / 27 / 94$ & 99 & CASS & $T$ & \\
\hline TE-105-AP-53 & $6 / 2 / 94$ & 101 & CASS & $\mathbf{T}$ & \\
\hline TE-105-AP-53 & $6 / 9 / 94$ & 101 & CASS & $\mathbf{T}$ & \\
\hline TE-105.AP.53 & $6 / 14 / 94$ & 98 & CASS & $T$ & \\
\hline TE-105.AP-53 & $6 / 16 / 94$ & 72 & CASS & $\mathbf{S}$ & Data point did not agree with local trend \\
\hline TE-105-AP-53 & $6 / 17 / 94$ & 72 & CASS & $\mathbf{s}$ & Data point did not agres with local trend \\
\hline TE-105.AP.53 & $6 / 20 / 94$ & 74 & CASS & $\mathbf{S}$ & Data point did not agree with local trand \\
\hline TE-105-AP.53 & $6 / 25 / 94$ & 100 & CASS & $T$ & \\
\hline TE-105-AP-53 & $7 / 2 / 94$ & 102 & CASS & $T$ & \\
\hline TE-105-AP-53 & $7 / 3 / 94$ & 100 & CASS & $\mathbf{T}$ & \\
\hline TE-105-AP.53 & $7 / 13 / 94$ & 100 & CASS & $T$ & \\
\hline TE-105-AP-53 & $7 / 19 / 94$ & 83 & CASS & $T$ & \\
\hline TE-105-AP-53 & $7 / 24 / 94$ & 98 & CASS & $T$ & \\
\hline TE-105-AP-53 & $7 / 31 / 94$ & 102 & CASS & $T$ & \\
\hline TE-105-AP-53 & $8 / 9 / 94$ & 103 & CASS & $\mathbf{T}$ & \\
\hline TE-105-AP-53 & $8 / 17 / 94$ & 103 & CASS & $\mathbf{T}$ & \\
\hline TE-105-AP-53 & $8 / 27 / 94$ & 100 & CASS & $T$ & \\
\hline TE-105-AP-53 & $9 / 3 / 94$ & 102 & CASS & $T$ & \\
\hline TE-105-AP-53 & $9 / 9 / 94$ & 104 & CASS & $\mathbf{T}$ & \\
\hline TE-105-AP-53 & 9/17/94 & 102 & CASS & $\mathbf{T}$ & \\
\hline TE-105-AP-53 & $9 / 23 / 94$ & 103 & CASS & $T$ & \\
\hline TE-105-AP.53 & $9 / 25 / 94$ & 105 & CASS & $\mathbf{T}$ & \\
\hline TE-105-AP-53 & $10 / 2 / 94$ & 105 & CASS & $\mathbf{T}$ & \\
\hline TE-105-AP-53 & $10 / 9 / 94$ & 105 & CASS & $\mathbf{T}$ & \\
\hline TE-105-AP-53 & $10 / 17 / 94$ & 105 & CASS & $\mathbf{T}$ & \\
\hline TE-105-AP-53 & $10 / 29 / 94$ & 105 & CASS & $T$ & \\
\hline TE-105-AP-53 & $11 / 4 / 84$ & 107 & CASS & $T$ & \\
\hline TE-105-AP-53 & $11 / 11 / 94$ & 107 & CASS & $\mathbf{T}$ & \\
\hline TE-105-AP-53 & $11 / 16 / 94$ & 107 & CASS & $\mathbf{T}$ & \\
\hline TE-105-AP-53 & $11 / 22 / 94$ & 108 & CASS & $\mathbf{T}$ & \\
\hline TE-105-AP-53 & $12 / 3 / 94$ & 102 & CASS & $T$ & \\
\hline TE-105-AP-53 & $12 / 4 / 94$ & 106 & CASS & $T$ & \\
\hline TE-105-AP-53 & $12 / 16 / 94$ & 100 & CASS & $T$ & \\
\hline TE-105-AP.53 & $12 / 19 / 94$ & 104 & CASS & $\mathbf{T}$ & \\
\hline TE-105-AP-53 & $12 / 29 / 94$ & 101 & CASS & $T$ & \\
\hline$T E-105-A P-53$ & 1/14/95 & 102 & CASS & $T$ & \\
\hline TE-105-AP-53 & $1 / 15 / 95$ & 102 & CASS & $\bar{T}$ & \\
\hline TE-105-AP-53 & $1 / 23 / 95$ & 107 & CASS & $\bar{T}$ & \\
\hline TE-105-AP-53 & 2/2/95 & 100 & CASS & $\mathbf{T}$ & \\
\hline TE-105-AP-53 & $2 / 10 / 95$ & 103 & CASS & $T$ & \\
\hline TE-105-AP-53 & $2 / 14 / 95$ & 110 & CASS & $T$ & \\
\hline TE-105-AP-53 & $2 / 23 / 95$ & 100 & CASS & $\mathbf{T}$ & \\
\hline TE-105-AP-53 & 3/2/95 & 105 & CASS & $\mathbf{T}$ & \\
\hline TE-105-AP-53 & 3/7/95 & 103 & CASS & $T$ & \\
\hline TE-105-AP-53 & $3 / 12 / 95$ & 89 & CASS & $\mathbf{T}$ & \\
\hline TE-105-AP-53 & $3 / 25 / 95$ & 99 & CASS & $T$ & \\
\hline TE-105-AP-53 & $3 / 30 / 95$ & 98 & CASS & $\mathbf{T}$ & \\
\hline
\end{tabular}




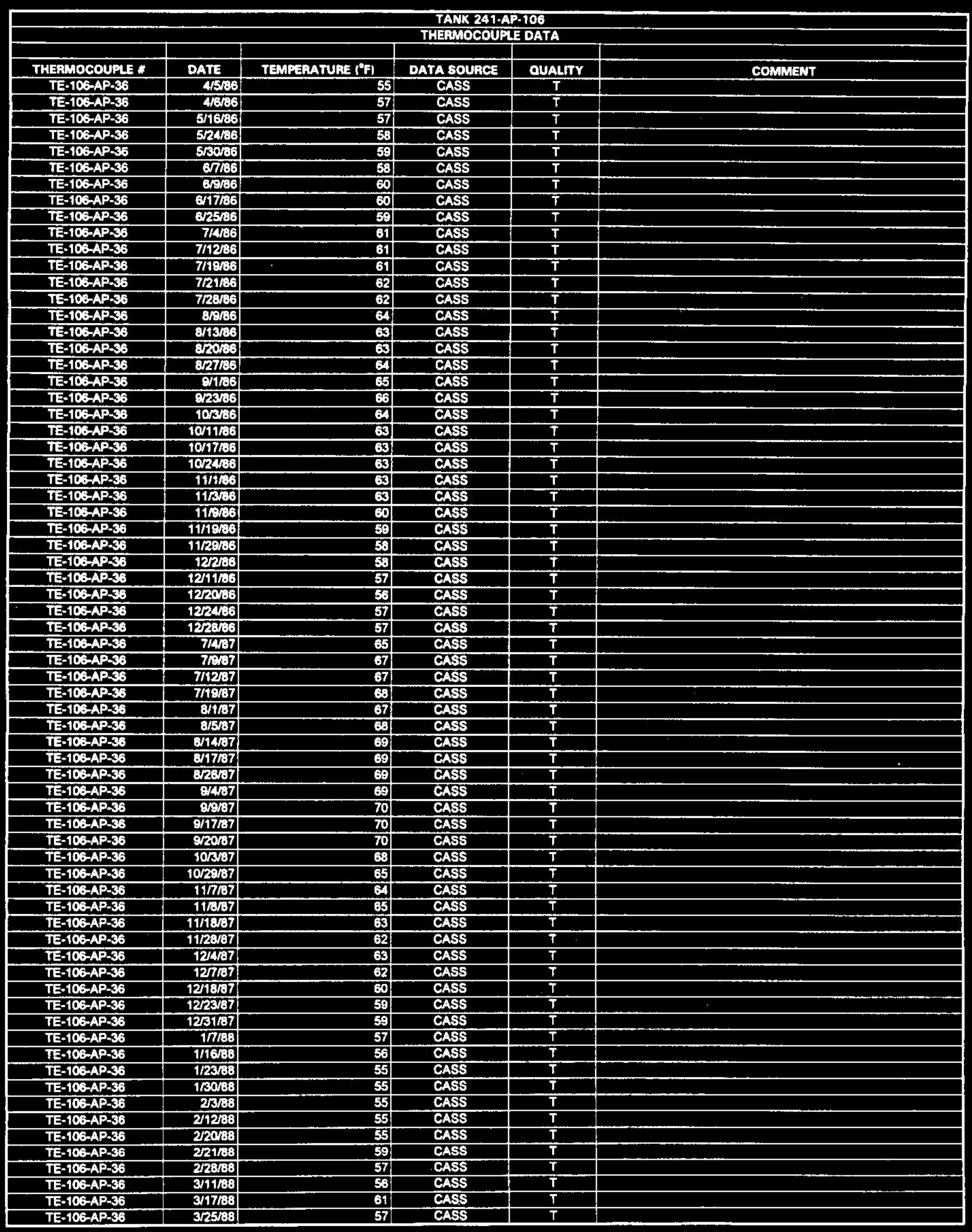

Data obteined from Computer Automated Surveillance Syatem (CASS), April 1995; and Surveillance Analysis Computer System (SACS), June 1995. 


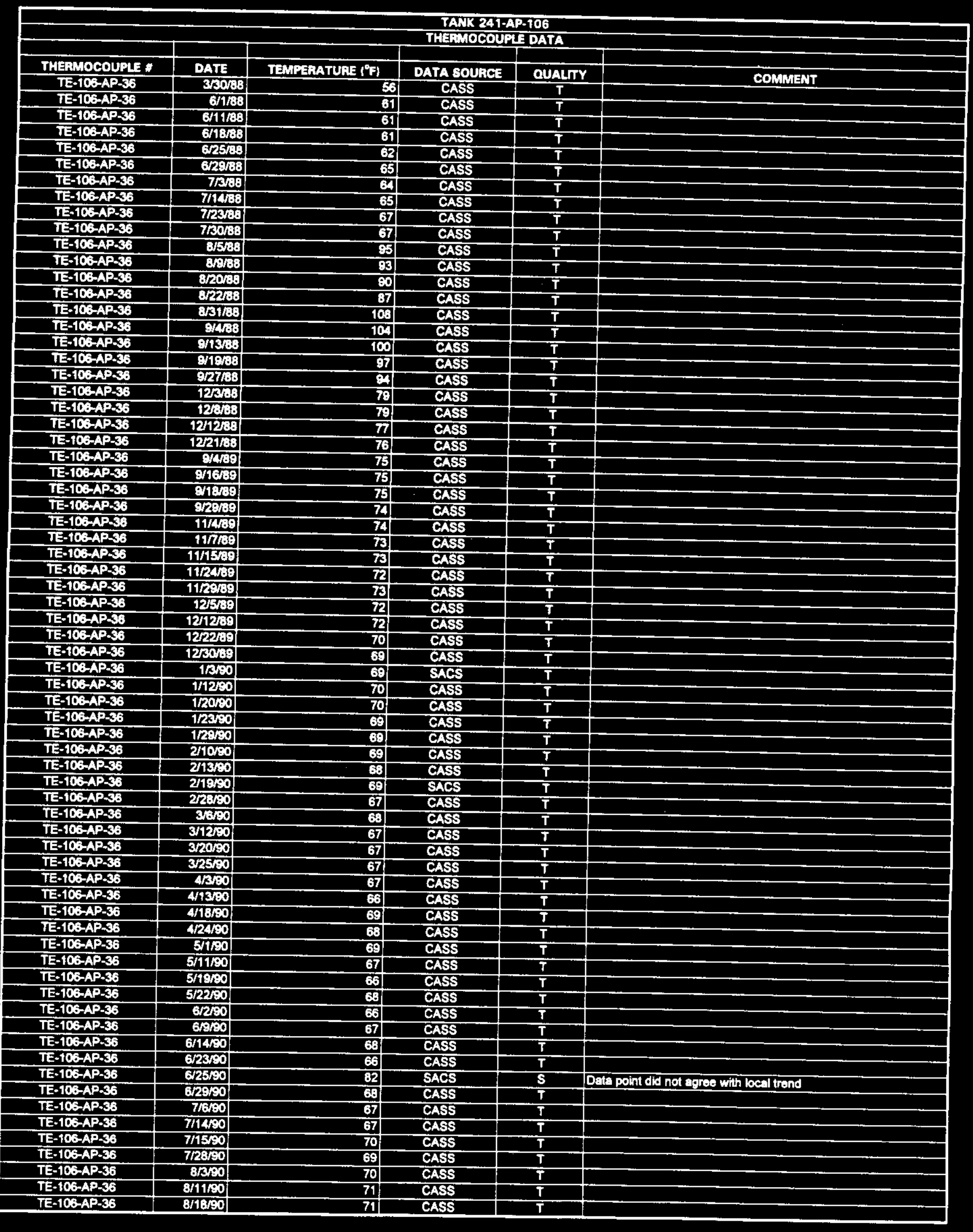




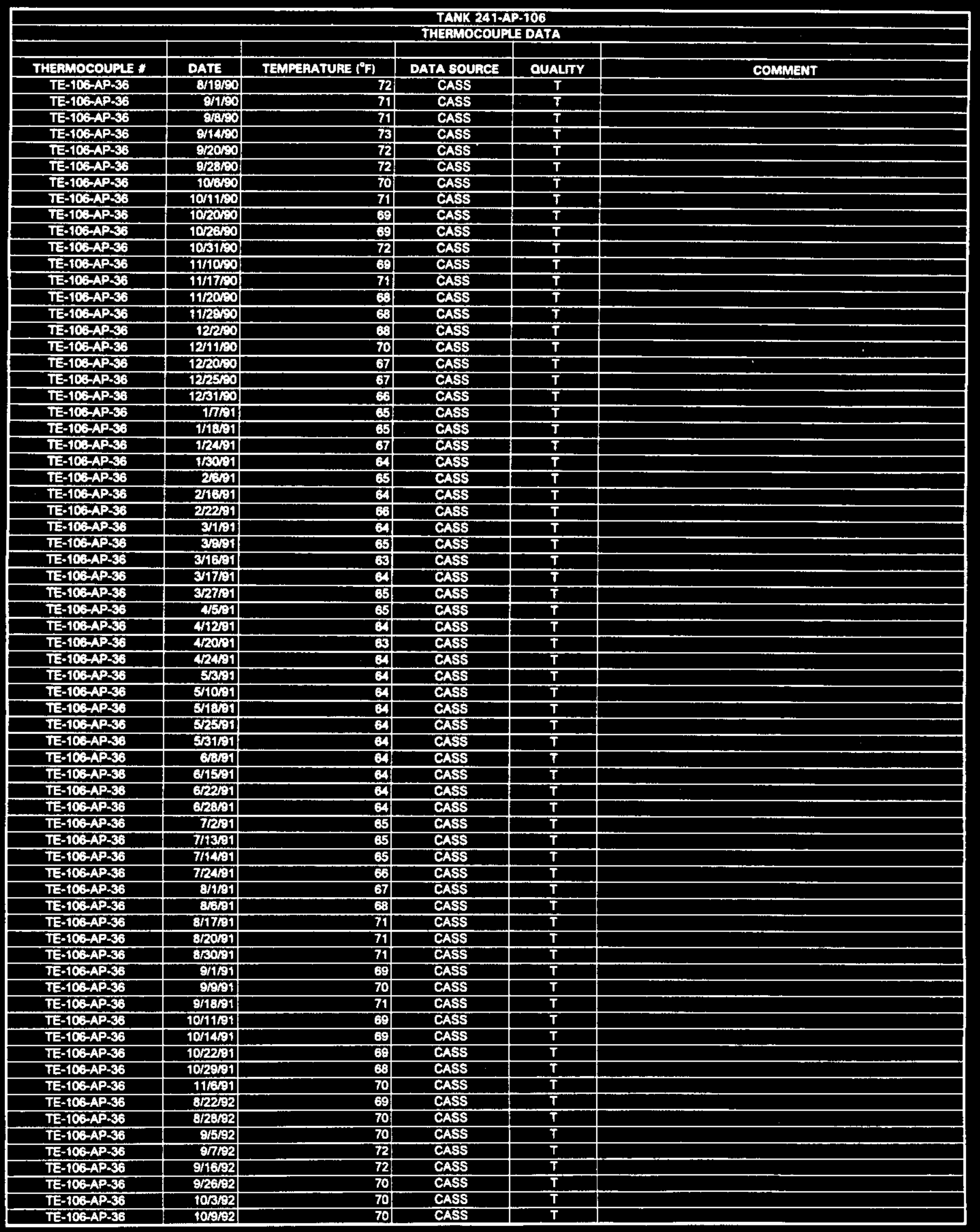




\begin{tabular}{|c|c|c|c|c|c|}
\hline \multicolumn{6}{|c|}{$\begin{array}{l}\text { TANK 241-AP-106 } \\
\text { THERAMOCOUPLE DATA }\end{array}$} \\
\hline & & & & & \\
\hline THEAHOCOUPLE * & DATE & TEMPERATURE $\left({ }^{\circ} \mathrm{F}\right)$ & DATA BOURCE & QUALTYY & COMMENT \\
\hline TE-106-AP-36 & $10 / 15192$ & 69 & CASS & $T$ & \\
\hline TE-106-AP-36 & $10 / 22192$ & 70 & CASS & $\bar{T}$ & \\
\hline TE-106-AP-36 & 10/31/92 & 70 & CASS & $T$ & \\
\hline TE-103-AP-36 & $11 / 6 / 92$ & 69 & CASS & $T$ & \\
\hline TE-106-AP-36 & $11 / 11 / 92$ & 68 & CASS & $T$ & \\
\hline TE-106-AP-36 & 11/20/92 & 68 & CASS & $T$ & \\
\hline TE-106AP-36 & $11 / 2 \mathrm{~B} / 92$ & 66 & CASS & $T$ & \\
\hline TE-106-AP-36 & $11 / 30 / 2$ & 66 & SACS & $T$ & \\
\hline TE-106-AP:36 & 121092 & 68 & CASS & $T$ & \\
\hline TE-106-AP-36 & $12 / 17 / 92$ & 66 & CASS & $T$ & \\
\hline TE-106-AP-36 & $12 / 26 \% 92$ & G] & CASS & $T$ & \\
\hline TE-106-AP-36 & $1230 / 92$ & 64 & CASS & $T$ & \\
\hline TE-106-AP-36 & 6/11/93 & 63 & SACS & $T$ & \\
\hline TE-106-AP-36 & E/RB/93 & 63 & SACS & $T$ & \\
\hline TE-106-AP-36 & 62893 & 63 & SACS & $T$ & \\
\hline TE-106-AP36 & 7 19/93 & 64 & SACS & $\bar{T}$ & \\
\hline TE-106-AP-36 & $7 / 15 \% 3$ & 69 & SACs & $T$ & \\
\hline TE-106-AP-36 & $7 / 1993$ & हA & SACS & $T$ & \\
\hline TE-106-AP-36 & $7 / 30 / 93$ & 63 & SACS & $T$ & \\
\hline TE-103-AP-33 & $8 / 393$ & 65 & SACS & $T$ & \\
\hline TE-106-APA36 & $8 / 12 / 93$ & 65 & SACS & $T$ & \\
\hline TE-106-AP.35 & $8 / 2403$ & E0 & SACS & $T$ & \\
\hline TE-106-AP-3S & 8/26ra3 & 68 & SACS & $T$ & \\
\hline TE-106-AP-36 & $10 / 15 / 93$ & 63 & SACS & $T$ & \\
\hline TE-106-AP-S6 & 102213 & 66 & SACS & $T$ & \\
\hline TE-106-AP-36 & $10 / 28 / 83$ & 65 & SACS & $T$ & \\
\hline TE-106-AP-36 & 111693 & 64 & SACS & $T$ & \\
\hline TE-103-AP-SB & $11 / 3000$ & 57 & SACS & $\mathbf{T}$ & \\
\hline IE-106-AP-36 & 12/14/33 & 6 & SACS & $T$ & \\
\hline TE-10B-APSB & 1220103 & Ea & SACS & $T$ & \\
\hline TE-10B-AP-33 & 1272703 & 66 & SACS & $T$ & \\
\hline TE-106-AP-36 & Tron & 61 & SACS & $T$ & \\
\hline TE-106-APS36 & 1/111/2 & 66 & SACS & $T$ & \\
\hline TE-106-APSB6 & T/18M & 64 & CASS & $\bar{T}$ & \\
\hline TE-106-AP-36 & 1/26194 & 66 & SACS & $T$ & \\
\hline TE-1OG-AP-33 & 2यक & 64 & CASS & $T$ & \\
\hline TE-106-AP- 36 & 211\% & 63 & CASS & $T$ & \\
\hline TE-103-AP-36 & याबल & 63 & CASS & $T$ & \\
\hline TE-106-AP-36 & 23694 & GA & CASS & $T$ & \\
\hline TE-106-AP-36 & 3/5\% & 65 & CASS & $T$ & \\
\hline TE-106-AP-36 & $3 / 10 \%$ & 64 & CASS & $T$ & \\
\hline TE-106-AP-36 & 31794 & 64 & CASS & $\bar{T}$ & \\
\hline TE-106-AP-36 & $3 / 2220$ & 63 & CASS & $T$ & \\
\hline TE-10G-AP-36 & 3 3ran & 63 & CASS & $\bar{T}$ & \\
\hline EE-106-AP-36 & arso4 & 66 & CASS & $\frac{T}{T}$ & \\
\hline TE-106-AP-36 & $4 / 1329$ & 64 & CASS & $\frac{T}{T}$ & \\
\hline TE-106-AP-S6 & $4 \sqrt{2094}$ & 65 & CASS & $\frac{T}{T}$ & \\
\hline TE-106-AP-36 & 4/299: & 65 & CASS & $\frac{T}{T}$ & \\
\hline TE-106-AP-36 & 5/29: & 64. & CASS & $\begin{array}{l}T \\
G\end{array}$ & \\
\hline TE-106-AP-36 & 59\%4 & 70 & SACS & $\frac{G}{S}$ & Dota point did not agree with local trend \\
\hline TE-10G-AP-36 & 5/11/84 & 84 & $\begin{array}{l}\text { CASS } \\
\text { CASS }\end{array}$ & $\frac{S}{T}$ & \\
\hline TE-10:AP-36 & S/169: & 100 & $\begin{array}{l}\text { CASS } \\
\text { CASS }\end{array}$ & $T$ & \\
\hline TE-106-AP-36 & $5 / 23194$ & 93 & $\begin{array}{l}\text { CASS } \\
\text { CASS }\end{array}$ & $\bar{T}$ & \\
\hline TE-106-AP-36 & 62194 & 95 & $\begin{array}{l}\text { CASS } \\
\text { CASS }\end{array}$ & $T$ & \\
\hline TE-106-AP-36 & E/Ba & 94 & $\begin{array}{l}\text { CASS } \\
\text { CASS }\end{array}$ & $T$ & \\
\hline TE-TOG-AP-36 & 6/14/94 & 92 & $\begin{array}{l}\text { CASS } \\
\text { CASS }\end{array}$ & $\mathbf{S}$ & Data point did not agree with local trend \\
\hline$\pi E-106-A P-36$ & 6/16/94 & \begin{tabular}{l|l}
65 \\
65
\end{tabular} & $\begin{array}{l}\text { CASS } \\
\text { CASS }\end{array}$ & $\mathbf{S}$ & Data point did not agree with local trend \\
\hline TE-106-AP-36 & 6/17as & 65 & $\begin{array}{l}\text { CASS } \\
\text { CASS }\end{array}$ & $\mathbf{S}$ & Defta point did not agree with local trend \\
\hline TE-106-AP-36 & $6 / 20194$ & $\frac{64}{94}$ & $\begin{array}{l}\text { CASS } \\
\text { CASS }\end{array}$ & $\mathbf{T}$ & \\
\hline $\begin{array}{l}\text { TE-106-AP-36 } \\
\text { TE-10S-AP-36 }\end{array}$ & 6/24/94 & $\begin{array}{l}94 \\
95\end{array}$ & CASS & $T$ & \\
\hline$\frac{\text { TE-106-AP-36 }}{\text { TE-106-AP-36 }}$ & $\frac{6 / 27 / 94}{7 / 3 / 94}$ & 95 & CASS & $\mathbf{T}$ & \\
\hline $\begin{array}{l}\text { TE-106-AP-36 } \\
\text { TE-106-AP-36 }\end{array}$ & Th1/39 & 64 & SACS & $S$ (G in SACS) & Deta point did not agree with local trend \\
\hline $\begin{array}{l}\text { TE-106-AP-36 } \\
\text { TE-106-AP-36 }\end{array}$ & 73009 & 92 & CASS & $\bar{T}$ & \\
\hline $\begin{array}{l}\frac{T E-106-A P-36}{} \\
\text { TE-106-AP-36 }\end{array}$ & 8/9/94 & se & CASS & $T$ & \\
\hline $\begin{array}{l}\text { TE-106-AP-36 } \\
\text { TE-106-AP-36 }\end{array}$ & 8/24/99 & 96 & CASS & $\mathbf{T}$ & \\
\hline $\begin{array}{l}\text { TE-106-AP-36 } \\
\text { TE-108-AP-36 }\end{array}$ & 10/1/34 & 99 & CASS & $\mathbf{T}$ & \\
\hline$\frac{\text { TE-106-AP-36 }}{\text { TE-10B-AP-36 }}$ & 10ray & 101 & CASS & $\mathbf{T}$ & \\
\hline$\frac{T E-106-A P-36}{T \text { TE-10B-AP-36 }}$ & $10 / 1219$ & 101 & CASS & $T$ & \\
\hline $\begin{array}{l}\text { TE-106-AP-36 } \\
\text { TE-106-AP-36 }\end{array}$ & 10/17\% & 102 & CASS & $\mathbf{T}$ & \\
\hline
\end{tabular}




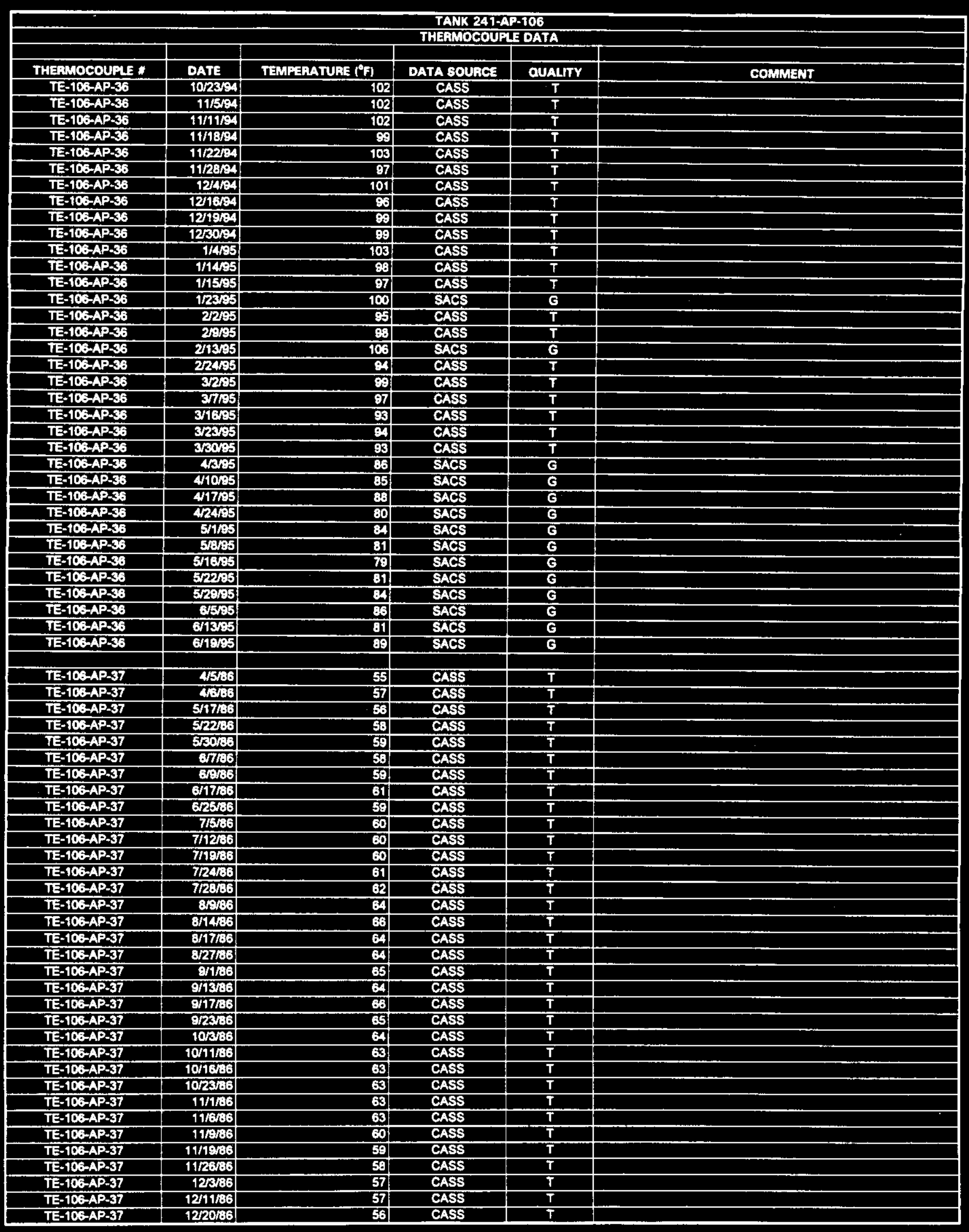




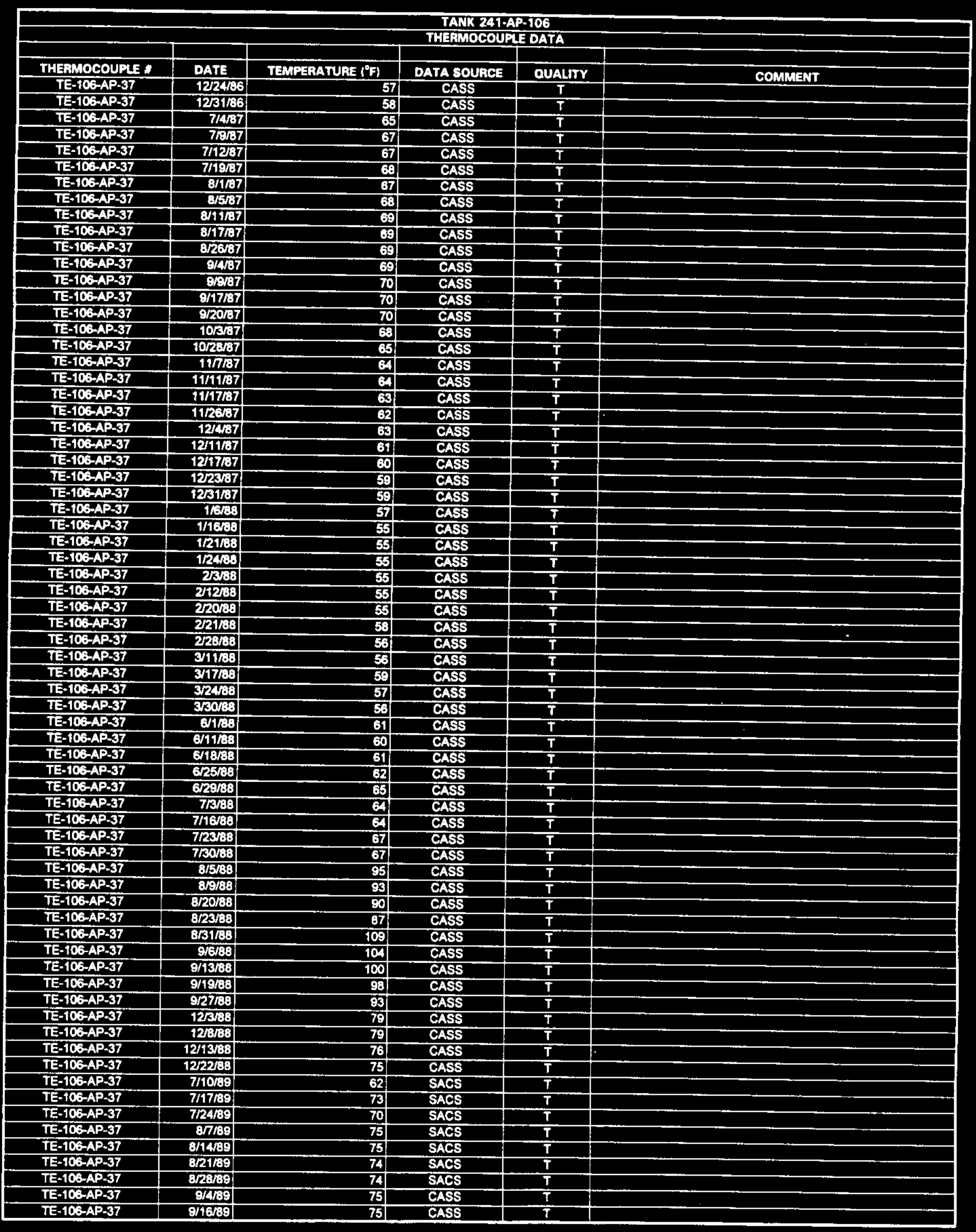




\begin{tabular}{|c|c|c|c|c|c|}
\hline \multicolumn{6}{|c|}{$\begin{array}{l}\text { TANK 241-AP-106 } \\
\text { THERMOCOUPLEATA }\end{array}$} \\
\hline THERMOCOUPLE * & DATE & TEMPERATURE $\left({ }^{\circ} \mathrm{F}\right)$ & DATA SOURCE & OUALTY & COMMENT \\
\hline TE-106-AP-37 & 9/18/89 & 75 & CASS & $T$ & \\
\hline TE-106-AP-37 & $8 / 29 / 89$ & 75 & CASS & $T$ & \\
\hline TE-106-AP-37 & $10 / 2169$ & 63 & SACS & $T$ & \\
\hline TE-106-AP-37 & $10 \times 9 / 69$ & 72 & SACS & $\bar{T}$ & \\
\hline TE-106-AP-37 & $10 / 16 / 39$ & 73 & SACS & $T$ & \\
\hline TE-106-AP-37 & $10 / 24 / 39$ & 75 & SACS & $T$ & \\
\hline TE-106-AP-37 & $11 / 4 / 69$ & 74 & CASS & $\bar{T}$ & \\
\hline TE-IOG-AP-37 & 11/7/69: & 74 & CASS & $T$ & \\
\hline TE-106.AP-37 & $11 / 18 / 89$ & 73 & CASS & $T$ & \\
\hline TE-10QAP-37 & $1 / 12369$ & 77 & CASS & $\mathbf{T}$ & \\
\hline TE-106-AP-37 & $11 / 2989$ & 74 & CASS & $T$ & \\
\hline TE-106-AP-37 & 25109 & 73 & CASS & $T$ & \\
\hline TE-106-AP-37 & $1251 / 39$ & 59 & SACS & $\mathbf{s}$ & Dats point did not stive with bocal trend \\
\hline TE-106-AP-37 & $12 / 12289$ & 72 & CASS & $\mathbf{T}$ & \\
\hline TE-106-AP-37 & $12 / 22189$ & 71 & CASS & $T$ & \\
\hline$T E-103-A P-37$ & $12 / 26 \%$ & 70 & CASS & $T$ & \\
\hline$T E=106-A P-37$ & 1/8100 & 70 & CASS & $T$ & \\
\hline TE-106-AP-37 & 1/1250 & 71 & CASS & $T$ & \\
\hline TE-106-AP-37 & I/2000 & 70 & CASS & $T$ & \\
\hline TE-103-AP-37 & $1 / 2300$ & 70 & CASS & $T$ & \\
\hline TE-10B-AP-37 & $2 / 1100$ & 70 & CASS & $\mathbf{T}$ & \\
\hline TE-10:AP-37 & 21000 & 70 & CASS & $T$ & \\
\hline TE-10B-AP-37 & $2 / 1350$ & 69 & CASS & $T$ & \\
\hline TE-106-AP-37 & 21800 & 69 & CASS & $T$ & \\
\hline TE-106-AP-37 & 2rak:0 & 63) & CASS & $T$ & \\
\hline TE-106-AP-37 & 3360 & 69 & CASS & $\mathbf{T}$ & \\
\hline TE-106-AP-37 & $3 / 12200$ & 63 & CASS & $\mathbf{T}$ & \\
\hline TE-10B-AP-37 & 32000 & 68 & CASS & $\mathbf{T}$ & \\
\hline TE-106-AP-37 & $3 / 25,0$ & 68 & CASS & $T$ & \\
\hline TE-106-AP-37 & 4860 & 68 & CASS & $\mathbf{T}$ & \\
\hline TE-103-AP-37 & A/1450 & 68 & CASS & $\mathbf{T}$ & \\
\hline TE-103-AP-37 & 4185 & 69 & CASS & $T$ & \\
\hline TE-106-AP-37 & 12400 & 68 & CASS & $\mathbf{T}$ & \\
\hline TE-106-AP-37 & SIINO & 69 & CASS & $T$ & \\
\hline TE-103-AP-37 & \$11180 & 67 & CASS & $T$ & \\
\hline TE-106-AP-37 & B/SEO & 66 & CASS & $\mathrm{T}$ & \\
\hline TE-100-AP-37 & इ2250 & 63 & CASS & $\bar{T}$ & \\
\hline TE-106-AP-37 & $6 / 2=0$ & E3 & CASS & $\mathbf{T}$ & \\
\hline TE-10G-AP-37 & 60180 & 67 & CASS & $T$ & \\
\hline TE-10:AP-37 & E11400 & 67 & CASS & $T$ & \\
\hline TE-108-AP-37 & 623100 & 86 & CASS & $T$ & \\
\hline TE-106-AP-37 & $6 / 2090$ & 68 & CASS & $T$ & \\
\hline$T E-106-A P-37$ & 7600 & 67 & CASS & $T$ & \\
\hline$T E-103-A P-37$ & 71410 & 67 & CASS & $T$ & \\
\hline TE-106-AP-37 & $715 \%$ & $70:$ & CASS & $T$ & \\
\hline TE-TOG-AP-37 & $7 / 27 / 50$ & 70 & CASS & $T$ & \\
\hline TE-103-AP-37 & B/3/so & 70 & CASS & $T$ & \\
\hline TE-106-AP-37 & $8 / 11 / 90$ & 71 & CASS & $T$ & \\
\hline TE-106-AP-37 & $8 / 18 / 90$ & 70 & CASS & $T$ & \\
\hline TE-106-AP-37 & 82250 & 71 & CASS & $\mathbf{T}$ & \\
\hline TE-106-AP-37 & $8 / 31 / 30$ & 71 & CASS & $\mathbf{T}$ & \\
\hline TE-106-AP-37 & 9/2:0 & 71 & CASS & $T$ & \\
\hline TE-106-AP-37 & 91400 & 71 & CASS & $T$ & \\
\hline TE-106-AP-37 & 8/17/00 & 72 & CASS & $T$ & \\
\hline TE-106-AP-37 & $9 / 28 / 00$ & 72 & CASS & $T$ & \\
\hline TE-106-AP-37 & $10 / 1 / 00$ & 711 & SACS & $\bar{T}$ & \\
\hline TE-10G-AP-37 & $10 / 11 \% 0$ & 71 & CASS & $\mathbf{T}$ & \\
\hline TE-106-AP-37 & $10 / 20100$ & 69 & CASS & $\mathbf{T}$ & \\
\hline TE-T06-AP-37 & $10 / 2800$ & 69 & CASS & $T$ & \\
\hline TE-106-AP-37 & $10 / 3150$ & 70 & CASS & $\mathrm{T}$ & \\
\hline TE-106-AP-37 & $11 / 590$ & 70 & SACS & $T$ & \\
\hline TE-10G-AP-37 & $11 / 1750$ & 711 & CASS & $T$ & \\
\hline TE-106-AP-37 & $11 / 19 / 90$ & 69 & SACS & $T$ & \\
\hline TE-106-AP-37 & $11 / 2960$ & 69 & CASS & $\mathbf{T}$ & \\
\hline TE-103AP.37 & 122200 & 69 & CASS & $T$ & \\
\hline TE-106-AP-37 & $12 / 1490$ & 69 & CASS & $T$ & \\
\hline TE-106-AP-37 & $12 / 2000$ & 68 & CASS & $T$ & \\
\hline TE-106-AP-37 & $12 / 2500$ & 68. & CASS & $T$ & \\
\hline TE-106-AP-37 & $1 / 5 / 1$ & 66 & CASS & $T$ & \\
\hline TE-106-AP-37 & 1/12/91 & 68: & CASS & $\mathbf{T}$ & \\
\hline
\end{tabular}




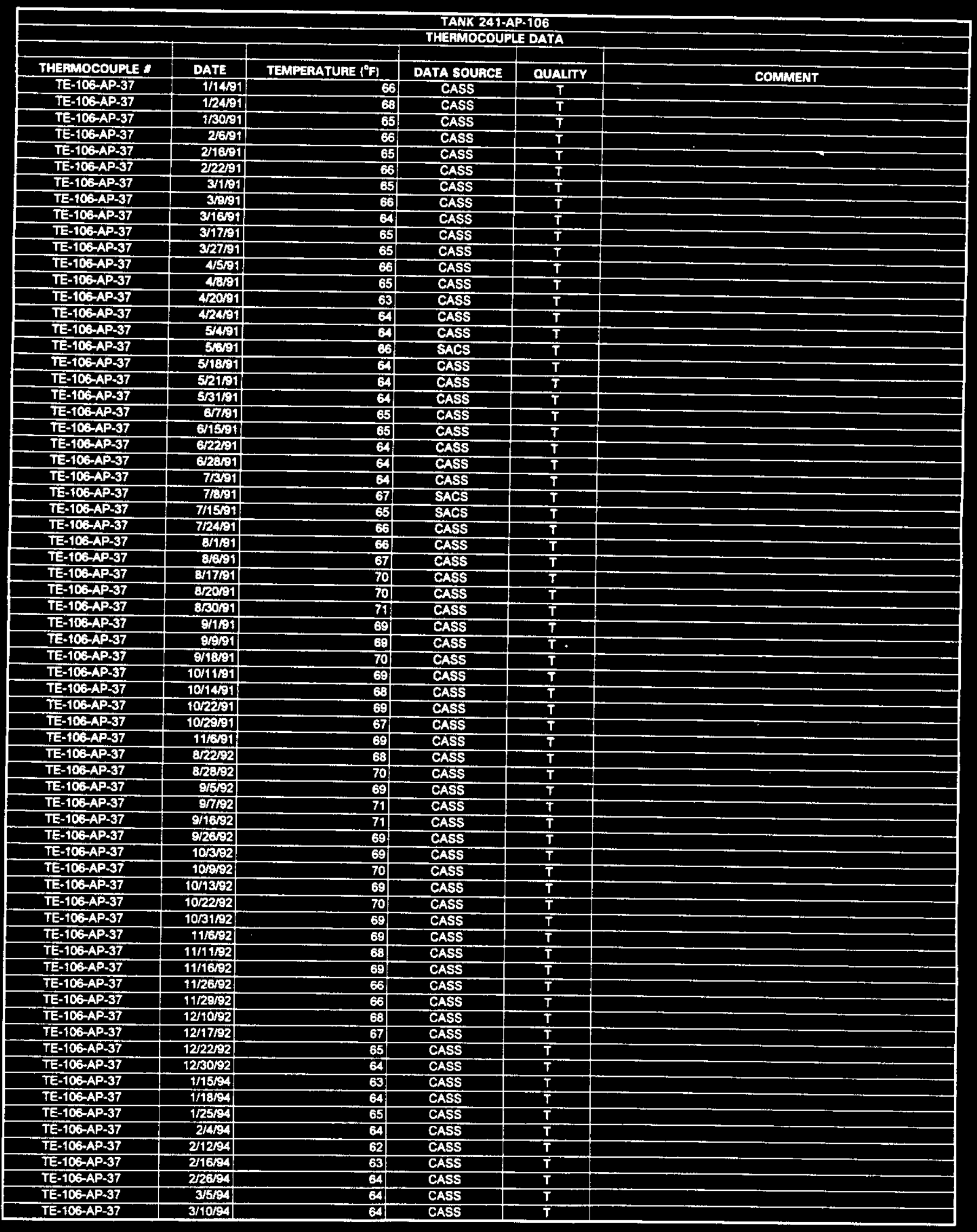




\begin{tabular}{|c|c|c|c|c|c|}
\hline \multicolumn{6}{|c|}{$\begin{array}{l}\text { TANK 241-AP-106 } \\
\text { THERMOCOUPLE DATA }\end{array}$} \\
\hline THERMOCOUPE * & DATE & TEMFERATURE ( $\left.{ }^{\circ} \mathrm{F}\right)$ & DATA SOUACE & QUALITY & COMMENT \\
\hline TE-106-AP-37 & $3 / 17 / 94$ & 64 & CASS & $T$ & \\
\hline TE-106-AP-37 & $3 / 2194$ & 63 & CASS & $\mathbf{T}$ & \\
\hline TE-106-AP-37 & $3 / 2864$ & 63 & CASS & $\bar{T}$ & \\
\hline TIE-106-AP-37 & $4 \sqrt[3134]{4}$ & 66 & CASS & $\mathbf{T}$ & \\
\hline TE-106-AP-37 & Q/1354 & 65 & CASS & $T$ & \\
\hline TE-106-AP-37 & AZOOS & 65 & CASS & $T$ & \\
\hline TE-106-AP-37 & 4289 & 65 & CASS & $\bar{T}$ & \\
\hline TE-106-AP-37 & S2204 & 6a & CASS & $\mathbf{T}$ & \\
\hline TE-106-AP-37 & B11\%4 & 85 & CASS & $\mathbf{s}$ & Data point did not apree with local trend \\
\hline TE-T06-AP-37 & S12M & 63 & CASS & $\mathbf{T}$ & \\
\hline RE-106-AP-37 & 5/16/94 & 100 & CASS & $\overline{\mathbf{T}}$ & \\
\hline TE-106-AP-37 & Sr2259 & 93 & CASS & $T$ & \\
\hline TE-106-AP-37 & 6r294 & 85 & CASS & $\mathbf{T}$ & \\
\hline TE-106-AP-37 & Gora & 83 & CASS & $\mathbf{T}$ & \\
\hline TE-106-AP-37 & 6/1494 & 82 & CASS & $\mathbf{T}$ & \\
\hline TE-106-AP-37 & 6/16/9 & 6] & CASS & $\mathbf{S}$ & Data point did not apree with local trend \\
\hline TE-106-AP-37 & E/17\%4 & GA & CASS & $\mathbf{S}$ & Data point did not saree with local trend \\
\hline TE-106-AP-37 & 8r2004 & 60 & CASS & $\mathbf{S}$ & Data point did not sgree with local trend \\
\hline TE-10:AP-37 & 6/25:94 & 83 & CASS & $\mathbf{T}$ & \\
\hline TE-106-AP-37 & 6/27\%a & 95 & CASS & $\mathbf{T}$ & \\
\hline TE-10G-AP-37 & 7man & 921 & CASS & $\mathbf{T}$ & \\
\hline TE-106-AP 37 & $730 \mathrm{OA}$ & 91 & CASS & $\mathbf{T}$ & \\
\hline TE-106-AP-37 & Brea & 86 & CASS & $T$ & \\
\hline TE-106-AP-37 & 8/2WA9 & 85 & CASS & $T$ & \\
\hline TE-106-AP-37 & 10/tion & 83 & CASS & $T$ & \\
\hline TE-10GAP-37 & 100rad & 100 & CASS & $T$ & \\
\hline TE-10G-AP-37 & 101250 & 104 & CASS & $T$ & \\
\hline TE-106-AP-37 & Toritis & 102 & CASS & $T$ & \\
\hline TE-106-AP-37 & $10 / 2350$ & 101 & CASS & $T$ & \\
\hline TE-103-AP-37 & TISBA & 102 & CASS & $\mathbf{T}$ & \\
\hline TE-10B-AP-37 & $11 / 1194$ & 103 & CAss & $T$ & \\
\hline TE-1DG-AP-37 & $11 / 16 \%$ & 100 & CASS & $\mathbf{T}$ & \\
\hline TE-106-AP-37 & $11 / 2200$ & 103 & CASS & $T$ & \\
\hline TE-106-AP-37 & $12 \pi \mathrm{sen}$ & 8 & CASS & $\mathbf{T}$ & \\
\hline TE-106-AP-37 & $12 / 49$ & 101 & CASS & $T$ & \\
\hline TE-106-AP:37 & 12/1694 & 83 & CASS & $T$ & \\
\hline TE-106-AP-37 & $12 / 189$ & 8 & CASS & $\bar{T}$ & \\
\hline TE-106-AP-37 & $12 / 31 \mathrm{A4}$ & 83 & CASS & $T$ & \\
\hline TE-106-AP-37 & 1/435) & 104 & CASS & $T$ & \\
\hline TE-106-AP-37 & $1 / 1403$ & 89 & CASS & $T$ & \\
\hline TE-106-AP-37 & $1 / 15>3$ & 89 & CASS & $T$ & \\
\hline TE-106-AP.37 & $1 / 2356$ & 100 & CASS & $\mathbf{T}$ & \\
\hline TE-T06-AP-37 & $2 / 2 / 35$ & 85 & CASS & $\mathbf{T}$ & \\
\hline TE-106-AP-37 & $2 \mathrm{ses}$ & 88 & CASS & $T$ & \\
\hline TE-106-AP-37 & 21495 & 109 & CASS & $\mathbf{T}$ & \\
\hline TE-106-AP-37 & 272465 & 99 & CASS & $T$ & \\
\hline TE-10G-AP-37 & $3 / 205$ & 8 & CASS & $T$ & \\
\hline TE-106-AP-37 & 3765 & 87 & CASS & $T$ & \\
\hline TE-106-AP-37 & $3 / 16 / 25$ & 92 & CASS & $T$ & \\
\hline TE-106-AP-37 & $3 / 2365$ & 89 & CASS & $T$ & \\
\hline TE-106-AP-37 & $3 / 30 \%$ & 93 & CASS & $T$ & \\
\hline & & & & & \\
\hline TE-106-AP-38 & 4/586 & 55 & CASS & $T$ & \\
\hline TE-106-AP-38 & $4 / 6 / 86$ & 57 & CASS & $T$ & \\
\hline TE-106-AP-38 & 5/16/26 & 57 & CASS & $T$ & \\
\hline TE-106-AP-38 & $5 \sqrt{24 / 66}$ & 58 & CASS & $T$ & \\
\hline TE-106-AP-33 & $5 / 30 / 36$ & 59 & CASS & $T$ & \\
\hline TE-106-AP-3B & 67/86 & 58 & CASS & $T$ & \\
\hline TE-103-AP-38 & 6/13/66 & 60 & CASS & $T$ & \\
\hline TE-106-AP-39 & 6/21/86 & 59 & CASS & $T$ & \\
\hline TE-106-AP-3B & 6/25/66 & 59 & CASS & $T$ & \\
\hline IE-106-AP-3B & 71406 & 61 & CASS & $T$ & \\
\hline TE-106-AP-38 & $7712 / 36$ & 60 & CASS & $T$ & \\
\hline TE-106-AP-3B & 7/19/66 & 61 & CASS & $\mathbf{T}$ & \\
\hline TE-106-AP-3B & $7 / 21 / 36$ & 62 & CASS & $T$ & \\
\hline TE-106-AP-3B & 7/28/B6 & 62 & CASS & $T$ & \\
\hline TE-106-AP-33 & $8 \% \mathrm{~B}$ & 64 & CASS & $T$ & \\
\hline TE-106-AP-33 & $8 / 1486$ & 63 & CASS & 7 & \\
\hline TE-106-AP-38 & $8 / 2356$ & 62. & CASS & $T$ & \\
\hline TE-106-AP-3B & $8 / 27 / 36$ & 6A & CASS & $\mathbf{T}$ & \\
\hline
\end{tabular}

Data obteined from Computer Automated Surveillance System (CASS), April 1995; and Surveillance Analysis Computer System (SACS), June 1995. 


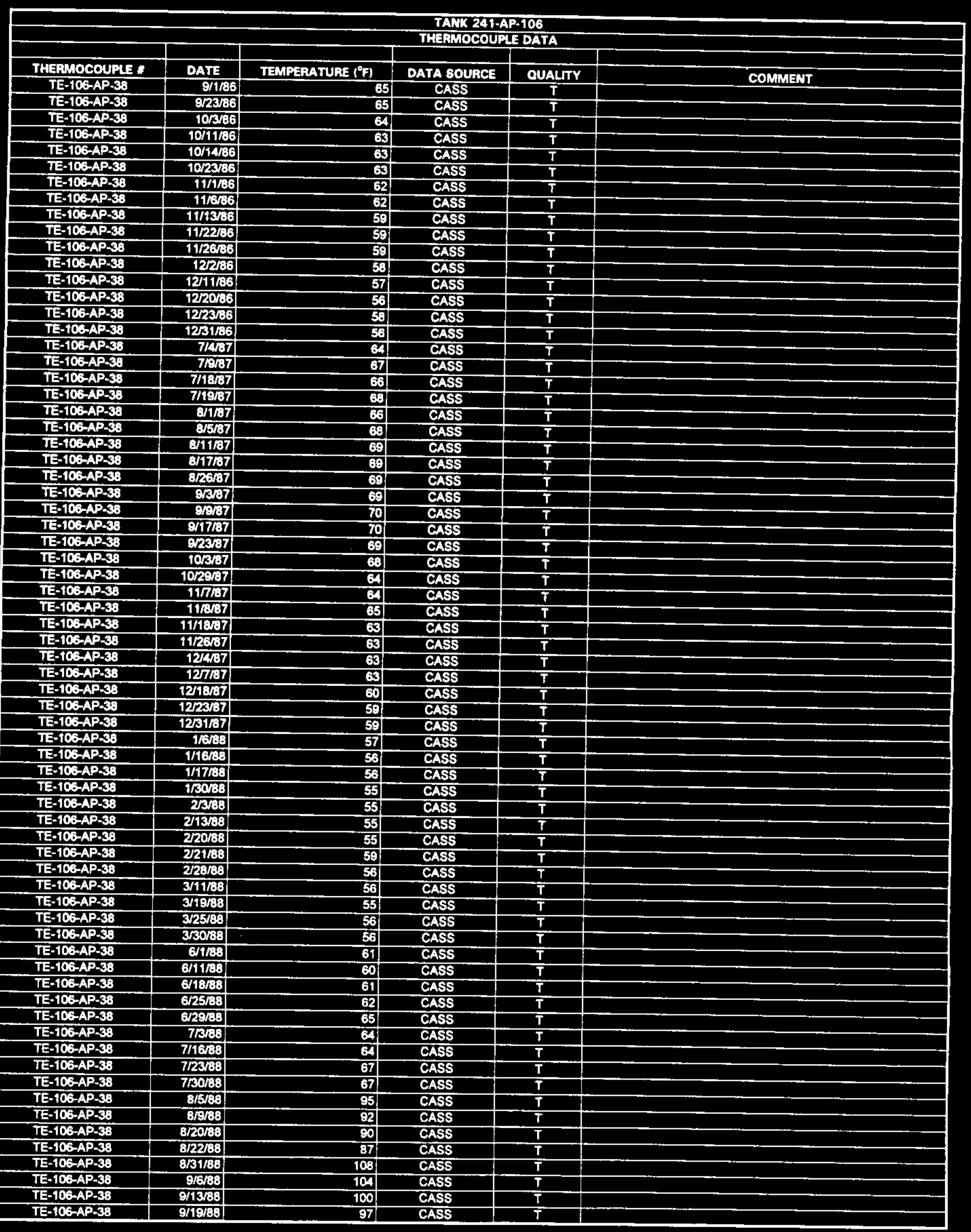




\begin{tabular}{|c|c|c|c|c|c|}
\hline \multicolumn{6}{|c|}{$\begin{array}{l}\text { TAKK 241-AP-106 } \\
\text { THEGOCOUPL DATA }\end{array}$} \\
\hline & & & & & \\
\hline THEAMOCOUPE * & DATE & TEMPERATURE $\left(^{\circ} \mathrm{F}\right)$ & DATA SOURCE & QUALITY & COMMENT \\
\hline TE-108-AP-33 & $9 / 2738$ & 94 & CASS & $T$ & \\
\hline TE-106-AP-33 & $1221 / 88$ & 80 & CASS & $T$ & \\
\hline TE-106-AP-3B & 12383 & 81 & CASS & $T$ & \\
\hline TE-106-AP-38 & $12 / 483$ & 77 & CASS & $T$ & \\
\hline TE-106-AP-38 & 12/21/38 & 78 & CASS & $T$ & \\
\hline TE-106-AP-33 & $94 / 89$ & 75 & CASS & $T$ & \\
\hline TE-TOB-AP-3B & 8/16/89 & 75 & CASS & $T$ & \\
\hline TE-108-AP-SB & $8 / 18 / 89$ & 75 & CASS & $T$ & \\
\hline TE-10S-AP-3B & 82969 & 75 & CASS & $T$ & \\
\hline TE-TOG-AP-33 & $11 / 469$ & 75 & CASS & $T$ & \\
\hline TE-106-APSBB & 117/30 & 75 & CASS & $\mathbf{T}$ & \\
\hline TE-106-AP-S3 & $11 / 1569$ & 74 & CASS & $T$ & \\
\hline$T E-106-A P-38$ & $11 / 2568$ & 73 & CASS & $T$ & \\
\hline TE-106-AP-30 & 11/23/69 & 74 & CASS & $T$ & \\
\hline TE-106-AP-38 & 1215\%9 & 73 & CASS & $\bar{T}$ & \\
\hline TE-106-AP-38 & 1212309 & 73 & CASS & $T$ & \\
\hline TE-10B-AP-SB & 12 233/69 & 71 & CASS & $T$ & \\
\hline TE-106-APS38 & 1230000 & 71 & CASS & $T$ & \\
\hline TE-106-AP-33 & 1/3600 & 73 & SACS & $\bar{T}$ & \\
\hline TE-106AP-33 & $1 / 12200$ & 71 & CASS & $T$ & \\
\hline TE-106-AP-3B & $1 / 2000$ & 72 & CASS & $\bar{T}$ & \\
\hline$T E-103-A P-38$ & $1 / 23100$ & 70 & CASS & $T$ & \\
\hline$\pi=106-A P-33$ & $2 \mu 100$ & 70 & CASS & $T$ & \\
\hline TE-106-AP-33 & 2/1000 & 70 & CASS & $\bar{T}$ & \\
\hline TE-106-AP-3B & $2 / 350$ & 69 & CASS & $T$ & \\
\hline TE-106-AP-33 & $2 / 21 / \infty 0$ & 69 & CASS & $T$ & \\
\hline TE-106-AP-38 & 222100 & 69 & CASS & $T$ & \\
\hline TE-106-AP-33 & 30 & 69 & CASS & $T$ & \\
\hline$T E-106-A P-3 B$ & $3 / 1250$ & 63 & CASS & $T$ & \\
\hline TE-TOE-APASB & $3 / 3000$ & 83 & CASS & $T$ & \\
\hline TE-10GAPB33 & $3 / 2500$ & 60 & CASS & $\bar{T}$ & \\
\hline TE-106-AP-38 & 4300 & 68 & CASS & $\mathbf{T}$ & \\
\hline TE-106-AP-3B & 4heo & 67 & CASS & $T$ & \\
\hline TE-105-AP-3B & 41850 & 70 & CASS & $T$ & \\
\hline TE-10G-AP-38 & 42450 & 63 & CASS & $T$ & \\
\hline TE-106-AP-3B & 5/160 & 69 & CASS & $\mathbf{T}$ & \\
\hline TE-106-AP-38 & 5000 & 83 & CASS & $\mathbf{T}$ & \\
\hline TE-106-AP-3B & S19\% & 66 & CASS & $T$ & \\
\hline TE-106-AP-38 & 5/2250 & 6 & CASS & $T$ & \\
\hline TE-100-AP-3B & 6riso & 6 & CASS & $T$ & \\
\hline$\overline{E-106-A P-33}$ & GEOO & 67 & CASS & $\bar{T}$ & \\
\hline TE-108-AP-38 & Grapo & 67 & CASS & $T$ & \\
\hline TE-10B-AP-3B & 62350 & 66 & CASS & $T$ & \\
\hline TE-103-AP-3B & 62500 & 80 & SACS & $\mathbf{s}$ & Onta point did not squee with beal trend \\
\hline TE-106-AP-33 & 6/29/90 & 67| & CASS & $T$ & \\
\hline TE-106-AP-33 & 7600 & 66 & CASS & $T$ & \\
\hline TE-106-AP-38 & 71450 & 66 & CASS & $T$ & \\
\hline TE-106-AP-38 & 7715190 & 70 & CASS & $T$ & \\
\hline TE-10G-AP-38 & 727100 & 67 & CASS & $T$ & \\
\hline TE-106-AP-3B & $8 / 3100$ & 68 & CASS & $T$ & \\
\hline TE-106-AP-33 & $8 / 11 / 30$ & 63 & CASS & $\bar{T}$ & \\
\hline TE-106-AP-38 & $8 / 18=0$ & 67 & CASS & $T$ & \\
\hline TE-10G-AP-33 & $8 / 2260$ & 68 & CASS & $\mathbf{T}$ & \\
\hline TE-106-AP-38 & 91/50 & 68 & CASS & $\bar{T}$ & \\
\hline TE-106-AP-3B & $8 / 200$ & 69 & CASS & $T$ & \\
\hline TE-10B-AP-3B & 8/1400 & 70 & CASS & $T$ & \\
\hline TE-106-AP-3B & 9/17/50 & 70 & CASS & $T$ & \\
\hline TE-103-AP-38 & $9 / 28 / 80$ & 70 & CASS & $T$ & \\
\hline TE-106-AP-33 & $10 / 3 / 50$ & 6 & CASS & $T$ & \\
\hline TE-106-AP-33 & $10 / 1150$ & 70 & CASS & $T$ & \\
\hline TE-106-AP-38 & $10 / 20180$ & 69 & CASS & $\bar{T}$ & \\
\hline TE-106-AP-38 & $10 / 26 \% 0$ & 70 & CASS & $T$ & \\
\hline TE-10E-AP-3B & $10 / 31 / 80$ & 71 & CASS & $T$ & \\
\hline TE-106-AP-38 & $11 / 10 / 80$ & 70 & CASS & $\mathbf{T}$ & \\
\hline TE-106-AP-33 & $11 / 17 / 60$ & 71 & CASS & $\mathbf{T}$ & \\
\hline TE-106-AP-318 & $11 / 2250$ & 68 & CASS & $T$ & \\
\hline TE-103-AP-38 & $11 / 29 \% 0$ & 69 & CASS & $T$ & \\
\hline TE-106-AP-38 & $1222 \% 0$ & 69 & CASS & $\bar{T}$ & \\
\hline TE-106-AP-38 & $12 / 11 / 00$ & 69 & CASS & $T$ & \\
\hline TE-106-AP-33 & $12 / 20 / 90$ & 69 & CASS & $\bar{T}$ & \\
\hline
\end{tabular}




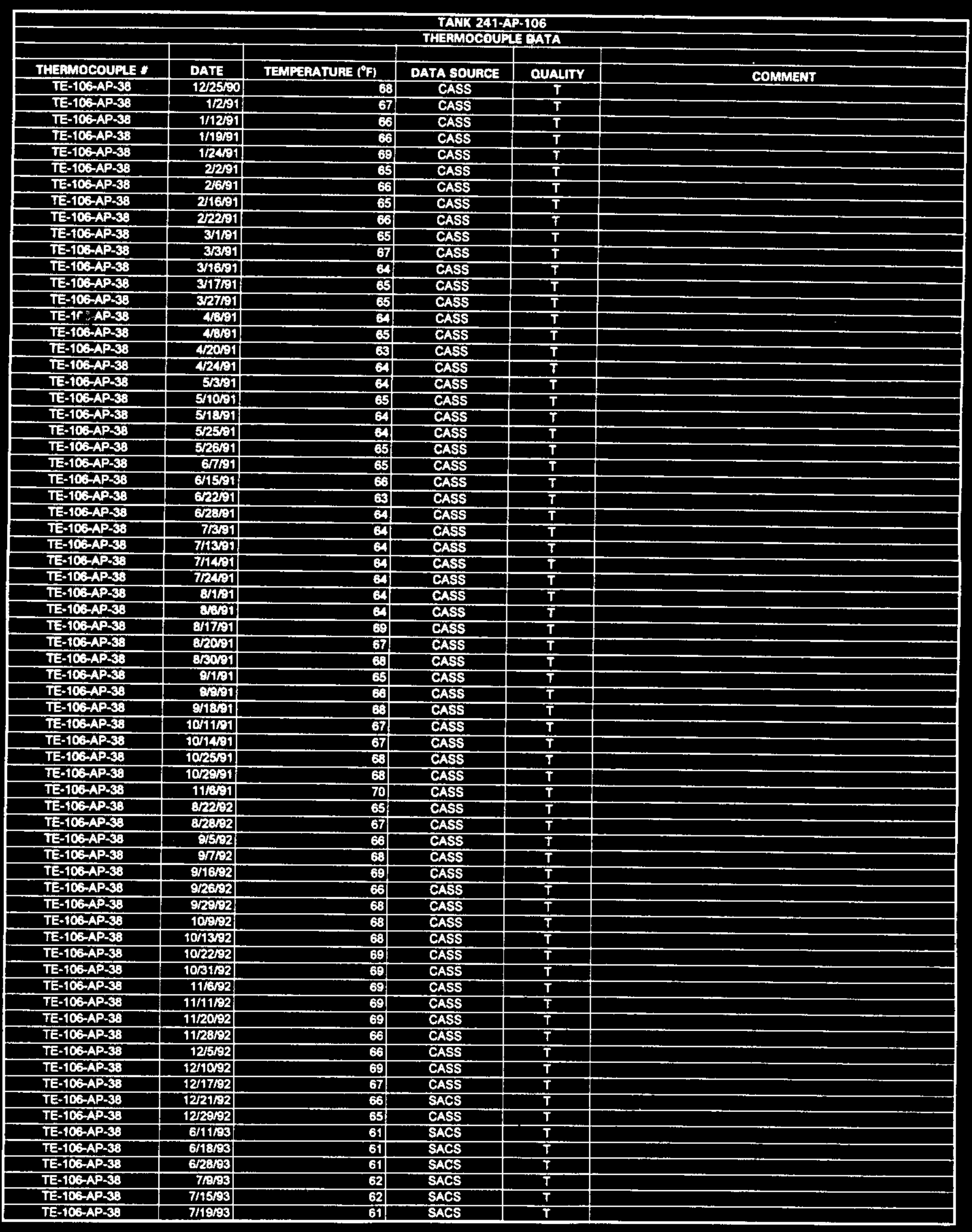




\begin{tabular}{|c|c|c|c|c|c|}
\hline \multicolumn{6}{|c|}{$\begin{array}{l}\text { TANR 241-AP-106 } \\
\text { THEANOCOUPLE DATA }\end{array}$} \\
\hline THERMOCOUPLE * & DATE & TEMPERATURE ( ${ }^{\circ} \mathrm{Fl}$ & DATA sOURCE & QUALITY & COMMENT \\
\hline TE-106-AP.38 & $7 / 30 / 93$ & 63 & SACS & $T$ & \\
\hline TE-106-AP-38 & B/693 & 63 & SACS & $T$ & \\
\hline TE-106-AP-39 & $8 / 1293$ & 62 & SACS & $\mathbf{T}$ & \\
\hline$T E-106-A P-38$ & 6/24r93 & 61 & SACS & $T$ & \\
\hline TE-106AP-3B & $10 / 1 / 93$ & 65 & SACS & $T$ & \\
\hline TE-10S-AP-38 & $10 / 1503$ & 67 & SACS & $T$ & \\
\hline TE-103-AP-SB & 1022293 & 65 & SACS & $T$ & \\
\hline TE-106-AP-38 & 1020103 & 63 & SACS & $\bar{T}$ & \\
\hline TE-103-AP-3B & $11 \mathrm{ses}$ & 62 & SACS & $T$ & \\
\hline TE-10.3-AP-38 & $11 / 3003$ & 59 & SACS & $\mathbf{T}$ & \\
\hline TE-103-AP-38 & $12 / 1493$ & 66 & SACS & $T$ & \\
\hline$T E-106-A P B 38$ & 1220193 & 65 & SACS & $T$ & \\
\hline TE-106-AP-38 & $12727: 3$ & 63 & SACS & $T$ & \\
\hline TE-TOS-AP-BB & $1 \times 3$ & 63 & SACS & $T$ & \\
\hline TE-106-AP-3B & $1 / 11 / 9$ & 67 & SACS & $T$ & \\
\hline TE-10G-AP-38 & IITEA & 65 & CASS & $T$ & \\
\hline TE-103-AP-38 & $1 / 2594$ & 65 & CASS & $T$ & \\
\hline TE-108-AP-38 & 2494 & 65 & CASS & $T$ & \\
\hline TE-106-AP-3B & $2 / 2 \%$ & 63 & CASS & $T$ & \\
\hline TE-103-AP-38 & $2 / 594$ & 65 & CASS & $\bar{T}$ & \\
\hline TE-106-AP-38 & 23694 & 64 & CASS & $T$ & \\
\hline TE-103-AP-33 & 3594 & 65 & CASS & $T$ & \\
\hline TE-103-AP-38 & 31004 & 65 & CASS & $T$ & \\
\hline$T=-103-A P-30$ & $318 \times 4$ & 86 & CASS & $\bar{T}$ & \\
\hline TE-103-APSB & $3 \sqrt{2204}$ & 63 & CASS & $T$ & \\
\hline TE-103-AP-38 & 3/2304 & 63 & CASS & $\bar{T}$ & \\
\hline TE-106-AP-33 & $4 \times 5$ & 6 & CASS & $T$ & \\
\hline TE-10A-AP-33 & 41394 & 65 & CASS & $T$ & \\
\hline TE-106-APA3 & 42094 & 85 & CASS & $\mathbf{T}$ & \\
\hline TE-106-APS3 & 4 rasu & 85 & CASS & $T$ & \\
\hline TE-106-APSB & sris & 64 & CASS & $T$ & \\
\hline TE-103-AP-38 & Stan & 70 & SACS & $\mathbf{G}$ & \\
\hline TE-160-AP-38 & \$11194 & 84. & CASS & 5 & Data point did not eqree with local trend \\
\hline TE-100-AP-BS & 51624 & 100 & CASS & $\bar{T}$ & \\
\hline$T E-103-A P-38$ & 572359 & 83 & CASS & $T$ & \\
\hline TE-10G-AP-38 & 625:4 & 85 & CASS & $T$ & \\
\hline EE-106-AP-3B & seren & 83 & CASS & $T$ & \\
\hline TE-106-NP-SB & Q/14\% & 91 & CASS & $\mathbf{T}$ & \\
\hline TE-103-AP-38 & 61624 & 64 & CASS & $\mathbf{S}$ & Dats point did not agree with loctl trend \\
\hline TE-163-AP-33 & 617\%9 & 64 & CASS & $\mathbf{S}$ & Dets point did not goree whth beal trend \\
\hline TE-106-AP-38 & 62004 & 84 & CASS & $\mathbf{s}$ & Data point did not cerres with beal trend \\
\hline TE-10G-AP-3B & $6 / 2494$ & 93 & CASS & $\bar{T}$ & \\
\hline TE-106-AP-33 & $6 / 2764$ & 9. & CASS & $T$ & \\
\hline TE-106-AP-38 & 73194 & 93 & CASS & $\mathbf{T}$ & \\
\hline TE-106-AP-33 & $7 / 25194$ & 62 & SACS & S(G in SACS) & Dota paint did not agree with locel trend \\
\hline TE-106-AP-38 & $7 / 30194$ & 88 & CASS & $T$ & \\
\hline TE-106-AP-38 & 8AS4 & 93 & CASS & $T$ & \\
\hline TE-106-AP-38 & $8 / 24 / 94$ & 91 & CASS & $T$ & \\
\hline$T E-106-A P-38$ & $10 / 1 / 9$ & 96 & CASS & $\mathbf{T}$ & \\
\hline TE-106-AP-SB & 108/94 & 89 & CASS & $T$ & \\
\hline TE-106-AP-38 & 10/15/94 & 89 & CASS & $T$ & \\
\hline TE-10S-AP-3B & $10 / 17 / 94$ & 101 & CASS & $\bar{T}$ & \\
\hline TE-106-AP-38 & $10 / 25 / 94$ & 100 & CASS & $T$ & \\
\hline TE-106-AP-3B & $11 / 524$ & 102 & CASS & $T$ & \\
\hline TE-10B-AP-36 & $11 / 11 / 9$ & 103 & CASS & $T$ & \\
\hline TE-106-AP-33 & $11 / 18 / 94$ & 100 & CASS & 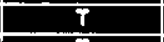 & \\
\hline TE-10G-AP-38 & $11 / 22194$ & 103 & CASS & $T$ & \\
\hline TE-100-AP-38 & $11 / 2984$ & 97 & CASS & $T$ & \\
\hline TE-1CG-AP-38 & $1214 \mathrm{~s}$ & 101. & CASS & $\mathbf{T}$ & \\
\hline TE-106-AP.88 & $12 / 1694$ & 96. & CASS & $\mathbf{T}$ & \\
\hline TE-10B-AP-38 & $12 / 19 / 94$ & 100 & CASS & $T$ & \\
\hline TE-106AP-3B & $1231 \% 4$ & 93 & CASS & $\mathbf{T}$ & \\
\hline TE-106-AP-3B & $1 / 495$ & 104 & CASS & $T$ & \\
\hline TE-105-AP-38 & $1 / 1495$ & 89 & CASS & $T$ & \\
\hline TE-106-AP-33 & $1 / 15 \times 5$ & 88 & CASS & $T$ & \\
\hline TE-106-AP-3B & $1 / 23155$ & 100 & CASS & $T$ & \\
\hline TE-106-AP-33 & $2 / 2195$ & 95 & CASS & $T$ & \\
\hline TE-106-AP-38 & $2 / 10 / 85$ & 98 & CASS & $T$ & \\
\hline TE-106-AP-33 & $2 / 4 / 55$ & 105 & CASS & $T$ & \\
\hline TE-106-AP-38 & $2 / 24 / 95$ & 94 & CASS & $T$ & \\
\hline
\end{tabular}




\begin{tabular}{|c|c|c|c|c|c|}
\hline \multicolumn{6}{|c|}{$\begin{array}{l}\text { TANK 241-APS106 } \\
\text { THEMMOCOUPLE DATA }\end{array}$} \\
\hline THERMOCOUPLE & DATE & TEMPERATUAE $\left({ }^{\circ} \mathrm{F}\right)$ & & & \\
\hline 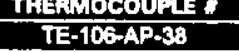 & DAIE & $\begin{array}{r}\text { HEMPIERATURE (MT) } \\
100\end{array}$ & $\frac{\text { DATA SOUACE }}{\text { CASS }}$ & $\frac{\text { OUALITY }}{T}$ & COMMENT \\
\hline TE-106-AP-3B & $3 \pi / 95$ & 97 & CASS & $\frac{T}{T}$ & \\
\hline TE-106-APS38 & 3/6ras & 83 & CASS & $\frac{T}{T}$ & \\
\hline TE-106-AP-38 & 3/23/95 & 100 & CASS & $T$ & \\
\hline TE-106-AP-38 & 3/30/95 & 94 & CASS & $T$ & \\
\hline TE-106-AP-33 & $4 / 3 / 55$ & 86 & SACS & $\mathbf{G}$ & \\
\hline TE-106-AP-38 & 4/10/25: & 85 & SACS & G & \\
\hline TE-106-AP-3B & 41705 & 80 & SACS & $G$ & \\
\hline TE-106-AP-3B & 4/2495 & 80 & SACS & G & \\
\hline TE-106-AP-38 & $5 / 195$ & 83 & SACS & $\mathbf{G}$ & \\
\hline TE-106-AP-38 & 5Bas & 81 & SACS & $\bar{G}$ & \\
\hline TE-106-AP-38 & $5 / 16 / 56$ & 79 & SACS & G & \\
\hline TE-106-AP-38 & 52235 & 81 & SACS & $G$ & \\
\hline$T E-106-A P-38$ & 5/29/35 & 84 & SACS & $\mathbf{G}$ & \\
\hline TE-106-AP-33 & 6/565 & 86 & SACS & G & \\
\hline TE-106-AP-38 & $6 / 13 / 95$ & 81 & SACS & G & \\
\hline TE-106-AP-3B & 6/19/95 & 89 & SACS & G & \\
\hline & & & & & \\
\hline TE-106-AP-39 & 45186 & 56 & CASS & $\mathbf{T}$ & \\
\hline TE-10B-AP-39 & 4636 & 57 & CASS & $\mathbf{T}$ & \\
\hline TE-106-AP-38 & 5/1E/86 & 57 & CASS & $\bar{T}$ & \\
\hline TE-106-AP-39 & $5 / 2466$ & 57 & CASS & $\mathbf{T}$ & \\
\hline TE-106-AP-39 & $5 / 30 / 86$ & 59 & CASS & $T$ & \\
\hline TE-105-AP-39 & 67/36 & 50 & CASS & $\bar{T}$ & \\
\hline TE-106-AP-39 & 6806 & 59 & CASS & $T$ & \\
\hline TE-106-AP-39 & $6 / 21 / 166$ & 59 & CASS & $T$ & \\
\hline TE-10B-AP- 39 & E/2565 & 59 & CASS & $T$ & \\
\hline TE-106-AP-39 & 74406 & 62 & CASS & $T$ & \\
\hline TE-106-APSBO & $7 / 2266$ & 60 & CASS & $T$ & \\
\hline TE-106-AP-38 & $718 / 36$ & 61 & CASS & $T$ & \\
\hline TE-106-AP-39 & $7 / 21 / 36$ & 62 & CASS & $\mathbf{T}$ & \\
\hline TE-105-AP-39 & 772806 & 62 & CASS & $\bar{T}$ & \\
\hline TE-106-AP-39 & 8086 & 6A & CASS & $T$ & \\
\hline TE-106-AP-39 & $8 / 1486$ & 69 & CASS & $T$ & \\
\hline TE-106-AP-39 & $8 / 23 / 66$ & 62 & CASS & $\mathbf{T}$ & \\
\hline TE-10E-AP-39 & $8 / 27156$ & 84 & CASS & $T$ & \\
\hline TE-10S-AP-39 & 91/36 & 65 & CASS & $T$ & \\
\hline TE-106-AP-38 & 9rab6 & 65. & CASS & $T$ & \\
\hline TE-106-AP-39 & 1003/36 & 64 & CASS & $T$ & \\
\hline TE-106-AP-39 & 10/8/86 & GA & CASS & $\mathbf{T}$ & \\
\hline TE-106-AP-39 & 10r1686 & 63 & CASS & $T$ & \\
\hline TE-106-AP-39 & $10 / 23 / 86$ & 63 & CASS & $\mathbf{T}$ & \\
\hline TE-106-AP-39 & $10 / 2686$ & 63 & CASS & $T$ & \\
\hline TE-106-AP-39 & $11 / 3 / 66$ & 63 & CASS & $T$ & \\
\hline TE-10B-AP-39 & $116 / 66$ & 60 & CASS & $T$ & \\
\hline TE-10G-AP-39 & $11 / 17 / 86$ & 69 & CASS & S & Data point did not agree with locel trend \\
\hline TE-10G-AP-30 & $11 / 22 / 86$ & 63 & CASS & $T$ & \\
\hline TE-106-AP-39 & $11 / 27 / 66$ & 58 & CASS & $T$ & \\
\hline TE-106-AP-39 & 122206 & 58 & CASS & $T$ & \\
\hline TE-10B-AP-39 & $12 / 1 / 36$ & 58 & CASS & $T$ & \\
\hline TE-106-AP-39 & 122006 & 56 & CASS & $T$ & \\
\hline TE-106-AP-39 & $12 / 23 / 36$ & 57 & CASS & $T$ & \\
\hline TE-106-AP-39 & $12 / 31 / 36$ & 56 & CASS & $T$ & \\
\hline TE-106-AP-39 & $7 / 4 / 87$ & 65 & CASS & $T$ & \\
\hline TE-106-AP-39 & 79 197 & 67 & CASS & $\bar{T}$ & \\
\hline TE-106-AP-39 & $7 / 12 / 37$ & 67 & CASS & $T$ & \\
\hline TE-106-AP-39 & $7 / 19 / 87$ & 68 & CASS & $T$ & \\
\hline TE-106-AP-39 & 8/167 & 66 & CASS & $\mathbf{T}$ & \\
\hline TE-106-AP-39 & $8 / 5 / 87$ & 68: & CASS & $T$ & \\
\hline TE-106-AP-39 & $8 / 44 / 87$ & 69 & CASS & $\mathbf{T}$ & \\
\hline TE-106-AP-39 & $8 / 17 / 67$ & 69 & CASS & $\bar{T}$ & \\
\hline TE-106-AP-39 & 8/26/87 & 69 & CASS & $T$ & \\
\hline TE-106-AP-39 & 9/4/87 & E8 & CASS & $T$ & \\
\hline TE-106-AP-39 & 9/8/37 & 70 & CASS & $\bar{T}$ & \\
\hline TE-106-AP-39 & 9/17/37 & 69 & CASS & $T$ & \\
\hline TE-106-AP-39 & 9/23/67 & 69 & CASS & $T$ & \\
\hline TE-106-AP-39 & 10/3/67 & 68 & CASS & $\bar{T}$ & \\
\hline TE-106-AP-39 & 10/27/37 & 65 & CASS & $\mathbf{T}$ & \\
\hline TE-106-AP-39 & 11/7/67 & 64 & CASS & $\mathbf{T}$ & \\
\hline TE-106-AP-39 & 11/11/37 & 64 & CASS & $\bar{T}$ & \\
\hline
\end{tabular}




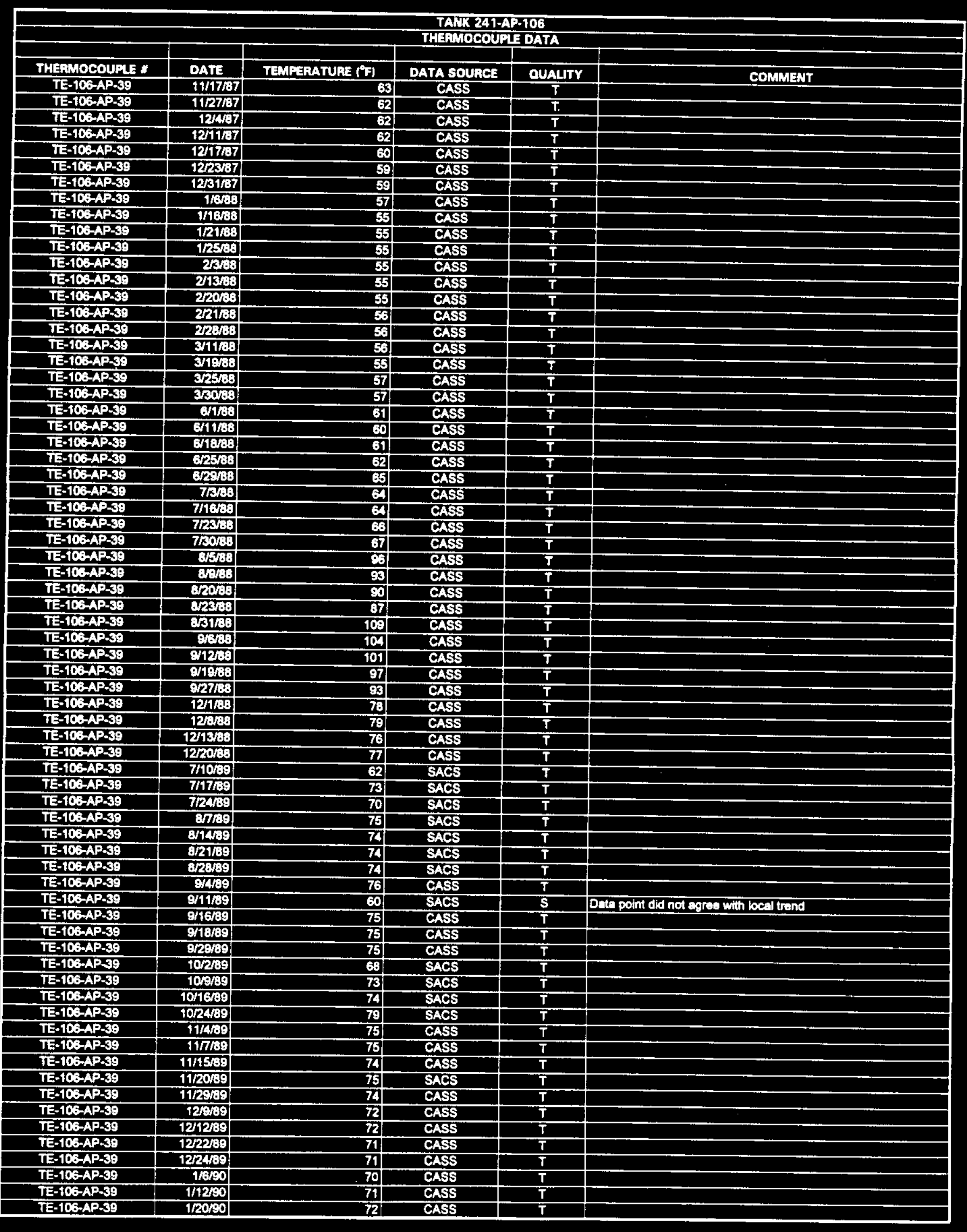




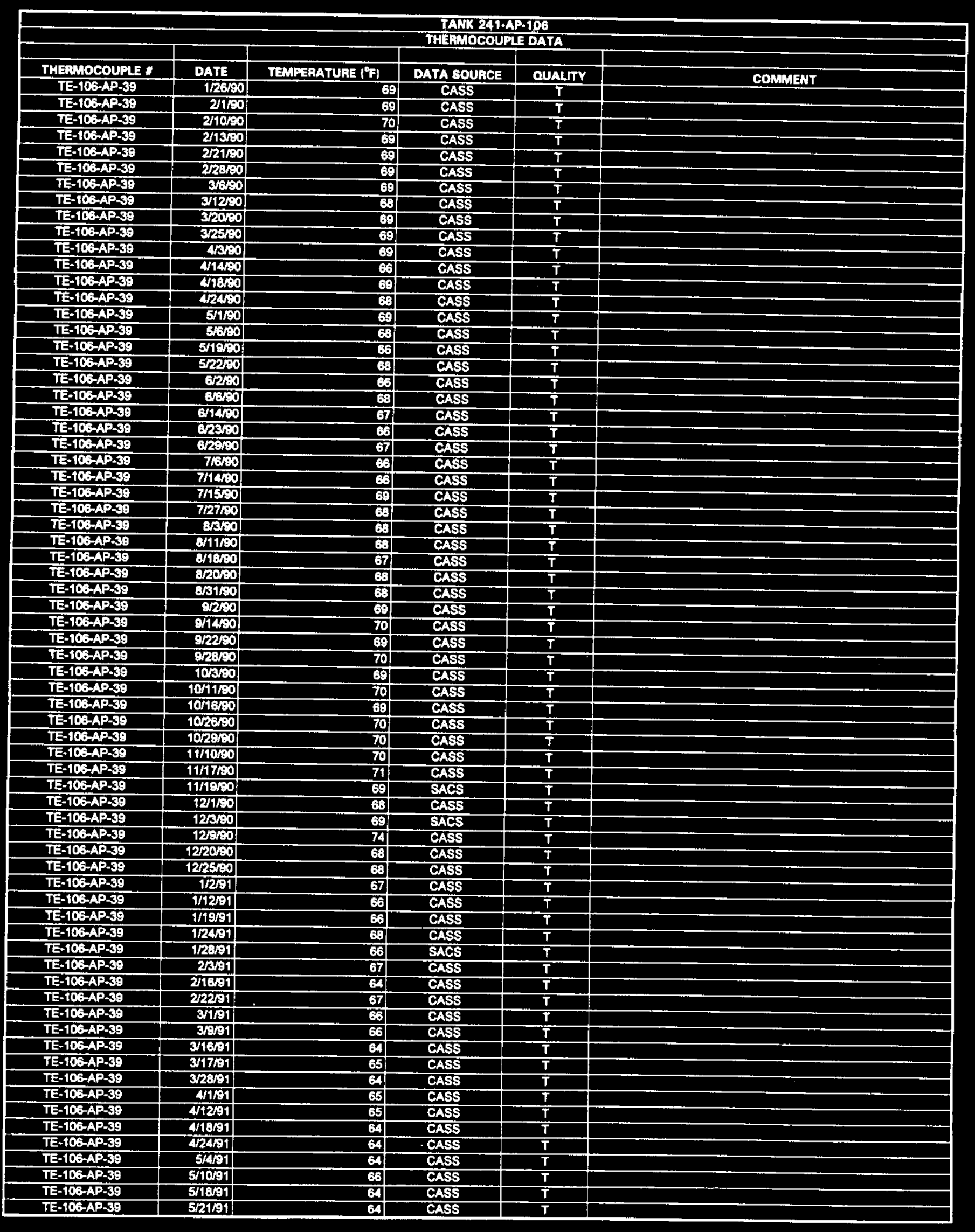




\begin{tabular}{|c|c|c|c|c|c|}
\hline \multicolumn{6}{|c|}{$\begin{array}{l}\text { TANK 241-AP-106 } \\
\text { THEFMOCOUFL DATA }\end{array}$} \\
\hline & & & & & \\
\hline THERHOCOUPE: & DATE & TEMFERATUAE $\left({ }^{\circ} F\right)$ & DATA SOUACE & QUALTY & COMMENT \\
\hline TE-106-AP-39 & $5 / 31 / 91$ & 64 & CASS & $T$ & \\
\hline TE-10B-AP-39 & 67191 & 65 & CASS & $T$ & \\
\hline TE-103-AP-39 & G/5ra1 & 66 & CASS & $T$ & \\
\hline TE-106APS39 & 6/18/81 & 64) & CASS & 7 & \\
\hline TE-10G-AP-39 & $\frac{6 / 28 / 91}{7 \times 201}$ & EA & $\begin{array}{l}\text { CASS } \\
\text { CASS }\end{array}$ & $\frac{T}{T}$ & \\
\hline TE-106-AP-30 & $\frac{7391}{7391}$ & 64 & $\begin{array}{l}\text { CASS } \\
\text { SACS }\end{array}$ & $\frac{T}{T}$ & \\
\hline & \begin{tabular}{r|}
73891 \\
$715 \times 1$
\end{tabular} & 64 & SACS & $T$ & \\
\hline$\frac{T E-106-A P-39}{T E-106-A P-39}$ & 7122201 & 68 & SACS & $T$ & \\
\hline TE-10B-AP-39 & 8/191 & GA & CASS & $T$ & \\
\hline TE-106-AP- 39 & B6O1 & 65 & CASS & $\mathbf{T}$ & \\
\hline TE-106-AP-39 & $8 / 17 \% 1$ & 68 & CASS & $T$ & \\
\hline TE-16S-AP-39 & $820 \% 1$ & 68 & CASS & $T$ & \\
\hline TE-106-AP-38 & B/sobal & 63 & CASS & $T$ & \\
\hline TE-103-AP-30 & 81/21 & 65 & CASS & $T$ & \\
\hline TE-106-APSB & Ese1 & 66 & CASS & $T$ & \\
\hline TE-106-AP.39 & gram & 68 & CASS & $T$ & \\
\hline TE-106-AP-30 & 10/1h1 & 67 & CASS & $\mathbf{T}$ & \\
\hline TE-106-AP-39 & $10 / 1401$ & 67 & CASS & $T$ & \\
\hline TE-103-AP-30 & 10/2501 & 83 & CASS & $\mathbf{T}$ & \\
\hline TE-103-AP-39 & 10/29a1 & 67 & CASS & $T$ & \\
\hline IE-106-AP-39 & 11391 & 69: & CASS & $\mathbf{T}$ & \\
\hline TE-106-AP-30 & 82292 & GA & CASS & $T$ & \\
\hline TE-106-AP-30 & $828 \% 2$ & 66 & CASS & $\mathbf{T}$ & \\
\hline TE-TOSAP-39 & $8 B \% 2$ & 65 & CASS & $\mathbf{T}$ & \\
\hline TE-106-AP-38 & Spo2 & 67 & CASS & $T$ & \\
\hline TE-106-AP-38 & 9/1682 & 63 & CASS & $T$ & \\
\hline TE-102-AP-39 & 82628 & 60 & CASS & $\mathbf{T}$ & \\
\hline TE-106-AP-30 & $10 / 32$ & 67 & CASS & $T$ & \\
\hline TE-106-AP-39 & $10 \mathrm{rat} 2$ & 67 & CASS & $T$ & \\
\hline TE-106-AP-30 & $10 / 1752$ & 66 & CASS & $T$ & \\
\hline TE-10B-AP-39 & $10 / 22292$ & 69 & CASS & $T$ & \\
\hline TE-106-AP-30 & $10 \sqrt{3132}$ & 63 & CASS & $T$ & \\
\hline TE-103APख39 & $11 / 3,2$ & 63 & CASS & $T$ & \\
\hline TE-10B-AP-38 & $11 / 11 / 02$ & 67 & CASS & $T$ & \\
\hline TE-106-AP-38 & T1rake2 & 63 & CASS & $T$ & \\
\hline TE-103AP-39 & $11 / 2352$ & 66 & CASS & $\mathbf{T}$ & \\
\hline TE-106-AP-39 & 123392 & 66 & CASS & $T$ & \\
\hline TE-106-APS30 & 121032 & 68 & CASS & $T$ & \\
\hline TE-106-AP-38 & 1211782 & 67 & CASS & $T$ & \\
\hline TE-106-AP-30 & 122625 & 65 & CASS & $\mathbf{T}$ & \\
\hline TE-106-AP-39 & $12 / 2782$ & 65 & CASS & $\mathbf{T}$ & \\
\hline TE-106-AP-39 & $1 / 15 \mathrm{~A}$ & Ga & CASS & $T$ & \\
\hline TE-106-AP-39 & $1 / 18 \mathrm{~m}$ & 65 & CASS & $\mathbf{T}$ & \\
\hline TE-10B-AP-39 & $1 / 25 \mathrm{~m}$ & 66 & CASS & $\mathbf{T}$ & \\
\hline TE-106-AP.39 & 2449 & 65 & CASS & $T$ & \\
\hline TE-106-AP-39 & 2/1194 & 63 & CASS & $T$ & \\
\hline TE-T0G-AP-39 & $2 / 15 \% 9$ & 64 & CASS & $T$ & \\
\hline TE-106-AP-39 & $2 / 2694$ & 64 & CASS & $T$ & \\
\hline TE-106-AP359 & $3 / 59$ & 65 & CASS & $T$ & \\
\hline TE-10S-AP-39 & 3/1004 & 65 & CASS & $T$ & \\
\hline TE-106-AP.39 & 3/17/94 & 65 & CASS & $T$ & \\
\hline TE-106-AP-39 & $3 / 22 / 34$ & 63. & CASS & $\mathbf{T}$ & \\
\hline TE-106-AP-39 & 3/23/94 & 63 & CASS & $T$ & \\
\hline TE-106-AP-39 & $4 / 3 / 4$ & 68 & CASS & $\bar{T}$ & \\
\hline TE-106-AP-39 & $4 / 1319$ & 64 & CASS & $T$ & \\
\hline TE-106-AP-39 & $4 \sqrt{2009}$ & 64. & CASS & $T$ & \\
\hline TE-106-AP-39 & 4/20194 & 65 & CASS & $T$ & \\
\hline TE-106-AP-39 & $5 / 294$ & 64 & CASS & $T$ & \\
\hline TE-106-AP-39 & 5/11/94 & 84. & CASS & $\mathbf{S}$ & Data point did not agree with local trend \\
\hline TE-106-AP-39 & 5/12/94 & 66 & CASS & $T$ & \\
\hline TE-106-AP-39 & 5/16/94 & 100 & CASS & $T$ & \\
\hline TE-106-AP-39 & 5/23/94 & 93 & CASS & $\mathbf{T}$ & \\
\hline TE-106-AP-39 & 6/2/94 & 85 & CASS & $\bar{T}$ & \\
\hline TE-106-AP-39 & 6/8/94 & 83 & CASS & $\mathbf{T}$ & \\
\hline TE-106-AP-39 & 6/14/94 & 91 & CASS & $T$ & \\
\hline TE-106-AP-39 & 6/16/94 & 64 & CASS & $\mathbf{s}$ & Data point did not egree with local trend \\
\hline TE-106-AP-39 & 6/17/89 & 64 & CASS & 8 & Data point dild not acree with beal trend \\
\hline TE-106-AP-39 & 6/20/84 & 64 & CASS & $\mathbf{S}$ & Data point did not erroe with bocal trend \\
\hline TE-106-AP-39 & G/2404 & 82 & CASS & $T$ & \\
\hline
\end{tabular}




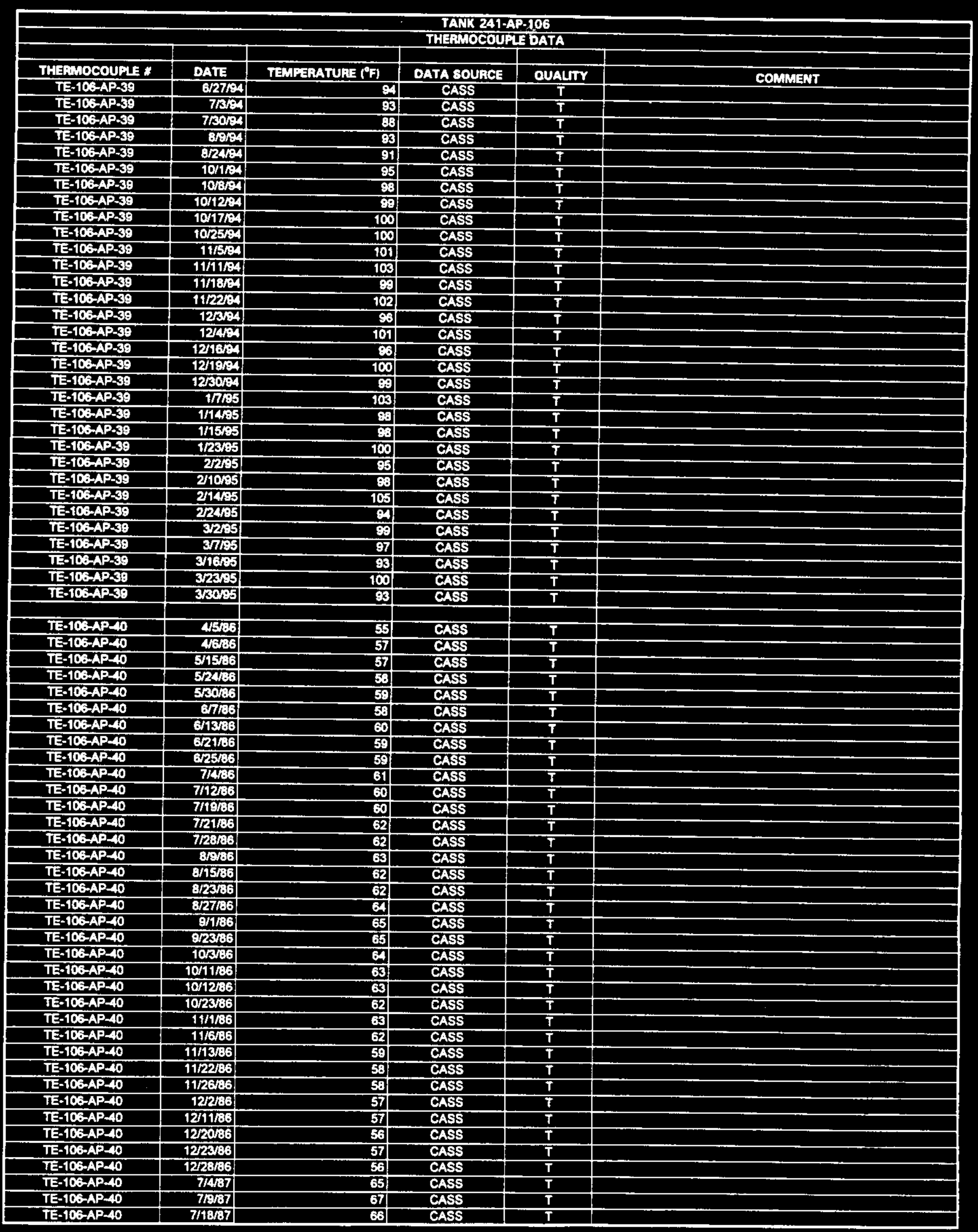




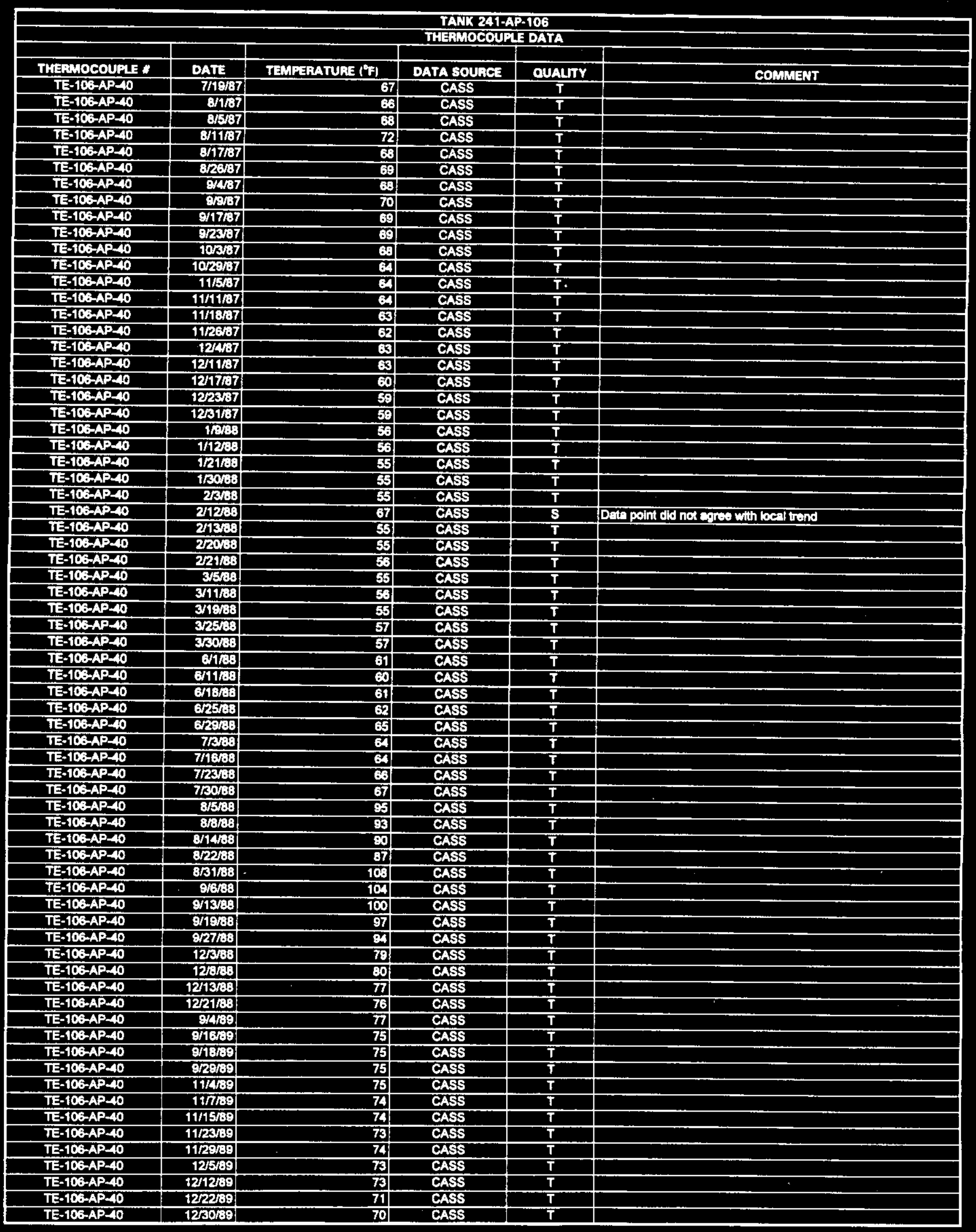




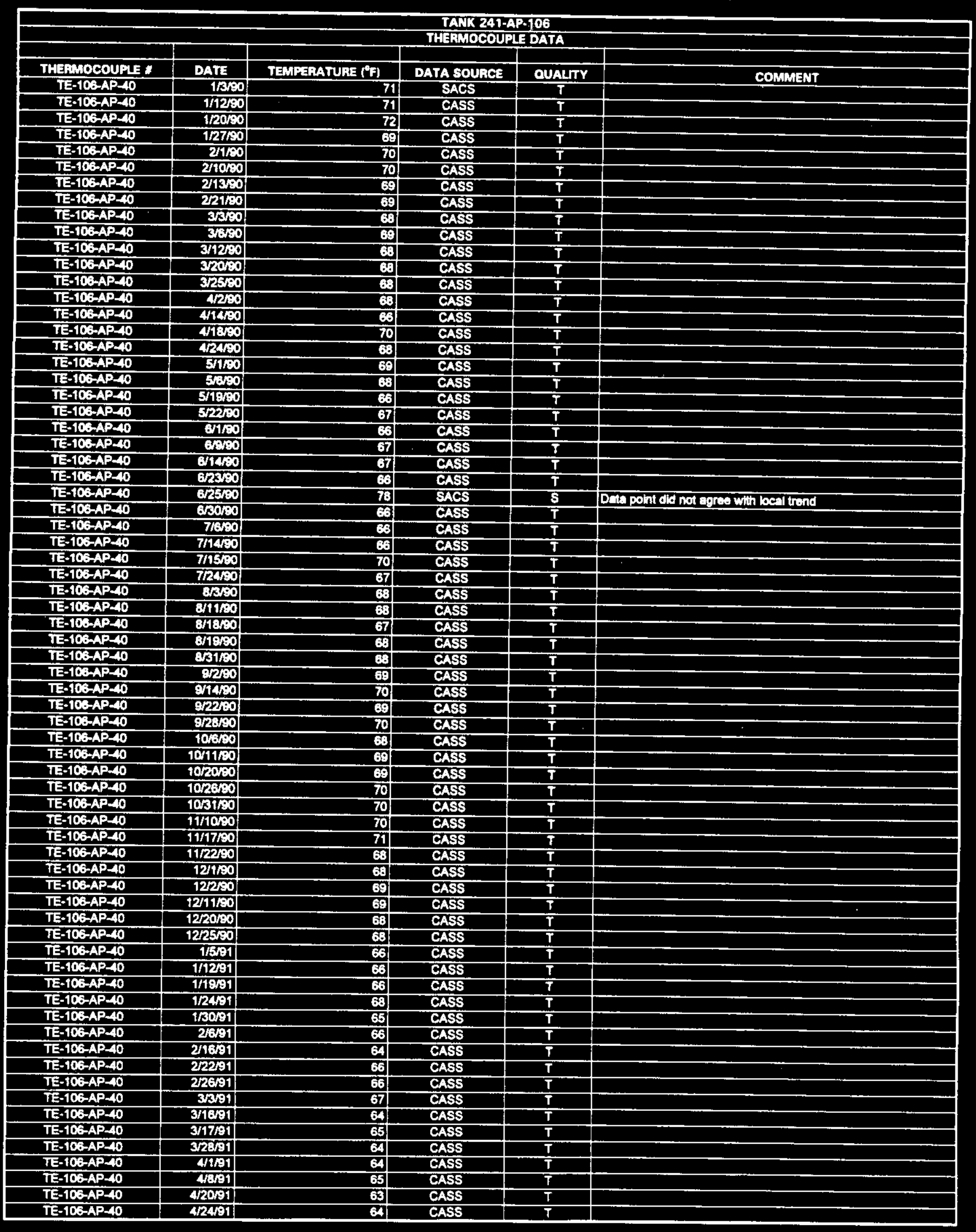




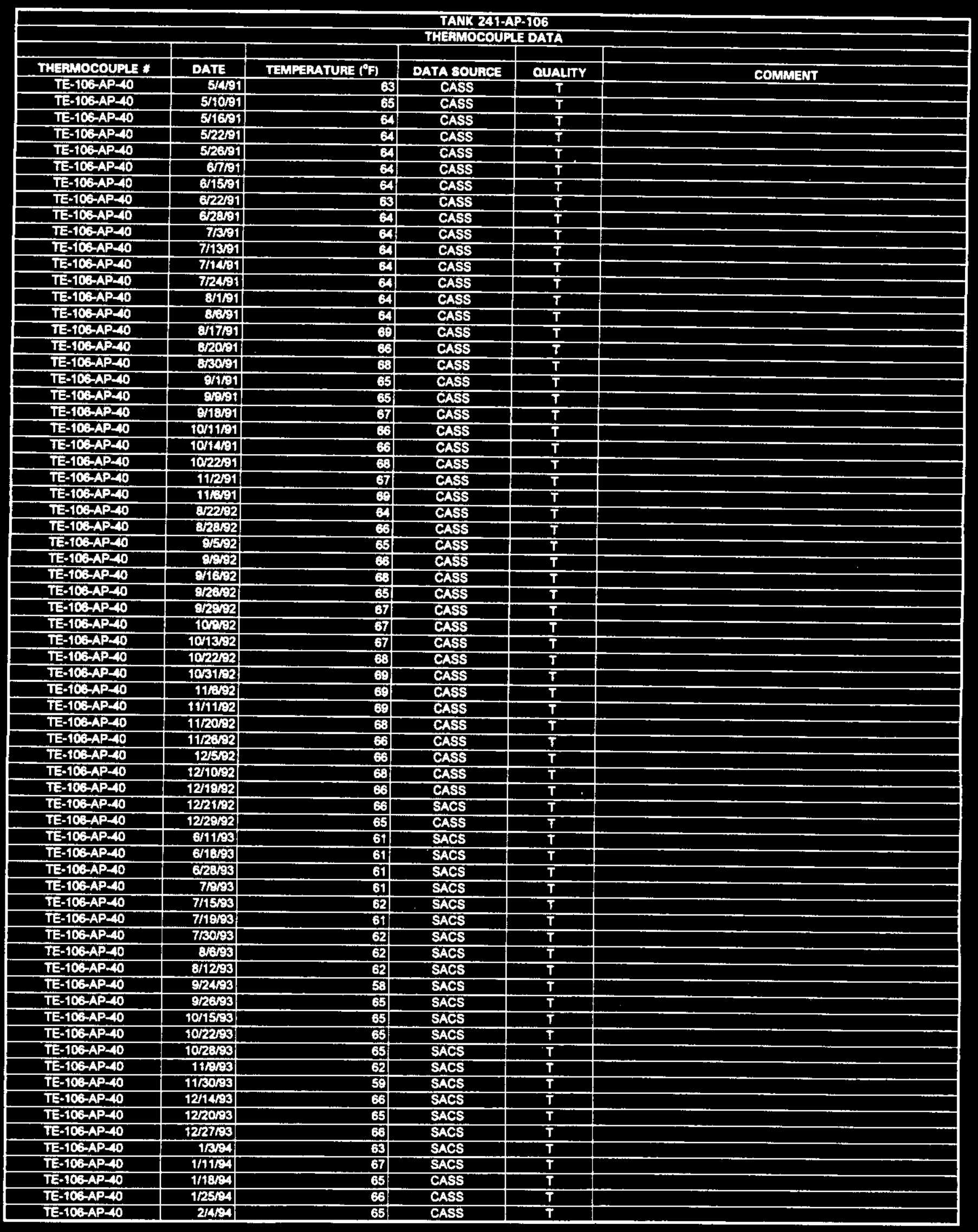

Data obtained from Computer Automated Surveislance Syatem (CASS), April 1995; and Surveillance Analysis Computer System (SACS), June 1995.

$$
\text { D-508 }
$$




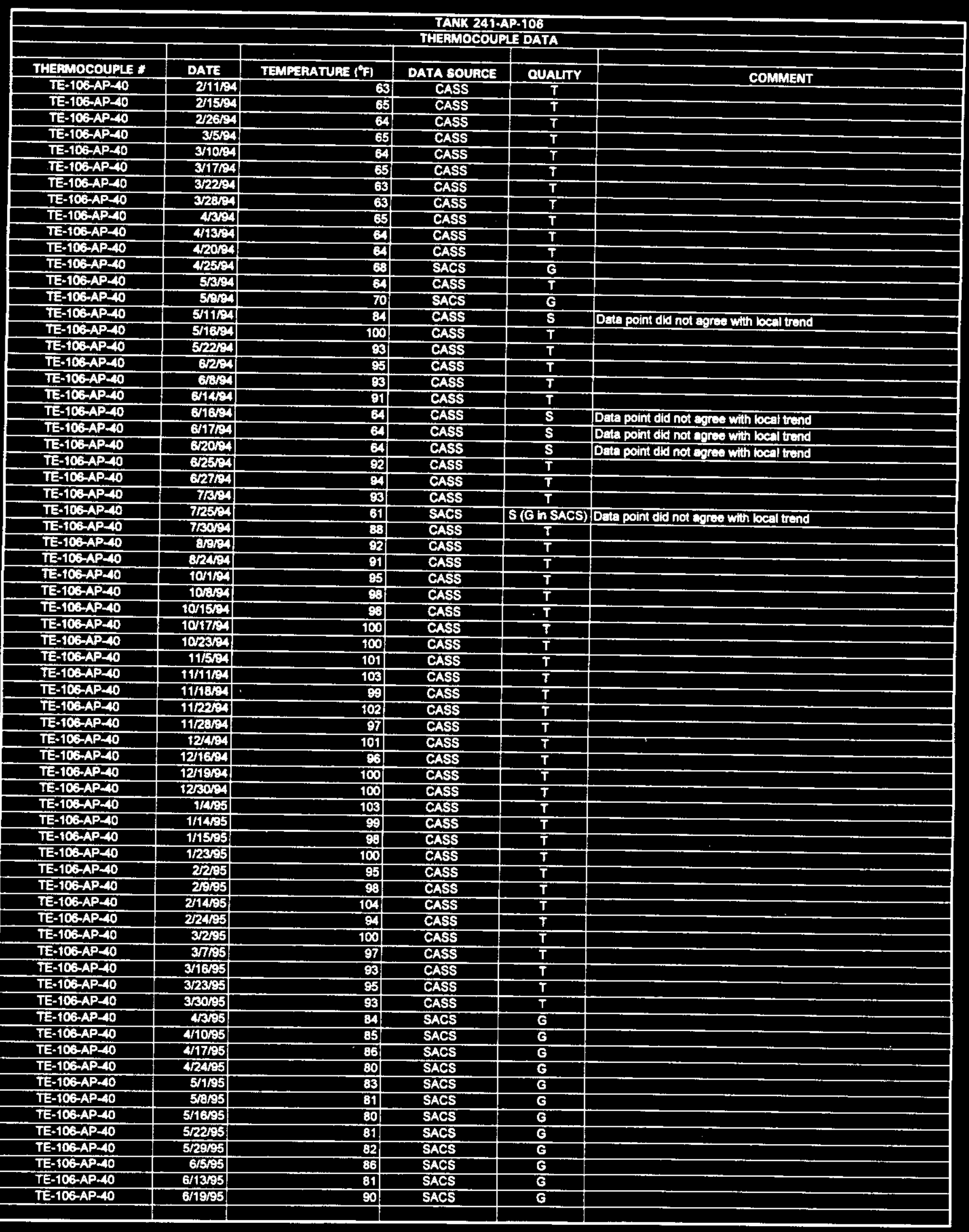




\begin{tabular}{|c|c|c|c|c|c|}
\hline \multicolumn{6}{|c|}{$\begin{array}{l}\text { TANK 241-AP-106 } \\
\text { THERMOCOUPLE DATA }\end{array}$} \\
\hline THERMOCOUPLE * & DATE & TEMPERATURE $\left({ }^{\circ} \mathrm{F}\right)$ & DATA sougce & OUAYTY & \\
\hline TE-106-AP-41 & $4 / 5 / 86$ & 55 & $\frac{\text { DAIA OODHCE }}{\text { CASS }}$ & $\frac{\text { QUALIY }}{T}$ & COMMENT \\
\hline TE-106-AP-AI & $46 / 66$ & 57 & CASS & $T$ & \\
\hline TE-106-AP-41 & 5/16/36 & 57 & CASS & $T$ & \\
\hline TE-106-AP-A1 & 5/2406: & $\mathbf{5 3}$ & CASS & $T$ & \\
\hline TE-106-AP-A1 & 533066 & 58 & CASS & $T$ & \\
\hline TE-106-AP-A1 & $67 / 36$ & $\mathbf{3 3}$ & CASS & $T$ & \\
\hline TE-103AP-A1 & erost & 60 & CASS & $T$ & \\
\hline TE-106-APA1 & $6 / 21 / 36$ & 50 & CASS & $T$ & \\
\hline TE-10B-AP-11 & 6/25/66 & 59 & CASS & $T$ & \\
\hline TE-106-AP-A1 & 71403 & 62 & CASS & $T$ & \\
\hline TE-106-AP-41 & 712/86 & 60 & CASS & $\mathbf{T}$ & \\
\hline TE-103-AP-41 & $77 \%$ & 80 & CASS & $T$ & \\
\hline TE-106-AP-41 & $7 / 21 / B 6$ & 62 & CASS & $T$ & \\
\hline$T E-10 B-A P-41$ & 720106 & 62 & CASS & $T$ & \\
\hline TE-106-APCA1 & Bab & 63 & CASS & $\mathbf{T}$ & \\
\hline TE-106-AP-A1 & 8/13/36 & 63 & CASS & $T$ & \\
\hline TE-TOB-AP-A1 & 123\%6 & 62 & CASS & $\mathbf{T}$ & \\
\hline TE-TOG-AP-A1 & $827 / 36$ & 63 & CASS & $\mathbf{T}$ & \\
\hline TE-108-AP-11 & Q1/30 & 6 & CASS & $\bar{T}$ & \\
\hline TE-106-AP-A1 & $8 / 23 / 36$ & 65 & CASS & $\bar{T}$ & \\
\hline$T E-106-A^{2}-11$ & TOMB & 61 & CASS & $\bar{T}$ & \\
\hline TE-103-AP-A1 & $10 / 11 / 36$ & 63 & CASS & $T$ & \\
\hline$T E-106-A P-11$ & $10 / 483$ & 63 & CASS & $\bar{T}$ & \\
\hline TE-106-AP-A1 & $10 / 223$ & 63 & CASS & $\bar{T}$ & \\
\hline TE-106-AP-AI & 11/1/66 & 63 & CASS & $T$ & \\
\hline TE-106-AP-41 & 11366 & 63 & CASS & $\bar{T}$ & \\
\hline TE-103-AP-41 & $11 / 13 \mathrm{~B} 6$ & 60 & CASS & $T$ & \\
\hline TE-106-APACA1 & 11/18:8 & 59 & CASS & $\bar{T}$ & \\
\hline$T E-106-A P-41$ & 11/23686 & 50 & CAss & $T$ & \\
\hline TE-10B-AP $=41$ & 12 22/36 & 58 & CASS & $T$ & \\
\hline TE-106-AP-AI & $12 / 1160$ & 57 & CASS & $\bar{T}$ & \\
\hline TE-106-AP-41 & 122006 & 36 & CASS & $T$ & \\
\hline TE-106-AP-A1 & $12 / 2466$ & 57 & CASS & $T$ & \\
\hline TE-IDE-AP-41 & 1222836 & 57 & CASS & $\bar{T}$ & \\
\hline TE-TOE-APA1 & 714867 & 65 & CASS & $T$ & \\
\hline$T E-106-A P A 1$ & 7807 & 67 & CASS & $T$ & \\
\hline TE-106AP-A1 & 718:87 & 63 & CASS & $T$ & \\
\hline TE-106-AP-A1 & 71607 & 67 & CASS & $T$ & \\
\hline TE-106-AP-A1 & 8/1307 & 6 & CASS & $\bar{T}$ & \\
\hline TE-103-AP-A1 & $8 \longdiv { 3 0 7 }$ & 63 & CASS & $T$ & \\
\hline TE-106-AP-A1 & $8 / 11 / 67$ & 69 & CASS & $\bar{T}$ & \\
\hline$T E-106-A P-4 !$ & $8 / 17 / 87$ & 69 & CASS & $T$ & \\
\hline TE-10B-AP-11 & $8 / 26 / 87$ & 69 & CASS & $T$ & \\
\hline$T E-106-A P-41$ & 9467 & 63 & CASS & $T$ & \\
\hline$T E-106-A P-41$ & 9\%/87 & 70 & CASS & $T$ & \\
\hline$T E-106-A P-41$ & 817/77 & 70 & CASS & $\mathbf{T}$ & \\
\hline TE-106-AP-A1 & g/23/67 & 69 & CASS & $T$ & \\
\hline$T E-106-A P-A 1$ & 10/1/87 & 68 & CASS & $T$ & \\
\hline TE-106-AP-A1 & $10 / 28 / 37$ & 65 & CASS & $T$ & \\
\hline TE-106-AP-A1 & $91 / \pi / 67$ & 64) & CASS & $T$ & \\
\hline TE-106-AP-A1 & 11/B/37 & 65 & CASS & $T$ & \\
\hline TE-106-AP-41 & $11 / 18 / 87$ & 64 & CASS & $\bar{T}$ & \\
\hline TE-10S-AP-A1 & $11 / 2687$ & 63 & CASS & $\bar{T}$ & \\
\hline TE-103-AP-41 & $12 / 4 / 87$ & 63 & CASS & $T$ & \\
\hline TE-106-AP-A1 & 127/87 & 63 & CASS & $T$ & \\
\hline TE-106-AP $=41$ & 12/18/87 & 60 & CASS & $T$ & \\
\hline TE-1OB-AP-A1 & 12/24/87 & 59 & CASS & $\overline{\mathbf{T}}$ & \\
\hline TE-10S-AP-41 & 12/3167 & 59 & CASS & $\bar{T}$ & \\
\hline TE-106-AP-AT & $1 / 7 \sqrt{3}$ & 57 & CASS & $T$ & \\
\hline$T E-103-A P-4$ & 1/16/88 & 56 & CASS & $T$ & \\
\hline TE-10B-AP-A1 & $1 / 1808$ & 56 & CASS & $\mathbf{T}$ & \\
\hline TE-1C6-AP-41 & $1 / 25 / 38$ & 50 & CASS & $T$ & \\
\hline TE-106-AP-41 & $2 / 183$ & 56 & CASS & $\bar{T}$ & \\
\hline TE-106-AP-41 & $2213 / 88$ & 55 & CASS & $T$ & \\
\hline TE-106-AP-41 & $2 / 20 / 88$ & 35 & CASS & $T$ & \\
\hline TE-106-AP-41 & $2 / 21 / 88$ & 57 & CASS & $T$ & \\
\hline$T E-10 B-A P-A 1$ & 2/2a/38 & 57. & CASS & $T$ & \\
\hline$T E-106-A P-41$ & $3 / 11 / 38$ & 57 & CASS & $\bar{T}$ & \\
\hline TE-106-AP-11 & $3 / 16 / 38$ & 56 & CASS & $T$ & \\
\hline TE-106-AP-A4 & $3 / 2538$ & 57 & CASS & $T$ & \\
\hline
\end{tabular}




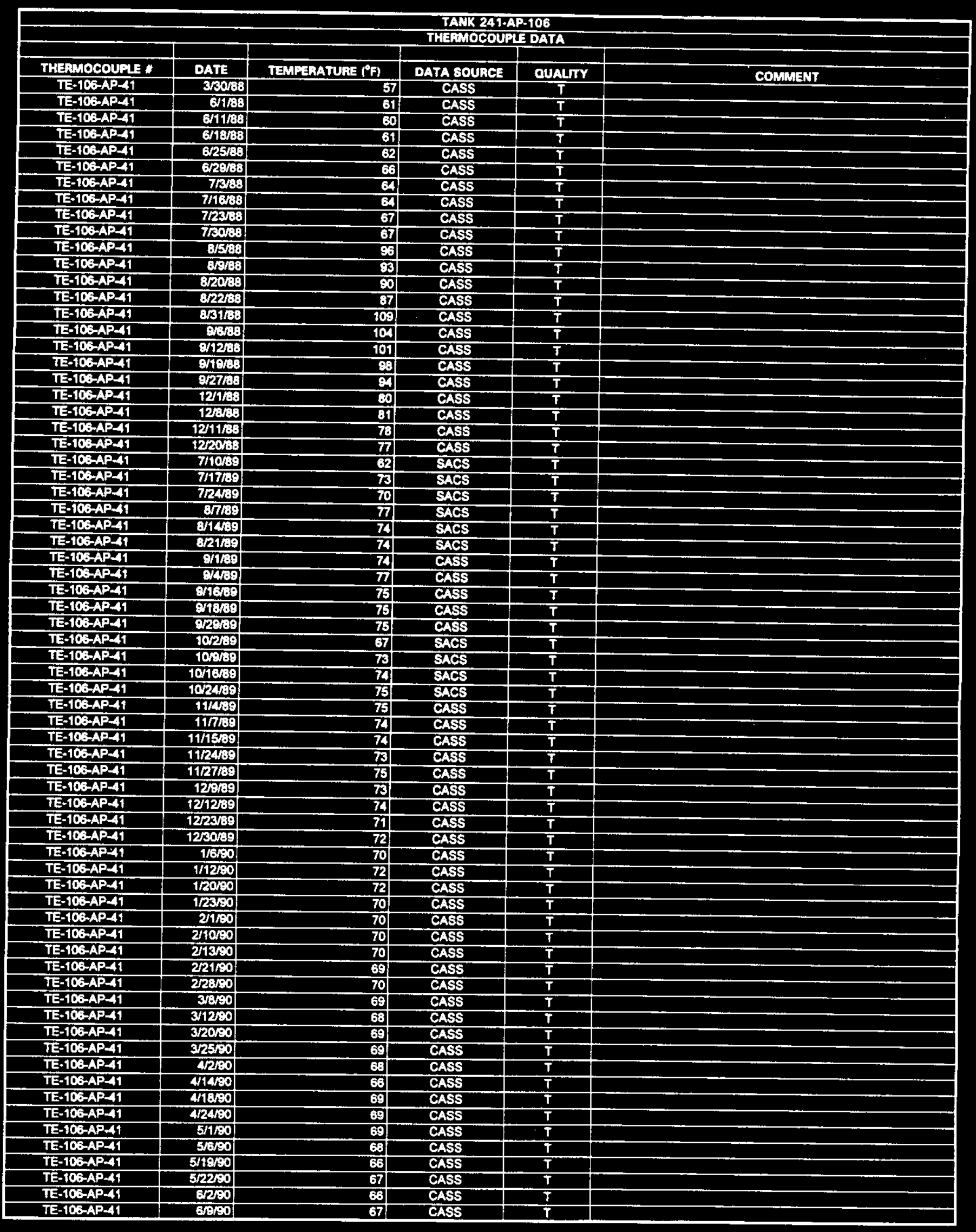




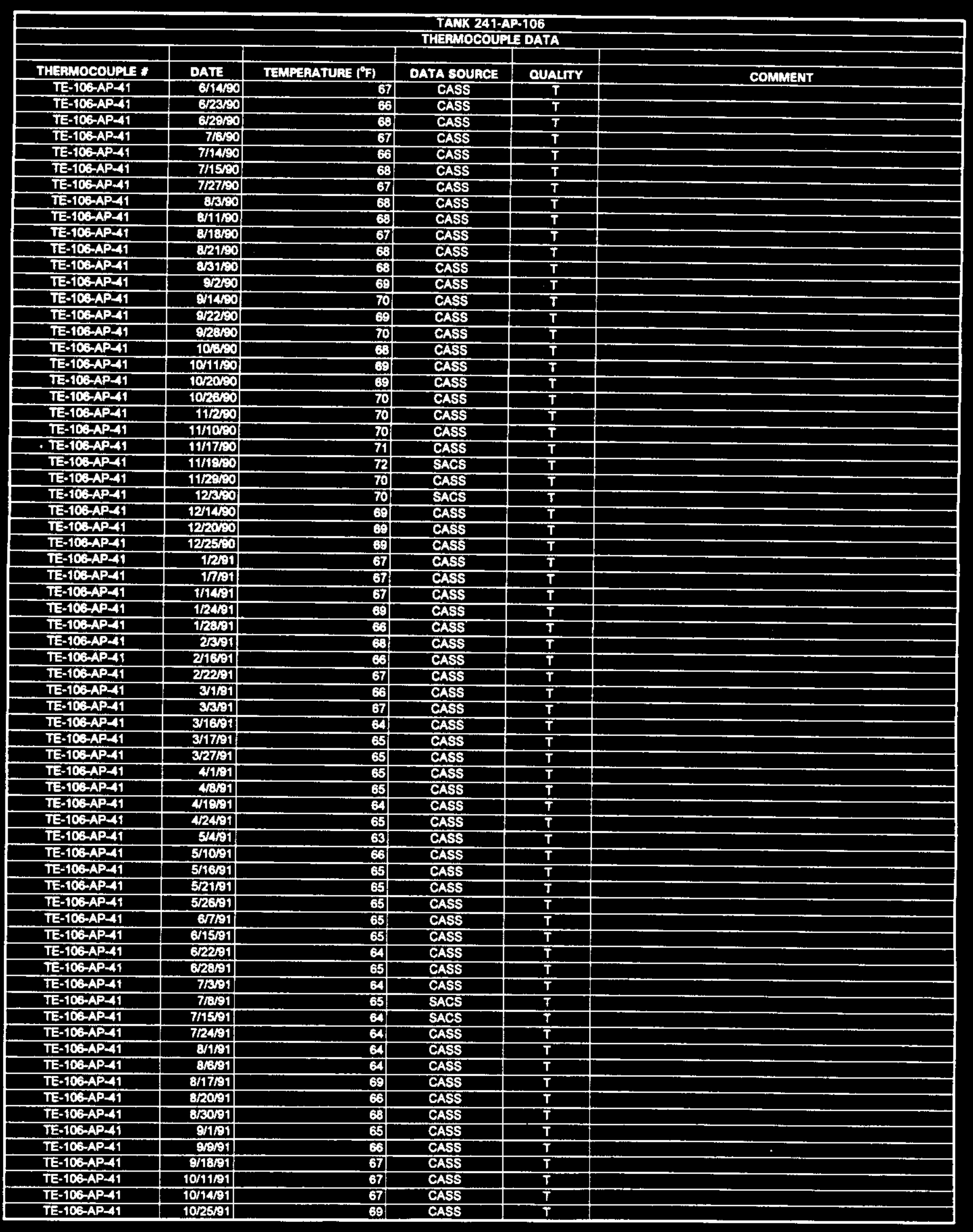

Date obtained from Computer Automated Survaillance Syatem (CASS), April 1995; and Surveillance Analyals Computer System (SACS), June 1995.

D-512 


\begin{tabular}{|c|c|c|c|c|c|}
\hline \multicolumn{6}{|c|}{$\begin{array}{l}\text { TALK 241-AP-106 } \\
\text { THEFMOCOUPLE DATA }\end{array}$} \\
\hline & & & & & \\
\hline THERMOCOUPE $*$ & DATE & TEMPERATUAE $\left(^{\circ} \mathrm{F}\right)$ & DATA SOURCE & OUALITY & COMMENT \\
\hline TE-106-AP-41 & $10 / 29 / 91$ & 68 & CASS & $T$ & \\
\hline TE-106-AP-A1 & 19/6/91 & 70 & CASS & $T$ & \\
\hline TE-106-AP-A1 & $8 / 22292$ & 6A & CASS & $\bar{T}$ & \\
\hline TE-106-AP-A1 & $8 / 28 / 92$ & 66 & CASS & $\mathbf{T}$ & \\
\hline TE-106-AP-A1 & $8 / 362$ & 65 & CASS & $T$ & \\
\hline TE-10G-AP-AT & 9/92 & 66 & CASS & $T$ & \\
\hline TE-106-AP-11 & 9/15/22 & 68 & CASS & $T$ & \\
\hline TE-106-AP-A1 & 8/26/92 & 65 & CASS & $T$ & \\
\hline TE-106-AP-A1 & 10/362 & 63 & CASS & $T$ & \\
\hline TE-106-AP-41 & $10 / 92$ & 67 & CASS & $T$ & \\
\hline TE-106-AP-11 & $10 / 13 / 92$ & 67 & CASS & $T$ & \\
\hline TE-106-AP-41 & $10 / 2262$ & 67 & CASS & $\bar{T}$ & \\
\hline$T E-106-A P-41$ & $10 / 2502$ & 69 & CASS & $T$ & \\
\hline TE-106-AP-41 & $11 / 6 / 2$ & 69 & CASS & $T$ & \\
\hline$T E-106-A P=-11$ & $19 / 11 / 92$ & 69 & CASS & $T$ & \\
\hline TE-10G-AP-A & $11 / 20192$ & 69 & CASS & $T$ & \\
\hline TE-106-AP-A1 & $11 / 28 / 92$ & 66 & CASS & $\mathbf{T}$ & \\
\hline TE-106-AP-19 & 123502 & 66 & CASS & $T$ & \\
\hline TE-106-AP-A1 & $12 / 1002$ & 68 & CASS & $T$ & \\
\hline TE-106-AP-11 & $1217 \% 2$ & 67 & CASS & $T$ & \\
\hline TE-106-AP-A1 & $12 / 26292$ & 65 & CASS & $T$ & \\
\hline TE-106-AP-41 & $1230 / 92$ & 65 & CASS & $\bar{T}$ & \\
\hline$T E-106-A P-A 1$ & $1 / 15 \%$ & 64 & CASS & $T$ & \\
\hline TE-106-AP-A1 & $1 / 189$ & 65 & CASS & $T$ & \\
\hline TE-TOG-AP-41 & $1 / 25 \%$ & 66 & CASS & $T$ & \\
\hline TE-106-AP-A1 & 21464 & 66 & CASS & $T$ & \\
\hline TE-105-AP-41 & $2 / 2 / 94$ & 6a & CASS & $\bar{T}$ & \\
\hline TE-106-AP-A1 & $2 / 16 / 9$ & 65 & CASS & $\mathbf{T}$ & \\
\hline$T E-106-A P-41$ & $2 \pi 29$ & 68 & CASS & $T$ & \\
\hline TE-106-AP-A1 & 35S4 & 66 & CASS & $T$ & . \\
\hline TE-106-AP-AT & 3/1004 & 86 & CASS & $T$ & \\
\hline IE-106-AP-A1 & $3 / 17 / 94$ & 60 & CASS & $\mathbf{T}$ & \\
\hline TE-106-AP-A1 & 3/2239 & GA & CASS & $\mathbf{T}$ & \\
\hline TE-106-AP-A1 & $3 / 3094$ & 63 & CASS & $T$ & \\
\hline TE-106-AP-A1 & 4304 & 66 & CASS & $T$ & $\%$ \\
\hline TE-106-AP-A1 & Q13/94 & 66 & CASS & $\mathbf{T}$ & \\
\hline TE-106-AP-41 & Troom & 65 & CASS & $T$ & \\
\hline TE-106AP 41 & 4290 & 65 & CASS & $T$ & \\
\hline TE-106-AP-A1 & $\sin 2$ & EA & CASS & $\bar{T}$ & \\
\hline TE-T06-AP-A1 & 5/11/34 & 8 & CASS & $\mathbf{S}$ & Data point did not epree with local trend \\
\hline TE-106-AP-A1 & $5 / 2 \%$ & 67) & CASS & $T$ & \\
\hline TE-106-AP-41 & 5/1604 & 100 & CASS & $T$ & \\
\hline TE-10B-AP-A1 & 5/23/34 & 93 & CASS & $\bar{T}$ & \\
\hline$T E-106-A P-41$ & 6/2/9 & 95 & CASS & $\mathbf{T}$ & \\
\hline TE-106-AP-41 & BBA & 93 & CASS & $\mathbf{T}$ & \\
\hline TE-106-AP-41 & 6/14/4/ & 91 & CASS & $T$ & \\
\hline TE-106-AP-A1 & 61694 & EA & CASS & $\mathbf{S}$ & Data point did not agrese with local trend \\
\hline TE-106-APA-A1 & 6/17/94 & 64 & CASS & $\mathbf{S}$ & Data point did not agree with local trend \\
\hline TE-103-AP-41 & 6/20194 & 64 & CASS & $\mathbf{S}$ & Data point did not agree with local trend \\
\hline TE-106-AP-41 & 6/25\%94 & 92 & CASS & $T$ & \\
\hline TE-106-AP-A1 & 6/27\%4 & 93 & CASS & $T$ & \\
\hline TE-106-AP-AT & $7 / 3 / 94$ & 93 & CASS & $T$ & \\
\hline TE-106-AP-11 & $730 \%$ & 88 & CASS & $\mathbf{T}$ & \\
\hline TE-106-AP-41 & 8 894 & 92 & CASS & $T$ & \\
\hline TE-106-AP-41 & $8 / 24 / 94$ & 80 & CASS & $T$ & \\
\hline TE-106-AP-41 & $10 / 1 / \%$ & 85 & CASS & $T$ & \\
\hline TE-106-AP-41 & 10/8/94 & 97 & CASS & $T$ & \\
\hline TE-106-AP-41 & $10 / 15 / 94$ & 88 & CASS & $\mathrm{T}$ & \\
\hline TE-106-AP-A1 & $10 / 1764$ & 100 & CASS & $\mathbf{T}$ & \\
\hline TE-106-AP-41 & $10 / 23194$ & 100 & CASS & $T$ & \\
\hline TE-106-AP-A1 & $11 / 5 \% 94$ & 101 & CASS & $T$ & \\
\hline TE-106-AP-41 & 11/11/6a & 103 & CASS & $T$ & \\
\hline TE-106-AP-41 & 11/1864 & 100 & CASS & $T$ & \\
\hline TE-106-AP-41 & $11 / 22294$ & 104 & CASS & $\mathbf{T}$ & \\
\hline TE-106-AP-41 & $1233 / 94$ & 97 & CASS & $T$ & \\
\hline TE-106-AP-41 & 12/494 & 102 & CASS & $T$ & \\
\hline TE-106-AP-A1 & 12/6\% & 86 & CASS & $T$ & \\
\hline TE-106-AP-A1 & $1219 \%$ & 100 & CASS & $T$ & \\
\hline TE-106-APA1 & 12/30/94 & 100 & CASS & $\mathbf{T}$ & \\
\hline TE-106-AP-A1 & $1 / 4205$ & 104 & CASS & $\bar{T}$ & \\
\hline
\end{tabular}




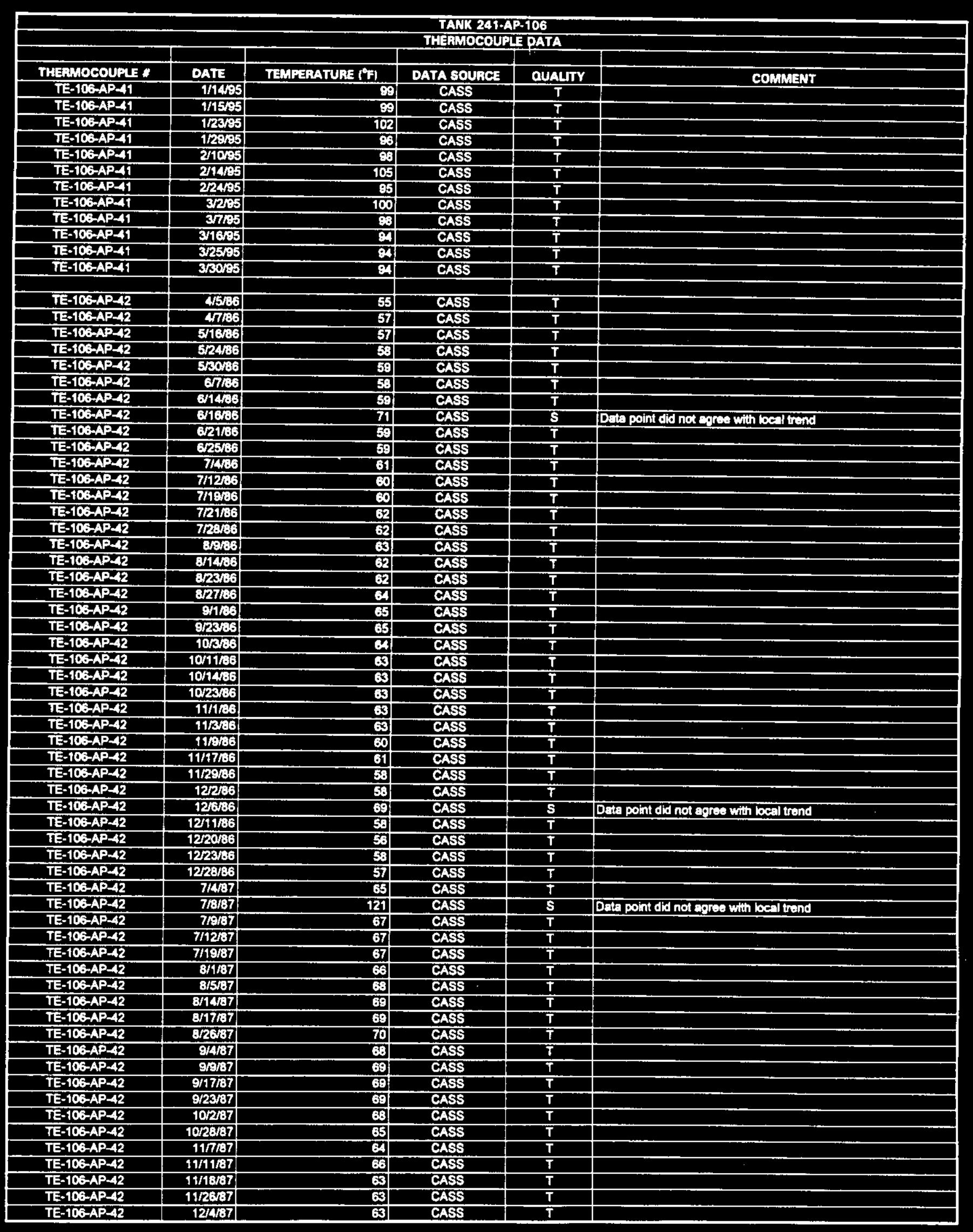

Data obtained from Computer Automated Surveillence Syotem (CASS), April 1995; and Surveillance Analysis Computer System (SACS), June 1995. 


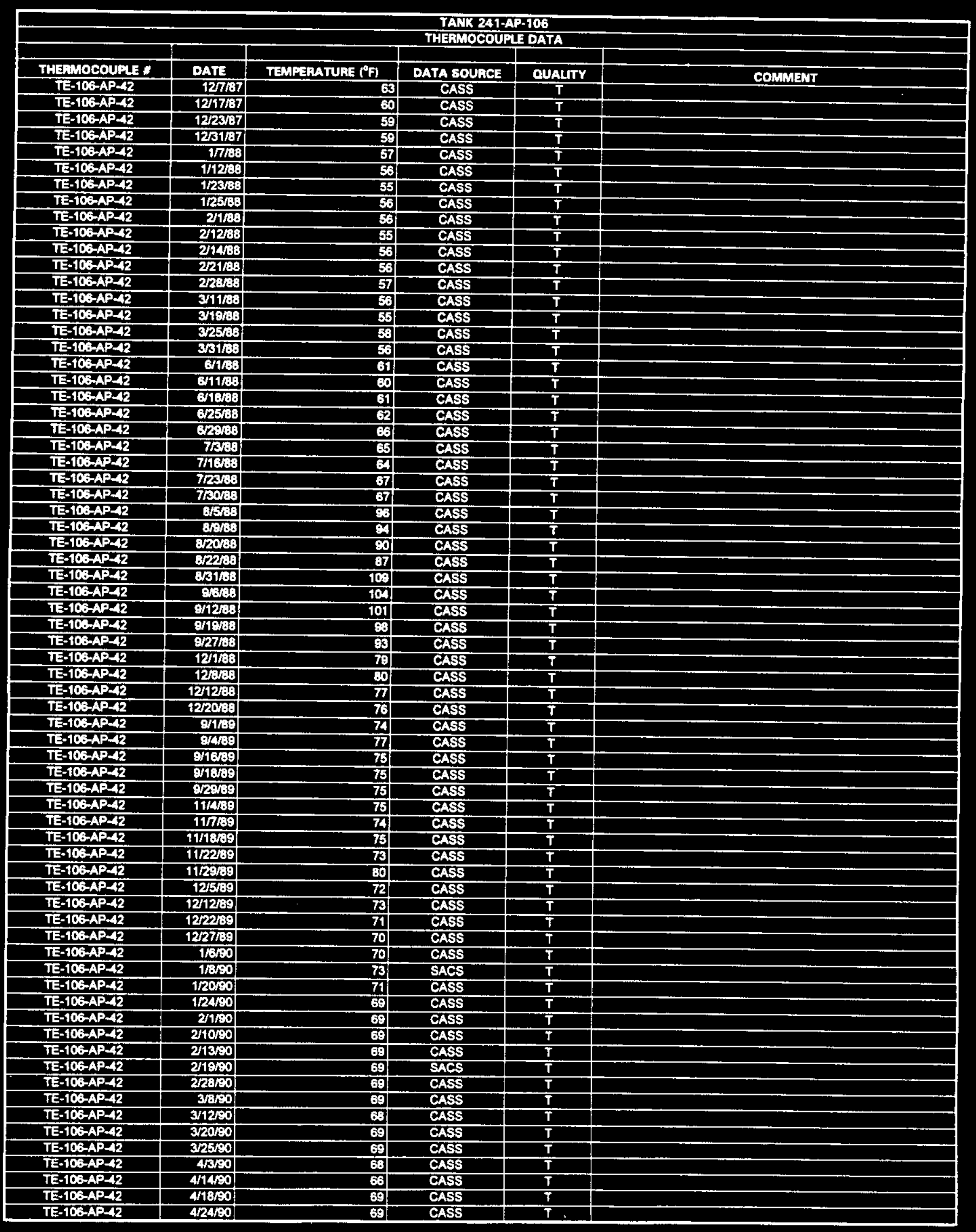




\begin{tabular}{|c|c|c|c|c|c|}
\hline \multicolumn{6}{|c|}{$\begin{array}{l}\text { TAKK 241-AP-106 } \\
\text { THIIMOCOUPLE DATA }\end{array}$} \\
\hline & & & & & \\
\hline 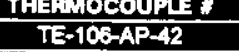 & $\begin{array}{l}\text { DAIE } \\
\text { SIISO }\end{array}$ & $\begin{array}{r}\text { TEMPEARATUAE }\left(0^{\circ}\right) \\
68\end{array}$ & $\frac{\text { DATA SOURCE }}{\text { CASS }}$ & OUALITY & COMMENT \\
\hline$T E-106-A P-42$ & $5 / 11 / 20$ & 68 & $\frac{\text { CASS }}{\text { CASS }}$ & $T$ & \\
\hline TE-106-AP-42 & 5/19so & 66 & $\begin{array}{l}\text { CASS } \\
\text { CASS }\end{array}$ & $\frac{T}{T}$ & \\
\hline$T E-106-A P-42$ & $5 \longdiv { 2 2 5 0 0 }$ & 67 & CASS & $\frac{T}{T}$ & \\
\hline TE-106-AP-A2 & 6/200 & 66 & CASS & $T$ & \\
\hline TE-10S-AP-A2 & G600 & 67 & CASS & $T$ & \\
\hline$T E-106-A P-42$ & 6/1400 & 68 & CASS & $T$ & \\
\hline TE-106-AP-42 & 6/2350 & 66 & CASS & $T$ & \\
\hline TE-106-AP-A2 & 625500 & 82 & SACS & $\mathbf{S}$ & Data point did not agree with hocal trend \\
\hline TE-10BAP-A2 & 8Z2900 & 68 & CASS & $\mathbf{T}$ & \\
\hline TE-106-AP-A2 & $76 \%$ & 66 & CASS & $T$ & \\
\hline TE-106-AP-A2 & $7 / 1480$ & E6 & CASS & $T$ & \\
\hline TE-103-AP-42 & 711600 & 68 & CASS & $\mathbf{T}$ & \\
\hline TE-10S-AP-42 & 727120 & 68 & CASS & $\mathbf{T}$ & \\
\hline TE-106-AP-42 & $8 / 3150$ & 68 & CASS & $\mathbf{T}$ & \\
\hline TE-106-AP-A2 & $8 / 11 \mathrm{AO}$ & 68 & CASS & $T$ & \\
\hline TE-106-AP-A2 & B/6E0 & 67 & CASS & $\mathbf{T}$ & \\
\hline TE-106-AP-12 & 8/1900 & 63 & CASS & $\mathbf{T}$ & \\
\hline TE-106-AP=2 & 83150 & 68 & CASS & $\mathbf{T}$ & \\
\hline TE-106-AP-42 & $8 / 200$ & 68 & CASS & $\mathbf{T}$ & \\
\hline TE-106-AP-12 & 9/400 & 70 & CASS & $T$ & \\
\hline TE-106AP-42 & $9 / 2200$ & 69 & CASS & $T$ & \\
\hline$T E-106-4 P-42$ & 8/2300 & 70 & CASS & $\mathbf{T}$ & \\
\hline TE-106-AP-A2 & $106 \% 0$ & 63 & CASS & $\bar{T}$ & \\
\hline$T E-106-A P-42$ & $10 / 11 \infty 0$ & 69 & CASS & $\bar{T}$ & \\
\hline TE-106-AP-42 & 10r2000 & 69 & CASS & $T$ & \\
\hline IE-100-AP-A2 & $10 / 2250$ & 69 & CASS & $T$ & \\
\hline TiE-106-AP-42 & $11 / 260$ & 68 & CASS & $T$ & \\
\hline$T E-106-A P-A 2$ & $11 / 10 / 00$ & 70 & CASS & $T$ & \\
\hline TE-106-AP-A2 & $11 / 17 \% 0$ & 71 & CASS & $\mathbf{T}$ & \\
\hline TE-106-AP-A2 & $11 / 2400$ & 68 & CASS & $T$ & \\
\hline$T E-106-A P-12$ & 11/29so & 69 & CASS & $T$ & \\
\hline TE-103-AP-A2 & $12 \pi \mathrm{ro}$ & (6) & CASS & $T$ & \\
\hline TE-10B-AP-A2 & 12000 & 70 & CASS & $T$ & \\
\hline$T E-10 B-A P-12$ & $12 / 2000$ & 60 & CASS & $T$ & \\
\hline TE-10B-AP-A2 & 1225300 & 60 & CASS & $\mathbf{T}$ & \\
\hline TE-1CB-AP-A2 & $1 / 2,81$ & 67 & CASS & $T$ & \\
\hline TE-106-AP-42 & 1/1201 & 86 & CASS & $T$ & \\
\hline$T E-103-A P-A_{2}$ & $1 / 189$ & 6 & CASS & $T$ & \\
\hline TE-106-AP-A2 & 1/2401 & 68 & CASS & $T$ & \\
\hline TE-106-AP-12 & 22291 & 65 & CASS & $T$ & \\
\hline TE-103-AP-12 & $2 \mathrm{~B} 3 \mathrm{a1}$ & 6 & CASS & $\mathbf{T}$ & \\
\hline TE-106-AP-A2 & 216:31 & 65 & CASS & $\mathbf{T}$ & \\
\hline TE-106-AP-A2 & $2 / 2201$ & 68 & CASS & $T$ & \\
\hline TE-10G-AP-42 & 3109 & 66 & CASS & $T$ & \\
\hline TE-106-AP-A2 & $3 / 3 / 91$ & 68 & CASS & $T$ & \\
\hline TE-106-AP- 12 & 3/1691 & 64 & CASS & $T$ & \\
\hline TE-106AP-12 & 3/17/91 & 65 & CASS & $T$ & \\
\hline$T E-106-A P-A 2$ & 3/27/31 & 65 & CASS & $T$ & \\
\hline TE-106-AP-A2 & $41 / 91$ & 65 & CASS & $T$ & \\
\hline TE-106-AP- 42 & $48 / 91$ & 65 & CASS & $T$ & \\
\hline$T E-103-A P-42$ & 4/1981 & 6A & CASS & $T$ & \\
\hline$T E-100-A P-42$ & 4/2491 & 65 & CASS & $T$ & \\
\hline TE-106-AP-12 & 54491 & 63 & CASS & $T$ & \\
\hline TE-106-AP-A2 & 5BS591 & 66 & CASS & $T$ & \\
\hline$T E-106-A P=42$ & S/1691 & 65 & CASS & $\mathbf{T}$ & \\
\hline$T E-106-A P-42$ & 5/21/91 & 65 & CASS & $T$ & \\
\hline TE-106-AP-A2 & $5 / 2601$ & 65 & CASS & $T$ & . \\
\hline TE-10 -AP- 22 & 67/21 & 6 & CASS & $\mathbf{T}$ & \\
\hline TE-106-AP-42 & 6/15/91 & 66 & CASS & $\mathbf{T}$ & \\
\hline TE-106-AP-42 & 6/22201 & EA & CASS & $T$ & \\
\hline TE-106-AP-A2 & 6/28/21 & 66 & CASS & $T$ & \\
\hline$T E-106-A P-A 2$ & 73181 & 64 & CASS & $\mathbf{T}$ & \\
\hline TE-106-AP-A2 & 771391 & 64 & CASS & $\bar{T}$ & \\
\hline$T E-106-A P-42$ & $7 / 14 / 91$ & EA & CASS & $T$ & \\
\hline$T E=106-A P-A 2$ & 712491 & 84 & CASS & $T$ & \\
\hline TE-106-AP-42 & 8/1/81 & 64 & CASS & $T$ & \\
\hline TE-106-AP-12 & B/6/91 & 64) & CASS & $T$ & \\
\hline TE-106-AP-A2 & $8 / 17 / 91$ & 69 & CASS & $T$ & \\
\hline TE-106-AP-A2 & $8 / 20 / 91$ & 66 & CASS & $T$ & \\
\hline
\end{tabular}




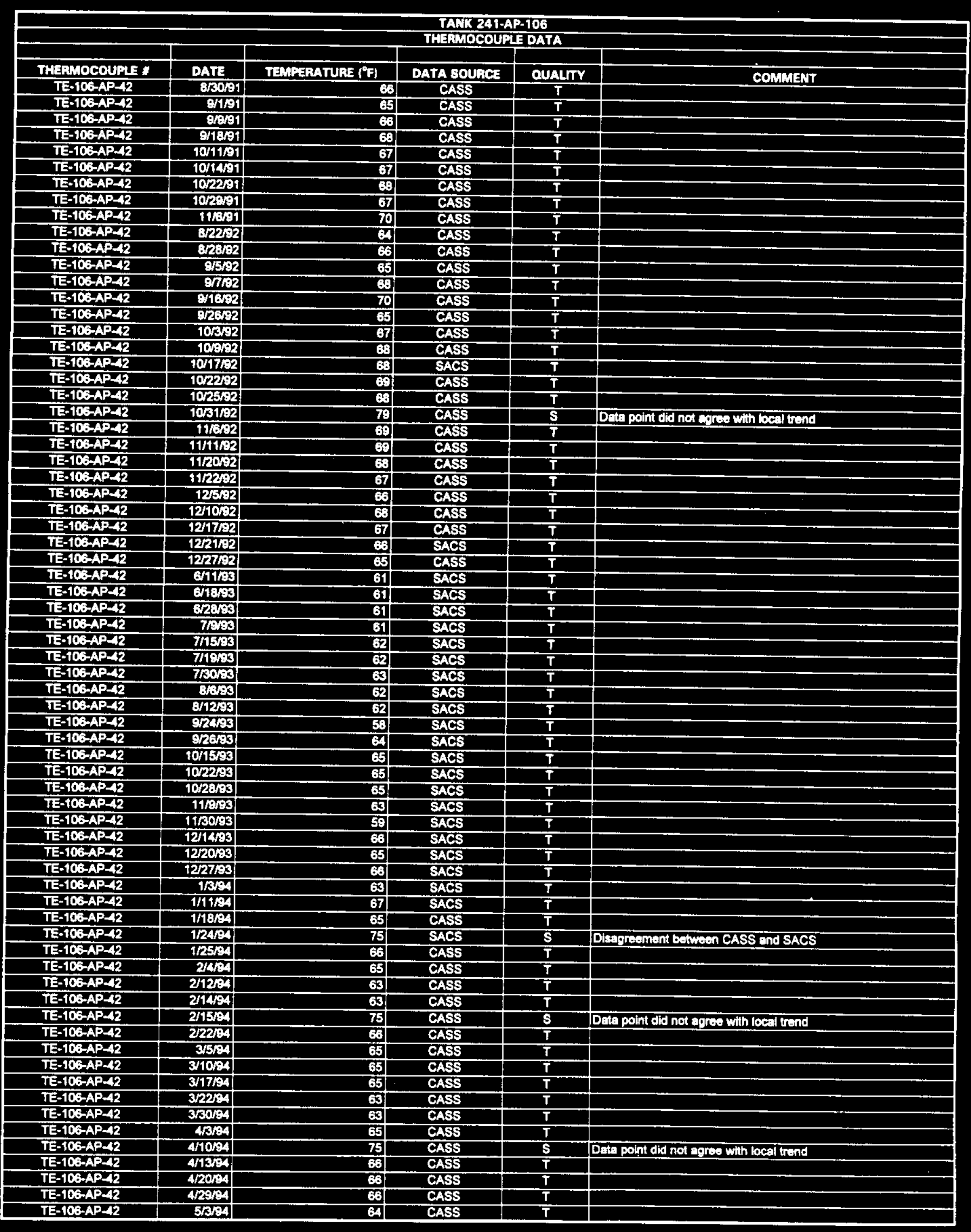




\begin{tabular}{|c|c|c|c|c|c|}
\hline \multicolumn{6}{|c|}{$\begin{array}{l}\text { TANK 241-AP.106 } \\
\text { THERMOCOUPLE DATA }\end{array}$} \\
\hline THEPMOCOUPE * & DATE & & & & \\
\hline TE-106-AP-42 & 5/964 & 70 & 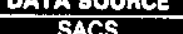 & DUALIY & COMMENT \\
\hline TE-106-AP-42 & S11/94 & 86 & & $\mathbf{6}$ & \\
\hline TE-106-AP-A2 & S/1894 & 100 & $\begin{array}{l}\text { CASS } \\
\text { CASS }\end{array}$ & $\mathbf{s}$ & Data point did not agree wih boal trond \\
\hline TE-TOG-AP-A2 & $5 / 2239$ & 83 & $\begin{array}{l}\text { CASS } \\
\text { CASS }\end{array}$ & $\mathrm{T}$ & \\
\hline TE-106-AP-A2 & $6 / 213$ & 95 & $\frac{\text { CASS }}{\text { CASS }}$ & $\frac{T}{T}$ & \\
\hline TE-106-AP-A2 & 68\% & 93 & CASS & $\frac{T}{T}$ & \\
\hline TE-106-AP- 42 & 6/14/94 & 91 & CASS & $\mathbf{T}$ & \\
\hline$T E-106-A P=-12$ & 6/16/94 & 69 & CASS & $\mathbf{S}$ & Data point did not agree with loeal trend \\
\hline TE-106-AP-42 & E/17\%A & 64 & CASS & $\mathbf{S}$ & Data point did not agree with locel trend \\
\hline TE-106-AP-12 & Ezore & 65 & CASS & $\mathbf{S}$ & Data point did not agres with local trond \\
\hline TE-106AP-A2 & 62404 & 83 & CASS & $T$ & \\
\hline TE-106-AP-A2 & 6/27/94 & 94 & CASS & $\mathbf{T}$ & \\
\hline TE-106-AP-42 & 7369 & 80 & CASS & $T$ & \\
\hline TE-106-AP-A2 & 72304 & 61 & SACS & S (Gin SACS) & Data point did not agree with local trend \\
\hline TE-106-AP-A2 & 73004 & 88 & CASS & $\mathbf{T}$ & \\
\hline$T E-106-A P-42$ & $\operatorname{sen}$ & 83 & CASS & $\mathbf{T}$ & \\
\hline TE-103-AP-42 & 82494 & 91 & CASS & $\mathbf{T}$ & \\
\hline TE-103-AP-A2 & 10/19 & $\mathbf{8 5}$ & CASS & $T$ & \\
\hline TE-106-AP-A2 & Torog & 97 & CASS & $T$ & \\
\hline TE-106AP-A2 & $10 / 158$ & 88 & CASS & $T$ & \\
\hline$T E-103-A P-A 2$ & 10/1\%4 & 100 & CASS & $T$ & \\
\hline IE-106-AP-12 & 10ras & 100 & CASS & $T$ & \\
\hline TE-T06-AP-A2 & $11 / 354$ & 101 & CASS & $T$ & \\
\hline TE-106-AP-A2 & $11 / 11 / 94$ & 105 & CASS & $\bar{T}$ & \\
\hline TE-106-APA2 & $11 / 18 \% 4$ & 100 & CASS & $T$ & \\
\hline TE-105-AP-A2 & $11 / 2204$ & 103 & CASS & $T$ & \\
\hline TE-106-AP-A2 & 11/289 & 87 & CASS & $T$ & \\
\hline TE-100-AP-A2 & 121494 & 101 & CASS & $T$ & \\
\hline TE-106-AP-A2 & 12160 & 86 & CASS & $\mathbf{T}$ & \\
\hline TE-106-AP-A2 & $121 \% 4$ & 100 & CASS & $T$ & \\
\hline TE-166-AP-A2 & $12130 \%$ & 8 & CASS & $T$ & \\
\hline TE-106-AP-A2 & $1 \pi / 85$ & 104 & CASS & $T$ & \\
\hline TE-106-AP- $=2$ & $1 / 1465$ & Q9. & CASS & $T$ & \\
\hline$T E-106-A P=-12$ & $1 / 16 \%$ & 8 & CASS & $\bar{T}$ & \\
\hline TE-103-AP-42 & $1 / 23105$ & 101 & CASS & $T$ & \\
\hline TE-106-AP- 42 & $22 / 35$ & 86 & CASS & $\bar{T}$ & \\
\hline$T E-106-A P-22$ & 2कs & 8 & CASS & $T$ & \\
\hline TE-106-AP-A2 & 21405 & 105 & CASS & $T$ & \\
\hline TE-10B-AP-12 & 22405 & 8 & CASS & $T$ & \\
\hline TE-10G-AP-12 & $3 / 205$ & 100 & CASS & $T$ & \\
\hline IE-106-AP-A2 & 3735 & 97 & CASS & $T$ & \\
\hline TE-106-AP-A2 & $3 / 16 / 95$ & 8 & CASS & $T$ & \\
\hline TE-10S-AP-A2 & $3 / 23 / 55$ & 103 & CASS & $T$ & \\
\hline IE-10:-AP-12 & $3 / 30 / 95$ & 8 & CASS & $T$ & \\
\hline$T E-106-A P-A 2$ & $4 \mathrm{BS3}$ & 85 & SACS & G & \\
\hline TE-106-AP-A2 & $4 / 10 / 85$ & 85 & SACS & $\bar{G}$ & \\
\hline$T E-106-A P-42$ & $4 / 1735$ & 87 & SACS & G & \\
\hline TE-106AP-A2 & 4/2405 & 80 & SACS & $\mathbf{G}$ & \\
\hline TE-106-AP-A2 & Sil/95 & 8 & SACS & $\bar{G}$ & \\
\hline TE-10S-AP-42 & 53as & 81 & SACS & $\bar{G}$ & \\
\hline TE-106-AP-A2 & 5/16/55 & 79 & SACS & G & \\
\hline$T E-106-A P-42$ & $5 / 22195$ & 80 & SACS & $\mathbf{G}$ & \\
\hline TE-106-AP-12 & 5/20/35 & 82 & SACS & 6 & \\
\hline TE-106-AP-12 & 65\%5 & 86 & SACS & $\mathbf{G}$ & \\
\hline TE-106-AP- 12 & 6/13/95 & 80 & SACS & G & \\
\hline TE-106-AP-A2 & 6/19/95 & 94 & SACS & G & \\
\hline TE-106-AP-A3 & $4 / 5386$ & 55 & CASS & $T$ & \\
\hline TE-10G-AP- 13 & $47 / 36$ & 57 & CASS & $T$ & \\
\hline$T E-100-A P-13$ & $5 / 1686$ & 57 & CASS & $T$ & \\
\hline TE-106-AP-43 & $5 / 22366$ & 59 & CASS & $T$ & \\
\hline TE-106-AP-13 & $5 / 30 / 86$ & 59 & CASS & $T$ & \\
\hline TE-106-AP-43 & $67 / 36$ & 53 & CASS & $T$ & \\
\hline TE-106-AP-43 & $6 / 13 / 66$ & 59 & CASS & $T$ & \\
\hline TE-106-AP-A3 & $6 / 16 / 86$ & 70 & CASS & $\mathbf{s}$ & Data point did not egree with local trend \\
\hline TE-106-AP-43 & $6 / 21 / 36$ & 59 & CASS & $T$ & \\
\hline TE-106-AP- 43 & $6 / 23 / 36$ & 59 & CASS & $\mathbf{T}$ & \\
\hline TE-106-APA $\sqrt{3}$ & 7466 & 61 & CASS & $T$ & \\
\hline TE-106-AP-43 & 7/12/36 & 60 & CASS & $T$ & \\
\hline TE-106-AP-43 & $7 / 16 / 66$ & 61 & CASS & $T$ & \\
\hline
\end{tabular}




\begin{tabular}{|c|c|c|c|c|c|}
\hline \multicolumn{6}{|c|}{$\begin{array}{l}\text { TAMK 241-AP-106 } \\
\text { THETHOCOUPE DATA }\end{array}$} \\
\hline & & & & & \\
\hline THERMOCOUPUE * & DATE & TEMPERATURE ${ }^{\circ} \mathrm{Fl}$ & DATA SOUACE & QUALITY & COMMENT \\
\hline TE-106-AP-43 & $7 / 19 \mathrm{B6}$ & 70 & CASS & 8 & Deta point did not agree with local trend \\
\hline TE-106-AP-43 & 712486 & 61 & CASS & $T$ & \\
\hline TE-106-AP-43 & $7 / 23186$ & 62 & CASS & $\bar{T}$ & \\
\hline TE-106-AP-43 & B/S/86 & 63 & CASS & $T$ & \\
\hline$T E-106-A P-43$ & 8/14/86 & 64 & CASS & $T$ & \\
\hline TE-106-AP-A3 & $8 / 1786$ & 63 & CASS & $T$ & \\
\hline $\mathrm{TE}-106-\mathrm{AP}-43$ & $8 / 2765$ & $\mathbf{6 4}$ & CASS & $\mathbf{T}$ & \\
\hline$T E-106-A P-43$ & $8 / 1 / 66$ & 66 & CASS & $\mathbf{T}$ & \\
\hline$T E-106-A P-A B$ & 8,23/86 & 65 & CASS & $T$ & \\
\hline TE-10:AP-13 & 103366 & 64 & CASS & $T$ & \\
\hline TE-106-AP-A3 & $1011 / 85$ & 63 & CASS & $T$ & \\
\hline TE-106-AP-43 & $10 / 1 / 86$ & 63 & CASS & $T$ & \\
\hline TE-106-AP-AB & $10 / 2486$ & 83 & CASS & $T$ & \\
\hline TE-106-AP-A3 & $11 / 1 / 66$ & 62 & CASS & $T$ & \\
\hline TE-106-AP-A3 & $11 / \mathrm{SAB}$ & 63 & CASS & $T$ & \\
\hline TE-103-AP-43 & 11/1086 & 59 & CASS & $T$ & \\
\hline$T E-103-A P=43$ & $11 / 18 / 06$ & 65 & CASS & $\mathbf{T}$ & \\
\hline TE-163-AP-43 & $11 / 2436$ & 58 & CASS & $T$ & \\
\hline$T E-106-A P-43$ & $12 / 2 / 36$ & 59 & CASS & T & \\
\hline$T E-103-A P-43$ & 12806 & 67 & CASS & $\mathbf{S}$ & Data point did not agree whith local trend \\
\hline TE-103-AP-43 & $1211 / 68$ & 57 & CASS & $T$ & \\
\hline TE-1CS-AP-43 & $12 / 6 / 80$ & 53 & CASS & $T$ & \\
\hline$T E-10 G-A P-43$ & 1212386 & 57 & CASS & $\mathbf{T}$ & \\
\hline TE-106-AP-43 & $1231 / 96$ & S3 & CASS & $\mathbf{T}$ & \\
\hline TE-103-AP-43 & 72467 & 85 & CASS & $T$ & \\
\hline TE-106-AP-A3 & 7287 & 67 & CASS & $T$ & \\
\hline TE-106AP-AB & $7 / 18 / 87$ & 63 & CASS & $T$ & \\
\hline TE-106-AP-43 & $7 / 1987$ & 60 & CASS & $T$ & \\
\hline TE-103-AP-43 & 81187 & 8 & CASS & $T$ & \\
\hline$T E-106-A P=43$ & $8 / 567$ & 83 & CASS & $\bar{T}$ & \\
\hline$T E-106-A P-43$ & B/467 & 89 & CASS & $T$ & \\
\hline TE-100-AP-43 & 81767 & 69 & CASS & $T$ & \\
\hline TE-10G-AP-A3 & ar2687 & 70 & CASS & $T$ & \\
\hline TE-103-AP-43 & 8467 & 68 & CASS. & $T$ & \\
\hline$T E-106-A P-13$ & 8/87 & 70 & CASS & $T$ & \\
\hline$T E-163-A P-43$ & 91767 & 69 & CASS & $T$ & \\
\hline TE-10S-AP-AS & aras7 & 69 & CASS & $T$ & \\
\hline TE-106-AP-43 & $10 / 187$ & 68 & CASS & $T$ & \\
\hline TE-103-AP-43 & 10rab7 & 65 & CASS & $T$ & \\
\hline TE-106-AP-43 & $11 / 6 / 37$ & E4 & CASS & $T$ & \\
\hline$T E-10 G-A P-43$ & $11 / 11 / 87$ & 65 & CASS & $T$ & \\
\hline TE-TCB-AP-43 & 11/18B & 63 & CASS & $T$ & \\
\hline TE-106-AP-43 & 11/26/37 & 62 & CASS & $T$ & \\
\hline$T E-103-A P-43$ & 122467 & 63 & CASS & $T$ & \\
\hline TE-106-AP-43 & $12 / 11 / 87$ & 63 & CASS & $T$ & \\
\hline TE-10B-AP-A3 & $12117 / 67$ & 60 & CASS & $T$ & \\
\hline$T E-106-A P-13$ & $12 / 23 / 37$ & 59 & CASS & $T$ & \\
\hline$T E-100-A P=43$ & $12 / 31 / 87$ & 59 & CASS & $T$ & \\
\hline$T E-106-A P-13$ & $1 / 7 / 38$ & 57 & CASS & $T$ & \\
\hline$T E-10 B-A P-43$ & $1 / 10 / 88$ & 56 & CASS & $T$ & \\
\hline E-10S-AP-A3 & $1 / 23 / 88$ & 55 & CASS & $T$ & \\
\hline TE-106-AP-43 & $1 / 27138$ & 55 & CASS & $T$ & \\
\hline$T E-106-A P-43$ & $2 / 388$ & 55 & CASS & $T$ & \\
\hline TE-10B-AP-A3 & $2 / 1288$ & 55 & CASS & $\bar{T}$ & \\
\hline TE-106-AP-43 & $2 / 1408$ & 56 & CASS & $T$ & \\
\hline TE-103-AP-43 & $2 / 21100$ & 57 & CASS & $\bar{T}$ & \\
\hline TE-106-AP-43 & 2/28/38 & 57 & CASS & $T$ & \\
\hline TE-106-AP-43 & $3 / 11 / 88$ & 57 & CASS & $T$ & \\
\hline$T E-106-A P-43$ & 3/19/88 & 55 & CASS & $T$ & \\
\hline$T E-106-A P-43$ & $3 / 25,38$ & 57 & CASS & $T$ & \\
\hline TE-106-AP-43 & $3 / 3088$ & 57 & CASS & $T$ & \\
\hline TE-106-AP-43 & $6 / 1 / B 8$ & 61 & CASS & $T$ & \\
\hline$T E-106-A P-43$ & 6r11/30 & 60 & CASS & $T$ & \\
\hline TE-106-AP-43 & $6 / 18 / 38$ & 61 & CASS & $T$ & \\
\hline TE-106-AP-43 & 6/25/89: & 62 & CASS & $T$ & \\
\hline$T E-106-A P-43$ & $6 / 29 / 88$ & 66 & CASS & $T$ & \\
\hline$T E-10 S-A P-43$ & $7 / 3 / 88$ & 64 & CASS & $T$ & \\
\hline$T E-106-A P-43$ & $7 / 16 / 88$ & 64 & CASS & $T$ & \\
\hline TE-106-AP-43 & $7 / 23 / 88$ & 67 & CASS & $T$ & \\
\hline$T E-103-A P-43$ & $7 / 30 / 38$ & 67 & CASS & $T$ & \\
\hline
\end{tabular}




\begin{tabular}{|c|c|c|c|c|c|}
\hline \multicolumn{6}{|c|}{$\begin{array}{l}\text { TANK 241-AP-106 } \\
\text { THEFiMOCOUPLE DATA }\end{array}$} \\
\hline & & & & & \\
\hline THEPMOCOUPL: * & DATE & TEMPERATURE $\left({ }^{\circ} \mathrm{F}\right)$ & DATA SOURCE & OUALTY & COMMENT \\
\hline TE-10G-AP-43 & $8 / 538$ & \&6 & CASS & $T$ & \\
\hline TE-10G-AP-43 & BNoB & 94 & CASS & $\bar{T}$ & \\
\hline$T E-10 B-A P-43$ & $8 / 20138$ & 80 & CASS & $\bar{T}$ & \\
\hline TE-106-AP-A3 & $8 / 2380$ & B7 & CASS & $T$ & \\
\hline TE-106-AP-A3 & B31/88 & 110 & CASS & $T$ & \\
\hline TE-106-AP-13 & $8 / 4 / 83$ & 105 & CASS & $T$ & \\
\hline$T E-10 S-A P-43$ & $9 / 13 / 88$ & 101 & CASS & $T$ & \\
\hline TE-10B-AP= 13 & 9/18\%8 & 100 & CASS & $T$ & \\
\hline TE-106-APAB & 8/27/38 & 93 & CASS & $\mathbf{T}$ & \\
\hline$T E-103-A P-43$ & 12/1/68 & 79 & CASS & $T$ & \\
\hline TE-TOS-AP-43 & 1223 & 80 & CASS & $\overline{\mathbf{T}}$ & \\
\hline TE-103-AP=43 & 1212/38 & 7 & CASS & $\bar{T}$ & \\
\hline TE.103AP-43 & 1212003 & 76 & CASS & $T$ & \\
\hline TE-106-AP-43 & $710 \%$ & 62 & SACS & $T$ & \\
\hline TE-106-AP-A3 & $7 / 17 / 69$ & 73 & SACS & $\bar{T}$ & \\
\hline TE-106-AP-43 & 72469 & 70 & SACS & $T$ & \\
\hline$\overline{E E-106-A P-43}$ & $87 \pi \theta^{2}$ & 74 & SACS & $T$ & \\
\hline TE-106-AP-4S & $8 / 14 / 89$ & 74 & SACS & $\bar{T}$ & \\
\hline$T E=106-A P=43$ & $8 / 2189$ & 74 & SACS & $\mathbf{T}$ & \\
\hline$T E-100 A P-43$ & \&1/ag & 74 & CASS & $T$ & \\
\hline TE-106AP-43 & 84/99 & 78 & CASS & $T$ & \\
\hline TE-103-AP-A3 & 816/39 & 76 & CASS & $T$ & \\
\hline TE-103-AP-43 & 8/18/89 & 75 & CASS & $\bar{T}$ & \\
\hline TE-106-AP-43 & 82969 & 75 & CASS & $\bar{T}$ & \\
\hline TE-106-AP-43 & 102209 & 68 & SACS & $\mathbf{T}$ & \\
\hline TE-106-AP-43 & Torog & 72 & SACS & $\bar{T}$ & \\
\hline$T E-106-A P-43$ & $10 / 1680$ & 74 & SACS & $T$ & \\
\hline TE-10G-AP-43 & $10 / 2489$ & 79 & SACS & $T$ & \\
\hline TE-106-AP-A3 & $11 / 4 \% 8$ & 75 & CASS & $T$ & \\
\hline$T E-103-A P-43$ & 11तrag & 80 & CASS & $T$ & \\
\hline$I E-106-A P-43$ & $11 / 1509$ & 74 & CASS & $T$ & \\
\hline TE-106-AP-43 & $11 / 25009$ & 73 & CASS & $T$ & \\
\hline TE-103-AP-43 & $11 / 2769$ & 74 & CASS & $T$ & \\
\hline$T E-103-A P-43$ & $11 / 2968$ & 83 & GASS & $\mathbf{S}$ & Dat a point did not arice with locel trand \\
\hline TE-103-AP-43 & $12 / 469$ & 74 & SACS & $T$ & \\
\hline TE-108-AP-43 & $12 / 1239$ & 74 & CASS & $T$ & \\
\hline$T E-106-A P-43$ & $\sqrt{223 \pi 89}$ & 71 & CASS & $\mathbf{T}$ & \\
\hline$T E-103-A P-43$ & 12 26369 & 71! & CASS & $\mathbf{T}$ & \\
\hline TE-103-AP-43 & $1 / 400$ & 70 & CASS & $\mathbf{T}$ & \\
\hline TE-106-AP-A3 & $1 / 1200$ & 71 & CASS & $T$ & \\
\hline TE-106-AP-43 & $1 / 17100$ & 75 & CASS & $T$ & \\
\hline TE-106-AP-43 & $1 / 26 \%$ & 69 & CASS & $\mathbf{T}$ & \\
\hline TE-106-AP-A3 & 21100 & 69 & CASS & $T$ & \\
\hline TE-106-AP-A3 & 2000 & 69 & CASS & $T$ & \\
\hline TE-106-AP-A3 & $2 / 13 \%$ & 69 & CASS & $T$ & \\
\hline$T E-106-A P-13$ & $2 / 8 / 50$ & 69 & CASS & $T$ & \\
\hline TE-106-AP-43 & $3 / 350$ & 69 & CASS & $T$ & \\
\hline$T E-106-A P-A 3$ & 36s0 & 69 & CASS & $T$ & \\
\hline$T E-103-A P-43$ & $3 / 1200$ & 68 & CASS & $T$ & \\
\hline TE-106-AP-A3 & 3/20/00 & 69 & CASS & $\mathbf{T}$ & \\
\hline TE-106-AP-A3 & $3 / 25 / 90$ & 69 & CASS & $\mathbf{T}$ & \\
\hline$T E-103-A P-43$ & ABSO & 68 & CASS & $T$ & \\
\hline TE-10B-AP-43 & 41400: & 60 & CASS & $T$ & \\
\hline TE-106-AP-43 & ATI8EO & 69 & CASS & $T$ & \\
\hline TE-106-AP-A3 & 42480 & 69 & CASS & $T$ & \\
\hline$T E-106-A P-43$ & $5 / 1 / 10$ & 68 & CASS & $T$ & \\
\hline$T E-10 B-A P-A 3$ & $5 / 6 / 90$ & 68 & CASS & $T$ & \\
\hline$T E-106-A P-43$ & 5/18/90 & 66 & CASS & $\mathbf{T}$ & \\
\hline$T E-106-A P-43$ & $5 / 22100$ & 63 & CASS & $T$ & \\
\hline TE-106-AP-43 & $6 / 200$ & 63 & CASS & $T$ & \\
\hline$T E-106-A P-43$ & GESO & 68 & CASS & $\mathbf{T}$ & \\
\hline TE-106-AP-A3 & G14a0 & 67 & CASS & $\mathbf{T}$ & \\
\hline TE-106-AP-A3 & 6/23/90 & 66 & CASS & $T$ & \\
\hline TE-106-AP-A3 & 6/2960 & 67 & CASS & $T$ & \\
\hline TE-106-AP-A3 & $7 / 6 / 90$ & 66 & CASS & $\bar{T}$ & \\
\hline$T E-106-A P-43$ & 71460 & 66 & CASS & $T$ & \\
\hline$T E-106-A P-43$ & $7 / 550$ & 68 & CASS & $T$ & \\
\hline TE-106-AP-43 & $7 / 27100$ & 68 & CASS & $T$ & \\
\hline TE-106-AP-43 & $8 / 3100$ & 68 & CASS & $T$ & \\
\hline$T E-103-A P-43$ & $8 / 800$ & 69 & CASS & $T$ & \\
\hline
\end{tabular}




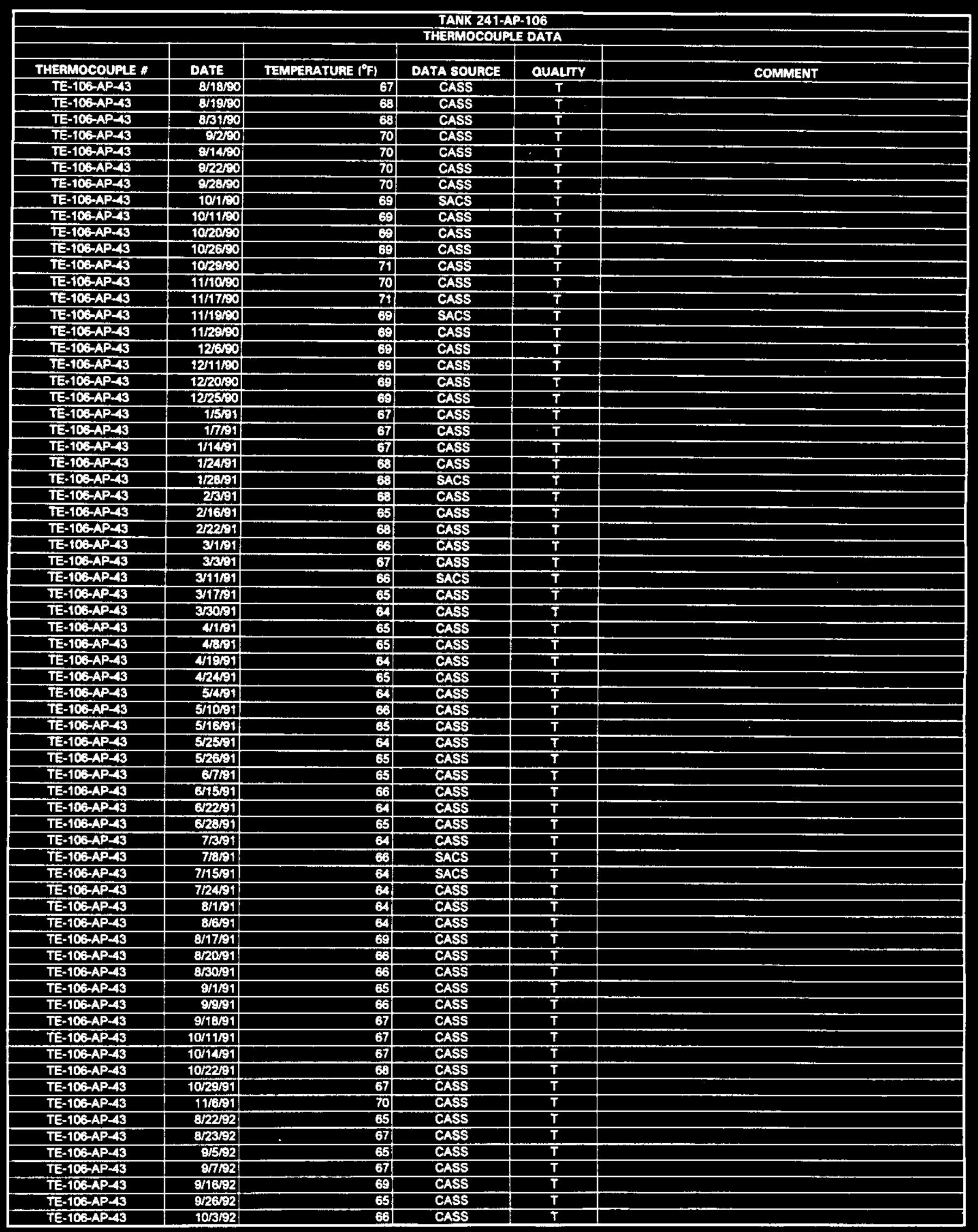




\begin{tabular}{|c|c|c|c|c|c|}
\hline \multicolumn{6}{|c|}{$\begin{array}{l}\text { TANR 241-AP-106 } \\
\text { THEAMOCOUPL DATA }\end{array}$} \\
\hline & & & & & \\
\hline THERMOCOUPLE * & DATE & TEMPERATUAE ( $\left.{ }^{\circ} \mathrm{F}\right)$ & DATA SOURCE & QUALTY & COMMENT \\
\hline TE-106-AP-43 & $10 \mathrm{seg}$ & 67 & CASS & $T$ & \\
\hline TE-TOG-AP-43 & $10 / 1592$ & 66 & CASS & $\mathbf{T}$ & \\
\hline$T E-10 S-A P-43$ & 102202 & 68 & CASS & $T$ & \\
\hline TE-10G-AP-43 & $10 / 27 / 92$ & 68 & CASS & $\mathbf{T}$ & \\
\hline TE-103-AP-43 & $10 \times 3152$ & Bo & CASS & $\mathbf{S}$ & Data point did not coree with local trend \\
\hline TE-1OA-AP-A3 & $11 / 628$ & 69 & CASS & $T$ & \\
\hline$T E-106-A P-43$ & $11 / 11 \omega 2$ & 69 & CASS & $\bar{T}$ & \\
\hline TE-10G-AP-43 & $11 / 2002$ & 68 & CASS & $T$ & \\
\hline$T E-10 B-A P-43$ & $11 / 22 / 92$ & 67 & CASS & $T$ & \\
\hline TE-TOSAPQA3 & 11/3002 & 86 & CASS & $\mathbf{T}$ & \\
\hline$\pi 5-106-A P=43$ & 121028 & 63 & CASS & $\mathbf{T}$ & \\
\hline TE-100-AP-43 & $12 / 192$ & 66 & CASS & $T$ & \\
\hline TE-106-AP-43 & $12 / 2602$ & 65 & CASS & $T$ & \\
\hline$T B-1 D B-A P-43$ & 12 rago2 & 65 & CASS & $T$ & \\
\hline TE-10G-AP-43 & V15BA & 64 & CASS & $\bar{T}$ & \\
\hline TE-103-AP-43 & $1 / 18 \% 4$ & 65 & CASS & $\mathbf{T}$ & \\
\hline TE-10SAP-A3 & $1 / 2504$ & 63 & CASS & $T$ & \\
\hline$T E-103-A P=3$ & $2 / 494$ & 63 & CASS & $\bar{T}$ & \\
\hline$T E-106-A P-43$ & $2 \pi / 8$ & 64 & CASS & $\mathbf{T}$ & \\
\hline$T E-106 A P-43$ & $2 / 1594$ & 76 & CASS & $\mathbf{S}$ & Deta point did not seree with beal trend \\
\hline$T E-103-A P=43$ & 2169 & 64 & CASS & $T$ & \\
\hline$\pi=-103-A P-43$ & 22234 & 66 & CASS & $\bar{T}$ & \\
\hline$T E-100 A P-43$ & $3 \sin$ & 66 & CASS & $\bar{T}$ & \\
\hline$T E-103-A P=3$ & 31004 & 65 & CASS & $T$ & \\
\hline$T E-103-A P-43$ & 3/7\%4 & 63 & CASS & $T$ & \\
\hline$T E-10 B-A P-43$ & $3 / 2494$ & 63 & CASS & $T$ & \\
\hline$T E-103-\mathrm{A}=43$ & $3 \times 004$ & 63 & CASS & $T$ & \\
\hline$T E-106-A P-43$ & $43 \mathrm{M}$ & 65 & CASS & $T$ & \\
\hline TE-1OSAP 43 & 4 tory & 74 & CASS & $\mathbf{S}$ & Dota point did not coree with local trend \\
\hline TE-10BAP-A3 & 41394 & 65 & CASS & $T$ & \\
\hline$T E-103-A P-43$ & Arasey. & 65 & CASS & $T$ & \\
\hline$T-103-A P-43$ & 4 ren & 65 & CASS & $T$ & \\
\hline $15 \cdot 103-A P-43$ & $52 \times 4$ & 64 & CASS & T & \\
\hline TE-106-AP-43 & 511/ & 85 & CASS & 5 & Oxta point did not atree with locel trend \\
\hline TE-10S-AP-AB & 51234 & 67 & CASS & $T$ & \\
\hline TE-100AP-43 & $5 / 6 \%$ & 89 & CASS & $T$ & \\
\hline$T E-108-A P=43$ & 5r27/84 & 82 & CASS & $T$ & \\
\hline$T E-103-A P-43$ & E2sal & 8 & CASS & $T$ & \\
\hline$T E-10-A P-43$ & $6 \times 5 \times 4$ & 93 & CASS & $T$ & \\
\hline TE-1DA-AP-A3 & 614t:4 & 81 & CASS & $T$ & \\
\hline$T E-106-A P-43$ & Grosen & 64 & CASS & $\mathbf{s}$ & Data point did not eqree with locel trend \\
\hline TE-103-AP-43 & 6/1784 & G4 & CASS & $\mathbf{S}$ & Dente point did not caree with beal trend \\
\hline$T E-103-A P-A 3$ & 620194 & 64) & CASS & $\mathbf{S}$ & Data point did not agree with local trend \\
\hline TE-10S-AP-43 & 6/25,94 & 82 & CASS & $T$ & \\
\hline$T E-103-A P-43$ & $6 / 2754$ & 83 & CASS & $T$ & \\
\hline TE-TOS-AP-A3 & $73 M 94$ & 93 & CASS & $T$ & \\
\hline TE-106-AP-43 & 7rors4 & 87 & CASS & $T$ & \\
\hline TE-106-AP-43 & 8919 & 92 & CASS & 7 & \\
\hline$T E-106-A P-43$ & $8 / 24 / 94$ & 90 & CASS & $\mathbf{T}$ & \\
\hline TE-106AP-43 & $10 / 1 / 94$ & 95 & CASS & $T$ & \\
\hline TE-106-AP-43 & 10brs4 & 97 & CASS & $T$ & \\
\hline TE-106-AP-A3 & $10 / 15 / 4$ & 98 & CASS & $T$ & \\
\hline$T E-103-A P=43$ & $10 / 17 / 94$ & 100 & CASS & $T$ & \\
\hline$T E-162-A P-A 3$ & $10 / 2594$ & 9 & CASS & $T$ & \\
\hline$T E-106-A P-43$ & 11594 & 101 & CASS & $T$ & \\
\hline TE-103-AP-43 & $11 / 11 / 4$ & 105 & CASS & $\mathbf{T}$ & \\
\hline$T E-103-A P-13$ & $11 / 18 \%$ & 100 & CASS & $T$ & \\
\hline TE-103-AP-13 & $11 / 22 / 94$ & 104 & CASS & $T$ & \\
\hline$T E-106-A P-43$ & $11 / 28 / 94$ & 8 & CASS & $T$ & \\
\hline TE-106AP-A3 & 12 ras & 102 & CASS & $T$ & \\
\hline TE-1OS-AP-A3 & $12 / 1694$ & 98 & CASS & $T$ & \\
\hline$T E-103-A P-43$ & 12/1994 & 100 & CASS & $T$ & \\
\hline$T E-106-A P-43$ & $12 / 30 / 94$ & 100 & CASS & $T$ & \\
\hline TE-106-AP-43 & $1 / 7 \%$ & 104 & CASS & $\bar{T}$ & \\
\hline$T E-103-A P-43$ & $1 / 14 / 85$ & 8 & CASS & $T$ & \\
\hline$T E-103-A P-43$ & $1 / 1595$ & 9 & CASS & $T$ & \\
\hline$T E-103-A P-43$ & $1 / 23 / 5$ & 101 & CASS & $T$ & \\
\hline$T E-103-A P-43$ & $2 / 2 \times 5$ & 86 & CASS & $T$ & \\
\hline$T E-1 B 6-A P-43$ & 2595 & 9日) & CASS & $T$ & \\
\hline$T E-106-A P-43$ & 21495 & 105 & CASS & $T$ & \\
\hline
\end{tabular}




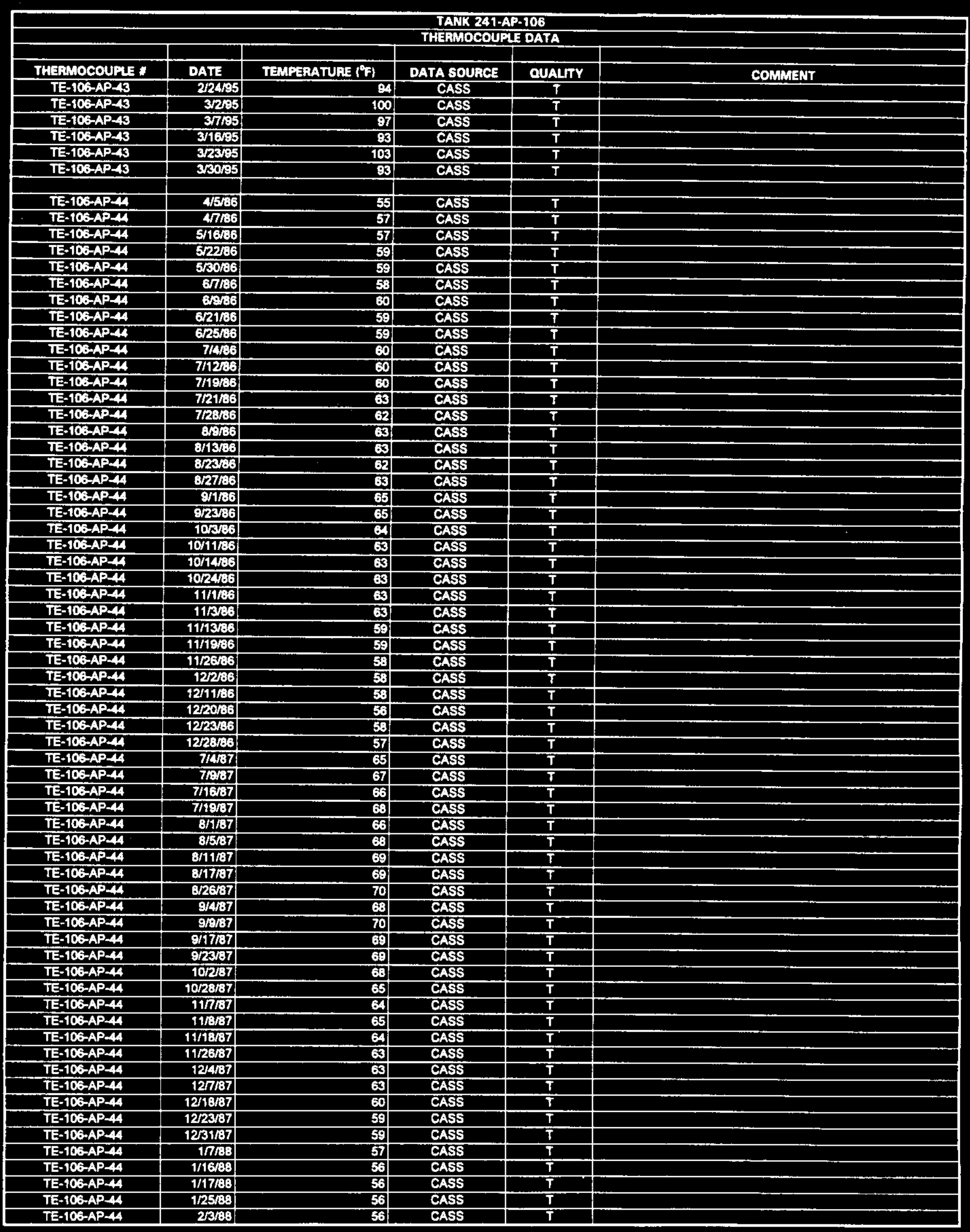




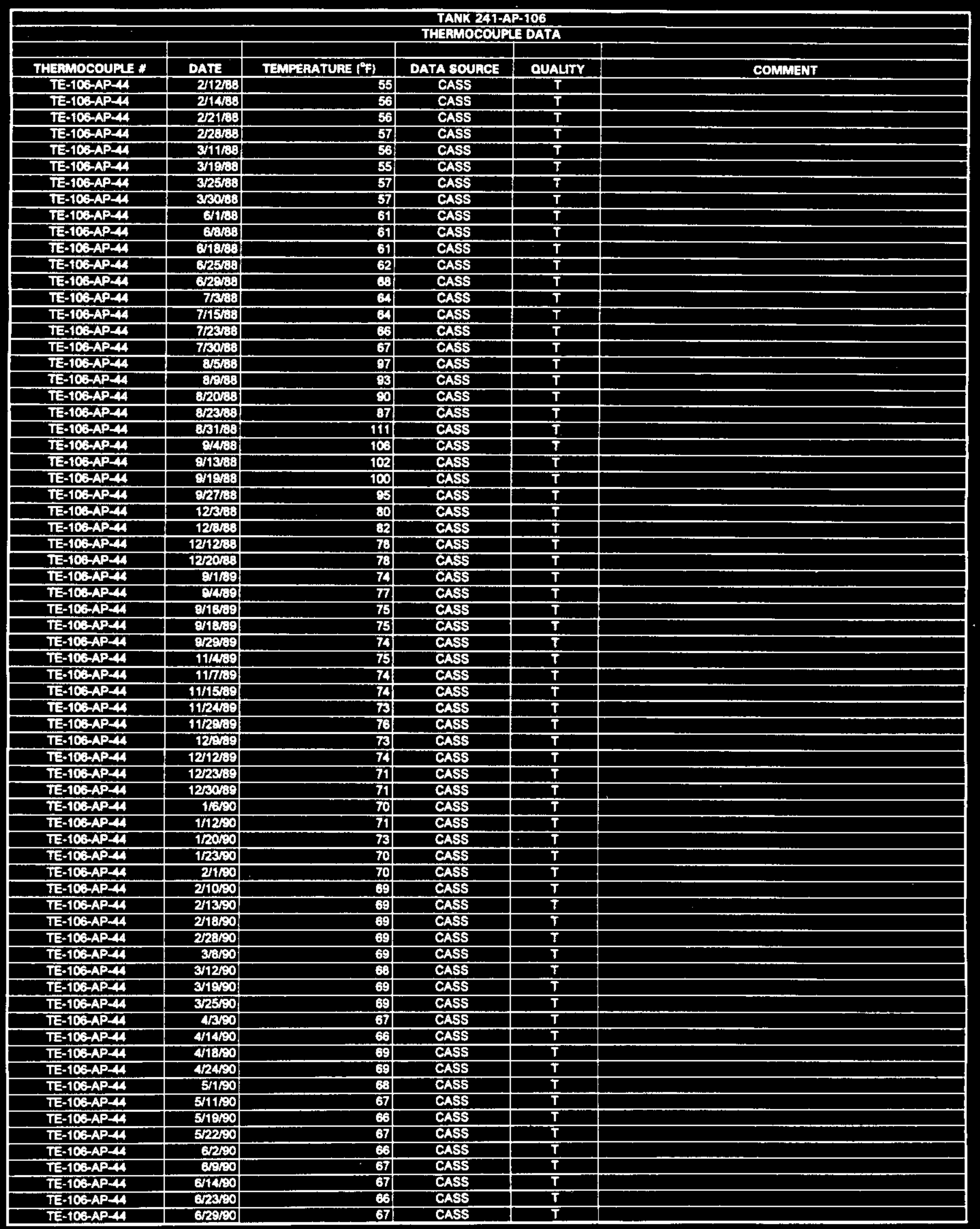




\begin{tabular}{|c|c|c|c|c|c|}
\hline \multicolumn{6}{|c|}{$\begin{array}{l}\text { TANK 241-AP-106 } \\
\text { THARAMOCOUPLE DATA }\end{array}$} \\
\hline & & & & & \\
\hline THERMOCOUPLE * & DATE & TEMPERATURE $\left({ }^{\circ} \mathrm{F}\right)$ & DATA SOUACE & QUALTY & COMMENT \\
\hline TE-106-AP-A4 & $7 / 490$ & 66 & CASS & $T$ & \\
\hline TE-106-AP-A4 & $7 / 1450$ & 66 & CASS & $T$ & \\
\hline TE-106-AP-A & $7 / 5 \times$ & 68 & CASS & $T$ & \\
\hline TE-106-AP-4 & 72710 & 67 & CASS & $T$ & \\
\hline $\begin{array}{l}T E-106-A P-44 \\
T E-106-A P-A 4\end{array}$ & $\begin{array}{l}8 / 3 / 00 \\
8 / 1 / 50\end{array}$ & 63 & $\frac{\text { CASS }}{\text { CASS }}$ & $\frac{T}{T}$ & \\
\hline$\frac{T E-106-A P-A 4}{T E-106-A P-44}$ & $\begin{array}{l}8 / 11 / 50 \\
8 / 48 / 80\end{array}$ & 67 & CASS & $\frac{1}{T}$ & \\
\hline$T E \cdot 103-A P-44$ & $8 / 19 \%$ & 63 & CASS & $T$ & \\
\hline TE-106-AP-A4 & 8/180 & 67 & CASS & $\bar{T}$ & \\
\hline$T E-106-A P-44$ & $8 / 2=0$ & 69 & CASS & $T$ & \\
\hline TE-106-AP-44 & g/400 & 70 & CASS & $T$ & \\
\hline TE-106-AP-A4 & $9 / 2250$ & 69 & CASS & $T$ & \\
\hline$T E-106-A P-44$ & 9/28/90 & 70 & CASS & $T$ & \\
\hline$T E-106-A P-4 A$ & $1016 \%$ & 68 & CASS & $\bar{T}$ & \\
\hline TE-106-APA4 & $10 / 11 \times 0$ & 69 & CASS & $\bar{T}$ & \\
\hline TE-106-AP-44 & 10/20/00 & 63 & CASS & $\bar{T}$ & \\
\hline TE-106-AP-44 & Taraso & 69 & CASS & $T$ & \\
\hline TE-106-AP-A4 & 10/31/00 & 70 & CASS & $T$ & \\
\hline TE-106-AP $=4$ & $11 / 10 / 00$ & 70 & CASS & $T$ & \\
\hline TE-106-AP-44 & $11 / 17 \% 0$ & 72 & CASS & $\bar{T}$ & \\
\hline TE-106AP-44 & $11 / 2200$ & 68 & CASS & $T$ & \\
\hline TE-106-AP-A4 & $11 / 2900$ & 69 & CASS & $T$ & \\
\hline TE-103-AP-4A & 126500 & 69 & CASS & $\bar{T}$ & \\
\hline$T E-106-A P-44$ & 12/460 & 60 & CASS & $T$ & \\
\hline$T E-106-A P-4$ & $12221 / 50$ & 68 & CASS & $T$ & \\
\hline$T E-10 B-A P-4$ & $12125 \% 0$ & 69 & CASS & $T$ & \\
\hline$\pi-106-A P-4 A$ & $1 / 131$ & 67 & CASS & $T$ & \\
\hline TE-106-AP-A & 1/12901 & 86 & CASS & $T$ & \\
\hline TE-106-AP-4 & $1 / 1801$ & 66 & CASS & $T$ & \\
\hline TE-100APCA & $1 / 2461$ & 69 & CASS & $T$ & \\
\hline$T E-10 B-A P-A$ & $2 / 291$ & 65 & CASS & $T$ & \\
\hline TE-1OS-AP-A & 2301 & 6 & CASS & $T$ & \\
\hline TE-106-AP- $\bar{A}$ & $2 \pi 601$ & 65 & CASS & $\bar{T}$ & \\
\hline TE-106-APA4 & $2 / 22301$ & 69 & CASS & $T$ & \\
\hline TE-106-AP $4 A$ & 3/191 & 65 & CASS & $\mathbf{T}$ & \\
\hline TE-106-AP-A4 & 3/399 & 68 & CASS & $T$ & \\
\hline TE-103-AP-44 & $3 / 16 / 1$ & 64 & CASS & $T$ & \\
\hline TE-106-AP-A & $3 / 17 / 91$ & 65 & CASS & $T$ & \\
\hline TE-106-AP-AA & $3 / 27131$ & 65 & CASS & $T$ & \\
\hline TE-106-AP-A4 & WG91 & 64 & CASS & $T$ & \\
\hline TE-106-AP-AA & $4 / 291$ & EA & CASS & $T$ & \\
\hline TE-106-AP-A4 & 4 ragon & 63. & CASS & $T$ & \\
\hline TE-10B-APCA & 42101 & 6 & CASS & $T$ & \\
\hline TE-106AP-A & 5xक1 & 63 & CASS & $T$ & \\
\hline$T E-106 \cdot A P-4 A$ & BBßa1 & 66 & CASS & $T$ & \\
\hline TE-10G-AP-A4 & 5/18/91 & 6A & CASS & $\bar{T}$ & \\
\hline$T E-106-A P-A$ & 5/25/91 & 64 & CASS & $T$ & \\
\hline TE-106-AP-4A & $5 / 26 / 91$ & EA & CASS & $T$ & \\
\hline TE-106-AP-A4 & 6/791 & 64 & CASS & $T$ & \\
\hline TE-106-AP-4A & 6/1501 & 65 & CASS & $T$ & \\
\hline TE-106-AP-A & 6/20\%91 & 64 & CASS & $T$ & \\
\hline TE-106-AP- 44 & $6 / 28 / 91$ & 65 & CASS & $T$ & \\
\hline$T E-10 G-A P-44$ & 73391 & 64 & CASS & $T$ & \\
\hline TE-106-AP-44 & 7/13/91 & 64 & CASS & $T$ & \\
\hline TE-106-AP-A4 & 7/1491 & 64 & CASS & $T$ & \\
\hline TE-106-AP-4A & $7 / 24 / 91$ & G) & CASS & $T$ & \\
\hline TE-106-AP-AM & $8 / 1 / 91$ & 64 & CASS & $T$ & \\
\hline TE-106-AP-A4 & $86 / 21$ & 64 & CASS & $T$ & \\
\hline TE-106-AP-44 & $8 / 17 / 91$ & 69 & CASS & $T$ & \\
\hline TE-106-AP-A4 & $8 / 20 / 91$ & 67 & CASS & $T$ & \\
\hline TE-106-AP-A4 & $8 / 30 / 91$ & 67 & CASS & $T$ & \\
\hline$T E-106-A P-44$ & 9/1/21 & 65 & CASS & $T$ & \\
\hline TE-106-AP-A4 & 9/8/91 & 65 & CASS & $\bar{T}$ & \\
\hline TE-10G-AP-4A & $9 / 18 / 91$ & 67 & CASS & $T$ & \\
\hline TE-10G-AP-44 & $10 / 11 / 91$ & 66 & CASS & $T$ & \\
\hline TE-106-AP-44 & $10 / 14 / 91$ & 66 & CASS & $\bar{T}$ & \\
\hline TE-106-AP-A4 & $10 / 25 / 91$ & 63 & CASS & $T$ & \\
\hline TE-106-AP-4 & $10 / 29 / 91$ & 68 & CASS & $\bar{T}$ & \\
\hline$T E-106-A P-44$ & $11 / 891$ & 70 & CASS & $T$ & \\
\hline TE-106-AP-44 & $8 / 2292$ & 64 & CASS & $\bar{T}$ & \\
\hline
\end{tabular}




\begin{tabular}{|c|c|c|c|c|c|}
\hline \multicolumn{6}{|c|}{$\begin{array}{l}\text { TANTK 241-AP-106 } \\
\text { THERRMOCOUPIE DATA }\end{array}$} \\
\hline & & & & & \\
\hline THERMOCOUPLE * & DATE & TEMPERATURE ( $\left.{ }^{\circ} \mathrm{F}\right)$ & DATA SOUACE & QUALTY & COMMENT \\
\hline TE-10G-APCA & $8 / 28+92$ & 66 & CASS & $T$ & \\
\hline$\frac{T E-106-A P-4}{T E-103-A P-4}$ & $\begin{array}{l}91502 \\
9722\end{array}$ & 65 & CASS & $T$ & \\
\hline$\frac{T E-106-A P-4}{\text { TE-106-AP-4A }}$ & $\begin{array}{r}\text { 87622 } \\
\text { 8/16\%2 }\end{array}$ & 68 & $\frac{\text { CASS }}{\text { CASS }}$ & $\frac{T}{T}$ & \\
\hline$\frac{T E-103-A P-44}{T E-106-A P-44}$ & 8/1692 & 65 & CASS & $T$ & \\
\hline TE-10B-AP-A4 & 10/3102 & 67 & CASS & $\mathbf{T}$ & \\
\hline TE-TOEAP-A & 10292 & 67 & CASS & $T$ & \\
\hline TE-10B-AP-A & 10/1782 & 86 & CASS & $\mathbf{T}$ & \\
\hline E-106-AP-4A & $10 / 2292$ & 68 & CASS & $T$ & \\
\hline TE-10B-AP-4A & $1031 / 2$ & 71 & CASS & $T$ & \\
\hline TE-10G-APCA & 111692 & 68 & CASS & $T$ & \\
\hline TE-10B-AP-4A & $11 / 11 / 92$ & 70 & CASS & $T$ & \\
\hline TE-106-AP-AA & $11 / 2092$ & 68 & CASS & $\mathbf{T}$ & \\
\hline$T E-10 B-A P-A$ & $11 / 2228$ & 67 & CASS & $T$ & \\
\hline TE-106-AP 44 & $125 \sqrt{32}$ & 86 & CASS & $\bar{T}$ & \\
\hline$\pi E-10640-4$ & $12 / 1002$ & 68 & CASS & $T$ & \\
\hline$T E-106-A P-4$ & $12 / 1732$ & 67 & CASS & $\mathbf{T}$ & \\
\hline TE-106-AP-A & 122692 & 65 & CASS & $T$ & \\
\hline TE-10B-AP-A & $12 / 30 \% 2$ & 65 & CASS & $\mathbf{T}$ & \\
\hline TE-106-AP-4A & 1/1/BM & 64 & CASS & $\bar{T}$ & \\
\hline TE-TCBAPA4 & 1/18\% & 66 & CASS & $T$ & \\
\hline TE-TOBAP-A & I/2594 & 63 & CASS & $T$ & \\
\hline IE-106-APAA & 2कWa & 68 & CASS & $T$ & \\
\hline$T E=106-A P=4$ & यारक्य & EA- & CASS & $\mathbf{T}$ & \\
\hline TE-106-APCA & याइल & 67 & CASS & $T$ & \\
\hline$T E-106-A P-4$ & 22229 & 67 & CASS & $T$ & \\
\hline TE-106-AP-4 & 3/BD & 66 & CASS & $\overline{\mathbf{T}}$ & \\
\hline TE-106-AP-4A & आ1004 & 66 & CASS & $\mathbf{T}$ & \\
\hline TE-TOBAP-44 & 3/179 & 63 & CASS & $T$ & \\
\hline$T E-103-A P-4$ & 3/20404 & $6 \mathbf{3}$ & CASS & $\bar{T}$ & \\
\hline$T=-106-A P-4$ & अ309 & 63 & CASS & $\mathbf{T}$ & \\
\hline TE-106-AP-4A & arka & 66 & CASS & $T$ & \\
\hline TE-103-AP-4A & Alag & 65 & CASS & $T$ & \\
\hline$\overline{E E-103-A P A 4}$ & 42004 & $E$ & CASS & $T$ & \\
\hline TE- TOB-AP-A & stand & 66 & CASS & $\bar{T}$ & \\
\hline TE-106-AP-AA & 5,2194 & 65 & CASS & $T$ & \\
\hline TE-TOB-AP-A & 511104 & 85 & CASS & $\mathbf{S}$ & Data point did not epree with local trend \\
\hline TE-10B-APA4 & STI20 & 67 & CASS & $\mathbf{T}$ & \\
\hline TE-100AP-4A & S16\% & 100 & CASS & $\mathbf{T}$ & \\
\hline TE-106AP-4A & $5,22 \%$ & 93 & CASS & $T$ & \\
\hline TE-10BAP-4A & $6 / 294$ & 95 & CASS & $\mathbf{T}$ & \\
\hline TE-10BAP-A & 6/OA & 8 & CASS & $\mathbf{T}$ & \\
\hline TE-10B-AP-4A & G/14a & 91 & CASS & $T$ & \\
\hline TE-106-AP-44 & 6/169 & 63 & CASS & $\mathbf{S}$ & Data point did not agree with beal trend \\
\hline TE-106-AP-44 & 6/17\% & 6a & CASS & $\mathbf{S}$ & Data point did not agree with loevil trend \\
\hline TE-106-APCA & 6/20/9a| & 65 & CASS & $\mathbf{S}$ & Data point did not Loree with local trend \\
\hline TE-106-AP-A4 & ER25: & 92 & CASS & $T$ & \\
\hline TE-10BAP-A & 627\% & 98 & CASS & $\bar{T}$ & \\
\hline TE-10G-AP-A & 7 sha & 83 & CASS & $T$ & \\
\hline TE-103-AP-4 & 73009 & 87 & CASS & $T$ & \\
\hline TE-106-AP-44 & 8\%9: & 82 & CASS & $T$ & \\
\hline TE-103-AP-44 & $8 / 2694$ & 80 & CASS & $\bar{T}$ & \\
\hline TE-106-AP-4 & 10/184 & 95 & CASS & $T$ & \\
\hline TE-103-AP-A & $106 / 4$ & 97 & CASS & $\mathbf{T}$ & \\
\hline TE-106-AP-4A & 10/1594 & 93 & CASS & $T$ & \\
\hline TE-106-AP-44 & $10 / 1794$ & 100 & CASS & $T$ & \\
\hline TE-103-AP-4 & $10 / 20 / 94$ & 89 & CASS & $T$ & \\
\hline TE-106-AP-A & $115 \%$ & 101 & CASS & $T$ & \\
\hline TE-10B-AP-4A & $11 / 11 / 94$ & 104 & CASS & $\mathbf{T}$ & \\
\hline TE-106-AP-4A & $11 / 18 / 94$ & 100 & CASS & $T$ & \\
\hline TE-10G-AP-A & $11 / 22 / 9$ & 103 & CASS & $\mathbf{T}$ & \\
\hline$T E-106-A P-44$ & $11 / 28 / 94$ & 88 & CASS & $T$ & \\
\hline TE-106-AP:AM & I21494 & 102 & CASS & $T$ & \\
\hline TE-106-AP-A & 121669 & 88 & CASS & $\mathbf{T}$ & \\
\hline TE-106-AP-A4 & $12 / 49 / 94$ & 100 & CASS & $T$ & \\
\hline TE-1OS-AP-4 & $12 / 30094$ & 100 & CASS & $T$ & \\
\hline TE-106-AP-4A & $1 / 7 \% 5$ & 104 & CASS & $T$ & \\
\hline TE-106-AP-A & 1/14/95 & 99 & CASS & $\bar{T}$ & \\
\hline TE-106-AP-4A & 1/16/95 & 88 & CASS & $T$ & \\
\hline TE-106-AP-AA & $1 / 2395$ & 102 & CASS & $T$ & \\
\hline
\end{tabular}




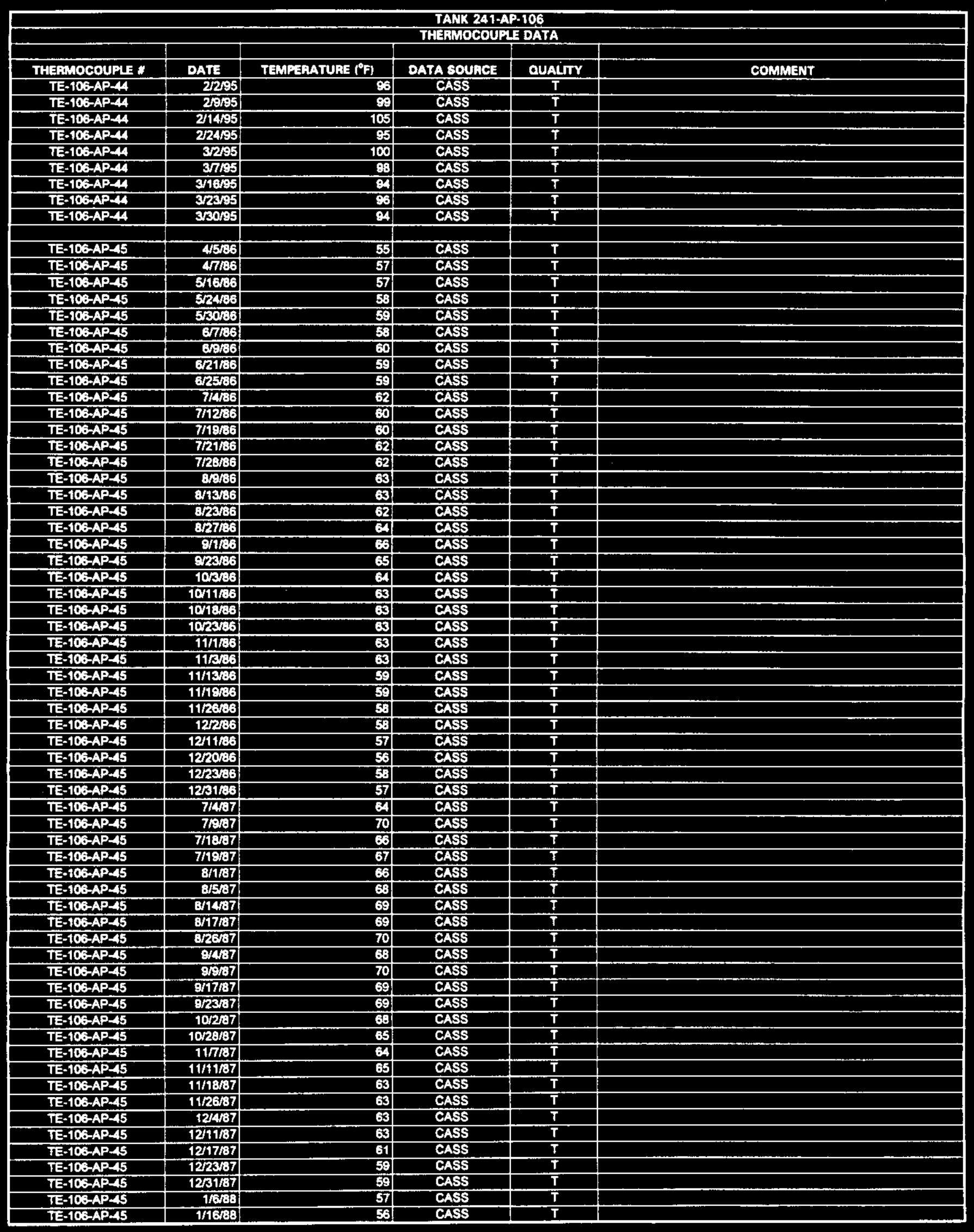




\begin{tabular}{|c|c|c|c|c|c|}
\hline \multicolumn{6}{|c|}{$\begin{array}{l}\text { TAKK 241-AP-106 } \\
\text { THEAWOCOUFL DATA }\end{array}$} \\
\hline & & & & & \\
\hline THEFHOCOUPLE * & DATE & TEMFERATUAE $\left({ }^{\circ} \mathrm{F}\right)$ & DATA SOURCE & OUALITY & COMMENT \\
\hline TE-106-AP-AS & $1 / 1773$ & 56 & CASS & $\bar{T}$ & \\
\hline TE-106-AP-AS & $1 / 25 / 68$ & 56 & CASS & $T$ & \\
\hline TE-10GAP-AS & $2 / 1 / 38$ & 56! & CASS & $T$ & \\
\hline TE-106-AP-AS & $2 \pi / 38$ & 55. & CASS & $T$ & \\
\hline TE-106-AP-45 & 271238 & 67) & CASS & $\mathbf{S}$ & Data point did not agree with locsi trand \\
\hline TE-106-AP-45 & $2 / 14 / 88$ & 56 & CASS & $\bar{T}$ & \\
\hline$T E=106-A P-45$ & 2/21/68 & 56 & CASS & $T$ & \\
\hline TE-106-AP-A5 & 22Z3603 & 57. & CASS & $T$ & \\
\hline$T E-10 B-A P A 5$ & $3 / 11 / 38$ & ES & CASS & $\mathbf{T}$ & \\
\hline TE-10B-AP-A5 & 3/1968 & 55 & CASS & $\bar{T}$ & \\
\hline TE-106-AP-AS & $3 / 2583$ & 57 & CASS & $T$ & \\
\hline TE-TOE-APAS & अ30/38 & 57 & CASS & $T$ & \\
\hline TE-106-AP-45 & 6/1/B8 & 61 & CASS & $\mathbf{T}$ & \\
\hline TE-103-AP-45 & Eन11B & 60 & CASS & $\bar{T}$ & \\
\hline TE-10E-AP-AS & G/18B & 61 & CASS & $T$ & \\
\hline TE-106-AP-AS & EREB3 & 62 & CASS & $\mathbf{T}$ & \\
\hline TE-TOS-AP-AS & ER2000 & 63 & CASS & $T$ & \\
\hline$T E-106-A P-45$ & 7300 & G & CASS & $\mathbf{T}$ & \\
\hline TE-106-AP-AS & $7 / 1468$ & E1 & CASS & $\bar{T}$ & \\
\hline$T E-106-A P-A 5$ & 7123138 & 67 & CASS & $T$ & \\
\hline TE-106-AP-AS & $7730 / 88$ & 67 & CASS & $\mathbf{T}$ & \\
\hline TE-103-AP-45 & $8 / 5 / 30$ & 80 & CASS & $\mathbf{T}$ & \\
\hline TE-100AP-AS & 8003 & M) & CASS & $T$ & \\
\hline TE-10AAPAS & 8/20\%8 & 80 & CASS & $T$ & \\
\hline$T E-106 A P-45$ & $8 / 22 / 68$ & 87 & CASS & $T$ & \\
\hline TE-103-AP-45 & 8/3108 & 118 & CASS & $\mathbf{T}$ & \\
\hline TE-106APAS & 8438 & 110 & CASS & $T$ & \\
\hline TE-10B-AP-AS & $8 / 13 / 38$ & TOM & CASS & $T$ & \\
\hline TE-103-AP-45 & Y1803 & 102 & CASS & $T$ & \\
\hline$\pi \in \cdot 106-A P-45$ & 927B3 & 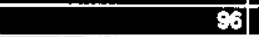 & CASS & $T$ & \\
\hline TE-106-AP-15 & 121B3 & 82 & CASS & $T$ & \\
\hline TE-106-APAS & $12 \% 3$ & 83 & CASS & $\bar{T}$ & \\
\hline TE-10 $3-A P-45$ & 12130 & 7 & CASS & $T$ & \\
\hline$T E-10 B-A P-45$ & 122163 & 78 & CASS & $\mathbf{T}$ & \\
\hline$T E-106-A P-45$ & 9169 & 74 & CASS & $T$ & \\
\hline TE-103-AP-45 & 2009 & 77 & CASS & $T$ & \\
\hline TE-103-AP-45 & 9/16\% & 73 & CASS & $T$ & \\
\hline TE-10G-AP-45 & G/18/39 & 75 & CASS & $\mathbf{T}$ & \\
\hline TE-103-AP-A5 & 9/2969 & 75 & CASS & $T$ & \\
\hline TE-10B-APA5 & $11 / 489$ & 75 & CASS & $T$ & \\
\hline TE-106AP-15 & $11 / 7 / 69$ & 75 & CASS & $\mathbf{T}$ & \\
\hline TE-106-AP-A5 & $11 / 15 B 89$ & 75 & CASS & $\mathbf{T}$ & \\
\hline TE-106-AP-15 & 11/23589 & 73 & CASS & $T$ & \\
\hline TE-10G-AP-A5 & $11 / 2989$ & 7 & CASS & $\bar{T}$ & \\
\hline TE-106-AP-45 & 12\%/69 & 73 & CASS & $T$ & \\
\hline TE-106-AP=AS & $12 / 1269$ & 74 & CASS & $T$ & \\
\hline TE-106-AP-45 & $12 / 23 / 89$ & 71 & CASS & 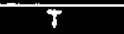 & \\
\hline TE-106-AP-AS & $12230 / 89$ & 71 & CASS & $\bar{T}$ & \\
\hline$T E-106-A P A S$ & $1 / 600$ & 70 & CASS & $T$ & \\
\hline TE-106-AP- -45 & $1 / 1200$ & 71 & CASES & $T$ & \\
\hline TE-106-AP-45 & $1 / 20 / 00$ & 73 & CASS & $\mathbf{T}$ & \\
\hline TE-106-AP-45 & $1 / 23 / 90$ & 70 & CASS & $T$ & \\
\hline TE-106-AP- 15 & $2 / 180$ & 70 & CASS & $T$ & \\
\hline TE-106-AP- 45 & 21080 & 69 & CASS & $T$ & \\
\hline TE-106-AP-45 & 2/13/50 & 70 & CASS & $T$ & \\
\hline TE-106-AP-45 & $2 / 21 / 90$ & 69 & CASS & $T$ & \\
\hline TE-106-AP-A5 & 23850 & 69 & CASS & $\mathbf{T}$ & \\
\hline TE-106-AP- $\overline{5}$ & $36 / 50$ & 70 & CASS & $T$ & \\
\hline$T E-106-A P=45$ & $3 / 12 / 00$ & 68 & CASS & $\mathbf{T}$ & \\
\hline TE-106-AP-AS & $3 / 20 / 50$ & 69 & CASS & $T$ & \\
\hline TE-106-AP- 15 & 3/25500 & 69 & CASS & $T$ & \\
\hline TE-106-AP-45 & 4390 & 67 & CASS & $T$ & \\
\hline TE-106-AP-AS & $4 / 14 / 50$ & 68 & CASS & $\mathbf{T}$ & \\
\hline TE-106-AP-45 & A/18/S0 & 69 & CASS & $T$ & \\
\hline TE-106-AP-AS & 42450 & 69 & CASS & 7 & \\
\hline TE-106-AP-45 & St1so & 68 & CASS & $T$ & \\
\hline . TE-106-AP-15 & $5 / 680$ & 68 & CASS & $T$ & \\
\hline TE-106-AP-A5 & \$/19/80 & 68 & CASS & $T$ & \\
\hline TE-TOSAPAS & 57250 & 67 & CASS & $T$ & \\
\hline TE-106-AP-45 & $6 / 2100$ & 66 & CASS & $T$ & \\
\hline
\end{tabular}




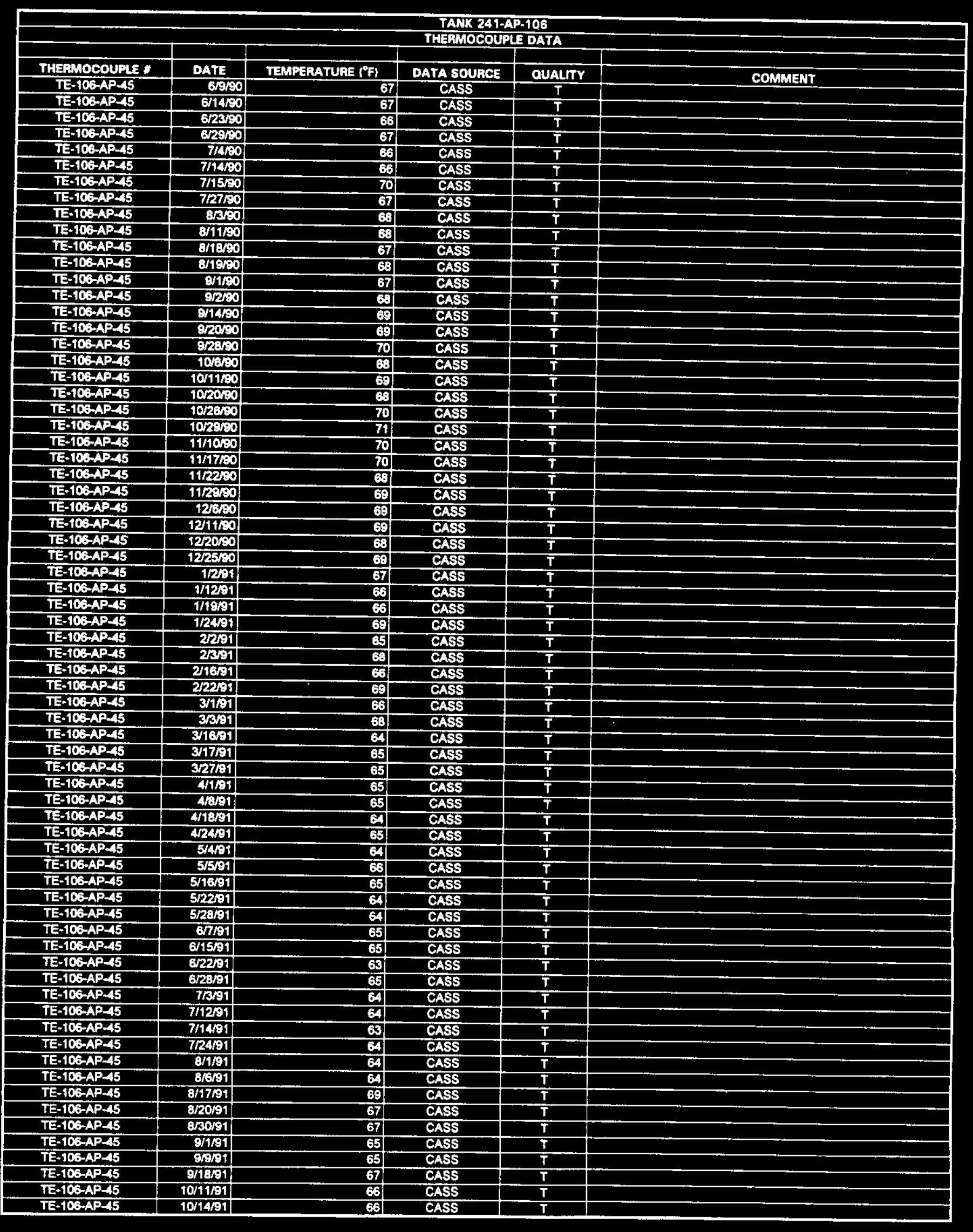

Data obtained from Computer Aunomated Surveillance Systom (CASS), Aprll 1995; and Survaillance Analysis Computer System (SACS), June 1995.

D-529 


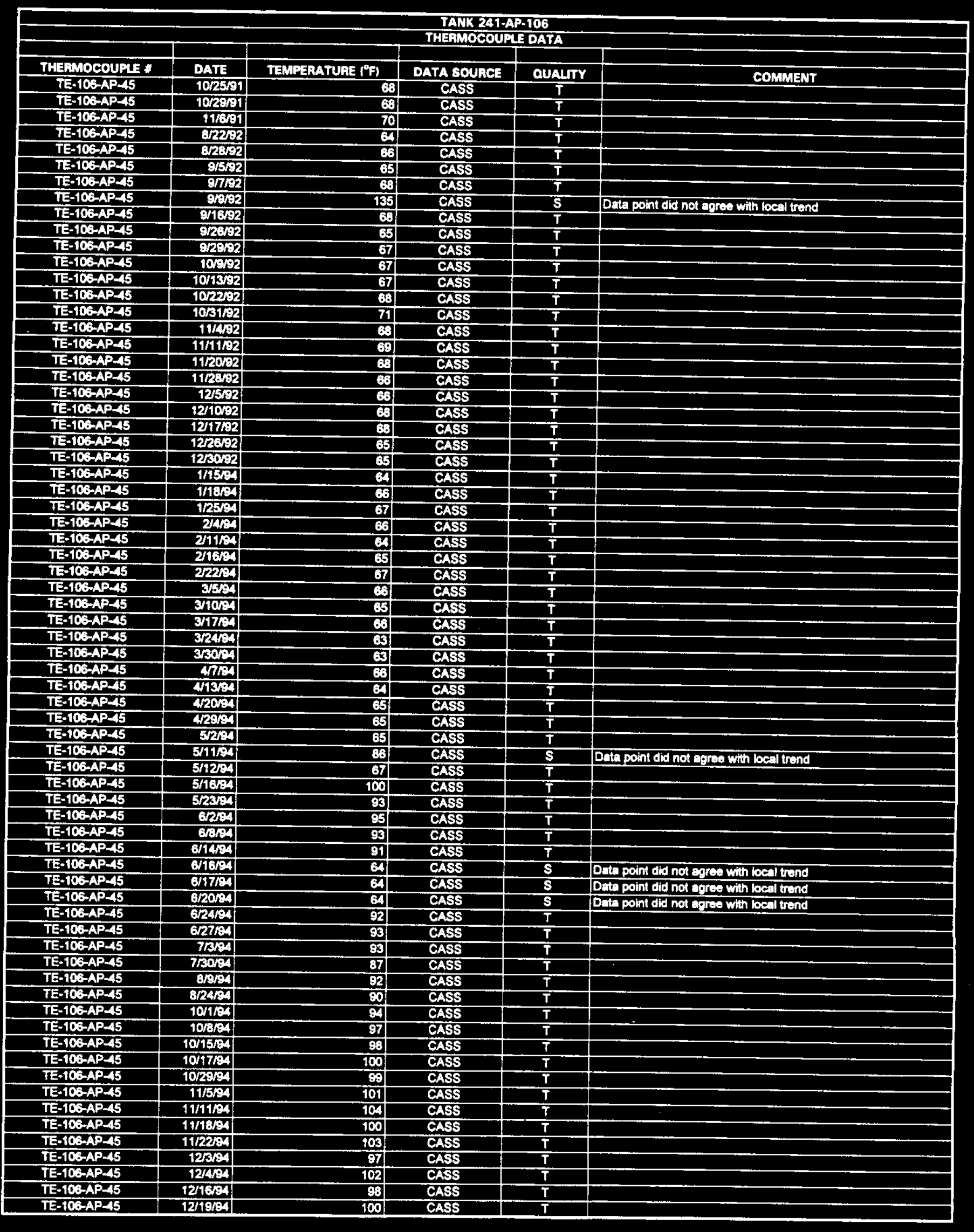

Data obteined from Computer Automated Surveillance Syatem (CASS), April 1995; and Surveillance Analysis Computer Syatem (SACS), June 1995. 


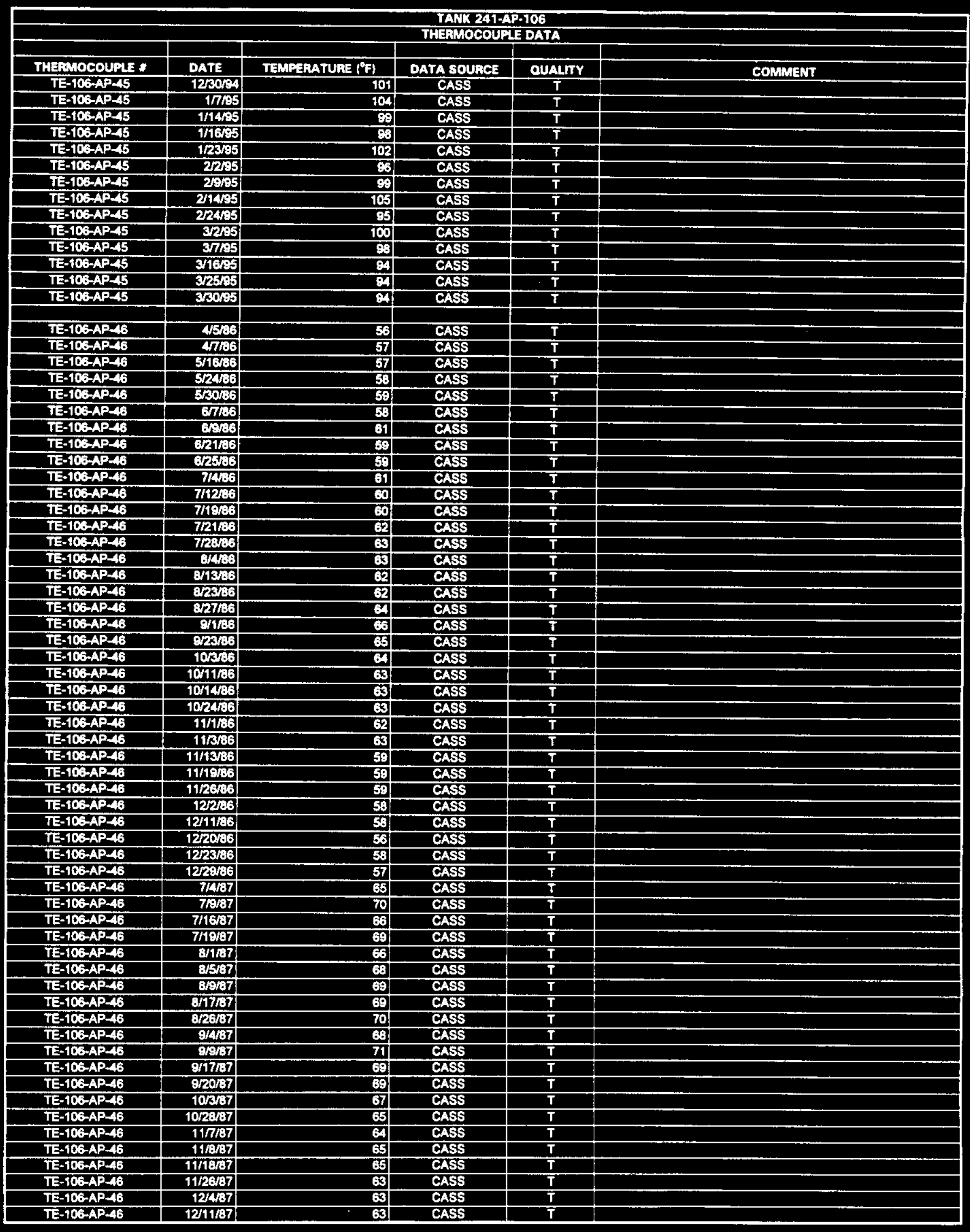




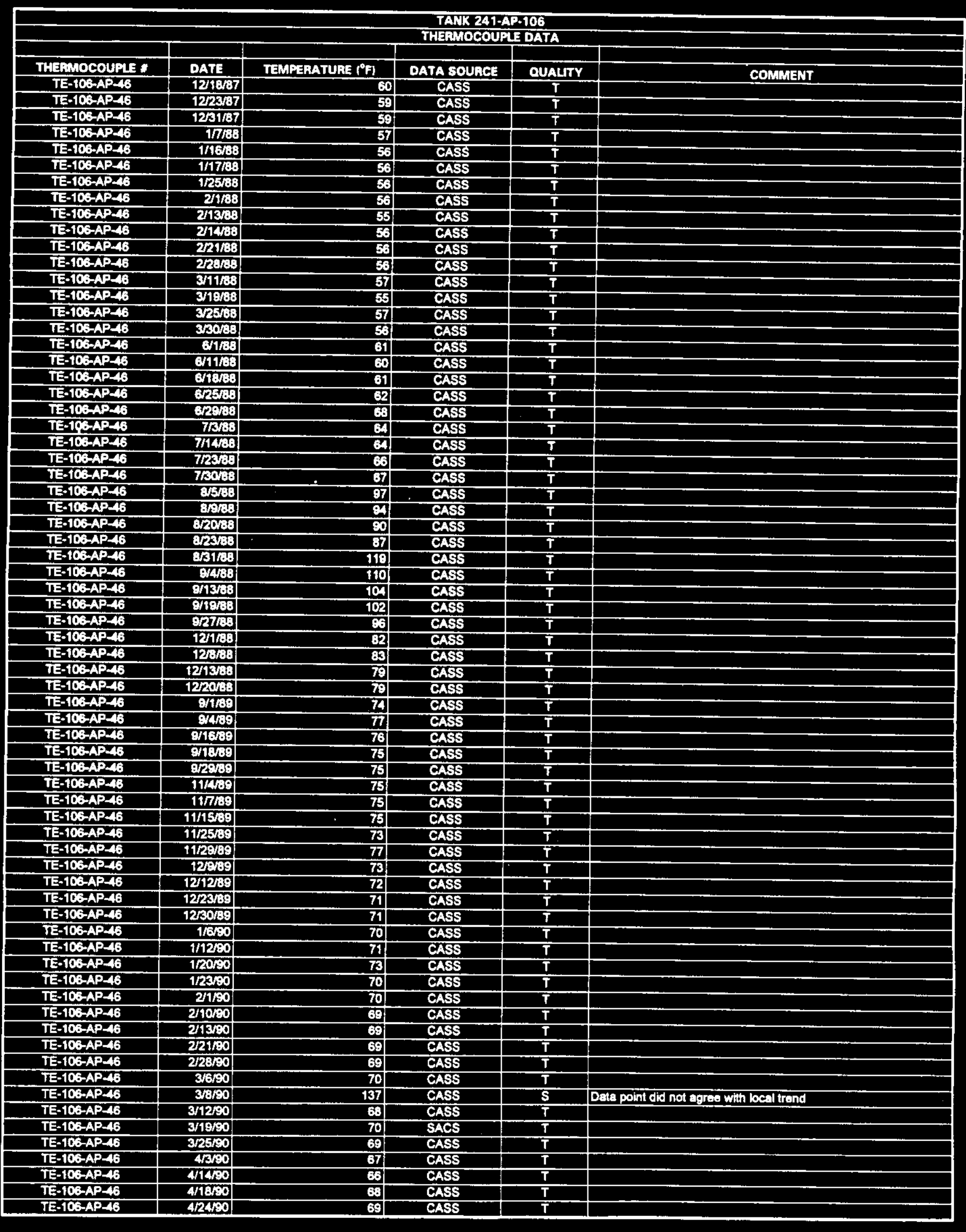

Date obtained from Computer Automated Survoillance System (CASS), April 1995; and Surveillence Analysis Computer Syatem (SACS), June 1995.

D-532 


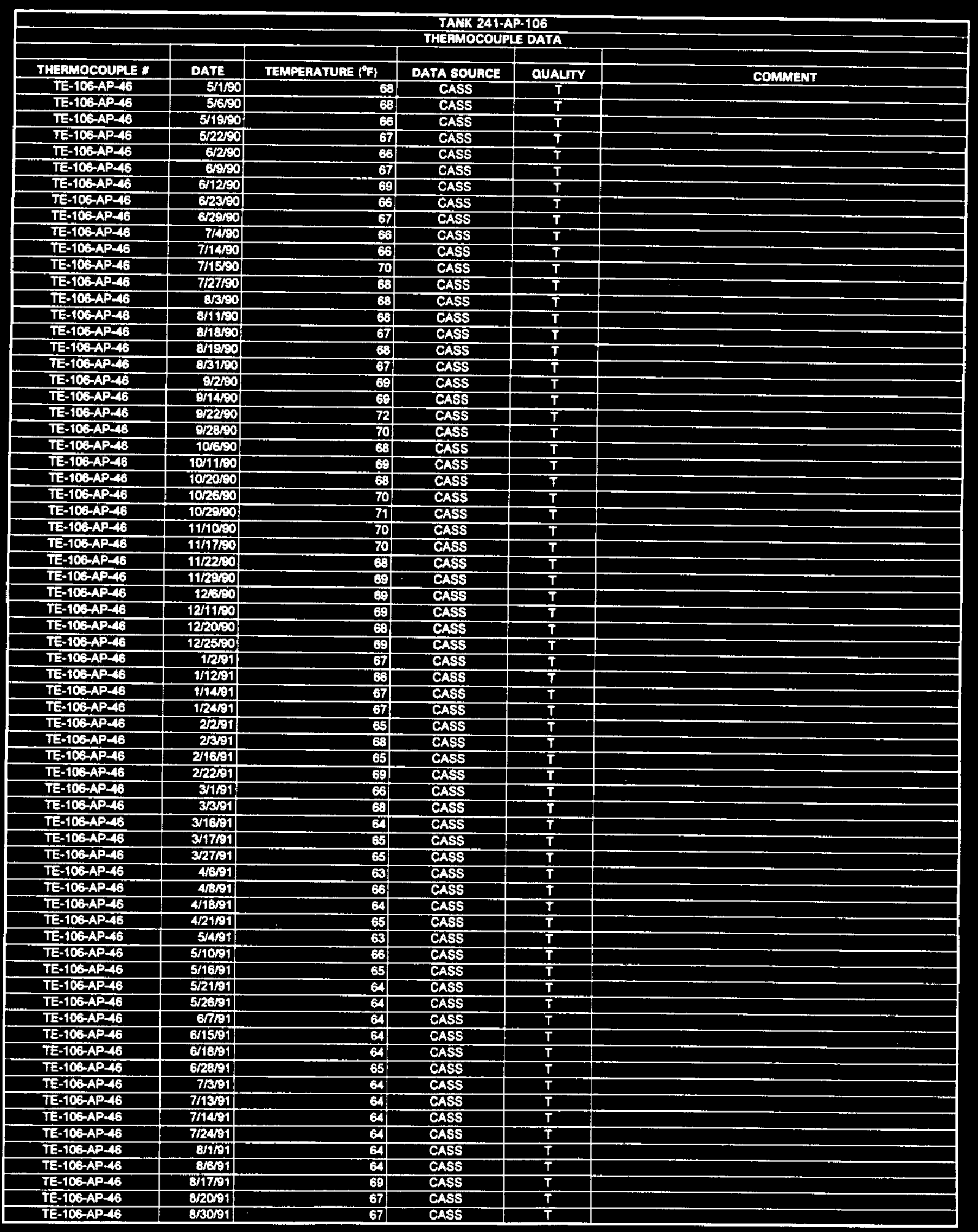

Data obtained from Computer Automated Surveillance System (CASS), April 1995; and Survaillance Analyais Computer System (SACS), June 1995.

D-533 


\begin{tabular}{|c|c|c|c|c|c|}
\hline \multicolumn{6}{|c|}{$\begin{array}{l}\text { TANK 241-AP-106 } \\
\text { THARROOCOUPLE DATA }\end{array}$} \\
\hline & & & & & \\
\hline THE:MOCOUPLE * & DATE & TEMPERATURE $\left({ }^{\circ} \mathrm{F}\right)$ & DATA SOURCE & QUALITY & COMMENT \\
\hline TE-106-AP-46 & 8/191 & 6-4 & CASS & $T$ & \\
\hline TE-106-AP-46 & Sos1 & 65 & CASS & $T$ & \\
\hline TE-10\%-AP-46 & 9/1801 & 67 & $\begin{array}{l}\text { CASS } \\
\text { CASS }\end{array}$ & $\frac{T}{T}$ & \\
\hline $\begin{array}{l}\text { TE-106-AP-16 } \\
\text { TE-106-AP } 46\end{array}$ & $\begin{array}{l}10 / 11 / 91 \\
10 / 1491\end{array}$ & $\frac{66}{66}$ & $\frac{\text { CASS }}{\text { CASS }}$ & $T$ & \\
\hline$\frac{T E-106-A P-16}{T E-106-A P=46}$ & $\frac{10 / 1491}{10 / 25+1}$ & $\frac{66}{68}$ & $\begin{array}{l}\text { CASS } \\
\text { CASS }\end{array}$ & $T$ & \\
\hline TE-106-AP-A6 & Ior2901 & 67 & CASS & $T$ & \\
\hline E-106-AP-46 & $116 / 91$ & 70 & CASS & $T$ & \\
\hline TE-106-AP-46 & $8 / 2202$ & 64 & CASS & $T$ & \\
\hline TE-106-AP-46 & $8 / 28192$ & 6 & CASS & $T$ & \\
\hline TE-103-AP-46 & E/SE2 & 65. & CASS & $T$ & \\
\hline TE-106-AP-46 & 8nra2 & 68 & CASS & $T$ & \\
\hline TE-103-AP-46 & 9R602 & 68 & CASS & $\mathbf{T}$ & \\
\hline TE-103-AP-46 & E2602 & 65: & CASS & $T$ & \\
\hline TE-106-AP-46 & 929292 & 66 & CASS & $\mathbf{T}$ & \\
\hline$T E-106-A P-46$ & 10002 & 67 & CASS & $T$ & \\
\hline TE-106-AP-48 & $10 / 1762$ & 66 & SACS & $\mathbf{T}$ & \\
\hline TE-TOG-AP-16 & 1022292 & 68 & CASS & $T$ & \\
\hline TE-106-AP 46 & $10 / 31 \% 2$ & 71 & CASS & $T$ & \\
\hline TE-106-AP-16 & 11,382 & 63 & CASS & $\mathbf{T}$ & \\
\hline TE-TOB-AP-A6 & $11 / 1 / / 2$ & 70 & CASS & $\bar{T}$ & \\
\hline TE-106APA & $11 / 20 \% 2$ & 68 & CASS & $T$ & \\
\hline$T E-106-A P-46$ & $11 / 2302$ & 66 & CASS & $\mathbf{T}$ & \\
\hline TE-106-AP-46 & $11 / 3002$ & 67 & SACS & $T$ & \\
\hline TE-106-AP-46 & $12 / 1002$ & 68 & CASS & $T$ & \\
\hline TE-106-AP-46 & 1217192 & 68 & CASS & $\bar{T}$ & \\
\hline TE-106-AP-AB & $12121 / 92$ & 68 & SACS & $T$ & \\
\hline TE-1C:A-APCA6 & 1213022 & 65 & CASS & $T$ & \\
\hline TE-106-AP-16 & E/163 & 61 & SACS & $\mathbf{T}$ & \\
\hline TE-106-AP-46 & 6/1803 & 61 & SACS & $\bar{T}$ & \\
\hline TE-106-AP-A6 & 622103 & 61 & SACS & $T$ & \\
\hline TE-106-AP- 16 & 7 1003 & 62 & SACS & $T$ & \\
\hline TE-TOS-AP-46 & 71503 & 62 & SACs & $\mathbf{T}$ & \\
\hline TE-106-AP-46 & 71803 & 61 & SACS & $\bar{T}$ & \\
\hline$T E-106-A P-46$ & 7130003 & 62 & SACS & $T$ & \\
\hline TE-106-AP-46 & $8 / 23$ & 62 & SACS & $\bar{T}$ & \\
\hline TE-106-AP-46 & $8 / 1203$ & 62 & SACS & $T$ & \\
\hline TE-106-AP- 16 & 924/93 & 58 & SACS & $T$ & \\
\hline TE-106-AP-4B & 92633 & 64 & SACS & $T$ & \\
\hline TE-106-AP-46 & $10 / 1503$ & 65 & SACS & $T$ & \\
\hline TE-103-AP-46 & $10 / 2223$ & 64 & SACS & $T$ & \\
\hline$T E-T 06-A P-46$ & $10 / 28 / 93$ & 65 & sAcs & $T$ & \\
\hline$T E-100-A P-46$ & $11 / 8 \% 3$ & EA & Sics & $T$ & \\
\hline TE-103-AP-4B & $11 / 3003$ & 59 & SACS & $T$ & \\
\hline TE-106-AP-46 & 1221493 & 66 & SACS & $T$ & \\
\hline$T E-106-A P-46$ & 122009 & 65 & SACS & $T$ & \\
\hline TE-106-AP-46 & 12/27/63 & 63 & SACS & $T$ & \\
\hline$T E-106-A P-46$ & 1/394 & 63 & SACS & $T$ & \\
\hline TE-106-AP-46 & 1/11/9 & 66 & SACS & $T$ & \\
\hline TE-106-AP-46 & $1 / 18 \% 4$ & 65 & CASS & $T$ & \\
\hline$T E-106-A P-46$ & $1 / 25194$ & 67 & CASS & $T$ & \\
\hline TE-106-AP-46 & 24804 & 66 & CASS & $T$ & \\
\hline TE-106-AP-46 & 2/69 & 64 & CASS & $T$ & \\
\hline TE-106-AP-46 & $2 / 5 / 4$ & 66 & CASS & $T$ & \\
\hline TE-106-AP- 16 & $2 / 22 / 4$ & 67 & CASS & $T$ & \\
\hline TE-106-AP-46 & 3/5/4 & 66 & CASS & $T$ & \\
\hline TE-TO6-AP-46 & 310\%9 & 66 & CASS & $T$ & \\
\hline TE-106-AP-46 & 3/17\%9 & 66 & CASS & $T$ & \\
\hline TE-106-AP-16 & 3/24/90 & 63 & CASS & $T$ & \\
\hline TE-106-AP 46 & 3/30/94 & 63 & CASS & $T$ & \\
\hline TE-106-AP-46 & काISA & 65 & CASS & $\mathbf{T}$ & \\
\hline TE-106-AP-A6 & $4 / 13 / 4$ & 66 & CASS & $\mathbf{T}$ & \\
\hline TE-106-AP-46 & $4 / 20 / 94$ & 65 & CASS & $T$ & \\
\hline TE-106-AP-46 & ARESM & 65 & CASS & $T$ & \\
\hline TE-106-AP-A6 & $5 / 2 / 94$ & 65 & SACS & 6 & \\
\hline TE-106-AP-46 & $5 / 11 / 94$ & 84 & CASS & $\mathbf{S}$ & Data point did not agroe with local trand \\
\hline TE-106-AP-46 & $5 / 128$ & 66. & CASS & $\mathbf{T}$ & \\
\hline TE-10B-AP-46 & 5/16/94 & 100 & CASS & $\mathbf{T}$ & \\
\hline TE-10S-AP-46 & $5 / 2354$ & 93 & CASS & $\bar{T}$ & \\
\hline TE-10G-AP-46 & $6 / 204$ & 95 & CASS & $\mathbf{T}$ & \\
\hline
\end{tabular}




\begin{tabular}{|c|c|c|c|c|c|}
\hline \multicolumn{6}{|c|}{$\begin{array}{l}\text { TANK 241-AP.106 } \\
\text { THERMOCOURLE DATA }\end{array}$} \\
\hline THEAMOCOUPL * & DATE & TEMPERATUAE $\left({ }^{\circ} \mathrm{F}\right)$ & DATA SOURCE & QUALTY & COMMENT \\
\hline TE-106-AP-46 & $6 / 8 / 94$ & 93 & CASS & $\mathbf{T}$ & \\
\hline TE-106-AP-46 & 6/14/94 & 91 & CASS & $\mathbf{T}$ & \\
\hline TE-106-AP-A6 & 6/16/98 & 63 & CASS & $\mathbf{S}$ & Data point did not agroe with local trend \\
\hline TE-106-AP-46 & G/17/9 & 64) & CASS & $\mathbf{S}$ & Data point did not agree with loenil trend \\
\hline TE-106-AP-46 & 620\%4 & 65 & CASS & $\mathbf{S}$ & Data point did not agree with locel trend \\
\hline TE-106-AP-46 & 6r20094 & 65 & SACS & S(G in SACS) & Data point did not agree with local trend \\
\hline TE-106-AP-46 & 6/2494 & 92 & CASS & $T$ & \\
\hline TE-106-AP-46 & 6/27/94 & 83 & CASS & $T$ & \\
\hline TE-106-AP-46 & IImen & 82 & CASS & $T$ & \\
\hline TE-106-AP-46 & 7/259A & 81 & SACS & S (G in SACS) & Data point did not agree with local trend \\
\hline$T E-106-A P-46$ & 73004 & 87 & CASS & $T$ & \\
\hline TE-106-AP-46 & 8POA & 92 & CASS & $\mathbf{T}$ & \\
\hline TE-106-AP-46 & 8/24/94 & 90 & CASS & $T$ & \\
\hline TE-106-AP-46 & $10 / 139$ & 94 & CASS & $\bar{T}$ & \\
\hline TE-106-AP-46 & 10ros & 97) & CASS & $\mathbf{T}$ & \\
\hline TE-106-AP-46 & 10/159 & 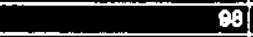 & CASS & $\mathbf{T}$ & \\
\hline TE-10B-AP-46 & 10/17\% & 100 & CASS & $\mathbf{T}$ & \\
\hline TE-103AP-46 & 10/29/94 & 8 & CASS & $T$ & \\
\hline TE-106-AP- 16 & $11 / 5 \%$ & 101 & CASS & $T$ & \\
\hline TE-106-AP-46 & $11 / 11 / 4$ & 105 & CASS & $\bar{T}$ & \\
\hline TE-106-AP-46 & $71 / 18 / 9$ & 100 & CASS & $T$ & \\
\hline TE-106-APA6 & $11 / 2204$ & 103 & CASS & $\mathbf{T}$ & \\
\hline TE-106-AP-46 & 11/298 & 97 & CASS & $\mathbf{T}$ & \\
\hline TE-10S-AP-46 & 12464 & 102 & CASS & $\mathbf{T}$ & \\
\hline TE-10B-AP=46 & $12 / 16 / 94$ & 87 & CASS & $T$ & \\
\hline TE-106-AP-46 & $12 / 190$ & 100 & CASS & $T$ & \\
\hline TE-106-AP- $=6$ & 12/30/9 & 100 & CASS & $\mathbf{T}$ & \\
\hline TE-106-AP-16 & $1 / 7 / 95$ & 100 & CASS & $\mathbf{T}$ & \\
\hline$T E-10 B-A P-46$ & 1/14605 & 8 & CASS & $T$ & \\
\hline TE-106-AP-46 & 1/15:5 & 8 & CASS & $\mathbf{T}$ & \\
\hline$T E-106-A P-46$ & $1 / 2365$ & 102 & CASS & $T$ & \\
\hline TE-106-AP-46 & $2 / 205$ & 8 & CASS & $\bar{T}$ & \\
\hline TE-106-AP-46 & $2 / 1005$ & 8 & CASS & $T$ & \\
\hline$T E-106-A P-46$ & 21405 & 105 & CASS & $T$ & \\
\hline$T E-106-A P-46$ & 2/24/65 & $\mathbf{9 4}$ & CASS & $T$ & \\
\hline TE-106-AP-A6 & $3 / 235$ & 100 & CASS & $T$ & \\
\hline TE-106-AP- 46 & $37 / 5$ & 83 & CASS & $T$ & \\
\hline$T E-106-A P-46$ & 3/1653 & 83 & CASS & $T$ & \\
\hline$T E-106-A P-16$ & $3 / 25 \% 5$ & 89 & CASS & $T$ & \\
\hline TE-106-AP-46 & 3/30/85 & 84 & CASS & $\bar{T}$ & \\
\hline TE-106-AP-46 & 4365 & 86 & SACS & $\mathbf{G}$ & \\
\hline TE-106-APA 16 & 41005 & 85 & SACS & G & \\
\hline TE-106-AP-46 & 4/1755 & 87 & SACS & G & \\
\hline TE-106-AP-46 & $4 / 24 / 85$ & 80 & SACS & G & \\
\hline$T E-106-A P-46$ & SI/SS & BA & SACS & $\mathbf{G}$ & \\
\hline$T E-106-A P-46$ & 5/6/85 & 81 & SACS & $\mathbf{G}$ & \\
\hline TE-106-AP-46 & 5/16/95 & 79 & SACS & $\mathbf{G}$ & \\
\hline TE-106-AP-46 & $5 / 2295$ & 80 & SACS & $\mathbf{G}$ & \\
\hline TE-106-AP-46 & 5/29\%5 & 82 & SACS & G & \\
\hline TE-106-AP-46 & 6/5/95 & 85 & SACS & G & \\
\hline TE-106-AP- 16 & 6/13/95 & 80 & SACS & G & \\
\hline TE-10S-AP-46 & 6/19/95 & 80 & SACS & $\bar{G}$ & \\
\hline TE-10G-AP-A7 & $4 / 5 / 86$ & 56 & CASS & $T$ & \\
\hline TE-106-AP-47 & $47 / 86$ & 57 & CASS & $T$ & \\
\hline TE-106-AP-47 & $5 / 16 / 86$ & 57 & CASS & $T$ & \\
\hline$T E-106-A P-47$ & $5 / 22186$ & 59 & CASS & $T$ & \\
\hline TE-106-AP-47 & $5 / 30 / 86$ & 59 & CASS & $T$ & \\
\hline TE-106-AP-A7 & $6 / 7 / 86$ & 58 & CASS & $T$ & \\
\hline TE-106-APA7 & 6/9/36 & 61 & CASS & $T$ & \\
\hline TE-106-AP- 47 & 6/21/36 & 59 & CASS & $T$ & \\
\hline TE-106-AP-47 & 6/25/36 & 59 & CASS & $\mathbf{T}$ & \\
\hline TE-106-AP-47 & $7 / 4 / 86$ & 62 & CASS & $\mathbf{T}$ & \\
\hline TE-106-AP $\triangle 17$ & $7 / 12 / 86$ & 60 & CASS & $T$ & \\
\hline$T E-106-A P-47$ & 7719/86 & 60 & CASS & $T$ & \\
\hline TE-10S-APAT & $7 / 21 / 86$ & 62 & CASS & $\mathbf{T}$ & \\
\hline TE-106-AP-47 & 7/28/86 & 63 & CASS & $T$ & \\
\hline TE-106-AP-A7 & $8 / 9 / 86$ & 63: & CASS & $\mathbf{T}$ & \\
\hline TE-10G-AP-A7 & $8 / 13 / 86$ & 63 & CASS & $T$ & \\
\hline TE-10B-AP-A7 & $8 / 23 / 86$ & 621 & CASS & $\mathbf{T}$ & \\
\hline
\end{tabular}




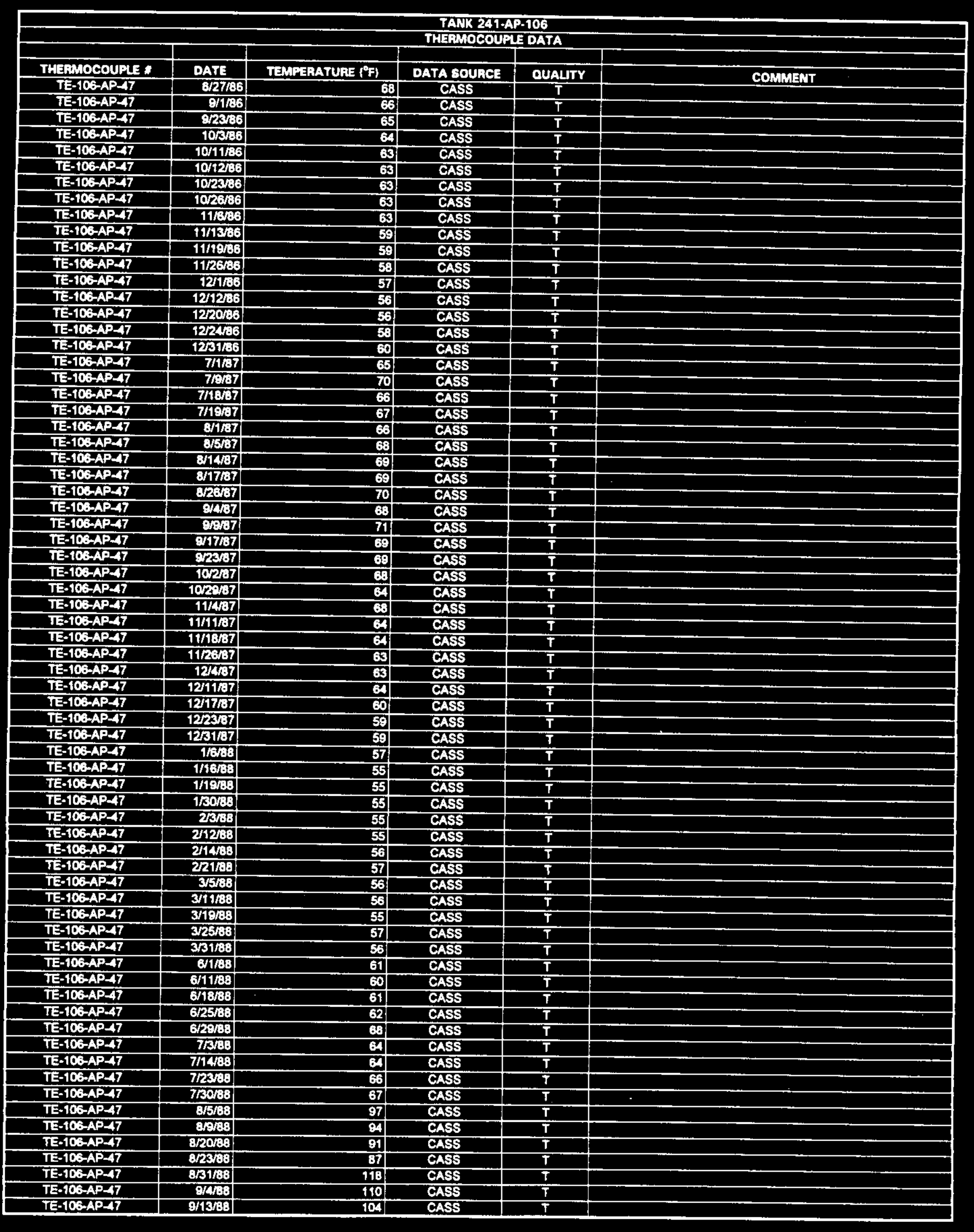

Data obtained from Computer Automated Surveillance System (CASS), April 1995; and Surveillance Analysis Computer System (SACS), June 1995.

D-536 


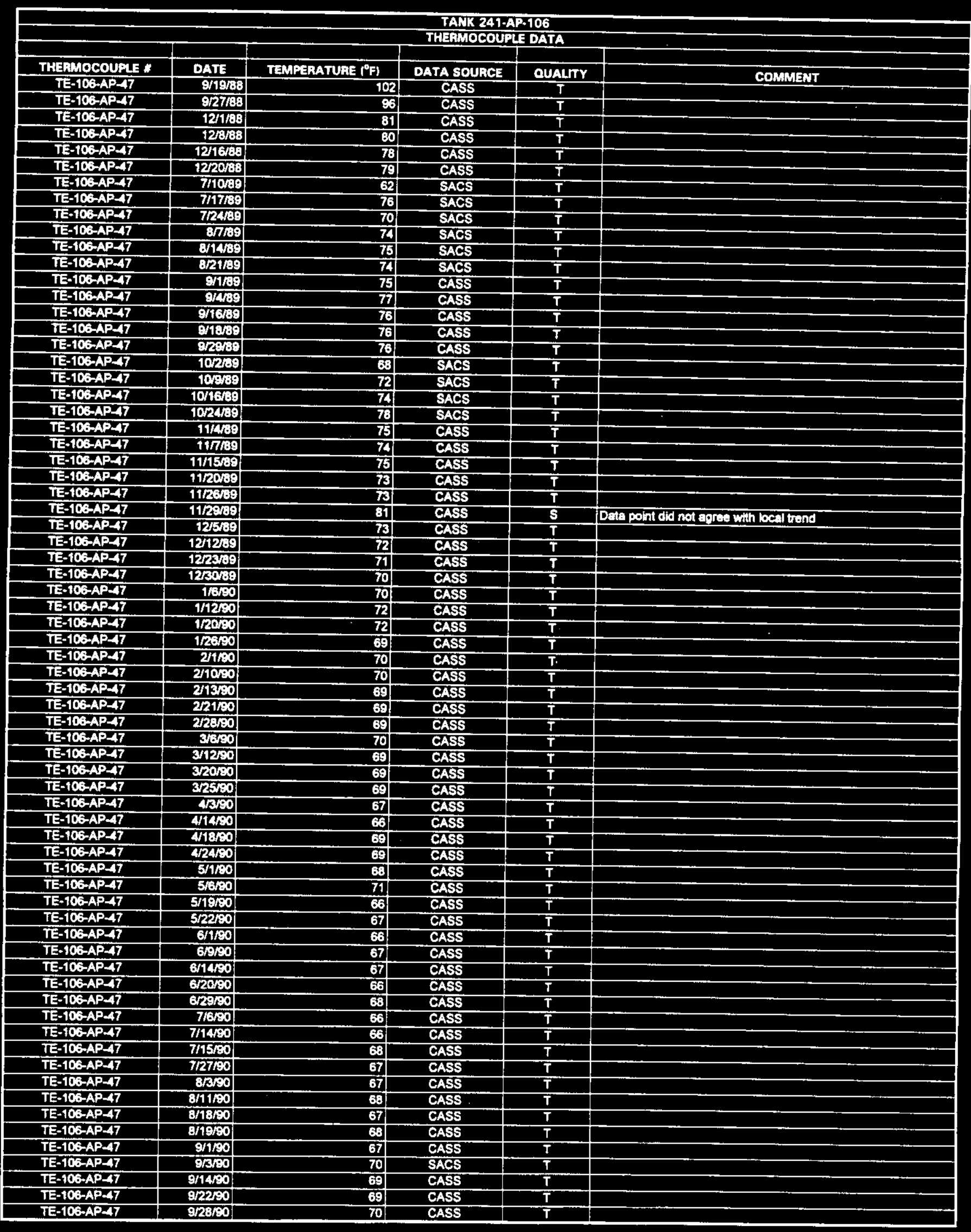

Data obtained from Computer Automated Surveillance Syatem (CASS), April 1995; and Surveillance Analysis Computer System (SACS), June 1995. D-537 


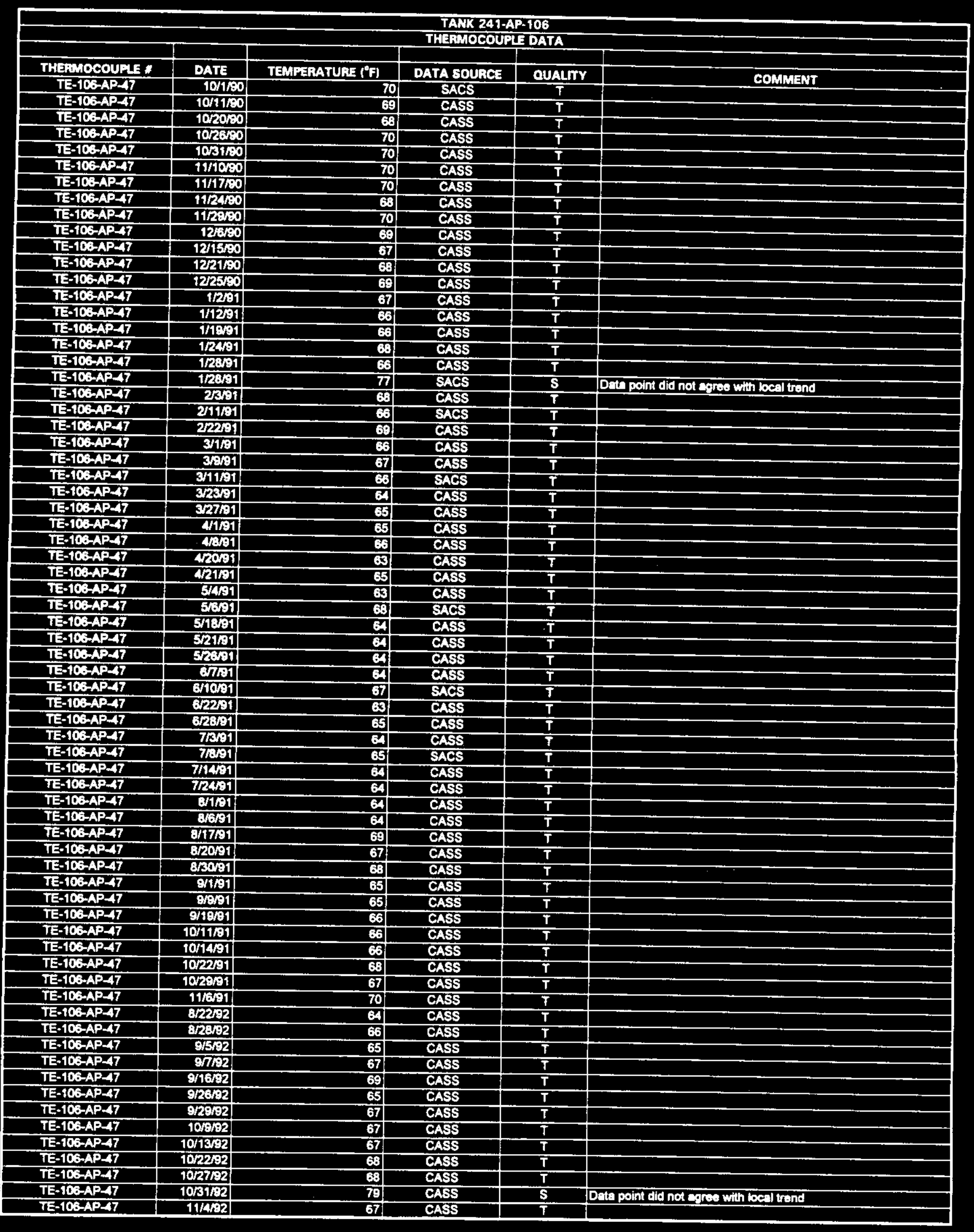




\begin{tabular}{|c|c|c|c|c|c|}
\hline \multicolumn{6}{|c|}{$\begin{array}{l}\text { TANK 241-AP-106 } \\
\text { THEAMOCOUAL DATA }\end{array}$} \\
\hline THERMOCOUPL : & DATE & TEMPEATUUE $t^{\circ} \mathrm{FI}$ & DATa solugce & & \\
\hline TE-10S-APA7 & $11 / 11 / 92$ & 69 & $\frac{\text { DATA SOULiCE }}{\text { CASS }}$ & $\frac{\text { QUALTY }}{T}$ & COMMENT \\
\hline $75-106-A P-47$ & $11 / 20 / 92$ & 68 & CASS & $T$ & \\
\hline TE-106-AP-47 & $11 / 2292$ & 66 & CASS & $T$ & \\
\hline TE-10S-AP-47 & $1216 / 92$ & 65 & CASS & $T$ & \\
\hline TE-106AP-A7 & $12 / 10 / 92$ & 67 & CASS & $T$ & \\
\hline TE-106-AP-A7 & $12 \pi 19 / 92$ & 67 & CASS & $T$ & \\
\hline TE-106-AP-A7 & $12 / 2692$ & 64 & CASS & $\bar{T}$ & \\
\hline TE-106-AP-A7 & $1230 / 92$ & 64 & CASS & $T$ & \\
\hline TE-106-AP-A7 & 1/15\% & 63 & CASS & $T$ & \\
\hline TE-106-AP-A7 & $1 / 21 \% 4$ & 64 & CASS & $T$ & \\
\hline TE-106-AP-A7 & $1 / 25 \%$ & 66 & CASS & $T$ & \\
\hline TE-106-AP-A7 & $2 / 4 / 94$ & 68 & CASS & $\bar{T}$ & \\
\hline$T E-106-A P-47$ & $2 / 12 / 94$ & 63 & CASS & $T$ & \\
\hline TE-106-AP-A7 & 2215/94 & 68 & CASS & $T$ & \\
\hline TE-100-AP-47 & 222394 & 67 & CASS & $T$ & \\
\hline TE-106-APA7 & 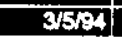 & 8 & CASS & $T$ & \\
\hline$T E-106-A P-47$ & अ/1094 & 65 & CASS & $T$ & \\
\hline TE-106-AP- 47 & 3/17\% & 66 & CASS & $T$ & \\
\hline TE-103-AP-47 & 3/26/94 & 63 & CASS & $T$ & \\
\hline TE-106-AP-47 & $3 \sqrt{3004}$ & 63 & CASS & $T$ & \\
\hline$T E-106-A P-A 7$ & $4 \pi \sqrt{3}$ & 65 & CASS & $T$ & \\
\hline TE-106-AP-A7 & 413,9 & 64 & CASS & $T$ & \\
\hline TE-106-AP-A7 & 4209 & 64 & CASS & $T$ & \\
\hline TE-106-AP-A7 & 42719 & 65 & CASS & $\bar{T}$ & \\
\hline TE-106-AP-17 & 5254 & 65 & CASS & $\bar{T}$ & \\
\hline TE-106-AP-47 & \$1104 & 8 & CASS & $\mathbf{S}$ & Data point did not arree with local trend \\
\hline - TE-106-AP-47 & $5 / 2 \%$ & 6 & CASS & 7 & \\
\hline TE-TDS-AP-A7 & s/169a & 100 & CASS & $\bar{T}$ & \\
\hline$T E-106-A P-47$ & $5 / 23 / 94$ & 83 & CASS & $T$ & \\
\hline TE-103-AP-A7 & 6/289 & est & CASS & $\bar{T}$ & \\
\hline TE-106-AP-17 & 6684 & 98 & CASS & $T$ & \\
\hline TE-106-AP-A7 & 6/169 & 81 & CASS & $\bar{T}$ & \\
\hline$T E-106-A P-A 7$ & G/16\% & 6. & CASS & $\mathbf{S}$ & Deta point did not arrea with local trend \\
\hline IE-106-AP- 47 & 6/17/94 & 64 & CASS & 8 & Data point did not coree with local trend \\
\hline$T E-106-A P-47$ & 6r2099 & 65 & CASS & $\mathbf{8}$ & Data point did not eqree with local trend \\
\hline$T E-106-A P-17$ & 6rata & 92 & CASS & $T$ & \\
\hline TE-106-AP-A7 & 62769 & at & CASS & $\bar{T}$ & \\
\hline$T E-106-A P=4$ & $7 / 390$ & 83 & CASS & $\bar{T}$ & \\
\hline TE-106-AP- $\triangle 7$ & $730 \%$ & 87 & CASS & $\mathbf{T}$ & \\
\hline TE-106-AP-47 & 8\%9| & 92 & CASS & $\mathbf{T}$ & \\
\hline TE-106-AP-A7 & 8/2469 & 80 & CASS & $T$ & \\
\hline TE-106-APA7 & 10/1\%9 & 94 & CASS & $\bar{T}$ & \\
\hline$T E-106-A P-47$ & 10894 & 97 & CASS & $T$ & \\
\hline TE-103-AP-47 & $70 / 12 / 94$ & 8 & CASS & $\bar{T}$ & \\
\hline TE-106-AP-A7 & $10 / 1794$ & 100 & CASS & $T$ & \\
\hline TE-10B-AP-A7 & $10 / 25 / 94$ & 9 & CASS & $T$ & \\
\hline TE-106-AP-A7 & $11 / 534$ & 9 & CASS & $T$ & \\
\hline TE-106-AP-A7 & $11 / 11 / 94$ & 104 & CASS & $\bar{T}$ & \\
\hline TE-106-APA7 & $11 / 18 / 94$ & 9 & CASS & $T$ & \\
\hline EE-106-AP- $\triangle 7$ & $11 / 22 / 94$ & 102 & CASS & $T$ & \\
\hline$T E-106-A P-47$ & $12 \mathrm{~K} / \mathrm{B}$ & 8 & CASS & $T$ & \\
\hline TE-106-AP-A7 & 12/4/94 & 100 & CASS & $T$ & \\
\hline TE-106-AP-A7 & $1216 \mathrm{ma}$ & 97 & CASS & $T$ & \\
\hline TE-106-AP-A7 & $12 / 19 / 94$ & 9 & CASS & $T$ & \\
\hline TE-106-AP-A7 & $12 / 30 / 9$ & 9 & CASS & $T$ & \\
\hline$T E-103-A P-47$ & $1 / 4 / 95$ & 104 & CASS & $\mathbf{T}$ & \\
\hline TE-106-AP-A7 & 1/14/95 & 99 & CASS & $T$ & \\
\hline TE-106-AP-47 & 1/15/95 & 98 & CASS & $T$ & \\
\hline TE-106-AP 47 & 1/23/95 & 101 & CASS & $T$ & \\
\hline TE-106-AP-A7 & $2 / 2 / 95$ & 95 & CASS & $T$ & \\
\hline TE-106-AP-A7 & $2 / 9195$ & 98 & CASS & $T$ & \\
\hline TE-10B-AP-A7 & 2/14/95 & 105 & CASS & $T$ & \\
\hline TE-106-AP-A7 & $2 / 24 / 95$ & 94 & CASS & $T$ & \\
\hline TE-106-AP-A7 & $3 / 2 / 5$ & 89 & CASS & $T$ & \\
\hline$T E-106-A P-47$ & 3/195 & 98 & CASS & $T$ & \\
\hline TE-106-AP-17 & $3 / 16 / 96$ & 93 & CASS & $\mathrm{T}$ & \\
\hline$T E-106-A P-17$ & $3 / 25 / 95$ & 94 & CASS & $\bar{T}$ & \\
\hline TE-106-AP-A7 & 3/30/95 & 9 & CASS & $T$ & \\
\hline TE-106-AP-18 & $45 / 86$ & 55 & CASS & $T$ & \\
\hline
\end{tabular}




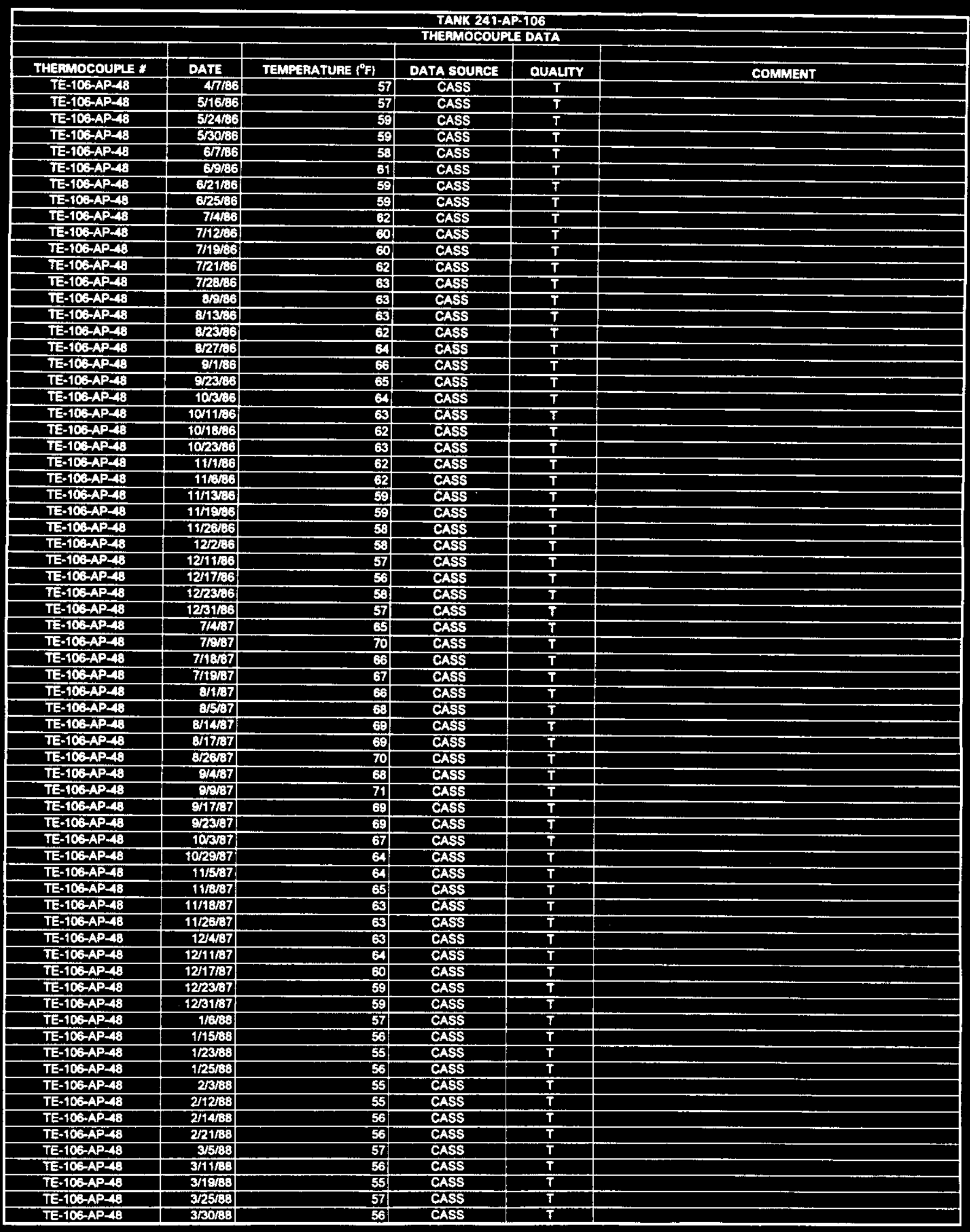




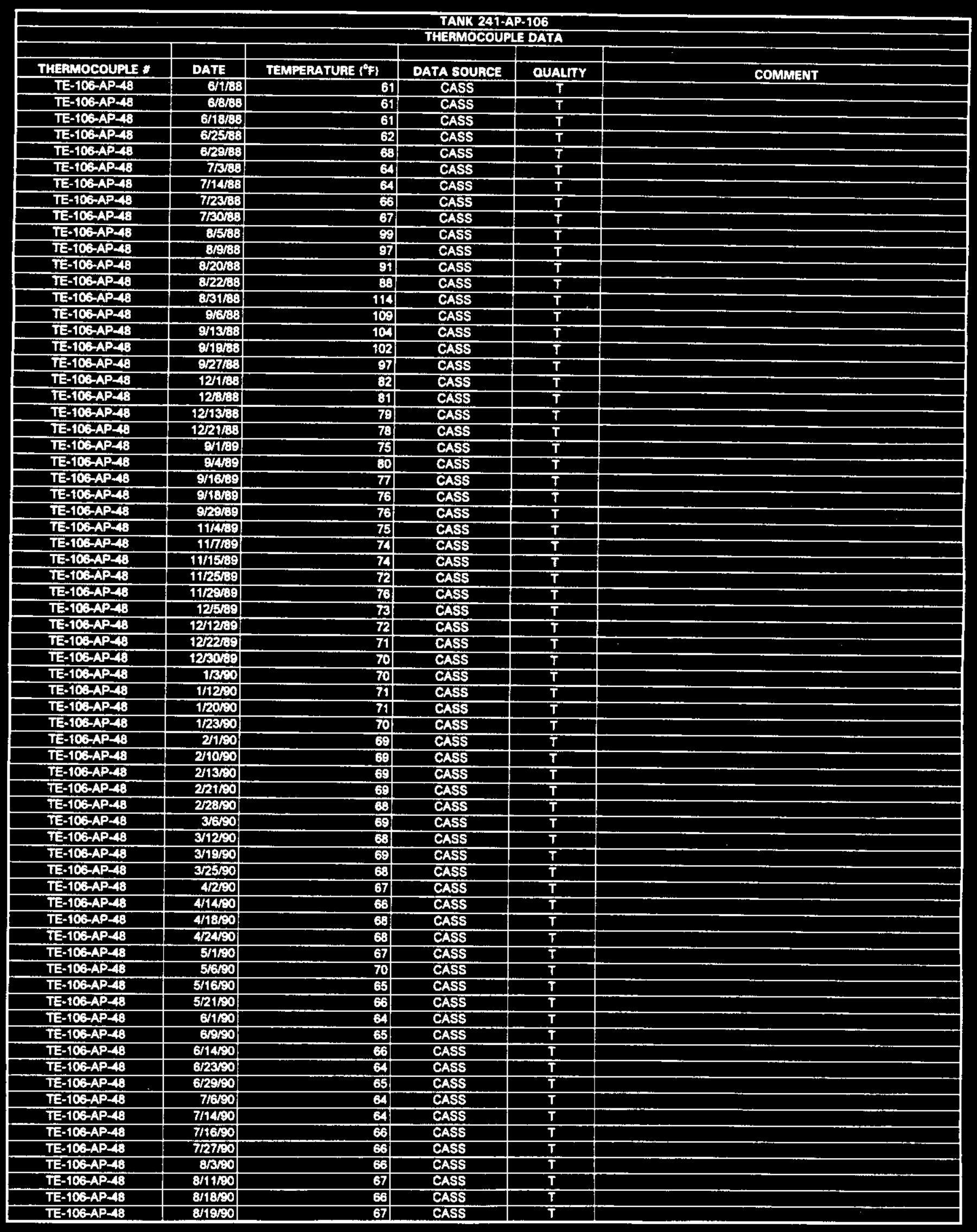




\begin{tabular}{|c|c|c|c|c|c|}
\hline \multicolumn{6}{|c|}{$\begin{array}{l}\text { TANK 241-AP-106 } \\
\text { THEAMOCOUPLE DATA }\end{array}$} \\
\hline THEFMOCOUPL * & DATE & TEMFERATURE $\left({ }^{\circ} \mathrm{F}\right)$ & DATA SOUACE & QUALTYY & COMMENT \\
\hline TE-106-AP-4B & $8 / 1 / 50$ & 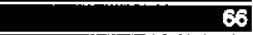 & CASS & $T$ & \\
\hline TE-106-AP-48 & $8 / 250$ & 67 & CASS & $T$ & \\
\hline TE-106-AP-48 & $8 / 14 / 50$ & 67 & CASS & $\mathbf{T}$ & \\
\hline TE-106-AP-4B & $8 / 17190$ & 69 & CASS & $T$ & \\
\hline$T E-106-A P-48$ & $928 \% 0$ & 69 & CASS & $T$ & \\
\hline TE-106-AP-4B & 10/60 & 67 & CASS & $T$ & \\
\hline TE-106-AP-4B & $10 / 10 / 80$ & 68 & CASS & $T$ & \\
\hline TE-106-AP-46 & $10 / 1800$ & 60 & CASS & $T$ & \\
\hline TE-106-AP-48 & $10 / 2250$ & 64 & CASS & $T$ & \\
\hline TE-106-AP- $4 B$ & $10 / 2900$ & 62 & CASS & $T$ & \\
\hline TE-106-AP-AB & $11 / 10 \%$ & 59 & CASS & $T$ & \\
\hline$T E \cdot 106-A P-4 B$ & $11 / 17 \% 0$ & 60 & CASS & $T$ & \\
\hline TE-106-AP-48 & $11 / 24100$ & 60 & CASS & $T$ & \\
\hline TE-106-AP=4B & $11 / 2900$ & 64 & CASS & $T$ & \\
\hline TE-106-AP-48 & 12600 & 65 & CASS & $\mathbf{T}$ & \\
\hline$T=-106-A P-46$ & $12 / 1150$ & 67 & CASS & $T$ & \\
\hline TE-106AP-48 & $12220 \% 0$ & 66 & CASS & $\mathbf{T}$ & \\
\hline$T=-106-A P-4 B$ & 1225500 & 67 & CASS & $\mathbf{T}$ & \\
\hline$T E-106-A P-48$ & $1 / 3 \mathrm{BO}$ & 63 & CASS & $T$ & \\
\hline TE-106APADB & $1 / 1201$ & 66 & CASS & $T$ & \\
\hline TE-106-AP-4B & 1/17/21 & 66 & CASS & $T$ & \\
\hline TE-10B-AP-AB & $1 / 22 / 91$ & 69 & CASS & $T$ & \\
\hline TE-106-AP-46 & $1 / 3001$ & 65 & CASS & $T$ & \\
\hline$T E-106-A P-48$ & 2 23:191 & 69 & CASS & $\mathbf{T}$ & \\
\hline TE-100-AP-48 & 2/691 & 85 & CASS & $T$ & \\
\hline TE-106-AP-43 & $2 / 22 \% 1$ & 69 & CASS & $T$ & \\
\hline$T E-106-A P-48$ & 3/161 & 63 & CASS & $T$ & \\
\hline TE-106-AP-4B & 3/291 & 6 & CASS & $\bar{T}$ & \\
\hline TE-106-AP-A6 & 31691 & GA & CASS & $\mathbf{T}$ & \\
\hline TE-103AP-AB & 3r23\%1 & G & CASS & $\mathbf{T}$ & \\
\hline$T E-106-A P-A B$ & 32761 & 8. & CASS & $T$ & \\
\hline IE-106-AP-48 & 41\%1 & 85 & CASS & $T$ & \\
\hline$\pi E-106-A P-48$ & कas & 63 & CASS & $\bar{T}$ & \\
\hline TE-106-AP-AB & A2001 & 63 & CASS & $T$ & \\
\hline TE-106-AP-AB & 42491 & 64 & CASS & $T$ & \\
\hline TE-106-AP-AB & TREO1 & 53 & CASS & $\mathbf{S}$ & Data point did not ceree wh locel trond \\
\hline TE-106-AP-4B & 54401 & 63 & CASS & $\bar{T}$ & \\
\hline TE-106-AP-48 & 5/1001 & 63 & CASS & $T$ & \\
\hline TE-106-AP-48 & इ16) & 64) & CASS & $T$ & \\
\hline TE-106-AP- 48 & 521/91 & 64 & CASS & $T$ & \\
\hline TE-TOG-AP-48 & $5 \sqrt{2631}$ & 64 & CASS & $T$ & \\
\hline TE-106AP-48 & 67/61 & 65 & CASS & $T$ & \\
\hline$T E-103-A P-4 B$ & G/15/21 & BA & CASS & $\bar{T}$ & \\
\hline TE-106-AP-AB & 622201 & 63 & CASS & $\bar{T}$ & \\
\hline$T E-106-A P-4 B$ & B/28191 & 65 & CASS & $T$ & \\
\hline TE-106-AP- 48 & 73301 & 64 & CASS & $T$ & \\
\hline TE-106-AP- 48 & 7113691 & 64 & CASS & $T$ & \\
\hline TE-106-AP 46 & 71491 & 64 & CASS & $T$ & \\
\hline TE-106-AP-40 & 712691 & 64) & CASS & $T$ & \\
\hline TE-103-AP-48 & $8 / 191$ & 64 & CASS & $T$ & \\
\hline$T E-106-A P-48$ & 8691 & 64 & CASS & $T$ & \\
\hline TE-100-AP-48 & 8/1791 & 67 & CASS & $T$ & \\
\hline TE-106-AP 48 & $8 / 2001$ & 67. & CASS & $T$ & \\
\hline TE-10B-AP- 18 & 8roos1 & 67 & CASS & $\bar{T}$ & \\
\hline TE-106-AP-AB & 8/101 & 64 & CASS & $T$ & \\
\hline TE-106-AP-18 & 8901 & 65 & CASS & $\bar{T}$ & \\
\hline TE-106-AP-48 & 91961 & 66 & CASS & $T$ & \\
\hline TE-106-AP- 48 & 10/19.91 & 66 & CASS & $T$ & \\
\hline TE-106-AP-AB & $10 / 14 / 81$ & 66 & CASS & $T$ & \\
\hline TE-106-AP-48 & $10 / 25391$ & 68 & CASS & $T$ & \\
\hline TE-106-AP-48 & 10r2901 & 68 & CASS & $T$ & \\
\hline TE-106-AP- 48 & 11/691 & 71 & CASS & $\mathbf{T}$ & \\
\hline TE-106-AP-AB & $8 / 22 / 92$ & BA & CASS & $T$ & \\
\hline$T E=106 A P=48$ & $8 / 2392$ & 83 & CASS & $T$ & \\
\hline TE-106-AP-AB & $9 / 592$ & 65 & CASS & $T$ & \\
\hline$T E-106-A P-48$ & 8/92 & 67 & CASS & $T$ & \\
\hline TE-106-AP-AB & 9/16/92 & 63 & CASS & $\bar{T}$ & \\
\hline TE-10B-APAB & 9/26192 & 63 & CASS & $T$ & \\
\hline TE-106-AP-AB & 9/29/92 & 67 & CASS & $T$ & \\
\hline$T E-103-A P-48$ & 10/9/2 & 67 & CASS & $T$ & \\
\hline
\end{tabular}

Data obtained from Computer Automated Surveillance System (CASS), April 1995; and Survellence Anelysis Computer System (SACS), June 1995. 


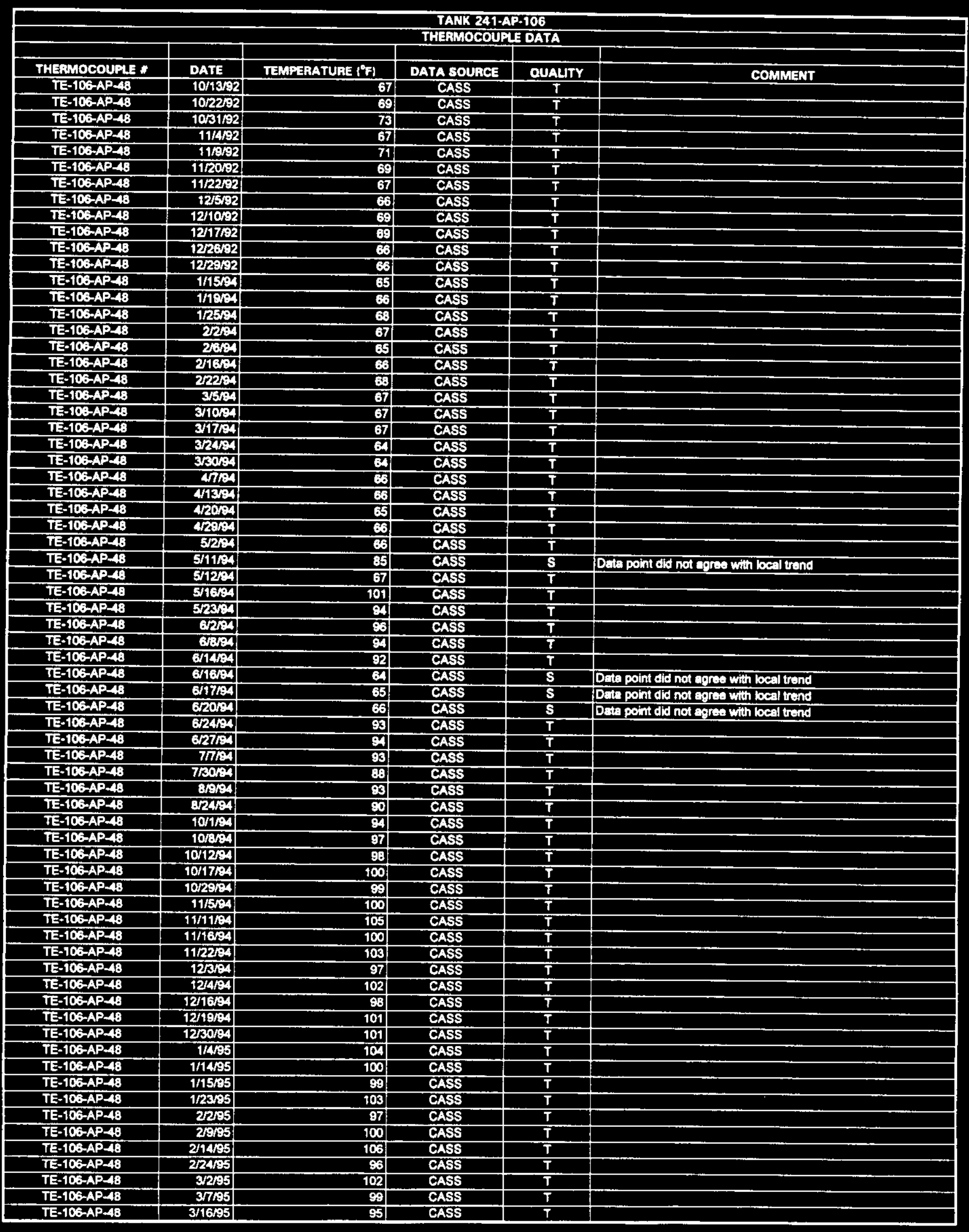

Data obtained from Computer Automated Surveillence System (CASS), April 1995; and Surveiltance Analysis Computer Syatem (SACS), June 1995.

D-543 


\begin{tabular}{|c|c|c|c|c|c|}
\hline \multicolumn{6}{|c|}{$\begin{array}{l}\text { TAKK 241-AP-106 } \\
\text { THEFMOCOUPL DATA }\end{array}$} \\
\hline & & & & & \\
\hline THERMOCOUPLE * & DATE & TEMPERATURE $\left({ }^{\circ} \mathrm{F}\right)$ & DATA SOURCE & QUALITY & COMMENT \\
\hline TE-106-AP-48 & $3 / 2505$ & 96 & CASS & $T$ & \\
\hline TE-106-AP-48 & $3 \sqrt{30 / 35}$ & 86 & CASS & $T$ & \\
\hline & & & & & \\
\hline $\begin{array}{l}\text { TE-106AP-19 } \\
\text { TE-10G-AP= } 49\end{array}$ & $\frac{4 / 5 / 36}{47 / 86}$ & $\frac{56}{57}$ & CASS & $T$ & \\
\hline TE-10G-AP- 49 & 4/7/36 & 57 & CASS & $T$ & \\
\hline TE-106-AP-49 & 5/1686 & 57 & CASS & $T$ & \\
\hline TE-106-AP-49 & $5 / 23 / 66$ & 59 & CASS & $T$ & \\
\hline$T E-10 B-A P-19$ & $5 / 30 / 36$ & 59 & CASS & $T$ & \\
\hline TE-10-AP-19 & $67 / 36$ & 5 & CASS & $\bar{T}$ & \\
\hline$T E-103-A P=A 9$ & 6006 & 81 & CASS & $T$ & \\
\hline TE-106-AP-49 & 6/21/6s & 59! & CASS & $\bar{T}$ & \\
\hline TE-TO3-AP-48 & $6 / 25363$ & 59 & CASS & $\mathbf{T}$ & \\
\hline TE-10G-AP-A9 & 7 7Le6 & 61 & CASS & $\bar{T}$ & \\
\hline TE-106-AP- 19 & 77268 & 60 & CASS & $T$ & \\
\hline TE-105-AP-49 & $77 B$ B & 60 & CASS & $\mathbf{T}$ & \\
\hline TE-106-AP-A9 & $7 / 21 / 86$ & 62 & CASS & $T$ & \\
\hline TE-106-AP-A8 & 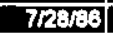 & $6 \mathbf{6 3}$ & CASS & $T$ & \\
\hline$T E-106-A P-A 9$ & BEB & 63 & CASS & $\mathbf{T}$ & \\
\hline TE-106-AP 69 & E/13/86 & 62 & CASS & $T$ & \\
\hline TE-106-AP=AB & $8 / 23168$ & 62 & CASS & $\mathbf{T}$ & \\
\hline$T E-106-A P=49$ & $8 / 2766$ & 61 & CASS & $T$ & \\
\hline TE-10B-AP-A9 & 8130 & 69 & CASS & $\mathbf{T}$ & \\
\hline$T E-10 B-A P-40$ & $9 / 2 \times 136$ & 64 & CASS & $\bar{T}$ & \\
\hline TE-106-AP-49 & $10 \mathrm{r} / \mathrm{se}$ & G1 & CASS & $\bar{T}$ & \\
\hline TE-106-APCAS & To110 & 63 & CASS & $\mathbf{T}$ & \\
\hline TE-106-AP-49 & 101830 & 62 & CASS & $\mathbf{T}$ & \\
\hline TE-106-AP-19 & Tar2236 & 64) & CASS & $T$ & \\
\hline$T E-10 B-A P-A 9$ & 102018 & 62 & CASS & $\mathbf{T}$ & \\
\hline$T E-103-A P=49$ & 111366 & 60 & CASS & $\mathbf{T}$ & \\
\hline TE-103AP-AO & $11 / 1358$ & 50 & CASS & $T$ & \\
\hline TE-103-AP- 19 & $11 / 2283$ & 53 & CASS & $\mathbf{T}$ & \\
\hline TE-106-AP-19 & $11 / 2686$ & 5 & CASS & $\mathbf{T}$ & \\
\hline TE-106-AP-AS & 122236 & 50 & CASS & $\bar{T}$ & \\
\hline TE-10B-AP=AB & 1221186 & 57 & CASS & $\mathbf{T}$ & \\
\hline TE-106-AP- 49 & 121736 & 56 & CASS & $\mathbf{T}$ & \\
\hline TE-10B-AP- 99 & 12123303 & 57 & CASS & $T$ & \\
\hline$T E-106-A P-49$ & 1212066 & 57 & CASS & $\bar{T}$ & \\
\hline TE-106-AP-49 & $7 / 1 / 87$ & 85 & CASS & $T$ & \\
\hline TE-106-AP-40 & 708 & 70 & CASS & $T$ & \\
\hline TE-106-AP- 69 & 712837 & 86 & CASS & $T$ & \\
\hline TE-106-AP-1989 & 71867 & 67 & CASS & $\mathbf{T}$ & \\
\hline TE-106AP- 19 & $8 / 67$ & 63 & CASS & $T$ & \\
\hline TE-106-AP-19 & 8/587 & 68 & CASS & $T$ & \\
\hline TE-106-AP- 49 & $8 / 11 / 67$ & 69 & CASS & $\mathbf{T}$ & \\
\hline TE-106-AP-A9 & $8 / 1767$ & 69 & CASS & $\bar{T}$ & \\
\hline TE-106-AP- 48 & $8 / 26 / 37$ & 69 & CASS & $T$ & \\
\hline TE-106-AP-A9 & 94/87 & 68 & CASS & $\bar{T}$ & \\
\hline TE-106-AP-49 & S987 & 71 & CASS & $T$ & \\
\hline TE-106-AP-A9 & 8/17/77 & 70 & CASS & $T$ & \\
\hline TE-10B-AP-49 & $9 / 23 / 37$ & 69 & CASS & $T$ & \\
\hline TE-106-AP-A9 & $10 / 3 / 67$ & 67 & CASS & $T$ & \\
\hline TE-103-AP-A9 & $10 / 29 / 87$ & 64 & CASS & $T$ & \\
\hline TE-103-AP=49 & $11 / 5 / 37$ & 6A & CASS & $T$ & \\
\hline TE-10S-AP-A9 & 11/8/87 & 65 & CASS & $T$ & \\
\hline TE-106-AP-49 & $11 / 16 / 87$ & 63 & CASS & $T$ & \\
\hline TE-106-AP-A9 & $11 / 26 / 87$ & 63 & CASS & $T$ & \\
\hline TE-106-AP-49 & 12/4/87 & 63 & CASS & $T$ & \\
\hline TE-106-AP-49 & $227 / 87$ & 63 & CASS & $\mathbf{T}$ & \\
\hline$T E-106-A P-49$ & 12/17/87 & 80 & CASS & $T$ & \\
\hline TE-1DG-APA9 & $12 / 23 / 67$ & 59 & CASS & $T$ & \\
\hline TE-106-AP-49 & 1213187 & 59 & CASS & $\bar{T}$ & \\
\hline TE-106-AP-49 & \begin{tabular}{ll|}
$1 / 688$ \\
\end{tabular} & 57 & CASS & $T$ & \\
\hline TE-106-AP- 49 & $1 / 11 / 88$ & 56 & CASS & $T$ & \\
\hline TE-106-AP-49 & $1 / 23 / 68$ & 5 & CASS & $\mathbf{T}$ & \\
\hline TE-106-AP-49 & $1 / 2568$ & 56 & CASS & $T$ & \\
\hline TE-106-AP- 19 & $21 / 38$ & 55 & CASS & $T$ & \\
\hline$\overline{H E-103-A P-A 9}$ & $2 / 283$ & 67 & CASS & $\mathbf{S}$ & Data point did not agree with locel trend \\
\hline TE-108-AP- 49 & $2 / 13 / 3$ & 55: & CASS & $T$ & \\
\hline$T E-106-A P-49$ & 214183 & 56 & CASS & $T$ & \\
\hline$T E-106-A P-19$ & $2 / 21 / 88$ & 56 & CASS & $T$ & \\
\hline
\end{tabular}




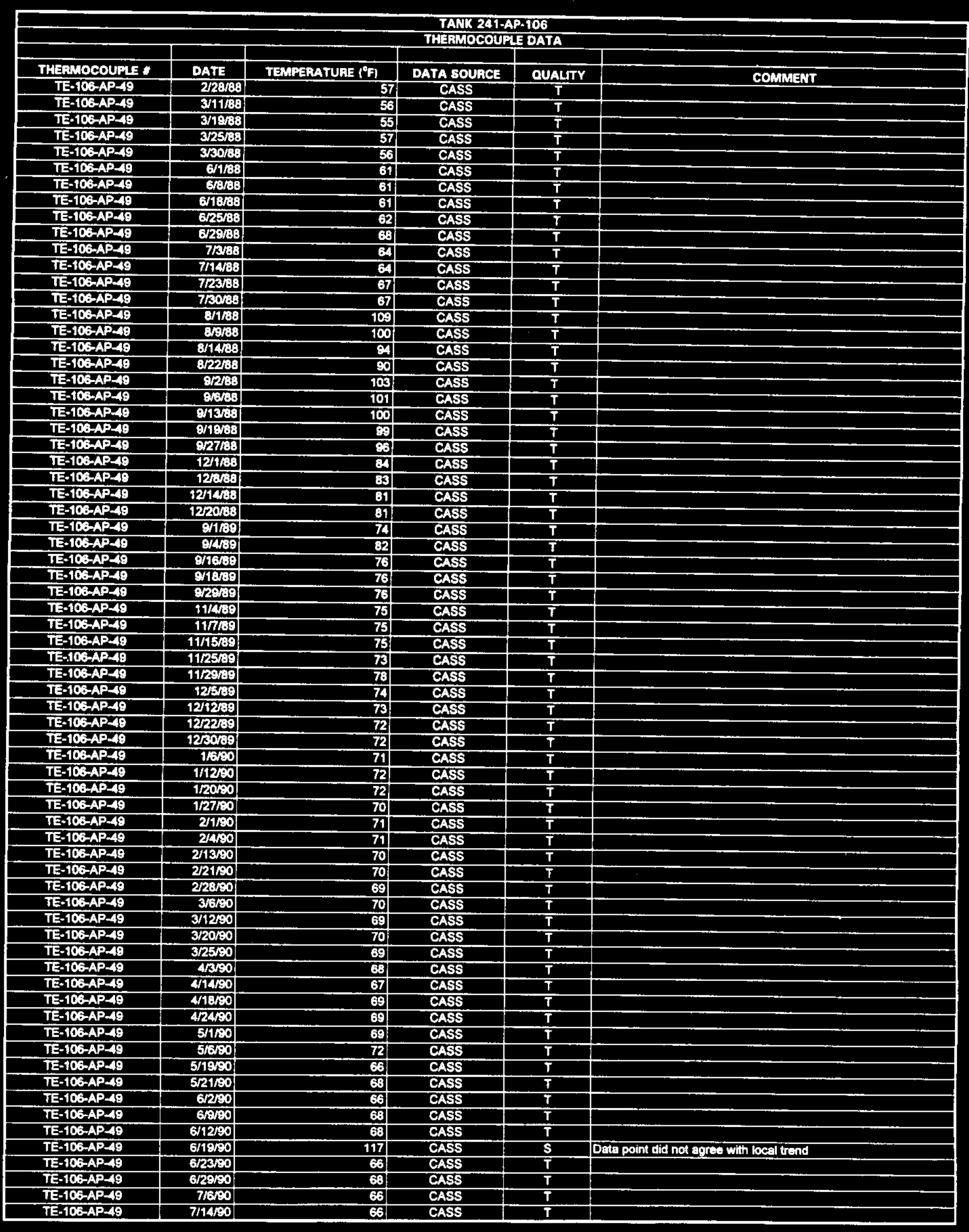




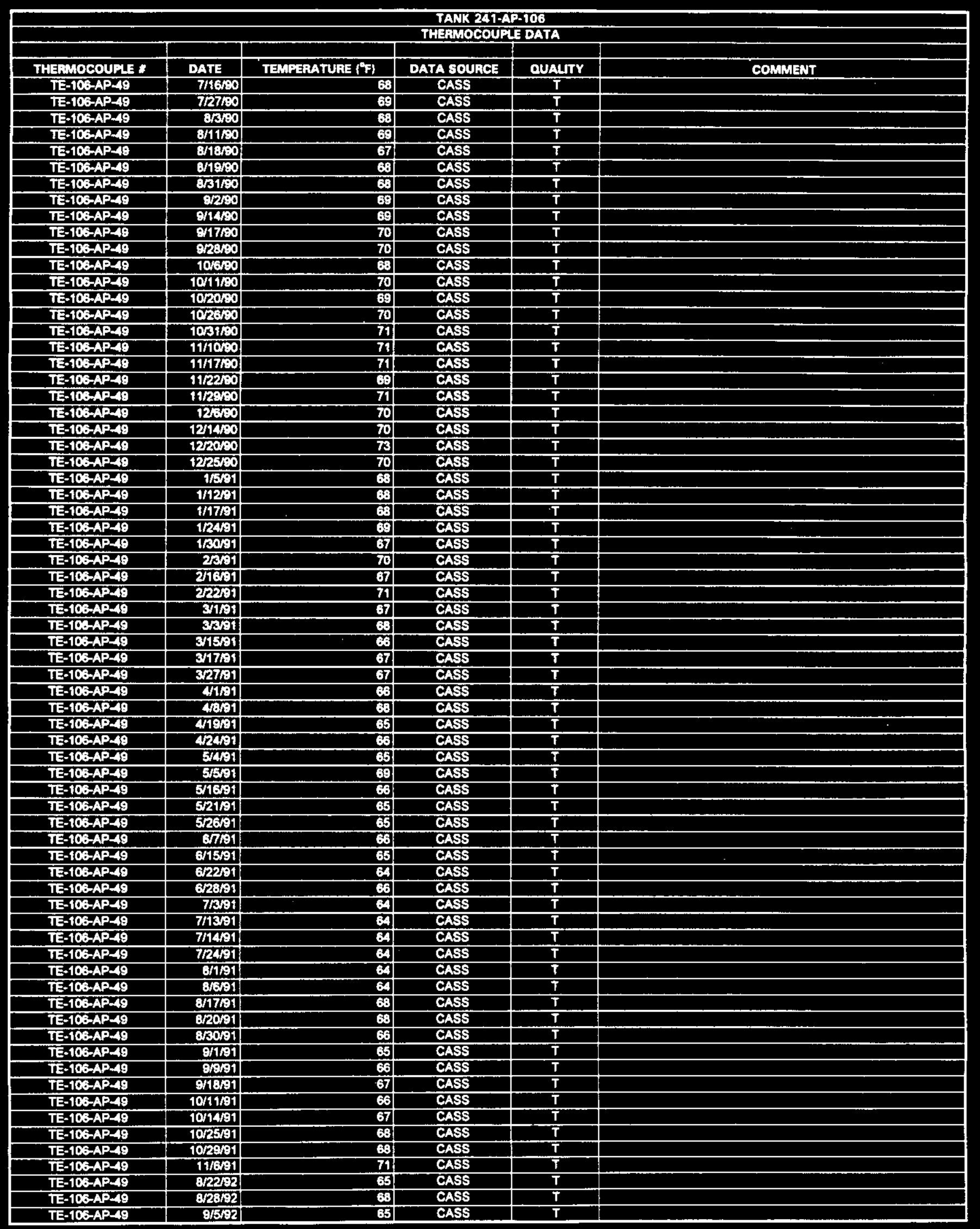

Data obtained from Computer Automated Surveillance System (CASS), April 1995; and Surveillance Analysis Computer System (SACS), Jurne 1995. 


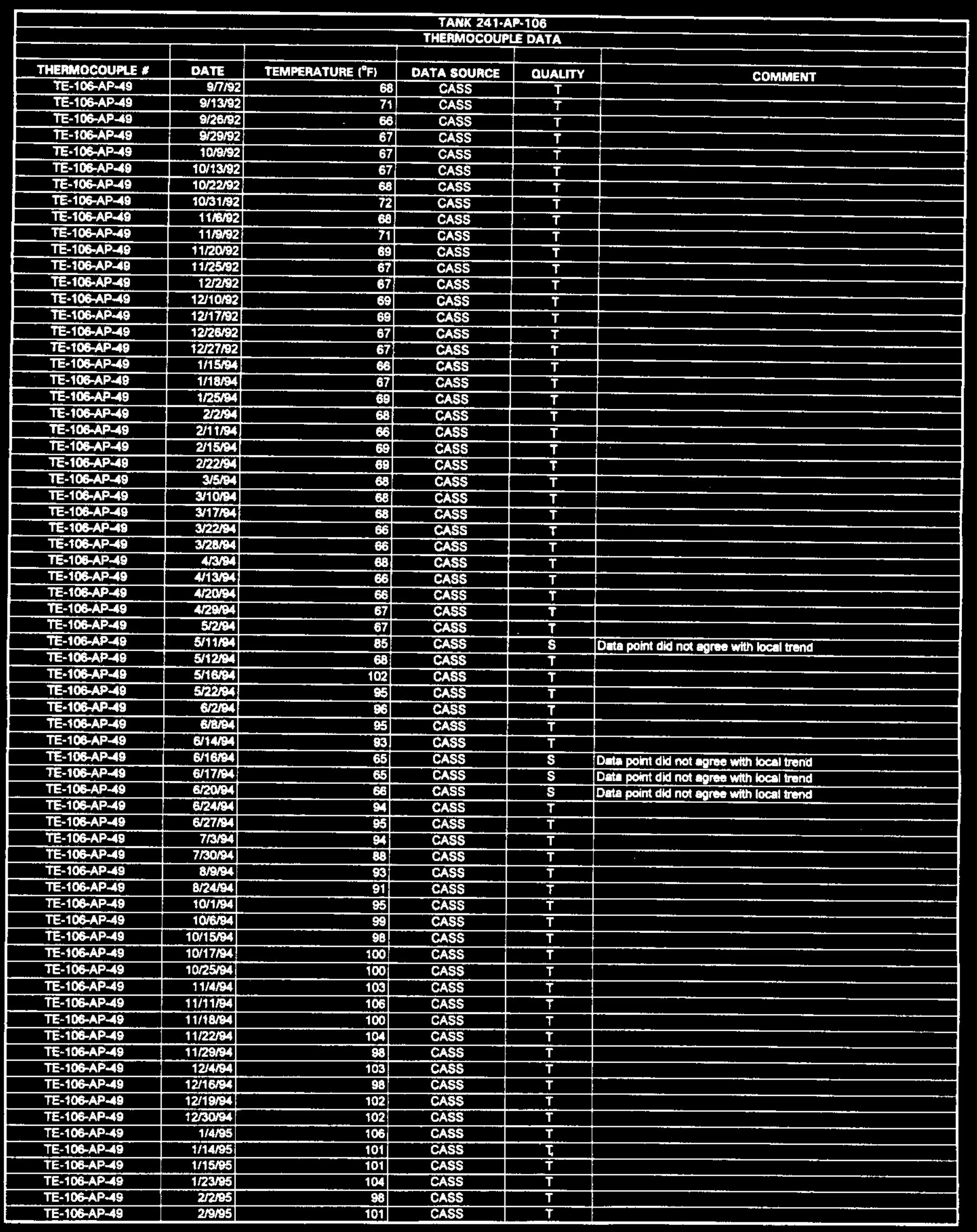




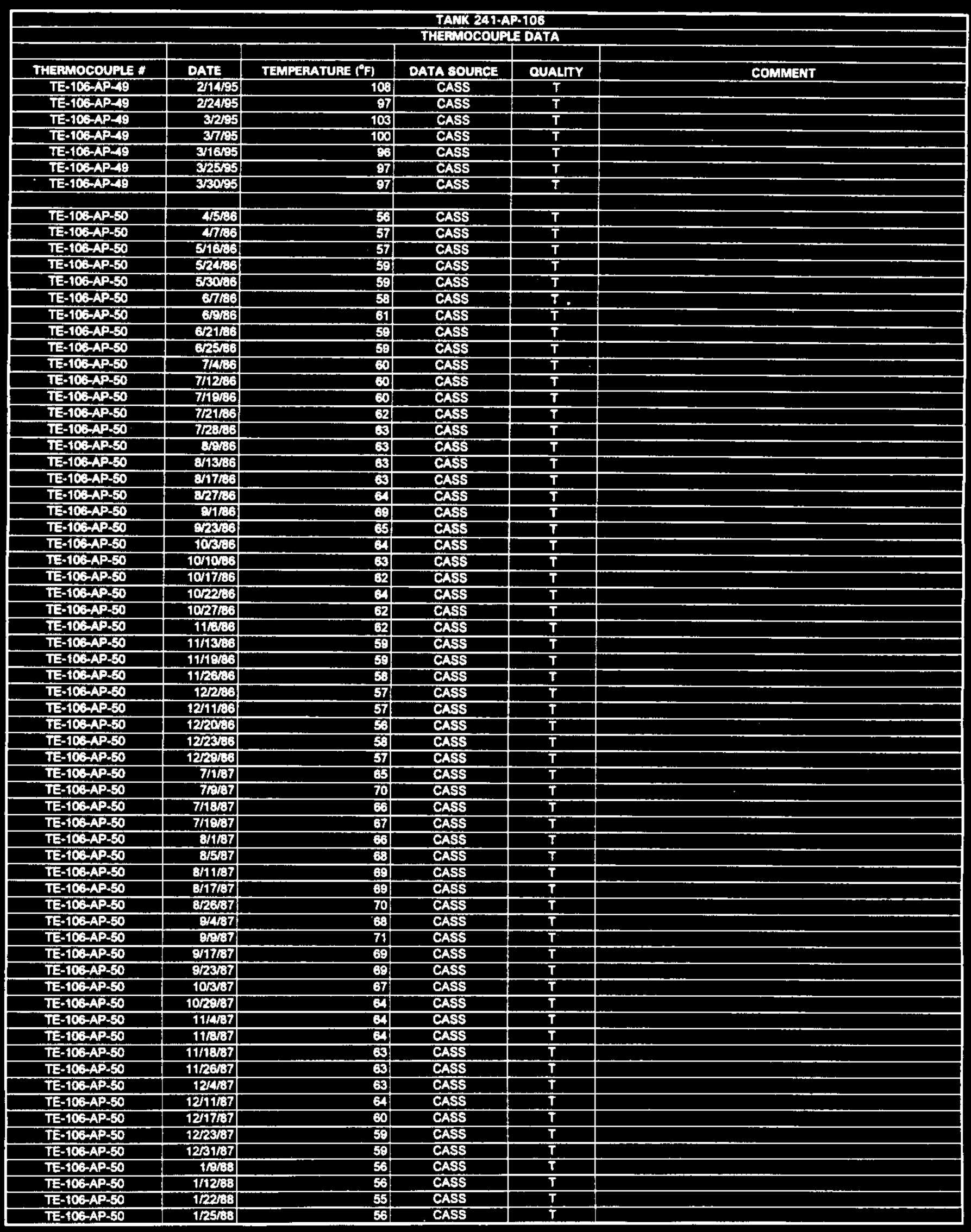

Date obtained from Computer Automated Surveillsnce System (CASS), April 1995; and Surveillance Analysis Computer Syatem (SACS), June 1995. 


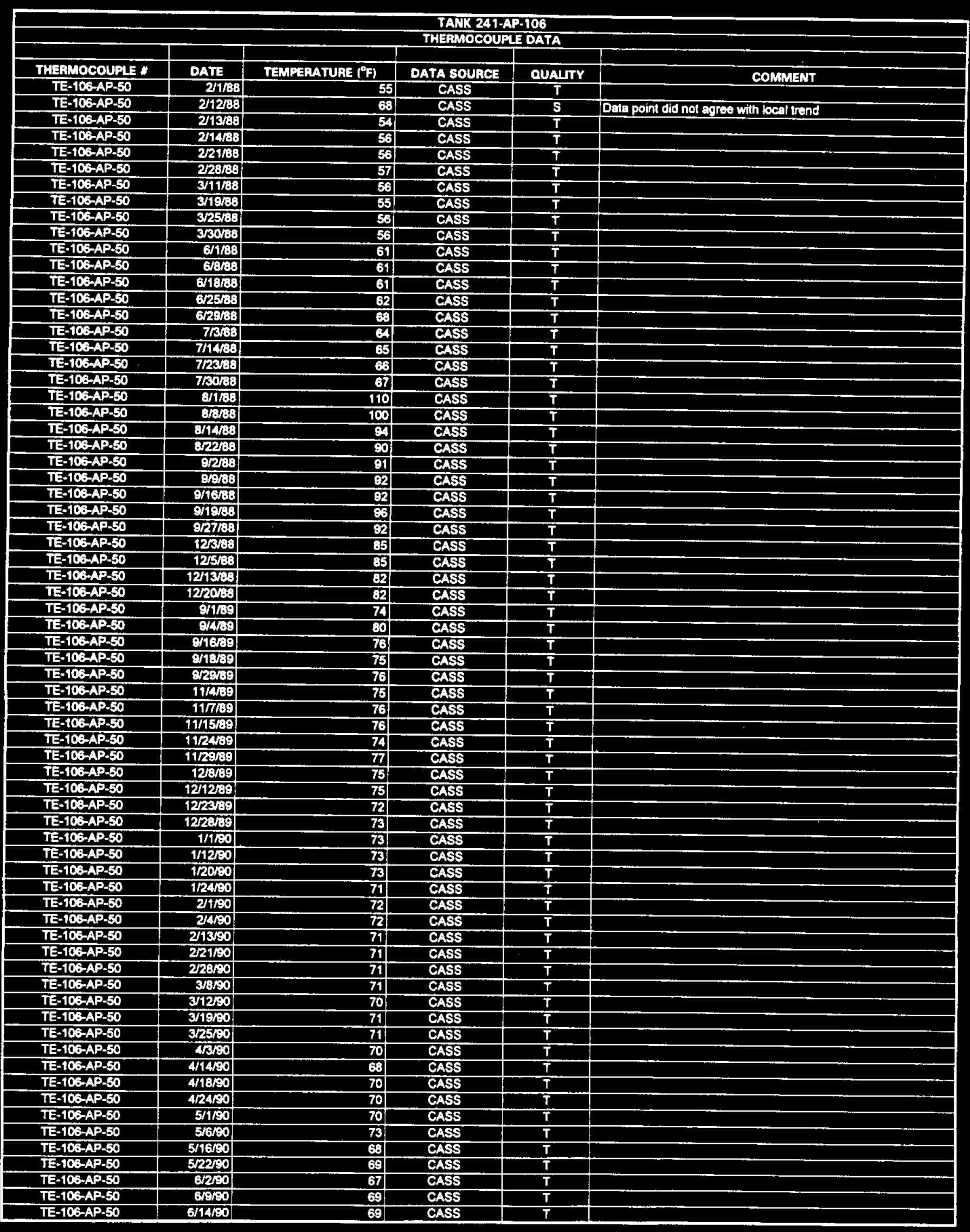




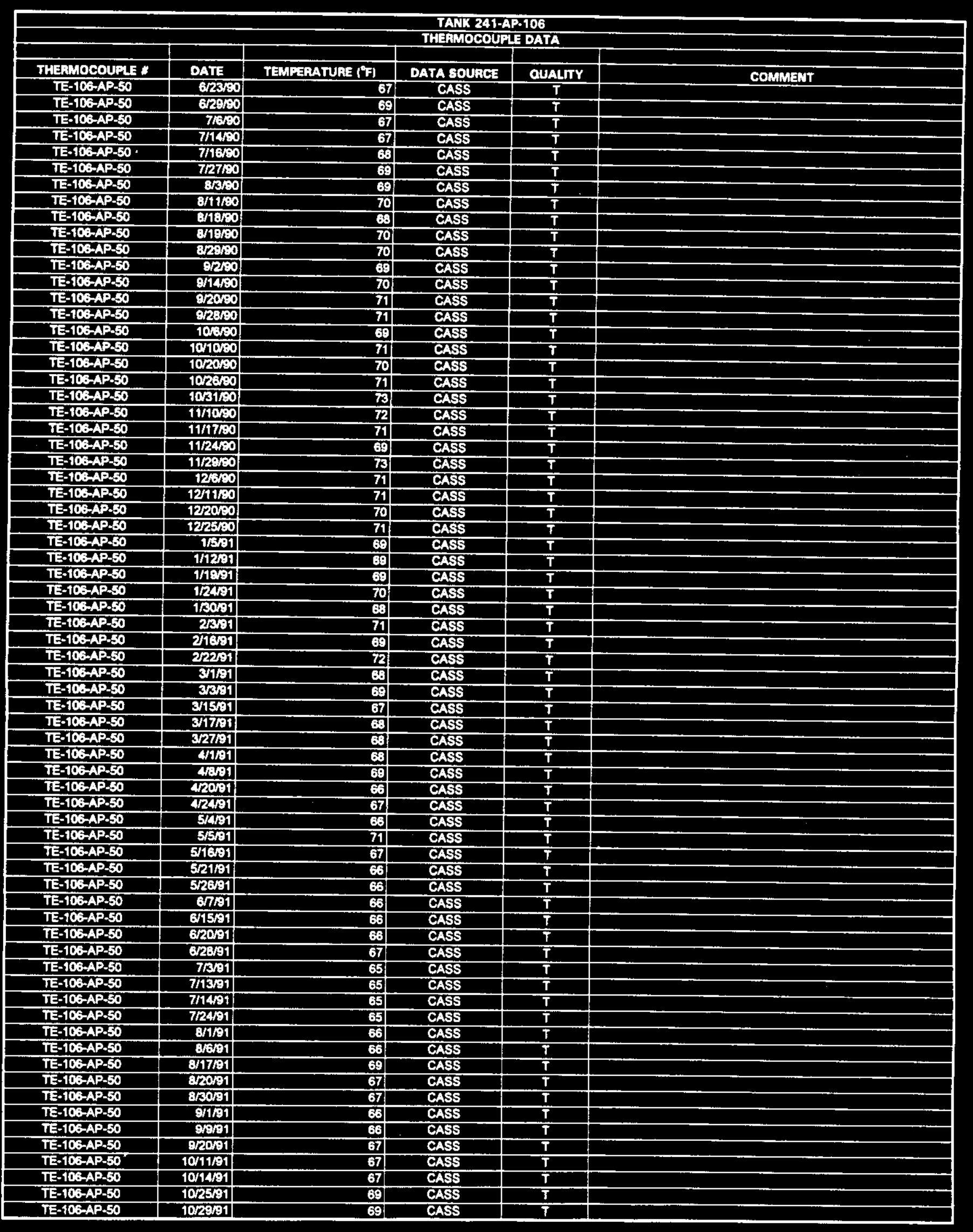

Data obtained from Computer Automated Surveillence System (CASS), April 1995; and Surveillance Analysis Computer Syatem (SACS), June 1995.

D-5 50 


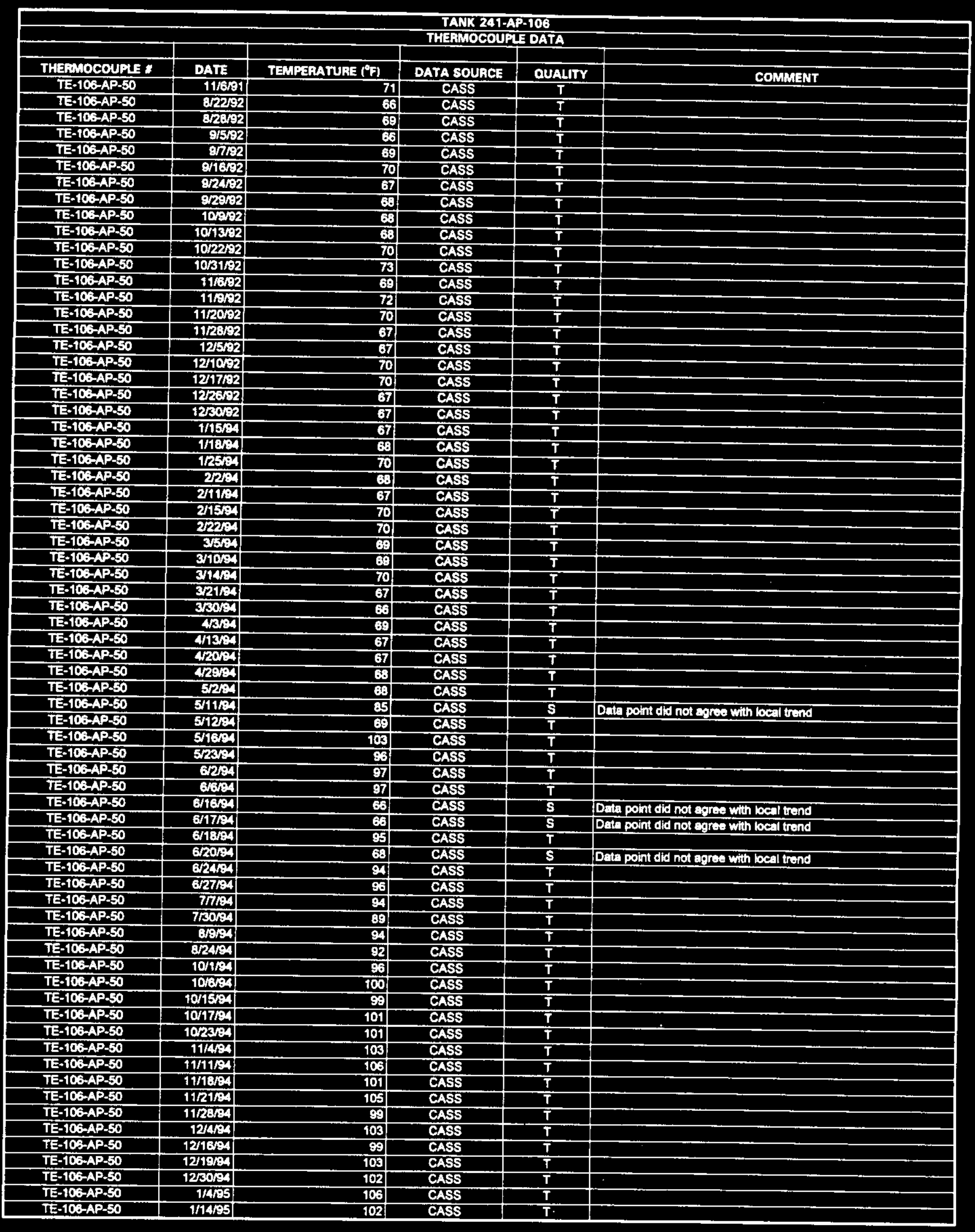

Date obtained from Computer Automated Surveillance System (CASS), April 1995; and Surveillance Analysis Computer System (SACS), June 1995.

D-551 


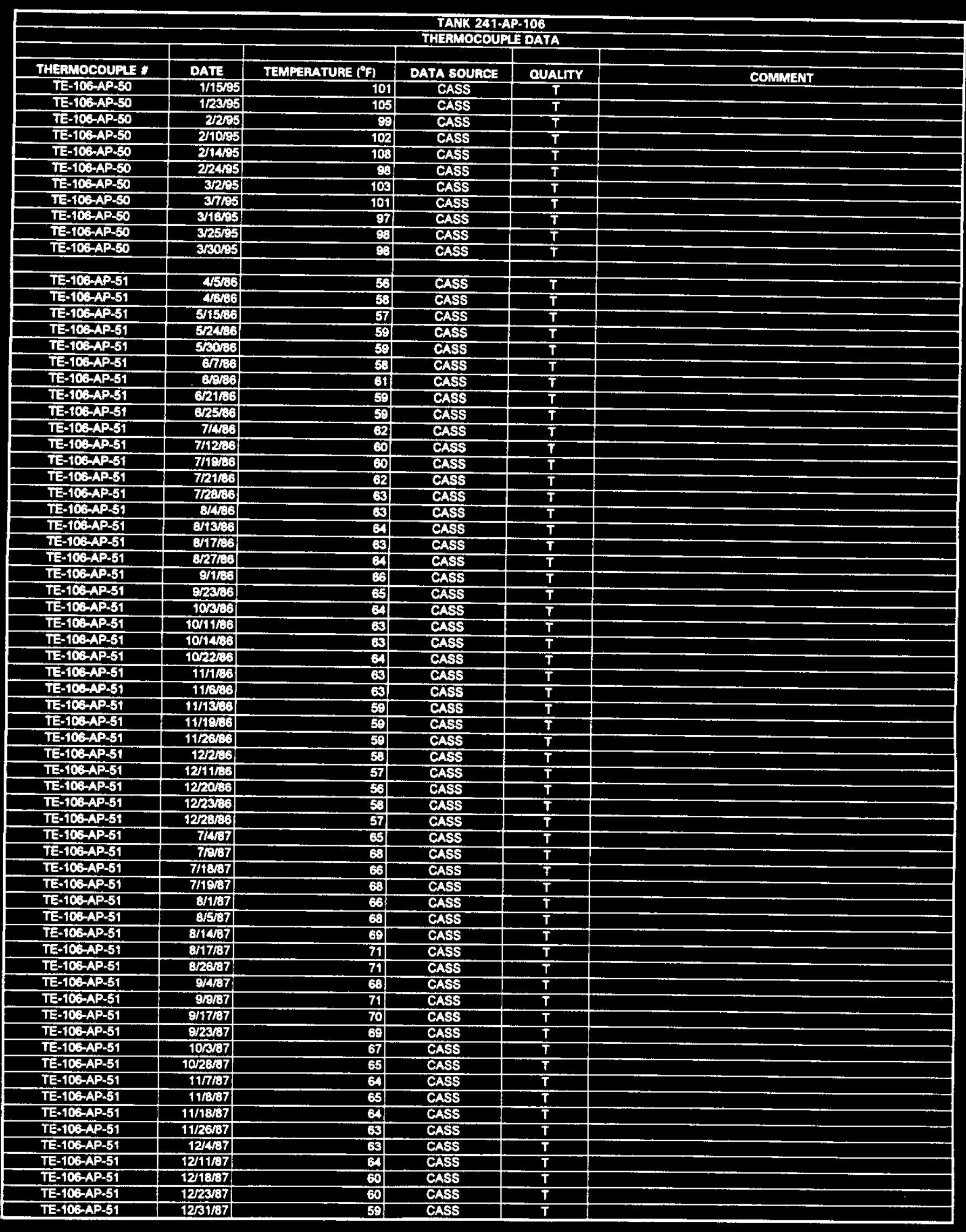

Data obtained from Computer Autometed Surveillance System (CASS), April 1995; ard Surveillance Analyais Computer Syatem (SACS), June 1995.

D-552 


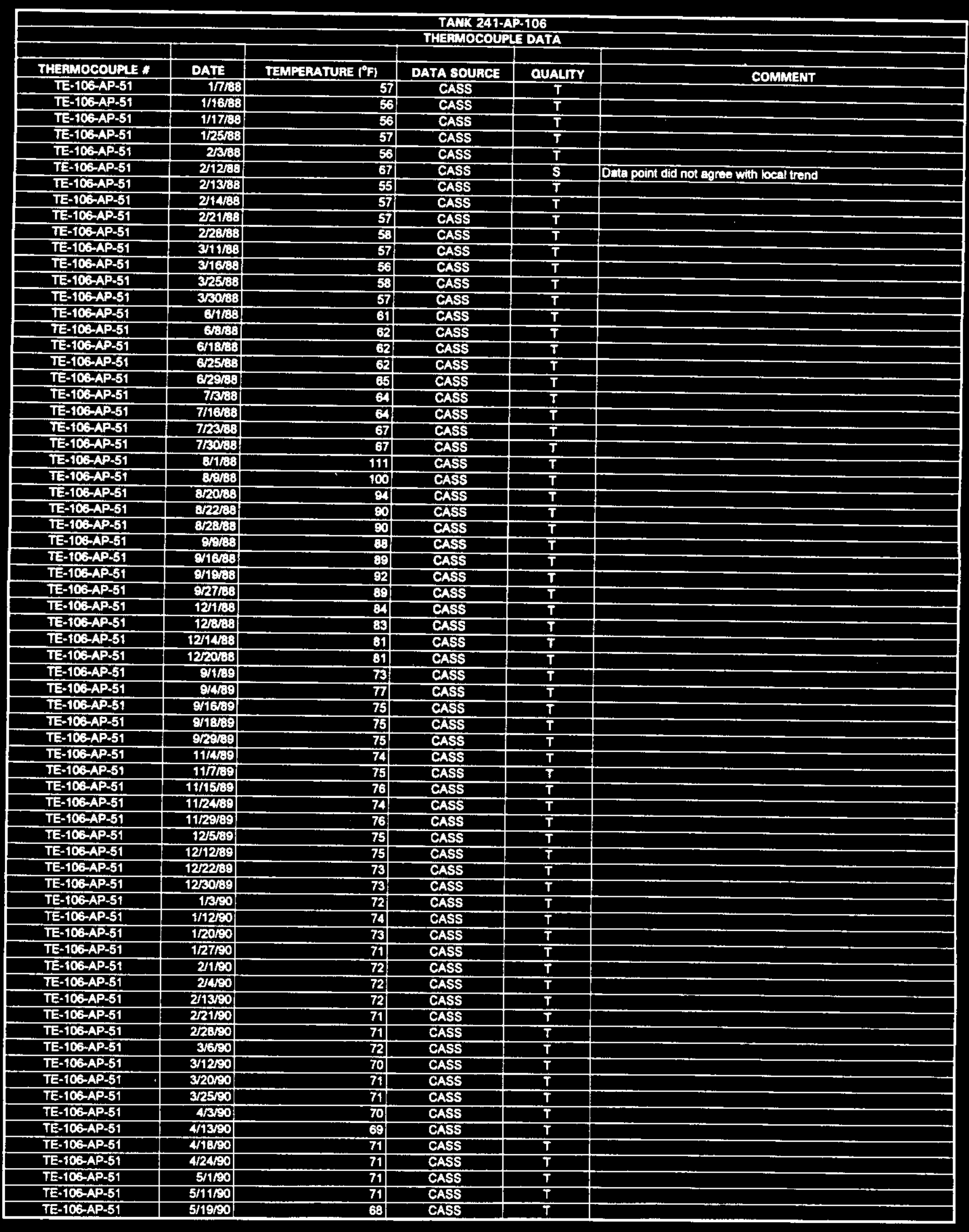

Data obteined from Computer Automated Surveillance Syatem (CASS), April 1995; and Surveillance Analysis Computer System (SACS), June 1995.

D-553 


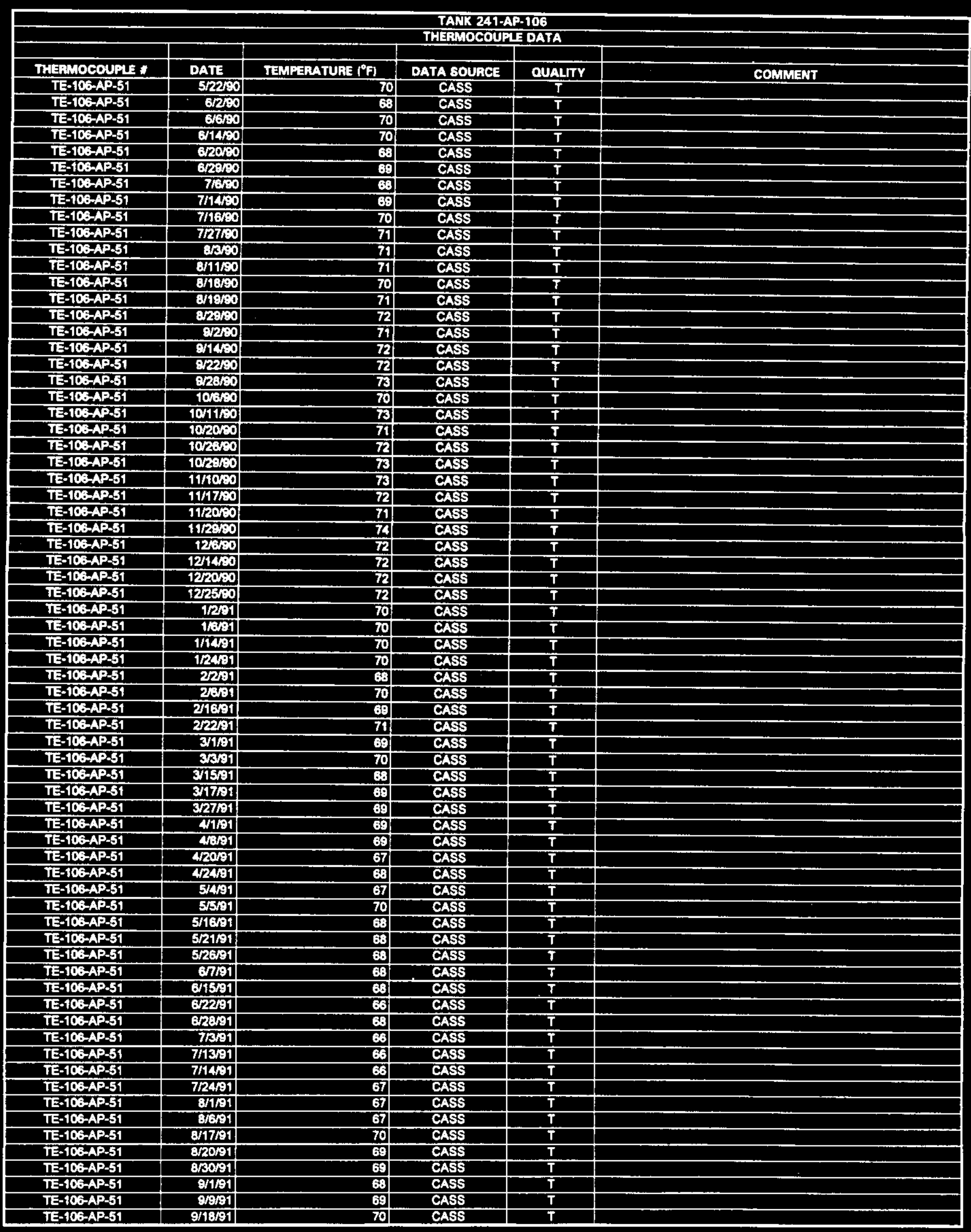




\begin{tabular}{|c|c|c|c|c|c|}
\hline \multicolumn{6}{|c|}{$\begin{array}{l}\text { TANK 261.AP-106 } \\
\text { THEAMOCOUAL DATA }\end{array}$} \\
\hline & & & & & \\
\hline THERMOCOUPLE * & DATE & TEMPERATURE $\left({ }^{\circ} \mathrm{F}\right)$ & DATA SOUACE & QUALTTY & COMMENT \\
\hline TE-106-AP-51 & $10 / 11 / 91$ & 69 & CASS & $T$ & \\
\hline TE-106-AP-51 & $10 / 44 / 91$ & 69 & CASS & $T$ & \\
\hline TE-106-AP-51 & 10/25/91 & 71 & CASS & $T$ & \\
\hline TE-106-AP-51 & 10/29\%1 & 71 & CASS & $T$ & \\
\hline TE-106-AP-51 & 11/6/81 & 72 & CASS & $T$ & \\
\hline TE-106-AP-S1 & $8 / 22292$ & 67 & CASS & $T$ & \\
\hline TE-106-AP-51 & $8 / 2862$ & 70 & CASS & $T$ & \\
\hline TE-106-AP-51 & $8 / 52$ & 67 & CASS & $T$ & \\
\hline TE-106-AP-51 & 87/92 & 79 & CASS & $T$ & \\
\hline TE-106-AP-51 & G/15\%2 & 70 & CASS & $T$ & \\
\hline TE-106-AP-51 & $9 / 26 / 2$ & 67 & CASS & $T$ & \\
\hline TE-106-AP.51 & \begin{tabular}{ll|}
$9 / 2992$ \\
\end{tabular} & 69 & CASS & $T$ & \\
\hline TE-106-AP-51 & 30/9192 & 70 & CASS & $T$ & \\
\hline TE-106-AP-61 & $10 / 13 / 92$ & 69 & CASS & $\bar{T}$ & \\
\hline TE-106-AP-51 & $10 / 22 \% 2$ & 70 & CASS & $T$ & \\
\hline TE-106-AP-51 & 10/31/92 & 72 & CASS & $T$ & \\
\hline TE-106-AP-51 & $11 / 6 / 22$ & 70 & CASS & $\bar{T}$ & \\
\hline TE-106-AP-51 & 11/A/02 & 72 & CASS & $T$ & \\
\hline TE-106-AP-S1 & $11 / 1692$ & 71 & CASS & $\bar{T}$ & \\
\hline TE-106-AP-51 & $11 / 28 / 02$ & 63 & CASS & $T$ & \\
\hline TE-108-AP-51 & $12 / 532$ & 69 & CASS & $T$ & \\
\hline TE-106-AP-51 & 12 rores & 71 & CASS & $T$ & \\
\hline TE-106-AP-51 & 121992 & 69 & CASS & 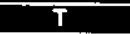 & \\
\hline TE-106-AP-51 & $12 / 2692$ & 68 & CASS & $T$ & \\
\hline TE-106-AP-51 & 1230002 & 68 & CASS & $T$ & \\
\hline TE-106-AP-51 & T/5m & 67 & CASS & $\bar{T}$ & \\
\hline TE-106-AP-51 & 11894 & 69 & CASS & $T$ & \\
\hline TE-106-AP-51 & $1 / 2504$ & 70 & CASS & $T$ & \\
\hline TE-106-APSS1 & $2 / 294$ & 69 & CASS & $T$ & \\
\hline$T E=100-A P-51$ & $2 / 213$ & 68 & CASS & $T$ & \\
\hline TE-106-AP-S1 & 2य15\% & 70 & CASS & $T$ & \\
\hline TE-106-AP-51 & $2 / 2294$ & 71 & CASS & $T$ & \\
\hline$T E-106-A p-51$ & $3 / 504$ & 70 & CASS & $\mathbf{T}$ & \\
\hline TE-106-AP-51 & 3/004 & 69 & CASS & $\bar{T}$ & \\
\hline TE-106-APS51 & 3/404 & 71 & CASS & $\bar{T}$ & \\
\hline TE-106-AP-51 & $3 \sqrt{2209}$ & 63 & CASS & $\mathbf{T}$ & \\
\hline TE-106-AP-51 & $323 \%$ & 6 & CASS & $T$ & \\
\hline TE-106-AP-51 & $43 \times 3$ & 70 & CASS & $T$ & \\
\hline TE-103-AP-51 & Q/139a & 69 & CASS & $\mathbf{T}$ & \\
\hline TE-108-AP-51 & 42004 & 69 & CASS & $T$ & \\
\hline EE-106-AP-51 & 420109 & 69 & CASS & $T$ & \\
\hline TE-106-AP-51 & 52204 & 69 & CASS & $\bar{T}$ & \\
\hline TE-106-AP-51 & 5/1184 & 85 & CASS & $\mathbf{S}$ & Deta point did not epree with local trend \\
\hline TE-106-AP-51 & 5/1294 & 70 & CASS & $\bar{T}$ & \\
\hline TE-106-APS1 & 5/16ha & 103 & CASS & $T$ & \\
\hline TE-106-APSS1 & 5/22294 & 56 & CASS & $\mathbf{T}$ & \\
\hline TE-106-AP-51 & 6/299- & 88 & CASS & $\bar{T}$ & \\
\hline TE-106-AP-51 & 6604 & 97 & CASS & $T$ & \\
\hline TE-106-AP-51 & G1404 & 95 & CASS & $T$ & \\
\hline TE-106-AP-51 & 6/1604 & 67 & GASS & $\mathbf{s}$ & Deta point did not egree with locsil trend \\
\hline TE-10G-AP-51 & $6 / 17194$ & 67 & GASS & $\overline{\mathbf{S}}$ & Data point did not agree with local trend \\
\hline TE-106-AP-51 & 6/20/94 & 68 & CASS & s & Data point did not agree with local trend \\
\hline TE-10E-AP-51 & 6/25/94 & 95 & CASS & $\bar{T}$ & \\
\hline TE-106-AP-51 & 6/27/34: & 97 & CASS & $T$ & \\
\hline TE-106-AP-S1 & $7 \pi / 94$ & 95 & CASS & $T$ & \\
\hline TE-103-AP-51 & 7 730199 & so & CASS & $T$ & \\
\hline TE-106-AP-51 & 8/9/94 & 95 & CASS & $T$ & \\
\hline$T E-106-A P-51$ & $8 / 24 / 9$ & 93 & CASS & $T$ & \\
\hline TE-100-AP-51 & 10/1/84 & 97 & CASS & $T$ & \\
\hline TE-106-AP-51 & $10 / 6 / 34$ & 10 & CASS & $T$ & \\
\hline TE-106-AP-51 & $10 / 15 / 94$ & 100 & CASS & $T$ & \\
\hline TE-106-AP-51 & $10 / 17 / 94$ & 102 & CASS & $\bar{T}$ & \\
\hline TE-106-AP-51 & $10 / 2994$ & 102 & CASS & $T$ & \\
\hline TE-106-AP-51 & 11/4\%94 & 105 & CASS & $T$ & \\
\hline TE-106-AP-51 & $11 / 11 / 94$ & 107 & CASS & $\bar{T}$ & \\
\hline TE-106-AP-51 & $11 / 1694$ & 103 & CASS & $T$ & \\
\hline TE-108-AP-51 & $11 / 21 / 94$ & 106 & CASS & $T$ & \\
\hline TE-106-AP-51 & $11 / 28 / 94$ & 100 & CASS & $T$ & \\
\hline TE-10S-AP-51 & 12/4/94 & 105 & CASS & $T$ & \\
\hline TE-106-AP-51 & 12/16/94 & 9 & CASS & $T$ & \\
\hline
\end{tabular}




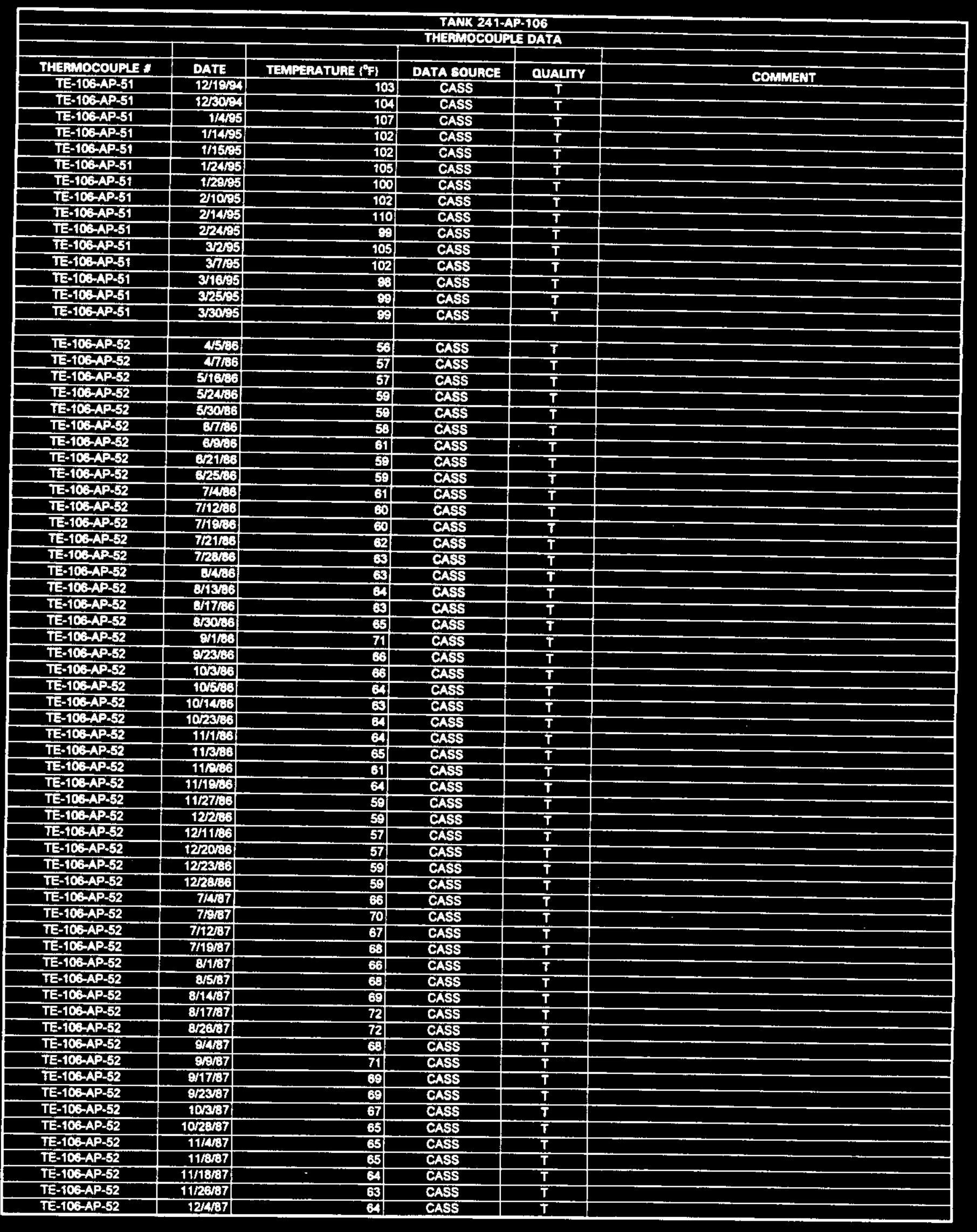




\begin{tabular}{|c|c|c|c|c|c|}
\hline \multicolumn{6}{|c|}{$\begin{array}{l}\text { TAKK 241-AP-106 } \\
\text { THERMOCOUPLE DATA }\end{array}$} \\
\hline THEMMOCOUPLE * & DATE & TEMPERATURE ("F) & DATA SOURCE & GUALTY & COMMENT \\
\hline TE-106-AP-52 & $12 / 11 / 87$ & 65 & CASS & $T$ & \\
\hline TE-106-AP-52 & $12 / 18 / 87$ & 60 & CASS & $T$ & \\
\hline TE-106-AP-52 & $12 / 24 / 87$ & 59 & CASS & $T$ & \\
\hline TE-106-AP-52 & 12/31/87 & 59 & CASS & $T$ & \\
\hline TE-106-AP-52 & 1/7/38 & 57 & CASS & $\mathbf{T}$ & \\
\hline TE-106-AP-52 & $1 / 16 / 8$ & 36 & CASS & $\mathbf{T}$ & \\
\hline TE-106-AP-52 & $1 / 198 / 88$ & 56 & CASS & $T$ & \\
\hline TE-106-AP-52 & $1 / 25 / 38$ & 57 & CASS & $\bar{T}$ & \\
\hline TE-105-AP-52 & $2 / 3 / 88$ & 56 & CASS & $T$ & \\
\hline TE-106-AP-52 & 211268 & 67 & CASS & $\mathbf{s}$ & Data point did not agree with tocel trend \\
\hline TE-106-AP-52 & $2 / 13 / 38$ & 53 & CASS & $T$ & \\
\hline TE-106-AP-52 & $2 / 1488$ & 57 & CASS & $T$ & \\
\hline TE-106-AP-52 & $2 / 21 / 38$ & 57 & CASS & $\bar{T}$ & \\
\hline TE-106-AP-52 & $2 / 23 / 3$ & 58 & CASS & $\mathbf{T}$ & \\
\hline TE-106-AP-52 & 3/11/88 & 57 & CASS & $\mathbf{T}$ & \\
\hline TE-106-AP-52 & 3/19/8 & 56 & CASS & $\mathbf{T}$ & \\
\hline TE-106-AP-52 & $3 / 25 / 38$ & 58 & CASS & $T$ & \\
\hline TE-106-AP-52 & $3 / 3078$ & 57 & CASS & $T$ & \\
\hline TE-106-AP-52 & 6/1/38 & 62 & CASS & $T$ & \\
\hline TE-106-AP-52 & $6 \% B$ & 62 & CASS & $\mathbf{T}$ & \\
\hline TE-106-APS.S2 & 6/18\%8 & 61 & CASS & $T$ & \\
\hline TE-106-AP-52 & 8/2538 & 62 & CASS & $T$ & \\
\hline TE-106-AP-52 & $6 / 2988$ & EA & CASS & $\mathbf{T}$ & \\
\hline TE-IOB-AP-52 & $7 / 3 / 68$ & 64 & CASS & $\bar{T}$ & \\
\hline TE-106-AP-52 & $7 / 1468$ & 65 & CASS & $\bar{T}$ & \\
\hline TE-106-AP-52 & $723 / 8$ & 66 & CASS & $T$ & \\
\hline TE-106-AP-52 & 715008 & 68 & CASS & $T$ & \\
\hline TE-106-AP-52 & 8/1/68 & 110 & CASS & $T$ & \\
\hline TE-106-AP-S2 & 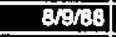 & 100 & CASS & $T$ & \\
\hline TE-106AP-52 & $8 / 168$ & था & CASS & $T$ & \\
\hline TE-106-AP-52 & $8 / 2228$ & $\infty$ & CASS & $T$ & \\
\hline TE-106-AP-52 & BzB68 & 80 & CASS & $T$ & \\
\hline TE-106-AP-52 & 8/9/38 & 87 & CASS & $T$ & \\
\hline TE-106-AP-52 & 9/13/83 & 87 & CASS & $\bar{T}$ & \\
\hline TE-106-AP-52 & 9ress & 80 & CASS & $T$ & \\
\hline TE-103-AP-52 & $8 / 27 / 38$ & 86 & CASS & $\mathbf{T}$ & \\
\hline TE-106-AP-52 & $12 \pi 168$ & 82 & CASS & $T$ & \\
\hline TE-106-AP-52 & $12 / 83$ & 82 & CASS & $T$ & \\
\hline TE-106-AP-52 & $12 / 468$ & 78 & CASS & $T$ & \\
\hline TE-106-AP-S2 & 1222068 & 78 & CASS & $\bar{T}$ & \\
\hline TE-106-AP-52 & $8 / 1 / 69$ & 72 & CASS & $T$ & \\
\hline TE-106-AP-52 & 9/489 & 7 & CASS & $\bar{T}$ & \\
\hline TE-106-AP-52 & 9/16/69 & 75 & CASS & $T$ & \\
\hline TE-106-AP-52 & 918Bo & 74 & CASS & $T$ & \\
\hline TE-106-AP-52 & 9/29189 & 74 & CASS & $T$ & \\
\hline TE-106-AP-52 & $11 / 4 / 89$ & 73 & CASS & $T$ & \\
\hline TE-106-AP-52 & 11/788 & 74 & CASS & $T$ & \\
\hline TE-106-AP-52 & 11/15/89 & 75 & CASS & $\mathbf{T}$ & \\
\hline TE-106-AP-52 & $11 / 23189$ & 73 & CASS & $T$ & \\
\hline TE-106-AP-52 & 11/29/89 & 74 & CASS & $T$ & \\
\hline TE-106-AP-52 & $12 / 8 / 89$ & 73 & CASS & $T$ & \\
\hline TE-106-AP-52 & 12/12/69 & 74 & CASS & $T$ & \\
\hline TE-106-AP-52 & 12/22/39 & 71 & CASS & $T$ & \\
\hline TE-106-AP-52 & $12 / 30 / 89$ & 71 & CASS & $T$ & \\
\hline TE-106-AP-52 & $1 / 3 / 90$ & 70 & SACS & $T$ & \\
\hline TE-106-AP-52 & $1 / 12200$ & 72 & CASS & $T$ & \\
\hline TE-106-AP-52 & $1 / 20 \% 0$ & 71 & CASS & $\mathbf{T}$ & \\
\hline TE-106-AP-52 & $1 / 23 / 90$ & 70 & CASS & $\mathbf{T}$ & \\
\hline TE-106-AP-52 & 21100 & 71 & CASS & $T$ & \\
\hline TE-106-AP-52 & 24480 & 79 & CASS & $T$ & \\
\hline TE-106-AP-52 & $2 / 13=0$ & 70 & CASS & $T$ & \\
\hline TE-106-AP-52 & $2 / 8 / 80$ & 70 & CASS & $T$ & \\
\hline TE-106-AP-52 & $228 \%$ & 69 & CASS & $T$ & \\
\hline TE-106-AP-52 & $3 / 600$ & 70 & CASS & $\mathbf{T}$ & \\
\hline TE-106-AP.52 & $3 / 12190$ & 69 & CASS & $T$ & \\
\hline TE-106-AP-52 & $3 / 20 / 80$ & 69 & CASS & $T$ & \\
\hline TE-106-AP-52 & $3 / 25 / 90$ & 70 & CASS & $\bar{T}$ & \\
\hline TE-106-AP-52 & $4 / 3 / 90$ & 69 & CASS & $\mathbf{T}$ & \\
\hline TE-106-AP-52 & $49 / 90$ & 68 & CASS & $T$ & \\
\hline TE-106-AP-52 & $8 / 18 / 00$ & 70 & CASS & $\mathbf{T}$ & \\
\hline
\end{tabular}




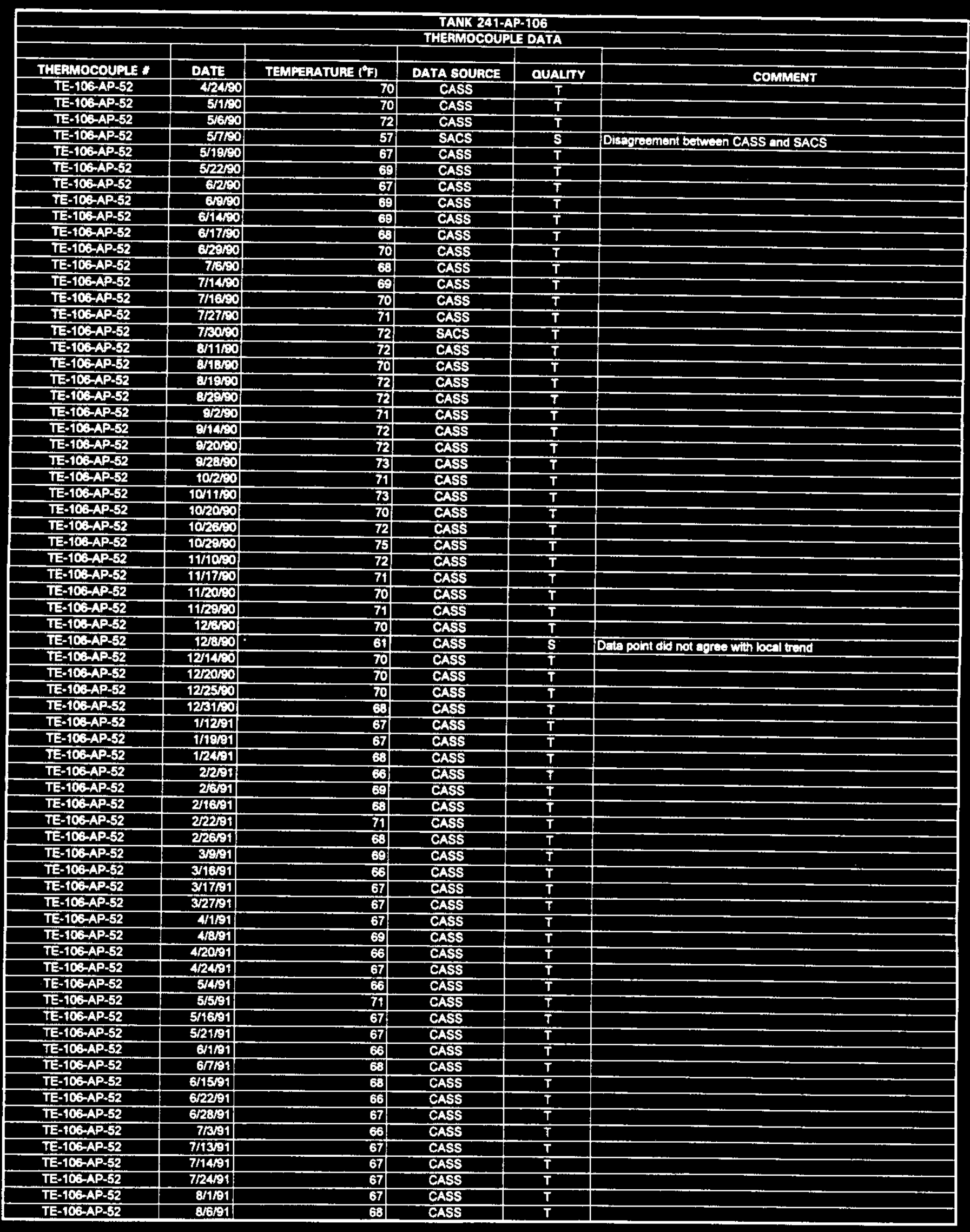




\begin{tabular}{|c|c|c|c|c|c|}
\hline \multicolumn{6}{|c|}{$\begin{array}{l}\text { TANK 241-AP-106 } \\
\text { THEFHOCOUFLE DATA }\end{array}$} \\
\hline & & & & & \\
\hline THERMOCOUPLE * & DATE & TEMPERATURE $\left({ }^{\circ} \mathrm{F}\right)$ & DATA SOURCE & QUALTY & COMMENT \\
\hline TE-106-AP.52 & $8 / 1791$ & 71 & CASS & $T$ & \\
\hline TE-106-AP-52 & $8 / 2091$ & 70 & CASS & $T$ & \\
\hline TE-106-AP.52 & 8/301 & 69 & CASS & $T$ & \\
\hline TE-106-AP-52 & 8/131 & 69 & CASS & $T$ & \\
\hline TE-106-AP-52 & gand & 68 & CASS & $T$ & \\
\hline TE-106-AP.52 & \&18/91 & 70 & CASS & $\bar{T}$ & \\
\hline TE-106.AP.52 & $10 / 11 \% 1$ & 69 & CASS & $\bar{T}$ & \\
\hline TE-106-AP-52 & 10/1401 & 60 & CASS & $\bar{T}$ & \\
\hline TE-106-AP-52 & $10 / 2201$ & 71 & CASS & $T$ & \\
\hline TE-106-AP-52 & 10/2981 & 70 & CASS & $\mathbf{T}$ & \\
\hline TE-106-AP-52 & $11 / 6 / 1$ & 72 & CASS & $T$ & \\
\hline TE-106-AP.52 & $8 / 2202$ & 67 & CASS & $T$ & \\
\hline TE-106-AP-52 & $823 \times 2$ & 69 & CASS & $T$ & \\
\hline$\pi=106-A P-52$ & OHB2 & 67 & CASS. & $T$ & \\
\hline TE-106-AP-52 & 87\%2 & 70 & CASS & $T$ & \\
\hline TE-103-AP-52 & $8 / 1692$ & 71 & CASS & $T$ & \\
\hline TE-106-AP-52 & 92862 & 67 & CASS & $T$ & \\
\hline TE-103-AP-52 & 820102 & 69 & CASS & $\mathbf{T}$ & \\
\hline TE-106-AP-52 & 10092 & 69 & CASS & $\mathbf{T}$ & \\
\hline TE-106-AP-52 & $10 / 17192$ & 69 & SACS & $T$ & \\
\hline TE-106-AP-52 & $10 / 2202$ & 70 & CASS & $T$ & \\
\hline TE-10\%-AP-52 & $1031 \% 2$ & 74 & CASS & $T$ & \\
\hline TE-103-AP-52 & $11 / 492$ & 70 & CASS & $T$ & \\
\hline TE-105-AP.52 & $11 / 3 / 2$ & 73 & CASS & $T$ & \\
\hline TE-106-AP-S2 & $11 / 1602$ & 71 & CASS & $T$ & \\
\hline TE-106-AP-52 & $11 / 22192$ & 68 & CASS & $T$ & \\
\hline TE-106-AP-52 & 12/36202 & 6 & CASS & $T$ & \\
\hline$T E-106-A P .52$ & $12 \mu 1092$ & 70 & CASS & $T$ & \\
\hline TE-106-AP-52 & 12/1762 & 6 & CASS & $T$ & \\
\hline EE-106-AP-52 & $1221 / 2$ & 63 & SACS & $T$ & \\
\hline TE-103-AP-52 & 12 2362 & 83 & SACS & $T$ & \\
\hline TE-106-AP-52 & $6 / 11103$ & 6 & SACS & $T$ & \\
\hline TE-106-AP-52 & $8 / 1803$ & 64 & SACS & $T$ & \\
\hline TE-106-AP-52 & 62203 & 6 & SACS & $T$ & \\
\hline$T E-106-A P-52$ & 7603 & 65 & SACS & $T$ & \\
\hline TE-106-AP-52 & $715 \times 3$ & 65 & SACS & $T$ & \\
\hline TE-10G-AP-52 & 71903 & 65 & SACS & $T$ & \\
\hline TE-106-AP-52 & $7130 / 93$ & 66 & SACS & $T$ & \\
\hline TE-106-AP-52 & $8 / 6=3$ & 66 & SACS & $T$ & \\
\hline TE-106-AP-52 & $8 / 12 / 33$ & 66 & SACS & $T$ & \\
\hline TE-106-AP-52 & $8 / 24 / 03$ & 61 & SACs & $\bar{T}$ & \\
\hline TE-10E-AP-52 & $8 / 26 / 93$ & 68 & SACS & $T$ & \\
\hline TE-10.5-AP-52 & $10 / 11 / 33$ & 69 & SACS & $T$ & \\
\hline TE-T06-AP-52 & $10 / 22393$ & 67 & SACS & $T$ & \\
\hline TE-106-AP-52 & $10 / 23 \% 3$ & 68 & SACS & $T$ & \\
\hline TE-106-AP-52 & $11 / 9 / 93$ & 63 & SACS & $T$ & \\
\hline TE-106-APS 52 & $11 / 30 / 93$ & 59 & SACS & $T$ & \\
\hline TE-108-AP-52 & 12/14/93 & 63 & SACS & $\mathbf{T}$ & \\
\hline TE-106-AP-52 & 122009 & 67 & SACS & $T$ & \\
\hline TE-106-AP-52 & $12 / 2793$ & 69 & SACS & $T$ & \\
\hline TE-106-AP.52 & $1 / 3 / 94$ & 65 & SACS & $T$ & \\
\hline TE-106-AP-52 & $1 / 11 / 2$ & 69 & SACS & $T$ & \\
\hline TE-106-AP-52 & $1 / 18 / 34$ & 68 & CASS & $\bar{T}$ & \\
\hline TE-106-AP-52 & $1 / 25 / 94$ & 69 & CASS & $T$ & \\
\hline TE.106-AP-52 & $2 / 5194$ & 67 & CASS & $T$ & \\
\hline TE-106-AP-52 & $2 / 1 / 94$ & 67 & CASS & $T$ & \\
\hline TE-106-AP-52 & 2/16/94 & 68 & CASS & $T$ & \\
\hline TE-106-AP-52 & 2122904 & 68 & CASS & $T$ & \\
\hline TE-TOG-AP-52 & $3 / 594$ & 69 & CASS & $T$ & \\
\hline TE-106-AP-52 & 3/10/94 & 63 & CASS & $\bar{T}$ & \\
\hline TE-106-AP-52 & $3 / 1494$ & 72 & CASS & $T$ & \\
\hline TE-106-AP-52 & $3 / 22294$ & 67 & CASS & $T$ & \\
\hline TE-106-AP-52 & $3 / 30 / 94$ & 67 & CASS & $T$ & \\
\hline TE-106-AP-52 & $47 / 24$ & 69 & CASS & $T$ & \\
\hline TE-106-AP-52 & $4 / 1359$ & 68 & CASS & $\frac{T}{T}$ & \\
\hline TE-106-AP-52 & 4/2004 & 68 & CASS & $\bar{T}$ & \\
\hline TE-106-AP-52 & $427 \%$ & 69 & CASS & $\bar{T}$ & \\
\hline TE-106-AP-52 & 5R29 & 68 & CASS & $T$ & \\
\hline TE-106-AP-52 & SI1194 & 85 & CASS & $\mathbf{S}$ & Data point did not agree with local trend \\
\hline TE-106-AP-52 & 5/1294 & 69 & CASS & $T$ & \\
\hline
\end{tabular}




\begin{tabular}{|c|c|c|c|c|c|}
\hline \multicolumn{6}{|c|}{$\begin{array}{l}\text { TAWK 241-AP-106 } \\
\text { THEHMOCOUPLE DATA }\end{array}$} \\
\hline & & & & & \\
\hline THERMOCOUPLE * & DATE & TEMPERATUAE $\left({ }^{\circ} \mathrm{F}\right\}$ & DATA SOURCE & OUALITY & COMMENT \\
\hline TE-106-AP-52 & 5/16ran & 103 & CASS & $T$ & \\
\hline TE-106-AP-52 & 523194 & 86 & CASS & $T$ & \\
\hline TE-106-AP-52 & 62294 & 8 & CASS & $\bar{T}$ & \\
\hline TE-106-AP-52 & 6/609 & 87 & CASS & $T$ & \\
\hline TE-106-AP-52 & E/1401 & 94 & CASS & $\mathbf{T}$ & \\
\hline TE-106-AP-52 & 6/1694 & 67 & CASS & $\mathbf{S}$ & Dete point did not agree with local trend \\
\hline TE-106-AP-52 & G/17/39 & 67 & CASS & $\mathbf{S}$ & Date point did not agree with local trend \\
\hline TE-106-AP-52 & Br20/94 & 68 & CASS & $\mathbf{s}$ & Data point did not agree with local trend \\
\hline TE-106-AP-52 & $820 \%$ & 65 & SACS & S(Gh SACS) & Data point did not agres with locel trend \\
\hline TE-106-AP-52 & 62404 & 8 & CASS & $T$ & \\
\hline TE-106-AP-52 & 627/24 & 97 & CASS & $\mathbf{T}$ & \\
\hline TE-106-AP-52 & $7 \mathrm{KM}$ & 8 & CASS & $\mathbf{T}$ & \\
\hline TE-106-AP-52 & 712594 & 65 & SACS & S(Gh SACS) & Data point did not agree with locel trend \\
\hline TE-106-AP-52 & 7309 & 91 & CASS & $T$ & \\
\hline TE-106-AP-52 & 8raga & 97 & CASS & $\overline{\mathbf{T}}$ & \\
\hline TE-103-AP-52 & 82494 & 85 & CASS & $T$ & \\
\hline TE-106-AP-52 & $10 / 1 / 4$ & 8. & CASS & $T$ & \\
\hline TE-106-AP-52 & 1069: & 102 & CASS & $T$ & \\
\hline$T E-106-A P-52$ & T0/15/4 & 101 & CASS & $\mathbf{T}$ & \\
\hline TE-106-AP-52 & 10/1784 & 103 & CASS & $T$ & \\
\hline TE-106-AP-52 & 102019 & 102 & CASS & $T$ & \\
\hline FE-106-AP-52 & 11/494 & 105 & CASS & $T$ & \\
\hline TE-106-AP-52 & $11 / 11 / 9$ & 109 & CASS & $T$ & \\
\hline TE-106-AP-52 & $11 / 18 / 4$ & 102 & CASS & $T$ & \\
\hline TE-106-AP-52 & $11 / 229$ & 105 & CASS & $T$ & \\
\hline TE-106-AP-52 & $11 / 3009$ & 103 & CASS & $T$ & \\
\hline TE-106-AP-S2 & 12/49 & 103 & CASS & $T$ & \\
\hline TE-106-AP-52 & $12 / 16 \%$ & 100 & CASS & $T$ & \\
\hline TE-10BAP-52 & 121804 & 102 & CASS & $T$ & \\
\hline TE-106-AP-52 & 123004 & 102 & CASS & $T$ & \\
\hline TE-106-AP-52 & $1 / 495$ & 107 & CASS & $T$ & \\
\hline TE-106-AP-52 & 1/1465 & 102 & CASS & $T$ & \\
\hline TE-106-AP-52 & $1 / 15 / 5$ & 102 & CASS & $T$ & \\
\hline TE-106-AP-52 & 1/24/55 & 103 & CASS & $T$ & \\
\hline TE-106-AP-52 & 21295 & क्ष & CASS & $\mathbf{T}$ & \\
\hline TE-106-AP-S2 & $2 \% 80$ & 102 & CASS & $\mathbf{T}$ & \\
\hline TE-106_AP-S2 & $2 / 16 \% 5$ & 112 & CASS & $T$ & \\
\hline TE-106-AP-52 & $2 / 23 \% 5$ & 100 & CASS & $T$ & \\
\hline TE-106-AP-52 & $3 / 203$ & 104 & CASS & $T$ & \\
\hline TE-106-AP-52 & $3 \pi / 25$ & 102 & CASS & $T$ & \\
\hline TE-106-AP:52 & अ16/5 & 8 & CASS & $T$ & \\
\hline TE-106-AP-52 & $3 / 2585$ & $\infty$ & CASS & $T$ & \\
\hline TE-106-AP-52 & $3 / 30 / 5$ & 88 & CASS & $T$ & \\
\hline TE-10G-AP-52 & $4 / 3 / 95$ & 87 & SACS & $\mathbf{G}$ & \\
\hline TE-106-AP-52 & 4/10/85 & 89 & SACS & G & \\
\hline TE-106-AP-52 & $417 \overline{8}$ & 91 & SACS & G & \\
\hline TE-106-AP-52 & 424195 & 83 & SACS & $\bar{G}$ & \\
\hline TE-106AP-52 & S1165 & 87 & SACS & G & \\
\hline TE-106-AP-52 & Shas & 83 & SACS & $\mathbf{G}$ & \\
\hline TE-106-AP-52 & 5/1695 & 81 & SACS & $\mathbf{G}$ & \\
\hline TE-106-AP-52 & $5 / 22295$ & 83 & SACS & G & \\
\hline TE-106-AP-52 & 5/29/95 & 80 & SACS & $\bar{G}$ & \\
\hline TE-106-AP-52 & 6/5/S5 & 86 & SACS & G & \\
\hline$T E-105 A P-52$ & 6/13/25 & 81 & SACS & 6 & \\
\hline TE-100-AP-52 & 6/19/95 & 91 & SACS & 6 & \\
\hline TE-106-AP-53 & 4586 & 56 & CASS & $T$ & \\
\hline TE-106-AP-53 & $46 / 86$ & 53 & CASS & $T$ & \\
\hline TE-TOG-AP-53 & $5 / 16 / 6$ & 57 & CASS & $T$ & \\
\hline TE-106-AP-53 & $5 / 24 / 86$ & 59 & CASS & $T$ & \\
\hline TE-106-AP-53 & $5 / 30 / 36$ & 59 & CASS & $T$ & \\
\hline TE-106-AP- 53 & $67 / 86$ & 58 & CASS & $T$ & \\
\hline TE-105-AP-53 & 6/968 & 61 & CASS & $T$ & \\
\hline TE-106-AP-53 & $6 / 21 / 36$ & 59 & CASS & $T$ & \\
\hline TE-106-AP-53 & $6 / 23 / 66$ & 69 & CASS & $\mathbf{S}$ & Data point did not agree with locil trend \\
\hline TE-106-AP-53 & 6/25/86 & 59 & CASS & $T$ & \\
\hline TE-106-AP-53 & $7 / 4 / 86$ & 61 & CASS & $T$ & \\
\hline TE-106-AP-53 & $7 / 1286$ & 60 & CASS & $\bar{T}$ & \\
\hline TE-106-AP-53 & $7 / 19 / 86$ & 60 & CASS & $T$ & \\
\hline TE-106-AP-53 & 7/21/36 & 62 & CASS & $T$ & \\
\hline
\end{tabular}

Date obtained from Compurer Automated Surveillance Syatem (CASS), April 1995; and Surveillance Analysis Computer System (SACS), June 1995. 


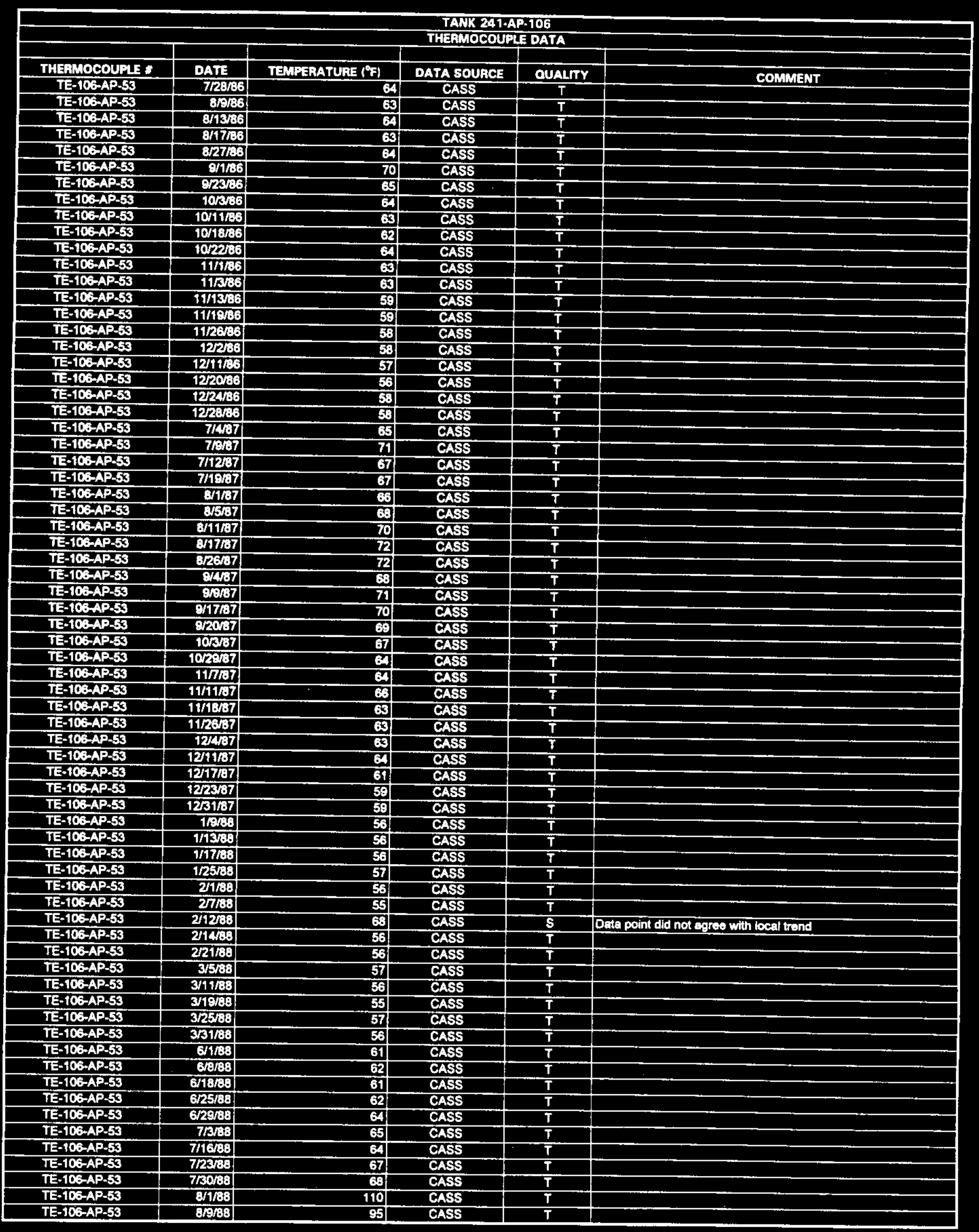




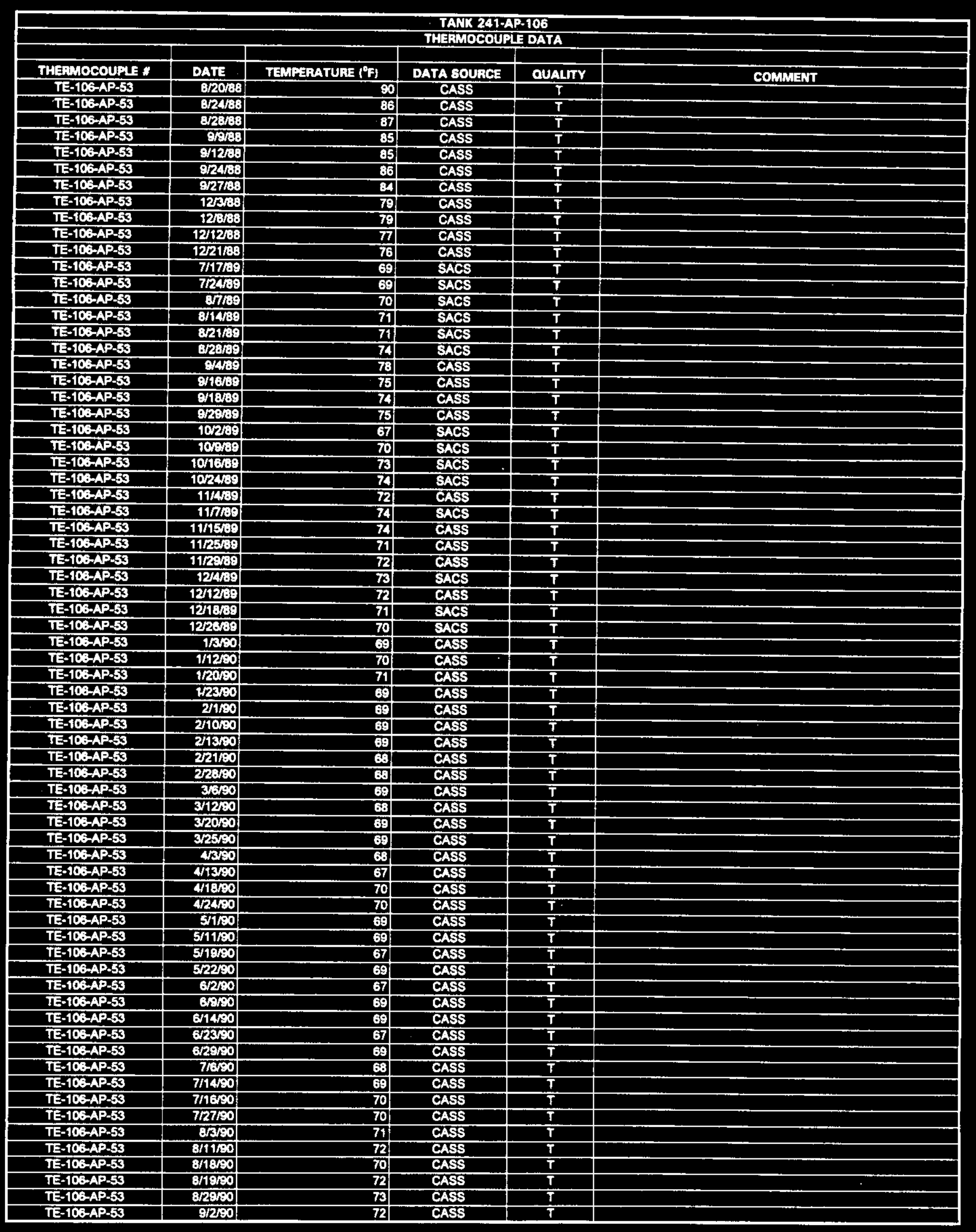




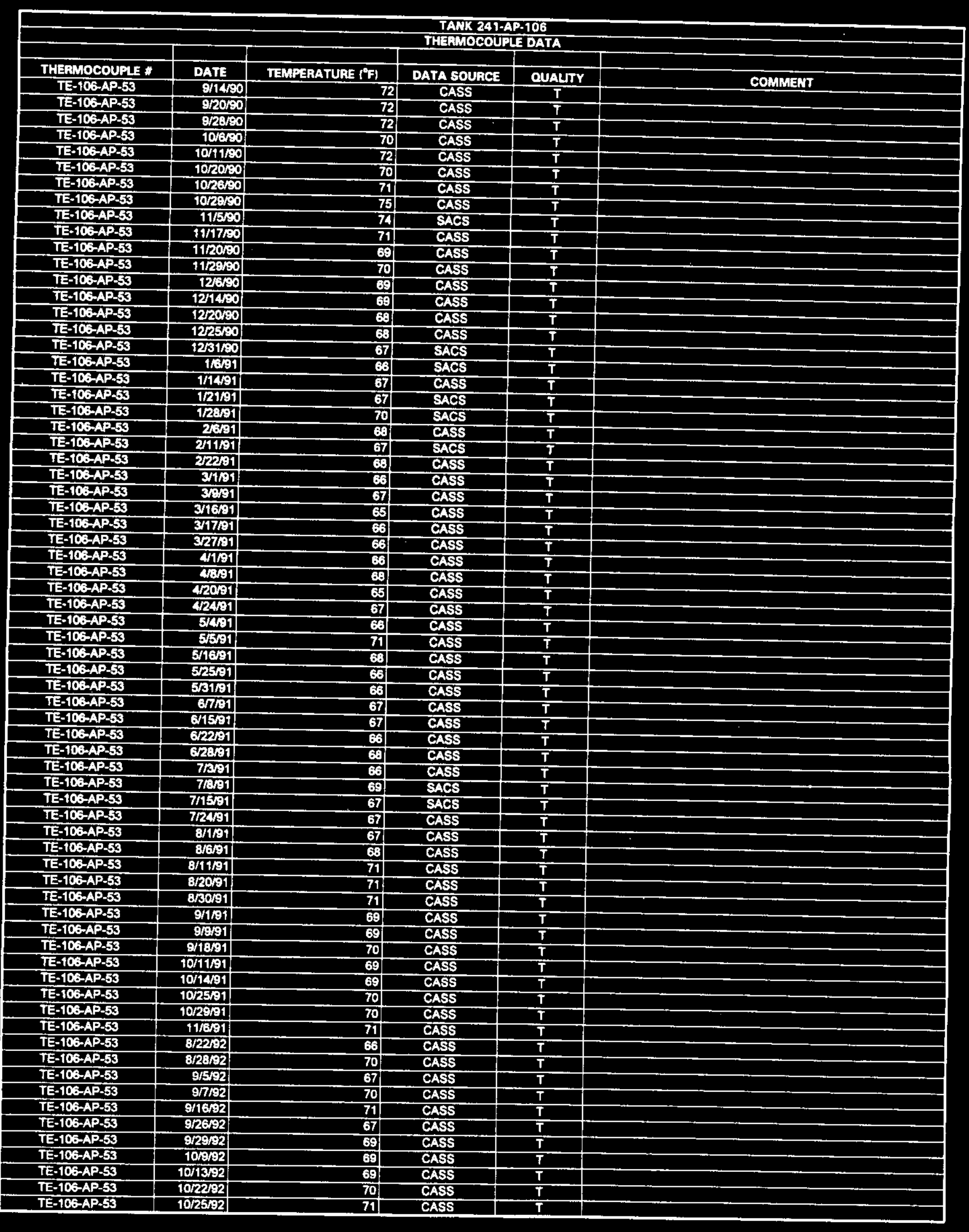




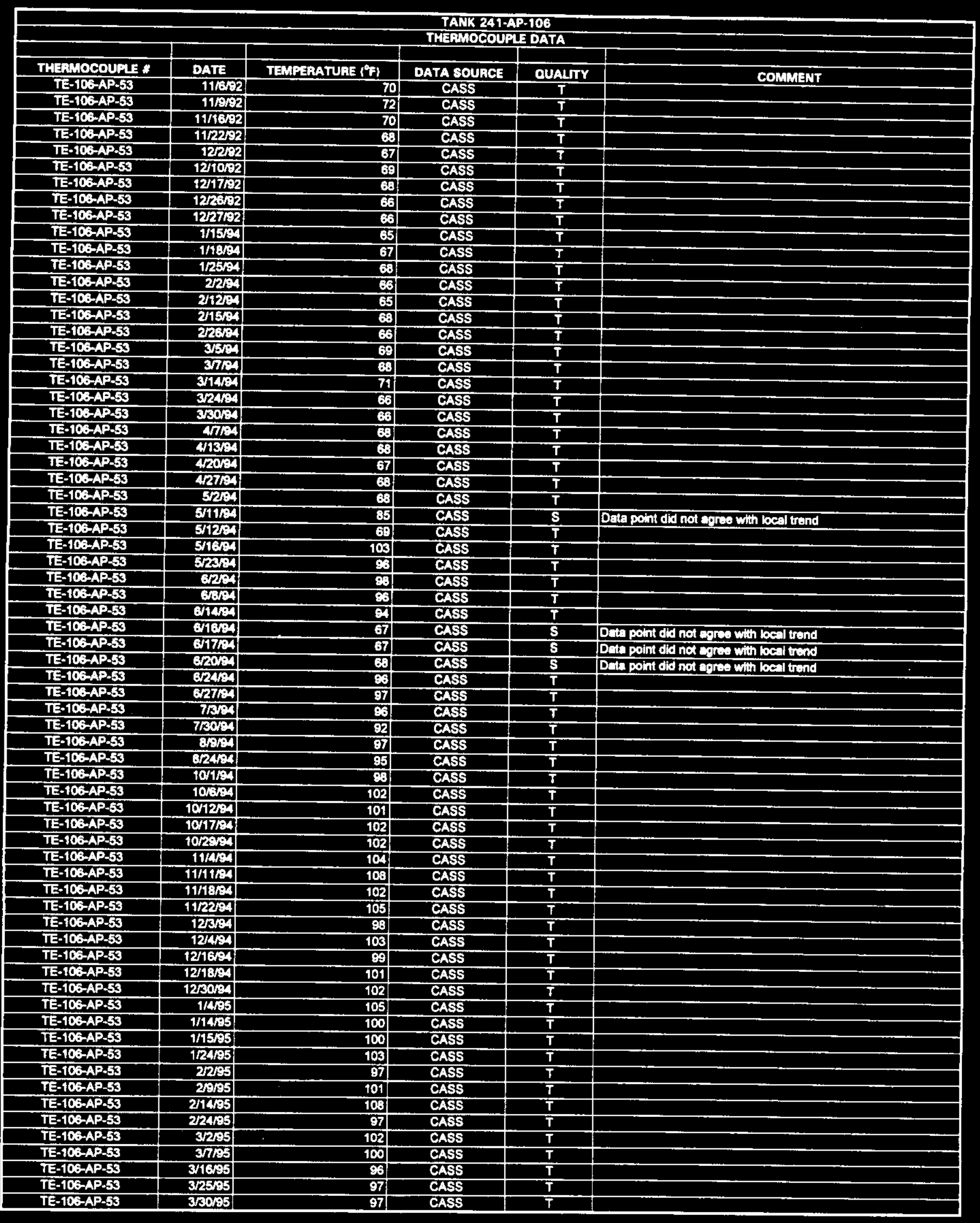




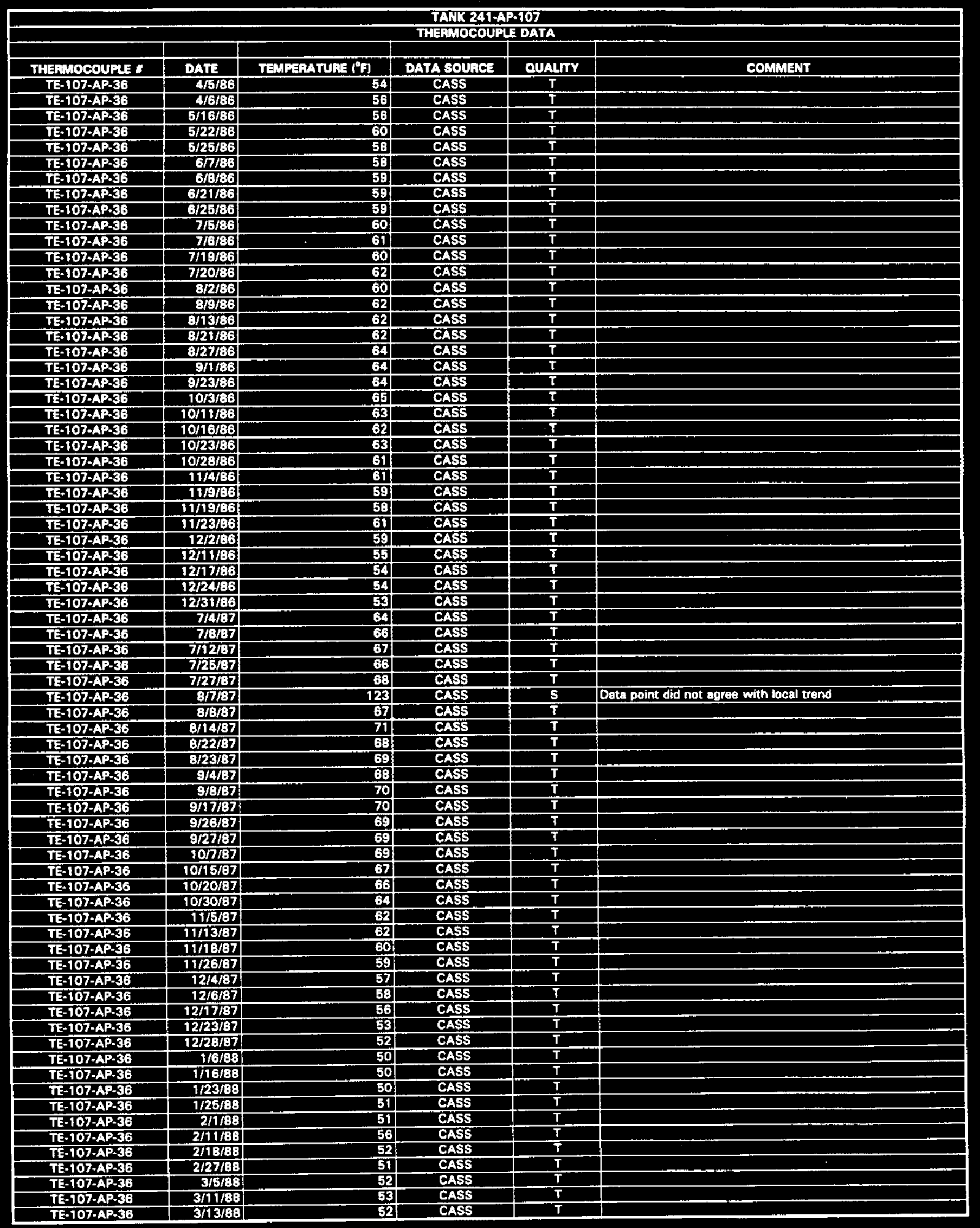

Data obtained from Computer Automated Surveillance System (CASS), April 1995; and Surveillance Analysis Computer System (SACS), June 1995. D-565 


\begin{tabular}{|c|c|c|c|c|c|}
\hline \multicolumn{6}{|c|}{$\begin{array}{l}\text { TANK 241-AP-107 } \\
\text { THERWOCOUFLE DATA }\end{array}$} \\
\hline & & & & & \\
\hline THERMOCOUPLE * & DATE & TEMPERATUAE $\left({ }^{\circ} \mathrm{F}\right)$ & DATA sOUACE & QUALTY & COMMENT \\
\hline $\begin{array}{l}\text { TE-107-AP-36 } \\
\text { TE-107-AP-36 }\end{array}$ & $3 / 25 / 88$ & 54 & CASS & $T$ & \\
\hline TE-107-AP-36 & $3 / 30 / 88$ & 54 & $\frac{\text { CASS }}{\text { CASS }}$ & $T$ & \\
\hline$\frac{T E-107-A P-36}{T E-107-A P-36}$ & \begin{tabular}{l|l|}
$6 / 1 / 88$ \\
$6 / 9 / 88$
\end{tabular} & \begin{tabular}{l|}
59 \\
60
\end{tabular} & $\frac{\text { CASS }}{\text { CASS }}$ & $\frac{T}{T}$ & \\
\hline$\frac{T E-107-A P-36}{T E-107-A P-36}$ & $6 / 18 / 38$ & 60 & $\frac{\text { CASS }}{\text { CASS }}$ & $\frac{T}{T}$ & \\
\hline TE-107-AP-36 & $6 / 25 / 88$ & 62 & CASS & $T$ & \\
\hline TE-107-AP-36 & $7 / 1 / 88$ & 64 & CASS & $T$ & \\
\hline TE-107-AP-36 & $7 / 3 / 88$ & 64 & CASS & $\bar{T}$ & \\
\hline$T E-107-A P-36$ & $7 / 13 / 88$ & 66) & CASS & $T$ & \\
\hline TE-107-AP-36 & $7 / 23 / 88$ & 68 & CASS & $T$ & \\
\hline TE-107-AP-36 & $7 / 27 / 83$ & 69 & CASS & $\bar{T}$ & \\
\hline TE-107-AP-36 & $8 / 5 / 68$ & 69 & CASS & $T$ & \\
\hline TE-107.AP-36 & $8 / 11 / 88$ & 70 & CASS & $\bar{T}$ & \\
\hline TE-107-AP-36 & $8 / 20 / 88$ & 70 & CASS & $\bar{T}$ & \\
\hline TE-107-AP-36 & $8 / 22 / 88$ & 70 & CASS & $T$ & \\
\hline TE-107.AP-36 & $9 / 2 / 88$ & 71 & CASS & $\bar{T}$ & \\
\hline TE-107-AP-36 & $8 / 9 / 98$ & 71 & CASS & $T$ & \\
\hline TE-107.AP-36 & $9 / 16 / 83$ & 70 & CASS & $\bar{T}$ & \\
\hline TE-107-AP-36 & $9 / 23 / 88$ & 69 & CASS & $T$ & \\
\hline TE-107.AP-36 & $9 / 27 / 88$ & 68 & CASS & $T$ & \\
\hline$T E-107-A P-36$ & 12/3/388 & 62 & CASS & $T$ & \\
\hline TE-107-AP-36 & $12 / 10 / 88$ & 61 & CASS & $T$ & \\
\hline TE-107-AP-36 & $12 / 11 / 88$ & 60 & CASS & $T$ & \\
\hline TE-107-AP-36 & $12 / 20 / 88$ & 62 & CASS & $T$ & \\
\hline TE-107-AP-36 & $9 / 2 / 89$ & 69 & CASS & $\mathbf{T}$ & \\
\hline TE-107-AP-36 & $9 / 9 / 89$ & 68 & CASS & $T$ & \\
\hline TE-107-AP-36 & 9/11/89 & 74 & CASS & $T$ & \\
\hline TE-107-AP+36 & 9/18/39 & 70 & CASS & $T$ & \\
\hline TE-107-AP.36 & 9/19/89 & 108 & CASS & $\mathbf{s}$ & Date point did not goree with local trend \\
\hline TE-107.AP-36 & 9/29/89 & 69 & CASS & $\mathbf{T}$ & \\
\hline TE-107-AP-36 & $11 / 3 / 69$ & 63) & CASS & $\mathbf{T}$ & \\
\hline TE-107-AP-36 & 11/7/89 & 64 & CASS & $\mathbf{T}$ & \\
\hline TE-107-AP-36 & $11 / 18 / 89$ & 62 & CASS & $\bar{T}$ & \\
\hline TE-107-AP-36 & $11 / 21 / 89$ & 60 & CASS & $T$ & \\
\hline TE-107.AP-36 & $11 / 26 / 89$ & 60 & CASS & $T$ & \\
\hline TE-T07-AP-36 & $12 / 5 / 69$ & SBI & CASS & $T$ & \\
\hline TE-107-AP-36 & $12 / 12 / 89$ & 57 & CASS & $\bar{T}$ & \\
\hline TE-107.AP-36 & $12 / 17 / 69$ & 53 & CASS & $\bar{T}$ & \\
\hline TE-107-AP-36 & $12 / 23 / 69$ & 52 & CASS & $\bar{T}$ & \\
\hline TE-107-AP-36 & $1 / 2 / 20$ & (52) & CASS & $T$ & \\
\hline TE-107-AP-36 & $1 / 13 / 90$ & B3 & CASS & $T$ & \\
\hline TE-107-AP-36 & 1/18/90 & 64 & CASS & $\mathbf{T}$ & \\
\hline TE-107-AP-36 & $1 / 2,6 / 90$ & 64 & CASS & $\bar{T}$ & \\
\hline TE-107-AP-36 & 1/29/80 & 65 & CASS & $\bar{T}$ & \\
\hline TE-107-AP-36 & 2/10/90 & 63 & CASS & $\mathbf{T}$ & \\
\hline TE-107-AP-36 & $2 / 17 / 90$ & 64 & CASS & $T$ & \\
\hline TE-107-AP-36 & $2 / 24 / 90$ & 65 & CASS & $T$ & \\
\hline TE-107.AP-36 & $3 / 3 / 90$ & 65 & CASS & $T$ & \\
\hline TE-107-AP-36 & $3 / 620$ & 86 & CASS & $T$ & \\
\hline TE-107.AP-36 & $3 / 17 / 90$ & 64 & CASS & $T$ & \\
\hline TE-107-AP-36 & $3 / 23 / 90$ & 69 & CASS & $T$ & \\
\hline TE-107-AP-36 & $3 / 29 / 90$ & 65 & CASS & $T$ & \\
\hline TE-107-AP-36 & $4 / 3 / 90$ & 66) & CASS & $\bar{T}$ & \\
\hline TE-107-AP-36 & $4 / 12 / 90$ & 65 & CASS & $\mathbf{T}$ & \\
\hline TE-107-AP-36 & $4 / 21 / 90$ & 64 & CASS & $\mathbf{T}$ & \\
\hline TE-107-AP-36 & $4 / 24 / 90$ & 65 & CASS & $T$ & \\
\hline TE-107-AP-36 & $4 / 30 / 90$ & 66 & CASS & T & \\
\hline TE-107-AP-36 & 5/9/50 & 102 & CASS & $\mathbf{s}$ & Data point did not agres with local trend \\
\hline$T E-107-A P-36$ & 5/11/90 & 65 & CASS & $T$ & \\
\hline TE-107-AP-36 & $5 / 17 / 80$ & 65 & CASS & $T$ & \\
\hline TE-107.AP-36 & $5 / 22190$ & 64 & CASS & $\mathbf{T}$ & \\
\hline TE-107-AP-36 & $6 / 2 / 90$ & 63 & CASS & $T$ & \\
\hline TE-107-AP-36 & 6/6/80 & 65 & CASS & $T$ & \\
\hline TE-107.AP.36 & $6 / 12 / 90$ & 66 & CASS & $T$ & \\
\hline TE-107-AP-36 & $6 / 21 / 90$ & 66 & CASS & $\bar{T}$ & \\
\hline TE-107-AP-36 & $6 / 29 / 90$ & 67 & CASS & $T$ & \\
\hline TE-107-AP-36 & $7 / 6 / 90$ & 66 & CASS & $T$ & \\
\hline TE-107-AP-36 & 7/12/90: & 68 & CASS & $T$ & \\
\hline TE-107-AP-36 & $7 / 16 / 90$ & 72 & CASS & $T$ & \\
\hline$T E-107-A P-36$ & $7 / 22 / 90$ & 70 & CASS & $T$ & \\
\hline$T E-107-A P-36$ & $8 / 3 / 90$ & 70 & CASS & $T$ & \\
\hline TE-107-AP-36 & $8 / 11 / 90$ & 72 & CASS & $T$ & \\
\hline$T=107-A P-36$ & $8 / 17 / 90$ & 72 & CASS & $T$ & \\
\hline
\end{tabular}

Data obtained from Computer Automated Surveillance Syatem (CASS), April 1995; and Surveillance Analysis Computer System (SACS), June 1995. D-566 


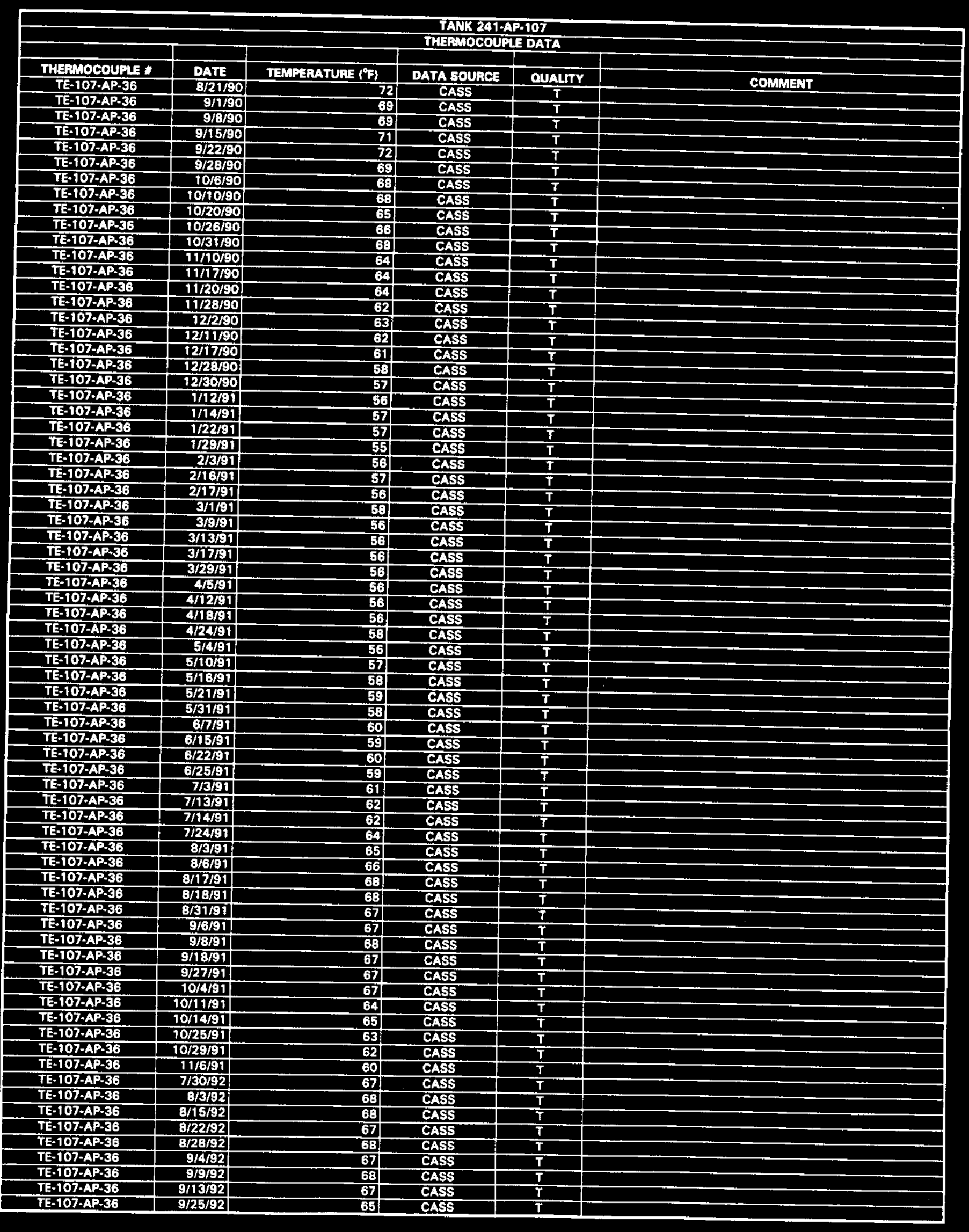

Data obteined from Computer Automated Surveillance Syatem (CASS), April 1995; and Surveillance Analyais Computer Syatem (SACS), June 1995. D-567 


\begin{tabular}{|c|c|c|c|c|c|}
\hline \multicolumn{6}{|c|}{$\begin{array}{l}\text { TANK 241-AP-107 } \\
\text { THERMOCOUPLE DATA }\end{array}$} \\
\hline & & & & & \\
\hline$\frac{\text { THEPMOCOUPLE }}{\text { TE-107-AP-36 }}$ & DATE & TEMPERATURE ("F) & DATA SOURCE & QUALTTY & COMMENT \\
\hline$\frac{\text { TE-107-AP-36 }}{\text { TE-107-AP-36 }}$ & $\frac{10 / 3 / 92}{10 / 8 / 82}$ & $\frac{66}{65}$ & CASS & $T$ & \\
\hline$\frac{1 E-107 \cdot A P-36}{T E-107 \cdot A P-36}$ & $\frac{10 / 9 / 92}{10 / 13 / 92}$ & 65 & CASS & $T$ & \\
\hline TE-107-AP-36 & $10 / 22 / 92$ & 67 & $\frac{\text { CASS }}{\text { CASS }}$ & $\frac{T}{T}$ & \\
\hline TE-107-AP-36 & $10 / 31 / 92$ & 61 & CASS & $T$ & \\
\hline TE-107-AP-36 & 11/4/92 & $\overline{62}$ & CASS & $\bar{T}$ & \\
\hline TE-107-AP-36 & $11 / 12 / 92$ & 80 & CASS & $T$ & \\
\hline TE-107.AP-36 & $11 / 17 / 92$ & 60 & CASS & $T$ & \\
\hline TE-107-AP-36 & $11 / 23 / 92$ & 58 & SACS & $T$ & \\
\hline TE-107-AP-36 & $11 / 29 / 92$ & 57 & CASS & $T$ & \\
\hline TE-107.AP-36 & $12 / 6 / 92$ & 58 & CASS & $T$ & \\
\hline TE-107-AP-36 & $12 / 19 / 92$ & 55 & CASS & $T$ & \\
\hline TE-107-AP-36 & $12 / 21 / 92$ & 56 & CASS & $T$ & \\
\hline TE-107-AP-36 & $12 / 30 / 92$ & 54 & CASS & $T$ & \\
\hline TE-107-AP-36 & $6 / 11 / 93$ & 58 & SACS & $T$ & \\
\hline TE-107.AP.36 & $6 / 18 / 93$ & 58 & SACS & $\mathbf{T}$ & \\
\hline TE-107.AP.36 & 8/28/931 & 60 & SACS & $\bar{T}$ & \\
\hline TE-107.AP-36 & $7 / 9 / 93$ & 60 & SACS & $T$ & \\
\hline IE-107-AP-36 & $7 / 15 / 93$ & 80 & SACS & $T$ & \\
\hline TE-107-AP-36 & $719 / 93$ & 60 & SACS & $\bar{T}$ & \\
\hline TE-107-AP.36 & $7 / 30 / 93$ & 62 & SACS & $T$ & \\
\hline IE-107.AP-36 & $8 / 6 / 93$ & 63 & SACS & $T$ & \\
\hline TE-107-AP-36 & $8 / 12 / 93$ & 63 & SACS & $T$ & \\
\hline TE-107.AP-36 & 9/24/33 & $\mathbf{5 3}$ & SACS & $\mathbf{s}$ & Date point did not agree with locel trend \\
\hline TE-107-AP-36 & 9/26/93 & 63 & SACS & $T$ & \\
\hline TE-107.AP.36 & $10 / 15 / 93$ & 61 & SACS & $T$ & \\
\hline TE-107.AP-36 & $10 / 22 / 93$ & 59 & SACS & $T$ & \\
\hline TE-107.AP.36 & 10/2:/93 & 60 & SACS & $T$ & \\
\hline TE-107-AP-36 & 11/9/93 & 56 & SACS & $T$ & \\
\hline TE-107-AP-36 & $11 / 30 / 93$ & 63 & SACS & $\bar{T}$ & \\
\hline TE-107-AP-36 & $12 / 14 / 83$ & 59 & SACS & $T$ & \\
\hline TE-107-AP-36 & $12 / 20 / 83$ & 56 & SACS & $T$ & \\
\hline TE-107-AP-36 & $12 / 27 / 93$ & S6] & SACS & $T$ & \\
\hline TE-107-AP-36 & $1 / 3 / 94$ & 54 & SACS & $\bar{T}$ & \\
\hline TE-107-AP-36 & $1 / 11 / 34$ & 58 & SACS & $T$ & \\
\hline TE-107-AP-36 & I/18/94 & 57 & CASS & $T$ & \\
\hline TE-107-AP-36 & $1 / 25 / 94$ & 56 & CASS & $T$ & \\
\hline TE-107-AP-36 & $2 / 4 / 94$ & 55 & CASS & $T$ & \\
\hline TE-107-AP-36 & $2 / 12 / 94$ & E4 & CASS & $\bar{T}$ & \\
\hline TE-107-AP-36 & $2 / 16 / 94$ & 57 & CASS & $\mathbf{T}$ & \\
\hline TE-107-AP-36 & $2 / 22 / 94$ & 56 & CASS & $T$ & \\
\hline TE-107-AP-36 & $3 / 5 / 94$ & 56 & CASS & $T$ & \\
\hline TE-107-AP-36 & $3 / 3 / 94$ & 57 & CASS & $\mathbf{T}$ & \\
\hline TE-107-AP-36 & $3 / 17 / 94$ & 56 & CASS & $T$ & \\
\hline TE-107-AP-36 & $3 / 25 / 94$ & 57 & CASS & $\mathbf{T}$ & \\
\hline TE-107-AP-36 & 3/29/94 & 56 & CASS & $T$ & \\
\hline TE-107-AP-36 & $4 / 9 / 94$ & 58: & CASS & $T$ & \\
\hline TE-107-AP-36 & 4/15/94 & 57 & CASS & $\mathbf{I}$ & \\
\hline TE-107-AP-36 & 4/22/94 & 58 & CASS & $T$ & \\
\hline TE-107-AP-36 & $4 / 29 / 94$ & 59 & CASS & $T$ & \\
\hline TE-107-AP-36 & $5 / 2 / 94$ & 59 & CASS & $T$ & \\
\hline TE-107-AP-36 & 5/14/34 & 59 & CASS & $\bar{T}$ & \\
\hline TE-107-AP-36 & 5/16/94 & 96 & CASS & $\mathbf{T}$ & \\
\hline TE-107-AP-36 & 5/22/84 & 91 & CASS & $T$ & \\
\hline TE-107-AP-36 & 6/2/94 & 92 & CASS & $T$ & \\
\hline TE-107-AP-36 & $6 / 9 / 94$ & 90 & CASS & $\mathbf{T}$ & \\
\hline TE-107.AP-36 & 6/14/94 & 90 & CASS & $\mathbf{T}$ & \\
\hline TE-107.AP-36 & 6/16/94 & 60 & CASS & $\mathbf{S}$ & Data point did not agree with local trend \\
\hline TE-107-AP-36 & 6/17/94 & 60 & CASS & $\mathbf{S}$ & Data point did not agree with local trend \\
\hline TE-107.AP-36 & $6 / 20 / 94$ & 62 & CASS & $\mathbf{S}$ & Dats point did not agrea with local trend \\
\hline TE-107-AP-36 & $6 / 20 / 94$ & 60 & SACS & $S$ (G in SACS) & Data point did not agree with local trend \\
\hline TE-107-AP-36 & $6 / 24 / 94$ & 93 & CASS & $\mathbf{T}$ & \\
\hline TE-107-AP-36 & 6/27/94 & 94 & CASS & $T$ & \\
\hline TE-107-AP-36 & $7 / 7 / 94$ & 93 & CASS & $\mathbf{T}$ & \\
\hline TE-107-AP-36 & 7/15/94 & 93 & CASS & $\mathbf{T}$ & \\
\hline TE-107-AP-36 & $7 / 20 / 94$ & 92 & CASS & $T$ & \\
\hline TE-107.AP-36 & $7 / 28 / 94$ & 93 & CASS & $\bar{T}$ & \\
\hline TE-107-AP-36 & $7 / 31 / 94$ & 97 & CASS & $\bar{T}$ & \\
\hline TE-107-AP-36 & $8 / 9 / 94$ & 97 & CASS & $\mathbf{T}$ & \\
\hline TE-107-AP-36 & $8 / 20 / 94$ & 97 & CASS & $T$ & \\
\hline TE-107-AP-36 & $8 / 22 / 94$ & 97 & CASS & $T$ & \\
\hline TE-107.AP.36 & 9/2/94 & 96 & CASS & $\mathbf{T}$ & \\
\hline TE-107-AP-36 & $9 / 10 / 94$ & 98 & CASS & $\bar{T}$ & \\
\hline
\end{tabular}




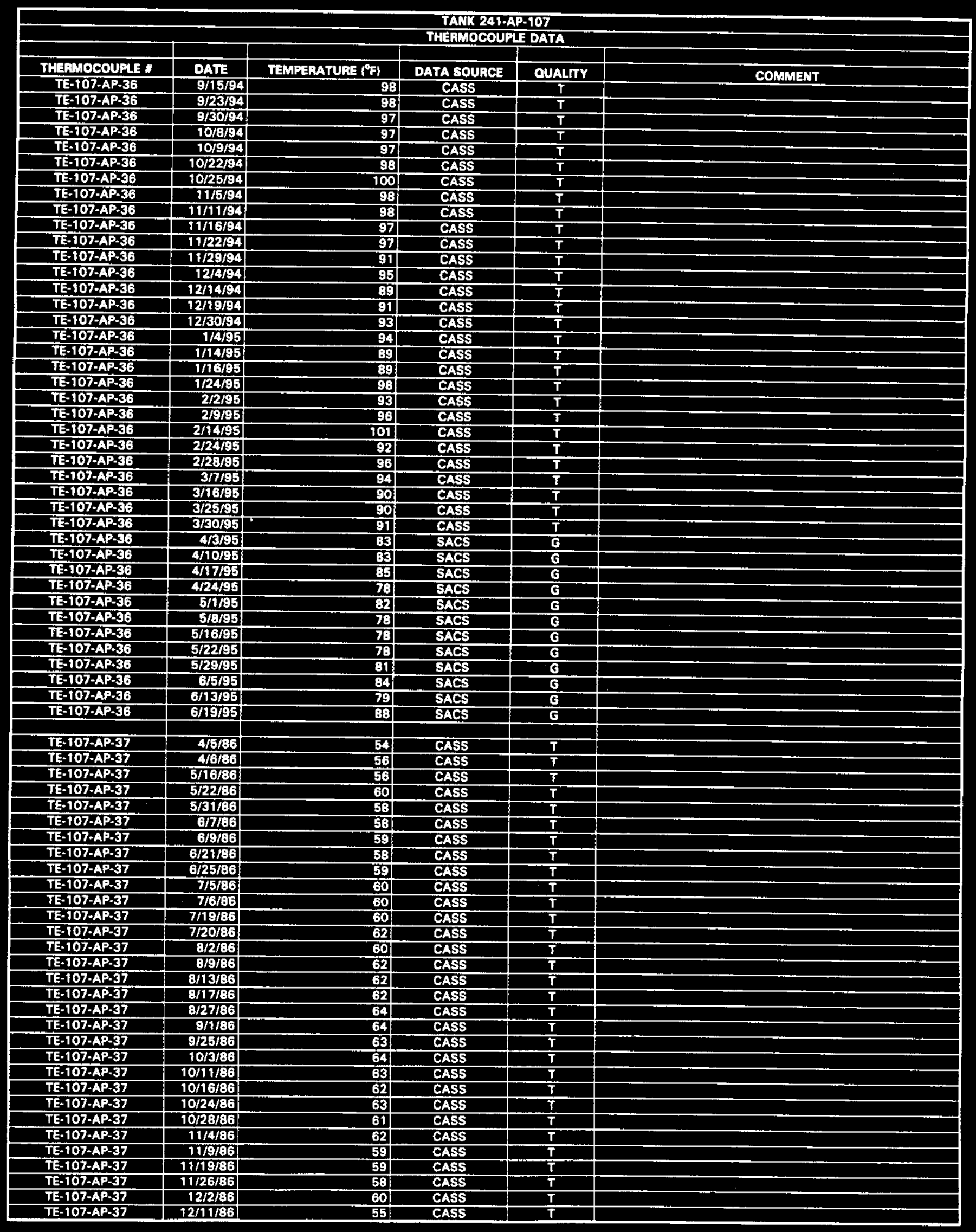




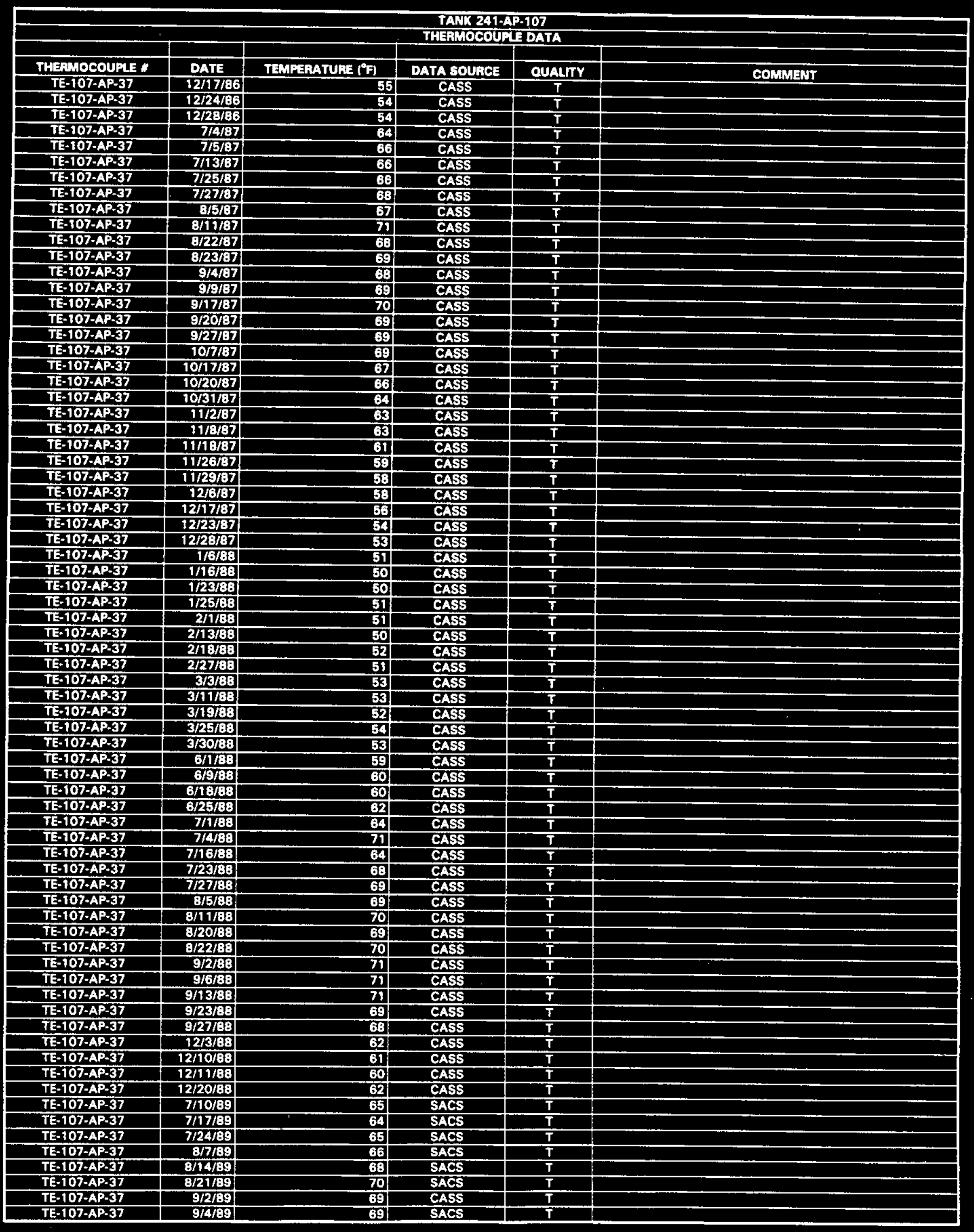




\begin{tabular}{|c|c|c|c|c|c|}
\hline \multicolumn{6}{|c|}{$\begin{array}{l}\text { TANK 241-AP-107 } \\
\text { THEFMOCOUPLE DATA }\end{array}$} \\
\hline & & & & & \\
\hline THEPMOCOUPLE * & DATE & TEMPERATURE ( $\left.{ }^{\circ} F\right)$ & DATA SOURCE & QUALTY & COMMENT \\
\hline TE-107-AP-37 & $9 / 11 / 89$ & 74 & CASS & $T$ & \\
\hline TE-107-AP-37 & 9/18/89 & 70 & CASS & $T$ & \\
\hline TE-107-AP-37 & 9/29/89 & 69 & CASS & $T$ & \\
\hline $\begin{array}{l}\text { TE-107-AP-37 } \\
\text { TE-107-AP-37 }\end{array}$ & $\begin{array}{l}10 / 2 / 89 \\
10 / 9 / 89\end{array}$ & $\frac{67}{67}$ & $\frac{\text { SACS }}{\text { SACS }}$ & $\frac{T}{T}$ & \\
\hline $\begin{array}{l}\text { TE-107-AP-37 } \\
\text { TE-107-AP-37 }\end{array}$ & \begin{tabular}{r|}
$10 / 9 / 89$ \\
$10 / 16 / 89$ \\
\end{tabular} & 67 & SACS & $\frac{T}{T}$ & \\
\hline TE-107-AP-37 & $10 / 24 / 89$ & 66 & SACS & $T$ & \\
\hline TE-107-AP-37 & $11 / 3 / 89$ & 63. & CASS & $T$ & \\
\hline TE-107-AP-37 & $11 / 11 / 39$ & 62 & CASS & $\bar{T}$ & \\
\hline TE-107-AP-37 & $11 / 16 / 89$ & 62 & CASS & $T$ & \\
\hline TE-107-AP-37 & $11 / 21 / 89$ & 60 & CASS & $T$ & \\
\hline TE-107-AP-37 & $11 / 26 / 89$ & 60 & CASS & $T$ & \\
\hline TE-107-AP-37 & $12 / 6 / 89$ & 57 & CASS & $\bar{T}$ & \\
\hline TE-107-AP-37 & 12 12/89 & 57 & CASS & $T$ & \\
\hline TE-107-AP-37 & 12/19/89 & 53 & CASS & $T$ & \\
\hline TE-107-AP-37 & $12 / 25 / 69$ & 52 & CASS & $\bar{T}$ & \\
\hline TE-107-AP-37 & $1 / 2 / 90$ & 52 & CASS & $\mathbf{T}$ & \\
\hline TE-107-AP-37 & $1 / 13 / 90$ & 53 & CASS & $T$ & \\
\hline TE-107-AP-37 & $1 / 18 / 90$ & 65 & CASS & $T$ & \\
\hline TE-107-AP-37 & $1 / 26 / 90$ & 65 & CASS & $T$ & \\
\hline TE-107-AP-37 & $1 / 29 / 90$ & 65 & CASS & $T$ & \\
\hline TE-107.AP-37 & $2 / 4 / 90$ & 64 & CASS & $T$ & \\
\hline TE-107-AP-37 & $2 / 17 / 90$ & 64 & CASS & $\bar{T}$ & \\
\hline TE-107-AP-37 & $2 / 24 / 90$ & 66 & CASS & $T$ & \\
\hline E-107-AP-37 & $3 / 3 / 90$ & 67 & CASS & $T$ & \\
\hline TE-107-AP-37 & $3 / 8 / 90$ & 69 & CASS & $T$ & \\
\hline TE-107-AP-37 & आ12/90 & 68 & CASS & $T$ & \\
\hline TE-107-AP-37 & $3 / 20 / 90$ & 67 & CASS & $T$ & \\
\hline TE-107-AP-37 & $3 / 29 / 90$ & 66 & CASS & $T$ & \\
\hline TE-107-AP-37 & $4 / 3 / 90$ & 66 & CASS & $\mathbf{T}$ & \\
\hline TE-107-AP-37 & $4 / 12 / 90$ & 66 & CASS & $T$ & \\
\hline TE-107-AP-37 & $4 / 15 / 90$ & 111 & CASS & $\mathbf{S}$ & Data point did not agree with local trend \\
\hline TE-107-AP-37 & $4 / 21 / 90$ & 64 & CASS & $T$ & \\
\hline TE-107-AP-37 & $4 / 26 / 90$ & 65 & CASS & $\mathbf{T}$ & \\
\hline TE-107-AP-37 & $4 / 30 / 30$ & 86 & CASS & $T$ & \\
\hline TE-107-AP-37 & $5 / 11 / 90$ & 65 & CASS & $T$ & \\
\hline TE-107-AP-37 & $5 / 17 / 90$ & 65 & CASS & $T$ & \\
\hline TE-107-AP-37 & $5 / 22 / 90$ & 65 & CASS & $T$ & \\
\hline TE-107-AP-37 & 6/2/30 & 63 & CASS & $T$ & \\
\hline TE-107-AP-37 & 6/6/80 & 64 & CASS & $T$ & \\
\hline TE-107-AP-37 & $6 / 11 / 90$ & 65 & CASS & $T$ & \\
\hline TE-107-AP-37 & $6 / 23 / 90$ & 63 & CASS & $T$ & \\
\hline IE-107-AP-37 & $6 / 28 / 90$ & 66 & CASS & $\mathbf{T}$ & \\
\hline TE-107-AP-37 & $7 / 6 / 90$ & 64 & CASS & $T$ & \\
\hline TE-107-AP-37 & 7/12/90 & 63 & CASS & $T$ & \\
\hline TE-107-AP-37 & $7 / 16 / 90$ & 70 & CASS & $\mathbf{T}$ & \\
\hline TE-107-AP-37 & $7 / 27 / 90$ & 68 & CASS & $T$ & \\
\hline TE-107-AP-37 & $8 / 3 / 90$ & 69 & CASS & $\mathbf{T}$ & \\
\hline TE-107-AP-37 & $8 / 11100$ & 70 & CASS & $\mathbf{T}$ & \\
\hline TE-107-AP-37 & $8 / 17 / 90$ & 70 & CASS & $\mathbf{T}$ & \\
\hline TE-107-AP-37 & $8 / 22 / 90$ & 70 & CASS & $\mathbf{T}$ & \\
\hline TE-107-AP-37 & $9 / 1 / 90$ & 68 & CASS & $\bar{T}$ & \\
\hline TE-107-AP-37 & $9 / 8 / 90$ & 68 & CASS & $T$ & \\
\hline TE-107-AP-37 & $9 / 14 / 90$ & 69 & CASS & $T$ & \\
\hline TE-107-AP-37 & $9 / 22 / 90$ & 71 & CASS & $T$ & \\
\hline TE-107-AP-37 & $9 / 28 / 90$ & 68 & CASS & $T$ & \\
\hline TE-107-AP-37 & $10 / 6 / 90$ & 67 & CASS & $\mathbf{T}$ & \\
\hline TE-107-AP-37 & $10 / 10 / 90$ & 68 & CASS & $T$ & \\
\hline TE-107-AP-37 & 10/20/90 & 65 & CASS & $\mathbf{T}$ & \\
\hline TE-107-AP-37 & $10 / 26 / 90$ & 66 & CASS & $T$ & \\
\hline TE-107-AP-37 & $10 / 28 / 90$ & 106 & CASS & $\mathbf{S}$ & Data point did not agree with local trend \\
\hline TE-107-AP-37 & $10 / 31 / 90$ & 88 & CASS & $\mathbf{T}$ & \\
\hline TE-107-AP-37 & $111 / 6 / 90$ & 66 & CASS & $\bar{T}$ & \\
\hline TE-107-AP-37 & $11 / 17 / 90$ & 64 & CASS & $\mathbf{T}$ & \\
\hline TE-107-AP-37 & $11 / 20 / 90$ & 65 & CASS & $\mathbf{T}$ & \\
\hline TE-107-AP-37 & $12 / 1 / 90$ & 63 & CASS & $\mathbf{T}$ & \\
\hline TE-107-AP-37 & $12 / 3 / 90$ & 69 & SACS & $\mathbf{T}$ & \\
\hline TE-107-AP-37 & $12 / 11 / 90$ & 64 & CASS & $\mathbf{T}$ & \\
\hline TE-107-AP-37 & $12 / 19 / 90$ & 63 & CASS & $\mathbf{T}$ & \\
\hline TE-107-AP-37 & $12 / 28 / 90$ & 60 & CASS & $T$ & \\
\hline TE-107-AP-37 & $12 / 30 / 90$ & 60 & CASS & $T$ & \\
\hline TE-107-AP-37 & $1 / 8 / 31$ & 59 & CASS & $T$ & \\
\hline TE-107-AP-37 & 1/17/91 & 59 & CASS & $\mathbf{T}$ & \\
\hline
\end{tabular}




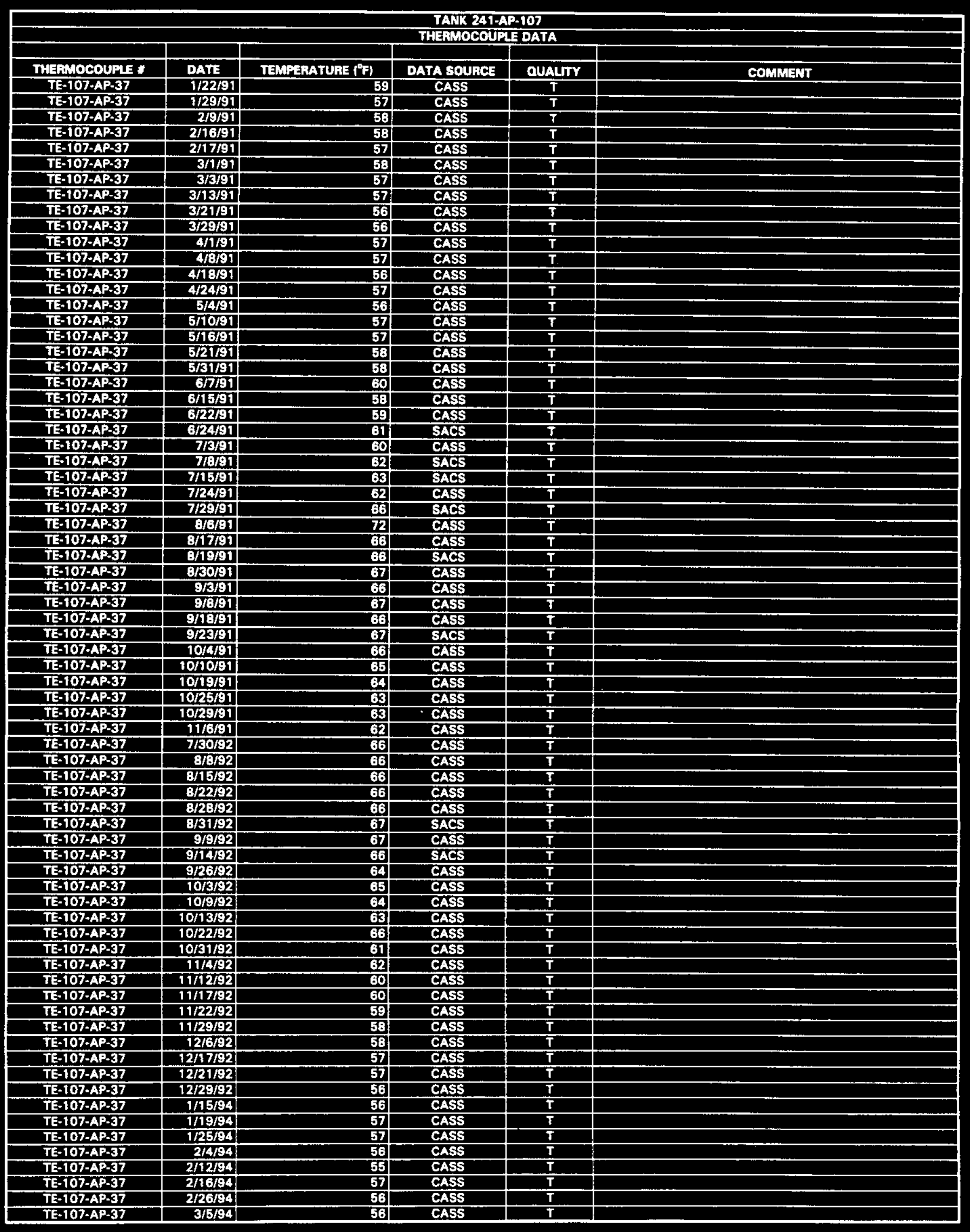

Data obtained from Computer Automated Surveillance System (CASS), April 1995; and Surveillance Analysis Computer Syotem (SACS), June 1995. D-572 


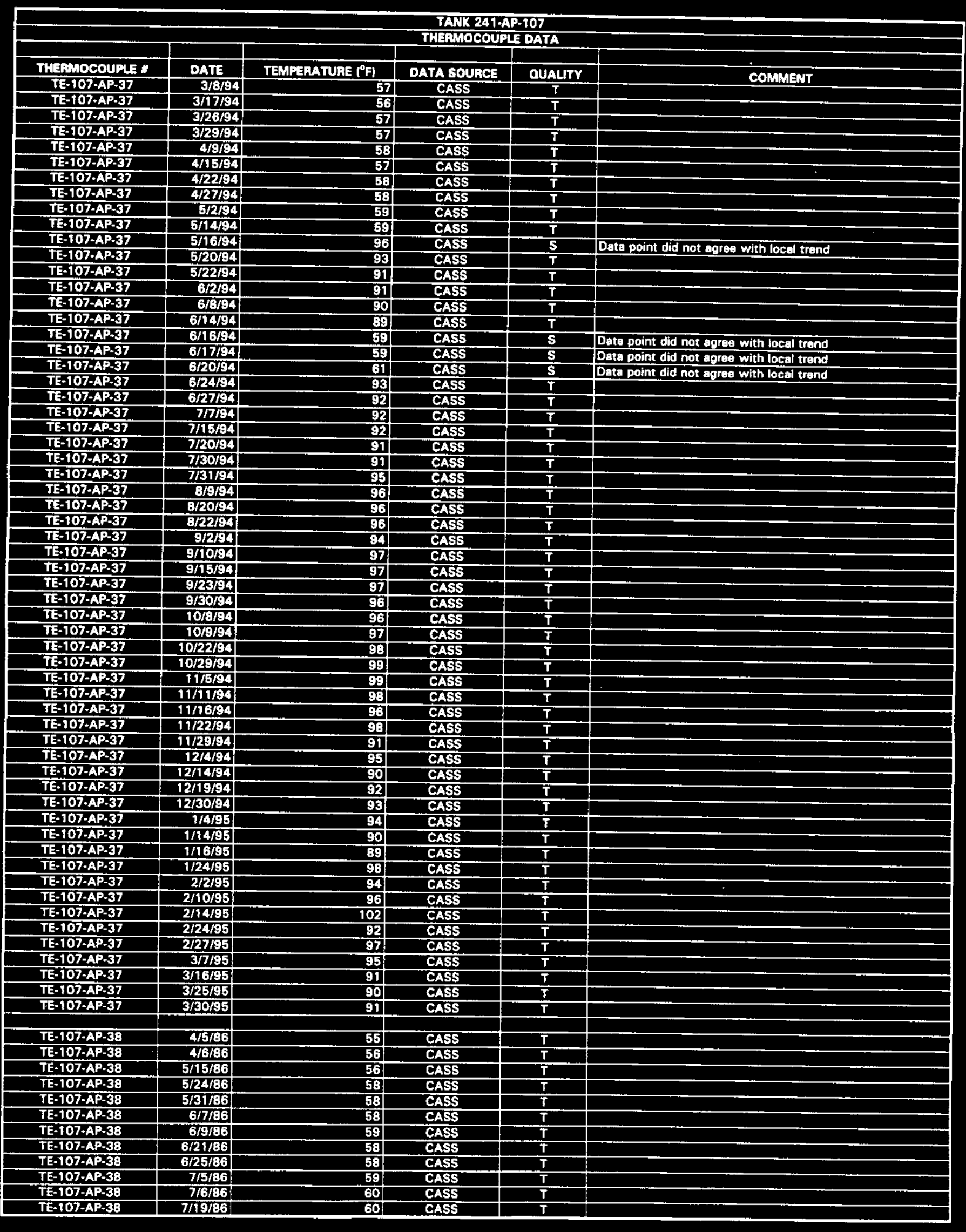

Date obtained from Computer Automated Surveillance System (CASS), April 1995; and Surveillance Analysis Computer System (SACS). June 1995. D-573 


\begin{tabular}{|c|c|c|c|c|c|}
\hline \multicolumn{6}{|c|}{$\begin{array}{l}\text { TANK 241-AP-107 } \\
\text { THEGMOCOUPLE DATA }\end{array}$} \\
\hline & & TEMPERATUAE $\left({ }^{\circ} \mathrm{F}\right)$ & DATA SOUACE & & \\
\hline$\frac{\text { THERMOCOUPLE * }}{\text { TE-107-AP-38 }}$ & $\frac{\text { DATE }}{7 / 20 / 86}$ & 82 & & QUALTY & COMMENT \\
\hline$\frac{\text { TE-107-AP-38 }}{\text { TE-107-AP-38 }}$ & \begin{tabular}{r|}
$7 / 20 / 86$ \\
$8 / 2 / 36$
\end{tabular} & $\frac{62}{60}$ & $\frac{\text { CASS }}{\text { CASS }}$ & $T$ & \\
\hline $\begin{array}{l}\text { TE-107-AP-38 } \\
\text { TE-107-AP-3B }\end{array}$ & $\frac{8 / 2 / 36}{8 / 3 / 86}$ & 63 & CASS & $T$ & \\
\hline TE-107-AP-3B & $8 / 3 / 86$ & 63 & CASS & $T$ & \\
\hline TE-107-AP-3B & $8 / 13 / 86$ & 62 & CASS & $T$ & \\
\hline TE-107-AP-38 & $8 / 17 / 86$ & 62 & CASS & $T$ & \\
\hline TE-107.AP-3B & $8 / 27 / 36$ & 64 & CASS & $T$ & \\
\hline TE-107-AP-38 & $9 / 2 / 86$ & 63 & CASS & $T$ & \\
\hline TE-107-AP-3B & $9 / 23 / 86$ & 64 & CASS & $\bar{T}$ & \\
\hline$T E \cdot 107-A P-38$ & $10 / 3 / 86$ & 64 & CASS & $\bar{T}$ & \\
\hline TE-107-AP-38 & $10 / 9 / 86$ & 63 & CASS & $\bar{T}$ & \\
\hline TE-107-AP-3B & $10 / 16 / 36$ & 62 & CASS & $T$ & \\
\hline TE-107-AP-38 & $10 / 24 / 86$ & 63 & CASS & $T$ & \\
\hline TE-107-AP-3B & $10 / 28 / 86$ & 67 & CASS & $T$ & \\
\hline TE-107-AP-3B & $1116 / 86$ & 60 & CASS & $\bar{T}$ & \\
\hline TE-107-AP-3B & 11/8/86 & 59: & CASS & $T$ & \\
\hline TE-107-AP-38 & $11 / 19 / 86$ & 59 & CASS & $T$ & \\
\hline TE-107.AP-3B & $11 / 29 / 66$ & 57 & CASS & $T$ & \\
\hline TE-107.AP-38 & $12 / 2 / B 6$ & 60 & CASS & $T$ & \\
\hline TE-107-AP-38 & $12 / 11 / 86$ & 56 & CASS & $T$ & \\
\hline TE-107-AP-38 & $12 / 17 / 86$ & 55 & CASS & $T$ & \\
\hline TE-107-AP-38 & $12 / 24 / 86$ & 55 & CASS & $\bar{T}$ & \\
\hline TE-107-AP-3B & $12 / 29 / 86$ & 54 & CASS & $\mathbf{T}$ & \\
\hline TE-107-AP-38 & $7 / 4 / 37$ & 84 & CASS & $T$ & \\
\hline TE-107-AP-38 & 7/5/37 & 66 & CASS & $T$ & \\
\hline TE-107-AP-38 & $7 / 13 / 67$ & 66 & CASS & $T$ & \\
\hline TE-107-AP-38 & $7 / 25 / 37$ & 66 & CASS & $\mathbf{T}$ & \\
\hline TE-107.AP-38 & $7 / 2.7 / 187$ & 68 & CASS & $T$ & \\
\hline TE-107-AP-38 & $8 / 5 / 67$ & 67 & CASS & $\bar{T}$ & \\
\hline TE-107-AP-3B & $8 / 11 / 87$ & 69 & CASS & $\bar{T}$ & \\
\hline TE-107-AP-3B & $8 / 22 / 37$ & 68 & CASS & $T$ & \\
\hline TE-107-AP-3B & $8 / 23 / 37$ & 69 & CASS & $\bar{T}$ & \\
\hline TE-107-AP-38 & $9 / 4 / 37$ & 68 & CASS & $T$ & \\
\hline TE-107-AP-3B & 9/9137 & 69 & CASS & $T$ & \\
\hline TE-107-AP-38 & 9/1787 & 70 & CASS & $T$ & \\
\hline TE-107-AP-38 & 9/26/87 & 68 & CASS & $T$ & \\
\hline TE-107-AP-3B & 9/27/87 & 68 & CASS & $T$ & \\
\hline TE-107-AP-38 & $10 / 7 / 87$ & 69 & CASS & $T$ & \\
\hline TE-107-AP-38 & $10 / 15 / 87$ & 6日 & CASS & $T$ & \\
\hline TE-107-AP-3B & $10 / 20 / 87$ & 60 & CASS & $T$ & \\
\hline TE-107-AP-38 & 10/30/87 & 65 & CASS & $T$ & \\
\hline IE-107-AP-3B & $11 / 2 / 37$ & 63 & CASS & $\mathbf{T}$ & \\
\hline TE-107-AP-38 & $11 / 8 / 87$ & 64 & CASS & $\bar{T}$ & \\
\hline TE-107-AP.38 & 11/18/87 & 61 & CASS & $\mathbf{T}$ & \\
\hline TE-107-AP-38 & $11 / 26 / 87$ & 60 & CASS & $\bar{T}$ & \\
\hline TE-107-AP-38 & $11 / 29 / 87$ & 59 & CASS & $\mathbf{T}$ & \\
\hline TE-107-AP-3B & $12 / 6 / 87$ & 59 & CASS & $T$ & \\
\hline TE-107.AP-3B & $12 / 17 / 87$ & 57 & CASS & $T$ & \\
\hline TE-107.AP-38 & $12 / 23 / 87$ & 54 & CASS & $\bar{T}$ & \\
\hline TE-107-AP-38 & 12/28/87 & 53 & CASS & $T$ & \\
\hline TE-107-AP-38 & $1 / 6 / 88$ & 52 & CASS & $T$ & \\
\hline TE-107-AP-38 & $1 / 16 / 8$ & 50। & CASS & $T$ & \\
\hline TE-107-AP-38 & $1 / 23 / 88$ & 50 & CASS & $T$ & \\
\hline TE-107-AP-38 & $1 / 25 / 88$ & 54 & CASS & $\mathbf{T}$ & \\
\hline TE-107-AP-3B & $2 / 3 / 68$ & 51 & CASS & $T$ & \\
\hline TE-107-AP-3B & $2 / 13 / 88$ & 50 & CASS & $T$ & \\
\hline TE-107-AP-38 & $2 / 18 / 88$ & 52 & CASS & $T$ & \\
\hline TE-107-AP-38 & $2 / 27 / 38$ & 51 & CASS & $\mathbf{T}$ & \\
\hline TE-107-AP-3B & $3 / 3 / 83$ & 52 & CASS & $T$ & \\
\hline$T=107-A P-3 B$ & $3 / 11 / 88$ & 53 & CASS & $T$ & \\
\hline TE-107-AP-3B & $3 / 13 / 88$ & 52 & CASS & $T$ & \\
\hline TE-107-AP-3B & $3 / 24 / 88$ & 67 & CASS & $\mathbf{S}$ & Data point did not agree with local trend \\
\hline TE-107-AP-38 & $3 / 25 / 88$ & 56 & CASS & $\mathbf{T}$ & \\
\hline TE-107-AP-3B & $3 / 30 / 88$ & 54 & CASS & $T$ & \\
\hline TE-107-AP-38 & $6 / 1 / 88$ & 60 & CASS & $\mathbf{T}$ & \\
\hline TE-107-AP-3B & $6 / 9 / 88$ & 60 & CASS & $T$ & \\
\hline TE-107-AP-38 & $6 / 18 / 8$ & 80 & CASS & $T$ & \\
\hline TE-107-AP-38 & $6 / 25 / 88$ & 62 & CASS & $\mathbf{T}$ & \\
\hline TE-107-AP-3B & $7 / 1 / 88$ & 64 & CASS & $\mathbf{T}$ & \\
\hline TE-T07-AP-38 & $7 / 9 / 88$ & 63 & CASS & $\mathbf{T}$ & \\
\hline TE-107-AP-38 & $7 / 16 / 88$ & 64 & CASS & $T$ & \\
\hline TE-107-AP-3B & $7 / 23 / 88$ & 68 & CASS & $T$ & \\
\hline TE-107-AP-3B & $7 / 27 / 88$ & 69 & CASS & $T$ & \\
\hline TE-107+AP-3B & $8 / 5 / 88$ & 69 & CASS & $\mathbf{T}$ & \\
\hline
\end{tabular}

Date obtained from Computer Automated Survellance Syatem (CASS), April 1995; and Surveillance Analysis Computer System (SACS), Juna 1995. D-574 


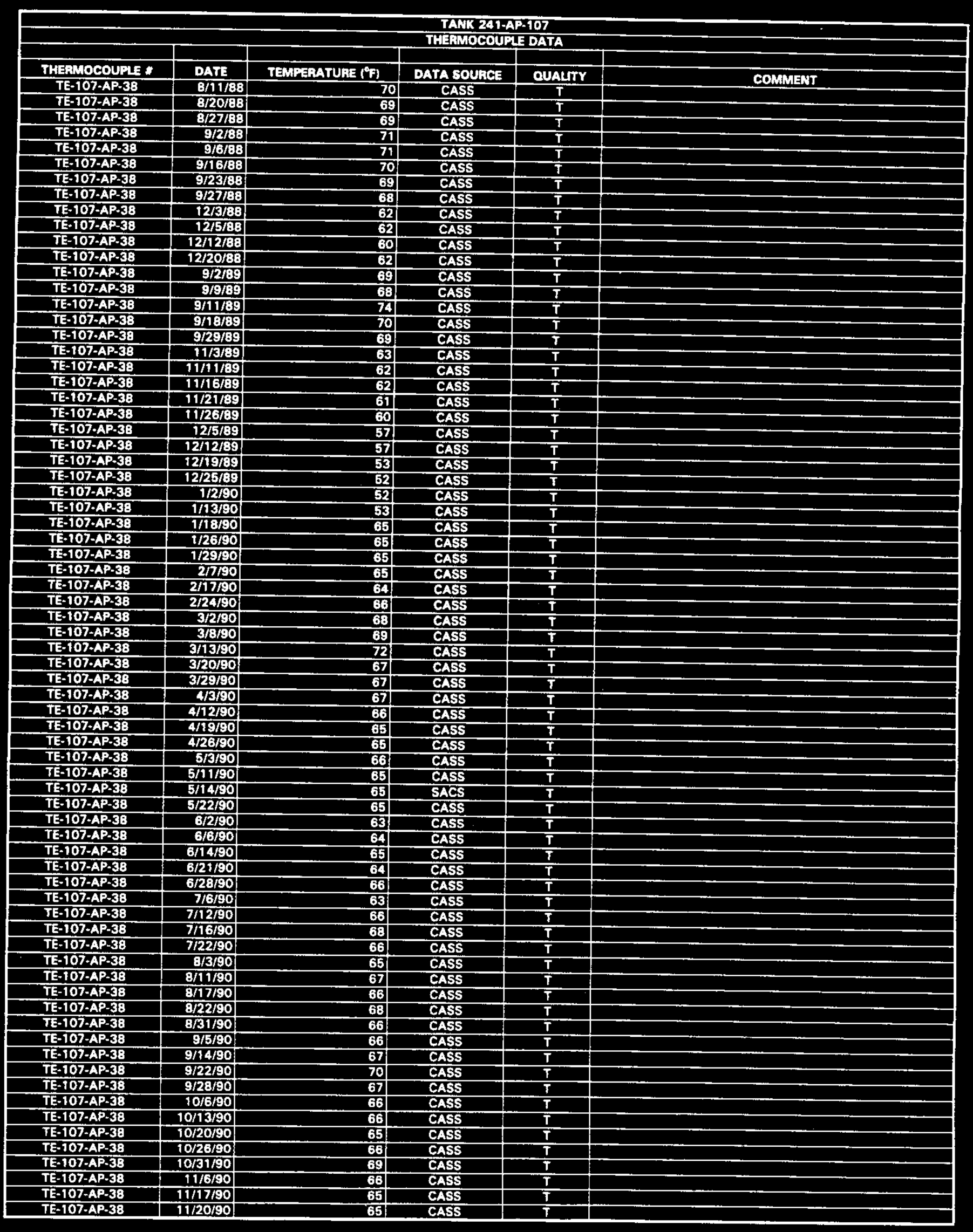

Date obteined from Computer Automated Surveillance Syatem (CASS), April 1995; and Surveillance Analysis Computer System (SACS), June 1995. D-575 


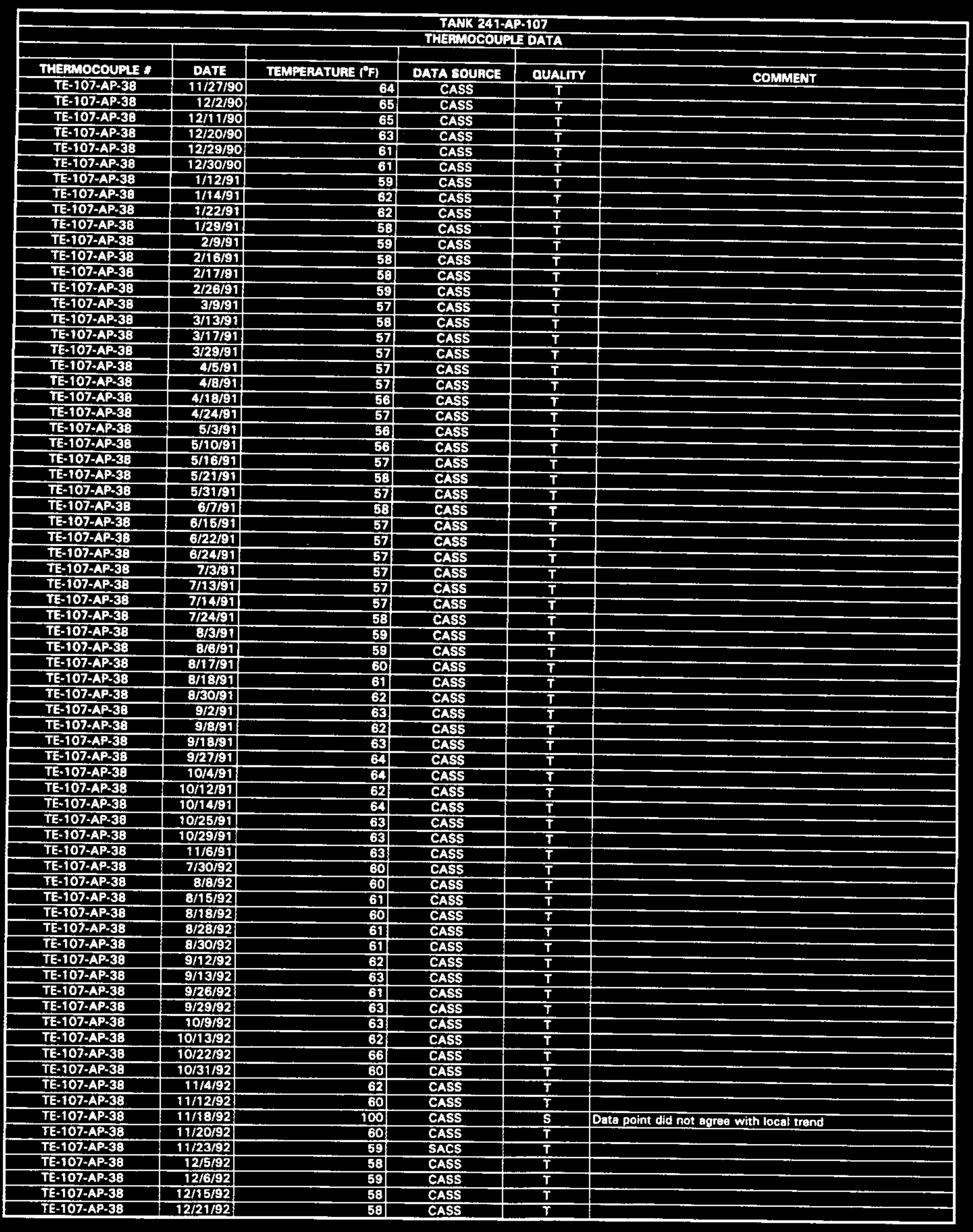




\begin{tabular}{|c|c|c|c|c|c|}
\hline \multicolumn{6}{|c|}{$\begin{array}{l}\text { TANK 241-AP-107 } \\
\text { THEFMOCOUFLF DATA }\end{array}$} \\
\hline & & & & & \\
\hline THERMOCOUPLE * & DATE & TEMPERATURE $\left({ }^{\circ} \mathrm{F}\right)$ & DATA SOUACE & OUALITY & COMMENT \\
\hline TE-107-AP-3B & $12 / 27 / 92$ & 57 & CASS & $T$ & \\
\hline TE-107.AP-38 & $6 / 11 / 93$ & 54 & SACS & $T$ & \\
\hline TE-107-AP-38 & $6 / 18 / 93$ & 53: & SACS & $\bar{T}$ & \\
\hline TE-107-AP-3B & $6 / 28 / 93$ & 55 & SACS & $\begin{array}{ll}\mathbf{T} \\
\end{array}$ & \\
\hline TE-107-AP-38 & $7 / 9 / 93$ & 55 & SACS & $\mathbf{T}$ & \\
\hline TE-107-AP-38 & $7 / 15 / 93$ & 56 & SACS & $T$ & \\
\hline TE-107-AP-38 & 7/19/93 & 55 & SACS & $T$ & \\
\hline TE-107-AP-38 & 7/30/93 & 57 & SACS & $\bar{T}$ & \\
\hline TE-107-AP-3B & $8 / 6 / 93$ & 56 & SACS & $T$ & \\
\hline TE-107-AP-38 & 8/12/93 & 56 & SACS & $T$ & \\
\hline TE-107-AP-38 & $9 / 24 / 93$ & 52 & SACS & $\bar{T}$ & \\
\hline TE-107.AP-38 & $9 / 26 / 93$ & 59 & SACS & $T$ & \\
\hline IE-107-AP-38 & $10 / 15 / 93$ & 60 & SACS & $T$ & \\
\hline TE-107-AP-39 & $10 / 22 / 93$ & 59 & SACS & $T$ & \\
\hline TE-107-AP-3B & $10 / 28 / 93$ & 60 & SACS & $T$ & \\
\hline TE-107-AP-38 & $11 / 9 / 93$ & 56 & SACS & $T$ & \\
\hline TE-107-AP-38 & $11 / 30 / 93$ & 63 & SACS & $T$ & \\
\hline IE-107-AP-38 & $12 / 14 / 93$ & 60 & SACS & $T$ & \\
\hline TE-107-AP-38 & $12 / 20 / 93$ & 67 & SACS & $T$ & \\
\hline TE-107-AP.3B & $12 / 27 / 93$ & 58 & SACS & $T$ & \\
\hline TE-107-AP-38 & $3 / 3 / 94$ & 56 & SACS & $\bar{T}$ & \\
\hline TE-107-AP-38 & $1 / 11 / 84$ & 59 & SACS & $T$ & \\
\hline TE-107-AP-38 & $1 / 18 / 94$ & 59 & CASS & $T$ & \\
\hline TE-107-AP-38 & $1 / 25 / 94$ & 58 & CASS & $\overline{\mathbf{T}}$ & \\
\hline TE-107-AP-38 & $2 / 2 / 94$ & 58 & CASS & $T$ & \\
\hline TE-107-AP-38 & $2 / 22194$ & 58 & CASS & $T$ & \\
\hline TE-107-AP-38 & $2 / 16 / 94$ & 58 & CASS & $T$ & \\
\hline TE-107-AP-38 & $2 / 22 / 94$ & 57) & CASS & $T$ & \\
\hline TE-107-AP-38 & $3 / 5 / 94$ & 57 & CASS & $T$ & \\
\hline TE-107-AP-38 & $3 / 10 / 94$ & 57 & CASS & $T$ & \\
\hline$T E \cdot 107-A P \cdot 3 B$ & $3 / 17 / 94$ & 57 & CASS & $T$ & \\
\hline TE-107-AP-38 & $3 / 26 / 94$ & 57 & CASS & $\bar{T}$ & \\
\hline TE-107-AP-38 & $3 / 29 / 94$ & 57 & CASS & $T$ & \\
\hline IE-107-AP-38 & $4 / 9 / 94$ & 58 & CASS & $\bar{T}$ & \\
\hline TE-107-AP-38 & $4 / 15 / 94$ & 56 & CASS & $T$ & \\
\hline TE-107-AP-38 & $4 / 22194$ & 57 & CASS & $T$ & \\
\hline TE-107-AP-38 & $4 / 27 / 94$ & 58 & CASS & $T$ & \\
\hline TE-107-AP-38 & $5 / 2 / 94$ & 58 & CASS & $T$ & \\
\hline TE-107-AP-38 & $5 / 14 / 94$ & 57. & CASS & $\mathbf{T}$ & \\
\hline TE-107-AP-38 & $5 / 16 / 94$ & 94 & CASS & $\mathbf{s}$ & Date point did not agree whth locel trend \\
\hline TE-107-APA38 & $5 / 20194$ & 93. & CASS & $T$ & \\
\hline TE-107-AP-38 & $5 / 22 / 94$ & 89 & CASS & $\bar{T}$ & \\
\hline TE-107-AP-38 & $6 / 2 / 94$ & so & CASS & $T$ & \\
\hline TE-107-AP-38 & $6 / 8 / 94$ & 87 & CASS & $T$ & \\
\hline TE-107-AP-3B & $6 / 16 / 94$ & 57 & CASS & $\mathbf{S}$ & Date point did not bgres with local trend \\
\hline IE-107-AP-38 & $6 / 17 / 94$ & 57 & CASS & $\mathbf{S}$ & Data point did not agree with local trend \\
\hline IE-107-AP-38 & $6 / 18 / 94$ & 86 & CASS & $T$ & \\
\hline TE-107-AP-38 & $6 / 20 / 94$ & 59 & CASS & $\mathbf{S}$ & Data point did not agree with local trend \\
\hline TE-107-AP-3B & $6 / 20 / 94$ & 57 & SACS & S [G in SACS] & Dasa point did not agree with local trend \\
\hline TE-107-AP-3B & $6 / 24 / 94$ & 89 & CASS & $T$ & \\
\hline TE-107-AP-38 & 6/27/94 & 89 & CASS & $T$ & \\
\hline TE-107-AP-38 & $7 / 7 / 64$ & 88 & CASS & $T$ & \\
\hline TE-107-AP-38 & $7 / 13 / 94$ & 88 & CASS & $T$ & \\
\hline TE-107.AP-38 & $7 / 20 / 94$ & 86 & CASS & $\mathbf{T}$ & \\
\hline TE-107-AP-38 & $7 / 28 / 94$ & 86 & CASS & $T$ & \\
\hline TE-107-AP-38 & $7 / 31 / 94$ & 89 & CASS & $T$ & \\
\hline TE-107-AP-38 & $8 / 9 / 94$ & 90 & CASS & $T$ & \\
\hline TE-107.AP-38 & $8 / 20 / 34$ & 89 & CASS & $T$ & \\
\hline$T E-107-A P-38$ & $8 / 22 / 94$ & 91 & CASS & $T$ & \\
\hline TE-107-AP-38 & 9/3/94 & 92 & CASS & $T$ & \\
\hline TE-107-AP-38 & $9 / 10 / 94$ & 93 & CASS & $T$ & \\
\hline TE-107-AP-3B & $9 / 15 / 94$ & 93) & CASS & $T$ & \\
\hline TE-107-AP-38 & $9 / 23 / 94$ & 93 & CASS & $\mathbf{T}$ & \\
\hline TE-107-AP-38 & $9 / 30 / 94$ & 96) & CASS & $T$ & \\
\hline TE-107-AP-38 & $10 / 8 / 94$ & 96 & CASS & $\bar{T}$ & \\
\hline TE-107-AP-38 & $10 / 12 / 94$ & 96 & CASS & $T$ & \\
\hline TE-107-AP.38 & $10 / 22 / 94$ & 98 & CASS & $T$ & \\
\hline TE-107-AP-38 & $10 / 28 / 94$ & 100 & CASS & $T$ & \\
\hline TE-107-AP-38 & $11 / 5 / 94$ & 99 & CASS & $T$ & \\
\hline TE-107-AP-38 & $11 / 11 / 94$ & 99 & CASS & $\mathbf{T}$ & \\
\hline TE-107-AP-38 & $11 / 16 / 94$ & 96 & CASS & $T$ & \\
\hline TE-107-AP-38 & $11 / 22 / 94$ & 98 & CASS & $\mathbf{T}$ & \\
\hline TE-107-AP-38 & $11 / 28 / 94$ & 92 & SACS & $\mathrm{G}$ & \\
\hline
\end{tabular}




\begin{tabular}{|c|c|c|c|c|c|}
\hline \multicolumn{6}{|c|}{$\begin{array}{l}\text { TANK 241-AP.107 } \\
\text { THERMOCOULE DATA }\end{array}$} \\
\hline THERMOCOUPLE * & DATE & TEMFERATUAE $\left\{^{\circ} \mathrm{F}\right\}$ & DATA sOURCE & OUALITY & COMMENT \\
\hline TE-107-AP-3B & $12 / 4 / 94$ & 95 & CASS & $T$ & \\
\hline TE-107-AP-3B & $12 / 14 / 94$ & 90 & CASS & $T$ & \\
\hline TE-107-AP-38 & $12 / 19 / 94$ & 92 & CASS & $T$ & \\
\hline TE-107-AP-3B & $12 / 30 / 94$ & 92 & CASS & $T$ & \\
\hline TE-107-AP-38 & $1 / 4 / 35$ & 94 & CASS & $T$ & \\
\hline TE-107-AP-38 & 1/14/95 & 80 & CASS & $T$ & \\
\hline TE-107-AP-38 & $1 / 16 / 95$ & 89 & CASS & $\mathbf{T}$ & \\
\hline TE-107-AP-38 & $1 / 24 / 95$ & 98 & CASS & $T$ & \\
\hline TE-107-AP-38 & $2 / 2 / 95$ & 95 & CASS & $\mathbf{T}$ & \\
\hline TE-107-AP-38 & $2 / 10 / 95$ & 87 & CASS & $\mathbf{T}$ & \\
\hline TE-107-AP-38 & $2 / 14 / 95$ & 103 & CASS & $T$ & \\
\hline TE-107-AP-38 & $2 / 24 / 95$ & 93 & CASS & $\mathbf{T}$ & \\
\hline TE-107.AP-38 & $2 / 27 / 95$ & 96 & CASS & $T$ & \\
\hline TE-107-AP-38 & $3 / 7 / 95$ & 95 & CASS & $T$ & \\
\hline TE-107.AP-38 & $3 / 16 / 95$ & 91 & CASS & $\mathbf{T}$ & \\
\hline TE-107.AP-38 & $3 / 25 / 95$ & 91 & CASS & 7 & \\
\hline TE-107-AP-38 & $3 / 30 / 95$ & 91 & CASS & $T$ & \\
\hline TE-107-AP-38 & 4/3/35 & 83 & SACS & G & \\
\hline IE-107-AP-38 & $4 \longdiv { 1 0 / 9 5 }$ & 83 & SACS & $\bar{G}$ & \\
\hline TE-107-AP-38 & 4/17/25 & 86 & SACS & $\mathbf{G}$ & \\
\hline TE-107-AP-3B & 4/24/95 & 78 & SACS & $\mathbf{G}$ & \\
\hline TE-107-AP-3B & $5 / 1 / 95$ & 82 & SACS & 6 & \\
\hline TE-107-AP-38 & $5 / 8 / 95$ & 78 & SACS & $\mathbf{G}$ & \\
\hline TE-107-AP-38 & $5 / 16 / 95$ & 78 & SACS & $\mathbf{G}$ & \\
\hline TE-107-AP-38 & $5 / 22 / 95$ & 78 & SACS & G & \\
\hline TE-107-AP-38 & $5 \longdiv { 2 9 / 9 5 }$ & BO & SACS & G & \\
\hline TE-107-AP-38 & $6 / 5 / 95$ & 84 & SACS & G & \\
\hline TE-107-AP-3B & 6/13/95 & 78 & SACS & G & \\
\hline TE-107-AP-3B & 6/19/95 & 87 & SACS & G & \\
\hline TE-107-AP-39 & $4 / 5 / 18$ & 55 & CASS & $T$ & \\
\hline TE-107-AP-39 & $4 / 7 / 66$ & 56 & CASS & $T$ & \\
\hline TE-107-AP-39 & $5 / 15 / 86$ & 56 & CASS & $T$ & \\
\hline TE-107-AP-39 & $5 / 24 / 86$ & 58 & CASS & $T$ & \\
\hline TE-107-AP-39 & $5 / 31 / 86$ & 58 & CASS & $\mathbf{T}$ & \\
\hline TE-107-AP-39 & $6 / 3 / 86$ & 58 & CASS & $\mathbf{T}$ & \\
\hline TE-107-AP-39 & 8/8/86 & 59 & CASS & $\bar{T}$ & \\
\hline TE-107-AP-39 & $6 / 21 / 86$ & 58 & CASS & $\bar{T}$ & \\
\hline TE-107.AP-3B & 6/25/36 & 53 & CASS & $T$ & \\
\hline TE-107-AP-39 & $7 / 5 / 86$ & 69 & CASS & $T$ & \\
\hline TE-107-AP-39 & $7 / 6 / 86$ & 60 & CASS & $T$ & \\
\hline TE-107-AP-39 & $7 / 18 / 86$ & 60 & CASS & $\bar{T}$ & \\
\hline TE-107.AP.39 & $7 / 20 / 86$ & 62: & CASS & $T$ & \\
\hline TE-107-AP-39 & $8 / 2 / 36$ & 60 & CASS & $T$ & \\
\hline TE-107-AP-39 & $8 / 3 / 66$ & 62 & CASS & $\bar{T}$ & \\
\hline TE-107-AP-39 & $8 / 13 / 86$ & 62 & CASS & $T$ & \\
\hline TE-107-AP-39 & $8 / 17 / 86$ & 86 & CASS & $T$ & \\
\hline TE-107.AP-39 & $8 / 27 / 86$ & 64 & CASS & $\mathbf{T}$ & \\
\hline TE-107-AP-39 & $9 / 3 / 86$ & 62 & CASS & $T$ & \\
\hline TE-107-AP-39 & $9 / 23 / 86$ & 64 & CASS & $T$ & \\
\hline TE-107-AP-39 & $10 / 3 / 86$ & 63 & CASS & $T$ & \\
\hline TE-107-AP-39 & $10 / 9 / 86$ & 108 & CASS & $\mathbf{S}$ & Data point did not agree with local trend \\
\hline TE-107-AP-39 & $10 / 11 / 86$ & 62 & CASS & $\mathbf{T}$ & \\
\hline TE-107-AP-39 & $10 / 16 / 36$ & 62 & CASS & $\mathbf{T}$ & \\
\hline TE-107.AP-39 & $10 / 24 / 86$ & 63. & CASS & $\bar{T}$ & \\
\hline TE-107-AP-39 & 10/26/36 & 62: & CASS & $\mathbf{T}$ & \\
\hline TE-107-AP-39 & $11 / 6 / B 6$ & 60 & CASS & $\bar{T}$ & \\
\hline TE-107.AP-39 & 11/9/BB & 59: & CASS & $T$ & \\
\hline TE-107.AP-39 & $11 / 19 / 86$ & 58 & CASS & $T$ & \\
\hline TE-107-AP-39 & $11 / 26 / 36$ & 57 & CASS & $\mathbf{T}$ & \\
\hline TE-107-AP-39 & $12 / 2 / 36$ & 60 & CASS & $T$ & \\
\hline TE-107-AP-39 & $12 / 11 / 86$ & 56 & CASS & $\mathbf{T}$ & \\
\hline TE-107-AP-39 & $12 / 17 / 36$ & 55 & CASS & $\mathbf{T}$ & \\
\hline TE-107-AP-39 & $12 / 24 / 86$ & 55 & CASS & $\mathbf{T}$ & \\
\hline TE-107-AP-39 & $12 / 28 / 86$ & 54 & CASS & $T$ & \\
\hline TE-107-AP-39 & $7 / 4 / 87$ & 64) & CASS & $\mathbf{T}$ & \\
\hline TE-107-AP-39 & $7 / 5 / 37$ & 66 & CASS & $T$ & \\
\hline TE-107-AP-39 & $7 / 13 / 87$ & 66 & CASS & $T$ & \\
\hline TE-107-AP-39 & $7 / 25 / 87$ & 66 & CASS & $T$ & \\
\hline TE-107-AP-39 & 7/27/87 & 68 & CASS & $T$ & \\
\hline TE-107-AP-39 & $8 / 5 / 87$ & 67 & CASS & $\mathbf{T}$ & \\
\hline TE-107-AP-39 & $8 / 14 / 87$ & 68 & CASS & $T$ & \\
\hline TE-107-AP-39 & $8 / 22 / 87$ & 68 & CASS & $\mathbf{T}$ & \\
\hline
\end{tabular}




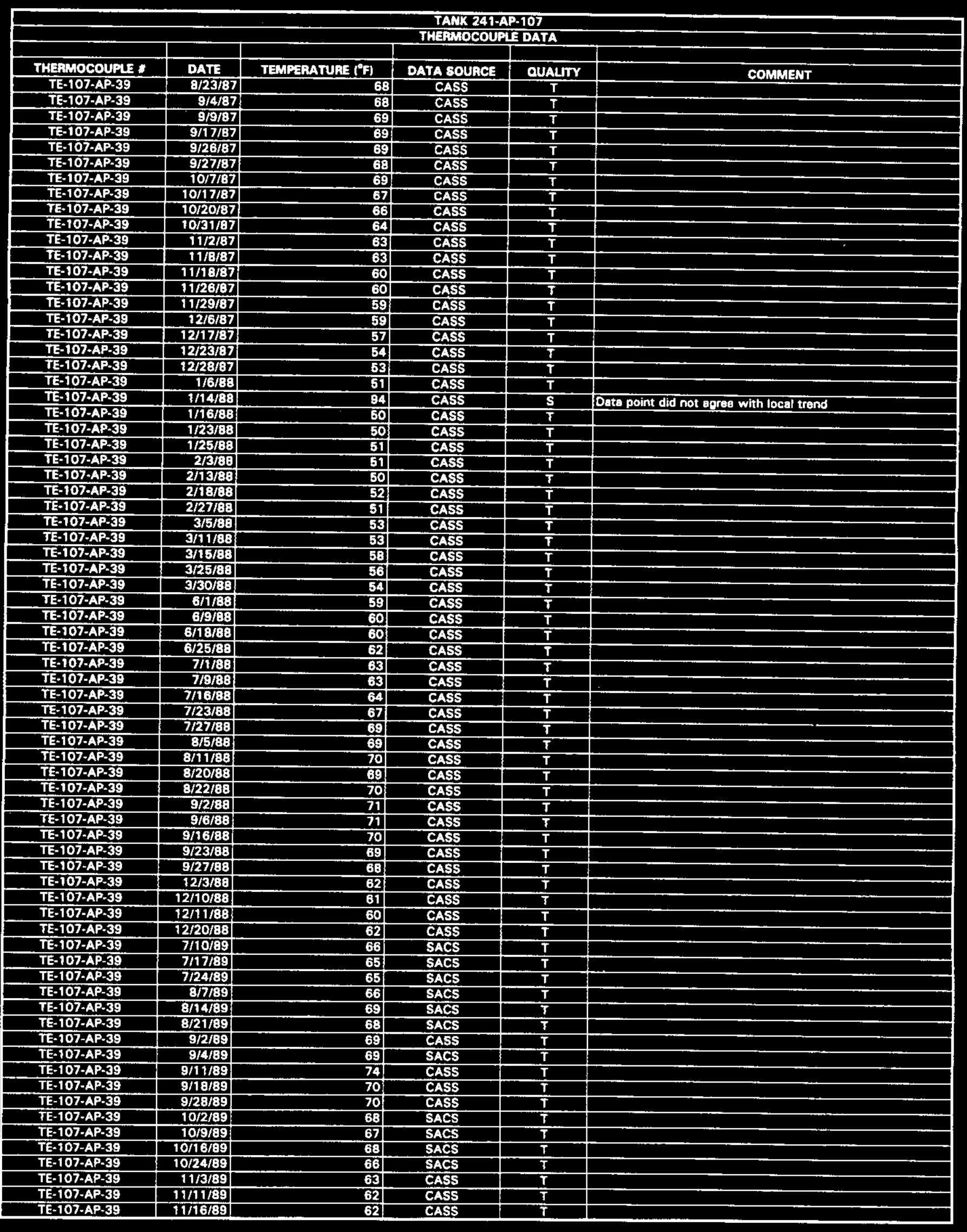

Oate obtained from Computer Automated Surveillance System (CASS), April 1995; and Surveillance Analysis Computer System (SACS), June 1995. 


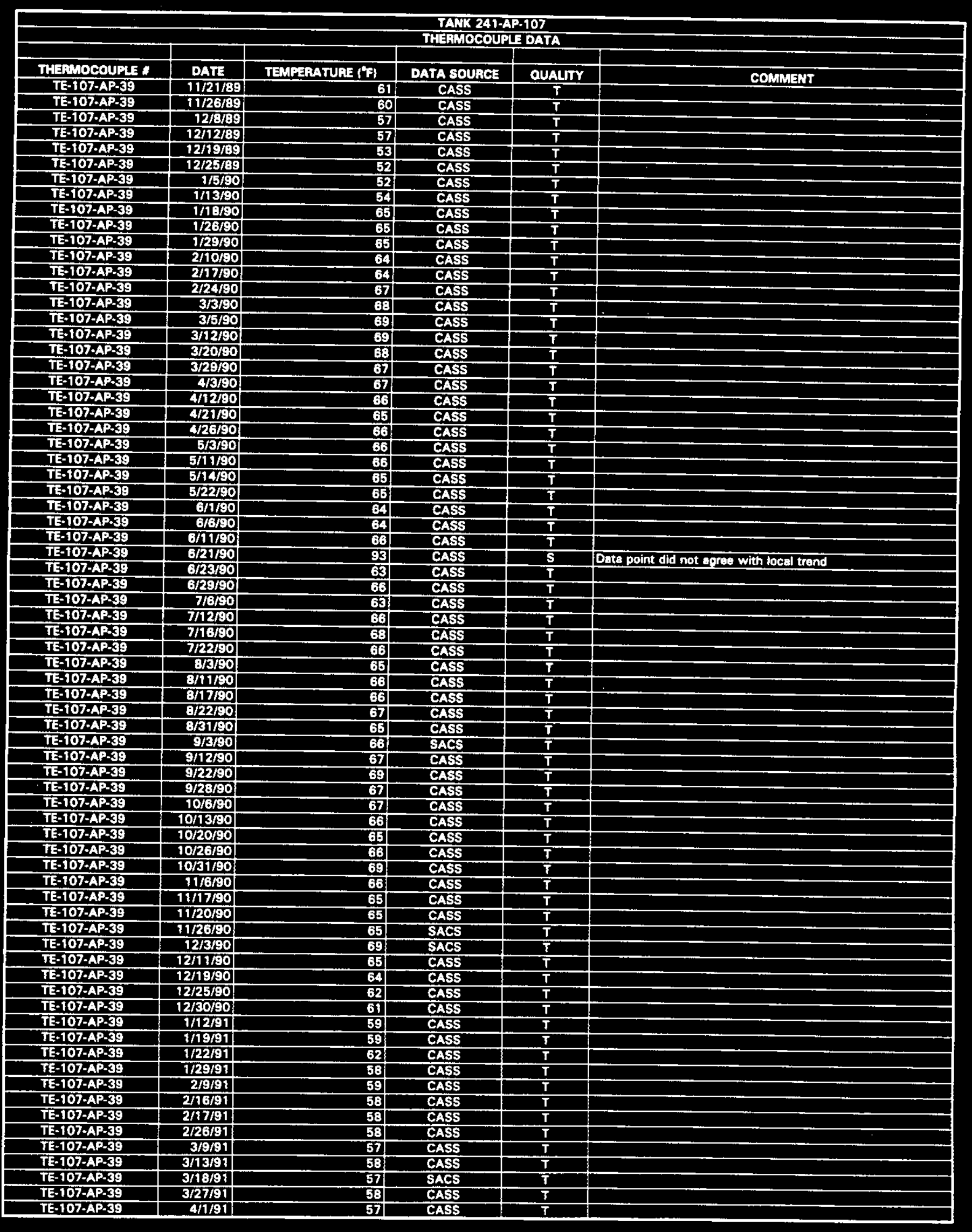

Date obteined from Computer Automated Surveillance System (CASS), April 1995; and Surveillance Analysis Computer Syatem (SACS), June 1995.

D-580 


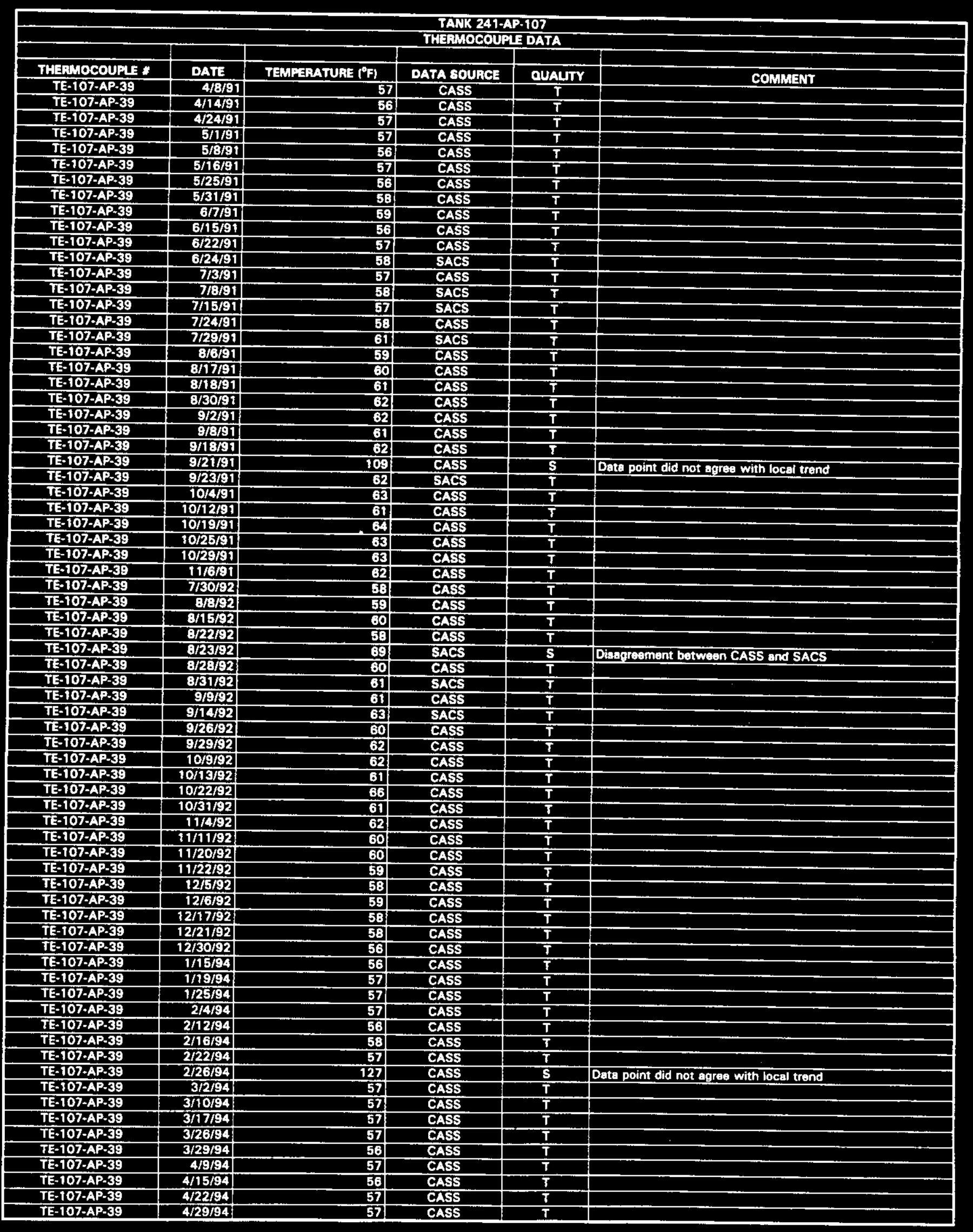




\begin{tabular}{|c|c|c|c|c|c|}
\hline & & & $\begin{array}{l}\text { TANK } 241-A \\
\text { THERimocoUP }\end{array}$ & $\frac{107}{\text { DATA }}$ & \\
\hline & & & & & 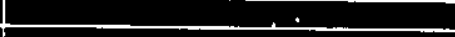 \\
\hline THERMOCOUPLE * & DATE & TEMPERATURE ( $\left.{ }^{\circ} \mathrm{F}\right)$ & DATA BOUACE & OUALTY & COMMENT \\
\hline TE-107-AP-39 & $5 / 2 / 94$ & 58 & CASS & $\mathbf{T}$ & \\
\hline TE-107-AP-39 & $5 / 14 / 94$ & 57 & CASS & $\mathbf{T}$ & \\
\hline TE-107-AP-39 & $5 / 16 / 94$ & 94 & CASS & $\mathbf{S}$ & Data point did not agree with local trend \\
\hline TE-107-AP-39 & $5 / 20 / 94$ & 91 & CASS & $T$ & \\
\hline TE-107-AP-39 & $5 / 22 / 94$ & 89 & CASS & $\mathbf{T}$ & \\
\hline TE-107-AP-39 & $6 / 2 / 94$ & 89 & CASS & $\mathbf{T}$ & \\
\hline TE-107-AP-39 & $6 / 8 / 94$ & 87. & CASS & $\bar{T}$ & \\
\hline TE-107-AP-39 & $6 / 14 / 94$ & 86 & CASS & $\bar{T}$ & \\
\hline TE-107-AP-39 & $6 / 16 / 94$ & 56 & CASS & $\mathbf{s}$ & Data point did not egres with local trend \\
\hline TE-107-AP-39 & $6 / 17 / 94$ & 56 & CASS & $\mathbf{S}$ & Data point did not agres with locsl trend \\
\hline TE-107-AP-39 & $6 / 20 / 94$ & 58 & CASS & $\mathbf{S}$ & Data point did not agree with local trend \\
\hline TE-107-AP-39 & $6 / 24 / 94$ & 89 & CASS & $\mathbf{T}$ & \\
\hline TE-107-AP-39 & $6 / 27 / 94$ & 88 & CASS & $\mathbf{T}$ & \\
\hline TE-107-AP-39 & $77 / 94$ & 87 & CASS & $\bar{T}$ & \\
\hline TE-107-AP-39 & $7 / 13 / 94$ & 87 & CASS & $T$ & \\
\hline TE-107-AP-39 & $7 / 18 / 94$ & 86 & CASS & $\bar{T}$ & \\
\hline TE-107-AP-39 & $7 / 24 / 94$ & 85 & CASS & $\bar{T}$ & \\
\hline TE-107-AP-39 & $7 / 31 / 94$ & 99 & CASS & $\mathbf{S}$ & Date point did not apres with local trend \\
\hline TE-107-AP-39 & $8 / 1 / 94$ & 87 & CASS & $\mathbf{T}$ & \\
\hline TE-107-AP-39 & $8 / 9 / 94$ & 91 & CASS & T & \\
\hline TE-107-AP-39 & $8 / 1794$ & 89 & CASS & $\mathbf{T}$ & \\
\hline TE-107-AP-39 & $8 / 22 / 94$ & 80 & CASS & $\mathbf{T}$ & \\
\hline TE-107-AP-39 & $9 / 3 / 94$ & 80. & CASS & $\mathbf{T}$ & \\
\hline TE-107-AP-39 & $9 / 10 / 94$ & 92 & CASS & $\mathbf{T}$ & \\
\hline TE-107-AP-39 & 9/15/94 & 92 & CASS & $T$ & \\
\hline TE-107-AP-39 & $9 / 23 / 94$ & 92 & CASS & $T$ & \\
\hline TE-107-AP-39 & $9 / 50 / 94$ & 96 & CASS & $\mathbf{T}$ & \\
\hline TE-107-AP-39 & 1018194 & 96 & CASS & $\mathbf{T}$ & \\
\hline TE-107-AP-39 & $10 / 12 / 94$ & 96 & CASS & $\mathbf{T}$ & \\
\hline TE-107-AP-39 & $10 / 22 / 94$ & 98 & CASS & $T$ & \\
\hline TE-107-AP-39 & $10 / 29 / 94$ & 99 & CASS & $T$ & \\
\hline TE-107-AP-39 & $11 / 5 / 94$ & 88 & CASS & $T$ & \\
\hline TE-107-AP-39 & $11 / 11 / 94$ & 89 & CASS & $T$ & \\
\hline TE-107-AP-39 & $11 / 14 / 94$ & 87 & CASS & $T$ & \\
\hline TE-107.AP-39 & $11 / 22 / 94$ & 97 & CASS & $T$ & \\
\hline$T E-107-A P-39$ & $11 / 29 / 94$ & 91 & CASS & $T$ & \\
\hline TE-107-AP-39 & $12 / 4 / 94$ & 95 & CASS & $T$ & \\
\hline TE-107-AP-39 & $12 / 14 / 94$ & 89 & CASS & $T$ & \\
\hline TE-107-AP-39 & $12 / 19 / 94$ & 92 & CASS & $T$ & \\
\hline TE-107-AP-39 & $12 / 30 / 94$ & 92 & CASS & $\bar{T}$ & \\
\hline TE-107-AP-39 & 1/4/95 & 94 & CASS & $T$ & \\
\hline TE-107-AP-39 & $1 / 14 / 95$ & 90 & CASS & $\bar{T}$ & \\
\hline TE-107-AP-39 & $3 / 16 / 95$ & 89 & CASS & $T$ & \\
\hline TE-107-AP-39 & $1 / 26 / 95$ & 98 & CASS & $T$ & \\
\hline TE-107-AP-39 & $2 / 2 / 95$ & 96 & CASS & $T$ & \\
\hline TE-107-AP-39 & $2 / 9 / 95$ & 98 & CASS & $\mathbf{T}$ & \\
\hline TE-107-AP-39 & $2 / 14 / 95$ & 104 & CASS & $T$ & \\
\hline TE-107-AP-39 & $2 / 24 / 95$ & 93 & CASS & $T$ & \\
\hline TE-107-AP-39 & 2/28/95 & 98 & CASS & $T$ & \\
\hline TE-107-AP-39 & . आ7/ब5 & 96 & CASS & $T$ & \\
\hline TE-107-AP-39 & $3 / 16 / 95$ & 91 & CASS & $T$ & \\
\hline TE-107-AP-39 & $3 / 25 / 95$ & 91 & CASS & $T$ & \\
\hline TE-107-AP-39 & $3 / 30 / 95$ & 91 & CASS & $T$ & \\
\hline & & & & & \\
\hline TE-107-AP-40 & $4 / 5 / 66$ & 54 & CASS & $T$ & \\
\hline TE-107-AP-40 & $4 / 7 / 86$ & 56 & CASS & $T$ & \\
\hline TE-107-AP-40 & $5 / 18 / 86$ & 56 & CASS & $T$ & \\
\hline TE-107-AP-40 & $5 / 24 / 86$ & 58 & CASS & $\mathbf{T}$ & \\
\hline TE-107-AP-40 & $5 / 25 / 86$ & 58 & CASS & $T$ & \\
\hline TE-107-AP-40 & $5 / 26 / 86$ & 123 & CASS & $\mathbf{S}$ & Data point did not agree with local trend \\
\hline TE-107-AP-40 & $6 / 4 / 86$ & 58 & CASS & $T$ & \\
\hline TE-107-AP-40 & 8/9/86: & 59 & CASS & $T$ & \\
\hline TE-107-AP-40 & 6/21/86 & 58 & CASS & $\mathbf{T}$ & \\
\hline TE-107-AP-40 & 6/25/86 & 58 & CASS & $T$ & \\
\hline TE-107.AP-40 & $7 / 5 / 86$ & 59 & CASS & $\bar{T}$ & \\
\hline TE-107-AP-40 & $7 / 6 / 86$ & 60 & CASS & $T$ & \\
\hline TE-107-AP-40 & $7 / 19 / 86$ & 60 & CASS & $T$ & \\
\hline TE-107-AP-40 & $7 / 20 / 86$ & 62 & CASS & $\mathbf{T}$ & \\
\hline TE-107-AP-40 & $7 / 31 / 86$ & 63 & CASS & $T$ & \\
\hline TE-107-AP-40 & $8 / 9 / 86$ & 62 & CASS & $T$ & \\
\hline TE-107-AP-40 & 8/16/86 & 61 & CASS & $T$ & \\
\hline TE-107-AP-40 & B/17/86 & 62 & CASS & $T$ & \\
\hline TE-107-AP-40 & $8 / 27 / 86$ & 64 & CASS & $\bar{T}$ & \\
\hline
\end{tabular}




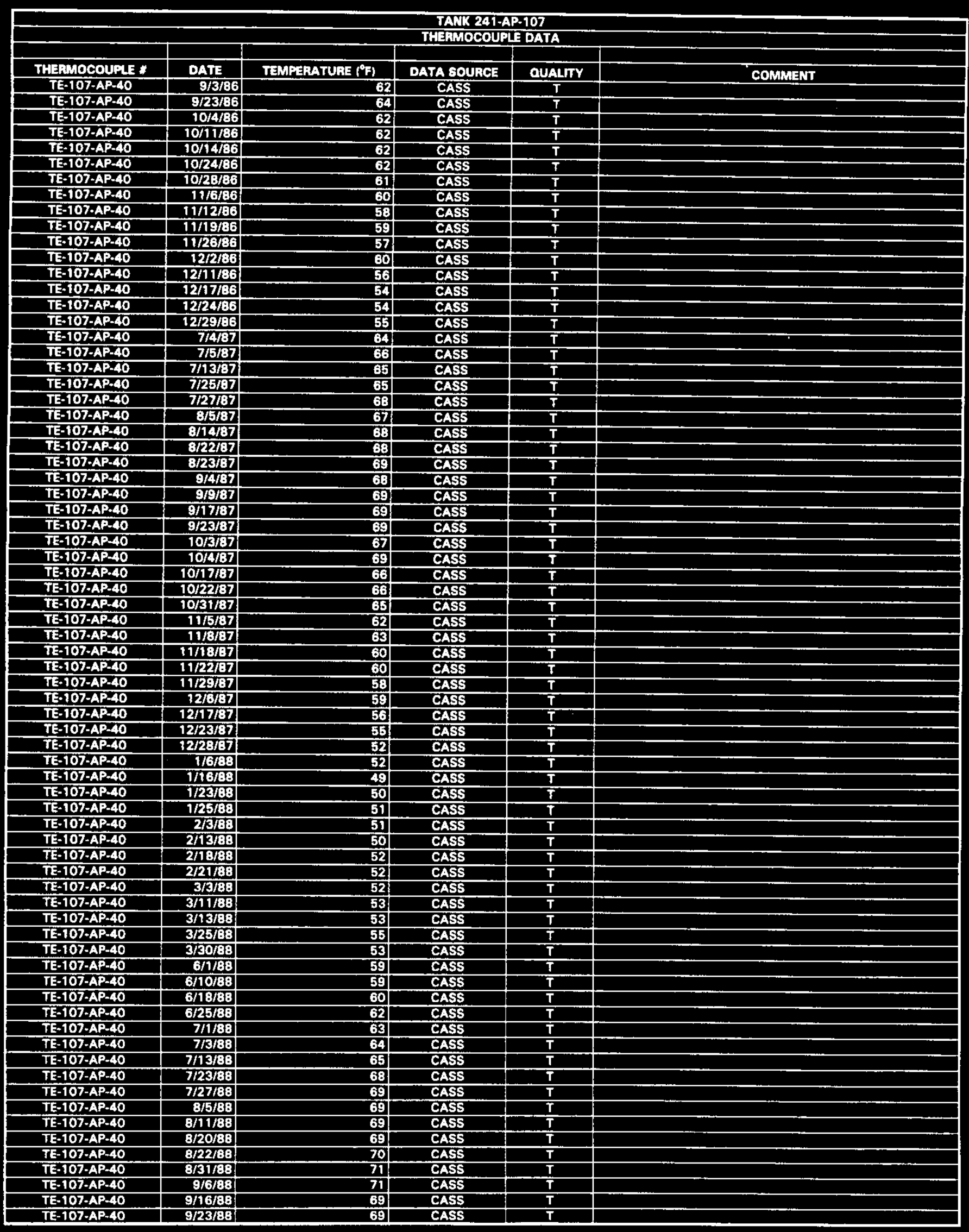

Data obtained from Computer Automated Surveillance Syotem (CASS), April 1995; and Surveillance Analysis Computer Syatem (SACS), June 1995. D-583 


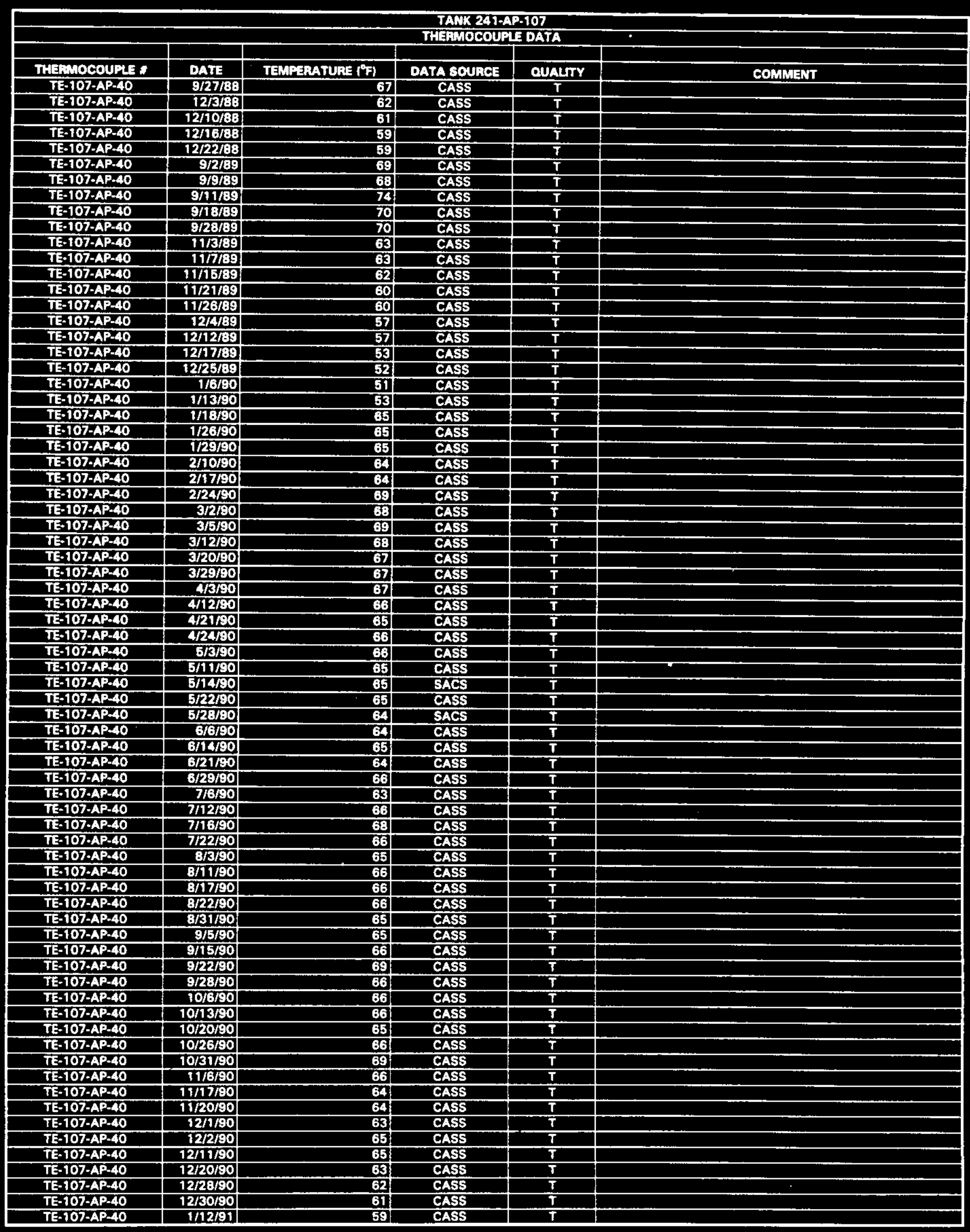

Data obtainad from Computer Automated Survallance Syatem (CASS), April 1995; and Survaillance Analysis Computer System (SACS), June 1995. 


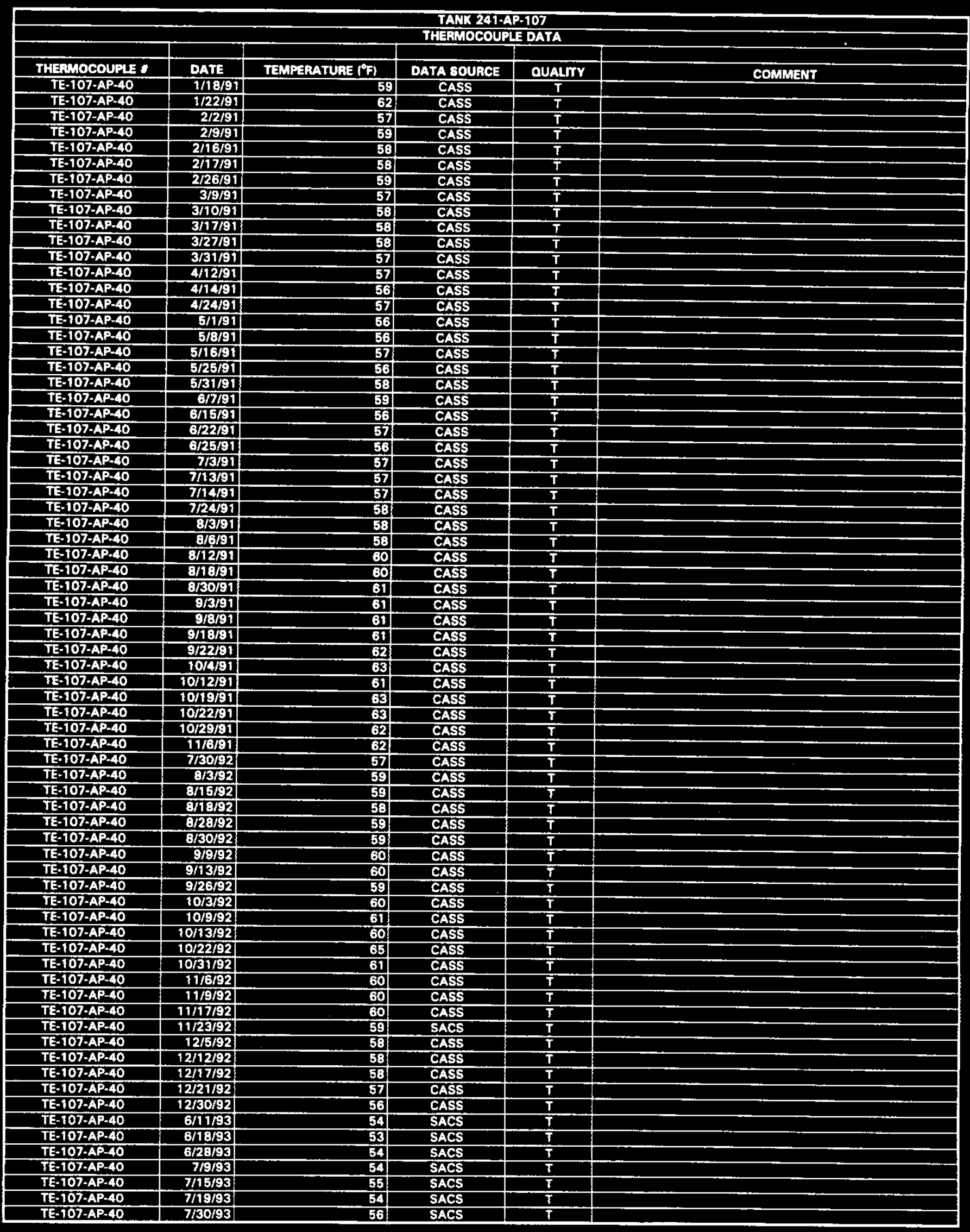




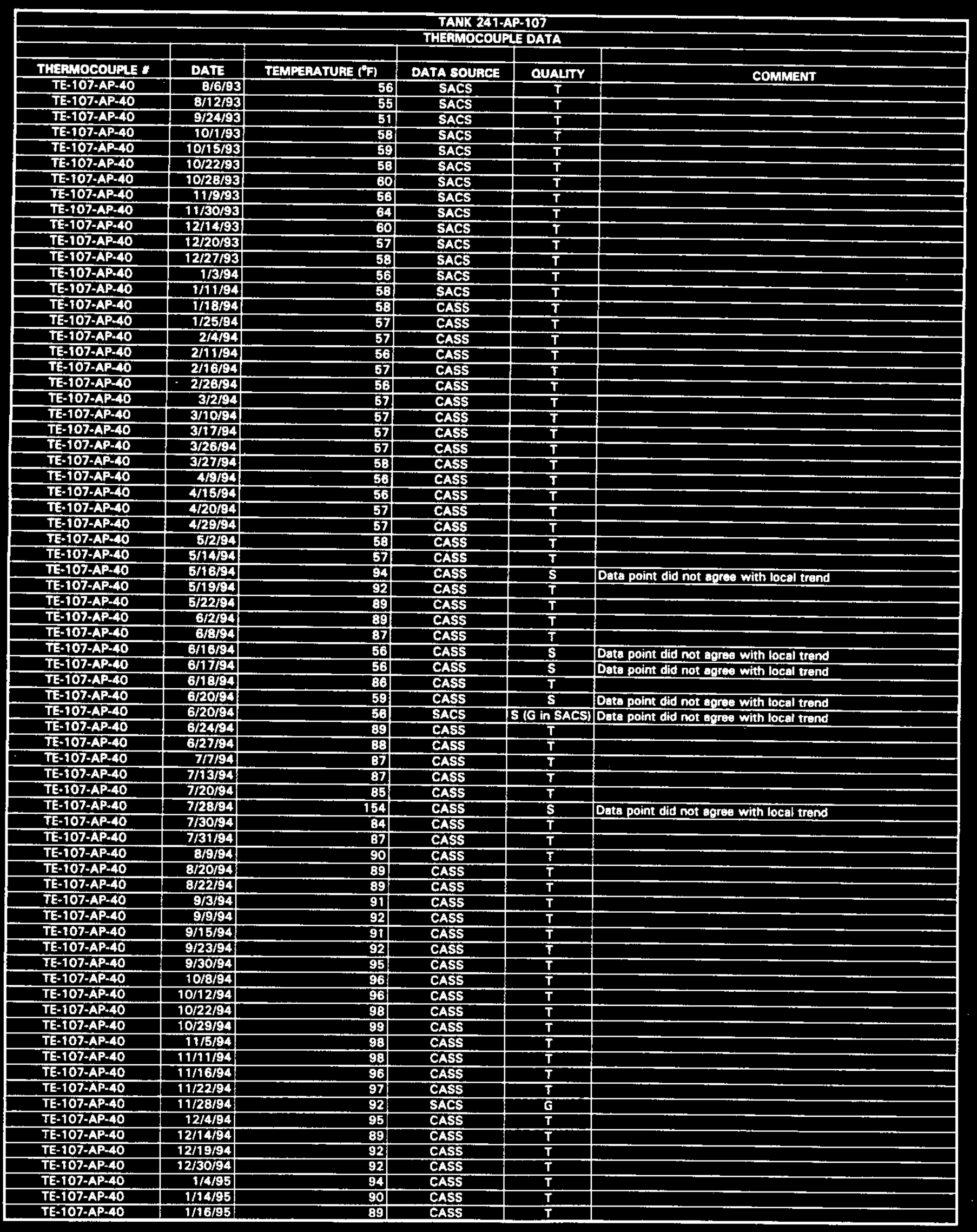




\begin{tabular}{|c|c|c|c|c|c|}
\hline \multicolumn{6}{|c|}{$\begin{array}{l}\text { TAWK 241-AP-107 } \\
\text { THERMOCOUPLE DATA }\end{array}$} \\
\hline THEEMOCOUPL : & DATE & TEMPERATUAE ("F) & DATA SOUACE & QUALTY & COMMENT \\
\hline IE-107-AP-40 & $1 / 26 / 95$ & 98 & CASS & $T$ & \\
\hline TE-107-AP-40 & $2 / 2 / 95$ & 96 & CASS & $\mathbf{T}$ & \\
\hline TE-107-AP-40 & $2 / 9 / 95$ & 98 & CASS & $T$ & \\
\hline TE-107-AP-40 & 2/14/95 & 103 & CASS & $\bar{T}$ & \\
\hline TE-107-AP-40 & $2 / 23 / 95$ & 128 & CASS & $\mathbf{S}$ & Data point did not agree with local trend \\
\hline TE-107-AP-40 & $2 / 24 / 95$ & 93 & CASS & $\bar{T}$ & \\
\hline TE-107-AP-40 & $2 / 27 / 95$ & 98 & CASS & $\mathbf{T}$ & \\
\hline TE-107-AP-40 & $3 / 7 / 55$ & 96 & CASS & $T$ & \\
\hline TE-107-AP-40 & $3 / 16 / 95$ & 91 & CASS & $T$ & \\
\hline TE-107-AP-40 & $3 / 25 / 95$ & 81 & CASS & $\mathbf{T}$ & \\
\hline TE-107-AP:40 & 3/30/95 & 91 & CASS & $\mathbf{T}$ & \\
\hline TE-107.AP-40 & $4 / 3 / 95$ & 82 & SACS & G & \\
\hline TE-107-AP-40 & $4 / 10 / 95$ & 84 & SACS & G & \\
\hline TE-107-AP-40 & $4 / 17 / 95$ & 85 & SACS & $\mathbf{G}$ & \\
\hline $\mathrm{TE}-107 \cdot A \mathrm{P}-40$ & a/24/85 & 78 & SACS & G & \\
\hline IE-107-AP-40 & $5 / 1 / 195$ & 82 & SACS & G & \\
\hline TE-107-AP-40 & $5 / 8 / 95$ & 79 & SACS & 6 & \\
\hline$T E-107-A P-40$ & 5/16/95 & 79 & SACS & G & \\
\hline TE-107.AP-40 & $5 / 22 / 95$ & 78 & SACS & G & \\
\hline TE-107.AP=40 & $5 / 29 / 95$ & 80 & SACS & G & \\
\hline TE-107-AP-40 & $8 / 5 / 95$ & 84 & SACS & $G$ & \\
\hline TE-107.AP-40 & 6/13/35 & 78 & SACS & G & \\
\hline TE-107-AP-40 & 6/19/95 & 88 & SACS & G & \\
\hline & & & & & \\
\hline TE-107-AP-41 & $4 / 5 / 86$ & 54 & CASS & $T$ & \\
\hline TE-107-AP-41 & $4 / 7 / 86$ & 57 & CASS & $\mathbf{T}$ & \\
\hline TE-107.AP-41 & $5 / 15 / 86$ & 56 & CASS & $\bar{T}$ & \\
\hline TE-107-AP-4I & $5 / 24 / 86$ & 58 & CASS & $T$ & \\
\hline TE-107-AP-41 & $5 / 25 / 36$ & 58 & CASS & $\bar{T}$ & \\
\hline TE-107-AP:41 & $6 / 4 / 36$ & 58 & CASS & $\mathbf{T}$ & \\
\hline$T E-107-A P-41$ & $6 / 8 / 86$ & 59: & CASS & $\mathbf{T}$ & \\
\hline$T E-107-A P-41$ & $6 / 21 / 86$ & 58 & CASS & $T$ & \\
\hline TE-107.AP-41 & $6 / 25 / 86$ & 38 & CASS & $T$ & \\
\hline TE-107-AP-41 & $7 / 5 / 36$ & 59 & CASS & $T$ & \\
\hline TE-107.AP-41 & $7 / 6 / 86$ & 60 & CASS & $T$ & \\
\hline TE-107-AP-41 & 7/19/86 & 59 & CASS & $T$ & \\
\hline TE-107-AP-41 & $7 / 20 / 86$ & 62 & CASS & $T$ & \\
\hline TE-107-AP-41 & $8 / 2 / 86$ & 60 & CASS & $\mathbf{T}$ & \\
\hline TE-107-AP+41 & $8 / 3 / 86$ & 63 & CASS & $\mathbf{T}$ & \\
\hline TE-107-AP-A1 & $8 / 13 / 86$ & 62 & CASS & $\bar{T}$ & \\
\hline TE-107-AP-41 & $8 / 17 / 86$ & 62 & CASS & $T$ & \\
\hline$T E-107-A P-41$ & $8 / 27 / 86$ & 64 & CASS & $T$ & \\
\hline TE-107-AP-41 & $9 / 1 / 86$ & 64 & CASS & $T$ & \\
\hline TE-107-AP-41 & 9/23/86 & 64 & CASS & $\mathbf{T}$ & \\
\hline TE-107.AP-41 & $10 / 2 / 36$ & 104 & CASS & s. & Data point did not agrae with local trend \\
\hline TE-107-AP-41 & $10 / 4 / 86$ & 62 & CASS & $\bar{T}$ & \\
\hline TE-107-AP-41 & $10 / 11 / 86$ & 62 & CASS & $T$ & \\
\hline TE-107-AP-41 & $10 / 14 / 86$ & 62 & CASS & $T$ & \\
\hline TE-107-AP-41 & $10 / 24 / 86$ & 63 & CASS & $T$ & \\
\hline TE-107-AP-41 & $11 / 1 / 86$ & 62 & CASS & $T$ & \\
\hline TE-107-AP-41 & $11 / 6 / 86$ & 60 & CASS & $T$ & \\
\hline TE-107-AP-41 & $11 / 7 / 36$ & 100 & CASS & $\overline{\mathbf{s}}$ & Data point did not agree with locel trend \\
\hline TE-107-AP-41 & $11 / 9 / 86$ & 59 & CASS & $T$ & \\
\hline TE-107-AP-41 & $11 / 19 / 86$ & 59 & CASS & $T$ & \\
\hline TE-107-AP-41 & $11 / 26 / 86$ & 59 & CASS & $\mathbf{T}$ & \\
\hline TE-107-AP-41 & $12 / 2 / 86$ & 60 & CASS & $T$ & \\
\hline TE-107-AP-41 & $12 / 11 / 86$ & 56 & CASS & $T$ & \\
\hline TE-107-AP-41 & $12 / 17 / 86$ & 55 & CASS & $T$ & \\
\hline TE-107-AP-41 & $12 / 24 / 86$ & 55 & CASS & $T$ & \\
\hline TE-107-AP-41 & $12 / 29 / 86$ & 55 & CASS & $T$ & \\
\hline TE-107-AP-41 & 7/4/87 & 64 & CASS & $T$ & \\
\hline TE-107-AP-41 & 7/5/87 & 66 & CASS & $\bar{T}$ & \\
\hline TE-107-AP-41 & 7/12/87 & 66 & CASS & $T$ & \\
\hline TE-107-AP-41 & $7 / 25 / 87$ & 65 & CASS & $T$ & \\
\hline TE-107-AP-41 & $\begin{array}{l}7 / 26 / 87 \\
\end{array}$ & 68 & CASS & $T$ & \\
\hline TE-107-AP-41 & $8 / 5 / 87$ & 67 & CASS & $T$ & \\
\hline TE-107-AP-41 & $8 / 14 / 87$ & 68 & CASS & $T$ & \\
\hline TE-107-AP-41 & $8 / 22 / 87$ & 68) & CASS & $T$ & \\
\hline TE-107-AP-41 & $8 / 23 / 87$ & 69 & CASS & $T$ & \\
\hline TE-107-AP-41 & $9 / 3 / 87$ & 68 & CASS & $T$ & \\
\hline TE-107-AP-41 & $9 / 9 / 87$ & 69 & CASS & $T$ & \\
\hline TE-107-AP-41 & 9/17/87 & 69 & CASS & $\bar{T}$ & \\
\hline TE-107-AP-41 & $9 / 26 / 87$ & 69 & CASS & $\bar{T}$ & \\
\hline
\end{tabular}




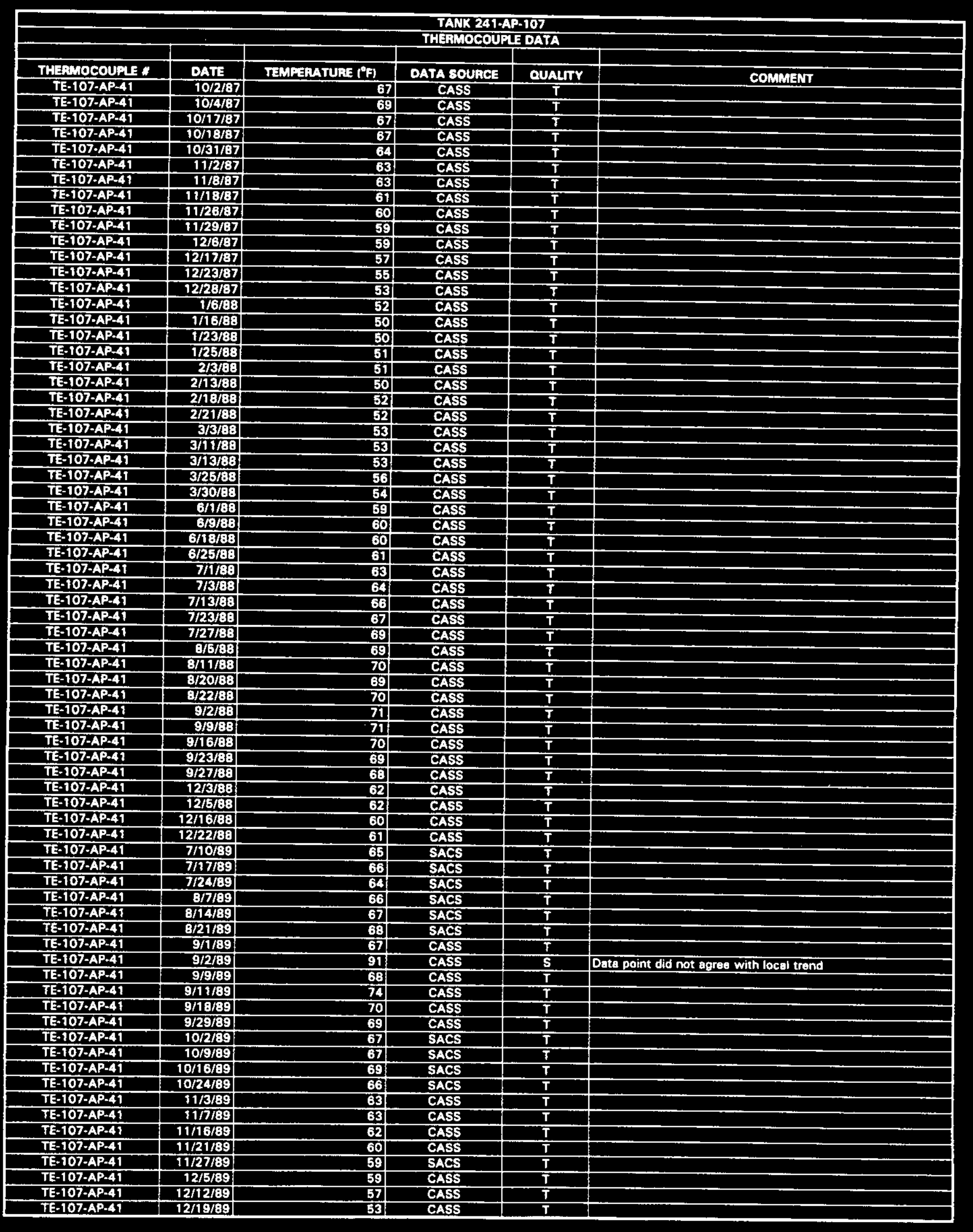

Data obtained from Computer Automated Surveillance System (CASS), April 1995; and Surveillance Analysis Computer Syatem (SACS), June 1995.

D -588 


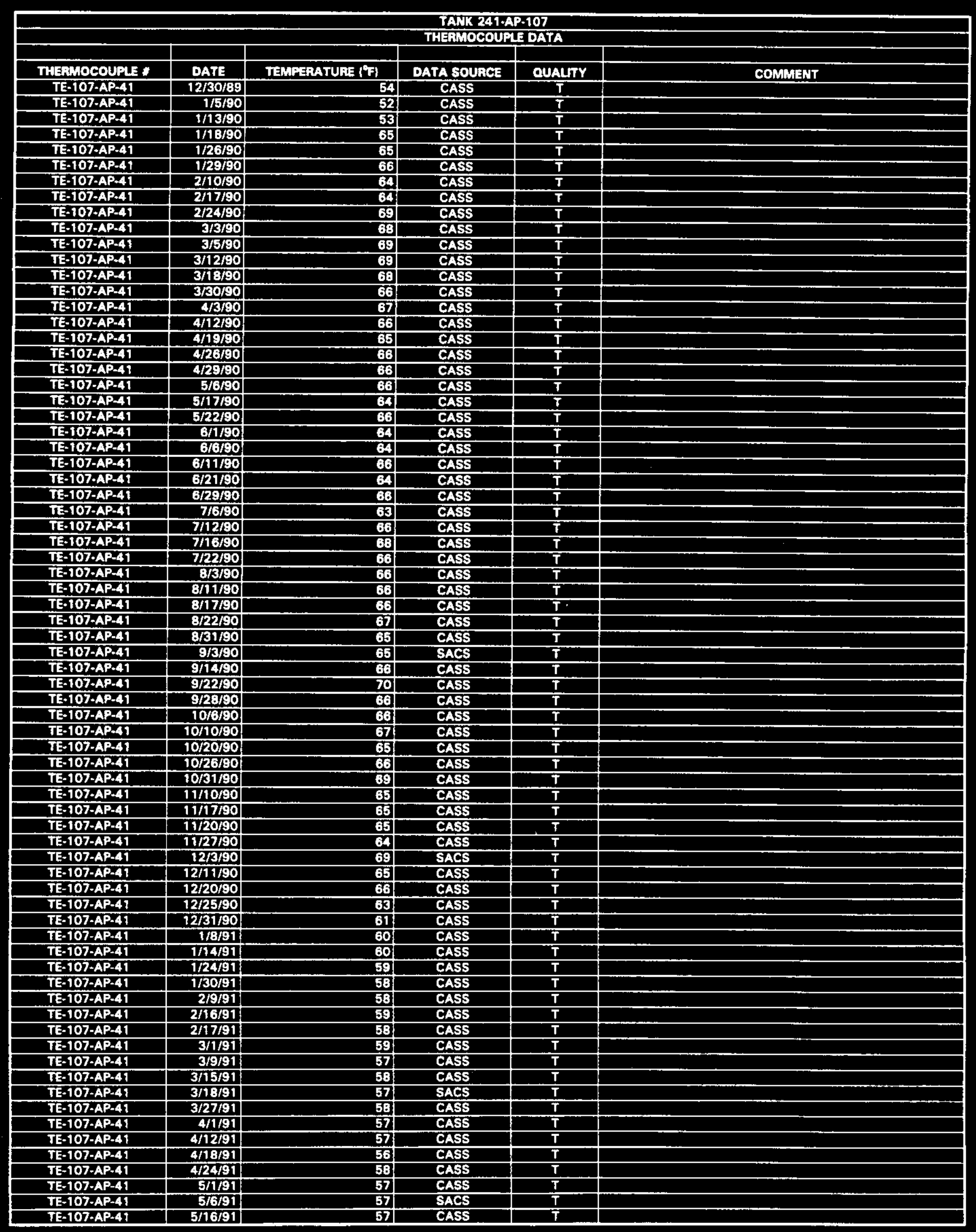




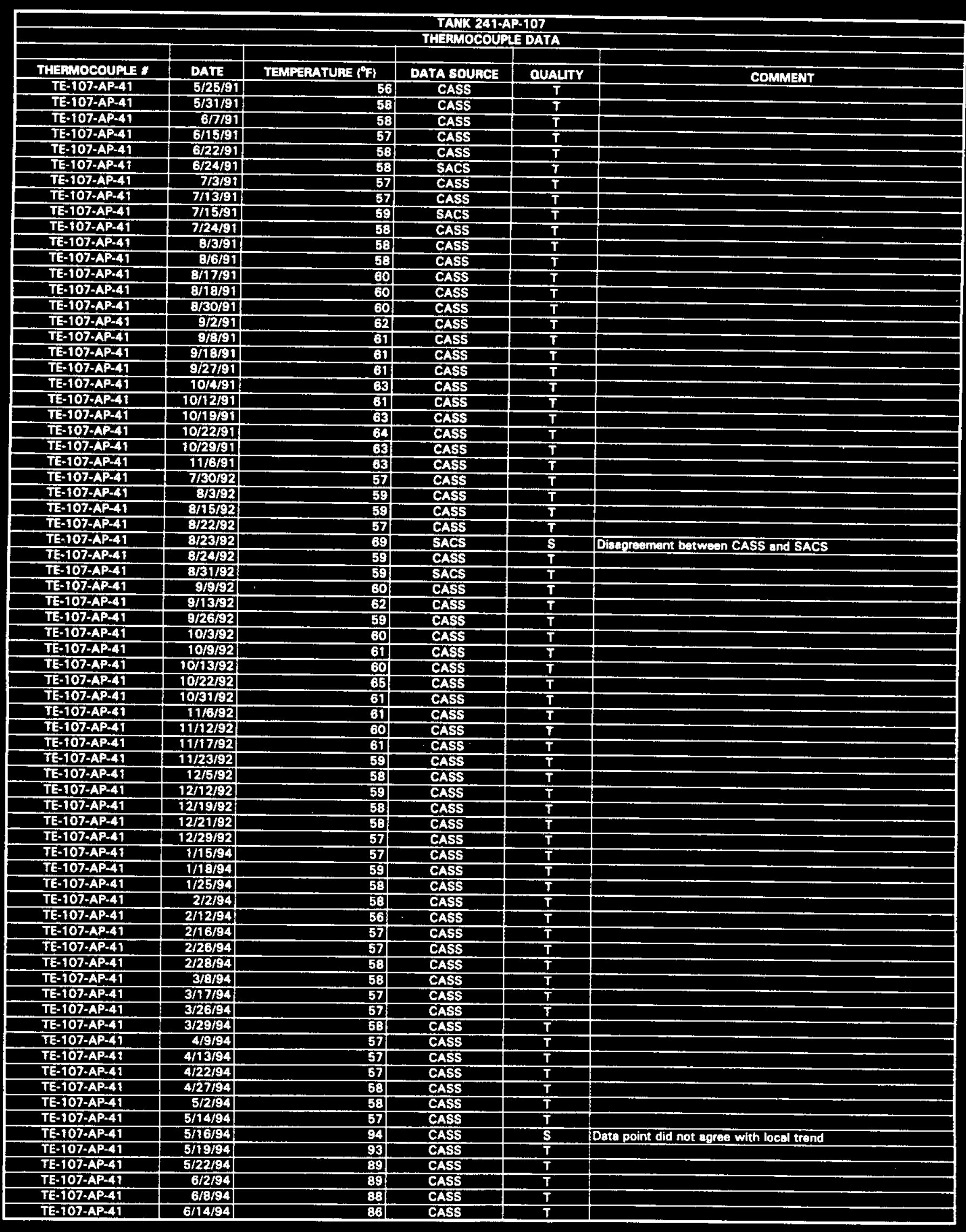

Data obtained from Computer Automated Surveillance System (CASS), April 1995; and Surveillance Anelysis Computer System (SACS), June 1995. D-590 


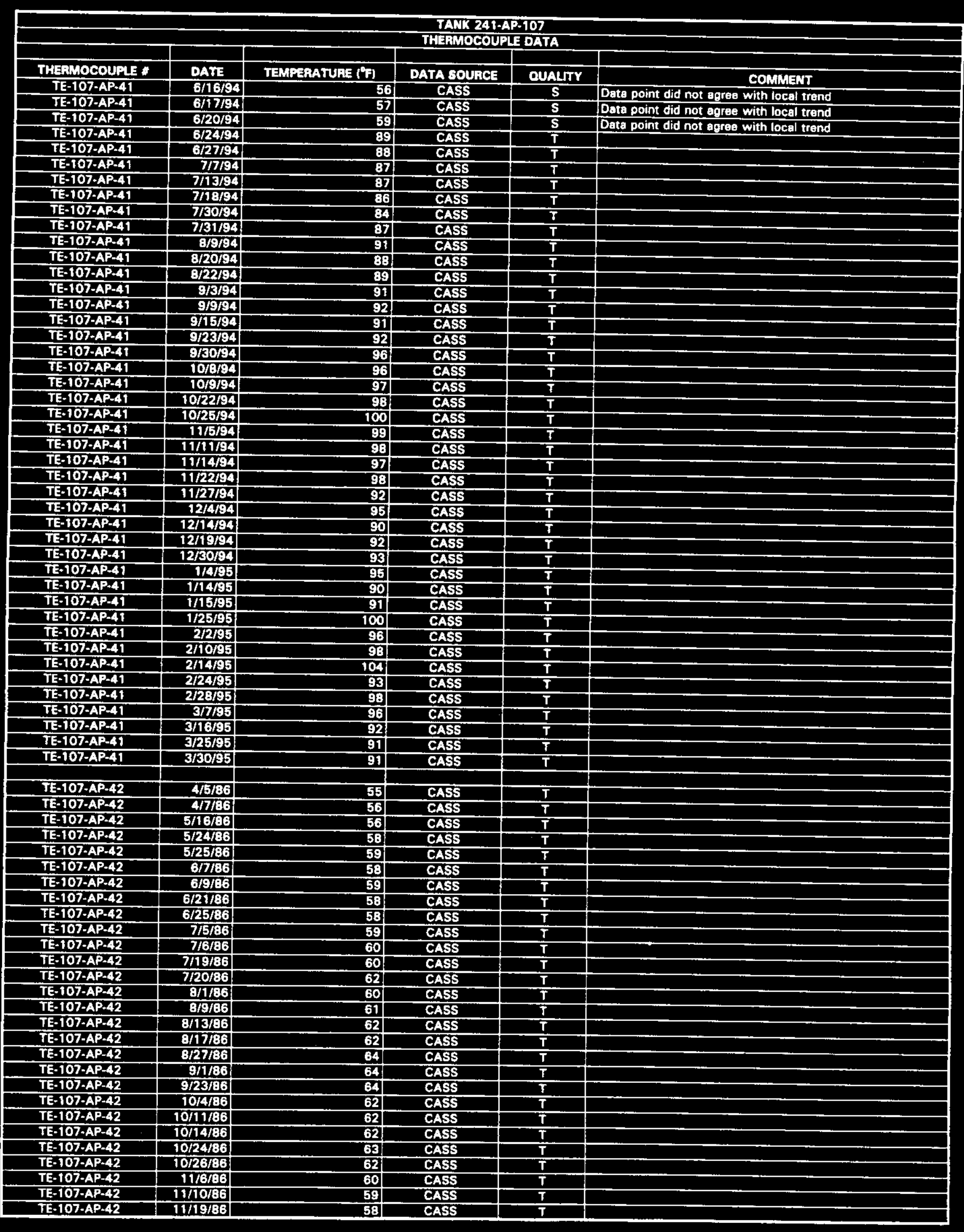

Data obtained from Computer Automated Surveillence System (CASS), April 1995; and Surveillance Analysis Computer System (SACS), June 1995. D-591 


\begin{tabular}{|c|c|c|c|c|c|}
\hline \multicolumn{6}{|c|}{$\begin{array}{l}\text { TANK 241.AP.107 } \\
\text { THERMOCOUPLEATA }\end{array}$} \\
\hline & & & DATA SOURCE & & \\
\hline$\frac{\text { TE-107-AP-42 }}{\text { TE-107-AP-42 }}$ & $11 / 26 / 86$ & 59 & $\frac{\text { CASS }}{\text { CASS }}$ & $T$ & \\
\hline$\frac{T E-107-A P-42}{T E-107-A P-42}$ & $\begin{array}{l}12 / 2 / 86 \\
2 / 11 / 86\end{array}$ & \begin{tabular}{l|}
61 \\
56
\end{tabular} & CASS & $T$ & \\
\hline TE-107-AP-42 & $\frac{12 / 11 / 86}{12 / 17 / 86}$ & $\frac{56}{55}$ & $\begin{array}{l}\text { CASS } \\
\text { CASS }\end{array}$ & $T$ & \\
\hline TE-107-AP=42 & $\frac{12 / 17 / 86}{12 / 24 / 86}$ & $\frac{55}{55}$ & $\begin{array}{l}\text { CASS } \\
\text { CASS }\end{array}$ & $T$ & \\
\hline TE-107-AP-42 & $12 / 29 / 66$ & 55 & $\begin{array}{l}\text { CASS } \\
\text { CASS }\end{array}$ & $\frac{T}{T}$ & \\
\hline TE-107-AP-42 & $7 / 4 / 87$ & 64 & CASS & $T$ & \\
\hline$\overline{T E}-107-A P-42$ & $7 / 5 / 87$ & 66 & CASS & $T$ & \\
\hline TE-107-AP-42 & $7 / 12 / 87$ & 66 & CASS & $T$ & \\
\hline TE-107-AP-42 & $7 / 25 / 87$ & 85 & CASS & $T$ & \\
\hline TE-107-AP-42 & $7 / 27 / 87$ & 68 & CASS & $T$ & \\
\hline TE-107.AP-42 & $8 / 5 / 87$ & 67 & CASS & $T$ & \\
\hline TE-107-AP-42 & $8 / 11 / 87$ & 69 & CASS & $T$ & \\
\hline TE-107-AP.42 & $8 / 22 / 87$ & 68 & CASS & $\bar{T}$ & \\
\hline TE-107.AP-42 & $8 / 23 / 37$ & 69 & CASS & $T$ & \\
\hline TE-107-AP-42 & $8 / 3 / 37$ & 68 & CASS & $T$ & \\
\hline TE-107-AP-42 & $9 / 9 / 87$ & 69 & CASS & $\bar{T}$ & \\
\hline TE-107-AP-42 & $8 / 17 / 87$ & 69 & CASS & $\mathbf{T}$ & \\
\hline TE-107-AP-42 & $8 / 26 / 87$ & 60 & CASS & $T$ & \\
\hline$T E-107-A P-42$ & $9 / 27 / 87$ & 67 & CASS & $T$ & \\
\hline TE-107-AP-42 & $10 / 4 / 87$ & 69 & CASS & $T$ & \\
\hline TE-107-AP-42 & $10 / 17 / 87$ & 67 & CASS & $T$ & \\
\hline$T E-107-A P-42$ & $10 / 22 / 87$ & 66 & CASS & $T$ & \\
\hline TE-107-AP-42 & $10 / 31 / 87$ & 64 & CASS & $T$ & \\
\hline$T E-107 \cdot A P-42$ & $11 / 3 / 87$ & 63 & CASS & $T$ & \\
\hline TE-107-AP-42 & 11/8/87 & 63 & CASS & $T$ & \\
\hline TE-107-AP-A2 & $11 / 16 / 87$ & 61 & CASS & $T$ & \\
\hline$T E-107-A P-42$ & $11 / 26 / 87$ & 60 & CASS & $\bar{T}$ & \\
\hline TE-107-AP-42 & $11 / 29 / 87$ & 59 & CASS & $\bar{T}$ & \\
\hline TE-107-AP-42 & $12 / 6 / 87$ & 59 & CASS & $\bar{T}$ & \\
\hline TE-107-AP-42 & $12 / 17 / 67$ & 57 & CASS & $T$ & \\
\hline TE-107-AP- 42 & $12 / 24 / 87$ & 56 & CASS & $\bar{T}$ & \\
\hline TE-107-AP-42 & $12 / 28 / 87$ & 53 & CASS & $\bar{T}$ & \\
\hline TE-107-AP-42 & $1 / 6 / 88$ & 52 & CASS & $T$ & \\
\hline TE-107-AP=42 & 1/16/88 & 50 & CASS & $T$ & \\
\hline TE-107-AP-42 & $1 / 23 / 88$ & 50 & CASS & $T$ & \\
\hline TE-107-AP-42 & $1 / 25 / 88$ & 51! & CASS & $T$ & \\
\hline$T E-107-A P-42$ & $2 / 3 / 38$ & 50 & CASS & $T$ & \\
\hline TE-107-AP-A2 & $2 / 13 / 88$ & 50 & CASS & $T$ & \\
\hline TE-107-AP-42 & $2 / 14 / 88$ & 53 & CASS & $T$ & \\
\hline TE-107-AP-42 & $2 / 21 / 88$ & 52 & CASS & $\bar{T}$ & \\
\hline TE-107-AP-42 & $3 / 3 / 68$ & 5 & CASS & $\bar{T}$ & \\
\hline TE-107-AP-42 & $3 / 11 / 89$ & 53 & CASS & $T$ & \\
\hline TE-107-AP-42 & $3 / 13 / 8$ & 52 & CASS & $T$ & \\
\hline$T E-107-A P-42$ & $3 / 24 / 88$ & 86 & CASS & $\mathbf{S}$ & Data point did not agree with local frend \\
\hline TE= 107-AP-42 & $3 / 25 / 8$ & 57 & CASS & $\bar{T}$ & \\
\hline TE-107-AP-42 & $3 / 30 / 88$ & 54 & CASS & $T$ & \\
\hline$T E-107-A P-42$ & $6 / 1 / 88$ & 5 & CASS & $\bar{T}$ & \\
\hline TE-107-AP-42 & 6/10/88 & 80: & CASS & $T$ & \\
\hline TE-107-AP-42 & 6/18/88 & 80 & CASS & $\bar{T}$ & \\
\hline TE-107-AP-42 & $6 / 25 / 88$ & 62 & CASS & $\bar{T}$ & \\
\hline TE-107-AP-42 & $7 / 1 / 38$ & 83 & CASS & $T$ & \\
\hline TE-107.AP-42 & $7 / 3 / 88$ & 64 & CASS & $\mathbf{T}$ & \\
\hline TE-107-AP-A2 & 7/13/88 & 65 & CASS & $T$ & \\
\hline TE-107.AP-42 & $7 / 23 / 88$ & 67 & CASS & $T$ & \\
\hline TE-107-AP-42 & $7 / 27 / 88$ & 69 & CASS & $T$ & \\
\hline TE-107-AP-42 & $8 / 5 / 88$ & 69 & CASS & $T$ & \\
\hline TE-107-AP-42 & $8 / 11 / 88$ & 70 & CASS & $T$ & \\
\hline TE-107-AP-42 & $8 / 20 / 88$ & 69 & CASS & $T$ & \\
\hline TE-107-AP-42 & $8 / 27 / 83$ & 69 & CASS & $T$ & \\
\hline TE-107-AP-42 & $9 / 2 / 88$ & 71 & CASS & $T$ & \\
\hline TE-107-AP-42 & $\begin{array}{ll}9 / 9 / 8 B \\
\end{array}$ & 71 & CASS & $T$ & \\
\hline TE-107-AP-42 & $9 / 16 / 88$ & 70 & CASS & $T$ & \\
\hline TE-107-AP-42 & 9/23/83 & 69 & CASS & $T$ & \\
\hline TE-107-AP-42 & $9 / 27 / 88$ & 68 & CASS & $T$ & \\
\hline TE-107-AP-42 & $12 / 3 / 88$ & 62 & CASS & $T$ & \\
\hline TE-107-AP-42 & $12 / 3 / 83$ & 61 & CASS & $T$ & \\
\hline TE-107-AP-42 & $12 / 11 / 88$ & 60 & CASS & $T$ & \\
\hline TE-107-AP-42 & $12 / 22 / 88$ & 60 & CASS & $T$ & \\
\hline TE-107-AP-42 & $9 / 2 / 89$ & 69 & CASS & $T$ & \\
\hline TE-107-AP-42 & 9/9/89 & 68 & CASS & $T$ & \\
\hline TE-107-AP-42 & $9 / 11 / 89$ & 74 & CASS & $\bar{T}$ & \\
\hline TE-107-AP-42 & $9 / 18 / 39$ & 70 & CASS & $T$ & \\
\hline
\end{tabular}




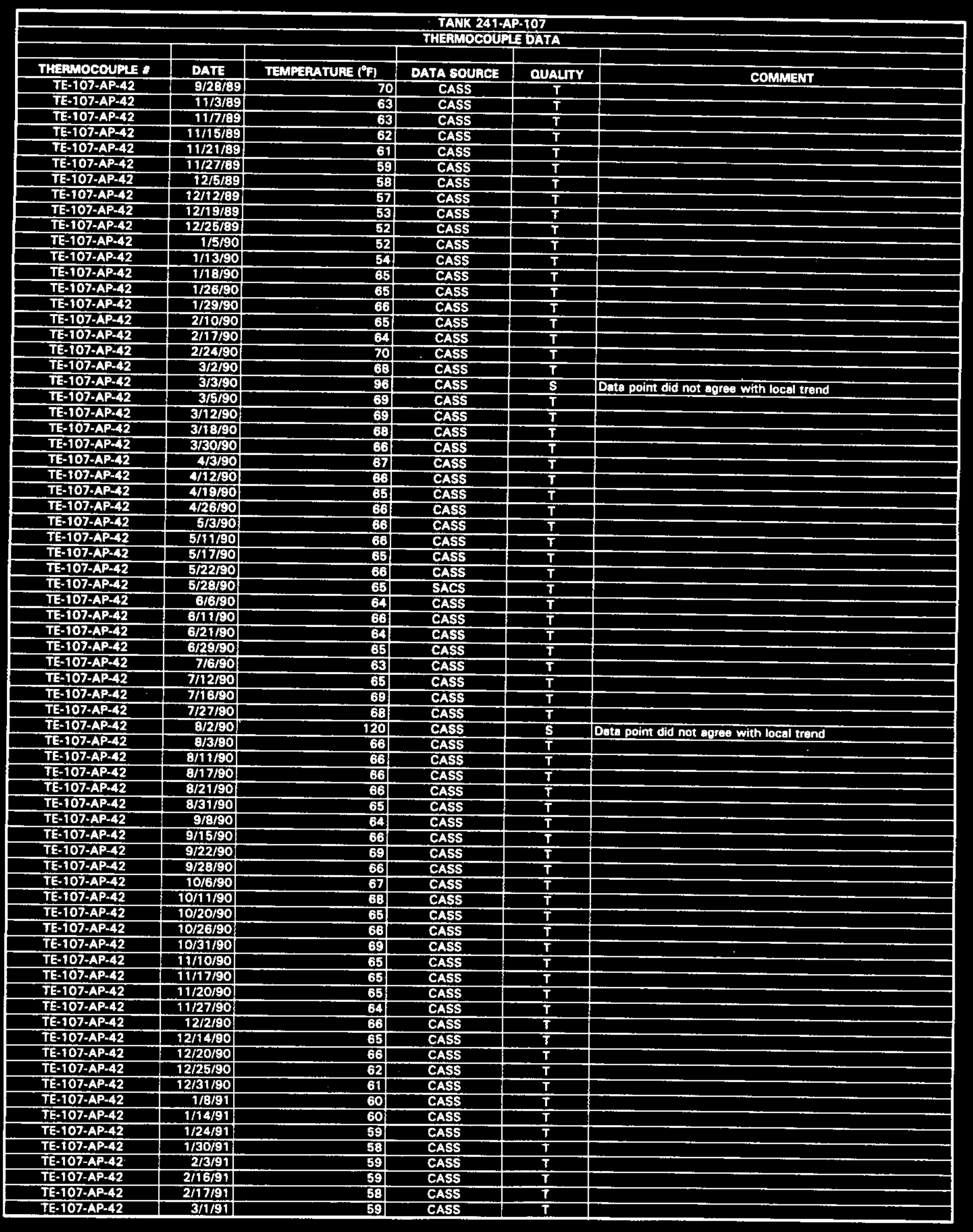

Data obtained from Computer Automated Survellance Syatem (CASS), April 1995; and Survallance Anatysis Computer System (SACS), June 1995.

D-593 


\begin{tabular}{|c|c|c|c|c|c|}
\hline \multicolumn{6}{|c|}{$\begin{array}{l}\text { TANK 24T-AP.107 } \\
\text { THERMOCOUFI DATA }\end{array}$} \\
\hline THERMOCOUPLE * & DATE & TEMPERATUAE ( $\left.{ }^{\circ} \mathrm{F}\right)$ & DATA SOUACE & oustry & \\
\hline TE-107-AP-42 & 3/9/91 & 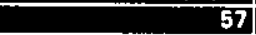 & CASS & $\frac{T}{T}$ & COMMENT \\
\hline TE-107-AP-42 & 3/15/91 & 58 & CASS & $T$ & \\
\hline TE-107.AP-42 & 3/17/91 & 57 & CASS & $T$ & \\
\hline TE-107-AP-42 & 3/27/91 & 58 & CASS & $T$ & \\
\hline TE-107-AP-42 & 4/1/91 & 57 & CASS & $T$ & \\
\hline TE-107-AP-42 & 4/12/91 & 57 & CASS & $\bar{T}$ & \\
\hline TE-107-AP-42 & $4 / 18 / 91$ & 56 & CASS & $\bar{T}$ & \\
\hline TE-107-AP-42 & $4 / 24 / 91$ & 57 & CASS & $T$ & \\
\hline TE-107.AP-42 & 5/1/91 & 57 & CASS & $T$ & \\
\hline TE-107-AP-42 & $5 / 10 / 91$ & 57 & CASS & $\mathbf{T}$ & \\
\hline TE-107-AP-42 & 5/16/91 & 57 & CASS & $T$ & \\
\hline TE-107-AP-42 & 5/25/31 & $\mathbf{5 6}$ & CASS & $T$ & \\
\hline TE-107-AP.42 & $5 / 31 / 91$ & 58 & CASS & $T$ & \\
\hline TE-107-AP-42 & $6 / 7 / 91$ & 57 & CASS & $\mathbf{T}$ & \\
\hline TE-107-AP-42 & 6/15/91 & 57. & CASS & $\mathbf{T}$ & \\
\hline$T E-107-A P-42$ & 6/22/91 & 58 & CASS & $\bar{T}$ & \\
\hline IE-107.AP-42 & 6/23/91 & .57 & CASS & $T$ & \\
\hline TE.107-AP-42 & $7 / 3 / 91$ & 57 & CASS & $\mathbf{T}$ & \\
\hline TE-107-AP-42 & $7 / 13 / 91$ & 57 & CASS & $T$ & \\
\hline TE-107-AP-A2 & 7/4/491 & 57 & CASS & $T$ & \\
\hline TE-107.AP-A2 & $7124 / 91$ & 58 & CASS & $\mathrm{T}$ & \\
\hline TE-107.AP-42 & $8 / 2 / 91$ & 58 & CASS & $T$ & \\
\hline TE-107.AP-42 & $8 / 3 / 91$ & 67 & CASS & $\mathbf{S}$ & Data point did not apree with locel trend \\
\hline TE-107.AP-42 & 8/6/91 & 58) & CASS & $T$ & \\
\hline TE-107.AP-42 & 8/17/91 & 60 & CASS & $T$ & \\
\hline TE-107-AP-A2 & 8/19/91 & 39. & CASS & $T$ & \\
\hline TE-107-AP-42 & $8 / 20 / 91$ & 129 & CASS & $\mathbf{S}$ & Data point did not agrea with local trend \\
\hline TE-107.AP-42 & $8 / 30 / 31$ & 60 & CASS & $\bar{T}$ & \\
\hline TE-107-AP-42 & $8 / 3 / 91$ & 81 & CASS & $\bar{T}$ & \\
\hline TE-107-AP-42 & 8/8/91 & 61 & CASS & $\mathbf{T}$ & \\
\hline TE-107-AP-42 & 9/18/91 & 61 & CASS & $\mathbf{T}$ & \\
\hline TE-107-AP-42 & $9 / 27 / 91$ & 63 & CASS & $T$ & \\
\hline TE-107-AP-42 & $10 / 4 / 91$ & 63 & CASS & $T$ & \\
\hline TE-107-AP-42 & 10/12/91 & 81 & CASS & $\mathbf{T}$ & \\
\hline$T E=107-A P-42$ & $10 / 19 / 91$ & 64 & CASS & $\mathbf{T}$ & \\
\hline TE-107-AP-42 & $10 / 25 / 91$ & 86 & CASS & $T$ & \\
\hline$T E-107-A P-42$ & 10/29/91 & 63 & CASS & $T$ & \\
\hline TE-107.AP-42 & $11 / 6 / 91$ & 64 & CASS & $\mathbf{T}$ & \\
\hline TE-107-AP-42 & $7 / 30 / 92$ & 58: & CASS & $\mathbf{T}$ & \\
\hline TE-107-AP-42 & $8 / 3 / 82$ & 59 & CASS & $T$ & \\
\hline TE-107.AP-42 & $8 / 15 / 92$ & 59 & CASS & $\bar{T}$ & \\
\hline$T E-107 \cdot A P-42$ & $8 / 22 / 32$ & 57 & CASS & $\mathbf{T}$ & \\
\hline IE-107-AP-42 & $8 / 24 / 92$ & 59 & CASS & $T$ & \\
\hline TE-107-AP-42 & $8 / 30 / 92$ & 59 & CASS & $T$ & \\
\hline TE-107-AP-A2 & 9/9/92 & 61 & CASS & $\mathbf{T}$ & \\
\hline$T E=107-A P-42$ & $8 / 13 / 92$ & 63 & CASS & $T$ & \\
\hline IE-107.AP-42 & $9 / 26 / 92$ & 58 & CASS & $T$ & \\
\hline TE-107-AP-42 & $10 / 3 / 92$ & 60 & CASS & $T$ & \\
\hline TE-107-AP-42 & $10 / 9 / 92$ & $6 !$ & CASS & $T$ & \\
\hline TE-107-AP-42 & $10 / 13 / 92$ & 60 & CASS & $T$ & \\
\hline TE-107.AP-42 & $10 / 22 / 92$ & 64 & CASS & $T$ & \\
\hline TE-107-AP-A2 & $10 / 29 / 92$ & 61 & CASS & $T$ & \\
\hline TE-107-AP-42 & $17 / 6 / 92$ & 61) & CASS & $\bar{T}$ & \\
\hline TE-107-AP-42 & $11 / 12 / 92$ & 60 & CASS & $\bar{T}$ & \\
\hline TE-107-AP-42 & $11 / 17 / 92$ & 61 & CASS & $\mathbf{T}$ & \\
\hline TE-107.AP-42 & $11 / 23 / 92$ & 59 & CAS5 & $\mathbf{I}$ & \\
\hline TE-107-AP-42 & $12 / 5 / 92$ & 58 & CASS & $T$ & \\
\hline TE-107-AP-42 & $12 / 10 / 92$ & 59 & CASS & $\bar{T}$ & \\
\hline TE-107-AP-42 & $12 / 14 / 92$ & 59 & SACS & $T$ & \\
\hline TE-107.AP-42 & $12 / 21 / 92$ & 58 & CASS & $T$ & \\
\hline TE-107-AP-42 & $12123 / 92$ & 98 & CASS & $\mathbf{S}$ & Dara point did not agree with tocal trend \\
\hline TE-107-AP-42 & $12 / 30 / 92$ & 57 & CASS & $T$ & \\
\hline TE-107.AP-42 & 6/11/93 & 54 & SACS & $\mathbf{T}$ & $\cdot$ \\
\hline TE-107-AP-42 & 6/18/93 & 53: & SACS & $\bar{T}$ & \\
\hline TE-107-AP-42 & 6/28/93 & 54 & SACS & $\mathbf{T}$ & \\
\hline TE-107.AP-42 & $7 / 9 / 93$ & $54 !$ & SACS & $\mathbf{T}$ & \\
\hline TE-107-AP-42 & $7 / 15 / 93$ & 55 & SACS & $\mathbf{T}$ & \\
\hline TE-107-AP-42 & $7 / 19 / 93$ & 55: & SACS & $\mathbf{T}$ & \\
\hline TE-107.AP-42 & $7 / 30 / 93$ & 56 & SACS & $T$ & \\
\hline TE-107-AP-42 & $8 / 6 / 83$ & 55 & SACS & $\bar{T}$ & \\
\hline$T E-107-A P-42$ & $8 / 12 / 93$ & 55 & SACS & $T$ & \\
\hline TE-107-AP-42 & $9 / 24 / 93$ & 52 & SACS & $\mathbf{T}$ & \\
\hline TE-107-AP-42 & 10/193 & 57 & SACS & $\mathbf{T}$ & \\
\hline
\end{tabular}




\begin{tabular}{|c|c|c|c|c|c|}
\hline \multirow{2}{*}{\multicolumn{6}{|c|}{$\begin{array}{l}\text { TANK 241-AP-107 } \\
\text { THEFMOCOUPLE DATA }\end{array}$}} \\
\hline & & & & & \\
\hline THERMOCOUPLE : & DATE. & TEMPERATURE $\left({ }^{\circ} \mathrm{F}\right)$ & DATA SOURCE & OUAUTY & COMMENT \\
\hline TE-107.AP:42 & $10 / 15 / 93$ & 58 & SACS & $T$ & \\
\hline TE-107-AP-42 & $10 / 22 / 93$ & 59 & SACS & $T$ & \\
\hline TE-107-AP-42 & 10/28/93 & 59 & SACS & $T$ & \\
\hline TE-107-AP-42 & 11/9/93 & 56 & SACS & $\mathbf{T}$ & \\
\hline TE-107-AP=42 & $11 / 30 / 93$ & 64 & SACS & $\mathbf{T}$ & \\
\hline TE-107-AP-42 & $12 / 14 / 93$ & 60 & SACS & $T$ & \\
\hline$T E-107-A P-42$ & $12 / 20 / 93$ & 58 & SACS & $T$ & \\
\hline IE-107.AP-42 & $12 / 27 / 93$ & 58 & SACS & $T$ & \\
\hline TE-107-AP-42 & $3 / 3 / 94$ & 56 & SACS & $\bar{T}$ & \\
\hline TE-107-AP-A2 & $1 / 11 / 94$ & 59 & SACS & $T$ & \\
\hline IE-107-AP-42 & 1/18/94 & 59 & CASS & $T$ & \\
\hline TE-107-AP-42 & $1 / 23 / 94$ & 57 & CASS & $T$ & \\
\hline TE-107-AP-42 & $2 / 4 / 94$ & 58 & CASS & $T$ & \\
\hline TE-107-AP-42 & $2 / 12 / 94$ & 56 & CASS & $T$ & \\
\hline TE-107-AP-42 & $2 / 16 / 94$ & 57 & CASS & $T$ & \\
\hline TE-107-AP-42 & $2 / 26 / 94$ & 57 & CASS & $T$ & \\
\hline TE-107-AP-42 & $3 / 5 / 94$ & 57 & CASS & $T$ & \\
\hline TE-107-AP-42 & $3 / 7 / 94$ & 58 & CASS & $T$ & \\
\hline TE-107-AP-42 & $3 / 17 / 94$ & 57 & CASS & $T$ & \\
\hline$T E-107-A P-42$ & $3 / 26 / 94$ & 57 & CASS & $\bar{T}$ & \\
\hline TE-107-AP-42 & $3 / 29 / 94$ & 58 & CASS & $T$ & \\
\hline$T E-107 \cdot A P-42$ & $4 / 8 / 94$ & 57 & CASS & $T$ & \\
\hline TE-107-AP-42 & $4 / 15 / 94$ & 57 & CASS & $T$ & \\
\hline TE-107-AP-42 & $4 / 22194$ & 57 & CASS & $T$ & \\
\hline TE-107-AP-42 & $4 / 27 / 94$ & 58 & CASS & $T$ & \\
\hline TE-107-AP-42 & $5 / 2 / 94$ & 59 & CASS & $T$ & \\
\hline TE-107-AP-42 & $5 / 14 / 94$ & 57 & CASS & $\bar{T}$ & \\
\hline TE-107-AP.42 & $5 / 16 / 94$ & 94 & CASS & $\overline{\mathbf{s}}$ & Data point did not sgree with local trend \\
\hline $\mathrm{TE}-107-A \mathrm{P}-42$ & $5 / 19 / 94$ & 93 & CASS & $\mathbf{T}$ & \\
\hline$T E \cdot 107 \cdot A P-42$ & $5 / 22 / 94$ & 88 & CASS & $\mathbf{T}$ & \\
\hline TE-107-AP-42 & $6 / 2 / 94$ & 89 & CASS & $T$ & \\
\hline TE-107-AP.A2 & 6/8/94 & 88 & CASS & $\overline{\mathbf{T}}$ & \\
\hline TE-107.AP-A2 & $6 / 16 / 94$ & G7 & CASS & $\mathbf{s}$ & Data point did not apree with local trend \\
\hline TE-107.AP-42 & 6/17/94 & 57 & CASS & $\mathrm{S}$ & Date point did not agres with local trend \\
\hline$T E=107-A P-42$ & $6 / 18 / 94$ & 86 & CASS & $\bar{T}$ & \\
\hline TE-107-AP-42 & 6/20/94 & 59 & CASS & $\mathbf{s}$ & Data poing did not agree with local trend \\
\hline TE-107-AP-42 & $6 / 20 / 34$ & 55) & SACS & S [G in SACS] & Dafa point did not agree with local trend \\
\hline TE-107-AP-42 & $6 / 24 / 94$ & 89 & CASS & $\mathrm{T}$ & \\
\hline TE-107-AP-42 & $6 / 27 / 94$ & 89 & CASS & $\bar{T}$ & \\
\hline TE-107-AP-42 & $7 / 7194$ & 67 & CASS & $T$ & \\
\hline TE-107-AP-42 & $7 / 76 / 94$ & 87 & CASS & $\mathbf{T}$ & \\
\hline TE-107-AP-42 & $7 / 18 / 84$ & 86 & CASS & $\mathbf{T}$ & \\
\hline TE-107.AP-42 & $7 / 24 / 94$ & 85 & CASS & $\mathbf{T}$ & \\
\hline TE-107-AP-42 & $7 / 31 / 94$ & 87 & CASS & $\bar{T}$ & \\
\hline TE-107-AP-42 & $8 / 9 / 94$ & 9i & CASS & $\mathbf{T}$ & \\
\hline TE-107-AP-A2 & $8 / 16 / 94$ & 147 & CASS & $\mathbf{S}$ & Data goint did not agree with local trend \\
\hline TE-107.AP-42 & $8 / 17 / 94$ & 89 & CASS & $\bar{T}$ & \\
\hline TE-107-AP-42 & $8 / 22 / 94$ & 89 & CASS & $T$ & \\
\hline TE-107-AP-42 & 9/3/S4 & 92 & CASS & $\bar{T}$ & \\
\hline TE-107-AP-42 & 9/9/94 & 93 & CASS & $T$ & \\
\hline$T E-107-A P=42$ & $9 / 15 / 94$ & 93 & CASS & $T$ & \\
\hline TE-107-AP-42 & 9/23/94 & 92 & CASS & $T$ & \\
\hline TE-107-AP-42 & $9 / 30 / 94$ & 96 & CASS & $T$ & \\
\hline TE-107-AP-42 & $10 / 8 / 94$ & 96 & CASS & $T$ & \\
\hline TE-107-AP-42 & $10 / 15 / 94$ & 96 & CASS & $\mathbf{T}$ & \\
\hline TE-107-AP-42 & $10 / 22 / 94$ & 98 & CASS & $T$ & \\
\hline TE-107-AP-42 & $10 / 25 / 94$ & 100 & CASS & $T$ & \\
\hline TE-107-AP-42 & $11 / 5 / 94$ & 99) & CASS & $T$ & \\
\hline RE-107-AP-4Z & $31 / 11 / 94$ & 98 & CASS & $T$ & \\
\hline TE-107-AP-42 & $11 / 14 / 94$ & 97 & CASS & $\mathbf{T}$ & \\
\hline TE-107-AP-42 & $11 / 22 / 94$ & 98 & CASS & $T$ & \\
\hline TE-107-AP-42 & $12 / 3 / 94$ & 91 & CASS & 7 & \\
\hline TE-107-AP-42 & $12 / 4 / 94$ & 95 & CASS & $T$ & \\
\hline TE-107-AP-42 & $12 / 14 / 94$ & 90 & CASS & $T$ & \\
\hline IE-107-AP-42 & $12 / 19 / 94$ & 92 & CASS & $T$ & \\
\hline TE-107-AP-42 & $12 / 31 / 34$ & 91 & CASS & $T$ & \\
\hline TE-107-AP.42 & $1 / 4 / 95$ & 95 & CASS & $\mathbf{T}$ & \\
\hline TE-107-AP-A2 & 1/14/95 & 80 & CASS & $T$ & \\
\hline TE-107-AP-A2 & $1 / 15 / 95$ & 81 & CASS & $T$ & \\
\hline TE-107.AP-42 & $1 / 25 / 95$ & 700 & CASS & $T$ & \\
\hline TE-307-AP-42 & $2 / 2 / 95$ & 96 & CASS & $\mathbf{T}$ & \\
\hline TE-107-AP-42 & $2 / 10 / 95$ & 98 & CASS & $T$ & \\
\hline$T E-107-A P-42$ & $2 / 14 / 95$ & 104 & CASS & $\mathbf{I}$ & \\
\hline
\end{tabular}




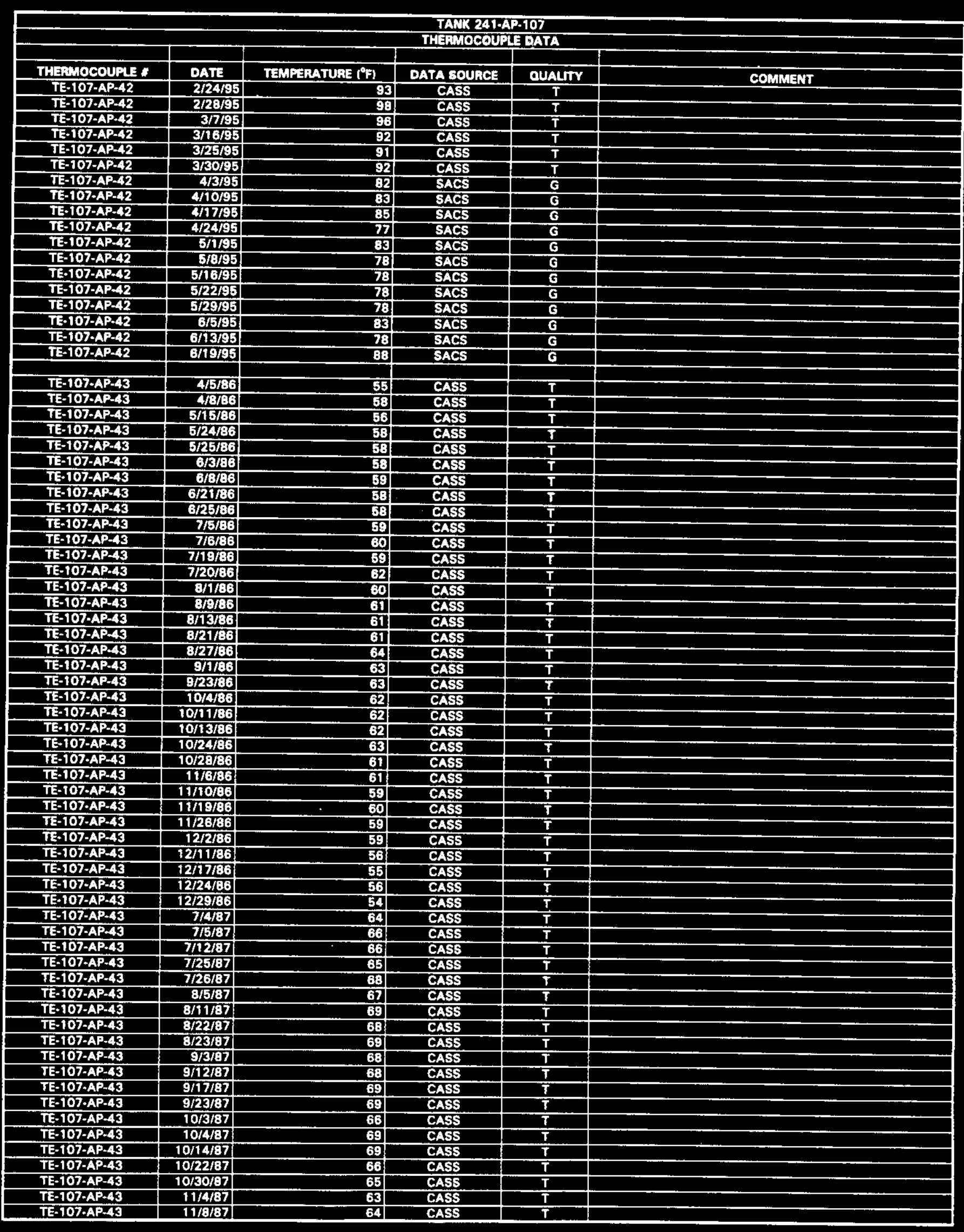

Data obtoined from Computer Automated Survoillance Syotem (CASS), April 1995; and Survellence Analyeis Computer System (SACSI, June 1995. D-596 


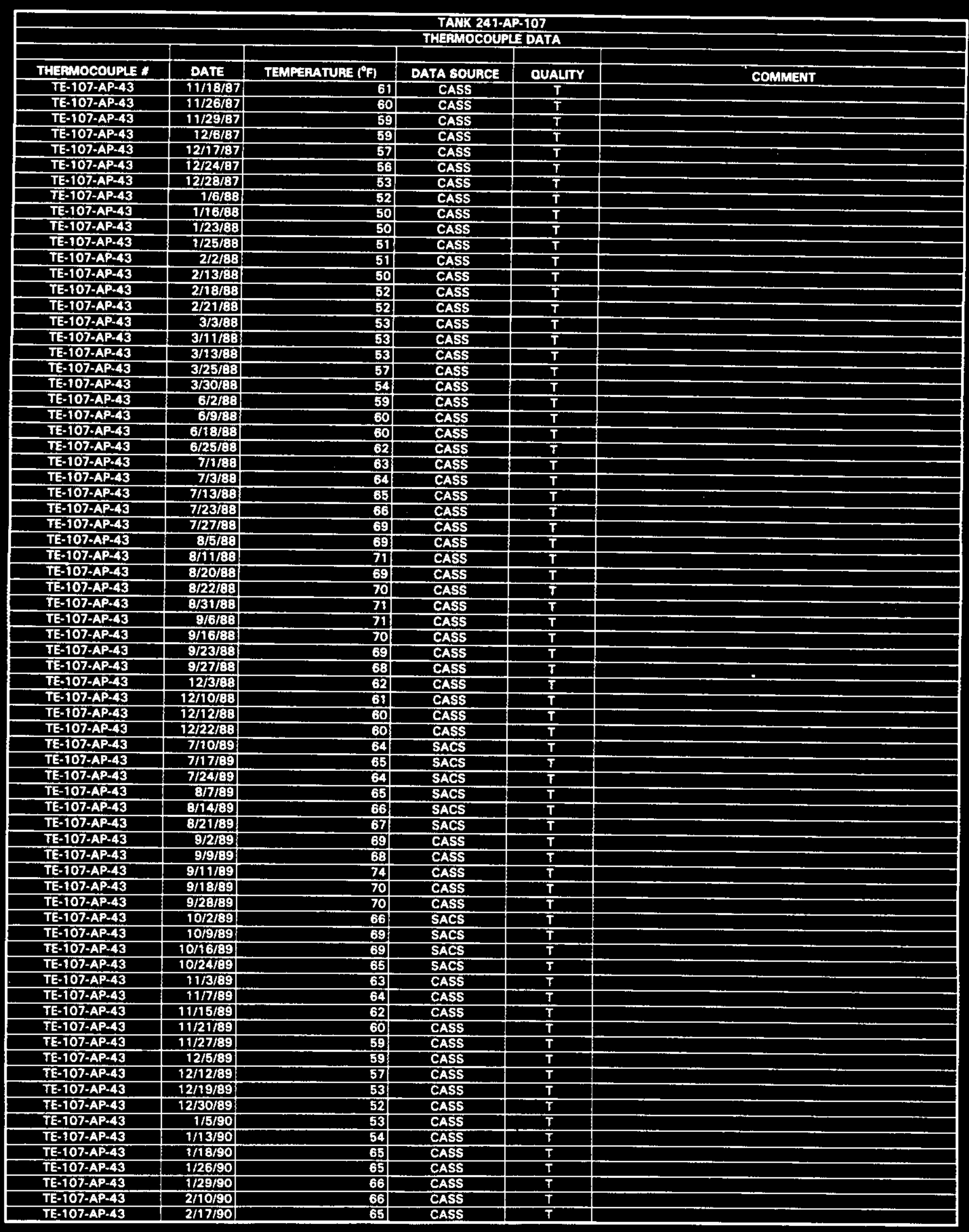




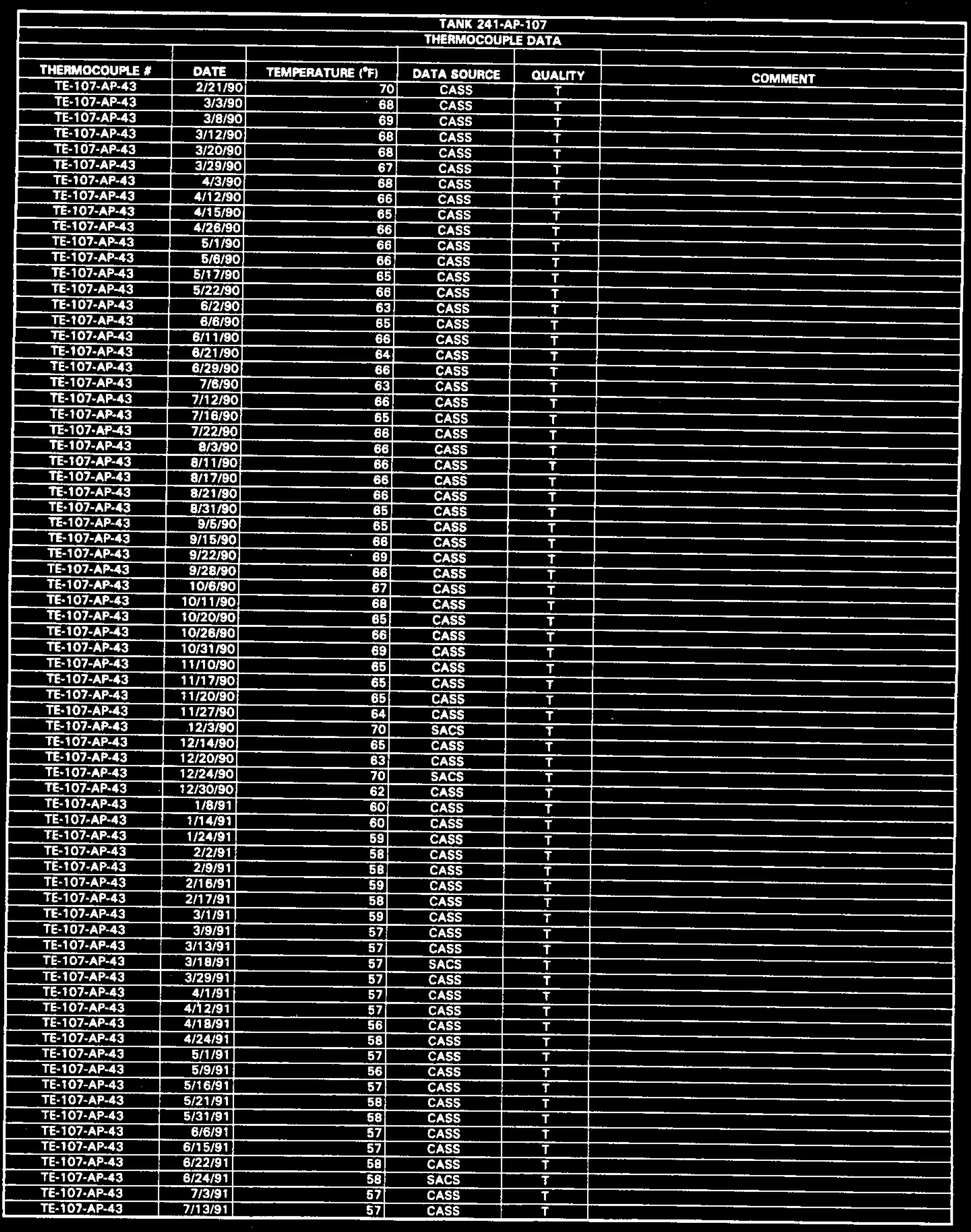

Data obteined from Computer Automated Surveillance Syatom (CASS), Aprll 1995; und Survaillance Analysis Computer System (SACS), June 1995.

$$
\text { D-598 }
$$




\begin{tabular}{|c|c|c|c|c|c|}
\hline \multicolumn{6}{|c|}{$\begin{array}{l}\text { TANK 241-AP-107 } \\
\text { THERMOCOUPLE DATA }\end{array}$} \\
\hline THERMOCOUPLE * & DATE & TEMPEAATUAE $\left({ }^{\circ} \mathrm{F}\right)$ & DATA SOUACE & & \\
\hline TE-107-AP-43 & $7 / 15 / 91$ & 59 & SACS & GUALIY & COMMENT \\
\hline TE-107-AP-43 & 7/24/91 & 58 & CASS & $T$ & \\
\hline TE-107-AP-43 & $8 / 3 / 91$ & 58 & CASS & $\frac{T}{T}$ & \\
\hline TE-107-AP-43 & $8 / 6 / 91$ & 881 & CASS & $\frac{T}{T}$ & \\
\hline TE-107-AP.43 & 8/17/91 & 60 & CASS & $T$ & \\
\hline TE-107-AP-43 & $8 / 20 / 91$ & 59 & CASS & $\bar{T}$ & \\
\hline TE-107-AP-43 & $8 / 30 / 91$ & 60 & CASS & $T$ & \\
\hline TE-107-AP-43 & 9/3/91 & 61 & CASS & $\bar{T}$ & \\
\hline TE-107-AP-43 & $9 / 8 / 91$ & 61 & CASS & $T$ & \\
\hline TE-107-AP-43 & 9/18/91 & 61 & CASS & $T$ & \\
\hline TE-107-AP-43 & $9 \longdiv { 2 7 9 1 }$ & 63 & CASS & $T$ & \\
\hline TE-107-AP-43 & 10/3/91 & 63 & CASS & $T$ & \\
\hline TE-107-AP-43 & $10 / 12 / 91$ & 61 & CASS & $T$ & \\
\hline TE-107-AP.43 & 10/19/91 & 63 & CASS & $\bar{T}$ & \\
\hline TE-107-AP-43 & $10 / 22 / 91$ & 64 & CASS & $T$ & \\
\hline IE-107-AP:43 & $10 / 29 / 91$ & 63 & CASS & $\mathbf{T}$ & \\
\hline$T E-107-A P-43$ & $11 / 6 / 91$ & 62 & CASS & $T$ & \\
\hline$T E-107 \cdot A P-43$ & $7 / 30 / 92$ & 58 & CASS & $\bar{T}$ & \\
\hline TE-107-AP-43 & $8 / 3 / 92$ & 59 & CASS & $\bar{T}$ & \\
\hline TE-107.AP-43 & $8 / 9 / 92$ & 58 & CASS & $T$ & \\
\hline TE-107-AP-43 & $8 / 22 / 92$ & 57 & CASS & $T$ & \\
\hline TE-107-AP-43 & $8 / 23 / 92$ & 71 & SACS & $\mathbf{S}$ & Disagreement between CASS and SACS \\
\hline$T E=107-A P-43$ & 8/24/92 & 59 & CASS & $\bar{T}$ & \\
\hline$T E-107-A P-43$ & $8 / 30 / 92$ & 58 & CASS & $\mathbf{T}$ & \\
\hline TE-107-AP-43 & 9/9/92 & 60 & CASS & $\mathbf{T}$ & \\
\hline TE-107-AP-43 & $9 / 13 / 92$ & 62 & CASS & $T$ & \\
\hline IE-107-AP-43 & $9 / 26 / 92$ & SB & CASS & $T$ & \\
\hline$T E-107-A P-43$ & 10/3/92 & 80 & CASS & $T$ & \\
\hline$T E-107-A P-43$ & $10 / 9 / 92$ & 60 & CASS & $T$ & \\
\hline TE-107-AP-43 & $10 / 13 / 92$ & 59 & CASS & $T$ & \\
\hline$T E-107-A P-43$ & $10 / 22 / 92$ & 64 & CASS & $T$ & \\
\hline TE-107-AP-43 & $10 / 29 / 92$ & 61 & CASS & $T$ & \\
\hline TE-107.AP-43 & $11 / 6 / 92$ & 60 & CASS & $T$ & \\
\hline TE-107-AP-43 & $11 / 12 / 92$ & 60 & CASS & $T$ & \\
\hline TE-107-AP-43 & $71 / 20 / 92$ & 60 & CASS & $T$ & \\
\hline TE-107-AP-43 & $11 / 23 / 92$ & 59 & CASS & $\mathbf{T}$ & \\
\hline TE-107-AP-43 & $12 / 5 / 92$ & 58 & CASS & $T$ & \\
\hline TE-107-AP-43 & $12 / 10 / 92$ & 59 & CASS & $\bar{T}$ & \\
\hline$T E-107-A P-43$ & $12 / 19 / 92$ & 50 & CASS & $T$ & \\
\hline TE-107-AP-43 & $12 / 21 / 92$ & 58) & CASS & $T$ & \\
\hline TE-107-AP-43 & $12 / 30 / 92$ & 57 & CASS & $\mathbf{T}$ & \\
\hline$T E-107-A P-43$ & $1 / 15 / 94$ & 57 & CASS & $\bar{T}$ & \\
\hline TE-107-AP-43 & $1 / 18 / 94$ & 59 & CASS & $\bar{T}$ & \\
\hline TE-107-AP-43 & $1 / 27 / 94$ & 57 & CASS & $T$ & \\
\hline TE-107-AP-43 & $2 / 4 / 94$ & 58 & CASS & $T$ & \\
\hline TE-107-AP-43 & $2 / 12 / 94$ & 56 & CASS & $T$ & \\
\hline TE-107-AP-43 & $2 / 16 / 94$ & Bg & CASS & $T$ & \\
\hline TE-107-AP-43 & $2 / 26 / 94$ & 57 & CASS & $T$ & \\
\hline TE-107-AP-43 & $3 / 5 / 94$ & 57 & CASS & T & \\
\hline TE-107.AP-43 & $3 / 6 / 94$ & 58 & CASS & $\mathbf{T}$ & \\
\hline TE-107-AP-43 & $3 / 17 / 94$ & 57 & CASS & $T$ & \\
\hline TE-107.AP-43 & $3 / 26 / 94$ & 58 & CASS & $\mathbf{T}$ & \\
\hline TE-107-AP-43 & $3 / 29 / 94$ & 57 & CASS & $\mathbf{T}$ & \\
\hline TE-107-AP-43 & $4 / 9 / 94$ & 57 & CASS & $T$ & \\
\hline TE-107-AP-43 & $4 / 15 / 94$ & 57 & CASS & $T$ & \\
\hline$T E-107 \cdot A P-43$ & $4 / 22 / 94$ & 57 & CASS & $T$ & \\
\hline TE-107-AP-43 & $4 / 27 / 94$ & 58 & CASS & $T$ & \\
\hline TE-107-AP-43 & $5 / 2 / 94$ & 60 & CASS & $\mathbf{T}$ & \\
\hline TE-107-AP-43 & $5 / 14 / 94$ & 57 & CASS & $T$ & \\
\hline TE-107.AP.43 & $5 / 16 / 94$ & 94 & CASS & $T$ & \\
\hline TE-107-AP-43 & $5 / 22 / 94$ & 88 & CASS & $T$ & \\
\hline $\mathrm{TE}-107 \cdot \mathrm{AP}-43$ & $6 / 2 / 94$ & 89 & CASS & $T$ & \\
\hline TE-107-AP-43 & 6/8/94 & 88 & CASS & $T$ & \\
\hline$T E-107-A P-43$ & $6 / 14 / 94$ & 86 & CASS & $T$ & \\
\hline TE-107-AP-43 & $6 / 16 / 94$ & 57 & CASS & $\mathbf{s}$ & Data point did not agres with local trend \\
\hline TE-107-AP-43 & $6 / 17 / 94$ & 57 & CASS & $\bar{s}$ & Data point did not egree with local trend \\
\hline TE-107-AP-43 & $6 / 20 / 94$ & 59 & CASS & s & Data point did not egree with local trend \\
\hline TE-107-AP-43 & $6 / 24 / 94$ & 89 & CASS & $\bar{T}$ & \\
\hline TE-107-AP-43 & $6 / 27 / 94$ & 88 & CASS & $\mathbf{T}$ & \\
\hline TE-107-AP-43 & $7 / 7194$ & B7 & CASS & $T$ & \\
\hline TE-107-AP-43 & $7 / 13 / 94$ & 87 & CASS & $\bar{T}$ & \\
\hline TE-107-AP-43 & $7 / 20 / 94$ & 85 & CASS & $T$ & \\
\hline TE-107-AP-43 & $7 / 30 / 94$ & 84 & CASS & $\mathbf{T}$ & \\
\hline
\end{tabular}




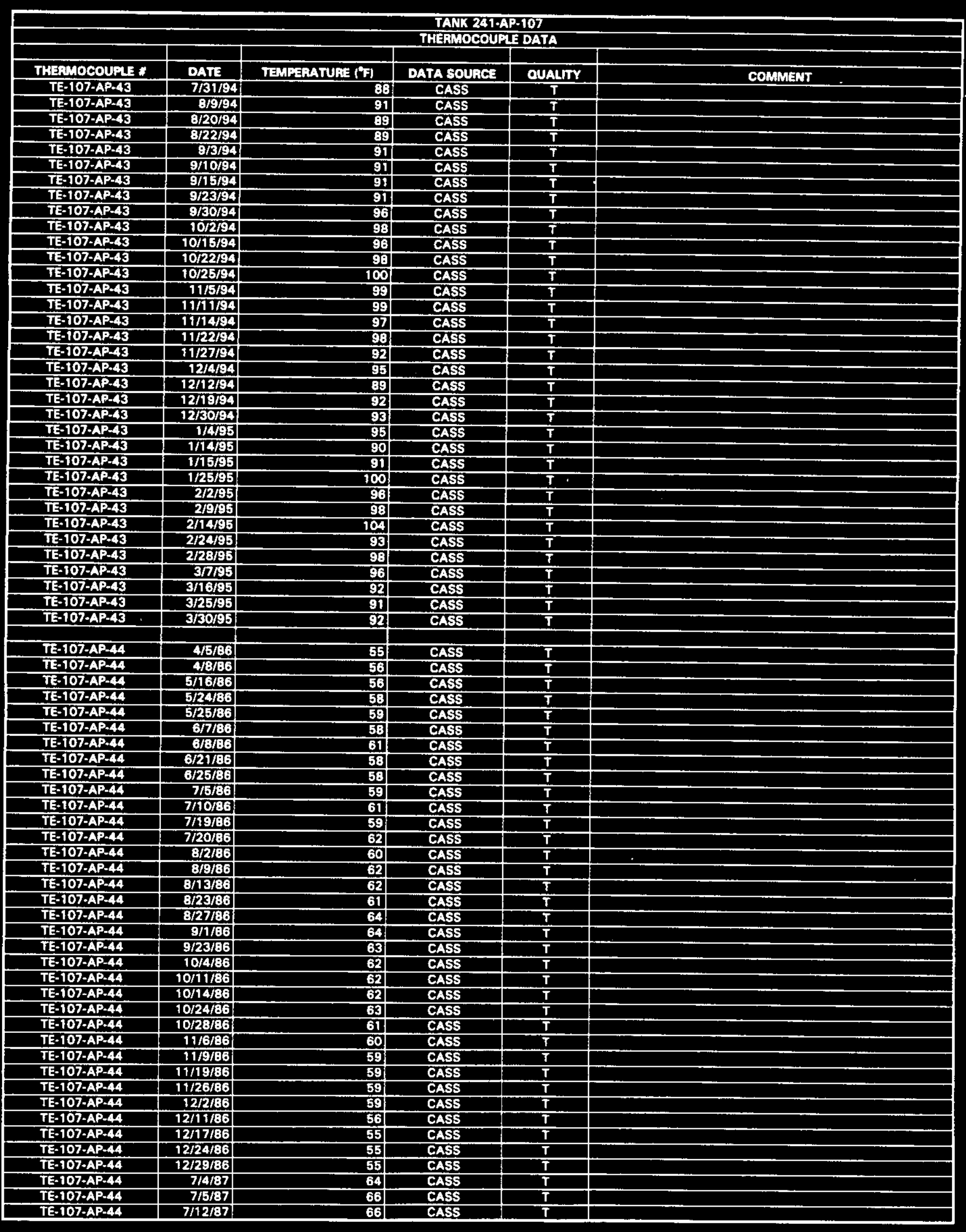

Data obtained from Computer Automated Surveillance Syatem (CASS), April 1995; and Surveillance Anelyais Computer Syatem (SACS), June 1995.

D-600 


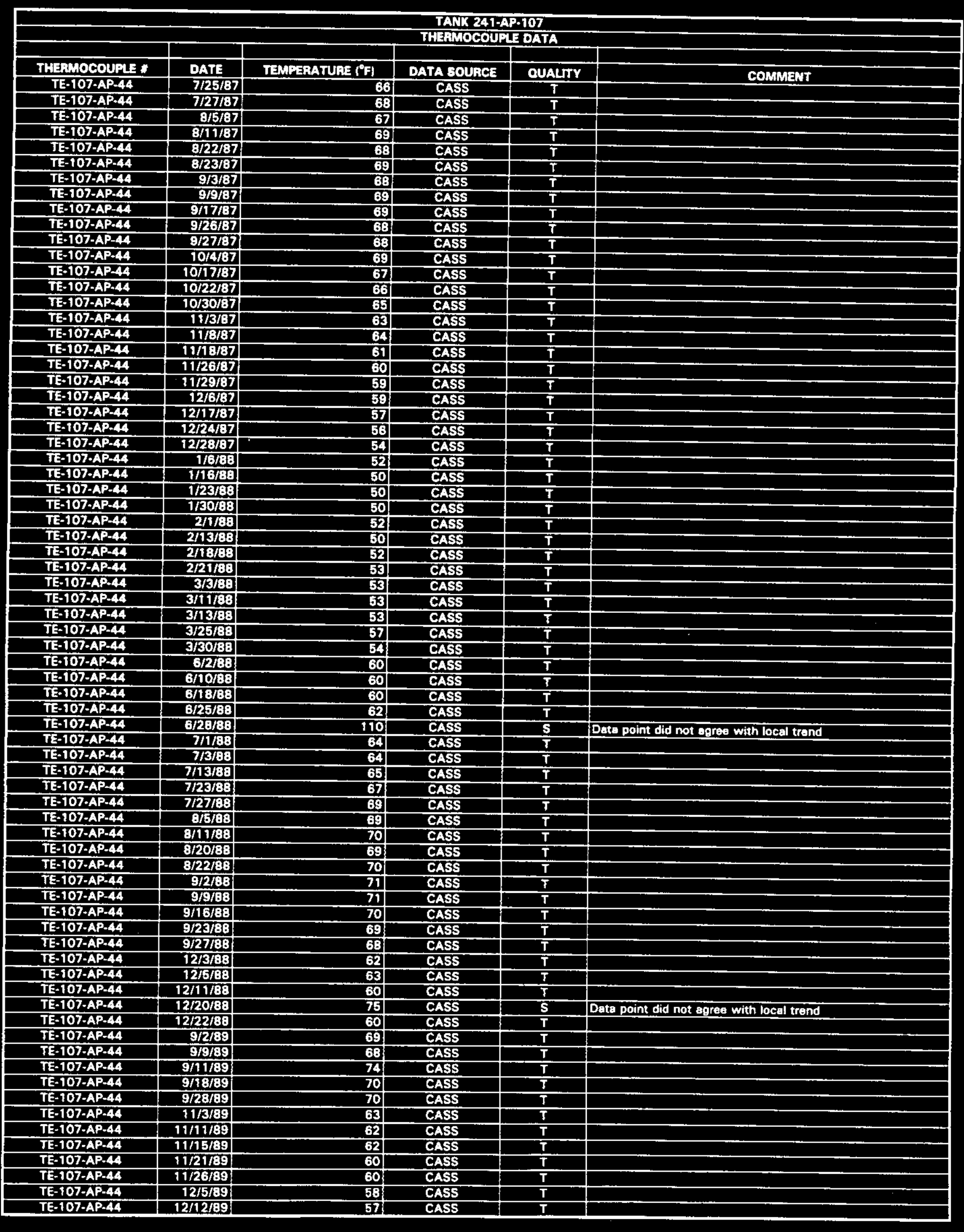

Data obtained from Computer Automated Survaillance System (CASS), April 1995; and Surveillance Analyais Computer System (SACS), June 1995.

D- 601 


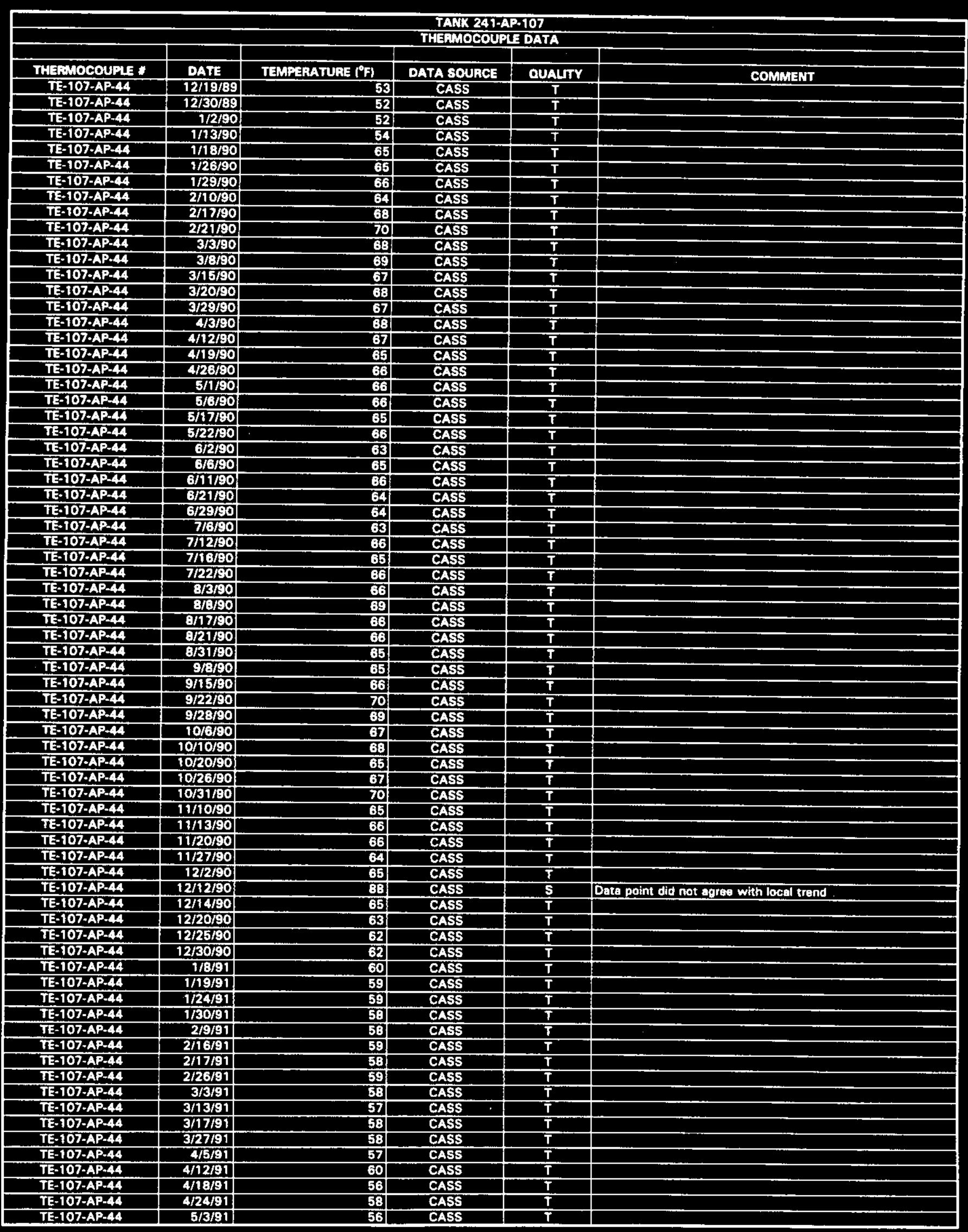




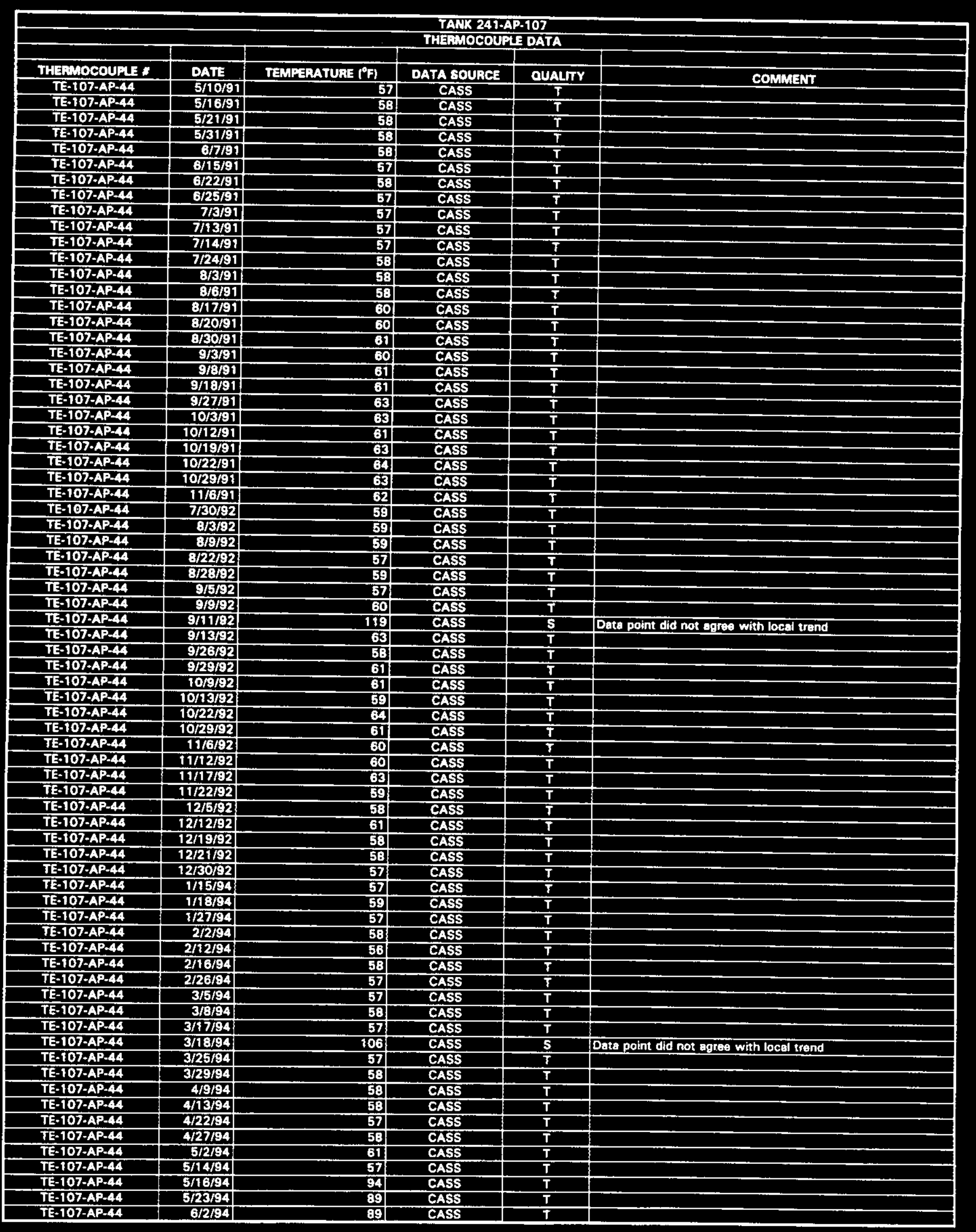




\begin{tabular}{|c|c|c|c|c|c|}
\hline \multicolumn{6}{|c|}{$\begin{array}{l}\text { TAKK 241-AP:-107 } \\
\text { THARMOCOUPLE DATA }\end{array}$} \\
\hline & & & & & \\
\hline THERAMOCOUPLE * & DATE & TEMFERATURE (FF) & DATA SOURCE & OUALITY & COMMENT \\
\hline TE-107-AP-44 & 6/8/94 & 88 & CASS & $\mathbf{T}$ & 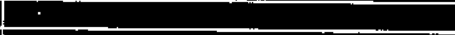 \\
\hline TE-107-AP-44 & 6/14/94 & 86 & CASS & $T$ & \\
\hline TE-107-AP-44 & 6/16/94 & 56 & CASS & $\mathbf{s}$ & Data point did not agree with local trend \\
\hline TE-107-AP-44 & 6/17/94 & 57 & CASS & $\mathbf{S}$ & Data point did not agree with local trend \\
\hline TE-107-AP-44 & 6/20/94 & 59 & CASS & $\mathbf{S}$ & Dare point did nol aprea with local trend \\
\hline TE-107-AP-44 & $6 / 24 / 94$ & 89 & CASS & $\mathbf{T}$ & \\
\hline TE-107-AP-44 & 6/27/94 & 88 & CASS & $\bar{T}$ & \\
\hline TE-107-AP-44 & $7 / 7 / 94$ & 87 & CASS & $\bar{T}$ & \\
\hline$T E=107 \cdot A P-44$ & $7 / 15 / 94$ & 87 & CASS & $T$ & \\
\hline TE-107-AP-44 & 7/20/94 & 85 & CASS & $\mathbf{T}$ & \\
\hline TE-107-AP-44 & $7 / 30 / 94$ & 84 & CASS & $T$ & \\
\hline TE-107-AP-44 & $731 / 24$ & 87 & CASS & $T$ & \\
\hline TE-107-AP-44 & $8 / 9 / 94$ & 91 & CASS & $\bar{T}$ & \\
\hline TE-107-AP-44 & $8 / 20 / 94$ & 80 & CASS & $T$ & \\
\hline TE-107-AP-44 & $8 / 22 / 94$ & 89 & CASS & $\mathbf{T}$ & \\
\hline TE-107.AP-44 & 9/3/94 & 82 & CASS & $\mathbf{T}$ & \\
\hline IE-107.AP-44 & 9/10/94 & 91 & CASS & $T$ & \\
\hline TE-107-AP-44 & 9/15/94 & 91 & CASS & $\mathbf{T}$ & \\
\hline TE-107-AP-44 & 9/19/94 & 148 & CASS & $\mathbf{S}$ & Data point did not agree with local trend \\
\hline TE.107-AP.44 & 9/23/94 & 911 & CASS & $T$ & \\
\hline TE-107-AP.44 & 9/30/94 & 96 & CASS & $\bar{T}$ & \\
\hline TE-107-AP-44 & 10/8/94 & 86 & CASS & $\mathbf{T}$ & \\
\hline TE-107-AP-44 & $10 / 15 / 94$ & 96 & CASS & $T$ & \\
\hline TE-107-AP-44 & $10 / 22 / 94$ & 88 & CASS & $\mathbf{T}$ & \\
\hline TE-107-AP-44 & $10 / 25 / 94$ & 100 & CASS & $T$ & \\
\hline TE-107-AP=44 & $11 / 5 / 94$ & 89: & CASS & $\mathbf{T}$ & \\
\hline TE-107-AP-44 & $11 / 11 / 94$ & 99 & CASS & $\mathbf{T}$ & \\
\hline TE-107-AP-44 & $11 / 1 / 4 / 94$ & 97 & CASS & $\mathbf{T}$ & \\
\hline TE-107-AP-44 & $11 / 22 / 94$ & 98 & CASS & $\mathbf{T}$ & \\
\hline TE-107.AP-44 & $11 / 26 / 94$ & 126 & CASS & $\mathbf{S}$ & Date point did not agree with local trand \\
\hline$T E-107-A P-44$ & $11 / 27 / 94$ & 92 & CASS & $\mathbf{T}$ & \\
\hline TE-107-AP-4A & $12 / 4 / 94$ & 95 & CASS & $\mathbf{T}$ & \\
\hline TE-107-AP-44 & $12 / 14 / 94$ & 89 & CASS & $\mathbf{T}$ & \\
\hline TE-107-AP-44 & $12 / 19 / 94$ & 92 & CASS & $\mathbf{T}$ & \\
\hline TE-107.AP-44 & $12 / 30 / 94$ & 92 & CASS & $T$ & \\
\hline TE-107-AP-44 & 1/4/S5 & 85 & CASS & $\bar{T}$ & \\
\hline IE-107-AP-44 & 1/14/95 & 90 & CASS & $T$ & \\
\hline TE-107-AP-44 & $1 / 15 / 95$ & 91 & CASS & $T$ & \\
\hline TE-107-AP-44 & $1 / 24 / 95$ & 102 & CASS & $T$ & \\
\hline IE-107-AP-4A & $2 / 2 / 95$ & 98 & CASS & $T$ & \\
\hline TE-107-AP-44 & $214 / 95$ & 129 & CASS & 5 & Data point did not egree with local trend \\
\hline TE-107-AP-44 & $2 / 10 / 35$ & 98 & CASS & $T$ & \\
\hline TE-107-AP-44 & 2/14/95 & 104 & CASS & $\bar{T}$ & \\
\hline TE-107-AP-44 & $2 / 24 / 95$ & 93 & CASS & $\bar{T}$ & \\
\hline TE-107-AP-44 & $2 / 28 / 95$ & as & CASS & $T$ & \\
\hline TE-107-AP.44 & $3 / 7 / 95$ & 96 & CASS & $T$ & \\
\hline TE-107.AP-44 & 3/16/95 & 92 & CASS & $\mathbf{T}$ & \\
\hline TE-107-AP-44 & 3/25/95 & 91 & CASS & $\bar{T}$ & \\
\hline JE-107-AP-44 & $3 / 30 / 95$ & 91 & CASS & $T$ & \\
\hline TE-107-AP-45 & $4 / 5 / 86$ & 54 & CASS & $\bar{T}$ & \\
\hline TE-107.AP.45 & $4 / 8 / 86$ & 57 & CASS & $T$ & \\
\hline TE-107-AP-45 & $5 / 16 / 86$ & 56 & CASS & $T$ & \\
\hline TE-107-AP-45 & $5 / 24 / 36$ & 58 & CASS & $T$ & \\
\hline TE-107-AP-45 & 5/25/36 & 58 & CASS & $T$ & \\
\hline TE-107-AP-45 & $6 / 4 / 86$ & 58 & CASS & $T$ & \\
\hline TE-107-AP-45 & $6 / 8 / 86$ & 59 & CASS & $T$ & \\
\hline TE-107-AP-45 & $6 / 21 / 86$ & 58 & CASS & $\bar{T}$ & \\
\hline TE-107-AP-45 & $6 / 25 / 86$ & 58 & CASS & $\bar{T}$ & \\
\hline TE-107-AP-45 & $7 / 5 / 86$ & 59 & CASS & $\mathbf{T}$ & \\
\hline TE-107-AP-45 & $7 / 10 / 86$ & 61 & CASS & $T$ & \\
\hline TE-107-AP-45 & 7/19/86 & 59 & CASS & $T$ & \\
\hline TE-107-AP-45 & $7120 / 86$ & 62 & CASS & $T$ & \\
\hline TE-107-AP-45 & $8 / 1 / 86$ & 60 & CASS & $\bar{T}$ & \\
\hline TE-107-AP-45 & $8 / 9 / 86$ & 62 & CASS & $T$ & \\
\hline TE-107-AP-45 & $8 / 13 / 86$ & 62 & CASS & $T$ & \\
\hline TE-107-AP-45 & $8 / 17 / 86$ & 98 & CASS & $\mathbf{S}$ & Date point did not agree with local trend \\
\hline TE-107.AP.45 & $8 / 21 / 86$ & 61 & CASS & $\mathbf{T}$ & \\
\hline TE-107-AP-45 & $8 / 27 / 36$ & 63 & CASS & $\mathbf{T}$ & \\
\hline TE-107-AP-45 & $9 / 3 / 366$ & 62: & CASS & $\bar{T}$ & \\
\hline TE-107-AP-45 & $9 / 23 / 86$ & 63 & CASS & $\mathbf{T}$ & \\
\hline TE-107-AP-45 & $10 / 4 / 86$ & 62 & CASS & $T$ & \\
\hline TE-107-AP-45 & $10 / 11 / 86$ & 62 & CASS & $\bar{T}$ & \\
\hline
\end{tabular}




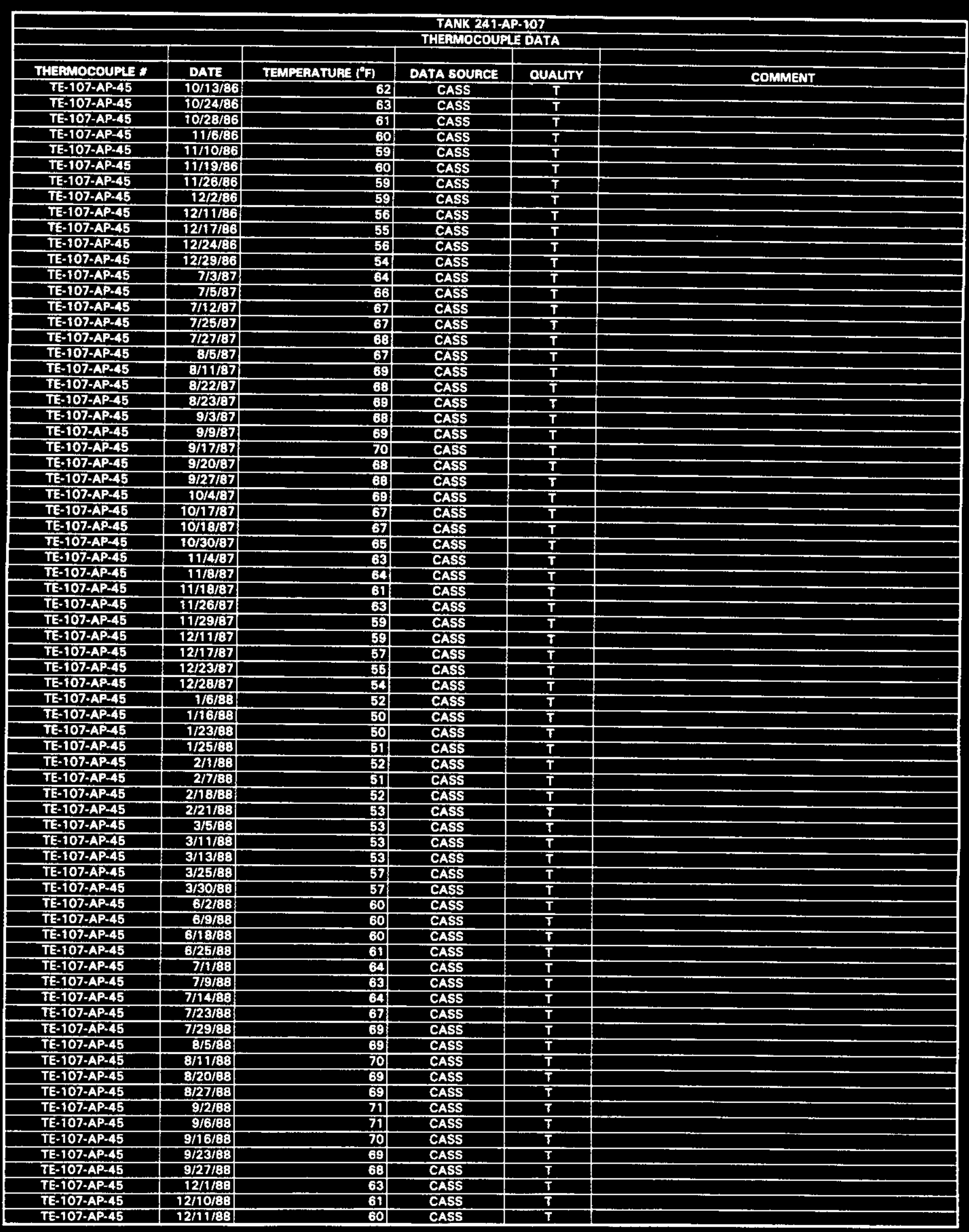

Data obtainad from Computer Automated Survaillance Syatem (CASS), April 1995; and Surveillance Analyais Computer Syatem (SACS), June 1995.

$$
\text { D- } 605
$$




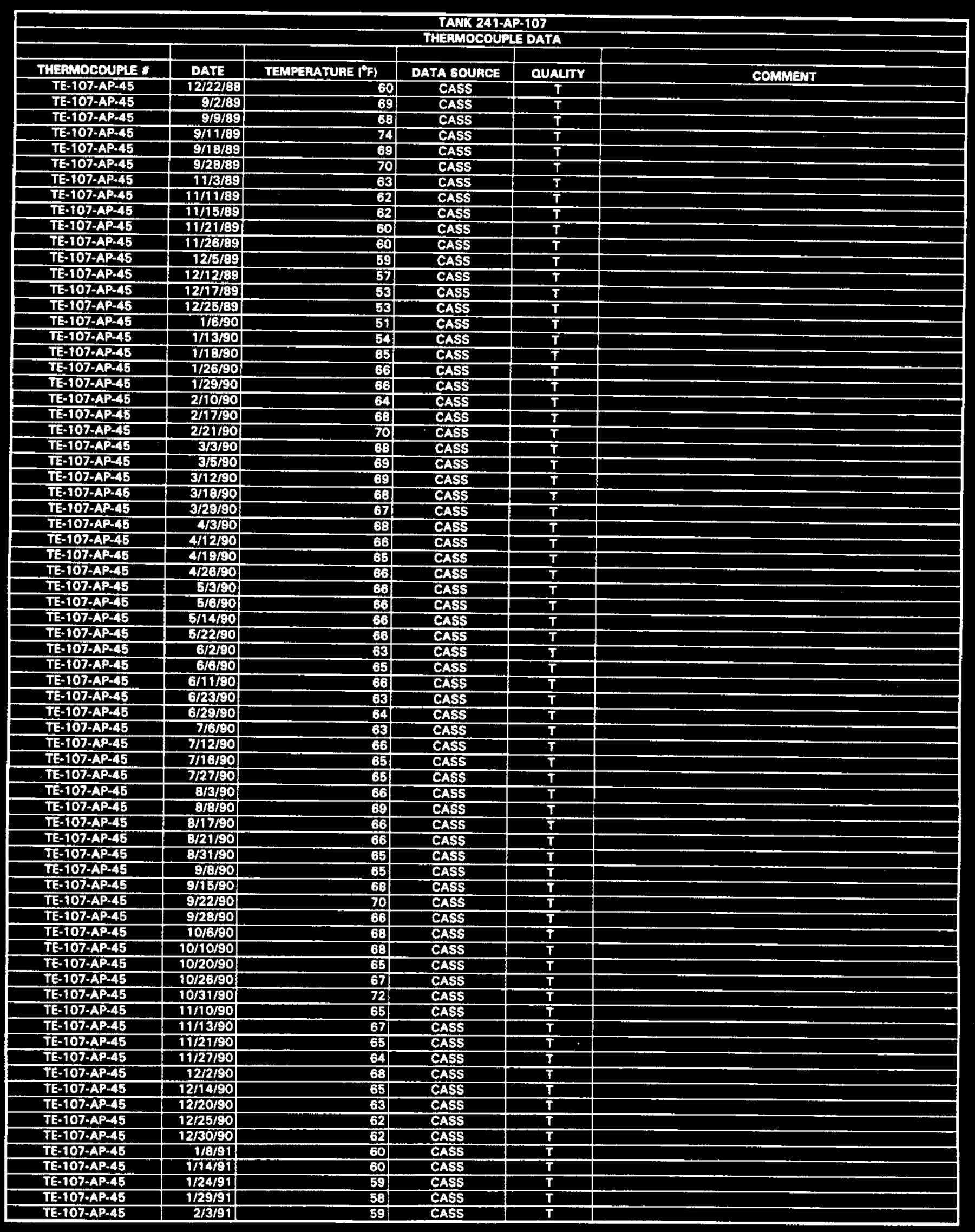




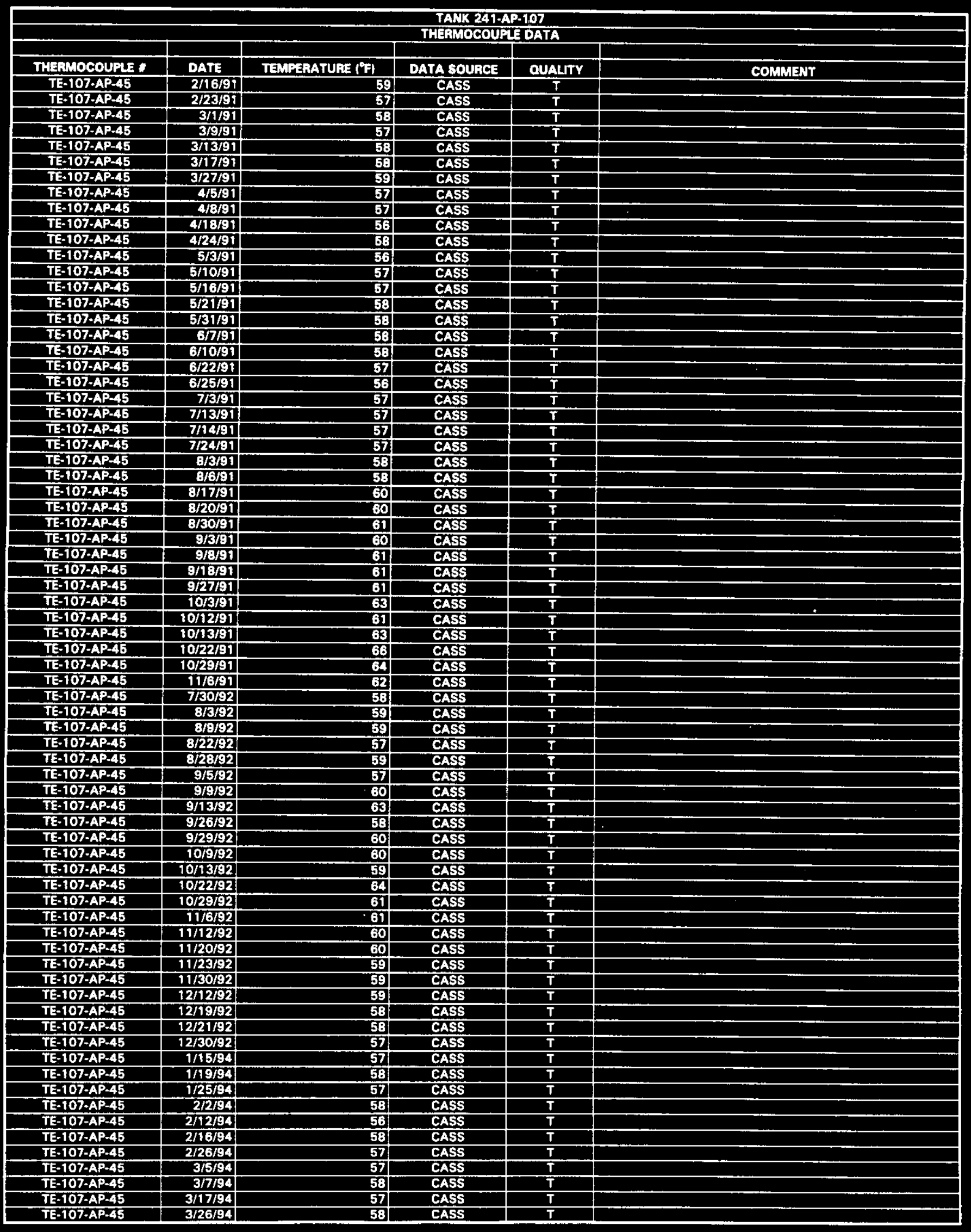




\begin{tabular}{|c|c|c|c|c|c|}
\hline \multirow{2}{*}{\multicolumn{6}{|c|}{$\begin{array}{l}\text { TANK 241-AP-107 } \\
\text { THERMOCOUALE DATA }\end{array}$}} \\
\hline & & & & & \\
\hline THERMOCOUPL $*$ & DATE & TEMPERATURE ( ${ }^{\circ} \mathrm{F}$ ) & DATA SOURCE & QUALTY & COMMENT \\
\hline TE-107-AP-45 & $3 / 29 / 94$ & 58 & CASS & $T$ & \\
\hline TE-107.AP-45 & $4 / 9 / 94$ & 58 & CASS & $\mathbf{T}$ & \\
\hline TE-107-AP-45 & 4/13/94 & 58 & CASS & $T$ & \\
\hline TE-107-AP-45 & $4 / 22 / 94$ & 57 & CASS & $\mathbf{T}$ & \\
\hline TE-107-AP.45 & 4/27/94 & 61 & CASS & $\mathbf{T}$ & \\
\hline$T E-107-A P-45$ & $5 / 2 / 94$ & 61 & CASS & $\mathbf{T}$ & \\
\hline TE-107-AP-45 & $5 / 14 / 94$ & 57 & CASS & $\mathbf{T}$ & \\
\hline TE-107-AP.45 & $5 / 16 / 94$ & 94 & CASS & $\mathbf{T}$ & \\
\hline TE-107-AP-AS & $5 / 23 / 94$ & 91 & CASS & $T$ & \\
\hline TE-107-AP-45 & $6 / 2 / 94$ & 89 & CASS & $T$ & \\
\hline TE-107-AP-45 & $6 / 894$ & 88 & CASS & $T$ & \\
\hline TE-107-AP-A5 & 6/14/94 & 86 & CASS & $\mathbf{T}$ & \\
\hline TE-107.AP-45 & 6/16/94 & 56 & CASS & $\mathbf{S}$ & Data point did not agres with local trend \\
\hline TE=107-AP-45 & $6 / 17 / 94$ & 57 & CASS & $\mathbf{S}$ & Date point did not agree with local trend \\
\hline TE-107.AP-45 & $6 / 20 / 94$ & 599 & CASS & $\mathbf{S}$ & Data point did not agree with local trend \\
\hline TE-107-AP-45 & 6/24/94 & 89 & CASS & $T$ & \\
\hline TE-107-AP-45 & 6/27/94 & 88 & CASS & $T$ & \\
\hline TE-107.AP-45 & $7 / 7 / 94$ & 87 & CASS & $\mathbf{T}$ & \\
\hline TE-107-AP-45 & $7 / 16 / 94$ & 87 & CASS & $T$ & \\
\hline TE-107-AP-45 & $7 / 20 / 94$ & 85 & CASS & $T$ & \\
\hline TE-107-AP-45 & $7130 / 94$ & 84 & CASS & $T$ & \\
\hline TE-107-AP-45 & $7 / 31 / 94$ & 87 & CASS & $T$ & \\
\hline TE-107-AP-45 & 8/9/94 & 91 & CASS & $\mathbf{T}$ & \\
\hline TE-107-AP-45 & $8 / 20 / 94$ & 88 & CASS & $T$ & \\
\hline TE-107-AP-46 & $8 / 22 / 94$ & 89 & CASS & $T$ & \\
\hline TE-107-AP-45 & $9 / 3 / 94$ & 80 & CASS & $\bar{T}$ & \\
\hline TE-107-AP-45 & $9 / 10 / 94$ & 91 & CASS & $T$ & \\
\hline TE-107-AP-45 & $9 / 15 / 94$ & 91 & CASS & $T$ & \\
\hline$T E-107 \cdot A P-45$ & $9 / 23 / 94$ & 91 & CASS & $T$ & \\
\hline TE-107-AP-45 & $9 / 30 / 94$ & 95 & CASS & $T$ & \\
\hline TE-107-AP-AS & $10 / 8 / 94$ & 96 & CASS & $T$ & \\
\hline TE-107.AP-45 & $10 / 12 / 94$ & 96 & CASS & $\bar{T}$ & \\
\hline TE-107-AP-45 & $10 / 22 / 94$ & 98 & CASS & $\mathbf{T}$ & \\
\hline TE-107-AP-45 & $10 / 25 / 94$ & 100 & CASS & $\mathbf{T}$ & \\
\hline TE-107-AP-45 & $11 / 5 / 94$ & 99 & CASS & $\mathbf{T}$ & \\
\hline TE-107-AP-45 & $11 / 11 / 34$ & 101 & CASS & $T$ & \\
\hline TE-107-AP-45 & $11 / 14 / 84$ & 97 & CASS & $T$ & \\
\hline TE-107-AP-45 & $71 / 22 / 94$ & 98 & CASS & $\mathbf{T}$ & \\
\hline TE-107-AP-45 & $11 / 27 / 94$ & 92 & CASS & $\mathbf{T}$ & \\
\hline TE-107-AP-45 & $12 / 4 / 94$ & 95 & CASS & $T$ & \\
\hline TE-107-AP-46 & $12 / 14 / 94$ & 89 & CASS & $\bar{T}$ & \\
\hline TE-107-AP-45 & $12 / 19 / 94$ & 92 & CASS & $T$ & \\
\hline TE-107-AP-45 & $12 / 30 / 94$ & 92 & CASS & $T$ & \\
\hline TE-107-AP-45 & $1 / 4 / 95$ & 94 & CASS & $\bar{T}$ & \\
\hline TE-107-AP-45 & $1 / 14 / 95$ & so: & CASS & $T$ & \\
\hline TE-107-AP-45 & $1 / 15 / 95$ & 91 & CASS & $T$ & \\
\hline TE-107-AP-45 & $1 / 25 / 95$ & 100 & CASS & $T$ & \\
\hline TE-107-AP-45 & $2 / 2 / 95$ & 96 & CASS & $T$ & \\
\hline TE-107-AP-45 & $2 / 9 / 95$ & 98 & CASS & $T$ & \\
\hline TE-107-AP-45 & $2 / 14 / 95$ & 104 & CASS & $T$ & \\
\hline TE-107-AP-45 & $2 / 24 / 95$ & 93 & CASS & $T$ & \\
\hline TE-107-AP-45 & $2 / 28 / 95$ & 98 & CASS & $T$ & \\
\hline IE-107-AP-45 & $3 / 7 / 95$ & 96 & CASS & $T$ & \\
\hline TE-107-AP-45 & $3 / 16 / 95$ & 91 & CASS & $T$ & \\
\hline TE-107-AP-45 & $3 / 25 / 95$ & 91 & CASS & $T$ & \\
\hline TE-107-AP-45 & $3 / 30 / 95$ & 91 & CASS & $T$ & \\
\hline & \begin{tabular}{l|}
$4 / 5 / 86$ \\
$4 / 2 / 86$
\end{tabular} & 55 & $\frac{\text { CASS }}{\text { CASS }}$ & $\frac{1}{T}$ & \\
\hline$\frac{\text { TE-107-AP-46 }}{\text { TE-107-AP-46 }}$ & \begin{tabular}{r|}
$4 / 8 / 86$ \\
$5 / 16 / 86$
\end{tabular} & 58 & $\frac{\text { CASS }}{\text { CASS }}$ & $\frac{1}{T}$ & \\
\hline TE-107-AP-46 & $5 / 24 / 86$ & 58 & CASS & $T$ & \\
\hline$T E-107-A P=46$ & $5 / 25 / 86$ & 等 & CASS & $T$ & \\
\hline TE-107-AP-46 & $6 / 4 / 86$ & 58 & CASS & $T$ & \\
\hline TE-107-AP-46 & $6 / 9 / 86$ & 5 & CASS & $T$ & \\
\hline TE-107-AP-46 & $6 / 21 / 86$ & 58 & CASS & $\bar{T}$ & \\
\hline TE-107-AP-46 & $6 / 25 / 86$ & 58 & CASS & $T$ & \\
\hline$T E-107-A P-46$ & $7 / 5 / 36$ & 59 & CASS & $T$ & \\
\hline TE-107.AP-46 & $7 / 6 / 86$ & 60 & CASS & $T$ & \\
\hline TE-107-AP-46 & $7719 / 86$ & 58 & CASS & $T$ & \\
\hline TE-107-AP-46 & $7 / 20 / 86$ & 62 & CASS & $\mathbf{T}$ & \\
\hline TE-107-AP-46 & $8 / 1 / 86$ & 60 & CASS & $T$ & \\
\hline TE-107-AP-46 & $8 / 9 / 96$ & 63 & CASS & 7 & \\
\hline TE-107-AP-46 & $8 / 13 / 86$ & 62 & CASS & $\bar{T}$ & \\
\hline
\end{tabular}




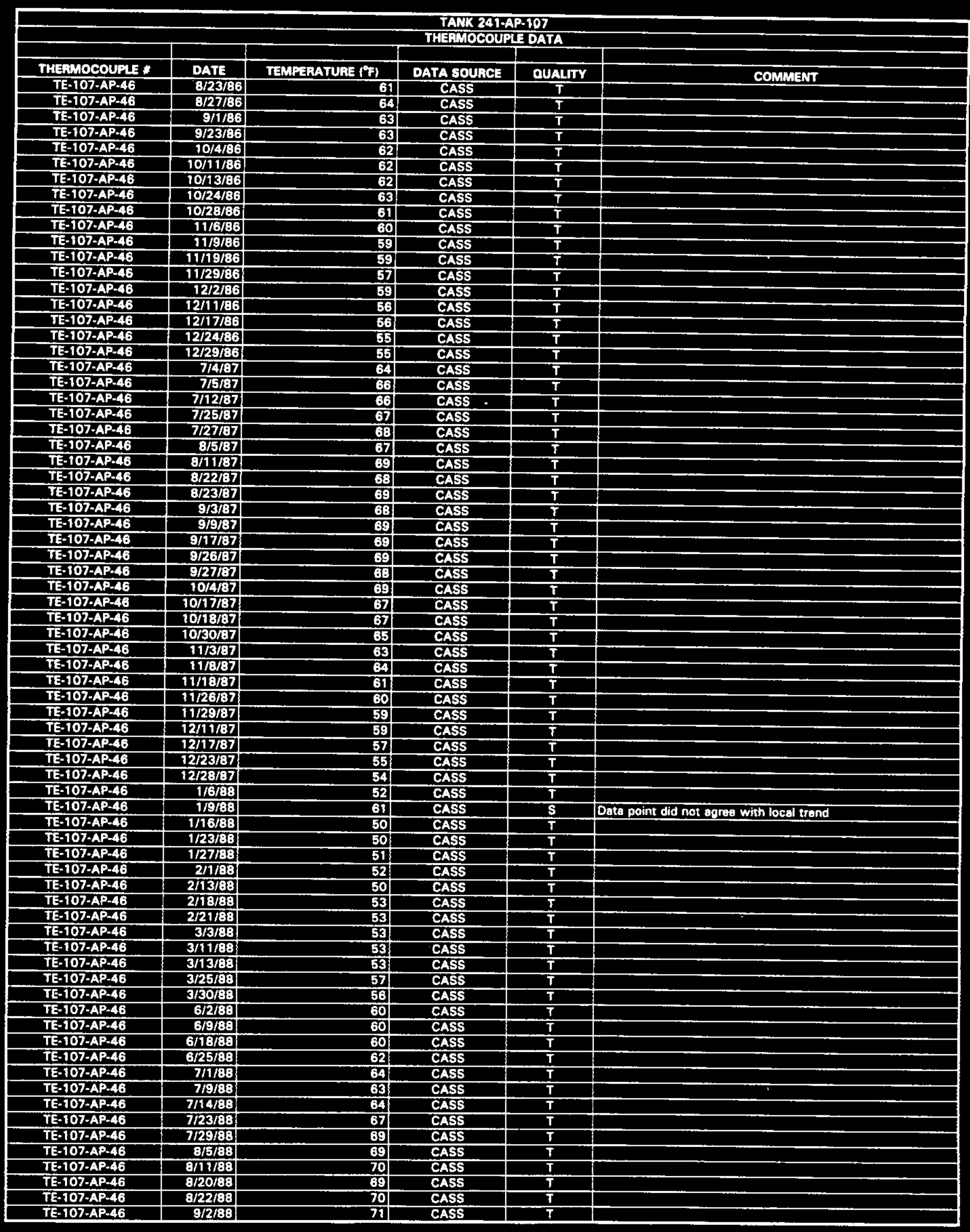




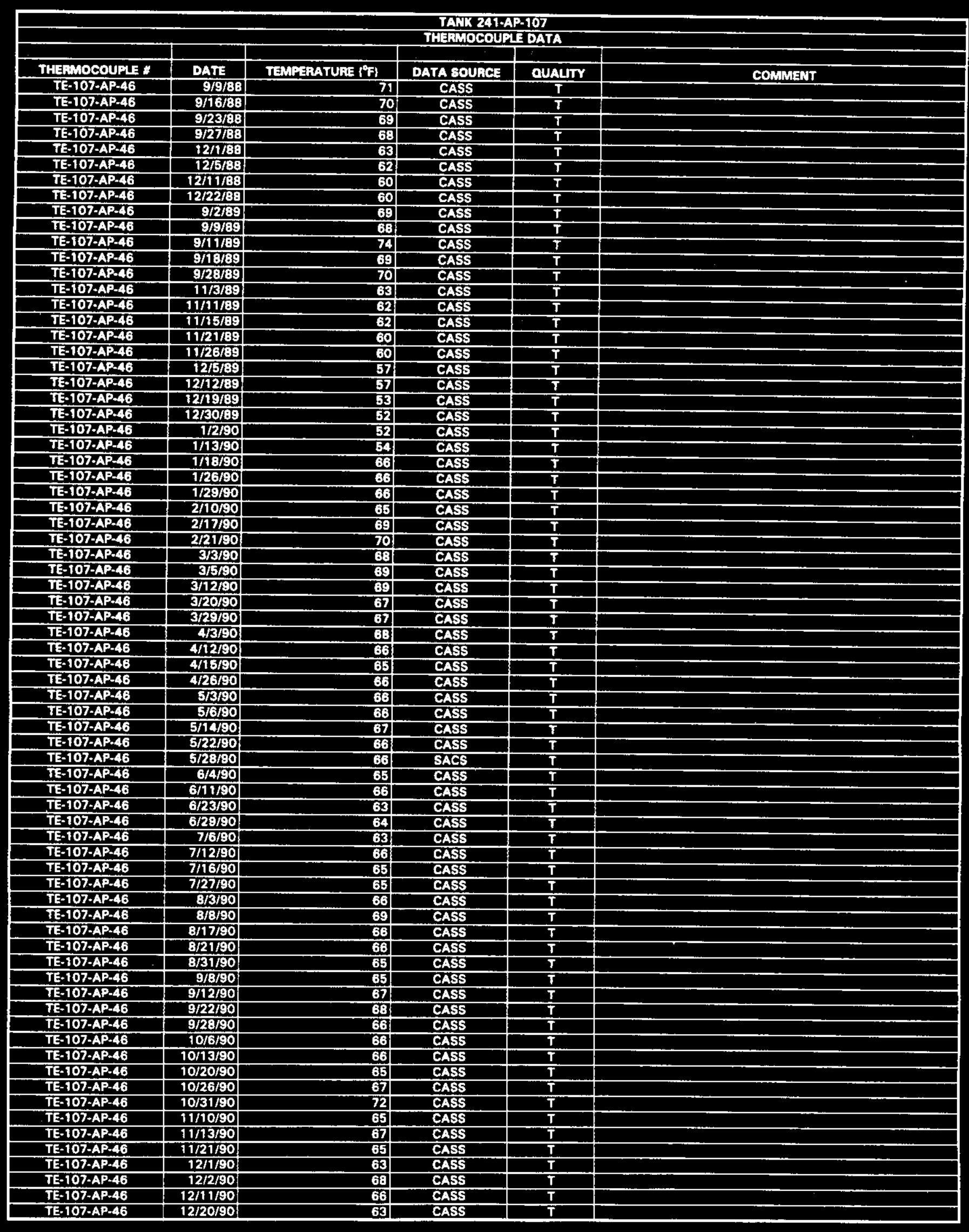

Date obteined from Computer Automated Surveillance System (CASS), April 1995; and Surveillance Analyais Computer System (SACS), June 1995. 


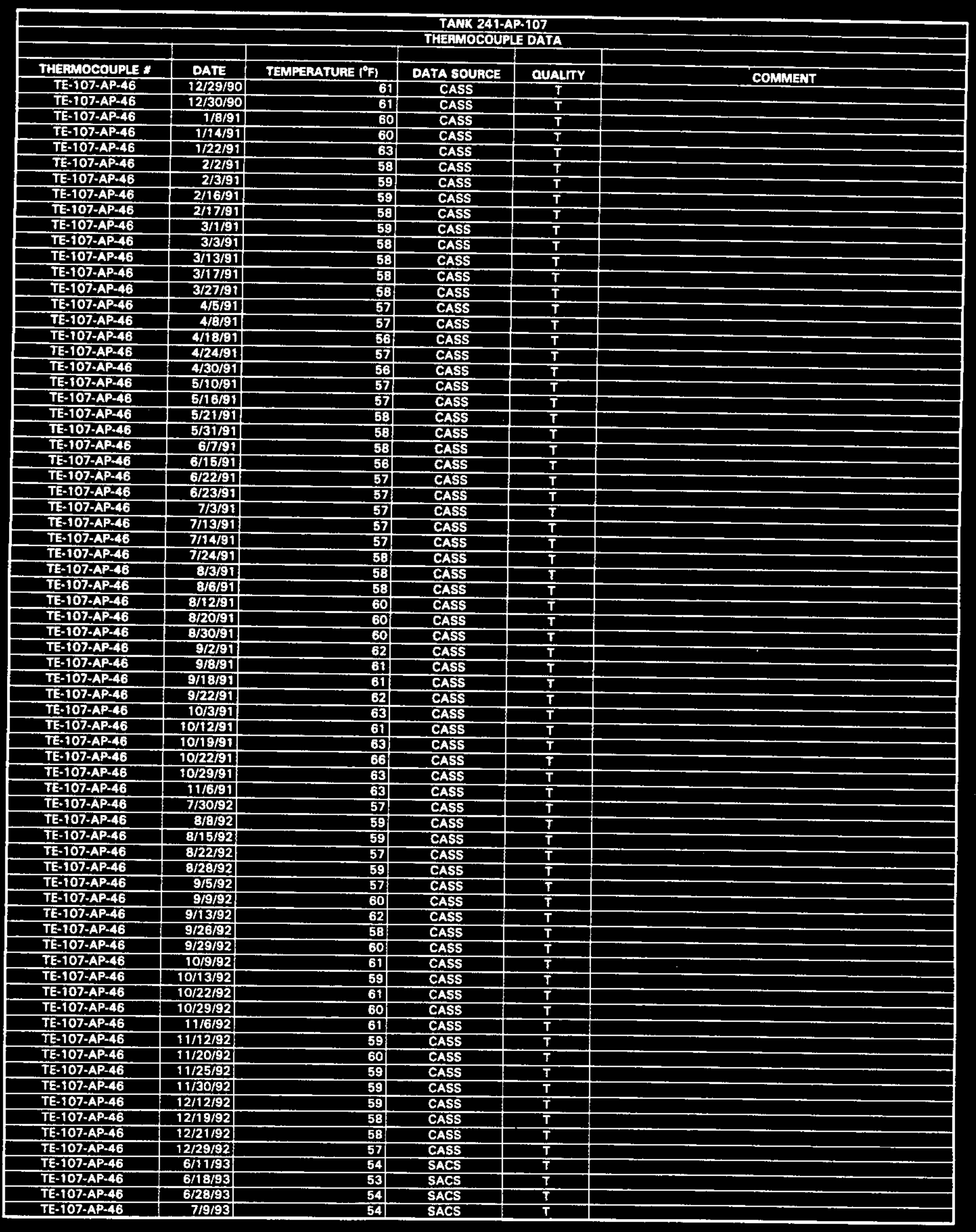

Date obtained from Computer Automated Surveillance System (CASS), April 1995; and Surveillance Analyais Computer Syatem (SACS), June 1995. D-611 


\begin{tabular}{|c|c|c|c|c|c|}
\hline \multicolumn{6}{|c|}{$\begin{array}{l}\text { TANK 241-AP.107 } \\
\text { THERMOCOUPLE DATA }\end{array}$} \\
\hline THEFMOCOUPLE : & DATE & TEMPERATUAE ( $\left.{ }^{\circ} \mathrm{F}\right)$ & DATA SOURCE & OUALTY & СОМMENT \\
\hline TE-107-AP-46 & $7 / 15 / 93$ & 55 & SACS & $T$ & ColMinEST \\
\hline TE-107-AP.46 & $7 / 19 / 93$ & 55 & SACS & $\bar{T}$ & \\
\hline TE-107-AP-46 & $7 / 30 / 93$ & 56 & SACS & $T$ & \\
\hline TE-107-AP-46 & $8 / 6 / 93$ & 55 & SACS & $\mathbf{T}$ & \\
\hline TE-107.AP-46 & $8 / 12 / 93$ & 55 & $\overline{\text { SACS }}$ & $\bar{T}$ & \\
\hline TE-107-AP-46 & $9 / 24 / 93$ & 52 & SACS & $\mathbf{T}$ & \\
\hline$T E-107-A P=46$ & 10/1//93 & 57 & SACS & $\mathbf{T}$ & \\
\hline TE-107-AP-46 & $10 / 15 / 93$ & 58 & SACS & $T$ & \\
\hline TE-107-AP-46 & $10 / 22 / 93$ & 59 & SACS & $T$ & \\
\hline TE-107.AP-46 & $10 / 28 / 93$ & 59 & SACS & $\mathbf{T}$ & \\
\hline TE-107-AP-46 & $11 / 9 / 93$ & 55 & SACS & $T$ & \\
\hline TE-107-AP-46 & $11 / 30 / 93$ & 64 & SACS & $T$ & \\
\hline TE-107-AP-46 & $12 / 14 / 33$ & 81 & SACS & $T$ & \\
\hline TE-107-AP-46 & $12 / 20 / 93$ & 57 & SACS & $T$ & \\
\hline TE-107-AP-46 & $12 / 27 / 93$ & 58 & SACS & $T$ & \\
\hline TE-107-AP-46 & $1 / 3 / 94$ & 56 & SACS & $\bar{T}$ & \\
\hline TE-107-AP-46 & $1 / 11 / 94$ & 59 & SACS & $\mathbf{T}$ & \\
\hline TE-107-AP-46 & 1/19/94 & 58 & CASS & $\overline{\mathbf{T}}$ & \\
\hline TE-107-AP.46 & $1 / 25 / 94$ & 58 & CASS & 7 & \\
\hline TE-107-AP-46 & $2 / 2 / 94$ & 58 & CASS & $\mathbf{T}$ & \\
\hline TE-107-AP-46 & $2 / 12 / 94$ & 56 & CASS & $T$ & \\
\hline TE-107-AP-46 & $2 / 16 / 94$ & B8 & CASS & $T$ & \\
\hline TE-107-AP-A6 & $2 / 28 / 94$ & 58 & CASS & $\mathbf{T}$ & \\
\hline$T E-107-A P-46$ & $2 / 28 / 94$ & 58 & SACS & $\bar{T}$ & \\
\hline$T E-107-A P-46$ & $3 / 10 / 94$ & 57 & CASS & $\mathbf{T}$ & \\
\hline TE-107-AP-46 & $3 / 17 / 94$ & 57 & CASS & $\mathbf{T}$ & \\
\hline TE:107-AP-46 & 3/26/94 & 58 & CASS & $\mathbf{T}$ & \\
\hline TE-107-AP-46 & $3 / 29 / 94$ & 59 & CASS & $\mathbf{T}$ & \\
\hline TE-107-AP-46 & 4/9/94 & 58 & CASS & $\bar{T}$ & \\
\hline TE-107-AP=46 & $9 / 15 / 94$ & 58: & CASS & $\bar{T}$ & \\
\hline TE-107-AP-46 & 4/22/94 & 57 & CASS & $\mathbf{T}$ & \\
\hline TE-107-AP-46 & 4/27/94 & 61 & CASS & $\mathbf{T}$ & \\
\hline TE-107-AP-46 & 5/2/94 & 61 & CASS & $\mathbf{T}$ & \\
\hline TE-107-AP-46 & 5/12/94 & 58 & CASS & $\mathbf{T}$ & \\
\hline TE-107-AP-46 & 5/16/94 & 94 & CASS & $\mathbf{T}$ & \\
\hline TE-107-AP-46 & 5/18/94 & 142 & CASS & $\mathbf{5}$ & Data point did not agree with local trend \\
\hline TE-107-AP-46 & 5/25/94 & 99 & CASS & $T$ & \\
\hline TE-107-AP-46 & 6/2/94 & 89 & CASS & $\mathbf{T}$ & \\
\hline TE-107-AP-46 & 6/8/94 & 88 & CASS & $\mathbf{T}$ & \\
\hline TE-107-AP-46 & 6/14/94 & 86 & CASS & $\mathbf{T}$ & \\
\hline TE-107-AP-46 & $6 / 16 / 94$ & 36: & CASS & $\mathbf{5}$ & Date point did not agree with local trond \\
\hline TE-107-AP-46 & 6/17/94 & 57 & CASS & $\mathbf{s}$ & Data point did not apree with focal trend \\
\hline TE-107-AP-46 & $6 / 20 / 94$ & 59 & CASS & $\mathbf{S}$ & Data point did not agree with local trend \\
\hline TE-107-AP-46 & $6 / 20 / 94$ & 55 & SACS & $S$ (G in SACS) & Date point did nol acree with local trend \\
\hline TE-107-AP-46 & 6/24/94 & 89 & CASS & $T$ & \\
\hline TE-107-AP-46 & $6 / 27 / 94$ & 88 & CASS & $T$ & \\
\hline TE-107-AP-46 & $7 / 7 / 94$ & 87 & CASS & $\mathbf{T}$ & \\
\hline TE-107-AP.46 & $7 / 16 / 94$ & 87 & CASS & $\mathbf{T}$ & \\
\hline TE-107-AP-46 & $7 / 20 / 94$ & 85 & CASS & $\mathbf{T}$ & \\
\hline TE-107.AP-46 & $7 / 30 / 94$ & 84 & CASS & $\mathbf{T}$ & \\
\hline TE-107-AP-46 & $7 / 31 / 94$ & 87 & CASS & $T$ & \\
\hline TE-107-AP-46 & $8 / 9 / 94$ & 92 & CASS & $\mathbf{T}$ & \\
\hline TE-107-AP-46 & $8 / 20 / 94$ & 88 & CASS & $T$ & \\
\hline TE-107-AP-46 & $8 / 22 / 94$ & 88 & CASS & $\mathbf{T}$ & \\
\hline TE-107-AP-46 & $9 / 3 / 94$ & 80 & CASS & $\mathbf{T}$ & \\
\hline TE-107-AP-46 & $9 / 9 / 94$ & 92 & CASS & $T$ & \\
\hline TE-107-AP-46 & $9 / 15 / 94$ & 91 & CASS & $T$ & \\
\hline TE-107-AP-46 & $9 / 23 / 94$ & 91 & CASS & $T$ & \\
\hline TE-107-AP-46 & $9 / 30 / 94$ & 95 & CASS & $T$ & \\
\hline TE-107-AP-46 & 10/8/94 & 96 & CASS & $\bar{T}$ & \\
\hline TE-107-AP-46 & $10 / 12 / 94$ & 96 & CASS & $\bar{T}$ & \\
\hline TE-107-AP-46 & $10 / 22 / 34$ & 98. & CASS & $T$ & \\
\hline TE-107-AP-46 & $10 / 29 / 94$ & 99? & CASS & $T$ & \\
\hline TE-107-AP-46 & $71 / 5 / 94$ & 991 & CASS & $T$ & \\
\hline TE-107-AP-46 & $11 / 11 / 94$ & 99 & CASS & $T$ & \\
\hline TE-107-AP-46 & $11 / 14 / 94$ & 97 & CASS & $T$ & \\
\hline TE-107-AP-46 & $11 / 22 / 94$ & 981 & CASS & $T$ & \\
\hline TE-107-AP-46 & $12 / 3 / 94$ & 91 & CASS & $T$ & \\
\hline TE-107-AP-46 & $12 / 4 / 94$ & 95 & CASS & $\bar{T}$ & \\
\hline TE-107-AP-46 & $12 / 12 / 94$ & 89 & CASS & $T$ & \\
\hline TE-107-AP-46 & $12 / 19 / 94$ & 92 & CASS & $T$ & \\
\hline$T E-107-A P-46$ & $12 / 30 / 94$ & 83 & CASS & $\bar{T}$ & \\
\hline TE-107-AP-46 & $1 / 4 / 95$ & 34 & CASS & $T$ & \\
\hline
\end{tabular}




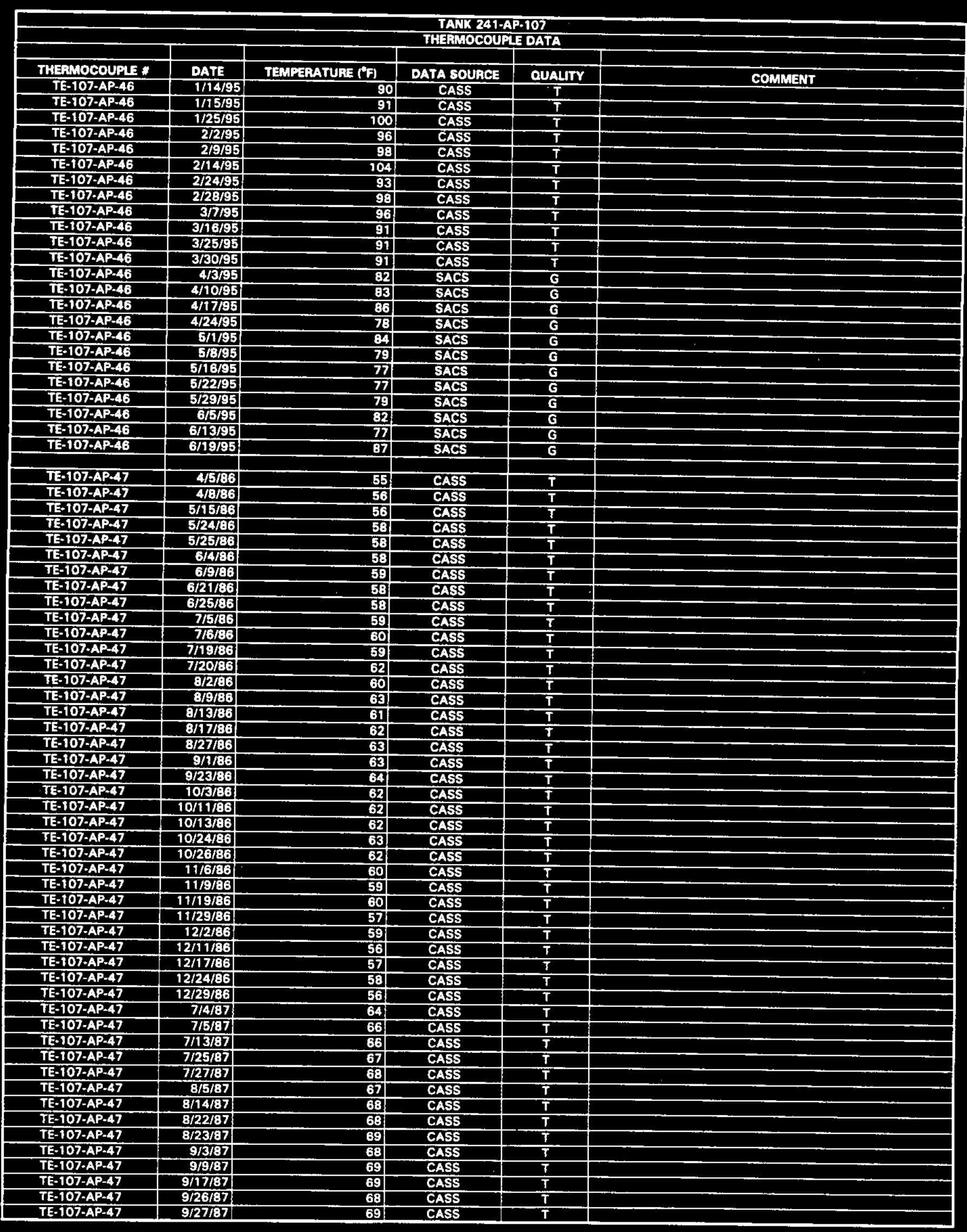




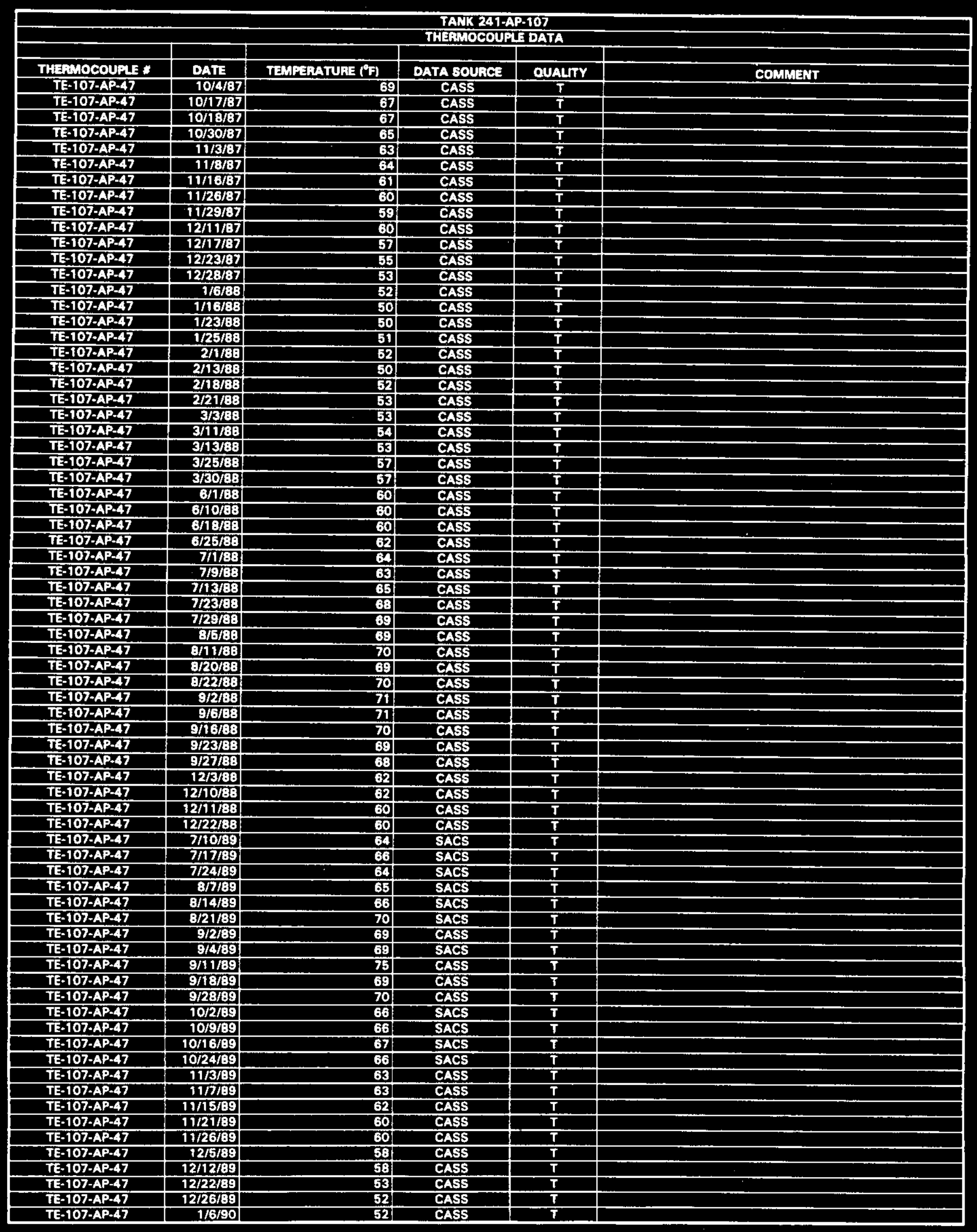




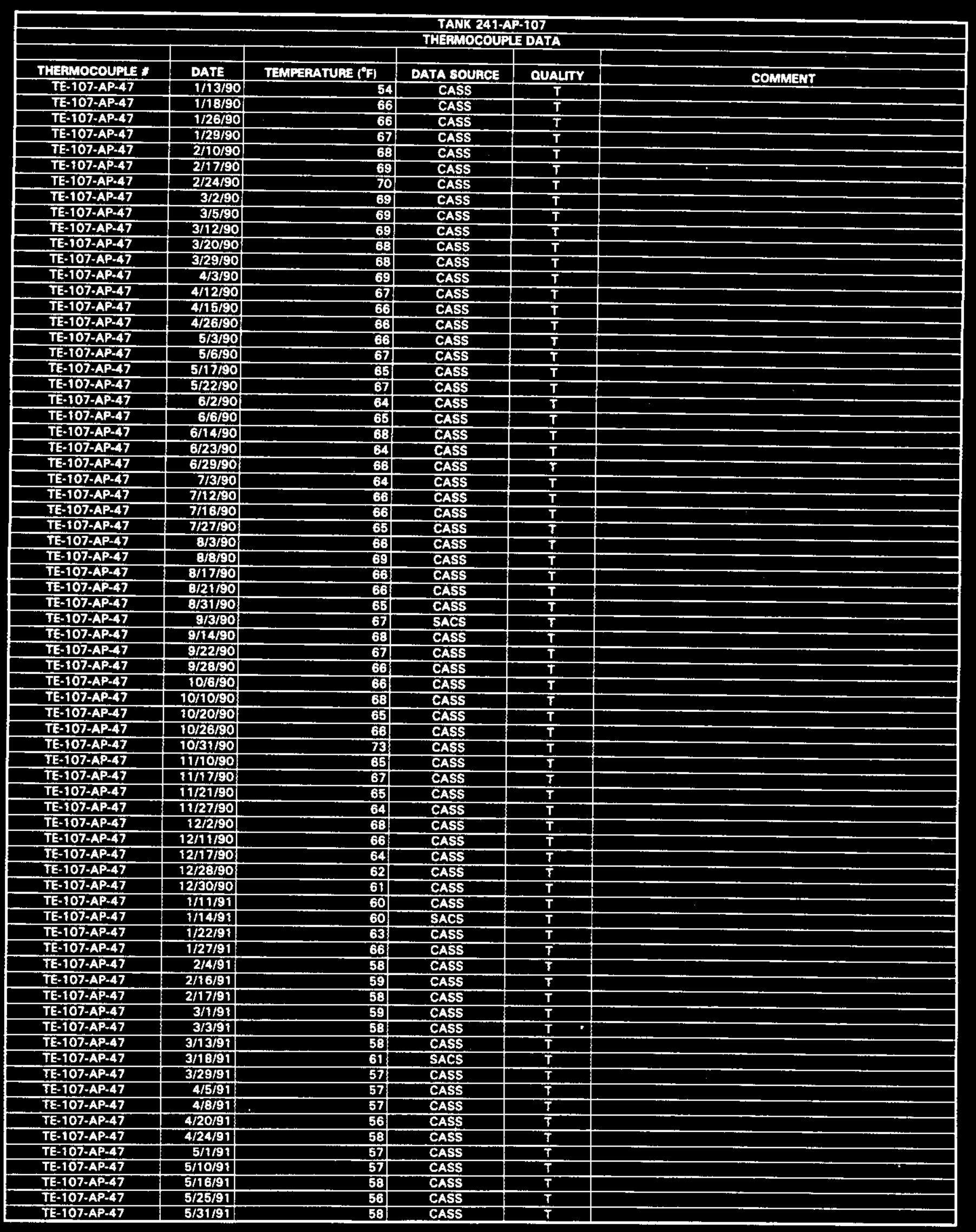




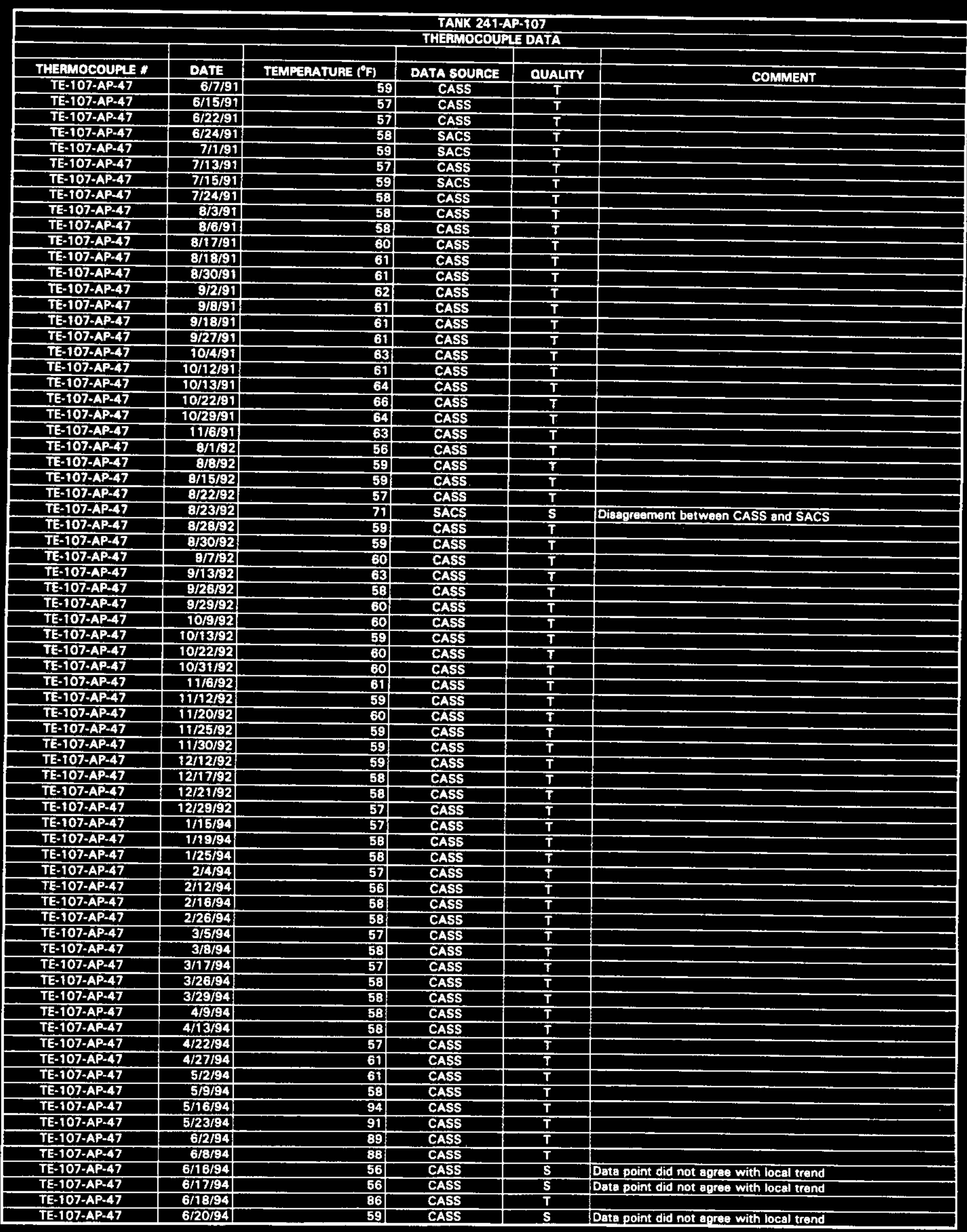

Data obtained from Computer Autometed Surveillance Systam (CASS), April 1995; and Surveillance Analysis Computer System (SACS), JUrie 1995.

D-616 


\begin{tabular}{|c|c|c|c|c|c|}
\hline \multicolumn{6}{|c|}{$\begin{array}{l}\text { TANKK 241-AP-107 } \\
\text { THEHIMOCOUPLE DATA }\end{array}$} \\
\hline & & & & & \\
\hline THERHMOCOUPE : & DATE & TEMPERATUAE $\left({ }^{\circ} \mathrm{F}\right)$ & DATA 8OURCE & OUALITY & COMMENT \\
\hline TE-107-AP-47 & $6 / 24 / 94$ & 89 & CASS & $T$ & \\
\hline $\begin{array}{l}\text { TE-107-AP-47 } \\
\text { TE-107-AP-47 }\end{array}$ & $\frac{6 / 27 / 94}{7 / 7 / 94}$ & 88 & $\frac{\text { CASS }}{\text { CASS }}$ & $\frac{T}{T}$ & \\
\hline & \begin{tabular}{r|}
$7 / 7 / 94$ \\
$7 / 16 / 94$
\end{tabular} & $\frac{87}{87}$ & $\frac{\text { CASS }}{\text { CASS }}$ & $\frac{T}{T}$ & \\
\hline$\frac{T E-107-A P-47}{T E-107-A P-47}$ & $7 / 20 / 94$ & 85 & CASS & $T$ & \\
\hline TE-107-AP-47 & $7 / 30 / 34$ & 84 & CASS & $T$ & \\
\hline TE-107-AP-47 & $7 / 31 / 94$ & 87 & CASS & $T$ & \\
\hline TE-107-AP-47 & $8 / 9 / 94$ & 91 & CASS & $T$ & \\
\hline TE-107-AP-47 & $8 / 20 / 94$ & 89 & CASS & $T$ & \\
\hline TE-107.AP-47 & $8 / 22 / 94$ & 88 & CASS & $\bar{T}$ & \\
\hline TE-107-AP-47 & $9 / 3 / 94$ & 80 & CASS & $\overline{\mathbf{T}}$ & \\
\hline TE-107-AP-47 & $9 / 9 / 94$ & 92 & CASS & $T$ & \\
\hline TE-107-AP-47 & 9/15/94 & 91 & CASS & $T$ & \\
\hline TE-107.AP-47 & 9/23/94 & 91 & CASS & $\frac{T}{T}$ & \\
\hline TE-107-AP-47 & 9/30/94 & 94 & CASS & $\mathbf{T}$ & \\
\hline TE-107-AP-47 & $10 / 8 / 94$ & 96 & CASS & $T$ & \\
\hline TE-107-AP-47 & 10/12/94 & 96 & CASS & $\mathbf{T}$ & \\
\hline TE-107-AP=47 & $10 / 22 / 94$ & 98 & CASS & $T$ & \\
\hline TE-107-AP-47 & $10 / 25 / 94$ & 101 & CASS & $T$ & \\
\hline TE-107-AP-47 & $11 / 5 / 94$ & 99 & CASS & $T$ & \\
\hline TE-107-AP-47 & $11 / 11 / 94$ & 98 & CASS & $T$ & \\
\hline TE-107-AP-47 & $11 / 14 / 94$ & 97 & CASS & $T$ & \\
\hline TE-107-AP-47 & $11 / 22 / 34$ & 98 & CASS & $T$ & \\
\hline TE-107-AP-47 & $12 / 3 / 34$ & 91 & CASS & $\bar{T}$ & \\
\hline TE-107-AP-47 & $12 / 4 / 94$ & 85: & CASS & $T$ & \\
\hline TE-107-AP.47 & $12 / 12 / 94$ & 89 & CASS & $T$ & \\
\hline TE-107.AP-47 & $12 / 19 / 94$ & 92 & CASS & $T$ & \\
\hline TE-107.AP-47 & $12 / 30 / 94$ & 93 & CASS & $T$ & \\
\hline TE-107.AP-47 & $1 / 4 / 95$ & 94 & CASS & $T$ & \\
\hline TE-107-AP-47 & //13/95 & 81 & CASS & $T$ & \\
\hline IT-107-AP-47 & MI5/95 & 911 & CASS & $\mathbf{T}$ & \\
\hline TE-107-AP-47 & $1 / 25 / 95$ & 100 & CASS & $\mathbf{T}$ & \\
\hline TE-107-AP-47 & $2 / 215$ & 96 & CASS & $T$ & \\
\hline TE-107-AP-47 & 2/9/95 & 98| & CASS & $T$ & \\
\hline TE-107-AP-47 & $2 / 14 / 95$ & 104 & CASS & $\bar{T}$ & \\
\hline$T E-107 \cdot A P-47$ & $2 / 24 / 95$ & 93 & CASS & $T$ & \\
\hline TE-107-AP.A7 & $2128 / 95$ & 98 & CASS & $T$ & \\
\hline$T E-107-A P-47$ & 3/9/95 & 86 & CASS & $\bar{T}$ & \\
\hline$T E-107 \cdot A P-47$ & 3/16/95 & 91 & CASS & $\bar{T}$ & \\
\hline TE-107-AP-47 & $3 / 25 / 95$ & 91 & CASS & $\bar{T}$ & \\
\hline TE-107-AP-47 & $3 / 30 / 95$ & 91) & CASS & $T$ & \\
\hline & & & & & \\
\hline$\overline{\mathrm{TE}}-107-A \mathrm{P}-48$ & $4 / 5 / 86$ & 50 & CASS & $\mathbf{s}$ & Disagreament with nearby thermocouples (woekly max) \\
\hline TE-107.AP-48 & A/9/86 & 56 & CASS & $\mathbf{S}$ & Disagreernent with naarby thermocouples (woskly max) \\
\hline TE-107-AP-48 & $5 / 17 / 86$ & 63. & CASS & $\mathbf{S}$ & Disapreament with nearby thermocouplas (weakly max) \\
\hline TE-107-AP-48 & $5 / 20 / 36$ & 69 & CASS & $\mathbf{S}$ & Disagreament with nearby thermocouplas (waekly max) \\
\hline TE-107-AP-48 & $5 / 31 / 86$ & 84 & CASS & $\mathbf{S}$ & Disegreement with nearby thermocouples (woekly max) \\
\hline TE-107-AP-48 & 6/1/86 & 84 & CASS & $\mathbf{S}$ & Disapreement with nearby thermocouples (woekly max) \\
\hline TE-107-AP-48 & $6 / 14 / 86$ & 80 & CASS & $\mathbf{S}$ & Disagreement with nearby thermocouples (woekly max) \\
\hline TE-107-AP-48 & $6 / 16 / 86$ & 77 & CASS & $\overline{\mathbf{S}}$ & Disagreement with nearby thermocouples (woekly max) \\
\hline TE-107-AP-48 & $6 / 24 / 86$ & 80 & CASS & $\mathbf{s}$ & Disagreament with nearby thermocouples (waekly max) \\
\hline TE-107-AP-A8 & $7 / 3 / 86$ & 7il & CASS & s & Disegreament with nearby thermocouples (waekly max) \\
\hline TE-107.AP-A8 & $7 / 10 / 86$ & 73 & CASS & $\mathbf{S}$ & Disagreement with noarby thermocouples (woekly mox) \\
\hline TE-107-AP-48 & 7/19/86 & 76 & CASS & $\mathbf{S}$ & Disagreament with narby thermocouples (woekly mox) \\
\hline TE-107-AP-48 & $7 / 21 / 86$ & 80 & CASS & $\mathbf{S}$ & Disagreament with nearby thermocouples (woekly max) \\
\hline TE-107-AP-48 & $8 / 2 / 86$ & 79 & CASS & $\mathbf{s}$ & Disagreament with nadrby thermocouplas (woekly max) \\
\hline$T E-107-A P-48$ & $8 / 9 / 86$ & 84 & CASS & $\mathbf{5}$ & Disagreannent with nearby thermocouplas (wookly max) \\
\hline TE-107.AP-48 & $8 / 10 / 86$ & 84 & CASS & $\mathbf{5}$ & Disagreantent with nearby thermocouples (weekly max) \\
\hline TE-107-AP-48 & $8 / 21 / 86$ & 81 & CASS & s & Disegreament with nearby thermocouplas (weekly max) \\
\hline TE-107-AP-48 & $8 / 28 / 86$ & 83 & CASS & $\mathbf{s}$ & Disagreament with nearby thermocouples (woekly max) \\
\hline TE-107-AP-48 & 9/3/86 & 76 & CASS & S & Disagreament with nearby thermocouples (woekly max) \\
\hline TE-107-AP-48 & 9/23/86 & 63 & CASS & $\mathbf{s}$ & Disagreement with rearby thermocouples (weekly max) \\
\hline TE-107-AP-48 & $9 / 29 / 86$ & 61 & CASS & $\mathbf{S}$ & Disagreement with neaby thermocouples (weakly mox) \\
\hline TE-107-AP-48 & 10/10/86 & 62 & CASS & $\mathbf{S}$ & Disagreement with nearby thermocouples (weekly max) \\
\hline TE-107-AP-48 & 10/16/86 & 55 & CASS & $\overline{\mathbf{S}}$ & Disagreement with nearby thermocouples (weekly max) \\
\hline TE-107.AP-48 & $10 / 24 / 86$ & 55 & CASS & S & Disagreement with nearby thermocouples (weekly max) \\
\hline TE-107-AP-48 & $10 / 26 / 86$ & 58! & CASS & $\mathbf{s}$ & Disagreement with nearby thermocouples (weakly max) \\
\hline TE-107-AP-48 & $11 / 4 / 86$ & 51 & CASS & $\mathbf{S}$ & Dissoreement with nearby thermocouples (woekly max) \\
\hline TE-107-AP-48 & $11 / 9 / 86$ & 44 & CASS & $\mathbf{S}$ & Disagreement with noarby thermocouples (woekly max) \\
\hline TE-107-AP-48 & $11 / 20 / 86$ & 51 & CASS & $\mathbf{S}$ & Disagreement with nearby thermocouples (waekly max) \\
\hline TE-107-AP-48 & $11 / 24 / 86$ & $\mathbf{5 3}$ & CASS & $\mathbf{s}$ & Disagreement with noarby thermocouplos (weekly max) \\
\hline TE-107.AP-48 & $11 / 30 / 86$ & 42 & CASS & $\mathbf{s}$ & Disagreement with nearby thermocouples (woekly max) \\
\hline TE-107-AP-48 & $12 / 8 / 86$ & 38 & CASS & $\mathbf{S}$ & Disagreement with nearby thermocouples (weekly max) \\
\hline
\end{tabular}


WHC-SD-WM-ER-315, Rev.

\begin{tabular}{|c|c|c|c|c|c|}
\hline \multirow{2}{*}{\multicolumn{6}{|c|}{$\begin{array}{l}\text { TANK 241-AP.107 } \\
\text { THEAMOCOUPIE DATA }\end{array}$}} \\
\hline & & & & & \\
\hline THERMOCOUPLE * & DATE & TEMPERATURE ("F) & DATA SOUACE & QUALITY & COMMENT \\
\hline TE-107-AP-48 & $12 / 20 / 86$ & 37 & CASS & $\mathrm{s}$ & Disagceement with nearby thermocouples (weekly $\mathrm{max}$ ) \\
\hline TE-107-AP-48 & $12 / 24 / 86$ & 39 & CASS & $\mathbf{S}$ & Disapreament with nearby thermocouples (weekly max) \\
\hline TE-107-AP-48 & $12 / 29 / 86$ & 39 & CASS & $\mathbf{S}$ & Disagreement with nearby thermocouples (weekly max) \\
\hline$T E-107 \cdot A P-48$ & $7 / 1 / 87$ & 85 & CASS & $\mathbf{s}$ & Disapreement with nearby thermocouples (woekly max) \\
\hline TE-107-AP-4B & 7/8/87 & 75 & CASS & $\mathbf{s}$ & Diagareement with nearby thermocouples (wookly max) \\
\hline TE-107-AP-48 & $7 / 15 / 87$ & 81 & CASS & $\mathbf{S}$ & Disagreement with nearby thermocouples (weekly mex) \\
\hline TE-107-AP-48 & $7 / 25 / 67$ & 80 & CASS & $\mathbf{S}$ & Disagrearnent with nearby thermocouples (wookly max) \\
\hline TE-107-AP-48 & $7 / 27 / 87$ & 79 & CASS & $\mathbf{s}$ & Diser reament with nearby tharmocouples (woekly max) \\
\hline TE-107-AP-48 & $8 / 8 / 87$ & 81 & CASS & $\mathbf{S}$ & Disagreernent with nearby thermocouples (woekly max) \\
\hline TE-107-AP-48 & $8 / 10 / 87$ & 82 & CASS & s & Disagraemant with noarby tharmocouples (woekly inax) \\
\hline TE-107-AP-48 & $8 / 22 / 87$ & 74 & CASS & $\overline{\mathbf{S}}$ & Disagreement with nearby tharmocouples (weakly max) \\
\hline IE-107-AP-4B & $8 / 28 / 97$ & 78 & CASS & $\mathbf{S}$ & Oisepreernent with nearby thermocouplas (weekly max) \\
\hline IE-107-AP-48 & $9 / 1 / 87$ & 84 & CASS & $\mathbf{s}$ & Diseproement whh noarby thermocouples (woekly max) \\
\hline TE-107-AP-48. & 9/8/87 & 78 & CASS & s & Disadroement with nearby thermocouples (woekly max) \\
\hline$T=107-A P-48$ & $9 / 13 / 87$ & $73 !$ & CASS & $\mathbf{s}$ & Disagreement with nearby tharmocouplas (wookly $\max$ ) \\
\hline TE-107-AP, 48 & $9 / 25 / 87$ & 72 & CASS & $\mathbf{s}$ & Diasproenemt with noarby tharmocouples (woekly max) \\
\hline TE-107-AP-4B & $10 / 3 / 87$ & 67 & CASS & $\mathbf{s}$ & Disaproement with nearby tharmocouplos (wookly max) \\
\hline TE-107-AP-48 & $10 / 8 / 87$ & 68 & CASS & $\mathbf{s}$ & Disagreement with nearby thormocouplos (weokly $\mathrm{max}$ ) \\
\hline TE-107-AP.48 & $10 / 12 / 87$ & 58 & CASS & $\mathbf{s}$ & Disegreemsmt with nasiby tharmocouples (weakly max) \\
\hline TE-107-AP-48 & $10 / 18 / 97$ & 55 & CASS & $\mathbf{5}$ & Disegreement with naarby tharmocouples (weekly max) \\
\hline TE-107-AP-48 & $10 / 31 / 87$ & 55 & CASS & $\mathbf{s}$ & Diegreement with nasorby thermocouplos (weokly max) \\
\hline TE-107.AP-48 & $11 / 2 / 87$ & 57 & CASS & $\mathbf{s}$ & Disagreverment with nearby thermocouples (weakly $\mathrm{max}$ ) \\
\hline TE-107-AP-4B & $111 / 13 / 37$ & 54 & CASS & $\bar{s}$ & Diasercesment with nearby thermocouples (weekty max) \\
\hline TE-107-AP-4B & $11 / 16 / 87$ & 46 & CASS & $\mathbf{s}$ & Disapreennent with nearby themocouples (woekly $\max$ ) \\
\hline$T E-107-A P-48$ & $11 / 22 / 87$ & 43 & CASS & $\mathbf{s}$ & Dieagroermant with noarby thermocouplos (woakly max) \\
\hline TE-107-AP-48 & $12 / 4 / 87$ & 45 & CASS & $\mathbf{s}$ & Disegroement with nosiby thaimocouples (woekky max) \\
\hline TE-107-AP-48 & $12 / 6 / 97$ & 47 & CASS & $\mathbf{S}$ & Diagorcement with nearby tharmocouples (weekly max) \\
\hline TE-107-AP-48 & $12 / 17 / 87$ & 37 & CASS & s & Diangrearnent with nearby thermocouplas (woekly max) \\
\hline TE-107-AP-48 & $12 / 21 / 87$ & 34 & CASS & S & Diaggreament whe nearby thermocouplas (woekly max) \\
\hline IE-107-AP-4B & 1/1/88 & 33 & CASS & 5 & Disepreament with nearby thermocouples (weokly max) \\
\hline TE-107-AP-48 & $1 / 6 / 88$ & 33 & CASS & 5 & Diserreament with nearby thermocouples (weokly max) \\
\hline TE-107-AP-48 & $1 / 13 / 88$ & 87 & CASS & $\mathbf{s}$ & Diedrevement with nearby thermocouplas (wookly $\max$ ) \\
\hline TE-107-AP-48 & $1 / 23 / 38$ & 37 & CASS & s & Diagdreement with noarby thermocouples (wookly max) \\
\hline TE-107-AP-48 & $1 / 29 / 88$ & 37 & CASS & $\mathbf{s}$ & Diseproement with nearby thermocouplos (weekly max) \\
\hline TE-107-AP-AB & $1 / 31 / 88$ & 35 & CASS & $\mathbf{S}$ & Divepresment with nearby thermocouples (wookly mex) \\
\hline TE-107-AP-4B & $2 / 13 / 88$ & 42 & CASS & $\mathbf{s}$ & Disegreerment with nearby tharmocouplos (wookly max) \\
\hline TE-107-AP-48 & $2 / 18 / 88$ & 42 & CASS & $\overline{\mathbf{s}}$ & Diacproement with nearby thermocouplas (weekly $m$ max) \\
\hline TE-107-AP-48 & $2 / 21 / 88$ & 44 & CASS & $\mathbf{s}$ & Disagreement with neaby thermocouplos (weekly max) \\
\hline TE-107-AP.48 & $3 / 3 / 68$ & 48 & CASS & $\mathbf{S}$ & 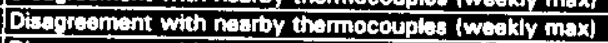 \\
\hline IE-107-AP-48 & 3/9/8 & 45 & CASS & $\mathbf{S}$ & Direpreament with neaby tharmocouples (weokly mex) \\
\hline TE-107-AP-48 & 3/19/88 & 46 & CASS & $\overline{\mathbf{s}}$ & Diangreament with nearby thermocouples (weakly max) \\
\hline TE-107-AP-48 & $3 / 26 / 88$ & 51 & CASS & $\mathbf{S}$ & Disagredment with nearby thermocouples (weekly max) \\
\hline TE-107-AP-48 & $3 / 30 / 8 \mathrm{~B}$ & 50 & CASS & S & Disepreament with noarby thermocouples (weakly max) \\
\hline TE-107-AP-48 & $6 / 1 / 8$ & 64 & CASS & $\mathbf{s}$ & Diespreement with nearby tharmocouples (weekly $\mathrm{max}$ ) \\
\hline TE-107-AP-48 & $6 / 5 / 38$ & 64) & CASS & $\bar{s}$ & Diserreenent with nearby thermocouples (woekly max) \\
\hline TE-107-AP-48 & $6 / 16 / 88$ & 76 & CASS & $\mathbf{s}$ & Disogreernent with nearby thermocouples (woekly max) \\
\hline TE-107-AP-48 & $6 / 25 / 38$ & 79 & CASS & $\mathbf{s}$ & Disegreament with noerby thermocouples (weekly max) \\
\hline TE-107-AP-48 & $7 / 2 / 88$ & 77 & CASS & $\mathbf{s}$ & Disegreament with nearby thermocouplas (weekly max) \\
\hline TE-107-AP-48 & $7 / 8 / 88$ & 79 & CASS & s & Disagreament with nearby thermocouples (woekly $\mathrm{max}$ ) \\
\hline TE-107-AP-48 & $7 / 71 / 88$ & 82 & CASS & $\mathbf{S}$ & Disegreament with nosiby thermocouples (woekly max) \\
\hline TE-107-AP-4B & $7 / 22 / 88$ & 85 & CASS & $\mathbf{S}$ & Disagraement with nearby thermocouples (weakly max) \\
\hline TE-107.AP-48 & $7 / 27 / 88$ & 90 & CASS & $\mathbf{S}$ & Disepreement with nearby thermocouples (waekly max) \\
\hline TE-107-AP-48 & $7 / 31 / 80$ & 85 & CASS & S & Disagrcement with nearby thermocouplas (wookly max) \\
\hline TE-107-AP-AB & $8 / 11 / 88$ & 82 & CASS & $\mathbf{s}$ & Disapreement with nearby thermocouplas (weekjy max) \\
\hline TE-107-AP-48 & $8 / 14 / 8$ & 79 & CASS & S & Disagreement with nestoy thermocouplas (woekly max) \\
\hline TE-107-AP-48 & $8 / 25 / 88$ & 82 & CASS & $\mathbf{s}$ & Dicegreement with noarby thermocouples (weekly max) \\
\hline TE-107-AP-48 & $8 / 29 / 89$ & 81 & CASS & $\mathbf{S}$ & Disepreement with nearby thermocouples (weokly max) \\
\hline TE-107-AP-48 & $9 / 5 / 88$ & 81 & CASS & $\mathbf{s}$ & Disegrcement with nearby thermocouples (weekly mox) \\
\hline TE-107-AP-48 & $9 / 15 / 88$ & 74 & CASS & $\frac{1}{\mathrm{~S}}$ & Diseproement with nearby tharmocouples (weekly $\mathrm{max}$ ) \\
\hline TE-107-AP-48 & 9/19/88 & 66 & CASS & $\mathbf{S}$ & Diasagreement with nearby thermocouples (weekly max) \\
\hline$T E-107-A P-48$ & $9 / 27 / 88$ & 66 & CASS & $\mathbf{S}$ & Diagprement with nearby thermocouples (weekly max) \\
\hline TE-107-AP-48 & 12/1/89 & 41 & CASS & $\mathbf{s}$ & Disagreentent with nearby thermocouples (weokly max) \\
\hline$T E-107-A P-48$ & $12 / 10 / 8$ & 44 & CASS & $\mathbf{s}$ & Disepreement with nearby thermocouplos (woekly max) \\
\hline TE-107-AP-48 & $12 / 13 / 38$ & 44 & CASS & $\mathbf{S}$ & Disaproement with nearby thermocouples (woekly mox) \\
\hline TE-107-AP-4B & $12 / 22 / 88$ & 37 & CASS & $\mathbf{s}$ & Disapresment with nearby thermocouples (woekly max) \\
\hline TE-107-AP-48 & 9/1/89 & 72 & CASS & $\mathbf{S}$ & Disapresment with noarby thermocouplas (woekly max) \\
\hline TE-107-AP-48 & 9/8/89 & 72 & CASS & $\mathbf{S}$ & Diagrreement with nearby thermocouples (weekly max) \\
\hline TE-107-AP-48 & $9 / 11 / 89$ & 75 & CASS & $\mathbf{s}$ & Disegreement with nearby thermocouples (wcekly max) \\
\hline TE-107-AP-48 & 9/18/89 & 70 & CASS & $\mathbf{s}$ & Disagrearment with naarby thermocouplea (weekly max) \\
\hline TE-107-AP-48 & $9 / 29 / 89$ & 71 & CASS & $\mathbf{S}$ & Disagreament with naarby thermocouplos (weakly max) \\
\hline TE-107-AP-4B & $11 / 4 / 89$ & 51 & CASS & $\mathbf{s}$ & Disagreernemt with nearby tharmocouples (weakly max) \\
\hline TE-107-AP.48 & 11/10/39 & 58: & CASS & $\mathbf{S}$ & Disaproerment with nearby thermocouples (woekly max) \\
\hline$T E=107-A P+48$ & $11 / 12 / 89$ & 561 & CASS & $\mathbf{5}$ & Disegreement with nearby thermocouples (woekly max) \\
\hline
\end{tabular}




\begin{tabular}{|c|c|c|c|c|c|}
\hline \multicolumn{6}{|c|}{$\begin{array}{l}\text { TANK 241-AP.107 } \\
\text { THEFMOCOUFLE DATA }\end{array}$} \\
\hline THERMOCOUFE \& & & & & & \\
\hline TE-107-AP-48 & DAUE & 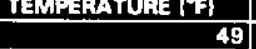 & $\frac{\text { DATA SOUACE }}{\text { CASS }}$ & $\frac{\text { QUALITY }}{\mathrm{S}}$ & COMMENT \\
\hline TE-107-AP-48 & $11 / 26 / 89$ & 47 & CASS & $\frac{\mathbf{s}}{\mathbf{s}}$ & $\begin{array}{l}\text { Oisagreement with nearby thermocouplas (weekly max) } \\
\text { Disagreement with nearby thermocouples (weekly max) }\end{array}$ \\
\hline TE-107-AP-48 & $12 / 8 / 39$ & 45 & CASS & $\mathbf{s}$ & Disagreement with nearby thermocouples (weakly max) \\
\hline TE-107.AP-4B & $12 / 10 / 89$ & 41 & CASS & $\mathbf{s}$ & Disagreement with nearby thermocouples (weekly $\mathrm{max}$ ) \\
\hline TE-107-AP-48 & $12 / 17 / 89$ & 39 & CASS & $\mathbf{S}$ & Disadreement with nearby thermocouples (weakly max) \\
\hline TE-107-AP-48 & 12/25/89 & 40 & CASS & $\mathbf{S}$ & Oisagroement with nearby thermocouples (weekly max) \\
\hline TE-107-AP-48 & $1 / 6 / 20$ & 44. & CASS & $\mathbf{s}$ & Disagreement with nearby thermocauples (weekly max) \\
\hline TE-107-AP-48 & $1 / 7 / 90$ & 47 & CASS & $\mathbf{s}$ & Disagreement with nearby tharmocouples (wookly max) \\
\hline TE-107-AP-48 & $1 / 16 / 90$ & 43 & CASS & $\mathbf{s}$ & Dissogresment with nearby thermocouplas (woekly $\mathrm{mox}$ ) \\
\hline TE-107-AP-48 & $1 / 23 / 20$ & 44 & CASS & $\mathbf{S}$ & Disagreement with nearby thermocouples (weokly $\mathrm{mex}$ ) \\
\hline TE-107-AP-48 & $1 / 29 / 90$ & 45 & CASS & $\mathbf{s}$ & Disedreement with neaby thermocouples (woekly max) \\
\hline TE-107-AP-48 & $2 / 10 / 90$ & 46 & CASS & $\mathbf{S}$ & Disegroement with neaby thermocouples (wedkly max \\
\hline TE-107-AP-48 & $2 / 11 / 30$ & 49 & CASS & $\mathbf{S}$ & Disagreament with nearby thermocouples (weakly max) \\
\hline TE-107-AP-48 & $2 / 24 / 90$ & 47 & CASS & $\mathbf{S}$ & Dinagreanent with nearby thermocouples (weekly max) \\
\hline TE-107-AP-48 & $3 / 3 / 90$ & 47 & CASS & $\mathbf{S}$ & Disagreament with nearby thermocouples (weakly max) \\
\hline TE-107-AP-48 & $3 / 10 / 90$ & 51 & CASS & $\mathbf{s}$ & Dieagreanent with nasiby thermocouples (wedkly max) \\
\hline TE-107.AP-48 & $3 / 37 / 90$ & 54 & CASS & $\mathbf{S}$ & Disegreement with nearby thermocouplos (weekly mex) \\
\hline TE-107-AP-48 & 3/19/90 & 66. & SACS & $T$ & \\
\hline TE-107-AP-48 & $3 / 22 / 90$ & 57 & CASS & $\mathbf{S}$ & Disagraement with naarby thermocouples (woekly $\mathrm{max}$ ) \\
\hline TE-107-AP-4B & $3 / 33 / 90$ & 38 & CASS & $\mathbf{5}$ & Disagreament with noarby thermocouples (woekly mox) \\
\hline TE-107-AP-4B & $4 / 7 / 90$ & 62 & CASS & $\mathbf{s}$ & Disagreemant with nastby thermocouples (woekly max) \\
\hline TE-107-AP-48 & $411 / 90$ & 63 & CASS & $\mathbf{s}$ & Diesgraement with naarby thermocouples (weakly max) \\
\hline TE=107-AP-48 & 4/20/90/ & 66 & CASS & $\mathbf{s}$ & Diagoreement with nearby thormocouples (woekly max) \\
\hline TE-107-AP-48 & $4 / 22100$ & 66 & CASS & $\mathbf{S}$ & Disegreement with nearby thermocouples (weokly max) \\
\hline TE-107-AP-48 & 5/5/30 & 67 & CASS & $\mathbf{S}$ & Diseqreement with nearby thermocouples (weakly max) \\
\hline TE-107-AP-4B & $5 / 11 / 30$ & 68 & CASS & $\mathbf{s}$ & Disegreament whith nosiby tharmocouplos (weakly max) \\
\hline TE-107-AP-48 & $5 / 17 / 90$ & 67 & CASS & $\mathbf{S}$ & Disudreernent whe nearby tharmocouplos (weekly max) \\
\hline TE-107-AP-48 & $5 / 22 / 90$ & 69 & CASS & $\mathbf{S}$ & Disagreernent with nearby thermocouples (weakly $\max$ ) \\
\hline TE-107-AP-48 & 6/2/200 & 64 & CASS & $\mathbf{S}$ & Disapreement with nearby thermocouples (weekly max) \\
\hline TE-107-AP-49 & 6/6/80 & 71 & CASS & $\mathbf{S}$ & Disepresenent with naarby thermocouples (wookly max) \\
\hline TE-107-AP-48 & 6/10/90 & 71 & CASS & $\mathbf{S}$ & Diagareement with nearby tharmocouples (weakly max) \\
\hline TE-107-AP-48 & $6 / 22 / 90$ & 78 & CASS & $\mathbf{s}$ & Disarreement with noerby thermocouples (weekly max) \\
\hline TE-107-AP-48 & $6 / 30 / 90$ & 81 & CASS & $\mathbf{s}$ & Disaqreamemt with noarby theimocouples (weekly max \\
\hline TE-107-AP-48 & $7 / 1 / 90$ & 80 & CASS & $\mathbf{s}$ & Dianpreemem with nearby tharmocouples (weekly max) \\
\hline TE-107-AP-48 & $7 / 12190$ & 91 & CASS & $\mathbf{s}$ & Dienoreement with nearby thermocouples (woekly max) \\
\hline TE-T07-AP-AB & $7 / 16 / 90$ & 88 & CASS & $\mathbf{S}$ & Disegreement with nearby tharmocouples (weekly max) \\
\hline TE-107-AP-48 & 7/23/90 & 50 & CASS & $\mathbf{S}$ & Disepresment with nearby thermocouples (weokly max) \\
\hline TE-107-AP-4B & 7/31/90 & 87 & CASS & $\mathbf{S}$ & Disapreement with noarby thermocouples (wookly max) \\
\hline TE-107-AP-48 & 8/8/90 & 90 & CASS & $\mathbf{s}$ & Distapreamem with noarby thermocouples (weekly mox) \\
\hline TE-107-AP-48 & $8 / 13 / 90$ & 88 & CASS & $\mathbf{S}$ & Disagrearment whe nearby thermocouples (weekly max) \\
\hline TE-107-AP-48 & $8 / 19 / 90$ & 82 & CASS & $\mathbf{S}$ & Disegreement with nearby thermocouples (weekly max) \\
\hline TE-107-AP-48 & $8 / 29 / 90$ & 77 & CASS & $\mathbf{S}$ & Disegreement with nearby thormocouples (weokly max) \\
\hline TE-107-AP-48 & $9 / 5 / 90$ & 78 & CASS & $\mathbf{S}$ & Diseqreement with nearby thermocouples (woekly max) \\
\hline TE-107-AP-4B & 9/12/90 & 79 & CASS & $\mathbf{S}$ & Disepreement with nearby thermocouples (woekly max) \\
\hline TE-107-AP-48 & 9/22/90 & 76 & CASS & $\mathbf{s}$ & Diagereament with noarby thermocouples (weakly max) \\
\hline TE-107.AP-48 & 9/25/90 & 76 & CASS & $\mathbf{S}$ & Diangreement with nearby thermocouples (weekly max) \\
\hline IE-107-AP-48 & $9 / 30 / 30$ & 71 & CASS & $\mathbf{S}$ & Disagreament with noarby thermocouples (woskly max) \\
\hline TE-107-AP-48 & $10 / 10 / 90$ & 64 & CASS & $\overline{\mathbf{s}}$ & Disagreement whth noarby thermocouples (weokly max) \\
\hline TE-107.AP-48 & $10 / 14 / 90$ & 60 & CASS & $\mathbf{S}$ & Disedreement with nearby thermocouplas (weakly max) \\
\hline TE-107-AP-48 & $10 / 26 / 90$ & 55 & CASS & $\mathbf{s}$ & Disagreament with nearby thermocouples (woekly max) \\
\hline TE-107-AP-48 & $10 / 31 / 30$ & 59 & CASS & $\mathbf{S}$ & Disegreement with neasby thermacouples (woekly max) \\
\hline TE-107-AP-48 & $11 / 10 / 90$ & 56 & CASS & $\mathbf{S}$ & Disegreament with nearby thermocouples (weekly max) \\
\hline TE-107-AP-48 & $11 / 13 / 90$ & 53 & CASS & $\mathbf{S}$ & Disapreament with rearby thermocouples (woekly max) \\
\hline TE-107-AP-48 & $11 / 24 / 90$ & 52 & CASS & $\mathbf{s}$ & Disagreament with nearby thermocouples (woekly max) \\
\hline$T E-107-A P-48$ & 11/25/90 & 53 & CASS & $\mathbf{S}$ & Disagreement with nearby thermocoupleas (woakly max) \\
\hline TE-107-AP-4B & $12 / 4 / 90$ & 45 & CASS & $\mathbf{S}$ & Disagreament with nearby thermocouples (weekly max) \\
\hline TE-107-AP-48 & $12 / 10 / 20$ & 41 & CASS & $\mathbf{S}$ & Diaagreement with nearby thermocouples (weekly max) \\
\hline TE-107-AP-48 & $12 / 18 / 90$ & 40 & CASS & $\mathbf{S}$ & Disagreement with nearby thermocouplas (weskly max) \\
\hline TE-107-AP-48 & $12 / 28 / 90$ & 30 & CASS & $\mathbf{s}$ & Disagreement with nearby thermocouples (woekly max) \\
\hline TE-107-AP-48 & $1 / 3 / 91$ & 29 & CASS & $\mathbf{S}$ & Disagreament with nearby thermocouplas (woekly max) \\
\hline TE-107-AP-48 & 1/11/97 & 33 & CASS & $\mathbf{s}$ & Diagareamant with nearby thermocouples (woekly max) \\
\hline TE-107-AP-48 & $1 / 15 / 91$ & 38 & CASS & $\mathbf{S}$ & Disegreement with noarby tharmocouples (weekly max) \\
\hline TE-107-AP-48 & $1 / 22 / 91$ & 32 & CASS & $\mathbf{S}$ & Disegreement with nearby thermocouples (waekly max) \\
\hline TE-107-AP-48 & $2 / 2191$ & 34 & CASS & $\mathbf{s}$ & Disagreament with nearby thermocouples (weakly max) \\
\hline TE-107-AP-48 & $2 / 5 / 91$ & 43 & CASS & $\mathbf{S}$ & Disegreement with nearby thermacouples (weakly max) \\
\hline TE-107-AP-48 & $2 / 15 / 91$ & 46 & CASS & $\mathbf{S}$ & Disegreement with nearby thermocouples (woekly max) \\
\hline TE-107-AP-48 & $2 / 20 / 91$ & 46 & CASS & $\mathbf{S}$ & Disagreement with nearby thermocouples (weekly max) \\
\hline TE-107-AP-4B & 3/1/91 & 46 & CASS & $\mathbf{s}$ & Disagreement with noarby thermocouples (weakly max) \\
\hline TE-107-AP-48 & $3 / 4 / 91$ & 45 & CASS & $\mathbf{s}$ & Disagreement with nearby thermocouples (weekly max) \\
\hline TE-107-AP-48 & $3 / 12 / 91$ & 44 & CASS & $\mathbf{S}$ & Diaggreament with nourby tharmocouples (wagkly $\mathrm{max}$ ) \\
\hline TE-107-AP-48 & $3 / 23 / 91$ & 47 & CASS & $\mathbf{s}$ & Fiaggreement with nasiby thermocouples (weekly max) \\
\hline TE-107-AP-48 & $3 / 24 / 91$ & 48 & CASS & $\mathbf{S}$ & Disapreement with nearby thermocouples (weekly max) \\
\hline TE-107-AP-48 & $4 / 5 / 91$ & 56 & CASS & $\mathbf{S}$ & Disagreament with nearby tharmacouples (woekly max) \\
\hline
\end{tabular}


WHC-SD-WM-ER-315, Rev.

\begin{tabular}{|c|c|c|c|c|c|}
\hline \multirow{2}{*}{\multicolumn{6}{|c|}{$\begin{array}{l}\text { TANK 241-AP-107 } \\
\text { THERMOCOUPII DATA }\end{array}$}} \\
\hline & & & & & \\
\hline THERMOCOUPLE * & DATE & TEMPERATURE $\left({ }^{\circ} \mathrm{F}\right)$ & DATA SOUACE & QUALTY & COMMENT \\
\hline TE-107.AP-48 & $4 / 13 / 91$ & 52 & CASS & $\mathbf{s}$ & Disagreament with nearby thermocouplas (woekly max) \\
\hline TE-107-AP-48 & 4/20/91 & 57 & CASS & $\mathbf{s}$ & Disagreennent with nearby thermocouples (woekly max) \\
\hline TE-107-AP-48 & 4/24/91 & 61 & CASS & $\bar{s}$ & Disagreement with nearby thermocouplas (woekly max) \\
\hline TE-107-AP-48 & $5 / 4 / 91$ & 61 & CASS & $\mathbf{s}$ & Disdareement with nearby thermocouples (woekly max) \\
\hline TE-107-AP-48 & 5/5/91 & 64 & CASS & $\mathbf{s}$ & Disepreement with nearby thermocouples (woekly max) \\
\hline TE-107-AP-48 & 5/16/91 & 67 & CASS & $\mathbf{S}$ & Disagreement with noarby thermocouples (weekly max) \\
\hline TE-107-AP-48 & $5 / 24 / 91$ & 64 & CASS & $\mathbf{S}$ & Disugreament with nearby thermocouples (woekly max) \\
\hline TE-107-AP-4B & $6 / 1 / 91$ & 67 & CASS & $\mathbf{S}$ & Diasgraement with nearby thermocouglas (weakly max) \\
\hline TE-107-AP-48 & 6/7/91 & 66 & CASS & $\mathbf{s}$ & Disagreament with rasiby thermocouplas (woekly max) \\
\hline TE-107.AP-4B & $6 / 11 / 91$ & 74 & CASS & $\mathbf{s}$ & Disegreemem with nearby thermocouples (woekly max) \\
\hline TE-107-AP-48 & 6/19/91 & 70 & CASS & $\mathbf{s}$ & Disagreament with nestby thermocouples (wookly max) \\
\hline TE-107.AP-48 & 6/24/91 & 68 & CASS & $\mathbf{s}$ & Disegroement with nasby thermocouples (wookly max) \\
\hline TE-107-AP-4B & $7 / 3 / 91$ & 78 & CASS & $\mathbf{s}$ & Diaegreament with nearby thermocouples (woakly max) \\
\hline TE-107-AP-4B & $7 / 13 / 91$ & B2 & CASS & $\mathbf{S}$ & Disapreement with nearby tharmocouples (woekly max) \\
\hline TE-107-AP-48 & $7 / 14 / 91$ & 82 & CASS & $\mathbf{S}$ & Disagreement with nearby thermocouples (weokly max) \\
\hline TE-107-AP-48 & $7 / 24 / 91$ & 84 & CASS & $\mathbf{S}$ & Disepreement with neabl by thermocouples (wookly max) \\
\hline$T E-107-A P-48$ & $8 / 3 / \mathbf{S 1}^{2}$ & 81 & CASS & $\mathbf{S}$ & Disegreement with neaby tharmocouples (weokly max) \\
\hline TE-107.AP-48 & 8/5/91 & 84 & CASS & $\mathbf{s}$ & Disagreament with noarby thermocouples (woekly max) \\
\hline TE-107-AP-48 & $8 / 17 / 91$ & 83 & CASS & $\mathbf{s}$ & Disagreement with nearty thermocouples (weekly max) \\
\hline TE-107-AP-48 & $8 / 19 / 91$ & 87 & CASS & $\mathbf{s}$ & Disagreament with neasby thermocouples (wookly max) \\
\hline TE-107-AP-48 & $8 / 31 / 91$ & 79 & CASS & $\overline{\mathbf{s}}$ & Disapreement with nearby thermocouples (weakly max) \\
\hline TE-107-AP-48 & $8 / 7 / 91$ & 7 & CASS & $\mathbf{s}$ & Dieagreament with neaby thermocouples (weckly max) \\
\hline TE-107-AP-A8 & 9/8/91 & 76 & CASS & $\mathbf{s}$ & Dieagreemont with neadby tharmocouplea (weakly max) \\
\hline TE-107-AP-48 & $9 / 18 / 91$ & 74 & CASS & 5 & Disagreament with nearby thormocouplas (wookly max) \\
\hline TE-107.AP-48 & 9/28/91 & 73 & CASS & $\mathbf{S}$ & Disegreement with noarby thermocouples (weokly max) \\
\hline TE-107.AP-48 & $10 / 1 / 91$ & 72 & CASS & $\mathbf{S}$ & Disedreement with nearby thermocouples (weakly max) \\
\hline TE-107-AP-48 & $10 / 791$ & 64 & CASS & 5 & Diasercement with nearby thermocouples (woekly mex) \\
\hline TE-107-AP-48 & $10 / 16 / 91$ & 66 & CASS & $\mathbf{s}$ & Diagapreement with nearby thermocouplas (woekly max) \\
\hline TE-107-AP-4B & $10 / 21 / 93$ & 59 & CASS & $\overline{\mathbf{s}}$ & Disapreenent whith nearby thermocouples (weakly max) \\
\hline TE-107-AP-48 & $10 / 27 / 91$ & 51 & CASS & $\mathbf{s}$ & Diasegresment with noarby thermocouples (weakly max) \\
\hline TE-107-AP-48 & $11 / 6 / 91$ & 46 & CASS & $\mathbf{s}$ & Diesdoreement with noarby thermocouples (weekly mox) \\
\hline TE-107-AP-48 & $8 / 1 / 32$ & 87 & CASS & $\mathbf{s}$ & 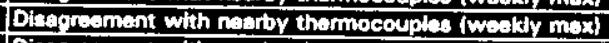 \\
\hline TE-107-AP-AB & $8 / 3 / 92$ & 85 & CASS & $\bar{s}$ & 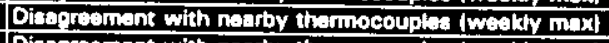 \\
\hline TE-107-AP-48 & $8 / 15 / 92$ & 89 & CASS & $\overline{\mathbf{s}}$ & Dialepreennent with nearby thermocouples (weekly max) \\
\hline TE-107-AP-48 & $8 / 18 / 82$ & 86 & CASS & $\mathbf{s}$ & Diagoreament with noarby thermocouples (wookly max) \\
\hline TE.107-AP.48 & $8 / 28 / 92$ & 78 & CASS & $\mathbf{s}$ & Disegroment with nearby thermocouples (weekly max) \\
\hline TE-107-AP-48 & $9 / 4 / 92$ & 80 & CASS & $\mathbf{s}$ & Diges reament with nearby tharmocouplos (woekly max) \\
\hline TE-107-AP-AB & 0/12/92 & 74 & CASS & $\mathbf{s}$ & Disepreament with nenby thermocouples (weakly mux \\
\hline TE-107-AP.48 & $9 / 13 / 92$ & 70 & CASS & $\mathbf{S}$ & Disergodment with neaby tharmocouplas (weekly max) \\
\hline TE-107-AP-48 & 9/23/92 & 72 & CASS & $\mathbf{S}$ & Diaser reament with nosiby thermocouples (wedkly max) \\
\hline TE-107-AP-48 & $10 / 2 / 92$ & 73 & CASS & $\mathrm{s}$ & Disedreemont with nearby thermocouplos (weekly max) \\
\hline TE-107-AP-48 & $10 / 4 / 92$ & 68 & CASS & $\mathbf{s}$ & 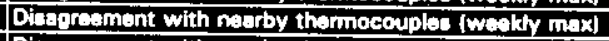 \\
\hline TE-107.AP-48 & $10 / 13 / 22$ & 68 & CASS & 5 & Disegreement with nearby theimocouples (weakly mox) \\
\hline TE-107-AP-48 & $10 / 20 / 92$ & 62 & CASS & $\mathbf{s}$ & Disedreement with nearby thermocouplas (woekly max) \\
\hline TE-107.AP-48 & $10 / 25 / 92$ & 59 & CASS & $\mathbf{s}$ & Disagreement with noarby thermocouples (woekly max) \\
\hline TE-107-AP-48 & $11 / 4 / 92$ & 54 & CASS & $\mathbf{s}$ & Disedrement with nearby thermocouples (weekly $\mathrm{max}$ ) \\
\hline TE-107-AP-4B & $11 / 3 / 92$ & 52. & CASS & $\mathbf{S}$ & Disagreement with nearby thermocouples (woekly max) \\
\hline TE-107-AP-4B & $81 / 16 / 92$ & 52 & CASS & 5 & Disagreement with neartby thermocouples (woekly max) \\
\hline TE-107-AP-48 & $11 / 22 / 92$ & 45 & CASS & $\mathbf{s}$ & Disaproement with nearby thermocouples (wookly max) \\
\hline TE-107-AP-48 & $12 / 2 / 92$ & 42 & CASS & $\mathbf{S}$ & Disagreement with nearby tharmocouples (weekly max) \\
\hline TE-107-AP-48 & $12 / 10 / 92$ & 38 & CASS & $\mathbf{5}$ & Disagreement with nearby thermocouples (weokly max) \\
\hline TE-107-AP-48 & $12 / 15 / 92$ & 38 & CASS & 5 & Disagreement with mearby thermocouples (woekly max) \\
\hline TE-107-AP-4B & $12 / 23 / 92$ & 39 & CASS & $\mathbf{s}$ & Disagreement with nearby thermocousples (weekly max) \\
\hline TE-107-AP-48 & $12 / 30 / 92$ & 37 & CASS & $\mathbf{s}$ & Disagreament with nearby thermocouples (weekly max) \\
\hline TE-107-AP-48 & $1 / 15 / 34$ & 44 & CASS & $\mathbf{S}$ & Oisegreement with nearby thermocouples (weekly max) \\
\hline TE-107-AP-48 & $1 / 18 / 94$ & 44 & CASS & $\mathbf{S}$ & Disagreement with nadrby tharmocouples (weekly max) \\
\hline$T E-107 \cdot A P-48$ & $1 / 25 / 34$ & 45: & CASS & $\mathbf{s}$ & Disegroement with neacby thermocouplas (wookly $\max$ ) \\
\hline TE-107.AP-48 & $2 / 2 / 94$ & $39:$ & CASS & $\mathbf{S}$ & Disegreemant with nearby themocouples (woekly max) \\
\hline TE-107-AP-48 & $2 / 12 / 94$ & 39 & CASS & 5 & Diaegreement with nearby thermocouples (weekly max) \\
\hline TE-107-AP-4B & $2 / 16 / 94$ & 48 & CASS & $\mathbf{s}$ & Diagareement with neaby thermocouples (weokly max) \\
\hline TE-107-AP-48 & $2 / 23 / 94$ & 46 & CASS & $\mathbf{s}$ & Disaproement with nearby thermocouplos (woekly max) \\
\hline TE-107-AP-48 & $3 / 4 / 94$ & \begin{tabular}{c|c}
$\mathbf{5 3}$ \\
\end{tabular} & CASS & $\mathbf{S}$ & Disupresment with nearby thermocouples (weekly max) \\
\hline TE-107-AP-48 & $3 / 10 / 94$ & 51 & CASS & $\mathbf{s}$ & Disagroement with nearby thermoccuples (woekly max) \\
\hline TE-107-AP-48 & 3/18/94 & 56 & CASS & $\mathbf{5}$ & Disagreament with nearby thermocouples (waekly max) \\
\hline TE-107-AP-48 & $3 / 26 / 94$ & 53 & CASS & $\mathbf{s}$ & Dieagreement with nearby thermocouples (woekly max) \\
\hline TE-107-AP-48 & $3 / 31 / 94$ & 61 & CASS & $\mathbf{s}$ & [Disegraement with nearby thermocouples (weekly max) \\
\hline TE-107-AP-48 & $4 / 3 / 94$ & 61 & CASS & $\mathbf{s}$ & Diasproement with nearby thermocouplas (weokly max) \\
\hline TE-107-AP-48 & $4 / 12 / 94$ & 64 & CASS & $\mathbf{S}$ & Disuproement with nearby thermocouplas (weokly max) \\
\hline TE-107-AP-48 & $4 / 19 / 94$ & 118 & CASS & $\mathbf{S}$ & Diseproement with nearby thermocouplas (wadkly max) \\
\hline TE-107-AP-4B & $4 / 27 / 94$ & 68 & CASS & $\mathbf{S}$ & Disagreement with nearby tharmocouplas (weekly max) \\
\hline TE-107-AP-4B & $5 / 7 / 94$ & 71 & CASS & $\mathbf{s}$ & Disegreament with nearby thermocouples (weekly max) \\
\hline TE-107-AP-48 & $5 / 11 / 94$ & 77 & CASS & $\mathbf{s}$ & Disapreament with naarby thermocouplas (wadkly max) \\
\hline TE-107-AP-48 & $5 / 16 / 94$ & 103 & CASS & $\mathbf{S}$ & Disarceament with nearby thermocouples (waekly max) \\
\hline
\end{tabular}




\begin{tabular}{|c|c|c|c|c|c|}
\hline \multicolumn{6}{|c|}{$\begin{array}{l}\text { TANK 241-AP.107 } \\
\text { THERMOCOUPIE DATA }\end{array}$} \\
\hline & & & & & \\
\hline THEFMOCOUPLE * & DATE & TEMPGRATURE $\left.{ }^{\circ} \bar{T}\right)$ & DATA SOURCE & oUALITY & COMMENT \\
\hline TE-107-AP-48 & $5 / 25 / 94$ & 104 & CASS & $\mathbf{S}$ & Disagreement with nasrby thermocouples (weekly max) \\
\hline TE-107-AP-4B & $6 / 4 / 94$ & 104 & CASS & $\mathbf{S}$ & Disagreement with nearby thermocouples (waekly max) \\
\hline TE-107-AP-48 & $6 / 11 / 94$ & 104 & CASS & $\mathbf{S}$ & Disagreament with nearby thermocouples (weakly $\max$ ) \\
\hline TE-107-AP-48 & $6 / 12 / 94$ & 104 & CASS & $\mathbf{S}$ & Disagraernent with nearby thermocouples (weekly max) \\
\hline TE-107-AP-48 & $6 / 24 / 94$ & 109 & CASS & $\mathbf{s}$ & Disagreement with nearby thermocouples (weekly max) \\
\hline TE-107-AP-48 & $6 / 30 / 94$ & 110 & CASS & $\mathbf{S}$ & Disagreement with nearby thermocouples (weekly max) \\
\hline TE-107.AP-49 & $7 / 9 / 94$ & 110 & CASS & $\mathbf{S}$ & Disacreament with nearby thermocouples (weakly max) \\
\hline TE-107-AP-48 & $7 / 16 / 94$ & 116 & CASS & $\mathbf{S}$ & Disagreement with nearby thermocouples (weekly max) \\
\hline TE-107-AP-48 & $7 / 23 / 94$ & 117 & CASS & $\mathbf{s}$ & Disagreament whh nesby thamocouples (weakly max) \\
\hline TE-107.AP-48 & $7 / 24 / 94$ & 118 & CASS & $\mathbf{S}$ & Disapreament with nearby tharmocouplas (wookly max) \\
\hline$T E-107+A P-48$ & $8 / 3 / 94$ & 116 & CASS & S & Disapreament with nearby thermocouples (weakly max) \\
\hline TE-107.AP-48 & $8 / 13 / 94$ & 112 & CASS & $\mathbf{S}$ & Disagreement with nasarby tharmocouples (weokly max) \\
\hline TE-107-AP.48 & $8 / 17 / 94$ & 113 & CASS & $\mathbf{S}$ & Disagreament with nastoy thermocouples (weakly max) \\
\hline TE-107-AP-48 & $8 / 21 / 94$ & 110 & CASS & $\mathbf{S}$ & Disagreemant with nearby themocouples (weakly max) \\
\hline TE-107-AP-48 & $9 / 3 / 94$ & 108 & CASS & $\mathbf{s}$ & Diagoreament with naarby tharmocouples (woekly max) \\
\hline TE-107-AP-48 & $9 / 9 / 94$ & 109 & CASS & 5 & Disagreament with nearby thermocouples (weakly max) \\
\hline TE-107-AP-48 & $9 / 15 / 94$ & 104 & CASS & $\mathbf{S}$ & Disegreemant with nearby thamocouplas (weakly max) \\
\hline TE-107-AP-48 & $9 / 23 / 94$ & 108 & CASS & $\mathbf{s}$ & Diaserreament with nearby tharmocouples (woekly max) \\
\hline TE-107-AP-48 & $9 / 29 / 94$ & 108 & CASS & $\mathbf{S}$ & Diagreament with naerby thermocouples (woakly max) \\
\hline TE-107-AP-48 & $10 / 2 / 94$ & 100 & CASS & $\mathbf{S}$ & Diagreament with nearby themocouples (woekly max) \\
\hline TE-107-AP-48 & $10 / 9 / 94$ & 86 & CASS & $\mathbf{s}$ & Diaagrearnant with nearby tharmocouplas (weokly max) \\
\hline TE-107-AP-48 & $10 / 17 / 94$ & 91 & CASS & $\mathbf{S}$ & Disagreement with nearby tharmocouples (weekly max) \\
\hline$T E=107-A P=48$ & $10 / 27 / 94$ & 90 & CASS & $\mathbf{S}$ & Disagreement with nearby thermocouples (weokly max) \\
\hline TE-107-AP-48 & $11 / 4 / 94$ & 83 & CAS5 & $\mathbf{s}$ & Disapreament with mastby themocouplas (weakly $\max$ ) \\
\hline TE-107-AP-48 & $11 / 11 / 94$ & 81 & CASS & $\mathbf{S}$ & Diagreament with noarby thamnocouples (weakly max) \\
\hline TE-107-AP-48 & $11 / 16 / 94$ & 78 & CASS & $\mathbf{S}$ & Disegreement with nearby thermocouples (weekly max) \\
\hline TE-107-AP-48 & $11 / 21 / 94$ & 76 & CASS & $\mathbf{S}$ & Diaagreement with nearby thermocouples (weakly max) \\
\hline TE-107-AP-48 & $12 / 2 / 94$ & 75 & CASS & $\mathbf{s}$ & Diasgrearnent with naoby tharmocouplas (wookly max) \\
\hline TE-107-AP-48 & $12 / 4 / 94$ & 71 & CASS & $\mathbf{s}$ & Diaggreament with noarby tharmocouples (weakly max) \\
\hline TE-107-AP-48 & $12 / 17 / 94$ & 69 & CASS & $\mathbf{s}$ & Disegresment with nastby tharmocouples (weakly $\max$ ) \\
\hline TE-107-AP-48 & $12 / 19 / 94$ & 77 & CASS & $\mathbf{S}$ & Disepreament with naerby tharmocouples (weakly max) \\
\hline TE-107-AP-48 & $12 / 27 / 94$ & 76 & CASS & $\mathbf{s}$ & Disagreement with nadby thermocouples (weekly max) \\
\hline TE-107-AP-4B & $1 / 4 / 55$ & 69 & CASS & $\mathbf{S}$ & Disagreernent with nearby themocouplas (weekly max) \\
\hline TE-107-AP-48 & $1 / 14 / 95$ & 75 & CASS & $\mathbf{s}$ & Diagrreemant with nearby tharmocouples (weakly max) \\
\hline TE-107-AP-48 & $1 / 16 / 95$ & 73 & CASS & $\mathbf{S}$ & Diangreament with nearby thermocouples (wookhy max) \\
\hline TE-107-AP-48 & $1 / 28 / 95$ & 76 & CASS & $\mathbf{s}$ & Disagreoment with noarby thermocouples (woakly max) \\
\hline TE-107-AP-48 & $2 / 205$ & 127 & CASS & 5 & Disegreermant with neaby thermocouplas (woekly max) \\
\hline TE-107-AP-48 & $2 / 965$ & 78 & CASS & $\mathbf{s}$ & Diagreemant with noarby tharmocouples (wakkly max) \\
\hline TE-107-AP.48 & $2 / 13 / 95$ & 77 & CASS & $\mathbf{S}$ & Disedreement with nearby thermocouplas (weakly max) \\
\hline TE-107-AP-48 & $2 / 25 / 95$ & 80 & CASS & $\mathbf{S}$ & Dinagresment with neaby tharmocouplas (wookly max) \\
\hline TE-107.AP-48 & $2 / 27 / 95$ & $\mathbf{8 3}$ & CASS & $\mathbf{S}$ & Diagrearmant with nearby tharmocouples (weekly max) \\
\hline TE-107.AP-48 & 39/95 & 78 & CASS & $\mathbf{S}$ & Diasprearinent with neaby tharmocouples (weakly max) \\
\hline TE-107.AP-48 & $3 / 18 / 95$ & 80 & CASS & $\mathbf{S}$ & Disegreement with nearby thermocouplas (woekly max) \\
\hline TE-107.AP-48 & $3 / 21 / 95$ & 127 & CASS & $\mathbf{S}$ & Diasgreament with nearby thermocouples (weakly max) \\
\hline TE-107.AP-48 & $3 / 30 / 95$ & 82 & CASS & $\underline{\mathbf{S}}$ & Disagreement with nearby tharmocouples (weekhy max) \\
\hline & & & & & \\
\hline TE-107-AP-49 & $4 / 5 / 86$ & 5 & CASS & $\mathbf{S}$ & Diasgreament with nearby tharmocouples (weakly max) \\
\hline TE-107-AP-49 & 4/9/86 & 55 & CASS & $\mathbf{s}$ & Diagoreement with nearby thermocouples (weakly max) \\
\hline TE-107-AP-49 & $5 / 17 / 86$ & 63 & CASS & $\mathbf{s}$ & Oisegreament with nasiby thermocouples (woekly max) \\
\hline TE-107-AP-49 & $5 / 20 / 86$ & 69 & CASS & $\mathbf{s}$ & Diragreement with nearby thermocouples (weakly max) \\
\hline TE-107-AP-49 & $5 / 31 / 86$ & 81 & CASS & $\mathbf{S}$ & Disagreement with nearby thermocouples (weokly max) \\
\hline TE-107-AP-49 & $6 / 2 / 86$ & 82 & CASS & $\mathbf{S}$ & Diagpreemant with nearby thermocouples (weakly max) \\
\hline TE-107-AP-49 & $6 / 14 / 86$ & 77 & CASS & $\mathbf{S}$ & Disagreement with nearby thermocouples (weekly max) \\
\hline TE-107-AP-49 & $6 / 16 / 86$ & 75 & CASS & $\mathbf{5}$ & Diaggreement with nearby thermocouples (weekly max) \\
\hline TE-107-AP-49 & $6 / 25 / 86$ & 76 & CASS & $\mathbf{S}$ & Disapreament with nearby thermocouples (weekly max) \\
\hline TE-107-AP-49 & $7 / 3 / 86$ & 70 & CASS & $\mathbf{s}$ & Diasareament with naarby thormacouplas (woekly $m a x$ ) \\
\hline TE-107-AP-49 & $7 / 7 / 86$ & 68 & CASS & $\mathbf{S}$ & Diagreament with nearby tharmocouples (waekly max) \\
\hline TE-107.AP-49 & $7 / 18 / 86$ & 66 & CASS & $\mathbf{S}$ & Disagreament with nearby tharmocouplas (weokly max) \\
\hline TE-107.AP-49 & $7 / 26 / 36$ & 75 & CASS & $\mathbf{s}$ & Diagareement with nearby thermocouples (weekly max) \\
\hline TE-107-AP-49 & $7 / 31 / 86$ & 73) & CASS & $\mathbf{s}$ & Disagreement with nearby tharmocouples (weekly max) \\
\hline TE-107-AP-49 & $8 / 8 / 86$ & 79 & CASS & $\mathbf{S}$ & Disegreament with nearby thermocouples (weakly max) \\
\hline TE-107.AP-49 & $8 / 11 / 86$ & 80 & CASS & $\mathbf{S}$ & Disapreament with nearby thermocouples (weakly max) \\
\hline TE-107-AP-49 & $8 / 17 / 86$ & 79 & CASS & $\mathbf{s}$ & Disagreement with nedrby thermocouples (weakly max) \\
\hline TE-107-AP-49 & $8 / 30 / 86$ & 72 & CASS & $\mathbf{S}$ & Diagrreement with nearby thermocouples (weekly max) \\
\hline TE-107-AP-49 & $9 / 3 / 86$ & 77 & CASS & $\mathbf{s}$ & Diasgreament with nearby thermocouples (woekly max) \\
\hline TE-107-AP-49 & $9 / 23 / 86$ & 62 & CASS & $\mathbf{5}$ & Disagreament with naarby thormocouples (weekly max) \\
\hline TE-107-AP-49 & $9 / 29 / 26$ & 60 & CASS & $\mathbf{5}$ & Disagreament with nearby tharmocouples (weekly max) \\
\hline TE-107-AP-49 & $10 / 8 / 86$ & 62 & CASS & $\mathbf{S}$ & Disagreement with nearby thermocouples (weekly max) \\
\hline TE-107-AP-49 & $10 / 12 / 86$ & 55 & CASS & $\mathbf{S}$ & Disagreement with nearby thermocouples (weekly max) \\
\hline TE-107-AP-49 & $10 / 24 / 86$ & 55 & CASS & $\mathbf{S}$ & Disagreament with nearby thermocouples (weakly $\mathrm{msx}$ ) \\
\hline TE-107-AP-49 & $10 / 26 / 86$ & 58 & CASS & $\mathbf{S}$ & Disegreement with nearby thermocouplas (weekly mex) \\
\hline TE-107-AP-49 & $11 / 5 / 86$ & 50 & CASS & $\mathbf{S}$ & Disagreement with nearby thermocouples (weakly $\mathrm{max}$ ) \\
\hline TE-107-AP.49 & $11 / 9 / 86$ & 44 & CASS & $\mathbf{S}$ & Disagreement with nearby thermocouples (weakly max) \\
\hline
\end{tabular}




\begin{tabular}{|c|c|c|c|c|c|}
\hline \multicolumn{6}{|c|}{$\begin{array}{l}\text { TANK 241-AP-107 } \\
\text { THIRMOCOUPEE DATA }\end{array}$} \\
\hline & & & & & \\
\hline THERMOCOUPL : & DATE & TEMPERATUAE $\left({ }^{\circ} \mathrm{F}\right)$ & DATA SOURCE & OUALITY & COMMENT \\
\hline TE-107-AP-49 & $11 / 20 / 66$ & 51 & CASS & $\mathbf{S}$ & Disagreement with nearby thermocouples (wookly max) \\
\hline TE-107-AP-49 & $19 / 24 / 86$ & 54 & CASS & $\mathbf{s}$ & Disagreement with nearby thermocouples (woekly max) \\
\hline TE-107-AP-49 & $12 / 1 / 36$ & 41 & CASS & $\mathbf{S}$ & Disagreament with nearby thermocouples (weakly max) \\
\hline TE-107-AP-49 & $12 / 8 / 86$ & 37 & CASS & $\overline{\mathbf{S}}$ & Dissarcement with nesiby thermocouples (wookly max) \\
\hline TE-107-AP-49 & $12 / 17 / 86$ & 37 & CASS & $\mathbf{s}$ & Disagreament with neaby thermocouples (weekly max) \\
\hline TE-107-AP-49 & $12 / 24 / 86$ & 39 & CASS & $\mathbf{S}$ & Disagreament with neaby thermocouples (woekly max) \\
\hline TE-107.AP-49 & $12 / 28 / 86$ & 39 & CASS & $\mathbf{S}$ & Disepreement with nearby thermocouplas (weakly max) \\
\hline TE-107.AP-48 & $7 / 1 / 87$ & 86 & CASS & $\overline{\mathbf{S}}$ & Diseproement with nestby thormocouples (weekly max) \\
\hline TE-107-AP.48 & $7 / 8 / 87$ & 75 & CASS & $\mathbf{S}$ & Disepreament whe neporty thermocouples (weakly max) \\
\hline TE-107-AP-49 & 7/15/87 & 81 & CASS & $\mathbf{S}$ & Disagreement with neaby thermocouples (woekly max) \\
\hline TE-107-AP-49 & 7125/87 & 79 & CASS & $\mathbf{s}$ & Disapresement with noarby tharmocouples (weekly max) \\
\hline TE-107-AP-49 & $7129 / 87$ & 78 & CASS & $\mathbf{s}$ & Diasproement with nearby thermocouples (weokly max) \\
\hline TE-107-AP.49 & $8 / 8 / 37$ & 80 & CASS & $\mathbf{s}$ & Disegreement with noarby thermocouples (weekly max) \\
\hline TE-107-AP-A9 & 8/9/87 & 82 & CASS & $\mathbf{S}$ & Disspresment with nearby thermocoupies (weekly max) \\
\hline TE-107.AP- 49 & $8 / 22 / 87$ & 73 & CASS & $\mathbf{s}$ & Deagreament with nearby thermocouples (woekly max) \\
\hline TE-107-AP-49 & $8 / 23 / 87$ & 78 & CASS & $\mathbf{s}$ & Oisagraement with nearby thermocouples (weokly max) \\
\hline TE-107.AP-49 & $9 / 1 / 37$ & 83 & CASS & S & Disepreement with nearby thermocouples (weakly max) \\
\hline TE-107.AP-49 & 9/8/A7 & 78 & CASS & $\mathbf{S}$ & Disedresment with nearby thermocouples (woekly max) \\
\hline TE-107.AP-49 & 8/13/87 & 73 & CASS & $\mathbf{S}$ & Disagreement with nearby tharmocouples (wookly max) \\
\hline TE-107-AP- $\overline{9}$ & 8/25/87 & 72 & CASS & $\mathbf{s}$ & Diasareemant with naarby thormocouples (wockly max) \\
\hline TE-107.AP-A9 & $10 / 3 / 67$ & 67 & CASS & $\overline{\mathbf{s}}$ & Disegroement with noarby thermocorples (weekby max) \\
\hline TE-107-AP-49 & $10 / 8 / 67$ & 69 & CASS & $\overline{\mathbf{5}}$ & Ditapreament with nearby tharmocouples (wookly max) \\
\hline TE-107-AP-49 & $10 / 12 / 87$ & 57 & CASS & $\mathbf{S}$ & Disapreament with nearby tharmocouples (woekly max) \\
\hline TE-107-AP-A9 & $10 / 18 / 87$ & 54 & CASS & $\overline{\mathbf{S}}$ & Dicapresment with nearby thormocouples (weekly max) \\
\hline TE-107-AP=-49 & $10 / 31 / 87$ & 55 & CASS & $\mathbf{S}$ & Disegroement with nearby thermocouples [weekly max] \\
\hline TE-107-AP-49 & $11 / 2 / 87$ & 57 & CASS & $\overline{\mathbf{S}}$ & Disarreement with noarby thermocouples (weakly max) \\
\hline TE-107.AP-49 & $11 / 13 / 87$ & 54 & CASS & $\mathbf{S}$ & Disespreement with neabby thermocouples (weokly max) \\
\hline TE-107-AP-49 & $11 / 16 / 37$ & 46 & CASS & $\mathbf{S}$ & Didagraement with naarby thermocouples (wookly max) \\
\hline TE-107.AP-49 & $11 / 25 / 87$ & 42 & CASS & $\mathbf{S}$ & Diearreoment with nearby thermocouples (woekly max) \\
\hline TE-107-AP-48 & $12 / 4 / 87$ & 45 & CASS & $\mathbf{S}$ & Diespreement with nearby thermocouplas (weekly max) \\
\hline TE-107.AP-48 & 12/6/37 & 47 & CASS & $\mathbf{S}$ & Diseproemam with nearby thermocouplos (weekly max) \\
\hline TE-107-AP-49 & $12 / 17 / 97$ & 37 & CASS & $\overline{\mathbf{S}}$ & Diaepreament with neaby thermocouphes (weekly max) \\
\hline TE-107.AP-49 & $12 / 21 / 87$ & 35 & CASS & $\mathbf{S}$ & Diagerseement with nesciby tharmocouples (woekty $\mathrm{max}$ ) \\
\hline TE-107-AP.49 & $1 / 1 / 8 \mathrm{~B}$ & 33 & CASS & $\mathbf{S}$ & Dienereoment with nearby thermocouples (wookly max) \\
\hline TE-107-AP-49 & 1/6/80 & 33 & CASS & $\mathbf{S}$ & 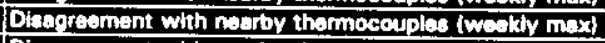 \\
\hline TE-107.AP-49 & $1 / 15 / 30$ & 38 & CASS & $\mathbf{S}$ & Diagryeement with noarby themocoupless (weakly max) \\
\hline TE-107-AP-49 & $1 / 23 / 88$ & 37 & CASS & $\mathbf{S}$ & Diespreament with nearby thermocouplas (weakly max) \\
\hline TE-107-AP-A9 & $1 / 29 / 88$ & 37 & CASS & $\mathbf{s}$ & Dicepreament with nearby thermocouples [weekly max) \\
\hline TE-107-AP-49 & $1 / 31 / 88$ & 35 & CASS & $\mathbf{s}$ & Diecricement with nearby thermocouphes (weekly max) \\
\hline TE-107-AP-49 & $2 / 13 / 98$ & 42 & CASS & $\mathbf{S}$ & Disedraement whih nearby theimocouplas (weokly max) \\
\hline TE-107-AP-49 & $2 / 15 / 88$ & 43 & CASS & $\overline{\mathbf{S}}$ & Disepresmant with nearby themocouples (wsekly $\max$ ) \\
\hline TE-107-AP-A9 & 2/21/88 & 45 & CASS & $\mathbf{s}$ & Dieserreament with neaby thormocouples (wedky max) \\
\hline TE-107-AP-49 & $3 / 3 / 88$ & 48: & CASS & $\overline{\mathbf{S}}$ & Disagresment with nearby thermocouplas (weakly max) \\
\hline TE-107-AP:48 & 3/9/38 & 45 & CASS & $\mathbf{S}$ & Diagrieament with nearby thermocouples (weokly max) \\
\hline TE-107-AP-49 & 3/19/88 & 47 & CASS & $\mathbf{s}$ & Distareement with nearby thermocouples (woekly max) \\
\hline TE-107-AP-A9 & $3 / 26 / 88$ & 51 & CASS & $\mathbf{S}$ & Disagreenent with nearby thermocouples (weekly max) \\
\hline TE-107-AP-49 & $3 / 30 / 88$ & 50 & CASS & $\mathbf{S}$ & Disagreement which nearby tharmocouples (weekly max) \\
\hline TE-107-AP-49 & 8/1/80 & 64 & CASS & $\mathbf{s}$ & Disapreement with neaph thempocouples (weekly max) \\
\hline$T E-107-A P-49$ & $8 / 5 / 88$ & 64 & CASS & $\mathbf{S}$ & Dibapreament with nearby thermocouples (weokly max) \\
\hline TE-107.AP.49 & $6 / 17 / 88$ & 75 & CASS & $\mathbf{s}$ & Disagreament with nearby thermocouples (waekly max) \\
\hline TE-107-AP-49 & $6 / 25 / 88$ & 78 & CASS & $\mathbf{S}$ & Disapreament with nastby tharmocouplas (wooksy max) \\
\hline TE-107-AP-49 & $7 / 2 / 88$ & 76 & CASS & $\mathbf{5}$ & Disagreament with nearby thermocouplas (woekly max) \\
\hline TE-107-AP-49 & 7/9/38 & 78 & CASS & $\mathbf{S}$ & Disagraement with nearby tharmocouplas (waekly max) \\
\hline TE-107-AP-49 & $7 / 11 / 88$ & 81 & CASS & $\mathbf{S}$ & Disagreement with nearby thermocouples (waekly mox) \\
\hline TE-107-AP-49 & $7 / 22 / 8$ & 84 & CASS & $\mathbf{S}$ & Disapraement with nearby tharmocouples (weekly max) \\
\hline TE-107-AP-49 & $7 / 27 / 88$ & 89 & CASS & $\mathbf{S}$ & Oisapresment with nearby tharmocouples (woekly mox) \\
\hline TE-107.AP-49 & 8/5/88 & 84 & CASS & $\mathbf{S}$ & Disagreament with nearby tharmocouples (weekly mex) \\
\hline TE-107-AP-49 & $8 / 11 / 88$ & 82 & CASS & $\mathbf{S}$ & Disagreament with nearby thermocouples (weekly max) \\
\hline TE-107-AP-49 & $8 / 14 / 88$ & 79 & CASS & $\mathbf{S}$ & Disapreament with nearby thermocouples (weekly max) \\
\hline TE-107-AP-49 & $8 / 25 / 88$ & 82 & CASS & $\mathbf{S}$ & Disagreoment with nearby thermocouples (wodkly max) \\
\hline TE-107-AP-49 & $8 / 29 / 88$ & 81 & CASS & $\mathbf{S}$ & Didegreement with noarby thermocouples (weekly max) \\
\hline TE-107-AP-49 & $9 / 5 / 88$ & 81 & CASS & $\mathrm{s}$ & Distagreement with nearby thermocouples (weekly max) \\
\hline TE-107-AP-49 & $9 / 15 / 88$ & 74 & CASS & $\mathbf{S}$ & Disagreement with nearby thermocouples (woekly max) \\
\hline TE-107-AP-49 & 9/19/8 & 66 & CASS & $\mathbf{s}$ & Disapreement with nearby thermocouplas (woekly max) \\
\hline TE-107-AP-49 & $9 / 30 / 88$ & 111 & CASS & $\mathbf{s}$ & Disegraement with nearby thermocouples (waekly max) \\
\hline TE-107-AP-49 & $12 / 1 / 88$ & 41 & CASS & $\mathbf{S}$ & Diasproement with nearby thermocouplas (woekly max) \\
\hline TE-107-AP-4Q & 12/10/88 & 44 & CASS & $\mathbf{S}$ & Disepreement with nearby thermocouples (woekly max) \\
\hline TE-107-AP-49 & $12 / 13 / 88$ & 45 & CASS & $\mathbf{s}$ & Diegrreement with nearby thermocouples (weekly mox) \\
\hline TE-107-AP-49 & $12 / 22 / 88$ & 38 & CASS & $\mathbf{S}$ & Disagraement with nearby thermocouples (weakly max) \\
\hline TE-107-AP-49 & $9 / 1 / 89$ & 72 & CASS & $\mathbf{S}$ & Disagreament with nearby thermocouples (woakty max) \\
\hline TE-107-AP-49 & $9 / 8 / 89$ & 72 & CASS & $\mathbf{S}$ & Disupreament with nearby thermocouples (weakiy max) \\
\hline TE-107-AP-49 & $9 / 11 / 89$ & 75 & CASS & $\mathbf{S}$ & Disapreament with nearby thamocouplas (woekly $\max$ ) \\
\hline TE-107-AP-49 & 9/18/89 & 70 & CASS & $\mathbf{S}$ & Disagreement with nearty thermocouples (weakly max) \\
\hline
\end{tabular}




\begin{tabular}{|c|c|c|c|c|c|}
\hline \multicolumn{6}{|c|}{$\begin{array}{l}\text { TANK 241.AP-107 } \\
\text { THERMOCOUPLE DATA }\end{array}$} \\
\hline & & & & & \\
\hline $\begin{array}{c}\text { THEABNOCOUPLE } \\
\text { TE-107-AP-49 }\end{array}$ & \begin{tabular}{l|} 
DATE \\
9/29/89
\end{tabular} & $\frac{\text { TEMPEAATUAE ("F) }}{71}$ & $\frac{\text { DATA SOUACE }}{\text { CASS }}$ & QUALITY & COMMENT \\
\hline TE-107-AP-49 & $11 / 4 / 89$ & 71 & $\frac{\text { CASS }}{\text { CASS }}$ & $\frac{s}{s}$ & \\
\hline TE-107-AP-49 & $11 / 11 / 89$ & 58 & CASS & S & $\begin{array}{l}\text { Oisegraement with nearby thermocouples (woekfy max) } \\
\text { Disegreement with nearby thermocouples (weekly max) }\end{array}$ \\
\hline TE-107-AP-49 & $11 / 12 / 89$ & 57. & CASS & $\mathbf{s}$ & Disapreement with nearby thermocouples (weakly max) \\
\hline TE-107-AP-49 & $11 / 21 / 89$ & 49 & CASS & $\mathbf{S}$ & Disagreament with nearby thermocouples (woekly max) \\
\hline TE-107-AP-49 & $11 / 26 / 89$ & 48 & CASS & $\mathbf{s}$ & Disagreement with nearby thermocouples (weekly max) \\
\hline TE-107-AP-49 & $12 / 8789$ & 45 & CASS & $\mathbf{S}$ & Disagreement with nearby thermocouples (weekly max) \\
\hline TE-107-AP-49 & $12 / 10 / 89$ & 42 & CASS & $\mathbf{s}$ & Disugreement with nearby thermocouplas (weekly max) \\
\hline TE-107-AP-49 & $12 / 17 / 89$ & 39 & CASS & $\mathbf{S}$ & Disagreement with nearby thermocouples (woekly max) \\
\hline TE-107-AP-49 & 12/25/99 & 40 & CASS & $\mathbf{S}$ & Disagreement with noarby thermocouplos (woekly max) \\
\hline TE-107-AP-48 & $1 / 6 / 30$ & 44 & CASS & $\mathbf{S}$ & Disegreament with nearby thermocouples (wooksy max) \\
\hline TE-107-AP-49 & $1 / 7 / 90$ & 46 & CASS & $\mathbf{S}$ & Disagreament with nearby thermocouples (weekly max) \\
\hline TE-107.AP-49 & $1 / 16 / 90$ & 44 & CASS & $\mathbf{S}$ & Disegreement with nearby thermocouples (woekly max) \\
\hline TE-107-AP-49 & $1 / 26 / 90$ & 44 & CASS & $\mathbf{S}$ & Disagreement with nearby thermoccouples (weekly max) \\
\hline TE-107-AP-49 & $2 / 3 / 90$ & Q4) & CASS & $\mathbf{S}$ & Disegreement with nearby thermocouples (woekly max) \\
\hline TE-107.AP-49 & $2 / 10 / 90$ & 46 & CASS & $\mathbf{S}$ & Disagreement with naarby thermocouples (weokly max) \\
\hline TE-107-AP-49 & $2 / 11 / 90$ & 48 & CASS & $\mathbf{s}$ & Disagreement with nearby thermocouples (weekhy max) \\
\hline TE-107-AP-49 & $2 / 24 / 90$ & 47 & CASS & $\mathbf{S}$ & Disepreement with nearby thermocouples (wookly max) \\
\hline TE-107-AP-49 & $3 / 3 / 90$ & 47 & CASS & $\mathbf{S}$ & Disagreament with nearby thermocouples (wookly max) \\
\hline TE-107-AP-49 & $3 / 10 / 90$ & 51: & CASS & $\mathbf{S}$ & Diagereament with nearby thermocouples (woekly max) \\
\hline TE-107-AP-49 & $3 / 17 / 90$ & 57 & CASS & $\mathbf{S}$ & Disegreernent with nearby tharmocouples (weekly max] \\
\hline TE-107-AP-49 & 3/22/90 & 57 & CASS & $\mathbf{S}$ & Diseqreement whe nosiby themocouples (woekly max) \\
\hline TE-107-AP-49 & $3 / 31 / 80$ & 58 & CASS & $\overline{\mathbf{S}}$ & Oisegreement with nearby thermocouples (woekly mex) \\
\hline TE-107-AP-49 & $4 / 7 / 90$ & 62 & CASS & $\mathbf{S}$ & Diagereement with nearby tharmocouples (weekly max) \\
\hline TE-107-AP-49 & 4/11/80 & 63) & CASS & $\mathbf{S}$ & Disgrgreament with nearby thermocouples (meekly mox) \\
\hline TE-107-AP-49 & 4/20/90 & 66 & CASS & S & Diasereement with nearby thermocouples (woekly max) \\
\hline TE-107-AP.49 & $4 / 22 / 80$ & 66 & CASS & $\mathbf{S}$ & Disepreament with nearby thermocouples (weakly max) \\
\hline TE-107-AP-49 & $5 / 5 / 90$ & 67 & CASS & $\mathbf{s}$ & Diacereement with nearby thembocouples (weakly max) \\
\hline TE-107-AP-49 & B/11/90 & 68 & CASS & $\mathbf{S}$ & Diedsreement with noartby thermocouples (woekly max) \\
\hline TE-107-AP-49 & 5/17/90 & 67 & CASS & $\mathbf{s}$ & Disagroement with noarby tharmocouplas (woekly max) \\
\hline TE-107-AP-49 & $5 / 22190$ & 69 & CASS & $\mathbf{S}$ & Disegreament with nearby tharmocouphes (weekly max) \\
\hline TE-107-AP-49 & 6/2/80 & 64 & CASS & $\mathbf{S}$ & Disegreement with nearby tharmacouples (weekly max) \\
\hline TE-107.AP-49 & 6/9/30 & 69 & CASS & $\mathbf{S}$ & Disagreement with nearby thermocouplas (weakly max) \\
\hline TE-107-AP-49 & $6 / 10 / 90$ & 71 & CASS & $\mathbf{s}$ & Diegoreoment whth nearby tharmocouplas (wookly max) \\
\hline IE-107-AP-49 & $6 / 22 / 90$ & 78 & CASS & $\mathbf{S}$ & Disagreement with noarby tharmocouplas (wookly max) \\
\hline TE-107-AP-49 & $6 / 30 / 90$ & 81 & CASS & $\mathbf{s}$ & Disepreement with nearby thermocouples (waekly max) \\
\hline TE-107.AP-49 & $7 / 190$ & 79 & CASS & $\mathbf{s}$ & Disapreament with noarby thermocouples (wopkly max) \\
\hline TE-107.AP-49 & $7 / 12 / 90$ & 80 & CASS & $\mathbf{S}$ & Disapreament with noarby theimocouples (weekly $\mathrm{max}$ ) \\
\hline TE-107.AP-49 & 7/16/90 & 87 & CASS & $\mathbf{s}$ & Diagorearmemt with nearby thermocouples (woekly $\mathrm{max}$ ) \\
\hline TE-107.AP-49 & $7 / 23 / 90$ & 89 & CASS & $\mathbf{s}$ & Disagreenent with neasby theimocouples (wedkly max) \\
\hline TE-107-AP-49 & $7 / 51 / 50$ & 86 & CASS & $\mathbf{s}$ & Disapreament with nearby thormocouples (weekly max) \\
\hline TE-107-AP-49 & $8 / 8 / 90$ & 90 & CASS & $\mathbf{S}$ & Diaspreament with nearby thermocouples (woekly max) \\
\hline TE-107.AP-49 & $8 / 13 / 90$ & 87 & CASS & $\mathbf{S}$ & Disapresment with nearby thermocouples (weakly max) \\
\hline TE-107-AP-49 & $8 / 19 / 90$ & 82 & CASS & $\mathbf{s}$ & Disapreament with nearby thermocouples (weakly max) \\
\hline TE-107-AP.49 & 8/29/50 & 77 & CASS & $\mathbf{s}$ & Disagraement with nearby thermocouples (wookly max) \\
\hline TE-107-AP-49 & 9/5/90 & 78 & CASS & $\mathbf{S}$ & Disapreement with nearby thermocouples (weakly max) \\
\hline TE-107.AP-49 & $9 / 12 / 90$ & 79 & CASS & $\mathbf{S}$ & Disagreement with nesrby thermocouples (woekky max) \\
\hline TE-107-AP-49 & 9/22/90 & 74 & CASS & $\mathbf{s}$ & Disagraement with naarby thermocouples (weakly max) \\
\hline$T E-107-A P-49$ & $9 / 25 / 90$ & 75 & CASS & $\mathbf{S}$ & Disegreement with nearby thermocouples (weakly max) \\
\hline TE-107-AP-49 & $9 / 30 / 90$ & 71 & CASS & $\mathbf{s}$ & Disagreement with nearby therinocouples (weakly max) \\
\hline TE-107-AP-49 & $10 / 9 / 90$ & 102 & CASS & $\mathbf{5}$ & Disapreement with nearby thermocouples (weekly max) \\
\hline IE-107-AP-49 & $10 / 14 / 90$ & 61 & CASS & $\mathbf{s}$ & Disapreement with nearby thermocouples (woeksy max) \\
\hline TE-107-AP-4Q & $10 / 26 / 90$ & 56 & CASS & $\mathbf{S}$ & Disapreament with nedpy themocouples (weekly max) \\
\hline TE-107-AP-49 & $10 / 31 / 90$ & 60 & CASS & $\mathbf{S}$ & Disagreament with nearby themocouples (weekly $m a x$ ) \\
\hline TE-107-AP-49 & $11 / 10 / 80$ & 56 & CASS & $\mathbf{S}$ & Disagreement with nearby thermocouples (weekly max) \\
\hline TE-107-AP-49 & $11 / 13 / 90$ & 53 & CASS & $\mathbf{S}$ & Disapreamant with nearby tharmocouples (waekly $\mathrm{max}$ ) \\
\hline TE-107-AP-49 & $11 / 24 / 90$ & 53 & CASS & $\mathbf{s}$ & Disagreamont with nearby thermocouples (weakly max) \\
\hline TE-107-AP-49 & $11 / 25 / 90$ & 53! & CASS & $\mathbf{s}$ & Disapreement with nearby thermocouples (weekly mox) \\
\hline TE-107-AP-49 & $12 / 4 / 90$ & 45 & CASS & $\mathbf{s}$ & Disegreement with nearby thermocouples (woekly $\mathrm{max}$ ) \\
\hline TE-107.AP-49 & $12 / 10 / 90$ & 42 & CASS & $\mathbf{S}$ & Diaspreament with nearby thermocouples (weakly max) \\
\hline TE-107-AP-49 & $12 / 18 / 90$ & 41 & CASS & $\mathbf{5}$ & Diasoreement with nearty thermocouples \{wogkly max \\
\hline TE-107-AP-49 & $12 / 28 / 90$ & 31 & CASS & $\mathbf{S}$ & Diasareement with nearty thermocouples (weakly max) \\
\hline TE-107-AP-49 & $1 / 3 / 91$ & 30 & CASS & $\mathbf{s}$ & Disapreement with neartby thermocouples (waekly max) \\
\hline TE-107-AP-49 & $1 / 12 / 91$ & 33 & CASS & $\mathbf{S}$ & Disagreement with nearby thermocouples (weekly max) \\
\hline TE-107-AP-49 & $1 / 15 / 91$ & 39 & CASS & $\mathbf{S}$ & Disagreament with nearby thermocouples (weekly max) \\
\hline TE-107-AP-49 & $1 / 22 / 91$ & 32 & CASS & $\mathbf{S}$ & Disegroement with nearby thermocouples (woekly max) \\
\hline TE-107-AP-49 & $2 / 2 / 91$ & 35 & CASS & $\mathbf{S}$ & Disegreement with nearby thermocouples (waekly max) \\
\hline TE-107.AP-49 & $2 / 5 / 91$ & 42 & CASS & $\mathbf{S}$ & Disegreament with nearby thermocouples (woekly max) \\
\hline TE-107-AP-49 & $2 / 16 / 91$ & 46 & CASS & $\mathbf{S}$ & Disagreament with nearby thermocouples (weekly max) \\
\hline TE-107-AP-49 & $2 / 20 / 91$ & 47 & CASS & $\mathbf{S}$ & Disagreament with nearby thermocouples (woekly max) \\
\hline TE-107-AP-49 & 3/1/91 & 46 & CASS & $\mathbf{S}$ & Disagreament with nearby thermocouples (weakly max) \\
\hline TE-107-AP-49 & 3/4/91 & 45 & CASS & $\mathbf{S}$ & Disagreement with nearby thermocouples (weekly max) \\
\hline TE-107-AP-49 & 3/12/91 & 44 & CASS & $\mathbf{S}$ & Disarreement with nearby thermocouplos (weakly max) \\
\hline
\end{tabular}




\begin{tabular}{|c|c|c|c|c|c|}
\hline \multicolumn{6}{|c|}{$\begin{array}{l}\text { TAWK 241-AP-107 } \\
\text { THEROMOCOUPLE DATA }\end{array}$} \\
\hline & & & & & \\
\hline THERMOCOUPLE : & DATE & TEMPERATURE ( ${ }^{\circ} \mathrm{F}$ ) & DATA SOURCE & QUALTYY & COMLENT \\
\hline$T E+107-A P-49$ & $3 / 23 / 91$ & 47 & CASS & $\mathbf{S}$ & Disapreement with nearby thermocouples (woekly $\max$ ) \\
\hline TE-107.AP-49 & $3 / 24 / 91$ & 48 & CASS & $\mathbf{s}$ & Disagreement with naarby thermocouplas (woekly max) \\
\hline TE-107-AP-49 & $4 / 5 / 91$ & 56- & CASS & $\mathbf{S}$ & Disagreement with nearby thermocouples (woekly max) \\
\hline TE-107.AP-49 & 4/13/91 & 52 & CASS & $\mathbf{S}$ & Disapreement with nearby thermocouples (woekly max) \\
\hline TE-107-AP-49 & 4/20/93 & 56 & CASS & $\mathbf{s}$ & Disapreament with nearby thermocouples (weakly max) \\
\hline TE-107.AP-49 & 4/24/91 & 61 & CASS & $\mathbf{S}$ & Diearreement with noarby thermocouples (weekly $\mathrm{max}$ ) \\
\hline TE-107-APCA9 & 5/4/91 & 60 & CASS & $\mathbf{S}$ & Disepreement with nearby thermocouples (weekly max) \\
\hline TE-107-AP-49 & $5 / 5 / 31$ & 63 & CASS & $\mathbf{S}$ & Disegreement whh noarby thermocouplos (waekly $\max$ ) \\
\hline TE-107-AP-49 & 5/16/91 & 66 & CASS & $\mathbf{s}$ & Dissoreament with nearby thermocouples (weekly $\max$ ) \\
\hline TE-107-AP-49 & 5/24/91: & 64. & CASS & $\mathbf{S}$ & Disagreament with noarby thermocouples (weakly max) \\
\hline TE-107-AP-49 & $6 / 1 / 81$ & 67 & CASS & $\mathbf{s}$ & Diseqreement with neaby thermocouples (weekly max) \\
\hline TE-107-AP-48 & 6/5/91 & 65 & CASS & $\mathbf{S}$ & Disegreement with nearby thermocouples (weokly max) \\
\hline TE:107-AP-49 & 6/11/91 & 74 & CASS & $\mathbf{S}$ & Dizepreement whe nearby thermocouples (wopkly max) \\
\hline TE-107-AP-49 & 8/19/91 & 69 & CASS & $\mathbf{S}$ & Diaspreament with nearby thermocouples (weakly max) \\
\hline TE-107-AP-49 & 6/24/91 & 6 & CASS & $\mathbf{s}$ & Diagaroement with nearby thormosouples (wreekly max) \\
\hline TE-107-AP.49 & $713 / 91$ & 78 & CASS & $\mathbf{s}$ & Disepreament with nearby thermocouples (weekly max) \\
\hline TE-107-AP-49 & $7 / 13 / 91$ & 82 & CASS & $\mathbf{5}$ & Diaspreament with neaby thermocouples (wodkly max) \\
\hline TE-107-AP-49 & $7 / 14 / 91$ & 82 & CASS & $\mathbf{s}$ & Diseproement with nearby thermocouphes (weekly max) \\
\hline TE-107-AP-49 & $7 / 24 / 91$ & 84 & CASS & $\mathbf{s}$ & Disarreament with naerby thormocouples (wookly max) \\
\hline IE-107.AP-49 & $8 / 3 / 91$ & 81 & CASS & $\mathbf{S}$ & Disepreament with nearby thermocouples (woekly max) \\
\hline TE-107-AP-49 & $8 / 5 / 91$ & 84 & CASS & $\mathbf{5}$ & 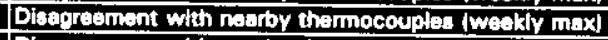 \\
\hline TE-107-AP- 49 & $8 / 17161$ & B4 & CASS & $\mathbf{S}$ & Disepreoment with nearby tharmocouples (weokly max) \\
\hline TE-107-AP- 19 & $8 / 20 / 91$ & 86 & CASS & $\mathbf{S}$ & Disegreement with neartby thermocouples (weekly max) \\
\hline TE-107-AP-49 & $8 / 30191$ & 79 & CASS & s & Diaserneement with neapy thermocouples (weokly max) \\
\hline TE-107-AP-49 & $9 / 7 / 91$ & 78 & CASS & $\mathbf{S}$ & Diasoreament with nearby thermocouplos (weakly max) \\
\hline TE-107-AP-49 & g/8/91 & 75 & CASS & $\mathbf{s}$ & Diaser roement with nearby thomocouplese (woekly max) \\
\hline TE-107.AP-49 & 9/18/31 & 74 & CASS & $\mathbf{s}$ & Diegrament with nearby tharmocouples (wookly max) \\
\hline TE-107-AP-4Q & 9/28/91 & 73 & CASS & $\mathbf{S}$ & Disugroement with nourby thermocouples (wodkjy max) \\
\hline TE-107-AP-49 & 10/1/91 & 72 & CASS & $\mathbf{S}$ & Diagareanemt with noarby thermocouplos (wedkly max) \\
\hline TE-107-AP-AS & 10/7191 & 63 & CASS & $\mathbf{s}$ & Disagroemant with masey theirmocousples (wepkly max) \\
\hline TE-107-AP-49 & 10/16/81 & 60 & CASS & s & Diecereement with nearby thermocouples (weekly max) \\
\hline TE-107-AP-49 & $10 / 21 / 61$ & 59 & CASS & $\mathbf{S}$ & Diegereoment with nosiby thormocouples (woekly max) \\
\hline TE-107-AP-49 & $10 / 27 / 93$ & 51 & CASS & $\mathbf{s}$ & Deaprearnent whi nadiby thermocouplas (waokly max) \\
\hline TE-107.AP-49 & $11 / 6 / 91$ & 46 & CASS & $\mathbf{s}$ & Digargeormant whth nuarby thermocouplas (woekly max) \\
\hline TE-107-AP-49 & 8/1/92 & 87 & CASS & $\mathbf{s}$ & Diespreemant with neaby tharmocouples (wodkly max) \\
\hline TE-107-AP-49 & $8 / 3 / 92$ & 85 & CASS & 5 & Ditapresiment with noarby tharmocouples (weekly max) \\
\hline TE-107-AP-49 & $8 / 15 / 92$ & 89 & CASS & $\mathbf{S}$ & Dispreament with nearby thermocouples (woekty max) \\
\hline TE-107-AP-49 & $8 / 19 / 92$ & 86 & CASS & $\mathbf{s}$ & Diedpreanent whth noaby thempocouplas (woekly $\mathrm{max}$ ) \\
\hline TE-107-AP-49 & 8/28/92 & 78 & CASS & $\mathbf{S}$ & Disapreament with neasby thermocouplas (woekly $\max$ ) \\
\hline TE-107-AP-49 & $9 / 492$ & 79 & CASS & $\mathbf{S}$ & Disgoresment with noarby thermocouples (woekly max) \\
\hline TE-107-AP-49 & $9 / 11 / 92$ & 74 & CASS & $\mathbf{s}$ & Diagareament with naarby thermocouplass (wookly max) \\
\hline TE-107-AP-49 & 8/13/92 & 70 & CASS & $\mathbf{S}$ & Detagreanent with naarby theimocouples (wockly max) \\
\hline TE-107-AP-49 & $8 / 23 / 92$ & 72 & CASS & $\mathbf{s}$ & Diengreement whth noarby the mocouples (weekly max) \\
\hline TE-107-AP-A9 & $10 / 2 / 92$ & 72 & CASS & $\mathbf{S}$ & Disegroement with neaby thermocouples (woekly max) \\
\hline TE-107-AP-49 & $10 / 4 / 92$ & 66 & CASS & $\mathbf{5}$ & Diseagresinent with nearby tharmocouples (weokly max) \\
\hline TE-107-AP-49 & $10 / 13 / 92$ & 68) & CASS & $\mathbf{5}$ & Diegereement with nederby thermocouples (weokly max) \\
\hline TE-107-AP-49 & $10 / 21 / 22$ & 62 & CASS & $\mathbf{S}$ & Diegereament with nearby thermocouplas (weekly max) \\
\hline$T E-107 \cdot A P-49$ & $10 / 25 / 92$ & 60 & CASS & $\mathbf{S}$ & Diseqpeement with nosiby tharmocouplos (weokhy max) \\
\hline TE-107-AP-49 & $1+/ 6 / 92$ & 53 & CASS & $\mathbf{s}$ & Diesgreement with noarby thermocouplas (woekly max) \\
\hline TE-107-AP-49 & $11 / 8 / 92$ & 52 & CASS & $\mathbf{S}$ & Diesgreemant with neseby thermocouples (weakly max) \\
\hline TIE-107-AP-49 & $11 / 16 / 92$ & 52 & CASS & $\mathbf{S}$ & Disapreement with nearby thermocouples (woekly max) \\
\hline TE-107-AP-49 & $11 / 23 / 92$ & 44 & CASS & $\mathbf{S}$ & Diasareament with nearby therimocouples (woekly max) \\
\hline TE-107-AP-49 & 12/2/92: & 42 & CASS & $\mathbf{S}$ & Dilgagreament with nedrby thernocouplas (wedkiy max) \\
\hline TE-107-AP-49 & $12 / 10 / 92$ & 39 & CASS & $\mathbf{s}$ & Dicagreement with nearby themocouples (weokly max) \\
\hline TE-107-AP-49 & $12 / 15 / 92$ & 39 & CASS & $\mathbf{S}$ & Oiasagreament with nearby thermocouples (woekly max) \\
\hline TE-107.AP-49 & $12 / 23192$ & 40 & CASS & $\mathbf{s}$ & Disagreement whe nearby themocouples (weokly max) \\
\hline TE-107.AP-49 & $12 / 30 / 92$ & 37 & CASS & $\mathbf{S}$ & Diacerrement with neaby thermocouplas (weakly max) \\
\hline TE-107.AP-49 & $1 / 15 / 94$ & 44 & CASS & $\mathbf{S}$ & Disagreament with nearby tharmocouples (weakly max) \\
\hline TE-107-AP-49 & $1 / 18 / 94$ & 44. & CASS & $\mathbf{s}$ & Disapreement with neachy thormocouples (weakly $\mathrm{max}$ ) \\
\hline TE-107-AP-49 & $1 / 25 / 94$ & 45 & CASS & $\mathbf{S}$ & Digagreement with nearby thermocouples (wookly $m a x$ ) \\
\hline TE-107-AP-49 & $2 / 2 / 94$ & 40 & CASS & $\mathbf{S}$ & Diagureament with nadrby thermocouples (weokly max) \\
\hline TE-107.AP-49 & $2 / 12 / 94$ & 39 & CASS & S & Dicegresment with naarby thermocougles (woekly max) \\
\hline TE-107-AP-49 & $2 / 16 / 94$ & 47 & CASS & $\mathbf{s}$ & Diagoreement with neser y themocouplas (wookly max) \\
\hline TE-107-AP-49 & $2 / 23 / 94$ & 46 & CASS & $\mathbf{s}$ & Disapreament with nearby thermocouples (woekly max) \\
\hline TE-107-AP-49 & $3 / 4 / 94$ & $\mathbf{5 3}$ & CASS & $\mathbf{s}$ & Disapreament with nearby thermocouples (waekly max) \\
\hline TE-107.AP.49 & $3710 / 94$ & 51 & CASS & $\mathbf{S}$ & Disepreement with nearby thormocouples (weakly max) \\
\hline$T E-107 \cdot A P-49$ & $3 / 18 / 94$ & 56 & CASS & $\mathbf{s}$ & Disaproement with noarby thermocouplas (woekly $\mathrm{max}$ ) \\
\hline TE-107-AP-49 & $3 / 26 / 94$ & 53 & CASS & $\mathbf{s}$ & Dispgreament with naarby thermocouples (weekly max) \\
\hline TE-107-AP-49 & $3 / 31 / 94$ & 61 & CASS & $\mathbf{S}$ & Disegreement with nearby thermocouplas (weakly max) \\
\hline TE-107-AP-49 & $4 / 3 / 94$ & 60 & CASS & $\mathbf{S}$ & Diasgreement with nearby thermocouples (woekly max) \\
\hline TE-107-AP-49 & $4 / 12 / 94$ & 64) & CASS & $\overline{\mathbf{S}}$ & Diagdgrement with noarby thermocouples (weakly max) \\
\hline TE-107-AP-49 & $4 / 19 / 94$ & 70 & CASS & $\mathbf{S}$ & Diagrreement with nearby tharmocouples (woekly max) \\
\hline TE-107-AP-49 & $4 / 27 / 94$ & 67) & CASS & $\mathbf{s}$ & Diss?reement with nearby tharmocouples (weakly mox) \\
\hline
\end{tabular}




\begin{tabular}{|c|c|c|c|c|c|}
\hline \multicolumn{6}{|c|}{$\begin{array}{l}\text { TANK 241-AP-107 } \\
\text { THERMOCOUPLE DATA }\end{array}$} \\
\hline & & & & & \\
\hline THERMOCOUPLE * & DATE & TEMPERATURE ( $\left.{ }^{\circ} \mathrm{F}\right)$ & DATA SOUACE & QUALTY & COMMENT \\
\hline TE-107-AP-49 & $5 / 7 / 94$ & 71 & CASS & $\mathbf{S}$ & Disagreement with nearby thermocouples (weskly max) \\
\hline TE-107.AP-49 & $5 / 9 / 94$ & 78 & CASS & 5 & Disagreement with nearby thermocouplas (weekly max) \\
\hline TE-107-AP-49 & $5 / 16 / 94$ & 103 & CASS & $\mathbf{S}$ & Disagreement with nearby tharmocouples (weakly max) \\
\hline TE-107-AP-49 & $5 / 23 / 94$ & 142 & CASS & $\mathbf{S}$ & Disedreement with nearby thermocouples (weakly max) \\
\hline TE-107-AP-49 & $6 / 4 / 94$ & 104 & CASS & $\mathbf{S}$ & Disepreement with nearby thempcouples (weakly max) \\
\hline TE-107-AP-49 & $6 / 11 / 94$ & 104 & CASS & 5 & Disagreament with nearby tharmocouples (weekly max) \\
\hline TE-107.AP-49 & $6 / 12 / 94$ & 104 & CASS & $\mathbf{S}$ & Disacreament with nearby tharmocouples (woekly max) \\
\hline TE-107.AP-49 & $6 / 23 / 94$ & 109 & CASS & $\mathbf{S}$ & Disapreament with nearby themocouples (weakly max) \\
\hline TE-107-AP-49 & $7 / 1 / 94$ & 109 & CASS & $\mathrm{S}$ & Disagreement with netrby themocougles (weakly max) \\
\hline TE-107.AP-49 & $7 / 9 / 94$ & 109 & CASS & $\mathbf{S}$ & Disagreament with nasiby tharmocouples (weakly max) \\
\hline TE-107-AP-49 & $7 / 15 / 94$ & 147 & CASS & $\mathbf{S}$ & Disagreement with naarby thermocouples (waokly max) \\
\hline TE-107-AP-49 & $7 / 23 / 94$ & 116 & CASS & $\mathbf{S}$ & Disegreement with naarby tharmocouples (weekly max) \\
\hline TE-107-AP-49 & $7 / 24 / 94$ & 117 & CAS5 & $\mathbf{s}$ & Diaspreement with nearby thermocouples (weakly max) \\
\hline TE-107-AP-49 & $8 / 3 / 94$ & 115 & CASS & 5 & Disagreament with nearby tharmocouples (woekly max) \\
\hline TE-107.AP-49 & 8/13/94 & 111 & CASS & $\mathrm{s}$ & Disegreament with naerby thermocouples (weakly max) \\
\hline TE-107-AP-49 & $8 / 17 / 94$ & 113 & CASS & 5 & Disapreement whth nearby tharmocouples (weakly max) \\
\hline TE-107-AP-49 & $8 / 21 / 94$ & 110 & CASS & $\mathbf{S}$ & Disarreement with nearby tharmocouples (woekly max) \\
\hline TE-107.AP-49 & $9 / 3 / 94$ & 108 & CASS & $\mathbf{S}$ & Diagoreament with nearby thermocouples (weekly max) \\
\hline TE-107-AP-49 & $9 / 9 / 94$ & 109 & CASS & $\mathbf{s}$ & Diaggreament with naarty thermocouplas (weekly max) \\
\hline TE-107-AP-49 & $9 / 15 / 94$ & 104 & CASS & $\mathbf{S}$ & Diatagreement with nearby thermocoupies (weekly max) \\
\hline TE-107-AP-49 & $9 / 23 / 94$ & 106 & CASS & $\mathbf{s}$ & Diagoreement with naarby tharmocouples (weekly max) \\
\hline TE-107-AP-49 & $9 / 25 / 94$ & 107 & CASS & $\mathbf{S}$ & Dinaprearnent with naarby thermocouples (weakhy max) \\
\hline TE-107-AP-49 & $10 / 2 / 94$ & 99 & CASS & $\mathbf{S}$ & Disegreement with noarby tharmocouples (weakly max) \\
\hline TE-107-AP-49 & $10 / 15 / 94$ & 131 & CASS & $\mathbf{S}$ & Disspreemont with nearby thermocouples (weakly $\max$ ) \\
\hline TE-107-AP-49 & $10 / 17 / 94$ & 91 & CASS & $\overline{\mathbf{S}}$ & Diagoreement with nearby thormocouples (weekly max) \\
\hline TE-107.AP-49 & $10 / 27 / 94$ & 90 & CASS & $\mathbf{S}$ & Disagreement with nearby tharmocouples (weokhy max) \\
\hline TE-107-AP-49 & $11 / 4 / 94$ & 84 & CASS & $\mathbf{S}$ & Disegreement with nearby thermocouples (weekly max) \\
\hline TE.107-AP.49 & $1 1 \longdiv { 1 1 / 9 4 }$ & 82 & CASS & $\mathbf{S}$ & Diaegreement with nearby tharmocouples (weakly max) \\
\hline TE-107-AP-49 & $11 / 16 / 84$ & 79 & CASS & $\mathbf{S}$ & Diagraement with narby tharmocouples (weekly max) \\
\hline TE-107-AP-49 & $11 / 26 / 94$ & 75 & CASS & $\mathbf{S}$ & Diagereament with nearby themocouples (weekhy max) \\
\hline TE-107-AP-49 & $11 / 29 / 94$ & 76 & CASS & $\mathbf{S}$ & Diaggreament with naarby thermocouplas (weeky max) \\
\hline TE-107-AP-49 & $12 / 4 / 94$ & 77 & CASS & $\mathbf{S}$ & Disagreament with nearby tharmocouples (woekly max) \\
\hline TE-107-AP-49 & $12 / 17 / 94$ & 69 & CASS & $\mathbf{S}$ & Diasereement with nearby tharmocouples (waekly max) \\
\hline TE-107-AP-49 & $12 / 19 / 94$ & 78 & CASS & $\mathbf{s}$ & Disarreament with nearby thermocouples (weakly max) \\
\hline TE-107-AP-49 & $12 / 27 / 94$ & 76 & CASS & $\mathbf{S}$ & Disapreament with naarby thermocouples (weokly max) \\
\hline TE-107-AP.49 & $1 / 7 / 95$ & 69 & CASS & $\mathbf{S}$ & Dieargeament with nearby thamocouplas (weekly max) \\
\hline TE-107-AP-49 & I/14/95 & 75 & CASS & $S$ & Diagoreement with nearby thormocouples (weakly $\mathrm{max}$ ) \\
\hline TE-107-AP-49 & $1 / 16 / 95$ & 74 & CASS & $\mathbf{S}$ & Diaspreament with nearby tharmocouples (weekly max) \\
\hline TE-107.AP-49 & $1 / 28 / 95$ & 75 & CASS & $\mathbf{s}$ & Diagoreamant with noarby thermocouplea (weakly max) \\
\hline TE-107-AP-49 & $2 / 3 / 95$ & 77 & CASS & $\mathbf{S}$ & Disegreament with naarby tharmocouples (weekly max) \\
\hline TE-107-AP-49 & $2 / 6 / 95$ & 79 & CASS & $\mathbf{s}$ & Diaegraement with naarby tharmocouples (weakly max) \\
\hline TE-107-AP-49 & $2 / 13 / 95$ & 77 & CASS & $\mathbf{S}$ & Diaspreament with naarby thormocouples (weakly max) \\
\hline TE-107-AP-49 & $2 / 25 / 95$ & 80 & CASS & $\mathbf{s}$ & Diagreement with narby thermocouplas (weakly max) \\
\hline TE-107-AP-49 & $2 / 27 / 95$ & 82 & CASS & $\$$ & Disepreament whth nodby themocouples (weekly max) \\
\hline TE-107-AP-49 & $3 / 9 / 95$ & 78 & CASS & $\mathbf{S}$ & Digagreement with nearby thamocouples (weakly max) \\
\hline TE-107-AP-49 & $3 / 18 / 95$ & 80 & CASS & $\mathbf{s}$ & Disagreement with neaby thermocouples (woekly max) \\
\hline TE-107-AP-49 & $3 / 20 / 95$ & 80 & CASS & $\mathbf{S}$ & Disapresment with naerby thermocouples (wookly max) \\
\hline TE-107-AP-49 & $3 / 30 / 95$ & 82 & CASS & $\mathbf{E}$ & Disagreament with nagrby thermocouplas (weakly max) \\
\hline & & & & & \\
\hline TE-107-AP-50 & $4 / 5 / 86$ & 55 & CASS & $\mathbf{T}$ & \\
\hline TE-107-AP-50 & $4 / 7 / 86$ & 56 & CASS & $T$ & \\
\hline TE-107-AP-50 & $5 / 16 / 86$ & 56 & CASS & $T$ & \\
\hline TE-107-AP-50 & $5 / 22 / 86$ & 59 & CASS & $T$ & \\
\hline TE-107-AP-50 & $5 / 25 / 86$ & 59 & CASS & $T$ & \\
\hline TE-107-AP-50 & $6 / 4 / 86$ & $\mathbf{5 B}$ & CASS & $T$ & \\
\hline TE-107-AP-50 & $6 / 9 / 86$ & 60 & CASS & $T$ & \\
\hline TE-107-AP-50 & $6 / 21 / 86$ & 58 & CASS & $T$ & \\
\hline TE-107-AP-50 & 6/25/86 & 58 & CASS & $T$ & \\
\hline TE-107-AP-50 & $7 / 5 / 86$ & 59 & CASS & $T$ & \\
\hline TE-107-AP-50 & $7 / 10 / 86$ & 61 & CASS & $T$ & \\
\hline TE-107-AP-50 & $7 / 19 / 86$ & 59 & CASS & $T$ & \\
\hline TE-107-AP-50 & $7 / 20 / 86$ & 63 & CASS & $T$ & \\
\hline TE-107.AP-50 & $8 / 2 / 66$ & 60 & CASS & $T$ & \\
\hline TE-107.AP-50 & 8/9/86 & 63 & CASS & $T$ & \\
\hline TE-107-AP-50 & $8 / 13 / 86$ & 62 & CASS & $T$ & \\
\hline TE-107-AP-SO & $8 / 17 / 86$ & 63 & CASS & $T$ & \\
\hline TE-107-AP-50 & $8 / 27 / 86$ & 64 & CASS & $T$ & \\
\hline TE-107-AP-50 & $9 / 1 / 86$ & 63 & CASS & $T$ & \\
\hline TE-107-AP-50 & 9/23/86 & 64 & CASS & $\mathbf{T}$ & \\
\hline TE-107-AP-50 & 9/29/86 & 62 & CASS & $T$ & \\
\hline TE-107-AP-50 & $10 / 11 / 86$ & 62 & CASS & $T$ & \\
\hline TE-107-AP-50 & $10 / 18 / 86$ & 61. & CASS & $T$ & \\
\hline TE-107-AP-50 & $10 / 24 / 86$ & 62. & CASS & $T$ & \\
\hline
\end{tabular}




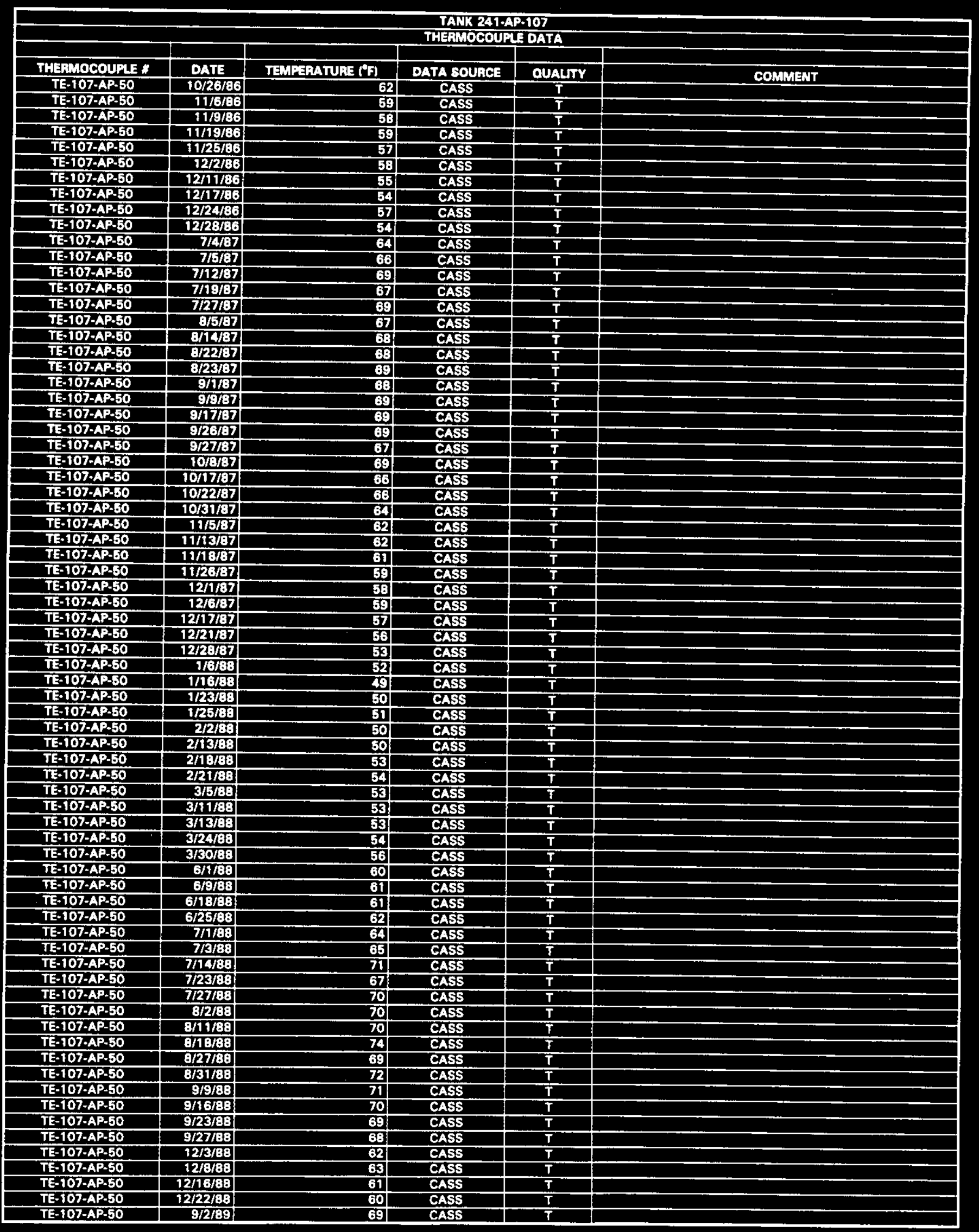




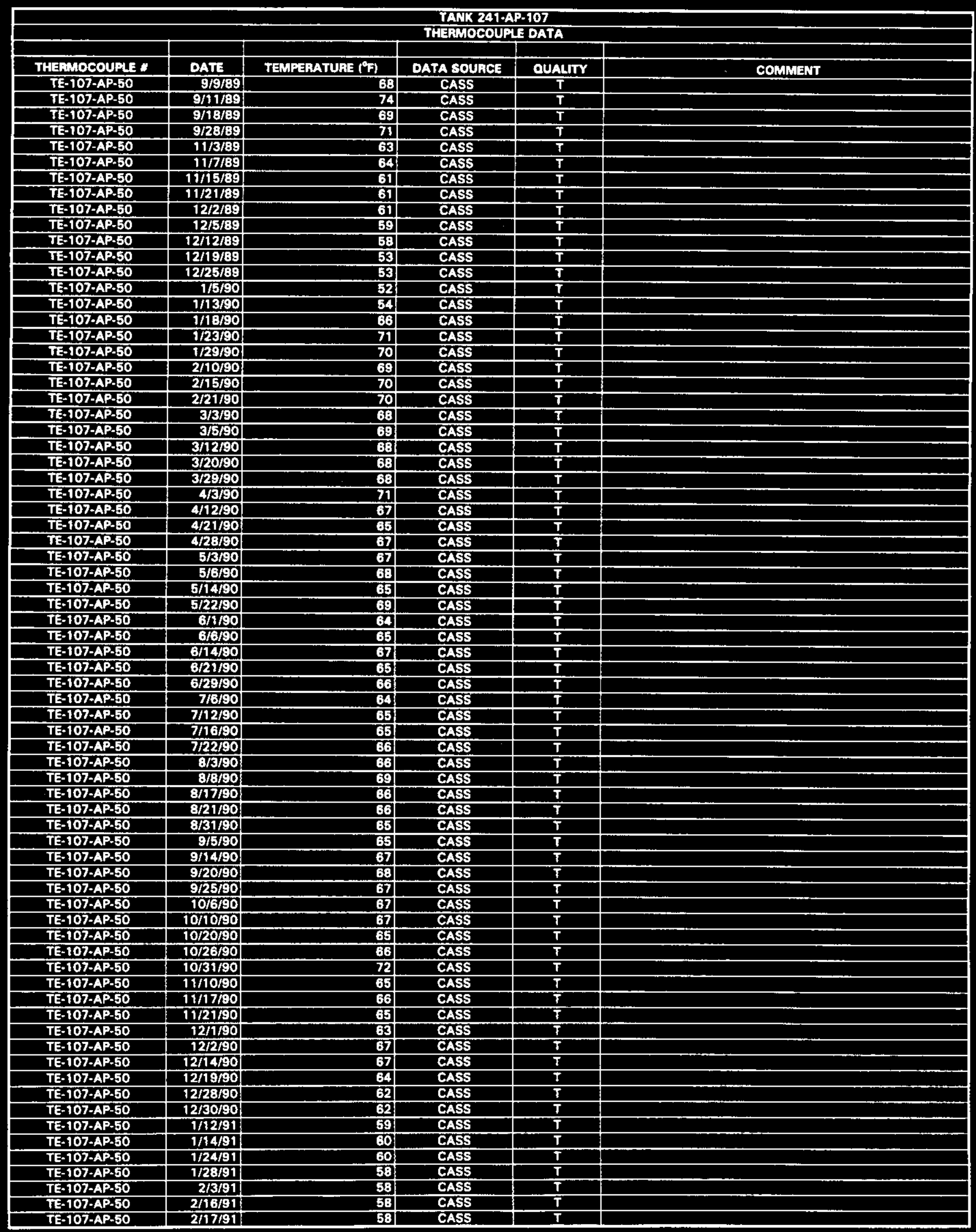




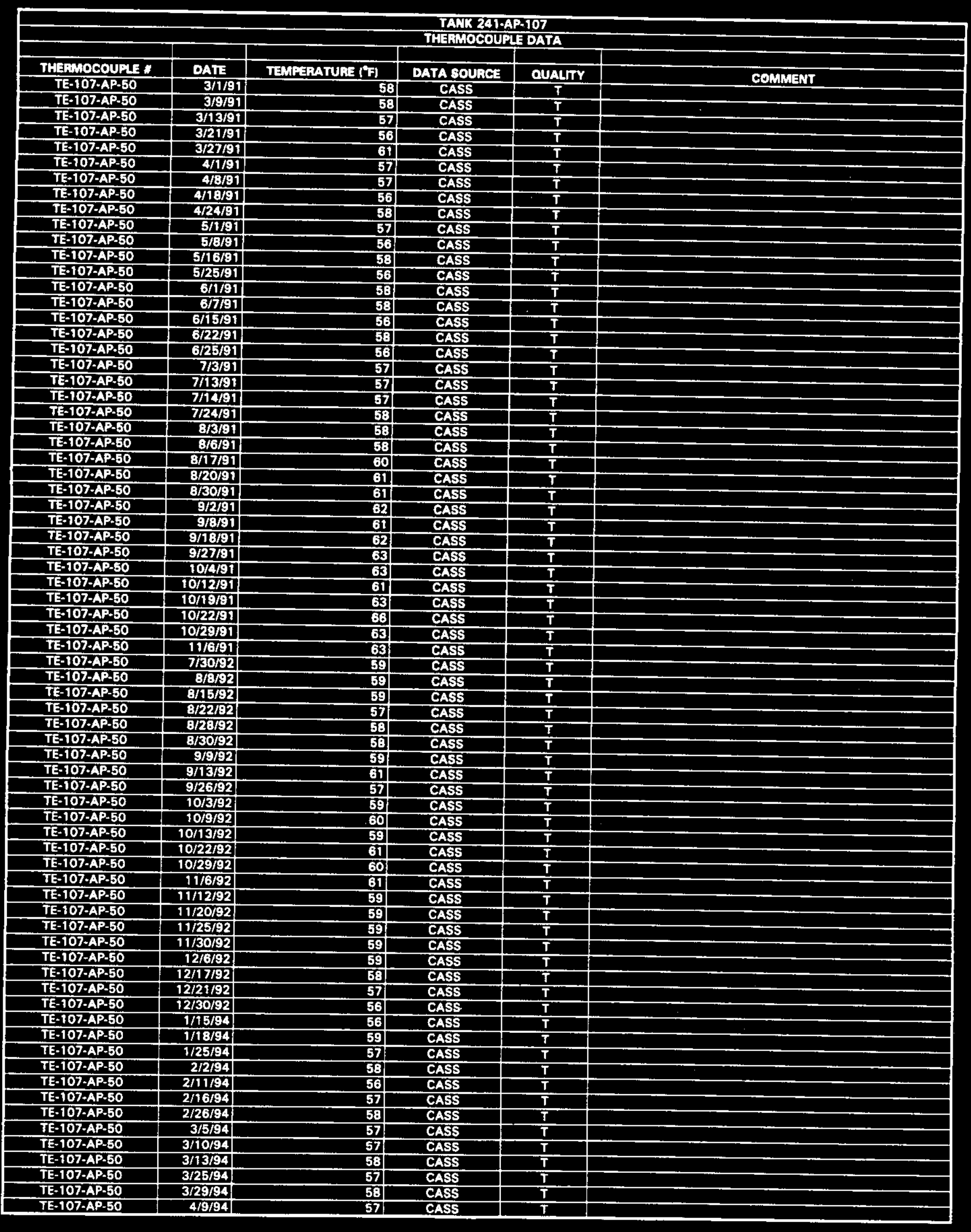

Date obtained from Computer Automated Surveillance Syatem (CASS), April 1995; and Survellance Analysis Computer System (SACS), June 1995.

D-628 


\begin{tabular}{|c|c|c|c|c|c|}
\hline \multicolumn{6}{|c|}{$\begin{array}{l}\text { TANK 241-AP-197 } \\
\text { THEFMOCOUPLE DATA }\end{array}$} \\
\hline THERMOCOUPLE & DATE & TKMPERATUES (PF) & DATA SOURCE & QuALTY & COMMENT \\
\hline TE-107-AP-50 & $4 / 13 / 94$ & 58 & CASS & $T$ & \\
\hline TE-107-AP-50 & $4 / 20 / 94$ & 58 & CASS & $\bar{T}$ & \\
\hline TE-107-AP-50 & $4 / 27 / 94$ & 61 & CASS & $T$ & \\
\hline TE-107.AP-50 & $5 / 2 / 94$ & 61 & CASS & $\mathbf{T}$ & \\
\hline TE-107-AP-50 & 5/9/94 & 58 & CASS & $T$ & \\
\hline TE-107-AP-50 & $5 / 76 / 94$ & 94 & CASS & $T$ & \\
\hline TE-107-AP-50 & $5 / 22 / 94$ & 89 & CASS & $T$ & \\
\hline TE-107-AP-50 & $6 / 2 / 94$ & 89 & CASS & $T$ & \\
\hline TE-107-AP-50 & 6/8/94 & 87 & CASS & $T$ & \\
\hline TE-107-AP-SO & $6 / 14 / 94$ & 86 & CASS & $T$ & \\
\hline TE-107-AP-50 & $6 / 16 / 94$ & 56 & CASS & $\mathbf{s}$ & Data point did not agres with local trend \\
\hline TE-107-AP-50 & $6 / 17 / 94$ & 56 & CASS & $\mathbf{S}$ & Data point did not agree with local trend \\
\hline TE-107.AP-50 & $6 / 20 / 94$ & 59: & CASS & 5 & Data point did not egree with locel trend \\
\hline TE-107-AP-50 & $6 / 24 / 94$ & 89 & CASS & $T$ & \\
\hline TE-107-AP-50 & $6 / 27 / 94$ & 92 & CASS & $\mathbf{T}$ & \\
\hline TE-107-AP-50 & $7 / 7 / 94$ & 87 & CASS & $\bar{T}$ & \\
\hline TE-107-AP-50 & $7 / 16 / 94$ & 87 & CASS & $T$ & \\
\hline TE-107-AP-50 & $7 / 18 / 94$ & 86 & CASS & $T$ & \\
\hline TE-107-AP-50 & $7 / 30 / 94$ & 84 & CASS & $T$ & \\
\hline TE.107-AP-SO & $7 / 31 / 94$ & 87 & CASS & $\mathbf{T}$ & \\
\hline TE-107.AP-50 & $8 / 9 / 94$ & 88 & CASS & $T$ & \\
\hline TE-107-AP.50 & 8/20/94 & 88 & CASS & $T$ & \\
\hline TE-107-AP-50 & $8 / 22 / 94$ & 89 & CASS & $T$ & \\
\hline TE-107-AP-50 & $9 / 3 / 94$ & 87 & CASS & $T$ & \\
\hline TE-107-AP-SO & $9 / 10 / 94$ & 90 & CASS & $T$ & \\
\hline TE-107-AP-50 & 9/15/94 & 91 & CASS & $T$ & \\
\hline TE-107-AP-50 & 9/23/94 & 81 & CASS & $T$ & \\
\hline TE-107-AP-50 & $10 / 1 / 94$ & 93 & CASS & $T$ & \\
\hline TE-107-AP-50 & $10 / 8 / 94$ & 95 & CASS & $T$ & \\
\hline TE-107-AP-50 & $10 / 15 / 94$ & 85 & CASS & $\mathbf{T}$ & \\
\hline TE-107.AP-50 & $10 / 22 / 94$ & 99 & CASS & $\mathbf{T}$ & \\
\hline TE-107-AP-50 & $10 / 26 / 94$ & 100 & CASS & $T$ & \\
\hline TE-107-AP.50 & $11 / 5 / 94$ & 88 & CASS & $T$ & \\
\hline TE-107-AP-SO & $11 / 11 / 94$ & 88 & CASS & $T$ & \\
\hline TE-107.AP.50 & $11 / 14 / 94$ & 87 & CASS & $T$ & \\
\hline TE-107-AP-50 & $11 / 22 / 84$ & 97 & CASS & $T$ & \\
\hline TE-107-AP-BO & $11 / 29 / 94$ & 91 & CASS & $T$ & \\
\hline TE-107-AP-50 & $12 / 4 / 84$ & 85 & CASS & $T$ & \\
\hline TE-107-AP-50 & $12 / 12 / 94$ & 89 & CASS & $T$ & \\
\hline TE-107-AP-50 & $12 / 19 / 94$ & 93 & CASS & $T$ & \\
\hline TE-107-AP-50 & $12 / 30 / 94$ & 92 & CASS & $\bar{T}$ & \\
\hline TE-107-AP-50 & $1 / 4 / 95$ & 94 & CASS & $T$ & \\
\hline TE-107-AP-50 & $1 / 13 / 95$ & 91 & CASS & $T$ & \\
\hline TE-107-AP-50 & $1 / 15 / 95$ & 90 & CASS & $\mathbf{T}$ & \\
\hline TE-107-AP-50 & $1 / 23 / 95$ & 100 & CASS & $T$ & \\
\hline TE-107-AP-50 & 2/2/95 & 96 & CASS & $T$ & \\
\hline TE-107-AP-50 & $2 / 9 / 95$ & 98 & CASS & $T$ & \\
\hline TE-107-AP-50 & $2 / 14 / 95$ & 104 & CASS & $T$ & \\
\hline TE-107-AP-S0 & $2 / 24 / 95$ & 93 & CASS & $T$ & \\
\hline TE-107-AP-SO & $2 / 28 / 95$ & 97 & CASS & $T$ & \\
\hline TE-107-AP-50 & $3 / 7 / 95$ & 96 & CASS & $T$ & \\
\hline TE-107-AP.50 & $3 / 16 / 95$ & 91 & CASS & $T$ & \\
\hline TE-107-AP-50 & $3 / 25 / 95$ & 91 & CA5s & $T$ & \\
\hline TE-107-AP-50 & $3 / 30 / 95$ & 91 & CASS & $\bar{T}$ & \\
\hline TE-107-AP-51 & $4 / 5 / 86$ & 46 & CASS & $\mathbf{S}$ & Disagreament with nearby thermocouples twegkly maxt \\
\hline TE-107-AP-51 & $4 / 9 / 86$ & 56 & CASS & $\mathbf{S}$ & Disagreament with nearby thermocouples (weakly max) \\
\hline TE-107-AP-51 & $5 / 17 / 8$ & 70 & CASS & $\mathbf{s}$ & Disagreament with nearby thermocouples (weakly max) \\
\hline TE-107-AP-51 & 5/18/86 & 83 & CASS & $\mathbf{S}$ & Disagreement with nearby themocouples (weekly max) \\
\hline TE-107-AP-51 & $5 / 31 / 86$ & 110 & CASS & S & Disagreement with nearby thermocouples (weekly max) \\
\hline TE-107-AP-51 & 6/1/86 & 111 & CASS & $\mathbf{S}$ & Disagreament with nearby themocouples (waekly max) \\
\hline TE-107-AP-51 & $6 / 14 / 86$ & 103 & CASS & $\mathbf{S}$ & Disagreement with nearby themocouples (weekly max) \\
\hline TE-107-AP-51 & 6/16/86 & 96 & CASS & $\mathbf{S}$ & Disagreament with nearty thermocouples (weakly $\mathrm{max}$ ) \\
\hline TE-107-AP-51 & $6 / 24 / 86$ & 102 & CASS & $\mathbf{s}$ & Disagreement with nearby thermocouplas (weekly $\mathrm{max}$ ) \\
\hline TE-107-AP-51 & $7 / 3 / 86$ & 86 & CASS & $\mathbf{S}$ & Disagreament with naarby tharmocouples (waskly max) \\
\hline TE-107-AP-51 & 710/86 & 89 & CASS & $\mathbf{S}$ & Disagreement with nearby thermocouples (weekly max) \\
\hline TE-107-AP-51 & $7 / 19 / 86$ & 93 & CASS & $\mathbf{S}$ & Disagreement with nesrby themocouples (weekly max) \\
\hline TE-107-AP-51 & $7 / 22 / 86$ & 100 & CASS & $\mathbf{s}$ & Disagreament with nearby thermocouples (weekly max) \\
\hline TE-107-AP-51 & $8 / 2 / 86$ & 100 & CASS & $\mathbf{S}$ & Disagreament with nearby thermocouplos (weekly max) \\
\hline TE-107.AP-51 & $8 / 9 / 86$ & 111 & CASS & $\mathbf{S}$ & Disagreament with nearby thermocouples (weekly max) \\
\hline TE-107-AP-51 & $8 / 10 / 86$ & 111 & CASS & $\mathbf{s}$ & Disagreement with nearby thermocouples (weekly max) \\
\hline TE-107-AP-51 & $8 / 23 / 86$ & 104 & CASS & $\mathbf{S}$ & Disagreement with nearby thermocouples (weekly max) \\
\hline TE-107-AP-51 & $8 / 28 / 86$ & 110 & CASS & $\mathbf{S}$ & Disagreement with nearby thermocouples (wadkly max) \\
\hline
\end{tabular}




\begin{tabular}{|c|c|c|c|c|c|}
\hline \multicolumn{6}{|c|}{$\begin{array}{l}\text { TANK 241-AP-107 } \\
\text { THEFIMOCOUFLE DATA }\end{array}$} \\
\hline & & & & & \\
\hline THERMOCOUPLE $*$ & DATE & TEMPERATURE ("F) & DATA SOURCE & QUALITY & COMMENT \\
\hline TE-107-AP-51 & 9/3/86 & 93 & CASS & $\mathbf{S}$ & Disagreement with nearby thermocouples (weekly max) \\
\hline TE-107-AP-5T & $9 / 23 / 86$ & 61 & CASS & $\mathbf{S}$ & Disagreament with naarby thermocouples (weekly $\max$ ) \\
\hline TE-107-AP-51 & 9/29/86 & 59 & CASS & $\mathbf{s}$ & Disagreament with nearby thermocouples (weekly max) \\
\hline TE-107-AP.51 & $10 / 8 / 86$ & 63 & CASS & $\mathbf{S}$ & Disegreement with nearby thermocouples (weekly max) \\
\hline TE-107-AP-51 & $70 / 12 / 86$ & 48 & CASS & $\mathbf{s}$ & Disegreement with nearby thermocouples (woekly mox) \\
\hline TE-107.AP-51 & $10 / 24 / 86$ & 50 & CASS & $\mathbf{S}$ & Disapreement with nearby thermocouples (woekly max) \\
\hline TE-107.AP-51 & $10 / 26 / 86$ & 55! & CASS & $\mathbf{s}$ & Dissagreement with nearby thermocouples (weekly max) \\
\hline TE-107-AP-51 & $11 / 5 / 86$ & 42 & CASS & $\mathbf{s}$ & Disegreament with nearby thermocouples (woekly max) \\
\hline TE-107-AP-51 & $11 / 9 / 86$ & 29 & CASS & $\mathbf{s}$ & Oisegreement with nearby thermocouples (woek ḱly max) \\
\hline TE-107-AP-51 & $11 / 20 / 86$ & 45 & CASS & $\mathbf{S}$ & Disapreement with noarby thermocouples (weakly mox) \\
\hline TE-107-AP-51 & $7 / 1 / 87$ & 106 & CASS & $\mathbf{s}$ & Disagreement with nearby tharmoccuples (woekly mex) \\
\hline TE-107.AP-51 & $7 / 8 / 87$ & 87 & CASS & $\mathbf{s}$ & Disagreanent with nearby tharmocouples iwoekly max f \\
\hline TE-107-AP-51 & $7 / 14 / 87$ & 99 & CASS & $\mathbf{s}$ & Disepresment whh nearby thermocouples (weakly max) \\
\hline TE-107-AP-51 & $7 / 25 / 87$ & 92 & CASS & $\mathbf{S}$ & Disepresmont with nearby thermocouples (weakly max) \\
\hline TE-107-AP-51 & $7 / 29 / 87$ & 91 & CASSS & $\mathbf{s}$ & Disegreement with naarby thermocouples (woekly max) \\
\hline TE-107.AP-51 & $8 / 8 / 87$ & 94 & CASS & $\mathbf{S}$ & Disepreement with nearby thermocouples (weekly max) \\
\hline TE-107-AP-51 & $8 / 10 / 87$ & 95 & CASS & $\overline{\mathbf{s}}$ & Direpreement with noarby thermocouplas (wookly max) \\
\hline TE-107-AP-51 & $8 / 22 / 87$ & 80 & CASS & $\mathbf{S}$ & Diasapresment with nearby thermocouplas (woekly max) \\
\hline TE-107-AP-51 & $8 / 27 / 87$ & 89 & CASS & $\mathbf{S}$ & Disagreement with naarby tharmocouplas (weakly max) \\
\hline TE-107-AP-51 & $9 / 1 / 87$ & 95 & CASSS & $\mathbf{s}$ & Disagreement with noarby thermocouples (woekly max) \\
\hline TE-107-AP-51 & 9/8/87 & 86 & CASS & $\mathbf{s}$ & Disaproement with nearby thermocouples (weakly max) \\
\hline TE-107-AP-51 & $9 / 13 / 87$ & 78 & CASS & $\mathbf{S}$ & Disagreement with nearby thermocouples (weekly max) \\
\hline TE-107-AP-5! & 9/25/87 & 75 & CASS & $\mathbf{S}$ & Disegreement with nearby thermocouples (weekly max) \\
\hline TE-107-AP-51 & $10 / 3 / 87$ & 88 & CASS & $\mathbf{S}$ & Disegraemant with noarby thermocouples (woekly max) \\
\hline TE-107-AP-51 & $10 / 8 / 87$ & 70 & CASS & $\mathbf{s}$ & Diseprearnent with nearby thermocouples (weekly mex) \\
\hline TE-107-AP-51 & $10 / 12 / 87$ & 53 & CASS & $\mathbf{S}$ & Disagrooment with nearby thermocouples (woekly max) \\
\hline TE-107-AP-6I & $10 / 18 / 87$ & 50 & CASS & $\mathbf{S}$ & Disagraement with nearby thermocouplos (weekly $\mathrm{max}$ ) \\
\hline TE-107-AP-51 & $10 / 31 / 87$ & 52 & CASS & $\mathbf{S}$ & Disagreerment with nearby thermocouples (woekly max) \\
\hline TE-107-AP-51 & $11 / 2 / 37$ & 53 & CASS & $\mathbf{s}$ & Diasegreement with nearby thermocouplas (wookly max) \\
\hline TE-107-AP-51 & $11 / 13 / 87$ & 51 & CASS & $\mathbf{s}$ & Disegreament with neasty tharmocouplos (woekly max) \\
\hline TE-107-AP-51 & $11 / 16 / 87$ & 42 & CASS & $\mathbf{s}$ & Diasogrement with noarby tharmocouplas (weekly max) \\
\hline TE-107-AP-51 & $11 / 22 / 87$ & 35 & CASS & $\mathbf{s}$ & Disagreerment with nearby thormocouples (woekly mox) \\
\hline TE-107-AP-51 & $12 / 5 / 87$ & 37 & CASS & $\mathbf{s}$ & Disepreement with noarby thermocouples (woekly max) \\
\hline TE-107-APS51 & $12 / 8 / 87$ & $4 \pi$ & CASS & $\mathbf{5}$ & Disapreament with nearby tharmocouplas (wookty max) \\
\hline TE-107-AP-51 & $12 / 16 / 87$ & 29 & CASS & $\mathbf{S}$ & Diasorcement with moarby tharmocouplas (woekly mox) \\
\hline TE-107-AP-51 & $12 / 23 / 97$ & 24 & CASS & $\mathbf{S}$ & Oisupreament with noarby tharmoccuples (weekly max) \\
\hline TE-107-AP.51 & $1 / 1 / 38$ & 24 & CASS & $\mathbf{5}$ & Disadreement with naarby thermocouplas (wookly max) \\
\hline IE-107-AP-51 & $1 / 8 / 88$ & 25 & CASS & $\mathbf{S}$ & Disaprosment with naarby thamocouples (woekly max) \\
\hline TE-107-AP-51 & 1/16/80 & 32 & CASS & $\mathbf{S}$ & Disarreament with nearby thermocouples (wedkly max) \\
\hline TE-107.AP-51 & $1 / 23 / 88$ & 30 & CASS & $\mathbf{s}$ & Disagreernemt with nearby thermocouples (weekly max) \\
\hline IE-107-AP-51 & $1 / 29 / 88$ & 29 & CASS & $\overline{5}$ & Disegroement with noerby thermocouples (weakly max) \\
\hline TE-107-AP-51 & $1 / 31 / 88$ & 29 & CASS & s & Digagreement with nearby thermocouples (weekly max) \\
\hline TE-107-AP-51 & $2 / 13 / 88$ & 39 & CASS & $\mathbf{s}$ & Disergeement with noarby thormocouples (weakly max) \\
\hline TE-107-AP-51 & $2 / 15 / 88$ & 39 & CASS & $\mathbf{S}$ & Disagraement with nearby thermocouples (weekly max) \\
\hline TE-107.AP-51 & $2 / 22 / 83$ & 39 & CASS & $\mathbf{S}$ & Disagreament with nearby thermocouplas (wedkly max) \\
\hline TE-107-APA51 & $3 / 3 / 68$ & 46 & CASS & $\bar{s}$ & Distgreament with nearby thermocouplas (waekly max) \\
\hline TE-107-AP-51 & $3 / 9 / 88$ & 42 & CASS & $\mathbf{S}$ & Disagresment with nearby thermocouples (woekly max) \\
\hline TE-107-AP-5I & 319/88 & 44 & CASS & $\mathbf{S}$ & Disegraement with nearty thermocoupless (woekly max) \\
\hline TE-107-AP-51 & $3 / 20 / 88$ & 51 & CASS & s & Disegreement with nearby thermocouples (weakly max) \\
\hline TE-107-AP-51 & $3 / 30 / 88$ & 46 & CASS & $\mathbf{s}$ & Disagreement with nearby thermocouples (weekly max) \\
\hline TE-107-AP-51 & $6 / 1 / 88$ & 70 & CASS & $\mathbf{s}$ & Disagreement with nearby thermocouples (weekly max) \\
\hline TE-107-AP-51 & $6 / 5 / 88$ & 70 & CASS & $\mathbf{s}$ & Oisegreament with nosiby thermocouplas (woekly max) \\
\hline TE-107-AP-51 & $6 / 16 / 88$ & 94 & CASS & $\mathbf{S}$ & Disagreement with nearby therrmocouplas (woekly max) \\
\hline IE-107-AP-51 & $6 / 25 / 88$ & 96 & CASS & $\mathbf{S}$ & Disagreement with nearby thermocouples (waekly max) \\
\hline TE-107-AP-51 & $712 / 88$ & 93. & CASS & s & Disagreement with nearby thermocouples (weekly max) \\
\hline TE-107-AP-51 & $7 / 9 / 88$ & 96 & CÁSS & $\mathbf{S}$ & Disagreement with nearby thermocouplos (weekly max) \\
\hline TE-107-AP-51 & $7 / 11 / 88$ & 99 & CASS & $\mathbf{5}$ & Disegreement with nearby thermocouples (woekly max) \\
\hline TE-107-AP-51 & $7 / 22 / 88$ & 105 & CASS & $\mathbf{S}$ & Disagreement with nearby thermocouples (woekly $\max$ ) \\
\hline TE-107-AP-51 & $7 / 27 / 88$ & 113 & CASS & $\mathbf{S}$ & Disegreement with nearby tharmocouplas (weekly max) \\
\hline TE-107-AP-51 & 7/31/88 & 103 & CASS & $\mathbf{S}$ & Disagreement with nearby thermocouplas (weekly max) \\
\hline TE-107-AP-51 & $8 / 11 / 88$ & 96 & CASS & $\overline{\mathbf{S}}$ & Diaagreament with nearby thermocouples (weekly max) \\
\hline TE-107-AP-51 & $8 / 14 / 88$ & 90 & CASS & $\mathbf{S}$ & Disagreament with nearby thermocouples (weekly mox) \\
\hline TE-107-AP-51 & 8/25/88 & 96 & CASS & $\mathbf{s}$ & Diasegreament with nearby thermocouples (woekly max) \\
\hline TE-107-AP-51 & $8 / 29 / 88$ & 94 & CASS & $\mathbf{s}$ & Disapreement with nearby thermocouplas (weekly max) \\
\hline TE-107-AP-51 & $9 / 5 / 88$ & 93 & CASS & $\mathbf{s}$ & Disagreement with nearby thermocouples (woekly max) \\
\hline TE-107-AP-51 & $9 / 15 / 88$ & 79 & CASS & $\mathbf{S}$ & Disapreement with nearby thermocouples (weakiy max) \\
\hline TE-107-AP-51 & $9 / 19 / 88$ & 67 & CASS & $\mathbf{S}$ & Disegreement with nearby theimocouplos (weekly max) \\
\hline TE-107-AP-51 & $9 / 2768$ & 64 & CASS & $\mathbf{S}$ & Disspreement with nearby thermocouples (weakly max) \\
\hline TE-107.AP-51 & $12 / 3 / 88$ & 21 & CASS & 5 & Diaegreement with nearby tharmocouples (woekly max) \\
\hline TE-107-AP-51 & $12 / 10 / 88$ & 28 & CASS & $\mathbf{s}$ & Disapreement with nearby thermocouples (woekly max) \\
\hline TE-107-AP-51 & $12 / 13 / 88$ & 30 & CASS & $\mathbf{s}$ & Disapreement with naarby thermocouples (weekly max) \\
\hline TE-107-AP-51 & $12 / 22 / 88$ & 17 & CASS & $\mathbf{S}$ & Disagreement with naarby thermocouples (weekfy max) \\
\hline TE-107-AP-51 & 9/1/89 & 78 & CASS & $\mathbf{s}$ & Disapreament with nearby thermocouplas (weekly max) \\
\hline
\end{tabular}




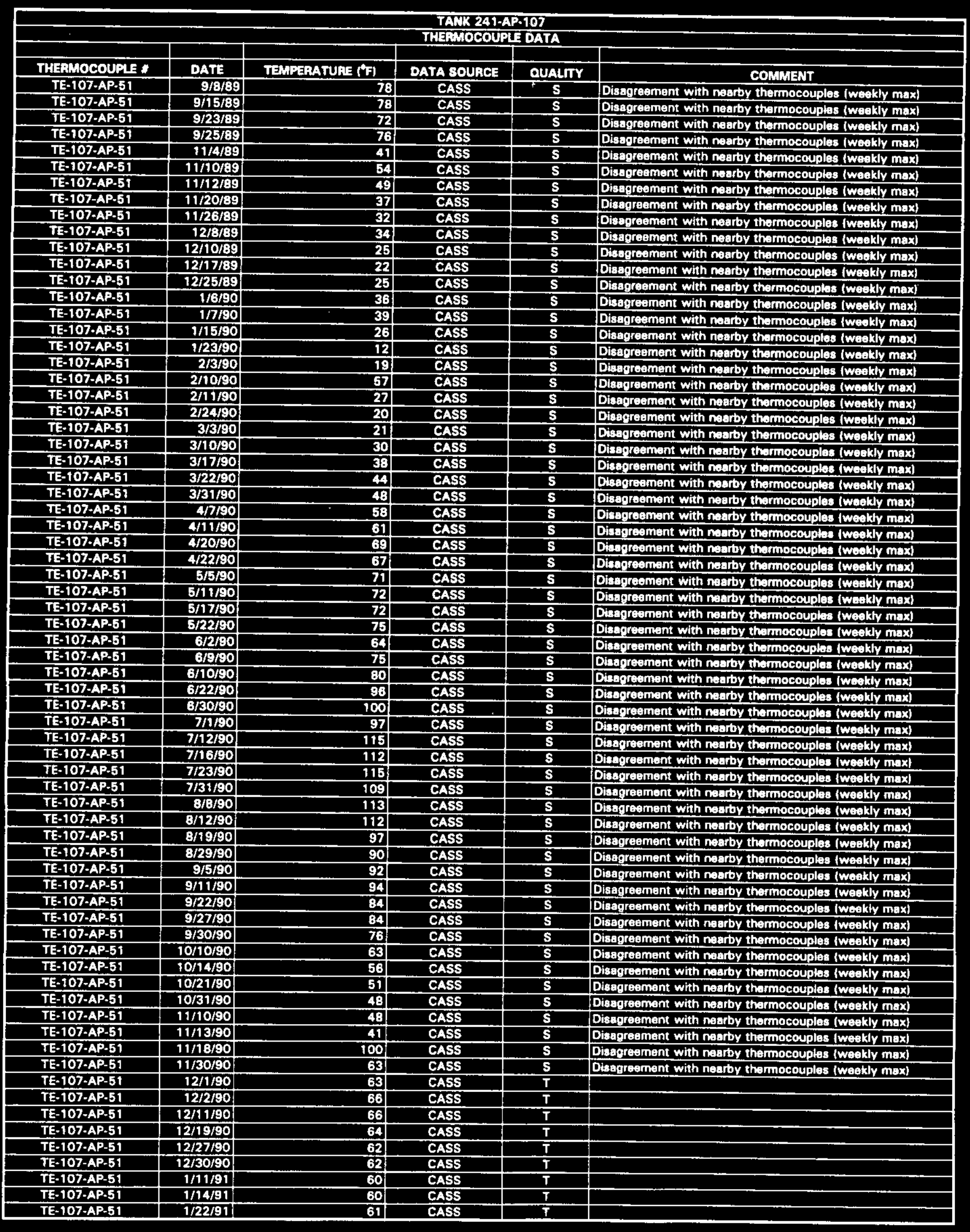




\begin{tabular}{|c|c|c|c|c|c|}
\hline \multicolumn{6}{|c|}{$\begin{array}{l}\text { TANK 241-AP-107 } \\
\text { THIFRMOCOUAIE DATA }\end{array}$} \\
\hline THEAMOCOUPLE * & DATE & TEMPERATUAE ( $\left.{ }^{\circ} \mathrm{F}\right)$ & DATA SOURCE & OUALITY & COMMENT \\
\hline TE-107-AP-51 & $1 / 30 / 91$ & 58 & CASS & $\mathbf{T}$ & \\
\hline TE-107.AP-51 & $2 / 3 / 91$ & 59 & CASS & $T$ & \\
\hline TE-107-AP-51 & $2 / 16 / 91$ & 59 & CASS & $T$ & \\
\hline TE-107-AP-51 & $2 / 17 / 91$ & 58 & CASS & $T$ & \\
\hline TE-107-AP-51 & 3/1/91 & 59 & CASS & $T$ & \\
\hline TE-107-AP-51 & $3 / 9 / 91$ & 59 & CASS & $T$ & \\
\hline TE-107-AP-51 & $3 / 13 / 91$ & 57 & CASS & $T$ & \\
\hline TE-107-AP-51 & $3 / 17 / 91$ & 57 & CASS & $T$ & \\
\hline TE-107-AP-51 & 3/27/91 & 59 & CASS & $\mathbf{T}$ & \\
\hline TE-107-AP-51 & $4 / 5 / 81$ & 58 & CASS & $T$ & \\
\hline TE-107-AP-51 & $4 / 12198$ & 57 & CASS & $T$ & \\
\hline TE-107-AP-51 & $4 / 18983$ & 56 & CASS & $T$ & \\
\hline TE-107-AP-51 & $4 / 24 / 91$ & 58 & CASS & $T$ & \\
\hline TE-107-AP-51 & $5 / 4 / 91$ & 68 & CASS & $\bar{T}$ & \\
\hline TE-107-AP.51 & 5/10/91 & 56 & CASS & $T$ & \\
\hline TE-107-AP-51 & 5/16/91 & 57 & CASS & $\bar{T}$ & \\
\hline TE-107-AP-51 & $5 / 21 / 81$ & 58 & CASS & $T$ & \\
\hline TE-107-AP-61 & 6/1/91 & $\mathbf{5 8}$ & CASS & $T$ & \\
\hline TE-107-AP-51 & $6 / 7 / 91$ & 57 & CASS & $\bar{T}$ & \\
\hline TE-107-AP-51 & $6 / 15 / 91$ & 57 & CASS & $T$ & \\
\hline TE-107-AP-51 & 6/22/91 & 59 & CASS & $\mathbf{T}$ & \\
\hline TE-107.AP-51 & $6 / 2491$ & 57 & CASS & $\bar{T}$ & \\
\hline TE-107-AP-51 & $7 / 3 / 91$ & 57 & CASS & $T$ & \\
\hline TE-107-AP-51 & $7 / 13 / 91$ & 57 & CASS & $T$ & \\
\hline TE-107-AP-51 & $7 / 14 / 91$ & 57 & CASS & $T$ & \\
\hline TE-107-AP-S1 & $7124 / 91$ & 58 & CASS & $T$ & \\
\hline TE-107-AP-51 & $8 / 3 / 91$ & 58 & CASS & $T$ & \\
\hline TE-107-AP-51 & $8 / 6 / 91$ & 58 & CASS & $\bar{T}$ & \\
\hline TE-107-AP-51 & 8/17/91 & 60 & CASS & $T$ & \\
\hline TE-107-AP-51 & 8/20/91 & 60 & CASS & $T$ & \\
\hline TE-107-AP-51 & $8 / 30 / 91$ & 61 & CASS & $\mathbf{T}$ & \\
\hline TE-107-AP-51 & $8 / 3 / 81$ & 61 & CASS & $\bar{T}$ & \\
\hline TE-107-AP-51 & $8 / 8 / 81$ & 61 & CASS & $T$ & \\
\hline TE-107-AP-51 & $9 / 16 / 81$ & 111 & CASS & $\mathbf{S}$ & Data point did not agree whi local trend \\
\hline TE-107-AP-51 & 9/18/91 & 62 & CASS & $T$ & \\
\hline TE-107-AP-51 & 9/27/91 & 63 & CASS & $T$ & \\
\hline TE-107-AP-51 & $10 / 4 / 91$ & 64 & CASS & $\mathbf{T}$ & \\
\hline TE-107-AP-51 & 10/12/91 & 61 & CASS & $\mathbf{T}$ & \\
\hline TE-107-AP-51 & $10 / 19 / 91$ & 64 & CASS & $\mathbf{T}$ & \\
\hline TE-107-AP-51 & $10 / 25 / 91$ & 63 & CASS & $T$ & \\
\hline TE-107-AP-51 & $10 / 29 / 91$ & 63 & CASS & $\bar{T}$ & \\
\hline TE-107-AP-51 & 11/6/91 & 62 & CASS & $\mathbf{T}$ & \\
\hline TE-107-AP-51 & $8 / 1 / 92$ & 56 & CASS & $\mathbf{T}$ & \\
\hline TE-107-AP-51 & $8 / 8 / 92$ & 59 & CASS & $\bar{T}$ & \\
\hline TE-107-AP-51 & $8 / 9 / 92$ & 59 & CASS & $\mathbf{T}$ & \\
\hline TE-107-AP-51 & $8 / 22 / 92$ & 57 & CASS & $\mathbf{T}$ & \\
\hline TE-107-AP-51 & $8 / 24 / 92$ & 59 & CASS & $\bar{T}$ & \\
\hline TE-107-AP-51 & 8/5/92 & 57 & CASS & $T$ & \\
\hline TE-107-AP-51 & 9/9/92 & 60 & CASS & $T$ & \\
\hline TE-107-AP-51 & $9 / 13 / 92$ & 60 & CASS & $T$ & \\
\hline TE-107-AP-51 & 9/26/82 & 58 & CASS & $T$ & \\
\hline TE-107-AP-51 & $10 / 3 / 92$ & 60 & CASS & $T$ & \\
\hline TE-107-AP-61 & $10 / 9 / 92$ & 61 & CASS & $T$ & \\
\hline TE-107-AP-51 & $10 / 12 / 92$ & 60 & CASS & $T$ & \\
\hline TE-107-AP-51 & $10 / 22 / 92$ & 80 & CASS & $T$ & \\
\hline TE-107-AP-51 & $10 / 31 / 92$ & 60 & CASS & $T$ & \\
\hline TE-107-AP-51 & $11 / 6 / 92$ & 61 & CASS & $\bar{T}$ & \\
\hline TE-107-AP-51 & $11 / 12 / 92$ & 80 & CASS & $\bar{T}$ & \\
\hline TE-107-AP-51 & $11 / 20 / 92$ & 61 & CASS & $T$ & \\
\hline TE-107-AP-51 & $11 / 28 / 92$ & 60 & CASS & $T$ & \\
\hline TE-107-AP-51 & $11 / 30 / 92$ & 60 & CASS & $T$ & \\
\hline TE-107-AP-51 & $12 / 10 / 92$ & 59 & CASS & $T$ & \\
\hline$T E-107-A P-51$ & $12 / 19 / 92$ & 58 & CASS & $T$ & \\
\hline TE-107-AP-51 & $12 / 21 / 92$ & 58 & CASS & $T$ & \\
\hline TE-107-AP-51 & $12 / 30 / 92$ & 57 & CASS & $T$ & \\
\hline TE-107-AP-51 & $1 / 15 / 94$ & 57 & CASS & $T$ & \\
\hline TE-107-AP-51 & $1 / 18 / 94$ & 59 & CASS & $\mathbf{T}$ & \\
\hline TE-107-AP-51 & $1 / 28 / 94$ & 57 & CASS & $T$ & \\
\hline TE-107-AP:51 & $2 / 2 / 94$ & 59 & CASS & $T$ & \\
\hline TE-107-AP-51 & $2 / 12 / 94$ & 56 & CASS & $T$ & \\
\hline
\end{tabular}




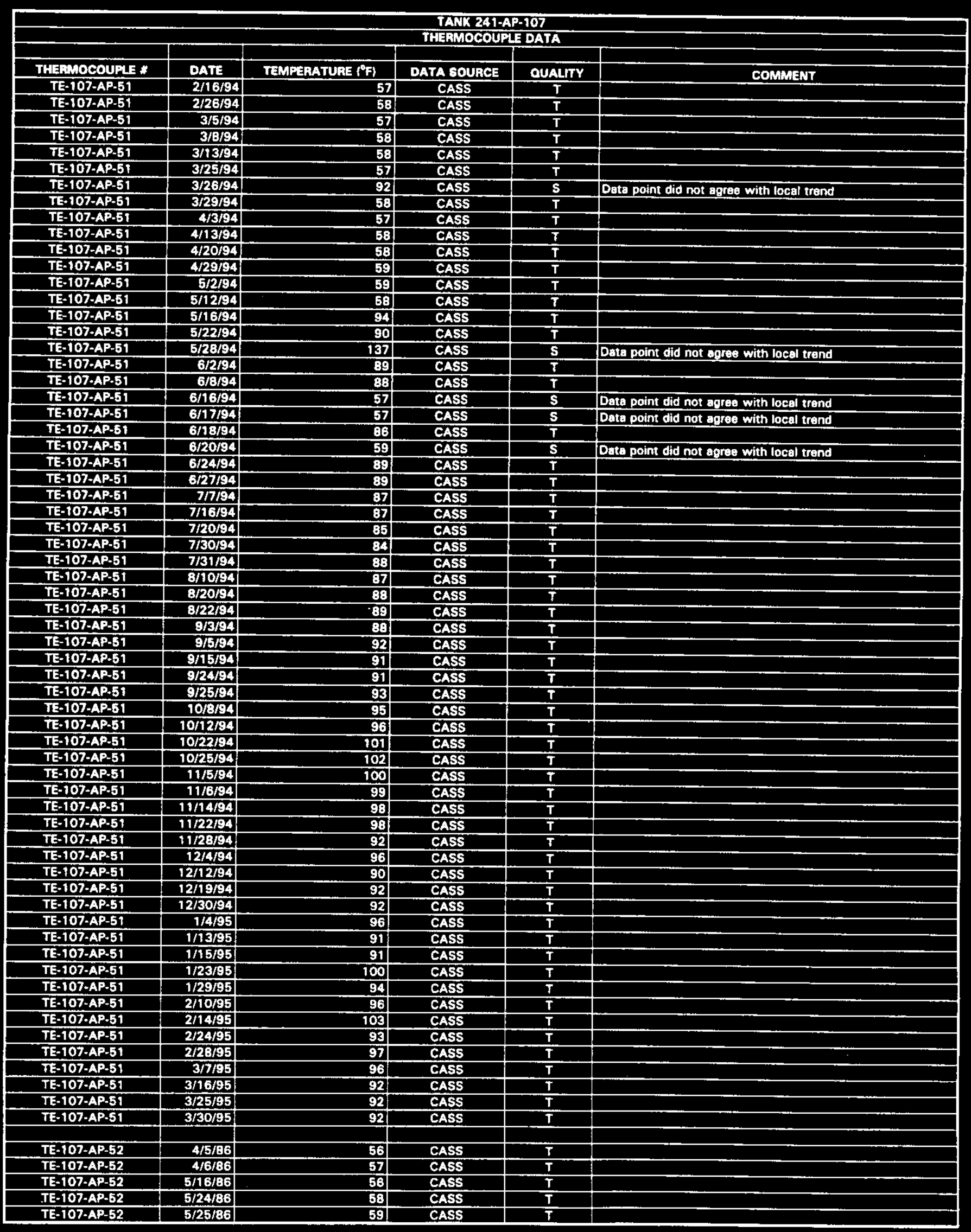




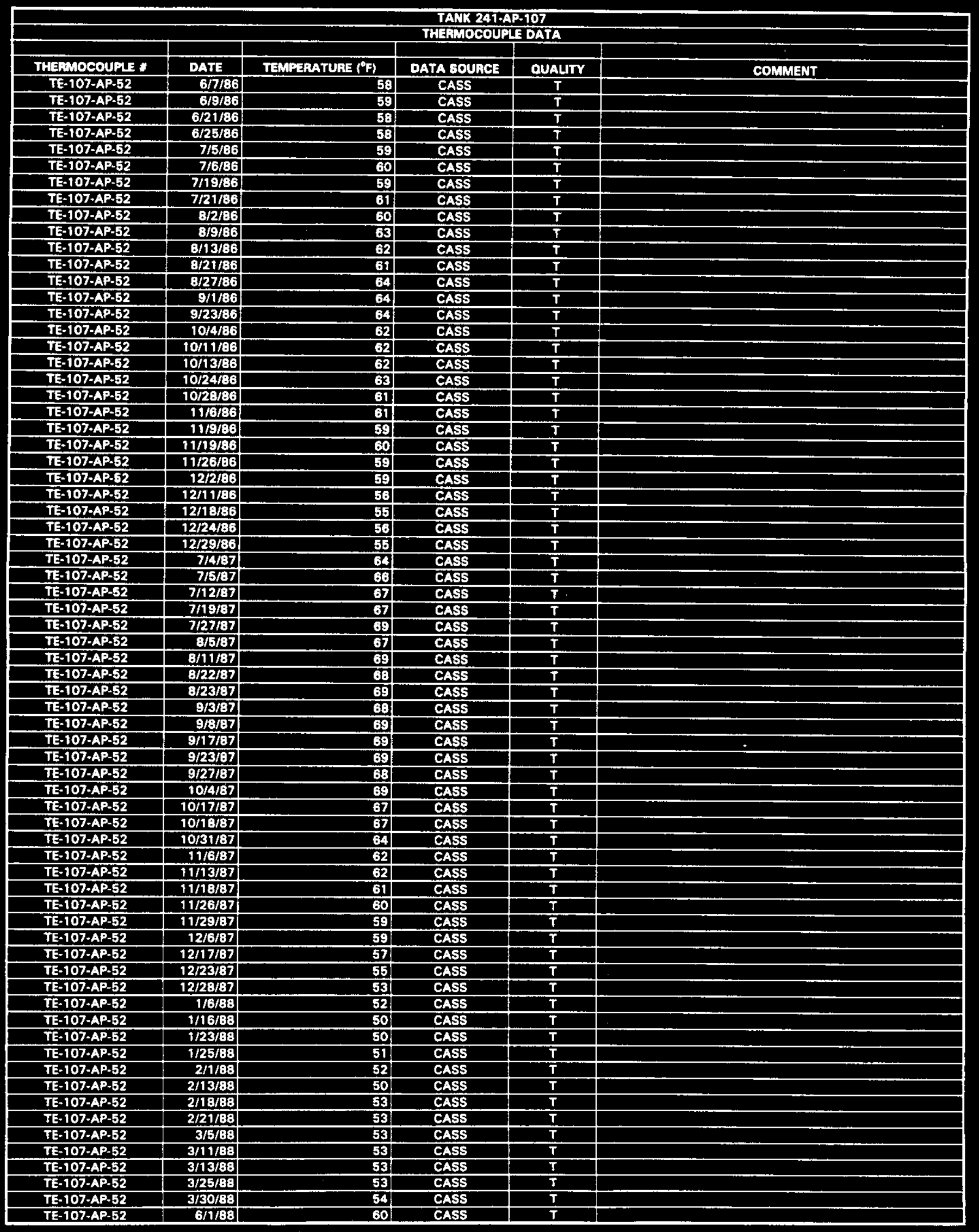




\begin{tabular}{|c|c|c|c|c|c|}
\hline \multicolumn{6}{|c|}{$\begin{array}{l}\text { TAWK 241.AP-107 } \\
\text { THEWMOCOUPL DATA }\end{array}$} \\
\hline THEAMOCOUPLE * & DATE & TEMPERATUAE $\left({ }^{\circ} \mathrm{F}\right)$ & DATA SOUACE & QUALITY & COMMENT \\
\hline TE-107-AP-52 & $6 / 9 / 88$ & 60 & CASS & $T$ & \\
\hline TE-107-AP-52 & $6 / 18 / 88$ & 60 & CASS & $\mathbf{T}$ & \\
\hline TE-107-AP-52 & $6 / 25 / 88$ & 62 & CASS & $\mathbf{T}$ & \\
\hline TE-107-AP.52 & $7 / 1 / 88$ & 64 & CASS & $\mathbf{T}$ & \\
\hline TE-107-AP-52 & $7 / 3 / 88$ & 64 & CASS & $T$ & \\
\hline TE-107-AP-52 & $7 / 13 / 88$ & 66 & CASS & $T$ & . \\
\hline TE-107-AP-52 & $7 / 23 / 88$ & 67 & CASS & $T$ & \\
\hline TE-107-AP-52 & $7 / 29 / 88$ & 69 & CASS & $\mathbf{T}$ & \\
\hline TE-107-AP-52 & $8 / 5 / 88$ & 70 & CASS & $\mathbf{T}$ & \\
\hline TE-107-AP-52 & $8 / 11 / 88$ & 70 & CASS & $\bar{T}$ & \\
\hline TE-107.AP-52 & $8 / 20 / 88$ & 69 & CASS & $T$ & \\
\hline TE-107-AP-52 & $8 / 22 / 88$ & 70 & CASS & $T$ & \\
\hline TE-107-AP-52 & $9 / 2 / 8 \mathrm{~B}$ & 71 & CASS & $T$ & \\
\hline TE-107-AP-52 & $9 / 9 / 88$ & 71 & CASS & $T$ & \\
\hline TE-107-AP-52 & $9 / 16 / 88$ & 70 & CASS & $T$ & \\
\hline TE-107-AP-52 & $9 / 23 / 88$ & 69 & CASS & $T$ & \\
\hline TE-107-AP-52 & $9 / 27 / 88$ & 68 & CASS & $T$ & \\
\hline TE.107-AP.52 & $12 / 3 / 88$ & 62 & CASS & $T$ & \\
\hline TE-107+AP-52 & $12 / 4 / 88$ & 88 & CASS & $\mathbf{s}$ & Data point did not agree with local trend \\
\hline TE-107-AP.52 & $12 / 8 / 88$ & 62 & CASS & $\bar{T}$ & \\
\hline TE-107-AP-52 & $12 / 16 / \mathrm{Bg}$ & 60 & CASS & $T$ & \\
\hline TE-107-AP-52 & $12 / 22 / 88$ & 60 & CASS & $T$ & \\
\hline TE-107+AP-52 & $9 / 2 / 89$ & 69 & CASS & $T$ & \\
\hline TE-107-AP-52 & $9 / 9 / 89$ & 68 & CASS & $T$ & \\
\hline TE-107.AP-52 & $9 / 11 / 89$ & 73 & CASS & $T$ & \\
\hline TE-107-AP-52 & $9 / 18 / 89$ & 69 & CASS & $T$ & \\
\hline TE-107-AP-52 & $9 / 28 / 89$ & 71 & CASS & $\mathbf{T}$ & \\
\hline TE-107-AP-52 & $11 / 3 / 89$ & 63 & CASS & $T$ & \\
\hline TE-107-AP-52 & $11 / 11 / 89$ & 86 & CASS & $T$ & \\
\hline TE-107-AP-52 & $11 / 15 / 99$ & 62 & CASS & $T$ & \\
\hline TE-107-AP-52 & $11 / 21 / 99$ & 62 & CASS & $T$ & \\
\hline TE-107-AP-52 & $11 / 27 / 89$ & 59 & CASS & $T$ & \\
\hline TE-107-AP-52 & $12 / 5 / 89$ & 59 & CASS & $T$ & \\
\hline TE-107-AP-52 & $12 / 12 / 89$ & 57 & CASS & $T$ & \\
\hline TE-107-AP-52 & $12 / 19 / 89$ & 53 & CASS & $T$ & \\
\hline TE-107-AP-52 & $12 / 30 / 89$ & 52 & CASS & $T$ & \\
\hline TE-107-AP-52 & $1 / 2 / 90$ & 52 & CASS & $T$ & \\
\hline TE-107-AP-52 & $1 / 13 / 90$ & 55 & CASS & $T$ & \\
\hline TE-107-AP-52 & $1 / 18 / 90$ & 72 & CASS & $\mathbf{T}$ & \\
\hline TE-107-AP-52 & $1 / 26 / 90$ & 70 & CASS & $\mathbf{T}$ & \\
\hline TE-107-AP-52 & $1 / 29 / 90$ & 69 & CASS & $\mathbf{T}$ & \\
\hline TE-107-AP-52 & $2 / 7 / 90$ & 70 & CASS & $\mathbf{T}$ & \\
\hline TE-107-AP-52 & $2 / 15 / 90$ & 71 & CASS & $T$ & \\
\hline TE-107-AP-52 & $2 / 24 / 90$ & 69 & CASS & $T$ & \\
\hline TE-107-AP-52 & $3 / 2 / 90$ & 68 & CASS & 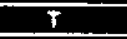 & \\
\hline TE-107-AP-52 & $3 / 5 / 90$ & 88 & CASS & $T$ & \\
\hline TE-107-AP-52 & $3 / 12 / 90$ & 68 & CASS & $T$ & \\
\hline TE-107-AP-52 & $3 / 20 / 90$ & 66 & CASS & $T$ & \\
\hline TE-107-AP-52 & $3 / 29 / 90$ & 66 & CASS & $T$ & \\
\hline TE-107-AP-52 & $4 / 3 / 90$ & 67 & CASS & $T$ & \\
\hline TE-107.AP-52 & $4 / 12 / 90$ & 65 & CASS & $\mathbf{T}$ & \\
\hline TE-107-AP-52 & $4 / 21 / 90$ & 64 & CASS & $T$ & \\
\hline TE-107-AP-52 & $4 / 26 / 90$ & 65 & CASS & $\mathbf{T}$ & \\
\hline TE-107-AP-52 & $4 / 29 / 90$ & 66 & CASS & $T$ & \\
\hline TE-107-AP-52 & $5 / 6 / 90$ & 66 & CASS & $\mathbf{T}$ & \\
\hline TE-107-AP-52 & $5 / 17 / 90$ & 65) & CASS & $\mathbf{T}$ & \\
\hline TE-107-AP-52 & $5 / 22 / 90$ & 66 & CASS & $\mathbf{T}$ & \\
\hline TE-107-AP-52 & $5 / 28 / 90$ & 64 & SACS & $\mathbf{T}$ & \\
\hline TE-107-AP-52 & $6 / 6 / 90$ & 65 & CASS & $T$ & \\
\hline TE-107-AP-52 & $6 / 14 / 90$ & 66 & CASS & $T$ & \\
\hline TE-107-AP-52 & $6 / 21 / 90$ & 65 & CASS & $T$ & \\
\hline TE-107-AP-52 & $6 / 29 / 90$ & 65 & CASS & $T$ & \\
\hline TE-107-AP-52 & $7 / 2 / 90$ & 65 & SACS & $T$ & \\
\hline TE-107-AP-52 & $7 / 12 / 90$ & 65 & CASS & $\mathbf{T}$ & \\
\hline TE-107-AP-52 & $7 / 16 / 90$ & 66 & CASS & $\mathbf{T}$ & \\
\hline TE-107-AP-52 & $7 / 22 / 90$ & 67 & CASS & $\mathbf{T}$ & \\
\hline TE-107-AP-52 & $8 / 3 / 90$ & 66 & CASS & $\mathbf{T}$ & \\
\hline TE-107-AP-52 & $8 / 11 / 90$ & 68 & CASS & $\mathbf{T}$ & \\
\hline TE-107-AP-52 & $8 / 17 / 90$ & 67 & CASS & $T$ & \\
\hline TE-107-AP-52 & $8 / 21 / 90$ & 67 & CASS & $T$ & \\
\hline
\end{tabular}




\begin{tabular}{|c|c|c|c|c|c|}
\hline \multicolumn{6}{|c|}{$\begin{array}{l}\text { TANK 241-AP-107 } \\
\text { THEAMOCOUPLE DATA }\end{array}$} \\
\hline THERMOCOUPE * & DATE & TEMPERATUAE $\left({ }^{\circ} \mathrm{F}\right)$ & DATA SOURCE & QUALITY & COMMENT \\
\hline TE-107-AP-52 & $8 / 31 / 90$ & 66 & CASS & $T$ & \\
\hline TE-107-AP-52 & 9/8/90 & 65 & CASS & $T$ & \\
\hline TE-107.AP-52 & 9/15/90 & 67 & CASS & $T$ & \\
\hline TE-107-AP-52 & $9 / 20 / 90$ & 69 & CASS & $\bar{T}$ & \\
\hline TE-107-AP-52 & $9 / 28 / 90$ & 67 & CASS & $T$ & \\
\hline TE-107.AP-52 & $10 / 6 / 90$ & 68 & CASS & $\mathbf{T}$ & \\
\hline TE-107-AP-52 & $10 / 10 / 90$ & 68 & CASS & $\bar{T}$ & \\
\hline TE-107-AP-52 & $10 / 17 / 90$ & 66 & CASS & $T$ & \\
\hline TE-107-AP-52 & $10 / 26 / 90$ & 67? & CASS & $T$ & \\
\hline TE-107-AP-52 & $10 / 31 / 90$ & 69 & CASS & $T$ & \\
\hline TE-107-AP-52 & $11 / 10 / 90$ & 65 & CASS & $\mathbf{T}$ & \\
\hline TE-107-AP-52 & $11 / 17 / 90$ & 68 & CASS & $\mathbf{T}$ & \\
\hline TE-107-AP-52 & $11 / 20 / 90$ & 66 & CASS & $\mathbf{T}$ & \\
\hline TE-107-AP-52 & $11 / 27 / 80$ & 64 & CASS & $\mathbf{T}$ & \\
\hline TE-107.AP-52 & $12 / 2 / 90$ & 66 & CASS & $T$ & \\
\hline TE-107.AP-52 & $12 / 11 / 90$ & 66 & CASS & $T$ & \\
\hline TE-107-AP-52 & $12 / 20 / 80$ & 63 & CASS & $T$ & \\
\hline TE-107-AP-52 & $12 / 27 / 80$ & 62 & CASS & $\bar{T}$ & \\
\hline TE-107-AP-52 & $12 / 30 / 90$ & 62 & CASS & $T$ & \\
\hline TE-107-AP-52 & 1/12/91 & 59 & CASS & $\mathbf{T}$ & \\
\hline TE-107-AP-52 & 1/1/4/91 & 60 & CASS & $\mathbf{T}$ & \\
\hline TE-107.AP-52 & $1 / 22 / 91$ & 60 & CASS & $\bar{T}$ & \\
\hline TE-107-AP-52 & $1 / 27 / 99$ & 58 & CASS & $T$ & \\
\hline TE-107-AP-52 & $2 / 3 / 91$ & 59 & CASS & $T$ & \\
\hline TE-107-AP-52 & $2 / 16 / 91$ & 59 & CASS & $\mathbf{T}$ & \\
\hline TE-107-AP-52 & $2 / 17 / 91$ & 58 & CASS & $\bar{T}$ & \\
\hline IE-107-AP-52 & 3/1/91 & 59 & CASS & $\bar{T}$ & \\
\hline TE-107-AP-52 & 3/9/91 & 59 & CASS & $T$ & \\
\hline TE-107-AP-52 & $3 / 13 / 91$ & 57 & CASS & $T$ & \\
\hline TE-107-AP-52 & 3/17/91 & 58 & CASS & $\bar{T}$ & \\
\hline TE-107-AP-52 & $3 / 27 / 91$ & 58 & CASS & $T$ & \\
\hline TE-107-AP-52 & $4 / 5 / 91$ & 58 & CASS & $T$ & \\
\hline TE-107-AP-62 & $4 / 12 / 91$ & 57 & CASS & $T$ & \\
\hline TE-107-AP-52 & 4/20/91 & 56 & CASS & $T$ & \\
\hline TE-107-AP-52 & $4 / 24 / 91$ & 59 & CASS & $T$ & \\
\hline TE-107.AP.52 & 5/4/91 & 56 & CASS & $T$ & \\
\hline TE-107-AP-52 & $5 / 10 / 91$ & 58 & CASS & $T$ & \\
\hline TE-107-AP-52 & $5 / 16 / 91$ & 58 & CASS & $\bar{T}$ & \\
\hline TE-107-AP-52 & $5 / 21 / 91$ & 59 & CASS & $T$ & \\
\hline TE-107-AP-52 & $5 / 31 / 91$ & 59 & CASS & $T$ & \\
\hline TE-107-AP-52 & $6 / 7 / 91$ & 58 & CASS & $T$ & \\
\hline TE-107-AP-52 & 6/15/91 & 58 & CASS & $\mathbf{T}$ & \\
\hline TE-107-AP-52 & $6 / 22 / 91$ & 59 & CASS & $T$ & \\
\hline TE-107-AP-52 & $6 / 25 / 91$ & 57 & CASS & $\mathbf{I}$ & \\
\hline IE-107-AP-52 & $7 / 3 / 91$ & 58 & CASS & $T$ & \\
\hline TE-107-AP-52 & $7 / 12 / 91$ & 59 & CASS & $T$ & \\
\hline TE-107-AP-52 & $7 / 14 / 91$ & 58 & CASS & $T$ & \\
\hline TE-107-AP-52 & $7 / 24 / 91$ & 59 & CASS & $\mathbf{T}$ & \\
\hline TE-107-AP-52 & $8 / 3 / 91$ & 59 & CASS & $\mathbf{T}$ & \\
\hline TE-107-AP-52 & $8 / 6 / 91$ & 60 & CASS & $\bar{T}$ & \\
\hline IE-107:AP-52 & 8/17/91 & 62 & CASS & $\mathbf{T}$ & \\
\hline TE.107.AP.52 & $8 / 20 / 91$ & 62 & CASS & $\mathbf{T}$ & \\
\hline TE-107-AP-52 & $8 / 30 / 91$ & 62 & CASS & $\bar{T}$ & \\
\hline TE-107-AP-52 & 9/3/91 & 62 & CASS & $\mathbf{T}$ & \\
\hline TE-107-AP-52 & 9/8/91 & 63 & CASS & $T$ & \\
\hline TE-107-AP-52 & $9 / 18 / 91$ & 64 & CASS & $T$ & \\
\hline TE-107-AP-52 & $9 / 27 / 91$ & 64 & CASS & $\mathbf{T}$ & \\
\hline IE-107-AP-52 & $10 / 4 / 91$ & 64 & CASS & $\mathbf{T}$ & \\
\hline TE-107-AP-52 & $10 / 12 / 91$ & 62 & CASS & $\bar{T}$ & \\
\hline TE-107-AP-52 & $10 / 19 / 91$ & 65 & CASS & $T$ & \\
\hline TE-107-AP-52 & $10 / 25 / 91$ & 64 & CASS & $\bar{T}$ & \\
\hline TE-107-AP-52 & $10 / 29 / 91$ & 64 & CASS & $T$ & \\
\hline TE-107-AP-52 & $11 / 6 / 91$ & 63. & CASS & $T$ & \\
\hline TE-107-AP-52 & 8/1/82 & 56. & CASS & $\mathbf{T}$ & \\
\hline TE-107-AP-52 & $8 / 3 / 92$ & 60 & CASS & $T$ & \\
\hline TE-107-AP-52 & $8 / 9 / 92$ & 59 & CASS & $\bar{T}$ & \\
\hline TE-107-AP-52 & $8 / 22 / 92$ & 57 & CASS & $T$ & \\
\hline TE-107-AP-52 & $8 / 28 / 92$ & 59 & CASS & $T$ & \\
\hline TE-107-AP-52 & $9 / 5 / 92$ & 57 & CASS & $T$ & \\
\hline TE-107-AP-52 & 9/9/92 & 60 & CASS & $\mathbf{T}$ & \\
\hline
\end{tabular}




\begin{tabular}{|c|c|c|c|c|c|}
\hline \multicolumn{6}{|c|}{$\begin{array}{l}\text { TANK 241-AP-107 } \\
\text { THEFMOCOUPLE DATA }\end{array}$} \\
\hline THERMOCOUPAE * & DATE & TEMPERATUAE ( $\left.{ }^{\circ} \mathrm{F}\right)$ & Data souRce & OLMITY & \\
\hline TE-107-AP-52 & $9 / 13 / 92$ & 60 & CASS & $T$ & \\
\hline TE-107-AP-52 & $9 / 26 / 92$ & 58 & CASS & $T$ & \\
\hline TE-107-AP-52 & $9 / 29 / 92$ & 61 & CASS & $\bar{T}$ & \\
\hline TE-107-AP-52 & $10 / 9 / 92$ & 69 & CASS & $T$ & \\
\hline TE-107-AP-52 & $10 / 13 / 92$ & 59 & CASS & $T$ & \\
\hline TE-107-AP-52 & $10 / 19 / 92$ & 61 & SACS & $T$ & \\
\hline TE-107-AP-52 & $10 / 29 / 92$ & 60 & CASS & $T$ & \\
\hline TE-107-AP-52 & $11 / 6 / 92$ & 62 & CASS & $T$ & \\
\hline TE-107-AP-52 & $11 / 9 / 92$ & 61 & CASS & $\mathbf{T}$ & \\
\hline TE-107-AP-52 & $11 / 20 / 92$ & 61 & CASS & $T$ & \\
\hline$T E-107-A P-52$ & $11 / 28 / 92$ & 60 & CASS & $T$ & \\
\hline TE-107-AP-52 & $11 / 30 / 92$ & 60 & CASS & $T$ & \\
\hline TE-107-AP-52 & $12 / 12 / 92$ & 59 & CASS & $T$ & \\
\hline TE-107-AP-52 & $12 / 19 / 92$ & 58 & CASS & $T$ & \\
\hline TE-107.AP-52 & $12 / 21 / 92$ & 58 & CASS & $T$ & \\
\hline TE-107-AP-52 & $12 / 30 / 92$ & 57 & CASS & $T$ & \\
\hline TE-107-AP-52 & $6 / 11 / 93$ & 54 & SACS & $T$ & \\
\hline TE-107-AP-52 & $8 / 18 / 93$ & 54 & SACS & $\bar{T}$ & \\
\hline TE-107-AP-52 & $6 / 28 / 93$ & 56 & SACS & $\mathbf{T}$ & \\
\hline TE-107-AP-52 & $7 / 9 / 93$ & 55 & SACS & $T$ & \\
\hline TE-107-AP-52 & $7 / 15 / 93$ & 56 & SACS & $T$ & \\
\hline TE-107-AP-52 & $7 / 19 / 93$ & 56 & SACS & $T$ & \\
\hline TE-107-AP-52 & $7 / 30 / 93$ & 57 & SACS & $\mathbf{T}$ & \\
\hline TE-107-AP-52 & $8 / 6 / 93$ & 57 & SACS & $T$ & \\
\hline TE-107.AP-52 & $8 / 12 / 93$ & 56 & SACS & $T$ & \\
\hline TE-107-AP-52 & $9 / 24 / 93$ & 57 & SACS & $T$ & \\
\hline TE-107.AP-52 & $10 / 1 / 93$ & 59 & SACS & $\bar{T}$ & \\
\hline TE-107-AP-52 & $10 / 15 / 93$ & 60 & SACS & $T$ & \\
\hline TE-107-AP-52 & $10 / 22 / 93$ & 59 & SACS & $\mathbf{T}$ & \\
\hline TE-107-AP-52 & $10 / 28 / 93$ & 60 & SACS & $\mathbf{T}$ & \\
\hline TE-107-AP-52 & $11 / 9 / 93$ & 57 & SACS & $T$ & \\
\hline TE-107.AP-52 & $11 / 30 / 93$ & 64 & SACS & $T$ & \\
\hline TE-107-AP-52 & $12 / 14 / 93$ & 80 & SACS & $T$ & \\
\hline TE-107-AP-52 & $12 / 20 / 93$ & $\mathbf{5 8}$ & SACS & $T$ & \\
\hline TE-107-AP-52 & $12 / 27 / 93$ & 86 & SACS & $\mathbf{T}$ & \\
\hline TE-107-AP-52 & $1 / 3 / 94$ & 56 & SACS & $\bar{T}$ & \\
\hline TE-107.AP-52 & $1 / 11 / 94$ & 60 & SACS & $\bar{T}$ & \\
\hline TE-107-AP-52 & $1 / 16 / 94$ & 60 & CASS & $\bar{T}$ & \\
\hline TE-107-AP-52 & $1 / 25 / 94$ & 136 & CASS & $\mathbf{s}$ & Data point did not agree with local trend \\
\hline TE-107-AP-52 & $1 / 28 / 94$ & 57 & CASS & $\bar{T}$ & \\
\hline TE-107.AP-52 & $2 / 4 / 94$ & 59 & CASS & $\mathbf{T}$ & \\
\hline TE-107-AP-52 & $2 / 11 / 94$ & 57 & CASS & $T$ & \\
\hline TE-107-AP-52 & $2 / 16 / 94$ & $\mathbf{5 8}$ & CASS & $T$ & \\
\hline TE-107-AP-52 & $2 / 26 / 94$ & 59 & CASS & $\mathbf{T}$ & \\
\hline TE-107-AP-52 & $3 / 5 / 94$ & 58 & CASS & $\mathbf{T}$ & \\
\hline TE-107-AP-52 & $3 / 7 / 94$ & 58 & CASS & $T$ & \\
\hline TE-107-AP-52 & 3/13/94 & 59 & CASS & $\bar{T}$ & \\
\hline TE-107-AP-52 & $3 / 25 / 94$ & $\mathbf{5 8}$ & CASS & $\bar{T}$ & \\
\hline TE-107-AP-52 & $3 / 29 / 94$ & $\mathbf{5 8}$ & CASS & $T$ & \\
\hline TE-107-AP-52 & $4 / 9 / 94$ & $\mathbf{5 8}$ & CASS & $T$ & \\
\hline TE-107-AP-52 & 4/13/94 & 59 & CASS & $\mathbf{T}$ & \\
\hline TE-107-AP-52 & $4 / 20 / 94$ & 69 & CASS & $\mathbf{T}$ & \\
\hline TE-107-AP-52 & 4/29/94 & 59 & CASS & $\bar{T}$ & \\
\hline TE-107-AP-52 & $5 / 2 / 94$ & 60 & CASS & $\mathbf{T}$ & \\
\hline TE-107-AP-52 & $5 / 14 / 94$ & 58 & CASS & $\bar{T}$ & \\
\hline TE-107-AP-52 & $5 / 16 / 94$ & 95 & CASS & $T$ & \\
\hline TE-107-AP-52 & $5 / 22 / 94$ & 90 & CASS & $\mathbf{T}$ & \\
\hline TE-107.AP-52 & $6 / 2 / 94$ & 90 & CASS & $\mathrm{T}$ & \\
\hline TE-107-AP-52 & $6 / 8 / 94$ & 88 & CASS & $\bar{T}$ & \\
\hline TE-107.AP-52 & $6 / 14 / 94$ & 87 & CASS & $\mathbf{T}$ & \\
\hline TE-107-AP-52 & $6 / 16 / 94$ & 57 & CASS & $\mathbf{s}$ & Data point did not agree with local trend \\
\hline TE-107-AP-52 & $6 / 17 / 94$ & 58 & CASS & $\bar{s}$ & Data point did not agree with local trend \\
\hline TE-107-AP-52 & $6 / 20 / 94$ & 80 & CASS & $\mathbf{s}$ & Data point did not agree with local trend \\
\hline TE-107-AP-52 & $6 / 20 / 94$ & 58 & SACS & $S$ (G in SACS) & Data point did not agree with local trend \\
\hline TE-107-AP-52 & $6 / 24 / 94$ & 90 & CASS & $\bar{T}$ & \\
\hline TE-107-AP-52 & $6 / 27 / 94$ & 90 & CASS & $T$ & \\
\hline TE-107-AP-52 & $7 / 3 / 94$ & 89 & CASS & $T$ & \\
\hline TE-107-AP-52 & $7 / 16 / 94$ & 89 & CASS & $T$ & \\
\hline TE-107.AP-52 & $7 / 20 / 94$ & 87 & CASS & $T$ & \\
\hline TE-107-AP-52 & $7 / 30 / 94$ & 86 & CASS & $T$ & \\
\hline
\end{tabular}




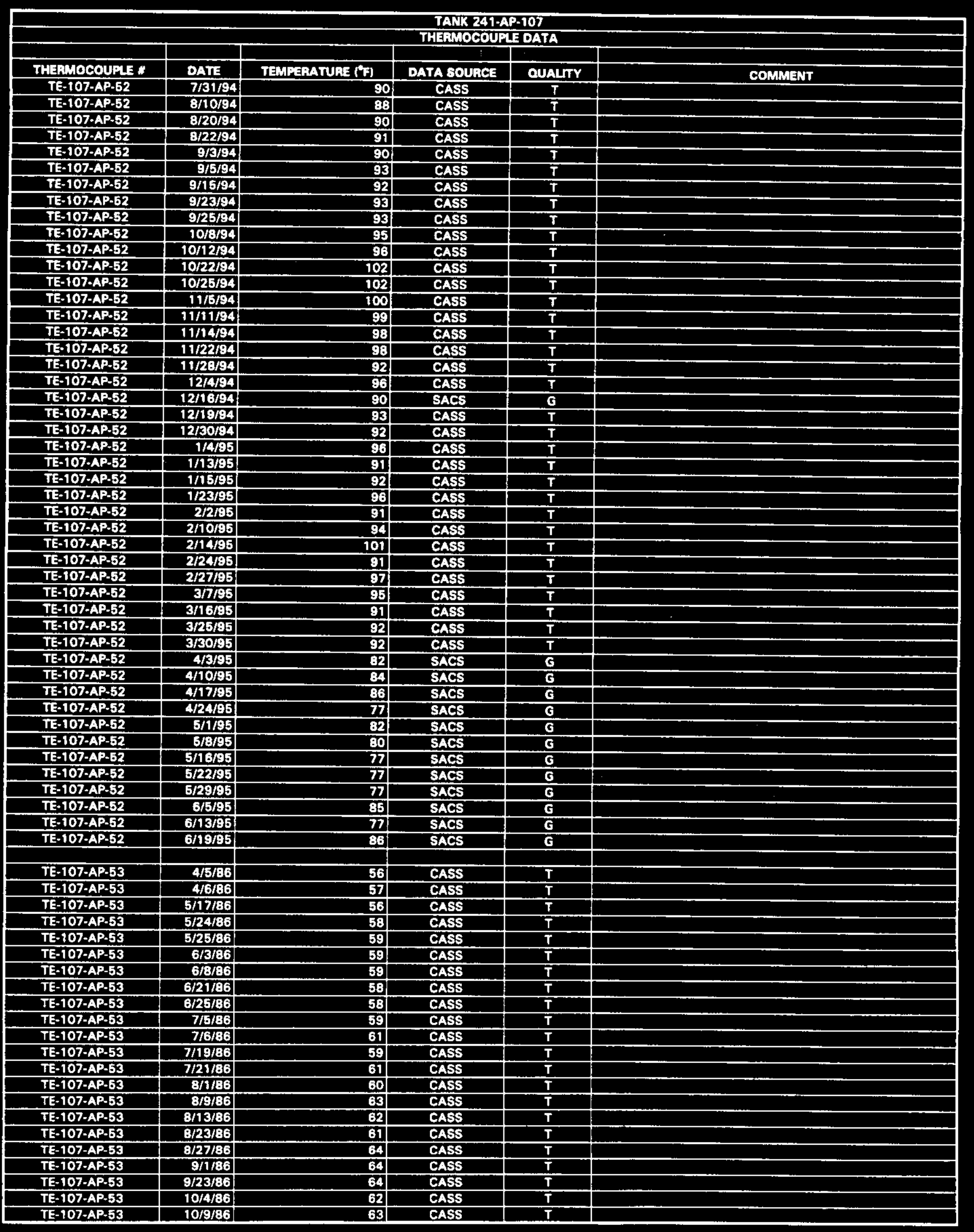

Data obtained from Computer Auromated Surveillance Syatem (CASS), April 1995; and Surveillance Analyais Computer System (SACS), June 1995.

$$
\text { D-638 }
$$




\begin{tabular}{|c|c|c|c|c|c|}
\hline \multicolumn{6}{|c|}{$\begin{array}{l}\text { TANK 241-AP-107 } \\
\text { THEFIMOCOUPLE DATA }\end{array}$} \\
\hline THERMOCOUPLE * & DATE & TEMPERATUAE $\left({ }^{\circ} \mathrm{F}\right)$ & DATA sOURCE & OUALTY & \\
\hline TE-107-AP-53 & $10 / 13 / 86$ & 62 & CASS & $T$ & Soming \\
\hline TE-107-AP-53 & $10 / 23 / 86$ & 64 & CASS & $T$ & \\
\hline TE-107.AP-53 & $10 / 28 / 86$ & 61 & CASS & $T$ & \\
\hline TE-107.AP-53 & $11 / 6 / 86$ & 60 & CASS & $T$ & \\
\hline TE-107-AP-53 & $11 / 10 / 86$ & 58 & CASS & $T$ & \\
\hline TE-107-AP-53 & $11 / 19 / 86$ & 59 & CASS & $T$ & \\
\hline TE-107-AP-53 & $31 / 29 / 86$ & 57 & CASS & $T$ & \\
\hline TE-107-AP-53 & $12 / 2 / 86$ & 59 & CASS & $T$ & \\
\hline TE-107-AP-53 & $12 / 11 / 86$ & 56 & CASS & $T$ & \\
\hline TE-107-AP-53 & $12 / 18 / 86$ & 55 & CASS & $T$ & \\
\hline TE-107-AP-53 & $12 / 24 / 86$ & 55 & CASS & $T$ & \\
\hline TE-107-AP-53 & $12 / 29 / 86$ & 54 & CASS & $T$ & \\
\hline TE-107.AP-53 & $7 / 4 / 87$ & 64 & CASS & $T$ & \\
\hline TE-107-AP-53 & $7 / 5 / 87$ & 67 & CASS & $T$ & \\
\hline TE-107-AP-53 & $7 / 12 / 87$ & 66 & CASS & $T$ & \\
\hline TE-107-AP-53 & $7 / 19 / 87$ & 68 & CASS & $T$ & \\
\hline TE-107-AP-53 & $7 / 27 / 87$ & 69 & CASS & $T$ & \\
\hline TE-107-AP-53 & 8/5/87 & 68 & CASS & $T$ & \\
\hline TE-107-AP-53 & $8 / 11 / 87$ & 69 & CASS & $T$ & \\
\hline TE-107-AP-53 & $8 / 22 / 87$ & 68 & CASS & $T$ & \\
\hline TE-107.AP-53 & $8 / 23 / 87$ & 69 & CASS & $T$ & \\
\hline TE-107.AP-53 & $9 / 4 / 87$ & 68 & CASS & $T$ & \\
\hline TE-107.AP-53 & $9 / 8 / 87$ & 69 & CASS & $T$ & \\
\hline TE-107-AP-53 & $9 / 17 / 87$ & 70 & CASS & $T$ & \\
\hline TE-107-AP-53 & 9/26/87 & 68 & CASS & $\bar{T}$ & \\
\hline TE-107.AP-53 & 9/27/87 & 68 & CASS & $\bar{T}$ & \\
\hline TE-107-AP-53 & $10 / 4 / 87$ & 69 & CASS & $\mathbf{T}$ & \\
\hline TE-107-AP-53 & 10/16/87 & 68 & CASS & $T$ & \\
\hline TE-107-AP-53 & $10 / 18 / 87$ & 67 & CASS & $T$ & \\
\hline TE-107-AP-S3 & 10/31/37 & 64 & CASS & $\bar{T}$ & \\
\hline TE-107-AP-53 & $11 / 6 / 87$ & 62 & CASS & $T$ & \\
\hline TE-107-AP-53 & $11 / 8 / 87$ & 63 & CASS & $T$ & \\
\hline TE-107-AP-53 & $11 / 18 / 87$ & 61 & CAS5 & $T$ & \\
\hline TE-107-AP-53 & $11 / 26 / 37$ & 60 & CASS & $\mathbf{T}$ & \\
\hline TE-107-AP-53 & $12 / 1 / 87$ & 59 & CASs & $T$ & \\
\hline TE-107-AP-53 & $12 / 6 / 87$ & 59 & CASS & $T$ & \\
\hline TE-107-AP-53 & $12 / 17 / 87$ & 67 & CASS & $T$ & \\
\hline TE-107-AP-53 & $12 / 20 / 87$ & 55 & CASS & $\bar{T}$ & \\
\hline TE-107-AP-53 & $12 / 28 / 87$ & 53 & CASS & $\bar{T}$ & \\
\hline TE-107-AP-53 & $1 / 6 / 88$ & 51 & CASS & $\mathbf{T}$ & \\
\hline TE-107-AP-53 & $1 / 16 / 88$ & 49 & CASS & $\mathbf{T}$ & \\
\hline TE-107-AP-53 & $1 / 19 / 88$ & 50 & CASS & $T$ & \\
\hline TE-107-AP-53 & $1 / 25 / 88$ & 51 & CASS & $\bar{T}$ & \\
\hline TE-107-AP-53 & $2 / 1 / 88$ & 52 & CASS & $\bar{T}$ & \\
\hline TE-107-AP-53 & $2 / 13 / 88$ & 50 & CASS & $T$ & \\
\hline TE-107-AP-53 & $2 / 18 / 88$ & 52 & CASS & $\mathbf{T}$ & \\
\hline TE-107.AP-53 & $2 / 21 / 88$ & 53 & CASS & $\mathbf{T}$ & \\
\hline TE-107-AP-53 & $3 / 5 / 88$ & 54 & CASS & $T$ & \\
\hline TE-107-AP-53 & 3/11/88 & 53 & CASS & $\bar{T}$ & \\
\hline TE-107-AP-53 & $3 / 13 / 88$ & 53 & CASS & $\bar{T}$ & \\
\hline TE-107-AP-53 & $3 / 25 / 68$ & 54 & CASS & $T$ & \\
\hline TE-107-AP-53 & $3 / 30 / 88$ & 55 & CASS & $T$ & \\
\hline TE-107.AP-53 & $6 / 1 / 88$ & 60 & CASS & $T$ & \\
\hline TE-107-AP-53 & 6/10/88 & 60 & CASS & $\bar{T}$ & \\
\hline TE-107-AP-53 & $6 / 12 / 88$ & 112 & CASS & $\mathbf{s}$ & Data point did not agree with local trend \\
\hline TE-107-AP-53 & $6 / 18 / 88$ & 60 & CASS & $T$ & \\
\hline TE-107.AP.53 & $6 / 25 / 38$ & 62 & CASS & $\bar{T}$ & \\
\hline TE-107-AP-53 & 6/29/38 & 65 & CASS & $\bar{T}$ & \\
\hline TE-107-AP-53 & 7/9/38 & 63 & CASS & $T$ & \\
\hline TE-107-AP-53 & $7 / 13 / 88$ & 66 & CASS & $T$ & \\
\hline TE-107-AP-53 & $7 / 23 / 88$ & 68 & CASS & $T$ & \\
\hline TE-107-AP-53 & $7 / 29 / 88$ & 69 & CASS & $T$ & \\
\hline TE-107-AP-53 & $8 / 5 / 88$ & 71 & CASS & $I$ & \\
\hline TE-107-AP-53 & $8 / 9 / 88$ & 71 & CASS & $T$ & \\
\hline TE-107-AP-53 & $8 / 20 / 88$ & 70 & CASS & $T$ & \\
\hline TE-107-AP-53 & $8 / 22 / 88$ & 70 & CASS & $\mathbf{T}$ & \\
\hline TE-107-AP-53 & $9 / 2 / 88$ & 71 & CASS & $T$ & \\
\hline TE-107-AP-53 & 9/9/8B & 71 & CASS & $\mathbf{T}$ & \\
\hline TE-107-AP-53 & $9 / 16 / 88$ & 70 & CASS & $\mathbf{T}$ & \\
\hline TE-107-AP-53 & 9/19/8B & 70 & CASS & $T$ & \\
\hline
\end{tabular}




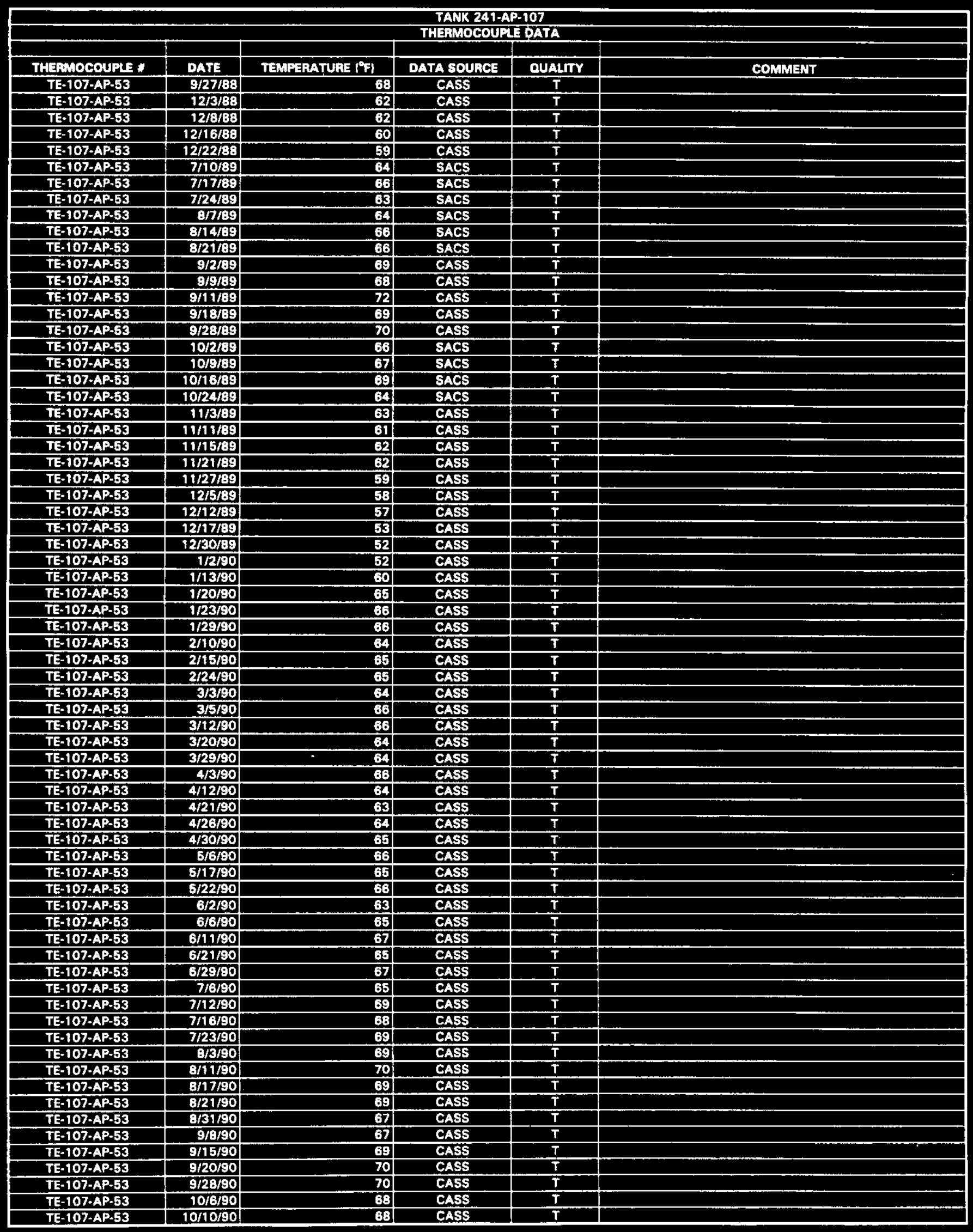




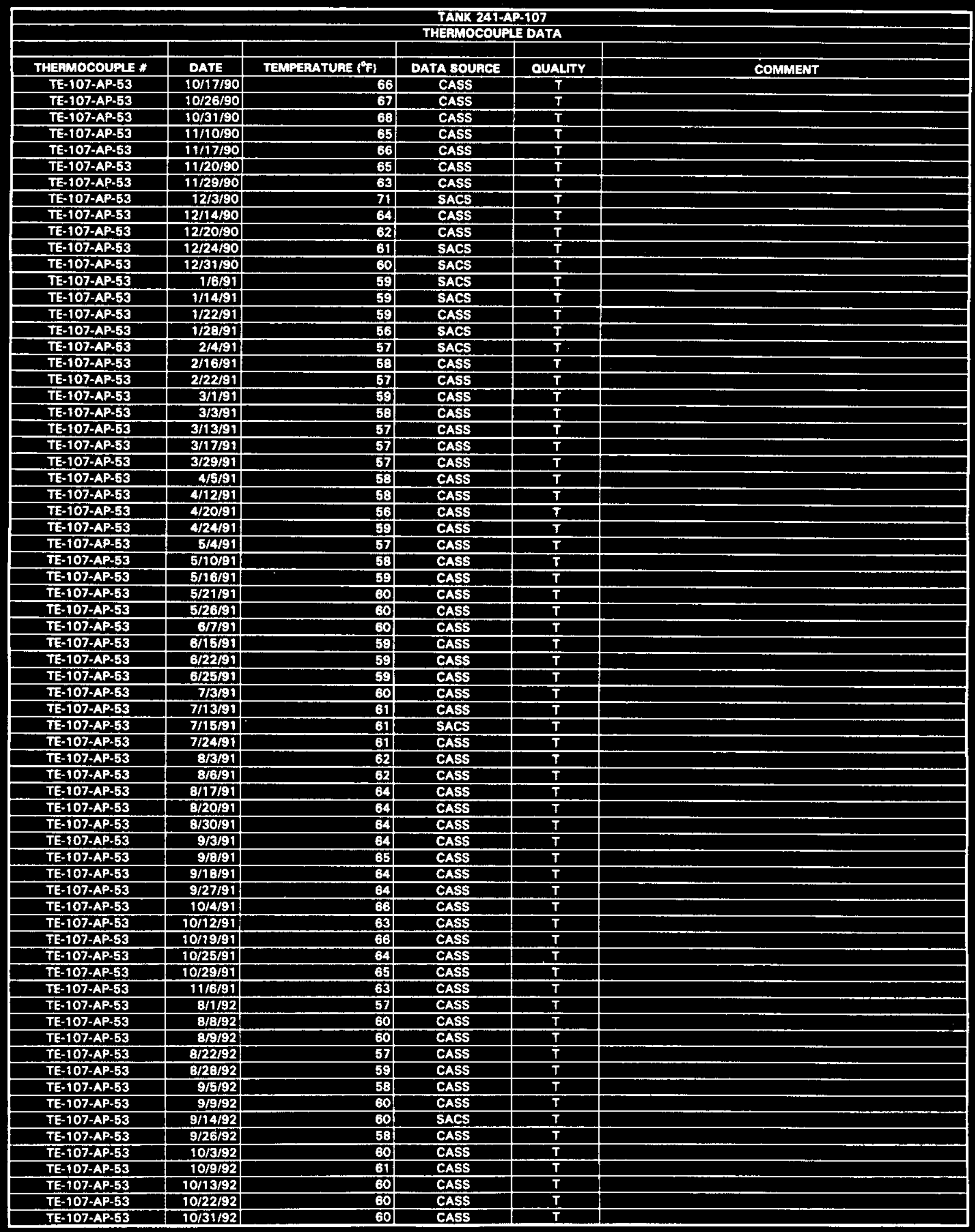




\begin{tabular}{|c|c|c|c|c|c|}
\hline \multicolumn{6}{|c|}{$\begin{array}{l}\text { TANK 241-AP-107 } \\
\text { THE:MOCOUFIE DATA }\end{array}$} \\
\hline THERMOCOUPLE * & DATE & TEMPERATURE (F) & DATA SOURCE & QUALTY & COMMENT \\
\hline TE-107-AP-53 & $11 / 6 / 92$ & 62 & CASS & $T$ & \\
\hline TE-107-AP-53 & $11 / 12 / 92$ & 60 & CASS & $T$ & \\
\hline TE-107-AP-53 & $11 / 20 / 92$ & 61 & CASS & $\bar{T}$ & \\
\hline TE-107-AP-53 & $11 / 28 / 92$ & 59 & CASS & $T$ & \\
\hline TE-107-AP-63 & $11 / 30 / 22$ & 59 & CASS & $T$ & \\
\hline TE-107-AP-53 & $12 / 30 / 92$ & 56 & CASS & $T$ & \\
\hline TE-107.AP.53 & $1 / 15 / 94$ & 57 & CASS & $T$ & \\
\hline TE-107-AP-53 & $1 / 20 / 94$ & 59 & CASS & $T$ & \\
\hline TE-107.AP-53 & $1 / 25 / 94$ & 58 & CASS & $T$ & \\
\hline TE-107-AP-53 & $2 / 4 / 94$ & 59 & CASS & $T$ & \\
\hline TE-107-AP-63 & 2/12/94 & 57 & CASS & $T$ & \\
\hline TE-107-AP-53 & $2 / 16 / 94$ & 59 & CASS & $T$ & \\
\hline TE-107-AP-53 & 3/29/94 & 60 & CASS & $T$ & \\
\hline TE-107-AP-53 & 4/9/94 & B9. & CASS & $T$ & \\
\hline TE-107-AP.53 & 4/13/94 & 60 & CASS & $\mathbf{T}$ & \\
\hline TE-107-AP-53 & $4 / 20 / 94$ & 61 & CASS & $\mathbf{T}$ & \\
\hline TE-107-AP-53 & $4 / 27 / 94$ & 61 & CASS & $T$ & \\
\hline TE-107-AP-53 & $5 / 4 / 94$ & 59 & CASS & $\bar{T}$ & \\
\hline TE-107-AP-53 & $5 / 14 / 94$ & 89 & CASS & $T$ & \\
\hline TE-107-AP-53 & $5 / 16 / 94$ & 95 & CASS & $T$ & \\
\hline IE-107-AP-53 & $5 / 22 / 94$ & 91 & CASS & $\bar{T}$ & \\
\hline TE-107-AP-63 & $6 / 2 / 94$ & 80 & CASS & $I$ & \\
\hline TE-107-AP-53 & 6/8/94 & 89 & CASS & $T$ & \\
\hline TE-107-AP-53 & 6/14/84 & 88 & CASS & $T$ & \\
\hline TE-107-AP-53 & $6 / 16 / 94$ & 59 & CASS & $\mathbf{s}$ & Date point did not apree with local trend \\
\hline TE-107-AP-53 & 6/17/94 & 59 & CASS & $\mathbf{S}$ & Data point did not apree with local trend \\
\hline$T E-107-A P-53$ & $8 / 17 / 94$ & 93 & CASS & $T$ & \\
\hline TE-107-AP-53 & 8/22/94 & 93 & CASS & $\mathbf{T}$ & \\
\hline TE-107-AP-E3 & $9 / 2 / 94$ & 91 & CASS & $\mathbf{T}$ & \\
\hline TE-107-AP-53 & $9 / 5 / 94$ & 95 & CASS & $T$ & \\
\hline TE-107.AP-53 & 9/15/94 & 83 & CASS & $\mathbf{T}$ & \\
\hline TE-107.AP-53 & $9 / 23 / 94$ & 94 & CASS & $T$ & \\
\hline TE-107-AP-53 & $9 / 25 / 94$ & 93 & CASS & $T$ & \\
\hline TE-107-AP-53 & $10 / 8 / 94$ & 95 & CASS & $T$ & \\
\hline TE-107-AP-53 & $10 / 12 / 94$ & 96 & CASS & $T$ & \\
\hline TE-107-AP-53 & $10 / 22 / 94$ & 101 & CASS & $T$ & \\
\hline TE-107-AP-53 & $10 / 25 / 94$ & 102 & CASS & $T$ & \\
\hline TE-107-AP-53 & $11 / 5 / 94$ & 100 & CASS & $T$ & \\
\hline TE-107-AP-53 & $11 / 11 / 94$ & 99 & CASS & $\mathbf{T}$ & \\
\hline TE-107-AP-53 & $11 / 14 / 34$ & 99 & CASS & $T$ & \\
\hline TE-107-AP-53 & $11 / 22 / 94$ & 88 & CASS & $T$ & \\
\hline TE-107-AP-53 & $11 / 28 / 94$ & 92 & CASS & $T$ & \\
\hline TE-107-AP-53 & $12 / 4 / 94$ & 86 & CASS & $\mathbf{T}$ & \\
\hline TE-107-AP-53 & $12 / 12 / 94$ & 90 & CASS & $T$ & \\
\hline TE-107-AP-53 & $12 / 19 / 94$ & 93 & CASS & $T$ & \\
\hline TE-107-AP-53 & $12 / 31 / 94$ & 92 & CASS & $\mathbf{T}$ & \\
\hline TE-107-AP-53 & $1 / 4 / 95$ & 96 & CASS & $T$ & \\
\hline TE-107-AP-53 & $1 / 13 / 95$ & 92 & CASS & $T$ & \\
\hline TE-107-AP-53 & 1/15/95 & 92 & CASS & $T$ & \\
\hline TE-107-AP-53 & $1 / 23 / 95$ & 94 & CASS & $T$ & \\
\hline TE-107-AP-53 & $2 / 3 / 95$ & 90 & CASS & $T$ & \\
\hline TE-107.AP-53 & $2 / 9 / 95$ & 94 & CASS & $T$ & \\
\hline TE-107-AP-53 & $2 / 14 / 95$ & 100 & CASS & $T$ & \\
\hline
\end{tabular}


WHC-SD-WM-ER-315, Rev. 0

\begin{tabular}{|c|c|c|c|c|c|}
\hline \multicolumn{6}{|c|}{$\begin{array}{l}\text { TANK 241-AP-107 } \\
\text { THEAMOCOUPLE DATA }\end{array}$} \\
\hline THERMOCOUPLE * & DATE & TEMPERATUAE $\left({ }^{\circ} \mathrm{F}\right)$ & DATA SOUACE & OUALTY & COMMENT \\
\hline TE-107-AP-53 & $2 / 24 / 95$ & 91. & CASS & $T$ & \\
\hline TE-107-AP-53 & 2/27/95 & 97 & CASS & $T$ & \\
\hline TE-107-AP-53 & $3 / 7 / 95$ & 95 & CASS & $T$ & \\
\hline TE-107-AP-53 & $3 / 16 / 95$ & 91 & CASS & $\bar{T}$ & \\
\hline TE-107-AP-53 & $3 / 25 / 95$ & 91 & CASS & $T$ & \\
\hline TE-107-AP-53 & $3 / 30 / 95$ & 92 & CASS & $\mathbf{T}$ & \\
\hline
\end{tabular}




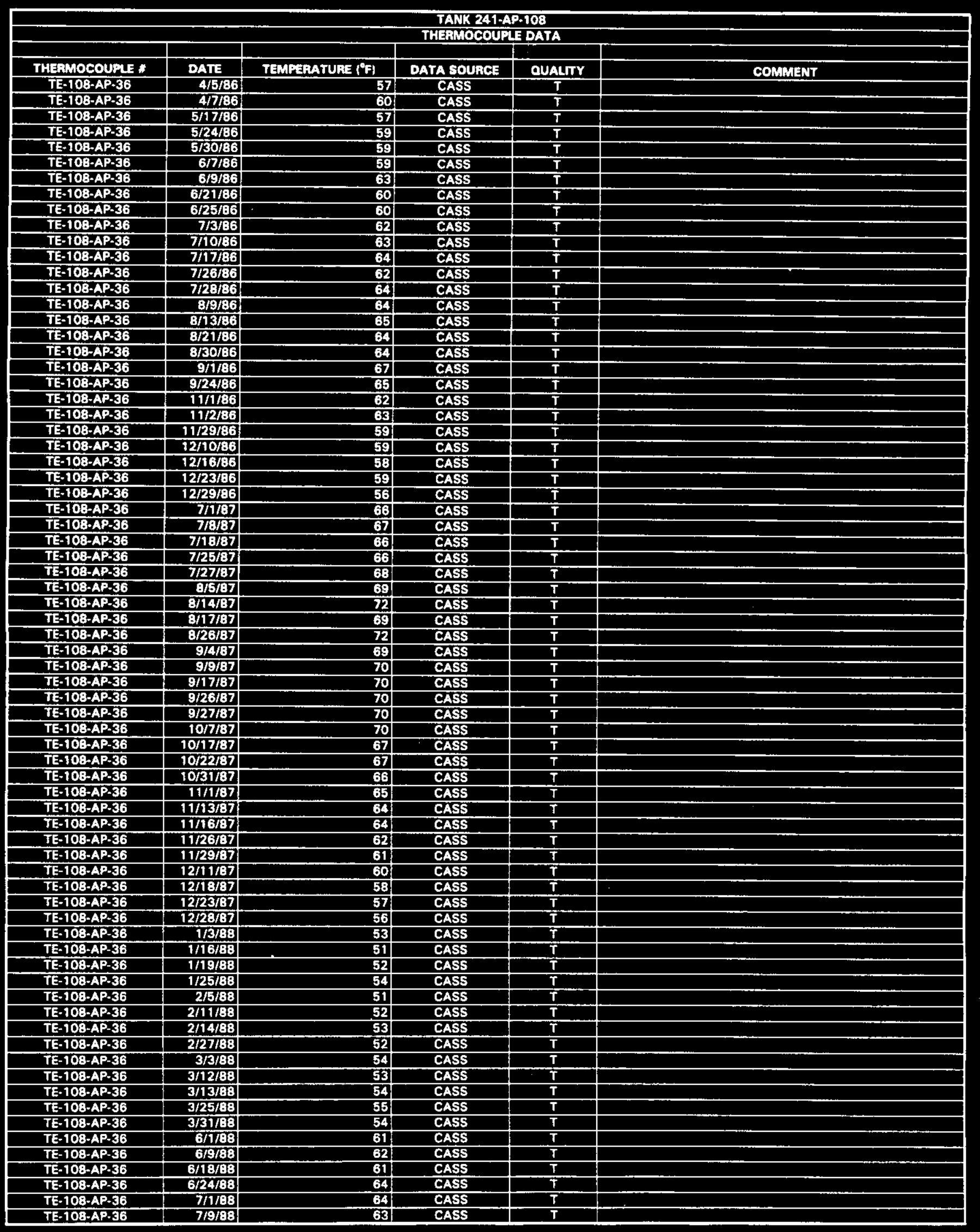




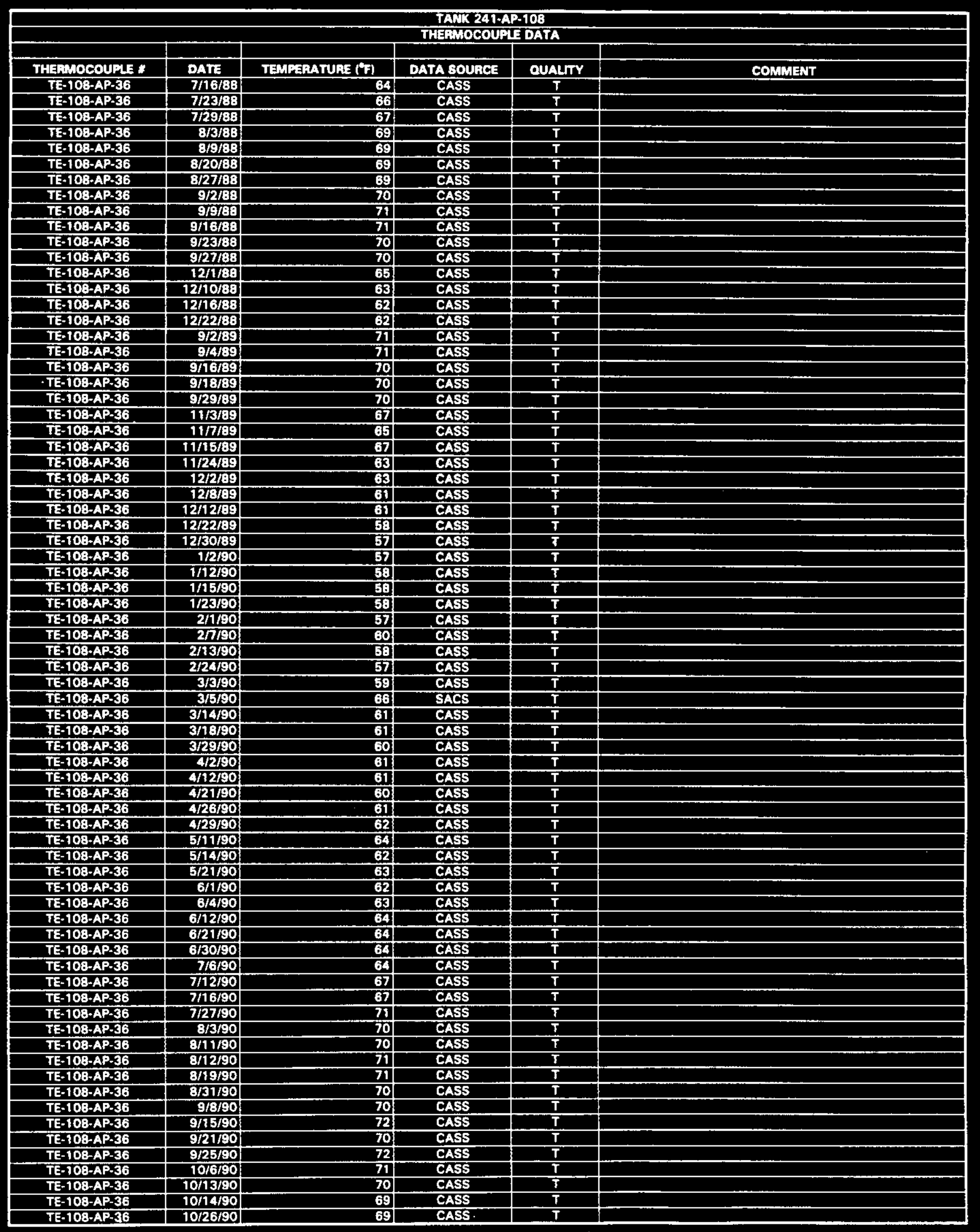




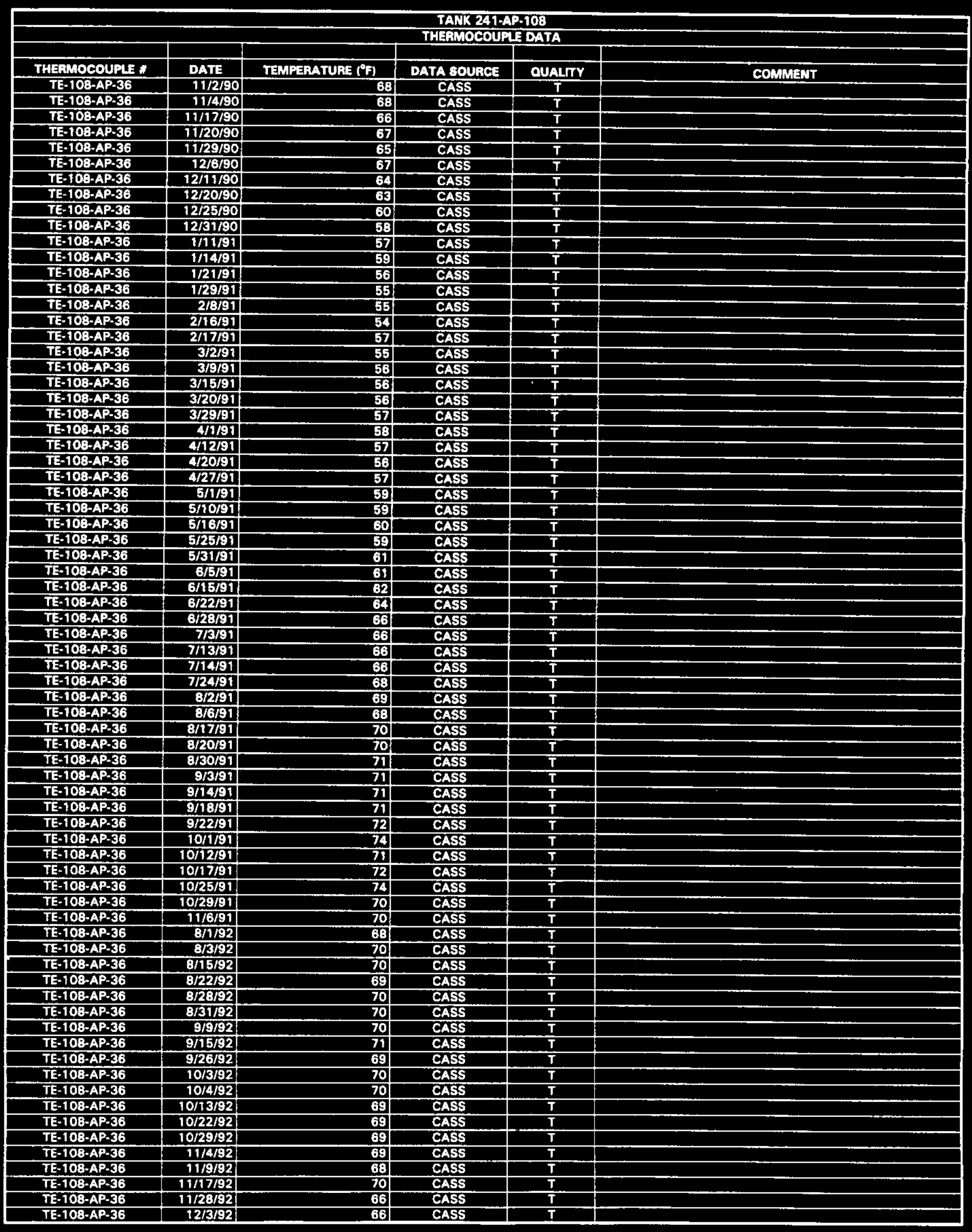

Data obtained from Computer Automated Surveillance System (CASS), April 1995; and Surveillance Analysis Computer Syatem (SACS), June 1995. 


\begin{tabular}{|c|c|c|c|c|c|}
\hline \multicolumn{6}{|c|}{$\begin{array}{l}\text { TANK 241-AP-108 } \\
\text { THEAROOCOUPLE DATA }\end{array}$} \\
\hline THEFMOCOUPIE * & DATE & TEMPERATUAE $\left({ }^{\circ} \mathrm{F}\right)$ & DATA SOURCE & OUALTY & COMMENT \\
\hline TE-108-AP-36 & $12 / 6 / 92$ & 69 & CASS & $T$ & 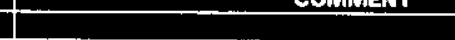 \\
\hline TE-108-AP-36 & $12 / 19 / 92$ & 66 & CASS & $\mathbf{T}$ & \\
\hline TE-108-AP-36 & $12 / 21 / 92$ & 65 & CASS & $T$ & \\
\hline TE-108-AP-36 & $12 / 30 / 82$ & 64 & CASS & $\mathbf{T}$ & \\
\hline TE-108-AP-36 & $6 / 4 / 93$ & 46 & SACS & $\mathbf{S}$ & Data point did nos agree with local trend \\
\hline TE-108-AP-36 & $6 / 11 / 93$ & 62 & SACS & $\bar{T}$ & \\
\hline TE-108.AP-36 & 6/18/93 & 61 & SACS & $\bar{T}$ & \\
\hline TE-108-AP-36 & $6 / 28 / 93$ & 62 & SACS & $T$ & \\
\hline TE-108-AP-36 & $7 / 9 / 93$ & 63 & SACS & $T$ & \\
\hline TE-108-AP-36 & $775 / 93$ & 62 & SACS & $T$ & \\
\hline TE-108-AP-36 & 7/19/93 & 63 & SACS & $\mathbf{T}$ & \\
\hline TE-10B-AP-36 & $7130 / 93$ & 64 & SACS & $\mathbf{T}$ & \\
\hline TE-10:-AP-36 & $8 / 6 / 93$ & 64 & SACS & $T$ & \\
\hline TE-108-AP-36 & $8 / 12 / 93$ & 64 & SACS & $T$ & \\
\hline TE-108-AP-36 & $8 / 24 / 93$ & 54 & SACS & $\mathbf{S}$ & Date point did not agree with local trend \\
\hline TE-102-AP-36 & T0/1/93 & 66 & SACS & $\bar{T}$ & \\
\hline TE-108-AP-36 & $10 / 15 / 93$ & 66 & SACS & $\bar{T}$ & \\
\hline TE-108-AP-36 & $10 / 22 / 93$ & 64 & SACS & $\mathbf{T}$ & \\
\hline TE-108-AP-36 & $10 / 28 / 93$ & 66. & SACS & $T$ & \\
\hline TE-108-AP-36 & $11 / 9 / 93$ & 62. & SACS & $T$ & \\
\hline TE-108-AP-36 & $11 / 30 / 93$ & 64) & SACS & $T$ & \\
\hline TE-108-AP-36 & $12 / 14 / 93$ & 86 & SACS & $\bar{T}$ & \\
\hline TE-108-AP-36 & $12 / 20 / 93$ & 62 & SACS & $\mathbf{T}$ & \\
\hline TE-108-AP-36 & $12 / 27 / 93$ & 64 & SACS & $\mathbf{T}$ & \\
\hline TE-108-AP-36 & $1 / 3 / 94$ & 61 & SACS & $\bar{T}$ & \\
\hline TE-10B-AP-36 & $1 / 17 / / 94$ & 64 & SACS & $\mathbf{T}$ & \\
\hline TE-108-AP-36 & $1 / 19 / 34$ & 64 & CASS & $T$ & \\
\hline TE-108-AP-36 & $1 / 28 / 94$ & 62: & CASS & $\mathbf{T}$ & \\
\hline TE-106-AP-36 & $2 / 4 / 94$ & 62 & CASS & $T$ & \\
\hline TE-108-AP-36 & $2 \pi / 94$ & 63 & SACS & G & \\
\hline TE-108-AP-36 & $2 / 16 / 94$ & 62 & CASS & $T$ & \\
\hline TE-108-AP-36 & 2/22/94 & 63 & CASS & $T$ & \\
\hline TE-108-AP-36 & $3 / 5 / 94$ & 65 & CASS & $\mathbf{I}$ & \\
\hline TE-10.AP-36 & 3/8/94 & 65 & CASS & $T$ & \\
\hline TE-108-AP-36 & $3 / 14 / 94$ & 67 & CASS & $\bar{T}$ & \\
\hline TE-108-AP-36 & $3 / 25 / 94$ & 65 & CASS & $\mathrm{T}$ & \\
\hline TE-108-AP-36 & $3 / 28 / 94$ & 63. & CASS & $T$ & \\
\hline TE-108-AP-36 & $4 / 9 / 94$ & 64 & CASS & $\mathbf{T}$ & \\
\hline TE-108-AP-36 & 4/15/94 & 65 & CASS & $\mathbf{T}$ & \\
\hline IE-108-AP-36 & 4/20/94 & 63. & CASS & $\mathbf{T}$ & \\
\hline TE-108-AP-36 & 4/29/94 & 64 & CASS & $T$ & \\
\hline TE-108-AP-36 & $5 / 1 / 94$ & 64 & CASS & $\mathbf{T}$ & \\
\hline TE-10B-AP-36 & $5 / 9 / 94$ & 86 & CASS & $\mathbf{T}$ & \\
\hline TE-108-AP+36 & 5/16/94 & 104 & CASS & $\mathbf{T}$ & \\
\hline TE-108-AP.36 & $5 / 22 / 94$ & 98 & CASS & $\mathbf{T}$ & \\
\hline TE-108-AP-36 & $6 / 2 / 94$ & 99 & CASS & $T$ & \\
\hline TE-108-AP-36 & $6 / 8 / 94$ & 98 & CASS & $\bar{T}$ & \\
\hline TE-108-AP-36 & $6 / 16 / 94$ & 63 & CASS & $\mathbf{S}$ & Data point did not eqgee with local trend \\
\hline TE-108-AP-36 & $6 / 17 / 94$ & 63 & CASS & $\mathbf{s}$ & Data point did not egree with tocal trend \\
\hline TE-108-AP-36 & 6/18/94 & 95 & CASS & $\mathbf{T}$ & \\
\hline TE-108-AP-36 & $6 / 20 / 94$ & 63 & CASS & $\mathrm{s}$ & Data point did not agree with local trend \\
\hline TE-108-AP-36 & 6/20/94 & 63: & SACS & S (G in SACS) & Dare point did not agree with local trend \\
\hline TE-108-AP-36 & $6 / 24 / 94$ & 98 & CASS & $\mathbf{T}$ & \\
\hline TE-108-AP-36 & $7 / 2 / 94$ & 98! & CASS & $T$ & \\
\hline TE-108-AP-36 & $7 / 7 / 94$ & 98 & CASS & $\mathbf{T}$ & \\
\hline TE-108-AP-36 & $7 / 10 / 94$ & 97 & CASS & $\mathbf{T}$ & \\
\hline TE-108-AP-36 & $7 / 18 / 94$ & 97 & CASS & $\mathbf{T}$ & \\
\hline TE-108-AP-36 & $7 / 30 / 94$ & 96 & CASS & $\mathbf{I}$ & \\
\hline TE-108-AP-36 & $7131 / 94$ & 100 & CASS & $\mathbf{T}$ & \\
\hline TE-108-AP-36 & $8 / 10 / 94$ & 100 & CASS & $\mathbf{T}$ & \\
\hline TE-108-AP-36 & 8/18/94 & 101 & CASS & $T$ & \\
\hline TE-108-AP-36 & $8 / 22 / 94$ & 101 & CASS & T & \\
\hline TE-108-AP-36 & $9 / 2 / 94$ & 102 & CASS & T & \\
\hline TE-108-AP-36 & 9/10/94 & 105 & CASS & $\bar{T}$ & \\
\hline TE-108-AP-36 & 9/15/94 & 104 & CASS & $T$ & \\
\hline TE-108-AP-36 & $9 / 23 / 94$ & 104 & CASS & $T$ & \\
\hline TE-108-AP-36 & $9 / 30 / 94$ & 104 & CASS & $T$ & \\
\hline TE-108-AP-36 & $10 / 6 / 94$ & 105 & CASS & $T$ & \\
\hline TE-108-AP-36 & $10 / 12 / 94$ & 104 & CASS & $T$ & \\
\hline TE-10B-AP-36 & $10 / 17 / 94$ & 105 & CASS & $T$ & \\
\hline TE-108-AP-36 & $10 / 25 / 94$ & 105 & CASS & $\mathbf{T}$ & \\
\hline TE-108-AP-36 & $11 / 5 / 84$ & 104 & CASS & $T$ & \\
\hline TE-108-AP-36 & $11 / 11 / 94$ & 104 & CASS & $\mathbf{T}$ & \\
\hline
\end{tabular}




\begin{tabular}{|c|c|c|c|c|c|}
\hline \multicolumn{6}{|c|}{$\begin{array}{l}\text { TANK 241-AP-108 } \\
\text { THEFIMOCOUPLE DATA }\end{array}$} \\
\hline & & & & & \\
\hline THERMOCOUPL * & DATE & TEMPERATUAE $\left({ }^{\circ} \mathrm{F}\right)$ & DATA SOURCE & OUALTY & COMMENT \\
\hline TE-108-AP-36 & $11 / 16 / 94$ & 103 & CASS & $T$ & \\
\hline TE-108-AP-36 & $11 / 22 / 94$ & 104 & CASS & $T$ & \\
\hline TE-108-AP-36 & $11 / 27 / 94$ & 103 & CASS & $T$ & \\
\hline TE-108-AP-36 & $12 / 4 / 94$ & 106 & CASS & $T$ & \\
\hline TE-108-AP-36 & $12 / 12 / 94$ & 101 & CASS & $\mathbf{T}$ & \\
\hline TE-108-AP-36 & $12 / 19 / 94$ & 104 & CASS & $\bar{T}$ & \\
\hline TE-108-AP-36 & $12 / 30 / 94$ & 104 & CASS & $\mathbf{T}$ & \\
\hline TE-108-AP-36 & $1 / 4 / 95$ & 109 & CASS & $T$ & \\
\hline TE-108-AP-36 & $1 / 1 / 4 / 95$ & 103 & CASS & $T$ & \\
\hline TE-108.AP-36 & $1 / 15 / 95$ & 104 & CASS & $T$ & \\
\hline TE-108-AP-36 & $1 / 23 / 95$ & 105 & CASS & $\mathbf{T}$ & \\
\hline TE-108.AP-36 & 1/30/35: & 101 & SACS & G & \\
\hline TE-108.AP-36 & $2 / 9 / 95$ & 100 & CASS & $\bar{T}$ & \\
\hline TE-108-AP-36 & $2 / 14 / 95$ & 106 & CASS & $\mathbf{T}$ & \\
\hline TE-108-AP-36 & $2 / 24 / 95$ & 95 & CASS & $T$ & \\
\hline TE-108-AP-36 & $3 / 2 / 35$ & 100 & CASS & $T$ & \\
\hline TE-108-AP-36 & $3 / 7 / 95$ & 98 & CASS & $T$ & \\
\hline TE-108-AP-36 & $3 / 16 / 95$ & 94 & CASS & $T$ & \\
\hline TE-108-AP-36 & $3 / 25 / 95$ & 95 & CASS & $\bar{T}$ & \\
\hline TE-108-AP-36 & $3 / 30 / 95$ & 96 & CASS & $T$ & \\
\hline TE-10B-AP-36 & $4 / 3 / 95$ & 86 & SACS & G & \\
\hline TE-108-AP-36 & $4 / 10 / 95$ & 88 & SACS & G & \\
\hline TE-10B-AP-36 & $4 / 17 / 95$ & 90 & SACS & G & \\
\hline TE-108:AP-36 & $4 / 24 / 95$ & 83 & SACS & $\mathbf{G}$ & \\
\hline TE-108-AP-36 & $5 / 1 / 95$ & 86 & SACS & G & \\
\hline TE-108-AP-36 & 5/3/95 & 86 & SACS & $G$ & \\
\hline TE-106-AP-36 & $5 / 16 / 95$ & 83 & SACS & G & \\
\hline TE-108-AP-36 & $5 / 22 / 95$ & 83 & SACS & G & \\
\hline TE-108-AP-36 & 5/29/95 & 84 & SACS & G & \\
\hline TE-108-AP-36 & $6 / 5 / 95$ & 88 & SACS & G & \\
\hline TE-108-AP-36 & $6 / 13 / 95$ & 86 & SACS & G & \\
\hline TE-106-AP-36 & 6/19/95 & 83 & SACS & $\mathbf{G}$ & \\
\hline & & & & & \\
\hline TE-108-AP-37 & $4 / 5 / 86$ & 57 & CASS & $\mathbf{T}$ & \\
\hline TE-108-AP-37 & $4 / 7 / 86$ & 60 & CASS & $T$ & \\
\hline TE-108-AP-37 & $5 / 17 / 86$ & 57 & CASS & $T$ & \\
\hline TE-108-AP-37 & 5/24/86 & 59 & CASS & $T$ & \\
\hline TE-108-AP-37 & $5 / 30 / 86$ & 59 & CASS & $\mathbf{T}$ & \\
\hline IE-108-AP-37 & $6 / 7 / 86$ & 59 & CASS & $\bar{T}$ & \\
\hline TE-108-AP-37 & $6 / 9 / 86$ & 63 & CASS & $T$ & \\
\hline TE-108-AP-37 & $6 / 21 / 86$ & 60 & CASS & $T$ & \\
\hline TE-10B-AP-37 & $6 / 25 / 86$ & 60 & CASS & $T$ & \\
\hline TE-108-AP-37 & $7 / 3 / 86$ & 62 & CASS & $\bar{T}$ & \\
\hline TE-108-AP-37 & 7/10/86 & 62 & CASS & $T$ & \\
\hline TE-108-AP-37 & 7/17/86 & 64 & CASS & $\mathbf{T}$ & \\
\hline TE-108.AP-37 & 7/26/36 & 62 & CASS & $T$ & \\
\hline TE-108-AP-37 & 7/28/36 & 64 & CASS & $T$ & \\
\hline TE-108-AP-37 & $8 / 9 / 36$ & 64 & CASS & $T$ & \\
\hline TE-108-AP-37 & $8 / 13 / 36$ & 65 & CASS & $\mathbf{T}$ & \\
\hline TE-108-AP-37 & 8/17/36 & 65 & CASS & $T$ & \\
\hline TE-108-AP-37 & $8 / 30 / 36$ & 64 & CASS & $\mathbf{T}$ & \\
\hline TE-108-AP-37 & $9 / 1 / 36$ & 65 & CASS & $T$ & \\
\hline TE-108-AP-37 & 9/24/36 & 64 & CASS & $\bar{T}$ & \\
\hline TE-108-AP-37 & $11 / 1 / 66$ & 62 & CASS & $T$ & \\
\hline TE-108-AP-37 & 91/4/86 & 62 & CASS & $T$ & \\
\hline TE-108-AP-37 & $11 / 29 / 86$ & 59 & CASS & $T$ & \\
\hline TE-108-AP-37 & $12 / 10 / 86$ & 59 & CASS & $T$ & \\
\hline TE-108-AP-37 & $12 / 16 / 86$ & 59 & CASS & $T$ & \\
\hline TE-108-AP-37 & $12 / 24 / 86$ & 57 & CASS & $T$ & \\
\hline TE-108-AP-37 & $12 / 29 / 86$ & 56: & CASS & $T$ & \\
\hline TE-108-AP-37 & $7 / 1 / 87$ & 66 & CASS & $\mathbf{T}$ & \\
\hline TE-108-AP-37 & 7/8/87 & 67 & CASS & $T$ & \\
\hline TE-108-AP-37 & 7/18/37 & 66 & CASS & $\bar{T}$ & \\
\hline TE-108-AP-37 & $7 / 19 / 87$ & 69 & CASS & $\mathbf{T}$ & \\
\hline TE-108-AP-37 & 7/27/87 & 68: & CASS & $T$ & \\
\hline TE-108-AP-37 & $8 / 5 / 87$ & 69. & CASS & $T$ & \\
\hline TE-108-AP-37 & $8 / 14 / 87$ & 72 & CASS & $T$ & \\
\hline TE-108-AP-37 & $8 / 17 / 87$ & 69 & CASS & $\bar{T}$ & \\
\hline TE-108-AP-37 & $8 / 22 / 87$ & 50 & CASS & 5 & Dara point did not aprea with local trend \\
\hline TE-108-AP-37 & $8 / 26 / 87$ & 72 & CASS & $\mathbf{T}$ & \\
\hline TE-108-AP-37 & 9/4/87 & 69 & CASS & $T$ & \\
\hline TE-108-AP-37 & 9/12/37 & 69 & CASS & $T$ & \\
\hline TE-108-AP-37 & 9/17/37 & 70 & CASS & $\mathbf{T}$ & \\
\hline
\end{tabular}




\begin{tabular}{|c|c|c|c|c|c|}
\hline \multicolumn{6}{|c|}{$\begin{array}{l}\text { TANK 241-AP-108 } \\
\text { THERMOCOULLE DATA }\end{array}$} \\
\hline & & & & & \\
\hline THERMOCOUPL * & DATE & TEMPERATURE ("F) & DATA SOURCE & OUALITY & COMMENT \\
\hline TE-108-AP-37 & $9 / 20 / 87$ & 70 & CASS & $T$ & \\
\hline TE-108-AP-37 & $9 / 27 / 87$ & 70 & CASS & $T$ & \\
\hline TE-108-AP-37 & $10 / 7 / 67$ & 70 & CASS & $T$ & \\
\hline TE-108-AP-37 & $10 / 16 / 37$ & 67 & CASS & $T$ & \\
\hline TE-108-AP-37 & $10 / 24 / 87$ & 66 & CASS & $T$ & \\
\hline TE-108-AP-37 & $10 / 31 / 87$ & 86 & CASS & $T$ & \\
\hline TE-108-AP-37 & $11 / 1 / 87$ & 65 & CASS & $T$ & \\
\hline TE-108-AP-37 & $11 / 13 / 87$ & 64 & CASS & $T$ & \\
\hline TE-108-AP-37 & $11 / 16 / 37$ & 64 & CASS & $\mathbf{T}$ & \\
\hline TE-108-AP-37 & $11 / 26 / 87$ & 64 & CASS & $\bar{T}$ & \\
\hline TE-108-AP-37 & $11 / 29 / 87$ & 61 & CASS & $T$ & \\
\hline TE-108-AP-37 & $12 / 6 / 87$ & 61 & CASS & $T$ & \\
\hline TE-108-AP-37 & $12 / 18 / 87$ & 58 & CASS & $\bar{T}$ & \\
\hline TE-108-AP-37 & $12 / 23 / 87$ & 57 & CASS & $T$ & \\
\hline TE-108-AP-37 & $12 / 28 / 87$ & $\mathbf{5 5}$ & CASS & $\bar{T}$ & \\
\hline TE-108-AP-37 & $1 / 7 / 88$ & 52 & CASS & $\bar{T}$ & \\
\hline TE-108-AP-37 & $1 / 16 / 88$ & 51 & CASS & $T$ & \\
\hline TE-10B-AP-37 & 1/21/88 & 53 & CASS & $T$ & \\
\hline TE-108-AP-37 & $1 / 25 / 88$ & 55 & CASS & $T$ & \\
\hline TE-108-AP-37 & $2 / 6 / 88$ & 51 & CASS & $T$ & \\
\hline TE-108-AP-37 & $2 / 7 / 88$ & 53 & CASS & $T$ & \\
\hline TE-108-AP.37 & $2 / 13 / 88$ & 66 & CASS & $\mathbf{s}$ & Data point did not egree with local trend \\
\hline TE-100-AP-37 & $2 / 14 / 88$ & 5 & CASS & $T$ & \\
\hline TE-108-AP-37 & $2 / 27 / 88$ & 52 & CASS & $T$ & \\
\hline IE.108-AP.37 & $3 / 3 / 88$ & 54 & CASS & $\mathbf{T}$ & \\
\hline TE-108-AP-37 & $3 / 12 / 88$ & 53 & CASS & $\mathbf{T}$ & \\
\hline TE-108-AP-37 & 3/13/80 & 54 & CASS & $\mathbf{T}$ & \\
\hline TE-108-AP-37 & $3 / 25 / 83$ & $\mathbf{5 5}$ & CASS & $\mathbf{T}$ & \\
\hline TE-106-AP-37 & $3 / 31 / 88$ & 54 & CASS & $\mathbf{T}$ & \\
\hline TE-108.AP.37 & $6 / 1 / 88$ & 60 & CASS & $T$ & \\
\hline TE-108-AP-37 & $6 / 8 / 88$ & 61 & CASS & $\mathbf{T}$ & \\
\hline TE-108-AP-37 & 6/18/88 & 61 & CASS & $\mathbf{T}$ & \\
\hline TE-108-AP-37 & 6/25/88 & 62 & CASS & $\mathbf{T}$ & \\
\hline TE-108-AP-37 & $7 / 1 / 68$ & 64 & CASS & $\mathbf{T}$ & \\
\hline TE-108-AP-37 & $7 / 9 / 68$ & 63 & CASS & $\mathbf{T}$ & \\
\hline TE-108-AP-37 & $7 / 16 / 88$ & 64 & CASS & $T$ & \\
\hline TE-108-AP-37 & $7 / 23 / 88$ & 66 & CASS & $T$ & \\
\hline TE-108-AP-37 & $7129 / 98$ & 67 & CASS & $\mathbf{T}$ & \\
\hline TE-108-AP-37 & $8 / 3 / 68$ & 69 & CASS & $T$ & \\
\hline TE-108-AP-37 & $8 / 9 / 68$ & 69 & CASS & $T$ & \\
\hline TE-108-AP-37 & $8 / 20 / 88$ & 69 & CASS & $T$ & \\
\hline TE-108-AP-37 & $8 / 22 / 88$ & 69 & CASS & $\mathbf{T}$ & \\
\hline TE-108-AP-37 & $9 / 2 / 88$ & 70 & CASS & $T$ & \\
\hline TE-108-AP-37 & $9 / 9 / 88$ & 71 & CASS & $T$ & \\
\hline TE-100-AP-37 & $9 / 16 / 88$ & 71 & CASS & $\bar{T}$ & \\
\hline TE-108-AP-37 & $9 / 23 / 88$ & 70 & CASS & $\bar{T}$ & \\
\hline TE-108-AP-37 & $9 / 27 / 88$ & 70 & CASS & $T$ & \\
\hline TE-108.AP.37 & $1213 / 88$ & 65 & CASS & $T$ & \\
\hline TE-108-AP-37 & $12 / 8 / 88$ & 64 & CASS & $\bar{T}$ & \\
\hline TE-108-AP-37 & $12 / 13 / 88$ & 63 & CASS & $\bar{T}$ & \\
\hline TE-108-AP-37 & $12 / 22 / 88$ & 62 & CASS & $\bar{T}$ & \\
\hline TE-108-AP-37 & 7/10/89 & 65 & SACS & $T$ & \\
\hline$T E-108-A P-37$ & $7 / 17 / 89$ & 65 & SACS & $T$ & \\
\hline TE-108-AP-37 & $7 / 24 / 89$ & 66 & SACS & $\mathbf{T}$ & \\
\hline TE-108-AP-37 & $8 / 7 / 89$ & 67 & SACS & $T$ & \\
\hline TE-108-AP-37 & $8 / 74 / 89$ & 68 & SACS & $T$ & \\
\hline TE-108-AP-37 & $8 / 21 / 89$ & 68 & SACS & $\bar{T}$ & \\
\hline TE-108-AP-37 & $9 / 2 / 89$ & 71 & CASS & $T$ & \\
\hline TE-108-AP-37 & $9 / 4 / 89$ & 72 & CASS & $T$ & \\
\hline TE-108-AP-37 & $9 / 16 / 89$ & 70 & CASS & $T$ & \\
\hline TE-108-AP-37 & 9/18/89 & 70 & CASS & $T$ & \\
\hline TE-108-AP-37 & 9/29/89 & 77 & CASS & $T$ & \\
\hline TE-108-AP-37 & $10 / 2 / 89$ & 67 & SACS & $\mathbf{T}$ & \\
\hline TE-108-AP-37 & $10 / 9 / 89$ & 67 & SACS & $\mathbf{T}$ & \\
\hline TE-108-AP-37 & $10 / 16 / 89$ & 68 & SACS & $T$ & \\
\hline TE-108-AP-37 & $10 / 24 / 89$ & 68 & SACS & $T$ & \\
\hline TE-108-AP-37 & T1/4/89 & 66 & CASS & $T$ & \\
\hline TE-108-AP-37 & $11 / 7 / 89$ & 65 & CASS & $T$ & \\
\hline TE-108-AP-37 & $11 / 19 / 89$ & 65 & CASS & $T$ & \\
\hline TE-108-AP-37 & $11 / 20 / 89$ & 64 & SACS & $\mathbf{T}$ & \\
\hline TE-108-AP-37 & $12 / 2 / 39$ & 63 & CASS & $T$ & \\
\hline TE-108-AP-37 & $12 / 4 / 89$ & $6 \mathbf{3 3}$ & SACS & $\mathbf{T}$ & \\
\hline TE-108-AP-37 & $12 / 12789$ & 81 & CASS & $T$ & \\
\hline
\end{tabular}

Data obtained from Computer Automated Surveillance System (CASS), April 1995; and Surveillance Analyais Computer System (SACS), June 1995.

D- 649 


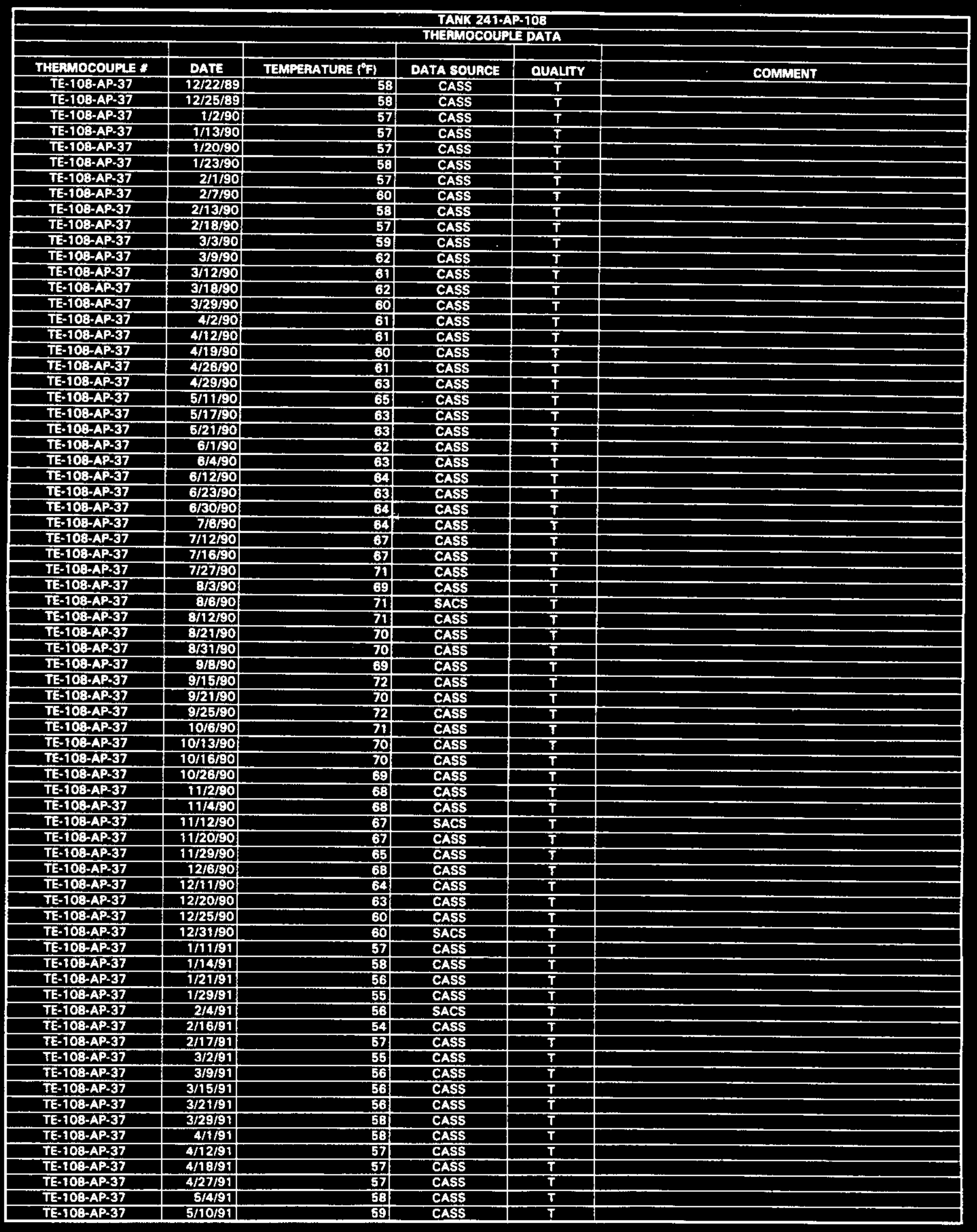




\begin{tabular}{|c|c|c|c|c|c|}
\hline \multicolumn{6}{|c|}{$\begin{array}{l}\text { TANK 241-AP-108 } \\
\text { THERMOCOUPLE DATA }\end{array}$} \\
\hline & & & & & \\
\hline$\frac{\text { THEAMOCOUPLE }}{\text { TE-108-AP-37 }}$ & DATE & TEMPERATUAE $\left({ }^{\circ} \mathrm{F}\right)$ & DATA SOUACE & QUALTY & COMMENT \\
\hline $\begin{array}{l}\text { TE-108-AP-37 } \\
\text { TE-108-AP-37 }\end{array}$ & \begin{tabular}{|l|}
$5 / 16 / 91$ \\
$5 / 25 / 91$
\end{tabular} & 60 & CASS & $T$ & \\
\hline $\begin{array}{l}\text { TE-108-AP-37 } \\
\text { TE-108-AP-37 }\end{array}$ & $\frac{5 / 25 / 91}{5 / 31 / 91}$ & 59 & CASS & $T$ & \\
\hline$\frac{T E-108-A P-37}{T E-108-A P-37}$ & $\frac{5 / 31 / 91}{6 / 3 / 91}$ & 61 & CASS & $T$ & \\
\hline TE-108-AP-37 & $6 / 3 / 91$ & 62 & SACS & $T$ & \\
\hline TE-108-AP-37 & $6 / 14 / 91$ & 105 & CASS & $\mathbf{s}$ & Data point did not agree with local trend \\
\hline TE-108-AP-37 & 6/15/91 & 62 & CASS & $T$ & \\
\hline TE-108-AP-37 & 6/22191 & 64 & CASS & $T$ & \\
\hline TE-108-AP-37 & 6/28/91 & 67. & CASS & $\mathbf{T}$ & \\
\hline TE-10B-AP-37 & $7 / 3 / 91$ & 66! & CASS & $\bar{T}$ & \\
\hline TE-108-AP-37 & $7 / 8 / 91$ & 68 & SACS & $T$ & \\
\hline TE-108-AP-37 & 7/15/91 & $\mathbf{6 6}$ & SACS & $\overline{\mathbf{T}}$ & \\
\hline TE-108-AP-37 & $7 / 24 / 91$ & 68 & CASS & $\mathbf{T}$ & \\
\hline TE-108-AP-37 & $8 / 3 / 91$ & 68 & CASS & $\mathbf{T}$ & \\
\hline TE-108-AP-37 & $8 / 6 / 91$ & 68. & CASS & $T$ & \\
\hline TE-108-AP-37 & 8/17/91 & 72 & CASS & $\mathbf{T}$ & \\
\hline TE-108-AP-37 & $8 / 20 / 91$ & 70 & CASS & $\mathbf{T}$ & \\
\hline TE-108-AP-37 & $8 / 30 / 91$ & 71 & CASS & $\mathbf{T}$ & \\
\hline TE-10B-AP-37 & $9 / 2 / 91$ & 71 & SACS & $\bar{T}$ & \\
\hline TE-108-AP-37 & $9 / 14 / 91$ & 7 & CASS & $T$ & \\
\hline TE-108-AP-37 & 9/15/91 & 71 & CASS & T & \\
\hline TE-108-AP-37 & $9 / 22 / 91$ & 71 & CASS & $\mathbf{T}$ & \\
\hline TE-108-AP-37 & $10 / 1 / 91$ & 74 & CASS & $\mathbf{T}$ & \\
\hline TE-108-AP-37 & 10/7/81 & 71 & SACS & $\mathbf{T}$ & \\
\hline TE-108-AP-37 & 10/3/91 & $6 \mathbf{3}$ & CASS & $\mathbf{s}$ & Data point did not agree with local trand \\
\hline TE-108-AP-37 & $10 / 17 / 91$ & 72 & CASS & $T$ & \\
\hline TE-108-AP-37 & $10 / 25 / 91$ & 72 & CASS & $T$ & \\
\hline TE-108-AP-37 & $10 / 28 / 91$ & 71 & CASS & $\mathbf{T}$ & \\
\hline TE-108-AP-37 & $11 / 6 / 91$ & 70 & CASS & $T$ & \\
\hline TE-108-AP-37 & $7 / 30 / 92$ & 69: & CASS & $\bar{T}$ & \\
\hline TE-108-AP-37 & $8 / 8 / 92$ & 69 & CASS & $\mathbf{T}$ & \\
\hline TE-108-AP-37 & $8 / 15 / 92$ & 70 & CASS & $\mathbf{T}$ & \\
\hline TE-106-AP-37 & $8 / 22 / 92$ & 69 & CASS & $\bar{T}$ & \\
\hline TE-108-AP-37 & $8 / 28 / 92$ & 71 & CASS & $\mathbf{T}$ & \\
\hline TE-108-AP-37 & $8 / 31 / 92$ & 70 & CASS & $\mathbf{T}$ & \\
\hline TE-10Q-AP-37 & 9/9/92 & 70 & CASS & $T$ & \\
\hline IE-108-AP-37 & 9/16/92 & 70 & CASS & $\bar{T}$ & \\
\hline TE-108-AP-37 & $9 / 26 / 92$ & 69 & CASS & $T$ & \\
\hline TE-108-AP-37 & $10 / 3 / 92$ & 69 & CASS & $T$ & \\
\hline TE-108-AP-37 & 10/8/92 & 70 & CASS & $\mathbf{T}$ & \\
\hline TE-108-AP-37 & $10 / 13 / 92$ & 69 & CASS & $T$ & \\
\hline TE-108-AP-37 & $10 / 22 / 92$ & $69 !$ & CASS & $\bar{T}$ & \\
\hline TE-108-AP-37 & $10 / 29 / 92$ & 69 & CASS & $\mathbf{T}$ & \\
\hline TE-108-AP-37 & $11 / 4 / 92$ & 69 & CASS & $T$ & \\
\hline TE-108-AP-37 & 11/9/92 & 69 & CASS & $T$ & \\
\hline TE-108-AP-37 & $11 / 17 / 92$ & 71 & CASS & $\mathrm{T}$ & \\
\hline TE-108-AP-37 & $11 / 23 / 92$ & 67 & CASS & $T$ & \\
\hline TE-108-AP-37 & $12 / 3 / 92$ & 66 & CASS & $\mathbf{T}$ & \\
\hline TE-108-AP-37 & $12 / 6 / 92$ & 67 & CASS & $T$ & \\
\hline TE-108-AP-37 & $12 / 19 / 92$ & 66 & CASS & $\mathbf{T}$ & \\
\hline TE-108-AP-37 & $12 / 21 / 92$ & 66 & CASS & $\mathbf{T}$ & \\
\hline TE-108-AP-37 & $12 / 27 / 92$ & 65 & CASS & $T$ & \\
\hline TE-108-AP-37 & $1 / 15 / 94$ & 62 & CASS & $\mathbf{T}$ & \\
\hline TE-108-AP-37 & $1 / 19 / 94$ & 64 & CASS & $\overline{\mathbf{T}}$ & \\
\hline TE-108-AP-37 & $1 / 28 / 94$ & 62 & CASS & $\mathbf{T}$ & \\
\hline TE-108-AP-37 & $2 / 2 / 94$ & 63 & CASS & $\mathbf{T}$ & \\
\hline TE-108-AP-37 & $2 / 11 / 94$ & 62 & CASS & $T$ & \\
\hline TE-108-AP-37 & $2 / 13 / 94$ & 63 & CASS & $\mathbf{T}$ & \\
\hline TE-108-AP-37 & $2 / 26 / 94$ & 62 & CASS & $\bar{T}$ & \\
\hline TE-108-AP-37 & $3 / 5 / 94$ & 66 & CASS & $T$ & \\
\hline TE-108-AP-37 & $3 / 8 / 94$ & 66 & CASS & $\mathbf{T}$ & \\
\hline TE-108-AP-37 & $3 / 14 / 94$ & 68 & CASS & $T$ & \\
\hline TE-108-AP-37 & $3 / 25 / 94$ & 64 & CASS & $T$ & \\
\hline TE-108-AP-37 & $4 / 2 / 94$ & 63 & CASS & $T$ & \\
\hline TE-108-AP-37 & $4 / 9 / 94$ & 64 & CASS & $\mathbf{T}$ & \\
\hline TE-108-AP-37 & $4 / 15 / 94$ & 64 & CASS & $T$ & \\
\hline TE-108-AP-37 & $4 / 22 / 94$ & 63 & CASS & $\mathbf{T}$ & \\
\hline TE-108-AP-37 & $4 / 29 / 94$ & 63 & CASS & $\bar{T}$ & \\
\hline TE-108-AP-37 & $5 / 1 / 94$ & 64 & CASS & $T$ & \\
\hline TE-106-AP-37 & $5 / 14 / 94$ & 64 & CASS & $T$ & \\
\hline TE-108-AP-37 & $5 / 16 / 94$ & 103 & CASS & $T$ & \\
\hline TE-10B-AP-37 & $5 / 22 / 94$ & 98 & CASS & $\mathbf{T}$ & \\
\hline TE-108-AP-37 & $6 / 2 / 94$ & 98 & CASS & $T$ & \\
\hline TE-108-AP-37 & $6 / 6 / 94$ & 98 & CASS & $T$ & \\
\hline
\end{tabular}




\begin{tabular}{|c|c|c|c|c|c|}
\hline \multicolumn{6}{|c|}{$\begin{array}{l}\text { TALK 391-AP-108 } \\
\text { THISMOCOUFL DATA }\end{array}$} \\
\hline THERMOCOUPLE \# & DATE & TEMPERATURE ("F) & DATA SOUACE & OUALTY & COMMENT \\
\hline TE-108-AP-37 & 6/17/94 & 108 & CASS & $T$ & \\
\hline TE-108-AP-37 & 6/20/94 & 63 & CASS & $\mathbf{S}$ & Data point did not agrae with local trend \\
\hline TE-108-AP-37 & 6/24/94 & 98 & CASS & $\mathbf{T}$ & \\
\hline TE-108.AP-37 & 6/26/94 & 144 & CASS & $\mathbf{S}$ & Data point did not agres with local trend \\
\hline TE-108-AP-37 & $6 / 27 / 94$ & 99 & CASS & $T$ & \\
\hline TE-108-AP-37 & $7 / 7 / 194$ & 98 & CASS & $\mathbf{T}$ & \\
\hline TE-108-AP-37 & $7 / 70 / 94$ & 97 & CASS & $T$ & \\
\hline TE-108-AP-37 & $778 / 94$ & 97 & CASS & $T$ & \\
\hline TE-108-AP-37 & $7130 / 94$ & 85 & CASS & $T$ & \\
\hline TE-108-AP-37 & $7 / 31 / 94$ & 100 & CASS & $\mathbf{T}$ & \\
\hline TE-108-AP-37 & $8 / 10 / 94$ & 99 & CASS & $\mathbf{T}$ & \\
\hline TE-10B-AP-37 & $8 / 18 / 34$ & 100 & CASS & $\mathbf{T}$ & \\
\hline TE-108-AP-37 & $8 / 21 / 94$ & 100 & CASS & $\mathbf{T}$ & \\
\hline TE-108-AP-37 & $9 / 2 / 94$ & 101 & CASS & $\mathbf{T}$ & \\
\hline TE-108-AP-37 & $9 / 10 / 94$ & 104 & CASS & $T$ & \\
\hline TE-108-AP-37 & $9 / 15 / 94$ & 103 & CASS & $\mathbf{T}$ & \\
\hline TE-108-AP.37 & $9 / 23 / 94$ & 104 & CASS & $\mathbf{T}$ & \\
\hline TE-108-AP-37 & 9/30/94 & 103 & CASS & $T$ & \\
\hline IE-108-AP-37 & $10 / 2 / 94$ & 105 & CASS & $T$ & \\
\hline TE-106-AP-37 & $10 / 12 / 94$ & 104 & CASS & $\mathbf{T}$ & \\
\hline TE-108-AP-37 & $10 / 17 / 94$ & 105 & CASS & $\mathbf{T}$ & \\
\hline TE-108-AP-37 & $10 / 25 / 94$ & 105 & CASS & $\mathbf{T}$ & \\
\hline TE-108-AP-37 & $11 / 5 / 94$ & 104 & CASS & $T$ & \\
\hline TE-108-AP-37 & $11 / 11 / 94$ & 104 & CASS & $\mathbf{I}$ & \\
\hline TE-108-AP-37 & $11 / 16 / 94$ & 103 & CASS & $T$ & \\
\hline TE-108-AP-37 & $11 / 22 / 94$ & 104 & CASS & $\bar{T}$ & \\
\hline TE-108-AP-37 & $11 / 28 / 94$ & 102 & CASS & $\mathbf{T}$ & \\
\hline TE-108-AP-37 & $12 / 4 / 94$ & 106 & CASS & $\mathbf{T}$ & \\
\hline TE-108-AP-37 & $12 / 12 / 94$ & 101 & CASS & $\bar{T}$ & \\
\hline TE-108-AP-37 & 12/19/94 & 104 & CASS & $T$ & \\
\hline TE-108-AP-37 & $12 / 30 / 34$ & 104 & CASS & $\mathbf{T}$ & \\
\hline TE-108-AP-S7 & $1 / 4 / 95$ & 109 & CASS & $T$ & \\
\hline IE-108-AP-37 & 1/14/95 & 103 & CASS & $\overline{\mathbf{T}}$ & \\
\hline TE-108.AP-37 & 1/15/95 & 104 & CASS & $\mathbf{T}$ & \\
\hline TE-108-AP-37 & 1/23/95 & 105 & CASS & $T$ & \\
\hline TE-108-AP-37 & $2 / 2 / 95$ & 98 & CASS & $T$ & \\
\hline TE-108-AP-37 & $219 / 95$ & 100 & CASS & $\mathbf{T}$ & \\
\hline TE-108-AP-37 & 2/14/95 & 106 & CASS & $T$ & \\
\hline TE-108-AP-37 & $2 / 24 / 95$ & 95 & CASS & $T$ & \\
\hline TE-108-AP-37 & $3 / 2 / 95$ & 100 & CASS & $\mathbf{T}$ & \\
\hline TE-108-AP-37 & $3 / 7 / 95$ & 98 & CASS & $T$ & \\
\hline TE-10:AP-AP-37 & $3 / 16 / 95$ & 94 & CASS & $T$ & \\
\hline TE-108-AP-37 & $3 / 25 / 95$ & 95 & CASS & $T$ & \\
\hline TE-108-AP-37 & 3/30/95 & 95 & CASS & $T$ & \\
\hline & & & & & \\
\hline TE-108-AP-38 & $4 / 5 / 86$ & 57 & CASS & $\bar{T}$ & \\
\hline TE-108-AP-3B & $4 / 7 / 86$ & 60 & CASS & $T$ & \\
\hline TE-108-AP-3B & 5/17/86 & 57 & CASS & $T$ & \\
\hline TE-108-AP-3B & 5/24/86 & 59 & CASS & $\mathbf{T}$ & \\
\hline TE-108AAP-3B & 5/30/86 & 59 & CASS & $T$ & \\
\hline TE-108-AP-3B & $6 / 7 / 86$ & 59 & CASS & $T$ & \\
\hline TE-108-AP-38 & 6/9/86 & 60 & CASS & $T$ & \\
\hline TE-108-AP-38 & $6 / 21 / 86$ & 60 & CASS & $T$ & \\
\hline TE-108-AP-38 & 6/25/36 & 60 & CASS & $\bar{T}$ & \\
\hline TE-108-AP-38 & $7 / 3 / 86$ & 62 & CASS & $\bar{T}$ & \\
\hline$T E-108-A P-38$ & 7/10/36 & 62 & CASS & $T$ & \\
\hline TE-108-AP-38 & $7 / 17 / 36$ & 63 & CASS & $T$ & \\
\hline TE-108-AP-38 & $7 / 26 / 36$ & 61 & CASS & $T$ & \\
\hline TE-108-AP-3B & $7 / 28 / 86$ & 64 & CASS & $\bar{T}$ & \\
\hline TE-108-AP-38 & B/9/36 & 64 & CASS & $T$ & \\
\hline$T E-108-A P \cdot 3 B$ & $8 / 13 / 36$ & 64 & CASS & $\bar{T}$ & \\
\hline TE-108-AP-38 & $8 / 17 / 86$ & 65 & CASS & $\mathbf{T}$ & \\
\hline TE-108-AP-38 & $8 / 29 / 36$ & 64 & CASS & $T$ & \\
\hline TE-108-AP-38 & $9 / 1 / 86$ & 65 & CASS & $T$ & \\
\hline TE-108-AP-38 & $9 / 24 / 86$ & 64 & CASS & $\bar{T}$ & \\
\hline TE-108-AP-3B & $11 / 1 / 86$ & 62 & CASS & $\mathbf{T}$ & \\
\hline TE-108-AP-3B & $11 / 2 / 36$ & 62 & CASS & $\mathbf{T}$ & \\
\hline TE-108-AP-38 & $11 / 29 / 36$ & 59 & CASS & $\mathbf{T}$ & \\
\hline TE-108-AP-3B & $12 / 10 / 36$ & 59 & CASS & $\mathbf{T}$ & \\
\hline TE-108-AP-38 & $12 / 16 / 36$ & 59 & CASS & $T$ & \\
\hline TE-108-AP-3B & $12 / 27 / 86$ & 56 & CASS & $T$ & \\
\hline TE-108-AP-38 & $12 / 28 / 86$ & 56: & CASS & $T$ & \\
\hline TE-108-AP-38 & $7 / 187$ & 66 & CASS & $I$ & \\
\hline
\end{tabular}

Dato obtained from Computer Automated Surveillance System (CASS), April 1995; and Surveillance Analysis Computer System (SACS), June 1995. 


\begin{tabular}{|c|c|c|c|c|c|}
\hline \multicolumn{6}{|c|}{$\begin{array}{l}\text { TATK 241-AP-108 } \\
\text { THEPMOCOUPL DATA }\end{array}$} \\
\hline THERMOCOUPLE * & DATE & TEMPERATURE ( $\left.{ }^{\circ} \mathrm{F}\right)$ & DATA SOURCE & QUALTYY & COMMENT \\
\hline TE-108-AP-3B & $7 / 8 / 87$ & 67 & CASS & $T$ & \\
\hline TE-10B-AP-3B & $7112 / 87$ & 82 & CASS & $\mathbf{S}$ & Data point did not agrea with local trend \\
\hline TE-108-AP-3B & $7 / 15 / 87$ & 66 & CASS & $\bar{T}$ & \\
\hline TE-10B-AP-38 & $7 / 19 / 87$ & 69 & CASS & $\mathbf{T}$ & \\
\hline TE-108-AP-3B & $7 / 27 / 87$ & 68 & CASS & $\bar{T}$ & \\
\hline TE-108-AP-38 & $8 / 5 / 87$ & 69 & CASS & $\bar{T}$ & \\
\hline TE-108-AP+3B & $8 / 14 / 87$ & 70 & CASS & $T$ & \\
\hline TE-108-AP-38 & $8 / 17 / 87$ & 70 & CASS & $T$ & \\
\hline TE-108-AP-3B & $8 / 26 / 87$ & 72 & CASS & $\mathbf{T}$ & \\
\hline TE-10:-AP-38 & 9/3/87 & 69 & CASS & $\mathbf{T}$ & \\
\hline TE-10B-AP-38 & $9 / 9 / 87$ & 71 & CASS & $T$ & \\
\hline TE-108-AP-38 & 9/14/87 & 70 & CASS & $T$ & \\
\hline TE-108-AP-3B & $9 / 20 / 87$ & 70 & CASS & $T$ & \\
\hline TE-108-AP-3B & $9 / 27 / 87$ & 70 & CASS & $T$ & \\
\hline TE-108-AP-38 & $10 / 7 / 87$ & 70 & CASS & $\mathbf{T}$ & \\
\hline TE-108-AP-3B & $10 / 16 / 87$ & 67 & CASS & $T$ & \\
\hline TE-108-AP-38 & $10 / 24 / 87$ & 67 & CASS & $T$ & \\
\hline TE-108-AP-38 & $10 / 27 / 87$ & 100 & CASS & $\mathbf{S}$ & Data point did not agres with local trend \\
\hline TE-108-AP-38 & $10731 / 87$ & 65 & CASS & $T$ & \\
\hline TE-108-AP-3B & $11 / 1 / 87$ & 65 & CASS & $T$ & \\
\hline TE-108-AP-3B & $11 / 13 / 87$ & 64 & CASS & $T$ & \\
\hline TE-108-AP-38 & $11 / 16 / 87$ & 63 & CASS & $T$ & \\
\hline TE-10B-AP-3B & $11 / 26 / 87$ & 64 & CASS & $T$ & \\
\hline TE-108-AP-3B & $11 / 29 / 87$ & 61 & CASS & $\mathbf{T}$ & \\
\hline TE-108-AP-3B & $12 / 6 / 87$ & 61 & CASS & $T$ & \\
\hline TE-108-AP-38 & $12 / 18 / 87$ & 58 & CASS & $T$ & \\
\hline TE-108-AP-38 & $12 / 23 / 87$ & 57 & CASS & $\mathbf{T}$ & \\
\hline TE-10B-AP-3B & $12 / 28 / 87$ & 56 & CASS & $T$ & \\
\hline TE-108-AP-38 & $1 / 7 / 68$ & 52 & CASS & $T$ & \\
\hline TE-108-AP-38 & 1/16/88 & 52 & CASS & $T$ & \\
\hline TE-108-AP-3B & $1 / 21 / 88$ & 54 & CASS & $T$ & \\
\hline TE-10B-AP-3B & $1 / 25 / 88$ & 56 & CASS & $T$ & \\
\hline TE-108-AP-38 & $2 / 2 / 88$ & 52 & CASS & $T$ & \\
\hline TE-108-AP-38 & $2 / 7 / 88$ & 53 & CASS & $T$ & \\
\hline TE-108-AP-38 & $2 / 14 / 88$ & 53 & CASS & $\bar{T}$ & \\
\hline TE-108-AP-3B & $2 / 27 / 88$ & 52 & CASS & $\mathbf{T}$ & \\
\hline TE-108-AP-38 & $3 / 3 / 88$ & 54 & CASS & $\mathbf{T}$ & \\
\hline TE-108-AP-38 & $3 / 12 / 88$ & 53 & CASS & $T$ & \\
\hline TE-108-AP-3B & $3 / 13 / 88$ & 54 & CASS & $T$ & \\
\hline TE-10B-AP-3B & 3/7/88 & 91 & CASS & $\mathbf{S}$ & Data point did not agree with local trend \\
\hline TE-108-AP-38 & $3 / 25 / 8$ & 55 & CASS & $T$ & \\
\hline TE-108-AP-38 & $3 / 31 / 88$ & 54 & CASS & $T$ & \\
\hline TE-108-AP-38 & $6 / 4 / 8$ & 59 & CASS & $\mathbf{T}$ & \\
\hline TE-108-AP-38 & $6 / 10 / 88$ & 60 & CASS & $\bar{T}$ & \\
\hline TE-108-AP-3B & $6 / 18 / 88$ & 61 & CASS & $\bar{T}$ & \\
\hline TE-108-AP-38 & $6 / 25 / 88$ & 62 & CASS & $\mathbf{T}$ & \\
\hline TE-108-AP-38 & $7 / 1 / 88$ & 63 & CASS & $\mathbf{T}$ & \\
\hline TE-100-AP-38 & $7 / 9 / 88$ & 63 & CASS & $\mathbf{T}$ & \\
\hline TE-108-AP-38 & $7 / 43 / 88$ & 66 & CASS & $\mathbf{T}$ & \\
\hline TE-10B-AP-3B & $7 / 23 / 88$ & 67 & CASS & $T$ & \\
\hline TE-10B-AP-38 & $7 / 29 / 88$ & 67 & CASS & $T$ & \\
\hline TE-108-AP-38 & 8/3/88 & 68 & CASS & $T$ & \\
\hline TE-108-AP-38 & $8 / 9 / 8$ & 69 & CASS & $\bar{T}$ & \\
\hline TE-108-AP-38 & $8 / 20 / 83$ & 69 & CASS & $T$ & \\
\hline TE-108-AP-3B & $8 / 22 / 88$ & 69 & CASS & $T$ & \\
\hline TE-108-AP-38 & $9 / 2 / 82$ & 70 & CASS & $T$ & \\
\hline TE-108-AP-3B & $9 / 9 / 62$ & 71 & CASS & $T$ & \\
\hline TE-108-AP-38 & $9 / 16 / 80$ & 71 & CASS & $T$ & \\
\hline TE-10B-AP-3B & $9 / 23 / 88$ & 70 & CASS & $T$ & \\
\hline TE-108-AP-38 & $9 / 27 / 88$ & 70 & CASS & $T$ & \\
\hline TE-108-AP-38 & $12 / 3 / 88$ & 65 & CASS & $T$ & \\
\hline TE-108-AP-3E & $12 / 8 / 88$ & 64 & CASS & $T$ & \\
\hline TE-108-AP-39 & $12 / 13 / 88$ & 63 & CASS & $T$ & \\
\hline TE-108-AP-38 & $12 / 22 / 88$ & 62 & CASS & $T$ & \\
\hline TE-108-AP-38 & $9 / 2 / 89$ & 70 & CASS & $T$ & \\
\hline TE-108-AP-38 & $9 / 7 / 89$ & 69 & CASS & $T$ & \\
\hline TE-108-AP-38 & $9 / 16 / 89$ & 71 & CASS & $T$ & \\
\hline TE-108-AP-38 & 9/18/89 & 70 & CASS & $T$ & \\
\hline TE-108-AP-38 & $9 / 29 / 89$ & 77 & CASS & $T$ & \\
\hline TE-108-AP-38 & $11 / 4 / 89$ & 66 & CASS & $T$ & \\
\hline TE-108-AP-38 & $11 / 7 / 89$ & 65 & CASS & $T$ & \\
\hline TE-108-AP-38 & $11 / 18 / 89$ & 65 & CASS & $T$ & \\
\hline TE-10E-AP-3B & $11 / 24 / 89$ & 63 & CASS & $\mathbf{T}$ & \\
\hline
\end{tabular}




\begin{tabular}{|c|c|c|c|c|c|}
\hline \multicolumn{6}{|c|}{$\begin{array}{l}\text { TANK 241-AP-108 } \\
\text { THEAMOCOUPLE DATA }\end{array}$} \\
\hline & & & & & \\
\hline THERMOCOUPLE * & DATE & TEMPERATUAE (*F) & DATA SOUACE & QUALTY & COMMENT \\
\hline TE-108-AP-38 & $12 / 2 / 89$ & 63 & CASS & $T$ & \\
\hline TE-10B-AP+38 & 12/8/89 & 62 & CASS & $T$ & \\
\hline TE-108-AP-38 & $12 / 12 / 89$ & 61 & CASS & $T$ & \\
\hline TE-108-AP-38 & $12 / 22 / 89$ & 58 & CASS & $T$ & \\
\hline TE-108-AP-38 & $12 / 25 / 89$ & 58 & CASS & $T$ & \\
\hline TE-108-AP-38 & $1 / 2 / 90$ & 57 & CASS & $\mathbf{T}$ & \\
\hline TE-108-AP-38 & $1 / 13 / 90$ & 57 & CASS & $T$ & \\
\hline TE-108-AP-38 & $1 / 15 / 90$ & 58 & CASS & $T$ & \\
\hline TE-108-AP-38 & $1 / 26 / 90$ & 58 & CASS & $T$ & \\
\hline TE-10Q-AP-38 & $2 / 1 / 90$ & 57 & CASS & $T$ & \\
\hline TE-108-AP-3B & $2 / 7 / 50$ & 60 & CASS & $T$ & \\
\hline TE-108-AP+38 & $2 / 13 / 90$ & 58 & CASS & $T$ & \\
\hline TE.108-AP-38 & $2 / 18 / 90$ & 57 & CASS & $T$ & \\
\hline TE-108-AP-38 & $2 / 20 / 90$ & 85 & CASS & $\mathbf{s}$ & Data point did not agree with local trend \\
\hline TE-108-AP-38 & $3 / 3 / 90$ & 59 & CASS & $T$ & \\
\hline TE-108-AP-38 & 3\%\%0 & 62 & CASS & $T$ & \\
\hline TE-108-AP-38 & आ12/90 & 61 & CASS & $T$ & \\
\hline TE-108-AP-3B & $3 / 20190$ & 61 & CASS & $T$ & \\
\hline TE-108-AP-38 & $3 / 29 / 90$ & 61 & CASS & $T$ & \\
\hline TE-108-AP-38 & $4 / 2 / 90$ & 61 & CASS & $T$ & \\
\hline TE-108.AP-38 & $4 / 2 / 90$ & 60 & CASS & $T$ & \\
\hline TE-108-AP-3B & $4 / 21 / 80$ & 60 & CASS & $T$ & \\
\hline TE-108-AP-38 & $4 / 26 / 90$ & 61 & CASS & $T$ & \\
\hline TE-10B-AP-3B & $4 / 29 / 90$ & 62 & CASS & $T$ & \\
\hline TE-10B-AP-3B & $5 / 11 / 90$ & 84 & CASS & $T$ & \\
\hline TE-108-AP-38 & $5 / 17 / 90$ & 63 & CASS & $T$ & \\
\hline TE-108-AP-38 & $5 / 21 / 90$ & 63 & CASS & $T$ & \\
\hline TE-108-AP-38 & $6 / 1 / 90$ & 63 & CASS & $T$ & \\
\hline TE-108-AP+38 & $6 / 2 / 90$ & 107 & CASS & $\mathbf{s}$ & Data point did not aprea with local trend \\
\hline TE-108.AP-38 & 6/O/90 & 64 & CASS & $\mathbf{T}$ & \\
\hline TE.10B-AP-38 & $6 / 12 / 90$ & 64 & CASS & $T$ & \\
\hline TE-108-AP-38 & $6 / 21 / 90$ & 64 & CASS & $\bar{T}$ & \\
\hline TE-108-AP-38 & $6 / 30 / 90$ & 64 & CASS & $T$ & \\
\hline TE-108-AP-3B & $7 / 6 / 90$ & 64 & CASS & $\bar{T}$ & \\
\hline TE-108-AP-38 & $7 / 12 / 90$ & 67 & CASS & $\mathbf{T}$ & \\
\hline TE-108-AP-3B & $7 / 16 / 90$ & 67 & CASS & $T$ & \\
\hline TE-108-AP-38 & $7 / 27 / 90$ & 71 & CASS & $T$ & \\
\hline TE-108-AP-38 & $7 / 30 / 90$ & 69 & SACS & $T$ & \\
\hline TE-108-AP-38 & $8 / 11 / 90$ & 69 & CASS & $T$ & \\
\hline TE-108-AP-38 & $8 / 17 / 90$ & 70 & CASS & $T$ & \\
\hline TE-106-AP-38 & E/19/90 & 71 & CASS & $T$ & \\
\hline TE-108-AP-3B & $9 / 1 / 90$ & 69 & CASS & $T$ & \\
\hline TE-108-AP-38 & 9/9/90 & 69 & CASS & $T$ & \\
\hline TE-102-AP-30 & $9 / 14 / 90$ & 71 & CASS & $\bar{T}$ & \\
\hline TE-108-AP-38 & $9 / 20 / 90$ & 70 & CASS & $\bar{T}$ & \\
\hline TE-108-AP-38 & $9 / 25 / 90$ & 72 & CASS & $T$ & \\
\hline TE-108-AP-38 & $10 / 6 / 90$ & 70 & CASS & $T$ & \\
\hline TE-10Q-AP-3B & $10 / 13 / 90$ & 69 & CASS & $T$ & \\
\hline TE-108-AP-3B & $10 / 16 / 90$ & 69 & CASS & $T$ & \\
\hline TE-108-AP-38 & $10 / 26 / 90$ & 70 & CASS & $T$ & \\
\hline TE-108-AP-38 & $10 / 31 / 90$ & 69 & CASS & $T$ & \\
\hline TE-108-AP-38 & $11 / 4 / 90$ & 68 & CASS & $T$ & \\
\hline TE.108-AP-3B & $11 / 17 / 90$ & 67 & CASS & $T$ & \\
\hline TE-108-AP-38 & $11 / 20 / 90$ & 67 & CASS & $T$ & \\
\hline TE-108-AP-.BB & $11 / 29 / 90$ & 65 & CASS & $T$ & \\
\hline$T E-108 \cdot A P-38$ & $12 / 6 / 90$ & 67 & CASS & $T$ & \\
\hline TE-108-AP-3B & $12 / 11 / 90$ & 65 & CASS & $T$ & \\
\hline TE-108-AP+38 & $12 / 20 / 90$ & 63 & CASS & $T$ & \\
\hline TE-108-AP-3B & $12 / 25 / 90$ & 60 & CASS & $T$ & \\
\hline TE-108-AP-38 & $12 / 30 / 90$ & 59 & CASS & $T$ & \\
\hline TE-108-AP-38 & $1 / 11 / 91$ & 57 & CASS & $T$ & \\
\hline TE-108-AP-38 & $1 / 14 / 91$ & 59 & CASS & $T$ & \\
\hline TE-108-AP-38 & $1 / 22 / 91$ & 56 & CASS & $T$ & \\
\hline TE-108-AP-3B & $1 / 29 / 91$ & 55 & CASS & $T$ & \\
\hline TE-108-AP-38 & $2 / 8 / 91$ & 55 & CASS & $T$ & \\
\hline TE-108-AP-38 & $2 / 15 / 91$ & 55 & CASS & $T$ & \\
\hline TE-108-AP-38 & $2 / 21 / 91$ & 56 & CASS & $T$ & \\
\hline TE-108-AP-38 & $3 / 2 / 91$ & 55 & CASS & $T$ & \\
\hline TE-108-AP-38 & $3 / 5 / 91$ & 57 & CASS & $T$ & \\
\hline TE-108-AP-38 & $3 / 15 / 91$ & 56 & CASS & $T$ & \\
\hline TE-108-AP-38 & $3 / 21 / 91$ & 56 & CASS & $T$ & \\
\hline TE-108-AP-38 & $3 / 29 / 91$ & 58 & CASS & $\mathbf{T}$ & \\
\hline TE-108-AP-38 & $4 / 1 / 91$ & 58 & CASS & $T$ & \\
\hline
\end{tabular}




\begin{tabular}{|c|c|c|c|c|c|}
\hline \multicolumn{6}{|c|}{$\begin{array}{l}\text { TAKK 241-AP-108 } \\
\text { THERMOCOUPIE DATA }\end{array}$} \\
\hline & & & & & \\
\hline THEAMOCOUPLE * & DATE & TEMPERATUAE ('F) & DATA SOURCE & QUALTY & COMMENT \\
\hline TE-108-AP-38 & $4 / 12 / 91$ & 57 & CASS & $T$ & 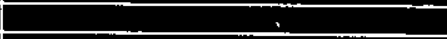 \\
\hline TE-10B-AP-38 & $4 / 18 / 91$ & 57 & CASS & $T$ & \\
\hline TE-108-AP-38 & $4 / 21 / 91$ & 58 & CASS & $\mathbf{T}$ & \\
\hline TE-108-AP-3B & $5 / 4 / 91$ & 58 & CASS & $T$ & \\
\hline TE-108-AP-3B & $5 / 10 / 91$ & 59 & CASS & $T$ & \\
\hline TE-108-AP-38 & $5 / 16 / 91$ & 61 & CASS & $T$ & \\
\hline TE-108-AP-38 & $5 / 25 / 91$ & 59 & CASS & $T$ & \\
\hline TE-108-AP-38 & $5 / 31 / 91$ & 61 & CASS & $\mathbf{T}$ & \\
\hline TE-108-AP-38 & 6/8/91 & 61 & CASS & $T$ & \\
\hline TE-108-AP-38 & 6/15/91 & 62 & CASS & $\bar{T}$ & \\
\hline TE-108-AP-38 & $6 / 22 / 91$ & 64 & CASS & $T$ & \\
\hline TE-108-AP-3B & $6 / 28 / 91$ & 67 & CASS & $T$ & \\
\hline TE-108-AP-3B & $7 / 3 / 91$ & 86 & CASS & $T$ & \\
\hline TE-108-AP-3B & $7 / 13 / 91$ & 66 & CASS & $\mathbf{T}$ & \\
\hline TE-108-AP-3B & $7 / 14 / 91$ & 66 & CASS & $T$ & \\
\hline TE-108-AP-3B & $7 / 24 / 91$ & 67 & CASS & $T$ & \\
\hline TE-108-AP-38 & $8 / 1 / 91$ & 69 & CASS & $T$ & \\
\hline TE-108-AP-3B & $8 / 6 / 91$ & 68 & CASS & $T$ & \\
\hline TE-10B-AP-38 & $8 / 32 / 91$ & 71 & CASS & $T$ & \\
\hline TE-108-AP-3B & $8 / 20 / 91$ & 70 & CASS & $T$ & \\
\hline TE-108-AP-38 & $8 / 30 / 91$ & 70 & CASS & $T$ & \\
\hline TE-108-AP-3B & 9/3/91 & 71 & CASS & $\bar{T}$ & \\
\hline TE-108-AP-38 & $9 / 12 / 91$ & 71 & CASS & $\mathbf{T}$ & \\
\hline TE-108-AP-38 & $9 / 21 / 91$ & 70 & CASS & $T$ & \\
\hline TE-108-AP-38 & $9 / 22 / 91$ & 73 & CASS & $T$ & \\
\hline TE-108-AP-3B & $10 / 1 / 91$ & 75 & CASS & $T$ & \\
\hline TE-108-AP-38 & $10 / 8 / 93$ & 62 & CASS & $\mathbf{s}$ & Data point did not agree with local trend \\
\hline TE-108-AP-3B & $10 / 12 / 91$ & 70 & CASS & $T$ & \\
\hline TE-108-AP-38 & $10 / 17 / 91$ & 72 & CASS & $T$ & \\
\hline TE-108-AP-3B & $10 / 25 / 91$ & 73 & CASS & $T$ & \\
\hline TE-108-AP-3B & $10 / 28 / 91$ & 70 & CASS & $T$ & \\
\hline TE-108-AP-38 & $11 / 6 / 91$ & 70 & CASS & $T$ & \\
\hline TE-108-AP-38 & $7 / 30 / 92$ & 69 & CASS & $T$ & \\
\hline TE-108-AP-3B & $8 / 8 / 92$ & 69 & CASS & $T$ & \\
\hline TE-10A-AP-3B & $8 / 11 / 92$ & 126 & CASS & $\mathbf{s}$ & Data point did not agree with local trend \\
\hline TE-108-AP-3B & $8 / 15 / 92$ & 69 & CASS & $T$ & \\
\hline TE+108-AP-3B & $8 / 22 / 92$ & 69 & CASS & $\mathbf{T}$ & \\
\hline TE-108-AP-3B & $8 / 28 / 92$ & 7 & CASS & $T$ & \\
\hline TE-108-AP-38 & 9/5/92 & 69 & CASS & $T$ & i. \\
\hline TE-10B-AP-3B & $9 / 12 / 92$ & 70 & CASS & $T$ & \\
\hline TE-108-AP-38 & $9 / 16 / 92$ & 70 & CASS & $\mathbf{T}$ & \\
\hline TE-108-AP-3B & $9 / 24 / 92$ & 69 & CASS & $\mathbf{T}$ & \\
\hline TE-108-AP-38 & $10 / 3 / 92$ & 69 & CASS & $\mathbf{T}$ & \\
\hline TE-108-AP-38 & $10 / 4 / 92$ & 69 & CASS & $\mathbf{T}$ & \\
\hline TE-108-AP-3B & $10 / 13 / 92$ & 69 & CASS & $T$ & \\
\hline TE-108-AP-38 & $10 / 22 / 92$ & 69 & CASS & $T$ & \\
\hline TE-108-AP-38 & $10 / 29 / 92$ & 69 & CASS & $T$ & \\
\hline TE-108-AP-38 & $11 / 4 / 92$ & 69 & CASS & $T$ & \\
\hline TE-10B-AP-38 & $11 / 9 / 92$ & 69 & CASS & $T$ & \\
\hline TE-108-AP-38 & $11 / 17 / 92$ & 71 & CASS & $T$ & \\
\hline TE-108-AP-38 & $11 / 25 / 92$ & 67 & CASS & $T$ & \\
\hline TE-108-AP-38 & $11 / 30 / 92$ & 67 & CASS & $T$ & \\
\hline TE-108-AP-38 & $12 / 12 / 92$ & 67 & CASS & $T$ & \\
\hline TE-108-AP-38 & $12 / 19 / 92$ & 66 & CASS & $T$ & \\
\hline TE-108-AP-38 & $12 / 21 / 92$ & 66 & CASS & $T$ & \\
\hline TE-108-AP-38 & $12 / 27 / 92$ & 65 & CASS & $T$ & \\
\hline TE-10B-AP-38 & $6 / 4 / 93$ & 44 & SACS & $\mathbf{S}$ & Data point did not agree with local trend \\
\hline TE-108-AP-38 & $6 / 11 / 93$ & 63 & SACS & $T$ & \\
\hline TE-108-AP-3B & $6 / 18 / 93$ & 61 & SACS & $T$ & \\
\hline TE-108-AP-3B & $6 / 28 / 93$ & 62 & SACS & $\mathbf{T}$ & \\
\hline TE-108-AP-3B & $7 / 9 / 93$ & 62 & SACS & $T$ & \\
\hline TE-108-AP-3B & $7 / 15 / 93$ & 61 & SACS & $\mathbf{T}$ & \\
\hline TE-108-AP-3B & $7 / 19 / 93$ & 63 & SACS & $\bar{T}$ & \\
\hline$T E-108-A P-3 B$ & $7 / 30 / 93$ & 64. & SACS & $\mathbf{T}$ & \\
\hline TE-108-AP-3B & $8 / 6 / 93$ & 64 & SACS & $T$ & \\
\hline TE-108-AP-38 & $8 / 12 / 93$ & 64) & SACS & $\mathbf{T}$ & \\
\hline TE-108-AP-3B & $9 / 24 / 93$ & 56 & SACS & $\mathbf{S}$ & Data point did not agree with local trend \\
\hline TE-108-AP-3B & $10 / 1 / 93$ & 651 & SACS & $T$ & \\
\hline TE-10B-AP-38 & $10 / 15 / 93$ & 66 & SACS & $\mathbf{T}$ & \\
\hline TE-108-AP-38 & $10 / 22 / 93$ & 64 & SACS & $T$ & \\
\hline TE-108-AP-38 & $10 / 28 / 93$ & 65 & SACS & $\bar{T}$ & \\
\hline TE-108-AP-38 & $11 / 9 / 93$ & 62 & SACS & $\bar{T}$ & \\
\hline TE-108-AP-3B & $11 / 30 / 93$ & 65 & SACS & $\mathbf{T}$ & \\
\hline
\end{tabular}




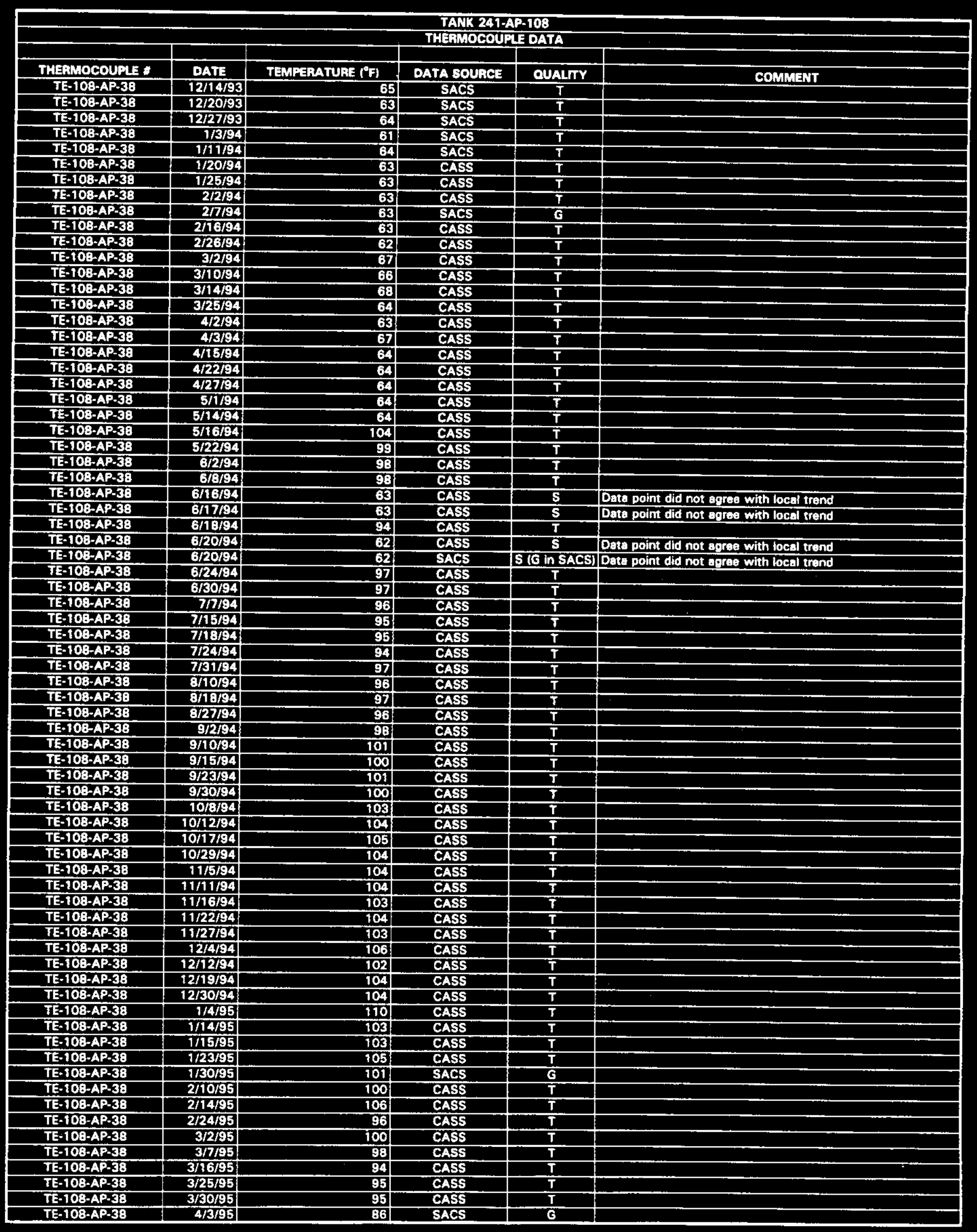




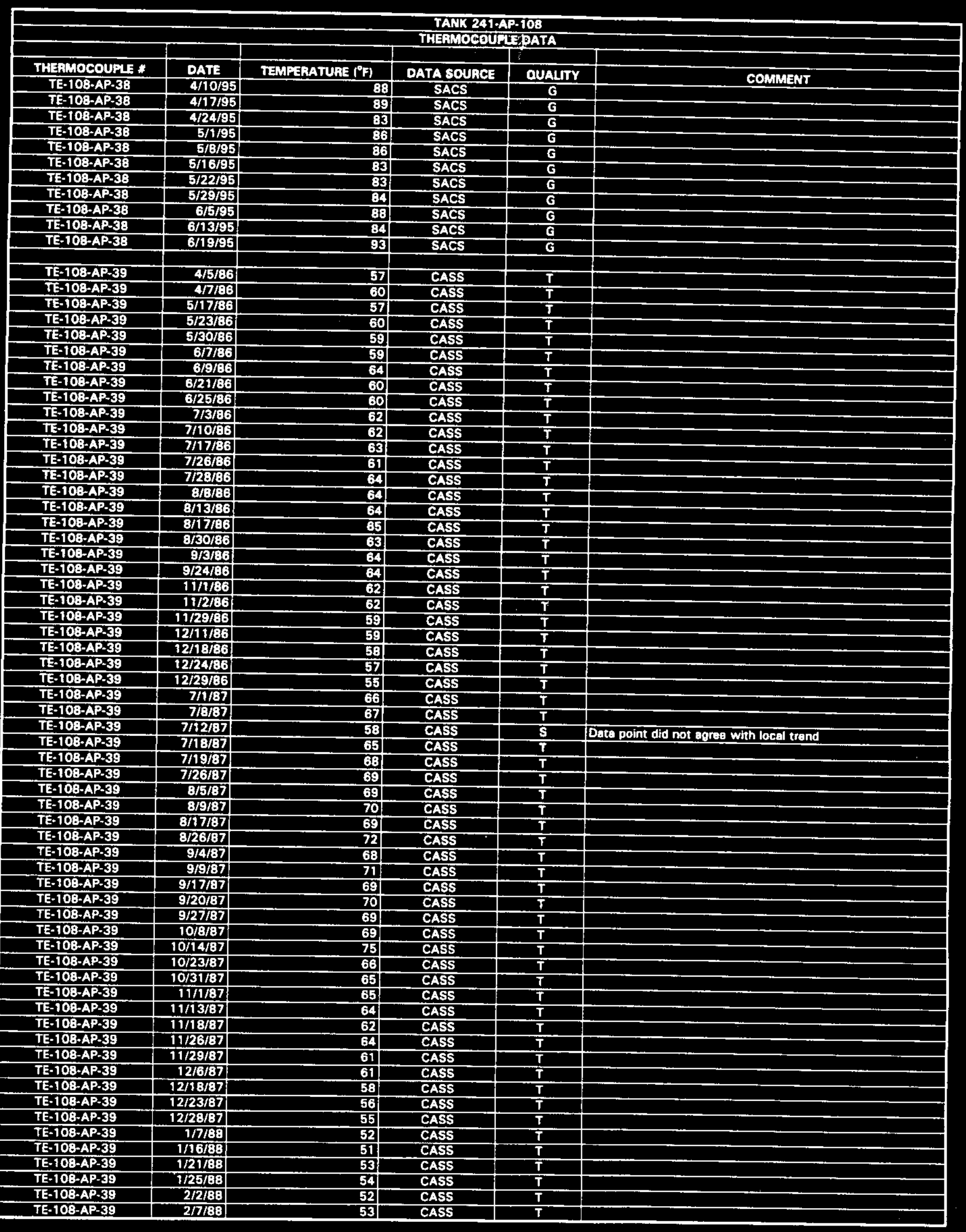

Date obtained from Computer Automated Surveillance System (CASS), April 1995; and Surveillance Analysis Computer System (SACS), June 1995. D- 657 


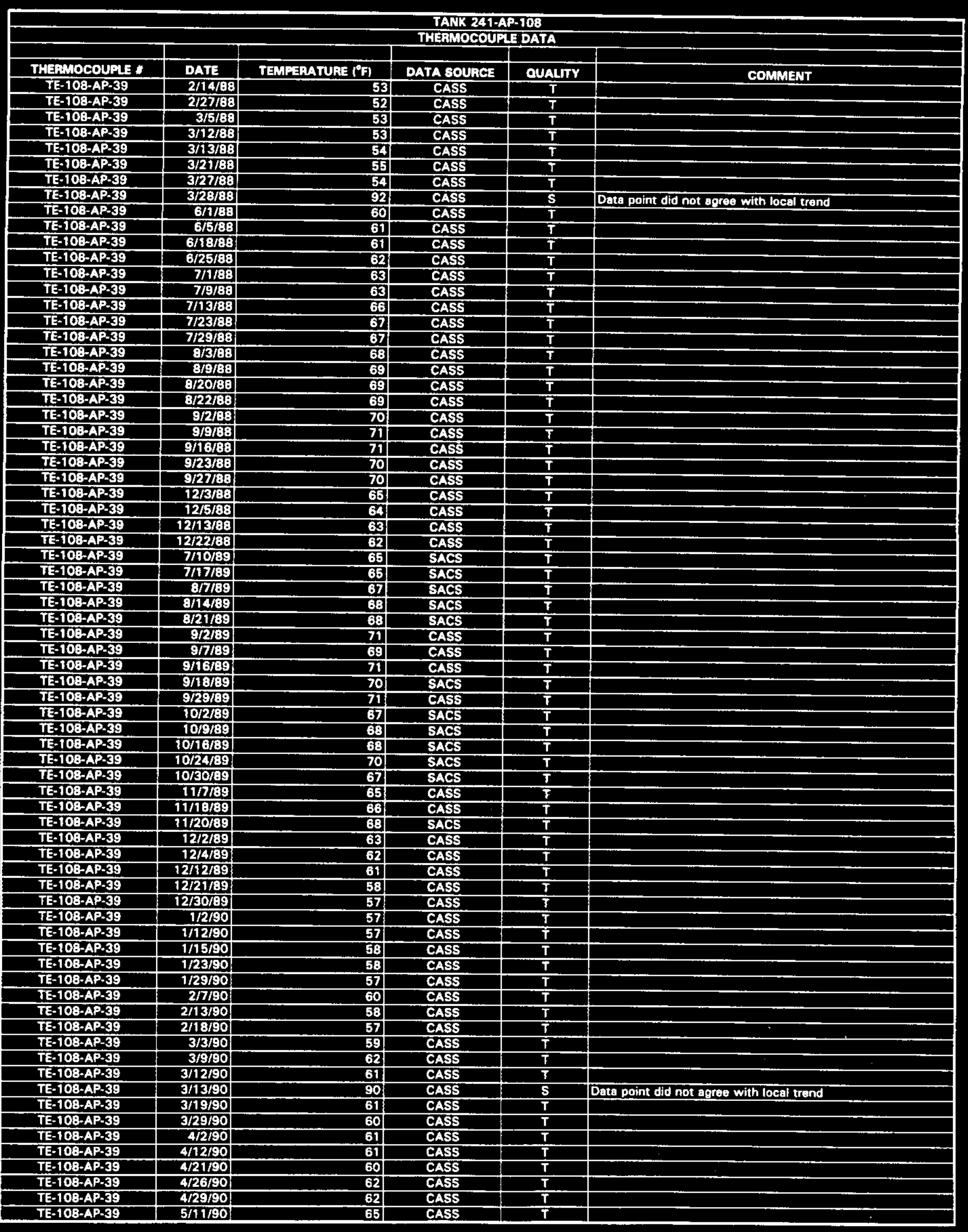




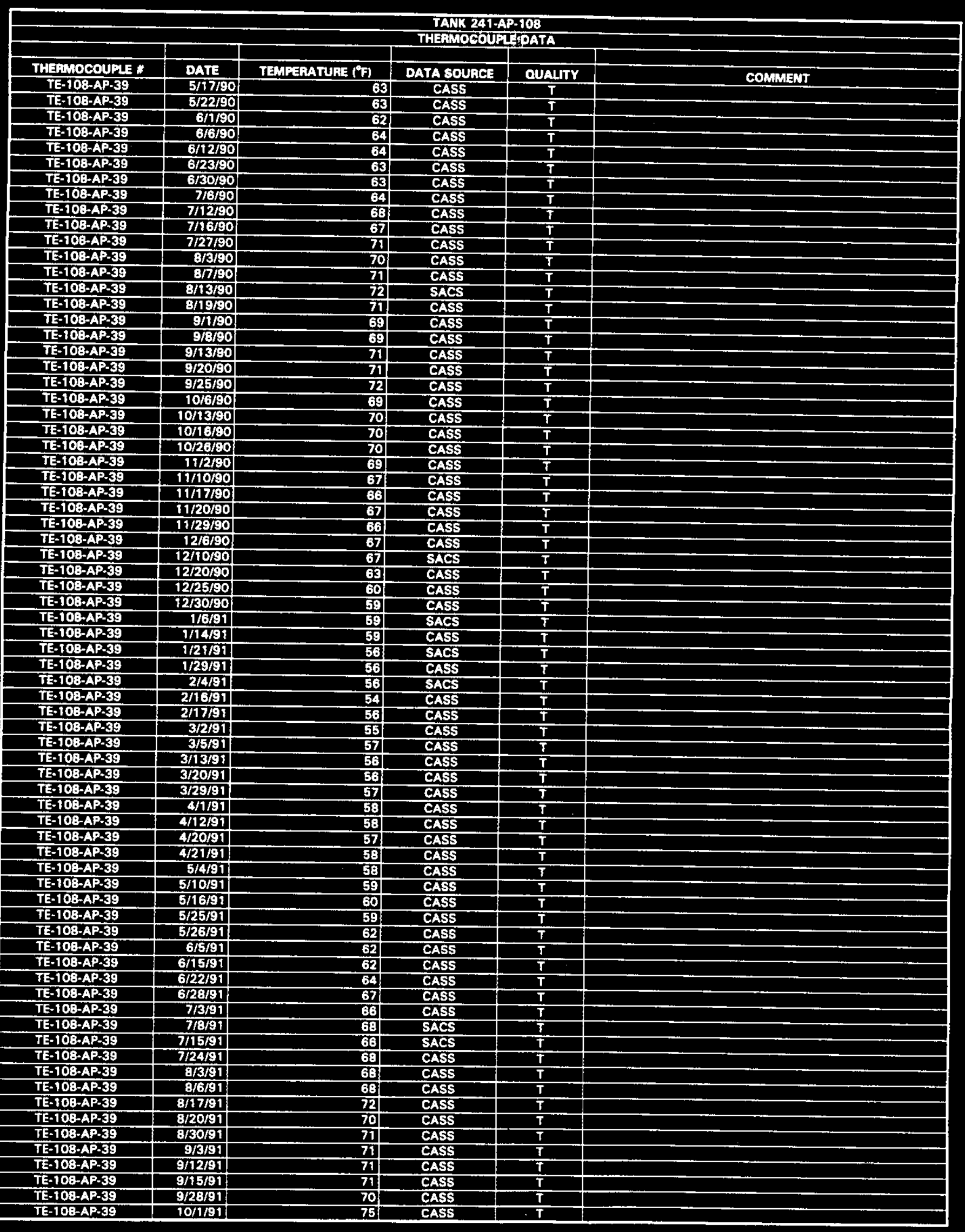

Data obtained from Computer Autometed Surveillance Syatem (CASS), April 1995; and Surveillance Analysis Computer System (SACS), June 1995. 


\begin{tabular}{|c|c|c|c|c|c|}
\hline \multicolumn{6}{|c|}{$\begin{array}{l}\text { TANK 241-AP-108 } \\
\text { THERMOCOUPLE DATA }\end{array}$} \\
\hline & & & & & \\
\hline THEAMOCOUPL * & DATE & TEMPERATUAE $\left({ }^{\circ} \mathrm{F}\right)$ & DATA SOUACE & QUALITY & COMMENT \\
\hline TE-108-AP-39 & $10 / 7 / 91$ & 71 & SACS & $T$ & \\
\hline TE-108-AP-39 & $10 / 8 / 91$ & 61 & CASS & $\mathbf{s}$ & Data point did not agrae with local trend \\
\hline TE-108-AP-39 & $10 / 14 / 91$ & 72 & CASS & $T$ & \\
\hline$T E-108-A P-39$ & $10 / 25 / 93$ & 73 & CASS & $T$ & \\
\hline TE-108-AP-39 & $10 / 28 / 91$ & 70 & CASS & $T$ & \\
\hline TE-108-AP-39 & $11 / 6 / 91$ & 69 & CASS & $T$ & \\
\hline TE-108-AP-39 & $7 / 30 / 92$ & 69 & CASS & $\bar{T}$ & \\
\hline TE-108-AP-39 & $8 / 8 / 92$ & 69 & CASS & $\bar{T}$ & \\
\hline TE-108-AP-39 & $8 / 15 / 92$ & 69 & CASS & $T$ & \\
\hline TE-108.AP-39 & $8 / 22 / 92$ & 68 & CASS & $T$ & \\
\hline TE-108-AP-39 & 8/28/92 & 71 & CASS & $T$ & \\
\hline TE-108-AP-39 & 9/4/92 & 69 & CASS & $\bar{T}$ & \\
\hline TE-108-AP-39 & 9/9/92 & 701 & CASS & $\bar{T}$ & \\
\hline TE-100-AP-39 & 9/6/92 & 69 & CASS & $T$ & \\
\hline TE-108-AP-39 & $9 / 21 / 92$ & 69 & CASS & $T$ & \\
\hline TE-108-AP-39 & $10 / 2192$ & 70 & CASS & $T$ & \\
\hline TE-108-AP-39 & $10 / 0 / 92$ & 68 & CASS & $\frac{1}{T}$ & \\
\hline TE-108-AP-39 & $10 / 13 / 92$ & 69 & CASS & $T$ & \\
\hline TE-108-AP-39 & $10 / 22 / 92$ & 69 & CASS & $T$ & \\
\hline TE-108-AP-39 & $10 / 29 / 92$ & 69 & CASS & $\mathbf{T}$ & \\
\hline TE-10Q-AP-39 & $11 / 7 / 92$ & 68 & CASS & $T$ & \\
\hline TE-10B-AP-39 & $11 / 9 / 92$ & 68: & CASS & $T$ & \\
\hline TE-108-AP-39 & $11 / 17 / 22$ & 70 & CASS & $T$ & \\
\hline TE-108-AP-38 & $11 / 25 / 92$ & 67 & CASS & $T$ & \\
\hline TE-108-AP-39 & $11 / 30 / 92$ & 67 & CASS & $\frac{1}{T}$ & \\
\hline TE-108-AP-39 & $12 / 12 / 92$ & 69 & CASS & $T$ & \\
\hline TE-108-AP-39 & $12119 / 92$ & 66 & CASS & $T$ & \\
\hline TE-108-AP-39 & $12 / 21 / 92$ & 86 & CASS & $T$ & \\
\hline TE-108-AP-39 & $12 / 27 / 32$ & 65 & CASS & $T$ & \\
\hline TE-108-AP-39 & 1/15/94 & 62 & CASS & $\bar{T}$ & \\
\hline TE-10Q-AP-39 & $1 / 19 / 94$ & 64 & CASS & $T$ & \\
\hline TE-108-AP-39 & 1/25/94 & 63 & CASS & $T$ & \\
\hline TE-108-AP-39 & $2 / 2 / 94$ & 63 & CASS & $T$ & \\
\hline TE-108-AP-39 & $2 / 11 / 94$ & 63 & CASS & $T$ & \\
\hline TE-109-AP-39 & $2 / 16 / 94$ & 63 & CASS & $\bar{T}$ & \\
\hline TE-100-AP-39 & $2 / 26 / 94$ & 63 & CASS & $T$ & \\
\hline TE-106-AP-39 & $3 / 5 / 94$ & 6) & CASS & $T$ & \\
\hline TE-108-AP-39 & $310 / 94$ & 66 & CASS & $\bar{T}$ & \\
\hline TE-108-AP-39 & $3 / 14 / 94$ & 68 & CASS & $T$ & \\
\hline TE-108-AP-39 & $3 / 25 / 94$ & 64 & CASS & $T$ & \\
\hline TE-108-AP-39 & $3 / 29 / 94$ & 64 & CASS & $T$ & \\
\hline TE-108-AP-39 & $4 / 8 / 94$ & 64) & CASS & $T$ & \\
\hline TE-106-AP-39 & $4 / 13 / 94$ & 63) & CASS & $T$ & \\
\hline TE-108-AP-39 & $4 / 22 / 94$ & 64 & CASS & $\frac{1}{T}$ & \\
\hline TE-108-AP-39 & $4 / 25 / 94$ & 64 & CASS & $T$ & \\
\hline TE-108-AP-39 & $5 / 2 / 94$ & 64 & CASS & $T$ & \\
\hline TE-108-AP-39 & $5 / 14 / 94$ & 64 & CASS & $T$ & \\
\hline TE-108-AP-39 & $5 / 16 / 94$ & 104 & CASS & $T$ & \\
\hline TE-108-AP-39 & $5 / 22 / 94$ & 99 & CASS & $T$ & \\
\hline TE-108-AP-39 & $6 / 2 / 94$ & 98 & CASS & $T$ & \\
\hline TE-108-AP-39 & $6 / 3 / 94$ & 97 & CASS & $T$ & \\
\hline TE-108-AP-39 & $6 / 14 / 94$ & 95 & CASS & $T$ & \\
\hline TE-108-AP-39 & $6 / 16 / 94$ & 63 & CASS & $\mathbf{s}$ & Data point did not agree with local trend \\
\hline TE-108-AP-39 & $6 / 17 / 144$ & 63 & CASS & $\mathbf{s}$ & Data point did not agree with local trend \\
\hline TE-108-AP-39 & $6 / 20 / 94$ & 62 & CASS & 5 & Data point did not agree with locel trend \\
\hline TE-108-AP-39 & $6 / 25 / 94$ & 96 & CASS & $\bar{T}$ & \\
\hline TE-102-AP-39 & $6 / 27 / 94$ & 98 & CASS & $\mathbf{T}$ & \\
\hline TE-108-AP-39 & $2 m 7 / 94$ & 96 & CASS & $T$ & \\
\hline TE-108-AP-39 & $7 / 15 / 94$ & 95 & CASS & $T$ & \\
\hline TE-108-AP-39 & $7 \longdiv { 1 8 / 9 4 }$ & 85 & CASS & $T$ & \\
\hline TE-108-AP-39 & $7 / 24 / 94$ & 93 & CASS & $T$ & \\
\hline TE-108-AP-39 & $7 / 31 / 94$ & 96 & CASS & $\mathbf{T}$ & \\
\hline TE-108-AP-39 & $8 / 9 / 94$ & 96 & CASS & $\bar{T}$ & \\
\hline TE-108-AP-39 & $8 / 18 / 94$ & 97 & CASS & $T$ & \\
\hline TE-108-AP-39 & $8 / 22 / 94$ & 96 & CASS & $\mathbf{T}$ & \\
\hline TE-108-AP-39 & $9 / 2 / 94$ & 97 & CASS & $T$ & \\
\hline TE-108-AP-39 & $9 / 10 / 94$ & 101 & CASS & $T$ & \\
\hline TE-108-AP-39 & $9 / 15 / 94$ & 100 & CASS & $\mathbf{T}$ & \\
\hline TE-108-AP-39 & $9 / 24 / 94$ & 100 & CASS & $\mathbf{T}$ & \\
\hline TE-108-AP-39 & $9 / 30 / 94$ & 100 & CASS & $\bar{T}$ & \\
\hline TE-108-AP-39 & $10 / 2 / 94$ & 104 & CASS & $\bar{T}$ & \\
\hline TE-108-AP-39 & $10 / 12 / 34$ & 104 & CASS & $T$ & \\
\hline TE-108-AP-39 & $10 / 17 / 94$ & 105 & CASS & $T$ & \\
\hline
\end{tabular}




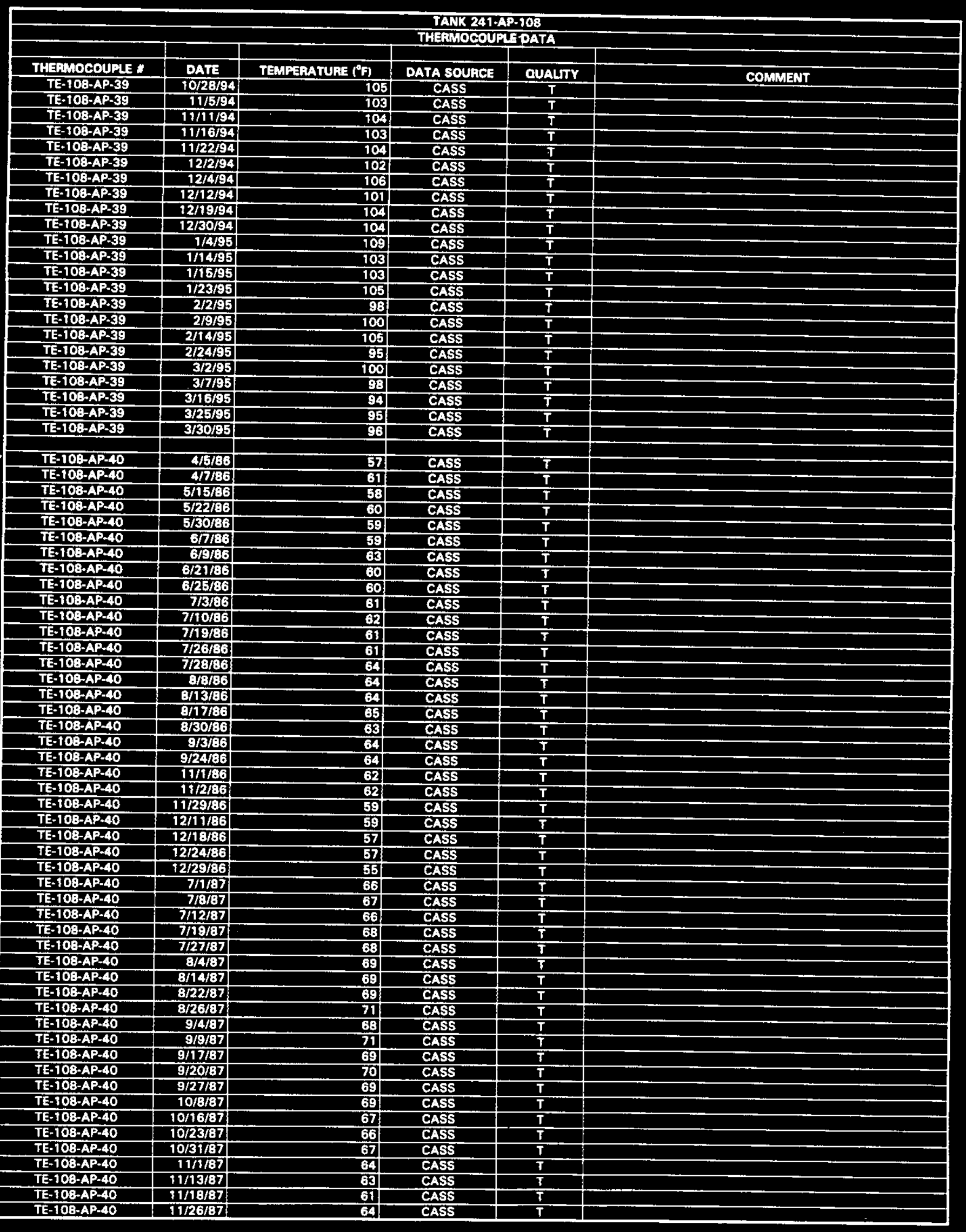

Data obtainad from Computer Automated Survaillance Syatem (CASS), April 1995; and Surveillance Analyais Computer Syatem (SACS), June 1995.

D-661 


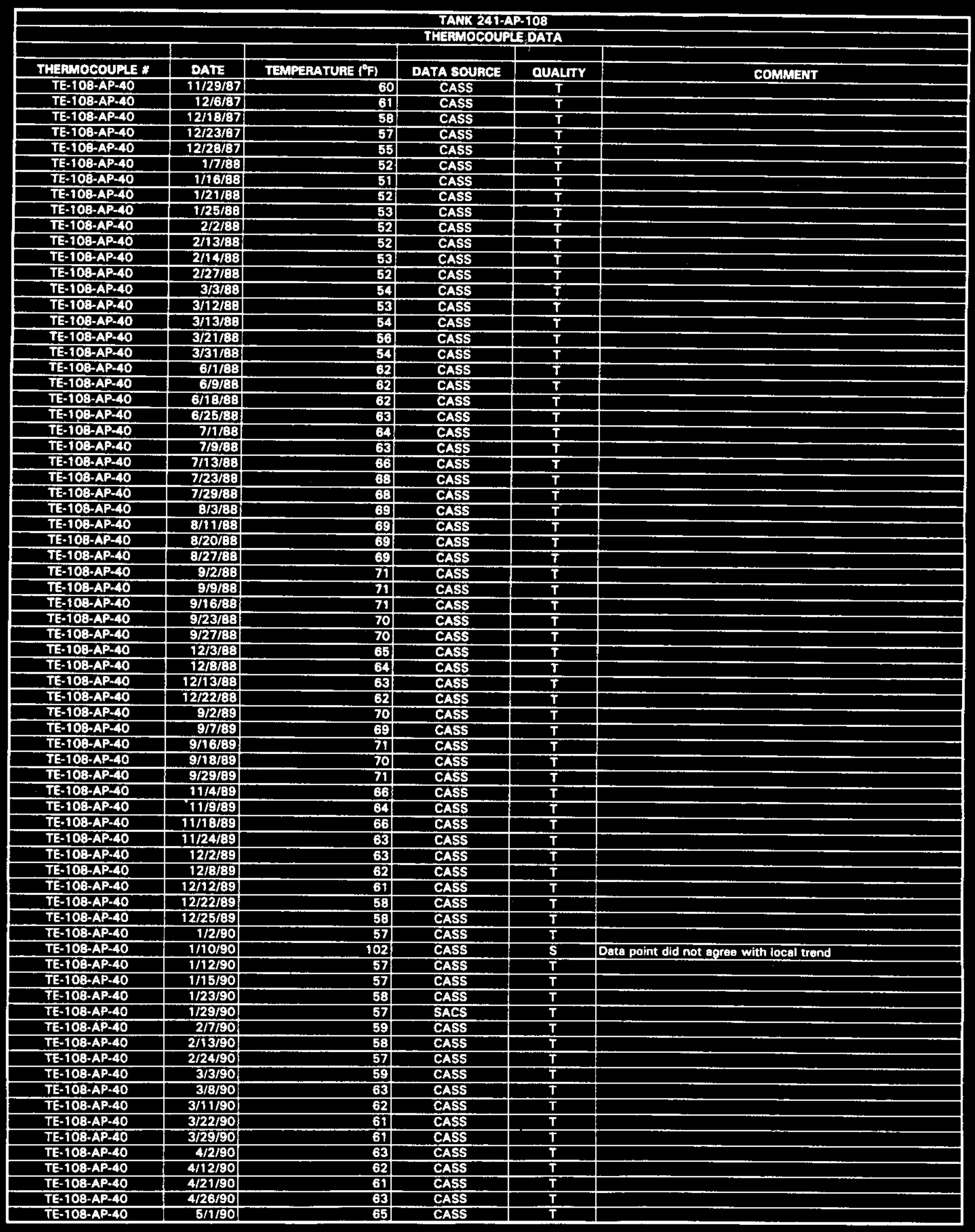




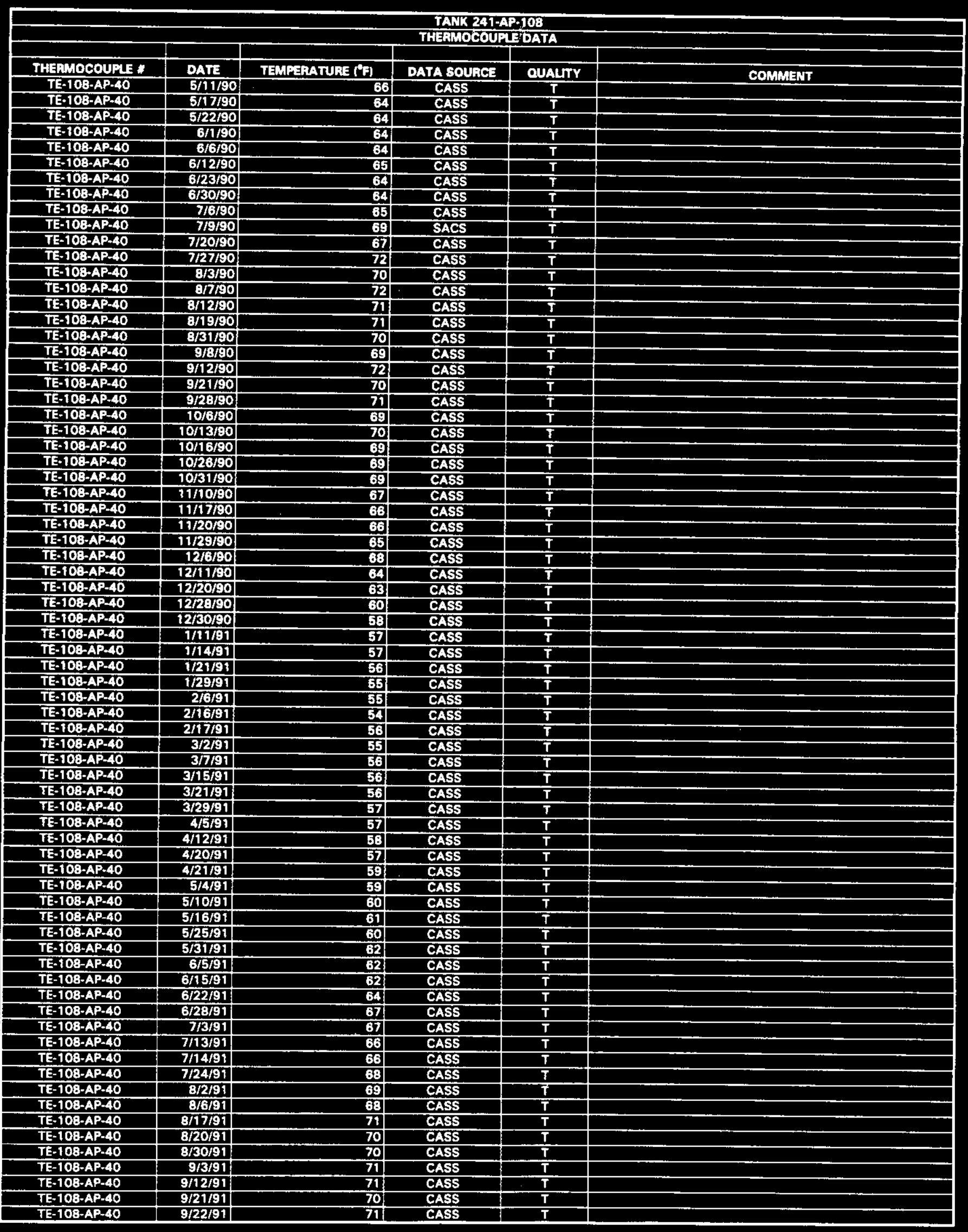

Data obtained trom Computer Automated Surveillance System (CASS), April 1995; and Surveillance Analysis Computer System (SACS), June 1995.

D- 663 


\begin{tabular}{|c|c|c|c|c|c|}
\hline \multicolumn{6}{|c|}{$\begin{array}{l}\text { TAKK 241-AP-108 } \\
\text { THERMOCOUPL DATA }\end{array}$} \\
\hline & & & & & \\
\hline THERMOCOUPLE * & DATE & TEMPERATUAE $\left.{ }^{\circ} \mathrm{F}\right)$ & DATA SOURCE & OUALTYY & COMMENT \\
\hline TE-108-AP-40 & $10 / 1 / 91$ & 73 & CASS & $T$ & \\
\hline TE-108-AP-40 & $10 / 8 / 91$ & 61 & CASS & $\mathbf{s}$ & Data point did not agree with local trend \\
\hline$T E-108-A P-40$ & $10 / 12 / 91$ & 70 & CAASS & $T$ & \\
\hline TE-10B-AP-40 & $10 / 16 / 91$ & 72 & CASS & $T$ & \\
\hline TE-108-AP-40 & $10 / 25 / 91$ & 74 & CASS & $T$ & \\
\hline TE-108-AP-40 & $10 / 28 / 91$ & 71 & CASS & $T$ & \\
\hline TE-108-AP-40 & $11 / 6 / 91$ & 68 & CASS & $T$ & \\
\hline$T E-108-A P-40$ & $7 / 30 / 92$ & 68 & CASS & $T$ & \\
\hline TE-108-AP-AO & $8 / 8 / 92$ & 68 & CASS & $T$ & \\
\hline TE-108-AP-40 & $8 / 75 / 92$ & 68 & CASS & $\mathbf{T}$ & \\
\hline TE-108-AP-40 & $8 / 22 / 92$ & 67 & CASS & $\bar{T}$ & \\
\hline TE-10B-AP-40 & $8 / 28 / 92$ & 68 & CASS & $T$ & \\
\hline TE-108-AP-40 & $9 / 5 / 92$ & 67 & CASS & $T$ & \\
\hline TE-108-AP-40 & 9/12/92 & 68 & CASS & $T$ & \\
\hline TE-10Q-AP-40 & $9 / 16 / 92$ & 69 & CASS & $\mathbf{T}$ & \\
\hline TE-108-AP-40 & $9 / 23 / 92$ & 68 & CASS & $T$ & \\
\hline TE-108-AP-40 & $10 / 2 / 92$ & 69 & CASS & $T$ & \\
\hline$T E-108-A P-40$ & $10 / 10 / 92$ & 67 & CASS & $T$ & \\
\hline TE-108-AP-AO & $10 / 13 / 92$ & 68 & CASS & $T$ & \\
\hline TE-108-AP-40 & $10 / 24 / 92$ & 68 & CASS & $T$ & \\
\hline TE-108-AP-40 & $10 / 29 / 92$ & 68 & CASS & $\mathbf{T}$ & \\
\hline TE-10B-AP-40 & $11 / 7 / 92$ & 69 & CASS & $\bar{T}$ & \\
\hline TE-108-AP-40 & $11 / 9 / 92$ & 6 & CASS & $\mathbf{T}$ & \\
\hline TE-108-AP-40 & $11 / 17 / 92$ & 70 & CASS & $T$ & \\
\hline TE-108-AP-AO & $11 / 28 / 92$ & 67 & CASS & $T$ & \\
\hline TE-108-AP-40 & $12 / 3 / 2 / 2$ & 67 & CASS & $T$ & \\
\hline TE-108-AP-40 & $12 / 12 / 92$ & 69 & CASS & $T$ & \\
\hline TE-108-AP-40 & $12 / 17 / 92$ & 67 & CASS & $\bar{T}$ & \\
\hline TE-108-AP-40 & $12 / 21 / 92$ & 66 & SACS & $T$ & \\
\hline TE-108-AP-40 & 1212792 & 66 & CASS & $T$ & \\
\hline TE-108-AP-40 & $6 / 4 / 93$ & 43 & SACS & $\mathbf{s}$ & Data point did not agree with local trend \\
\hline TE-108-AP-40 & $6 / 11 / 93$ & 61 & SACS & $T$ & \\
\hline TE-108-AP-40 & $6 / 18 / 93$ & 60 & SACS & $T$ & \\
\hline TE-108.AP-40 & 8/28/93 & 61 & SACS & $T$ & \\
\hline TE-108-AP-40 & $7 / 9 / 93$ & 61 & SACS & $T$ & \\
\hline TE-108-AP-40 & $7 / 15 / 93$ & 61 & SACS & $T$ & \\
\hline TE-108-AP-40 & $7 / 19 / 93$ & 62 & SACS & $T$ & \\
\hline TE-10B-AP-40 & $7 / 30 / 93$ & 63 & SACS & $\mathbf{T}$ & \\
\hline TE-108-AP-40 & $8 / 6 / 93$ & 62 & SACS & $T$ & \\
\hline TE-108-AP-40 & $8 / 12 / 93$ & 62 & SACS & $T$ & \\
\hline TE-10B-AP-40 & $9 / 24 / 93$ & 58 & SACS & $\mathbf{T}$ & \\
\hline TE-108-AP-40 & $10 / 1 / 93$ & 64 & SACS & $T$ & \\
\hline TE-108-AP-40 & $10 / 15 / 93$ & 65 & SACS & $\mathbf{T}$ & \\
\hline TE-108-AP-40 & $10 / 22 / 93$ & 64 & SACS & $\mathbf{T}$ & \\
\hline TE-108-AP-40 & $10 / 28 / 93$ & 66 & SACS & $T$ & \\
\hline TE-108-AP-40 & $11 / / 9 / 93$ & 61 & SACS & $T$ & \\
\hline TE-108-AP-40 & $11 / 30 / 93$ & 64 & SACS & $T$ & \\
\hline TE-108-AP-40 & $12 / 14 / 93$ & 66 & SACS & $T$ & \\
\hline TE-106-AP-40 & $12 / 20 / 93$ & 63 & SACS & $T$ & \\
\hline TE-108-AP-40 & $12 / 27 / 93$ & 65 & SACS & $T$ & \\
\hline$T E=10 \mathrm{~B}-\mathrm{AP}=40$ & $1 / 3 / 94$ & 62 & SACS & $T$ & \\
\hline TE-10QAP-40 & $1 / 11 / 94$ & 66 & SACS & $T$ & \\
\hline TE-108-AP-40 & $1 / 20 / 94$ & 64) & CASS & $T$ & \\
\hline TE-108-AP-40 & $1 / 25 / 94$ & 63 & CASS & $\mathbf{T}$ & \\
\hline TE-108-AP-40 & $2 / 4 / 94$ & 63 & CASS & $T$ & \\
\hline TE-108-AP-40 & $2 / 7 / 94$ & 66 & SACS & $\mathbf{G}$ & \\
\hline TE-108-AP-40 & $2 / 16 / 94$ & 63 & CASS & $T$ & \\
\hline TE-108-AP-40 & $2 / 26 / 94$ & 64 & CASS & $T$ & \\
\hline TE-108-AP-40 & $3 / 5 / 94$ & 66 & CASS & $T$ & \\
\hline$T E=108-A P+40$ & $3 / 10 / 94$ & 66 & CASS & $T$ & \\
\hline TE-108-AP-40 & 3/17/94 & 65 & CASS & $\mathbf{T}$ & \\
\hline TE-10B-AP-40 & $3 / 25 / 94$ & 65 & CASS & $\bar{T}$ & \\
\hline TE-108.AP.40 & $3 / 29 / 94$ & 64 & CASS & $T$ & \\
\hline TE-108-AP-40 & $4 / 9 / 94$ & 64 & CASS & $T$ & \\
\hline TE-108-AP-40 & $4 / 11 / 94$ & 64 & SACS & $\mathbf{G}$ & \\
\hline TE-108-AP-40 & $4 / 22 / 94$ & 64 & CASS & $T$ & \\
\hline TE-108-AP-40 & $4 / 27 / 94$ & 64 & CASS & $T$ & \\
\hline TE-108-AP-40 & $5 / 1 / 94$ & 64 & CASS & $T$ & \\
\hline TE-108-AP-40 & $5 / 14 / 94$ & 64 & CASS & $T$ & \\
\hline TE-10B-AP-40 & $5 / 16 / 94$ & 104 & CASS & $T$ & \\
\hline TE-10B-AP-40 & $5 / 22 / 94$ & 99 & CASS & $T$ & \\
\hline TE-108-AP-40 & $6 / 2 / 94$ & 89 & CASS & $T$ & \\
\hline TE-108-AP-40 & 6/8/94 & 97 & CASS & $T$ & \\
\hline
\end{tabular}




\begin{tabular}{|c|c|c|c|c|c|}
\hline \multicolumn{6}{|c|}{$\begin{array}{l}\text { TANK 241-AP-108 } \\
\text { THEFMOCOUPLE DATA }\end{array}$} \\
\hline & & & & & \\
\hline THERMOCOUPLE \# & DATE & TEMPERATURE ( $\left.{ }^{\circ} \mathrm{F}\right)$ & DATA SOURCE & OUALITY & COMMENT \\
\hline TE-10B-AP-40 & 6/16/94 & 63 & CASS & $\overline{\mathbf{S}}$ & Data point did not agree with local trend \\
\hline TE-108-AP-40 & 6/17/94 & 63 & CASS & $\mathbf{S}$ & Data point did not agree with local trend \\
\hline TE-108-AP.40 & $6 / 18 / 94$ & 94! & CASS & $\mathbf{T}$ & \\
\hline TE-108-AP-40 & $6 / 20 / 94$ & 62 & CASS & $\mathbf{S}$ & Data point did not agree with local trand \\
\hline TE-108-AP-40 & $6 / 20 / 94$ & 62 & SACS & S [O in SACS & Data point did not agree with local trend \\
\hline TE-108-AP-40 & $6 \longdiv { 2 4 / 9 4 }$ & 97 & CASS & $T$ & \\
\hline TE-108-AP-40 & 6/27/94 & 98: & CASS & $T$ & \\
\hline TE-10B-AP-40 & $7 / 7 / 194$ & 96 & CASS & $T$ & \\
\hline TE-108-AP-40 & $7 / 15 / 94$ & 95 & CASS & $T$ & \\
\hline TE-108-AP-40 & $7 / 20 / 94$ & 93 & CASS & $\mathbf{T}$ & \\
\hline TE-10B-AP-40 & $7 / 24 / 94$ & 93 & CASS & $\mathbf{T}$ & \\
\hline TE-108-AP-40 & $7 / 31 / 94$ & 96 & CASS & $\mathbf{T}$ & \\
\hline TE.108-AP-40 & 8/10/84 & 85 & CASS & $\bar{T}$ & \\
\hline TE-108-AP-40 & $8 / 18 / 94$ & 97. & CASS & $T$ & \\
\hline$T E-108-A P-40$ & $8 / 21 / 94$ & 96 & CASS & $\mathbf{T}$ & \\
\hline IE-108-AP-40 & $9 / 3 / 94$ & 96 & CASS & $T$ & \\
\hline TE-108-AP-40 & $9 / 10 / 94$ & 100 & CASS & $T$ & \\
\hline TE-108-AP-40 & $9 / 15 / 94$ & 991 & CASS & $T$ & \\
\hline TE-108-AP-40 & $9 / 23 / 94$ & 100 & CASS & $T$ & \\
\hline TE-10B-AP-40 & $9 / 30 / 94$ & 100 & CASS & $T$ & \\
\hline TE- $108-A P-40$ & $10 / 2 / 94$ & 103 & CASS & $T$ & \\
\hline TE-10B-AP-40 & $10 / 15 / 94$ & 103 & CASS & 7 & \\
\hline TE-108-AP-40 & $10 / 17 / 94$ & 305 & CASS & $T$ & \\
\hline TE-108-AP-40 & $10 / 25 / 94$ & 104 & CASS & $T$ & \\
\hline TE-10B-APACO & $11 / 5 / 94$ & 103 & CASS & $\bar{T}$ & \\
\hline TE-108-AP-40 & $11 / 11 / 94$ & 105 & CASS & $\bar{T}$ & \\
\hline TE-10B-AP-40 & $11 / 16 / 94$ & 102 & CASS & $\mathbf{T}$ & \\
\hline TE-10B-AP-40 & $11 / 22 / 94$ & 104 & CASS & $T$ & \\
\hline TE-108-AP-40 & $12 / 2 / 94$ & 102 & CASS & $T$ & \\
\hline TE-108-AP-40 & $12 / 4 / 94$ & 106 & CASS & $\bar{T}$ & \\
\hline TE-108-AP-40 & $12 / 12 / 94$ & 101 & CASS & $\mathbf{T}$ & \\
\hline$T E-10 B-A P=40$ & $12 / 19 / 94$ & 104 & CASS & $T$ & \\
\hline TE-108-AP-40 & $12 / 30 / 94$ & 104 & CASS & $T$ & \\
\hline TE-108-AP-40 & $1 / 4 / 95$ & 109 & CASS & $T$ & \\
\hline TE-108-AP-40 & 1/14/95 & 103 & CASS & $T$ & \\
\hline TE-108-AP-40 & $1 / 15 / 95$ & 103 & CASS & $T$ & \\
\hline TE-108-AP-40 & $1 / 23 / 95$ & 105 & CASS & $T$ & \\
\hline TE-108-AP-40 & $1 / 30 / 95$ & 100 & SACS & $\mathbf{G}$ & \\
\hline TE-108-AP-40 & $2 / 9 / 95$ & 100 & CASS & $T$ & \\
\hline TE-108-AP-40 & $2 / 14 / 95$ & 105 & CASS & $T$ & \\
\hline TE-108-AP-40 & $2 / 24 / 96$ & 95 & CASS & $T$ & \\
\hline TE-108-AP-40 & $3 / 2 / 95$ & 100 & CASS & $T$ & \\
\hline TE-108-AP-40 & 37195 & 88 & CASS & $T$ & \\
\hline TE-109.AP-40 & $3 / 16 / 95$ & 94 & CASS & $T$ & \\
\hline TE-10B-AP-40 & $3 / 25 / 95$ & 94 & CASS & $T$ & \\
\hline TE-108-AP-40 & $3 / 30 / 95$ & 95 & CASS & $T$ & \\
\hline TE-10Q-AP-40 & $4 / 3 / 95$ & 86 & SACS & G & \\
\hline TE-108-AP-40 & $4 / 10 / 95$ & 90 & SACS & G & \\
\hline TE-108-AP-40 & $4 / 17 / 95$ & 87 & SACS & G & \\
\hline TE-108-AP-40 & $4 / 24 / 95$ & 83 & SACS & $\bar{G}$ & \\
\hline$T E-108-A P-40$ & $5 / 1 / 95$ & 85 & SACS & G & \\
\hline TE-108-AP-40 & 5/8/95 & B7 & SACS & $G$ & \\
\hline$T E-108-A P-40$ & 5/16/95 & B4 & SACS & $G$ & \\
\hline TE-108-AP-40 & 5/22/95 & 84 & SACS & 6 & \\
\hline TE-108-AP-40 & 5/29/95 & 84 & SACS & G & \\
\hline TE-108-AP-40 & $6 / 5 / 95$ & BB & SACS & $\bar{G}$ & \\
\hline TE-108-AP-40 & $6 / 13 / 95$ & 84 & SACS & $\mathbf{G}$ & \\
\hline TE-108-AP-40 & 6/19/95 & 93 & SACS & $\mathbf{G}$ & \\
\hline TE-108-AP-41 & $4 / 5 / 86$ & 57 & CASS & $T$ & \\
\hline TE-108-AP-41 & $4 / 7 / 86$ & 60 & CASS & $\frac{1}{T}$ & \\
\hline TE-108-AP-41 & $5 / 16 / 86$ & 58 & CASS & $T$ & \\
\hline TE-108-AP-41 & $5 / 24 / 86$ & 60 & CASS & $T$ & \\
\hline TE-108-AP-41 & $5 / 25 / 86$ & 60 & CASS & $T$ & \\
\hline TE-108-AP-41 & $677 / 86$ & 59 & CASS & $\mathbf{T}$ & \\
\hline TE-108-AP-41 & $6 / 9 / 86$ & 64) & CASS & $T$ & \\
\hline TE-108-AP-41 & $6 / 21 / 86$ & 60 & CASS & $T$ & \\
\hline TE-108-AP-41 & 6/25/36 & 60 & CASS & $T$ & \\
\hline TE-108-AP-4T & $7 / 5 / 86$ & 61 & CASS & $\mathbf{T}$ & \\
\hline TE-108-AP-41 & $7 / 10 / 86$ & 62 & CASS & $\mathbf{T}$ & \\
\hline TE-108-AP-41 & 7/17/86 & 62 & CASS & $T$ & \\
\hline TE-108-AP-41 & 7/26/B6 & 62 & CASS & $T$ & \\
\hline TE-108-AP-41 & 7/28/86 & 64 & CASS & $\mathbf{T}$ & \\
\hline
\end{tabular}




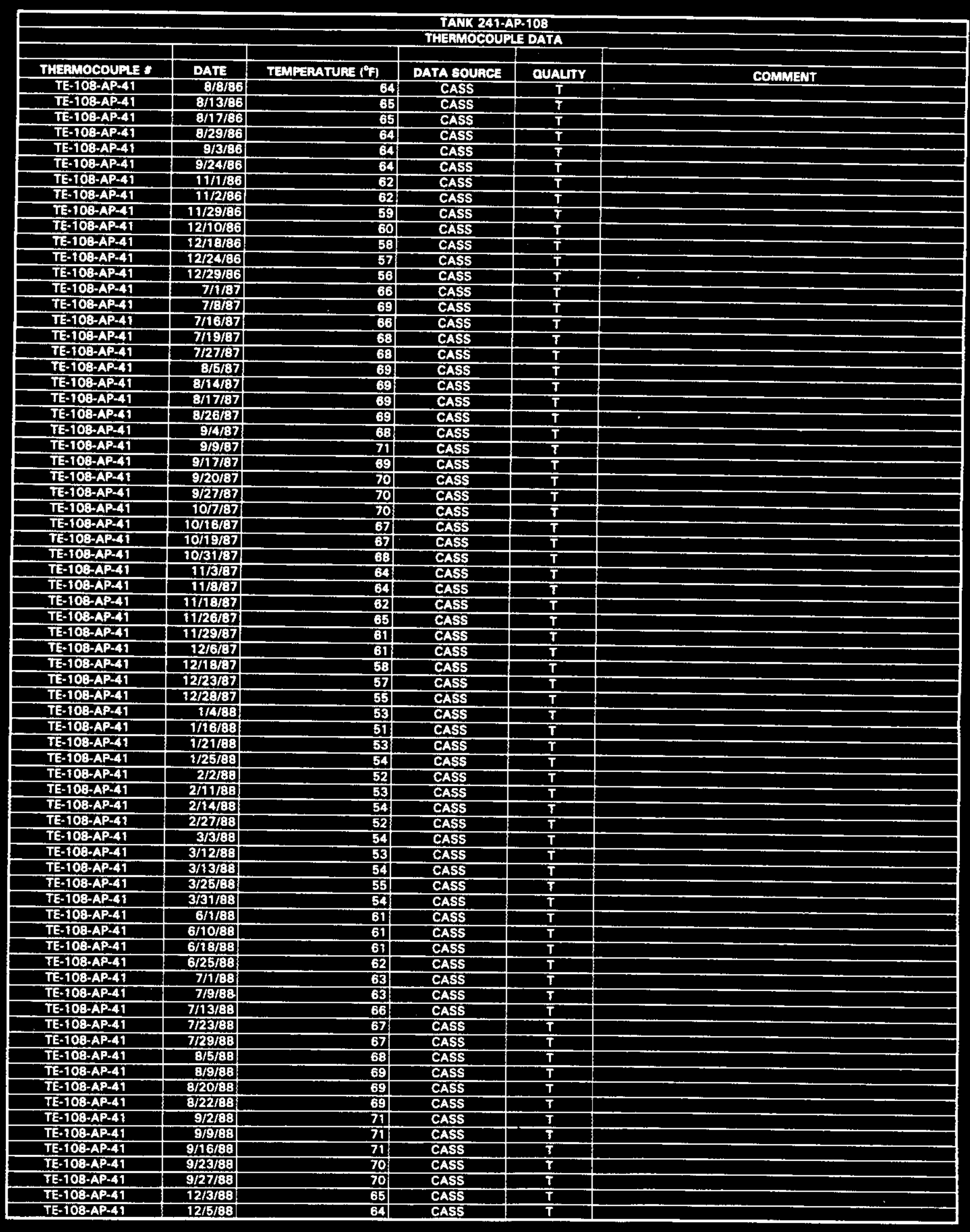

Date obteined from Computer Automated Surveillance System (CASS), April 1995; and Surveillence Analyais Computer System (SACS), June 1995. D- 666 


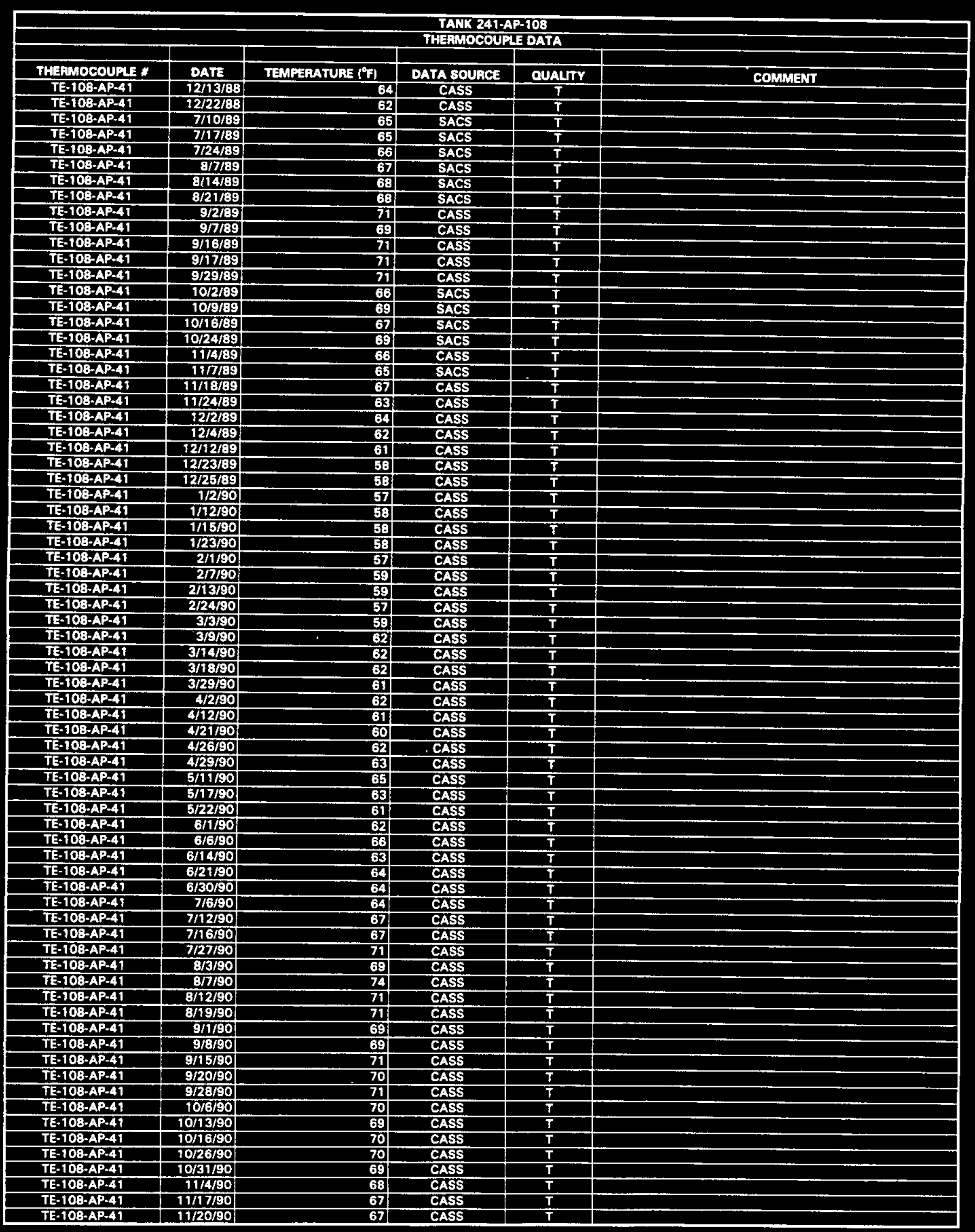




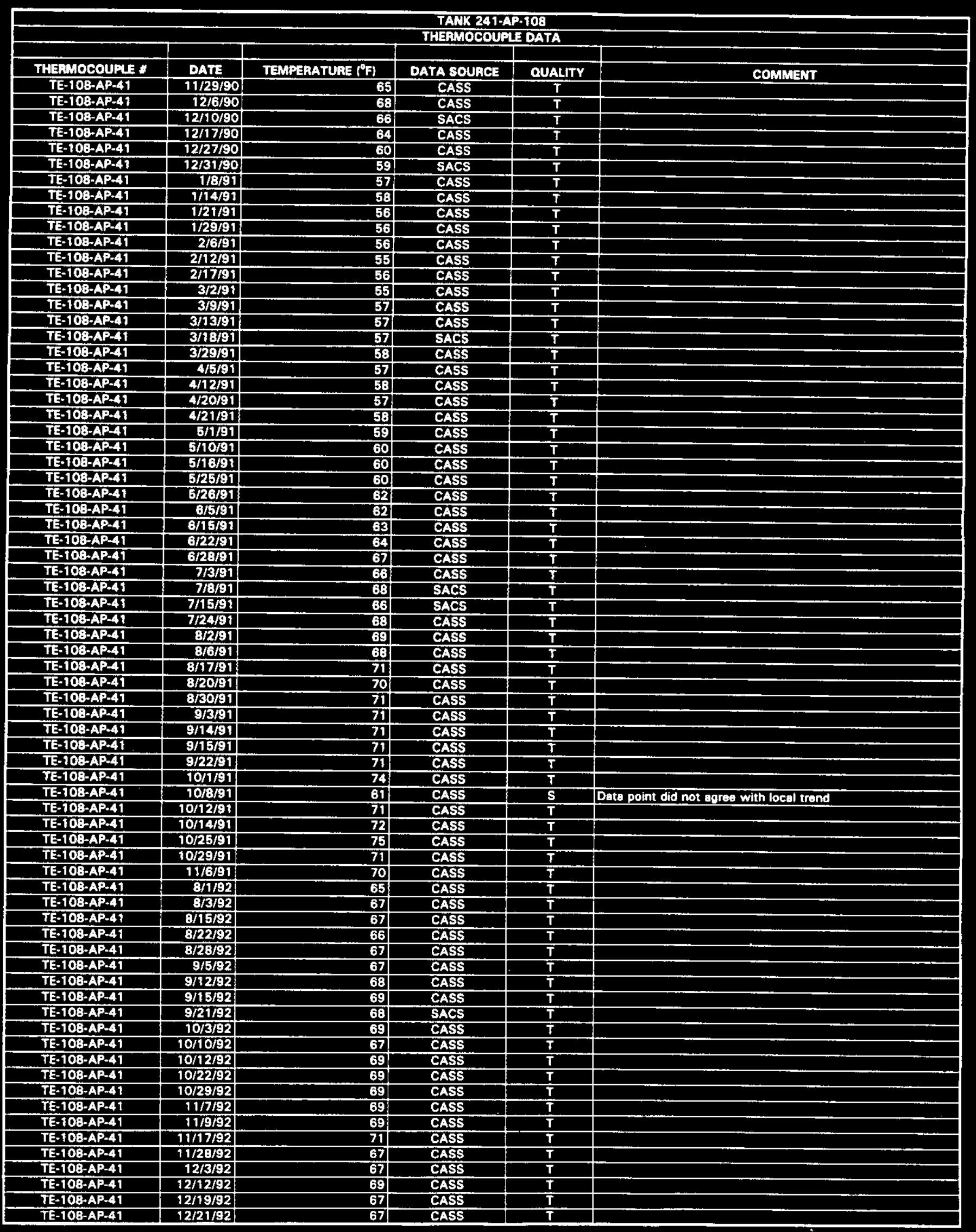




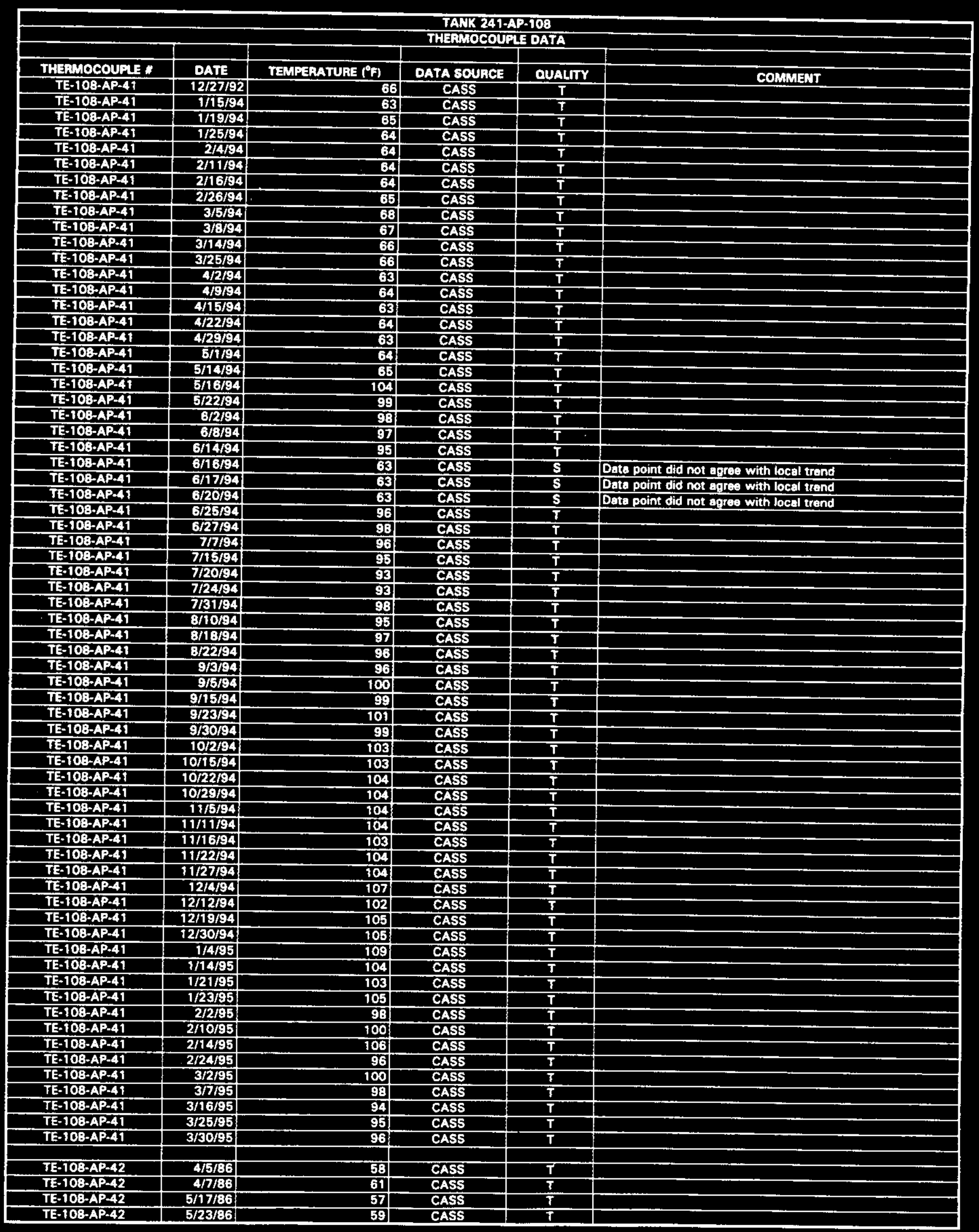




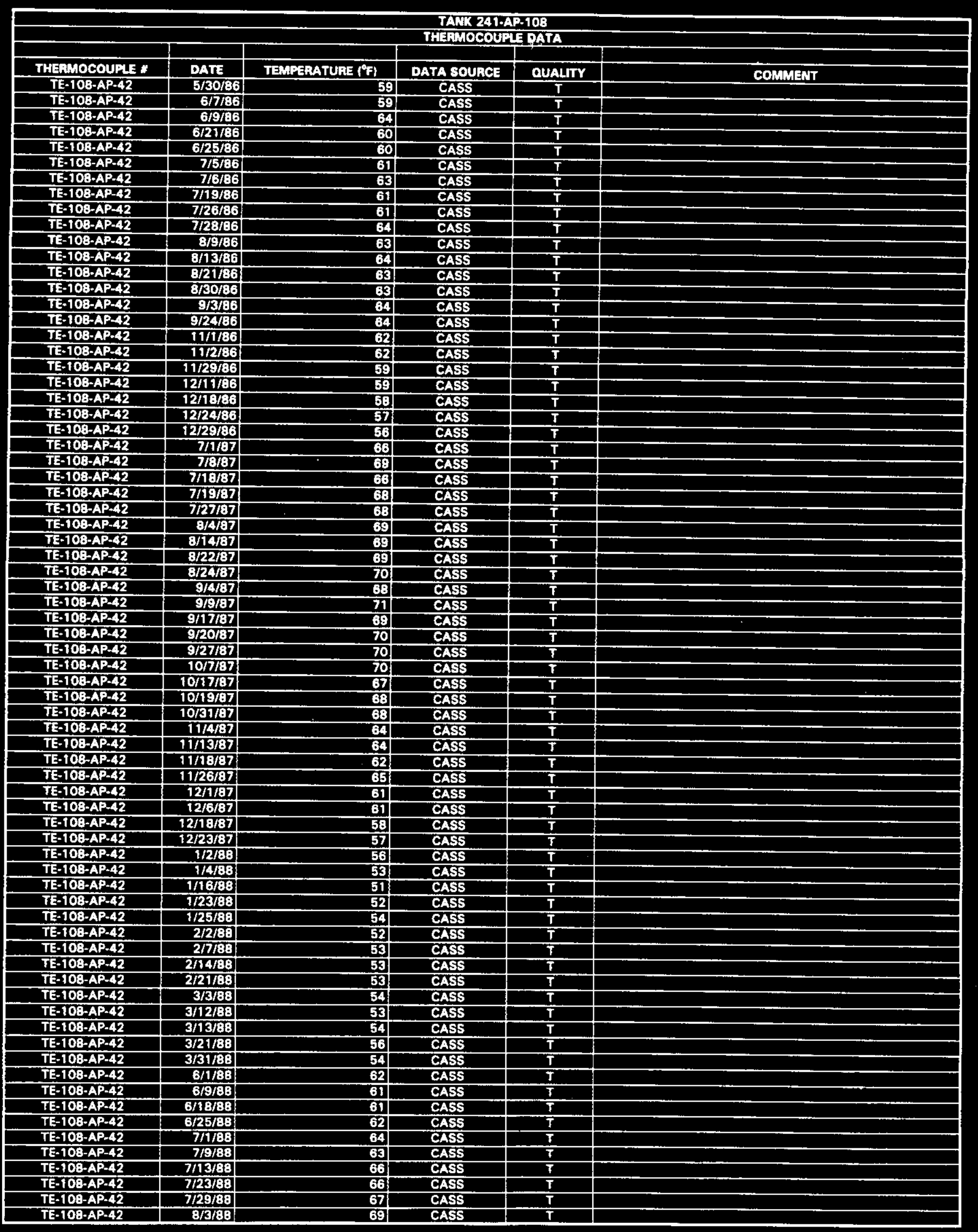




\begin{tabular}{|c|c|c|c|c|c|}
\hline \multicolumn{6}{|c|}{$\begin{array}{l}\text { TAMK 241-AP-108 } \\
\text { THEAMOCOUFTE DATA }\end{array}$} \\
\hline & & & & & \\
\hline THERMOCOUPLE * & DATE & TEMPERATUAE $\left({ }^{\circ} \mathrm{F}\right)$ & DATA SOURCE & QUALITY & COMMENT \\
\hline TE-108-AP-42 & $8 / 9 / 88$ & 69 & CASS & $T$ & \\
\hline TE-108-AP-42 & $8 / 20 / 88$ & 69 & CASS & $T$ & \\
\hline TE-108-AP-42 & $8 / 27 / 88$ & 68 & CASS & $T$ & \\
\hline TE-108-AP-42 & $9 / 2 / 88$ & 71 & CASS & $T$ & \\
\hline TE-108-AP-42 & 9/9/8B & 71 & CASS & $T$ & \\
\hline TE- $108-A P=42$ & $9 / 16 / 88$ & 71 & CASS & $T$ & \\
\hline$T E-108-A P-42$ & $9 / 23 / 88$ & 69 & CASS & $T$ & \\
\hline TE-108-AP-42 & $9 / 27 / 88$ & 70 & CASS & $T$ & \\
\hline TE-108-AP-42 & $12 / 3 / 88$ & 65 & CASS & $\bar{T}$ & \\
\hline TE-108-AP-42 & $12 / 5 / 88$ & 64 & CASS & $T$ & \\
\hline TE-10B-AP-42 & $12 / 16 / 88$ & 63 & CASS & $\bar{T}$ & \\
\hline TE-108-AP-42 & $12 / 22 / 88$ & 62 & CASS & $\mathbf{T}$ & \\
\hline TE-108-AP-42 & $9 / 2 / 89$ & 72 & CASS & $T$ & \\
\hline TE-108-AP-42 & 8/7/39 & 69 & CASS & $T$ & \\
\hline TE-108-AP-42 & $9 / 16 / 89$ & 71 & CASS & $T$ & \\
\hline TE-10D-AP-42 & $9 / 17 / 89$ & 71 & CASS & $T$ & \\
\hline TE-108-AP-42 & $9 / 29 / 89$ & 71 & CASS & $\mathbf{T}$ & $\cdot$ \\
\hline$T E-108-A P-42$ & $11 / 4 / 89$ & 66 & CASS & $T$ & \\
\hline TE-108-AP-42 & $11 / 7 / 89$ & 65 & CASS & $T$ & \\
\hline TE-108-AP-42 & 11/18/a9 & 67 & CASS & $T$ & \\
\hline TE-108-AP-42 & $12 / 2 / 89$ & 64 & CASS & $T$ & \\
\hline TE-10Q-AP-42 & $12 / 4 / 89$ & 63 & CASS & $T$ & \\
\hline TE-108-AP-42 & $12 / 12 / 89$ & 61 & CASS & $T$ & \\
\hline TE-108-AP-42 & $12 / 23 / 89$ & 58 & CASS & $T$ & \\
\hline TE-108-AP-42 & $12 / 25 / 89$ & 58 & CASS & $T$ & \\
\hline TE-108-AP-42 & $1 / 2 / 90$ & 58 & CASS & $T$ & \\
\hline TE-108-AP-42 & $1 / 12 / 90$ & 58 & CASS & $T$ & \\
\hline$T E-108-A P-42$ & $3 / 15 / 90$ & 58 & CASS & $T$ & \\
\hline TE-108-AP-42 & $1 / 23 / 90$ & 58 & CASS & $T$ & \\
\hline TE-102-AP-42 & 27190 & 58 & CASS & $T$ & \\
\hline TE-108-AP-42 & $2 / 7 / 90$ & 59 & CASS & $\mathbf{T}$ & \\
\hline $\mathrm{TE}-108-A P-42$ & $2 / 13 / 90$ & 59 & CASS & $T$ & \\
\hline TE-108-AP-42 & $2 / 24 / 90$ & 57 & CASS & $T$ & \\
\hline$T E-108-A P-42$ & $3 / 3 / 90$ & 59 & CASS & $T$ & \\
\hline TE-108-AP-42 & $3 / 9 / 90$ & 62 & CASS & $T$ & \\
\hline TE-108-AP-42 & $3 / 14 / 90$ & 62 & CASS & $T$ & \\
\hline TE-108-AP-42 & 318/90 & 62 & CASS & $T$ & \\
\hline TE-108-AP-42 & $3 / 29 / 90$ & 60 & CASS & $\mathbf{T}$ & \\
\hline TE-108-AP-42 & $4 / 2 / 90$ & 61 & CASS & $T$ & \\
\hline TE-10B-AP-42 & $4 / 12 / 90$ & 62 & CASS & $T$ & \\
\hline TE-108-AP-42 & $4 / 21 / 90$ & 60 & CASS & $T$ & \\
\hline TE-108-AP-42 & $4 / 26 / 90$ & 62 & CASS & $T$ & \\
\hline TE-108-AP-42 & $4 / 29 / 50$ & 63 & CASS & $T$ & \\
\hline TE-10B-AP-42 & $5 / 11 / 90$ & 65 & CASS & $T$ & \\
\hline TE-10Q-AP-42 & $5 / 17 / 90$ & 63 & CASS & $\mathbf{T}$ & \\
\hline$T E-108-A P-42$ & $5 / 22 / 90$ & 61 & CASS & $T$ & \\
\hline TE-108-AP-42 & $6 / 1 / 90$ & 62 & CASS & $T$ & \\
\hline$T E-108-A P-42$ & $6 / 6 / 90$ & 66 & CASS & $T$ & \\
\hline TE-108-AP-42 & $6 / 12 / 90$ & 63 & CASS & $T$ & \\
\hline TE-108-AP-42 & $6 / 21 / 90$ & 64 & CASS & $T$ & \\
\hline TE-108-AP-42 & $6 / 30 / 90$ & 63 & CASS & $T$ & \\
\hline TE-108-AP-42 & $7 / 6 / 90$ & 64 & CASS & $T$ & \\
\hline TE-108-AP-42 & $7 / 9 / 90$ & 69 & SACS & $T$ & \\
\hline TE-10B-AP-42 & $7 / 21 / 90$ & 66 & CASS & $T$ & \\
\hline TE-108-AP-42 & $7 / 27 / 90$ & 71 & CASS & $T$ & \\
\hline TE-108-AP-42 & $8 / 3 / 90$ & 70 & CASS & $T$ & \\
\hline TE-108-AP-42 & $8 / 7 / 90$ & 72 & CASS & $T$ & \\
\hline$T E-108-A P-42$ & $8 / 77 / 90$ & 70 & CASS & $T$ & \\
\hline TE-108-AP-42 & $8 / 19 / 90$ & 71 & CASS & $\bar{T}$ & \\
\hline TE-108-AP-42 & $9 / 1 / 90$ & 69 & CASS & $T$ & \\
\hline TE-108-AP-42 & 9/a/90 & 69 & CASS & $T$ & \\
\hline TE-108-AP-42 & $9 / 15 / 90$ & 71 & CASS & $T$ & \\
\hline TE-108-AP-42 & $9 / 22 / 90$ & 69 & CASS & $T$ & \\
\hline$T E-108-A P-42$ & $9 / 28 / 90$ & 71 & CASS & $T$ & \\
\hline TE-108-AP-42 & $10 / 6 / 90$ & 70 & CASS & $T$ & \\
\hline TE-108-AP-42 & $70 / 13 / 90$ & 70 & CASS & $T$ & \\
\hline TE-108-AP-42 & $10 / 16 / 90$ & 70 & CASS & $\mathbf{T}$ & \\
\hline TE-108-AP-42 & $10 / 25 / 90$ & 99 & CASS & 5 & Data point did not agres with local trend \\
\hline TE-10B-AP-42 & $10 / 26 / 90$ & 69 & CASS & $T$ & \\
\hline TE-108-AP-42 & $10 / 31 / 90$ & 69 & CASS & $\bar{T}$ & \\
\hline TE-10B-AP-42 & $11 / 4 / 90$ & 68: & CASS & $\mathbf{T}$ & \\
\hline TE-108-AP-42 & $11 / 17 / 90$ & 67 & CASS & $\mathbf{T}$ & \\
\hline
\end{tabular}




\begin{tabular}{|c|c|c|c|c|c|}
\hline \multicolumn{6}{|c|}{$\begin{array}{l}\text { TANKK 241-AP-108 } \\
\text { THERMOCOUPLE MATA }\end{array}$} \\
\hline & & & & & \\
\hline THEAMOCOUPLE * & DATE & TEMPERATUAE $\left({ }^{\circ} \mathrm{F}\right\}$ & DATA SOURCE & OUAUTY & COMMENT \\
\hline TE-108-AP-42 & $11 / 20 / 90$ & 67 & CASS & $T$ & \\
\hline TE-106-AP-42 & $11 / 29 / 90$ & 65 & CASS & $T$ & \\
\hline TE-108-AP-42 & $12 / 6 / 90$ & 66 & CASS & $T$ & \\
\hline TE-108-AP-42 & $12 / 11 / 20$ & 64 & CASS & $T$ & \\
\hline TE-108-AP-42 & $12 / 20 / 90$ & 63 & CASS & $T$ & \\
\hline TE-10B-AP-42 & $12 / 27 / 90$ & 60 & CASS & $T$ & \\
\hline TE-10B-AP-42 & $12 / 30 / 90$ & 59 & CASS & $\bar{T}$ & \\
\hline TE-108-AP-42 & $1 / 11 / 91$ & 57 & CASS & $T$ & \\
\hline TE-10B-AP-42 & $1 / 14 / 91$ & 58 & CASS & $\bar{T}$ & \\
\hline TE-108-AP-42 & $1 / 24 / 91$ & 56 & CASS & $T$ & \\
\hline TE-108-AP-42 & 1/29/81 & 56 & CASS & $\mathrm{T}$ & \\
\hline TE-108-AP-42 & $2 / 6 / 91$ & 56 & CASS & $T$ & \\
\hline TE-108-AP-42 & $2 / 15 / 91$ & 55 & CASS & $T$ & \\
\hline TE-108-AP-42 & $2 / 17 / 91$ & 57 & CASS & $\mathbf{T}$ & \\
\hline TE-108-AP-42 & $3 / 2 / 91$ & 55 & CASS & $T$ & \\
\hline TE-108-AP-42 & 3/7/91 & 57 & CASS & $T$ & \\
\hline TE-108-AP-42 & $3 / 13 / 91$ & 57 & CASS & $T$ & \\
\hline TE-108-AP-42 & $3 / 23 / 91$ & 56 & CASS & $T$ & \\
\hline TE-108-AP-42 & 3/29/91 & 58 & CASS & $\mathbf{T}$ & \\
\hline$T E-108-A P-42$ & $4 / 1 / 91$ & 58 & CASS & $\mathbf{T}$ & \\
\hline TE-108-AP-42 & 4/12/91 & 57 & CASS & $T$ & \\
\hline TE-108-AP-A2 & 4/20/91 & 57. & CASS & $T$ & \\
\hline TE-108-AP-42 & $4 / 21 / 91$ & 581 & CASS & $T$ & \\
\hline TE-108-AP-42 & $5 / 1 / 91$ & 59 & CASS & $T$ & \\
\hline TE-108-AP-42 & $5 / 10 / 91$ & 59 & CASS & $T$ & \\
\hline TE-108-AP-42 & $5 / 16 / 81$ & 63 & CASS & $\mathbf{T}$ & \\
\hline TE-108-AP-42 & $5 / 25 / 91$ & 59 & CASS & $\mathbf{T}$ & \\
\hline TE-10B-AP-42 & $5 / 26 / 91$ & 62 & CASS & $T$ & \\
\hline TE-108.AP-42 & 6/5/91 & 61 & CASS & $\mathrm{T}$ & \\
\hline TE-108-AP-42 & $6 / 15 / 91$ & 62 & CASS & $\bar{T}$ & \\
\hline TE-106-AP-42 & $6 / 22 / 91$ & 64 & CASS & $\bar{T}$ & \\
\hline TE-108-AP-42 & $6 / 28 / 91$ & 67 & CASS & $T$ & \\
\hline TE-108-AP-42 & $7 / 3 / 91$ & 66 & CASS & 7 & \\
\hline TE-108-AP-42 & $7 / 13 / 91$ & 66 & CASS & $T$ & \\
\hline TE-108-AP-42 & $7 / 14 / 91$ & 66 & CASS & $\bar{T}$ & \\
\hline TE-108-AP-A2 & $7 / 24 / 91$ & 68 & CASS & $\bar{T}$ & \\
\hline TE-108-AP-42 & $8 / 291$ & 69 & CASS & $T$ & \\
\hline$T E+108-A P-42$ & $8 / 6 / 91$ & 68 & CASS & $T$ & \\
\hline$T E-108-A P-42$ & $8 / 17 / 91$ & 71 & CASS & $\bar{T}$ & \\
\hline$T E-108-A P=42$ & $8 / 20 / 91$ & 70 & CASS & $T$ & \\
\hline TE-108.AP-42 & $8 / 30 / 91$ & 70 & CASS & $\mathbf{T}$ & \\
\hline TE-108-AP-42 & 9/3/91 & 73 & CASS & $T$ & \\
\hline TE-108-AP-42 & 9/12/91 & 71 & CASS & $\mathrm{T}$ & \\
\hline TE-10B-AP-42 & $9 / 18 / 91$ & 72 & CASS & $\mathrm{T}$ & \\
\hline TE-108-AP-42 & $9 / 22 / 91$ & 71 & CASS & $\mathbf{T}$ & \\
\hline TE-108-AP-42 & $10 / 1 / 91$ & 74 & CASS & $T$ & \\
\hline TE-108-AP-42 & $10 / 8 / 91$ & 61 & CASS & $\mathbf{S}$ & Data point did not agree with local trend \\
\hline TE-108-AP-42 & 10/10/91 & 71 & CASS & $T$ & \\
\hline TE-108-AP-42 & $10 / 1791$ & 72. & CASS & $\mathbf{T}$ & \\
\hline TE-108-AP-42 & $10 / 25 / 91$ & 75 & CASS & $\bar{T}$ & \\
\hline TE-108-AP-42 & $10 / 28 / 91$ & 7iा & CASS & $\mathbf{T}$ & \\
\hline$T E-108-A P-42$ & $11 / 6 / 91$ & 70 & CASS & $\mathbf{T}$ & \\
\hline TE-108-AP-42 & $7 / 30 / 92$ & 66 & CASS & $\mathbf{T}$ & \\
\hline TE-108-AP-42 & $8 / 8 / 92$ & 66 & CASS & $\mathbf{T}$ & \\
\hline TE-108-AP-42 & $8 / 15 / 92$ & 67 & CASS & $\mathbf{T}$ & $\cdot$ \\
\hline TE-108-AP-42 & $8 / 20 / 92$ & 68 & CASS & $T$ & \\
\hline TE-108-AP-42 & $8 / 29 / 92$ & 66 & CASS & $\mathbf{T}$ & \\
\hline TE-108-AP-42 & $8 / 31 / 92$ & 67 & CASS & $\bar{T}$ & \\
\hline TE-108-AP-42 & $9 / 12 / 92$ & 68 & CASS & $\mathbf{T}$ & \\
\hline TE-108-AP-42 & $9 / 16 / 92$ & 68 & CASS & $\mathbf{T}$ & \\
\hline TE-108-AP-42 & $9 / 26 / 92$ & 67 & CASS & $\mathbf{T}$ & \\
\hline TE-108-AP-A2 & $10 / 3 / 92$ & 68 & CASS & $T$ & \\
\hline TE- $108-A P-42$ & $10 / 10 / 92$ & 67 & CASS & $\bar{T}$ & \\
\hline TE-108-AP-42 & $10 / 13 / 92$ & 68 & CASS & $\bar{T}$ & \\
\hline TE-108-AP-42 & $10 / 24 / 92$ & 69 & CASS & $T$ & \\
\hline TE-108-AP-42 & $10 / 29 / 92$ & 69 & CASS & $\mathbf{T}$ & \\
\hline TE-108-AP-42 & $11 / 4 / 92$ & 69 & CASS & $\mathbf{T}$ & \\
\hline TE-108-AP-A2 & $11 / 9 / 92$ & 69 & CASS & $\mathbf{T}$ & \\
\hline TE-108-AP-42 & $11 / 17 / 92$ & 71 & CASS & $T$ & \\
\hline TE-108-AP-42 & $11 / 28 / 92$ & 67 & CASS & $T$ & \\
\hline TE-108-AP-42 & $12 / 3 / 92$ & 67 & CASS & $T$ & \\
\hline TE-108-AP-42 & $12 / 12 / 92$ & 69 & CASS & $T$ & \\
\hline$T E-108-A P-42$ & $12 / 19 / 92$ & 67 & CASS & $\bar{T}$ & \\
\hline
\end{tabular}

Data obtained from Computer Automated Surveillance Syatem (CASS), April 1995; and Surveillance Analyals Computer Syatom (SACS), June 1995. 


\begin{tabular}{|c|c|c|c|c|c|}
\hline \multicolumn{6}{|c|}{$\begin{array}{l}\text { TANK 24T-AP-108 } \\
\text { THËMOOOUPLE DATA }\end{array}$} \\
\hline & & & & & \\
\hline THERMOCOUPLE & DATE & TEMPERATUAE $\left({ }^{\circ} \mathrm{F}\right)$ & DATA SOURCE & OUALTY & COMMENT \\
\hline TE-10D-AP-42 & $12 / 21 / 92$ & 67 & SACS & $T$ & \\
\hline TE-108-AP-42 & $12 / 30 / 92$ & 66 & CASS & $T$ & \\
\hline TE-108-AP-42 & $6 / 4 / 93$ & 44 & SACS & $\mathbf{S}$ & Data point did not agree with local trend \\
\hline$T E-10 B-A P-42$ & $6 / 11 / 93$ & 61 & SACS & $\bar{T}$ & \\
\hline TE-108-AP-42 & $6 / 18 / 93$ & 60 & SACS & $\bar{T}$ & \\
\hline TE-108-AP-42 & $6 / 28 / 93$ & 61 & SACS & $\mathbf{T}$ & \\
\hline TE-108-AP-42 & $7 / 9 / 93$ & 61 & SACS & $T$ & \\
\hline TE-108-AP-42 & $7 / 15 / 93$ & 61 & SACS & $\mathbf{T}$ & \\
\hline TE-108-AP-42 & $7 / 19 / 93$ & 62 & SACS & $\bar{T}$ & \\
\hline TE-108-AP-42 & $7 / 30 / 93$ & 62 & SACS & $T$ & \\
\hline$T E-108-A P-42$ & $8 / 6 / 93$ & 62 & SACS & $\bar{T}$ & \\
\hline TE-108-AP-42 & $8 / 12 / 93$ & 62 & SACS & $T$ & \\
\hline$T E-108-A P=42$ & $9 / 24 / 93$ & 54 & SACS & 5 & Data point did not agree with local trend \\
\hline TE-108-AP-42 & $10 / 1 / 93$ & 63 & SACS & $T$ & \\
\hline TE-108-AP-42 & $10 / 15 / 93$ & 65 & SACS & $T$ & \\
\hline TE-108-AP-42 & $10 / 22 / 93$ & 64 & SACS & $T$ & \\
\hline TE-108-AP-42 & $10 / 28 / 93$ & 65 & SACS & $\bar{T}$ & \\
\hline TE-108-AP-42 & $11 / 9 / 93$ & 62 & SACS & $T$ & \\
\hline TE-108-AP-42 & $11 / 30 / 93$ & 65 & SACS & $T$ & \\
\hline TE-102-AP- 42 & $12 / 14 / 93$ & 66 & SACS & $T$ & \\
\hline TE-10Q-AP-42 & $12 / 20 / 93$ & 64 & SACS & $T$ & \\
\hline TE-10D-AP-A2 & $12 / 27 / 93$ & 64 & SACS & $T$ & \\
\hline TE-108-AP-42 & $1 / 3 / 94$ & 62 & SACS & $T$ & \\
\hline TE-108-AP-42 & $1 / 11 / 94$ & 66 & SACS & $T$ & \\
\hline TE-102-AP-42 & $1 / 20 / 94$ & 64 & CASS & $T$ & \\
\hline TE-108-AP-42 & $1 / 25 / 94$ & 64 & CASS & $T$ & \\
\hline TE-108-AP-42 & $2 / 2 / 94$ & 64 & CASS & $\mathbf{T}$ & \\
\hline$T E-108-A P-42$ & $2 / 7 / 94$ & 64 & SACS & $\mathbf{G}$ & \\
\hline TE-108-AP-42 & $2 / 15 / 94$ & 64 & CASS & $T$ & \\
\hline TE-108-AP-42 & 2126194 & 63 & CASS & $\mathbf{T}$ & \\
\hline TE-108-AP-42 & $2 / 29 / 94$ & 67 & CASS & $T$ & \\
\hline$T E-102-A P-42$ & $3 / 10 / 94$ & 66 & CASS & $T$ & \\
\hline TE-108-AP-42 & 317/94 & 65 & CASS & $T$ & \\
\hline TE-108-AP-42 & $3 / 25 / 94$ & 65 & CASS & $T$ & \\
\hline TE-108-AP-42 & 32794 & 64 & CASS & $T$ & \\
\hline TE-108-AP-42 & $4 / 9 / 94$ & 64 & CASS & $T$ & \\
\hline TE-108-AP-42 & $4 / 13 / 94$ & 84 & CASS & $T$ & \\
\hline TE-108-AP- 12 & $4 / 22 / 94$ & 65 & CASS & $T$ & \\
\hline TE-108-AP-42 & $4 / 25 / 94$ & 64 & CASS & $T$ & \\
\hline TE-108-AP-42 & $5 / 2 / 94$ & 64 & CASS & $T$ & \\
\hline TE-108-AP-42 & $5 / 9 / 94$ & 64 & CASS & $\mathbf{T}$ & \\
\hline TE-108-AP-42 & $5 / 16 / 94$ & 104 & CASS & $T$ & \\
\hline TE-108-AP-42 & $5 / 23 / 94$ & 98 & CASS & $T$ & \\
\hline TE-108-AP+42 & $6 / 2 / 94$ & 98 & CASS & $T$ & \\
\hline$T E-108-A P-42$ & $6 / 8 / 94$ & 98 & CASS & $T$ & \\
\hline TE-108-AP-42 & $6 / 14 / 94$ & 95 & CASS & $T$ & \\
\hline TE-108-AP-42 & $6 / 16 / 94$ & 63 & CASS & 5 & Data point did not agree with local trend \\
\hline TE-108-AP-42 & $6 / 17 / 94$ & 63 & CASS & $\mathbf{S}$ & Data point did not agree with local trend \\
\hline TE-108-AP-42 & $6 / 20 / 94$ & 62 & CASS & S & Data point did not agree with local trend \\
\hline$T E-108-A P-42$ & $6 / 20 / 94$ & 62 & SACS & $S$ (G in SACS] & Data point did not agree with local trend \\
\hline TE-108-AP-42 & $6 / 25 / 94$ & 97 & CASS & $T$ & \\
\hline TE-108-AP-42 & $6 / 30 / 94$ & 97 & CASS & $T$ & \\
\hline TE-10B-AP-42 & $7 / 7 / 94$ & 96 & CASS & $T$ & \\
\hline TE-10B-AP-42 & $7 / 15 / 94$ & 96 & CASS & $T$ & \\
\hline TE-108-AP-42 & $7 / 17 / 94$ & 94 & CASS & $T$ & \\
\hline$T E-108-A P-42$ & $7 / 24 / 94$ & 931 & CASS & $\bar{T}$ & \\
\hline TE-108-AP-42 & $7 / 31 / 94$ & 100 & CASS & $\mathbf{T}$ & \\
\hline TE-108-AP-42 & $8 / 9 / 94$ & 96 & CASS & $T$ & \\
\hline TE-108-AP-42 & $8 / 18 / 94$ & 97 & CASS & $T$ & \\
\hline TE-108-AP-42 & $8 / 22 / 94$ & 97 & CASS & $T$ & \\
\hline TE-108-AP- 42 & $9 / 3 / 94$ & 97 & CASS & $T$ & \\
\hline TE-10B-AP-42 & $9 / 5 / 94$ & 100 & CASS & $T$ & \\
\hline TE-108-AP-42 & $9 / 15 / 94$ & 99 & CASS & $T$ & \\
\hline TE-108-AP- 42 & $9 / 23 / 94$ & 100 & CASS & $T$ & \\
\hline TE-108-AP-42 & $9 / 30 / 94$ & 99 & CASS & $T$ & \\
\hline TE-108-AP-42 & $10 / 2 / 94$ & 103 & CASS & $T$ & \\
\hline TE-108-AP-42 & $10 / 15 / 94$ & 102 & CASS & $T$ & \\
\hline TE-108-AP-42 & $10 / 22 / 94$ & 104 & CASS & $T$ & \\
\hline$T E-108-A P-42$ & $10 / 29 / 94$ & 104 & CASS & $T$ & \\
\hline TE-108-AP-42 & $11 / 5 / 94$ & 104 & CASS & $T$ & \\
\hline TE-108-AP-42 & $11 / 11 / 94$ & 103 & CASS & $T$ & \\
\hline TE-108-AP-42 & $11 / 16 / 94$ & 103 & CASS & $T$ & \\
\hline TE-10B-AP-42 & $11 / 22 / 94$ & 104 & CASS & $T$ & \\
\hline
\end{tabular}




\begin{tabular}{|c|c|c|c|c|c|}
\hline \multicolumn{6}{|c|}{$\begin{array}{l}\text { TANK 241APP108 } \\
\text { THEFWOCOUFLE BATA }\end{array}$} \\
\hline THEPMOCOUPLE * & DATE & TEMPERATUAE $\left({ }^{\circ} \mathbf{F}\right)$ & DATA SOUACE & OUALTY & \\
\hline TE-108-AP-42 & $12 / 3 / 94$ & 103 & CASS & $T$ & Cominemt \\
\hline TE-108-AP-42 & $12 / 4 / 94$ & 108 & CASS & $\mathbf{T}$ & \\
\hline TE-108-AP-42 & $12 / 12 / 94$ & 103 & CASS & $\mathbf{T}$ & \\
\hline TE-106-AP-42 & $12 / 19 / 94$ & 105 & CASS & $T$ & \\
\hline TE-108-AP-42 & $12 / 30 / 94$ & 106 & CASS & $\mathbf{T}$ & \\
\hline TE-108-AP-42 & $1 / 4 / 95$ & 110 & CASS & $T$ & \\
\hline TE-108-AP-42 & 1/13/95 & 105 & CASS & $T$ & \\
\hline TE-108-AP-42 & $1 / 15 / 95$ & 105 & CASS & $\mathbf{T}$ & \\
\hline TE-108-AP-42 & 1/23/95 & 106 & CASS & $\mathbf{T}$ & \\
\hline TE-108-AP:A2 & $1 / 30 / 95$ & 100 & SACS & G & \\
\hline TE-108-AP-42 & $2 / 10 / 95$ & 100 & CASS & $T$ & \\
\hline TE-108-AP-42 & $2 / 14 / 95$ & 106 & CASS & $\bar{T}$ & \\
\hline TE-108-AP-42 & $2 / 24 / 95$ & 96 & CASS & $\bar{T}$ & \\
\hline TE-108-AP-42 & $3 / 2 / 95$ & 101 & CASS & $T$ & \\
\hline TE-10B-AP-42 & 3/7/95 & 98 & CASS & $\mathbf{T}$ & \\
\hline TE-108-AP-42 & $3 / 16 / 95$ & 94 & CASS & $T$ & \\
\hline TE-108-AP-42 & 3/25/95 & 95 & CASS & $T$ & \\
\hline TE-108-AP-42 & 3/30/95 & 86 & CASS & $T$ & \\
\hline TE-108-AP.42 & $4 / 3 / 95$ & 86 & SACS & $\mathbf{G}$ & \\
\hline TE-108-AP-42 & $4 / 10 / 95$ & 89 & SACS & $\mathbf{G}$ & \\
\hline TE-108-AP-42 & 4/17/95 & 90 & SACS & $\bar{G}$ & \\
\hline TE-108-AP-42 & 4/24/95 & 86 & SACS & G & \\
\hline TE-108-AP-42 & $5 / 1 / 95$ & 86 & SACS & $G$ & \\
\hline TE-106-AP-42 & $5 / 8 / 95$ & 87 & SACS & G & \\
\hline TE-10B-AP-42 & 5/16/95 & 84 & SACS & $\mathbf{G}$ & \\
\hline TE-108-AP-42 & $5 / 22 / 95$ & 84 & SACS & G & \\
\hline TE-108-AP-42 & 5/29/95 & 83 & SACS & G & \\
\hline TE-10B-AP-42 & $6 / 5 / 95$ & 88 & SACS & G & \\
\hline TE-108-AP-42 & 6/13/35 & 83 & SACS & G & \\
\hline TE-100-AP-42 & $6 / 19 / 95$ & 93 & SACS & G & \\
\hline TE-108-AP-43 & $4 / 5 / 86$ & 57 & CASS & $T$ & \\
\hline TE-108-AP-43 & $4 / 7 / 86$ & 59 & CASS & $T$ & \\
\hline TE-108-AP-43 & $5 / 15 / 36$ & 58 & CASS & $\mathbf{T}$ & \\
\hline TE-108-AP-43 & $5 / 22 / 86$ & 60 & CASS & $T$ & \\
\hline$T E-108-A P-43$ & 5/30/86 & 59 & CASS & $T$ & \\
\hline$T E=100-A P_{-43}$ & $6 / 7 / 36$ & 5 & CASS & $T$ & \\
\hline TE-108-AP-43 & 6/9/86 & 64 & CASS & $T$ & \\
\hline TE-108-AP-4B & 6/21/36 & 80 & CASS & $T$ & \\
\hline TE-108-AP-43 & $6 / 25 / 86$ & 60 & CASS & $T$ & \\
\hline TE-108-AP-43 & $7 / 5186$ & 61 & CASS & $T$ & \\
\hline$T E-108-A P=43$ & $7 / 10 / 86$ & 62 & CASS & $\mathbf{T}$ & \\
\hline TE-108-AP-43 & $7 / 19 / 86$ & 61 & CASS & $T$ & \\
\hline TE-100-AP-43 & $7 / 26 / 86$ & 61 & CASS & $T$ & \\
\hline TE-108-AP.A3 & $7 / 28 / 86$ & 64| & CASS & $T$ & \\
\hline TE-108-AP-43 & $8 / 9 / 86$ & 64 & CASS & $\mathbf{T}$ & \\
\hline TE-108:AP-43 & 8/13/86 & 64 & CASS & $\mathbf{T}$ & \\
\hline TE-108-AP-43 & $8 / 21 / 86$ & 63 & CASS & $T$ & \\
\hline IE-108-AP-43 & $8 / 30 / 86$ & 63 & CASS & $T$ & \\
\hline TE-108-AP-43 & $9 / 3 / 86$ & 64 & CASS & $T$ & \\
\hline TE-108-AP-43 & $9 / 24 / 86$ & 64 & CASS & $T$ & \\
\hline TE-108-AP-43 & $11 / 1 / 86$ & 62 & CASS & $T$ & \\
\hline TE-108-AP-43 & $11 / 3 / 36$ & 62 & CASS & $T$ & \\
\hline TE-10B-AP-43 & $11 / 29 / 86$ & 59 & CASS & $T$ & \\
\hline TE-108-AP-43 & $12 / 11 / 86$ & 59 & CASS & $T$ & \\
\hline TE-108-AP-43 & $12 / 18 / 86$ & 58 & CASS & $T$ & \\
\hline TE-108-AP-43 & $12 / 24 / 86$ & 57 & CASS & $\mathbf{T}$ & \\
\hline TE-108-AP.43 & $12 / 29 / 86$ & 56 & CASS & $\mathbf{T}$ & \\
\hline TE-108-AP-43 & 7/1/87 & 67 & CASS & $\mathbf{T}$ & \\
\hline TE-108-AP-43 & 7/8/87 & 69 & CASS & $T$ & \\
\hline TE-108-AP-43 & 7/14/87 & 66 & CASS & $\mathbf{T}$ & \\
\hline TE-108-AP-43 & 7/15/87 & 126 & CASS & $\mathbf{s}$ & Data point did not agree with local trend \\
\hline TE-108-AP-43 & 7/19/97 & 69 & CASS & $T$ & \\
\hline TE-108-AP-43 & $7 / 27 / 87$ & 68 & CASS & $\mathbf{T}$ & \\
\hline TE-108-AP-43 & $8 / 5 / 87$ & 69 & CASS & $\mathbf{T}$ & \\
\hline TE-108-AP-43 & $8 / 14 / 87$ & 70 & CASS & $\bar{T}$ & \\
\hline TE-108-AP-43 & $8 / 22 / 97$ & 68 & CASS & $T$ & \\
\hline TE-10B-AP-43 & $8 / 26 / 67$ & 69 & CASS & $T$ & \\
\hline TE-108-AP-43 & $9 / 4 / 87$ & 68 & CASS & $T$ & \\
\hline$T E-108-A P-43$ & 9/9/87 & 69 & CASS & $T$ & \\
\hline TE-108-AP-43 & $9 / 17 / 87$ & 69 & CASS & $T$ & \\
\hline TE-108-AP-43 & 9/20/37 & 70 & CASS & $T$ & \\
\hline TE-108-AP-43 & 9/27/87 & 70 & CASS & $\mathbf{T}$ & \\
\hline
\end{tabular}




\begin{tabular}{|c|c|c|c|c|c|}
\hline \multicolumn{6}{|c|}{$\begin{array}{l}\text { TANK 241.AP.108 } \\
\text { THEAMOCOUME DATA }\end{array}$} \\
\hline & & & & & \\
\hline 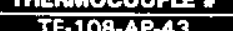 & $\frac{0412}{100707}$ & TGMPIRATULIE (MT) & DATA SOURCE & QUALITY & COMMENT \\
\hline$T E-108-A P=43$ & $\frac{10 / 1 / 8 / 2}{10 / 9 / 87}$ & 70 & CASS & $\mathbf{T}$ & \\
\hline TE-108-AP-43 & \begin{tabular}{r|}
$10 / 9 / 87$ \\
$10 / 16 / 87$
\end{tabular} & 109 & CASS & $\mathbf{s}$ & Data point did not agree with local trend \\
\hline TE-108-AP-4S & $\begin{array}{l}10 / 16 / 87 \\
10 / 19 / 87\end{array}$ & 67 ? & $\frac{\text { CASS }}{\text { CASS }}$ & $T$ & \\
\hline TE-10B-AP-43 & $\frac{10 / 19 / 67}{10 / 31 / 87}$ & 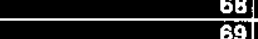 & $\frac{\text { CASS }}{\text { CASS }}$ & $T$ & \\
\hline TE-108-AP-43 & $11 / 4 / 87$ & 64 & $\frac{\text { CASS }}{\text { CASS }}$ & $T$ & \\
\hline TE-108-AP-43 & $11 / 8 / 87$ & 64 & $\begin{array}{l}\text { CASS } \\
\text { CASS }\end{array}$ & $T$ & \\
\hline TE-108-AP-43 & $11 / 18 / 87$ & 62 & $\frac{\text { CASS }}{\text { CASS }}$ & $\frac{T}{T}$ & \\
\hline TE-108-AP-43 & $11 / 26 / 87$ & 65! & CASS & $\bar{T}$ & \\
\hline TE-108-AP-43 & $12 / 1 / 87$ & 61 & CASS & $T$ & \\
\hline TE-108-AP-43 & $12 / 6 / 87$ & 60 & CASS & $\mathbf{T}$ & \\
\hline TE-108-AP-43 & $12 / 18 / 87$ & 58 & CASS & $\mathbf{T}$ & \\
\hline TE-108-AP-43 & $12 / 23 / 87$ & 57 & CASS & $T$ & \\
\hline TE-108-AP-43 & $1 / 2 / 88$ & 56 & CASS & $T$ & \\
\hline TE-108-AP-43 & $1 / 4 / 88$ & 53 & CASS & $T$ & \\
\hline TE-108-AP-43 & $1 / 15 / 88$ & 52 & CASS & $T$ & \\
\hline TE+108-AP-43 & 1/23/a8 & 52 & CASS & $\mathbf{T}$ & \\
\hline TE-108-AP-43 & $1 / 25 / 83$ & 54 & CASS & $T$ & \\
\hline TE-108-AP-43 & $2 / 2 / 88$ & 52 & CASS & $T$ & \\
\hline TE-10B-AP-43 & $2 / 13 / 88$ & 52 & CASS & $T$ & \\
\hline TE-108-AP-43 & $2 / 14 / 88$ & 53 & CASS & $T$ & \\
\hline TE-108-AP-43 & $2 / 21 / 88$ & 53 & CASS & $\mathbf{T}$ & \\
\hline TE-10B-AP-43 & $3 / 3 / 8$ & 54 & CASS & $T$ & \\
\hline TE-108-AP-43 & आ12/88 & 53 & CASS & $T$ & \\
\hline$T E-108-A P-43$ & आ1388 & 54 & CASS & $T$ & \\
\hline TE-108-AP-43 & $3 / 25 / 88$ & 55 & CASS & $T$ & \\
\hline TE-108-AP-43 & $3 / 31 / 88$ & 54 & CASS & $\bar{T}$ & \\
\hline TE-10B-AP-43 & 6/1/8a & 62 & CASS & $T$ & \\
\hline TE-108-AP-43 & $6 / 10 / 88$ & 61 & CASS & $\mathbf{T}$ & \\
\hline TE-108-AP-43 & 6/18/88 & 61 & CASS & $T$ & \\
\hline TE-108-AP-43 & $8 / 25 / 88$ & 62 & CASS & $T$ & \\
\hline TE-108-AP-43 & 6/29/8s & 70 & CASS & $T$ & \\
\hline TE-10D-AP-43 & 7/9/89 & 63 & CASS & $T$ & \\
\hline TE-108-AP-43 & 713/88 & 66 & CASS & $\bar{T}$ & \\
\hline TE-108-AP-43 & $7 / 23 / 88$ & 66 & CASS & $\bar{T}$ & \\
\hline TE-108-AP-43 & $7 / 29 / 8 a$ & 67 & CASS & $T$ & \\
\hline TE-108-AP-43 & $8 / 3 / 38$ & 69 & CASS & $\mathbf{T}$ & \\
\hline TE-10B-AP-43 & $8 / 9 / 89$ & 69 & CASS & $T$ & \\
\hline TE-108-AP-43 & 8/20/88 & 69 & CASS & $T$ & \\
\hline TE-108-AP-43 & $8 / 23 / 8 B$ & 120 & CASS & $\mathbf{S}$ & Data point did not arges with local trend \\
\hline TE-108-AP-43 & $8 / 27 / 8 \mathrm{~B}$ & 68 & CASS & $T$ & \\
\hline TE-108-AP-43 & $9 / 2 / 88$ & 69 & CASS & $T$ & \\
\hline TE-100-AP-43 & 9/9/8B & 71 & CASS & $T$ & \\
\hline TE-108-AP-43 & 9/16/88 & 71 & CASS & $T$ & \\
\hline TE-108-AP.43 & $9 / 23 / 88$ & 69 & CASS & $T$ & \\
\hline TE-108-AP-43 & $9 / 27 / 88$ & 70 & CASS & $T$ & \\
\hline TE-108-AP-43 & 12/7/88 & 66 & CASS & $T$ & \\
\hline TE-108-AP-43 & 12/8/88 & 64 & CASS & $\bar{T}$ & \\
\hline TE-108-AP-43 & 12/16/88 & 62 & CASS & $T$ & \\
\hline TE-108-AP-43 & $12 / 20 / 88$ & 63 & CASS & $T$ & \\
\hline TE-108-AP-43 & $7 / 10 / 89$ & 64 & SACS & $\mathbf{T}$ & \\
\hline TE-108-AP-43 & $7 / 17 / 89$ & 65 & SACS & $\bar{T}$ & \\
\hline TE-108-AP-43 & $7 / 24 / 89$ & 66 & SACS & $T$ & \\
\hline TE-108-AP-43 & $8 / 7 / 89$ & 66 & SACS & $T$ & \\
\hline TE-108-AP-43 & $8 / 14 / 89$ & 68 & SACS & $T$ & \\
\hline TE-10B-AP.43 & $8 / 21 / 89$ & 68 & SACS & $T$ & \\
\hline TE-108-AP-43 & $9 / 2 / 89$ & 71 & CASS & $T$ & \\
\hline TE-108-AP-43 & $9 / 7 / 89$ & 69 & CASS & $\bar{T}$ & \\
\hline $\mathrm{TE}-108-\mathrm{AP}-43$ & 9/16/89 & 71 & CASS & $T$ & \\
\hline TE-108-AP-43 & $9 / 18 / 89$ & 70 & CASS & $\mathbf{T}$ & \\
\hline TE-108-AP-43 & 9/29/Bg & 71 & CA5S & $T$ & \\
\hline TE-108-AP-43 & $10 / 2 / 89$ & 67 & SACS & $T$ & \\
\hline TE-10B-AP-43 & $10 / 9 / 89$ & 68 & SACS & $T$ & \\
\hline TE-10B-AP-43 & $10 / 16 / 89$ & 68 & SACS & $T$ & \\
\hline TE-108-AP-43 & $10 / 24 / 89$ & 72 & SACS & $\bar{T}$ & \\
\hline TE-108-AP-43 & $11 / 4 / 89$ & 66 & CASS & $\bar{T}$ & \\
\hline TE-108-AP-43 & $11 / 7 / 89$ & 65 & CASS & $T$ & \\
\hline TE-108-AP-43 & $11 / 18 / 89$ & 67 & CASS & $T$ & \\
\hline TE-108-AP-43 & $11 / 20 / 89$ & 64 & SACS & $\bar{T}$ & \\
\hline TE-108-AP-43 & $12 / 2 / 89$ & 66 & CASS & $T$ & \\
\hline TE-108-AP-43 & $12 / 8 / 89$ & 62 & CASS & $T$ & \\
\hline TE-108-AP-43 & 12/12/89 & 61 & CASS & $\bar{T}$ & \\
\hline TE-10B-AP-43 & $12 / 22 / 89$ & 58 & CASS & $\bar{T}$ & \\
\hline
\end{tabular}




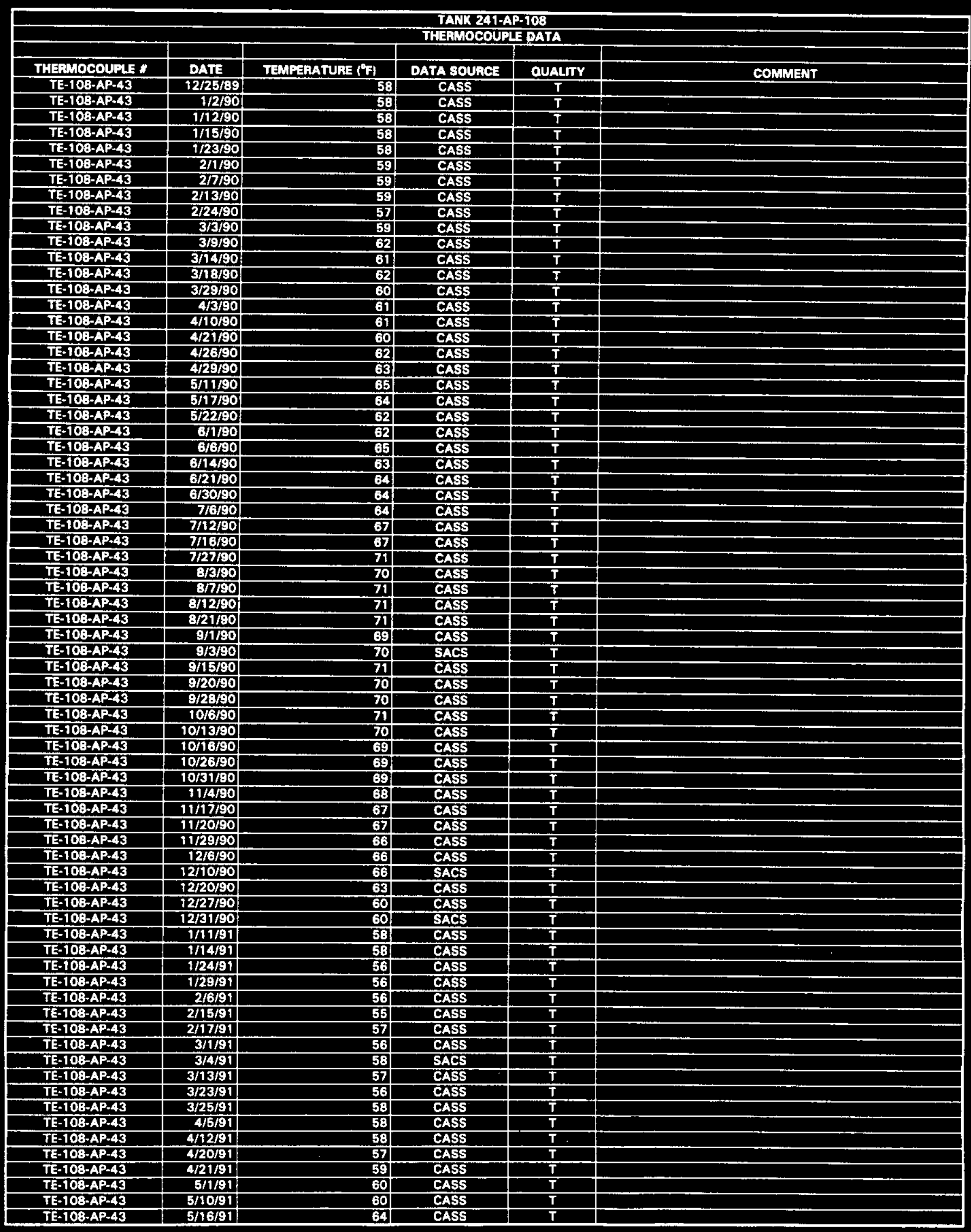




\begin{tabular}{|c|c|c|c|c|c|}
\hline \multicolumn{6}{|c|}{$\begin{array}{l}\text { TAKK 2GI-AP.108 } \\
\text { THEAMOCOUPLE DATA }\end{array}$} \\
\hline THERMOCOUPL * & DATE & TEMPERATURE ( $\left.{ }^{\circ} \mathrm{F}\right)$ & DATA SOURCE & OUAUTY & COMMENT \\
\hline TE-108-AP-43 & $5 / 25 / 91$ & 60 & CASS & $\bar{T}$ & \\
\hline TE-108-AP-43 & 5/26/91 & 63 & CASS & $\mathbf{T}$ & \\
\hline TE-108-AP-43 & 6/5/91 & 63 & CASS & $T$ & \\
\hline TE-108-AP-43 & 6/15/91 & 63 & CASS & $T$ & \\
\hline IE-108-AP-43 & $6 / 22 / 91$ & 65 & CASS & $T$ & \\
\hline TE-108-AP-43 & 6/28/91 & 68 & CASS & $T$ & \\
\hline TE-108-AP-43 & $7 / 3 / 91$ & 67 & CASS & $T$ & \\
\hline TE-108-AP-43 & $7 / 8 / 91$ & 69 & SACS & $T$ & \\
\hline TE-108-AP-43 & 7/14/91 & 67 & CASS & $\mathbf{T}$ & \\
\hline TE-10B-AP-43 & $7 / 24 / 91$ & 68 & CASS & $\mathbf{T}$ & \\
\hline TE-106-AP-43 & $8 / 2 / 91$ & 69 & CASS & $T$ & \\
\hline TE-108-AP-43 & $8 / 6 / 91$ & 69 & CASS & $T$ & \\
\hline TE-108-AP-43 & $8 / 17 / 91$ & 71 & CASS & $\mathbf{T}$ & \\
\hline TE-108-AP-43 & $8 / 18 / 91$ & 71 & CASS & $\mathbf{T}$ & \\
\hline TE-106-AP-43 & $8 / 30 / 91$ & 71 & CASS & $\mathbf{T}$ & \\
\hline TE-108-AP-43 & $9 / 7 / 91$ & 70 & CASS & $\mathbf{T}$ & \\
\hline TE-10B-AP-43 & 9/8/91 & 72 & CASS & $\mathbf{T}$ & \\
\hline TE-109-AP-43 & 9/18/91 & 73 & CASS & $\mathbf{T}$ & \\
\hline TE-108-AP-43 & 9/22/91 & 71 & CASS & $\mathbf{T}$ & \\
\hline IE-108-AP-43 & $10 / 1 / 91$ & 76 & CASS & $\mathbf{T}$ & \\
\hline TE-108-AP-43 & 10/8/91 & 61 & CASS & $\mathbf{S}$ & Data point did not agree with local trend \\
\hline TE-108-AP-43 & $10 / 10 / 91$ & 72 & CASS & $\mathbf{T}$ & \\
\hline TE-108-AP-43 & $10 / 17 / 91$ & 73 & CASS & $\mathbf{T}$ & \\
\hline TE-108-AP-43 & $10 / 25 / 31$ & 75 & CASS & $\mathbf{T}$ & \\
\hline TE-108-AP-43 & $10 / 28 / 91$ & 71 & CASS & $\mathbf{T}$ & \\
\hline TE-108-AP-43 & 11/6/91 & 70 & CASS & $\mathbf{T}$ & \\
\hline TE-108-AP-43 & $7 / 30 / 92$ & 66 & CASS & $\mathbf{T}$ & \\
\hline TE-108-AP-43 & $8 / 8 / 92$ & 67 & CASS & $\bar{T}$ & \\
\hline TE-108-AP-43 & $8 / 15 / 92$ & 67 & CASS & $\mathbf{T}$ & \\
\hline TE-108-AP-43 & $8 / 20 / 92$ & 68 & CASS & $T$ & \\
\hline TE-108-AP-43 & $8 / 28 / 92$ & 68 & CASS & $\mathbf{T}$ & \\
\hline TE-108-AP-43 & $9 / 5 / 92$ & 68: & CASS & $\bar{T}$ & \\
\hline TE-108-AP-43 & 9/12/92 & 67 & CASS & $\bar{T}$ & \\
\hline TE-108-AP-43 & $9 / 16 / 92$ & 68 & CASS & $\mathbf{T}$ & \\
\hline TE-108-AP-43 & 9/21/92 & 67 & SACS & $\mathbf{T}$ & \\
\hline TE-103-AP-43 & $10 / 3 / 22$ & 68 & CASS & $T$ & \\
\hline TE-103-AP-43 & 10/9/s2 & 67 & CASS & $T$ & \\
\hline TE-108-AP-43 & $10 / 22 / 92$ & 68 & CASS & $\mathbf{T}$ & \\
\hline TE-10B-AP-43 & $10 / 24 / 82$ & 69 & CASS & $\mathbf{T}$ & \\
\hline TE-108-AP-43 & $10 / 29 / 92$ & 70 & CASS & $\bar{T}$ & \\
\hline TE-109-AP-43 & $11 / 7 / 92$ & 69 & CASS & $\mathbf{T}$ & \\
\hline TE-109.AP-43 & 11/9/92 & 89 & CASS & $\mathbf{T}$ & \\
\hline TE-108-AP-43 & $11 / 17 / 92$ & 71 & CASS & $\bar{T}$ & \\
\hline TE-108-AP-43 & $11 / 28 / 92$ & 67 & CASS & $T$ & \\
\hline TE-10B-AP-43 & $12 / 3 / 92$ & 67 & CASS & $T$ & \\
\hline IE-108-AP-43 & $12 / 12 / 92$ & 69 & CASS & $\mathbf{T}$ & \\
\hline TE-108-AP-43 & $12 / 19 / 92$ & 67 & CASS & $T$ & \\
\hline TE-108-AP-43 & $12 / 21 / 92$ & 66 & CASS & $\mathbf{T}$ & \\
\hline TE-108-AP-43 & $12 / 30 / 92$ & 66 & CASS & $T$ & \\
\hline TE-108-AP-43 & 1/15/94 & 63. & CASS & $\mathbf{T}$ & \\
\hline TE-TOS-AP-43 & $1 / 20 / 94$ & 64) & CASS & $\mathbf{T}$ & \\
\hline TE-108-AP-43 & 1/25/94 & 64: & CASS & $T$ & \\
\hline TE-108-AP-43 & $2 / 2 / 94$ & 64 & CASS & $T$ & \\
\hline TE-108-AP-43 & $2 / 11 / 94$ & 63 & CASS & $T$ & \\
\hline TE-108-AP-43 & $2 / 77 / 94$ & 63 & CASS & $T$ & \\
\hline TE-108-AP-43 & $2 / 22 / 94$ & 64: & CASS & $\mathbf{T}$ & \\
\hline TE-108-AP-43 & $2 / 28 / 94$ & 65 & CASS & $T$ & \\
\hline IE-108-AP-43 & $3 / 8 / 94$ & 65 & CASS & $T$ & \\
\hline TE-108-AP-43 & $3 / 17 / 94$ & 64 & CASS & $\mathbf{T}$ & \\
\hline TE-108-AP-43 & $3 / 25 / 94$ & 65 & CASS & $T$ & \\
\hline TE-108-AP-43 & $3 / 29 / 94$ & 64) & CASS & $T$ & \\
\hline TE-10B-AP-43 & $4 / 9 / 94$ & 65 & CASS & $\mathbf{T}$ & \\
\hline TE-108-AP-43 & $4 / 15 / 94$ & 65 & CASS & $\mathbf{T}$ & \\
\hline TE-108-AP-43 & $4 / 20 / 94$ & 64 & CASS & $\mathbf{T}$ & \\
\hline TE-108-AP-43 & 4/29/94 & 64 & CASS & $\mathbf{T}$ & \\
\hline TE-108-AP-43 & 5/4/94 & 64 & CASS & $\mathbf{T}$ & \\
\hline TE-108-AP-43 & $5 / 9 / 94$ & 65 & CASS & $T$ & \\
\hline TE-108-AP-43 & $5 / 16 / 94$ & 104 & CASS & $T$ & \\
\hline TE-108-AP-43 & $5 / 23 / 94$ & 99 & CASS & $T$ & \\
\hline TE-108-AP-43 & $6 / 2 / 94$ & 99 & CASS & $T$ & \\
\hline TE-108-AP-43 & $6 / 8 / 94$ & 98 & CASS & $T$ & \\
\hline TE-108-AP-43 & $6 / 14 / 94$ & 95 & CASS & $\mathbf{T}$ & \\
\hline TE-108-AP-43 & $6 / 16 / 94$ & 63 & CASS & $\mathbf{S}$ & Daka point did not eqree with locel trend \\
\hline
\end{tabular}




\begin{tabular}{|c|c|c|c|c|c|}
\hline \multirow{2}{*}{\multicolumn{6}{|c|}{$\begin{array}{l}\text { TANK 341-AP-108 } \\
\text { THIFAWOGOUFLE BATA }\end{array}$}} \\
\hline & & & & & \\
\hline THEFMOCOUPL * & DATE & TEMFERATUAE $\left({ }^{\circ} F\right)$ & DATA SOUACE & QUALTY & COMMENT \\
\hline TE-108-AP-43 & $6 / 17 / 94$ & 63 & CASS & $\mathrm{s}$ & Date point did not agree with local trend \\
\hline TE-108-AP-43 & 6/20/94 & 63 & CASS & $\mathbf{s}$ & Data point did not agree with local trend \\
\hline$T E-108-A P-43$ & 6/24/94 & 98 & CASS & $\mathbf{T}$ & \\
\hline TE-108-AP-43 & $6 / 27 / 94$ & 99 & CASS & $T$ & \\
\hline TE-108-AP-43 & $7 / 7 / 94$ & 97 & CASS & $T$ & \\
\hline TE-108-AP-43 & $7 / 15 / 94$ & 96 & CASS & $\mathbf{T}$ & \\
\hline TE-108-AP-43 & $7 / 78 / 94$ & 95 & CASS & $\mathbf{T}$ & \\
\hline TE-108-AP-43 & $7 / 28 / 94$ & 93 & CASS & $T$ & \\
\hline TE-108-AP:43 & 7/31/94 & 100 & CASS & $\mathbf{T}$ & \\
\hline TE-108-AP-43 & $8 / 10 / 94$ & 96 & CASS & $\mathbf{T}$ & \\
\hline TE-108-AP-43 & $8 / 18 / 94$ & 97 & CASS & $T$ & \\
\hline TE-108-AP-43 & $8 / 22 / 94$ & 88 & CASS & $\mathbf{T}$ & \\
\hline TE-108-AP-43 & $9 / 3 / 94$ & 97 & CASS & $\mathbf{T}$ & \\
\hline$T E-108-A P-43$ & $9 / 10 / 94$ & 101 & CASS & $\mathbf{T}$ & \\
\hline$T E-108-A P-43$ & 9/15/94 & 100 & CASS & $\mathrm{T}$ & \\
\hline TE-108-AP-43 & 9/23/94 & 101 & CASS & $T$ & \\
\hline TE-108-AP-43 & $9 / 30 / 94$ & 100 & CASS & $T$ & \\
\hline TE-108-AP.43 & $10 / 2 / 94$ & 103 & CASS & $T$ & \\
\hline TE-108-AP-43 & $10 / 16 / 94$ & 102 & CASS & $T$ & \\
\hline TE-108-AP.43 & $10 / 17 / 94$ & 104 & CASS & $T$ & \\
\hline TE-108-AP-43 & $10 / 29 / 94$ & 104 & CASS & $\bar{T}$ & \\
\hline TE-103-AP-43 & $11 / 5 / 94$ & 304 & CASS & $T$ & \\
\hline TE-102-AP-43 & $11 / 11 / 34$ & 103 & CASS & $T$ & \\
\hline TE-10B.AP-43 & $11 / 16 \mathrm{BS4}$ & 103 & CASS & $T$ & \\
\hline TE-108-AP.A3 & $11 / 22194$ & 104 & CASS & $\mathbf{T}$ & \\
\hline TE-108-AP-A3 & $12 / 3 / 94$ & 104 & CASS & $T$ & \\
\hline TE-108-AP-43 & $12 / 4 / 94$ & 108 & CASS & $T$ & \\
\hline TE-108-AP-43 & $12 / 12 / 94$ & 103 & CASS & $\mathbf{T}$ & \\
\hline TE-108-AP-43 & 12/19/94 & 105 & CASS & $\mathbf{T}$ & \\
\hline TE-108-AP-45 & $12 / 30 / 94$ & 106 & CASS & $\mathbf{T}$ & \\
\hline TE-108-AP-43 & 1/4/85 & 110 & CASS & $T$ & \\
\hline TE-108-AP-43 & 1/14/85 & 104 & CASS & $\mathbf{T}$ & \\
\hline TE-108-AP-A3 & V/15/95 & 105 & CASS & $\mathbf{T}$ & \\
\hline TE-108-AP-43 & $1 / 23 / 95$ & 105 & CASS & $T$ & \\
\hline TE-108-AP-A3 & 2/2/95 & 88 & CASS & $\mathbf{T}$ & \\
\hline TE-100-AP-43 & $2 / 6 / 95$ & 101 & CASS & $T$ & \\
\hline TE-108-AP-43 & $2 / 14 / 95$ & 106 & CASS & $\mathbf{T}$ & \\
\hline TE-108-AP-43 & $2 / 24 / 95$ & 96 & CASS & $T$ & \\
\hline TE-108-AP-43 & $3 / 2 / 95$ & 101 & CASS & $T$ & \\
\hline IE-108-AP-43 & $3 / 2 / 35$ & 98 & CASS & $T$ & \\
\hline TE-100-AP-43 & $3 / 16 / 95$ & 94 & CASS & $\mathbf{T}$ & \\
\hline TE-100-AP-43 & $3 / 25 / 95$ & 94 & CASS & $T$ & \\
\hline$T E-108-A P-43$ & $3 / 30 / 95$ & 96 & CASS & $\bar{T}$ & \\
\hline & & & & & \\
\hline TE-108-AP-44 & 4/5/86 & 58 & CASS & $\mathbf{T}$ & \\
\hline TE-106-AP-44 & $4 / 6 / 86$ & 59 & CASS & $\mathbf{T}$ & \\
\hline TE-106-AP-44 & $5 / 17 / 86$ & 57 & CASS & $T$ & \\
\hline TE-108-AP-44 & $5 / 22 / 96$ & 6i & CASS & $T$ & \\
\hline TE-108-AP-44 & $5 / 30 / 66$ & 59 & CASS & $\bar{T}$ & \\
\hline TE-108-AP-44 & $6 / 7 / 86$ & 59) & CASS & $T$ & \\
\hline TE-108-AP-44 & 6/9/86 & 64 & CASS & $T$ & \\
\hline$T E-108-A P-44$ & $6 / 21 / 86$ & 60 & CASS & $\bar{T}$ & \\
\hline TE-108-AP-44 & $6 / 25 / 86$ & 60 & CASS & $T$ & \\
\hline TE-10Q-AP-44 & $7 / 3 / 86$ & 62 & CASS & $T$ & \\
\hline TE-108-AP-44 & $7 / 10 / 36$ & 62 & CASS & $T$ & \\
\hline TE-108-AP-44 & 7/19/96 & 61) & CASS & $T$ & \\
\hline TE-108-AP-44 & $7 / 26 / 86$ & 61 & CASS & $\mathbf{T}$ & \\
\hline TE-108-AP-44 & $712 \mathrm{~B} / 86$ & 64 & CASS & $\mathbf{T}$ & \\
\hline TE-108-AP.44 & 8/9/86 & 65 & CASS & $\bar{T}$ & \\
\hline TE-108-AP-44 & $8 / 13 / 86$ & 65 & CASS & $T$ & \\
\hline TE-108-AP-44 & $8 / 17 / 86$ & 64 & CASS & $\mathbf{T}$ & \\
\hline TE-108-AP-44 & $8 / 30 / 86$ & 63 & CASS & $T$ & \\
\hline TE-100-AP-44 & $9 / 3 / 86$ & 64 & CASS & $T$ & \\
\hline TE-100-AP-44 & $8 / 24 / 86$ & 64 & CASS & $\mathbf{T}$ & \\
\hline$T E-108-A P-44$ & $11 / 1 / 86$ & 62 & CASS & $T$ & \\
\hline TE-108-AP-44 & $11 / 2 / 86$ & 62 & CASS & $T$ & \\
\hline TE-108-AP-44 & $11 / 29 / 86$ & 59 & CASS & $\mathbf{T}$ & \\
\hline$T E-108-A P-44$ & $12 / 11 / \mathrm{A6}$ & 59 & CASS & $\mathbf{T}$ & \\
\hline TE-108-AP-44 & $12 / 18 / 86$ & 58 & CASS & $\mathbf{T}$ & \\
\hline TE-108-AP-44 & $12 / 24 / 66$ & 57! & CASS & $\mathbf{T}$ & \\
\hline TE-108-AP-44 & $12 / 29 / 86$ & 56 & CASS & $T$ & \\
\hline TE-108-AP-44 & $7 / 1 / 87$ & 66 & CASS & $T$ & \\
\hline TE-108-AP-44 & $7 / 8 / 87$ & 70. & CASS & $T$ & \\
\hline
\end{tabular}




\begin{tabular}{|c|c|c|c|c|c|}
\hline \multicolumn{6}{|c|}{$\begin{array}{l}\text { TANK 241-AP-108 } \\
\text { THERMOCOUPLE DATA }\end{array}$} \\
\hline THERMOCOUML * & DATE & TEMPERATURE ( $\left.{ }^{\circ} F\right)$ & DATA SOUACE & OUALTY & COMAIFNT \\
\hline TE-108-AP-44 & $7 / 12 / 87$ & r. & CASS & $T$ & COMMENT \\
\hline TE-108-AP-44 & $7 / 19 / 87$ & 69 & CASS & $\bar{T}$ & \\
\hline TE-10B-AP-44 & 7/27/37 & 68 & CASS & $T$ & \\
\hline TE-108-AP-44 & $8 / 5 / 37$ & 69 & CASS & $T$ & \\
\hline$T E-108-A P-44$ & $8 / 14 / 87$ & 69 & CASS & $T$ & \\
\hline TE-108-AP-44 & 8/22/87 & 68 & CASS & $T$ & \\
\hline TE-108-AP-44 & $8 / 26 / 37$ & 69 & CASS & $\bar{T}$ & \\
\hline TE-108-AP-44 & $9 / 4 / 87$ & 68 & CASS & $T$ & \\
\hline TE-108-AP-44 & $9 / 3 / 87$ & 69 & CASS & $T$ & \\
\hline TE-108-AP-44 & $9 / 17 / 87$ & 69 & CASS & $T$ & \\
\hline TE-108-AP-44 & 9/20/87 & 70 & CASS & $\bar{T}$ & \\
\hline TE-108-AP-44 & 9/27/87 & 70 & CASS & $T$ & \\
\hline TE-10B-AP-44 & $10 / 7 / 87$ & 70 & CASS & $T$ & \\
\hline TE-108-AP-44 & $10 / 16 / 87$ & 67 & CASS & $T$ & \\
\hline TE-108-AP-44 & $10 / 19 / 87$ & 68 & CASS & $T$ & \\
\hline TE-108-AP-44 & $10 / 31 / 87$ & 69 & CASS & $T$ & \\
\hline TE-10B-AP-44 & $11 / 4 / 87$ & 64 & CASS & $T$ & \\
\hline TE-108-AP-44 & $11 / 13 / 87$ & 64 & CASS & $\bar{T}$ & \\
\hline TE-108-AP-44 & $11 / 18 / 67$ & 62 & CASS & $T$ & \\
\hline$T E-108-A P-44$ & $11 / 26 / 87$ & 65 & CASS & $T$ & \\
\hline TE-108-AP-44 & $12 \pi / 87$ & 61 & CASS & $\bar{T}$ & \\
\hline TE-108-AP-44 & $12 / 6 / 87$ & 61 & CASS & $T$ & \\
\hline TE-108-AP-44 & $12 / 18 / 87$ & 58 & CASS & $\mathrm{T}$ & \\
\hline TE-108-AP-44 & $12 / 23 / 87$ & 57 & CASS & $\bar{T}$ & \\
\hline TE-108-AP-44 & $1 / 2 / 88$ & 57 & CASS & $T$ & \\
\hline TE-108-AP-44 & $1 / 7 / 88$ & 57 & CASS & $T$ & \\
\hline TE-108-AP-44 & $1 / 16 / 83$ & 52 & CASS & $T$ & \\
\hline TE-108-AP-44 & $1 / 23 / 8$ & 52 & CASS & $T$ & \\
\hline TE-108-AP-44 & $1 / 25 / 88$ & 53 & CASS & $\bar{T}$ & \\
\hline$T E-108-A P-44$ & $2 / 2 / 88$ & 53 & CASS & $T$ & \\
\hline$T E-108-A P-44$ & $2 / 11 / 88$ & 53 & CASS & $T$ & \\
\hline TE-108-AP-44 & $2 / 14 / 88$ & 54 & CASS & $\mathbf{T}$ & \\
\hline TE-108-AP-44 & $2 / 23 / 88$ & 53 & CASS & $T$ & \\
\hline TE-108-AP-44 & $3 / 3 / 88$ & 54 & CASS & $\mathbf{T}$ & \\
\hline TE-108-AP-44 & $3 / 12 / 38$ & $\mathbf{5 3}$ & CASS & $T$ & \\
\hline TE-108-AP-44 & $3 / 13 / 8$ & 54 & CASS & $T$ & \\
\hline TE-108-AP-44 & $3 / 21 / 68$ & 56 & CASS & $T$ & \\
\hline TE-108-AP-44 & 3/31/80 & 54! & CASS & $\mathbf{T}$ & \\
\hline TE-108-AP-44 & $6 / 1 / 83$ & 60 & CASS & $T$ & \\
\hline TE-108-AP-44 & $6 / 10 / 88$ & 61 & CASS & $\mathbf{T}$ & \\
\hline TE-108-AP-44 & $6 / 18 / 88$ & 61 & CASS & $\bar{T}$ & \\
\hline TE-108-AP-44 & 6/25/88 & 62 & CASS & $T$ & \\
\hline TE-108-AP-44 & $7 / 1 / 88$ & 64 & CASS & $T$ & \\
\hline TE-108-AP-44 & $7 / 9 / 88$ & 63 & CASS & $T$ & \\
\hline TE-108-AP-44 & $7 / 13 / 88$ & 66 & CASS & $T$ & \\
\hline TE-108-AP-44 & $7 / 23 / 83$ & 66 & CASS & $T$ & \\
\hline TE-108-AP-44 & $7 / 29 / 88$ & 67 & CASS & $T$ & \\
\hline TE-108-AP-44 & $8 / 3 / 83$ & 153 & CASS & $\mathbf{S}$ & Data point did not agree with local trend \\
\hline TE-108-AP-44 & $8 / 5 / 88$ & 68 & CASS & $\bar{T}$ & \\
\hline TE-108-AP-44 & $8 / 9 / 88$ & 69 & CASS & $T$ & \\
\hline TE-108-AP-44 & $8 / 20 / 88$ & 69 & CASS & $\mathbf{T}$ & \\
\hline TE-108-AP-44 & $8 / 22 / 88$ & 70 & CASS & $T$ & \\
\hline TE-108-AP-44 & 9/2/88 & 70 & CASS & $\mathbf{T}$ & \\
\hline TE-10B-AP-44 & 9/9/88 & 71 & CASS & $T$ & \\
\hline TE-108-AP-44 & 9/16/88 & 71 & CASS & $\mathbf{T}$ & \\
\hline TE-108-AP-A4 & 9/23/88 & 69 & CASS & $T$ & \\
\hline TE-108-AP-44 & 9/27/83 & 70 & CASS & $\bar{T}$ & \\
\hline TE-108-AP-44 & $12 / 1 / 88$ & 66 & CASS & $\mathbf{T}$ & \\
\hline TE-108-AP-44 & $12 / 8 / 88$ & 64 & CASS & $T$ & \\
\hline TE-108-AP-44 & $12 / 9 / 88$ & 97 & CASS & $\mathbf{S}$ & Data point did not agree with local trend \\
\hline TE-108-AP-44 & $12 / 16 / 88$ & 63 & CASS & $T$ & \\
\hline TE-108-AP-44 & $12 / 22 / 88$ & 62 & CASS & $T$ & \\
\hline TE-10B-AP-44 & $9 / 2 / 89$ & 71 & CASS & $T$ & \\
\hline TE-108-AP-44 & 9/7/39 & 69 & CASS & $\mathbf{T}$ & \\
\hline TE-10B-AP-44 & 9/16/89 & 70 & CASS & $\bar{T}$ & \\
\hline TE-108-AP-44 & $9 / 23 / 89$ & 69 & CASS & $T$ & \\
\hline TE-108-AP-44 & 9/29/89 & 71 & CASS & $T$ & \\
\hline TE-108-AP-44 & $11 / 4 / 89$ & 66 & CASS & $T$ & \\
\hline TE-108-AP-44 & $\begin{array}{ll}11 / 7 / 39 \\
\end{array}$ & 65 & CASS & $\mathbf{T}$ & \\
\hline TE-108-AP-44 & $11 / 18 / 89$ & 67 & CASS & $\bar{T}$ & \\
\hline TE-108-AP-44 & $11 / 24 / 89$ & 63 & CASS & $\mathbf{T}$ & \\
\hline TE-108-AP-44 & 12/2/89 & 65 & CASS & $\mathbf{T}$ & \\
\hline TE-108-AP-44 & $12 / 8 / 89$ & 62 & CASS & $\mathbf{T}$ & \\
\hline
\end{tabular}




\begin{tabular}{|c|c|c|c|c|c|}
\hline \multicolumn{6}{|c|}{$\begin{array}{l}\text { TANK 241-AP-108 } \\
\text { THEAMOCOUPLE DATA }\end{array}$} \\
\hline THERMOCOUPLE * & DATE & TEMPERATUAE $\left({ }^{\circ} F\right)$ & DATA SOURCE & GUALITY & COMMENT \\
\hline TE-108-AP-44 & $12 / 12 / 89$ & 61 & CASS & $T$ & \\
\hline TE-108.AP.44 & $12 / 23 / 89$ & 58 & CASS & $T$ & \\
\hline TE-10B-AP-44 & $12 / 30 / 89$ & 58 & CASS & $T$ & \\
\hline TE-108-AP-44 & $1 / 2 / 90$ & 58 & CASS & $T$ & \\
\hline TE-108-AP-44 & $7 / 12 / 90$ & 59 & CASS & $\mathbf{T}$ & \\
\hline TE-108-AP-44 & $1 / 15 / 90$ & 58 & CASS & $\bar{T}$ & \\
\hline TE-10B-AP-44 & $1 / 23 / 90$ & 58 & CASS & $T$ & \\
\hline TE-108-AP-44 & $2 / 1 / 190$ & 57 & CASS & $\bar{T}$ & \\
\hline TE-108-AP-44 & $2 / 7 / 90$ & 59 & CASS & $\bar{T}$ & \\
\hline TE-10Q-AP-44 & $2 / 13 / 90$ & 59 & CASS & $\mathbf{T}$ & \\
\hline TE-108-AP-44 & 2/24/90 & 58 & CASS & $\mathbf{T}$ & \\
\hline TE-108-AP-44 & $3 / 3 / 90$ & 60 & CASS & $T$ & \\
\hline TE-108-AP-44 & 3/9/90 & 62 & CASS & $\bar{T}$ & \\
\hline TE-108-AP-44 & $3 / 16 / 90$ & 61 & CASS & $T$ & \\
\hline TE-108-AP-44 & $3 / 18 / 90$ & 63 & CASS & $T$ & \\
\hline TE-108-AP-4A & $3 / 29 / 90$ & 84 & CASS & $\mathbf{S}$ & Data point did not agree with local trend \\
\hline TE-108-AP-44 & $3 / 30 / 30$ & 60 & CASS & $\bar{T}$ & \\
\hline TE-108-AP-44 & $4 / 2 / 90$ & 62 & CASS & $\mathbf{T}$ & \\
\hline TE-108-AP-44 & 4/12/80 & 63 & CASS & $\mathbf{T}$ & \\
\hline TE.10:-AP.44 & 4/21/90 & 60 & CASS & $\mathbf{T}$ & \\
\hline TE-108-AP-44 & $4 / 26 / 90$ & 63 & CASS & $\bar{T}$ & \\
\hline TE-108-AP-44 & $4 / 29 / 90$ & 63 & CASS & $\mathbf{T}$ & \\
\hline TE-108-AP-44 & $5 / 11 / 90$ & 86 & CASS & $\mathbf{T}$ & \\
\hline TE-108-AP-44 & $5 / 17 / 30$ & 63 & CASS & $T$ & \\
\hline TE-108-AP-44 & 5/21/30 & 63 & CASS & $T$ & \\
\hline TE-108-AP-44 & $6 / 1 / 90$ & 62 & CASS & $\mathbf{T}$ & \\
\hline TE-108-AP-44 & $6 / 6 / 90$ & 65 & CASS & $\bar{T}$ & \\
\hline TE-108-AP-44 & 6/14/90 & 63 & CASS & $T$ & \\
\hline TE-108-AP-44 & $6 / 21 / 90$ & 64 & CASS & $T$ & \\
\hline TE-106-AP-44 & 6/30/90 & 63 & CASS & $\mathbf{T}$ & \\
\hline TE-108-AP-44 & $7 / 6 / 50$ & 64 & CASS & $\mathbf{T}$ & \\
\hline TE-108-AP-44 & 7/12/80 & 68 & CASS & $\bar{T}$ & \\
\hline TE-108-AP-44 & $7 / 6 / 80$ & 68 & CASS & $\bar{T}$ & \\
\hline TE-108-AP-44 & $7 / 27 / 90$ & 69 & CASS & $\mathbf{T}$ & \\
\hline TE-108-AP-44 & $8 / 3 / 90$ & 70 & CASS & $\mathbf{T}$ & \\
\hline TE-108-AP-44 & $8 / 11 / 90$ & 69 & CASS & $T$ & \\
\hline$T E-108-A P-44$ & $8 / 12 / 90$ & 71 & CASS & $T$ & \\
\hline TE-108-AP-44 & $8 / 19 / 90$ & 711 & CASS & $\mathbf{T}$ & \\
\hline TE-10:AP.44 & $8 / 1 / 90$ & 69 & CASS & $T$ & \\
\hline TE-108-AP.44 & 9/8/90 & 69 & CASS & $\bar{T}$ & \\
\hline$T E-108-A P-44$ & 9/5/50 & 71 & CASS & $\bar{T}$ & \\
\hline TE-108-AP-44 & $9 / 20 / 90$ & 71 & CASS & $T$ & \\
\hline TE-108.AP-44 & $9 / 28 / 90$ & 70 & CASS & $T$ & \\
\hline TE-108-AP-44 & $10 / 6 / 90$ & 70 & CASS & $\bar{T}$ & \\
\hline$T E-108-A P-44$ & $10 / 13 / 90$ & 70 & CASS & $T$ & \\
\hline TE-108-AP-44 & $10 / 16 / 90$ & 69 & CASS & $T$ & \\
\hline$T E-108-A P-44$ & $10 / 26 / 90$ & 69 & CASS & $\bar{T}$ & \\
\hline TE-106-AP-44 & $11 / 2 / 90$ & 68 & CASS & $T$ & \\
\hline TE-108-AP-44 & $11 / 4 / 90$ & 68 & CASS & $T$ & \\
\hline TE-108-AP-44 & $11 / 17 / 90$ & 67 & CASS & $T$ & \\
\hline TE-108-AP-44 & $11 / 20 / 90$ & 67 & CASS & $\mathbf{T}$ & \\
\hline TE-108-AP-44 & $11 / 29 / 90$ & 65 & CASS & $T$ & \\
\hline TE-108-AP-44 & $12 / 6 / 90$ & 66 & CASS & $T$ & \\
\hline TE-108-AP-44 & $12 / 14 / 90$ & 65 & CASS & $\mathbf{T}$ & \\
\hline TE-108-AP-44 & $12 / 20 / 90$ & 64 & CASS & $T$ & \\
\hline TE-108-AP-44 & $12 / 27 / 90$ & 60 & CASS & $T$ & \\
\hline TE-108-AP-44 & $12 / 30 / 90$ & 59 & CASS & $T$ & \\
\hline TE-108-AP-44 & $1 / 11 / 91$ & 57 & CASS & $T$ & \\
\hline TE-108-AP-44 & $1 / 14 / 91$ & 58 & CASS & $T$ & \\
\hline TE-108-AP-44 & $1 / 24 / 91$ & 56 & CASS & $T$ & \\
\hline TE-108-AP-44 & $1 / 30 / 91$ & 55 & CASS & $\bar{T}$ & \\
\hline TE-108-AP-44 & $2 / 6 / 91$ & 56 & CASS & $T$ & \\
\hline TE-108-AP-44 & $2 / 16 / 91$ & 55 & CASS & $\bar{T}$ & \\
\hline TE-108.AP-44 & $2 / 17 / 91$ & 56 & CASS & $T$ & \\
\hline$T E-108-A P-44$ & $3 / 2 / 91$ & 55 & CASS & $\bar{T}$ & \\
\hline TE-108-AP-44 & $3 / 9 / 91$ & 57 & CASS & $T$ & \\
\hline$T E-108-A P-44$ & $3 / 13 \% 1$ & 57 & CASS & $\mathbf{T}$ & \\
\hline TE-108-AP-44 & $3 / 23 / 91$ & 56 & CASS & $T$ & \\
\hline TE-108-AP-44 & $3 / 25 / 91$ & 58 & CASS & $T$ & \\
\hline TE-108-AP-44 & $4 / 1 / 91$ & 59 & CASS & $T$ & \\
\hline TE-108-AP-44 & $4 / 12 / 91$ & 58 & CASS & $T$ & \\
\hline$T E-108 \cdot A P \cdot 44$ & $4 / 20 / 91$ & 57 & CASS & $T$ & \\
\hline TE-108-AP-44 & 4/27/91 & 57 & CASS & $T$ & \\
\hline
\end{tabular}




\begin{tabular}{|c|c|c|c|c|c|}
\hline \multicolumn{6}{|c|}{$\begin{array}{l}\text { TAKK 241.AP. } 108 \\
\text { THEAMOCOUPIE DATA }\end{array}$} \\
\hline THERMOCOUPL * & DATE & TEMPERATURE $\left({ }^{\circ} F\right)$ & DATA SOURCE & ouALTY & COMUEYT \\
\hline TE-108-AP-44 & $5 / 1 / 91$ & (2) & CASS & $T$ & \\
\hline TE-108-AP-44 & $5 / 5 / 91$ & 71 & CASS & $\mathbf{s}$ & Data point did not agree with local trend \\
\hline TE-108-AP-44 & $5 / 10 / 91$ & 59 & CASS & $T$ & \\
\hline TE-108-AP-44 & $5 / 16 / 91$ & 64 & CASS & $T$ & \\
\hline$T E-108-A P-44$ & $5 / 25 / 91$ & 69 & CASS & $T$ & \\
\hline TE-108-AP-44 & $5 / 31 / 91$ & 61 & CASS & $T$ & \\
\hline TE-108-AP-44 & $6 / 5 / 91$ & 62 & CASS & $T$ & \\
\hline TE-108-AP-44 & $6 / 15 / 91$ & 63 & CASS & $T$ & \\
\hline TE-108-AP-44 & $6 / 22 / 91$ & 64 & CASS & $T$ & \\
\hline TE-108-AP-44 & $6 / 28 / 91$ & 67 & CASS & $T$ & \\
\hline$T E-108-A P-44$ & $7 / 3 / 91$ & 66 & CASS & $T$ & \\
\hline TE-108-AP-44 & $7 / 13 / 91$ & 86 & CASS & $T$ & \\
\hline TE-108-AP-44 & $7 / 14 / 91$ & 66 & CASS. & $T$ & \\
\hline TE-108-AP-44 & $7 / 24 / 91$ & 68 & CASS & $\mathrm{T}$ & \\
\hline TE-108-AP-44 & 8/2/91 & 69 & CASS & $\bar{T}$ & \\
\hline TE-108-AP-44 & $8 / 6 / 91$ & 68 & CASS & $T$ & \\
\hline TE-10B-AP-44 & $8 / 12 / 91$ & 71 & CASS & $\bar{T}$ & \\
\hline TE-10B-AP-44 & $8 / 18 / 91$ & 71 & CASS & $\bar{T}$ & \\
\hline TE-10B-AP-44 & $8 / 30 / 91$ & 70 & CASS & $T$ & \\
\hline TE-108-AP-44 & $9 / 3 / 91$ & 70 & CASS & $\bar{T}$ & \\
\hline$T E-108-A P-44$ & $9 / 8 / 91$ & 72 & CASS & $T$ & \\
\hline TE-10B-AP-44 & $9 / 18 / 91$ & 73 & CASS & $\bar{T}$ & \\
\hline TE-108-AP-44 & $9 / 22 / 97$ & 71 & CASS & $T$ & \\
\hline TE-10B-AP-44 & 10/1/91 & 72 & CASS & $T$ & \\
\hline TE-108-AP-44 & 10/3/91 & 61 & CASS & s & Data point did not apree with local trand \\
\hline TE-108-AP-44 & $10 / 12 / 91$ & 71 & CASS & $\bar{T}$ & \\
\hline TE-108-AP-44 & $10 / 17 / 91$ & 73 & CASS & $T$ & \\
\hline TE-108-AP-44 & $10 / 25 / 91$ & 72 & CASS & $\bar{T}$ & \\
\hline TE-108-AP-44 & $10 / 28 / 91$ & 71 & CASS & $\bar{T}$ & \\
\hline TE-108-AP-44 & $11 / 6 / 91$ & 71 & CASS & $T$ & \\
\hline$T E-108-A P-44$ & $7 / 30 / 92$ & 66 & CASS & $T$ & \\
\hline TE-108-AP-44 & 8/8/22 & 67 & CASS & $T$ & \\
\hline TE-106-AP.44 & $8 / 15 / 92$ & 67, & CASS & $T$ & \\
\hline TE-108-AP-44 & $8 / 20 / 92$ & 68 & CASS & $T$ & \\
\hline TE-108-AP-44 & $8 / 28 / 92$ & 68. & CASS & T & \\
\hline$T E-108-A P-44$ & $8 / 31 / 92$ & 67 & CASS & $\mathbf{T}$ & \\
\hline TE-108-AP.44 & 9/9/92 & 67 & CASS & $T$ & \\
\hline TE-108-AP-44 & $9 / 16 / 92$ & 67 & CASS & $\mathbf{T}$ & \\
\hline TE-108-AP-44 & $9 / 26 / 92$ & 66 & CASS & $\mathbf{T}$ & \\
\hline TE-108-AP-44 & $10 / 3 / 92$ & 67 & CASS & $\mathbf{T}$ & \\
\hline TE-108-AP-44 & $10 / 10 / 92$ & 66 & CASS & $T$ & \\
\hline TE-106-AP.44 & $10 / 12 / 92$ & 68 & CASS & $T$ & \\
\hline TE-108-AP-44 & $10 / 24 / 92$ & 69 & CASS & $T$ & \\
\hline TE-108-AP-44 & $10 / 29 / 92$ & 70 & CASS & $T$ & \\
\hline TE-108-AP-44 & $11 / 7 / 92$ & 69 & CASS & $T$ & \\
\hline TE-108.AP-44 & $11 / 9 / 92$ & 69 & CASS & $T$ & \\
\hline TE-108-AP-44 & $11 / 17 / 92$ & 71 & CASS & $T$ & \\
\hline TE-108-AP-44 & $11 / 25 / 92$ & 68 & CASS & $T$ & \\
\hline TE-108-AP-44 & $12 / 3 / 92$ & 67 & CASS & $T$ & \\
\hline TE-108-AP-44 & $12 / 12 / 92$ & 69 & CASS & $\bar{T}$ & \\
\hline TE-108-AP-44 & $12 / 19 / 92$ & 67 & CASS & $T$ & \\
\hline TE-108-AP-44 & $12 / 21 / 92$ & 66 & CASS & $T$ & \\
\hline TE-108-AP-44 & $12 / 30 / 92$ & 66 & CASS & $\bar{T}$ & \\
\hline TE-108-AP-44 & $1 / 15 / 94$ & 63 & CASS & $T$ & \\
\hline TE-108-AP-44 & $1 / 20 / 94$ & 64 & CASS & $T$ & \\
\hline TE-108-AP-44 & $1 / 25 / 94$ & 64 & CASS & $T$ & \\
\hline TE-108-AP-44 & $2 / 4 / 94$ & 64 & CASS & $\bar{T}$ & \\
\hline TE-108-AP-44 & $2 / 12 / 94$ & 69 & CASS & $T$ & \\
\hline TE-108-AP-44 & $2 / 16 / 94$ & 63 & CASS & $T$ & \\
\hline TE-108-AP+44 & $2 / 26 / 94$ & 63 & CASS & $T$ & \\
\hline TE-108-AP-44 & $2 / 28 / 94$ & 65 & CASS & T & \\
\hline TE-108-AP-44 & $3 / 10 / 94$ & 64 & CASS & $T$ & \\
\hline TE-108-AP-44 & $3 / 14 / 94$ & 65 & CASS & $T$ & \\
\hline TE-108-AP-44 & $3 / 25 / 94$ & 65 & CASS & $\bar{T}$ & \\
\hline TE-108-AP-44 & $3 / 29 / 94$ & 64 & CASS & $T$ & \\
\hline TE-10B-AP-44 & $4 / 9 / 94$ & 64 & CASS & $T$ & \\
\hline TE-108-AP-44 & $4 / 15 / 94$ & 65 & CASS & $T$ & \\
\hline TE-108-AP-44 & $4 / 20 / 94$ & 64 & CASS & $T$ & \\
\hline TE-108-AP-44 & $4 / 27 / 94$ & 65 & CASS & $T$ & \\
\hline TE-10B-AP-44 & $5 / 4 / 94$ & 64 & CASS & $\bar{T}$ & \\
\hline TE-108-AP-44 & $5 / 8 / 94$ & 66 & CASS & $T$ & \\
\hline TE-108-AP-44 & $5 / 16 / 94$ & 104 & CASS & $T$ & \\
\hline TE-10B-AP-44 & $5 / 22 / 94$ & 99 & CASS & $\bar{T}$ & \\
\hline
\end{tabular}




\begin{tabular}{|c|c|c|c|c|c|}
\hline \multicolumn{6}{|c|}{$\begin{array}{l}\text { TANK 241-AP.108 } \\
\text { THERMOCOUFLE DATA }\end{array}$} \\
\hline THERMOCOUPLE & DATE & TEMPERATUAE $\left({ }^{\circ} \mathrm{F}\right)$ & DATA SOURCE & OUALTY & COMMENT \\
\hline TE-108-AP-44 & $6 / 2 / 94$ & 99 & CASS & $T$ & \\
\hline TE-10B-AP-44 & $6 / 8 / 94$ & 98 & CASS & $T$ & \\
\hline TE-108-AP-44 & $6 / 14 / 94$ & 95 & CASS & $T$ & \\
\hline TE-108-AP-44 & 6/16/94 & 63 & CASS & $\mathbf{S}$ & Data point did not agree with local trend \\
\hline TE-108-AP-44 & 6/17/94 & 63 & CASS & $\mathbf{S}$ & Data point did not agree with local trend \\
\hline TE-108-AP-44 & 6/20/94 & 63 & CASS & $\overline{\mathbf{S}}$ & Data point did not agree with local frend \\
\hline TE-108-AP-44 & $6 / 24 / 94$ & 98 & CASS & $\bar{T}$ & \\
\hline TE-108-AP-44 & 6/27/94 & 99 & CASS & $\mathbf{T}$ & \\
\hline TE-10B-AP-44 & $7 / 7 / 94$ & 97 & CASS & $\mathbf{T}$ & \\
\hline TE-108-AP-44 & $7715 / 94$ & 95 & CASS & $T$ & \\
\hline TE-108-AP-44 & $7718 / 94$ & 95 & CASS & $\mathbf{T}$ & \\
\hline TE-108-AP-44 & $7 / 28 / 94$ & 93 & CASS & $\mathbf{T}$ & \\
\hline TE-108-AP-44 & $7 / 31 / 34$ & 100 & CASS & $\bar{T}$ & \\
\hline TE-108-AP-44 & $8 / 10 / 94$ & 96 & CASS & $\mathbf{T}$ & \\
\hline TE-108-AP-44 & $8 / 18 / 94$ & 97 & CASS & $T$ & \\
\hline TE-106-AP-44 & $8 / 22194$ & 98 & CASS & $T$ & \\
\hline TE-108-AP-44 & $9 / 3 / 94$ & 97 & CASS & $T$ & \\
\hline TE-108-AP-44 & $9 / 10 / 94$ & 101 & CASS & $T$ & \\
\hline TE-108-AP-44 & $9 / 15 / 94$ & 100 & CASS & $T$ & \\
\hline TE-108-AP-44 & $9 / 23 / 94$ & 100 & CASS & $T$ & \\
\hline TE-10:AP-44 & $9 / 30 / 94$ & 100 & CASS & $T$ & \\
\hline TE-108-AP-44 & $10 / 2 / 94$ & 103 & CASS & $T$ & \\
\hline TE-108-AP-A4 & $10 / 12 / 94$ & 102 & CASS & $T$ & \\
\hline TE-108.AP-44 & $10 / 22 / 94$ & 103 & CASS & $\bar{T}$ & \\
\hline TE-103-AP-44 & $10 / 25 / 94$ & 145 & CASS & $\mathbf{S}$ & Data point did not agree with local trend \\
\hline TE-108-AP-44 & $10 / 29 / 94$ & 104 & CASS & $T$ & \\
\hline$T E-108-A P-4$ & $11 / 5 / 94$ & 104 & CASS & $\bar{T}$ & \\
\hline TE-10B-AR-44 & $11 / 11 / 94$ & 104 & CASS & $T$ & \\
\hline TE-108-AP-A4 & $11 / 16 / 94$ & 104 & CASS & $\bar{T}$ & \\
\hline$\overline{I E-108-A P-44}$ & $11 / 22 / 94$ & 104 & CASS & $\mathbf{T}$ & \\
\hline TE-108-AP-44 & $11 / 27 / 94$ & 104 & CASS & $T$ & \\
\hline TE-108-AP-44 & $12 / 4 / 34$ & 109 & CASS & $\mathrm{T}$ & \\
\hline TE-108-AP-44 & $12 / 12 / 94$ & 104 & CASS & $\bar{T}$ & \\
\hline TE-108-AP-44 & $12 / 19 / 94$ & 105 & CASS & $T$ & \\
\hline TE-108-AP-44 & $12 / 30 / 94$ & 106 & CASS & $T$ & \\
\hline IE-108-APA4 & $1 / 4 / 95$ & 111 & CASS & $T$ & \\
\hline TE-108-AP-44 & 1/13/95 & 105 & CASS & $\mathbf{T}$ & \\
\hline TE-108.AP-A4 & $1 / 15 / 25$ & 106 & CASS & $T$ & \\
\hline TE-108-AP-44 & $1 / 23 / 25$ & 105 & CASS & $\bar{T}$ & \\
\hline TE-108-AP-44 & $2 / 2 / 95$ & 98 & CASS & $\mathbf{T}$ & \\
\hline TE-108-AP-44 & $2 / 6 / 95$ & 101 & CASS & $T$ & \\
\hline TE-10:-AP-44 & 2/14/95 & 100 & CASS & $T$ & \\
\hline TE-108-AP-44 & 2/24/95 & 96 & CASS & $T$ & \\
\hline TE-108-AP-44 & $3 / 2 / 95$ & 100 & CASS & $T$ & \\
\hline TE-108-AP-44 & $3 / 7 / 95$ & 98 & CASS & $T$ & \\
\hline TE-108-AP-44 & 3/16/95 & 94 & CASS & $T$ & \\
\hline TE-108-AP-44 & $3 / 25 / 95$ & 94 & CASS & $T$ & \\
\hline TE-108-AP-44 & $3 / 30 / 95$ & 96 & CASS & $I$ & \\
\hline TE-102AP-5 & & $\rightarrow$ & & & \\
\hline TE-108-AP-45 & $4 / 5 / 86$ & 57 & CASS & $T$ & \\
\hline$\frac{\text { TE-108-AP-45 }}{\text { TE-108-AP-45 }}$ & $4 / 6 / 86$ & 59 & CASS & $T$ & \\
\hline $\begin{array}{l}\text { TE-108-AP-45 } \\
T E-108-A P-45\end{array}$ & 5/17/86 & $\frac{57}{60}$ & CASS & $T$ & \\
\hline $\begin{array}{l}\text { TE-108-AP-45 } \\
\text { TE-108-AP-45 }\end{array}$ & $5 / 24 / 86$ & $\frac{60}{59}$ & CASS & $T$ & \\
\hline$\frac{\text { TE-108-AP-45 }}{\text { TE-108-AP-45 }}$ & $5 / 30 / 86$ & $\frac{59}{59}$ & CASS & $T$ & \\
\hline $\begin{array}{l}\text { TE-108-AP-45 } \\
\text { TE-10B-AP-45 }\end{array}$ & 6/7/86: & $\frac{59}{64}$ & CASS & $\frac{T}{T}$ & \\
\hline$\frac{\text { TE-108-AP-45 }}{\text { TE-108-AP-45 }}$ & $\begin{array}{r}\text { 6/9/86 } \\
6 / 21 / 36\end{array}$ & 64 & $\begin{array}{l}\text { CASS } \\
\text { CASS }\end{array}$ & $\frac{T}{T}$ & \\
\hline TE-108-AP-45 & 6/25/86 & 60 & CASS & $T$ & \\
\hline TE-108-AP-45 & $7 / 3 / 66$ & 62 & CASS & $\mathbf{T}$ & \\
\hline TE-108-AP-A5 & $7 / 10 / 86$ & 62 & CASS & $T$ & \\
\hline TE-108-AP-45 & 7/19/36 & 61 & CASS & $\bar{T}$ & \\
\hline TE-108-AP-45 & 7/26/36 & 61 & CASS & $T$ & \\
\hline TE-108-AP-45 & $7 / 28 / 86$ & 63 & CASS & $T$ & \\
\hline TE-108-AP-45 & 8/9/86 & 64 & CASS & $T$ & \\
\hline TE-108-AP-45 & $8 / 13 / 36$ & 64 & CASS & $\mathbf{T}$ & \\
\hline TE-108-AP-45 & $8 / 17 / 86$ & 65 & CASS & $T$ & \\
\hline TE-108-AP-45 & $8 / 30 / 86$ & 63 & CASS & $T$ & \\
\hline TE-108-AP-45 & $9 / 3 / 36$ & $64 \mid$ & CASS & $T$ & \\
\hline TE-108-AP-45 & 9/24/86 & 64 & CASS & $T$ & \\
\hline$T E-108-A P-45$ & $11 / 1 / 86$ & 62 & CASS & $T$ & \\
\hline TE-108-AP-45 & $11 / 2 / 86$ & 62: & CASS & $\bar{T}$ & \\
\hline$T E-108 \cdot A P \cdot 45$ & $11 / 29 / 86$ & 59! & CASS & $\bar{T}$ & \\
\hline TE-108-AP-45 & $12 / 10 / 86$ & 59 & CASS & $\mathbf{T}$ & \\
\hline
\end{tabular}




\begin{tabular}{|c|c|c|c|c|c|}
\hline \multicolumn{6}{|c|}{$\begin{array}{l}\text { TANK 241-AP-108 } \\
\text { THERMOCOUPLE DATA }\end{array}$} \\
\hline & & & & & \\
\hline THE:HOCOUPL * & DATE & TEMPERATUAE $\left({ }^{\circ} F\right\}$ & DATA SOURCE & QUALTYY & COMMENT \\
\hline TE-108-AP-45 & $12 / 18 / 86$ & 58 & CASS & $T$ & \\
\hline TE-10B-AP-45 & $12 / 24 / 86$ & $5 B$ & CASS & $T$ & \\
\hline TE-108-AP-45 & $12 / 29 / 86$ & 56 & CASS & $T$ & \\
\hline TE-108-AP-45 & $7 / 1 / 87$ & 66 & CASS & $T$ & \\
\hline TE-108-AP-45 & $7 / 8 / 87$ & 69 & CASS & $T$ & \\
\hline TE-108-AP-45 & $7 / 12 / 87$ & 66 & CASS & $T$ & \\
\hline TE-108-AP-45 & $7 / 19 / 87$ & 71 & CASS & $T$ & \\
\hline TE-108-AP-45 & $7 / 26 / 87$ & 69 & CASS & $\bar{T}$ & \\
\hline TE-108-AP-45 & $8 / 5 / 87$ & 69 & CASS & $T$ & \\
\hline IE-108-AP-45 & $8 / 14 / 87$ & 70 & CASS & $T$ & \\
\hline TE-108-AP-45 & $8 / 22 / 87$ & 68 & CASS & $T$ & \\
\hline TE-108-AP-45 & $8 / 26 / 87$ & 69 & CASS & $T$ & \\
\hline TE-10:-AP-45 & $9 / 4 / 87$ & 68 & CASS & $T$ & \\
\hline TE-108-AP-45 & $9 / 8 / 87$ & 69 & CASS & $\bar{T}$ & \\
\hline TE-108-AP-45 & $9 / 17 / 87$ & 69 & CASS & $T$ & \\
\hline TE-108-AP-45 & 9/20/87 & 70 & CASS & $T$ & \\
\hline TE-108-AP-45 & 9/27/87 & 70 & CASS & $\mathbf{T}$ & \\
\hline TE-108.AP-45 & $10 / 7 / 87$ & 70 & CASS & $T$ & \\
\hline TE-108-AP-45 & $10 / 16 / 87$ & 67 & CASS & $T$ & \\
\hline TE-108-AP-45 & $10 / 19 / 87$ & 88 & CASS & $T$ & \\
\hline TE-108-AP-45 & $10 / 31 / 87$ & 68 & CASS & $\bar{T}$ & \\
\hline TE-108-AP-45 & $11 / 1 / 87$ & 66 & CASS & $\mathbf{T}$ & \\
\hline TE-108-AP-A5 & $11 / 6 / 87$ & 97. & CASS & $\mathbf{S}$ & Data point did not agree with local trend \\
\hline TE-108-AP-45 & $19 / 13 / 87$ & 63 & CASS & $\bar{T}$ & \\
\hline TE-10B-AP-45 & $11 / 18 / 87$ & 62 & CASS & $T$ & \\
\hline TE-108-AP-45 & $11 / 26 / 97$ & 65 & CASS & $T$ & \\
\hline TE-108-AP-45 & $11 / 29 / 87$ & 63 & CASS & $T$ & \\
\hline TE-108-AP-45 & $12 / 6 / 87$ & 61 & CASS & $T$ & \\
\hline$T E \cdot 108-A P-45$ & $12 / 18 / 87$ & 59 & CASS & $T$ & \\
\hline TE-108-AP-45 & $12 / 23 / 87$ & 57 & CASS & $T$ & \\
\hline TE-108-AP-45 & $1 / 2 / 88$ & 57 & CASS & $T$ & \\
\hline$T E-108-A P-45$ & $1 / 4 / 88$ & 53 & CASS & $T$ & \\
\hline TE-108-AP-45 & $1 / 16 / 88$ & 52 & CASS & $T$ & \\
\hline TE-108-AP-A5 & $1 / 22 / 83$ & 52 & CASS & $T$ & \\
\hline TE-108-AP-45 & $1 / 25 / 88$ & $\mathbf{5 3}$ & CASS & $T$ & \\
\hline TE-108-APAS5 & $2 / 2 / 88$ & 53 & CASS & $T$ & \\
\hline TE-108-AP-45 & 2/11/83 & $5 \mathbf{5 3}$ & CASS & $T$ & \\
\hline TE-108-AP-45 & $2 / 14 / 68$ & 54 & CASS & $T$ & \\
\hline TE-108-AP-45 & $2127 / 88$ & 52 & CASS & $\bar{T}$ & \\
\hline TE-108-AP-45 & $3 / 3 / 88$ & 54 & CASS & $\bar{T}$ & \\
\hline TE-108-AP-45 & 3/12/88 & 53 & CASS & $T$ & \\
\hline TE-108-AP-45 & $3 / 13 / 88$ & 54 & CASS & $\mathbf{T}$ & \\
\hline TE-108-AP-45 & $3 / 25 / 88$ & SE & CASS & 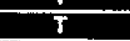 & \\
\hline TE-108-AP-45 & 3/31/88 & 54 & CASS & $T$ & \\
\hline TE-108-AP-45 & $6 / 1 / 88$ & 60 & CASS & $T$ & \\
\hline TE-108-AP-45 & 6/8/89 & 62 & CASS & $\bar{T}$ & \\
\hline TE-108-AP-45 & $6 / 18 / 88$ & 61 & CASS & $\mathbf{T}$ & \\
\hline TE-108-AP-45 & $6 / 25 / 88$ & 62 & CASS & $T$ & \\
\hline TE-108-AP-45 & $6 / 26 / 88$ & 120 & CASS & $\mathbf{s}$ & Data point did not agree with locel trend \\
\hline TE-108-AP-45 & 6/27/88 & 120 & CASS & $\mathbf{s}$ & Date point did not agree with locel trend \\
\hline TE-108-AP-45 & $7 / 1 / 88$ & 64 & CASS & $T$ & \\
\hline TE-108-AP-45 & $7 / 9 / 88$ & 63 & CASS & $\bar{T}$ & \\
\hline TE-108-AP-45 & $7 / 76 / 88$ & 64 & CASS & $\mathbf{T}$ & \\
\hline TE-108-AP-45 & 7/23/88 & 66 & CASS & $\bar{T}$ & \\
\hline TE-108-AP-45 & $7 / 29 / 68$ & 67 & CASS & $T$ & \\
\hline TE-108-AP-45 & $8 / 3 / 8 B$ & 69 & CASS & $T$ & \\
\hline TE-108-AP-45 & $8 / 9 / 68$ & 69 & CASS & $T$ & \\
\hline TE-108-AP+45 & $8 / 15 / 88$ & 118 & CASS & $\mathbf{s}$ & Data point did not agree whith local trend \\
\hline TE-108-AP-45 & $8 / 20 / 88$ & 69 & CASS & $\bar{T}$ & \\
\hline TE-108-AP-45 & $8 / 22 / 88$ & 69 & CASS & $T$ & \\
\hline TE-108-AP-45 & $8 / 31 / 88$ & 70 & CASS & $T$ & \\
\hline TE-108-AP-45 & $9 / 9 / 88$ & 71 & CASS & $T$ & \\
\hline TE-108-AP-45 & $9 / 16 / 8$ & 71 & CASS & $T$ & \\
\hline TE-108-AP-45 & $9 / 23 / 88$ & 69 & CASS & $T$ & \\
\hline TE-108-AP-45 & $9 / 27 / 88$ & 70 & CASS & $T$ & \\
\hline TE-108-AP-45 & $12 / 1 / 88$ & 66 & CASS & $T$ & \\
\hline TE-108-AP-45 & $12 / 8 / 88$ & 64 & CASS & $T$ & \\
\hline TE-108-AP-45 & $12 / 13 / 88$ & 63 & CASS & $T$ & \\
\hline TE-108-AP-45 & $12 / 22 / 89$ & 63 & CASS & $T$ & \\
\hline TE-108-AP-45 & $9 / 2 / 89$ & 71 & CASS & $T$ & \\
\hline TE-108-AP-45 & $9 / 7 / 89$ & 69 & CASS & $T$ & \\
\hline TE-108-AP-45 & $9 / 16 / 89$ & 70 & CASS & $T$ & \\
\hline TE-108-AP-45 & 9/18/89 & 70 & CASS & $\mathbf{T}$ & \\
\hline
\end{tabular}




\begin{tabular}{|c|c|c|c|c|c|}
\hline \multicolumn{6}{|c|}{$\begin{array}{l}\text { TANK 241-AP-108 } \\
\text { THERMOCOUFE DATA }\end{array}$} \\
\hline THERMOCOUPLE * & DATE & TEMPERATURE ( $\left.{ }^{\circ} F\right)$ & DATA SOUACE & OUALITY & COMMENT \\
\hline TE-108-AP-45 & 9/29/89 & 71 & CASS & $T$ & \\
\hline TE-108-AP-45 & $11 / 4 / 89$ & 66 & CASS & $T$ & \\
\hline TE-108-AP-45 & $11 / 7 / 89$ & 65 & CASS & $T$ & \\
\hline TE-108-AP-45 & $11 / 18 / 89$ & 67 & CASS & $T$ & \\
\hline$T E-108-A P-45$ & $11 / 24 / 89$ & 63 & CASS & $T$ & \\
\hline TE-108-AP-45 & $12 / 2 / 89$ & 66 & CASS & $T$ & \\
\hline TE-108-AP-45 & $12 / 8 / 89$ & 61 & CASS & $T$ & \\
\hline TE-108-AP-45 & $12 / 12 / 39$ & 61 & CASS & $\mathbf{T}$ & \\
\hline TE-108-AP-45 & $12 / 22 / 89$ & 58 & CASS & $T$ & \\
\hline TE-108-AP-45 & $12 / 30 / 89$ & 58 & CASS & $\mathbf{T}$ & \\
\hline TE-108-AP-45 & $1 / 2 / 90$ & 59 & CASS & $T$ & \\
\hline TE-108-AP-45 & $1 / 12 / 90$ & 59 & CASS & $T$ & \\
\hline$I E-108-A P-45$ & $1 / 20 / 90$ & 58 & CASS & $\bar{I}$ & \\
\hline TE-108-AP-45 & $1 / 23 / 90$ & 58 & CASS & $\bar{T}$ & \\
\hline TE-108-AP-45 & $2 / 1 / 80$ & 58 & CASS & $T$ & \\
\hline IE-108-AP-45 & $2 / 4 / 90$ & 59 & CASS & $T$ & \\
\hline TE-108-AP-45 & 2/15/90 & 58 & CASS & $T$ & \\
\hline TE-108-AP-45 & $2 / 24 / 90$ & 57 & CASS & $\mathrm{T}$ & \\
\hline TE-108-AP-45 & $3 / 3 / 90$ & 69 & CASS & $\mathbf{T}$ & \\
\hline TE-108-AP-45 & $3 / 9 / 90$ & 62 & CASS & $T$ & \\
\hline TE-108-AP-45 & $3 / 14 / 90$ & 61 & CASS & $\mathbf{T}$ & \\
\hline TE-108-AP-45 & $3 / 18 / 90$ & 62 & CASS & $T$ & \\
\hline TE-108-AP-45 & $3 / 29 / 90$ & 62 & CASS & $T$ & \\
\hline TE-108-AP-45 & $4 / 3 / 80$ & 61 & CASS & $T$ & \\
\hline$T E-108-A P-45$ & $4 / 12 / 90$ & 62 & CASS & $T$ & \\
\hline TE-108-AP-45 & $4 / 21 / 30$ & 60 & CASS & $T$ & \\
\hline TE-108-AP.45 & $4 / 26 / 200$ & 63 & CASS & $\mathbf{T}$ & \\
\hline TE-108-AP-45 & 4/29/90 & 63 & CASS & $\bar{T}$ & \\
\hline TE-10B-AP-45 & $5 / 11 / 90$ & 66 & CASS & $T$ & \\
\hline$T E-108-A P-45$ & 5/17/80 & 63 & CASS & $\bar{T}$ & \\
\hline TE-106-AP-45 & $5 / 21 / 90$ & 63 & CASS & $T$ & \\
\hline TE-108-AP-45 & $6 / 1 / 90$ & 63 & CASS & $T$ & \\
\hline TE-108-AP-45 & $6 / 6 / 90$ & 64 & CASS & $\mathbf{T}$ & \\
\hline TE-108-AP-45 & $6 / 12 / 90$ & 64 & CASS & $\mathbf{T}$ & \\
\hline TE-108-AP-45 & $6 / 21 / 30$ & 64 & CASS & $T$ & \\
\hline TE-108-AP-45 & $6 / 30 / 90$ & 63 & CASS & $T$ & \\
\hline TE-108-AP-45 & $7 / 6 / 90$ & 64 & CASS & $T$ & \\
\hline TE-108-AP-45 & $7 / 12 / 90$ & 67 & CASS & $\bar{T}$ & \\
\hline TE-108-AP-A5 & $7 / 16 / 90$ & 68 & CASS & $\mathbf{T}$ & \\
\hline TE-108-AP-45 & $7 / 27 / 90$ & 69 & CASS & $\bar{T}$ & \\
\hline TE-108-AP-45 & $8 / 3 / 30$ & 70 & CASS & $\mathbf{T}$ & \\
\hline TE-108-AP-45 & $8 / 7 / 90$ & 70 & CASS & $T$ & \\
\hline TE-102-AP-45 & $8 / 12 / 90$ & 7 & CASS & $\bar{T}$ & \\
\hline TE-108-AP-45 & $8 / 19 / 90$ & 71 & CASS & 7 & \\
\hline TE-100-AP-45 & $9 / 1 / 90$ & 69 & CASS & $T$ & \\
\hline TE-10BAP-45 & 9/8/90 & 69 & CASS & $T$ & \\
\hline TE-108-AP-45 & 9/12/30: & 71 & CASS & $T$ & \\
\hline TE-10B-AP-45 & $9 / 20 / 90$ & 70 & CASS & $\mathbf{T}$ & \\
\hline TE-108-AP-45 & $9 / 28 / 90$ & 70 & CASS & $T$ & \\
\hline TE-108-AP-45 & $10 / 6 / 90$ & 70 & CASS & $T$ & \\
\hline TE-108-AP-4S & $10 / 13 / 90$ & 69 & CASS & $\mathbf{T}$ & \\
\hline TE-108-AP+45 & $10 / 20 / 90$ & 68 & CASS & $\bar{T}$ & \\
\hline TE-108-AP-45 & $10 / 26 / 90$ & 69 & CASS & $T$ & \\
\hline TE-108-AP-45 & $10 / 31 / 90$ & 71 & CASS & $\bar{T}$ & \\
\hline TE-108-AP-45 & $11 / 4 / 90$ & 68 & CASS & $T$ & \\
\hline TE-108-AP-45 & $11 / 17 / 80$ & 67 & CASS & $T$ & \\
\hline TE-108-AP-45 & $11 / 20 / 90$ & 67 & CASS & $\bar{T}$ & \\
\hline TE-108-AP-45 & $11 / 29 / 80$ & 65 & CASS & $T$ & \\
\hline TE-108-AP-45 & $12 / 6 / 90$ & 66 & CASS & $T$ & \\
\hline TE-108-AP-45 & $12 / 11 / 90$ & 65 & CASS & $T$ & \\
\hline$T E-108-A P-45$ & $12 / 20 / 90$ & 63 & CASS & $T$ & \\
\hline TE-108-AP-45 & $12 / 27 / 90$ & 80 & CASS & $T$ & \\
\hline TE-108-AP-45 & $12 / 30 / 90$ & 59 & CASS & $\mathbf{T}$ & \\
\hline TE-TOB-AP-45 & $1 / 11 / 91$ & 58 & CASS & $\mathbf{T}$ & \\
\hline TE-108-AP-45 & $1 / 14 / 91$ & 58 & CASS & $T$ & \\
\hline TE-108-AP-45 & $1 / 21 / 91$ & 56) & CASS & $T$ & \\
\hline$T E-108-A P-45$ & $1 / 29 / 91$ & 55 & CASS & $T$ & \\
\hline TE-108-AP-45 & $2 / 6 / 91$ & 56 & CASS & $\mathbf{T}$ & \\
\hline TE-108-AP-45 & $2 / 7 / 91$ & 91 & CASS & $\mathbf{s}$ & Data point did not agree with local trend \\
\hline TE-108-AP-45 & $2 / 16 / 91$ & 55: & CASS & $\mathbf{T}$ & \\
\hline TE-108-AP-45 & $2 / 17 / 191$ & 56 & CASS & $T$ & \\
\hline TE-108-AP-45 & $3 / 2 / 91$ & $\mathbf{5 5}$ & CASS & $\mathbf{T}$ & \\
\hline TE-108-AP-45 & 3/3/91 & 60 ! & CAASS & $T$ & \\
\hline
\end{tabular}




\begin{tabular}{|c|c|c|c|c|c|}
\hline \multicolumn{6}{|c|}{$\begin{array}{l}\text { TANK 241-AP-108 } \\
\text { THEFMOCOUPLE DATA }\end{array}$} \\
\hline & & & & & \\
\hline THERMOCOUPLE : & DATE & TEMPERATUAE $\left({ }^{\circ} \mathrm{F}\right)$ & DATA SOURCE & QUALITY & COMMENT \\
\hline TE-108-AP-45 & $3 / 13 / 91$ & 58 & CASS & $T$ & \\
\hline TE-108-AP-45 & $3 / 23 / 91$ & 56 & CASS & $T$ & \\
\hline TE-108-AP-45 & $3 / 25 / 91$ & 58 & CASS & $\bar{T}$ & \\
\hline TE-108-AP-45 & $4 / 1 / 91$ & 58 & CASS & $\mathbf{T}$ & \\
\hline TE-108-AP-45 & $4 / 12 / 91$ & 58 & CASS & $T$ & \\
\hline TE-108-AP-45 & 4/20/91 & 57 & CASS & $\mathbf{T}$ & \\
\hline TE-108-AP-45 & $4 / 21 / 91$ & 58 & CASS & $T$ & \\
\hline TE-10B-AP-45 & $5 / 4 / 91$ & 58 & CASS & $\mathbf{T}$ & \\
\hline TE-108-AP-45 & 5/10/91 & 60 & CASS & $T$ & \\
\hline TE-108-AP-45 & 5/16/91 & 64 & CASS & $\mathbf{T}$ & \\
\hline TE-108-AP-45 & 5/25/91 & 59 & CASS & $\mathbf{T}$ & \\
\hline TE-108-AP-45 & $5 / 31 / 91$ & 61 & CASS & $T$ & \\
\hline TE-10B-AP-45 & $6 / 5 / 91$ & 62 & CASS & $\mathbf{T}$ & \\
\hline TE-108-AP-45 & $6 / 15 / 91$ & 63 & CASS & $T$ & \\
\hline TE-108-AP-45 & $6 / 22 / 91$ & 64) & CASS & $T$ & \\
\hline TE-108-AP-45 & $6 / 28 / 91$ & 67 & CASS & $T$ & \\
\hline TE-108-AP-45 & $7 / 3 / 91$ & 66 & CASS & $\mathbf{T}$ & \\
\hline TE-108-AP-45 & 7/13/91 & 66 & CASS & $T$ & \\
\hline TE-108-AP-45 & $7 / 14 / 91$ & 66 & CASS & $\mathbf{T}$ & \\
\hline TE-10:-AP-45 & $7 / 24 / 91$ & 68 & CASS & $\mathbf{T}$ & \\
\hline TE-10Q-AP-45 & $8 / 3 / 91$ & 68 & CASS & $\overline{\mathbf{T}}$ & \\
\hline TE-108-AP-A5 & $8 / \mathrm{a} / \mathrm{s} 1$ & 68: & CASS & $T$ & \\
\hline TE-10B-AP-A5 & $8 / 17 / 31$ & 71 & CASS & $T$ & \\
\hline TE-108-AP-45 & $8 / 18 / 91$ & 71 & CASS & $\mathbf{T}$ & \\
\hline TE-108-AP-45 & $8 / 30 / 91$ & 70 & CASS & $T$ & \\
\hline TE-108-AP-45 & 8/3/21 & 72 & CASS & $T$ & \\
\hline TE-108-AP-45 & $9 / 14 / 91$ & 71 & CASS & $\mathbf{T}$ & \\
\hline TE-108-AP-45 & 8/18/91 & 71 & CASS & $\mathbf{T}$ & \\
\hline TE-108-AP-45 & 9/22/91 & 71 & CASS & $\mathbf{T}$ & \\
\hline TE-108-AP-45 & 10/1/91 & 73 & CASS & $\mathbf{T}$ & \\
\hline TE-108-AP-45 & 10/8/91 & 61 & CASS & $\mathbf{s}$ & Data point did not agree with local trend \\
\hline TE-108-AP-45 & $10 / 12 / 91$ & 71 & CASS & $\mathbf{T}$ & \\
\hline TE-10B-AP-45 & 10/17/91 & 73 & CASS & $\mathbf{T}$ & \\
\hline TE-108+AP-45 & $10 / 25 / 91$ & 72 & CASS & $T$ & \\
\hline TE-108-AP-45 & $10 / 28 / 91$ & 71 & CASS & $\mathbf{T}$ & \\
\hline TE-108-AP-45 & $11 / 6 / 91$ & 71 & CASS & $\mathbf{T}$ & \\
\hline TE-108-AP-45 & $7 / 30 / 92$ & 67 & CASS & $T$ & \\
\hline TE-108-AP-45 & $8 / 3 / 92$ & 67 & CASS & $\mathbf{T}$ & \\
\hline TE-108-AP-45 & 8/15/92 & 67. & CASS & $\mathbf{T}$ & \\
\hline TE-108-AP-45 & $8 / 20 / 92$ & 68 & CASS & $\mathbf{T}$ & \\
\hline TE-108.AP-45 & $8 / 28 / 92$ & 68 & CASS & $\mathbf{T}$ & \\
\hline TE-108-AP-45 & $9 / 5 / 92$ & 66 & CASS & $\mathbf{T}$ & \\
\hline TE-108-AP-45 & $9 / 12 / 92$ & 67 & CASS & $\mathbf{T}$ & \\
\hline TE-108-AP-45 & 9/16/92 & 67 & CASS & $\mathbf{T}$ & \\
\hline TE-108-AP-45 & $9 / 26 / 92$ & 86 & CASS & $T$ & \\
\hline TE-108-AP-45 & $10 / 3 / 92$ & 67 & CASS & $\mathbf{T}$ & \\
\hline TE-10B-AP-45 & $10 / 10 / 92$ & 66 & CASS & $T$ & \\
\hline TE-108-AP-45 & $10 / 17 / 92$ & 68 & CASS & $T$ & \\
\hline TE-108-AP-45 & $10 / 18 / 92$ & 69 & CASS & $\mathbf{T}$ & \\
\hline TE-108-AP-45 & $10 / 29 / 92$ & 70 & CASS & $T$ & \\
\hline$T E-108-A P+45$ & $11 / 4 / 92$ & 69 & CASS & $\mathbf{T}$ & \\
\hline TE-108-AP-45 & $11 / 9 / 92$ & 69 & CASS & $\mathbf{T}$ & \\
\hline TE-108-AP-45 & $11 / 17 / 92$ & 71 & CASS & $T$ & \\
\hline TE-108-AP-45 & $11 / 28 / 92$ & 67 & CASS & $\bar{T}$ & \\
\hline TE-108-AP-45 & $12 / 3 / 92$ & 67 & CASS & $T$ & \\
\hline TE-108-AP-45 & $12 / 12 / 92$ & 70 & CASS & $T$ & \\
\hline TE-108-AP-45 & $12 / 19 / 92$ & 67 & CASS & $T$ & \\
\hline IE-108-AP-45 & $12121 / 92$ & 66 & CASS & $T$ & \\
\hline TE-10B-AP-45 & $12 / 30 / 92$ & 66 & CASS & $\mathbf{T}$ & \\
\hline TE-108-AP-45 & $1 / 15 / 94$ & 63 & CASS & $\bar{T}$ & \\
\hline TE-108-AP-45 & $1 / 20 / 94$ & 64 & CASS & $\bar{T}$ & \\
\hline TE-108-AP-45 & $1 / 25 / 94$ & 64 & CASS & $T$ & \\
\hline TE-108-AP-45 & $2 / 4 / 94$ & 64 & CASS & $T$ & \\
\hline TE-108-AP-45 & $2 / 11 / 94$ & 64 & CASS & $T$ & \\
\hline TE-108-AP-45 & $2 / 15 / 94$ & 64 & CASS & $T$ & \\
\hline TE-108-AP-45 & $2 / 22 / 94$ & 64 & CASS & $T$ & \\
\hline TE-108-AP-45 & $2 / 28 / 94$ & 65 & CASS & $T$ & \\
\hline TE-108-AP-45 & $3 / 10 / 94$ & 64 & CASS & $T$ & \\
\hline TE-108-AP-45 & $3 / 17 / 94$ & 64 & CASS & $T$ & \\
\hline TE-108-AP-45 & $3 / 26 / 94$ & 63 & CASS & $T$ & \\
\hline TE-108-AP-45 & 3/29/94 & 64 & CASS & $T$ & \\
\hline TE-108-AP-45 & $4 / 9 / 94$ & 66 & CASS & $\bar{T}$ & \\
\hline TE-108-AP-45 & $4 / 15 / 94$ & 65 & CASS & $T$ & \\
\hline
\end{tabular}




\begin{tabular}{|c|c|c|c|c|c|}
\hline \multicolumn{6}{|c|}{$\begin{array}{l}\text { TANK 241-AP-108 } \\
\text { THEAMOCOUFLE DATA }\end{array}$} \\
\hline & & & & & \\
\hline THERMOCOUPL * & DATE & TEMFERATUAE $\left({ }^{\circ} F\right)$ & DATA SOURCE & QUALITY & COMMENT \\
\hline TE-108-AP-45 & $4 / 20 / 94$ & 64 & CASS & $T$ & \\
\hline TE-108-AP-45 & $4 / 29 / 94$ & 64 & CASS & $T$ & \\
\hline TE-108-AP-45 & $5 / 4 / 94$ & 64 & CASS & $\mathbf{T}$ & \\
\hline TE-108-AP-45 & $5 / 9 / 94$ & 65 & CASS & $\mathrm{T}$ & \\
\hline TE-108-AP-45 & $5 / 16 / 94$ & 104 & CASS & $T$ & \\
\hline TE-109-AP-45 & $5 / 23 / 94$ & 99 & CASS & $\mathbf{T}$ & \\
\hline TE-108-AP-45 & $6 / 2 / 94$ & 99 & CASS & $T$ & \\
\hline TE-108-AP-45 & $6 / 8 / 94$ & 98 & CASS & $\bar{T}$ & \\
\hline TE-108-AP-45 & $6 / 14 / 94$ & 85 & CASS & $\mathbf{T}$ & \\
\hline TE-108-AP-45 & $6 / 16 / 94$ & 63 & CASS & $\mathbf{S}$ & Date point did not agres with locesl trend \\
\hline TE-108-AP-45 & $6 / 17 / 94$ & 63 & CASS & $\mathbf{S}$ & Data point did not agree with tocal trend \\
\hline TE-108-AP-45 & $6 / 20 / 94$ & 63 & CASS & $\mathbf{S}$ & Data point did not agree with local trend \\
\hline TE-108-AP-45 & $6 / 24 / 94$ & 88 & CASS & $\mathbf{T}$ & \\
\hline TE-108-AP-45 & $6 / 30 / 94$ & 98 & CASS & $\mathbf{T}$ & \\
\hline TE-108-AP-45 & $7 / 794$ & 97 & CASS & $\mathbf{T}$ & \\
\hline TE-108-AP-45 & $7 / 15 / 94$ & 96 & CASS & $\mathbf{T}$ & \\
\hline TE-10:-AP-45 & $7 / 8 / 94$ & 95 & CASS & $T$ & \\
\hline TE-10A-AP-45 & $7 / 26 / 94$ & 93 & CASS & $T$ & \\
\hline TE-108-AP-45 & $7 / 31 / 94$ & 100 & CASS & $\mathbf{T}$ & \\
\hline TE-108-AP-45 & $8 / 10 / 94$ & 96 & CASS & $T$ & \\
\hline TE-108-AP-45 & $8 / 18 / 94$ & 97 & CASS & $T$ & \\
\hline TE-108-AP-45 & $8 / 22 / 94$ & 98 & CASS & $T$ & \\
\hline TE-108-AP-45 & $9 / 3 / 94$ & 98 & CASS & $T$ & \\
\hline$T E-108-A P-45$ & $9 / 10 / 94$ & 101 & CASS & $T$ & \\
\hline$T E-108-A P-45$ & $9 / 15 / 94$ & 100 & CASS & $T$ & \\
\hline TE-108-AP-46 & $9 / 23 / 94$ & 100 & CASS & $T$ & \\
\hline TE-108-AP-46 & $9 / 30 / 94$ & 100 & CASS & $\mathbf{T}$ & \\
\hline TE-108-AP-45 & 10/8/94 & 101 & CASS & $T$ & \\
\hline$T E+108-A P=45$ & $10 / 15 / 94$ & 101 & CASS & $\bar{T}$ & \\
\hline TE-10Q-AP-45 & $10 / 17 / 94$ & 103 & CASS & $T$ & \\
\hline TE+108-AP-45 & $10 / 29 / 94$ & 104 & CASS & $T$ & \\
\hline$T E-108-A P-46$ & $11 / 5 / 94$ & 104 & CASS & $T$ & \\
\hline TE-10B-AP-45 & $11 / 11 / 94$ & 104 & CASS & $T$ & \\
\hline TE-108-AP-45 & $11 / 18 / 94$ & 104 & CASS & $T$ & \\
\hline TE-108-AP-45 & $11 / 22 / 94$ & 104 & CASS & $T$ & \\
\hline TE-10B-AP-45 & $11 / 27 / 94$ & 105 & CASS & $T$ & \\
\hline TE-10B-AP-45 & $12 / 4 / 94$ & 108 & CASS & $T$ & \\
\hline TE-108-AP-45 & $12 / 12 / 94$ & 104 & CASS & $\bar{T}$ & \\
\hline TE-10B-AP-45 & $12 / 18 / 94$ & 106 & CASS & $\bar{T}$ & $\cdot$ \\
\hline TE-10b-AP-45 & $12 / 31 / 94$ & 106 & CASS & $T$ & \\
\hline TE-108-AP-45 & $1 / 4 / 95$ & 111 & CASS & $T$ & \\
\hline TE-10B-AP-45 & $1 / 13 / 95$ & 105 & CASS & $T$ & \\
\hline TE-108-AP-45 & 1/15/95 & 106 & CASS & $T$ & \\
\hline TE-108-AP-45 & 1/23/95 & 105 & CASS & $T$ & \\
\hline TE-108-AP-45 & $2 / 2 / 95$ & 9B & CASS & $\mathbf{T}$ & \\
\hline TE-108-AP-45 & $2 / 6 / 95$ & 101 & CASS & $T$ & \\
\hline TE-108-AP-45 & $2 / 14 / 95$ & 108 & CASS & $T$ & \\
\hline TE-108-AP-45 & $2 / 24 / 95$ & 96 & CASS & $T$ & \\
\hline TE-108-AP-45 & $3 / 2 / 95$ & 101 & CASS & $T$ & \\
\hline TE-10A-AP-45 & $3 / 7 / 95$ & 98 & CASS & $T$ & \\
\hline TE-108-AP-45 & $3 / 16 / 95$ & 94 & CASS & $T$ & \\
\hline TE-108-AP-45 & $3 / 25 / 85$ & 94 & CASS & $T$ & \\
\hline TE-108-AP-45 & $3 / 30 / 95$ & 96 & CASS & $T$ & \\
\hline & & & & & \\
\hline TE-108-AP-46 & $4 / 5 / 86$ & 58 & CASS & $T$ & \\
\hline TE-108-AP-46 & $477 / 86$ & 59 & CASS & $T$ & \\
\hline TE-108-AP-46 & $5 / 16 / 86$ & 58 & CASS & $T$ & \\
\hline TE-108-AP-46 & $5 / 24 / 86$ & 60 & CASS & $T$ & \\
\hline TE-108-AP-46 & $5 / 30 / 86$ & 59 & CASS & $T$ & \\
\hline TE-108-AP-46 & $6 / 7 / 86$ & 59 & CASS & $T$ & \\
\hline TE-108-AP-46 & $6 / 9 / 86$ & 60 & CASS & $T$ & \\
\hline$T E-108-A P-46$ & $6 / 21 / 86$ & 60 & CASS & $T$ & \\
\hline TE-108-AP-46 & $6 / 25 / 86$ & 60 & CASS & $T$ & \\
\hline TE-108-AP-46 & $7 / 3 / 86$ & 62 & CASS & $T$ & \\
\hline TE-108-AP-46 & $7 / 4 / 86$ & 107 & CASS & $\mathbf{s}$ & Data point did not agrea with local trand \\
\hline TE-108-AP-46 & $7 / 10 / 86$ & 62 & CASS & $\bar{T}$ & \\
\hline TE-108-AP-46 & $7 / 19 / 86$ & 61 & CASS & $T$ & \\
\hline TE-108-AP-46 & $7 / 26 / 86$ & 61 & CASS & $T$ & \\
\hline TE-108-AP-46 & $7 / 28 / 96$ & 63 & CASS & $\mathbf{T}$ & \\
\hline TE-108-AP-46 & B/9/86 & 64 & CASS & $\mathbf{T}$ & \\
\hline TE-108-AP-46 & $8 / 13 / 86$ & 64 & CASS & $\mathbf{T}$ & \\
\hline TE-108-AP-46 & $8 / 17 / 86$ & 65 & CASS & $\mathbf{T}$ & \\
\hline TE-108-AP-46 & $8 / 30 / 86$ & 63 & CASS & $\mathbf{T}$ & \\
\hline
\end{tabular}




\begin{tabular}{|c|c|c|c|c|c|}
\hline \multicolumn{6}{|c|}{ TANK 241-AP-108 } \\
\hline & & & THERMOCOU & DATA & \\
\hline THERMOCOUPE * & DATE & TEMPERATURE ( $\left.{ }^{\circ} \mathrm{F}\right)$ & DATA SOURCE & GUALTY & СОММЕNT \\
\hline TE-108-AP-46 & $9 / 3 / 86$ & 64 & $\frac{\text { CASS }}{\text { CANOE }}$ & $\frac{T}{T}$ & \\
\hline$T E-108-A P-46$ & $9 / 24 / 86$ & 64 & CASS & $\bar{T}$ & \\
\hline TE-108-AP-46 & $11 / 1 / 86$ & 62 & CASS & $T$ & \\
\hline TE-108-AP-46 & $19 / 5 / 66$ & 61 & CASS & $T$ & \\
\hline TE-108-AP-46 & $11 / 29 / 86$ & 59 & CASS & $T$ & \\
\hline TE-108-AP-46 & $12 / 10 / 86$ & 59 & CASS & $T$ & \\
\hline TE-108-AP-46 & $12 / 18 / 86$ & 58 & CASS & $\bar{T}$ & \\
\hline TE-108-AP-46 & $12 / 23 / 66$ & 57 & CASS & $T$ & \\
\hline TE-108-AP.46 & $12 / 29 / 86$ & 56 & CASS & $T$ & \\
\hline TE-108-AP.46 & $7 / 1 / 87$ & 65 & CASS & $\bar{T}$ & \\
\hline TE-106-AP.46 & $7 / 8 / 87$ & 70 & CASS & $T$ & \\
\hline TE-108-AP-46 & $7 / 12 / 87$ & 66 & CASS & $T$ & \\
\hline TE-108-AP-46 & $7 / 19 / 87$ & 71 & CASS & $\bar{T}$ & \\
\hline TE-108-AP-46 & $7 / 26 / 67$ & 69 & CASS & $\bar{T}$ & \\
\hline TE-108-AP-46 & $8 / 5 / 67$ & 69 & CASS & $T$ & \\
\hline TE-108-AP-46 & $8 / 14 / 87$ & 69 & CASS & $T$ & \\
\hline TE-108-AP-46 & $8 / 22 / 87$ & 68 & CASS & $T$ & \\
\hline TE-108-AP-46 & $8 / 26 / 87$ & 69 & CASS & $\bar{T}$ & \\
\hline TE-108-AP-46 & 9/4/87 & 68 & CASS & $T$ & \\
\hline TE-10B-AP-46 & $9 / 8 / 87$ & 69 & CASS & $\bar{T}$ & \\
\hline TE-108-AP-46 & $9 / 17 / 87$ & 69 & CASS & $T$ & \\
\hline TE-108-AP-A6 & $9 / 20 / 87$ & 70 & CASS & $T$ & \\
\hline TE-108-AP-46 & $9 / 27 / 87$ & 71 & CASS & $T$ & \\
\hline TE-108-AP-46 & $10 / 8 / 87$ & 69 & CASS & $\bar{T}$ & \\
\hline TE-108-AP-46 & $10 / 16 / 87$ & 67 & CASS & $\bar{T}$ & \\
\hline TE-108-AP-46 & $10 / 19 / 87$ & 68 & CASS & $T$ & \\
\hline TE-108-AP-48 & $10 / 31 / 87$ & 68 & CASS & $T$ & \\
\hline TE-10B-AP-46 & $19 / 1 / 87$ & 65 & CASS & $\bar{T}$ & \\
\hline TE-10B-AP-46 & $11 / 11 / 87$ & 64 & CASS & $T$ & \\
\hline TE-108-AP-46 & $31 / 18 / 87$ & 62 & CASS & $T$ & \\
\hline TE-108-AP-46 & $11 / 26 / 87$ & 64 & CASS & $T$ & \\
\hline TE-10:-AP-46 & $11 / 29 / 87$ & 61 & CASS & $T$ & \\
\hline TE-108-AP.46 & $12 / 6 / 87$ & 61 & CASS & $T$ & \\
\hline TE-108-AP-46 & $12 / 18 / 87$ & 59 & CASS & $T$ & \\
\hline TE-108-AP-46 & $12 / 23 / 87$ & 58 & CASS & $T$ & \\
\hline TE-108-AP-46 & $1 / 2 / 83$ & 57 & CASS & $\bar{T}$ & \\
\hline TE-108-AP-46 & $1 / 6 / 8 B$ & 53 & CASS & $T$ & \\
\hline TE-10B-AP-46 & $1 / 18 / 88$ & 52 & CASS & $T$ & \\
\hline TE-108-AP-46 & $1 / 23 / 88$ & 52 & CASS & $T$ & \\
\hline TE-10B-AP-46 & 1/25/88 & $\mathbf{5 3}$ & CASS & $T$ & \\
\hline TE-108-AP-46 & $2 / 2 / 83$ & 52 & CASS & $T$ & \\
\hline TE-108-AP-A6 & $2 / 11 / 8$ & 53 & CASS & $T$ & \\
\hline TE-108-AP-46 & $2 / 14 / 83$ & 54 & CASS & $\bar{T}$ & \\
\hline TE-10B-AP-46 & $2 / 27 / 68$ & 53 & CASS & $\bar{T}$ & \\
\hline TE-108-AP-46 & $3 / 3 / 83$ & 54 & CASS & $\mathbf{T}$ & \\
\hline TE-108-AP-46 & $3 / 10 / 88$ & 54 & CASS & $T$ & \\
\hline TE-TOB-AP-46 & $3 / 13 / 38$ & 55 & CASS & $T$ & \\
\hline TE-10B-AP-46 & $3 / 21 / 88$ & 56 & CASS & $T$ & \\
\hline TE-TOQ-AP-46 & $3 / 31 / 88$ & 54 & CASS & $\bar{T}$ & \\
\hline TE-108-AP-46 & $8 / 1 / 88$ & 61 & CASS & $T$ & \\
\hline TE-108-AP-46 & 6/9/88 & 63 & CASS & $T$ & \\
\hline TE-108-AP-46 & $6 / 18 / 8$ & 62 & CASS & $T$ & \\
\hline TE-108-AP-46 & $6 / 25 / 8$ & 63 & CASS & $T$ & \\
\hline TE-108-AP-46 & 7 m/68 & 65 & CASS & $\bar{T}$ & \\
\hline TE-108-AP-46 & 7/9/8 & 64 & CASS & $T$ & \\
\hline TE-10B-AP-46 & $713 / \mathrm{a}$ & 66 & CASS & $T$ & \\
\hline TE-10B-AP-46 & $7 / 23 / 88$ & 69 & CASS & $\bar{T}$ & \\
\hline TE-108-AP-46 & $7 / 29 / 88$ & 69 & CASS & $T$ & \\
\hline TE-108-AP-46 & $8 / 3 / 88$ & 70 & CASS & $T$ & \\
\hline TE-10B-AP-46 & $8 / 9 / 88$ & 70 & CASS & $\bar{T}$ & \\
\hline TE-10B-AP-46 & $8 / 20 / 83$ & 69 & CASS & $T$ & \\
\hline TE-108-AP-46 & $8 / 22 / 8$ & 70 & CASS & $T$ & \\
\hline TE-108-AP-46 & 9/2/88 & 71 & CASS & $T$ & \\
\hline TE-108-AP-46 & $9 / 9 / 88$ & 73 & CASS & $\bar{T}$ & \\
\hline TE-108-AP-46 & $9 / 16 / 8$ & 72 & CASS & $\bar{T}$ & \\
\hline TE-108-AP-46 & $9 / 23 / 8$ & 70 & CASS & $T$ & \\
\hline TE-108-AP-46 & $9 / 27 / 83$ & 71 & CASS & $T$ & \\
\hline TE-108-AP-46 & $12 / 3 / 88$ & 66 & CASS & $T$ & \\
\hline TE-108-AP-46 & $12 / 8 / 88$ & 65 & CASS & $T$ & \\
\hline TE-108-AP-46 & $12 / 16 / 88$ & 64 & CASS & $T$ & \\
\hline TE-108-AP-46 & $12 / 22 / 88$ & 64 & CASS & $T$ & \\
\hline TE-108-AP-46 & $9 / 2 / 89$ & 72 & CASS & $\bar{T}$ & \\
\hline TE-10B-AP-46 & $9 / 7 / 89$ & 69 & CASS & $T$ & \\
\hline
\end{tabular}

Data obteined from Computer Automated Surveillance System (CASS), April 1995; and Survelllence Analysis Computer System (SACS), June 1995.

D-687 


\begin{tabular}{|c|c|c|c|c|c|}
\hline \multicolumn{6}{|c|}{$\begin{array}{l}\text { TAKK 241-AP-108 } \\
\text { THERMOCOUPLE DATA }\end{array}$} \\
\hline THEAMOCOUPLE * & DATE & TEMPEAATUAE $\left({ }^{\circ} F\right)$ & DATA SOURCE & OUALITY & соMMENT \\
\hline TE-10B-AP-46 & $9 / 16 / 89$ & 70 & CASS & $T$ & \\
\hline TE-108-AP-46 & $9 / 18 / 89$ & 71 & CASS & $\bar{T}$ & \\
\hline TE-10B-AP-46 & $9 / 29 / 89$ & 71 & CASS & $T$ & \\
\hline TE-108-AP-46 & 11/4/69 & 66 & CASS & $\bar{T}$ & \\
\hline TE-108-AP-46 & 11/7/89 & 65 & CASS & $\bar{T}$ & \\
\hline TE-1008-AP-46 & $11 / 18 / 89$ & 67 & CASS & $T$ & \\
\hline TE-108-AP-46 & $11 / 24 / 89$ & 63 & CASS & $T$ & \\
\hline TE-108-AP-46 & $12 / 2 / 69$ & 66 & CASS & $T$ & \\
\hline TE-108-AP-46 & $12 / 4 / 89$ & 63 & CASS & $T$ & \\
\hline TE-108-AP-46 & $12 / 12 / 89$ & 61 & CASS & $T$ & \\
\hline TE-108-AP-46 & $12 / 18 / 89$ & 59 & CASS & $T$ & \\
\hline TE-108-AP-46 & $12 / 30 / 89$ & 58 & CASS & $T$ & \\
\hline TE-108-AP-46 & $1 / 2 / 90$ & 58 & CASS & $T$ & \\
\hline TE-10B-AP-46 & $1 / 12 / 90$ & 58 & CASS & $T$ & \\
\hline TE-108-AP-46 & $1 / 20 / 50$ & 60 & CASS & $\bar{T}$ & \\
\hline TE-108-AP-46 & $1 / 26 / 90$ & 58 & CASS & $T$ & \\
\hline TE:108-AP-46 & $2 / 1180$ & 58 & CASS & $T$ & \\
\hline TE-108-AP-46 & $2 / 7 / 190$ & 59 & CASS & $\bar{T}$ & \\
\hline$T E-108-A P-46$ & $2 / 13 / 90$ & 59 & CASS & $\bar{T}$ & \\
\hline TE-108-AP-46 & $2 / 24 / 90$ & 57 & CASS & 7 & \\
\hline TE-108-AP-46 & $3 / 3 / 90$ & 60 & CASS & $\bar{T}$ & \\
\hline TE-10B-AP-46 & $3 / 8 / 90$ & 63 & CASS & $\mathbf{T}$ & \\
\hline TE-108-AP-46 & $3 / 12 / 90$ & 62 & SACS & $T$ & \\
\hline TE-108-AP-46 & $3 / 18 / 90$ & 63 & CASS & $\mathbf{T}$ & \\
\hline TE-108-AP-46 & $3 / 29 / 90$ & 62 & CASS & $\bar{T}$ & \\
\hline TE-108-AP-46 & $4 / 3 / 30$ & 62 & CASS & $T$ & \\
\hline TE-108-AP-46 & $4 / 2 / 90$ & 62 & CASS & $T$ & \\
\hline TE-TOB-AP-46 & $4 / 21 / 90$ & 62 & CASS & $T$ & \\
\hline TE-108-AP-46 & 4/26/90 & 64 & CASS & $T$ & \\
\hline TE-108-AP-46 & $4 / 29 / 20$ & 64 & CASS & $T$ & \\
\hline TE-10Q-AP-46 & $5 / 6 / 90$ & 65 & CASS & $\mathbf{T}$ & \\
\hline TE-108-AP-46 & 5/17/90 & 65 & CASS & $\mathbf{T}$ & \\
\hline TE-108-AP-46 & $5 / 21 / 90$ & 65 & CASS & $\bar{T}$ & \\
\hline TE-103-AP-46 & $6 / 1 / 80$ & 64 & CASS & $T$ & \\
\hline TE-106-AP-46 & $6 / 6 / 90$ & 67 & CASS & $T$ & \\
\hline TE-108-AP-46 & $6 / 7190$ & 109 & CASS & s & Data point did not agree with local trand \\
\hline TE-10B-AP-46 & $6 / 12 / 90$ & 66 & CASS & $T$ & \\
\hline TE-10:AP.46 & 6/21/90 & 65 & CASS & $T$ & \\
\hline TE-108-AP.46 & 6/30/30 & 64 & CASS & $T$ & \\
\hline TE-108-AP-46 & $7 / 6 / 90$ & 65 & CASS & $\mathbf{T}$ & \\
\hline TE-108-AP-46 & $7 / 12 / 90$ & 69 & CASS & $\bar{T}$ & \\
\hline TE-108-AP-46 & $7 / 16 / 90$ & 69 & CASS & I & \\
\hline TE- $108, A$ P-46 & $7 / 27 / 90$ & 69 & CASS & $T$ & \\
\hline$T E-108-A P-46$ & $8 / 3 / 90$ & 68 & CASS & $T$ & \\
\hline TE-10:-AP-46 & $8 / 7 / 90$ & 71 & CASS & $T$ & \\
\hline TE-108-AP-46 & $8 / 12 / 90$ & 71 & CASS & $\mathbf{T}$ & \\
\hline TE-106-AP-46 & 8/19/90 & 71 & CASS & $T$ & \\
\hline$T E-108-A P-46$ & $9 / 1 / 190$ & 69 & CASS & $T$ & \\
\hline TE-108-AP-46 & 9/8/90 & 69 & CASS & $T$ & \\
\hline TE-108-AP-46 & 9/15/90 & 72 & CASS & $\overline{\mathbf{T}}$ & \\
\hline TE-108-AP-46 & $9 / 20 / 90$ & 71 & CASS & $T$ & \\
\hline TE-108-AP-46 & 9/28/90 & 70 & CASS & $\bar{T}$ & \\
\hline TE-108-AP-46 & $10 / 6 / 90$ & 70 & CASS & $\mathbf{T}$ & \\
\hline$T E-108-A P-46$ & $10 / 13 / 90$ & 70 & CASS & $\mathbf{T}$ & \\
\hline$T E-108-A P-46$ & $10 / 16 / 90$ & 69 & CASS & $T$ & \\
\hline TE-108-AP-46 & $10 / 26 / 90$ & 70 & CASS & $T$ & \\
\hline TE-108-AP-46 & $10 / 31 / 90$ & 70 & CASS & $\mathbf{T}$ & \\
\hline TE-108-AP-46 & $17 / 4 / 90$ & 68 & CASS & $T$ & \\
\hline TE-108-AP-46 & $11 / 17 / 90$ & 67 & CASS & $T$ & \\
\hline TE-108.AP.46 & $11 / 20 / 90$ & 67 & CASS & $T$ & \\
\hline$T E-108-A P-46$ & $11 / 29 / 90$ & 65 & CASS & $T$ & \\
\hline TE-108-AP-46 & $12 / 6 / 90$ & 66 & CASS & $\bar{T}$ & \\
\hline TE-108-AP-46 & $12 / 14 / 90$ & 64 & CASS & 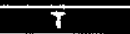 & \\
\hline TE-10B-AP-46 & $12 / 20 / 90$ & 63 & CASS & $T$ & \\
\hline TE-10B-AP-46 & $12 / 27 / 90$ & 60 & CASS & $\mathbf{T}$ & \\
\hline TE-108-AP-46 & $12 / 30 / 90$ & 59 & CASS & $\bar{T}$ & \\
\hline TE-108-AP-46 & 1/11/81 & 58 & CASS & $T$ & \\
\hline$T E+108 \cdot A P \cdot 46$ & $1 / 18 / 91$ & 57 & CASS & $T$ & \\
\hline TE-108-AP-46 & $1 / 26 / 91$ & 56 & CASS & $T$ & \\
\hline TE-108-AP-46 & $1 / 30 / 91$ & 55 & CASS & $\bar{T}$ & \\
\hline TE-108-AP-46 & $2 / 6 / 31$ & 56 & CASS & $\mathbf{T}$ & \\
\hline TE-108-AP-46 & 2/16/91 & 55 & CASS & $\bar{T}$ & \\
\hline TE-108-AP-46 & $2 / 17 / 91$ & 57 & CASS & $\mathbf{T}$ & \\
\hline
\end{tabular}




\begin{tabular}{|c|c|c|c|c|c|}
\hline \multicolumn{6}{|c|}{$\begin{array}{l}\text { TANK 241-AP-108 } \\
\text { THEAMOCOUILE DATA }\end{array}$} \\
\hline THEAMOCOUPE & DATE & TEMPERATUAE $\left({ }^{\circ} \mathrm{F}\right.$ ) & DATA SOURCE & ouALTY & COMMENT \\
\hline TE-108-AP-46 & $3 / 1 / 91$ & 56 & CASS & $T$ & \\
\hline TE-108-AP-46 & $3 / 5 / 91$ & 57 & CASS & $T$ & \\
\hline TE-108-AP-46 & $3 / 16 / 91$ & 56 & CASS & $T$ & \\
\hline TE-108-AP-46 & $3 / 23 / 91$ & 56 & CASS & $\bar{T}$ & \\
\hline TE-108-AP-46 & $3 / 27 / 91$ & 58 & CASS & $\mathbf{T}$ & \\
\hline TE-108-AP-46 & $4 / 1 / 81$ & 59 & CASS & $\mathbf{T}$ & \\
\hline TE-108-AP-46 & 4/12/91 & 58: & CASS & $T$ & \\
\hline TE-108-AP-46 & $4 / 20 / 91$ & 57 & CASS & $T$ & \\
\hline TE-108-AP-46 & $4 / 27 / 91$ & 58 & CASS & $T$ & \\
\hline TE-108-AP-46 & $5 / 1 / 91$ & 61 & CASS & $T$ & \\
\hline TE-108-AP-46 & 5/10/91 & 60 & CASS & $T$ & \\
\hline TE-108-AP-46 & 5/16/91 & 64 & CASS & $\mathbf{T}$ & \\
\hline TE-108-AP-46 & 5/25/91 & 60 & CASS & $T$ & \\
\hline TE-106-AP-46 & $5 / 26 / 91$ & 63 & CASS & $T$ & \\
\hline TE-108-AP-46 & $6 / 5 / 91$ & 62 & CASS & $T$ & \\
\hline TE-108-AP-46 & $6 / 15 / 91$ & 63 & CASS & $T$ & \\
\hline TE-108-AP-46 & $6 / 22 / 91$ & 65 & CASS & $T$ & \\
\hline TE-108-AP-46 & 6/28/91 & 68 & CASS & $\bar{T}$ & \\
\hline TE-108-AP-46 & $7 / 3 / 91$ & 67 & CASS & $T$ & \\
\hline TE-108-AP-46 & $7 / 3 / 21$ & 67: & CASS & $T$ & \\
\hline$T E-108-A P-46$ & $7 / 14 / 91$ & 67 & CASS & $\bar{T}$ & \\
\hline TE-108-AP-46 & $7 / 24 / 91$ & 69 & CASS & $T$ & \\
\hline TE-108-AP-46 & $8 / 2 / 91$ & 70 & CASS & $\bar{T}$ & \\
\hline TE-108-AP-46 & $8 / 6 / 91$ & 69 & CASS & $\bar{T}$ & \\
\hline TE-102-AP.46 & $8 / 12 / 97$ & 71 & CASS & $T$ & \\
\hline TE-108-AP-46 & $8 / 20 / 91$ & 71 & CASS & 7 & \\
\hline$T E-108-A P-46$ & 8/30/91 & 71 & CASS & $T$ & \\
\hline TE-108:AP-46 & $9 / 3 / 91$ & 72 & CASS & $T$ & \\
\hline TE-108-AP-A6 & 9/8/91 & 72 & CASS & $\mathbf{T}$ & \\
\hline TE-108-AP-46 & 9/18/91 & 74 & CASS & $T$ & \\
\hline TE-10B-AP-46 & 9/22/91 & 72 & CASS & $\mathbf{T}$ & \\
\hline TE-108-AP-46 & 10/1/90 & 72 & CASS & $\bar{T}$ & \\
\hline TE-108-AP-AB & 10/3/91 & 61 & CASS & $\mathbf{S}$ & Data point did not agree with local trand \\
\hline TE-108-AP-46 & $10 / 10 / 91$ & 72 & CASS & $T$ & \\
\hline TE-108-AP-46 & $10 / 17 / 91$ & 73 & CASS & $\mathbf{T}$ & \\
\hline TE-108-AP-46 & $10 / 25 / 91$ & 72 & CASS & $T$ & \\
\hline TE-108-AP-46 & $10 / 28 / 91$ & 71 & CASS & $T$ & \\
\hline TE-10:AP-46 & $11 / 6 / 91$ & 70 & CASS & $\mathbf{T}$ & \\
\hline TE-108-AP-46 & $7 / 30 / 92$ & 67 & CASS & $\bar{T}$ & \\
\hline TE-108-AP-46 & $8 / 3 / 92$ & 68 & CASS & $\mathbf{T}$ & \\
\hline TE-108-AP-46 & 8/15/A2 & 67 & CASS & $T$ & \\
\hline TE-10B-AP-46 & $8 / 20 / 92$ & 68 & CASS & $\mathbf{T}$ & \\
\hline TE-10B-AP.46 & $8 / 28 / 92$ & 68 & CASS & $\mathbf{T}$ & \\
\hline TE-108-AP-46 & $8 / 31 / 92$ & 67 & CASS & $\mathbf{T}$ & \\
\hline TE-108-AP-46 & 9/12/92 & 67 & CASS & $\bar{T}$ & \\
\hline$T E-108-A P-46$ & 8/16/92 & 67 & CASS & $T$ & \\
\hline TE-108-AP-46 & 9/26/92 & 66 & CASS & $\mathbf{T}$ & \\
\hline TE-106.AP-46 & $10 / 2 / 92$ & 68 & CASS & $T$ & \\
\hline TE-108-AP-46 & $10 / 10 / 92$ & 66 & CASS & $\mathbf{T}$ & \\
\hline TE-108-AP-46 & $10 / 17 / 92$ & 69 & CASS & $\mathbf{T}$ & \\
\hline TE-108-AP-46 & $10 / 22 / 92$ & 69 & CASS & $\mathbf{T}$ & \\
\hline TE-108-AP-46 & $10 / 29 / 92$ & 70 & CASS & $T$ & \\
\hline TE-108-AP-46 & 11/7/92 & 69 & CASS & $\mathbf{T}$ & \\
\hline TE-108-AP-46 & 11/9/92 & 69 & CASS & $\mathbf{T}$ & \\
\hline TE-108-AP-46 & $11 / 17 / 92$ & 69 & CASS & $T$ & \\
\hline TE-108-AP-46 & $11 / 28 / 92$ & 68 & CASS & $\mathbf{T}$ & \\
\hline TE-108-AP-46 & $12 / 3 / 92$ & 67 & CASS & $\mathbf{T}$ & \\
\hline TE-108·AP-46 & $12 / 12 / 92$ & 68 & CASS & $\mathbf{T}$ & \\
\hline TE-108-AP-46 & $12 / 17 / 92$ & 67 & CASS & $T$ & \\
\hline TE-108-AP-46 & $12 / 21 / 92$ & 67 & SACS & $\mathrm{T}$ & \\
\hline TE-108-AP-46 & $12 / 30 / 92$ & 66 & CASS & $T$ & \\
\hline TE-108-AP-46 & 6/4/93 & 43 & SACS & $\mathbf{S}$ & Data point did not agree with local trend \\
\hline TE-108-AP-46 & $6 / 11 / 93$ & 61 & SACS & $T$ & \\
\hline TE-108-AP-46 & $6 / 18 / 93$ & 60 & SACS & $T$ & \\
\hline TE-108-AP-46 & $6 / 28 / 93$ & 61 & SACS & $T$ & \\
\hline$T E-108-A P-46$ & $7 / 9 / 93$ & 61 & SACS & $T$ & \\
\hline TE-108-AP-46 & $7 / 15 / 93$ & 61 & SACS & $T$ & \\
\hline TE-108-AP-46 & $7 / 19 / 93$ & 61 & SACS & $T$ & \\
\hline TE-108-AP-46 & $7 / 30 / 93$ & 62 & SACS & $T$ & \\
\hline TE-108-AP-A6 & $8 / 6 / 93$ & 62 & SACS & $T$ & \\
\hline TE-108-AP-46 & B/12/93 & 62 & SACS & $T$ & \\
\hline TE-108-AP-46 & $9 / 24 / 93$ & 56 & SACS & $T$ & \\
\hline TE-108-AP-46 & $10 / 1 / 33$ & 63 & SACS & $T$ & \\
\hline
\end{tabular}




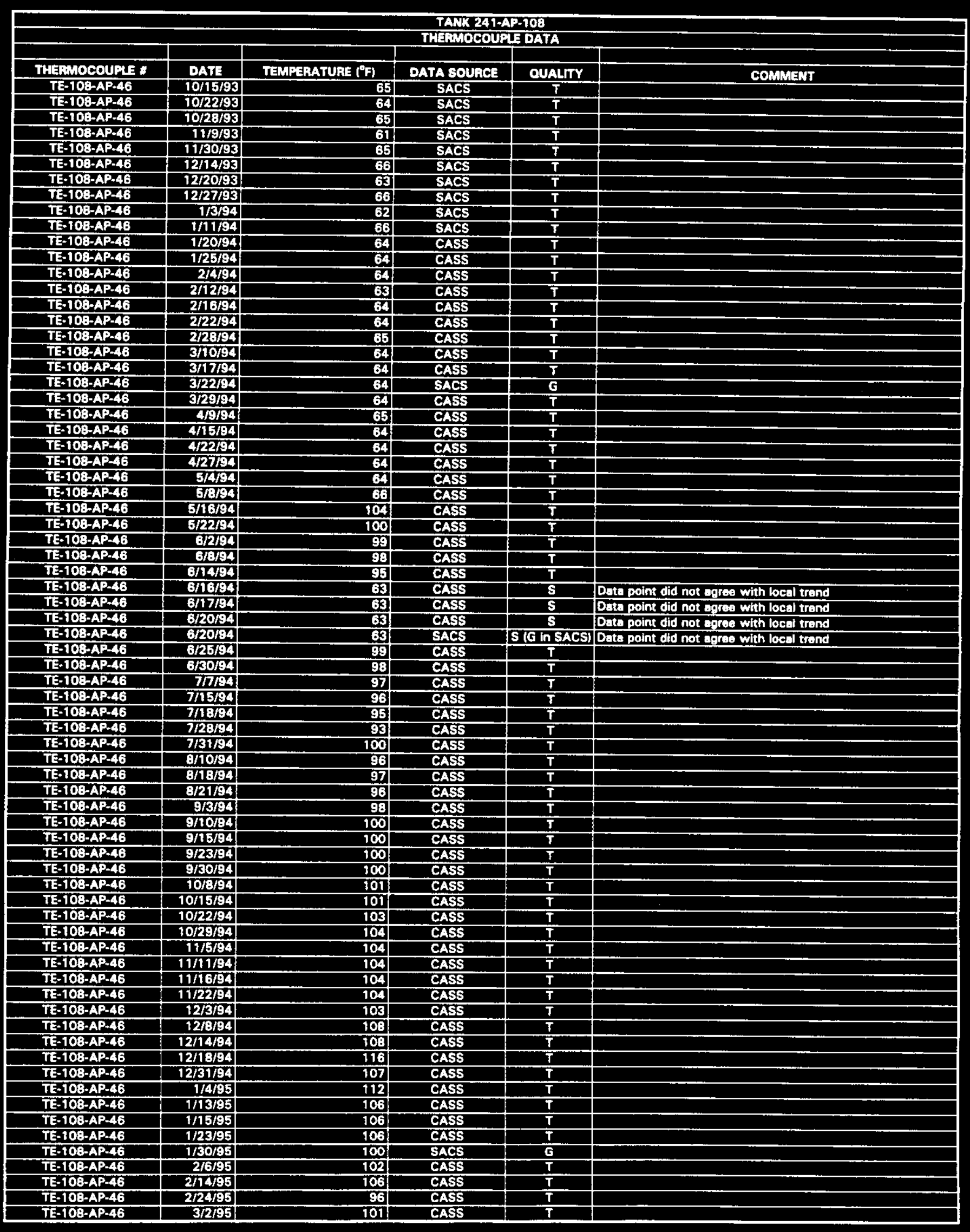

Data obtained from Computer Automated Surveillance Syatem (CASS), April 1995; and Surveillance Analysis Computer Syatem (SACS), June 1995.

$$
\text { D- } 690
$$




\begin{tabular}{|c|c|c|c|c|c|}
\hline \multicolumn{6}{|c|}{$\begin{array}{l}\text { TANK 241-AP-108 } \\
\text { THEAMOCOUFLE DATA }\end{array}$} \\
\hline & & TEMPERATURE ("F) & DATA BOUACE & & \\
\hline$\frac{\text { THERMOCOUPLE }}{\text { TE-TOB-AP-46 }}$ & \begin{tabular}{c|} 
DATE \\
$3 / 7 / 95$
\end{tabular} & 99 & $\frac{\text { DATA BOULiCE }}{\text { CASS }}$ & $\frac{\text { QUALTY }}{T}$ & COMMENT \\
\hline TE-108-AP-46 & $3 / 16 / 95$ & 95 & CASS & $T$ & \\
\hline TE-103-AP-46 & $3 / 25 / 95$ & 95 & CASS & $T$ & \\
\hline TE-108-AP-46 & $3 / 30 / 95$ & 96 & CASS & $T$ & \\
\hline TE-108-AP-46 & $4 / 3 / 95$ & 86 & SACS & $\mathbf{G}$ & \\
\hline TE-108-AP-46 & $4 / 10 / 95$ & 89 & SACS & G & \\
\hline TE-108-AP-46 & $4 / 17 / 95$ & 88 & SACS & G & \\
\hline TE-108-AP-4B & 4/24/95 & 85 & SACS & $G$ & \\
\hline TE-108-AP-46 & 5/1/95 & 86 & SACS & $\mathbf{G}$ & \\
\hline TE-108-AP-46 & $5 / 8 / 95$ & 90 & SACS & $\mathbf{G}$ & \\
\hline TE-108-AP-46 & 5/16/95 & 85 & SACS & G & \\
\hline TE-108-AP-46 & 5/22135 & 84 & SACS & G & \\
\hline TE-106-AP-46 & $5 / 29 / 95$ & 84 & SACS & G & \\
\hline TE-108-AP-46 & $6 / 5 / 95$ & 88 & SACS & $G$ & $\cdot$ \\
\hline TE-10B-AP-46 & 6/13/95 & 83 & SACS & $\mathbf{G}$ & \\
\hline TE-108-AP-46 & 6/19/95 & 98 & SACS & $\mathbf{G}$ & \\
\hline & & & & & \\
\hline TE-10B-AP-47 & $4 / 5 / 86 /$ & 58 & CASS & $T$ & \\
\hline TE-108-AP-47 & $4 / 7 / 86$ & 59 & CASS & $T$ & \\
\hline TE-108-AP-47 & 5/17/86 & 57 & CASS & $T$ & \\
\hline TE-108-AP-47 & $5 / 24 / 86$ & 60 & CASS & $T$ & \\
\hline TE-108-AP-47 & $5 / 30 / 86$ & 59) & CASS & $T$ & \\
\hline TE-108-AP-47 & $6 / 3 / 86$ & 128 & CASS & $\mathbf{S}$ & Data point did not agree with local trend \\
\hline$T E-108-A P-47$ & $6 / 7 / 86$ & 59 & CASS & $\mathbf{T}$ & \\
\hline TE-108-AP-47 & 6/9/86 & 80 & CASS & $\mathbf{T}$ & \\
\hline TE-108-AP-47 & 6/21/86 & 60 & CASS & $T$ & \\
\hline TE-108-AP-47 & 6/25/86 & 60 & CASS & $\bar{T}$ & \\
\hline TE-108-AP-47 & $7 / 3 / 86$ & 62 & CASS & $\bar{T}$ & \\
\hline TE-108-AP-47 & $7 / 10 / 86$ & 62 & CASS & $\mathbf{T}$ & \\
\hline TE-108-AP-47 & 7/19/86 & 61 & CASS & $\mathbf{T}$ & \\
\hline$T E-108-A P-47$ & $7 / 23 / 86$ & 120 & CASS & $\mathbf{s}$ & Dota point did not egres with local trend \\
\hline TE-102-AP:A7 & $7 / 26 / 96$ & 61 & CASS & $\mathbf{T}$ & \\
\hline TE-10Q-AP-47 & $7729 / 36$ & 63 & CASS & $\mathbf{T}$ & \\
\hline TE-108-AP-47 & $8 / 8 / 36$ & 64 & CASS & $\mathbf{T}$ & \\
\hline TE-108-AP-47 & $8 / 13 / 38$ & 64 & CASS & $\bar{T}$ & \\
\hline TE-108-AP-47 & $8 / 17 / 36$ & 66 & CASS & $\mathbf{T}$ & \\
\hline TE-108-AP-47 & $8 / 30 / 86$ & 63 & CASS & $\bar{T}$ & \\
\hline TE-108-AP-47 & $9 / 3 / 36$ & 64 & CASS & $T$ & \\
\hline TE-108-AP-47 & $9 / 24 / 66$ & 64) & CASS & $\mathbf{T}$ & \\
\hline TE-108-AP-47 & $11 / 1 / 86$ & 62 & CASS & $\mathbf{T}$ & \\
\hline TE-108-AP-47 & $11 / 2 / 86$ & 62 & CASS & $T$ & \\
\hline TE-108-AP-47 & $11 / 29 / 86$ & 59 & CASS & $\mathbf{T}$ & \\
\hline TE-108-AP-47 & $12 / 11 / 86$ & 59 & CASS & $\mathbf{T}$ & \\
\hline TE-108-AP-47 & $12 / 17 / 86$ & 58 & CASS & $T$ & \\
\hline TE-108-APA47 & $12 / 24 / 36$ & 57 & CASS & $T$ & \\
\hline TE-108-AP-47 & $12 / 29 / 36$ & 56 & CASS & $T$ & \\
\hline TE-108-AP-47 & $7 / 1 / 87$ & 65 & CASS & $T$ & \\
\hline TE-108-AP-47 & $7 / 8 / 87$ & 70 & CASS & $T$ & \\
\hline TE-108-AP-47 & $7 / 12 / 87$ & 66 & CASS & $T$ & \\
\hline TE-108-AP-47 & $7 / 19 / 87$ & 71 & CASS & $T$ & \\
\hline TE-108-AP-47 & 7127/87 & 67 & CASS & $\bar{T}$ & \\
\hline TE-108-AP-47 & $8 / 5 / 87$ & 69 & CASS & $\mathbf{T}$ & \\
\hline TE-108-AP-47 & $8 / 14 / 87$ & 69 & CASS & $T$ & \\
\hline TE-106-AP-47 & $8 / 22 / 87$ & 67 & CASS & $\mathbf{T}$ & \\
\hline TE-108-AP-47 & $8 / 26 / 87$ & 69 & CASS & $T$ & \\
\hline$T E-108-A P-47$ & $9 / 4 / 87$ & 68 & CASS & $T$ & \\
\hline TE-108-AP-47 & $9 / 8 / 37$ & 71 & CASS & $\mathbf{T}$ & \\
\hline TE-108-AP-47 & 9/17/87 & 69 & CASS & $T$ & \\
\hline TE-108-AP-47 & 9/20/87 & 70 & CASS & $\mathbf{T}$ & \\
\hline TE-108-AP-47 & 9/27/87 & 69 & CASS & $T$ & \\
\hline TE-108-AP-47 & $10 / 7 / 87$ & 70 & CASS & $T$ & \\
\hline TE-108-AP-47 & $10 / 16 / 87$ & 67 & CASS & $T$ & \\
\hline TE-108-AP-47 & $\begin{array}{l}10 / 19 / 87 \\
\end{array}$ & 68 & CASS & $T$ & \\
\hline$T E-108-A P-47$ & $10 / 31 / 67$ & 68 & CASS & $T$ & \\
\hline TE-108-AP-47 & $11 / 1 / 87$ & 65 & CASS & $T$ & \\
\hline TE-108-AP-47 & $11 / 13 / 87$ & 64 & CASS & $\mathbf{T}$ & \\
\hline TE-108-AP-47 & $11 / 18 / 87$ & 62 & CASS & $\mathbf{T}$ & \\
\hline TE-108-AP-47 & $11 / 26 / 87$ & 62 & CASS & $T$ & \\
\hline TE-108-AP-47 & 11/29/87 & 61 & CASS & $\bar{T}$ & \\
\hline TE-108-AP-47 & \begin{tabular}{|l|}
$12 / 6 / 87$ \\
\end{tabular} & 61 & CASS & $\mathbf{T}$ & \\
\hline TE-108-AP-47 & $12 / 18 / 87$ & 58 & CASS & $T$ & \\
\hline TE-108-AP-47 & $12 / 23 / 87$ & 57 & CASS & $\bar{T}$ & \\
\hline TE-108-AP-47 & $12 / 28 / 87$ & 55 & CASS & $\mathbf{I}$ & \\
\hline
\end{tabular}




\begin{tabular}{|c|c|c|c|c|c|}
\hline \multicolumn{6}{|c|}{ TANK 241-AP.108 } \\
\hline & & & & & \\
\hline THEAMOCOUPLE * & DATE & TEMPERATURE ( $\mathrm{F} F)$ & DATA SOURCE & QUALITY & COMMENT \\
\hline TE-108-AP-47 & $1 / 4 / 88$ & 53 & CASS & $T$ & \\
\hline TE-108-AP-47 & $1 / 16 / 88$ & 51 & CASS & $\mathrm{T}$ & \\
\hline TE-108-AP-47 & $1 / 21 / 88$ & 52 & CASS & $T$ & \\
\hline TE-108-AP-47 & $1 / 25 / 88$ & 53 & CASS & $\bar{T}$ & \\
\hline TE-10:-AP-47 & $2 / 2 / 88$ & 52 & CASS & $\mathbf{T}$ & \\
\hline TE-108-AP.47 & $2 / 11 / 88$ & 53 & CASS & $T$ & \\
\hline TE-10B-AP-47 & $2 / 14 / 88$ & 54 & CASS & $\mathbf{T}$ & \\
\hline TE-108-AP-47 & 2/27/88 & 52 & CASS & $T$ & \\
\hline TE-108-AP-47 & $3 / 3 / 88$ & 54 & CASS & $\mathbf{T}$ & \\
\hline TE-108-AP-47 & $3 / 10 / 88$ & 54 & CASS & $\mathbf{T}$ & \\
\hline TE-108-AP-47 & 3/13/88 & 54 & CASS & $\mathbf{T}$ & \\
\hline TE-108-AP-47 & $3 / 24 / 88$ & 56 & CASS & $\mathbf{T}$ & \\
\hline TE-108-AP-47 & $3 / 31 / 88$ & 54 & CASS & $\bar{T}$ & \\
\hline TE-108-AP-47 & $6 / 1 / 88$ & 60 & CASS & $\mathbf{T}$ & \\
\hline TE-108-AP-47 & $6 / 10 / 8$ & 61 & CASS & $T$ & \\
\hline IE-108-AP-47 & $6 / 16 / 88$ & 71 & CASS & $\overline{\mathbf{s}}$ & Data point did not agree with local trend \\
\hline TE-108-AP-47 & $6 / 18 / 88$ & 61 & CASS & $T$ & \\
\hline TE-108-AP-47 & $6 / 25 / 88$ & 62 & CASS & $T$ & \\
\hline TE-108-AP-47 & $7 / 1 / 88$ & 66 & CASS & $T$ & \\
\hline TE-108-AP-47 & $7 / 9 / 88$ & 63. & CASS & $T$ & \\
\hline TE-108-AP-47 & $7 / 16 / 88$ & 64 & CASS & $T$ & \\
\hline TE-108-AP-47 & $7 / 23 / 83$ & 68 & CASS & $T$ & \\
\hline TE-108-AP-47 & 7/29/83 & 68 & CASS & $T$ & \\
\hline TE-108-AP-47 & $8 / 3 / 88$ & 69 & CASS & $\bar{T}$ & \\
\hline TE-108-AP-47 & $8 / 9 / 88$ & 69! & CASS & $T$ & \\
\hline TE-108-AP.47 & $8 / 20 / 88$ & 69 & CASS & $T$ & \\
\hline TE-108-AP-47 & $8 / 22 / 38$ & 69: & CASS & $T$ & \\
\hline$T E-108-A P-47$ & $8 / 2 / 88$ & 71 & CASS & $T$ & \\
\hline TE-10A-AP-47 & 9/9/88 & 71 & CASS & $T$ & \\
\hline TE-108-AP-47 & $9 / 16 / 88$ & 71 & CASS & $\bar{T}$ & \\
\hline TE-108-AP-47 & $9 / 23 / 68$ & 70 & CASS & $T$ & \\
\hline TE-108.AP-47 & 9/27/88 & 70 & CASS & $T$ & \\
\hline TE-108-AP-47 & $12 / 3 / 68$ & 67 & CASS & $\bar{T}$ & \\
\hline TE-108.AP-47 & $12 / 5 / 8$ & 65 & CASS & $T$ & \\
\hline TE-108-AP-47 & 12/13/88 & 63 & CASS & $T$ & \\
\hline TE-108-AP-47 & $12 / 22 / 88$ & 62 & CASS & $T$ & \\
\hline TE-108-AP-47 & $7 / 10 / 89$ & 64 & SACS & $T$ & \\
\hline TE-106-AP.47 & $7 / 17 / 69$ & 65 & SACS & $T$ & \\
\hline TE-108-AP-47 & $7 / 24 / 69$ & 66 & SACS & $T$ & \\
\hline TE-108-AP-47 & $8 / 7 / 69$ & 67 & SACS & $T$ & \\
\hline TE-108-AP-47 & $8 / 14 / 69$ & 69 & SACS & $T$ & \\
\hline TE-108-AP-47 & $8 / 21 / 69$ & 68 & SACS & $\bar{T}$ & \\
\hline TE-108-AP-47 & $9 / 2 / 89$ & 71 & CASS & $T$ & \\
\hline TE-108-AP-47 & $9 / 7 / 89$ & 69 & CASS & $\bar{T}$ & \\
\hline TE-108-AP-47 & $9 / 16 / 89$ & 70 & CASS & $T$ & \\
\hline TE-108-AP-47 & 9/18/89 & 71 & CASS & $T$ & \\
\hline TE-108-AP-47 & 9/29/89 & 71 & CASS & $T$ & \\
\hline TE-108-AP-47 & $10 / 2 / 89$ & 67 & SACS & $T$ & \\
\hline TE-108-AP.47 & $10 / 9 / 89$ & 69 & SACS & $T$ & \\
\hline TE-108-AP-47 & $10 / 16 / 89$ & 68 & SACS & $T$ & \\
\hline TE-108-AP-47 & $10 / 24 / 89$ & 71 & SACS & $T$ & \\
\hline TE-108.AP-47 & $11 / 4 / 89$ & 66 & CASS & $T$ & \\
\hline TE-108-AP-47 & $11 / 7 / 69$ & 65 & SACS & $\bar{T}$ & \\
\hline TE-108-AP-47 & $11 / 18 / 89$ & 67 & CASS & $\bar{T}$ & \\
\hline TE-108-AP-47 & $11 / 20 / 89$ & 66 & SACS & $T$ & \\
\hline TE-108.AP-47 & $12 / 2 / 69$ & 66 & CASS & $T$ & \\
\hline$T E-108-A P-47$ & $12 / 4 / 89$ & 62 & CASS & $T$ & \\
\hline TE-108-AP-47 & $12 / 12 / 89$ & 61 & CASS & $T$ & \\
\hline TE-108-AP-47 & 12/22/89 & 58 & CASS & $T$ & \\
\hline TE-108-AP-47 & $12 / 30 / 89$ & 57 & CASS & $T$ & \\
\hline TE-108-AP-47 & $1 / 2 / 90$ & 58 & CASS & $T$ & \\
\hline TE-108-AP-47 & 1/13/90 & 57 & CASS & $T$ & \\
\hline TE-108-AP-47 & $1 / 15 / 90$ & 58 & CASS & $T$ & \\
\hline TE-108-AP-47 & $1 / 23 / 90$ & 58 & CASS & $\bar{T}$ & \\
\hline TE-108-AP-47 & $2 / 1 / 90$ & 58 & CASS & $T$ & \\
\hline TE-108-AP-47 & $2 / 4 / 90$ & 59 & CASS & $T$ & \\
\hline TE-108-AP-47 & $2 / 13 / 90$ & 59 & CASS & $T$ & \\
\hline TE-108-AP-47 & $2 / 18 / 90$ & 57 & CASS & $T$ & \\
\hline TE-108-AP-47 & $3 / 3 / 90$ & 59: & CASS & $T$ & \\
\hline TE-108-AP-47 & $3 / 9 / 90$ & 62 & CASS & $T$ & \\
\hline$T E-106-A P-47$ & $3 / 12 / 90$ & 91 & CASS & $\mathbf{s}$ & Data point did not agrae with local trand \\
\hline TE-108-AP-47 & $3 / 14 / 90$ & 61 & CASS & $T$ & \\
\hline TE-108-AP-47 & $3 / 22 / 90$ & 61 & CASS & $\mathbf{T}$ & \\
\hline
\end{tabular}

Date obteined from Computer Automated Surveillence Syatem (CASS), April 1995; and Surveillance Analysis Computer System (SACS), June 1995.

D-692 


\begin{tabular}{|c|c|c|c|c|c|}
\hline \multicolumn{6}{|c|}{$\begin{array}{l}\text { TANK 241-AP-108 } \\
\text { THERMOCOUPLE DATA }\end{array}$} \\
\hline THERMOCOUPL * & DATE & TEMPERATURE ( $\left.{ }^{\circ} \mathrm{F}\right)$ & DATA SOUACE & OYUALTY & COMYENT \\
\hline TE-108-AP-47 & $3 / 29 / 90$ & 61 & CASS & $T$ & 年 \\
\hline TE-108-AP-47 & $4 / 3 / 90$ & 61 & CASS & $T$ & \\
\hline IE-108-AP-47 & $4 / 12 / 90$ & 62 & CASS & $\bar{T}$ & \\
\hline$T E-108 \times A P-47$ & $4 / 21 / 90$ & 60 & CASS & $T$ & \\
\hline TE-10B-AP-47 & $4 / 26 / 90$ & 63 & CASS & $\bar{T}$ & \\
\hline TE-108-AP-47 & $5 / 3 / 20$ & 61 & CASS & $T$ & \\
\hline TE-108-AP-47 & $5 / 11 / 90$ & 62 & CASS & $T$ & \\
\hline TE-TOB-AP-47 & $5 / 17 / 90$ & 63 & CASS & $T$ & \\
\hline TE-108-AP-47 & $5 / 21 / 90$ & 63 & CASS & $T$ & \\
\hline TE-108-AP-47 & $6 / 1 / 90$ & 63 & CASS & $\bar{T}$ & \\
\hline$T E-108-A P-47$ & $6 / 6 / 90$ & 67 & CASS & $T$ & \\
\hline TE-108-AP-47 & $6 / 12 / 90$ & 64 & CASS & $T$ & \\
\hline TE-108-AP-47 & $6 / 21 / 90$ & 63 & CASS & $T$ & \\
\hline TE-108-AP-47 & $6 / 30 / 90$ & 63 & CASS & $T$ & \\
\hline TE-108-AP.47 & $7 / 6 / 90$ & 64 & CASS & $T$ & \\
\hline$T=-108 \cdot A P-47$ & $7 / 12 / 90$ & 67 & CASS & $T$ & \\
\hline TE-100-AP-47 & $716 / 90$ & 68 & CASS & $\bar{T}$ & \\
\hline TE-108-AP-47 & $7 / 27 / 90$ & 69 & CASS & $\bar{T}$ & \\
\hline$T E-108 \cdot A P-47$ & $8 / 3 / 90$ & 88 & CASS & $T$ & \\
\hline TE-10B-AP-47 & $8 / 7 / 90$ & 70 & CASS & $T$ & \\
\hline TE-108-AP-47 & $8 / 12 / 90$ & 71 & CASS & $T$ & \\
\hline TE-108-AP-47 & $8 / 19 / 90$ & 72 & CASS & $\bar{T}$ & \\
\hline TE-108-AP-47 & 9/1/s0: & 69 & CASS & $T$ & \\
\hline TE-108-AP-47 & 9/B/SO & 69 & CASS & $T$ & \\
\hline TE-108-AP-47 & $9 / 15 / 90$ & 71 & CASS & $T$ & \\
\hline$T E-108-A P-47$ & 9/20/90 & 71 & CASS & $\mathbf{T}$ & \\
\hline TE-108-AP-47 & $9 / 28 / 90$ & 70 & CASS & $\bar{T}$ & \\
\hline$T E-108-A P-47$ & $10 / 6 / 90$ & 70 & CASS & $\bar{T}$ & \\
\hline TE-108-AP-47 & $10 / 10 / 90$ & 87 & CASS & $\mathbf{s}$ & Data point did not agrea with local trend \\
\hline TE-108-AP-47 & $10 / 13 / 90$ & 70 & CASS & $\bar{T}$ & \\
\hline$T E-108-A P-47$ & $10 / 16 / 90$ & 70 & CASS & $T$ & \\
\hline TE-108-AP-47 & $10 / 26 / 50$ & 70 & CASS & $T$ & \\
\hline TE-108-AP-47 & $10 / 31 / 80$ & 70 & CASS & $T$ & \\
\hline TE-108-AP-47 & $11 / 10 / 90$ & 67 & CASS & $T$ & \\
\hline$T=-108 \cdot A P-47$ & $11 / 17 / 90$ & 67 & CASS & $\mathbf{T}$ & \\
\hline$T E \cdot 108-A P \cdot 47$ & $11 / 20 / 80$ & 67 & CASS & $T$ & \\
\hline TE-108-AP-47 & $11 / 29 / 80$ & 65 & CASS & $\mathbf{T}$ & \\
\hline TE-108-AP-47 & $12 / 6 / 90$ & $66\}$ & CASS & $\bar{T}$ & \\
\hline TE-108-AP-47 & $12 / 10 / 90$ & 86 & SACS & $\bar{T}$ & \\
\hline TE-108-AP-47 & $12 / 20 / 90$ & 63 & CASS & $T$ & \\
\hline TE-108-AP-47 & $12 / 28 / 90$ & 60 & CASS & $T$ & \\
\hline TE-108-AP-47 & 12/31/80 & 59 & SACS & $\mathbf{T}$ & \\
\hline TE-108-AP-47 & $1 / 11 / 81$ & 57 & CASS & $\bar{T}$ & \\
\hline TE-108-AP-47 & $1 / 14 / 91$ & 58 & CASS & $T$ & \\
\hline TE-108-AP-47 & $1 / 21 / 91$ & 57 & SACS & $T$ & \\
\hline TE-108-AP-47 & $1 / 25 / 91$ & 83 & CASS & $\overline{\mathbf{S}}$ & Data point did not agree with locel trend \\
\hline TE-108-AP-47 & $1 / 29 / 91$ & 56 & CASS & $T$ & \\
\hline TE-108-AP-47 & $2 / 6 / 91$ & 56 & CASS & $T$ & \\
\hline TE-108-AP-47 & $2 / 12 / 91$ & 55 & CASS & $T$ & \\
\hline TE-108-AP-47 & $2 / 17 / 93$ & 56 & CASS & $\mathbf{T}$ & \\
\hline$T E-108-A P-47$ & $2 / 25 / 91$ & 57 & SACS & $T$ & \\
\hline TE-108-AP-47 & $3 / 5 / 91$ & 57 & CASS & $\bar{T}$ & \\
\hline TE-108-AP-47 & $3 / 13 / 91$ & 57 & CASS & $T$ & \\
\hline TE-108-AP-47 & $3 / 21 / 91$ & 56 & CASS & $T$ & \\
\hline TE-108-AP-47 & $3 / 25 / 93$ & 58 & CASS & $T$ & \\
\hline TE-108-AP-47 & 4/1/91 & 58 & CASS & $\bar{T}$ & \\
\hline TE-108-AP-47 & $4 / 12 / 91$ & 58 & CASS & $T$ & \\
\hline TE-108-AP-47 & $4 / 18 / 91$ & 57 & CASS & $T$ & \\
\hline TE-10B-AP-47 & 4/27/91 & 57 & CASS & $\mathbf{T}$ & \\
\hline TE-108-AP-47 & $5 / 4 / 91$ & 58 & CASS & $T$ & \\
\hline TE-108-AP-47 & $5 / 6 / 91$ & 60 & SACS & $T$ & \\
\hline TE-10B-AP-47 & $5 / 16 / 91$ & 64 & CASS & $T$ & \\
\hline TE-108-AP-47 & $5 / 25 / 91$ & 59 & CASS & $T$ & \\
\hline TE-108-AP-47 & $5 / 26 / 91$ & 65 & CASS & $T$ & \\
\hline TE-108-AP-47 & $6 / 5 / 91$ & 62 & CASS & $T$ & \\
\hline TE-10B-AP-47 & $6 / 15 / 91$ & 62 & CASS & $T$ & \\
\hline TE-108-AP-47 & $6 / 22 / 91$ & 64 & CASS & $T$ & \\
\hline TE-10B-AP-47 & $6 / 28 / 91$ & 67 & CASS & $\mathbf{T}$ & \\
\hline$T E-108-A P-47$ & $7 / 3 / 91$ & 66 & CASS & $T$ & \\
\hline TE-108-AP-47 & $7 / 8 / 91$ & 71 & SACS & $\bar{T}$ & \\
\hline TE-108-AP-47 & $7 / 15 / 91$ & 66 & SACS & $T$ & \\
\hline TE-108-AP-47 & $7 / 24 / 91$ & 68 & CASS & $T$ & \\
\hline TE-108-AP-47 & $8 / 2 / 91$ & 69 & CASS & $\bar{T}$ & \\
\hline
\end{tabular}

Data obtained from Computer Automated Surveillance System \{CASS\}, April 1995; and Surveillance Analysis Computer System (SACS\}, Jume 1995.

D- 693 


\begin{tabular}{|c|c|c|c|c|c|}
\hline \multicolumn{6}{|c|}{$\begin{array}{l}\text { TANK 241-AP-108 } \\
\text { THERIMOCOUPLI DATA }\end{array}$} \\
\hline & & & & & \\
\hline THERMOCOUPL $*$ & DATE & TEMPERATURE $\left({ }^{\circ} \mathrm{F}\right\}$ & DATA SOURCE & OUALTYY & COMMENT \\
\hline TE-108-AP-47 & $8 / 6 / 91$ & 68 & CASS & $T$ & \\
\hline TE-108-AP-47 & $8 / 17 / 91$ & 71 & CASS & $\mathbf{T}$ & \\
\hline TE-108-AP-47 & $8 / 19 / 91$ & 71 & SACS & $T$ & \\
\hline TE-108-AP-47 & $8 / 30 / 91$ & 69 & CASS & $T$ & \\
\hline TE-108-AP-47 & $9 / 3 / 91$ & 7 & CASS & $\mathbf{T}$ & \\
\hline TE-108-AP-47 & 9/12/91 & 71 & CASS & $\mathbf{T}$ & \\
\hline TE-108-AP-47 & 9/78/91 & 75 & CASS & $T$ & \\
\hline TE-108-AP-47 & 9/26/91 & 70 & CASS & $T$ & - \\
\hline TE-108-AP-A7 & 10/1/91 & 72 & CASS & $\bar{T}$ & \\
\hline TE-108-AP-47 & 10/8/91 & 61 & CASS & $\mathbf{S}$ & Data point did not agree with local trend \\
\hline TE-108-AP-47 & $10 / 10 / 91$ & 72 & CASS & $\mathbf{T}$ & \\
\hline TE-106-AP-47 & $10 / 14 / 91$ & 73 & CASS & $\mathbf{T}$ & \\
\hline$T E-108-A P-47$ & $10 / 21 / 93$ & 72 & SACS & $T$ & \\
\hline IE-10D-AP-47 & $10 / 28 / 91$ & 71 & CASS & $T$ & \\
\hline TE-108-AP-47 & $11 / 6 / 81$ & 70 & CASS & $\mathbf{T}$ & \\
\hline TE-108-AP-47 & $7 / 30 / 92$ & 67 & CASS & $\mathbf{T}$ & \\
\hline TE-108-AP=47 & 8/3/92 & 68 & CASS & $T$ & \\
\hline TE-10:-AP-47 & $8 / 15 / 92$ & 67 & CASS & $\bar{T}$ & \\
\hline TE-108-AP-47 & $8 / 20 / 92$ & 68 & CASS & $T$ & \\
\hline TE-102-AP-47 & $8 / 28 / 92$ & 67 & CASS & $\mathbf{T}$ & \\
\hline$T E-108-A P=47$ & $8 / 5 / 92$ & 66 & CASS & $\mathbf{T}$ & \\
\hline TE-108-AP-47 & 9/12/92 & 67: & CASS & $T$ & \\
\hline TE-108-AP-47 & $9 / 16 / 92$ & 67 & CASS & $T$ & \\
\hline TE-106-AP-47 & 9/21/32 & 67 & SACS & $T$ & \\
\hline TE-108-AP-47 & $10 / 3 / 32$ & 68 & CASS & $T$ & \\
\hline TE-108-AP-47 & $10 / 10 / 92$ & 66 & CASS & $\bar{T}$ & \\
\hline$T E-108-A P-47$ & $10 / 17 / 92$ & 69 & CASS & $T$ & \\
\hline TE-108-AP-47 & 10/22/92 & 70 & CASS & $T$ & \\
\hline TE-10B-AP-47 & $10 / 29 / 92$ & 70 & CASS & $T$ & \\
\hline TE-108-AP-47 & $11 / 7 / 92$ & 69 & CASS & $T$ & \\
\hline TE-108-AP-47 & $11 / 9 / 92$ & 69 & CASS & $\bar{T}$ & \\
\hline TE-10B-AP-47 & $11 / 17 / 92$ & 69 & CASS & $\mathbf{T}$ & \\
\hline TE-108-AP-47 & $11 / 25 / 92$ & 68 & CASS & $T$ & \\
\hline TE-108-AP-47 & $11 / 30 / 92$ & 69 & CASS & $\bar{T}$ & \\
\hline TE-108-AP-A7 & $12 / 12 / 92$ & 68 & CASS & $\bar{T}$ & \\
\hline TE-108-AP-A7 & $12 / 17 / 92$ & 67 & CASS & $T$ & \\
\hline$T E-108-A P-47$ & $12 / 21 / 92$ & 66 & CASS & $T$ & \\
\hline TE-108-AP-47 & $12 / 30 / 92$ & 66 & CASS & $T$ & \\
\hline$T E-108-A P-47$ & 1/15/94 & 63 & CASS & $T$ & \\
\hline TE-108-AP.47 & $1 / 20 / 94$ & 64 & CASS & $\bar{T}$ & \\
\hline TE-108-AP-47 & 1/25/94 & 64 & CASS & $T$ & \\
\hline TE-10:AP-47 & $2 / 4 / 94$ & 64| & CASS & $T$ & \\
\hline TE-108-AP-A7 & $2 / 11 / 94$ & 63 & CASS & $T$ & \\
\hline TE-108.AP-47 & $2 / 15 / 94$ & 64 & CASS & $T$ & \\
\hline TE-108-AP-47 & $2 / 22 / 94$ & 63 & CASS & $T$ & \\
\hline TE-10:AP-47 & $3 / 5 / 94$ & 63 & CASS & $T$ & \\
\hline TE-108-AP-47 & $3 / 6 / 94$ & B9 & CASS & $\mathbf{S}$ & Data point did not agree with locel trend \\
\hline TE-108-AP-47 & $3 / 10 / 94$ & 64 & CASS & $T$ & \\
\hline TE-108-AP-47 & 3/17/94 & 64 & CASS & $T$ & \\
\hline TE-108-AP-47 & $3 / 25 / 94$ & 631 & CASS & $\mathbf{T}$ & \\
\hline TE-108-AP-47 & $3 / 29 / 94$ & 63) & CASS & $\mathbf{T}$ & \\
\hline TE-108-AP-47 & $4 / 9 / 94$ & 65 & CASS & $T$ & \\
\hline TE-108-AP-47 & $4 / 13 / 94$ & 64) & CASS & $T$ & \\
\hline TE-108-AP-47 & $4 / 22 / 94$ & 65 & CASS & $\mathbf{T}$ & \\
\hline TE-108-AP-47 & $4 / 27 / 94$ & 64 & CASS & $T$ & \\
\hline TE-108-AP-47 & $5 / 4 / 94$ & 64 & CASS & $\mathbf{T}$ & \\
\hline TE-108-AP-47 & $5 / 14 / 94$ & 64 & CASS & $T$ & \\
\hline$T E-108-A P-47$ & $5 / 16 / 94$ & 104 & CASS & $\mathbf{T}$ & \\
\hline TE-108-AP-47 & $5 / 22 / 94$ & 100 & CASS & $T$ & \\
\hline TE-108-AP-47 & $6 / 2 / 94$ & 99 & CASS & $T$ & \\
\hline TE-108-AP-47 & 6/8/94 & 98 & CASS & $\mathbf{T}$ & \\
\hline TE-108-AP-47 & $6 / 14 / 94$ & 85 & CASS & $T$ & \\
\hline TE-108-AP-47 & 6/16/94 & 63 & CASS & $\mathbf{S}$ & Data point did not agres with local trend \\
\hline TE-108-AP-47 & $6 / 17 / 94$ & 63 & CASS & $\mathbf{s}$ & Data point did not agrea with local trend \\
\hline TE-108-AP-47 & $6 / 20 / 94$ & 63 & CASS & $\mathbf{S}$ & Data point did not agrea with local trend \\
\hline TE-108-AP-47 & $6 / 25 / 94$ & 100 & CASS & $T$ & \\
\hline TE-108-AP-47 & $6 / 27 / 94$ & 97 & CASS & $T$ & \\
\hline TE-108-AP-47 & $6 / 29 / 94$ & 151 & CASS & $\mathbf{S}$ & Data point did not agree with local trend \\
\hline TE-108-AP-47 & $7 / 7 / 94$ & 97 & CASS & $T$ & \\
\hline TE-108-AP-47 & $7 / 15 / 94$ & 96 & CASS & $\mathbf{T}$ & \\
\hline TE-108-AP-47 & $7 / 18 / 94$ & 95 & CASS & $T$ & \\
\hline TE-108-AP-47 & $7 / 24 / 94$ & 94 & CASS & $T$ & \\
\hline TE-108-AP-47 & $7 / 31 / 94$ & 100 & CASS & $T$ & \\
\hline
\end{tabular}




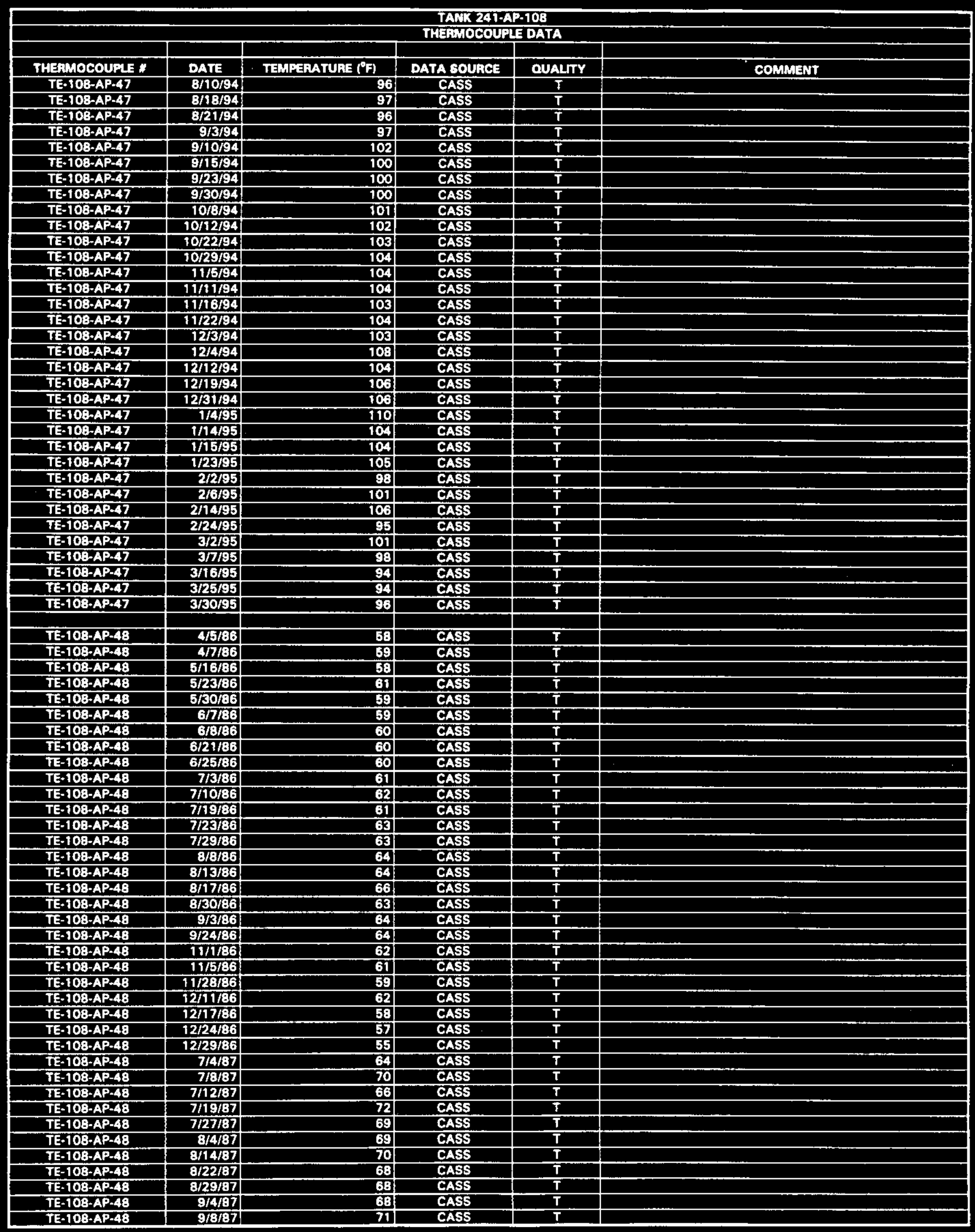




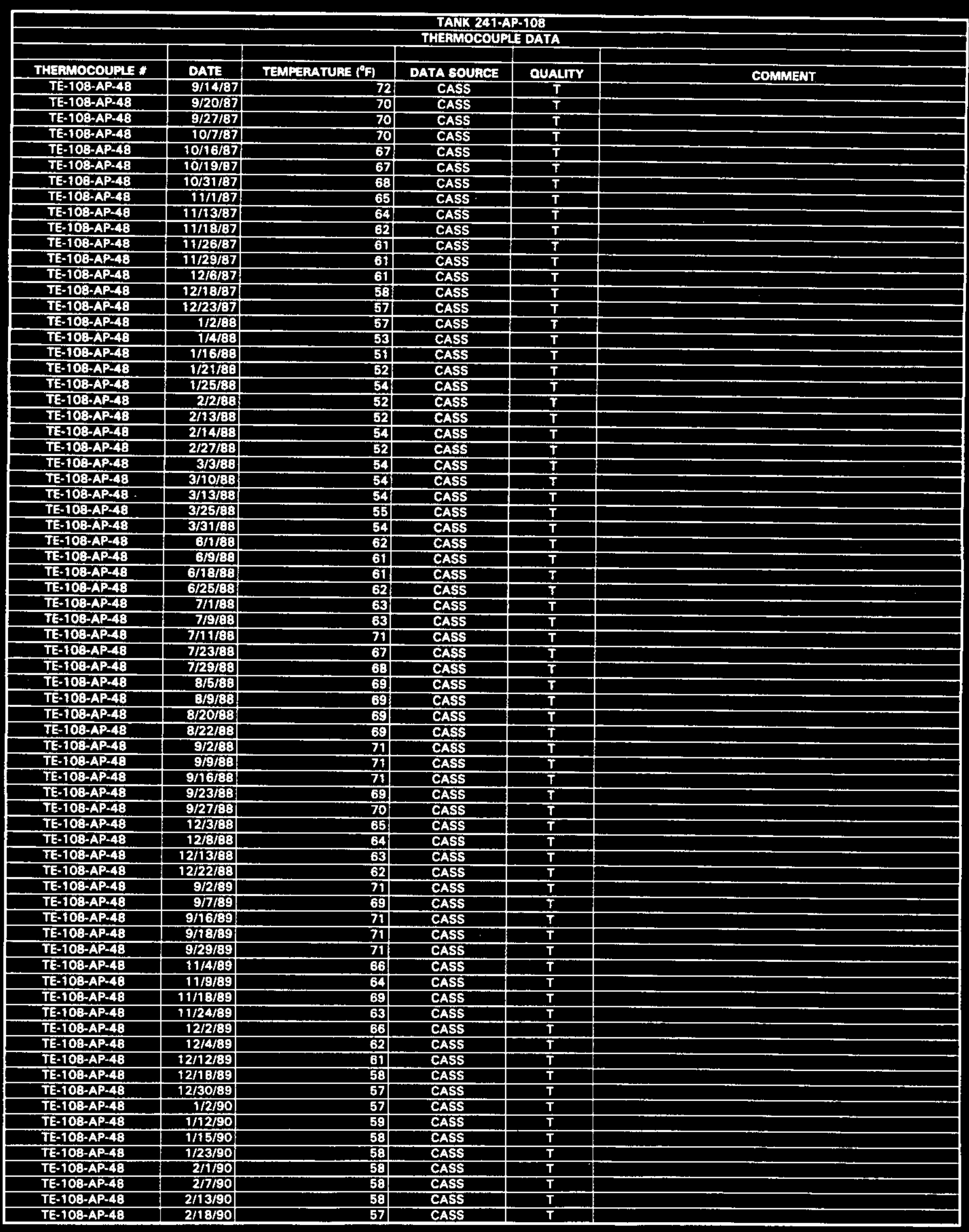




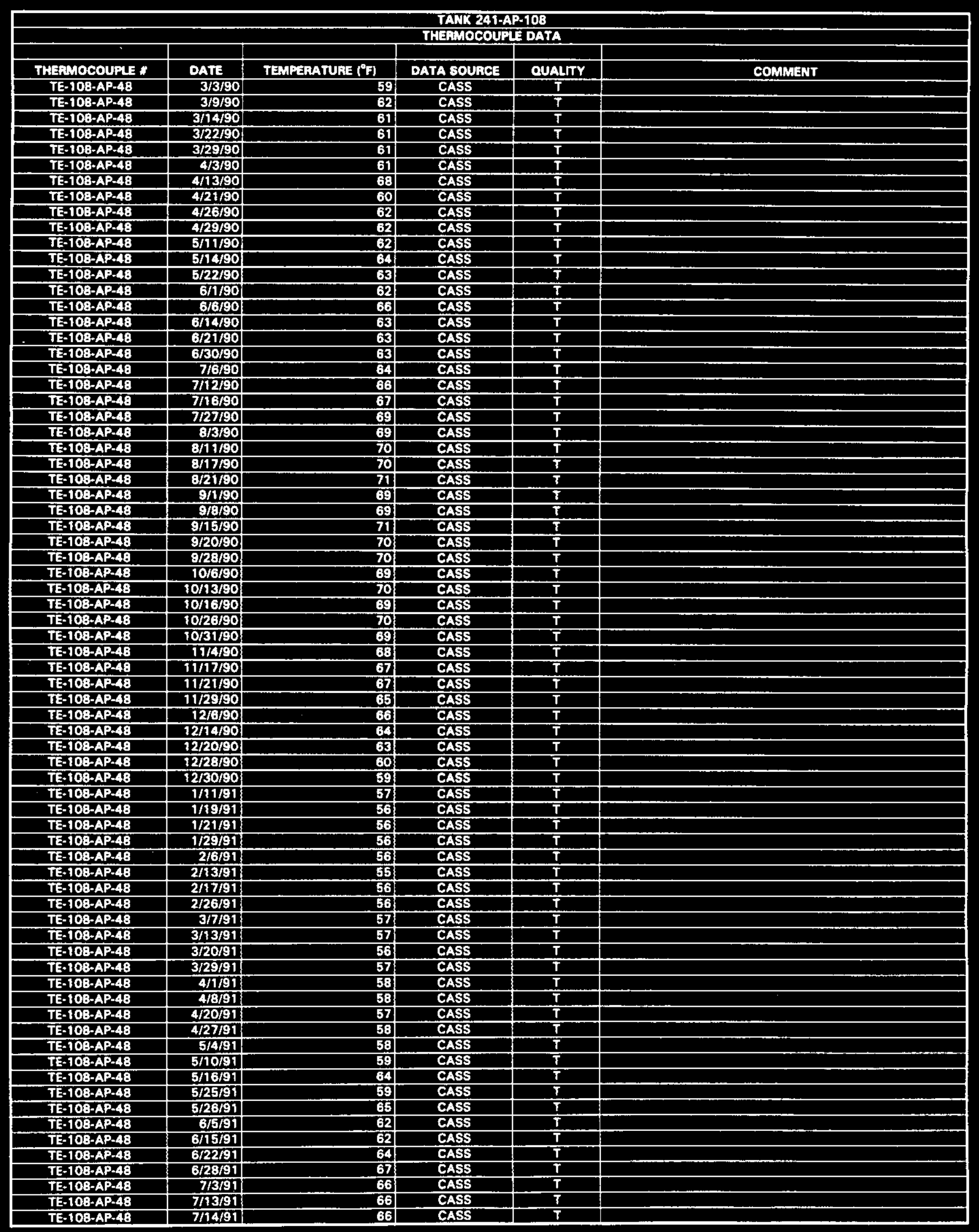

Data obtained from Computer Automated Surveillance Syatem (CASS), April 1995; and Surveillance Analysis Computer System (SACS), June 1995.

D-697 


\begin{tabular}{|c|c|c|c|c|c|}
\hline \multicolumn{6}{|c|}{$\begin{array}{l}\text { TANK 241-AP-108 } \\
\text { THEFMOCOUPLEDATA }\end{array}$} \\
\hline THERMOCOUAE : & & & & & \\
\hline TE-TOB-AP-48 & $\frac{\text { DAYE }}{\text { 7/24/91 }}$ & 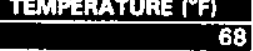 & $\frac{\text { DATA SOULiCE }}{\text { CASS }}$ & $\frac{\text { OUAUTY }}{T}$ & COMMENT \\
\hline TE-108-AP-48 & $8 / 2 / 91$ & 69 & CASS & $T$ & \\
\hline TE-108-AP-48 & $8 / 6 / 91$ & 68. & CASS & $\mathbf{T}$ & \\
\hline TE-108-AP-4B & $8 / 17 / 91$ & 71 & CASS & $T$ & \\
\hline TE-108-AP-48 & $8 / 20 / 91$ & 70 & CASS & $\mathbf{T}$ & \\
\hline TE-10B-AP-4B & $8 / 30 / 91$ & 70 & CASS & $\mathbf{T}$ & \\
\hline TE-108-AP-48 & $9 / 3 / 91$ & 71 & CASS & $\mathbf{T}$ & \\
\hline TE-108-AP-48 & $9 / 8 / 91$ & 72 & CASS & $\mathbf{T}$ & \\
\hline TE-108-AP-48 & 9/18/91 & 76 & CASS & $T$ & \\
\hline TE-108-AP-48 & 9/22/91 & 71 & CASS & $T$ & \\
\hline$T E-100-A P-48$ & $9 / 30 / 91$ & 72 & CASS & $T$ & \\
\hline TE-106-AP-48 & 10/8/91 & 62 & CASS & $\underline{\mathbf{S}}$ & Data point did not agrae with local trend \\
\hline TE-10B-AP-48 & $10 / 10 / 91$ & 72 & CASS & $\mathbf{T}$ & \\
\hline TE-108-AP-48 & $10 / 17 / 97$ & 73 & CASS & $\mathbf{T}$ & \\
\hline TE-108-AP-48 & $10 / 25 / 91$ & 71 & CASS & $\mathbf{T}$ & \\
\hline TE-108-AP-48 & $10 / 28 / 91$ & 71 & CASS & $T$ & \\
\hline TE-108-APAB & $11 / 6 / 91$ & 70 & CASS & $\mathbf{T}$ & \\
\hline TE-100-AP-48 & 7/30/92 & 67 & CASS & T & \\
\hline TE-108-AP-48 & $8 / 3 / 92$ & 67 & CASS & $T$ & \\
\hline TE-108-AP-48 & $8 / 15 / 92$ & 67 & CASS & $T$ & \\
\hline TE-108-AP-48 & $8 / 20 / 92$ & 68 & CASS & $T$ & \\
\hline TE-108-AP-48 & $8 / 28 / 92$ & 67 & CASS & $T$ & \\
\hline TE-108-AP-AB & 9/5/92 & 66 & CASS & $T$ & \\
\hline TE-102-AP-48 & 9/12/92 & 67 & CASS & $T$ & \\
\hline TE-106-AP-48 & 9/15/92 & 68 & CASS & $\bar{T}$ & \\
\hline TE-108-AP-48 & 9/26/92 & 66 & CASS & $\mathbf{T}$ & \\
\hline TE-108-AP-48 & 10/2/92 & 68 & CASS & $T$ & \\
\hline TE-103-AP-48 & $10 / 5 / 82$ & 67 & CASS & $\mathbf{T}$ & \\
\hline TE-108-AP-48 & $10 / 17 / 92$ & 68 & CASS & $\bar{T}$ & \\
\hline TE-108-AP-48 & $10 / 22 / 92$ & 69 & CASS & $T$ & \\
\hline TE-100-AP-48 & $10 / 28 / 92$ & 70 & CASS & $T$ & \\
\hline TE-108-AP-48 & $11 / 4 / 82$ & 69 & CASS & $T$ & \\
\hline TE-108-AP-48 & $11 / 11 / 92$ & 70 & CASS & $T$ & \\
\hline TE-108-AP-48 & $11 / 17 / 92$ & 67 & CASS & $T$ & \\
\hline TE-108-AP-48 & $11 / 25 / 92$ & 68 & CASS & $T$ & \\
\hline TE-10B-AP-AB & $11 / 30 / 92$ & 68 & CASS & $\bar{T}$ & \\
\hline TE-10B-AP-AB & $12 / 12 / 92$ & 68 & CASS & $\bar{T}$ & \\
\hline TE-108-AP.48 & 12/19/92 & 66 & CASS & $T$ & \\
\hline TE-108-AP-48 & $12 / 21 / 92$ & 66 & CASS & $T$ & \\
\hline TE-108-AP- 18 & $12 / 30 / 22$ & 66 & CASS & $T$ & \\
\hline TE-108-AP. 48 & $1 / 15 / 94$ & 63 & CASS & $T$ & \\
\hline TE-108-AP-48 & $1 / 20 / 94$ & 64 & CASS & $\bar{T}$ & \\
\hline TE.108-AP-4B & $1 / 25 / 94$ & 64 & CASS & $T$ & \\
\hline TE-108-AP-48 & $2 / 4 / 94$ & 64 & CASS & $\bar{T}$ & \\
\hline TE-108AP-48 & $2 / 13 / 94$ & 64 & CASS & $T$ & \\
\hline TE-108.AP-48 & $2 / 16 / 94$ & 64 & CASS & $\bar{T}$ & \\
\hline TE-108-AP-48 & $2 / 23 / 94$ & 63 & CASS & $T$ & \\
\hline TE-108-AP-48 & $3 / 5 / 94$ & 64 & CASS & $\bar{T}$ & \\
\hline TE-108-AP-4B & $3 / 10 / 94$ & 64 & CASS & $T$ & \\
\hline TE-108-AP-48 & $3 / 17 / 94$ & 64 & CASS & $T$ & \\
\hline TE-108-AP-48 & $3 / 25 / 94$ & 65 & CASS & $\bar{T}$ & \\
\hline TE-108-AP-48 & $3 / 29 / 94$ & 63 & CASS & $T$ & \\
\hline TE-108-AP-48 & $4 / 9 / 94$ & 65 & CASS & $T$ & \\
\hline TE-108-AP-48 & $4 / 13 / 94$ & 64 & CASS & $\mathbf{T}$ & \\
\hline TE-108-AP-4B & $4 / 22 / 94$ & 64 & CASS & $\mathbf{T}$ & \\
\hline TE-108-AP-48 & $4 / 27 / 94$ & 64 & CASS & $\mathbf{I}$ & \\
\hline TE-108-AP+48 & $5 / 2 / 94$ & 64 & CASS & $\bar{T}$ & \\
\hline TE-108-AP-48 & $5 / 8 / 94$ & 66 & CASS & $\mathbf{T}$ & \\
\hline TE-108-AP-48 & $5 / 16 / 94$ & 104 & CASS & $T$ & \\
\hline TE-108-AP-48 & $5 / 23 / 94$ & 99 & CASS & $T$ & \\
\hline TE-108-AP-48 & $6 / 2 / 94$ & 98 & CASS & $\bar{T}$ & \\
\hline TE-108-AP-48 & 6/8/94 & 98 & CASS & $T$ & \\
\hline TE-108-AP-48 & $6 / 16 / 94$ & 63 & CASS & $\mathbf{s}$ & Dats point did not agree with local trend \\
\hline TE-108-AP-48 & $6 / 17 / 94$ & 63 & CASS & $\mathbf{S}$ & Date point did not apree with local trend \\
\hline TE-108-AP-4B & $6 / 18 / 94$ & 95 & CASS & $\mathbf{T}$ & \\
\hline TE-108-AP-48 & $6 / 20 / 94$ & 63. & CASS & $\mathbf{s}$ & Data point did not agree with local trend \\
\hline TE-108-AP-48 & $6 / 25 / 94$ & 100 & CASS & $T$ & \\
\hline TE-10B-AP-4B & $6 / 27 / 94$ & 97 & CASS & $\mathbf{T}$ & \\
\hline TE-108-AP-48 & $7 / 7 / 94$ & 97 & CASS & $T$ & \\
\hline TE-108-AP-48 & $7 / 15 / 94$ & 96. & CASS & $T$ & \\
\hline TE-108-AP-48 & $7 / 20 / 94$ & 94 & CASS & $\mathbf{T}$ & \\
\hline TE-108-AP-48 & $7 / 24 / 94$ & 94 & CASS & $\mathbf{T}$ & \\
\hline TE-10B-AP-48 & $7 / 31 / 94$ & 97. & CASS & $T$ & \\
\hline
\end{tabular}




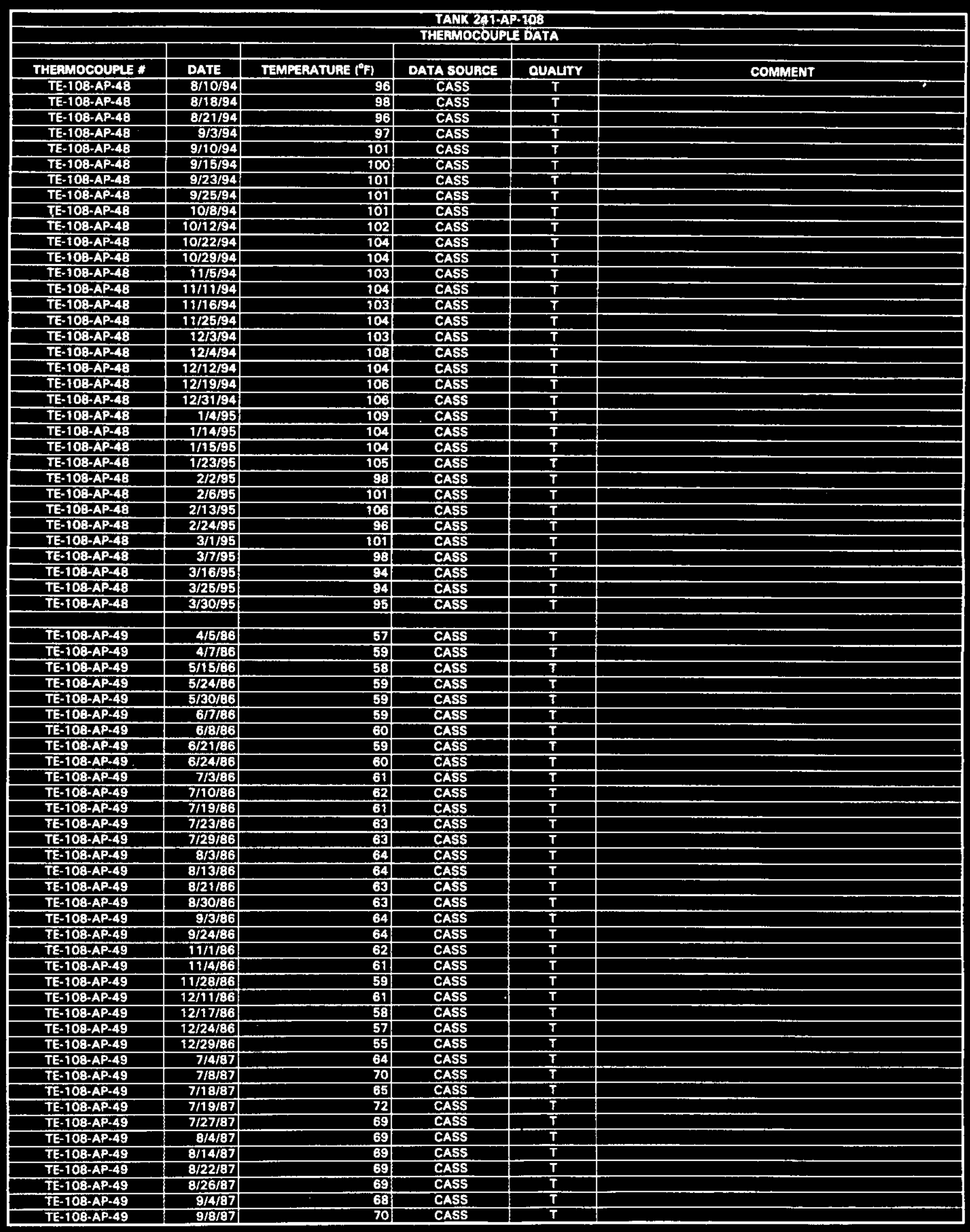

Data obtained from Computer Automated Surveillance Syatem (CASS), April 1995; and Surveillanca Analysis Computer System (SACS), June 1995. 


\begin{tabular}{|c|c|c|c|c|c|}
\hline \multicolumn{6}{|c|}{$\begin{array}{l}\text { TANK 241-AP-108 } \\
\text { THEFWOCOUPL DATA }\end{array}$} \\
\hline & & & & & \\
\hline THERMOCOUPLE $\#$ & DATE & TEMPERATURE $\left({ }^{\circ} \mathrm{F}\right)$ & DATA SOURCE & OUALITY & COMMENT \\
\hline TE-108.AP-49 & 9/14/87 & 72 & CASS & $T$ & \\
\hline TE-108-AP-49 & $9 / 20 / 87$ & 70 & CASS & $\bar{T}$ & \\
\hline TE-108-AP-49 & 9/27/87 & 70 & CASS & $\bar{T}$ & \\
\hline TE-108-AP-49 & $10 / 8 / 87$ & 69 & CASS & $T$ & \\
\hline TE-108-AP-49 & $10 / 16 / 87$ & 67 & CASS & $T$ & \\
\hline$T E-108-A P-49$ & $10 / 19 / 87$ & 67 & CASS & $T$ & \\
\hline TE-108-AP-49 & $10 / 31 / 87$ & 64 & CASS & $T$ & \\
\hline TE-108-AP-49 & $11 / 1 / 87$ & 64 & CASS & $T$ & \\
\hline TE-108-AP.49 & $11 / 8 / 87$ & 64 & CASS & $T$ & \\
\hline TE-108-AP-49 & 11/16/87 & 62 & CASS & $T$ & \\
\hline TE-108-AP-49 & $11 / 26 / 87$ & 61 & CASS & $\bar{T}$ & \\
\hline TE-108-AP-49 & $11 / 29 / 87$ & 61 & CASS & $T$ & \\
\hline TE-108-AP-49 & 12/6/a7 & 61 & CASS & $T$ & \\
\hline TE-108-AP-49 & $12 / 18 / 87$ & 58 & CASS & $T$ & \\
\hline TE-108-AP-49 & $12 / 23 / 37$ & 56 & CASS & $T$ & \\
\hline TE-108-AP-49 & 1/2/83 & 56 & CASS & $T$ & \\
\hline TE-108-AP-49 & $1 / 4 / 88$ & 53 & CASS & $T$ & \\
\hline TE-108-AP-49 & $1 / 16 / 88$ & ह1 & CASS & $T$ & \\
\hline TE-108-AP-49 & $1 / 17 / 88$ & 52 & CASS & $T$ & \\
\hline TE-108-AP-49 & $1 / 25 / 88$ & 53 & CASS & $T$ & \\
\hline TE-108-AP-49 & $2 / 2 / 88$ & 52 & CASS & $T$ & \\
\hline TE-108-AP-49 & $2 / 13 / 8$ & 52 & CASS & $T$ & \\
\hline TE-108-AP-49 & $2 / 4 / 88$ & 54 & CASS & $T$ & \\
\hline TE-108-AP-49 & $2 / 27 / 88$ & 52 & CASS & $T$ & \\
\hline TE-108-AP-49 & $3 / 3 / 68$ & 54 & CASS & $T$ & \\
\hline TE-108-AP-49 & 3/10/88 & 54 & CASS & $T$ & \\
\hline TE-108-AP-49 & $3 / 13 / 88$ & 54 & CASS & $T$ & \\
\hline TE-108-AP-49 & $3 / 24 / 88$ & 56 & CASS & $T$ & \\
\hline TE-108-AP-49 & 3/28/8日 & 54 & CASS & $T$ & \\
\hline TE-103-AP-49 & $6 / 1 / 88$ & 62 & CASS & $T$ & \\
\hline TE-108-AP-A9 & 6/10/8s & 61 & CASS & $T$ & \\
\hline TE-10B-AP-A9 & $6 / 18 / 88$ & 61 & CASS & $T$ & \\
\hline TE-108-AP-49 & $6 / 25 / 88$ & 62 & CASS & $T$ & \\
\hline TE-108-AP-49 & $7 / 1 / 83$ & 64 & CASS & $T$ & \\
\hline TE-108-AP-A9 & $7 / 9 / 88$ & 63 & CASS & $\mathbf{T}$ & \\
\hline TE-108-AP-A9 & $7 / 13 / 88$ & 66 & CASS & $\bar{T}$ & \\
\hline TE-108-AP-49 & $7 / 23 / 88$ & 67 & CASS & $T$ & \\
\hline TE-108-AP-49 & $7129 / 88$ & 68 & CASS & $T$ & \\
\hline TE-108-AP-49 & $8 / 3 / 88$ & 69 & CASS & $T$ & \\
\hline TE-108-AP-49 & $8 / 9 / 88$ & 69 & CASS & $T$ & \\
\hline TE-108-AP-4B & 8/20/83 & 69 & CASS & $T$ & \\
\hline$T E-108-A P-49$ & $8 / 27 / 88$ & 68 & CASS & $T$ & \\
\hline TE-108-AP-49 & $8 / 31 / 88$ & 69 & CASS & $T$ & \\
\hline$T E-108-A P+49$ & $9 / 9 / 88$ & 71 & CASS & $T$ & \\
\hline TE-108-AP-49 & $9 / 16 / 38$ & 71 & CASS & $T$ & \\
\hline TE-108-AP-49 & $9 / 23 / 8$ & 69 & CASS & $T$ & \\
\hline TE-108-AP-49 & $9 / 27 / 88$ & 70 & CASS & $T$ & \\
\hline TE-108-AP-49 & $12 / 3 / 88$ & 65 & CASS & $T$ & \\
\hline TE-108-AP-49 & $12 / 8 / 8 B$ & 64 & CASS & $T$ & \\
\hline TE-108-AP-49 & $12 / 13 / 88$ & 63 & CASS & $T$ & \\
\hline$T E-108-A P-49$ & $12 / 20 / 88$ & 63 & CASS & $T$ & \\
\hline TE-108-AP-49 & $8 / 2 / 89$ & 69 & CASS & $T$ & \\
\hline TE-108-AP-AS & $9 / 5789$ & 69 & CASS & $T$ & \\
\hline TE-10B-AP-49 & 9/16/eg & 71 & CASS & $\bar{T}$ & \\
\hline TE-108-AP-49 & $9 / 18 / 89$ & 71 & CASS & $T$ & \\
\hline TE-108-AP-49 & $9 / 29 / 89$ & 72 & CASS & $T$ & \\
\hline TE-108-AP-49 & $11 / 4 / 89$ & 66 & CASS & $T$ & \\
\hline TE-108-AP-49 & $11 / 8 / 89$ & 64 & CASS & $\bar{T}$ & \\
\hline$T E-108-A P-49$ & $11 / 18 / 89$ & 69 & CASS & $T$ & \\
\hline TE-108-AP-49 & $11 / 24 / 89$ & 63 & CASS & $T$ & \\
\hline TE-108-AP-49 & $12 / 2 / 89$ & 66 & CASS & $T$ & \\
\hline TE-108-AP-49 & $12 / 4 / 89$ & 62 & CASS & $T$ & \\
\hline TE-10B-AP-49 & $12 / 12 / 89$ & 61 & CASS & $T$ & \\
\hline TE-108-AP-49 & $12 / 21 / 89$ & 58 & CASS & $\bar{T}$ & \\
\hline TE-108-AP-49 & $12 / 30 / 89$ & 57 & CASS & $\bar{T}$ & \\
\hline TE-108-AP-49 & $1 / 2 / 90$ & 58 & CASS & $T$ & \\
\hline TE-108-AP-49 & $1 / 11 / 90$ & 95 & CASS & $\mathbf{S}$ & Data point did not agres with local trend \\
\hline TE-108-AP-49 & $1 / 12 / 90$ & 58 & CASS & $\bar{T}$ & \\
\hline TE-108-AP-49 & $1 / 15 / 90$ & 58 & CASS & $T$ & \\
\hline TE-108-AP-49 & $1 / 21 / 90$ & 66 & CASS & $\mathbf{s}$ & Data point did not agree with local trend \\
\hline TE-106-AP-49 & $1 / 23 / 90$ & 58 & CASS & $\bar{T}$ & \\
\hline TE-108-AP.49 & $2 / 1 / 90$ & 58 & CASS & $T$ & \\
\hline TE-10B-AP-49 & $2 / 4 / 90$ & 59 & CASS & $\mathbf{T}$ & \\
\hline
\end{tabular}

Data obtained from Computer Automated Survelliance System (CASS), April 1995; and Surveillance Analyats Computer System (SACS), June 1995.

$$
\text { D-700 }
$$




\begin{tabular}{|c|c|c|c|c|c|}
\hline \multicolumn{6}{|c|}{$\begin{array}{l}\text { TANK 241-AP-108 } \\
\text { THERWOCOUPLE DATA }\end{array}$} \\
\hline & & & & & \\
\hline THERMOCOUPUE * & DATE & TEMPERATURE ( $\left.{ }^{\circ} \mathrm{F}\right)$ & DATA SOURCE & QUALITY & COMMENT \\
\hline TE-108-AP-49 & $2 / 15 / 90$ & 58 & CASS & $T$ & \\
\hline TE-10B-AP-4Q & $2 / 18 / 90$ & 57 & CASS & $\mathbf{T}$ & \\
\hline TE-108-AP-49 & $3 / 3 / 90$ & 59 & CASS & $T$ & \\
\hline TE-108-AP-49 & $3 / 9 / 90$ & 62 & CASS & $T$ & \\
\hline TE-108-AP-49 & $3 / 14 / 90$ & 61: & CASS & $T$ & \\
\hline TE-108-AP-49 & $3 / 1 \mathrm{~B} / 90$ & 62 & CASS & $T$ & \\
\hline TE-108-AP-49 & $3 / 29 / 90$ & 61 & CASS & $T$ & \\
\hline TE-108-AP-49 & $4 / 2 / 90$ & 62 & CASS & $\mathbf{T}$ & \\
\hline TE-108-AP-49 & $4 / 12 / 90$ & 62 & CASS & $T$ & \\
\hline TE-108-AP-49 & 4/21/90 & 60 & CASS & $T$ & \\
\hline TE-108-AP-49 & $4 / 26 / 90$ & 63 & CASS & $T$ & \\
\hline TE-108-AP-49 & $5 / 3 / 90$ & 62 & CASS & $T$ & \\
\hline TE-108-AP-49 & $5 / 6 / 90$ & 63 & CASS & $T$ & \\
\hline TE-10B-AP-49 & $5 / 17 / 190$ & 64 & CASS & $T$ & \\
\hline TE-108-AP-49 & $5 / 22190$ & 62 & CASS & $T$ & \\
\hline TE-10B-AP-AG & $6 / 1 / 90$ & 63 & CASS & $T$ & \\
\hline TE-108.AP-49 & 6/6/90 & 66 & CASS & $\bar{T}$ & \\
\hline TE-108-AP-49 & $6 / 14 / 90$ & 64 & CASS & $T$ & \\
\hline TE-108-AP-49 & $6 / 21 / 90$ & 64 & CASS & $T$ & \\
\hline TE-108-AP-49 & $6 / 30 / 90$ & 64 & CASS & $T$ & \\
\hline TE-108-AP-49 & $7 / 6 / 90$ & 64 & CASS & $T$ & \\
\hline TE-108-AP-49 & 7/14/90 & 66 & CASS & $T$ & \\
\hline TE-108-AP-49 & $7 / 16 / 90$ & 69 & CASS & $T$ & \\
\hline TE-108-AP-49 & $7 / 27 / 90$ & 69 & CASS & $\bar{T}$ & \\
\hline$T E-108-A P-49$ & $8 / 3 / 90$ & 69 & CASS & $T$ & \\
\hline TE-108-AP-49 & $8 / 11 / 90$ & 70 & CASS & $\bar{T}$ & \\
\hline TE-108-AP-49 & $8 / 17 / 30$ & 70 & CASS & $\bar{I}$ & \\
\hline TE-108-AP-49 & $8 / 19 / 90$ & 72 & CASS & $T$ & \\
\hline TE-108-AP-49 & $9 / 1 / 90$ & 69 & CASS & $\bar{T}$ & \\
\hline TE-106-AP-49 & $9 / 8 / 90$ & 69 & CASS & $T$ & \\
\hline TE-108-AP-49 & $9 / 15 / 90$ & 73 & CASS & $T$ & \\
\hline TE-108-AP-49 & $9 / 20 / 90$ & 71 & CASS & $T$ & \\
\hline TE-108.AP-49 & $9 / 27 / 80$ & 72 & CASS & $T$ & \\
\hline TE-10B-AP-49 & $10 / 1 / 90$ & 71 & CASS & $T$ & \\
\hline TE-108-AP- 49 & $10 / 10 / 90$ & 721 & CASS & $T$ & \\
\hline TE-108-AP-49 & 10/16/90 & 69 & CASS & $\mathrm{T}$ & \\
\hline$T E-108-A P-49$ & $10 / 26 / 90$ & 70 & CASS & $T$ & \\
\hline TE-10B-AP-AS & $10 / 31 / 90$ & 70 & CASS & $\mathbf{T}$ & \\
\hline TE-108-AP-49 & $11 / 10 / 90$ & 67 & CASS & $T$ & \\
\hline TE-108-AP-49 & $11 / 17 / 90$ & 67 & CASS & $\bar{T}$ & \\
\hline$T \mathrm{TE}-108-A P-49$ & $11 / 21 / 90$ & 66 & CASS & $T$ & \\
\hline TE-10B-AP-49 & $11 / 29 / 90$ & 65 & CASS & $\bar{T}$ & \\
\hline TE-108-AP-49 & $12 / 6 / 90$ & 86 & CASS & $T$ & \\
\hline TE-108-AP-49 & $12 / 14 / 90$ & 64 & CASS & $T$ & \\
\hline TE-108-A.P-49 & $12 / 20 / 90$ & 63 & CASS & $T$ & \\
\hline TE-10B-AP-49 & $12 / 28 / 90$ & 60 & CASS & $T$ & \\
\hline TE-108-AP-49 & $12 / 30 / 90$ & 59 & CASS & $T$ & \\
\hline TE-108-AP-49 & $7 / 11 / 91$ & 58 & CASS & $\bar{T}$ & \\
\hline TE-108-AP-49 & $1 / 19 / 91$ & 56 & CASS & $T$ & \\
\hline TE-108-AP-49 & $1 / 21 / 91$ & 56 & CASS & $\bar{T}$ & \\
\hline TE-108-AP-49 & 1/29/91 & 56 & CASS & $T$ & \\
\hline TE-108-AP-49 & $2 / 3 / 91$ & 56 & CASS & $\bar{T}$ & \\
\hline TE-108-AP-49 & $2 / 16 / 97$ & 54 & CASS & $T$ & \\
\hline TE-108-AP-A9 & $2 / 17 / 91$ & 56 & CASS & $T$ & \\
\hline TE-108-AP-49 & $2 / 26 / 91$ & 57 & CASS & $T$ & \\
\hline TE-108-AP-49 & $3 / 7 / 91$ & 57 & CASS & $T$ & \\
\hline TE-108-AP-49 & 3/13/91 & 57 & CASS & $T$ & \\
\hline TE-108-AP-49 & $3 / 20 / 91$ & 56 & CASS & $T$ & \\
\hline TE-108-AP-49 & $3 / 25 / 91$ & 58 & CASS & $T$ & \\
\hline TE-108-AP-49 & $4 / 5 / 91$ & 57 & CASS & $T$ & \\
\hline TE-108-AP-49 & $4 / 12 / 91$ & 58 & CASS & $T$ & \\
\hline TE-108-AP-49 & $4 / 20 / 91$ & 57 & CASS & 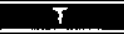 & \\
\hline TE-108-AP-49 & $4 / 21 / 91$ & 59 & CASS & $T$ & \\
\hline TE-108-AP-49 & $5 / 1 / 91$ & 59 & CASS & $\mathbf{T}$ & \\
\hline TE-108-AP-49 & $5 / 2 / 91$ & 73 & CASS & $\mathbf{s}$ & Data point did not agree with local trend \\
\hline$T E-10 B-A P-49$ & $5 / 10 / 91$ & 60 & CASS & $T$ & \\
\hline TE-10B-AP-49 & $5 / 16 / 91$ & 64 & CASS & $\bar{T}$ & \\
\hline TE-108-AP-49 & $5 / 25 / 91$ & 60 & CASS & $\mathbf{T}$ & \\
\hline TE-108-AP-49 & $5 / 26 / 91$ & 65: & CASS & $\mathbf{T}$ & \\
\hline TE-108-AP-49 & 6/5/91 & 62 & CASS & $\mathbf{T}$ & \\
\hline TE-108-AP-49 & 6/10/91 & 117 & CASS & $\mathbf{S}$ & Data point did not sgree with local trend \\
\hline TE-108-AP-49 & $6 / 15 / 91$ & 63 & CASS & $\mathbf{T}$ & \\
\hline TE-108-AP-49 & 6/22/91 & 65 & CASS & $\bar{T}$ & \\
\hline
\end{tabular}




\begin{tabular}{|c|c|c|c|c|c|}
\hline \multicolumn{6}{|c|}{$\begin{array}{l}\text { TANK 241-AP-108 } \\
\text { THEAMOCOUML DATA }\end{array}$} \\
\hline & & & & & \\
\hline THERMOCOUPLE * & DATE & TEMPERATUAE $\left.f^{\circ} \mathrm{F}\right\}$ & DATA SOUACE & OUALTY & COMMENT \\
\hline TE-108-AP-49 & $6 / 23 / 91$ & 68 & CASS & $T$ & \\
\hline TE-108-AP-49 & $7 / 3 / 91$ & 68 & CASS & $T$ & \\
\hline TE-108-AP-49 & $7 / 13 / 91$ & 67 & CASS & $\mathbf{T}$ & \\
\hline TE-108-AP-49 & $7 / 14 / 91$ & 67 & CASS & $\mathbf{T}$ & \\
\hline TE-108-AP-49 & $7 / 24 / 91$ & 69 & CASS & $\mathbf{T}$ & \\
\hline TE-108-AP-49 & $8 / 2 / 91$ & 70 & CASS & $T$ & \\
\hline TE-108-AP-49 & $8 / 6 / 91$ & 69 & CASS & $T$ & \\
\hline TE-108-AP-49 & $8 / 17 / 91$ & 72 & CASS & $T$ & \\
\hline TE-108-AP-49 & $8 / 20 / 91$ & 71 & CASS & $T$ & \\
\hline TE-108-AP-49 & $8 / 30 / 91$ & 71 & CASS & $T$ & \\
\hline TE-10B-AP-49 & $9 / 3 / 91$ & 71 & CASS & $\mathbf{T}$ & \\
\hline TE-10B-AP-49 & 9/8/91 & $7 \sqrt{2}$ & CASS & $T$ & \\
\hline TE-108-AP-49 & 9/18/91 & 76 & CASS & $T$ & \\
\hline$T E=10 \mathrm{~B}-\mathrm{AP}-49$ & $9 / 22 / 91$ & 71 & CASS & $\mathbf{T}$ & \\
\hline TE-108-AP-49 & $10 / 1 / 91$ & 73 & CASS & $\mathrm{T}$ & \\
\hline TE-108-AP-49 & $10 / 8 / 91$ & 61 & CASS & $\mathbf{S}$ & Date point did not agres whi local trend \\
\hline TE-109-AP-49 & $10 / 10 / 81$ & 72 & CASS & $T$ & \\
\hline TE-108-AP-49 & $10 / 17 / 91$ & 7 & CASS & $\bar{T}$ & \\
\hline TE-108-AP-49 & $10 / 25 / 91$ & 72 & CASS & $T$ & \\
\hline TE-108-AP-49 & $10 / 28 / 91$ & 71 & CASS & $\mathbf{T}$ & \\
\hline TE-108-AP-49 & $11 / 8 / 91$ & 70 & CASS & $T$ & \\
\hline TE-108-AP-49 & $7 / 30 / 22$ & 67 & CASS & $\mathbf{T}$ & \\
\hline TE-108-AP-49 & $8 / 3 / 92$ & 66 & CASS & $\bar{T}$ & \\
\hline TE-108-AP-49 & $8 / 15 / 92$ & 68 & CASS & $\mathbf{T}$ & \\
\hline TE-108-AP-49 & $8 / 20 / 92$ & 69 & CASS & $T$ & \\
\hline TE-108-AP-49 & $8 / 28 / 92$ & 67 & CASS & $\mathbf{T}$ & \\
\hline TE-108-AP-49 & $8 / 31 / 92$ & 66 & CASS & $\mathbf{T}$ & \\
\hline TE-108-AP-A9 & 9/12/92 & 66 & CASS & $\bar{T}$ & \\
\hline TE-108-AP-A9 & $9 / 16 / 92$ & 67 & CASS & $\mathbf{T}$ & \\
\hline TE-108-AP-A9 & 9/26/92 & 66 & CASS & $T$ & \\
\hline TE-10B-AP-49 & $10 / 2 / 92$ & 68 & CASS & $\mathbf{T}$ & \\
\hline TE-103-AP-49 & $10 / 5 / 92$ & 67 & CASS & $\bar{T}$ & \\
\hline TE-108-AP-49 & $10 / 13 / S_{2}$ & 60 & CASS & $\bar{T}$ & \\
\hline TE-108-AP-49 & $10 / 22 / 92$ & 70 & CASS & $\bar{T}$ & \\
\hline TE-108-AP-49 & $10 / 29 / 92$ & 70 & CASS & $T$ & \\
\hline TE-108-AP-49 & $11 / 4 / 92$ & 69 & CASS & $T$ & \\
\hline TE-108-AP-49 & $11 / 9 / 92$ & 69 & CASS & $T$ & \\
\hline$T E-108-A P-49$ & $11 / 17 / 92$ & 68 & CASS & $T$ & \\
\hline TE-108-AP-49 & $11 / 25 / 92$ & 68 & CASS & $T$ & \\
\hline TE-108-AP-49 & $11 / 30 / 92$ & 88 & CASS & $T$ & \\
\hline TE-108-AP-49 & $12 / 6 / 92$ & 68 & CASS & $T$ & \\
\hline TE-108-AP-49 & $12 / 19 / 92$ & 66 & CASS & $T$ & \\
\hline TE-108.AP-49 & $12 / 25 / 92$ & 65 & CASS & $\mathbf{T}$ & \\
\hline TE-108-AP-49 & $12127 / 92$ & 66 & CASS & $T$ & \\
\hline TE-108-AP-49 & $1 / 15 / 94$ & 63 & CASS & $T$ & \\
\hline TE-108-AP-49 & $1 / 20 / 94$ & 64 & CASS & $T$ & \\
\hline TE-108-AP-49 & $1 / 25 / 94$ & 64 & CASS & $\bar{T}$ & \\
\hline TE-108-AP-49 & $2 / 4 / 94$ & 63 & CASS & $T$ & \\
\hline TE-108-AP-49 & $2 / 11 / 94$ & 63 & CASS & $T$ & \\
\hline TE-108-AP-49 & $2 / 16 / 94$ & 64 & CASS & $T$ & \\
\hline TE-108-AP-49 & $2 / 22 / 94$ & 63 & CASS & $T$ & \\
\hline TE-108-AP-49 & $3 / 5 / 94$ & 63 & CASS & $T$ & \\
\hline TE-108-AP-49 & $3 / 10 / 94$ & 64 & CASS & $\mathrm{T}$ & \\
\hline TE-108-AP-49 & $3 / 17 / 94$ & 64 & CASS & $T$ & \\
\hline TE-108-AP-49 & $3 / 25 / 94$ & 65 & CASS & $\mathrm{T}$ & \\
\hline TE-108-AP-49 & $3 / 28 / 94$ & 102 & CASS & $\mathbf{S}$ & Data point did not agree with local trend \\
\hline TE-108-AP-49 & $4 / 2 / 94$ & 62 & CASS & $\mathbf{T}$ & \\
\hline TE-108-AP-49 & $4 / 9 / 94$ & 64 & CASS & $T$ & \\
\hline TE-108-AP-49 & $4 / 15 / 94$ & 63 & CASS & $T$ & \\
\hline TE-108-AP-49 & $4 / 22 / 94$ & 64 & CASS & $\mathbf{T}$ & \\
\hline TE-108-AP-49 & $4 / 27 / 94$ & 64 & CASS & $\bar{T}$ & \\
\hline TE-108-AP-49 & $5 / 2 / 94$ & 64 & CASS & $\mathbf{T}$ & \\
\hline TE-108-AP-49 & $5 / 14 / 94$ & 64 & CASS & $\mathbf{T}$ & \\
\hline TE-108-AP-49 & $5 / 16 / 94$ & 104 & CASS & $T$ & \\
\hline$T E=108-A P-49$ & $5 / 23 / 94$ & 100 & CASS & $\bar{T}$ & \\
\hline TE-108-AP-49 & $6 / 2 / 94$ & 99 & CASS & $T$ & \\
\hline TE-108-AP-49 & $6 / 8 / 94$ & 98 & CASS & $\bar{T}$ & \\
\hline TE-108-AP-49 & 6/14/94 & 95 & CASS & $\bar{T}$ & \\
\hline TE-108-AP-49 & $6 / 16 / 94$ & 63 & CASS & $\mathbf{S}$ & Data point did not agree with tocal trend \\
\hline TE-10B-AP-49 & 6/17/94 & 63 & CASS & $\mathbf{s}$ & Date point did not agree. with locel trend \\
\hline TE-108-AP-49 & 6/20/94 & 63 & CASS & $\mathbf{s}$ & Data point did not aprae with local trend \\
\hline TE-108-AP-49 & $6 / 25 / 94$ & 100 & CASS & $T$ & \\
\hline TE-10B-AP-49 & $6 / 30 / 94$ & 97 & CASS & $T$ & \\
\hline
\end{tabular}

Data obtained from Computer Automated Surveillance System (CASS), April 1995; and Survaillance Analysis Computer System (SACS), June 1995.

D-702 


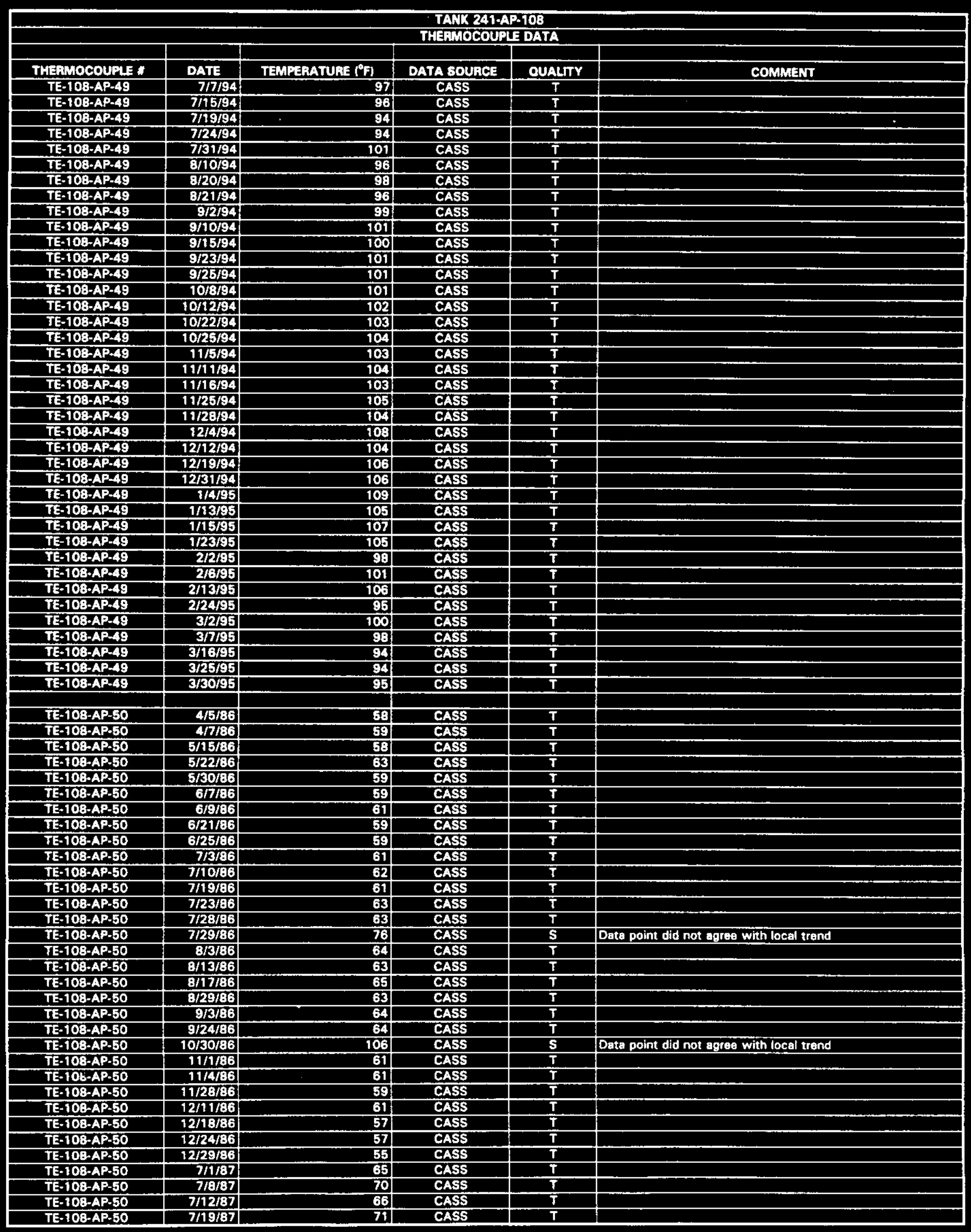




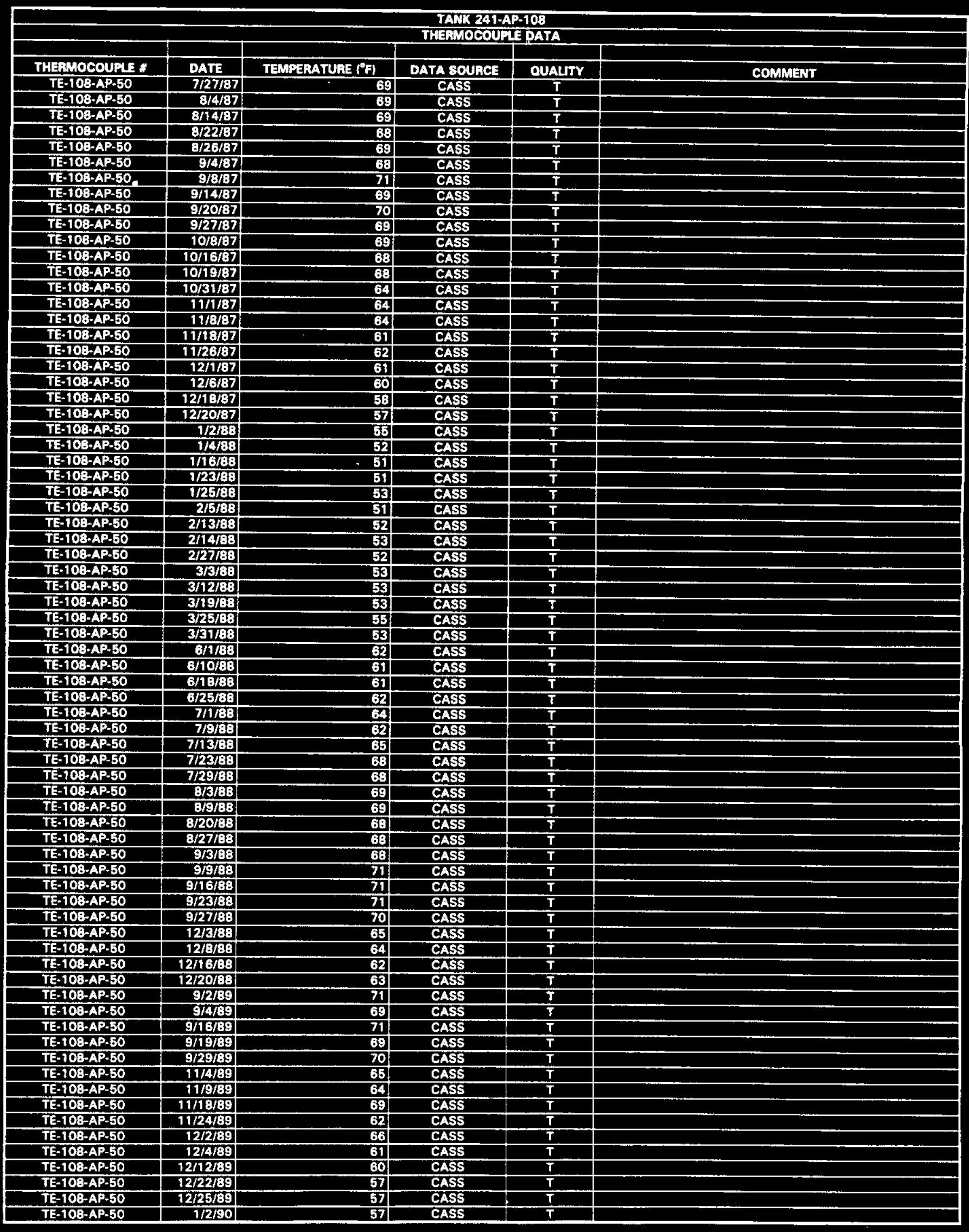




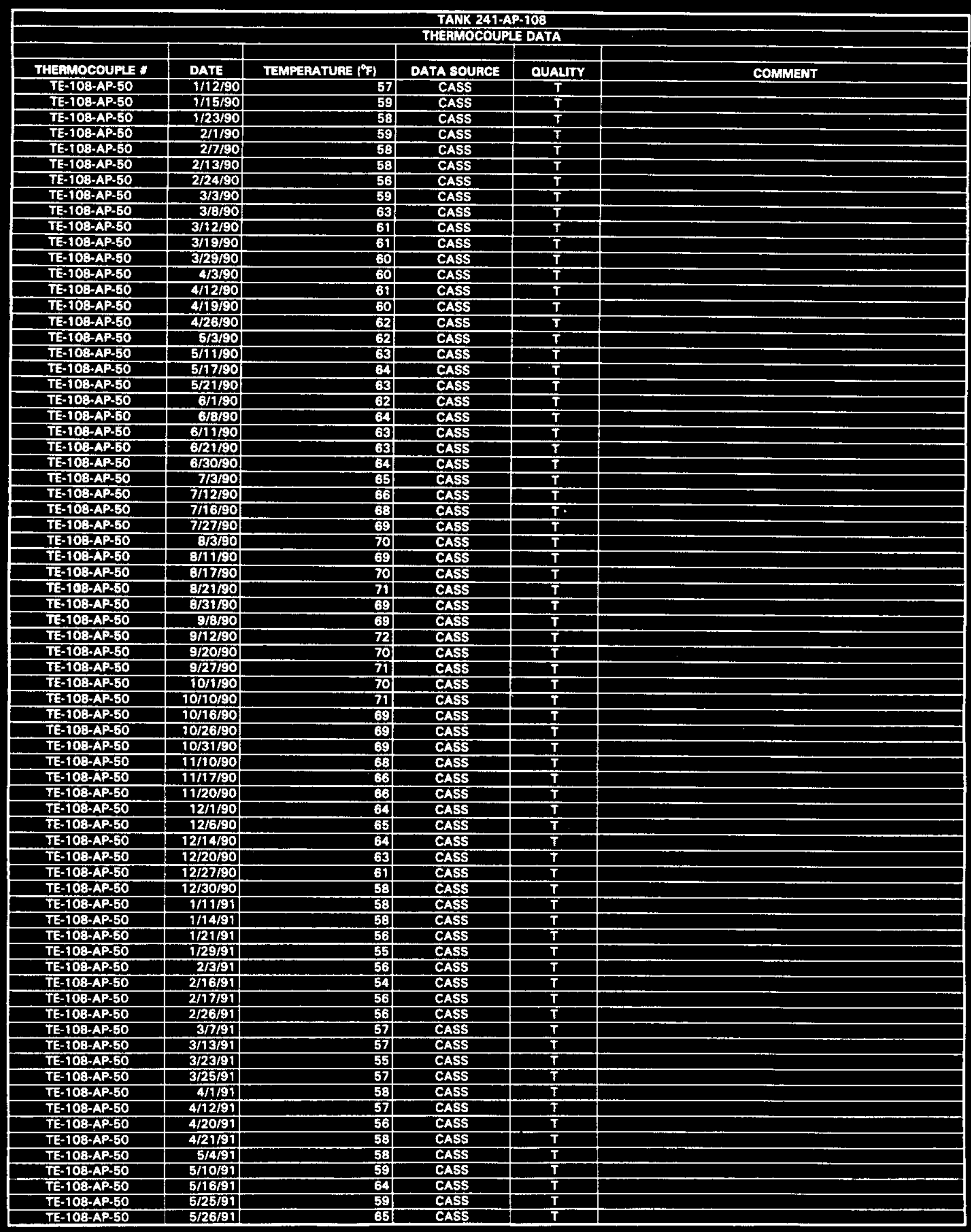




\begin{tabular}{|c|c|c|c|c|c|}
\hline \multicolumn{6}{|c|}{$\begin{array}{l}\text { TAKK 241-AP-108 } \\
\text { THEMMOCOUFLE DATA }\end{array}$} \\
\hline & & & & & \\
\hline$\frac{\text { THERMOCOUPE : }}{\text { TE-108-AP-50 }}$ & $\frac{\text { DATE }}{6 / 5 / 91}$ & TEMPERATUAE $\left({ }^{\circ} \mathrm{F}\right)$ & DATA SOUACE & QUALITY & COMMENT \\
\hline $\begin{array}{l}\text { TE-108-AP-50 } \\
\text { TE-108-AP-50 }\end{array}$ & $\frac{6 / 5 / 91}{6 / 15 / 91}$ & \begin{tabular}{|c|}
63 \\
63
\end{tabular} & CASS & $T$ & \\
\hline$\frac{\text { TE-108-AP-50 }}{\text { TE-108-AP-50 }}$ & $\begin{array}{l}6 / 15 / 91 \\
6 / 22 / 91\end{array}$ & $\frac{63}{65}$ & CASS & $T$ & \\
\hline TE-108-AP-50 & $6 / 28 / 91$ & 65 & $\frac{\text { CASS }}{\text { CASS }}$ & $\frac{T}{T}$ & \\
\hline TE-108-AP-50 & $7 / 3 / 91$ & 67 & CASS & $T$ & \\
\hline TE-108-AP-50 & $7 / 13 / 91$ & 66 & CASS & $\mathbf{T}$ & \\
\hline TE-108-AP.SO & 7/14/91 & 66 & CAASS & $T$ & \\
\hline TE-108-AP-50 & $7 / 24 / 93$ & 68 & CASS & $\bar{T}$ & \\
\hline TE-108-AP-50 & $8 / 2 / 91$ & 69 & CASS & $\bar{T}$ & \\
\hline TE-108-AP-50 & $8 / 6 / 91$ & 68 & CASS & $\mathbf{T}$ & \\
\hline TE-108-AP-50 & $8 / 17 / 91$ & 71 & CASS & $T$ & \\
\hline TE-10B-AP-50 & $8 / 18 / 91$ & 72 & CASS & $T$ & \\
\hline TE-10Q-AP-SO & $8 / 30 / 91$ & 71 & CASS & $T$ & \\
\hline TE-108-AP-50 & 9/3/9i & 71 & CASS & $\mathbf{T}$ & \\
\hline TE-108-AP.50 & 9/8/9? & 72 & CASS & $T$ & \\
\hline TE-108-AP-50 & $9 / 18 / 91$ & 76 & CASS & $\bar{T}$ & \\
\hline TE-108-AP-BO & $9 / 22191$ & 77 & CASS & $T$ & \\
\hline TE-108-AP-BO & $9 / 30 / 91$ & 73 & CASS & $T$ & \\
\hline TE-108-AP.50 & 10/3/91 & 61 & CASS & $\mathbf{S}$ & Dota point did not apree with local trend \\
\hline TE-108-AP.50 & $10 / 10 / 91$ & 72 & CASS & $T$ & \\
\hline TE-108-AP-50 & 10/19/91 & 72 & CASS & $T$ & \\
\hline TE-10:AP:50 & 10/25/91 & 72 & CASS & $T$ & \\
\hline TE-108-AP-BO & $10 / 28 / 91$ & 71 & CASS & $\bar{T}$ & \\
\hline TE-108-AP.5O & $11 / 6 / 92$ & 70 & CASS & $T$ & \\
\hline TE-108-AP-50 & $7 / 30 / 92$ & 67 & CASS & $T$ & \\
\hline TE-108-APS50 & $8 / 3 / 92$ & 67 & CASS & $\mathbf{T}$ & \\
\hline TE-108-AP-50 & $8 / 15 / 92$ & 67 & CASS & $\bar{T}$ & \\
\hline TE-108-AP-50 & $8 / 20 / 92$ & 69 & CASS & $\mathbf{T}$ & \\
\hline TE-108-AP-50 & $8 / 28 / 92$ & 67 & CASS & $T$ & \\
\hline TE-103-AP-50 & $8 / 31 / 92$ & 66 & CASS & $\mathbf{T}$ & \\
\hline IE-108-AP.50 & $8 / 12 / 92$ & 86 & CASS & $T$ & \\
\hline IE-108-AP-50 & $9 / 16 / 92$ & 67 & CASS & $\mathbf{T}$ & \\
\hline TE-108-AP-SO & $9 / 23 / 92$ & 86 & CASS & $T$ & \\
\hline TE-108-AP-50 & $10 / 3 / 92$ & 87 & CASS & $T$ & \\
\hline TE-108-AP.50 & $10 / 5 / 92$ & 68 & CASS & $\bar{T}$ & \\
\hline TE-108-AP-50 & $10 / 13 / 92$ & 68 & CASS & $T$ & \\
\hline TE-108-AP-50 & $10 / 22 / 92$ & 69 & CASS & $\bar{T}$ & \\
\hline TE-10Q-AP-50 & $10 / 29 / 92$ & 70 & CASS & $T$ & \\
\hline TE-108-AP-50 & $11 / 4 / 92$ & 69 & CASS & $T$ & \\
\hline TE-108-AP-50 & $11 / 9 / 92$ & 69 & CASS & $T$ & \\
\hline TE-108-AP.50 & $11 / 17 / 92$ & 68 & CASS & $T$ & \\
\hline TE-108-AP-50 & $91 / 25 / 92$ & 69 & CASS & $\mathrm{T}$ & \\
\hline TE-10B-AP-50 & $11 / 30 / 92$ & 69 & CASS & $\mathbf{T}$ & \\
\hline TE-108-AP-50 & $12 / 10 / 92$ & 67 & CASS & $T$ & \\
\hline TE-108-AP-50 & $12 / 19 / 92$ & 66 & CASS & $T$ & \\
\hline TE-108-AP-50 & $12 / 25 / 92$ & 65 & CASS & $T$ & \\
\hline TE-10B-AP-50 & $12 / 27 / 92$ & 66 & CASS & $T$ & \\
\hline TE-10B-AP-50 & T/15/94 & 63 & CASS & $\bar{T}$ & \\
\hline TE-108-AP-50 & $1 / 20 / 94$ & 64 & CASS & $T$ & \\
\hline TE-108-AP-50 & $1 / 25 / 94$ & 64 & CASS & $\mathbf{T}$ & \\
\hline TE-108-AP-50 & $2 / 2 / 94$ & 64 & CASS & $T$ & \\
\hline TE-108-AP-50 & $2 / 19 / 94$ & 63 & CASS & $T$ & \\
\hline TE-108-AP-50 & $2 / 15 / 94$ & 64 & CASS & $T$ & \\
\hline TE-108-AP-50 & $2 / 22 / 94$ & 86 & CASS & $T$ & \\
\hline TE-10Q-AP-50 & $3 / 5 / 94$ & 63 & CASS & $\mathbf{T}$ & \\
\hline TE-108-AP-50 & $3 / 10 / 94$ & 64 & CASS & $\mathbf{T}$ & \\
\hline TE-108-AP-50 & $3 / 17 / 94$ & 64 & CASS & $\mathbf{T}$ & \\
\hline TE-108-AP-50 & $3 / 25 / 94$ & 64 & CASS & $T$ & \\
\hline TE-108-AP-50 & $3 / 29 / 94$ & 63 & CASS & $\bar{T}$ & \\
\hline TE-10B-AP-50 & $4 / 9 / 94$ & 64 & CASS & $T$ & \\
\hline TE-108-AP-50 & $4 / 13 / 94$ & 64 & CASS & $T$ & \\
\hline TE-108-AP-50 & $4 / 20 / 94$ & 64 & CASS & $\mathbf{T}$ & \\
\hline TE-108-AP-50 & $4 / 29 / 94$ & 64 & CASS & $\bar{T}$ & \\
\hline TE-108-AP-50 & $5 / 2 / 94$ & 64 & CASS & $\mathbf{T}$ & \\
\hline TE-108-AP-SO & $5 / 8 / 94$ & 66 & CASS & $T$ & \\
\hline TE-TOOA-AP-50 & 5/16/94 & 104 & CASS & $T$ & \\
\hline TE-108-AP-50 & 5/22/94 & 100 & CASS & $T$ & \\
\hline TE-108-AP-5O & $6 / 2 / 94$ & 99 & CASS & $T$ & \\
\hline TE-108-AP-50 & $6 / 8 / 94$ & 98 & CASS & $T$ & \\
\hline TE-108-AP-50 & $6 / 16 / 94$ & 83 & CASS & s & Data point did not agrea with local trend \\
\hline$T E-108-A P-50$ & 6/17/94 & 63 & CASS & $\mathbf{S}$ & Data point did not agree with lacal trend \\
\hline TE-108-AP-50 & $6 / 18 / 94$ & 95 & CASS & $\mathbf{T}$ & \\
\hline TE-108-AP-50 & 6/20/94 & 63 & CASS & $\mathbf{S}$ & Data point did nat arree with local trend \\
\hline
\end{tabular}




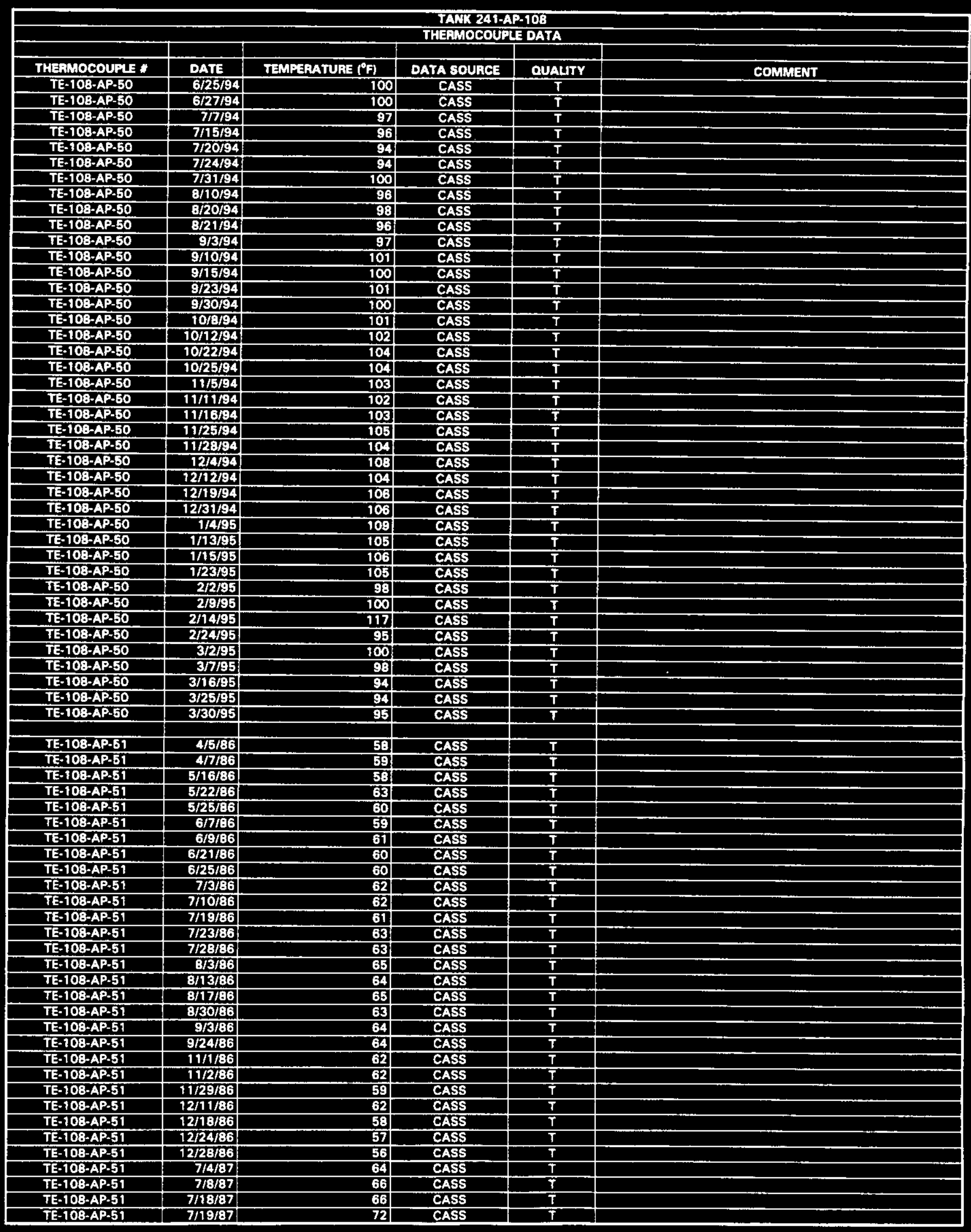




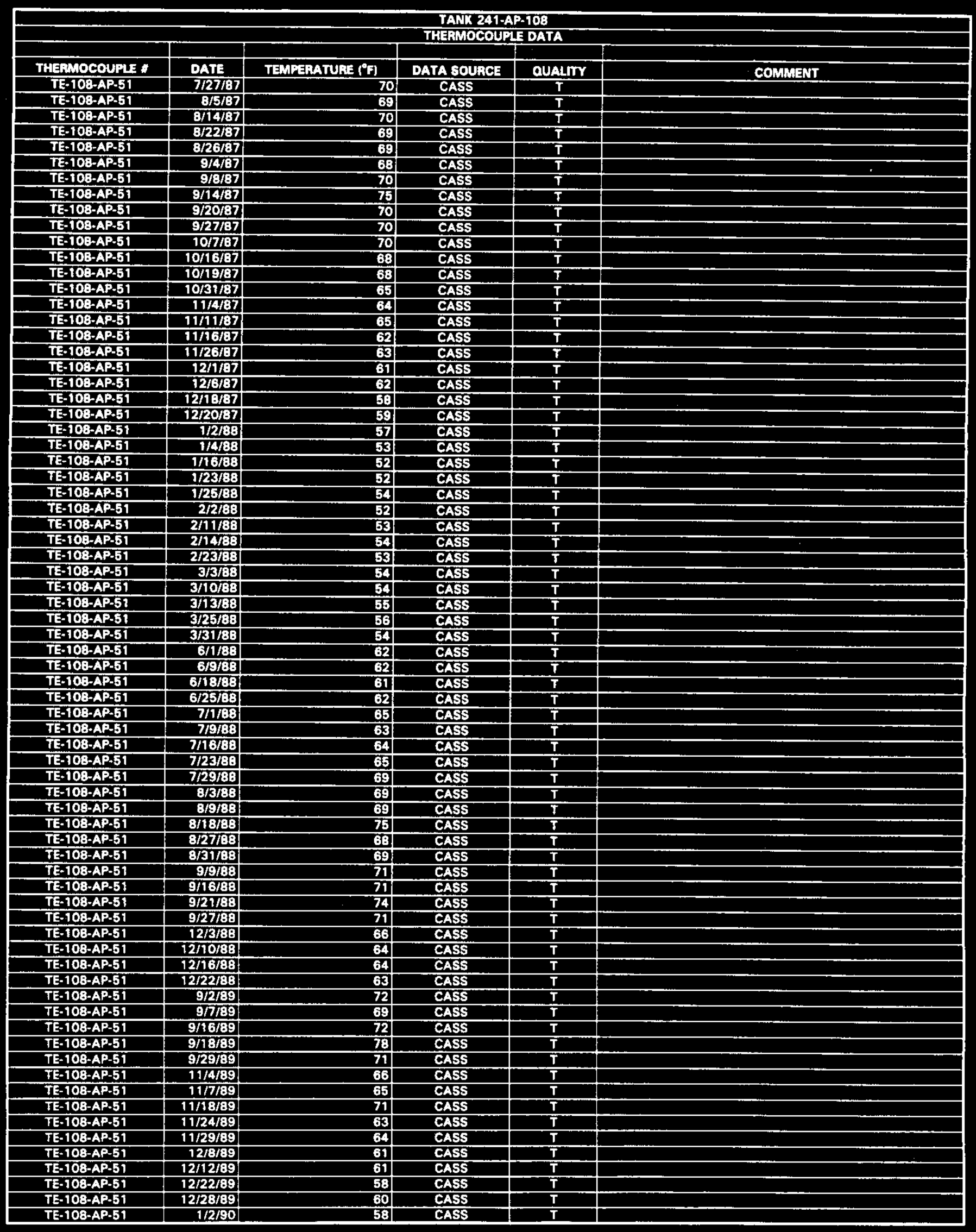

Data obtained from Computer Automated Surveillance Syatem (CASS), April 1995; and Surveillonce Analysis Computer Syatem (SACS), June 1995. 


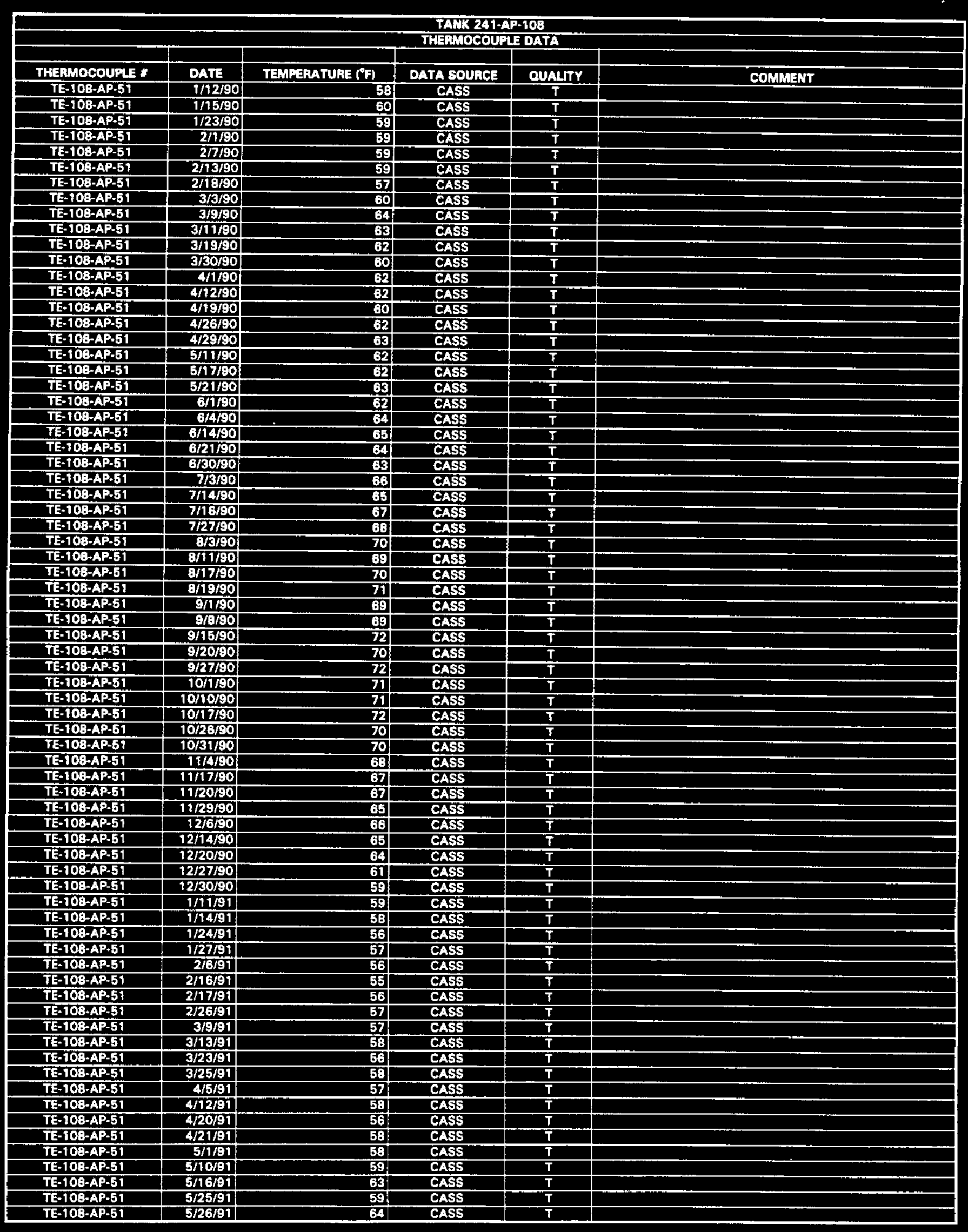




\begin{tabular}{|c|c|c|c|c|c|}
\hline \multicolumn{6}{|c|}{$\begin{array}{l}\text { TAKK 241.AP-108 } \\
\text { THERMOCOUPIE DATA }\end{array}$} \\
\hline & & & & & \\
\hline THEAMOCOUPLE $*$ & DATE & TEMPERATURE ( ${ }^{\circ}$ ) & DATA SOUACE & QUALTTY & COMMENT \\
\hline TE-108-AP-51 & $6 / 5 / 91$ & 62 & CASS & $T$ & \\
\hline TE-108-AP.51 & 6/14/91 & 65 & CASS & $T$ & \\
\hline TE-108-AP-51 & $6 / 22 / 91$ & 68 & CASS & $T$ & \\
\hline TE-108-AP-51 & 6/28/91 & 69 & CASS & $\mathbf{T}$ & \\
\hline IE-108-AP-51 & $7 / 3 / 91$ & 67 & CASS & $\mathbf{T}$ & \\
\hline TE-100-AP-51 & 7/13/91 & 66 & CASS & $\mathbf{T}$ & \\
\hline TE-108-AP-51 & 7/14/91 & 66 & CASS & $T$ & \\
\hline TE-108-AP-5I & 7124/81 & 69 & CASS & $T$ & \\
\hline TE-108-AP-51 & $8 / 2 / 91$ & 69 & CASS & $\mathbf{T}$ & \\
\hline TE-108-AP551 & B/6/91 & 68 & CASS & $\mathbf{T}$ & \\
\hline TE-108-AP-51 & $8 / 17 / 91$ & 71 & CASS & $\mathbf{T}$ & \\
\hline TE-108-AP-51 & 8/18/91 & 72 & CASS & $\mathbf{T}$ & \\
\hline TE-108-AP-51 & $8 / 30 / 93$ & 70 & CASS & $\mathbf{T}$ & \\
\hline TE-108-AP-51 & $9 / 3 / 91$ & 71 & CASS & $\mathbf{T}$ & \\
\hline TE-108-AP.51 & $9 / 11 / 91$ & 72 & CASS & $T$ & \\
\hline TE-108.AP-51 & 9/18/91 & 76 & CASS & $T$ & \\
\hline TE-108-AP-51 & 9/22/91 & 71 & CASS & $\mathbf{T}$ & \\
\hline TE-108-AP-51 & 9/30/91 & 73 & CASS & $\mathbf{T}$ & \\
\hline TE-106-AP-51 & $10 / 8 / 91$ & 61 & CASS & $\mathbf{s}$ & Data point did not agree with local trand \\
\hline TE-108-AP-51 & 10/2/91 & 72 & CASS & $T$ & \\
\hline TE-106-AP-51 & $10 / 1791$ & 74 & CASS & $T$ & \\
\hline TE-108-AP-51 & $10 / 25 / 91$ & $7 \mathbf{7 3}$ & CASS & $T$ & \\
\hline TE-108-AP.51 & $10 / 29 / 91$ & 72 & CASS & $\mathbf{T}$ & \\
\hline TE-108-AP-51 & $11 / 6 / 91$ & 71 & CASS & $\mathbf{T}$ & \\
\hline TE-10:APPS1 & $8 / 1 / 92$ & 65 & CASS & $\mathbf{T}$ & \\
\hline TE-106-AP-51 & $8 / 3 / 92$ & 67 & CASS & $\mathbf{T}$ & \\
\hline TE-10B-AP-51 & $8 / 9 / 92$ & 68 & CASS & $\mathbf{T}$ & \\
\hline TE-108.AP-51 & $8 / 20 / 92$ & 69 & CASS & $\mathbf{T}$ & \\
\hline TE-108-AP-51 & $8 / 24 / 92$ & 67 & CASS & $\bar{T}$ & \\
\hline TE-108-AP-51 & $8 / 31 / 92$ & 67. & CASS & $\mathbf{T}$ & \\
\hline TE-108-AP-51 & $9 / 12 / 92$ & 67 & CASS & $\mathbf{T}$ & \\
\hline TE-108-AP-51 & 9/16/92 & 68 & CASS & $\mathbf{T}$ & \\
\hline TE-108-AP-51 & 9/26/92 & 66 & CASS & $\mathbf{T}$ & \\
\hline TE-TOS-AP-51 & 10/3/92 & 68 & CASS & $\mathbf{T}$ & \\
\hline TE-10:AP-51 & $10 / 5 / 92$ & 69 & CASS & $\mathbf{T}$ & \\
\hline TE-10B-AP-51 & $10 / 13 / 92$ & 69 & CASS & $\mathbf{T}$ & \\
\hline TE-102-AP.51 & 10/22/92 & 70 & CASS & $\mathbf{T}$ & \\
\hline TE-103-AP-51 & $10 / 29 / 92$ & 72 & CASS & $\mathbf{T}$ & \\
\hline TE-108-AP-51 & $11 / 7 / 92$ & 69 & CASS & $\mathbf{T}$ & \\
\hline TE-106-AP-51 & $11 / 9 / 92$ & 69 & CASS & $\mathbf{T}$ & \\
\hline TE-108-AP-51 & $11 / 20 / 32$ & 68 & CASS & $T$ & \\
\hline TE-108-AP-51 & $11 / 25 / 92$ & 69 & CASS & $T$ & \\
\hline TE-108-AP-51 & $11 / 30 / 92$ & 69 & CASS & $T$ & \\
\hline TE-108-AP-51 & $12 / 10 / 92$ & 67 & CASS & $T$ & \\
\hline TE-108-AP-51 & $12 / 19 / 92$ & 86 & CASS & $T$ & \\
\hline TE-106-AP-51 & $12 / 21 / 92$ & 66 & CASS & $T$ & \\
\hline TE-10B-AP-51 & $12 / 30 / 92$ & 66 & CASS & $T$ & \\
\hline TE-108-AP-51 & $1 / 15 / 94$ & 64 & CASS & $T$ & \\
\hline TE-108.AP-51 & $1 / 20 / 94$ & 64 & CASS & $T$ & \\
\hline TE-108-AP-51 & $1 / 28 / 94$ & 63 & CASS & $T$ & \\
\hline TE-108-AP-51 & $2 / 2 / 94$ & 65 & CASS & $T$ & \\
\hline TE-108-AP-51 & $2 / 11 / 94$ & 64 & CASS & $T$ & \\
\hline TE-108-AP-51 & $2 / 15 / 94$ & 64 & CASS & $T$ & \\
\hline TE-108-AP-51 & $2 / 22 / 94$ & 64 & CASS & $T$ & \\
\hline TE-108-AP-51 & $2 / 28 / 94$ & 66 & CASS & $T$ & \\
\hline TE-108-AP-51 & $3 / 10 / 94$ & 64 & CASS & $T$ & \\
\hline TE-108-AP-51 & $3 / 17 / 94$ & 64 & CASS & $T$ & \\
\hline TE-108-AP-51 & $3 / 25 / 94$ & 65 & CASS & $\mathbf{T}$ & \\
\hline TE-108-AP-51 & $4 / 2 / 94$ & 63 & CASS & $T$ & \\
\hline TE-108-AP-51 & 4/9/94 & 64 & CASS & $T$ & \\
\hline TE-108-AP-51 & 4/15/94 & 64 & CASS & $T$ & \\
\hline TE-108-AP-51 & 4/20/94 & 65 & CASS & $\mathbf{T}$ & \\
\hline TE-108-AP-51 & $4 / 29 / 94$ & 65 & CASS & $T$ & \\
\hline TE-108-AP-51 & $5 / 2 / 94$ & 64 & CASS & $\mathbf{T}$ & \\
\hline TE-108-AP-51 & $5 / 8 / 94$ & 66 & CASS & $\bar{T}$ & \\
\hline TE-103-AP-51 & $5 / 16 / 94$ & 104 & CASS & $\mathbf{T}$ & \\
\hline TE-108-AP-51 & 5/23/94 & 99 & CASS & $\mathbf{T}$ & \\
\hline TE-10B-AP-51 & $6 / 2 / 94$ & 99 & CASS & $\mathbf{T}$ & \\
\hline TE-108-AP-51 & $6 / 8 / 94$ & 99 & CASS & $T$ & \\
\hline TE-108-AP-51 & 6/16/94 & 64 & CASS & $\mathbf{s}$ & Date point did not agree with local trend \\
\hline TE-108-AP-51 & 6/17/94 & 64 & CASS & $\mathbf{S}$ & Data point did not agree with local trend \\
\hline TE-108-AP-51 & 6/18/94 & 95 & CASS & $T$ & \\
\hline TE-108-AP-51 & 6/20/94 & 63. & CASS & $\mathbf{s}$ & Data point did not angee with local trend \\
\hline
\end{tabular}




\begin{tabular}{|c|c|c|c|c|c|}
\hline \multicolumn{6}{|c|}{$\begin{array}{l}\text { TANK 241-AP-108 } \\
\text { TH }\end{array}$} \\
\hline & & & & & \\
\hline THEPMOCOUPIE & DATE & TEMPERATUAE ("F) & DATA SOURCE & QUALITY & COMMENT \\
\hline TE-108-AP-51 & $6 / 25 / 94$ & 100 & CASS & $T$ & \\
\hline TE-108-AP-51 & 6/27/94 & 100 & CASS & $T$ & \\
\hline TE-108-AP-51 & $7 / 7194$ & 97 & CASS & $T$ & \\
\hline TE-108-AP-51 & $7 / 13 / 94$ & 96 & CASS & $T$ & \\
\hline TE-108-AP-51 & $7 / 20 / 94$ & 94 & CASS & $T$ & \\
\hline TE-108-AP-51 & $7 / 30 / 94$ & 83 & CASS & $T$ & \\
\hline TE-108-AP-51 & $7 / 31 / 94$ & 97 & CASS & $T$ & \\
\hline TE-108-AP-51 & $8 / 9 / 94$ & 98 & CASS & $T$ & \\
\hline TE-108-AP-51 & $8 / 11 / 94$ & 157 & CASS & 5 & Date point did not agree with local trend \\
\hline TE-108-AP-51 & $8 / 20 / 94$ & 98 & CASS & $T$ & \\
\hline TE-10:AP-51 & $8 / 27 / 94$ & 96 & CASS & $T$ & \\
\hline TE-108-AP-51 & $9 / 3 / 94$ & 98 & CASS & $T$ & \\
\hline TE-T08-AP-51 & $9 / 10 / 94$ & 101 & CASS & $\bar{T}$ & \\
\hline TE-108-AP-51 & 9/15/94 & 100 & CASS & $T$ & \\
\hline TE-108-AP-51 & $9 / 23 / 94$ & 101 & CASS & $T$ & \\
\hline TE-108-AP-51 & $9 / 30 / 94$ & 100 & CASS & $\begin{array}{r}T \\
\end{array}$ & \\
\hline TE-108-AP.51 & $10 / 8 / 94$ & 101 & CASS & $\mathbf{T}$ & \\
\hline TE-10B-AP-51 & $10 / 12 / 94$ & 102 & CASS & $T$ & \\
\hline TE-108-AP-51 & $10 / 20 / 94$ & 103 & CASS & $\bar{T}$ & \\
\hline TE-108-AP-5I & $10 / 29 / 94$ & 104 & CASS & $\bar{T}$ & \\
\hline TE-108-AP-5T & $11 / 5 / 94$ & 104 & CASS & $\mathbf{T}$ & \\
\hline TE-108-AP-51 & $11 / 11 / 94$ & 103 & CASS & $\bar{T}$ & \\
\hline TE-102-AP:51 & $11 / 16 / 94$ & 104 & CASS & $\mathbf{T}$ & \\
\hline TE-108-AP-51 & $11 / 25 / 94$ & 105 & CASS & $\mathbf{T}$ & \\
\hline TE-108-AP-51 & $12 / 3 / 94$ & 104 & CASS & $\overline{\mathbf{T}}$ & \\
\hline TE-108-AP-51 & $12 / 4 / 94$ & 109 & CASS & $\bar{T}$ & \\
\hline TE-108-AP-51 & $12 / 12 / 94$ & 105 & CASS & $T$ & \\
\hline TE-108-AP-51 & $12 / 19 / 94$ & 105 & CASS & $T$ & \\
\hline TE-108-AP-51 & $12 / 31 / 94$ & 107 & CASS & $T$ & \\
\hline TE-108-AP-51 & $1 / 4 / 95$ & 110 & CASS & $T$ & \\
\hline TE-108-AP-51 & 1/13/85 & 107 & CASS & $\bar{T}$ & \\
\hline TE-108-AP-51 & 1/15/95 & 108 & CASS & $T$ & \\
\hline TE-108-AP-51 & 1/23/95 & 106 & CASS & $T$ & \\
\hline TE-108-AP-51 & $2 / 3 / 95$ & 98 & CASS & $T$ & \\
\hline TE-108.AP-51 & $2 / 6 / 95$ & 101 & CASS & $\mathbf{T}$ & \\
\hline TE-108-AP-51 & $2 / 14 / 95$ & 106 & CASS & $T$ & \\
\hline TE-108-AP-51 & $2 / 24 / 95$ & 96 & CASS & $T$ & \\
\hline TE-108-AP-51 & $3 / 2 / 95$ & 101 & CASS & $\bar{T}$ & \\
\hline TE-108-AP-51 & $3 / 7 / 95$ & 98 & CASS & $\mathbf{T}$ & \\
\hline TE-108-AP-51 & 3/16/95 & 95 & CASS & $T$ & \\
\hline TE-108-AP-51 & 3/23/95 & 95 & CASS & $\mathbf{T}$ & \\
\hline TE-108-AP-51 & $3 / 30 / 95$ & 97 & CASS & $T$ & \\
\hline & & & & & \\
\hline TE-10:-AP-52 & 4/5/86 & 58 & CASS & $T$ & \\
\hline TE-108-AP-52 & $4 / 7 / 86$ & 59 & CASS & $T$ & \\
\hline TE-108-AP-52 & $5 / 17 / 86$ & 57 & CASS & $T$ & \\
\hline TE-108-AP-52 & $5 / 22 / 86$ & 64 & CASS & $\bar{T}$ & \\
\hline TE-108-AP-52 & $5 / 25 / 86$ & 60 & CASS & $\bar{T}$ & \\
\hline TE-108-AP-52 & $6 / 7 / 86$ & 59 & CASS & $T$ & \\
\hline TE-108-AP-52 & 6/9/86 & 61 & CASS & $T$ & \\
\hline TE-108-AP-52 & 6/19/86 & 60 & CASS & $T$ & \\
\hline TE-108-AP-52 & $6 / 22 / 86$ & 60 & CASS & $T$ & \\
\hline TE-108-AP-52 & $711 / 86$ & 61 & CASS & $\bar{T}$ & \\
\hline TE-108-AP-52 & $7 / 29 / 86$ & 633 & CASS & $\bar{T}$ & \\
\hline TE-108-AP-52 & $8 / 17 / 86$ & 65 & CASS & $T$ & \\
\hline TE-108-AP-52 & $7 / 1 / 87$ & 65. & CASS & $T$ & \\
\hline$T E-108-A P-52$ & $7 / 8 / 87$ & 66 & CASS & $T$ & \\
\hline TE-108-AP-52 & $7 / 12 / 87$ & 66 & CASS & $T$ & \\
\hline TE-108-AP-52 & 7/19/a7 & 72 & CASS & $T$ & \\
\hline TE-108-AP-52 & 7/27/87 & 70 & CASS & $\mathbf{T}$ & \\
\hline TE-108-AP-52 & $8 / 4 / 87$ & 69 & CASS & $T$ & \\
\hline TE-108-AP-52 & $8 / 74 / 87$ & 70 & CASS & $T$ & \\
\hline TE-108-AP-52 & $8 / 22 / 87$ & 70 & CASS & $T$ & \\
\hline TE-108-AP-52 & $8 / 26 / 87$ & 69 & CASS & $T$ & \\
\hline TE-108-AP-52 & 9/4/87 & 69 & CASS & $T$. & \\
\hline TE-108-AP-52 & $9 / 8 / 87$ & 70 & CASS & $T$ & \\
\hline TE-108-AP-52 & $9 / 17 / 87$ & 69 & CASS & $\bar{T}$ & \\
\hline TE-108-AP-52 & 9/20/87 & 70 & CASS & $T$ & \\
\hline TE-108-AP-52 & $9 / 27 / 87$ & 70 & CASS & $T$ & \\
\hline TE-108-AP-52 & $10 / 7 / 87$ & 70 & CASS & $T$ & \\
\hline TE-108-AP-52 & $10 / 16 / 87$ & 68 & CASS & $T$ & \\
\hline TE-108-AP-52 & $10 / 19 / 87$ & 68 & CASS & $\mathbf{T}$ & \\
\hline TE-108-AP-52 & $10 / 31 / 87$ & 65 & CASS & $T$ & \\
\hline
\end{tabular}




\begin{tabular}{|c|c|c|c|c|c|}
\hline \multicolumn{6}{|c|}{$\begin{array}{l}\text { TALK 241-AP-108 } \\
\text { THIAHOCOUPLEDATA }\end{array}$} \\
\hline & & & & & \\
\hline THERMOCOUPLE * & DATE & TEMPERATUAE ("F) & DATA BOURCE & OUALITY & COMMENT \\
\hline TE-108-AP-52 & $11 / 1 / 87$ & 66 & CASS & $\mathbf{T}$ & \\
\hline TE-108-AP-52 & $11 / 8 / 87$ & 65 & CASS & $T$ & \\
\hline$\frac{T E-108-A P-52}{T E-10: A P D}$ & $11 / 18 / 87$ & 62 & CASS & $T$ & \\
\hline $\begin{array}{l}\text { TE-108-AP-52 } \\
\text { TE-108-AP-52 }\end{array}$ & $\frac{11 / 26 / 87}{11 / 29 / 87}$ & 63 & $\frac{\text { CASS }}{\text { CASS }}$ & $\frac{T}{T}$ & \\
\hline TE-108-AP-52 & 12/6/97 & 83 & CASS & $\mathbf{s}$ & Data point did not agree with local trend \\
\hline TE-108-AP-52 & $12 / 11 / 87$ & 59 & CASS & $\bar{T}$ & \\
\hline TE-108-AP-62 & $12 / 18 / 87$ & 58 & CASS & $\mathbf{T}$ & \\
\hline TE-108-AP-52 & $12 / 23 / 87$ & 57 & CASS & $T$ & \\
\hline TE-108-AP-52 & $12 / 28 / 87$ & 55 & CASS & $T$ & \\
\hline TE-108-AP-52 & $1 / 4 / 88$ & 53 & CASS & $\bar{T}$ & \\
\hline TE-108-AP-52 & $1 / 16 / 88$ & 5? & CASS & $T$ & \\
\hline TE-108-AP-52 & 1/25/88 & 52 & CASS & $T$ & \\
\hline TE-108-AP-52 & 1/25/88 & 53 & CASS & $T$ & \\
\hline TE-108-AP-52 & $2 / 2 / 88$ & 5 & CASS & $T$ & \\
\hline TE-108-AP-52 & $2 / 11 / 88$ & 53 & CASS & $T$ & \\
\hline TE-108-AP-52 & $2 / 14 / 88$ & 54 & CASS & $\bar{T}$ & \\
\hline TE-108-AP-52 & $2 / 23 / 88$ & 53 & CASS & $\bar{T}$ & \\
\hline TE-106-AP-52 & $3 / 3 / 88$ & 54) & CASS & $\mathbf{T}$ & \\
\hline TE-108-AP-52 & $3 / 10 / 88$ & 54 & CASS & $\bar{T}$ & \\
\hline TE-10B-AP-52 & $3 / 13 / 88$ & 54 & CASS & $T$ & \\
\hline TE-108-AP-52 & $3 / 25 / 88$ & 57 & CASS & $T$ & \\
\hline$T E-108-A P-52$ & 3/31/88 & 54 & CASS & $T$ & \\
\hline TE-10:-AP-52 & $6 / 1 / 88$ & 62 & CASS & $T$ & \\
\hline TE-108-AP-52 & 6//8/38 & 621 & CASS & $T$ & \\
\hline TE-108-AP-52 & $6 / 18 / 88$ & 61 & CASS & $\bar{T}$ & \\
\hline TE-108-AP-52 & $6 / 25 / 88$ & 82 & CASS & $\bar{T}$ & \\
\hline TE-10:AP:52 & $77 / 1 / 88$ & 64 & CASS & $T$ & \\
\hline TE-108-AP-52 & 7/9/83 & 63 & CASS & $T$ & \\
\hline TE-108-AP-52 & $7 / 13 / 88$ & 65 & CASS & $T$ & \\
\hline TE-108-AP-52 & $7 / 23 / 88$ & 6 & CASS & $T$ & \\
\hline TE-108-AP.52 & $7 / 29 / 88$ & 68 & CASS & $\bar{T}$ & \\
\hline TE-108-AP-52 & B/3/8B & 69 & CASS & $T$ & \\
\hline TE-108-AP-52 & $8 / 9 / 88$ & 69 & CASS & $T$ & \\
\hline TE-108-AP-52 & $8 / 20 / 89$ & 69 & CASS & $\bar{T}$ & \\
\hline TE-108-AP-52 & $8 / 22 / 83$ & 69 & CASS & $T$ & \\
\hline$T E-108-A P \cdot 52$ & $8 / 31 / 88$ & 69 & CASS & $\bar{T}$ & \\
\hline TE-103-AP-52 & $9 / 9 / 88$ & 71 & CASS & $T$ & \\
\hline TE-10:-AP-52 & $9 / 16 / 88$ & 71 & CASS & $T$ & \\
\hline TE-108-AP-52 & 9/23/88 & 70 & CASS & $T$ & \\
\hline TE-108-AP-52 & $9 / 27 / 89$ & 70 & CASS & $T$ & \\
\hline TE-108-AP-52 & $12 / 1 / 88$ & 66) & CASS & $T$ & \\
\hline TE-109-AP-52 & $12 / 10 / 88$ & 64 & CASS & $\bar{T}$ & \\
\hline TE-108-AP-52 & $12 / 16 / 88$ & 64 & CASS & $T$ & \\
\hline TE-108-AP-52 & $12 / 22 / 88$ & 62 & CASS & $T$ & \\
\hline$T E-108-A P-52$ & $9 / 2 / 89$ & 72 & CASS & $T$ & \\
\hline TE-108-AP-52 & $9 / 7 / 89$ & 69 & CASS & $T$ & \\
\hline TE-108-AP-52 & $9 / 16 / 89$ & 70 & CASS & $T$ & \\
\hline TE-108-AP-52 & 9/18/89 & 70 & CASS & $T$ & \\
\hline IE-108-AP-52 & 9/29/89 & 71 & CASS & $T$ & \\
\hline TE-108-AP-52 & $11 / 3 / 69$ & 66 & CASS & $\bar{T}$ & \\
\hline TE-10B-AP-52 & $11 / 7 / 89$ & 65 & CASS & $T$ & \\
\hline TE-108-AP-52 & $11 / 18 / 89$ & 70 & CASS & $T$ & \\
\hline TE-108-AP-52 & $11 / 24 / 89$ & 63 & CASS & $T$ & \\
\hline TE-108-AP-52 & $12 / 2 / 89$ & 66 & CASS & $T$ & \\
\hline TE-108-AP-52 & $12 / 8 / 89$ & 61 & CASS & $T$ & \\
\hline TE-108-AP-52 & $12 / 12 / 89$ & 61 & CASS & $T$ & \\
\hline TE-108-AP-52 & $12 / 22 / 89$ & 58 & CASS & $T$ & \\
\hline TE-108-AP-52 & $12 / 28 / 89$ & 59 & CASS & $T$ & \\
\hline TE-108-AP-52 & $1 / 2190$ & 57 & CASS & $T$ & \\
\hline TE-108-AP-52 & $1 / 12 / 90$ & 59 & CASS & $T$ & \\
\hline TE-108-AP-52 & $1 / 15 / 90$ & 59 & CASS & $T$ & \\
\hline TE-108-AP-52 & $1 / 26 / 90$ & 58 & CASS & $\bar{T}$ & \\
\hline TE-108-AP-52 & $2 / 1 / 90$ & 59 & CASS & $T$ & \\
\hline TE-108-AP-52 & $2 / 3 / 90$ & 98 & CASS & $\mathbf{s}$ & Data point did not apree with local trend \\
\hline TE-108-AP-52 & $2 / 7 / 50$ & 59 & CASS & $\bar{T}$ & \\
\hline TE-100.AP-52 & $2 / 12 / 90$ & 71 & SACS & $\mathbf{s}$ & Data point did not agrae with local trend \\
\hline TE-108-AP-52 & $2 / 13 / 90$ & 59 & CASS & $T$ & \\
\hline TE-108-AP-52 & $2 / 18 / 90$ & 57 & CASS & $T$ & \\
\hline TE-108-AP-52 & $3 / 3 / 90$ & 61 & CASS & $T$ & \\
\hline TE-108-AP-52 & $3 / 8 / 90$ & 66 & CASS & $\mathbf{T}$ & \\
\hline$T E-108-A P-52$ & $3 / 11 / 90$ & 64 & CASS & $\bar{T}$ & \\
\hline TE-108-AP-52 & $3 / 18 / 90$ & 63 & CASS & $T$ & \\
\hline
\end{tabular}




\begin{tabular}{|c|c|c|c|c|c|}
\hline \multicolumn{6}{|c|}{$\begin{array}{l}\text { TAKK 241-AP-108 } \\
\text { THEFROCOUPLE DATA }\end{array}$} \\
\hline THERMOCOUPE * & DATE & TEMFERATURE $\left({ }^{\circ} \mathrm{F}\right)$ & DATA SOURCE & QUALTY & COMMENT \\
\hline TE-108-AP-52 & $3 / 26 / 90$ & 97 & CASS & $\mathbf{S}$ & Data point did not agree with local trend \\
\hline TE-108-AP-52 & $3 / 27 / 90$ & 97 & CASS & $\mathbf{S}$ & Data point did not agree with local trend \\
\hline TE-108-AP-52 & $3 / 30 / 30$ & 60 & CASS & $T$ & \\
\hline TE-10B-AP-52 & $9 / 3 / 90$ & 61 & CASS & $\mathbf{I}$ & \\
\hline TE-108-AP-52 & 4/12/90 & 62 & CASS & $T$ & \\
\hline TE-108-AP-52 & $4 / 21 / 90$ & 60 & CASS & $T$ & \\
\hline TE-108-AP-52 & 4/26/90 & 62. & CASS & $\mathbf{T}$ & \\
\hline TE-108-APD2 & $5 / 3 / 90$ & 62 & CASS & $T$ & \\
\hline TE-108-AP-52 & $5 / 11 / 90$ & 62 & CASS & $T$ & \\
\hline TE-108-AP-52 & $5 / 17 / 90$ & 62 & CASS & $T$ & \\
\hline TE-108-AP-52 & $5 / 22 / 90$ & 62 & CASS & $\bar{T}$ & \\
\hline TE-108-AP-52 & $6 / 1 / 90$ & 62 & CASS & $T$ & \\
\hline TE-108-AP-52 & $6 / 8 / 90$ & 65. & CASS & $T$ & \\
\hline TE-108-AP-52 & $6 / 14 / 90$ & 64 & CASS & $\mathbf{T}$ & \\
\hline TE-108.AP-52 & 6/21/90 & 63 & CASS & $T$ & \\
\hline TE-108-AP-52 & $6 / 30 / 90$ & 63 & CASS & $\mathbf{T}$ & \\
\hline TE-108-AP-52 & $7 / 3 / 90$ & 66 & CASS & $\mathbf{T}$ & \\
\hline TE-108-AP-52 & $7 / 12 / 90$ & 67 & CASS & $\bar{T}$ & \\
\hline TE-108-AP-52 & $7 / 16 / 90$ & 68 & CASS & $T$ & \\
\hline TE-108-AP-52 & $7 / 27 / 90$ & 68 & CASS & $T$ & \\
\hline TE-108-AP-52 & $7 / 30 / 90$ & 71 & SACS & $\mathbf{T}$ & \\
\hline TE-108.AP-52 & $8 / 9 / 90$ & 70 & CASS & $\mathrm{T}$ & \\
\hline TE-108-AP-52 & 8/17/90 & 70 & CASS & $\bar{T}$ & \\
\hline TE-108-AP-52 & $8 / 21 / 90$ & 70 & CASS & $T$ & \\
\hline TE-108.AP-52 & $9 / 1 / 90$ & 69 & CASS & $\mathbf{T}$ & \\
\hline TE-108-AP-52 & $9 / 8 / 90$ & 69 & CASS & $\mathbf{T}$ & $\therefore$ \\
\hline TE-108-AP-52 & 9/15/30 & 72 & CASS & $\mathbf{T}$ & \\
\hline TE-108-AP-52 & $9 / 20 / 90$ & 70 & CASS & $\mathbf{T}$ & \\
\hline TE-106-AP-52 & $9 / 27 / 80$ & 72 & CASS & $\mathbf{T}$ & \\
\hline TE-108-AP-52 & 10/1/80 & 72 & CASS & $\mathbf{T}$ & \\
\hline TE-108-AP-52 & $10 / 10 / 90$ & 71 & CASS & $T$ & \\
\hline TE-10B-AP-52 & $10 / 20 / 90$ & 68 & CASS & $T$ & \\
\hline TE-10B-AP-52 & $10 / 26 / 90$ & 701 & CASS & $\bar{T}$ & \\
\hline TE-108-AP-52 & $10 / 31 / 90$ & 70 & CASS & $T$ & \\
\hline TE-106-AP-52 & $11 / 10 / 80$ & 68 & CASS & $T$ & \\
\hline TE-106-AP-52 & $11 / 17 / 80$ & 67 & CASS & $T$ & \\
\hline TE-108.AP-52 & $11 / 20 / 90$ & 67 & CASS & $T$ & \\
\hline TE-10:-AP-52 & $11 / 22 / 90$ & 106 & CASS & $\bar{s}$ & Data point did not agree with local trend \\
\hline TE-108-AP-52 & $11 / 29 / 90$ & 65 & CASS & $\mathbf{T}$ & \\
\hline TE-108-AP-52 & $12 / 6 / 30$ & 68 & CASS & $\mathbf{T}$ & \\
\hline TE-108-AP-52 & $12 / 14 / 90$ & 86 & CASS & $\mathbf{T}$ & \\
\hline TE-108-AP-52 & $12 / 20 / 90$ & 64 & CASS & $\bar{T}$ & \\
\hline TE-108-AP-52 & $12 / 27 / 90$ & 62 & CASS & $T$ & \\
\hline TE-108-AP-52 & $12 / 30 / 90$ & 60 & CASS & $\mathbf{T}$ & \\
\hline TE-108-AP-52 & $1 / 11 / 91$ & 59 & CASS & $T$ & \\
\hline TE-108-AP-52 & $1 / 1 / 4 / 91$ & 58 & CASS & $\mathbf{T}$ & \\
\hline TE-100-AP-52 & $1 / 26 / 91$ & 56 & CASS & $\mathbf{T}$ & \\
\hline TE-108-AP-52 & 1/27/91 & 57 & CASS & $\mathbf{T}$ & \\
\hline TE-108-AP-52 & $2 / 6 / 91$ & 56 & CASS & $T$ & \\
\hline TE-108-AP-52 & $2 / 15 / 91$ & 55: & CASS & $\mathbf{T}$ & \\
\hline TE-108-AP-52 & $2 / 17 / 81$ & 56. & CASS & $\mathbf{T}$ & \\
\hline TE-108-AP-52 & $3 / 2 / 91$ & 57. & CASS & $\mathbf{T}$ & \\
\hline TE-108-AP-52 & 3/9/91 & 58: & CASS & $\mathbf{T}$ & \\
\hline TE-108-AP-52 & $3 / 13 / 91$ & 58 & CASS & $\bar{I}$ & \\
\hline TE-106-AP-52 & 3/23/91 & 56 & CASS & $\mathbf{T}$ & \\
\hline TE-108-AP-52 & 3/27/91 & 581 & CASS & $T$ & \\
\hline TE-108-AP-52 & $4 / 5 / 91$ & 57 & CASS & $\mathbf{T}$ & \\
\hline TE-108-AP-52 & $4 / 12 / 91$ & 58 & CASS & $\mathbf{T}$ & \\
\hline TE-108-AP.52 & $4 / 18 / 91$ & 57 & CASS & $T$ & \\
\hline TE-108-AP-52 & 4/21/91 & 58 & CASS & $\mathbf{T}$ & \\
\hline TE-108-AP-52 & $5 / 4 / 91$ & 59 & CASS & $\mathbf{T}$ & \\
\hline TE-108-AP-52 & $5 / 10 / 91$ & 60 & CASS & $\mathbf{T}$ & \\
\hline TE-108-AP-52 & $5 / 16 / 91$ & 60 & CASS & $\bar{T}$ & \\
\hline TE-108-AP-52 & $5 / 25 / 91$ & 61 & CASS & $T$ & \\
\hline TE-108-AP-52 & 5/26/91 & 65 & CASS & $T$ & \\
\hline TE-108-AP-52 & $6 / 5 / 91$ & 62: & CASS & $\bar{T}$ & \\
\hline TE-108-AP-52 & 6/15/91 & 64 & CASS & $T$ & \\
\hline TE-108-AP-52 & $6 / 22 / 91$ & 66 & CASS & $T$ & \\
\hline$T E=108-A P=52$ & $6 / 28 / 91$ & 69 & CASS & $T$ & \\
\hline TE-108-AP-52 & $7 / 3 / 91$ & 68 & CASS & $T$ & \\
\hline TE-108-AP-52 & 7/13/91 & 67 & CASS & $T$ & \\
\hline TE-108-AP-52 & $7 / 14 / 91$ & 67 & CASS & $\mathrm{T}$ & \\
\hline TE-108-AP-52 & $7 / 24 / 81$ & 68 & CASS & $\bar{T}$ & \\
\hline
\end{tabular}

Data obtained from Computer Automated Surveillance Syatem (CASS), April 1995; and Survelltance Analyais Computer System (SACS), June 1995. 


\begin{tabular}{|c|c|c|c|c|c|}
\hline \multicolumn{6}{|c|}{$\begin{array}{l}\text { TANK 241.AP-108 } \\
\text { THEAMOCOUPLI DATA }\end{array}$} \\
\hline & & & & & \\
\hline THËHOCOUPLE * & DATE & TEMPERATURE ( $\left.{ }^{\circ} \mathrm{F}\right)$ & DATA SOURCE & OUALTY & COMMENT \\
\hline TE-108-AP-52 & $8 / 2 / 91$ & 69 & CASS & $T$ & \\
\hline TE-108-AP-52 & $8 / 6 / 91$ & 68 & CASS & $\mathbf{T}$ & \\
\hline TE-108-AP-52 & 8/12/91 & 71 & CASS & $T$ & \\
\hline TE-108-AP-52 & $8 / 18 / 91$ & 71 & CASS & $T$ & \\
\hline TE-108-AP 52 & $8 / 30 / 91$ & 70 & CASS & $\mathbf{T}$ & \\
\hline TE-108-AP-52 & 9/3/91 & 71 & CASS & $T$ & \\
\hline TE-108-AP-52 & 9/8/91 & 71 & CASS & $\mathbf{T}$ & \\
\hline TE-108-AP-52 & 9/18/91 & 74 & CASS & $\mathbf{T}$ & \\
\hline TE-10:AP-52 & 9/22/91 & 71 & CASS & $T$ & \\
\hline TE-108-AP-52 & $10 / 1 / 91$ & 73 & CASS & $\mathbf{T}$ & \\
\hline TE-108-AP-52 & 10/8/91 & 62 & CASS & $\mathbf{s}$ & Data point did not agree with local trend \\
\hline TE-108-AP-52 & 10/12/91 & 73 & CASS & $\mathbf{T}$ & \\
\hline TE-108-AP-52 & $10 / 17 / 91$ & 75 & CASS & $\mathbf{T}$ & \\
\hline TE-108-AP-52 & $10 / 25 / 91$ & 73 & CASS & $\mathbf{T}$ & \\
\hline TE-108-AP-52 & $10 / 28 / 91$ & 72 & CASS & $\mathbf{T}$ & \\
\hline TE-10:-AP-52 & 11/6/9? & 72 & CASS & $T$ & \\
\hline TE-108-AP-52 & $8 / 1 / 22$ & 65 & CASS & $T$ & \\
\hline TE-108-AP-52 & $8 / 8 / 92$ & 67 & CASS & $T$ & \\
\hline TE-108-AP-52 & $8 / 15 / 92$ & 67 & CASS & $\mathbf{T}$ & \\
\hline TE-108-AP-52 & $8 / 20 / 92$ & 69 & CASS & $\mathbf{T}$ & \\
\hline TE-108-AP-52 & $8 / 28 / 92$ & 88 & CASS & $T$ & \\
\hline TE-108-AP-52 & $8 / 31 / 92$ & 67 & CASS & $\mathbf{T}$ & \\
\hline TE-108-AP-62 & 9/2/92 & 67 & CASS & $T$ & \\
\hline TE-100-AP-52 & 8/16/32 & 6 & CASS & $T$ & \\
\hline TE-108-AP-52 & 9/26/32 & 66 & CASS & $\mathbf{T}$ & \\
\hline TE-108-AP-52 & $10 / 3 / 92$ & 68 & CASS & $T$ & \\
\hline TE-108-AP-52 & $10 / 5 / 92$ & 69 & CASS & $\mathbf{T}$ & \\
\hline TE-108-AP-B2 & $10 / 13 / 92$ & 69 & CASS & $\mathbf{T}$ & \\
\hline TE-108-AP-52 & $10 / 22 / 92$ & 70 & CASS & $\mathbf{T}$ & \\
\hline TE-108-AP-52 & $10 / 29 / 92$ & 71 & CASS & $T$ & \\
\hline TE-108-AP-52 & $11 / 7 / 92$ & 69 & CASS & $T$ & \\
\hline TE-108-AP-52 & $11 / 9 / 92$ & 69 & CASS & $T$ & \\
\hline TE-106-AP-52 & $11 / 10 / 92$ & 91 & CASS & $\mathbf{S}$ & Qute point did not egree whe local trend \\
\hline TE-108-AP-52 & $11 / 17 / 92$ & 70 & CASS & $\bar{T}$ & \\
\hline TE-108-AP-52 & $11 / 20 / 92$ & 68 & CASS & $\mathbf{T}$ & \\
\hline TE-10:AP.52 & $11 / 30 / 22$ & 68 & CASS & $T$ & \\
\hline TE-108-AP-52 & 12/12/92 & 68 & CASS & $T$ & \\
\hline TE-106-AP-52 & 12/17/92 & 67 & CASS & $T$ & \\
\hline TE-10:AP-52 & $12 / 21 / 82$ & 68 & SACS & $T$ & \\
\hline TE-108-AP-52 & $12130 / 92$ & 86 & CASS & $\mathbf{T}$ & \\
\hline TE-108-AP-52 & $6 / 4 / 93$ & 44 & SACS & 5 & Data point did not agree with local trend \\
\hline TE-108.AP-52 & $8 / 11 / 93$ & 80 & SACS & $T$ & \\
\hline TE-108-AP-52 & $6 / 18 / 93$ & 60 & SACS & $T$ & \\
\hline TE-10:-AP-52 & 6/28/93 & 61 & SACS & $\bar{T}$ & \\
\hline TE-108-AP-52 & $7 / 9 / 93$ & 61 & SACS & $\mathrm{T}$ & \\
\hline TE-108-AP+52 & $7 / 15 / 93$ & 61 & SACS & $T$ & \\
\hline TE-108-AP-52 & 719/93 & 62 & SACS & $T$ & \\
\hline TE-10B-AP-52 & $730 / 93$ & 62 & SACS & $T$ & \\
\hline TE-108-AP-52 & $8 / 6 / 93$ & 62 & SACS & $T$ & \\
\hline TE-108-AP-52 & $8 / 12 / 93$ & 61 & SACS & $\mathbf{T}$ & \\
\hline TE-108-AP-52 & 9/24/93 & 60 & SACS & $T$ & \\
\hline TE-108.AP-52 & $10 / 1 / \mathrm{s}$ & 63 & SACS & $T$ & \\
\hline IE-108-AP-52 & $10 / 15 / 93$ & 65 & SACS & $T$ & \\
\hline TE-108-AP-52 & $10 / 22 / 93$ & 64 & SACS & $\bar{T}$ & \\
\hline TE-108-AP-52 & $10 / 28 / 93$ & 65 & SACS & $T$ & \\
\hline TE-108-AP-52 & 11/9/93 & 62 & SACS & $T$ & \\
\hline TE-108-AP-52 & $11 / 30 / 93$ & 67 & SACS & $T$ & \\
\hline TE-108-AP-52 & $12 / 14 / 93$ & 67 & SACS & $T$ & \\
\hline TE-108-AP-52 & $12 / 20 / 93$ & 63 & SACS & $\mathbf{T}$ & \\
\hline TE-108-AP-52 & $12 / 27 / 93$ & 63 & SACS & $\mathbf{T}$ & \\
\hline TE-108-AP-52 & $1 / 3 / 94$ & 62 & SACS & $\mathbf{T}$ & \\
\hline TE-108-AP-52 & $1 / 31 / 94$ & 65 & SACS & $\mathbf{T}$ & \\
\hline TE-108-AP-52 & $1 / 20 / 94$ & 64 & CASS & $\mathbf{T}$ & \\
\hline TE-108-AP-52 & $1 / 28 / 94$ & 63 & CASS & $\mathbf{T}$ & \\
\hline TE-108-AP-52 & $2 / 4 / 94$ & 64 & CASS & $\mathbf{T}$ & \\
\hline TE-108-AP-52 & $2 / 11 / 34$ & 64 & CASS & $T$ & \\
\hline TE-108-AP-52 & $2 / 15 / 94$ & 64 & CASS & $\mathbf{T}$ & \\
\hline TE-108-AP-52 & $2 / 22 / 94$ & 64 & CASS & $\mathbf{T}$ & \\
\hline TE-108-AP-52 & $2128 / 94$ & 65 & CASS & $\bar{T}$ & \\
\hline TE-108-AP-52 & $3 / 8 / 94$ & 65 & CASS & $T$ & \\
\hline TE-108-AP-52 & $3 / 17 / 94$ & 64 & CASS & $\mathbf{T}$ & \\
\hline TE-108-AP-52 & $3 / 26 / 94$ & 63 & CASS & $T$ & \\
\hline TE-108-AP-52 & $3 / 31 / 94$ & 63 & CASS & $T$ & \\
\hline
\end{tabular}




\begin{tabular}{|c|c|c|c|c|c|}
\hline \multicolumn{6}{|c|}{$\begin{array}{l}\text { TANK 241-AP-108 } \\
\text { THERWOCOUPLE DATA }\end{array}$} \\
\hline & & & & & \\
\hline THERMOCOUPE * & DATE & TEMPERATUAE $\left({ }^{\circ} \mathrm{F}\right)$ & DATA SOURCE & QUALTY & COMMENT \\
\hline TE-108-AP.52 & $4 / 9 / 94$ & 65 & CASS & $T$ & \\
\hline TE-108-AP-52 & /15/94 & 64 & CASS & $\mathbf{T}$ & \\
\hline TE-108-AP-52 & $4 / 20 / 94$ & 64 & CASS & $\mathbf{T}$ & \\
\hline TE-108-AP-52 & 4/29/94 & 64 & CASS & $\bar{T}$ & \\
\hline TE-108-AP-52 & $5 / 2 / 94$ & 64 & SACS & $G$ & \\
\hline TE-106-AP-52 & $5 / 8 / 94$ & 66 & CASS & $\mathbf{T}$ & \\
\hline TE-108-AP-52 & 5/16/94 & 104 & CASS & $\mathbf{T}$ & \\
\hline TE-108-AP-52 & 5/23/94 & 99 & CASS & $\mathbf{T}$ & \\
\hline TE-102-APE52 & $6 / 2 / 94$ & 98 & CASS & $\mathbf{T}$ & \\
\hline TE-108-AP-52 & 6/8/94 & 98 & CASS & $\mathbf{T}$ & \\
\hline TE-108-AP-52 & $6 / 14 / 94$ & 85 & CASS & $T$ & \\
\hline TE-108-AP-52 & 6/16/94 & 63 & CASS & $\mathbf{S}$ & Data point did not agree with local trend \\
\hline TE-108-AP-52 & 6/17/94 & 63) & CASS & $\mathbf{S}$ & Data point did not egres with local trend \\
\hline TE-108-AP-52 & 6/20/94 & 63 & CASS & $\mathbf{s}$ & Data point did not agree with local trand \\
\hline TE-108-AP-52 & $6 / 20 / 94$ & 61 & SACS & S (G in SACS) & Oata point did not agree with local trand \\
\hline TE-108-AP-52 & 6/25/94 & 100 & CASS & $T$ & \\
\hline TE-108-AP-52 & $6 / 27194$ & 102 & CASS & $T$ & \\
\hline TE-108-AP-52 & $7 / 7 / 194$ & 97 & CASS & $T$ & \\
\hline TE-108-AP-52 & $7115 / 94$ & 96 & CASS & $\mathbf{T}$ & \\
\hline TE-108-AP-52 & $7 / 18 / 94$ & 85 & CASS & $T$ & \\
\hline TE-108-AP-52 & $7 / 30 / 94$ & 93 & CASS & $T$ & \\
\hline TE-108-AP-52 & $7 / 31 / 94$ & 96 & CASS & $\mathbf{T}$ & \\
\hline TE-106.AP-52 & $8 / 9 / 94$ & 98 & CASS & $\mathbf{T}$ & \\
\hline TE-106-AP-52 & $8 / 20 / 94$ & 88 & CASS & $\bar{T}$ & \\
\hline TE-108-AP-52 & $8 / 21 / 94$ & 96 & CASS & $\bar{T}$ & \\
\hline TE-108-AP-52 & $9 / 3 / 94$ & 98 & CASS & $T$ & \\
\hline TE-108-AP-52 & $9 / 10 / 94$ & 101 & CASS & $\mathbf{T}$ & \\
\hline TE-108-AP-52 & $9 / 15 / 94$ & 100 & CASS & $T$ & \\
\hline TE-108-AP-52 & 9/23/94 & 101 & CASS & $\bar{T}$ & \\
\hline TE-108-AP-52 & $9 / 30 / 94$ & 100 & CASS & $\bar{T}$ & \\
\hline TE-108-AP-52 & $10 / 8 / 34$ & 101 & CASS & $\mathbf{T}$ & \\
\hline TE-108-AP-52 & $10 / 12 / 94$ & 102 & CASS & $\bar{T}$ & \\
\hline TE-108-AP-52 & $10 / 22 / 94$ & 105 & CASS & $T$ & \\
\hline TE-108-AP-52 & $10 / 29 / 94$ & 104 & CASS & $T$ & \\
\hline TE-108-AP-52 & $11 / 5 / 94$ & 104 & CASS & $T$ & \\
\hline TE-10B-AP-52 & $11 / 11 / 94$ & 103 & CASS & $T$ & \\
\hline TE-108-AP-52 & $11 / 16 / 94$ & 104 & CASS & $T$ & \\
\hline TE-108-AP-52 & $11 / 25 / 94$ & 105 & CASS & $\mathbf{T}$ & \\
\hline TE-108-AP-52 & $12 / 3 / 94$ & 104 & CASS & $T$ & \\
\hline TE-108-AP-52 & $12 / 4 / 94$ & 109 & CASS & $\mathbf{T}$ & \\
\hline TE-108-AP-52 & $12 / 12 / 94$ & 106 & CASS & $T$ & \\
\hline TE-108-AP-52 & $12 / 19 / 94$ & 107 & CASS & $T$ & \\
\hline TE-108-AP-52 & $12 / 30 / 94$ & 106 & CASS & $T$ & \\
\hline TE-108-AP-S2 & $1 / 4 / 95$ & 110 & CASS & $T$ & \\
\hline TE-108-AP-52 & $1 / 13 / 95$ & 107 & CASS & $T$ & \\
\hline TE-108-AP-52 & 1/15/95 & 108 & CASS & $\mathbf{T}$ & \\
\hline TE-108-AP-52 & $1 / 23 / 95$ & 106 & CASS & $\mathbf{T}$ & \\
\hline TE-108-AP-52 & 1/30/95 & 100 & SACS & $\mathbf{G}$ & \\
\hline TE-108-AP-52 & $2 / 6 / 95$ & 101 & CASS & $T$ & \\
\hline TE-10B-AP-52 & $2 / 14 / 95$ & 106 & CASS & $T$ & \\
\hline TE-108-AP-52 & $2 / 24 / 95$ & 96 & CASS & $T$ & \\
\hline TE-108-AP-52 & $3 / 2 / 95$ & 101 & CASS & $T$ & \\
\hline TE-108-AP-52 & $3 / 7 / 95$ & 98 & CASS & $T$ & \\
\hline TE-108-AP-52 & $3 / 16 / 95$ & 128 & CASS & $\mathbf{S}$ & Date point did not agree with locel trend \\
\hline TE-108-AP-52 & $3 / 17 / 95$ & 93 & CASS & $\bar{T}$ & \\
\hline TE-108-AP-52 & $3 / 25 / 95$ & 95 & CASS & $\bar{T}$ & \\
\hline TE-108-AP-52 & $3 / 30 / 85$ & 96 & CASS & $\mathbf{T}$ & \\
\hline TE-108-AP-52 & $4 / 3 / 95$ & 85 & SACS & $\mathbf{G}$ & \\
\hline TE-108-AP-52 & $4 / 10 / 95$ & 87 & SACS & G & \\
\hline TE-108-AP-52 & $4 / 17 / 95$ & 88 & SACS & G & \\
\hline TE-108-AP-52 & $4 / 24 / 95$ & 81 & SACS & $\mathbf{G}$ & \\
\hline TE-108-AP-52 & $5 / 1 / 95$ & 86 & SACS & $\mathbf{G}$ & \\
\hline TE-108-AP-52 & $5 / 8 / 95$ & 85 & SACS & $\mathbf{G}$ & \\
\hline TE-108-AP-52 & $5 / 16 / 95$ & 84 & SACS & G & \\
\hline TE-108-AP-52 & $5 / 22 / 95$ & 84 & SACS & G & \\
\hline TE-108-AP-52 & $5 / 29 / 95$ & 84 & SACS & G & \\
\hline TE-108-AP-52 & $6 / 5 / 95$ & 89 & SACS & G & \\
\hline TE-108-AP-52 & $6 / 13 / 95$ & 84 & SACS & $\mathbf{G}$ & \\
\hline TE-108-AP-52 & 6/19/95 & 101 & SACS & G & \\
\hline & & & & & \\
\hline TE-108-AP-53 & $4 / 5 / 86$ & 58 & CASS & $T$ & \\
\hline TE-108-AP-53 & $4 / 7 / 86$ & 59 & CASS & $T$ & \\
\hline TE-108-AP-53 & $5 / 17 / 86$ & 57 & CASS & $\mathbf{T}$ & \\
\hline
\end{tabular}

Data obtained from Computer Automated Surveillance System (CASS), April 1995; and Surveillance Analysis Computer System (SACS), June 1995.

$$
\text { D-715 }
$$




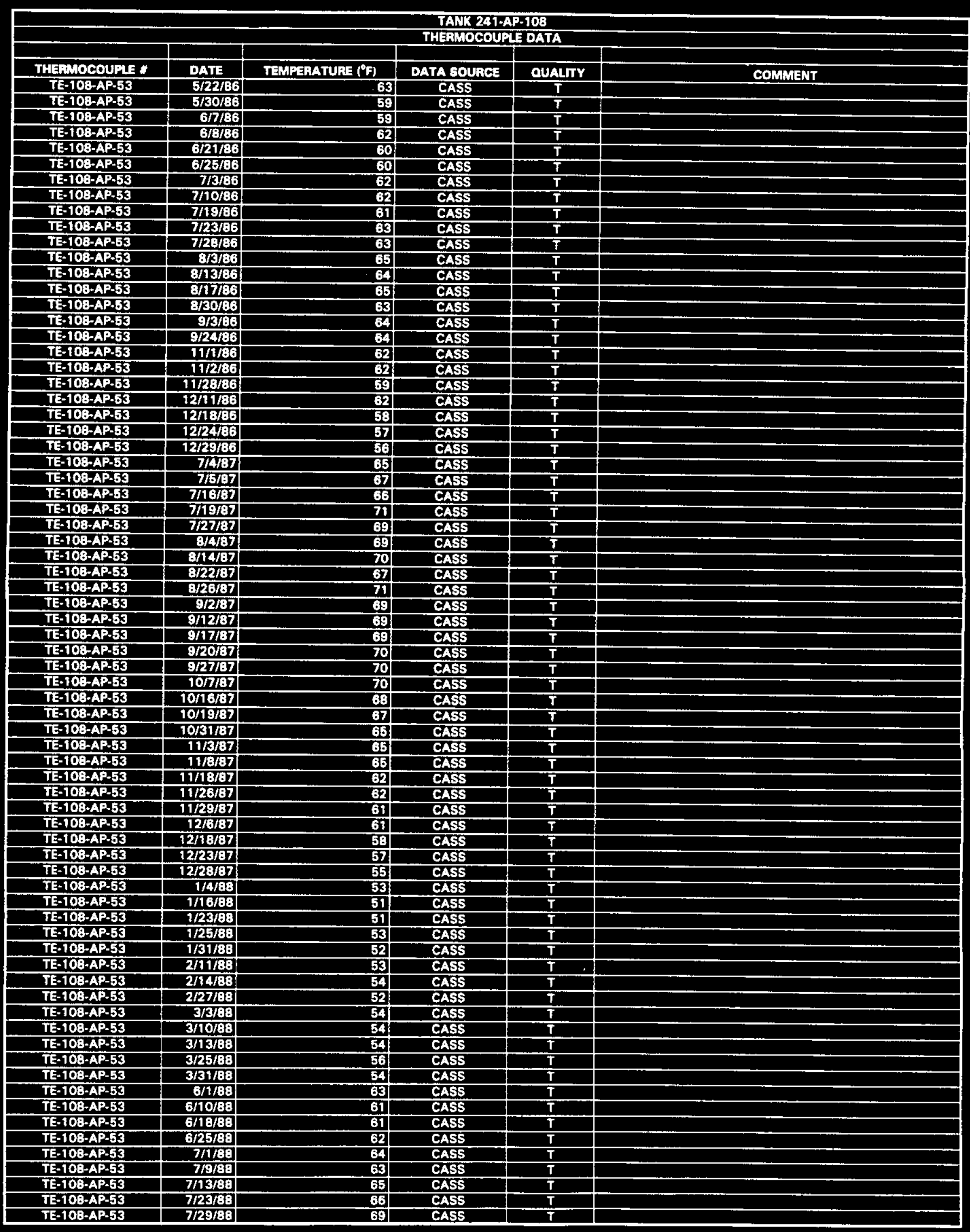




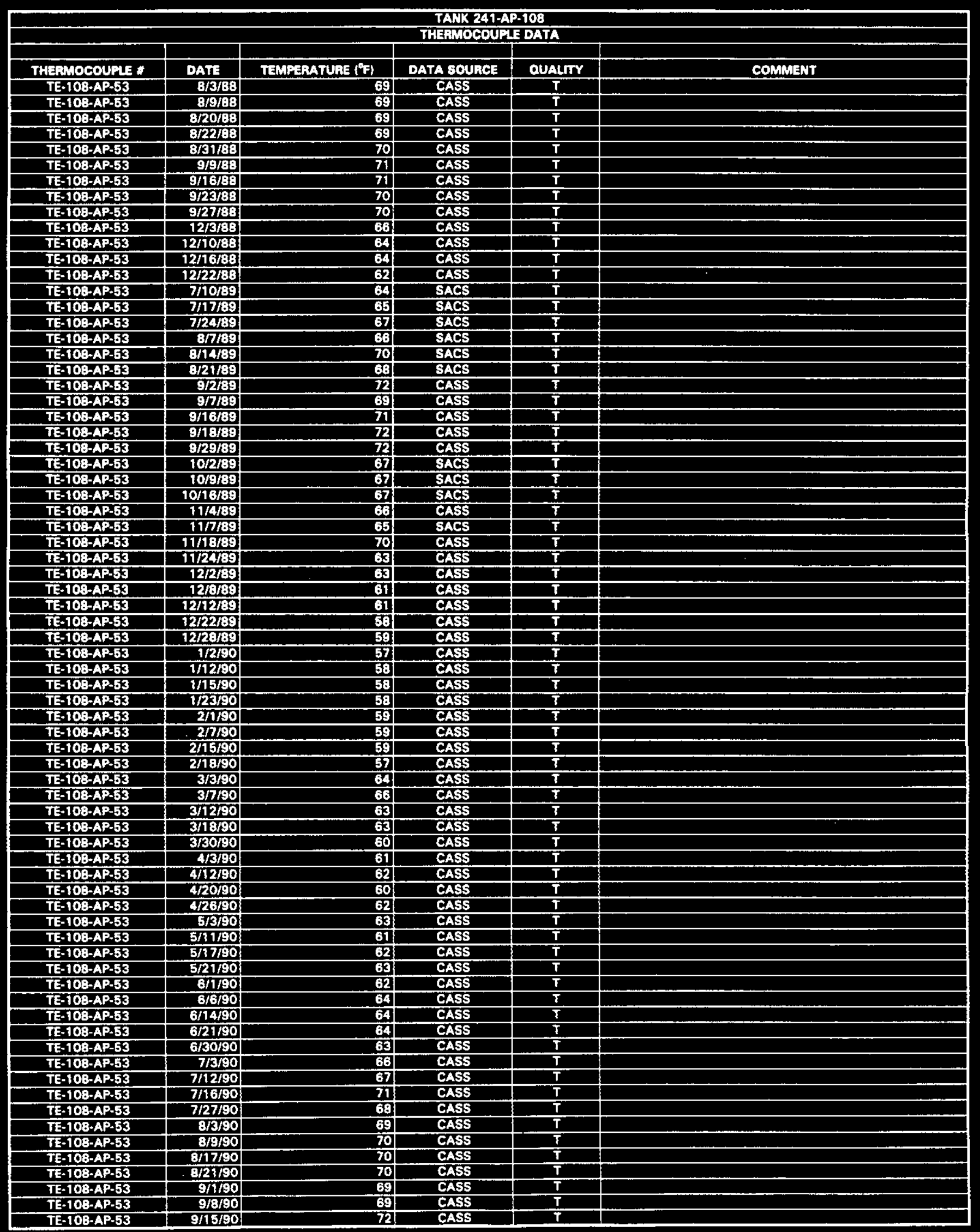




\begin{tabular}{|c|c|c|c|c|c|}
\hline \multicolumn{6}{|c|}{$\begin{array}{l}\text { TAWK 241-AP-108 } \\
\text { THEWOOCOUPIE DATA }\end{array}$} \\
\hline & & TEMPERATUAE $\left({ }^{\circ} \mathrm{F}\right)$ & & & \\
\hline$\frac{\text { THEBMOCOUPLE } *}{\text { TE-108-AP-53 }}$ & $\frac{\text { DATE }}{9 / 22 / 90}$ & & DATA SOUACE & OUALTY & COMMENT \\
\hline IE-108-AP-53 & $\frac{9 / 22 / 90}{9 / 27 / 90}$ & $\frac{69}{72}$ & CASS & $\mathbf{T}$ & \\
\hline$\frac{\text { TE-10B-AP-53 }}{\text { TE-108-AP-53 }}$ & 9/27/90 & 72 & CASS & $\mathbf{T}$ & \\
\hline TE-108-AP-53 & $10 / 6 / 90$ & 71 & CASS & $\mathbf{T}$ & \\
\hline TE-108-AP-53 & $10 / 13 / 90$ & 69 & CASS & $T$ & \\
\hline TE-108-AP-53 & $10 / 16 / 80$ & 70 & CASS & $\mathbf{T}$ & \\
\hline TE-108-AP-53 & $10 / 26 / 90$ & 70 & CASS & $\mathbf{T}$ & \\
\hline TE-108-AP-53 & $10 / 31 / 30$ & 70 & CASS & $\mathbf{T}$ & \\
\hline TE-108-AP-53 & $11 / 10 / 90$ & 88 & CASS & $\mathbf{T}$ & \\
\hline TE-108-AP-53 & $11 / 17 / 90$ & 67 & CASS & $T$ & \\
\hline TE-108.AP-53 & $11 / 20 / 90$ & 67 & CASS & $T$ & \\
\hline TE-108-AP-53 & $11 / 29 / 90$ & 65 & CASS & $\mathbf{T}$ & \\
\hline TE-108-AP-53 & 12/6/90 & 66 & CASS & $\mathbf{T}$ & \\
\hline TE-108-AP-53 & $12 / 10 / 80$ & 68 & SACS & $\mathbf{T}$ & \\
\hline TE-108-AP-53 & $12 / 20 / 90$ & 64 & CASS & $\mathbf{T}$ & \\
\hline TE-10B-AP-53 & $12 / 27 / 90$ & 61 & CASS & $\mathbf{T}$ & \\
\hline TE-108-AP-53 & $12 / 30 / 90$ & 59 & CASS & $\mathbf{T}$ & \\
\hline TE-108-AP.53 & $12 / 31 / 80$ & 71 & SACS & $\mathbf{S}$ & Date point did not egree with local trend \\
\hline TE-10:-AP-53 & $1 / 11 / 91$ & 58 & CASS & $T$ & \\
\hline TE-108-AP-53 & 1/14/91 & 59 & SACS & $\mathbf{T}$ & \\
\hline TE-108-AP-53 & $1 / 26 / 91$ & 56 & CASS & $T$ & \\
\hline TE-106-AP-53 & $1 / 29 / 91$ & 57 & CASS & $T$ & \\
\hline TE-108-AP-5K & $2 / 6 / 91$ & 56 & CASS & $T$ & \\
\hline TE-108-AP-53 & 2/16/91 & 55 & CASS & $\mathbf{T}$ & \\
\hline TE-108-AP-53 & $2 / 17 / 91$ & 56 & CASS & $T$ & \\
\hline TE-108-AP-53 & $3 / 1 / 21$ & 58 & CASS & $\mathbf{T}$ & \\
\hline TE-108-AP-53 & $3 / 3 / 31$ & 59 & CASS & $\bar{T}$ & \\
\hline TE-108-AP-53 & $3 / 12 / 91$ & 69. & CASS & $T$ & \\
\hline TE-108-AP-53 & $3 / 21 / 91$ & 57 & CASS & $\mathbf{T}$ & \\
\hline TE-108-AP-53 & $3 / 25 / 91$ & 59 & CASS & $T$ & \\
\hline TE-108-AP-5B & $4 / 1 / 91$ & S9 & SACS & $T$ & \\
\hline TE-108.AP-53 & 4/12/91 & 88: & CASS & $T$ & \\
\hline TE-108-AP-53 & 4/20/9? & 57 & CASS & $T$ & \\
\hline TE-108-AP-53 & 4/26/91 & 88 & CASS & $T$ & \\
\hline TE-108-AP-53 & 5/4/91 & 59 & CASS & $\bar{T}$ & \\
\hline TE-108-AP-53 & $5 / 10 / 91$ & 60 & CASS & $\mathbf{T}$ & \\
\hline TE-108-AP-53 & 5/16/91 & 60 & CASS & $T$ & \\
\hline TE-108-AP-53 & 5/24/91 & 63 & CASS & $\mathbf{T}$ & \\
\hline TE-10B-APS53 & $5 / 26 / 91$ & 661 & CASS & $\mathbf{T}$ & \\
\hline TE-108-AP-53 & $6 / 5 / 91$ & 63 & CASS & $T$ & \\
\hline TE-108-AP-53 & $6 / 15 / 91$ & 62 & CASS & $T$ & \\
\hline TE-108-AP-53 & $6 / 22 / 91$ & 66 & CASS & $T$ & \\
\hline TE-108-AP-53 & $6 / 28 / 91$ & 67 & CASS & $T$ & \\
\hline TE-108-AP-53 & $7 / 3 / 91$ & 69 & CASS & $T$ & \\
\hline TE-108-AP-53 & $7 / 13 / 91$ & 67 & CASS & $\mathbf{T}$ & \\
\hline TE-108-AP-53 & $7 / 14 / 91$ & 67 & CASS & $\mathbf{T}$ & \\
\hline TE-108-AP-63 & 7/24/91 & 67 & CASS & $\mathbf{T}$ & \\
\hline TE-108-AP-53 & $8 / 2 / 91$ & 69 & CASS & $T$ & \\
\hline TE-108-AP-53 & $8 / 6 / 91$ & 68) & CASS & $T$ & \\
\hline TE-108-AP-53 & $8 / 17 / 91$ & 72 & CASS & $T$ & \\
\hline TE-108-AP-53 & $8 / 18 / 93$ & 71 & CASS & $\bar{T}$ & \\
\hline TE-108-AP-53 & 8/30/91 & 70 & CASS & $T$ & \\
\hline TE-108-AP-53 & $9 / 3 / 91$ & 71 & CASS & $\mathbf{T}$ & \\
\hline TE-108-AP-53 & 9/8/91 & 72 & CASS & $T$ & \\
\hline TE-10B-AP-53 & 9/18/91 & 74 & CASS & $T$ & \\
\hline TE-108-AP-53 & $9 / 22 / 91$ & 71 & CASS & $T$ & \\
\hline TE-108-AP-53 & $9 / 30 / 91$ & 72 & CASS & $T$ & \\
\hline TE-108-AP-53 & $10 / 6 / 91$ & 74 & CASS & $\mathbf{T}$ & \\
\hline TE-108-AP-53 & $10 / 17 / 91$ & 74 & CASS & $T$ & \\
\hline TE-108-AP-53 & $10 / 21 / 91$ & 74 & SACS & $T$ & \\
\hline TE-10B-AP-53 & $10 / 2 \mathrm{E} / 91$ & 73 & SACS & $T$ & \\
\hline TE-108-AP-53 & $11 / 6 / 91$ & 71 & CASS & $T$ & \\
\hline TE-108-APAS3 & $8 / 1 / 92$ & 65 & CASS & $T$ & \\
\hline TE-108-AP-53 & $8 / 3 / 92$ & 68 & CASS & $T$ & \\
\hline TE-106-AP-53 & $8 / 15 / 92$ & 67 & CASS & $\mathbf{T}$ & \\
\hline TE-108-AP-53 & $8 / 20 / 92$ & 68 & CASS & $\bar{T}$ & \\
\hline TE-106-AP-53 & $8 / 28 / 92$ & 68 & CASS & $T$ & \\
\hline TE-10:AP-53 & $8 / 31 / 92$ & 67 & CASS & $\mathbf{T}$ & \\
\hline TE-108-AP-53 & $9 / 9 / 92$ & 67 & CASS & $T$ & \\
\hline TE-108-AP-53 & 9/16/92 & 68 & CASS & $\bar{T}$ & \\
\hline TE-108-AP-53 & 9/21/92 & 67 & SACS & $T$ & \\
\hline TE-108-AP-53 & $10 / 3 / 92$ & 68 & CASS & $T$ & \\
\hline TE-108-AP-53 & $10 / 5 / 92$ & 69 & CASS & $T$ & \\
\hline TE-108-AP-53 & $10 / 13 / 92$ & 69 & CASS & $\mathbf{T}$ & \\
\hline
\end{tabular}




\begin{tabular}{|c|c|c|c|c|c|}
\hline \multicolumn{6}{|c|}{$\begin{array}{l}\text { TANK 241-AP-108 } \\
\text { THERMOCOUPLE DATA }\end{array}$} \\
\hline & & & & & \\
\hline THEAMOCOUPLE \# & DATE & TEMPERATUAE $\left({ }^{\circ} \mathrm{F}\right)$ & DATA SOUACE & OUALITY & COMMENT \\
\hline TE-108-AP-53 & $10 / 22 / 92$ & 71 & CASS & $T$ & \\
\hline TE-108-AP-53 & $10 / 29 / 92$ & 71 & CASS & $T$ & \\
\hline TE-108-AP-53 & $11 / 7 / 92$ & 69 & CASS & $\bar{T}$ & \\
\hline TE-108-AP-53 & $11 / 9 / 92$ & 89 & CASS & $\mathbf{s}$ & Data point did not egree with locel trend \\
\hline TE-108-AP-53 & $11 / 12 / 92$ & 68 & CASS & $T$ & \\
\hline TE-108-AP-53 & $11 / 47 / 92$ & 69 & CASS & $T$ & \\
\hline TE-108-AP-53 & $11 / 28 / 92$ & 68 & CASS & $\mathbf{T}$ & \\
\hline TE-108-AP-53 & $11 / 30 / 92$ & 68 & CASS & $\mathbf{T}$ & \\
\hline TE-108-AP-53 & $12 / 12 / 92$ & 68 & CASS & $\mathbf{T}$ & \\
\hline TE-108-AP-53 & $12 / 17 / 92$ & 66 & CASS & $T$ & \\
\hline TE-10B.AP-53 & $12 / 21 / 92$ & 66 & CASS & $T$ & \\
\hline TE-108.AP-53 & $12 / 30 / 92$ & 66 & CASS & $T$ & \\
\hline TE-108-AP-53 & $1 / 15 / 94$ & 63 & CASS & $\bar{T}$ & \\
\hline TE-108-AP-53 & $1 / 20 / 94$ & 64 & CASS & $\mathbf{T}$ & \\
\hline TE-108-AP-53 & $1 / 28 / 94$ & 63 & CASS & $T$ & \\
\hline TE-108-AP-53 & $2 / 2 / 94$ & 64 & CASS & $\mathbf{T}$ & \\
\hline TE-108-AP-53 & $2 / 111 / 94$ & 64 & CASS & $\bar{T}$ & \\
\hline TE-108-AP-53 & $2 / 16 / 94$ & 64 & CASS & $\mathbf{T}$ & \\
\hline TE-108-AP-53 & $2 / 22 / 94$ & 64 & CASS & $\mathbf{T}$ & \\
\hline TE-108-AP-53 & $3 / 6 / 94$ & 64 & CASS & $T$ & \\
\hline TE-108-AP-53 & $3 / 8 / 94$ & 65 & CASS & $\mathbf{T}$ & \\
\hline TE-108-AP-53 & $3 / 17 / 94$ & 64| & CASS & $\mathbf{I}$ & \\
\hline TE-108-AP-53 & $3 / 26 / 94$ & 63 & CASS & $\mathbf{T}$ & \\
\hline TE-108-AP-53 & $4 / 2 / 94$ & 63 & CASS & $\mathbf{T}$ & \\
\hline TE-108-AP-53 & $4 / 9 / 94$ & 66 & CASS &. $\mathrm{T}$ & \\
\hline TE-108-AP-53 & $4 / 15 / 94$ & 64 & CASS & $T$ & \\
\hline TE-108-AP-53 & $4 / 20 / 94$ & 64 & CASS & $T$ & \\
\hline TE-108-AP-53 & 4/29/94 & 65 & CASS & $T$ & \\
\hline TE-108-APA53 & $5 / 2 / 94$ & 64 & CASS & $\mathbf{T}$ & \\
\hline TE-108-AP-53 & $5 / 8 / 94$ & 66 & CASS & $\mathbf{T}$ & \\
\hline TE-108-AP-53 & $5 / 16 / 94$ & 104 & CASS & $T$ & \\
\hline TE-108-AP-53 & $5 / 22 / 94$ & 111 & CASS & $\bar{T}$ & \\
\hline TE-108-AP-53 & $6 / 2 / 94$ & 99 & CASS & $T$ & \\
\hline TE-108-AP-53 & $6 / 8 / 94$ & 96) & CASS & $\mathbf{T}$ & \\
\hline TE-108-AP-53 & 6/14/94 & 95 & CASS & $T$ & \\
\hline TE-108-AP=53 & 6/16/94 & 63) & CASS & $\mathbf{S}$ & Data point did not agree with local trend \\
\hline TE-108-AP-53 & 6/17/94 & 63) & CASS & $\mathbf{S}$ & Data point did not agree with locsl trend \\
\hline TE-10B-AP-53 & 6/20/94 & 63 & CASS & $\mathbf{S}$ & Data point did not apree with local trend \\
\hline TE-108-AP.53 & $6 / 25 / 94$ & 100 & CASS & $\bar{T}$ & \\
\hline TE-108.AP-53 & 6/27/94 & 99 & CASS & $\mathbf{T}$ & \\
\hline TE-108-AP-53 & $7 / 7 / 94$ & 97 & CASS & $T$ & \\
\hline TE-108-AP-53 & 7/15/94 & 96 & CASS & $\mathbf{T}$ & \\
\hline TE-108-AP-53 & 719794 & 95 & CASS & $\mathbf{T}$ & \\
\hline TE-108-AP-53 & $7 / 30 / 94$ & 93 & CASS & $T$ & \\
\hline TE-10Q-AP-53 & $7 / 31 / 34$ & 97 & CASS & $T$ & \\
\hline TE-108-AP-53 & $8 / 9 / 94$ & 98 & CASS & $T$ & \\
\hline TE-108-AP-53 & $8 / 18 / 94$ & 98 & CASS & $T$ & \\
\hline TE-108-AP-53 & $8 / 21 / 94$ & 96 & CASS & $T$ & \\
\hline TE-108-AP-53 & $9 / 3 / 94$ & 96 & CASS & $\mathbf{T}$ & \\
\hline TE-108-AP-53 & $9 / 5 / 34$ & 103 & CASS & $T$ & \\
\hline TE-108-AP-53 & $9 / 15 / 94$ & 100 & CASS & $T$ & \\
\hline TE-108-AP-53 & 9/23/94 & 101 & CASS & $T$ & \\
\hline TE-108-AP-53 & 9/25/94 & 100 & CASS & $T$ & \\
\hline TE-108-AP-53 & 10/2/94 & 102 & CASS & $T$ & \\
\hline TE-108-AP-53 & 10/9/94 & 105 & CASS & $T$ & \\
\hline TE-108-AP-53 & $10 / 22 / 94$ & 103 & CASS & $T$ & \\
\hline TE-108-AP-53 & $10 / 29 / 94$ & 104 & CASS & $T$ & \\
\hline TE-108-AP-53 & $11 / 5 / 94$ & 103 & CASS & $\mathrm{T}$ & \\
\hline TE-108-AP-53 & $11 / 11 / 94$ & 108 & CASS & $T$ & \\
\hline TE-108-AP-53 & $11 / 16 / 94$ & 104 & CASS & $T$ & \\
\hline TE-108-AP-53 & $11 / 25 / 94$ & 105 & CASS & $T$ & \\
\hline TE-108-AP-53 & $11 / 28 / 94$ & 104 & CASS & $T$ & \\
\hline TE-108-AP-53 & $12 / 4 / 94$ & 108 & CASS & $\mathrm{T}$ & \\
\hline TE-108-AP-53 & $12 / 12 / 94$ & 105 & CASS & $T$ & \\
\hline TE-103-AP-53 & $12 / 19 / 94$ & 106 & CASS & $\mathbf{T}$ & \\
\hline TE-108-AP-53 & $12 / 31 / 94$ & 105 & CASS & $\mathbf{T}$ & \\
\hline TE-108-AP-53 & $1 / 4 / 95$ & 110 & CASS & $\mathbf{T}$ & \\
\hline TE-108-AP-53 & $1 / 13 / 95$ & 106 & CASS & $T$ & \\
\hline TE-108-AP-53 & $1 / 15 / 95$ & 105 & CASS & $T$ & \\
\hline TE-108-AP-53 & $1 / 23 / 95$ & 106 & CASS & $T$ & \\
\hline TE-108-AP-53 & $2 / 3 / 95$ & 98 & CASS & $T$ & \\
\hline TE-108-AP-53 & $2 / 6 / 95$ & 101 & CASS & $T$ & \\
\hline TE-108-AP-53 & $2 / 14 / 95$ & 106 & CASS & $\mathbf{I}$ & \\
\hline
\end{tabular}


WHC-SD-WM-ER-315, Rev, 0

\begin{tabular}{|c|c|c|c|c|c|}
\hline \multicolumn{6}{|c|}{$\begin{array}{l}\text { TANK 241.AP.108 } \\
\text { THEAMOCOUPLE DATA }\end{array}$} \\
\hline THEPMOCOUPLE \# & DATE & TEMPERATURE ("F) & DATA SOURCE & OUALTY & COMMEST \\
\hline TE-108-AP-53 & $2 / 24 / 95$ & 96 & CASS & $T$ & \\
\hline TE-108-AP-53 & $3 / 3 / 95$ & 101 & CASS & $T$ & \\
\hline TE-108-AP-53 & $3 / 7 / 95$ & 98 & CASS & $T$ & \\
\hline TE-108-AP-53 & 3/16/95 & 94 & CASS & $\bar{T}$ & \\
\hline TE-108-AP-53 & $3 / 25 / 95$ & 95 & CASS & $\mathbf{T}$ & \\
\hline TE-108-AP-53 & $3 / 30 / 95$ & 96 & CASS & $T$ & \\
\hline
\end{tabular}


Fils View/Frint Document Cover Sheet

This document was retrieved from the Boeing ISEARCH System.

Accession \#: D196051039

Document \#: SD-WM-ER-315

Title/Desc:

SUPPORTING DOCUMENT FOR THE SOUTHEAST QUANDRANT HISTORICAL TANK CONTENT ESTIMATE REPORT FOR AP

TANK FARM [VOL II SEC 1 OF 2 PAGE E-1 TO E-390] 
THIS DOCUMENT WAS TOO LARGE TO SCAN AS A SINGLE DOCUMENT. IT REQUIRED BREAKING INTO SMALLER SECTIONS. DOCUMENT NUMBER: $S D-\omega m-\varepsilon R-315$ (Vol II) SECTION: 1 OF 2 TITLE: Southeast Quadrant Historical Man k

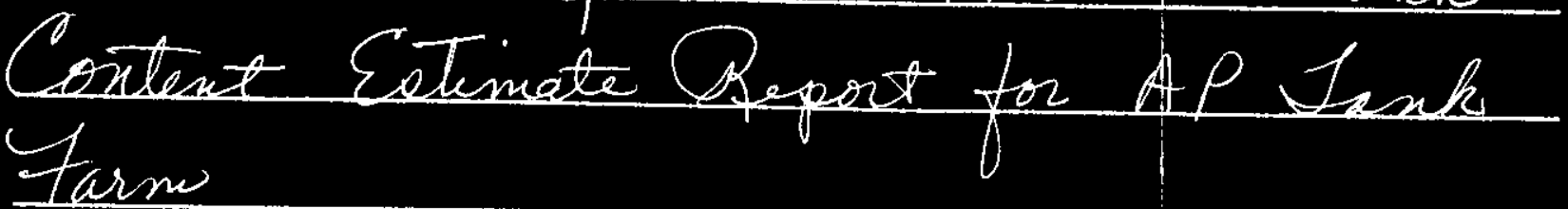
AUTHOR: LA Gaddis/SD Consort RECIPIENT:

DATE: $\quad 9 / 13 / 95$

KEYWORDS:

REFERENCES: 
THIS PAGE INTENTIONALLY
LEFT BLANK 


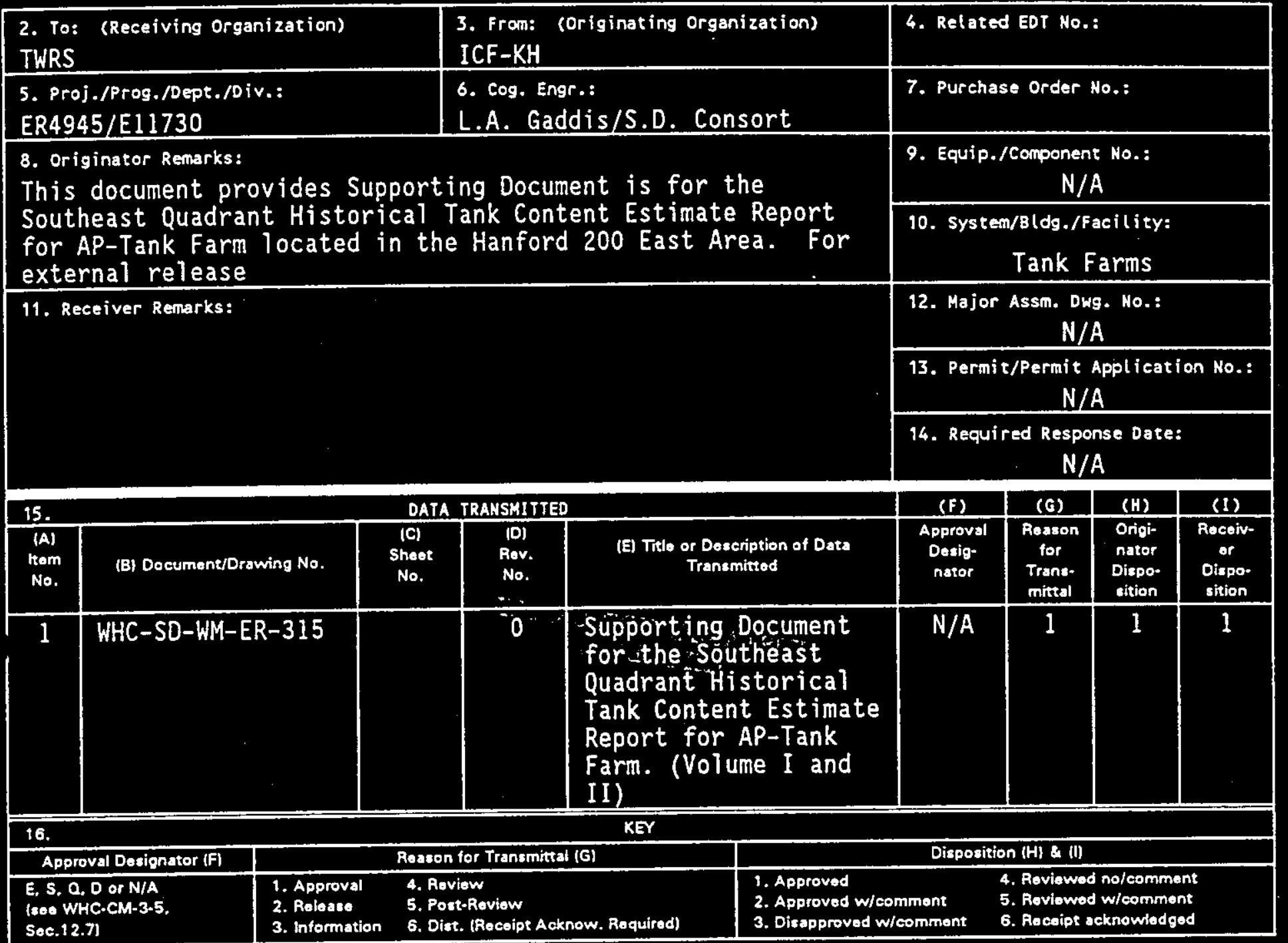

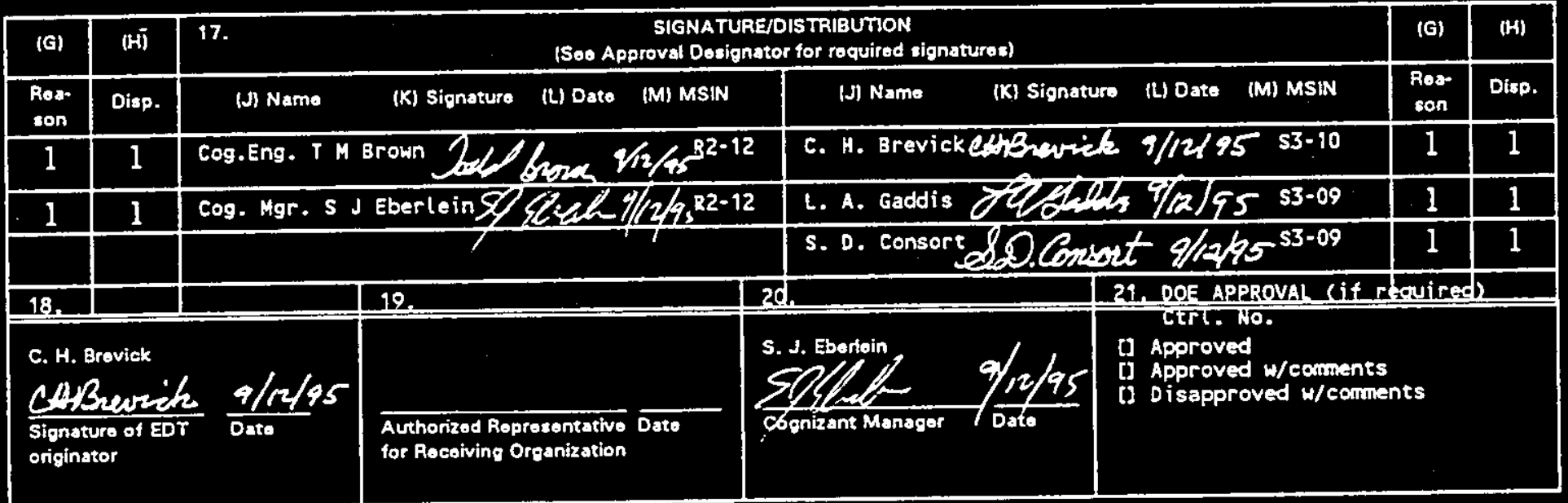

BD-7400-172-2 (04/94) GEF097 


\section{TEIS PAGS INTENTIONALIT LWFT BLANK}




\begin{tabular}{|c|c|c|}
\hline $\begin{array}{l}\text { 2. Title } \\
\text { Supporting Document for the Southeast Quadrant } \\
\text { Historical Tank Content Estimate Report for AP- } \\
\text { Tank Farm. (Volume I and II) }\end{array}$ & $\begin{array}{l}\text { 3. Number } \\
\text { WHC-SD-WM-ER-315 }\end{array}$ & $\begin{array}{c}\text { 4. Rev No. } \\
0\end{array}$ \\
\hline $\begin{array}{l}\text { 5. Key Hords } \\
\text { Southeast Quadrant, Historical Tank Content } \\
\text { Estimate, Historical Characterization, Tank Farms, } \\
\text { Tank Waste Level, Tank Aerial Photos, In-Tank } \\
\text { Photo, Tank Layer Model. Estimated Composite } \\
\text { Inventories, Riser Locations, Tank Layering Model. } \\
\text { Supernatant Mixing Model. }\end{array}$ & $\begin{array}{l}\text { 6. Author } \\
\text { Name: CHBrevick/LAGadd } \\
\text { SOCons } \\
\text { SAhnewnelzurs } \\
\text { Organization/charge code } \\
\text { Facilities and Techn }\end{array}$ & $\begin{array}{l}\text { So. Coniont } \\
400 / \text { E } 11730 \\
2 \text { al Support }\end{array}$ \\
\hline
\end{tabular}

\section{Abstract}

Historical Tank Content Estimate of the Southeast Quadrant provides historical evaluations on a tank by tank basis of the radioactive mixed wastes stored in the underground double-shell tanks of the Hanford 200 East and West Areas. This report summarizes historical information such as waste history, temperature profiles, psychrometric data, tank integrity, inventory estimates and tank level history on a tank by tank basis. Tank Farm aerial photos and in-tank photos of each tank are provided. A brief description of instrumentation methods used for waste tank surveillance are included. Components of the data management effort, such as Waste Status and Transaction Record Summary, Tank Layer Model, Supernatant Mixing Model, Defined Waste Types, and Inventary Estimates which generate these tank content estimates, are also given in this report.

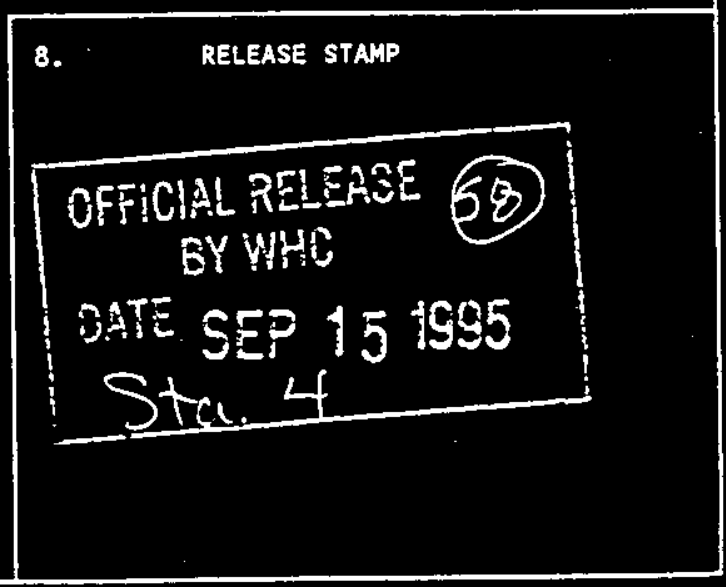




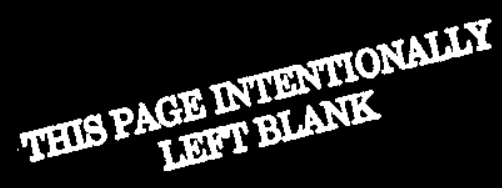




\title{
SUPPORTING DOCUMENT FOR THE HISTORICAL TANK CONTENT ESTIMATE FOR
}

\section{AP TANK FARM VOLUME II WORK ORDER E11730}

\author{
Prepared for \\ Westinghouse Hanford Company \\ September 1995
}

Prepared by

ICF Kaiser Hanford Company

Richland, Washington 


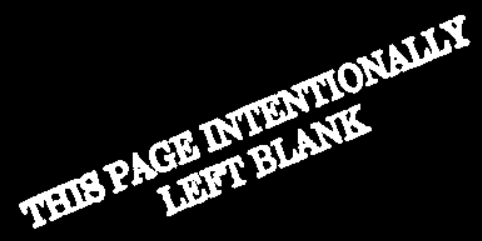




\section{APPENDIX E}

\section{Surface Level Graphs and Data}



WHC-SD-WM-ER-315, Rev . 0

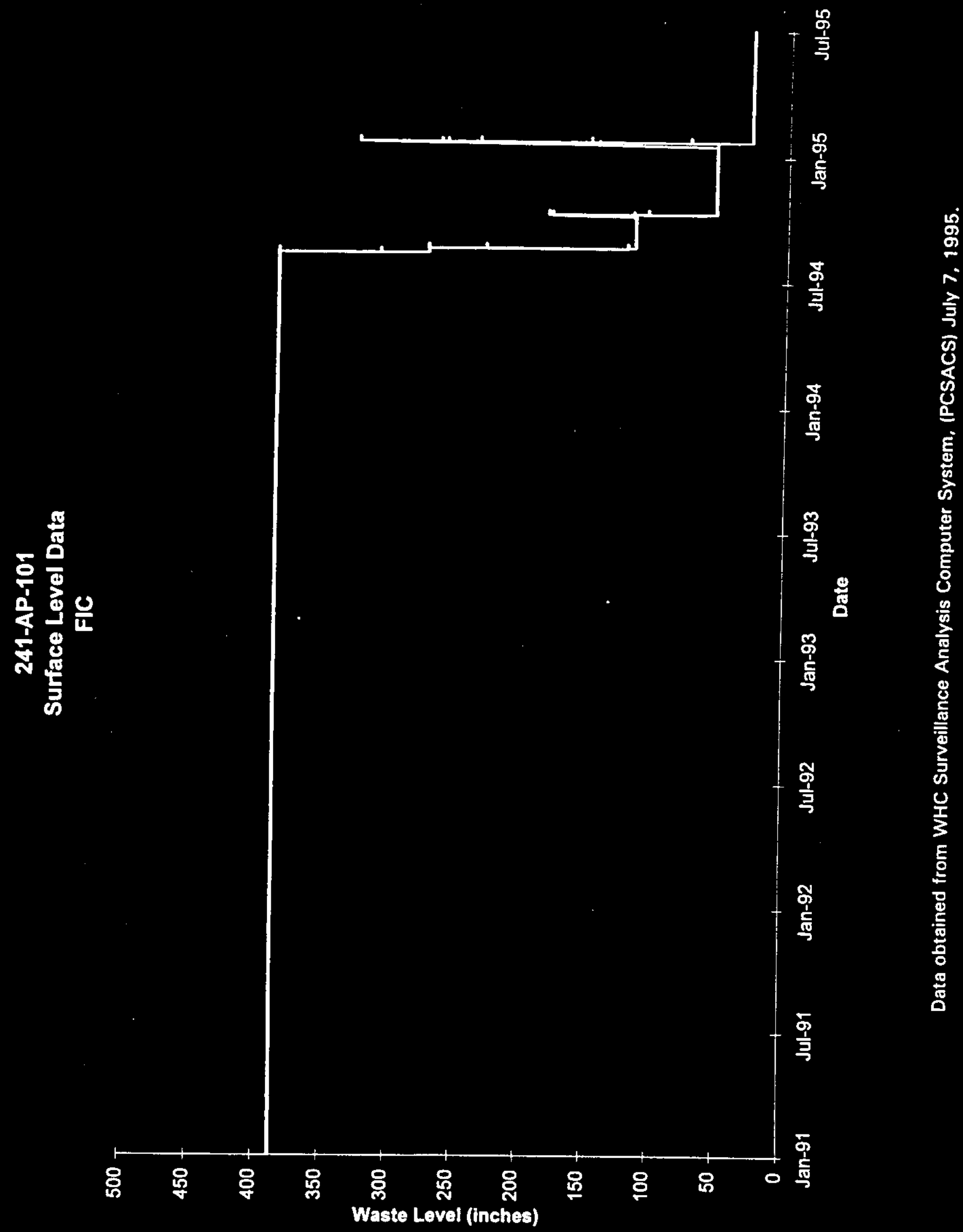


WHC-SD-WM-ER-315， Rev , 0

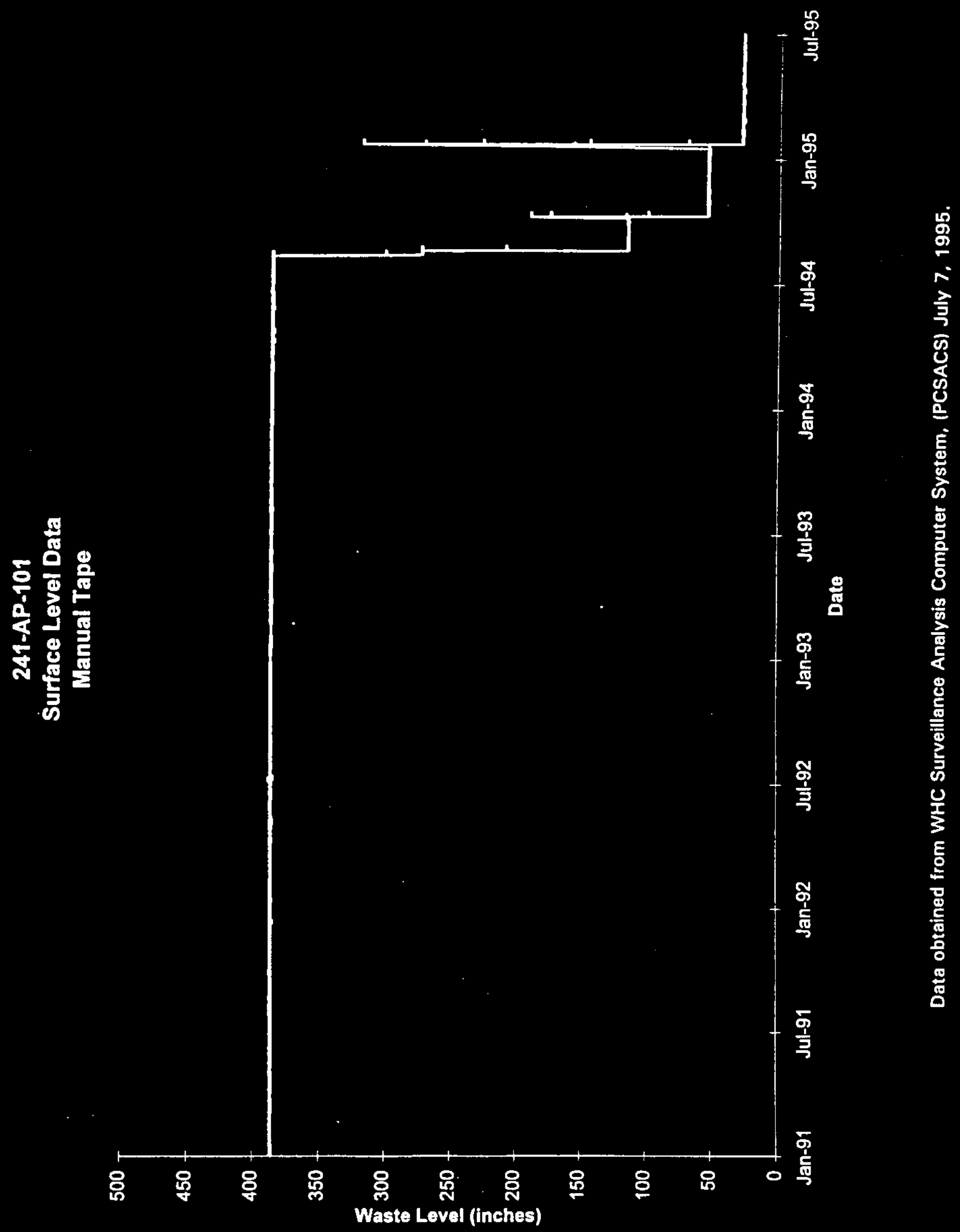

E-2 
WHC-SD-WM-ER-315，Rev. 0

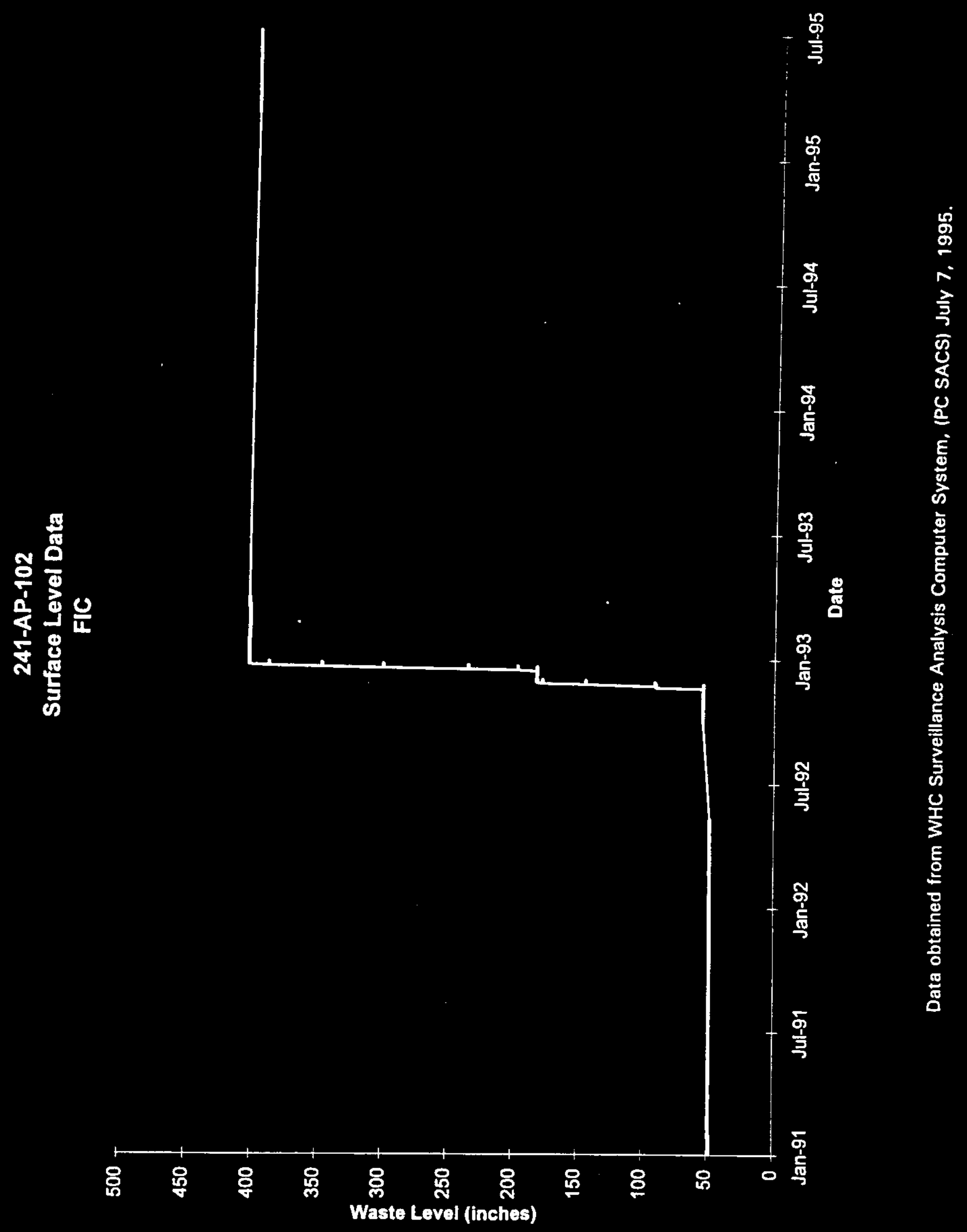

E-3 
WHC-SD-WM-ER-315，Rev. 0

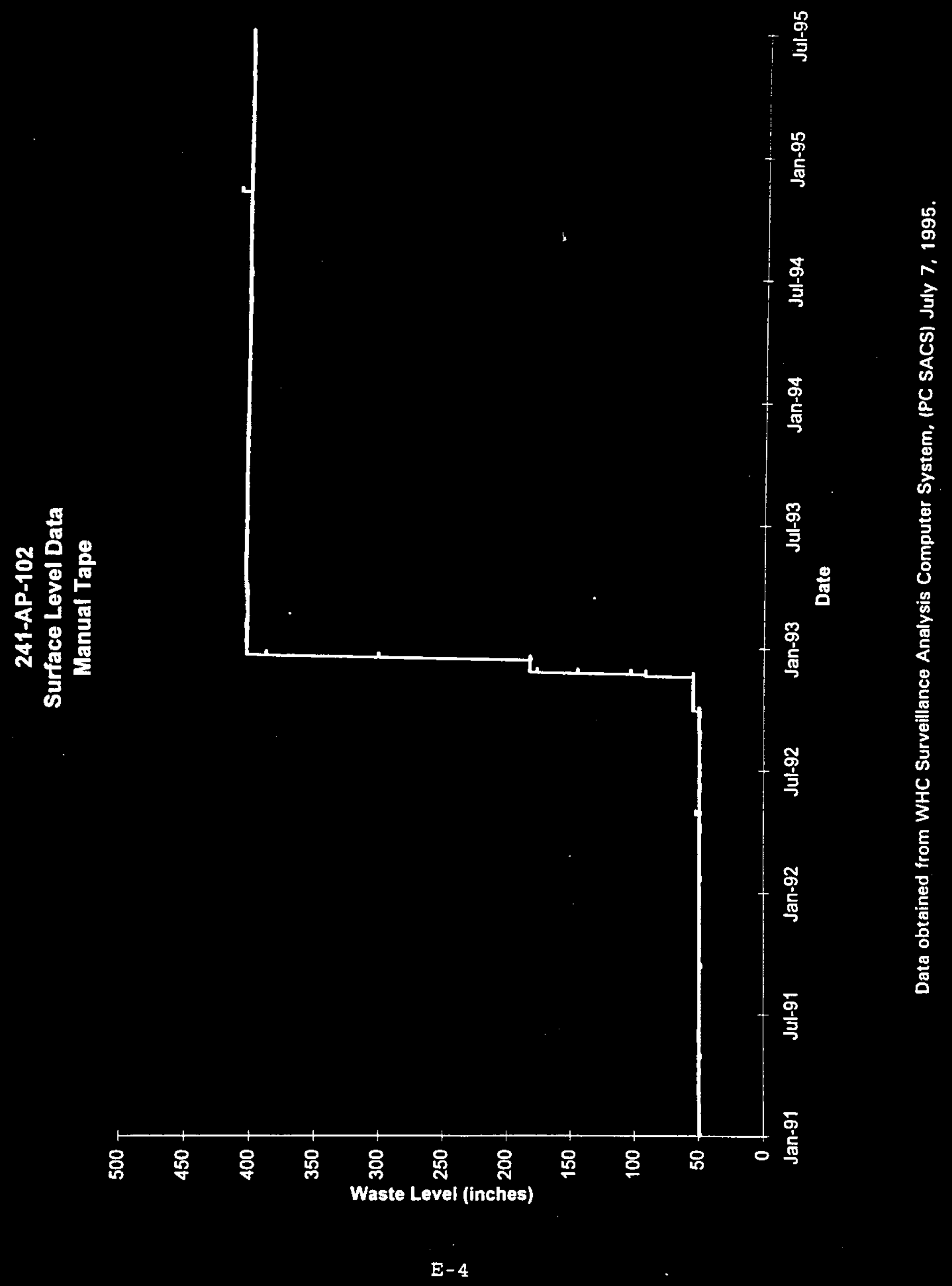


WHC-SD-WM-ER-315, Rev. 0

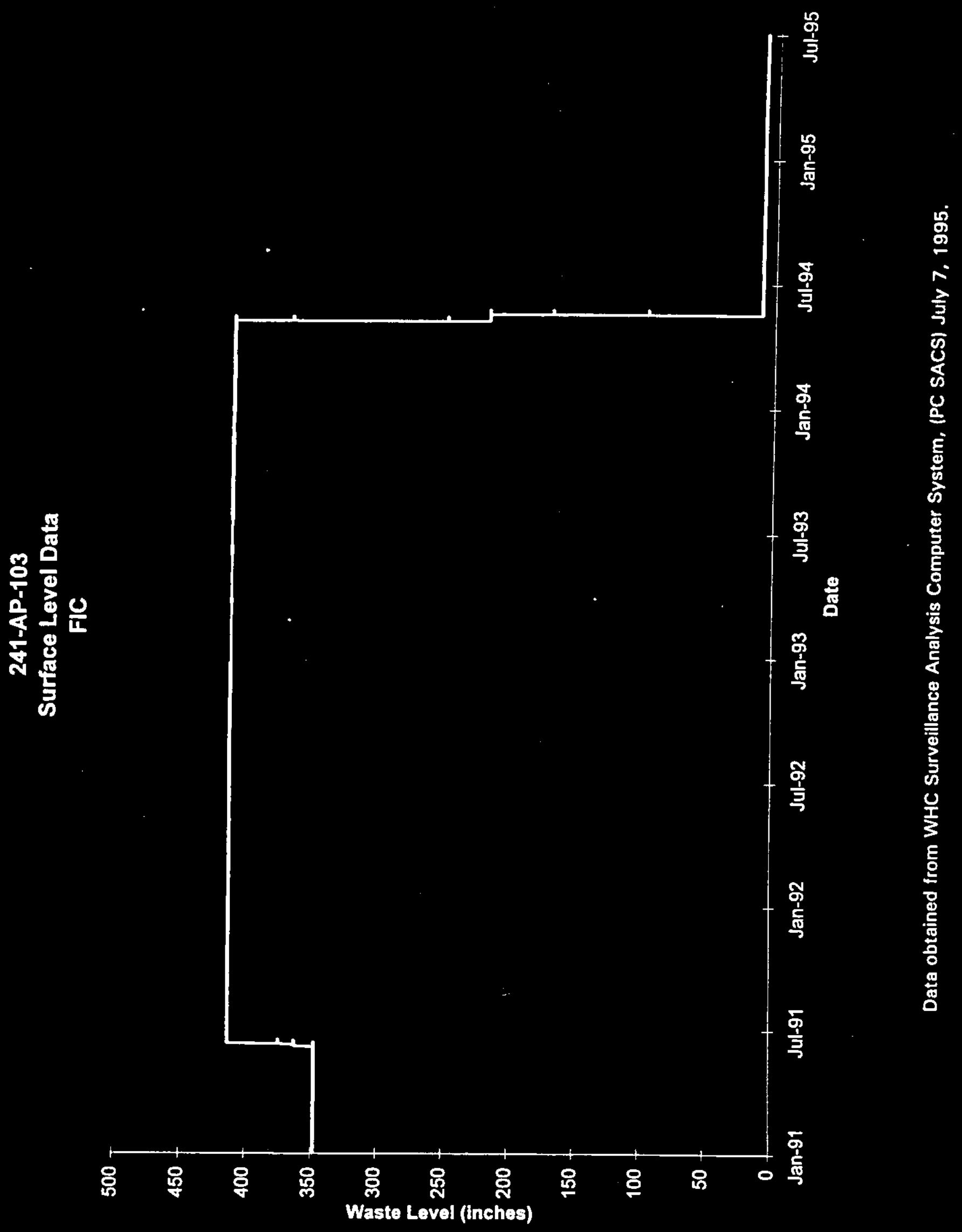

E-5 
WHC-SD-WM-ER-315, Rev. 0

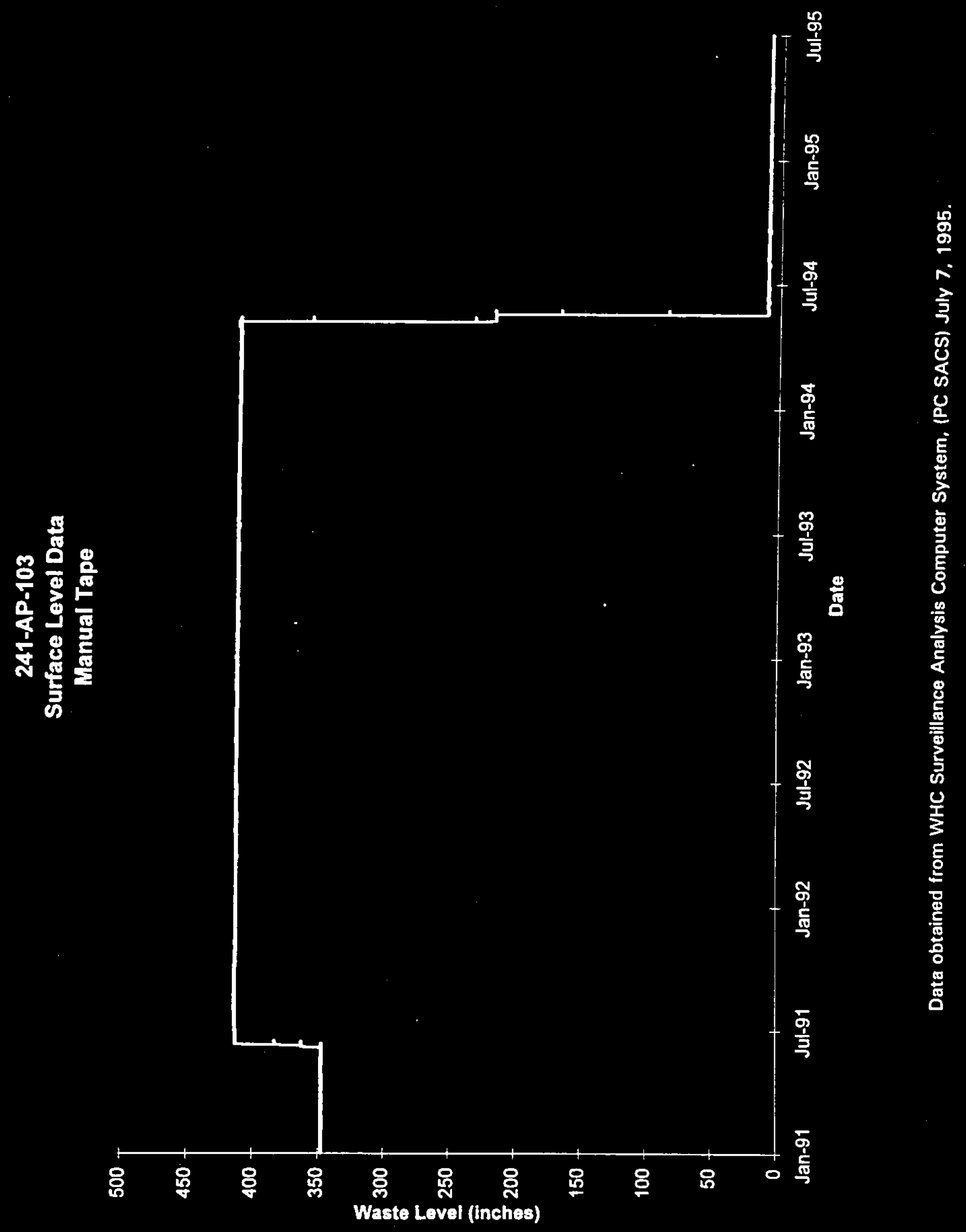

$E-6$ 


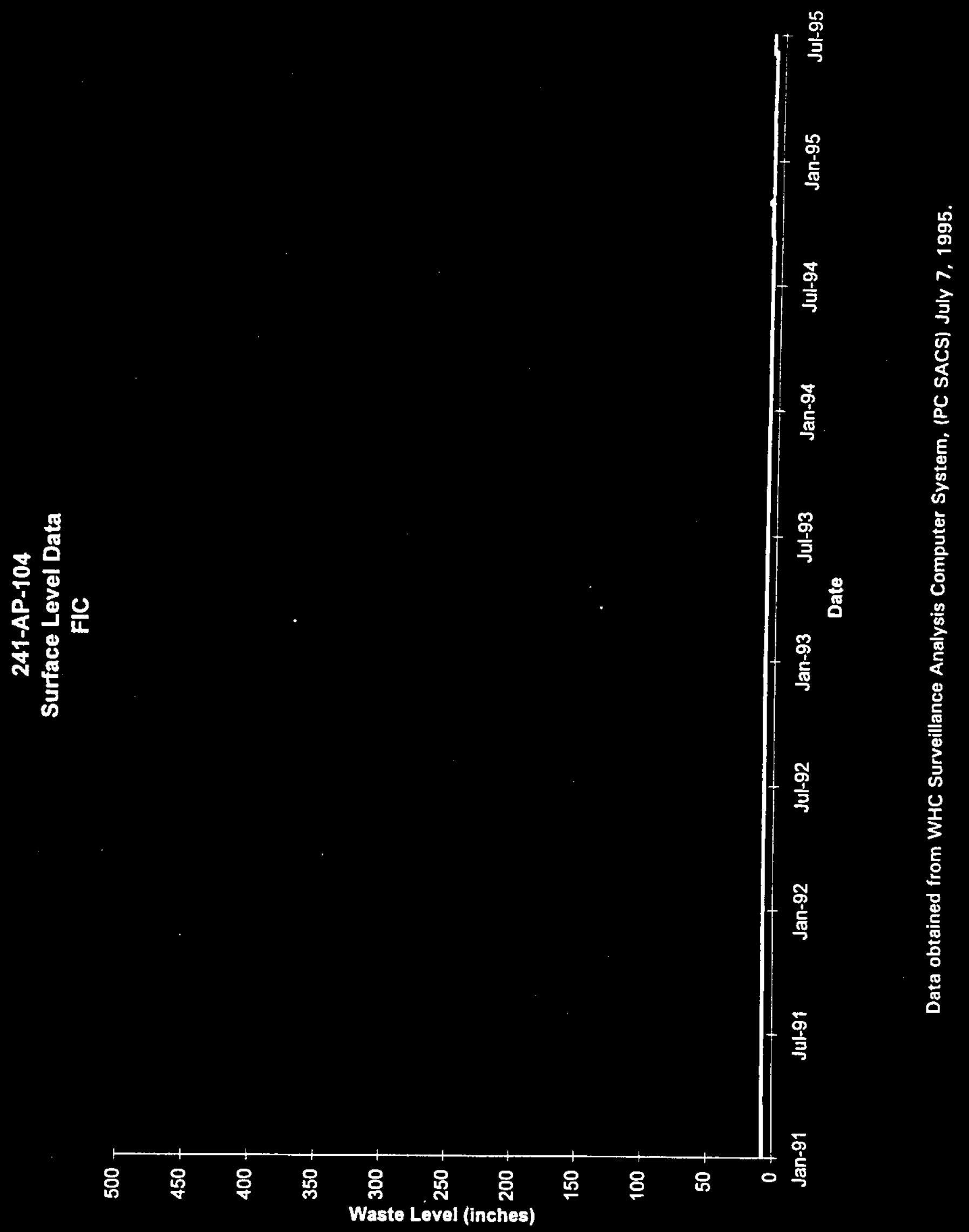


WHC-SD-WM-ER-315, Rev. 0

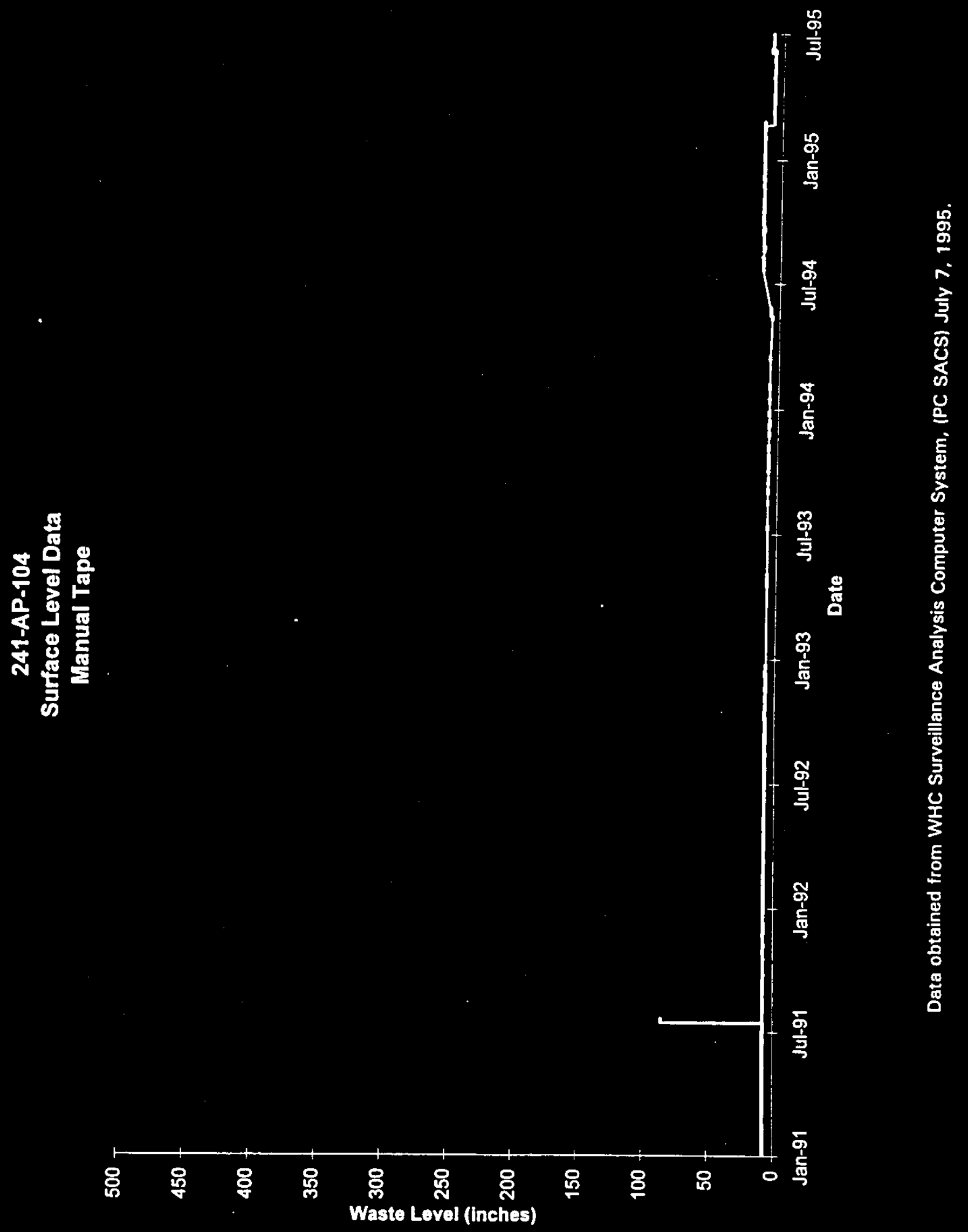

E-8 
WHC-SD-WM-ER-315, Rev . 0

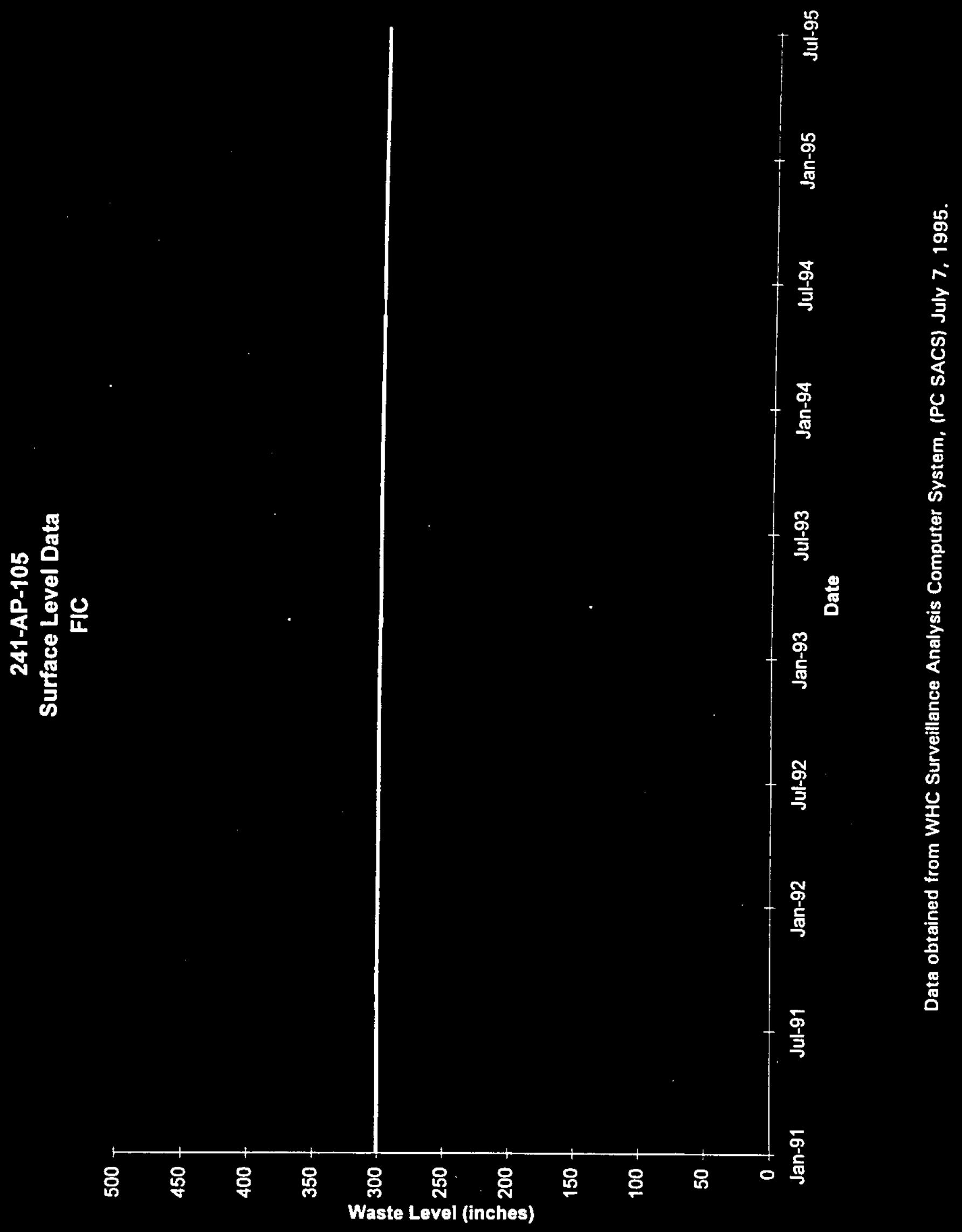

E-9 
WHC-SD-WM-ER-315, Rev. 0

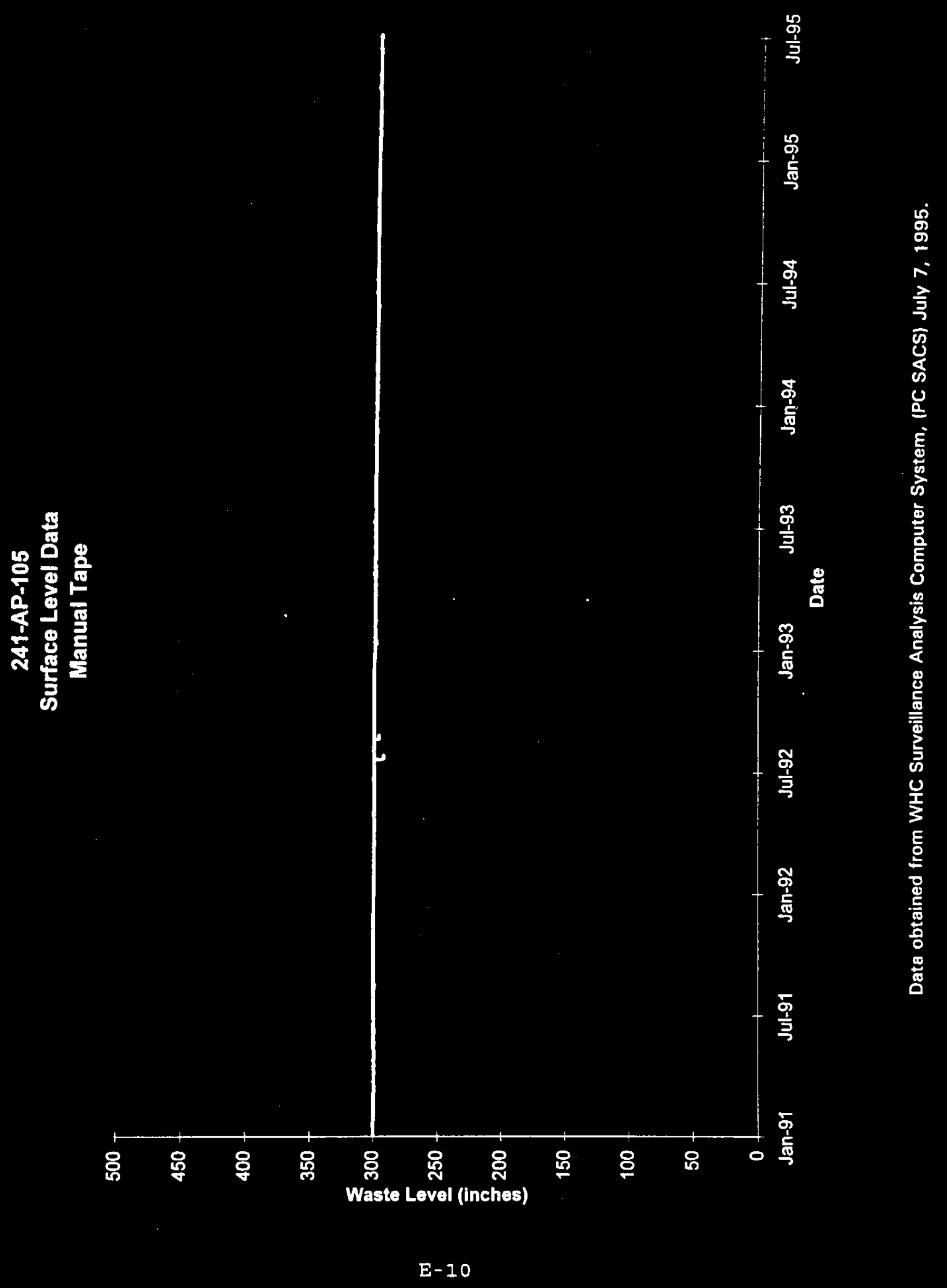


WHC-SD-WM-ER-315， Rev . 0

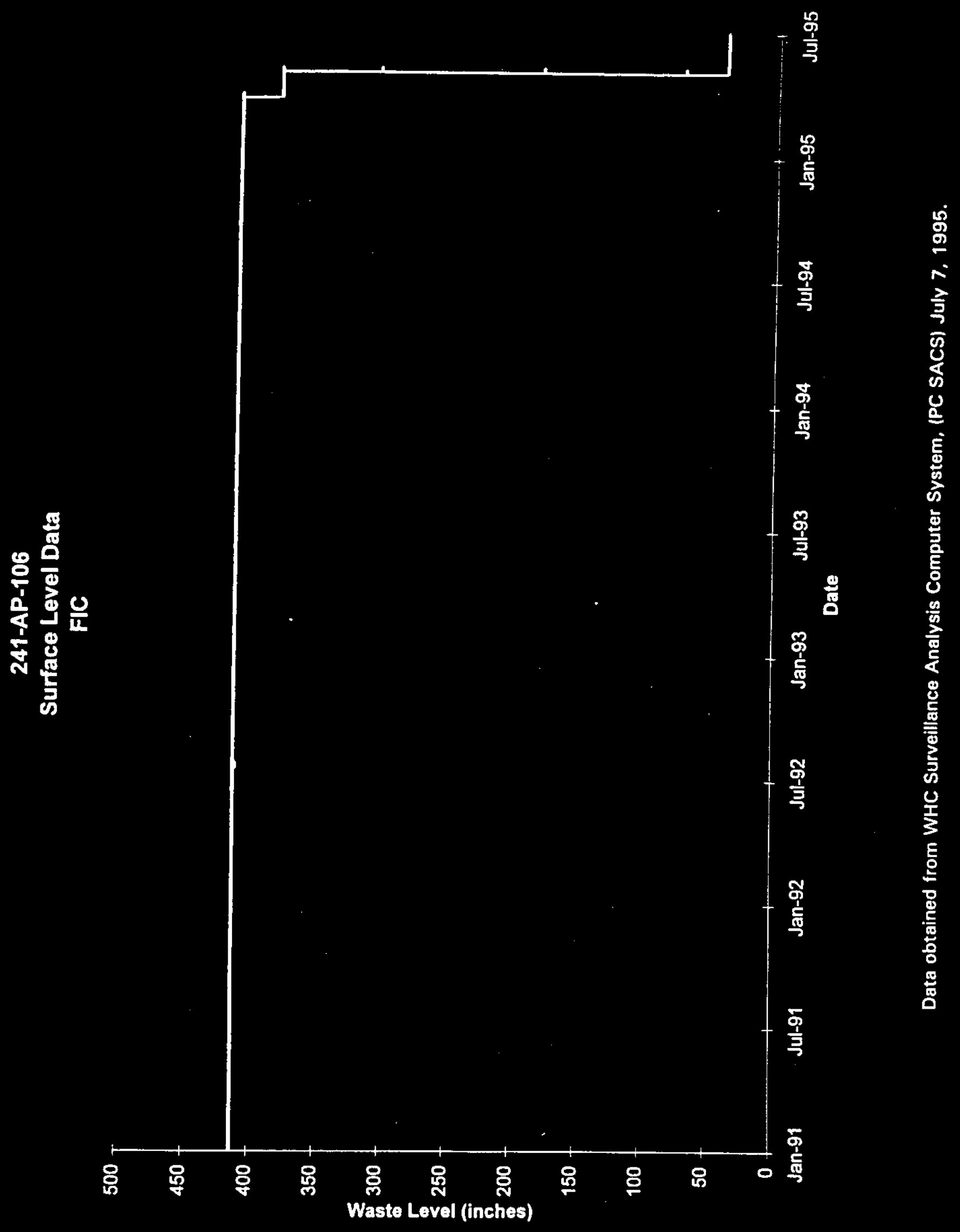

E-11 
WHC-SD-WM-ER-3I5， Rev . 0

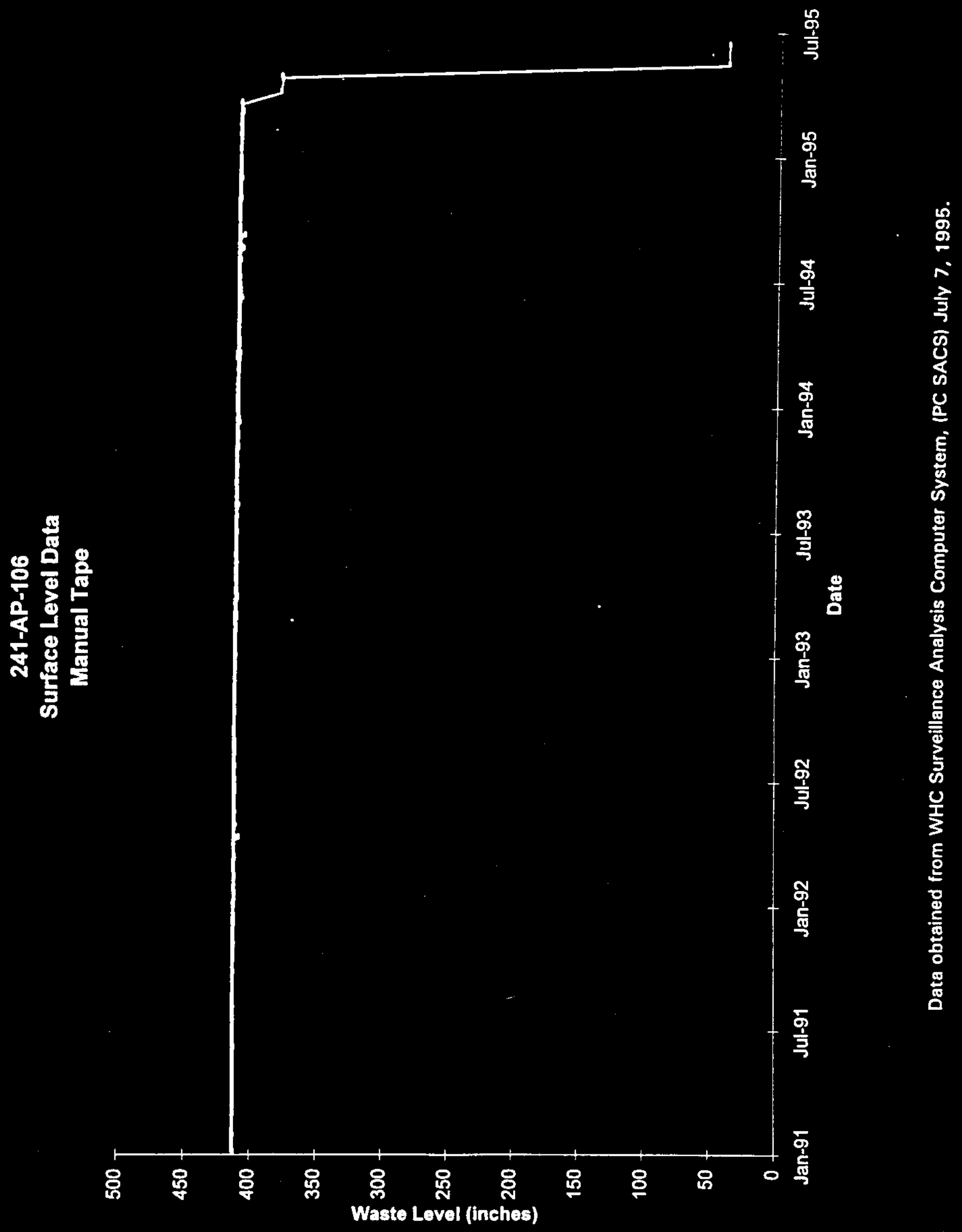


WHC-SD-WM-ER-315, Rev . 0

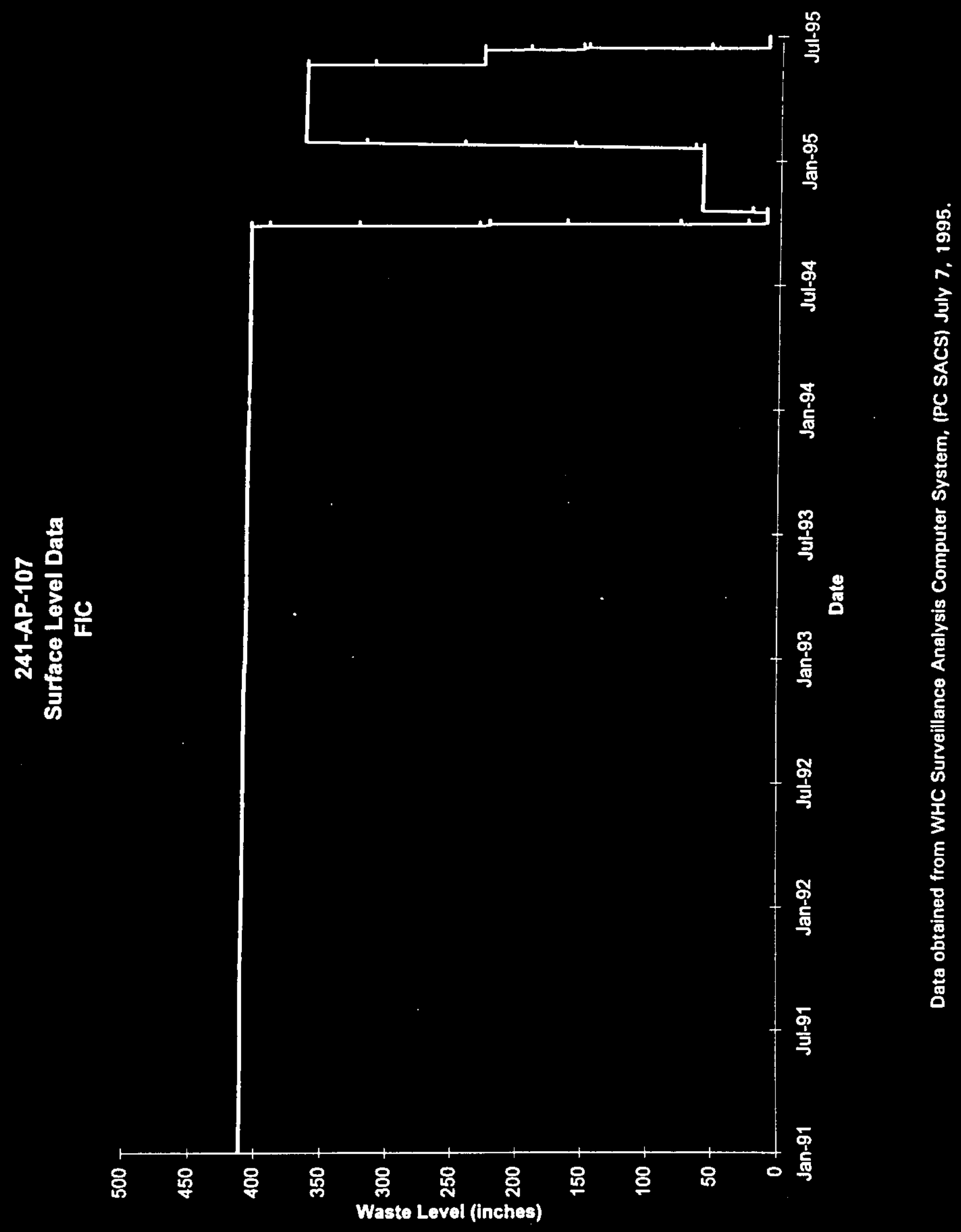


WHC-SD-WM-ER-315, Rev. 0

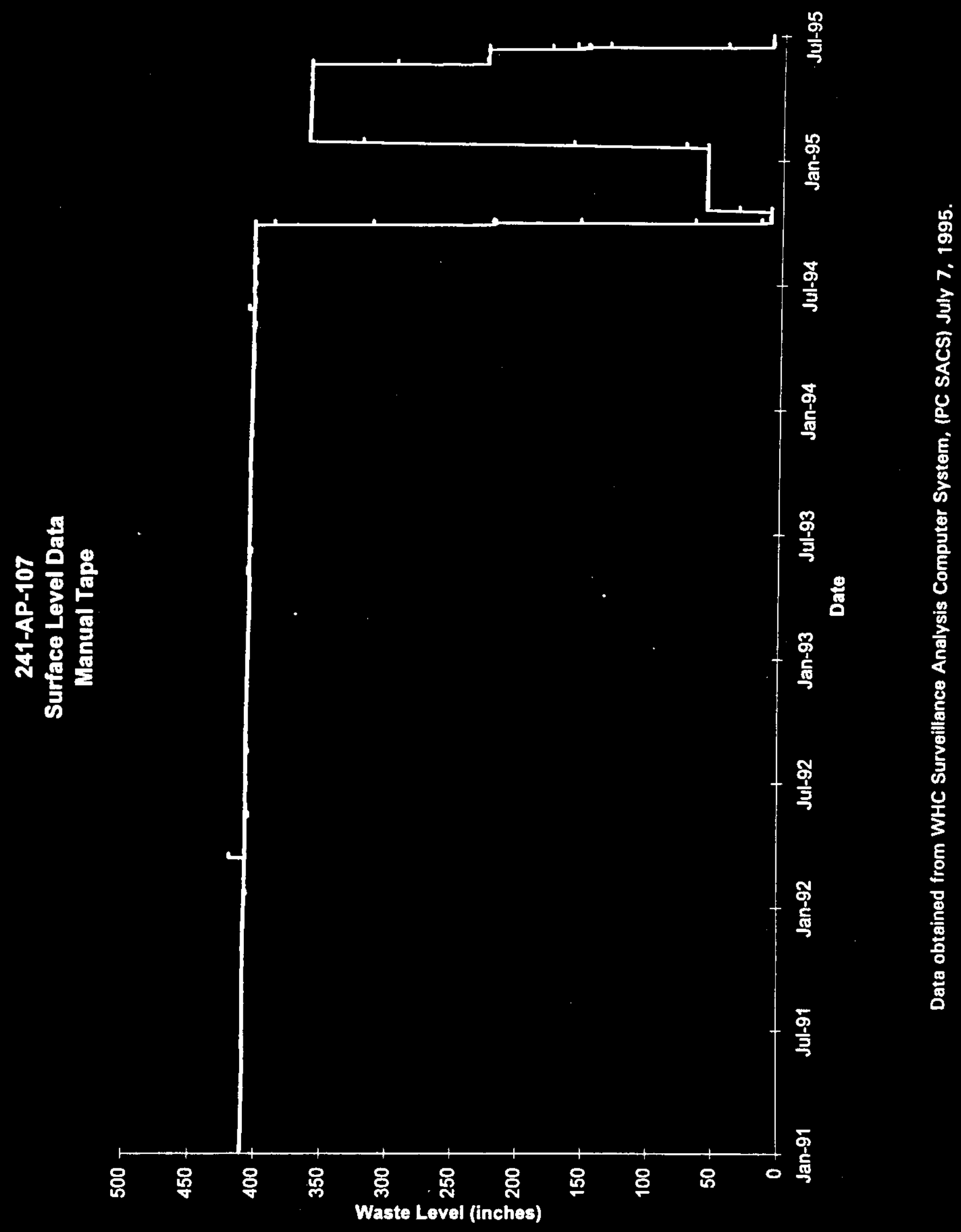

E-14 


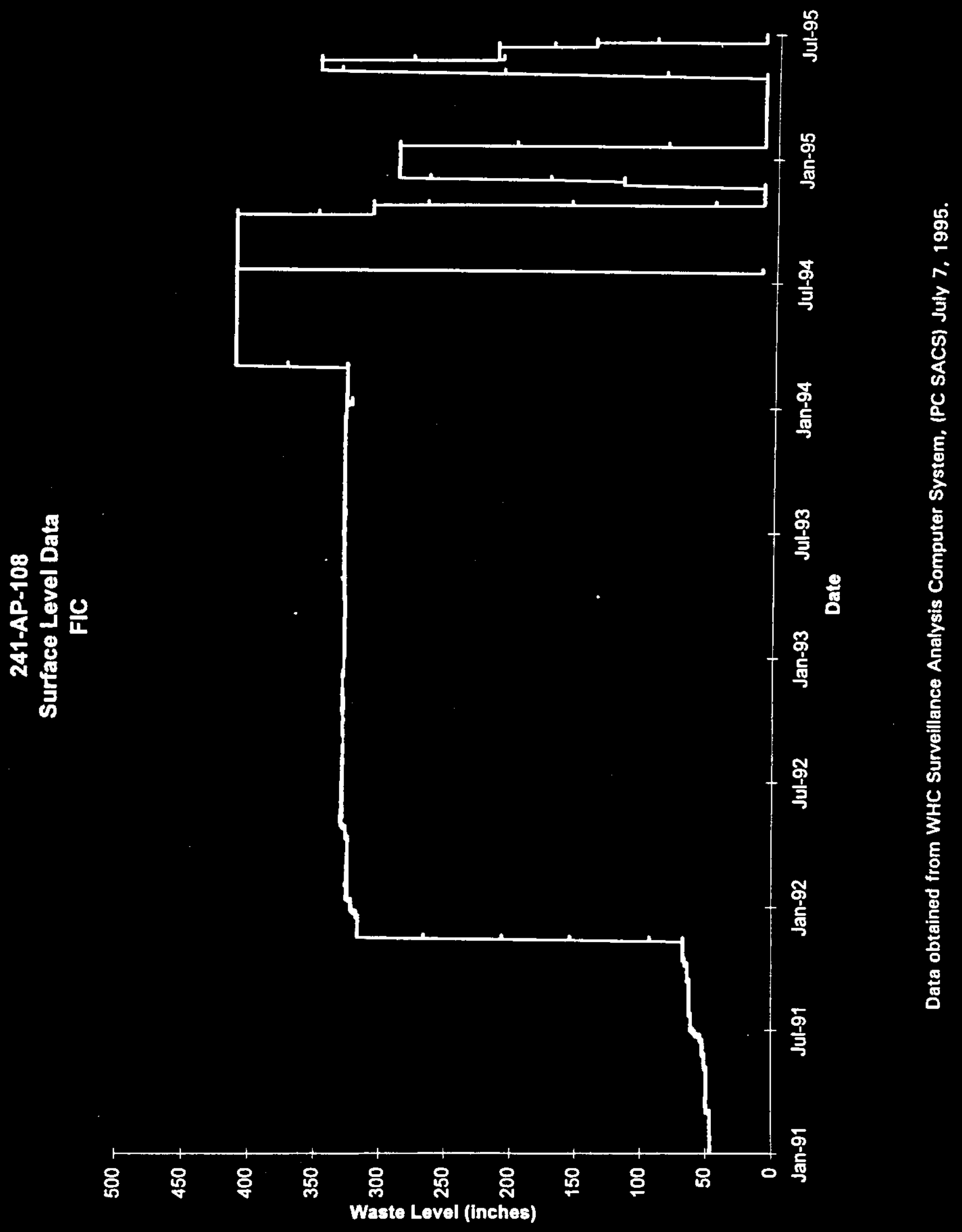

E-15 
WHC-SD-WM-ER-315, Rev. 0

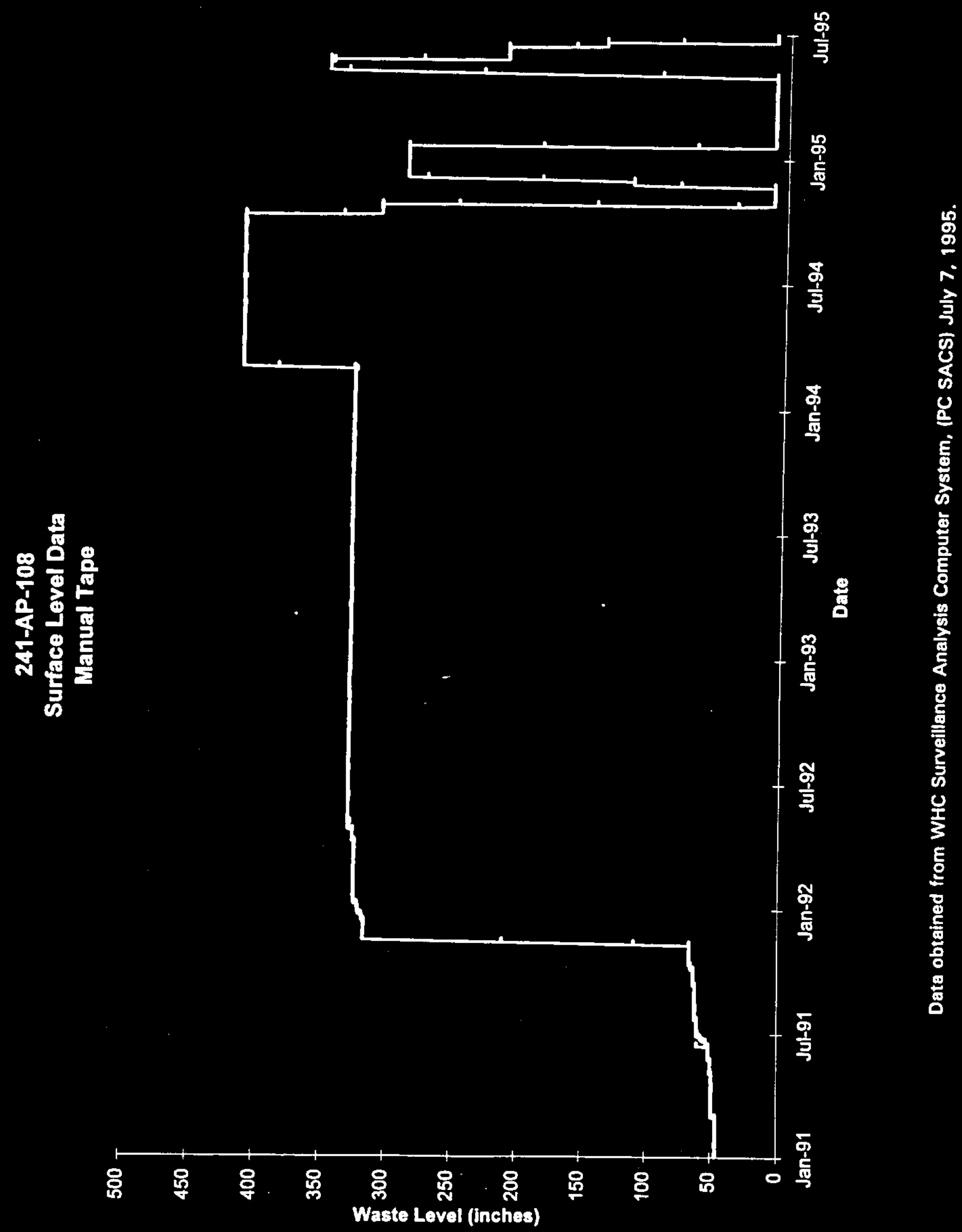

E-16 


\begin{tabular}{|c|c|c|c|c|}
\hline \multicolumn{5}{|c|}{ 241-AP-101 } \\
\hline \multicolumn{5}{|c|}{ Surface Lavel Data } \\
\hline Date & Type of Measurement & Leval (Inches) & Data Quality & Comments \\
\hline $1 / 1 / 910: 00$ & Manual Tape & 386.25 & Transcribed & \\
\hline 1/1/91 7:03 & Auto FIC & 386.8 & Good & \\
\hline $1 / 2 / 910: 00$ & Manual Tape & 386.5 & Transcribed & \\
\hline $1 / 2 / 917: 02$ & Auto FIC & 386.80 & Good & \\
\hline $1 / 3 / 910: 00$ & Manual Tape & 386.5 & Transcribed & \\
\hline $1 / 3 / 917: 03$ & Auto FIC & 386.8 & Good & \\
\hline $1 / 4 / 910: 00$ & Manual Tape & 386.50 & Transcribed & \\
\hline $1 / 4 / 917: 02$ & Auto FIC & 386.80 & Good & \\
\hline $1 / 5 / 910: 00$ & Manual Tape & 386.50 & Transcribed & \\
\hline 1/5/91 7:02 & Auto FIC & 386.8 & Good & \\
\hline $1 / 6 / 910: 00$ & Manual Tape & 386.5 & Transcribed & \\
\hline $1 / 6 / 917: 02$ & Auto FIC & 386.80 & Good & \\
\hline $1 / 7 / 910: 00$ & Manual Tape & 386.50 & Transcribed & \\
\hline $1 / 7 / 917: 02$ & Auto FIC & 386.8 & Good & \\
\hline $1 / 8 / 910: 00$ & Manual Tape & 386.50 & Transcribed & \\
\hline $1 / 8 / 917: 03$ & Auto FIC & 386.80 & Good & \\
\hline 1/9/91 0:00 & Manual Tape & 386.5 & Transcribed & \\
\hline $1 / 9 / 917: 02$ & Auto FIC & 386.8 & Good & \\
\hline $1 / 10 / 910: 00$ & Manual Tape & 386.5 & Transcribed & \\
\hline $1 / 10 / 917: 02$ & Auto FIC & 386.80 & Good & \\
\hline $1 / 11 / 910: 00$ & Manual Tape & 386.25 & Transcribed & \\
\hline $1 / 11 / 917: 03$ & Auto FIC & 386.8 & Good & \\
\hline $1 / 12 / 910: 00$ & Manual Tape & 386.50 & Transcribed & \\
\hline $1 / 12 / 917: 02$ & Auto FIC & 386.80 & Good & \\
\hline $1 / 13 / 910: 00$ & Manual Tape & 386.50 & Transçribed & \\
\hline $1 / 13 / 917: 03$ & Auto FIC & 386.8 & Good & \\
\hline $1 / 14 / 91 \quad 0: 00$ & Manual Tape & 386.5 & Transcribed & \\
\hline $1 / 14 / 917: 04$ & Auto FIC & 386.80 & Good & \\
\hline $1 / 15 / 910: 00$ & Manual Tape & 386.5 & Transcribed & \\
\hline $1 / 15 / 917: 03$ & Auto FIC & 386.8 & Good & \\
\hline $1 / 16 / 910: 00$ & Manual Tape & 386.50 & Transcribed & \\
\hline $1 / 16 / 917: 04$ & Auto FIC & 386.80 & Good & \\
\hline $1 / 17 / 910: 00$ & Manual Tape & 386.50 & Transcribed & \\
\hline $1 / 17 / 917: 04$ & Auto FIC & 386.8 & Good & \\
\hline $1 / 18 / 910: 00$ & Manual Tape & 386.5 & Transcribed & \\
\hline $1 / 18 / 917: 04$ & Auto FIC & 386.80 & Good & \\
\hline $1 / 19 / 910: 00$ & Manual Tape & 386.5 & Transcribed & \\
\hline $1 / 19 / 917: 04$ & Auto FIC & 386.7 & Good & \\
\hline $1 / 20 / 910: 00$ & Manual Tape & 386.50 & Transcribed & \\
\hline $1 / 20 / 917: 04$ & Auto FIC & 386.70 & Good & \\
\hline $1 / 21 / 910: 00$ & Manual Tape & 386.50 & Transcribed & \\
\hline $1 / 21 / 917: 04$ & Auto FIC & 386.7 & Good & \\
\hline $1 / 22 / 910: 00$ & Manual Tape & 386.5 & Transcribed & \\
\hline $1 / 22 / 917: 04$ & Auto FIC & 386.70 & Good & \\
\hline 1/23/91 0:00 & Manual Tape & 386.5 & Transcribed & \\
\hline $1 / 23 / 917: 04$ & Auto FIC & 386.7 & Good & \\
\hline $1 / 24 / 910: 00$ & Manual Tape & 386.5 & Transcribed & \\
\hline $1 / 24 / 917: 04$ & Auto FIC & 386.70 & Good & \\
\hline
\end{tabular}

Data obtained from WHC Surveillance Analysis Computer System, (PC SACS) July 7, 1995. 


\begin{tabular}{|c|c|c|c|c|}
\hline \multicolumn{5}{|c|}{ 241-AP-101 } \\
\hline \multicolumn{5}{|c|}{ Surface Level Data } \\
\hline Date & Type of Measurement & Level (Inches) & Data Quality & Comments \\
\hline $1 / 25 / 910: 00$ & Manual Tape & 386.5 & Transcribed & \\
\hline $1 / 25 / 917: 03$ & Auto FIC & 386.7 & Good & \\
\hline $1 / 26 / 910: 00$ & Manual Tape & 386.50 & Transcribed & \\
\hline $1 / 26 / 917: 03$ & Auto FIC & 386.70 & Good & \\
\hline $1 / 27 / 910: 00$ & Manual Tape & 386.50 & Transcribed & \\
\hline $1 / 27 / 917: 03$ & Auto FIC & 386.7 & Good & \\
\hline 1/28/91 0:00 & Manual Tape & 386.50 & Transcribed & \\
\hline $1 / 28 / 916: 03$ & Auto FIC & 386.70 & Good & \\
\hline $1 / 29 / 910: 00$ & Manual Tape & 386.5 & Transcribed & \\
\hline $1 / 29 / 91 \quad 7: 02$ & Auto FIC & 386.7 & Good & \\
\hline $1 / 30 / 910: 00$ & Manual FIC & 386.70 & Transcribed & \\
\hline 1/30/91 0:00 & Manual Tape & 386.50 & Transcribed & \\
\hline $1 / 31 / 910: 00$ & Manual Tape & 386.5 & Transcribed & \\
\hline $1 / 31 / 917: 02$ & Auto FIC & 386.70 & Good & \\
\hline 2/1/910:00 & Manual Tape & 386.50 & Transcribed & \\
\hline $2 / 1 / 917: 02$ & Auto FIC & 386.7 & Good & \\
\hline $2 / 2 / 910: 00$ & Manual Tape & 386.50 & Transcribed & \\
\hline 2/2/91 7:04 & Auto FIC & 386.70 & Good & \\
\hline 2/3/910:00 & Manual Tape & 386.5 & Transcribed & \\
\hline $2 / 3 / 917: 03$ & Auto FIC & 386.7 & Good & \\
\hline $2 / 4 / 910: 00$ & Manual Tape & 386.5 & Transçribed & \\
\hline $2 / 4 / 917: 03$ & Auto FIC & 386.70 & Good & \\
\hline $2 / 5 / 910: 00$ & Manual Tepe & 386.50 & Transcribed & \\
\hline $2 / 5 / 917: 04$ & Auto FIC & 386.7 & Good & \\
\hline $2 / 6 / 910: 00$ & Manual Tape & 386.50 & Transcribed & \\
\hline $2 / 6 / 917: 04$ & Auto FIC & 386.70 & Good & \\
\hline $2 / 7 / 910: 00$ & Manual Tape & 386.50 & Transcribed & \\
\hline $2 / 7 / 917: 04$ & Auto FIC & 386.7 & Good & \\
\hline $2 / 8 / 910: 00$ & Manual Tape & 386.5 & Transcribed & \\
\hline $2 / 8 / 917: 04$ & Auto FIC & 386.70 & Good & \\
\hline $2 / 9 / 910: 00$ & Manual Tape & 386.50 & Transcribed & \\
\hline $2 / 9 / 917: 04$ & Auto FIC & 386.7 & - Good & \\
\hline $2 / 10 / 910: 00$ & Manual Tape & 386.5 & Transcribed & \\
\hline $2 / 10 / 917: 03$ & Auto FIC & 386.70 & Good & \\
\hline $2 / 11 / 910: 00$ & Manual Tape & 386.50 & Transcribed & \\
\hline 2/11/91 7:04 & Auto FIC & 386.7 & Good & \\
\hline $2 / 12 / 910: 00$ & Manual Tape & 386.5 & Transcribed & \\
\hline 2/12/91 7:04 & Auto FIC & 386.70 & Good & \\
\hline $2 / 13 / 910: 00$ & Manual Tape & 386.5 & Transcribed & \\
\hline $2 / 13 / 917: 04$ & Auto FIC & 386.7 & Good & \\
\hline $2 / 14 / 910: 00$ & Manual Tape & 386.50 & Transcribed & \\
\hline $2 / 14 / 917: 04$ & Auto FIC & 386.70 & Good & \\
\hline $2 / 15 / 910: 00$ & Manual Tape & 386.50 & Transcribed & \\
\hline $2 / 15 / 917: 03$ & Auto FIC & 386.7 & Good & \\
\hline $2 / 16 / 910: 00$ & Manual Tape & 386.5 & Transcribed & \\
\hline $2 / 16 / 917: 02$ & Auto FIC & 386.70 & Good & \\
\hline $2 / 17 / 910: 00$ & Manual Tape & 386.50 & Transcribed & \\
\hline $2 / 17 / 917: 02$ & Auto FIC & 386.7 & Good & \\
\hline
\end{tabular}

Data obtained from WHC Surveillance Analysis Computer System, (PC SACS) July 7, 1995. 


\begin{tabular}{|c|c|c|c|c|}
\hline \multicolumn{5}{|c|}{ 241-AP-101 } \\
\hline \multicolumn{5}{|c|}{ Surface Level Data } \\
\hline Date & Type of Measurement & Leval (Inches) & Data Quality & Comments \\
\hline 2/18/91 0:00 & Manual Tape & 386.5 & Transcribed & \\
\hline $2 / 18 / 917: 03$ & Auto FIC & 386.70 & Good & \\
\hline 2/19/910:00 & Manual Tape & 386.5 & Transcribed & \\
\hline 2/19/91 7:02 & Auto FIC & 386.7 & Good & \\
\hline $2 / 20 / 910: 00$ & Manual Tape & 386.50 & Transcribed & \\
\hline 2/20/91 7:03 & Auto FIC & 386.70 & Good & \\
\hline $2 / 21 / 910: 00$ & Manual Tape & 386.50 & Transcribed & \\
\hline $2 / 21 / 917: 02$ & Auto FIC & 386.7 & Good & \\
\hline $2 / 22 / 910: 00$ & Manual Tape & 386.5 & Transcribed & \\
\hline $2 / 22 / 917: 02$ & Auto FIC & 386.70 & Good & \\
\hline $2 / 23 / 910: 00$ & Manual Tape & 386.50 & Transcribed & \\
\hline $2 / 23 / 917: 02$ & Auto FIC & 386.7 & Good & \\
\hline $2 / 24 / 910: 00$ & Manual Tape & 386.50 & Transcribed & \\
\hline $2 / 24 / 917: 03$ & Auto FIC & 386.70 & Good & \\
\hline $2 / 25 / 910: 00$ & Manual Tape & 386.5 & Transcribed & \\
\hline $2 / 25 / 917: 03$ & Auto FIC & 386.7 & Good & \\
\hline $2 / 26 / 910: 00$ & Manual Tape & 386.5 & Transcribed & \\
\hline $2 / 26 / 917: 03$ & Auto FIC & 386.7 & Good & \\
\hline $2 / 27 / 910: 00$ & Manual Tape & 386.50 & Transcribed & \\
\hline $2 / 27 / 917: 02$ & Auto FIC & 386.70 & Good & \\
\hline $2 / 28 / 910: 00$ & Manual Tape & 386.50 & Transcribed & \\
\hline $2 / 28 / 917: 02$ & Auto FIC & 386.7 & Good & \\
\hline 3/1/91 0:00 & Manual Tape & 386.50 & Transcribed & \\
\hline $3 / 1 / 917: 02$ & Auto FIC & 386.7 & Good & \\
\hline $3 / 2 / 910: 00$ & Manual Tape & 386.5 & Transcribed & \\
\hline $3 / 2 / 917: 02$ & Auto FIC & 386.70 & Good & \\
\hline $3 / 3 / 910: 00$ & Manual Tape & 386.5 & Transcribed & \\
\hline $3 / 3 / 917: 03$ & Auto FIC & 386.7 & Good & \\
\hline $3 / 4 / 910: 00$ & Manual Tape & 386.50 & Transcribed & \\
\hline $3 / 4 / 917: 03$ & Auto FIC & 386.70 & Good & \\
\hline $3 / 5 / 910: 00$ & Manual Tape & 386.50 & Transcribed & \\
\hline $3 / 5 / 917: 03$ & Auto FIC & 386.7 & Good & \\
\hline $3 / 6 / 910: 00$ & Manual Tape & 386.5 & Transcribed & \\
\hline $3 / 6 / 917: 02$ & Auto FIC & 386.70 & Good & \\
\hline $3 / 7 / 910: 00$ & Manual Tape & 386.25 & Transćribed & \\
\hline $3 / 7 / 917: 02$ & Auto FIC & 386.70 & Good & \\
\hline $3 / 8 / 910: 00$ & Manual Tape & 386.25 & Transcribed & \\
\hline $3 / 8 / 917: 02$ & Auto FIC & 386.6 & Good & \\
\hline $3 / 9 / 910: 00$ & Manual Tape & 386.25 & Transcribed & \\
\hline $3 / 9 / 917: 02$ & Auto FIC & 386.60 & Good & \\
\hline $3 / 10 / 910: 00$ & Manual Tape & 386.25 & Transcribed & \\
\hline $3 / 10 / 917: 02$ & Auto FIC & 386.6 & Good & \\
\hline $3 / 11 / 910: 00$ & Manual Tape & 386.25 & Transçribed & \\
\hline $3 / 11 / 917: 02$ & Auto FIC & 386.60 & Good & \\
\hline $3 / 12 / 910: 00$ & Manual Tape & 386.25 & Transcribed & \\
\hline $3 / 12 / 917: 03$ & Auto FIC & 386.60 & Good & \\
\hline $3 / 13 / 910: 00$ & Manual Tape & 386.25 & Transcribed & \\
\hline $3 / 13 / 917: 03$ & Auto FIC & 386.6 & Good & \\
\hline
\end{tabular}

Data obtained from WHC Surveillance Analysis Computer System, (PC SACS) July 7, 1995. 


\begin{tabular}{|c|c|c|c|c|}
\hline \multicolumn{5}{|c|}{ 241-AP-101 } \\
\hline \multicolumn{5}{|c|}{ Surface Level Data } \\
\hline Date & Type of Mesasurement & Level (Inches) & Data Quality & Comments \\
\hline $3 / 14 / 910: 00$ & Manual Tape & 386.25 & Transcribed & \\
\hline $3 / 14 / 917: 03$ & Auto FIC & 386.60 & Good & \\
\hline $3 / 15 / 910: 00$ & Manual Tape & 386.25 & Transcribed & \\
\hline 3/15/91 7:03 & Auto FIC & 386.60 & Good & \\
\hline $3 / 16 / 910: 00$ & Manual Tape & 386.25 & Transcribed & \\
\hline $3 / 16 / 917: 03$ & Auto FIC & 386.6 & Good & \\
\hline $3 / 17 / 910: 00$ & Manual Tape & 386.25 & Transcribed & \\
\hline $3 / 17 / 917: 02$ & Auto FIC & 386.60 & Good & \\
\hline $3 / 18 / 910: 00$ & Manual Tape & 386.25 & Transcribed & \\
\hline $3 / 18 / 917: 03$ & Auto FIC & 386.60 & Good & \\
\hline $3 / 19 / 910: 00$ & Manual Tape & 386.25 & Transcribed & \\
\hline 3/19/91 7:03 & Auto FIC & 386.6 & Good & \\
\hline $3 / 20 / 910: 00$ & Manual Tape & 386.25 & Transcribed & \\
\hline $3 / 20 / 917: 02$ & Auto FIC & 386.60 & Good & \\
\hline $3 / 21 / 910: 00$ & Manual Tape & 386.50 & Transcribed & \\
\hline $3 / 21 / 917: 03$ & Auto FIC & 386.6 & Good & \\
\hline $3 / 22 / 910: 00$ & Manual Tape & 386.5 & Transcribed & \\
\hline $3 / 22 / 917: 02$ & Auto FIC & 386.60 & Good & \\
\hline $3 / 23 / 910: 00$ & Manual Tape & 386.5 & Transcribed & \\
\hline $3 / 23 / 917: 03$ & Auto FIC & 386.6 & Good & \\
\hline $3 / 24 / 910: 00$ & Manual Tape & 386.50 & Transcribed & \\
\hline $3 / 24 / 917: 03$ & Auto FIC & 386.60 & Good & \\
\hline $3 / 25 / 910: 00$ & Manual Tape & 386.50 & Transeribed & \\
\hline $3 / 25 / 917: 03$ & Auto FIC & 386.6 & Good & \\
\hline $3 / 26 / 910: 00$ & Manual Tape & 386.5 & Transcribed & \\
\hline $3 / 26 / 917: 03$ & Auto FIC & 386.90 & Good & \\
\hline $3 / 27 / 910: 00$ & Manual Tape & 386.5 & Transcribed & \\
\hline $3 / 27 / 917: 03$ & Auto FIC & 386.6 & Good & \\
\hline $3 / 28 / 910: 00$ & Manual Tape & 386.50 & Transcribed & \\
\hline $3 / 28 / 917: 03$ & Auto FIC & 386.60 & Good & \\
\hline $3 / 29 / 910: 00$ & Manual Tape & 386.5 & Transcribed & \\
\hline $3 / 29 / 917: 03$ & Auto FIC & 386.6 & Good & \\
\hline $3 / 30 / 910: 00$ & Manual Tape & 386.5 & Transcribed & \\
\hline $3 / 30 / 917: 03$ & Auto FIC & 386.60 & Good & \\
\hline $3 / 31 / 910: 00$ & Manual Tape & 386.5 & Transcribed & \\
\hline $3 / 31 / 917: 03$ & Auto FIC & 386.6 & Good & \\
\hline 4/1/91 $0: 00$ & Manual Tape & 386.50 & Transeribed & \\
\hline 4/1/91 7:03 & Auto FIC & 386.60 & Good & \\
\hline $4 / 2 / 910: 00$ & Manual Tape & 386.5 & Transcribed & \\
\hline $4 / 2 / 917: 03$ & Auto FIC & 386.6 & Gopd & \\
\hline $4 / 3 / 910: 00$ & Manual Tape & 386.5 & Transcribed & \\
\hline 4/3/91 7:03 & Auto FIC & 386.60 & Good & \\
\hline $4 / 4 / 910: 00$ & Manual Tape & 386.25 & Transcribed & \\
\hline 4/4/91 7:03 & Auto FIC & 386.6 & Good & \\
\hline $4 / 5 / 910: 00$ & Manual Tape & 386.25 & Transcribed & \\
\hline $4 / 5 / 917: 03$ & Auto FIC & 386.60 & Good & \\
\hline $4 / 6 / 910: 00$ & Manual Tape & 386.50 & Transcribed & \\
\hline $4 / 6 / 917: 03$ & Auto FIC & 386.6 & Good & \\
\hline
\end{tabular}

Data obtained from WHC Surveillance Analysis Computer System, (PC SACS) July 7, 1995. 


\begin{tabular}{|c|c|c|c|c|}
\hline \multicolumn{5}{|c|}{ 241-AP-101 } \\
\hline & 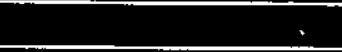 & Jrface Level Dat & & \\
\hline Date & Type of Measurement & Leval (inches) & Data Quality & Comments \\
\hline 4/7/91 $0: 00$ & Manual Tape & 386.25 & Transcribed & \\
\hline $4 / 7 / 917: 03$ & Auto FIC & 386.60 & Good & \\
\hline $4 / 8 / 910: 00$ & Manual Tape & 386.25 & Transcribed & \\
\hline 4/8/91 7:04 & Auto FIC & 386.6 & Good & \\
\hline 4/9/910:00 & Manual Tape & 386.25 & Transcribed & \\
\hline $4 / 9 / 917: 03$ & Auto FIC & 386.60 & Good & \\
\hline $4 / 10 / 910: 00$ & Manual Tape & 386.50 & Transcribed & \\
\hline 4/10/917:03 & Auto FIC & 386.6 & Good & \\
\hline $4 / 11 / 910: 00$ & Manual Tape & 386.5 & Transcribed & \\
\hline $4 / 11 / 917: 03$ & Auto FIC & 386.60 & Good & \\
\hline 4/12/910:00 & Manual Tape & 386.25 & Transcribed & \\
\hline 4/12/91 7:03 & Auto FIC & 386.6 & Good & \\
\hline 4/13/91 0:00 & Manual Tape & 386.5 & Transcribed & \\
\hline $4 / 13 / 917: 04$ & Auto FIC & 386.60 & Good & \\
\hline $4 / 14 / 910: 00$ & Manual Tape & 386.50 & Transcribed & \\
\hline $4 / 14 / 917: 03$ & Auto FIC & 386.6 & Good & \\
\hline $4 / 15 / 910: 00$ & Manual Tape & 386.50 & Transcribed & \\
\hline $4 / 15 / 917: 03$ & Auto FIC & 386.60 & Good & \\
\hline $4 / 16 / 910: 00$ & Manual Tape & 386.5 & Transcribed & \\
\hline $4 / 16 / 910: 00$ & Manual FIC & 386.6 & Good & \\
\hline $4 / 17 / 91 \quad 0: 00$ & Manual Tape & 386.25 & Transcribed & \\
\hline $4 / 17 / 917: 03$ & Auto FIC & 386.6 & Good & \\
\hline $4 / 18 / 910: 00$ & Manual Tape & 386.25 & Transcribed & \\
\hline $4 / 18 / 917: 06$ & Auto FIC & 386.60 & Good & \\
\hline $4 / 19 / 910: 00$ & Manual Tape & 386.25 & Transcribed & \\
\hline $4 / 19 / 917: 05$ & Auto FIC & 386.6 & Good & \\
\hline $4 / 20 / 910: 00$ & Manual Tape & 386.5 & Transcribed & \\
\hline $4 / 20 / 917: 06$ & Auto FIC & 386.60 & Good & \\
\hline 4/21/91 $0: 00$ & Manual Tape & 386.5 & Transcribed & \\
\hline $4 / 21 / 917: 05$ & Auto FIC & 386.6 & Good & \\
\hline 4/22/910:00 & Manual Tape & 386.5 & Transcribed & \\
\hline 4/22/91 7:06 & Auto FIC & 386.60 & Good & \\
\hline $4 / 23 / 910: 00$ & Manual Tape & 386.5 & Transcribed & \\
\hline 4/23/917:06 & Auto FIC & 386.6 & Good & \\
\hline 4/24/910:00 & Manual Tape & 386.5 & Transcribed & \\
\hline 4/24/91 7:05 & Auto FIC & 386.60 & Good & \\
\hline $4 / 25 / 910: 00$ & Manual Tape & 386.5 & Transcribed & \\
\hline 4/25/91 7:05 & Auto FIC & 386.6 & Good & \\
\hline $4 / 26 / 910: 00$ & Manual Tape & 386.5 & Transcribed & \\
\hline 4/26/91 7:05 & Auto FIC & 386.60 & Good & \\
\hline 4/27/91 0:00 & Manual Tape & 386.5 & Transcribed & \\
\hline 4/27/91 7:05 & Auto FIC & 386.6 & Good & \\
\hline $4 / 28 / 910: 00$ & Manual Tape & 386.25 & Transcribed & \\
\hline 4/28/91 7:06 & Auto FIC & 386.60 & Good & \\
\hline 4/29/91 0:00 & Manual Tape & 386.25 & Transcribed & \\
\hline 4/29/91 7:05 & Auto FIC & 386.6 & Good & \\
\hline 4/30/910:00 & Manual Tape & 386.5 & Transcribed & \\
\hline 4/30/91 7:06 & Auto FIC & 386.60 & Good & \\
\hline
\end{tabular}

Data obtained from WHC Surveillance Analysis Computer System, (PC SACS) July 7, 1995. 


\begin{tabular}{|c|c|c|c|c|}
\hline \multicolumn{5}{|c|}{ 241-AP-101 } \\
\hline Date & Type of Measurement & Leval (Inches) & Date Quality & Comments \\
\hline $5 / 1 / 910: 00$ & Manual Tape & 386.5 & Transcribed & \\
\hline $5 / 1 / 917: 05$ & Auto FIC & 386.6 & Good & \\
\hline $5 / 2 / 910: 00$ & Manual Tape & 386.5 & Transcribed & \\
\hline $5 / 2 / 917: 05$ & Auto FIC & 386.60 & Good & \\
\hline $5 / 3 / 910: 00$ & Manual Tape & 386.5 & Transcribed & \\
\hline $5 / 3 / 917: 06$ & Auto FIC & 386.6 & Good & \\
\hline $5 / 4 / 910: 00$ & Manual Tape & 386.50 & Transcribed & \\
\hline $5 / 4 / 917: 05$ & Auto FIC & 386.60 & Good & \\
\hline $5 / 5 / 910: 00$ & Manual Tape & 386.5 & Transcribed & \\
\hline $5 / 5 / 917: 05$ & Auto FIC & 386.6 & Good & \\
\hline $5 / 6 / 910: 00$ & Manual Tape & 386.50 & Transcribed & \\
\hline $5 / 6 / 917: 05$ & Auto FIC & 386.60 & Good & \\
\hline $5 / 7 / 910: 00$ & Manual Tape & 386.5 & Transcribed & \\
\hline $5 / 7 / 917: 05$ & Auto FIC & 386.6 & Good & \\
\hline $5 / 8 / 910: 00$ & Manual Tape & 386.50 & Transcribed & \\
\hline $5 / 8 / 917: 05$ & Auto FIC & 386.60 & Good & \\
\hline $5 / 9 / 910: 00$ & Manual Tape & 386.5 & Transcribed & \\
\hline 5/9/91 7:05 & Auto FIC & 386.6 & Good & \\
\hline $5 / 10 / 910: 00$ & Manual Tape & 386.50 & Transcribed & \\
\hline $5 / 10 / 917: 05$ & Auto FIC & 386.60 & Good & \\
\hline $5 / 11 / 910: 00$ & Manual Tape & 386.25 & Transcribed & \\
\hline $5 / 11 / 917: 05$ & Auto FIC & 386.6 & Good & \\
\hline $5 / 12 / 910: 00$ & Manual Tape & 386.50 & Transcribed & \\
\hline 5/12/91 7:05 & Auto FIC & 386.60 & Good & \\
\hline $5 / 13 / 910: 00$ & Manual Tape & 386.5 & Transcribed & \\
\hline $5 / 13 / 917: 05$ & Auto FIC & 386.6 & Good & \\
\hline 5/14/91 0:00 & Manual Tape & 386.50 & Transcribed & \\
\hline $5 / 14 / 91 \quad 7: 05$ & Auto FIC & 386.60 & Good & \\
\hline $5 / 15 / 910: 00$ & Manual Tape & 386.5 & Transcribed & . \\
\hline $5 / 15 / 917: 05$ & Auto FIC & 386.6 & Good & \\
\hline $5 / 16 / 910: 00$ & Manual Tape & 386.50 & Transcribed & \\
\hline $5 / 16 / 917: 06$ & Auto FIC & 386.60 & Good & \\
\hline $5 / 17 / 91 \quad 0: 00$ & Manual Tape & 386.5 & Transcribed & \\
\hline $5 / 17 / 917: 06$ & Auto FIC & 386.6 & Good & \\
\hline 5/18/91 0:00 & Manual Tape & 386.25 & Transcribed & \\
\hline $5 / 18 / 917: 06$ & Auto FIC & 386.60 & Good & \\
\hline $5 / 19 / 910: 00$ & Manual Tape & 386.25 & Transcribed & \\
\hline $5 / 19 / 917: 05$ & Auto FIC & 386.6 & Good & \\
\hline $5 / 20 / 910: 00$ & Manual Tape & 386.25 & Transcribed & \\
\hline $5 / 20 / 917: 06$ & Auto FIC & 386.60 & Good & \\
\hline $5 / 21 / 910: 00$ & Manual Tape & 386.25 & Transcribed & \\
\hline $5 / 21 / 91 \quad 7: 06$ & Auto FIC & 386.6 & Good & \\
\hline $5 / 22 / 910: 00$ & Manual Tape & 386.25 & Transcribed & \\
\hline $5 / 22 / 917: 05$ & Auto FIC & 386.60 & Good & \\
\hline 5/23/91 $0: 00$ & Manual Tape & 386.25 & Transcribed & \\
\hline $5 / 23 / 917: 05$ & Auto FIC & 386.6 & Good & \\
\hline 5/24/91 0:00 & Manual Tape & 386.25 & Transcribed & \\
\hline $5 / 24 / 917: 05$ & Auto FIC & 386.60 & Good & \\
\hline
\end{tabular}

Data obtained from WHC Surveillance Analysis Computer System, (PC SACS) July 7, 1995. 


\begin{tabular}{|c|c|c|c|c|}
\hline \multicolumn{5}{|c|}{ 241-AP-101 } \\
\hline \multicolumn{5}{|c|}{ Surface Level Data } \\
\hline Date & Type of Measurement & Level (Inches) & Data Quality & Comments \\
\hline $5 / 25 / 910: 00$ & Manual Tape & 386.25 & Transcribed & \\
\hline $5 / 25 / 917: 05$ & Auto FIC & 386.5 & Good & \\
\hline $5 / 26 / 910: 00$ & Manual Tape & 386.25 & Transcribed & \\
\hline 5/26/91 7:05 & Auto FIC & 386.50 & Good & \\
\hline $5 / 27 / 910: 00$ & Manual Tape & 386.25 & Transcribed & \\
\hline $5 / 27 / 917: 05$ & Auto FIC & 386.5 & Good & \\
\hline $5 / 28 / 910: 00$ & Manual Tape & 386.25 & Transcribed & \\
\hline $5 / 28 / 917: 05$ & Auto FIC & 386.50 & Good & \\
\hline $5 / 29 / 910: 00$ & Manual Tape & 386.5 & Transcribed & \\
\hline $5 / 29 / 917: 05$ & Auto FIC & 386.5 & Good & \\
\hline $5 / 30 / 910: 00$ & Manual Tape & 386.25 & Transcribed & \\
\hline $5 / 30 / 917: 05$ & Auto FIC & 386.50 & Good & \\
\hline $5 / 31 / 910: 00$ & Manual Tape & 386.25 & Transcribed & \\
\hline $5 / 31 / 917: 05$ & Auto FIC & 386.5 & Good & \\
\hline $6 / 1 / 910: 00$ & Manual Tape & 386.25 & Transcribed & \\
\hline $6 / 1 / 916: 05$ & Auto FIC & 386.50 & Good & \\
\hline $6 / 2 / 910: 00$ & Manual Tape & 386.25 & Transcribed & \\
\hline $6 / 2 / 913: 05$ & Auto FIC & 386.5 & Good & \\
\hline 6/3/91 0:00 & Manual Tape & 386.25 & Transcribed & \\
\hline $6 / 3 / 917: 05$ & Auto FIC & 386.50 & Good & \\
\hline $6 / 4 / 910: 00$ & Manual Tape & 386 & Transcribed & \\
\hline $6 / 4 / 917: 05$ & Auto FIC & 386.7 & Good & \\
\hline $6 / 5 / 910: 00$ & Manual Tape & 386.25 & Transcribed & \\
\hline $6 / 5 / 917: 05$ & Auto FIC & 386.70 & Good & \\
\hline $6 / 6 / 910: 00$ & Manual Tape & 386.5 & Transcribed & \\
\hline $6 / 6 / 917: 06$ & Auto FIC & 386.8 & Good & \\
\hline 6/7/91 0:00 & Manual Tape & 386.25 & Transcribed & \\
\hline 6/7/91 7:05 & Auto FIC & 386.80 & Good & \\
\hline $6 / 8 / 910: 00$ & Manual Tape & 386.5 & Transcribed & $\therefore$ \\
\hline 6/8/91 2:05 & Auto FIC & 386.7 & Good & \\
\hline 6/9/91 0:00 & Manual FIC & 386.80 & Transcribed & \\
\hline $6 / 9 / 910: 00$ & Manual Tape & 386.50 & Transcribed & \\
\hline $6 / 9 / 917: 05$ & Auto FIC & 0.00 & Suspect & Data not included in graph \\
\hline $6 / 10 / 910: 00$ & Manual Tape & 386.25 & Transcribed & \\
\hline $6 / 10 / 915: 05$ & Auto FIC & 386.2 & Good & \\
\hline $6 / 11 / 910: 00$ & Manual Tape & 386.25 & Transcribed & \\
\hline $6 / 11 / 912: 05$ & Auto FIC & 386.20 & Good & \\
\hline $6 / 12 / 910: 00$ & Manual Tape & 386.25 & Transcribed & \\
\hline $6 / 12 / 914: 05$ & Auto FIC & 386.2 & Good & \\
\hline $6 / 13 / 910: 00$ & Manual Tape & 386.25 & Transcribed & \\
\hline $6 / 13 / 917: 05$ & Auto FIC & 0.00 & Suspect & Data not included in graph \\
\hline $6 / 14 / 910: 00$ & Manual Tape & 386.25 & Transcribed & \\
\hline $6 / 14 / 917: 05$ & Auto FIC & 0 & Suspect & Data not included in graph \\
\hline $6 / 15 / 910: 00$ & Manual Tape & 386.25 & Transcribed & \\
\hline $6 / 15 / 917: 05$ & Auto FIC & 0.00 & Suspect & Data not included in graph \\
\hline 6/16/91 0:00 & Manual Tape & 386.25 & Transcribed & \\
\hline $6 / 16 / 910: 00$ & Manual FIC & 386.2 & Gopd & \\
\hline $6 / 16 / 917: 05$ & Auto FIC & 0 & Suspect & Data not included in graph \\
\hline
\end{tabular}

Data obtained from WHC Surveillance Analysis Computer System, (PC SACS) July 7, 1995. 


\begin{tabular}{|c|c|c|c|c|}
\hline \multicolumn{5}{|c|}{ 241-AP-101 } \\
\hline \multicolumn{5}{|c|}{ Surface Level Data } \\
\hline Date & Type of Measurement & Lovel (Inches) & Data Quality & Comments \\
\hline $6 / 17 / 910: 00$ & Manual Tape & 386.25 & Transcribed & \\
\hline $6 / 17 / 910: 00$ & Manual FIC & 386.20 & Good & \\
\hline $6 / 17 / 917: 05$ & Auto FIC & 0.00 & Suspect & Data not included in graph \\
\hline $6 / 18 / 910: 00$ & Manual Tape & 386.25 & Transcribed & \\
\hline 6/18/91 0:00 & Manual FIC & 386.2 & Good & \\
\hline $6 / 18 / 917: 05$ & Auto FIC & 0 & Suspect & Data not included in graph \\
\hline $6 / 19 / 910: 00$ & Manual Tape. & 386.25 & Transcribed & \\
\hline 6/19/91 1:05 & Auto FIC & 386.40 & Good & \\
\hline $6 / 20 / 910: 00$ & Manual Tape & 386.25 & Transcribed & \\
\hline $6 / 20 / 917: 05$ & Auto FIC & 386.5 & Good & \\
\hline $6 / 21 / 910: 00$ & Manual Tape & 386.25 & Transcribed & \\
\hline $6 / 21 / 917: 06$ & Auto FIC & 386.50 & Good & \\
\hline $6 / 22 / 910: 00$ & Manual Tape & 386.25 & Transcribed & \\
\hline $6 / 22 / 913: 06$ & Auto FIC & 386.5 & Good & \\
\hline $6 / 23 / 910: 00$ & Manual Tape & 386.5 & Transcribed & \\
\hline $6 / 23 / 917: 05$ & Auto FIC & 386.50 & Good & \\
\hline $6 / 24 / 910: 00$ & Manual Tape & 386.5 & Transcribed & \\
\hline $6 / 24 / 915: 05$ & Auto FIC & 386.5 & Good & \\
\hline $6 / 25 / 910: 00$ & Manual Tape & $386: 50$ & Transcribed & \\
\hline $6 / 25 / 917: 06$ & Auto FIC & 386.50 & Good & \\
\hline $6 / 26 / 910: 00$ & Manual Tape & 386.50 & Transcribed & \\
\hline $6 / 26 / 911: 03$ & Auto FIC & 386.5 & Good & \\
\hline 6/27/91 0:00 & Manual Tape & 386.5 & Transcribed & \\
\hline $6 / 27 / 910: 00$ & Manual FIC & 386.50 & Good & \\
\hline $6 / 28 / 910: 00$ & Manual Tape & 386.50 & Transcribed & \\
\hline $6 / 28 / 917: 03$ & Auto FIC & 386.50 & Good & \\
\hline $6 / 29 / 910: 00$ & Manual Tape & 386.25 & Transcribed & \\
\hline $6 / 29 / 916: 02$ & Auto FIC & 386.5 & Good & \\
\hline $6 / 30 / 910: 00$ & Manual Tape & 386.25 & Transcribed & \\
\hline $6 / 30 / 910: 00$ & Manual FIC & 386.5 & Good & \\
\hline $7 / 1 / 910: 00$ & Manual Tape & 386.25 & Transcribed & \\
\hline $7 / 1 / 910: 00$ & Manual FIC & 386.50 & Good & \\
\hline $7 / 2 / 910: 00$ & Manual Tape & 386.25 & Transcribed & \\
\hline $7 / 2 / 917: 06$ & Auto FIC & 386.50 & Good & \\
\hline $7 / 3 / 910: 00$ & Manual Tape & 386.25 & Transcribed & \\
\hline $7 / 3 / 917: 06$ & Auto FIC & 386.5 & Good & \\
\hline $7 / 4 / 910: 00$ & Manual Tape & 386.25 & Transcribed & \\
\hline $7 / 4 / 910: 00$ & Manual FIC & 386.5 & Good & \\
\hline $7 / 5 / 910: 00$ & Manual Tape & 386.25 & Transcribed & \\
\hline $7 / 5 / 910: 00$ & Manual FIC & 386.50 & Good & \\
\hline $7 / 6 / 910: 00$ & Manual Tape & 386.25 & Transcribed & \\
\hline $7 / 6 / 910: 00$ & Manual FIC & 386.5 & Good & \\
\hline $7 / 7 / 910: 00$ & Manual Tape & 386.25 & Transcribed & \\
\hline $7 / 7 / 910: 00$ & Manual FIC & 386.50 & Good & \\
\hline $7 / 8 / 910: 00$ & Manual Tape & 386.25 & Transcribed & \\
\hline $7 / 8 / 910: 00$ & Manual FIC & 386.5 & Good & \\
\hline $7 / 9 / 910: 00$ & Manual Tape & 386.25 & Transcribed & \\
\hline 7/9/91 0:00 & Manual FIC & 386.50 & Good & \\
\hline
\end{tabular}

Data obtained from WHC Surveillance Analysis Computer System, (PC SACS) July 7, 1995. 


\begin{tabular}{|c|c|c|c|c|}
\hline \multicolumn{5}{|c|}{ 241-AP-101 } \\
\hline \multicolumn{5}{|c|}{ Surface Lavel Data } \\
\hline Dato & Type of Measurement & Level (Inches) & Data Quality & Comments \\
\hline $7 / 10 / 910: 00$ & Manual Tape & 386.25 & Transcribed & \\
\hline $7 / 10 / 910: 00$ & Manual FIC & 386.5 & Good & \\
\hline $7 / 11 / 910: 00$ & Manual Tape & 386.25 & Transcribed & \\
\hline $7 / 11 / 917: 06$ & Auto FIC & 386.50 & Good & \\
\hline $7 / 12 / 910: 00$ & Manual Tape & 386.00 & Transcribed & \\
\hline $7 / 12 / 917: 06$ & Auto FIC & 386.5 & Good & \\
\hline $7 / 13 / 910: 00$ & Manual Tape & 386.25 & Transcribed & \\
\hline 7/13/91 7:06 & Auto FIC & 386.5 & Good & \\
\hline $7 / 14 / 910: 00$ & Manual Tape & 386.25 & Transcribed & \\
\hline $7 / 14 / 912: 06$ & Auto FIC & 386.50 & Good & \\
\hline $7 / 15 / 910: 00$ & Manual Tape & 386 & Transcribed & \\
\hline $7 / 15 / 917: 06$ & Auto FIC & 386.5 & Good & \\
\hline $7 / 16 / 910: 00$ & Manual Tape & 386.25 & Transcribed & \\
\hline $7 / 16 / 910: 00$ & Manual FIC & 386.5 & Good & \\
\hline $7 / 17 / 910: 00$ & Manual Tape & 386.25 & Transcribed & \\
\hline $7 / 17 / 910: 05$ & Auto FIC & 386.50 & Good & \\
\hline $7 / 18 / 910: 00$ & Manual Tape & 386.25 & Transcribed & \\
\hline $7 / 18 / 910: 00$ & Manual FIC & 386.5 & Good & \\
\hline $7 / 19 / 910: 00$ & Manual Tape & 386.25 & Transcribed & \\
\hline $7 / 19 / 917: 05$ & Auto FIC & 386.50 & Good & \\
\hline $7 / 20 / 910: 00$ & Manual Tape & 386.25 & Transcribed & \\
\hline $7 / 20 / 912: 05$ & Auto FIC & 386.5 & Good & \\
\hline $7 / 21 / 910: 00$ & Manual Tape & 386.25 & Transcribed & \\
\hline $7 / 21 / 91 \quad 1: 05$ & Auto FIC & 386.50 & Good & \\
\hline $7 / 22 / 910: 00$ & Manual Tape & 386.25 & Transcribed & \\
\hline $7 / 22 / 910: 00$ & Manual FIC & 386.5 & Good & \\
\hline $7 / 23 / 910: 00$ & Manual Tape & 386.25 & Transcribed & \\
\hline $7 / 23 / 910: 05$ & Auto FIC & 386.5 & Good & \\
\hline 7/24/91 0:00 & Manual Tape & 386.00 & Transcribed & \\
\hline 7/24/91 7:05 & Auto FIC & 386.50 & Good & \\
\hline $7 / 25 / 910: 00$ & Manual Tape & 386.25 & Transcribed & \\
\hline $7 / 25 / 911: 03$ & Auto FIC & 386.5 & Good & \\
\hline $7 / 26 / 910: 00$ & Manual Tape & 386.50 & Transcribed & \\
\hline $7 / 26 / 910: 00$ & Manual FIC & 386.5 & Good & \\
\hline $7 / 27 / 910: 00$ & Manual Tape & 386.25 & Transcribed & \\
\hline $7 / 27 / 910: 00$ & Manual FIC & 386.5 & Good & \\
\hline $7 / 28 / 910: 00$ & Manual Tape & 386.25 & Transcribed & \\
\hline $7 / 28 / 91 \quad 0: 00$ & Manual FIC & 386.50 & Good & \\
\hline $7 / 29 / 910: 00$ & Manual Tape & 386.5 & Transcribed & \\
\hline $7 / 29 / 910: 00$ & Manual FIC & 386.5 & Good & \\
\hline 7/30/91 0:00 & Manual Tape & 386.50 & Transcribed & \\
\hline $7 / 30 / 917: 02$ & Auto FIC & 386.50 & Good & \\
\hline $7 / 31 / 910: 00$ & Manual Tape & 386.5 & Transcribed & \\
\hline $7 / 31 / 917: 02$ & Auto FIC & 386.5 & Good & \\
\hline $8 / 1 / 910: 00$ & Manual Tape & 386.50 & Transcribed & \\
\hline $8 / 1 / 917: 02$ & Auto FIC & 386.5 & Good & \\
\hline $8 / 2 / 910: 00$ & Manual Tape & 386.25 & Transcribed & \\
\hline $8 / 2 / 917: 02$ & Auto FIC & 386.50 & Good & \\
\hline
\end{tabular}

Data obtained from WHC Surveillance Analysis Computer System, (PC SACS) July 7, 1995. 


\begin{tabular}{|c|c|c|c|c|}
\hline \multicolumn{5}{|c|}{ 241-AP-101 } \\
\hline \multicolumn{5}{|c|}{ Surface Level Data } \\
\hline Date & Type of Measurement & Level (Inches) & Data Quality & Comments \\
\hline $8 / 3 / 910: 00$ & Manual Tape & 386.50 & Transcribed & \\
\hline $8 / 3 / 917: 02$ & Auto FIC & 386.5 & Good & \\
\hline $8 / 4 / 910: 00$ & Manual Tape & 386.5 & Transeribed & \\
\hline $8 / 4 / 917: 02$ & Auto FIC & 386.50 & Good & \\
\hline $8 / 5 / 910: 00$ & Manual Tape & 386.25 & Transcribed & \\
\hline $8 / 5 / 917: 02$ & Auto FIC & 386.5 & Good & \\
\hline $8 / 6 / 910: 00$ & Manual Tape & 386.25 & Transcribed & \\
\hline $8 / 6 / 917: 02$ & Auto FIC & 386.50 & Good & \\
\hline $8 / 7 / 910: 00$ & Manual Tape & 386.50 & Transcribed & \\
\hline $8 / 7 / 917: 06$ & Auto FIC & 386.50 & Good & \\
\hline $8 / 8 / 910: 00$ & Manual Tape & 386.25 & Transcribed & \\
\hline $8 / 8 / 917: 06$ & Auto FIC & 386.5 & Good & \\
\hline $8 / 9 / 910: 00$ & Manual Tape & 386.25 & Transcribed & \\
\hline $8 / 9 / 917: 04$ & Auto FIC & 386.5 & Good & \\
\hline $8 / 10 / 910: 00$ & Manual Tape & 386.25 & Transcribed & \\
\hline $8 / 10 / 917: 04$ & Auto FIC & 386.50 & Good & \\
\hline $8 / 11 / 910: 00$ & Manual Tape & 386.25 & Transcribed & \\
\hline $8 / 11 / 917: 04$ & Auto FIC & 386.5 & Good & \\
\hline $8 / 12 / 910: 00$ & Manual Tape & 386.25 & Transcribed & \\
\hline $8 / 12 / 917: 04$ & Auto FIC & 386.50 & Good & \\
\hline $8 / 13 / 910: 00$ & Manual Tape & 386.25 & Transcribed & \\
\hline $8 / 13 / 917: 04$ & Auto FIC & 386.50 & . Good & \\
\hline $8 / 14 / 910: 00$ & Manual Tape & 386.25 & Transcribed & \\
\hline $8 / 14 / 917: 04$ & Auto FIC & 386.5 & Good & \\
\hline 8/15/91 0:00 & Manual Tape & 386.25 & Transcribed & \\
\hline $8 / 15 / 917: 03$ & Auto FIC & 386.50 & Good & \\
\hline $8 / 16 / 910: 00$ & Manual Tape & 386.25 & Transcribed & \\
\hline $8 / 16 / 910: 00$ & Manual FIC & 386.50 & Good & \\
\hline $8 / 17 / 910: 00$ & Manual Tape & 386.50 & Transcribed & \\
\hline $8 / 17 / 917: 02$ & Auto FIC & 386.5 & Good & \\
\hline $8 / 18 / 910: 00$ & Manual Tape & 386.5 & Transcribed & \\
\hline $8 / 18 / 917: 03$ & Auto FIC & 386.50 & Good & \\
\hline $8 / 19 / 910: 00$ & Manual Tape & 386.25 & Transcribed & \\
\hline $8 / 19 / 917: 02$ & Auto FIC & 386.5 & Good & \\
\hline $8 / 20 / 910: 00$ & Manual Tape & 386.25 & Transcribed & \\
\hline $8 / 20 / 917: 04$ & Auto FIC & 386.50 & Good & \\
\hline $8 / 21 / 910: 00$ & Manual Tape & 386.25 & Transcribed & \\
\hline $8 / 21 / 917: 03$ & Auto FIC & 386.5 & Good & \\
\hline $8 / 22 / 910: 00$ & Manual Tape & 386.25 & Transcribed & \\
\hline $8 / 22 / 917: 03$ & Auto FIC & 386.50 & Good & \\
\hline $8 / 23 / 910: 00$ & Manual Tape & 386.25 & Transcribed & \\
\hline $8 / 23 / 917: 02$ & Auto FIC & 386.5 & Good & \\
\hline $8 / 24 / 910: 00$ & Manual Tape & 386.25 & Transcribed & \\
\hline $8 / 24 / 917: 02$ & Auto FIC & 386.50 & Good & \\
\hline $8 / 25 / 910: 00$ & Manual Tape & 386.25 & Transcribed & \\
\hline $8 / 25 / 917: 02$ & Auto FIC & 386.5 & Good & \\
\hline $8 / 26 / 910: 00$ & Manual Tape & 386.25 & Transcribed & \\
\hline $8 / 26 / 917: 03$ & Auto FIC & 386.50 & Good & \\
\hline
\end{tabular}

Data obtained from WHC Surveillance Analysis Computer System, (PC SACS) July 7, 1995. 


\begin{tabular}{|c|c|c|c|c|}
\hline \multicolumn{5}{|c|}{ 241-AP-101 } \\
\hline \multicolumn{5}{|c|}{ Surface Level Data } \\
\hline Date & Type of Measurement & Level (lnches) & Date Quality & Comments \\
\hline $8 / 27 / 910: 00$ & Manual Tape & 386.25 & Transcribed & \\
\hline $8 / 27 / 917: 03$ & Auto FIC & 386.5 & Good & \\
\hline $8 / 28 / 910: 00$ & Manual Tape & 386.25 & Transcribed & \\
\hline $8 / 28 / 917: 03$ & Auto FIC & 386.50 & Good & \\
\hline $8 / 29 / 910: 00$ & Manual Tape & 386.25 & Transcribed & \\
\hline $8 / 29 / 917: 02$ & Auto FIC & 386.5 & Good & \\
\hline $8 / 30 / 910: 00$ & Manual Tape & 386 & Transcribed & \\
\hline $8 / 30 / 916: 02$ & Auto FIC & 386.50 & Good & \\
\hline $8 / 31 / 910: 00$ & Manual Tape & 386.25 & Transcribed & \\
\hline $8 / 31 / 917: 02$ & Auto FIC & 386.5 & Good & \\
\hline $9 / 1 / 910: 00$ & Manual Tape & 386.25 & Transcribed & \\
\hline $9 / 1 / 917: 02$ & Auto FIC & 386.50 & Good & \\
\hline $9 / 2 / 910: 00$ & Manual Tape & 386.25 & Transcribed & \\
\hline $9 / 2 / 917: 02$ & Auto FIC & 386.5 & Good & \\
\hline $9 / 3 / 910: 00$ & Manual Tape & 386.25 & Transcribed & \\
\hline $9 / 3 / 917: 02$ & Auto FIC & 386.50 & Good & \\
\hline $9 / 4 / 910: 00$ & Manual Tape & 386.25 & Transcribed & \\
\hline $9 / 4 / 917: 03$ & Auto FIC & 386.5 & Good & \\
\hline $9 / 5 / 910: 00$ & Manual Tape & 386.25 & Transcribed & \\
\hline $9 / 5 / 917: 02$ & Auto FIC & 386.50 & Good & \\
\hline $9 / 6 / 910: 00$ & Manual Tape & 386.25 & Transcribed & \\
\hline $9 / 6 / 917: 02$ & Auto FIC & 386.5 & Good & \\
\hline $9 / 7 / 910: 00$ & Manual Tape & 386.25 & Transcribed & \\
\hline $9 / 7 / 917: 02$ & Auto FIC & 386.5 & Good & \\
\hline $9 / 8 / 910: 00$ & Manual Tape & 386.25 & Transcribed & \\
\hline $9 / 8 / 917: 02$ & Auto FIC & 386.50 & Good & \\
\hline $9 / 9 / 910: 00$ & Manual Tape & 386.25 & Transcribed & \\
\hline $9 / 9 / 917: 03$ & Auto FIC & 386.50 & Good & \\
\hline 9/10/91 0:00 & Manual Tape & 386.25 & Transcribed & \\
\hline $9 / 10 / 917: 02$ & Auto FIC & 386.5 & Good & \\
\hline 9/11/910:00 & Manual Tape & 386.25 & Transcribed & \\
\hline 9/11/91 7:03 & Auto FIC & 386.5 & Good & \\
\hline 9/12/91 0:00 & Manual Tape & 386.25 & Transçribed & \\
\hline $9 / 12 / 917: 02$ & Auto FIC & 386.50 & Good & \\
\hline 9/13/910:00 & Manual Tape & 386.25 & Transcribed & \\
\hline $9 / 13 / 917: 02$ & Auto FIC & 386.5 & Good & \\
\hline 9/14/91 0:00 & Manual Tape & 386.25 & Transcribed & \\
\hline $9 / 14 / 917: 03$ & Auto FIC & 386.50 & Good & \\
\hline 9/15/91 0:00 & Manual Tape & 386.25 & Transcribed & \\
\hline $9 / 15 / 917: 02$ & Auto FIC & 386.5 & Good & \\
\hline $9 / 16 / 910: 00$ & Manual Tape & 386.25 & Transcribed & \\
\hline $9 / 16 / 917: 03$ & Auto FIC & 386.50 & Good & \\
\hline 9/17/91 0:00 & Manual Tape & 386.25 & Transcribed & \\
\hline $9 / 17 / 917: 03$ & Auto FIC & 386.5 & Good & \\
\hline $9 / 18 / 910: 00$ & Manual Tape & 386.25 & Transcribed & \\
\hline $9 / 18 / 917: 03$ & Auto FIC & 386.50 & Good & \\
\hline 9/19/91 0:00 & Manual Tape & 386.25 & Transcribed & \\
\hline $9 / 19 / 917: 03$ & Auto FIC & 386.5 & Good & \\
\hline
\end{tabular}

Data obtained from WHC Surveillance Analysis Computer System, (PC SACS) July 7, 1995. 


\begin{tabular}{|c|c|c|c|c|}
\hline \multicolumn{5}{|c|}{ 241-AP-101 } \\
\hline \multicolumn{5}{|c|}{ Surface Level Data } \\
\hline Date & Type of Measurement & Level (Inches) & Data Quality & Comments \\
\hline $9 / 20 / 910: 00$ & Manual Tape. & 386.25 & Transcribed & \\
\hline $9 / 20 / 917: 02$ & Auto FIC & 386.50 & Good & \\
\hline 9/21/910:00 & Manual Tape & 386.25 & Transcribed & \\
\hline 9/21/91 7:02 & Auto FIC & 386.5 & Good & \\
\hline 9/22/910:00 & Manual Tape & 386.25 & Transćribed & \\
\hline $9 / 22 / 917: 03$ & Auto FIC & 386.50 & Good & \\
\hline $9 / 23 / 91 \quad 0: 00$ & Manual Tape & 386.25 & Transcribed & \\
\hline $9 / 23 / 917: 03$ & Auto FIC & 386.5 & Good & \\
\hline $9 / 24 / 910: 00$ & Manual Tape & 386.50 & Transcribed & \\
\hline $9 / 24 / 917: 02$ & Auto FIC & 386.50 & Good & \\
\hline $9 / 25 / 910: 00$ & Manual Tape & 386.5 & Transcribed & \\
\hline $9 / 25 / 917: 03$ & Auto FIC & 386.5 & Good & \\
\hline $9 / 26 / 910: 00$ & Manual Tape & 386.25 & Transcribed & \\
\hline $9 / 26 / 917: 03$ & Auto FIC & 386.50 & Good & \\
\hline $9 / 27 / 910: 00$ & Manual Tape & 386.25 & Transcribed & \\
\hline $9 / 27 / 917: 02$ & Auto FIC & 386.5 & Good & \\
\hline 9/28/91 0:00 & Manual Tape & 386.25 & Transcribed & \\
\hline $9 / 28 / 917: 02$ & Auto FIC & 386.50 & Good & \\
\hline $9 / 29 / 910: 00$ & Manual Tape & 386.25 & Transcribed & \\
\hline 9/29/91 7:03 & Auto FIC & 386.5 & Good & \\
\hline 9/30/910:00 & Manual Tape & 386.25 & Transcribed & \\
\hline $9 / 30 / 917: 03$ & Auto FIC & 386.50 & Goid & \\
\hline $10 / 1 / 910: 00$ & Manual Tape & 386.25 & Transcribed & \\
\hline $10 / 1 / 917: 02$ & Auto FIC & 386.5 & Good & \\
\hline $10 / 2 / 910: 00$ & Manual Tape & 386.25 & Transcribed & \\
\hline $10 / 2 / 917: 02$ & Auto FIC & 386.50 & Good & \\
\hline $10 / 3 / 910: 00$ & Manual Tape & 386.25 & Transcribed & \\
\hline $10 / 3 / 917: 02$ & Auto FIC & 386.5 & Good & \\
\hline $10 / 4 / 910: 00$ & Manual Tape & 386.25 & Transcribed & \\
\hline $10 / 4 / 917: 03$ & Auto FIC & 386.50 & Gopd & \\
\hline $10 / 5 / 910: 00$ & Manual Tape & 386.25 & Transcribed & \\
\hline $10 / 5 / 910: 00$ & Manual FIC & 386.5 & Good & \\
\hline $10 / 6 / 91 \quad 0: 00$ & Manual Tape & 386.25 & Transcribed & \\
\hline $10 / 6 / 917: 03$ & Auto FIC & 386.5 & Good & \\
\hline $10 / 7 / 910: 00$ & Manual Tape & 386.25 & Transcribed & \\
\hline $10 / 7 / 917: 02$ & Auto FIC & 386.50 & Good & \\
\hline $10 / 8 / 910: 00$ & Manual Tape & 386.25 & Transcribed & \\
\hline $10 / 8 / 91 \quad 7: 02$ & Auto FIC & 386.5 & Good & \\
\hline $10 / 9 / 910: 00$ & Manual Tape & 386.25 & Transcribed & \\
\hline $10 / 9 / 917: 02$ & Auto FIC & 386.50 & Good & \\
\hline $10 / 10 / 910: 00$ & Manual Tape & 386.25 & Transcribed & \\
\hline $10 / 10 / 917: 02$ & Auto FIC & 386.5 & Good & \\
\hline $10 / 11 / 910: 00$ & Manual Tape & 386.25 & Transcribed & \\
\hline 10/11/91 7:02 & Auto FIC & 386.50 & Good & \\
\hline $10 / 12 / 91 \quad 0: 00$ & Manual Tape & 386.25 & Transcribed & \\
\hline $10 / 12 / 917: 02$ & Auto FIC & 386.5 & Good & \\
\hline $10 / 13 / 910: 00$ & Manual Tape & 386.25 & Transcribed & \\
\hline 10/13/91 7:03 & Auto FIC & 386.50 & Good & \\
\hline
\end{tabular}

Data obtained from WHC Surveillance Analysis Computer System, (PC SACS) July 7, 1995. 


\begin{tabular}{|c|c|c|c|c|}
\hline \multicolumn{5}{|c|}{ 241-AP-101 } \\
\hline \multicolumn{5}{|c|}{ Surface Level Data } \\
\hline Date & Type of Measurement & Level (Inches) & Data Quality & Comments \\
\hline 10/14/91 0:00 & Manual Tape & 386.25 & Transcribed & \\
\hline $10 / 14 / 917: 02$ & Auto FIC & 386.5 & Good & \\
\hline $10 / 15 / 910: 00$ & Manual Tape & 386.25 & Transcribed & \\
\hline $10 / 15 / 917: 02$ & Auto FIC & 386.50 & Good & \\
\hline $10 / 16 / 910: 00$ & Manual Tape & 386.25 & Transcribed & \\
\hline $10 / 16 / 917: 03$ & Auto FIC & 386.5 & Good & \\
\hline $10 / 17 / 910: 00$ & Manual Tape & 386.25 & Transcribed & \\
\hline $10 / 17 / 917: 05$ & Auto FIC & 386.40 & Good & \\
\hline $10 / 18 / 910: 00$ & Manual Tape & 386.25 & Transcribed & \\
\hline $10 / 18 / 917: 06$ & Auto FIC & 386.4 & Good & \\
\hline $10 / 19 / 910: 00$ & Manual Tape & 386.25 & Transcribed & \\
\hline $10 / 19 / 917: 06$ & Auto FIC & 386.40 & Good & \\
\hline $10 / 20 / 910: 00$ & Manual Tape & 386.25 & Transcribed & \\
\hline $10 / 20 / 917: 02$ & Auto FIC & 386.4 & Good & \\
\hline $10 / 21 / 910: 00$ & Manual Tape & 386.25 & Transcribed & \\
\hline $10 / 21 / 917: 02$ & Auto FIC & 386.40 & Good & \\
\hline 10/22/91 $0: 00$ & Manual Tape & 386.25 & Transcribed & \\
\hline $10 / 22 / 917: 03$ & Auto FIC & 386.4 & Good & \\
\hline $10 / 23 / 910: 00$ & Manual Tape & 386.25 & Transcribed & \\
\hline $10 / 23 / 917: 02$ & Auto FIC & 386.40 & Good & \\
\hline $10 / 24 / 91 \quad 0: 00$ & Manual Tape & 386.25 . & Transcribed & \\
\hline $10 / 24 / 917: 02$ & Auto FIC & 386.4 & Good & \\
\hline $10 / 25 / 910: 00$ & Manual Tape & 386.25 & Transcribed & \\
\hline $10 / 25 / 917: 03$ & Auto FIC & 386.40 & Good & \\
\hline $10 / 26 / 910: 00$ & Manual Tape & 386.25 & Transcribed & \\
\hline $10 / 26 / 917: 03$ & Auto FIC & 386.4 & Good & \\
\hline $10 / 27 / 910: 00$ & Manual Tape & 386.25 & Transcribed & \\
\hline $10 / 27 / 917: 02$ & Auto FIC & 386.40 & Good & \\
\hline $10 / 28 / 910: 00$ & Manual Tape & 386.25 & Transcribed & \\
\hline 10/28/91 7:02 & Auto FIC & 386.40 & Good & \\
\hline 10/29/91 0:00 & Manual Tape & 386.25 & Transcribed & \\
\hline $10 / 29 / 917: 03$ & Auto FIC & 386.4 & Good & \\
\hline $10 / 30 / 910: 00$ & Manual Tape & 386.25 & Transcribed & \\
\hline $10 / 30 / 917: 03$ & Auto FIC & 386.40 & Good & \\
\hline 10/31/910:00 & Manual Tape & 386.25 & Transcribed & \\
\hline $10 / 31 / 917: 03$ & Auto FIC & 386.4 & Good & \\
\hline $11 / 1 / 910: 00$ & Manual Tape & 386.25 & Transcribed & \\
\hline $11 / 1 / 917: 02$ & Auto FIC & 386.40 & Good & \\
\hline $11 / 2 / 910: 00$ & Manual Tape & 386.25 & Transcribed & \\
\hline $11 / 2 / 917: 03$ & Auto FIC & 386.4 & Good & \\
\hline $11 / 3 / 910: 00$ & Manual Tape & 386.25 & Transcribed & \\
\hline $11 / 3 / 917: 03$ & Auto FIC & 386.40 & Good & \\
\hline $11 / 4 / 910: 00$ & Manual Tape & 386.25 & Transcribed & \\
\hline $11 / 4 / 917: 02$ & Auto FIC & 386.4 & Good & \\
\hline $11 / 5 / 910: 00$ & Manual Tape & 386.25 & Transcribed & \\
\hline $11 / 5 / 917: 03$ & Auto FIC & 386.40 & Good & \\
\hline $11 / 6 / 910: 00$ & Manual Tape & $386.25^{\circ}$ & Transcribed & \\
\hline $11 / 6 / 917: 03$ & Auto FIC & 386.4 & Good & \\
\hline
\end{tabular}

Data obtained from WHC Surveillance Analysis Computer System, (PC SACS) July 7, 1995. 


\begin{tabular}{|c|c|c|c|c|}
\hline \multicolumn{5}{|c|}{ 241-AP-101 } \\
\hline \multicolumn{5}{|c|}{ Surface Leval Data } \\
\hline Date & Type of Measurement & Level (Inches) & Data Quality & Comments \\
\hline $11 / 7 / 910: 00$ & Manual Tape & 386.25 & Transcribed & \\
\hline $11 / 7 / 917: 02$ & Auto FIC & 386.40 & Good & \\
\hline $11 / 8 / 910: 00$ & Manual Tape & 386.25 & Transcribed & \\
\hline $11 / 8 / 917: 03$ & Auto FIC & 386.4 & Good & \\
\hline $11 / 9 / 910: 00$ & Manual Tape & 386.25 & Transcribed & \\
\hline 11/9/91 7:02 & Auto FIC & 386.40 & Good & \\
\hline $11 / 10 / 910: 00$ & Manual Tape & 386.25 & Transcribed & \\
\hline $11 / 10 / 917: 03$ & Auto FIC & 386.4 & Good & \\
\hline $11 / 11 / 910: 00$ & Manual Tape & 386.25 & Transcribed & \\
\hline $11 / 11 / 916: 03$ & Auto FIC & 386.40 & Good & \\
\hline 11/12/91 0:00 & Manual Tape & 386.25 & Transcribed & \\
\hline $11 / 12 / 917: 03$ & Auto FIC & 386.4 & Good & \\
\hline $11 / 13 / 910: 00$ & Manual Tape & 386.25 & Transcribed & \\
\hline $11 / 13 / 917: 03$ & Auto FIC & 386.40 & Good & \\
\hline $11 / 14 / 910: 00$ & Manual Tape & 386.25 & Transcribed & \\
\hline $11 / 14 / 917: 03$ & Auto FIC & 386.4 & Good & \\
\hline $11 / 15 / 910: 00$ & Manual Tape & 386.25 & Transcribed & \\
\hline $11 / 15 / 917: 03$ & Auto FIC & 386.40 & Good & \\
\hline $11 / 16 / 910: 00$ & Manual Tape & 386.25 & Transcribed & \\
\hline $11 / 16 / 917: 03$ & Auto FIC & 386.4 & Good & \\
\hline $11 / 17 / 910: 00$ & Manual Tape & 386.25 & Transcribed & \\
\hline $11 / 17 / 917: 02$ & Auto FIC & 386.40 & Good & \\
\hline $11 / 18 / 910: 00$ & Manual Tape & 386.25 & Transcribed & \\
\hline $11 / 18 / 917: 03$ & Auto FIC & 386.4 & Good & \\
\hline $11 / 19 / 910: 00$ & Manual Tape & 386.25 & Transcribed & \\
\hline $11 / 19 / 917: 03$ & Auto FIC & $386 . \overline{40}$ & Good & \\
\hline $11 / 20 / 910: 00$ & Manual Tape & 386.25 & Transcribed & \\
\hline $11 / 20 / 917: 03$ & Auto FIC & 386.4 & Good & \\
\hline $11 / 21 / 910: 00$ & Manual Tape & 386.25 & Transcribed & \\
\hline $11 / 21 / 917: 02$ & Auto FIC & 386.40 & Good & \\
\hline $11 / 22 / 910: 00$ & Manual Tape & 386.25 & Transcribed & \\
\hline $11 / 22 / 917: 03$ & Auto FIC & 386.4 & Good & \\
\hline $11 / 23 / 910: 00$ & Manual Tape & 386.25 & Transcribed & \\
\hline $11 / 23 / 917: 02$ & Auto FIC & 386.40 & Good & \\
\hline $11 / 24 / 910: 00$ & Manual Tape & 386.25 & Transcribed & \\
\hline $11 / 24 / 917: 03$ & Auto FIC & 386.4 & Good & \\
\hline $11 / 25 / 910: 00$ & Manua! Tape & 386.25 & Transcribed & \\
\hline $11 / 25 / 917: 03$ & Auto FIC & 386.40 & Good & \\
\hline $11 / 26 / 910: 00$ & Manual FIC & 386.40 & Transcribed & \\
\hline $11 / 26 / 910: 00$ & Manual Tape & 386.00 & Transcribed & \\
\hline $11 / 27 / 910: 00$ & Manual Tape & 386.25 & Transcribed & \\
\hline $11 / 27 / 917: 03$ & Auto FIC & 386.4 & Good & \\
\hline $11 / 28 / 910: 00$ & Manual Tape & 386.25 & Transcribed & \\
\hline $11 / 28 / 917: 03$ & Auto FIC & 386.40 & Good & \\
\hline $11 / 29 / 910: 00$ & Manual Tape & 386.25 & Transcribed & \\
\hline $11 / 29 / 917: 03$ & Auto FIC & 386.4 & Good & \\
\hline $11 / 30 / 910: 00$ & Manual Tape & 386.25 & Transcribed & \\
\hline $11 / 30 / 910: 00$ & Manual FIC & 386.7 & Good & \\
\hline
\end{tabular}

Data obtained from WHC Surveillance Analysis Computer System, (PC SACS) July 7, 1995. 


\begin{tabular}{|c|c|c|c|c|}
\hline \multicolumn{5}{|c|}{ 241-AP-101 } \\
\hline \multicolumn{5}{|c|}{ Surface Level Data } \\
\hline Date & Type of Measurement & Level (Inches) & Data Quality & Comments \\
\hline $11 / 30 / 917: 03$ & Auto FIC & 386.40 & Good & \\
\hline $12 / 1 / 910: 00$ & Manual Tape & 386.25 & Transcribed & \\
\hline $12 / 1 / 917: 03$ & Auto FIC & 386.4 & Good & \\
\hline 12/2/910:00 & Manual Tape & 386.25 & Transcribed & \\
\hline $12 / 2 / 917: 03$ & Auto FIC & 386.40 & Good & \\
\hline $12 / 3 / 910: 00$ & Manual Tape & 386 & Transcribed & \\
\hline 12/3/917:03 & Auto FIC & 386.4 & Good & \\
\hline $12 / 4 / 910: 00$ & Manual Tape & 386 & Transcribed & \\
\hline $12 / 4 / 917: 03$ & Auto FIC & 386.40 & Good & \\
\hline $12 / 5 / 910: 00$ & Manual Tape & 386.00 & Transcribed & \\
\hline $12 / 5 / 917: 03$ & Auto FIC & 386.4 & Good & \\
\hline $12 / 6 / 910: 00$ & Manual Tape & 386 & Transcribed & \\
\hline $12 / 6 / 917: 04$ & Auto FIC & 386.40 & Good & \\
\hline $12 / 7 / 910: 00$ & Manual Tape & 386.25 & Transcribed & \\
\hline $12 / 7 / 917: 03$ & Auto FIC & 386.4 & Good & \\
\hline $12 / 8 / 910: 00$ & Manual Tape & 386.25 & Transcribed & \\
\hline $12 / 8 / 917: 03$ & Auto FIC & 386.40 & Good & \\
\hline $12 / 9 / 910: 00$ & Manual Tape & 385 & Transcribed & \\
\hline $12 / 9 / 917: 03$ & Auto FIC & 386.4 & Good & \\
\hline $12 / 10 / 910: 00$ & Manual Tape & 386.25 & Transcribed & \\
\hline $12 / 10 / 917: 03$ & Auto FIC & 386.30 & Good & \\
\hline $12 / 11 / 910: 00$ & Manual Tape & 386.25 . & Transcribed & \\
\hline $12 / 11 / 917: 02$ & Auto FIC & 386.3 & Good & \\
\hline $12 / 12 / 910: 00$ & Manual Tape & 386.25 & Transcribed & \\
\hline $12 / 12 / 917: 03$ & Auto FIC & 386.30 & Good & \\
\hline $12 / 13 / 910: 00$ & Manual Tape & 386.25 & Transcribed & \\
\hline $12 / 13 / 917: 05$ & Auto FIC & 386.3 & Good & \\
\hline $12 / 14 / 910: 00$ & Manual Tape & 386.25 & Transcribed & \\
\hline $12 / 14 / 917: 02$ & Auto FIC & 386.30 & Good & \\
\hline $12 / 15 / 910: 00$ & Manual Tape & 386.25 & Transcribed & \\
\hline 12/15/91 7:02 & Auto FIC & 386.3 & Good & \\
\hline $12 / 16 / 910: 00$ & Manual Tape & 386.25 & Transcribed & \\
\hline $12 / 16 / 917: 03$ & Auto FIC & 386.30 & Good & \\
\hline $12 / 17 / 910: 00$ & Manual Tape & 386.25 & Transcribed & \\
\hline $12 / 17 / 917: 06$ & Auto FIC & 386.3 & Good & \\
\hline $12 / 18 / 910: 00$ & Manual Tape & 386.25 & Transcribed & \\
\hline $12 / 18 / 917: 03$ & Auto FIC & 386.30 & Good & \\
\hline $12 / 19 / 910: 00$ & Manual Tape & 386.25 & Transcribed & \\
\hline $12 / 19 / 917: 03$ & Auto FIC & 386.3 & Good & \\
\hline $12 / 20 / 910: 00$ & Manual Tape & 386.00 & Transcribed & \\
\hline $12 / 20 / 917: 02$ & Auto FIC & 386.3 & Good & \\
\hline $12 / 21 / 910: 00$ & Manual Tape & 386 & Transcribed & . \\
\hline $12 / 21 / 917: 03$ & Auto FIC & 386.30 & Good & \\
\hline $12 / 22 / 910: 00$ & Manual Tape & 386.25 & Transcribed & \\
\hline $12 / 22 / 917: 05$ & Auto FIC & 386.3 & Good & \\
\hline $12 / 23 / 910: 00$ & Manual Tape & 386.25 & Transcribed & \\
\hline $12 / 23 / 917: 06$ & Auto FIC & 386.30 & Good & \\
\hline $12 / 24 / 910: 00$ & Manual Tape & 386.125 & Transcribed & \\
\hline
\end{tabular}

Data obtained from WHC Surveillance Analysis Computer System, (PC SACS) July 7, 1995. 


\begin{tabular}{|c|c|c|c|c|}
\hline \multicolumn{5}{|c|}{ 241-AP-101 } \\
\hline \multicolumn{5}{|c|}{ Surface Level Data } \\
\hline Dato & Type of Messurement & Level (Inches) & Data Quality & Comments \\
\hline $12 / 24 / 917: 03$ & Auto FIC & 386.3 & Good & \\
\hline 12/25/910:00 & Manual Tape & 386.25 & Transcribed & \\
\hline $12 / 25 / 917: 03$ & Auto FIC & 386.30 & Good & \\
\hline $12 / 26 / 910: 00$ & Manual Tape & 386.25 & Transcribed & \\
\hline $12 / 26 / 917: 03$ & Auto FIC & 386.3 & Good & \\
\hline $12 / 27 / 910: 00$ & Manual Tape & 386.25 & Transcribed & \\
\hline $12 / 27 / 91 \quad 7: 03$ & Auto FIC & 386.30 & Good & \\
\hline 12/28/91 0:00 & Manual Tape & 386.25 & Transcribed & \\
\hline $12 / 28 / 91 \quad 7: 02$ & Auto FIC & 386.3 & Good & \\
\hline $12 / 29 / 910: 00$ & Manual Tape & 386.25 & Transcribed & \\
\hline $12 / 29 / 917: 03$ & Auto FIC & 386.30 & Good & \\
\hline 12/30/910:00 & Manual Tape & 386.25 & Transcribed & \\
\hline $12 / 30 / 917: 03$ & Auto FIC & 386.3 & Good & \\
\hline 12/31/910:00 & Manual Tape & 386.25 & Transcribed & \\
\hline $12 / 31 / 917: 03$ & Auto FIC & 386.30 & Good & \\
\hline $1 / 1 / 920: 00$ & Manual Tape & 386.25 & Transcribed & \\
\hline $1 / 1 / 927: 03$ & Auto FIC & 386.3 & Good & \\
\hline $1 / 2 / 920: 00$ & Manual Tape & 386 & Transcribed & \\
\hline $1 / 2 / 927: 02$ & Auto FIC & 386.30 & Good & \\
\hline $1 / 3 / 920: 00$ & Manual Tape & 386.25 & Transcribed & \\
\hline $1 / 3 / 927: 04$ & Auto FIC & 386.3 & Good & \\
\hline $1 / 4 / 920: 00$ & Manual Tape & 386.25 & Transcribed & \\
\hline $1 / 4 / 927: 03$ & Auto FIC & 386.30 & Good & \\
\hline $1 / 5 / 920: 00$ & Manual Tape & 386 & Transcribed & \\
\hline 1/5/92 7:03 & Auto FIC & 386.3 & Good & \\
\hline $1 / 6 / 920: 00$ & Manual Tape & 386.00 & Transcribed & \\
\hline $1 / 6 / 927: 03$ & Auto FIC & 386.30 & Good & \\
\hline $1 / 7 / 920: 00$ & Manual Tape & 386 & Transcribed & \\
\hline $1 / 7 / 927: 04$ & Auto FIC & 386.3 & Good & \\
\hline $1 / 8 / 920: 00$ & Manual Tape & 386.00 & Transcribed & \\
\hline $1 / 8 / 927: 03$ & Auto FIC & 386.30 & Good & \\
\hline $1 / 9 / 920: 00$ & Manual Tape & 386 & Transcribed & \\
\hline $1 / 9 / 927: 03$ & Auto FIC & 386.3 & Good & \\
\hline $1 / 10 / 92 \quad 0: 00$ & Manual Tape & 385.50 & Transcribed & \\
\hline $1 / 10 / 927: 04$ & Auto FIC & 386.30 & Good & \\
\hline $1 / 11 / 920: 00$ & Manual Tape & 386 . & Transcribed & \\
\hline $1 / 11 / 927: 04$ & Auto FIC & 386.3 & Good & \\
\hline $1 / 12 / 920: 00$ & Manual Tape & 386.00 & Transcribed & \\
\hline $1 / 12 / 920: 00$ & Manual FIC & 386.30 & Good & \\
\hline $1 / 13 / 920: 00$ & Manual Tape & 386.25 & Transcribed & \\
\hline $1 / 13 / 920: 00$ & Manual FIC & 386.3 & Good & \\
\hline $1 / 14 / 920: 00$ & Manual Tape & 386.00 & Transcribed & \\
\hline $1 / 14 / 920: 00$ & Manual FIC & 386.30 & Good & \\
\hline $1 / 15 / 920: 00$ & Manual Tape & 386 & Transcribed & \\
\hline $1 / 15 / 927: 03$ & Auto FIC & 386.30 & Good & \\
\hline $1 / 16 / 920: 00$ & Manual Tape & 386.00 & Transcribed & \\
\hline $1 / 16 / 927: 04$ & Auto FIC & 386.3 & Good & \\
\hline $1 / 17 / 920: 00$ & Manual Tape & 386 & Transcribed & \\
\hline
\end{tabular}

Data obtained from WHC Surveillance Analysis Computer System, (PC SACS) July 7, 1995. 


\begin{tabular}{|c|c|c|c|c|}
\hline \multicolumn{5}{|c|}{ 241-AP:101 } \\
\hline \multicolumn{5}{|c|}{ Surface Level Data } \\
\hline Date & Type of Measurement & Level (Inches) & Data Quality & Comments \\
\hline $1 / 17 / 927: 04$ & Auto FIC & 386.30 & Good & \\
\hline $1 / 18 / 920: 00$ & Manual Tape & 386.00 & Transcribed & \\
\hline $1 / 18 / 927: 04$ & Auto FIC & 386.3 & Good & \\
\hline $1 / 19 / 920: 00$ & Manual Tape & 386 & Transcribed & \\
\hline $1 / 19 / 927: 03$ & Auto FIC & 386.30 & Good & \\
\hline $1 / 20 / 920: 00$ & Manual Tape & 386.00 & Transcribed & \\
\hline $1 / 20 / 927: 03$ & Auto FIC & 386.3 & Good & \\
\hline $1 / 21 / 920: 00$ & Manual Tape & 386 & Transcribed & \\
\hline $1 / 21 / 927: 03$ & Auto FIC & 386.30 & Good & \\
\hline $1 / 22 / 920: 00$ & Manual Tape & 386.00 & Transcribed & \\
\hline $1 / 22 / 927: 06$ & Auto FIC & 386.3 & Good & \\
\hline $1 / 23 / 920: 00$ & Manual Tape & 386 & Transcribed & \\
\hline $1 / 23 / 927: 03$ & Auto FIC & 386.30 & Good & \\
\hline $1 / 24 / 920: 00$ & Manual Tape & 386.00 & Transcribed & \\
\hline $1 / 24 / 927: 02$ & Auto FIC & 386.3 & Good & \\
\hline $1 / 25 / 920: 00$ & Manual Tape & 386.25 & Transcribed & \\
\hline $1 / 25 / 927: 03$ & Auto FIC & 386.30 & Good & \\
\hline $1 / 26 / 920: 00$ & Manual Tape & 386.25 & Transcribed & \\
\hline $1 / 26 / 926: 03$ & Auto FIC & 386.2 & Good & \\
\hline $1 / 27 / 920: 00$ & Manual Tape & 386.25 & Transcribed & \\
\hline $1 / 27 / 927: 02$ & Auto FIC & 386.20 & Good & \\
\hline $1 / 28 / 920: 00$ & Manual Tape & 386.25 & Transcribed & \\
\hline $1 / 28 / 927: 03$ & Auto FIC & . 386.3 & Good & \\
\hline $1 / 29 / 920: 00$ & Manual Tape & 386.25 & Transcribed & \\
\hline $1 / 29 / 927: 03$ & Auto FIC & 386.30 & Good & \\
\hline $1 / 30 / 920: 00$ & Manual Tape & 386.25 & Transcribed & \\
\hline $1 / 30 / 927: 03$ & Auto FIC & 386.2 & Good & \\
\hline $1 / 31 / 920: 00$ & Manual Tape & 386.25 & Transcribed & \\
\hline $1 / 31 / 927: 03$ & Auto FIC & 386.20 & Good & \\
\hline $2 / 1 / 920: 00$ & Manual Tape & 386.25 & Transcribed & \\
\hline $2 / 1 / 927: 02$ & Auto FIC & 386.2 & Good & \\
\hline $2 / 2 / 920: 00$ & Manual Tape & 386.25 & Transcribed & \\
\hline $2 / 2 / 927: 03$ & Auto FIC & 386.20 & Good & \\
\hline $2 / 3 / 920: 00$ & Manual Tape & 386.25 & Transcribed & \\
\hline $2 / 3 / 927: 03$ & Auto FIC & 386.2 & Good & \\
\hline $2 / 4 / 920: 00$ & Manual Tape & 386.25 & Transcribed & \\
\hline $2 / 4 / 927: 03$ & Auto FIC & 386.20 & Good & \\
\hline $2 / 5 / 920: 00$ & Manual Tape & 386.25 & Transcribed & \\
\hline $2 / 5 / 92 \quad 7: 02$ & Auto FIC & 386.2 & Good & \\
\hline $2 / 6 / 920: 00$ & Manual Tape & 386 & Transcribed & \\
\hline $2 / 6 / 920: 00$ & Manual FIC & 386.2 & Good & \\
\hline $2 / 7 / 920: 00$ & Manual Tape & 386.00 & Transcribed & \\
\hline $2 / 7 / 920: 00$ & Manual FIC & 386.20 & Good & \\
\hline $2 / 8 / 920: 00$ & Manual Tape & 386 & Transcribed & \\
\hline $2 / 8 / 927: 04$ & Auto FIC & 386.20 & Good & \\
\hline 2/9/92 0:00 & Manual Tape & 386.00 & Transcribed & \\
\hline 2/9/92 7:03 & Auto FIC & 386.20 & Good & \\
\hline $2 / 10 / 920: 00$ & Manual Tape & 386 & Transcribed & \\
\hline
\end{tabular}

Data obtained from WHC Surveillance Analysis Computer System, (PC SACS) July 7, 1995. 


\begin{tabular}{|c|c|c|c|c|}
\hline \multicolumn{5}{|c|}{ 241-AP-101 } \\
\hline \multicolumn{5}{|c|}{ Surface Level Data } \\
\hline Date & Type of Measurement & Level (Inches) & Data Quality & Comments \\
\hline $2 / 10 / 927: 04$ & Auto FIC & 386.20 & Good & . \\
\hline $2 / 11 / 920: 00$ & Manual Tape & 386.00 & Transcribed & \\
\hline $2 / 11 / 927: 03$ & Auto FIC & 386.2 & Good & \\
\hline $2 / 12 / 920: 00$ & Manual Tape & 386 & Transcribed & \\
\hline $2 / 12 / 927: 03$ & Auto FIC & 386.20 & Good & \\
\hline $2 / 13 / 920: 00$ & Manual Tape & 386.00 & Transcribed & \\
\hline 2/13/92 7:03 & Auto FIC & 386.2 & Good & \\
\hline $2 / 14 / 920: 00$ & Manual Tape & 386 & Transcribed & \\
\hline $2 / 14 / 927: 03$ & Auto FIC & 386.2 & Good & \\
\hline $2 / 15 / 920: 00$ & Manual Tape & 386.00 & Transcribed & \\
\hline 2/15/92 7:03 & Auto FIC & 386.20 & Good & \\
\hline $2 / 16 / 920: 00$ & Manual Tape & 386 & Transcribed & \\
\hline $2 / 16 / 927: 03$ & Auto FIC & 386.20 & Good & \\
\hline $2 / 17 / 920: 00$ & Manual Tape & 386.00 & Transcribed & \\
\hline $2 / 17 / 927: 03$ & Auto FIC & 386.2 & Good & \\
\hline $2 / 18 / 920: 00$ & Manual Tape & 386 & Transcribed & \\
\hline $2 / 18 / 927: 03$ & Auto FIC & 386.20 & Good & \\
\hline 2/19/92 0:00 & Manual Tape & 386.00 & Transcribed & \\
\hline $2 / 19 / 927: 03$ & Auto FIC & 386.20 & Good & \\
\hline $2 / 20 / 920: 00$ & Manual Tape & 386 & Transcribed & \\
\hline $2 / 20 / 927: 03$ & Auto FIC & 386.2 & Good & \\
\hline $2 / 21 / 920: 00$ & Manual Tape & 386.00 & Transcribed & \\
\hline $2 / 21 / 927: 03$ & Auto FIC & 386.20 & Good & \\
\hline $2 / 22 / 920: 00$ & Manual Tape & 386 & Transcribed & \\
\hline $2 / 22 / 927: 03$ & Auto FIC & 386.2 & Good & \\
\hline $2 / 23 / 920: 00$ & Manual Tape & 386.00 & Transcribed & \\
\hline $2 / 23 / 927: 03$ & Auto FIC & 386.20 & Good & \\
\hline $2 / 24 / 920: 00$ & Manual Tape & 386 & Transcribed & \\
\hline $2 / 24 / 927: 03$ & Auto FIC & 386.2 & Good & \\
\hline $2 / 25 / 920: 00$ & Manual Tape & 386.00 & Transcribed & \\
\hline $2 / 25 / 927: 04$ & Auto FIC & 386.20 & Good & \\
\hline $2 / 26 / 920: 00$ & Manual Tape & 386 & Transcribed & \\
\hline $2 / 26 / 927: 03$ & Auto FIC & 386.2 & Good & \\
\hline 2/27/920:00 & Manual Tape & 386.00 & Transcribed & \\
\hline 2/27/92 7:04 & Auto FIC & 386.20 & Good & \\
\hline 2/28/92 0:00 & Manual Tape & 386 & Transcribed & \\
\hline $2 / 28 / 927: 04$ & Auto FIC & 386.20 & - Good & \\
\hline $2 / 29 / 920: 00$ & Manual Tape & 386.00 & Transcribed & \\
\hline $2 / 29 / 927: 03$ & Auto FIC & 386.2 & Good & \\
\hline $3 / 1 / 920: 00$ & Manual Tape & 386 & Transcribed & \\
\hline $3 / 1 / 927: 03$ & Auto FIC & 386.20 & Good & \\
\hline $3 / 2 / 920: 00$ & Manual Tape & 386.00 & Transcribed & \\
\hline $3 / 2 / 927: 03$ & Auto FIC & 386.2 & Good & \\
\hline $3 / 3 / 920: 00$ & Manual Tape & 386 & Transcribed & \\
\hline $3 / 3 / 927: 04$ & Auto FIC & 386.20 & Good & \\
\hline 3/4/92 0:00 & Manual Tape & 386.00 & Transcribed & \\
\hline $3 / 4 / 927: 04$ & Auto FIC & 386.2 & Good & \\
\hline $3 / 5 / 920: 00$ & Manual Tape & 386 & Transcribed & \\
\hline
\end{tabular}

Data obtained from WHC Surveillance Analysis Computer System, (PC SACS) July 7, 1995. 


\begin{tabular}{|c|c|c|c|c|}
\hline \multicolumn{5}{|c|}{ 241-AP-101 } \\
\hline \multicolumn{5}{|c|}{ Surface Level Data } \\
\hline Date & Type of Measurement & Level (Inches) & Data Quality & Comments \\
\hline $3 / 5 / 927: 03$ & Auto FIC & 386.20 & Good & \\
\hline $3 / 6 / 920: 00$ & Manual Tape & 386.00 & Transcribed & \\
\hline $3 / 6 / 927: 03$ & Auto FIC & 386.2 & Good & \\
\hline 3/7/92 0:00 & Manual Tape & 386 & Transcribed & \\
\hline $3 / 7 / 927: 02$ & Auto FIC & 386.20 & Good & \\
\hline $3 / 8 / 920: 00$ & Manual Tape & 386.00 & Transcribed & \\
\hline $3 / 8 / 927: 02$ & Auto FIC & 386.2 & Good & \\
\hline $3 / 9 / 920: 00$ & Manual Tape & 386 & Transcribed & \\
\hline $3 / 9 / 927: 02$ & Auto FIC & 386.20 & Good & \\
\hline $3 / 10 / 92 \quad 0: 00$ & Manual Tape & 386.00 & Transcribed & \\
\hline $3 / 10 / 927: 03$ & Auto FIC & 386.2 & Good & \\
\hline $3 / 11 / 920: 00$ & Manual Tape & 386 & Transcribed & \\
\hline $3 / 11 / 927: 03$ & Auto FIC & 386.20 & Good & \\
\hline $3 / 12 / 920: 00$ & Manual Tape & 386.00 & Transcribed & \\
\hline $3 / 12 / 927: 03$ & Auto FIC & 386.2 & Good & \\
\hline $3 / 13 / 920: 00$ & Manual Tape & 386 & Transcribed & \\
\hline $3 / 13 / 927: 03$ & Auto FIC & 386.20 & Good & \\
\hline $3 / 14 / 920: 00$ & Manual Tape & 386.00 & Transcribed & \\
\hline $3 / 14 / 927: 03$ & Auto FIC & 386.2 & Good & \\
\hline $3 / 15 / 920: 00$ & Manual Tape & 386 & Transcribed & \\
\hline $3 / 15 / 927: 06$ & Auto FIC & 386.20 & Good & \\
\hline $3 / 16 / 920: 00$ & Manual Tape & 386.00 & Transcribed & \\
\hline $3 / 16 / 927: 03$ & Auto FIC & 386.2 & Good & \\
\hline $3 / 17 / 920: 00$ & Manual Tape & 386 & Transcribed & \\
\hline $3 / 17 / 927: 03$ & Auto FIC & 386.20 & Good & \\
\hline $3 / 18 / 920: 00$ & Manual Tape & 386.00 & Transcribed & \\
\hline $3 / 18 / 927: 03$ & Auto FIC & 386.2 & Good & \\
\hline $3 / 19 / 920: 00$ & Manual Tape & 386 & Transcribed & \\
\hline $3 / 19 / 926: 02$ & Auto FIC & 386.20 & Good & \\
\hline $3 / 20 / 920: 00$ & Manual Tape & 386.00 & Transcribed & \\
\hline $3 / 20 / 927: 02$ & Auto FIC & 386.2 & Good & \\
\hline $3 / 21 / 920: 00$ & Manual Tape & 386 & Transcribed & \\
\hline $3 / 21 / 927: 03$ & Auto FIC & 386.20 & Good & \\
\hline $3 / 22 / 920: 00$ & Manual Tape & 386.00 & Transcribed & \\
\hline $3 / 22 / 927: 02$ & Auto FIC & 386.2 & Good & \\
\hline $3 / 23 / 920: 00$ & Manual Tape & 386 & Transcribed & \\
\hline $3 / 23 / 927: 02$ & Auto FIC & 386.20 & Good & \\
\hline $3 / 24 / 920: 00$ & Manual Tape & 386.00 & Transcribed & \\
\hline $3 / 24 / 927: 02$ & Auto FIC & 386.2 & Good & \\
\hline $3 / 25 / 920: 00$ & Manual Tape & 386 & Transcribed & \\
\hline $3 / 25 / 927: 03$ & Auto FIC & 386.20 & Good & \\
\hline $3 / 26 / 920: 00$ & Manual Tape & 386.00 & Transcribed & \\
\hline $3 / 26 / 927: 03$ & Auto FIC & 386.20 & Good & \\
\hline $3 / 27 / 920: 00$ & Manual Tape & 386 & Transcribed & \\
\hline $3 / 27 / 927: 02$ & Auto FIC & 386.2 & Good & \\
\hline $3 / 28 / 920: 00$ & Manual Tape & 386 & Transcribed & \\
\hline $3 / 28 / 927: 03$ & Auto FIC & 386.20 & Good & \\
\hline $3 / 29 / 920: 00$ & Manual Tape & 386.00 & Transcribed & \\
\hline
\end{tabular}

Data obtained from WHC Surveillance Analysts Computer System, (PC SACS) July 7, 1995. 


\begin{tabular}{|c|c|c|c|c|}
\hline \multicolumn{5}{|c|}{ 241-AP-101 } \\
\hline \multicolumn{5}{|c|}{ Surface Levol Data } \\
\hline Date & Type of Measurement & Level (Inches) & Data Quality & Comments \\
\hline $3 / 29 / 927: 03$ & Auto FIC & 386.2 & Good & \\
\hline $3 / 30 / 920: 00$ & Manual Tape & 386 & Transçribed & \\
\hline $3 / 30 / 927: 03$ & Auto FIC & 386.20 & Good & \\
\hline $3 / 31 / 920: 00$ & Manual Tape & 386.00 & Transcribed & \\
\hline $3 / 31 / 927: 03$ & Auto FIC & 386.20 & Good & \\
\hline $4 / 1 / 920: 00$ & Manual Tape & 386 & Transcribed & \\
\hline 4/1/92 7:03 & Auto FIC & 386.2 & Good & \\
\hline 4/2/920:00 & Manual Tape & 386.00 & Transcribed & \\
\hline 4/2/92 7:03 & Auto FIC & 386.2 & Good & \\
\hline $4 / 3 / 920: 00$ & Manual Tape & 386 & Transcribed & \\
\hline $4 / 3 / 927: 03$ & Auto FIC & 386.20 & Good & \\
\hline $4 / 4 / 920: 00$ & Manual Tape & 386.00 & Transcribed & \\
\hline 4/4/92 7:03 & Auto FIC & 386.2 & Good & \\
\hline $4 / 5 / 920: 00$ & Manual Tape & 386 & Transcribed & \\
\hline $4 / 5 / 927: 03$ & Auto FIC & 386.10 & Good & \\
\hline $4 / 6 / 920.00$ & Manual Tape & 386.00 & Transcribed & \\
\hline $4 / 6 / 927: 03$ & Auto FIC & 386.1 & Good & \\
\hline $4 / 7 / 920: 00$ & Manual Tape & 385.5 & Transcribed & \\
\hline $4 / 7 / 927: 03$ & Auto FIC & 386.10 & Good & \\
\hline $4 / 8 / 920: 00$ & Manual Tape & 386.00 & Transcribed & \\
\hline 4/8/92 5:02 & Auto FIC & 386.10 & Good & \\
\hline $4 / 9 / 920: 00$ & Manual Tape & 385.75 & Transcribed & \\
\hline 4/9/92 7:03 & Auto FIC & 386.1 & Good & \\
\hline $4 / 10 / 920: 00$ & Manual Tape . & 385.75 & Transcribed & \\
\hline $4 / 10 / 927: 03$ & Auto FIC & 386.1 & Good & \\
\hline $4 / 11 / 920: 00$ & Manual Tape & 386 & Transcribed & \\
\hline $4 / 11 / 927: 03$ & Auto FIC & 386.10 & Good & \\
\hline $4 / 12 / 920: 00$ & Manual Tape & 386.00 & Transcribed & \\
\hline $4 / 12 / 925: 03$ & Auto FIC & 386.1 & Good & \\
\hline 4/13/920:00 & Manual Tape & 386 & Transcribed & \\
\hline 4/13/92 7:03 & Auto FIC & 386.1 & Good & \\
\hline $4 / 14 / 920: 00$ & Manual Tape & 386.00 & Transcribed & \\
\hline $4 / 14 / 927: 03$ & Auto FIC & 386.10 & Good & \\
\hline 4/15/92 0:00 & Manual Tape & 386 & Transcribed & \\
\hline $4 / 15 / 927: 02$ & Auto FIC & 386.10 & Good & \\
\hline 4/16/92 0:00 & Manual Tape & 386.00 & Transcribed & \\
\hline 4/16/92 7:03 & Auto FIC & 386.1 & Good & \\
\hline $4 / 17 / 920: 00$ & Manual Tape & 385.75 & Transcribed & \\
\hline 4/17/92 7:03 & Auto FIC & 386.10 & Good & \\
\hline $4 / 18 / 920: 00$ & Manual Tape & 386.00 & Transcribed & \\
\hline $4 / 18 / 927: 02$ & Auto FIC & 386.10 & Good & \\
\hline $4 / 19 / 920: 00$ & Manual Tape & 386 & Transcribed & \\
\hline $4 / 19 / 927: 02$ & Auto FIC & 386.1 & Good & \\
\hline 4/20/92 0:00 & Manual Tape & 386.00 & Transcribed & \\
\hline $4 / 20 / 927: 03$ & Auto FIC & 386.10 & Good & \\
\hline 4/21/92 0:00 & Manual Tape & 386 & Transcribed & \\
\hline $4 / 21 / 927: 03$ & Auto FIC & 386.1 & - Good & \\
\hline $4 / 22 / 920: 00$ & Manual Tape & 386.00 & Transcribed & \\
\hline
\end{tabular}

Data obtained from WHC Surveillance Analysis Computer System, (PC SACS) July 7, 1995. 


\begin{tabular}{|c|c|c|c|c|}
\hline \multicolumn{5}{|c|}{ 241-AP-101 } \\
\hline \multicolumn{5}{|c|}{ Surface Level Data } \\
\hline Date & Type of Measurement & Level (Inches) & Data Quality & Comments \\
\hline $4 / 22 / 927: 02$ & Auto FIC & 386.10 & Good & \\
\hline $4 / 23 / 920: 00$ & Manual Tape & 386 & Transcribed & \\
\hline 4/23/92 7:03 & Auto FIC & 386.1 & Good & \\
\hline $4 / 24 / 920: 00$ & Manual Tape & 386.00 & Transcribed & \\
\hline $4 / 24 / 927: 03$ & Auto FIC & 386.10 & Good & \\
\hline $4 / 25 / 920: 00$ & Manual Tape & 386 & Transcribed & \\
\hline 4/25/92 7:04 & Auto FIC & 386.1 & Good & \\
\hline $4 / 26 / 920: 00$ & Manual Tape & 386.00 & Transcribed & \\
\hline $4 / 26 / 927: 03$ & Auto FIC & 386.1 & Good & \\
\hline $4 / 27 / 920: 00$ & Manual Tape & 385.25 & Transcribed & \\
\hline $4 / 27 / 927: 04$ & Auto FIC & 386.10 & Good & \\
\hline $4 / 28 / 920: 00$ & Manual Tape & 385.25 & Transcribed & \\
\hline $4 / 28 / 927: 03$ & Auto FIC & 386.1 & Good & \\
\hline $4 / 29 / 920: 00$ & Manual Tape & 386 & Transcribed & \\
\hline $4 / 29 / 927: 03$ & Auto FIC & 386.10 & Good & \\
\hline $4 / 30 / 920: 00$ & Manual Tape & 386 & Transcribed & \\
\hline $4 / 30 / 92$ 7:03 & Auto FIC & 386.1 & Good & \\
\hline $5 / 1 / 920: 00$ & Manual Tape & 386.00 & Transcribed & \\
\hline $5 / 1 / 927: 03$ & Auto FIC & 386.10 & Good & \\
\hline $5 / 2 / 920: 00$ & Manual Tape & 386 & Transcribed & \\
\hline $5 / 2 / 922: 03$ & Auto FIC & 386.1 & Good & \\
\hline $5 / 3 / 920: 00$ & Manual Tape & 386.00 & Transcribed & \\
\hline $5 / 3 / 927: 03$ & Auto FIC & 386.10 & Good & \\
\hline $5 / 4 / 920: 00$ & Manual Tape & 385 & Transcribed & \\
\hline $5 / 4 / 92$ 7:03 & Auto FIC & 386.10 & Good & \\
\hline $5 / 5 / 920: 00$ & Manual Tape & 385.00 & Transcribed & \\
\hline $5 / 5 / 927: 04$ & Auto FIC & 386.1 & Good & \\
\hline $5 / 6 / 920: 00$ & Manual Tape & 385.75 & Transcribed & \\
\hline $5 / 6 / 927: 03$ & Auto FIC & 386.10 & Good & \\
\hline $5 / 7 / 920: 00$ & Manual Tape & 385.75 & Transcribed & \\
\hline $5 / 7 / 927: 04$ & Auto FIC & 386.1 & Good & \\
\hline $5 / 8 / 920: 00$ & Manual Tape & 385.75 & Transcribed & \\
\hline 5/8/92 7:03 & Auto FIC & 386.10 & Good & \\
\hline $5 / 9 / 920: 00$ & Manual Tape & 386.00 & Transcribed & \\
\hline $5 / 9 / 920: 00$ & Manual FIC & 386.1 & Good & \\
\hline $5 / 10 / 920: 00$ & Manual Tape & 386 & Transcribed & \\
\hline $5 / 10 / 926: 03$ & Auto FIC & 386.1 & Good & \\
\hline $5 / 11 / 920: 00$ & Manual Tape & 386.00 & Transcribed & \\
\hline $5 / 11 / 920: 00$ & Manual FIC & 386.10 & Good & \\
\hline $5 / 12 / 920: 00$ & Manual Tape & 386 & Transcribed & \\
\hline $5 / 12 / 927: 03$ & Auto FIC & 386.10 & Good & \\
\hline $5 / 13 / 920: 00$ & Manual Tape & 385.75 & Transcribed & \\
\hline $5 / 13 / 927: 03$ & Auto FIC & 386.1 & Good & \\
\hline $5 / 14 / 920: 00$ & Manual Tape & 385.75 & Transcribed & \\
\hline $5 / 14 / 927: 03$ & Auto FIC & 386.10 & Good & \\
\hline $5 / 15 / 920: 00$ & Manual Tape & 386.00 & Transcribed & \\
\hline $5 / 15 / 927: 03$ & Auto FIC & 386.1 & Good & \\
\hline $5 / 16 / 920: 00$ & Manual Tape & 386 & Transcribed & \\
\hline
\end{tabular}

Data obtained from WHC Surveillance Analysis Computer System, (PC SACS) July 7, 1995. 


\begin{tabular}{|c|c|c|c|c|}
\hline \multicolumn{5}{|c|}{ 241-AP-101 } \\
\hline \multicolumn{5}{|c|}{ Surface Level Data } \\
\hline Date & Type of Measurement & Level (Inches) & Data Quality & Comments \\
\hline $5 / 16 / 927: 03$ & Auto FIC & 386.10 & Good & \\
\hline $5 / 17 / 920: 00$ & Manual Tape & 386.00 & Transcribed & \\
\hline $5 / 17 / 927: 02$ & Auto FIC & 386.1 & Good & \\
\hline $5 / 18 / 920: 00$ & Manual Tape & 386 & Transcribed & \\
\hline $5 / 18 / 927: 03$ & Auto FIC & 386.10 & Good & \\
\hline $5 / 19 / 920: 00$ & Manual Tape & 386.00 & Transcribed & \\
\hline $5 / 19 / 927: 03$ & Auto FIC & 386.1 & Good & \\
\hline $5 / 20 / 920: 00$ & Manual Tape & 386 & Transcribed & \\
\hline $5 / 20 / 927: 03$ & Auto FIC & 386.10 & Good & \\
\hline $5 / 21 / 920: 00$ & Manual Tape & 386.00 & Transcribed & \\
\hline $5 / 21 / 927: 03$ & Auto FIC & 386.1 & Good & \\
\hline $5 / 22 / 920: 00$ & Manual Tape & 386 & Transcribed & \\
\hline $5 / 22 / 927: 02$ & Auto FIC & 386.10 & Good & \\
\hline $5 / 23 / 920: 00$ & Manual Tape & 386.00 & Transcribed & \\
\hline $5 / 23 / 927: 03$ & Auto FIC & 386.1 & Good & \\
\hline $5 / 24 / 920: 00$ & Manual Tape & 386 & Transcribed & \\
\hline $5 / 24 / 927: 03$ & Auto FIC & 386.10 & Good & \\
\hline $5 / 25 / 920: 00$ & Manual Tape & 386.00 & Transcribed & \\
\hline $5 / 25 / 927: 03$ & Auto FIC & 386.1 & Good & \\
\hline $5 / 26 / 920: 00$ & Manual Tape & 386 & Transcribed & \\
\hline $5 / 26 / 920: 00$ & Manual FIC & 386.1 & Good & \\
\hline $5 / 27 / 920: 00$ & Manual Tape & 386.00 & Transcribed & \\
\hline $5 / 27 / 927: 03$ & Auto FIC & 386.10 & Good & \\
\hline $5 / 28 / 920: 00$ & Manual Tape & 386 & Transcribed & \\
\hline $5 / 28 / 927: 02$ & Auto FIC & 386.1 & Good & \\
\hline $5 / 29 / 920: 00$ & Manual Tape & 386.00 & Transcribed & \\
\hline $5 / 29 / 927: 02$ & Auto FIC & 386.10 & Good & \\
\hline $5 / 30 / 920: 00$ & Manual Tape & 386 & Transcribed & \\
\hline $5 / 30 / 927: 03$ & Auto FIC & 386.1 & Good & \\
\hline $5 / 31 / 920: 00$ & Manual Tape & 386.00 & Transcribed & \\
\hline $5 / 31 / 927: 03$ & Auto FIC & 386.10 & Good & \\
\hline $6 / 1 / 920: 00$ & Manual Tape & 386 & Transcribed & \\
\hline $6 / 1 / 927: 03$ & Auto FIC & 386.1 & Good & \\
\hline $6 / 2 / 920: 00$ & Manual Tape & 386.00 & Transcribed & \\
\hline $6 / 2 / 927: 03$ & Auto FIC & 386.10 & Good & \\
\hline $6 / 3 / 920: 00$ & Manual Tape & 386 & Transcribed & \\
\hline $6 / 3 / 927: 03$ & Auto FIC & 386.10 & Good & \\
\hline $6 / 4 / 920: 00$ & Manual Tape & 386.00 & Transcribed & \\
\hline $6 / 4 / 927: 03$ & Auto FIC & 386.1 & Good & \\
\hline $6 / 5 / 920: 00$ & Manual Tape & 386 & Transcribed & \\
\hline $6 / 5 / 927: 02$ & Auto FIC & 386.1 & Good & \\
\hline $6 / 6 / 920: 00$ & Manual Tape & 386.00 & Transcribed & \\
\hline $6 / 6 / 927: 03$ & Auto FIC & 386.10 & Good & \\
\hline $6 / 7 / 920: 00$ & Manual Tape & 386 & Transcribed & \\
\hline 6/7/92 7:03 & Auto FIC & 386.1 & Good & \\
\hline $6 / 8 / 920: 00$ & Manual Tape & 386.00 & Transcribed & \\
\hline 6/8/92 7:03 & Auto FIC & 386.10 & Good & \\
\hline $6 / 9 / 920: 00$ & Manual Tape & 385.5 & Transcribed & \\
\hline
\end{tabular}

Data obtained from WHC Surveillance Analysis Computer System, (PC SACS) July 7, 1995. 


\begin{tabular}{|c|c|c|c|c|}
\hline \multicolumn{5}{|c|}{ 241-AP-101 } \\
\hline \multicolumn{5}{|c|}{ Surface Leval Data } \\
\hline Date & Type of Measurement & Level (Inches) & Data Quality & Comments \\
\hline $6 / 9 / 927: 03$ & Auto FIC & 386.1 & Good & \\
\hline $6 / 10 / 920: 00$ & Manual Tape & 386.00 & Transcribed & \\
\hline $6 / 10 / 927: 03$ & Auto FIC & 386.10 & Good & \\
\hline $6 / 11 / 920: 00$ & Manual Tape & 385.5 & Transcribed & \\
\hline $6 / 11 / 927: 03$ & Auto FIC & 386.1 & Good & \\
\hline $6 / 12 / 920: 00$ & Manual Tape & 385.50 & Transcribed & \\
\hline $6 / 12 / 927: 05$ & Auto FIC & 386.1 & Good & \\
\hline $6 / 13 / 920: 00$ & Manual Tape & 385.5 & Transcribed & \\
\hline 6/13/92 7:05 & Auto FIC & 386.10 & Good & \\
\hline $6 / 14 / 920: 00$ & Manual Tape & 385.75 & Transcribed & \\
\hline $6 / 14 / 920: 00$ & Manual FIC & 386.10 & Good & \\
\hline $6 / 15 / 920: 00$ & Manual Tope & 386 & Transcribed & \\
\hline $6 / 15 / 927: 03$ & Auto FIC & 386.10 & Good & \\
\hline $6 / 16 / 920: 00$ & Manual Tape & 386.00 & Transcribed & \\
\hline $6 / 16 / 927: 03$ & Auto FIC & 386.1 & Good & \\
\hline $6 / 17 / 920: 00$ & Manual Tape & 386 & Transcribed & \\
\hline $6 / 17 / 927: 03$ & Auto FIC & 386.10 & Good & \\
\hline $6 / 18 / 920: 00$ & Manual Tape & 385.50 & Transcribed & \\
\hline $6 / 18 / 927: 03$ & Auto FIC & 386.1 & Good & \\
\hline $6 / 19 / 920: 00$ & Manual Tape & 386 & Transcribed & \\
\hline $6 / 19 / 927: 02$ & Auto FIC & 386.10 & Good & \\
\hline $6 / 20 / 920: 00$ & Manual Tape & 386.00 & Transcribed & \\
\hline $6 / 20 / 92 \quad 7: 02$ & Auto FIC & 386.1 & Good & \\
\hline $6 / 21 / 920: 00$ & Manual Tape & 386 & Transcribed & \\
\hline $6 / 21 / 927: 03$ & Auto FIC & 386.10 & Good & \\
\hline 6/22/92 0:00 & Manual Tape & 386.00 & Transçribed & \\
\hline $6 / 22 / 927: 03$ & Auto FIC & 386.10 & Good & \\
\hline $6 / 23 / 920: 00$ & Manual Tape & 386 & Transcribed & \\
\hline $6 / 23 / 927: 03$ & Auto FIC & 386.1 & Good & \\
\hline $6 / 24 / 920: 00$ & Manual Tape & 386.00 & Transcribed & \\
\hline $6 / 24 / 927: 03$ & Auto FIC & 386.10 & Good & \\
\hline $6 / 25 / 92 \quad 0: 00$ & Manual Tape & 386 & Transcribed & \\
\hline $6 / 25 / 927: 03$ & Auto FIC & 386.1 & Good & \\
\hline 6/26/92 0:00 & Manual Tape & 386.00 & Transcribed & \\
\hline $6 / 26 / 927: 02$ & Auto FIC & 386.10 & Good & \\
\hline $6 / 27 / 920: 00$ & Manual Tape & 386 & Transcribed & \\
\hline $6 / 27 / 927: 03$ & Auto FIC & 386.1 & Good & \\
\hline $6 / 28 / 920: 00$ & Manual Tape & 386.00 & Transcribed & \\
\hline $6 / 28 / 927: 06$ & Auto FIC & 386.00 & Good & \\
\hline $6 / 29 / 920: 00$ & Manual Tape & 385.5 & Transcribed & \\
\hline $6 / 29 / 927: 06$ & Auto FIC & 386.1 & Good & \\
\hline $6 / 30 / 92 \quad 0: 00$ & Manual Tape & 385.50 & Transcribed & \\
\hline $6 / 30 / 927: 06$ & Auto FIC & 386.1 & Good & \\
\hline $7 / 1 / 920: 00$ & Manual Tape & 385.75 & Transcribed & \\
\hline $7 / 1 / 920: 00$ & Manual FIC & 386.1 & Good & \\
\hline $7 / 2 / 920: 00$ & Manual Tape & 385.75 & Transcribed & \\
\hline $7 / 2 / 920: 00$ & Manual FIC & 386.10 & Good & \\
\hline $7 / 3 / 920: 00$ & Manual Tape. & 386 & Transcribed & \\
\hline
\end{tabular}

Data obtained from WHC Surveillance Analysis Computer System, (PC SACS) July 7, 1995. 


\begin{tabular}{|c|c|c|c|c|}
\hline \multicolumn{5}{|c|}{ 241-AP-101 } \\
\hline \multicolumn{5}{|c|}{ Surface Level Data } \\
\hline Date & Type of Measurement & Leval (Inches) & Data Quality & Comments \\
\hline $7 / 3 / 927: 06$ & Auto FIC & 386.10 & Good & \\
\hline $7 / 4 / 920: 00$ & Manual Tape & 387.00 & Transcribed & \\
\hline 7/4/92 7:06 & Auto FIC & 386.1 & Good & \\
\hline $7 / 5 / 920: 00$ & Manual Tape & 386 & Transcribed & \\
\hline $7 / 5 / 927: 06$ & Auto FIC & 386.10 & Good & \\
\hline $7 / 6 / 920: 00$ & Manual Tape & 386.00 & Transcribed & \\
\hline $7 / 6 / 927: 06$ & Auto FIC & 386.1 & Good & \\
\hline $7 / 7 / 920: 00$ & Manual Tape & 384.5 & Transcribed & \\
\hline $7 / 7 / 927: 06$ & Auto FIC & 386.10 & Good & \\
\hline $7 / 8 / 920: 00$ & Manual Tape & 386.00 & Transcribed & \\
\hline $7 / 8 / 927: 06$ & Auto FIC & 386.1 & Good & \\
\hline $7 / 9 / 920: 00$ & Manual Tape & 386 & Transcribed & \\
\hline $7 / 9 / 927: 06$ & Auto FIC & 386.10 & Good & \\
\hline $7 / 10 / 920: 00$ & Manual Tape & 386.00 & Transcribed & \\
\hline $7 / 10 / 927: 06$ & Auto FIC & 386.1 & Good & \\
\hline 7/11/92 0:00 & Manual Tape & 386 & Transcribed & \\
\hline 7/11/92 7:06 & Auto FIC & 386.10 & Good & \\
\hline $7 / 12 / 920: 00$ & Manual Tape & 386.00 & Transcribed & \\
\hline 7/12/92 7:06 & Auto FIC & 386.1 & Good & \\
\hline $7 / 13 / 920: 00$ & Manual Tape & 386 & Transcribed & \\
\hline $7 / 13 / 927: 06$ & Auto FIC & 386.10 & Good & \\
\hline $7 / 14 / 920: 00$ & Manual Tape & 386.00 & Transcribed & \\
\hline $7 / 14 / 927: 06$ & Auto FIC & 386.1 & Good & \\
\hline $7 / 15 / 920: 00$ & Manual Tape & 386 & Transcribed & \\
\hline $7 / 15 / 927: 06$ & Auto FIC & 386.10 & Good & \\
\hline $7 / 16 / 920: 00$ & Manual Tape & 386.00 & Transcribed & \\
\hline $7 / 16 / 927: 06$ & Auto FIC & 386.1 & Good & \\
\hline $7 / 17 / 92 \quad 0: 00$ & Manual Tape & 386 & Transcribed & \\
\hline $7 / 17 / 927: 06$ & Auto FIC & 386.10 & Good & \\
\hline $7 / 18 / 920: 00$ & Manual Tape & 386.00 & Transcribed & \\
\hline 7/18/92 7:06 & Auto FIC & 386.1 & Good & \\
\hline $7 / 19 / 920: 00$ & Manual Tape & 386 & Transcribed & \\
\hline $7 / 19 / 927: 06$ & Auto FIC & 386.10 & Good & \\
\hline $7 / 20 / 920: 00$ & Manual Tape & 386.00 & Transcribed & \\
\hline $7 / 20 / 927: 06$ & Auto FIC & 386.1 & Good & \\
\hline $7 / 21 / 920: 00$ & Manual Tape & 385.75 & Transcribed & \\
\hline 7/21/92 7:05 & Auto FIC & 386.10 & Good & \\
\hline $7 / 22 / 920: 00$ & Manual Tape & 385.75 & Transcribed & \\
\hline $7 / 22 / 927: 06$ & Auto FIC & 386.10 & Good & \\
\hline $7 / 23 / 920: 00$ & Manual FIC & 386.1 & Transcribed & \\
\hline $7 / 23 / 92 \quad 0: 00$ & Manual Tape & 385.75 & Transcribed & \\
\hline $7 / 24 / 920: 00$ & Manual Tape & 385.75 & Transcribed & \\
\hline $7 / 24 / 927: 05$ & Auto FIC & 386.1 & Good & \\
\hline $7 / 25 / 920: 00$ & Manual Tape & 385.75 & Transcribed & \\
\hline $7 / 25 / 927: 06$ & Auto FIC & 386.10 & Good & \\
\hline $7 / 26 / 920: 00$ & Manual Tape & 385.75 & Transcribed & \\
\hline $7 / 26 / 927: 06$ & Auto FIC & 386.1 & Good & \\
\hline $7 / 27 / 920: 00$ & Manual Tape & 385.75 & Transcribed & \\
\hline
\end{tabular}

Data obtained from WHC Surveillance Analysis Computer System, (PC SACS) July 7, 1995. 


\begin{tabular}{|c|c|c|c|c|}
\hline \multicolumn{5}{|c|}{ 241-AP-101 } \\
\hline \multicolumn{5}{|c|}{ - Surface Level Data } \\
\hline Date & Type of Measurement & Level (Inches) & Data Quality & Comments \\
\hline $7 / 27 / 927: 05$ & Auto FIC & 386.10 & Good & \\
\hline $7 / 28 / 920: 00$ & Manual Tape & 386.00 & Transcribed & \\
\hline $7 / 28 / 927: 06$ & Auto FIC & 386.10 & Good & \\
\hline $7 / 29 / 920: 00$ & Manual Tape & 386 & Transcribed & \\
\hline $7 / 29 / 927: 06$ & Auto FIC & 386.1 & Good & \\
\hline $7 / 30 / 920: 00$ & Manual Tape & 386.00 & Transcribed & \\
\hline $7 / 30 / 927: 06$ & Auto FIC & 386.10 & Good & \\
\hline $7 / 31 / 920: 00$ & Manual Tape & 386 & Transcribed & \\
\hline $7 / 31 / 927: 05$ & Auto FIC & 386.1 & Good & \\
\hline $8 / 1 / 920: 00$ & Manual Tape & 386.00 & Transcribed & \\
\hline 8/1/92 7:06 & Auto FIC & 386.10 & Good & \\
\hline $8 / 2 / 920: 00$ & Manual Tape & 386 & Transcribed & \\
\hline $8 / 2 / 927: 06$ & Auto FIC & 386.1 & Good & \\
\hline $8 / 3 / 920: 00$ & Manual Tape & 386.00 & Transcribed & \\
\hline $8 / 3 / 927: 06$ & Auto FIC & 386.10 & Good & \\
\hline $8 / 4 / 920: 00$ & Manual Tape & 386 & Transcribed & \\
\hline $8 / 4 / 927: 06$ & Auto FIC & 386.1 & Good & \\
\hline $8 / 5 / 920: 00$ & Manual Tape & 386.00 & Transcribed & \\
\hline $8 / 5 / 927: 05$ & Auto FIC & 386.10 & Good & \\
\hline $8 / 6 / 920: 00$ & Manual Tape & 386 & Transcribed & \\
\hline $8 / 6 / 927: 05$ & Auto FIC & 386.1 & Good & \\
\hline $8 / 7 / 920: 00$ & Manual Tape & 386.00 & Transcribed & \\
\hline $8 / 7 / 927: 05$ & Auto FIC & 386.10 & Good & \\
\hline $8 / 8 / 920: 00$ & Manual Tape & 386 & Transcribed & \\
\hline 8/8/92 7:06 & Auto FIC & 386.1 & Good & \\
\hline $8 / 9 / 920: 00$ & Manual Tape & 386.00 & Transcribed & \\
\hline $8 / 9 / 927: 06$ & Auto FIC & 386.10 & Good & \\
\hline $8 / 10 / 920: 00$ & Manual Tape & 386 & Transcribed & \\
\hline $8 / 10 / 927: 05$ & Auto FIC & 386.1 & Good & \\
\hline $8 / 11 / 920: 00$ & Manual Tape & 386.00 & Transcribed & \\
\hline $8 / 11 / 927: 06$ & Auto FIC & 386.10 & Good & \\
\hline $8 / 12 / 920: 00$ & Manual Tape & 385.5 & Transcribed & \\
\hline $8 / 12 / 927: 06$ & Auto FIC & 386.1 & Good & \\
\hline 8/13/92 0:00 & Manual Tape & 385.50 & Transcribed & \\
\hline $8 / 13 / 927: 06$ & Auto FIC & 386.10 & Good & \\
\hline $8 / 14 / 920: 00$ & Manual Tape & 386 & Transcribed & \\
\hline $8 / 14 / 927: 06$ & Auto FIC & 386.1 & Good & \\
\hline $8 / 15 / 920: 00$ & Manual Tape & 385.75 & Transcribed & \\
\hline $8 / 15 / 927: 06$ & Auto FIC & 386.10 & Good & \\
\hline $8 / 16 / 920: 00$ & Manual Tape & 385.75 & Transcribed & \\
\hline $8 / 16 / 927: 06$ & Auto FIC & 386.1 & Good & \\
\hline $8 / 17 / 920: 00$ & Manual Tape & 385.75 & Transcribed & \\
\hline $8 / 17 / 927: 06$ & Auto FIC & 386.10 & Good & \\
\hline $8 / 18 / 920: 00$ & Manual Tape & 385.75 & Transcribed & \\
\hline $8 / 18 / 927: 06$ & Auto FIC & 386.1 & Good & \\
\hline $8 / 19 / 920: 00$ & Manual Tape & 386:00 & Transcribed & \\
\hline $8 / 19 / 927: 06$ & Auto FIC & 386.10 & Good & \\
\hline $8 / 20 / 920: 00$ & Manual Tape & 386 & Transcribed & \\
\hline
\end{tabular}

Data obtained from WHC Surveillance Analysis Computer System, (PC SACS) July 7, 1995. 


\begin{tabular}{|c|c|c|c|c|}
\hline \multicolumn{5}{|c|}{ 241-AP-101 } \\
\hline \multicolumn{5}{|c|}{ Surface Level Data } \\
\hline Date & Type of Measurement & Level (Inches) & Data Quality & Comments \\
\hline $8 / 20 / 927: 06$ & Auto FIC & 386.1 & Good & \\
\hline $8 / 21 / 92 \quad 0: 00$ & Manual Tape & 386.00 & Transcribed & \\
\hline $8 / 21 / 927: 05$ & Auto FIC & 386.10 & Good & \\
\hline $8 / 22 / 920: 00$ & Manual Tape & 386 & Transcribed & \\
\hline $8 / 22 / 927: 04$ & Auto FIC & 386.1 & Good & \\
\hline 8/23/92 0:00 & Manual Tape & 386.00 & Transcribed & \\
\hline $8 / 23 / 927: 04$ & Auto FIC & 386.10 & Good & \\
\hline $8 / 24 / 920: 00$ & Manual Tape & 386 & Transcribed & \\
\hline $8 / 24 / 927: 04$ & Auto FIC & 386.1 & Good & \\
\hline $8 / 25 / 920: 00$ & Manual Tape & 386.00 & Transcribed & \\
\hline $8 / 25 / 927: 04$ & Auto FIC & 386.10 & Good & \\
\hline $8 / 26 / 920: 00$ & Manual Tape & 386 & Transcribed & \\
\hline $8 / 26 / 927: 03$ & Auto FIC & 386.1 & Good & \\
\hline 8/27/92 0:00 & Manual Tape & 385.25 & Transcribed & \\
\hline $8 / 27 / 92$ 7:04 & Auto FIC & 386.10 & Good & \\
\hline $8 / 28 / 920: 00$ & Manual Tape & 386 & Transcribed & \\
\hline $8 / 28 / 927: 02$ & Auto FIC & 386.1 & Good & \\
\hline $8 / 29 / 920: 00$ & Manual Tape & 386 & Transcribed & \\
\hline $8 / 29 / 927: 02$ & Auto FIC & 386.10 & Good & \\
\hline $8 / 30 / 920: 00$ & Manual Tape & 386.00 & Transcribed & \\
\hline $8 / 30 / 927: 02$ & Auto FIC & 386 & Good & \\
\hline $8 / 31 / 920: 00$ & Manual Tape & 386 & Transcribed & \\
\hline $8 / 31 / 927: 02$ & Auto FIC & 386 & Good & \\
\hline $9 / 1 / 920: 00$ & Manual Tape & 386.00 & Transcribed & \\
\hline 9/1/92 7:02 & Auto FIC & 386.00 & Good & \\
\hline $9 / 2 / 920: 00$ & Manual Tape & 386 & Transcribed & \\
\hline $9 / 2 / 927: 02$ & Auto FIC & 386 & Good & \\
\hline 9/3/92 0:00 & Manual Tape & 385.5 & Transcribed & \\
\hline $9 / 3 / 927: 04$ & Auto FIC & 386.00 & Good & \\
\hline $9 / 4 / 920: 00$ & Manual Tape & 386.00 & Transcribed & \\
\hline 9/4/92 7:04 & Auto FIC & 386 & Good & \\
\hline $9 / 5 / 920: 00$ & Manual Tape & 386 & Transcribed & \\
\hline $9 / 5 / 927: 04$ & Auto FIC & 386.00 & Good & \\
\hline $9 / 6 / 920: 00$ & Manual Tape & 386.00 & Transcribed & \\
\hline $9 / 6 / 927: 04$ & Auto FIC & 386 & Good & \\
\hline 9/7/92 0:00 & Manual Tape & 386 & Transcribed & \\
\hline 9/7/92 7:05 & Auto FIC & 386.00 & Good & \\
\hline $9 / 8 / 920: 00$ & Manual Tape & 386.00 & Transcribed & \\
\hline 9/8/92 7:05 & Auto FIC & 386 & Good & \\
\hline $9 / 9 / 920: 00$ & Manual Tape & 386 & Transcribed & \\
\hline $9 / 9 / 927: 04$ & Auto FIC & 386.00 & Good & \\
\hline $9 / 10 / 920: 00$ & Manual Tape & 386.00 & Transcribed & \\
\hline $9 / 10 / 927: 03$ & Auto FIC & 386 & Good & \\
\hline 9/11/92 0:00 & Manual Tape & 386 & Transcribed & \\
\hline $9 / 11 / 920: 00$ & Manual FIC & 386.00 & Good & \\
\hline 9/12/92 0:00 & Manual Tape & 386.00 & Transcribed & \\
\hline $9 / 12 / 920: 00$ & Manua! FIC & 486 & Suspect & Data not included in graph \\
\hline $9 / 13 / 920: 00$ & Manual Tape & 386 & Transcribed & \\
\hline
\end{tabular}

Data obtained from WHC Surveillance Analysis Computer System, (PC SACS) July 7, 1995. 


\begin{tabular}{|c|c|c|c|c|}
\hline \multicolumn{5}{|c|}{ 241-AP-101 } \\
\hline \multicolumn{5}{|c|}{ Surface Level Data } \\
\hline Date & Type of Measurement & Lavol (Inches) & Data Quality & Comments \\
\hline $9 / 13 / 920: 00$ & Manual FIC & 386.00 & Good & \\
\hline $9 / 14 / 920: 00$ & Manual Tape & 386.00 & Transcribed & \\
\hline $9 / 14 / 920: 00$ & Manual FIC & 386 & Good & \\
\hline $9 / 15 / 920: 00$ & Manual Tape & 386 & Transcribed & \\
\hline $9 / 15 / 920: 00$ & Manual FIC & 386.00 & Good & \\
\hline $9 / 16 / 920: 00$ & Manual Tape & 386.00 & Transcribed & \\
\hline $9 / 16 / 920: 00$ & Manual FIC & 386 & Good & \\
\hline $9 / 17 / 920: 00$ & Manual Tape & 386 & Transcribed & \\
\hline $9 / 17 / 927: 06$ & Auto FIC & 386.00 & Good & \\
\hline $9 / 18 / 920: 00$ & Manual Tape & 386.00 & Transcribed & \\
\hline 9/18/92 7:06 & Auto FIC & 386 & Good & \\
\hline $9 / 19 / 920: 00$ & Manual Tape & 386 & Transcribed & \\
\hline $9 / 19 / 920: 00$ & Manual FIC & 386.00 & Good & \\
\hline $9 / 20 / 920: 00$ & Manual Tape & 386.00 & Transcribed & \\
\hline $9 / 20 / 920: 00$ & Manual FIC & 386 & Good & \\
\hline $9 / 21 / 920: 00$ & Manual Tape & 386 & Transcribed & \\
\hline $9 / 21 / 927: 06$ & Auto FIC & 386.00 & Good & \\
\hline $9 / 22 / 920: 00$ & Manual Tape & 386.00 & Transcribed & \\
\hline $9 / 22 / 927: 03$ & Auto FIC & 386 & Good & \\
\hline $9 / 23 / 920: 00$ & Manual Tape & 386 & Transcribed & \\
\hline $9 / 23 / 927: 03$ & Auto FIC & 386.00 & Good & \\
\hline $9 / 24 / 920: 00$ & Manual Tape & 386.00 & Transcribed & \\
\hline $9 / 24 / 927: 03$ & Auto FIC & 386 & Good & \\
\hline $9 / 25 / 920: 00$ & Manual Tape & 386 & Transcribed & \\
\hline $9 / 25 / 927: 03$ & Auto FIC & 386.00 & Good & \\
\hline $9 / 26 / 920: 00$ & Manual Tape & 386.00 & Transcribed & \\
\hline $9 / 26 / 927: 02$ & Auto FIC & 386 & Good & \\
\hline $9 / 27 / 920: 00$ & Manual Tape & 386 & Transcribed & \\
\hline $9 / 27 / 927: 02$ & Auto FIC & 386.00 & Good & \\
\hline $9 / 28 / 920: 00$ & Manual Tape & 385.75 & Transcribed & \\
\hline $9 / 28 / 927: 02$ & Auto FIC & 386 & Good & \\
\hline $9 / 29 / 920: 00$ & Manual Tepe & 385.75 & Transcribed & \\
\hline $9 / 29 / 927: 03$ & Auto FIC & 386.00 & Good & \\
\hline $9 / 30 / 920: 00$ & Manual Tape & 385.75 & Transcribed & \\
\hline $9 / 30 / 927: 02$ & Auto FIC & 386 & Good & \\
\hline $10 / 1 / 920: 00$ & Manual Tape & 386.00 & Transcribed & \\
\hline $10 / 1 / 927: 03$ & Auto FIC & 386.00 & Good & \\
\hline $10 / 2 / 920: 00$ & Manual Tape & 386 & Transcribed & \\
\hline $10 / 2 / 927: 02$ & Auto FIC & 386.00 & Good & \\
\hline $10 / 3 / 920: 00$ & Manual Tape & 386.00 & Transcribed & \\
\hline $10 / 3 / 927: 03$ & Auto FIC & 386 & Good & \\
\hline $10 / 4 / 920: 00$ & Manual Tape & 386 & Transcribed & \\
\hline $10 / 4 / 927: 03$ & Auto FIC & 386 & Good & \\
\hline $10 / 5 / 920: 00$ & Manual Tape & 386.00 & Transcribed & \\
\hline $10 / 5 / 927: 03$ & Auto FIC & 386.00 & Good & \\
\hline $10 / 6 / 920: 00$ & Manual Tape & 386.00 & Transcribed & \\
\hline $10 / 6 / 927: 03$ & Auto FIC & 386 & Good & \\
\hline $10 / 7 / 920: 00$ & Manual Tape & 385.75 & Transcribed & \\
\hline
\end{tabular}

Data obtained from WHC Surveillance Analysis Computer System, (PC SACS) July 7, 1995. 


\begin{tabular}{|c|c|c|c|c|}
\hline \multicolumn{5}{|c|}{ 241-AP-101 } \\
\hline \multicolumn{5}{|c|}{ Surface Level Data } \\
\hline Date & Type of Measurement & Leval (Inches) & Data Quality & Comments \\
\hline 10/7/92 0:00 & Manual FIC & 386.00 & Good & \\
\hline $10 / 8 / 920: 00$ & Manual Tape & 385.75 & Transcribed & \\
\hline 10/8/92 7:02 & Auto FIC & 386.00 & Good & \\
\hline 10/9/92 0:00 & Manual Tape & 385.75 & Transcribed & \\
\hline $10 / 9 / 927: 03$ & Auto FIC & 386 & Good & \\
\hline $10 / 10 / 920: 00$ & Manual Tape & 385.75 & Transcribed & \\
\hline $10 / 10 / 927: 03$ & Auto FIC & 386.00 & Good & \\
\hline $10 / 11 / 920: 00$ & Manual Tape & 385.75 & Transcribed & \\
\hline $10 / 11 / 927: 03$ & Auto FIC & 386 & Good & \\
\hline $10 / 12 / 920: 00$ & Manual Tape & 385.75 & Transcribed & \\
\hline $10 / 12 / 920: 00$ & Manual FIC & 386 & Good & \\
\hline $10 / 13 / 920: 00$ & Manual Tape & 386 & Transcribed & \\
\hline $10 / 13 / 920: 00$ & Manual FIC & 386.00 & Good & \\
\hline $10 / 14 / 920: 00$ & Manual Tape & 386.00 & Transcribed & \\
\hline $10 / 14 / 927: 03$ & Auto FIC & 386.00 & Good & \\
\hline $10 / 15 / 920: 00$ & Manual Tape & 386.00 & Transcribed & \\
\hline 10/15/92 7:04 & Auto FIC & 386 & Good & \\
\hline $10 / 16 / 920: 00$ & Manual Tape & 386 & Transcribed & \\
\hline $10 / 16 / 927: 03$ & Auto FIC & 386.00 & Good & \\
\hline $10 / 17 / 920: 00$ & Manual Tape & 386.00 & Transcribed & \\
\hline $10 / 17 / 927: 03$ & Auto FIC & 386 & Good & \\
\hline $10 / 18 / 920: 00$ & Manual Tape & 386.00 & Transcribed & \\
\hline $10 / 18 / 927: 03$ & Auto FIC & 386.00 & Good & \\
\hline $10 / 19 / 920: 00$ & Manual Tape & 386 & Transeribed & \\
\hline $10 / 19 / 927: 03$ & Auto FIC & 386 & Good & \\
\hline $10 / 20 / 920: 00$ & Manual Tape & 386.00 & Transcribed & \\
\hline $10 / 20 / 927: 03$ & Auto FIC & 386.00 & Good & \\
\hline $10 / 21 / 920: 00$ & Manual Tape & 386 & Transcribed & \\
\hline $10 / 21 / 927: 03$ & Auto FIC & 386 & Good & \\
\hline $10 / 22 / 920: 00$ & Manual Tape & 386 & Transcribed & \\
\hline $10 / 22 / 92 \quad 0: 00$ & Manual FIC & 386 & Good & \\
\hline $10 / 23 / 92 \quad 0: 00$ & Manual Tape & 385.75 & Transcribed & \\
\hline $10 / 23 / 920: 00$ & Manual FIC & 386.00 & Good & \\
\hline $10 / 24 / 920: 00$ & Manual Tape & 385.75 & Transcribed & \\
\hline $10 / 24 / 920: 00$ & Manual FIC & 386 & Good & \\
\hline $10 / 24 / 927: 03$ & Auto FIC & 386.00 & Good & \\
\hline $10 / 25 / 920: 00$ & Manual Tape & 385.75 & Transcribed & \\
\hline $10 / 25 / 927: 03$ & Auto FIC & 386 & Good & \\
\hline $10 / 26 / 920: 00$ & Manual Tape & 385.75 & Transcribed & \\
\hline $10 / 26 / 920: 00$ & Manual FIC & 386.00 & Good & \\
\hline $10 / 27 / 920: 00$ & Manual Tape & 385.75 & Transcribed & \\
\hline $10 / 27 / 927: 04$ & Auto FIC & 386.00 & Good & \\
\hline $10 / 28 / 920: 00$ & Manual Tape & 385.75 & Transcribed & \\
\hline $10 / 28 / 927: 03$ & Auto FIC & 386 & Good & \\
\hline $10 / 29 / 920: 00$ & Manual Tape & 385.75 & Transcribed & \\
\hline $10 / 29 / 927: 03$ & Auto FIC & 386.00 & Good & \\
\hline $10 / 30 / 920: 00$ & Manual Tape & 385.75 & Transcribed & \\
\hline $10 / 30 / 927: 03$ & Auto FIC & 386 & Good & \\
\hline
\end{tabular}

Data obtained from WHC Surveillance Analysis Computer System, (PC SACS) July 7, 1995. 


\begin{tabular}{|c|c|c|c|c|}
\hline \multicolumn{5}{|c|}{ 241-AP-101 } \\
\hline \multicolumn{5}{|c|}{ Surface Leval Data } \\
\hline Date & Type of Measurement & Level (Inches) & Data Quality & Comments \\
\hline $10 / 31 / 920: 00$ & Manual Tape & 385.75 & Transcribed & \\
\hline $10 / 31 / 927: 03$ & Auto FIC & 386.00 & Good & \\
\hline $11 / 1 / 920: 00$ & Manual Tape & 385.75 & Transcribed & \\
\hline $11 / 1 / 927: 03$ & Auto FIC & 386 & Good & \\
\hline $11 / 2 / 920: 00$ & Manual Tape & 385.75 & Transcribed & \\
\hline $11 / 2 / 927: 03$ & Auto FIC & 386.00 & Good & \\
\hline $11 / 3 / 920: 00$ & Manual Tape & 386 & Transcribed & \\
\hline $11 / 3 / 927: 03$ & Auto FIC & 386 & Good & \\
\hline $11 / 4 / 920: 00$ & Manual Tape & 385.75 & Transcribed & \\
\hline $11 / 4 / 927: 03$ & Auto FIC & 386.00 & Good & \\
\hline $11 / 5 / 920: 00$ & Manual Tape & 386.00 & Transcribed & \\
\hline $11 / 5 / 920: 00$ & Manual FIC & 386 & Good & \\
\hline $11 / 6 / 920: 00$ & Manual Tape & 386 & Transcribed & \\
\hline $11 / 6 / 920: 00$ & Manual FIC & 386.00 & Good & \\
\hline $11 / 7 / 920: 00$ & Manual Tape & 386.00 & Transcribed & \\
\hline $11 / 7 / 920: 00$ & Manual FIC & 386 & Good & \\
\hline $11 / 8 / 920: 00$ & Manual Tape & 386 & Transcribed & \\
\hline $11 / 8 / 920: 00$ & Manual FIC & 386.00 & Good & \\
\hline $11 / 9 / 920: 00$ & Manual Tape & 386.00 & Transcribed & \\
\hline $11 / 9 / 920: 00$ & Manual FIC & 386 & Good & \\
\hline $11 / 10 / 920: 00$ & Manual Tape & 386 & Transcribed & \\
\hline $11 / 10 / 927: 03$ & Auto FIC & 386 & Good & \\
\hline $11 / 11 / 920: 00$ & Manual Tape & 386.00 & Transeribed & \\
\hline $11 / 11 / 927: 04$ & Auto FIC & 386.00 & Good & \\
\hline $11 / 12 / 920: 00$ & Manual Tape & 385.75 & Transcribed & \\
\hline $11 / 12 / 927: 03$ & Auto FIC & 386 & Good & \\
\hline $11 / 13 / 920: 00$ & Manual Tape & 385.75 & Transcribed & \\
\hline $11 / 13 / 927: 04$ & Auto FIC & 386.00 & Good & \\
\hline $11 / 14 / 920: 00$ & Manual Tape & 385.75 & Transcribed & \\
\hline $11 / 14 / 927: 04$ & Auto FIC & 386 & Good & \\
\hline $11 / 15 / 920: 00$ & Manual Tape & 385.75 & Transcribed & \\
\hline $11 / 15 / 927: 04$ & Auto FIC & 386.00 & Good & \\
\hline $11 / 16 / 920: 00$ & Manual Tape & 385.75 & Transcribed & \\
\hline $11 / 16 / 927: 04$ & Auto FIC & 386 & Good & \\
\hline $11 / 17 / 920: 00$ & Manual Tape & 385.75 & Transcribed & \\
\hline $11 / 17 / 927: 04$ & Auto FIC & 386.00 & Good & \\
\hline $11 / 18 / 920: 00$ & Manual Tape & 386.00 & Transcribed & \\
\hline $11 / 18 / 927: 02$ & Auto FIC & 386 & Good & \\
\hline $11 / 19 / 920: 00$ & Manual Tape & 385.75 & Transcribed & \\
\hline $11 / 19 / 927: 02$ & Auto FIC & 386.00 & Good & \\
\hline $11 / 20 / 920: 00$ & Manual Tape & 385.75 & Transcribed & \\
\hline $11 / 20 / 927: 03$ & Auto FIC & 386 & Good & \\
\hline $11 / 21 / 920: 00$ & Manual Tape & 385.75 & Transcribed & \\
\hline $11 / 21 / 927: 02$ & Auto FIC & 386.00 & Good & \\
\hline $11 / 22 / 920: 00$ & Manual Tape & 385.75 & Transcribed & \\
\hline $11 / 22 / 927: 03$ & Auto FIC & 386 & Good & \\
\hline $11 / 23 / 920: 00$ & Manual Tape & 385.75 & Transcribed & \\
\hline $11 / 23 / 927: 03$ & Auto FIC & 386.00 & Good & \\
\hline
\end{tabular}

Data obtained from WHC Surveillance Analysis Computer System, (PC SACS) July 7, 1995. 


\begin{tabular}{|c|c|c|c|c|}
\hline \multicolumn{5}{|c|}{ 241-AP-101 } \\
\hline \multicolumn{5}{|c|}{ Surface Level Data } \\
\hline Date & Type of Measurement & Level (Inches) & Data Quality & Comments \\
\hline $11 / 24 / 920: 00$ & Manual Tape & 385.75 & Transcribed & \\
\hline $11 / 24 / 927: 03$ & Auto FIC & 386 & Good & \\
\hline $11 / 25 / 920: 00$ & Manual Tape & 385.75 & Transcribed & \\
\hline $11 / 25 / 927: 03$ & Auto FIC & 386.00 & Good & \\
\hline $11 / 26 / 920: 00$ & Manual Tape & 385.75 & Transcribed & \\
\hline $11 / 26 / 927: 03$ & Auto FIC & 386 & Good & \\
\hline 11/27/92 0:00 & Manual Tape & 385.75 & Transcribed & \\
\hline $11 / 27 / 927: 03$ & Auto FIC & 386.00 & Good & \\
\hline $11 / 28 / 920: 00$ & Manual Tape & 385.75 & Transcribed & \\
\hline $11 / 28 / 927: 03$ & Auto FIC & 386 & Good & \\
\hline $11 / 29 / 920: 00$ & Manual Tape & 385.75 & Transcribed & \\
\hline $11 / 29 / 927: 03$ & Auto FIC & 386.00 & Good & \\
\hline $11 / 30 / 920: 00$ & Manual Tape & 385.75 & Transcribed & \\
\hline $11 / 30 / 920: 00$ & Manual FIC & 386.00 & Good & \\
\hline $12 / 1 / 920: 00$ & Manual Tape & 385.75 & Transcribed & \\
\hline $12 / 1 / 927: 03$ & Auto FIC & 386 & Good & \\
\hline $12 / 2 / 920: 00$ & Manual Tape & $\mathbf{3 8 5 . 7 5}$ & Transcribed & \\
\hline $12 / 2 / 927: 03$ & Auto FIC & 386.00 & Good & \\
\hline $12 / 3 / 920: 00$ & Manual Tape & 385.75 & Transcribed & \\
\hline $12 / 3 / 927: 03$ & Auto FIC & 386 & Good & \\
\hline $12 / 4 / 920: 00$ & Manual Tape & 385.75 & Transcribed & \\
\hline $12 / 4 / 927: 03$ & Auto FIC & 386.00 & Good & \\
\hline $12 / 5 / 920: 00$ & Manual Tape & 385.75 & Transcribed & \\
\hline $12 / 5 / 920: 00$ & Manual FIC & 386 & Good & \\
\hline $12 / 6 / 920: 00$ & Manual Tape & 385.75 & Transcribed & \\
\hline $12 / 6 / 927: 02$ & Auto FIC & 386.00 & Good & \\
\hline $12 / 7 / 920: 00$ & Manual Tape & 385.75 & Transcribed & \\
\hline $12 / 7 / 927: 03$ & Auto FIC & 386 & Good & \\
\hline $12 / 8 / 920: 00$ & Manual Tape & $385: 75$ & Transcribed & \\
\hline $12 / 8 / 927: 03$ & Auto FIC & 386.00 & Good & \\
\hline $12 / 9 / 920: 00$ & Manual Tape & 385.75 & Transcribed & \\
\hline $12 / 9 / 927: 03$ & Auto FIC & 386 & Good & \\
\hline $12 / 10 / 920: 00$ & Manual Tape & 385.75 & Transcribed & \\
\hline $12 / 10 / 927: 03$ & Auto FIC & 386 & Good & \\
\hline $12 / 11 / 920: 00$ & Manual Tape & 385.75 & Transcribed & \\
\hline $12 / 11 / 927: 03$ & Auto FIC & 386.00 & Good & \\
\hline $12 / 12 / 920: 00$ & Manual Tape & 385.75 & Transcribed & \\
\hline $12 / 12 / 920: 00$ & Manual FIC & 386 & Good & \\
\hline $12 / 13 / 920: 00$ & Manual Tape & 385.75 & Transcribed & \\
\hline $12 / 13 / 927: 04$ & Auto FIC & 386.00 & Good & \\
\hline $12 / 14 / 920: 00$ & Manual Tape & 385.75 & Transcribed & \\
\hline $12 / 14 / 927: 03$ & Auto FIC & 386 & Good & \\
\hline $12 / 15 / 920: 00$ & Manual Tape & 385.75 & Transcribed & \\
\hline $12 / 15 / 927: 03$ & Auto FIC & 386.00 & Good & \\
\hline $12 / 16 / 920: 00$ & Manual Tape & 385.75 & Transcribed & \\
\hline $12 / 16 / 927: 04$ & Auto FIC & 385.9 & Good & \\
\hline $12 / 17 / 920: 00$ & Manual Tape & 385.75 & Transcribed & \\
\hline $12 / 17 / 927: 03$ & Auto FIC & 385.90. & Good & \\
\hline
\end{tabular}

Data obtained from WHC Surveillance Analysis Computer System, (PC SACS) July 7, 1995. 


\begin{tabular}{|c|c|c|c|c|}
\hline \multicolumn{5}{|c|}{ 241-AP-101 } \\
\hline & & urface Level Dat & . & \\
\hline Date & Type of Measurement & Level'(Inches) & Data Quality & Comments \\
\hline $12 / 18 / 920: 00$ & Manual Tape & 385.75 & Transcribed & \\
\hline $12 / 18 / 927: 03$ & Auto FIC & 385.9 & Good & \\
\hline $12 / 19 / 920: 00$ & Manual Tape & 385.75 & Transcribed & \\
\hline $12 / 19 / 927: 03$ & Auto FIC & 385.90 & Good & \\
\hline $12 / 20 / 920: 00$ & Manual Tape & 385.75 & Transcribed & \\
\hline $12 / 20 / 927: 04$ & Auto FIC & 385.9 & Good & \\
\hline $12 / 21 / 927: 04$ & Auto FIC & 385.9 & Good & \\
\hline $12 / 22 / 920: 00$ & Manual FIC & 385.90 & Transcribed & \\
\hline $12 / 23 / 927: 04$ & Auto FIC & 385.90 & Good & \\
\hline $12 / 24 / 927: 03$ & Auto FIC & 385.90 & Good & \\
\hline $12 / 25 / 927: 03$ & Auto FIC & 385.9 & Good & \\
\hline $12 / 26 / 927: 03$ & Auto FIC & 385.9 & Good & \\
\hline $12 / 27 / 927: 03$ & Auto FIC & 385.90 & Good & \\
\hline $12 / 28 / 927: 03$ & Auto FIC & 385.9 & Good & \\
\hline $12 / 29 / 920: 00$ & Manual FIC & 385.9 & Good & \\
\hline $12 / 30 / 927: 03$ & Auto FIC & 385.90 & Good & \\
\hline $12 / 31 / 927: 03$ & Auto FIC & 385.9 & Good & \\
\hline $1 / 1 / 937: 04$ & Auto FIC & 385.90 & Good & \\
\hline $1 / 2 / 930: 00$ & Manual FIC & 385.90 & Good & \\
\hline 1/3/93 0:00 & Manual FIC & 385.9 & Good & \\
\hline 1/4/93 0:00 & Manual FIC & 385.90 & Good & \\
\hline 1/4/93 0:00 & Manual Tape & 385.75 & Good & \\
\hline $1 / 5 / 930: 00$ & Manual FIC & 385.9 & Good & \\
\hline $1 / 6 / 930: 00$ & Manual FIC & 385.90 & Good & \\
\hline $1 / 7 / 930: 00$ & Manual FIC & 385.9 & Good & \\
\hline 1/7/930:00 & Manual Tape & . 385.75 & Good & \\
\hline 1/8/93 0:00 & Manual Tape & 354.00 & Suspect & Data not included in graph \\
\hline $1 / 8 / 930: 00$ & Manual FIC & 385.9 & Good & \\
\hline 1/9/93 0:00 & Manual Tape & 354.50 & Suspect & Data not included in graph \\
\hline 1/9/93 0:00 & Manual FIC & 385.90 & Good & \\
\hline $1 / 10 / 930: 00$ & Manual FIC & 385.9 & Good & \\
\hline $1 / 10 / 930: 00$ & Manual Tape & 386 & Good & \\
\hline $1 / 11 / 930: 00$ & Manual FIC & 385.90 & Good & \\
\hline $1 / 11 / 930: 00$ & Manual Tape & 386.00 & Good & \\
\hline $1 / 12 / 930: 00$ & Manual Tape & 385.75 & Good & \\
\hline 1/12/93 7:06 & Auto FIC & 385.90 & Good & \\
\hline $1 / 13 / 930: 00$ & Manual Tape & 385.75 & Good & \\
\hline $1 / 13 / 937: 06$ & Auto FIC & 385.9 & Good & \\
\hline $1 / 14 / 930: 00$ & Manual Tape & 385.75 & Good & \\
\hline $1 / 14 / 935: 03$ & Auto FIC & 385.90 & Good & \\
\hline $1 / 15 / 930: 00$ & Manual Tape & 385.75 & Good & \\
\hline $1 / 15 / 937: 03$ & Auto FIC & 385.90 & Good & \\
\hline $1 / 16 / 930: 00$ & Manual FIC & 385.9 & Good & \\
\hline $1 / 16 / 930: 00$ & Manual Tape & 385.50 & Good & \\
\hline $1 / 17 / 930: 00$ & Manual FIC & 385.90 & Good & \\
\hline 1/17/930:00 & Manual Tape & 385.25 & Good & \\
\hline $1 / 18 / 930: 00$ & Manual FIC & 385.85 & Good & \\
\hline $1 / 18 / 930: 00$ & Manual Tape & 385.25 & Good & \\
\hline
\end{tabular}

Data obtained from WHC Surveillance Analysis Computer System, (PC SACS) July 7, 1995. 


\begin{tabular}{|c|c|c|c|c|}
\hline \multicolumn{5}{|c|}{ 241-AP-101 } \\
\hline & & Irface Level Dat & & \\
\hline Date & Type of Measurement & Lovel (Inches) & Data Quality. & Comments \\
\hline $1 / 19 / 930: 00$ & Manual FIC & 385.85 & Good & \\
\hline $1 / 19 / 930: 00$ & Manual Tape & 385.50 & Good & \\
\hline $1 / 20 / 930: 00$ & Manual FIC & 385.8 & Good & \\
\hline $1 / 20 / 930: 00$ & Manual Tape & 385.50 & Good & \\
\hline $1 / 21 / 930: 00$ & Manual Tape & 385.5 & Good & \\
\hline $1 / 21 / 937: 02$ & Auto FIC & 385.80 & Good & \\
\hline $1 / 22 / 930: 00$ & Manual Tape & 385.50 & Good & \\
\hline 1/22/93 7:04 & Auto FIC & 385.8 & Good & \\
\hline $1 / 23 / 930: 00$ & Manual Tape & 382.00 & Suspect & Data not included in graph \\
\hline $1 / 23 / 937: 03$ & Auto FIC & 385.80 & Good & \\
\hline $1 / 24 / 937: 03$ & Auto FIC & 385.8 & Good & \\
\hline $1 / 25 / 937: 03$ & Auto FIC & 385.80 & Good & \\
\hline $1 / 26 / 937: 04$ & Auto FIC & 385.8 & Good & \\
\hline $1 / 27 / 937: 04$ & Auto FIC & 385.80 & Good & \\
\hline $1 / 28 / 930: 00$ & Manual Tape & 385.5 & Good & \\
\hline $1 / 28 / 937: 04$ & Auto FIC & 385.80 & Good & \\
\hline $1 / 29 / 937: 04$ & Auto FIC & 385.8 & Good & \\
\hline $1 / 30 / 937: 03$ & Auto FIC & 385.80 & Good & \\
\hline $1 / 31 / 937: 04$ & Auto FIC & 385.80 & Good & \\
\hline $2 / 1 / 937: 04$ & Auto FIC & 385.8 & Good & \\
\hline $2 / 2 / 937: 04$ & Auto FIC & 385.80 & Good & \\
\hline $2 / 3 / 93 \quad 0: 00$ & Manual FIC & 385.80 & Good & \\
\hline 2/4/93 7:04 & Auto FIC & 385.80 & Good & \\
\hline 2/5/93 7:04 & Auto FIC & 385.8 & Good & \\
\hline $2 / 6 / 937: 04$ & Auto FIC & 385.80 & Good & \\
\hline $2 / 7 / 93$ 7:03 & Auto FIC & 385.8 & Good & \\
\hline $2 / 8 / 937: 04$ & Auto FIC & 385.80 & Good & \\
\hline 2/9/93 7:04 & Auto FIC & 385.8 & Good & \\
\hline $2 / 10 / 937: 04$ & Auto FIC & 385.80 & Good & \\
\hline $2 / 11 / 937: 04$ & Auto FIC & 385.8 & Good & \\
\hline $2 / 12 / 937: 03$ & Auto FIC & 385.80 & Good & \\
\hline $2 / 13 / 937: 03$ & Auto FIC & 385.8 & Good & \\
\hline $2 / 14 / 937: 04$ & Auto FIC & 385.80 & Good & \\
\hline $2 / 15 / 937: 04$ & Auto FIC & 385.8 & Good & \\
\hline $2 / 16 / 937: 02$ & Auto FIC & 385.8 & Good & \\
\hline $2 / 17 / 930: 00$ & Manual FIC & 385.8 & Good & \\
\hline $2 / 18 / 937: 03$ & Auto FIC & 385.80 & Good & \\
\hline $2 / 19 / 930: 00$ & Manual Tape & 385.50 & Good & \\
\hline 2/19/93 7:02 & Auto FIC & 385.80 & Good & \\
\hline $2 / 20 / 930: 00$ & Manual Tape & 385.50 & Good & \\
\hline $2 / 20 / 937: 03$ & Auto FIC & 385.8 & Good & \\
\hline $2 / 21 / 930: 00$ & Manual Tape & 385.5 & Good & \\
\hline $2 / 21 / 937: 04$ & Auto FIC & 385.80 & Good & \\
\hline $2 / 22 / 930: 00$ & Manual Tape & 385.75 & Good & \\
\hline $2 / 22 / 937: 04$ & Auto FIC & 385.80 & Good & \\
\hline $2 / 23 / 930: 00$ & Manual Tape & 385.50 & Good & \\
\hline $2 / 23 / 937: 03$ & Auto FIC & 385.80 & Good & \\
\hline $2 / 24 / 930: 00$ & Manual Tape & 385.5 & Good & \\
\hline
\end{tabular}

Data obtained from WHC Surveillance Analysis Computer System, (PC SACS) July 7, 1995. 


\begin{tabular}{|c|c|c|c|c|}
\hline \multicolumn{5}{|c|}{ 241-AP-101 } \\
\hline \multicolumn{5}{|c|}{ Surface Level Data } \\
\hline Date & Type of Measurement & Leval. (Inches) & Data Quality & Comments \\
\hline $2 / 24 / 937: 04$ & Auto FIC & 385.8 & Good & \\
\hline $2 / 25 / 930: 00$ & Manual Tape & 385.50 & Good & \\
\hline $2 / 25 / 937: 03$ & Auto FIC & 385.80 & Good & \\
\hline $2 / 26 / 930: 00$ & Manual Tape & 385.50 & Good & \\
\hline $2 / 26 / 937: 03$ & Auto FIC & 385.8 & Good & \\
\hline $2 / 27 / 930: 00$ & Manual Tape & 385.5 & Good & \\
\hline $2 / 27 / 937: 04$ & Auto FIC & 385.80 & Good & \\
\hline $2 / 28 / 930: 00$ & Manual Tape & 385.50 & Good & \\
\hline $2 / 28 / 937: 03$ & Auto FIC & 385.80 & Good & \\
\hline $3 / 1 / 930: 00$ & Manual Tape & 385.50 & Good & \\
\hline 3/1/93 7:03 & Auto FIC & 385.8 & Good & \\
\hline $3 / 2 / 930: 00$ & Manual Tape & 385.5 & Good & \\
\hline $3 / 2 / 937: 04$ & Auto FIC & 385.8 & Good & \\
\hline $3 / 3 / 93 \quad 0: 00$ & Manual Tape & 385.50 & Good & \\
\hline $3 / 3 / 93 \quad 7: 03$ & Auto FIC & 385.80 & Good & \\
\hline 3/4/93 0:00 & Manual Tape & 385.50 & Good & \\
\hline $3 / 4 / 937: 04$ & Auto FIC & 385.80 & Good & \\
\hline $3 / 5 / 930: 00$ & Manual Tape & 385.5 & Good & \\
\hline $3 / 5 / 937: 04$ & Auto FIC & 385.8 & Good & \\
\hline 3/6/93 0:00 & Manual Tape & 385.50 & Good & \\
\hline $3 / 6 / 937: 03$ & Auto FIC & 385.80 & Good & \\
\hline $3 / 7 / 930: 00$ & Manual Tape & 385.50 & Good & \\
\hline $3 / 7 / 937: 03$ & Auto FIC & 385.8 & Good & \\
\hline $3 / 8 / 93 \quad 0: 00$ & Manual Tape & 385.5 & Good & \\
\hline $3 / 8 / 937: 03$ & Auto FIC & 385.80 & Good & \\
\hline $3 / 9 / 930: 00$ & Manual Tape & 385.50 & Good & \\
\hline $3 / 9 / 937: 04$ & Auto FIC & 385.80 & Good & \\
\hline $3 / 10 / 930: 00$ & Manual Tape & $385.75^{\circ}$ & Good & \\
\hline $3 / 10 / 93$ 7:05 & Auto FIC & 385.8 & Good & \\
\hline $3 / 11 / 930: 00$ & Manual Tape & 385.75 & Good & \\
\hline $3 / 11 / 937: 04$ & Auto FIC & 385.80 & Good & \\
\hline $3 / 12 / 93 \quad 0: 00$ & Manual Tape & 385.50 & Good & \\
\hline $3 / 12 / 93$ 7:04 & Auto FIC & 385.80 & Good & \\
\hline $3 / 13 / 930: 00$ & Manual Tape & 385.50 & Good & \\
\hline $3 / 13 / 937: 03$ & Auto FIC & 385.80 & Good & \\
\hline 3/14/93 0:00 & Manual Tape & 385.75 . & Good & \\
\hline $3 / 14 / 937: 04$ & Auto FIC & 385.7 & Good & \\
\hline $3 / 15 / 930: 00$ & Manual Tape & 385.50 & Good & \\
\hline $3 / 15 / 937: 03$ & Auto FIC & 385.80 & Good & \\
\hline $3 / 16 / 930: 00$ & Manual Tape & 385.50 & Good & \\
\hline $3 / 16 / 93 \quad 7: 04$ & Auto FIC & 385.8 & Good & \\
\hline $3 / 17 / 930: 00$ & Manual Tape & 385.5 & Good & \\
\hline $3 / 17 / 937: 03$ & Auto FIC & 385.70 & Good & \\
\hline $3 / 18 / 930: 00$ & Manual Tape & 385.50 & Good & \\
\hline $3 / 18 / 93 \quad 7: 04$ & Auto FIC & 385.7 & Good & \\
\hline $3 / 19 / 930: 00$ & Manual Tape & 385.50 & Good & \\
\hline $3 / 19 / 937: 03$ & Auto FIC & 385.7 & Good & \\
\hline $3 / 20 / 930: 00$ & Manual Tape & 385.5 & Good & \\
\hline
\end{tabular}

Data obtained from WHC Surveillance Analysis Computer System, (PC SACS) July 7, 1995. 


\begin{tabular}{|c|c|c|c|c|}
\hline \multicolumn{5}{|c|}{ 241-AP-101 } \\
\hline \multicolumn{5}{|c|}{ Surface Level Date } \\
\hline Date & Type of Measurement & Levol (Inches) & Data Quality & Comments \\
\hline $3 / 20 / 93$ 7:04 & Auto FIC & 385.70 & Good & \\
\hline $3 / 21 / 930: 00$ & Manual Tape & 385.75 & Good & \\
\hline 3/21/93 7:04 & Auto FIC & 385.70 & Good & \\
\hline $3 / 22 / 93 \quad 0: 00$ & Manual Tape & 385.00 & Good & \\
\hline $3 / 22 / 937: 03$ & Auto FIC & 385.70 & Good & \\
\hline $3 / 23 / 930: 00$ & Manual Tape & 385.5 & Good & \\
\hline $3 / 23 / 937: 03$ & Auto FIC & 385.7 & Good & \\
\hline $3 / 24 / 930: 00$ & Manual Tape & 385.50 & Good & \\
\hline $3 / 24 / 93 \quad 7: 04$ & Auto FIC & 385.70 & Good & \\
\hline $3 / 25 / 930: 00$ & Manual Tape & 385.50 & Good & \\
\hline $3 / 25 / 937: 03$ & Auto FIC & 385.8 & Good & \\
\hline $3 / 26 / 930: 00$ & Manual Tape & 385.5 & Good & \\
\hline $3 / 26 / 937: 03$ & Auto FIC & 385.70 & Good & \\
\hline $3 / 27 / 930: 00$ & Manual Tape & 385.50 & Good & \\
\hline $3 / 27 / 937: 03$ & Auto FIC & 385.7 & Good & \\
\hline $3 / 28 / 93 \quad 0: 00$ & Manual Tape & 385.50 & Good & \\
\hline $3 / 28 / 937: 03$ & Auto FIC & 385.7 & Good & \\
\hline $3 / 29 / 93 \quad 0: 00$ & Manual Tape & 385.5 & Good & \\
\hline $3 / 29 / 937: 03$ & Auto FIC & 385.7 & Good & \\
\hline $3 / 30 / 930: 00$ & Manual Tope & 385.50 & Good & \\
\hline $3 / 30 / 93$ 7:03 & Auto FIC & 385.7 & Good & \\
\hline $3 / 31 / 930: 00$ & Manual Tape & 385.50 & Good & \\
\hline $3 / 31 / 937: 03$ & Auto FIC & 385.7 & Good & \\
\hline 4/1/93 7:03 & Auto FIC & 385.7 & Good & \\
\hline $4 / 1 / 93 \quad 10: 03$ & Manual Tape & 385.5 & Good & \\
\hline 4/2/93 7:06 & Auto FIC & 385.70 & Good & \\
\hline $4 / 2 / 93 \quad 10: 03$ & Manual Tape & 385.50 & Good & \\
\hline 4/3/93 7:06 & Auto FIC & 385.70 & Good & \\
\hline $4 / 3 / 9310: 03$ & Manual Tape & 385.50 & Good & \\
\hline $4 / 4 / 937: 05$ & Auto FIC & 385.7 & Good & \\
\hline $4 / 4 / 93 \quad 10: 03$ & Manual Tape & 385.5 & Good & \\
\hline $4 / 5 / 937: 06$ & Auto FIC & 385.7 & Good & \\
\hline $4 / 5 / 93 \quad 10: 03$ & Manual Tape & 385.75 & Good & \\
\hline 4/6/93 7:06 & Auto FIC & 385.7 & Good & \\
\hline $4 / 6 / 9310: 03$ & Manual Tape & 385.50 & Good & \\
\hline $4 / 7 / 937: 06$ & Auto FIC & 385.7 & Good & \\
\hline $4 / 7 / 93 \quad 10: 03$ & Manual Tape & 385.5 & Good & \\
\hline $4 / 8 / 937: 06$ & Auto FIC & 385.7 & Good & \\
\hline $4 / 8 / 93 \quad 10: 03$ & Manual Tape & 385.50 & Good & \\
\hline 4/9/93 7:06 & Auto FIC & 385.7 & Good & \\
\hline $4 / 9 / 93 \quad 10: 03$ & Manual Tape & 385.50 & Good & \\
\hline 4/10/93 7:05 & Auto FIC & 385.7 & Good & \\
\hline $4 / 10 / 93 \quad 10: 03$ & Manual Tape & 385.5 & Good & \\
\hline $4 / 11 / 937: 06$ & Auto FIC & 385.70 & Good & \\
\hline $4 / 11 / 93 \quad 10: 03$ & Manual Tape & 385.50 & Good & \\
\hline $4 / 12 / 937: 06$ & Auto FIC & 385.70 & Good & \\
\hline $4 / 12 / 9310: 03$ & Manual Tape & $385: 50$ & Good & \\
\hline $4 / 13 / 937: 06$ & Auto FIC & 385.7 & Good & \\
\hline
\end{tabular}

Data obtained from WHC Surveillance Analysis Computer System, (PC SACS) July 7, 1995. 


\begin{tabular}{|c|c|c|c|c|}
\hline \multicolumn{5}{|c|}{ 241-AP-101 } \\
\hline \multicolumn{5}{|c|}{ Surface Level Data } \\
\hline Date & Type of Measurement & Level (Inches) & Data Quality & Commants \\
\hline 4/13/93 10:03 & Manual Tape & 385.5 & Good & \\
\hline $4 / 14 / 937: 06$ & Auto FIC & 385.70 & Good & \\
\hline $4 / 14 / 93 \quad 10: 03$ & Manual Tape & 385.50 & Good & \\
\hline 4/15/93 7:03 & Auto FIC & 385.70 & Good & \\
\hline 4/15/93 10:03 & Manual Tape & 385.50 & Good & \\
\hline $4 / 16 / 937: 06$ & Auto FIC & 385.7 & Good & \\
\hline 4/16/93 10:03 & Manual Tape & 385.5 & Good & \\
\hline 4/17/93 7:05 & Auto FIC & 385.70 & Good & \\
\hline $4 / 17 / 93 \quad 10: 03$ & Manual Tape & 385.50 & Good & \\
\hline 4/18/93 7:06 & Auto FIC & 385.70 & Good & \\
\hline $4 / 18 / 93 \quad 10: 03$ & Manual Tape & 385.5 & Good & \\
\hline 4/19/93 7:06 & Auto FIC & 385.7 & Good & \\
\hline $4 / 19 / 93 \quad 10: 03$ & Manual Tape & 385.50 & Good & \\
\hline $4 / 20 / 930: 00$ & Manual FIC & 385.70 & Good & \\
\hline 4/20/93 10:03 & Manual Tape & 385.25 & Good & \\
\hline 4/21/93 0:00 & Manual FIC & 385.7 & Good & \\
\hline 4/21/93 10:03 & Manual Tape & 385.50 & Good & \\
\hline $4 / 22 / 937: 06$ & Auto FIC & 385.70 & Good & \\
\hline $4 / 22 / 93 \quad 10: 03$ & Manual Tape & 385.25 & Good & \\
\hline $4 / 23 / 937: 06$ & Auto FIC & 385.7 & Good & \\
\hline $4 / 23 / 9310: 03$ & Manual Tape & 385.25 & Good & \\
\hline $4 / 24 / 937: 03$ & Auto FIC & 385.70 & Good & \\
\hline $4 / 24 / 93 \quad 10: 03$ & Manual Tape & 385.50 & Good & \\
\hline 4/25/93 7:06 & Auto FIC & 385.7 & Good & \\
\hline 4/25/93 10:03 & Manual Tape & 385.50 & Good & \\
\hline 4/26/93 7:06 & Auto FIC & 385.7 & Good & \\
\hline $4 / 26 / 93 \quad 10: 03$ & Manual Tape & 385.50 & Good & \\
\hline 4/27/93 7:06 & Auto FIC & 385.70 & Good & \\
\hline 4/27/93 10:03 & Manual Tape & 385.50 & Good & \\
\hline 4/28/93 7:03 & Auto FIC & 385.7 & Good & \\
\hline 4/28/93 10:03 & Manual Tape & 385.50 & Good & \\
\hline $4 / 29 / 93 \quad 7: 03$ & Auto FIC & 385.70 & Good & $\cdot$ \\
\hline $4 / 29 / 93 \quad 10: 03$ & Manual Tape & 385.5 & Good & \\
\hline $4 / 30 / 937: 06$ & Auto FIC & 385.7 & Good & \\
\hline $4 / 30 / 93 \quad 10: 03$ & Manual Tape & 385.50 & Good & \\
\hline $5 / 1 / 937: 05$ & Auto FIC & 385.70 & Good & \\
\hline $5 / 1 / 93 \quad 10: 05$ & Manual Tape & 385.50 & Good & \\
\hline $5 / 2 / 937: 06$ & Auto FIC & 385.7 & Good & \\
\hline $5 / 2 / 93 \quad 10: 05$ & Manual Tape & 385.5 & Good & \\
\hline 5/3/93 7:06 & Auto FIC & 385.70 & Good & \\
\hline $5 / 3 / 93 \quad 10: 05$ & Manual Tape & 385.50 & Good & \\
\hline $5 / 4 / 93 \quad 10: 05$ & Manual FIC & 385.7 & Good & \\
\hline $5 / 4 / 93 \quad 10: 05$ & Manual Tape & 385.50 & Good & \\
\hline $5 / 5 / 93 \quad 10: 05$ & Manual FIC & $385: 70$ & Good & \\
\hline $5 / 5 / 93 \quad 10: 05$ & Manual Tape & 385.5 & Good & \\
\hline $5 / 6 / 93 \quad 10: 05$ & Manual FIC & 385.7 & Good & \\
\hline $5 / 6 / 93 \quad 10: 05$ & Manual Tape & 385.50 & Good & \\
\hline $5 / 7 / 93 \quad 10: 05$ & Manual FIC & 385.70 & Good & \\
\hline
\end{tabular}

Data obtained from WHC Surveillance Analysis Computer System, (PC SACS) July 7, 1995. 


\begin{tabular}{|c|c|c|c|c|}
\hline \multicolumn{5}{|c|}{ 241-AP-101 } \\
\hline \multicolumn{5}{|c|}{ Surface Level Data } \\
\hline Date & Type of Measurement & Lovel (Inches) & Data Quality & Comments \\
\hline $5 / 7 / 93 \quad 10: 05$ & Manual Tape & 385.00 & Good & \\
\hline $5 / 8 / 93 \quad 10: 05$ & Manual FIC : & 385.7 & Good & \\
\hline $5 / 8 / 93 \quad 10: 05$ & Manual Tape & 385 & Good & \\
\hline $5 / 9 / 93 \quad 10: 05$ & Manual FIC & 385.70 & Good & \\
\hline $5 / 9 / 93 \quad 10: 05$ & Manual Tape & 385.00 & Good & \\
\hline $5 / 10 / 9310: 05$ & Manual FIC & 385.7 & Good & \\
\hline $5 / 10 / 93 \quad 10: 05$ & Manual Tape & 385.00 & Good & \\
\hline $5 / 11 / 937: 06$ & Auto FIC & 385.7 & Good & \\
\hline $5 / 11 / 93 \quad 10: 05$ & Manual Tape & 385.5 & Good & \\
\hline $5 / 12 / 937: 06$ & Auto FIC & 385.70 & Good & \\
\hline $5 / 12 / 93 \quad 10: 05$ & Manual Tape & 385.50 & Good & \\
\hline $5 / 13 / 93 \quad 10: 05$ & Manual FIC & 385.70 & Good & \\
\hline $5 / 13 / 9310: 05$ & Manual Tape & 385.25 & Good & \\
\hline $5 / 14 / 93 \quad 10: 05$ & Manual FIC & 385.70 & Good & \\
\hline $5 / 14 / 93 \quad 10: 05$ & Manual Tape & 385.25 & Good & \\
\hline $5 / 15 / 937: 06$ & Auto FIC & 385.70 & Good & \\
\hline $5 / 15 / 93 \quad 10: 05$ & Manual Tape & 385.50 & Good & \\
\hline $5 / 16 / 937: 05$ & Auto FIC & 385.7 & Good & \\
\hline $5 / 16 / 93 \quad 10: 05$ & Manual Tape & 385.50 & Good & \\
\hline $5 / 17 / 93 \quad 7: 03$ & Auto FIC & 385.70 & Good & \\
\hline $5 / 17 / 93 \quad 10: 05$ & Manual Tape & 385.5 & Good & \\
\hline $5 / 18 / 937: 03$ & Auto FIC & 385.7 & Good & \\
\hline $5 / 18 / 93 \quad 10: 05$ & Manual Tape & 385.50 & Good & \\
\hline $5 / 19 / 937: 03$ & Auto FIC & 385.7 & Good & \\
\hline $5 / 19 / 93 \quad 10: 05$ & Manual Tape & 385.50 & Good & \\
\hline $5 / 20 / 937: 03$ & Auto FIC & 385.70 & Good & \\
\hline $5 / 20 / 9310: 05$ & Manual Tape & 385.5 & Good & \\
\hline $5 / 21 / 930: 00$ & Manual FIC & 385.7 & Good & \\
\hline $5 / 21 / 9310: 05$ & Manual Tape & 385.50 & Good & \\
\hline $5 / 22 / 937: 03$ & Auto FIC & 385.7 & Good & \\
\hline $5 / 22 / 93 \quad 10: 05$ & Manual Tape & 385.50 & Good & \\
\hline $5 / 23 / 937: 04$ & Auto FIC & 385.70 & Good & \\
\hline $5 / 23 / 93 \quad 10: 05$ & Manual Tape & 385.5 & Good & \\
\hline $5 / 24 / 937: 03$ & Auto FIC & 385.7 & Good & \\
\hline $5 / 24 / 93 \quad 10: 05$ & Manual Tape & 385.50 & Good & \\
\hline $5 / 25 / 937: 03$ & Auto FIC & 385.70 & Good & \\
\hline $5 / 25 / 93 \quad 10: 05$ & Manual Tape & 385.5 & Good & \\
\hline $5 / 26 / 937: 03$ & Auto FIC & 385.70 & Good & \\
\hline $5 / 26 / 93 \quad 10: 05$ & Manual Tape & 385.50 & Good & \\
\hline $5 / 27 / 937: 03$ & Auto FIC & 385.7 & Good & \\
\hline $5 / 27 / 93 \quad 10: 05$ & Manual Tape & 385.50 & Good & \\
\hline $5 / 28 / 937: 03$ & Auto FIC & 385.70 & Good & \\
\hline $5 / 28 / 93 \quad 10: 05$ & Manual Tape & 385.5 & Good & \\
\hline $5 / 29 / 937: 05$ & Auto FIC & 385.7 & Good & \\
\hline $5 / 29 / 93 \quad 10: 05$ & Manual Tape & 385.50 & Good & \\
\hline 5/30/93 7:05 & Auto FIC & 385.70 & Good & \\
\hline $5 / 30 / 93 \quad 10: 05$ & Manual Tape & 385.50 & Good & \\
\hline $5 / 31 / 937: 06$ & Auto FIC & 385.7 & Gopd & \\
\hline
\end{tabular}

Data obtained from WHC Surveillance Analysis Computer System, (PC SACS) July 7, 1995. 


\begin{tabular}{|c|c|c|c|c|}
\hline \multicolumn{5}{|c|}{ 241-AP-101 } \\
\hline \multicolumn{5}{|c|}{ Surface Leval Data } \\
\hline Date & Type of Measurement & Level (Inches) & Data Quality & Comments \\
\hline $5 / 31 / 93 \quad 10: 05$ & Manual Tape & 385.5 & Good & \\
\hline $6 / 1 / 937: 06$ & Auto FIC & 385.70 & Good & \\
\hline $6 / 1 / 93 \quad 10: 06$ & Manual Tape & 385.70 & Good & \\
\hline $6 / 2 / 937: 06$ & Auto FIC & 385.7 & Good & \\
\hline $6 / 2 / 93 \quad 10: 06$ & Manual Tape & 385.25 & Good & \\
\hline $6 / 3 / 937: 06$ & Auto FIC & 385.70 & Good & \\
\hline $6 / 3 / 93 \quad 10: 06$ & Manual Tape & 385.5 & Good & \\
\hline $6 / 4 / 937: 06$ & Auto FIC & 385.7 & Good & \\
\hline $6 / 4 / 93 \quad 10: 06$ & Manual Tape & 385.50 & Good & \\
\hline 6/5/93 7:06 & Auto FIC & 385.70 & Good & \\
\hline $6 / 5 / 93 \quad 10: 06$ & Manual Tape & 385.50 & Good & \\
\hline 6/6/93 7:05 & Auto FIC & 385.70 & Good & \\
\hline $6 / 6 / 93 \quad 10: 06$ & Manual Tape & 385.5 & Good & \\
\hline $6 / 7 / 93 \quad 7: 05$ & Auto FIC & 385.7 & Good & \\
\hline $6 / 7 / 93 \quad 10: 06$ & Manual Tape & 385.50 & Good & \\
\hline $6 / 8 / 937: 05$ & Auto FIC & 385.70 & Good & \\
\hline 6/8/93 10:06 & Manual Tape & 385.50 & Good & \\
\hline $6 / 9 / 937: 05$ & Auto FIC & 385.7 & Good & \\
\hline $6 / 9 / 93 \quad 10: 06$ & Manual Tape & 385.5 & Good & \\
\hline $6 / 10 / 937: 04$ & Auto FIC & 385.70 & Good & \\
\hline $6 / 10 / 93 \quad 10: 05$ & Manual Tape & $\cdot \mathbf{3 8 5 . 5 0}$ & Good & \\
\hline $6 / 11 / 937: 05$ & Auto FIC & 385.7 & Good & \\
\hline $6 / 11 / 93 \quad 10: 05$ & Manual Tape & 385.50 & Good & \\
\hline $6 / 12 / 937: 05$ & Auto FIC & 385.70 & Good & \\
\hline $6 / 12 / 93 \quad 10: 05$ & Manual Tape & 385.5 & Good & \\
\hline $6 / 13 / 937: 04$ & Auto FIC & 385.70 & Good & \\
\hline $6 / 13 / 93 \quad 10: 05$ & Manual Tape & 385.50 & Good & \\
\hline $6 / 14 / 937: 05$ & Auto FIC & 385.7 & Good & \\
\hline $6 / 14 / 93 \quad 10: 05$ & Manual Tape & 385.50 & Good & \\
\hline $6 / 15 / 937: 05$ & Auto FIC & 385.70 & Good & \\
\hline $6 / 15 / 93 \quad 10: 05$ & Manual Tape & 385.50 & Good & \\
\hline $6 / 16 / 937: 05$ & Auto FIC & 385.7 & Good & \\
\hline $6 / 16 / 93 \quad 10: 05$ & Manual Tape & 385.5 & Good & \\
\hline 6/17/93 7:05 & Auto FIC & 385.70 & Good & \\
\hline $6 / 17 / 93 \quad 10: 05$ & Manual Tape & 385.50 & Good & \\
\hline $6 / 18 / 937: 06$ & Auto FIC & 385.7 & Good & \\
\hline $6 / 18 / 93 \quad 10: 05$ & Manual Tape & 385:50 & Good & \\
\hline $6 / 19 / 937: 06$ & Auto FIC & 385.70 & Good & \\
\hline $6 / 19 / 93 \quad 10: 05$ & Manual Tape & 385.5 & Good & \\
\hline $6 / 20 / 93$ 7:06 & Auto FIC & 385.70 & Good & \\
\hline $6 / 20 / 93 \quad 10: 05$ & Manual Tape & 385.50 & Good & \\
\hline $6 / 21 / 937: 06$ & Auto FIC & 385.7 & Good & \\
\hline $6 / 21 / 93 \quad 10: 05$ & Manual Tape & 385.50 & Good & \\
\hline $6 / 22 / 937: 06$ & Auto FIC & 385.70 & Good & \\
\hline $6 / 22 / 93 \quad 10: 05$ & Manual Tape & 385.50 & Good & \\
\hline $6 / 23 / 93 \quad 7: 06$ & Auto FIC & 385.7 & Good & \\
\hline $6 / 23 / 93 \quad 10: 05$ & Manual Tape & 385.5 & Good & \\
\hline $6 / 24 / 937: 06$ & Auto FIC & $385.70^{\circ}$ & Good & \\
\hline
\end{tabular}

Data obtained from WHC Surveillance Analysis Computer System, (PC SACS) July 7, 1995. 


\begin{tabular}{|c|c|c|c|c|}
\hline \multicolumn{5}{|c|}{ 241-AP-101 } \\
\hline & & Urface Level Dat & & \\
\hline Date & Type of Measurement & Lovel (Inches) & Data Quality & Comments \\
\hline $6 / 24 / 93 \quad 10: 05$ & Manual Tape & 385.50 & Good & \\
\hline $6 / 25 / 937: 03$ & Auto FIC & 385.7 & Good & \\
\hline $6 / 25 / 93 \quad 10: 05$ & Manual Tape & 385.50 & Good & \\
\hline $6 / 26 / 937: 03$ & Auto FIC & 385.70 & Good & \\
\hline $6 / 26 / 93 \quad 10: 05$ & Manual Tape & 385.50 & Good & \\
\hline $6 / 27 / 937: 03$ & Auto FIC & 385.7 & Good & \\
\hline $6 / 27 / 9310: 05$ & Manual Tape & 385.5 & Good & \\
\hline $6 / 28 / 937: 03$ & Auto FIC & 385.70 & Good & \\
\hline $6 / 28 / 93 \quad 10: 05$ & Manual Tape & 385.50 & Good & \\
\hline $6 / 29 / 937: 04$ & Auto FIC & 385.7 & Good & \\
\hline $6 / 29 / 93 \quad 10: 05$ & Manual Tape & 385.50 & Good & \\
\hline $6 / 30 / 937: 03$ & Auto FIC & 385.70 & Good & \\
\hline $6 / 30 / 93 \quad 10: 05$ & Manual Tape & 385.50 & Good & \\
\hline 7/1/93 7:06 & Auto FIC & 385.7 & Good & \\
\hline $7 / 1 / 93 \quad 10: 06$ & Manual Tape & 385.75 & Good & \\
\hline $7 / 2 / 937: 06$ & Auto FIC & 385.70 & Good & \\
\hline $7 / 2 / 93 \quad 10: 06$ & Manual Tape & 385.50 & Good & \\
\hline 7/3/93 7:03 & Auto FIC & 385.75 & Good & \\
\hline $7 / 3 / 93 \quad 10: 06$ & Manual Tape & 385.50 & Good & \\
\hline 7/4/93 7:04 & Auto FIC & 385.75 & Good & \\
\hline $7 / 4 / 93 \quad 10: 06$ & Manual Tape & 385.50 & Good & \\
\hline $7 / 5 / 937: 03$ & Auto FIC & 385.75 & Good & \\
\hline $7 / 5 / 9310: 06$ & Manual Tape & 385.5 & Good & \\
\hline $7 / 6 / 93 \quad 7: 03$ & Auto FIC & 385.7 & Good & \\
\hline $7 / 6 / 93 \quad 10: 06$ & Manual Tape & 385.50 & Good & \\
\hline $7 / 7 / 937: 04$ & Auto FIC & 385.70 & Good & \\
\hline $7 / 7 / 93 \quad 10: 06$ & Manual Tape & 385.50 & Good & \\
\hline $7 / 8 / 937: 04$ & Auto FIC & 385.7 & Good & \\
\hline $7 / 8 / 93 \quad 10: 06$ & Manual Tape & 385.25 & Good & \\
\hline 7/9/93 7:05 & Auto FIC & 385.70 & Good & \\
\hline $7 / 9 / 93 \quad 10: 06$ & Manual Tape & 385.5 & Good & \\
\hline $7 / 10 / 937: 03$ & Auto FIC & 385.7 & Good & \\
\hline $7 / 10 / 93 \quad 10: 06$ & Manual Tape & 385.50 & Good & \\
\hline $7 / 11 / 937: 03$ & Auto FIC & 385.70 & Good & \\
\hline $7 / 11 / 93 \quad 10: 06$ & Manual Tape & 385.50 & Good & \\
\hline $7 / 12 / 937: 02$ & Auto FIC & 385.7 & Good & \\
\hline $7 / 12 / 93 \quad 10: 06$ & Manual Tape & 385.5 & Good & \\
\hline $7 / 13 / 937: 03$ & Auto FIC & 385.70 & Good & \\
\hline $7 / 13 / 93 \quad 10: 06$ & Manual Tape & 385.50 & Good & \\
\hline $7 / 14 / 937: 06$ & Auto FIC & 385.7 & Good & \\
\hline $7 / 14 / 93 \quad 10: 06$ & Manual Tape & 385.50 & Good & \\
\hline $7 / 15 / 937: 06$ & Auto FIC & 385.70 & Good & \\
\hline $7 / 15 / 93 \quad 10: 06$ & Manual Tape & 385.50 & Good & \\
\hline $7 / 16 / 937: 06$ & Auto FIC & 385.7 & Good & \\
\hline $7 / 16 / 93 \quad 10: 06$ & Manual Tape & 385.5 & Good & \\
\hline $7 / 17 / 937: 07$ & Auto FIC & 385.70 & Good & \\
\hline $7 / 17 / 93 \quad 10: 06$ & Manual Tape & 385.50 & Good & \\
\hline $7 / 18 / 937: 06$ & Auto FIC & 385.7 & Good & \\
\hline
\end{tabular}

Data obtained from WHC Surveillance Analysis Computer System, (PC SACS) July 7, 1995. 


\begin{tabular}{|c|c|c|c|c|}
\hline \multicolumn{5}{|c|}{ 241-AP-101 } \\
\hline \multicolumn{5}{|c|}{ Surface Level Data } \\
\hline Date & Type of Measurement & Level (Inches) & Data Quality & Comments \\
\hline $7 / 18 / 93 \quad 10: 06$ & Manual Tape & 385.50 & Good & \\
\hline $7 / 19 / 937: 03$ & Auto FIC & 385.70 & Good & \\
\hline $7 / 19 / 93 \quad 10: 06$ & Manual Tape & 385.50 & Good & \\
\hline $7 / 20 / 937: 03$ & Auto FIC & 385.7 & Good & \\
\hline $7 / 20 / 93 \quad 10: 06$ & Manual Tape & 385.5 & Good & \\
\hline $7 / 21 / 937: 03$ & Auto FIC & 385.7 & Good & \\
\hline $7 / 21 / 93 \quad 10: 06$ & Manual Tape & 385.50 & Good & \\
\hline $7 / 22 / 937: 03$ & Auto FIC & 385.7 & Good & \\
\hline $7 / 22 / 93 \quad 10: 06$ & Manual Tape & 385.25 & Good & \\
\hline 7/23/93 7:03 & Auto FIC & 385.70 & Good & \\
\hline $7 / 23 / 93 \quad 10: 06$ & Manual Tape & 385.50 & Good & \\
\hline $7 / 24 / 937: 03$ & Auto FIC & 385.7 & Good & \\
\hline $7 / 24 / 93 \quad 10: 06$ & Manual Tape & 385.25 & Good & \\
\hline $7 / 25 / 93 \quad 7: 04$ & Auto FIC & 385.70 & Good & \\
\hline $7 / 25 / 93 \quad 10: 06$ & Manual Tape & 385.25 & Good & \\
\hline $7 / 26 / 937: 03$ & Auto FIC & 385.70 & Good & \\
\hline $7 / 26 / 9310: 06$ & Manual Tape & 385.25 & Good & \\
\hline $7 / 27 / 937: 03$ & Auto FIC & 385.7 & Good & \\
\hline 7/27/93 10:06 & Manual Tape & 385.25 & Good & \\
\hline $7 / 28 / 930: 00$ & Manual Tape & 385.25 & Good & \\
\hline $7 / 28 / 93 \quad 7: 04$ & Auto FIC & 385.70 & Good & \\
\hline 7/29/93 0:00 & Manual Tape & 385.25 & Good & \\
\hline $7 / 29 / 937: 03$ & Auto FIC & 385.7 & Good & \\
\hline $7 / 30 / 930: 00$ & Manual Tape & $385.50^{\circ}$ & Good & \\
\hline 7/30/93 7:03 & Auto FIC & 385.70 & Good & \\
\hline $7 / 31 / 930: 00$ & Manual Tape & 385.50 & Good & \\
\hline 7/31/93 7:03 & Auto FIC & 385.70 & Good & \\
\hline $8 / 1 / 930: 00$ & Manual Tape & 385.5 & Good & \\
\hline 8/1/93 7:03 & Auto FIC & 385.7 & Good & \\
\hline 8/2/93 0:00 & Manual Tape & 385.75 & Good & \\
\hline $8 / 2 / 937: 06$ & Auto FIC & 385.70 & Good & \\
\hline $8 / 3 / 930: 00$ & Manual Tape & 385.00 & Good & \\
\hline $8 / 3 / 937: 03$ & Auto FIC & 385.7 & Good & \\
\hline 8/4/93 0:00 & Manual Tape & 385.5 & Good & \\
\hline $8 / 4 / 937: 03$ & Auto FIC & 385.7 & Good & \\
\hline $8 / 5 / 930: 00$ & Manual Tape & 385.75 & Good & \\
\hline $8 / 5 / 937: 04$ & Auto FIC & 385.7 & Good & \\
\hline 8/6/93 0:00 & Manual Tape & 385.50 & Good & \\
\hline $8 / 6 / 937: 04$ & Auto FIC & 385.7 & Good & \\
\hline $8 / 7 / 930: 00$ & Manual Tape & 385.5 & Good & \\
\hline 8/7/93 7:04 & Auto FIC & 385.7 & Good & \\
\hline $8 / 8 / 930: 00$ & Manual Tape & 385.50 & Good & \\
\hline 8/8/93 7:04 & Auto FIC & 385.7 & Good & \\
\hline $8 / 9 / 930: 00$ & Manual Tape & 385.50 & Good & \\
\hline $8 / 9 / 937: 04$ & Auto FIC & 385.7 & Good & \\
\hline $8 / 10 / 930: 00$ & Manual Tape & 385.5 & Good & \\
\hline $8 / 10 / 937: 04$ & Auto FIC & 385.70 & Good & \\
\hline $8 / 11 / 930: 00$ & Manual Tape & 385.50 & Good & \\
\hline
\end{tabular}

Data obtained from WHC Surveillance Analysis Computer System, (PC SACS) July 7, 1995. 


\begin{tabular}{|c|c|c|c|c|}
\hline \multicolumn{5}{|c|}{ 241-AP-101 } \\
\hline \multicolumn{5}{|c|}{ Surface Level Data } \\
\hline Date & Type of Measurement & Level (Inches) & Data Quality & Comments \\
\hline $8 / 11 / 937: 04$ & Auto FIC & 385.7 & Good & \\
\hline $8 / 12 / 930: 00$ & Manual Tape & 385.50 & Good & \\
\hline $8 / 12 / 937: 04$ & Auto FIC & 385.7 & Good & \\
\hline $8 / 13 / 930: 00$ & Manual Tape & 385.5 & Good & \\
\hline $8 / 13 / 937: 04$ & Auto FIC & 385.7 & Good & \\
\hline $8 / 14 / 930: 00$ & Manual Tape & 385.50 & Good & \\
\hline $8 / 14 / 937: 03$ & Auto FIC & 385.70 & Good & \\
\hline $8 / 15 / 930: 00$ & Manual Tape & 385.75 & Good & \\
\hline 8/15/93 7:04 & Auto FIC & 385.70 & Good & \\
\hline $8 / 16 / 930: 00$ & Manual Tape & 385.75 & Good & \\
\hline $8 / 16 / 937: 04$ & Auto FIC & 385.7 & Good & \\
\hline $8 / 17 / 930: 00$ & Manual Tape & 385.50 & Good & \\
\hline $8 / 17 / 937: 04$ & Auto FIC & 385.70 & Good & \\
\hline $8 / 18 / 930: 00$ & Manual Tape & $3 \overline{85.50}$ & Good & \\
\hline $8 / 18 / 937: 03$ & Auto FIC & 385.70 & Good & \\
\hline $8 / 19 / 930: 00$ & Manual Tape & 385.50 & Good & \\
\hline $8 / 19 / 937: 04$ & Auto FIC & 385.7 & Good & \\
\hline $8 / 20 / 930: 00$ & Manual Tape & 385.50 & Good & \\
\hline $8 / 20 / 937: 04$ & Auto FIC & 385.7 & Good & \\
\hline $8 / 21 / 930: 00$ & Manual Tape & 385.50 & Good & \\
\hline $8 / 21 / 937: 04$ & Auto FIC & 385.70 & Good & \\
\hline $8 / 22 / 93 \quad 0: 00$ & Manual Tape & 385.50 & Good & \\
\hline $8 / 22 / 937: 04$ & Auto FIC & 385.7 & Good & \\
\hline $8 / 23 / 930: 00$ & Manual Tape & 385.25 & Good & \\
\hline $8 / 23 / 937: 04$ & Auto FIC & 385.70 & Good & \\
\hline $8 / 24 / 930: 00$ & Manual Tape & 385.50 & Gopd & \\
\hline $8 / 24 / 937: 04$ & Auto FIC & 385.7 & Good & \\
\hline $8 / 25 / 930: 00$ & Manual Tape & 385.5 & Good & \\
\hline $8 / 25 / 937: 04$ & Auto FIC & 385.70 & Good & \\
\hline $8 / 26 / 93 \quad 0: 00$ & Manual Tape & 385.50 & Good & \\
\hline $8 / 26 / 937: 04$ & Auto FIC & 385.7 & Good & \\
\hline $8 / 27 / 93 \quad 7: 05$ & Auto FIC & 385.70 & Good & \\
\hline $8 / 28 / 937: 04$ & Auto FIC & 385.70 & Good & \\
\hline $8 / 29 / 937: 03$ & Auto FIC & 385.7 & Good & \\
\hline $8 / 30 / 930: 00$ & Manual Tape & 385,50 & Good & \\
\hline $8 / 30 / 937: 04$ & Auto FIC & 385.70 & Good & \\
\hline $8 / 31 / 930: 00$ & Manual Tape & 385.5 & Good & \\
\hline $8 / 31 / 937: 04$ & Auto FIC & 385.7 & Good & \\
\hline 9/1/93 0:00 & Manual Tape & 385.75 & Good & \\
\hline $9 / 1 / 937: 04$ & Auto FIC & 385.70 & Good & \\
\hline $9 / 2 / 930: 00$ & Manual Tape & 385.75 & Good & \\
\hline $9 / 2 / 937: 04$ & Auto FIC & 385.70 & Good & \\
\hline $9 / 3 / 930: 00$ & Manual Tape & 385.75 & Good & \\
\hline $9 / 3 / 937: 04$ & Auto FIC & 385.7 & Good & \\
\hline 9/4/93 0:00 & Manual Tape & 385.75 & Good & \\
\hline $9 / 4 / 937: 04$ & Auto FIC & 385.70 & Good & \\
\hline $9 / 5 / 930: 00$ & Manual Tape & 385.75 & Good & \\
\hline $9 / 5 / 937: 04$ & Auto FIC & 385.7 & Good & \\
\hline
\end{tabular}

Data obtained from WHC Surveillance Analysis Computer System, (PC SACS) July 7, 1995. 


\begin{tabular}{|c|c|c|c|c|}
\hline \multicolumn{5}{|c|}{ 241-AP-101 } \\
\hline \multicolumn{5}{|c|}{ Surface Level Data } \\
\hline Date & Type of Measurement & Leval (Inchas) & Data Quality & Comments \\
\hline $9 / 6 / 930: 00$ & Manual Tape & 385.75 & Good & \\
\hline 9/6/93 7:04 & Auto FIC & 385.70 & Good & \\
\hline 9/7/93 0:00 & Manual Tape & 385.75 & Good & \\
\hline $9 / 7 / 937: 04$ & Auto FIC & 385.7 & Good & \\
\hline 9/8/93 0:00 & Manual Tape & 385.75 & Good & \\
\hline 9/8/93 7:04 & Auto FIC & 385.70 & Good & \\
\hline $9 / 9 / 930: 00$ & Manual Tape & 385.75 & Good & \\
\hline 9/9/93 7:04 & Auto FIC & 385.70 & Good & \\
\hline $9 / 10 / 930: 00$ & Manual Tape & 385.75 & Good & \\
\hline $9 / 10 / 937: 04$ & Auto FIC & 385.7 & Good & \\
\hline $9 / 11 / 930: 00$ & Manual Tape & 385.75 & Good & \\
\hline $9 / 11 / 937: 04$ & Auto FIC & 385.70 & Good & \\
\hline $9 / 12 / 930: 00$ & Manual Tape & 385.75 & Good & \\
\hline $9 / 12 / 937: 04$ & Auto FIC & 385.70 & Good & \\
\hline $9 / 13 / 930: 00$ & Manual Tape & 385.50 & Good & \\
\hline 9/13/93 7:04 & Auto FIC & 385.7 & Good & \\
\hline $9 / 14 / 930: 00$ & Manual Tape & 385.50 & Good & \\
\hline $9 / 14 / 937: 04$ & Auto FIC & 385.70 & Good & \\
\hline $9 / 15 / 930: 00$ & Manual Tape & 385.5 & Good & \\
\hline $9 / 15 / 937: 04$ & Auto FIC & 385.7 & Good & \\
\hline $9 / 16 / 930: 00$ & Manual Tape & 385.25 & Good & \\
\hline $9 / 16 / 937: 04$ & Auto FIC & 385.70 & Good & \\
\hline $9 / 17 / 930: 00$ & Manual Tape & 385.50 & Good & \\
\hline $9 / 17 / 937: 04$ & Auto FIC & 385.7 & Good & \\
\hline 9/18/93 0:00 & Manual Tape & 385.5 & Good & \\
\hline $9 / 18 / 937: 04$ & Auto FIC & 385.70 & Good & \\
\hline 9/19/93 0:00 & Manual Tape & 385.50 & Good & \\
\hline 9/19/93 7:04 & Auto FIC & 385.7 & Good & \\
\hline $9 / 20 / 930: 00$ & Manual Tape & 385.50 & Good & \\
\hline $9 / 20 / 937: 04$ & Auto FIC & 385.70 & Good & \\
\hline $9 / 21 / 930: 00$ & Manual Tape & 385.5 & Good & \\
\hline $9 / 21 / 937: 04$ & Auto FIC & 385.7 & Good & \\
\hline $9 / 22 / 930: 00$ & Manual Tape & 385.50 & Good & \\
\hline $9 / 22 / 937: 04$ & Auto FIC & 385.70 & Good & \\
\hline $9 / 23 / 930: 00$ & Manual Tape & 385.50 & Good & \\
\hline $9 / 23 / 937: 04$ & Auto FIC & 385.7 & Good & \\
\hline $9 / 24 / 930: 00$ & Manual Tape & 385.5 & Good & \\
\hline $9 / 24 / 937: 04$ & Auto FIC & 385.70 & Good & \\
\hline $9 / 25 / 930: 00$ & Manual Tape & 385.50 & Good & \\
\hline $9 / 25 / 937: 04$ & Auto FIC & 385.7 & Good & \\
\hline $9 / 26 / 93 \quad 0: 00$ & Manual Tape & $\mathbf{3 8 5 . 5 0}$ & Good & \\
\hline $9 / 26 / 937: 04$ & Auto FIC & 385.70 & Good & \\
\hline 9/27/93 0:00 & Manual Tape & 385.5 & Good & \\
\hline $9 / 27 / 937: 04$ & Auto FIC & 385.7 & Good & \\
\hline $9 / 28 / 930: 00$ & Manual Tape & 385.50 & Good & \\
\hline $9 / 28 / 937: 04$ & Auto FIC & 385.70 & Good & \\
\hline 9/29/93 0:00 & Manual Tape & 385.50 & Good & \\
\hline $9 / 29 / 937: 04$ & Auto FIC & 385.7 & Good & \\
\hline
\end{tabular}

Data obtained from WHC Surveillance Analysis Computer System, (PC SACS) July 7, 1995. 


\begin{tabular}{|c|c|c|c|c|}
\hline \multicolumn{5}{|c|}{ 241-AP-101 } \\
\hline \multicolumn{5}{|c|}{ Surface Leval Data } \\
\hline Date & Type of Measurement & Level (Inches) & Data Quality & Comments \\
\hline $9 / 30 / 930: 00$ & Manual Tape & 385.5 & Good & \\
\hline $9 / 30 / 937: 03$ & Auto FIC & 385.70 & Good & \\
\hline 10/1/93 0:00 & Manual Tape & 385:50 & Good & \\
\hline $10 / 1 / 937: 06$ & Auto FIC & 385.7 & Good & \\
\hline $10 / 2 / 930: 00$ & Manual Tape & 385.50 & Good & \\
\hline $10 / 2 / 937: 05$ & Auto FIC & 385.70 & Good & \\
\hline $10 / 3 / 930: 00$ & Manual Tape & 385.75 & Good & \\
\hline 10/3/93 7:06 & Auto FIC & 385.7 & Good & \\
\hline $10 / 4 / 930: 00$ & Manual Tape & 385.50 & Good & \\
\hline $10 / 4 / 937: 06$ & Auto FIC & 385.70 & Good & \\
\hline $10 / 5 / 930: 00$ & Manual Tape & 385.50 & Good & \\
\hline $10 / 5 / 937: 06$ & Auto FIC & 385.7 & Good & \\
\hline $10 / 6 / 930: 00$ & Manual Tape & 385.5 & Good & \\
\hline $10 / 6 / 937: 06$ & Auto FIC & 385.70 & Good & \\
\hline $10 / 7 / 930: 00$ & Manual Tape & 385.50 & Good & \\
\hline $10 / 7 / 937: 05$ & Auto FIC & 385.7 & Good & \\
\hline $10 / 8 / 93 \quad 0: 00$ & Manual Tape & 385.50 & Good & \\
\hline $10 / 8 / 937: 04$ & Auto FIC & 385.70 & Good & \\
\hline $10 / 9 / 93 \quad 0: 00$ & Manual Tape & 385.5 & Good & \\
\hline $10 / 9 / 937: 03$ & Auto FIC & 385.7 & Good & \\
\hline $10 / 10 / 930: 00$ & Manual Tape & 385.50 & Good & \\
\hline $10 / 10 / 936: 04$ & Auto FIC & 385.70 & Good & \\
\hline $10 / 11 / 930: 00$ & Manual Tape & 385.50 & Good & \\
\hline $10 / 11 / 937: 03$ & Auto FIC & 385.7 & Good & \\
\hline $10 / 12 / 930: 00$ & Manual Tape & 385.5 & Good & \\
\hline $10 / 12 / 937: 04$ & Auto FIC & 385.70 & Good & \\
\hline 10/13/93 0:00 & Manual Tape & 385.50 & Good & \\
\hline $10 / 13 / 937: 03$ & Auto FIC & 385.70 & Good & \\
\hline $10 / 14 / 930: 00$ & Manual Tape & 385.50 & Good & \\
\hline $10 / 14 / 937: 04$ & Auto FIC & 385.7 & Good & \\
\hline $10 / 15 / 930: 00$ & Manual Tape & 385.50 & Good & \\
\hline $10 / 15 / 937: 04$ & Auto FIC & 385.70 & Good & \\
\hline $10 / 16 / 930: 00$ & Manual Tape & 385.50 & Good & \\
\hline $10 / 16 / 937: 04$ & Auto FIC & 385.7 & Good & \\
\hline $10 / 17 / 930: 00$ & Manual Tape & 385.5 & Good & \\
\hline $10 / 17 / 937: 03$ & Auto FIC & 385.7 & Good & \\
\hline $10 / 18 / 930: 00$ & Manual Tape & 385:50 & Good & \\
\hline $10 / 18 / 937: 04$ & Auto FIC & 385.70 & Good & \\
\hline $10 / 19 / 930: 00$ & Manual Tape & 385.50 & Good & \\
\hline $10 / 19 / 937: 04$ & Auto FIC & 385.7 & Good & \\
\hline $10 / 20 / 930: 00$ & Manual Tape & 385.5 & Good & \\
\hline $10 / 20 / 937: 03$ & Auto FIC & 385.70 & Good & \\
\hline $10 / 21 / 930: 00$ & Manual Tape & 385.50 & Good & \\
\hline $10 / 21 / 937: 04$ & Auto FIC & 385.7 & Good & \\
\hline $10 / 22 / 930: 00$ & Manual Tape & 385.50 & Good & \\
\hline $10 / 22 / 937: 04$ & Auto FIC & 385.70 & Good & \\
\hline $10 / 23 / 930: 00$ & Manual Tape & 385.5 & - Good & \\
\hline $10 / 23 / 937: 04$ & Auto FIC & 385.7 & Good & \\
\hline
\end{tabular}

Data obtained from WHC Surveillance Analysis Computer System, (PC SACS) July 7, 1995. 


\begin{tabular}{|c|c|c|c|c|}
\hline \multicolumn{5}{|c|}{ 241-AP-101 } \\
\hline \multicolumn{5}{|c|}{ Surface Level Data } \\
\hline Date & Type of Measurement & Level-(linches) & Data Quality & Comments \\
\hline $10 / 24 / 930: 00$ & Manual Tape & 385.50 & Good & \\
\hline 10/24/93 7:04 & Auto FIC & 385.70 & Good & \\
\hline $10 / 25 / 930: 00$ & Manual Tape & 385.50 & Good & \\
\hline $10 / 25 / 93 \quad 7: 04$ & Auto FIC & 385.7 & Good & \\
\hline $10 / 26 / 930: 00$ & Manual Tape & 385.5 & Good & \\
\hline $10 / 26 / 936: 03$ & Auto FIC & 385.70 & Good & \\
\hline $10 / 27 / 930: 00$ & Manual Tape & 385.50 & Good & \\
\hline $10 / 27 / 93 \quad 7: 04$ & Auto FIC & 385.7 & Good & \\
\hline $10 / 28 / 930: 00$ & Manual Tape & 385.50 & Good & \\
\hline $10 / 28 / 937: 04$ & Auto FIC & 385.70 & Good & \\
\hline $10 / 29 / 930: 00$ & Manual Tape & 385.5 & Good & \\
\hline $10 / 29 / 936: 03$ & Auto FIC & 385.7 & Good & \\
\hline $10 / 30 / 930: 00$ & Manual Tape & 385.50 & Good & \\
\hline 10/30/93 7:04 & Auto FIC & 385.70 & Good & \\
\hline $10 / 31 / 930: 00$ & Manual Tape & 385.50 & Good & \\
\hline $10 / 31 / 937: 06$ & Auto FIC & 385.7 & Good & \\
\hline $11 / 1 / 930: 00$ & Manual Tape & 385.5 & Good & \\
\hline $11 / 1 / 937: 06$ & Auto FIC & 385.70 & Good & \\
\hline $11 / 2 / 930: 00$ & Manual Tape & 385.50 & Good & \\
\hline $11 / 2 / 937: 04$ & Auto FIC & 385.7 & Good & \\
\hline $11 / 3 / 930: 00$ & Manual Tape & 385.50 & Good & \\
\hline $11 / 3 / 937: 06$ & Auto FIC & 385.70 & Good & \\
\hline $11 / 4 / 930: 00$ & Manual Tape & 385.5 & Good & \\
\hline $11 / 4 / 937: 04$ & Auto FIC & 385.7 & Good & \\
\hline $11 / 5 / 930: 00$ & Manual Tape & 385.50 & Good & \\
\hline $11 / 5 / 937: 06$ & Auto FIC & 385.70 & Good & \\
\hline $11 / 6 / 930: 00$ & Manual Tape & 385.50 & Good & \\
\hline $11 / 6 / 937: 06$ & Auto FIC & 385.7 & Good & \\
\hline $11 / 7 / 930: 00$ & Manual Tape & 385.5 & Good & \\
\hline $11 / 7 / 937: 06$ & Auto FIC & 385.70 & Good & \\
\hline $11 / 8 / 930: 00$ & Manual Tape & 385.50 & Good & \\
\hline 11/8/93 7:06 & Auto FIC & 385.7 & Good & \\
\hline $11 / 9 / 930: 00$ & Manual Tape & 385.5 & Good & \\
\hline 11/9/93 7:03 & Auto FIC & 385.70 & Good & \\
\hline $11 / 10 / 930: 00$ & Manual Tape & 385.50 & Good & \\
\hline $11 / 10 / 937: 04$ & Auto FIC & 385.7 & Good & \\
\hline $11 / 11 / 930: 00$ & Manual Tape & 385.50 & Good & \\
\hline $11 / 11 / 937: 04$ & Auto FIC & 385.70 & Good & \\
\hline $11 / 12 / 930: 00$ & Manual Tape & 385.5 & Good & \\
\hline $11 / 12 / 937: 04$ & Auto FIC & 385.7 & Good & \\
\hline $11 / 13 / 930: 00$ & Manual Tape & 385.50 & Good & \\
\hline $11 / 13 / 937: 04$ & Auto FIC & 385.70 & Good & \\
\hline $11 / 14 / 930: 00$ & Manual Tape & 385.50 & Good & \\
\hline $11 / 14 / 936: 04$ & Auto FIC & 385.7 & Good & \\
\hline $11 / 15 / 930: 00$ & Manual Tape & 385.5 & Good & \\
\hline $11 / 15 / 937: 04$ & Auto FIC & 385.70 & Good & \\
\hline $11 / 16 / 930: 00$ & Manual Tape & 385.50 & Good & \\
\hline $11 / 16 / 937: 03$ & Auto FIC & 385.7 & Good & \\
\hline
\end{tabular}

Data obtained from WHC Surveillance Analysis Computer System, (PC SACS) July 7, 1995. 


\begin{tabular}{|c|c|c|c|c|}
\hline \multicolumn{5}{|c|}{ 241-AP-101 } \\
\hline \multicolumn{5}{|c|}{ Surface Levol Data } \\
\hline Date & Type of Measuremept & Level (Inches) & Data Quality & Comments \\
\hline $11 / 17 / 930: 00$ & Manual Tape & 385.50 & Good & \\
\hline $11 / 17 / 937: 04$ & Auto FIC & 385.70 & Good & \\
\hline $11 / 18 / 93 \quad 0: 00$ & Manual Tape & 385.5 & Good & \\
\hline 11/18/93 7:04 & Auto FIC & 385.7 & Good & \\
\hline $11 / 19 / 930: 00$ & Manual Tape & 385.75 & Good & \\
\hline $11 / 19 / 937: 04$ & Auto FIC & 385.70 & Good & \\
\hline $11 / 20 / 930: 00$ & Manual Tape & 385.50 & Good & \\
\hline $11 / 20 / 937: 04$ & Auto FIC & 385.7 & Good & \\
\hline $11 / 21 / 930: 00$ & Manual Tape & 385.50 & Good & \\
\hline $11 / 21 / 937: 04$ & Auto FIC & 385.70 & Good & \\
\hline $11 / 22 / 930: 00$ & Manual Tape & 385.50 & Good & \\
\hline $11 / 22 / 937: 04$ & Auto FIC & 385.6 & Good & \\
\hline $11 / 23 / 930: 00$ & Manual Tape & 385.50 & Good & \\
\hline $11 / 23 / 937: 04$ & Auto FIC & 385.6 & Good & \\
\hline $11 / 24 / 930: 00$ & Manual Tape & 385.50 . & Good & \\
\hline $11 / 24 / 937: 04$ & Auto FIC & 385.60 & Good & \\
\hline $11 / 25 / 930: 00$ & Manual Tape & 385.5 & Good & \\
\hline $11 / 25 / 937: 04$ & Auto FIC & 385.6 & Good & \\
\hline $11 / 26 / 930: 00$ & Manual Tape & 385.50 & Good & \\
\hline $11 / 26 / 937: 04$ & Auto FIC & 385.60 & Good & \\
\hline $11 / 27 / 930: 00$ & Manual Tape & 385.50 & Good & \\
\hline $11 / 27 / 937: 04$ & Auto FIC & 385.6 & Good & \\
\hline $11 / 28 / 930: 00$ & Manual Tape & 385.50 & Good & \\
\hline $11 / 28 / 937: 03$ & Auto FIC & $385.60^{\circ}$ & Good & \\
\hline $11 / 29 / 930: 00$ & Manual Tape & 385.5 & Good & \\
\hline $11 / 29 / 937: 04$ & Auto FIC & 385.6 & Good & \\
\hline $11 / 30 / 930: 00$ & Manual Tape & 385.50 & Good & \\
\hline $11 / 30 / 93$ 7:03 & Auto FIC & 385.60 & Good & \\
\hline $12 / 1 / 930: 00$ & Manual Tape & 385.50 & Good & \\
\hline $12 / 1 / 937: 04$ & Auto FIC & 385.6 & Good & \\
\hline $12 / 2 / 930: 00$ & Manual Tape & 385.5 & Good & \\
\hline $12 / 2 / 937: 04$ & Auto FIC & 385.60 & Good & \\
\hline $12 / 3 / 930: 00$ & Manual Tape & 385.50 & Good & \\
\hline $12 / 3 / 937: 05$ & Auto FIC & 385.6 & Good & \\
\hline $12 / 4 / 930: 00$ & Manual Tape & 385.50 & Good & \\
\hline $12 / 4 / 937: 05$ & Auto FIC & 385.60 & Good & \\
\hline $12 / 5 / 930: 00$ & Manual Tape & 385.5 & Good & \\
\hline 12/5/93 7:04 & Auto FIC & 385.6 & Good & \\
\hline $12 / 6 / 930: 00$ & Manual Tape & 384.50 & Suspect & Data not included in graph \\
\hline $12 / 6 / 937: 05$ & Auto FIC & 385.60 & Good & \\
\hline $12 / 7 / 930: 00$ & Manual Tape & 396.00 & Suspect & Data not included in graph \\
\hline $12 / 7 / 937: 04$ & Auto FIC & 385.6 & Good & \\
\hline $12 / 8 / 930: 00$ & Manual Tape & 385.25 & Good & \\
\hline $12 / 8 / 937: 06$ & Auto FIC & 385.60 & Good & \\
\hline $12 / 9 / 930: 00$ & Manual Tape & 385.50 & Good & \\
\hline $12 / 9 / 937: 04$ & Auto FIC & 385.6 & Good & \\
\hline $12 / 10 / 930: 00$ & Manual Tape & 385.75 & Good & \\
\hline $12 / 10 / 936: 05$ & Auto FIC & 385.60 & Good & \\
\hline
\end{tabular}

Data obtained from WHC Surveillance Analysis Computer System, (PC SACS) July 7, 1995. 


\begin{tabular}{|c|c|c|c|c|}
\hline \multicolumn{5}{|c|}{ 241-AP-101 } \\
\hline \multicolumn{5}{|c|}{ Surface Level Data } \\
\hline Date & Type of Measurement & Level (Inches) & Data Quality & Comments \\
\hline $12 / 11 / 930: 00$ & Manual Tape & 385.5 & Good & \\
\hline $12 / 11 / 937: 06$ & Auto FIC & 385.6 & Good & \\
\hline $12 / 12 / 930: 00$ & Manual Tape & 385.25 & Good & \\
\hline $12 / 12 / 937: 06$ & Auto FIC & 385.60 & Good & \\
\hline $12 / 13 / 930: 00$ & Manual Tape & 385 & Good & \\
\hline $12 / 13 / 937: 06$ & Auto FIC & 385.6 & Good & \\
\hline $12 / 14 / 930: 00$ & Manual Tape & 385.00 & Good & \\
\hline $12 / 14 / 937: 06$ & Auto FIC & 385.60 & Good & \\
\hline $12 / 15 / 930: 00$ & Manual Tape & 385.25 & Good & \\
\hline 12/15/93 7:06 & Auto FIC & 385.6 & Good & \\
\hline 12/16/93 0:00 & Manual Tape & 385.25 & Good & \\
\hline $12 / 16 / 937: 06$ & Auto FIC & 385.60 & Good & \\
\hline $12 / 17 / 930: 00$ & Manual Tape & 385.5 & Good & \\
\hline $12 / 17 / 937: 05$ & Auto FIC & 385.6 & Good & \\
\hline $12 / 18 / 930: 00$ & Manual Tape & 385.25 & Good & \\
\hline $12 / 18 / 937: 06$ & Auto FIC & 385.60 & Good & \\
\hline $12 / 19 / 930: 00$ & Manual Tape & 384.50 & Suspect & Data not included in graph \\
\hline $12 / 19 / 937: 06$ & Auto FIC & 385.6 & Good & \\
\hline $12 / 20 / 930: 00$ & Manual Tape & 385.5 & Good & \\
\hline $12 / 20 / 937: 06$ & Auto FIC & 385.60 & Good & \\
\hline $12 / 21 / 930: 00$ & Manual Tape & 385.50 & Good & \\
\hline $12 / 21 / 937: 06$ & Auto FIC & 385.6 & Good & \\
\hline $12 / 22 / 930: 00$ & Manual Tape & 385.50 & Good & \\
\hline $12 / 22 / 937: 05$ & Auto FIC & 385.60 & Good & \\
\hline $12 / 23 / 930: 00$ & Manual Tape & 385.5 & Good & \\
\hline $12 / 23 / 936: 06$ & Auto FIC & 385.6 & Good & \\
\hline $12 / 24 / 930: 00$ & Manual Tape & 385.50 & Good & \\
\hline $12 / 24 / 937: 06$ & Auto FIC & 385.60 & Good & \\
\hline $12 / 25 / 930: 00$ & Manual Tape & 385.50 & Good & \\
\hline $12 / 25 / 937: 06$ & Auto FIC & 385.6 & Good & \\
\hline $12 / 26 / 930: 00$ & Manual Tape & 385.5 & Good & \\
\hline $12 / 26 / 937: 06$ & Auto FIC & 385.60 & Good & \\
\hline $12 / 27 / 930: 00$ & Manual Tape & 385.50 & Good & \\
\hline $12 / 27 / 937: 06$ & Auto FIC & 385.6 & Good & \\
\hline $12 / 28 / 93 \quad 0: 00$ & Manual Tape & 385.50 & Good & \\
\hline 12/28/93 7:06 & Auto FIC & 385.60 & Good & \\
\hline $12 / 29 / 930: 00$ & Manual Tape & 385.5 & Good & \\
\hline $12 / 29 / 937: 06$ & Auto FIC & 385.6 & Good & \\
\hline $12 / 30 / 930: 00$ & Manual Tape & 385.50 & Good & \\
\hline $12 / 30 / 937: 05$ & Auto FIC & 385.60 & Good & \\
\hline $12 / 31 / 930: 00$ & Manual Tape & 385.50 & Good & \\
\hline $12 / 31 / 937: 06$ & Auto FIC & 385.6 & Good & \\
\hline 1/1/940:00 & Manual Tape & 385.50 & Good & \\
\hline 1/1/94 7:06 & Auto FIC & 385.60 & Good & \\
\hline $1 / 2 / 940: 00$ & Manual Tape & 385.50 & Good & \\
\hline $1 / 2 / 947: 06$ & Auto FIC & 385.60 & Good & \\
\hline $1 / 3 / 940: 00$ & Manual Tape & 385.50 & Good & . \\
\hline $1 / 3 / 947: 07$ & Auto FIC & 385.6 & Good & \\
\hline
\end{tabular}

Data obtained from WHC Surveillance Analysis Computer System, (PC SACS) July 7, 1995. 


\begin{tabular}{|c|c|c|c|c|}
\hline \multicolumn{5}{|c|}{ 241-AP-101 } \\
\hline \multicolumn{5}{|c|}{ Surface Lovel Data } \\
\hline Date & Type of Measurement & Level (Inches) & Data Ouality & Comments \\
\hline $1 / 4 / 940: 00$ & Manual Tape & 385.50 & Good & \\
\hline $1 / 4 / 947: 07$ & Auto FIC & 385.60 & Good & \\
\hline 1/5/940:00 & Manual Tape & 385.50 & Goad & \\
\hline $1 / 5 / 947: 06$ & Auto FIC & 385.6 & Good & \\
\hline $1 / 6 / 940: 00$ & Manual Tape & 385.50 & Good & \\
\hline $1 / 6 / 947: 06$ & Auto FIC & 385.60 & Good & \\
\hline $1 / 7 / 94 \quad 0: 00$ & Manual Tape & 385.25 & Good & \\
\hline $1 / 7 / 947: 07$ & Auto FIC & 385.6 & Good & \\
\hline $1 / 8 / 940: 00$ & Manual Tape & 385.25 & Good & \\
\hline $1 / 8 / 947: 06$ & Auto FIC & 385.60 & Good & \\
\hline $1 / 9 / 940: 00$ & Manual Tape & 385.25 & Good & \\
\hline $1 / 9 / 947: 06$ & Auto FIC & 385.6 & Good & \\
\hline 1/10/940:00 & Manual Tape & 385.25 & Good & \\
\hline $1 / 10 / 947: 07$ & Auto FIC & 385.50 & Good & \\
\hline $1 / 11 / 940: 00$ & Manual Tape & 385.25 & Good & \\
\hline $1 / 11 / 947: 07$ & Auto FIC & 385.5 & Good & \\
\hline $1 / 12 / 940: 00$ & Manual Tape & $385.50^{\circ}$ & Good & \\
\hline $1 / 12 / 947: 06$ & Auto FIC & 385.50 & Good & \\
\hline $1 / 13 / 940: 00$ & Manual Tape & 385.25 & Good & \\
\hline $1 / 13 / 945: 07$ & Auto FIC & 385.5 & Good & \\
\hline $1 / 14 / 940: 00$ & Manual Tape & 385.25 & Good & \\
\hline $1 / 14 / 947: 06$ & Auto FIC & 385.50 & Good & \\
\hline $1 / 15 / 940: 00$ & Manual Tape & 385.25 & Good & \\
\hline $1 / 15 / 945: 07$ & Auto FIC & 385.5 & Good & \\
\hline $1 / 16 / 940: 00$ & Manual Tape & 385.25 . & Good & \\
\hline $1 / 16 / 947: 07$ & Auto FIC & 385.50 & Good & \\
\hline $1 / 17 / 940: 00$ & Manual Tape & 385.25 & Good & \\
\hline $1 / 17 / 947: 07$ & Auto FIC & 385.5 & Good & \\
\hline $1 / 18 / 940: 00$ & Manual Tape & 385.25 & Good & \\
\hline $1 / 18 / 947: 07$ & Auto FIC & 385.50 & Good & \\
\hline $1 / 19 / 940: 00$ & Manual Tape & 385.25 & Good & \\
\hline $1 / 19 / 947: 07$ & Auto FIC & 385.5 & Good & \\
\hline $1 / 20 / 940: 00$ & Manual Tape & 385.25 & Good & \\
\hline $1 / 20 / 947: 07$ & Auto FIC & $385.50^{\circ}$ & Good & \\
\hline 1/21/940:00 & Manual Tape & 385.25 & Good & \\
\hline $1 / 21 / 947: 07$ & Auto FIC & 385.5 & Good & \\
\hline $1 / 22 / 940: 00$ & Manual Tape & 385.50 & Good & \\
\hline $1 / 22 / 947: 08$ & Auto FIC & 385.50 & Good & \\
\hline $1 / 23 / 940: 00$ & Manual Tape & 385.5 & Good & \\
\hline $1 / 23 / 947: 07$ & Auto FIC & 385.5 & Good & \\
\hline $1 / 24 / 940: 00$ & Manual Tape & . $\mathbf{3 8 5 . 5 0}$ & Good & \\
\hline $1 / 24 / 947: 08$ & Auto FIC & 385.50 & Good & \\
\hline $1 / 24 / 9414: 00$ & Manual Tape & 383.75 & Suspect & Data not included in graph \\
\hline $1 / 25 / 940: 00$ & Manual Tape & 385.5 & Good & \\
\hline $1 / 25 / 947: 07$ & Auto FIC & 385.5 & Good & \\
\hline $1 / 26 / 940: 00$ & Manual Tape & 385.50 & Good & \\
\hline $1 / 26 / 947: 06$ & Auto FIC & 385.50 & Good & \\
\hline $1 / 27 / 940: 00$ & Manual Tape & 385.50 & Good & \\
\hline
\end{tabular}

Data obtained from WHC Surveillance Analysis Computer System, (PC SACS) July 7, 1995. 


\begin{tabular}{|c|c|c|c|c|}
\hline \multicolumn{5}{|c|}{ 241-APr101 } \\
\hline \multicolumn{5}{|c|}{ Surface Level Data } \\
\hline Date. & Type of Measurement & Level (Inches) & Data Quality & Comments \\
\hline $1 / 27 / 946: 06$ & Auto FIC & 385.5 & Good & \\
\hline $1 / 28 / 940: 00$ & Manual Tape & 385.5 & Good & \\
\hline $1 / 28 / 947: 07$ & Auto FIC & $385: 50$ & Good & \\
\hline $1 / 29 / 940: 00$ & Manual Tape & 385.50 & Good & \\
\hline $1 / 29 / 947: 07$ & Auto FIC & 385.5 & Good & \\
\hline 1/30/940:00 & Manual Tape & 385.50 & Good & \\
\hline $1 / 30 / 947: 06$ & Auto FIC & 385.50 & Good & \\
\hline $1 / 31 / 940: 00$ & Manual Tape & 385.5 & Good & \\
\hline $1 / 31 / 947: 07$ & Auto FIC & 385.5 & Good & \\
\hline $2 / 1 / 940: 00$ & Manual Tape & 385.50 & Good & \\
\hline 2/1/94 7:06 & Auto FIC & 385.50 & Good & \\
\hline 2/2/94 0:00 & Manual Tape & 385.50 & Good & \\
\hline $2 / 2 / 947: 07$ & Auto FIC & 385.5 & Good & \\
\hline 2/3/94 0:00 & Manual Tape & 385.25 & Good & \\
\hline $2 / 3 / 947: 07$ & Auto FIC & 385.50 & Good & \\
\hline $2 / 4 / 940.00$ & Manual Tape & 385.25 & Good & \\
\hline $2 / 4 / 947: 07$ & Auto FIC & 385.5 & Good & \\
\hline $2 / 5 / 940: 00$ & Manual Tape & 385.50 & Good & \\
\hline $2 / 5 / 947: 07$ & Auto FIC & 385.50 & Good & \\
\hline $2 / 6 / 940: 00$ & Manual Tape & 385.5 & Good & \\
\hline $2 / 6 / 947: 07$ & Auto FIC & 385.5 & Good & \\
\hline $2 / 7 / 940: 00$ & Manual Tape & 385.25 & Good & \\
\hline 2/7/94 7:06 & Auto FIC & 385.50 & Good & \\
\hline $2 / 8 / 940: 00$ & Manual Tape. & 385.25 & Good & \\
\hline 2/8/94 7:07 & Auto FIC & 385.5 & Good & \\
\hline $2 / 9 / 940: 00$ & Manual Tape & 385.25 & Good & \\
\hline $2 / 9 / 94 \quad 7: 04$ & Auto FIC & 385.50 & Good & \\
\hline $2 / 10 / 940: 00$ & Manual Tape & 385.25 & Good & \\
\hline 2/10/94 7:04 & Auto FIC & 385.5 & Good & \\
\hline $2 / 11 / 940: 00$ & Manual Tape & 385.50 & Good & \\
\hline $2 / 11 / 947: 06$ & Auto FIC & 385.50 & Good & \\
\hline $2 / 11 / 9414: 00$ & Manual Tape & 385.25 & Good & \\
\hline $2 / 12 / 940: 00$ & Manual Tape & 385.25 & Good & \\
\hline $2 / 12 / 947: 03$ & Auto FIC & 385.5 & Good & \\
\hline 2/13/94 0:00 & Manual Tape & 385.25 & Good & \\
\hline $2 / 13 / 947: 04$ & Auto FIC & 385.50 & Good & \\
\hline $2 / 14 / 940: 00$ & Manual Tape & 385.5 & Good & \\
\hline $2 / 14 / 947: 04$ & Auto FIC & 385.5 & Good & \\
\hline $2 / 14 / 9414: 00$ & Manual Tape & 385.25 & Good & \\
\hline $2 / 15 / 940: 00$ & Manual Tape & 385.25 & Good & \\
\hline 2/15/94 7:04 & Auto FIC & 385.50 & Good & \\
\hline 2/16/940:00 & Manual Tape & 385.25 & Good & \\
\hline 2/16/94 7:03 & Auto FIC & 385.5 & Good & \\
\hline $2 / 17 / 940: 00$ & Manual Tape & 385.50 & Good & \\
\hline $2 / 17 / 947: 04$ & Auto FIC & 385.50 & Good & \\
\hline $2 / 18 / 940: 00$ & Manual Tape & 385.25 & Good & \\
\hline $2 / 18 / 947: 06$ & Auto FIC & 385.5 & Good & \\
\hline $2 / 19 / 940: 00$ & Manual Tape & $385.25^{\circ}$ & Good & \\
\hline
\end{tabular}

Data obtained from WHC Surveillance Analysis Computer System, (PC SACS) July 7, 1995. 


\begin{tabular}{|c|c|c|c|c|}
\hline \multicolumn{5}{|c|}{ 241-AP-101 } \\
\hline \multicolumn{5}{|c|}{ Surface Lovel Data } \\
\hline Date & Type of Measurement & Level (Inched) & Data Quality & Comments \\
\hline $2 / 19 / 947: 06$ & Auto FIC & 385.50 . & Good & \\
\hline $2 / 20 / 940: 00$ & Manual Tape & 385.25 & Good & \\
\hline $2 / 20 / 947: 07$ & Auto FIC & 385.5 & Good & \\
\hline $2 / 21 / 940: 00$ & Manual Tape & 385.25 & Good & \\
\hline $2 / 21 / 947: 06$ & Auto FIC & 385.50 & Good & \\
\hline $2 / 22 / 94 \quad 0: 00$ & Manual Tape & 385.25 & Good & \\
\hline $2 / 22 / 947: 05$ & Auto FIC & 385.5 & Good & \\
\hline $2 / 23 / 940: 00$ & Manual Tape & 385.25 & Good & \\
\hline $2 / 23 / 947: 06$ & Auto FIC & 385.50 & Good & \\
\hline $2 / 24 / 940: 00$ & Manual Tape & 385.00 & Good & \\
\hline $2 / 24 / 947: 06$ & Auto FIC & 385.5 & Good & \\
\hline $2 / 25 / 940: 00$ & Manual Tape & 385.25 & Good & \\
\hline $2 / 25 / 946: 06$ & Auto FIC & 385.50 & Good & \\
\hline $2 / 26 / 940: 00$ & Manual Tape & 385.25 & Good & \\
\hline $2 / 26 / 947: 07$ & Auto FIC & 385.5 & Good & \\
\hline $2 / 27 / 940: 00$ & Manual Tape & $385: 25$ & Good & \\
\hline $2 / 27 / 947: 07$ & Auto FIC & 385.50 & Good & \\
\hline $2 / 28 / 940: 00$ & Manual Tape & 385.75 & Good & \\
\hline $2 / 28 / 946: 06$ & Auto FIC & 385.5 & Good & \\
\hline $3 / 1 / 940: 00$ & Manual Tape & 385.25 & Good & \\
\hline $3 / 1 / 947: 04$ & Auto FIC & 385.50 & Good & \\
\hline $3 / 2 / 940: 00$ & Manual Tape & 385.25 & Good & \\
\hline $3 / 2 / 947: 06$ & Auto FIC & 385.5 & Good & \\
\hline $3 / 3 / 94 \quad 0: 00$ & Manual Tape & 385 & Good & \\
\hline $3 / 3 / 947: 06$ & Auto FIC & 385.50 & Good & \\
\hline $3 / 4 / 940: 00$ & Manual Tape & 385.25 & Good & \\
\hline $3 / 4 / 947: 04$ & Auto FIC & 385.5 & Good & \\
\hline $3 / 5 / 940: 00$ & Manual Tape & 385.25 & Good & \\
\hline $3 / 5 / 947: 05$ & Auto FIC & 385.50 & Good & \\
\hline $3 / 6 / 940: 00$ & Manual Tape & 385.25 & Good & \\
\hline $3 / 6 / 947: 05$ & Auto FIC & 385.5 & Good & \\
\hline $3 / 7 / 940: 00$ & Manual Tape & 385.25 & Good & \\
\hline $3 / 7 / 947: 05$ & Auto FIC & 385.50 & Good & \\
\hline $3 / 8 / 940: 00$ & Manual Tape & 385.25 & Good & \\
\hline $3 / 8 / 947: 04$ & Auto FIC & 385.5 & Good & \\
\hline $3 / 9 / 940: 00$ & Manual Tape & 385.25 & Good & \\
\hline $3 / 9 / 947: 04$ & Auto FIC & 385.50 & Good & \\
\hline $3 / 10 / 940: 00$ & Manual Tape & 385.25 & Good & \\
\hline $3 / 10 / 947: 05$ & Auto FIC & 385.5 & Good & \\
\hline $3 / 11 / 940: 00$ & Manual Tape & 385.25 & Good & \\
\hline $3 / 11 / 947: 06$ & Auto FIC & 385.50 & Good & \\
\hline $3 / 12 / 940: 00$ & Manual Tape & 385.25 & Good & \\
\hline $3 / 12 / 947: 06$ & Auto FIC & 385.5 & Good & \\
\hline $3 / 13 / 940: 00$ & Manual Tape & 385.25 & Good & \\
\hline $3 / 13 / 947: 05$ & Auto FIC & 385.50 & Good & \\
\hline $3 / 14 / 940: 00$ & Manual FIC & 385.50 & Good & \\
\hline $3 / 14 / 940: 00$ & Manua! Tape & 385.25 & Good & \\
\hline 3/15/94 0:00 & Manual Tape & 385.25 & Good & \\
\hline
\end{tabular}

Data obtained from WHC Surveillance Analysis Computer System, (PC SACS) July 7, 1995. 


\begin{tabular}{|c|c|c|c|c|}
\hline \multicolumn{5}{|c|}{ 241-AP:101 } \\
\hline \multicolumn{5}{|c|}{ Surface Level Data } \\
\hline Date & Type of Measurement & Level (Inches) & Data Quality & Comments \\
\hline $3 / 15 / 947: 05$ & Auto FIC & 385.5 & Good & \\
\hline $3 / 16 / 940: 00$ & Manual Tape & 385.25 & Good & \\
\hline $3 / 16 / 947: 04$ & Auto FIC & 385.50 & Good & \\
\hline $3 / 17 / 940: 00$ & Manual Tape & 385.5 & Good & \\
\hline $3 / 17 / 947: 05$ & Auto FIC & 385.5 & Good & \\
\hline $3 / 18 / 940: 00$ & Manual Tape & 385.25 & Good & \\
\hline $3 / 18 / 947: 05$ & Auto FIC & 385.50 & Good & \\
\hline $3 / 19 / 940: 00$ & Manual Tape & 385.25 & Good & \\
\hline $3 / 19 / 947: 04$ & Auto FIC & $385 . \overline{5}$ & Good & \\
\hline $3 / 20 / 940: 00$ & Manual Tape & 385.25 & Good & \\
\hline $3 / 20 / 947: 05$ & Auto FIC & 385.50 & Good & \\
\hline $3 / 21 / 940: 00$ & Manual Tape & 385.5 & Good & \\
\hline $3 / 21 / 947: 05$ & Auto FIC & 385.5 & Good & \\
\hline $3 / 22 / 940: 00$ & Manual Tape & 385.50 & Good & \\
\hline $3 / 22 / 947: 04$ & Auto FIC & 385.50 & Good & \\
\hline $3 / 23 / 940: 00$ & Manual FIC & 385.50 & Good & \\
\hline $3 / 23 / 94 \quad 0: 00$ & Manual Tape . & 385.00 & Good & \\
\hline $3 / 24 / 940: 00$ & Manual Tape & 385 & Good & \\
\hline $3 / 24 / 947: 04$ & Auto FIC & 385.50 & Good & \\
\hline $3 / 25 / 940: 00$ & Manual Tape & 385.00 & Good & \\
\hline $3 / 25 / 947: 05$ & Auto FIC & 385.4 & Good & \\
\hline $3 / 26 / 940: 00$ & Manual Tape & 385.00 & Good & \\
\hline $3 / 26 / 947: 04$ & Auto FIC & 385.40 & Good & \\
\hline $3 / 27 / 940: 00$ & Manual Tape & 385 & Good & \\
\hline $3 / 27 / 947: 04$ & Auto FIC & 385.4 & Good & \\
\hline $3 / 28 / 940: 00$ & Manual Tape & 385.50 & Good & \\
\hline $3 / 28 / 947: 04$ & Auto FIC & 385.50 & Good & \\
\hline $3 / 29 / 940: 00$ & Manual Tape & 385.50 & Good & \\
\hline $3 / 29 / 947: 03$ & Auto FIC & 385.5 & Good & \\
\hline $3 / 30 / 940: 00$ & Manual Tape & 385.5 & Good & \\
\hline $3 / 30 / 947: 03$ & Auto FIC & 385.40 & Good & \\
\hline $3 / 31 / 940: 00$ & Manual Tape & 385.50 & Good & \\
\hline $3 / 31 / 947: 03$ & Auto FIC & 385.4 & Good & \\
\hline $4 / 1 / 940: 00$ & Manual Tape & 385.50 & Good & \\
\hline 4/1/940:04 & Auto FIC & 385.40 & Good & \\
\hline $4 / 2 / 940: 00$ & Manual Tape & 385.5 & Good & \\
\hline 4/2/94 7:07 & Auto FIC & 385.4 & Good & \\
\hline $4 / 3 / 94 \quad 0: 00$ & Manual Tape & 385.50 & Good & \\
\hline $4 / 3 / 947: 03$ & Auto FIC & 385.40 & Good & \\
\hline $4 / 4 / 940: 00$ & Manual Tape & 385.50 & Good & \\
\hline 4/4/94 7:03 & Auto FIC & 385.4 & Good & \\
\hline $4 / 5 / 940: 00$ & Manual Tape & 385.25 & Good & \\
\hline $4 / 5 / 947: 03$ & Auto FIC & 385.4 & Good & \\
\hline $4 / 6 / 94 \quad 0: 00$ & Manual Tape & 385.50 & Good & \\
\hline 4/6/94 7:06 & Auto FIC & 385.40 & Good & \\
\hline 4/7/940:00 & Manual Tape & 384.25 & Good & \\
\hline $4 / 7 / 947: 07$ & Auto FIC & 385.40 & Good & \\
\hline $4 / 8 / 94 \quad 0: 00$ & Manual Tape & 385.25 & Good & \\
\hline
\end{tabular}

Data obtained from WHC Surveillance Analysis Computer System, (PC SACS) July 7, 1995. 


\begin{tabular}{|c|c|c|c|c|}
\hline \multicolumn{5}{|c|}{ 241-AP-101 } \\
\hline \multicolumn{5}{|c|}{ Surface Level Data } \\
\hline Date & Type of Measurement & Leval.(Inches) & Data Quality & Comments \\
\hline $4 / 8 / 947: 03$ & Auto FIC & 385.4 & Good & \\
\hline 4/9/940:00 & Manual Tape & 385.25 & Good & \\
\hline 4/9/94 7:07 & Auto FIC & 385.40 & Good & \\
\hline 4/10/940:00 & Manual Tape & 385.25 & Good & \\
\hline $4 / 10 / 947: 03$ & Auto FIC & 385.40 & Good & \\
\hline $4 / 11 / 940: 00$ & Manual Tape & $\mathbf{3 8 5 . 2 5}$ & Good & \\
\hline $4 / 11 / 947: 03$ & Auto FIC & 385.4 & Good & \\
\hline $4 / 12 / 940: 00$ & Manual Tape & 385.25 & Good & \\
\hline $4 / 12 / 947: 03$ & Auto FIC & 385.4 & Good & \\
\hline $4 / 13 / 940: 00$ & Manual Tape & 385.25 & Good & \\
\hline $4 / 13 / 947: 03$ & Auto FIC & 385.40 & Good & \\
\hline $4 / 14 / 940: 00$ & Manual Tape & 385.5 & Good & \\
\hline $4 / 14 / 947: 03$ & Auto FIC & 385.4 & Good & \\
\hline 4/15/94 0:00 & Manual Tape & 385.25 & Good & \\
\hline $4 / 15 / 947: 03$ & Auto FIC & 385.40 & Good & \\
\hline 4/16/940:00 & Manual Tape & 385.50 & Good & \\
\hline 4/16/94 7:03 & Auto FIC & 385.4 & Good & \\
\hline $4 / 17 / 940: 00$ & Manual Tape & 385.5 & Good & \\
\hline $4 / 17 / 947: 03$ & Auto FIC & 385.40 & Good & \\
\hline 4/18/94 0:00 & Manual Tape & 385.50 & Good & \\
\hline 4/18/94 7:03 & Auto FIC & 385.4 & Good & \\
\hline 4/19/940:00 & Manual Tape & 385.50 & Good & \\
\hline $4 / 19 / 946: 06$ & Auto FIC & 385.40 & Good & \\
\hline 4/20/94 0:00 & Manual Tape & 385.25 & Good & \\
\hline $4 / 20 / 947: 04$ & Auto FIC & 385.4 & Good & \\
\hline $4 / 21 / 940: 00$ & Manual Tape & 384.00 & Good & \\
\hline 4/21/94 7:05 & Auto FIC & 385.40 & - Good & \\
\hline $4 / 22 / 940: 00$ & Manual Tape & $385.50^{\circ}$ & Good & \\
\hline $4 / 22 / 947: 04$ & Auto FIC & 385.4 & Good & \\
\hline 4/23/94 0:00 & Manual Tape & 385.5 & Good & \\
\hline $4 / 23 / 947: 05$ & Auto FIC & 385.4 & Good & \\
\hline $4 / 24 / 940: 00$ & Manual Tape & 385.50 & Good & \\
\hline $4 / 24 / 947: 04$ & Auto FIC & 385.4 & Good & \\
\hline $4 / 25 / 940: 00$ & Manual Tape & 385.25 & Good & \\
\hline 4/25/94 7:04 & Auto FIC & . 385.40 & Good & \\
\hline 4/26/94 0:00 & Manual Tape & 385.25 & Good & \\
\hline $4 / 26 / 947: 07$ & Auto FIC & 385.4 & Good & \\
\hline $4 / 27 / 940: 00$ & Manual Tape & 385.50 & Good & \\
\hline $4 / 27 / 947: 04$ & Auto FIC & 385.4 & Good & \\
\hline $4 / 28 / 940: 00$ & Manual Tape & 385.25 & Good & \\
\hline $4 / 28 / 947: 05$ & Auto FIC & 385.4 & Good & \\
\hline $4 / 29 / 940: 00$ & Manual Tape & 385.25 & Good & \\
\hline $4 / 29 / 947: 04$ & Auto FIC & 385.4 & Good & \\
\hline $4 / 30 / 940: 00$ & Manual Tape & 385.25 & Good & \\
\hline $4 / 30 / 947: 05$ & Auto FIC & 385.4 & Good & \\
\hline 5/1/94 0:00 & Manual Tape & 385.25 & Good & \\
\hline 5/1/94 7:05 & Auto FIC & 385.40 & Good & \\
\hline $5 / 2 / 940: 00$ & Manual Tape & 385.25 & Good & \\
\hline
\end{tabular}

Data obtained from WHC Surveillance Analysis Computer System, (PC SACS) July 7, 1995. 


\begin{tabular}{|c|c|c|c|c|}
\hline \multicolumn{5}{|c|}{ 241-AP-101 } \\
\hline \multicolumn{5}{|c|}{ Surface Level Date } \\
\hline Date & Type of Measurement & Level (Inches) & Date Quality & Comments \\
\hline $5 / 2 / 947: 05$ & Auto FIC & 385.4 & Good & \\
\hline $5 / 3 / 940: 00$ & Manual Tape & 385.25 & Good & \\
\hline $5 / 3 / 947: 05$ & Auto FIC & 385.4 & Good & \\
\hline $5 / 4 / 940: 00$ & Manual Tape & 385.25 & Good & \\
\hline $5 / 4 / 947: 05$ & Auto FIC & 385.4 & Good & \\
\hline $5 / 5 / 940: 00$ & Manual Tape & 385.5 & Good & \\
\hline $5 / 5 / 947: 03$ & Auto FIC & 385.40 & Good & \\
\hline $5 / 6 / 940: 00$ & Manual Tape & 385.25 & Good & \\
\hline $5 / 6 / 947: 03$ & Auto FIC & 385.4 & Good & \\
\hline $5 / 7 / 940: 00$ & Manual Tape & 385.25 & Good & \\
\hline $5 / 7 / 947: 03$ & Auto FIC & 385.4 & Good & \\
\hline $5 / 8 / 940: 00$ & Manual Tape & 385.25 & Good & \\
\hline $5 / 8 / 947: 03$ & Auto FIC & 385.4 & Good & \\
\hline $5 / 9 / 940: 00$ & Manual Tape & 385.25 & Good & \\
\hline $5 / 9 / 947: 03$ & Auto FIC & 385.40 & Good & \\
\hline $5 / 10 / 940: 00$ & Manual Tape & 385.25 & Good & \\
\hline $5 / 10 / 947: 03$ & Auto FIC & 385.4 & Good & \\
\hline $5 / 11 / 940: 00$ & Manual Tape & 385.25 & Good & \\
\hline $5 / 11 / 947: 03$ & Auto FIC & 385.4 & Good & \\
\hline $5 / 12 / 94 \quad 0: 00$ & Manual Tape & 385.25 & Good & \\
\hline $5 / 12 / 946: 03$ & Auto FIC & 385.4 & Good & \\
\hline $5 / 13 / 940: 00$ & Manual Tape & 385.50 & Good & \\
\hline $5 / 13 / 947: 03$ & Auto FIC & 385.40 & Good & \\
\hline $5 / 14 / 940: 00$ & Manual Tape & 385.25 & Good & \\
\hline $5 / 14 / 947: 03$ & Auto FIC & 385.4 & Good & \\
\hline $5 / 15 / 940: 00$ & Manual Tape & 385.25 & Good & \\
\hline $5 / 15 / 947: 06$ & Auto FIC & 385.4 & Good & \\
\hline $5 / 16 / 940: 00$ & Manual Tape & 385.50 & Good & \\
\hline $5 / 16 / 947: 02$ & Auto FIC & 385.4 & Good & \\
\hline $5 / 17 / 940: 00$ & Manual Tape & 385.25 & Good & \\
\hline $5 / 17 / 947: 03$ & Auto FIC & 385.40 & Good & \\
\hline $5 / 18 / 940: 00$ & Manual Tape & 385.25 & Good & \\
\hline $5 / 18 / 947: 03$ & Auto FIC & 385.4 & Good & \\
\hline $5 / 19 / 940: 00$ & Manual Tape & 385.25 & Good & \\
\hline $5 / 19 / 947: 03$ & Auto FIC & 385.4 & Good & \\
\hline $5 / 20 / 940: 00$ & Manual Tape & 385.25 & Good & \\
\hline $5 / 20 / 947: 03$ & Auto FIC & 385.4 & Good & \\
\hline $5 / 21 / 940: 00$ & Manual Tape & 385.25 & Good & \\
\hline $5 / 21 / 947: 05$ & Auto FIC & 385.40 & Good & \\
\hline $5 / 22 / 940: 00$ & Manual Tape & 385.25 & Good & \\
\hline $5 / 22 / 947: 05$ & Auto FIC & 385.4 & Good & \\
\hline $5 / 23 / 940: 00$ & Manual Tape & 385.25 & Good & \\
\hline 5/23/94 7:04 & Auto FIC & 385.4 & Good & \\
\hline $5 / 24 / 940: 00$ & Manual Tape & 385.50 & Good & \\
\hline 5/24/94 7:04 & Auto FIC & 385.4 & Good & \\
\hline $5 / 25 / 940: 00$ & Manual Tape & 384.50 & Good & \\
\hline $5 / 25 / 947: 04$ & Auto FIC & 385.40 & Good & \\
\hline $5 / 26 / 940: 00$ & Manual Tape & 384.50 & Good & \\
\hline
\end{tabular}

Data abtained from WHC Surveillance Analysis Computer System, (PC SACS) July 7, 1995. 


\begin{tabular}{|c|c|c|c|c|}
\hline \multicolumn{5}{|c|}{ 241-AP-101 } \\
\hline \multicolumn{5}{|c|}{ Surface Level Data } \\
\hline Date & Type of Measurement & Leval (Inches) & Data Quality & Comments \\
\hline $5 / 26 / 946: 05$ & Auto FIC & 385.4 & Good & \\
\hline $5 / 27 / 940: 00$ & Manual Tape & 385.25 & Good & \\
\hline $5 / 27 / 947: 03$ & Auto FIC & 385.4 & Good & \\
\hline $5 / 28 / 940: 00$ & Manual Tape & 385.25 & Good & \\
\hline $5 / 28 / 947: 03$ & Auto FIC & 385.4 & Good & \\
\hline $5 / 29 / 940: 00$ & Manual Tape & 385.25 & Good & \\
\hline $5 / 29 / 947: 03$ & Auto FIC & 385.40 & Good & \\
\hline $5 / 30 / 940: 00$ & Manual FIC & 385.4 & Good & \\
\hline $5 / 30 / 94 \quad 0: 00$ & Manual Tape & 385.25 & Good & \\
\hline $5 / 30 / 947: 02$ & Auto FIC & 385.4 & Good & \\
\hline $5 / 31 / 940: 00$ & Manual FIC & 385.40 & Good & \\
\hline $5 / 31 / 940: 00$ & Manual Tape & 385.25 & Good & \\
\hline $5 / 31 / 947: 03$ & Auto FIC & 385.4 & Good & \\
\hline $6 / 1 / 940: 00$ & Manual Tape & 385.25 & Good & \\
\hline $6 / 1 / 947: 03$ & Auto FIC & 385.4 & Good & \\
\hline $6 / 2 / 94 \quad 0: 00$ & Manual Tape & 385.00 & Good & \\
\hline $6 / 2 / 947: 03$ & Auto FIC & 385.40 & Good & \\
\hline $6 / 3 / 940: 00$ & Manual Tape & 359.25 & Suspect & Data not included in graph \\
\hline $6 / 3 / 940: 00$ & Manual FIC & 385.40 & Good & \\
\hline $6 / 4 / 940: 00$ & Manual Tape & 385.00 & Good & \\
\hline $6 / 4 / 947: 04$ & Auto FIC & 385.4 & Good & \\
\hline $6 / 5 / 940: 00$ & Manual Tape & 385.00 & Good & \\
\hline $6 / 5 / 947: 03$ & Auto FIC & 385.4 & Good & \\
\hline $6 / 6 / 94 \quad 0: 00$ & Manual Tape & 385 & Good & \\
\hline $6 / 6 / 947: 06$ & Auto FIC & 385.4 & Good & \\
\hline $6 / 7 / 940: 00$ & Manual Tape & 385.50 & Good & \\
\hline $6 / 7 / 947: 02$ & Auto FIC & 385.40 & Good & \\
\hline $6 / 8 / 940: 00$ & Manual Tape & 385.25 & Good & \\
\hline 6/8/94 7:03 & Auto FIC & 385.4 & Good & \\
\hline $6 / 9 / 940: 00$ & Manual FIC & 385.45 & Good & \\
\hline $6 / 9 / 940: 00$ & Manual Tape & 385.25 & Good & \\
\hline $6 / 10 / 940: 00$ & Manual Tape & 385.25 & Good & \\
\hline $6 / 10 / 947: 04$ & Auto FIC & 385.4 & Good & \\
\hline $6 / 11 / 940: 00$ & Manual Tape & 385.25 & Good & \\
\hline $6 / 11 / 947: 04$ & Auto FIC & 385.4 & Good & \\
\hline $6 / 12 / 940: 00$ & Manual Tape & 385.25 & Good & \\
\hline $6 / 12 / 947: 03$ & Auto FIC & 385.40 & Good & \\
\hline $6 / 13 / 940: 00$ & Manual Tape & 385.25 & Good & \\
\hline $6 / 13 / 947: 02$ & Auto FIC & 385.4 & Good & \\
\hline $6 / 14 / 940: 00$ & Manual Tape & 385.00 & Good & \\
\hline $6 / 14 / 947: 03$ & Auto FIC & 385.4 & Good & \\
\hline $6 / 15 / 94 \quad 0: 00$ & Manual Tape & 385.25 & Good & \\
\hline $6 / 15 / 947: 03$ & Auto FIC & 385.4 & Good & \\
\hline $6 / 16 / 940: 00$ & Manual Tape & 385.25 & Good & \\
\hline $6 / 16 / 947: 03$ & Auto FIC & 385.40 & Good & \\
\hline $6 / 17 / 940: 00$ & Manual Tape & 385.25 & Good & \\
\hline $6 / 17 / 947: 03$ & Auto FIC & 385.4 & Good & \\
\hline $6 / 18 / 940: 00$ & Manual Tape & 385.5 & Good & \\
\hline
\end{tabular}

Data obtained from WHC Surveillance Analysis Computer System, (PC SACS) July 7, 1995. 


\begin{tabular}{|c|c|c|c|c|}
\hline \multicolumn{5}{|c|}{ 241-AP-101 } \\
\hline \multicolumn{5}{|c|}{ Surface Level Data } \\
\hline Date & Type of Maasurement & Level (Inches) & Data Quality & Comments \\
\hline $6 / 18 / 947: 04$ & Auto FIC & 385.4 & Good & \\
\hline $6 / 19 / 940: 00$ & Manual Tape & 385.75 & Good & \\
\hline $6 / 19 / 947: 04$ & Auto FIC & 385.4 & Good & \\
\hline $6 / 20 / 940: 00$ & Manual Tape & 385.50 & Good & \\
\hline $6 / 20 / 947: 04$ & Auto FIC & 385.40 & Good & \\
\hline $6 / 21 / 940: 00$ & Manual Tape & 385.25 & Good & \\
\hline $6 / 21 / 947: 04$ & Auto FIC & 385.4 & Good & \\
\hline $6 / 22 / 940: 00$ & Manual Tape & 385.25 & Good & \\
\hline $6 / 22 / 947: 03$ & Auto FIC & 385.4 & Good & \\
\hline $6 / 23 / 940: 00$ & Manual Tape & 385.25 & Good & \\
\hline $6 / 23 / 947: 05$ & Auto FIC & 385.40 & Good & \\
\hline $6 / 24 / 940: 00$ & Manual Tape & 385.25 & Good & \\
\hline $6 / 24 / 947: 06$ & Auto FIC & 385.40 & Good & \\
\hline $6 / 25 / 940: 00$ & Manual Tape & 385.25 & Good & \\
\hline $6 / 25 / 947: 05$ & Auto FIC & 385.4 & Good & \\
\hline $6 / 26 / 940: 00$ & Manual Tape & 385.25 & Good & \\
\hline $6 / 26 / 947: 06$ & Auto FIC & 385.4 & Good & \\
\hline $6 / 27 / 940: 00$ & Manual Tape & 385.25 & Good & \\
\hline $6 / 27 / 947: 06$ & Auto FIC & 385.40 & Good & \\
\hline $6 / 28 / 940: 00$ & Manual Tape & 385.25 & Good & \\
\hline 6/28/94 7:06 & Auto FIC & 385.40 & Good & \\
\hline $6 / 29 / 940: 00$ & Manual Tape & 385.25 & Good & \\
\hline $6 / 29 / 947: 03$ & Auto FIC & 385.4 & Good & \\
\hline $6 / 30 / 940: 00$ & Manual Tape & 385.25 & Good & \\
\hline $6 / 30 / 947: 04$ & Auto FIC & 385.4 & Good & \\
\hline $7 / 1 / 940: 00$ & Manual Tape & 385.25 & Good & \\
\hline 7/1/94 7:04 & Auto FIC & 385.40 & Good & \\
\hline $7 / 2 / 940: 00$ & Manual Tape & 385.00 & Good & \\
\hline $7 / 2 / 947: 04$ & Auto FIC & 385.40 & Good & \\
\hline $7 / 3 / 940: 00$ & Manual Tape & 385.25 & Good & \\
\hline $7 / 3 / 947: 04$ & Auto FIC & 385.4 & Good & \\
\hline $7 / 4 / 940: 00$ & Manual Tape & 385.25 & Good & \\
\hline $7 / 4 / 947: 04$ & Auto FIC & 385.4 & Good & \\
\hline $7 / 5 / 940: 00$ & Manual Tape & 385.25 & Good & \\
\hline $7 / 5 / 947: 03$ & Auto FIC & 385.40 & Good & \\
\hline $7 / 6 / 94 \quad 0: 00$ & Manual Tape & 385 & Good & \\
\hline $7 / 6 / 94 \quad 7: 05$ & Auto FIC & 385.4 & Good & \\
\hline $7 / 7 / 940: 00$ & Manual Tape & 385.25 & Good & \\
\hline $7 / 7 / 947: 03$ & Auto FIC & 385.4 & Good & \\
\hline $7 / 8 / 94 \quad 0: 00$ & Manual Tape & 385.25 & Good & \\
\hline $7 / 8 / 947: 03$ & Auto FIC & 385.40 & Good & \\
\hline 7/9/94 0:00 & Manual Tape & 385.25 & Good & \\
\hline 7/9/94 7:03 & Auto FIC & 385.4 & Good & \\
\hline 7/10/94 0:00 & Manual Tape & 385.25 & Good & \\
\hline 7/10/94 7:03 & Auto FIC & 385.4 & Good & \\
\hline $7 / 11 / 940: 00$ & Manual Tape & 385.50 & Good & \\
\hline 7/11/94 7:03 & Auto FIC & 385.4 & Good & \\
\hline $7 / 12 / 940: 00$ & Manual Tape & 385 & Good & \\
\hline
\end{tabular}

Data obtained from WHC Surveillance Analysis Computer System, (PC SACS) July 7, 1995. 


\begin{tabular}{|c|c|c|c|c|}
\hline \multicolumn{5}{|c|}{ 241-AP-101 } \\
\hline \multicolumn{5}{|c|}{ Surface Level Data } \\
\hline Date & Type of Measurement & Level (inches) & Data Quality & Comments \\
\hline 7/12/94 7:03 & Auto FIC & 385.4 & Good & \\
\hline 7/13/94 0:00 & Manual Tape & 385.25 & Good & \\
\hline $7 / 13 / 947: 03$ & Auto FIC & 385.40 & Good & \\
\hline $7 / 14 / 940: 00$ & Manual Tape & 385.25 & - Good & \\
\hline $7 / 14 / 947: 04$ & Auto FIC & 385.4 & Good & \\
\hline $7 / 15 / 940: 00$ & Manual Tape & 385 & Good & \\
\hline $7 / 15 / 947: 04$ & Auto FIC & 385.4 & Good & \\
\hline $7 / 16 / 940: 00$ & Manual Tape & 385.50 & Good & \\
\hline $7 / 16 / 947: 04$ & Auto FIC & 385.4 & Good & \\
\hline $7 / 17 / 940: 00$ & Manual Tape & 385.50 & Good & \\
\hline 7/17/947:04 & Auto FIC & 385.4 & Good & \\
\hline $7 / 18 / 940: 00$ & Manual FIC & 385.4 & Good & \\
\hline $7 / 18 / 940: 00$ & Manual Tape & 385.5 & Good & \\
\hline $7 / 19 / 940: 00$ & Manual FIC & 385.4 & Good & \\
\hline $7 / 19 / 940: 00$ & Manual Tape & 385.25 & Good & \\
\hline $7 / 19 / 947: 03$ & Auto FIC & 385.40 & Good & \\
\hline $7 / 20 / 940: 00$ & Manual FIC & 385.4 & Good & \\
\hline $7 / 20 / 940: 00$ & Manual Tape & 385.25 & Good & \\
\hline $7 / 20 / 947: 03$ & Auto FiC & 385.4 & Good & \\
\hline $7 / 21 / 940: 00$ & Manual FIC & 385.40 & Good & \\
\hline $7 / 21 / 940: 00$ & Manual Tape & 385.25 & Good & \\
\hline $7 / 21 / 947: 03$ & Auto FIC & 385.4 & Good & \\
\hline $7 / 22 / 940: 00$ & Manual Tape & 385.25 & Good & \\
\hline $7 / 22 / 947: 03$ & Auto FIC & 385.4 & Good & . \\
\hline $7 / 23 / 940: 00$ & Manual Tape & 385.25 & Good & \\
\hline $7 / 23 / 947: 03$ & Auto FIC & 385.40 & Good & \\
\hline $7 / 24 / 940: 00$ & Manual Tape & 385.25 & Good & \\
\hline $7 / 24 / 947: 03$ & Auto FIC & 385.4 & Good & \\
\hline $7 / 25 / 940: 00$ & Manual Tape & 385.00 & Good & \\
\hline $7 / 25 / 947: 03$ & Auto FIC & 385.4 & Good & \\
\hline $7 / 26 / 947: 03$ & Auto FIC & 385.4 & Good & \\
\hline $7 / 26 / 9412: 00$ & Manual Tape & 385.00 & Good & \\
\hline $7 / 27 / 947: 03$ & Auto FIC & 385.40 & Good & \\
\hline $7 / 27 / 94 \quad 12: 00$ & Manual Tape & 385 & Good & \\
\hline $7 / 28 / 947: 04$ & Auto FIC & 385.4 & Good & \\
\hline $7 / 28 / 9412: 00$ & Manual Tape & 385.25 & Good & \\
\hline $7 / 29 / 947: 04$ & Auto FIC & 385.4 & Good & \\
\hline $7 / 29 / 9412: 00$ & Manual Tape & 385.25 & Good & \\
\hline $7 / 30 / 947: 04$ & Auto FIC & 385.4 & Good & \\
\hline $7 / 30 / 9412: 00$ & Manual Tape & 378 & Suspect & Data not included in graph \\
\hline $7 / 31 / 9412: 00$ & Manual Tape & 385.25 & Good & \\
\hline $7 / 31 / 9423: 03$ & Auto FIC & 385.40 & Good & \\
\hline $8 / 1 / 947: 03$ & Auto FIC & 385.4 & Good & \\
\hline $8 / 1 / 9412: 00$ & Manual Tape & 385.00 & Good & \\
\hline $8 / 2 / 947: 03$ & Auto FIC & 385.4 & Good & \\
\hline $8 / 2 / 9412: 00$ & Manual Tape & 385.25 & Good & \\
\hline $8 / 3 / 947: 03$ & Auto FIC & 385.40 & Good & \\
\hline $8 / 3 / 9412: 00$ & Manual Tape & 385.25 & Good & \\
\hline
\end{tabular}

Data obtained from WHC Surveillance Analysis Computer System, (PC SACS) July 7, 1995. 


\begin{tabular}{|c|c|c|c|c|}
\hline \multicolumn{5}{|c|}{ 241-AP-101 } \\
\hline \multicolumn{5}{|c|}{ Surface Level Data } \\
\hline Date & Type of Measurement & Level (Inches) & Data Quality & Comments \\
\hline $8 / 4 / 947: 02$ & Auto FIC & 0 & Suspect & Data not included in graph \\
\hline $8 / 4 / 9412: 00$ & Manual FIC & 385.4 & Good & \\
\hline $8 / 4 / 9412: 00$ & Manual Tape & 385.25 & Good & \\
\hline 8/5/94 7:03 & Auto FIC & 385.4 & Good & \\
\hline $8 / 5 / 9412: 00$ & Manual Tape & 385.25 & Good & \\
\hline $8 / 6 / 947: 03$ & Auto FIC & 385.40 & Good & \\
\hline $8 / 6 / 9412: 00$ & Manual Tape & 385.25 & Good & \\
\hline $8 / 7 / 947: 04$ & Auto FIC & 385.4 & Good & \\
\hline $8 / 7 / 9412: 00$ & Manual Tape & 385.25 & Good & \\
\hline $8 / 8 / 947: 12$ & Auto FIC & 0 & Suspect & Data not included in graph \\
\hline $8 / 8 / 9412: 00$ & Manual FIC & 385.50 & Good & \\
\hline $8 / 9 / 947: 06$ & Auto FIC & 385.4 & Good & \\
\hline $8 / 9 / 9412: 00$ & Manual Tape & 385.50 & Good & \\
\hline 8/10/94 7:04 & Auto FIC & 385.40 & Good & \\
\hline $8 / 10 / 9412: 00$ & Manual Tape & 385.25 & Good & \\
\hline $8 / 11 / 947: 04$ & Auto FIC & 385.4 & Good & \\
\hline $8 / 11 / 9412: 00$ & Manual Tape & 385.25 & Good & \\
\hline $8 / 12 / 947: 04$ & Auto FIC & 308.6 & Good & \\
\hline $8 / 12 / 9412: 00$ & Manual Tape & 299.50 & Good & \\
\hline $8 / 13 / 947: 03$ & Auto FIC & 272.1 & Good & \\
\hline $8 / 13 / 9412: 00$ & Manual Tape & 272.00 & Good & - \\
\hline $8 / 14 / 947: 06$ & Auto FIC & 272.10 & Good & \\
\hline $8 / 14 / 9412: 00$ & Manual Tape & 272.00 & Good & \\
\hline $8 / 15 / 947: 06$ & Auto FIC & 272.1 & Gopd & \\
\hline $8 / 15 / 9412: 00$ & Manual Tape & 272 & Good & \\
\hline $8 / 16 / 947: 06$ & Auto FIC & 272.10 & Good & \\
\hline $8 / 16 / 9412: 00$ & Manual Tape & 272.00 & Good & \\
\hline $8 / 17 / 947: 06$ & Auto FIC & 272.1 & Good & \\
\hline $8 / 17 / 9412: 00$ & Manual Tape & 271.75 & Good & \\
\hline $8 / 18 / 947: 06$ & Auto FIC & 272.10 & Good & \\
\hline $8 / 18 / 9412: 00$ & Manual Tape & 272 & Good & \\
\hline 8/19/946:06 & Auto FIC & 272.1 & Good & \\
\hline $8 / 19 / 9412: 00$ & Manual Tape & 272.00 & Good & \\
\hline $8 / 20 / 94 \quad 7: 04$ & Auto FIC & 228.30 & Good & \\
\hline $8 / 20 / 9412: 00$ & Manual Tape & 208.00 & Good & \\
\hline $8 / 21 / 947: 04$ & Auto FIC & 120.8 & Good & \\
\hline $8 / 21 / 9412: 00$ & Manual Tape & 115 & Good & \\
\hline $8 / 22 / 946: 04$ & Auto FIC & 115 & Good & $\cdot$ \\
\hline $8 / 22 / 9412: 00$ & Manual Tape & 114.75 & Good & \\
\hline $8 / 23 / 947: 04$ & Auto FIC & 115.00 & Good & \\
\hline $8 / 23 / 9412: 00$ & Manual Tape & 114.50 & Good & \\
\hline $8 / 24 / 947: 04$ & Auto FIC & 115 & Good & \\
\hline $8 / 24 / 9412: 00$ & Manual Tape & 114.5 & Good & \\
\hline $8 / 25 / 947: 03$ & Auto FIC & 115.00 & Good & \\
\hline $8 / 25 / 9412: 00$ & Manual Tape & 114.50 & Good & \\
\hline $8 / 26 / 947: 06$ & Auto FIC & 115 & Good & \\
\hline $8 / 26 / 9412: 00$ & Manual Tape & 114.50 & Good & \\
\hline $8 / 27 / 947: 04$ & Auto FIC & 115.00 & Good & \\
\hline
\end{tabular}

Data obtained from WHC Surveillance Analysis Computer System, (PC SACS) July 7, 1995. 


\begin{tabular}{|c|c|c|c|c|}
\hline \multicolumn{5}{|c|}{ 241-AP-101 } \\
\hline \multicolumn{5}{|c|}{ Surface Level Data } \\
\hline Date & Type of Measurement & Levol (Inchea) & Data Quality & Comments \\
\hline $8 / 27 / 9412: 00$ & Manual Tape & 115 & Good & \\
\hline $8 / 28 / 947: 04$ & Auto FIC & 115 & Good & \\
\hline $8 / 28 / 9412: 00$ & Manual Tape & 114.75 & Good & \\
\hline $8 / 29 / 947: 04$ & Auto FIC & 115.00 & Good & \\
\hline $8 / 29 / 9412: 00$ & Manual Tape & 115.00 & Good & \\
\hline $8 / 30 / 947: 04$ & Auto FIC & 115 & Good & \\
\hline $8 / 30 / 9412: 00$ & Manual Tape & 115 & Good & \\
\hline $8 / 31 / 947: 04$ & Auto FIC & 115.00 & Good & \\
\hline $8 / 31 / 9412: 00$ & Manual Tape & 114.75 & Good & \\
\hline 9/1/94 7:04 & Auto FIC & 115 & Good & \\
\hline $9 / 1 / 9412: 00$ & Manual Tape & 115 & Good & \\
\hline $9 / 2 / 947: 06$ & Auto FIC & 115 & Good & \\
\hline $9 / 2 / 9412: 00$ & Manual Tape & 114.75 & Good & \\
\hline $9 / 3 / 9412: 00$ & Manual Tape & 114.75 & Good & $\cdot$ \\
\hline 9/3/94 23:04 & Auto FIC & 115.00 & Good & \\
\hline $9 / 4 / 947: 04$ & Auto FIC & 115 & Good & \\
\hline $9 / 4 / 9412: 00$ & Manual Tape & 115.00 & Good & \\
\hline $9 / 5 / 947: 04$ & Auto FIC & 115.00 & Good & \\
\hline $9 / 5 / 9412: 00$ & Manual Tape & 114.75 & Good & \\
\hline $9 / 6 / 947: 03$ & Auto FIC & 115 & Good & \\
\hline $9 / 6 / 9412: 00$ & Manual Tape & 114.75 & Good & \\
\hline $9 / 7 / 947: 03$ & Auto FIC & 115.00 & Good & \\
\hline $9 / 7 / 9412: 00$ & Manual Tape & $114.75^{\circ}$ & Good & \\
\hline $9 / 8 / 947: 04$ & Auto FIC & 115 & Good & \\
\hline $9 / 8 / 9412: 00$ & Manual Tape & 114.75 & Good & \\
\hline $9 / 9 / 947: 03$ & Auto FIC & 115.00 & Good & \\
\hline $9 / 9 / 9412: 00$ & Manual Tape & 114.75 & Good & \\
\hline $9 / 10 / 947: 04$ & Auto FIC & 115 & Good & \\
\hline $9 / 10 / 9412: 00$ & Manual Tape & $114: 75$ & Good & \\
\hline $9 / 11 / 947: 03$ & Auto FIC & 115.00 & Good & \\
\hline $9 / 11 / 9412: 00$ & Manual Tape & 114.75 & Good & \\
\hline $9 / 12 / 947: 03$ & Auto FIC & 115 & Good & \\
\hline $9 / 12 / 9412: 00$ & Manual Tape & 114.75 & Good & \\
\hline $9 / 13 / 947: 02$ & Auto FIC & 115.00 & Good & \\
\hline $9 / 13 / 94 \quad 12: 00$ & Manual Tape & 114.75 & Good & \\
\hline $9 / 14 / 947: 03$ & Auto FIC & 115 & Good & \\
\hline $9 / 14 / 9412: 00$ & Manual Tape & 114.75 & Good & \\
\hline $9 / 15 / 947: 03$ & Auto FIC & 115.00 & Good & \\
\hline $9 / 15 / 9412: 00$ & Manual Tape & 114.75 & Good & \\
\hline $9 / 16 / 947: 02$ & Auto FIC & 115 & Good & \\
\hline $9 / 16 / 9412: 00$ & Manual Tape & 114.75 & Good & \\
\hline $9 / 17 / 947: 03$ & Auto FIC & 115.00 & Good & \\
\hline $9 / 17 / 9412: 00$ & Manual Tape & 114.50 & Good & \\
\hline $9 / 18 / 947: 02$ & Auto FIC & 115 & Good & \\
\hline $9 / 18 / 9412: 00$ & Manual Tape & 114.75 & Good & \\
\hline $9 / 19 / 947: 03$ & Auto FIC & 115.00 & Good & \\
\hline $9 / 19 / 9412: 00$ & Manual Tape & . 114.75 & Good & \\
\hline $9 / 20 / 947: 03$ & Auto FIC & 115 & Good & \\
\hline
\end{tabular}

Data obtained from WHC Surveillance Analysis Computer System, (PC SACS) July 7, 1995. 


\begin{tabular}{|c|c|c|c|c|}
\hline \multicolumn{5}{|c|}{ 241-AP-101 } \\
\hline & & urface Level Date & . & \\
\hline Date & Type of Measurement & Leval (Inches) & Data Quality & Commants \\
\hline $9 / 20 / 9412: 00$ & Manual Tape & 114.75 & - Good & \\
\hline $9 / 21 / 947: 04$ & Auto FIC & $115.00^{\circ}$ & Good & \\
\hline $9 / 21 / 9412: 00$ & Manual Tape & 114.75 & Good & \\
\hline $9 / 22 / 947: 03$ & Auto FIC & 115 & Good & \\
\hline $9 / 22 / 9412: 00$ & Manual Tape & 114.75 & Good & \\
\hline $9 / 23 / 947: 04$ & Auto FIC & 115.10 & Good & \\
\hline $9 / 23 / 9412: 00$ & Manual Tape & 114.75 & Good & \\
\hline $9 / 24 / 947: 04$ & Auto FIC & 115.1 & Good & \\
\hline $9 / 24 / 9412: 00$ & Manual Tape & 114.75 & Good & \\
\hline $9 / 25 / 947: 03$ & Auto FIC & 115.00 & Good & \\
\hline $9 / 25 / 9412: 00$ & Manual Tape & 114.75 & Good & \\
\hline $9 / 26 / 947: 04$ & Auto FIC & 115 & Good & \\
\hline $9 / 26 / 9412: 00$ & Manual Tape & 114.75 & Good & \\
\hline $9 / 27 / 947: 04$ & Auto FIC & 115.00 & Good & \\
\hline $9 / 27 / 9412: 00$ & Manual Tape & 115 & Good & \\
\hline $9 / 28 / 947: 03$ & Auto FIC & 115 & Good & \\
\hline $9 / 28 / 9412: 00$ & Manual Tape & 115.00 & Good & \\
\hline $9 / 29 / 947: 04$ & Auto FIC & 115.00 & Good & \\
\hline $9 / 29 / 9412: 00$ & Manual Tape & 114.5 & Good & \\
\hline $9 / 30 / 947: 04$ & Auto FIC & 115 & Good & \\
\hline $9 / 30 / 9412: 00$ & Manual Tape & 114.75 & Good & \\
\hline $10 / 1 / 947: 06$ & Auto FIC & 115.00 & Good & \\
\hline $10 / 1 / 9412: 00$ & Manual Tape & 114.75 & Good & \\
\hline $10 / 2 / 947: 05$ & Auto FIC & 115 & Good & \\
\hline $10 / 2 / 9412: 00$ & Manual Tape & 11.4 .75 & Good & \\
\hline $10 / 3 / 947: 04$ & Aluto FIC & .115 .00 & Good & \\
\hline $10 / 3 / 9412: 00$ & Manual Tape & 114.75 & Good & \\
\hline $10 / 4 / 947: 04$ & Auto FIC & 115.9 & Good & \\
\hline $10 / 4 / 9412: 00$ & Manual Tape & 115.75 & Good & \\
\hline $10 / 5 / 947: 04$ & Auto FIC & 116.5 & Good & \\
\hline $10 / 5 / 9412: 00$ & Manual Tape & 115.75 & Good & \\
\hline $10 / 6 / 947: 04$ & Auto FIC & 116.5 & Good & \\
\hline $10 / 6 / 9412: 00$ & Manual Tape & 116.50 & Good & \\
\hline $10 / 7 / 946: 04$ & Auto FIC & 116.50 & Good & \\
\hline $10 / 7 / 9412: 00$ & Manual Tape & 116.50 & . Good & \\
\hline $10 / 8 / 947: 03$ & Auto FIC & 178.1 & Good & \\
\hline $10 / 8 / 94 \quad 12: 00$ & Manual Tape & 189 & Good & \\
\hline $10 / 9 / 947: 06$ & Auto FIC & 181.00 & Good & \\
\hline $10 / 9 / 9412: 00$ & Manual Tape & 173.75 & Good & \\
\hline $10 / 10 / 947: 05$ & Auto FIC & 105.4 & Good & \\
\hline $10 / 10 / 9412: 00$ & Manual Tape & 99.50 & Good & \\
\hline $10 / 11 / 947: 03$ & Auto FIC & 54.20 & Good & \\
\hline $10 / 11 / 9412: 00$ & Manual Tape & 54.00 & Good & \\
\hline $10 / 12 / 947: 06$ & Auto FIC & 54.4 & Good & \\
\hline $10 / 12 / 9412: 00$ & Manual Tape & 54.25 & Good & \\
\hline $10 / 13 / 947: 05$ & Auto FIC & 54.40 & Good & \\
\hline $10 / 13 / 9412: 00$ & Manual Tape & 54.25 & Good & \\
\hline $10 / 14 / 947: 06$ & Auto FIC & 54.4 & Good & \\
\hline
\end{tabular}

Data obtained from WHC Surveillance Analysis Computer System, (PC SACS) July 7, 1995. 


\begin{tabular}{|c|c|c|c|c|}
\hline \multicolumn{5}{|c|}{ 241-AP-101 } \\
\hline \multicolumn{5}{|c|}{ Surface Level Data } \\
\hline Date & Type of Moasurement & Level (Inches) & Data Quality & Comments \\
\hline $10 / 14 / 9412: 00$ & Manual Tape & 54.00 & Good & \\
\hline $10 / 15 / 947: 06$ & Auto FIC & 54.40 & Good & \\
\hline $10 / 15 / 9412: 00$ & Manual Tape & 54.00 & Good & \\
\hline $10 / 16 / 947: 06$ & Auto FIC & 54.4 & Good & \\
\hline $10 / 16 / 9412: 00$ & Manual Tape & 54.25 & Good & \\
\hline $10 / 17 / 947: 06$ & Auto FIC & 54.40 & Good & \\
\hline $10 / 17 / 9412: 00$ & Manual Tape & 54.00 & Good & \\
\hline $10 / 18 / 947: 06$ & Auto FIC & 54.4 & Good & \\
\hline $10 / 18 / 9412: 00$ & Manual Tape & 54.25 & Good & \\
\hline $10 / 19 / 947: 06$ & Auto FIC & 54.40 & Good & \\
\hline $10 / 19 / 9412: 00$ & Manual Tape & 54.25 & Good & \\
\hline $10 / 20 / 945: 07$ & Auto FIC & 54.4 & Good & \\
\hline $10 / 20 / 9412: 00$ & Manual Tape & 54.25 & Good & \\
\hline $10 / 21 / 947: 06$ & Auto FIC & 54.40 & Good & \\
\hline $10 / 21 / 9412: 00$ & Manual Tape & 54.25 & Good & \\
\hline $10 / 22 / 94 \quad 12: 00$ & Manual Tape & 54.00 & Good & \\
\hline $10 / 22 / 9423: 06$ & Auto FIC & 54.3 & Good & \\
\hline $10 / 23 / 947: 06$ & Auto FIC & 54.40 & Good & \\
\hline $10 / 23 / 9412: 00$ & Manual Tape & 54.25 & Good & \\
\hline $10 / 24 / 947: 06$ & Auto FIC & 54.3 & Good & \\
\hline $10 / 24 / 9412: 00$ & Manual Tape & 54.25 & Good & \\
\hline $10 / 25 / 947: 06$ & Auto FIC & 54.30 & Good & \\
\hline $10 / 25 / 9412: 00$ & Manual Tape & 54.25 & Good & \\
\hline $10 / 26 / 947: 06$ & Auto FIC & 54.3 & Good & \\
\hline $10 / 26 / 9412: 00$ & Manual Tape & 54.25 & Good & \\
\hline $10 / 27 / 947: 06$ & Auto FIC & 54.30 & Good & \\
\hline $10 / 27 / 9412: 00$ & Manual Tape & 54.50 & Good & \\
\hline $10 / 28 / 947: 04$ & Auto FIC & 54.3 & Good & \\
\hline $10 / 28 / 9412: 00$ & Manual Tape & 53.50 & Good & \\
\hline $10 / 29 / 9412: 00$ & Manual FIC & 54.4 & Good & \\
\hline $10 / 29 / 9412: 00$ & Manual Tape & 54.25 & Good & \\
\hline $10 / 30 / 9412: 00$ & Manual Tape & 54.00 & Good & \\
\hline $10 / 30 / 9423: 04$ & Auto FIC & 54.3 & Good & \\
\hline $10 / 31 / 947: 05$ & Auto FIC & 54.3 & Good & \\
\hline $10 / 31 / 9412: 00$ & Manual Tape & 54.00 & Good & ; \\
\hline $11 / 1 / 9412: 00$ & Manual Tape & 54.00 & Good & \\
\hline $11 / 1 / 9423: 04$ & Auto FIC & 54.30 & Good & \\
\hline $11 / 2 / 947: 03$ & Auto FIC & 54.3 & Good & \\
\hline $11 / 2 / 9412: 00$ & Manual Tape & 54.25 & Good & \\
\hline $11 / 3 / 947: 04$ & Auto FIC & 54.30 & Good & \\
\hline $11 / 3 / 9412: 00$ & Manual Tape & 54.00 & Good & \\
\hline $11 / 4 / 947: 03$ & Auto FIC & 54.3 & Good & \\
\hline $11 / 4 / 9412: 00$ & Manual Tape & 54.25 & Good & \\
\hline $11 / 5 / 947: 03$ & Auto FIC & 54.3 & Good & \\
\hline $11 / 5 / 9412: 00$ & Manual Tape & 54.25 & Good & \\
\hline 11/6/94 7:04 & Auto FIC & 54.30 & Good & \\
\hline $11 / 6 / 9412: 00$ & Manual Tape & 54.25 & Good & \\
\hline $11 / 7 / 947: 04$ & Auto FIC & 54.3 & Good & \\
\hline
\end{tabular}

Data obtained from WHC Surveillance Analysis Computer System, (PC SACS) July 7, 1995. 


\begin{tabular}{|c|c|c|c|c|}
\hline \multicolumn{5}{|c|}{ 241-AP-101 } \\
\hline \multicolumn{5}{|c|}{ Surface Level Data } \\
\hline Date & Type of Measurement & Level (inches) & Data Quality & Comments \\
\hline $11 / 7 / 9412: 00$ & Manual Tape & 54.5 & Good & \\
\hline $11 / 8 / 947: 03$ & Auto FIC & 54.30 & Good & \\
\hline $11 / 8 / 9412: 00$ & Manual Tape & 54.50 & Good & \\
\hline $11 / 9 / 947: 05$ & Auto FIC & 54.3 & Good & \\
\hline $11 / 9 / 9412: 00$ & Manual Tape & 54.25 & Good & \\
\hline $11 / 10 / 947: 03$ & Auto FIC & 54.3 & Good & \\
\hline $11 / 10 / 9412: 00$ & Manual Tape & 54.25 & Good & \\
\hline $11 / 11 / 947: 03$ & Auto FIC & 54.30 & Good & \\
\hline $11 / 11 / 9412: 00$ & Manual Tape & 54.00 & Good & \\
\hline $11 / 12 / 947: 03$ & Auto FIC & 54.3 & Good & \\
\hline $11 / 12 / 9412: 00$ & Manual Tape & 54.25 & Good & \\
\hline $11 / 13 / 947: 04$ & Auto FIC & 54.30 & Good & \\
\hline $11 / 13 / 9412: 00$ & Manual Tape & 53.50 & Good & \\
\hline $11 / 14 / 947: 03$ & Auto FIC & 54.3 & Good & \\
\hline $11 / 14 / 9412: 00$ & Manual Tape & 54.25 & Good & \\
\hline $11 / 15 / 947: 04$ & Auto FIC & 54.30 & Good & \\
\hline $11 / 15 / 9412: 00$ & Manual Tape ' & 54.25 & Good & \\
\hline $11 / 16 / 947: 06$ & Auto FIC & 54.3 & Good & \\
\hline $11 / 16 / 9412: 00$ & Manual Tape & 54.25 & Good & \\
\hline $11 / 17 / 947: 04$ & Auto FIC & 54.30 & Good & \\
\hline $11 / 17 / 9412: 00$ & Manual Tape & 54.25 & Good & \\
\hline $11 / 18 / 947: 04$ & Auto FIC & 54.3 & Good & \\
\hline $11 / 18 / 9412: 00$ & Manual Tape & 54.25 & Good & \\
\hline $11 / 19 / 947: 03$ & Auto FIC & 54.30 & Good & \\
\hline $11 / 19 / 9412: 00$ & Manual Tape & 54 & Good & \\
\hline $11 / 20 / 947: 03$ & Auto FIC & 54.3 & Good & \\
\hline $11 / 21 / 947: 04$ & Auto FIC & 0 & Suspect & Data not included in graph \\
\hline $11 / 21 / 9412: 00$ & Manual FIC & 54.3 & Good & \\
\hline $11 / 21 / 9412: 00$ & Manual Tape & 54.25 & Good & \\
\hline $11 / 22 / 947: 03$ & Auto FIC & 54.30 & Good & \\
\hline $11 / 22 / 9412: 00$ & Manual Tape & 54.25 & Good & \\
\hline $11 / 23 / 9412: 00$ & Manual FIC & 54.3 & Good & \\
\hline $11 / 23 / 9412: 00$ & Manual Tape & 54 & Good & \\
\hline $11 / 24 / 9412: 00$ & Manual Tape & 54.25 & Good & \\
\hline $11 / 24 / 9423: 03$ & Auto FIC & 54.3 & Good & \\
\hline $11 / 25 / 9412: 00$ & Manual Tape & 54.00 & Good & \\
\hline $11 / 25 / 9418: 04$ & Auto FIC & 54.30 & Good & \\
\hline $11 / 26 / 947: 03$ & Auto FIC & 54.3 & Good & \\
\hline $11 / 26 / 9412: 00$ & Manual Tape & 54 & Good & \\
\hline $11 / 27 / 942: 04$ & Auto FIC & 54.3 & Good & \\
\hline $11 / 27 / 9412: 00$ & Manual Tape & 54.25 & Good & \\
\hline $11 / 28 / 940: 03$ & Auto FIC & 54.30 & Good & \\
\hline $11 / 28 / 9412: 00$ & Manual Tape & 54.25 & Good & \\
\hline $11 / 29 / 945: 03$ & Auto FIC & 54.3 & Good & \\
\hline $11 / 29 / 9412: 00$ & Manual Tape & 54 & Good & \\
\hline $11 / 30 / 947: 03$ & Auto FIC & 54.30 & Gopd & \\
\hline $11 / 30 / 9412: 00$ & Manual Tape & 54.25 & Good & \\
\hline $12 / 1 / 944: 04$ & Auto FIC & 54.3 & Good & \\
\hline
\end{tabular}

Data obtained from WHC Surveillance Analysis Computer System, (PC SACS) July 7, 1995. 


\begin{tabular}{|c|c|c|c|c|}
\hline \multicolumn{5}{|c|}{ 241-AP-101 } \\
\hline \multicolumn{5}{|c|}{ Surface Level Data } \\
\hline Date & Type of Measurement & Leval (Inches) & Data Quality & Comments \\
\hline $12 / 1 / 9412: 00$ & Manual Tape & 54.00 & Good & \\
\hline $12 / 2 / 947: 03$ & Auto FIC & 54.30 & Good & \\
\hline $12 / 2 / 9412: 00$ & Manual Tape & 54 & Good & \\
\hline $12 / 3 / 942: 04$ & Auto FIC & 54.3 & Good & \\
\hline $12 / 3 / 9412: 00$ & Manual Tape & 54.00 & Good & \\
\hline $12 / 4 / 947: 03$ & Auto FIC & 54.30 & Good & \\
\hline $12 / 4 / 9412: 00$ & Manual Tape & 54.00 & Good & \\
\hline $12 / 5 / 947: 03$ & Auto FIC & 54.3 & Good & \\
\hline $12 / 5 / 9412: 00$ & Manual Tape & 54 & Good & \\
\hline $12 / 6 / 947: 04$ & Auto FIC & 0.00 & Suspect & Data not included in graph \\
\hline $12 / 6 / 9412: 00$ & Manual FIC & 54.30 & Good & \\
\hline $12 / 6 / 9412: 00$ & Manual Tape & 54.00 & Good & \\
\hline $12 / 7 / 947: 03$ & Auto FiC & 0 & Suspect & Data not included in graph \\
\hline $12 / 7 / 9412: 00$ & Manual FIC & 54.3 & Good & \\
\hline $12 / 7 / 9412: 00$ & Manual Tape & 54.13 & Good & \\
\hline $12 / 8 / 947: 10$ & Auto FIC & 0.00 & Suspect & Data not included in graph \\
\hline $12 / 8 / 9412: 00$ & Manual FIC & 54.30 & Good & \\
\hline $12 / 8 / 9412: 00$ & Manual Tape & 54 & Good & \\
\hline $12 / 9 / 947: 03$ & Auto FIC & 54.3 & Good & \\
\hline $12 / 9 / 9412: 00$ & Manual Tape & 54.25 & Good & \\
\hline $12 / 10 / 947: 04$ & Auto FIC & 0.00 & Suspect & Data not included in graph \\
\hline $12 / 10 / 9412: 00$ & Manual FIC & 54.30 & Good & \\
\hline $12 / 10 / 9412: 00$ & Manual Tape & 54.25 & Good & \\
\hline $12 / 11 / 947: 04$ & Auto FIC & 0 & Suspect & Data not included in graph \\
\hline $12 / 11 / 9412: 00$ & Manual FIC & 54.3 & Good & \\
\hline $12 / 11 / 9412: 00$ & Manual Tape & 54.25 & Good & \\
\hline $12 / 12 / 947: 03$ & Auto FIC & 0.00 & Suspect & Data not included in graph \\
\hline $12 / 12 / 9412: 00$ & Manual FIC & 54.3 & Good & \\
\hline $12 / 12 / 9412: 00$ & Manual Tape & 54.20 & Good & \\
\hline $12 / 13 / 947: 03$ & Auto FIC & 0 & Suspect & Data not included in graph \\
\hline $12 / 13 / 9412: 00$ & Manual FIC & 54.30 & Good & \\
\hline $12 / 13 / 9412: 00$ & Manual Tape & 54.25 & Good & \\
\hline $12 / 14 / 947: 04$ & Auto FIC & 54.30 & Good & \\
\hline $12 / 14 / 9412: 00$ & Manual Tape & 54.13 & Good & \\
\hline $12 / 15 / 945: 03$ & Auto FIC & 54.3 & Good & \\
\hline $12 / 15 / 9412: 00$ & Manual Tape & 54.00 & Good & \\
\hline $12 / 16 / 946: 03$ & Auto FIC & 54.30 & Good & \\
\hline $12 / 16 / 9412: 00$ & Manual Tape & $\mathbf{5 3 . 7 5}$ & Good & \\
\hline $12 / 17 / 947: 06$ & Auto FIC & 54.3 & Good & \\
\hline $12 / 17 / 9412: 00$ & Manual Tape & 54.00 & Good & \\
\hline $12 / 18 / 947: 04$ & Auto FIC & 54.30 & Good & \\
\hline $12 / 18 / 9412: 00$ & Manual Tape & 54.25 & Good & \\
\hline $12 / 19 / 947: 04$ & Auto FIC & 54.3 & Good & \\
\hline $12 / 19 / 9412: 00$ & Manual Tape & 54.00 & Good & \\
\hline $12 / 20 / 947: 04$ & Auto FIC & 0.00 & Suspect & Data not included in graph \\
\hline $12 / 20 / 9411: 03$ & Manual Tape & 54.00 & Good & \\
\hline $12 / 21 / 947: 04$ & Auto FIC & 0 & Suspect & Data not included in graph \\
\hline 12/21/9411:03 & Manual Tape & 54.00 & Good & \\
\hline
\end{tabular}

Data obtained from WHC Surveillance Analysis Computer System, (PC SACS) July 7, 1995. 


\begin{tabular}{|c|c|c|c|c|}
\hline \multicolumn{5}{|c|}{ 241-AP-101 } \\
\hline \multicolumn{5}{|c|}{ Surface Level Data } \\
\hline Date & Type of Measurement & Level (Inches) & Data Quality & Comments \\
\hline $12 / 22 / 947: 05$ & Auto FIC & 54.3 & Good & \\
\hline $12 / 22 / 9412: 00$ & Manual Tape & 54.25 & Good & \\
\hline 12/23/94 7:03 & Auto FIC & 54.3 & Good & \\
\hline $12 / 23 / 9412: 00$ & Manual Tape & 54.00 & Good & \\
\hline $12 / 24 / 947: 04$ & Auto FIC & 54.30 & Good & \\
\hline $12 / 24 / 9412: 00$ & Manual Tape & 53.50 & Good & \\
\hline $12 / 25 / 947: 03$ & Auto FIC & 54.3 & Good & \\
\hline $12 / 25 / 9412: 00$ & Manual Tape & 54 & Good & \\
\hline $12 / 26 / 947: 03$ & Auto FIC & 54.3 & Good & \\
\hline $12 / 26 / 9412: 00$ & Manual Tape & 57.00 & Suspect & Data not included in graph \\
\hline $12 / 27 / 947: 04$ & Auto FIC & 54.30 & Good & \\
\hline $12 / 27 / 9412: 00$ & Manual Tape & 54.25 & Good & \\
\hline $12 / 28 / 947: 04$ & Auto FIC & 54.3 & Good & \\
\hline $12 / 28 / 9412: 00$ & Manual Tape & 54 & Good & \\
\hline $12 / 29 / 947: 04$ & Auto FIC & 54.30 & Good & \\
\hline $12 / 29 / 9412: 00$ & Manual Tape & 54.00 & Good & \\
\hline $12 / 30 / 947: 04$ & Auto FIC & 54.3 & Good & \\
\hline $12 / 30 / 9412: 00$ & Manual Tape & 54.00 & Good & \\
\hline $12 / 31 / 947: 04$ & Auto FIC & 54.30 & Good & \\
\hline $12 / 31 / 9412: 00$ & Manual Tape & 54.25 & Good & \\
\hline 1/1/95 7:04 & Auto FIC & 54.3 & Good & \\
\hline $1 / 1 / 95 \quad 12: 00$ & Manual Tape & 54.25 & Good & \\
\hline 1/2/95 7:03 & Auto FIC & 54.30 & Good & \\
\hline $1 / 2 / 95 \quad 12: 00$ & Manual Tape & 54.25 & Good & \\
\hline 1/3/95 7:02 & Auto FIC & 54.3 & Good & \\
\hline $1 / 3 / 9512: 00$ & Manual Tape & 53.75 & Good & \\
\hline 1/4/95 7:03 & Auto FIC & 54.30 & Good & \\
\hline $1 / 4 / 9512: 00$ & Manual Tape & 54.25 & Good & \\
\hline $1 / 5 / 95$ 7:04 & Auto FIC & 54.3 & Good & \\
\hline $1 / 5 / 95 \quad 12: 00$ & Manual Tape & 54.25 & Good & \\
\hline $1 / 6 / 957: 04$ & Auto FIC & 54.30 & Good & \\
\hline $1 / 6 / 95 \quad 12: 00$ & Manual Tape & 54.00 & Good & \\
\hline $1 / 7 / 957: 04$ & Auto FIC & 54.3 & Good & \\
\hline $1 / 7 / 9512: 00$ & Manual Tape & 54.00 & Good & \\
\hline $1 / 8 / 95 \quad 12: 00$ & Manual Tape & 54.00 & Good & \\
\hline $1 / 8 / 95 \quad 23: 03$ & Auto FIC & 54.30 & Good & \\
\hline 1/9/95 7:05 & Auto FIC & 54.3 & Good & \\
\hline $1 / 9 / 9512: 00$ & Manual Tape & 53.00 & Good & \\
\hline $1 / 10 / 957: 06$ & Auto FIC & 54.3 & Good & \\
\hline $1 / 10 / 9512: 00$ & Manual Tape & 53.00 & Good & \\
\hline $1 / 11 / 957: 07$ & Auto FIC & 54.30 & Good & \\
\hline $1 / 11 / 95 \quad 12: 00$ & Manual Tape & 54.25 & Good & \\
\hline $1 / 12 / 957: 07$ & Auto FIC & 54.3 & Good & \\
\hline $1 / 12 / 95 \quad 12: 00$ & Manual Tape & 53.00 & Good & \\
\hline $1 / 13 / 956: 07$ & Auto FIC & 54.30 & Good & \\
\hline $1 / 13 / 9512: 00$ & Manual Tape & 53.00 & Good & \\
\hline $1 / 14 / 957: 07$ & Auto FIC & 54.3 & Good & \\
\hline $1 / 14 / 95 \quad 12: 00$ & Manual Tape & 54.00 & Good & \\
\hline
\end{tabular}

Data obtained from WHC Surveillance Analysis Computer System, (PC SACS) July 7, 1995. 


\begin{tabular}{|c|c|c|c|c|}
\hline \multicolumn{5}{|c|}{ 241-AP-101 } \\
\hline & & Urface Level Dat & & \\
\hline Date & Type of Measurement & Level (Inches) & Data Quality & Comments \\
\hline $1 / 15 / 95 \quad 7: 06$ & Auto FIC & 54.3 & Good & \\
\hline $1 / 15 / 9512: 00$ & Manual Tape & 54 & Gopd & \\
\hline 1/16/95 7:08 & Auto FIC & 54.20 & Good & \\
\hline $1 / 16 / 9512: 00$ & Manual Tape & 54.00 & Good & \\
\hline 1/17/95 6:07 & Auto FIC & 54.3 & Good & \\
\hline $1 / 17 / 95 \quad 12: 00$ & Manual Tape & 54.00 & Good & \\
\hline $1 / 18 / 957: 07$ & Auto FIC & 54.20 & Good & \\
\hline $1 / 18 / 95 \quad 12: 00$ & Manual Tape & 54 & Good & \\
\hline $1 / 19 / 957: 07$ & Auto FIC & 54.3 & Good & \\
\hline $1 / 19 / 9512: 00$ & Manual Tape & 54.00 & Good & \\
\hline $1 / 20 / 957: 03$ & Auto FIC & 143.70 & Good & \\
\hline $1 / 20 / 9512: 00$ & Manual FIC & 143.70 & Good & \\
\hline $1 / 20 / 9512: 00$ & Manual Tape & 156.25 & Good & \\
\hline $1 / 21 / 957: 04$ & Auto FIC & 259.1 & Good & \\
\hline $1 / 21 / 95 \quad 12: 00$ & Manual FIC & 263.80 & Good & \\
\hline $1 / 21 / 9512: 00$ & Manual Tape & 270.00 & Good & \\
\hline 1/22/95 7:03 & Auto FIC & 326.00 & Good & \\
\hline $1 / 22 / 9512: 00$ & Manual Tape & 317.25 & Good & \\
\hline $1 / 23 / 957: 03$ & Auto FIC & 234 & Good & \\
\hline $1 / 23 / 9512: 00$ & Manual Tape & 226.00 & Good & \\
\hline $1 / 24 / 957: 04$ & Auto FIC & 149.60 & Good & \\
\hline $1 / 24 / 9512: 00$ & Manual Tape & 144.00 & Good & \\
\hline $1 / 25 / 95 \quad 7: 03$ & Auto FIC & 74.2 & Good & \\
\hline $1 / 25 / 95 \quad 12: 00$ & Manual Tape & 69.25 & Good & \\
\hline $1 / 26 / 957: 03$ & Auto FIC & 28.10 & Good & \\
\hline $1 / 26 / 95 \quad 12: 00$ & Manual Tape & 28.00 & Good & \\
\hline $1 / 27 / 957: 03$ & Auto FIC & 28.2 & Good & \\
\hline $1 / 27 / 9512: 00$ & Manual Tape & 28.00 & Good & \\
\hline $1 / 28 / 957: 04$ & Auto FIC & 28.20 & Good & \\
\hline $1 / 28 / 95 \quad 12: 00$ & Manual Tape & 28.00 & Good & \\
\hline $1 / 29 / 957: 04$ & Auto FIC & 28.2 & Good & \\
\hline $1 / 29 / 95 \quad 12: 00$ & Manual Tape & 28.25 & Good & \\
\hline $1 / 30 / 957: 04$ & Auto FIC & 28.20 & Good & \\
\hline $1 / 30 / 95 \quad 12: 00$ & Manual Tape & 28.25 & Good & \\
\hline $1 / 31 / 957: 03$ & Auto FIC & 28.2 & Good & \\
\hline $1 / 31 / 9512: 00$ & Manual Tape & 28.00 & Good & \\
\hline 2/1/95 7:03 & Auto FIC & 28.20 & Good & \\
\hline $2 / 1 / 9512: 00$ & Manual Tape & 28.00 & Good & \\
\hline $2 / 2 / 957: 04$ & Auto FIC & 28.2 & Good & \\
\hline $2 / 2 / 9512: 00$ & Manual Tape & 28.00 & Good & \\
\hline $2 / 3 / 95$ 7:03 & Auto FIC & 28.20 & Good & \\
\hline $2 / 3 / 9512: 00$ & Manual Tape & 28.00 & Good & \\
\hline 2/4/95 7:04 & Auto FIC & 28.2 & Good & \\
\hline $2 / 4 / 9512: 00$ & Manual Tape & 28.25 & Good & \\
\hline 2/5/95 7:03 & Auto FIC & 28.20 & Good & \\
\hline $2 / 5 / 9512: 00$ & Manual Tape & 28.25 & Good & \\
\hline 2/6/95 7:03 & Auto FIC & 28.2 & Good & \\
\hline $2 / 6 / 9512: 00$ & Manual Tape & 27.25 & Suspect & Data not included in graph \\
\hline
\end{tabular}

Data obtained from WHC Surveillance Analysis Computer System, (PC SACS) July 7, 1995. 


\begin{tabular}{|c|c|c|c|c|}
\hline \multicolumn{5}{|c|}{ 241-AP-101 } \\
\hline \multicolumn{5}{|c|}{ Surface Level Data } \\
\hline Date & Type of Masaurement & Level (Inches) & Data Quality & Comments \\
\hline 2/7/95 7:03 & Auto FIC & 28.20 & Good & \\
\hline $2 / 7 / 9512: 00$ & Manual Tape & 27.75 & Good & \\
\hline 2/8/95 7:04 & Auto FIC & 28.2 & Good & \\
\hline $2 / 8 / 95 \quad 12: 00$ & Manual Tape & 28.00 & Good & \\
\hline 2/9/95 7:06 & Auto FIC & 28.20 & Good & \\
\hline $2 / 9 / 9512: 00$ & Manual Tape & 28.50 & Good & \\
\hline $2 / 10 / 957: 06$ & Auto FIC & 28.2 & Good & \\
\hline $2 / 10 / 9512: 00$ & Manual Tape & 27.75 & Good & \\
\hline $2 / 11 / 957: 07$ & Auto FIC & 28.20 & Good & \\
\hline 2/11/95 12:00 & Manual Tape & 28.50 & Good & \\
\hline $2 / 12 / 957: 06$ & Auto FIC & 28.3 & Good & \\
\hline 2/12/95 12:00 & Manual Tape & 28.25 & Good & \\
\hline $2 / 13 / 957: 05$ & Auto FIC & 28.20 & Good & \\
\hline $2 / 13 / 95 \quad 12: 00$ & Manual Tape & 26 & Suspect & Data not included in graph \\
\hline $2 / 14 / 957: 06$ & Auto FIC & 28.2 & Good & \\
\hline $2 / 14 / 9512: 00$ & Manual Tape & 28 & Good & \\
\hline $2 / 15 / 957: 07$ & Auto FIC & 28.20 & Good & \\
\hline $2 / 15 / 9512: 00$ & Manual Tape & 28 & Good & \\
\hline $2 / 16 / 957: 06$ & Auto FIC & 28.2 & Good & \\
\hline $2 / 16 / 9512: 00$ & Manual Tape & 28 & Good & \\
\hline $2 / 17 / 957: 06$ & Auto FIC & 28.2 & Good & \\
\hline $2 / 17 / 9512: 00$ & Manual Tape & 28 & Good & $\cdot$ \\
\hline 2/18/95 7:06 & Auto FIC & 28.2 & Good & \\
\hline 2/18/95 12:00 & Manual Tape & 28 & Good & \\
\hline 2/19/95 7:06 & Auto FIC & 28.20 & Good & \\
\hline $2 / 19 / 95 \quad 12: 00$ & Manual Tape & 28 & Good & \\
\hline 2/20/95 7:07 & Auto FIC & 28.2 & Good & . \\
\hline $2 / 20 / 9512: 00$ & Manual Tape & 28 & Good & \\
\hline $2 / 21 / 957: 06$ & Auto FIC & 28.2 & Good & \\
\hline $2 / 21 / 9512: 00$ & Manual Tape & 28.25 & Good & \\
\hline $2 / 22 / 957: 06$ & Auto FIC & 28.20 & Good & \\
\hline $2 / 22 / 9512: 00$ & Manual Tape & 28.25 & Good & \\
\hline 2/23/95 7:07 & Auto FIC & 28.3 & Good & \\
\hline $2 / 23 / 95 \quad 12: 00$ & Manual Tape & 28 & Good & \\
\hline $2 / 24 / 957: 06$ & Auto FIC & 28.2 & Good & \\
\hline $2 / 24 / 95 \quad 12: 00$ & Manual Tape & 28.25 & Good & \\
\hline $2 / 25 / 957: 06$ & Auto FIC & 28.20 & Good & \\
\hline $2 / 25 / 9512: 00$ & Manual Tape & 28.25 & Good & \\
\hline $2 / 26 / 956: 07$ & Auto FIC & 28.2 & Good & \\
\hline $2 / 26 / 95 \quad 12: 00$ & Manual Tape & 27.5 & Good & \\
\hline $2 / 27 / 957: 06$ & Auto FIC & 28.2 & Good & \\
\hline $2 / 27 / 9512: 00$ & Manual Tape & 27 & Good & \\
\hline 2/28/95 5:07 & Auto FIC & 28.20 & Good & \\
\hline 2/28/95 12:00 & Manual Tape & 28 & Good & \\
\hline $3 / 1 / 957: 06$ & Auto FIC & 28.2 & Good & \\
\hline $3 / 1 / 95 \quad 12: 00$ & Manual Tape & 28 & Good & \\
\hline $3 / 2 / 957: 06$ & Auto FIC & 28.2 & Good & \\
\hline $3 / 2 / 9512: 00$ & Manual Tape & 28 & Good & \\
\hline
\end{tabular}

Data obtained from WHC Surveillance Analysis Computer System, (PC SACS) July 7, 1995. 


\begin{tabular}{|c|c|c|c|c|}
\hline \multicolumn{5}{|c|}{ 241-AP-101 } \\
\hline \multicolumn{5}{|c|}{ Surface Leval Data } \\
\hline Date & Type of Measurement & Level (Inches) & Data Quality & Comments \\
\hline 3/3/95 7:06 & Auto FIC & 28.20 & Good & \\
\hline $3 / 3 / 95 \quad 12: 00$ & Manual Tape & 28 & Good & \\
\hline $3 / 4 / 957: 06$ & Auto FIC & 28.2 & Good & \\
\hline $3 / 4 / 9512: 00$ & Manual Tape & 28 & Good & \\
\hline $3 / 5 / 957: 06$ & Auto FIC & 28.2 & Good & \\
\hline $3 / 5 / 9512: 00$ & Manual Tape & 28 & Good & \\
\hline $3 / 6 / 957: 06$ & Auto FIC & 28.20 & Good & \\
\hline $3 / 6 / 9512: 00$ & Manual Tape & 28.125 & Good & \\
\hline 3/7/95 7:06 & Auto FIC & 28.2 & Good & \\
\hline $3 / 7 / 9512: 00$ & Manual Tape & 28.25 & Good & \\
\hline $3 / 8 / 957: 03$ & Auto FIC & 28.2 & Good & \\
\hline $3 / 8 / 9512: 00$ & Manual Tape & 28 & Good & \\
\hline 3/9/95 7:03 & Auto FIC & 28.20 & Good & \\
\hline $3 / 9 / 95 \quad 12: 00$ & Manual Tape & 28 & Good & \\
\hline $3 / 10 / 957: 02$ & Auto FIC & 28.2 & Good & \\
\hline $3 / 10 / 9512: 00$ & Manual Tape & 28 & Good & \\
\hline $3 / 11 / 957: 03$ & Auto FIC & 28.2 & Good & \\
\hline $3 / 11 / 9512: 00$ & Manual Tape & 28 & Good & \\
\hline $3 / 12 / 957: 03$ & Auto FIC & 28.20 & Good & \\
\hline $3 / 12 / 95 \quad 12: 00$ & Manual Tape & 28 & Good & \\
\hline $3 / 13 / 957: 02$ & Auto FIC & 28.2 & Good & \\
\hline $3 / 13 / 9512: 00$ & Manual Tape & 28 & Good & \\
\hline $3 / 14 / 957: 03$ & Auto FIC & 28.2 & Good & \\
\hline $3 / 14 / 95 \quad 12: 00$ & Manual Tape & 28 & Good & \\
\hline $3 / 15 / 957: 05$ & Auto FIC & 28.20 & Good & \\
\hline $3 / 15 / 95 \quad 12: 00$ & Manual Tape & 28 & Good & \\
\hline $3 / 16 / 957: 03$ & Auto FIC & 28.2 & Good & \\
\hline $3 / 16 / 95 \quad 12: 00$ & Manual Tape & 28 & Good & \\
\hline $3 / 17 / 957: 03$ & Auto FIC & 28.2 & Good & \\
\hline $3 / 17 / 9512: 00$ & Manual Tape & 28 & Good & \\
\hline 3/18/95 7:03 & Auto FIC & 28.20 & Good & \\
\hline $3 / 18 / 95 \quad 12: 00$ & Manual Tape & 28 & Good & \\
\hline $3 / 19 / 957: 03$ & Auto FIC & 28.2 & Good & \\
\hline $3 / 19 / 9512: 00$ & Manual Tape & 28 & Good & \\
\hline $3 / 20 / 956: 03$ & Auto FIC & 28.2 & Good & \\
\hline $3 / 20 / 95 \quad 1.2: 00$ & Manual Tape & 28 & Good & \\
\hline $3 / 21 / 957: 03$ & Auto FIC & 28.20 & Good & \\
\hline $3 / 21 / 95 \quad 12: 00$ & Manual Tape & 28 & Good & \\
\hline $3 / 22 / 957: 02$ & Auto FIC & 28.2 & Good & \\
\hline $3 / 22 / 9512: 00$ & Manual Tape & 28 & Good & \\
\hline $3 / 23 / 957: 03$ & Auto FIC & 28.2 & Good & \\
\hline $3 / 23 / 9512: 00$ & Manual Tape & 28 & Good & \\
\hline $3 / 24 / 957: 03$ & Auto FIC & 28.20 & Good & \\
\hline $3 / 24 / 95 \quad 12: 00$ & Manual Tape & 28 & Good & \\
\hline $3 / 25 / 957: 02$ & Auto FIC & 28.2 & Good & \\
\hline $3 / 25 / 95$ 12:00 & Manual Tape & 28 & Good & \\
\hline $3 / 26 / 957: 02$ & Auto FIC & 28.2 & Good & \\
\hline $3 / 26 / 9512: 00$ & Manual Tape & 27.35 & Good & \\
\hline
\end{tabular}

Data obtained from WHC Surveillance Analysis Computer System, (PC SACS) July 7, 1995. 


\begin{tabular}{|c|c|c|c|c|}
\hline \multicolumn{5}{|c|}{ 241-AP-101 } \\
\hline \multicolumn{5}{|c|}{ Surface Level Data } \\
\hline Date & Type of Measurement & Leval (Inches) & Data Quality & Comments \\
\hline $3 / 27 / 957: 03$ & Auto FIC & 28.20 & Good & \\
\hline $3 / 27 / 9512: 00$ & Manual Tape & 28 & Good & \\
\hline $3 / 28 / 957: 03$ & Auto FIC & 28.2 & Good & \\
\hline $3 / 28 / 95 \quad 12: 00$ & Manual Tape & 28 & Good & \\
\hline $3 / 29 / 957: 03$ & Auto FIC & 28.2 & Good & \\
\hline $3 / 29 / 9512: 00$ & Manual Tape & 28 & Good & \\
\hline $3 / 30 / 957: 03$ & Auto FIC & 28.20 & Good & \\
\hline $3 / 30 / 9512: 00$ & Manual Tape & 28 & Good & \\
\hline $3 / 31 / 957: 04$ & Auto FIC & 28.2 & Good & \\
\hline $3 / 31 / 95 \quad 12: 00$ & Manual Tape & 28 & Good & \\
\hline 4/1/95 7:02 & Auto FIC & 28.2 & Good & \\
\hline $4 / 1 / 9512: 00$ & Manual Tape & 28 & Good & \\
\hline 4/2/95 7:03 & Auto FIC & 28.20 & Good & \\
\hline $4 / 2 / 95 \quad 12: 00$ & Manual Tape & 28 & Good & \\
\hline $4 / 3 / 957: 03$ & Auto FIC & 28.2 & Good & \\
\hline $4 / 3 / 9512: 00$ & Manual Tape & 28 & Good & \\
\hline 4/4/95 7:03 & Auto FIC & 28.2 & Good & \\
\hline $4 / 4 / 9512: 00$ & Manual Tape & 28 & Good & \\
\hline 4/5/95 7:03 & Auto FIC & 28.20 & Good & \\
\hline $4 / 5 / 95 \quad 12: 00$ & Manual Tape & 28 & Good & \\
\hline 4/6/95 7:03 & Auto FIC & 28.2 & Good & \\
\hline $4 / 6 / 9512: 00$ & Manual Tape & 28 & Good & \\
\hline 4/7/95 7:03 & Auto FIC & 28.2 & Good & \\
\hline 4/7/95 12:00 & Manual Tape & 28 & Good & . \\
\hline 4/8/95 7:02 & Auto FIC & 28.2 & Good & \\
\hline $4 / 8 / 9512: 00$ & Manual Tape & 28 & Good & \\
\hline 4/9/95 12:00 & Manual Tape & 28 & Good & \\
\hline 4/9/95 23:03 & Auto FIC & 28.20 & Good & \\
\hline 4/10/95 7:02 & Auto FIC & 28.2 & Good & \\
\hline $4 / 10 / 95 \quad 12: 00$ & Manual Tape & 28 & Good & \\
\hline 4/11/95 7:04 & Auto FIC & 28.2 & Good & \\
\hline 4/11/95 12:00 & Manual Tape & 27 & Good & \\
\hline 4/12/95 7:03 & Auto FIC & 28.20 & Good & \\
\hline $4 / 12 / 95 \quad 12: 00$ & Manual Tape & 28 & Good & \\
\hline $4 / 13 / 957: 05$ & Auto FIC & 28.2 & Good & \\
\hline $4 / 13 / 9512: 00$ & Manual Tape & 28 & Good & \\
\hline $4 / 14 / 957: 03$ & Auto FIC & 28.2 & Good & \\
\hline $4 / 14 / 95 \quad 12: 00$ & Manual Tape & 28 & Good & \\
\hline $4 / 15 / 957: 03$ & Auto FIC & 28.20 & Good & \\
\hline $4 / 15 / 95 \quad 12: 00$ & Manual Tape & 28 & Good & \\
\hline 4/16/95 7:03 & Auto FIC & 28.2 & Good & \\
\hline $4 / 16 / 9512: 00$ & Manual Tape & 28 & Good & \\
\hline 4/17/95 7:03 & Auto FIC & 28.2 & Good & \\
\hline $4 / 17 / 9512: 00$ & Manual Tape & 400 & Suspect & Data not included in graph \\
\hline $4 / 18 / 957: 03$ & Auto FIC & 28.20 & Good & \\
\hline $4 / 18 / 9512: 00$ & Manual Tape & 28 & Good & \\
\hline $4 / 19 / 957: 03$ & Auto FIC & 28.2 & Good & \\
\hline $4 / 19 / 9512: 00$ & Manual Tape & 28 & Good & \\
\hline
\end{tabular}

Data obtained from WHC Surveillance Analysis Computer System, (PC SACS) July 7, 1995. 


\begin{tabular}{|c|c|c|c|c|}
\hline \multicolumn{5}{|c|}{ 241-AP-101 } \\
\hline \multicolumn{5}{|c|}{ Surface Leval Date } \\
\hline Date & Type of Measurement & Level (Inches) & Data Quality & Comments \\
\hline 4/20/95 7:03 & Auto FIC & 28.2 & Good & \\
\hline $4 / 20 / 9512: 00$ & Manual Tape & 28 & Good & \\
\hline $4 / 21 / 957: 03$ & Auto FIC & 28.20 & Good & \\
\hline $4 / 21 / 9512: 00$ & Manual Tape & 28 & Good & \\
\hline $4 / 22 / 957: 03$ & Auto FIC & 28.2 & Good & \\
\hline $4 / 22 / 9512: 00$ & Manual Tape & 28 & Good & \\
\hline $4 / 23 / 957: 03$ & Auto FIC & 28.2 & Good & \\
\hline $4 / 23 / 9512: 00$ & Manual Tape & 28.5 & Good & \\
\hline $4 / 24 / 957: 03$ & Auto FIC & 28.20 & Good & \\
\hline 4/25/95 7:03 & Auto FIC & 28.2 & Good & \\
\hline $4 / 25 / 9512: 00$ & Manual Tape & 28 & Good & \\
\hline $4 / 26 / 957: 03$ & Auto FIC & 28.2 & Good & \\
\hline 4/26/95 12:00 & Manual Tape & 28 & Good & \\
\hline $4 / 27 / 957: 03$ & Auto FIC & 28.20 & Good & \\
\hline $4 / 27 / 9512: 00$ & Manual Tape & 28 & Good & \\
\hline $4 / 28 / 957: 03$ & Auto FIC & 28.2 & Good & \\
\hline $4 / 28 / 9512: 00$ & Manual Tape & 28 & Good & \\
\hline $4 / 29 / 957: 03$ & Auto FIC & 28.2 & Good & \\
\hline $4 / 29 / 95 \quad 12: 00$ & Manua! Tape & 28.25 & Good & \\
\hline $4 / 30 / 957: 03$ & Auto FIC & 28.20 & Good & \\
\hline $4 / 30 / 9512: 00$ & Manual Tape & 28.25 & Good & \\
\hline 5/1/95 7:03 & Auto FIC & 28.2 & Good & \\
\hline $5 / 1 / 9512: 00$ & Manual Tape & 28 & Good & \\
\hline $5 / 2 / 957: 02$ & Auto FIC & 28.20 & Good & \\
\hline $5 / 2 / 95 \quad 12: 00$ & Manual Tape & 28 & Good & \\
\hline $5 / 3 / 957: 03$ & Auto FIC & 28.2 & Good & \\
\hline $5 / 3 / 95 \quad 12: 00$ & Manual Tape & 28 & Good & \\
\hline $5 / 4 / 957: 04$ & Auto FIC & 28.2 & Good & \\
\hline $5 / 4 / 9512: 00$ & Manual Tape & 28 & Good & \\
\hline 5/5/95 7:04 & Auto FIC & 28.20 & Good & \\
\hline $5 / 5 / 9512: 00$ & Manual Tape & 28 & Good & \\
\hline $5 / 6 / 957: 03$ & Auto FIC & 28.2 & Good & \\
\hline $5 / 6 / 9512: 00$ & Manual Tape & 28 & Good & \\
\hline $5 / 7 / 957: 04$ & Auto FIC & 28.20 & Good & \\
\hline $5 / 7 / 95 \quad 12: 00$ & Manual Tape & 28 & Good & \\
\hline $5 / 8 / 957: 04$ & Auto FIC & 28.2 & Good & \\
\hline 5/8/95 12:00 & Manual Tape & 28 & Good & \\
\hline $5 / 9 / 95$ 7:04 & Auto FIC & 28.20 & Good & \\
\hline $5 / 9 / 9512: 00$ & Manual Tape & 28 & Good & \\
\hline $5 / 10 / 957: 04$ & Auto FIC & 28.2 & Good & \\
\hline $5 / 10 / 9512: 00$ & Manual Tape & 28 & Good & \\
\hline $5 / 11 / 957: 03$ & Auto FIC & 28.20 & Good & \\
\hline $5 / 11 / 9512: 00$ & Manual Tape & 28 & Good & \\
\hline $5 / 12 / 957: 06$ & Auto FIC & 28.20 & Good & \\
\hline $5 / 12 / 95 \quad 12: 00$ & Manual Tape & 28 & Good & \\
\hline $5 / 13 / 957: 03$ & Auto FIC & 28.2 & Good & \\
\hline $5 / 13 / 9512: 00$ & Manual Tape & 28.25 & Good & \\
\hline $5 / 14 / 957: 03$ & Auto FIC & 28.20 & Good & \\
\hline
\end{tabular}

Data obtained from WHC Surveillance Analysis Computer System, (PC SACS) July 7, 1995. 


\begin{tabular}{|c|c|c|c|c|}
\hline \multicolumn{5}{|c|}{ 241-AP-101 } \\
\hline \multicolumn{5}{|c|}{ Surface Level Data } \\
\hline Date & Type of Measurement & Level (Inches) & Data Quality & Comments \\
\hline $5 / 14 / 9512: 00$ & Manual Tape & 28 & Good & \\
\hline $5 / 15 / 957: 03$ & Auto FIC & 28.2 & Good & \\
\hline $5 / 15 / 9512: 00$ & Manual Tape & 28 & Good & \\
\hline $5 / 16 / 957: 03$ & Auto FIC & 28.20 & Good & \\
\hline $5 / 16 / 9512: 00$ & Manual Tape & 28 & Good & \\
\hline $5 / 17 / 957: 02$ & Auto FIC & 28.2 & Good & \\
\hline $5 / 17 / 9512: 00$ & Manual Tape & 28 & Good & \\
\hline 5/18/95 $6: 02$ & Auto FIC & 28.20 & Good & \\
\hline $5 / 18 / 9512: 00$ & Manual Tape & 28 & Good & \\
\hline $5 / 19 / 957: 05$ & Auto FIC & 28.2 & Good & \\
\hline $5 / 19 / 95 \quad 12: 00$ & Manual Tape & 28 & Good & \\
\hline $5 / 20 / 957: 05$ & Auto FIC & 28.20 & Good & \\
\hline $5 / 20 / 9512: 00$ & Manual Tape & 28 & Good & \\
\hline 5/21/95 7:05 & Auto FIC & 28.2 & Good & \\
\hline $5 / 21 / 9512: 00$ & Manual Tape & 28 & Good & \\
\hline $5 / 22 / 957: 05$ & Auto FIC & 28.20 & Good & \\
\hline $5 / 22 / 9512: 00$ & Manual Tape & 28 & Good & \\
\hline $5 / 23 / 957: 11$ & Auto FIC & 0 & Suspect & Data not included in graph \\
\hline $5 / 23 / 9512: 00$ & Manual FIC & 28.2 & Good & \\
\hline $5 / 23 / 9512: 00$ & Manual Tape & 28 & Good & \\
\hline $5 / 24 / 957: 10$ & Auto FIC & 0.00 & Suspect & Data not included in graph \\
\hline $5 / 24 / 9512: 00$ & Manual FIC & 28.2 & Good & \\
\hline $5 / 24 / 95 \quad 12: 00$ & Manual Tape & 28 & Good & \\
\hline $5 / 25 / 957: 10$ & Auto FIC & 0 & Suspect & Data not included in graph \\
\hline $5 / 25 / 95 \quad 12: 00$ & Manual FIC & 28.20 & Good & \\
\hline $5 / 25 / 95 \quad 12: 00$ & Manual Tape & 28 & Good & \\
\hline $5 / 26 / 957: 04$ & Auto FIC & 28.20 & Good & \\
\hline $5 / 26 / 95 \quad 12: 00$ & Manual Tape & 28 & Good & \\
\hline $5 / 27 / 95 \quad 7: 05$ & Auto FIC & 28.1 & Good & \\
\hline $5 / 27 / 9512: 00$ & Manual Tape & 27.75 & Good & \\
\hline $5 / 28 / 95 \quad 7: 05$ & Auto FIC & 28.20 & Good & \\
\hline $5 / 28 / 95 \quad 12: 00$ & Manual Tape & 27.75 & Good & \\
\hline $5 / 29 / 957: 05$ & Auto FIC & 28.2 & Good & \\
\hline $5 / 29 / 95 \quad 12: 00$ & Manual Tape & 27.75 & Good & \\
\hline $5 / 30 / 957: 05$ & Auto FIC & 28.20 & Good & \\
\hline $5 / 30 / 9512: 00$ & Manual Tape & 27.75 & Good & \\
\hline $5 / 31 / 957: 05$ & Auto FIC & 28.2 & Good & \\
\hline $5 / 31 / 9512: 00$ & Manual Tape & 28 & Good & \\
\hline $6 / 1 / 957: 05$ & Auto FIC & 28.20 & Good & \\
\hline $6 / 1 / 95 \quad 12: 00$ & Manual Tape & 28 & Good & \\
\hline $6 / 2 / 95 \quad 7: 05$ & Auto FIC & 28.2 & Good & \\
\hline $6 / 2 / 95 \quad 12: 00$ & Manual Tape & 28 & Good & \\
\hline $6 / 3 / 95 \quad 12: 00$ & Manual Tape & 28 & Good & \\
\hline $6 / 3 / 9523: 05$ & Auto FIC & 28.20 & Good & \\
\hline $6 / 4 / 95 \quad 7: 05$ & Auto FIC & 28.2 & Good & \\
\hline $6 / 4 / 95 \quad 12: 00$ & Manual Tape & 28 & Good & \\
\hline $6 / 5 / 957: 05$ & Auto FIC & 28.20 & Good & \\
\hline $6 / 5 / 9512: 00$ & Manual Tape & 28 & Good & \\
\hline
\end{tabular}

Data obtained from WHC Surveillance Analysis Computer System, (PC SACS) July 7, 1995. 


\begin{tabular}{|c|c|c|c|c|}
\hline \multicolumn{5}{|c|}{ 241-AP-101 } \\
\hline \multicolumn{5}{|c|}{ Surface Level Data } \\
\hline Date & Type of Measurement & Level (Inches) & Data Quality & Comments \\
\hline $6 / 6 / 957: 05$ & Auto FIC & 28.2 & Good & \\
\hline $6 / 6 / 95 \quad 12: 00$ & Manual Tape & 28 & Good & \\
\hline $6 / 7 / 95 \quad 7: 05$ & Auto FIC & 28.20 & Good & \\
\hline $6 / 7 / 95 \quad 12: 00$ & Manual Tape & 28 & Good & \\
\hline 6/8/95 7:05 & Auto FIC & 28.2 & Good & \\
\hline $6 / 8 / 95 \quad 12: 00$ & Manual Tape & 28 & Good & \\
\hline 6/9/95 7:05 & Auto FIC & 28.20 & Good & \\
\hline $6 / 9 / 95 \quad 12: 00$ & Manual Tape & 28 & Good & \\
\hline $6 / 10 / 957: 05$ & Auto FIC & 28.2 & Good & \\
\hline $6 / 10 / 9512: 00$ & Manual Tape & 28 & Good & \\
\hline $6 / 11 / 95$ 7:05 & Auto FIC & 28.20 & Good & \\
\hline $6 / 11 / 95 \quad 12: 00$ & Manual Tape & 28 & Good & \\
\hline $6 / 12 / 957: 05$ & Auto FIC & 28.2 & Good & \\
\hline $6 / 12 / 95 \quad 12: 00$ & Manual Tape & 28 & Good & \\
\hline $6 / 13 / 957: 05$ & Auto FIC & 28.10 & Good & \\
\hline $6 / 13 / 9512: 00$ & Manual Tape & 28 & Good & \\
\hline $6 / 14 / 957: 05$ & Auto FIC & 28.2 & Good & \\
\hline $6 / 14 / 9512: 00$ & Manual Tape & 28 & Good & \\
\hline 6/15/95 7:05 & Auto FIC & 28.20 & Good & \\
\hline $6 / 15 / 9512: 00$ & Manual Tape & 28 & Good & \\
\hline $6 / 16 / 957: 05$ & Auto FIC & 28.2 & Good & \\
\hline $6 / 16 / 9512: 00$ & Manual Tape & 28 & Good & \\
\hline $6 / 17 / 957: 05$ & Auto FIC & 28.20 & Good & \\
\hline $6 / 17 / 9512: 00$ & Manual Tape & 28 & Good & \\
\hline $6 / 18 / 957: 05$ & Auto FIC & 28.2 & Good & \\
\hline $6 / 18 / 95 \quad 12: 00$ & Manual Tape & 28 & Good & \\
\hline $6 / 19 / 957: 06$ & Auto FIC & 28.10 & Good & \\
\hline $6 / 19 / 9512: 00$ & Manual Tape & 28 & Good & \\
\hline $6 / 20 / 95$ 7:06 & Auto FIC & 28.2 & Good & \\
\hline $6 / 20 / 9512: 00$ & Manual Tape & 28 & Good & \\
\hline $6 / 21 / 957: 06$ & Auto FIC & 28.20 & Good & \\
\hline $6 / 21 / 95 \quad 12: 00$ & Manual Tape & 28 & Good & \\
\hline $6 / 22 / 957: 06$ & Auto FIC & 28.2 & Good & \\
\hline $6 / 22 / 9512: 00$ & Manual Tape & 28 & Good & \\
\hline $6 / 23 / 957: 06$ & Auto FIC & 28.20 & Good & \\
\hline $6 / 23 / 9512: 00$ & Manual Tape & 28 & Good & \\
\hline $6 / 24 / 957: 06$ & Auto FIC & 28.20 & Good & \\
\hline $6 / 24 / 9512: 00$ & Manual Tape & 28 & Good & \\
\hline $6 / 25 / 95$ 7:05 & Auto FIC & 28.2 & Good & \\
\hline $6 / 25 / 9512: 00$ & Manual Tape & 28 & Good & \\
\hline $6 / 26 / 957: 05$ & Auto FIC & 28.20 & Good & \\
\hline $6 / 26 / 95 \quad 12: 00$ & Manual Tape & 28 & Good & \\
\hline $6 / 27 / 957: 06$ & Auto FIC & 28.2 & Good & \\
\hline $6 / 27 / 9512: 00$ & Manual Tape & 28 & Good & \\
\hline $6 / 28 / 957: 05$ & Auto FIC & 28.20 & Good & \\
\hline $6 / 28 / 95 \quad 12: 00$ & Manual Tape & 28 & Good & \\
\hline $6 / 29 / 957: 06$ & Auto FIC & 28.2 & Good & \\
\hline $6 / 29 / 95 \quad 12: 00$ & Manual Tape & 28 & Good & \\
\hline
\end{tabular}

Data obtained from WHC Surveillance Analysis Computer System, (PC SACS) July 7, 1995. 
WHC-SD-WM-ER-315, Rev, 0

\begin{tabular}{|c|c|c|c|c|}
\hline \multicolumn{5}{|c|}{ 241-AP-101 } \\
\hline Date & Type of Measurement & Level (Inches) & Data Quality & Comments \\
\hline $6 / 30 / 957: 06$ & Auto FIC & 28.20 & Good & \\
\hline $6 / 30 / 9512: 00$ & Manual Tape & 28 & Transcribed & \\
\hline
\end{tabular}

Data obtained from WHC Surveillance Analysis Computer System, (PC SACS) July 7, 1995.

E-85 


\begin{tabular}{|c|c|c|c|c|}
\hline \multicolumn{5}{|c|}{ 241-AP-102 } \\
\hline \multicolumn{5}{|c|}{ Surface Level Data } \\
\hline Date & Type of Measurement & Level (Inches) & Data Quality & Comments \\
\hline $1 / 1 / 910: 00$ & Manual Tape & 49.25 & Transcribed & \\
\hline 1/1/91 7:03 & Auto FIC & 48.40 & Good & \\
\hline $1 / 2 / 910: 00$ & Manual Tape & 49.25 & Transcribed & \\
\hline 1/2/91 7:02 & Auto FIC & 48.4 & Good & \\
\hline $1 / 3 / 91 \quad 0: 00$ & Manual Tape & 49.50 & Transcribed & \\
\hline 1/3/917:03 & Auto FIC & 48.4 & Good & \\
\hline $1 / 4 / 910: 00$ & Manual Tape & 49.5 & Transcribed & \\
\hline $1 / 4 / 917: 02$ & Auto FIC & 48.40 & Good & \\
\hline 1/5/91 0:00 & Manual Tape & 49.5 & Transcribed & \\
\hline $1 / 5 / 917: 02$ & Auto FIC & 48.4 & Good & \\
\hline $1 / 6 / 910: 00$ & Manual Tape & 49.50 & Transcribed & \\
\hline $1 / 6 / 917: 02$ & Auto FIC & 48.4 & Good & \\
\hline 1/7/91 0:00 & Manual Tape & 49.5 & Transcribed & \\
\hline $1 / 7 / 917: 02$ & Auto FIC & 48.40 & Good & \\
\hline $1 / 8 / 910: 00$ & Manual Tape & 49.50 & Transcribed & \\
\hline $1 / 8 / 917: 03$ & Auto FIC & 48.40 & Good & \\
\hline $1 / 9 / 910: 00$ & Manual Tape & 49.50 & Transcribed & \\
\hline $1 / 9 / 917: 02$ & Auto FIC & 48.40 & Good & \\
\hline 1/10/910:00 & Manual Tape & 49.5 & Transcribed & \\
\hline $1 / 10 / 917: 02$ & Auto FIC & 48.4 & Good & \\
\hline $1 / 11 / 910: 00$ & Manual Tape & 49.50 & Transcribed & \\
\hline $1 / 11 / 917: 03$ & Auto FIC & 48.4 & Good & \\
\hline $1 / 12 / 910: 00$ & Manual Tape & 49.50 & Transcribed & \\
\hline $1 / 12 / 917: 02$ & Auto FIC & 48.40 & Good & \\
\hline $1 / 13 / 910: 00$ & Manual Tape & 49.5 & Transcribed & \\
\hline $1 / 13 / 917: 03$ & Auto FIC & 48.50 & Good & \\
\hline $1 / 14 / 910: 00$ & Manual Tape & 49.50 & Transcribed & \\
\hline $1 / 14 / 917: 04$ & Auto FIC & 48.4 & Good & \\
\hline $1 / 15 / 910: 00$ & Manual Tape & 49.5 & Transcribed & \\
\hline $1 / 15 / 917: 03$ & Auto FIC & 48.40 & Good & 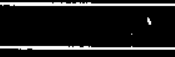 \\
\hline $1 / 16 / 910: 00$ & Manual Tape & 49.5 & Transcribed & \\
\hline $1 / 16 / 917: 04$ & Auto FIC & 48.40 & Good & \\
\hline $1 / 17 / 910: 00$ & Manual Tape & 49.50 & Transcribed & \\
\hline $1 / 17 / 917: 04$ & Auto FIC & 48.4 & Good & \\
\hline $1 / 18 / 910: 00$ & Manual Tape & 49.5 & Transcribed & \\
\hline $1 / 18 / 917: 04$ & Auto FIC & 48.5 & Good & \\
\hline $1 / 19 / 910: 00$ & Manual Tape & 49.50 & Transcribed & \\
\hline $1 / 19 / 917: 04$ & Auto FIC & 48.4 & Good & \\
\hline $1 / 20 / 910: 00$ & Manual Tape & 49.50 & Transcribed & \\
\hline $1 / 20 / 917: 04$ & Auto FIC & 48.40 & Good & \\
\hline $1 / 21 / 910: 00$ & Manual Tape & 49.5 & Transcribed & \\
\hline $1 / 21 / 917: 04$ & Auto FIC & 48.40 & Good & \\
\hline $1 / 22 / 910: 00$ & Manual Tape & 49.5 & Transcribed & \\
\hline $1 / 22 / 917: 04$ & Auto FIC & 48.4 & Good & \\
\hline $1 / 23 / 910: 00$ & Manual Tape & 49.50 & Transcribed & \\
\hline $1 / 23 / 917: 04$ & Auto FIC & 48.40 & Good & \\
\hline $1 / 24 / 910: 00$ & Manual Tape & 49.50 & Transcribed & \\
\hline $1 / 24 / 917: 04$ & Auto FIC & 48.40 & Good & \\
\hline
\end{tabular}

Data obtained from WHC Surveillance Analysis Computer System, (PC SACS) July 7, 1995. 


\begin{tabular}{|c|c|c|c|c|}
\hline \multicolumn{5}{|c|}{ 241-AP-102 } \\
\hline & & Urface Level Dat & & \\
\hline Date & Type of Measurement & Leval (Inches) & Data Quality & Comments \\
\hline $1 / 25 / 910: 00$ & Manual Tape & 49.50 & Transcribed & \\
\hline $1 / 25 / 917: 03$ & Auto FIC & 48.40 & Good & \\
\hline 1/26/910:00 & Manual Tape & 49.50 & Transcribed & \\
\hline $1 / 26 / 917: 03$ & Auto FIC & 48.8 & Good & \\
\hline $1 / 27 / 910: 00$ & Manual Tape & 49.75 & Transcribed & \\
\hline $1 / 27 / 917: 03$ & Auto FIC & 48.7 & Good & \\
\hline $1 / 28 / 910: 00$ & Manual Tape & 49.75 & Transcribed & \\
\hline $1 / 28 / 916: 03$ & Auto FIC & 48.70 & Good & \\
\hline $1 / 29 / 910: 00$ & Manual Tape & 49.75 & Transcribed & \\
\hline $1 / 29 / 917: 02$ & Auto FIC & 48.7 & Good & \\
\hline $1 / 30 / 910: 00$ & Manual FIC & 48.70 & Transcribed & \\
\hline $1 / 30 / 910: 00$ & Manual Tape & 49.50 & Transcribed & \\
\hline $1 / 31 / 910: 00$ & Manual Tape & 49.75 & Transcribed & \\
\hline $1 / 31 / 917: 02$ & Auto FIC & 48.7 & Good & \\
\hline $2 / 1 / 910: 00$ & Manual Tape & 49.75 & Transcribed & \\
\hline 2/1/91 7:02 & Auto FIC & 48.70 & Good & \\
\hline $2 / 2 / 910: 00$ & Manual Tape & 49.75 & Transcribed & \\
\hline $2 / 2 / 917: 04$ & Auto FIC & 48.70 & Good & \\
\hline $2 / 3 / 910: 00$ & Manual Tape & 49.75 & Transcribed & \\
\hline 2/3/91 7:03 & Auto FIC & 48.70 & Good & \\
\hline $2 / 4 / 910: 00$ & Manual Tape & 49.75 & Transcribed & \\
\hline 2/4/91 7:03 & Auto FIC & 48.7 & Good & \\
\hline $2 / 5 / 910: 00$ & Manual Tape & 49.75 & Transcribed & \\
\hline $2 / 5 / 917: 04$ & Auto FIC & 48.7 & Good & \\
\hline $2 / 6 / 91 \quad 0: 00$ & Manual Tape & 49.75 & Transćribed & \\
\hline $2 / 6 / 917: 04$ & Auto FIC & 48.70 & Good & \\
\hline 2/7/910:00 & Manual Tape & 49.5 & Transcribed & \\
\hline 2/7/91 7:04 & Auto FIC & 48.7 & Good & \\
\hline $2 / 8 / 910: 00$ & Manual Tape & 49.50 & Transcribed & \\
\hline $2 / 8 / 917: 04$ & Auto FIC & 48.7 & Good & \\
\hline $2 / 9 / 910: 00$ & Manual Tape & 49.25 & Transcribed & \\
\hline $2 / 9 / 917: 04$ & Auto FIC & 48.60 & Good & \\
\hline $2 / 10 / 910: 00$ & Manual Tape & 49.5 & Transcribed & \\
\hline $2 / 10 / 917: 03$ & Auto FIC & 48.60 & Good & \\
\hline $2 / 11 / 910: 00$ & Manual Tape & 49.50 & Transcribed & \\
\hline $2 / 11 / 917: 04$ & Auto FIC & 48.70 & Good & \\
\hline $2 / 12 / 910: 00$ & Manual Tape & 49.5 & Transcribed & \\
\hline $2 / 12 / 917: 04$ & Auto FIC & 48.7 & Good & \\
\hline $2 / 13 / 910: 00$ & Manual Tape & 49.50 & Transcribed & \\
\hline $2 / 13 / 917: 04$ & Auto FIC & 48.8 & Good & \\
\hline $2 / 14 / 910: 00$ & Manual Tape & 49.50 & Transcribed & \\
\hline $2 / 14 / 917: 04$ & Auto FIC & 48.70 & Good & \\
\hline $2 / 15 / 910: 00$ & Manual Tape & 49.5 & Transcribed & \\
\hline $2 / 15 / 917: 03$ & Auto FIC & 48.7 & Good & \\
\hline $2 / 16 / 910: 00$ & Manual Tape & 49.75 & Transcribed & \\
\hline $2 / 16 / 917: 02$ & Auto FIC & 48.7 & Good & \\
\hline $2 / 17 / 910: 00$ & Manual Tape & 49.75 & Transcribed & \\
\hline $2 / 17 / 917: 02$ & Auto FIC & 48.6 & Good & \\
\hline
\end{tabular}

Data obtained from WHC Surveillance Analysis Computer System, (PC SACS) July 7, 1995. 


\begin{tabular}{|c|c|c|c|c|}
\hline \multicolumn{5}{|c|}{ 241-AP-102 } \\
\hline \multicolumn{5}{|c|}{ Surface Level Date } \\
\hline Date & Type of Measurement & Level (Inches) & Data Quality & Comments \\
\hline $2 / 18 / 910: 00$ & Manual Tape & 49.75 & Transcribed & \\
\hline $2 / 18 / 917: 03$ & Auto FIC & 48.60 & Good & \\
\hline 2/19/91 0:00 & Manual Tape & 49.75 & Transcribed & \\
\hline 2/19/91 7:02 & Auto FIC & 48.8 & Good & \\
\hline 2/20/91 0:00 & Manual Tape & 49.75 & Transeribed & \\
\hline $2 / 20 / 917: 03$ & Auto FIC & 48.7 & Good & \\
\hline $2 / 21 / 910: 00$ & Manual Tape & 49.75 & Transcribed & \\
\hline $2 / 21 / 917: 02$ & Auto FIC & 48.60 & Good & \\
\hline 2/22/910:00 & Manual Tape & 49.75 & Transcribed & \\
\hline $2 / 22 / 917: 02$ & Auto FIC & 48.60 & Good & \\
\hline $2 / 23 / 910: 00$ & Manual Tape & 49.75 & Transcribed & \\
\hline $2 / 23 / 917: 02$ & Auto FIC & 48.70 & Good & \\
\hline $2 / 24 / 910: 00$ & Manual Tape & 49.75 & Transcribed & \\
\hline $2 / 24 / 917: 03$ & Auto FIC & 48.7 & Good & \\
\hline $2 / 25 / 910: 00$ & Manual Tape & 49.75 & Transcribed & \\
\hline 2/25/91 7:03 & Auto FIC & 48.50 & Good & \\
\hline $2 / 26 / 910: 00$ & Manual Tape & 49.75 & Transcribed & \\
\hline $2 / 26 / 917: 03$ & Auto FIC & 48.60 & Good & \\
\hline $2 / 27 / 910: 00$ & Manual Tape & 49.75 & Transcribed & $\cdot$ \\
\hline $2 / 27 / 917: 02$ & Auto FIC & 48.6 & Good & \\
\hline $2 / 28 / 910: 00$ & Manual Tape & 49.75 & Transcribed & \\
\hline $2 / 28 / 917: 02$ & Auto FIC & 48.7 & Good & \\
\hline 3/1/91 0:00 & Manual Tape & 49.50 & Transcribed & \\
\hline $3 / 1 / 917: 02$ & Auto FIC & 48.60 & Good & \\
\hline $3 / 2 / 910: 00$ & Manual Tape & 49.75 & Transcribed & \\
\hline $3 / 2 / 917: 02$ & Auto FIC & 48.60 & Good & \\
\hline $3 / 3 / 910: 00$ & Manual Tape . & 49.75 & Transcribed & \\
\hline $3 / 3 / 910: 00$ & Manual FIC & 48.60 & Good & \\
\hline $3 / 3 / 917: 03$ & Auto FIC & 0 & Suspect & Data not included in graph \\
\hline $3 / 4 / 910: 00$ & Manual Tape & 49.75 & Transcribed & \\
\hline $3 / 4 / 917: 03$ & Auto FIC & 48.60 & Good & \\
\hline $3 / 5 / 910: 00$ & Manual Tape & 50 & Transcribed & \\
\hline $3 / 5 / 917: 03$ & Auto FIC & 48.60 & Good & \\
\hline $3 / 6 / 910: 00$ & Manual Tape & 50.00 & Transcribed & \\
\hline $3 / 6 / 917: 02$ & Auto FIC & 48.6 & Good & \\
\hline $3 / 7 / 910: 00$ & Manual Tape & 50 & Transcribed & \\
\hline $3 / 7 / 917: 02$ & Auto FIC & 48.60 & Good & \\
\hline $3 / 8 / 910: 00$ & Manual Tape & 49.50 & Transcribed & \\
\hline $3 / 8 / 917: 02$ & Auto FIC & 48.60 & Good & \\
\hline $3 / 9 / 910: 00$ & Manual Tape & 49.5 & Transcribed & \\
\hline $3 / 9 / 91 \quad 7: 02$ & Auto FIC & 48.6 & Good & \\
\hline $3 / 10 / 910: 00$ & Manual Tape & 49.50 & Transcribed & \\
\hline $3 / 10 / 917: 02$ & Auto FIC & 48.6 & Good & \\
\hline $3 / 11 / 910: 00$ & Manual Tape & 49.75 & Transcribed & \\
\hline $3 / 11 / 917: 02$ & Auto FIC & 48.6 & Good & \\
\hline $3 / 12 / 910: 00$ & Manual Tape & 49.75 & Transcribed & \\
\hline $3 / 12 / 917: 03$ & Auto FIC & 48.60 & Good & \\
\hline $3 / 13 / 910: 00$ & Manual Tape & 49.75 & Transcribed & \\
\hline
\end{tabular}

Data obtained from WHC Surveillance Analysis Computer System, (PC SACS) July 7, 1995. 


\begin{tabular}{|c|c|c|c|c|}
\hline \multicolumn{5}{|c|}{ 241-AP-102 } \\
\hline \multicolumn{5}{|c|}{ Surface Level Data } \\
\hline Date & Type of Measurement & Level (Inches) & Data Quality & Comments \\
\hline $3 / 13 / 917: 03$ & Auto FIC & 48.60 & Good & \\
\hline 3/14/91 0:00 & Manual Tape & 49.75 & Transcribed & \\
\hline $3 / 14 / 917: 03$ & Auto FIC & 48.6 & Good & \\
\hline $3 / 15 / 910: 00$ & Manual Tape & 49.75 & Transcribed & \\
\hline $3 / 15 / 917: 03$ & Auto FIC & $48.60^{\circ}$ & Good & \\
\hline $3 / 16 / 910: 00$ & Manual Tape & 49.75 & Transcribed & \\
\hline $3 / 16 / 917: 03$ & Auto FIC & 48.60 & Good & \\
\hline $3 / 17 / 910: 00$ & Manual Tape & 49.75 & Transcribed & \\
\hline $3 / 17 / 917: 02$ & Auto FIC & 48.60 & Good & \\
\hline $3 / 18 / 910: 00$ & Manual Tape & 49.75 & Transcribed & \\
\hline $3 / 18 / 917: 03$ & Auto FIC & 48.6 & Good & \\
\hline $3 / 19 / 910: 00$ & Manual Tape & 49.75 & Transcribed & \\
\hline $3 / 19 / 917: 03$ & Auto FIC & 48.6 & Good & \\
\hline $3 / 20 / 910: 00$ & Manual Tape & 49.75 & Transcribed & \\
\hline $3 / 20 / 917: 02$ & Auto FIC & 48.60 & Good & \\
\hline $3 / 21 / 910: 00$ & Manual Tape & 49.75 & Transcribed & \\
\hline $3 / 21 / 917: 03$ & Auto FIC & 48.60 & Good & \\
\hline $3 / 22 / 910: 00$ & Manual Tape & 49.75 & Transcribed & \\
\hline $3 / 22 / 917: 02$ & Auto FIC & 48.6 & Good & \\
\hline $3 / 23 / 910: 00$ & Manual Tape & 49.75 & Transcribed & \\
\hline $3 / 23 / 917: 03$ & Auto FIC & 48.6 & Good & \\
\hline $3 / 24 / 910: 00$ & Manual Tape & 49.75 & Transcribed & \\
\hline $3 / 24 / 917: 03$ & Auto FIC & 48.60 & Good & $\cdot$ \\
\hline $3 / 25 / 910: 00$ & Manual Tape & 49.75 & Transcribed & \\
\hline $3 / 25 / 910: 00$ & Manual FIC & 48.4 & Good & \\
\hline $3 / 25 / 917: 03$ & Auto FIC & 0.00 & Suspect & Data not included in graph \\
\hline $3 / 26 / 910: 00$ & Manual Tape & 49.75 & Transcribed & \\
\hline $3 / 26 / 910: 00$ & Manual FIC & 48.6 & Good & \\
\hline $3 / 26 / 917: 03$ & Auto FIC & 0 & Suspect & Data not included in graph \\
\hline $3 / 27 / 910: 00$ & Manual Tape & 49.75 & Transcribed & \\
\hline $3 / 27 / 910: 00$ & Manual FIC & 48.60 & Good & \\
\hline $3 / 27 / 917: 03$ & Auto FIC & 0 & Suspect & Data not included in graph \\
\hline $3 / 28 / 910: 00$ & Manual Tape & 49.75 & Transcribed & \\
\hline $3 / 28 / 914: 03$ & Auto FIC & 48.60 & Good & \\
\hline $3 / 29 / 910: 00$ & Manual Tape & 49.75 & Transcribed & \\
\hline $3 / 29 / 910: 00$ & Manual FIC & 48.60 & Good & \\
\hline $3 / 29 / 917: 03$ & Auto FIC & 0 & Suspect & Data not included in graph \\
\hline $3 / 30 / 910: 00$ & Manual Tape & 49.75 & Transcribed & \\
\hline $3 / 30 / 910: 00$ & Manual FIC & 48.6 & Good & \\
\hline 3/30/91 7:03 & Auto FIC & 0 & Suspect & Data not included in graph \\
\hline $3 / 31 / 910: 00$ & Manual Tape & 49.75 & Transcribed & \\
\hline $3 / 31 / 910: 00$ & Manual FIC & 48.6 & Good & \\
\hline $3 / 31 / 917: 03$ & Auto FIC & 0.00 & Suspect & Data not included in graph \\
\hline 4/1/910:00 & Manual Tape & 49.75 & Transcribed & \\
\hline $4 / 1 / 910: 00$ & Manual FIC & 48.6 & Good & \\
\hline 4/1/917:05 & Manual FIC & 48.60 & Good & \\
\hline 4/2/910:00 & Manual Tape & 49.75 & Transcribed & \\
\hline $4 / 2 / 910: 00$ & Manual FIC & 48.60 & Good & \\
\hline
\end{tabular}

Data obtained from WHC Surveillance Analysis Computer System, (PC SACS) July 7, 1995. 


\begin{tabular}{|c|c|c|c|c|}
\hline \multicolumn{5}{|c|}{ 241-AP-102 } \\
\hline \multicolumn{5}{|c|}{ Surface Loval Data } \\
\hline Date & Type of Measurement & Level (Inches) & Data Quality & Comments \\
\hline $4 / 2 / 917: 05$ & Manual FIC & 48.6 & Good & \\
\hline $4 / 3 / 910: 00$ & Manual Tape & 49.50 & Transcribed & \\
\hline $4 / 3 / 910: 00$ & Manual FIC & 48.6 & Good & \\
\hline 4/3/91 7:05 & Manual FIC & 48.55 & Good & \\
\hline 4/4/91 0:00 & Manual Tape & 49.25 & Transcribed & \\
\hline 4/4/91 0:00 & Manual FIC & 48.60 & Good & \\
\hline $4 / 4 / 917: 05$ & Manual FIC & 48.55 & Good & \\
\hline $4 / 5 / 910: 00$ & Manual Tape & 49.25 & Transcribed & \\
\hline $4 / 5 / 910: 00$ & Manual FIC & 48.6 & Good & \\
\hline $4 / 5 / 917: 05$ & Manual FIC & 48.6 & Good & \\
\hline $4 / 6 / 910: 00$ & Manuel Tape & 49.50 & Transcribed & \\
\hline $4 / 6 / 910: 00$ & Manual FIC & 48.6 & Good & \\
\hline $4 / 6 / 917: 05$ & Manual FIC & 48.60 & Good & \\
\hline $4 / 7 / 910: 00$ & Manual Tape & 49.5 & Transcribed & \\
\hline 4/7/91 0:00 & Manual FIC & 48.60 & Good & \\
\hline $4 / 7 / 917: 05$ & Manual FIC & 48.6 & Good & \\
\hline $4 / 8 / 910: 00$ & Manual Tape & 49.5 & Transcribed & \\
\hline 4/8/91 0:00 & Manual FIC & 48.6 & Good & \\
\hline 4/9/91 0:00 & Manual FIC & 48.60 & Good & \\
\hline $4 / 9 / 917: 05$ & Manual FIC & 48.60 & Good & \\
\hline 4/10/91 0:00 & Manual Tape & 49.5 & Transcribed & \\
\hline $4 / 10 / 910: 00$ & Manual FIC & 48.6 & Good & \\
\hline 4/10/91 7:05 & Manual FIC & 48.6 & Good & \\
\hline 4/11/91 0:00 & Manual Tape & 49.50 & Transcribed & \\
\hline $4 / 11 / 917: 05$ & Manual FIC & 0.00 & Suspect & Data not included in graph \\
\hline $4 / 12 / 910: 00$ & Manual Tape & 49.50 & Transcribed & \\
\hline $4 / 12 / 910: 00$ & Manual FIC & 48.55 & Good & \\
\hline 4/12/91 7:05 & Manual FIC & 48.55 & Good & \\
\hline 4/13/91 0:00 & Manual Tape. & 49.50 & Transcribed & \\
\hline $4 / 13 / 910: 00$ & Manual FIC & 48.55 & Good & \\
\hline 4/13/91 7:05 & Manual FIC & 48.55 & Good & \\
\hline 4/14/910:00 & Manual Tape & 49.75 & Transcribed & \\
\hline $4 / 14 / 910: 00$ & Manual FIC & 48.55 & Good & \\
\hline 4/14/91 7:05 & Manual FIC & 48.55 & Good & \\
\hline $4 / 15 / 910: 00$ & Manual Tape & 49.75 & Transcribed & \\
\hline 4/15/910:00 & Manual FIC & 48.55 & Good & \\
\hline $4 / 15 / 917: 05$ & Manual FIC & 48.55 & Good & \\
\hline $4 / 16 / 910: 00$ & Manual Tape & 49.75 & Transcribed & \\
\hline $4 / 16 / 910: 00$ & Manual FIC & 48.55 & Good & \\
\hline $4 / 16 / 917: 05$ & Manual FIC & 48.6 & Good & \\
\hline $4 / 17 / 910: 00$ & Manual Tape & 49.50 & Transcribed & \\
\hline $4 / 17 / 910: 00$ & Manual FIC & $48: 6$ & Good & \\
\hline $4 / 17 / 917: 05$ & Manual FIC & 48.60 & Good & \\
\hline 4/18/91 0:00 & Manual Tape & 49.50 & Transcribed & \\
\hline $4 / 18 / 910: 00$ & Manual FIC & 48.60 & Good & \\
\hline $4 / 18 / 917: 05$ & Manual FIC & 48.6 & Good & \\
\hline $4 / 19 / 910: 00$ & Manual Tape & 49.50 & Transcribed & \\
\hline 4/19/910:00 & Manual FIC & 48.6 & Good & \\
\hline
\end{tabular}

Data obtained from WHC Surveillance Analysis Computer System, (PC SACS) July 7, 1995. 


\begin{tabular}{|c|c|c|c|c|}
\hline \multicolumn{5}{|c|}{ 241-AP-102 } \\
\hline \multicolumn{5}{|c|}{ Surface Level Data } \\
\hline Date & Type of Measurement & Level (Inches) & Data Quality & Comments \\
\hline 4/19/91 7:05 & Manual FIC & 48.60 & Good & \\
\hline $4 / 20 / 910: 00$ & Manual Tape & 49.5 & Transcribed & \\
\hline $4 / 20 / 910: 00$ & Manual FIC & 48.60 & Good & \\
\hline $4 / 20 / 917: 05$ & Manual FIC & 48.55 & Good & \\
\hline $4 / 21 / 910: 00$ & Manual Tape & 49.5 & Transcribed & \\
\hline $4 / 21 / 910: 00$ & Manual FIC & 48.6 & Good & \\
\hline $4 / 21 / 917: 05$ & Manual FIC & 48.60 & Good & \\
\hline $4 / 22 / 910: 00$ & Manual Tape & 49.50 & Transcribed & \\
\hline $4 / 22 / 910: 00$ & Manual FIC & 48.55 & Good & \\
\hline 4/22/91 7:05 & Manual FIC & 48.55 & Good & \\
\hline $4 / 23 / 910: 00$ & Manual Tape & 49.50 & Transcribed & \\
\hline $4 / 23 / 910: 00$ & Manual FIC & 48.55 & Good & \\
\hline 4/23/91 7:05 & Manual FIC & 48.55 & Good & \\
\hline $4 / 24 / 910: 00$ & Manual Tape & 49.75 & Transcribed & \\
\hline $4 / 24 / 910: 00$ & Manual FIC & 48.55 & Good & \\
\hline $4 / 24 / 917: 05$ & Auto FIC & 0.00 & Suspect & Deta not included in graph \\
\hline 4/24/917:05 & Manual FIC & 48.55 & Good & \\
\hline $4 / 25 / 910: 00$ & Manual Tape & 49.75 & Transcribed & \\
\hline $4 / 25 / 910: 00$ & Manual FIC & 48.55 & Good & \\
\hline 4/25/91 7:05 & Auto FIC & 0.00 & Suspect & Data not included in graph \\
\hline $4 / 26 / 910: 00$ & Manual Tape & 49.75 & Transcribed & \\
\hline $4 / 26 / 910: 00$ & Manual FIC & 48.55 & Good & \\
\hline 4/26/91 7:05 & Auto FIC & 0 & Suspect & Data not included in graph \\
\hline $4 / 27 / 910: 00$ & Manual Tape & 48.5 & Transcribed & \\
\hline 4/27/91 $0: 00$ & Manual FIC & 48.55 & Good & \\
\hline $4 / 27 / 917: 05$ & Auto FIC & 0 & Suspect & Data not included in graph \\
\hline $4 / 28 / 910: 00$ & Manual Tape & 49.50 & Transcribed & \\
\hline $4 / 28 / 910: 00$ & Manual FIC & 48.55 & Good & \\
\hline 4/28/91 7:06 & Auto FIC & 0.00 & Suspect & Data not included in graph \\
\hline $4 / 29 / 910: 00$ & Manual Tape & 49.50 & Transcribed & \\
\hline $4 / 29 / 910: 00$ & Manual FIC & 48.55 & Good & \\
\hline $4 / 29 / 917: 05$ & Auto FIC & 0.00 & Suspect & Data not included in graph \\
\hline $4 / 30 / 910: 00$ & Manual Tape & 49.50 & Transcribed & \\
\hline 4/30/917:06 & Auto FIC & 48.5 & Good & \\
\hline $5 / 1 / 910: 00$ & Manual Tape & 49.50 & Transcribed & \\
\hline $5 / 1 / 917: 05$ & Auto FIC & 48.5 & Good & \\
\hline $5 / 2 / 910: 00$ & Manual Tape & 49.50 & Transcribed & \\
\hline $5 / 2 / 917: 05$ & Auto FIC & 48.50 & Good & \\
\hline $5 / 3 / 910: 00$ & Manual Tape & $49: 5$ & Transcribed & \\
\hline $5 / 3 / 917: 06$ & Auto FIC & 48.5 & Good & \\
\hline $5 / 4 / 910: 00$ & Manual Tape & 49.5 & Transcribed & \\
\hline $5 / 4 / 917: 05$ & Auto FIC & 48.5 & Good & \\
\hline $5 / 5 / 910: 00$ & Manual Tape & 49.50 & Transcribed & \\
\hline $5 / 5 / 917: 05$ & Auto FIC & 48.50 & Good & \\
\hline $5 / 6 / 910: 00$ & Manual Tape & 49.50 & Transcribed & \\
\hline $5 / 6 / 917: 05$ & Auto FIC & 48.50 & Good & \\
\hline $5 / 7 / 910: 00$ & Manual Tape & 49.50 & Transcribed & \\
\hline $5 / 7 / 917: 05$ & Auto FIC & 48.50 & Good & \\
\hline
\end{tabular}

Data obtained from WHC Surveillance Analysis Computer System, (PC SACS) July 7, 1995. 


\begin{tabular}{|c|c|c|c|c|}
\hline \multicolumn{5}{|c|}{ 241-AP-102 } \\
\hline \multicolumn{5}{|c|}{ Surface Level Data } \\
\hline Date & Type of Measurement & Level (Inchas) & Data Quality & Comments \\
\hline $5 / 8 / 910: 00$ & Manual Tape & 49.5 & Transcribed & \\
\hline $5 / 8 / 917: 05$ & Auto FIC & 48.5 & Good & \\
\hline $5 / 9 / 910: 00$ & Manual Tape & 49.5 & Transcribed & \\
\hline $5 / 9 / 917: 05$ & Auto FIC & 48.5 & Good & \\
\hline $5 / 10 / 910: 00$ & Manual Tape & 49.50 & Transcribed & \\
\hline $5 / 10 / 917: 05$ & Auto FIC & 48.50 & Good & \\
\hline $5 / 11 / 910: 00$ & Manual Tape & 49.50 & Transcribed & \\
\hline $5 / 11 / 917: 05$ & Auto FIC & 48.50 & Good & \\
\hline $5 / 12 / 910: 00$ & Manual Tape & 49.5 & Transcribed & \\
\hline $5 / 12 / 917: 05$ & Auto FIC & 48.5 & Good & \\
\hline $5 / 13 / 910: 00$ & Manual Tape & 49.50 & Transcribed & \\
\hline $5 / 13 / 917: 05$ & Auto FIC & 48.50 & Good & \\
\hline $5 / 14 / 910: 00$ & Manual Tape & 49.5 & Transcribed & \\
\hline $5 / 14 / 917: 05$ & Auto FIC & 48.50 & Good & \\
\hline $5 / 15 / 910: 00$ & Manual Tape & 49.5 & Transcribed & \\
\hline $5 / 15 / 917: 05$ & Auto FIC & 48.5 & Good & . \\
\hline $5 / 16 / 910: 00$ & Manual Tape & 49.50 & Transcribed & \\
\hline $5 / 16 / 917: 06$ & Auto FIC & 48.5 & Good & \\
\hline $5 / 17 / 910: 00$ & Manual Tape & 49.50 & Transcribed & \\
\hline $5 / 17 / 917: 06$ & Auto FIC & 48.5 & Good & \\
\hline $5 / 18 / 910: 00$ & Manual Tape & 49.50 & Transcribed & \\
\hline $5 / 18 / 917: 06$ & Auto FIC & 48.5 & Good & \\
\hline $5 / 19 / 910: 00$ & Manual Tape & 49.50 & Transcribed & \\
\hline $5 / 19 / 917: 05$ & Auto FIC & 48.50 & Good & \\
\hline $5 / 20 / 910: 00$ & Manual Tape & 49.50 & Transcribed & \\
\hline $5 / 20 / 917: 06$ & Auto FIC & 48.50 & Good & \\
\hline $5 / 21 / 910: 00$ & Manual Tape & 49.5 & Transcribed & \\
\hline $5 / 21 / 917: 06$ & Auto FIC & 48.50 & Good & \\
\hline $5 / 22 / 910: 00$ & Manual Tape & 49.75 & Transcribed & \\
\hline $5 / 22 / 917: 05$ & Auto FIC & 48.5 & Good & \\
\hline $5 / 23 / 910: 00$ & Manual Tape & 49.50 & Transcribed & \\
\hline $5 / 23 / 917: 05$ & Auto FIC & 48.50 & Good & \\
\hline $5 / 24 / 910: 00$ & Manual Tape & 49.50 & Transcribed & \\
\hline $5 / 24 / 917: 05$ & Auto FIC & 48.5 & Good & \\
\hline $5 / 25 / 910: 00$ & Manual Tape & 49.75 & Transcribed & \\
\hline $5 / 25 / 917: 05$ & Auto FIC & 48.5 & Good & \\
\hline $5 / 26 / 910: 00$ & Manual Tape & 49.75 & Transcribed & \\
\hline $5 / 26 / 917: 05$ & Auto FIC & 48.50 & Good & \\
\hline 5/27/91 0:00 & Manual Tape & 49.75 & Transcribed & \\
\hline $5 / 27 / 917: 05$ & Auto FIC & 48.5 & Good & \\
\hline $5 / 28 / 910: 00$ & Manual Tape & 49.75 & Transcribed & \\
\hline 5/28/91 7:05 & Auto FIC & 48.5 & Good & \\
\hline $5 / 29 / 910: 00$ & Manual Tape & 49.50 & Transeribed & \\
\hline 5/29/91 7:05 & Auto FIC & 48.50 & Good & \\
\hline $5 / 30 / 910: 00$ & Manual Tape & 49.5 & Transcribed & \\
\hline $5 / 30 / 917: 05$ & Auto FIC & 48.50 & Good & \\
\hline $5 / 31 / 910: 00$ & Manual Tape & 49.50 & Transcribed & \\
\hline $5 / 31 / 917: 05$ & Auto FIC & 48.50 & Good & \\
\hline
\end{tabular}

Data obtained from WHC Surveillance Analysis Computer System, (PC SACS) July 7, 1995. 


\begin{tabular}{|c|c|c|c|c|}
\hline \multicolumn{5}{|c|}{ 241-AP-102 } \\
\hline & & Irface Level Dat & & \\
\hline Date & Type of Measurement & Lavel (Inches) & Data Quality & Comments \\
\hline $6 / 1 / 910: 00$ & Manual Tape & 49.5 & Transcribed & \\
\hline 6/1/91 6:05 & Auto FIC & 48.5 & Good & \\
\hline $6 / 2 / 910: 00$ & Manual Tape & 49.5 & Transcribed & \\
\hline $6 / 2 / 913: 05$ & Auto FIC & 48.5 & Good & \\
\hline $6 / 3 / 910: 00$ & Manual Tape & 49.5 & Transcribed & \\
\hline $6 / 3 / 917: 05$ & Auto FIC & 48.50 & Good & \\
\hline $6 / 4 / 910: 00$ & Manual Tape & 49.75 & Transcribed & \\
\hline $6 / 4 / 917: 05$ & Auto FIC & 48.5 & Good & \\
\hline $6 / 5 / 910: 00$ & Manual Tape & 49.5 & Transcribed & \\
\hline $6 / 5 / 917: 05$ & Auto FIC & 48.50 & Good & \\
\hline $6 / 6 / 91 \quad 0: 00$ & Manual Tape & 49.50 & Transcribed & \\
\hline $6 / 6 / 917: 06$ & Auto FIC & 48.50 & Good & \\
\hline $6 / 7 / 910: 00$ & Manual Tape & 49.5 & Transcribed & \\
\hline $6 / 7 / 917: 05$ & Auto FIC & 48.5 & Good & \\
\hline 6/8/91 $0: 00$ & Manual Tape & 49.5 & Transcribed & \\
\hline $6 / 8 / 917: 05$ & Auto FIC & 48.50 & Good & \\
\hline $6 / 9 / 910: 00$ & Manual Tape & 49.5 & Transcribed & \\
\hline $6 / 9 / 917: 05$ & Auto FIC & 48.50 & Good & \\
\hline $6 / 10 / 910: 00$ & Manual Tape & 49.50 & Transcribed & \\
\hline $6 / 10 / 917: 06$ & Auto FIC & 48.5 & Good & \\
\hline $6 / 11 / 910: 00$ & Manual Tape & 49.50 & Transcribed & \\
\hline $6 / 11 / 917: 05$ & Auto FIC & 48.5 & Good & \\
\hline $6 / 12 / 910: 00$ & Manual Tape & 49.50 & Transcribed & \\
\hline $6 / 12 / 917: 04$ & Auto FIC & 48.5 & Good & \\
\hline 6/13/91 0:00 & Manual Tape & 49.50 & Transcribed & \\
\hline $6 / 13 / 917: 05$ & Auto FIC & $\mathbf{4 8 . 5 0}$ & Good & \\
\hline $6 / 14 / 910: 00$ & Manual Tape & 49.5 & Transcribed & \\
\hline $6 / 14 / 917: 05$ & Auto FIC & 48.50 & Good & \\
\hline $6 / 15 / 910: 00$ & Manual Tape & 49.5 & Transcribed & \\
\hline $6 / 15 / 917: 05$ & Auto FIC & 48.50 & Good & \\
\hline $6 / 16 / 910: 00$ & Manual Tape & 49.5 & Transcribed & \\
\hline $6 / 16 / 917: 05$ & Auto FIC & 48.50 & Good & \\
\hline $6 / 17 / 910: 00$ & Manual Tape & 49.50 & Transcribed & \\
\hline $6 / 17 / 917: 05$ & Auto FIC & 48.5 & Good & \\
\hline $6 / 18 / 910: 00$ & Manual Tape & 49.5 & Transcribed & \\
\hline $6 / 18 / 917: 05$ & Auto FIC & 48.5 & Good & \\
\hline $6 / 19 / 910: 00$ & Manual Tape & 49.75 & Transcribed & \\
\hline $6 / 19 / 917: 05$ & Auto FIC & 48.50 & Good & \\
\hline $6 / 20 / 910: 00$ & Manual Tape & 49.75 & Transcribed & \\
\hline $6 / 20 / 917: 05$ & Auto FIC & 48,5 & Good & \\
\hline $6 / 21 / 910: 00$ & Manual Tape & 49.75 & Transcribed & \\
\hline $6 / 21 / 917: 06$ & Auto FIC & 48.50 & Good & \\
\hline $6 / 22 / 910: 00$ & Manual Tape & 49.75 & Transcribed & \\
\hline $6 / 22 / 913: 06$ & Auto FIC & 48.50 & Good & \\
\hline $6 / 23 / 910: 00$ & Manual Tape & 49.75 & Transcribed & \\
\hline $6 / 23 / 917: 05$ & Auto FIC & 48.5 & Good & \\
\hline $6 / 24 / 910: 00$ & Manual Tape & 49.5 & Transcribed & \\
\hline $6 / 24 / 915: 05$ & Auto FIC & 48.50 & Good & \\
\hline
\end{tabular}

Data obtained from WHC Surveillance Analysis Computer System, (PC SACS) July 7, 1995. 


\begin{tabular}{|c|c|c|c|c|}
\hline \multicolumn{5}{|c|}{ 241-AP-102 } \\
\hline \multicolumn{5}{|c|}{ Surface Level Data } \\
\hline Dare & Type of Measurement & Level (Inches) & Data Quality & Comments \\
\hline $6 / 25 / 910: 00$ & Manual Tape & 49.50 & Transcribed & \\
\hline $6 / 25 / 917: 06$ & Auto FIC & 48.50 & Good & \\
\hline $6 / 26 / 910: 00$ & Manual Tape & 49.5 & Transcribed & \\
\hline $6 / 26 / 911: 03$ & Auto FIC & 48.5 & - Good & \\
\hline $6 / 27 / 910: 00$ & Manual FIC & 48.50 & Transcribed & \\
\hline $6 / 27 / 910: 00$ & Manual Tape & 49.5 & Transcribed & \\
\hline $6 / 28 / 910: 00$ & Manual Tape & 49.50 & Transcribed & \\
\hline $6 / 28 / 917: 03$ & Auto FIC & 48.50 & Good & \\
\hline 6/29/91 0:00 & Manual Tape & 49.50 & Transcribed & \\
\hline $6 / 29 / 916: 02$ & Auto FIC & 48.5 & Good & \\
\hline $6 / 30 / 910: 00$ & Manual FIC & 48.60 & Transcribed & \\
\hline $6 / 30 / 910: 00$ & Manual Tape & 49.50 & Transcribed & \\
\hline $7 / 1 / 910: 00$ & Manual FIC & 48.5 & Transcribed & \\
\hline $7 / 1 / 910: 00$ & Manual Tape & 49.50 & Transcribed & \\
\hline $7 / 2 / 910: 00$ & Manual FIC & 48.5 & Transcribed & \\
\hline $7 / 2 / 910: 00$ & Manual Tape & 49.5 & Transcribed & \\
\hline 7/2/917:06 & Auto FIC & 48.5 & Good & \\
\hline $7 / 3 / 910: 00$ & Manual FIC & 48.50 & Transeribed & \\
\hline $7 / 3 / 910: 00$ & Manual Tape & 49.5 & Transeribed & \\
\hline $7 / 3 / 917: 06$ & Auto FIC & 48.50 & Good & \\
\hline $7 / 4 / 910: 00$ & Manual Tape & 49.50 & Transcribed & \\
\hline 7/4/910:00 & Manual FIC & 48.50 & Good & \\
\hline $7 / 5 / 910: 00$ & Manual Tape & 49.50 & Transcribed & \\
\hline $7 / 5 / 910: 00$ & Manual FIC & 48.5 & Good & \\
\hline $7 / 6 / 910: 00$ & Manual Tape & 49.50 & Transcribed & \\
\hline $7 / 6 / 910: 00$ & Manual FIC & 48.5 & Good & \\
\hline $7 / 7 / 910: 00$ & Manual Tape & 49.50 & Transcribed & \\
\hline $7 / 7 / 910: 00$ & Manual FIC & 48.55 & Good & \\
\hline $7 / 8 / 910: 00$ & Manual Tape & 49.5 & Transcribed & \\
\hline 7/8/91 0:00 & Manual FIC & 48.56 & Good & \\
\hline $7 / 9 / 910: 00$ & Manual Tape & 49.5 & Transcribed & \\
\hline $7 / 9 / 910: 00$ & Manual FIC & 48.6 & Good & \\
\hline $7 / 10 / 910: 00$ & Manual Tape & 49.50 & Transcribed & \\
\hline $7 / 10 / 910: 00$ & Manual FIC & 48.5 & Good & \\
\hline $7 / 11 / 910: 00$ & Manual Tape & 49.50 & Transcribed & \\
\hline $7 / 11 / 917: 06$ & Auto FIC & 48.50 & Good & \\
\hline $7 / 12 / 910: 00$ & Manual Tape & 49.50 & Transcribed & \\
\hline $7 / 12 / 917: 06$ & Auto FIC & 48.50 & Good & \\
\hline $7 / 13 / 910: 00$ & Manual Tape & 49.5 & Transcribed & \\
\hline $7 / 13 / 917: 06$ & Auto FIC & 48.5 & Good & \\
\hline 7/14/910:00 & Manual Tape & 49.5 & Transcribed & \\
\hline $7 / 14 / 912: 06$ & Auto FIC & 48.5 & Good & \\
\hline $7 / 15 / 910: 00$ & Manual Tape & 49.50 & Transcribed & \\
\hline $7 / 15 / 917: 06$ & Auto FIC & 48.50 & Good & \\
\hline 7/16/91 $0: 00$ & Manual Tape & 49.50 & Transcribed & \\
\hline $7 / 16 / 910: 00$ & Manual FIC & 48.50 & Good & \\
\hline $7 / 17 / 910: 00$ & Manual Tape & 49.50 & Transcribed & \\
\hline $7 / 17 / 910: 05$ & Auto FIC & 48.5 & Good & 2 \\
\hline
\end{tabular}

- Data obtained from WHC Surveillance Analysis Computer System, (PC SACS) July 7, 1995. 


\begin{tabular}{|c|c|c|c|c|}
\hline \multicolumn{5}{|c|}{ 241-AP-102 } \\
\hline \multicolumn{5}{|c|}{ Surface Level Data } \\
\hline Date & Typa of Measurement & Level (Inches) & Data Quality & Comments \\
\hline 7/18/910:00 & Manual Tape & 49.5 & Transcribed & \\
\hline $7 / 18 / 910: 00$ & Manual FIC & 48.5 & Good & \\
\hline 7/19/91 0:00 & Manual Tape & 49.50 & Transcribed & \\
\hline 7/19/91 7:05 & Auto FIC & 48.5 & Good & \\
\hline $7 / 20 / 910: 00$ & Manual Tape & 49.50 & Transcribed & \\
\hline $7 / 20 / 912: 05$ & Auto FIC & 48.50 & Good & \\
\hline $7 / 21 / 910: 00$ & Manual Tape & 49.50 & Transcribed & \\
\hline $7 / 21 / 91 \quad 1: 05$ & Auto FIC & 48.50 & Good & \\
\hline $7 / 22 / 910: 00$ & Manual Tape & 49.5 & Transcribed & \\
\hline $7 / 22 / 910: 00$ & Manual FIC & 48.5 & Good & \\
\hline 7/23/91 0:00 & Manual Tape & 49.50 & Transcribed & \\
\hline 7/23/910:05 & Auto FIC & 48.50 & Good & \\
\hline 7/24/91 0:00 & Manual Tape & 49.50 & Transcribed & \\
\hline $7 / 24 / 917: 05$ & Auto FIC & 48.5 & Good & \\
\hline $7 / 25 / 910: 00$ & Manual Tape & 49.50 & Transcribed & \\
\hline $7 / 25 / 911: 03$ & Auto FIC & 48.5 & Good & \\
\hline $7 / 26 / 910: 00$ & Manual Tape & 49.5 & Transcribed & \\
\hline $7 / 26 / 91 \quad 0: 00$ & Manual FIC & 48.50 & Good & \\
\hline 7/27/91 $0: 00$ & Manual Tape & $\mathbf{4 9 . 5 0}$ & Transcribed & \\
\hline $7 / 27 / 910: 00$ & Manual FIC & 48.50 & Good & \\
\hline $7 / 28 / 910: 00$ & Manual Tape & 49.5 & Transcribed & \\
\hline $7 / 28 / 910: 00$ & Manual FIC & 48.5 & Good & \\
\hline $7 / 29 / 910: 00$ & Manual Tape & 49.75 & Transcribed & \\
\hline 7/29/91 0:00 & Manual FIC & $\mathbf{4 8 . 5 0}$ & Good & \\
\hline 7/30/91 0:00 & Manual Tape & 49.75 & Transcribed & \\
\hline $7 / 30 / 91 \quad 7: 02$ & Auto FIC & 48.50 & Good & \\
\hline $7 / 31 / 910: 00$ & Manual Tape & 49.50 & Transcribed & \\
\hline 7/31/91 7:02 & Auto FIC & 48.5 & Good & \\
\hline $8 / 1 / 910: 00$ & Manual Tape & 49.75 & Transcribed & \\
\hline $8 / 1 / 917: 02$ & Auto FIC & 48.5 & Good & \\
\hline $8 / 2 / 910: 00$ & Manual Tape & 49.50 & Transcribed & \\
\hline $8 / 2 / 917: 02$ & Auto FIC & 48.50 & Good & \\
\hline $8 / 3 / 910: 00$ & Manual Tape & 49.50 & Transcribed & \\
\hline $8 / 3 / 917: 02$ & Auto FIC & 48.50 & Good & \\
\hline $8 / 4 / 910: 00$ & Manual Tape & 49.5 & Transcribed & \\
\hline $8 / 4 / 917: 02$ & Auto FIC & 48.5 & Good & \\
\hline $8 / 5 / 910: 00$ & Manual Tape & 49.5 & Transcribed & \\
\hline $8 / 5 / 917: 02$ & Auto FIC & 48.5 & Good & \\
\hline $8 / 6 / 910: 00$ & Manual Tape & 49.50 & Transcribed & \\
\hline $8 / 6 / 917: 02$ & Auto FIC & 48.5 & Good & \\
\hline $8 / 7 / 910: 00$ & Manual Tape & 49.50 & Transcribed & \\
\hline $8 / 7 / 917: 06$ & Auto FIC & 48.50 & Good & \\
\hline $8 / 8 / 910: 00$ & Manual Tape & 49.50 & Transcribed & \\
\hline $8 / 8 / 917: 06$ & Auto FIC & 48.5 & Good & \\
\hline $8 / 9 / 910: 00$ & Manual Tape & 49.5 & Transcribed & \\
\hline $8 / 9 / 917: 04$ & Auto FIC & 48.5 & Good & \\
\hline $8 / 10 / 910: 00$ & Manual Tape & 49.5 & Transcribed & \\
\hline $8 / 10 / 917: 04$ & Auto FIC & 48.50 & Good & \\
\hline
\end{tabular}

Data obtained from WHC Surveillance Analysis Computer System, (PC SACS) July 7, 1995. 


\begin{tabular}{|c|c|c|c|c|}
\hline \multicolumn{5}{|c|}{ 241-AP-102 } \\
\hline \multicolumn{5}{|c|}{ Surface Lavel Data } \\
\hline Date & Type of Measurement & Laval (Inches) & Data Quality & Comments \\
\hline $8 / 11 / 910: 00$ & Manual Tape & 49.50 & Transcribed & \\
\hline $8 / 11 / 917: 04$ & Auto FIC & 48.50 & Good & \\
\hline $8 / 12 / 910: 00$ & Manual Tape & 49.50 & Transcribed & \\
\hline $8 / 12 / 917: 04$ & Auto FIC & 48.5 & Good & . \\
\hline $8 / 13 / 910: 00$ & Manual Tape & 49.5 & Transcribed & \\
\hline $8 / 13 / 917: 04$ & Auto FIC & 48.5 & Good & \\
\hline $8 / 14 / 910: 00$ & Manual Tape & 49.50 & Transcribed & \\
\hline $8 / 14 / 917: 04$ & Auto FIC & 48.5 & Good & \\
\hline $8 / 15 / 910: 00$ & Manual Tape & 49.50 & Transcribed & \\
\hline $8 / 15 / 917: 03$ & Auto FIC & 48.50 & Good & \\
\hline $8 / 16 / 910: 00$ & Manual FIC & 48.50 & Transcribed & \\
\hline $8 / 16 / 910: 00$ & Manual Tape & 49.5 & Transcribed & \\
\hline $8 / 17 / 910: 00$ & Manual Tape & 49.5 & Transcribed & \\
\hline $8 / 17 / 917: 02$ & Auto FIC & 48.5 & Good & \\
\hline $8 / 18 / 910: 00$ & Manual Tape & 49.50 & Transcribed & \\
\hline $8 / 18 / 917: 03$ & Auto FIC & 48.5 & Good & \\
\hline $8 / 19 / 910: 00$ & Manual Tape & 49.50 & Transcribed & \\
\hline $8 / 19 / 917: 02$ & Auto FIC & 48.50 & Good & \\
\hline $8 / 20 / 910: 00$ & Manual Tape & 49.50 & Transcribed & \\
\hline $8 / 20 / 917: 04$ & Auto FIC & 48.50 & Good & \\
\hline $8 / 21 / 910: 00$ & Manual Tape & 49.5 & Transcribed & \\
\hline $8 / 21 / 917: 03$ & Auto FIC & 48.50 & Good & \\
\hline $8 / 22 / 910: 00$ & Manual Tape & 49.5 & Transcribed & \\
\hline $8 / 22 / 917: 03$ & Auto FIC & 48.5 & Good & \\
\hline $8 / 23 / 910: 00$ & Manual Tape & 49.50 & Transcribed & \\
\hline $8 / 23 / 917: 02$ & Auto FIC & 48.5 & Good & \\
\hline $8 / 24 / 910: 00$ & Manual Tape & 49.25 & Transcribed & \\
\hline $8 / 24 / 917: 02$ & Auto FIC & 48.50 & Good & \\
\hline $8 / 25 / 910: 00$ & Manual Tape & 49.5 & Transcribed & \\
\hline $8 / 25 / 917: 02$ & Auto FIC & 48.5 & Good & . \\
\hline $8 / 26 / 910: 00$ & Manual Tape & 49.50 & Transcribed & \\
\hline $8 / 26 / 917: 03$ & Auto FIC & 48.5 & Good & \\
\hline $8 / 27 / 910: 00$ & Manual Tape & 49.5 & Transcribed & \\
\hline $8 / 27 / 917: 03$ & Auto FIC & 48.50 & Good & \\
\hline $8 / 28 / 910: 00$ & Manual Tape & 49.5 & Transcribed & \\
\hline $8 / 28 / 917: 03$ & Auto FIC & 48.50 & Good & \\
\hline $8 / 29 / 910: 00$ & Manual Tape & 49.50 & Transcribed & \\
\hline $8 / 29 / 91 \quad 7: 02$ & Auto FIC & 48.50 & Good & \\
\hline $8 / 30 / 910: 00$ & Manual Tape & 49.50 & Transcribed & \\
\hline $8 / 30 / 916: 02$ & Auto FIC & 48.5 & Good & \\
\hline $8 / 31 / 910: 00$ & Manual Tape & 49.50 & Transcribed & \\
\hline $8 / 31 / 917: 02$ & Auto FIC & 48.5 & Good & \\
\hline $9 / 1 / 910: 00$ & Manual Tape & 49.50 & Transcribed & \\
\hline $9 / 1 / 917: 02$ & Auto FIC & 48.50 & Good & \\
\hline $9 / 2 / 910: 00$ & Manual Tape & 49.50 & Transcribed & \\
\hline 9/2/91 7:02 & Auto FIC & 48.5 & Good & \\
\hline $9 / 3 / 910: 00$ & Manual Tape & 49.5 & Transcribed & \\
\hline $9 / 3 / 917: 02$ & Auto FIC & 48.5 & Good & \\
\hline
\end{tabular}

Data obtained from WHC Surveillance Analysis Computer System, (PC SACS) July 7, 1995. 


\begin{tabular}{|c|c|c|c|c|}
\hline \multicolumn{5}{|c|}{ 241-AP-102 } \\
\hline \multicolumn{5}{|c|}{ Surface Level Data } \\
\hline Date & Type of Measurement & Level (Inches) & Data Quality & Comments \\
\hline $9 / 4 / 910: 00$ & Manual Tape & 49.50 & Transcribed & \\
\hline $9 / 4 / 917: 03$ & Auto FIC & 48.5 & Good & \\
\hline 9/5/91 0:00 & Manual Tape & 49.50 & Transcribed & \\
\hline $9 / 5 / 917: 02$ & Auto FIC & 48.50 & Good & \\
\hline 9/6/91 0:00 & Manual Tape & 49.5 & Transcribed & \\
\hline $9 / 6 / 917: 02$ & Auto FIC & 48.50 & Good & \\
\hline $9 / 7 / 910: 00$ & Manual Tape & 49.50 & Transcribed & \\
\hline $9 / 7 / 917: 02$ & Auto FIC & 48.5 & Good & \\
\hline 9/8/91 0:00 & Manual Tape & 49.50 & Transcribed & \\
\hline 9/8/91 7:02 & Auto FIC & 48.50 & Good & \\
\hline 9/9/91 0:00 & Manual Tape & 49.5 & Transcribed & \\
\hline 9/9/91 7:03 & Auto FIC & 48.50 & Good & \\
\hline $9 / 10 / 910: 00$ & Manual Tape & 48.5 & Transcribed & \\
\hline $9 / 10 / 917: 02$ & Auto FIC & 48.5 & Good & \\
\hline $9 / 11 / 910: 00$ & Manual Tape & 48.50 & Transcribed & \\
\hline $9 / 11 / 917: 03$ & Auto FIC & 48.5 & Good & \\
\hline $9 / 12 / 910: 00$ & Manual Tape & 49 & Transcribed & \\
\hline $9 / 12 / 917: 02$ & Auto FIC & 48.5 & Good & \\
\hline 9/13/91 0:00 & Manual Tape & .49 .50 & Transcribed & \\
\hline $9 / 13 / 917: 02$ & Auto FIC & 48.5 & Good & \\
\hline $9 / 14 / 910: 00$ & Manual Tape & 49.50 & Transcribed & \\
\hline $9 / 14 / 917: 03$ & Auto FIC & 48.5 & Good & \\
\hline $9 / 15 / 910: 00$ & Manual Tape & 49.5 & Transcribed & \\
\hline $9 / 15 / 917: 02$ & Auto FIC & 48.50 & Good & \\
\hline $9 / 16 / 910: 00$ & Manual Tape & 49.5 & Transcribed & \\
\hline $9 / 16 / 917: 03$ & Auto FIC & 48.4 & Good & \\
\hline 9/17/91 0:00 & Manual Tape & 49.50 & Transcribed & \\
\hline $9 / 17 / 917: 03$ & Auto FIC & 48.5 & Good & \\
\hline $9 / 18 / 910: 00$ & Manual Tape & 49.5 & Transcribed & \\
\hline 9/18/91 7:03 & Auto FIC & 48.4 & Good & \\
\hline 9/19/91 0:00 & Manual Tape & 49.50 & Transcribed & \\
\hline $9 / 19 / 917: 03$ & Auto FIC & 48.40 & Good & \\
\hline $9 / 20 / 910: 00$ & Manual Tape & 49.50 & Transcribed & \\
\hline $9 / 20 / 917: 02$ & Auto FIC & 48.4 & Good & \\
\hline $9 / 21 / 910: 00$ & Manual Tape & 49.5 & Transcribed & \\
\hline $9 / 21 / 917: 02$ & Auto FIC & 48.4 & Good & \\
\hline $9 / 22 / 910: 00$ & Manual Tape & 49.5 & Transcribed & \\
\hline $9 / 22 / 917: 03$ & Auto FIC & 48.4 & Good & \\
\hline $9 / 23 / 910: 00$ & Manual Tape & 49.50 & Transcribed & \\
\hline $9 / 23 / 917: 03$ & Auto FIC & 48.40 & Good & \\
\hline $9 / 24 / 910: 00$ & Manual Tape & 49.50 & Transcribed & \\
\hline $9 / 24 / 917: 02$ & Auto FIC & 48.4 & Good & \\
\hline $9 / 25 / 910: 00$ & Manual Tape & 49.5 & Transcribed & \\
\hline $9 / 25 / 917: 03$ & Auto FIC & 48.4 & Good & \\
\hline $9 / 26 / 910: 00$ & Manual Tape & 49.50 & Transcribed & \\
\hline $9 / 26 / 917: 03$ & Auto FIC & 48.4 & Good & \\
\hline $9 / 27 / 910: 00$ & Manual Tape & 49.50 & Transcribed & \\
\hline $9 / 27 / 917: 02$ & Auto FIC & 48.40 & Good & \\
\hline
\end{tabular}

Data obtained from WHC Surveillance Analysis Computer System, (PC SACS) July. 7, 1995. 


\begin{tabular}{|c|c|c|c|c|}
\hline \multicolumn{5}{|c|}{ 241-AP-102 } \\
\hline \multicolumn{5}{|c|}{ Surface Level Data } \\
\hline Date & Type of Measurement & Level (Inches) & Data Quality & Comments \\
\hline $9 / 28 / 910: 00$ & Manual Tape & 49.5 & Transcribed & \\
\hline $9 / 28 / 917: 02$ & Auto FIC & 48.4 & Good & \\
\hline 9/29/910:00 & Manual Tape & 49.50 & Transcribed & \\
\hline $9 / 29 / 917: 03$ & Auto FIC & 48.4 & Good & \\
\hline $9 / 30 / 910: 00$ & Manual Tape & 49.50 & Transcribed & \\
\hline $9 / 30 / 917: 03$ & Auto FIC & 48.4 & Good & \\
\hline $10 / 1 / 910: 00$ & Manual Tape & 49.5 & Transcribed & \\
\hline $10 / 1 / 917: 02$ & Auto FIC & 48.40 & Good & \\
\hline $10 / 2 / 910: 00$ & Manual Tape & 49.5 & Transcribed & \\
\hline $10 / 2 / 917: 02$ & Auto FIC & 48.4 & Good & \\
\hline $10 / 3 / 910: 00$ & Manual Tape & 49.50 & Transcribed & \\
\hline $10 / 3 / 917: 02$ & Auto FIC & 48.4 & Good & \\
\hline $10 / 4 / 910: 00$ & Manual Tape & 49.50 & Transcribed & \\
\hline $10 / 4 / 917: 03$ & Auto FIC & 48.4 & Good & \\
\hline 10/5/91 0:00 & Manual Tape & 49.50 & Transcribed & \\
\hline $10 / 5 / 910: 00$ & Manual FIC & 48.4 & Good & \\
\hline $10 / 6 / 910: 00$ & Manual Tape & 49.5 & Transcribed & \\
\hline $10 / 6 / 917: 03$ & Auto FIC & 48.40 & Good & \\
\hline $10 / 7 / 910: 00$ & Manual Tape & 49.5 & Transcribed & \\
\hline $10 / 7 / 917: 02$ & Auto FIC & 48.4 & Good & \\
\hline 10/8/910:00 & Manual Tape & 49.50 & Transcribed & \\
\hline $10 / 8 / 917: 02$ & Auto FIC & 48.4 & Good & \\
\hline $10 / 9 / 910: 00$ & Manual Tape & 49.50 & Transcribed & \\
\hline $10 / 9 / 917: 02$ & Auto FIC & 48.4 & Good & \\
\hline $10 / 10 / 910: 00$ & Manual Tape & 49.5 & Transcribed & \\
\hline $10 / 10 / 917: 02$ & Auto FIC & 48.40 & Good & \\
\hline $10 / 11 / 910: 00$ & Manual Tape & 49.50 & Transcribed & \\
\hline $10 / 11 / 917: 02$ & Auto FIC & 48.4 & Good & \\
\hline $10 / 12 / 910: 00$ & Manual Tape & 49.50 & Transcribed & \\
\hline $10 / 12 / 917: 02$ & Auto FIC & 48.4 & Good & \\
\hline $10 / 13 / 910: 00$ & Manual Tape & 49.5 & Transçribed & \\
\hline $10 / 13 / 917: 03$ & Auto FIC & 48.4 & Good & \\
\hline $10 / 14 / 910: 00$ & Manual Tape & 49.5 & Transcribed & \\
\hline $10 / 14 / 917: 02$ & Auto FIC & 48.40 & Good & \\
\hline $10 / 15 / 910: 00$ & Manual Tape & 49.50 & Transcribed & \\
\hline $10 / 15 / 917: 02$ & Auto FIC & 48.4 & Good & \\
\hline $10 / 16 / 910: 00$ & Manual Tape & 49.50 & Transcribed & \\
\hline $10 / 16 / 917: 03$ & Auto FIC & 48.4 & Good & \\
\hline $10 / 17 / 910: 00$ & Manual Tape & 49.50 & Transcribed & \\
\hline $10 / 17 / 917: 05$ & Auto FIC & 48.40 & Good & \\
\hline $10 / 18 / 910: 00$ & Manual Tape & 49.50 & Transcribed & \\
\hline $10 / 18 / 917: 06$ & Auto FIC & 48.40 & Good & \\
\hline $10 / 19 / 910: 00$ & Manual Tape & 49.5 & Transcribed & \\
\hline $10 / 19 / 917: 06$ & Auto FIC & 48.4 & Good & \\
\hline $10 / 20 / 910: 00$ & Manual Tape & 49.5 & Transcribed & \\
\hline $10 / 20 / 917: 02$ & Auto FIC & 48.4 & Good & \\
\hline $10 / 21 / 910: 00$ & Manual Tape & 49.50 & Transcribed & \\
\hline $10 / 21 / 917: 02$ & Auto FIC & 48.40 & Good & \\
\hline
\end{tabular}

Data obtained from WHC Surveillance Analysis Computer System, (PC SACS) July 7, 1995. 


\begin{tabular}{|c|c|c|c|c|}
\hline \multicolumn{5}{|c|}{ 241-AP-102 } \\
\hline Date & Type of Measurement & Level (Inches) & Data Quality & Comments \\
\hline$\frac{\text { Date }}{10 / 22 / 910: 00}$ & Manual Tape & 49.50 & Transcribed & \\
\hline $\begin{array}{l}10 / 22 / 910: 00 \\
10 / 22 / 917: 03\end{array}$ & Auto FIC & 48.40 & Good & \\
\hline 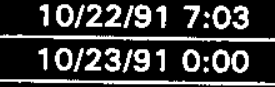 & Manual Tape & 49.50 & Transcribed & \\
\hline$\frac{10 / 23 / 91}{0: 00}$ & Auto FIC & 48.4 & Good & \\
\hline $\begin{array}{ll}10 / 23 / 91 & 7: 02 \\
10 / 24 / 91 & 0: 00\end{array}$ & Manual Tape & 49.5 & Transcribed & \\
\hline $\begin{array}{ll}10 / 24 / 91 & 0: 00 \\
10 / 24 / 91 & 7: 02 \\
\end{array}$ & Auto FIC & 48.4 & Good & \\
\hline$\frac{10 / 24 / 917: 02}{10 / 25 / 910: 00}$ & Manual Tape & 49.5 & Transcribed & \\
\hline $\begin{array}{l}10 / 25 / 910: 00 \\
10 / 25 / 917: 03\end{array}$ & Auto FIC & 48.40 & Good & \\
\hline 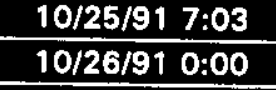 & Manual Tape & 49.50 & Transcribed & \\
\hline $\begin{array}{ll}10 / 26 / 91 & 0: 00 \\
10 / 26 / 91 & 7: 03\end{array}$ & Auto FIC & 48.30 & Good & \\
\hline $10 / 27 / 910: 00$ & Manual Tape & 49.50 & Transcribed & \\
\hline $10 / 27 / 917: 02$ & Auto FIC & 48.3 & Good & \\
\hline $10 / 28 / 910: 00$ & Manual Tape & 49.5 & Transcribed & \\
\hline $10 / 28 / 917: 02$ & Auto FIC & 48.3 & Good & \\
\hline $10 / 29 / 910: 00$ & Manual Tape & 49.50 & Transcribed & \\
\hline $10 / 29 / 917: 03$ & Auto FIC & 48.30 & Good & \\
\hline $10 / 30 / 910: 00$ & Manual Tape & 49.25 & Transcribed & \\
\hline $10 / 30 / 917: 03$ & Auto FIC & 48.30 & Good & \\
\hline $10 / 31 / 910: 00$ & Manual Tape & 49.25 & Transcribed & \\
\hline $10 / 31 / 917: 03$ & Auto FIC & 48.3 & Good & \\
\hline $11 / 1 / 910: 00$ & Manual Tape & 49.50 & Transcribed & \\
\hline $11 / 1 / 917: 02$ & Auto FIC & 48.3 & Good & \\
\hline $11 / 2 / 910: 00$ & Manual Tape & 49.25 & Transcribed & \\
\hline $11 / 2 / 917: 03$ & Auto FIC & 48.30 & Good & \\
\hline $11 / 3 / 910: 00$ & Manual Tape & 49.5 & Transcribed & \\
\hline $11 / 3 / 917: 03$ & Auto FIC & 48.30 & Good & \\
\hline $11 / 4 / 910: 00$ & Manual Tape & 49.50 & Transcribed & \\
\hline $11 / 4 / 917: 02$ & Auto FIC & 48.3 & Good & \\
\hline 11/5/910:00 & Manual Tape & 49.50 & Transcribed & \\
\hline $11 / 5 / 917: 03$ & Auto FIC & 48.3 & Good & \\
\hline $11 / 6 / 910: 00$ & Manual Tape & 49.25 & Transcribed & \\
\hline $11 / 6 / 917: 03$ & Auto FIC & 48.30 & Good & \\
\hline $11 / 7 / 910: 00$ & Manual Tape & 49.5 & Transcribed & \\
\hline $11 / 7 / 917: 02$ & Auto FIC & 48.40 & Good & \\
\hline 11/8/91 0:00 & Manual Tape & 49.5 & Transcribed & \\
\hline $11 / 8 / 917: 03$ & Auto FIC & 48.4 & Good & \\
\hline $11 / 9 / 910: 00$ & Manual Tape & 49.50 & Transcribed & \\
\hline 11/9/91 7:02 & Auto FIC & 48.4 & Good & \\
\hline 11/10/910:00 & Manual Tape & 49.50 & Transcribed & \\
\hline $11 / 10 / 917: 03$ & Auto FIC & 48.30 & Good & \\
\hline $11 / 11 / 910: 00$ & Manual Tape & 49.5 & Transcribed & \\
\hline $11 / 11 / 916: 03$ & Auto FIC & 48.30 & Good & \\
\hline $11 / 12 / 910: 00$ & Manual Tape & 49.50 & Transcribed & \\
\hline $11 / 12 / 917: 03$ & Auto FIC & 48.3 & Good & \\
\hline $11 / 13 / 910: 00$ & Manual Tape & 49.50 & Transcribed & \\
\hline $11 / 13 / 917: 03$ & Auto FIC & 48.3 & Good & \\
\hline $11 / 14 / 910: 00$ & Manual Tape & 49.5 & Transcribed & \\
\hline $11 / 14 / 917: 03$ & Auto FIC & 48.30 & Good & \\
\hline
\end{tabular}

Data obtained from WHC Surveillance Analysis Computer System, (PC SACS) July 7, 1995. 


\begin{tabular}{|c|c|c|c|c|}
\hline \multicolumn{5}{|c|}{ 241-AP-102 } \\
\hline \multicolumn{5}{|c|}{ Surface Level Data } \\
\hline Date & Type of Messurement & Leval (Inches) & Data Quality & Comments \\
\hline $11 / 15 / 910: 00$ & Manual Tape & 49.50 & Transcribed & \\
\hline $11 / 15 / 917: 03$ & Auto FIC & 48.60 & Good & \\
\hline $11 / 16 / 910: 00$ & Manual Tape & 49.50 & Transcribed & \\
\hline $11 / 16 / 917: 03$ & Auto FIC & 48.5 & Good & \\
\hline $11 / 17 / 910: 00$ & Manual Tape & 49.5 & Transcribed & \\
\hline $11 / 17 / 917: 02$ & Auto FIC & 48.5 & Good & \\
\hline $11 / 18 / 910: 00$ & Manual Tape & 49.50 & Transcribed & \\
\hline $11 / 18 / 917: 03$ & Auto FIC & 48.60 & Good & \\
\hline $11 / 19 / 910: 00$ & Manual Tape & 49.50 & Transcribed & \\
\hline $11 / 19 / 917: 03$ & Auto FIC & 48.60 & Good & \\
\hline $11 / 20 / 910: 00$ & Manual Tape & 49.50 & Transcribed & \\
\hline $11 / 20 / 917: 03$ & Auto FIC & 48.5 & Good & \\
\hline $11 / 21 / 910: 00$ & Manual Tape & 49.5 & Transcribed & \\
\hline $11 / 21 / 917: 02$ & Auto FIC & 48.5 & Good & \\
\hline $11 / 22 / 910: 00$ & Manual Tape & 49.5 & Transcribed & \\
\hline $11 / 22 / 917: 03$ & Auto FIC & 48.50 & Good & \\
\hline $11 / 23 / 910: 00$ & Manual Tape & 49.50 & Transcribed & \\
\hline $11 / 23 / 917: 02$ & Auto FIC & 48.50 & Good & \\
\hline $11 / 24 / 910: 00$ & Manual Tape & 49.50 & Transcribed & \\
\hline $11 / 24 / 917: 03$ & Auto FIC & 48.5 & Good & \\
\hline $11 / 25 / 910: 00$ & Manual Tape & 49.5 & Transcribed & \\
\hline $11 / 25 / 917: 03$ & Auto FIC & 48.5 & Good & \\
\hline $11 / 26 / 910: 00$ & Manual Tape & 49.50 & Transcribed & \\
\hline $11 / 26 / 910: 00$ & Manual FIC & 48.4 & Good & \\
\hline $11 / 27 / 910: 00$ & Manual Tape & 49.50 & Transcribed & \\
\hline $11 / 27 / 917: 03$ & Auto FIC & 48.50 & Good & \\
\hline $11 / 28 / 910: 00$ & Manual Tape & 49.50 & Transcribed & \\
\hline $11 / 28 / 917: 03$ & Auto FIC & 48.50 & Good & \\
\hline $11 / 29 / 910: 00$ & Manual Tape & 49.5 & Transcribed & \\
\hline $11 / 29 / 917: 03$ & Auto FIC & 48.5 & Good & \\
\hline $11 / 30 / 910: 00$ & Manual Tape & 49.5 & Transcribed & \\
\hline $11 / 30 / 917: 03$ & Auto FIC & 48.5 & Good & \\
\hline $12 / 1 / 910: 00$ & Manual Tape & 49.50 & Transcribed & \\
\hline 12/1/91 7:03 & Auto FIC & 48.50 & Good & \\
\hline 12/2/910:00 & Manual Tape & 49.50 & Transcribed & \\
\hline $12 / 2 / 917: 03$ & Auto FIC & 48.50 & Good & \\
\hline $12 / 3 / 910: 00$ & Manual Tape & 49.5 & Transcribed & \\
\hline $12 / 3 / 917: 03$ & Auto FIC & 48.5 & Good & \\
\hline $12 / 4 / 910: 00$ & Manual Tape & 49.50 & Transcribed & \\
\hline $12 / 4 / 917: 03$ & Auto FIC & 48.5 & Good & \\
\hline $12 / 5 / 910: 00$ & Manual Tape & 49.50 & Transcribed & \\
\hline $12 / 5 / 917: 03$ & Auto FIC & 48.50 & Good & \\
\hline $12 / 6 / 910: 00$ & Manual Tape & . 49.50 & Transcribed & \\
\hline $12 / 6 / 917: 04$ & Auto FIC & 48.50 & Good & \\
\hline $12 / 7 / 910: 00$ & Manual Tape & 49.75 & Transcribed & \\
\hline $12 / 7 / 917: 03$ & Auto FIC & 48.5 & Good & \\
\hline $12 / 8 / 910: 00$ & Manual Tape & 49.5 & Transcribed & \\
\hline $12 / 8 / 917: 03$ & Auto FIC & 48.5 & Good & \\
\hline
\end{tabular}

Data obtained from WHC Surveillance Analysis Computer System, (PC SACS) July 7, 1995. 


\begin{tabular}{|c|c|c|c|c|}
\hline \multicolumn{4}{|c|}{ 241-AP-102 } & \\
\hline$\frac{\text { Date }}{12 / 9 / 910: 00}$ & Manual Tape & 49.50 & Transcribed & \\
\hline $\begin{array}{l}12 / 9 / 910: 00 \\
12 / 9 / 917: 03\end{array}$ & Auto FIC & 48.60 & Good & \\
\hline $12 / 10 / 910: 00$ & Manual Tape & 49.50 & Transcribed & \\
\hline $12 / 10 / 917: 03$ & Auto FIC & 48.50 & Good & \\
\hline $12 / 11 / 910: 00$ & Manual Tape & 49,5 & Transcribed & \\
\hline $12 / 11 / 917: 02$ & Auto FIC & 48.5 & Good & \\
\hline $12 / 12 / 910: 00$ & Manual Tape & 49.50 & Transcribed & \\
\hline $12 / 12 / 917: 03$ & Auto FIC & 48.5 & Good & \\
\hline $12 / 13 / 910: 00$ & Manual Tape & 49.50 & Transcribed & \\
\hline $12 / 13 / 917: 05$ & Auto FIC & 48.50 & Good & \\
\hline $12 / 14 / 910: 00$ & Manual Tape & 49.50 & Transcribed & \\
\hline $12 / 14 / 917: 02$ & Auto FIC & 48.50 & Good & \\
\hline $12 / 15 / 910: 00$ & Manual Tape & 49.50 & Transcribed & \\
\hline $12 / 15 / 917: 02$ & Auto FIC & 48.4 & Good & \\
\hline $12 / 16 / 910: 00$ & Manual Tape & 49.5 & Transcribed & \\
\hline $12 / 16 / 917: 03$ & Auto FIC & 48.5 & Good & \\
\hline $12 / 17 / 910: 00$ & Manual Tape & 49.5 & Transcribed & \\
\hline $12 / 17 / 917: 06$ & Auto FIC & 48.50 & Good & \\
\hline $12 / 18 / 910: 00$ & Manual Tape & 49.50 & Transcribed & \\
\hline $12 / 18 / 917: 03$ & Auto FIC & 48.50 & Good & \\
\hline $12 / 19 / 910: 00$ & Manual Tape & 49.50 & Transcribed & \\
\hline $12 / 19 / 917: 03$ & Auto FIC & 48,5 & Good & \\
\hline $12 / 20 / 910: 00$ & Manual Tape & 49.50 & Transcribed & \\
\hline $12 / 20 / 917: 02$ & Auto FIC & 48.5 & Good & \\
\hline $12 / 21 / 910: 00$ & Manual Tape & 49.5 & Transcribed & \\
\hline $12 / 21 / 91 \quad 7: 03$ & Auto FIC & 48.50 & Good & \\
\hline $12 / 22 / 910: 00$ & Manual Tape & 49.50 & Transcribed & \\
\hline $12 / 22 / 917: 05$ & Auto FIC & 48.50 & Good & \\
\hline $12 / 23 / 910: 00$ & Manual Tape & 49.50 & Transcribed & \\
\hline $12 / 23 / 917: 06$ & Auto FIC & 48.5 & Good & \\
\hline $12 / 24 / 910: 00$ & Manual Tape & 49.5 & Transcribed & \\
\hline $12 / 24 / 917: 03$ & Auto FIC & 48.4 & Good & \\
\hline $12 / 25 / 910: 00$ & Manual Tape & 49.5 & Transcribed & \\
\hline $12 / 25 / 917: 03$ & Auto FIC & 48.50 & Good & \\
\hline $12 / 26 / 910: 00$ & Manual Tape & 49.50 & Transcribed & \\
\hline $12 / 26 / 917: 03$ & Auto FIC & 48.50 & Good & \\
\hline $12 / 27 / 910: 00$ & Manual Tape & 49.50 & Transcribed & \\
\hline $12 / 27 / 917: 03$ & Auto FIC & 48.5 & Good & \\
\hline $12 / 28 / 910: 00$ & Manual Tape & 49.50 & Transcribed & \\
\hline $12 / 28 / 917: 02$ & Auto FIC & 48.4 & Good & \\
\hline $12 / 29 / 910: 00$ & Manual Tape & 49.25 & Transcribed & \\
\hline 12/29/91 7:03 & Auto FIC & 48.40 & Good & \\
\hline $12 / 30 / 910: 00$ & Manual Tape & 49.5 & Transcribed & \\
\hline $12 / 30 / 917: 03$ & Auto FIC & 48.50 & Good & \\
\hline $12 / 31 / 910: 00$ & Manual Tape & 49.50 & Transcribed & \\
\hline $12 / 31 / 917: 03$ & Auto FIC & 48.50 & Good & \\
\hline 1/1/92 0:00 & Manual Tape & 49.50 & Transcribed & \\
\hline 1/1/92 7:03 & Auto FIC & 48.4 & Good & \\
\hline
\end{tabular}

Data obtained from WHC Surveillance Analysis Computer System, (PC SACS) July 7, 1995. 


\begin{tabular}{|c|c|c|c|c|}
\hline \multicolumn{5}{|c|}{ 241-AP-102 } \\
\hline \multicolumn{5}{|c|}{ Surface Leval Data } \\
\hline Date & Type of Measurement & Level (Inchas) & Data Quality & Comments \\
\hline $1 / 2 / 920: 00$ & Manual Tape & 49.25 & Transcribed & \\
\hline $1 / 2 / 927: 02$ & Auto FIC & 48.4 & Good & \\
\hline 1/3/92 0:00 & Manual Tape & 49.50 & Transcribed & \\
\hline $1 / 3 / 927: 04$ & Auto FIC & 48.40 & Good & \\
\hline $1 / 4 / 920: \overline{00}$ & Manual Tape & 49.50 & Transcribed & \\
\hline $1 / 4 / 927: 03$ & Auto FIC & 48.40 & Good & \\
\hline 1/5/92 7:03 & Auto FIC & 48.4 & Good & \\
\hline 1/6/92 0:00 & Manual Tape & 49.50 & Transcribed & \\
\hline 1/6/92 7:03 & Auto FIC & 48.5 & Good & \\
\hline 1/7/92 0:00 & Manual Tape & 49.5 & Transcribed & \\
\hline 1/7/92 7:04 & Auto FIC & 48.40 & Good & \\
\hline 1/8/92 0:00 & Manual Tape & 49.5 & Transcribed & \\
\hline $1 / 8 / 927: 03$ & Auto FIC & 48.40 & Good & \\
\hline 1/9/92 0:00 & Manual Tape & 49.50 & Transcribed & \\
\hline $1 / 9 / 927: 03$ & Auto FIC & 48.40 & Good & \\
\hline $1 / 10 / 920: 00$ & Manual Tape & 49.50 & Transcribad & \\
\hline $1 / 10 / 927: 04$ & Auto FIC & 48.5 & Good & \\
\hline $1 / 11 / 920: 00$ & Manual Tape & 49.5 & Transcribed & \\
\hline $1 / 11 / 927: 04$ & Auto FIC & 48.4 & Good & \\
\hline $1 / 12 / 920: 00$ & Manual FIC & 48.4 & Good & \\
\hline $1 / 13 / 920: 00$ & Manual FIC & 48.50 & Good & \\
\hline $1 / 14 / 920: 00$ & Manual Tape & 49.50 & Transcribed & \\
\hline $1 / 14 / 920: 00$ & Manual FIC & 48.50 & Good & \\
\hline $1 / 15 / 920: 00$ & Manual Tape & 49.50 & Transcribed & \\
\hline $1 / 15 / 927: 03$ & Auto FIC & 48.40 & Good & \\
\hline $1 / 16 / 920: 00$ & Manual Tape & 49.50 & Transcribed & \\
\hline $1 / 16 / 927: 04$ & Auto FIC & 48.40 & Good & \\
\hline $1 / 17 / 920: 00$ & Manual Tape & 49.5 & Transcribed & \\
\hline $1 / 17 / 927: 04$ & Auto FIC & 48.4 & Good & \\
\hline $1 / 18 / 927: 04$ & Auto FIC & 48.4 & Good & \\
\hline $1 / 19 / 927: 03$ & Auto FIC & 48.4 & Good & \\
\hline $1 / 20 / 920: 00$ & Manual Tape & 48.5 & Transcribed & \\
\hline $1 / 20 / 927: 03$ & Auto FIC & 48.50 & Good & \\
\hline $1 / 21 / 927: 03$ & Auto FIC & 48.40 & Good & \\
\hline $1 / 22 / 920: 00$ & Manual Tape & 49.25 & Transcribed & \\
\hline $1 / 22 / 927: 06$ & Auto FIC & 48.40 & Good & \\
\hline $1 / 23 / 920: 00$ & Manual Tape & 49.25 & Transcribed & \\
\hline $1 / 23 / 927: 03$ & Auto FIC & 48.4 & Good & \\
\hline $1 / 24 / 920: 00$ & Manual Tape & 49.5 & Transcribed & \\
\hline $1 / 24 / 92 \quad 7: 02$ & Auto FIC & 48.4 & Good & \\
\hline 1/25/92 0:00 & Manual Tape & 49.50 & Transcribed & \\
\hline $1 / 25 / 927: 03$ & Auto FIC & 48.4 & Good & \\
\hline $1 / 26 / 920: 00$ & Manual Tape & 49.50 & Transcribed & \\
\hline 1/26/92 6:03 & Auto FIC & 48.40 & Good & \\
\hline $1 / 27 / 920: 00$ & Manual Tape & 49.50 & Transcribed & \\
\hline $1 / 27 / 927: 02$ & Auto FIC & 48.40 & Good & \\
\hline $1 / 28 / 920: 00$ & Manual Tape & 49.5 & Transcribed & \\
\hline $1 / 28 / 927: 03$ & Auto FIC & 48.5 & Good & \\
\hline
\end{tabular}

Data obtained from WHC Surveillance Analysis Computer System, (PC SACS) July 7, 1995. 


\begin{tabular}{|c|c|c|c|c|}
\hline \multicolumn{5}{|c|}{ 241-AP-102 } \\
\hline \multicolumn{5}{|c|}{ Surface Level Data } \\
\hline Date & Type of Measurement & Level (Inches) & Data Quality & Comments \\
\hline $1 / 29 / 920: 00$ & Manual Tape & 49.5 & Transcribed & \\
\hline $1 / 29 / 927: 03$ & Auto FIC & 48.4 & Good & \\
\hline $1 / 30 / 920: 00$ & Manual Tape & 49.50 & Transcribed & \\
\hline $1 / 30 / 927: 03$ & Auto FIC & 48.40 & Good & \\
\hline $1 / 31 / 920: 00$ & Manual Tape & 49.50 & Transcribed & \\
\hline $1 / 31 / 927: 03$ & Auto FIC & 48.40 & Good & \\
\hline $2 / 1 / 920: 00$ & Manual Tape & 49.50 & Transcribed & \\
\hline 2/1/92 7:02 & Auto FIC & 48.4 & Good & \\
\hline $2 / 2 / 920: 00$ & Manual Tape & 49.50 & Transcribed & \\
\hline $2 / 2 / 927: 03$ & Auto FIC & 48.4 & Good & \\
\hline $2 / 3 / 920: 00$ & Manual Tape & 49.5 & Transcribed & \\
\hline 2/3/92 7:03 & Auto FIC & 48.40 & Good & \\
\hline $2 / 4 / 920: 00$ & Manual Tape & 49.50 & Transcribed & \\
\hline $2 / 4 / 927: 03$ & Auto FIC & 48.4 & Good & \\
\hline $2 / 5 / 920: 00$ & Manual Tape & 49.5 & Transcribed & \\
\hline $2 / 5 / 927: 02$ & Auto FIC & 48.4 & Good & \\
\hline $2 / 6 / 920: 00$ & Manual Tape & 49.50 & Transcribed & \\
\hline $2 / 6 / 920: 00$ & Manual FIC & 48.40 & Good & \\
\hline $2 / 7 / 920: 00$ & Manual Tape & 49.25 & Transcribed & \\
\hline $2 / 7 / 92 \quad 0: 00$ & Manual FIC & 48.4 & Good & \\
\hline $2 / 8 / 920: 00$ & Manual Tape & 49.5 & Transcribed & \\
\hline $2 / 8 / 927: 04$ & Auto FIC & 48.40 & Good & \\
\hline 2/9/92 0:00 & Manual Tape & 49.50 & Transcribed & \\
\hline 2/9/92 7:03 & Auto FIC & 48.40 & Good & \\
\hline $2 / 10 / 920: 00$ & Manual Tape & 49.50 & Transcribed & \\
\hline $2 / 10 / 927: 04$ & Auto FIC & 48.40 & Good & \\
\hline $2 / 11 / 920: 00$ & Manual Tape & 49.50 & Transcribed & \\
\hline $2 / 11 / 927: 03$ & Auto FIC & 48.3 & Good & \\
\hline $2 / 12 / 920: 00$ & Manual Tape & 49.50 & Transcribed & \\
\hline 2/12/92 7:03 & Auto FIC & 48.4 & Good & \\
\hline $2 / 13 / 920: 00$ & Manual Tape & 49.5 & Transcribed & \\
\hline $2 / 13 / 927: 03$ & Auto FIC & 48.30 & Good & \\
\hline $2 / 14 / 920: 00$ & Manual Tape & 49.5 & Transcribed & \\
\hline $2 / 14 / 927: 03$ & Auto FIC & 48.30 & Good & \\
\hline $2 / 15 / 920: 00$ & Manual Tape & 49.50 & Transcribed & \\
\hline $2 / 15 / 927: 03$ & Auto FIC & 48.30 & Good & \\
\hline $2 / 16 / 920: 00$ & Manual Tape & 49.25 & Transcribed & \\
\hline $2 / 16 / 927: 03$ & Auto FIC & 48.3 & Good & \\
\hline $2 / 17 / 920: 00$ & Manual Tape & 49.25 & Transcribed & \\
\hline $2 / 17 / 927: 03$ & Auto FIC & 48.4 & Good & \\
\hline $2 / 18 / 920: 00$ & Manual Tape & 49.25 & Transcribed & \\
\hline $2 / 18 / 927: 03$ & Auto FIC & 48.40 & Good & \\
\hline $2 / 19 / 920: 00$ & Manual Tape & 49.5 & Transcribed & \\
\hline $2 / 19 / 927: 03$ & Auto FIC & 48.3 & Good & \\
\hline $2 / 20 / 920: 00$ & Manual Tape & 49.5 & Transcribed & \\
\hline $2 / 20 / 927: 03$ & Auto FIC & 48.6 & Good & \\
\hline $2 / 21 / 920: 00$ & Manual Tape & 49.25 & Transcribed & \\
\hline $2 / 21 / 927: 03$ & Auto FIC & 48.40 & Good & \\
\hline
\end{tabular}

Data obtained from WHC Surveillance Analysis Computer System, (PC SACS) July 7, 1995. 


\begin{tabular}{|c|c|c|c|c|}
\hline \multicolumn{5}{|c|}{ 241-AP-102 } \\
\hline \multicolumn{5}{|c|}{ Surface Level Data } \\
\hline Date & Type of Measurement & Level (Inches) & Data Quality & Comments \\
\hline $2 / 22 / 920: 00$ & Manual Tape & 49.5 & Transcribed & $\cdot$ \\
\hline $2 / 22 / 927: 03$ & Auto FIC & 48.40 & Good & \\
\hline $2 / 23 / 920: 00$ & Manual Tape & 49.25 & Transcribed & \\
\hline $2 / 23 / 927: 03$ & Auto FIC & 48.30 & Good & \\
\hline $2 / 24 / 920: 00$ & Manual Tape & 49.25 & Transcribed & \\
\hline $2 / 24 / 927: 03$ & Auto FIC & 48.3 & Good & \\
\hline $2 / 25 / 920: 00$ & Manual Tape & 49.25 & Transcribed & \\
\hline $2 / 25 / 927: 04$ & Auto FIC & 48.3 & Good & \\
\hline $2 / 26 / 92 \quad 0: 00$ & Manual Tape & 49.25 & Transcribed & \\
\hline $2 / 26 / 927: 03$ & Auto FIC & 48.30 & Good & \\
\hline $2 / 27 / 920: 00$ & Manual Tape & 49.25 & Transcribed & \\
\hline $2 / 27 / 927: 04$ & Auto FIC & 48.3 & Good & \\
\hline $2 / 28 / 920: 00$ & Manual Tape & 49.25 & Transcribed & \\
\hline $2 / 28 / 927: 04$ & Auto FIC & 48.3 & Good & \\
\hline $2 / 29 / 920: 00$ & Manual Tape & 49.25 & Transcribed & \\
\hline $2 / 29 / 927: 03$ & Auto FIC & 48.30 & Good & \\
\hline $3 / 1 / 920: 00$ & Manual Tape & 49.25 & Transcribed & \\
\hline $3 / 1 / 927: 03$ & Auto FIC & 48.30 & Good & \\
\hline $3 / 2 / 920: 00$ & Manual Tape & 49.5 & Transcribed & \\
\hline $3 / 2 / 927: 03$ & Auto FIC & 48.3 & Good & \\
\hline $3 / 3 / 920: 00$ & Manual Tape & 49.50 & Transcribed & \\
\hline $3 / 3 / 927: 04$ & Auto FIC & 48.3 & Good & \\
\hline $3 / 4 / 920: 00$ & Manual Tape & 49.50 & Transcribed & \\
\hline $3 / 4 / 927: 04$ & Auto FIC & 48.30 & Good & \\
\hline $3 / 5 / 920: 00$ & Manual Tape & 49.5 & Transcribed & \\
\hline $3 / 5 / 927: 03$ & Auto FIC & 48.30 & Good & \\
\hline $3 / 6 / 920: 00$ & Manual Tape & 49.50 & Transcribed & \\
\hline $3 / 6 / 927: 03$ & Auto FIC & 48.3 & Good & \\
\hline $3 / 7 / 920: 00$ & Manual Tape & 49.5 & Transcribed & \\
\hline $3 / 7 / 92$ 7:02 & Auto FIC & 48.4 & Good & \\
\hline $3 / 8 / 920: 00$ & Manual Tape & 49.5 & Transcribed & \\
\hline $3 / 8 / 927: 02$ & Auto FIC & 48.30 & Good & \\
\hline $3 / 9 / 920: 00$ & Manual Tape & 49.5 & Transcribed & \\
\hline $3 / 9 / 927: 02$ & Auto FIC & 48.30 & Good & \\
\hline $3 / 10 / 920: 00$ & Manual Tape & 49.25 & Transcribed & \\
\hline $3 / 10 / 927: 03$ & Auto FIC & 48.3 & Good & \\
\hline $3 / 11 / 920: 00$ & Manual Tape & 49.50 & Transcribed & \\
\hline $3 / 11 / 927: 03$ & Auto FIC & 48.3 & Good & \\
\hline $3 / 12 / 920: 00$ & Manual Tape & 49.50 & Transcribed & \\
\hline $3 / 12 / 927: 03$ & Auto FIC & 48.30 & Good & \\
\hline $3 / 13 / 920: 00$ & Manual Tape & 49.50 & Transcribed & \\
\hline $3 / 13 / 927: 03$ & Auto FIC & 48.30 & Good & \\
\hline $3 / 14 / 920: 00$ & Manual Tape & 49.5 & Transcribed & \\
\hline $3 / 14 / 927: 03$ & Auto FIC & 48.3 & Good & \\
\hline $3 / 15 / 920: 00$ & Manual Tape & 49.5 & Transcribed & \\
\hline $3 / 15 / 927: 06$ & Auto FIC & 48.3 & Good & \\
\hline $3 / 16 / 920: 00$ & Manual Tape & 49.50 & Transcribed & \\
\hline $3 / 16 / 927: 03$ & Auto FIC & 48.3 & Good & \\
\hline
\end{tabular}

Data obtained from WHC Surveillance Analysis Computer System, (PC SACS) July 7, 1995. 


\begin{tabular}{|c|c|c|c|c|}
\hline \multicolumn{5}{|c|}{ 241-AP-102 } \\
\hline \multicolumn{5}{|c|}{ Surface Level Data } \\
\hline Date & Type of Measurement & Level (Inches) & Data Quality & Comments \\
\hline $3 / 17 / 920: 00$ & Manual Tape & 49.50 & Transcribed & \\
\hline $3 / 17 / 927: 03$ & Auto FIC & 48.30 & Good & \\
\hline $3 / 18 / 920: 00$ & Manual Tape & 49.50 & Transcribed & \\
\hline $3 / 18 / 927: 03$ & Auto FIC & 48.30 & Good & \\
\hline $3 / 19 / 920: 00$ & Manual Tape & 49.50 & Transcribed & \\
\hline $3 / 19 / 926: 02$ & Auto FIC & 48.3 & Good & \\
\hline $3 / 20 / 920: 00$ & Manual Tape & 49.50 & Transcribed & \\
\hline $3 / 20 / 927: 02$ & Auto FIC & 48.3 & Good & \\
\hline $3 / 21 / 920: 00$ & Manual Tape & 49.25 & Transcribed & \\
\hline $3 / 21 / 927: 03$ & Auto FIC & 48.40 & Good & \\
\hline $3 / 22 / 92 \quad 0: 00$ & Manual Tape & 49.50 & Transcribed & \\
\hline $3 / 22 / 927: 02$ & Auto FIC & 48.3 & Good & \\
\hline $3 / 23 / 920: 00$ & Manual Tape & 49.5 & Transcribed & \\
\hline $3 / 23 / 927: 02$ & Auto FIC & 48.3 & Good & \\
\hline $3 / 24 / 920: 00$ & Manual Tape & 49.25 & Transcribed & \\
\hline $3 / 24 / 927: 02$ & Auto FIC & 48.30 & Good & \\
\hline $3 / 25 / 920: 00$ & Manual Tape & 49.50 & Transcribed & \\
\hline $3 / 25 / 927: 03$ & Auto FIC & 48.30 & Good & \\
\hline $3 / 26 / 927: 03$ & Auto FIC & 48.3 & Good & \\
\hline $3 / 27 / 927: 02$ & Auto FIC & 48.7 & Good & \\
\hline $3 / 28 / 92 \quad 0: 00$ & Manual Tape & 49.50 & Transcribed & \\
\hline $3 / 28 / 927: 03$ & Auto FIC & 48.50 & Good & \\
\hline $3 / 29 / 920: 00$ & Manual Tape & 49.5 & Transcribed & \\
\hline $3 / 29 / 927: 03$ & Auto FIC & 48.50 & Good & \\
\hline $3 / 30 / 920: 00$ & Manual Tape & 49.50 & Transcribed & \\
\hline $3 / 30 / 927: 03$ & Auto FIC & 48.5 & Good & \\
\hline $3 / 31 / 920: 00$ & Manual Tape & 49.00 & Transcribed & \\
\hline $3 / 31 / 927: 03$ & Auto FIC & 48.5 & Good & \\
\hline $4 / 1 / 92 \quad 0: 00$ & Manual Tape & 49.75 & Transcribed & \\
\hline $4 / 1 / 92$ 7:03 & Auto FIC & 48.50 & Good & \\
\hline $4 / 2 / 92 \quad 0: 00$ & Manual Tape & 49 & Transcribed & \\
\hline 4/2/92 7:03 & Auto FIC & 48.50 & Good & \\
\hline 4/3/92 0:00 & Manual Tape & 49 & Transcribed & \\
\hline 4/3/92 7:03 & Auto FIC & 48.5 & Gopd & \\
\hline 4/4/92 0:00 & Manual Tape & 49.00 & Transcribed & \\
\hline 4/4/92 7:03 & Auto FIC & 48.5 & Good & \\
\hline 4/5/92 0:00 & Manual Tape & 49.00 & Transcribed & \\
\hline $4 / 5 / 927: 03$ & Auto FIC & 48.5 & Good & \\
\hline 4/6/92 0:00 & Manual Tape & 49.25 & Transcribed & \\
\hline $4 / 6 / 927: 03$ & Auto FIC & 48.50 & Good & \\
\hline $4 / 7 / 92 \quad 0: 00$ & Manual Tape & 49.00 & Transcribed & \\
\hline $4 / 7 / 927: 03$ & Auto FIC & 48.50 & Good & \\
\hline $4 / 8 / 92 \quad 0: 00$ & Manual Tape & 49.50 & Transćribed & \\
\hline 4/8/92 5:02 & Auto FIC & 48.5 & Good & \\
\hline 4/9/92 0:00 & Manual Tape & 49.25 & Transcribed & \\
\hline 4/9/92 7:03 & Auto FIC & 48.5 & Good & \\
\hline $4 / 10 / 920: 00$ & Manual Tape & 49.25 & Transcribed & \\
\hline $4 / 10 / 927: 03$ & Auto FIC & 48.60 & Good & \\
\hline
\end{tabular}

Data obtained from WHC Surveillance Analysis Computer System, (PC SACS) July 7, 1995. 


\begin{tabular}{|c|c|c|c|c|}
\hline \multicolumn{5}{|c|}{ 241-AP-102 } \\
\hline & & Urface Level Dat & 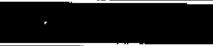 & \\
\hline Date & Type of Measurement & Level (Inches) & Data Quality & Comments \\
\hline $4 / 11 / 920: 00$ & Manual Tape & 49.50 & Transcribed & \\
\hline $4 / 11 / 927: 03$ & Auto FIC & 48.5 & Good & \\
\hline 4/12/92 0:00 & Manual Tape & 49.5 & Transcribed & \\
\hline 4/12/92 5:03 & Auto FIC & 48.6 & Good & \\
\hline $4 / 13 / 92 \quad 0: 00$ & Manual Tape & 49.5 & Transcribed & \\
\hline $4 / 13 / 927: 03$ & Auto FIC & 48.5 & Good & \\
\hline $4 / 14 / 92 \quad 0: 00$ & Manual Tape & 49.50 & Transcribed & \\
\hline $4 / 14 / 927: 03$ & Auto FIC & 48.50 & Good & \\
\hline 4/15/92 0:00 & Manual Tape & 49.50 & Transcribed & \\
\hline $4 / 15 / 927: 02$ & Auto FIC & 48.50 & Good & \\
\hline $4 / 16 / 920: 00$ & Manual Tape & 49.50 & Transcribed & \\
\hline $4 / 16 / 927: 03$ & Auto FIC & 48.5 & Good & \\
\hline $4 / 17 / 920: 00$ & Manual Tape & 49.50 & Transcribed & \\
\hline 4/17/92 7:03 & Auto FIC & 48.5 & Good & \\
\hline $4 / 18 / 92 \quad 0: 00$ & Manual Tape & 49.5 & Transcribed & \\
\hline $4 / 18 / 927: 02$ & Auto FIC & 48.50 & Good & \\
\hline $4 / 19 / 92 \quad 0: 00$ & Manual Tape & 49.5 & Transcribed & \\
\hline $4 / 19 / 927: 02$ & Auto FIC & 48.50 & Good & \\
\hline $4 / 20 / 920: 00$ & Manual Tape & 49.50 & Transcribed & \\
\hline $4 / 20 / 927: 03$ & Auto FIC & 48.5 & Good & \\
\hline $4 / 21 / 920: 00$ & Manual Tape & 49.50 & Transcribed & \\
\hline $4 / 21 / 927: 03$ & Auto FIC & 48.5 & Good & . \\
\hline $4 / 22 / 920: 00$ & Manual Tape & 49.50 & Transcribed & \\
\hline $4 / 22 / 927: 02$ & Auto FIC & 48.50 & Good & \\
\hline $4 / 23 / 920: 00$ & Manual Tape & 49.5 & Transcribed & \\
\hline $4 / 23 / 927: 03$ & Auto FIC & 48.5 & Good & \\
\hline $4 / 24 / 920: 00$ & Manual Tape & 49.5 & Transcribed & \\
\hline $4 / 24 / 927: 03$ & Auto FIC & 48.5 & Good & \\
\hline $4 / 25 / 920: 00$ & Manual Tape & 49.50 & Transcribed & \\
\hline $4 / 25 / 927: 04$ & Auto FIC & 48.50 & Good & \\
\hline $4 / 26 / 920: 00$ & Manual Tape & 49.50 & Transeribed & \\
\hline $4 / 26 / 927: 03$ & Auto FIC & 48.50 & Good & \\
\hline $4 / 27 / 920: 00$ & Manual Tape & 49 & Transcribed & \\
\hline $4 / 27 / 927: 04$ & Auto FIC & 48.5 & Good & \\
\hline $4 / 28 / 920: 00$ & Manual Tape & 52.25 & Transcribed & \\
\hline $4 / 28 / 927: 03$ & Auto FIC & 48.5 & Good & \\
\hline $4 / 29 / 920: 00$ & Manual Tape & 49.50 & Transcribed & \\
\hline $4 / 29 / 927: 03$ & Auto FIC & 48.50 & Good & \\
\hline $4 / 30 / 920: 00$ & Manual Tape & 49.25 & Transcribed & \\
\hline $4 / 30 / 927: 03$ & Auto FIC & 48.50 & Good & \\
\hline $5 / 1 / 92 \quad 0: 00$ & Manual Tape & 49.50 & Transcribed & \\
\hline $5 / 1 / 927: 03$ & Auto FIC & 48.5 & Good & \\
\hline $5 / 2 / 920: 00$ & Manual Tape & 49.50 & Transcribed & \\
\hline $5 / 2 / 922: 03$ & Auto FIC & 48.5 & Good & \\
\hline $5 / 3 / 920: 00$ & Manual Tape & 49.5 & Transcribed & \\
\hline $5 / 3 / 927: 03$ & Auto FIC & 48.50 & Good & \\
\hline $5 / 4 / 920: 00$ & Manual Tape & 49.50 & Transcribed & \\
\hline $5 / 4 / 927: 03$ & Auto FIC & 48.50 & Good & \\
\hline
\end{tabular}

Data obtained from WHC Surveillance Analysis Computer System, (PC SACS) July 7, 1995. 
WHC-SD-WM-ER-315, Rev. 0

\begin{tabular}{|c|c|c|c|c|}
\hline \multicolumn{5}{|c|}{ 241-AP-102 } \\
\hline & & urface Level Dat: & & \\
\hline Date & Type of Measurement & Level (Inches) & Data Quality & Comments \\
\hline $5 / 5 / 920: 00$ & Manual Tape & 49.50 & Transcribed & \\
\hline 5/5/92 7:04 & Auto FIC & 48.50 & Good & \\
\hline $5 / 6 / 920: 00$ & Manual Tape & 49.5 & Transcribed & \\
\hline $5 / 6 / 927: 03$ & Auto FIC & 48.5 & Good & \\
\hline $5 / 7 / 920: 00$ & Manual Tape & 49.50 & Transcribed & \\
\hline $5 / 7 / 927: 04$ & Auto FIC & 0 & Suspect & Data not included in graph \\
\hline 5/8/92 0:00 & Manual Tape & 49.50 & Transcribed & \\
\hline 5/8/92 7:03 & Auto FIC & 0.00 & Suspect & Data not included in graph \\
\hline $5 / 9 / 920: 00$ & Manual Tape & 49.5 & Transcribed & \\
\hline $5 / 10 / 920: 00$ & Manual Tape & 49.50 & Transcribed & \\
\hline $5 / 11 / 920: 00$ & Manual Tape & 49.50 & Transcribed & \\
\hline $5 / 12 / 920: 00$ & Manual Tape & 49.5 & Transcribed & \\
\hline $5 / 12 / 927: 03$ & Auto FIC & 0 & Suspect & Data not included in graph \\
\hline $5 / 13 / 920: 00$ & Manual Tape & 49.5 & Transcribed & \\
\hline 5/13/92 7:03 & Auto FIC & 0 & Suspect & Data not included in graph \\
\hline $5 / 14 / 920: 00$ & Manual Tape & 49.50 & Transcribed & \\
\hline $5 / 14 / 927: 03$ & Auto FIC & 0.00 & Suspect & Data not included in graph \\
\hline $5 / 15 / 920: 00$ & Manual Tape & 49.50 & Transcribed & \\
\hline 5/15/92 7:03 & Auto FIC & 0.00 & Suspect & Data not included in graph \\
\hline $5 / 16 / 920: 00$ & Manual Tape & 49.50 & Transcribed & \\
\hline $5 / 16 / 927: 03$ & Auto FIC & 0 & Suspect & Data not included in graph \\
\hline $5 / 17 / 920: 00$ & Manual Tape & 49.5 & Transcribed & \\
\hline $5 / 17 / 927: 02$ & Auto FIC & 0 & Suspect & Data not included in graph \\
\hline 5/18/92 0:00 & Manual Tape . & 49.5 & Transcribed & \\
\hline $5 / 18 / 927: 03$ & Auto FIC & 0.00 & Suspect & Data not included in graph \\
\hline $5 / 19 / 920: 00$ & Manual Tape & 49.50 & Transcribed & \\
\hline $5 / 19 / 927: 03$ & Auto FIC & 0.00 & Suspect & Data not included in graph \\
\hline $5 / 20 / 920: 00$ & Manual Tape & 49.50 & Transcribed & \\
\hline $5 / 20 / 927: 03$ & Auto FIC & 0.00 & Suspect & Data not included in graph \\
\hline $5 / 21 / 920: 00$ & Manual Tape & 49.5 & Transcribed & \\
\hline $5 / 21 / 927: 03$ & Auto FIC & 0 & Suspect & Data not included in graph \\
\hline $5 / 22 / 920: 00$ & Manual Tape & 49.50 & Transcribed & \\
\hline $5 / 22 / 927: 02$ & Auto FIC & 0 & Suspect & Data not included in graph \\
\hline $5 / 23 / 920: 00$ & Manual Tape & 49.50 & Transcribed & \\
\hline $5 / 23 / 927: 03$ & Auto FIC & 0.00 & Suspect & Data not included in graph \\
\hline $5 / 24 / 920: 00$ & Manual Tape & 49.5 & Transcribed & \\
\hline $5 / 24 / 927: 03$ & Auto FIC & 0.00 & Suspect & Data not included in graph \\
\hline $5 / 25 / 92 \quad 0: 00$ & Manual Tape & 49.5 & Transcribed & \\
\hline $5 / 25 / 927: 03$ & Auto FIC & 0 & Suspect & Data not included in graph \\
\hline $5 / 26 / 92 \quad 0: 00$ & Manual Tape & 49.50 & Transcribed & \\
\hline $5 / 27 / 920: 00$ & Manual Tape & 49.50 & Transcribed & \\
\hline $5 / 27 / 927: 03$ & Auto FIC & 0.00 & Suspect & Data not included in graph \\
\hline $5 / 28 / 920: 00$ & Manual Tape & 49.50 & Transcribed & \\
\hline $5 / 28 / 927: 02$ & Auto FIC & 0.00 & Suspect & Data not included in graph \\
\hline $5 / 29 / 920: 00$ & Manual Tape & 49.5 & Transcribed & \\
\hline $5 / 29 / 927: 02$ & Auto FIC & 0 & Suspect & Data not included in graph \\
\hline $5 / 30 / 920: 00$ & Manual Tape & 49.5 & Transcribed & \\
\hline $5 / 30 / 927: 03$ & Auto FIC & 0 & Suspect & Data not included in graph \\
\hline
\end{tabular}

Data obtained from WHC Surveillance Analysis Computer System, (PC SACS) July 7, 1995. 
WHC-SD-WM-ER-315, Rev. 0

\begin{tabular}{|c|c|c|c|c|}
\hline \multicolumn{5}{|c|}{ 241-AP-102 } \\
\hline \multicolumn{5}{|c|}{ Surface Level Data } \\
\hline Date & Type of Measurement & Level (Inches) & Data Quality & Comments \\
\hline $5 / 31 / 920: 00$ & Manual Tape & 49.25 & Transcribed & \\
\hline $5 / 31 / 927: 03$ & Auto FIC & 0.00 & Suspect & Data not included in graph \\
\hline 6/1/92 0:00 & Manual Tape & 49.50 & Transcribed & \\
\hline 6/1/92 7:03 & Auto FIC & 0.00 & Suspect & Data not included in graph \\
\hline 6/2/92 0:00 & Manual Tape & 49.25 & Transcribed & \\
\hline 6/2/92 7:03 & Auto FIC & 0 & Suspect & Data not included in graph \\
\hline $6 / 3 / 920: 00$ & Manual Tape & 49.25 & Transcribed & \\
\hline $6 / 3 / 927: 03$ & Auto FIC & 0 & Suspect & Data not included in graph \\
\hline $6 / 4 / 920: 00$ & Manual Tape & 49.25 & Transcribed & \\
\hline 6/4/92 7:03 & Auto FIC & 0.00 & Suspect & Data not included in graph \\
\hline $6 / 5 / 920: 00$ & Manual Tape & 49.25 & Transcribed & \\
\hline 6/5/92 7:02 & Auto FIC & 0.00 & Suspect & Data not included in graph \\
\hline $6 / 6 / 92 \quad 0: 00$ & Manual Tape & 49.5 & Transcribed & \\
\hline 6/6/92 7:03 & Auto FIC & 0 & Suspect & Data not included in graph \\
\hline 6/7/920:00 & Manual Tape & 49.50 & Transcribed & \\
\hline 6/7/92 7:03 & Auto FIC & 0 & Suspect & Data not included in graph \\
\hline $6 / 8 / 920: 00$ & Manual Tape & 49.25 & Transcribed & \\
\hline 6/8/92 7:03 & Auto FIC & 0.00 & Suspect & Data not included in graph \\
\hline 6/9/92 0:00 & Manual Tape & 49.50 & Transcribed & \\
\hline 6/9/92 7:03 & Auto FIC & 0.00 & Suspect & Data not included in graph \\
\hline $6 / 10 / 920: 00$ & Manual Tape & 49.5 & Transcribed & \\
\hline $6 / 10 / 927: 03$ & Auto FIC & 0 & Suspect & Data not included in graph \\
\hline $6 / 11 / 920: 00$ & Manual Tape & 49.5 & Transcribed & \\
\hline $6 / 11 / 927: 03$ & Auto FIC & 0 & Suspect & Data not included in graph \\
\hline $6 / 12 / 920: 00$ & Manual Tape & 49.50 & Transcribed & \\
\hline $6 / 12 / 927: 05$ & Auto FIC & 0.00 & Suspect & Data not included in graph \\
\hline $6 / 13 / 920: 00$ & Manual Tape & 49.50 & Transcribed & \\
\hline $6 / 13 / 927: 05$ & Auto FIC & 0.00 & Suspect & Data not included in graph \\
\hline $6 / 14 / 920: 00$ & Manual Tape & 49.5 & Transcribed & \\
\hline $6 / 15 / 92 \quad 0: 00$ & Manual Tape & 49.50 & Transcribed & \\
\hline $6 / 15 / 927: 03$ & Auto FIC & 0 & Suspect & Data not included in graph \\
\hline $6 / 16 / 920: 00$ & Manual Tape & 49.50 & Transcribed & \\
\hline $6 / 16 / 927: 03$ & Auto FIC & 0 & Suspect & Data not included in graph \\
\hline $6 / 17 / 920: 00$ & Manual Tape & 49.5 & Transcribed & \\
\hline $6 / 17 / 927: 03$ & Auto FIC & 0.00 & Suspect & Data not included in graph \\
\hline $6 / 18 / 920: 00$ & Manual Tape & 49.5 & Transcribed & \\
\hline $6 / 18 / 927: 03$ & Auto FIC & 0.00 & Suspect & Data not included in graph \\
\hline $6 / 19 / 920: 00$ & Manual Tape & 49.50 & Transcribed & \\
\hline $6 / 19 / 927: 02$ & Auto FIC & 0 & Suspect & Data not included in graph \\
\hline $6 / 20 / 920: 00$ & Manual Tape & 49.50 & Transcribed & \\
\hline $6 / 20 / 927: 02$ & Auto FIC & 0 & Suspect & Data not included in graph \\
\hline $6 / 21 / 920: 00$ & Manual Tape & 49.50 & Transcribed & \\
\hline $6 / 21 / 927: 03$ & Auto FIC & 0.00 & Suspect & Data not included in graph \\
\hline $6 / 22 / 920: 00$ & Manual Tape & 49.5 & Transcribed & \\
\hline $6 / 22 / 927: 03$ & Auto FIC & 0.00 & Suspect & Data not included in graph \\
\hline $6 / 23 / 920: 00$ & Manual Tape & 49.25 & Transcribed & \\
\hline $6 / 23 / 927: 03$ & Auto FIC & 0 & Suspect & Data not included in graph \\
\hline $6 / 24 / 920: 00$ & Manual Tape & 49.25 & Transcribed & \\
\hline
\end{tabular}

Data obtained from WHC Surveillance Analysis Computer System, (PC SACS) July 7, 1995. 


\begin{tabular}{|c|c|c|c|c|}
\hline \multicolumn{5}{|c|}{ 241-AP-102 } \\
\hline \multicolumn{5}{|c|}{ Surface Level Data } \\
\hline Date & Type of Measurement & Level (Inches) & Data Quality & Comments \\
\hline $6 / 24 / 927: 03$ & Auto FIC & 0 & Suspect & Data not included in graph \\
\hline $6 / 25 / 920: 00$ & Manual Tape & 49.25 & Transcribed & \\
\hline $6 / 25 / 927: 03$ & Auto FIC & 0.00 & Suspect & Data not included in graph \\
\hline $6 / 26 / 920: 00$ & Manual Tape & 49.5 & Transcribed & \\
\hline $6 / 26 / 927: 02$ & Auto FIC & 0.00 & Suspect & Data not included in graph \\
\hline $6 / 27 / 920: 00$ & Manual Tape & 49.50 & Transcribed & \\
\hline 6/27/92 7:03 & Auto FIC & 0 & Suspect & Data not included in graph \\
\hline $6 / 28 / 920: 00$ & Manual Tape & 49.50 & Transcribed & \\
\hline $6 / 28 / 927: 06$ & Auto FIC & 0 & Suspect & Data not included in graph \\
\hline $6 / 29 / 920: 00$ & Manual Tape & $\cdot 49.5$ & Transcribed & \\
\hline $6 / 29 / 927: 06$ & Auto FIC & 0.00 & Suspect & Data not included in graph \\
\hline $6 / 30 / 920: 00$ & Manual Tape & 49.5 & Transcribed & \\
\hline $6 / 30 / 927: 06$ & Auto FIC & 0.00 & Suspect & Data not included in graph \\
\hline 7/1/92 0:00 & Manual Tape & 49.50 & Transcribed & \\
\hline $7 / 2 / 920: 00$ & Manual Tape & 49.50 & Transcribed & \\
\hline $7 / 3 / 920: 00$ & Manual Tape & 49.50 & Transcribed & \\
\hline $7 / 3 / 927: 06$ & Auto FIC & 0 & Suspect & Data not included in graph \\
\hline $7 / 4 / 920: 00$ & Manual Tape & 49.5 & Transcribed & \\
\hline $7 / 4 / 927: 06$ & Auto FIC & 0 & Suspect & Data not included in graph \\
\hline $7 / 5 / 920: 00$ & Manual Tape & 49.5 & Transcribed & \\
\hline $7 / 5 / 927: 06$ & Auto FIC & $0 . \overline{00}$ & Suspect & Data not included in graph \\
\hline $7 / 6 / 920: 00$ & Manual Tape & 49.25 & Transcribed & \\
\hline 7/6/92 7:06 & Auto FIC & 0.00 & Suspect & Data not included in graph \\
\hline $7 / 7 / 920: 00$ & Manual Tape & 49.50 & Transcribed & \\
\hline 7/7/92 7:06 & Auto FIC & 0 & Suspect & Data not included in graph \\
\hline $7 / 8 / 920: 00$ & Manual Tape & 49.50 & Transcribed & \\
\hline 7/8/92 7:06 & Auto FIC & 0 & Suspect & Data not included in graph \\
\hline $7 / 9 / 920: 00$ & Manual Tape & 49.50 & Transcribed & \\
\hline 7/9/92 7:06 & Auto FIC & $0 . \overline{00}$ & Suspect & Data not included in graph \\
\hline $7 / 10 / 920: 00$ & Manual Tape & $49: 5$ & Transcribed & \\
\hline $7 / 10 / 927: 06$ & Auto FIC & 0.00 & Suspect & Data not included in graph \\
\hline $7 / 11 / 920: 00$ & Manual Tape & 49.50 . & Transcribed & \\
\hline $7 / 11 / 927: 06$ & Auto FIC & 0 & Suspect & Data not included in graph \\
\hline $7 / 12 / 920: 00$ & Manual Tape & 49.50 & Transcribed & \\
\hline 7/12/92 7:06 & Auto FIC & 0 & Suspect & Data not included in graph \\
\hline 7/13/92 0:00 & Manual Tape & 49.50 & Transcribed & \\
\hline $7 / 13 / 927: 06$ & Auto FIC & 0 & Suspect & Data not included in graph \\
\hline $7 / 14 / 920: 00$ & Manual Tape & 49.5 & Transcribed & \\
\hline $7 / 14 / 927: 06$ & Auto FIC & 0.00 & Suspect & Data not included in graph \\
\hline $7 / 15 / 920: 00$ & Manual Tape & 49.5 & Transcribed & \\
\hline $7 / 15 / 927: 06$ & Auto FIC & 0.00 & Suspect & Data not included in graph \\
\hline $7 / 16 / 920: 00$ & Manual Tape & 49.5 & Transcribed & \\
\hline $7 / 16 / 927: 06$ & Auto FIC & 0 & Suspect & Data not included in graph \\
\hline $7 / 17 / 920: 00$ & Manual Tape & 49.50 & Transcribed & \\
\hline $7 / 17 / 927: 06$ & Auto FIC & 0.00 & Suspect & Data not included in graph \\
\hline $7 / 18 / 920: 00$ & Manual Tape & 49.50 & Transcribed & \\
\hline $7 / 18 / 927: 06$ & Auto FIC & 0.00 & Suspect & Data not included in graph \\
\hline $7 / 19 / 920: 00$ & Manual Tape & 49.50 & Transcribed & \\
\hline
\end{tabular}

Data obtained from WHC Surveillance Analysis Computer System, (PC SACS) July 7, 1995. 
WHC-SD-WM-ER-315, Rev. 0

\begin{tabular}{|c|c|c|c|c|}
\hline \multicolumn{5}{|c|}{ 241-AP-102 } \\
\hline \multicolumn{5}{|c|}{ Surface Level Data } \\
\hline Date & Type of Measurement & Level (Inches) & Data Quality & Comments \\
\hline $7 / 19 / 927: 06$ & Auto FIC & 0 & Suspect & Data not included in graph \\
\hline $7 / 20 / 920: 00$ & Manual Tape & 49.5 & Transcribed & \\
\hline $7 / 20 / 927: 06$ & Auto FIC & 0 & Suspect & Data not included in graph \\
\hline $7 / 21 / 920: 00$ & Manual Tape & 49.5 & Transcribed & \\
\hline $7 / 21 / 927: 05$ & Auto FIC & 0 & Suspect & Data not included in graph \\
\hline $7 / 22 / 920: 00$ & Manual Tape & 49.50 & Transcribed & \\
\hline $7 / 22 / 927: 06$ & Auto FIC & 0.00 & Suspect & Data not included in graph \\
\hline $7 / 23 / 920: 00$ & Manual Tape & 49.50 & Transcribed & \\
\hline $7 / 24 / 920: 00$ & Manual Tape & 49.5 & Transcribed & \\
\hline $7 / 24 / 927: 05$ & Auto FIC & 0.00 & Suspect & Data not included in graph \\
\hline $7 / 25 / 920: 00$ & Manual Tape & 49.50 & Transcribed & \\
\hline 7/25/92 7:06 & Auto FIC & 0 & Suspect & Data not included in graph \\
\hline 7/26/92 0:00 & Manual Tape & 49.50 & Transcribed & \\
\hline $7 / 26 / 92 / 7: 06$ & Auto FIC & 0 & Suspect & Data not included in graph \\
\hline $7 / 27 / 920: 00$ & Manual Tape & 49.5 & Transcribed & \\
\hline 7/27/92 7:05 & Auto FIC & 0.00 & Suspect & Data not included in graph \\
\hline $7 / 28 / 920: 00$ & Manual Tape & 49.5 & Transcribed & \\
\hline 7/28/92 7:06 & Auto FIC & 0.00 & Suspect & Data not included in graph \\
\hline $7 / 29 / 920: 00$ & Manual Tape & 49.50 & Transcribed & \\
\hline $7 / 29 / 927: 06$ & Auto FIC & 0 & Suspect & Data not included in graph \\
\hline $7 / 30 / 920: 00$ & Manual Tape & 49.50 & Transcribed & \\
\hline $7 / 30 / 927: 06$ & Auto FIC & 0 & Suspect & Data not included in graph \\
\hline $7 / 31 / 920: 00$ & Manual Tape & 49.50 & Transcribed & \\
\hline $7 / 31 / 927: 05$ & Auto FIC & 0.00 & Suspect & Data not included in graph \\
\hline $8 / 1 / 920: 00$ & Manual Tape & 49.5 & Transcribed & \\
\hline $8 / 1 / 927: 06$ & Auto FIC & 0.00 & Suspect & Data not included in graph \\
\hline $8 / 2 / 920: 00$ & Manual Tape & 49.5 & Transcribed & \\
\hline $8 / 2 / 927: 06$ & Auto FIC & 0 & Suspect & Data not included in graph \\
\hline $8 / 3 / 920: 00$ & Manual Tape & 49.50 & Transcribed & \\
\hline $8 / 3 / 927: 06$ & Auto FIC & 0 & Suspect & Data not included in graph \\
\hline $8 / 4 / 920: 00$ & Manual Tape & 49.50 & Transcribed & \\
\hline $8 / 4 / 927: 06$ & Auto FIC & 0.00 & Suspect & Data not included in graph \\
\hline $8 / 5 / 920: 00$ & Manual Tape & 49.5 & Transcribed & \\
\hline $8 / 5 / 927: 05$ & Auto FIC & 0.00 & Suspect & Data not included in graph \\
\hline $8 / 6 / 920: 00$ & Manual Tape & 49.50 & Transcribed & \\
\hline $8 / 6 / 927: 05$ & Auto FIC & 0 & Suspect & Data not included in graph \\
\hline $8 / 7 / 920: 00$ & Manual Tape & 49.50 & Transcribed & \\
\hline $8 / 7 / 927: 05$ & Auto FIC & 0 & Suspect & Data not included in graph \\
\hline $8 / 8 / 920: 00$ & Manual Tape & 49.5 & Transcribed & \\
\hline $8 / 8 / 927: 06$ & Auto FIC & 0.00 & Suspect & Data not included in graph \\
\hline $8 / 9 / 920: 00$ & Manual Tape & 49.5 & Transcribed & \\
\hline $8 / 9 / 927: 06$ & Auto FIC & 0.00 & Suspect & Data not included in graph \\
\hline $8 / 10 / 920: 00$ & Manual Tape & 49.50 & Transcribed & \\
\hline $8 / 10 / 927: 05$ & Auto FIC & 0 & Suspect & Data not included in graph \\
\hline $8 / 11 / 920: 00$ & Manual Tape & 49.50 & Transcribed & \\
\hline $8 / 11 / 927: 06$ & Auto FIC & 0 & Suspect & Data not included in graph \\
\hline $8 / 12 / 920: 00$ & Manual Tape & 49.50 & Transcribed & \\
\hline $8 / 12 / 927: 06$ & Auto FIC & 0.00 & Suspect & Data not included in graph \\
\hline
\end{tabular}

Data obtained from WHC Surveillance Analysis Computer System, (PC SACS) July 7, 1995. 
WHC-SD-WM-ER-315, Rev. 0

\begin{tabular}{|c|c|c|c|c|}
\hline \multicolumn{5}{|c|}{ 241-AP-102 } \\
\hline \multicolumn{5}{|c|}{ Surface Level Data } \\
\hline Date & Type of Measurement & Level (Inchias) & Data Quality & Comments \\
\hline $8 / 13 / 920: 00$ & Manual Tape & 49.5 & Transcribed & \\
\hline $8 / 13 / 927: 06$ & Auto FIC & 0.00 & Suspect & Data not included in graph \\
\hline $8 / 14 / 920: 00$ & Manual Tape & 49.5 & Transcribed & \\
\hline $8 / 14 / 927: 06$ & Auto FIC & 0 & Suspect & Data not included in graph \\
\hline $8 / 15 / 920: 00$ & Manual Tape & 49.50 & Transcribed & \\
\hline $8 / 15 / 927: 06$ & Auto FIC & 0 & Suspect & Data not included in graph \\
\hline $8 / 16 / 920: 00$ & Manual Tape & 49.50 & Transcribed & \\
\hline $8 / 16 / 927: 06$ & Auto FIC & 0.00 & Suspect & Data not included in graph \\
\hline $8 / 17 / 920: 00$ & Manual Tape & 49.5 & Transcribed & \\
\hline $8 / 17 / 927: 06$ & Auto FIC & 0.00 & Suspect & Data not included in graph \\
\hline $8 / 18 / 920: 00$ & Manual Tape & 49.50 & Transcribed & \\
\hline $8 / 18 / 927: 06$ & Auto FIC & 0 & Suspect & Data not included in graph \\
\hline $8 / 19 / 920: 00$ & Manual Tape & 49.50 & Transcribed & \\
\hline $8 / 19 / 927: 06$ & Auto FIC & 0 & Suspect & Data not included in graph \\
\hline $8 / 20 / 920: 00$ & Manual Tape & 49.5 & Transcribed & \\
\hline $8 / 20 / 927: 06$ & Auto FIC & 0.00 & Suspect & Data not included in graph \\
\hline $8 / 21 / 920: 00$ & Manual Tape & 49.5 & Transcribed & \\
\hline $8 / 21 / 927: 05$ & Auto FIC & 0.00 & Suspect & Data not included in graph \\
\hline $8 / 22 / 920: 00$ & Manual Tape & 49.50 & Transcribed & \\
\hline $8 / 22 / 927: 04$ & Auto FIC & 0 & Suspect & Data not included in graph \\
\hline $8 / 23 / 920: 00$ & Manual Tape & 49.50 & Transcribed & \\
\hline $8 / 23 / 927: 04$ & Auto FIC & 0 & Suspect & Data not included in graph \\
\hline $8 / 24 / 920: 00$ & Manual Tape & 49.25 & Transcribed & \\
\hline $8 / 24 / 927: 04$ & Auto FIC & 0.00 & Suspect & Data not included in graph \\
\hline $8 / 25 / 920: 00$ & Manual Tape & 49.5 & Transcribed & \\
\hline $8 / 25 / 927: 04$ & Auto FIC & 0.00 & Suspect & Data not included in graph \\
\hline $8 / 26 / 920: 00$ & Manual Tape & 49 & Transcribed & \\
\hline $8 / 26 / 927: 03$ & Auto FIC & 0 & Suspect & Data not included in graph \\
\hline $8 / 27 / 920: 00$ & Manual Tape & 49.75 & Transcribed & \\
\hline $8 / 27 / 92 \quad 7: 04$ & Auto FIC & 0 & Suspect & Data not included in graph \\
\hline $8 / 28 / 920: 00$ & Manual Tape & 49.75 & Transcribed & \\
\hline $8 / 28 / 927: 02$ & Auto FIC & 0.00 & Suspect & Data not included in graph \\
\hline $8 / 29 / 920: 00$ & Manual Tape & 49.75 & Transcribed & \\
\hline $8 / 29 / 92 \quad 7: 02$ & Auto FIC & 0.00 & Suspect & Data not included in graph \\
\hline $8 / 30 / 920: 00$ & Manual Tape & 49.75 & Transcribed & \\
\hline $8 / 30 / 927: 02$ & Auto FIC & 0 & Suspect & Data not included in graph \\
\hline $8 / 31 / 920: 00$ & Manual Tape & 49.75 & Transcribed & \\
\hline $8 / 31 / 927: 02$ & Auto FIC & 0 & Suspect & Data not included in graph \\
\hline 9/1/92 0:00 & Manual Tape & 49.75 & Transcribed & \\
\hline $9 / 1 / 927: 02$ & Auto FIC & 0.00 & Suspect & Data not included in graph \\
\hline 9/2/92 0:00 & Manual Tape & 49.75 & Transcribed & \\
\hline $9 / 2 / 927: 02$ & Auto FIC & 0.00 & Suspect & Data not included in graph \\
\hline $9 / 3 / 920: 00$ & Manual Tape & 50.00 & Transcribed & \\
\hline $9 / 3 / 927: 04$ & Auto FIC & 0 & Suspect & Data not included in graph \\
\hline 9/4/92 0:00 & Manual Tape & 49.75 & Transcribed & \\
\hline $9 / 4 / 927: 04$ & Auto FIC & 0 & Suspect & Data not included in graph \\
\hline 9/5/92 0:00 & Manual Tape & 49.75 & Transcribed & \\
\hline 9/5/92 7:04 & Auto FIC & 0.00 & Suspect & Data not included in graph \\
\hline
\end{tabular}

Data obtained from WHC Surveillance Analysis Computer System, (PC SACS) July 7, 1995. 


\begin{tabular}{|c|c|c|c|c|}
\hline \multicolumn{5}{|c|}{ 241-AP-102 } \\
\hline \multicolumn{5}{|c|}{ Surface Level Data } \\
\hline Date & Type of Measurement & Lovel (Inches) & Data Quality & Comments \\
\hline 9/6/92 0:00 & Manual Tape & 49.75 & Transcribed & \\
\hline $9 / 6 / 927: 04$ & Auto FIC & 0.00 & Suspect & Data not included in graph \\
\hline $9 / 7 / 92 \quad 0: 00$ & Manual Tape & 49.75 & Transcribed & \\
\hline $9 / 7 / 927: 05$ & Auto FIC & 0 & Suspect & Data not included in graph \\
\hline $9 / 8 / 920: 00$ & Manual Tape & 49.75 & Transcribed & \\
\hline $9 / 8 / 927: 05$ & Auto FIC & 0 & Suspect & Data not included in graph \\
\hline $9 / 9 / 920: 00$ & Manual Tape & 49.75 & Transcribed & \\
\hline 9/9/92 7:04 & Auto FIC & 0.00 & Suspect & Data not included in graph \\
\hline $9 / 10 / 920: 00$ & Manual Tape & 49.75 & Transcribed & \\
\hline $9 / 10 / 927: 03$ & Auto FIC & 0 & Suspect & Data not included in graph \\
\hline $9 / 11 / 920: 00$ & Manual Tepe & 49.75 & Transcribed & \\
\hline $9 / 12 / 920: 00$ & Manual Tape & 50.00 & Transcribed & \\
\hline $9 / 13 / 920: 00$ & Manual Tape & 50.00 & Transcribed & \\
\hline $9 / 14 / 920: 00$ & Manual Tape & 49.75 & Transcribed & \\
\hline 9/15/92 0:00 & Manual Tape & 49.75 & Transcribed & \\
\hline $9 / 16 / 920: 00$ & Manual Tape & 49.75 & Transcribed & \\
\hline $9 / 17 / 920: 00$ & Manual Tape & 49.75 & Transcribed & \\
\hline $9 / 17 / 927: 06$ & Auto FIC & 0 & Suspect & Data not included in graph \\
\hline $9 / 18 / 920: 00$ & Manual Tape & 49.75 & Transcribed & \\
\hline $9 / 18 / 927: 06$ & Auto FIC & 0.00 & Suspect & Data not included in graph \\
\hline $9 / 19 / 920: 00$ & Manual Tape & 49.75 & Transcribed & \\
\hline $9 / 20 / 920: 00$ & Manual Tape & 49.75 & Transcribed & \\
\hline 9/21/92 0:00 & Manual Tape & 49.25 & Transcribed & \\
\hline $9 / 21 / 927: 06$ & Auto FIC & 0.00 & Suspect & Data not included in graph \\
\hline $9 / 22 / 920: 00$ & Manual Tape & 49.25 & Transcribed & \\
\hline $9 / 22 / 927: 03$ & Auto FIC & 0 & Suspect & Data not included in graph \\
\hline $9 / 23 / 920: 00$ & Manual Tape & 50.00 & Transcribed & \\
\hline $9 / 23 / 927: 03$ & Auto FIC & 0 & Suspect & Data not included in graph \\
\hline $9 / 24 / 920: 00$ & Manual Tape & 50.00 & Transcribed & \\
\hline $9 / 24 / 927: 03$ & Auto FIC & 0.00 & Suspect & Data not included in graph \\
\hline $9 / 25 / 920: 00$ & Manual Tape & 50.00 & Transcribed & \\
\hline $9 / 25 / 927: 03$ & Auto FIC & 0.00 & Suspect & Data not included in graph \\
\hline $9 / 26 / 920: 00$ & Manual Tape & 50 & Transcribed & \\
\hline $9 / 26 / 927: 02$ & Auto FIC & 0 & Suspect & Data not included in graph \\
\hline $9 / 27 / 920: 00$ & Manual Tape & 50.00 & Transcribed & \\
\hline $9 / 27 / 927: 02$ & Auto FIC & 0 & Suspect & Data not included in graph \\
\hline $9 / 28 / 920: 00$ & Manual Tape & 49.5 & Transcribed & \\
\hline 9/28/92 7:02 & Auto FIC & 0.00 & Suspect & Data not included in graph \\
\hline 9/29/92 0:00 & Manual Tape & 49.50 & Transcribed & \\
\hline $9 / 29 / 927: 03$ & Auto FIC & 0.00 & Suspect & Data not included in graph \\
\hline $9 / 30 / 920: 00$ & Manual Tape & 50.00 & Transcribed & \\
\hline $9 / 30 / 927: 02$ & Auto FIC & 0 & Suspect & Data not included in graph \\
\hline $10 / 1 / 920: 00$ & Manual Tape & 54.5 & Transcribed & \\
\hline $10 / 1 / 927: 03$ & Auto FIC & 0 & Suspect & Data not included in graph \\
\hline $10 / 2 / 920: 00$ & Manual FIC & 54.2 & Transcribed & \\
\hline $10 / 2 / 920: 00$ & Manual Tape & 54.25 & Transcribed & \\
\hline $10 / 2 / 927: 02$ & Auto FIC & 0.00 & Suspect & Data not included in graph \\
\hline $10 / 3 / 920: 00$ & Manual FIC & 54.20 & Transcribed & \\
\hline
\end{tabular}

Data obtained from WHC Surveillance Analysis Computer System, (PC SACS) July 7, 1995. 


\begin{tabular}{|c|c|c|c|c|}
\hline \multicolumn{5}{|c|}{$\begin{array}{cc}\text { 241-AP-102 } \\
\text { Surface Level Data }\end{array}$} \\
\hline & Type of Measurement & Level (Inches) & Data Quality & Comments \\
\hline $\begin{array}{c}\text { Date } \\
10 / 3 / 920: 00\end{array}$ & Manual Tape & 54.50 & Transcribed & \\
\hline $\begin{array}{l}10 / 3 / 920: 00 \\
10 / 3 / 927: 03\end{array}$ & Auto FIC & 0 & Suspect & Data not included in graph \\
\hline$\frac{10 / 3 / 927: 03}{10 / 4 / 920: 00}$ & Manual FIC & 54.20 & Transcribed & \\
\hline $\begin{array}{l}10 / 4 / 920: 00 \\
10 / 4 / 920: 00\end{array}$ & Manual Tape & 54.50 & Transcribed & \\
\hline $\begin{array}{l}10 / 4 / 920: 00 \\
10 / 4 / 927: 03\end{array}$ & Auto FIC & 0 & Suspect & Data not included in graph \\
\hline$\frac{10 / 4 / 927: 03}{10 / 5 / 920: 00}$ & Manual Tape & 54.50 & Transcribed & \\
\hline $\begin{array}{l}10 / 5 / 920: 00 \\
10 / 5 / 920: 00\end{array}$ & Manual FIC & 54.20 & Good & \\
\hline $\begin{array}{l}10 / 5 / 920: 00 \\
10 / 5 / 92 \quad 7: 03\end{array}$ & Auto FIC & 0.00 & Suspect & Data not included in graph \\
\hline $\begin{array}{l}10 / 5 / 927: 03 \\
10 / 6 / 920: 00\end{array}$ & Manual Tape & 54.5 & Transcribed & \\
\hline $\begin{array}{l}10 / 6 / 920: 00 \\
10 / 6 / 92 \quad 7: 03\end{array}$ & Auto FIC & 54.20 & Good & \\
\hline$\frac{10 / 6 / 927: 03}{10 / 7 / 92 \quad 0: 00}$ & Manual Tape & 54.50 & Transcribed & \\
\hline $\begin{array}{lll}10 / 7 / 92 & 0: 00 \\
10 / 7 / 92 & 0: 00\end{array}$ & Manual FIC & 54.20 & Good & \\
\hline $\begin{array}{ll}10 / 8 / 92 & 0: 00 \\
10 / 8 / 92 & 3: 03 \\
\end{array}$ & Auto FIC & 54.2 & Good & \\
\hline$\frac{10 / 8 / 923: 03}{10 / 9 / 920: 00}$ & Manual Tape & 54.5 & Transcribed & \\
\hline$\frac{10 / 9 / 920: 00}{10 / 9 / 927: 03}$ & Auto FIC & 54.2 & Good & \\
\hline$\frac{10 / 9 / 927: 03}{10 / 10 / 920: 00}$ & Manual FIC & 54.2 & Good & \\
\hline$\frac{10 / 10 / 920: 00}{10 / 10 / 927: 03}$ & Auto FIC & 0 & Suspect & Data not included in graph \\
\hline$\frac{10 / 10 / 927: 03}{10 / 11 / 920: 00}$ & Manual Tape & 54.5 & Transcribed & \\
\hline $10 / 11 / 924: 02$ & Auto FIC & 54.20 & Good & . \\
\hline $10 / 12 / 920: 00$ & Manual FIC & 54.2 & Good & \\
\hline $10 / 13 / 920: 00$ & Manual FIC & 54.20 & Good & \\
\hline $10 / 14 / 920: 00$ & Manual Tape & 54.50 & Transcribed & \\
\hline $10 / 14 / 922: 03$ & Auto FIC & 54.20 & Good & \\
\hline $10 / 15 / 920: 00$ & Manual Tape & 54.50 & Transcribed & \\
\hline $10 / 15 / 925: 04$ & Auto FIC & 54.2 & Good & \\
\hline $10 / 16 / 920: 00$ & Manual Tape & 54.50 & Transcribed & \\
\hline $10 / 16 / 927: 03$ & Auto FIC & 54.2 & Good & \\
\hline $10 / 17 / 920: 00$ & Manual Tape & 54.5 & Transcribed & \\
\hline $10 / 17 / 920: 00$ & Manual FIC & 54.20 & Good & \\
\hline $10 / 17 / 927: 03$ & Auto FIC & 0.00 & Suspect & Data not included in graph \\
\hline $10 / 18 / 920: 00$ & Manual Tape & 54.5 & Transcribed & \\
\hline $10 / 18 / 920: 00$ & Manual FIC & $54: 2$ & Good & \\
\hline $10 / 18 / 927: 03$ & Auto FIC & 0 & Suspect & Data not included in graph \\
\hline $10 / 19 / 920: 00$ & Manual Tape & 54.50 & Transcribed & \\
\hline $10 / 19 / 920: 00$ & Manual FIC & 54.20 & Good & \\
\hline $10 / 19 / 927: 03$ & Auto FIC & 0 & Suspect & Data not included in graph \\
\hline $10 / 20 / 920: 00$ & Manual Tape & 54.50 & Transcribed & \\
\hline $10 / 20 / 920: 00$ & Manual FIC & 54.2 & Good & \\
\hline $10 / 20 / 927: 03$ & Auto FIC & 0.00 & Suspect & Data not included in graph \\
\hline $10 / 21 / 920: 00$ & Manual Tape & 54.5 & Transcribed & \\
\hline $10 / 21 / 920: 00$ & Manual FIC & 54.2 & Good & \\
\hline $10 / 21 / 927: 03$ & Auto FIC & 0.00 & Suspect & Data not included in graph \\
\hline $10 / 22 / 920: 00$ & Manual Tape & 54.50 & Transcribed & \\
\hline $10 / 22 / 920: 00$ & Manual FIC & 54.20 & Good & \\
\hline $10 / 23 / 920: 00$ & Manual Tape & 54.50 & Transcribed & \\
\hline $10 / 23 / 920: 00$ & Manual FIC & 54.20 & Good & \\
\hline $10 / 24 / 920: 00$ & Manual Tape & 54.5 & Transcribed & \\
\hline
\end{tabular}

Data obtained from WHC Surveillance Analysis Computer System, (PC SACS) July 7, 1995. 


\begin{tabular}{|c|c|c|c|c|}
\hline \multicolumn{5}{|c|}{ 241-AP-102 } \\
\hline \multicolumn{5}{|c|}{ Surface Level Data } \\
\hline Date & Type of Measurement & Level (Inches) & Data Quality & Comments \\
\hline $10 / 24 / 920: 00$ & Manual FIC & 54.20 & Good & \\
\hline $10 / 24 / 927: 03$ & Auto FIC & 0 & Suspect & Data not included in graph \\
\hline $10 / 25 / 920: 00$ & Manual Tape & 54.5 & Transcribed & \\
\hline 10/25/92 0:00 & Manual FIC & 54.20 & Good & \\
\hline $10 / 25 / 927: 03$ & Auto FIC & 0 & Suspect & Data not included in graph \\
\hline $10 / 26 / 920: 00$ & Manual Tape & 54.50 & Transcribed & \\
\hline $10 / 26 / 920: 00$ & Manual FIC & 54.2 & Good & \\
\hline $10 / 27 / 920: 00$ & Manual Tape & 54.50 & Transcribed & \\
\hline $10 / 27 / 920: 00$ & Manual FIC & 54.2 & Good & \\
\hline $10 / 27 / 927: 04$ & Auto FIC & 0 & Suspect & Data not included in graph \\
\hline $10 / 28 / 920: 00$ & Manual Tape & 54.50 & Transcribed & \\
\hline $10 / 28 / 920: 00$ & Manual FIC & 54.20 & Good & \\
\hline $10 / 28 / 927: 03$ & Auto FIC & 0.00 & Suspect & Data not included in graph \\
\hline $10 / 29 / 920: 00$ & Manual Tape & 54.5 & Transcribed & \\
\hline $10 / 29 / 920: 00$ & Manual FIC & 54.20 & Good & \\
\hline $10 / 29 / 927: 03$ & Auto FIC & 0.00 & Suspect & Data not included in graph \\
\hline $10 / 30 / 920: 00$ & Manual Tape & 54.5 & Transcribed & \\
\hline $10 / 30 / 920: 00$ & Manual FIC & 54.2 & Good & \\
\hline $10 / 30 / 927: 03$ & Auto FIC & 0 & Suspect & Data not included in graph \\
\hline $10 / 31 / 920: 00$ & Manual Tape & 54.50 & Transçribed & \\
\hline $10 / 31 / 920: 03$ & Auto FIC & $54: 1$ & Good & \\
\hline 11/1/92 0:00 & Manual Tape & 54.50 & Transcribed & \\
\hline $11 / 1 / 920: 00$ & Manual FIC & 54.10 & Good & \\
\hline $11 / 1 / 927: 03$ & Auto FIC & 0.00 & Suspect & Data not included in graph \\
\hline $11 / 2 / 920: 00$ & Manual Tape & 54.5 & Transcribed & \\
\hline $11 / 2 / 920: 00$ & Manual FIC & 54.10 & Good & \\
\hline $11 / 2 / 927: 03$ & Auto FIC & 0 & Suspect & Data not included in graph \\
\hline $11 / 3 / 920: 00$ & Manual Tape & $\mathbf{5 4 . 5 0}$ & Transcribed & \\
\hline $11 / 3 / 920: 00$ & Manual FIC & 54.1 & Good & \\
\hline $11 / 3 / 927: 03$ & Auto FIC & 0 & Suspect & Data not included in graph \\
\hline $11 / 4 / 920: 00$ & Manual Tape & 54.50 & Transcribed & \\
\hline $11 / 4 / 920: 00$ & Manual FiC & 54.1 & Good & \\
\hline $11 / 4 / 927: 03$ & Auto FIC & 0.00 & Suspect & Data not included in graph \\
\hline $11 / 5 / 920: 00$ & Manual Tape & 54.5 & Transcribed & \\
\hline $11 / 5 / 920: 00$ & Manual FIC & 54.10 & Good & \\
\hline $11 / 6 / 920: 00$ & Manual Tape & 54.5 & Transcribed & \\
\hline $11 / 6 / 920: 00$ & Manual FIC & 54.10 & Good & \\
\hline $11 / 7 / 920: 00$ & Manual Tape & 54.50 & Transcribed & \\
\hline $11 / 7 / 920: 00$ & Manual FIC & 54.1 & Good & \\
\hline $11 / 8 / 920: 00$ & Manual Tape & 54.50 & Transcribed & \\
\hline $11 / 8 / 920: 00$ & Manual FIC & 54.2 & Good & \\
\hline $11 / 9 / 920: 00$ & Manual Tape & 54.5 & Transcribed & \\
\hline $11 / 9 / 920: 00$ & Manual FIC & 54.1 & Good & \\
\hline $11 / 10 / 920: 00$ & Manual Tape & 54.5 & Transcribed & \\
\hline $11 / 10 / 926: 04$ & Auto FIC & 54.10 & Good & \\
\hline $11 / 11 / 920: 00$ & Manual Tape & 54.50 & Transcribed & \\
\hline $11 / 11 / 920: 00$ & Manual FIC & 54.1 & Good & \\
\hline $11 / 11 / 927: 04$ & Auto FIC & 0 & Suspect & Data not included in graph \\
\hline
\end{tabular}

Data obtained from WHC Surveillance Analysis Computer System, (PC SACS) July 7, 1995. 
WHC-SD-WM-ER-315, Rev. 0

\begin{tabular}{|c|c|c|c|c|}
\hline \multicolumn{5}{|c|}{ 241-AP-102 } \\
\hline \multicolumn{5}{|c|}{ Surface Level Data } \\
\hline Date & Type of Measurement & Leval (Inches) & Data Quality & Comments \\
\hline $11 / 12 / 920: 00$ & Manual Tape & 54.50 & Transcribed & \\
\hline $11 / 12 / 920: 00$ & Manual FIC & 54.10 & Good & \\
\hline $11 / 12 / 927: 03$ & Auto FIC & 0.00 & Suspect & Data not included in graph \\
\hline $11 / 13 / 920: 00$ & Manual Tape & 54.50 & Transcribed & \\
\hline $11 / 13 / 920: 00$ & Manual FIC & 54.10 & Good & \\
\hline 11/13/92 7:04 & Auto FIC & 0 & Suspect & Data not included in graph \\
\hline $11 / 14 / 920: 00$ & Manual Tape & 54.5 & Transcribed & \\
\hline $11 / 14 / 920: 00$ & Manual FIC & 54.2 & Good & \\
\hline $11 / 14 / 927: 04$ & Auto FIC & 0 & Suspect & Data not included in graph \\
\hline $11 / 15 / 920: 00$ & Manual Tape & 54.50 & Transcribed & \\
\hline $11 / 15 / 920: 00$ & Manual FIC & 54.1 & Good & \\
\hline $11 / 15 / 927: 04$ & Auto FIC & 0.00 & Suspect & Data not included in graph \\
\hline $11 / 16 / 920: 00$ & Manual Tape & 54.50 & Transcribed & \\
\hline $11 / 16 / 920: 00$ & Manual FIC & 54.10 & Good & \\
\hline $11 / 16 / 927: 04$ & Auto FIC & 0.00 & Suspect & Data not included in graph \\
\hline $11 / 17 / 920: 00$ & Manual Tape & 54.5 & Transcribed & \\
\hline $11 / 17 / 920: 00$ & Manual FIC & 54.10 & Good & \\
\hline $11 / 17 / 927: 04$ & Auto FIC & 0 & Suspect & Data not included in graph \\
\hline $11 / 18 / 920: 00$ & Manual Tape & 54.5 & Transcribed & \\
\hline $11 / 18 / 920: 00$ & Manual FIC & 54.1 & Good & \\
\hline $11 / 18 / 927: 02$ & Auto FIC & 0 & Suspect & Data not included in graph \\
\hline $11 / 19 / 920: 00$ & Manual Tape & 54.50 & Transcribed & \\
\hline 11/19/92 0:00 & Manual FIC & 54.1 & Good & \\
\hline 11/19/92 7:02 & Auto FIC & 0 & Suspect & Data not included in graph \\
\hline 11/20/920:00 & Manual Tape & 54.50 & Transcribed & \\
\hline 11/20/92 6:03 & Auto FIC & 54.10 & Good & \\
\hline $11 / 21 / 920: 00$ & Manual Tape & 91.50 & Transcribed & \\
\hline $11 / 21 / 920: 00$ & Manual FIC & 90.60 & Good & \\
\hline 11/21/92 7:02 & Auto FIC & 0 & Suspect & Data not included in graph \\
\hline $11 / 22 / 920: 00$ & Manual Tape & 91.5 & Transcribed & \\
\hline $11 / 22 / 920: 00$ & Manual FIC & 91.10 & Good & \\
\hline $11 / 22 / 927: 03$ & Auto FIC & 0 & Suspect & Data not included in graph \\
\hline $11 / 23 / 920: 00$ & Manual Tape & 91.5 & Transcribed & \\
\hline $11 / 23 / 920: 00$ & Manual FIC & 91.10 & Good & \\
\hline $11 / 23 / 927: 03$ & Auto FIC & 0 & Suspect & Data not included in graph \\
\hline $11 / 24 / 920: 00$ & Manual Tape & 103.25 & Transcribed & \\
\hline $11 / 24 / 927: 03$ & Auto FIC & 0.00 & Suspect & Data not included in graph \\
\hline $11 / 25 / 920: 00$ & Manual Tape & 144.50 & Transcribed & \\
\hline $11 / 25 / 920: 00$ & Manual FIC & 144 & Good & \\
\hline $11 / 25 / 927: 03$ & Auto FIC & 0 & Suspect & Data not included in graph \\
\hline $11 / 26 / 920: 00$ & Manual Tape & 176.5 & Transcribed & \\
\hline $11 / 26 / 92 \quad 0: 00$ & Manual FIC & 177.4 & Good & \\
\hline $11 / 26 / 927: 03$ & Auto FIC & 0 & Suspect & Data not included in graph \\
\hline $11 / 27 / 920: 00$ & Manual Tape & 182.00 & Transeribed & \\
\hline $11 / 27 / 920: 00$ & Manual FIC & 181.70 & Good & \\
\hline $11 / 27 / 927: 03$ & Auto FIC & 0 & Suspect & Data not included in graph \\
\hline $11 / 28 / 920: 00$ & Manual Tape & 182.00 & Transcribed & \\
\hline $11 / 28 / 920: 00$ & Manual FIC & 181.70 & Good & \\
\hline
\end{tabular}

Data obtained from WHC Surveillance Analysis Computer System, (PC SACS) July 7, 1995. 


\begin{tabular}{|c|c|c|c|c|}
\hline \multicolumn{5}{|c|}{ 241-AP-102 } \\
\hline \multicolumn{5}{|c|}{ Surface Laval Data } \\
\hline Date & Type of Measurement & Level (Inches) & Data Quality & Comments \\
\hline $11 / 28 / 927: 03$ & Auto FIC & 0.00 & Suspect & Data not included in graph \\
\hline $11 / 29 / 920: 00$ & Manual Tape & 182 & Transcribed & \\
\hline $11 / 29 / 920: 00$ & Manual FIC & 181.7 & Good & \\
\hline $11 / 29 / 927: 03$ & Auto FIC & 0 & Suspect & Data not included in graph \\
\hline $11 / 30 / 920: 00$ & Manual Tape & 182 & Transcribed & \\
\hline $11 / 30 / 920: 00$ & Manual FIC & 181.7 & Good & \\
\hline 12/1/92 0:00 & Manual Tape & 182.00 & Transcribed & \\
\hline $12 / 1 / 921: 03$ & Auto FIC & 181.6 & Good & \\
\hline $12 / 2 / 920: 00$ & Manual Tape & 182.00 & Transcribed & \\
\hline $12 / 2 / 920: 00$ & Manual FIC & 181.60 & Good & \\
\hline $12 / 2 / 926: 03$ & Auto FIC & 181.6 & Good & \\
\hline $12 / 3 / 920: 00$ & Manual Tape & 182.00 & Transcribed & \\
\hline $12 / 3 / 920: 00$ & Manual FIC & 181.60 & Good & \\
\hline $12 / 3 / 925: 03$ & Auto FIC & 181.60 & Good & \\
\hline $12 / 4 / 920: 00$ & Manual Tape & 182 & Transcribed & \\
\hline 12/4/92 0:00 & Manual FIC & 181.7 & Good & \\
\hline $12 / 4 / 927: 03$ & Auto FIC & 0 & Suspect & Data not included in graph \\
\hline $12 / 5 / 920: 00$ & Manual Tape & 182 & Transcribed & \\
\hline $12 / 5 / 920: 00$ & Manual FIC & 181.6 & Good & \\
\hline $12 / 6 / 920: 00$ & Manual Tape & 182.00 & Transcribed & \\
\hline $12 / 6 / 92 \quad 0: 00$ & Manual FIC & 181.60 & Good & \\
\hline $12 / 6 / 927: 02$ & Auto FIC & 0 & Suspect & Data not included in graph \\
\hline $12 / 7 / 920: 00$ & Manual Tape & 182.00 & Transcribed & . \\
\hline $12 / 7 / 920: 00$ & Manual FIC & 181.60 & Good & \\
\hline $12 / 7 / 927: 03$ & Auto FIC & 0 & Suspect & Data not included in graph \\
\hline $12 / 8 / 920: 00$ & Manual Tape & 182 & Transcribed & \\
\hline $12 / 8 / 920: 00$ & Manual FIC & 181.6 & Good & \\
\hline $12 / 8 / 927: 03$ & Auto FIC & 0.00 & Suspect & Data not included in graph \\
\hline $12 / 9 / 920: 00$ & Manual Tape & 182.00 & Transcribed & \\
\hline $12 / 9 / 927: 03$ & Auto FIC & 181.6 & Good & \\
\hline $12 / 10 / 920: 00$ & Manual Tape & 182.00 & Transcribed & \\
\hline $12 / 10 / 920: 00$ & Manual FIC & 181.6 & Good & \\
\hline $12 / 10 / 927: 03$ & Auto FIC & 0 & Suspect & Data not included in graph \\
\hline 12/11/92 0:00 & Manual Tape & 182 & Transcribed & \\
\hline $12 / 11 / 927: 03$ & Auto FIC & 181.6 & Good & \\
\hline $12 / 12 / 920: 00$ & Manual Tape & 182 & Transcribed & \\
\hline $12 / 13 / 920: 00$ & Manual Tape & 181.50 & Transcribed & \\
\hline $12 / 13 / 927: 04$ & Auto FIC & 0.00 & Suspect & Data not included in graph \\
\hline 12/14/92 0:00 & Manual Tape & 181.75 & Transcribed & \\
\hline $12 / 14 / 920: 00$ & Manual FIC & $181: 50$ & Good & \\
\hline $12 / 14 / 927: 03$ & Auto FIC & 0 & Suspect & Data not included in graph \\
\hline $12 / 15 / 927: 03$ & Auto FIC & 196.4 & Good & \\
\hline $12 / 16 / 922: 02$ & Auto FIC & 234.3 & Good & \\
\hline $12 / 17 / 920: 00$ & Manual Tape & 300.00 & Transcribed & \\
\hline 12/17/92 0:00 & Manual FIC & 299.30 & Good & \\
\hline $12 / 17 / 927: 03$ & Auto FIC & 0.00 & Suspect & Data not included in graph \\
\hline $12 / 18 / 920: 00$ & Manual FIC & 346.60 & Good & \\
\hline $12 / 18 / 927: 03$ & Auto FIC & 42.60 & Suspect & Data not included in graph \\
\hline
\end{tabular}

Data obtained from WHC Surveillance Analysis Computer System, (PC SACS) July 7, 1995. 


\begin{tabular}{|c|c|c|c|c|}
\hline \multicolumn{5}{|c|}{ 241-AP-102 } \\
\hline \multicolumn{5}{|c|}{ Surface Leval Data } \\
\hline Date & Type of Mesasurement & Leval (Inches) & Data Quality & Comments \\
\hline $12 / 19 / 920: 00$ & Manual Tape & 387 & Transcribed & \\
\hline $12 / 19 / 920: 00$ & Manual FIC & 386.9 & Good & \\
\hline $12 / 19 / 927: 03$ & Auto FIC & 82.3 & Suspect & Data not included in graph \\
\hline $12 / 20 / 920: 00$ & Manual Tape & 402.25 & Transcribed & $\cdot$ \\
\hline $12 / 20 / 927: 04$ & Auto FIC & 402 & Good & \\
\hline $12 / 21 / 926: 03$ & Auto FIC & 402.00 & Good & \\
\hline $12 / 22 / 920: 00$ & Manual FIC & 402 & Good & \\
\hline $12 / 23 / 927: 04$ & Auto FIC & 402 & Good & \\
\hline $12 / 24 / 927: 03$ & Auto FIC & 402 & Good & \\
\hline $12 / 25 / 922: 03$ & Auto FIC & 401.90 & Good & \\
\hline $12 / 26 / 920: 00$ & Manual FIC & 401.90 & Good & \\
\hline $12 / 26 / 927: 03$ & Auto FIC & 0.00 & Suspect & Data not included in graph \\
\hline $12 / 27 / 920: 00$ & Manual FIC & 401.90 & Good & \\
\hline $12 / 27 / 927: 03$ & Auto FIC & 0 & Suspect & Data not included in graph \\
\hline $12 / 28 / 920.00$ & Manual FIC & 401.9 & Good & \\
\hline $12 / 28 / 927: 03$ & Auto FIC & 0 & Suspect & Data not included in graph \\
\hline $12 / 29 / 920: 00$ & Manual Tape & 402.25 & Transcribed & \\
\hline 12/29/92 0:00 & Manual FIC & 401.9 & Good & \\
\hline $12 / 30 / 920: 00$ & Manual FIC & 401.90 & Good & $x_{1}$ \\
\hline $12 / 30 / 927: 03$ & Auto FIC & 0 & Suspect & Data not included in graph \\
\hline $12 / 31 / 920: 00$ & Manual FIC & 401.90 & Good & \\
\hline $12 / 31 / 927: 03$ & Auto FIC & 0.00 & Suspect & Data not inciuded in graph \\
\hline $1 / 1 / 930: 00$ & Manual FIC & 401.9 & Good & \\
\hline $1 / 1 / 937: 04$ & Auto FIC & 0.00 & Suspect & Data not included in graph \\
\hline $1 / 2 / 930: 00$ & Manual FIC & 401.9 & Good & \\
\hline $1 / 2 / 930: 00$ & Manual Tape & 402.25 & Good & \\
\hline $1 / 3 / 930: 00$ & Manual FIC & 401.9 & Good & \\
\hline $1 / 4 / 930: 00$ & Manual FIC & 401.90 & Good & \\
\hline $1 / 4 / 930: 00$ & Manual Tape & 402.00 & Good & \\
\hline $1 / 5 / 930: 00$ & Manual FIC & 401.90 & Good & \\
\hline $1 / 5 / 930: 00$ & Manual Tape & 402.25 & Good & \\
\hline $1 / 6 / 930: 00$ & Manual FIC & 401.95 & Good & \\
\hline 1/6/93 0:00 & Manual Tape & 402.5 & Good & \\
\hline $1 / 7 / 930: 00$ & Manual FIC & 401.95 & Good & \\
\hline $1 / 7 / 930: 00$ & Manual Tape & 402.25 & Good & \\
\hline $1 / 8 / 930: 00$ & Manual FIC & 401.95 & Good & \\
\hline 1/8/93 0:00 & Manual Tape & 402.00 & Good & \\
\hline $1 / 9 / 930: 00$ & Manual Tape & 402.00 & Good & \\
\hline $1 / 10 / 930: 00$ & Manual FIC & 402.00 & Good & \\
\hline $1 / 10 / 930: 00$ & Manual Tape & 402 & Good & \\
\hline $1 / 11 / 930: 00$ & Manual FIC & 402.00 & Good & \\
\hline $1 / 11 / 930.00$ & Manual Tape & 402.25 & Good & \\
\hline $1 / 12 / 930: 00$ & Manual Tape & 402.00 & Good & \\
\hline $1 / 12 / 937: 06$ & Auto FIC & 402 & Gopd & \\
\hline $1 / 13 / 930: 00$ & Manual Tape & 402.00 & Good & \\
\hline $1 / 13 / 937: 06$ & Auto FIC & 402 & Good & \\
\hline $1 / 14 / 930: 00$ & Manual Tape & 402.25 & Good & \\
\hline $1 / 14 / 931: 02$ & Auto FIC & 402.10 & Good & \\
\hline
\end{tabular}

Data obtained from WHC Surveillance Analysis Computer System, (PC SACS) July 7, 1995. 
WHC-SD-WM-ER-315， Rev. 0

\begin{tabular}{|c|c|c|c|c|}
\hline \multicolumn{5}{|c|}{$\frac{241-A P-102}{\text { Surface Level Data }}$} \\
\hline \multicolumn{5}{|c|}{ Surface Level Data } \\
\hline Date & Type of Measurement & Level (Inches) & Data Quality & Comments \\
\hline $1 / 15 / 930: 00$ & Manual FIC & 402.1 & Good & \\
\hline $1 / 15 / 930: 00$ & Manual Tape & 402.25 & Good & \\
\hline $1 / 15 / 937: 03$ & Auto FIC & 0 & Suspect & Data not included in graph \\
\hline $1 / 16 / 930: 00$ & Manual FIC & 402.10 & Good & \\
\hline $1 / 16 / 930: 00$ & Manual Tape & 402.00 & Good & \\
\hline $1 / 17 / 930: 00$ & Manual FIC & 402.10 & Good & \\
\hline $1 / 17 / 930: 00$ & Manual Tape & 402.25 & Good & \\
\hline 1/18/93 0:00 & Manual FIC & 402.1 & Good & \\
\hline $1 / 18 / 930: 00$ & Manual Tape & 402 & Good & \\
\hline $1 / 19 / 930: 00$ & Manual FIC & 402.1 & Good & \\
\hline $1 / 19 / 930: 00$ & Manual Tape & 402.25 & Good & \\
\hline $1 / 20 / 930: 00$ & Manual FIC & 402.10 & Good & \\
\hline $1 / 20 / 930: 00$ & Manual Tape & 402.25 & Good & \\
\hline $1 / 21 / 930: 00$ & Manual Tape & 402.25 & Good & \\
\hline 1/21/937:02 & Auto FIC & 402.2 & Good & \\
\hline 1/22/93 0:00 & Manual Tape & 402.25 & Good & \\
\hline $1 / 22 / 937: 04$ & Auto FIC & 0.00 & Suspect & Data not included in graph \\
\hline $1 / 23 / 930: 00$ & Manual FIC & 401.80 & Good & \\
\hline $1 / 23 / 930: 00$ & Manual Tape & 402.00 & Good & \\
\hline 1/23/93 6:03 & Auto FIC & 400.80 & Suspect & Data not included in graph \\
\hline 1/24/93 0:00 & Manual Tape & 402.00 & Good & \\
\hline 1/24/93 7:03 & Auto FIC & 0 & Suspect & Data not included in graph \\
\hline $1 / 25 / 930: 00$ & Manual FIC & 401.80 & Good & \\
\hline $1 / 25 / 930: 00$ & Manual Tape & 401.75 & Good & \\
\hline 1/25/93 7:03 & Auto FIC & 0 & Suspect & Data not included in graph \\
\hline $1 / 26 / 930: 00$ & Manual FIC & 401.8 & Good & \\
\hline $1 / 26 / 930: 00$ & Manual Tape & 402 & Good & \\
\hline $1 / 26 / 937: 04$ & Auto FIC & 0 & Suspect & Data not included in graph \\
\hline $1 / 27 / 930: 00$ & Manual FIC & 401.7 & Good & \\
\hline $1 / 27 / 930: 00$ & Manual Tape & 402 & Good & \\
\hline $1 / 27 / 937: 04$ & Auto FIC & 0.00 & Suspect & Data not included in graph \\
\hline $1 / 28 / 930: 00$ & Manual FIC & 401.70 & Good & \\
\hline $1 / 28 / 930: 00$ & Manual Tape & 402.50 & Good & \\
\hline $1 / 28 / 937: 04$ & Auto FIC & 0.00 & Suspect & Data not included in graph \\
\hline $1 / 29 / 930: 00$ & Manual FIC & 401.70 & Good & \\
\hline $1 / 29 / 930: 00$ & Manual Tape & 402.25 & Good & \\
\hline $1 / 29 / 937: 04$ & Auto FIC & 0.00 & Suspect & Data not included in graph \\
\hline $1 / 30 / 930: 00$ & Manual FIC & 401.7 & Good & \\
\hline $1 / 30 / 930: 00$ & Manual Tape & 402.5 & Good & \\
\hline $1 / 30 / 937: 03$ & Auto FIC & 0 & Suspect & Data not included in graph \\
\hline $1 / 31 / 930: 00$ & Manual FIC & 401.70 & Good & \\
\hline $1 / 31 / 930: 00$ & Manual Tape & 402.50 & Good & \\
\hline $1 / 31 / 937: 04$ & Auto FIC & 0 & Suspect & Data not included in graph \\
\hline $2 / 1 / 93 \quad 0: 00$ & Manual FIC & $401.70^{\circ}$ & Good & \\
\hline $2 / 1 / 93 \quad 0: 00$ & Manual Tape & 402.00 & Good & \\
\hline $2 / 1 / 937: 04$ & Auto FIC & 0 & Suspect & Data not included in graph \\
\hline $2 / 2 / 930: 00$ & Manual FIC & 401.7 & Good & \\
\hline $2 / 2 / 930: 00$ & Manual Tape & 402 & Good & \\
\hline
\end{tabular}

Data obtained from WHC Surveillance Analysis Computer System, (PC SACS) July 7, 1995. 
WHC-SD-WM-ER-315, Rev. 0

\begin{tabular}{|c|c|c|c|c|}
\hline \multicolumn{5}{|c|}{ 241-AP-102 } \\
\hline \multicolumn{5}{|c|}{ Surface Level Data } \\
\hline Date & Type of Measurement & Level (Inches) & Data Quality & Comments \\
\hline $2 / 2 / 937: 04$ & Auto FIC & 0.00 & Suspect & Data not included in graph \\
\hline $2 / 3 / 930: 00$ & Manual FIC & 401.65 & Good & \\
\hline 2/3/93 0:00 & Manual Tape & 402.25 & Good & \\
\hline 2/4/93 0:00 & Manual FIC & 401.60 & Good & \\
\hline $2 / 4 / 930: 00$ & Manual Tape & 402.25 & Good & \\
\hline 2/4/93 7:04 & Auto FIC & 0.00 & Suspect & Data not included in graph \\
\hline $2 / 5 / 930: 00$ & Manual FIC & 401.40 & Good & \\
\hline $2 / 5 / 930: 00$ & Manual Tape & 402.00 & Good & \\
\hline 2/5/93 7:04 & Auto FIC & 0 & Suspect & Data not included in graph \\
\hline $2 / 6 / 930: 00$ & Manual FIC & 401.40 & Good & \\
\hline $2 / 6 / 930: 00$ & Manual Tape & 401.75 & Good & \\
\hline $2 / 6 / 937: 04$ & Auto FIC & 0 & Suspect & Data not included in graph \\
\hline $2 / 7 / 930: 00$ & Manual FIC & 401.4 & Good & \\
\hline $2 / 7 / 930: 00$ & Manual Tape & 401.75 & Good & \\
\hline 2/7/93 7:03 & Auto FIC & 0 & Suspect & Data not included in graph \\
\hline $2 / 8 / 930: 00$ & Manual FIC & 401.4 & Good & \\
\hline $2 / 8 / 930: 00$ & Manual Tape & 401.75 & Good & \\
\hline $2 / 8 / 937: 04$ & Auto FIC & 0.00 & Suspect & Data not included in graph \\
\hline $2 / 9 / 930: 00$ & Manual FIC & 401.30 & Good & \\
\hline 2/9/93 0:00 & Manual Tape & 401.75 & Good & \\
\hline 2/9/93 7:04 & Auto FIC & 0 & Suspect & Data not included in graph \\
\hline $2 / 10 / 930: 00$ & Manual FIC & 401.35 & Good & \\
\hline $2 / 10 / 930: 00$ & Manual Tape & 401.75 & Good & \\
\hline $2 / 10 / 937: 04$ & Auto FIC & 0 & Suspect & Data not included in graph \\
\hline $2 / 11 / 930: 00$ & Manual FIC & 401.4 & Good & \\
\hline 2/11/93 0:00 & Manual Tape & 401.75 & Good & \\
\hline $2 / 11 / 937: 04$ & Auto FIC & 0.00 & Suspect & Data not included in graph \\
\hline $2 / 12 / 930: 00$ & Manual FIC & 401.40 & Good & \\
\hline 2/12/93 0:00 & Manual Tape & 401.50 & Good & \\
\hline $2 / 12 / 937: 03$ & Auto FIC & 0.00 & Suspect & Data not included in graph \\
\hline $2 / 13 / 930: 00$ & Manual FIC & 0.00 & Suspect & Data not included in graph \\
\hline 2/13/93 0:00 & Manual Tape & 401.25 & Good & \\
\hline 2/13/93 7:03 & Auto FIC & 0 & Suspect & Data not included in graph \\
\hline $2 / 14 / 930: 00$ & Manual FIC & 401.3 & Good & $\cdot$ \\
\hline $2 / 14 / 930: 00$ & Manual Tape & 401.25 & Good & \\
\hline $2 / 14 / 937: 04$ & Auto FIC & 0 & Suspect & Data not included in graph \\
\hline 2/15/93 0:00 & Manual FIC & 401.3 & Good & \\
\hline 2/15/93 0:00 & Manual Tape & 401.5 & Good & \\
\hline 2/15/93 7:04 & Auto FIC & 0 & Suspect & Data not included in graph \\
\hline $2 / 16 / 930: 00$ & Manual FIC & 401.30 & Good & \\
\hline $2 / 16 / 930: 00$ & Manual Tape & 401.25 & Good & \\
\hline $2 / 16 / 937: 02$ & Auto FIC & 0.00 & Suspect & Data not included in graph \\
\hline 2/17/93 0:00 & Manual FIC & 401.30 & Good & \\
\hline 2/17/930:00 & Manual Tape & 401.50 & Good & \\
\hline $2 / 18 / 930: 00$ & Manual FIC & 0.00 & Suspect & Data not included in graph \\
\hline $2 / 18 / 930: 00$ & Manual Tape & 401.50 & Good & \\
\hline $2 / 18 / 937: 03$ & Auto FIC & 0.00 & Suspect & Data not included in graph \\
\hline $2 / 19 / 930: 00$ & Manual Tape & 401.5 & Good & \\
\hline
\end{tabular}

Data obtained from WHC Surveillance Analysis Computer System, (PC SACS) July 7, 1995. 
WHC-SD-WM-ER-315, Rev. 0

\begin{tabular}{|c|c|c|c|c|}
\hline \multicolumn{5}{|c|}{ 241-AP-102 } \\
\hline \multicolumn{5}{|c|}{ Surface Leval Data } \\
\hline Date & Type of Measurement & Lovel (Inches) & Data Quality & Comments \\
\hline 2/19/93 7:02 & Auto FIC & 0 & Suspect & Data not included in graph \\
\hline $2 / 20 / 930: 00$ & Manual FIC & 401.30 & Good & \\
\hline $2 / 20 / 930: 00$ & Manual Tape & 401.5 & Good & \\
\hline $2 / 20 / 937: 03$ & Auto FIC & 0 & Suspect & Data not included in graph \\
\hline $2 / 21 / 930: 00$ & Manual FIC & 401.3 & Good & \\
\hline $2 / 21 / 930: 00$ & Manual Tape & 401.50 & Good & \\
\hline $2 / 21 / 937: 04$ & Auto FIC & 0.00 & Suspect & Data not included in graph \\
\hline $2 / 22 / 930: 00$ & Manual FIC & 401.3 & Good & \\
\hline $2 / 22 / 930: 00$ & Manual Tape & $401: 50$ & Good & \\
\hline 2/22/93 7:04 & Auto FIC & 0 & Suspect & Data not included in graph \\
\hline $2 / 23 / 930: 00$ & Manusl FIC & 401.30 & Good & \\
\hline $2 / 23 / 930: 00$ & Manual Tape & 401.75 & Good & \\
\hline $2 / 23 / 937: 03$ & Auto FIC & 0 & Suspect & Data not included in graph \\
\hline $2 / 24 / 930: 00$ & Manual FIC & 401.30 & Good & \\
\hline $2 / 24 / 93 \quad 0: 00$ & Manual Tape & 401.75 & Good & \\
\hline $2 / 24 / 937: 04$ & Auto FIC & 0.00 & Suspect & Data not included in graph \\
\hline $2 / 25 / 930: 00$ & Manual FIC & 401.30 & Good & \\
\hline $2 / 25 / 930: 00$ & Manual Tape & 402 & Good & \\
\hline $2 / 25 / 937: 03$ & Auto FIC & 0.00 & Suspect & Data not included in graph \\
\hline $2 / 26 / 93 \quad 0: 00$ & Manual FIC & 401.25 & Good & \\
\hline $2 / 26 / 930: 00$ & Manual Tape & 401.75 & Good & \\
\hline $2 / 26 / 937: 03$ & Auto FIC & 0 & Suspect & Data not included in graph \\
\hline $2 / 27 / 930: 00$ & Manual FIC & 401.2 & Good & \\
\hline $2 / 27 / 930: 00$ & Manual Tape & 402.00 & Good & \\
\hline $2 / 27 / 937: 04$ & Auto FIC & 0 & Suspect & Data not included in graph \\
\hline $2 / 28 / 930: 00$ & Manual FIC & $401: 20$ & Good & \\
\hline $2 / 28 / 930: 00$ & Manual Tape & 402 & Good & \\
\hline $2 / 28 / 937: 03$ & Auto FIC & 0 & Suspect & Data not included in graph \\
\hline $3 / 1 / 93 \quad 0: 00$ & Manual FIC & 401.20 & Good & \\
\hline 3/1/93 0:00 & Manual Tape & 401.50 & Good & \\
\hline $3 / 1 / 937: 03$ & Auto FIC & 0.00 & Suspect & Data not included in graph \\
\hline 3/2/93 0:00 & Manual FIC & 0 & Suspect & Data not included in graph \\
\hline $3 / 2 / 930: 00$ & Manual Tape & 401.50 & Good & \\
\hline $3 / 2 / 937: 04$ & Auto FIC & 0.00 & Suspect & Data not included in graph \\
\hline $3 / 3 / 930: 00$ & Manual FIC & 401.2 & Good & \\
\hline $3 / 3 / 930: 00$ & Manual Tape & 401.75 & Good & \\
\hline $3 / 3 / 937: 03$ & Auto FIC & 0 & Suspect & Data not included in graph \\
\hline $3 / 4 / 930: 00$ & Manual FIC & 401.30 & Good & \\
\hline $3 / 4 / 930: 00$ & Manual Tape & 401.75 & Good & \\
\hline 3/4/93 7:04 & Auto FIC & 0 & Suspect & Data not included in graph \\
\hline $3 / 5 / 930: 00$ & Manual FIC & 401.35 & Good & \\
\hline $3 / 5 / 930: 00$ & Manual Tape & 401.50 & Good & \\
\hline $3 / 5 / 937: 04$ & Auto FIC & 0.00 & Suspect & Data not included in graph \\
\hline $3 / 6 / 930: 00$ & Manual FIC & 401.4 & Good & \\
\hline $3 / 6 / 93 \quad 0: 00$ & Manual Tape & 401.50 & Good & \\
\hline $3 / 6 / 937: 03$ & Auto FIC & 0 & Suspect & Data not included in graph \\
\hline 3/7/93 0:00 & Manual FIC & 401.35 & Good & \\
\hline $3 / 7 / 930: 00$ & Manual Tape & 401.75 & Good & \\
\hline
\end{tabular}

Data obtained from WHC Surveillance Analysis Computer System, (PC SACS) July 7, 1995. 


\begin{tabular}{|c|c|c|c|c|}
\hline \multicolumn{5}{|c|}{ 241-AP-102 } \\
\hline \multicolumn{5}{|c|}{ Surface Level Data } \\
\hline Date & Type of Measurement & Leval (Inches) & Data Quality & Comments \\
\hline $3 / 7 / 937: 03$ & Auto FIC & 0 & Suspect & Data not included in graph \\
\hline $3 / 8 / 930: 00$ & Manual FIC & 401.40 & Good & \\
\hline $3 / 8 / 93 \quad 0: 00$ & Manual Tape & 401.75 & Good & \\
\hline 3/8/93 7:03 & Auto FIC & 0.00 & Suspect & Data not included in graph \\
\hline $3 / 9 / 930: 00$ & Manual FIC & 401.40 & Good & \\
\hline $3 / 9 / 930: 00$ & Manual Tape & 401.75 & Good & \\
\hline $3 / 9 / 937: 04$ & Auto FIC & 0.00 & Suspect & Data not included in graph \\
\hline $3 / 10 / 930: 00$ & Manual FIC & 401.45 & Good & \\
\hline $3 / 10 / 930: 00$ & Manual Tape & 401.50 & Good & \\
\hline $3 / 10 / 937: 05$ & Auto FIC & 0 & Suspect & Data not included in graph \\
\hline $3 / 11 / 930: 00$ & Manual FIC & 401.5 & Good & \\
\hline $3 / 11 / 930: 00$ & Manual Tape & 401.75 & Good & \\
\hline $3 / 11 / 937: 04$ & Auto FIC & 0 & Suspect & Data not included in graph \\
\hline $3 / 12 / 930: 00$ & Manual FIC & 401.5 & Good & \\
\hline $3 / 12 / 930: 00$ & Manual Tape & 401.75 & Good & \\
\hline $3 / 12 / 937: 04$ & Auto FIC & 0 & Suspect & Data not included in graph \\
\hline $3 / 13 / 930: 00$ & Manual FIC & 401.50 & Good & \\
\hline $3 / 13 / 93 \quad 0: 00$ & Manual Tape & 402.00 & Good & \\
\hline $3 / 13 / 937: 03$ & Auto FIC & 0.00 & Suspect & Data not included in graph \\
\hline $3 / 14 / 930: 00$ & Manual FIC & 401.60 & Good & \\
\hline $3 / 14 / 930: 00$ & Manual Tape & 402.00 & Good & \\
\hline $3 / 14 / 93 \quad 7: 04$ & Auto FIC & 0.00 & Suspect & Data not included in graph \\
\hline $3 / 15 / 930: 00$ & Manual FIC & $401: 60$ & Good & \\
\hline $3 / 15 / 93 \quad 0: 00$ & Manual Tape & 402 & Good & \\
\hline 3/15/93 7:03 & Auto FIC & 0 & Suspect & Data not included in graph \\
\hline $3 / 16 / 930: 00$ & Manual FIC & 401.5 & Good & \\
\hline $3 / 16 / 930: 00$ & Manual Tape & 402 & Good & \\
\hline $3 / 16 / 937: 04$ & Auto FIC & 0 & Suspect & Data not included in graph \\
\hline 3/17/93 0:00 & Manual FIC & 0 & Suspect & Data not included in graph \\
\hline $3 / 17 / 930: 00$ & Manual Tape & 401.25 & Good & \\
\hline $3 / 17 / 937: 03$ & Auto FIC & 0.00 & Suspect & Data not included in graph \\
\hline $3 / 18 / 930: 00$ & Manual FIC & 0.00 & Suspect & Data not included in graph \\
\hline $3 / 18 / 930: 00$ & Manual Tape & 401.50 & Good & \\
\hline $3 / 18 / 93 \quad 7: 04$ & Auto FIC & 0 & Suspect & Data not included in graph \\
\hline $3 / 19 / 930: 00$ & Manual FIC & 401.90 & Good & \\
\hline $3 / 19 / 930: 00$ & Manual Tape & 401.25 & Good & \\
\hline $3 / 19 / 937: 03$ & Auto FIC & 0 & Suspect & Data not included in graph \\
\hline $3 / 20 / 930: 00$ & Manual FIC & 0 & Suspect & Data not included in graph \\
\hline 3/20/93 0:00 & Manual Tape & 401.25 & Good & \\
\hline $3 / 20 / 937: 04$ & Auto FIC & 0.00 & Suspect & Data not included in graph \\
\hline $3 / 21 / 930: 00$ & Manual FIC & 402.00 & Good & \\
\hline $3 / 21 / 930: 00$ & Manual Tape & 402.25 & Good & \\
\hline $3 / 21 / 937: 04$ & Auto FIC & 0 & Suspect & Data not included in graph \\
\hline $3 / 22 / 930: 00$ & Manual FIC & 402.00 & Good & \\
\hline $3 / 22 / 930: 00$ & Manual Tape & 402.00 & Good & \\
\hline $3 / 22 / 937: 03$ & Auto FIC & 0 & Suspect & Data not included in graph \\
\hline $3 / 23 / 930: 00$ & Manual FIC & 402 & Good & \\
\hline $3 / 23 / 930: 00$ & Manual Tape & 401.75 & Good & \\
\hline
\end{tabular}

Data obtained from WHC Surveillance Analysis Computer System, (PC SACS) July 7, 1995. 
WHC-SD-WM-ER-315, Rev. 0

\begin{tabular}{|c|c|c|c|c|}
\hline \multicolumn{5}{|c|}{ 241-AP-102 } \\
\hline \multicolumn{5}{|c|}{$\begin{array}{l}\text { Surface Level Data } \\
\text { Tyne of Measurement }\end{array}$} \\
\hline Date & Type of Measurement & Level (Inches) & Data Quality & Comments \\
\hline $3 / 23 / 937: 03$ & Auto FIC & 0.00 & Suspect & Data not included in graph \\
\hline $3 / 24 / 930: 00$ & Manual FIC & 0 & Suspect & Data not included in graph \\
\hline $3 / 24 / 93 \quad 0: 00$ & Manual Tape & 402 & Good & \\
\hline $3 / 24 / 937: 04$ & Auto FIC & 0 & Suspect & Data not included in graph \\
\hline 3/25/93 0:00 & Manual Tape & 401.50 & Good & \\
\hline $3 / 25 / 937: 03$ & Auto FIC & 0 & Suspect & Data not included in graph \\
\hline $3 / 26 / 930: 00$ & Manual Tape & 401.75 & Good & \\
\hline $3 / 26 / 93 \quad 7: 03$ & Auto FIC & 0.00 & Suspect & Data not included in graph \\
\hline $3 / 27 / 93 \quad 0: 00$ & Manual Tape & 401.75 & Good & \\
\hline $3 / 27 / 937: 03$ & Auto FIC & 0.00 & Suspect & Data not included in graph \\
\hline $3 / 28 / 930: 00$ & Manual Tape & 401.75 & Good & \\
\hline $3 / 28 / 937: 03$ & Auto FIC & 0 & Suspect & Data not included in graph \\
\hline $3 / 29 / 93 \quad 0: 00$ & Manual Tape & 402 & Good & \\
\hline $3 / 29 / 93$ 7:03 & Auto FIC & 0 & Suspect & Data not included in graph \\
\hline $3 / 30 / 930: 00$ & Manual Tape & 402.50 & Good & \\
\hline $3 / 30 / 937: 03$ & Auto FIC & 0 & Suspect & Data not included in graph \\
\hline $3 / 31 / 930: 00$ & Manual Tape & 402.75 & Good & \\
\hline $3 / 31 / 937: 03$ & Auto FIC & 0.00 & Suspect & Data not included in graph \\
\hline 4/1/93 10:03 & Manual Tape & 402.5 & Good & \\
\hline $4 / 2 / 93 \quad 10: 03$ & Manual Tape & 402.5 & Good & \\
\hline 4/3/93 10:03 & Manual Tape & 402.75 & Good & \\
\hline $4 / 4 / 93 \quad 10: 03$ & Manual Tape & 402.50 & Good & \\
\hline $4 / 5 / 93 \quad 10: 03$ & Manual Tape & 402.50 & Good & \\
\hline $4 / 6 / 93 \quad 10: 03$ & Manual Tape & 402.5 & Good & \\
\hline $4 / 7 / 93 \quad 10: 03$ & Manual Tape & 402.5 & Good & \\
\hline $4 / 8 / 93 \quad 10: 03$ & Manual Tape & 402.75 & Good & \\
\hline 4/9/93 10:03 & Manual Tape & 402.75 & Good & \\
\hline $4 / 10 / 93 \quad 10: 03$ & Manual Tape & 402.75 & Good & \\
\hline $4 / 11 / 93 \quad 10: 03$ & Manual Tape & 402.75 & Good & \\
\hline $4 / 12 / 93 \quad 10: 03$ & Manual Tape & 402.75 & Good & \\
\hline $4 / 13 / 9310: 03$ & Manual Tape & $402 . \overline{75}$ & Good & \\
\hline 4/14/93 10:03 & Manual Tape & 402.75 & Good & \\
\hline $4 / 15 / 93 \quad 10: 03$ & Manual Tape & 402.75 & Good & \\
\hline 4/16/93 10:03 & Manual Tape & 402.75 & Good & \\
\hline 4/17/93 10:03 & Manual Tape & 402.75 & Good & \\
\hline 4/18/93 10:03 & Manual Tape & 402.75 & Good & \\
\hline 4/19/93 10:03 & Manual Tape & 402.75 & Good & \\
\hline $4 / 20 / 9310: 03$ & Manual Tape & 403.00 & Good & \\
\hline $4 / 21 / 93 \quad 10: 03$ & Manual Tape & 403.00 & Good & \\
\hline $4 / 22 / 93 \quad 10: 03$ & Manual Tape & 402.75 & Good & \\
\hline $4 / 23 / 93 \quad 10: 03$ & Manual Tape & 402.75 & Good & \\
\hline 4/24/93 10:03 & Manual Tape & 403 & Good & \\
\hline $4 / 25 / 93 \quad 10: 03$ & Manual Tape & 403.00 & Good & \\
\hline $4 / 26 / 93 \quad 10: 03$ & Manual Tape & 403.00 & Good & \\
\hline $4 / 27 / 93 \quad 10: 03$ & Manual Tape & 403 & Good & \\
\hline $4 / 28 / 93 \quad 10: 03$ & Manual Tape & 403.00 & Good & \\
\hline $4 / 29 / 93 \quad 10: 03$ & Manual Tape & 403.00 & Good & \\
\hline $4 / 30 / 9310: 03$ & Manual Tape & 403 & Good & \\
\hline
\end{tabular}

Data obtained from WHC Surveillance Analysis Computer System, (PC SACS) July 7, 1995. 


\begin{tabular}{|c|c|c|c|c|}
\hline \multicolumn{5}{|c|}{ 241-AP-102 } \\
\hline & & Urface Level Date & & \\
\hline Date & Type of Measurement & Level (Inches) & Data Quality & Comments \\
\hline $5 / 1 / 93 \quad 10: 03$ & Manual Tape & 403 & Good & \\
\hline $5 / 2 / 93 \quad 10: 03$ & Manual Tape & 403.00 & Good & \\
\hline $5 / 3 / 9310: 03$ & Manual Tape & 403.00 & Good & \\
\hline $5 / 4 / 93 \quad 10: 03$ & Manual Tape & 403.00 & Good & \\
\hline $5 / 5 / 93 \quad 10: 03$ & Manual Tape & 403 & Good & \\
\hline $5 / 6 / 93 \quad 10: 03$ & Manual Tape & 403 & Good & \\
\hline $5 / 7 / 93 \quad 10: 03$ & Manual Tape & 403.00 & Good & \\
\hline $5 / 8 / 93 \quad 10: 03$ & Manual Tape & 403.00 & Good & \\
\hline $5 / 9 / 93 \quad 10: 03$ & Manual Tape & 403 & Good & \\
\hline $5 / 10 / 9310: 03$ & Manual Tape & 403.00 & Good & \\
\hline 5/11/93 10:03 & Manual Tape & 403.00 & Good & \\
\hline 5/12/93 10:03 & Manual Tape & 402.75 & Good & \\
\hline $5 / 13 / 9310: 03$ & Manual Tape & 403 & Good & \\
\hline 5/14/93 10:03 & Manual Tape & 403.00 & Good & \\
\hline $5 / 15 / 93 \quad 10: 03$ & Manual Tape & 402.75 & Good & \\
\hline $5 / 16 / 9310: 03$ & Manual Tape & 402.75 & Good & \\
\hline 5/17/93 7:03 & Auto FIC & 0.00 & Suspect & Data not included in graph \\
\hline $5 / 17 / 93 \quad 10: 03$ & Manual Tape & 402.75 & Good & \\
\hline $5 / 18 / 937: 03$ & Auto FIC & 0 & Suspect & Data not included in graph \\
\hline $5 / 18 / 93 \quad 10: 03$ & Manual Tape & 402.75 & Good & \\
\hline $5 / 19 / 937: 03$ & Auto FIC & 0 & Suspect & Dato not included in graph \\
\hline $5 / 19 / 93 \quad 10: 03$ & Manual Tape & 402.75 & Good & \\
\hline $5 / 20 / 937: 03$ & Auto FIC & 0.00 & Suspect & Data not included in graph \\
\hline $5 / 20 / 9310: 03$ & Manual Tape & 402.75 & Good & \\
\hline $5 / 21 / 9310: 03$ & Manual Tape & 402.75 & Good & \\
\hline $5 / 22 / 93 \quad 10: 03$ & Manual Tape & 402.75 & Good & \\
\hline $5 / 23 / 9310: 03$ & Manual Tape & 402.75 & Good & \\
\hline $5 / 24 / 93 \quad 10: 03$ & Manual Tape & 402.75 & Good & \\
\hline $5 / 25 / 93 \quad 10: 03$ & Manual Tape & 402.5 & Good & \\
\hline $5 / 26 / 93 \quad 10: 03$ & Manual Tape & 402.75 & Good & \\
\hline $5 / 27 / 9310: 03$ & Manual Tape & 402.50 & Good & \\
\hline $5 / 28 / 93 \quad 10: 03$ & Manual Tape & 402.75 & Good & \\
\hline $5 / 29 / 9310: 03$ & Manual Tape & 402.75 & Good & \\
\hline $5 / 30 / 9310: 03$ & Manual Tape & 402.75 & Good & \\
\hline $5 / 31 / 9310: 03$ & Manual Tape & 402.75 & Good & \\
\hline $6 / 1 / 93 \quad 10: 00$ & Manual Tape & 402.50 & Good & \\
\hline $6 / 2 / 9310: 00$ & Manual Tape & 402.50 & Good & \\
\hline $6 / 3 / 93 \quad 10: 00$ & Manual Tape & 402.5 & Good & \\
\hline $6 / 4 / 93 \quad 10: 00$ & Manual Tape & 402.75 & Good & \\
\hline $6 / 5 / 93 \quad 10: 00$ & Manual Tape & 402.75 & Good & \\
\hline $6 / 6 / 9310: 00$ & Manual Tape & 402.50 & Good & \\
\hline $6 / 7 / 9310: 00$ & Manual Tape & 402.50 & Good & \\
\hline $6 / 8 / 93 \quad 10: 00$ & Manual Tape & 402.5 & Good & \\
\hline $6 / 9 / 9310: 00$ & Manual Tape & 402.50 & Good & \\
\hline $6 / 10 / 9310: 00$ & Manual Tape & 402.50 & Good & \\
\hline $6 / 11 / 9310: 00$ & Manual Tape & 402.5 & Good & \\
\hline $6 / 12 / 93 \quad 10: 00$ & Manual Tape & 402.5 & Good & \\
\hline $6 / 13 / 93 \quad 10: 00$ & Manual Tape & 402.50 & Good & \\
\hline
\end{tabular}

Data obtained from WHC Surveillance Analysis Computer System, (PC SACS) July 7, 1995. 


\begin{tabular}{|c|c|c|c|c|}
\hline \multicolumn{5}{|c|}{ 241-AP-102 } \\
\hline \multicolumn{5}{|c|}{ Surface Level Data } \\
\hline Date & Type of Measurement & Level (Inches) & Data Quality & Comments \\
\hline $6 / 14 / 93 \quad 10: 00$ & Manual Tape & 402.50 & Good & \\
\hline $6 / 15 / 9310: 00$ & Manual Tape & 402.50 & Good & \\
\hline $6 / 16 / 93 \quad 10: 00$ & Manual Tape & 402.25 & Good & \\
\hline 6/17/93 10:00 & Manual Tape & 402.5 & Good & \\
\hline $6 / 18 / 93 \quad 10: 00$ & Manual Tape & 402.50 & Good & \\
\hline $6 / 19 / 93 \quad 10: 00$ & Manual Tape & 402.50 & Good & \\
\hline $6 / 20 / 93 \quad 10: 00$ & Manual Tape & 402.5 & Good & \\
\hline $6 / 21 / 9310: 00$ & Manual Tape & 402.50 & Good & \\
\hline $6 / 22 / 93 \quad 10: 00$ & Manual Tape & 402.50 & Good & \\
\hline $6 / 23 / 93 \quad 10: 00$ & Manual Tape & 402.5 & Good & \\
\hline $6 / 24 / 93 \quad 10: 00$ & Manual Tape & 402.5 & Good & \\
\hline $6 / 25 / 93 \quad 10: 00$ & Manual Tape & 402.50 & Good & \\
\hline $6 / 26 / 93 \quad 10: 00$ & Manual Tape & 402.50 & Good & \\
\hline $6 / 27 / 93 \quad 10: 00$ & Manual Tape & 402.50 & Good & \\
\hline $6 / 28 / 93 \quad 10: 00$ & Manual Tape & 402.5 & Good & \\
\hline $6 / 29 / 93 \quad 10: 00$ & Manual Tape & 402.5 & Good & \\
\hline $6 / 30 / 93 \quad 10: 00$ & Manual Tape & 402.50 & Good & \\
\hline $7 / 1 / 93 \quad 10: 00$ & Manual Tape & 402.50 & Good & \\
\hline $7 / 2 / 9310: 00$ & Manual Tape & 402.5 & Good & \\
\hline $7 / 3 / 93 \quad 10: 00$ & Manual Tape & 402.50 & Good & \\
\hline $7 / 4 / 9310: 00$ & Manual Tape & 402.50 & Good & \\
\hline 7/5/93 10:00 & Manual Tape & 402.5 & Good & \\
\hline $7 / 6 / 93 \quad 10: 00$ & Manual Tape & 402.5 & Good & \\
\hline $7 / 7 / 9310: 00$ & Manual Tape & 401.75 & Good & \\
\hline 7/8/93 10:00 & Manual Tape & 402.50 & Good & \\
\hline $7 / 9 / 9310: 00$ & Manual Tape & 402.50 & Good & \\
\hline $7 / 10 / 93 \quad 10: 00$ & Manual Tape & 402.5 & Good & \\
\hline $7 / 11 / 93 \quad 10: 00$ & Manual Tape & 402.25 & Good & \\
\hline $7 / 11 / 93 \quad 15: 04$ & Auto FIC & 0 & Suspect & Data not included in graph \\
\hline $7 / 12 / 93 \quad 10: 00$ & Manual Tape & 402.25 & Good & \\
\hline $7 / 12 / 93 \quad 15: 04$ & Auto FIC & 0 & Suspect & Data not included in graph \\
\hline $7 / 13 / 93 \quad 10: 00$ & Manual Tape & 402.25 & Good & \\
\hline $7 / 13 / 93 \quad 15: 04$ & Auto FIC & 0.00 & Suspect & Data not included in graph \\
\hline $7 / 14 / 93 \quad 10: 00$ & Manual Tape & 402.25 & Good & \\
\hline $7 / 14 / 93 \quad 15: 04$ & Auto FIC & 0.00 & Suspect & Data not included in graph \\
\hline $7 / 15 / 9310: 00$ & Manual Tape & 402.25 & Good & \\
\hline $7 / 15 / 93 \quad 15: 04$ & Auto FIC & 0 & Suspect & Data not included in graph \\
\hline $7 / 16 / 93 \quad 10: 00$ & Manual Tape & 402.25 & Good & \\
\hline 7/16/93 15:04 & Auto FIC & 0 & Suspect & Data not included in graph \\
\hline $7 / 17 / 93 \quad 10: 00$ & Manual Tape & 402.25 & Good & \\
\hline $7 / 17 / 9315: 04$ & Auto FIC & 0 & Suspect & Data not included in graph \\
\hline $7 / 18 / 93 \quad 10: 00$ & Manual Tape & 402.25 & Good & \\
\hline 7/18/93 15:04 & Auto FIC & 0.00 & Suspect & Data not included in graph \\
\hline 7/19/93 10:00 & Manual Tape & 402.50 & Good & \\
\hline 7/19/93 15:04 & Auto FIC & 0 & Suspect & Data not included in graph \\
\hline $7 / 20 / 93 \quad 10: 00$ & Manual Tape & 402.25 & Good & \\
\hline $7 / 20 / 93 \quad 15: 04$ & Auto FIC & 0 & Suspect & Data not included in graph \\
\hline 7/21/93 10:00 & Manual Tape & 402.25 & Good & \\
\hline
\end{tabular}

Data obtained from WHC Surveillance Analysis Computer System, (PC SACS) July 7, 1995. 


\begin{tabular}{|c|c|c|c|c|}
\hline \multicolumn{5}{|c|}{ 241-AP:102 } \\
\hline \multicolumn{5}{|c|}{ Surface Level Data } \\
\hline Date & Type of Measurement & Level (Inches) & Data Quality & Comments \\
\hline $7 / 21 / 93 \quad 15: 04$ & Auto FIC & 0 & Suspect & Data not included in graph \\
\hline $7 / 22 / 9310: 00$ & Manual Tape & 402.25 & Good & \\
\hline 7/22/93 15:04. & Auto FIC & 0.00 & Suspect & Data not included in graph \\
\hline $7 / 23 / 93$ 10:00 & Manual Tape & 402 & Good & \\
\hline $7 / 23 / 93 \quad 15: 04$ & Auto FIC & 0.00 & Suspect & Data not included in graph \\
\hline $7 / 24 / 93 \quad 10: 00$ & Manual Tape & 402 & Good & \\
\hline $7 / 24 / 93 \quad 15: 04$ & Auto FIC & 0 & Suspect & Data not included in graph \\
\hline $7 / 25 / 93 \quad 10: 00$ & Manual Tape & 402.25 & Good & \\
\hline $7 / 25 / 93 \quad 15: 04$ & Auto FIC & 0 & Suspect & Data not included in graph \\
\hline $7 / 26 / 9310: 00$ & Manual Tape & 402.25 & Good & \\
\hline $7 / 26 / 9315: 04$ & Auto FIC & 0.00 & Suspect & Data not included in graph \\
\hline $7 / 27 / 9310: 00$ & Manual Tape & 402.50 & Good & \\
\hline $7 / 27 / 93 \quad 15: 04$ & Auto FIC & 0 & Suspect & Data not included in graph \\
\hline $7 / 28 / 930: 00$ & Manual Tape & 402.50 & Good & \\
\hline $7 / 28 / 9315: 04$ & Auto FIC & 0 & Suspect & Data not included in graph \\
\hline $7 / 29 / 930: 00$ & Manual Tape & 402.5 & Good & \\
\hline $7 / 29 / 937: 03$ & Auto FIC & 0.00 & Suspect & Data not included in graph \\
\hline $7 / 30 / 930: 00$ & Manual Tape & 402.5 & Good & \\
\hline $7 / 30 / 937: 03$ & Auto FIC & 0.00 & Suspect & Data not included in graph \\
\hline $7 / 31 / 930: 00$ & Manual Tape & 402.25 & Good & \\
\hline $7 / 31 / 937: 03$ & Auto FIC & 0 & Suspect & Data not included in graph \\
\hline $8 / 1 / 930: 00$ & Manual Tape & 402.25 & Good & \\
\hline $8 / 1 / 937: 03$ & Auto FIC & 0 & Suspect & Data not included in graph \\
\hline $8 / 2 / 930: 00$ & Manual Tape & 402.50 & Good & \\
\hline $8 / 2 / 937: 06$ & Auto FIC & 0.00 & Suspect & Data not included in graph \\
\hline $8 / 3 / 930: 00$ & Manual Tape & 402.5 & Good & \\
\hline $8 / 3 / 937: 03$ & Auto FIC & 0.00 & Suspect & Data not included in graph \\
\hline $8 / 4 / 930: 00$ & Manual Tape & 402.25 & Good & \\
\hline $8 / 4 / 937: 03$ & Auto FIC & 0 & Suspect & Data not included in graph \\
\hline $8 / 5 / 930: 00$ & Manual Tape & 402.25 & Good & \\
\hline $8 / 5 / 937: 04$ & Auto FIC & 0 & Suspect & Data not included in graph \\
\hline $8 / 6 / 930: 00$ & Manual Tape & 402.25 & Good & \\
\hline $8 / 6 / 9315: 04$ & Auto FIC & 0.00 & Suspect & Data not included in graph \\
\hline $8 / 7 / 93 \quad 0: 00$ & Manual Tape & 402.5 & Good & \\
\hline $8 / 7 / 937: 04$ & Auto FIC & 0.00 & Suspect & Data not included in graph \\
\hline $8 / 8 / 930: 00$ & Manual Tape & 402.50 & Good & \\
\hline $8 / 8 / 937: 04$ & Auto FIC & 0 & Suspect & Data not included in graph \\
\hline $8 / 9 / 930: 00$ & Manual Tape & 402.50 & Good & \\
\hline $8 / 9 / 937: 04$ & Auto FIC & 0 & Suspect & Data not included in graph \\
\hline $8 / 10 / 930: 00$ & Manual Tape & 402.25 & Good & \\
\hline $8 / 10 / 937: 04$ & Auto FIC & 0.00 & Suspect & Data not included in graph \\
\hline $8 / 11 / 930: 00$ & Manual Tape & 402.25 & Good & . \\
\hline $8 / 11 / 937: 04$ & Auto FIC & 0.00 & Suspect & Data not included in graph \\
\hline $8 / 12 / 930: 00$ & Manual Tape & 402.25 & Good & \\
\hline $8 / 12 / 937: 04$ & Auto FIC & 0 & Suspect & Data not included in graph \\
\hline $8 / 13 / 930: 00$ & Manual Tape & 402.25 & Good & \\
\hline $8 / 13 / 937: 04$ & Auto FIC & 0 & Suspect & Data not included in graph \\
\hline $8 / 14 / 930: 00$ & Manual Tape & 402.25 & Good & \\
\hline
\end{tabular}

Data obtained from WHC Surveillance Analysis Computer System, (PC SACS) July 7, 1995. 
WHC-SD-WM-ER-315, Rev. 0

\begin{tabular}{|c|c|c|c|c|}
\hline \multicolumn{5}{|c|}{ 241-AP-102 } \\
\hline \multicolumn{5}{|c|}{ Surface Lovel Data } \\
\hline Date & Type of Maasurement & Level (Inches) & Data Quality & Comments \\
\hline $8 / 14 / 937: 03$ & Auto FIC & 0.00 & Suspect & Data not included in graph \\
\hline $8 / 15 / 930: 00$ & Manual Tape & 402.25 & Good & \\
\hline $8 / 15 / 93$ 7:04 & Auto FIC & 0.00 & Suspect & Data not included in graph \\
\hline $8 / 16 / 930: 00$ & Manual Tape & 402.25 & Good & \\
\hline $8 / 16 / 937: 04$ & Auto FIC & 0 & Suspect & Data not included in graph \\
\hline $8 / 17 / 930: 00$ & Manual Tape & 402.25 & Good & \\
\hline $8 / 17 / 937: 04$ & Auto FIC & 0.00 & Suspect & Data not included in graph \\
\hline $8 / 18 / 930: 00$ & Manual Tape & 402.50 & Good & \\
\hline $8 / 18 / 937: 03$ & Auto FIC & 0.00 & Suspect & Data not included in graph \\
\hline $8 / 19 / 930: 00$ & Manual Tape & 402.25 & Good & \\
\hline $8 / 19 / 937: 04$ & Auto FIC & 0 & Suspect & Data not included in graph \\
\hline $8 / 20 / 930: 00$ & Manual Tape & 402.25 & Good & \\
\hline $8 / 20 / 937: 04$ & Auto FIC & 0 & Suspect & Data not included in graph \\
\hline $8 / 21 / 930: 00$ & Manual Tape & 402.25 & Good & \\
\hline $8 / 21 / 937: 04$ & Auto FIC & 0.00 & Suspect & Data not included in graph \\
\hline $8 / 22 / 930: 00$ & Manual Tape & 402.25 & Good & \\
\hline 8/22/93 7:04 & Auto FIC & 0.00 & Suspect & Data not included in graph \\
\hline $8 / 23 / 930: 00$ & Manual Tape & 402.5 & Good & \\
\hline $8 / 23 / 937: 04$ & Auto FIC & 0 & Suspect & Data not included in graph \\
\hline $8 / 24 / 930: 00$ & Manual Tape & 402.25 & Good & \\
\hline $8 / 24 / 937: 04$ & Auto FIC & 0 & Suspect & Data not included in graph \\
\hline $8 / 25 / 930: 00$ & Manual Tape & 402 & Good & \\
\hline $8 / 25 / 937: 04$ & Auto FIC & 0.00 & Suspect & Data not included in graph \\
\hline $8 / 26 / 930: 00$ & Manual Tape & 402.00 & Good & \\
\hline $8 / 26 / 937: 04$ & Auto FIC & 0.00 & Suspect & Data not included in graph \\
\hline $8 / 27 / 930: 00$ & Manual Tape & 402.25 & Good & \\
\hline $8 / 27 / 937: 05$ & Auto FIC & 0 & Suspect & Data not included in graph \\
\hline $8 / 28 / 930: 00$ & Manual Tape & 402.25 & Good & \\
\hline $8 / 28 / 937: 04$ & Auto FIC & 0 & Suspect & Data not included in graph \\
\hline $8 / 29 / 930: 00$ & Manual Tape & 402.25 & Good & \\
\hline $8 / 29 / 937: 03$ & Auto FIC & 0.00 & Suspect & Data not included in graph \\
\hline $8 / 30 / 930: 00$ & Manual Tape & 402.25 & Good & \\
\hline $8 / 30 / 937: 04$ & Auto FIC & 0.00 & Suspect & Data not included in graph \\
\hline $8 / 31 / 930: 00$ & Manual Tape & $402 . \overline{25}$ & Good & \\
\hline $8 / 31 / 937: 04$ & Auto FIC & 0 & Suspect & Data not included in graph \\
\hline 9/1/93 0:00 & Manual Tape & 402.25 & Good & \\
\hline 9/1/93 7:04 & Auto FIC & 0 & Suspect & Data not included in graph \\
\hline 9/2/93 0:00 & Manual Tape & 402.50 & Good & \\
\hline $9 / 2 / 937: 04$ & Auto FIC & 0.00 & Suspect & Data not included in graph \\
\hline $9 / 3 / 930: 00$ & Manual Tape & 402.5 & Good & \\
\hline 9/3/93 7:04 & Auto FIC & 0.00 & Suspect & Data not included in graph \\
\hline 9/4/93 0:00 & Manual Tape & 402.5 & Good & \\
\hline 9/4/93 7:04 & Auto FIC & 0 & Suspect & Data not included in graph \\
\hline 9/5/93 0:00 & Manual Tape & 402.50 & Good & \\
\hline $9 / 5 / 937: 04$ & Auto FIC & 0 & Suspect & Data not included in graph \\
\hline $9 / 6 / 930: 00$ & Manual Tape & 402.5 & Good & \\
\hline $9 / 6 / 937: 04$ & Auto FIC & 0.00 & Suspect & Data not included in graph \\
\hline $9 / 7 / 930: 00$ & Manual Tape & 402.50 & Good & \\
\hline
\end{tabular}

Data obtained from WHC Surveillance Analysis Computer System, (PC SACS) July 7, 1995. 


\begin{tabular}{|c|c|c|c|c|}
\hline \multicolumn{5}{|c|}{ 241-AP-102 } \\
\hline \multicolumn{5}{|c|}{ Surface Level Data } \\
\hline Date & Type of Measurement & Level (Inches) & Data Quality & Comments \\
\hline $9 / 7 / 937: 04$ & Auto FIC & 0.00 & Suspect & Data not included in graph \\
\hline $9 / 8 / 930: 00$ & Manual Tape & 402.5 & Good & \\
\hline 9/8/93 7:04 & Auto FIC & 0 & Suspect & Data not included in graph \\
\hline $9 / 9 / 930: 00$ & Manual Tape & 402.25 & Good & \\
\hline $9 / 9 / 937: 04$ & Auto FIC & 0 & Suspect & Data not included in graph \\
\hline 9/10/93 0:00 & Manual Tape & 402.25 & Good & \\
\hline 9/10/93 7:04 & Auto FIC & 0.00 & Suspect & Data not included in graph \\
\hline $9 / 11 / 930: 00$ & Manual Tape & 402.25 & Good & \\
\hline 9/11/93 7:04 & Auto FIC & 0.00 & Suspect & Data not included in graph \\
\hline $9 / 12 / 930: 00$ & Manual Tape & 402.25 & Good & \\
\hline $9 / 12 / 937: 04$ & Auto FIC & 0 & Suspect & Data not included in graph \\
\hline $9 / 13 / 930: 00$ & Manual Tape & 402.25 & Good & \\
\hline $9 / 13 / 937: 04$ & Auto FIC & 0 & Suspect & Data not included in graph \\
\hline $9 / 14 / 930: 00$ & Manual Tape & 402.25 & Good & \\
\hline $9 / 14 / 937: 04$ & Auto FIC & 0.00 & Suspect & Data not included in graph \\
\hline $9 / 15 / 93 \quad 0: 00$ & Manual Tape & 402.25 & Good & \\
\hline 9/15/93 7:04 & Auto FIC & 0.00 & Suspect & Data not included in graph \\
\hline 9/16/93 0:00 & Manual Tape & 402.25 & Good & \\
\hline $9 / 16 / 937: 04$ & Auto FIC & 0 & Suspect & Data not included in graph \\
\hline 9/17/93 0:00 & Manual Tape & 402.25 & Good & \\
\hline $9 / 17 / 937: 04$ & Auto FIC & 0 & Suspect & Data not included in graph \\
\hline $9 / 18 / 930: 00$ & Manual Tape & 402.25 & Good & \\
\hline 9/18/93 7:04 & Auto FIC & 0.00 & Suspect & Data not included in graph \\
\hline 9/19/93 0:00 & Manual Tape & 402.25 & Good & \\
\hline 9/19/93 7:04 & Auto FIC & 0.00 & Suspect & Data not included in graph \\
\hline $9 / 20 / 930: 00$ & Manual Tape & 402.25 & Good & \\
\hline 9/20/93 7:04 & Auto FIC & 0 & Suspect & Data not included in graph \\
\hline $9 / 21 / 930: 00$ & Manual Tape & 402.25 & Good & \\
\hline $9 / 21 / 937: 04$ & Auto FIC & 0 & Suspect & Data not included in graph \\
\hline 9/22/93 0:00 & Manual Tape & 402.25 & Good & . \\
\hline $9 / 22 / 937: 04$ & Auto FIC & 0.00 & Suspect & Data not included in graph \\
\hline $9 / 23 / 930: 00$ & Manual Tape & 402.25 & Good & \\
\hline $9 / 23 / 937: 04$ & Auto FIC & 0.00 & Suspect & Data not included in graph \\
\hline 9/24/93 0:00 & Manual Tape & 402.25 & Good & \\
\hline $9 / 24 / 937: 04$ & Auto FIC & 0 & Suspect & Data not included in graph \\
\hline $9 / 25 / 930: 00$ & Manual Tape & 402:25 & Good & \\
\hline $9 / 25 / 937: 04$ & Auto FIC & 0 & Suspect & Data not included in graph \\
\hline $9 / 26 / 930: 00$ & Manual Tape & 402.25 & Good & \\
\hline $9 / 26 / 937: 04$ & Auto FIC & 0.00 & Suspect & Data not included in graph \\
\hline $9 / 27 / 930: 00$ & Manual Tape & 402.25 & Good & \\
\hline 9/27/93 7:04 & Auto FIC & 0.00 & Suspect & Data not included in graph \\
\hline $9 / 28 / 93 \quad 0: 00$ & Manual Tape & 402.25 & Good & \\
\hline $9 / 28 / 937: 04$ & Auto FIC & 0 & Suspect & Data not included in graph \\
\hline $9 / 29 / 93 \quad 0: 00$ & Manual Tape & 402.25 & Good & \\
\hline $9 / 29 / 937: 04$ & Auto FIC & 0 & Suspect & Data not included in graph \\
\hline 9/30/93 0:00 & Manual Tape & 402.25 & Good & \\
\hline 9/30/93 7:03 & Auto FIC & 0 & Suspect & Data not included in graph \\
\hline $10 / 1 / 930: 00$ & Manual Tape & 402.25 & Good & \\
\hline
\end{tabular}

Data obtained from WHC Surveillance Analysis Computer System, (PC SACS) July 7, 1995. 
WHC-SD-WM-ER-315, Rev, 0

\begin{tabular}{|c|c|c|c|c|}
\hline \multicolumn{5}{|c|}{ 241-AP-102 } \\
\hline \multicolumn{5}{|c|}{ Surface Lovel Data } \\
\hline Date & Type of Measurement & Level (Inches) & Data Quality & Comments \\
\hline 10/1/93 7:06 & Auto FIC & 0.00 & Suspect & Data not included in graph \\
\hline $10 / 2 / 930: 00$ & Manual Tape & 402.25 & Good & \\
\hline $10 / 2 / 937: 05$ & Auto FIC & 0.00 & Suspect & Data not included in graph \\
\hline $10 / 3 / 930: 00$ & Manual Tape & 402.25 & Good & \\
\hline $10 / 3 / 937: 06$ & Auto FIC & 0 & Suspect & Data not included in graph \\
\hline $10 / 4 / 930: 00$ & Manual Tape & 402.25 & Good & \\
\hline $10 / 4 / 937: 06$ & Auto FIC & 0.00 & Suspect & Data not included in graph \\
\hline $10 / 5 / 930: 00$ & Manual Tape & 402.25 & Good & \\
\hline 10/5/93 7:06 & Auto FIC & 0.00 & Suspect & Data not included in graph \\
\hline 10/6/93 0:00 & Manual Tape & 402.25 & Good & \\
\hline 10/6/93 7:06 & Auto FIC & 0.00 & Suspect & Data not included in graph \\
\hline $10 / 7 / 930: 00$ & Manual Tape & 402.25 & Good & \\
\hline $10 / 7 / 937: 05$ & Auto FIC & 0 & Suspect & Data not included in graph \\
\hline $10 / 8 / 930: 00$ & Manual Tape & 402 & Good & \\
\hline $10 / 8 / 937: 04$ & Auto FIC & 0.00 & Suspect & Data not included in graph \\
\hline 10/9/93 0:00 & Manual Tape & 402 & Good & \\
\hline $10 / 9 / 937: 03$ & Auto FIC & 0.00 & Suspect & Data not included in graph \\
\hline $10 / 10 / 930: 00$ & Manual Tape & 402 & Good & \\
\hline $10 / 11 / 930: 00$ & Manual Tape & 402.00 & Good & \\
\hline $10 / 11 / 937: 03$ & Auto FIC & 0 & Suspect & Data not included in graph \\
\hline 10/12/93 0:00 & Manual Tape & 402.00 & Good & \\
\hline $10 / 12 / 937: 04$ & Auto FIC & 0 & Suspect & Data not included in graph \\
\hline $10 / 13 / 930: 00$ & Manual Tape & 401.75 & Good & \\
\hline $10 / 13 / 937: 03$ & Auto FIC & 0.00 & Suspect & Data not included in graph \\
\hline $10 / 14 / 93 \quad 0: 00$ & Manual Tape & 402 & Good & \\
\hline $10 / 14 / 937: 04$ & Auto FIC & 0.00 & Suspect & Data not included in graph \\
\hline $10 / 15 / 93 \quad 0: 00$ & Manual Tape & 402 & Good & \\
\hline $10 / 15 / 93 \quad 7: 04$ & Auto FIC & 0 & Suspect & Data not included in graph \\
\hline $10 / 16 / 93 \quad 0: 00$ & Manual Tape & 402 & Good & \\
\hline $10 / 16 / 937: 04$ & Auto FIC & 0 & Suspect & Data not included in graph \\
\hline $10 / 17 / 930: 00$ & Manual Tape & 402.00 & Good & \\
\hline 10/17/93 7:03 & Auto FIC & 0 & Suspect & Data not included in graph \\
\hline $10 / 18 / 930: 00$ & Manual Tape & 402 & Good & \\
\hline $10 / 18 / 937: 04$ & Auto FIC & 0.00 & Suspect & Data not included in graph \\
\hline $10 / 19 / 930: 00$ & Manual Tape & 402.00 & Good & \\
\hline $10 / 19 / 937: 04$ & Auto FIC & 0.00 & Suspect & Data not included in graph \\
\hline $10 / 20 / 930: 00$ & Manual Tape & 402.00 & Good & \\
\hline $10 / 20 / 937: 03$ & Auto FIC & 0 & Suspect & Data not included in graph \\
\hline $10 / 21 / 930: 00$ & Manual Tape & 402.00 & Good & \\
\hline $10 / 21 / 937: 04$ & Auto FIC & 0.00 & Suspect & Data not included in graph \\
\hline $10 / 22 / 930: 00$ & Manual Tape & 402 & Good & \\
\hline $10 / 22 / 937: 04$ & Auto FIC & 0.00 & Suspect & Data not included in graph \\
\hline $10 / 23 / 930: 00$ & Manual Tape & 402.00 & Good & \\
\hline $10 / 23 / 937: 04$ & Auto FIC & 0 & Suspect & Data not included in graph \\
\hline $10 / 24 / 930: 00$ & Manual Tape & 401.75 & Good & \\
\hline $10 / 24 / 937: 04$ & Auto FIC & 0 & Suspect & Data not included in graph \\
\hline 10/25/930:00 & Manual Tape & 402.00 & Good & \\
\hline $10 / 25 / 937: 04$ & Auto FIC & 0.00 & Suspect & Data not included in graph \\
\hline
\end{tabular}

Data obtained from WHC Surveillance Analysis Computer System, (PC SACS) July 7, 1995. 
WHC-SD-WM-ER-315, Rev. 0

\begin{tabular}{|c|c|c|c|c|}
\hline \multicolumn{5}{|c|}{ 241-AP-102 } \\
\hline \multicolumn{5}{|c|}{ Type of Megarurface Leval Data } \\
\hline Date & Type of Measurement & Leval (Inches) & Data Quality & Comments \\
\hline $10 / 26 / 930: 00$ & Manual Tape & 402 & Good & \\
\hline $10 / 26 / 937: 04$ & Auto FIC & 0.00 & Suspect & Data not included in graph \\
\hline $10 / 27 / 930: 00$ & Manual Tape & 402.00 & Good & \\
\hline $10 / 27 / 937: 04$ & Auto FIC & 0.00 & Suspect & Data not included in graph \\
\hline $10 / 28 / 930: 00$ & Manual Tape & 401.75 & Good & \\
\hline $10 / 28 / 937: 04$ & Auto FIC & 0 & Suspect & Data not included in graph \\
\hline $10 / 29 / 930: 00$ & Manual Tape & 401.75 & Good & \\
\hline $10 / 29 / 937: 04$ & Auto FIC & 0 & Suspect & Data not included in graph \\
\hline $10 / 30 / 930: 00$ & Manual Tape & 402.00 & Good & \\
\hline $10 / 30 / 937: 04$ & Auto FIC & 0.00 & Suspect & Data not included in graph \\
\hline $10 / 31 / 930: 00$ & Manual Tape & 402 & Good & \\
\hline 10/31/93 7:06 & Auto FIC & 0.00 & Suspect & Data not included in graph \\
\hline $11 / 1 / 930: 00$ & Manual Tape & 402.00 & Good & \\
\hline $11 / 1 / 937: 06$ & Auto FIC & 0 & Suspect & Data not included in graph \\
\hline $11 / 2 / 930: 00$ & Manual Tape & 402.00 & Good & \\
\hline $11 / 2 / 937: 04$ & Auto FIC & 0 & Suspect & Data not included in graph \\
\hline $11 / 3 / 930: 00$ & Manual Tape & 402 & Good & \\
\hline $11 / 3 / 937: 06$ & Auto FIC & 0.00 & Suspect & Data not included in graph \\
\hline 11/4/93 0:00 & Manual Tape & 402.00 & Good & \\
\hline $11 / 4 / 937: 04$ & Auto FIC & 0.00 & Suspect & Data not included in graph \\
\hline $11 / 5 / 930: 00$ & Manual Tape & 402 & Good & \\
\hline 11/5/93 7:06 & Auto FIC & 0 & Suspect & Data not included in graph \\
\hline $11 / 6 / 930: 00$ & Manual Tape & 402.00 & Good & \\
\hline $11 / 6 / 93 \cdot 7: 06$ & Auto FIC & 0 & Suspect & Data not included in graph \\
\hline $11 / 7 / 930: 00$ & Manual Tape & 402.00 & Good & \\
\hline $11 / 7 / 937: 06$ & Auto FIC & 0.00 & Suspect & Data not included in graph \\
\hline $11 / 8 / 930: 00$ & Manual Tape & 402 & Good & \\
\hline $11 / 8 / 937: 06$ & Auto FIC & 0.00 & Suspect & Data not included in graph \\
\hline $11 / 9 / 930: 00$ & Manual Tape & 402.00 & Good & \\
\hline $11 / 9 / 937: 03$ & Auto FIC & 0 & Suspect & Data not included in graph \\
\hline $11 / 10 / 930: 00$ & Manual Tape & 402.00 & Good & \\
\hline $11 / 10 / 937: 04$ & Auto FIC & 0 & Suspect & Data not included in graph \\
\hline $11 / 11 / 930: 00$ & Manual Tape & 401.75 & Good & \\
\hline $11 / 11 / 937: 04$ & Auto FIC & 0.00 & Suspect & Data not included in graph \\
\hline $11 / 12 / 930: 00$ & Manual Tape & 402.00 & Good & \\
\hline 11/12/93 7:04 & Auto FIC & 0.00 & Suspect & Data not included in graph \\
\hline $11 / 13 / 930: 00$ & Manual Tape & 402 & Good & \\
\hline $11 / 13 / 937: 04$ & Auto FIC & 0 & Suspect & Data not included in graph \\
\hline $11 / 14 / 930: 00$ & Manual Tape & 402 & Good & \\
\hline $11 / 14 / 937: 04$ & Auto FIC & 0.00 & Suspect & Data not included in graph \\
\hline $11 / 15 / 930: 00$ & Manual Tape & 401.75 & Good & \\
\hline $11 / 15 / 937: 04$ & Auto FIC & 0.00 & Suspect & Data not included in graph \\
\hline $11 / 16 / 930: 00$ & Manual Tape & 401.75 & Good & \\
\hline $11 / 16 / 937: 03$ & Auto FIC & 0 & Suspect & Data not included in graph \\
\hline $11 / 17 / 930: 00$ & Manual Tape & 401.75 & Good & \\
\hline $11 / 17 / 937: 04$ & Auto FIC & 0 & Suspect & Data not included in graph \\
\hline $11 / 18 / 930: 00$ & Manual Tape & $401: 50$ & Good & \\
\hline $11 / 18 / 937: 04$ & Auto FIC & 0.00 & Suspect & Data not included in graph \\
\hline
\end{tabular}

Data obtained from WHC Surveillance Analysis Computer System, (PC SACS) July 7, 1995. 


\begin{tabular}{|c|c|c|c|c|}
\hline \multicolumn{5}{|c|}{ 241-AP-102 } \\
\hline \multicolumn{5}{|c|}{ Surface Level Data } \\
\hline Date & Type of Measurement & Leval (Inches) & Data Quality & Comments \\
\hline $11 / 19 / 930: 00$ & Manual Tape & 401.25 & Good & \\
\hline $11 / 19 / 937: 04$ & Auto FIC & 0.00 & Suspect & Data not included in graph \\
\hline $11 / 20 / 930: 00$ & Manual Tape & $401.50^{\circ}$ & Good & \\
\hline $11 / 20 / 937: 04$ & Auto FIC & 0 & Suspect & Data not included in graph \\
\hline $11 / 21 / 930: 00$ & Manual Tape & 401.5 & Good & \\
\hline $11 / 21 / 937: 04$ & Auto FIC & 0 & Suspect & Data not included in graph \\
\hline $11 / 22 / 930: 00$ & Manual Tape & 402.00 & Good & \\
\hline $11 / 22 / 937: 04$ & Auto FIC & 0.00 & Suspect & Data not included in graph \\
\hline $11 / 23 / 930: 00$ & Manual Tape & 401.75 & Good & \\
\hline $11 / 23 / 937: 04$ & Auto FIC & 0.00 & Suspect & Data not included in graph \\
\hline $11 / 24 / 930: 00$ & Manual Tape & 461.75 & Suspect & Data not included in graph \\
\hline $11 / 24 / 937: 04$ & Auto FIC & 0 & Suspect & Data not included in graph \\
\hline $11 / 25 / 930: 00$ & Manual Tape & 401.75 & Good & \\
\hline $11 / 25 / 937: 04$ & Auto FIC & 0 & Suspect & Data not included in graph \\
\hline $11 / 26 / 930: 00$ & Manual Tape & 401.75 & Good & \\
\hline $11 / 26 / 937: 04$ & Auto FIC & 0.00 & Suspect & Data not included in graph \\
\hline $11 / 27 / 930: 00$ & Manual Tape & 401.75 & Good & \\
\hline $11 / 27 / 937: 04$ & Auto FIC & 0.00 & Suspect & Data not included in graph \\
\hline $11 / 28 / 930: 00$ & Manual Tape & 401.50 & Good & \\
\hline $11 / 28 / 937: 03$ & Auto FIC & 0 & Suspect & Data not included in graph \\
\hline $11 / 29 / 930: 00$ & Manual Tape & 401.5 & Good & \\
\hline $11 / 29 / 937: 04$ & Auto FIC & 0 & Suspect & Data not included in graph \\
\hline $11 / 30 / 930: 00$ & Manual Tape & 401.75 & Good & \\
\hline $11 / 30 / 937: 03$ & Auto FIC & 0.00 & Suspect & Data not included in graph \\
\hline $12 / 1 / 930: 00$ & Manual Tape & 401.75 & Good & \\
\hline 12/1/93 7:04 & Auto FIC & 0.00 & Suspect & Data not included in graph \\
\hline $12 / 2 / 930: 00$ & Manual Tape & 401.75 & Good & \\
\hline 12/2/93 7:04 & Auto FIC & 0 & Suspect & Data not included in graph \\
\hline $12 / 3 / 930: 00$ & Manual Tape & 401.75 & Good & \\
\hline $12 / 3 / 937: 05$ & Auto FIC & 0 & Suspect & Data not included in graph \\
\hline $12 / 4 / 930: 00$ & Manual Tape & 401.75 & Good & \\
\hline $12 / 4 / 937: 05$ & Auto FIC & 0.00 & Suspect & Data not included in graph \\
\hline $12 / 5 / 930: 00$ & Manual Tape & 401.75 & Good & \\
\hline $12 / 5 / 937: 04$ & Auto FIC & 0.00 & Suspect & Data not included in graph \\
\hline $12 / 6 / 930: 00$ & Manual Tape & 401.75 & Good & \\
\hline 12/6/93 7:05 & Auto FIC & 0 & Suspect & Data not included in graph \\
\hline $12 / 7 / 930: 00$ & Manual Tape & 401.75 & Good & \\
\hline $12 / 7 / 937: 04$ & Auto FIC & 0 & Suspect & Data not included in graph \\
\hline 12/8/93 0:00 & Manual Tape & 401.5 & Good & \\
\hline $12 / 8 / 937: 06$ & Auto FIC & 0.00 & Suspect & Data not included in graph \\
\hline $12 / 9 / 930: 00$ & Manual Tape & 401.5 & Good & \\
\hline $12 / 9 / 937: 04$ & Auto FIC & 0.00 & Suspect & Data not included in graph \\
\hline $12 / 10 / 930: 00$ & Manual Tape & 401.50 & Good & \\
\hline $12 / 11 / 930: 00$ & Manual Tape & 401.5 & Good & \\
\hline $12 / 11 / 937: 06$ & Auto FIC & 0 & Suspect & Data not included in graph \\
\hline $12 / 12 / 930: 00$ & Manual Tape & 401.25 & Good & \\
\hline $12 / 12 / 937: 06$ & Auto FIC & 0 & Suspect & Data not included in graph \\
\hline $12 / 13 / 930: 00$ & Manual Tape & 401.50 & Good & \\
\hline
\end{tabular}

Data obtained from WHC Surveillance Analysis Computer System, (PC SACS) July 7, 1995. 
WHC-SD-WM-ER-315, Rev. 0

\begin{tabular}{|c|c|c|c|c|}
\hline \multicolumn{5}{|c|}{ 241-AP-102 } \\
\hline \multicolumn{5}{|c|}{ Surface Leval Data } \\
\hline Date & Type of Measurement & Levol (Inches) & Data Quality & Comments \\
\hline 12/13/93 7:06 & Auto FIC & 0.00 & Suspect & Data not included in graph \\
\hline 12/14/93 0:00 & Manual Tape & 401.5 & Good & \\
\hline 12/14/93 7:06 & Auto FIC & 0.00 & Suspect & Data not included in graph \\
\hline 12/15/93 0:00 & Manual Tape & 401.75 & Good & \\
\hline 12/15/93 7:06 & Auto FIC & 0 & Suspect & Data not included in graph \\
\hline $12 / 16 / 930: 00$ & Manual Tape & 401.50 & Good & \\
\hline 12/16/93 7:06 & Auto FIC & 0 & Suspect & Data not included in graph \\
\hline 12/17/93 0:00 & Manual Tape & 401.5 & Good & \\
\hline $12 / 17 / 937: 05$ & Auto FIC & 0.00 & Suspect & Data not included in graph \\
\hline 12/18/93 0:00 & Manual Tape & 401.50 & Good & \\
\hline $12 / 18 / 937: 06$ & Auto FIC & 0.00 & Suspect & Data not included in graph \\
\hline $12 / 19 / 930: 00$ & Manual Tape & 401.50 & Good & \\
\hline 12/19/93 7:06 & Auto FIC & 0 & Suspect & Data not included in graph \\
\hline 12/20/93 0:00 & Manual Tape & 401.5 & Good & \\
\hline $12 / 20 / 937: 06$ & Auto FIC & 0 & Suspect & Data not included in graph \\
\hline 12/21/93 0:00 & Manual Tape & 401.50 & Good & \\
\hline 12/21/93 7:06 & Auto FIC & 0.00 & Suspect & Data not included in graph \\
\hline 12/22/93 0:00 & Manual Tape & 401.50 & Good & \\
\hline 12/22/93 7:05 & Auto FIC & 0.00 & Suspect & Data not included in graph \\
\hline $12 / 23 / 930: 00$ & Manual Tape & 401.5 & Good & \\
\hline $12 / 23 / 937: 04$ & Auto FIC & 0 & Suspect & Data not included in graph \\
\hline $12 / 24 / 930: 00$ & Manual Tape & 401.50 & Good & \\
\hline 12/24/93 7:06 & Auto FIC & .0 & Suspect & Data not included in graph \\
\hline 12/25/93 0:00 & Manual Tape & 401.5 & Good & \\
\hline $12 / 25 / 937: 06$ & Auto FIC & 0.00 & Suspect & Data not included in graph \\
\hline $12 / 26 / 930: 00$ & Manual Tape & 401.5 & Good & \\
\hline $12 / 26 / 93$ 7:06 & Auto FIC & 0.00 & Suspect & Data not included in graph \\
\hline $12 / 27 / 930: 00$ & Manual Tape & 401.50 & Good & \\
\hline $12 / 27 / 93 \quad 7: 06$ & Auto FIC & 0 & Suspect & Data not included in graph \\
\hline $12 / 28 / 930: 00$ & Manual Tape & 401.50 & Good & \\
\hline $12 / 28 / 937: 06$ & Auto FIC & 0 & Suspect & Data not included in graph \\
\hline $12 / 29 / 930: 00$ & Manual Tape & 401.50 & Good & \\
\hline $12 / 29 / 937: 06$ & Auto FIC & 0.00 & Suspect & Data not included in graph \\
\hline $12 / 30 / 930: 00$ & Manual Tape & 401.50 & Good & \\
\hline $12 / 30 / 937: 05$ & Auto FIC & 0.00 & Suspect & Data not included in graph \\
\hline $12 / 31 / 930: 00$ & Manual Tape & 401.25 & Good & \\
\hline 12/31/93 7:06 & Auto FIC & 0 & Suspect & Data not included in graph \\
\hline $1 / 1 / 940: 00$ & Manual Tape & 401.5 & Good & \\
\hline 1/1/94 7:06 & Auto FIC & 0 & Suspect & Data not included in graph \\
\hline $1 / 2 / 940: 00$ & Manual Tape & 401.5 & Good & \\
\hline $1 / 2 / 947: 06$ & Auto FIC & 0.00 & Suspect & Data not included in graph \\
\hline $1 / 3 / 940: 00$ & Manual Tape & 401.50 & Good & \\
\hline $1 / 3 / 947: 07$ & Auto FIC & 0.00 & Suspect & Data not included in graph \\
\hline $1 / 4 / 940: 00$ & Manual Tape & 401.50 & Good & \\
\hline $1 / 4 / 947: 07$ & Auto FIC & 0 & Suspect & Data not included in graph \\
\hline $1 / 5 / 940: 00$ & Manual Tape & 401.25 & Good & \\
\hline $1 / 5 / 947: 06$ & Auto FIC & 0 & Suspect & Data not included in graph \\
\hline $1 / 6 / 940: 00$ & Manual Tape & 401.25 & Good & \\
\hline
\end{tabular}

Data obtained from WHC Surveillance Analysis Computer System, (PC SACS) July 7, 1995. 
WHC-SD-WM-ER-315, Rev. 0

\begin{tabular}{|c|c|c|c|c|}
\hline \multicolumn{5}{|c|}{ 241-AP-102 } \\
\hline \multicolumn{5}{|c|}{ Surface Level Data } \\
\hline Date & Type of Measurement & Level (Inches) & Data Quality & Comments \\
\hline $1 / 6 / 947: 06$ & Auto FIC & 0.00 & Suspect & Data not included in graph \\
\hline $1 / 7 / 940: 00$ & Manual Tape & 401.25 & Good & \\
\hline $1 / 7 / 947: 07$ & Auto FIC & 0.00 & Suspect & Data not included in graph \\
\hline $1 / 8 / 940: 00$ & Manual Tape & 401.25 . & Good & \\
\hline $1 / 8 / 947: 06$ & Auto FIC & 0.00 & Suspect & Data not included in graph \\
\hline $1 / 9 / 940: 00$ & Manual Tape & 401.25 & Good & \\
\hline 1/9/94 7:06 & Auto FIC & 0 & Suspect & Data not included in graph \\
\hline $1 / 10 / 940: 00$ & Manual Tape & 401.5 & Good & \\
\hline $1 / 10 / 947: 07$ & Auto FIC & 0 & Suspect & Data not included in graph \\
\hline $1 / 11 / 940: 00$ & Manual Tape & 401.50 & Good & \\
\hline $1 / 11 / 947: 07$ & Auto FIC & 0 & Suspect & Data not included in graph \\
\hline $1 / 12 / 940: 00$ & Manual Tape & 401.25 & Good & \\
\hline $1 / 12 / 947: 06$ & Auto FIC & 0.00 & Suspect & Data not included in graph \\
\hline $1 / 13 / 940: 00$ & Manual Tape & 401.5 & Good & \\
\hline $1 / 13 / 947: 04$ & Auto FIC & 0.00 & Suspect & Data not included in graph \\
\hline $1 / 14 / 940: 00$ & Manual Tape & 401.25 & Good & \\
\hline $1 / 14 / 947: 06$ & Auto FIC & 0 & Suspect & Data not included in graph \\
\hline $1 / 15 / 940: 00$ & Manual Tape & 401.50 & Good & \\
\hline $1 / 15 / 947: 04$ & Auto FIC & 0 & Suspect & Data not included in graph \\
\hline $1 / 16 / 940: 00$ & Manual Tape & 401.5 & Good & \\
\hline $1 / 16 / 947: 07$ & Auto FIC & 0.00 & Suspect & Data not included in graph \\
\hline $1 / 17 / 940: 00$ & Manual Tape & 401.25 & Good & \\
\hline $1 / 17 / 947: 07$ & Auto FIC & 0.00 & Suspect & Data not included in graph \\
\hline $1 / 18 / 940: 00$ & Manual Tape & 401.25 & Good & \\
\hline $1 / 18 / 947: 07$ & Auto FIC & 0.00 & Suspect & Data not included in graph \\
\hline $1 / 19 / 940: 00$ & Manual Tape & 401.25 & Good & \\
\hline $1 / 19 / 947: 07$ & Auto FIC & 0 & Suspect & Data not included in graph \\
\hline $1 / 20 / 940: 00$ & Manual Tape & 401.25 & Good & \\
\hline $1 / 20 / 947: 07$ & Auto FIC & 0 & Suspect & Data not included in graph \\
\hline $1 / 21 / 940: 00$ & Manual Tape & 401.25 & Good & \\
\hline $1 / 21 / 947: 07$ & Auto FIC & 0.00 & Suspect & Data not included in graph \\
\hline $1 / 22 / 940: 00$ & Manual Tape & 401.25 & Good & \\
\hline $1 / 22 / 947: 08$ & Auto FIC & 0 & Suspect & Data not included in graph \\
\hline $1 / 23 / 940: 00$ & Manual Tape & 401.25 & Good & \\
\hline $1 / 23 / 947: 07$ & Auto FIC & 0 & Suspect & Data not included in graph \\
\hline $1 / 24 / 940: 00$ & Manual Tape & $401: 25$ & Good & \\
\hline $1 / 24 / 947: 08$ & Auto FIC & 0.00 & Suspect & Data not included in graph \\
\hline $1 / 25 / 940: 00$ & Manual Tape & 401.25 & Good & \\
\hline $1 / 25 / 947: 07$ & Auto FIC & 0.00 & Suspect & Data not included in graph \\
\hline $1 / 26 / 940: 00$ & Manual Tape & 401.25 & Good & \\
\hline $1 / 26 / 947: 06$ & Auto FIC & 0 & Suspect & Data not included in graph \\
\hline $1 / 27 / 940: 00$ & Manual Tape & 401.25 & Good & \\
\hline $1 / 27 / 947: 04$ & Auto FIC & 0 & Suspect & Data not included in graph \\
\hline $1 / 28 / 940: 00$ & Manual Tape & 401.25 & Good & \\
\hline $1 / 28 / 947: 07$ & Auto FIC & 0.00 & Suspect & Data not included in graph \\
\hline $1 / 29 / 940: 00$ & Manual Tape & 401.25 & Good & \\
\hline $1 / 29 / 947: 07$ & Auto FIC & 0.00 & Suspect & Data not included in graph \\
\hline $1 / 30 / 940: 00$ & Manual Tape & 401.25 & Good & \\
\hline
\end{tabular}

Data obtained from WHC Surveillance Analysis Computer System, (PC SACS) July 7, 1995. 


\begin{tabular}{|c|c|c|c|c|}
\hline \multicolumn{5}{|c|}{ 241-AP-102 } \\
\hline \multicolumn{5}{|c|}{ Surface Level Data } \\
\hline Date & Type of Measurement & Lovel (Inches) & Data Quality & Comments \\
\hline $1 / 30 / 947: 06$ & Auto FIC & 0 & Suspect & Data not included in graph \\
\hline 1/31/940:00 & Manual Tape & 401 & Good & \\
\hline $1 / 31 / 947: 07$ & Auto FIC : & 0 & Suspect & Data not included in graph \\
\hline $2 / 1 / 940: 00$ & Manual Tape & 401.25 & Good & \\
\hline 2/1/94 7:06 & Auto FIC & 0.00 & Suspect & Data not included in graph \\
\hline $2 / 2 / 940: 00$ & Manual Tepe & 401.25 & Good & \\
\hline $2 / 2 / 947: 07$ & Auto FIC & 0.00 & Suspect & Data not included in graph \\
\hline $2 / 3 / 940: 00$ & Manual Tape & 401.25 & Good & \\
\hline $2 / 3 / 947: 07$ & Auto FIC & 0.00 & Suspect & Data not included in graph \\
\hline $2 / 4 / 940: 00$ & Manual Tape & 401.25 & Good & \\
\hline $2 / 4 / 947: 07$ & Auto FIC & 0 & Suspect & Data not included in graph \\
\hline $2 / 5 / 940: 00$ & Manual Tape & 401.25 & Good & \\
\hline $2 / 5 / 947: 07$ & Auto FIC & 0 & Suspect & Data not included in graph \\
\hline $2 / 6 / 940: 00$ & Manual Tape & 401.25 & Good & \\
\hline $2 / 6 / 947: 07$ & Auto FIC & 0.00 & Suspect & Data not included in graph \\
\hline $2 / 7 / 940: 00$ & Manual Tape & 401.25 & Good & \\
\hline $2 / 7 / 947: 06$ & Auto FIC & 0 & Suspect & Data not included in graph \\
\hline $2 / 8 / 940: 00$ & Manual Tape & 401.25 & Good & \\
\hline $2 / 8 / 947: 07$ & Auto FIC & 0 & Suspect & Data not included in graph \\
\hline $2 / 9 / 940: 00$ & Manual Tape & 401.25 & Good & \\
\hline 2/9/94 7:04 & Auto FIC & 0.00 & Suspect & Data not included in graph \\
\hline $2 / 10 / 940: 00$ & Manual Tape & 401.25 & Good & \\
\hline $2 / 10 / 947: 04$ & Auto FIC & 0.00 & Suspect & Date not included in graph \\
\hline $2 / 11 / 940: 00$ & Manual Tape & 401.25 & Good & \\
\hline $2 / 11 / 947: 06$ & Auto FIC & 0 & Suspect & Data not included in graph \\
\hline $2 / 12 / 940: 00$ & Manual Tape & 401.25 & Good & \\
\hline $2 / 12 / 947: 03$ & Auto FIC & 0 & Suspect & Data not included in graph \\
\hline $2 / 13 / 940: 00$ & Manual Tape & 401.25 & Good & \\
\hline $2 / 13 / 947: 04$ & Auto FIC & 0 & Suspect & Data not included in graph \\
\hline $2 / 14 / 940: 00$ & Manual Tape & 401.25 & Good & \\
\hline $2 / 14 / 947: 04$ & Auto FIC & 0.00 & Suspect & Data not included in graph \\
\hline $2 / 15 / 940: 00$ & Manual Tape & 401.25 & Good & \\
\hline 2/15/94 7:04 & Auto FIC & 0.00 & Suspect & Data not included in graph \\
\hline $2 / 16 / 940: 00$ & Manual Tape & 401 & Good & \\
\hline $2 / 16 / 947: 03$ & Auto FIC & 0 & Suspect & Data not included in graph \\
\hline $2 / 17 / 940: 00$ & Manual Tape & 401.25 & Good & \\
\hline $2 / 17 / 947: 04$ & Auto FIC & 0.00 & Suspect & Data not included in graph \\
\hline 2/18/94 0:00 & Manual Tape & 401.25 & Good & \\
\hline $2 / 18 / 947: 06$ & Auto FIC & 0.00 & Suspect & Data not included in graph \\
\hline $2 / 19 / 940: 00$ & Manual Tape & 401.25 & Good & \\
\hline 2/19/94 7:06 & Auto FIC & 0 & Suspect & Data not included in graph \\
\hline $2 / 20 / 940: 00$ & Manual Tape & 401.25 & Good & \\
\hline $2 / 20 / 947: 07$ & Auto FIC & 0 & Suspect & Data not included in graph \\
\hline $2 / 21 / 940: 00$ & Manual Tape & 401 & Good & \\
\hline $2 / 21 / 947: 06$ & Auto FIC & 0.00 & Suspect & Data not included in graph \\
\hline $2 / 22 / 940: 00$ & Manual Tape & 401 & Good & \\
\hline $2 / 22 / 947: 05$ & Auto FIC & 0.00 & Suspect & Data not included in graph \\
\hline $2 / 23 / 940: 00$ & Manual Tape & 401.00 & Good & \\
\hline
\end{tabular}

Data obtained from WHC Surveillance Analysis Computer System, (PC SACS) July 7, 1995. 
WHC-SD-WM-ER-315, Rev. 0

\begin{tabular}{|c|c|c|c|c|}
\hline \multicolumn{5}{|c|}{ 241-AP-102 } \\
\hline \multicolumn{5}{|c|}{. Surface Level Data } \\
\hline Date & Type of Measurement & Leval (Inches) & Data Quality & Comments \\
\hline $2 / 23 / 947: 06$ & Auto FIC & 0.00 & Suspect & Data not included in graph \\
\hline $2 / 24 / 940: 00$ & Manual Tape & 401 & Good & \\
\hline $2 / 24 / 947: 06$ & Auto FIC & 0 & Suspect & Data not included in graph \\
\hline $2 / 25 / 940: 00$ & Manual Tape & 401.25 & Good & \\
\hline $2 / 26 / 940: 00$ & Manual Tape & 401.00 & Good & \\
\hline $2 / 26 / 947: 07$ & Auto FIC & 0 & Suspect & Data not included in graph \\
\hline $2 / 27 / 940: 00$ & Manual Tape & 401.00 & Good & \\
\hline $2 / 27 / 947: 07$ & Auto FIC & 0.00 & Suspect & Data not included in graph \\
\hline $2 / 28 / 9417: 04$ & Menual Tape & 400.75 & Good & \\
\hline $3 / 1 / 940: 00$ & Manual Tape & 401 & Good & \\
\hline $3 / 1 / 947: 04$ & Auto FIC & 0.00 & Suspect & Data not included in graph \\
\hline $3 / 2 / 947: 06$ & Auto FIC & 0 & Suspect & Data not included in graph \\
\hline $3 / 2 / 9417: 04$ & Manual Tape & 401.00 & Good & \\
\hline $3 / 3 / 940: 00$ & Manual Tape & 401 & Good & \\
\hline $3 / 3 / 947: 06$ & Auto FIC & 0 & Suspect & Data not included in graph \\
\hline $3 / 4 / 940: 00$ & Manual Tape & 400.75 & Good & \\
\hline 3/4/94 7:04 & Auto FIC & 0.00 & Suspect & Data not included in graph \\
\hline $3 / 5 / 940: 00$ & Manual Tape & 400.75 & Good & \\
\hline $3 / 5 / 947: 05$ & Auto FIC & 0.00 & Suspect & Data not included in graph \\
\hline $3 / 6 / 940: 00$ & Manual Tape & 400.75 & Good & \\
\hline 3/6/94 7:05 & Auto FIC & 0 & Suspect & Data not included in graph \\
\hline $3 / 7 / 940: 00$ & Manual Tape & 400.75 & Good & \\
\hline $3 / 7 / 94$ 7:05 & Auto FIC & 0 & Suspect & Data not included in graph \\
\hline $3 / 8 / 940: 00$ & Manual Tape & 401.00 & Good & \\
\hline 3/8/94 7:04 & Auto FIC & 0.00 & Suspect & Data not included in graph \\
\hline 3/9/94 0:00 & Manual Tape & 401 & Good & \\
\hline 3/9/94 7:04 & Auto FIC & 0.00 & Suspect & Data not included in graph \\
\hline $3 / 10 / 940: 00$ & Manual Tape & 401.00 & Good & \\
\hline $3 / 10 / 947: 05$ & Auto FIC & 0 & Suspect & Data not included in graph \\
\hline $3 / 11 / 940: 00$ & Manual Tape & 401 & Good & \\
\hline $3 / 11 / 947: 06$ & Auto FIC & 0 & Suspect & Data not included in graph \\
\hline $3 / 12 / 940: 00$ & Manual Tape & 401 & Good & \\
\hline 3/12/94 7:06 & Auto FIC & 0.00 & Suspect & Data not included in graph \\
\hline $3 / 13 / 940: 00$ & Manual Tape & 401.00 & Good & \\
\hline 3/13/94 7:05 & Auto FIC & 0 & Suspect & Data not included in graph \\
\hline $3 / 14 / 940: 00$ & Manual Tape & 401.00 & Good & \\
\hline $3 / 15 / 940: 00$ & Manual Tape & 401 & Good & \\
\hline 3/15/94 7:05 & Auto FIC & 0 & Suspect & Data not included in graph \\
\hline $3 / 16 / 940: 00$ & Manual Tape & 401 & Good & \\
\hline $3 / 16 / 947: 04$ & Auto FIC & 0.00 & Suspect & Data not included in graph \\
\hline 3/17/94 0:00 & Manual Tape & 401.00 & Good & \\
\hline $3 / 17 / 947: 05$ & Auto FIC & 0.00 & Suspect & Data not included in graph \\
\hline 3/18/94 0:00 & Manual Tape & 401 & Good & \\
\hline $3 / 18 / 947: 05$ & Auto FIC & 0 & Suspect & Data not included in graph \\
\hline $3 / 19 / 940: 00$ & Manual Tape & 401.00 & Good & \\
\hline 3/19/94 7:04 & Auto FIC & 0 & Suspect & Data not included in graph \\
\hline $3 / 20 / 940: 00$ & Manual Tape & 401.00 & Good & \\
\hline $3 / 20 / 947: 05$ & Auto FIC & 0.00 & Suspect & Data not included in graph \\
\hline
\end{tabular}

Data obtained from WHC Surveillance Analysis Computer System, (PC SACS) July 7, 1995. 
WHC-SD-WM-ER-315, Rev. 0

\begin{tabular}{|c|c|c|c|c|}
\hline \multicolumn{5}{|c|}{ 241-AP-102 } \\
\hline \multicolumn{5}{|c|}{ Surface Level Data } \\
\hline Date & Type of Measurement & Level (Inches) & Data Quality & Comments \\
\hline $3 / 21 / 940: 00$ & Manual Tape & 401 & Good & \\
\hline $3 / 21 / 947: 05$ & Auto FIC & 0 & Suspect & Data not included in graph \\
\hline $3 / 22 / 940: 00$ & Manual Tape & 401 & Good & \\
\hline $3 / 22 / 947: 04$ & Auto FIC & 0 & Suspect & Data not included in graph \\
\hline $3 / 23 / 940: 00$ & Manual Tape & 401.00 & Good & \\
\hline $3 / 24 / 940: 00$ & Manual Tape & 401 & Good & \\
\hline $3 / 24 / 947: 04$ & Auto FIC & 0.00 & Suspect & Data not included in graph \\
\hline $3 / 25 / 940: 00$ & Manual Tape & 401.00 & Good & \\
\hline $3 / 25 / 947: 05$ & Auto FIC & 0.00 & Suspect & Data not included in graph \\
\hline $3 / 26 / 940: 00$ & Manual Tape & 401 & Good & \\
\hline $3 / 26 / 947: 04$ & Auto FIC & 0 & Suspect & Data not included in graph \\
\hline 3/27/940:00 & Manual Tape & 401.00 & Good & \\
\hline $3 / 27 / 947: 04$ & Auto FIC & 0 & Suspect & Data not included in graph \\
\hline $3 / 28 / 940: 00$ & Manual Tape & 401.00 & Good & \\
\hline $3 / 28 / 947: 04$ & Auto FIC & 0.00 & Suspect & Data not included in graph \\
\hline $3 / 29 / 940: 00$ & Manual Tape & 401 & Good & \\
\hline $3 / 29 / 947: 03$ & Auto FIC & 0.00 & Suspect & Data not included in graph \\
\hline $3 / 30 / 940: 00$ & Manual Tape & 401 & Good & \\
\hline $3 / 30 / 947: 03$ & Auto FIC & 0 & Suspect & Data not included in graph \\
\hline $3 / 31 / 940: 00$ & Manual Tape & 401.00 & Good & \\
\hline $3 / 31 / 947: 03$ & Auto FIC & 0.00 & Suspect & Data not included in graph \\
\hline $4 / 1 / 940: 00$ & Manual Tape & 401 & Good & \\
\hline $4 / 2 / 940: 00$ & Manual Tape & 401.00 & Good & \\
\hline $4 / 2 / 947: 07$ & Auto FIC & 0.00 & Suspect & Data not included in graph \\
\hline $4 / 3 / 940: 00$ & Manual Tape & 401 & Good & \\
\hline 4/3/94 7:03 & Auto FIC & 0 & Suspect & Data not included in graph \\
\hline 4/4/94 0:00 & Manual Tape & 401.00 & Good & \\
\hline 4/4/94 7:03 & Auto FIC & 0. & Suspect & Data not included in graph \\
\hline $4 / 5 / 940: 00$ & Manual Tape & 401.00 & Good & \\
\hline $4 / 5 / 947: 03$ & Auto FIC & 0.00 & Suspect & Data not included in graph \\
\hline 4/6/94 0:00 & Manual Tape & 401.00 & Good & \\
\hline $4 / 6 / 947: 06$ & Auto FIC & 0.00 & Suspect & Data not included in graph \\
\hline $4 / 7 / 940: 00$ & Manual Tape & 401 & Good & \\
\hline $4 / 7 / 947: 07$ & Auto FIC & 0 & Suspect & Data not included in graph \\
\hline $4 / 8 / 94 \quad 0: 00$ & Manual Tape & 401 & Good & \\
\hline $4 / 8 / 947: 03$ & Auto FIC & 0 & Suspect & Data not included in graph \\
\hline 4/9/94 0:00 & Manual Tape & 401.00 & Good & \\
\hline 4/9/94 7:07 & Auto FIC & 0.00 & Suspect & Data not included in graph \\
\hline $4 / 10 / 940: 00$ & Manual Tape & 401.00 & Good & \\
\hline $4 / 10 / 947: 03$ & Auto FIC & 0.00 & Suspect & Data not included in graph \\
\hline $4 / 11 / 940: 00$ & Manual Tape & 401 & Good & \\
\hline $4 / 11 / 947: 03$ & Auto FIC & 0 & Suspect & Data not included in graph \\
\hline $4 / 12 / 940: 00$ & Manual Tape & $401: 00$ & Good & \\
\hline $4 / 12 / 947: 03$ & Auto FIC & 0 & Suspect & Data not included in graph \\
\hline 4/13/94 0:00 & Manual Tape & 401.00 & Good & \\
\hline $4 / 13 / 947: 03$ & Auto FIC & 0 & Suspect & Data not included in graph \\
\hline $4 / 14 / 940: 00$ & Manual Tape & 401 & Good & \\
\hline $4 / 14 / 947: 03$ & Auto FIC & 0.00 & Suspect & Data not included in graph \\
\hline
\end{tabular}

Data obtained from WHC Surveillance Analysis Computer System, (PC SACS) July 7, 1995. 
WHC-SD-WM-ER-315, Rev. 0

\begin{tabular}{|c|c|c|c|c|}
\hline \multicolumn{5}{|c|}{ 241-AP-102 } \\
\hline \multicolumn{5}{|c|}{ Surface Level Data } \\
\hline Date & Type of Measurement & Level (Inches) & Data Quality & Comments \\
\hline $4 / 15 / 940: 00$ & Manual Tape ' & 400.75 & Good & \\
\hline 4/15/94 7:03 & Auto FIC & 0.00 & Suspect & Data not included in graph \\
\hline $4 / 16 / 940: 00$ & Manual Tape & 401.00 & Good & \\
\hline $4 / 16 / 947: 03$ & Auto FIC & 0 & Suspect & Data not included in graph \\
\hline 4/17/940:00 & Manual Tape & 401.00 & Good & \\
\hline 4/17/94 7:03 & Auto FIC & 0.00 & Suspect & Data not included in graph \\
\hline $4 / 18 / 940: 00$ & Manual Tape & 401.00 & Good & \\
\hline 4/18/947:03 & Auto FIC & 0.00 & Suspect & Data not included in graph \\
\hline $4 / 19 / 940: 00$ & Manual Tape & 401 & Good & \\
\hline $4 / 20 / 940: 00$ & Manual Tape & 401 & Good & \\
\hline $4 / 20 / 947: 04$ & Auto FIC & 0 & Suspect & Data not included in graph \\
\hline $4 / 21 / 940: 00$ & Manual Tape & 401 & Good & \\
\hline 4/21/94 7:05 & Auto FIC & 0 & Suspect & Data not included in graph \\
\hline 4/22/940:00 & Manual Tape & 401.00 & Good & \\
\hline $4 / 22 / 947: 04$ & Auto FIC & 0.00 & Suspect & Data not included in graph \\
\hline 4/23/940:00 & Manual Tape & 401.00 & Good & \\
\hline $4 / 23 / 947: 05$ & Auto FIC & 0.00 & Suspect & Data not included in graph \\
\hline 4/24/94 0:00 & Manual Tape & 401.00 & Good & \\
\hline $4 / 24 / 947: 04$ & Auto FIC & 0 & Suspect & Data not included in graph \\
\hline $4 / 25 / 940: 00$ & Manual Tape & 400.75 & Good & \\
\hline 4/25/94 7:04 & Auto FIC & 0 & Suspect & Data not included in graph \\
\hline $4 / 26 / 940: 00$ & Manual Tape & 400.75 & Good & \\
\hline $4 / 26 / 947: 07$ & Auto FIC & 0.00 & Suspect & Data not included in graph \\
\hline $4 / 27 / 940: 00$ & Manual Tape & 400.75 & Good & \\
\hline $4 / 27 / 947: 04$ & Auto FIC & 0.00 & Suspect & Data not included in graph \\
\hline $4 / 28 / 947: 05$ & Auto FIC & 0 & Suspect & Data not included in graph \\
\hline 4/28/94 13:05 & Manual Tape & 401.00 & Good & \\
\hline 4/29/940:00 & Manual Tape & 401 & Good & \\
\hline $4 / 29 / 947: 04$ & Auto FIC & 0 & Suspect & Data not included in graph \\
\hline $4 / 30 / 940: 00$ & Manual Tape & 401.00 & Good & \\
\hline $4 / 30 / 947: 05$ & Auto FIC & 0.00 & Suspect & Data not included in graph \\
\hline $5 / 1 / 947: 05$ & Auto FIC & 0.00 & Suspect & Data not included in graph \\
\hline $5 / 1 / 9413: 05$ & Manual Tape & 401.00 & Good & \\
\hline $5 / 2 / 947: 05$ & Auto FIC & 0 & Suspect & Data not included in graph \\
\hline $5 / 2 / 94 \quad 13: 05$ & Manual Tape & 401 & Good & \\
\hline $5 / 3 / 94 \quad 0: 00$ & Manual Tape & 401.25 & Good & \\
\hline $5 / 3 / 947: 05$ & Auto FIC & 0 & Suspect & Data not included in graph \\
\hline $5 / 4 / 940: 00$ & Manual Tape & 400.75 & Good & \\
\hline 5/4/94 7:05 & Auto FIC & 0.00 & Suspect & Data not included in graph \\
\hline $5 / 5 / 94 \quad 0: 00$ & Manual Tape & 400.75 & Good & \\
\hline $5 / 5 / 947: 03$ & Auto FIC & 0.00 & Suspect & Data not included in graph \\
\hline $5 / 6 / 94 \quad 0: 00$ & Manual Tape & 401.00 & Good & \\
\hline $5 / 6 / 947: 03$ & Auto FIC & 0 & Suspect & Data not included in graph \\
\hline $5 / 7 / 940: 00$ & Manual Tape & 401 & Good & \\
\hline $5 / 7 / 947: 03$ & Auto FIC & 0 & Suspect & Data not included in graph \\
\hline $5 / 8 / 940: 00$ & Manual Tape & 401 & Good & \\
\hline $5 / 8 / 947: 03$ & Auto FIC & 0.00 & Suspect & Data not included in graph \\
\hline $5 / 9 / 940: 00$ & Manual Tape & 401.00 & Good & \\
\hline
\end{tabular}

Data obtained from WHC Surveillance Analysis Computer System, (PC SACS) July 7, 1995. 
WHC-SD-WM-ER-315, Rev. 0

\begin{tabular}{|c|c|c|c|c|}
\hline \multicolumn{5}{|c|}{$\frac{241-A P-102}{\text { Surface Level Data }}$} \\
\hline \multicolumn{5}{|c|}{ Surface Level Data } \\
\hline Date & Type of Measurement & Level (Inches) & Data Quality & Comments \\
\hline $5 / 9 / 947: 03$ & Auto FIC & 0.00 & Suspect & Data not included in graph \\
\hline $5 / 10 / 940: 00$ & Manual Tape & 400.75 & Good & \\
\hline $5 / 10 / 947: 03$ & Auto FIC & 0 & Suspect & Data not included in graph \\
\hline $5 / 11 / 940: 00$ & Manual Tape & 400.75 . & Good & \\
\hline $5 / 11 / 947: 03$ & Auto FIC & 0 & Suspect & Data not included in graph \\
\hline $5 / 12 / 940: 00$ & Manual Tape & 400.5 & Good & \\
\hline $5 / 13 / 940: 00$ & Manual Tape & 400.75 & Good & \\
\hline $5 / 13 / 947: 03$ & Auto FIC & 0.00 & Suspect & Data not included in graph \\
\hline $5 / 14 / 940: 00$ & Manual Tape & 400.75 & Good & \\
\hline $5 / 14 / 947: 03$ & Auto FIC & 0.00 & Suspect & Data not included in graph \\
\hline $5 / 15 / 940: 00$ & Manual Tape & 400.75 & Good & \\
\hline $5 / 15 / 947: 06$ & Auto FIC & 0 & Suspect & Data not included in graph \\
\hline $5 / 16 / 940: 00$ & Manual Tape & 400.75 & Good & \\
\hline $5 / 16 / 947: 02$ & Auto FIC & 0 & Suspect & Data not included in graph \\
\hline $5 / 17 / 940: 00$ & Manual Tape & 400.75 & Good & \\
\hline $5 / 17 / 947: 03$ & Auto FIC & 0 & Suspect & Data not included in graph \\
\hline $5 / 18 / 940: 00$ & Manual Tape & 400.50 & Good & \\
\hline $5 / 18 / 947: 03$ & Auto FIC & 0.00 & Suspect & Data not included in graph \\
\hline $5 / 19 / 940: 00$ & Manual Tape & 400.75 & Good & \\
\hline $5 / 19 / 947: 03$ & Auto FIC & 0.00 & Suspect & Data not included in graph \\
\hline $5 / 20 / 940: 00$ & Manual Tape & 400.75 & Good & \\
\hline $5 / 20 / 947: 03$ & Auto FIC & 0 & Suspect & Data not included in graph \\
\hline $5 / 21 / 940: 00$ & Manual Tape & 400.75 & Good & \\
\hline $5 / 21 / 947: 05$ & Auto FIC & 0 & Suspect & Data not included in graph \\
\hline $5 / 22 / 940: 00$ & Manual Tape & 400.75 & Good & \\
\hline $5 / 22 / 947: 05$ & Auto FIC & 0.00 & Suspect & Deta not included in graph \\
\hline $5 / 23 / 940: 00$ & Manual Tape & 309.25 & Suspect & Data not included in graph \\
\hline $5 / 23 / 947: 04$ & Auto FIC & 0.00 & Suspect & Data not included in graph \\
\hline $5 / 24 / 940: 00$ & Manual Tape & 400.75 & Good & \\
\hline $5 / 24 / 947: 04$ & Auto FIC & 0 & Suspect & Data not included in graph \\
\hline $5 / 25 / 940: 00$ & Manual Tape & 401 & Good & \\
\hline $5 / 25 / 947: 04$ & Auto FIC & 0 & Suspect & Data not included in graph \\
\hline $5 / 26 / 940: 00$ & Manual Tape & 400.75 & Good & \\
\hline $5 / 27 / 940: 00$ & Manual Tape & 401.00 & Good & \\
\hline $5 / 27 / 947: 03$ & Auto FIC & 0.00 & Suspect & Data not included in graph \\
\hline $5 / 28 / 940: 00$ & Manual Tape & 400.75 & Good & \\
\hline $5 / 28 / 947: 03$ & Auto FIC & $0 . \overline{00}$ & Suspect & Data not included in graph \\
\hline $5 / 29 / 940: 00$ & Manual Tape & 400 & Good & \\
\hline $5 / 29 / 947: 03$ & Auto FIC & 0 & Suspect & Data not included in graph \\
\hline $5 / 30 / 940: 00$ & Manual Tape & 400.25 & Good & \\
\hline $5 / 30 / 947: 02$ & Auto FIC & 0 & Suspect & Data not included in graph \\
\hline $5 / 31 / 940: 00$ & Manual Tape & 400.50 & Good & \\
\hline $5 / 31 / 947: 03$ & Auto FIC & 0.00 & Suspect & Data not included in graph \\
\hline $6 / 1 / 940: 00$ & Manual Tape & 400.75 & Good & \\
\hline $6 / 1 / 947: 03$ & Auto FIC & 0.00 & Suspect & Data not included in graph \\
\hline $6 / 2 / 940: 00$ & Manual Tape & 400.5 & Good & \\
\hline $6 / 2 / 947: 03$ & Auto FIC & 0 & Suspect & Data not included in graph \\
\hline $6 / 3 / 94 \quad 0: 00$ & Manual FIC & 0.00 & Suspect & Data not included in graph \\
\hline
\end{tabular}

Data obtained from WHC Surveillance Analysis Computer System, (PC SACS) July 7, 1995. 


\begin{tabular}{|c|c|c|c|c|}
\hline \multicolumn{5}{|c|}{ 241-AP-102 } \\
\hline \multicolumn{5}{|c|}{ Surface Leval Data } \\
\hline Date & Type of Measurement & Level (Inches) & Date Quality & Comments \\
\hline $6 / 3 / 940: 00$ & Manual Tape & 400.75 & Good & \\
\hline 6/4/94 0:00 & Manual Tape & 400.75 & Good & \\
\hline $6 / 4 / 947: 04$ & Auto FIC & 0.00 & Suspect & Data not included in graph \\
\hline $6 / 5 / 940: 00$ & Manual Tape & 400.75 & Good & \\
\hline $6 / 5 / 947: 03$ & Auto FIC & 0.00 & Suspect & Data not included in graph \\
\hline $6 / 6 / 940: 00$ & Manual Tape & 400.5 & Good & \\
\hline 6/6/94 7:06 & Auto FIC & 0 & Suspect & Data not included in graph \\
\hline $6 / 7 / 940: 00$ & Manual Tape & 400.75 & Good & \\
\hline $6 / 7 / 947: 02$ & Auto FIC & 0 & Suspect & Data not included in graph \\
\hline $6 / 8 / 940: 00$ & Manual Tape & 400.75 & Good & \\
\hline $6 / 8 / 947: 03$ & Auto FIC & 0 & Suspect & Data not included in graph \\
\hline $6 / 9 / 940: 00$ & Manual FIC & 0.00 & Suspect & Data not included in graph \\
\hline 6/9/94 0:00 & Manual Tape & 400.75 & Good & \\
\hline $6 / 10 / 940: 00$ & Manual Tape & 401 & Good & \\
\hline $6 / 10 / 947: 04$ & Auto FIC & 0.00 & Suspect & Data not included in graph \\
\hline $6 / 11 / 940: 00$ & Manual Tape & 401.25 & Good & \\
\hline $6 / 11 / 947: 04$ & Auto FIC & 0.00 & Suspect & Data not included in graph \\
\hline $6 / 12 / 940: 00$ & Manual Tape & 401.00 & Good & \\
\hline $6 / 12 / 947: 03$ & Auto FIC & 0 & Suspect & Data not included in graph \\
\hline $6 / 13 / 940: 00$ & Manual Tape & 400.75 & Good & \\
\hline $6 / 13 / 947: 02$ & Auto FIC & 0 & Suspect & Data not included in graph \\
\hline $6 / 14 / 940: 00$ & Menual Tape & 401 & Good & \\
\hline $6 / 14 / 947: 03$ & Auto FIC & 0.00 & Suspect & Data not included in graph \\
\hline 6/15/94 0:00 & Manual Tape & 400.75 & Good & \\
\hline $6 / 15 / 947: 03$ & Auto FIC & 0.00 & Suspect & Data not included in graph \\
\hline $6 / 16 / 940: 00$ & Manual Tape & 400.75 & Good & \\
\hline $6 / 16 / 947: 03$ & Auto FIC & 0 & Suspect & Data not included in graph \\
\hline $6 / 17 / 940: 00$ & Manual Tape & 400.50 & Good & \\
\hline $6 / 1.7 / 947: 03$ & Auto FIC & 0 & Suspect & Data not included in graph \\
\hline 6/18/94 0:00 & Manual Tape & 400.5 & Good & \\
\hline $6 / 18 / 947: 04$ & Auto FIC & 0.00 & Suspect & Data not included in graph \\
\hline $6 / 19 / 940: 00$ & Manual Tape & 400.75 & Good & \\
\hline $6 / 19 / 947: 04$ & Auto FIC & 0.00 & Suspect & Data not included in graph \\
\hline $6 / 20 / 940: 00$ & Manual Tape & 400.75 & Good & \\
\hline $6 / 20 / 947: 04$ & Auto FIC & 0 & Suspect & Data not included in graph \\
\hline $6 / 21 / 940: 00$ & Manual Tape & 400.75 & Good & \\
\hline 6/21/94 7:04 & Auto FIC & 0 & Suspect & Data not included in graph \\
\hline $6 / 22 / 940: 00$ & Manual Tape & 400.75 & Good & \\
\hline $6 / 22 / 947: 03$ & Auto FIC & 0.00 & Suspect & Data not included in graph \\
\hline $6 / 23 / 940: 00$ & Manual Tape & 400.75 & Good & \\
\hline $6 / 23 / 947: 05$ & Auto FIC & 0 & Suspect & Data not included in graph \\
\hline $6 / 24 / 940: 00$ & Manual Tape & 400.75 & Good & \\
\hline $6 / 24 / 947: 06$ & Auto FIC & 0 & Suspect & Data not included in graph \\
\hline $6 / 25 / 94 \quad 0: 00$ & Manual Tape & 400.75 & Good & \\
\hline $6 / 25 / 947: 05$ & Auto FIC & 0.00 & Suspect & Data not included in graph \\
\hline $6 / 26 / 94 \quad 0: 00$ & Manual Tape & 400.75 & Good & \\
\hline $6 / 26 / 947: 06$ & Auto FIC & 0.00 & Suspect & Data not included in graph \\
\hline $6 / 27 / 940: 00$ & Manual Tape & 400.75 & Good & \\
\hline
\end{tabular}

Data obtained from WHC Surveillance Analysis Computer System;, (PC SACS) July 7, 1995. 


\begin{tabular}{|c|c|c|c|c|}
\hline \multicolumn{5}{|c|}{ 241-AP-102 } \\
\hline Date & Type of Measurement & Leval (Inches) & Data Quality & Comments \\
\hline $6 / 27 / 947: 06$ & Auto FIC & 0.00 & Suspect & Data not included in graph \\
\hline $6 / 28 / 940: 00$ & Manual Tape & 400.75 & Good & \\
\hline $6 / 28 / 947: 06$ & Auto FIC & 0 & Suspect & Data not included in graph \\
\hline $6 / 29 / 940: 00$ & Manual Tape & 400.75 & Good & \\
\hline $6 / 29 / 947: 03$ & Auto FIC & 0.00 & Suspect & Data not included in graph \\
\hline $6 / 30 / 94 \quad 0: 00$ & Manual Tape & 401 & Good & \\
\hline $6 / 30 / 947: 04$ & Auto FIC & 0.00 & Suspect & Data not included in graph \\
\hline 7/1/940:00 & Manual Tape & 400.75 & Good & \\
\hline $7 / 1 / 947: 04$ & Auto FIC & 0 & Suspect & Data not included in graph \\
\hline $7 / 2 / 940: 00$ & Manua! Tape & 400.75 & Good & \\
\hline $7 / 2 / 947: 04$ & Auto FIC & 0 & Suspect & Data not included in graph \\
\hline $7 / 3 / 940: 00$ & Manual Tape & 400.75 & Good & \\
\hline $7 / 3 / 947: 04$ & Auto FIC & 0.00 & Suspect & Data not included in graph \\
\hline $7 / 4 / 940: 00$ & Manual Tape & 400.75 & Good & \\
\hline $7 / 4 / 947: 04$ & Auto FIC & 0.00 & Suspect & Data not included in graph \\
\hline $7 / 5 / 940: 00$ & Manual Tape & 400.75 & Good & \\
\hline $7 / 5 / 947: 03$ & Auto FIC & 0 & Suspect & Data not included in graph \\
\hline $7 / 6 / 940: 00$ & Manual Tape & 400.00 & Good & \\
\hline $7 / 6 / 947: 05$ & Auto FIC & 0 & Suspect & Data not included in graph \\
\hline 7/7/940:00 & Manual Tape & 400 & Good & \\
\hline $7 / 7 / 947: 03$ & Auto FIC & 0.00 & Suspect & Data not included in graph \\
\hline 7/8/940:00 & Manual Tape & 400.25 & Good & \\
\hline $7 / 8 / 947: 03$ & Auto FIC & 0.00 & Suspect & Data not included in graph \\
\hline $7 / 9 / 940: 00$ & Manual Tape & 400.75 & Good & \\
\hline $7 / 9 / 947: 03$ & Auto FIC & 0 & Suspect & Data not included in graph \\
\hline $7 / 10 / 940: 00$ & Manual Tape & 400.75 & Good & \\
\hline $7 / 10 / 947: 03$ & Auto FIC & 0 & Suspect & Data not included in graph \\
\hline $7 / 11 / 940: 00$ & Manual Tape & 400.30 & Good & \\
\hline $7 / 11 / 947: 03$ & Auto FIC & 0.00 & Suspect & Data not included in graph \\
\hline 7/12/940:00 & Manual Tape & 400.25 & Good & \\
\hline 7/12/94 7:03 & Auto FIC & 0.00 & Suspect & Data not included in graph \\
\hline $7 / 13 / 947: 03$ & Auto FIC & 0.00 & Suspect & Data not included in graph \\
\hline $7 / 13 / 94$ 18:52 & Manual Tape & $\mathbf{4 0 0 . 7 5}$ & Good & \\
\hline $7 / 14 / 9414: 06$ & Manual Tape & 400.75 & Good & \\
\hline $7 / 15 / 9410: 01$ & Manual Tape & 400.75 & Good & \\
\hline $7 / 16 / 947: 10$ & Manual Tape & 400.75 & Good & \\
\hline 7/17/945:07 & Manual Tape & 400.75 & Good & \\
\hline $7 / 18 / 943: 28$ & Manual Tape & 400.75 & Good & \\
\hline $7 / 19 / 940: 00$ & Manual Tape & 400.75 & Good & \\
\hline 7/19/946:59 & Manual Tape & 400.75 & Good & \\
\hline $7 / 20 / 940: 00$ & Manual Tape & 385.25 & Suspect & Data not included in graph \\
\hline $7 / 20 / 9412: 59$ & Manual Tape & 400.75 & Good & \\
\hline $7 / 21 / 94 \quad 14: 39$ & Manual Tape & 400.75 & Good & \\
\hline $7 / 22 / 947: 35$ & Manual Tape & 400.75 & Good & \\
\hline $7 / 23 / 9411: 46$ & Manual Tape & 400.75 & Good & \\
\hline $7 / 24 / 94 \quad 14: 44$ & Manual Tape & 400.75 & Good & \\
\hline $7 / 25 / 9421: 45$ & Manual Tape & 400.75 & Good & \\
\hline $7 / 26 / 94 \quad 15: 26$ & Manual Tape & 400.75 & Good & \\
\hline
\end{tabular}

Data obtained from WHC Surveillance Analysis Computer System, (PC SACS) July 7, 1995. 


\begin{tabular}{|c|c|c|c|c|}
\hline \multicolumn{5}{|c|}{ 241-AP-102 } \\
\hline \multicolumn{5}{|c|}{ Surface Level Data } \\
\hline Date & Type of Measurement & Level (Inches) & Data Quality & Comments \\
\hline $7 / 27 / 94 \quad 0: 00$ & Manual Tape & 400.75 & Good & \\
\hline $7 / 28 / 940: 19$ & Manual Tape & 400.75 & Good & \\
\hline $7 / 29 / 945: 10$ & Manual Tape & 401.00 & Good & \\
\hline $7 / 30 / 9412: 00$ & Manual Tape & 400.75 & Good & \\
\hline $7 / 31 / 9415: 09$ & Manual Tape & 400.75 & Good & \\
\hline $8 / 1 / 9412: 00$ & Manual Tape & 401.00 & Good & \\
\hline $8 / 2 / 9412: 00$ & Manual Tape & 400.75 & Good & \\
\hline $8 / 3 / 9414: 30$ & Manual Tape & 400.75 & Good & \\
\hline $8 / 4 / 9415: 02$ & Manual Tape & 400.75 & Good & \\
\hline $8 / 5 / 9419: 08$ & Manual Tape & 400.75 & Good & \\
\hline $8 / 6 / 9414: 34$ & Manual Tape & 400.75 & Good & \\
\hline $8 / 7 / 9412: 51$ & Manual Tape & 400.75 & Good & \\
\hline $8 / 8 / 9412: 00$ & Manual Tape & 400.75 & Good & \\
\hline $8 / 9 / 9421: 12$ & Manual Tape & 401.00 & Good & \\
\hline $8 / 10 / 9416: 49$ & Manual Tape & 401.00 & Good & \\
\hline $8 / 11 / 9421: 44$ & Manual Tape & 401 & Good & \\
\hline $8 / 12 / 9412: 00$ & Manual Tape & 401 & Good & \\
\hline $8 / 13 / 9414: 43$ & Manual Tape & 400.75 & Good & \\
\hline $8 / 14 / 9413: 28$ & Manual Tape & 401.00 & Good & \\
\hline $8 / 15 / 9413: 01$ & Manual Tape & 400.75 & Good & \\
\hline $8 / 16 / 9420: 43$ & Manual Tape & 400.75 & Good & \\
\hline $8 / 17 / 9416: 17$ & Manual Tape & 400.75 & Good & \\
\hline $8 / 18 / 9416: 45$ & Manual Tape & 400.75 & Good & \\
\hline $8 / 19 / 9423: 14$ & Manual Tape & 400.75 & Good & \\
\hline $8 / 20 / 9415: 19$ & Manual Tape & 400.75 & Good & \\
\hline $8 / 21 / 9411: 52$ & Manual Tape & $400: 75$ & Good & \\
\hline $8 / 22 / 9413: 12$ & Manual Tape & 400.75 & Good & \\
\hline $8 / 23 / 9419: 52$ & Manual Tape & 400.75 & Good & \\
\hline $8 / 24 / 947: 15$ & Manual Tape & 400.75 & Good & \\
\hline $8 / 25 / 9415: 00$ & Manual Tape & 400.75 & Good & \\
\hline $8 / 26 / 9414: 42$ & Manual Tape & 400.75 & Good & \\
\hline $8 / 27 / 9414: 45$ & Manual Tape & 401 & Good & \\
\hline $8 / 28 / 9415: 03$ & Manual Tape & 401.00 & Good & \\
\hline $8 / 29 / 9412: 00$ & Manual Tape & 400.50 & Good & \\
\hline $8 / 30 / 94 \quad 14: 59$ & Manual Tape & 400.75 & Good & \\
\hline $8 / 31 / 9415: 27$ & Manual Tape & 400.75 & Good & \\
\hline $9 / 1 / 9414: 52$ & Manual Tape & 400.75 & Good & \\
\hline $9 / 2 / 9414: 08$ & Manual Tape & 400.75 & Good & \\
\hline $9 / 3 / 9414: 20$ & Manual Tape & 400.75 & Good & \\
\hline $9 / 4 / 9414: 40$ & Manual Tape & 400.75 & Good & \\
\hline $9 / 5 / 94 \quad 14: 07$ & Manual Tape & 400.75 & Good & \\
\hline $9 / 6 / 9413: 52$ & Manual Tape & 401 & Good & \\
\hline $9 / 7 / 9414: 57$ & Manual Tape & $401: 00$ & Good & \\
\hline $9 / 8 / 9414: 43$ & Manual Tape & 401.00 & Good & \\
\hline $9 / 9 / 9423: 27$ & Manual Tape & 401.00 & Good & \\
\hline $9 / 10 / 9414: 54$ & Manual Tape & 401 & Good & \\
\hline $9 / 11 / 9415: 23$ & Manual Tape & 401 & Good & \\
\hline $9 / 12 / 9412: 00$ & Manual Tape & 401.00 & Good & \\
\hline
\end{tabular}

Data obtained from WHC Surveillance Analysis Computer System, (PC SACS) July 7, 1995. 


\begin{tabular}{|c|c|c|c|c|}
\hline \multicolumn{5}{|c|}{ 241-AP-102 } \\
\hline \multicolumn{5}{|c|}{ Surface Level Data } \\
\hline Date & Type of Measurement & Level (Inches) & Data Quality & Comments \\
\hline 9/12/94 15:34 & Manual Tape & 401.00 & Good & \\
\hline $9 / 13 / 9414: 42$ & Manual Tape & 401 & Good & \\
\hline $9 / 14 / 9412: 49$ & Manual Tape & 401.00 & Good & \\
\hline $9 / 15 / 9413: 53$ & Manual Tape & 401 & Good & \\
\hline $9 / 16 / 9413: 06$ & Manual Tape & 401 & Good & \\
\hline $9 / 17 / 9412: 00$ & Manual Tape & 400.75 & Good & \\
\hline $9 / 18 / 9415: 13$ & Manual Tape & 400.75 & Good & \\
\hline $9 / 19 / 9414: 53$ & Manual Tape & 400.75 & Good & \\
\hline $9 / 20 / 9414: 44$ & Manual Tape & 401 & Good & \\
\hline $9 / 21 / 9414: 13$ & Manual Tape & 401.00 & Good & \\
\hline $9 / 22 / 9414: 35$ & Manual Tape & 400.50 & Good & \\
\hline $9 / 23 / 9412: 50$ & Manual Tape & 400.5 & Good & \\
\hline $9 / 24 / 9412: 00$ & Manual Tape & 401 & Good & \\
\hline $9 / 24 / 9418: 24$ & Manual Tape & 0 & Suspect & Data not included in graph \\
\hline $9 / 25 / 94 \quad 17: 12$ & Manual Tape & 400.75 & Good & \\
\hline $9 / 26 / 9416: 28$ & Manual Tape & 400.75 & Good & \\
\hline $9 / 27 / 9415: 35$ & Manual Tape & 400.75 & Good & \\
\hline $9 / 28 / 9414: 43$ & Manual Tape & 400.75 & Good & \\
\hline $9 / 29 / 94 \quad 14: 42$ & Manual Tape & 400.75 & Good & $\cdot$ \\
\hline $9 / 30 / 9414: 36$ & Manual Tape & 401.00 & Good & \\
\hline $10 / 1 / 9415: 01$ & Manual Tape & 401.00 & Good & \\
\hline $10 / 2 / 9412: 55$ & Manual Tape & 401 & Good & \\
\hline $10 / 3 / 9417: 53$ & Manual Tape & 400.75 & Good & \\
\hline $10 / 4 / 9413: 49$ & Manual Tape & 400.75 & Good & \\
\hline $10 / 5 / 94 \quad 15: 27$ & Manual Tape & $400.75^{\prime}$ & Good & \\
\hline $10 / 6 / 94 \quad 15: 26$ & Manual Tape & 400.5 & Good & \\
\hline $10 / 7 / 947: 21$ & Manual Tape & 400.75 & Good & \\
\hline $10 / 8 / 9412: 37$ & Manual Tape & 400.75 & Good & \\
\hline $10 / 9 / 9413: 33$ & Manual Tape & 400.75 & Good & \\
\hline $10 / 10 / 9414: 38$ & Manual Tape & 400.75 & Good & \\
\hline $10 / 11 / 9414: 01$ & Manual Tape & 400.75 & Good & \\
\hline $10 / 12 / 9417: 19$ & Manual Tape & 400.75 & Good & \\
\hline $10 / 13 / 9422: 50$ & Manual Tape & 400.75 & Good & \\
\hline $10 / 14 / 9416: 12$ & Manual Tape & 400.75 & Good & \\
\hline $10 / 15 / 9420: 30$ & Manual Tape & 400.75 & Good & \\
\hline $10 / 16 / 9415: 21$ & Manual Tape & 400.75 & Good & $\cdot$ \\
\hline $10 / 17 / 9415: 45$ & Manual Tape & 400.75 & Good & \\
\hline $10 / 18 / 9415: 35$ & Manual Tape & 400.50 & Good & \\
\hline $10 / 19 / 94 \quad 14: 31$ & Manual Tape & 400.75 & Good & \\
\hline $10 / 20 / 9415: 59$ & Manual Tape & 400.75 & Good & \\
\hline $10 / 21 / 9415: 12$ & Manual Tape & 400.75 & Good & \\
\hline $10 / 22 / 944: 15$ & Manual Tape & 400.75 & Good & \\
\hline $10 / 23 / 9412: 00$ & Manual Tape & 400.75 & Good & \\
\hline $10 / 23 / 9416: 23$ & Manual Tape & 0.00 & Suspect & Data not included in graph \\
\hline $10 / 24 / 94 \quad 11: 39$ & Manual Tape & 412.75 & Suspect & Data not included in graph \\
\hline $10 / 24 / 9412: 00$ & Manual Tape & 400.75 & Good & \\
\hline $10 / 25 / 949: 50$ & Manual Tape & 400.75 & Good & \\
\hline $10 / 26 / 9414: 44$ & Manual Tape & 400.75 & Good & \\
\hline
\end{tabular}

Data obtained from WHC Surveillance Analysis Computer System, (PC SACS) July 7, 1995. 


\begin{tabular}{|c|c|c|c|c|}
\hline \multicolumn{5}{|c|}{ 241-AP-102 } \\
\hline \multicolumn{5}{|c|}{ Surface Level Data } \\
\hline Date & Type of Measurement & Level (Inches) & Data Quality & Comments \\
\hline 10/27/94 13:06 & Manual Tape & 400.75 & Good & \\
\hline $10 / 28 / 9412: 25$ & Manual Tape & 400.75 & Good & \\
\hline $10 / 29 / 9420: 41$ & Manual Tape & 400.75 & Good & \\
\hline $10 / 30 / 9412: 00$ & Manual Tape & 400.75 & Good & \\
\hline $10 / 31 / 949: 29$ & Manual Tape & 400.75 & Good & \\
\hline $11 / 1 / 94 \quad 15: 02$ & Manual Tape & 400.75 & Good & \\
\hline $11 / 2 / 9415: 20$ & Manual Tape & 400.75 & Good & \\
\hline $11 / 3 / 9415: 10$ & Manual Tape & 400.75 & Good & \\
\hline $11 / 4 / 9415: 16$ & Manual Tape & 400.75 & Good & \\
\hline $11 / 5 / 9414: 26$ & Manual Tape & 400.75 & Good & \\
\hline 11/6/94 10:16 & Manual Tape & 400.75 & Good & \\
\hline $11 / 7 / 9412: 00$ & Manual Tape & 407.75 & Good & \\
\hline $11 / 8 / 9417: 09$ & Manual Tape & 400.50 & Good & \\
\hline $11 / 9 / 94 \quad 17: 28$ & Manual Tape & 400.75 & Good & \\
\hline $11 / 10 / 9414: 05$ & Manual Tape & 400.75 & Good & \\
\hline $11 / 11 / 9414: 53$ & Manual Tape & 400.75 & Good & \\
\hline $11 / 12 / 946: 10$ & Manual Tape & 400.5 & Good & \\
\hline 11/13/94 15:10 & Manual Tape & 400.75 & Good & \\
\hline $11 / 14 / 947: 12$ & Manual Tape & $400: 75$ & Good & \\
\hline $11 / 15 / 94 \quad 15: 26$ & Manual Tape & 400.75 & Good & \\
\hline $11 / 16 / 947: 02$ & Manual Tape & 400.74 & Good & \\
\hline $11 / 17 / 9412: 00$ & Manual Tape & 400.75 & Good & \\
\hline $11 / 18 / 9413: 52$ & Manual Tape & 400.75 & Good & \\
\hline $11 / 19 / 9412: 00$ & Manual Tape & 400.50 & Good & \\
\hline $11 / 20 / 949: 32$ & Manual Tape & 400.50 & Good & \\
\hline $11 / 21 / 9414: 56$ & Manual Tape & 400.75 & Good & \\
\hline $11 / 22 / 9415: 26$ & Manual Tape & 400.5 & Good & \\
\hline $11 / 23 / 94 \quad 15: 12$ & Manual Tape & 400.50 & Good & \\
\hline $11 / 24 / 9415: 24$ & Manual Tape & 400.50 & Good & \\
\hline $11 / 25 / 9412: 55$ & Manual Tape & 400.75 & Good & \\
\hline $11 / 26 / 9415: 15$ & Manual Tape & 400.50 & Good & \\
\hline $11 / 27 / 9414: 43$ & Manual Tape & 400.5 & Good & \\
\hline $11 / 28 / 9415: 14$ & Manual Tape & 400.75 & Good & \\
\hline $11 / 29 / 9415: 34$ & Manual Tape & 400.75 & Good & \\
\hline $11 / 30 / 9418: 51$ & Manual Tape & 400.75 & Good & \\
\hline $12 / 1 / 94 \quad 15: 30$ & Manual Tape & $400: 75$ & Good & \\
\hline $12 / 2 / 94 \quad 15: 22$ & Manual Tape & 400.75 & Good & \\
\hline $12 / 3 / 9416: 57$ & Manual Tape & 400.5 & Good & \\
\hline $12 / 4 / 9417: 18$ & Manual Tape & 400.50 & Good & \\
\hline $12 / 5 / 9414: 19$ & Manual Tape & 400.50 & Good & \\
\hline $12 / 6 / 947: 28$ & Manual Tape & 400.25 & Suspect & Data not included in graph \\
\hline $12 / 6 / 9412: 00$ & Manual Tape & 400.00 & Good & \\
\hline $12 / 7 / 947: 28$ & Manual Tape & 400.5 & Good & \\
\hline $12 / 8 / 9412: 00$ & Manual Tape & 400.50 & Good & \\
\hline 12/8/94 15:16 & Manual Tape & 400.75 & Suspect & Data not included in graph \\
\hline $12 / 9 / 9417: 53$ & Manual Tape & 400.50 & Good & \\
\hline 12/10/94 13:17 & Manual Tape & 400.5 & Good & \\
\hline $12 / 11 / 9418: 13$ & Manual Tape & 400.50 & Good & \\
\hline
\end{tabular}

Data obtained from WHC Surveillance Analysis Computer System, (PC SACS) July 7, 1995. 


\begin{tabular}{|c|c|c|c|c|}
\hline \multicolumn{5}{|c|}{ 241-AP-102 } \\
\hline \multicolumn{5}{|c|}{ Surface Level Data } \\
\hline Date & Type of Measurement & Level (Inches) & Data Quality & Comments \\
\hline $12 / 12 / 9420: 23$ & Manual Tape & 400.50 & Good & \\
\hline $12 / 13 / 9412: 00$ & Manual Tape & 401.00 & Good & \\
\hline $12 / 13 / 94 \quad 14: 08$ & Manual Tape & 400.75 & Suspect & Data not included in graph \\
\hline $12 / 14 / 9412: 24$ & Manual Tape & 400.50 & Good & . \\
\hline $12 / 15 / 9412: 00$ & Manual Tape & 400.50 & Good & \\
\hline $12 / 15 / 9414: 30$ & Manual Tape & 400.75 & Suspect & Data not included in graph \\
\hline $12 / 16 / 9412: 00$ & Manual Tape & 400.5 & Good & \\
\hline $12 / 16 / 9413: 04$ & Manual Tape & 400.75 & Suspect & Data not included in graph \\
\hline $12 / 17 / 9414: 54$ & Manual Tape & 400.5 & Good & \\
\hline $12 / 18 / 9415: 40$ & Manual Tape & 400.5 & Good & \\
\hline $12 / 19 / 9414: 29$ & Manual Tape & 400.5 & Good & \\
\hline $12 / 20 / 9412: 00$ & Manual Tape & 400.50 & Good & \\
\hline $12 / 20 / 94 \quad 14: 51$ & Manual Tape & 400.75 & Suspect & Data not included in graph \\
\hline $12 / 21 / 9415: 11$ & Manual Tape & 400.50 & Good & \\
\hline $12 / 22 / 9413: 43$ & Manual Tape & 400.5 & Gopd & \\
\hline $12 / 23 / 9412: 00$ & Manual Tape & 400.25 & Good & \\
\hline $12 / 23 / 9415: 24$ & Manual Tape & 400.5 & Suspect & Data not included in graph \\
\hline 12/24/94 11:17 & Manual Tape & 400.50 & Good & \\
\hline $12 / 25 / 9414: 34$ & Manual Tape & 400.50 & Good & \\
\hline $12 / 26 / 94 \quad 14: 53$ & Manual Tape & 400.50 & Good & \\
\hline 12/27/9421:23 & Manual Tape & 400.5 & Good & \\
\hline $12 / 28 / 9412: 00$ & Manual Tape & 400.25 & Good & \\
\hline $12 / 28 / 9416: 01$ & Manual Tape & 400.50 & Suspect & Data not included in graph \\
\hline $12 / 29 / 9415: 28$ & Manual Tape & 400.25 & Good & \\
\hline $12 / 30 / 942: 49$ & Manual Tape & 400.5 & Good & \\
\hline $12 / 31 / 9414: 24$ & Manual Tape & 400.50 & Good & \\
\hline $1 / 1 / 95 \quad 15: 50$ & Manual Tape & 400.50 & Good & \\
\hline $1 / 2 / 9513: 49$ & Manual Tape & 400.5 & Good & \\
\hline $1 / 3 / 9514: 18$ & Manual Tape & 400.5 & Good & \\
\hline $1 / 4 / 9514: 43$ & Manual Tape & 400.50 & Good & \\
\hline $1 / 5 / 95 \quad 15: 12$ & Manual Tape & 400.50 & Good & \\
\hline $1 / 6 / 95 \quad 13: 48$ & Manual Tape & 400.50 & Good & \\
\hline $1 / 7 / 9514: 19$ & Manual Tape & 400.5 & Good & \\
\hline $1 / 8 / 95 \quad 14: 39$ & Manual Tape & 400.25 & Good & \\
\hline 1/9/95 15:28 & Manual Tape & 400.25 & Good & \\
\hline $1 / 10 / 95 \quad 14: 36$ & Manual Tape & 400.25 & Good & \\
\hline $1 / 11 / 95 \quad 14: 27$ & Manual Tape & 400.25 & Good & \\
\hline $1 / 12 / 95 \quad 14: 26$ & Manual Tape & 400.25 & Good & \\
\hline 1/13/95 12:00 & Manual Tape & 400.00 & Good & \\
\hline $1 / 13 / 95 \quad 14: 46$ & Manual Tape & 400.25 & Suspect & Data not included in graph \\
\hline $1 / 14 / 95 \quad 12: 00$ & Manual Tape & 400.5 & Good & \\
\hline $1 / 15 / 95 \quad 12: 00$ & Manual Tape & 400.50 & Good & \\
\hline $1 / 15 / 95 \quad 14: 34$ & Manual Tape & 401.50 & Suspect & Data not included in graph \\
\hline $1 / 16 / 9512: 00$ & Manual Tape & 400.50 & Good & \\
\hline $1 / 16 / 95 \quad 14: 39$ & Manual Tape & 30.75 & Suspect & Data not included in graph \\
\hline $1 / 17 / 95 \quad 12: 00$ & Manual Tape & 400.50 & Good & \\
\hline $1 / 17 / 95 \quad 14: 54$ & Manual Tape & 400.25 & Suspect & Data not included in graph \\
\hline $1 / 18 / 95 \quad 14: 49$ & Manual Tepe & 400.25 & Good & \\
\hline
\end{tabular}

Data obtained from WHC Surveillance Analysis Computer Systern, (PC SACS) July 7, 1995. 


\begin{tabular}{|c|c|c|c|c|}
\hline \multicolumn{5}{|c|}{ 241-AP-102 } \\
\hline \multicolumn{5}{|c|}{ 'Surface Leval Data } \\
\hline Dato & Type of Measurement & Level (Inches) & Data Quality & Comments \\
\hline $1 / 19 / 95 \quad 14: 38$ & Manual Tape & 400.25 & Good & \\
\hline $1 / 20 / 9512: 00$ & Manual Tape & 400.25 & Good & \\
\hline $1 / 20 / 9514: 52$ & Manual Tape & 400 & Suspect & Data not included in graph \\
\hline 1/21/95 15:02 & Manual Tape & 400.25 & Good & \\
\hline $1 / 22 / 95 \quad 13: 35$ & Manual Tape & 400.25 & Good & \\
\hline $1 / 23 / 95 \quad 12: 00$ & Manual Tape & 400.00 & Good & \\
\hline $1 / 23 / 95 \quad 14: 27$ & Manual Tape & 400.25 & Suspect & Data not included in graph \\
\hline $1 / 24 / 9512: 00$ & Manual Tape & 400.00 & Good & \\
\hline $1 / 24 / 95 \quad 14: 53$ & Manual Tape & 400.25 & Suspect & Data not included in graph \\
\hline $1 / 25 / 95 \quad 14: 40$ & Manual Tape & 400.5 & Good & \\
\hline $1 / 26 / 95 \quad 14: 51$ & Manual Tape & 400.50 & Good & \\
\hline $1 / 27 / 9514: 15$ & Manual Tape & 400.25 & Good & \\
\hline $1 / 28 / 9515: 13$ & Manual Tape & 400.25 & Good & \\
\hline $1 / 29 / 95 \quad 12: 00$ & Manual Tape & 400.25 & Good & \\
\hline $1 / 29 / 95 \quad 14: 18$ & Manual Tape & 0.00 & Suspect & Data not included in graph \\
\hline $1 / 30 / 9515: 14$ & Manual Tape . & 400.25 & Good & \\
\hline $1 / 31 / 9512: 00$ & Manual Tape & 400.25 & Good & \\
\hline $1 / 31 / 9514: 53$ & Manual Tape & 400.50 & Suspect & Data not included in graph \\
\hline 2/1/95 14:50 & Manual Tape & 400.25 & Good & \\
\hline 2/2/95 15:11 & Manual Tape & 400.25 & Good & \\
\hline $2 / 3 / 95 \quad 14: 52$ & Manual Tape & 400.25 & Good & \\
\hline $2 / 4 / 9514: 18$ & Manual Tape & 400.25 & Good & \\
\hline 2/5/95 2:13 & Manual Tape & 400.50 & Suspect & Data not included in graph \\
\hline 2/5/95 12:00 & Manual Tape & 400.25 & Good & \\
\hline 2/6/95 11:17 & Manual Tape & 400.24 & Suspect & Data not included in graph \\
\hline 2/6/95 12:00 & Manual Tape & 400.25 & Good & \\
\hline $2 / 7 / 951: 56$ & Manual Tape & 400.5 & Suspect & Data not included in graph \\
\hline $2 / 7 / 95 \quad 12: 00$ & Manual Tape & 400.25 & Good & \\
\hline 2/8/95 14:30 & Manual Tape & $400: 25$ & Good & \\
\hline $2 / 9 / 95 \quad 14: 21$ & Manual Tape & 400.25 & Good & \\
\hline $2 / 10 / 95 \quad 15: 00$ & Manual Tape & 400.25 & Good & \\
\hline $2 / 11 / 95 \quad 16: 00$ & Manual Tape & 400.25 & Good & \\
\hline $2 / 12 / 9512: 00$ & Manual Tape & 400.25 & Good & \\
\hline $2 / 12 / 95 \quad 13: 14$ & Manual Tape & 400.50 & Suspect & Data not included in graph \\
\hline 2/13/95 18:21 & Manual Tape & 400.25 & Good & \\
\hline $2 / 14 / 9514: 34$ & Manual Tape & 400.25 & Good & \\
\hline 2/15/95 15:11 & Manual Tape & 400.25 & Good & \\
\hline 2/16/95 14:20 & Manual Tape & 400.25 & Good & \\
\hline 2/17/95 15:00. & Manual Tape & 400.25 & Good & \\
\hline 2/18/95 12:00 & Manual Tape & 400.00 & Good & \\
\hline 2/19/95 12:30 & Manual Tape & 400.25 & Good & \\
\hline $2 / 20 / 9512: 55$ & Manual Tape & 400.25 & Good & \\
\hline $2 / 21 / 95 \quad 13: 26$ & Manual Tape & 400 & Good & \\
\hline 2/22/95 13:19 & Manual Tape & 400.00 & Good & \\
\hline 2/23/95 9:40 & Manual Tape & 400.00 & Good & \\
\hline 2/24/95 15:01 & Manual Tape & 400.00 & Good & \\
\hline $2 / 25 / 9512: 00$ & Manual Tape & 400 & Good & \\
\hline $2 / 25 / 95 \quad 14: 07$ & Manual Tape & 0.00 & Suspect & Data not included in graph \\
\hline
\end{tabular}

Data obtained from WHC Surveillance. Analysis Computer System, (PC SACS) July 7, 1995. 


\begin{tabular}{|c|c|c|c|c|}
\hline & & 241-AP-102 & & \\
\hline \multicolumn{5}{|c|}{ Surface Lovel Data } \\
\hline Date & Type of Messurement & Level (Inches) & Data Quality & Comments \\
\hline $2 / 26 / 9512: 00$ & Manual Tape & 400.00 & Good & \\
\hline $2 / 26 / 9514: 26$ & Manual Tape & 27.50 & Suspect & Data not included in graph \\
\hline $2 / 27 / 9512: 00$ & Manual Tape & 381.75 & Suspect & Data not included in graph \\
\hline 2/27/95 23:05 & Manual Tape & 400.25 & Good & \\
\hline $2 / 28 / 9514: 58$ & Manual Tape & 400.25 & Good & . \\
\hline $3 / 1 / 95 \quad 13: 49$ & Manual Tape & 400.25 & Good & \\
\hline $3 / 2 / 95 \quad 13: 56$ & Manual Tape & 400.12 & Good & \\
\hline $3 / 3 / 95 \quad 12: 00$ & Manual Tape & 400.25 & Good & \\
\hline $3 / 3 / 95 \quad 17: 06$ & Auto FIC & 399.9 & Suspect & Data not included in graph \\
\hline $3 / 4 / 957: 06$ & Auto FIC & 0 & Suspect & Data not included in graph \\
\hline $3 / 4 / 9512: 00$ & Manual Tape & 400.25 & Good & \\
\hline $3 / 5 / 957: 06$ & Auto FIC & 0.00 & Suspect & Data not included in graph \\
\hline $3 / 5 / 95 \quad 12: 00$ & Manual Tape & 400.25 & Good & \\
\hline $3 / 6 / 95$ 7:06 & Auto FIC & 0.00 & Suspect & Data not included in graph \\
\hline $3 / 6 / 9512: 00$ & Manual Tape & 400.125 & Good & \\
\hline $3 / 7 / 957: 06$ & Auto FIC & 0 & Suspect & Data not included in graph \\
\hline $3 / 7 / 9512: 00$ & Manual FIC & 399.80 & Good & \\
\hline $3 / 7 / 95 \quad 12: 00$ & Manual Tape & 400.00 & Good & \\
\hline $3 / 8 / 957: 03$ & Auto FIC & 399.8 & Good & \\
\hline $3 / 8 / 9512: 00$ & Manual Tape & 400.25 & Good & \\
\hline $3 / 9 / 95 \quad 1: 03$ & Auto FIC & 399.80 & Good & \\
\hline $3 / 9 / 95 \quad 12: 00$ & Manual Tape & 400.00 & Good & \\
\hline $3 / 10 / 957: 02$ & Auto FIC & 399.80 & Good & \\
\hline $3 / 10 / 95 \quad 12: 00$ & Manual Tape & 400 & Good & \\
\hline $3 / 11 / 957: 03$ & Auto FIC & 399.8 & Good & \\
\hline $3 / 11 / 95 \quad 12: 00$ & Manual Tape & $400: 00$ & Good & \\
\hline $3 / 12 / 957: 03$ & Auto FIC & 399.8 & Good & \\
\hline $3 / 12 / 9512: 00$ & Manual Tape & 400.00 & Good & \\
\hline $3 / 13 / 9567: 02$ & Auto FIC & 399.80 & Good & \\
\hline $3 / 13 / 95 \quad 12: 00$ & Manual Tape & 399.50 & Good & \\
\hline $3 / 14 / 956: 03$ & Auto FIC & 399.80 & Good & \\
\hline $3 / 14 / 9512: 00$ & Manual Tape & 399.5 & Good & \\
\hline $3 / 15 / 95$ 7:05 & Auto FIC & 399.8 & Good & \\
\hline $3 / 15 / 95 \quad 12: 00$ & Manual Tape & 400.5 & Good & \\
\hline $3 / 16 / 957: 03$ & Auto FIC & 399.8 & Good & \\
\hline $3 / 16 / 95 \quad 12: 00$ & Manual Tape & 400.00 & Good & \\
\hline $3 / 17 / 955: 03$ & Auto FIC & 399.80 & Good & \\
\hline $3 / 17 / 95 \quad 12: 00$ & Manual Tape & 400.25 & Good & \\
\hline $3 / 18 / 95$ 7:03 & Auto FIC & 399.8 & Good & \\
\hline $3 / 18 / 95 \quad 12: 00$ & Manual Tape & 400.25 & Good & \\
\hline $3 / 19 / 957: 03$ & Auto FIC & 399.8 & Good & \\
\hline $3 / 19 / 95 \quad 12: 00$ & Manual Tape & 400 & Good & \\
\hline $3 / 20 / 953: 03$ & Auto FIC & 399.8 & Good & \\
\hline $3 / 20 / 9512: 00$ & Manual Tape & 400.50 & Good & \\
\hline $3 / 21 / 957: 03$ & Auto FIC & 399.80 & Good & \\
\hline $3 / 21 / 9512: 00$ & Manual Tape & 400 & Good & \\
\hline $3 / 22 / 95$ 7:02 & Auto FIC & 399.80 & Good & \\
\hline $3 / 22 / 95 \quad 12: 00$ & Manual Tape & 400 & Good & \\
\hline
\end{tabular}

Data obtained from WHC Surveillance Analysis Computer System, (PC SACS) July 7, 1995. 
WHC-SD-WM-ER-315, Rev. 0

\begin{tabular}{|c|c|c|c|c|}
\hline \multicolumn{5}{|c|}{ 241-AP-102 } \\
\hline \multicolumn{5}{|c|}{$\begin{array}{l}\text { Surface Level Data } \\
\text { Type of }\end{array}$} \\
\hline Date & Type of Measurement & Level (Inches) & Data Quality & Comments \\
\hline $3 / 23 / 957: 03$ & Auto FIC & 399.8 & Good & \\
\hline $3 / 23 / 9512: 00$ & Manual Tape & 400 & Good & \\
\hline $3 / 24 / 957: 03$ & Auto FIC & 99.80 & Suspect & Data not included in graph \\
\hline $3 / 24 / 9512: 00$ & Manual FIC & 399.8 & Good & \\
\hline $3 / 24 / 9512: 00$ & Manual Tape & 400 & Good & \\
\hline 3/25/95 7:02 & Auto FIC & 399.80 & Good & \\
\hline $3 / 25 / 95 \quad 12: 00$ & Manual Tape & 400 & Good & \\
\hline $3 / 26 / 957: 02$ & Auto FIC & 399.8 & Good & \\
\hline $3 / 26 / 95 \quad 12: 00$ & Manual Tape & 400 & Good & \\
\hline $3 / 27 / 956: 03$ & Auto FIC & 399.8 & Good & \\
\hline $3 / 27 / 95 \quad 12: 00$ & Manual Tape & 400 & Good & \\
\hline $3 / 28 / 957: 03$ & Auto FIC & 399.80 & Good & \\
\hline $3 / 28 / 95 \quad 12: 00$ & Manual Tape & 400.00 & Good & \\
\hline $3 / 29 / 956: 02$ & Auto FIC & 399.80 & Good & \\
\hline $3 / 29 / 95 \quad 12: 00$ & Manual Tape & 400 & Good & \\
\hline $3 / 30 / 956: 03$ & Auto FIC & 399.8 & Good & \\
\hline $3 / 30 / 95 \quad 12: 00$ & Manual Tape & 400 & Good & \\
\hline $3 / 31 / 957: 04$ & Auto FIC & 399.8 & Good & \\
\hline $3 / 31 / 95 \quad 12: 00$ & Manual Tape & 400 & Good & \\
\hline 4/1/95 7:02 & Auto FIC & 399.80 & Good & \\
\hline $4 / 1 / 95 \quad 12: 00$ & Manual Tape & 400 & Good & \\
\hline $4 / 2 / 957: 03$ & Auto FIC & 399.80 & Good & \\
\hline 4/2/95 12:00 & Manual Tape & 400 & Good & \\
\hline $4 / 3 / 957: 03$ & Auto FIC & 399.8 & Good & \\
\hline $4 / 3 / 9512: 00$ & Manual Tape & 400.00 & Good & \\
\hline 4/4/95 7:03 & Auto FIC & 399.7 & Good & \\
\hline 4/4/95 12:00 & Manual Tape & 400 & Good & \\
\hline 4/5/95 7:03 & Auto FIC & 399.70 & Good & \\
\hline $4 / 5 / 95 \quad 12: 00$ & Manual Tape & 400 & Good & \\
\hline 4/6/95 7:03 & Auto FIC & 399.70 & Good & \\
\hline $4 / 6 / 95 \quad 12: 00$ & Manual Tape & 400 & Good & \\
\hline 4/7/95 7:03 & Auto FIC & 399.7 & Good & \\
\hline $4 / 7 / 95 \quad 12: 00$ & Manual Tape & 400 & Good & \\
\hline 4/8/95 7:02 & Auto FIC & 399.7 & Good & \\
\hline 4/8/95 12:00 & Manual Tape & 400 & Good & \\
\hline 4/9/95 12:00 & Manual Tape & 400.00 & Good & \\
\hline 4/9/95 23:03 & Auto FIC & 399.70 & Good & \\
\hline $4 / 10 / 957: 02$ & Auto FIC & 399.70 & Good & \\
\hline $4 / 10 / 95 \quad 12: 00$ & Manual Tape & 400 & Good & \\
\hline $4 / 11 / 956: 03$ & Auto FIC & 399.7 & Good & \\
\hline $4 / 11 / 95 \quad 12: 00$ & Manual Tape & 400 & Good & \\
\hline 4/12/95 7:03 & Auto FIC & 399.7 & Good & \\
\hline $4 / 12 / 9512: 00$ & Manual Tape & 400 & Good & \\
\hline 4/13/95 7:05 & Auto FIC & 0.00 & Suspect & Data not included in graph \\
\hline $4 / 13 / 95 \quad 12: 00$ & Manual FIC & 399.7 & Good & \\
\hline 4/13/95 12:00 & Manual Tape & 400 & Good & \\
\hline $4 / 14 / 957: 03$ & Auto FIC & 399.70 & Good & \\
\hline 4/14/95 12:00 & Manual Tape & 399.75 & Good & \\
\hline
\end{tabular}

Data obtained from WHC Surveillance Analysis Computer System, (PC SACS) July 7, 1995. 


\begin{tabular}{|c|c|c|c|c|}
\hline \multicolumn{5}{|c|}{ 241-AP.102 } \\
\hline \multicolumn{5}{|c|}{ Surface Level Data } \\
\hline Date & Type of Measurement & Leval (Inches) & Data Quality & Comments \\
\hline $4 / 15 / 957: 03$ & Auto FIC & 399.7 & Good & \\
\hline $4 / 15 / 9512: 00$ & Manual Tape & 400.00 & Good & \\
\hline $4 / 16 / 957: 03$ & Auto FIC & 399.7 & Good & \\
\hline $4 / 16 / 9512: 00$ & Manual Tape & 400 & Good & \\
\hline $4 / 17 / 957: 03$ & Auto FIC & 399.70 & Good & \\
\hline 4/17/95 12:00 & Manual Tape & 28 & Suspect & Data not included in graph \\
\hline 4/18/95 7:03 & Auto FIC & 399.70 & Good & \\
\hline $4 / 18 / 95 \quad 12: 00$ & Manual Tape & 400 & Good & \\
\hline 4/19/95 7:03 & Auto FIC & 399.7 & Good & \\
\hline 4/19/95 12:00 & Manual Tape & 400 & Good & \\
\hline $4 / 20 / 956: 03$ & Auto FIC & 399.7 & Good & \\
\hline $4 / 20 / 9512: 00$ & Manual Tape & 400 & Good & \\
\hline 4/21/95 7:03 & Auto FIC & 399.70 & Good & \\
\hline $4 / 21 / 9512: 00$ & Manual Tape & 400.00 & Good & \\
\hline $4 / 22 / 957: 03$ & Auto FIC & 399.70 & Good & \\
\hline 4/22/95 12:00 & Manual Tape & 400 & Good & \\
\hline $4 / 23 / 956: 03$ & Auto FIC & 399.7 & Good & \\
\hline $4 / 23 / 9512: 00$ & Manuel Tape & 399.25 & Good & \\
\hline $4 / 24 / 956: 03$ & Auto FIC & 399.7 & Good & \\
\hline $4 / 24 / 9512: 00$ & Manual Tape & 399.75 & Good & \\
\hline $4 / 25 / 957: 03$ & Auto FIC & 399.70 & Good & \\
\hline $4 / 25 / 9512: 00$ & Manual Tape & 400 & Good & \\
\hline $4 / 26 / 957: 03$ & Auto FIC & 399.70 & Good & \\
\hline $4 / 26 / 9512: 00$ & Manual Tape & 400 & Good & \\
\hline 4/27/95 4:03 & Auto FIC & 399.7 & Good & \\
\hline $4 / 27 / 9512: 00$ & Manual Tape & 400.00 & Good & \\
\hline $4 / 28 / 957: 03$ & Auto FIC & 0 & Suspect & Data not included in graph \\
\hline $4 / 28 / 95 \quad 12: 00$ & Manual FIC & 399.70 & Good & \\
\hline $4 / 28 / 95 \quad 12: 00$ & Manual Tape & 400 & Good & \\
\hline 4/29/95 7:03 & Auto FIC & 0.00 & Suspect & Data not included in graph \\
\hline $4 / 29 / 95 \quad 12: 00$ & Manual FIC & 399.65 & Good & \\
\hline $4 / 29 / 9512: 00$ & Manual Tape & 400.25 & Good & \\
\hline $4 / 30 / 956: 02$ & Auto FIC & 399.70 & Good & \\
\hline $4 / 30 / 9512: 00$ & Manual Tape & 400.25 & Good & \\
\hline $5 / 1 / 950: 03$ & Auto FIC & 399.70 & Good & \\
\hline $5 / 1 / 9512: 00$ & Manual Tape & 400 & Good & \\
\hline $5 / 2 / 957: 02$ & Auto FIC & 399.7 & Good & \\
\hline $5 / 2 / 9512: 00$ & Manual Tape & 400 & Good & \\
\hline $5 / 3 / 957: 03$ & Auto FIC & 399.7 & Good & \\
\hline $5 / 3 / 9512: 00$ & Manual Tape & 399.75 & Good & \\
\hline $5 / 4 / 95$ 7:04 & Auto FIC & 399.70 & Good & \\
\hline $5 / 4 / 95 \quad 12: 00$ & Manual Tape & 400 & Good & \\
\hline $5 / 5 / 956: 04$ & Auto FIC & 399.7 & Good & \\
\hline $5 / 5 / 9512: 00$ & Manual Tape & 400 & Good & \\
\hline $5 / 6 / 957: 03$ & Auto FIC & 399.7 & Good & \\
\hline $5 / 6 / 95 \quad 12: 00$ & Manual Tape & 400 & Good & \\
\hline $5 / 7 / 95 \quad 7: 04$ & Auto FIC & 399.70 & Good & \\
\hline $5 / 7 / 9512: 00$ & Manual Tape & 400 & Good & \\
\hline
\end{tabular}

Data obtained from WHC Surveillance Analysis Computer System, (PC SACS) July 7, 1995. 


\begin{tabular}{|c|c|c|c|c|}
\hline \multicolumn{5}{|c|}{ 241-AP-102 } \\
\hline & & Irface Level Dat: & & \\
\hline Date & Type of Measurement & Level (Inches) & Data Quality & Comments \\
\hline $5 / 8 / 95 \quad 4: 04$ & Auto FIC & 399.70 & Good & \\
\hline $5 / 8 / 9512: 00$ & Manual Tape & 400 & Good & \\
\hline $5 / 9 / 955: 04$ & Auto FIC & 399.7 & Good & \\
\hline $5 / 9 / 9512: 00$ & Manual Tape & 400.00 & Good & \\
\hline $5 / 10 / 95$ 7:04 & Auto FIC & 399.7 & Good & \\
\hline $5 / 10 / 9512: 00$ & Manual Tape & 400 & Good & \\
\hline $5 / 11 / 957: 03$ & Auto FIC & 399.70 & Good & \\
\hline $5 / 11 / 95 \quad 12: 00$ & Manual Tape & 400 & Good & \\
\hline $5 / 12 / 956: 06$ & Auto FIC & 399.70 & Good & \\
\hline $5 / 12 / 9512: 00$ & Manual Tape & 400 & Good & \\
\hline $5 / 13 / 952: 03$ & Auto FIC & 399.70 & Good & \\
\hline $5 / 13 / 9512: 00$ & Manual Tape & 400 & Good & \\
\hline 5/14/95 5:03 & Auto FIC & 399.7 & Good & \\
\hline $5 / 14 / 95 \quad 12: 00$ & Manual Tape & 400 & Good & \\
\hline $5 / 15 / 957: 03$ & Auto FIC & 399.7 & Good & \\
\hline $5 / 15 / 95 \quad 12: 00$ & Manual Tape & 399.75 & Good & \\
\hline $5 / 16 / 956: 03$ & Auto FIC & 399.60 . & Good & \\
\hline $5 / 16 / 95 \quad 12: 00$ & Manual Tape & 399.5 & Good & \\
\hline 5/17/95 7:02 & Auto FIC & 399.60 & Good & \\
\hline $5 / 17 / 95 \quad 12: 00$ & Manual Tape & 400 & Good & \\
\hline $5 / 18 / 956: 02$ & Auto FIC & 399.6 & Good & \\
\hline $5 / 18 / 95 \quad 12: 00$ & Manual Tape & 399.75 & Good & \\
\hline $5 / 19 / 957: 05$ & Auto FIC & 399.6 & Good & \\
\hline $5 / 19 / 95 \quad 12: 00$ & Manual Tape & 399.5 & Good & \\
\hline $5 / 20 / 957: 05$ & Auto FIC & 399.60 & Good & \\
\hline $5 / 20 / 9512: 00$ & Manual Tape & 399.5 & Good & \\
\hline $5 / 21 / 957: 05$ & Auto FIC & 399.60 & Good & \\
\hline $5 / 21 / 9512: 00$ & Manual Tape & 399.50 & Good & \\
\hline $5 / 22 / 957: 05$ & Auto FIC & 399.6 & Good & \\
\hline $5 / 22 / 9512: 00$ & Manual Tape & 399.75 & Good & \\
\hline $5 / 23 / 957: 11$ & Auto FIC & 0 & Suspect & Data not included in graph \\
\hline $5 / 23 / 9512: 00$ & Manual FIC & 399.6 & Good & \\
\hline $5 / 23 / 9512: 00$ & Manual Tape & 399.75 & Good & \\
\hline $5 / 24 / 957: 10$ & Auto FIC & 0.00 & Suspect & Data not included in graph \\
\hline $5 / 24 / 9512: 00$ & Manual FIC & 399.6 & Good & \\
\hline $5 / 24 / 95 \quad 12: 00$ & Manual Tape & 400 & Good & \\
\hline $5 / 25 / 957: 10$ & Auto FIC & 0.00 & Suspect & Data not included in graph \\
\hline $5 / 25 / 9512: 00$ & Manual FIC & 399.60 & Good & \\
\hline $5 / 25 / 9512: 00$ & Manual Tape & 400 & Good & \\
\hline $5 / 26 / 955: 04$ & Auto FIC & 399.6 & Good & \\
\hline $5 / 26 / 95 \quad 12: 00$ & Manual Tape & 400 & Good & \\
\hline 5/27/95 5:05 & Auto FIC & 399.6 & Good & \\
\hline $5 / 27 / 9512: 00$ & Manual Tape & 400.00 & Good & \\
\hline 5/28/95 5:05 & Auto FIC & 399.60 & Good & \\
\hline $5 / 28 / 9512: 00$ & Manual Tape & 399.25 & Good & \\
\hline $5 / 29 / 957: 05$ & Auto FIC & 399.60 & Good & \\
\hline $5 / 29 / 95 \quad 12: 00$ & Manual Tape & 399.25 & Good & \\
\hline $5 / 30 / 957: 05$ & Auto FIC & 399.6 & Good & \\
\hline
\end{tabular}

Data obtained from WHC Surveillance Analysis Computer System, (PC SACS) July 7, 1995. 


\begin{tabular}{|c|c|c|c|c|}
\hline \multicolumn{5}{|c|}{ 241-AP-102 } \\
\hline & & urface Level Date & & \\
\hline Date & Type of Measuremant & Level (Inches) & Data Quallty & Comments \\
\hline $5 / 30 / 9512: 00$ & Manual Tape & 396.5 & Suspect & Data not included in graph \\
\hline $5 / 31 / 957: 05$ & Auto FIC & 399.6 & Good & \\
\hline $5 / 31 / 9512: 00$ & Manual Tape & 396.5 & Suspect & Data not included in graph \\
\hline $6 / 1 / 957: 05$ & Auto FIC & 399.60 & Good & \\
\hline $6 / 1 / 9512: 00$ & Manual Tape & 396.5 & Suspect & Data not included in graph \\
\hline $6 / 2 / 957: 05$ & Auto FIC & 399.60 & Good & \\
\hline $6 / 2 / 9512: 00$ & Manual Tape & 396.50 & Suspect & Data not included in graph \\
\hline $6 / 3 / 9512: 00$ & Manual Tape & 400 & Good & \\
\hline 6/3/95 23:05 & Auto FIC & 399.6 & Good & \\
\hline $6 / 4 / 957: 05$ & Auto FIC & 399.6 & Good & \\
\hline $6 / 4 / 9512: 00$ & Manual Tape & 399.75 & Good & \\
\hline $6 / 5 / 956: 05$ & Auto FIC & 399.60 & Good & \\
\hline $6 / 5 / 9512: 00$ & Manual Tape & 400 & Good & \\
\hline $6 / 6 / 957: 05$ & Auto FIC & 399.60 & Good & \\
\hline $6 / 6 / 9512: 00$ & Manual Tape & 399.5 & Good & \\
\hline $6 / 7 / 95 \quad 7: 05$ & Auto FIC & 0 & Suspect & Data not included in graph \\
\hline $6 / 7 / 95 \quad 12: 00$ & Manual FIC & 399.60 & Good & \\
\hline $6 / 7 / 95 \quad 12: 00$ & Manual Tape & 399.75 & Good & \\
\hline 6/8/95 7:05 & Auto FIC & 399.6 & Good & \\
\hline $6 / 8 / 95 \quad 12: 00$ & Manual Tape & 399,75 & Good & \\
\hline 6/9/95 6:05 & Auto FIC & 399.60 & Good & \\
\hline $6 / 9 / 9512: 00$ & Manual Tape & 399.25 & Good & \\
\hline $6 / 10 / 957: 05$ & Auto FIC & 399.60 & Good & \\
\hline $6 / 10 / 9512: 00$ & Manual Tape & 400 & Good & \\
\hline $6 / 11 / 957: 05$ & Auto FIC & 399.6 & Good & \\
\hline $6 / 11 / 9512: 00$ & Manual Tape & 400 & Good & \\
\hline $6 / 12 / 956: 05$ & Auto FIC & 399.6 & Good & \\
\hline $6 / 12 / 95 \quad 12: 00$ & Manual Tape & 400 & Good & \\
\hline 6/13/95 7:05 & Auto FIC & $399: 60$ & Good & \\
\hline $6 / 13 / 95 \quad 12: 00$ & Manual Tape & 400 & Good & \\
\hline $6 / 14 / 957: 05$ & Auto FIC & 0.00 & Suspect & Data not included in graph \\
\hline $6 / 14 / 9512: 00$ & Manual FIC & 399.60 & Good & \\
\hline $6 / 14 / 95 \quad 12: 00$ & Manual Tape & 400.00 & Good & \\
\hline $6 / 15 / 95 \quad 1: 05$ & Auto FIC & 399.6 & Good & \\
\hline $6 / 15 / 9512: 00$ & Manual Tape & 399.75 & Good & \\
\hline $6 / 16 / 957: 05$ & Auto FIC & 399.6 & Good & \\
\hline $6 / 16 / 9512: 00$ & Manual Tape & 399.75 & Good & \\
\hline $6 / 17 / 957: 05$ & Auto FIC & 399.60 & Good & \\
\hline $6 / 17 / 95 \quad 12: 00$ & Manual Tape & 400 & Good & \\
\hline 6/18/95 7:05 & Auto FIC & 399.60 & Good & \\
\hline $6 / 18 / 95 \quad 12: 00$ & Manual Tape & 400 & Good & \\
\hline $6 / 19 / 954: 05$ & Auto FIC & 399.6 & Good & \\
\hline $6 / 19 / 95 \quad 12: 00$ & Manual Tape & 399.75 & Good & \\
\hline $6 / 20 / 957: 06$ & Auto FIC & 399.6 & Good & \\
\hline $6 / 20 / 9512: 00$ & Manual Tape & 399.75 & Good & \\
\hline $6 / 21 / 957: 06$ & Auto FIC & $399: 60$ & Good & \\
\hline $6 / 21 / 95 \quad 12: 00$ & Manual Tape & 400 & Good & \\
\hline $6 / 22 / 957: 06$ & Auto FIC & 399.60 & Good & \\
\hline
\end{tabular}

Data obtained from WHC Surveillance Analysis Computer System, (PC SACS) July 7, 1995. 


\begin{tabular}{|c|c|c|c|c|}
\hline \multicolumn{5}{|c|}{ 241-AP-102 } \\
\hline \multicolumn{5}{|c|}{ Surface Level Data } \\
\hline Date & Type of Measurement & Lovel (Inches) & Data Quality & Comments \\
\hline $6 / 22 / 9512: 00$ & Manual Tape & 400 & Good & \\
\hline $6 / 23 / 95 \quad 7: 06$ & Auto FIC & 399.6 & Good & \\
\hline $6 / 23 / 9512: 00$ & Manual Tape & 412 & Suspect & Data not included in graph \\
\hline $6 / 24 / 957: 06$ & Auto FIC & 399.6 & Good & \\
\hline $6 / 24 / 9512: 00$ & Manual Tape & 400 & Good & $\cdot$ \\
\hline $6 / 25 / 957: 05$ & Auto FIC & 399.60 & Good & \\
\hline $6 / 25 / 9512: 00$ & Manual Tape & 400 & Good & \\
\hline $6 / 26 / 957: 05$ & Auto FIC & 399.60 & Good & \\
\hline $6 / 26 / 9512: 00$ & Manual Tape & 400.00 & Good & \\
\hline $6 / 27 / 957: 06$ & Auto FIC & 399.6 & Good & \\
\hline $6 / 27 / 9512: 00$ & Manual Tape & 400 & Good & \\
\hline $6 / 28 / 957: 05$ & Auto FIC & 399.6 & Good & \\
\hline $6 / 28 / 9512: 00$ & Manual Tape & 400 & Good & \\
\hline $6 / 29 / 957: 06$ & Auto FIC & 399.60 & Suspect & Data not included in graph \\
\hline $6 / 29 / 95 \quad 12: 00$ & Manual Tape & 400 & Good & \\
\hline $6 / 30 / 957: 06$ & Auto FIC & 399.60 & Suspect & Data not included in graph \\
\hline $6 / 30 / 9512: 00$ & Manual Tape & 400 & Transcribed & \\
\hline
\end{tabular}




\begin{tabular}{|c|c|c|c|c|}
\hline \multicolumn{5}{|c|}{ 241-AP-103 } \\
\hline \multicolumn{5}{|c|}{ Surface Level Data } \\
\hline Date & Type of Measurement & Level (Inches) & Data Quality & Comments \\
\hline $1 / 1 / 910: 00$ & Manual Tape & 347.50 & Good & \\
\hline $1 / 1 / 917: 03$ & Auto FIC & 347.8 & Good & \\
\hline $1 / 2 / 910: 00$ & Manual Tape & 347.5 & Good & \\
\hline $1 / 2 / 917: 02$ & Auto FIC & 347.80 & Good & \\
\hline $1 / 3 / 910: 00$ & Manual Tape & 347.75 & Good & \\
\hline 1/3/91 7:03 & Auto FIC & 347.8 & Good & \\
\hline 1/4/910:00 & Manual Tape & 347.75 & Good & \\
\hline $1 / 4 / 917: \overline{02}$ & Auto FIC & 347.8 & Good & \\
\hline $1 / 5 / 91 \quad 0: 00$ & Manual Tape & 347.50 & Good & \\
\hline $1 / 5 / 917: 02$ & Auto FIC & 347.80 & Good & \\
\hline $1 / 6 / 910: 00$ & Manual Tape & 347.50 & Good & \\
\hline 1/6/91 7:02 & Auto FIC & 347.8 & Good & \\
\hline 1/7/910:00 & Manual Tape & 347.50 & Good & \\
\hline $1 / 7 / 917: 02$ & Auto FIC & 347.8 & Good & \\
\hline $1 / 8 / 910: 00$ & Manual Tape & 347.50 & Good & \\
\hline 1/8/91 7:03 & Auto FIC & 347.80 & Good & \\
\hline 1/9/91 0:00 & Manual Tape & 347.5 & Good & \\
\hline 1/9/91 7:02 & Auto FIC & 347.8 & Good & \\
\hline $1 / 10 / 910: 00$ & Manual Tape & 347.50 & Good & \\
\hline $1 / 10 / 917: 02$ & Auto FIC & 347.8 & Good & \\
\hline $1 / 11 / 910: 00$ & Manual Tape & 347.50 & Good & \\
\hline $1 / 11 / 917: 03$ & Auto FIC & 347.80 & Good & \\
\hline $1 / 12 / 910: 00$ & Manual Tape & 347.5 & Good & \\
\hline $1 / 12 / 917: 02$ & Auto FIC & 347.8 & Good & \\
\hline $1 / 13 / 910: 00$ & Manual Tape & 347.5 & Good & \\
\hline $1 / 13 / 917: 03$ & Auto FIC & 347.8 & Good & \\
\hline $1 / 14 / 910: 00$ & Manual Tape & 347.5 & Good & \\
\hline $1 / 14 / 917: 04$ & Auto FIC & 347.80 & Good & \\
\hline $1 / 15 / 910: 00$ & Manual Tape & 347.5 & Good & \\
\hline $1 / 15 / 917: 03$ & Auto FIC & 347.80 & Good & \\
\hline $1 / 16 / 910: 00$ & Manual Tape & 347.5 & Good & \\
\hline 1/16/91 7:04 & Auto FIC & 347.80 & Good & \\
\hline $1 / 17 / 910: 00$ & Manual Tape & 347.5 & Good & \\
\hline $1 / 17 / 917: 04$ & Auto FIC & 347.80 & Good & \\
\hline $1 / 18 / 910: 00$ & Manual Tape & 347.50 & Good & \\
\hline $1 / 18 / 917: 04$ & Auto FIC & 347.80 & Good & \\
\hline 1/19/91 0:00 & Manual Tape & 347.50 & Good & \\
\hline $1 / 19 / 917: 04$ & Auto FIC & 347.8 & Good & \\
\hline $1 / 20 / 910: 00$ & Manual Tape & 347.50 & Good & \\
\hline $1 / 20 / 917: 04$ & Auto FIC & 347.8 & Good & \\
\hline $1 / 21 / 910: 00$ & Manual Tape & 347.50 & Good & \\
\hline $1 / 21 / 917: 04$ & Auto FIC & 347.80 & Good & \\
\hline $1 / 22 / 910: 00$ & Manual Tape & 347.50 & Good & \\
\hline $1 / 22 / 91 \quad 7: 04$ & Auto FIC & 347.8 & Good & \\
\hline $1 / 23 / 910: 00$ & Manual Tape & 347.75 & Good & \\
\hline $1 / 23 / 917: 04$ & Auto FIC & 347.80 & Good & \\
\hline $1 / 24 / 910: 00$ & Manual Tape & 347.75 & Good & \\
\hline $1 / 24 / 917: 04$ & Auto FIC & 347.80 & Good & \\
\hline
\end{tabular}

Data obtained from WHC Surveillance Analysis Computer System, (PC SACS) July 7, 1995. 


\begin{tabular}{|c|c|c|c|c|}
\hline \multicolumn{5}{|c|}{ 241-AP-103 } \\
\hline \multicolumn{5}{|c|}{ Surface Level Data } \\
\hline Date & Type of Measurement & Lovel (Inches) & Data Quality & Comments \\
\hline $1 / 25 / 910: 00$ & Manual Tape & $\mathbf{3 4 7 . 7 5}$ & Good & \\
\hline $1 / 25 / 917: 03$ & Auto FIC & 347.8 & Good & \\
\hline $1 / 26 / 910: 00$ & Manual Tape & $34 \overline{7.5}$ & Good & \\
\hline $1 / 26 / 917: 03$ & Auto FIC & 347.80 & Good & \\
\hline $1 / 27 / 910: 00$ & Manual Tape & 347.5 & Good & \\
\hline $1 / 27 / 917: 03$ & Auto FIC & 347.80 & Good & \\
\hline $1 / 28 / 910: 00$ & Manual Tape & 347.50 & Good & \\
\hline $1 / 28 / 916: 03$ & Auto FIC & 347.8 & Good & \\
\hline $1 / 29 / 910: 00$ & Manual Tape & 347.50 & Good & \\
\hline 1/29/91 7:02 & Auto FIC & 347.8 & Good & \\
\hline $1 / 30 / 910: 00$ & Manual FIC & 347.80 & Good & \\
\hline $1 / 30 / 910: 00$ & Manual Tape & 347.5 & Good & \\
\hline $1 / 31 / 910: 00$ & Manual Tape & 347.50 & Good & \\
\hline $1 / 31 / 917: 02$ & Auto FIC & 347.80 & Good & \\
\hline $2 / 1 / 910: 00$ & Manual Tape & 347.50 & Good & \\
\hline 2/1/91 7:02 & Auto FIC & 347.8 & Good & \\
\hline $2 / 2 / 910: 00$ & Manual Tape & 347.5 & Good & \\
\hline 2/2/91 7:04 & Auto FIC & 347.80 & Good & \\
\hline $2 / 3 / 910: 00$ & Manual Tape & 347.5 & Good & \\
\hline $2 / 3 / 917: 03$ & Auto FIC & 347.80 & Good & \\
\hline $2 / 4 / 910: 00$ & Manual Tape & 347.5 & Good & \\
\hline 2/4/91 7:03 & Auto FIC & 347.7 & Good & \\
\hline $2 / 5 / 910: 00$ & Manual Tape & 347.50 & Good & \\
\hline 2/5/91 7:04 & Auto FIC & 347.7 & Gopd & \\
\hline $2 / 6 / 910: 00$ & Manual Tape & 347 & Good & \\
\hline 2/6/91 7:04 & Auto FIC & 347.7 & Good & \\
\hline 2/7/91 0:00 & Manual Tape & 347.25 & Good & \\
\hline $2 / 7 / 917: 04$ & Auto FIC & 347.70 & Good & \\
\hline $2 / 8 / 910: 00$ & Manual Tape & 347.25 & Good & \\
\hline $2 / 8 / 917: 04$ & Auto FIC & 347.7 & Good & \\
\hline $2 / 9 / 910: 00$ & Manual Tape & 347.25 & Good & \\
\hline 2/9/91 7:04 & Auto FIC & 347.70 & Good & \\
\hline $2 / 10 / 910: 00$ & Manual Tape & 347.50 & Good & \\
\hline $2 / 10 / 917: 03$ & Auto FIC & 347.70 & Good & \\
\hline $2 / 11 / 910: 00$ & Manual Tape & 347.50 & Good & \\
\hline $2 / 11 / 917: 04$ & Auto FIC & 347.7 & Good & \\
\hline $2 / 12 / 910: 00$ & Manual Tape & 347.50 & Good & \\
\hline 2/12/91 7:04 & Auto FIC & 347.70 & Good & \\
\hline $2 / 13 / 910: 00$ & Manual Tape & 347.5 & Good & \\
\hline $2 / 13 / 917: 04$ & Auto FIC & 347.7 & Good & \\
\hline $2 / 14 / 910: 00$ & Manual Tape & 347.50 & Good & \\
\hline $2 / 14 / 917: 04$ & Auto FIC & 347.7 & Good & \\
\hline $2 / 15 / 910: 00$ & Manual Tape & 347.25 & Good & \\
\hline 2/15/91 7:03 & Auto FIC & 347.70 & Good & \\
\hline $2 / 16 / 910: 00$ & Manual Tape & 347.25 & Good & \\
\hline $2 / 16 / 917: 02$ & Auto FIC & 347.70 & Good & \\
\hline 2/17/91 0:00 & Manual Tape & 347.25 & Good & \\
\hline $2 / 17 / 917: 02$ & Auto FIC & 347.70 & Good & \\
\hline
\end{tabular}

Data obtained from WHC Surveillance Analysis Computer System, (PC SACS) July 7, 1995. 


\begin{tabular}{|c|c|c|c|c|}
\hline \multicolumn{5}{|c|}{ 241-AP-103 } \\
\hline \multicolumn{5}{|c|}{ Surface Lovel Data } \\
\hline Date & Type of Measuremeat & Level (Inches) & Data Quality & Comments \\
\hline $2 / 18 / 910: 00$ & Manual Tape & 347.25 & Good & \\
\hline 2/18/91 7:03 & Auto FIC & 347.70 & Good & \\
\hline $2 / 19 / 910: 00$ & Manual Tape & 347.25 & Good & \\
\hline $2 / 19 / 917: 02$ & Auto FIC & $\mathbf{3 4 7 . 7 0}$ & Good & \\
\hline $2 / 20 / 910: 00$ & Manual Tape & 347.25 & Good & \\
\hline $2 / 20 / 917: 03$ & Auto FIC & $\mathbf{3 4 7 . 7 0}$ & Good & \\
\hline $2 / 21 / 910: 00$ & Manual Tape & 347.5 & Good & \\
\hline $2 / 21 / 917: 02$ & Auto FIC & 347.70 & Good & \\
\hline $2 / 22 / 910: 00$ & Manual Tape & 347.50 & Good & \\
\hline $2 / 22 / 917: 02$ & Auto FIC & 347.7 & Good & \\
\hline $2 / 23 / 910: 00$ & Manual Tape & 347.75 & Good & \\
\hline $2 / 23 / 917: 02$ & Auto FIC & $\mathbf{3 4 7 . 7}$ & Good & \\
\hline $2 / 24 / 910: 00$ & Manual Tape & 347.75 & Good & \\
\hline $2 / 24 / 917: 03$ & Auto FIC & 347.7 & Good & \\
\hline $2 / 25 / 910: 00$ & Manual Tape & 347.5 & Good & \\
\hline $2 / 25 / 917: 03$ & Auto FIC & 347.7 & Good & \\
\hline $2 / 26 / 910: 00$ & Manual Tape & 347.75 & Good & \\
\hline $2 / 26 / 917: 03$ & Auto FIC & 347.70 & Good & \\
\hline $2 / 27 / 910: 00$ & Manual Tape & 347.75 & Good & \\
\hline $2 / 27 / 917: 02$ & Auto FIC & 347.70 & Good & \\
\hline $2 / 28 / 910: 00$ & Manual Tape & 347.75 & Good & \\
\hline $2 / 28 / 917: 02$ & Auto FIC & 347.7 & Good & \\
\hline $3 / 1 / 910: 00$ & Manual Tape & 347.5 & Good & \\
\hline $3 / 1 / 917: 02$ & Auto FIC & $347.70^{\circ}$ & Good & \\
\hline $3 / 2 / 910: 00$ & Manual Tape & 347.5 & Good & \\
\hline $3 / 2 / 917: 02$ & Auto FIC & 347.70 & Good & \\
\hline $3 / 3 / 910: 00$ & Manual Tape & 347.50 & Good & \\
\hline $3 / 3 / 917: 03$ & Auto FIC & 347.7 & Good & \\
\hline $3 / 4 / 910: 00$ & Manual Tape & 347.50 & Good & \\
\hline $3 / 4 / 917: 03$ & Auto FIC & 347.7 & Good & \\
\hline $3 / 5 / 910: 00$ & Manual Tape & . 347.50 & Good & \\
\hline $3 / 5 / 917: 03$ & Auto FIC & 347.70 & Good & \\
\hline $3 / 6 / 910: 00$ & Manual Tape & 347.25 & Good & \\
\hline $3 / 6 / 917: 02$ & Auto FIC & 347.70 & Good & \\
\hline $3 / 7 / 910: 00$ & Manual Tape & 347.25 & Good & \\
\hline $3 / 7 / 917: 02$ & Auto FIC & 347.7 & Good & \\
\hline $3 / 8 / 910: 00$ & Manual Tape & 347.25 & Good & \\
\hline $3 / 8 / 917: 02$ & Auto FIC & 347.70 & Good & \\
\hline $3 / 9 / 910: 00$ & Manual Tape & 347.5 & Good & \\
\hline $3 / 9 / 917: 02$ & Auto FIC & 347.70 & Good & \\
\hline $3 / 10 / 910: 00$ & Manual Tape & 347.5 & Good & \\
\hline $3 / 10 / 917: 02$ & Auto FIC & 347.70 & Good & \\
\hline $3 / 11 / 910: 00$ & Manual Tape & 347.5 & Good & \\
\hline $3 / 11 / 917: 02$ & Auto FIC & 347.70 & Good & \\
\hline $3 / 12 / 910: 00$ & Manual Tape & 347.5 & Good & \\
\hline $3 / 12 / 917: 03$ & Auto FIC & 347.7 & Good & \\
\hline $3 / 13 / 910: 00$ & Manual Tape & 347.5 & Good & \\
\hline $3 / 13 / 917: 03$ & Auto FIC & 347.70 & Good & \\
\hline
\end{tabular}

Data obtained from WHC Surveillance Analysis Computer System, (PC SACS) July 7, 1995. 


\begin{tabular}{|c|c|c|c|c|}
\hline \multicolumn{5}{|c|}{ 241-AP-103 } \\
\hline \multicolumn{5}{|c|}{ Surface Level Data } \\
\hline Date & Type of Measurement & Level (Inches) & Data Quality & Comments \\
\hline $3 / 14 / 910: 00$ & Manual Tape & 347.5 & Good & \\
\hline $3 / 14 / 917: 03$ & Auto FIC & 347.7 & Good & \\
\hline $3 / 15 / 910: 00$ & Manual Tape & 347.5 & Good & \\
\hline $3 / 15 / 917: 03$ & Auto FIC & 347.7 & Good & \\
\hline $3 / 16 / 910: 00$ & Manual Tape & 347.25 & Good & \\
\hline $3 / 16 / 917: 03$ & Auto FIC & 347.70 & Good & \\
\hline $3 / 17 / 910: 00$ & Manual Tape & 347.25 & Good & \\
\hline $3 / 17 / 917: 02$ & Auto FIC & 347.7 & Good & \\
\hline $3 / 18 / 910: 00$ & Manual Tape & 347.25 & Good & \\
\hline $3 / 18 / 917: 03$ & Auto FIC & 347.7 & Good & \\
\hline $3 / 19 / 910: 00$ & Manual Tape & 347.25 & Good & \\
\hline $3 / 19 / 917: 03$ & Auto FIC & 347.60 & Good & \\
\hline $3 / 20 / 910: 00$ & Manual Tape & 347.25 & Good & \\
\hline $3 / 20 / 917: 02$ & Auto FIC & 347.6 & Good & \\
\hline $3 / 21 / 910: 00$ & Manual Tape & 347.25 & Good & \\
\hline $3 / 21 / 917: 03$ & Auto FIC & 347.60 & Good & \\
\hline $3 / 22 / 910: 00$ & Manual Tape & 347.25 & Good & \\
\hline $3 / 22 / 917: 02$ & Auto FIC & 347.60 & Good & \\
\hline $3 / 23 / 910: 00$ & Manual Tape & 347.25 & Good & \\
\hline $3 / 23 / 917: 03$ & Auto FIC & 347.6 & Good & \\
\hline $3 / 24 / 910: 00$ & Manual Tape & 347.5 & Good & \\
\hline $3 / 24 / 917: 03$ & Auto FIC & 347.60 & Good & \\
\hline $3 / 25 / 910: 00$ & Manual Tape & 347.5 & Good & \\
\hline $3 / 25 / 917: 03$ & Auto FIC & 347.60 & Good & \\
\hline $3 / 26 / 910: 00$ & Manual Tape & 347.50 & Good & \\
\hline $3 / 26 / 917: 03$ & Auto FIC & 347.60 & Good & \\
\hline $3 / 27 / 910: 00$ & Manual Tape & 347.5 & Good & \\
\hline $3 / 27 / 917: 03$ & Auto FIC & 347.6 & Good & \\
\hline $3 / 28 / 910: 00$ & Manual Tape & 347.5 & Good & \\
\hline $3 / 28 / 917: 03$ & Auto FIC & 347.6 & Good & \\
\hline $3 / 29 / 910: 00$ & Manual Tape & 347.5 & Good & \\
\hline $3 / 29 / 917: 03$ & Auto FIC & 347.60 & Good & \\
\hline $3 / 30 / 910: 00$ & Manual Tape & 347.50 & Good & \\
\hline $3 / 30 / 917: 03$ & Auto FIC & 347.6 & Good & \\
\hline 3/31/910:00 & Manual Tape & 347.50 & Good & \\
\hline $3 / 31 / 917: 03$ & Auto FIC & 347.60 & Good & \\
\hline $4 / 1 / 910: 00$ & Manual Tape & 347.5 & Good & \\
\hline $4 / 1 / 917: 03$ & Auto FIC & 347.60 & Good & \\
\hline $4 / 2 / 910: 00$ & Manual Tape & 347.50 & Good & \\
\hline $4 / 2 / 91 \quad 7: 03$ & Auto FIC & 347.6 & Good & \\
\hline $4 / 3 / 910: 00$ & Manual Tape & 347.5 & Good & \\
\hline $4 / 3 / 917: 03$ & Auto FIC & 347.6 & Good & \\
\hline $4 / 4 / 910: 00$ & Manual Tape & 347.25 & Good & \\
\hline $4 / 4 / 917: 03$ & Auto FIC & 347.60 & Good & \\
\hline $4 / 5 / 910: 00$ & Manual Tape & 347.25 & Good & \\
\hline $4 / 5 / 917: 03$ & Auto FIC & 347.6 & Good & \\
\hline $4 / 6 / 910: 00$ & Manual Tape & 347.25 & Good & \\
\hline $4 / 6 / 917: 03$ & Auto FIC & 347.60 & Good & \\
\hline
\end{tabular}

Data obtained from WHC Surveillance Analysis Computer System, (PC SACS) July 7, 1995. 


\begin{tabular}{|c|c|c|c|c|}
\hline \multicolumn{5}{|c|}{ 241-AP-103 } \\
\hline \multicolumn{5}{|c|}{ Surface Level Data } \\
\hline Date & Type of Measurement & Level (Inches) & Data Quality & Comments \\
\hline $4 / 7 / 910: 00$ & Manual Tape & 347.25 & Good & \\
\hline $4 / 7 / 917: 03$ & Auto FIC & 347.6 & Good & \\
\hline $4 / 8 / 910: 00$ & Manual Tape & 347.25 & Good & \\
\hline 4/8/91 7:04 & Auto FIC & 347.6 & Good & \\
\hline $4 / 9 / 910: 00$ & Manual Tape & 347.25 & Good & \\
\hline $4 / 9 / 917: 03$ & Auto FIC & 347.60 & Good & \\
\hline 4/10/91 0:00 & Manual Tape & 347.25 & Good & \\
\hline $4 / 10 / 917: 03$ & Auto FIC & 347.60 & Good & \\
\hline $4 / 11 / 910: 00$ & Manual Tape & 347.25 & Good & \\
\hline 4/11/917:03 & Auto FIC & 347.6 & Good & \\
\hline $4 / 12 / 910: 00$ & Manual Tape & 347.25 & Good & \\
\hline $4 / 12 / 917: 03$ & Auto FIC & 347.6 & Good & \\
\hline $4 / 13 / 910: 00$ & Manual Tape & 347.25 & Good & \\
\hline $4 / 13 / 917: 04$ & Auto FIC & 347.6 & Good & \\
\hline 4/14/91 0:00 & Manual Tape & 347.50 & Good & \\
\hline 4/14/91 7:03 & Auto FIC & 347.60 & Good & \\
\hline 4/15/910:00 & Manual Tape & 347.5 & Good & \\
\hline $4 / 15 / 917: 03$ & Auto FIC & 347.60 & Good & \\
\hline $4 / 16 / 910: 00$ & Manual FIC & 347.6 & Good & \\
\hline 4/16/91 0:00 & Manual Tape & 347.5 & Good & \\
\hline $4 / 17 / 910: 00$ & Manual Tape & $\mathbf{3 4 7 . 2 5}$ & Good & \\
\hline 4/17/91 7:03 & Auto FIC & 347.6 & Good & \\
\hline $.4 / 18 / 910: 00$ & Manual Tape & 347.25 & Good & \\
\hline 4/18/91 7:06 & Auto FIC & 347.60 & Good & \\
\hline 4/19/910:00 & Manual Tape & 347.25 & Good & \\
\hline 4/19/91 7:05 & Auto FIC & 347.60 & Good & \\
\hline $4 / 20 / 910: 00$ & Manual Tape & 347.25 & Good & \\
\hline 4/20/917:06 & Auto FIC & 347.60 & Good & \\
\hline $4 / 21 / 910: 00$ & Manual Tape & 347.25 & Good & \\
\hline $4 / 21 / 917: 05$ & Auto FIC & 347.6 & Good & \\
\hline $4 / 22 / 910: 00$ & Manual Tape & 347.25 & Good & \\
\hline $4 / 22 / 917: 06$ & Auto FIC & 347.6 & Good & \\
\hline $4 / 23 / 910: 00$ & Manual Tape & 347.25 & Good & \\
\hline $4 / 23 / 917: 06$ & Auto FIC & 347.60 & Good & \\
\hline 4/24/91 0:00 & Manual Tape & 347.50 & Good & \\
\hline 4/24/91 7:05 & Auto FIC & 347.60 & Good & \\
\hline 4/25/91 0:00 & Manual Tape & 347.5 & Good & \\
\hline $4 / 25 / 917: 05$ & Auto FIC & 347.60 & Good & \\
\hline 4/26/91 $0: 00$ & Manual Tape & 347.50 & Good & \\
\hline 4/26/91 7:05 & Auto FIC & 347.60 & Good & \\
\hline 4/27/91 0:00 & Manual Tape & 347.50 & Good & \\
\hline 4/27/91 7:05 & Auto FIC & 347.6 & Good & \\
\hline $4 / 28 / 910: 00$ & Manual Tape & 347.5 & Good & \\
\hline $4 / 28 / 917: 06$ & Auto FIC & 347.6 & Good & \\
\hline 4/29/91 0:00 & Manual Tape & 347.5 & Good & \\
\hline 4/29/91 7:05 & Auto FIC & 347.60 & Good & \\
\hline 4/30/910:00 & Manual Tape & 347.5 & Good & \\
\hline 4/30/91 7:06 & Auto FIC & 347.6 & Good & \\
\hline
\end{tabular}

Data obtained from WHC Surveillance Analysis Computer System, (PC SACS) July 7, 1995. 


\begin{tabular}{|c|c|c|c|c|}
\hline \multicolumn{4}{|c|}{ 241-AP-103 } & \\
\hline & Type of Measurement & Level (lnchea) & Data Quality & Comments \\
\hline$\frac{\text { Date }}{5 / 1 / 910: 00}$ & Manual Tape & 347.50 & Good & \\
\hline $\begin{array}{ll}5 / 1 / 91 & 0: 00 \\
5 / 1 / 91 & 7: 05\end{array}$ & Auto FIC & 347.60 & Good & \\
\hline $\begin{array}{ll}5 / 1 / 917: 05 \\
5 / 2 / 910: 00\end{array}$ & Manual Tape & 347.5 & Good & \\
\hline $\begin{array}{ll}5 / 2 / 91 & 0: 00 \\
5 / 2 / 91 & 7: 05\end{array}$ & Auto FIC & 347.60 & Good & \\
\hline $\begin{array}{lll}5 / 2 / 91 & 7: 05 \\
5 / 3 / 91 & 0: 00\end{array}$ & Manual Tape & 347.5 & Good & \\
\hline $\begin{array}{ll}5 / 3 / 91 & 0: 00 \\
5 / 3 / 91 & 7: 06 \\
\end{array}$ & Auto FIC & 347.60 & Good & \\
\hline $\begin{array}{lll}5 / 3 / 91 & 7: 06 \\
5 / 4 / 91 & 0: 00\end{array}$ & Manual Tape & 347.50 & Good & \\
\hline $\begin{array}{lll}5 / 4 / 91 & 0: 00 \\
5 / 4 / 91 & 7: 05 \\
\end{array}$ & Auto FIC & 347.60 & Good & \\
\hline $\begin{array}{l}5 / 4 / 917: 05 \\
5 / 5 / 910: 00\end{array}$ & Manual Tape & 347.50 & Good & \\
\hline $\begin{array}{l}5 / 5 / 910: 00 \\
5 / 5 / 917: 05\end{array}$ & Auto FIC & 347.6 & Good & \\
\hline $\begin{array}{l}5 / 5 / 917: 05 \\
5 / 6 / 910: 00 \\
\end{array}$ & Manual Tape & 347.50 & Good & \\
\hline $\begin{array}{lll}5 / 6 / 91 & 0: 00 \\
5 / 6 / 91 & 7: 05 \\
\end{array}$ & Auto FIC & 347.60 & Good & \\
\hline $\begin{array}{ll}5 / 6 / 91 & 7: 05 \\
5 / 7 / 91 & 0: 00\end{array}$ & Manual Tape & 347.5 & Good & \\
\hline $\begin{array}{ll}5 / 7 / 91 & 0: 00 \\
5 / 7 / 91 & 7: 05\end{array}$ & Auto FIC & 347.6 & Good & \\
\hline $\begin{array}{lll}5 / 7 / 91 & 7: 05 \\
5 / 8 / 91 & 0: 00\end{array}$ & Manual Tape & 347.50 & Good & \\
\hline $\begin{array}{l}5 / 8 / 910: 00 \\
5 / 8 / 917: 05\end{array}$ & Auto FIC & 347.60 & Good & \\
\hline $\begin{array}{ll}5 / 8 / 917: 05 \\
5 / 9 / 91 & 0: 00\end{array}$ & Manual Tape & 347.5 & Good & \\
\hline $\begin{array}{ll}5 / 9 / 91 & 0: 00 \\
5 / 9 / 91 & 7: 05\end{array}$ & Auto FIC & 347.60 & Good & \\
\hline $\begin{array}{c}5 / 9 / 917: 05 \\
5 / 10 / 910: 00\end{array}$ & Manual Tape & 347.5 & Good & - \\
\hline $\begin{array}{l}5 / 10 / 910: 00 \\
5 / 10 / 91 \quad 7: 05 \\
\end{array}$ & Auto FIC & 347.6 & Good & \\
\hline $5 / 11 / 910: 00$ & Manual Tape & 347.50 & Good & \\
\hline $5 / 11 / 917: 05$ & Auto FIC & 347.60 & Good & \\
\hline $5 / 12 / 910: 00$ & Manual Tape & 347.25 & Good & \\
\hline $5 / 12 / 917: 05$ & Auto FIC & 347.60 & Good & \\
\hline $5 / 13 / 910: 00$ & Manual Tape & 347.25 & Good & \\
\hline $5 / 13 / 917: 05$ & Auto FIC & 347.6 & Good & \\
\hline $5 / 14 / 910: 00$ & Manual Tape & 347.25 & Good & \\
\hline $5 / 14 / 917: 05$ & Auto FIC & 347.6 & Good & \\
\hline $5 / 15 / 910: 00$ & Manual Tape & 347.25 & Good & \\
\hline $5 / 15 / 917: 05$ & Auto FIC & 347.60 & Good & \\
\hline $5 / 16 / 910: 00$ & Manual Tape & 347.25 & Good & \\
\hline $5 / 16 / 917: 06$ & Auto FIC & 347.60 & Good & \\
\hline 5/17/91 0:00 & Manual Tape & 347.75 & Good & \\
\hline $5 / 17 / 917: 06$ & Auto FIC & 347.60 & Good & \\
\hline $5 / 18 / 910: 00$ & Manual Tape & 347.25 & Good & \\
\hline $5 / 18 / 917: 06$ & Auto FIC & 347.6 & Good & \\
\hline $5 / 19 / 910: 00$ & Manual Tape & 347.25 & Good & \\
\hline $5 / 19 / 917: 05$ & Auto FIC & 347.6 & Good & \\
\hline 5/20/91 0:00 & Manual Tape & 347.25 & Good & \\
\hline $5 / 20 / 917: 06$ & Auto FIC & 347.60 & Good & \\
\hline $5 / 21 / 910: 00$ & Manual Tape & 347.25 & Good & \\
\hline $5 / 21 / 917: 06$ & Auto FIC & 347.6 & Good & \\
\hline $5 / 22 / 910: 00$ & Manual Tape & 347.5 & Good & \\
\hline $5 / 22 / 917: 05$ & Auto FIC & 347.6 & Gopd & \\
\hline 5/23/91 0:00 & Manual Tape & 347.25 & Good & \\
\hline $5 / 23 / 917: 05$ & Auto FIC & 347.6 & Good & \\
\hline $5 / 24 / 910: 00$ & Manual Tape & 347.25 & Good & \\
\hline $5 / 24 / 917: 05$ & Auto FIC & 347.60 & Good & \\
\hline
\end{tabular}

Data obtained from WHC Surveillance Analysis Computer System, (PC SACS) July 7, 1995. 


\begin{tabular}{|c|c|c|c|c|}
\hline \multicolumn{5}{|c|}{ 241-AP-103 } \\
\hline \multicolumn{5}{|c|}{ Surface Lovel Data } \\
\hline Date & Type of Measurement & Level (Inches) & Data Quality & Comments \\
\hline $5 / 25 / 910: 00$ & Manual Tape & 347.25 & Good & \\
\hline $5 / 25 / 917: 05$ & Auto FIC & 347.60 & Good & \\
\hline $5 / 26 / 910: 00$ & Manual Tape & 347.25 & Good & \\
\hline $5 / 26 / 917: 05$ & Auto FIC & 347.6 & Good & \\
\hline $5 / 27 / 910: 00$ & Manual Tape & 347.25 & Good & \\
\hline $5 / 27 / 917: 05$ & Auto FIC & 347.60 & Good & \\
\hline $5 / 28 / 910: 00$ & Manual Tape & 347.25 & Good & \\
\hline $5 / 28 / 917: 05$ & Auto FIC & 347.6 & Good & \\
\hline $5 / 29 / 910: 00$ & Manual Tape & 347.25 & Good & \\
\hline $5 / 29 / 917: 05$ & Auto FIC & 347.6 & Good & \\
\hline $5 / 30 / 910: 00$ & Manual Tape & 347.25 & Good & \\
\hline 5/30/91 7:05 & Auto FIC & 347.6 & Good & \\
\hline $5 / 31 / 910: 00$ & Manual Tape & 347.25 & Good & \\
\hline $5 / 31 / 917: 05$ & Auto FIC & 347.60 & Good & \\
\hline $6 / 1 / 910: 00$ & Manual Tape & 347.25 & Good & \\
\hline $6 / 1 / 916: 05$ & Auto FIC & 347.60 & Good & \\
\hline 6/2/91 0:00 & Manual Tape & 347.25 & Good & \\
\hline 6/2/913:05 & Auto FIC & 347.60 & Good & \\
\hline $6 / 3 / 910: 00$ & Manual Tape & 347.25 & Good & \\
\hline 6/3/91 7:05 & Auto FIC & 347.60 & Good & \\
\hline $6 / 4 / 910: 00$ & Manual Tape & 347.5 & Good & \\
\hline 6/4/91 7:05 & Auto FIC & 347.60 & Good & \\
\hline $6 / 5 / 910: 00$ & Manual Tape & 347.25 & Good & \\
\hline 6/5/91 7:05 & Auto FIC & 347.60 & Good & \\
\hline 6/6/91 0:00 & Manual Tape & 347.25 & Good & \\
\hline $6 / 6 / 917: 06$ & Auto FIC & 347.6 & Good & \\
\hline $6 / 7 / 910: 00$ & Manual Tape & 347.25 & Good & \\
\hline 6/7/91 7:05 & Auto FIC & 347.60 & Good & \\
\hline 6/8/91 0:00 & Manual-Tape & 362.25 & Good & \\
\hline 6/8/91 7:05 & Auto FIC & 362.5 & Good & \\
\hline 6/9/91 0:00 & Manual Tape & 362.5 & Good & \\
\hline 6/9/91 7:05 & Auto FIC & 362.6 & Good & \\
\hline $6 / 10 / 910: 00$ & Manual Tape & 362.50 & Good & \\
\hline $6 / 10 / 917: 06$ & Auto FIC & 362.70 & Good & \\
\hline $6 / 11 / 910: 00$ & Manual Tape & 382.75 & Good & \\
\hline $6 / 11 / 917: 05$ & Auto FIC & 374.5 & Good & \\
\hline $6 / 12 / 910: 00$ & Manual Tape & 412.50 & Good & \\
\hline $6 / 12 / 917: 04$ & Auto FIC & 412.80 & Good & \\
\hline $6 / 13 / 910: 00$ & Manual Tape & 412.50 & Good & \\
\hline $6 / 13 / 917: 05$ & Auto FIC & 412.80 & Good & \\
\hline $6 / 14 / 910: 00$ & Manual Tape & 412.5 & Good & \\
\hline $6 / 14 / 917: 05$ & Auto FIC & 412.8 & Good & \\
\hline $6 / 15 / 91 \quad 0: 00$ & Manual Tape & 412.50 & Good & \\
\hline $6 / 15 / 917: 05$ & Auto FIC & 412.80 & Good & \\
\hline $6 / 16 / 910: 00$ & Manual Tape & 412.50 & Good & \\
\hline $6 / 16 / 917: 05$ & Auto FIC & 412.8 & Good & \\
\hline $6 / 17 / 910: 00$ & Manual Tape & 412.5 & Good & \\
\hline $6 / 17 / 917: 05$ & Auto FIC & 412.80 & Good & \\
\hline
\end{tabular}

Data obtained from WHC Surveillance Analysis Computer System, (PC SACS) July 7, 1995. 


\begin{tabular}{|c|c|c|c|c|}
\hline \multicolumn{5}{|c|}{ 241-AP-103 } \\
\hline \multicolumn{5}{|c|}{ Surface Lovel Data } \\
\hline Date & Type of Measurement & Level (Inches) & Data Quality & Comments \\
\hline $6 / 18 / 910: 00$ & Manual Tape & 412.5 & Good & \\
\hline $6 / 18 / 917: 05$ & Auto FIC & 412.80 & Good & \\
\hline $6 / 19 / 910: 00$ & Manual Tape & 412.5 & Good & \\
\hline $6 / 19 / 917: 05$ & Auto FIC & 41.2 .80 & Good & \\
\hline $6 / 20 / 910: 00$ & Manual Tape & 412.75 & Good & \\
\hline $6 / 20 / 917: 05$ & Auto FIC & 412.8 & Good & \\
\hline $6 / 21 / 910: 00$ & Manual Tape & 412.75 & Good & \\
\hline $6 / 21 / 917: 06$ & Auto FIC & 412.80 & Good & \\
\hline $6 / 22 / 910: 00$ & Manual Tape & 412.75 & Good & \\
\hline $6 / 22 / 913: 06$ & Auto FIC & 412.80 & Good & \\
\hline $6 / 23 / 910: 00$ & Manual Tape & 412.75 & Good & \\
\hline $6 / 23 / 917: 05$ & Auto FIC & 412.8 & Good & \\
\hline $6 / 24 / 910: 00$ & Manual Tape & 412.75 & Good & \\
\hline $6 / 24 / 915: 05$ & Auto FIC & 412.8 & Good & \\
\hline $6 / 25 / 910: 00$ & Manual Tape & 412.75 & Good & \\
\hline $6 / 25 / 917: 06$ & Auto FIC & 412.8 & Good & \\
\hline $6 / 26 / 910: 00$ & Manual Tape & 412.75 & Good & \\
\hline $6 / 26 / 911: 03$ & Auto FIC & 412.80 & Good & \\
\hline $6 / 27 / 910: 00$ & Manual FIC & 412.8 & Transcribed & \\
\hline $6 / 27 / 910: 00$ & Manual Tape & 412.75 & Good & \\
\hline $6 / 28 / 910: 00$ & Manual Tape & 412.75 & Good & \\
\hline $6 / 28 / 917: 03$ & Auto FIC & 412.8 & Good & \\
\hline $6 / 29 / 910: 00$ & Manual Tape & 413.00 & Good & \\
\hline $6 / 29 / 916: 02$ & Auto FIC & 412.8 & Good & \\
\hline $6 / 30 / 910: 00$ & Manual FIC & 412.8 & Good & \\
\hline $6 / 30 / 910: 00$ & Manual Tape & 413 & Good & \\
\hline $7 / 1 / 910: 00$ & Manual FIC & 412.8 & Good & \\
\hline 7/1/91 0:00 & Manual Tape & 413 & Good & \\
\hline 7/2/91 0:00 & Manual Tape & 413 & Good & \\
\hline 7/2/91 7:06 & Auto FIC & 412.80 & Good & \\
\hline $7 / 3 / 910: 00$ & Manual Tape & 413.00 & Good & \\
\hline $7 / 3 / 917: 06$ & Auto FIC & 412.80 & Good & \\
\hline $7 / 4 / 910: 00$ & Manual FIC & 412.8 & Good & \\
\hline $7 / 4 / 910: 00$ & Manual Tape & 413 & Good & \\
\hline $7 / 5 / 910: 00$ & Manual FIC & 412.80 & Good & \\
\hline $7 / 5 / 910: 00$ & Manual Tape & 413.00 & Good & \\
\hline $7 / 6 / 910: 00$ & Manual FIC & 412.80 & Good & \\
\hline $7 / 6 / 910: 00$ & Manual Tape & 41.3 .00 & Good & \\
\hline $7 / 7 / 910: 00$ & Manual FIC & 412.80 & Good & \\
\hline $7 / 7 / 910: 00$ & Manual Tape & 413 & Good & \\
\hline $7 / 8 / 910: 00$ & Manual FIC & 412.80 & Good & \\
\hline $7 / 8 / 910: 00$ & Manual Tape & 413.00 & Good & \\
\hline $7 / 9 / 910: 00$ & Manual FIC & 412.8 & Good & \\
\hline $7 / 9 / 910: 00$ & Manual Tape & 413.00 & Good & \\
\hline $7 / 10 / 910: 00$ & Manual FIC & 412.8 & Good & \\
\hline $7 / 10 / 910: 00$ & Manual Tape & 413 & Good & \\
\hline $7 / 11 / 910: 00$ & Manual Tape & 413.25 & Good & \\
\hline $7 / 11 / 917: 06$ & Auto FIC & 412.80 & Good & \\
\hline
\end{tabular}

Data obtained from WHC Surveillance Analysis Computer System, (PC SACS) July 7, 1995. 


\begin{tabular}{|c|c|c|c|c|}
\hline & & $\begin{array}{l}\text { urface Level Date } \\
\text { Level (Inches) }\end{array}$ & & \\
\hline Date & Type of Measurement & Leval (Inches) & Data Quality & Comments \\
\hline 7/12/91 0:00 & Manual Tape & 413.25 & Good & \\
\hline 7/12/91 7:06 & Auto FIC & 412.8 & Good & \\
\hline 7/13/91 0:00 & Manual Tape & 413.25 & Good & \\
\hline 7/13/91 7:06 & Auto FIC & 412.80 & Good & \\
\hline $7 / 14 / 910: 00$ & Manual Tape & 413.25 & Good & \\
\hline 7/14/912:06 & Auto FIC & 412.8 & Good & \\
\hline $7 / 15 / 910: 00$ & Manual Tape & 413.00 & Good & \\
\hline $7 / 15 / 917: 06$ & Auto FIC & 412.8 & Good & \\
\hline $7 / 16 / 910: 00$ & Manual FIC & 412.8 & Transcribed & \\
\hline $7 / 16 / 910: 00$ & Manual Tape & 413.00 & Good & \\
\hline $7 / 17 / 910: 00$ & Manual Tape & 413 & Good & \\
\hline $7 / 17 / 910: 05$ & Auto FIC & $412 . \overline{80}$ & Good & \\
\hline $7 / 18 / 910: 00$ & Manual FIC & 412.80 & Transcribed & \\
\hline $7 / 18 / 910: 00$ & Manual Tape & 413.25 & Good & \\
\hline $7 / 19 / 910: 00$ & Manual Tape & 413.25 & Good & \\
\hline 7/19/91 7:05 & Auto FIC & 412.8 & Good & \\
\hline $7 / 20 / 910: 00$ & Manual Tape & 413.25 & Good & \\
\hline $7 / 20 / 912: 05$ & Auto FIC & 412.80 & Good & \\
\hline $7 / 21 / 910: 00$ & Manual Tape & 413.25 & Good & \\
\hline $7 / 21 / 91 \quad 1: 05$ & Auto FIC & 412.8 & Good & \\
\hline $7 / 22 / 910: 00$ & Manual FIC & 412.80 & Good & \\
\hline $7 / 22 / 910: 00$ & Manual Tape & 413.25 & Good & \\
\hline $7 / 23 / 910: 00$ & Manual Tape & 413.25 & Good & \\
\hline $7 / 23 / 910: 05$ & Auto FIC & 412.8 & Good & \\
\hline $7 / 24 / 91 \quad 0: 00$ & Manual Tape & 413.00 & Good & \\
\hline $7 / 24 / 917: 05$ & Auto FIC & 412.80 & Good & \\
\hline $7 / 25 / 910: 00$ & Manual Tape & 413.00 & Good & \\
\hline $7 / 25 / 91 \quad 1: 03$ & Auto FIC & 412.8 & Good & \\
\hline $7 / 26 / 910: 00$ & Manual FIC & 412.80 & Good & \\
\hline $7 / 26 / 910: 00$ & Manual Tape & 413.25 & Good & \\
\hline $7 / 27 / 910: 00$ & Manual FIC & 412.80 & Good & \\
\hline $7 / 27 / 910: 00$ & Manual Tape & 413 & Good & \\
\hline $7 / 28 / 910: 00$ & Manual FIC & 412.80 & Good & \\
\hline $7 / 28 / 910: 00$ & Manual Tape & 413 & Good & \\
\hline $7 / 29 / 910: 00$ & Manual FIC & 412.8 & Good & \\
\hline $7 / 29 / 910: 00$ & Manual Tape & 413.50 & Good & \\
\hline $7 / 30 / 910: 00$ & Manual Tape & 413.50 & Good & \\
\hline $7 / 30 / 917: 02$ & Auto FIC & 412.80 & Good & \\
\hline $7 / 31 / 910: 00$ & Manual Tape & 413.5 & Good & \\
\hline $7 / 31 / 91 \quad 7: 02$ & Auto FIC & 412.8 & Good & \\
\hline $8 / 1 / 910: 00$ & Manual Tape & 413.25 & Good & \\
\hline $8 / 1 / 917: 02$ & Auto FIC & 412.80 & Good & \\
\hline $8 / 2 / 910: 00$ & Manual Tape & 413.25 & Good & \\
\hline $8 / 2 / 917: 02$ & Auto FIC & 412.8 & Good & \\
\hline $8 / 3 / 910: 00$ & Manual Tape & 413.5 & Good & \\
\hline $8 / 3 / 917: 02$ & Auto FIC & 412.8 & Good & \\
\hline $8 / 4 / 910: 00$ & Manual Tape & 413.25 & Good & \\
\hline $8 / 4 / 917: 02$ & Auto FIC & 412.80 & Good & \\
\hline
\end{tabular}

Data obtained from WHC Surveillance Analysis Computer System, (PC SACS) July 7, 1995. 


\begin{tabular}{|c|c|c|c|c|}
\hline \multicolumn{5}{|c|}{ 241-AP-103 } \\
\hline \multicolumn{5}{|c|}{ Surface Level-Data } \\
\hline Date & Type of Measurement & Level (Inches) & Data Quality & Comments \\
\hline $8 / 5 / 910: 00$ & Manual Tape & 413.00 & Good & \\
\hline $8 / 5 / 917: 02$ & Auto FIC & 412.80 & Good & \\
\hline $8 / 6 / 910: 00$ & Manual Tape & 413.00 & Good & \\
\hline $8 / 6 / 917: 02$ & Auto FIC & 412.80 & Good & \\
\hline $8 / 7 / 910: 00$ & Manual Tape & 413.25 & Good & \\
\hline $8 / 7 / 917: 06$ & Auto FIC & 412.80 & Good & \\
\hline $8 / 8 / 910: 00$ & Manual Tape & 413.25 & Good & \\
\hline 8/8/91 7:06 & Auto FIC & 412.8 & Good & \\
\hline $8 / 9 / 910: 00$ & Manual Tape & 413.25 & Good & \\
\hline $8 / 9 / 917: 04$ & Auto FIC & 412.8 & Good & \\
\hline $8 / 10 / 910: 00$ & Manual Tape & 413.25 & Good & \\
\hline $8 / 10 / 917: 04$ & Auto FIC & 412.80 & Good & \\
\hline $8 / 11 / 910: 00$ & Manual Tape & 413.25 & Good & \\
\hline $8 / 11 / 917: 04$ & Auto FIC & 412.8 & Good & \\
\hline $8 / 12 / 910: 00$ & Manual Tape & 413.5 & Good & \\
\hline $8 / 12 / 917: 04$ & Auto FIC & 412.80 & Good & \\
\hline $8 / 13 / 910: 00$ & Manual Tape & 413.25 & Good & \\
\hline $8 / 13 / 917: 04$ & Auto FIC & 412.8 & Good & \\
\hline $8 / 14 / 910: 00$ & Manual Tape & 413.25 & Good & \\
\hline $8 / 14 / 917: 04$ & Auto FIC & 412.80 & Good & \\
\hline $8 / 15 / 910: 00$ & Manual Tape & 413.25 & Good & \\
\hline $8 / 15 / 917: 03$ & Auto FIC & 412.8 & Good & \\
\hline $8 / 16 / 910: 00$ & Manual FIC & 412.8 & Transcribed & \\
\hline $8 / 16 / 910: 00$ & Manual Tape & 413.25 & Good & \\
\hline $8 / 17 / 910: 00$ & Manual Tape & 413.25 & Good & \\
\hline $8 / 17 / 917: 02$ & Auto FIC & 412.8 & Good & \\
\hline $8 / 18 / 910: 00$ & Manual Tape & 413.25 & Good & \\
\hline $8 / 18 / 917: 03$ & Auto FIC & 412.8 & Good & \\
\hline 8/19/91 0:00 & Manual Tape & 413.25 & Good & \\
\hline $8 / 19 / 917: 02$ & Auto FIC & 412.80 & Good & \\
\hline $8 / 20 / 910: 00$ & Manual Tape & 413.25 & Good & \\
\hline $8 / 20 / 917: 04$ & Auto FIC & $\begin{array}{r}412.80 \\
\end{array}$ & Good & \\
\hline $8 / 21 / 910: 00$ & Manual Tape & 413.5 & Good & \\
\hline $8 / 21 / 917: 03$ & Auto FIC & 412.8 & Good & \\
\hline $8 / 22 / 910: 00$ & Manual Tape & 413.50 & Good & \\
\hline $8 / 22 / 917: 03$ & Auto FIC & 412.8 & Good & \\
\hline $8 / 23 / 910: 00$ & Manual Tape & 413.25 & Good & \\
\hline $8 / 23 / 917: 02$ & Auto FIC & 412.8 & Good & \\
\hline $8 / 24 / 910: 00$ & Manual Tape & 413.25 & Good & \\
\hline $8 / 24 / 917: 02$ & Auto FIC & 412.80 & Good & \\
\hline $8 / 25 / 910: 00$ & Manual Tape & 413.25 & Good & \\
\hline $8 / 25 / 917: 02$ & Auto FIC & 412.80 & Good & \\
\hline 8/26/910:00 & Manual Tape & 413.25 & Good & \\
\hline $8 / 26 / 917: 03$ & Auto FIC & 412.80 & Good & \\
\hline $8 / 27 / 910: 00$ & Manual Tape & 413.25 & Good & \\
\hline $8 / 27 / 917: 03$ & Auto FIC & 412.80 & Good & \\
\hline $8 / 28 / 910: 00$ & Manual Tape & 413.25 & Good & \\
\hline $8 / 28 / 917: 03$ & Auto FIC & 412.8 & Good & \\
\hline
\end{tabular}

Data obtained from WHC Surveillance Analysis Computer System, (PC SACS) July 7, 1995. 


\begin{tabular}{|c|c|c|c|c|}
\hline \multicolumn{5}{|c|}{ 241-AP-103 } \\
\hline & & urface Leval Date & & \\
\hline Date & Type of Measurement & Level (Inches) & Data Quality & Comments \\
\hline $8 / 29 / 910: 00$ & Manual Tape & 413.25 & Good & \\
\hline $8 / 29 / 917: 02$ & Auto FIC & 412.80 & Good & \\
\hline $8 / 30 / 910: 00$ & Manual Tape & 413.25 & Good & \\
\hline $8 / 30 / 916: 02$ & Auto FIC & 412.8 & Good & \\
\hline $8 / 31 / 910: 00$ & Manual Tape & 413.25 & Good & \\
\hline $8 / 31 / 917: 02$ & Auto FIC & 412.80 & Good & \\
\hline 9/1/910:00 & Manual Tape & 413.25 & Good & \\
\hline $9 / 1 / 917: 02$ & Auto FIC & 412.80 & Good & \\
\hline 9/2/91 0:00 & Manual Tape & 413.25 & Good & \\
\hline $9 / 2 / 917: 02$ & Auto FIC & 412.80 & Good & \\
\hline $9 / 3 / 910: 00$ & Manual Tape & 413.25 & Good & \\
\hline 9/3/91 7:02 & Auto FIC & 412.8 & Good & \\
\hline 9/4/91 0:00 & Manual Tape & 413.25 & Good & \\
\hline $9 / 4 / 917: 03$ & Auto FIC & 412.8 & Good & \\
\hline $9 / 5 / 910: 00$ & Manual Tape & 413.25 & Good & \\
\hline $9 / 5 / 917: 02$ & Auto FIC & 412.8 & Good & \\
\hline 9/6/91 0:00 & Manual Tape & 413.25 & Good & \\
\hline $9 / 6 / 917: 02$ & Auto FIC & 412.80 & Good & \\
\hline $9 / 7 / 910: 00$ & Manual Tape & 413.25 & Good & \\
\hline $9 / 7 / 917: 02$ & Auto FIC & 412.8 & Good & \\
\hline $9 / 8 / 910: 00$ & Manual Tape & 413.25 & Good & \\
\hline $9 / 8 / 917: 02$ & Auto FIC & 412.80 & Good & \\
\hline 9/9/91 0:00 & Manual Tape & 413.25 & Good & \\
\hline 9/9/91 7:03 & Auto FIC & 412.8 & Good & \\
\hline $9 / 10 / 910: 00$ & Manual Tape & 413.25 & Good & \\
\hline $9 / 10 / 917: 02$ & Auto FIC & 412.80 & Good & \\
\hline $9 / 11 / 910: 00$ & Manual Tape & 413.25 & Good & \\
\hline $9 / 11 / 917: 03$ & Auto FIC & 412.8 & Good & \\
\hline $9 / 12 / 91 \quad 0: 00$ & Manual Tape & 413.25 & Good & \\
\hline $9 / 12 / 917: 02$ & Auto FIC & 412.80 & Good & \\
\hline 9/13/91 0:00 & Manual Tape & 413.25 & Good & \\
\hline $9 / 13 / 917: 02$ & Auto FIC & 412.80 & Good & \\
\hline $9 / 14 / 910: 00$ & Manual Tape & 413.25 & Good & \\
\hline 9/14/91 7:03 & Auto FIC & 412.80 & Good & \\
\hline 9/15/91 0:00 & Manual Tape & 413.25 & Good & \\
\hline $9 / 15 / 917: 02$ & Auto FIC & 412.8 & Good & \\
\hline $9 / 16 / 910: 00$ & Manual Tape & 413.25 & Good & \\
\hline 9/16/91 7:03 & Auto FIC & 412.80 & Good & \\
\hline $9 / 17 / 910: 00$ & Manual Tape & 413.25 & Good & \\
\hline $9 / 17 / 917: 03$ & Auto FIC & 412.8 & Good & \\
\hline $9 / 18 / 910: 00$ & Manual Tape & 413 & Good & \\
\hline 9/18/91 7:03 & Auto FIC & 412.80 & Good & \\
\hline $9 / 19 / 910: 00$ & Manual Tape & 413 & Good & \\
\hline $9 / 19 / 917: 03$ & Auto FIC & 412.8 & Good & \\
\hline $9 / 20 / 910: 00$ & Manual Tape & 413.00 & Good & \\
\hline $9 / 20 / 917: 02$ & Auto FIC & 412.80 & Good & \\
\hline 9/21/91 0:00 & Manual Tape & 413.00 & Good & \\
\hline $9 / 21 / 917: 02$ & Auto FIC & 412.8 & Good & \\
\hline
\end{tabular}

Data obtained from WHC Surveiliance Analysis Computer System, (PC SACS) July 7, 1995. 


\begin{tabular}{|c|c|c|c|c|}
\hline \multicolumn{5}{|c|}{ Surface Level Data } \\
\hline Date & Type of Measurement & Leval (Inches) & Data Quality & Comments \\
\hline $9 / 22 / 910: 00$ & Manual Tape & 413 & Good & \\
\hline $9 / 22 / 917: 03$ & Auto FIC & 412.8 & Good & \\
\hline $9 / 23 / 910: 00$ & Manual Tape & 413.25 & Good & \\
\hline $9 / 23 / 917: 03$ & Auto FIC & 412.8 & Good & \\
\hline $9 / 24 / 910: 00$ & Manual Tape & 413.00 & Good & \\
\hline $9 / 24 / 917: 02$ & Auto FIC & 412.80 & Good & \\
\hline $9 / 25 / 910: 00$ & Manual Tape & 413.25 & Good & \\
\hline $9 / 25 / 917: 03$ & Auto FIC & 412.80 & Good & \\
\hline 9/26/91 0:00 & Manual Tape & 413.25 & Good & \\
\hline 9/26/91 7:03 & Auto FIC & 412.80 & Good & \\
\hline 9/27/91 0:00 & Manual Tape & 413.25 & Good & \\
\hline $9 / 27 / 917: 02$ & Auto FIC & 412.8 & Good & \\
\hline $9 / 28 / 910: 00$ & Manual Tape & 413.25 & Good & \\
\hline $9 / 28 / 917: 02$ & Auto FIC & 412.80 & Good & \\
\hline 9/29/91 0:00 & Manual Tape & 413.25 & Good & \\
\hline $9 / 29 / 917: 03$ & Auto FIC & 412.8 & Good & \\
\hline $9 / 30 / 910: 00$ & Manual Tape & 413.25 & Good & \\
\hline $9 / 30 / 917: 03$ & Auto FIC & 412.80 & Good & \\
\hline $10 / 1 / 910: 00$ & Manual Tape & 413.25 & Good & \\
\hline $10 / 1 / 917: 02$ & Auto FIC & 412.80 & Good & \\
\hline $10 / 2 / 910: 00$ & Manual Tape & 413.25 & Good & \\
\hline $10 / 2 / 917: 02$ & Auto FIC & 412.80 & Good & \\
\hline $10 / 3 / 910: 00$ & Manual Tape & 413.00 & Good & \\
\hline $10 / 3 / 917: 02$ & Auto FIC & 412.80 & Good & \\
\hline 10/4/910:00 & Manual Tape & 412.50 & Good & \\
\hline 10/4/91 7:03 & Auto FIC & 412.8 & Good & \\
\hline $10 / 5 / 910: 00$ & Manual FIC & 412.8 & Good & \\
\hline 10/5/91 0:00 & Manual Tape & 412.75 & Good & \\
\hline $10 / 6 / 910: 00$ & Manual Tape & 412.75 & Good & \\
\hline $10 / 6 / 917: 03$ & Auto FIC & 412.80 & Good & \\
\hline $10 / 7 / 910: 00$ & Manual Tape & 412.50 & Good & \\
\hline $10 / 7 / 917: 02$ & Auto FIC & 412.8 & Good & \\
\hline $10 / 8 / 910: 00$ & Manual Tape & 412.50 & Good & \\
\hline $10 / 8 / 917: 02$ & Auto FIC & 412.80 & Good & \\
\hline $10 / 9 / 910: 00$ & Manual Tape & 412.75 & Good & \\
\hline $10 / 9 / 917: 02$ & Auto FIC & 412.8 & Good & \\
\hline $10 / 10 / 910: 00$ & Manual Tape & 412.75 & Good & \\
\hline $10 / 10 / 917: 02$ & Auto FIC & 412.80 & Good & \\
\hline $10 / 11 / 910: 00$ & Manual Tape & 412.75 & Good & \\
\hline $10 / 11 / 917: 02$ & Auto FIC & 412.8 & Good & \\
\hline $10 / 12 / 910: 00$ & Manual Tape & 412.75 & Good & \\
\hline $10 / 12 / 917: 02$ & Auto FIC & 412.8 & Good & \\
\hline $10 / 13 / 910: 00$ & Manual Tape & 412.75 & Good & \\
\hline $10 / 13 / 917: 03$ & Auto FIC & 412.80 & Good & \\
\hline $10 / 14 / 910: 00$ & Manual Tape & 412.50 & Good & \\
\hline $10 / 14 / 917: 02$ & Auto FIC & 412.8 & Good & \\
\hline $10 / 15 / 91 \quad 0: 00$ & Manual Tape & 412.50 & Good & \\
\hline $10 / 15 / 917: 02$ & Auto FIC & 412.80 & Good & \\
\hline
\end{tabular}

Data obtained from WHC Surveillance Analysis Computer System, (PC SACS) July 7, 1995. 


\begin{tabular}{|c|c|c|c|c|}
\hline \multicolumn{5}{|c|}{ 241-AP-103 } \\
\hline \multicolumn{5}{|c|}{ Surface Level Data } \\
\hline Date & Type of Measurement & Level (Inches) & Date Quality & Comments \\
\hline $10 / 16 / 910: 00$ & Manual Tape & 412.50 & Good & \\
\hline $10 / 16 / 917: 03$ & Auto FIC & 412.8 & Good & \\
\hline $10 / 17 / 910: 00$ & Manual Tape & 412.5 & Good & \\
\hline $10 / 17 / 917: 05$ & Auto FIC & 412.80 & Good & \\
\hline $10 / 18 / 910: 00$ & Manual Tape & 412.5 & Good & \\
\hline $10 / 18 / 917: 06$ & Auto FIC & 412.80 & Good & \\
\hline $10 / 19 / 91 \quad 0: 00$ & Manual Tape & 412.75 & Good & \\
\hline $10 / 19 / 917: 06$ & Auto FIC & 412.8 & Good & \\
\hline $10 / 20 / 910: 00$ & Manual Tape & 412.75 & Good & \\
\hline $10 / 20 / 917: 02$ & Auto FIC & 412.80 & Good & \\
\hline $10 / 21 / 910: 00$ & Manual Tape & 412.75 & Good & \\
\hline $10 / 21 / 917: 02$ & Auto FIC & 412.80 & Good & \\
\hline 10/22/910:00 & Manual Tape & 412.50 & Good & \\
\hline $10 / 22 / 917: 03$ & Auto FIC & 412.8 & Good & \\
\hline $10 / 23 / 910: 00$ & Manual Tape & 412.50 & Good & \\
\hline $10 / 23 / 917: 02$ & Auto FIC & 412.80 & Good & \\
\hline $10 / 24 / 910: 00$ & Manual Tape & 412.5 & Good & \\
\hline $10 / 24 / 917: 02$ & Auto FIC & 412.8 & Good & \\
\hline $10 / 25 / 910: 00$ & Manual Tape & 412.5 & Good & \\
\hline $10 / 25 / 917: 03$ & Auto FIC & 412.80 & Good & \\
\hline $10 / 26 / 910: 00$ & Manual Tape & 41.2 .50 & Good & \\
\hline $10 / 26 / 917: 03$ & Auto FIC & 412.70 & Good & \\
\hline $10 / 27 / 910: 00$ & Manual Tape & 412.25 & Good & \\
\hline $10 / 27 / 917: 02$ & Auto FIC & 412.7 & Good & \\
\hline $10 / 28 / 910: 00$ & Manual Tape & 412.50 & Good & \\
\hline $10 / 28 / 917: 02$ & Auto FIC & 412.7 & Good & \\
\hline 10/29/910:00 & Manual Tape & 412.5 & Good & \\
\hline $10 / 29 / 917: 03$ & Auto FIC & 412.7 & Good & \\
\hline $10 / 30 / 910: 00$ & Manual Tape & 412.5 & Good & \\
\hline $10 / 30 / 917: 03$ & Auto FIC & 412.7 & Good & \\
\hline $10 / 31 / 910: 00$ & Manual Tape & 412.5 & Good & \\
\hline $10 / 31 / 917: 03$ & Auto FIC & 412.70 & Good & \\
\hline $11 / 1 / 910: 00$ & Manual Tape & 412.5 & Good & \\
\hline $11 / 1 / 917: 02$ & Auto FIC & 412.70 & Good & \\
\hline $11 / 2 / 910: 00$ & Manual Tape & 412.25 & Good & \\
\hline $11 / 2 / 917: 03$ & Auto FIC & 412.70 & Good & \\
\hline $11 / 3 / 910: 00$ & Manual Tape & 412.25 & Good & \\
\hline $11 / 3 / 917: 03$ & Auto FIC & $41.2: 70$ & Good & \\
\hline $11 / 4 / 910: 00$ & Manual Tape & $\begin{array}{r}412.50 \\
\end{array}$ & Good & \\
\hline $11 / 4 / 917: 02$ & Auto FIC & 412.70 & Good & \\
\hline $11 / 5 / 910: 00$ & Manual Tape & 412.75 & Good & \\
\hline $11 / 5 / 917: 03$ & Auto FIC & 412.70 & Good & \\
\hline $11 / 6 / 910: 00$ & Manual Tape & 412.75 & Good & \\
\hline $11 / 6 / 917: 03$ & Auto FIC & 412.7 & Good & \\
\hline $11 / 7 / 910: 00$ & Manual Tape & 412.50 & Good & \\
\hline $11 / 7 / 917: 02$ & Auto FIC & 412.7 & Good & \\
\hline $11 / 8 / 910: 00$ & Manual Tape & 412.5 & Good & \\
\hline $11 / 8 / 917: 03$ & Auto FIC & 412.7 & Good & \\
\hline
\end{tabular}

Data obtained from WHC Surveillance Analysis Computer System, (PC SACS) July 7, 1995. 


\begin{tabular}{|c|c|c|c|c|}
\hline \multicolumn{5}{|c|}{ 241-AP-103 } \\
\hline \multicolumn{5}{|c|}{ Surface Level Date } \\
\hline Date & Type of Measurement & Level (Inches) & Data Quality & Comments \\
\hline $11 / 9 / 910: 00$ & Manual Tape & 412.50 & Good & \\
\hline $11 / 9 / 917: 02$ & Auto FIC & 412.7 & . Good & \\
\hline $11 / 10 / 910: 00$ & Manual Tape & 412.5 & Good & \\
\hline 11/10/91 7:03 & Auto FIC & 412.70 & Good & \\
\hline $11 / 11 / 910: 00$ & Manual Tape ". & 412.5 & Good & \\
\hline $11 / 11 / 916: 03$ & Auto FIC & 412.70 & Good & \\
\hline 11/12/910:00 & Manual Tape & 412.5 & Good & \\
\hline $11 / 12 / 917: 03$ & Auto FIC & 412.70 & Good & \\
\hline $11 / 13 / 910: 00$ & Manual Tape & 412.5 & Good & \\
\hline $11 / 13 / 917: 03$ & Auto FIC & 412.70 & Good & \\
\hline $11 / 14 / 910: 00$ & Manual Tape & 412.25 & Good & \\
\hline $11 / 14 / 917: 03$ & Auto FIC & 412.7 & Good & \\
\hline $11 / 15 / 910: 00$ & Manual Tape & 412.50 & Good & \\
\hline $11 / 15 / 917: 03$ & Auto FIC & 412.7 & Good & \\
\hline $11 / 16 / 910: 00$ & Manual Tape & 412.5 & Good & \\
\hline 11/16/91 7:03 & Auto FIC & 412.70 & Good & \\
\hline $11 / 17 / 910: 00$ & Manual Tape & 412.50 & Good & \\
\hline $11 / 17 / 917: 02$ & Auto FIC & 412.70 & Good & \\
\hline $11 / 18 / 910: 00$ & Manual Tape & 412.5 & Good & \\
\hline $11 / 18 / 917: 03$ & Auto FIC & 412.7 & Good & \\
\hline $11 / 19 / 910: 00$ & Manual Tape & 412.50 & Good & \\
\hline $11 / 19 / 917: 03$ & Auto FIC & 412.70 & Good & \\
\hline $11 / 20 / 910: 00$ & Manual Tape & 412.5 & Good & \\
\hline $11 / 20 / 917: 03$ & Auto FIC & 412.7 & Good & \\
\hline $11 / 21 / 910: 00$ & Manual Tape & 412.50 & Good & \\
\hline $11 / 21 / 917: 02$ & Auto FIC & 412.7 & Good & \\
\hline $11 / 22 / 910: 00$ & Manual Tape & 412.50 & Good & \\
\hline 11/22/91 7:03 & Auto FIC & 412.70 & Good & \\
\hline 11/23/91 0:00 & Manual Tape & 412.5 & Good & \\
\hline $11 / 23 / 917: 02$ & Auto FIC & 412.7 & Good & \\
\hline $11 / 24 / 910: 00$ & Manual Tape & 412.50 & Good & \\
\hline $11 / 24 / 917: 03$ & Auto FIC & 412.70 & Good & \\
\hline $11 / 25 / 910: 00$ & Manual Tape & 412.5 & Good & \\
\hline $11 / 25 / 917: 03$ & Auto FIC & 412.7 & Good & \\
\hline $11 / 26 / 910: 00$ & Manual FIC & 412.50 & Transcribed & \\
\hline $11 / 26 / 910: 00$ & Manual Tape & 412.5 & Good & \\
\hline $11 / 27 / 910: 00$ & Manual Tape & 412.50 & Good & \\
\hline $11 / 27 / 917: 03$ & Auto FIC & 412.70 & Good & \\
\hline $11 / 28 / 910: 00$ & Manual Tape & 412.5 & Good & \\
\hline $11 / 28 / 917: 03$ & Auto FIC & 412.7 & Good & \\
\hline $11 / 29 / 910: 00$ & Manual Tape & 412.5 & Good & \\
\hline $11 / 29 / 917: 03$ & Auto FIC & 412.70 & Good & \\
\hline $11 / 30 / 910: 00$ & Manual Tape & 412.50 & Good & \\
\hline 11/30/91 7:03 & Auto FIC & 412.70 & Good & \\
\hline $12 / 1 / 910: 00$ & Manual Tape & 412.50 & Good & \\
\hline $12 / 1 / 917: 03$ & Auto FIC & 412.70 & Good & \\
\hline 12/2/91 0:00 & Manual Tape & 412.5 & Good & \\
\hline $12 / 2 / 917: 03$ & Auto FIC & 412.70 & Good & \\
\hline
\end{tabular}

Data obtained from WHC Surveillance Analysis Computer System, (PC SACS) July 7, 1995. 


\begin{tabular}{|c|c|c|c|c|}
\hline \multicolumn{5}{|c|}{ 241-AP-103 } \\
\hline \multicolumn{5}{|c|}{ Surface Level Data } \\
\hline Date & Type of Measurement & Level (Inches) & Data Quality & Comments \\
\hline $12 / 3 / 910: 00$ & Manual Tape & 412.50 & Good & \\
\hline $12 / 3 / 917: 03$ & Auto FIC & 412.7 & Good & \\
\hline $12 / 4 / 910: 00$ & Manual Tape & 412.5 & Good & \\
\hline $12 / 4 / 917: 03$ & Auto FIC & 412.70 & Good & \\
\hline $12 / 5 / 910: 00$ & Manual Tape & 412.5 & Good & \\
\hline 12/5/91 7:03 & Auto FIC & 412.7 & Good & \\
\hline $12 / 6 / 910: 00$ & Manual Tape & 412.50 & Good & \\
\hline $12 / 6 / 917: 04$ & Auto FIC & 412.7 & Good & \\
\hline $12 / 7 / 910: 00$ & Manual Tape & 412.5 & Good & \\
\hline $12 / 7 / 917: 03$ & Auto FIC & 412.70 & Good & \\
\hline 12/8/91 0:00 & Manual Tape & 412.50 & Good & \\
\hline $12 / 8 / 917: 03$ & Auto FIC & 412.70 & Good & \\
\hline $12 / 9 / 910: 00$ & Manual Tape & 412.50 & Good & \\
\hline $12 / 9 / 917: 03$ & Auto FIC & 41.2 .70 & Good & \\
\hline $12 / 10 / 910: 00$ & Manual Tape & 412.5 & Good & \\
\hline $12 / 10 / 917: 03$ & Auto FIC & 412.7 & Good & \\
\hline $12 / 11 / 910: 00$ & Manual Tape & 412.50 & Good & \\
\hline $12 / 11 / 917: 02$ & Auto FIC & 412.70 & Good & \\
\hline 12/12/910:00 & Manual Tape & 412.5 & Good & \\
\hline 12/12/91 7:03 & Auto FIC & 412.7 & Good & \\
\hline $12 / 13 / 910: 00$ & Manual Tape & 412.50 & Good & \\
\hline $12 / 13 / 917: 05$ & Auto FIC & 412.7 & Good & \\
\hline $12 / 14 / 910: 00$ & Manual Tape & 412.50 & Good & \\
\hline $12 / 14 / 917: 02$ & Auto FIC & 412.70 & Good & \\
\hline $12 / 15 / 910: 00$ & Manual Tape & 412.5 & Good & \\
\hline $12 / 15 / 917: 02$ & Auto FIC & 412.7 & Good & \\
\hline $12 / 16 / 910: 00$ & Manual Tape & 412.50 & Good & \\
\hline $12 / 16 / 917: 03$ & Auto FIC & 412.7 & Good & \\
\hline 12/17/91 0:00 & Manual Tape & 412.5 & Good & \\
\hline $12 / 17 / 917: 06$ & Auto FIC & 412.70 & Good & \\
\hline $12 / 18 / 910: 00$ & Manual Tape & 412.5 & Good & \\
\hline $12 / 18 / 917: 03$ & Auto FIC & 412.7 & Good & \\
\hline $12 / 19 / 910: 00$ & Manual Tape & 412.50 & Good & \\
\hline $12 / 19 / 917: 03$ & Auto FIC & 412.70 & Good & \\
\hline $12 / 20 / 910: 00$ & Manual Tape & 412.5 & Good & \\
\hline $12 / 20 / 917: 02$ & Auto FIC & 412.70 & Good & \\
\hline $12 / 21 / 910: 00$ & Manual Tape & 412.50 & Good & \\
\hline $12 / 21 / 917: 03$ & Auto FIC & 412.7 & Good & \\
\hline $12 / 22 / 910: 00$ & Manual Tape & 412,50 & Good & \\
\hline $12 / 22 / 917: 05$ & Auto FIC & 412.60 & Good & \\
\hline $12 / 23 / 910: 00$ & Manual Tape & 412.5 & Good & \\
\hline $12 / 23 / 917: 06$ & Auto FIC & 412.60 & Good & \\
\hline $12 / 24 / 910: 00$ & Manual Tape & 412.50 & Good & \\
\hline $12 / 24 / 917: 03$ & Auto FIC & 412.60 & Good & \\
\hline $12 / 25 / 910: 00$ & Manual Tape & 412.50 & Good & \\
\hline $12 / 25 / 917: 03$ & Auto FIC & 412.7 & Good & \\
\hline $12 / 26 / 910: 00$ & Manual Tape & 412.5 & Good & \\
\hline $12 / 26 / 917: 03$ & Auto FIC & 412.7 & Good & \\
\hline
\end{tabular}

Data obtained from WHC Surveillance Analysis Computer System, (PC SACS) July 7, 1995. 


\begin{tabular}{|c|c|c|c|c|}
\hline \multicolumn{5}{|c|}{ 241-AP-103 } \\
\hline \multicolumn{5}{|c|}{ Surface Level Data } \\
\hline Date & Type of Messurement & Level (Inches) & Data Quality & Comments \\
\hline $12 / 27 / 910: 00$ & Manual Tape & 412.50 & Good & \\
\hline $12 / 27 / 917: 03$ & Auto FIC & 412.7 & Good & \\
\hline $12 / 28 / 910: 00$ & Manual Tape & 412.25 & Good & \\
\hline $12 / 28 / 917: 02$ & Auto FIC & 412.6 & Good & \\
\hline $12 / 29 / 910: 00$ & Manual Tape & 412.25 & Good & \\
\hline 12/29/91 7:03 & Auto FIC & 412.6 & Good & \\
\hline $12 / 30 / 910: 00$ & Manual Tape & 412.25 & Good & \\
\hline $12 / 30 / 917: 03$ & Auto FIC & 412.70 & Good & \\
\hline $12 / 31 / 910: 00$ & Manual Tape & 412.25 & Good & \\
\hline $12 / 31 / 917: 03$ & Auto FIC & 412.60 & Good & \\
\hline $1 / 1 / 920: 00$ & Manual Tape & 412.50 & Good & \\
\hline 1/1/92 7:03 & Auto FIC & 412.7 & Good & \\
\hline $1 / 2 / 920: 00$ & Manual Tape & 412.50 & Good & \\
\hline $1 / 2 / 927: 02$ & Auto FIC & 412.7 & Good & \\
\hline $1 / 3 / 920: 00$ & Manual Tape & 412.25 & Good & \\
\hline 1/3/92 7:04 & Auto FIC & 412.60 & Good & \\
\hline 1/4/92 0:00 & Manual Tape & 412.50 & Good & \\
\hline $1 / 4 / 927: 03$ & Auto FIC & 412.60 & Good & \\
\hline $1 / 5 / 920: 00$ & Manual Tape & 412.5 & Good & \\
\hline 1/5/92 7:03 & Auto FIC & 412.60 & Good & \\
\hline $1 / 6 / 920: 00$ & Manual Tape & 412.50 & Good & \\
\hline $1 / 6 / 927: 03$ & Auto FIC & 412.6 & Good & \\
\hline $1 / 7 / 920: 00$ & Manual Tape & 412.50 & Good & \\
\hline $1 / 7 / 927: 04$ & Auto FIC & 412.6 & Good & \\
\hline $1 / 8 / 920: 00$ & Manual Tape & 412.5 & Good & \\
\hline 1/8/92 7:03 & Auto FIC & 412.6 & Good & \\
\hline 1/9/92 0:00 & Manual Tape & 412.50 & Good & \\
\hline 1/9/92 7:03 & Auto FIC & 41.2 .60 & Good & \\
\hline $1 / 10 / 920: 00$ & Manual Tape & 412.25 & Good & \\
\hline $1 / 10 / 927: 04$ & Auto FIC & 412.6 & Good & \\
\hline $1 / 11 / 920: 00$ & Manual Tape & 412.5 & Good & \\
\hline $1 / 11 / 927: 04$ & Auto FIC & 412.60 & Good & \\
\hline $1 / 12 / 920: 00$ & Manual FIC & 412.6 & Good & \\
\hline $1 / 12 / 920: 00$ & Manual Tape & $412 . \overline{5}$ & Good & \\
\hline $1 / 13 / 920: 00$ & Manual FIC & 412.60 & Good & \\
\hline $1 / 13 / 920: 00$ & Manual Tape & 412.25 & Good & \\
\hline $1 / 14 / 920: 00$ & Manual FIC & 412.6 & Good & \\
\hline $1 / 14 / 920: 00$ & Manual Tape & 412.25 & Good & \\
\hline $1 / 15 / 920: 00$ & Manual Tape & 412.25 & Good & \\
\hline $1 / 15 / 927: 03$ & Auto FIC & 412.60 & Good & \\
\hline $1 / 16 / 920: 00$ & Manual Tape & 412.5 & Good & \\
\hline $1 / 16 / 927: 04$ & Auto FIC & 412.6 & Good & \\
\hline $1 / 17 / 920: 00$ & Manual Tape & 412.5 & Good & \\
\hline $1 / 17 / 927: 04$ & Auto FIC & 412.60 & Good & \\
\hline 1/18/92 0:00 & Manual Tape & 412.25 & Good & \\
\hline $1 / 18 / 927: 04$ & Auto FIC & 412.6 & Good & \\
\hline $1 / 19 / 920: 00$ & Manual Tape & 412.25 & Good & \\
\hline $1 / 19 / 927: 03$ & Auto FIC & 412.60 & Good & \\
\hline
\end{tabular}

Data obtained from WHC Surveillance Analysis Computer System, (PC SACS) July 7, 1995. 


\begin{tabular}{|c|c|c|c|c|}
\hline \multicolumn{5}{|c|}{ 241-AP-103 } \\
\hline \multicolumn{5}{|c|}{ Surface Leval Data } \\
\hline Date & Type of Measurement & Lovel (Inches) & Data Quality & Comments \\
\hline $1 / 20 / 920: 00$ & Manual Tape & 412.50 & Good & \\
\hline $1 / 20 / 927: 03$ & Auto FIC & 412.6 & Good & \\
\hline $1 / 21 / 920: 00$ & Manual Tape & 412.50 & Good & \\
\hline $1 / 21 / 927: 03$ & Auto FIC & 412.60 & Good & \\
\hline $1 / 22 / 920: 00$ & Manual Tape & 412.5 & Good & \\
\hline $1 / 22 / 927: 06$ & Auto FIC & 412.60 & Good & \\
\hline $1 / 23 / 920: 00$ & Manual Tape & 412.50 & Good & \\
\hline $1 / 23 / 927: 03$ & Auto FIC & 412.6 & Good & \\
\hline $1 / 24 / 920: 00$ & Manual Tape & 412.5 & Good & \\
\hline $1 / 24 / 927: 02$ & Auto FIC & 412.60 & Good & \\
\hline $1 / 25 / 920: 00$ & Manual Tape & 412.5 & Good & \\
\hline $1 / 25 / 927: 03$ & Auto FIC & 412.6 & Good & \\
\hline 1/26/92 0:00 & Manual Tape & 412.50 & Good & \\
\hline $1 / 26 / 926: 03$ & Auto FIC & 412.60 & Good & \\
\hline 1/27/92 0:00 & Manual Tape & 412.5 & Good & \\
\hline $1 / 27 / 927: 02$ & Auto FIC & 412.6 & Good & \\
\hline $1 / 28 / 920: 00$ & Manual Tape & 412.5 & Good & \\
\hline $1 / 28 / 927: 03$ & Auto FIC & 412.60 & Good & \\
\hline $1 / 29 / 920: 00$ & Manual Tape & 412.50 & Good & \\
\hline $1 / 29 / 927: 03$ & Auto FIC & 412.60 & Good & \\
\hline $1 / 30 / 920: 00$ & Manual Tape & 412.50 & Good & \\
\hline $1 / 30 / 927: 03$ & Auto FIC & 412.6 & Good & \\
\hline $1 / 31 / 920: 00$ & Manual Tape & 412.50 & Good & \\
\hline $1 / 31 / 927: 03$ & Auto FIC & 412.6 & Good & \\
\hline $2 / 1 / 920: 00$ & Manual Tape & 412.5 & Good & \\
\hline $2 / 1 / 927: 02$ & Auto FIC & 412.6 & Good & \\
\hline $2 / 2 / 920: 00$ & Manual Tape & 412.5 & Good & \\
\hline $2 / 2 / 927: 03$ & Auto FIC & 412.60 & Good & \\
\hline $2 / 3 / 920: 00$ & Manual Tape & 412.5 & Good & \\
\hline 2/3/92 7:03 & Auto FIC & 412.60 & Good & \\
\hline $2 / 4 / 920: 00$ & Manual Tape & 412.5 & Good & \\
\hline 2/4/92 7:03 & Auto FIC & 412.6 & Good & \\
\hline 2/5/92 0:00 & Manual Tape & 412.5 & Good & \\
\hline 2/5/92 7:02 & Auto FIC & 412.6 & Good & \\
\hline $2 / 6 / 920: 00$ & Manual FIC & 412.6 & Good & \\
\hline $2 / 6 / 920: 00$ & Manual Tape & 412.50 & Good & \\
\hline $2 / 7 / 920: 00$ & Manual FIC & 412.6 & Good & \\
\hline $2 / 7 / 920: 00$ & Manual Tape & 412.25 & Good & \\
\hline $2 / 8 / 920: 00$ & Manual Tape & 412.25 & Good & \\
\hline $2 / 8 / 927: 04$ & Auto FIC & 412.6 & Good & \\
\hline $2 / 9 / 920: 00$ & Manual Tape & 412.25 & Good & \\
\hline 2/9/92 7:03 & Auto FIC & 412.60 & Good & \\
\hline $2 / 10 / 920: 00$ & Manual Tape & 412.25 & Good & \\
\hline $2 / 10 / 927: 04$ & Auto FIC & 412.5 & Good & \\
\hline $2 / 11 / 920: 00$ & Manual Tape & 412.25 & Good & \\
\hline $2 / 11 / 927: 03$ & Auto FIC & 412.60 & Good & \\
\hline $2 / 12 / 920: 00$ & Manual Tape & 412.25 & Good & \\
\hline $2 / 12 / 927: 03$ & Auto FIC & 412.60 & Good & \\
\hline
\end{tabular}

Data obtained from WHC Surveillance Analysis Computer System, (PC SACS) July 7, 1995. 


\begin{tabular}{|c|c|c|c|c|}
\hline \multicolumn{5}{|c|}{ 241-AP 193} \\
\hline \multicolumn{5}{|c|}{, Surface Level Data } \\
\hline Date & Type of Measurement & Lovel (Inches) & Data Quality & Comments \\
\hline $2 / 13 / 920: 00$ & Manual Tape & 412.25 & Good & \\
\hline $2 / 13 / 927: 03$ & Auto FIC & 412.6 & Good & \\
\hline $2 / 14 / 920: 00$ & Manual Tape & 412.25 & Good & \\
\hline $2 / 14 / 927: 03$ & Auto FIC & 412.6 & Good & \\
\hline $2 / 15 / 920: 00$ & Manual Tape & 412.25 & Good & \\
\hline $2 / 15 / 92$ 7:03 & Auto FIC & 412.60 & Good & \\
\hline $2 / 16 / 920: 00$ & Manual Tape & 412.25 & Good & \\
\hline $2 / 16 / 927: 03$ & Auto FIC & 412.60 & Good & \\
\hline $2 / 17 / 920: 00$ & Manual Tape & 412.25 & Good & \\
\hline $2 / 17 / 927: 03$ & Auto FIC & 412.60 & Good & \\
\hline $2 / 18 / 920: 00$ & Manual Tape & 412.25 & Good & \\
\hline $2 / 18 / 927: 03$ & Auto FIC & 412.6 & Good & \\
\hline $2 / 19 / 920: 00$ & Manual Tape & 412.25 & Good & \\
\hline $2 / 19 / 927: 03$ & Auto FIC & 412.5 & Good & \\
\hline $2 / 20 / 920: 00$ & Manual Tape & 412.25 & Good & \\
\hline $2 / 20 / 927: 03$ & Auto FIC & 412.60 & Good & \\
\hline $2 / 21 / 920: 00$ & Manual Tape & 412.25 & Good & \\
\hline $2 / 21 / 927: 03$ & Auto FIC & 412.5 & Good & \\
\hline $2 / 22 / 920: 00$ & Manual Tape & 412.25 & Good & \\
\hline $2 / 22 / 927: 03$ & Auto FIC & 412.60 & Good & \\
\hline $2 / 23 / 920: 00$ & Manual Tape & 412.25 & Good & \\
\hline $2 / 23 / 927: 03$ & Auto FIC & 412.50 & Good & \\
\hline $2 / 24 / 920: 00$ & Manual Tape & 412.50 & Good & \\
\hline $2 / 24 / 927: 03$ & Auto FIC & 412.50 & Good & \\
\hline $2 / 25 / 920: 00$ & Manual Tape & 412.5 & Good & \\
\hline $2 / 25 / 927: 04$ & Auto FIC & 412.5 & Good & \\
\hline $2 / 26 / 920: 00$ & Manual Tape & 412.5 & Good & \\
\hline $2 / 26 / 927: 03$ & Auto FIC & 412.50 & Good & \\
\hline $2 / 27 / 920: 00$ & Manual Tape & 412.5 & Good & \\
\hline $2 / 27 / 927: 04$ & Auto FIC & 412.50 & Good & \\
\hline $2 / 28 / 920: 00$ & Manual Tape & 412.5 & Good & \\
\hline $2 / 28 / 927: 04$ & Auto FIC & 412.5 & Good & \\
\hline $2 / 29 / 920: 00$ & Manual Tape & 412.50 & Good & \\
\hline $2 / 29 / 927: 03$ & Auto FIC & 412.50 & Good & \\
\hline $3 / 1 / 920: 00$ & Manual Tape & 412.50 & Good & \\
\hline $3 / 1 / 927: 03$ & Auto FIC & 412.5 & Good & \\
\hline $3 / 2 / 920: 00$ & Manual Tape & 412.25 & Good & \\
\hline $3 / 2 / 927: 03$ & Auto FIC & 412.5 & Good & \\
\hline $3 / 3 / 92 \quad 0: 00$ & Manual Tape & 412.25 & Good & \\
\hline $3 / 3 / 927: 04$ & Auto FIC & 412.5 & Good & \\
\hline $3 / 4 / 920: 00$ & Manual Tape & 412.25 & Good & \\
\hline $3 / 4 / 927: 04$ & Auto FIC & 412.50 & Good & \\
\hline $3 / 5 / 92 \quad 0: 00$ & Manual Tape & 412.25 & Good & \\
\hline $3 / 5 / 927: 03$ & Auto FIC & 412.5 & Good & \\
\hline $3 / 6 / 920: 00$ & Manual Tape & 412.25 & Good & \\
\hline $3 / 6 / 927: 03$ & Auto FIC & $41,2.50$ & Good & \\
\hline $3 / 7 / 920: 00$ & Manual Tape & 412.25 & Good & \\
\hline $3 / 7 / 927: 02$ & Auto FIC & 412.50. & Good & \\
\hline
\end{tabular}

Data obtained from WHC Surveillance Analysis Computer System, (PC SACS) July 7, 1995. 


\begin{tabular}{|c|c|c|c|c|}
\hline \multicolumn{5}{|c|}{ 241-AP-103 } \\
\hline \multicolumn{5}{|c|}{ Surface Leval Data } \\
\hline Date & Type of Measurement & Lovel (Inches) & Data Quality & Comments \\
\hline $3 / 8 / 920: 00$ & Manual Tape & 412.25 & Good & \\
\hline $3 / 8 / 927: 02$ & Auto FIC & 412.50 & Good & \\
\hline 3/9/92 0:00 & Manual Tape & 412.00 & Good & \\
\hline 3/9/92 7:02 & Auto FIC & 412.50 & Good & \\
\hline $3 / 10 / 920: 00$ & Manual Tape & 411.75 & Good & \\
\hline $3 / 10 / 927: 03$ & Auto FIC & 412.5 & Good & \\
\hline $3 / 11 / 920: 00$ & Manual Tape & 412.25 & Good & \\
\hline 3/11/92 7:03 & Auto FIC & 412.50 & Good & \\
\hline $3 / 12 / 920: 00$ & Manual Tape & 412.25 & Good & \\
\hline 3/12/92 7:03 & Auto FIC & 412.5 & Good & \\
\hline $3 / 13 / 920: 00$ & Manual Tape & 412 & Good & \\
\hline $3 / 13 / 927: 03$ & Auto FIC & 412.50 & Good & \\
\hline $3 / 14 / 920: 00$ & Manual Tape & 412.25 & Good & \\
\hline $3 / 14 / 927: 03$ & Auto FIC & 412.50 & Good & \\
\hline $3 / 15 / 920: 00$ & Manual Tape & 412.25 & Good & \\
\hline $3 / 15 / 927: 06$ & Auto FIC & 412.5 & Good & \\
\hline $3 / 16 / 920: 00$ & Manual Tape & 412.25 & Good & \\
\hline $3 / 16 / 927: 03$ & Auto FIC & 412.50 & Good & \\
\hline 3/17/92 0:00 & Manual Tape & 412.25 & Good & \\
\hline $3 / 17 / 927: 03$ & Auto FIC & 412.5 & Good & \\
\hline $3 / 18 / 920: 00$ & Manual Tape & 412.25 & Good & \\
\hline $3 / 18 / 927: 03$ & Auto FIC & 412.5 & Good & \\
\hline 3/19/92 0:00 & Manual Tape & 412.00 & Good & \\
\hline $3 / 19 / 926: 02$ & Auto FIC & 412.50 & Gopd & \\
\hline $3 / 20 / 920: 00$ & Manual Tape & 412 & Good & \\
\hline $3 / 20 / 927: 02$ & Auto FIC & 412.5 & Good & \\
\hline $3 / 21 / 920: 00$ & Manual Tape & 412.25 & Good & \\
\hline $3 / 21 / 927: 03$ & Auto FIC & 412.50 & Good & \\
\hline $3 / 22 / 920: 00$ & Manual Tape & 412.25 & Good & \\
\hline $3 / 22 / 927: 02$ & Auto FIC & 412.50 & Good & \\
\hline $3 / 23 / 920: 00$ & Manual Tape & 412.25 & Good & \\
\hline $3 / 23 / 927: 02$ & Auto FIC & 412.50 & Good & \\
\hline $3 / 24 / 920: 00$ & Manual Tape & 412,25 & Good & \\
\hline $3 / 24 / 92$ 7:02 & Auto FIC & 412.50 & Good & \\
\hline $3 / 25 / 920: 00$ & Manual Tape & 412.50 & Good & \\
\hline $3 / 25 / 927: 03$ & Auto FIC & 412.5 & Good & \\
\hline $3 / 26 / 920: 00$ & Manual Tape & 412.50 & Good & \\
\hline $3 / 26 / 927: 03$ & Auto FIC & 412.5 & Good & \\
\hline $3 / 27 / 920: 00$ & Manual Tape & 412.25 & Good & \\
\hline $3 / 27 / 927: 02$ & Auto FIC & 412.5 & Good & \\
\hline $3 / 28 / 920: 00$ & Manual Tape & 412.25 & Good & \\
\hline $3 / 28 / 927: 03$ & Auto FIC & 412.5 & Good & \\
\hline $3 / 29 / 92 \quad 0: 00$ & Manual Tape & 412.5 & Good & \\
\hline $3 / 29 / 927: 03$ & Auto FIC & 412.50 & Good & \\
\hline $3 / 30 / 920: 00$ & Manual Tape & 412.50 & Good & \\
\hline $3 / 30 / 927: 03$ & Auto FIC & 412.50 & Good & \\
\hline $3 / 31 / 920: 00$ & Manual Tape & 412.25 & Good & \\
\hline $3 / 31 / 927: 03$ & Auto FIC & 412.50 & Good & \\
\hline
\end{tabular}

Data obtained from WHC Surveillance Analysis Computer System. (PC SACS) July 7, 1995. 


\begin{tabular}{|c|c|c|c|c|}
\hline \multicolumn{5}{|c|}{ 241-AP-103 } \\
\hline & & urface Level Date & & \\
\hline Date & Type of Measurement & Level (Inches) & Data Quality & Comments \\
\hline $4 / 1 / 920: 00$ & Manual Tape & 412.25 & Good & \\
\hline 4/1/92 7:03 & Auto FIC & 412.50 & Good & \\
\hline $4 / 2 / 920: 00$ & Manual Tape & 412.25 & Good & \\
\hline $4 / 2 / 927: 03$ & Auto FIC & 41.2 .50 & Good & \\
\hline $4 / 3 / 920: 00$ & Manual Tape & 412.25 & Good & \\
\hline $4 / 3 / 927: 03$ & Auto FIC & 412.5 & Good & \\
\hline 4/4/92 0:00 & Manual Tape & 412.25 & Good & \\
\hline 4/4/92 7:03 & Auto FIC & 412.5 & Good & \\
\hline 4/5/92 0:00 & Manual Tape & 412.25 & Good & \\
\hline 4/5/92 7:03 & Auto FIC & 412.5 & Good & \\
\hline $4 / 6 / 920: 00$ & Manual Tape & 412.25 & Good & \\
\hline $4 / 6 / 927: 03$ & Auto FIC & 412.40 & Good & \\
\hline 4/7/920:00 & Manual Tape & 412.25 & Good & \\
\hline 4/7/92 7:03 & Auto FIC & 412.5 & Good & \\
\hline 4/8/92 0:00 & Manual Tape & 412.5 & Good & \\
\hline 4/8/92 5:02 & Auto FIC & 412.50 & Good & \\
\hline 4/9/92 0:00 & Manual Tape & 412 & Good & \\
\hline 4/9/92 7:03 & Auto FIC & 412.50 & Good & \\
\hline $4 / 10 / 920: 00$ & Manual Tape & 412.5 & Good & \\
\hline $4 / 10 / 927: 03$ & Auto FIC & 412.50 & Good & \\
\hline $4 / 11 / 920: 00$ & Manual Tape & 41.2 .25 & Good & \\
\hline 4/11/92 7:03 & Auto FIC & 412.50 & Good & \\
\hline 4/12/92 0:00 & Manual Tape & 412.25 & Good & \\
\hline 4/12/92 5:03 & Auto FIC & 412.6 & Good & \\
\hline 4/13/92 0:00 & Manual Tape & 412.25 & Good & \\
\hline $4 / 13 / 927: 03$ & Auto FIC & 412.5 & Good & \\
\hline 4/14/92 0:00 & Manual Tape & 412.25 & Good & \\
\hline $4 / 14 / 927: 03$ & Auto FIC & 412.50 & Good & \\
\hline $4 / 15 / 920: 00$ & Manual Tape & 412.25 & Good & \\
\hline $4 / 15 / 927: 02$ & Auto FIC & 412.50 & Good & \\
\hline $4 / 16 / 920: 00$ & Manual Tape & 412.25 & Good & \\
\hline 4/16/92 7:03 & Auto FIC & 412.50 & Good & \\
\hline $4 / 17 / 920: 00$ & Manual Tape & 412.75 & Good & \\
\hline $4 / 17 / 927: 03$ & Auto FIC & 412.5 & Good & \\
\hline 4/18/92 0:00 & Manual Tape & 412.25 & Good & \\
\hline $4 / 18 / 92$ 7:02 & Auto FIC & 412.50 & Good & \\
\hline 4/19/92 0:00 & Manual Tape & 412.25 & Good & \\
\hline 4/19/92 7:02 & Auto FIC & 412.5 & Good & \\
\hline 4/20/92 0:00 & Manual Tape & 412.25 & Good & \\
\hline $4 / 20 / 927: 03$ & Auto FIC & 412.5 & Good & \\
\hline $4 / 21 / 920: 00$ & Manual Tape & 412.25 & Good & \\
\hline $4 / 21 / 92 \quad 7: 03$ & Auto FIC & 412.50 & Good & \\
\hline $4 / 22 / 920: 00$ & Manual Tape & 412.25 & Good & \\
\hline $4 / 22 / 927: 02$ & Auto FIC & 412.50 & Good & \\
\hline $4 / 23 / 920: 00$ & Manual Tape & 412.25 & Good & \\
\hline $4 / 23 / 927: 03$ & Auto FIC & 412.50 & Good & \\
\hline $4 / 24 / 920: 00$ & Manual Tape & 412.25 & Good & \\
\hline $4 / 24 / 927: 03$ & Auto FIC & 412.5 & Good & \\
\hline
\end{tabular}

Data obtained from WHC Surveillance Analysis Computer System, (PC SACS) July 7, 1995. 


\begin{tabular}{|c|c|c|c|c|}
\hline \multicolumn{5}{|c|}{ 241-AP-103 } \\
\hline \multicolumn{5}{|c|}{ Surface Level Data } \\
\hline Date & Type of Measurement & Level (Inches) & Data Quality & Comments \\
\hline $4 / 25 / 920: 00$ & Manual Tape & 412.25 & Good & \\
\hline $4 / 25 / 927: 04$ & Auto FIC & 412.5 & Good & \\
\hline $4 / 26 / 920: 00$ & Manual Tape & 412.25 & Good & \\
\hline $4 / 26 / 927: 03$ & Auto FIC & 412.50 & Good & \\
\hline $4 / 27 / 920: 00$ & Manual Tape & 412.25 & Good & \\
\hline 4/27/92 7:04 & Auto FIC & 412.50 & Good & \\
\hline $4 / 28 / 920: 00$ & Manual Tape & 412.25 & Good & \\
\hline $4 / 28 / 927: 03$ & Auto FIC & 412.50 & Good & \\
\hline $4 / 29 / 92 \quad 0: 00$ & Manual Tape & 412 & Good & \\
\hline $4 / 29 / 927: 03$ & Auto FIC & 412.50 & Good & \\
\hline $4 / 30 / 92$ 0:00 & Manual Tape & 412.25 & Good & \\
\hline $4 / 30 / 927: 03$ & Auto FIC & 412.50 & Good & \\
\hline $5 / 1 / 920: 00$ & Manual Tape & 412.25 & Good & \\
\hline $5 / 1 / 927: 03$ & Auto FIC & 412.5 & Good & \\
\hline $5 / 2 / 920: 00$ & Manual FIC & 412.25 & Transcribed & \\
\hline $5 / 2 / 920: 00$ & Manual Tape & 412.25 & Good & \\
\hline $5 / 2 / 922: 03$ & Auto FIC & 412.5 & Good & \\
\hline $5 / 3 / 920: 00$ & Manual Tape & 412.25 & Good & \\
\hline $5 / 3 / 927: 03$ & Auto FIC & 412.5 & Good & \\
\hline $5 / 4 / 920: 00$ & Manual Tape & 412.25 & Good & \\
\hline $5 / 4 / 927: 03$ & Auto FIC & 412.50 & Good & \\
\hline $5 / 5 / 920: 00$ & Manual Tape & 412.25 & Good & \\
\hline $5 / 5 / 927: 04$ & Auto FIC & 412.50 & Good & \\
\hline $5 / 6 / 920: 00$ & Manual Tape & 412.25 & Good & \\
\hline $5 / 6 / 927: 03$ & Auto FIC & 412.50 & Good & \\
\hline $5 / 7 / 920: 00$ & Manual Tape & 412.25 & Good & \\
\hline $5 / 7 / 927: 04$ & Auto FIC & 412.50 & Good & \\
\hline $5 / 8 / 920: 00$ & Manual Tape & 412 & Good & \\
\hline 5/8/92 7:03 & Auto FIC & 412.5 & Good & \\
\hline $5 / 9 / 92 \quad 0: 00$ & Manual Tape & 412.25 & Good & \\
\hline $5 / 10 / 920: 00$ & Manual Tape & 412.25 & Good & \\
\hline $5 / 10 / 926: 03$ & Auto FIC & 412.5 & Good & \\
\hline $5 / 11 / 920: 00$ & Manual FIC & 412.50 & Good & \\
\hline $5 / 11 / 920: 00$ & Manual Tape & 412 & Good & \\
\hline $5 / 12 / 920: 00$ & Manual Tape & 412.25 & Good & \\
\hline $5 / 12 / 927: 03$ & Auto FIC & 412.5 & Good & \\
\hline $5 / 13 / 920: 00$ & Manual Tape & 412.25 & Good & \\
\hline $5 / 13 / 927: 03$ & Auto FIC & 412.5 & Good & \\
\hline $5 / 14 / 920: 00$ & Manual Tape & 412.25 & Good & \\
\hline $5 / 14 / 927: 03$ & Auto FIC & 412.50 & Good & \\
\hline $5 / 15 / 920: 00$ & Manual Tape & 412.25 & Good & \\
\hline $5 / 15 / 927: 03$ & Auto FIC & 412.5 & Good & \\
\hline $5 / 16 / 920: 00$ & Manual Tape & 412.25 & Good & \\
\hline $5 / 16 / 927: 03$ & Auto FIC & 412.50 & Good & \\
\hline $5 / 17 / 920: 00$ & Manual Tape & 412.25 & Good & \\
\hline $5 / 17 / 927: 02$ & Auto FIC & 412.50 & Good & \\
\hline $5 / 18 / 920: 00$ & Manual Tape & 412.25 & Good & \\
\hline $5 / 18 / 927: 03$ & Auto FIC & 412.50 & Good & \\
\hline
\end{tabular}

Data obtained from WHC Surveillance Analysis Computer System, (PC SACS) July 7, 1995. 


\begin{tabular}{|c|c|c|c|c|}
\hline \multicolumn{5}{|c|}{ 241-AP-103 } \\
\hline & & urface Level. Dat & & \\
\hline Date & Type of Measurement & Level (Inches) & Data Quality & Comments \\
\hline $5 / 19 / 920: 00$ & Manual Tape & 412.25 & Good & \\
\hline $5 / 19 / 927: 03$ & Auto FIC & 412.5 & Good & \\
\hline $5 / 20 / 920: 00$ & Manual Tape & 412.25 & Good & \\
\hline $5 / 20 / 927: 03$ & Auto FIC & $412 . \overline{5}$ & Good & \\
\hline $5 / 21 / 920: 00$ & Manual Tape & 412.25 & Good & \\
\hline $5 / 21 / 927: 03$ & Auto FIC & 412.5 & Good & \\
\hline $5 / 22 / 920: 00$ & Manual Tape & 412.25 & Good & \\
\hline $5 / 22 / 927: 02$ & Auto FIC & 412.5 & Good & \\
\hline $5 / 23 / 920: 00$ & Manual Tape & 412.25 & Good & \\
\hline $5 / 23 / 927: 03$ & Auto FIC & 412.40 & Good & \\
\hline $5 / 24 / 920: 00$ & Manual Tape & 412.25 & Good & \\
\hline $5 / 24 / 927: 03$ & Auto FIC & 412.40 & Good & \\
\hline $5 / 25 / 920: 00$ & Manual Tape & 412.25 & Good & \\
\hline $5 / 25 / 927: 03$ & Auto FIC & $412: 40$ & Good & \\
\hline $5 / 26 / 920: 00$ & Manual FIC & 412.40 & Good & \\
\hline $5 / 26 / 920: 00$ & Manual Tape & 412.25 & Good & \\
\hline $5 / 27 / 920: 00$ & Manual Tape & 412.25 & Good & \\
\hline $5 / 27 / 927: 03$ & Auto FIC & 412.50 & Good & \\
\hline $5 / 28 / 920: 00$ & Manual Tape & 412.25 & Good & \\
\hline $5 / 28 / 927: 02$ & Auto FIC & 412.4 & Good & \\
\hline $5 / 29 / 920: 00$ & Manual Tape & 412.25 & Good & \\
\hline $5 / 29 / 927: 02$ & Auto FIC & 412.4 & Good & \\
\hline $5 / 30 / 920: 00$ & Manual Tape & 412.25 & Good & \\
\hline $5 / 30 / 927: 03$ & Auto FIC & 412.4 & Good & \\
\hline $5 / 31 / 920: 00$ & Manual Tape & 412.25 & Good & \\
\hline $5 / 31 / 927: 03$ & Auto FIC & 412.40 & Good & \\
\hline $6 / 1 / 920: 00$ & Manual Tape & 412.25 & Good & \\
\hline $6 / 1 / 927: 03$ & Auto FIC & 412.4 & Good & \\
\hline $6 / 2 / 920: 00$ & Manual Tape & 412.25 & Good & \\
\hline $6 / 2 / 927: 03$ & Auto FIC & 412.40 & Good & \\
\hline $6 / 3 / 920: 00$ & Manual Tape & 412.25 & Good & \\
\hline $6 / 3 / 927: 03$ & Auto FIC & 412.40 & Good & \\
\hline $6 / 4 / 920: 00$ & Manual Tape & 412.25 & Good & \\
\hline 6/4/92 7:03 & Auto FIC & 412.40 & Good & \\
\hline $6 / 5 / 920: 00$ & Manual Tape & 412.25 & Good & \\
\hline $6 / 5 / 927: 02$ & Auto FIC & 412.4 & Good & \\
\hline $6 / 6 / 920: 00$ & Manual Tape & 412.25 & Good & \\
\hline $6 / 6 / 927: 03$ & Auto FIC & 412.4 & Good & \\
\hline $6 / 7 / 920: 00$ & Manual Tape & 412.25 & Good & \\
\hline $6 / 7 / 927: 03$ & Auto FIC & 412.4 & Good & \\
\hline $6 / 8 / 920: 00$ & Manual Tape & 412.25 & Good & \\
\hline $6 / 8 / 927: 03$ & Auto FIC & 412.40 & Good & \\
\hline $6 / 9 / 920: 00$ & Manual Tape & 412.5 & Good & \\
\hline $6 / 9 / 927: 03$ & Auto FIC & 412.4 & Good & \\
\hline $6 / 10 / 920: 00$ & Manual Tape & 412.25 & Good & \\
\hline $6 / 10 / 927: 03$ & Auto FIC & 412.30 & Good & \\
\hline $6 / 11 / 920: 00$ & Manual Tape & 412.00 & Good & \\
\hline $6 / 11 / 927: 03$ & Auto FIC & 412.40 & Good & \\
\hline
\end{tabular}

Data obtained from WHC Surveillance Analysis Computer System, (PC SACS) July 7, 1995. 


\begin{tabular}{|c|c|c|c|c|}
\hline \multicolumn{5}{|c|}{ 241-AP-103 } \\
\hline \multicolumn{5}{|c|}{ Surface Level Data } \\
\hline Date & Type of Measurement & Level (Inches) & Data Quality & Comments \\
\hline $6 / 12 / 920: 00$ & Manual Tape & 412 & Good & \\
\hline $6 / 12 / 927: 05$ & Auto FIC & 412.40 & Good & \\
\hline $6 / 13 / 920: 00$ & Manual Tape & 412.00 & Good & \\
\hline $6 / 13 / 927: 05$ & Auto FIC & 412.40 & Good & \\
\hline $6 / 14 / 920: 00$ & Manual FIC & 412.40 & Transcribed & \\
\hline $6 / 14 / 920: 00$ & Manual Tape & 412.25 & Good & \\
\hline $6 / 15 / 920: 00$ & Manual Tape & 412.25 & Good & \\
\hline $6 / 15 / 927: 03$ & Auto FIC & 412.4 & Good & \\
\hline $6 / 16 / 920: 00$ & Manual Tape & 412.25 & Good & \\
\hline $6 / 16 / 927: 03$ & Auto FIC & 412.40 & Good & \\
\hline $6 / 17 / 920: 00$ & Manual Tape & 412.25 & Good & \\
\hline $6 / 17 / 927: 03$ & Auto FIC & 412.4 & Good & \\
\hline $6 / 18 / 920: 00$ & Manual Tape & 412.25 & Good & \\
\hline $6 / 18 / 927: 03$ & Auto FIC & 412.4 & Good & \\
\hline $6 / 19 / 920: 00$ & Manual Tape & 412 & Good & \\
\hline $6 / 19 / 927: 02$ & Auto FIC & 412.40 & Good & \\
\hline $6 / 20 / 92 \quad 0: 00$ & Manual Tape & 412 & Good & \\
\hline $6 / 20 / 927: 02$ & Auto FIC & 412.4 & Good & \\
\hline $6 / 21 / 920: 00$ & Manual Tape & 412.25 & Good & \\
\hline $6 / 21 / 927: 03$ & Auto FIC & 412.40 & Good & \\
\hline $6 / 22 / 920: 00$ & Manual Tape & 412.25 & Good & \\
\hline $6 / 22 / 927: 03$ & Auto FIC & 412.40 & Good & \\
\hline $6 / 23 / 920: 00$ & Manual Tape & 412.25 & Good & \\
\hline $6 / 23 / 927: 03$ & Auto FIC & 412.4 & Good & \\
\hline $6 / 24 / 920: 00$ & Manual Tape & 412.25 & Good & \\
\hline $6 / 24 / 927: 03$ & Auto FIC & 412.4 & Good & \\
\hline $6 / 25 / 920: 00$ & Manual Tape & 412.5 & Good & \\
\hline $6 / 25 / 927: 03$ & Auto FIC & 41.2 .40 & Good & \\
\hline $6 / 26 / 920: 00$ & Manual Tape & 412.25 & Good & \\
\hline $6 / 26 / 927: 02$ & Auto FIC & 412.4 & Good & \\
\hline $6 / 27 / 920: 00$ & Manual Tape & 412.50 & Good & \\
\hline $6 / 27 / 927: 03$ & Auto FIC & 412.4 & Good & \\
\hline $6 / 28 / 920: 00$ & Manual Tape & 412.5 & Good & \\
\hline $6 / 28 / 927: 06$ & Auto FIC & 412.20 & Good & \\
\hline $6 / 29 / 920: 00$ & Manual Tape & 412.25 & Good & \\
\hline $6 / 29 / 92$ 7:06 & Auto FIC & 412.4 & Good & \\
\hline $6 / 30 / 920: 00$ & Manual Tape & 412.5 & Good & \\
\hline $6 / 30 / 92$ 7:06 & Auto FIC & 412.40 & Good & \\
\hline $7 / 1 / 920: 00$ & Manual FIC & 412.4 & Good & \\
\hline $7 / 1 / 920: 00$ & Manual Tape & 412.5 & Good & \\
\hline $7 / 2 / 920: 00$ & Manual FIC & 412.4 & Good & \\
\hline $7 / 2 / 920: 00$ & Manual Tape & 412.25 & Good & \\
\hline $7 / 3 / 92 \quad 0: 00$ & Manual Tape & 412.25 & Good & \\
\hline $7 / 3 / 927: 06$ & Auto FIC & 412.40 & Good & \\
\hline $7 / 4 / 920: 00$ & Manual Tape & 41.2 .25 & Good & \\
\hline $7 / 4 / 927: 06$ & Auto FIC & 412.40 & Good & \\
\hline $7 / 5 / 92 \quad 0: 00$ & Manual Tape & 412.25 & Good & \\
\hline $7 / 5 / 927: 06$ & Auto FIC & 412.40 & Good & \\
\hline
\end{tabular}

Data obtained from WHC Surveiliance Analysis Computer System, (PC SACS) July 7, 1995. 


\begin{tabular}{|c|c|c|c|c|}
\hline \multicolumn{5}{|c|}{ 241-AP-103 } \\
\hline \multicolumn{5}{|c|}{ Surface Level Data } \\
\hline Date & Type of Measurement & Level (Inches) & Data Quality & Comments \\
\hline $7 / 6 / 920: 00$ & Manual Tape & 412.25 & Good & \\
\hline $7 / 6 / 927: 06$ & Auto FIC & 412.4 & Good & \\
\hline $7 / 7 / 920: 00$ & Manual Tape & 412.25 & Good & \\
\hline $7 / 7 / 927: 06$ & Auto FIC & 412.40 & Good & \\
\hline $7 / 8 / 920: 00$ & Manual Tape & 412.25 & Good & \\
\hline $7 / 8 / 927: 06$ & Auto FIC & 412.4 & Good & \\
\hline $7 / 9 / 920: 00$ & Manual Tape & 412.25 & Good & \\
\hline 7/9/92 7:06 & Auto FIC & 412.40 & Good & \\
\hline $7 / 10 / 920: 00$ & Manual Tape & 412.25 & Good & \\
\hline $7 / 10 / 927: 06$ & Auto FIC & 412.40 & Good & \\
\hline 7/11/92 0:00 & Manual Tape & 412.25 & Good & \\
\hline 7/11/92 7:06 & Auto FIC & 412.40 & Good & \\
\hline $7 / 12 / 920: 00$ & Manual Tape & 412.25 & Good & \\
\hline $7 / 12 / 927: 06$ & Auto FIC & 412.4 & Good & \\
\hline $7 / 13 / 920: 00$ & Manual Tape & 412.25 & Good & \\
\hline $7 / 13 / 927: 06$ & Auto FIC & 412.4 & Good & \\
\hline $7 / 14 / 920: 00$ & Manual Tape & 412.25 & Good & \\
\hline $7 / 14 / 92 \quad 7: 06$ & Auto FIC & 412.4 & Good & \\
\hline $7 / 15 / 920: 00$ & Manual Tape & 412.25 & Good & \\
\hline $7 / 15 / 927: 06$ & Auto FIC & 412.40 & Good & \\
\hline $7 / 16 / 920: 00$ & Manual Tape & 412.25 & Good & \\
\hline $7 / 16 / 927: 06$ & Auto FIC & 412.4 & Good & \\
\hline $7 / 17 / 920: 00$ & Manual Tape & 412.25 & Good & \\
\hline $7 / 17 / 927: 06$ & Auto FIC & 41,$2 ; 40$ & Good & \\
\hline $7 / 18 / 920: 00$ & Manual Tape & 412.25 & Good & \\
\hline 7/18/92 7:06 & Auto FIC & 412.40 & Good & \\
\hline 7/19/92 0:00 & Manual Tape & 412.25 & Good & \\
\hline $7 / 19 / 927: 06$ & Auto FIC & 412.4 & Good & \\
\hline $7 / 20 / 920: 00$ & Manual Tape & 412.25 & Good & \\
\hline $7 / 20 / 927: 06$ & Auto FIC & 412.40 & Good & \\
\hline $7 / 21 / 920: 00$ & Manual Tape & 412.25 & Good & \\
\hline $7 / 21 / 927: 05$ & Auto FIC & 412.40 & Good & \\
\hline $7 / 22 / 920: 00$ & Manual Tape & 412.25 & Good & \\
\hline $7 / 22 / 92$ 7:06 & Auto FIC & 412.4 & Good & \\
\hline $7 / 23 / 920: 00$ & Manual FIC & 412.4 & Transcribed & \\
\hline $7 / 23 / 920: 00$ & Manual Tape & 412.25 & Good & \\
\hline $7 / 24 / 920: 00$ & Manual Tape & 412.25 & Good & \\
\hline $7 / 24 / 927: 05$ & Auto FIC & 412.40 & Good & \\
\hline $7 / 25 / 920: 00$ & Manual Tape & 412.25 & Good & \\
\hline $7 / 25 / 927: 06$ & Auto FIC & 412.40 & Good & \\
\hline $7 / 26 / 920: 00$ & Manual Tape & 412.25 & Good & \\
\hline $7 / 26 / 927: 06$ & Auto FIC & 412.4 & Good & \\
\hline $7 / 27 / 920: 00$ & Manual Tape & 412.25 & Good & \\
\hline $7 / 27 / 927: 05$ & Auto FIC & 412.40 & Good & \\
\hline $7 / 28 / 92 \quad 0: 00$ & Manual Tape & 412.25 & Good & \\
\hline $7 / 28 / 927: 06$ & Auto FIC & 412.4 & Good & \\
\hline $7 / 29 / 92 \quad 0: 00$ & Manual Tape & 412.25 & Good & \\
\hline $7 / 29 / 927: 06$ & Auto FIC & 412.4 & Good & \\
\hline
\end{tabular}

Data obtained from WHC Surveillance Analysis Computer System, (PC SACS) July 7, 1995. 


\begin{tabular}{|c|c|c|c|c|}
\hline \multicolumn{5}{|c|}{ 241-AP-103 } \\
\hline \multicolumn{5}{|c|}{ Surface Lovel Data } \\
\hline Date & Type of Measurement & Level (Inches) & Data Quality & Comments \\
\hline $7 / 30 / 920: 00$ & Manual Tape & 412.25 & Good & \\
\hline $7 / 30 / 927: 06$ & Auto FIC & 412.40 & Good & \\
\hline $7 / 31 / 920: 00$ & Manual Tape & 412.25 & Good & \\
\hline $7 / 31 / 927: 05$ & Auto FIC & 412.4 & Good & \\
\hline $8 / 1 / 920: 00$ & Manual Tape & .412 .25 & Good & \\
\hline $8 / 1 / 927: 06$ & Auto FIC & 412.4 & Good & \\
\hline $8 / 2 / 920: 00$ & Manual Tape & 412.25 & Good & \\
\hline $8 / 2 / 927: 06$ & Auto FIC & 412.4 & Good & \\
\hline $8 / 3 / 920: 00$ & Manual Tape & 412.25 & Good & \\
\hline $8 / 3 / 927: 06$ & Auto FIC & 412.40 & Good & \\
\hline $8 / 4 / 920: 00$ & Manual Tape & 412.25 & Good & \\
\hline $8 / 4 / 927: 06$ & Auto FIC & 412.4 & Good & \\
\hline $8 / 5 / 920: 00$ & Manual Tape & 412.25 & Good & \\
\hline $8 / 5 / 927: 05$ & Auto FIC & 412.40 & Good & \\
\hline $8 / 6 / 920: 00$ & Manual Tape & 412 & Good & \\
\hline $8 / 6 / 927: 05$ & Auto FIC & 412.4 & Good & \\
\hline $8 / 7 / 920: 00$ & Manual Tape & 412 & Good & \\
\hline $8 / 7 / 927: 05$ & Auto FIC & 412.4 & Good & \\
\hline $8 / 8 / 920: 00$ & Manual Tape & 412.00 & Good & \\
\hline $8 / 8 / 927: 06$ & Auto FIC & 412.40 & Good & \\
\hline $8 / 9 / 92 \quad 0: 00$ & Manual Tape & 412.25 & Good & \\
\hline $8 / 9 / 927: 06$ & Auto FIC & 412.4 & Good & \\
\hline $8 / 10 / 920: 00$ & Manual Tape & 412.25 . & Good & \\
\hline $8 / 10 / 927: 05$ & Auto FIC & 412.4 & Good & \\
\hline $8 / 11 / 920: 00$ & Manual Tape & 412.25 & Good & \\
\hline $8 / 11 / 927: 06$ & Auto FIC & 412.40 & Good & \\
\hline $8 / 12 / 920: 00$ & Manual Tape & 412 & Good & \\
\hline $8 / 12 / 927: 06$ & Auto FIC & 412.4 & Good & \\
\hline $8 / 13 / 920: 00$ & Manual Tape & 412 & Good & \\
\hline $8 / 13 / 927: 06$ & Auto FIC & 412.4 & Good & $\therefore$ \\
\hline $8 / 14 / 920: 00$ & Manual Tape & 412.25 & Good & \\
\hline $8 / 14 / 927: 06$ & Auto FIC & 412.4 & Good & \\
\hline $8 / 15 / 920: 00$ & Manual Tape & 412 & Good & \\
\hline $8 / 15 / 927: 06$ & Auto FIC & 412.40 & Good & \\
\hline $8 / 16 / 920: 00$ & Manual Tape & 412.00 & Good & \\
\hline $8 / 16 / 927: 06$ & Auto FIC & 412.40 & Good & \\
\hline $8 / 17 / 920: 00$ & Manual Tape & 412.00 & Good & \\
\hline $8 / 17 / 927: 06$ & Auto FIC & $41,2: 40$ & Good & \\
\hline $8 / 18 / 920: 00$ & Manual Tape & 412.25 & Good & \\
\hline $8 / 18 / 927: 06$ & Auto FIC & 0 & Suspect & Data not included in graph \\
\hline $8 / 19 / 920: 00$ & Manual Tape & 412.25 & Good & \\
\hline $8 / 19 / 927: 06$ & Auto FIC & 0 & Suspect & Data not included in graph \\
\hline $8 / 20 / 920: 00$ & Manual Tape & 412.00 & Good & \\
\hline $8 / 20 / 927: 06$ & Auto FIC & 0 & Suspect & Data not included in graph \\
\hline $8 / 21 / 920: 00$ & Manual Tape & 412 & Good & \\
\hline $8 / 21 / 927: 05$ & Auto FIC & 0 & Suspect & Data not included in graph \\
\hline $8 / 22 / 920: 00$ & Manusl FIC & 412.40 & Good & \\
\hline $8 / 22 / 920: 00$ & Manual Tape & 412 & Good & \\
\hline
\end{tabular}

Data obtained from WHC Surveillance Analysis Computer System, (PC SACS) July 7, 1995. 


\begin{tabular}{|c|c|c|c|c|}
\hline \multicolumn{5}{|c|}{ 241-AP-103 } \\
\hline \multicolumn{5}{|c|}{ Surface Level Data } \\
\hline Date & Type of Measurement & Lovel (Inches) & Data Cuality & Comments \\
\hline $8 / 22 / 927: 04$ & Auto FIC & 0 & Suspect & Data not included in graph \\
\hline $8 / 23 / 920: 00$ & Manual Tape & 412 & Good & \\
\hline $8 / 23 / 927: 04$ & Auto FIC & 412.40 & Good & \\
\hline $8 / 24 / 920: 00$ & Manual Tape & 412.25 & Good & \\
\hline $8 / 24 / 927: 04$ & Auto FIC & 412.40 & Good & \\
\hline $8 / 25 / 920: 00$ & Manual Tape & 412.00 & Good & \\
\hline $8 / 25 / 927: 04$ & Auto FIC & 412.40 & Good & \\
\hline $8 / 26 / 920: 00$ & Manual Tape & 412 & Good & \\
\hline $8 / 26 / 927: 03$ & Auto FIC & 412.4 & Good & \\
\hline $8 / 27 / 920: 00$ & Manual Tape & 412.25 & Good & \\
\hline $8 / 27 / 927: 04$ & Auto FIC & 412.4 & Good & \\
\hline $8 / 28 / 920: 00$ & Manual Tape & 412.75 & Good & \\
\hline $8 / 28 / 927: 02$ & Auto FIC & 412.4 & Good & \\
\hline $8 / 29 / 920: 00$ & Manual Tape & 412.25 & Good & \\
\hline $8 / 29 / 927: 02$ & Auto FIC & 412.40 & Good & \\
\hline $8 / 30 / 920: 00$ & Manual Tape & 412.25 & Good & \\
\hline $8 / 30 / 927: 02$ & Auto FIC & 412.4 & Good & \\
\hline $8 / 31 / 920: 00$ & Manual Tape & 412.25 & Good & \\
\hline $8 / 31 / 927: 02$ & Auto FIC & 412.40 & Good & \\
\hline $9 / 1 / 920: 00$ & Manual Tape & 412.25 & Good & \\
\hline $9 / 1 / 927: 02$ & Auto FIC & 412.40 & Good & \\
\hline $9 / 2 / 92 \quad 0: 00$ & Manual Tape & 412.25 & Good & \\
\hline $9 / 2 / 927: 02$ & Auto FIC & 412.4 & Good & \\
\hline $9 / 3 / 920: 00$ & Manual Tape & 412.00 & Good & \\
\hline $9 / 3 / 927: 04$ & Auto FIC & 412.4 & Good & \\
\hline 9/4/92 0:00 & Manual Tape & 412.25 & Good & \\
\hline $9 / 4 / 927: 04$ & Auto FIC & 412.40 & Good & \\
\hline $9 / 5 / 92 \quad 0: 00$ & Manual Tape & 412.25 & Good & \\
\hline $9 / 5 / 927: 04$ & Auto FIC & 412.4 & Good & \\
\hline $9 / 6 / 920: 00$ & Manual Tape & 412.25 & Good & \\
\hline $9 / 6 / 927: 04$ & Auto FIC & 412.40 & Good & \\
\hline $9 / 7 / 920: 00$ & Manual Tape & 412.25 & Good & \\
\hline $9 / 7 / 927: 05$ & Auto FIC & 412.4 & Good & \\
\hline $9 / 8 / 92 \quad 0: 00$ & Manual Tape & 412.25 & Good & \\
\hline 9/8/92 7:05 & Auto FIC & 412.4 & Good & \\
\hline $9 / 9 / 920: 00$ & Manual Tape & 412.25 & Good & \\
\hline 9/9/92 7:04 & Auto FIC & 412.40 & Good & \\
\hline $9 / 10 / 920: 00$ & Manual Tape & 412.25 & Good & \\
\hline $9 / 10 / 927: 03$ & Auto FIC & 412.4 & Good & \\
\hline $9 / 11 / 920: 00$ & Manual FIC & 412.4 & Good & \\
\hline $9 / 11 / 920: 00$ & Manual Tape & 412.25 & Good & \\
\hline $9 / 12 / 920: 00$ & Manual FIC & 412.40 & Good & \\
\hline $9 / 12 / 920: 00$ & Manual Tape & 412.25 & Good & \\
\hline 9/13/92 0:00 & Manual FIC & 412.4 & Good & \\
\hline $9 / 13 / 920: 00$ & Manual Tape & 412.25 & Good & \\
\hline $9 / 14 / 920: 00$ & Manual FIC & 412.40 & Good & \\
\hline 9/14/92 0:00 & Manual Tape & 412.25 & Good & \\
\hline $9 / 15 / 920: 00$ & Manual FIC & 412.40 & Good & \\
\hline
\end{tabular}

Data obtained from WHC Surveillance Analysis Computer System, (PC SACS) July 7, 1995. 


\begin{tabular}{|c|c|c|c|c|}
\hline \multicolumn{5}{|c|}{ 241-AP-103 } \\
\hline Surface Level. Data & Type of Measurement & Level (Inches) & Data Quality & Comments \\
\hline$\frac{\text { Date }}{9 / 15 / 920: 00}$ & Manual Tape & 412.25 & Good & \\
\hline $\begin{array}{l}9 / 15 / 920: 00 \\
9 / 16 / 920: 00\end{array}$ & Manual FIC & 412.4 & Good & \\
\hline $\begin{array}{l}9 / 16 / 920: 00 \\
9 / 16 / 920: 00\end{array}$ & Manual Tape & 412.25 & Good & \\
\hline $\begin{array}{l}9 / 16 / 92 \quad 0: 00 \\
9 / 17 / 920: 00\end{array}$ & Manual Tape & 412 & Good & \\
\hline $\begin{array}{l}9 / 17 / 920: 00 \\
9 / 17 / 927: 06\end{array}$ & Auto FIC & 412.4 & Good & \\
\hline $\begin{array}{l}9 / 17 / 92 \quad 7: 06 \\
9 / 18 / 92 \quad 0: 00\end{array}$ & Manual Tape & 412.00 & Good & \\
\hline $\begin{array}{l}9 / 18 / 920: 00 \\
9 / 18 / 927: 06\end{array}$ & Auto FIC & 412.40 & Good & \\
\hline $\begin{array}{ll}9 / 19 / 92 & 0: 00 \\
9 / 20 / 92 & 0: 00\end{array}$ & Manual FIC & 412.4 & Good & \\
\hline $\begin{array}{l}9 / 20 / 920: 00 \\
9 / 20 / 920: 00\end{array}$ & Manual Tape & 412.00 & Good & \\
\hline $9 / 21 / 920: 00$ & Manual Tape & 412.00 & Good & \\
\hline $9 / 21 / 927: 06$ & Auto FIC & 412.4 & Good & \\
\hline $9 / 22 / 920: 00$ & Manual Tape & 412 & Good & \\
\hline $9 / 22 / 927: 03$ & Auto FIC & 412.40 & Good & \\
\hline $9 / 23 / 920: 00$ & Manual Tape & 412.25 & Good & \\
\hline 9/23/92 7:03 & Auto FIC & 412.4 & Good & \\
\hline 9/24/92 0:00 & Manual Tape & 412.25 & Good & \\
\hline $9 / 24 / 927: 03$ & Auto FIC & 412.40 & Good & \\
\hline $9 / 25 / 920: 00$ & Manual Tape & 412 & Good & \\
\hline $9 / 25 / 927: 03$ & Auto FIC & 412.4 & Good & \\
\hline $9 / 26 / 920: 00$ & Manual Tape & 412.25 & Good & \\
\hline 9/26/92 7:02 & Auto FIC & 412.4 & Good & \\
\hline $9 / 27 / 920: 00$ & Manual Tape & 412.25 & Good & \\
\hline 9/27/92 7:02 & Auto FIC & 412.40 & Good & \\
\hline $9 / 28 / 920: 00$ & Manual Tape & 412.25 & Good & \\
\hline $9 / 28 / 927: 02$ & Auto FIC & 412.4 & Good & \\
\hline $9 / 29 / 920: 00$ & Manual Tape & 412.25 & Good & \\
\hline $9 / 29 / 927: 03$ & Auto FIC & 412.5 & Good & \\
\hline $9 / 30 / 920: 00$ & Manual Tape & 412.25 & Good & \\
\hline 9/30/92 7:02 & Auto FIC & 412.40 & Good & \\
\hline 10/1/92 0:00 & Manual Tape & 412.25 & Good & \\
\hline $10 / 1 / 927: 03$ & Auto FIC & 412.4 & Good & \\
\hline 10/2/92 0:00 & Manual Tape & 412.00 & Good & \\
\hline $10 / 2 / 927: 02$ & Auto FIC & 412.50 & Good & \\
\hline $10 / 3 / 920: 00$ & Manual Tape & 412 & Good & \\
\hline $10 / 3 / 927: 03$ & Auto FIC & 412.5 & Good & \\
\hline $10 / 4 / 920: 00$ & Manual Tape & 412.00 & Good & \\
\hline $10 / 4 / 927: 03$ & Auto FIC & 412.4 & Good & \\
\hline $10 / 5 / 920: 00$ & Manual Tape & 412.25 & Good & \\
\hline $10 / 5 / 927: 03$ & Auto FIC & 412.50 & Good & \\
\hline $10 / 6 / 920: 00$ & Manual Tape & 412.25 & Good & \\
\hline $10 / 6 / 927: 03$ & Auto FIC & 412.50 & Good & \\
\hline $10 / 7 / 920: 00$ & Manual FIC & 412.50 & Good & \\
\hline $10 / 7 / 92 \quad 0: 00$ & Manual Tape & 412.00 & Good & \\
\hline 10/8/92 0:00 & Manual Tape & 412 & Good & \\
\hline $10 / 8 / 927: 02$ & Auto FIC & 412.4 & Good & \\
\hline 10/9/92 0:00 & Manual Tape & 412 & Good & \\
\hline
\end{tabular}

Data obtained from WHC Surveillance Analysis Computer System, (PC SACS) July 7, 1995. 


\begin{tabular}{|c|c|c|c|c|}
\hline \multicolumn{5}{|c|}{ 241-AP-103 } \\
\hline & & urface Level Dat & & \\
\hline Date & Type of Measurement & Level (Inches) & Data Quality & Comments \\
\hline $10 / 9 / 927: 03$ & Auto FIC & 412.50 & Good & \\
\hline $10 / 10 / 920: 00$ & Manual Tape & 41.2 .25 & Good & \\
\hline 10/10/92 7:03 & Auto FIC & 412.4 & Good & \\
\hline $10 / 11 / 920: 00$ & Manual Tape & 412.25 & Good & \\
\hline $10 / 11 / 927: 03$ & Auto FIC & 412.4 & Good & \\
\hline $10 / 12 / 920: 00$ & Manual FIC & 412.4 & Good & \\
\hline $10 / 12 / 920: 00$ & Manual Tape & 412 & Good & \\
\hline $10 / 13 / 920: 00$ & Manual FIC & 412.50 & Good & \\
\hline $10 / 13 / 920: 00$ & Manual Tape & 412.00 & Good & \\
\hline $10 / 14 / 920: 00$ & Manual Tape & 412.00 & Good & \\
\hline 10/14/92 7:03 & Auto FIC & 412.4 & Good & \\
\hline $10 / 15 / 920: 00$ & Manual Tape & 412.00 & Good & \\
\hline $10 / 15 / 927: 04$ & Auto FIC & 412.4 & Good & \\
\hline $10 / 16 / 920: 00$ & Manual Tape & 412.00 & Good & \\
\hline $10 / 16 / 927: 03$ & Auto FIC & 412.40 & Good & \\
\hline $10 / 17 / 920: 00$ & Manual Tape & 412 & Good & \\
\hline $10 / 17 / 927: 03$ & Auto FIC & 412.40 & Good & \\
\hline $10 / 18 / 920: 00$ & Manual Tape & 412.00 & Good & \\
\hline $10 / 18 / 927: 03$ & Auto FIC & 412.4 & Good & \\
\hline $10 / 19 / 920: 00$ & Manual Tape & 412 & Good & \\
\hline $10 / 19 / 927: 03$ & Auto FIC & 412.40 & Good & \\
\hline $10 / 20 / 920: 00$ & Manual Tape & 412.00 & Good & \\
\hline $10 / 20 / 927: 03$ & Auto FIC & 412.4 & Good & \\
\hline $10 / 21 / 920: 00$ & Manual Tape & 412.00 & Good & \\
\hline $10 / 21 / 927: 03$ & Auto FIC & 412.40 & Good & \\
\hline $10 / 22 / 920: 00$ & Manual FIC & 412.40 & Good & \\
\hline $10 / 22 / 920: 00$ & Manual Tape & 412 & Good & \\
\hline $10 / 23 / 920: 00$ & Manual FIC & 412.40 & Good & \\
\hline $10 / 23 / 920: 00$ & Manual Tape & 412.00 & Good & \\
\hline $10 / 24 / 920: 00$ & Manual Tape & 412 & Good & \\
\hline $10 / 24 / 927: 03$ & Auto FIC & 412.30 & Good & \\
\hline $10 / 25 / 92 \quad 0: 00$ & Manual Tape & 412.00 & Good & \\
\hline $10 / 25 / 927: 03$ & Auto FIC & 412.30 & Good & \\
\hline $10 / 26 / 920: 00$ & Manual FIC & 412.30 & Good & \\
\hline $10 / 26 / 920: 00$ & Manual Tape & 412.00 & Good & \\
\hline $10 / 27 / 920: 00$ & Manual Tape & 412 & Good & \\
\hline $10 / 27 / 927: 04$ & Auto FIC & 412.30 & Good & \\
\hline $10 / 28 / 920: 00$ & Manual Tape & 412 & Good & \\
\hline $10 / 28 / 927: 03$ & Auto FIC & 412.3 & Good & \\
\hline $10 / 29 / 92 \quad 0: 00$ & Manual Tape & 412 & Good & \\
\hline $10 / 29 / 927: 03$ & Auto FIC & 412.3 & Good & \\
\hline $10 / 30 / 920: 00$ & Manual Tape & 412.00 & Good & \\
\hline $10 / 30 / 927: 03$ & Auto FIC & 412.3 & Good & \\
\hline $10 / 31 / 920: 00$ & Manual Tape & 412 & Good & \\
\hline $10 / 31 / 927: 03$ & Auto FIC & 412.3 & Good & \\
\hline $11 / 1 / 920: 00$ & Manual Tape & 412.00 & Good & \\
\hline $11 / 1 / 927: 03$ & Auto FIC & 412.30 & Good & \\
\hline $11 / 2 / 920: 00$ & Manual Tape & 412.00 & Good & \\
\hline
\end{tabular}

Data obtained from WHC Surveillance Analysis Computer System, (PC SACS) July 7, 1995. 


\begin{tabular}{|c|c|c|c|c|}
\hline \multicolumn{5}{|c|}{ 241-AP-103 } \\
\hline \multicolumn{5}{|c|}{ Surface Level Data } \\
\hline Date & Type of Measurement & Level (Inches) & Data Quality & Comments \\
\hline $11 / 2 / 927: 03$ & Auto FIC & $412.30^{\circ}$ & Good & \\
\hline $11 / 3 / 920: 00$ & Manual Tape & 412 & Good & \\
\hline $11 / 3 / 927: 03$ & Auto FIC & 412.30 & Good & \\
\hline $11 / 4 / 92 \quad 0: 00$ & Manual Tape & 412.00 & Good & \\
\hline $11 / 4 / 927: 03$ & Auto FIC & 412.30 & Good & \\
\hline $11 / 5 / 920: 00$ & Manual FIC & 412.30 & Good & \\
\hline $11 / 5 / 920: 00$ & Manual Tape & 412 & Good & \\
\hline $11 / 6 / 920: 00$ & Manual FIC & 412.3 & Good & \\
\hline $11 / 6 / 920: 00$ & Manual Tape & 412 & Good & \\
\hline $11 / 7 / 920: 00$ & Manual FIC & 412.3 & Good & \\
\hline $11 / 7 / 920: 00$ & Manual Tape & 412.00 & Good & \\
\hline $11 / 8 / 920: 00$ & Manual FIC & 412.35 & Good & \\
\hline $11 / 8 / 920: 00$ & Manual Tope & 412 & Good & \\
\hline $11 / 9 / 920: 00$ & Manual FIC & 412.3 & Good & \\
\hline $11 / 9 / 920: 00$ & Manual Tape & 412 & Good & \\
\hline $11 / 10 / 920: 00$ & Manual Tape & 412.00 & Good & \\
\hline $11 / 10 / 927: 03$ & Auto FIC & 412.30 & Good & \\
\hline $11 / 11 / 920: 00$ & Manual Tape & 412.25 & Good & \\
\hline $11 / 11 / 927: 04$ & Auto FIC & 412.3 & Good & \\
\hline $11 / 12 / 920: 00$ & Manual Tape & 412.25 & Good & \\
\hline $11 / 12 / 927: 03$ & Auto FIC & 412.3 & Good & \\
\hline $11 / 13 / 920: 00$ & Manual Tape & 412.25 & Good & \\
\hline $11 / 13 / 927: 04$ & Auto FIC & 412.3 & Good & \\
\hline $11 / 14 / 920: 00$ & Manual Tape & 412.25 & Good & \\
\hline $11 / 14 / 927: 04$ & Auto FIC & 412.3 & Good & \\
\hline $11 / 15 / 920: 00$ & Manual Tape & 412 & Good & \\
\hline $11 / 15 / 927: 04$ & Auto FIC & 412.30 & Good & \\
\hline $11 / 16 / 920: 00$ & Manual Tape & 412 & Good & \\
\hline $11 / 16 / 927: 04$ & Auto FIC & 412.30 & Good & \\
\hline $11 / 17 / 920: 00$ & Manual Tape & 411.75 & Good & \\
\hline $11 / 17 / 927: 04$ & Auto FIC & 412.30 & Good & \\
\hline $11 / 18 / 920: 00$ & Manual Tape & 412 & Good & \\
\hline $11 / 18 / 927: 02$ & Auto FIC & 412.30 & Good & \\
\hline $11 / 19 / 920: 00$ & Manual Tape & 412.00 & Good & \\
\hline $11 / 19 / 927: 02$ & Auto FIC & 412.3 & Good & \\
\hline $11 / 20 / 920: 00$ & Manual Tape & 412.25 & Good & \\
\hline $11 / 20 / 927: 03$ & Auto FIC & 412.3 & Good & \\
\hline $11 / 21 / 920: 00$ & Manual Tape & 412 & Good & \\
\hline $11 / 21 / 927: 02$ & Auto FIC & 412.3 & Good & \\
\hline $11 / 22 / 920: 00$ & Manual Tape & 412.25 & Good & \\
\hline $11 / 22 / 927: 03$ & Auto FIC & 412.30 & Good & \\
\hline $11 / 23 / 920: 00$ & Manual Tape & 412.25 & Good & \\
\hline $11 / 23 / 927: 03$ & Auto FIC & 412.30 & Good & \\
\hline $11 / 24 / 920: 00$ & Manual Tape & 412 & Good & \\
\hline $11 / 24 / 927: 03$ & Auto FIC & 412.30 & Good & \\
\hline $11 / 25 / 920: 00$ & Manual Tape & 412 & Good & \\
\hline $11 / 25 / 927: 03$ & Auto FIC & 412.30 & Good & \\
\hline $11 / 26 / 920: 00$ & Manual Tape & 412.00 & Good & \\
\hline
\end{tabular}

Data obtained from WHC Surveillance Analysis Computer System, (PC SACS) July 7, 1995. 


\begin{tabular}{|c|c|c|c|c|}
\hline \multicolumn{4}{|c|}{ 241-AP-103 } & \\
\hline Surface Level Data & Type of Measurement & Level (Inches) & Data Quality & Comments \\
\hline$\frac{\text { Date }}{11 / 26 / 927: 03}$ & Auto FIC & 412.3 & Good & \\
\hline $\begin{array}{l}11 / 26 / 927: 03 \\
11 / 27 / 920: 00\end{array}$ & Manual Tape & 412 & Good & \\
\hline$\frac{11 / 27 / 920: 00}{11 / 27 / 927: 03}$ & Auto FIC & 412.3 & Good & \\
\hline$\frac{11 / 27 / 927: 03}{11 / 28 / 920: 00}$ & Manual Tape & 41.2 .00 & Good & \\
\hline $\begin{array}{l}11 / 28 / 920: 00 \\
11 / 28 / 927: 03\end{array}$ & Auto FIC & $\cdot 412.30$ & Good & \\
\hline$\frac{11 / 28 / 927: 03}{11 / 29 / 920: 00}$ & Manual Tape & 412 & Good & \\
\hline $\begin{array}{l}11 / 29 / 920: 00 \\
11 / 29 / 92 \quad 7: 03\end{array}$ & Auto FIC & 412.3 & Good & \\
\hline $\begin{array}{l}11 / 29 / 92 \quad 7: 03 \\
11 / 30 / 920: 00\end{array}$ & Manual FIC & 412.30 & Good & \\
\hline $\begin{array}{l}11 / 30 / 920: 00 \\
11 / 30 / 920: 00\end{array}$ & Manual Tape & 412 & Good & \\
\hline$\frac{11 / 30 / 920: 00}{12 / 1 / 920: 00}$ & Manual Tape & 412.00 & Good & \\
\hline $\begin{array}{l}12 / 1 / 920: 00 \\
12 / 1 / 927: 03\end{array}$ & Auto FIC & 412.30 & Good & \\
\hline 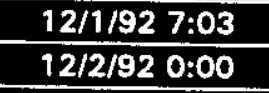 & Manual Tape & 412 & Good & \\
\hline 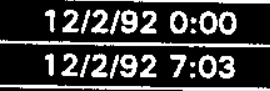 & Auto FIC & 412.30 & Good & \\
\hline$\frac{12 / 2 / 92 \quad 7: 03}{12 / 3 / 920: 00}$ & Manual Tape & 412.00 & Good & \\
\hline$\frac{12 / 3 / 920: 00}{12 / 3 / 927: 03}$ & Auto FIC & 412.3 & Good & \\
\hline$\frac{12 / 3 / 927: 03}{12 / 4 / 920: 00}$ & Manual Tape & 412.00 & Good & \\
\hline $\begin{array}{l}12 / 4 / 920: 00 \\
12 / 4 / 92 \quad 7: 03\end{array}$ & Auto FIC & 412.20 & Good & \\
\hline$\frac{12 / 4 / 927: 03}{12 / 5 / 92 \quad 0: 00}$ & Manual FIC & 412.30 & Good & \\
\hline$\frac{12 / 5 / 92}{12 / 5 / 92} 0: 000$ & Manual Tape & 412.00 & Good & \\
\hline$\frac{12 / 5 / 92}{12 / 6 / 92} 0: 000$ & Manual Tape & 412.25 & Good & \\
\hline $\begin{array}{ll}12 / 6 / 92 & 0: 00 \\
12 / 6 / 92 & 7: 02\end{array}$ & Auto FIC & 412.20 & Good & \\
\hline $\begin{array}{ll}12 / 6 / 92 & 7: 02 \\
12 / 7 / 92 & 0: 00\end{array}$ & Manual Tape & 412 & Good & \\
\hline $\begin{array}{l}12 / 7 / 920: 00 \\
12 / 7 / 92 \quad 7: 03\end{array}$ & Auto FIC & 412.3 & Good & \\
\hline$\frac{12 / 7 / 92 \quad 7: 03}{12 / 8 / 92 \quad 0: 00}$ & Manual Tape & 412 & Good & \\
\hline $12 / 8 / 927: 03$ & Auto FIC & 412.20 & Good & \\
\hline $12 / 9 / 920: 00$ & Manual Tape & 412 & Good & \\
\hline $12 / 9 / 927: 03$ & Auto FIC & 412.3 & Good & \\
\hline $12 / 10 / 920: 00$ & Manual Tape & 412 & Good & \\
\hline $12 / 10 / 927: 03$ & Auto FIC & 412.30 & Good & \\
\hline $12 / 11 / 920: 00$ & Manual Tape & 412 & Good & \\
\hline $12 / 11 / 927: 03$ & Auto FIC & 412.20 & Good & \\
\hline $12 / 12 / 920: 00$ & Manual FIC & 412.20 & Good & \\
\hline $12 / 12 / 920: 00$ & Manual Tape & 412.00 & Good & \\
\hline $12 / 13 / 920: 00$ & Manual Tape & 412.00 & Good & \\
\hline $12 / 13 / 927: 04$ & Auto FIC & 412.2 & Good & \\
\hline $12 / 14 / 920: 00$ & Manual Tape & 412.00 & Good & \\
\hline $12 / 14 / 927: 03$ & Auto FIC & 412.20 & Good & \\
\hline $12 / 15 / 920: 00$ & Manual Tape & 411.75 & Good & \\
\hline $12 / 15 / 925: 03$ & Auto FIC & 412.2 & Good & \\
\hline $12 / 16 / 920: 00$ & Manual Tape & 411.75 & Good & \\
\hline $12 / 16 / 927: 04$ & Auto FIC & 0 & Suspect & Data not included in graph \\
\hline $12 / 17 / 920: 00$ & Manual Tape & 412.00 & Good & \\
\hline $12 / 17 / 927: 03$ & Auto FIC & 0.00 & Suspect & Data not included in graph \\
\hline $12 / 18 / 920: 00$ & Manual Tape & 412 & Good & \\
\hline $12 / 18 / 927: 03$ & Auto FIC & 0 & Suspect & Data not included in graph \\
\hline $12 / 19 / 920: 00$ & Manual Tape & 411.75 & Good & \\
\hline $12 / 19 / 927: 03$ & Auto FIC & 0.00 & Suspect & Data not included in graph \\
\hline $12 / 20 / 920: 00$ & Manual Tape & 412 & Good & \\
\hline
\end{tabular}

Data obtained from WHC Surveillance Analysis Computer System, (PC SACS) July 7. 1995. 
WHC-SD-WM-ER-315, Rev. 0

\begin{tabular}{|c|c|c|c|c|}
\hline \multicolumn{5}{|c|}{ 241-AP-103 } \\
\hline \multicolumn{5}{|c|}{ Surface Level Data } \\
\hline Date & Type of Measurement & Level (inches) & Data Quality & Comments \\
\hline $12 / 20 / 927: 04$ & Auto FIC & 0.00 & Suspect & Data not included in graph \\
\hline $12 / 21 / 920: 00$ & Manual Tape & 412 & Good & \\
\hline 12/21/92 7:04 & Auto FIC & 0 & Suspect & Data not included in graph \\
\hline $12 / 22 / 920: 00$ & Manual Tape & 412.00 & Good & \\
\hline $12 / 23 / 920: 00$ & Manual Tape . & 412 & Good & \\
\hline $12 / 23 / 927: 04$ & Auto FIC & 0.00 & Suspect & Data not included in graph \\
\hline $12 / 24 / 920: 00$ & Manual Tape & 412 & Good & \\
\hline $12 / 24 / 927: 03$ & Auto FIC & 0.00 & Suspect & Data not included in graph \\
\hline $12 / 25 / 920: 00$ & Manual Tape & 412 & Good & \\
\hline $12 / 25 / 927: 03$ & Auto FIC & 0 & Suspect & Data not included in graph \\
\hline $12 / 26 / 920: 00$ & Manual Tape & 411.75 & Good & \\
\hline $12 / 26 / 927: 03$ & Auto FIC & 0.00 & Suspect & Data not included in graph \\
\hline $12 / 27 / 920: 00$ & Manual Tape & 412.50 & Good & \\
\hline $12 / 27 / 927: 03$ & Auto FIC & 0 & Suspect & Data not included in graph \\
\hline $12 / 28 / 920: 00$ & Manual Tape & 412.00 & Good & \\
\hline $12 / 28 / 927: 03$ & Auto FIC & 0 & Suspect & Data not included in graph \\
\hline $12 / 29 / 920: 00$ & Manual Tape & 412.00 & Good & \\
\hline $12 / 30 / 920: 00$ & Manual Tape & 41.2 .00 & Good & \\
\hline $12 / 30 / 927: 03$ & Auto FIC & 0.00 & Suspect & Data not included in graph \\
\hline $12 / 31 / 920: 00$ & Manual Tape & 412 & Good & \\
\hline 12/31/92 7:03 & Auto FIC & 0.00 & Suspect & Data not included in graph \\
\hline 1/1/93 0:00 & Manual Tape & 412 & Good & \\
\hline 1/1/93 7:04 & Auto FIC & 0.00 & Suspect & Data not included in graph \\
\hline 1/2/93 0:00 & Manual Tape & 412.00 & Good & \\
\hline $1 / 3 / 930: 00$ & Manual Tape & 412 & Good & \\
\hline 1/4/93 0:00 & Manual Tape & 412 & Good & \\
\hline 1/5/930:00 & Manual Tape & 412 & Good & \\
\hline $1 / 6 / 930: 00$ & Manual Tape & 412.00 & Good & \\
\hline $1 / 7 / 930: 00$ & Manual Tape & 412.00 & Good & \\
\hline 1/8/93 0:00 & Manual Tape & 412 & Good & \\
\hline 1/9/93 0:00 & Manual Tape & 412.00 & Good & \\
\hline $1 / 10 / 930: 00$ & Manual Tape & 412 & Good & \\
\hline $1 / 11 / 930: 00$ & Manual Tape & 411.75 & Good & \\
\hline 1/12/93 0:00 & Manual Tape & 411.75 & Good & \\
\hline $1 / 12 / 937: 06$ & Auto FIC & 0.00 & Suspect & Data not included in graph \\
\hline $1 / 13 / 930: 00$ & Manual Tape & 411.75 & Good & \\
\hline 1/13/93 7:06 & Auto FIC & 0 & Suspect & Data not included in graph \\
\hline $1 / 14 / 930: 00$ & Manual Tape & 411.75 & Good & \\
\hline $1 / 14 / 937: 03$ & Auto FIC & 0 & Suspect & Data not included in graph \\
\hline $1 / 15 / 930: 00$ & Manual Tape & 412.00 & Good & \\
\hline $1 / 15 / 937: 03$ & Auto FIC & 0 & Suspect & Data not included in graph \\
\hline $1 / 16 / 930: 00$ & Manual Tape & 411.75 & Good & \\
\hline $1 / 17 / 930: 00$ & Manual Tape & 412 & Good & \\
\hline $1 / 18 / 930: 00$ & Manual Tape & 411.75 & Good & \\
\hline $1 / 19 / 930: 00$ & Manual Tape & 411.75 & Good & \\
\hline $1 / 20 / 930: 00$ & Manual Tape & 412 & Good & \\
\hline $1 / 21 / 930: 00$ & Manual Tape & 412.00 & Good & \\
\hline $1 / 21 / 937: 02$ & Auto FIC & 0.00 & Suspect & Data not included in graph \\
\hline
\end{tabular}

Data obtained from WHC Surveillance Analysis Computer System, (PC SACS) July 7, 1995. 


\begin{tabular}{|c|c|c|c|c|}
\hline \multicolumn{5}{|c|}{ 241-AP-103 } \\
\hline & & Urface Level Date & & \\
\hline Date & Type of Measurement & Level (Inches) & Data Quality & Comments \\
\hline $1 / 22 / 930: 00$ & Manual Tape & 412 & Good & \\
\hline $1 / 22 / 937: 04$ & Auto FIC & 0.00 & Suspect & Data not included in graph \\
\hline $1 / 23 / 930: 00$ & Manual FIC & 385.8 & Suspect & Data not included in graph \\
\hline $1 / 23 / 930: 00$ & Manual Tape & 411.75 & Good & \\
\hline 1/23/93 7:03 & Auto FIC & 0.00 & Suspect & Data not included in graph \\
\hline $1 / 24 / 930: 00$ & Manual Tape & 412.00 & Good & \\
\hline $1 / 24 / 937: 03$ & Auto FIC & 0.00 & Suspect & Data not included in graph \\
\hline 1/25/93 0:00 & Manual Tape & 411.75 & Good & \\
\hline $1 / 25 / 937: 03$ & Auto FIC & 0.00 & Suspect & Data not included in graph \\
\hline $1 / 26 / 930: 00$ & Manual Tape & 412 & Good & \\
\hline $1 / 26 / 93 \quad 7: 04$ & Auto FIC & 0 & Suspect & Data not included in graph \\
\hline $1 / 27 / 930: 00$ & Manual Tape & 411.75 & Good & \\
\hline $1 / 27 / 937: 04$ & Auto FIC & 0 & Suspect & Data not included in graph \\
\hline $1 / 28 / 930: 00$ & Manual Tape & 412 & Good & \\
\hline $1 / 28 / 937: 04$ & Auto FIC & 0 & Suspect & Data not included in graph \\
\hline 1/29/93 0:00 & Manual Tape & 411.75 & Good & \\
\hline $1 / 29 / 937: 04$ & Auto FIC & 0 & Suspect & Data not included in graph \\
\hline $1 / 30 / 930: 00$ & Manual Tape & 412 & Good & \\
\hline $1 / 30 / 937: 03$ & Auto FIC & 0.00 & Suspect & Data not included in graph \\
\hline 1/31/93 0:00 & Manual Tape & 412.00 & Good & \\
\hline $1 / 31 / 937: 04$ & Auto FIC & 0.00 & Suspect & Data not included in graph \\
\hline $2 / 1 / 930: 00$ & Manual Tape & 412.00 & Good & \\
\hline $2 / 1 / 937: 04$ & Auto FIC & 0.00 & Suspect & Data not included in graph \\
\hline $2 / 2 / 930: 00$ & Manual Tape & 412 & Good & \\
\hline 2/2/93 7:04 & Auto FIC & 0.00 & Suspect & Data not included in graph \\
\hline 2/3/93 0:00 & Manual Tape & 412 & Good & \\
\hline $2 / 4 / 930: 00$ & Manual Tape & 412.00 & Good & \\
\hline 2/4/93 7:04 & Auto FIC & 0 & Suspect & Data not included in graph \\
\hline $2 / 5 / 930: 00$ & Manual Tape & 411.75 & Good & \\
\hline 2/5/93 7:04 & Auto FIC & 0 & Suspect & Data not included in graph \\
\hline $2 / 6 / 930: 00$ & Manual Tape & 411.75 & Good & \\
\hline $2 / 6 / 937: 04$ & Auto FIC & 0 & Suspect & Data not included in graph \\
\hline 2/7/930:00 & Manual Tape & 411.75 & Good & \\
\hline $2 / 7 / 937: 03$ & Auto FIC & 0.00 & Suspect & Data not included in graph \\
\hline $2 / 8 / 93$ 0:00 & Manual Tape & 411.75 & Good & \\
\hline 2/8/93 7:04 & Auto FIC & 0 & Suspect & Data not included in graph \\
\hline 2/9/93 0:00 & Manual Tape & 411.75 & Good & \\
\hline 2/9/93 7:04 & Auto FIC & 0.00 & Suspect & Data not included in graph \\
\hline $2 / 10 / 930: 00$ & Manual Tape & 411.75 & Good & \\
\hline 2/10/93 7:04 & Auto FIC & 0.00 & Suspect & Data not included in graph \\
\hline $2 / 11 / 930: 00$ & Manual Tape & 411.75 & Good & \\
\hline 2/11/93 7:04 & Auto FIC & 0.00 & Suspect & Data not included in graph \\
\hline $2 / 12 / 930: 00$ & Manual Tape & 411.50 & Good & \\
\hline 2/12/93 7:03 & Auto FIC & 0.00 & Suspect & Data not included in graph \\
\hline $2 / 13 / 93 \quad 0: 00$ & Manual Tape & 411.75 & Good & \\
\hline 2/13/93 7:03 & Auto FIC & 0 & Suspect & Data not included in graph \\
\hline $2 / 14 / 930: 00$ & Manual Tape & 411.75 & Good & \\
\hline 2/14/93 7:04 & Auto FIC & 0 & Suspect & Data not included in graph \\
\hline
\end{tabular}

Data obtained from WHC Surveillance Analysis Computer System, (PC SACS) July 7, 1995. 
WHC-SD-WM-ER-315, Rev. 0

\begin{tabular}{|c|c|c|c|c|}
\hline \multicolumn{5}{|c|}{ 241-AP-103 } \\
\hline & & urface Level Dat & & \\
\hline Date & Type of Measurement & Level (Inches) & Data Quality & Comments \\
\hline 2/15/93 0:00 & Manual Tape & 411.75 & Good & \\
\hline 2/15/93 7:04 & Auto FIC & $a$ & Suspect & Data not included in graph \\
\hline 2/16/93 0:00 & Manual Tape & 411.75 & Good & \\
\hline $2 / 16 / 937: 02$ & Auto FIC & 0 & Suspect & Data not included in graph \\
\hline 2/17/93 0:00 & Manual Tape & 411.75 & Good & \\
\hline 2/18/93 0:00 & Manual Tape & 411.75 & Good & \\
\hline 2/18/93 7:03 & Auto FIC & 0.00 & Suspect & Data not included in graph \\
\hline 2/19/93 0:00 & Manual Tape & 411.75 & Good & \\
\hline 2/19/93 7:02 & Auto FIC & 0.00 & Suspect & Data not included in graph \\
\hline $2 / 20 / 930: 00$ & Manual Tape & 411.75 & Good & \\
\hline 2/20/93 7:03 & Auto FIC & 0.00 & Suspect & Data not included in graph \\
\hline $2 / 21 / 930: 00$ & Manual Tape & 411.75 & Good & \\
\hline $2 / 21 / 937: 04$ & Auto FIC & 0.00 & Suspect & Data not included in graph \\
\hline $2 / 22 / 930: 00$ & Manual Tape & 411.75 & Good & \\
\hline 2/22/93 7:04 & Auto FIC & 0 & Suspect & Data not included in graph \\
\hline $2 / 23 / 930: 00$ & Manual Tape & 411.5 & Good & \\
\hline $2 / 23 / 937: 03$ & Auto FIC & 0 & Suspect & Data not included in graph \\
\hline $2 / 24 / 930: 00$ & Manual Tape & 411.75 & Good & \\
\hline 2/24/93 7:04 & Auto FIC & 0.00 & Suspect & Data not included in graph \\
\hline $2 / 25 / 930: 00$ & Manual Tape & 411.5 & Good & \\
\hline $2 / 25 / 937: 03$ & Auto FIC & 0 & Suspect & Data not included in graph \\
\hline $2 / 26 / 930: 00$ & Manual Tape & 411.75 & Good & \\
\hline $2 / 26 / 937: 03$ & Auto FIC & 0.00 & Suspect & Data not included in graph \\
\hline $2 / 27 / 930: 00$ & Manual Tape & 411.75 & Good & \\
\hline 2/27/93 7:04 & Auto FIC & 0.00 & Suspect & Data not included in graph \\
\hline $2 / 28 / 930: 00$ & Manual Tape & 411.5 & Good & \\
\hline $2 / 28 / 93 \quad 7: 03$ & Auto FIC & 0.00 & Suspect & Data not included in graph \\
\hline 3/1/93 0:00 & Manual Tape & 41.1 .50 & Good & \\
\hline 3/1/93 7:03 & Auto FIC & 0.00 & Suspect & Data not included in graph \\
\hline 3/2/93 0:00 & Manual Tape & 411.5 & Good & \\
\hline 3/2/93 7:04 & Auto FIC & 0 & Suspect & Data not included in graph \\
\hline $3 / 3 / 930: 00$ & Manual Tape & 411.75 & Good & \\
\hline 3/3/93 7:03 & Auto FIC & 0 & Suspect & Data not included in graph \\
\hline 3/4/93 0:00 & Manual Tape & 411.75 & Good & \\
\hline $3 / 4 / 937: 04$ & Auto FIC & 0 & Suspect & Data not included in graph \\
\hline 3/5/93 0:00 & Manual Tape & 411.75 & Good & \\
\hline $3 / 5 / 937: 04$ & Auto FIC & $\overline{0}$ & Suspect & Data not included in graph \\
\hline $3 / 6 / 930: 00$ & Manual Tape & 411.75 & Good & \\
\hline $3 / 6 / 937: 03$ & Auto FIC & 0.00 & Suspect & Data not included in graph \\
\hline $3 / 7 / 930: 00$ & Manual Tape & 411.75 & Good & \\
\hline $3 / 7 / 937: 03$ & Auto FIC & 0.00 & Suspect & Data not included in graph \\
\hline 3/8/93 0:00 & Manual Tape & 411.75 & Good & \\
\hline $3 / 8 / 937: 03$ & Auto FIC & 0.00 & Suspect & Data not included in graph \\
\hline $3 / 9 / 930: 00$ & Manual Tape & 411.75 & Good & \\
\hline $3 / 9 / 937: 04$ & Auto FIC & 0.00 & Suspect & Data not included in graph \\
\hline $3 / 10 / 930: 00$ & Manual Tape & 411.75 & Good & \\
\hline 3/10/93 7:05 & Auto FIC & 0 & Suspect & Data not included in graph \\
\hline $3 / 11 / 930: 00$ & Manual Tape & 411.75 & Good & \\
\hline
\end{tabular}

Data obtained from WHC Surveillance Analysis Computer System, (PC SACS) July 7, 1995. 
WHC-SD-WM-ER-315, Rev. 0

\begin{tabular}{|c|c|c|c|c|}
\hline \multicolumn{5}{|c|}{ 241-AP-103 } \\
\hline & & urface Level Date & & \\
\hline Date & Type of Measurement & Lovel (Inches) & Data Quality & Comments \\
\hline $3 / 11 / 937: 04$ & Auto FIC & 0 & Suspect & Data not included in graph \\
\hline $3 / 12 / 93 \quad 0: 00$ & Manual Tape & 411.75 & Good & \\
\hline $3 / 12 / 937: 04$ & Auto FIC & 0.00 & Suspect & Data not included in graph \\
\hline $3 / 13 / 930: 00$ & Manual Tape & 411.75 & Good & \\
\hline $3 / 13 / 937: 03$ & Auto FIC & 0 & Suspect & Data not included in graph \\
\hline $3 / 14 / 93 \quad 0: 00$ & Manual Tape & 411.75 & Good & \\
\hline $3 / 14 / 937: 04$ & Auto FIC & 0.00 & Suspect & Data not included in graph \\
\hline $3 / 15 / 930: 00$ & Manual Tape & 411.75 & Good & \\
\hline $3 / 15 / 937: 03$ & Auto FIC & 0.00 & Suspect & Data not included in graph \\
\hline $3 / 16 / 930: 00$ & Manual Tape & 411.75 & Good & \\
\hline $3 / 16 / 937: 04$ & Auto FIC & 0.00 & Suspect & Data not included in graph \\
\hline $3 / 17 / 930: 00$ & Manual Tape & 411.5 & Good & \\
\hline $3 / 17 / 937: 03$ & Auto FIC & 0.00 & Suspect & Data not included in graph \\
\hline $3 / 18 / 930: 00$ & Manual Tape & 411.50 & Good & \\
\hline $3 / 18 / 937: 04$ & Auto FIC & 0.00 & Suspect & Data not included in graph \\
\hline $3 / 19 / 930: 00$ & Manual FIC & 412.00 & Good & \\
\hline $3 / 19 / 930: 00$ & Manusl Tape & 411.75 & Good & \\
\hline $3 / 19 / 937: 03$ & Auto FIC & 0 & Suspect & Data not included in graph \\
\hline $3 / 20 / 930: 00$ & Manual Tape & 411.50 & Good & \\
\hline $3 / 20 / 937: 04$ & Auto FIC & 0 & Suspect & Data not included in graph \\
\hline $3 / 21 / 930: 00$ & Manual Tape & 411.75 & Good & \\
\hline $3 / 21 / 937: 04$ & Auto FIC & 0 & Suspect & Data not included in graph \\
\hline $3 / 22 / 930: 00$ & Manual Tape & 411.75 & Good & \\
\hline $3 / 22 / 937: 03$ & Auto FIC & 0 & Suspect & Data not included in graph \\
\hline $3 / 23 / 930: 00$ & Manual Tape & 411.75 & Good & \\
\hline $3 / 23 / 937: 03$ & Auto FIC & 0.00 & Suspect & Data not included in graph \\
\hline $3 / 24 / 930: 00$ & Manual Tape & 411.75 & Good & \\
\hline $3 / 24 / 937: 04$ & Auto FIC & 0.00 & Suspect & Data not included in graph \\
\hline $3 / 25 / 930: 00$ & Manual Tape & 411.75 & Good & $\cdot$ \\
\hline $3 / 25 / 937: 03$ & Auto FIC & 0.00 & Suspect & Data not included in graph \\
\hline $3 / 26 / 93 \quad 0: 00$ & Manual Tape & 411.75 & Good & \\
\hline $3 / 26 / 937: 03$ & Auto FIC & 0.00 & Suspect & Data not included in graph \\
\hline $3 / 27 / 930: 00$ & Manual Tape & 411.75 & Good & \\
\hline $3 / 27 / 937: 03$ & Auto FIC & 0 & Suspect & Data not included in graph \\
\hline $3 / 28 / 930: 00$ & Manual Tape & 411.75 & Good & \\
\hline $3 / 28 / 937: 03$ & Auto FIC & 0.00 & Suspect & Data not included in graph \\
\hline $3 / 29 / 93 \quad 0: 00$ & Manual Tape & 411.75 & Good & \\
\hline $3 / 29 / 937: 03$ & Auto FIC & 0 & Suspect & Data not included in graph \\
\hline $3 / 30 / 930: 00$ & Manual Tape & 411.75 & Good & \\
\hline $3 / 30 / 937: 03$ & Auto FIC & 0 & Suspect & Data not included in graph \\
\hline $3 / 31 / 930: 00$ & Manual Tape & 411.75 & Good & \\
\hline 3/31/93 7:03 & Auto FIC & 0.00 & Suspect & Data not included in graph \\
\hline 4/1/93 10:00 & Manual Tape & 411.75 & Good & \\
\hline $4 / 2 / 93 \quad 10: 00$ & Manual Tape & 411.75 & Good & \\
\hline 4/3/93 10:00 & Manual Tape & 411.75 & Good & \\
\hline $4 / 4 / 9310: 00$ & Manual Tape & 411.75 & Good & \\
\hline $4 / 5 / 93 \quad 10: 00$ & Manual Tape & 411.75 & Good & \\
\hline $4 / 6 / 93 \quad 10: 00$ & Manual Tape & 411.75 & Good & \\
\hline
\end{tabular}

Data obtained from WHC Surveillance Analysis Computer System, (PC SACS) July 7, 1995. 


\begin{tabular}{|c|c|c|c|c|}
\hline \multicolumn{5}{|c|}{ 241-AP-103 } \\
\hline Surface Level Date & Type of Measurement & Level (Inches) & Data Quality & Comments \\
\hline$\frac{\text { Date }}{4 / 7 / 9310: 00}$ & Manual Tape & 411.75 & Good & \\
\hline $\begin{array}{ll}4 / 7 / 93 & 10: 00 \\
4 / 8 / 93 & 10: 00 \\
\end{array}$ & Manual Tape & 411.75 & Good & \\
\hline $\begin{array}{lll}4 / 8 / 93 & 10: 00 \\
4 / 9 / 93 & 10: 00 \\
\end{array}$ & Manual Tape & 411.75 & Good & \\
\hline $\begin{array}{c}4 / 9 / 93 \quad 10: 00 \\
4 / 10 / 93 \quad 10: 00\end{array}$ & Manual Tape & 411.75 & Good & \\
\hline $\begin{array}{l}4 / 10 / 93 \quad 10: 00 \\
4 / 11 / 93 \quad 10: 00\end{array}$ & Manual Tape & 411.75 & Good & \\
\hline $\begin{array}{ll}4 / 11 / 93 & 10: 00 \\
4 / 12 / 93 & 10: 00\end{array}$ & Manual Tape & 411.75 & Good & \\
\hline $\begin{array}{l}4 / 12 / 93 \quad 10: 00 \\
4 / 13 / 93 \quad 10: 00\end{array}$ & Manual Tape & 411.75 & Good & \\
\hline $\begin{array}{ll}4 / 13 / 93 & 10: 00 \\
4 / 14 / 93 & 10: 00\end{array}$ & Manual Tape & 411.75 & Good & \\
\hline $\begin{array}{l}4 / 14 / 93 \quad 10: 00 \\
4 / 15 / 93 \quad 10: 00\end{array}$ & Manual Tape & 411.5 & Good & \\
\hline $\begin{array}{ll}4 / 15 / 93 & 10: 00 \\
4 / 16 / 93 & 10: 00\end{array}$ & Manual Tape & 411.5 & Good & \\
\hline $\begin{array}{ll}4 / 16 / 93 & 10: 00 \\
4 / 17 / 93 & 10: 00\end{array}$ & Manual Tape & 411.5 & Good & \\
\hline $4 / 18 / 93 \quad 10: 00$ & Manual Tape & 411.5 & Good & \\
\hline $4 / 19 / 93 \quad 10: 00$ & Manual Tape & 411.75 & Good & \\
\hline $4 / 20 / 9310: 00$ & Manual Tape & 411.75 & Good & \\
\hline $4 / 21 / 9310: 00$ & Manual Tape & $411: 75$ & Good & \\
\hline $4 / 22 / 93 \quad 10: 00$ & Manual Tape & 411.75 & Good & \\
\hline $4 / 23 / 9310: 00$ & Manual Tape & 411.75 & Good & \\
\hline $4 / 24 / 93 \quad 10: 00$ & Manual Tape & 411.75 & Good & \\
\hline $4 / 25 / 93 \quad 10: 00$ & Manual Tape & 411.75 & Good & \\
\hline $4 / 26 / 93 \quad 10: 00$ & Manual Tape & 411.75 & Good & \\
\hline $4 / 27 / 9310: 00$ & Manual Tape & 411.75 & Good & \\
\hline $4 / 28 / 93 \quad 10: 00$ & Manual Tape & 411.75 & Good & \\
\hline $4 / 29 / 93 \quad 10: 00$ & Manual Tape & 411.75 & Good & \\
\hline $4 / 30 / 9310: 00$ & Manual Tape & 411.75 & Good & \\
\hline $5 / 1 / 9310: 00$ & Manual Tape & 411.75 & Good & \\
\hline $5 / 2 / 93 \quad 10: 00$ & Manua! Tape & 411.75 & Good & \\
\hline $5 / 3 / 93 \quad 10: 00$ & Manual Tape & 411.75 & Good & \\
\hline $5 / 4 / 9310: 00$ & Manual Tape & 411.75 & Good & \\
\hline $5 / 5 / 9310: 00$ & Manual Tape & 411.75 & Good & \\
\hline $5 / 6 / 93 \quad 10: 00$ & Manual Tape & 411.75 & Good & \\
\hline $5 / 7 / 93 \quad 10: 00$ & Manual Tape & 411.75 & Good & \\
\hline $5 / 8 / 93 \quad 10: 00$ & Manual Tape & 41.1 .75 & Good & \\
\hline 5/9/93 10:00 & Manual Tape & 411.75 & Good & \\
\hline $5 / 10 / 93 \quad 10: 00$ & Manual Tape & 411.75 & Good & \\
\hline $5 / 11 / 93 \quad 10: 00$ & Manual Tape & 411.5 & Good & \\
\hline $5 / 12 / 9310: 00$ & Manual Tape & 411.75 & Good & \\
\hline $5 / 13 / 93 \quad 10: 00$ & Manual Tape & 411.75 & Good & \\
\hline $5 / 14 / 9310: 00$ & Manual Tape & 411.75 & Good & \\
\hline $5 / 15 / 93 \quad 10: 00$ & Manual Tape & 411.75 & Good & \\
\hline $5 / 16 / 93 \quad 10: 00$ & Manual Tape & 411.75 & Good & \\
\hline $5 / 17 / 937: 03$ & Auto FIC & 0.00 & Suspect & Data not included in graph \\
\hline 5/17/93 10:00 & Manual Tape & 411.75 & Good & \\
\hline $5 / 18 / 937: 03$ & Auto FIC & 0.00 & Suspect & Data not included in graph \\
\hline 5/18/93 10:00 & Manual Tape & 411.75 & Good & \\
\hline $5 / 19 / 937: 03$ & Auto FIC & 0.00 & Suspect & Data not included in graph \\
\hline $5 / 19 / 93 \quad 10: 00$ & Manual Tape & 411.75 & Good & \\
\hline $5 / 20 / 937: 03$ & Auto FIC & 0 & Suspect & Data not included in graph \\
\hline $5 / 20 / 9310: 00$ & Manual Tape & 411.75 & Good & \\
\hline
\end{tabular}

Data obtained from WHC Surveillance Analysis Computer System, (PC SACS) July 7, 1995. 


\begin{tabular}{|c|c|c|c|c|}
\hline \multicolumn{5}{|c|}{ 241-AP-103 } \\
\hline \multicolumn{5}{|c|}{ Surface Level Data } \\
\hline Date & Type of Measurement & Leval (Inches) & Data Quality & Comments \\
\hline $5 / 21 / 9310: 00$ & Manual Tape & 411.75 & Good & \\
\hline $5 / 22 / 9310: 00$ & Manual Tape & 411.75 & Good & \\
\hline $5 / 23 / 93 \quad 10: 00$ & Manual Tape & 411.75 & Good & \\
\hline $5 / 24 / 93 \quad 10: 00$ & Manual Tape & 411.75 & Good & \\
\hline $5 / 25 / 9310: 00$ & Manual Tape & 411.75 & Good & \\
\hline $5 / 26 / 9310: 00$ & Manual Tape & 411.75 & Good & \\
\hline $5 / 27 / 93 \quad 10: 00$ & Manual FIC & 411.90 & Good & \\
\hline $5 / 27 / 9310: 00$ & Manual Tape & 411.75 & Good & \\
\hline $5 / 28 / 93 \quad 10: 00$ & Manual FIC & 411.90 & Good & \\
\hline $5 / 28 / 9310: 00$ & Manual Tape & 411.75 & Good & \\
\hline $5 / 29 / 93 \quad 10: 00$ & Manual Tape & 411.75 & Good & \\
\hline $5 / 30 / 93 \quad 10: 00$ & Manual Tape & 411.75 & Good & \\
\hline $5 / 31 / 93 \quad 10: 00$ & Manual Tape & 411.75 & Good & \\
\hline $6 / 1 / 9310: 00$ & Manual Tape & 411.75 & Good & \\
\hline $6 / 2 / 9310: 00$ & Manual Tape & 411.50 & Good & \\
\hline $6 / 3 / 93 \quad 10: 00$ & Manual Tape & 411.5 & Good & \\
\hline $6 / 4 / 93 \quad 10: 00$ & Manual FIC & 411.9 & Good & \\
\hline $6 / 4 / 93 \quad 10: 00$ & Manual Tape & 411.5 & Good & \\
\hline $6 / 5 / 9310: 00$ & Manual Tape & 411.75 & Good & \\
\hline $6 / 6 / 93 \quad 10: 00$ & Manual Tape & 411.5 & Good & \\
\hline $6 / 7 / 93 \quad 10: 00$ & Manual Tape & 411.50 & Good & \\
\hline $6 / 8 / 9310: 00$ & Manual Tape & 411.50 & Good & \\
\hline $6 / 9 / 93$ 10:00 & Manual Tape & 411.50 & Good & \\
\hline $6 / 10 / 93 \quad 10: 00$ & Manual Tape & 411.50 & Good & \\
\hline $6 / 11 / 93 \quad 10: 00$ & Manual Tape & 411.75 & Good & \\
\hline $6 / 12 / 93 \quad 10: 00$ & Manual Tape & 411.75 & Good & \\
\hline $6 / 13 / 93 \quad 10: 00$ & Manual Tape & 411.75 & Good & \\
\hline $6 / 14 / 93 \quad 10: 00$ & Manual Tape & 411.75 & Good & \\
\hline $6 / 15 / 9310: 00$ & Manual Tape & $411: 75$ & Good & \\
\hline $6 / 16 / 93 \quad 10: 00$ & Manual Tape & 411.75 & Good & \\
\hline $6 / 17 / 9310: 00$ & Manual Tape & 411.75 & Good & \\
\hline $6 / 18 / 93 \quad 10: 00$ & Manual Tape & 411.75 & Good & \\
\hline $6 / 19 / 93$ 10:00 & Manual Tape & 411.75 & Good & \\
\hline $6 / 20 / 93 \quad 10: 00$ & Manual Tape & 411.75 & Good & \\
\hline $6 / 21 / 93 \quad 10: 00$ & Manual Tape & 411.75 & Good & \\
\hline $6 / 22 / 93 \quad 10: 00$ & Manual Tape & 411.75 & Good & \\
\hline $6 / 23 / 93 \quad 10: 00$ & Manual Tape & 411.75 & Good & \\
\hline $6 / 24 / 9310: 00$ & Manual Tape & 411.75 & Good & \\
\hline $6 / 25 / 93 \quad 10: 00$ & Manual Tape & 411.75 & Good & \\
\hline $6 / 26 / 93 \quad 10: 00$ & Manual Tape & 411.75 & Good & \\
\hline $6 / 27 / 93 \quad 10: 00$ & Manual Tape & 411.75 & Good & \\
\hline $6 / 28 / 93 \quad 10: 00$ & Manual Tape & 411.75 & Good & \\
\hline $6 / 29 / 9310: 00$ & Manual Tape & 411.75 & Good & \\
\hline $6 / 30 / 93 \quad 10: 00$ & Manual Tape & 411.75 & Good & \\
\hline $7 / 1 / 93 \quad 10: 00$ & Manual Tape & 411.75 & Good & \\
\hline $7 / 2 / 9310: 00$ & Manual Tape & 411.75 & Good & \\
\hline $7 / 3 / 93 \quad 10: 00$ & Manual Tape & 411.75 & Good & \\
\hline 7/4/93 10:00 & Manual Tape & 411.75 & Good & \\
\hline
\end{tabular}

Data abtained from WHC Surveillance Analysis Computer System, (PC SACS) July 7, 1995. 


\begin{tabular}{|c|c|c|c|c|}
\hline \multicolumn{5}{|c|}{$\begin{array}{c}\text { 241-AP-108 } \\
\text { Surface Level Data }\end{array}$} \\
\hline & & Irface Level Date & & \\
\hline Date & Type of Measurement & Levol (Inches) & Data Quality & Comments \\
\hline $7 / 5 / 93 \quad 10: 00$ & Manual Tape & 411.75 & Good & \\
\hline $7 / 6 / 93 \quad 10: 00$ & Manual Tape & 411.75 & Good & \\
\hline $7 / 7 / 93 \quad 10: 00$ & Manual Tape & 411.25 & Good & \\
\hline $7 / 8 / 93 \quad 10: 00$ & Manual Tape & 411.00 & Good & \\
\hline $7 / 9 / 9310: 00$ & Manual Tape & 411.5 & Good & \\
\hline $7 / 10 / 93 \quad 10: 00$ & Manual Tape & 411.75 & Good & \\
\hline $7 / 111 / 93 \quad 10: 00$ & Manual Tape & 411.75 & Good & \\
\hline $7 / 11 / 93$ 15:04 & Auto FIC & 0 & Suspect & Data not included in graph \\
\hline $7 / 12 / 93 \quad 10: 00$ & Manual Tape & 411.75 & Good & \\
\hline $7 / 12 / 9315: 04$ & Auto FIC & 0 & Suspect & Data not included in graph \\
\hline $7 / 13 / 93 \quad 10: 00$ & Manual Tape & 411.75 & Good & \\
\hline $7 / 13 / 93 \quad 15: 04$ & Auto FIC & 0 & Suspect & Data not included in graph \\
\hline $7 / 14 / 9310: 00$ & Manual Tape & 411.75 & Good & \\
\hline $7 / 14 / 9315: 04$ & Auto FIC & 0.00 & Suspect & Data not included in graph \\
\hline $7 / 15 / 93 \quad 10: 00$ & Manual Tape & 411.75 & Good & \\
\hline $7 / 15 / 93 \quad 15: 04$ & Auto FIC & 0.00 & Suspect & Data not included in graph \\
\hline $7 / 16 / 93 \quad 10: 00$ & Manual Tape & 411.75 & Good & \\
\hline $7 / 16 / 93 \quad 15: 04$ & Auto FIC & 411.80 & Suspect & Data not included in graph \\
\hline 7/17/93 7:07 & Auto FIC & 411.80 & Good & \\
\hline $7 / 17 / 93 \quad 10: 00$ & Manual Tape & 411.75 & Good & \\
\hline $7 / 18 / 937: 06$ & Auto FIC & 411.8 & Good & \\
\hline $7 / 18 / 9310: 00$ & Manual Tape & 411.75 & Good & \\
\hline $7 / 19 / 937: 03$ & Auto FIC & 411.80 & Good & \\
\hline $7 / 19 / 93 \quad 10: 00$ & Manual Tape & 411.75 & Good & \\
\hline $7 / 20 / 937: 03$ & Auto FIC & 411.8 & Good & \\
\hline $7 / 20 / 9310: 00$ & Manual Tape & 411.5 & Good & \\
\hline $7 / 21 / 937: 03$ & Auto FIC & 411.8 & Good & \\
\hline $7 / 21 / 9310: 00$ & Manual Tape & 411.75 & Good & \\
\hline $7 / 22 / 937: 03$ & Auto FIC & 411.80 & Good & \\
\hline $7 / 22 / 9310: 00$ & Manual Tape & 411.75 & Good & \\
\hline $7 / 23 / 937: 03$ & Auto FIC & 411.9 & Good & \\
\hline $7 / 23 / 93 \quad 10: 00$ & Manual Tape & 411.75 & Good & \\
\hline $7 / 24 / 937: 03$ & Auto FIC & 411.80 & Good & \\
\hline $7 / 24 / 93 \quad 10: 00$ & Manual Tape & 411.50 & Good & \\
\hline $7 / 25 / 937: 04$ & Auto FIC & 411.80 & Good & \\
\hline $7 / 25 / 93 \quad 10: 00$ & Manual Tape & 411.5 & Good & \\
\hline $7 / 26 / 937: 03$ & Auto FIC & 411.80 & Good & \\
\hline $7 / 26 / 93 \quad 10: 00$ & Manual Tape & 411.5 & Good & \\
\hline $7 / 27 / 937: 03$ & Auto FIC & 411.90 & Good & \\
\hline $7 / 27 / 93 \quad 10: 00$ & Manual Tape & 411.50 & Good & \\
\hline $7 / 28 / 930: 00$ & Manual Tape & 411.5 & Good & \\
\hline $7 / 28 / 937: 04$ & Auto FIC & 411.9 & Good & \\
\hline $7 / 29 / 930: 00$ & Manual Tape & $41.1: 50$ & Good & \\
\hline $7 / 29 / 93 \quad 7: 03$ & Auto FIC & 411.8 & Good & \\
\hline $7 / 30 / 930: 00$ & Manual Tape & 411.50 & Good & \\
\hline 7/30/93 7:03 & Auto FIC & 411.9 & Good & \\
\hline $7 / 31 / 930: 00$ & Manual Tape & 411.50 & Good & \\
\hline $7 / 31 / 937: 03$ & Auto FIC & 411.80 & Good & \\
\hline
\end{tabular}

Data obtained from WHC Surveillance Analysis Computer System, (PC SACS) July 7, 1995. 


\begin{tabular}{|c|c|c|c|c|}
\hline \multicolumn{5}{|c|}{ 241-AP-103 } \\
\hline \multicolumn{5}{|c|}{ Surface Level Date } \\
\hline Date & Type of Measurement & Level (Inches) & Data Quality & Comments \\
\hline $8 / 1 / 930: 00$ & Manual Tape & 411.75 & Good & \\
\hline $8 / 1 / 937: 03$ & Auto FIC & 411.90 & Good & \\
\hline $8 / 2 / 930: 00$ & Manual Tape & 411.75 & Good & \\
\hline $8 / 2 / 937: 06$ & Auto FIC & 411.80 & Good & \\
\hline $8 / 3 / 930: 00$ & Manual Tape & 411.5 & Good & \\
\hline $8 / 3 / 937: 03$ & Auto FIC & 411.90 & Good & \\
\hline $8 / 4 / 930: 00$ & Manual Tape & 411.75 & Good & \\
\hline $8 / 4 / 937: 03$ & Auto FIC & 411.8 & Good & \\
\hline $8 / 5 / 930: 00$ & Manual Tape & 411.75 & Good & \\
\hline $8 / 5 / 937: 04$ & Auto FIC & 411.90 & Good & \\
\hline $8 / 6 / 930: 00$ & Manual Tape & 411.50 & Good & \\
\hline $8 / 6 / 937: 04$ & Auto FIC & 411.9 & Good & \\
\hline $8 / 7 / 930: 00$ & Manual Tape & 411.75 & Good & \\
\hline $8 / 7 / 937: 04$ & Auto FIC & 411.9 & Good & \\
\hline $8 / 8 / 930: 00$ & Manual Tape & $411: 50$ & Good & \\
\hline 8/8/93 7:04 & Auto FIC & 411.80 & Good & \\
\hline $8 / 9 / 930: 00$ & Manual Tape & 411.50 & Good & \\
\hline $8 / 9 / 937: 04$ & Auto FIC & 411.9 & Good & \\
\hline $8 / 10 / 930: 00$ & Manual Tape & 411.5 & Good & \\
\hline $8 / 10 / 937: 04$ & Auto FIC & 411.90 & Good & \\
\hline $8 / 11 / 930: 00$ & Manual Tape & 411.75 & Good & \\
\hline $8 / 11 / 93$ 7:04 & Auto FIC & 411.90 & Good & \\
\hline $8 / 12 / 930: 00$ & Manual Tape & 411.75 & Good & \\
\hline $8 / 12 / 937: 04$ & Auto FIC & 411.80 & Good & \\
\hline $8 / 13 / 930: 00$ & Manual Tape & 411.5 & Good & \\
\hline $8 / 13 / 937: 04$ & Auto FIC & 411.80 & Good & \\
\hline $8 / 14 / 930: 00$ & Manual Tape & 411.75 & Good & \\
\hline $8 / 14 / 937: 03$ & Auto FIC & 411.8 & Good & \\
\hline $8 / 15 / 930: 00$ & Manual Tape & 411.75 & Good & \\
\hline $8 / 15 / 93$ 7:04 & Auto FIC & 411.8 & Good & \\
\hline $8 / 16 / 930: 00$ & Manual Tape & 411.75 & Good & \\
\hline $8 / 16 / 937: 04$ & Auto FIC & 411.8 & Good & \\
\hline $8 / 17 / 930: 00$ & Manual Tape & 411.5 & Good & \\
\hline $8 / 17 / 937: 04$ & Auto FIC & 411.8 & Good & \\
\hline $8 / 18 / 930: 00$ & Manual Tape & 411.5 & Good & \\
\hline $8 / 18 / 937: 03$ & Auto FIC & 411.80 & Good & \\
\hline $8 / 19 / 930: 00$ & Manual Tape & 411.50 & Good & \\
\hline $8 / 19 / 937: 04$ & Auto FIC & 411.80 & Good & \\
\hline $8 / 20 / 930: 00$ & Manual Tape & 411.50 & Good & \\
\hline $8 / 20 / 937: 04$ & Auto FIC & 411.80 & Good & \\
\hline $8 / 21 / 930: 00$ & Manual Tape & 411.50 & Good & \\
\hline $8 / 21 / 937: 04$ & Auto FIC & 411.80 & Good & \\
\hline $8 / 22 / 930: 00$ & Manual Tape & 411.75 & Good & \\
\hline $8 / 22 / 937: 04$ & Auto FIC & 411.80 & Good & \\
\hline $8 / 23 / 93 \quad 0: 00$ & Manual Tape & 411.75 & Good & \\
\hline $8 / 23 / 937: 04$ & Auto FIC & 411.80 & Good & \\
\hline $8 / 24 / 930: 00$ & Manual Tape & 411.75 & Good & \\
\hline $8 / 24 / 937: 04$ & Auto FIC & 411.80 & Good & \\
\hline
\end{tabular}

Data obtained from WHC Surveillance Analysis Computer System, (PC SACS) July 7, 1995. 


\begin{tabular}{|c|c|c|c|c|}
\hline \multicolumn{5}{|c|}{ 241-AP-103 } \\
\hline \multicolumn{5}{|c|}{ Surface Level Data } \\
\hline Date & Type of Measurement & Lovel (Inches) & Data Quality & Comments \\
\hline $8 / 25 / 930: 00$ & Manual Tape & 411.5 & Good & \\
\hline $8 / 25 / 93 \quad 7: 04$ & Auto FIC & 411.8 & Good & \\
\hline $8 / 26 / 930: 00$ & Manual Tape & 411 & Good & \\
\hline $8 / 26 / 937: 04$ & Auto FIC & 411.7 & Good & \\
\hline $8 / 27 / 930: 00$ & Manual Tape & 411.75 & Good & \\
\hline 8/27/93 7:05 & Auto FIC & 411.8 & Good & \\
\hline $8 / 28 / 930: 00$ & Manual Tape & 411.5 & Good & \\
\hline $8 / 28 / 937: 04$ & Auto FIC & 411.8 & Good & \\
\hline $8 / 29 / 930: 00$ & Manual Tape & 411.50 & Good & \\
\hline $8 / 29 / 937: 03$ & Auto FIC & 411.8 & Good & \\
\hline $8 / 30 / 930: 00$ & Manual Tape & 411.50 & Good & \\
\hline $8 / 30 / 937: 04$ & Auto FIC & 411.80 & Good & \\
\hline $8 / 31 / 930: 00$ & Manual Tape & 411.5 & Good & \\
\hline $8 / 31 / 937: 04$ & Auto FIC & 411.80 & Good & \\
\hline 9/1/93 0:00 & Manual Tape & 411.75 & Good & \\
\hline $9 / 1 / 937: 04$ & Auto FIC & 411.8 & Good & \\
\hline $9 / 2 / 930: 00$ & Manual Tape & 411.5 & Good & \\
\hline 9/2/93 7:04 & Auto FIC & 411.80 & Good & \\
\hline $9 / 3 / 930: 00$ & Manual Tape & 411.5 & Good & \\
\hline $9 / 3 / 937: 04$ & Auto FIC & 411,80 & Good & \\
\hline 9/4/93 0:00 & Manual Tape & 411.50 & Good & $\cdot$ \\
\hline 9/4/93 7:04 & Auto FIC & 411.8 & Good & \\
\hline $9 / 5 / 930: 00$ & Manual Tape & 411.50 & Good & \\
\hline $9 / 5 / 937: 04$ & Auto FIC & 411.8 & Good & \\
\hline $9 / 6 / 930: 00$ & Manual Tape & 411.75 & Good & \\
\hline $9 / 6 / 937: 04$ & Auto FIC & 411.8 & Good & \\
\hline $9 / 7 / 930: 00$ & Manual Tape & 411.75 & Good & \\
\hline 9/7/93 7:04 & Auto FIC & 411.80 & Good & \\
\hline $9 / 8 / 930: 00$ & Manual Tape & 41.1 .75 & Good & \\
\hline $9 / 8 / 93 \quad 7: 04$ & Auto FIC & 411.8 & Good & \\
\hline 9/9/93 0:00 & Manual Tape & 411.75 & Good & \\
\hline 9/9/93 7:04 & Auto FIC & 411.80 & Good & \\
\hline 9/10/93 0:00 & Manual Tape & 411.75 & Good & \\
\hline $9 / 10 / 937: 04$ & Auto FIC & 411.80 & Good & \\
\hline 9/11/93 0:00 & Manual Tape & 411.75 & Good & \\
\hline 9/11/93 7:04 & Auto FIC & 411.80 & Good & \\
\hline $9 / 12 / 930: 00$ & Manual Tape & 411,75 & Good & \\
\hline $9 / 12 / 937: 04$ & Auto FIC & 411.80 & Good & \\
\hline $9 / 13 / 930: 00$ & Manual Tape & 411.75 & Good & \\
\hline $9 / 13 / 937: 04$ & Auto FIC & 411.8 & Good & \\
\hline $9 / 14 / 930: 00$ & Manual Tape & 411.75 & Good & \\
\hline 9/14/93 7:04 & Auto FIC & 411.8 & Good & \\
\hline $9 / 15 / 930: 00$ & Manual Tape & 411.75 & Good & \\
\hline 9/15/93 7:04 & Auto FIC & 411.8 & Good & \\
\hline 9/16/93 0:00 & Manual Tape & 411.75 & Good & \\
\hline $9 / 16 / 937: 04$ & Auto FIC & 411.8 & Good & \\
\hline 9/17/93 0:00 & Manual Tape & 411.75 & Good & \\
\hline $9 / 17 / 93$ 7:04 & Auto FIC & 411.80 & Good & \\
\hline
\end{tabular}

Data obtained from WHC Surveillance Analysis Computer System, (PC SACS) July 7, 1995. 


\begin{tabular}{|c|c|c|c|c|}
\hline & Type of Measurement & Leval (Inches) & Data Quality & Comments \\
\hline $\begin{array}{c}\text { Date } \\
9 / 18 / 930: 00\end{array}$ & Manual Tape & 411.75 & Good & \\
\hline $\begin{array}{l}9 / 18 / 93 \quad 0: 00 \\
9 / 18 / 93 \quad 7: 04\end{array}$ & Auto FIC & 411.80 & Good & \\
\hline $\begin{array}{l}9 / 18 / 93 \quad 7: 04 \\
9 / 19 / 930: 00\end{array}$ & Manual Tape & 411.75 & Good & \\
\hline $\begin{array}{l}9 / 19 / 930: 00 \\
9 / 19 / 937: 04\end{array}$ & Auto FIC & 411.80 & Good & \\
\hline $\begin{array}{l}9 / 19 / 937: 04 \\
9 / 20 / 930: 00\end{array}$ & Manual Tape & 411.50 & Good & \\
\hline $\begin{array}{l}9 / 20 / 930: 00 \\
9 / 20 / 937: 04\end{array}$ & Auto FIC & 411.80 & Good & \\
\hline$\frac{9 / 20 / 937: 04}{9 / 21 / 930: 00}$ & Manual Tape & 411.5 & Good & \\
\hline $\begin{array}{l}9 / 21 / 930: 00 \\
9 / 21 / 937: 04\end{array}$ & Auto FIC & 411.80 & Good & \\
\hline$\frac{9 / 21 / 937: 04}{9 / 22 / 937: 04}$ & Auto FIC & 411.8 & Good & \\
\hline$\frac{9 / 22 / 937: 04}{9 / 23 / 930: 00}$ & Manual Tape & 411.50 & Good & \\
\hline $9 / 23 / 937: 04$ & Auto FIC & 411.70 & Good & \\
\hline 9/24/93 0:00 & Manual Tape & 411.25 & Good & \\
\hline 9/24/93 7:04 & Auto FIC & 411.7 & Good & \\
\hline 9/25/93 0:00 & Manual Tape & 411.25 & Good & \\
\hline $9 / 25 / 937: 04$ & Auto FIC & 411.7 & Good & \\
\hline $9 / 26 / 930: 00$ & Manual Tape & 411.25 & Good & \\
\hline 9/26/93 7:04 & Auto FIC & 411.70 & Good & \\
\hline $9 / 27 / 930: 00$ & Manual Tape & 411.25 & Good & \\
\hline $9 / 27 / 937: 04$ & Auto FIC & 411.70 & Good & \\
\hline $9 / 28 / 93 \quad 0: 00$ & Manual Tape & 411.25 & Good & \\
\hline 9/28/93 7:04 & Auto FIC & 411.7 & Good & \\
\hline $9 / 29 / 930: 00$ & Manual Tape & 411.50 & Good & \\
\hline 9/29/93 7:04 & Auto FIC & 411.7 & Good & \\
\hline $9 / 30 / 930: 00$ & Manual Tape & 411.25 & Good & \\
\hline $9 / 30 / 937: 03$ & Auto FIC & 411.7 & Good & \\
\hline $10 / 1 / 930: 00$ & Manual Tape & 411.25 & Good & \\
\hline $10 / 1 / 937: 06$ & Auto FIC & 411.70 & Good & \\
\hline $10 / 2 / 93 \quad 0: 00$ & Manual Tape & 411.25 & Good & \\
\hline $10 / 2 / 937: 05$ & Auto FIC & 411.70 & Good & \\
\hline $10 / 3 / 930: 00$ & Manual Tape & 411.25 & Good & \\
\hline $10 / 3 / 937: 06$ & Auto FIC & 411.80 & Good & \\
\hline $10 / 4 / 930: 00$ & Manual Tape & 411.5 & Good & \\
\hline $10 / 4 / 937: 06$ & Auto FIC & 411.8 & Good & \\
\hline $10 / 5 / 930: 00$ & Manual Tape & 411.50 & Good & \\
\hline $10 / 5 / 937: 06$ & Auto FIC & 411.8 & Good & \\
\hline $10 / 6 / 930: 00$ & Manual Tape & 411.5 & Good & \\
\hline $10 / 6 / 937: 06$ & Auto FIC & 411.80 & Good & \\
\hline $10 / 7 / 93 \quad 0: 00$ & Manual Tape & 411.50 & Good & \\
\hline $10 / 7 / 937: 05$ & Auto FIC & 411.80 & Good & \\
\hline $10 / 8 / 930: 00$ & Manual Tape & 411.50 & Good & \\
\hline $10 / 8 / 937: 04$ & Auto FIC & 411.80 & Good & \\
\hline $10 / 9 / 930: 00$ & Manual Tape & 411.25 & Good & \\
\hline $10 / 9 / 937: 03$ & Auto FIC & 411.80 & Good & \\
\hline $10 / 10 / 93 \quad 0: 00$ & Manual Tape & 411.25 & Good & \\
\hline 10/10/93 6:04 & Auto FIC & 411.80 & Good & \\
\hline 10/11/93 0:00 & Manual Tape & 411.5 & Good & \\
\hline $10 / 11 / 937: 03$ & Auto FIC & 411.80 & Good & \\
\hline $10 / 12 / 930: 00$ & Manual Tape & 411.5 & Good & \\
\hline
\end{tabular}

Data obtained from WHC Surveillance Analysis Computer System, (PC SACS) July 7, 1995. 


\begin{tabular}{|c|c|c|c|c|}
\hline \multicolumn{5}{|c|}{ 241-AP-103 } \\
\hline \multicolumn{5}{|c|}{ Surface Level Data } \\
\hline Date & Type of Measurement & Level (Inches) & Data Quality & Comments \\
\hline $10 / 12 / 937: 04$ & Auto FIC & 411.8 & Good & \\
\hline $10 / 13 / 930: 00$ & Manual Tape & 411.50 & Good & \\
\hline $10 / 13 / 937: 03$ & Auto FIC & 411.80 & Good & \\
\hline 10/14/93 0:00 & Manual Tape & 411.5 & Good & \\
\hline 10/14/93 7:04 & Auto FIC & 411.9 & Good & \\
\hline $10 / 15 / 930: 00$ & Manual Tape & 411.50 & Good & \\
\hline $10 / 15 / 937: 04$ & Auto FIC & 411.8 & Good & \\
\hline $10 / 16 / 930: 00$ & Manual Tape & 411.50 & Good & \\
\hline $10 / 16 / 937: 04$ & Auto FIC & 411.80 & Good & \\
\hline $10 / 17 / 930: 00$ & Manual Tape & $411 . \overline{5}$ & Good & \\
\hline $10 / 17 / 937: 03$ & Auto FIC & 411.8 & Good & \\
\hline $10 / 18 / 930: 00$ & Manual Tape & 411.50 & Good & \\
\hline 10/18/93 7:04 & Auto FIC & 411.80 & Good & \\
\hline 10/19/93 0:00 & Manual Tape & 411.5 & Good & \\
\hline 10/19/93 7:04 & Auto FIC & 411.80 & Good & \\
\hline $10 / 20 / 930: 00$ & Manual Tape & 411.50 & Good & \\
\hline $10 / 20 / 937: 03$ & Auto FIC & 411.70 & Good & \\
\hline $10 / 21 / 930: 00$ & Manual Tape & 411.50 & Good & \\
\hline $10 / 21 / 937: 04$ & Auto FIC & 411.70 & Good & \\
\hline $10 / 22 / 930: 00$ & Manual Tape & 411.5 & Good & \\
\hline 10/22/93 7:04 & Auto FIC & 411.7 & Good & \\
\hline $10 / 23 / 930: 00$ & Manual Tape & 411.50 & Good & \\
\hline 10/23/93 7:04 & Auto FIC & 411.70 & Good & \\
\hline $10 / 24 / 930: 00$ & Manual Tape & 411.50 & Good & \\
\hline $10 / 24 / 937: 04$ & Auto FIC & 411.7 & Good & \\
\hline $10 / 25 / 93 \quad 0: 00$ & Manual Tape & 411.5 & Good & \\
\hline $10 / 25 / 937: 04$ & Auto FIC & 411.7 & Good & \\
\hline $10 / 26 / 930: 00$ & Manual Tape & 411.50 & Good & \\
\hline $10 / 26 / 936: 03$ & Auto FIC & 411.70 & Good & \\
\hline $10 / 27 / 930: 00$ & Manual Tape & 411.50 & Good & \\
\hline $10 / 27 / 937: 04$ & Auto FIC & 411.7 & Good & \\
\hline 10/28/93 0:00 & Manual Tape & 411.5 & Good & \\
\hline 10/28/93 7:04 & Auto FIC & 411.8 & Good & \\
\hline $10 / 29 / 930: 00$ & Manual Tape & 411.50 & Good & \\
\hline $10 / 29 / 936: 03$ & Auto FIC & 411.6 & Good & \\
\hline $10 / 30 / 930: 00$ & Manual Tape & 411.5 & Good & \\
\hline $10 / 30 / 937: 04$ & Auto FIC & 411.6 & Good & \\
\hline $10 / 31 / 93 \quad 0: 00$ & Manual Tape & 411.5 & Good & \\
\hline $10 / 31 / 937: 06$ & Auto FIC & 411.60 & Good & \\
\hline $11 / 1 / 937: 06$ & Auto FIC & 411.60 & Good & \\
\hline $11 / 2 / 930: 00$ & Manual Tape & 411.25 & Good & \\
\hline $11 / 2 / 937: 04$ & Auto FIC & 411.6 & Good & \\
\hline $11 / 3 / 930: 00$ & Manual Tape & 411.50 & Good & \\
\hline $11 / 3 / 937: 06$ & Auto FIC & 411.60 & Good & \\
\hline $11 / 4 / 930: 00$ & Manual Tape & 411.25 & Good & \\
\hline $11 / 4 / 937: 04$ & Auto FIC & 411.60 & Good & \\
\hline $11 / 5 / 930: 00$ & Manual Tape & 411.25 & Good & \\
\hline $11 / 5 / 937: 06$ & Auto FIC & 411.60 & Good & \\
\hline
\end{tabular}

Date obtained from WHC Surveillance Analysis Computer System, (PC SACS) July 7, 1995. 


\begin{tabular}{|c|c|c|c|c|}
\hline \multicolumn{5}{|c|}{ 241-AP-103 } \\
\hline \multicolumn{5}{|c|}{ Surface Lavel Data } \\
\hline Date & Type of Measurement & Leval (Inches) & Data Quality & Comments \\
\hline $11 / 6 / 930: 00$ & Manual Tape & 411.25 & Good & \\
\hline $11 / 6 / 937: 06$ & Auto FIC & 411.7 & Good & \\
\hline $11 / 7 / 930: 00$ & Manual Tape & 411.25 & Good & \\
\hline $11 / 7 / 937: 06$ & Auto FIC & 411.8 & Good & \\
\hline $11 / 8 / 930: 00$ & Manual Tape & 411.25 & Good & \\
\hline $11 / 8 / 937: 06$ & Auto FIC & 411.8 & Good & \\
\hline 11/9/93 0:00 & Manual Tape & 411.25 & Good & \\
\hline $11 / 9 / 937: 03$ & Auto FIC & 411.7 & Good & \\
\hline $11 / 10 / 930: 00$ & Manual Tape & 411.25 & Good & \\
\hline $11 / 10 / 937: 04$ & Auto FIC & 411.7 & Good & \\
\hline $11 / 11 / 930: 00$ & Manual Tape & 411.25 & Good & \\
\hline $11 / 11 / 937: 04$ & Auto FIC & 411.80 & Good & \\
\hline $11 / 12 / 930: 00$ & Manual Tape & 411.25 & Good & \\
\hline $11 / 12 / 937: 04$ & Auto FIC & 411.80 & Good & \\
\hline $11 / 13 / 930: 00$ & Manual Tape & 411.25 & Good & \\
\hline $11 / 13 / 937: 04$ & Auto FIC & 411.8 & Good & \\
\hline $11 / 14 / 930: 00$ & Manual Tape & 411.25 & Good & \\
\hline $11 / 14 / 936: 04$ & Auto FIC & 411.5 & Good & \\
\hline $11 / 15 / 930: 00$ & Manual Tape & 411.25 & Good & \\
\hline $11 / 15 / 937: 04$ & Auto FIC & 411.5 & Good & \\
\hline $11 / 16 / 930: 00$ & Manual Tape & 411.25 & Good & \\
\hline $11 / 16 / 937: 03$ & Auto FIC & 411.80 & Good & \\
\hline $11 / 17 / 930: 00$ & Manual Tape & 411.25 & Good & \\
\hline $11 / 17 / 937: 04$ & Auto FIC & 411.80 & Good & \\
\hline $11 / 18 / 937: 04$ & Auto FIC & 411.8 & Good & \\
\hline $11 / 19 / 937: 04$ & Auto FIC & 411.80 & Good & \\
\hline $11 / 20 / 937: 04$ & Auto FIC & 411.8 & Good & \\
\hline $11 / 21 / 937: 04$ & Auto FIC & 411.8 & Good & \\
\hline $11 / 22 / 930: 00$ & Manual Tape & $4 \uparrow 1.25$ & Good & \\
\hline $11 / 22 / 937: 04$ & Auto FIC & 411.80 & Good & \\
\hline $11 / 23 / 930: 00$ & Manual Tape & 411.25 & Good & \\
\hline $11 / 23 / 937: 04$ & Auto FIC & 411.8 & Good & \\
\hline $11 / 24 / 930: 00$ & Manual Tape & 411.25 & Good & \\
\hline $11 / 24 / 937: 04$ & Auto FIC & 411.80 & Good & \\
\hline $11 / 25 / 930: 00$ & Manual Tape & 411.25 & Good & \\
\hline $11 / 25 / 937: 04$ & Auto FIC & 411.80 & Good & \\
\hline $11 / 26 / 930: 00$ & Manual Tape & 411.25 & Good & \\
\hline $11 / 26 / 937: 04$ & Auto FIC & 411.9 & Good & \\
\hline $11 / 27 / 930: 00$ & Manual Tape & 411.25 & Good & \\
\hline $11 / 27 / 937: 04$ & Auto FIC & 411.80 & Good & \\
\hline $11 / 28 / 930: 00$ & Manual Tape & 411.25 & Good & \\
\hline $11 / 28 / 937: 03$ & Auto FIC & 411.80 & Good & \\
\hline $11 / 29 / 930: 00$ & Manual Tape & 411.25 & Good & \\
\hline $11 / 29 / 93 \quad 7: 04$ & Auto FIC & 411.8 & Good & \\
\hline $11 / 30 / 930: 00$ & Manual Tape & 411.25 & Good & \\
\hline $11 / 30 / 937: 03$ & Auto FIC & 411.8 & Good & \\
\hline $12 / 1 / 930: 00$ & Manual Tape & 411.25 & Good & \\
\hline $12 / 1 / 937: 04$ & Auto FIC & 411.7 & Good & \\
\hline
\end{tabular}

Data obtained from WHC Surveillance Analysis Computer System, (PC SACS) July 7, 1995. 


\begin{tabular}{|c|c|c|c|c|}
\hline \multicolumn{5}{|c|}{ 241-AP-103 } \\
\hline \multicolumn{5}{|c|}{ Surface Level Data } \\
\hline Date & Type of Measurement & Level (Inches) & Data Quality & Comments \\
\hline $12 / 2 / 930: 00$ & Manual Tape & 411.25 & Good & \\
\hline $12 / 2 / 937: 04$ & Auto FIC & 411.7 & Good & \\
\hline $12 / 3 / 930: 00$ & Manual Tape & 411.25 & Good & \\
\hline $12 / 3 / 937: 05$ & Auto FIC & 411.80 & Good & \\
\hline $12 / 4 / 930: 00$ & Manual Tape & 411.25 & Good & \\
\hline $12 / 4 / 937: 05$ & Auto FIC & 411.8 & Good & \\
\hline $12 / 5 / 930: 00$ & Manual Tape & 411.25 & Good & \\
\hline $12 / 5 / 937: 04$ & Auto FIC & 411.80 & Good & \\
\hline $12 / 6 / 930: 00$ & Manual Tape & 411.00 & Good & \\
\hline $12 / 6 / 937: 05$ & Auto FIC & 411.80 & Good & \\
\hline $12 / 7 / 930: 00$ & Manual Tape & 411 & Good & \\
\hline $12 / 7 / 93 \quad 7: 04$ & Auto FIC & 411.8 & Good & \\
\hline $12 / 8 / 930: 00$ & Manual Tape & 411.25 & Good & \\
\hline $12 / 8 / 937: 06$ & Auto FIC & 411.40 & Good & \\
\hline $12 / 9 / 930: 00$ & Manual Tape & 411 & Good & \\
\hline $12 / 9 / 937: 04$ & Auto FIC & 411.4 & Good & \\
\hline $12 / 10 / 930: 00$ & Manual Tape & 41.1 .00 & Good & \\
\hline 12/10/93 6:05 & Auto FIC & 411.40 & Good & \\
\hline $12 / 11 / 930: 00$ & Manual Tape & 411.00 & Good & \\
\hline 12/11/93 7:06 & Auto FIC & 411.40 & Good & \\
\hline $12 / 12 / 930: 00$ & Manual Tape & 411.5 & Good & \\
\hline $12 / 12 / 937: 06$ & Auto FIC & 411.40 & Good & \\
\hline $12 / 13 / 930: 00$ & Manual Tape & 411 & Good & \\
\hline $12 / 13 / 937: 06$ & Auto FIC & 411.40 & Good & \\
\hline $12 / 14 / 930: 00$ & Manual Tape & 411.25 & Good & \\
\hline $12 / 14 / 937: 06$ & Auto FIC & 411.4 & Good & \\
\hline $12 / 15 / 93 \quad 0: 00$ & Manual Tape & 411.25 & Good & \\
\hline $12 / 15 / 937: 06$ & Auto FIC & 411.4 & Good & \\
\hline $12 / 16 / 930: 00$ & Manual Tape & 411.25 & Good & \\
\hline $12 / 16 / 937: 06$ & Auto FIC & 411.4 & Good & \\
\hline $12 / 17 / 930: 00$ & Manual Tape & 411.00 & Good & \\
\hline $12 / 17 / 937: 05$ & Auto FIC & 411.4 & Good & \\
\hline $12 / 18 / 930: 00$ & Manual Tape & 411.25 & Good & \\
\hline $12 / 18 / 937: 06$ & Auto FIC & 411.40 & Good & \\
\hline $12 / 19 / 930: 00$ & Manual Tape & 411.00 & Good & \\
\hline $12 / 19 / 937: 06$ & Auto FIC & 411.4 & Good & \\
\hline $12 / 20 / 930: 00$ & Manual Tape & 411 & Good & \\
\hline $12 / 20 / 937: 06$ & Auto FIC & 411.40 & Good & \\
\hline $12 / 21 / 930: 00$ & Manual Tape & 411.00 & Good & \\
\hline $12 / 21 / 937: 06$ & Auto FIC & 411.40 & Good & \\
\hline $12 / 22 / 930: 00$ & Manual Tape & 411.25 & Good & \\
\hline $12 / 22 / 93 \quad 7: 05$ & Auto FIC & 411.40 & Good & \\
\hline $12 / 23 / 930: 00$ & Manual Tape & 411.25 & Good & \\
\hline $12 / 23 / 936: 06$ & Auto FIC & 411.40 & Good & \\
\hline $12 / 24 / 930: 00$ & Manua! Tape & 411.25 & Good & \\
\hline $12 / 24 / 937: 06$ & Auto FIC & 411.60 & Good & \\
\hline $12 / 25 / 930: 00$ & Manual Tape & 411.25 & Good & \\
\hline $12 / 25 / 937: 06$ & Auto FIC & 411.3 & Good & \\
\hline
\end{tabular}

Data obtained from WHC Surveillance Analysis Computer System, (PC SACS) July 7, 1995. 


\begin{tabular}{|c|c|c|c|c|}
\hline \multicolumn{5}{|c|}{ 241-AP-103 } \\
\hline & Type of Measurement & Level (Inches) & Data Quality & Comments \\
\hline$\frac{\text { Date }}{12 / 26 / 930: 00}$ & Manual Tape & 411.25 & Good & \\
\hline $\begin{array}{l}12 / 26 / 930: 00 \\
12 / 26 / 937: 06\end{array}$ & Auto FIC & 411.30 & Good & \\
\hline$\frac{12 / 26 / 937: 06}{12 / 27 / 930: 00}$ & Manual Tape & 411.25 & Good & \\
\hline$\frac{12 / 27 / 930: 00}{12 / 27 / 937: 06}$ & Auto FIC & 411.5 & Good & \\
\hline & Manual Tape & 411.25 & Good & \\
\hline $12 / 28 / 937: 06$ & Auto FIC & 411.5 & Good & \\
\hline $12 / 29 / 930: 00$ & Manual Tape & 411.00 & Good & \\
\hline $12 / 29 / 937: 06$ & Auto FIC & 411.30 & Good & \\
\hline $12 / 30 / 930: 00$ & Manual Tape & 411 & Good & \\
\hline $12 / 30 / 937: 05$ & Auto FIC & 411.30 & Good & \\
\hline $12 / 31 / 930: 00$ & Manual Tape & 411 & Good & \\
\hline 12/31/93 7:06 & Auto FIC & 411.3 & Good & \\
\hline 1/1/940:00 & Manual Tape & 411.00 & Good & \\
\hline 1/1/947:06 & Auto FIC & 411.3 & Good & \\
\hline 1/2/940:00 & Manual Tape & 411.00 & Good & \\
\hline $1 / 2 / 947: 06$ & Auto FIC & 411.3 & Good & \\
\hline $1 / 3 / 940: 00$ & Manual Tape & 411 & Good & \\
\hline $1 / 3 / 947: 07$ & Auto FIC & 411.30 & Good & \\
\hline $1 / 4 / 940: 00$ & Manual Tape & 411.00 & Good & \\
\hline $1 / 4 / 947: 07$ & Auto FIC & 411.30 & Good & \\
\hline $1 / 5 / 940: 00$ & Manual Tape & 411 & Good & \\
\hline $1 / 5 / 947: 06$ & Auto FIC & 411.40 & Good & \\
\hline $1 / 6 / 940: 00$ & Manual Tape & 411 & Good & \\
\hline $1 / 6 / 947: 06$ & Auto FIC & 411.30 & Good & \\
\hline $1 / 7 / 940: 00$ & Manual Tape & 411.00 & Good & \\
\hline $1 / 7 / 947: 07$ & Auto FIC & 411.3 & Good & \\
\hline $1 / 8 / 940: 00$ & Manual Tape & 411.00 & Good & \\
\hline 1/8/94 7:06 & Auto FIC & 411.3 & Good & \\
\hline $1 / 9 / 940: 00$ & Manual Tape & 411 & Good & \\
\hline $1 / 9 / 947: 06$ & Auto FIC & 411.30 & Good & \\
\hline $1 / 10 / 940: 00$ & Manual Tape & 411 & Good & \\
\hline $1 / 10 / 947: 07$ & Auto FIC & 411.30 & Good & \\
\hline $1 / 11 / 940: 00$ & Manual Tape & 411.00 & Good & \\
\hline $1 / 11 / 947: 07$ & Auto FIC & 411.5 & Good & \\
\hline 1/12/940:00 & Manual Tape & 411.00 & Good & \\
\hline $1 / 12 / 947: 06$ & Auto FIC & 411.50 & Good & \\
\hline $1 / 13 / 940: 00$ & Manual Tape & 411.25 & Good & \\
\hline $1 / 13 / 945: 07$ & Auto FIC & 411.30 & Good & \\
\hline $1 / 14 / 940: 00$ & Manual Tape & 411 & Good & \\
\hline $1 / 14 / 947: 06$ & Auto FIC & 411.3 & Good & \\
\hline $1 / 15 / 940: 00$ & Manual Tape & 411.25 & Good & \\
\hline $1 / 15 / 945: 07$ & Auto FIC & 411.30 & Good & \\
\hline $1 / 16 / 940: 00$ & Manual Tape & 411.00 & Good & \\
\hline $1 / 16 / 947: 07$ & Auto FIC & 411.2 & Good & \\
\hline $1 / 17 / 940: 00$ & Manual Tape & 411.00 & Good & \\
\hline $1 / 17 / 947: 07$ & Auto FIC & 411.30 & Good & \\
\hline $1 / 18 / 940: 00$ & Manual Tape & 411.00 & Good & \\
\hline $1 / 18 / 947: 07$ & Auto FIC & 411.50 & Good & \\
\hline
\end{tabular}

Data obtained from WHC Surveillance Analysis Computer System, (PC SACS) July 7, 1995. 


\begin{tabular}{|c|c|c|c|c|}
\hline \multicolumn{5}{|c|}{ 241-AP-103 } \\
\hline \multicolumn{5}{|c|}{ Surface Level Data } \\
\hline Date & Type of Measurement & Level (Inches) & Data Quality & Comments \\
\hline 1/19/940:00 & Manual Tape & 411 & Good & \\
\hline $1 / 19 / 947: 07$ & Auto FIC & 411.5 & Good & \\
\hline $1 / 20 / 940: 00$ & Manual Tape & 411 & Good & \\
\hline $1 / 20 / 947: 07$ & Auto FIC & $411 . \overline{50}$ & Good & \\
\hline $1 / 21 / 940: 00$ & Manual Tape & 411.00 & Good & \\
\hline $1 / 21 / 947: 07$ & Auto FIC & 411.5 & Good & \\
\hline $1 / 22 / 940: 00$ & Manual Tape & 411.00 & Good & \\
\hline $1 / 22 / 947: 08$ & Auto FIC & 411.5 & Good & \\
\hline $1 / 23 / 940: 00$ & Manual Tape & 411 & Good & \\
\hline $1 / 23 / 947: 07$ & Auto FIC & 411.50 & Good & \\
\hline $1 / 24 / 940: 00$ & Manual Tape & 411.00 & Good & \\
\hline $1 / 24 / 947: 08$ & Auto FIC & 411.5 & Good & \\
\hline $1 / 25 / 940: 00$ & Manual Tape & 411.00 & Good & \\
\hline $1 / 25 / 947: 07$ & Auto FIC & 411.50 & Good & \\
\hline $1 / 26 / 940: 00$ & Manual Tape & 411.00 & Good & \\
\hline $1 / 26 / 947: 06$ & Auto FIC & 411.5 & Good & \\
\hline $1 / 27 / 940: 00$ & Manual Tape & 411 & Good & \\
\hline $1 / 27 / 946: 06$ & Auto FIC & 411.5 & Good & \\
\hline $1 / 28 / 940: 00$ & Manual Tape & 411 & Good & \\
\hline $1 / 28 / 947: 07$ & Auto FIC & 411.50 & Good & \\
\hline $1 / 29 / 940: 00$ & Manual Tape & 411.00 & Good & \\
\hline $1 / 29 / 947: 07$ & Auto FIC & 411.50 & Good & \\
\hline $1 / 30 / 940: 00$ & Manual Tape & 411.00 & Good & \\
\hline $1 / 30 / 947: 06$ & Auto FIC & 411.40 & Good & \\
\hline $1 / 31 / 940: 00$ & Manual Tape & 411 & Good & \\
\hline $1 / 31 / 947: 07$ & Auto FIC & 411.40 & Good & \\
\hline $2 / 1 / 940: 00$ & Manual Tape & 411.00 & Good & \\
\hline 2/1/94 7:06 & Auto FIC & 411.4 & Good & \\
\hline $2 / 2 / 940: 00$ & Manual Tape & 411.00 & Good & \\
\hline $2 / 2 / 947: 07$ & Auto FIC & 411.4 & Good & \\
\hline $2 / 3 / 940: 00$ & Manual Tape & 411 & Good & \\
\hline $2 / 3 / 947: 07$ & Auto FIC & 411.4 & Good & \\
\hline $2 / 4 / 940: 00$ & Manual Tape & 411 & Good & \\
\hline $2 / 4 / 947: 07$ & Auto FIC & 411.40 & Good & \\
\hline $2 / 5 / 940: 00$ & Manual Tape & 411 & Good & \\
\hline $2 / 5 / 947: 07$ & Auto FIC & $411: 40$ & Good & \\
\hline $2 / 6 / 940: 00$ & Manual Tape & 411 & Good & \\
\hline $2 / 6 / 947: 07$ & Auto FIC & 411.40 & Good & \\
\hline $2 / 7 / 940: 00$ & Manual Tape & 411.00 & Good & \\
\hline $2 / 7 / 947: 06$ & Auto FIC & 411.4 & Good & \\
\hline $2 / 8 / 940: 00$ & Manual Tape & 411.00 & Good & \\
\hline $2 / 8 / 947: 07$ & Auto FIC & 411.4 & Good & \\
\hline 2/9/94 0:00 & Manual Tape & 411.00 & Good & \\
\hline $2 / 9 / 947: 04$ & Auto FIC & 411.1 & Good & \\
\hline $2 / 10 / 940: 00$ & Manual Tape & 411 & Good & \\
\hline $2 / 10 / 947: 04$ & Auto FIC & 411.1 & Good & \\
\hline $2 / 11 / 940: 00$ & Manual Tape & 411 & Good & \\
\hline $2 / 11 / 947: 06$ & Auto FIC & 411.1 & Good & \\
\hline
\end{tabular}

Data obtained from WHC Surveillance Analysis Computer System, (PC SACS) July 7, 1995. 


\begin{tabular}{|c|c|c|c|c|}
\hline \multicolumn{5}{|c|}{ 241-AP-103 } \\
\hline \multicolumn{5}{|c|}{ Surface Level Data } \\
\hline Date & Type of Measurement & Level (Inches) & Data Quality & Comments \\
\hline $2 / 12 / 940: 00$ & Manual Tape & 411.00 & Good & \\
\hline 2/12/94 7:03 & Auto FIC & 411.30 & Good & \\
\hline $2 / 13 / 940: 00$ & Manual Tape & 411 & Good & \\
\hline 2/13/94 7:04 & Auto FIC & 411.10 & Good & \\
\hline $2 / 14 / 940: 00$ & Manual Tape & 411.00 & Good & \\
\hline 2/14/94 7:04 & Auto FIC & 411.10 & Good & \\
\hline $2 / 15 / 940: 00$ & Manual Tape & 411.00 & Good & \\
\hline 2/15/94 7:04 & Auto FIC & 411.10 & Good & \\
\hline $2 / 16 / 940: 00$ & Manual Tape & 411 & Good & \\
\hline $2 / 16 / 947: 03$ & Auto FIC & 411.10 & Good & \\
\hline 2/17/94 0:00 & Manual Tape & 411 & Good & \\
\hline $2 / 17 / 947: 04$ & Auto FIC & 411.1 & Good & \\
\hline $2 / 18 / 940: 00$ & Manual Tape & 411 & Good & \\
\hline $2 / 18 / 947: 06$ & Auto FIC & 411.10 & Good & \\
\hline $2 / 19 / 940: 00$ & Manual Tape & 411.00 & Good & \\
\hline $2 / 19 / 947: 06$ & Auto FIC & 411.1 & Good & \\
\hline $2 / 20 / 940: 00$ & Manual Tape & 411.00 & Good & \\
\hline $2 / 20 / 947: 07$ & Auto FIC & 411.2 & Good & \\
\hline $2 / 21 / 940: 00$ & Manual Tape & 411.00 & Good & \\
\hline $2 / 21 / 947: 06$ & Auto FIC & 411.20 & Good & \\
\hline $2 / 22 / 940: 00$ & Manual Tape & 411 & Good & \\
\hline $2 / 22 / 947: 05$ & Auto FIC & 411.2 & Good & \\
\hline $2 / 23 / 940: 00$ & Manual Tape & 411 & Good & \\
\hline $2 / 23 / 947: 06$ & Auto FIC & 411.20 & Good & \\
\hline $2 / 24 / 940: 00$ & Manual Tape & 410.75 & Good & \\
\hline $2 / 24 / 947: 06$ & Auto FIC & 411.20 & Good & \\
\hline $2 / 25 / 940: 00$ & Manual Tape & 411.00 & Good & \\
\hline $2 / 25 / 946: 06$ & Auto FIC & 411.3 & Good & \\
\hline $2 / 26 / 940: 00$ & Manual Tape & 411 & Good & \\
\hline $2 / 26 / 947: 07$ & Auto FIC & 411.30 & Good & \\
\hline $2 / 27 / 940: 00$ & Manual Tape & 411.00 & Good & \\
\hline $2 / 27 / 947: 07$ & Auto FIC & 411.3 & Good & \\
\hline $2 / 28 / 940: 00$ & Manual Tape & 411.00 & Good & \\
\hline $2 / 28 / 946: 06$ & Auto FIC & 411.30 & Good & \\
\hline $3 / 1 / 940: 00$ & Manual Tape & 411 & Good & \\
\hline $3 / 1 / 947: 04$ & Auto FIC & 411.40 & Good & \\
\hline $3 / 2 / 940: 00$ & Manual Tape & 411 & Good & \\
\hline $3 / 2 / 947: 06$ & Auto FIC & 411.4 & Good & \\
\hline $3 / 3 / 940: 00$ & Manual Tape & 411.25 & Good & \\
\hline $3 / 3 / 947: 06$ & Auto FIC & 411.4 & Good & \\
\hline $3 / 4 / 940: 00$ & Manual Tape & 410.75 & Good & \\
\hline 3/4/94 7:04 & Auto FIC & 411.4 & Good & \\
\hline $3 / 5 / 940: 00$ & Manual Tape & 410.75 & Good & \\
\hline $3 / 5 / 947: 05$ & Auto FIC & 411.1 & Good & \\
\hline $3 / 6 / 940: 00$ & Manual Tape & 410.5 & Good & \\
\hline $3 / 6 / 947: 05$ & Auto FIC & 411.10 & Good & \\
\hline $3 / 7 / 940: 00$ & Manual Tape & 411 & Good & \\
\hline $3 / 7 / 947: 05$ & Auto FIC & 411.10 & Good & \\
\hline
\end{tabular}

Data obtained from WHC Surveillance Analysis Computer System, (PC SACS) July 7, 1995. 


\begin{tabular}{|c|c|c|c|c|}
\hline \multicolumn{5}{|c|}{ 241-AP-103 } \\
\hline \multicolumn{5}{|c|}{ Surface Level Data } \\
\hline Date & Type of Measurement & Level (Inches) & Data Quality & Comments \\
\hline $3 / 8 / 940: 00$ & Manual Tape & 411.00 & Good & \\
\hline $3 / 8 / 947: 04$ & Auto FIC & 411.10 & Good & \\
\hline 3/9/94 0:00 & Manual Tape & 410.75 & Good & \\
\hline $3 / 9 / 947: 04$ & Auto FIC & 411.40 & Good & \\
\hline $3 / 10 / 940: 00$ & Manual Tape & $411: 00$ & Good & \\
\hline $3 / 10 / 947: 05$ & Auto FIC & 411.4 & Good & \\
\hline $3 / 11 / 940: 00$ & Manual Tape & 411 & Good & \\
\hline $3 / 11 / 947: 06$ & Auto FIC & 411.4 & Good & \\
\hline $3 / 12 / 940: 00$ & Manual Tape & 411 & Good & \\
\hline $3 / 12 / 947: 06$ & Auto FIC & 411.40 & Good & \\
\hline $3 / 13 / 940: 00$ & Manual Tape & 411.00 & Good & \\
\hline $3 / 13 / 947: 05$ & Auto FIC & 411.4 & Good & \\
\hline $3 / 14 / 940: 00$ & Auto FIC & 411.40 & Good & \\
\hline $3 / 14 / 940: 00$ & Manual Tape & 410.75 & Good & \\
\hline $3 / 15 / 940: 00$ & Manual Tape & 410.75 & Good & \\
\hline 3/15/94 7:05 & Auto FIC & 411,40 & Good & \\
\hline $3 / 16 / 940: 00$ & Manual Tape & 411.25 & Good & \\
\hline $3 / 16 / 94 \quad 7: 04$ & Auto FIC & 411.4 & Good & \\
\hline $3 / 17 / 940: 00$ & Manual Tape & 411 & Good & \\
\hline $3 / 17 / 947: 05$ & Auto FIC & 411.40 & Good & \\
\hline 3/18/94 0:00 & Manual Tape & 410.75 & Good & \\
\hline 3/18/94 7:05 & Auto FIC & 411:40 & Good & \\
\hline $3 / 19 / 940: 00$ & Manual Tape & 410.75 & Good & \\
\hline $3 / 19 / 947: 04$ & Auto FIC & 411.4 & Good & \\
\hline $3 / 20 / 940: 00$ & Manual Tape & 410.75 & Good & \\
\hline $3 / 20 / 947: 05$ & Auto FIC & 411.40 & Good & \\
\hline $3 / 21 / 940: 00$ & Manual Tape & 410.75 & Good & \\
\hline $3 / 21 / 947: 05$ & Auto FIC & 411.4 & Good & \\
\hline $3 / 22 / 940: 00$ & Manual Tape & 410.75 & Good & \\
\hline $3 / 22 / 947: 04$ & Auto FIC & 411.4 & Good & \\
\hline $3 / 23 / 940: 00$ & Manual FIC & 411.40 & Good & \\
\hline $3 / 23 / 940: 00$ & Manual Tape & 410.75 & Good & \\
\hline $3 / 24 / 940: 00$ & Manual Tape & 410.75 & Good & \\
\hline $3 / 24 / 947: 04$ & Auto FIC & 411.30 & Good & \\
\hline $3 / 25 / 940: 00$ & Manual Tape & 410.75 & Good & \\
\hline $3 / 25 / 947: 05$ & Auto FIC & 411.30 & Good & \\
\hline $3 / 26 / 940: 00$ & Manual Tape & 410.75 & Good & \\
\hline $3 / 26 / 947: 04$ & Auto FIC & 411.30 & Good & \\
\hline $3 / 27 / 940: 00$ & Manual Tape & 41.0 .75 & Good & \\
\hline $3 / 27 / 947: 04$ & Auto FIC & 411.30 & Good & \\
\hline $3 / 28 / 940: 00$ & Manual Tape & 410.75 & Good & \\
\hline $3 / 28 / 947: 04$ & Auto FIC & 411.30 & Good & \\
\hline $3 / 29 / 940: 00$ & Manual Tape & 410.75 & Good & \\
\hline $3 / 29 / 947: 03$ & Auto FIC & 411.3 & Good & \\
\hline $3 / 30 / 940: 00$ & Manual Tape & 410.75 & Good & \\
\hline $3 / 30 / 947: 03$ & Auto FIC & 411.3 & Good & \\
\hline 3/31/94 0:00 & Manual Tape & 410.75 & Good & \\
\hline $3 / 31 / 947: 03$ & Auto FIC & 411.30 & Good & \\
\hline
\end{tabular}

Data obtained from WHC Surveillance Analysis Computer System, (PC SACS) July 7, 1995. 


\begin{tabular}{|c|c|c|c|c|}
\hline \multicolumn{4}{|c|}{ 241-AP-103 } & \\
\hline Date & Type of Measurement & Level (Inches) & Data Quality & Comments \\
\hline $4 / 1 / 940: 00$ & Manual Tape & 410.75 & Good & \\
\hline 4/1/94 0:04 & Auto FIC & 411.3 & Good & \\
\hline $4 / 2 / 940: 00$ & Manual Tape & 411 & Good & \\
\hline 4/2/94 7:07 & Auto FIC & 411.30 & Good & \\
\hline 4/3/94 0:00 & Manual Tape & 411.00 & Good & \\
\hline 4/3/947:03 & Auto FIC & 411.30 & Good & \\
\hline $4 / 4 / 940: 00$ & Manual Tape & 411.00 & Good & \\
\hline $4 / 4 / 947: 03$ & Auto FIC & 411.3 & Good & \\
\hline $4 / 5 / 940: 00$ & Manual Tape & 411.00 & Good & \\
\hline 4/5/94 7:03 & Auto FIC & 411.30 & Good & \\
\hline $4 / 6 / 940: 00$ & Manual Tape & 410.75 & Good & \\
\hline $4 / 6 / 947: 06$ & Auto FIC & 411.30 & Good & \\
\hline 4/7/940:00 & Manual Tape & 410.75 & Good & \\
\hline 4/7/94 7:07 & Auto FIC & 411.3 & Good & \\
\hline $4 / 8 / 940: 00$ & Manual Tape & 410.75 & Good & \\
\hline $4 / 8 / 947: 03$ & Auto FIC & 411.3 & Good & \\
\hline $4 / 9 / 940: 00$ & Manual Tape & 410.75 & Good & \\
\hline $4 / 9 / 947: 07$ & Auto FIC & 411.3 & Good & \\
\hline $4 / 10 / 940: 00$ & Manual Tape & 410.75 & Good & \\
\hline 4/10/947:03 & Auto FIC & 411.3 & Good & \\
\hline $4 / 11 / 940: 00$ & Manual Tape & 410.75 & Good & \\
\hline 4/11/947:03 & Auto FIC & 411.30 & Good & \\
\hline 4/12/940:00 & Manual Tape . & 410.75 & Good & \\
\hline 4/12/94 7:03 & Auto FIC & 411.30 & Good & \\
\hline $4 / 13 / 940: 00$ & Manual Tape & 410.75 & Good & \\
\hline 4/13/94 7:03 & Auto FIC & 411.30 & Good & \\
\hline $4 / 14 / 940: 00$ & Manual Tape & 410.75 & Good & \\
\hline 4/14/94 7:03 & Auto FIC & 411.30 & Good & \\
\hline $4 / 15 / 940: 00$ & Manual Tape & 410.50 & Good & \\
\hline 4/15/94 7:03 & Auto FIC & 411.30 & Good & \\
\hline 4/16/940:00 & Manual Tape & 410.75 & Good & \\
\hline 4/16/94 7:03 & Auto FIC & 411.3 & Good & \\
\hline $4 / 17 / 940: 00$ & Manual Tape & 410.75 & Good & \\
\hline $4 / 17 / 947: 03$ & Auto FIC & 411.3 & Good & \\
\hline $4 / 18 / 940: 00$ & Manual Tape & 410.75 & Good & \\
\hline 4/18/94 7:03 & Auto FIC & 411.3 & Good & \\
\hline 4/19/940:00 & Manual Tape & 410.75 & Good & \\
\hline 4/19/94 6:06 & Auto FIC & 411.3 & Good & \\
\hline 4/20/94 0:00 & Manual Tape & 410.75 & Good & \\
\hline 4/20/94 7:04 & Auto FIC & 411.30 & Good & \\
\hline $4 / 21 / 940: 00$ & Manual Tape & 410.75 & Good & \\
\hline 4/21/94 7:05 & Auto FIC & 411.30 & Good & \\
\hline 4/22/94 0:00 & Manual Tape & 410.75 & Good & \\
\hline 4/22/94 7:04 & Auto FIC & 411.00 & Good & \\
\hline $4 / 23 / 940: 00$ & Manual Tape & 410.75 & Good & \\
\hline $4 / 23 / 947: 05$ & Auto FIC & 411.00 & Good & \\
\hline $4 / 24 / 940: 00$ & Manual Tape & 410.75 & Good & \\
\hline $4 / 24 / 947: 04$ & Auto FIC & 411.00 & Good & \\
\hline
\end{tabular}

Data obtained from WHC Surveillance Analysis Computer System, (PC SACS) July 7, 1995. 


\begin{tabular}{|c|c|c|c|c|}
\hline \multicolumn{5}{|c|}{ 241-AP-103 } \\
\hline \multicolumn{5}{|c|}{ Surface Level Date } \\
\hline Date & Type of Measurement & Leval (Inches) & Data Quality & Comments \\
\hline $4 / 25 / 940: 00$ & Manual Tape & 410.75 & Good & \\
\hline 4/25/94 7:04 & Auto FIC & 411 & Good & \\
\hline 4/26/94 0:00 & Manual Tape & 411.75 & Good & \\
\hline 4/26/94 7:07 & Auto FIC & 411 & Good & \\
\hline $4 / 27 / 940: 00$ & Manual Tape & 411.75 & Good & \\
\hline $4 / 27 / 947: 04$ & Auto FIC & 411.00 & Good & \\
\hline $4 / 28 / 940: 00$ & Manual Tape & 411.75 & Good & \\
\hline $4 / 28 / 947: 05$ & Auto FIC & 411 & Good & \\
\hline $4 / 29 / 94 \quad 0: 00$ & Manual Tape & 411 & Good & \\
\hline 4/29/94 7:04 & Auto FIC & 411.00 & Good & \\
\hline $4 / 30 / 940: 00$ & Manual Tape & 411.75 & Good & \\
\hline 4/30/94 7:05 & Auto FIC & 411.00 & Good & \\
\hline 5/1/94 0:00 & Manual Tape & 411.00 & Good & \\
\hline 5/1/94 7:05 & Auto FIC & 411 & Good & \\
\hline $5 / 2 / 940: 00$ & Manual Tape & 411 & Good & \\
\hline 5/2/94 7:05 & Auto FIC & 411,00 & Gobd & \\
\hline $5 / 3 / 940: 00$ & Manual Tape & 410.75 & Good & \\
\hline 5/3/94 7:05 & Auto FIC & 411 & Good & \\
\hline $5 / 4 / 940: 00$ & Manual Tape & 410.75 & Good & \\
\hline 5/4/94 7:05 & Auto FIC & 411 & Good & \\
\hline 5/5/94 0:00 & Manual Tape & 410.75 & Good & \\
\hline 5/5/94 7:03 & Auto FIC & 411.00 & Good & \\
\hline $5 / 6 / 940: 00$ & Manual Tape & 356.25 & Good & \\
\hline $5 / 6 / 947: 03$ & Auto FIC & 367.10 & Good & \\
\hline $5 / 7 / 940: 00$ & Manual Tape & $232: 25$ & Good & \\
\hline 5/7/94 7:03 & Auto FIC & 249.20 & Good & \\
\hline 5/8/94 0:00 & Manual Tape & 217.00 & Good & \\
\hline $5 / 8 / 947: 03$ & Auto FIC & 217.20 & Good & \\
\hline $5 / 9 / 940: 00$ & Manual Tape & 217.00 & Good & \\
\hline $5 / 9 / 947: 03$ & Auto FIC & 217.20 & Good & \\
\hline $5 / 10 / 940: 00$ & Manual Tape & 216.75 & Good & \\
\hline $5 / 10 / 947: 03$ & Auto FIC & 217.2 & Good & \\
\hline $5 / 11 / 940: 00$ & Manual Tape & 217.00 & Good & \\
\hline $5 / 11 / 947: 03$ & Auto FIC & 217.2 & Good & \\
\hline $5 / 12 / 940: 00$ & Manual Tape & 216.50 & Good & \\
\hline $5 / 12 / 946: 03$ & Auto FIC & 217.2 & Good & \\
\hline $5 / 13 / 940: 00$ & Manual Tape & 217.00 & Good & \\
\hline $5 / 13 / 947: 03$ & Auto FIC & 217.2 & Good & \\
\hline $5 / 14 / 940: 00$ & Manual Tape & 216.5 & Good & \\
\hline $5 / 14 / 947: 03$ & Auto FIC & 217.20 & Good & \\
\hline $5 / 15 / 940: 00$ & Manual Tape & 216.75 & Good & \\
\hline $5 / 15 / 947: 06$ & Auto FIC & 21.7 .20 & Good & \\
\hline 5/16/94 0:00 & Manual Tape & 216.75 & Good & \\
\hline $5 / 16 / 947: 02$ & Auto FIC & 217.20 & Good & \\
\hline $5 / 17 / 940: 00$ & Manual Tape & 216.75 & Good & \\
\hline $5 / 17 / 947: 03$ & Auto FIC & 217.20 & Good & \\
\hline $5 / 18 / 940: 00$ & Manual Tape & 217 & Good & \\
\hline $5 / 18 / 947: 03$ & Auto FIC & 217.2 & Good & \\
\hline
\end{tabular}

Data obtained from WHC Surveillance Analysis Computer System, (PC SACS) July 7, 1995. 


\begin{tabular}{|c|c|c|c|c|}
\hline \multicolumn{5}{|c|}{ 241-AP-103 } \\
\hline \multicolumn{5}{|c|}{ Surface Levol Data } \\
\hline Date & Type of Measurement & Level (Inches) & Data Quality & Comments \\
\hline $5 / 19 / 940: 00$ & Manual Tape & 166.50 & Good & \\
\hline $5 / 19 / 947: 03$ & Auto FIC & 169.1 & Good & \\
\hline $5 / 20 / 940: 00$ & Manual Tape & 85.00 & Good & \\
\hline $5 / 20 / 947: 03$ & Auto FIC & 96.40 & Good & \\
\hline $5 / 21 / 940: 00$ & Manual Tape & 9.75 & Good & \\
\hline $5 / 21 / 947: 05$ & Auto FIC & 10 & Good & \\
\hline $5 / 22 / 940: 00$ & Manual Tape & 9.75 & Good & \\
\hline $5 / 22 / 947: 05$ & Auto FIC & 10.00 & Good & \\
\hline $5 / 23 / 940: 00$ & Manual Tape & 9.00 & Good & \\
\hline $5 / 23 / 947: 04$ & Auto FIC & 10.00 & Good & \\
\hline $5 / 24 / 940: 00$ & Manual Tape & 9.75 & Good & \\
\hline $5 / 24 / 947: 04$ & Auto FIC & 10 & Good & \\
\hline $5 / 25 / 947: 04$ & Auto FIC & 10.00 & Good & \\
\hline $5 / 26 / 940: 00$ & Manual Tape & 9.50 & Good & \\
\hline $5 / 26 / 946: 05$ & Auto FIC & 10 & Good & \\
\hline $5 / 27 / 94 \quad 0: 00$ & Manual Tape & 9.75 & Good & \\
\hline $5 / 27 / 947: 03$ & Auto FIC & 10 & Good & \\
\hline $5 / 28 / 940: 00$ & Manual Tape & 9.50 & Good & \\
\hline $5 / 28 / 947: 03$ & Auto FIC & 10.00 & Good & \\
\hline $5 / 29 / 940: 00$ & Manual Tape & 9.5 & Good & \\
\hline $5 / 29 / 947: 03$ & Auto FIC & 10 & Good & \\
\hline $5 / 30 / 940: 00$ & Manual FIC & 10 & Good & \\
\hline $5 / 30 / 940: 00$ & Manual Tape & 9.50 & Good & \\
\hline $5 / 30 / 947: 02$ & Auto FIC & 10.00 & Good & \\
\hline $5 / 31 / 940: 00$ & Manual FIC & 10 & Good & \\
\hline $5 / 31 / 94 \quad 0: 00$ & Manual Tape & 10.00 & Good & \\
\hline $5 / 31 / 947: 03$ & Auto FIC & 10.00 & Good & \\
\hline $6 / 1 / 940: 00$ & Manual Tape & 9.75 & Good & \\
\hline 6/1/94 7:03 & Auto FIC & 10.00 & Good & \\
\hline $6 / 2 / 940: 00$ & Manual Tape & 9.75 & Good & \\
\hline $6 / 2 / 947: 03$ & Auto FIC & 10.00 & Good & \\
\hline $6 / 3 / 940: 00$ & Manual FIC & 10.00 & Good & \\
\hline $6 / 3 / 940: 00$ & Manual Tape & 9.50 & Good & \\
\hline $6 / 4 / 94 \quad 0: 00$ & Manual Tape & 9.5 & Good & \\
\hline $6 / 4 / 947: 04$ & Auto FIC & 10 & Good & \\
\hline $6 / 5 / 940: 00$ & Manual Tape & 9.5 & Good & \\
\hline 6/5/94 7:03 & Auto FIC & 10 & Good & \\
\hline $6 / 6 / 940: 00$ & Manual Tape & 9.00 & Good & \\
\hline $6 / 6 / 947: 06$ & Auto FIC & 10 & Good & \\
\hline $6 / 7 / 940: 00$ & Manual Tape & 9.50 & Good & \\
\hline $6 / 7 / 947: 02$ & Auto FIC & 10 & Good & \\
\hline $6 / 8 / 94 \quad 0: 00$ & Manual Tape & 9.5 & Good & \\
\hline $6 / 8 / 947: 03$ & Auto FIC & 10.00 & Good & \\
\hline 6/9/94 0:00 & Manual FIC & 10 & Good & \\
\hline $6 / 9 / 940: 00$ & Manual Tape & 9.50 & Good & \\
\hline $6 / 10 / 940: 00$ & Manual Tape & 9.50 & Good & \\
\hline $6 / 10 / 947: 04$ & Auto FIC & 10.00 & Good & \\
\hline $6 / 11 / 940: 00$ & Manual Tape & 10 & Good & \\
\hline
\end{tabular}

Data obtained from WHC Surveillance Analysis Computer System, (PC SACS) July 7, 1995. 


\begin{tabular}{|c|c|c|c|c|}
\hline \multicolumn{5}{|c|}{ 241-AP-108 } \\
\hline \multicolumn{5}{|c|}{ Surface Level Data } \\
\hline Date & Type of Measurement & Level (Inches) & Data Quality & Comments \\
\hline $6 / 11 / 947: 04$ & Auto FIC & 10.00 & Good & \\
\hline $6 / 12 / 940: 00$ & Manual Tape & 9.75 & Good & \\
\hline $6 / 12 / 947: 03$ & Auto FIC & 10.00 & Good & \\
\hline $6 / 13 / 940: 00$ & Manual Tape & 9.75 & Good & \\
\hline $6 / 13 / 947: 02$ & Auto FIC & 10 & Good & \\
\hline $6 / 14 / 940: 00$ & Manual Tapo & 9.75 & Good & \\
\hline $6 / 14 / 947: 03$ & Auto FIC & 10.00 & Good & \\
\hline $6 / 15 / 940: 00$ & Manual Tape & 9.75 & Good & \\
\hline $6 / 15 / 947: 03$ & Auto FIC & 10 & Good & \\
\hline $6 / 16 / 940: 00$ & Manual Tapo & 9.50 & Good & \\
\hline $6 / 16 / 947: 03$ & Auto FIC & 10.00 & Good & \\
\hline $6 / 17 / 940: 00$ & Manual Tape & 9.75 & Good & \\
\hline $6 / 17 / 947: 03$ & Auto FIC & 10 & Good & \\
\hline $6 / 18 / 940: 00$ & Manual Tape & 9.75 & Good & \\
\hline $6 / 18 / 947: 04$ & Auto FIC & 10 & Good & \\
\hline $6 / 19 / 940: 00$ & Manual Tape & 9.75 & Good & \\
\hline $6 / 19 / 947: 04$ & Auto FIC & 10.10 & Good & \\
\hline $6 / 20 / 940: 00$ & Manual Tape & 9.75 & Good & \\
\hline $6 / 20 / 947: 04$ & Auto FIC & 10 & Good & \\
\hline $6 / 21 / 940: 00$ & Manual Tape & 9.75 & Good & \\
\hline $6 / 21 / 947: 04$ & Auto FIC & 10.00 & Good & \\
\hline $6 / 22 / 940: 00$ & Manual Tape & 9.75 & Good & \\
\hline $6 / 22 / 947: 03$ & Auto FIC & 10.00 & Good & \\
\hline $6 / 23 / 940: 00$ & Manual Tape & 9.50 & Good & \\
\hline $6 / 23 / 947: 05$ & Auto FIC & 10 & Good & \\
\hline $6 / 24 / 940: 00$ & Manual Tape & 9.75 & Good & \\
\hline $6 / 24 / 947: 06$ & Auto FIC & 10.00 & Good & \\
\hline $6 / 25 / 940: 00$ & Manual Tape & 9.75 & Good & \\
\hline 6/25/94 7:05 & Auto FIC & 10 & Good & \\
\hline $6 / 26 / 940: 00$ & Manual Tape & 9.25 & Good & \\
\hline $6 / 26 / 947: 06$ & Auto FIC & 10.00 & Good & \\
\hline $6 / 27 / 940: 00$ & Manual Tape & 9.50 & Good & \\
\hline $6 / 27 / 947: 06$ & Auto FIC & 10.00 & Good & \\
\hline $6 / 28 / 940: 00$ & Manual Tape & 9.75 & Good & \\
\hline $6 / 28 / 947: 06$ & Auto FIC & 10 & Good & \\
\hline $6 / 29 / 940: 00$ & Manual Tape & 9.75 & Good & \\
\hline $6 / 29 / 947: 03$ & Auto FIC & 10.00 & Good & \\
\hline $6 / 30 / 940: 00$ & Manual Tape & 9.5 & Good & \\
\hline $6 / 30 / 947: 04$ & Auto FIC & 10 & Good & \\
\hline $7 / 1 / 940: 00$ & Manual Tape & 9.75 & Good & \\
\hline $7 / 1 / 947: 04$ & Auto FIC & 10 & Good & \\
\hline $7 / 2 / 940: 00$ & Manual Tape & 9.50 & Good & \\
\hline 7/2/94 7:04 & Auto FIC & 9.90 & Good & \\
\hline $7 / 3 / 940: 00$ & Manual Tape & 9.50 & Good & \\
\hline $7 / 3 / 947: 04$ & Auto FIC & 9.9 & Good & \\
\hline $7 / 4 / 940: 00$ & Manual Tape & 9.75 & Good & \\
\hline $7 / 4 / 947: 04$ & Auto FIC & 9.90 & Good & \\
\hline $7 / 5 / 940: 00$ & Manual Tape & 9.75 & Good & \\
\hline
\end{tabular}

Data obtained from WHC Surveillance Analysis Computer System, (PC SACS) July 7, 1995. 


\begin{tabular}{|c|c|c|c|c|}
\hline \multicolumn{5}{|c|}{ 241-AP-103 } \\
\hline & & Urface Lovel Dat & & \\
\hline Date & Type of Measurement & Leval (Inchea) & Data Quality & Comments \\
\hline $7 / 5 / 947: 03$ & Auto FIC & 9.90 & Good & \\
\hline $7 / 6 / 940: 00$ & Manual Tape & 9.50 & Good & \\
\hline 7/6/94 7:05 & Auto FIC & 9.9 & Good & \\
\hline $7 / 7 / 940: 00$ & Manual Tape & 9.50 & Good & \\
\hline 7/7/94 7:03 & Auto FIC & 9.90 & Good & \\
\hline $7 / 8 / 940: 00$ & Manual Tape & 9.75 & Good & \\
\hline $7 / 8 / 947: 03$ & Auto FIC & 9.90 & Good & \\
\hline $7 / 9 / 940: 00$ & Manual Tape & 9.75 & Good & \\
\hline $7 / 9 / 947: 03$ & Auto FIC & 9.9 & Good & \\
\hline $7 / 10 / 940: 00$ & Manual Tape & 9.50 & Good & \\
\hline 7/10/94 7:03 & Auto FIC & 9.90 & Good & \\
\hline $7 / 11 / 940: 00$ & Manual Tape & 9.75 & Good & \\
\hline 7/11/94.7:03 & Auto FIC & 9.9 & Good & \\
\hline $7 / 12 / 940: 00$ & Manual Tape & 9.75 & Good & \\
\hline $7 / 12 / 947: 03$ & Auto FIC & 9.9 & Good & \\
\hline $7 / 13 / 940: 00$ & Manual Tape & 9.75 & Good & \\
\hline $7 / 13 / 947: 03$ & Auto FIC & 9.90 & Good & \\
\hline $7 / 14 / 940: 00$ & Manual Tape & 9.75 & Good & \\
\hline $7 / 1.4 / 947: 04$ & Auto FIC & 9.9 & Good & \\
\hline $7 / 15 / 940: 00$ & Manual Tape & 9.75 & Good & \\
\hline $7 / 15 / 947: 04$ & Auto FIC & 9.90 & Good & \\
\hline $7 / 16 / 940: 00$ & Manual Tape & 9.5 & Good & \\
\hline $7 / 16 / 947: 04$ & Auto FIC & 9.90 & Good & \\
\hline $7 / 17 / 940: 00$ & Manual Tape & 9.50 & Good & \\
\hline 7/17/94 7:04 & Auto FIC & 9.90 & Good & \\
\hline $7 / 18 / 940: 00$ & Manual FIC & 9.90 & Good & \\
\hline $7 / 18 / 940: 00$ & Manual Tape & 9.75 & Good & \\
\hline $7 / 19 / 940: 00$ & Manual FIC & 9.9 & Good & \\
\hline $7 / 19 / 940: 00$ & Manual Tape & 9.75 & Good & \\
\hline $7 / 19 / 947: 03$ & Auto FIC & 9.90 & Good & \\
\hline $7 / 20 / 940: 00$ & Manual FIC & 9.90 & Good & \\
\hline $7 / 20 / 940: 00$ & Manual Tape & 9.75 & Good & \\
\hline $7 / 20 / 947: 03$ & Auto FIC & 9.9 & Good & \\
\hline $7 / 21 / 94 \quad 0: 00$ & Manual Tape & 9.75 & Good & \\
\hline $7 / 21 / 947: 03$ & Auto FIC & 9.9 & Good & \\
\hline $7 / 22 / 940: 00$ & Manual Tape & 9.75 & Good & \\
\hline $7 / 22 / 947: 03$ & Auto FIC & 10.2 & Good & \\
\hline $7 / 23 / 940: 00$ & Manual Tape & 9.75 & Good & \\
\hline $7 / 23 / 947: 03$ & Auto FIC & 10.2 & Good & \\
\hline $7 / 24 / 94 \quad 0: 00$ & Manual Tape & 9.75 & Good & \\
\hline $7 / 24 / 947: 03$ & Auto FIC & 10.20 & Good & \\
\hline $7 / 25 / 940: 00$ & Manual Tape & 9.75 & Good & \\
\hline $7 / 25 / 947: 03$ & Auto FIC & 10.20 & Good & \\
\hline $7 / 26 / 947: 03$ & Auto FIC & 10.20 & Good & \\
\hline $7 / 26 / 94 \quad 12: 00$ & Manual Tape & 9.75 & Good & \\
\hline $7 / 27 / 947: 03$ & Auto FIC & 10.20 & Good & \\
\hline $7 / 27 / 9412: 00$ & Manual Tape & 9.50 & Good & \\
\hline $7 / 28 / 947: 04$ & Auto FIC & 10.2 & Good & \\
\hline
\end{tabular}

Data obtained from WHC Surveillance Analysis Computer System, (PC SACS) July 7, 1995. 


\begin{tabular}{|c|c|c|c|c|}
\hline \multicolumn{5}{|c|}{ 241-AP-103 } \\
\hline \multicolumn{5}{|c|}{ Surface Level Data } \\
\hline Dato & Type of Measurement & Level (Inchea) & Data Quality & Comments \\
\hline $7 / 28 / 94 \quad 12: 00$ & Manual Tape & 9.5 & Good & \\
\hline 7/29/94 7:04 & Auto FIC & 10.20 & Good & \\
\hline $7 / 29 / 9412: 00$ & Manual Tape & 9.5 & Good & \\
\hline $7 / 30 / 947: 04$ & Auto FIC & 10,2 & Gobd & \\
\hline $7 / 30 / 9412: 00$ & Manual Tape & 9.00 & Good & \\
\hline $7 / 31 / 9412: 00$ & Manual Tape & 9.50 & Good & \\
\hline $7 / 31 / 9423: 03$ & Auto FIC & 10.20 & Good & \\
\hline $8 / 1 / 947: 03$ & Auto FIC & 10.20 & Good & \\
\hline $8 / 1 / 9412: 00$ & Manual Tape & 9.75 & Good & \\
\hline $8 / 2 / 947: 03$ & Auto FIC & 10.2 & Good & \\
\hline $8 / 2 / 9412: 00$ & Manual Tape & 9.75 & Good & \\
\hline $8 / 3 / 947: 03$ & Auto FIC & 10.20 & Good & \\
\hline $8 / 3 / 9412: 00$ & Manual Tape & 9.75 & Good & \\
\hline $8 / 4 / 947: 02$ & Auto FIC & 0.00 & Suspect & Data not included in graph \\
\hline $8 / 4 / 9412: 00$ & Manual FIC & 10.20 & Good & \\
\hline $8 / 4 / 9412: 00$ & Manual Tape & 9.75 & Good & \\
\hline $8 / 5 / 947: 03$ & Auto FIC & 10.2 & Good & \\
\hline $8 / 5 / 9412: 00$ & Manual Tape & 9.75 & Good & \\
\hline $8 / 6 / 947: 03$ & Auto FIC & 10.10 & Good & \\
\hline $8 / 6 / 9412: 00$ & Manual Tape & 9.75 & Good & \\
\hline $8 / 7 / 947: 04$ & Auto FIC & 10,1 & Good & \\
\hline $8 / 7 / 9412: 00$ & Manual Tape & 9.75 & Good & \\
\hline $8 / 8 / 947: 12$ & Auto FIC & 0.00 & -Suspect & Data not included in graph \\
\hline $8 / 8 / 9412: 00$ & Manual FIC & 10.15 & Good & \\
\hline $8 / 8 / 9412: 00$ & Manual Tape & 10.00 & Good & \\
\hline $8 / 9 / 947: 06$ & Auto FIC & 10.1 & Good & \\
\hline $8 / 9 / 9412: 00$ & Manual Tape & 9.75 & Good & \\
\hline $8 / 10 / 947: 04$ & Auto FIC & 10.1 & Good & \\
\hline $8 / 10 / 9412: 00$ & Manual Tape & 9.50 & Good & . \\
\hline $8 / 11 / 947: 04$ & Auto FIC & 10.10 & Good & \\
\hline $8 / 11 / 9412: 00$ & Manual Tape & 9.75 & Good & \\
\hline $8 / 12 / 947: 04$ & Auto FIC & 10.1 & Good & \\
\hline $8 / 12 / 9412: 00$ & Manual Tape & 9.50 & Good & \\
\hline 8/13/94 7:03 & Auto FIC & 10.10 & Good & \\
\hline $8 / 13 / 9412: 00$ & Manual Tape & 9.50 & Good & \\
\hline $8 / 14 / 947: 06$ & Auto FIC & 10.1 & Good & \\
\hline $8 / 14 / 9412: 00$ & Manual Tape & 9.75 & Good & \\
\hline $8 / 15 / 947: 06$ & Auto FIC & 10.1 & Good & \\
\hline $8 / 15 / 9412: 00$ & Manual Tape & 9.5 & Good & \\
\hline $8 / 16 / 947: 06$ & Auto FIC & 10.10 & Good & \\
\hline $8 / 16 / 9412: 00$ & Manual Tape & 9.75 & Good & \\
\hline $8 / 17 / 947: 06$ & Auto FIC & 10.10 & Good & \\
\hline $8 / 17 / 9412: 00$ & Manual Tape & 9.75 & Good & \\
\hline $8 / 18 / 947: 06$ & Auto FIC & 10.10 & Good & \\
\hline $8 / 18 / 9412: 00$ & Manual Tape & 9.75 & Good & \\
\hline $8 / 19 / 946: 06$ & Auto FIC & 10.10 & Good & \\
\hline $8 / 19 / 9412: 00$ & Manual Tape & 9.75 & Good & \\
\hline $8 / 20 / 947: 04$ & Auto FIC & 10.10 & Good & \\
\hline
\end{tabular}

Data obtained from WHC Surveillance Analysis Computer System, (PC SACS) July 7, 1995. 


\begin{tabular}{|c|c|c|c|c|}
\hline \multicolumn{5}{|c|}{ 241-AP-103 } \\
\hline \multicolumn{5}{|c|}{ Surface Level Data } \\
\hline Date & Type of Measurement & Level (Inches) & Dața Quality & Comments \\
\hline $8 / 20 / 9412: 00$ & Manual Tape & 9.75 & Good & \\
\hline $8 / 21 / 947: 04$ & Auto FIC & 10.1 & Good & \\
\hline $8 / 21 / 9412: 00$ & Manual Tape & 9.75 & Good & \\
\hline $8 / 22 / 946: 04$ & Auto FIC & 10.1 & Good & \\
\hline $8 / 22 / 9412: 00$ & Manual Tape & 9.5 & Good & \\
\hline $8 / 23 / 947: 04$ & Auto FIC & 10.1 & Good & \\
\hline $8 / 23 / 9412: 00$ & Manual Tape & 9.75 & Good & \\
\hline $8 / 24 / 947: 04$ & Auto FIC & 10.1 & Good & \\
\hline $8 / 24 / 9412: 00$ & Manual Tape & 9.75 & Good & \\
\hline $8 / 25 / 947: 03$ & Auto FIC & 10.1 & Good & \\
\hline $8 / 25 / 9412: 00$ & Manual Tape & 9.75 & Good & \\
\hline $8 / 26 / 947: 06$ & Auto FIC & 10.10 & Good & \\
\hline $8 / 26 / 9412: 00$ & Manual Tape & 9.75 & Good & \\
\hline $8 / 27 / 947: 04$ & Auto FIC & 10.00 & Good & \\
\hline $8 / 27 / 9412: 00$ & Manual Tape & 9.75 & Good & \\
\hline $8 / 28 / 947: 04$ & Auto FIC & 10.00 & Good & \\
\hline $8 / 28 / 9412: 00$ & Manual Tape & 9.5 & Good & \\
\hline $8 / 29 / 947: 04$ & Auto FIC & 10 & Good & \\
\hline $8 / 29 / 9412: 00$ & Manual Tape & 9.50 & Good & \\
\hline $8 / 30 / 947: 04$ & Auto FIC & 10.10 & Good & \\
\hline $8 / 30 / 94 \quad 12: 00$ & Manual Tape & 9.5 & Good & \\
\hline $8 / 31 / 947: 04$ & Auto FIC & 10.10 & Good & \\
\hline $8 / 31 / 9412: 00$ & Manual Tape & 9.5 & Good & \\
\hline $9 / 1 / 947: 04$ & Auto FIC & 10 & Good & \\
\hline $9 / 1 / 9412: 00$ & Manual Tape & 9.5 & Good & \\
\hline $9 / 2 / 947: 06$ & Auto FIC & 10.00 & Good & \\
\hline $9 / 2 / 9412: 00$ & Manual Tape & 9.50 & Good & \\
\hline $9 / 3 / 9412: 00$ & Manual Tape & 9.5 & Good & \\
\hline $9 / 3 / 9423: 04$ & Auto FIC & 10 & Good & \\
\hline $9 / 4 / 947: 04$ & Auto FIC & 10 & Good & \\
\hline $9 / 4 / 9412: 00$ & Manual Tape & 9.5 & Good & \\
\hline $9 / 5 / 947: 04$ & Auto FIC & 10.00 & Good & \\
\hline $9 / 5 / 9412: 00$ & Manual Tape & 9.50 & Good & \\
\hline $9 / 6 / 947: 03$ & Auto FIC & 10 & Good & \\
\hline $9 / 6 / 9412: 00$ & Manual Tape & 9.75 & Good & \\
\hline 9/7/94 7:03 & Auto FIC & 10.00 & Good & \\
\hline $9 / 7 / 9412: 00$ & Manual Tape & 9.75 & Good & \\
\hline $9 / 8 / 947: 04$ & Auto FIC & 10.00 & Good & \\
\hline $9 / 8 / 9412: 00$ & Manual Tape & 9.5 & Good & \\
\hline 9/9/94 7:03 & Auto FIC & 10 & Good & \\
\hline $9 / 9 / 94 \quad 12: 00$ & Manual Tape & 9.5 & Good & \\
\hline $9 / 10 / 947: 04$ & Auto FIC & 10.00 & Good & \\
\hline $9 / 10 / 9412: 00$ & Manual Tape & 9.5 & Good & \\
\hline $9 / 11 / 947: 03$ & Auto FIC & 10.1 & Good & \\
\hline $9 / 11 / 9412: 00$ & Manual Tape & 9.50 & Good & \\
\hline $9 / 12 / 947: 03$ & Auto FIC & 10.00 & Good & \\
\hline $9 / 12 / 9412: 00$ & Manual Tape & 9.75 & Good & \\
\hline $9 / 13 / 947: 02$ & Auto FIC & 10.10 & Good & \\
\hline
\end{tabular}

Data obtained from WHC Surveillance Analysis Computer System, (PC SACS) July 7, 1995. 


\begin{tabular}{|c|c|c|c|c|}
\hline \multicolumn{5}{|c|}{ 241-AP-103 } \\
\hline \multicolumn{5}{|c|}{ Surface Level Data } \\
\hline Date & Type of Measurement & Lovel (Inchos) & Data Quality & Comments \\
\hline $9 / 13 / 9412: 00$ & Manual Tape & 9.50 & Good & \\
\hline $9 / 14 / 947: 03$ & Auto FIC & 10 & Good & \\
\hline $9 / 14 / 9412: 00$ & Manual Tape & 9.5 & Good & \\
\hline $9 / 15 / 947: 03$ & Auto FIC & 10.00 & Good & \\
\hline $9 / 15 / 9412: 00$ & Manual Tape & 9.75 & Good & \\
\hline $9 / 16 / 947: 02$ & Auto FIC & 10 & Good & \\
\hline $9 / 16 / 9412: 00$ & Manual Tape & 9.75 & Good & \\
\hline $9 / 17 / 947: 03$ & Auto FIC & 10 & Good & \\
\hline $9 / 17 / 9412: 00$ & Manual Tape & 8.75 & Good & \\
\hline $9 / 18 / 947: 02$ & Auto FIC & 10.00 & Good & \\
\hline $9 / 18 / 9412: 00$ & Manual Tape & 9.5 & Good & \\
\hline $9 / 19 / 947: 03$ & Auto FIC & 10 & Good & \\
\hline $9 / 19 / 9412,00$ & Manual Tape & 9.88 & Good & \\
\hline $9 / 20 / 947: 03$ & Auto FIC & 10.00 & Good & \\
\hline $9 / 20 / 9412: 00$ & Manual Tape & 9.5 & Good & \\
\hline $9 / 21 / 947: 04$ & Auto FIC & 10.00 & Good & \\
\hline $9 / 21 / 9412,00$ & Manual Tape & 9.50 & Good & \\
\hline $9 / 22 / 947: 03$ & Auto FIC & 10 & Good & \\
\hline $9 / 22 / 9412: 00$ & Manual Tape & 9.25 & Good & \\
\hline $9 / 23 / 947: 04$ & Auto FIG & 10.00 & Good & \\
\hline $9 / 23 / 9412: 00$ & Manual Tape & 9.5 & Good & . \\
\hline $9 / 24 / 947: 04$ & Auto FIC & 10.00 & Good & \\
\hline $9 / 24 / 9412,00$ & Manual Tape & 9.50 & Good & \\
\hline $9 / 25 / 947: 03$ & Auto FIC & 10 & Good & \\
\hline $9 / 25 / 9412: 00$ & Manual Tape & 9.25 & Good & \\
\hline $9 / 26 / 947: 04$ & Auto FIC & 10.00 & Good & \\
\hline $9 / 26 / 9412: 00$ & Manual Tape & 9.25 & Good & \\
\hline $9 / 27 / 947: 04$ & Auto FIC & 10 & Good & \\
\hline $9 / 27 / 9412: 00$ & Manual Tape & 9.25 & Good & \\
\hline $9 / 28 / 947: 03$ & Auto FIC & 10 & Good & \\
\hline $9 / 28 / 94 \quad 12: 00$ & Manual Tape & 8.5 & Suspect & Data not included in graph \\
\hline $9 / 29 / 947: 04$ & Auto FIC & 10.00 & Good & \\
\hline $9 / 29 / 9412: 00$ & Manual Tape & 9.50 & Good & \\
\hline $9 / 30 / 947: 04$ & Auto FIC & 10 & Good & \\
\hline $9 / 30 / 9412: 00$ & Manual Tape & 9.5 & Good & \\
\hline $10 / 1 / 947: 06$ & Auto FIC & 10.00 & Good & \\
\hline $10 / 1 / 9412: 00$ & Manual Tape & 9.5 & Good & \\
\hline $10 / 2 / 947: 05$ & Auto FIC & 10.00 & Good & \\
\hline $10 / 2 / 9412: 00$ & Manual Tape & 9.5 & Good & \\
\hline 10/3/94 7:04 & Auto FIC & 10 & Good & \\
\hline $10 / 3 / 9412: 00$ & Manual Tape & 9.5 & Good & \\
\hline $10 / 4 / 947: 04$ & Auto FIC & 9.90 & Good & \\
\hline $10 / 4 / 9412: 00$ & Manual Tape & 9.25 & Good & \\
\hline $10 / 5 / 947: 04$ & Auto FIC & 10 & Good & \\
\hline $10 / 5 / 9412: 00$ & Manual Tape & 9.25 & Good & \\
\hline $10 / 6 / 947: 04$ & Auto FIC & 10.00 & Good & \\
\hline $10 / 6 / 94 \quad 12: 00$ & Manual Tape & 9.50 & Good & \\
\hline $10 / 7 / 946: 04$ & Auto FIC & 10.00 & Good & \\
\hline
\end{tabular}

Data obtained from WHC Surveillance Analysis Computer System, (PC SACS) July 7, 1995. 


\begin{tabular}{|c|c|c|c|c|}
\hline \multicolumn{5}{|c|}{ 241-AP-103 } \\
\hline \multicolumn{5}{|c|}{ Surface Leval Data } \\
\hline Date & Type of Measurement & Level (Inchea) & Data Ouality & Comments \\
\hline $10 / 7 / 9412: 00$ & Manual Tape & 9.50 & Good & \\
\hline $10 / 8 / 947: 03$ & Auto FIC & 10 & Good & \\
\hline $10 / 8 / 9412: 00$ & Manual Tape & 9.5 & Good & \\
\hline $10 / 9 / 947: 06$ & Auto FIC & 10.00 & Good & \\
\hline $10 / 9 / 9412: 00$ & Manual Tape & 9.5 & - Good & \\
\hline $10 / 10 / 947: 05$ & Auto FIC & 10 & Good & \\
\hline $10 / 10 / 9412: 00$ & Manual Tape & 9.5 & Good & \\
\hline $10 / 11 / 947: 03$ & Auto FIC & 9.9 & Good & \\
\hline $10 / 11 / 9412: 00$ & Manual Tape & 9.5 & Good & \\
\hline 10/12/94 7:06 & Auto FIC & 9.90 & Good & \\
\hline $10 / 12 / 9412: 00$ & Manual Tape & 9.5 & Good & \\
\hline $10 / 13 / 947: 05$ & Auto FIC & 9.9 & Good & \\
\hline $10 / 13 / 9412: 00$ & Manual Tape & 9.50 & Good & \\
\hline $10 / 14 / 947: 06$ & Auto FIC & 9.90 & Good & \\
\hline $10 / 14 / 9412: 00$ & Manual Tape & 9.5 & Good & \\
\hline $10 / 15 / 947: 06$ & Auto FIC & 9.90 & Good & \\
\hline $10 / 15 / 9412: 00$ & Manual Tape & 9.5 & Good & \\
\hline $10 / 16 / 947: 06$ & Auto FIC & 9.9 & Good & \\
\hline $10 / 16 / 94 \quad 12: 00$ & Manual Tape & 9.50 & Good & \\
\hline $10 / 17 / 947: 06$ & Auto FIC & 9.90 & Good & \\
\hline $10 / 17 / 9412: 00$ & Manual Tape & 9.25 & Good & \\
\hline $10 / 18 / 947: 06$ & Auto FIC & 9.90 & Good & \\
\hline $10 / 18 / 9412: 00$ & Manual Tape & 9.5 & Good & \\
\hline $10 / 19 / 947: 06$ & Auto FIC & 9.9 & Good & \\
\hline $10 / 19 / 9412: 00$ & Manual Tape & 9.50 & Good & \\
\hline $10 / 20 / 945: 07$ & Auto FIC & 9.90 & Good & \\
\hline $10 / 20 / 9412: 00$ & Manual Tape & 9.5 & Good & \\
\hline $10 / 21 / 947: 06$ & Auto FIC & 9.9 & Good & \\
\hline $10 / 21 / 94 \quad 12: 00$ & Manual-Tape & 9.5 & Good & \\
\hline $10 / 22 / 9412: 00$ & Manual Tape & 9.25 & Good & \\
\hline $10 / 22 / 9423: 06$ & Auto FIC & 9.9 & Good & \\
\hline $10 / 23 / 947: 06$ & Auto FIC & 9.90 & Good & \\
\hline $10 / 23 / 9412: 00$ & Manual Tape & 9.5 & Good & \\
\hline $10 / 24 / 947: 06$ & Auto FIC & 9.9 & Good & \\
\hline $10 / 24 / 9412: 00$ & Manual Tape & 9.25 & Good & \\
\hline $10 / 25 / 947: 06$ & Auto FIC & 9.90 & Good & \\
\hline $10 / 25 / 9412: 00$ & Manual Tape & 9.5 & Good & \\
\hline $10 / 26 / 947: 06$ & Auto FIC & 9.90 & Good & \\
\hline $10 / 26 / 9412: 00$ & Manual Tape & 9.5 & Good & \\
\hline $10 / 27 / 947: 06$ & Auto FIC & 9.9 & Good & \\
\hline $10 / 27 / 94 \quad 12: 00$ & Manual Tape & 9.5 & Good & \\
\hline $10 / 28 / 947: 04$ & Auto FIC & 9.90 & Good & \\
\hline $10 / 28 / 9412: 00$ & Manual Tape & 9.25 & Good & \\
\hline $10 / 29 / 9412: 00$ & Manual FIC & 9.8 & Good & \\
\hline $10 / 29 / 9412: 00$ & Manual Tape & 9.5 & Good & \\
\hline $10 / 30 / 9412: 00$ & Manual Tape & 9.50 & Good & \\
\hline $10 / 30 / 9423: 04$ & Auto FIC & 9.8 & Good & \\
\hline $10 / 31 / 947: 05$ & Auto FIC & 9.80 & Good & \\
\hline
\end{tabular}

Data obtained from WHC Surveillance Analysis Computer System, (PC SACS) July 7, 1995. 


\begin{tabular}{|c|c|c|c|c|}
\hline \multicolumn{5}{|c|}{ 241-AP-103 } \\
\hline \multicolumn{5}{|c|}{ Surface Levol Date } \\
\hline Date & Type of Measurement & Level (Inchea) & Data Quality & Comments \\
\hline $10 / 31 / 9412: 00$ & Manual Tape & 9.25 & Good & \\
\hline $11 / 1 / 9412: 00$ & Manual Tape & 9.50 & Good & \\
\hline $11 / 1 / 94$ 23:04 & Auto FIC & 9.80 & Good & \\
\hline $11 / 2 / 947: 03$ & Auto FIC & 9.8 & Good & \\
\hline $11 / 2 / 9412: 00$ & Manual Tape & 9.25 & Good & \\
\hline $11 / 3 / 947: 04$ & Auto FIC & 9.80 & Good & \\
\hline $11 / 3 / 9412: 00$ & Manual Tape & 9.5 & Good & \\
\hline 11/4/947:03 & Auto FIC & 9.8 & Good & \\
\hline $11 / 4 / 9412: 00$ & Manual Tape & 9.25 & Good & \\
\hline $11 / 5 / 947: 03$ & Auto FIC & 9.8 & Good & \\
\hline $11 / 5 / 9412: 00$ & Manual Tape & 9.25 & Good & \\
\hline $11 / 6 / 947: 04$ & Auto FIC & 9.80 & Good & \\
\hline $11 / 6 / 9412: 00$ & Manual Tape & 9.25 & Good & \\
\hline $11 / 7 / 947: 04$ & Auto FIC & 9.8 & Good & \\
\hline $11 / 7 / 94 \quad 12: 00$ & Manual Tape & 9.25 & Good & \\
\hline $11 / 8 / 947: 03$ & Auto FIC & 9.80 & Good & $\cdot$ \\
\hline $11 / 8 / 9412: 00$ & Manual Tape & 9.25 & Good & \\
\hline $11 / 9 / 947: 05$ & Auto FIC & 9.80 & Good & \\
\hline $11 / 9 / 9412: 00$ & Manual Tape & 9.25 & Good & \\
\hline $11 / 10 / 947: 03$ & Auto FIC & 9.8 & Good & \\
\hline $11 / 10 / 9412: 00$ & Manual Tape & 9.25 & Good & \\
\hline $11 / 11 / 947: 03$ & Auto FIC & 9.80 & Good & \\
\hline $11 / 11 / 9412: 00$ & Manual Tape & 9.25 & Good & \\
\hline $11 / 12 / 947: 03$ & Auto FIC & 9.8 & Good & \\
\hline $11 / 12 / 9412: 00$ & Manual Tape & 9.50 & Good & \\
\hline $11 / 13 / 947: 04$ & Auto FIC & 9.8 & Good & \\
\hline $11 / 13 / 9412: 00$ & Manual Tape & 9.25 & Good & \\
\hline $11 / 14 / 947: 03$ & Auto FIC & 9.70 & Good & \\
\hline $11 / 14 / 9412: 00$ & Manual Tape & 9.50 & Good & \\
\hline $11 / 15 / 947: 04$ & Auto FIC & 9.7 & Good & \\
\hline $11 / 15 / 9412: 00$ & Manual Tape & 9.5 & Good & \\
\hline $11 / 16 / 947: 06$ & Auto FIC & 9.80 & Good & \\
\hline $11 / 16 / 9412: 00$ & Manual Tape & 9.50 & Good & \\
\hline $11 / 17 / 947: 04$ & Auto FIC & 9.70 & Good & \\
\hline $11 / 17 / 9412: 00$ & Manual Tape & 9.50 & Good & \\
\hline $11 / 18 / 947: 04$ & Auto FIC & 9.7 & Good & \\
\hline $11 / 18 / 9412 ; 00$ & Manual Tape & 9.5 & Good & \\
\hline $11 / 19 / 947: 03$ & Auto FIC & 9.70 & Good & \\
\hline $11 / 19 / 9412: 00$ & Manual Tape & 9.50 & Good & \\
\hline $11 / 20 / 947: 03$ & Auto FIC & 9.70 & Good & \\
\hline $11 / 21 / 947: 04$ & Auto FIC & 9.7 & Good & \\
\hline $11 / 21 / 9412: 00$ & Manual Tape & 9.5 & Good & \\
\hline $11 / 22 / 947: 03$ & Auto FIC & 9.70 & Good & \\
\hline $11 / 22 / 9412: 00$ & Manual Tape & 9.25 & Good & \\
\hline $11 / 23 / 9412: 00$ & Manual FIC & 9.7 & Good & \\
\hline $11 / 23 / 9412: 00$ & Manual Tape & 9.25 & Good & \\
\hline $11 / 24 / 9412: 00$ & Manual Tape & 9.25 & Good & \\
\hline $11 / 24 / 9423: 03$ & Auto FIC & 9.7 & Good & \\
\hline
\end{tabular}

Data obtained from WHC Surveillance Analysis Computer System, (PC SACS) July 7, 1995. 


\begin{tabular}{|c|c|c|c|c|}
\hline \multicolumn{5}{|c|}{ 241.AP-103 } \\
\hline \multicolumn{5}{|c|}{ Surface Lovel Data } \\
\hline Date & Type of Measurement & Level (Inchea) & Data Quality & Comments \\
\hline $11 / 25 / 9412: 00$ & Manual Tape & 9.25 & Good & \\
\hline $11 / 25 / 9423: 03$ & Auto FIC & 9.7 & Good & \\
\hline $11 / 26 / 947: 03$ & Auto FIC & 9.70 & Good & \\
\hline $11 / 26 / 9412: 00$ & Manual Tape & 9.26 & Good & \\
\hline $11 / 27 / 94 \quad 7: 04$ & Auto FIC & 9.7 & Good & \\
\hline $11 / 27 / 94 \quad 12: 00$ & Manual Tape & 9.25 & Good & \\
\hline $11 / 28 / 947: 04$ & Auto FIC & 9.70 & Good & \\
\hline $11 / 28 / 9412: 00$ & Manual Tape & 9.25 & Good & \\
\hline $11 / 29 / 947: 04$ & Auto FIC & 9.70 & Good & \\
\hline $11 / 29 / 9412: 00$ & Manual Tape & 9.25 & Good & \\
\hline 11/30/94 7:03 & Auto FIC & 9.7 & Good & \\
\hline $11 / 30 / 9412: 00$ & Manual Tape & 9.25 & Good & \\
\hline $12 / 1 / 946: 05$ & Auto FIC & 9.70 & Good & \\
\hline $12 / 1 / 9412: 00$ & Manual Tape & 9.25 & Good & . \\
\hline $12 / 2 / 947: 03$ & Auto FIC & 9.7 & Good & \\
\hline $12 / 2 / 9412: 00$ & Manual Tape & 9.25 & Good & \\
\hline $12 / 3 / 946: 04$ & Auto FIC & 9.70 & Good & \\
\hline $12 / 3 / 9412: 00$ & Manual Tape & 9.25 & Good & \\
\hline $12 / 4 / 947: 03$ & Auto FIC & 9.70 & Good & \\
\hline $12 / 4 / 9412: 00$ & Manual Tape & 9.00 & Good & \\
\hline $12 / 5 / 947: 03$ & Auto FIC & 9.7 & Good & \\
\hline $12 / 5 / 9412: 00$ & Manual Tape & 9 & Good & \\
\hline $12 / 6 / 947: 04$ & Auto FIC & 9.7 & Good & \\
\hline $12 / 6 / 94 \quad 12: 00$ & Manual Tape & 9.25 & Good & \\
\hline $12 / 7 / 947: 03$ & Auto FIC & 9.7 & Good & \\
\hline $12 / 7 / 94 \quad 12: 00$ & Manual Tape & 9.25 & Good & \\
\hline $12 / 8 / 945: 03$ & Auto FIC & 9.70 & Good & \\
\hline $12 / 8 / 94 \quad 12: 00$ & Manual Tape & 9.25 & Good & \\
\hline $12 / 9 / 947: 03$ & Auto FIC & 9.70 & Good & \\
\hline $12 / 9 / 9412: 00$ & Manual Tape & 9.25 & Good & \\
\hline $12 / 10 / 947: 04$ & Auto FIC & 9.7 & Good & \\
\hline $12 / 10 / 9412: 00$ & Manual Tape & 9.25 & Good & \\
\hline $12 / 11 / 947: 04$ & Auto FIC & 9.70 & Good & \\
\hline $12 / 11 / 9412: 00$ & Manual Tape & 9.25 & Good & \\
\hline $12 / 12 / 947: 03$ & Auto FIC & 9.70 & Good & \\
\hline $12 / 12 / 9412: 00$ & Manual Tape & 9.25 & Good & \\
\hline $12 / 13 / 947: 03$ & Auto FIC & 9.6 & Good & \\
\hline $12 / 13 / 9412: 00$ & Manual Tape & 9 & Good & \\
\hline $12 / 14 / 947: 04$ & Auto FIC & 9.7 & Good & \\
\hline $12 / 14 / 9412: 00$ & Manual Tape & 9.25 & Good & \\
\hline $12 / 15 / 947: 03$ & Auto FIC & 9.6 & Good & \\
\hline $12 / 15 / 9412: 00$ & Manual Tape & 9.25 & Good & \\
\hline $12 / 16 / 947: 03$ & Auto FIC & 9.60 & Good & \\
\hline $12 / 16 / 9412: 00$ & Manual Tape & 9.25 & Good & \\
\hline $12 / 17 / 947: 06$ & Auto FIC & 9.60 & Good & \\
\hline $12 / 17 / 9412: 00$ & Manual Tape & 9.5 & Good & \\
\hline $12 / 18 / 947: 04$ & Auto FIC & 9.60 & Good & \\
\hline $12 / 18 / 9412: 00$ & Manual Tape & 9.25 & Good & \\
\hline
\end{tabular}

Data obtained from WHC Surveillance Analysis Computer System, (PC SACS) July 7, 1995. 


\begin{tabular}{|c|c|c|c|c|}
\hline \multicolumn{5}{|c|}{ 241-AP-103 } \\
\hline \multicolumn{5}{|c|}{ Surface Leval Data } \\
\hline Date & Type of Measurement & Level (inches) & Data Quality & Comments \\
\hline $12 / 19 / 947: 04$ & Auto FIC & 9.60 & Good & \\
\hline $12 / 19 / 9412: 00$ & Manual Tape & 9.25 & Good & \\
\hline $12 / 20 / 947: 04$ & Auto FIC & 9.70 & Good & \\
\hline $12 / 20 / 9412: 00$ & Manual Tape & 108 & Suspect & Data not included in graph \\
\hline $12 / 21 / 947: 04$ & Auto FIC & 9.7 & Good & \\
\hline $12 / 21 / 9412: 00$ & Manual Tape & 9.00 & Good & \\
\hline $12 / 22 / 947: 05$ & Auto FIC & 9.8 & Good & \\
\hline $12 / 22 / 9412,00$ & Manual Tape & 9.25 & Good & \\
\hline $12 / 23 / 947: 03$ & Auto FIC & 9.70 & Good & \\
\hline $12 / 23 / 9412: 00$ & Manual Tape & 9.25 & Good & \\
\hline $12 / 24 / 947: 04$ & Auto FIC & 9.7 & Good & \\
\hline $12 / 24 / 9412: 00$ & Manual Tape & 9.25 & Good & \\
\hline $12 / 25 / 947: 03$ & Auto FIC & 9.70 & Good & \\
\hline $12 / 25 / 9412: 00$ & Manual Tape & 9.25 & Good & \\
\hline $12 / 26 / 947: 03$ & Auto FIC & 9.70 & Good & \\
\hline $12 / 26 / 9412: 00$ & Manual Tape & 9 & Good & \\
\hline $12 / 27 / 947: 04$ & Auto FIC & 9.70 & Good & \\
\hline $12 / 27 / 9412: 00$ & Manual Tape & 9.25 & Good & \\
\hline $12 / 28 / 947: 04$ & Auto FIC & 9.70 & Good & \\
\hline $12 / 28 / 9412: 00$ & Manual Tape & 9.25 & Good & \\
\hline $12 / 29 / 947: 04$ & Auto FIC & 9.7 & Good & \\
\hline $12 / 29 / 9412: 00$ & Manual Tape & 9.25 & Good & \\
\hline $12 / 30 / 947: 04$ & Auto FIC & 9.7 & Good & \\
\hline $12 / 30 / 9412: 00$ & Manual Tape & 108.25 & Suspect & Data not included in graph \\
\hline $12 / 31 / 947: 04$ & Auto FIC & 9.70 & Good & \\
\hline 12/31/94 12:00 & Manual Tape & 9.25 & Good & \\
\hline 1/1/95 7:04 & Auto FIC & 9.7 & Good & \\
\hline $1 / 1 / 9512: 00$ & Manual Tape & 9.25 & Good & \\
\hline 1/2/95 7:03 & Auto FIC & 9.7 & Good & \\
\hline $1 / 2 / 9512: 00$ & Manual Tape & 9.25 & Good & \\
\hline 1/3/95 7:02 & Auto FIC & 9.70 & Good & \\
\hline $1 / 3 / 95 \quad 12: 00$ & Manual Tape & 9.25 & Good & \\
\hline $1 / 4 / 957: 03$ & Auto FIC & 9.70 & Good & \\
\hline $1 / 4 / 95 \quad 12: 00$ & Manual Tape & 9.25 & Good & \\
\hline 1/5/95 7:04 & Auto FIC & 9.70 & Good & \\
\hline $1 / 5 / 95 \quad 12: 00$ & Manual Tape & 9.25 & Good & \\
\hline $1 / 6 / 957: 04$ & Auto FIC & 9.70 & Good & \\
\hline $1 / 6 / 9512: 00$ & Manual Tape & 9.25 & Good & \\
\hline $1 / 7 / 95 \quad 7: 04$ & Auto FIC & 9.6 & Good & \\
\hline $1 / 7 / 9512: 00$ & Manual Tape & 9.25 & Good & \\
\hline $1 / 8 / 9512: 00$ & Manual Tape & 9.00 & Good & \\
\hline $1 / 8 / 9523: 03$ & Auto FIC & 9.7 & Good & \\
\hline 1/9/95 7:05 & Auto FIC & 9.7 & Good & \\
\hline $1 / 9 / 95 \quad 12: 00$ & Manual Tape & 9.25 & Good & \\
\hline $1 / 10 / 957: 06$ & Auto FIC & 9.7 & Good & \\
\hline $1 / 10 / 9512: 00$ & Manual Tape & 9.25 & Good & \\
\hline $1 / 11 / 957: 07$ & Auto FIC & 9.7 & Good & \\
\hline $1 / 11 / 95 \quad 12: 00$ & Manual Tape & 9.25 & Good & \\
\hline
\end{tabular}

Data obtained from WHC Surveillance Analysis Computer System, (PC SACS) July 7, 1995. 


\begin{tabular}{|c|c|c|c|c|}
\hline \multicolumn{5}{|c|}{ 241-AP-103 } \\
\hline & & urface Level Date & & \\
\hline Date & Type of Measurement & Level (thches) & Data Quality & Comments \\
\hline $1 / 12 / 957: 07$ & Auto FIC & 9.70 & Good & \\
\hline $1 / 12 / 95 \quad 12: 00$ & Manual Tape & 9.25 & Good & \\
\hline $1 / 13 / 956: 07$ & Auto FIC & 9.70 & Good & \\
\hline $1 / 13 / 9512: 00$ & Manual Tape & 9.25 & Good & \\
\hline $1 / 14 / 957: 07$ & Auto FIC & 9.70 & Good & \\
\hline 1/14/95 12:00 & Manual Tape & 9.25 & Good & \\
\hline $1 / 15 / 957: 06$ & Auto FIC & 9.7 & Good & \\
\hline $1 / 15 / 9512: 00$ & Manual Tape & 9 & Good & \\
\hline $1 / 16 / 957: 08$ & Auto FIC & 9.70 & Good & \\
\hline $1 / 16 / 9512: 00$ & Manual Tape & 9.25 & Good & \\
\hline 1/17/95 6:07 & Auto FIC & 9.70 & Good & \\
\hline $1 / 17 / 95 \quad 12: 00$ & Manual Tape & 9.25 & Good & \\
\hline $1 / 18 / 957: 07$ & Auto FIC & 9.7 & Good & \\
\hline $1 / 18 / 95 \quad 12: 00$ & Manual Tape & 9.25 & Good & \\
\hline $1 / 19 / 957: 07$ & Auto FIC & 9.70 & Good & \\
\hline $1 / 19 / 9512: 00$ & Manual Tape & 9.25 & Good & \\
\hline $1 / 20 / 957: 03$ & Auto FIC & 9.70 & Good & \\
\hline $1 / 20 / 95 \quad 12: 00$ & Manual Tape & 9.25 & Good & \\
\hline $1 / 21 / 957: 04$ & Auto FIC & 9.70 & Good & \\
\hline $1 / 21 / 95 \quad 12: 00$ & Manual Tape & 9.25 & Good & \\
\hline $1 / 22 / 957: 03$ & Auto FIC & 9.7 & Good & \\
\hline $1 / 22 / 95 \quad 12: 00$ & Manual Tape & 9.25 & Good & \\
\hline $1 / 23 / 957: 03$ & Auto FIC & 9.70 & Good & \\
\hline $1 / 23 / 95 \quad 12: 00$ & Manual Tape & 9.25 & Good & \\
\hline $1 / 24 / 957: 04$ & Auto FIC & 9.60 & Good & \\
\hline $1 / 24 / 9512: 00$ & Manual Tape & 9 & Good & \\
\hline $1 / 25 / 957: 03$ & Auto FIC & 9.70 & Good & \\
\hline $1 / 25 / 95 \quad 12: 00$ & Manual Tape & 9.25 & Good & \\
\hline $1 / 26 / 957: 03$ & Auto FIC & 9.60 & Good & \\
\hline $1 / 26 / 95 \quad 12: 00$ & Manual Tape & 9.00 & Good & \\
\hline $1 / 27 / 957: 03$ & Auto FIC & 9.6 & Good & \\
\hline $1 / 27 / 9512: 00$ & Manual Tape & 9.25 & Good & \\
\hline $1 / 28 / 957: 04$ & Auto FIC & 9.7 & Good & \\
\hline $1 / 28 / 95 \quad 12: 00$ & Manual Tape & 9.25 & Good & \\
\hline $1 / 29 / 957: 04$ & Auto FIC & 9.7 & Good & \\
\hline $1 / 29 / 95 \quad 12: 00$ & Manual Tape & 9.25 & Good & \\
\hline $1 / 30 / 957: 04$ & Auto FIC & 9.7 & Good & \\
\hline $1 / 30 / 9512: 00$ & Manual Tape & 9.25 & Good & \\
\hline $1 / 31 / 957: 03$ & Auto FIC & 9.8 & Good & \\
\hline $1 / 31 / 95 \quad 12: 00$ & Manual Tape & 9.25 & Good & \\
\hline 2/1/95 7:03 & Auto FIC & 9.8 & Good & \\
\hline $2 / 1 / 95 \quad 12: 00$ & Manual Tape & 9.25 & Good & \\
\hline $2 / 2 / 957: 04$ & Auto FIC & 9.80 & Good & \\
\hline $2 / 2 / 95 \quad 12: 00$ & Manual Tape & 9.25 & Good & \\
\hline 2/3/95 7:03 & Auto FIC & 9.8 & Good & \\
\hline $2 / 3 / 95 \quad 12: 00$ & Manual Tape & 9.25 & Good & \\
\hline $2 / 4 / 957: 04$ & Auto FIC & 9.80 & Good & \\
\hline $2 / 4 / 95 \quad 12: 00$ & Manual Tape & 9.25 & Good & \\
\hline
\end{tabular}

Data obtained from WHC Surveillance Analysis Computer System, (PC SACS) July 7, 1995. 


\begin{tabular}{|c|c|c|c|c|}
\hline \multicolumn{5}{|c|}{ 241-AP-103 } \\
\hline \multicolumn{5}{|c|}{ Surface Level Data } \\
\hline Date & Type of Measurement & Loval (Inchea) & Data Quality. & Comment: \\
\hline $2 / 5 / 957: 03$ & Auto FIC & 9.80 & Good & \\
\hline $2 / 5 / 9512: 00$ & Manual Tape & 9.75 & Suspect & Data not included in graph \\
\hline 2/6/95 7:03 & Auto FIC & 9.80 & Good & \\
\hline $2 / 6 / 9512: 00$ & Manual Tape & 8.5 & Suspect & Data not included in graph \\
\hline $2 / 7 / 957: 03$ & Auto FIC & 9.80 & Good & \\
\hline $2 / 7 / 95 \quad 12: 00$ & Manual Tape & .9 & Good & \\
\hline $2 / 8 / 957: 04$ & Auto FIC & 9.8 & Good & \\
\hline $2 / 8 / 9512: 00$ & Manual Tape & 8.75 & Good & \\
\hline $2 / 9 / 957: 06$ & Auto FIC & 9.80 & Good & 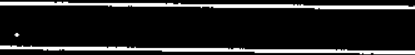 \\
\hline $2 / 9 / 9512: 00$ & Manual Tape & 9.25 & Good & \\
\hline $2 / 10 / 957: 06$ & Auto FIC & 9.8 & Good & \\
\hline $2 / 10 / 9512: 00$ & Manual Tape & 9 & Good & \\
\hline $2 / 11 / 957: 07$ & Auto FIC & 9.8 & Good & \\
\hline $2 / 11 / 9512: 00$ & Manual Tape. & 9.00 & Good & \\
\hline 2/12/95 7:06 & Auto FIC & 9.80 & Good & \\
\hline $2 / 12 / 9512: 00$ & Manual Tape & 9.00 & Good & \\
\hline $2 / 13 / 957: 05$ & Auto FIC & 9.80 & Good & \\
\hline $2 / 13 / 9512: 00$ & Manual Tape & 9.25 & Good & \\
\hline $2 / 14 / 957: 06$ & Auto FIC & 9.8 & Good & \\
\hline 2/14/95 12:00 & Manual Tape & 9.25 & Good & \\
\hline 2/15/95 7:07 & Auto FIC & 9.7 & Good & \\
\hline $2 / 15 / 9512: 00$ & Manual Tape & 9.25 & Good & \\
\hline $2 / 16 / 957: 06$ & Auto FIC & 9.7 & Good & \\
\hline $2 / 16 / 95 \quad 12: 00$ & Manual Tape & 9.25 & Good & \\
\hline 2/17/95 7:06 & Auto FIC & 9.70 & Good & \\
\hline $2 / 17 / 9512: 00$ & Manual Tape & 9.25 & Good & \\
\hline $2 / 18 / 957: 06$ & Auto FIC & 9.70 & Good & \\
\hline $2 / 18 / 9512: 00$ & Manual Tape & 9 & Good & \\
\hline $2 / 19 / 957: 06$ & Auto FIC & 9.7 & Good & \\
\hline 2/19/95 12:00 & Manual Tape & 9.25 & Good & \\
\hline $2 / 20 / 957: 07$ & Auto FIC & 9.70 & Good & \\
\hline $2 / 20 / 9512: 00$ & Manual Tape & 9.25 & Good & \\
\hline $2 / 21 / 957: 06$ & Auto FIC & 9.7 & Good & \\
\hline $2 / 21 / 9512: 00$ & Manual Tape & 9 & Good & \\
\hline $2 / 22 / 957: 06$ & Auto FIC & 9.70 & Good & \\
\hline 2/22/95 12:00 & Manual Tape & 9.00 & Good & \\
\hline $2 / 23 / 95 \quad 7: 07$ & Auto FIC & 9.7 & Good & \\
\hline $2 / 23 / 95 \quad 12: 00$ & Manual Tape & 9.00 & Good & \\
\hline $2 / 24 / 957: 06$ & Auto FIC & 9.7 & Good & \\
\hline $2 / 24 / 9512: 00$ & Manual Tape & 9.00 & Good & \\
\hline $2 / 25 / 957: 06$ & Auto FIC & 9.70 & Good & \\
\hline $2 / 25 / 95 \quad 12: 00$ & Manual Tape & 9 & Good & \\
\hline $2 / 26 / 956: 07$ & Auto FIC & 9.60 & Good & \\
\hline $2 / 26 / 9512: 00$ & Manual Tape & 9 & Good & \\
\hline 2/27/95 7:06 & Auto FIC & 9.70 & Good & \\
\hline $2 / 27 / 95 \quad 12: 00$ & Manual Tape & 8.25 & Suspect & Data not included in graph \\
\hline 2/28/95 5:07 & Auto FIC & 9.70 & Good & \\
\hline 2/28/95 12:00 & Manual Tape & 9.25 & Good & \\
\hline
\end{tabular}

Data obtained from WHC Surveillance Analysis Computer System, (PC SACS) July 7, 1995. 


\begin{tabular}{|c|c|c|c|c|}
\hline \multicolumn{5}{|c|}{ 241-AP-103 } \\
\hline \multicolumn{5}{|c|}{ Surface Levol Data } \\
\hline Date & Type of Measurement & Levol (Inches) & Date Quality & Comments \\
\hline 3/1/95 7:06 & Auto FIC & 9.80 & Good & \\
\hline 3/1/95 12:00 & Manual Tape & 9.25 & Good & \\
\hline $3 / 2 / 95 \quad 7: 06$ & Auto FIC & 9.7 & Good & \\
\hline $3 / 2 / 95 \quad 12: 00$ & Manual Tape & 9 & Good & \\
\hline 3/3/95 7:06 & Auto FIC & 9.60 & Good & \\
\hline $3 / 3 / 9512: 00$ & Manual Tape & 9.25 & Good & \\
\hline $3 / 4 / 957: 06$ & Auto FIC & 9.6 & Good & \\
\hline $3 / 4 / 95 \quad 12: 00$ & Manual Tape & 9.25 & Good & \\
\hline 3/5/95 7:06 & Auto FIC & 9.7 & Good & \\
\hline $3 / 5 / 95 \quad 12: 00$ & Manual Tape & 9.25 & Good & \\
\hline 3/6/95 7:06 & Auto FIC & 9.70 & Good & \\
\hline $3 / 6 / 9512: 00$ & Manual Tape & 9.125 & Good & \\
\hline $3 / 7 / 957: 06$ & Auto FIC & 9.6 & Good & \\
\hline $3 / 7 / 9512: 00$ & Manual Tape & 9.25 & Good & \\
\hline $3 / 8 / 957: 03$ & Auto FIC & 9.60 & Good & \\
\hline $3 / 8 / 95 \quad 12: 00$ & Menual Tape & 9.00 & Good & \\
\hline $3 / 9 / 95$ 7:03 & Auto FIC & 9.6 & Good & \\
\hline $3 / 9 / 95 \quad 12: 00$ & Manual Tape & 9.00 & Good & \\
\hline $3 / 10 / 957: 02$ & Auto FIC & 9.60 & Good & \\
\hline $3 / 10 / 9512: 00$ & Manual Tape & 9.25 & Good & \\
\hline $3 / 11 / 957: 03$ & Auto FIC & 9.6 & Good & \\
\hline $3 / 11 / 9512: 00$ & Manual Tape & 8.75 & Good & \\
\hline $3 / 12 / 957: 03$ & Auto FIC & 9.6 & Good & \\
\hline $3 / 12 / 95 \quad 12: 00$ & Manual Tape & 9.25 & Good & \\
\hline $3 / 13 / 957: 02$ & Auto FIC & 9.60 & Good & \\
\hline $3 / 13 / 95 \quad 12: 00$ & Manual Tape & 8.75 & Good & \\
\hline $3 / 14 / 957: 03$ & Auto FIC & 9.60 & Good & \\
\hline $3 / 14 / 9512: 00$ & Manual Tape & 8.75 & Good & \\
\hline 3/15/95 7:05 & Auto FIC & 9.70 & Good & \\
\hline $3 / 15 / 9512: 00$ & Manual Tape & 9 & Good & \\
\hline $3 / 16 / 957: 03$ & Auto FIC & 9.6 & Good & \\
\hline $3 / 16 / 95 \quad 12: 00$ & Manual Tape & 9 & Good & \\
\hline $3 / 17 / 957: 03$ & Auto FIC & 9.60 & Good & \\
\hline $3 / 17 / 95 \quad 12: 00$ & Manual Tape & 9.25 & Good & \\
\hline $3 / 18 / 95 \quad 7: 03$ & Auto FIC & 9.60 & Good & \\
\hline $3 / 18 / 9512: 00$ & Manual Tape & 9 & Good & \\
\hline 3/19/95 7:03 & Auto FIC & 9.6 & Good & \\
\hline $3 / 19 / 95 \quad 12: 00$ & Manual Tape & 9.00 & Good & \\
\hline $3 / 20 / 956: 03$ & Auto FIC & 9.6 & Good & \\
\hline 3/20/95 12:00 & Manual Tape & 9.25 & Good & \\
\hline $3 / 21 / 95 \quad 7: 03$ & Auto FIC & 9.6 & Good & \\
\hline $3 / 21 / 9512: 00$ & Manual Tape & 9 & Good & \\
\hline $3 / 22 / 957: 02$ & Auto FIC & 9.60 & Good & \\
\hline $3 / 22 / 9512: 00$ & Manual Tape & 9 & Good & \\
\hline $3 / 23 / 957: 03$ & Auto FIC & 9.6 & Good & \\
\hline $3 / 23 / 9512: 00$ & Manual Tape & 9 & Good & \\
\hline $3 / 24 / 957: 03$ & Auto FIC & 9.70 & Good & \\
\hline $3 / 24 / 9512: 00$ & Manual Tape & 9.25 & Good & \\
\hline
\end{tabular}

Data obtained from WHC Surveillance Analysis Computer System, (PC SACS) July 7, 1995. 


\begin{tabular}{|c|c|c|c|c|}
\hline \multicolumn{5}{|c|}{ 241-AP-103 } \\
\hline \multicolumn{5}{|c|}{ Surface Level Data } \\
\hline Date & Type of Measurement & Level (Inches) & Data Ouality & Comments \\
\hline $3 / 25 / 957: 02$ & Auto FIC & 9.60 & $\therefore$ Good & \\
\hline $3 / 25 / 95 \quad 12: 00$ & Manual Tape & 9.00 & Good & \\
\hline $3 / 26 / 957: 02$ & Auto FIC & 9.60 & Good & \\
\hline $3 / 26 / 95 \quad 12: 00$ & Manual Tape & 9 & Good & \\
\hline $3 / 27 / 95 \quad 7: 03$ & Auto FIC & 9.6 & Good & \\
\hline $3 / 27 / 9512: 00$ & Manual Tape & 9.00 & Good & \\
\hline $3 / 28 / 957: 03$ & Auto FIC & 9.60 & Good & \\
\hline $3 / 28 / 9512: 00$ & Manual Tape & 9 & Good & \\
\hline $3 / 29 / 957: 03$ & Auto FIC & 9.6 & Good & \\
\hline $3 / 29 / 95 \quad 12: 00$ & Manual Tape & $\mathbf{9}$ & Good & \\
\hline $3 / 30 / 957: 03$ & Auto FIC & 9.60 & Good & \\
\hline $3 / 30 / 9512: 00$ & Manual Tape & 9 & Good & \\
\hline $3 / 31 / 957: 04$ & Auto FIC & 9.6 & Good & \\
\hline $3 / 31 / 9512: 00$ & Manual Tape & 9 & Good & \\
\hline $4 / 1 / 957: 02$ & Auto FIC & 9.6 & Good & \\
\hline $4 / 1 / 9512: 00$ & Manual Tape & 9 & Good & \\
\hline 4/2/95 7:03 & Auto FIC & 9.60 & Good & \\
\hline $4 / 2 / 9512: 00$ & Manual Tape & 9.00 & Good & \\
\hline $4 / 3 / 957: 03$ & Auto FIC & 9.6 & Good & \\
\hline $4 / 3 / 9512: 00$ & Manual Tape & 9 & Good & \\
\hline 4/4/95 7:03 & Auto FIC & 9.60 & Good & \\
\hline $4 / 4 / 95 \quad 12: 00$ & Manual Tape & 9.00 & Good & \\
\hline $4 / 5 / 957: 03$ & Auto FIC & 9.6 & Good & \\
\hline $4 / 5 / 9512: 00$ & Manual Tape & 9.25 & Good & \\
\hline 4/6/95 7:03 & Auto FIC & 9.60 & Good & . \\
\hline 4/6/95 12:00 & Manual Tape & 9 & Good & \\
\hline $4 / 7 / 957: 03$ & Auto FIC & 9.6 & Good & \\
\hline $4 / 7 / 9512: 00$ & Manual Tape & 9.00 & Good & 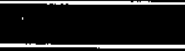 \\
\hline 4/8/95 7:02 & Auto FIC & $9.60^{\circ}$ & Good & $\cdot$ \\
\hline $4 / 8 / 95 \quad 12: 00$ & Manual Tape & 9 & Good & \\
\hline $4 / 9 / 95 \quad 12: 00$ & Manual Tape & 9 & Good & \\
\hline 4/9/95 23:03 & Auto FIC & 9.60 & Good & \\
\hline 4/10/95 7:02 & Auto FIC & 9.6 & Good & \\
\hline $4 / 10 / 9512: 00$ & Manual Tape & 9 & Good & \\
\hline 4/11/95 7:04 & Auto FIC & 9.60 & Good & \\
\hline 4/11/95 12:00 & Manual Tape & 9 & Good & \\
\hline $4 / 12 / 957: 03$ & Auto FIC & 9.6 & Good & \\
\hline $4 / 12 / 95 \quad 12: 00$ & Manual Tape & 9 & Good & \\
\hline $4 / 13 / 957: 05$ & Auto FIC & 9.6 & Good & \\
\hline 4/13/95 12:00 & Manual Tape & 9.00 & Good & \\
\hline 4/14/95 7:03 & Auto FIC & 9.6 & Good & \\
\hline $4 / 14 / 9512: 00$ & Manual Tape & 9 & Good & \\
\hline $4 / 15 / 957: 03$ & Auto FIC & 9.6 & Good & \\
\hline 4/15/95 12:00 & Manual Tape & 9 & Good & \\
\hline 4/16/95 7:03 & Auto FIC & 9.60 & Good & \\
\hline $4 / 16 / 9512: 00$ & Manual Tape & 8.5 & Good & \\
\hline 4/17/95 7:03 & Auto FIC & 9.50 & Good & \\
\hline $4 / 17 / 95 \quad 12: 00$ & Manual Tape & 9 & Good & \\
\hline
\end{tabular}

Data obtained from WHC Surveillance Analysis Computer System, (PC SACS) July 7, 1995. 


\begin{tabular}{|c|c|c|c|c|}
\hline \multicolumn{5}{|c|}{$\begin{array}{c}\text { 241-AP-103 } \\
\text { Surface Level Data }\end{array}$} \\
\hline & & Arface Level Date & & \\
\hline Date & Type of Measurement & Level (Inches) & Data Quality & Comments \\
\hline $4 / 18 / 957: 03$ & Auto FIC & 9.5 & Good & \\
\hline $4 / 18 / 9512: 00$ & Manual Tape & 9 & Good & \\
\hline 4/19/95 7:03 & Auto FIC & 9.5 & Good & \\
\hline 4/19/95 12:00 & Manual Tape & 9.00 & Good & \\
\hline $4 / 20 / 957: 03$ & Auto FIC & 9.50 & Good & \\
\hline $4 / 20 / 9512: 00$ & Manual Tape & 9 & Good & \\
\hline 4/21/95 7:03 & Auto FIC & 9.50 & Good & \\
\hline 4/21/95 12:00 & Manual Tape & 9 & Good & \\
\hline 4/22/95 7:03 & Auto FIC & 9.50 & Good & \\
\hline 4/22/95 12:00 & Manual Tape & 9 & Good & \\
\hline $4 / 23 / 957: 03$ & Auto FIC & 9.50 & Good & \\
\hline 4/23/95 12:00 & Manual Tape & 9 & Good & \\
\hline 4/24/95 7:03 & Auto FIC & 9.50 & Good & \\
\hline $4 / 25 / 957: 03$ & Auto FIC & 9.5 & Good & \\
\hline 4/25/95 12:00 & Manual Tape & 9 & Good & \\
\hline $4 / 26 / 957: 03$ & Auto FIC & 9.4 & Good & \\
\hline $4 / 26 / 9512: 00$ & Manual Tape & 9.00 & Good & \\
\hline 4/27/95 7:03 & Auto FIC & 9.4 & Good & \\
\hline $4 / 27 / 9512: 00$ & Manual Tape & 9 & Good & \\
\hline 4/28/95 7:03 & Auto FIC & 9.4 & Good & \\
\hline $4 / 28 / 95 \quad 12: 00$ & Manual Tape & 9 & Good & \\
\hline $4 / 29 / 957: 03$ & Auto FIC & 9.40 & Good & \\
\hline $4 / 29 / 9512: 00$ & Manual Tape & 9.25 & Good & \\
\hline 4/30/95 7:03 & Auto FIC & 9.4 & Good & \\
\hline 4/30/95 12:00 & Manual Tape & 9.25 & Good & \\
\hline 5/1/95 7:03 & Auto FIC & 9.40 & Good & \\
\hline $5 / 1 / 9512: 00$ & Manual Tape & 9 & Good & \\
\hline $5 / 2 / 957: 02$ & Auto FIC & 9.40 & Good & \\
\hline $5 / 2 / 95 \quad 12: 00$ & Manual Tape & 9.00 & Good & \\
\hline $5 / 3 / 957: 03$ & Auto FIC & 9.40 & Good & \\
\hline $5 / 3 / 9512: 00$ & Manual Tape & 9 & Good & \\
\hline 5/4/95 7:04 & Auto FIC & 9.40 & Good & \\
\hline $5 / 4 / 95 \quad 12: 00$ & Manual Tape & 9 & Good & \\
\hline $5 / 5 / 957: 04$ & Auto FIC & 9.4 & Good & \\
\hline $5 / 5 / 95 \quad 12: 00$ & Manual Tape & 9 & Good & \\
\hline 5/6/95 7:03 & Auto FIC & 9.50 & Good & \\
\hline $5 / 6 / 95 \quad 12: 00$ & Manual Tape & 9 & Good & \\
\hline $5 / 7 / 957: 04$ & Auto FIC & 9.4 & Good & \\
\hline $5 / 7 / 95 \quad 12: 00$ & Manual Tape & 9 & Good & \\
\hline 5/8/95 7:04 & Auto FIC & 9.4 & Good & \\
\hline $5 / 8 / 95 \quad 12: 00$ & Manual Tape & 9.00 & Good & \\
\hline 5/9/95 7:04 & Auto FIC & 9.4 & Good & \\
\hline $5 / 9 / 9512: 00$ & Manual FIC & 9.5 & Good & \\
\hline $5 / 9 / 9512: 00$ & Manual Tape & 9 & Good & \\
\hline $5 / 10 / 95$ 7:04 & Auto FIC & 0 & Suspect & Data not included in graph \\
\hline $5 / 10 / 9512: 00$ & Manual FIC & 9.45 & Good & \\
\hline $5 / 10 / 9512: 00$ & Manual Tape & 9 & Good & \\
\hline $5 / 11 / 957: 03$ & Auto FIC & 9.40 & Good & \\
\hline
\end{tabular}

Data obtained from WHC Surveillance Analysis Computer System, (PC SACS) July 7, 1995. 


\begin{tabular}{|c|c|c|c|c|}
\hline \multicolumn{5}{|c|}{ 241-AP-103 } \\
\hline \multicolumn{5}{|c|}{ Surface Lovel Dato } \\
\hline Date & Type of Measurement & Leval (Inchoa) & Data Oualiky & Comments \\
\hline $5 / 11 / 9512: 00$ & Manual Tape & 9 & Good & \\
\hline $5 / 12 / 957: 06$ & Auto FIC & 9.4 & Good & \\
\hline $5 / 12 / 9512: 00$ & Manual Tape & 9 & Good & \\
\hline $5 / 13 / 957: 03$ & Auto FIC & 9.4 & Good & \\
\hline $5 / 13 / 9512: 00$ & Manual Tape & 9.00 & Good & \\
\hline $5 / 14 / 957: 03$ & Auto FIC & 9.40 & Good & \\
\hline $5 / 14 / 9512: 00$ & Manual Tape & 9 & Good & \\
\hline $5 / 15 / 957: 03$ & Auto FIC & 9.40 & Good & \\
\hline $5 / 15 / 9512: 00$ & Manual Tape & 9 & - Good & \\
\hline 5/16/95 7:03 & Auto FIC & 9.50 & Good & \\
\hline $5 / 16 / 9512: 00$ & Manual Tape & 9 & Good & \\
\hline $5 / 17 / 957: 02$ & Auto FIC & 9.50 & Good & \\
\hline $5 / 17 / 9512: 00$ & Manual Tape & 9 & Good & \\
\hline $5 / 18 / 956: 02$ & Auto FIC & 9.4 & Good & \\
\hline $5 / 18 / 95 \quad 12: 00$ & Manual Tape & 9 & Good & \\
\hline $5 / 19 / 957: 05$ & Auto FIC & 9.4 & Good & \\
\hline $5 / 19 / 95 \quad 12: 00$ & Manual Tape & 9.00 & Good & \\
\hline $5 / 20 / 95 \quad 7: 05$ & Auto FIC & 9.50 & Good & \\
\hline $5 / 20 / 9512: 00$ & Manual Tape & 9 & Good & \\
\hline $5 / 21 / 957: 05$ & Auto FIC & 9.4 & Good & \\
\hline $5 / 21 / 9512: 00$ & Manual Tape & 9 & Good & \\
\hline $5 / 22 / 957: 05$ & Auto FIC & 9.4 & Good & \\
\hline $5 / 22 / 9512,00$ & Manual Tape & 9 & Good & \\
\hline $5 / 23 / 95 \quad 7: 11$ & Auto FIC & 0.00 & Suspect & Data not included in graph \\
\hline $5 / 23 / 95122: 00$ & Manual FIC & 9.40 & Good & \\
\hline $5 / 23 / 9512: 00$ & Manual Tape & 9.00 & Good & \\
\hline $5 / 24 / 95 \quad 7: 10$ & Auto FIC & 0 & Suspect & Data not included in graph \\
\hline $5 / 24 / 9512: 00$ & Manual FIC & 9.40 & Good & \\
\hline $5 / 24 / 9512: 00$ & Manual Tape & 9 & Good & \\
\hline $5 / 25 / 957: 10^{\circ}$ & Auto FIC & 0.00 & Suspect & Data not included in graph \\
\hline $5 / 25 / 95 \quad 12: 00$ & Manual FIC & 9.45 & Good & \\
\hline $5 / 25 / 95 \quad 12 ; 00$ & Manual Tape & 9 & Good & \\
\hline $5 / 26 / 95 \quad 7: 04$ & Auto FIC & 9.50 & Good & \\
\hline $5 / 26 / 9512: 00$ & Manual Tape & 9 & Good & \\
\hline $5 / 27 / 95 \quad 7: 05$ & Auto FIC & 9.40 & Good & \\
\hline $5 / 27 / 9512: 00$ & Manual Tape & 9 & Good & \\
\hline $5 / 28 / 95 \quad 7: 05$ & Auto FIC & 9.5 & Good & \\
\hline $5 / 28 / 95 \quad 12: 00$ & Manual Tape & 9.25 & Good & \\
\hline $5 / 29 / 957: 05$ & Auto FIC & 9.4 & Good & \\
\hline $5 / 29 / 95 \quad 12: 00$ & Manual Tape & 9.25 & Good & \\
\hline $5 / 30 / 957: 05$ & Auto FIC & 9.4 & Good & \\
\hline $5 / 30 / 9512: 00$ & Manual Tape & 9.25 & Good & \\
\hline $5 / 31 / 957: 05$ & Auto FIC & 9.4 & Good & \\
\hline $5 / 31 / 95 \quad 12: 00$ & Manual Tape & 9 & Good & \\
\hline $6 / 1 / 95 \quad 7: 05$ & Auto FIC & 9.4 & Good & \\
\hline $6 / 1 / 95 \quad 12: 00$ & Manual Tape & 9 & Good & \\
\hline 6/2/95 7:05 & Auto FIC & 9.40 & Good & \\
\hline $6 / 2 / 95 \quad 12: 00$ & Manual Tape & 9.00 & Good & \\
\hline
\end{tabular}

Data obtained from WHC Surveiliance Analysis Computer System, (PC SACS) July 7, 1995. 


\begin{tabular}{|c|c|c|c|c|}
\hline \multicolumn{5}{|c|}{ 241-AP-103 } \\
\hline \multicolumn{5}{|c|}{ Surface Leval Data } \\
\hline Date & Type of Messurement & Level (Inches) & Data Quality & Comments \\
\hline $6 / 3 / 9512: 00$ & Manual Tape & 9 & Good & \\
\hline $6 / 3 / 9523: 05$ & Auto FIC & 9.40 & Good & \\
\hline 6/4/95 7:05 & Auto FIC & 9.4 & Good & \\
\hline $6 / 4 / 9512: 00$ & Manual Tape & 9 & Good & \\
\hline $6 / 5 / 957: 05$ & Auto FIC & 9.40 & Good & \\
\hline $6 / 5 / 9512: 00$ & Manual Tape & 9 & Good & \\
\hline 6/6/95 7:05 & Auto FIC & 9.40 & Good & \\
\hline $6 / 6 / 9512: 00$ & Manual Tape & 9.00 & Good & \\
\hline 6/7/95 7:05 & Auto FIC & 9.40 & Good & \\
\hline $6 / 7 / 95 \quad 12: 00$ & Manual Tape & 9 & Good & \\
\hline $6 / 8 / 957: 05$ & Auto FIC & 9.4 & Good & \\
\hline $6 / 8 / 95 \quad 12: 00$ & Manual Tape & 9 & Good & \\
\hline 6/9/95 7:05 & Auto FIC & 9.4 & Good & \\
\hline $6 / 9 / 9512: 00$ & Manual Tapa & 9 & Good & \\
\hline $6 / 10 / 957: 05$ & Auto FIC & 9.4 & Good & \\
\hline $6 / 10 / 9512: 00$ & Manual Tape & 9.25 & Good & \\
\hline $6 / 11 / 957: 05$ & Auto FIC & 9.4 & Good & \\
\hline $6 / 11 / 9512: 00$ & Manual Tape & 9 & Good & \\
\hline 6/12/95 7:05 & Auto FIC & 9.4 & Good & \\
\hline $6 / 12 / 9512: 00$ & Manual Tape & 9.00 & Good & \\
\hline 6/13/95 7:05 & Auto FIC & 9.40 & Good & \\
\hline $6 / 13 / 9512: 00$ & Manual Tape & 9 & Good & \\
\hline 6/14/95 7:05 & Auto FIC & 9.4 & Good & \\
\hline $6 / 14 / 9512: 00$ & Manual Tape & 9 & Good & \\
\hline $6 / 15 / 957: 05$ & Auto FIC & 9.40 & Good & \\
\hline $6 / 15 / 9512: 00$ & Manual Tape & 9 & Good & \\
\hline $6 / 16 / 95$ 7:05 & Auto FIC & 9.40 & Good & \\
\hline 6/16/95 12:00 & Manual Tape & 9.00 & Good & \\
\hline $6 / 17 / 957: 05$ & Auto FIC & 9.4 & Good & \\
\hline $6 / 17 / 9512: 00$ & Manual Tape & 9 & Good & \\
\hline $6 / 18 / 957: 05$ & Auto FIC & 9.40 & Good & \\
\hline $6 / 18 / 9512: 00$ & Manual Tape & 9 & Good & \\
\hline $6 / 19 / 957: 06$ & Auto FIC & 9.40 & Good & \\
\hline $6 / 19 / 95 \quad 12: 00$ & Manual Tape & 9 & Good & \\
\hline $6 / 20 / 957: 06$ & Auto FIC & 9.40 & Good & \\
\hline $6 / 20 / 9512: 00$ & Manual Tape & 9.00 & Good & \\
\hline $6 / 21 / 957: 06$ & Auto FIC & 9.4 & Good & \\
\hline $6 / 21 / 95 \quad 12: 00$ & Manual Tape & 9 & Good & \\
\hline 6/22/95 7:06 & Auto FIC & 9.4 & Good & \\
\hline $6 / 22 / 9512: 00$ & Manual Tape & 9 & Good & \\
\hline $6 / 23 / 957: 06$ & Auto FIC & 9.4 & Good & \\
\hline $6 / 23 / 9512: 00$ & Manual Tape & 9 & Good & \\
\hline $6 / 24 / 957: 06$ & Auto FIC & 9.4 & Good & \\
\hline $6 / 24 / 9512: 00$ & Manual Tape & 9.00 & Good & \\
\hline $6 / 25 / 957: 05$ & Auto FIC & 9.4 & Good & \\
\hline $6 / 25 / 9512: 00$ & Manual Tape & 9 & Good & \\
\hline $6 / 26 / 957: 05$ & Auto FIC & 9.40 & Good & \\
\hline $6 / 26 / 9512: 00$ & Manual Tape & 9 & Good & \\
\hline
\end{tabular}

Data obtained from WHC Surveillance Analysis Computer System, (PC SACS) July 7, 1995. 
247-AP-103

Surface Level Data

\begin{tabular}{|c|c|c|c|c|}
\hline Date & Type of Measurement & Lovel (Inches) & Data Ouality & Comments \\
\hline $6 / 27 / 957: 06$ & Auto FIC & 9.40 & Good & \\
\hline $6 / 27 / 9512: 00$ & Manual Tape & 9 & Good & \\
\hline $6 / 28 / 957: 05$ & Auto FIC & 9.4 & Good & \\
\hline $6 / 28 / 9512: 00$ & Manual Tape & 9.00 & Good & \\
\hline $6 / 29 / 957: 06$ & Auto FIC & 9.40 & Good & \\
\hline $6 / 29 / 9512: 00$ & Manual Tape & 9 & Good & \\
\hline $6 / 30 / 957: 06$ & Auto FIC & 9.40 & Good & \\
\hline $6 / 30 / 9512: 00$ & Manual Tape & 9 & Transcribad & \\
\hline
\end{tabular}

Data obtained from WHC Surveillance Analysis Computer System, (PC SACS) July 7, 1995. 


\begin{tabular}{|c|c|c|c|c|}
\hline \multicolumn{5}{|c|}{ 241-AP-104 } \\
\hline \multicolumn{5}{|c|}{ Surface Level Data } \\
\hline Date & Type of Measurement & Level (Inchee) & Data Quality & Comments \\
\hline $1 / 1 / 910: 00$ & Manual Tape & 7.75 & Transcribed & \\
\hline 1/1/91 7:03 & Auto FIC & 7.70 & Good & \\
\hline 1/2/91 0:00 & Manual Tape & 7.75 & Transcribed & \\
\hline 1/2/91 7:02 & Auto FIC & 7.7 & Good & \\
\hline $1 / 3 / 910: 00$ & Manual Tape & 8 & Transcribed & \\
\hline $1 / 3 / 917: 03$ & Auto FIC & 7.7 & Good & \\
\hline 1/4/91 0:00 & Manual Tape & 8 & Transcribed & \\
\hline 1/4/91 7:02 & Auto FIC & 7.70 & Good & \\
\hline $1 / 5 / 910: 00$ & Manual Tape & 7.75 & Transcribed & \\
\hline $1 / 5 / 917: 02$ & Auto FIC & 7.7 & Good & \\
\hline $1 / 6 / 910: 00$ & Manual Tape & 7.75 & Transcribed & \\
\hline $1 / 6 / 917: 02$ & Auto FIC & 7.7 & Good & \\
\hline $1 / 7 / 910: 00$ & Manual Tape & 7.75 & Transcribed & \\
\hline $1 / 7 / 917: 02$ & Auto FIC & 7.70 & Good & \\
\hline $1 / 8 / 910: 00$ & Manual Tape & 7.75 & Transcribed & \\
\hline $1 / 8 / 917: 03$ & Auto FIC & 7.7 & Good & \\
\hline $1 / 9 / 910: 00$ & Manual Tape & 7.75 & Transcribed & \\
\hline 1/9/91 7:02 & Auto FIC & 7.70 & Good & \\
\hline $1 / 10 / 910: 00$ & Manual Tape & 7.75 & Transcribed & \\
\hline $1 / 10 / 91.7: 02$ & Auto FIC & 7.70 & Good & \\
\hline $1 / 11 / 910: 00$ & Manual Tape & 7.75 & Transcribed & \\
\hline $1 / 11 / 917: 03$ & Auto FIC & 7.70 & Good & \\
\hline $1 / 12 / 910: 00$ & Manual Tape & 7.75 & Transcribed & \\
\hline 1/12/91 7:02 & Auto FIC & 7.7 & Good & \\
\hline $1 / 13 / 910: 00$ & Manual Tape & 7.75 & Transcribed & \\
\hline $1 / 13 / 917: 03$ & Auto FIC & 7.70 & Good & \\
\hline $1 / 14 / 910: 00$ & Manual Tape & 8.00 & Transcribed & \\
\hline $1 / 14 / 917: 04$ & Auto FIC & 7.7 & Good & \\
\hline $1 / 15 / 91 \quad 0: 00$ & Manual Tape & 8.00 & Transcribed & \\
\hline $1 / 15 / 917: 03$ & Auto FIC & 7.80 & Good & \\
\hline $1 / 16 / 910: 00$ & Manual Tape & 8.25 & Transcribed & \\
\hline $1 / 16 / 917: 04$ & Auto FIC & 7.70 & Good & \\
\hline $1 / 17 / 910: 00$ & Manual Tape & 8.25 & Transcribed & \\
\hline $1 / 17 / 917: 04$ & Auto FIC & 7.7 & Good & \\
\hline $1 / 18 / 91 \quad 0: 00$ & Manual Tape & 8.25 & Transcribed & \\
\hline $1 / 18 / 917: 04$ & Auto FIC & 7.7 & Good & \\
\hline $1 / 19 / 910: 00$ & Manual Tape & 8 & Transcribed & \\
\hline $1 / 19 / 917: 04$ & Auto FIC & 7.70 & Good & \\
\hline $1 / 20 / 910: 00$ & Manual Tape & 8 & Transcribed & \\
\hline $1 / 20 / 917: 04$ & Auto FIC & 7.7 & Good & \\
\hline $1 / 21 / 910: 00$ & Manual Tape & 7.75 & Transcribed & \\
\hline $1 / 21 / 917: 04$ & Auto FIC & 7.70 & Good & \\
\hline $1 / 22 / 910: 00$ & Manual Tape & $\mathbf{7 . 7 5}$ & Transcribed & \\
\hline $1 / 22 / 917: 04$ & Auto FIC & 7.70 & Good & \\
\hline $1 / 23 / 910: 00$ & Manual Tape & 7.75 & Transcribed & \\
\hline $1 / 23 / 917: 04$ & Auto FIC & 7.7 & Good & \\
\hline 1/24/91 0:00 & Manual Tape & 8.00 & Transcribed & \\
\hline $1 / 24 / 917: 04$ & Auto FIC & 7.7 & Good & \\
\hline
\end{tabular}

Data obtained from WHC Surveillance Analysis Computer System, (PC SACS) July 7, 1995. 


\begin{tabular}{|c|c|c|c|c|}
\hline \multicolumn{5}{|c|}{ 241-AP-104 } \\
\hline \multicolumn{5}{|c|}{ Surface Lavel Data } \\
\hline Date & Type of Measuremont & Lavel (Inchea) & Data Quality & Comments \\
\hline $1 / 25 / 910: 00$ & Manual Tape & 8.00 & Transcribed & \\
\hline $1 / 25 / 917: 03$ & Auto FIC & 7.7 & Good & \\
\hline $1 / 26 / 910: 00$ & Manual Tape & 8.00 & Transcribed & \\
\hline $1 / 26 / 917: 03$ & Auto FIC & 7.7 & Good & \\
\hline $1 / 27 / 910: 00$ & Manual Tape & $\mathbf{7 . 7 5}$ & Transcribed & \\
\hline $1 / 27 / 917: 03$ & Auto FIC & 7.7 & Good & \\
\hline $1 / 28 / 910: 00$ & Manual Tapo & 7.75 & Transcribed & \\
\hline $1 / 28 / 916: 03$ & Auto FIC & 7.70 & Good & \\
\hline $1 / 29 / 91 \quad 0: 00$ & Manual Tape & 7.75 & Trenscribed & \\
\hline $1 / 29 / 917: 02$ & Auto FIC & 7.7 & Good & \\
\hline $1 / 30 / 910: 00$ & Manual FIC & 7.7 & Transcribed & \\
\hline 1/30/910:00 & Manual Tape & 7.75 & Transcribed & \\
\hline $1 / 31 / 910: 00$ & Manual Tape & 7.75 & Transcribed & \\
\hline 1/31/91 7:02 & Auto FIC & 7.70 & Good & \\
\hline $2 / 1 / 910: 00$ & Manual Tape & 7.75 & Transcribed & \\
\hline $2 / 1 / 917: 02$ & Auto FIC & 7.7 & Good & \\
\hline $2 / 2 / 910: 00$ & Manual Tape & 7.75 & Transcribed & \\
\hline 2/2/91 7:04 & Auto FIC & 7.70 & Good & \\
\hline $2 / 3 / 910.00$ & Manual Tape & 7.75 & Transcribed & \\
\hline 2/3/917:03 & Auto FIC & 7.7 & Good & \\
\hline 2/4/91 0:00 & Manual Tape & 7.75 & Transcribed & \\
\hline $2 / 4 / 917: 03$ & Auto FIC & 7.70 & Good & \\
\hline $2 / 5 / 910: 00$ & Manual Tape & 7.75 & Transcribed & \\
\hline $2 / 5 / 917: 04$ & Auto FIC & 7.70 & Good & \\
\hline $2 / 6 / 910: 00$ & Manual Tape & 7.50 & Transcribed & \\
\hline $2 / 6 / 917: 04$ & Auto FIC & 7.70 & Good & \\
\hline $2 / 7 / 910: 00$ & Manual Tape & 7.5 & Transcribad & \\
\hline 2/7/91 7:04 & Auto FIC & 7.70 & Good & \\
\hline 2/8/910:00 & Manual Tape & 7.5 & Transcribed & \\
\hline 2/8/91 7:04 & Auto FIC & 7.7 & Good & \\
\hline 2/9/91 0:00 & Manual Tape & 7.75 & Transcribed & \\
\hline 2/9/91 7:04 & Auto FIC & 7.80 & Good & \\
\hline $2 / 10 / 910: 00$ & Manual Tape & 7.75 & Transcribed & \\
\hline $2 / 10 / 917: 03$ & Auto FIC & 7.7 & Good & \\
\hline $2 / 11 / 910: 00$ & Manual Tape & 7.75 & Transcribed & \\
\hline $2 / 11 / 917: 04$ & Auto FIC & 7.70 & Good & \\
\hline 2/12/910:00 & Manual Tape & 7.75 & Transcribed & \\
\hline $2 / 12 / 917: 04$ & Auto FIC & 7.70 & Good & \\
\hline $2 / 13 / 910: 00$ & Manual Tape & 7.75 & Transcribed & \\
\hline $2 / 13 / 917: 04$ & Auto FIC & 7.70 & Good & \\
\hline $2 / 14 / 910: 00$ & Manual Tape & 7.75 & Transcribed & \\
\hline 2/14/91 7:04 & Auto FIC & 7.7 & Good & \\
\hline $2 / 15 / 910: 00$ & Manual Tape & 7.75 & Transcribed & \\
\hline $2 / 15 / 917: 03$ & Auto FIC & 7.70 & Good & \\
\hline $2 / 16 / 91 \quad 0: 00$ & Manual Tape & 7.75 & Transcribed & \\
\hline $2 / 16 / 917: 02$ & Auto FIC & 7.70 & Good & \\
\hline $2 / 17 / 910: 00$ & Manual Tape & 7.75 & Transcribed & \\
\hline $2 / 17 / 917: 02$ & Auto FIC & 7.70 & Good & \\
\hline
\end{tabular}

Data obtained from WHC Surveillance Analysis Computer System, (PC SACS) July 7, 1995. 


\begin{tabular}{|c|c|c|c|c|}
\hline & \multicolumn{4}{|c|}{ Sufface Level Data } \\
\hline Date & Type of Measurement & Level (Inches) & Data Quality & Comments \\
\hline 2/18/910:00 & Manual Tape & 7.75 & Transcribed & \\
\hline $2 / 18 / 917: 03$ & Auto FIC & 7.70 & Good & \\
\hline 2/19/91 0:00 & Manual Tape & 7.75 & Transcribed & \\
\hline $2 / 19 / 917: 02$ & Auto FIC & 7.7 & Good & \\
\hline 2/20/91 0:00 & Manual Tape & 7.75 & Transcribed & \\
\hline $2 / 20 / 917: 03$ & Auto FIC & 7.7 & Good & \\
\hline $2 / 21 / 910: 00$ & Manual Tape & 7.75 & Transcribed & \\
\hline $2 / 21 / 917: 02$ & Auto FIC & 7.7 & Good & \\
\hline 2/22/910:00 & Manual Tape & 7.75 & Transcribed & \\
\hline $2 / 22 / 917: 02$ & Auto FIC & 7.70 & Good & \\
\hline $2 / 23 / 910: 00$ & Manual Tape & 7.75 & Transcribed & 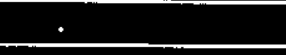 \\
\hline $2 / 23 / 917: 02$ & Auto FIC : & 7.7 & Good & \\
\hline $2 / 24 / 910: 00$ & Manual Tape & 7.75 & Transcribed & \\
\hline $2 / 24 / 917: 03$ & Auto FIC & 7.70 & Good & \\
\hline 2/25/91 0:00 & Manual Tape & 7.75 & Transcribed & \\
\hline $2 / 25 / 917: 03$ & Auto FIC & 7.70 & Good & \\
\hline $2 / 26 / 910: 00$ & Manual Tape & 7.75 & Transcribed & \\
\hline $2 / 26 / 917: 03$ & Auto FIC & 7.7 & Good & \\
\hline $2 / 27 / 910: 00$ & Manual Tape & 7.75 & Transcribed & \\
\hline $2 / 27 / 917: 02$ & Auto FIC & 7.70 & Good & \\
\hline $2 / 28 / 910: 00$ & Manual Tape & 7.75 & Transcribed & \\
\hline $2 / 28 / 917: 02$ & Auto FIC & 7.7 & Good & \\
\hline $3 / 1 / 910: 00$ & Manual Tape & 7.75 & Transcribed & \\
\hline $3 / 1 / 917: 02$ & Auto FIC & 7.70 & Good & \\
\hline $3 / 2 / 910: 00$ & Manual Tape & 7.75 & Transcribed & \\
\hline $3 / 2 / 917: 02$ & Auto FIC & 7.7 & Good & \\
\hline $3 / 3 / 910: 00$ & Manual Tape & 7.75 & Transcribed & \\
\hline $3 / 3 / 917: 03$ & Auto FIC & 7.70 & Good & \\
\hline $3 / 4 / 910: 00$ & Manual Tape & 7.5 & Transcribed & \\
\hline $3 / 4 / 917: 03$ & Auto FIC & 7.70 & Good & \\
\hline $3 / 5 / 910: 00$ & Manual Tape, & 7.75 & Transcribed & 1 \\
\hline $3 / 5 / 917: 03$ & Auto FIC & 7.70 & Good & \\
\hline $3 / 6 / 910: 00$ & Manual Tape & 7.75 & Transcribed & \\
\hline $3 / 6 / 917: 02$ & Auto FIC & 7.7 & Good & \\
\hline $3 / 7 / 910: 00$ & Manual Tape & 7.75 & Transcribed & \\
\hline $3 / 7 / 917: 02$ & Auto FIC & 7.7 & Good & \\
\hline $3 / 8 / 910: 00$ & Manual Tape & 7.75 & Transcribed & \\
\hline 3/8/91 7:02 & Auto FIC & 7.70 & Good & \\
\hline $3 / 9 / 910: 00$ & Manual Tape & 7.75 & Transcribed & \\
\hline $3 / 9 / 917: 02$ & Auto FIC & 7.7 & Good & \\
\hline $3 / 10 / 910: 00$ & Manual Tape & 7.50 & Transcribed & \\
\hline $3 / 10 / 917: 02$ & Auto FIC & 7.70 & Good & \\
\hline $3 / 11 / 910: 00$ & Manual Tape & 7.75 & Transcribed & \\
\hline $3 / 11 / 917: 02$ & Auto FIC & 7.7 & Good & \\
\hline $3 / 12 / 910: 00$ & Manual Tape & 7.75 & Transcribed & \\
\hline $3 / 12 / 917: 03$ & Auto FIC & 7.7 & Good & \\
\hline $3 / 13 / 910: 00$ & Manual Tape & 7.75 & Transcribed & \\
\hline $3 / 13 / 917: 03$ & Auto FIC & 7.70 & Good & \\
\hline
\end{tabular}

Data obtained from WHC Surveillance Analysis Computer System, (PC SACS) July 7, 1995. 


\begin{tabular}{|c|c|c|c|c|}
\hline \multicolumn{5}{|c|}{ 241-AP-104 } \\
\hline & & Ifface Level Dat & & \\
\hline Date & Type of Measurement & Level (Inches) & Data Quality & Comments \\
\hline $3 / 14 / 910: 00$ & Manual Tape & 7.75 & Transcribed & \\
\hline $3 / 14 / 917: 03$ & Auto FIC & 7.7 & Good & \\
\hline $3 / 15 / 910: 00$ & Manual Tape & 7.75 & Transcribed & \\
\hline $3 / 15 / 917: 03$ & Auto FIC & 7.7 & Good & \\
\hline $3 / 16 / 910: 00$ & Manual Tape & 7.75 & Transcribed & \\
\hline $3 / 16 / 917: 03$ & Auto FIC & 7.7 & Good & \\
\hline $3 / 17 / 910: 00$ & Manual Tape & 7.75 & Transcribed & \\
\hline $3 / 17 / 917: 02$ & Auto FIC & 7.7 & Good & \\
\hline $3 / 18 / 910: 00$ & Manual Tape & 7.75 & Transcribed & \\
\hline $3 / 18 / 917: 03$ & Auto FIC & 7.80 & Good & \\
\hline $3 / 19 / 910: 00$ & Manual Tape & 7.75 & Transcribed & \\
\hline $3 / 19 / 917: 03$ & Auto FIC & 7.80 & Good & \\
\hline $3 / 20 / 910: 00$ & Manual Tape & 7.75 & Transcribed & \\
\hline $3 / 20 / 917: 02$ & Auto FIC & 7.8 & Good & \\
\hline $3 / 21 / 910: 00$ & Manual Tape & 7.75 & Transcribed & \\
\hline $3 / 21 / 917: 03$ & Auto FIC & 7.80 & Good. & \\
\hline $3 / 22 / 910: 00$ & Manual Tape & 7.75 & Transcribed & \\
\hline $3 / 22 / 917: 02$ & Auto FIC & 7.80 & Good & \\
\hline $3 / 23 / 910: 00$ & Manual Tape & 7.75 & Transcribed & \\
\hline $3 / 23 / 917: 03$ & Auto FIC & 7.80 & Good & \\
\hline $3 / 24 / 910: 00$ & Manual Tape & 7.75 & Transcribed & \\
\hline $3 / 24 / 917: 03$ & Auto FIC & 7.80 & Good & \\
\hline $3 / 25 / 910: 00$ & Manual Tape & 7.75 & Transeribed & \\
\hline $3 / 25 / 917: 03$ & Auto FIC & 7.80 & Good & \\
\hline $3 / 26 / 910: 00$ & Manual Tape & 7.75 & Transcribed & \\
\hline $3 / 26 / 917: 03$ & Auto FIC & 7.8 & Good & \\
\hline $3 / 27 / 910: 00$ & Manual Tape & 7.75 & Transcribed & \\
\hline $3 / 27 / 917: 03$ & Auto FIC & 7.80 & Good & \\
\hline $3 / 28 / 910: 00$ & Manual Tape & 7.75 & Transcribed & \\
\hline $3 / 28 / 917: 03$ & Auto FIC & 7.80 & Good & \\
\hline $3 / 29 / 910: 00$ & Manual Tape & 7.75 & Transcribed & \\
\hline $3 / 29 / 917: 03$ & Auto FIC & 7.8 & Good & \\
\hline $3 / 30 / 910: 00$ & Manual Tape & 7.75 & Transcribed & \\
\hline $3 / 30 / 917: 03$ & Auto FIC & 7.8 & Good & \\
\hline $3 / 31 / 910: 00$ & Manual Tape & 7.75 & Transcribed & \\
\hline $3 / 31 / 917: 03$ & Auto FIC & 7.80 & Good & \\
\hline $4 / 1 / 910: 00$ & Manual Tape & 7.75 & Transcribed & \\
\hline $4 / 1 / 917: 03$ & Auto FIC & 7.8 & Good & \\
\hline $4 / 2 / 910: 00$ & Manual Tape & 7.75 & Transcribed & \\
\hline 4/2/91 7:03 & Auto FIC & 7.80 & Good & \\
\hline 4/3/91 0:00 & Manual Tape & 7.75 & Transcribed & \\
\hline $4 / 3 / 917: 03$ & Auto FIC & 7.80 & Good & \\
\hline 4/4/91 0:00 & Manual Tape & 7.75 & Transcribed & \\
\hline 4/4/91 7:03 & Auto FIC & 7.7 & Good & \\
\hline $4 / 5 / 910: 00$ & Manual Tape & 7.75 & Transcribed & \\
\hline 4/5/91 7:03 & Auto FIC & 7.70 & Good & \\
\hline $4 / 6 / 910: 00$ & Manual Tape & 7.75 & Transcribed & \\
\hline $4 / 6 / 917: 03$ & Auto FIC & 7.7 & Good & \\
\hline
\end{tabular}

Data obtained from WHC Surveillance Analysis Computer System, (PC SACS) July 7, 1995. 


\begin{tabular}{|c|c|c|c|c|}
\hline \multicolumn{5}{|c|}{ 241-AP-104 } \\
\hline & . & Ufface Level Da & & \\
\hline Date & Type of Measurement & Lovel (Inches) & Data Quality & Comments \\
\hline $4 / 7 / 910: 00$ & Manual Tape & 7.5 & Transcribed & \\
\hline $4 / 7 / 917: 03$ & Auto FIC & 7.60 & Good & \\
\hline $4 / 8 / 910: 00$ & Manual Tape & 7.5 & Transcribed & \\
\hline 4/8/91 7:04 & Auto FIC & 7.6 & Good & \\
\hline 4/9/91 0:00 & Manual Tape & 7.50 & Transcribed & \\
\hline 4/9/91 7:03 & Auto FIC & 7.60 & Good & \\
\hline 4/10/91 0:00 & Manual Tape & 7.75 & Transcribed & \\
\hline 4/10/91 7:03 & Auto FIC & 7.6 & Good & \\
\hline $4 / 11 / 910: 00$ & Manual Tape & 7.75 & Transcribed & \\
\hline 4/11/917:03 & Auto FIC & 7.60 & Good & \\
\hline $4 / 12 / 910: 00$ & Manual Tape & 7.75 & Transcribed & \\
\hline $4 / 12 / 917: 03$ & Auto FIC & 7.6 & Good & \\
\hline $4 / 13 / 910: 00$ & Manual Tape & 7.75 & Transeribed & \\
\hline 4/13/91 7:04 & Auto FIC & 7.60 & Good & \\
\hline 4/14/910:00 & Manual Tape & 7.75 & Transcribed & \\
\hline $4 / 14 / 917: 03$ & Auto FIC & 7.6 & Good & \\
\hline $4 / 15 / 910: 00$ & Manual Tape & 7.75 & Transcribed & \\
\hline 4/15/91 7:03 & Auto FIC & 7.60 & Good & \\
\hline 4/16/91 0:00 & Manual FIC & 7.60 & Transcribed & \\
\hline 4/16/91 0:00 & Manual Tape & 7.75 & Transeribed & \\
\hline $4 / 17 / 910: 00$ & Manual Tape & $\mathbf{7 . 5}$ & Transcribed & \\
\hline 4/17/91 7:03 & Auto FIC & 7.60 & Good & \\
\hline 4/18/91 0:00 & Manual Tape & 7.5 & Transcribed & \\
\hline $4 / 18 / 917: 06$ & Auto FIC & 7.6 & Good & \\
\hline 4/19/91 0:00 & Manual Tape & 7.50 & Transcribed & \\
\hline $4 / 19 / 917: 05$ & Auto FIC & 7.60 & Good & \\
\hline $4 / 20 / 910: 00$ & Manual Tape & 7.5 & Transcribed & \\
\hline 4/20/91 7:06 & Auto FIC & 7.6 & Good & \\
\hline $4 / 21 / 910: 00$ & Manual Tape & 7.50 & Transcribed & \\
\hline $4 / 21 / 917: 05$ & Auto FIC & 7.60 & Good & \\
\hline $4 / 22 / 910: 00$ & Manual Tape & 7.50 & Transcribed & \\
\hline $4 / 22 / 91 \quad 7: 06$ & Auto FIC & 7.6 & Good & \\
\hline $4 / 23 / 910: 00$ & Manual Tape & 7.50 & Transcribed & \\
\hline 4/23/91 7:06 & Auto FIC & 7.6 & Good & \\
\hline 4/24/91 0:00 & Manual Tape & 7.75 & Transcribed & \\
\hline $4 / 24 / 917: 05$ & Auto FIC & 7.6 & Good & \\
\hline $4 / 25 / 910: 00$ & Manual Tape & 7.75 & Transcribed & \\
\hline 4/25/91 7:05 & Auto FIC & 7.60 & Good & \\
\hline 4/26/91 $0: 00$ & Manual Tape & 7.75 & Transcribed & \\
\hline 4/26/91 7:05 & Auto FIC & 7.60 & Good & \\
\hline 4/27/91 0:00 & Manual Tape & 7.75 & Transcribed & \\
\hline $4 / 27 / 917: 05$ & Auto FIC & 7.60 & Good & \\
\hline $4 / 28 / 910: 00$ & Manual Tape & 7.75 & Transcribed & \\
\hline $4 / 28 / 917: 06$ & Auto FIC & 7.60 & Good & \\
\hline $4 / 29 / 910: 00$ & Manual Tape & 7.75 & Transcribed & \\
\hline $4 / 29 / 917: 05$ & Auto FIC & 7.6 & Good & \\
\hline 4/30/91 $0: 00$ & Manual Tape & 7.75 & Transcribed & \\
\hline 4/30/91 7:06 & Auto FIC & 7.6 & Good & \\
\hline
\end{tabular}

Data obtained from WHC Surveillance Analysis Computer System, (PC SACS) July 7, 1995. 


\begin{tabular}{|c|c|c|c|c|}
\hline \multicolumn{5}{|c|}{ 241-AP-104 } \\
\hline \multicolumn{5}{|c|}{ Surface Lovel Data } \\
\hline Date & Type of Measurement & Lavel (Inchoo) & Data Quality & Comments \\
\hline $5 / 1 / 910: 00$ & Manual Tape & 7.75 & Transcribed & \\
\hline $5 / 1 / 917: 05$ & Auto FIC & 7.6 & Good & \\
\hline $5 / 2 / 910: 00$ & Manual Tape & 7.75 & Transcribed & \\
\hline $5 / 2 / 917: 05$ & Auto FIC & 7.60 & Good & \\
\hline $5 / 3 / 910: 00$ & Manual Tape & 7.75 & Transcribed & \\
\hline $5 / 3 / 917: 06$ & Auto FIC & 7.6 & Good & \\
\hline $5 / 4 / 910: 00$ & Manual Tape & $\mathbf{7 . 7 5}$ & Transcribed & \\
\hline $5 / 4 / 917: 05$ & Auto FIC & 7.60 & Good & \\
\hline 5/5/91 0:00 & Manual Tape & 7.75 & Transcribed & \\
\hline $5 / 5 / 917: 05$ & Auto FIC & 7.60 & Good & \\
\hline $5 / 6 / 910: 00$ & Manual Tape & 7.75 & Transcribed & \\
\hline $5 / 6 / 917: 05$ & Auto FIC & 7.6 & Good & \\
\hline $5 / 7 / 910: 00$ & Manual Tape & 7.75 & Transcribed & \\
\hline $5 / 7 / 917: 05$ & Auto FIC & 7.60 & Good & \\
\hline $5 / 8 / 910: 00$ & Manual Tape & 7.75 & Transcribed & \\
\hline 5/8/91 7:05 & Auto FIC & 7.6 & Good & \\
\hline $5 / 9 / 910: 00$ & Manual Tape & 7.75 & Transcribed & . \\
\hline 5/9/91 7:05 & Auto FIC & 7.60 & Good & \\
\hline $5 / 10 / 910: 00$ & Manual Tape & 7.75 & Transcribed & \\
\hline $5 / 10 / 917: 05$ & Auto FIC & 7.60 & Good & \\
\hline $5 / 11 / 910: 00$ & Manual Tape & 7.50 & Transcribed & \\
\hline $5 / 11 / 917: 05$ & Auto FIC & 7.60 & Good & \\
\hline 5/12/91 0:00 & Manual Tapo & 7.75 & Transcribed & \\
\hline $5 / 12 / 917: 05$ & Auto FIC & 7.60 & Good & \\
\hline $5 / 13 / 910: 00$ & Manual Tape & 7.75 & Transcribed & \\
\hline 5/13/91 7:05 & Auto FIC & 7.60 & Good & \\
\hline 5/14/91 0:00 & Manual Tape & 7.75 & Transcribed & \\
\hline $5 / 14 / 917: 05$ & Auto FIC & 7.6 & Good & \\
\hline $5 / 15 / 910: 00$ & Manual Tape & 7.75 & Transcribed & \\
\hline $5 / 15 / 917: 05$ & Auto FIC & 7.70 & Good & \\
\hline $5 / 16 / 910: 00$ & Manual Tape & 7.75 & Transcribed & \\
\hline $5 / 16 / 917: 06$ & Auto FIC & 7.60 & Good & . \\
\hline $5 / 17 / 910: 00$ & Manual Tape & 7.75 & Transcribed & \\
\hline $5 / 17 / 917: 06$ & Auto FIC & 7.6 & Good & \\
\hline 5/18/91 0:00 & Manual Tapo & 7.75 & Transcribed & \\
\hline $5 / 18 / 917: 06$ & Auto FIC & 7.70 & Good & \\
\hline 5/19/91 0:00 & Manual Tape & 7.75 & Transcribed & \\
\hline 5/19/91 7:05 & Auto FIC & 7.7 & Good & \\
\hline $5 / 20 / 910: 00$ & Manual Tape & 7.75 & Transcribed & \\
\hline $5 / 20 / 917: 06$ & Auto FIC & 7.70 & Good & \\
\hline $5 / 21 / 910: 00$ & Manual Tape & 7.75 & Transcribed & \\
\hline $5 / 21 / 917: 06$ & Auto FIC & 7.7 & Good & \\
\hline $5 / 22 / 910: 00$ & Manual Tape & 7.75 & Transcribed & \\
\hline $5 / 22 / 917: 05$ & Auto FIC & 7.70 & Good & \\
\hline $5 / 23 / 910: 00$ & Manual Tape & 7.75 & Transcribed & \\
\hline $5 / 23 / 917: 05$ & Auto FIC & 7.7 & Good & \\
\hline 5/24/91 0:00 & Manual Tape & 7.75 & Transcribed & \\
\hline $5 / 24 / 917: 05$ & Auto FIC & 7.7 & Good & \\
\hline
\end{tabular}

Data obtained from WHC Surveillance Analysis Computer System, (PC SACS) July 7, 1995. 


\begin{tabular}{|c|c|c|c|c|}
\hline \multicolumn{5}{|c|}{ 241-AP-104 } \\
\hline \multicolumn{5}{|c|}{ Surface Level Data } \\
\hline Date & Type of Messurement & Level (Inches) & Data Quality & Comments \\
\hline $5 / 25 / 910: 00$ & Manual Tape & 7.75 & Transcribed & \\
\hline 5/25/91 7:05 & Auto FIC & 7.7 & Good & \\
\hline $5 / 26 / 910: 00$ & Manual Tape & 7.75 & Transcribed & \\
\hline $5 / 26 / 917: 05$ & Auto FIC & 7.70 & Good & \\
\hline $5 / 27 / 910: 00$ & Manual Tape & 7.75 & Transcribed & \\
\hline $5 / 27 / 917: 05$ & Auto FIC & 7.7 & Good & \\
\hline $5 / 28 / 910: 00$ & Manual Tape & 7.75 & Transcribed & \\
\hline $5 / 28 / 917: 05$ & Auto FIC & 7.7 & Good & \\
\hline $5 / 29 / 910: 00$ & Manual Tape & 7.75 & Transcribed & \\
\hline $5 / 29 / 917: 05$ & Auto FIC & 7.7 & Good & \\
\hline $5 / 30 / 910: 00$ & Manual Tape & 7.75 & Transcribed & \\
\hline $5 / 30 / 917: 05$ & Auto FIC & 7.7 & Good & \\
\hline $5 / 31 / 910: 00$ & Manual Tape & 7.75 . & Transcribed & \\
\hline $5 / 31 / 917: 05$ & Auto FIC & 7.70 & Good & \\
\hline $6 / 1 / 910: 00$ & Manual Tape & 7.75 & Transcribed & \\
\hline 6/9/91 6:05 & Auto FIC & 7.70 & Good & \\
\hline $6 / 2 / 910: 00$ & Manual Tape & $\mathbf{7 . 7 5}$ & Transcribed & \\
\hline 6/2/91 3:05 & Auto FIC & 7.70 & Good & \\
\hline $6 / 3 / 910: 00$ & Manual Tape & 7.75 & Transcribed & - \\
\hline $6 / 3 / 917: 05$ & Auto FIC & 7.7 & Good & \\
\hline $6 / 4 / 910: 00$ & Manual Tape & 7.75 & Transçribed & \\
\hline 6/4/917:05 & Auto FIC & 7.70 & Good & \\
\hline $6 / 5 / 910: 00$ & Manual Tape & 7.75 & Transcribed & \\
\hline $6 / 5 / 917: 05$ & Auto FIC & 7.7 & Good & \\
\hline $6 / 6 / 91 \quad 0: 00$ & Manual Tape & 7.50 & Transcribed & \\
\hline $6 / 6 / 917: 06$ & Auto FIC & 7.70 & Good & \\
\hline $6 / 7 / 910: 00$ & Manual Tape & $\mathbf{7 . 7 5}$ & Transcribed & \\
\hline $6 / 7 / 917: 05$ & Auto FIC & 7.70 & Good & \\
\hline $6 / 8 / 910: 00$ & Manual Tape & 7.5 & Transcribed & \\
\hline $6 / 8 / 917: 05$ & Auto FIC & 7.70 & Good & \\
\hline $6 / 9 / 910: 00$ & Manual Tape & 7.50 & Transcribed & \\
\hline $6 / 9 / 917: 05$ & Auto FIC & 7.7 & Good & \\
\hline $6 / 10 / 910: 00$ & Manual Tape & 7.75 & Transcribed & \\
\hline $6 / 10 / 917: 06$ & Auto FIC & 7.70 & Good & \\
\hline $6 / 11 / 910: 00$ & Manual Tape & 7.75 & Transcribed & \\
\hline $6 / 11 / 917: 05$ & Auto FIC & 7.70 & Good & \\
\hline $6 / 12 / 910: 00$ & Manual Tape & 7.75 & Transcribed & \\
\hline $6 / 12 / 917: 04$ & Auto FIC & 7.70 & Good & \\
\hline $6 / 13 / 910: 00$ & Manual Tape & 7.50 & Transcribed & \\
\hline $6 / 13 / 917: 05$ & Auto FIC & 7.70 & Good & \\
\hline $6 / 14 / 910: 00$ & Manual Tape & 7.50 & Transcribed & \\
\hline $6 / 14 / 917: 05$ & Auto FIC & 7.70 & Good & \\
\hline $6 / 15 / 910: 00$ & Manual Tape. & 7.5 & Transcribed & \\
\hline $6 / 15 / 917: 05$ & Auto FIC & 7.7 & Good & \\
\hline $6 / 16 / 910: 00$ & Manual Tape & 7.5 & Transcribed & \\
\hline $6 / 16 / 917: 05$ & Auto FIC & 7.7 & Good & \\
\hline $6 / 17 / 910: 00$ & Manual Tape & 7.5 & Transcribed & \\
\hline $6 / 17 / 917: 05$ & Auto FIC & 7.70 & Good & \\
\hline
\end{tabular}

Data obtained from WHC Surveillance Analysis Computer System, (PC SACS) July 7, 1995. 


\begin{tabular}{|c|c|c|c|c|}
\hline \multicolumn{5}{|c|}{$\begin{array}{c}\text { 241-AP-104 } \\
\text { Surface Level Data } \\
\end{array}$} \\
\hline Date & Type of Measurement & Lovel (Inchea) & Data Quality & Comments \\
\hline $6 / 18 / 910: 00$ & Manual Tape & 7.50 & Transcribed & \\
\hline $6 / 18 / 917: 05$ & Auto FIC & 7.7 & Good & \\
\hline 6/19/910:00 & Manual Tape & 7.75 & Transcribed & \\
\hline 6/19/91 7:05 & Auto FIC & 7.70 & Good & \\
\hline $6 / 20 / 910: 00$ & Manual Tape & 7.75 & Transcribed & \\
\hline $6 / 20 / 917: 05$ & Auto FIC & 7.70 & Good & \\
\hline $6 / 21 / 910: 00$ & Manual Tape & 7.75 & Transcribed & \\
\hline $6 / 21 / 917: 06$ & Auto FIC & 7.70 & Good & \\
\hline $6 / 22 / 910: 00$ & Manual Tape & 7.75 & Transcribed & \\
\hline $6 / 22 / 913: 06$ & Auto FIC & 7.7 . & Good & \\
\hline $6 / 23 / 910: 00$ & Manual Tape & 7.75 & Transcribed & \\
\hline $6 / 23 / 917: 05$ & Auto FIC & 7.7 & Good & \\
\hline $6 / 24 / 910: 00$ & Manual Tape & 7.75 & Transcribed & \\
\hline $6 / 24 / 915: 05$ & Auto FIC & 7.7 & Good & \\
\hline $6 / 25 / 910: 00$ & Manual Tape & 7.75 & Transcribed & \\
\hline $6 / 25 / 917: 06$ & Auto FIC & 7.7 & Good & \\
\hline $6 / 26 / 910: 00$ & Manual Tape & 7.75 & Transcribed & \\
\hline $6 / 26 / 911: 03$ & Auto FIC & 7.7 & Good & \\
\hline $6 / 27 / 910: 00$ & Manual FIC & 7.70 & Transcribed & \\
\hline $6 / 27 / 910: 00$ & Manual Tape & 7.75 & Transeribed & \\
\hline 6/28/91 0:00 & Manual Tape & 7.75 & Transcribed & \\
\hline $6 / 28 / 917: 03$ & Auto FIC & 7.60 & Good & \\
\hline $6 / 29 / 910: 00$ & Manual Tape & 7.75 & Transcribed & \\
\hline $6 / 29 / 916: 02$ & Auto FIC & 7.60 & Good & \\
\hline $6 / 30 / 91 \quad 0: 00$ & Manual FIC & 7.60 & Transcribed & \\
\hline $6 / 30 / 910: 00$ & Manual Tape & 7.75 & Transcribed & \\
\hline $7 / 1 / 910: 00$ & Manual FIC & 7.6 & Transcribed & \\
\hline 7/1/91,0:00 & Manual Tape & 7.5 & Transcribed & \\
\hline $7 / 2 / 910: 00$ & Manual Tape & 7.75 & Transcribed & \\
\hline $7 / 2 / 917: 06$ & Auto FIC & 7.6 & Good & \\
\hline $7 / 3 / 910: 00$ & Manual Tape & 7.75 & Transcribed & \\
\hline $7 / 3 / 917: 06$ & Auto FIC & $7 . \overline{60}$ & Good & \\
\hline $7 / 4 / 910: 00$ & Manual FIC & 7.6 & Transcribed & \\
\hline $7 / 4 / 910: 00$ & Manual Tape & 7.50 & Transcribed & \\
\hline $7 / 5 / 910: 00$ & Manual FIC & 7.6 & Transcribed & \\
\hline $7 / 5 / 910: 00$ & Manual Tape & 7.50 & Transcribed & \\
\hline $7 / 6 / 91 \quad 0: 00$ & Manual FIC & 7.6 & Transcribed & \\
\hline $7 / 6 / 910: 00$ & Manual Tape & 7.50 & Transcribed & \\
\hline $7 / 7 / 910: 00$ & Manual FIC & 7.6 & Transcribed & \\
\hline $7 / 7 / 910: 00$ & Manual Tape & 7.5 & Transcribed & \\
\hline $7 / 8 / 910: 00$ & Manual FIC & 7.6 & Transcribed & \\
\hline $7 / 8 / 91 \quad 0: 00$ & Manual Tape & 7.50 & Transcribed & \\
\hline $7 / 9 / 910: 00$ & Manual FIC & 7.60 & Transcribed & \\
\hline $7 / 9 / 910: 00$ & Manual Tape & 7.50 & Transćribed & \\
\hline $7 / 10 / 910: 00$ & Manual FIC & 7.6 & Transcribed & \\
\hline $7 / 10 / 910: 00$ & Manual Tape & 7.50 & Transcribed & \\
\hline $7 / 11 / 910: 00$ & Manual Tape & 7.5 & Transcribed & \\
\hline $7 / 11 / 917: 06$ & Auto FIC & 7.60 & Good & \\
\hline
\end{tabular}

Data obtained from WHC Surveillance Analysis Computer System, (PC SACS) July 7, 1995. 


\begin{tabular}{|c|c|c|c|c|}
\hline \multicolumn{5}{|c|}{ 247-AP-104 } \\
\hline & & Urface Level Dat & & \\
\hline Date & Type of Measurement & Leval (Inches) & Data Quality & Commonts \\
\hline $7 / 12 / 910: 00$ & Manual Tape & 7.50 & Transcribed & \\
\hline $7 / 12 / 917: 06$ & Auto FIC & 7.6 & Good & \\
\hline $7 / 13 / 910: 00$ & Manual Tape & 7.75 & Transcribed & \\
\hline $7 / 13 / 917: 06$ & Auto FIC & 7.60 & Good & \\
\hline 7/14/91 0:00 & Manual Tape & 7.75 & Transcribed & \\
\hline 7/14/91 2:06 & Auto FIC & 7.60 & Good & \\
\hline $7 / 15 / 910: 00$ & Manual Tape & 7.75 & Transcribed & \\
\hline $7 / 15 / 917: 06$ & Auto FIC & 7.6 & Good & \\
\hline $7 / 16 / 910: 00$ & Manual FIC & 7.60 & Transcribed & \\
\hline $7 / 16 / 910: 00$ & Manual Tape & 7.75 & Transcribed & \\
\hline $7 / 17 / 910: 00$ & Manual Tape & 84.75 & Transcribed & \\
\hline $7 / 17 / 910: 05$ & Auto FIC & 7.6 & Good & \\
\hline $7 / 18 / 910: 00$ & Manual FIC & 7.60 & Transcribed & \\
\hline $7 / 18 / 910: 00$ & Manual Tape & 7.75 & Transcribed & \\
\hline $7 / 19 / 910: 00$ & Manual Tape & 7.75 & Transcribed & \\
\hline $7 / 19 / 917: 05$ & Auto FIC & 7.6 & Good & \\
\hline $7 / 20 / 910: 00$ & Manual Tape & 7.75 & Transeribed & \\
\hline $7 / 20 / 912: 05$ & Auto FIC & 7.60 & Good & \\
\hline $7 / 21 / 910: 00$ & Manual Tape & 7.75 & Transcribed & \\
\hline 7/21/91 1:05 & Auto FIC & 7.60 & Good & \\
\hline $7 / 22 / 91 \quad 0: 00$ & Manual FIC & 7.60 & Transcribed & \\
\hline $7 / 22 / 910: 00$ & Manual Tape & 7.75 & Transcribed & \\
\hline $7 / 23 / 910: 00$ & Manual Tape & 7.75 & Transcribed & \\
\hline $7 / 23 / 910: 05$ & Auto FIC & 7.60 & Good & \\
\hline $7 / 24 / 910: 00$ & Manual Tape & 7.75 & Transcribed & \\
\hline $7 / 24 / 917: 05$ & Auto FIC & 7.6 & Good & \\
\hline $7 / 25 / 910: 00$ & Manual Tape & 7.75 & Transeribed & \\
\hline $7 / 25 / 91 \quad 1: 03$ & Auto FIC & 7.6 & Good & \\
\hline $7 / 26 / 910: 00$ & Manual FIC & 7.60 & Traniscribed & \\
\hline $7 / 26 / 910: 00$ & Manual Tape & 7.75 & Transcribed & ? \\
\hline $7 / 27 / 910: 00$ & Manual FIC & 7.6 & Transcribed & \\
\hline $7 / 27 / 91 \quad 0: 00$ & Manual Tape . & 7.75 & Transeribed & \\
\hline $7 / 28 / 910: 00$ & Manual FIC & 7.60 & Transcribed & \\
\hline $7 / 28 / 910: 00$ & Manual Tape & 7.75 & Transcribed & \\
\hline 7/29/91 0:00 & Manual FIC & 7.6 & Transcribed & \\
\hline $7 / 29 / 910: 00$ & Manual Tape & 7.75 & Transcribed & \\
\hline $7 / 30 / 910: 00$ & Manual Tape & 7.75 & Transcribed & \\
\hline $7 / 30 / 91 \quad 7: 02$ & Auto FIC & 7.60 & Good & \\
\hline $7 / 31 / 91 \quad 0: 00$ & Manual Tape & 7.75 & Transcribed & \\
\hline 7/31/91 7:02 & Auto FIC & 7.60 & Good & \\
\hline $8 / 1 / 910: 00$ & Manual Tape & 7.50 & Transcribed & \\
\hline $8 / 1 / 917: 02$ & Auto FIC & 7.6 & Good & \\
\hline $8 / 2 / 910: 00$ & Manual Tape & 7.75 & Transcribed & \\
\hline $8 / 2 / 917: 02$ & Auto FIC & 7.6 & Good & \\
\hline $8 / 3 / 910: 00$ & Manual Tape & 7.5 & Transcribed & \\
\hline $8 / 3 / 917: 02$ & Auto FIC & 7.6 & Good & \\
\hline $8 / 4 / 910: 00$ & Manual Tape & 7.50 & Transcribed & \\
\hline $8 / 4 / 917: 02$ & Auto FIC & 7.60 & Good & \\
\hline
\end{tabular}

Data obtained from WHC Surveillance Analysis Computer System, (PC SACS) July 7, 1995. 


\begin{tabular}{|c|c|c|c|c|}
\hline \multicolumn{5}{|c|}{ 241-AP-104 } \\
\hline Date & Type of Measurement & Lavel (Inchas) & Data Quality & Commonts \\
\hline $8 / 5 / 910: 00$ & Manual Tape & 7.5 & Transcribed & \\
\hline $8 / 5 / 917: 02$ & Auto FIC & 7.60 & Good & \\
\hline $8 / 6 / 910: 00$ & Manual Tape & 7.5 & Transcribed & \\
\hline $8 / 6 / 917: 02$ & Auto FIC & 7.60 & Good & \\
\hline 8/7/910:00 & Manual Tape & 7.75 & Transcribed & \\
\hline $8 / 7 / 917: 06$ & - Auto FIC & 7.60 & Good & \\
\hline $8 / 8 / 910: 00$ & Manual Tape & 7.75 & Transcribed & \\
\hline $8 / 8 / 917: 06$ & Auto FIC & 7.6 & Good & \\
\hline $8 / 9 / 910: 00$ & Manual Tape & 7.75 & Transcribed & \\
\hline $8 / 9 / 917: 04$ & Auto FIC & 7.6 & Good & \\
\hline $8 / 10 / 910: 00$ & Manual Tape & 7.75 & Transcribed & \\
\hline $8 / 10 / 917: 04$ & Auto FIC & 7.60 & Good & \\
\hline $8 / 11 / 910: 00$ & Manual Tape & 7.75 & Transcribed & \\
\hline 8/11/91 7:04 & Auto FIC & 7.6 & Good & \\
\hline $8 / 12 / 910: 00$ & Manual Tape & 7.50 & Transcribed & \\
\hline $8 / 12 / 917: 04$ & Auto FIC & 7.6 . & Good & \\
\hline $8 / 13 / 910: 00$ & Manual Tape & 7.50 & Transcribed & \\
\hline $8 / 13 / 917: 04$ & Auto FIC & 7.6 & Good & \\
\hline $8 / 14 / 910: 00$ & Manual Tape & 7.50 & Transcribed & \\
\hline $8 / 14 / 917: 04$ & Auto FIC & 7.60 & Good & \\
\hline $8 / 15 / 910: 00$ & Manual Tape & 7.75 & Transcribed & \\
\hline $8 / 15 / 917: 03$ & Auto FIC & 7.60 & Good & \\
\hline $8 / 16 / 910: 00$ & Manual FIC & 7.60 & Transcribed & \\
\hline $8 / 16 / 910: 00$ & Manual Tape & 7.50 & Transcribed & \\
\hline $8 / 17 / 910: 00$ & Manual Tape & 7.75 & Transcribed & \\
\hline $8 / 17 / 917: 02$ & Auto FIC & 7.6 & Good & \\
\hline $8 / 18 / 910: 00$ & Manual Tape & 7.75 & Transcribed & \\
\hline $8 / 18 / 917: 03$ & Auto FIC & 7.60 & Good & \\
\hline $8 / 19 / 910: 00$ & Manual Tape & 7.75 & Transcribed & \\
\hline $8 / 19 / 917: 02$ & Auto FIC & 7.6 & Good & \\
\hline 8/20/910:00 & Manual Tape & 7.75 & Transcribed & \\
\hline $8 / 20 / 917: 04$ & Auto FIC & 7.6 & Good. & \\
\hline $8 / 21 / 910: 00$ & Manual Tape . & 7.75 & Transcribed & \\
\hline $8 / 21 / 917: 03$ & Auto FIC & 7.60 & Good & \\
\hline $8 / 22 / 910: 00$ & Manual Tape & 7.75 & Transcribed & \\
\hline $8 / 22 / 917: 03$ & Auto FIC & 7.6 & Good & \\
\hline $8 / 23 / 910: 00$ & Manual Tape & 7.75 & Transcribed & \\
\hline $8 / 23 / 917: 02$ & Auto FIC & 7.6 & Good & \\
\hline $8 / 24 / 910: 00$ & Manual Tape & 7.75 & Transcribed & \\
\hline $8 / 24 / 917: 02$ & Auto FIC & 7.60 & Good & \\
\hline $8 / 25 / 910: 00$ & Manual Tape & 7.75 & Transcribed & \\
\hline $8 / 25 / 917: 02$ & Auto FIC & 7.60 & Good & $\cdot$ \\
\hline $8 / 26 / 910: 00$ & Manual Tape & 7.75 & Transcribed & \\
\hline $8 / 26 / 917: 03$ & Auto FIC & 7.60 & Good & \\
\hline $8 / 27 / 910: 00$ & Manual Tape & 7.75 & Transçribed & \\
\hline $8 / 27 / 917: 03$ & Auto FIC & 7.6 & Good & \\
\hline $8 / 28 / 910: 00$ & Manual Tape & 7.75 & Transcribed & \\
\hline $8 / 28 / 917: 03$ & Auto FIC & 7.60 & Good & \\
\hline
\end{tabular}

Data obtained from WHC Surveitlance Analysis Computer System, (PC SACS) July 7, 1995. 


\begin{tabular}{|c|c|c|c|c|}
\hline \multicolumn{5}{|c|}{ 241-AP-104 } \\
\hline \multicolumn{5}{|c|}{ Surface Level Data } \\
\hline Date & Type of Measurement & Leval (Inches) & Data Cuallity & Commants \\
\hline 8/29/91 0:00 & Manual Tape & 7.50 & Transcribed & \\
\hline $8 / 29 / 917: 02$ & Auto FIC & 7.6 & Good & \\
\hline $8 / 30 / 910: 00$ & Manual Tape & 7.75 & Transcribed & \\
\hline $8 / 30 / 916: 02$ & Auto FIC & 7.6 & Good & \\
\hline $8 / 31 / 910: 00$ & Manual Tape & 7.75 & Transcribed & \\
\hline $8 / 31 / 917: 02$ & Auto FIC & 7.60 & Good & \\
\hline $9 / 1 / 910: 00$ & Manual Tape & 7.75 & Transcribed & \\
\hline $9 / 1 / 917: 02$ & Auto FIC & 7.6 & Good & \\
\hline $9 / 2 / 910: 00$ & Manual Tape & 7.75 & Transcribed & \\
\hline $9 / 2 / 917: 02$ & Auto FIC & 7.60 & Good & \\
\hline 9/3/910:00 & Manual Tape & 7.75 & Transcribed & \\
\hline $9 / 3 / 917: 02$ & Auto FIC & 7.50 & Good & \\
\hline $9 / 4 / 910: 00$ & Manual Tape & 7.75 & Transcribed & \\
\hline $9 / 4 / 917: 03$ & Auto FIC & 7.60 & Good & \\
\hline $9 / 5 / 910: 00$ & Manual Tape & 7.75 & Transcribed & \\
\hline $9 / 5 / 917: 02$ & Auto FIC & 7.60 & Good & \\
\hline $9 / 6 / 910: 00$ & Manual Tape & 7.50 & Transcribed & \\
\hline $9 / 6 / 917: 02$ & Auto FIC & 7.6 & Good & \\
\hline $9 / 7 / 910: 00$ & Manual Tape & 7.50 & Transcribed & \\
\hline $9 / 7 / 917: 02$ & Auto FIC & 7.50 & Good & \\
\hline $9 / 8 / 910: 00$ & Manual Tape & 7.50 & Transcribed & \\
\hline $9 / 8 / 917: 02$ & Auto FIC & 7.50 & Good & \\
\hline $9 / 9 / 91 \quad 0: 00$ & Manual Tape & 7.50 & Transcribed & \\
\hline $9 / 9 / 917: 03$ & Auto FIC & 7.50 & Good & \\
\hline $9 / 10 / 910: 00$ & Manual Tape & 7.5 & Transcribed & \\
\hline $9 / 10 / 917: 02$ & Auto FIC & 7.50 & Good & \\
\hline $9 / 11 / 910: 00$ & Manual Tapo & 7.5 & Transcribed & \\
\hline $9 / 11 / 917: 03$ & Auto FIC & 7.5 & Good & \\
\hline 9/12/91 0:00 & Manual Tape & 7.50 & Transcribed & \\
\hline 9/12/91 7:02 & Auto FIC & 7.5 & Good & \\
\hline 9/13/91 0:00 & Manual Tape & 7.75 & Transcribed & \\
\hline $9 / 13 / 917: 02$ & Auto FIC & 7.5 & Good & \\
\hline $9 / 14 / 910: 00$ & Manual Tape & 7.50 & Transcribed & \\
\hline $9 / 14 / 917: 03$ & Auto FIC & 7.5 & Good & \\
\hline 9/15/91 0:00 & Manual Tape & 7.5 & Transcribed & \\
\hline $9 / 15 / 917: 02$ & Auto FIC & 7.50 & Good & \\
\hline $9 / 16 / 910: 00$ & Manual Tape & 7.5 & Transcribed & \\
\hline $9 / 16 / 917: 03$ & Auto FIC & 7.5 & Good & \\
\hline $9 / 17 / 910: 00$ & Manual Tape & 7.5 & Transcribed & \\
\hline 9/17/91 7:03 & Auto FIC & 7.5 & Good & \\
\hline $9 / 18 / 910: 00$ & Manual Tape & 7.50 & Transcribed & \\
\hline $9 / 18 / 917: 03$ & Auto FIC & 7.50 & Good & \\
\hline 9/19/91 0:00 & Manual Tape & 7.50 & Transcribed & \\
\hline 9/19/91 7:03 & Auto FIC & 7.50 & Good & \\
\hline $9 / 20 / 910: 00$ & Manual Tape & 7.25 & Transcribed & \\
\hline $9 / 20 / 917: 02$ & Auto FIC & 7.5 & Good & \\
\hline $9 / 21 / 910: 00$ & Manual Tape & 7.25 & Transcribed & \\
\hline $9 / 21 / 917: 02$ & Auto FIC & 7.50 & Good & \\
\hline
\end{tabular}

Data obtained from WHC Surveillance Analysis Computer System, (PC SACS) July 7, 1995. 


\begin{tabular}{|c|c|c|c|c|}
\hline \multicolumn{5}{|c|}{ 241-AP-104 } \\
\hline \multicolumn{5}{|c|}{ Surface Level Data } \\
\hline Date & Type of Measurement & Level (Inches) & Data Quality & Comments \\
\hline 9/22/91 0:00 & Manual Tape & 7.25 & Transcribed & \\
\hline 9/22/91 7:03 & Auto FIC & 7.5 & Good & \\
\hline $9 / 23 / 910: 00$ & Manual Tape & 7.25 & Transcribed & \\
\hline $9 / 23 / 917: 03$ & Auto FIC & 7.50 & Good & \\
\hline $9 / 24 / 910: 00$ & Manual Tape & 7.50 & Transcribed & \\
\hline $9 / 24 / 917: 02$ & Auto FIC & 7.50 & Good & \\
\hline $9 / 25 / 910: 00$ & Manual Tape & 7.5 & Transcribed & \\
\hline $9 / 25 / 917: 03$ & Auto FIC & 7.50 & Good & \\
\hline 9/26/91 0:00 & Manual Tape & 7.5 & Transcribed & \\
\hline 9/26/91 7:03 & Auto FIC & 7.50 & Good & \\
\hline $9 / 27 / 910: 00$ & Manual Tape & 7.5 & Transcribed & - \\
\hline $9 / 27 / 917: 02$ & Auto FIC & 7.5 & Good & \\
\hline $9 / 28 / 910: 00$ & Manual Tape & 7.5 & Transcribed & \\
\hline $9 / 28 / 917: 02$ & Auto FIC & 7.50 & Good & \\
\hline $9 / 29 / 910: 00$ & Manual Tape & 7.50 & Transcribed & \\
\hline $9 / 29 / 917: 03$ & Auto FIC & 7.50 & Good & \\
\hline $9 / 30 / 910: 00$ & Manual Tape & 7.50 & Transcribed & \\
\hline 9/30/91 7:03 & Auto FIC & 7.5 & Good & \\
\hline $10 / 1 / 91.0: 00$ & Manual Tape & 7.5 & Transcribed & \\
\hline $10 / 1 / 917: 02$ & Auto FIC & 7.5 & Good & \\
\hline $10 / 2 / 910: 00$ & Manual Tape & 7.5 & Transcribed & \\
\hline $10 / 2 / 917: 02$ & Auto FIC & 7.50 & Good & \\
\hline $10 / 3 / 910: 00$ & Manual Tape & 7.50 & Transcribed & \\
\hline $10 / 3 / 917: 02$ & Auto FIC & 7.50 & Good & \\
\hline $10 / 4 / 910: 00$ & Manual Tape & 7.50 & Transcribed & \\
\hline $10 / 4 / 917: 03$ & Auto FIC & 7.5 & Good & \\
\hline $10 / 5 / 910: 00$ & Manual FIC & 7.50 & Transcribed & \\
\hline $10 / 5 / 910: 00$ & Manual Tape & 7.50 & Transeribed & \\
\hline $10 / 6 / 910: 00$ & Manual Tape & 7.50 & Transcribed & \\
\hline 10/6/91 7:03 & Auto FIC & 7.50 & Good & \\
\hline $10 / 7 / 910: 00$ & Manual Tape & 7.50 & Transeribed & \\
\hline $10 / 7 / 917: 02$ & Auto FIC & 7.5 & Good & $\cdot$ \\
\hline $10 / 8 / 910: 00$ & Manual Tape & 7.50 & Transcribed & \\
\hline $10 / 8 / 917: 02$ & Auto FIC & 7.5 & Good & \\
\hline $10 / 9 / 910: 00$ & Manual Tape & 7.5 & Transcribed & \\
\hline $10 / 9 / 91 \quad 7: 02$ & Auto FIC & 7.50 & Good & \\
\hline 10/10/91 $0: 00$ & Manual Tape & 7.5 & Transcribed & \\
\hline $10 / 10 / 917: 02$ & Auto FIC & 7.50 & Good & \\
\hline $10 / 11 / 910: 00$ & Manual Tape & 7.5 & Transcribed & \\
\hline 10/11/917:02 & Auto FIC & 7.5 & Good & \\
\hline $10 / 12 / 910: 00$ & Manual Tape & 7.5 & Transcribed & \\
\hline $10 / 12 / 917: 02$ & Auto FIC & 7.50 & Good & \\
\hline $10 / 13 / 910: 00$ & Manual Tape & 7.50 & Transcribed & \\
\hline $10 / 13 / 917: 03$ & Auto FIC & 7.50 & Good & \\
\hline $10 / 14 / 910: 00$ & Manual Tape & 7.25 & Transcribed & \\
\hline $10 / 14 / 917: 02$ & Auto FIC & 7.5 & Good & \\
\hline $10 / 15 / 910: 00$ & Manual Tape & 7.50 & Transcribed & \\
\hline $10 / 15 / 917: 02$ & Auto FIC & 7.5 & Good & \\
\hline
\end{tabular}

Data obtained from WHC Surveillance Analysis Computer System, (PC SACS) July 7, 1995. 


\begin{tabular}{|c|c|c|c|c|}
\hline \multicolumn{5}{|c|}{ 241-AP-104 } \\
\hline & & Urface Loval Dat & & \\
\hline Date & Type of Measurement & Levol (lnched) & Data Quallty & Comments \\
\hline $10 / 16 / 910: 00$ & Manual Tape & 7.5 & Transcribed & \\
\hline 10/16/91 7:03 & Auto FIC & 7.5 & Good & \\
\hline $10 / 17 / 910: 00$ & Manual Tape & 7.5 & Transcribed & \\
\hline $10 / 17 / 917: 05$ & Auto FIC & 7.50 & Good & \\
\hline $10 / 18 / 910: 00$ & Manual Tape & 7.5 & Transcribed & \\
\hline $10 / 18 / 917: 06$ & Auto FIC & 7.50 & Good & \\
\hline $10 / 19 / 910: 00$ & Manual Tape & 7.50 & Transcribed & \\
\hline 10/19/91 7:06 & Auto FIC & 7.50 & Good & \\
\hline $10 / 20 / 910: 00$ & Manual Tape & 7.50 & Transcribed & \\
\hline $10 / 20 / 917: 02$ & Auto FIC & 7.50 & Good & \\
\hline $10 / 21 / 910: 00$ & Manual Tape & 7.50 & Transeribed & \\
\hline 10/21/91 7:02 & Auto FIC & 7.5 & Good & \\
\hline $10 / 22 / 910: 00$ & Manual Tape & 7.50 & Transcribed & \\
\hline $10 / 22 / 917: 03$ & Auto FIC & 7.50 & Good & \\
\hline $10 / 23 / 910: 00$ & Manual Tape & 7.50 & Transcribed & \\
\hline $10 / 23 / 917: 02$ & Auto FIC & 7.50 & Good & \\
\hline $10 / 24 / 910: 00$ & Manual Tape & 7.5 & Transcribed & \\
\hline $10 / 24 / 917: 02$ & Auto FIC & 7.5 & Good & \\
\hline $10 / 25 / 910: 00$ & Manual Tape & 7.5 & Transcribed & \\
\hline $10 / 25 / 917: 03$ & Auto FIC & 7.5 & Good & \\
\hline $10 / 26 / 910: 00$ & Manual Tape & 7.5 & Transcribed & \\
\hline 10/26/91 7:03 & Auto FIC & 7.5 & Good & \\
\hline $10 / 27 / 910: 00$ & Manual Tape & $\mathbf{7 . 7 5}$ & Transcribed & \\
\hline $10 / 27 / 917: 02$ & Auto FIC & 7.50 & Good & \\
\hline $10 / 28 / 91 \quad 0: 00$ & Manual Tape & 7.5 & Transcribed & \\
\hline $10 / 28 / 917: 02$ & Auto FIC & 7.50 & Good & \\
\hline 10/29/91 0:00 & Manual Tape & 7.50 & Transcribed & \\
\hline $10 / 29 / 917: 03$ & Auto FIC & 7.4 & Good & \\
\hline $10 / 30 / 910: 00$ & Manual Tape & 7.50 & Transcribed & \\
\hline $10 / 30 / 917: 03$ & Auto FIC & 7.4 & Good & \\
\hline $10 / 31 / 910: 00$ & Manual Tape & 7.50 & Transcribed & \\
\hline $10 / 31 / 917: 03$ & Auto FIC & 7.4 & Good & \\
\hline $11 / 1 / 910: 00$ & Manual Tape & 7.50 & Transcribed & \\
\hline $11 / 1 / 917: 02$ & Auto FIC & 7.4 & Good & \\
\hline $11 / 2 / 910: 00$ & Manual Tape & 7.5 & Transcribed & \\
\hline $11 / 2 / 917: 03$ & Auto FIC & 7.40 & Good & \\
\hline $11 / 3 / 910: 00$ & Manual Tape & 7.50 & Transcribed & \\
\hline $11 / 3 / 917: 03$ & Auto FIC & 7.4 & Good & \\
\hline $11 / 4 / 910: 00$ & Manual Tape & 7.5 & Transcribed & \\
\hline $11 / 4 / 917: 02$ & Auto FIC & 7.4 & Good & \\
\hline $11 / 5 / 910: 00$ & Manual Tape & 7.25 & Transcribed & \\
\hline $11 / 5 / 917: 03$ & Auto FIC & 7.40 & Good & \\
\hline $11 / 6 / 910: 00$ & Manual Tape & 7.5 & Transcribed & \\
\hline $11 / 6 / 917: 03$ & Auto FIC & 7.4 & Good & \\
\hline $11 / 7 / 910: 00$ & Manual Tape & 7.50 & Transcribed & \\
\hline $11 / 7 / 917: 02$ & Auto FIC & 7.40 & Good & \\
\hline $11 / 8 / 910: 00$ & Manual Tape & 7.50 & Transcribed & \\
\hline $11 / 8 / 917: 03$ & Auto FIC & 7.4 & Good & \\
\hline
\end{tabular}

Data obtained from WHC Surveillance Analysis Computer System, (PC SACS) July 7, 1995. 


\begin{tabular}{|c|c|c|c|c|}
\hline \multicolumn{5}{|c|}{ 241-AP-104 } \\
\hline \multicolumn{5}{|c|}{ Surface Level Data } \\
\hline Date & Type of Measurement & Lovel, (linched & Data Quality & Comments \\
\hline $11 / 9 / 910: 00$ & Manual Tape & 7.5 & Transcribed & \\
\hline $11 / 9 / 917: 02$ & Auto FIC & 7.4 & Good & \\
\hline $11 / 10 / 910: 00$ & Manual Tape & 7.50 & Transcribed & \\
\hline $11 / 10 / 917: 03$ & Auto FIC & 7.4 & Good & \\
\hline $11 / 11 / 910: 00$ & Manual Tape & 7.50 & Transcribed & \\
\hline $11 / 11 / 916: 03$ & Auto FIC & 7.40 & Good & \\
\hline $11 / 12 / 910: 00$ & Manual Tape & 7.50 & Transcribed & \\
\hline $11 / 12 / 917: 03$ & Auto FIC & 7.40 & Good & \\
\hline $11 / 13 / 910: 00$ & Manual Tape & 7.50 & Transcribed & \\
\hline $11 / 13 / 917: 03$ & Auto FIC & 7.4 & Good & \\
\hline $11 / 14 / 910: 00$ & Manual Tape & 7.5 & Transcribed & \\
\hline $11 / 14 / 917: 03$ & Auto FIC & 7.40 & Good & \\
\hline $11 / 15 / 910: 00$ & Manual Tape & 7.5 & Trenscribed & \\
\hline $11 / 15 / 917: 03$ & Auto FIC & 7.4 & Good & \\
\hline $11 / 16 / 910: 00$ & Manual Tape & 7.50 & Transcribed & \\
\hline $11 / 16 / 917: 03$ & Auto FIC & 7.40 & Good & \\
\hline $11 / 17 / 910: 00$ & Manual Tape & 7.50 & Transcribed & \\
\hline $11 / 17 / 917: 02$ & Auto FIC & 7.4 & Good & \\
\hline $11 / 18 / 910: 00$ & Manual Tape & 7.50 & Transcribed & \\
\hline $11 / 18 / 917: 03$ & Auto FIC & 7.40 & Good & \\
\hline $11 / 19 / 910: 00$ & Manual Tape & .7 .50 & Transcribed & \\
\hline $11 / 19 / 917: 03$ & Auto FIC & 7.40 & Good & \\
\hline $11 / 20 / 910: 00$ & Manual Tape & 7.50 & Transcribed & \\
\hline $11 / 20 / 917: 03$ & Auto FIC & 7.40 & Good & \\
\hline $11 / 21 / 910: 00$ & Manual Tape & 7.75 & Transcribed & \\
\hline $11 / 21 / 917: 02$ & Auto FIC & 7.40 & Good & \\
\hline $11 / 22 / 910: 00$ & Manual Tape & 7.5 & Transcribed & \\
\hline $11 / 22 / 917: 03$ & Auto FIC & 7.40 & Good & \\
\hline $11 / 23 / 910: 00$ & Manual Tape & 7.5 & Transcribed & \\
\hline $11 / 23 / 917: 02$ & Auto FIC & 7.40 & Good & \\
\hline $11 / 24 / 910: 00$ & Manual Tape & 7.5 & Transcribed & \\
\hline $11 / 24 / 917: 03$ & Auto FIC & 7.40 & Good & \\
\hline $11 / 25 / 910: 00$ & Manual Tape & 7.5 & Transcribed & \\
\hline $11 / 25 / 917: 03$ & Auto FIC & 7.40 & Good & \\
\hline $11 / 26 / 910: 00$ & Manual FIC & 7.4 & Transcribed & \\
\hline $11 / 26 / 910: 00$ & Manual Tape & 7.25 & Transcribed & \\
\hline $11 / 27 / 910: 00$ & Manual Tape & 7.25 & Transcribed & \\
\hline $11 / 27 / 917: 03$ & Auto FIC & 7.4 & Good & \\
\hline $11 / 28 / 910: 00$ & Manual Tape & 7.25 & Transcribed & \\
\hline $11 / 28 / 917: 03$ & Auto FIC & 7.40 & Good & \\
\hline $11 / 29 / 910: 00$ & Manual Tape & 7.50 & Transcribed & \\
\hline $11 / 29 / 917: 03$ & Auto FIC & 7.4 & Good & \\
\hline 11/30/91 0:00 & Manual Tape & 7.5 & Transcribed & \\
\hline $11 / 30 / 917: 03$ & Auto FIC & 7.40 & Good & \\
\hline $12 / 1 / 910: 00$ & Manual Tape & 7.5 & Transcribed & \\
\hline $12 / 1 / 917: 03$ & Auto FIC & 7.4 & Good & \\
\hline 12/2/91 0:00 & Manual Tape & 7.50 & Transcribed & \\
\hline $12 / 2 / 917: 03$ & Auto FIC & 7.4 & Good & \\
\hline
\end{tabular}

Data obtained from WHC Surveillance Analysis Computer System, (PC SACS) July 7, 1995. 


\begin{tabular}{|c|c|c|c|c|}
\hline \multicolumn{5}{|c|}{ 241-AP-104 } \\
\hline \multicolumn{5}{|c|}{ Surface Level Data } \\
\hline Date & Type of Measurement & Level (Inches) & Data Qualfity & Comments \\
\hline 12/3/91 0:00 & Manual Tape & 7.25 & Transcribed & \\
\hline $12 / 3 / 917: 03$ & Auto FIC & 7.4 & Good & \\
\hline 12/4/91 0:00 & Manual Tape & 7.50 & Transcribed & \\
\hline $12 / 4 / 917: 03$ & Auto FIC & 7.40 & Good & \\
\hline $12 / 5 / 910: 00$ & Manual Tape & 7.50 & Transcribed & \\
\hline $12 / 5 / 917: 03$ & Auto FIC & 7.4 & Good & \\
\hline 12/6/91 0:00 & Manual Tapo & 7.25 & Transcribed & \\
\hline $12 / 6 / 917: 04$ & Auto FIC & 7.4 & Good & - \\
\hline $12 / 7 / 910: 00$ & Manual Tape & 7.5 & Transcribed & \\
\hline $12 / 7 / 917: 03$ & Auto FIC & 7.4 & Good & \\
\hline 12/8/91 0:00 & Manual Tape & 7.50 & Transcribed & \\
\hline $12 / 8 / 917: 03$ & Auto FIC & 7.4 & Good & \\
\hline 12/9/91 $0: 00$ & Manual Tape & 7.50 & Transcribed & \\
\hline $12 / 9 / 917: 03$ & Auto FIC & 7.40 & Good & \\
\hline $12 / 10 / 910: 00$ & Manual Tape & 7.5 & Transcribed & \\
\hline $12 / 10 / 917: 03$ & Auto FIC & 7.40 & Good & \\
\hline 12/11/910:00 & Manual Tape & 7.5 & Transcribed & \\
\hline $12 / 11 / 917: 02$ & Auto FIC & 7.40 & Good & \\
\hline 12/12/91 0:00 & Manual Tape & 7.50 & Transcribed & \\
\hline $12 / 12 / 917: 03$ & Auto FIC & 7.4 & Good & \\
\hline $12 / 13 / 910: 00$ & Manual Tape & 7.50 & Transcribed & \\
\hline $12 / 13 / 917: 05$ & Auto FIC & 7.40 & Good & \\
\hline 12/14/91 0:00 & Manual Tape & 7.50 & Transcribed & \\
\hline $12 / 14 / 917: 02$ & Auto FIC & 7.40 & Good & \\
\hline 12/15/91 0:00 & Manual Tape & 7.50 & Transeribed & \\
\hline $12 / 15 / 917: 02$ & Auto FIC & 7.40 & Good & \\
\hline $12 / 16 / 910: 00$ & Manual Tape & 7.5 & Transcribed & \\
\hline $12 / 16 / 917: 03$ & Auto FIC & 7.40 & Good & \\
\hline 12/17/91 0:00 & Manual Tape & 7.50 & Transcribed & \\
\hline $12 / 17 / 917: 06$ & Auto FIC & 7.4 & Good & \\
\hline $12 / 18 / 910: 00$ & Manual Tape & 7.5 & Trangeribed & \\
\hline $12 / 18 / 917: 03$ & Auto FIC & 7.4 & Good & \\
\hline $12 / 19 / 910: 00$ & Manual Tape & 7.50 & Transcribed & \\
\hline $12 / 19 / 917: 03$ & Auto FIC & 7.40 & Good & \\
\hline $12 / 20 / 910: 00$ & Manual Tape & 7.50 & Transcribed & \\
\hline $12 / 20 / 917: 02$ & Auto FIC & 7.40 & Good & \\
\hline $12 / 21 / 910: 00$ & Manual Tape & 7.5 & Transcribed & \\
\hline $12 / 21 / 917: 03$ & Auto FIC & 7.40 & Good & \\
\hline $12 / 22 / 910: 00$ & Manual Tape & 7.50 & Transcribed & \\
\hline $12 / 22 / 917: 05$ & Auto FIC & 7.4 & Good & \\
\hline $12 / 23 / 91 \quad 0: 00$ & Manual Tape & 7.5 & Transcribed & \\
\hline $12 / 23 / 917: 06$ & Auto FIC & 7.4 & Good & \\
\hline $12 / 24 / 910: 00$ & Manual Tape & 7.5 & Transcribed & \\
\hline $12 / 24 / 917: 03$ & Auto FIC & 7.40 & Good & \\
\hline $12 / 25 / 910: 00$ & Manual Tape & 7.5 & Transcribed & \\
\hline $12 / 25 / 917: 03$ & Auto FIC & 7.4 & Good & \\
\hline $12 / 26 / 910: 00$ & Manual Tape & 7.50 & Transcribed & \\
\hline $12 / 26 / 917: 03$ & Auto FIC & 7.40 & Good & \\
\hline
\end{tabular}

Data obtained from WHC Surveillance Analysis Computer System, (PC SACS) July 7, 1995. 


\begin{tabular}{|c|c|c|c|c|}
\hline \multicolumn{5}{|c|}{ 241-AP-104 } \\
\hline \multicolumn{5}{|c|}{ Surface Level Data } \\
\hline Date & Type of Measurement & Level (Inches) & Data Quality & Comments \\
\hline $12 / 27 / 910: 00$ & Manual Tape & 7.5 & Transcribed & \\
\hline $12 / 27 / 91 \quad 7: 03$ & Auto FIC & 7.4 & Good & \\
\hline $12 / 28 / 910: 00$ & Manual Tape & 7.50 & Transcribed & \\
\hline $12 / 28 / 917: 02$ & Auto FIC & 7.50 & Good & \\
\hline $12 / 29 / 91.0: 00$ & Manual Tape & 7.50 & Transeribed & \\
\hline $12 / 29 / 917: 03$ & Auto FIC & 7.40 & Good & \\
\hline $12 / 30 / 910: 00$ & Manual Tape & 7.50 & Transcribed & \\
\hline $12 / 30 / 917: 03$ & Auto FIC & 7.3 & Good & \\
\hline $12 / 31 / 910: 00$ & Manual Tape & 7.5 & Transcribed & \\
\hline $12 / 31 / 917: 03$ & Auto FIC & 7.30 & Good & \\
\hline $1 / 1 / 920: 00$ & Manual Tape & 7.5 & Transcribed & \\
\hline 1/1/92 7:03 & Auto FIC & 7.30 & Good & \\
\hline $1 / 2 / 920: 00$ & Manual Tape & 7.50 & Transcribed & \\
\hline $1 / 2 / 927: 02$ & Auto FIC & 7.3 & Good & \\
\hline $1 / 3 / 920: 00$ & Manual Tape & 7.25 & Transcribed & \\
\hline $1 / 3 / 927: 04$ & Auto FIC & 7.30 & Good & \\
\hline $1 / 4 / 920: 00$ & Manual Tape & 7.50 & Transcribed & \\
\hline $1 / 4 / 927: 03$ & Auto FIC & 7.3 & Good & \\
\hline $1 / 5 / 920: 00$ & Manual Tape & 7.5 & Transcribed & \\
\hline $1 / 5 / 927: 03$ & Auto FIC & $\cdot 7.3$ & Good & \\
\hline $1 / 6 / 92 \quad 0: 00$ & Manual Tape & 7.25 & Transeribed & \\
\hline $1 / 6 / 927: 03$ & Auto FIC & 7.30 & Good & \\
\hline $1 / 7 / 920: 00$ & Manual Tape & 7.5 & Transcribed & \\
\hline $1 / 7 / 927: 04$ & Auto FIC & 7.3 & Good & \\
\hline $1 / 8 / 920: 00$ & Manual Tape & 7.50 & Transcribed & \\
\hline 1/8/92 7:03 & Auto FIC & 7.30 & Good & \\
\hline $1 / 9 / 920: 00$ & Manual Tape & 7.25 & Transcribed & \\
\hline 1/9/92 7:03 & Auto FIC & 7.3 & Good & \\
\hline $1 / 10 / 920: 00$ & Manual Tape & 7.50 & Transcribed & \\
\hline $1 / 10 / 927: 04$ & Auto FIC & 7.3 & Good & \\
\hline $1 / 11 / 920: 00$ & Manual Tape & 7.25 & Transcribed & \\
\hline $1 / 11 / 927: 04$ & Auto FIC & 7.30 & Good & \\
\hline $1 / 12 / 920: 00$ & Manual FIC & 7.3 & Transeribed & \\
\hline $1 / 12 / 920: 00$ & Manual Tape & 7.5 & Transcribed & \\
\hline $1 / 13 / 920: 00$ & Manual FIC & 7.3 & Transcribed & \\
\hline $1 / 13 / 920: 00$ & Manual Tape & 7.5 & Transcribed & \\
\hline $1 / 14 / 920: 00$ & Manual FIC & 7.30 & Transcribed & \\
\hline $1 / 14 / 920: 00$ & Manual Tape & 7.50 & Transcribed & \\
\hline $1 / 15 / 920: 00$ & Manual Tape & 7.25 & Transcribed & \\
\hline 1/15/92 7:03 & Auto FIC & 7.3 & Good & \\
\hline $1 / 16 / 920: 00$ & Manual Tape & 7.25 & Transcribed & \\
\hline $1 / 16 / 927: 04$ & Auto FIC & 7.3 & Good & \\
\hline $1 / 17 / 920: 00$ & Manual Tape & 7.50 & Transcribed & \\
\hline $1 / 17 / 927: 04$ & Auto FIC & 7.30 & Good & \\
\hline $1 / 18 / 920: 00$ & Manual Tape & 7.25 & Transeribed & \\
\hline $1 / 18 / 927: 04$ & Auto FIC & 7.3 & Good & \\
\hline $1 / 19 / 920: 00$ & Manual Tape & 7.25 & Transcribed & \\
\hline $1 / 19 / 927: 03$ & Auto FIC & 7.30 & Good & \\
\hline
\end{tabular}

Data obtained from WHC Surveillance Analysis Computer System, (PC SACS) July 7, 1995. 


\begin{tabular}{|c|c|c|c|c|}
\hline \multicolumn{5}{|c|}{ 241-AP-104 } \\
\hline \multicolumn{5}{|c|}{ Surface Level Data } \\
\hline Date & Type of Measurement & Lavel (inches) & Data Quality & Comments \\
\hline $1 / 20 / 920: 00$ & Manual Tape & 7.25 & Transcribed & \\
\hline $1 / 20 / 927: 03$ & Auto FIC & 7.3 & Good & \\
\hline $1 / 21 / 920: 00$ & Manual Tape & 7.25 & Transcribed & \\
\hline 1/21/92 7:03 & Auto FIC & 7.3 & Good & \\
\hline 1/22/92 0:00 & Manual Tape & 7.25 & Transcribed & \\
\hline 1/22/92 7:06 & Auto FIC & 7.3 & Good & \\
\hline $1 / 23 / 920: 00$ & Manual Tape & 7.25 & Transcribed & \\
\hline $1 / 23 / 927: 03$ & Auto FIC & 7.3 & Good & \\
\hline $1 / 24 / 920: 00$ & Manual Tape & 7.25 & Transcribed & \\
\hline $1 / 24 / 927: 02$ & Auto FIC & 7.3 & Good & \\
\hline $1 / 25 / 920: 00$ & Manual Tape & 7.25 & Transcribed & \\
\hline $1 / 25 / 927: 03$ & Auto FIC & 7.3 & Good & \\
\hline $1 / 26 / 920: 00$ & Manual Tape & 7.25 & Transcribed & \\
\hline $1 / 26 / 926: 03$ & Auto FIC & 7.30 & Good & \\
\hline $1 / 27 / 920: 00$ & Manual Tape & 7.50 & Transcribed & \\
\hline $1 / 27 / 927: 02$ & Auto FIC & 7.30 & Good & \\
\hline $1 / 28 / 920: 00$ & Manual Tape & 7.5 & Transcribed & \\
\hline 1/28/92 7:03 & Auto FIC & 7.30 & Good & \\
\hline $1 / 29 / 920: 00$ & Manual Tape & 7.25 & Transcribed & \\
\hline 1/29/92 7:03 & Auto FIC & 7.3 & Good & $\cdot$ \\
\hline $1 / 30 / 920: 00$ & Manual Tape & 7.50 & Transcribed & \\
\hline $1 / 30 / 927: 03$ & Auto FIC & 7.3 & Good & \\
\hline $1 / 31 / 920: 00$ & Manual Tape & 7.25 & Transcribed . & \\
\hline 1/31/92 7:03 & Auto FIC & 7.30 & Good & \\
\hline $2 / 1 / 920: 00$ & Manual Tape & 7.50 & Transcribed & \\
\hline 2/1/92 7:02 & Auto FIC & 7.30 & Good & \\
\hline $2 / 2 / 920: 00$ & Manual Tape & 7.5 & Transcribed & \\
\hline 2/2/92 7:03 & Auto FIC & 7.30 & Good & \\
\hline $2 / 3 / 920: 00$ & Manual Tape & 7.50 & Transcribed & \\
\hline $2 / 3 / 927: 03$ & Auto FIC & 7.30 & Good & \\
\hline $2 / 4 / 920: 00$ & Manual Tape & 7.5 & Transcribed & \\
\hline $2 / 4 / 927: 03$ & Auto FIC & 7.30 & Good & \\
\hline $2 / 5 / 920: 00$ & Manual Tape & 7.50 & Transciribed & \\
\hline $2 / 5 / 92 \quad 7: 02$ & Auto FIC & 7.3 & Good & \\
\hline $2 / 6 / 920: 00$ & Manual FIC & 7.30 & Transcribed & \\
\hline $2 / 6 / 920: 00$ & Manual Tape & 7.50 & Transcribed & \\
\hline $2 / 7 / 920: 00$ & Manual FIC & 7.30 & Transcribed & \\
\hline $2 / 7 / 920: 00$ & Manual Tape & 7.5 & Transcribed & \\
\hline $2 / 8 / 920: 00$ & Manual Tape & 7.25 & Transcribed & \\
\hline 2/8/92 7:04 & Auto FIC & 7.30 & Good & \\
\hline $2 / 9 / 920: 00$ & Manual Tape & 7.25 & Transcribed & \\
\hline $2 / 9 / 927: 03$ & Auto FIC & 7.30 & Good & \\
\hline $2 / 10 / 920: 00$ & Manual Tape & 7.25 & Transcribed & \\
\hline $2 / 10 / 927: 04$ & Auto FIC & 7.3 & Good & \\
\hline $2 / 11 / 920: 00$ & Manual Tape & 7.50 & Transcribed & \\
\hline $2 / 11 / 927: 03$ & Auto FIC & 7.30 & Good & \\
\hline 2/12/92 0:00 & Manual Tape & 7.5 & Transcribed & \\
\hline 2/12/92 7:03 & Auto FIC & 7.3 & Good & \\
\hline
\end{tabular}

Data obtained from WHC Surveillance Analysis Computer System, (PC SACS) July 7, 1995. 


\begin{tabular}{|c|c|c|c|c|}
\hline \multicolumn{5}{|c|}{ 241-AP-104 } \\
\hline & & Irface Level Da & & \\
\hline Date & Type of Measurement & Level (Inches) & Data Quality & Comments \\
\hline $2 / 13 / 920: 00$ & Manual Tape & 7.50 & Transcribed & \\
\hline $2 / 13 / 927: 03$ & Auto FIC & 7.3 & Good & \\
\hline $2 / 14 / 920: 00$ & Manual Tape & 7.50 & Transcribed & \\
\hline 2/14/92 7:03 & Auto FIC & 7.3 & Good & \\
\hline $2 / 15 / 920: 00$ & Manual Tape & 7.5 & Transcribed & \\
\hline $2 / 15 / 927: 03$ & Auto FIC & 7.30 & Good & \\
\hline $2 / 16 / 920: 00$ & Manual Tape & 7.25 & Transcribed & \\
\hline $2 / 16 / 927: 03$ & Auto FIC & 7.3 & Good & \\
\hline $2 / 17 / 920: 00$ & Manual Tape & 7.25 & Transcribed & \\
\hline $2 / 17 / 927: 03$ & Auto FIC & 7.3 & Good & \\
\hline $2 / 18 / 920: 00$ & Manual Tape & 7.5 & Transcribed & \\
\hline 2/18/92 7:03 & Auto FIC & 7.30 & Good & \\
\hline $2 / 19 / 920: 00$ & Manual Tape & 7.25 & Transcribed & \\
\hline $2 / 19 / 927: 03$ & Auto FIC & 7.30 & Good & \\
\hline $2 / 20 / 920: 00$ & Manual Tape & 7.25 & Transcribed. & \\
\hline $2 / 20 / 927: 03$ & Auto FIC & 7.3 & Good & \\
\hline $2 / 21 / 920: 00$ & Manual Tape & 7.5 & Transcribed & \\
\hline $2 / 21 / 927: 03$ & Auto FIC & 7.3 & Good & \\
\hline $2 / 22 / 920: 00$ & Manual Tape & 7.25 & Transcribed & \\
\hline $2 / 22 / 927: 03$ & Auto FIC & 7.30 & Good & \\
\hline $2 / 23 / 920: 00$ & Manual Tape & 7.50 & Transcribed & \\
\hline $2 / 23 / 927: 03$ & Auto FIC & 7.3 & Good & \\
\hline $2 / 24 / 920: 00$ & Manual Tape & 7.50 & Transcribed & \\
\hline $2 / 24 / 927: 03$ & Auto FIC & 7.3 & Good & \\
\hline $2 / 25 / 920: 00$ & Manual Tape & 7.5 & Transcribed & \\
\hline $2 / 25 / 927: 04$ & Auto FIC & 7.30 & Good & \\
\hline 2/26/92 0:00 & Manual Tape & $\mathbf{7 . 5 0}$ & Transcribed & \\
\hline $2 / 26 / 927: 03$ & Auto FIC & 7.3 & Good & \\
\hline $2 / 27 / 920: 00$ & Manual Tape & 7.50 & Transcribed & \\
\hline 2/27/92 7:04 & Auto FIC & 7.30 & Good & \\
\hline $2 / 28 / 920: 00$ & Manual Tape & 7.50 & Transcribed & \\
\hline $2 / 28 / 927: 04$ & Auto FIC & 7.30 & Good & \\
\hline $2 / 29 / 920: 00$ & Manual Tape & 7.50 & Transcribed & \\
\hline $2 / 29 / 927: 03$ & Auto FIC & 7.3 & Good & \\
\hline $3 / 1 / 920: 00$ & Manual Tape & 7.5 & Transcribed & \\
\hline $3 / 1 / 927: 03$ & Auto FIC & 7.30 & Good & \\
\hline $3 / 2 / 92 \quad 0: 00$ & Manual Tape & 7.5 & Transcribed & \\
\hline $3 / 2 / 927: 03$ & Auto FIC & 7.3 & Good & \\
\hline 3/3/92 0:00 & Manual Tape & 7.5 & Transcribed & \\
\hline $3 / 3 / 92$ 7:04 & Auto FIC & 7.30 & Good & \\
\hline $3 / 4 / 92 \quad 0: 00$ & Manual Tape & 7.25 & Transcribed & \\
\hline $3 / 4 / 927: 04$ & Auto FIC & 7.30 & Good & \\
\hline $3 / 5 / 92 \quad 0: 00$ & Manual Tape & 7.25 & Transcribed & \\
\hline $3 / 5 / 927: 03$ & Auto FIC & 7.30 & Good & \\
\hline $3 / 6 / 920: 00$ & Manual Tape & 7.25 & Transcribed & \\
\hline $3 / 6 / 927: 03$ & Auto FIC & 7.3 & Good & \\
\hline $3 / 7 / 920: 00$ & Manual Tape & 7.50 & Transcribed & \\
\hline $3 / 7 / 92$ 7:02 & Auto FIC & 7.30 & Good & \\
\hline
\end{tabular}

Data obtained from WHC Surveillance Analysis Computer System, (PC SACS) July 7, 1995. 


\begin{tabular}{|c|c|c|c|c|}
\hline \multicolumn{5}{|c|}{ 241-AP-104 } \\
\hline \multicolumn{5}{|c|}{ Surface Level Data } \\
\hline Date & Type of Measurement & Level (Inches) & Data Quality & Comments \\
\hline $3 / 8 / 920: 00$ & Manual Tape & 7.5 & Transcribed & \\
\hline $3 / 8 / 927: 02$ & Auto FIC & 7.3 & Good & \\
\hline $3 / 9 / 92$ 0:00 & Manual Tape & 7.375 & Transcribed & \\
\hline $3 / 9 / 927: 02$ & Auto FIC & 7.3 & Good & \\
\hline $3 / 10 / 920: 00$ & Manual Tape & 7.50 & Transcribed & \\
\hline $3 / 10 / 927: 03$ & Auto FIC & 7.3 & Good & \\
\hline $3 / 11 / 920: 00$ & Manual Tape & 7.50 & Transcribad & \\
\hline $3 / 11 / 927: 03$ & Auto FIC & 7.30 & Good & \\
\hline $3 / 12 / 920: 00$ & Manual Tape & 7.5 & Transcribed & \\
\hline $3 / 12 / 927: 03$ & Auto FIC & 7.30 & Good & \\
\hline $3 / 13 / 92$ 0:00 & Manual Tape & 7.50 & Transcribed & \\
\hline $3 / 13 / 927: 03$ & Auto FIC & 7.30 & Good & \\
\hline $3 / 14 / 920: 00$ & Manual Tape & 7.50 & Transcribed & \\
\hline $3 / 14 / 927: 03$ & Auto FIC & 7.3 & Good & \\
\hline $3 / 15 / 920: 00$ & Manual Tape & 7.50 & Transcribed & \\
\hline $3 / 15 / 927: 06$ & Auto FIC & 7.30 & Good & \\
\hline $3 / 16 / 920: 00$ & Manual Tape & 7.5 & Transcribed & \\
\hline $3 / 16 / 927: 03$ & Auto FIC & 7.30 & Good & \\
\hline $3 / 17 / 920: 00$ & Manual Tape & 7.5 & Transcribed & \\
\hline $3 / 17 / 927: 03$ & Auto FIC & 7.30 & Good & \\
\hline $3 / 18 / 920: 00$ & Manual Tape & 7.25 & Transcribed & \\
\hline $3 / 18 / 927: 03$ & Auto FIC & 7.3 & Good & \\
\hline 3/19/92 0:00 & Manual Tape & 7.25 & Transcribed & \\
\hline $3 / 19 / 926: 02$ & Auto FIC & 7.30 & Good & \\
\hline $3 / 20 / 920: 00$ & Manual Tape & 7.50 & Transcribed & \\
\hline $3 / 20 / 927: 02$ & Auto FIC & 7.3 & Good & \\
\hline $3 / 21 / 920: 00$ & Manual Tape & 7.50 & Transcribed & \\
\hline $3 / 21 / 927: 03$ & Auto FIC & 7.3 & Good & \\
\hline $3 / 22 / 920: 00$ & Manual Tape & 7.25 & Transcribed & \\
\hline $3 / 22 / 927: 02$ & Auto FIC & 7.30 & Good & \\
\hline $3 / 23 / 920: 00$ & Manual Tape & 7.25 & Transcribed & \\
\hline $3 / 23 / 927: 02$ & Auto FIC & 7.30. & Good & \\
\hline $3 / 24 / 920: 00$ & Manual Tape & 7.25 & Transcribed & \\
\hline $3 / 24 / 92$ 7:02 & Auto FIC & 7.30 & Good & \\
\hline $3 / 25 / 920: 00$ & Manual Tape & 7.25 & Transcribed & \\
\hline $3 / 25 / 927: 03$ & Auto FIC & 7.3 & Good & \\
\hline $3 / 26 / 920: 00$ & Manual Tape & 7.25 & Transcribed & \\
\hline $3 / 26 / 927: 03$ & Auto FIC & 7.3 & Good & \\
\hline $3 / 27 / 920: 00$ & Manual Tape & 7.50 & Transcribed & \\
\hline $3 / 27 / 927: 02$ & Auto FIC & 7.30 & Good & \\
\hline $3 / 28 / 920: 00$ & Manual Tape & 7.5 & Transcribed & \\
\hline $3 / 28 / 927: 03$ & Auto FIC & 7.30 & Good & \\
\hline $3 / 29 / 920: 00$ & Manual Tape & 7.50 & Transeribed & \\
\hline $3 / 29 / 927: 03$ & Auto FIC & 7.30 & Good & \\
\hline $3 / 30 / 920: 00$ & Manual Tape & 7.5 & Transcribed & \\
\hline $3 / 30 / 927: 03$ & Auto FIC & 7.3 & Good & \\
\hline $3 / 31 / 920: 00$ & Manual Tape & 7.5 & Transcribed & \\
\hline $3 / 31 / 927: 03$ & Auto FIC & 7.3 & Good & \\
\hline
\end{tabular}

Data obtained from WHC Surveillance Analysis Computer System, (PC SACS) July 7, 1995. 


\begin{tabular}{|c|c|c|c|c|}
\hline \multicolumn{5}{|c|}{ 241-AP-104 } \\
\hline \multicolumn{5}{|c|}{ Surface Leval Data } \\
\hline Date & Type of Measurement & Lovel (Inchios) & Data Quality & Comments \\
\hline $4 / 1 / 920: 00$ & Manual Tape & 7.25 & Transcribed & \\
\hline 4/1/92 7:03 & Auto FIC & 7.3 & Good & \\
\hline 4/2/92 0:00 & Manual Tape & 7.5 & Transcribed & \\
\hline $4 / 2 / 927: 03$ & Auto FIC & 7.3 & Good & \\
\hline 4/3/92 0:00 & Manual Tape & 7.50 & Transcribed & \\
\hline 4/3/92 7:03 & Auto FIC & 7.30 & Good & \\
\hline $4 / 4 / 920: 00$ & Manual Tape & 7.5 & Transcribed & \\
\hline $4 / 4 / 927: 03$ & Auto FIC & 0.00 & Suspect & Data not included in graph \\
\hline $4 / 5 / 920: 00$ & Manual Tape & 7.50 & Transcribed & \\
\hline $4 / 5 / 927: 03$ & Auto FIC & 0.00 & Suspect & Data not included in graph \\
\hline $4 / 6 / 920: 00$ & Manual Tape & $\mathbf{7 . 5}$ & Transcribed & \\
\hline $4 / 6 / 927: 03$ & Auto FIC & 0.00 & Suspect & Data not included in graph \\
\hline $4 / 7 / 920.00$ & Manual Tape & 7.25 & Transcribed & \\
\hline $4 / 7 / 927: 03$ & Auto FIC & 0.00 & Suspect & Data not includad in graph \\
\hline 4/8/92 0:00 & Manual Tape & 7.25 & Transcribed & \\
\hline $4 / 9 / 920: 00$ & Manual Tape & 7.25 & Transcribed & \\
\hline 4/9/92 7:03 & Auto FIC & 0.00 & Suspect & Data not included in graph \\
\hline $4 / 10 / 920: 00$ & Manual Tape & 7.25 & Transcribed & \\
\hline 4/10/92 7:03 & Auto FIC & 0.00 & Suspect & Data not included in graph \\
\hline 4/11/92 0:00 & Manual Tape & 7.25 & Transcribed & \\
\hline 4/11/92 7:03 & Auto FIC & 0 & Suspect & Data not included in graph \\
\hline $4 / 12 / 920: 00$ & Manual FIC & 7.3 & Transcribed & \\
\hline 4/12/92 0:00 & Manual Tape & 7.25 & Transcribed & \\
\hline $4 / 13 / 920: 00$ & Manual FIC & 7.3 & Transcribed & \\
\hline 4/13/92 0:00 & Manual Tape & 7.25 & Transcribed & \\
\hline $4 / 13 / 927: 03$ & Auto FIC & 0 & Suspect & Data not included in graph \\
\hline $4 / 14 / 920: 00$ & Manual Tape & 7.25 & Transcribed & \\
\hline $4 / 14 / 927: 03$ & Auto FIC & 0 & Suspect & Data not included in graph \\
\hline $4 / 15 / 920: 00$ & Manual FIC & 7.30 & Transcribed & \\
\hline $4 / 15 / 920: 00$ & Manual Tape & 7.5 & Transcribed & \\
\hline 4/15/92 7:02 & Auto FIC & 0 & Suspect & Data not included in graph \\
\hline 4/16/92 0:00 & Manual Tape & 7.5 & Transcribed & \\
\hline 4/16/92 7:03 & Auto FIC & 0.00 & Suspect & Data not included in graph \\
\hline $4 / 17 / 920: 00$ & Manual Tape & 7.25 & Transcribed & \\
\hline $4 / 17 / 927: 03$ & Auto FIC & 0.00 & Suspect & Data not included in graph \\
\hline $4 / 18 / 920: 00$ & Manual Tape : & 7.25 & Transcribed & \\
\hline 4/18/92 7:02 & Auto FIC & 0.00 & Suspect & Data not included in graph \\
\hline 4/19/920:00 & Manual Tape & 7.25 & Transcribed & \\
\hline $4 / 19 / 927: 02$ & Auto FIC & 0 & Suspect & Data not included in graph \\
\hline $4 / 20 / 920: 00$ & Manual Tape & 7.25 & Transcribed & \\
\hline $4 / 20 / 927: 03$ & Auto FIC & 0 & Suspect & Data not included in graph \\
\hline $4 / 21 / 920: 00$ & Manual FIC & 7.3 & Transcribed & \\
\hline $4 / 21 / 920: 00$ & Manual Tape & 7.25 & Transcribed & \\
\hline $4 / 21 / 927: 03$ & Auto FIC & 0.00 & Suspect & Data not included in graph \\
\hline 4/22/92 0:00 & Manual Tape & 7.25 & Transcribed & - \\
\hline $4 / 22 / 927: 02$ & Auto FIC & 7.20 & Good & \\
\hline $4 / 23 / 920: 00$ & Manual Tape & 7.25 & Transcribed & \\
\hline $4 / 23 / 927: 03$ & Auto FIC & 7.2 & Good & \\
\hline
\end{tabular}

Data obtained from WHC Surveillance Analysis Computer System, (PC SACS) July 7, 1995. 


\begin{tabular}{|c|c|c|c|c|}
\hline \multicolumn{5}{|c|}{ 241-AP-104 } \\
\hline & & Ifface Level Data & & \\
\hline Date & Type of Measurement & Level (Inches) & Data Quality & Comments \\
\hline $4 / 24 / 920: 00$ & Manual Tape & 7.25 & Transcribed & \\
\hline 4/24/92 7:03 & Auto FIC & 7.20 & Good & \\
\hline 4/25/92 0:00 & Manual Tape & 7.25 & Transcribed & \\
\hline $4 / 25 / 92$ 7:04 & Auto FIC & 7.20 & Good & \\
\hline 4/26/92 0:00 & Manual Tape & 7.25 & Transcribed & \\
\hline 4/26/92 7:03 & Auto FIC & 7.2 & Good & \\
\hline $4 / 27 / 920: 00$ & Manual Tape & 7.50 & Transcribed & \\
\hline $4 / 27 / 927: 04$ & Auto FIC & 7.2 & Good & \\
\hline $4 / 28 / 920: 00$ & Manual Tape & 7.25 & Transcribed & \\
\hline 4/28/92 7:03 & Auto FIC & 7.2 & Good & \\
\hline 4/29/92 0:00 & Manual Tape & 7.25 & Transcribed & \\
\hline 4/29/92 7:03 & Auto FIC & 7.2 & Good & \\
\hline $4 / 30 / 920: 00$ & Manual Tape & 7.25 & Transcribed & \\
\hline 4/30/92 7:03 & Auto FIC & 7.20 & Good & \\
\hline $5 / 1 / 920: 00$ & Manual Tape & 7.50 & Transcribed & \\
\hline $5 / 1 / 927: 03$ & Auto FIC & 7.2 & Good & \\
\hline $5 / 2 / 920: 00$ & Manual Tape & 7.50 & Transcribed & \\
\hline $5 / 2 / 922: 03$ & Auto FIC & 7.20 & Good & \\
\hline $5 / 3 / 920: 00$ & Manual Tape & 7.25 & Transcribed & \\
\hline $5 / 3 / 927: 03$ & Auto FIC & 7.2 & Good & \\
\hline $5 / 4 / 920: 00$ & Manual Tape & 7.50 & Transcribed & \\
\hline 5/4/92 7:03 & Auto FIC & 7.20 & Good & \\
\hline 5/5/92 0:00 & Manual Tape & 7.5 & Transcribed & \\
\hline $5 / 5 / 927: 04$ & Auto FIC & 7.20 & Good & \\
\hline $5 / 6 / 920: 00$ & Manual Tape & 7.50 & Transcribed & \\
\hline 5/6/92 7:03 & Auto FIC & 7.2 & Good & \\
\hline $5 / 7 / 920: 00$ & Manual Tape & 7.50 & Transcribed & \\
\hline $5 / 7 / 927: 04$ & Auto FIC & 7.20 & Good & \\
\hline $5 / 8 / 920: 00$ & Manual Tape & 7.5 & Transcribed & \\
\hline $5 / 8 / 927: 03$ & Auto FIC & 7.2 & Good & \\
\hline $5 / 9 / 920: 00$ & Manual FIC & 7.25 & Transcribed & \\
\hline $5 / 9 / 920: 00$ & Manual Tape & 7.5 & Transcribed & \\
\hline $5 / 10 / 920: 00$ & Manual Tape & 7.5 & Transcribed & \\
\hline $5 / 10 / 926: 03$ & Auto FIC & 7.2 & Good & \\
\hline $5 / 11 / 920: 00$ & Manual FIC & 7.25 & Transcribed & \\
\hline $5 / 11 / 920: 00$ & Manual Tape & 7.25 & Transeribed & \\
\hline $5 / 12 / 920: 00$ & Manual Tape & 7.25 & Transcribed & \\
\hline $5 / 12 / 927: 03$ & Auto FIC & 7.20 & Good & \\
\hline $5 / 13 / 920: 00$ & Manual Tape & 7.25 & Transcribed & \\
\hline $5 / 13 / 927: 03$ & Auto FIC & 7.2 & Good & \\
\hline $5 / 14 / 920: 00$ & Manual Tape & 7.25 & Transcribed & \\
\hline $5 / 14 / 927: 03$ & Auto FIC & 7.20 & Good & \\
\hline $5 / 15 / 920: 00$ & Manual Tape & 7.25 & Transcribed & \\
\hline $5 / 15 / 927: 03$ & Auto FIC & 7.2 & Good & \\
\hline $5 / 16 / 920: 00$ & Manual Tape & 7.25 & Transcribed & \\
\hline $5 / 16 / 927: 03$ & Auto FIC & 7.2 & Good & \\
\hline $5 / 17 / 920: 00$ & Manual Tape & 7.25 & Transcribed & \\
\hline $5 / 17 / 927: 02$ & Auto FIC & 7.20 & Good & \\
\hline
\end{tabular}

Data obtained from WHC Surveillance Analysis Computer System, (PC SACS) July 7, 1995. 


\begin{tabular}{|c|c|c|c|c|}
\hline \multicolumn{5}{|c|}{ 241-AP-104 } \\
\hline \multicolumn{5}{|c|}{ Surface Level Data } \\
\hline Date & Type of Measuroment & Levol (Inches) & Data Quality & Comments \\
\hline $5 / 18 / 920: 00$ & Manual Tape & 7.25 & Transcribed & \\
\hline $5 / 18 / 927: 03$ & Auto FIC & 7.20 & Good & \\
\hline $5 / 19 / 920: 00$ & Manual Tape & 7.25 & Transcribed & \\
\hline $5 / 19 / 927: 03$ & Auto FIC & 7.20 & Good & \\
\hline $5 / 20 / 920: 00$ & Manual Tape & 7.25 & Transcribed & \\
\hline $5 / 20 / 927: 03$ & Auto FIC & 7.20 & Good & \\
\hline $5 / 21 / 920: 00$ & Manual Tape & 7.25 & Transcribed & \\
\hline $5 / 21 / 927: 03$ & Auto FIC & 7.20 & Good & \\
\hline $5 / 22 / 920: 00$ & Manual Tape & 7.25 & Transcribed & \\
\hline $5 / 22 / 927: 02$ & Auto FIC & 7.2 & Good & \\
\hline $5 / 23 / 920: 00$ & Manual Tape & 7.25 & Transcribed & \\
\hline $5 / 23 / 927: 03$ & Auto FIC & 7.20 & Good & \\
\hline $5 / 24 / 920: 00$ & Manual Tape & 7.25 & Transcribed & \\
\hline $5 / 24 / 927: 03$ & Auto FIC & 7.2 & Good & \\
\hline $5 / 25 / 920: 00$ & Manual Tape & 7.25 & Transcribed & \\
\hline $5 / 25 / 927: 03$ & Auto FIC & 7.2 & Good & \\
\hline $5 / 26 / 920: 00$ & Manual FIC & 7.20 & Transcribed & \\
\hline $5 / 26 / 920: 00$ & Manual Tape & 7.25 & Transcribed & \\
\hline $5 / 27 / 920: 00$ & Manual Tape & 7.25 & Transcribed & \\
\hline $5 / 27 / 927: 03$ & Auto FIC & 7.20 & Good & \\
\hline $5 / 28 / 920: 00$ & Manual Tape & 7.25 & Transcribed & \\
\hline $5 / 28 / 927: 02$ & Auto FIC & 7.20 & Good & \\
\hline $5 / 29 / 920: 00$ & Manual Tape & 7.25 & Transcribed & \\
\hline $5 / 29 / 927: 02$ & Auto FIC & 7.20 & Good & \\
\hline $5 / 30 / 920: 00$ & Manual Tape & 7.25 & Transcribed & \\
\hline $5 / 30 / 927: 03$ & Auto FIC & 7.2 & Good & \\
\hline $5 / 31 / 920: 00$ & Manual Tape & 7.25 & Transcribed & \\
\hline $5 / 31 / 927: 03$ & Auto FIC & 7.20 & Good & \\
\hline $6 / 1 / 920: 00$ & Manual Tape & 7.25 & Transcribed & \\
\hline $6 / 1 / 927: 03$ & Auto FIC & 7.20 & Good & \\
\hline $6 / 2 / 92 \quad 0: 00$ & Manual Tape & 7.25 & Transcribed & \\
\hline $6 / 2 / 927: 03$ & Auto FIC & 7.20 & Good & \\
\hline $6 / 3 / 92 \quad 0: 00$ & Manual Tape & 7.25 & Transcribed & \\
\hline $6 / 3 / 927: 03$ & Auto FIC & 7.20 & Good & \\
\hline $6 / 4 / 920: 00$ & Manual Tape & 7.25 & Transcribed & \\
\hline $6 / 4 / 927: 03$ & Auto FIC & 7.2 & Good & \\
\hline $6 / 5 / 920: 00$ & Manual Tape & 7.25 & Transcribed & \\
\hline $6 / 5 / 927: 02$ & Auto FIC & 7.20 & Good & \\
\hline $6 / 6 / 920: 00$ & Manual Tape & 7.25 & Transcribed & \\
\hline $6 / 6 / 927: 03$ & Auto FIC & 7.2 & Good & \\
\hline $6 / 7 / 920: 00$ & Manual Tape & 7.25 & Transcribed & \\
\hline $6 / 7 / 927: 03$ & Auto FIC & 7.2 & Good & \\
\hline $6 / 8 / 92$ 0:00 & Manual Tape & 7.25 & Transcribed & \\
\hline $6 / 8 / 927: 03$ & Auto FIC & 7.2 & Good & \\
\hline 6/9/92 0:00 & Manual Tape & 7.50 & Transcribed & \\
\hline 6/9/92 7:03 & Auto FIC & 7.2 & Good & \\
\hline $6 / 10 / 920: 00$ & Manual Tape & 7.5 & Transcribed & \\
\hline $6 / 10 / 927: 03$ & Auto FIC & 7.2 & Good & \\
\hline
\end{tabular}

Data obtained from WHC Surveillance Analysis Computer System, (PC SACS) July 7, 1995. 


\begin{tabular}{|c|c|c|c|c|}
\hline \multicolumn{5}{|c|}{ 241-AP-104 } \\
\hline & & Ifrace Leval Dats & & \\
\hline Date & Type of Measurement & Level (Inchea) & Data Quality & Comments \\
\hline $6 / 11 / 920: 00$ & Manual Tape & 7.5 & Transcribed & \\
\hline $6 / 11 / 927: 03$ & Auto FIC & 7.2 & Good & \\
\hline $6 / 12 / 920: 00$ & Manual Tape & 7.5 & Transcribed & \\
\hline $6 / 12 / 927: 05$ & Auto FIC & 7.20 & Good & \\
\hline $6 / 13 / 920: 00$ & Manual Tape & 7.5 & Transcribed & \\
\hline $6 / 13 / 927: 05$ & Auto FIC & 7.20 & Good & \\
\hline $6 / 14 / 920: 00$ & Manual FIC & 7.2 & Transcribed & \\
\hline $6 / 14 / 920: 00$ & Manuạl Tape & 7.25 & Transcribed & \\
\hline $6 / 15 / 920: 00$ & Manual Tape & 7.50 & Transcribed & \\
\hline $6 / 15 / 927: 03$ & Auto FIC & 7.20 & Good & \\
\hline $6 / 16 / 920: 00$ & Manual Tape & 7.25 & Transcribed & \\
\hline $6 / 16 / 927: 03$ & Auto FIC & 7.2 & Good & \\
\hline $6 / 17 / 920: 00$ & Manual Tape & 7.50 & Transcribed & \\
\hline $6 / 17 / 927: 03$ & Auto FIC & 7.20 & Good & \\
\hline $6 / 18 / 920: 00$ & Manual Tape & 7.50 & Transcribed & \\
\hline $6 / 18 / 927: 03$ & Auto FIC & 7.20 & Good & \\
\hline $6 / 19 / 920: 00$ & Manual Tape & 7.50 & Transcribed & \\
\hline $6 / 19 / 927: 02$ & Auto FIC & 7.2 & Good & \\
\hline $6 / 20 / 920: 00$ & Manual Tape & 7.50 & Transcribed & \\
\hline $6 / 20 / 927: 02$ & Auto FIC & 7.2 & Good & \\
\hline $6 / 21 / 920: 00$ & Manual Tape & 7.5 & Transcribed & \\
\hline $6 / 21 / 927: 03$ & Auto FIC & 7.20 & Good & \\
\hline $6 / 22 / 920: 00$ & Manual Tape & 7.5 & Transcribed & \\
\hline $6 / 22 / 927: 03$ & Auto FIC & 7.20 & Good & \\
\hline $6 / 23 / 920: 00$ & Manual Tape & 7.5 & Transcribed & \\
\hline $6 / 23 / 927: 03$ & Auto FIC & 7.20 & Good & \\
\hline $6 / 24 / 920: 00$ & Manual Tape & 7.50 & Transcribed & \\
\hline $6 / 24 / 927: 03$ & Auto FIC & 7.2 & Good & \\
\hline $6 / 25 / 920: 00$ & Manual Tape & 7.25 & Transcribed & \\
\hline $6 / 25 / 927: 03$ & Auto FIC & 7.2 & Good & \\
\hline $6 / 26 / 920: 00$ & Manual Tape & 7.25 & Transcribed & \\
\hline $6 / 26 / 927: 02$ & Auto FIC & 7.20 & Good & \\
\hline $6 / 27 / 920: 00$ & Manual Tape & 7.50 & Transcribed & \\
\hline $6 / 27 / 927: 03$ & Auto FIC & 7.2 & Good & \\
\hline $6 / 28 / 920: 00$ & Manual Tape & 7.5 & Transcribed & \\
\hline $6 / 28 / 927: 06$ & Auto FIC & 7.20 & Good & \\
\hline $6 / 29 / 920: 00$ & Manual Tape & 7.50 & Transcribed & \\
\hline $6 / 29 / 927: 06$ & Auto FIC & 7.20 & Good & \\
\hline $6 / 30 / 920: 00$ & Manual Tape & 7.50 & Transcribed & \\
\hline $6 / 30 / 927: 06$ & Auto FIC & 7.20 & Good & \\
\hline $7 / 1 / 92$ 0:00 & Manual FIC & 7.20 & Transcribed & \\
\hline 7/1/92 0:00 & Manual Tape & 7.50 & Transcribed & \\
\hline $7 / 2 / 920: 00$ & Manual FIC & 7.20 & Transcribed & \\
\hline $7 / 2 / 920: 00$ & Manual Tape & 7.5 & Transcribed & \\
\hline $7 / 3 / 92$ 0:00 & Manual Tape & 7.25 & Transcribed & \\
\hline $7 / 3 / 927: 06$ & Auto FIC & 7.20 & Good & \\
\hline $7 / 4 / 920: 00$ & Manual Tape & 7.25 & Transcribed & \\
\hline $7 / 4 / 927: 06$ & Auto FIC & 7.2 & Good & \\
\hline
\end{tabular}

Data obtained from WHC Surveillance Analysis Computer System, (PC SACS) July 7, 1995. 


\begin{tabular}{|c|c|c|c|c|}
\hline Date & Type of Measuroment & Level (Inches) & Data Quality & Comments \\
\hline $7 / 5 / 920: 00$ & Manual Tape & 7.25 & Transcribed & \\
\hline $7 / 5 / 92 \quad 7: 06$ & Auto FIC & 7.20 & Good & \\
\hline $7 / 6 / 920: 00$ & Manual Tape & 7.25 & Transcribed & \\
\hline $7 / 6 / 927: 06$ & Auto FIC & 7.20 & Good & \\
\hline $7 / 7 / 920: 00$ & Manual Tape & 7.25 & Transcribed & \\
\hline $7 / 7 / 92 \quad 7: 06$ & Auto FIC & 7.20 & Good & \\
\hline $7 / 8 / 920: 00$ & Manual Tape & 7.25 & Transcribed & \\
\hline $7 / 8 / 927: 06$ & Auto FIC & 7.2 & Good & \\
\hline $7 / 9 / 92 \quad 0: 00$ & Manual Tape & 7.25 & Transcribed & \\
\hline $7 / 9 / 927: 06$ & Auto FIC & 7.2 & Good & \\
\hline $7 / 10 / 920: 00$ & Manual Tape & 7.25 & Transcribed & \\
\hline 7/10/92 7:06 & Auto FIC & 7.20 & Good & \\
\hline 7/11/92 0:00 & Manual Tape & 7.25 & Transcribed & \\
\hline $7 / 11 / 927: 00$ & Auto FIC & 7.2 & Good & \\
\hline $7 / 12 / 920: 00$ & Manual Tape & 7.50 & Transcribed & \\
\hline $7 / 12 / 927: 06$ & Auto FIC & 7.2 & Good & \\
\hline $7 / 13 / 920: 00$ & Manual Tape & 7.25 & Transcribed & \\
\hline $7 / 13 / 927: 06$ & Auto FIC & 7.20 & Good & \\
\hline $7 / 14 / 920: 00$ & Manual Tape & 7.25 & Transcribed & \\
\hline $7 / 14 / 927: 06$ & Auto FIC & 7.20 & Good & \\
\hline $7 / 15 / 920: 00$ & Manual Tape & 7.25 & Transcribed & \\
\hline $7 / 15 / 92 \quad 7: 06$ & Auto FIC & 7.20 & Good & \\
\hline $7 / 16 / 920: 00$ & Manual Tape & 7.25 & Transcribed & \\
\hline $7 / 16 / 927: 06$ & Auto FIC & 7.2 & Good & \\
\hline $7 / 17 / 920: 00$ & Manual Tape & 7.25 & Transcribed & \\
\hline $7 / 17 / 927: 06$ & Auto FIC & 7.2 & Good & \\
\hline $7 / 18 / 920: 00$ & Manual Tape & 7.25 & Transcribed & \\
\hline $7 / 18 / 927: 06$ & Auto FIC & 7.2 & Good & \\
\hline $7 / 19 / 920: 00$ & Manual Tape & 7.25 & Transcribed & \\
\hline $7 / 19 / 927: 06$ & Auto FIC & 7.2 & Good & \\
\hline $7 / 20 / 920: 00$ & Manusl Tape & 7.25 & Transcribed & \\
\hline $7 / 20 / 927: 06$ & Auto FIC & 7.2 & Good & \\
\hline $7 / 21 / 920: 00$ & Manual Tape & 7.25 & Transcribed & \\
\hline $7 / 21 / 927: 05$ & Auto FIC & 7.20 & Good & \\
\hline $7 / 22 / 920: 00$ & Manual Tape & 7.00 & Transcribed & \\
\hline $7 / 22 / 927: 06$ & Auto FIC & 7.20 & Good & \\
\hline $7 / 23 / 920: 00$ & Manual FIC & 7.20 & Transcribed & \\
\hline $7 / 23 / 920.00$ & Manual Tape & 7.00 & Transcribed & \\
\hline $7 / 24 / 920: 00$ & Manual Tape & 7.25 & Transcribed & \\
\hline 7/24/92 7:05 & Auto FIC & 7.20 & Good & \\
\hline $7 / 25 / 920: 00$ & Manual Tape & 7.25 & Transcribed & \\
\hline $7 / 25 / 927: 06$ & Auto FIC & 7.20 & Good & \\
\hline $7 / 26 / 920: 00$ & Manual Tape & 7.25 & Transcribed & \\
\hline $7 / 26 / 927: 06$ & Auto FIC & 7.20 & Good & \\
\hline $7 / 27 / 920: 00$ & Manual Tape & 7.25 & Transcribed & \\
\hline $7 / 27 / 927: 05$ & Auto FIC & 7.20 & Good & \\
\hline $7 / 28 / 920: 00$ & Manual Tape & 7.25 & Transcribed & \\
\hline $7 / 28 / 927: 06$ & Auto FIC & 7.2 & Good & \\
\hline
\end{tabular}

Data obtained from WHC Surveillance Analysis Computer System, (PC SACS) July 7, 1995. 


\begin{tabular}{|c|c|c|c|c|}
\hline \multicolumn{5}{|c|}{ 241-AP-104 } \\
\hline & & urface Level Dat & & \\
\hline Date & Type of Moasurement & Level (Inchios) & Data Qualty & Comments \\
\hline $7 / 29 / 920: 00$ & Manual Tape. & 7.25 & Transcribed & \\
\hline $7 / 29 / 927: 06$ & Auto FIC & 7.2 & Good & \\
\hline $7 / 30 / 920: 00$ & Manual Tape & 7.25 & Transcribed & \\
\hline $7 / 30 / 92$ 7:06 & Auto FIC & 7.20 & Good & \\
\hline $7 / 31 / 920: 00$ & Manual Tape & 7.25 & Transcribed & \\
\hline 7/31/92 7:05 & Auto FIC & 7.20 & Good & \\
\hline $8 / 1 / 920: 00$ & Manual Tape & 7.25 & Transcribed & \\
\hline $8 / 1 / 927: 06$ & Auto FIC & 7.20 & Good & \\
\hline $8 / 2 / 920: 00$ & Manual Tape & 7.25 & Transcribed & \\
\hline $8 / 2 / 927: 06$ & Auto FIC & 7.2 & Good & \\
\hline $8 / 3 / 920: 00$ & Manual Tape & 7.25 & Transcribed & \\
\hline $8 / 3 / 927: 06$ & Auto FIC & 7.2 & Good & \\
\hline $8 / 4 / 920: 00$ & Manual Tape & 7.25 & Transcribed & \\
\hline 8/4/92 7:06 & Auto FIC & 7.20 & Good & \\
\hline $8 / 5 / 92 \quad 0: 00$ & Manual Tape & 7.25 & Transcribed & \\
\hline $8 / 5 / 927: 05$ & Auto FIC & 7.2 & Good & \\
\hline $8 / 6 / 920: 00$ & Manual Tape & 7.25 & Transcribed & \\
\hline $8 / 6 / 927: 05$ & Auto FIC & 7.20 & Good & \\
\hline $8 / 7 / 920: 00$ & Manual Tape & 7.25 & Transcribed & \\
\hline $8 / 7 / 927: 05$ & Auto FIC & 7.20 & Good & \\
\hline $8 / 8 / 920: 00$ & Manual Tape & 7.25 & Transcribed & \\
\hline $8 / 8 / 927: 06$ & Auto FIC & 7.20 & Good & \\
\hline $8 / 9 / 920: 00$ & Manual Tape & 7.25 & Transcribed & \\
\hline $8 / 9 / 927: 06$ & Auțo FIC & 7.20 & Good & \\
\hline $8 / 10 / 920: 00$ & Manual Tape & 7 & Transcribed & \\
\hline $8 / 10 / 927: 05$ & Auto FIC & 7.20 & Good & \\
\hline $8 / 11 / 920: 00$ & Manual Tape & 7 & Transcribed & \\
\hline $8 / 11 / 927: 06$ & Auto FIC & 7.20 & Good & \\
\hline $8 / 12 / 920: 00$ & Manual Tape & 7.25 & Transcribed & \\
\hline $8 / 12 / 927: 06$ & Auto FIC & 7.2 & Good & \\
\hline $8 / 13 / 920: 00$ & Manual Tape & 7.25 & Transcribed & \\
\hline $8 / 13 / 927: 06$ & Auto FIC & 7.2 & Good & \\
\hline $8 / 14 / 920: 00$ & Manual Tape & 7.00 & Transcribed & \\
\hline $8 / 14 / 927: 06$ & Auto FIC & 7.20 & Good & \\
\hline $8 / 15 / 920: 00$ & Manual Tape & 7.50 & Transcribed & \\
\hline $8 / 15 / 927: 06$ & Auto FIC & 7.2 & Good & \\
\hline $8 / 16 / 920: 00$ & Manual Tape & 7.50 & Transcribed & \\
\hline $8 / 16 / 927: 06$ & Auto FIC & 7.20 & Good & \\
\hline $8 / 17 / 920: 00$ & Manual Tape & 7.50 & Transcribed & \\
\hline $8 / 17 / 927: 06$ & Auto FIC & 7.20 & Good & \\
\hline $8 / 18 / 920: 00$ & Manual Tape & 7.5 & Transcribed & \\
\hline $8 / 18 / 927: 06$ & Auto FIC & 7.2 & Good & \\
\hline $8 / 19 / 920: 00$ & Manual Tape & 7.25 & Transcribed & \\
\hline $8 / 19 / 927: 06$ & Auto FIC & 7.20 & Good & \\
\hline $8 / 20 / 920: 00$ & Manual Tape & 7.25 & Transcribed & \\
\hline $8 / 20 / 927: 06$ & Auto FIC & 7.20 & Good & \\
\hline $8 / 21 / 920: 00$ & Manual Tape & 7.25 & Transcribed & \\
\hline $8 / 21 / 927: 05$ & Auto FIC & 7.2 & Good & \\
\hline
\end{tabular}

Data obtained from WHC Surveillance Analysis Computer System, (PC SACS) July 7, 1995. 


\begin{tabular}{|c|c|c|c|c|}
\hline \multicolumn{5}{|c|}{ 241-AP-104 } \\
\hline \multicolumn{5}{|c|}{ Surface Leval Data } \\
\hline Date & Type of Measurement & Leval (Inches) & Data Quality & Comments \\
\hline $8 / 22 / 920: 00$ & Manual Tape & 7.25 & Transcribed & \\
\hline $8 / 22 / 927: 04$ & Auto FIC & 7.2 & Good & \\
\hline $8 / 23 / 920: 00$ & Manual Tape & 7.25 & Transcribed & \\
\hline $8 / 23 / 927: 04$ & Auto FIC & 7.20 & Good & \\
\hline $8 / 24 / 920: 00$ & Manual Tape & 7.25 & Transcribed & \\
\hline $8 / 24 / 92 \quad 7: 04$ & Auto FIC & 7.20 & Good & \\
\hline $8 / 25 / 920: 00$ & Manual Tape & 7.25 & Transcribed & \\
\hline $8 / 25 / 927: 04$ & Auto FIC & 7.20 & Good & \\
\hline $8 / 26 / 927: 03$ & Auto FIC & 7.2 & Good & \\
\hline $8 / 27 / 927: 04$ & Auto FIC & 7.2 & Good & \\
\hline $8 / 28 / 920: 00$ & Manual Tape & 7.25 & Transcribed & \\
\hline $8 / 28 / 927: 02$ & Auto FIC & 7.2 & Good & \\
\hline $8 / 29 / 927: 02$ & Auto FIC & 7.2 & Good & \\
\hline $8 / 30 / 927: 02$ & Auto FIC & 7.20 & Good & \\
\hline $8 / 31 / 920: 00$ & Manual Tape & 7 & Transcribed & \\
\hline $8 / 31 / 927: 02$ & Auto FIC & 7.20 & Good & \\
\hline 9/1/92 0:00 & Manual Tape & 7.00 & Transcribed & \\
\hline 9/1/92 7:02 & Auto FIC & 7.2 & Good & \\
\hline $9 / 2 / 920: 00$ & Manual Tape & 7 & Transcribed & \\
\hline $9 / 2 / 927: 02$ & Auto FIC & 7.20 & Good & \\
\hline $9 / 3 / 920: 00$ & Manual Tape & 7.25 & Transcribed & \\
\hline $9 / 3 / 927: 04$ & Auto FIC & $7.20^{\circ}$ & Good & \\
\hline 9/4/92 0:00 & Manual Tape & 7.00 & Transcribed & \\
\hline $9 / 4 / 927: 04$ & Auto FIC & 7.20 & Good & \\
\hline $9 / 5 / 920: 00$ & Manual Tape & 7.00 & Transcribed & \\
\hline $9 / 5 / 927: 04$ & Auto FIC & 7.2 & Good & \\
\hline 9/6/92 0:00 & Manual Tape & 7.00 & Transcribed & \\
\hline 9/6/92 7:04 & Auto FIC & 7.2 & Good & \\
\hline 9/7/92 0:00 & Manual Tape & 7 & Transcribed & \\
\hline $9 / 7 / 927: 05$ & Auto FIC & 7.20 & Good & \\
\hline 9/8/92 0:00 & Manual Tape & 7.00 & Transcribed & \\
\hline $9 / 8 / 927: 05$ & Auto FIC & 7.2 & Good & \\
\hline 9/9/92 0:00 & Manual Tape & 7 & Transcribed & \\
\hline $9 / 9 / 927: 04$ & Auto FIC & 7.20 & Good & \\
\hline $9 / 10 / 920: 00$ & Manual Tape & 7.25 & Transcribed & \\
\hline $9 / 10 / 927: 03$ & Auto FIC & 7.20 & Good & \\
\hline $9 / 11 / 920: 00$ & Manual FIC & 7.20 & Transcribed & \\
\hline $9 / 11 / 920: 00$ & Manual Tape & 7 & Transcribed & \\
\hline $9 / 12 / 920: 00$ & Manual FIC & 7.2 & Transcribed & \\
\hline $9 / 12 / 920: 00$ & Manual Tape & 7.00 & Transcribed & \\
\hline 9/13/92 0:00 & Manual FIC & 7.1 & Transcribed & \\
\hline $9 / 13 / 920: 00$ & Manual Tape & 7.00 & Transcribed & \\
\hline $9 / 14 / 920: 00$ & Manual FIC & 7.20 & Transcribed & \\
\hline $9 / 14 / 920: 00$ & Manual Tape & 7 & Transcribed & \\
\hline $9 / 15 / 920: 00$ & Manual FIC & 7.2 & Transcribed & \\
\hline $9 / 15 / 920: 00$ & Manual Tape & 7.00 & Transcribed & \\
\hline $9 / 16 / 920: 00$ & Manual FIC & 7.20 & Transcribed & \\
\hline $9 / 16 / 920: 00$ & Manual Tape & 7 & Transcribed & \\
\hline
\end{tabular}

Data obtained from WHC Surveillance Analysis Computer System, (PC SACS) July 7, 1995. 


\begin{tabular}{|c|c|c|c|c|}
\hline \multicolumn{5}{|c|}{ 241-AP-104 } \\
\hline & & Ifface Level Dat: & & \\
\hline Date & Type of Measurement & Leval (Inches) & Data Quality & Comments \\
\hline $9 / 17 / 920: 00$ & Manual Tape & 7.00 & Transcribed & \\
\hline $9 / 17 / 927: 06$ & Auto FIC & 7.2 & Good & \\
\hline $9 / 18 / 920: 00$ & Manual Tape & 7.00 & Transcribed & \\
\hline $9 / 18 / 927: 06$ & Auto FIC & 7.20 & Good & \\
\hline $9 / 19 / 920: 00$ & Manual FIC & 7.2 & Transcribed & \\
\hline $9 / 19 / 920: 00$ & Manual Tape & 7 & Transcribed & \\
\hline $9 / 20 / 920: 00$ & Manual FIC & 7.20 & Transcribed & \\
\hline $9 / 20 / 920: 00$ & Manual Tape & 7 & Transcribed & \\
\hline $9 / 21 / 920: 00$ & Manual Tape & 7 & Transcribed & \\
\hline $9 / 21 / 927: 06$ & Auto FIC & 7.20 & Good & \\
\hline 9/22/92 0:00 & Menual Tape & 7.00 & Transcribed & \\
\hline 9/22/92 7:03 & Auto FIC & 7.2 & Good & \\
\hline $9 / 23 / 920: 00$ & Manual Tape & 7.00 & Transcribed & \\
\hline $9 / 23 / 927: 03$ & Auto FIC & 7.20 & Good & \\
\hline $9 / 24 / 920: 00$ & Manual Tape & 7.00 & Transcribed & \\
\hline $9 / 24 / 927: 03$ & Auto FIC . & 7.20 & Good & \\
\hline $9 / 25 / 920: 00$ & Manual Tape & 7.00 & Transcribed & \\
\hline $9 / 25 / 927: 03$ & Auto FIC & 7.2 & Good & \\
\hline $9 / 26 / 920: 00$ & Manual Tape & 7 & Transcribed & \\
\hline $9 / 26 / 927: 02$ & Auto FIC & 7.20 & Good & \\
\hline $9 / 27 / 920: 00$ & Manual Tape & 7 & Transcribed & \\
\hline $9 / 27 / 927: 02$ & Auto FIC & 7.20 & Good & \\
\hline $9 / 28 / 920: 00$ & Manual Tape & 6.75 & Transcribed & \\
\hline $9 / 28 / 927: 02$ & Auto FIC & 7.2 & Good & \\
\hline $9 / 29 / 920: 00$ & Manual Tape & 6.75 & Transcribed & \\
\hline $9 / 29 / 927: 03$ & Auto FIC & 7.2 & Good & \\
\hline $9 / 30 / 920: 00$ & Manual Tape & 6.75 & Transcribed & \\
\hline $9 / 30 / 927: 02$ & Auto FIC & 7.2 & Good & \\
\hline $10 / 1 / 920: 00$ & Manual Tape & 7.00 & Transcribed & \\
\hline $10 / 1 / 927: 03$ & Auto FIC & 7.20 & Good & \\
\hline $10 / 2 / 920: 00$ & Manual Tape & 7 & Transcribed & \\
\hline $10 / 2 / 927: 02$ & Auto FIC & 7.20 & Good & \\
\hline $10 / 3 / 920: 00$ & Manual Tape & 7 & Transćribed & \\
\hline $10 / 3 / 927: 03$ & Auto FIC & 7.20 & Good & \\
\hline $10 / 4 / 920: 00$ & Manual Tape & 7.00 & Transcribed & \\
\hline $10 / 4 / 927: 03$ & Auto FIC & 7.20 & Good & \\
\hline $10 / 5 / 920: 00$ & Manual Tape & 7.25 & Transcribed & \\
\hline $10 / 5 / 927: 03$ & Auto FIC & 7.2 & Good & \\
\hline $10 / 6 / 920: 00$ & Manual Tape & 7.25 & Transcribed & \\
\hline $10 / 6 / 927: 03$ & Auto FIC & 7.20 & Good & \\
\hline $10 / 7 / 920: 00$ & Manual FIC & 7.2 & Transcribed & \\
\hline $10 / 7 / 920: 00$ & Manual Tape & 7.25 & Transcribed & \\
\hline $10 / 8 / 920: 00$ & Manual Tape & 7.25 & Transcribed & \\
\hline $10 / 8 / 927: 02$ & Auto FIC & 7.2 & Good & \\
\hline $10 / 9 / 920: 00$ & Manual Tape & 7.50 & Transcribed & \\
\hline 10/9/92 7:03 & Auto FIC & 7.2 & Good & \\
\hline $10 / 10 / 920: 00$ & Manual Tape & 7.25 & Transcribed & \\
\hline $10 / 10 / 927: 03$ & Auto FIC & 7.2 & Good & \\
\hline
\end{tabular}

Data obtained from WHC Surveillance Analysis Computer System, (PC SACS) July 7, 1995. 


\begin{tabular}{|c|c|c|c|c|}
\hline \multicolumn{5}{|c|}{ 241-AP-104 } \\
\hline \multicolumn{5}{|c|}{ Surface Level Data } \\
\hline Date & Type of Measurement & Level (Inchea) & Data Quality & Comments \\
\hline $10 / 11 / 920: 00$ & Manual Tape & 7.25 & Transcribed & \\
\hline 10/11/92 7:03 & Auto FIC & 7.2 & Good & \\
\hline $10 / 12 / 920: 00$ & Manual FIC & 7.2 & Transcribed & \\
\hline $10 / 12 / 920: 00$ & Manual Tape & 7.25 & Transeribed & \\
\hline $10 / 13 / 920: 00$ & Manual FIC & 7.20 & Transcribed & \\
\hline $10 / 13 / 920: 00$ & Manual Tape & 7.25 & Transcribed & \\
\hline $10 / 14 / 920: 00$ & Manual Tape & 7.25 & Transcribed & \\
\hline $10 / 14 / 927: 03$ & Auto FIC & 7.2 & Good & \\
\hline $10 / 15 / 920: 00$ & Manual Tape & 7.25 & Transcribed & \\
\hline $10 / 15 / 927: 04$ & Auto FIC & 7.2 & Good & \\
\hline $10 / 16 / 920: 00$ & Manual Tape & 7.25 & Transcribed & \\
\hline $10 / 16 / 927: 03$ & Auto FIC & 7.20 & Good & \\
\hline $10 / 17 / 920: 00$ & Manual Tape & 7.25 & Transcribed & \\
\hline $10 / 17 / 927: 03$ & Auto FIC & 7.2 & Good & \\
\hline $10 / 18 / 920: 00$ & Manual Tape & 7.25 & Transcribed & \\
\hline $10 / 18 / 927: 03$ & Auto FIC & 7.20 & Good & \\
\hline $10 / 19 / 920: 00$ & Manual Tape & 7.00 & Transcribed & \\
\hline $10 / 19 / 927: 03$ & Auto FIC & 7.2 & Good & \\
\hline $10 / 20 / 920: 00$ & Manual Tape & 7 & Transcribed & \\
\hline $10 / 20 / 927: 03$ & Auto FIC & 7.20 & Good & \\
\hline $10 / 21 / 920: 00$ & Manual Tape & 7 & Transeribed & \\
\hline 10/21/92 7:03 & Auto FIC & 7.20 & Good & \\
\hline $10 / 22 / 920: 00$ & Manual FIC & 7.2 & Transcribed & \\
\hline $10 / 22 / 920: 00$ & Manual Tape & 7.00 & Transcribed & \\
\hline $10 / 23 / 920: 00$ & Manual FIC & 7.20 & Transcribed & \\
\hline $10 / 23 / 920: 00$ & Manual Tape & 7.00 & Transcribed & \\
\hline $10 / 24 / 920: 00$ & Manual Tape & 7.00 & Transcribed & \\
\hline $10 / 24 / 927: 03$ & Auto FIC & 7.20 & Good & \\
\hline $10 / 25 / 920: 00$ & Manual Tape & 7.00 & Transcribed & \\
\hline $10 / 25 / 927: 03$ & Auto FIC & 7.20 & Good & \\
\hline $10 / 26 / 920: 00$ & Manual FIC & 7.20 & Transcribed & \\
\hline $10 / 26 / 920: 00$ & Manual Tape & 7.00 & Transcribed & \\
\hline $10 / 27 / 920: 00$ & Manual Tape & 7.00 & Transcribed & \\
\hline $10 / 27 / 927: 04$ & Auto FIC & 7.20 & Good & \\
\hline $10 / 28 / 920: 00$ & Manual Tape & 7.00 & Transcribed & \\
\hline $10 / 28 / 927: 03$ & Auto FIC & 7.20 & Good & \\
\hline $10 / 29 / 920: 00$ & Manual Tape & 7 & Transcribed & \\
\hline $10 / 29 / 927: 03$ & Auto FIC & 7.20 & Good & \\
\hline $10 / 30 / 920: 00$ & Manual Tape & 7 & Transcribed & \\
\hline $10 / 30 / 927: 03$ & Auto FIC & 7.20 & Good & \\
\hline $10 / 31 / 920: 00$ & Manual Tape & 7.00 & Transcribed & \\
\hline 10/31/92 7:03 & Auto FIC & 7.2 & Good & \\
\hline $11 / 1 / 920: 00$ & Manual Tape & 7 & Transcribed & \\
\hline $11 / 1 / 927: 03$ & Auto FIC & 7.20 & Good & \\
\hline $11 / 2 / 920: 00$ & Manual Tape & 7.00 & Transcribed & \\
\hline $11 / 2 / 927: 03$ & Auto FIC & 7.2 & Good & . \\
\hline $11 / 3 / 920: 00$ & Manual Tape & 7.25 & Transcribed & \\
\hline $11 / 3 / 927: 03$ & Auto FIC & 7.2 & Good & \\
\hline
\end{tabular}

Data obtained from WHC Surveillance Analysis Computer System, (PC SACS) July 7, 1995. 


\begin{tabular}{|c|c|c|c|c|}
\hline \multicolumn{5}{|c|}{ 241-AP-104 } \\
\hline \multicolumn{5}{|c|}{ Surface Level Data } \\
\hline Date & Type of Measurement & Level (Inches) & Data Quality & Comments \\
\hline $11 / 4 / 920: 00$ & Manual Tape & 7.25 & Transcribed & \\
\hline $11 / 4 / 92$ 7:03 & Auto FIC & 7.2 & Good & \\
\hline $11 / 5 / 920: 00$ & Manual FIC & 7.20 & Transcribed & \\
\hline $11 / 5 / 920: 00$ & Manual Tape & 7 & Transeribed & \\
\hline $11 / 6 / 920: 00$ & Manual FIC & 7.2 & Transcribed & \\
\hline $11 / 6 / 920: 00$ & Manual Tape & 7 & Transcribed & \\
\hline $11 / 7 / 920: 00$ & Manual FIC & 7.20 & Transcribed & \\
\hline $11 / 7 / 920: 00$ & Manual Tape & 7.00 & Transcribed & \\
\hline $11 / 8 / 920: 00$ & Manual FIC & 7.20 & Transcribed & \\
\hline $11 / 8 / 920: 00$ & Manual Tape & 7.00 & Transcribed & \\
\hline $11 / 9 / 920: 00$ & Manual FIC & 7.2 & Transcribed & \\
\hline $11 / 9 / 920: 00$ & Manual Tape & 7.00 & Transcribed & \\
\hline $11 / 10 / 920: 00$ & Manual Tape & 7 & Transcribed & \\
\hline $11 / 10 / 927: 03$ & Auto FIC & 7.20 & Good & \\
\hline $11 / 11 / 920: 00$ & Manual Tape & 7 & Transcribed & \\
\hline $11 / 11 / 927: 04$ & Auto FIC & 7.2 & Good & \\
\hline $11 / 12 / 920: 00$ & Manual Tape & 7.00 & Transcribed & \\
\hline $11 / 12 / 927: 03$ & Auto FIC & 7.2 & Good & \\
\hline $11 / 13 / 920: 00$ & Manual Tape & 7.00 & Transcribed & \\
\hline $11 / 13 / 927: 04$ & Auto FIC & 7.20 & Good & \\
\hline $11 / 14 / 920: 00$ & Manual Tape & 6.75 & Transcribed & \\
\hline $11 / 14 / 927: 04$ & Auto FIC & 7.2 & Good & \\
\hline $11 / 15 / 920.00$ & Manual Tape & 7.00 & Transcribed & \\
\hline 11/15/92 7:04 & Auto FIC & 7.20 & Good & \\
\hline $11 / 16 / 920: 00$ & Manual Tape & 7 & Transcribed & \\
\hline $11 / 16 / 927: 04$ & Auto FIC & 7.2 & Good & \\
\hline $11 / 17 / 920: 00$ & Manual Tape & 7.25 & Transcribed & \\
\hline $11 / 17 / 927: 04$ & Auto FIC & 7.20 & Good & \\
\hline $11 / 18 / 927: 02$ & Auto FIC & 7.2 & Good & \\
\hline $11 / 19 / 927: 02$ & Auto FIC & 7.20 & Good & \\
\hline $11 / 20 / 927: 03$ & Auto FIC & 7.20 & Good & \\
\hline $11 / 21 / 927: 02$ & Auto FIC & 7.2 & Good & \\
\hline $11 / 22 / 927: 03$ & Auto FIC & 7.20 & Good & \\
\hline $11 / 23 / 927: 03$ & Auto FIC & 7.2 & Gopd & \\
\hline $11 / 24 / 920: 00$ & Manual Tape & 6.75 & Transcribed & \\
\hline $11 / 24 / 927: 03$ & Auto FIC & 7.2 & Good & \\
\hline $11 / 25 / 920: 00$ & Manual Tape & 6.75 & Transcribed & \\
\hline $11 / 25 / 927: 03$ & Auto FIC & 7.20 & Good & \\
\hline $11 / 26 / 927: 03$ & Auto FIC & 7.20 & Good & \\
\hline $11 / 27 / 927: 03$ & Auto FIC & 7.20 & Good & \\
\hline $11 / 28 / 927: 03$ & Auto FIC & 7.2 & Good & \\
\hline $11 / 29 / 927: 03$ & Auto FIC & 7.2 & Good & \\
\hline $11 / 30 / 920: 00$ & Manual FIC & 7.20 & Transćribed & \\
\hline $12 / 1 / 920: 00$ & Manual Tape & 7 & Transcribed & \\
\hline $12 / 1 / 927: 03$ & Auto FIC & 7.20 & Good & \\
\hline $12 / 2 / 927: 03$ & Auto FIC & 7.20 & Good & \\
\hline $12 / 3 / 927: 03$ & Auto FIC & 7.2 & Good & \\
\hline $12 / 4 / 927: 03$ & Auto FIC & 7.2 & Good & \\
\hline
\end{tabular}

Data obtained from WHC Surveillance Analysis Computer System, (PC SACS) July 7, 1995. 


\begin{tabular}{|c|c|c|c|c|}
\hline \multicolumn{5}{|c|}{ 241-AP-104 } \\
\hline \multicolumn{5}{|c|}{ Surface Level Data } \\
\hline Date & Type of Measurement & Level (lnches) & Data Quality & Cornments \\
\hline $12 / 5 / 920: 00$ & Manual FIC & 7.20 & Transcribed & \\
\hline $12 / 6 / 920: 00$ & Manual Tape ' & 7 & Transcribed & \\
\hline $12 / 6 / 927: 02$ & Auto FIC & 7.20 & Good & \\
\hline $12 / 7 / 920: 00$ & Manual Tape & 7.25 & Transcribed & \\
\hline $12 / 7 / 927: 03$ & Auto FIC & 7.2 & Good & \\
\hline 12/8/92 0:00 & Manual FIC & 7.4 & Transcribed & \\
\hline $12 / 8 / 920: 00$ & Manual Tape & 7.00 & Transcribed & . \\
\hline $12 / 8 / 927: 03$ & Auto FIC & 0.30 & Suspect & Data not included in graph \\
\hline $12 / 9 / 927: 03$ & Auto FIC & 7.10 & Good & $\cdot$ \\
\hline $12 / 10 / 927: 03$ & Auto FIC & 7.1 & Good & \\
\hline $12 / 11 / 927: 03$ & Auto FIC & 7.1 & Good & \\
\hline $12 / 12 / 920: 00$ & Manual FIC & 7.2 & Transcribed & \\
\hline $12 / 12 / 920: 00$ & Manual Tape & 7.25 & Transcribed & \\
\hline $12 / 13 / 927: 04$ & Auto FIC & 7.10 & Good & \\
\hline $12 / 14 / 927: 03$ & Auto FIC & 7.10 & Good & \\
\hline $12 / 15 / 927: 03$ & Auto FIC & 7.1 & Good & \\
\hline $12 / 16 / 927: 04$ & Auto FIC & 7.1 & Good & \\
\hline $12 / 17 / 927: 03$ & Auto FIC & 7.10 & Good & \\
\hline $12 / 18 / 927: 03$ & Auto FIC & 7.1 & Good & \\
\hline $12 / 19 / 927: 03$ & Auto FIC & 7.10 & Good & $\dot{-}$ \\
\hline $12 / 20 / 920: 00$ & Manual Tape & 7.00 & Transcribed & \\
\hline $12 / 20 / 927: 04$ & Auto FIC & 7.10 & Good & \\
\hline $12 / 21 / 927: 04$ & Auto FIC & 7.1 & Good & \\
\hline $12 / 22 / 920: 00$ & Manual FIC & 7.10 & Transcribed & \\
\hline $12 / 23 / 927: 04$ & Auto FIC & 7.1 & Good & \\
\hline $12 / 24 / 927: 03$ & Auto FIC & 7.1 & Good & \\
\hline $12 / 25 / 927: 03$ & Auto FIC & 7.10 & Good & \\
\hline $12 / 26 / 927: 03$ & Auto FIC & 7.10 & Good & \\
\hline $12 / 27 / 927: 03$ & Auto FIC & 7.10 & Good & \\
\hline 12/28/92 7:03 & Auto FIC & 7.10 & Good & \\
\hline $12 / 29 / 920: 00$ & Manual FIC & 7.1 & Transcribed & \\
\hline $12 / 30 / 927: 03$ & Auto FIC & $7.10^{\circ}$ & Good & \\
\hline $12 / 31 / 927: 03$ & Auto FIC & 7.1 & Good & \\
\hline $1 / 1 / 93$ 7:04 & Auto FIC & 7.10 & Good & \\
\hline $1 / 2 / 930: 00$ & Manual FIC & 7.1 & Good & \\
\hline 1/3/93 0:00 & Manual FIC & 7.10 & Good & \\
\hline $1 / 4 / 930: 00$ & Manual FIC & 7.1 & Good & \\
\hline $1 / 5 / 930: 00$ & Manual FIC & 7.10 & Good & \\
\hline $1 / 6 / 93 \quad 0: 00$ & Manual FIC & 7.10 & Good & \\
\hline 1/7/93 0:00 & Manual FIC & 7.1 & Good & \\
\hline 1/8/93 0:00 & Manual FIC & 7.10 & Good & \\
\hline 1/9/93 0:00 & Manual FIC & 7.10 & Good & \\
\hline $1 / 10 / 930: 00$ & Manual FIC & 7.1 & Good & \\
\hline $1 / 11 / 930: 00$ & Manual FIC & 7.1 & Good & \\
\hline $1 / 12 / 937: 06$ & Auto FIC & 7.10 & Good & \\
\hline $1 / 13 / 937: 06$ & Auto FIC & 7.1 & Good & \\
\hline $1 / 14 / 93$ 7:03 & Auto FIC & 7.1 & Good & \\
\hline $1 / 15 / 937: 03$ & Auto FIC & 7.10 & Good & \\
\hline
\end{tabular}

Data obtained from WHC Surveillance Analysis Computer System, (PC SACS) July 7, 1995. 


\begin{tabular}{|c|c|c|c|c|}
\hline \multicolumn{5}{|c|}{ 241-AP-104 } \\
\hline \multicolumn{5}{|c|}{ Surface Level Data } \\
\hline Date & Type of Measurement & Lovel (Incheas) & Date Quality & Comments \\
\hline $1 / 16 / 930: 00$ & Manual FIC & 7.10 & Good & \\
\hline $1 / 17 / 930: 00$ & Manual FIC & 7.1 & Good & \\
\hline 1/18/93 0:00 & Manual FIC & 7.10 & Good & \\
\hline $1 / 19 / 930: 00$ & Manual FIC & 7.10 & Good & \\
\hline $1 / 20 / 930: 00$ & Manual FIC & 7.10 & Good & \\
\hline $1 / 21 / 937: 02$ & Auto FIC & 7.1 & Good & \\
\hline $1 / 22 / 937: 04$ & Auto FIC & 7.1 & Good & \\
\hline $1 / 23 / 937: 03$ & Auto FIC & 7.10 & Good & \\
\hline $1 / 24 / 937: 03$ & Auto FIC & 7.1 & Good & \\
\hline $1 / 25 / 937: 03$ & Auto FIC & 7.1 & Good & \\
\hline $1 / 26 / 937: 04$ & Auto FIC & 7.10 & Good & \\
\hline $1 / 27 / 937: 04$ & Auto FIC & 7.10 & Good & \\
\hline $1 / 28 / 937: 04$ & Auto FIC & 6.90 & Good & \\
\hline $1 / 29 / 937: 04$ & Auto FIC & 6.90 & Good & \\
\hline $1 / 30 / 937: 03$ & Auto FIC & 6.90 & Good & \\
\hline $1 / 31 / 937: 04$ & Auto FIC & 6.9 & Good & \\
\hline 2/1/93 7:04 & Auto FIC & 6.9 & Good & \\
\hline $2 / 2 / 937: 04$ & Auto FIC & 6.90 & Good & \\
\hline $2 / 3 / 930: 00$ & Manual FIC & 6.90 & Good & \\
\hline 2/4/93 7:04 & Auto FIC & 6.90 & Good & \\
\hline 2/5/93 7:04 & Auto FIC & 6.9 & Good & \\
\hline $2 / 6 / 937: 04$ & Auto FIC & 6.9 & Good & \\
\hline $2 / 7 / 937: 03$ & Auto FIC & 6.90 & Good & \\
\hline $2 / 8 / 93 \quad 7: 04$ & Auto FIC & 6.9 & Good & \\
\hline $2 / 9 / 937: 04$ & Auto FIC & 6.90 & Good & \\
\hline $2 / 10 / 937: 04$ & Auto FIC & 6.90 & Good & \\
\hline $2 / 11 / 937: 04$ & Auto FIC & 6.9 & Good & \\
\hline $2 / 12 / 937: 03$ & Auto FIC & 6.9 & Good & \\
\hline $2 / 13 / 937: 03$ & Auto FIC & 6.90 & Good & \\
\hline $2 / 14 / 93$ 7:04 & Auto FIC & 6.9 & Good & \\
\hline 2/15/93 7:04 & Auto FIC & 6.9 & Good & \\
\hline $2 / 16 / 937: 02$ & Auto FIC & 6.90 & Good & \\
\hline $2 / 17 / 93$ 0:00 & Manual FIC & 6.9 & Good & \\
\hline 2/18/93 7:03 & Auto FIC & 6.9 & Good & \\
\hline $2 / 19 / 937: 02$ & Auto FIC & 6.9 & Good & \\
\hline $2 / 20 / 937: 03$ & Auto FIC & 6.90 & Good & \\
\hline $2 / 21 / 937: 04$ & Auto FIC & 6.90 & Good & \\
\hline 2/22/93 7:04 & Auto FIC & 6.9 & Good & \\
\hline $2 / 23 / 937: 03$ & Auto FIC & 6.90 & Good & \\
\hline $2 / 24 / 937: 04$ & Auto FIC & 6.90 & Good & \\
\hline $2 / 25 / 937: 03$ & Auto FIC & 6.9 & Good & \\
\hline $2 / 26 / 937: 03$ & Auto FIC & 6.90 & Good & \\
\hline $2 / 27 / 937: 04$ & Auto FIC & 6.90 & Good & \\
\hline $2 / 28 / 937: 03$ & Auto FIC & 6.9 & Good & \\
\hline $3 / 1 / 937: 03$ & Auto FIC & 6.90 & Good & \\
\hline $3 / 2 / 937: 04$ & Auto FIC & 6.90 & Good & \\
\hline $3 / 3 / 937: 03$ & Auto FIC & 6.90 & Good & \\
\hline $3 / 4 / 937: 04$ & Auto FIC & 6.90 & Good & \\
\hline
\end{tabular}

Data obtained from WHC Surveillance Analysis Computer System, (PC SACS) July 7, 1995. 


\begin{tabular}{|c|c|c|c|c|}
\hline \multicolumn{5}{|c|}{ 241-AP-104 } \\
\hline & & urface Levol Dat & & \\
\hline Date & Type of Moasurement & Level (Inches) & Date Quality & Comments \\
\hline $3 / 5 / 937: 04$ & Auto FIC & 6.9 & Good & \\
\hline $3 / 6 / 937: 03$ & Auto FIC & 6.9 & Good & \\
\hline $3 / 7 / 937: 03$ & Auto FIC & 6.9 & Good & \\
\hline $3 / 8 / 937: 03$ & Auto FIC & 6.90 & Good & \\
\hline $3 / 9 / 937: 04$ & Auto FIC & 6.9 & Good & \\
\hline $3 / 10 / 937: 05$ & Auto FIC & 6.9 & Good & \\
\hline $3 / 11 / 937: 04$ & Auto FIC & 6.9 & Good & \\
\hline $3 / 12 / 937: 04$ & Auto FIC & 6.9 & Good & \\
\hline $3 / 13 / 937: 03$ & Auto FIC & 6.9 & Good & \\
\hline $3 / 14 / 937: 04$ & Auto FIC & 6.90 & Good & \\
\hline $3 / 15 / 937: 03$ & Auto FIC & 6.90 & Good & \\
\hline $3 / 16 / 937: 04$ & Auto FIC & 6.9 & Good & \\
\hline $3 / 17 / 937: 03$ & Auto FIC & 6.90 & Good & \\
\hline $3 / 18 / 937: 04$ & Auto FIC & 6.90 & Good & \\
\hline $3 / 19 / 93$ 7:03 & Auto FIC & 6.9 & Good & \\
\hline $3 / 20 / 937: 04$ & Auto FIC & 6.90 & Good & 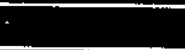 \\
\hline $3 / 21 / 93$ 7:04 & Auto FIC & 6.9 & Good & \\
\hline $3 / 22 / 937: 03$ & Auto FIC & 6.9 & Good & \\
\hline $3 / 23 / 937: 03$ & Auto FIC & 6.90 & Good & \\
\hline $3 / 24 / 937: 04$ & Auto FIC & 6.90 & Good & \\
\hline $3 / 25 / 937: 03$ & Auto FIC & 6.90 & Good & \\
\hline $3 / 26 / 93 \quad 7: 03$ & Auto FIC & 6.9 & Good & \\
\hline $3 / 27 / 93 \quad 7: 03$ & Auto FIC & 6.90 & Good & \\
\hline $3 / 28 / 937: 03$ & Auto FIC & 6.90 & Good & \\
\hline $3 / 29 / 93$ 7:03 & Auto FIC & 6.9 & Good & \\
\hline $3 / 30 / 937: 03$ & Auto FIC & 6.90 & Good & \\
\hline $3 / 31 / 93$ 7:03 & Auto FIC & 6.9 & Good & - \\
\hline 4/1/93 7:03 & Auto FIC & 6.9 & Good & \\
\hline $4 / 2 / 937: 06$ & Auto FIC & 6.9 & Good & \\
\hline 4/3/93 7:06 & Auto FIC & 6.90 & Good & \\
\hline $4 / 4 / 937: 05$ & Auto FIC & 6.90 & Good & \\
\hline $4 / 5 / 937: 06$ & Auto FIC & 6.9 & Good & \\
\hline $4 / 6 / 937: 06$ & Auto FIC & 6.9 & Good & \\
\hline $4 / 7 / 937: 06$ & Auto FIC & 6.9 & Good & \\
\hline 4/8/93 7:06 & Auto FIC & 6.90 & Good & \\
\hline 4/9/93 7:06 & Auto FIC & 6.9 & Good & \\
\hline 4/10/93 7:05 & Auto FIC & 6.9 & Good & \\
\hline 4/11/93 7:06 & Auto FIC & 6.90 & Good & \\
\hline 4/12/93 7:06 & Auto FIC & 6.90 & Good & \\
\hline $4 / 13 / 937: 06$ & Auto FIC & 6.9 & Good & \\
\hline 4/14/93 7:06 & Auto FIC & 6.90 & Good & \\
\hline 4/15/93 7:03 & Auto FIC & 6.90 & Good & \\
\hline $4 / 16 / 937: 06$ & Auto FIC & 6.90 & Good & \\
\hline 4/17/93 7:05 & Auto FIC & 6.9 & Good & \\
\hline $4 / 17 / 93 \quad 10: 03$ & Manual Tape & 7.00 & Good & \\
\hline $4 / 18 / 937: 06$ & Auto FIC & 6.9 & Good & \\
\hline $4 / 18 / 93 \quad 10: 03$ & Manual Tape & 7.00 & Good & \\
\hline $4 / 19 / 93 \quad 7: 06$ & Auto FIC & 6.9 & Good & \\
\hline
\end{tabular}

Data obtained from WHC Surveillance Analysis Computer System, (PC SACS) July 7, 1995. 
WHC-SD-WM-ER-315, Rev. 0

\begin{tabular}{|c|c|c|c|c|}
\hline \multicolumn{5}{|c|}{ 241-AP-104 } \\
\hline & & If & & \\
\hline Date & Type of Measurement & Level (Inches) & Data Quality & Comments \\
\hline 4/19/93 10:03 & Manual Tape & 7.00 & Good & \\
\hline $4 / 20 / 930: 00$ & Manual FIC & 6.9 & Good & \\
\hline $4 / 20 / 93 \quad 10: 03$ & Manual Tape & 7 & Good & \\
\hline $4 / 21 / 93 \quad 0: 00$ & Manual FIC & 6.9 & Good & \\
\hline $4 / 21 / 93 \quad 10: 03$ & Manual Tape & 7.00 & Good & \\
\hline $4 / 22 / 937: 06$ & Auto FIC & 6.90 & Good & \\
\hline $4 / 23 / 937: 06$ & Auto FIC & 6.90 & Good & \\
\hline $4 / 23 / 93$ 10:03 & Manual Tape & 7.00 & Good & \\
\hline $4 / 24 / 937: 05$ & Auto FIC & 6.9 & Good & \\
\hline $4 / 24 / 93 \quad 10: 03$ & Manual Tape & 7.00 & Good & \\
\hline $4 / 25 / 937: 06$ & Auto FIC & 6.9 & Good & \\
\hline $4 / 26 / 937: 06$ & Auto FIC & 6.90 & Good & \\
\hline 4/27/93 7:06 & Auto FIC & 6.90 & Good & \\
\hline $4 / 27 / 93 \quad 10: 03$ & Manual Tape & 7 & Good & \\
\hline 4/28/93 7:03 & Auto FIC & 6.9 & Good & \\
\hline $4 / 28 / 9310: 03$ & Manual Tape & 7 & Good & \\
\hline $4 / 29 / 937: 03$ & Auto FIC & 6.90 & Good & \\
\hline $4 / 30 / 937: 06$ & Auto FIC & 6.90 & Good & \\
\hline 5/1/93 7:05 & Auto FIC & 6.9 & Good & \\
\hline $5 / 1 / 93 \quad 10: 05$ & Manual Tape & 7 & Good & \\
\hline $5 / 2 / 937: 06$ & Auto FIC & 6.90 & Good & \\
\hline $5 / 3 / 937: 06$ & Auto FIC & 6.90 & Good & \\
\hline $5 / 4 / 937: 05$ & Auto FIC & 6.9 & Good & \\
\hline $5 / 5 / 937: 05$ & Auto FIC & 6.9 & Good. & \\
\hline 5/6/93 7:05 & Auto FIC & 6.90 & Good & \\
\hline 5/7/93 7:05 & Auto FIC & 6.9 & Good & \\
\hline $5 / 8 / 937: 05$ & Auto FIC & 6.9 & Good & \\
\hline 5/9/93 7:05 & Auto FIC & 6.90 & Good & \\
\hline $5 / 10 / 937: 05$ & Auto FIC & 6.90 & Good & \\
\hline $5 / 11 / 937: 06$ & Auto FIC & 6.9 & Good & \\
\hline $5 / 12 / 937: 06$ & Auto FIC & 6.9 & Good & \\
\hline $5 / 13 / 937: 05$ & Auto FIC & 6.9 & Good & $\cdot$ \\
\hline 5/14/93 7:05 & Auto FIC & 6.90 & Good & \\
\hline 5/15/93 7:06 & Auto FIC & 6.90 & Good & \\
\hline $5 / 16 / 937: 05$ & Auto FIC & 6.90 & Good & \\
\hline $5 / 17 / 937: 03$ & Auto FIC & 6.90 & Good & \\
\hline $5 / 18 / 937: 03$ & Auto FIC & 6.9 & Good & \\
\hline $5 / 19 / 937: 03$ & Auto FIC & 6.90 & Good & \\
\hline $5 / 20 / 937: 03$ & Auto FIC & 6.9 & Good & \\
\hline $5 / 21 / 93 \quad 0: 00$ & Manual FIC & 6.9 & Good & \\
\hline $5 / 22 / 937: 03$ & Auto FIC & 6.9 & Good & \\
\hline $5 / 23 / 937: 04$ & Auto FIC & 6.9 & Good & \\
\hline $5 / 24 / 937: 03$ & Auto FIC & 6.9 & Good & \\
\hline $5 / 25 / 937: 03$ & Auto FIC & 6.90 & Good & \\
\hline $5 / 26 / 937: 03$ & Auto FIC & 6.9 & Good & \\
\hline $5 / 27 / 937: 03$ & Auto FIC & 6.90 & Good & \\
\hline $5 / 28 / 937: 03$ & Auto FIC & 6.9 & Good & \\
\hline $5 / 29 / 937: 05$ & Auto FIC & 6.8 & Good & \\
\hline
\end{tabular}

Data obtained from WHC Surveillance Analysis Computer System, (PC SACS) July 7, 1995. 


\begin{tabular}{|c|c|c|c|c|}
\hline \multicolumn{5}{|c|}{ 241-AP-104 } \\
\hline \multicolumn{5}{|c|}{ Surface Level Data } \\
\hline Date & Type of Measurement & Level (Inches) & Data Quality & Comments \\
\hline $5 / 30 / 937: 05$ & Auto FIC & 6.8 & Good & \\
\hline $5 / 31 / 937: 06$ & Auto FIC & 6.9 & Good & \\
\hline $6 / 1 / 937: 06$ & Auto FIC & 6.9 & Good & \\
\hline $6 / 2 / 937: 06$ & Auto FIC & 6.90 & Good & \\
\hline $6 / 3 / 937: 06$ & Auto FIC & 6.9 & Good & \\
\hline 6/4/93 7:06 & Auto FIC & 6.90 & Good & \\
\hline $6 / 5 / 937: 06$ & Auto FIC & 6.90 & Good & \\
\hline $6 / 6 / 937: 05$ & Auto FIC & 6.9 & Good & \\
\hline $6 / 7 / 937: 05$ & Auto FIC & 6.9 & Good & \\
\hline $6 / 8 / 937: 05$ & Auto FIC & 6.90 & Good & \\
\hline $6 / 9 / 937: 05$ & Auto FIC & 6.8 & Good & \\
\hline $6 / 10 / 937: 04$ & Auto FIC & 6.8 & Good & \\
\hline $6 / 11 / 937: 05$ & Auto FIC & 6.80 & Good & \\
\hline 6/12/93 7:05 & Auto FIC & 6.80 & Good & \\
\hline $6 / 13 / 93 \quad 7: 04$ & Auto FIC & 6.8 & Good & \\
\hline $6 / 14 / 937: 05$ & Auto FIC & 6.80 & Good & \\
\hline $6 / 15 / 93$ 7:05 & Auto FIC & 6.80 & Good & \\
\hline $6 / 16 / 937: 05$ & Auto FIC & 6.8 & Good & \\
\hline $6 / 17 / 937: 05$ & Auto FIC & 6.90 & Good & \\
\hline $6 / 18 / 937: 06$ & Auto FIC & 6.9 & Good & \\
\hline $6 / 19 / 937: 06$ & Auto FIC & 6.9 & Good & \\
\hline $6 / 20 / 937: 06$ & Auto FIC & 6.90 & Good & \\
\hline $6 / 21 / 937: 06$ & Auto FIC & 6.85 & Good & \\
\hline $6 / 22 / 937: 06$ & Auto FIC & 6.85 & Good & \\
\hline $6 / 23 / 937: 06$ & Auto FIC & 6.8 & Good & \\
\hline $6 / 24 / 937: 06$ & Auto FIC & 6.80 & Good & \\
\hline $6 / 25 / 93 \quad 7: 03$ & Auto FIC & 6.8 & Good & \\
\hline $6 / 26 / 937: 03$ & Auto FIC & 6.8 & Good & \\
\hline $6 / 27 / 93 \quad 7: 04$ & Auto FIC & 6.80 & Good & \\
\hline $6 / 28 / 937: 03$ & Auto FIC & 6.8 & Good & \\
\hline $6 / 29 / 93 \quad 7: 04$ & Auto FIC & 6.8 & Good & \\
\hline $6 / 30 / 93 \quad 7: 03$ & Auto FIC & 6.85 & Good & \\
\hline 7/1/93 7:06 & Auto FIC & 6.85 & Good & \\
\hline $7 / 2 / 937: 06$ & Auto FIC & 6.85 & Good & \\
\hline $7 / 3 / 937: 03$ & Auto FIC & 6.85 & Good & \\
\hline $7 / 4 / 937: 04$ & Auto FIC & 6.85 & Good & \\
\hline $7 / 5 / 93 \quad 7: 03$ & Auto FIC & 6.85 & Good & \\
\hline 7/6/93 7:03 & Auto FIC & 6.80 & Good & \\
\hline 7/7/93 7:04 & Auto FIC & 6.80 & Good & \\
\hline 7/8/93 7:04 & Auto FIC & 6.80 & Good & \\
\hline $7 / 9 / 93$ 7:05 & Auto FIC & 6.8 & Good & \\
\hline $7 / 10 / 937: 03$ & Auto FIC & 6.8 & Good & \\
\hline $7 / 10 / 93$ 10:06 & Manual Tape & 7 & Good & \\
\hline $7 / 11 / 937: 03$ & Auto FIC & 6.8 & Good & \\
\hline $7 / 11 / 93 \quad 10: 06$ & Manual Tape & 7.00 & Good & \\
\hline $7 / 12 / 937: 02$ & Auto FIC & 6.80 & Good & \\
\hline $7 / 13 / 937: 03$ & Auto FIC & 6.8 & Good & \\
\hline 7/14/93 7:06 & Auto FIC & 6.80 & Good & \\
\hline
\end{tabular}

Data obtained from WHC Surveillance Analysis Computer System, (PC SACS) July 7, 1995. 


\begin{tabular}{|c|c|c|c|c|}
\hline \multicolumn{5}{|c|}{ 241-AP-104 } \\
\hline & & Ifface Level Dat & & \\
\hline Date & Type of Measurement & Leval (Inchioe) & Data Quality & Commants \\
\hline $7 / 15 / 937: 06$ & Auto FIC & 6.8 & Good & \\
\hline 7/16/93 7:06 & Auto FIC & 6.8 & Good & \\
\hline $7 / 17 / 937: 07$ & Auto FIC & 6.80 & Good & \\
\hline $7 / 18 / 937: 06$ & Auto FIC & 6.80 & Good & . \\
\hline $7 / 19 / 937: 03$ & Auto FIC & 6.80 & Good & \\
\hline $7 / 20 / 937: 03$ & Auto FIC & 6.8 & Good & \\
\hline $7 / 21 / 937: 03$ & Auto FIC & 6.80 & Good & \\
\hline $7 / 22 / 937: 03$ & Auto FIC & 6.8 & Good & \\
\hline $7 / 23 / 937: 03$ & Auto FIC & 6.80 & Good & \\
\hline $7 / 24 / 937: 03$ & Auto FIC & 6.8 & Good & \\
\hline $7 / 25 / 937: 04$ & Auto FIC & 6.8 & Good & \\
\hline $7 / 26 / 937: 03$ & Auto FIC & 6.80 & Good & \\
\hline $7 / 27 / 937: 03$ & Auto FIC & 6.8 & Good & \\
\hline $7 / 28 / 937: 04$ & Auto FIC & 6.8 & Good & \\
\hline $7 / 29 / 937: 03$ & Auto FIC & 6.80 & Good & \\
\hline $7 / 30 / 937: 03$ & Auto FIC & 6.80 & Good & \\
\hline $7 / 31 / 937: 03$ & Auto FIC & 6.8 & Good & \\
\hline $8 / 1 / 937: 03$ & Auto FIC & 6.80 & Good & \\
\hline $8 / 2 / 937: 06$ & Auto FIC & 6.8 & Good & \\
\hline $8 / 3 / 937: 03$ & Auto FIC & 6.80 & Good & \\
\hline 8/4/93 7:03 & Auto FIC & 6.80 & Good & \\
\hline $8 / 5 / 937: 04$ & Auto FIC & 6.80 & Good & \\
\hline $8 / 6 / 937: 04$ & Auto FIC & 6.8 & Good & \\
\hline $8 / 7 / 937: 04$ & Auto FIC & 6.8 & Good & \\
\hline $8 / 8 / 937: 04$ & Auto FIC & 6.80 & Good & \\
\hline $8 / 9 / 937: 04$ & Auto FIC & 6.80 & Good & \\
\hline $8 / 10 / 937: 04$ & Auto FIC & 6.8 & Good & \\
\hline $8 / 11 / 937: 04$ & Auto FIC & 6.8 & Good & \\
\hline $8 / 12 / 937: 04$ & Auto FIC & 6.8 & Good & \\
\hline $8 / 13 / 937: 04$ & Auto FIC & 6.80 & Good & \\
\hline $8 / 14 / 937: 03$ & Auto FIC & 6.80 & Good & \\
\hline $8 / 15 / 937: 04$ & Auto FIC & 6.80 & Good & \\
\hline $8 / 16 / 937: 04$ & Auto FIC & 6.8 & Good & \\
\hline $8 / 17 / 930: 00$ & Manual Tape & 7 & Good & \\
\hline $8 / 17 / 937: 04$ & Auto FIC & 6.8 & Good & \\
\hline $8 / 18 / 937: 03$ & Auto FIC & 6.80 & Good & \\
\hline $8 / 19 / 937: 04$ & Auto FIC & 6.80 & Good & \\
\hline $8 / 20 / 937: 04$ & Auto FIC & 6.80 & Good & \\
\hline $8 / 21 / 937: 04$ & Auto FIC & 6.8 & Good & \\
\hline $8 / 22 / 937: 04$ & Auto FIC & 6.80 & Good & \\
\hline $8 / 23 / 93 \quad 7: 04$ & Auto FIC & 6.8 & Good & \\
\hline $8 / 24 / 937: 04$ & Auto FIC & 6.80 & Good & \\
\hline $8 / 25 / 93.7: 04$ & Auto FIC & 6.80 & Good & \\
\hline $8 / 26 / 937: 04$ & Auto FIC & 6.8 & Good & \\
\hline $8 / 27 / 937: 05$ & Auto FIC & 6.8 & Good & \\
\hline $8 / 28 / 937: 04$ & Auto FIC & 6.8 & Good & \\
\hline $8 / 29 / 937: 03$ & Auto FIC & 6.80 & Good & \\
\hline $8 / 30 / 937: 04$ & Auto FIC & 6.8 & Good & \\
\hline
\end{tabular}

Data obtained from WHC Surveiliance Analysis Computer System, (PC SACS) July 7, 1995. 


\begin{tabular}{|c|c|c|c|c|}
\hline \multicolumn{5}{|c|}{ 241-AP-104 } \\
\hline \multicolumn{5}{|c|}{ Surface Level Data } \\
\hline Date & Type of Measurement & Level (Inchea) & Data Quality & Commonts \\
\hline $8 / 31 / 937: 04$ & Auto FIC & 6.80 & Good & \\
\hline 9/1/93 7:04 & Auto FIC & 6.8 & Good & \\
\hline $9 / 2 / 930: 00$ & Manual Tape & 7.00 & Good & \\
\hline $9 / 2 / 937: 04$ & Auto FIC & 6.80 & Good & \\
\hline $9 / 3 / 930: 00$ & Manual Tape & 7 & Good & \\
\hline $9 / 3 / 937: 04$ & Auto FIC & 6.8 & Good & \\
\hline 9/4/93 0:00 & Manual Tape & 7 & Good & \\
\hline 9/4/93 7:04 & Auto FIC & 6.80 & Good & \\
\hline $9 / 5 / 937: 04$ & Auto FIC & 6.80 & Good & \\
\hline 9/6/93 7:04 & Auto FIC & 6.8 & Good & \\
\hline 9/7/93 7:04 & Auto FIC & 6.80 & Good & \\
\hline 9/8/93 7:04 & Auto FIC & 6.8 & Good & \\
\hline 9/9/93 7:04 & Auto FIC & 6.80 & Good & \\
\hline $9 / 10 / 937: 04$ & Auto FIC & 6.8 & Good & \\
\hline 9/11/93 7:04 & Auto FIC & 6.8 & Good & \\
\hline $9 / 12 / 93 \quad 7: 04$ & Auto FIC & 6.8 & Good & \\
\hline $9 / 13 / 937: 04$ & Auto FIC & 6.8 & Good & \\
\hline $9 / 14 / 937: 04$ & Auto FIC & 6.80 & Good & \\
\hline 9/15/93 0:00 & Manual Tape & 6.75 & Good & \\
\hline 9/15/93 7:04 & Auto FIC & 6.80 & Good & \\
\hline 9/16/93 0:00 & Manual Tape & 6.75 & Good & \\
\hline 9/16/93 7:04 & Auto FIC & 6.80 & Good & \\
\hline 9/17/93 0:00 & Manual Tape & 6.75 & Good & \\
\hline $9 / 17 / 937: 04$ & Auto FIC & 6.8 & Good & \\
\hline 9/18/93 7:04 & Auto FIC & 6.80 & Good & \\
\hline $9 / 19 / 930: 00$ & Manual Tape & 6.75 & Good & \\
\hline 9/19/93 $7: 04$ & Auto FIC & 6.80 & Good & \\
\hline $9 / 20 / 930: 00$ & Manual Tape & 6.75 & Good & \\
\hline $9 / 20 / 93 \quad 7: 04$ & Auto FIC & 6.80 & Good & . \\
\hline $9 / 21 / 93 \quad 7: 04$ & Auto FIC & 6.80 & Good & \\
\hline $9 / 22 / 937: 04$ & Auto FIC & 6.80 & Good & \\
\hline $9 / 23 / 93 \quad 7: 04$ & Auto FIC & 6.8 & Good & \\
\hline 9/24/93 7:04 & Auto FIC & 6.8 & Good & \\
\hline $9 / 25 / 93 \quad 7: 04$ & Auto FIC & 6.80 & Good & \\
\hline $9 / 26 / 93 \quad 7: 04$ & Auto FIC & 6.8 & Good & . \\
\hline $9 / 27 / 937: 04$ & Auto FIC & 6.8 & Good & \\
\hline $9 / 28 / 937: 04$ & Auto FIC & 6.8 & Good & \\
\hline $9 / 29 / 937: 04$ & Auto FIC & 6.8 & Good & \\
\hline 9/30/93 7:03 & Auto FIC & 6.80 & Good & \\
\hline 10/1/93 7:06 & Auto FIC & 6.80 & Good & \\
\hline $10 / 2 / 930: 00$ & Manual Tape & 6.75 & Good & \\
\hline $10 / 2 / 937: 05$ & Auto FIC & 6.8 & Good & \\
\hline $10 / 3 / 930: 00$ & Manual Tape & 7.00 & Good & \\
\hline $10 / 3 / 937: 06$ & Auto FIC & 6.8 & Good & \\
\hline $10 / 4 / 930: 00$ & Manual Tape & 7 & Good & \\
\hline $10 / 4 / 937: 06$ & Auto FIC & 6.80 & Good & \\
\hline $10 / 5 / 930: 00$ & Manual Tape & 6.75 & Good & \\
\hline $10 / 5 / 937: 06$ & Auto FIC & 6.8 & Good & \\
\hline
\end{tabular}

Data obtained from WHC Surveillance Analysis Computer System, (PC SACS) July 7, 1995. 


\begin{tabular}{|c|c|c|c|c|}
\hline \multicolumn{5}{|c|}{ 241-AP-104 } \\
\hline \multicolumn{5}{|c|}{ Surface Lêval Data } \\
\hline Date & Type of Measurement & Level (Inches) & Data Quality & Comments \\
\hline $10 / 6 / 930: 00$ & Manual Tape & 6.75 & Good & \\
\hline $10 / 6 / 937: 06$ & Auto FIC & 6.80 & Good & \\
\hline $10 / 7 / 930: 00$ & Manual Tape & 7 & Good & \\
\hline $10 / 7 / 937: 05$ & Auto FIC & 6.8 & Good & \\
\hline 10/8/93 0:00 & Manual Tape & 6.75 & Good & \\
\hline $10 / 8 / 937: 04$ & Auto FIC & 6.70 & Good & \\
\hline $10 / 9 / 937: 03$ & Auto FIC & 6.70 & Good & \\
\hline $10 / 10 / 936: 04$ & Auto FIC & 6.70 & Good & \\
\hline $10 / 11 / 930: 00$ & Manual Tape & 6.75 & Good & \\
\hline 10/11/93 7:03 & Auto FIC & 6.70 & Good & \\
\hline $10 / 12 / 930: 00$ & Manual Tape & 6.75 & Good & \\
\hline $10 / 12 / 937: 04$ & Auto FIC & 6.7 & Good & \\
\hline $10 / 13 / 930: 00$ & Manual Tape & 7 & Good & \\
\hline $10 / 13 / 937: 03$ & Auto FIC & 6.7 & Good & \\
\hline $10 / 14 / 930: 00$ & Manual Tape & 7.00 & Good & \\
\hline 10/14/93 7:04 & Auto FIC & 6.7 & Good & \\
\hline $10 / 15 / 930: 00$ & Manual Tape & 7.00 & Good & \\
\hline $10 / 15 / 937: 04$ & Auto FIC & 6.70 & Good & \\
\hline $10 / 16 / 930: 00$ & Manual Tape & 7 & Good & \\
\hline $10 / 16 / 937: 04$ & Auto FIC & 6.70 & Good & \\
\hline 10/17/93 0:00 & Manual Tape & 6.75 & Good & \\
\hline $10 / 17 / 937: 03$ & Auto FIC & 6.7 & Good & \\
\hline $10 / 18 / 930: 00$ & Manual Tape & 6.75 & Good & \\
\hline $10 / 18 / 93 \quad 7: 04$ & Auto FIC & 6.70 & Good & \\
\hline $10 / 19 / 930: 00$ & Manual Tape & 6.75 & Good & \\
\hline $10 / 19 / 937: 04$ & Auto FIC & 6.7 & Good & \\
\hline $10 / 20 / 937: 03$ & Auto FIC & 6.70 & Good & \\
\hline 10/21/93 7:04 & Auto FIC & 6.70 & Good & \\
\hline $10 / 22 / 93 \quad 7: 04$ & Auto FIC & 6.7 & Good & \\
\hline $10 / 23 / 937: 04$ & Auto FIC & 6.70 & Good & \\
\hline $10 / 24 / 930: 00$ & Manual Tape & 6.75 & Good & \\
\hline $10 / 24 / 93 \quad 7: 04$ & Auto FIC & 6.70 & Good & \\
\hline $10 / 25 / 937: 04$ & Auto FIC & 6.70 & Good & \\
\hline $10 / 26 / 936: 03$ & Auto FIC & 6.7 & Good & \\
\hline $10 / 27 / 93 \quad 7: 04$ & Auto FIC & 6.70 & Good & \\
\hline $10 / 28 / 93 \quad 7: 04$ & Auto FIC & 6.7 & Good & \\
\hline $10 / 29 / 936: 03$ & Auto FIC & 6.7 & Good & \\
\hline $10 / 30 / 937: 04$ & Auto FIC & 6.70 & Good & \\
\hline 10/31/93 7:06 & Auto FIC & 6.70 & Good & \\
\hline $11 / 1 / 937: 06$ & Auto FIC & 6.70 & Good & \\
\hline $11 / 2 / 937: 04$ & Auto FIC & 6.70 & Good & \\
\hline $11 / 3 / 937: 06$ & Auto FIC & 6.70 & Good & \\
\hline $11 / 4 / 930: 00$ & Manual Tape & 7.00 & Good & \\
\hline $11 / 4 / 937: 04$ & Auto FIC & 6.7 & Good & \\
\hline $11 / 5 / 930: 00$ & Manual Tape & 7.00 & Good & \\
\hline $11 / 5 / 937: 06$ & Auto FIC & 6.7 & Good & \\
\hline 11/6/93 0:00 & Manual Tape & 7 & Good & \\
\hline $11 / 6 / 937: 06$ & Auto FIC & 6.7 & Good & \\
\hline
\end{tabular}

Data obtained from WHC Surveillance Analysis Computer System, (PC SACS) July 7, 1995. 


\begin{tabular}{|c|c|c|c|c|}
\hline \multicolumn{5}{|c|}{ 241-AP-104 } \\
\hline \multicolumn{5}{|c|}{ Surface Level Data } \\
\hline Date & Type of Measurement & Lovel (Inchoo) & Data Quality & Comments \\
\hline $11 / 7 / 930: 00$ & Manual Tape & 7.00 & Good & \\
\hline $11 / 7 / 937: 06$ & Auto FIC & 6.7 & Good & \\
\hline 11/8/93 7:06 & Auto FIC & 6.80 & Good & \\
\hline $11 / 9 / 937: 03$ & Auto FIC & 6.80 & Good & \\
\hline $11 / 10 / 937: 04$ & Auto FIC & 6.7 & Good & \\
\hline $11 / 11 / 937: 04$ & Auto FIC & 6.70 & Good & \\
\hline $11 / 12 / 937: 04$ & Auto FIC & 6.70 & Good & \\
\hline $11 / 13 / 937: 04$ & Auto FIC & 6.70 & Good & \\
\hline $11 / 14 / 936: 04$ & Auto FIC & 6.7 & Good & \\
\hline $11 / 15 / 937: 04$ & Auto FIC & 6.70 & Good & \\
\hline $11 / 16 / 937: 03$ & Auto FIC & 6.80 & Good & \\
\hline $11 / 17 / 937: 04$ & Auto FIC & 6.70 & Good & \\
\hline $11 / 18 / 937: 04$ & Auto FIC & 6.7 & Good & \\
\hline $11 / 19 / 937: 04$ & Auto FIC & 6.7 & Good & \\
\hline $11 / 20 / 937: 04$ & Auto FIC & 6.70 & Good & \\
\hline $11 / 21 / 937: 04$ & Auto FIC & 6.70 & Good & \\
\hline $11 / 22 / 937: 04$ & Auto FIC & 6.7 & Good & \\
\hline $11 / 23 / 937: 04$ & Auto FIC & 6.7 & Good & \\
\hline $11 / 24 / 930: 00$ & Manual Tape & 6.75 & Good & \\
\hline $11 / 24 / 937: 04$ & Auto FIC & 6.7 & Good & \\
\hline $11 / 25 / 930: 00$ & Manual Tape & 6.75 & Good & \\
\hline $11 / 25 / 937: 04$ & Auto FIC & 6.70 & Good & \\
\hline $11 / 26 / 930: 00$ & Manual Tape & 6.75 & Good & \\
\hline $11 / 26 / 937: 04$ & Auto FIC & 6.70 & Good & \\
\hline $11 / 27 / 937: 04$ & Auto FIC & 6.70 & Good & \\
\hline $11 / 28 / 937: 03$ & Auto FIC & 6.70 & Good & \\
\hline $11 / 29 / 937: 04$ & Auto FIC & 6.7 & Good & \\
\hline $11 / 30 / 937: 03$ & Auto FIC & 6.70 & Good & \\
\hline $12 / 1 / 937: 04$ & Auto FIC & 6.70 & Good & \\
\hline $12 / 2 / 937: 04$ & Auto FIC & 6.70 & Good & \\
\hline $12 / 3 / 937: 05$ & Auto FIC & 6.6 & Good & \\
\hline $12 / 4 / 937: 05$ & Auto FIC & 6.6 & Good & \\
\hline $12 / 5 / 937: 04$ & Auto FIC & 6.6 & Good & \\
\hline $12 / 6 / 930: 00$ & Manual Tape & 7.00 & Good & \\
\hline $12 / 6 / 93 \quad 7: 05$ & Auto FIC & 6.6 & Good & \\
\hline $12 / 7 / 937: 04$ & Auto FIC & 6.6 & Good & \\
\hline $12 / 8 / 930: 00$ & Manual Tape & 6.75 & Good & \\
\hline $12 / 8 / 937: 06$ & Auto FIC & 6.60 & Good & \\
\hline $12 / 9 / 930: 00$ & Manual Tape & 6.50 & Good & \\
\hline $12 / 9 / 937: 04$ & Auto FIC & 6.6 & Good & \\
\hline $12 / 10 / 930: 00$ & Manual Tape & 6.25 & Good & \\
\hline $12 / 10 / 936: 05$ & Auto FIC & 6.60 & Good & \\
\hline $12 / 11 / 930: 00$ & Manual Tape & 6.50 & Good & \\
\hline 12/11/93 7:06 & Auto FIC & 6.6 & Good & \\
\hline $12 / 12 / 930: 00$ & Manual Tape & 6.25 & Good & \\
\hline $12 / 12 / 937: 06$ & Auto FIC & 6.6 & Good & \\
\hline $12 / 13 / 930: 00$ & Manual Tape & 6.5 & Good & \\
\hline $12 / 13 / 937: 06$ & Auto FIC & 6.6 & Good & \\
\hline
\end{tabular}

Data obtained from WHC Surveillance Analysis Computer System, (PC SACS) July 7, 1995. 


\begin{tabular}{|c|c|c|c|c|}
\hline \multicolumn{5}{|c|}{ 241-AP-104 } \\
\hline Date & Type of Measurement & Level (Inchea) & Data Quality & Comments \\
\hline 12/14/930:00 & Manual Tape & 6.50 & Good & \\
\hline $12 / 14 / 937: 06$ & Auto FIC & 6.6 & . Good & \\
\hline 12/15/93 7:06 & Auto FIC & 6.60 & Good & \\
\hline $12 / 16 / 937: 06$ & Auto FIC & 6.60 & Good & \\
\hline $12 / 17 / 937: 05$ & Auto FIC & 6.6 & Good & \\
\hline $12 / 18 / 937: 06$ & Auto FIC & 6.60 & Good & \\
\hline 12/19/93 7:06 & Auto FIC & 6.60 & Good & \\
\hline $12 / 20 / 937: 06$ & Auto FIC & 6.60 & Good & \\
\hline $12 / 21 / 937: 06$ & Auto FIC & 6.60 & Good & \\
\hline 12/22/93 7:05 & Auto FIC & 6.60 & Good & \\
\hline 12/23/93 6:06 & Auto FIC & 6.60 & Good & \\
\hline 12/24/93 0:00 & Manual Tape & 6.75 & Good & \\
\hline 12/24/93 7:06 & Auto FIC & 6.6 & Good & . \\
\hline $12 / 25 / 93 \quad 0: 00$ & Manual Tape & 6.75 & Good & \\
\hline $12 / 25 / 937: 06$ & Auto FIC & 6.6 & Good & \\
\hline 12/26/93 0:00 & Manual Tape & 6.75 & Good & \\
\hline $12 / 26 / 937: 06$ & Auto FIC & 6.60 & Good & \\
\hline 12/27/93 7:06 & Auto FIC & 6.6 & Good & \\
\hline $12 / 28 / 937: 06$ & Auto FIC & 6.60 & Good & \\
\hline $12 / 29 / 937: 06$ & Auto FIC & 6.6 & Good & \\
\hline 12/30/93 7:05 & Auto FIC & 6.6 & Good & \\
\hline 12/31/93 7:06 & Auto FIC & 6.5 & Good & \\
\hline $1 / 1 / 940: 00$ & Manual Tape & 0 & Suspect & Data not included in graph \\
\hline $1 / 1 / 947: 06$ & Auto FIC & 6.50 & Good & \\
\hline $1 / 2 / 940: 00$ & Manual Tape & 0.00 & Suspect & Data not included in graph \\
\hline $1 / 2 / 947: 06$ & Auto FIC & 6.50 & Good & \\
\hline $1 / 3 / 940: 00$ & Manual Tape & 0.00 & Suspect & Data not included in graph \\
\hline $1 / 3 / 947: 07$ & Auto FIC & 6.50 & Good & \\
\hline $1 / 4 / 940: 00$ & Manual Tape & 0 & Suspect & Data not included in graph \\
\hline $1 / 4 / 947: 07$ & Auto FIC & 6.50 & Good & \\
\hline $1 / 5 / 940: 00$ & Manual Tape & 0 & Suspect & Data not included in graph \\
\hline $1 / 5 / 947: 06$ & Auto FIC & 6.5 & Good & \\
\hline $1 / 6 / 940: 00$ & Manual Tape & 0.00 & Suspect & Data not included in graph \\
\hline 1/6/94 7:06 & Auto FIC & 6.50 & Good & \\
\hline 1/7/940:00 & Manual Tape & 0 & Suspect & Data not included in graph \\
\hline $1 / 7 / 947: 07$ & Auto FIC & 6.50 & Good & \\
\hline $1 / 8 / 940: 00$ & Manual Tape & 0.00 & Suspect & Data not included in graph \\
\hline 1/8/94 7:06 & Auto FIC & 6.5 & Good & \\
\hline 1/9/940:00 & Manual Tape ' & 0.00 & Suspect & Data not included in graph \\
\hline $1 / 9 / 947: 06$ & Auto FIC & 6.5 & Good & \\
\hline $1 / 10 / 940: 00$ & Manual Tape & 0.00 & Suspect & Data not included in graph \\
\hline $1 / 10 / 947: 07$ & Auto FIC & 6.50 & Good & \\
\hline $1 / 11 / 940: 00$ & Manual Tape & 0 & Suspect & Data not included in graph \\
\hline $1 / 11 / 947: 07$ & Auto FIC & 6.50 & Good & \\
\hline 1/12/940:00 & Manual Tape & 6.75 & Good & \\
\hline $1 / 12 / 947: 06$ & Auto FIC & 6.5 & Good & \\
\hline $1 / 13 / 940: 00$ & Manual Tape & 0.00 & Suspect & Data not included in graph \\
\hline $1 / 13 / 945: 07$ & Auto FIC & 6.50 & Good & \\
\hline
\end{tabular}

Data obtained from WHC Surveillance Analysis Computer System, (PC SACS) July 7, 1995. 


\begin{tabular}{|c|c|c|c|c|}
\hline \multicolumn{5}{|c|}{ 241-AP-104 } \\
\hline \multicolumn{5}{|c|}{ Surface Level Data } \\
\hline Date & Type of Measurement & Level (Inchos) & Data Quality & Comments \\
\hline $1 / 14 / 940: 00$ & Manual Tape & 6.75 & Good & \\
\hline $1 / 14 / 947: 06$ & Auto FIC & 6.5 & Good & \\
\hline $1 / 15 / 940: 00$ & Manual Tape & 0.00 & Suspect & Data not included in graph \\
\hline $1 / 15 / 945: 07$ & Auto FIC & 6.5 & Good & \\
\hline $1 / 16 / 940: 00$ & Manuel Tape & 0.00 & Suspect & Data not included in graph \\
\hline $1 / 16 / 947: 07$ & Auto FIC & 6.5 & Good & \\
\hline $1 / 17 / 940: 00$ & Manual Tape & 0 & Suspect & Data not included in graph \\
\hline $1 / 17 / 947: 07$ & Auto FIC & 6.50 & Good & \\
\hline $1 / 18 / 940: 00$ & Manual Tape & 0.00 & Suspect & Data not included in graph \\
\hline $1 / 18 / 947: 07$ & Auto FIC & 6.50 & Good & \\
\hline $1 / 19 / 940: 00$ & Manual Tape & 0.00 & Suspect & Data not included in graph \\
\hline $1 / 19 / 947: 07$ & Auto FIC & 6.50 & Good & \\
\hline $1 / 20 / 940: 00$ & Manual Tape & 0 & Suspect & Data not included in graph \\
\hline $1 / 20 / 947: 07$ & Auto FIC & 6.5 & Good & \\
\hline $1 / 21 / 940: 00$ & Manual Tape & 0.00 & Suspect & Data not included in graph \\
\hline $1 / 21 / 947: 07$ & Auto FIC & 6.50 & Good & \\
\hline $1 / 22 / 940: 00$ & Manual Tape & 0.00 & Suspect & Data not included in graph \\
\hline 1/22/94 7:08 & Auto FIC & 6.50 & Good & \\
\hline $1 / 23 / 94 \quad 0: 00$ & Manual Tape & 0 & Suspect & Data not included in graph \\
\hline $1 / 23 / 947: 07$ & Auto FIC & 6.50 & Good & \\
\hline $1 / 24 / 940: 00$ & Manual Tape & 0.00 & Suspect & Data not included in graph \\
\hline $1 / 24 / 947: 08$ & Auto FIC & 6.50 & Good & \\
\hline $1 / 25 / 940: 00$ & Manual Tape & 0.00 & Suspect & Data not included in graph \\
\hline $1 / 25 / 947: 07$ & Auto FIC & 6.5 & Good & \\
\hline $1 / 26 / 940: 00$ & Manual Tape & 0.00 & Suspect & Data not included in graph \\
\hline $1 / 26 / 947: 06$ & Auto FIC & 6.50 & Good & \\
\hline $1 / 27 / 940: 00$ & Manual Tape & 0 & Suspect & Data not included in graph \\
\hline $1 / 27 / 946: 06$ & Auto FIC & 6.5 & Good & \\
\hline $1 / 28 / 940: 00$ & Manual Tape & 0 & Suspect & Data not included in graph \\
\hline $1 / 28 / 947: 07$ & Auto FIC & 6.5 & Good & \\
\hline $1 / 29 / 940: 00$ & Manual Tape/ & 0 & Suspect & Data not included in graph \\
\hline $1 / 29 / 947: 07$ & Auto FIC & 6.5 & Good & \\
\hline $1 / 30 / 940: 00$ & Manual Tape & 0.00 & Suspect & Data not included in graph \\
\hline $1 / 30 / 947: 06$ & Auto FIC & 6.5 & Good & \\
\hline $1 / 31 / 940: 00$ & Manual Tape & 6.75 & Good & \\
\hline $1 / 31 / 947: 07$ & Auto FIC & 6.50 & Good & \\
\hline $2 / 1 / 940: 00$ & Manual Tape & 6.75 & Good & \\
\hline $2 / 1 / 947: 06$ & Auto FIC & 6.5 & Good & \\
\hline $2 / 2 / 940: 00$ & Manual Tape & 0 & Suspect & Data not included in graph \\
\hline 2/2/94 7:07 & Auto FIC & 6.50 & Good & \\
\hline 2/3/94 0:00 & Manual Tape & 6.75 & Good & \\
\hline 2/3/94 7:07 & Auto FIC & 6.50 & Good & \\
\hline $2 / 4 / 940: 00$ & Manual Tape & 0.00 & Suspect & Data not included in graph \\
\hline 2/4/94 7:07 & Auto FIC & 6.5 & Good & \\
\hline $2 / 5 / 940: 00$ & Manual Tape & 0 & Suspect & Data not included in graph \\
\hline 2/5/94 7:07 & Auto FIC & 6.5 & Good & \\
\hline $2 / 6 / 940: 00$ & Manual Tape & 0.00 & Suspect & Data not included in graph \\
\hline $2 / 6 / 947: 07$ & Auto FIC & 6.50 & Good & \\
\hline
\end{tabular}

Data obtained from WHC Surveillance Analysis Computer System, (PC SACS) July 7, 1995. 
WHC-SD-WM-ER-315, Rev, 0

\begin{tabular}{|c|c|c|c|c|}
\hline \multicolumn{5}{|c|}{$\quad 241-A P-104$} \\
\hline \multicolumn{5}{|c|}{ Surface Level Data } \\
\hline Date & Type of Measurement & Level (Inchos) & Data Quality & Comments \\
\hline $2 / 7 / 940: 00$ & Manual Tape & 0.00 & Suspect & Data not included in graph \\
\hline $2 / 7 / 947: 06$ & Auto FIC & 6.50 & Good & \\
\hline 2/8/94 0:00 & Manual Tape & 0.00 & Suspect & Data not included in graph \\
\hline $2 / 8 / 947: 07$ & Auto FIC & 6.50 & Good & . \\
\hline $2 / 9 / 940: 00$ & Manual Tape & 0.00 & Suspect & Data not included in graph \\
\hline $2 / 9 / 947: 04$ & Auto FIC & 6.50 & Good & \\
\hline $2 / 10 / 940: 00$ & Manual Tape & 0.00 & Suspect & Data not included in graph \\
\hline 2/10/94 7:04 & Auto FIC & 6.5 & Good & \\
\hline $2 / 11 / 940: 00$ & Manual Tape & 0 & Suspect & Data not included in graph \\
\hline $2 / 11 / 947: 06$ & Auto FIC & 6.50 & Good & \\
\hline $2 / 12 / 940: 00$ & Manual Tape & 0.00 & Suspect & Data not included in graph \\
\hline $2 / 12 / 947: 03$ & Auto FIC & 6.50 & Good & \\
\hline $2 / 13 / 940: 00$ & Manual Tape & 0.00 & Suspect & Data not included in graph \\
\hline $2 / 13 / 947: 04$ & Auto FIC & 6.50 & Good & \\
\hline $2 / 14 / 940: 00$ & Manual Tape : & 0.00 & Suspect & Data not included in graph \\
\hline 2/14/94 7:04 & Auto FIC & 6.50 & Good & 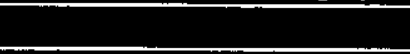 \\
\hline $2 / 15 / 940: 00$ & Manual Tape & 0 & Suspect & Data not included in graph \\
\hline $2 / 15 / 947: 04$ & Auto FIC & 6.5 & Good & \\
\hline $2 / 16 / 940: 00$ & Manual Tape & 0 & Suspect & Data not included in graph \\
\hline $2 / 16 / 947: 03$ & Auto FIC & 6.5 & Good & \\
\hline $2 / 17 / 940: 00$ & Manual Tape & 0.00 & Suspect & Data not included in graph \\
\hline $2 / 17 / 947: 04$ & Auto FIC & 6.5 & Good & \\
\hline 2/18/940:00 & Manual Tape & 0 & Suspect & Data not included in graph \\
\hline 2/18/94 7:06 & Auto FIC & 6.50 & Good & - \\
\hline $2 / 19 / 940 ; 00$ & Manual Tape & 0 & Suspect & Data not included in graph \\
\hline $2 / 19 / 947: 06$ & Auto FIC & 6.50 & Good & \\
\hline $2 / 20 / 940: 00$ & Manual Tape & 0 & Suspect & Data not included in graph \\
\hline $2 / 20 / 947: 07$ & Auto FIC & 6.50 & Good & \\
\hline $2 / 21 / 940: 00$ & Manual Tape & 0 & Suspect & Data not included in graph \\
\hline $2 / 21 / 947: 06$ & Auto FIC & 6.5 & Good & \\
\hline $2 / 22 / 940: 00$ & Manual Tape & 0 & Suspect & Data not included in graph \\
\hline $2 / 22 / 947: 05$ & Auto FIC & 6.50 & Good & 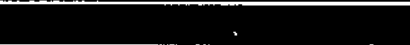 \\
\hline $2 / 23 / 940: 00$ & Manual. Tape & 0.00 & Suspect & Data not included in graph \\
\hline $2 / 23 / 947: 06$ & Auto FIC & 6.5 & Good & \\
\hline $2 / 24 / 940: 00$ & Manual Tape & 0.00 & Suspect & Data not included in graph \\
\hline 2/24/94 7:06 & Auto FIC & 6.5 & Good & \\
\hline $2 / 25 / 940: 00$ & Manual Tape & 0.00 & Suspect & Data not included in graph \\
\hline 2/25/94 6:06 & Auto FIC & 6.50 & Good & \\
\hline $2 / 26 / 940: 00$ & Manual Tape & 0.00 & Suspect & Data not included in graph \\
\hline $2 / 26 / 947: 07$ & Auto FIC & 6.5 & Good & \\
\hline $2 / 27 / 940: 00$ & Manual Tape & 0.00 & Suspect & Data not included in graph \\
\hline $2 / 27 / 947: 07$ & Auto FIC & 6.5 & Good & \\
\hline $2 / 28 / 940: 00$ & Manual Tape & 0.00 & Suspect & Data not included in graph \\
\hline $2 / 28 / 946: 06$ & Auto FIC & 6.50 & Good & \\
\hline $3 / 1 / 940: 00$ & Manual Tape & 0.00 & Suspect & Data not included in graph \\
\hline $3 / 1 / 947: 04$ & Auto FIC & 6.50 & Good & \\
\hline $3 / 2 / 940: 00$ & Manual Tape & 0.00 & Suspect & Data not included in graph \\
\hline $3 / 2 / 947: 06$ & Auto FIC & 6.5 & Good & \\
\hline
\end{tabular}

Data obtained from WHC Surveillance Analysis Computer System, (PC SACS) July 7, 1995. 
WHC-SD-WM-ER-3I5, Rev. 0

\begin{tabular}{|c|c|c|c|c|}
\hline \multicolumn{5}{|c|}{ 241-AP-104 } \\
\hline & & Urface Level Dat & & \\
\hline Date & Type of Measurement & Level (Inches) & Data Quality & Comments \\
\hline $3 / 3 / 940: 00$ & Manual Tape & 0 & Suspect & Data not included in graph \\
\hline $3 / 3 / 947: 06$ & Auto FIC & 6.50 & Good & \\
\hline $3 / 4 / 940: 00$ & Manual Tape & 0 & Suspect & Data not included in graph \\
\hline $3 / 4 / 947: 04$ & Auto FIC & 6.50 & Good & \\
\hline $3 / 5 / 940: 00$ & Manual Tapo & 0.00 & Suspect & Data not included in graph \\
\hline $3 / 5 / 94$ 7:05 & Auto FIC & 6.5 & Good & \\
\hline $3 / 6 / 940: 00$ & Manual Tape & 0 & Suspect & Data not included in graph \\
\hline $3 / 6 / 947: 05$ & Auto FIC & 6.50 & Good & \\
\hline $3 / 7 / 94 \quad 0: 00$ & Manual Tape & 0 & Suspect & Data not included in graph \\
\hline $3 / 7 / 947: 05$ & Auto FIC & 6.5 & Good & \\
\hline 3/8/94 0:00 & Manual Tape & 0.00 & Suspect & Data not included in graph \\
\hline $3 / 8 / 947: 04$ & Auto FIC & 6.5 & Good & \\
\hline $3 / 9 / 940: 00$ & Manual Tape & 0.00 & Suspect & Data not included in graph \\
\hline $3 / 9 / 947: 04$ & Auto FIC & 6.50 & Good & \\
\hline $3 / 10 / 940.00$ & Manual Tape & 0.00 & Suspect & Data not included in graph \\
\hline $3 / 10 / 947: 05$ & Auto FIC & 6.50 & Good & \\
\hline $3 / 11 / 940: 00$ & Manual Tape & 0.00 & Suspect & Data not included in graph \\
\hline $3 / 11 / 947: 06$ & Auto FIC & 6.5 & Good & \\
\hline $3 / 12 / 940: 00$ & Manual Tape & 0 & Suspect & Data not included in graph \\
\hline $3 / 12 / 947: 06$ & Auto FIC & 6.50 & Good & \\
\hline $3 / 13 / 940: 00$ & Manual Tape & 0 & Suspect & Data not included in graph \\
\hline 3/13/94 7:05 & Auto FIC & 6.5 & Good & \\
\hline $3 / 14 / 940: 00$ & Manual Tape & 0.00 & Suspect & Data not included in graph \\
\hline $3 / 14 / 940: 00$ & Manual FIC & 6.50 & Good & \\
\hline $3 / 15 / 940: 00$ & Manual Tape & 6.75 & Good & \\
\hline $3 / 15 / 947: 05$ & Auto FIC & 6.5 & Good & \\
\hline $3 / 16 / 940: 00$ & Manual Tape & 0.00 & Suspect & Data not included in graph \\
\hline $3 / 16 / 947: 04$ & Auto FIC & 6.50 & Good & \\
\hline $3 / 17 / 940: 00$ & Manual Tape & 0.00 & Suspect & Data not included in graph \\
\hline $3 / 17 / 947: 05$ & Auto FIC & 6.50 & Good & \\
\hline $3 / 18 / 940: 00$ & Manual Tape & 0.00 & Suspect & Data not included in graph \\
\hline $3 / 18 / 947: 05$ & Auto FIC & 6.5 & Good & \\
\hline $3 / 19 / 940: 00$ & Manual Tape & 0.00 & Suspect & Data not included in graph \\
\hline $3 / 19 / 947: 04$ & Auto FIC & 6.5 & Good & \\
\hline $3 / 20 / 940: 00$ & Manual Tape & 0 & Suspect & Data not included in graph \\
\hline $3 / 20 / 947: 05$ & Auto FIC & 6.50 & Good & \\
\hline $3 / 21 / 940: 00$ & Manual Tape & 0.00 & Suspect & Data not included in graph \\
\hline $3 / 21 / 947: 05$ & Auto FIC & 6.5 & Good & \\
\hline $3 / 22 / 940: 00$ & Manual Tape & 0.00 & Suspect & Data not included in graph \\
\hline $3 / 22 / 947: 04$ & Auto FIC & 6.5 & Good & \\
\hline $3 / 23 / 940: 00$ & Manual Tape & 0 & Suspect & Data not included in graph \\
\hline $3 / 23 / 940: 00$ & Manual FIC & 6.50 & Good & \\
\hline $3 / 24 / 940: 00$ & Manual Tape & 0 & Suspect & Data not included in graph \\
\hline $3 / 24 / 947: 04$ & Auto FIC & 6.5 & Good & . \\
\hline $3 / 25 / 940: 00$ & Manual Tape & 0 & Suspect & Data not included in graph \\
\hline $3 / 25 / 947: 05$ & Auto FIC & 6.50 & Good & \\
\hline $3 / 26 / 940: 00$ & Manual Tape & 0.00 & Suspect & Data not included in graph \\
\hline $3 / 26 / 947: 04$ & Auto FIC & 6.50 & Good & \\
\hline
\end{tabular}

Data obtained from WHC Surveillance Analysis Computer System, (PC SACS) July 7, 1995. 
WHC-SD-WM-ER-315, Rev. 0

\begin{tabular}{|c|c|c|c|c|}
\hline \multicolumn{5}{|c|}{ 241-AP-104 } \\
\hline \multicolumn{5}{|c|}{ Surface Leval Data } \\
\hline Date & Type of Measurement & Loval (Inchea) & Data Quality & Comments \\
\hline $3 / 27 / 940: 00$ & Manual Tape & 0.00 & Suspect & Data not included in graph \\
\hline $3 / 27 / 947: 04$ & Auto FIC & 6.50 & Good & \\
\hline $3 / 28 / 940: 00$ & Manual Tape & 0.00 & Suspect & Data not included in graph \\
\hline $3 / 28 / 947: 04$ & Auto FIC & 6.50 & Good & \\
\hline $3 / 29 / 940: 00$ & Manual Tape & 0 & Suspect & Data not included in graph \\
\hline $3 / 29 / 947: 03$ & Auto FIC & 6.50 & Good & \\
\hline $3 / 30 / 947: 03$ & Auto FIC & 6.5 & Good & \\
\hline $3 / 31 / 947: 03$ & Auto FIC & 6.50 & Good & \\
\hline $4 / 1 / 94 \quad 0: 04$ & Auto FIC & 6.5 & Good & \\
\hline 4/2/94 7:07 & Auto FIC & 6.5 & Good & \\
\hline $4 / 3 / 947: 03$ & Auto FIC & 6.5 & Good & \\
\hline 4/4/94 7:03 & Auto FIC & 6.50 & Good & \\
\hline $4 / 5 / 947: 03$ & Auto FIC & 6.50 & Good & \\
\hline $4 / 6 / 947: 06$ & Auto FIC & 6.5 & Good & \\
\hline 4/7/94 7:07 & Auto FIC & 6.5 & Good & \\
\hline 4/8/94 7:03 & Auto FIC & 6.50 & Good & \\
\hline 4/9/94 7:07 & Auto FIC & 6.50 & Good & \\
\hline $4 / 10 / 947: 03$ & Auto FIC & 6.50 & Good & \\
\hline $4 / 11 / 947: 03$ & Auto FIC & 6.50 & Good & \\
\hline 4/12/94 7:03 & Auto FIC & 6.50 & Good & \\
\hline $4 / 13 / 947: 03$ & Auto FIC & 6.5 & Good & \\
\hline $4 / 14 / 947: 03$ & Auto FIC & 6.5 & Good & \\
\hline 4/15/94 7:03 & Auto FIC & 6.50 & Good & \\
\hline $4 / 16 / 947: 03$ & Auto FIC & 6.5 & Good & \\
\hline $4 / 17 / 947: 03$ & Auto FIC & 6.5 & Good & \\
\hline $4 / 18 / 947: 03$ & Auto FIC & 6.5 & Good & \\
\hline $4 / 19 / 946: 06$ & Auto FIC & 6.50 & Good & \\
\hline $4 / 20 / 947: 04$ & Auto FIC & 6.50 & Good & \\
\hline $4 / 21 / 947: 05$ & Auto FIC & 6.50 & Good & \\
\hline $4 / 22 / 947: 04$ & Auto FIC & 6.5 & Good & \\
\hline $4 / 23 / 947: 05$ & Auto FIC & 6.5 & Good & \\
\hline 4/24/94 7:04 & Auto FIC & 6.50 & Good & \\
\hline 4/25/94 7:04 & Auto FIC & 6.50 & Good & \\
\hline $4 / 26 / 947: 07$ & Auto FIC & 6.50 & Good & \\
\hline $4 / 27 / 947: 04$ & Auto FIC & 6.50 & Good & \\
\hline 4/28/94 7:05 & Auto FIC & 6.5 & Good & \\
\hline $4 / 29 / 947: 04$ & Auto FIC & 6.5 & Good & \\
\hline $4 / 30 / 940: 00$ & Manual Tape & .0 & Suspect & Data not included in graph \\
\hline 4/30/94 7:05 & Auto FIC & 6.50 & Good & \\
\hline $5 / 1 / 947: 05$ & Auto FIC & 6.5 & Good & \\
\hline $5 / 2 / 947: 05$ & Auto FIC & 6.50 & Good & \\
\hline $5 / 3 / 947: 05$ & Auto FIC & 6.5 & Good & \\
\hline $5 / 4 / 947: 05$ & Auto FIC & 6.5 & Good & \\
\hline $5 / 5 / 947: 03$ & Auto FIC & 6.5 & Good & \\
\hline $5 / 6 / 947: 03$ & Auto FIC & 6.50 & Good & \\
\hline $5 / 7 / 947: 03$ & Auto FIC & 6.50 & Good & \\
\hline $5 / 8 / 947: 03$ & Auto FIC & 6.50 & Good & \\
\hline $5 / 9 / 947: 03$ & Auto FIC & 6.5 & Good & \\
\hline
\end{tabular}

Data obtained from WHC Surveillance Analysis Computer System, (PC SACS) July 7, 1995. 


\begin{tabular}{|c|c|c|c|c|}
\hline \multicolumn{5}{|c|}{ 241-AP-104 } \\
\hline \multicolumn{5}{|c|}{ Surface Leval Data } \\
\hline Date & Type of Measurement & Level (Inchea) & Data Qually & Comments \\
\hline $5 / 10 / 947: 03$ & Auto FIC & 6.50 & Good & \\
\hline $5 / 11 / 947: 03$ & Auto FIC & 6.50 & Good & \\
\hline $5 / 12 / 946: 03$ & Auto FIC & 6.5 & Good & \\
\hline $5 / 13 / 940: 00$ & Manual Tape & $\mathbf{5 . 5}$ & Good & \\
\hline $5 / 13 / 947: 03$ & Auto FIC & 6.50 & Good & \\
\hline $5 / 14 / 947: 03$ & Auto FIC & 6.60 & Good & \\
\hline $5 / 15 / 947: 06$ & Auto FIC & 6.5 & Good & \\
\hline $5 / 16 / 947: 02$ & Auto FIC & 6.5 & Good & \\
\hline $5 / 17 / 947: 03$ & Auto FIC & 6.5 & Good & \\
\hline $5 / 18 / 947: 03$ & Auto FIC & 6.5 & Good & \\
\hline $5 / 19 / 940: 00$ & Manual Tape & 6.75 & Good & \\
\hline $5 / 19 / 947: 03$ & Auto FIC & 6.5 & Good & \\
\hline $5 / 20 / 940: 00$ & Manual Tape & 6.50 & Good & \\
\hline $5 / 20 / 947: 03$ & Auto FIC & 6.50 & Good & \\
\hline $5 / 21 / 947: 05$ & Auto FIC & 6.5 & Good & \\
\hline $5 / 22 / 940: 00$ & Manual Tape & 6.75 & Good & \\
\hline $5 / 22 / 947: 05$ & Auto FIC & 6.5 & Good & \\
\hline $5 / 23 / 940: 00$ & Manual Tape & 6.75 & Good & \\
\hline $5 / 23 / 947: 04$ & Auto FIC & 6.50 & Good & \\
\hline $5 / 24 / 947: 04$ & Auto FIC & 6.5 & Good & \\
\hline $5 / 25 / 947: 04$ & Auto FIC & 6.50 & Good & \\
\hline $5 / 26 / 940: 00$ & Manual Tape & 6.75 & Good & \\
\hline $5 / 26 / 946: 05$ & Auto FIC & 6.5 & Good & \\
\hline $5 / 27 / 947: 03$ & Auto FIC & 6.50 & Good & \\
\hline $5 / 28 / 947: 03$ & Auto FIC & 6.50 & Good & \\
\hline $5 / 29 / 947: 03$ & Auto FIC & $\mathbf{6 . 5 0}$ & Good & \\
\hline $5 / 30 / 940: 00$ & Manual FIC & 6.50 & Good & \\
\hline $5 / 30 / 947: 02$ & Auto FIC & 6.5 & Good & \\
\hline $5 / 31 / 940: 00$ & Manual FIC & 6.50 & Good & \\
\hline $5 / 31 / 947: 03$ & Auto FIC & 6.5 & Good & \\
\hline $6 / 1 / 940: 00$ & Manual Tape & 0.00 & Suspect & Data not included in graph \\
\hline $6 / 1 / 947: 03$ & Auto FIC & 6.50 & Good & \\
\hline $6 / 2 / 940: 00$ & Manual Tape & 0.00 & Suspect & Data not included in graph \\
\hline $6 / 2 / 947: 03$ & Auto FIC & 6.5 & Good & \\
\hline $6 / 3 / 940: 00$ & Manual Tape & 0 & Suspect & Data not included in graph \\
\hline $6 / 3 / 940: 00$ & Manual FIC & 6.5 & Good & \\
\hline $6 / 4 / 940: 00$ & Manual Tape & 0 & Suspect & Data not included in graph \\
\hline $6 / 4 / 947: 04$ & Auto FIC & 6.5 & Good & \\
\hline $6 / 5 / 940: 00$ & Manual Tape & 0 & Suspect & Data not included in graph \\
\hline $6 / 5 / 947: 03$ & Auto FIC & 6.5 & Good & \\
\hline $6 / 6 / 940: 00$ & Manual Tape & 0 & Suspect & Data not included in graph \\
\hline $6 / 6 / 947: 06$ & Auto FIC & 6.5 & Good & \\
\hline $6 / 7 / 940: 00$ & Manual Tape & 0.00 & Suspect & Data not included in graph \\
\hline $6 / 7 / 947: 02$ & Auto FIC & 6.50 & Good & \\
\hline 6/8/94 0:00 & Manual Tape & 0 & Suspect & Data not included in graph \\
\hline $6 / 8 / 947: 03$ & Auto FIC & 6.50 & Good & \\
\hline $6 / 9 / 940: 00$ & Manual Tape & 0.00 & Suspect & Data not included in graph \\
\hline $6 / 9 / 940: 00$ & Manual FIC & 6.5 & Good & \\
\hline
\end{tabular}

Data obtained from WHC Surveillance Analysis Computer System, (PC SACS) July 7, 1995. 
WHC-SD-WM-ER-315, Rev. 0

\begin{tabular}{|c|c|c|c|c|}
\hline \multicolumn{5}{|c|}{ 241-AP-104 } \\
\hline & & urface Level Dat & & \\
\hline Date & Type of Moasurement & Level (Inches) & Data Quality & Comments \\
\hline $6 / 10 / 940: 00$ & Manual Tape & 0 & Suspect & Data not included in graph \\
\hline $6 / 10 / 947: 04$ & Auto FIC & 6.50 & Good & \\
\hline $6 / 11 / 940: 00$ & Manual Tape & 0.00 & Suspect & Data not included in graph \\
\hline $6 / 11 / 947: 04$ & Auto FIC & 6.5 & Good & \\
\hline $6 / 12 / 940: 00$ & Manual Tape & 0.00 & Suspect & Data not included in graph \\
\hline $6 / 12 / 947: 03$ & Auto FIC & 6.5 & Good & \\
\hline $6 / 13 / 940: 00$ & Manual Tape & 0.00 & Suspect & Data not included in graph \\
\hline $6 / 13 / 947: 02$ & Auto FIC & 6.5 & Good & \\
\hline 6/14/940:00 & Manual Tape & 0.00 & Suspect & Data not included in graph \\
\hline $6 / 14 / 947: 03$ & Auto FIC & 6.50 & Good & \\
\hline $6 / 15 / 940: 00$ & Manual Tape & 0.00 & Suspect & Data not included in graph \\
\hline $6 / 15 / 947: 03$ & Auto FIC & 6.50 & Good & \\
\hline $6 / 16 / 940: 00$ & Manual Tape & 0 & Suspect & Data not included in graph \\
\hline $6 / 16 / 947: 03$ & Auto FIC & 6.5 & Good & \\
\hline 6/17/94 0:00 & Manual Tape & 0 & Suspect & Data not included in graph \\
\hline $6 / 17 / 947: 03$ & Auto FIC & 6.5 & Good & \\
\hline $6 / 18 / 940: 00$ & Manual Tape & 0 & Suspect & Data not included in graph \\
\hline $6 / 18 / 947: 04$ & Auto FIC & 6.5 & Good & \\
\hline 6/19/94 0:00 & Manual Tape & 0 & Suspect & Data not included in graph \\
\hline $6 / 19 / 947: 04$ & Auto FIC & 6.5 & Good & \\
\hline $6 / 20 / 940: 00$ & Manual Tape & 0 & Suspect & Data not included in graph \\
\hline $6 / 20 / 947: 04$ & Auto FIC & 6.5 & Good & \\
\hline $6 / 21 / 947: 04$ & Auto FIC & 6.5 & Good & \\
\hline $6 / 22 / 947: 03$ & Auto FIC & 6.5 & Good & \\
\hline $6 / 23 / 947: 05$ & Auto FIC & 6.50 & Good & \\
\hline $6 / 24 / 947: 06$ & Auto FIC & 6.50 & Good & \\
\hline $6 / 25 / 947: 05$ & Auto FIC & 6.50 & Good & \\
\hline $6 / 26 / 947: 06$ & Auto FIC & 6.50 & Good & \\
\hline $6 / 27 / 947: 06$ & Auto FIC & 6.50 & Good & \\
\hline $6 / 28 / 947: 06$ & Auto FIC & 6.50 & Good & \\
\hline $6 / 29 / 947: 03$ & Auto FIC & 6.5 & Good & \\
\hline $6 / 30 / 947: 04$ & Auto FIC & 6.50 & Good & \\
\hline $7 / 1 / 947: 04$ & Auto FIC & 6.5 & Good & \\
\hline $7 / 2 / 947: 04$ & Auto FIC & 6.50 & Good & \\
\hline 7/3/94 7:04 & Auto FIC & 6.5 & Good & \\
\hline 7/4/94 7:04 & Auto FIC & 6.5 & Good & \\
\hline 7/5/94 7:03 & Auto FIC & 6.5 & Good & \\
\hline $7 / 6 / 947: 05$ & Auto FIC & 6.50 & Good & \\
\hline $7 / 7 / 947: 03$ & Auto FIC & 6.5 & Good & \\
\hline $7 / 8 / 947: 03$ & Auto FIC & 6.5 & Good & \\
\hline $7 / 9 / 947: 03$ & Auto FIC & 6.5 & Good & \\
\hline $7 / 10 / 947: 03$ & Auto FIC & 6.50 & Good & \\
\hline $7 / 11 / 947: 03$ & Auto FIC & 6.5 & Good & \\
\hline $7 / 12 / 947: 03$ & Auto FIC & 6.50 & Good & \\
\hline $7 / 13 / 947: 03$ & Auto FIC & 6.50 & Good & \\
\hline $7 / 14 / 947: 04$ & Auto FIC & 6.50 & Good & \\
\hline $7 / 15 / 947: 04$ & Auto FIC & 6.5 & Good & \\
\hline $7 / 16 / 947: 04$ & Auto FIC & 6.5 & Good & \\
\hline
\end{tabular}

Data obtained from WHC Surveillance Analysis Computer System, (PC SACS) July 7, 1995. 


\begin{tabular}{|c|c|c|c|c|}
\hline \multicolumn{5}{|c|}{ 241-AP-104 } \\
\hline \multicolumn{5}{|c|}{ Surface Level Data } \\
\hline Date & Type of Measurement & Leval (Inchea) & Data Quality & Comments \\
\hline $7 / 17 / 947: 04$ & Auto FIC & 6.50 & Good & \\
\hline $7 / 18 / 940: 00$ & Manual FIC & 6.50 & Good & \\
\hline $7 / 19 / 947: 03$ & Auto FIC & 6.5 & Good & \\
\hline $7 / 20 / 940: 00$ & Manual Tape & 26.25 & Suspect & Data not included in graph \\
\hline $7 / 20 / 940: 00$ & Manual FIC & 6.5 & Good & \\
\hline $7 / 20 / 947: 03$ & Auto FIC & 6.50 & Good & \\
\hline $7 / 21 / 947: 03$ & Auto FIC & 6.5 & Good & \\
\hline $7 / 22 / 940: 00$ & Manual Tape & 25 & Suspect & Data not included in graph \\
\hline $7 / 22 / 947: 03$ & Auto FIC & 6.6 & Good & \\
\hline $7 / 23 / 940: 00$ & Manual Tape & 13 & Good & \\
\hline $7 / 23 / 947: 03$ & Auto FIC & 6.50 & Good & \\
\hline $7 / 24 / 947: 03$ & Auto FIC & 6.5 & Good & \\
\hline $7 / 25 / 947: 03$ & Auto FIC & 6.5 & Good & \\
\hline $7 / 26 / 947: 03$ & Auto FIC & 6.50 & Good & \\
\hline $7 / 27 / 947: 03$ & Auto FIC & 6.50 & Good & \\
\hline $7 / 28 / 94 \quad 7: 04$ & Auto FIC & 6.5 & Good & \\
\hline $7 / 29 / 947: 04$ & Auto FIC & 6.50 & Good & \\
\hline $7 / 29 / 9412: 00$ & Manual Tape & 25 & Suspect & Data not included in graph \\
\hline 7/30/94 7:04 & Auto FIC & 6.50 & Good & \\
\hline $7 / 30 / 9412: 00$ & Manual Tape & 12.75 & Good & \\
\hline $7 / 31 / 9412: 00$ & Manual Tape & 13 & Good & \\
\hline 7/31/94 23:03 & Auto FIC & 6.5 & Good & \\
\hline $8 / 1 / 947: 03$ & Auto FIC & 6.50 & Good & \\
\hline $8 / 1 / 9412: 00$ & Manual Tape & 13.00 & Good & \\
\hline $8 / 2 / 947: 03$ & Auto FIC & 6.5 & Good & \\
\hline $8 / 2 / 9412: 00$ & Manual Tapa & 13.00 & Good & \\
\hline $8 / 3 / 947: 03$ & Auto FIC & 6.5 & Good & \\
\hline $8 / 3 / 9412: 00$ & Manual Tape & 13.00 & Good & \\
\hline $8 / 4 / 947: 02$ & Auto FIC & 0.00 & Suspect & Data not included in graph \\
\hline $8 / 4 / 9412: 00$ & Manual FIC & 6.40 & Good & \\
\hline $8 / 4 / 9412: 00$ & Manual Tape & 13.00 & Good & \\
\hline $8 / 5 / 947: 03$ & Auto FIC & 6.40 & Good & \\
\hline $8 / 5 / 9412: 00$ & Manual Tape & 13.00 & Good & \\
\hline $8 / 6 / 947: 03$ & Auto FIC & 6.4 & Good & \\
\hline $8 / 6 / 9412: 00$ & Manual FIC & 13 & Suspect & Data not included in graph \\
\hline $8 / 6 / 9412: 00$ & Manual Tape & 13 & Good & \\
\hline $8 / 7 / 947: 04$ & Auto FIC & 6.4 & Good & \\
\hline $8 / 7 / 9412: 00$ & Manual Tape & 13 & Good & \\
\hline $8 / 8 / 947: 12$ & Auto FIC & 0.00 & Suspect & Data not included in graph \\
\hline $8 / 8 / 9412: 00$ & Manual Tape & 13.38 & Good & \\
\hline 8/9/94 7:06 & Auto FIC & 6.4 & Good & \\
\hline $8 / 10 / 947: 04$ & Auto FIC & 6.4 & Good & \\
\hline $8 / 11 / 94$ 7:04 & Auto FIC & 6.50 & Good & \\
\hline $8 / 12 / 947: 04$ & Auto FIC & 6.5 & Good & \\
\hline $8 / 13 / 947: 03$ & Auto FIC & 6.4 & Good & \\
\hline $8 / 14 / 947: 06$ & Auto FIC & 6.40 & Good & \\
\hline $8 / 15 / 947: 06$ & Auto FIC & 6.4 & Good & \\
\hline $8 / 16 / 947: 06$ & Auto FIC & 6.4 & Good & \\
\hline
\end{tabular}

Data obtained from WHC Surveillance Analysis Computer System, (PC SACS) July 7, 1995. 


\begin{tabular}{|c|c|c|c|c|}
\hline \multicolumn{5}{|c|}{ 247-AP-104 } \\
\hline \multicolumn{5}{|c|}{ Surface Level Data } \\
\hline Date & Type of Measurement & Level (Inchea) & Data Quality & Comments \\
\hline $8 / 17 / 947: 06$ & Auto FIC & 6.4 & Good & \\
\hline $8 / 18 / 947: 06$ & Auto FIC & 6.40 & Good & \\
\hline $8 / 19 / 946: 06$ & Auto FIC & 6.40 & Good & \\
\hline $8 / 19 / 9412: 00$ & Manual Tape & 12.75 & Good & \\
\hline $8 / 20 / 947: 04$ & Auto FIC & 6.40 & Good & \\
\hline $8 / 20 / 9412: 00$ & Manual Tape & 13 & Good & \\
\hline $8 / 21 / 947: 04$ & Auto FIC & 6.4 & Good & \\
\hline $8 / 21 / 9412: 00$ & Manual Tape & 13.00 & Good & \\
\hline $8 / 22 / 946: 04$ & Auto FIC & 6.40 & Good & \\
\hline $8 / 22 / 9412: 00$ & Manual Tape & 13 & Good & \\
\hline $8 / 23 / 947: 04$ & Auto FIC & 6.40 & Good & \\
\hline $8 / 23 / 9412: 00$ & Manual Tape & 12.25 & Good & \\
\hline $8 / 24 / 947: 04$ & Auto FIC & 6.40 & Good & \\
\hline $8 / 24 / 9412: 00$ & Manual Tape & 13 & Good & \\
\hline $8 / 25 / 947: 03$ & Auto FIC & 6.40 & Good & \\
\hline $8 / 25 / 9412: 00$ & Manual Tape & 13.00 & Good & \\
\hline $8 / 26 / 947: 06$ & Auto FIC & 6.4 & Good & \\
\hline $8 / 26 / 9412: 00$ & Manual Tape & 13.00 & Good & \\
\hline $8 / 27 / 947: 04$ & Auto FIC & 6.4 & Good & \\
\hline $8 / 27 / 9412: 00$ & Manual Tape & 13 & Good & \\
\hline $8 / 28 / 947: 04$ & Auto FIC & 6.4 & Good & \\
\hline $8 / 28 / 9412: 00$ & Manual Tape & 13 & Good & \\
\hline $8 / 29 / 947: 04$ & Auto FIC & 6.4 & Good & \\
\hline $8 / 29 / 9412: 00$ & Manual Tape & 13 & Good & \\
\hline $8 / 30 / 947: 04$ & Auto FIC & 6.4 & Good & \\
\hline $8 / 30 / 9412: 00$ & Manual Tape & 13 & Good & \\
\hline $8 / 31 / 947: 04$ & Auto FIC & 6.40 & Good & \\
\hline $8 / 31 / 9412: 00$ & Manual Tape & 13.00 & Good & \\
\hline 9/1/94 7:04 & Auto FIC & 6.40 & Good & \\
\hline $9 / 1 / 9412: 00$ & Manual Tape & 13.00 & Good & \\
\hline $9 / 2 / 947: 06$ & Auto FIC & 6.30 & Good & \\
\hline $9 / 2 / 9412: 00$ & Manual Tape & 13 & Good & \\
\hline $9 / 3 / 9412: 00$ & Manual Tape & .13 & Good & \\
\hline $9 / 3 / 9423: 04$ & Auto FIC & 6.40 & Good & \\
\hline 9/4/94 7:04 & Auto FIC & 6.4 & Good & \\
\hline $9 / 4 / 9412: 00$ & Manual Tape & 13.00 & Good & \\
\hline 9/5/94 7:04 & Auto FIC & 6.40 & Good & \\
\hline $9 / 5 / 9412: 00$ & Manual Tape & 13 & Good & \\
\hline $9 / 6 / 947: 03$ & Auto FIC & 6.40 & Good & \\
\hline $9 / 6 / 9412: 00$ & Manual Tape & 13.00 & Good & \\
\hline $9 / 7 / 947: 03$ & Auto FIC & 6.4 & Good & \\
\hline $9 / 7 / 9412: 00$ & Manual Tape & 13 & Good & \\
\hline $9 / 8 / 947: 04$ & Auto FIC & 6.4 & Good & \\
\hline $9 / 8 / 9412: 00$ & Manual Tape & 13 & Good & \\
\hline 9/9/94 7:03 & Auto FIC & 6.40 & Good & \\
\hline $9 / 9 / 9412: 00$ & Manual Tape & 13 & Good & \\
\hline $9 / 10 / 947: 04$ & Auto FIC & 6.4 & Good & \\
\hline $9 / 10 / 9412: 00$ & Manual Tape & 13.00 & Good & \\
\hline
\end{tabular}

Data obtained from WHC Surveillance Analysis Computer System, (PC SACS) July 7, 1995. 


\begin{tabular}{|c|c|c|c|c|}
\hline Date & Type of Measurement & Level (Inches) & Data Quality & Comments \\
\hline $9 / 11 / 947: 03$ & Auto FIC & 6.4 & Good & \\
\hline $9 / 11 / 9412: 00$ & Manual Tape & 13 & Good & \\
\hline $9 / 12 / 947: 03$ & Auto FIC & 6.40 & Good & \\
\hline $9 / 12 / 9412: 00$ & Manual Tape & 13 & Good & \\
\hline $9 / 13 / 947: 02$ & Auto FIC & 6.80 & Good & \\
\hline $9 / 13 / 94 \quad 12: 00$ & Manual Tape & 13.50 & Good & \\
\hline $9 / 14 / 947: 03$ & Auto FIC & 7.2 & Good & \\
\hline $9 / 14 / 9412: 00$ & Manual Tape & 13.50 & Good & \\
\hline $9 / 15 / 947: 03$ & Auto FIC & 0 & Suspect & Data not included in graph \\
\hline $9 / 15 / 9412: 00$ & Manual FIC & 7.3 & Good & \\
\hline $9 / 15 / 9412: 00$ & Manual Tape & 13.50 & Good & \\
\hline $9 / 16 / 947: 02$ & Auto FIC & 0 & Suspect & Data not included in graph \\
\hline $9 / 16 / 9412: 00$ & Menual FIC & 7.30 & Good & \\
\hline $9 / 16 / 9412: 00$ & Manual Tape & 13.50 & Good & \\
\hline 9/17/94 7:03 & Auto FIC & 0 & Suspect & Data not included in graph \\
\hline $9 / 17 / 9412: 00$ & Manual FIC & 7.3 & Good & \\
\hline $9 / 17 / 9412: 00$ & Manual Tape & 13.25 & Good & \\
\hline $9 / 18 / 947: 02$ & Auto FIC & 0.00 & Suspect & Data not included in graph \\
\hline $9 / 18 / 9412: 00$ & Manual FIC & 6.80 & Good & \\
\hline $9 / 19 / 947: 03$ & Auto FIC & 7.30 & Good & \\
\hline $9 / 19 / 9412: 00$ & Manual Tape & 12.25 & Good & \\
\hline $9 / 20 / 947: 03$ & Auto FIC & 0 & Suspect & Data not included in graph \\
\hline $9 / 20 / 9412: 00$ & Manual FIC & 7.50 & Good & \\
\hline $9 / 20 / 9412: 00$ & Manual Tape & 13.25 & Good & \\
\hline $9 / 21 / 947: 04$ & Auto FIC & 0 & Suspect & Data nat included in graph \\
\hline $9 / 21 / 9412: 00$ & Manual FIC & $\mathbf{7 . 4 5}$ & Good & \\
\hline $9 / 21 / 9412: 00$ & Manual Tape & 13.25 & Good & \\
\hline $9 / 22 / 947: 03$ & Auto FIC & 0.00 & Suspect & Data not included in graph \\
\hline $9 / 22 / 9412: 00$ & Manual FIC & 7.40 & Good & \\
\hline $9 / 22 / 9412: 00$ & Manual Tape & 13.25 & Good & \\
\hline $9 / 23 / 947: 04$ & Auto FIC & 0 & Suspect & Data not included in graph \\
\hline $9 / 23 / 9412: 00$ & Manual FIC & 7.30 & Good & \\
\hline $9 / 23 / 9412: 00$ & Manual Tape & 13.25 & Good & \\
\hline $9 / 24 / 947: 04$ & Auto FIC & 0.00 & Suspect & Data not included in graph \\
\hline $9 / 24 / 9412: 00$ & Manual FIC & 7.45 & Good & \\
\hline $9 / 24 / 9412: 00$ & Manual Tape & 13.25 & Good & \\
\hline $9 / 25 / 947: 03$ & Auto FIC & 0.00 & Suspect & Data not included in graph \\
\hline $9 / 25 / 9412: 00$ & Manual FIC & 7.45 & Good & \\
\hline $9 / 25 / 9412: 00$ & Manual Tape & 13.25 & Good & \\
\hline $9 / 26 / 947: 04$ & Auto FIC & 0 & Suspect & Data not included in graph \\
\hline $9 / 26 / 9412: 00$ & Manual FIC & 7.3 & Good & \\
\hline $9 / 26 / 9412: 00$ & Manual Tape . & 13.125 & Good & \\
\hline $9 / 27 / 947: 04$ & Auto FIC & 0 & Suspect & Data not included in graph \\
\hline $9 / 27 / 9412: 00$ & Manual Tape & 12.50 & Suspect & Data not included in graph \\
\hline $9 / 27 / 9412: 00$ & Manual FIC & 7.45 & Good & \\
\hline $9 / 28 / 947: 03$ & Auto FIC & 7.40 & Good & \\
\hline $9 / 28 / 9412: 00$ & Manual Tape & 13.5 & Good & \\
\hline $9 / 29 / 947: 04$ & Auto FIC & 7.40 & Good & \\
\hline
\end{tabular}

Data obtained from WHC Surveillance Analysis Computer System, (PC SACS) July 7, 1995. 


\begin{tabular}{|c|c|c|c|c|}
\hline \multicolumn{5}{|c|}{ 241-AP-104 } \\
\hline & & Urface Level Date & & \\
\hline Date & Type of Measurement & Level (Inches) & Data Quality & Comments \\
\hline $9 / 29 / 94 \quad 12: 00$ & Manual Tape & 13.5 & Good & \\
\hline $9 / 30 / 947: 04$ & Auto FIC & 7.5 & Good & \\
\hline $9 / 30 / 9412: 00$ & Manual Tape & 13.25 & Good & \\
\hline $10 / 1 / 947: 06$ & Auto FIC & 7.5 & Good & \\
\hline $10 / 1 / 9412 ; 00$ & Manual Tape & 13.25 & Good & \\
\hline $10 / 2 / 947: 05$ & Auto FIC & 7.50 & Good & \\
\hline $10 / 2 / 9412: 00$ & Manual Tape & 13.25 & Good & \\
\hline $10 / 3 / 947: 04$ & Auto FIC & 7.5 & Good & \\
\hline $10 / 3 / 9412: 00$ & Manual Tape & 13.50 & Good & \\
\hline $10 / 4 / 947: 04$ & Auto FIC & 7.50 & Good & \\
\hline $10 / 5 / 947: 04$ & Auto FIC & 7.60 & Good & \\
\hline $10 / 5 / 9412: 00$ & Manual Tape & 13.25 & Good & \\
\hline $10 / 6 / 947: 04$ & Auto FIC & 6.70 & Good & \\
\hline $10 / 6 / 9412: 00$ & Manual Tape & 13.5 & Good & \\
\hline $10 / 7 / 946: 04$ & Auto FIC & 6.90 & Good & \\
\hline $10 / 7 / 9412: 00$ & Manual Tape & 13.5 & Good & \\
\hline $10 / 8 / 947: 03$ & Auto FIC & 7.00 & Good & \\
\hline $10 / 8 / 94 \quad 12: 00$ & Manual Tape & 13.25 & Good & \\
\hline $10 / 9 / 947: 06$ & Auto FIC & 7.00 & Good & \\
\hline $10 / 9 / 94 \quad 12: 00$ & Manual Tape & 13.25 & Good & \\
\hline $10 / 10 / 947: 05$ & Auto FIC & 7 & Good & . \\
\hline $10 / 10 / 9412: 00$ & Manual Tape & 13.25 & Good & \\
\hline $10 / 11 / 947: 03$ & Auto FIC & 7 & Good & \\
\hline $10 / 11 / 9412: 00$ & Manual Tape & 13.25 & Good & \\
\hline $10 / 12 / 947: 06$ & Auto FIC & 7 & Good & \\
\hline $10 / 12 / 9412: 00$ & Manual Tape & 13.25 & Good & \\
\hline $10 / 13 / 947: 05$ & Auto FIC & 7.00 & Good & \\
\hline $10 / 13 / 9412: 00$ & Manual Tape & 13.25 & Good & \\
\hline $10 / 14 / 947: 06$ & Auto FIC & 7 & Good & \\
\hline $10 / 14 / 9412: 00$ & Manual Tape & 13.25 & Good & \\
\hline $10 / 15 / 947: 06$ & Auto FIC & 7 & Good & \\
\hline $10 / 15 / 9412: 00$ & Manual Tape & 13.25 & Good & \\
\hline $10 / 16 / 947: 06$ & Auto FIC & 7 & Good & \\
\hline $10 / 16 / 9412: 00$ & Manual Tape & 13.5 & Good & \\
\hline $10 / 17 / 947: 06$ & Auto FIC & 7 & Good & \\
\hline $10 / 17 / 94 \quad 12: 00$ & Manual Tape & 13.00 & Good & \\
\hline $10 / 18 / 947: 06$ & Auto FIC & 7 & Good & \\
\hline $10 / 18 / 94 \quad 12: 00$ & Manual Tape & 13 & Good & \\
\hline $10 / 19 / 947: 06$ & Auto FIC & 7 & Good & \\
\hline $10 / 19 / 9412: 00$ & Manual Tape & 13.25 & Good & \\
\hline $10 / 20 / 945: 07$ & Auto FIC & 7.00 & Good & \\
\hline $10 / 20 / 9412: 00$ & Manual Tape & 13.25 & Good & \\
\hline $10 / 21 / 947: 06$ & Auto FIC & 7.00 & Good & \\
\hline $10 / 21 / 9412: 00$ & Manual Tape & 13.25 & Good & $\bullet$ \\
\hline $10 / 22 / 94 \quad 12: 00$ & Manual Tape & 13 & Good & \\
\hline $10 / 22 / 9423: 06$ & Auto FIC & 7.00 & Good & \\
\hline $10 / 23 / 947: 06$ & Auto FIC & 7 & Good & \\
\hline $10 / 23 / 9412: 00$ & Manual Tape & 13 & Good & \\
\hline
\end{tabular}

Data obtained from WHC Surveillance Analysis Computer System, (PC SACS) July 7, 1995. 


\begin{tabular}{|c|c|c|c|c|}
\hline \multirow{2}{*}{\multicolumn{5}{|c|}{$\begin{array}{c}\text { 241-AP-104 } \\
\text { Surfoce Level Date }\end{array}$}} \\
\hline & & & & \\
\hline Date & Type of Moasurement & Level (Incheo) & Data Quality & Comments \\
\hline $10 / 24 / 947: 06$ & Auto FIC & 7.00 & Good & \\
\hline $10 / 24 / 9412: 00$ & Manual Tape & 13.25 & Good & \\
\hline $10 / 25 / 947: 06$ & Auto FIC & 7.1 & Good & \\
\hline $10 / 25 / 9412: 00$ & Manual Tape & 13.5 & Good & \\
\hline $10 / 26 / 947: 06$ & Auto FIC & 6.80 & Good & \\
\hline $10 / 26 / 9412: 00$ & Manual Tape & 13.50 & Good & \\
\hline $10 / 27 / 947: 06$ & Auto FIC & 6.70 & Good & \\
\hline $10 / 27 / 9412: 00$ & Manual Tape & 13.5 & Good & \\
\hline $10 / 28 / 947: 04$ & Auto FIC & 6.7 & Good & \\
\hline $10 / 28 / 9412: 00$ & Manual Tape & 13.5 & Good & \\
\hline $10 / 29 / 9412: 00$ & Manual FIC & 8.2 & Good & \\
\hline $10 / 29 / 9412: 00$ & Manual Tape & 13.25 & Good & \\
\hline $10 / 30 / 9412: 00$ & Manual Tape & 13.25 & Good & \\
\hline $10 / 30 / 9423: 04$ & Auto FIC & 8.00 & Good & \\
\hline 10/31/94 7:05 & Auto FIC & 8.50 & Good & \\
\hline $10 / 31 / 9412: 00$ & Manual Tape & 13 & Good & \\
\hline $11 / 1 / 9412: 00$ & Manual Tape & 13.25 & Good & \\
\hline $11 / 1 / 9423: 04$ & Auto FIC & 16.1 & Suspect & Data not included in graph \\
\hline $11 / 2 / 947: 03$ & Auto FIC & 9.4 & Suspect & Data not included in graph \\
\hline $11 / 2 / 94 \quad 12: 00$ & Manual FIC & 6.9 & Good & \\
\hline $11 / 2 / 9412: 00$ & Manual Tape & 13.25 & Good & \\
\hline $11 / 3 / 947: 04$ & Auto FIC & 0.00 & Suspect & Data not included in graph \\
\hline $11 / 3 / 9412: 00$ & Manual FIC & 9.55 & Suspect & Data not included in graph \\
\hline $11 / 3 / 9412: 00$ & Manual Tape & 13.25 & Good & \\
\hline $11 / 4 / 947: 03$ & Auto FIC & 0.00 & Suspect & Data not included in graph \\
\hline $11 / 4 / 9412: 00$ & Manual FIC & 8.80 & Suspect & Data not included in graph \\
\hline $11 / 4 / 9412: 00$ & Manual Tape & 13.25 & Good & \\
\hline $11 / 5 / 947: 03$ & Auto FIC & 0 & Suspect & Data not included in graph \\
\hline $11 / 5 / 9412: 00$ & Manual FIC & 9.00 & Suspect & Data not included in graph \\
\hline $11 / 5 / 9412: 00$ & Manual Tape & 13.25 & Good & \\
\hline $11 / 6 / 947: 04$ & Auto FIC & 0.00 & Suspect & Data not included in graph \\
\hline $11 / 6 / 9412: 00$ & Manual FIC & 9.00 & Suspect & Data not included in graph \\
\hline $11 / 6 / 9412: 00$ & Manual Tape & 13.25 & Good & \\
\hline $11 / 7 / 947: 04$ & Auto FIC & 0.00 & Suspect & Data not included in graph \\
\hline $11 / 7 / 9412: 00$ & Manual FIC & 9.40 & Suspect & Data not included in graph \\
\hline $11 / 7 / 9412: 00$ & Manual Tape & 13 & Good & \\
\hline $11 / 8 / 947: 03$ & Auto FIC & 0.00 & Suspect & Data not included in graph \\
\hline $11 / 8 / 9412: 00$ & Manual FIC & 8.90 & Suspect & Data not included in graph \\
\hline $11 / 8 / 9412: 00$ & Manual Tape & 13 & Good & \\
\hline $11 / 9 / 947: 05$ & Auto FIC & .0 & Suspect & Data not included in graph \\
\hline $11 / 9 / 94 \quad 12: 00$ & Manual Tape & 13.25 & Good & \\
\hline $11 / 10 / 947: 03$ & Auto FIC & 12.50 & Suspect & Data not included in graph \\
\hline $11 / 10 / 9412: 00$ & Manual Tape & 13.25 & Good & \\
\hline $11 / 11 / 947: 03$ & Auto FIC & 0.00 & Suspect & Data not included in graph \\
\hline $11 / 11 / 9412: 00$ & Manual Tape & 13 & Good & \\
\hline $11 / 12 / 947: 03$ & Auto FIC & 11.5 & Suspect & Data not included in graph \\
\hline $11 / 12 / 9412: 00$ & Manual Tape & 13.25 & Good & \\
\hline $11 / 13 / 947: 04$ & Auto FIC & 13.20 & Suspect & Data not included in graph \\
\hline
\end{tabular}

Data obtained from WHC Surveillance Analysis Computer System, (PC SACS) July 7, 1995. 


\begin{tabular}{|c|c|c|c|c|}
\hline \multicolumn{5}{|c|}{ 241-AP-104 } \\
\hline \multicolumn{5}{|c|}{ Surface Level Data } \\
\hline Date & Type of Measurement & Level (Inches) & Data Quality & Commants \\
\hline $11 / 13 / 9412: 00$ & Manual Tape & 13.25 & Good & \\
\hline $11 / 14 / 947: 03$ & Auto FIC & 9.60 & Suspect & Data not included in graph \\
\hline $11 / 14 / 9412: 00$ & Manual Tape & 13 & Good & \\
\hline $11 / 15 / 947: 04$ & Auto FIC & 6.60 & Good & \\
\hline $11 / 15 / 9412: 00$ & Manual Tape & 13.00 & Good & \\
\hline $11 / 16 / 947: 06$ & Auto FIC & 6.60 & Good & \\
\hline $11 / 16 / 9412: 00$ & Manual Tape & 13 & Good & \\
\hline $11 / 17 / 947: 04$ & Auto FIC & 10.60 & Suspect & Data not included in graph \\
\hline $11 / 17 / 9412: 00$ & Manual FIC & 6.60 & Good & \\
\hline $11 / 17 / 9412: 00$ & Manual Tape & 13 & Good & \\
\hline $11 / 18 / 947: 04$ & Auto FIC & 6.7 & Good & \\
\hline $11 / 18 / 9412: 00$ & Manual Tape & 13.25 & Good & \\
\hline $11 / 19 / 947: 03$ & Auto FIC & 6.70 & Good & \\
\hline $11 / 19 / 9412: 00$ & Manual Tape & 13.00 & Good & \\
\hline $11 / 20 / 947: 03$ & Auto FIC & 16.2 & Suspect & Data not included in graph \\
\hline $11 / 21 / 947: 04$ & Auto FIC & 6.6 & Good & \\
\hline $11 / 21 / 9412: 00$ & Manual FIC & 6.60 & Good & \\
\hline $11 / 21 / 9412: 00$ & Manual Tape & 13.25 & Good & \\
\hline $11 / 22 / 947: 03$ & Auto FIC & $1.2: 1$ & Suspect & Data not included in graph \\
\hline $11 / 22 / 9412: 00$ & Manual Tape & 297.25 & Suspect & Data not included in graph \\
\hline $11 / 22 / 9412: 00$ & Manual FIC & 6.60 & Good & \\
\hline $11 / 23 / 9412: 00$ & Manual FIC & 6.6 & Good & \\
\hline $11 / 23 / 9412: 00$ & Manual Tape & 13.5 & Good & \\
\hline $11 / 24 / 9412: 00$ & Manual Tape & 13.25 & Good & \\
\hline $11 / 24 / 9423: 03$ & Auto FIC & 6.6 & Good & \\
\hline $11 / 25 / 9412: 00$ & Manual Tape & 13.25 & Good & \\
\hline $11 / 25 / 9423: 03$ & Auto FIC & 6.60 & Good & \\
\hline $11 / 26 / 947: 03$ & Auto FIC & 6.6 & Good & \\
\hline $11 / 26 / 9412: 00$ & Manual Tape & 13.25 & Good & \\
\hline $11 / 27 / 947: 04$ & Auto FIC & 6.6 & Good & \\
\hline $11 / 27 / 9412: 00$ & Manual Tape & 13.25 & Good & \\
\hline $11 / 28 / 947: 04$ & Auto FIC & 6.6 & Good & \\
\hline $11 / 28 / 9412: 00$ & Manual Tape & 13.25 & Good & \\
\hline $11 / 29 / 947: 04$ & Auto FIC & 6.60 & Good & \\
\hline $11 / 29 / 9412: 00$ & Manual Tape & 13 & Good & \\
\hline $11 / 30 / 947: 03$ & Auto FIC & 6.60 & Good & \\
\hline $11 / 30 / 9412: 00$ & Manual Tape & 13 & Good & \\
\hline $12 / 1 / 946: 05$ & Auto FIC & 6.60 & Good & \\
\hline $12 / 1 / 9412: 00$ & Manual Tape & 13.00 & Good & \\
\hline $12 / 2 / 947: 03$ & Auto FIC & 6.60 & Good & \\
\hline $12 / 2 / 9412: 00$ & Manual Tape & 13.00 & Good & \\
\hline $12 / 3 / 946: 04$ & Auto FIC & 7.7 & Suspect & Data not included in graph \\
\hline $12 / 3 / 9412: 00$ & Manual FIC & 6.6 & Good & \\
\hline $12 / 3 / 9412: 00$ & Manual Tape & 13 & Good & \\
\hline $12 / 4 / 947: 03$ & Auto FIC & 6.60 & Good & \\
\hline $12 / 4 / 9412: 00$ & Manual Tape & 13 & Good & \\
\hline $12 / 5 / 947: 03$ & Auto FIC & 8.90 & Suspect & Data not included in graph \\
\hline $12 / 5 / 9412: 00$ & Manual FIC & 6.6 & Good & \\
\hline
\end{tabular}

Data obtained from WHC Surveillance Analysis Computer System, (PC SACS) July 7, 1995. 


\begin{tabular}{|c|c|c|c|c|}
\hline \multicolumn{5}{|c|}{ 241-AP-104 } \\
\hline \multicolumn{5}{|c|}{ Surface Level Data } \\
\hline Date & Type of Measurement & Lovel (Inches) & Data Quality & Comments \\
\hline $12 / 5 / 9412: 00$ & Manual Tape & 13.5 & Good & \\
\hline $12 / 6 / 947: 04$ & Auto FIC & 6.60 & Good & \\
\hline $12 / 6 / 9412: 00$ & Manual Tape. & 13.25 & Good & \\
\hline $12 / 7 / 947: 03$ & Auto FIC & 6.60 & Good & \\
\hline $12 / 7 / 9412: 00$ & Manual Tape & 13.125 & Good & \\
\hline $12 / 8 / 945: 03$ & Auto FIC & 7.9 & Suspect & Data not included in graph \\
\hline $12 / 8 / 9412: 00$ & Manual FIC & 6.6 & Good & \\
\hline $12 / 9 / 947: 03$ & Auto FIC & 0 & Suspect & Data not included in graph \\
\hline $12 / 9 / 94 \quad 12: 00$ & Manual Tape & 13.25 & Good & \\
\hline $12 / 10 / 947: 04$ & Auto FIC & 0 & Suspect & Data not included in graph \\
\hline 12/10/94 12:00 & Manual Tape & 13.25 & Good & \\
\hline 12/11/94 7:04 & Auto FIC & 0 & Suspect & Data not included in graph \\
\hline 12/11/94 12:00 & Manual Tape & 13.125 & Good & \\
\hline $12 / 12 / 947: 03$ & Auto FIC & 0.00 & Suspect & Data not included in graph \\
\hline $12 / 12 / 9412: 00$ & Manual Tape & 13.00 & Good & \\
\hline $12 / 13 / 947: 03$ & Auto FIC & 6.70 & Good & \\
\hline $12 / 13 / 9412: 00$ & Manual Tape & 13.00 & Good & \\
\hline $12 / 14 / 947: 04$ & Auto FIC & 6.7 & Good & \\
\hline $12 / 14 / 9412: 00$ & Manual Tape & 13 & Good & \\
\hline $12 / 15 / 947: 03$ & Auto FIC & 6.7 & Good & \\
\hline $12 / 15 / 9412: 00$ & Manual Tape & 13.00 & Good & \\
\hline $12 / 16 / 947: 03$ & Auto FIC & 6.6 & Good & \\
\hline $12 / 16 / 9412: 00$ & Manual Tape & 13 & Good & \\
\hline $12 / 17 / 947: 06$ & Auto FIC & 6.70 & Good & \\
\hline 12/17/94 12:00 & Manual Tape & 13 & Good & \\
\hline $12 / 18 / 945: 03$ & Auto FIC & 9.10 & Suspect & Data not included in graph \\
\hline $12 / 18 / 9412: 00$ & Manual FIC & 6.7 & Good & \\
\hline $12 / 18 / 9412: 00$ & Manual Tape & 13 & Good & \\
\hline $12 / 19 / 947: 04$ & Auto FIC & 6.70 & Good & \\
\hline $12 / 19 / 9412: 00$ & Manual Tape & 12.75 & Good & \\
\hline 12/20/94 7:04 & Auto FIC & 6.7 & Good & \\
\hline $12 / 20 / 9412: 00$ & Manual Tape & 13.25 & Good & \\
\hline $12 / 21 / 947: 04$ & Auto FIC & 6.70 & Good & \\
\hline $12 / 21 / 9412: 00$ & Manual Tape & 13.25 & Good & \\
\hline $12 / 22 / 947: 05$ & Auto FIC & 6.7 & Good & \\
\hline $12 / 22 / 9412: 00$ & Manual Tape & 13 & Good & \\
\hline $12 / 23 / 947: 03$ & Auto FIC & 6.70 & Good & \\
\hline $12 / 23 / 9412: 00$ & Manual Tape & 13.00 & Good & \\
\hline $12 / 24 / 947: 04$ & Auto FIC & 6.7 & Good & \\
\hline $12 / 24 / 9412: 00$ & Manual Tape & 13 & Good & \\
\hline $12 / 25 / 947: 03$ & Auto FIC & 6.70 & Good & \\
\hline $12 / 25 / 94 \quad 12: 00$ & Manual Tape & 13.00 & Good & \\
\hline $12 / 26 / 947: 03$ & Auto FIC & 6.7 & Good & \\
\hline $12 / 26 / 9412: 00$ & Manual Tape & 13.00 & Good & \\
\hline $12 / 27 / 947: 04$ & Auto FIC & 6.7 & Good & \\
\hline $12 / 27 / 9412: 00$ & Manual Tape & 13.00 & Good & \\
\hline $12 / 28 / 947: 04$ & Auto FIC & 6.80 & Good & \\
\hline $12 / 28 / 9412: 00$ & Manual Tape & 13.00 & Good & \\
\hline
\end{tabular}

Data obtained from WHC Surveillance Analysis Computer System, (PC SACS) July 7, 1995. 


\begin{tabular}{|c|c|c|c|c|}
\hline \multicolumn{5}{|c|}{ 241-AP-104 } \\
\hline \multicolumn{5}{|c|}{ Surface Level Data } \\
\hline Date & Type of Messurement & Level (Inches) & Data Quality & Comments \\
\hline $12 / 29 / 947: 04$ & Auto FIC & 6.8 & Good & \\
\hline $12 / 29 / 9412: 00$ & Manual Tape & 13 & Good & \\
\hline $12 / 30 / 947: 04$ & Auto FIC & 6.50 & Good & \\
\hline $12 / 30 / 9412: 00$ & Manual Tape & 13 & Good & \\
\hline $12 / 31 / 947: 04$ & Auto FIC & 6.5 & Good & \\
\hline $12 / 31 / 9412: 00$ & Manual Tape & 13.25 & Good & \\
\hline 1/1/95 7:04 & Auto FIC & 6.50 & Good & \\
\hline $1 / 1 / 95 \quad 12: 00$ & Manual Tape & 13.25 & Good & \\
\hline 1/2/95 7:03 & Auto FIC & 6.5 & Good & \\
\hline $1 / 2 / 9512: 00$ & Manual Tape & 13.25 & Good & \\
\hline 1/3/95 7:02 & Auto FIC & 6.50 & Good & \\
\hline $1 / 3 / 9512: 00$ & Manual Tape & 12.5 & Suspect & Data not included in graph \\
\hline $1 / 4 / 957: 03$ & Auto FIC & 6.50 & Good & \\
\hline $1 / 4 / 9512: 00$ & Manual Tape & 13.5 & Good & \\
\hline $1 / 5 / 957: 04$ & Auto FIC & 6.5 & Good & \\
\hline $1 / 5 / 95 \quad 12: 00$ & Manual Tape & 13.5 & Good & - \\
\hline $1 / 6 / 957: 04$ & Auto FIC & 6.50 & Good & \\
\hline $1 / 6 / 95 \quad 12: 00$ & Manual Tape & 13.50 & Good & \\
\hline 1/7/95 7:04 & Auto FIC & 6.5 & Good & \\
\hline $1 / 7 / 95 \quad 12: 00$ & Manual Tape & 13.25 & Good & \\
\hline $1 / 8 / 95 \quad 12: 00$ & Manual Tape & 12.25 & Suspect & Data not included in graph \\
\hline 1/8/95 23:03 & Auto FIC & 6.5 & Good & \\
\hline 1/9/95 7:05 & Auto FIC & 6.5 & Good & \\
\hline $1 / 9 / 9512: 00$ & Manual Tape & 13.00 & Good & \\
\hline 1/10/95 7:06 & Auto FIC & 6.5 & Good & . \\
\hline $1 / 10 / 9512: 00$ & Manual Tape & 13.00 & Good & \\
\hline $1 / 11 / 957: 07$ & Auto FIC & 6.50 & Good & $\dot{.}$ \\
\hline $1 / 11 / 9512: 00$ & Manual Tape & 13 & Good & \\
\hline $1 / 12 / 957: 07$ & Auto FIC & 6.50 & Good & \\
\hline 1/12/95 12:00 & Manual Tape & 13.00 & Good & \\
\hline $1 / 13 / 956: 07$ & Auto FIC & 6.50 & Good & $\cdot$ \\
\hline $1 / 13 / 9512: 00$ & Manual Tape & 13.00 & Good & \\
\hline $1 / 14 / 95 \quad 7: 07$ & Auto FIC & 7.3 & Suspect & Data not included in graph \\
\hline $1 / 14 / 9512: 00$ & Manual FIC & 6.55 & Good & $\cdot$ \\
\hline $1 / 14 / 95 \quad 12: 00$ & Manual Tape & 13 & Good & \\
\hline $1 / 15 / 957: 06$ & Auto FIC & 6.50 & Good & \\
\hline $1 / 15 / 95 \quad 12: 00$ & Manual Tape & 13 & Good & \\
\hline 1/16/95 7:08 & Auto FIC & 6.5 & Good & \\
\hline $1 / 16 / 95 \quad 12: 00$ & Manual Tape & 13.00 & Good & \\
\hline $1 / 17 / 956: 07$ & Auto FIC & 6.50 & Good & \\
\hline $1 / 17 / 9512: 00$ & Manual Tape & 13 & Good & \\
\hline $1 / 18 / 957: 07$ & Auto FIC & 6.50 & Good & \\
\hline $1 / 18 / 9512: 00$ & Manual Tape & 13 & Good & \\
\hline $1 / 19 / 957: 07$ & Auto FIC & 6.5 & Good & \\
\hline $1 / 19 / 95 \quad 12: 00$ & Manual Tape & 13.00 & Good & \\
\hline $1 / 20 / 957: 03$ & Auto FIC & 6.5 & Good & \\
\hline 1/20/95 $12: 00$ & Manual Tape & 13 & Good & \\
\hline $1 / 21 / 957: 04$ & Auto FIC & 6.5 & Good & \\
\hline
\end{tabular}

Data obtained from WHC Surveillance Analysis Computer System, (PC SACS) July 7, 1995. 


\begin{tabular}{|c|c|c|c|c|}
\hline \multicolumn{5}{|c|}{ 241-AP-104 } \\
\hline & & Irface Level Date & & \\
\hline Date & Type of Measurement & Lovel (Inches) & Data Quality & Comments \\
\hline $1 / 21 / 9512: 00$ & Manual Tape & 13 & Good & \\
\hline $1 / 22 / 957: 03$ & Auto FIC & 6.50 & Good & \\
\hline 1/22/95 12:00 & Manual Tape & 13.00 & Good & \\
\hline $1 / 23 / 957: 03$ & Auto FIC & 6.50 & Good & \\
\hline $1 / 23 / 9512: 00$ & Manual Tape & 13.00 & Good & \\
\hline $1 / 24 / 957: 04$ & Auto FIC & 6.5 & Good & \\
\hline $1 / 24 / 9512: 00$ & Manual Tape & 13 & Good & \\
\hline 1/25/95 7:03. & Auto FIC & 6.5 & Good & \\
\hline $1 / 25 / 95 \quad 12: 00$ & Manual Tape & 13 & Good & \\
\hline $1 / 26 / 957: 03$ & Auto FIC & 6.50 & Good & \\
\hline $1 / 26 / 95 \quad 12: 00$ & Manual Tape & 13.00 & Good & - \\
\hline $1 / 27 / 957: 03$ & Auto FIC & 6.5 & Good & \\
\hline $1 / 27 / 9512: 00$ & Manual Tape & 13.00 & Good & \\
\hline $1 / 28 / 957: 04$ & Auto FIC & 6.50 & Good & \\
\hline $1 / 28 / 9512: 00$ & Manual Tape & 13.00 & Good & \\
\hline $1 / 29 / 957: 04$ & Auto FIC & 6.50 & Good & \\
\hline $1 / 29 / 95 \quad 12: 00$ & Manual Tape & 13.00 & Good & \\
\hline $1 / 30 / 957: 04$ & Auto FIC & 6.6 & Good & \\
\hline $1 / 30 / 9512: 00$ & Manual Tape & 13 & Good & \\
\hline $1 / 31 / 957: 03$ & Auto FIC & 6.50 & Good & \\
\hline $1 / 31 / 95 \quad 12: 00$ & Manual Tape & 13 & Good & \\
\hline 2/1/95 7:03 & Auto FIC & 8.60 & Good & \\
\hline 2/1/95 12:00 & Manual Tape & 13.00 & Good & \\
\hline 2/2/95 7:04 & Auto FIC & 6.5 & Good & \\
\hline 2/2/95 12:00 & Manual Tape & 13.00 & Good & \\
\hline 2/3/95 7:03 & Auto FIC & 6.50 & Good & \\
\hline 2/3/95 12:00 & Manual Tape & 13.00 & Good & \\
\hline 2/4/95 7:04 & Auto FIC & 6.60 & Good & \\
\hline 2/4/95 12:00 & Manual Tape & 13 & Good & \\
\hline 2/5/95 7:03 & Auto FIC & 6.60 & Good & \\
\hline 2/5/95 12:00 & Manual Tape & 13 & Good & $\therefore$ \\
\hline 2/6/95 7:03 & Auto FIC & 6.60 & Good & \\
\hline $2 / 6 / 95 \quad 12: 00$ & Manual Tape & 13.25 & Good & \\
\hline 2/7/95 7:03 & Auto FIC & 6.60 & Good & \\
\hline $2 / 7 / 9512: 00$ & Manual Tape & 13.00 & Good & \\
\hline $2 / 8 / 957: 04$ & Auto FIC & 6.60 & Good & \\
\hline $2 / 8 / 95 \quad 12: 00$ & Manual Tape & 13 & Good & \\
\hline 2/9/95 7:06 & Auto FIC & 6.6 & Good & \\
\hline $2 / 9 / 95 \quad 12: 00$ & Manual Tape & 13 & Good & \\
\hline $2 / 10 / 95$ 7:06 & Auto FIC & 6.6 & Good & \\
\hline $2 / 10 / 9512: 00$ & Manual Tape & 13 & Good & \\
\hline $2 / 11 / 957: 07$ & Auto FIC & 6.6 & Good & \\
\hline 2/11/95 12:00 & Manual Tape & .13 & Good & \\
\hline $2 / 12 / 957: 06$ & Auto FIC & 6.60 & Good & \\
\hline $2 / 12 / 95 \quad 12: 00$ & Manual Tape & 13 & Good & \\
\hline $2 / 13 / 957: 05$ & Auto FIC & 6.6 & Good & \\
\hline $2 / 13 / 95 \quad 12: 00$ & Manual Tape & 13.25 & Good & \\
\hline $2 / 14 / 957: 06$ & Auto FIC & 6.6 & Good & \\
\hline
\end{tabular}

Data obtained from WHC Surveillance Analysis Computer System, (PC SACS) July 7, 1995. 


\begin{tabular}{|c|c|c|c|c|}
\hline \multicolumn{5}{|c|}{ 241-AP-104 } \\
\hline \multicolumn{5}{|c|}{ Surface Lavel Data } \\
\hline Date & Type of Mosaurement & Level (Inches) & Data Quality & Comments \\
\hline 2/14/95 12:00 & Manual Tape & 13.25 & Good & \\
\hline $2 / 15 / 957: 07$ & Auto FIC & 6.6 & Good & \\
\hline $2 / 15 / 95 \quad 12: 00$ & Manual Tape & 13 & Good & \\
\hline 2/16/95 7:06 & Auto FIC & 6.60 & Good & \\
\hline $2 / 16 / 9512: 00$ & Manual Tape & 13 & Good & \\
\hline 2/17/95 7:06 & Auto FIC & 6.6 & Good & \\
\hline $2 / 17 / 9512: 00$ & Manual Tape & 13.00 & Good & \\
\hline $2 / 18 / 957: 06$ & Auto FIC & 6.60 & Good & \\
\hline $2 / 18 / 9512: 00$ & Manual Tape & 13.00 & Good & \\
\hline 2/19/95 7:06 & Auto FIC & 6.60 & Good & \\
\hline $2 / 19 / 9512: 00$ & Manual Tape & 13.00 & Good & \\
\hline $2 / 20 / 95 \quad 7: 07$ & Auto FIC & 6.60 & Good & \\
\hline $2 / 20 / 9512: 00$ & Manual Tape & 13.00 & Good & \\
\hline $2 / 21 / 957: 06$ & Auto FIC & 6.60 & Good & \\
\hline $2 / 21 / 9512: 00$ & Manual Tape & 13 & Good & \\
\hline 2/22/95 7:06 & Auto FIC & 6.6 & Good & \\
\hline $2 / 22 / 95 \quad 12: 00$ & Manual Tape & 13 & Good & \\
\hline $2 / 23 / 957: 07$ & Auto FIC & 6.60 & Good & \\
\hline $2 / 23 / 9512: 00$ & Manual Tape & 4 & Suspect & Data not included in graph \\
\hline 2/24/95 1:06 & Auto FIC & 6.70 & Good & \\
\hline $2 / 24 / 9512: 00$ & Manual Tape & 6.50 & Good & \\
\hline $2 / 25 / 957: 06$ & Auto FIC & 6.9 & Suspect & Data not included in graph \\
\hline $2 / 25 / 95 \quad 12: 00$ & Manual FIC & 6.70 & Good & \\
\hline $2 / 26 / 956: 07$ & Auto FIC & 6.7 & Good & \\
\hline $2 / 26 / 9512: 00$ & Manual Tape & 6.5 & Good & \\
\hline $2 / 27 / 957: 06$ & Auto FIC & 6.70 & Good & \\
\hline $2 / 27 / 95 \quad 12: 00$ & Manual Tape & 6.5 & Good & \\
\hline $2 / 28 / 955: 07$ & Auto FIC & 6.7 & Good & \\
\hline $2 / 28 / 95 \quad 12: 00$ & Manual Tape & 4 & Suspect & Data not included in graph \\
\hline 3/1/95 7:06 & Auto FIC & 6.70 & Good & \\
\hline $3 / 1 / 95 \quad 12: 00$ & Manual Tape & 6.50 & Good & \\
\hline $3 / 2 / 957: 06$ & Auto FIC & 6.70 & Good & \\
\hline $3 / 2 / 95 \quad 12: 00$ & Manual Tape & 6.5 & Good & \\
\hline 3/3/95 7:06 & Auto FIC & 6.7 & Good & \\
\hline $3 / 3 / 95 \quad 12: 00$ & Manual Tape & 6.50 & Good & \\
\hline 3/4/95 7:06 & Auto FIC & 6.7 & Good & \\
\hline $3 / 4 / 95 \quad 12: 00$ & Manuat Tape & 6.50 & Good & \\
\hline $3 / 5 / 957: 06$ & Auto FIC & 6.7 & Good & \\
\hline $3 / 5 / 95 \quad 12: 00$ & Manual Tape & 6.5 & Good & \\
\hline $3 / 6 / 95 \quad 7: 06$ & Auto FIC & 6.70 & Good & \\
\hline $3 / 6 / 95 \quad 12: 00$ & Manual Tape & 6.5 & Good & \\
\hline $3 / 7 / 95$ 7:06 & Auto FIC & 6.8 & Good & \\
\hline 3/7/95 12:00 & Manual Tape & 6.5 & Good & \\
\hline $3 / 8 / 957: 03$ & Auto FIC & 6.70 & Good & \\
\hline $3 / 8 / 9512: 00$ & Manual Tape & 6.50 & Good & \\
\hline 3/9/95 7:03 & Auto FIC & 6.7 & Good & \\
\hline 3/9/95 12:00 & Manual Tape & 6.5 & Good & \\
\hline $3 / 10 / 957: 02$ & Auto FIC & 6.7 & Good & \\
\hline
\end{tabular}

Data obtained from WHC Surveillance Analysis Computer System, (PC SACS) July 7, 1995. 


\begin{tabular}{|c|c|c|c|c|}
\hline \multicolumn{5}{|c|}{ 241-AP-104 } \\
\hline & & Uface Level Dat & & \\
\hline Date & Type of Measurement & Level (Inchea) & Data Quality & Comments \\
\hline $3 / 10 / 95 \quad 12: 00$ & Manual Tape & 6.5 & Good & \\
\hline $3 / 11 / 957: 03$ & Auto FIC & 6.70 & Good & \\
\hline $3 / 11 / 9512: 00$ & Manual Tape & 6.75 & Good & \\
\hline $3 / 12 / 957: 03$ & Auto FIC & 6.6 & Good & \\
\hline $3 / 12 / 95 \quad 12: 00$ & Manual Tape & 6.5 & Good & \\
\hline $3 / 13 / 957: 02$ & Auto FIC & 6.50 & Good & \\
\hline $3 / 13 / 95 \quad 12: 00$ & Manual Tape & 6.50 & Good & \\
\hline $3 / 14 / 957: 03$ & Auto FIC & 6.6 & Good & \\
\hline $3 / 14 / 9512: 00$ & Manual Tape & 6.50 & Good & \\
\hline 3/15/95 7:05 & Auto FIC & 6.60 & Good & \\
\hline $3 / 15 / 9512: 00$ & Manual Tape & 6.5 & Good & \\
\hline $3 / 16 / 95 \quad 7: 03$ & Auto FIC & 6.6 & Good & \\
\hline $3 / 16 / 9512: 00$ & Manual Tape & 6.5 & Good & \\
\hline 3/17/95 7:03 & Auto FIC & 6.6 & Good & \\
\hline $3 / 17 / 9512: 00$ & Manual Tape & 6.50 & Good & \\
\hline $3 / 18 / 957: 03$ & Auto FIC & 6.6 & Good & \\
\hline $3 / 18 / 9512: 00$ & Manual Tape & 6.5 & Good & \\
\hline $3 / 19 / 95$ 7:03 & Auto FIC & 6.60 & Good & \\
\hline 3/19/95 12:00 & Manual Tape & 6.50 & Good & \\
\hline $3 / 20 / 956: 03$ & Auto FIC & 6.60 & Good & \\
\hline $3 / 20 / 95 \quad 12: 00$ & Manual Tape & 6.50 & Good & \\
\hline $3 / 21 / 957: 03$ & Auto FIC & 6.6 & Good & \\
\hline $3 / 21 / 9512: 00$ & Manual Tape & 6.5 & Good & \\
\hline $3 / 22 / 957: 02$ & Auto FIC & 6.60 & Good & \\
\hline $3 / 22 / 95.12: 00$ & Manual Tape & 6.5 & Good & \\
\hline $3 / 23 / 95 \quad 7: 03$ & Auto FIC & 6.6 & Good & \\
\hline $3 / 23 / 95 \quad 12: 00$ & Manual Tape & 6.5 & Good & \\
\hline $3 / 24 / 957: 03$ & Auto FIC & 6.60 & Good & \\
\hline $3 / 24 / 9512: 00$ & Manual Tape & 6.50 & Good & \\
\hline $3 / 25 / 95$ 7:02 & Auto FIC & 6.60 & Good & \\
\hline $3 / 25 / 9512: 00$ & Manual Tape & 6.5 & Good & \\
\hline $3 / 26 / 95 \quad 7: 02$ & Auto FIC & 6.60 & Good & \\
\hline $3 / 26 / 95 \quad 12: 00$ & Manual Tape & 6.5 & Good & \\
\hline $3 / 27 / 957: 03$ & Auto FIC & 6.6 & Good & \\
\hline $3 / 27 / 95 \quad 12: 00$ & Manual Tape & 6.50 & Good & \\
\hline $3 / 28 / 957: 03$ & Auto FIC & 6.60 & Good & \\
\hline $3 / 28 / 95 \quad 12: 00$ & Manual Tape & 6.50 & Good & \\
\hline $3 / 29 / 957: 03$ & Auto FIC & 6.6 & Good & \\
\hline $3 / 29 / 9512: 00$ & Manual Tape & 6.50 & Good & \\
\hline 3/30/95 7:03 & Auto FIC & 6.60 & Good & \\
\hline $3 / 30 / 95 \quad 12: 00$ & Manual Tape & 6.5 & Good & \\
\hline $3 / 31 / 957: 04$ & Auto FIC & 6.60 & Good & \\
\hline $3 / 31 / 9512: 00$ & Manual Tape & 6.50 & Good & \\
\hline 4/1/95 7:02 & Auto FIC & 6.6 & Good & \\
\hline $4 / 1 / 95 \quad 12: 00$ & Manual Tape & 6.5 & Good & \\
\hline 4/2/95 7:03 & Auto FIC & 6.6 & Good & \\
\hline 4/2/95 $12: 00$ & Manual Tape & 6.50 & Good & \\
\hline 4/3/95 7:03 & Auto FIC & 6.6 & Good & \\
\hline
\end{tabular}

Data obtained from WHC Surveillance Analysis Computer System, (PC SACS) July 7, 1995. 


\begin{tabular}{|c|c|c|c|c|}
\hline \multicolumn{5}{|c|}{ 247-AP-104 } \\
\hline \multicolumn{5}{|c|}{ Surface Level Data } \\
\hline Date & Type of Measurement & Lavel (Inches) & Data Quality & Comments \\
\hline $4 / 3 / 95 \quad 12: 00$ & Manual Tape & 6.5 & Good & \\
\hline 4/4/95 7:03 & Auto FIC & 6.60 & Good & \\
\hline $4 / 4 / 95 \quad 12: 00$ & Manual Tape & 6.75 & Good & \\
\hline 4/5/95 7:03 & Auto FIC & 6.60 & Good & \\
\hline $4 / 5 / 95 \quad 12: 00$ & Manual Tape & 6.5 & Good & \\
\hline 4/6/95 7:03 & Auto FIC & 6.4 & Good & \\
\hline $4 / 6 / 95 \quad 12: 00$ & Manual Tape & 6.5 & Good & \\
\hline 4/7/95 7:03 & Auto FIC & 6.40 & Good & \\
\hline $4 / 7 / 9512: 00$ & Manual Tape & 6.50 & Good & \\
\hline 4/8/95 7:02 & Auto FIC & 6.4 & Good & \\
\hline $4 / 9 / 95 \quad 12: 00$ & Manual Tape & 6.5 & Good & \\
\hline 4/9/95 23:03 & Auto FIC & 6.4 & Good & \\
\hline $4 / 10 / 957: 02$ & Auto FIC & 6.40 & Good & \\
\hline $4 / 10 / 9512: 00$ & Manual Tape & 6.50 & Good & \\
\hline $4 / 11 / 957: 04$ & Auto FIC & 6.40 & Good & \\
\hline $4 / 11 / 9512: 00$ & Manual Tape & 6.50 & Good & \\
\hline 4/12/95 7:03 & Auto FIC & 6.40 & Good & \\
\hline 4/12/95 12:00 & Manual Tape & 6.5 & Good & \\
\hline 4/13/95 7:05 & Auto FIC & 6.4 & Good & \\
\hline $4 / 13 / 9512: 00$ & Manual Tape & 6.5 & Good & \\
\hline 4/14/95 7:03 & Auto FIC & 6.4 & Good & \\
\hline $4 / 14 / 95 \quad 12: 00$ & Manual Tape & 6.50 & Good & \\
\hline 4/15/95 7:03 & Auto FIC & 6.4 & Good & \\
\hline $4 / 15 / 9512: 00$ & Manual Tape & 6.50 & Good & \\
\hline 4/16/95 7:03 & Auto FIC & 6.40 & Good & \\
\hline $4 / 16 / 95 \quad 12: 00$ & Manual Tape & 6.5 & Good & \\
\hline $4 / 17 / 957: 03$ & Auto FIC & 6.4 & Good & \\
\hline $4 / 17 / 9512: 00$ & Manual Tape & 6.50 & Good & \\
\hline 4/18/95 7:03 & Auto FIC & 6.40 & Good & $\cdot$ \\
\hline 4/18/95 12:00 & Manual Tape & 6.5 & Good & \\
\hline 4/19/95 7:03 & Auto FIC & 6.4 & Good & \\
\hline 4/19/95 12:00 & Manual Tape & 6.50 & Good & \\
\hline $4 / 20 / 957: 03$ & Auto FIC & 6.40 & Good & \\
\hline $4 / 20 / 9512: 00$ & Manual Tape & 6.5 & Good & \\
\hline 4/21/95 7:03 & Auto FIC & 6.4 & Good & \\
\hline 4/21/95 12:00 & Manual Tape & 6.50 & Good & \\
\hline 4/22/95 7:03 & Auto FIC & 6.4 & Good & \\
\hline $4 / 22 / 95 \quad 12: 00$ & Manual Tape & 6.5 & Good & \\
\hline $4 / 23 / 957: 03$ & Auto FIC & 6.40 & Good & \\
\hline $4 / 23 / 9512: 00$ & Manual Tape & 6.5 & Good & \\
\hline 4/24/95 7:03 & Auto FIC & 6.40 & Good & \\
\hline $4 / 24 / 9512: 00$ & Manual Tape & 6.5 & Good & \\
\hline $4 / 25 / 957: 03$ & Auto FIC & 6.4 & Good & \\
\hline $4 / 25 / 9512: 00$ & Manual Tape & 6.5 & Good & \\
\hline 4/26/95 7:03 & Auto FIC & 6.40 & Good & \\
\hline 4/26/95 12:00 & Manual Tape & 6.50 & Good & \\
\hline $4 / 27 / 957: 03$ & Auto FIC & 6.4 & Good & \\
\hline 4/27/95 12:00 & Manual Tape & 18.50 & Suspect & Data not included in graph \\
\hline
\end{tabular}

Data obtained from WHC Surveiliance Analysis Computer Systern, (PC SACS) July 7, 1995. 


\begin{tabular}{|c|c|c|c|c|}
\hline \multicolumn{5}{|c|}{ 241-AP-104 } \\
\hline \multicolumn{5}{|c|}{ Surface Leval Data } \\
\hline Date & Type of Measurement & Level finchea) & Data Cuality & Comments \\
\hline 4/28/95 7:03 & Auto FIC & 6.40 & Good & \\
\hline $4 / 28 / 95 \quad 12: 00$ & Manual Tape & 18.5 & Suspect & Data not included in graph \\
\hline $4 / 29 / 957: 03$ & Auto FIC & 6.40 & Good & \\
\hline $4 / 29 / 9512: 00$ & Manual Tape & 6.75 & Good & \\
\hline 4/30/95 7:03 & Auto FIC & 6.40 & Good & \\
\hline 4/30/95 $12: 00$ & Manual Tape & 6.75 & Good & \\
\hline 5/1/95 7:03 & Auto FIC & 6.40 & Good & \\
\hline $5 / 1 / 9512: 00$ & Manual Tape & 6.5 & Good & \\
\hline 5/2/95 7:02 & Auto FIC & 6.40 & Good & \\
\hline $5 / 2 / 9512: 00$ & Manual Tape & 6.50 & Good & \\
\hline 5/3/95 7:03 & Auto FIC & 6.40 & Good & \\
\hline $5 / 3 / 9512: 00$ & Manual Tape & 6.50 & Good & \\
\hline 5/4/95 7:04 & Auto FIC & 6.4 & Good & \\
\hline $5 / 4 / 95 \quad 12: 00$ & Manual Tape & 6.5 & Good & \\
\hline 5/5/95 7:04 & Auto FIC & 6.4 & Good & \\
\hline $5 / 5 / 95 \quad 12: 00$ & Manual Tape & 6.50 & Good & \\
\hline 5/6/95 7:03 & Auto FIC & 6.4 & Good & \\
\hline $5 / 6 / 9512: 00$ & Manual Tape & 6.5 & Good & \\
\hline 5/7/95 7:04 & Auto FIC & 6.4 & Good & \\
\hline $5 / 7 / 95 \quad 12: 00$ & Manual Tape & 6.50 & Good & \\
\hline 5/8/95 7:04 & Auto FIC & 6.4 & Good & \\
\hline $5 / 8 / 95 \quad 12: 00$ & Manual Tape & 6.75 & Good & \\
\hline 5/9/95 7:04 & Auto FIC & 6.4 & Good & \\
\hline $5 / 9 / 95 \quad 12: 00$ & Manual Tape & 6.75 & Good & \\
\hline $5 / 10 / 957: 04$ & Auto FIC & 6.40 & Good & \\
\hline $5 / 10 / 95 \quad 12: 00$ & Manual Tape & 6.5 & Good & \\
\hline $5 / 11 / 957: 03$ & Auto FIC & 6.4 & Good & \\
\hline $5 / 11 / 9512: 00$ & Manual Tape & 6.5 & Good & \\
\hline 5/12/95 7:06 & Auto FIC & 6.4 & Good & \\
\hline $5 / 12 / 95 \quad 12: 00$ & Manual Tape & 6.5 & Good & \\
\hline $5 / 13 / 957: 03$ & Auto FIC & 6.40 & Good & \\
\hline $5 / 13 / 95 \quad 12: 00$ & Manual Tape & 6.50 & Good & \\
\hline $5 / 14 / 9567: 03$ & Auto FIC & 6.40 & Good & \\
\hline $5 / 14 / 95 \quad 12: 00$ & Manual Tape & 6.5 & Good & \\
\hline $5 / 15 / 957: 03$ & Auto FIC & 6.40 & Good & \\
\hline $5 / 15 / 9512: 00$ & Manual Tape & 6.5 & Good & \\
\hline $5 / 16 / 957: 03$ & Auto FIC & 6.40 & Good & \\
\hline $5 / 16 / 95 \quad 12: 00$ & Manual Tape & 6.5 & Good & \\
\hline $5 / 17 / 957: 02$ & Auto FIC & 6.40 & Good & \\
\hline $5 / 17 / 95 \quad 12: 00$ & Manual Tape & 6.5 & Good & \\
\hline $5 / 18 / 956: 02$ & Auto FIC & 6.4 & Good & \\
\hline $5 / 18 / 95 \quad 12: 00$ & Manual Tape & 6.5 & Good & \\
\hline $5 / 19 / 95 \quad 7: 05$ & Auto FIC & 6.40 & Good & \\
\hline $5 / 19 / 95 \quad 12: 00$ & Manual Tape & 6.25 & Good & \\
\hline $5 / 20 / 957: 05$ & Auto FIC & 6.4 & Good & \\
\hline $5 / 20 / 95 \quad 12: 00$ & Manual Tape & 6.50 & Good & \\
\hline $5 / 21 / 957: 05$ & Auto FIC & 6.40 & Good & \\
\hline $5 / 21 / 95 \quad 12: 00$ & Manual Tape & 6.50 & Good & \\
\hline
\end{tabular}

Data obtained from WHC Surveillance Analysis Computer System, (PC SACS) July 7, 1995. 
WHC-SD-WM-ER-315, Rev. 0

\begin{tabular}{|c|c|c|c|c|}
\hline \multirow{2}{*}{\multicolumn{5}{|c|}{$\begin{array}{c}\text { 241-AP-104 } \\
\text { Surface Level Data }\end{array}$}} \\
\hline & & Ifface Level Dat & & \\
\hline Date & Type of Measurement & Level (inches) & Data Quality & Comments \\
\hline $5 / 22 / 957: 05$ & Auto FIC & 6.5 & Good & \\
\hline $5 / 22 / 9512: 00$ & Manual Tape & 6.50 & Good & \\
\hline $5 / 23 / 957: 11$ & Auto FIC & 0 & Suspect & Data not included in graph \\
\hline $5 / 23 / 9512: 00$ & Manual FIC & 6.50 & Good & \\
\hline $5 / 23 / 95 \quad 12: 00$ & Manual Tape & 6.50 & Good & \\
\hline $5 / 24 / 957: 10$ & Auto FIC & 0 & Suspect & Data not included in graph \\
\hline $5 / 24 / 95 \quad 12: 00$ & Manual FIC & 6.5 & Good & \\
\hline $5 / 24 / 95 \quad 12: 00$ & Manual Tape & 6.75 & Good & \\
\hline $5 / 25 / 957: 10$ & Auto FIC & 0.00 & Suspect & Data not included in graph \\
\hline $5 / 25 / 9512: 00$ & Manual FIC & 6.50 & Good & \\
\hline $5 / 25 / 9512: 00$ & Manual Tape & 6.75 & Good & \\
\hline $5 / 26 / 957: 04$ & Auto FIC & 6.5 & Good & \\
\hline $5 / 26 / 9512: 00$ & Manual Tape & 6.5 & Good & \\
\hline $5 / 27 / 957: 05$ & Auto FIC & 6.5 & Good & \\
\hline $5 / 27 / 9512: 00$ & Manual Tape & 6.75 & Good & \\
\hline 5/28/95 7:05 & Auto FIC & 6.50 & Good & \\
\hline $5 / 28 / 9512: 00$ & Manual Tape & 6.75 & Good & \\
\hline 5/29/95 7:05 & Auto FIC & 6.5 & Good & \\
\hline $5 / 29 / 9512: 00$ & Manual Tape & 6.75 & Good & \\
\hline $5 / 30 / 957: 05$ & Auto FIC & 6.5 & Good & \\
\hline $5 / 30 / 95 \quad 12: 00$ & Manual Tape & 6.75 & Good & \\
\hline $5 / 31 / 957: 05$ & Auto FIC & 6.50 & Good & \\
\hline $5 / 31 / 9512: 00$ & Manual Tape & 6.50 & Good & \\
\hline $6 / 1 / 957: 05$ & Auto FIC & 6.50 & Good & \\
\hline $6 / 1 / 9512: 00$ & Manual Tape & 6.50 & Good & \\
\hline $6 / 2 / 957: 05$ & Auto FIC & 6.50 & Good & \\
\hline $6 / 2 / 9512: 00$ & Manual Tape & 6.5 & Good & \\
\hline $6 / 3 / 95 \quad 12: 00$ & Manual Tape & 6.50 & Good & \\
\hline 6/3/95 23:05 & Auto FIC & 6.50 & Good & \\
\hline $6 / 4 / 957: 05$ & Auto FIC & 6.5 & Good & \\
\hline $6 / 4 / 95 \quad 12: 00$ & Manual Tape & 6.50 & Good & \\
\hline $6 / 5 / 957: 05$ & Auto FIC & $6 . \overline{50}$ & Good & \\
\hline $6 / 5 / 9512: 00$ & Manual Tape & 6.5 & Good & \\
\hline $6 / 6 / 95 \quad 7: 05$ & Auto FIC & 6.50 & Good & \\
\hline $6 / 6 / 95 \quad 12: 00$ & Manual Tape & 6.25 & Good & \\
\hline $6 / 7 / 957: 05$ & Auto FIC & 8.10 & Good & \\
\hline $6 / 7 / 95 \quad 12: 00$ & Manual Tape & 9.00 & Good & \\
\hline $6 / 8 / 95 \quad 7: 05$ & Auto FIC & 8.1 & Good & \\
\hline $6 / 8 / 95 \quad 12: 00$ & Manual Tape & 8.00 & Good & \\
\hline 6/9/95 7:05 & Auto FIC & 8.1 & Good & \\
\hline $6 / 9 / 9512: 00$ & Manual Tape & 8 & Good & \\
\hline $6 / 10 / 957: 05$ & Auto FIC & 8.1 & Good & \\
\hline $6 / 10 / 95 \quad 12: 00$ & Manual Tape & 8 & Good & \\
\hline $6 / 11 / 957: 05$ & Auto FIC & 8.1 & Good & \\
\hline $6 / 11 / 9512: 00$ & Manual Tape & 8 & Good & \\
\hline $6 / 12 / 957: 05$ & Auto FIC & 8.10 & Good & \\
\hline $6 / 12 / 95 \quad 12: 00$ & Manual Tape & 8.25 & Good & \\
\hline $6 / 13 / 957: 05$ & Auto FIC & 8.1 & Good & \\
\hline
\end{tabular}

Data obtained from WHC Surveillance Analysis Computer System, (PC SACS) July 7, 1995. 


\begin{tabular}{|c|c|c|c|c|}
\hline \multicolumn{5}{|c|}{ 241-AP-104 } \\
\hline \multicolumn{5}{|c|}{ Surface Levol Date } \\
\hline Date & Type of Measurement & Level (Inchea) & Data Quality & Comments \\
\hline $6 / 13 / 9512: 01$ & Manual Tape & 8 & Good & \\
\hline $6 / 14 / 957: 05$ & Auto FIC & 8.1 & Good & \\
\hline $6 / 14 / 9512: 01$ & Manual Tape & 8.25 & Good & \\
\hline $6 / 15 / 957: 05$ & Auto FIC & 8.1 & Good & \\
\hline $6 / 15 / 9512: 00$ & Manual Tape & 8.25 & Good & \\
\hline $6 / 16 / 957: 05$ & Auto FIC & 8.10 & Good & \\
\hline $6 / 16 / 9512: 00$ & Manual Tape & 8.00 & Good & \\
\hline $6 / 17 / 957: 05$ & Auto FIC & 8.10 & Good & . \\
\hline $6 / 17 / 9512: 00$ & Manual Tape & 8.25 & Good & \\
\hline $6 / 18 / 957: 05$ & Auto FIC & 8.10 & Good & \\
\hline $6 / 18 / 9512: 00$ & Manual Tape & 8.25 & Good & \\
\hline $6 / 19 / 957: 06$ & Auto FIC & 8.1 & Good & \\
\hline $6 / 19 / 9512: 00$ & Manual Tape & 8.00 & Good & \\
\hline $6 / 20 / 957: 06$ & Auto FIC & 8.10 & Good & \\
\hline $6 / 20 / 9512: 00$ & Manual Tape & 8 & Good & \\
\hline $6 / 21 / 957: 06$ & Auto FIC & 8.10 & Good & \\
\hline $6 / 21 / 9512: 00$ & Manual Tape & 8 & Good & \\
\hline $6 / 22 / 95 \quad 7: 06$ & Auto FIC & 8.10 & Good & \\
\hline $6 / 22 / 9512: 00$ & Manual Tape & 8 & Good & \\
\hline $6 / 23 / 957: 06$ & Auto FIC & 8.10 & Good & \\
\hline $6 / 23 / 9512: 00$ & Manual Tape & 8.00 & Good & \\
\hline $6 / 24 / 957: 06$ & Auto FIC & 8.10 & Good & \\
\hline $6 / 24 / 9512: 00$ & Manual Tape & $\cdot 8$ & Good & \\
\hline $6 / 25 / 957: 05$ & Auto FIC & 8.1 & Good & \\
\hline $6 / 25 / 9512: 00$ & Manual Tape & 8.00 & Good & \\
\hline $6 / 26 / 957: 05$ & Auto FIC & 8.1 & Good & \\
\hline $6 / 26 / 9512: 00$ & Manual Tape & 8 & Good & \\
\hline $6 / 27 / 957: 06$ & Auto FIC & 8.1 & Good & \\
\hline $6 / 27 / 95 \quad 12: 00$ & Manual Tape & 8 & Good & \\
\hline $6 / 28 / 95 \quad 7: 05$ & Auto FIC & 8.1 & Good & \\
\hline $6 / 28 / 9512: 00$ & Manual Tape & 8 & Good & \\
\hline $6 / 29 / 957: 06$ & Auto FIC & 8.1 & Good & \\
\hline $6 / 29 / 95 \quad 12: 00$ & Manual Tape & 8 & Good & \\
\hline $6 / 30 / 957: 06$ & Auto FIC & 8.10 & Good & \\
\hline $6 / 30 / 9512: 00$ & Manual Tape & 8 & Transcribed & \\
\hline
\end{tabular}

Data obtained from WHC Surveillance Analysis Compurter System, (PC SACS) July 7, 1995. 


\begin{tabular}{|c|c|c|c|c|}
\hline \multicolumn{5}{|c|}{ 241-AP-105 } \\
\hline & & Irface Level Dat & & \\
\hline Date & Type of Measurement & Leval (Inchea) & Data Quality & Comments \\
\hline $1 / 1 / 910: 00$ & Manual Tape & 300.25 & Transcribed & \\
\hline $1 / 1 / 917: 03$ & Auto FIC & 300.60 & Good & \\
\hline $1 / 2 / 910: 00$ & Manual Tape & 300.25 & Transeribed & \\
\hline $1 / 2 / 917: 02$ & Auto FIC & 300.60 & Good & \\
\hline $1 / 3 / 910: 00$ & Manual Tape & 300.25 & Transcribed & \\
\hline $1 / 3 / 917: 03$ & Auto FIC & 300.60 & Good & \\
\hline 1/4/910:00 & Manual Tape & 300.25 & Transeribed & \\
\hline 1/4/91 7:02 & Auto FIC & 300.6 & Good & \\
\hline $1 / 5 / 910: 00$ & Manual Tape & 300.25 & Transcribed & \\
\hline 1/5/91 7:02 & Auto FIC & 300.6 & Good & \\
\hline 1/6/91 0:00 & Manual Tape & 300.25 & Transcribed & \\
\hline $.1 / 6 / 917: 02$ & Auto FIC & 300.5 & Good & \\
\hline $1 / 7 / 910: 00$ & Manual Tape & 300.25 & Transcribed & \\
\hline 1/7/91 7:02 & Auto FIC & 300.5 & Good & \\
\hline 1/8/91 $0: 00$ & Manual Tape & 300.25 & Transcribed & \\
\hline 1/8/91 7:03 & Auto FIC & 300.50 & Good & \\
\hline 1/9/91 0:00 & Manual Tape & 300.25 & Transeribed & \\
\hline 1/9/91 7:02 & Auto FIC & 300.50 & Good & \\
\hline $1 / 10 / 910: 00$ & Manual Tape & 300.25 & Transcribed & \\
\hline $1 / 10 / 917: 02$ & Auto FIC & 300.5 & Good & \\
\hline $1 / 11 / 910: 00$ & Manual Tape & 300.25 & Transcribed & \\
\hline $1 / 11 / 917: 03$ & Auto FIC & 300.50 & Good & \\
\hline $1 / 12 / 910: 00$ & Manual Tape & 300.25 & Transcribed & \\
\hline $1 / 12 / 917: 02$ & Auto FIC & 300.5 & Good & \\
\hline $1 / 13 / 910: 00$ & Manual Tape , & 300.25 & Transcribed & \\
\hline 1/13/91 7:03 & Auto FIC & 300.50 & Good & \\
\hline $1 / 14 / 910: 00$ & Manual Tape & 300.25 & Transcribed & \\
\hline $1 / 14 / 917: 04$ & Auto FIC & 300.50 & Good & \\
\hline $1 / 15 / 910: 00$ & Manual Tape & 300.25 & Transcribed & \\
\hline $1 / 15 / 917: 03$ & Auto FIC & 300.50 & Good & \\
\hline $1 / 16 / 910: 00$ & Manual Tape & .300 .50 & Transcribed & \\
\hline $1 / 16 / 917: 04$ & Auto FIC & 300.5 & Good & \\
\hline $1 / 17 / 910: 00$ & Manual Tape & 300.25 & Transcribed & \\
\hline $1 / 17 / 917: 04$ & Auto FIC & 300.5 & Good & \\
\hline $1 / 18 / 910: 00$ & Manual Tape & 300.25 & Transcribed & \\
\hline $1 / 18 / 917: 04$ & Auto FIC & 300.50 & Good & \\
\hline $1 / 19 / 910: 00$ & Manual Tape & 300.25 & Transcribed & \\
\hline $1 / 19 / 917: 04$ & Auto FIC & 300.5 & Good & \\
\hline $1 / 20 / 910: 00$ & Manual Tape & 300.25 & Transcribed & \\
\hline $1 / 20 / 917: 04$ & Auto FIC & 300.50 & Good & \\
\hline $1 / 21 / 910: 00$ & Manual Tape & 300.25 & Transcribed & \\
\hline 1/21/91 7:04 & Auto FIC & 300.5 & Good & \\
\hline $1 / 22 / 910: 00$ & Manual Tape & 300.25 & Transcribed & \\
\hline $1 / 22 / 917: 04$ & Auto FIC & 300.50 & Good & \\
\hline $1 / 23 / 910: 00$ & Manual Tape & 300.25 & Transcribed & \\
\hline $1 / 23 / 917: 04$ & Auto FIC & 300.5 & Good & \\
\hline $1 / 24 / 910: 00$ & Manual Tape & 300.00 & Transcribed & \\
\hline $1 / 24 / 917: 04$ & Auto FIC & 300.5 & Good & \\
\hline
\end{tabular}

Data obtained from WHC Surveillance Analysis Computer System, (PC SACS) July 7, 1995. 


\begin{tabular}{|c|c|c|c|c|}
\hline \multicolumn{5}{|c|}{ 241-AP-105 } \\
\hline & & Urface Level Dat & & \\
\hline Date & Type of Measurement & Level (Inches) & Data Quality & Comments \\
\hline 1/25/91 0:00 & Manual Tape & 300.00 & Transcribed & \\
\hline $1 / 25 / 917: 03$ & Auto FIC & 300.4 & Good & \\
\hline $1 / 26 / 910: 00$ & Manual Tape & 300.00 & Transcribed & \\
\hline $1 / 26 / 917: 03$ & Auto FIC & 300.40 & Good & \\
\hline $1 / 27 / 910: 00$ & Manual Tape & 300.25 & Transcribed & \\
\hline $1 / 27 / 917: 03$ & Auto FIC & 300.40 & Good & \\
\hline $1 / 28 / 910: 00$ & Manual Tape & 300 & Transcribed & \\
\hline $1 / 28 / 916: 03$ & Auto FIC & 300.4 & Good & \\
\hline $1 / 29 / 910: 00$ & Manual Tape & 300 & Transcribed & \\
\hline $1 / 29 / 917: 02$ & Auto FIC & 300.40 & Good & \\
\hline 1/30/91 0:00 & Manual Tape & 300.25 & Transcribed & \\
\hline 1/30/910:00 & Manual FIC & 300.50 & Good & \\
\hline $1 / 31 / 910: 00$ & Manual Tape & 300 & Transcribed & \\
\hline $1 / 31 / 917: 02$ & Auto FIC & 300.40 & Good & \\
\hline 2/1/91 0:00 & Manual Tape & 300 & Transcribed & \\
\hline 2/1/91 7:02 & Auto FIC & 300.40 & Good & \\
\hline 2/2/91 0:00 & Manual Tape & 300 & Transcribed & \\
\hline 2/2/91 7:04 & Auto FIC & 300.40 & Good & \\
\hline 2/3/91 0:00 & Manual Tape & 300 & Transcribed & \\
\hline $2 / 3 / 917: 03$ & Auto FIC & 300.40 & Good & \\
\hline 2/4/910:00 & Manual Tape & 300.00 & Transcribed & \\
\hline 2/4/91 7:03 & Auto FIC & 300.4 & Good & \\
\hline 2/5/91 0:00 & Manual Tape & 300.00 & Transcribed & \\
\hline 2/5/91 7:04 & Auto FIC & 300.40 & Good & \\
\hline 2/6/91 0:00 & Manual Tape & 300 & Transcribed & \\
\hline 2/6/91 7:04 & Auto FIC & 300.4 & Good & \\
\hline 2/7/91 0:00 & Manual Tape & 300 & Transcribed & \\
\hline 2/7/91 7:04 & Auto FIC & 300.4 & Good & \\
\hline 2/8/91 0:00 & Manual Tape & 300.00 & Transcribed & \\
\hline 2/8/91 7:04 & Auto FIC & 300.40 & Good & \\
\hline 2/9/91 0:00 & Manual Tape & 300 & Transcribed & \\
\hline $2 / 9 / 917: 04$ & Auto FIC & 300.4 & Good & \\
\hline 2/10/910:00 & Manual Tape & 300 & Transcribed & \\
\hline $2 / 10 / 917: 03$ & Auto FIC & 300.4 & Good & \\
\hline $2 / 11 / 910: 00$ & Manual Tape & 300.00 & Transcribed & \\
\hline $2 / 11 / 917: 04$ & Auto FIC & 300.4 & Good & \\
\hline $2 / 12 / 910: 00$ & Manual Tape & $300.00^{\circ}$ & Transcribed & \\
\hline $2 / 12 / 917: 04$ & Auto FIC & 300.40 & Good & \\
\hline $2 / 13 / 910: 00$ & Manual Tape & 300 & Transcribed & \\
\hline $2 / 13 / 917: 04$ & Auto FIC & 300.4 & Good & \\
\hline $2 / 14 / 910: 00$ & Manual Tape & 299.75 & Transcribed & \\
\hline $2 / 14 / 917: 04$ & Auto FIC & 300.40 & Good & \\
\hline $2 / 15 / 910: 00$ & Manual Tape & 299.75 & Transcribed & \\
\hline 2/15/91 7:03 & Auto FIC & 300.40 & Good & \\
\hline $2 / 16 / 910: 00$ & Manual Tape & 299.75 & Transcribed & \\
\hline 2/16/91 7:02 & Auto FIC & 300.4 & Good & \\
\hline $2 / 1.7 / 910: 00$ & Manual Tape & 299.75 & Transcribed & \\
\hline $2 / 17 / 917: 02$ & Auto FIC & 300.40 & Goad & \\
\hline
\end{tabular}

Data obtained from WHC Surveillance Analysis Computer System, (PC SACS) July 7, 1995. 


\begin{tabular}{|c|c|c|c|c|}
\hline \multicolumn{5}{|c|}{ 241-AP-105 } \\
\hline & & urface Level Dat & . & \\
\hline Date & Type of Measurement & Level (Inchios) & Data Quality & Comments \\
\hline $2 / 18 / 910: 00$ & Manual Tape & 299.75 & Transcribed & \\
\hline $2 / 18 / 917: 03$ & Auto FIC & 300.4 & Good & \\
\hline $2 / 19 / 910: 00$ & Manual Tape & 299.75 & Transcribed & \\
\hline $2 / 19 / 91 \quad 7: 02$ & Auto FIC & 300.40 & Good & \\
\hline $2 / 20 / 910: 00$ & Manual Tape & 299.75 & Transcribed & \\
\hline $2 / 20 / 91 \quad 7: 03$ & Auto FIC & 300.4 & Good & \\
\hline $2 / 21 / 910: 00$ & Manual Tape & 299.75 & Transcribed & \\
\hline $2 / 21 / 917: 02$ & Auto FIC & 300.40 & Good & \\
\hline $2 / 22 / 910: 00$ & Manual Tape & 300.00 & Transcribed & \\
\hline $2 / 22 / 917: 02$ & Auto FIC & 300.4 & Good & \\
\hline $2 / 23 / 910: 00$ & Manual Tape & 300.00 & Transcribed & \\
\hline $2 / 23 / 917: 02$ & Auto FIC & 300.3 & Good & \\
\hline $2 / 24 / 910: 00$ & Manual Tape & 300.00 & Transcribed & \\
\hline $2 / 24 / 917: 03$ & Auto FIC & 300.3 & Good & $\cdot$ \\
\hline $2 / 25 / 910: 00$ & Manual Tape & 300 & Transcribed & \\
\hline $2 / 25 / 917: 03$ & Auto FIC & 300.3 & Good & \\
\hline $2 / 26 / 910: 00$ & Manual Tape & 300 & Transcribed & \\
\hline $2 / 26 / 917: 03$ & Auto FIC & 300.30 & Good & \\
\hline $2 / 27 / 910: 00$ & Manual Tape & 300 & Transcribed & \\
\hline $2 / 27 / 917: 02$ & Auto FIC & 300.3 & Good & \\
\hline $2 / 28 / 91 \quad 0: 00$ & Manual Tape & 300.00 & Transcribed & \\
\hline $2 / 28 / 917: 02$ & Auto FIC & 300.3 & Good & \\
\hline $3 / 1 / 91 \quad 0: 00$ & Manual Tape & 299.75 & Transcribed & \\
\hline 3/1/91 7:02 & Auto FIC & 300.3 & Good & \\
\hline $3 / 2 / 910: 00$ & Manual Tape & 299.75 & Transcribed & \\
\hline $3 / 2 / 917: 02$ & Auto FIC & 300.3 & Good & \\
\hline $3 / 3 / 910: 00$ & Manual Tape & 299.75 & Transcribed & \\
\hline $3 / 3 / 917: 03$ & Auto FIC & 300.3 & Good & \\
\hline $3 / 4 / 910: 00$ & Manual Tape & 299.75 & Transcribed & \\
\hline $3 / 4 / 917: 03$ & Auto FIC & 300.30 & Good & \\
\hline $3 / 5 / 910: 00$ & Manual Tape & 299.75 & Transcribed & \\
\hline $3 / 5 / 917: 03$ & Auto FIC & 300.30 & Good & \\
\hline $3 / 6 / 910: 00$ & Manual Tape & 299.75 & Transcribed & \\
\hline $3 / 6 / 917: 02$ & Auto FIC & 300.3 & Good & \\
\hline $3 / 7 / 910: 00$ & Manual Tape & 299.75 & Transcribed & \\
\hline $3 / 7 / 917: 02$ & Auto FIC & 300.30 & Good & \\
\hline $3 / 8 / 910: 00$ & Manual Tape & 299.75 & Transcribed & \\
\hline $3 / 8 / 917: 02$ & Auto FIC & 300.30 & Good & \\
\hline 3/9/91 0:00 & Manual Tape & 299.50 & Transcribed & \\
\hline $3 / 9 / 917: 02$ & Auto FIC & 300.30 & Good & \\
\hline 3/10/91 0:00 & Manual Tape & 299.5 & Transcribed & \\
\hline $3 / 10 / 917: 02$ & Auto FIC & 300.3 & Good & \\
\hline $3 / 11 / 910: 00$ & Manual Tape & 299.5 & Transcribed & \\
\hline $3 / 11 / 917: 02$ & Auto FIC & 300.3 & Good & \\
\hline 3/12/91 0:00 & Manual Tape & 299.50 & Transcribed & \\
\hline $3 / 12 / 917: 03$ & Auto FIC & 300.30 & Good & \\
\hline $3 / 13 / 910: 00$ & Manual Tape & 299.50 & Transcribed & \\
\hline $3 / 13 / 917: 03$ & Auto FIC & 300.3 & Good & \\
\hline
\end{tabular}

Data obtained from WHC Surveillance Analysis Computer System, (PC SACS) July 7, 1995. 


\begin{tabular}{|c|c|c|c|c|}
\hline \multicolumn{5}{|c|}{ 241-AP-105 } \\
\hline \multicolumn{5}{|c|}{ Surface Lavel Data } \\
\hline Date & Type of Measurement & Leval (Inohes) & Data Quality & Comments \\
\hline 3/14/91 0:00 & Manual Tape & 299.5 & Transcribed & \\
\hline 3/14/917:03 & Auto FIC & 300.30 & Good & \\
\hline $3 / 15 / 91 \quad 0: 00$ & Manual Tape & 299.75 & Transcribed & \\
\hline $3 / 15 / 917: 03$ & Auto FIC & 300.30 & Good & \\
\hline $3 / 16 / 910: 00$ & Manual Tape & 299.75 & Transcribed & \\
\hline $3 / 16 / 917: 03$ & Auto FIC & 300.30 & Good & \\
\hline $3 / 17 / 910: 00$ & Manual Tape : & 299.75 & Transcribed & \\
\hline 3/17/91 7:02 & Auto FIC & 300.3 & Good & \\
\hline 3/18/91 0:00 & Manual Tape & 299.75 & Transcribed & \\
\hline $3 / 18 / 917: 03$ & Auto FIC & 300.30 & Good & \\
\hline 3/19/91 0:00 & Manual Tape & 299.75 & Transcribed & \\
\hline $3 / 19 / 917: 03$ & Auto FIC & 300.3 & Good & \\
\hline $3 / 20 / 910: 00$ & Manual Tape & 299.75 & Transcribed. & \\
\hline $3 / 20 / 917: 02$ & Auto FIC & 300.3 & Good & \\
\hline $3 / 21 / 910: 00$ & Manual Tape & 299.75 & Transcribed & \\
\hline $3 / 21 / 917: 03$ & Auto FIC & 300.3 & Good & \\
\hline $3 / 22 / 910: 00$ & Manual Tape & 299.75 & Transcribed & \\
\hline $3 / 22 / 917: 02$ & Auto FIC & 300.30 & Good & \\
\hline $3 / 23 / 910: 00$ & Manual Tape & 299.75 & Transçibed & \\
\hline $3 / 23 / 917: 03$ & Auto FIC & 300.30 & Good & \\
\hline $3 / 24 / 910: 00$ & Manual Tape & 299.75 & Transcribed & \\
\hline $3 / 24 / 917: 03$ & Auto FIC & 300.3 & Good & \\
\hline $3 / 25 / 910: 00$ & Manual Tape & 299.75 & Transcribed & \\
\hline $3 / 25 / 917: 03$ & Auto FIC & 300.30 & Good & \\
\hline $3 / 26 / 910: 00$ & Manual Tape & 299.75 & Transcribed & \\
\hline $3 / 26 / 917: 03$ & Auto FIC & 300.30 & Good & \\
\hline $3 / 27 / 91.0: 00$ & Manual Tape & 299.75 & Transcribed & \\
\hline $3 / 27 / 917: 03$ & Auto FIC & 300.30 & Good & \\
\hline $3 / 28 / 910: 00$ & Manual Tape & 299.75 & Transcribed & \\
\hline $3 / 28 / 917: 03$ & Auto FIC & 300.30 & Good & \\
\hline $3 / 29 / 910: 00$ & Manual Tape & 299.75 & Transcribed & \\
\hline $3 / 29 / 917: 03$ & Auto FIC & 300.30 & Good & \\
\hline $3 / 30 / 910: 00$ & Manual Tape & 299.75 & Transcribed & \\
\hline $3 / 30 / 917: 03$ & Auto FIC & 300.30 & Good & \\
\hline $3 / 31 / 910: 00$ & Manual Tape & 299.75 & Transcribed & \\
\hline $3 / 31 / 917: 03$ & Auto FIC & 300.30 & Good & \\
\hline $4 / 1 / 910: 00$ & Manual Tape & $299: 75$ & Transcribed & \\
\hline 4/1/91 7:03 & Auto FIC & 300.3 & Good & \\
\hline $4 / 2 / 910: 00$ & Manual Tape & 299.75 & Transcribed & \\
\hline $4 / 2 / 917: 03$ & Auto FIC & 300.3 & Good & \\
\hline $4 / 3 / 910: 00$ & Manual Tape & 299.75 & Transcribed & \\
\hline 4/3/91 7:03 & Auto FIC & 300.30 & Good & \\
\hline 4/4/910:00 & Manual Tape & 299.5 & Transcribed & \\
\hline 4/4/91 7:03 & Auto FIC & 300.3 & Good & \\
\hline $4 / 5 / 910: 00$ & Manual Tape & 299.50 & Transcribed & \\
\hline 4/5/917:03 & Auto FIC & 300.30 & Good & \\
\hline $4 / 6 / 910: 00$ & Manual Tape & 299.75 & Transcribed & \\
\hline $4 / 6 / 91 \quad 7: 03$ & Auto FIC & 300.3 & Good & \\
\hline
\end{tabular}

Data obtained from WHC Surveillance Analysis Computer System, (PC SACS) July 7, 1995. 


\begin{tabular}{|c|c|c|c|c|}
\hline \multicolumn{5}{|c|}{ 241-AP-105 } \\
\hline \multicolumn{5}{|c|}{ Surface Level Data } \\
\hline Date & Type of Measurement & Level (Inches) & Data Quality & Comments \\
\hline $4 / 7 / 910: 00$ & Manual Tape & 299.5 & Transcribed & \\
\hline $4 / 7 / 917: 03$ & Auto FIC & 300.30 & Good & \\
\hline $4 / 8 / 910: 00$ & Manual Tape. & 299.50 & Transcribed & \\
\hline $4 / 8 / 917: 04$ & Auto FIC & 300.2 & Good & \\
\hline $4 / 9 / 910: 00$ & Manual Tapo & 299.75 & Transcribed & \\
\hline $4 / 9 / 917: 03$ & Auto FIC & 300.20 & Good & \\
\hline $4 / 10 / 910: 00$ & Manual Tape & 299.75 & Transcribed & \\
\hline $4 / 10 / 917: 03$ & Auto FIC & 300.20 & Good & \\
\hline $4 / 11 / 910: 00$ & Manual Tape & 299.75 & Transcribed & \\
\hline $4 / 11 / 917: 03$ & Auto FIC & 300.2 & Good & \\
\hline 4/12/910:00 & Manual Tape & 299.50 & Transcribed & \\
\hline $4 / 12 / 917: 03$ & Auto FIC & 300.2 & Good & \\
\hline $4 / 13 / 910: 00$ & Manual Tape & 299.50 & Transcribed & \\
\hline 4/13/91 7:04 & Auto FIC & 300.2 & Good & \\
\hline $4 / 14 / 910: 00$ & Manual Tape & 299.75 & Transcribed & \\
\hline $4 / 14 / 917: 03$ & Auto FIC & 300.20 & Good & \\
\hline $4 / 15 / 910: 00$ & Manual Tape & 299.75 & Transcribed & \\
\hline $4 / 15 / 917: 03$ & Auto FIC & 300.2 & Good & \\
\hline $4 / 16 / 910: 00$ & Manual Tape & 299.75 & Transcribed & \\
\hline 4/16/91 0:00 & Manual FIC & 300.30 & Good & \\
\hline $4 / 17 / 910: 00$ & Manual Tape & 299.50 & Transcribed & \\
\hline $4 / 17 / 917: 03$ & Auto FIC & 300.2 & Good & \\
\hline $4 / 18 / 910: 00$ & Manual Tape & 299.75 & Transcribed & \\
\hline $4 / 18 / 917: 06$ & Auto FIC & 300.20 & Good & \\
\hline $4 / 19 / 910: 00$ & Manual Tape & 299.75 & Transcribed & \\
\hline $4 / 19 / 917: 05$ & Auto FIC & 300.20 & Good & \\
\hline $4 / 20 / 910: 00$ & Manual Tape & $299.75^{\circ}$ & Transcribed & \\
\hline $4 / 20 / 917: 06$ & Auto FIC & 300.2 & Good & \\
\hline $4 / 21 / 910: 00$ & Manual Tape & 299.75 & Transcribed & \\
\hline $4 / 21 / 917: 05$ & Auto FIC & 300.20 & Good & $\cdot$ \\
\hline $4 / 22 / 91 \quad 0: 00$ & Manual Tape & 299.75 & Transcribed & \\
\hline $4 / 22 / 917: 06$ & Auto FIC. & 300.20 & Good & \\
\hline $4 / 23 / 910: 00$ & Manual Tape & 299.75 & Transcribed & \\
\hline 4/23/91 7:06 & Auto FIC & 300.20 & Good & \\
\hline $4 / 24 / 910: 00$ & Manual Tape & 299.75. & Transcribed & \\
\hline $4 / 24 / 917: 05$ & Auto FIC & 300.20 & Good & \\
\hline $4 / 25 / 910: 00$ & Manual Tape & 299.50 & Transcribed & \\
\hline $4 / 25 / 917: 05$ & Auto FIC & 300.20 & Good & \\
\hline $4 / 26 / 91 \quad 0: 00$ & Manual Tape & 299.75 & Transcribed & \\
\hline $4 / 26 / 917: 05$ & Auto FIC & 300.2 & Good & \\
\hline $4 / 27 / 910: 00$ & Manual Tape & 299.75 & Transeribed & \\
\hline $4 / 27 / 917: 05$ & Auto FIC & 300.20 & Good & \\
\hline $4 / 28 / 910: 00$ & Manual Tape & 299.50 & Transcribed & \\
\hline $4 / 28 / 917: 06$ & Auto FIC & $300.20^{\circ}$ & Good & \\
\hline $4 / 29 / 910: 00$ & Manual Tape & 299.5 & Transcribed & \\
\hline $4 / 29 / 917: 05$ & Auto FIC & 300.20 & Good & \\
\hline $4 / 30 / 910: 00$ & Manual Tape & 299.50 & Transcribed & \\
\hline $4 / 30 / 917: 06$ & Auto FIC & 300.2 & Good & \\
\hline
\end{tabular}

Data obtained from WHC Surveillance Analysis Computer System, (PC SACS) July 7, 1995. 


\begin{tabular}{|c|c|c|c|c|}
\hline \multicolumn{5}{|c|}{ 241-AP-105 } \\
\hline \multicolumn{5}{|c|}{ Surface Level Data } \\
\hline Date & Type of Moasurement & Level (Inches) & Data Quality & Comments \\
\hline $5 / 1 / 910: 00$ & Manual Tape & 299.50 & Transcribed & \\
\hline $5 / 1 / 917: 05$ & Auto FIC & 300.20 & Good & \\
\hline $5 / 2 / 910: 00$ & Manual Tape & 299.50 & Transcribed & \\
\hline $5 / 2 / 917: 05$ & Auto FIC & 300.20 & Good & \\
\hline $5 / 3 / 910: 00$ & Manual Tape & 299.5 & Transcribed & \\
\hline $5 / 3 / 917: 06$ & Auto FIC & 300.2 & Good & \\
\hline $5 / 4 / 910: 00$ & Manual Tape & 299.25 & Transcribed & \\
\hline $5 / 4 / 917: 05$ & Auto FIC & 300.2 & Good & \\
\hline $5 / 5 / 910: 00$ & Manual Tape & 299.5 & Transcribed & \\
\hline $5 / 5 / 91 \quad 7: 05$ & Auto FIC & 300.2 & Good & \\
\hline $5 / 6 / 910: 00$ & Manual Tape & 299.50 & Transcribed & \\
\hline $5 / 6 / 917: 05$ & Auto FIC & 300.20 & Good & \\
\hline $5 / 7 / 910: 00$ & Manual Tape & 299.5 & Transcribed & \\
\hline $5 / 7 / 917: 05$ & Auto FIC & 300.2 & Good & \\
\hline $5 / 8 / 910: 00$ & Manual Tape & 299.5 & Transcribed & \\
\hline $5 / 8 / 917: 05$ & Auto FIC & 300.20 & Good & \\
\hline $5 / 9 / 910: 00$ & Manual Tape & 299.5 & Transcribed & \\
\hline $5 / 9 / 917: 05$ & Auto FIC & 300.20 & Good & \\
\hline $5 / 10 / 910: 00$ & Manual Tape & 299.50 & Transcribed & \\
\hline $5 / 10 / 917: 05$ & Auto FIC & 300.20 & Good & \\
\hline $5 / 11 / 910: 00$ & Manual Tape & 299.50 & Transcribed & \\
\hline $5 / 11 / 917: 05$ & Auto FIC & 300.2 & Good & \\
\hline $5 / 12 / 910: 00$ & Manual Tape & 299.75 & Transcribed & \\
\hline $5 / 12 / 917: 05$ & Auto FIC & 300.2 & Good & \\
\hline $5 / 13 / 910: 00$ & Manual Tape: & 299.75 & Transcribed & \\
\hline $5 / 13 / 917: 05$ & Auto FIC & 300.2 & Good & \\
\hline $5 / 14 / 910: 00$ & Manual Tape & 299.75 & Transcribed & \\
\hline $5 / 14 / 917: 05$ & Auto FIC & 300.2 & Good & \\
\hline $5 / 15 / 910: 00$ & Manual Tape & 299.75 & Transcribed & \\
\hline $5 / 15 / 917: 05$ & Auto FIC & 300.2 & Good & \\
\hline $5 / 16 / 910: 00$ & Manual Tape & 299.75 & Transcribed & \\
\hline $5 / 16 / 917: 06$ & Auto FIC & 300.20 & Gaod & \\
\hline $5 / 17 / 910: 00$ & Manual Tape & 299.75 & Transcribed & \\
\hline $5 / 17 / 917: 06$ & Auto FIC & 300.2 & Good & \\
\hline $5 / 18 / 910: 00$ & Manual Tape & 299.5 & Transcribed & \\
\hline $5 / 18 / 917: 06$ & Auto FIC & 300.2 & Good & \\
\hline $5 / 19 / 910: 00$ & Manual Tape & 299.5 & Transcribed & \\
\hline $5 / 19 / 917: 05$ & Auto FIC & 300.20 & Good & \\
\hline $5 / 20 / 910: 00$ & Manual Tape & 299.75 & Transcribed & \\
\hline $5 / 20 / 917: 06$ & Auto FIC & 300.20 & Good & \\
\hline $5 / 21 / 910: 00$ & Manual Tape & 299.75 & Transcribed & \\
\hline $5 / 21 / 917: 06$ & Auto FIC & 300.20 & Good & \\
\hline $5 / 22 / 910: 00$ & Manual Tape & 299.75 & Transcribed & \\
\hline $5 / 22 / 917: 05$ & Auto FIC & 300.2 & Good & \\
\hline $5 / 23 / 910: 00$ & Manual Tape & 299.75 & Transcribed & \\
\hline $5 / 23 / 917: 05$ & Auto FIC & 300.20 & Good & \\
\hline $5 / 24 / 910: 00$ & Manual Tape & 299.75 & Transcribed & \\
\hline $5 / 24 / 917: 05$ & Auto FIC & 300.2 & Good & \\
\hline
\end{tabular}

Data obtained from WHC Surveillance Analysis Computer System, (PC SACS) July 7, 1995. 


\begin{tabular}{|c|c|c|c|c|}
\hline \multicolumn{5}{|c|}{ 241-AP-105 } \\
\hline & & Irfaca Level Date & & \\
\hline Date & Type of Measurement & Level (inches) & Data Quality & Comments \\
\hline $5 / 25 / 910: 00$ & Manual Tape & 299.75 & Transcribed & \\
\hline $5 / 25 / 917: 05$ & Auto FIC & 300.2 & Good & \\
\hline $5 / 26 / 910: 00$ & Manual Tape & 299.75 & Transcribed & \\
\hline $5 / 26 / 917: 05$ & Auto FIC & 300.20 & Good & \\
\hline $5 / 27 / 910: 00$ & Manual Tape & 299.75 & Transcribed & \\
\hline $5 / 27 / 917: 05$ & Auto FIC & 300.20 & Good & \\
\hline $5 / 28 / 910: 00$ & Manual Tape & 299.75 & Transcribed & \\
\hline $5 / 28 / 917: 05$ & Auto FIC & 300.2 & Good & \\
\hline $5 / 29 / 910: 00$ & Manual Tape & 299.75 & Transcribed & \\
\hline $5 / 29 / 917: 05$ & Auto FIC & 300.20 & Good & \\
\hline $5 / 30 / 910: 00$ & Manual Tape & 299.75. & Transcribed & \\
\hline $5 / 30 / 917: 05$ & Auto FIC & 300.2 & Good & \\
\hline $5 / 31 / 910: 00$ & Manual Tape & 299.75 & Transcribed & \\
\hline $5 / 31 / 917: 05$ & Auto FIC & 300.20 & Good & \\
\hline $6 / 1 / 910: 00$ & Manual Tape & 299.75 & Transcribed & \\
\hline 6/1/91 6:05 & Auto FIC & 300.2 & Good & \\
\hline $6 / 2 / 910: 00$ & Manual Tape & 299.75 & Transcribed & \\
\hline 6/2/913:05 & Auto FIC & 300.20 & Good & \\
\hline 6/3/91 0:00 & Manual Tape & 299.75 & Transcribed & \\
\hline $6 / 3 / 917: 05$ & Auto FIC & 300.2 & Good & \\
\hline $6 / 4 / 910: 00$ & Manual Tape & 299.5 & Transcribed & \\
\hline 6/4/91 7:05 & Auto FIC & 300.2 & Good & \\
\hline 6/5/91 0:00 & Manual Tape & 299.50 & Transcribed & \\
\hline 6/5/91 7:05 & Auto FIC & 300.20 & Good & \\
\hline $6 / 6 / 910: 00$ & Manual Tape & 299.25 & Transcribed & \\
\hline 6/6/91 7:06 & Auto FIC & 300.20 & Good & \\
\hline 6/7/91 0:00 & Manual Tape & 299.5 & Transcribed & \\
\hline $6 / 7 / 917: 05$ & Auto FIC & 300.20 & Good & \\
\hline 6/8/91 0:00 & Manual Tape & 299.75 & Transcribed & \\
\hline $6 / 8 / 917: 05$ & Auto FIC & 300.2 & Good & \\
\hline 6/9/91 0:00 & Manual Tape & 299.75 & Transcribed & \\
\hline $6 / 9 / 917: 05$ & Auto FIC & 300.2 & Good & \\
\hline $6 / 10 / 910: 00$ & Manual Tape & 299.5 & Transcribed & \\
\hline $6 / 10 / 917: 06$ & Auto FIC & 300.20 & Good & \\
\hline $6 / 11 / 910: 00$ & Manual Tape & 299.5 & Transcribed & \\
\hline $6 / 11 / 917: 05$ & Auto FIC & 300.20 & Good & \\
\hline $6 / 12 / 910: 00$ & Manual Tape & 299.5 & Transcribed & \\
\hline $6 / 12 / 917: 04$ & Auto FIC & 300.20 & Good & \\
\hline $6 / 13 / 910: 00$ & Manual Tape & 299.75 & Transcribed & \\
\hline $6 / 13 / 917: 05$ & Auto FIC & 300.20 & Good & \\
\hline $6 / 14 / 910: 00$ & Manual Tape & 299.75 & Transcribed & \\
\hline $6 / 14 / 917: 05$ & Auto FIC & 300.2 & Good & \\
\hline $6 / 15 / 910: 00$ & Manual Tape & 299.75 & Transcribed & \\
\hline $6 / 15 / 917: 05$ & Auto FIC & 300.20 & Good & \\
\hline $6 / 16 / 910: 00$ & Manual Tape & 299.75 & Transcribed & \\
\hline $6 / 16 / 91 \quad 7: 05$ & Auto FIC & 300.2 & Good & \\
\hline $6 / 17 / 910: 00$ & Manual Tape & 299.50 & Transcribed & \\
\hline $6 / 17 / 917: 05$ & Auto FIC & 300.20 & Good & \\
\hline
\end{tabular}

Data obtained from WHC Surveillance Analysis Computer System, (PC SACS) July 7, 1995. 


\begin{tabular}{|c|c|c|c|c|}
\hline \multicolumn{5}{|c|}{ 241-AP-105 } \\
\hline \multicolumn{5}{|c|}{ Surface Levol Data } \\
\hline Date & Type of Measurement & Leval (Inchies) & Data Quality & Comments \\
\hline $6 / 18 / 910: 00$ & Manual Tape & 299.5 & Transcribed & \\
\hline $6 / 18 / 917: 05$ & Auto FIC & 300.20 & Good & \\
\hline $6 / 19 / 910: 00$ & Manual Tape & 299.50 & Transcribed & \\
\hline $6 / 19 / 917: 05$ & Auto FIC & 300.20 & Good & \\
\hline $6 / 20 / 910: 00$ & Manual Tape & 299.5 & Transcribed & \\
\hline 6/20/91 7:05 & Auto FIC & 300.2 & Good & \\
\hline $6 / 21 / 910: 00$ & Manual Tape & 299.5 & Transcribed & \\
\hline $6 / 21 / 917: 06$ & Auto FIC & 300.2 & Good & \\
\hline $6 / 22 / 910: 00$ & Manual Tape & 299.50 & Transcribed & \\
\hline 6/22/913:06 & Auto FIC & 300.20 & Good & \\
\hline $6 / 23 / 910: 00$ & Manual Tape & 299.50 & Transcribed & \\
\hline $6 / 23 / 917: 05$ & Auto FIC & 300.20 & Good & \\
\hline $6 / 24 / 910: 00$ & Manual Tape & 299.5 & Transcribed & \\
\hline $6 / 24 / 915: 05$ & Auto FIC & 300.2 & Good & \\
\hline $6 / 25 / 910: 00$ & Manual Tape & 299.50 & Transcribed & \\
\hline $6 / 25 / 917: 06$ & Auto FIC & 300.20 & - Good & \\
\hline $6 / 26 / 910: 00$ & Manual Tape & $299.75^{\circ}$ & Transcribed & \\
\hline $6 / 26 / 911: 03$ & Auto FIC & 300.20 & Good & \\
\hline $6 / 27 / 910: 00$ & Manual Tape & 299.75 & Transcribed & \\
\hline 6/27/91 0:00 & Manual FIC & 300.2 & Good & \\
\hline $6 / 28 / 910: 00$ & Manual Tape & 299.75 & Transcribed & \\
\hline $6 / 28 / 917: 03$ & Auto FIC & 300.2 & Good & \\
\hline $6 / 29 / 91 \quad 0: 00$ & Manual Tape & 299.75 & Transcribed & \\
\hline $6 / 29 / 916: 02$ & Auto FIC & 300.20 & Good & \\
\hline $6 / 30 / 910: 00$ & Manual Tape & 299.75. & Transcribed & \\
\hline 6/30/91 0:00 & Manual FIC & 300.3 & Good & \\
\hline $7 / 1 / 910: 00$ & Manual Tape & 299.75 & Transcribed & \\
\hline $7 / 1 / 910: 00$ & Manual FIC & 300.30 & Good & \\
\hline 7/2/91 0:00 & Manual Tape & 299.75 & Transcribed & \\
\hline $7 / 2 / 917: 06$ & Auto FIC & 300.2 & Good & \\
\hline $7 / 3 / 910: 00$ & Manual Tape & 299.75 & Transcribed & \\
\hline $7 / 3 / 917: 06$ & Auto FIC & 300.20 & Good & \\
\hline $7 / 4 / 910: 00$ & Manual Tape & 299.75 & Transcribed & \\
\hline $7 / 4 / 910: 00$ & Manual FIC & 300.30 & Good & \\
\hline $7 / 5 / 910: 00$ & Manual Tape & 299.75 & Transcribed & \\
\hline $7 / 5 / 910: 00$ & Manual FIC & 300.3 & Good & \\
\hline $7 / 6 / 910: 00$ & Manual Tape & 299.75 & Transcribed & \\
\hline 7/6/91 $0: 00$ & Manual FIC & 300.3 & Good & \\
\hline $7 / 7 / 910: 00$ & Manual Tape & 299.75 & Transcribed & \\
\hline $7 / 7 / 910: 00$ & Manuai FIC & 300.30 & Good & \\
\hline $7 / 8 / 910: 00$ & Manual Tape & 299.75 & Transcribed & \\
\hline $7 / 8 / 910: 00$ & Manual FIC & 300.3 & Good & \\
\hline $7 / 9 / 910: 00$ & Manual Tape & 299.75 & Transcribed & \\
\hline $7 / 9 / 910: 00$ & Manual FIC & 300.3 & Good & \\
\hline 7/10/91 0:00 & Manual Tape & 299.75 & Transcribed & \\
\hline $7 / 10 / 910: 00$ & Manual FIC & 300.30 & Good & \\
\hline 7/11/91 0:00 & Manual Tape & 299.75 & Transcribed & \\
\hline $7 / 11 / 917: 06$ & Auto FIC & 300.20 & Good & \\
\hline
\end{tabular}

Data obtained from WHC Surveillance Analysis Computer System, (PC SACS) July 7, 1995. 


\begin{tabular}{|c|c|c|c|c|}
\hline \multicolumn{5}{|c|}{ 241-AP.105 } \\
\hline \multicolumn{5}{|c|}{ Surface Level Data } \\
\hline Date & Type of Measuremont & Lovel (inches) & Data Quality & Comments \\
\hline $7 / 12 / 910: 00$ & Manual Tape & 299.75 & Transcribed & \\
\hline 7/12/917:06 & Auto FIC & 300.20 & Good & \\
\hline $7 / 13 / 910: 00$ & Manual Tape & 299.75 & Transcribed & \\
\hline 7/13/91 7:06 & Auto FIC & 300.3 & Good & \\
\hline $7 / 14 / 910: 00$ & Manual Tape & 299.75 & Transcribed & \\
\hline $7 / 14 / 912: 06$ & Auto FIC & 300.3 & Good & \\
\hline 7/15/910:00 & Manual Tape & 299.75 & Transcribed & \\
\hline $7 / 15 / 917: 06$ & Auto FIC & 300.30 & Good & \\
\hline $7 / 16 / 910: 00$ & Manual FIC & 300.30 & Transcribed & \\
\hline $7 / 16 / 910: 00$ & Manual Tape & 299.50 & Transcribed & \\
\hline $7 / 17 / 910: 00$ & Manual Tape & 299.50 & Transcribed & \\
\hline $7 / 17 / 910: 05$ & Auto FIC & 300.30 & Good & \\
\hline $7 / 18 / 910: 00$ & Manual Tapo & 299.75 & Transcribed & \\
\hline $7 / 18 / 910: 00$ & Manual FIC & 300.3 & Good & \\
\hline $7 / 19 / 910: 00$ & Manual Tape & 299.75 & Transcribed & \\
\hline $7 / 19 / 917: 05$ & Auto FIC & 300.30 & Good & \\
\hline $7 / 20 / 910: 00$ & Manual Tape & 299.75 & Transcribed & \\
\hline $7 / 20 / 912: 05$ & Auto FIC & 300.3 & Good & \\
\hline $7 / 21 / 910: 00$ & Manual Tape & 299.75 & Transcribed & \\
\hline $7 / 21 / 911: 05$ & Auto FIC & 300.3 & Good & \\
\hline $7 / 22 / 910: 00$ & Manual Tape & 299.75 & Transcribed & \\
\hline $7 / 22 / 910: 00$ & Manual FIC & 300.3 & Good & \\
\hline $7 / 23 / 910: 00$ & Manual Tape & 299.75 & Transcribed & \\
\hline $7 / 23 / 910: 05$ & Auto FIC & 300.30 & Good. & \\
\hline $7 / 24 / 910: 00$ & Manual Tape & 299.75 & Transcribed & \\
\hline $7 / 24 / 917: 05$ & Auto FIC & 300.30 & Good & \\
\hline $7 / 25 / 910: 00$ & Manual Tape & 299.75 & Transcribed & \\
\hline $7 / 25 / 91 \quad 1: 03$ & Auto FIC & 300.30 & Good & \\
\hline $7 / 26 / 910: 00$ & Manual Tape & 299.75 & Transcribed & \\
\hline $7 / 26 / 910: 00$ & Manual FIC & 300.3 & Good & \\
\hline $7 / 27 / 910: 00$ & Manual Tape & 299.75 & Transcribed & \\
\hline $7 / 27 / 910: 00$ & Manual FIC & 300.30 & Good & \\
\hline $7 / 28 / 910: 00$ & Manual Tape & 299.75 & Transcribed & \\
\hline $7 / 28 / 910: 00$ & Manual FIC & 300.30 & Good & \\
\hline $7 / 29 / 910: 00$ & Manual Tape & 299.75 & Transcribed & \\
\hline $7 / 29 / 910: 00$ & Manual FIC & 300.30 & Good & \\
\hline $7 / 30 / 910: 00$ & Manual Tape & 299.75 & Transcribed & \\
\hline $7 / 30 / 917: 02$ & Auto FIC & 300.3 & Good & \\
\hline $7 / 31 / 910: 00$ & Manual Tape & 299.75 & Transcribed & \\
\hline $7 / 31 / 91 \quad 7: 02$ & Auto FIC & 300.3 & Good & \\
\hline $8 / 1 / 910: 00$ & Manual Tape & 299.75 & Transcribed & \\
\hline $8 / 1 / 917: 02$ & Auto FIC & 300.30 & Good & \\
\hline $8 / 2 / 910: 00$ & Manual Tape & 299.75 & Transcribed & \\
\hline $8 / 2 / 917: 02$ & Auto FIC & 300.3 & Good & \\
\hline $8 / 3 / 910: 00$ & Manual Tape & 299.75 & Transcribed & \\
\hline $8 / 3 / 917: 02$ & Auto FIC & 300.3 & Good & \\
\hline $8 / 4 / 910: 00$ & Manual Tape & 299.75 & Transcribed & \\
\hline $8 / 4 / 917: 02$ & Auto FIC & 300.30 & Good & \\
\hline
\end{tabular}

Data obtained from WHC Surveillance Analysis Computer System, (PC SACS) July 7, 1995. 


\begin{tabular}{|c|c|c|c|c|}
\hline \multicolumn{5}{|c|}{ 241-AP-105 } \\
\hline & & Urface Level Dat & & \\
\hline Date & Type of Measurement & Level (Inchea) & Data Quality & Comments \\
\hline $8 / 5 / 910: 00$ & Manual Tape & 299.5 . & Transcribed & \\
\hline $8 / 5 / 917: 02$ & Auto FIC & 300.30 & Good & \\
\hline $8 / 6 / 910: 00$ & Manual Tape , & 299.75 & Transcribed & \\
\hline $8 / 6 / 917: 02$ & Auto FIC & 300.30 & Good & \\
\hline $8 / 7 / 910: 00$ & Manual Tape & 299.75 & Transcribed & \\
\hline $8 / 7 / 917: 06$ & Auto FIC & 300.3 & Good & \\
\hline $8 / 8 / 910: 00$ & Manual Tape & 299.75 & Transcribed & \\
\hline $8 / 8 / 917: 06$ & Auto FIC & 300.3 & Good & \\
\hline 8/9/91 0:00 & Manual Tape & 299.75 & Transcribed & \\
\hline $8 / 9 / 917: 04$ & Auto FIC & 300.30 & Good & \\
\hline $8 / 10 / 910: 00$ & Manual Tape & 299.75 & Transcribed & \\
\hline $8 / 10 / 917: 04$ & Auto FIC & 300.30 & Good & \\
\hline $8 / 11 / 910: 00$ & Manual Tape & 299.75 & Transcribed & \\
\hline $8 / 11 / 91 / 7: 04$ & Auto FIC & 300.3 & Good & \\
\hline $8 / 12 / 91 \quad 0: 00$ & Manual Tape & 298.75 & Transcribed & \\
\hline $8 / 12 / 917: 04$ & Auto FIC & 300.3 & Good & \\
\hline $8 / 13 / 910: 00$ & Manual Tape & 298.75 & Transeribed & \\
\hline $8 / 13 / 917: 04$ & Auto FIC & 300.30 & Good & \\
\hline $8 / 14 / 910: 00$ & Manual Tape & 299.75 & Transcribed & \\
\hline $8 / 14 / 917: 04$ & Auto FIC & 300.30 & Good & \\
\hline $8 / 15 / 910: 00$ & Manual Tape & 299.75 & Transeribed & \\
\hline $8 / 15 / 917: 03$ & Auto FIC & 300.3 & Good & \\
\hline $8 / 16 / 910: 00$ & Manual Tape & 299.75 & Transcribed & \\
\hline $8 / 16 / 91 \quad 0: 00$ & Manual FIC & 300.30 & Good & \\
\hline $8 / 17 / 910: 00$ & Manual Tape & 300.00 & Transcribed & \\
\hline $8 / 17 / 917: 02$ & Auto FIC & 300.30 & Good & \\
\hline $8 / 18 / 910: 00$ & Manual Tape & 299.75 & Transcribed - & . \\
\hline $8 / 18 / 917: 03$ & Ayto FIC & 300.30 & Good & \\
\hline $8 / 19 / 910: 00$ & Manual Tape & 299.75 & Transeribed & \\
\hline $8 / 19 / 917: 02$ & Auto FIC & 300.30 & Good & \\
\hline $8 / 20 / 910: 00$ & Manual Tape & 299.75 & Transcribed & \\
\hline $8 / 20 / 917: 04$ & Auto FIC & 300.30 & Good & \\
\hline $8 / 21 / 910: 00$ & Manual Tape & 299.75 & Transcribed & \\
\hline $8 / 21 / 917: 03$ & Auto FIC & 300.3 & Good & \\
\hline $8 / 22 / 910: 00$ & Manual Tape & 299.75 . & Transcribed & \\
\hline $8 / 22 / 917: 03$ & Auto FIC & 300.30 & Good & \\
\hline $8 / 23 / 910: 00$ & Manual Tape & 299.75 & Transcribed & \\
\hline $8 / 23 / 917: 02$ & Auto FIC & 300.3 & Good & \\
\hline $8 / 24 / 910: 00$ & Manual Tape & 299.75 & Transcribed & \\
\hline $8 / 24 / 917: 02$ & Auto FIC & 300.3 & Good & \\
\hline $8 / 25 / 910: 00$ & Manual Tape & 299.75 & Transcribed & \\
\hline $8 / 25 / 917: 02$ & Auto FIC & 300.30 & Good & \\
\hline $8 / 26 / 910: 00$ & Manual Tape & 299.75 & Transcribed & \\
\hline $8 / 26 / 917: 03$ & Auto FIC & 300.30 & Good & \\
\hline $8 / 27 / 910: 00$ & Manual Tape & 299.75 & Transcribed & \\
\hline $8 / 27 / 917: 03$ & Auto FIC & 300.4 & Good & \\
\hline $8 / 28 / 910: 00$ & Manual Tape & 299.75 & Transcribed & \\
\hline $8 / 28 / 917: 03$ & Auto FIC & 300.4 & Good & \\
\hline
\end{tabular}

Data obtained from WHC Surveillance Analysis Computer System, (PC SACS) July 7, 1995. 


\begin{tabular}{|c|c|c|c|c|}
\hline \multicolumn{5}{|c|}{ 241-AP-105 } \\
\hline \multicolumn{5}{|c|}{ Surface Level Data } \\
\hline Date & Type of Measurement & Leval (Incheal & Data Quality & Commants \\
\hline $8 / 29 / 910: 00$ & Manual Tape & 299.75 & Transcribed & \\
\hline $8 / 29 / 917: 02$ & Auto FIC & 300.40 & Good & \\
\hline $8 / 30 / 910: 00$ & Manual Tape & 299.75 & Transcribed & \\
\hline $8 / 30 / 916: 02$ & Auto FIC & 300.40 & Good & \\
\hline $8 / 31 / 910: 00$ & Manual Tape & 299.75 & Transcribed & \\
\hline $8 / 31 / 917: 02$ & Auto FIC & 300.40 & Good & \\
\hline 9/1/91 0:00 & Manual Tape & 299.75 & Transcribed & \\
\hline 9/1/91 7:02 & Auto FIC & 300.4 & Good & \\
\hline 9/2/91 0:00 & Manual Tape & 299.75 & Transcribed & \\
\hline 9/2/91 7:02 & Auto FIC & 300.40 & Good & \\
\hline 9/3/91 0:00 & Manual Tape & 299.75 & Transcribed & \\
\hline 9/3/91 7:02 & Auto FIC & 300.4 & Good & \\
\hline $9 / 4 / 910: 00$ & Manual Tape & 299.75 & Transcribed & \\
\hline 9/4/91 7:03 & Auto FIC & 300.4 & Good & \\
\hline $9 / 5 / 910: 00$ & Manual Tape & 299.75 & Transcribed & \\
\hline $9 / 5 / 917: 02$ & Auto FIC & 300.4 & Good & \\
\hline $9 / 6 / 910: 00$ & Manual Tape & 299.75 & Transcribed & \\
\hline $9 / 6 / 917: 02$ & Auto FIC & 300.40 & Good & \\
\hline $9 / 7 / 910: 00$ & Manual Tape & 299.50 & Transcribed & \\
\hline 9/7/91 7:02 & Auto FIC & 300.40 & Good & \\
\hline 9/8/910:00 & Manual Tape & 299.75 & Transcribed & \\
\hline $9 / 8 / 917: 02$ & Auto FIC & 300.40 & Good & \\
\hline 9/9/91 0:00 & Manual Tape & 299.75 & Transcribed. & \\
\hline 9/9/91 7:03 & Auto FIC & 300.40 & Good & \\
\hline $9 / 10 / 910: 00$ & Manual Tape & 299.75 & Transcribed & \\
\hline $9 / 10 / 917: 02$ & Auto FIC & 300.4 & Good & \\
\hline $9 / 11 / 910: 00$ & Manual Tape & 299.75 & Transcribed & \\
\hline 9/11/91 7:03 & Auto FIC & 300.40 & Good & \\
\hline $9 / 12 / 910: 00$ & Manual Tape & 299.75 & Transcribed & \\
\hline $9 / 12 / 917: 02$ & Auto FIC & 300.4 & Good & \\
\hline 9/13/91 0:00 & Manual Tape & 299.75 & Transcribed & \\
\hline $9 / 13 / 91 \quad 7: 02$ & Auto FIC & 300.40 & Good & 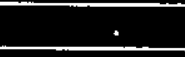 \\
\hline 9/14/91 $0: 00$ & Manual Tape & 299.75 & Transcribed & \\
\hline $9 / 14 / 917: 03$ & Auto FIC & 300.4 & Good & \\
\hline $9 / 15 / 910: 00$ & Manual Tape & 299.75 & Transcribed & \\
\hline $9 / 15 / 917: 02$ & Auto FIC & 300.4 & Good & \\
\hline 9/16/91 0:00 & Manual Tape & 299.75 & Transcribed & \\
\hline $9 / 16 / 917: 03$ & Auto FIC & $300: 40$ & Good & \\
\hline $9 / 17 / 910: 00$ & Manual Tape & 299.75 & Transcribed & \\
\hline $9 / 17 / 917: 03$ & Auto FIC & 300.4 & Good & \\
\hline $9 / 18 / 910: 00$ & Manual Tape & 299.75 & Transcribed & \\
\hline $9 / 18 / 917: 03$ & Auto FIC & 300.4 & Good & \\
\hline $9 / 19 / 910: 00$ & Manual Tape & 299.75 & Transcribed & \\
\hline $9 / 19 / 917: 03$ & Auto FIC & 300.4 & Good & \\
\hline $9 / 20 / 910: 00$ & Manual Tape & 299.75 & Transcribed & \\
\hline $9 / 20 / 917: 02$ & Auto FIC & 300.4 & Good & \\
\hline $9 / 21 / 910: 00$ & Manual Tape & 299.75 & Transcribed & \\
\hline $9 / 21 / 917: 02$ & Auto FIC & 300.40 & Good & \\
\hline
\end{tabular}

Data obtained from WHC Surveillance Analysis Computer System, (PC SACS) July 7, 1995. 


\begin{tabular}{|c|c|c|c|c|}
\hline \multicolumn{5}{|c|}{ 241-AP-105 } \\
\hline \multicolumn{5}{|c|}{ Surface Lovel Data } \\
\hline Date & Type of Measurement & Loval tinches\} & Data Quality & Comments \\
\hline 9/22/91 0:00 & Manual Tape & 299.75 & Transcribed & \\
\hline $9 / 22 / 917: 03$ & Auto FIC & 300.3 & Good & \\
\hline $9 / 23 / 910: 00$ & Manual Tape & 299.75 & Transcribed & \\
\hline $9 / 23 / 917: 03$ & Auto FIC & 300.3 & Good & \\
\hline 9/24/910:00 & Manual Tape: & 299.75 & Transcribed & \\
\hline 9/24/91 7:02 & Auto FIC & 300.3 & Good & \\
\hline $9 / 25 / 910: 00$ & Manual Tape & 299.75 & Transcribed & \\
\hline $9 / 25 / 917: 03$ & Auto FIC & 300.30 & Good & \\
\hline 9/26/910:00 & Manual Tape & 299.75 & Transcribed & \\
\hline $9 / 26 / 917: 03$ & Auto FIC & 300.30 & Good & \\
\hline $9 / 27 / 910: 00$ & Manual Tape & 299.75 & Transcribed & \\
\hline $9 / 27 / 917: 02$ & - Auto FIC & 300.30 & Good & \\
\hline $9 / 28 / 910: 00$ & Manual Tape & 299.75 & Transcribed & \\
\hline $9 / 28 / 917: 02$ & Auto FIC & 300.40 & Good & \\
\hline 9/29/910:00 & Manual Tape & 299.75 & Transcribed & \\
\hline 9/29/91 7:03 & Auto FIC & 300.3 & Good & \\
\hline 9/30/91 0:00 & Manual Tape & 299.75 & Transcribed & \\
\hline 9/30/91 7:03 & Auto FIC & 300.30 & Good & \\
\hline 10/1/91 0:00 & Manual Tape & 299.75 & Transcribed & \\
\hline $10 / 1 / 917: 02$ & Auto FIC & 300.30 & Good & \\
\hline $10 / 2 / 91 \quad 0: 00$ & Manual Tape & 299.75 & Transcribed & \\
\hline $10 / 2 / 917: 02$ & Auto FIC & 300.30 & Good & \\
\hline $10 / 3 / 910: 00$ & Manual Tape & 299.75 & Transcribed & \\
\hline 10/3/91 7:02 & Auto FIC & 300.3 & Good & \\
\hline $10 / 4 / 91 \quad 0: 00$ & Manual Tape & 299.75 & Transcribed & \\
\hline $10 / 4 / 917: 03$ & Auto FIC & 300.30 & Good & \\
\hline 10/5/91 0:00 & Manual Tape & 299.75 & Transcribed & \\
\hline 10/5/91 0:00 & Manual FIC & 300.3 & Good & \\
\hline $10 / 6 / 910: 00$ & Manual Tape & 299.75 & Transćribed & \\
\hline $10 / 6 / 917: 03$ & Auto FIC & 300.30 & Good & \\
\hline $10 / 7 / 910: 00$ & Manual Tape & 299.75 & Transcribed & \\
\hline $10 / 7 / 917: 02$ & Auto FIC & 300.30 & Good & \\
\hline $10 / 8 / 910: 00$ & Manual Tape & 299.75 & Transcribed & \\
\hline $10 / 8 / 917: 02$ & Auto FIC & 300.30 & Good & \\
\hline $10 / 9 / 910: 00$ & Manual Tape & 299.75 & Transcribed & \\
\hline $10 / 9 / 917: 02$ & Auto FIC & 300.30 & Good & \\
\hline $10 / 10 / 910: 00$ & Manual Tape & 299.75 & Transcribed & \\
\hline $10 / 10 / 917: 02$ & Auto FIC & 300.30 & Good & \\
\hline $10 / 11 / 910: 00$ & Manual Tape & 299.75 & Transcribed & \\
\hline $10 / 11 / 917: 02$ & Auto FIC & 300.30 & Good & \\
\hline $10 / 12 / 910: 00$ & Manual Tape & 299.75 & Transcribed & \\
\hline $10 / 12 / 917: 02$ & Auto FIC & 300.30 & Good & \\
\hline $10 / 13 / 910: 00$ & Manual Tape & 299.75 & Transcribed & \\
\hline $10 / 13 / 91 \quad 7: 03$ & Auto FIC & 300.30 & Good & \\
\hline $10 / 14 / 910: 00$ & Manual Tape & 299.75 & Transcribed & \\
\hline 10/14/91 7:02 & Auto FIC & 300.30 & Good & \\
\hline $10 / 15 / 91 \quad 0: 00$ & Manual Tape & 299.75 & Transcribed & \\
\hline $10 / 15 / 917: 02$ & Auto FIC & 300.3 & Good & \\
\hline
\end{tabular}

Data obtained from WHC Surveillance Analysis Computer System, (PC SACS) July 7, 1995. 


\begin{tabular}{|c|c|c|c|c|}
\hline \multicolumn{5}{|c|}{ 241-AP-105 } \\
\hline \multicolumn{5}{|c|}{ Surface Leval Data } \\
\hline Date & Type of Measurement & Level (Inchea) & Data Quality & Comments \\
\hline $10 / 16 / 910: 00$ & Manual Tape & 299.75 & Transcribed & \\
\hline $10 / 16 / 917: 03$ & Auto FIC & 300.3 & Good & \\
\hline $10 / 17 / 910: 00$ & Manual Tape & 299.75 & Transcribed & \\
\hline $10 / 17 / 917: 05$ & Auto FIC & 300.30 & Good & \\
\hline $10 / 18 / 910: 00$ & Manual Tape & 299.75 & Transcribed & \\
\hline 10/18/91 7:06 & Auto FIC & 300.30 & Good & \\
\hline $10 / 19 / 910: 00$ & Manual Tape & 299.76 & Transcribed & \\
\hline $10 / 19 / 917: 06$ & Auto FIC & 300.30 & Good & \\
\hline $10 / 20 / 910: 00$ & Manual Tape & 299.75 & Transcribed & \\
\hline $10 / 20 / 917: 02$ & Auto FIC & 300.30 & Good & \\
\hline $10 / 21 / 910: 00$ & Manual Tape & 299.75 & Transcribed & \\
\hline $10 / 21 / 917: 02$ & Auto FIC & 300.30 & Good & \\
\hline $10 / 22 / 910: 00$ & Manual Tape & 299.75 & Transcribed & \\
\hline $10 / 22 / 917: 03$ & Auto FIC & 300.3 & Good & \\
\hline $10 / 23 / 910: 00$ & Manual Tape & 299.75 & Transcribed & \\
\hline $10 / 23 / 917: 02$ & Auto FIC & 300.3 & Good & \\
\hline $10 / 24 / 910: 00$ & Manual Tape & 299.75 & Transcribed & \\
\hline $10 / 24 / 917: 02$ & Auto FIC & 300.30 & Good & \\
\hline $10 / 25 / 910: 00$ & Manual Tape & 299.75 & Transcribed & \\
\hline $10 / 25 / 917: 03$ & Auto FIC & 300.3 & Good & \\
\hline $10 / 26 / 910: 00$ & Manual Tape & 299.75 & Transcribed & \\
\hline $10 / 26 / 917: 03$ & Auto FIC & 300.3 & Good & \\
\hline $10 / 27 / 910: 00$ & Manual Tape & $299: 75$ & Transcribed & \\
\hline $10 / 27 / 917: 02$ & Auto FIC & 300.3 & Good & \\
\hline $10 / 28 / 910: 00$ & Manual Tape & 299.75 . & Transcribed & \\
\hline $10 / 28 / 917: 02$ & Auto FIC & 300.30 & Good & \\
\hline $10 / 29 / 91 \quad 0: 00$ & Manual Tape & 299.75 & Transcribed & \\
\hline $10 / 29 / 917: 03$ & Auto FIC & 300.3 & Good & \\
\hline $10 / 30 / 910: 00$ & Manual Tape & 299.75 & Transcribed & \\
\hline $10 / 30 / 917: 03$ & Auto FIC & 300.2 & Good & \\
\hline $10 / 31 / 91 \quad 0: 00$ & Manual Tape & 299.75 & Transcribed & \\
\hline $10 / 31 / 917: 03$ & Auto FIC & 300.20 & Good & \\
\hline $11 / 1 / 910: 00$ & Manual Tape & 299.75 & Transcribed & \\
\hline $11 / 1 / 917: 02$ & Auto FIC & 300.20 & Good & \\
\hline $11 / 2 / 910: 00$ & Manual Tape & 299.75 & Transcribed & \\
\hline $11 / 2 / 917: 03$ & Auto FIC & 300.2 & Good & \\
\hline $11 / 3 / 910: 00$ & Manual Tape & 299.75 & Transcribed & \\
\hline $11 / 3 / 917: 03$ & Auto FIC & 300.2 & Good & \\
\hline $11 / 4 / 910: 00$ & Manual Tape & 299.75 & Transcribed & \\
\hline $11 / 4 / 917: 02$ & Auto FIC & 300.2 & Good & \\
\hline $11 / 5 / 910: 00$ & Manual Tape & 299.75 & Transcribed & \\
\hline $11 / 5 / 917: 03$ & Auto FIC & 300.2 & Good & \\
\hline $11 / 6 / 910: 00$ & Manual Tape & 299.75 & Transcribed & \\
\hline $11 / 6 / 917: 03$ & Auto FIC & 300.2 & Good & \\
\hline $11 / 7 / 910: 00$ & Manual Tape & 299.75 & Transcribed & \\
\hline $11 / 7 / 917: 02$ & Auto FIC & 300.2 & Good & \\
\hline 11/8/91 0:00 & Manual Tape & 299.75 & Transcribed & \\
\hline $11 / 8 / 917: 03$ & Auto FIC & 300.20 & Good & \\
\hline
\end{tabular}

Data obtained from WHC Surveillance Analysis Computer System, (PC SACS) July 7, 1995. 


\begin{tabular}{|c|c|c|c|c|}
\hline \multicolumn{5}{|c|}{ 241-AP-105 } \\
\hline & & urface Level Dat & & \\
\hline Date & Type of Measurement & Leval (Inchos) & Data Quality & Comments \\
\hline $11 / 9 / 910: 00$ & Manual Tape & 299.75 & Transcribed & \\
\hline 11/9/91 7:02 & Auto FIC & 300.20 & Good & \\
\hline $11 / 10 / 910: 00$ & Manual Tape & 299.75 & Transcribed & \\
\hline $11 / 10 / 917: 03$ & Auto FIC & 300.2 & Good & \\
\hline $11 / 11 / 910: 00$ & Manual Tape & 299.75 & Transcribed & \\
\hline $11 / 11 / 916: 03$ & Auto FIC & 300.2 & Good & \\
\hline $11 / 12 / 910: 00$ & Manual Tape & 299.75 & Transcribed & \\
\hline $11 / 12 / 917: 03$ & Auto FIC & 300.20 & Good & \\
\hline $11 / 13 / 910: 00$ & Manual Tape & 299.75 & Transcribed & \\
\hline 11/13/91 7:03 & Auto FIC & 300.2 & Good & \\
\hline 11/14/910:00. & Manual Tape & 299.75 & Transcribed & \\
\hline $11 / 14 / 917: 03$ & Auto FIC & 300.20 & Good & \\
\hline $11 / 15 / 910: 00$ & Manual Tape & 299.75 & Transcribed & \\
\hline $11 / 15 / 917: 03$ & Auto FIC & 300.2 & Good & \\
\hline $11 / 16 / 910: 00$ & Manual Tape & 299.75 & Transcribed & \\
\hline $11 / 16 / 917: 03$ & Auto FIC & 300.2 & Good & \\
\hline $11 / 17 / 910: 00$ & Manual Tape & 299.75 & Transcribed & \\
\hline $11 / 17 / 917: 02$ & Auto FIC & 300.2 & Good & \\
\hline $11 / 18 / 910: 00$ & Manual Tape & 299.75 & Transcribed & \\
\hline $11 / 18 / 917: 03$ & Auto FIC & 300.20 & Good & \\
\hline $11 / 19 / 910: 00$ & Manual Tape & 299.75 & Transcribed & \\
\hline $11 / 19 / 917: 03$ & Auto FIC & 300.20 & Good & \\
\hline $11 / 20 / 910: 00$ & Manual Tape & 299.75 & Transcribed & \\
\hline $11 / 20 / 917: 03$ & Auto FIC & 300.20 & Good & \\
\hline $11 / 21 / 910: 00$ & Manual Tape & 299.75 & Transcribed & \\
\hline $11 / 21 / 917: 02$ & Auto FIC & 300.20 & Good & \\
\hline $11 / 22 / 910: 00$ & Manual Tapo & 299.75 & Transçribod & \\
\hline $11 / 22 / 917: 03$ & Auto FIC & 300.2 & Good & \\
\hline $11 / 23 / 910: 00$ & Manual Tape & 299.75 & Transcribed & \\
\hline $11 / 23 / 917: 02$ & Auto FIC & 300.20 & Good & \\
\hline $11 / 24 / 910: 00$ & Manual Tape & 299.75 & Transcribed & \\
\hline $11 / 24 / 917: 03$ & Auto FIC & 300.2 & Good & \\
\hline $11 / 25 / 910: 00$ & Manual Tape & 299.75 & Transcribed & \\
\hline $11 / 25 / 917: 03$ & Auto FIC & 300.2 & Good & \\
\hline $11 / 26 / 910: 00$ & Manual Tape & 299.50 & Transcribed & \\
\hline $11 / 26 / 910: 00$ & Manual FIC & 300.20 & Good & \\
\hline $11 / 27 / 910: 00$ & Manual Tape & 299.50 & Transcribed & \\
\hline $11 / 27 / 917: 03$ & Auto FIC & 300.20 & Good & \\
\hline $11 / 28 / 910: 00$ & Manual Tape & 299.50 & Transcribed & \\
\hline $11 / 28 / 917: 03$ & Auto FIC & 300.10 & Good & \\
\hline $11 / 29 / 910: 00$ & Manual Tape & 299.50 & Transcribed & \\
\hline $11 / 29 / 917: 03$ & Auto FIC & 300.1 & Good & \\
\hline $11 / 30 / 910: 00$ & Manual Tape & 299.50 & Transcribed & \\
\hline $11 / 30 / 917: 03$ & Auto FIC & 300.1 & Good & \\
\hline $12 / 1 / 910: 00$ & Manual Tape & 299.50 & Transcribed & \\
\hline $12 / 1 / 917: 03$ & Auto FIC & 300.10 & Good & \\
\hline $12 / 2 / 910: 00$ & Manual Tape & 299.50 & Transcribed & \\
\hline $12 / 2 / 917: 03$ & Auto FIC & 300.10 & Good & \\
\hline
\end{tabular}

Data obtained from WHC Surveillance Analysis Computer System, (PC SACS) July 7, 1995. 


\begin{tabular}{|c|c|c|c|c|}
\hline \multicolumn{5}{|c|}{ 241-AP-105 } \\
\hline & & Ufface Level Dat & & \\
\hline Date & Type of Moasurement & Levol (Inches) & Data Quality & Comments \\
\hline $12 / 3 / 910: 00$ & Manual Tape & 299.5 . & Transcribed & \\
\hline $12 / 3 / 917: 03$ & Auto FIC & 300.10 & Good & \\
\hline $12 / 4 / 910: 00$ & Manual Tape & 299.50 & Transcribed & \\
\hline $12 / 4 / 917: 03$ & Auto FIC & 300.10 & Good & \\
\hline $12 / 5 / 910: 00$ & Manual Tape & 299.5 & Transcribed & \\
\hline $12 / 5 / 917: 03$ & Auto FIC & 300.1 & Good & \\
\hline $12 / 6 / 910: 00$ & Manual Tape & 299.50 & Transeribed & \\
\hline $12 / 6 / 917: 04$ & Auto FIC & 300.10 & Good & \\
\hline $12 / 7 / 910: 00$ & Manual Tape & 299.5 & Transcribed & \\
\hline $12 / 7 / 917: 03$ & Auto FIC & 300.10 & Good & \\
\hline $12 / 8 / 910: 00$ & Manual Tape & 299.5 & Transcribed & \\
\hline $12 / 8 / 917: 03$ & Auto FIC & 300.1 & Good & \\
\hline $12 / 9 / 910: 00$ & Manual Tape & 299.5 & Transcribed & \\
\hline $12 / 9 / 917: 03$ & Auto FIC & 300.1 & Good & \\
\hline 12/10/910:00 & Manual Tape & 299.50 & Transcribed & \\
\hline $12 / 10 / 917: 03$ & Auto FIC & $300: 10$ & Good & \\
\hline $12 / 11 / 910: 00$ & Manual Tape & . 299.50 & Transcribed & \\
\hline $12 / 11 / 917: 02$ & Auto FIC & 300.10 & Good & \\
\hline $12 / 12 / 910: 00$ & Manual Tape & 299.5 & Transcribed & \\
\hline $12 / 12 / 917: 03$ & Auto FIC & 300.10 & Good & \\
\hline $12 / 13 / 910: 00$ & Manual Tape & 299.5 & Transcribed & \\
\hline $12 / 13 / 917: 05$ & Auto FIC & 300.1 & Good & \\
\hline $12 / 14 / 910: 00$ & Manual Tape & 299.50 & Transcribed & \\
\hline 12/14/917:02 & Auto FIC & 300.10 & Good & \\
\hline $12 / 15 / 910: 00$ & Manual Tape & 299.50 & Transcribed & \\
\hline $12 / 15 / 917: 02$ & Auto FIC & 300.10 & Good & \\
\hline $12 / 16 / 910: 00$ & Manual Tape & $299.50^{\circ}$ & Transcribed & \\
\hline $12 / 16 / 917: 03$ & Auto FIC & 300.10 & Good & \\
\hline $12 / 17 / 910: 00$ & Manual Tape & 299.50 & Transcribed & \\
\hline $12 / 17 / 917: 06$ & Auto FIC & 300.10 & Good & \\
\hline $12 / 18 / 910: 00$ & Manual Tape & 299.50 & Transcribed & \\
\hline $12 / 18 / 917: 03$ & Auto FIC & 300.10 & Goad & \\
\hline $12 / 19 / 910: 00$ & Manual Tape & 299.5 & Transcribed & \\
\hline $12 / 19 / 917: 03$ & Auto FIC & 300 & Good & \\
\hline $12 / 20 / 910: 00$ & Manual Tape & 299.5 & Transcribed & \\
\hline $12 / 20 / 917: 02$ & Auto FIC & 300 & Good & \\
\hline $12 / 21 / 910: 00$ & Manual Tape & 299.50 & Transcribed & \\
\hline $12 / 21 / 917: 03$ & Auto FIC & 300 & Good & \\
\hline $12 / 22 / 910: 00$ & Manual Tape & 299.5 & Transcribed & \\
\hline $12 / 22 / 917: 05$ & Auto FIC & 300 & Good & \\
\hline $12 / 23 / 910: 00$ & Manual Tape & 299.5 & Transcribed & \\
\hline $12 / 23 / 917: 06$ & Auto FIC & 300 & Good & \\
\hline $12 / 24 / 910: 00$ & Manual Tape & 299.5 & Transcribed & \\
\hline $12 / 24 / 917: 03$ & Auto FIC & 300.00 & Good & \\
\hline $12 / 25 / 910: 00$ & Manual Tape & 299.50 & Transcribed & \\
\hline $12 / 25 / 917: 03$ & Auto FIC & 300.00 & Good & \\
\hline $12 / 26 / 910: 00$ & Manual Tape & 299.50 & Transcribed & \\
\hline $12 / 26 / 917: 03$ & Auto FIC & 300 & Good & \\
\hline
\end{tabular}

Data obtained from WHC Surveillance Analysis Computer System, (PC SACS) July 7, 1995. 


\begin{tabular}{|c|c|c|c|c|}
\hline \multicolumn{5}{|c|}{ 241-AP-105 } \\
\hline \multicolumn{5}{|c|}{ Surface Level Data } \\
\hline Date & Type of Measurement & Leval (Inchea) & Data Quality & Comments \\
\hline 12/27/91 0:00 & Manual Tape & 299.50 & Transcribed & \\
\hline $12 / 27 / 917: 03$ & Auto FIC & 300 & Good & \\
\hline 12/28/91 0:00 & Manual Tape & 299.5 & Transcribed & \\
\hline $12 / 28 / 917: 02$ & Auto FIC & 300.00 & Good & \\
\hline 12/29/910:00 & Manual Tape & 299.5 & Transcribed & \\
\hline $12 / 29 / 917: 03$ & Auto FIC & 300.00 & Good & \\
\hline $12 / 30 / 910: 00$ & Manual Tape & 299.25 & Transcribed & \\
\hline $12 / 30 / 917: 03$ & Auto FIC & 300.00 & Good & \\
\hline 12/31/910:00 & Manual Tape & 299.25 & Transcribed & \\
\hline $12 / 31 / 917: 03$ & Auto FIC & 300 & Good & \\
\hline $1 / 1 / 920: 00$ & Manual Tape & 299.50 & Transcribed & \\
\hline 1/1/92 7:03 & Auto FIC & 300.00 & Good & \\
\hline $1 / 2 / 920: 00$ & Manual Tape & 299.50 & Transcribed & \\
\hline 1/2/92 7:02 & Auto FIC & 300.00 & Good & \\
\hline $1 / 3 / 920: 00$ & Manual Tape & 299.25 & Transcribed & \\
\hline $1 / 3 / 927: 04$ & Auto FIC & 300 & Good & - \\
\hline 1/4/92 0:00 & Manual Tape & 299.5 & Transcribed & \\
\hline 1/4/92 7:03 & Auto FIC & 300.00 & Good & \\
\hline 1/5/92 0:00 & Manual Tape & 299.5 & Transcribed & \\
\hline $1 / 5 / 927: 03$ & Auto FIC. & 300 & Good & \\
\hline $1 / 6 / 920: 00$ & Manual Tape & 299.50 & Transcribed & \\
\hline $1 / 6 / 927: 03$ & Auto FIC & 300.00 & Good & \\
\hline $1 / 7 / 920: 00$ & Manual Tape & 299.5 & Transcribed & \\
\hline 1/7/92 7:04 & Auto FIC & 300 & Good & \\
\hline $1 / 8 / 920: 00$ & Manual Tape & 299.50 & Transcribed & \\
\hline 1/8/92 7:03 & Auto FIC & 300.00 & Good & \\
\hline 1/9/92 0:00 & Manual Tape & 299.25 & Transcribed & \\
\hline 1/9/92 7:03 & Auto FIC & 300 & Good & \\
\hline $1 / 10 / 920: 00$ & Manual Tape & 299.5 & Transcribed & \\
\hline 1/10/92 7:04 & Auto FIC & 300 & Good & \\
\hline $1 / 11 / 920: 00$ & Manual Tape & 299.25 & Transcribed & \\
\hline $1 / 11 / 927: 04$ & Auto FIC & 300 & Good & \\
\hline $1 / 12 / 920: 00$ & Manual Tape & 299.25 & Transcribed & \\
\hline $1 / 12 / 920: 00$ & Manual FIC & 299.9 & Good & \\
\hline 1/13/92 0:00 & Manual Tape & 299.25 & Transcribed & \\
\hline $1 / 13 / 920: 00$ & Manual FIC & 299.9 & Good & \\
\hline $1 / 14 / 920: 00$ & Manual Tape & 299.25 & Transcribed & \\
\hline $1 / 14 / 920: 00$ & Manual FIC & 299.9 & Good & \\
\hline $1 / 15 / 920: 00$ & Manual Tape & 299.25 & Transcribed & \\
\hline $1 / 15 / 927: 03$ & Auto FIC & 299.90 & Good & \\
\hline $1 / 16 / 920: 00$ & Manual Tape & 299.25 & Transcribed & \\
\hline $1 / 16 / 927: 04$ & Auto FIC & 299.9 & Good & \\
\hline $1 / 17 / 920: 00$ & Manual Tape & 299.50 & Transcribed & \\
\hline $1 / 17 / 927: 04$ & Auto FIC & 299.9 & Good & \\
\hline $1 / 18 / 920: 00$ & Manual Tape & 299.5 & Transcribed & \\
\hline $1 / 18 / 927: 04$ & Auto FIC & 299.90 & Good & \\
\hline $1 / 19 / 920: 00$ & Manual Tape & 299.25 & Transcribed & \\
\hline 1/19/92 7:03 & Auto FIC & 299.90 & Good & \\
\hline
\end{tabular}

Data obtained from WHC Surveillance Analysis Computer System, (PC SACS) July 7, 1995. 


\begin{tabular}{|c|c|c|c|c|}
\hline \multicolumn{5}{|c|}{ 241-AP-105 } \\
\hline & & Urface Level Dat & & \\
\hline Date & Type of Measurement & Level (Inchea) & Data Quality & Comments \\
\hline $1 / 20 / 920: 00$ & Manual Tape & 299.25 & Transcribed & \\
\hline $1 / 20 / 927: 03$ & Auto FIC & 299.90 & Good & \\
\hline $1 / 21 / 920: 00$ & Manual Tape & 299.50 & Transcribed & \\
\hline $1 / 21 / 927: 03$ & Auto FIC & 299.90 & Good & \\
\hline $1 / 22 / 920: 00$ & Manual Tape ' & 299.25 & Transcribed & \\
\hline $1 / 22 / 927: 06$ & Auto FIC & 299.9 & Good & \\
\hline $1 / 23 / 920: 00$ & Manual Tape & 299.25 & Transcribed & \\
\hline $1 / 23 / 927: 03$ & Auto FIC & 299.9 & Good & \\
\hline $1 / 24 / 920: 00$ & Manual Tape & 299.25 & Transcribed & \\
\hline $1 / 24 / 927: 02$ & Auto FIC & 299.9 & Good & \\
\hline $1 / 25 / 920: 00$ & Manual Tape & 299.25 & Transcribed & \\
\hline $1 / 25 / 927: 03$ & Auto FIC & 299.9 & Good & \\
\hline $1 / 26 / 920: 00$ & Manual Tape & 299.25 & Transcribed & \\
\hline $1 / 26 / 926: 03$ & Auto FIC & 299.9 & Good & \\
\hline $1 / 27 / 920: 00$ & Manual Tape & 299.25 & Transcribed & \\
\hline $1 / 27 / 927: 02$ & Auto FIC & 299.90 & Good & \\
\hline $1 / 28 / 920: 00$ & Manual Tape & 299.25 & Transcribed & \\
\hline $1 / 28 / 927: 03$ & Auto FIC & 299.90 & Good & \\
\hline $1 / 29 / 920: 00$ & Manual Tape & 299.25 & Transcribed & \\
\hline $1 / 29 / 927: 03$ & Auto FIC & 299.9 & Good & \\
\hline $1 / 30 / 920: 00$ & Manual Tape & 299.25 & Transcribed & \\
\hline $1 / 30 / 927: 03$ & Auto FIC & 299.90 & Good & \\
\hline $1 / 31 / 920: 00$ & Manual Tape & $299: 25$ & Transcribed & \\
\hline $1 / 31 / 927: 03$ & Auto FIC & 299.9 & Good & \\
\hline $2 / 1 / 920: 00$ & Manual Tape & 299.25 & Transcribed & \\
\hline $2 / 1 / 927: 02$ & Auto FIC & 299.90 & Good & \\
\hline $2 / 2 / 920: 00$ & Manual Tape & 299.25 & Transcribed & \\
\hline 2/2/92 7:03 & Auto FIC & 299.8 & Good & \\
\hline $2 / 3 / 92 \quad 0: 00$ & Manual Tape & 299.25 & Transcribed & \\
\hline $2 / 3 / 927: 03$ & Auto FIC & 299.80 & Good & \\
\hline $2 / 4 / 920: 00$ & Manual Tape & 299.25 & Transcribed & \\
\hline $2 / 4 / 927: 03$ & Auto FIC & 299.8 & Good & \\
\hline $2 / 5 / 92$ 0:00 & Manual Tape & 299.25 & Transcribed & \\
\hline 2/5/92 7:02 & Auto FIC & 299.8 & Good & \\
\hline $2 / 6 / 920: 00$ & Manual Tape & 299.25 & Transcribed & \\
\hline $2 / 6 / 920: 00$ & Manual FIC & 299.8 & Good & \\
\hline 2/7/92 0:00 & Manual Tape & 299.25 & Transcribed & \\
\hline $2 / 7 / 920: 00$ & Manual FIC & 299.8 & Good & \\
\hline $2 / 8 / 920: 00$ & Manual Tape & 299.25 & Transcribed & \\
\hline $2 / 8 / 927: 04$ & Auto FIC & 299.8 & Good & \\
\hline $2 / 9 / 920: 00$ & Manual Tape & 299.25 & Transcribed & \\
\hline 2/9/92 7:03 & Auto FIC & 299.80 & Good & \\
\hline $2 / 10 / 920: 00$ & Manual Tape & 299.25 & Transćribed & \\
\hline $2 / 10 / 927: 04$ & Auto FIC & 299.80 & Good & \\
\hline $2 / 11 / 920: 00$ & Manual Tape & 299.25 & Transcribed & \\
\hline $2 / 11 / 927: 03$ & Auto FIC & 299.80 & Good & \\
\hline $2 / 12 / 920: 00$ & Manual Tape & 299.25 & Transcribed & \\
\hline $2 / 12 / 927: 03$ & Auto FIC & 299.8 & Good & \\
\hline
\end{tabular}

Data obtained from WHC Surveillance Analysis Computer System, (PC SACS) July 7, 1995. 


\begin{tabular}{|c|c|c|c|c|}
\hline \multicolumn{5}{|c|}{ 241-AP-105 } \\
\hline & & Irface Level Da & & \\
\hline Dato & Type of Moasurement & Leval (Inchea) & Data Quality & Comments \\
\hline 2/13/92 0:00 & Manual Tape & 299.00 & Transcribed & \\
\hline $2 / 13 / 927: 03$ & Auto FIC & 299.80 & Good & \\
\hline $2 / 14 / 920: 00$ & Manual Tape & 299.25 & Transcribed & \\
\hline $2 / 14 / 927: 03$ & Auto FIC & 299.8 & Good & \\
\hline $2 / 15 / 920: 00$ & Manual Tape & 299.25 & Transcribed & \\
\hline $2 / 15 / 927: 03$ & Auto FIC & 299.80 & Good & \\
\hline $2 / 16 / 920: 00$ & Manual Tape & 299.25 & Transcribed & . \\
\hline 2/16/92 7:03 & Auto FIC & 299.80. & Good & \\
\hline $2 / 17 / 920: 00$ & Manual Tape & 299.25 & Transcribed & \\
\hline $2 / 17 / 927: 03$ & Auto FIC & 299.8 & Good & \\
\hline $2 / 18 / 920: 00$ & Manual Tape & 299.25 & Transcribed & \\
\hline 2/18/92 7:03 & Auto FIC & 299.8 & Good & \\
\hline $2 / 19 / 920: 00$ & Manual Tape & 299.25 & Transcribed & \\
\hline 2/19/92 7:03 & Auto FIC & 299.80 & Good & \\
\hline $2 / 20 / 920: 00$ & Manual Tape & 299.25 & Transcribed & \\
\hline $2 / 20 / 927: 03$ & Auto FIC & 299.80 & Good & \\
\hline 2/21/92 0:00 & Manual Tape & 299.25 & Transcribed & \\
\hline 2/21/92 7:03 & Auto FIC & 299.80 & Good & \\
\hline $2 / 22 / 920: 00$ & Manual Tape & 299.25 & Transcribed & \\
\hline $2 / 22 / 927: 03$ & Auto FIC & 299.80 & Good & \\
\hline $2 / 23 / 920: 00$ & Manual Tape & 299.25 & Transcribed & \\
\hline $2 / 23 / 927: 03$ & Auto FIC & 299.8 & Good & \\
\hline $2 / 24 / 920: 00$ & Manual Tapo & 299.25 & Transcribed & \\
\hline 2/24/92 7:03 & Auto FIC & 299.80 & Good & \\
\hline $2 / 25 / 920: 00$ & Manual Tape & 299.25 . & Transcribed & \\
\hline $2 / 25 / 927: 04$ & Auto FIC & 299.80 & Good & \\
\hline $2 / 26 / 920: 00$ & Manual Tape & 299.25 & Transcribed & \\
\hline $2 / 26 / 927: 03$ & Auto FIC & 299.8 & Good & \\
\hline $2 / 27 / 920: 00$ & Maniual Tape & 299.25 & Transcribed & \\
\hline $2 / 27 / 927: 04$ & Auto FIC & 299.8 & Good & \\
\hline $2 / 28 / 920: 00$ & Manual Tape & 299.25 & Transcribed & \\
\hline $2 / 28 / 927: 04$ & Auto FIC & 299.80 & Good & \\
\hline $2 / 29 / 920: 00$ & Manual Tape & 299.25 & Transcribed & \\
\hline $2 / 29 / 927: 03$ & Auto FIC & 299.8 & Good & \\
\hline $3 / 1 / 920: 00$ & Manual Tape & 299.25 & Transcribed & \\
\hline $3 / 1 / 92$ 7:03 & Auto FIC & 299.8 & Good & \\
\hline $3 / 2 / 920: 00$ & Manual Tape & 299.25 & Transcribed & \\
\hline 3/2/92 7:03 & Auto FIC & 299.8 & Good & \\
\hline $3 / 3 / 920: 00$ & Manual Tape & 299.25 & Transcribed & \\
\hline $3 / 3 / 927: 04$ & Auto FIC & $299: 80$ & Good & \\
\hline 3/4/92 0:00 & Manual Tape & . 299.25 & Transcribed & \\
\hline $3 / 4 / 92$ 7:04 & Auto FIC & 299.70. & Good & \\
\hline $3 / 5 / 920: 00$ & Manual Tape & 299.25 & Transcribed & \\
\hline 3/5/92 7:03 & Auto FIC & 299.70 & Good & \\
\hline $3 / 6 / 920: 00$ & Manual Tape & 299.25 & Transcribed & \\
\hline $3 / 6 / 927: 03$ & Auto FIC & 299.70 & Good & \\
\hline $3 / 7 / 920: 00$ & Manual Tape & 299.25 & Transcribed & \\
\hline $3 / 7 / 92$ 7:02 & Auto FIC & 299.70 & Good & \\
\hline
\end{tabular}

Data obtained from WHC Surveillance Analysis Computer System, (PC SACS) July 7, 1995. 


\begin{tabular}{|c|c|c|c|c|}
\hline \multicolumn{5}{|c|}{ 241-AP-105 } \\
\hline \multicolumn{5}{|c|}{ Surface Leval Data } \\
\hline Date & Type of Measurement & Level (Inchea) & Data Quality & Comments \\
\hline $3 / 8 / 92 \quad 0: 00$ & Manual Tape & 299.25 & Transcribed & \\
\hline $3 / 8 / 927: 02$ & Auto FIC & 299.70 & Good & \\
\hline $3 / 9 / 920: 00$ & Manual Tape & 299 & Transcribed & \\
\hline $3 / 9 / 927: 02$ & Auto FIC & 299.70. & Good & \\
\hline $3 / 10 / 920: 00$ & Manual Tape & 298.75 & Transcribed & \\
\hline $3 / 10 / 927: 03$ & Auto FIC & 299.70 & Good & \\
\hline $3 / 11 / 920: 00$ & Manual Tape & 299.25 & Transcribed & \\
\hline $3 / 11 / 927: 03$ & Auto FIC & 299.7 & Good & \\
\hline $3 / 12 / 920: 00$ & Manual Tape & 299.00 & Transcribed & \\
\hline $3 / 12 / 92$ 7:03 & Auto FIC & 299.7 & Good & \\
\hline $3 / 13 / 920: 00$ & Manual Tape & 298.75 & Transcribed & \\
\hline $3 / 13 / 927: 03$ & Auto FIC & 299.7 & Good & \\
\hline $3 / 14 / 920: 00$ & Manual Tape & 299.00 & Transcribed & \\
\hline $3 / 14 / 927: 03$ & Auto FIC & 299.7 & Good & \\
\hline $3 / 15 / 920: 00$ & Manual Tape & 299.00 & Transcribed & \\
\hline $3 / 15 / 927: 06$ & Auto FIC & 299.70 & Good & \\
\hline $3 / 16 / 920: 00$ & Manual Tape & 299 & Transcribed & \\
\hline $3 / 16 / 927: 03$ & Auto FIC & 299.70 & Good & \\
\hline $3 / 17 / 920: 00$ & Manual Tape & 299 & Transcribed & \\
\hline $3 / 17 / 927: 03$ & Auto FIC & 299.7 & Good & \\
\hline $3 / 18 / 920: 00$ & Manual Tape & 299 & Transcribed & \\
\hline $3 / 18 / 927: 03$ & Auto FIC & 299.7 & Good & \\
\hline $3 / 19 / 920: 00$ & Manual Tape & 299 & Transcribed & \\
\hline $3 / 19 / 926: 02$ & Auto FIC & 299.70 & Good & \\
\hline $3 / 20 / 920: 00$ & Manual Tape & 299 & Transcribed & \\
\hline $3 / 20 / 927: 02$ & Auto FIC & 299.70 & Good & \\
\hline $3 / 21 / 920: 00$ & Manual Tape & 299 & Transcribed & \\
\hline $3 / 21 / 927: 03$ & Auto FIC & 299.7 & Good. & \\
\hline $3 / 22 / 920: 00$ & Manual Tape & 299 & Transcribed & \\
\hline $3 / 22 / 927: 02$ & Auto FIC & 299.7 & Good & \\
\hline $3 / 23 / 92 \quad 0: 00$ & Manual Tape & 299 & Transcribed & \\
\hline $3 / 23 / 927: 02$ & Auto FIC & 299.7 & Good & \\
\hline $3 / 24 / 920: 00$ & Manual Tape & 299 & Transcribed & \\
\hline $3 / 24 / 927: 02$ & Auto FIC & 299.70 & Good & \\
\hline $3 / 25 / 920: 00$ & Manual Tape & 299 & Transcribed & \\
\hline $3 / 25 / 927: 03$ & Auto FIC & 299.70 & Good & \\
\hline $3 / 26 / 920: 00$ & Manual Tape & 299 & Transcribed & \\
\hline $3 / 26 / 927: 03$ & Auto FIC & 299.70 & Good & \\
\hline $3 / 27 / 92 \quad 0: 00$ & Manual Tape & 299 & Transcribed & \\
\hline $3 / 27 / 927: 02$ & Auto FIC & 299.70 & Good & \\
\hline $3 / 28 / 920: 00$ & Manual Tape & 299.00 & Transeribed & \\
\hline $3 / 28 / 927: 03$ & Auto FIC & 299.7 & Good & \\
\hline $3 / 29 / 920: 00$ & Manual Tape & 299.00 & Transcribed & \\
\hline $3 / 29 / 927: 03$ & Auto FIC & 299.70 & Good & \\
\hline $3 / 30 / 920: 00$ & Manual Tape & 299.00 & Transcribed & \\
\hline $3 / 30 / 927: 03$ & Auto FIC & 299.7 & Good & \\
\hline $3 / 31 / 920: 00$ & Manual Tape & 299.00 & Transcribed & \\
\hline $3 / 31 / 927: 03$ & Auto FIC & 299.7 & Good & \\
\hline
\end{tabular}

Data obtained from WHC Surveillance Analysis Computer System, (PC SACS) July 7, 1995. 


\begin{tabular}{|c|c|c|c|c|}
\hline \multicolumn{5}{|c|}{ 241-AP-105 } \\
\hline \multicolumn{5}{|c|}{ Surface Level Data } \\
\hline Date & Type of Measurement & Loval (Inches) & Data Quality & Comments \\
\hline $4 / 1 / 920: 00$ & Manual Tape & 299.00 & Transcribed & \\
\hline 4/1/92 7:03 & Auto FIC & 299.7 & Good & \\
\hline 4/2/92 0:00 & Manual Tape & 299.00 & Transcribed & \\
\hline 4/2/92 7:03 & Auto FIC & 299.70 & Good & \\
\hline 4/3/92 0:00 & Manual Tape & 299 & Transcribed & \\
\hline 4/3/92 7:03 & Auto FIC & 299.70 & Good & \\
\hline 4/4/92 0:00 & Manual Tape & 298.75 & Transeribed & \\
\hline 4/4/92 7:03 & Auto FIC & 299.7 & Good & \\
\hline 4/5/92 0:00 & Manual Tape & 298.75 & Transcribed & \\
\hline 4/5/92 7:03 & Auto FIC & 299.70 & Good & \\
\hline $4 / 6 / 920: 00$ & Manual Tape & 299.00 & Transcribed & \\
\hline $4 / 6 / 927: 03$ & Auto FIC & 299.6 & Good & \\
\hline $4 / 7 / 920: 00$ & Manual Tape & 299 & Transeribed & \\
\hline $4 / 7 / 927: 03$ & Auto FIC & 299.70 & Good & \\
\hline $4 / 8 / 920: 00$ & Manual Tape & 299 & Transcribed & \\
\hline 4/8/92 5:02 & Auto FIC & 299.70 & Good & \\
\hline $4 / 9 / 920: 00$ & Manual Tape & 299 & Transcribed & \\
\hline $4 / 9 / 927: 03$ & Auto FIC & 299.70 & Good & \\
\hline $4 / 10 / 920: 00$ & Manual Tape & 299.00 & Transeribed & \\
\hline $4 / 10 / 927: 03$ & Auto FIC & 299.7 & Good & \\
\hline $4 / 11 / 920: 00$ & Manual Tape & 299 & Transcribed & \\
\hline $4 / 11 / 927: 03$ & Auto FIC & 299.7 & Good & \\
\hline $4 / 12 / 920: 00$ & Manual Tape & 299 & Transcribed & \\
\hline $4 / 12 / 925: 03$ & Auto FIC & 299.7 & Good & \\
\hline $4 / 13 / 920: 00$ & Manual Tape & 299 & Transcribed & \\
\hline 4/13/92 7:03 & Auto FIC & 299.7 & Good & \\
\hline $4 / 14 / 920: 00$ & Manual Tape & 299.00 & Transcribed & \\
\hline $4 / 14 / 927: 03$ & Auto FIC & 299.70 & Good & \\
\hline $4 / 15 / 920: 00$ & Manual Tape & 298.75 & Transcribed & \\
\hline 4/15/92 7:02 & Auto FIC & 299.70 & Good & \\
\hline $4 / 16 / 92 \quad 0: 00$ & Manual Tape & 298.75 & Transcribed & \\
\hline $4 / 16 / 927: 03$ & Auto FIC & 299.70 & Good & \\
\hline $4 / 17 / 920: 00$ & Manual Tape & 299.00 & Transcribed & \\
\hline 4/17/92 7:03 & Auto FIC & 299.7 & Good & \\
\hline 4/18/92 0:00 & Manual Tape & 299.00 & Transcribed & \\
\hline 4/18/92 7:02 & Auto FIC & 299.70 & Good & \\
\hline $4 / 19 / 920: 00$ & Manual Tape & 299 & Transcribed & \\
\hline $4 / 19 / 927: 02$ & Auto FIC & 299.7 & Good & \\
\hline $4 / 20 / 920: 00$ & Manual Tape & 299 & Transcribed & \\
\hline $4 / 20 / 927: 03$ & Auto FIC & 299.70 & Good & \\
\hline $4 / 21 / 920: 00$ & Manual Tapo & 299.00 & Transcribed & \\
\hline $4 / 21 / 927: 03$ & Auto FIC & 299.7 & Good & \\
\hline $4 / 22 / 920: 00$ & Manual Tape & 299.00 & Transcribed & \\
\hline $4 / 22 / 927: 02$ & Auto FIC & 299.70 & Good & \\
\hline $4 / 23 / 920: 00$ & Manual Tape & 299 & Transcribed & \\
\hline $4 / 23 / 927: 03$ & Auto FIC & 299.60 & Good & \\
\hline 4/24/92 0:00 & Manual Tape & 299 & Transcribed & \\
\hline $4 / 24 / 927: 03$ & Auto FIC & 299.6 & Good & \\
\hline
\end{tabular}

Data obtained from WHC Surveillance Analysis Computer System, (PC SACS) July 7, 1995. 


\begin{tabular}{|c|c|c|c|c|}
\hline \multicolumn{5}{|c|}{ 241-AP-105 } \\
\hline \multicolumn{5}{|c|}{ Surface Level Data } \\
\hline Date & Type of Measurement & Level (Inchea) & Data Quality & Commants \\
\hline $4 / 25 / 920: 00$ & Manual Tape & 299 & Transcribed & \\
\hline $4 / 25 / 927: 04$ & Auto FIC & 299.70 & Good & \\
\hline $4 / 26 / 920: 00$ & Manual Tape & 299.00 & Transcribed & \\
\hline $4 / 26 / 927: 03$ & Auto FIC & 299.70 & Good & \\
\hline $4 / 27 / 920: 00$ & Manual Tape & 299.5 & Transcribed & \\
\hline $4 / 27 / 927: 04$ & Auto FIC & 299.70 & Good & \\
\hline $4 / 28 / 920: 00$ & Manual Tape & 299 & Transcribed & \\
\hline 4/28/92 7:03 & Auto FIC & 299.70 & Good & \\
\hline $4 / 29 / 920: 00$ & Manual Tape & 299 & Transcribed & \\
\hline $4 / 29 / 927: 03$ & Auto FIC & 299.7 & Good & \\
\hline $4 / 30 / 92 \quad 0: 00$ & Manual Tape & 299 & Transcribed & \\
\hline $4 / 30 / 927: 03$ & Auto FIC & 299.60 & Good & \\
\hline $5 / 1 / 920: 00$ & Manual Tape & 299.00 & Transcribed & \\
\hline $5 / 1 / 927: 03$ & Auto FIC & 299.60 & Good & \\
\hline $5 / 2 / 920: 00$ & Manual Tape & 299 & Transcribed & \\
\hline 5/2/92 2:03 & Auto FIC & 299.6 & Good & \\
\hline $5 / 3 / 920: 00$ & Manual Tape & 299 & Transcribed & \\
\hline $5 / 3 / 927: 03$ & Auto FIC & 299.6 & Good & \\
\hline $5 / 4 / 920: 00$ & Manual Tape & 299.00 & Transcribed & \\
\hline $5 / 4 / 927: 03$ & Auto FIC & 299.60 & Good & \\
\hline $5 / 5 / 920: 00$ & Manual Tape & 299.00 & Transcribed & \\
\hline $5 / 5 / 927: 04$ & Auto FIC & 299.6 & Good & \\
\hline $5 / 6 / 920: 00$ & Manual Tape & 299 & Transcribed & \\
\hline $5 / 6 / 927: 03$ & Auto FIC & 299.6 & Good & \\
\hline $5 / 7 / 920: 00$ & Manual Tape & 299.00 & Transcribed & \\
\hline $5 / 7 / 927: 04$ & Auto FIC & 299.60 & Good & \\
\hline $5 / 8 / 920: 00$ & Manual Tape & 299 & Transcribed & \\
\hline $5 / 8 / 92$ 7:03 & Auto FIC & 299.6 & Good & \\
\hline $5 / 9 / 920: 00$ & Manual Tape & 299.00 & Transcribed & \\
\hline $5 / 9 / 920: 00$ & Manual FIC & 299.60 & Good & \\
\hline $5 / 10 / 920: 00$ & Manual Tape & 299 & Transcribed & \\
\hline $5 / 10 / 926: 03$ & Auto FIC & 299.6 & Good & \\
\hline $5 / 11 / 920: 00$ & Manual Tape & 299 & Transcribed & \\
\hline $5 / 11 / 920: 00$ & Manual FIC & 299.6 & Good & \\
\hline $5 / 12 / 920: 00$ & Manual Tape & 299 & Transcribed & \\
\hline $5 / 12 / 927: 03$ & Auto FIC & 299.60 & Good & \\
\hline $5 / 13 / 920: 00$ & Manual Tape & 299.00 & Transçribed & \\
\hline $5 / 13 / 927: 03$ & Auto FIC & 299.6 & Good & \\
\hline $5 / 14 / 920: 00$ & Manual Tape & 299.00 & Transcribed & \\
\hline $5 / 14 / 927: 03$ & Auto FIC & 299.60 & Good & \\
\hline $5 / 15 / 920: 00$ & Manual Tape & 299 & Transcribed & \\
\hline $5 / 15 / 927: 03$ & Auto FIC & 299.60 & Good & \\
\hline $5 / 16 / 920: 00$ & Manual Tape & 299.00 & Transcribed & \\
\hline $5 / 16 / 927: 03$ & Auto FIC & 299.6 & Good & \\
\hline $5 / 17 / 920: 00$ & Manual Tape & 299 & Transcribed & \\
\hline $5 / 17 / 927: 02$ & Auto FIC & 299.60 & Good & \\
\hline $5 / 18 / 920: 00$ & Manual Tape & 299.00 & Transcribed & \\
\hline $5 / 18 / 927: 03$ & Auto FIC & 299.6 & Good & \\
\hline
\end{tabular}

Data obtained from WHC Surveillance Analysis Computer System, (PC SACS) July 7, 1995. 


\begin{tabular}{|c|c|c|c|c|}
\hline \multicolumn{5}{|c|}{ 241-AP-105 } \\
\hline \multicolumn{5}{|c|}{ Surface Leval Data } \\
\hline Date & Type of Measurement & Leval (Inches) & Data Quality & Comments \\
\hline $5 / 19 / 920: 00$ & Manual Tape & 299.00 & Transcribed & \\
\hline $5 / 19 / 927: 03$ & Auto FIC & 299.60 & Good & \\
\hline $5 / 20 / 920: 00$ & Manual Tape & $299.00^{\circ}$ & Transcribed & \\
\hline $5 / 20 / 927: 03$ & Auto FIC & 299.60 & Good & \\
\hline $5 / 21 / 920: 00$ & Manual Tape ' & 299.00 & Transcribed & \\
\hline $5 / 21 / 927: 03$ & Auto FIC & 299.6 & Good & \\
\hline $5 / 22 / 920: 00$ & Manual Tape & 299.00 & Transcribed & \\
\hline $5 / 22 / 927: 02$ & Auto FIC & 299.6 & Good & \\
\hline $5 / 23 / 920: 00$ & Manual Tape & 299.00 & Transcribed & \\
\hline $5 / 23 / 927: 03$ & Auto FIC & 299.60 & Good & \\
\hline $5 / 24 / 920: 00$ & Manual Tape & 299 & Transcribed & \\
\hline $5 / 24 / 927: 03$ & Auto FIC & 299.60 & Good & \\
\hline $5 / 25 / 920.00$ & Manual Tape & 299 & Transcribed & \\
\hline $5 / 25 / 927: 03$ & Auto FIC & 299.6 & Good & \\
\hline $5 / 26 / 920: 00$ & Manual Tapo & 299 & Transcribed & \\
\hline $5 / 26 / 920: 00$ & Manual FIC & 299.60 & Good & \\
\hline $5 / 27 / 920: 00$ & Manual Tape & 299 & Transeribed & \\
\hline $5 / 27 / 927: 03$ & Auto FIC & 299.60 & Good & \\
\hline $5 / 28 / 920: 00$ & Manual Tape & 299 & Transcribed & \\
\hline $5 / 28 / 927: 02$ & Auto FIC & 299.6 & Good & \\
\hline $5 / 29 / 92 \quad 0: 00$ & Manual Tapo & 299.00 & Transcribed & \\
\hline $5 / 29 / 927: 02$ & Auto FIC & 299.6 & Good & \\
\hline $5 / 30 / 920: 00$ & Manual Tape & 299.00 & Transcribed & \\
\hline $5 / 30 / 927: 03$ & Auto FIC & 299.60 & Good & \\
\hline $5 / 31 / 920: 00$ & Manual Tape & 299 & Transcribed & \\
\hline $5 / 31 / 927: 03$ & Auto FIC & 299.6 & Good & \\
\hline $6 / 1 / 920: 00$ & Manual Tape & 299 & Transcribed & \\
\hline 6/1/92 7:03 & Auto FIC & 299.6 & Good & \\
\hline $6 / 2 / 920: 00$ & Manual Tape & 299.00 & Transcribed & \\
\hline $6 / 2 / 927: 03$ & Auto FIC & 299.60 & Good & \\
\hline $6 / 3 / 920: 00$ & Manual Tape & 299 & Transcribed & \\
\hline $6 / 3 / 927: 03$ & Auto FIC & 299.60 & Good & \\
\hline $6 / 4 / 92$ 0:00 & Manual Tape & 299 & Transcribed & \\
\hline $6 / 4 / 927: 03$ & Auto FIC & 299.60 & Good & \\
\hline $6 / 5 / 92 \quad 0: 00$ & Manual Tape & 299.00 & Transcribed & \\
\hline $6 / 5 / 927: 02$ & Auto FIC & 299.60 & Good & \\
\hline $6 / 6 / 920: 00$ & Manual Tape & 299 & Transcribed & \\
\hline $6 / 6 / 927: 03$ & Auto FIC & 299.6 & Good & \\
\hline $6 / 7 / 920: 00$ & Manual Tape & 299 & Transcribed & \\
\hline $6 / 7 / 927: 03$ & Auto FIC & 299.60 & Good & \\
\hline $6 / 8 / 920: 00$ & Manual Tape & 299 & Transcribed & \\
\hline $6 / 8 / 927: 03$ & Auto FIC & 299.60 & Good & \\
\hline $6 / 9 / 920: 00$ & Manual Tape & 298.75 & Transcribed & \\
\hline $6 / 9 / 927: 03$ & Auto FIC & 299.60 & Good & \\
\hline $6 / 10 / 920: 00$ & Manual Tape & 299.00 & Transcribed & \\
\hline $6 / 10 / 927: 03$ & Auto FIC & 299.60 & Good & \\
\hline $6 / 11 / 920: 00$ & Manual Tape & 299.00 & Transcribed & \\
\hline $6 / 11 / 927: 03$ & Auto FIC & 299.6 & Good & \\
\hline
\end{tabular}

Data obtained from WHC Surveillance Analysis Computer System, (PC SACS) July 7, 1995. 


\begin{tabular}{|c|c|c|c|c|}
\hline \multicolumn{5}{|c|}{ 241-AP-105 } \\
\hline \multicolumn{5}{|c|}{ Surface Level-Data } \\
\hline Date & Type of Measurement & Lavel (Inches) & Data Quality & Comments \\
\hline $6 / 12 / 920: 00$ & Manual Tape & 298.75 & Transcribed & \\
\hline 6/12/92 7:05 & Auto FIC & 299.60 & Good & \\
\hline $6 / 13 / 920: 00$ & Manual Tape & 299 & Transcribed & \\
\hline $6 / 13 / 92$ 7:05 & Auto FIC & 299.60 & Good & \\
\hline $6 / 14 / 920: 00$ & Manual Tape & 299 & Transcribed & \\
\hline $6 / 14 / 920: 00$ & Manual FIC & 299.6 & Good & \\
\hline $6 / 15 / 920: 00$ & Manual Tape & 299.00 & Transcribed & \\
\hline $6 / 15 / 927: 03$ & Auto FIC & 299.60 & Good & \\
\hline $6 / 16 / 920: 00$ & Manual Tape & 299.00 & Transcribed & \\
\hline $6 / 16 / 927: 03$ & Auto FIC & 299.60 & Good & \\
\hline $6 / 17 / 920: 00$ & Manual Tape & 299.25 & Transcribed & \\
\hline $6 / 17 / 92 \quad 7: 03$ & Auto FIC & 299.60 & Good & \\
\hline $6 / 18 / 92 \quad 0: 00$ & Manual Tape & 298.75 & Transcribed & \\
\hline $6 / 18 / 927: 03$ & Auto FIC & 299.60 & Good & \\
\hline $6 / 19 / 92 \quad 0: 00$ & Manual Tape & 299 & Transcribed & \\
\hline $6 / 19 / 927: 02$ & Auto FIC & 299.6 & Good & \\
\hline $6 / 20 / 920: 00$ & Manual Tape & 299.00 & Transcribed & \\
\hline $6 / 20 / 927: 02$ & Auto FIC & 299.60 & Good & \\
\hline $6 / 21 / 920: 00$ & Manual Tape & 299 & Transcribed & \\
\hline $6 / 21 / 927: 03$ & Auto FIC & 299.6 & Good & \\
\hline $6 / 22 / 920: 00$ & Manual Tape & 299.00 & Transcribed & \\
\hline $6 / 22 / 927: 03$ & Auto FIC & 299.6 & Good & \\
\hline $6 / 23 / 920: 00$ & Manual Tape & 299.00 & Transeribed & \\
\hline $6 / 23 / 927: 03$ & Auto FIC & 299.60 & Good & \\
\hline $6 / 24 / 920: 00$ & Manual Tape & 299 & Transcribed & \\
\hline $6 / 24 / 927: 03$ & Auto FIC & 299.6 & Good & \\
\hline $6 / 25 / 92 \quad 0: 00$ & Manual Tape & 299.00 & Transcribed & \\
\hline $6 / 25 / 927: 03$ & Auto FIC & 299.60 & Good & \\
\hline $6 / 26 / 920: 00$ & Manual Tape & 299 & Transcribed & \\
\hline $6 / 26 / 927: 02$ & Auto FIC & 299.6 & Good & \\
\hline $6 / 27 / 92 \quad 0: 00$ & Manual Tape & 299 & Transcribed & \\
\hline $6 / 27 / 927: 03$ & Auto FIC & 299.60 & Good & \\
\hline $6 / 28 / 920: 00$ & Manual Tape & 298.75 & Transcribed & \\
\hline $6 / 28 / 927: 06$ & Auto FIC & 299.6 & Good & \\
\hline $6 / 29 / 920: 00$ & Manual Tape & 299 & Transcribed & \\
\hline $6 / 29 / 927: 06$ & Auto FIC & 299.6 & Good & \\
\hline $6 / 30 / 920: 00$ & Manual Tape & 299 & Transcribed & \\
\hline $6 / 30 / 927: 06$ & Auto FIC & 299.60 & Good & \\
\hline $7 / 1 / 920: 00$ & Manual Tape & 299 & Transcribed & \\
\hline $7 / 1 / 920: 00$ & Manual FIC & 299.6 & Good & \\
\hline $7 / 2 / 920: 00$ & Manual Tape & 299 & Transcribed & \\
\hline $7 / 2 / 920: 00$ & Manual FIC & 299.6 & Good & \\
\hline $7 / 3 / 920: 00$ & Manual Tape & 299 & Transcribed & \\
\hline $7 / 3 / 927: 06$ & Auto FIC & 299.6 & Good & \\
\hline $7 / 4 / 920: 00$ & Manual Tape & 299.00 & Transcribed & \\
\hline $7 / 4 / 927: 06$ & Auto FIC & 299.6 & Good & \\
\hline $7 / 5 / 920: 00$ & Manual Tape & 299.00 & Transcribed & \\
\hline $7 / 5 / 927: 06$ & Auto F1C & 299.6 & Good & \\
\hline
\end{tabular}

Data obtained from WHC Surveillance Analysis Computer System, (PC SACS) July 7, 1995. 


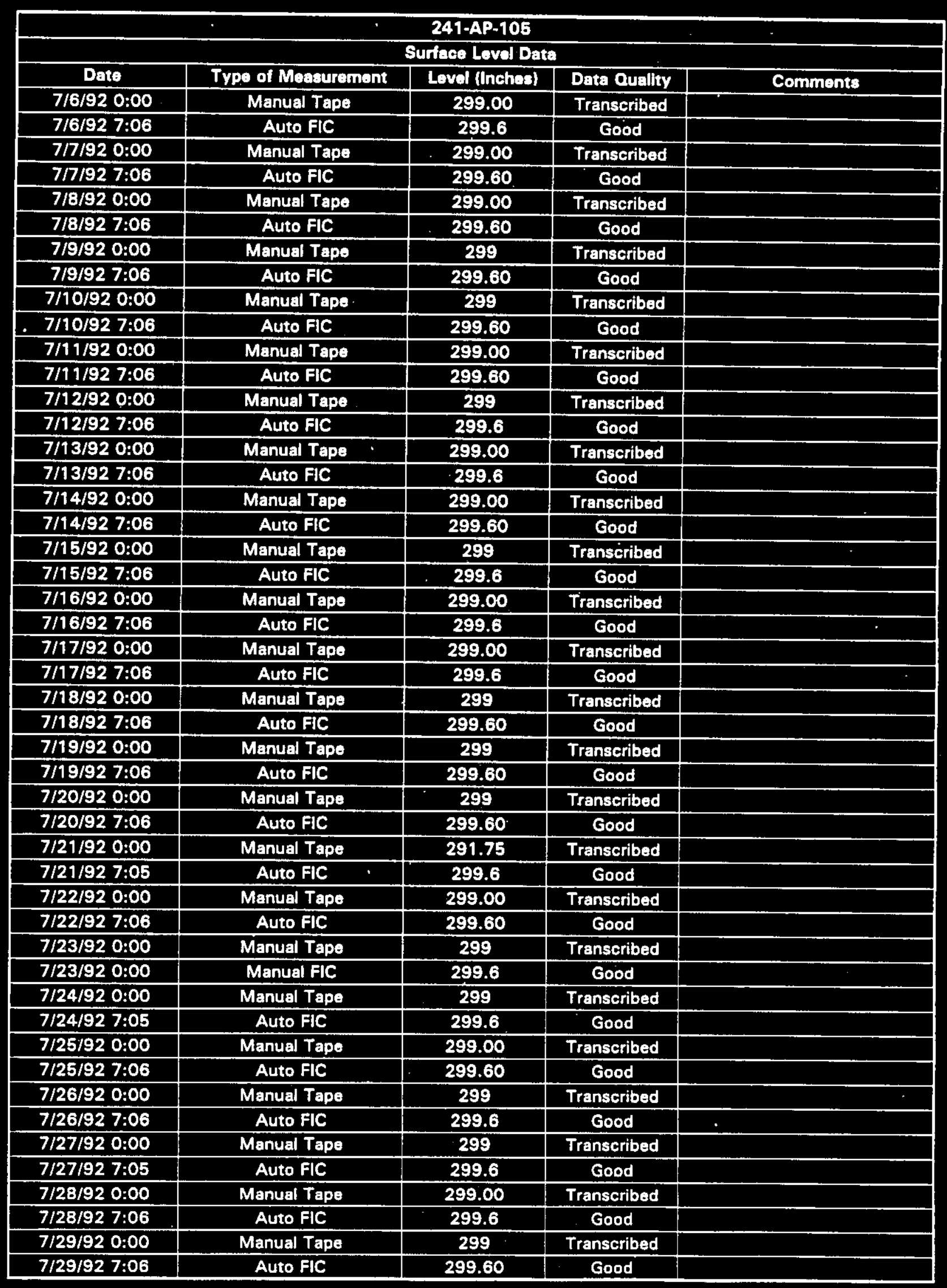

Data obtained from WHC Surveillance Analysis Computer System, (PC SACS) July 7, 1995. 


\begin{tabular}{|c|c|c|c|c|}
\hline \multicolumn{5}{|c|}{ 241-AP:105 } \\
\hline & & Urface Lovel Dat & & \\
\hline Date & Type of Measurement & Level (Inches) & Date Quality & Comments \\
\hline $7 / 30 / 920: 00$ & Manual Tape & 299.00 & Transcribed & \\
\hline $7 / 30 / 927: 06$ & Auto FIC & 299.60 & Good & \\
\hline $7 / 31 / 920: 00$ & Manual Tape & 299.00 & Transcribed & \\
\hline 7/31/92 7:05 & Auto FIC & 299.60 & Good & \\
\hline $8 / 1 / 920: 00$ & Manual Tape & 299.25 & Transcribed & \\
\hline $8 / 1 / 927: 06$ & Auto FIC & 299.70 & Good & \\
\hline $8 / 2 / 920: 00$ & Manual Tape & 299.00 & Transcribed & \\
\hline $8 / 2 / 927: 06$ & Auto FIC & 299.6 & Good & \\
\hline $8 / 3 / 920: 00$ & Manual Tape & 299 & Transcribed & \\
\hline $8 / 3 / 927: 06$ & Auto FIC & 299.7 & Good & \\
\hline $8 / 4 / 920: 00$ & Manual Tape & 299 & Transcribed & \\
\hline 8/4/92 7:06. & Auto FIC & 299.7 & Good & \\
\hline $8 / 5 / 920: 00$ & Manual Tape & 299.00 & Transcribed & \\
\hline $8 / 5 / 927: 05$ & Auto FIC & 299.6 & Good & \\
\hline $8 / 6 / 920: 00$ & Manual Tape & 299 & Transcribed & \\
\hline $8 / 6 / 927: 05$ & Auto FIC & 299.70 & Good & \\
\hline $8 / 7 / 920: 00$ & Manual Tape & 299.00 & Transcribed & \\
\hline $8 / 7 / 927: 05$ & Auto FIC & 299.70. & Good & \\
\hline $8 / 8 / 920: 00$ & Manual Tape & 299.00 & Transcribed & \\
\hline $8 / 8 / 927: 06$ & Auto FIC & 299.60 & Good & \\
\hline $8 / 9 / 920: 00$ & Manual Tape & 299 & Transcribed & \\
\hline 8/9/92 7:06 & Auto FIC & 299.60 & Good & \\
\hline $8 / 10 / 920: 00$ & Manual Tape & 299 & Transcribed & \\
\hline $8 / 10 / 927: 05$ & Auto FIC & 299.6 & Good & \\
\hline $8 / 11 / 920: 00$ & Manual Tape & 299.00 & Transcribed & \\
\hline $8 / 11 / 927: 06$ & Auto FIC & 299.70 & Good & \\
\hline $8 / 12 / 920: 00$ & Manual Tape & 298.75 & Transcribed & \\
\hline $8 / 12 / 927: 06$ & Auto FIC & 299.70 & Good & \\
\hline $8 / 13 / 920: 00$ & Manual Tape & 298.75 & Transcribed & \\
\hline $8 / 13 / 927: 06$ & Auto FIC & 299.70 & Good & \\
\hline $8 / 14 / 920: 00$ & Manual Tape & 299.00 & Transcribed & \\
\hline $8 / 14 / 927: 06$ & Auto FIC & 299.70 & Good & \\
\hline $8 / 15 / 920: 00$ & Manual Tape & 299 & Transcribed & \\
\hline $8 / 15 / 927: 06$ & Auto FIC & 299.70 & Good & \\
\hline $8 / 16 / 920: 00$ & Manual Tape & 299 & Transcribed & \\
\hline $8 / 16 / 927: 06$ & Auto FIC & 299.7 & Good & \\
\hline $8 / 17 / 920: 00$ & Manual Tape & 299.00 & Transcribed & \\
\hline $8 / 17 / 927: 06$ & Auto FIC & 299.70 & Good & \\
\hline $8 / 18 / 920: 00$ & Manual Tape & 299 & Transcribed & \\
\hline $8 / 18 / 927: 06$ & Auto FIC & 299.7 & Good & \\
\hline $8 / 19 / 920: 00$ & Manual Tape & 299 & Transcribed & \\
\hline $8 / 19 / 927: 06$ & Auto FIC & 299.7 & Good & \\
\hline $8 / 20 / 920: 00$ & Manual Tape & 295.25 & Transcribed & \\
\hline $8 / 20 / 927: 06$ & Auto FIC & 299.70 & Good & \\
\hline $8 / 21 / 920: 00$ & Manual Tape & 299.00 & Transcribed & \\
\hline $8 / 21 / 927: 05$ & Auto FIC & 299.7 & Good & \\
\hline $8 / 22 / 920: 00$ & Manual Tape & 299.00 & Transcribed & \\
\hline $8 / 22 / 927: 04$ & Auto FIC & 299.70 & Good & \\
\hline
\end{tabular}

Data obtained from WHC Surveillance Analysis Computer System, (PC SACS) July 7, 1995. 


\begin{tabular}{|c|c|c|c|c|}
\hline \multicolumn{5}{|c|}{ 241-AP-105 } \\
\hline \multicolumn{5}{|c|}{ Surface Lovel Data } \\
\hline Date & Type of Moasurement & Level (Inches) & Date Quality & Comments \\
\hline $8 / 23 / 920: 00$ & Manual Tape & 299.00 & Transcribed & \\
\hline $8 / 23 / 927: 04$ & Auto FIC & 299.7 & Good & \\
\hline $8 / 24 / 920: 00$ & Manual Tape & 299 & Transcribed & \\
\hline $8 / 24 / 927: 04$ & Auto FIC & 299.7 & Good & \\
\hline $8 / 25 / 920: 00$ & Manual Tape & 299 & Transcribed & \\
\hline $8 / 25 / 927: 04$ & Auto FIC & 299.7 & Good & \\
\hline $8 / 26 / 920: 00$ & Manual Tape & 299.00 & Transcribed & \\
\hline $8 / 26 / 927: 03$ & Auto FIC & 299.70 & Good & \\
\hline $8 / 27 / 920: 00$ & Manual Tape & 298.75 & Transcribed & \\
\hline $8 / 27 / 927: 04$ & Auto FIC & 299.70 & Good & \\
\hline $8 / 28 / 920: 00$ & Manual Tape & 299.00 & Transcribed & \\
\hline $8 / 28 / 927: 02$ & Auto FIC & 299.70 & Good & \\
\hline $8 / 29 / 920: 00$ & Manual Tape & 299.00 & Transcribed & \\
\hline $8 / 29 / 927: 02$ & Auto FIC & 299.7 & Good & \\
\hline $8 / 30 / 920: 00$ & Manual Tape & 299 & Transcribed & \\
\hline $8 / 30 / 927: 02$ & Auto FIC & 299.7 & Good & \\
\hline $8 / 31 / 920: 00$ & Manual Tape & 299.00 & Transcribed & \\
\hline $8 / 31 / 927: 02$ & Auto FIC & 299.70 & Good & \\
\hline $9 / 1 / 920: 00$ & Manual Tape & 299.00 & Transcribed & \\
\hline $9 / 1 / 927: 02$ & Auto FIC & 299.7 & Good & \\
\hline $9 / 2 / 92 \quad 0: 00$ & Manual Tape & 299 & Transcribed & \\
\hline $9 / 2 / 927: 02$ & Auto FIC & 299.7 & Good & \\
\hline $9 / 3 / 920: 00$ & Manual Tape & 299 & Transcribed & \\
\hline $9 / 3 / 927: 04$ & Auto FIC & 299.7 & Good & \\
\hline $9 / 4 / 920: 00$ & Manual Tape & 299 & Transcribed & \\
\hline 9/4/92 7:04 & Auto FIC & 299.7 & Good & \\
\hline $9 / 5 / 920: 00$ & Manual Tape & 299 & Transcribed & \\
\hline $9 / 5 / 927: 04$ & Auto FIC & 299.70 & Good & \\
\hline $9 / 6 / 920: 00$ & Manual Tape & 299 & Transćribed & \\
\hline $9 / 6 / 927: 04$ & Auto FIC & 299.6 & Good & \\
\hline $9 / 7 / 920: 00$ & Manual Tape & 299.00 & Transcribed & \\
\hline $9 / 7 / 92 \quad 7: 05$ & Auto FIC. & 299.60 & Good & \\
\hline $9 / 8 / 920: 00$ & Manual Tape & 299 & Transcribed & \\
\hline $9 / 8 / 92 \quad 7: 05$ & Auto FIC & 299.70 & Good & \\
\hline 9/9/92 0:00 & Manual Tape & 299.00 & Transcribed & \\
\hline $9 / 9 / 927: 04$ & Auto FIC & 299.60 & Good & \\
\hline $9 / 10 / 920: 00$ & Manual Tape & 299 & Transçribed & \\
\hline $9 / 10 / 927: 03$ & Auto FIC & 299.60 & Good & \\
\hline $9 / 11 / 920: 00$ & Manual Tape & 299.00 & Transcribed & \\
\hline $9 / 11 / 920: 00$ & Manual FIC & 299.6 & Good & \\
\hline $9 / 12 / 920: 00$ & Manual Tape & 299 & Transcribed & \\
\hline $9 / 12 / 920: 00$ & Manual FIC & 299.60 & Good & \\
\hline 9/13/92 0:00 & Manual Tape & 299 & Transcribed & \\
\hline $9 / 13 / 920: 00$ & Manual FIC & 299.6 & Good & \\
\hline $9 / 14 / 920: 00$ & Manual Tape & 299.00 & Transcribed & \\
\hline $9 / 14 / 920: 00$ & Manual FIC & 299.60 & Good & \\
\hline $9 / 15 / 920: 00$ & Manual Tape & 299 & Transcribed & \\
\hline $9 / 15 / 920: 00$ & Manual FIC & 299.60 & Good & \\
\hline
\end{tabular}

Data obtained from WHC Surveillance Analysis Computer System, (PC SACS) July 7, 1995. 


\begin{tabular}{|c|c|c|c|c|}
\hline \multicolumn{5}{|c|}{ 241-AP-105 } \\
\hline Date & Type of Measurement & Level (Inchos) & Data Quality & Comments \\
\hline $9 / 16 / 920: 00$ & Manual Tape & 299.00 & Transcribed & \\
\hline $9 / 16 / 920: 00$ & Manual FIC & 299.6 & - Good & \\
\hline $9 / 17 / 920: 00$ & Manual Tape & 299.00 & Transcribed & \\
\hline $9 / 17 / 927: 06$ & Auto FIC & 299.60 & Good & \\
\hline $9 / 18 / 920: 00$ & Manual Tape & 299 & Transcribed & \\
\hline 9/18/92 7:06 & Auto FIC & 299.60 & Good & : \\
\hline $9 / 19 / 920: 00$ & Manual Tape & 299 & Transcribed & \\
\hline 9/19/92 0:00 & Manual FIC & 299.6 & Good & \\
\hline $9 / 20 / 920: 00$ & Manual Tape & 299.00 & Transcribed & \\
\hline $9 / 20 / 920: 00$ & Manual FIC & 299.60 & Good & \\
\hline $9 / 21 / 920: 00$ & Manual Tape & 299.00 & Transcribed & \\
\hline $9 / 21 / 927: 06$ & Auto FIC & 299.6 & Good & \\
\hline 9/22/92 0:00 & Manual Tape & 299.00 & Transcribad & \\
\hline $9 / 22 / 927: 03$ & Auto FIC & 299.60 & Good & \\
\hline $9 / 23 / 92 \quad 0: 00$ & Manual Tape & 299.00 & Transcribed & \\
\hline 9/23/92 7:03 & Auto FIC & 299.60 & Good & \\
\hline $9 / 24 / 920: 00$ & Manual Tape & 299.00 & Transeribed & \\
\hline $9 / 24 / 927: 03$ & Auto FIC & 299.60 & Good & \\
\hline $9 / 25 / 920: 00$ & Manual Tape & 299 & Transcribed & \\
\hline $9 / 25 / 927: 03$ & Auto FIC & $299.60^{\circ}$ & Good & \\
\hline 9/26/92 0:00 & Manual Tape & 299.00 & Transcribed & \\
\hline $9 / 26 / 927: 02$ & Auto FIC & 299.6 & Good & \\
\hline $9 / 27 / 920: 00$ & Manual Tape & 299 & Transcribed & \\
\hline $9 / 27 / 927: 02$ & Auto FIC & 299.6 & Good & \\
\hline $9 / 28 / 920: 00$ & Manual Tape & 299.00 & Transcribed & \\
\hline $9 / 28 / 927: 02$ & Auto FIC & 299.6 & Good & \\
\hline $9 / 29 / 920: 00$ & Manual Tapa & 299.00 & Transcribed & \\
\hline $9 / 29 / 927: 03$ & Auto FIC & 299.6 & Good & \\
\hline $9 / 30 / 920: 00$ & Manual Tape & 299.00 & Transcribed & \\
\hline $9 / 30 / 927: 02$ & Auto FIC & 299.6 & Good & \\
\hline $10 / 1 / 920: 00$ & Manual Tape & 299 & Transcribed & \\
\hline $10 / 1 / 927: 03$ & Auto FIC & 299.6 & Good & \\
\hline $10 / 2 / 920: 00$ & Manual Tape & 299 & Transcribed & \\
\hline $10 / 2 / 927: 02$ & Auto FIC & 299.60 & Good & \\
\hline $10 / 3 / 920: 00$ & Manual Tape & 299.00 & Transcribed & \\
\hline 10/3/92 7:03 & Auto FIC & 299.60 & Good & \\
\hline $10 / 4 / 920: 00$ & Manual Tape & 299 & Transcribed & \\
\hline $10 / 4 / 927: 03$ & Auto FIC & 299.6 & Good & \\
\hline $10 / 5 / 920: 00$ & Manual Tape & 299.00 & Transcribed & \\
\hline $10 / 5 / 927: 03$ & Auto FIC & 299.60 & Good & \\
\hline $10 / 6 / 920: 00$ & Manual Tape & 299 & Transcribed & \\
\hline $10 / 6 / 927: 03$ & Auto FIC & 299.60 & Good & \\
\hline $10 / 7 / 920: 00$ & Manual Tape & 298.75 & Transcribed & \\
\hline $10 / 7 / 920: 00$ & Manual FIC & 299.6 & Good & \\
\hline $10 / 8 / 920: 00$ & Manual Tape & 298.75 & Transcribed & \\
\hline $10 / 8 / 927: 02$ & Auto FIC & 299.6 & Good & \\
\hline $10 / 9 / 920: 00$ & Manual Tape & 299 & Transcribed & \\
\hline $10 / 9 / 927: 03$ & Auto FIC & 299.60 & Good & \\
\hline
\end{tabular}

Data obtained from WHC Surveillance Analysis Computer System, (PC SACS) July 7, 1995. 


\begin{tabular}{|c|c|c|c|c|}
\hline \multicolumn{5}{|c|}{ 241-AP-105 } \\
\hline \multicolumn{5}{|c|}{ Surface Level Data } \\
\hline Date & Type of Measurement & Level (Inches) & Data Quality & Comments \\
\hline 10/10/92 0:00 & Manual Tape . & 299 & Transcribed & \\
\hline 10/10/92 7:03 & Auto FIC & 299.6 & Good & \\
\hline $10 / 11 / 920: 00$ & Manual Tape & 299.00 & Transcribed & \\
\hline $10 / 11 / 927: 03$ & Auto FIC & 299.60 & Good & \\
\hline $10 / 12 / 920: 00$ & Manual Tape & 299 & Transcribed & \\
\hline 10/12/92 0:00 & Manual FIC & 299.6 & Good & \\
\hline $10 / 13 / 920: 00$ & Manual Tape & 299 & Transcribed & \\
\hline $10 / 13 / 920: 00$ & Manual FIC & 299.60 & Good & \\
\hline $10 / 14 / 920: 00$ & Manual Tape & 299 & Transcribed & \\
\hline $10 / 14 / 927: 03$ & Auto FIC & 299.6 & Good & \\
\hline $10 / 15 / 920: 00$ & Manual Tape & 299 & Transcribed & \\
\hline $10 / 15 / 927: 04$ & Auto FIC & 299.60 & Good & \\
\hline $10 / 16 / 920: 00$ & Manual Tape & 299 & Transcribed & \\
\hline $10 / 16 / 927: 03$ & Auto FIC & 299.6 & Good & \\
\hline $10 / 17 / 920: 00$ & Manual Tape & 299.00 & Transcribed & \\
\hline 10/17/92 7:03 & Auto FIC & 299.6 & Good & \\
\hline $10 / 18 / 920: 00$ & Manual Tape & 299 & Transcribed & \\
\hline $10 / 18 / 927: 03$ & Auto FIC & 299.60 & Good & \\
\hline $10 / 19 / 920: 00$ & Manual Tape & 299 & Transcribed & \\
\hline 10/19/92 7:03 & Auto FIC & 299.60 & Good & \\
\hline 10/20/92 0:00 & Manual Tape & 299 & Transcribed & \\
\hline $10 / 20 / 927: 03$ & Auto FIC & 299.6 & Good & \\
\hline 10/21/920:00 & Manual Tape & 299 & Transcribed & \\
\hline $10 / 21 / 927: 03$ & Auto FIC & 299.6 & Good & \\
\hline $10 / 22 / 920: 00$ & Manual Tape & 299.00 & Transcribed & \\
\hline $10 / 22 / 920: 00$ & Manual FIC & 299.60 & Good & \\
\hline $10 / 23 / 920.00$ & Manual Tape & 299.00 & Transcribed & \\
\hline $10 / 23 / 920: 00$ & Manual FIC & 299.6 & Good & \\
\hline $10 / 24 / 920: 00$ & Manual Tape & 299.00 & Transcribed & \\
\hline $10 / 24 / 927: 03$ & Auto FIC & 299.6 & Good & \\
\hline $10 / 25 / 920: 00$ & Manual Tape & 299:00 & Transcribed & \\
\hline $10 / 25 / 927: 03$ & Auto FIC & 299.6 & Good & \\
\hline $10 / 26 / 920: 00$ & Manual Tape & 299 & Transcribed & \\
\hline $10 / 26 / 920: 00$ & Manual FIC & 299.60 & Good & \\
\hline 10/27/920:00 & Manual Tape & 299.00 & Transcribed & \\
\hline $10 / 27 / 927: 04$ & Auto FIC & 299.60 & Good & \\
\hline $10 / 28 / 920: 00$ & Manual Tape & 299 & Transcribed & \\
\hline $10 / 28 / 927: 03$ & Auto FIC & 299.6 & Good & \\
\hline $10 / 29 / 920: 00$ & Manual Tape & 299 & Transcribed & \\
\hline $10 / 29 / 927: 03$ & Auto FIC & 299.6 & Good & \\
\hline $10 / 30 / 920: 00$ & Manual Tape & 299.00 & Transcribed & \\
\hline $10 / 30 / 927: 03$ & Auto FIC & 299.60 & Good & \\
\hline $10 / 31 / 920: 00$ & Manual Tape & 299.00 & Transcribed & \\
\hline $10 / 31 / 927: 03$ & Auto FIC & 299.60 & Good & \\
\hline $11 / 1 / 920: 00$ & Manual Tape & 299 & Transcribed & \\
\hline $11 / 1 / 927: 03$ & Auto FIC & 299.50 & Good & \\
\hline $11 / 2 / 920: 00$ & Manual Tape & 299 & Transcribed & \\
\hline $11 / 2 / 927: 03$ & Auto FIC & 299.5 & Good & \\
\hline
\end{tabular}

Data obtained from WHC Surveillance Analysis Computer System, (PC SACS) July 7, 1995. 


\begin{tabular}{|c|c|c|c|c|}
\hline \multicolumn{5}{|c|}{ 241-AP-105 } \\
\hline & & Urface Leval Dat: & & \\
\hline Date & Type of Measurement & Lovel (Inchea) & Data Quality & Comments \\
\hline $11 / 3 / 920: 00$ & Manual Tape & 299.00 & Transcribed & \\
\hline $11 / 3 / 927: 03$ & Auto FIC & 299.5 & Good & \\
\hline $11 / 4 / 920: 00$ & Manual Tape & 299.00 & Transcribed & \\
\hline $11 / 4 / 927: 03$ & Auto FIC & 299.5 & Good & \\
\hline $11 / 5 / 920: 00$ & Manual Tape & 299.00 & Transcribed & \\
\hline $11 / 5 / 920: 00$ & Manual FIC & 299.50 & Good & \\
\hline $11 / 6 / 920: 00$ & Manual Tape & 299 & Transcribed & \\
\hline $11 / 6 / 920: 00$ & Manual FIC & 299.5 & Good & \\
\hline $11 / 7 / 920: 00$ & Manual Tape & 299 & Transcribed & \\
\hline $11 / 7 / 920: 00$ & Manual FIC & 299.50 & Good & \\
\hline $11 / 8 / 920: 00$ & Manual Tape & 299 & Transcribed & \\
\hline $11 / 8 / 920: 00$ & Manual FIC & 299.5 & Good & \\
\hline $11 / 9 / 920: 00$ & Manual Tape & 299 & Transcribed & \\
\hline $11 / 9 / 920: 00$ & Manual FIC & 299.5 & Good & \\
\hline $11 / 10 / 920: 00$ & Manual Tape : & 299 & Transcribed & \\
\hline $11 / 10 / 927: 03$ & Auto FIC & 299.50 & Good & \\
\hline $11 / 11 / 920: 00$ & Manual Tape & 299.00 & Transcribed & \\
\hline $11 / 11 / 927: 04$ & Auto FIC & 299.5 & Good & \\
\hline $11 / 12 / 920: 00$ & Manual Tape & 299.00 & Transcribed & . \\
\hline $11 / 12 / 927: 03$ & Auto FIC & 299.50 & Good & \\
\hline $11 / 13 / 920: 00$ & Manual Tape & 299.00 & Transcribed & \\
\hline $11 / 13 / 927: 04$ & Auto FIC & 299.50 & Good & \\
\hline $11 / 14 / 920: 00$ & Manual Tape & 299 & Transcribed & \\
\hline $11 / 14 / 927: 04$ & Auto FIC & 299.50 & Good & \\
\hline $11 / 15 / 920: 00$ & Manual Tape & 298.75 & Transcribed & \\
\hline $11 / 15 / 927: 04$ & Auto FIC & 299.50 & Good & \\
\hline $11 / 16 / 920: 00$ & Manual Tapo & 299 & Transcribed & \\
\hline $11 / 16 / 927: 04$ & Auto FIC & 299.50 & Good & \\
\hline $11 / 17 / 920: 00$ & Manual Tape & 298.75 & Transcribed & \\
\hline $11 / 17 / 927: 04$ & Auto FIC & 299.5 & Good & \\
\hline $11 / 18 / 92 \quad 0: 00$ & Manual Tape & 299 & Transcribed & \\
\hline $11 / 18 / 927: 02$ & Auto FIC & 299.5 & Good & \\
\hline $11 / 19 / 920: 00$ & Manual Tape & 298.75 & Transcribed & \\
\hline $11 / 19 / 927: 02$ & Auto FIC & 299.50 & Good & \\
\hline $11 / 20 / 920: 00$ & Manual Tape & 298.75 & Transcribed & \\
\hline $11 / 20 / 927: 03$ & Auto FIC & 299.5 & Good & \\
\hline $11 / 21 / 92 \quad 0: 00$ & Manual Tape & 298.75 & Transcribed & \\
\hline $11 / 21 / 927: 02$ & Auto FIC & 299.5 & Good & \\
\hline $11 / 22 / 920: 00$ & Manual Tape & 299.00 & Transcribed & \\
\hline $11 / 22 / 927: 03$ & Auto FIC & 299.50 & Good & \\
\hline $11 / 23 / 920: 00$ & Manual Tape & 299.00 & Transcribed & \\
\hline $11 / 23 / 927: 03$ & Auto FIC & 299.40 & Good & \\
\hline $11 / 24 / 920: 00$ & Manual Tape & 299.00 & Transcribed & \\
\hline $11 / 24 / 927: 03$ & Auto FIC & 299.40 & Good & \\
\hline $11 / 25 / 920: 00$ & Manual Tape & 299.00 & Transcribed & \\
\hline $11 / 25 / 927: 03$ & Auto FIC & 299.4 & Good & \\
\hline $11 / 26 / 920: 00$ & Manual Tape & 299 & Transcribed & \\
\hline $11 / 26 / 927: 03$ & Auto FIC & 299.40 & Good & \\
\hline
\end{tabular}

Data obtained from WHC Surveillance Analysis Computer System, (PC SACS) July 7, 1995. 


\begin{tabular}{|c|c|c|c|c|}
\hline \multicolumn{5}{|c|}{ 241-AP-105 } \\
\hline \multicolumn{5}{|c|}{ Surface Level Data } \\
\hline Date & Type of Moasurement & Level (Inches) & Data Quality & Comments \\
\hline $11 / 27 / 920: 00$ & Manual Tape & 299.00 & Transcribed & \\
\hline $11 / 27 / 927: 03$ & Auto FIC & 299.40 & Good & \\
\hline $11 / 28 / 920: 00$ & Manual Tape & 299.00 & Transcribed & \\
\hline $11 / 28 / 927: 03$ & Auto FIC & 299.40 & Good & \\
\hline $11 / 29 / 920: 00$ & Manual Tape & 299.00 & Transcribed & \\
\hline $11 / 29 / 927: 03$ & Auto FIC & 299.40 & Good & \\
\hline $11 / 30 / 920: 00$ & Manual Tape & 299.00 & Transeribed & \\
\hline $11 / 30 / 920: 00$ & Manual FIC & 299.40 & Good & \\
\hline $12 / 1 / 920: 00$ & Manual Tape & 298.75 & Transcribed & \\
\hline $12 / 1 / 927: 03$ & Auto FIC & 299.40 & Good & \\
\hline $12 / 2 / 920: 00$ & Manual Tape & 298.75 & Transcribed & \\
\hline $12 / 2 / 927: 03$ & Auto FIC & 299.40 & Good & \\
\hline $12 / 3 / 920: 00$ & Manual Tape & 298.75 & Transcribed & \\
\hline $12 / 3 / 927: 03$ & Auto FIC & 299.4 & Good & \\
\hline $12 / 4 / 920: 00$ & Manual Tape & $298.75^{\circ}$ & Transcribed & \\
\hline $12 / 4 / 927: 03$ & Auto FIC & 299.40 & Good & \\
\hline $12 / 5 / 920: 00$ & Manual Tape & 298.75 & Transcribed & \\
\hline $12 / 5 / 920: 00$ & Manual FIC & 299.40 & Good & \\
\hline $12 / 6 / 92 \quad 0: 00$ & Manual Tape & 298.75 & Transcribed & \\
\hline $12 / 6 / 927: 02$ & Auto FIC & 299.4 & Good & $\dot{.}$ \\
\hline $12 / 7 / 920: 00$ & Manual Tape & 298.75 & Transcribed & \\
\hline $12 / 7 / 927: 03$ & Auto FIC & 299.40 & Good & \\
\hline $12 / 8 / 920.00$ & Manual Tape & 298.75 & Transcribed & \\
\hline $12 / 8 / 927: 03$ & Auto FIC & 299.3 & Good & \\
\hline $12 / 9 / 920: 00$ & Manual Tape & 298.75 & Transcribed & \\
\hline $12 / 9 / 927: 03$ & Auto FIC & 299.30 & Good & \\
\hline $12 / 10 / 920: 00$ & Manual Tape & 298.75 & Transcribed & \\
\hline $12 / 10 / 927: 03$ & Auto FIC & 299.3 & Good & \\
\hline $12 / 11 / 920: 00$ & Manual Tape & 298.75 & Transcribed & \\
\hline $12 / 11 / 927: 03$ & Auto FIC & 299.30 & Good & \\
\hline $12 / 12 / 920: 00$ & Manual Tape & 298.75 & Transcribed & \\
\hline $12 / 12 / 920: 00$ & Manual FIC & 299.30 & Good & \\
\hline $12 / 13 / 920: 00$ & Manual Tape & $298: 75$ & Transcribed & \\
\hline $12 / 13 / 927: 04$ & Auto FIC & 299.3 & Good & \\
\hline $12 / 14 / 920: 00$ & Manual Tape & 298.75 & Transcribed & \\
\hline $12 / 14 / 927: 03$ & Auto FIC & 299.30 & Good & \\
\hline $12 / 15 / 920: 00$ & Manual Tape & 298.75 & Transcribed & \\
\hline $12 / 15 / 927: 03$ & Auto FIC & 299.3 & Good & \\
\hline $12 / 16 / 920: 00$ & Manual Tape & 298.75 & Transcribed & \\
\hline $12 / 16 / 927: 04$ & Auto FIC & 299.30 & Good & \\
\hline $12 / 17 / 920: 00$ & Manual Tape & 298.75 & Transcribed & \\
\hline $12 / 17 / 927: 03$ & Auto FIC & 299.3 & Good & \\
\hline $12 / 18 / 920: 00$ & Manual Tape & 298.50 & Transcribed & \\
\hline $12 / 18 / 927: 03$ & Auto FIC & 299.3 & Good & \\
\hline $12 / 19 / 920: 00$ & Manual Tape & 298.75 & Transcribed & \\
\hline $12 / 19 / 927: 03$ & Auto FIC & 299.30 & Good & \\
\hline $12 / 20 / 920: 00$ & Manual Tape & 298.50 & Transcribed & \\
\hline $12 / 20 / 927: 04$ & Auto FIC & 299.30 & Good & \\
\hline
\end{tabular}

Data obtained from WHC Surveillance Analysis Computer System, (PC SACS) July 7, 1995. 


\begin{tabular}{|c|c|c|c|c|}
\hline \multicolumn{5}{|c|}{ 241-AP 105} \\
\hline & & Iface Lovel Dat & & \\
\hline Date & Type of Measurement & Level (inchos) & Data Quality & Comments \\
\hline $12 / 21 / 920: 00$ & Manual Tape & 298.50 & Transcribed & \\
\hline $12 / 21 / 927: 04$ & Auto FIC & 299.30 & Good & \\
\hline $12 / 22 / 920: 00$ & Manual Tape & 298.50 & Transcribed & \\
\hline $12 / 22 / 920: 00$ & Manual FIC & 299.2 & Good & \\
\hline $12 / 23 / 920: 00$ & Manual Tape & 298.5 & Transcribed & \\
\hline $12 / 23 / 927: 04$ & Auto FIC & 299.2 & Good & \\
\hline $12 / 24 / 920: 00$ & Manual Tape & 298.50 & Transcribed & \\
\hline $12 / 24 / 927: 03$ & Auto FIC & 299.3 & Good & \\
\hline $12 / 25 / 920: 00$ & Manual Tape & 298.50 & Transcribed & \\
\hline $12 / 25 / 927: 03$ & Auto FIC & 299.20 & Good & \\
\hline $12 / 26 / 920: 00$ & Manual Tape & 298.25 & Transcribed & \\
\hline $12 / 26 / 927: 03$ & Auto FIC & 299.2 & Good & \\
\hline 12/27/92 7:03 & Auto FIC & 299.2 & Good & \\
\hline $12 / 28 / 920: 00$ & Manual Tape & 298.5 & Transcribed & \\
\hline $12 / 28 / 927: 03$ & Auto FIC & 299.20 & Good & \\
\hline $12 / 29 / 920: 00$ & Manual Tape & 298.50 & Transcribed & \\
\hline $12 / 29 / 920: 00$ & Manual FIC & 299.20 & Good & \\
\hline $12 / 30 / 920: 00$ & Manual Tape & 298.50 & Transcribed & \\
\hline $12 / 30 / 927: 03$ & Auto FIC & 299.2 & Good & \\
\hline $12 / 31 / 920: 00$ & Manual Tape & 298.25 & Transcribed & \\
\hline $12 / 31 / 927: 03$ & Auto FIC & 299.20 & Good & \\
\hline 1/1/93 0:00 & Manual Tape & 298.5 & Good & \\
\hline $1 / 1 / 937: 04$ & Auto FIC & 299.2 & Good & \\
\hline 1/2/93 0:00 & Manual FIC & 299.2 & Good & \\
\hline 1/2/93 0:00 & Manual Tape & 298.25 & Good & \\
\hline $1 / 3 / 930: 00$ & Manual FIC & 299.2 & Good & \\
\hline $1 / 3 / 930: 00$ & Manual Tape & 298.50 & Good & \\
\hline $1 / 4 / 930: 00$ & Manuạl FIC & 299.20 & Good & \\
\hline $1 / 4 / 930: 00$ & Manual Tape & 298.5 & Good & \\
\hline $1 / 5 / 930: 00$ & Manual FIC & 299.2 & Good & \\
\hline $1 / 5 / 93 \quad 0: 00$ & Manual Tape & 298.5 & Good & \\
\hline 1/6/93 0:00 & Manual FIC & 299.1 & Good & \\
\hline $1 / 6 / 930: 00$ & Manual Tape & 298.50 & Good & \\
\hline 1/7/93 0:00 & Manual FIC & 299.15 & Good & \\
\hline $1 / 7 / 930: 00$ & Manual Tape & 298.50 & Good & \\
\hline $1 / 8 / 930: 00$ & Manual FIC & 299.1 & Good & \\
\hline 1/8/93 0:00 & Manual Tape & 297.25 & Good & \\
\hline 1/9/93 0:00 & Manual FIC & 299.1 & Good & \\
\hline $1 / 9 / 930: 00$ & Manual Tape & 298.5 & Good & \\
\hline $1 / 10 / 930: 00$ & Manual FIC & 299.10 & Good & \\
\hline $1 / 10 / 930: 00$ & Manual Tape & 298.5 & Good & \\
\hline 1/11/93 0:00 & Manual FIC & 299.10 & Good & \\
\hline $1 / 11 / 930: 00$ & Manual Tape & 298.25 & Good & \\
\hline $1 / 12 / 930: 00$ & Manual Tape & 298.50 & Good & \\
\hline $1 / 12 / 937: 06$ & Auto FIC & 299.10 & Good & \\
\hline $1 / 13 / 930: 00$ & Manual Tape & 298,50 & Good & \\
\hline $1 / 13 / 937: 06$ & Auto FIC & 299.10 & Good & \\
\hline 1/14/930:00 & Manual Tape & 298.5 & Good & \\
\hline
\end{tabular}

Data obtained from WHC Surveillance Analysis Computer System, (PC SACS) July 7, 1995. 


\begin{tabular}{|c|c|c|c|c|}
\hline \multicolumn{5}{|c|}{ 241-AP-105 } \\
\hline Date & Type of Moasurement & Lavel (Inches) & Data Quality & Comments \\
\hline $1 / 14 / 937: 03$ & Auto FIC & 299.10 & Good & \\
\hline $1 / 15 / 93 \quad 0: 00$ & Manual Tape & 298.50 & Good & \\
\hline $1 / 15 / 937: 03$ & Auto FIC & 299.1 & Good & \\
\hline $1 / 16 / 930: 00$ & Manual FIC & 299.10 & Good & \\
\hline $1 / 16 / 930: 00$ & Manual Tape & 298.25 & Good & \\
\hline $1 / 17 / 930: 00$ & Manual FIC & 299.10 & Good & \\
\hline $1 / 17 / 930: 00$ & Manual Tape & 298.25 & Good & \\
\hline 1/18/93 0:00 & Manual FIC & 299.10 & Good & \\
\hline $1 / 18 / 930: 00$ & Manual Tape & 298.5 & Good & \\
\hline $1 / 19 / 930: 00$ & Manual FIC & 299.10 & Good & \\
\hline $1 / 19 / 930: 00$ & Manual Tape & 298.25 & Good & \\
\hline $1 / 20 / 930: 00$ & Manual FIC & 299.1 & Good & \\
\hline $1 / 20 / 930: 00$ & Manual Tape & 298.50 & Good & \\
\hline $1 / 21 / 930: 00$ & Manual Tape & 298.5 & Good & \\
\hline $1 / 21 / 937: 02$ & Auto FIC & 299 & Good & \\
\hline $1 / 22 / 930: 00$ & Manual Tape & 298.50 & Good & \\
\hline $1 / 22 / 93 \quad 7: 04$ & Auto FIC & 299 & Good & \\
\hline 1/23/93 0:00 & Manual Tape & 298.5 & Good & \\
\hline $1 / 23 / 937: 03$ & Auto FIC & 299 & Good & \\
\hline $1 / 24 / 930: 00$ & Manual Tape & 298.50 & Good & \\
\hline $1 / 24 / 937: 03$ & Auto FIC & 299 & Good & \\
\hline $1 / 25 / 930: 00$ & Manual Tape ' & 298.00 & Good & \\
\hline $1 / 25 / 937: 03$ & - Auto FIC & 299.00 & Good & \\
\hline $1 / 26 / 930: 00$ & Manual Tape & 298:25 & Good & \\
\hline $1 / 26 / 937: 04$ & Auto FIC & 299 & Good & \\
\hline $1 / 27 / 930: 00$ & Manual Tape & 298.50 & Good & \\
\hline 1/27/93 7:04 & Auto FIC & 299.00 & Good & \\
\hline $1 / 28 / 930: 00$ & Manual Tape & 298.25 . & Good & \\
\hline $1 / 28 / 937: 04$ & Auto FIC & 299.00 & Good ' & \\
\hline $1 / 29 / 930: 00$ & Manual Tape & 298.5 & Good & . \\
\hline $1 / 29 / 937: 04$ & Auto FIC & 299 & Good & \\
\hline 1/30/93 7:03 & Auto FIC & 299.00 & Good & \\
\hline $1 / 31 / 930: 00$ & Manual Tape & 298.5 & Good & \\
\hline $1 / 31 / 937: 04$ & Auto FIC & 299 & Good & \\
\hline $2 / 1 / 937: 04$ & Auto FIC & 299.00 & Good & \\
\hline $2 / 2 / 937: 04$ & Auto FIC & 299.00 & Good & \\
\hline $2 / 3 / 930: 00$ & Manual FIC & 299 & Good & \\
\hline $2 / 4 / 937: 04$ & Auto FIC & 299 & Good & \\
\hline $2 / 5 / 937: 04$ & Auto FIC & 299.00 & Good & \\
\hline $2 / 6 / 937: 04$ & Auto FIC & 299.00 & Good & \\
\hline $2 / 7 / 937: 03$ & Auto FIC & 299.00 & Good & \\
\hline $2 / 8 / 930: 00$ & Manual Tape & 298.00 & Good & \\
\hline $2 / 8 / 937: 04$ & Auto FIC & 299.00 & Good & \\
\hline $2 / 9 / 930: 00$ & Manual Tape & . 297.75 & Good & \\
\hline $2 / 9 / 93 \quad 7: 04$ & Auto FIC & 299.00 & Good & \\
\hline $2 / 10 / 930: 00$ & Manual Tape & 298.25 & Good & \\
\hline 2/10/93 7:04 & Auto FIC & 299.00 & Good & \\
\hline $2 / 11 / 930: 00$ & Manual Tape & 298.25 & Good & \\
\hline
\end{tabular}

Data obtained from WHC Surveillance Analysis Computer System, (PC SACS) July 7, 1995. 


\begin{tabular}{|c|c|c|c|c|}
\hline \multicolumn{5}{|c|}{ 241-AP-105 } \\
\hline \multicolumn{5}{|c|}{ Surface Level Data } \\
\hline Date & Type of Measurement & Lovel (Inchea) & Data Qualíy & Comments \\
\hline $2 / 11 / 937: 04$ & Auto FIC & 299 & Good & \\
\hline $2 / 12 / 930: 00$ & Manual Tape & 298.25 & Good & \\
\hline $2 / 12 / 937: 03$ & Auto FIC & 298.90 & Good & \\
\hline $2 / 13 / 930: 00$ & Manual Tape & 298.25 & Good & \\
\hline $2 / 13 / 937: 03$ & Auto FIC & 298.90 & Good & \\
\hline $2 / 14 / 930: 00$ & Manual Tape & 298.25 & Good & \\
\hline $2 / 14 / 937: 04$ & Auto FIC & 298.9 & Good & \\
\hline $2 / 15 / 930: 00$ & Manual Tape & 298.25 & Good & \\
\hline $2 / 15 / 937: 04$ & Auto FIC & 298.9 & Good & \\
\hline $2 / 16 / 930: 00$ & Manual Tape & 298.25 & Good & \\
\hline $2 / 16 / 937: 02$ & Auto FIC & 298.9 & Good & \\
\hline $2 / 17 / 930: 00$ & Manual FIC . & 298.9 & Good & \\
\hline $2 / 17 / 930: 00$ & Manual Tape & 298.25 & Good & \\
\hline $2 / 18 / 930: 00$ & Manual Tape & 298.25 & Good & \\
\hline $2 / 18 / 93$ 7:03 & Auto FIC & 299.00 & Good & \\
\hline $2 / 19 / 930: 00$ & Manual Tape & 298.25 & Good & \\
\hline $2 / 19 / 93 \quad 7: 02$ & Auto FIC & 298.90 & Good & \\
\hline $2 / 20 / 930: 00$ & Manual FIC & 298.25 & Good & \\
\hline $2 / 20 / 930: 00$ & Manual Tape & 298.25 & Good & \\
\hline $2 / 20 / 937: 03$ & Auto FIC & 298.90 & Good & \\
\hline $2 / 21 / 930: 00$ & Manual Tape & 298.25 & Good & \\
\hline $2 / 21 / 937: 04$ & Auto FIC & 298.90 & Good & \\
\hline $2 / 22 / 93 \quad 0: 00$ & Manual Tape & 298.25 & Good & \\
\hline $2 / 22 / 937: 04$ & Auto FIC & 298.9 & Good & \\
\hline $2 / 23 / 930: 00$ & Manual Tape & 298.5 & Good & \\
\hline 2/23/93 7:03 & Auto FIC & 298.90 & Good & \\
\hline $2 / 24 / 930: 00$ & Manual Tape & 298.25 & Good & \\
\hline $2 / 24 / 93 \quad 7: 04$ & Auto FIC & 298.90 & Good & \\
\hline $2 / 25 / 930: 00$ & Manual Tape & 298.00 & Good & \\
\hline $2 / 25 / 937: 03$ & Auto FIC & 298.8 & Good & \\
\hline $2 / 26 / 93 \quad 0: 00$ & Manual Tape & 298.25 & Good & \\
\hline $2 / 26 / 937: 03$ & Auto FIC & 298.7 & Good & \\
\hline $2 / 27 / 930: 00$ & Manual Tape & 298.00 & Good & \\
\hline $2 / 27 / 937: 04$ & Auto FIC & 298.8 & Good & \\
\hline $2 / 28 / 930: 00$ & Manual Tape & 298.00 & Good & \\
\hline $2 / 28 / 937: 03$ & Auto FIC & 298.8 & Good & \\
\hline $3 / 1 / 930: 00$ & Manual Tape & 298 & Good & \\
\hline $3 / 1 / 937: 03$ & Auto FIC & 298.80 & Good & \\
\hline $3 / 2 / 930: 00$ & Manual Tape & 298 & Good & \\
\hline $3 / 2 / 937: 04$ & Auto FIC & 298.8 & Good & \\
\hline $3 / 3 / 930: 00$ & Manual Tape & 298.25 & Good & \\
\hline $3 / 3 / 937: 03$ & Auto FIC & 298.8 & Good & \\
\hline $3 / 4 / 93 \quad 0: 00$ & Manual Tape & 298.00 & Good & \\
\hline $3 / 4 / 937: 04$ & Auto FIC & 298.80 & Good & \\
\hline $3 / 5 / 930: 00$ & Manual Tape & 298.25 & Good & \\
\hline $3 / 5 / 937: 04$ & Auto FIC & 298.80 & Good & \\
\hline $3 / 6 / 93$ 0:00 & Manual Tape & 298.25 & Good & \\
\hline $3 / 6 / 937: 03$ & Auto FIC & 298.8 & Good & \\
\hline
\end{tabular}

Data obtained from WHC Surveillance Analysis Computer System, (PC SACS) July 7, 1995. 


\begin{tabular}{|c|c|c|c|c|}
\hline \multicolumn{5}{|c|}{ 241-AP-105 } \\
\hline \multicolumn{5}{|c|}{ Surface Lavel Data } \\
\hline Date & Type of Measurement & Level (Inchea) & Data Quality & Comments \\
\hline $3 / 7 / 930: 00$ & Manual Tape & 298.25 & Good & \\
\hline $3 / 7 / 93 \quad 7: 03$ & Auto FIC & 298.8 & Good & \\
\hline $3 / 8 / 930: 00$ & Manual Tape & 298.25 & Good & \\
\hline $3 / 8 / 937: 03$ & Auto FIC & 298.80 & Good & \\
\hline $3 / 9 / 930: 00$ & Manual Tape & 298.25 & Good & \\
\hline $3 / 9 / 937: 04$ & Auto FIC & 298.80 & Good & \\
\hline 3/10/93 0:00 & Manual Tape & 298.25 & Good & \\
\hline 3/10/93 7:05 & Auto FIC & 298.80 & Good & \\
\hline $3 / 11 / 930: 00$ & Manual Tape & 298 & Good & \\
\hline 3/11/93 7:04 & Auto FIC & 298.80 & Good & \\
\hline $3 / 12 / 930: 00$ & Manual Tape & 298.25 & Good & \\
\hline $3 / 12 / 937: 04$ & Auto FIC & 298.80 & Good & \\
\hline $3 / 13 / 930: 00$ & Manual Tape & 298.25 & Good & \\
\hline $3 / 13 / 937: 03$ & Auto FIC & 298.8 & Good & \\
\hline $3 / 14 / 930: 00$ & Manual Tape & 298.25 & Good & \\
\hline $3 / 14 / 937: 04$ & Auto FIC & 298.8 & Good & \\
\hline $3 / 15 / 930: 00$ & Manual Tape & 298.25 & Good & \\
\hline $3 / 15 / 937: 03$ & Auto FIC & 298.80 & Good & \\
\hline $3 / 16 / 930: 00$ & Manual Tape & 298.25 & Good & \\
\hline $3 / 16 / 937: 04$ & Auto FIC & 298.8 & Good & \\
\hline $3 / 17 / 93 \quad 0: 00$ & Manual Tape & 298 & Good & \\
\hline $3 / 17 / 937: 03$ & Auto FIC & 298.70 & Good & \\
\hline $3 / 18 / 930: 00$ & Manual Tape & 298 & Good & \\
\hline $3 / 18 / 937: 04$ & Auto FIC & 298.70 & Good & \\
\hline $3 / 19 / 930: 00$ & Manual Tape & 298 & Good & \\
\hline $3 / 19 / 937: 03$ & Auto FIC & 298.7 & Good & \\
\hline 3/20/93 0:00 & Manual Tape & 298.00 & Good & \\
\hline $3 / 20 / 937: 04$ & Auto FIC & 298.70 & Good & \\
\hline $3 / 21 / 930: 00$ & Manual Tape & 298.13 & Good & \\
\hline $3 / 21 / 937: 04$ & Auto FIC & 298.70 & Good & \\
\hline $3 / 22 / 93 \quad 0: 00$ & Manual Tape & 298 & Good & \\
\hline $3 / 22 / 937: 03$ & Auto FIC & 298.70 & Good & \\
\hline $3 / 23 / 93 \quad 0: 00$ & Manual Tape & 298 & Good & \\
\hline $3 / 23 / 937: 03$ & Auto FIC & 298.7 & Good & \\
\hline $3 / 24 / 930: 00$ & Manual Tape & 298.00 & Good & \\
\hline $3 / 24 / 937: 04$ & Auto FIC & 298.7 & Good & \\
\hline $3 / 25 / 93 \quad 0: 00$ & Manual Tape & 298.00 & Good & \\
\hline $3 / 25 / 937: 03$ & Auto FIC & 298.7 & Good & \\
\hline $3 / 26 / 930: 00$ & Manual Tape & 298 & Good & \\
\hline $3 / 26 / 937: 03$ & Auto FIC & 298.70 & Good & \\
\hline $3 / 27 / 930: 00$ & Manual Tape & 298.00 & Good & \\
\hline $3 / 27 / 937: 03$ & Auto FIC & 298.70 & Good & \\
\hline $3 / 28 / 930: 00$ & Manual Tape & 298 & Good & \\
\hline 3/28/93 7:03 & Auto FIC & 298.7 & Good & \\
\hline $3 / 29 / 930: 00$ & Manual Tape & 298.00 & Good & \\
\hline $3 / 29 / 937: 03$ & Auto FIC & 298.7 & Good & \\
\hline $3 / 30 / 930: 00$ & Manual Tape & 298.25 & Good & \\
\hline $3 / 30 / 937: 03$ & Auto FIC & 298.70 & Good & \\
\hline
\end{tabular}

Data obtained from WHC Surveillance Analysis Computer System, (PC SACS) July 7, 1995. 


\begin{tabular}{|c|c|c|c|c|}
\hline \multicolumn{5}{|c|}{ 241-APa105 } \\
\hline & & ufface Level Dat & & \\
\hline Date & Type of Measurement & Level (Inchea) & Data Quality & Comments \\
\hline 3/31/93 0:00 & Manual Tape & 298.25 & Good & \\
\hline $3 / 31 / 93$ 7:03 & Auto FIC & 298.70 & Good & \\
\hline 4/1/93 7:03 & Auto FIC & 298.70 & Good & \\
\hline $4 / 1 / 9310: 03$ & Manual Tape & 298 & Good & \\
\hline 4/2/93 7:06 & Auto FIC & 298.7 & Good & \\
\hline 4/2/93 10:03 & Manual Tape & 298.25 & Good & \\
\hline 4/3/93 7:06 & Auto FIC & 298.70 & Good & \\
\hline $4 / 3 / 93 \quad 10: 03$ & Manual Tape & 298.25 & Good & \\
\hline 4/4/93 7:05 & Auto FIC & 298.7 & Good & \\
\hline $4 / 4 / 93 \quad 10: 03$ & Manual Tape & 298 & Good & \\
\hline $4 / 5 / 937: 06$ & Auto FIC & 298.7 & Good & \\
\hline $4 / 5 / 93 \quad 10: 03$ & Manual Tape & 298 & Good & \\
\hline 4/6/93 7:06 & Auto FIC & 298.70 & Good & \\
\hline $4 / 6 / 93,10: 03$ & Manual Tape & 298.00 & Good & \\
\hline 4/7/93 7:06 & Auto FIC & $\begin{array}{l}298.7 \\
\end{array}$ & Good & \\
\hline $4 / 7 / 93 \quad 10: 03$ & Manual Tape & 298.00 & Good & \\
\hline 4/8/93 7:06 & Auto FIC & 298.7 & Good & \\
\hline 4/8/93 10:03 & Manual Tape & 298 & Good & \\
\hline 4/9/93 7:06 & Auto FIC & 298.7 & Good & \\
\hline $4 / 9 / 93 \quad 10: 03$ & Manual Tape & 298 & Good & \\
\hline 4/10/93 7:05 & Auto FIC & 298.7 & Good & \\
\hline $4 / 10 / 93 \quad 10: 03$ & Manual Tape & 298 & Good & \\
\hline $4 / 11 / 937: 06$ & Auto FIC & 298.70 & Good & \\
\hline $4 / 11 / 93 \quad 10: 03$ & Manual Tape & 298.00 & Good & \\
\hline $4 / 12 / 937: 06$ & Auto FIC & 298.70 & Good & \\
\hline $4 / 12 / 93 \quad 10: 03$ & Manual Tape & 298 & Good & \\
\hline $4 / 13 / 937: 06$ & Auto FIC & 298.70 & Good & \\
\hline $4 / 13 / 9310: 03$ & Manual Tape & 298.00 & Good & \\
\hline 4/14/93 7:06 & Auto FIC & 298.7 & Good & \\
\hline 4/14/93 10:03 & Manual Tape & 298.25 & Good & \\
\hline $4 / 15 / 937: 03$ & - Auto FIC & 298.7 & Good & \\
\hline $4 / 15 / 9310: 03$ & Manual Tape & 298 & Good & \\
\hline $4 / 16 / 937: 06$ & Auto FIC & 298.7 & Good & \\
\hline $4 / 16 / 93 \quad 10: 03$ & Manual Tape & 298 & Good & \\
\hline $4 / 17 / 937: 05$ & Auto FIC & 298.70 & Good & \\
\hline $4 / 17 / 93 \quad 10: 03$ & Manual Tape & 298.00 & Good & \\
\hline $4 / 18 / 937: 06$ & Auto FIC & 298.70 & Good & \\
\hline $4 / 18 / 93 \quad 10: 03$ & Manual Tape & 298.00 & Good & \\
\hline $4 / 19 / 937: 06$ & Auto FIC & 298.7 & Good & \\
\hline $4 / 19 / 93 \quad 10: 03$ & Manual Tape & 298 & Good & \\
\hline $4 / 20 / 930: 00$ & Manual FIC & 298.70 & Good & \\
\hline $4 / 20 / 93 \quad 10: 03$ & Manual Tape & 298.75 & Good & \\
\hline 4/21/93 0:00 & Manual FIC & 298.70 & Good & \\
\hline $4 / 21 / 93 \quad 10: 03$ & Manual Tape & 298 & Good & \\
\hline 4/22/93 7:06 & Auto FIC & 298.7 & Good & \\
\hline $4 / 22 / 93 \quad 10: 03$ & Manual Tape & 298 & Good & \\
\hline $4 / 23 / 937: 06$ & Auto FIC & 298.70 & Good & \\
\hline $4 / 23 / 93 \quad 10: 03$ & Manual Tape & 298 & Good & \\
\hline
\end{tabular}

Data obtained from WHC Surveillance Analysis Computer System, (PC SACS) July 7, 1995. 


\begin{tabular}{|c|c|c|c|c|}
\hline \multicolumn{5}{|c|}{ 241-AP-105 } \\
\hline \multicolumn{5}{|c|}{ Surface Level Data } \\
\hline Date & Type of Measurement & Leval (Inchea) & Data Quality & Comments \\
\hline $4 / 24 / 937: 05$ & Auto FIC & 298.70 & Good & \\
\hline $4 / 24 / 93 \quad 10: 03$ & Manual Tape & 298.00 & Good & \\
\hline $4 / 25 / 937: 06$ & Auto FIC & 298.70 & Good & \\
\hline 4/25/93 10:03 & Manual Tape & 298 & Good & \\
\hline $4 / 26 / 937: 06$ & Auto FIC & 298.7 & Good & \\
\hline $4 / 26 / 93 \quad 10: 03$ & Manual Tape & 298.00 & Good & \\
\hline 4/27/93 7:06 & Auto FIC & 298.6 & Good & \\
\hline 4/27/93 10:03 & Manual Tape & 298.00 & Good & \\
\hline $.4 / 28 / 937: 03$ & Auto FIC & 298.6 & Good & \\
\hline 4/28/93 10:03 & Manual Tape & 298 & Good & \\
\hline $4 / 29 / 937: 03$ & Auto FIC & 298.60 & Good & \\
\hline 4/29/93 10:03 & Manual Tape & 298.00 & Good & \\
\hline $4 / 30 / 937: 06$ & Auto FIC & 298.6 & Good & \\
\hline 4/30/93 10:03 & Manual Tape & 298.00 & Good & \\
\hline $5 / 1 / 937: 05$ & Auto FIC & 298.6 & Good & \\
\hline $5 / 1 / 9310: 05$ & Manual Tape & 298.70 & Good & \\
\hline $5 / 2 / 937: 06$ & Auto FIC & 298.7 & Good & \\
\hline $5 / 2 / 93 \quad 10: 05$ & Manual Tape & 298.70 & Good & \\
\hline $5 / 3 / 937: 06$ & Auto FIC & 298.7 & Good & \\
\hline $5 / 4 / 93 \quad 10: 05$ & Manual FIC & 298.6 & Good & \\
\hline $5 / 4 / 93 \quad 10: 05$ & Manual Tape & 298.7 & Good & \\
\hline $5 / 5 / 93 \quad 10: 05$ & Manual FIC & 298.60 & Good & \\
\hline 5/5/93 10:05 & Manual Tape & 298.70 & Good & \\
\hline $5 / 6 / 93 \quad 10: 05$ & Manual FIC & 298.6 & Good & \\
\hline $5 / 6 / 93 \quad 10: 05$ & Manual Tape & 298.7 & Good & \\
\hline $5 / 7 / 93 \quad 10: 05$ & Manual FIC & 298.6 & Good & \\
\hline $5 / 7 / 93 \quad 10: 05$ & Manual Tape & 298.70 & Good & \\
\hline 5/8/93 10:05 & Manual FIC & 298.60 & Good & \\
\hline 5/8/93 10:05 & Manual Tape & 298.7 & Good & \\
\hline 5/9/93 10:05 & Manual FIC & 298.60 & Good & \\
\hline $5 / 9 / 93 \quad 10: 05$ & Manual Tape & $\cdot 298.7$ & Good & \\
\hline $5 / 10 / 9310: 05$ & Manual FIC & 298.60 & Good & \\
\hline $5 / 10 / 93 \quad 10: 05$ & Manual Tape & 298.70 & Good & \\
\hline $5 / 11 / 937: 06$ & Auto FIC & 298.7 & Good & \\
\hline $5 / 11 / 9310: 05$ & Manual Tape & 298.70 & Good & \\
\hline $5 / 12 / 937: 06$ & Auto FIC & 298.60 & Good & \\
\hline $5 / 12 / 9310: 05$ & Manual Tape & 298.7 & Good & \\
\hline $5 / 13 / 93 \quad 10: 05$ & Manual FIC & 298.65 & Good & \\
\hline $5 / 13 / 93 \quad 10: 05$ & Manual Tape & 298.25 & Good & \\
\hline $5 / 14 / 93 \quad 10: 05$ & Manual FIC & 298.70 & Good & \\
\hline $5 / 14 / 93 \quad 10: 05$ & Manual Tape & 298 & Good & \\
\hline 5/15/93 7:06 & Auto FIC & 298.6 & Good & \\
\hline $5 / 15 / 93 \quad 10: 05$ & Manual Tape & 298 & Good & \\
\hline $5 / 16 / 937: 05$ & Auto FIC & 298.7 & Good & \\
\hline $5 / 16 / 93 \quad 10: 05$ & Manual Tape & 298.00 & Good & \\
\hline $5 / 17 / 93$ 7:03 & Auto FIC & 298.70 & Good & \\
\hline $5 / 17 / 93 \quad 10: 05$ & Manual Tape & 298 & Good & \\
\hline $5 / 18 / 937: 03$ & Auto FIC & 298.70 & Good & \\
\hline
\end{tabular}

Data obtained from WHC Surveillance Analysis Computer System, (PC SACS) July 7, 1995. 


\begin{tabular}{|c|c|c|c|c|}
\hline \multicolumn{5}{|c|}{ 241-AP,105 } \\
\hline \multicolumn{5}{|c|}{ Surface Level Data } \\
\hline Date & Type of Measurement & Levol (Inches) & Data Quality & Comments \\
\hline $5 / 18 / 93 \quad 10: 05$ & Manual Tape & 298 & Good & \\
\hline $5 / 19 / 937: 03$ & Auto FIC & 298.70 & Good & \\
\hline $5 / 19 / 93 \quad 10: 05$ & Manual Tape & 298 & Good & \\
\hline $5 / 20 / 937: 03$ & Auto FIC & 298.7 & Good & \\
\hline $5 / 20 / 9310: 05$ & Manual Tape & 298 & Good & \\
\hline $5 / 21 / 930: 00$ & Manual FIC & 298.7 & Good & \\
\hline $5 / 21 / 93 \quad 10: 05$ & Manual Tape & 297.75 & Good & \\
\hline $5 / 22 / 93$ 7:03 & Auto FIC & 298.70 & Good & \\
\hline $5 / 22 / 93 \quad 10: 05$ & Manual Tape & 297.75 & Good & \\
\hline $5 / 23 / 937: 04$ & Auto FIC & 298.7 & Good & \\
\hline $5 / 23 / 93 \quad 10: 05$ & Manual Tape & 297.75 & Good & \\
\hline $5 / 24 / 937: 03$ & Auto FIC & 298.7 & Good & \\
\hline $5 / 24 / 93 \quad 10: 05$ & Manual Tape & 297.75 & Good & \\
\hline $5 / 25 / 937: 03$ & Auto FIC & 298.70 & Good & \\
\hline $5 / 25 / 93 \quad 10: 05$ & Manual Tape & 297.75 & Good & \\
\hline $5 / 26 / 937: 03$ & Auto FIC & 298.7 & Good & \\
\hline $5 / 26 / 93 \quad 10: 05$ & Manual Tape & 298 & Good & \\
\hline $5 / 27 / 937 ; 03$ & Auto FIC & 298.70 & Good & \\
\hline 5/27/93 10:05 & Manual Tape & 298 & Good & \\
\hline $5 / 28 / 937: 03$ & Auto FIC & 298.70 & Good & \\
\hline $5 / 28 / 93 \quad 10: 05$ & Manual Tape & 298 & Good & \\
\hline $5 / 29 / 93 \quad 7: 05$ & Auto FIC & 298.70 & Good & \\
\hline $5 / 29 / 93$ 10:05 & Manual Tape & 298.00 & Good & \\
\hline $5 / 30 / 937: 05$ & Auto FIC & 298.70 & Good & \\
\hline $5 / 30 / 93$ 10:05 & Manual Tape & 298.00 & Good & \\
\hline $5 / 31 / 937: 06$ & Auto FIC & 298.7 & Good & \\
\hline $5 / 31 / 93$ 10:05 & Manual Tape & 298.25 & Good & \\
\hline 6/1/93 7:06 & Auto FIC & 298.70 & Good & \\
\hline $6 / 1 / 93 \quad 10: 06$ & Manual Tape & 298.00 & Good & \\
\hline $6 / 2 / 93$ 7:06 & Auto FIC & 298.70 & Good & \\
\hline $6 / 2 / 93 \quad 10: 06$ & Manual Tape & 298.00 & Good & \\
\hline $6 / 3 / 937: 06$ & Auto FIC & 298.70 & Good & \\
\hline $6 / 3 / 93 \quad 10: 06$ & Manual Tape & 298.00 & Good & \\
\hline 6/4/93 7:06 & Auto FIC & 298.7 & Good & \\
\hline $6 / 4 / 9310: 06$ & Manual Tape & 298.00 & Good & \\
\hline $6 / 5 / 937: 06$ & Auto FIC & 298.70 & Good & \\
\hline $6 / 5 / 93 \quad 10: 06$ & Manual Tape & 298.00 & Good & \\
\hline $6 / 6 / 937: 05$ & Auto FIC & 298.70 & Good & \\
\hline $6 / 6 / 93 \quad 10: 06$ & Manual Tape & 298 & Good & \\
\hline $6 / 7 / 937: 05$ & Auto FIC & 298.7 & Good & \\
\hline $6 / 7 / 93 \quad 10: 06$ & Manual Tape & 298 & Good & \\
\hline 6/8/93 7:05 & Auto FIC & 298.70 & Good & \\
\hline $6 / 8 / 93 \quad 10: 06$ & Manual Tape & 298.00 & Good & \\
\hline $6 / 9 / 937: 05$ & Auto FIC & 298.70 & Good & \\
\hline $6 / 9 / 93 \quad 10: 06$ & Manual Tape & 298 & Good & \\
\hline $6 / 10 / 937: 04$ & Auto FIC & 298.70 & Good & \\
\hline $6 / 10 / 9310: 06$ & Manual Tape & 298.00 & Good & \\
\hline $6 / 11 / 937: 05$ & Auto FIC & 298.7 & Good & \\
\hline
\end{tabular}

Data obtained from WHC Surveillance Analysis Computer System, (PC SACS) July 7, 1996. 


\begin{tabular}{|c|c|c|c|c|}
\hline \multicolumn{5}{|c|}{ 241-AP-105 } \\
\hline \multicolumn{5}{|c|}{ Surface Level Date } \\
\hline Date & Type of Measurement & Leval (Inchea) & Data Quality & Comments \\
\hline $6 / 11 / 93 \quad 10: 06$ & Manual Tape & 298.00 & Good & \\
\hline $6 / 12 / 93$ 7:05 & Auto FIC & 298.70 & Good & \\
\hline $6 / 12 / 93 \quad 10: 06$ & Manual Tape & 298 & Good & \\
\hline $6 / 13 / 937: 04$ & Auto FIC & $298: 70$ & Good & \\
\hline 6/13/93 10:06 & Manual Tape & 298.00 & Good & \\
\hline $6 / 14 / 93$ 7:05 & Auto FIC & 298.7 & Good & \\
\hline $6 / 14 / 93 \quad 10: 06$ & Manual Tape & 298.25 & Good & \\
\hline $6 / 15 / 937: 05$ & Auto FIC & 298.70 & Good & \\
\hline $6 / 15 / 93$ 10:06 & Manual Tape & 298.00 & Good & . \\
\hline $6 / 16 / 937: 05$ & Auto FIC & 298.7 & Good & \\
\hline $6 / 16 / 93 \quad 10: 06$ & Manual Tape & 298.00 & Good & \\
\hline $6 / 17 / 937: 05$ & Auto FIC & 298.7 & Good & \\
\hline $6 / 17 / 93 \quad 10: 06$ & Manual Tape & 298 & Good & \\
\hline $6 / 18 / 93$ 7:06 & Auto FIC & 298.70 & Good & \\
\hline $6 / 18 / 9310: 06$ & Manual Tape & 298.00 & Good & \\
\hline $6 / 19 / 937: 06$ & Auto FIC & 298.70 & Good & \\
\hline $6 / 19 / 93 \quad 10: 06$ & Manual Tape & 298 & Good & - \\
\hline $6 / 20 / 937: 06$ & Auto FIC & 298.7 & Good & \\
\hline $6 / 20 / 93 \quad 10: 06$ & Manual Tape & 298.00 & Good & \\
\hline $6 / 21 / 937: 06$ & Auto FIC & 298.7 & Good & \\
\hline $6 / 21 / 93 \quad 10: 06$ & Manual Tape & 298:00 & Good & \\
\hline $6 / 22 / 937: 06$ & Auto FIC & 298.70 & Good & \\
\hline $6 / 22 / 93 \quad 10: 06$ & Manual Tape & 298.00 & Good & \\
\hline $6 / 23 / 937: 06$ & Auto FIC & 298.7 & Good & \\
\hline $6 / 23 / 9310: 06$ & Manual Tape & 298.00 & Good & \\
\hline $6 / 24 / 937: 06$ & Auto FIC & 298.70 & Good & \\
\hline 6/24/93 10:06 & Manual Tape & 298.00 & Good & \\
\hline $6 / 25 / 937: 03$ & Auto FIC & 298.7 & Good & \\
\hline $6 / 25 / 93 \quad 10: 06$ & Manual Tape & 298 & Good & \\
\hline $6 / 26 / 937: 03$ & Auto FIC & 298.7 & Good & \\
\hline $6 / 26 / 93 \quad 10: 06$ & Manual Tape & 298.25 & Good & \\
\hline $6 / 27 / 937: 04$ & Auto FIC & 298.70 & Good & \\
\hline $6 / 27 / 93 \quad 10: 06$ & Manual Tape & 298.25 & Good & \\
\hline $6 / 28 / 937: 03$ & Auto FIC & 298.70 & Good & \\
\hline $6 / 28 / 93 \quad 10: 06$ & Manual Tape & 298.25 & Good & \\
\hline $6 / 29 / 937: 04$ & Auto FIC & 298.7 & Good & \\
\hline $6 / 29 / 93 \quad 10: 06$ & Manual Tape & 298 & Good & \\
\hline $6 / 30 / 937: 03$ & Auto FIC & 298.70 & Good & \\
\hline $6 / 30 / 93 \quad 10: 06$ & Manual Tape & 298.25 & Good & \\
\hline 7/1/93 7:06 & Auto FIC & 298.7 & Good & \\
\hline $7 / 1 / 93 \quad 10: 06$ & Manual Tape & 298 & Good & \\
\hline $7 / 2 / 937: 06$ & Auto FIC & 298.7 & Good & \\
\hline $7 / 2 / 93 \quad 10: 06$ & Manual Tape & 298.00 & Good & \\
\hline $7 / 3 / 937: 03$ & Auto FIC & 298.70 & Good & \\
\hline $7 / 3 / 93 \quad 10: 06$ & Manual Tape & 298 & Good & \\
\hline 7/4/93 7:04 & Auto FIC & 298.70 & Good & \\
\hline $7 / 4 / 93 \quad 10: 06$ & Manual Tape & 298 & Good & \\
\hline 7/5/93 7:03 & Auto FIC & 298.70 & Good & \\
\hline
\end{tabular}

Data obtained from WHC Surveillance Analysis Computer System, (PC SACS) July 7, 1995. 


\begin{tabular}{|c|c|c|c|c|}
\hline \multicolumn{5}{|c|}{ 241-AP-105 } \\
\hline \multicolumn{5}{|c|}{ Surface Level Data } \\
\hline Date & Type of Measurement & Level (Inches) & Data Quality & Comments \\
\hline $7 / 5 / 93 \quad 10: 06$ & Manual Tape & 298 & Good & \\
\hline 7/6/93 7:03 & Auto FIC & 298.7 & Good & \\
\hline $7 / 6 / 93 \quad 10: 06$ & Manual Tape & 298.25 & Good & \\
\hline $7 / 7 / 93 \quad 7: 04$ & Auto FIC & 298.7 & Good & \\
\hline $7 / 7 / 93 \quad 10: 06$ & Manual Tape & 298.75 & Good & \\
\hline 7/8/93 7:04 & Auto FIC & 298.7 & Good & \\
\hline $7 / 8 / 9310: 06$ & Manual Tape & 298 & Good & \\
\hline 7/9/93 7:05 & Auto FIC & 298.7 & Good & \\
\hline $7 / 9 / 93 \quad 10: 06$ & Manual Tape & 298 & Good & \\
\hline $7 / 10 / 937: 03$ & Auto FIC & 298.7 & Good & \\
\hline $7 / 10 / 93 \quad 10: 06$ & Manual Tape & 298.00 & Good & \\
\hline $7 / 11 / 937: 03$ & Auto FIC & 298.70 & Good & \\
\hline $7 / 11 / 9310: 06$ & Manual Tape & 298 & Good & \\
\hline 7/12/93 7:02 & Auto FIC & 298.70 & Good & \\
\hline $7 / 12 / 9310: 06$ & Manual Tape & 298 & Good & \\
\hline $7 / 13 / 937: 03$ & Auto FIC & 298.7 & Good & \\
\hline $7 / 13 / 9310: 06$ & Manual Tape & 298.00 & Good & \\
\hline $7 / 14 / 937: 06$ & Auto FIC & 298.70 & Good & \\
\hline 7/14/93 10:06 & Manual Tape & 298.00 & Good & \\
\hline 7/15/93 7:06 & Auto FIC & 298.7 & Good & \\
\hline $7 / 15 / 93 \quad 10: 06$ & Manual Tape & 298.00 & Good & \\
\hline $7 / 16 / 937: 06$ & Auto FIC & 298.7 & Good & \\
\hline $7 / 16 / 93 \quad 10: 06$ & Manual Tape & 298.00 & Good & \\
\hline 7/17/93 7:07 & Auto FIC & 298.70 & Good & \\
\hline $7 / 17 / 9310: 06$ & Manual Tape & 298.00 & Good & \\
\hline $7 / 18 / 937: 06$ & Auto FIC & 298.70 & Good & \\
\hline $7 / 18 / 9310: 06$ & Manual Tape & 298.00 & Good & \\
\hline $7 / 19 / 937: 03$ & Auto FIC & 298.7 & Good & \\
\hline $7 / 19 / 9310: 06$ & Manual Tape & 298.00 & Good & \\
\hline $7 / 20 / 930: 00$ & Manual Tape : & 294.00 & Suspect & Data not included in graph \\
\hline $7 / 20 / 937: 03$ & Auto FIC & 298.70 & Good & \\
\hline $7 / 20 / 93 \quad 10: 06$ & Manual Tape & 294 & Suspect & Data not included in graph \\
\hline $7 / 21 / 937: 03$ & Auto FIC & 298.70 & Good & \\
\hline $7 / 21 / 9310: 06$ & Manual Tape & 298 & Good & \\
\hline $7 / 22 / 937: 03$ & Auto FIC & 298.70 & Good & \\
\hline $7 / 22 / 9310: 06$ & Manual Tape & 298 & Good & \\
\hline 7/23/93 7:03 & Auto FIC & 298.70 & Good & \\
\hline $7 / 23 / 9310: 06$ & Manual Tape & 298.00 & Good & \\
\hline $7 / 24 / 937: 03$ & Auto FIC & 298.70 & Good & \\
\hline $7 / 24 / 9310: 06$ & Manual Tape & 298 & Good & \\
\hline $7 / 25 / 937: 04$ & Auto FIC & 298.7 & Good & \\
\hline $7 / 25 / 93 \quad 10: 06$ & Manual Tape & 298.00 & Good & \\
\hline $7 / 26 / 937: 03$ & Auto FIC & 298.7 & Good & \\
\hline $7 / 26 / 93 \quad 10: 06$ & Manual Tape & 298.00 & Good & \\
\hline 7/27/93 7:03 & Auto FIC & 298.70 & Good & \\
\hline $7 / 27 / 93 \quad 10: 06$ & Manual Tape & 298 & Good & \\
\hline $7 / 28 / 93 \quad 0: 00$ & Manual Tape & 298.00 & Good & \\
\hline $7 / 28 / 937: 04$ & Auto FIC & 298.7 & Good & \\
\hline
\end{tabular}

Data obtained from WHC Surveillance Analysis Computer System, (PC SACS) July 7, 1995. 


\begin{tabular}{|c|c|c|c|c|}
\hline \multicolumn{5}{|c|}{ 241-AP-105 } \\
\hline \multicolumn{5}{|c|}{ Surface Level Data } \\
\hline Date & Type of Measurement & Leval (Inchea) & Data Quality & Cornments \\
\hline $7 / 29 / 930: 00$ & Manual Tape & 298 & Good & . \\
\hline $7 / 29 / 937: 03$ & Auto FIC & 298.7 & Good & \\
\hline $7 / 30 / 930: 00$ & Manual Tape & 298 & Good & \\
\hline $7 / 30 / 937: 03$ & Auto FIC & 298.7 & Good & \\
\hline 7/31/93 0:00 & Manual Tapo & 298.00 & Good & \\
\hline $7 / 31 / 937: 03$ & Auto FIC & 298.70 & Good & \\
\hline $8 / 1 / 930: 00$ & Manual Tape & 298 & Good & \\
\hline $8 / 1 / 937: 03$ & Auto FIC & 298.70 & Good & \\
\hline $8 / 2 / 930: 00$ & Manual Tape & 298.25 & Good & \\
\hline $8 / 2 / 937: 06$ & Auto FIC & 298.70 & Good & \\
\hline $8 / 3 / 930: 00$ & Manual Tape & 298 & Good & \\
\hline $8 / 3 / 937: 03$ & Auto FIC & 298.70 & Good & \\
\hline $8 / 4 / 930: 00$ & Manual Tape & 298.00 & Good & \\
\hline $8 / 4 / 937: 03$ & Auto FIC & 298.70 & Good & \\
\hline $8 / 5 / 930: 00$ & Manual Tape & 298.00 & Good & \\
\hline $8 / 5 / 937: 04$ & Auto FIC & 298.7 & Good & \\
\hline $8 / 6 / 930: 00$ & Manual Tape & 298.00 & Good & \\
\hline $8 / 6 / 937: 04$ & Auto FIC & 298.7 & Good & \\
\hline $8 / 7 / 930: 00$ & Manual Tape & 298.25 & Good & \\
\hline $8 / 7 / 937: 04$ & Auto FIC & 298.80 & Good & \\
\hline $8 / 8 / 930: 00$ & Manual Tape & 298.25 & Good & \\
\hline $8 / 8 / 937: 04$ & Auto FIC & 298.80 & Good & \\
\hline $8 / 9 / 930: 00$ & Manual Tape & 298.25 & Good & \\
\hline $8 / 9 / 937: 04$ & Auto FIC & 298.7 & Good & \\
\hline $8 / 10 / 930: 00$ & Manual Tape & 298 & Good & \\
\hline $8 / 10 / 937: 04$ & Auto FIC & 298.8 & Good & \\
\hline $8 / 11 / 930: 00$ & Manual Tape & 298.00 & Good & \\
\hline $8 / 11 / 937: 04$ & Auto FIC & 298.8 & Good & \\
\hline $8 / 12 / 930: 00$ & Manual Tape & 298 & Good & \\
\hline $8 / 12 / 937: 04$ & Auto FIC & 298.8 & Good & \\
\hline $8 / 13 / 930: 00$ & Manual Tape & 298 & Good & \\
\hline $8 / 13 / 937: 04$ & Auto FIC & 298.80 & Good & \\
\hline $8 / 14 / 930: 00$ & Manual Tape & 298.25 . & Good & \\
\hline $8 / 14 / 937: 03$ & Auto FIC & 298.8 & Good & \\
\hline $8 / 15 / 930: 00$ & Manual Tape & 298.25 & Good & \\
\hline $8 / 15 / 937: 04$ & Auto FIC & 298.80 & Good & \\
\hline $8 / 16 / 930: 00$ & Manual Tape & 298.25 & Good & \\
\hline $8 / 16 / 937: 04$ & Auto FIC & 298.80 & Good & \\
\hline $8 / 17 / 930: 00$ & Manual Tapo & 298.25 & Good & \\
\hline $8 / 17 / 937: 04$ & Auto FIC & 299 & Good & \\
\hline $8 / 18 / 930: 00$ & Manual Tape & 298.25 & Good & \\
\hline $8 / 18 / 937: 03$ & Auto FIC & $298.80^{\circ}$ & Good & \\
\hline $8 / 19 / 930: 00$ & Manual Tape & 298.25 & Good & \\
\hline $8 / 19 / 93 \quad 7: 04$ & Auto FIC & 298.80 & Good & \\
\hline $8 / 20 / 930: 00$ & Manual Tape & 298.25 & Good & \\
\hline $8 / 20 / 937: 04$ & Auto FIC & 298.80 & Good & \\
\hline $8 / 21 / 930: 00$ & Manual Tape & 298.25 & Good & \\
\hline $8 / 21 / 937: 04$ & Auto FIC & 298.80 & Good & \\
\hline
\end{tabular}

Data obtained from WHC Surveillance Analysis Computer System, (PC SACS) July 7, 1995. 


\begin{tabular}{|c|c|c|c|c|}
\hline \multicolumn{5}{|c|}{$241-4 P: 105$} \\
\hline & & Ufface Lavel Dat & & \\
\hline Date & Type of Measuroment & Leval (Inches) & Data Quality & Comments \\
\hline $8 / 22 / 930: 00$ & Manual Tape & 298 & Good & \\
\hline $8 / 22 / 937: 04$ & Auto FIC & 298.8 & Good & \\
\hline $8 / 23 / 930: 00$ & Manual Tape & 298.25 & Good & \\
\hline $8 / 23 / 937: 04$ & Auto FIC & 298.80 & Good & \\
\hline $8 / 24 / 930: 00$ & Manual Tape & 298.00 & Good & \\
\hline $8 / 24 / 937: 04$ & Auto FIC & 298.80 & Good & \\
\hline 8/25/93 0:00 & Manual Tape & 298.25 & Good & \\
\hline $8 / 25 / 937: 04$ & Auto FIC & 298.8 & Good & \\
\hline $8 / 26 / 930: 00$ & Manual Tape & 298.25 & Good & \\
\hline $8 / 26 / 937: 04$ & Auto FIC & 298.80 & Good & \\
\hline $8 / 27 / 937: 05$ & Auto FIC & 298.8 & Good & \\
\hline $8 / 28 / 930: 00$ & Manual Tape & 298 & Good & \\
\hline $8 / 28 / 937: 04$ & Auto FIC & 298.80 & Good & \\
\hline $8 / 29 / 93 \quad 0: 00$ & Manual Tape & 298.00 & Good & \\
\hline $8 / 29 / 93 \quad 7: 03$ & Auto FIC & 298.8 & Good & \\
\hline $8 / 30 / 930: 00$ & Manual Tape & 298.00 & Good & \\
\hline $8 / 30 / 937: 04$ & Auto FIC & 298.8 & Good & \\
\hline $8 / 31 / 930: 00$ & Manual Tape & 298 & Gopd & \\
\hline $8 / 31 / 937: 04$ & Auto FIC & 298.8 & Good & \\
\hline 9/1/93 0:00 & Manual Tape & 298.25 & Good & \\
\hline 9/1/93 7:04 & Auto FIC & 298.80 & Good & \\
\hline $9 / 2 / 93 \quad 0: 00$ & Manual Tape & 298.25 & Good & \\
\hline $9 / 2 / 937: 04$ & Auto FIC & 298.80 & Good & \\
\hline 9/3/93 0:00 & Manual Tape & 298.25 & Good & \\
\hline $9 / 3 / 937: 04$ & Auto FIC & 298.8 & Good & \\
\hline $9 / 4 / 930: 00$ & Manual Tape & 298.5 & Good & \\
\hline $9 / 4 / 937: 04$ & Auto FIC & 298.80 & Good & \\
\hline 9/5/93 0:00 & Manual Tape & 298.5 & Good & \\
\hline $9 / 5 / 937: 04$ & Auto FIC & 298.8 & Good & \\
\hline $9 / 6 / 930: 00$ & Manual Tape & 298.25 & Good & \\
\hline $9 / 6 / 937: 04$ & Auto FIC & 298.90 & Good & \\
\hline 9/7/93 0:00 & Manual Tape & 298.25 & Good & \\
\hline $9 / 7 / 937: 04$ & Auto F1C & 298.80 & Good & \\
\hline $9 / 8 / 930: 00$ & Manual Tape & 298.25 & Good & \\
\hline 9/8/93 7:04 & Auto FIC & 298.80 & Good & \\
\hline 9/9/93 0:00 & Manual Tape & 298.25 & Good & \\
\hline 9/9/93 7:04 & Auto FIC & 298.80 & Good & \\
\hline 9/10/93 0:00 & Manual Tape & 298.25 & Good & \\
\hline $9 / 10 / 937: 04$ & Auto FIC & 298.80 & Good & \\
\hline $9 / 11 / 930: 00$ & Manual Tape & 298.25 & Good & \\
\hline $9 / 11 / 937: 04$ & Auto FIC & 298.80 & Good & \\
\hline $9 / 12 / 930: 00$ & Manual Tape & 298.25 & Good & \\
\hline $9 / 12 / 937: 04$ & Auto FIC & 298.8 & Good & \\
\hline $9 / 13 / 930: 00$ & Manual Tape & 298.00 & Good & \\
\hline 9/13/93 7:04 & Auto FIC & 298.8 & Good & \\
\hline $9 / 14 / 930: 00$ & Manual Tape & 298 & Good & \\
\hline $9 / 14 / 937: 04$ & Auto FIC & 298.80 & Good & \\
\hline $9 / 15 / 930: 00$ & Manual Tape & 298 & Good & \\
\hline
\end{tabular}

Data obtained from WHC Surveillance Analysis Computer System, (PC SACS) July 7, 1995. 


\begin{tabular}{|c|c|c|c|c|}
\hline \multicolumn{5}{|c|}{ 241-AP-105 } \\
\hline & & Urface Level Dat & & \\
\hline Date & Type of Measurement & Level (Inches) & Data Quality & Comments \\
\hline $9 / 15 / 93$ 7:04 & Auto FIC & 298.8 & Good & \\
\hline $9 / 16 / 930: 00$ & Manual Tape & 298.00 & Good & \\
\hline $9 / 16 / 937: 04$ & Auto FIC & 298.80 & Good & \\
\hline $9 / 17 / 930: 00$ & Manual Tape & 298 & Good & \\
\hline $9 / 17 / 937: 04$ & Auto FIC & 298.80 & Good & \\
\hline $9 / 18 / 930: 00$ & Manual Tape & 298 & Good & \\
\hline $9 / 18 / 937: 04$ & Auto FIC & 298.8 & Good & \\
\hline $9 / 19 / 930: 00$ & Manual Tape & 298 & Good & \\
\hline $9 / 19 / 937: 04$ & Auto FIC & 298.80 & Good & \\
\hline $9 / 20 / 930: 00$ & Manual Tape & 298.00 & Good & \\
\hline $9 / 20 / 937: 04$ & Auto FIC & 298.80 & Good & \\
\hline $9 / 21 / 930: 00$ & Manual Tape & 298.00 & Good & \\
\hline $9 / 21 / 937: 04$ & Auto FIC & 298.80 & Good & \\
\hline $9 / 22 / 930: 00$ & Manual Tape & 298.00 & Good & \\
\hline $9 / 22 / 937: 04$ & Auto FIC & 298.8 & Good & \\
\hline $9 / 23 / 930: 00$ & Manual Tape & 298 & Good & \\
\hline 9/23/93 7:04 & Auto FIC & 298.8 & Good & \\
\hline 9/24/93 0:00 & Manual Tape & $298.00^{\circ}$ & Good & \\
\hline $9 / 24 / 937: 04$ & Auto FIC & 298.8 & Good & \\
\hline $9 / 25 / 930: 00$ & Manual Tape & 298 & Good & \\
\hline 9/25/93 7:04 & Auto FIC & 298.8 & Good & \\
\hline $9 / 26 / 930: 00$ & Manual Tape & 298 & Good & \\
\hline $9 / 26 / 937: 04$ & Auto FIC & 298.8 & Good & \\
\hline $9 / 27 / 930: 00$ & Manual Tape & 298.00 & Good & \\
\hline 9/27/93 7:04 & Auto FIC & 298.8 & Good & \\
\hline $9 / 28 / 930: 00$ & Manual Tape & 298.00 & Good & \\
\hline 9/28/93 7:04 & Auto FIC & 298.7 & Good & \\
\hline $9 / 29 / 93 \quad 0: 00$ & Manual Tape & 298.00 & Good & \\
\hline $9 / 29 / 937: 04$ & Auto FIC & 298.7 & Good & \\
\hline $9 / 30 / 930.00$ & Manual Tape & 298 & Good & \\
\hline 9/30/93 7:03 & Auto FIC & 298.80 & Good & \\
\hline $10 / 1 / 930: 00$ & Manual Tape & 298.00 & Good & \\
\hline $10 / 1 / 937: 06$ & Auto FIC & 298.80 & Good & \\
\hline $10 / 2 / 930: 00$ & Manual Tape & 298.00 & Good & \\
\hline $10 / 2 / 937: 05$ & Auto FIC & 298.8 & Good & \\
\hline $10 / 3 / 930: 00$ & Manual Tape & 298 & Good & \\
\hline $10 / 3 / 937: 06$ & Auto FIC & 298.80 & Good & \\
\hline $10 / 4 / 930: 00$ & Manual Tape & 298.00 & Good & \\
\hline $10 / 4 / 937: 06$ & Auto FIC & 298.80 & Good & \\
\hline $10 / 5 / 930: 00$ & Manual Tape & 298.00 & Good & \\
\hline $10 / 5 / 937: 06$ & Auto FIC & 298.80 & Good & \\
\hline $10 / 6 / 930: 00$ & Manual Tape & 298 & Good & \\
\hline $10 / 6 / 937: 06$ & Auto FIC & 298.8 & Good & \\
\hline $10 / 7 / 930: 00$ & Manual Tape & 298 & Good & \\
\hline $10 / 7 / 937: 05$ & Auto FIC & 298.70 & Good & \\
\hline $10 / 8 / 937: 04$ & Auto FIC & 298.70 & Good & \\
\hline $10 / 9 / 930: 00$ & Manual Tape & 298 & Good & \\
\hline $10 / 9 / 93 \quad 7: 03$ & Auto FIC & 298.70 & Good & \\
\hline
\end{tabular}

Data obtained from WHC Surveillance Analysis Computer System, (PC SACS) July 7, 1995. 


\begin{tabular}{|c|c|c|c|c|}
\hline \multicolumn{5}{|c|}{ 241-AP105 } \\
\hline & & urface Level Dat & & \\
\hline Date & Type of Moasurement & Leval (Inchea) & Data Quality & Comments \\
\hline 10/10/930:00 & Manual Tape & 298.00 & Good & \\
\hline $10 / 10 / 936: 04$ & Auto FIC & 298.70 & Good & \\
\hline $10 / 11 / 937: 03$ & Auto FIC & 298.7 & Good & \\
\hline $10 / 12 / 937: 04$ & Auto FIC & 298.70 & Good & \\
\hline 10/13/93 0:00 & Manual Tape & 298 & Good & \\
\hline $10 / 13 / 937: 03$ & Auto FIC & 298.80 & Good & \\
\hline $10 / 14 / 930: 00$ & Manual Tape & 298 & Good & \\
\hline $10 / 14 / 937: 04$ & Auto FIC & 298.70 & Good & \\
\hline $10 / 15 / 930: 00$ & Manual Tape & 298.00 & Good & \\
\hline $10 / 15 / 93$ 7:04 & Auto FIC & 298.7 & Good & \\
\hline $10 / 16 / 930: 00$ & Manual Tape & 298.00 & Good & \\
\hline $10 / 16 / 937: 04$ & Auto FIC & 298.7 & Good & \\
\hline $10 / 17 / 930: 00$ & Manual Tape & 298.00 & Good & \\
\hline $10 / 17 / 937: 03$ & Auto FIC & 298.7 & Good & \\
\hline $10 / 18 / 930: 00$ & Manual Tape & 298 & Good & \\
\hline $10 / 18 / 937: 04$ & Auto FIC & 298.70 & Good & \\
\hline $10 / 19 / 930: 00$ & Manual Tape & 298 & Good & \\
\hline $10 / 19 / 937: 04$ & Auto FIC & 298.7 & Good & \\
\hline $10 / 20 / 930: 00$ & Manual Tape & 298 & Good & \\
\hline $10 / 20 / 937: 03$ & Auto FIC & 298.7 & Good & \\
\hline $10 / 21 / 930: 00$ & Manual Tape & 298.00 & Good & \\
\hline $10 / 21 / 937: 04$ & Auto FIC & 298.7 & Good & \\
\hline $10 / 22 / 930: 00$ & Manual Tape & 298.00 & Good & \\
\hline $10 / 22 / 937: 04$ & Auto FIC & 298.7 & Good & \\
\hline $10 / 23 / 930: 00$ & Manual Tape & 298 & Good & \\
\hline $10 / 23 / 937: 04$ & Auto FIC & 298.7 & Good & \\
\hline $10 / 24 / 930: 00$ & Manual Tape & 298 & Good & \\
\hline $10 / 24 / 937: 04$ & Auto FIC & 298.70 & Good & \\
\hline $10 / 25 / 930: 00$ & Manual Tape & 298 & Good & \\
\hline $10 / 25 / 937: 04$ & Auto FIC & 298.7 & Good & \\
\hline $10 / 26 / 93 \quad 0: 00$ & Manual Tape & 298.00 & Good & \\
\hline $10 / 26 / 936: 03$ & Auto FIC & 298.7 & Good & \\
\hline $10 / 27 / 930: 00$ & Manual Tape & 298.00 & Good & \\
\hline $10 / 27 / 937: 04$ & Auto FIC & 298.7 & Good & \\
\hline $10 / 28 / 930: 00$ & Manual Tape & 298.00 & Good & \\
\hline $10 / 28 / 937: 04$ & Auto FIC & 298.70 & Good & \\
\hline $10 / 29 / 93 \quad 0: 00$ & Manual Tape & 298.00 & Good & \\
\hline $10 / 29 / 936: 03$ & Auto FIC & 298.7 & Good & \\
\hline $10 / 30 / 930: 00$ & Manual Tape & 298.00 & Good & \\
\hline $10 / 30 / 937: 04$ & Auto FIC & 298.70 & Good & \\
\hline $10 / 31 / 930: 00$ & Manual Tape & 298 & Good & \\
\hline $10 / 31 / 937: 06$ & Auto FIC & 298.70 & Good & \\
\hline $11 / 1 / 930: 00$ & Manual Tapo & 298.25 & Good & \\
\hline $11 / 1 / 937: 06$ & Auto FIC & 298.70 & Good & \\
\hline $11 / 2 / 930: 00$ & Manual Tape & 298 & Good & \\
\hline $11 / 2 / 93 \quad 7: 04$ & Auto FIC & 298.70 & Good & \\
\hline $11 / 3 / 930: 00$ & Manual Tape & 298 & Good & \\
\hline $11 / 3 / 937: 06$ & Auto FIC & 298.7 & Good & \\
\hline
\end{tabular}

Data obtained from WHC Surveillance Analysis Computer System, (PC SACS) July 7, 1995. 


\begin{tabular}{|c|c|c|c|c|}
\hline \multicolumn{5}{|c|}{ 241-AP-105 } \\
\hline Date & Type of Measurement & Level (Inches) & Data Ouality & Comments \\
\hline $11 / 4 / 930: 00$ & Manual Tape & 298.00 & Good & \\
\hline 11/4/93 7:04 & Auto FIC & 298.7 & Good & \\
\hline $11 / 5 / 930: 00$ & Manual Tape . & 298 & Good & \\
\hline $11 / 5 / 937: 06$ & Auto FIC & 298.6 & Good & \\
\hline $11 / 6 / 930: 00$ & Manual Tape & 298.00 & Good & \\
\hline $11 / 6 / 937: 06$ & Auto FIC & 298.6 & Good & \\
\hline $11 / 7 / 930: 00$ & Manual Tape & 298.00 & Good & \\
\hline $11 / 7 / 937: 06$ & Auto FIC & 298.60 & Good & \\
\hline $11 / 8 / 930: 00$ & Manual Tape & 298 & Good & \\
\hline $11 / 8 / 937: 06$ & Auto FIC & 298.60 & Good & \\
\hline $11 / 9 / 930: 00$ & Manual Tape & 298 & Good & \\
\hline $11 / 9 / 937: 03$ & Auto FIC & 298.60 & Good & \\
\hline $11 / 10 / 930: 00$ & Manual Tape & 298.00 & Good & \\
\hline $11 / 10 / 937: 04$ & Auto FIC & 298.60 & Good & \\
\hline $11 / 11 / 930: 00$ & Manual Tape & 298.00 & Good & \\
\hline $11 / 11 / 937: 04$ & Auto FIC & 298.60 & Good & \\
\hline $11 / 12 / 930: 00$ & Manual Tape & 298 & Good & \\
\hline $11 / 12 / 937: 04$ & Auto FIC & 298.6 & Good & \\
\hline 11/13/930:00 & Manual Tape & 298 & Good & \\
\hline 11/13/93 7:04 & Auto FIC & 298.60 & Good & \\
\hline $11 / 14 / 930: 00$ & Manual Tape & 298.00 & Good & \\
\hline $11 / 14 / 936: 04$ & Auto FIC & 298.60 & Good & \\
\hline $11 / 15 / 930: 00$ & Manual Tape & 298.00 & Good & \\
\hline $11 / 15 / 937: 04$ & Auto FIC & 298.60 & Good & \\
\hline $11 / 16 / 930: 00$ & Manual Tape & 298 & Good & \\
\hline $11 / 16 / 937: 03$ & Auto FIC & 298.60 & Good & \\
\hline 11/17/930:00 & Manual Tape & 298 & Good & \\
\hline $11 / 17 / 937: 04$ & Auto FIC & 298.6 & Good & \\
\hline $11 / 18 / 930: 00$ & Manual Tape & 297.75 & Good & \\
\hline 11/18/93 7:04 & Auto FIC & 298.6 & Good & \\
\hline $11 / 19 / 930: 00$ & Manual Tape & 297.5 & Good & \\
\hline $11 / 19 / 937: 04$ & Auto FIC & 298.60 & Good & \\
\hline $11 / 20 / 930: 00$ & Manual Tape & 298 & Good & \\
\hline $11 / 20 / 937: 04$ & Auto FIC & 298.6 & Good & \\
\hline $11 / 21 / 930: 00$ & Manual Tape & 298.00 & Good & \\
\hline $11 / 21 / 937: 04$ & Auto FIC & 298.6 & Good & \\
\hline $11 / 22 / 930: 00$ & Manual Tape & 298 & Good & \\
\hline $11 / 22 / 937: 04$ & Auto FIC & 298.6 & Good & \\
\hline $11 / 23 / 930: 00$ & Manual Tape & 298.00 & Good & \\
\hline $11 / 23 / 937: 04$ & Auto FIC & 298.6 & Good & \\
\hline $11 / 24 / 930: 00$ & Manual Tape & 297,75 & Good & \\
\hline $11 / 24 / 937: 04$ & Auto FIC & 298.6 & Good & \\
\hline 11/25/93 0:00 & Manual Tape & 298 & Good & \\
\hline $11 / 25 / 937: 04$ & Auto FIC & 298.5 & Good & \\
\hline $11 / 26 / 930: 00$ & Manual Tape & 298 & Good & \\
\hline $11 / 26 / 937: 04$ & Auto FIC & 298.50 & Good & \\
\hline $11 / 27 / 930: 00$ & Manual Tape & 298.00 & Good & \\
\hline $11 / 27 / 937: 04$ & Auto FIC & 298.50 & Good & \\
\hline
\end{tabular}

Data obtained from WHC Surveillance Analysis Computer System, (PC SACS) July 7, 1995. 


\begin{tabular}{|c|c|c|c|c|}
\hline \multicolumn{5}{|c|}{$\begin{array}{c}\text { 241-AP-105 } \\
\text { Surface Leval Data }\end{array}$} \\
\hline & $\cdot$ & Ifface Loval Date & & \\
\hline Date & Type of Measurement & Level (Inches) & Data Quality & Comments \\
\hline $11 / 28 / 930: 00$ & Manual Tape & 298.00 & Good & \\
\hline 11/28/93 7:03 & Auto FIC & 298.5 & Good & \\
\hline 11/29/93 0:00 & Manual Tape & 298 & Good & \\
\hline $11 / 29 / 937: 04$ & Auto FIC & 298.50 & Good & \\
\hline $11 / 30 / 930: 00$ & Manual Tape & 298.00 & Good & \\
\hline 11/30/93 7:03 & Auto FIC & 298.50 & Good & \\
\hline $12 / 1 / 930: 00$ & Manual Tape & 298.00 & Good & \\
\hline $12 / 1 / 937: 04$ & Auto FIC & 298.5 & Good & \\
\hline $12 / 2 / 930: 00$ & Manual Tape & 298 & Good & \\
\hline $12 / 2 / 937: 04$ & Auto FIC & 298.5 & Good & \\
\hline $12 / 3 / 93 \quad 0: 00$ & Manual Tape & 298.00 & Good & \\
\hline $12 / 3 / 93$ 7:05 & Auto FIC & 298.50 & Good & \\
\hline $12 / 4 / 93 \quad 0: 00$ & Manual Tape & 298 & Good & \\
\hline $12 / 4 / 93 \quad 7: 05$ & Auto FIC & 298.50 & Good & \\
\hline $12 / 5 / 930: 00$ & Manual Tape & 298.00 & Good & \\
\hline $12 / 5 / 937: 04$ & Auto FIC & 298.5 & Good & \\
\hline $12 / 6 / 930: 00$ & Manual Tape & 297.75 & Good & \\
\hline $12 / 6 / 937: 05$ & Auto FIC & 298.50 & Good & \\
\hline $12 / 7 / 930: 00$ & Manual Tape & 298.00 & Good & \\
\hline $12 / 7 / 937: 04$ & Auto FIC & 298.50 & Good & \\
\hline $12 / 8 / 930: 00$ & Manual Tape & 298 & Good & \\
\hline $12 / 8 / 937: 06$ & Auto FIC & 298.5 & Good & \\
\hline $12 / 9 / 930: 00$ & Manual Tape & 298.00 & Good & \\
\hline $12 / 9 / 937: 04$ & Auto FIC & 298.50 & Good & \\
\hline $12 / 10 / 930: 00$ & Manual Tape & 298.00 & Good & \\
\hline $12 / 10 / 936: 05$ & Auto FIC & 298.50 & Good & \\
\hline $12 / 11 / 930: 00$ & Manual Tape & 238.00 & Suspect & Data not included in graph \\
\hline $12 / 11 / 937: 06$ & Auto FIC & 298.5 & Good & \\
\hline $12 / 12 / 930: 00$ & Manual Tape & $297: 75$ & Good & \\
\hline $12 / 12 / 937: 06$ & Auto FIC & 298.5 & Good & \\
\hline $12 / 13 / 930: 00$ & Manual Tape & 298 & Good & \\
\hline $12 / 13 / 937: 06$ & Auto FIC & 298.50 & Good & \\
\hline $12 / 14 / 930: 00$ & Manual Tape . & 298 & Good & \\
\hline $12 / 14 / 937: 06$ & Auto FIC & 298.5 & Good & \\
\hline $12 / 15 / 930: 00$ & Manual Tape & 297.75 & Good & \\
\hline $12 / 15 / 937: 06$ & Auto FIC & 298.5 & Good & \\
\hline $12 / 16 / 930: 00$ & Manual Tape & 297.75 & Good & \\
\hline $12 / 16 / 937: 06$ & Auto FIC & 298.4 & Good & \\
\hline $12 / 17 / 930: 00$ & Manual Tape & 298 & Good & \\
\hline $12 / 17 / 937: 05$ & Auto FIC & 298.4 & Good & \\
\hline 12/18/93 0:00 & Manual Tape & 298 & Good & \\
\hline $12 / 18 / 937: 06$ & Auto FIC & 298.40 & Good & \\
\hline $12 / 19 / 930: 00$ & Manual Tape & 297.75 & Good & \\
\hline $12 / 19 / 937: 06$ & Auto FIC & 298.40 & Good & \\
\hline $12 / 20 / 930: 00$ & Manual Tape & 297.75 & Good & \\
\hline $12 / 20 / 937: 06$ & Auto FIC & $298: 40$ & Good & \\
\hline $12 / 21 / 930: 00$ & Manual Tape & 297.75 & Good & \\
\hline $12 / 21 / 937: 06$ & Auto FIC & 298.40 & Good & \\
\hline
\end{tabular}

Data obtained from WHC Surveillance Analysis Computer System, (PC SACS) July 7, 1995. 


\begin{tabular}{|c|c|c|c|c|}
\hline \multicolumn{5}{|c|}{ 241-AP-105 } \\
\hline \multicolumn{5}{|c|}{ Surface Leval Dato } \\
\hline Date & Type of Measurement & Level (Inches) & Data Quality & Comments \\
\hline $12 / 22 / 930: 00$ & Manual Tape & 297.75 & Good & \\
\hline $12 / 22 / 937: 05$ & Auto FIC & 298.4 & Good & \\
\hline $12 / 23 / 930: 00$ & Manual Tape & 297.75 & Good & \\
\hline $12 / 23 / 936: 06$ & Auto FIC & 298.40 & Good & \\
\hline $12 / 24 / 930: 00$ & Manual Tape & 297.75 & Good & \\
\hline $12 / 24 / 937: 06$ & Auto FIC & 298.40 & Good & \\
\hline $12 / 25 / 930: 00$ & Manual Tape & 297.75 & Good & \\
\hline $12 / 25 / 937: 06$ & Auto FIC & 298.4 & Good & \\
\hline $12 / 26 / 930: 00$ & Manual Tape & 297.75 & Good & \\
\hline $12 / 26 / 937: 06$ & Auto FIC & 298.4 & Good & \\
\hline $12 / 27 / 930: 00$ & Manual Tape & 297.75 & Good & \\
\hline $12 / 27 / 937: 06$ & Auto FIC & 298.4 & Good & \\
\hline $12 / 28 / 930: 00$ & Manual Tape & 297.75 & Good & \\
\hline $12 / 28 / 937: 06$ & Auto FIC & 298.4 & Good & \\
\hline $12 / 29 / 930: 00$ & Manual Tape & 297.75 & Good & \\
\hline $12 / 29 / 937: 06$ & Auto FIC & 298.40 & Good & \\
\hline 12/30/93 0:00 & Manual Tape & 297.75 & Good & \\
\hline 12/30/93 7:05 & Auto FIC & 298.40. & Good & 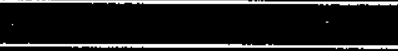 \\
\hline $12 / 31 / 930: 00$ & Manual Tape & 298.00 & Good & \\
\hline $12 / 31 / 937: 06$ & Auto FIC & 298.40 & Good & \\
\hline $1 / 1 / 940: 00$ & Manual Tape & 297.75 & Good & \\
\hline $1 / 1 / 947: 06$ & Auto FIC & 298.40 & Good & \\
\hline $1 / 2 / 940: 00$ & Manual Tape & 297.75 & Good & \\
\hline $1 / 2 / 947: 06$ & Auto FIC & 298.4 & Good & \\
\hline $1 / 3 / 940: 00$ & Manual Tape & 297.75 & Good & \\
\hline $1 / 3 / 947: 07$ & Auto FIC & 298.40 & Good & \\
\hline $1 / 4 / 940: 00$ & Manual Tape & $297.75^{\circ}$ & Good & \\
\hline $1 / 4 / 947: 07$ & Auto FIC & 298.40 & Good & \\
\hline $1 / 5 / 940: 00$ & Manual Tape & 297.75 & Good & \\
\hline $1 / 5 / 947: 06$ & Auto FIC & 298.40 & Good & \\
\hline $1 / 6 / 940: 00$ & Manual Tape & 297.75 & Good & \\
\hline 1/6/94 7:06 & Auto FIC & 298.30 & Good & \\
\hline $1 / 7 / 940: 00$ & Manual Tape & 297.75 & Good & \\
\hline $1 / 7 / 947: 07$ & Auto FIC & 298.30 & Good & \\
\hline $1 / 8 / 940: 00$ & Manual Tape & 297.75 & Good & \\
\hline $1 / 8 / 947: 06$ & Auto FIC & 298.3 & Good & \\
\hline $1 / 9 / 940: 00$ & Manual Tape & 297.75 & Good & \\
\hline $1 / 9 / 947: 06$ & Auto FIC & 298.3 & Good & \\
\hline $1 / 10 / 940: 00$ & Manual FIC & 298.40 & Good & \\
\hline $1 / 10 / 940: 00$ & Manual Tape & 297.75 & Good & \\
\hline $1 / 10 / 947: 07$ & Auto FIC & 0.00 & Suspect & Data not included in graph \\
\hline $1 / 11 / 940: 00$ & Manual FIC & 298.3 & Good & \\
\hline $1 / 11 / 940: 00$ & Manual Tape & 297.75 & Good & \\
\hline $1 / 11 / 947: 07$ & Auto FIC & 0.00 & Suspect & Data not included in graph \\
\hline $1 / 12 / 940: 00$ & Manual FIC & 298.35 & Good & \\
\hline $1 / 12 / 940: 00$ & Manual Tape & 297.75 & Good & \\
\hline $1 / 12 / 947: 06$ & Auto FIC & 0 & Suspect & Data not included in graph \\
\hline $1 / 13 / 940: 00$ & Manual FIC & 298.30 & Good & \\
\hline
\end{tabular}

Data obtained from WHC Surveillance Analysis Computer System, (PC SACS) July 7, 1995. 


\begin{tabular}{|c|c|c|c|c|}
\hline \multicolumn{5}{|c|}{$\begin{array}{c}\text { 241-AP\$O5 } \\
\text { Surface Lovel Dato }\end{array}$} \\
\hline$\frac{\text { Date }}{1 / 13 / 940: 00}$ & Manual Tape & 298 & Good & \\
\hline$\frac{1 / 13 / 940: 00}{1 / 13 / 947: 04}$ & Auto FIC & 0 & Suspect & Data not included in graph \\
\hline$\frac{1 / 13 / 947: 04}{1 / 14 / 940: 00}$ & Manual FIC & 298.35 & Good & \\
\hline$\frac{1 / 14 / 940: 00}{1 / 14 / 940: 00}$ & Manual Tape & 297.75 & Good & \\
\hline$\frac{1 / 14 / 940: 00}{1 / 14 / 947: 06}$ & Auto FIC & 0 & Suspect & Data not included in graph \\
\hline $1 / 15 / 940: 00$ & Manual FIC & 298.35 & Good & \\
\hline $1 / 15 / 940: 00$ & Manual Tape & 297.50 & Good & \\
\hline $1 / 15 / 947: 04$ & Auto FIC & 0 & Suspect & Data not included in graph \\
\hline $1 / 16 / 940: 00$ & Manual FIC & 298.30 & Good & \\
\hline $1 / 16 / 940: 00$ & Manual Tape & 297.50 & Good & \\
\hline $1 / 16 / 947: 07$ & Auto FIC & 0.00 & Suspect & Data not included in graph \\
\hline $1 / 17 / 940: 00$ & Manual FIC & 298.35 & Good & . \\
\hline 1/17/940:00 & Manual Tape & 297.75 & Good & \\
\hline $1 / 17 / 947: 07$ & Auto FIC & 0.00 & Suspect & Data not included in graph \\
\hline $1 / 18 / 940: 00$ & Manual FIC & 298.3 & Good & \\
\hline - $1 / 18 / 940: 00$ & Manual Tape & 297.75 & Good & \\
\hline $1 / 18 / 947: 07$ & Auto FIC & 0 & Suspect & Data not included in graph \\
\hline $1 / 19 / 940: 00$ & Manual FIC & 298.30 & Good & \\
\hline $1 / 19 / 940: 00$ & Manual Tape & 297.75 & Good & \\
\hline 1/19/94 7:07 & Auto FIC & 0.00 & Suspect & Data not included in graph \\
\hline $1 / 20 / 940: 00$ & Manual FIC & 298.3 & Good & \\
\hline $1 / 20 / 940: 00$ & Manual Tape & 297.75 & Good & \\
\hline $1 / 20 / 947: 07$ & Auto FIC & 0.00 & Suspect & Data not included in graph \\
\hline $1 / 21 / 940: 00$ & Manual FIC & 298.30 & Good & \\
\hline $1 / 21 / 940: 00$ & Manual Tape & 297.75 & Good & \\
\hline $1 / 21 / 947: 07$ & Auto FIC & 0.00 & Suspect & Data not included in graph \\
\hline $1 / 22 / 940: 00$ & Manual FIC & 298.30 & Good & \\
\hline $1 / 22 / 940: 00$ & Manual Tape & 297.75 & Good & \\
\hline 1/22/947:08 & Auto FIC & 0 & Suspect & Data not included in graph \\
\hline $1 / 23 / 940: 00$ & Manual FIC & 298.30 & Good & \\
\hline $1 / 23 / 940: 00$ & Manual Tape & 297.5 & Good & \\
\hline $1 / 23 / 947: 07$ & Auto FIC & 0.00 & Suspect & Data not included in graph \\
\hline 1/24/940:00 & Manual FIC & 298.3 & Good & \\
\hline $1 / 24 / 940: 00$ & Manual Tape & 297.75 & Good & \\
\hline $1 / 24 / 947: 08$ & Auto FIC & 0.00 & Suspect & Data not included in graph \\
\hline $1 / 25 / 940: 00$ & Manual FIC & 298.3 & Good & \\
\hline $1 / 25 / 940: 00$ & Manual Tape & 297.75 & Good & \\
\hline $1 / 25 / 947: 07$ & Auto FIC & .0 & Suspect & Data not included in graph \\
\hline $1 / 26 / 940: 00$ & Manual FIC & $\begin{array}{l}.298 .30 \\
\end{array}$ & Good & \\
\hline $1 / 26 / 940: 00$ & Manual Tape & 297.75 & Good & \\
\hline $1 / 26 / 947: 06$ & Auto FIC & 0 & Suspect & Data not included in graph \\
\hline $1 / 27 / 94 \quad 0: 00$ & Manual Tape & 297.75 & Good & \\
\hline $1 / 27 / 9423: 04$ & Auto FIC & 298.20 & Good & $\cdot$ \\
\hline $1 / 28 / 940: 00$ & Manual Tape & 297.50 & Good & \\
\hline $1 / 28 / 947: 07$ & Auto FIC & 298.2 & Good & \\
\hline $1 / 29 / 940: 00$ & Manual Tape & 297.75 & Good & \\
\hline $1 / 29 / 947: 07$ & Auto FIC & 298.30 & Good & \\
\hline $1 / 30 / 940: 00$ & Manual Tape & 297.75 & - Good & \\
\hline
\end{tabular}

Data obtained from WHC Surveillance Analysis Computer System, (PC SACS) July 7, 1995. 


\begin{tabular}{|c|c|c|c|c|}
\hline \multicolumn{5}{|c|}{ 241-AP-105 } \\
\hline & & urface Level Dat & & \\
\hline Date & Type of Measurement & Level (Inchea) & Data Ouality & Comments \\
\hline $1 / 30 / 947: 06$ & Auto FIC & 298.20 & Good & \\
\hline $1 / 31 / 940: 00$ & Manual Tape & 297.5 & Good & \\
\hline $1 / 31 / 947: 07$ & Auto FIC & 298.20 & Good & \\
\hline $2 / 1 / 940: 00$ & Manual Tape & 297.5 & Good & \\
\hline $2 / 1 / 947: 06$ & Auto FIC & 298.2 & Good & \\
\hline $2 / 2 / 940: 00$ & Manual Tape & 297.75 & Good & \\
\hline $2 / 2 / 947: 07$ & Auto FIC & 298.20 & Good & \\
\hline $2 / 3 / 940: 00$ & Manual Tape & 297.75 & Good & \\
\hline $2 / 3 / 947: 07$ & Auto FIC & 298.20 & Good & \\
\hline $2 / 4 / 940: 00$ & Manual Tape & 297.75 & Good & \\
\hline $2 / 4 / 947: 07$ & Auto FIC & 298.2 & Good & \\
\hline $2 / 5 / 940: 00$ & Manual Tape & 297.50 & Good & \\
\hline $2 / 5 / 947: 07$ & Auto FIC & 298.20 & Good & \\
\hline $2 / 6 / 940: 00$ & Manual Tape & 297.75 & Good & \\
\hline $2 / 6 / 947: 07$ & Auto FIC & 298.20 & Good & \\
\hline $2 / 7 / 940: 00$ & Manual Tape & 297.5 & Good & \\
\hline $2 / 7 / 947: 06$ & Auto FIC & 298.20 & Good & \\
\hline $2 / 8 / 940: 00$ & Manual Tape & 297.50 & Good & \\
\hline $2 / 8 / 947: 07$ & Auto FIC & 298.2 . & Good & \\
\hline $2 / 9 / 940: 00$ & Manual Tape & 297.50 & Good & \\
\hline $2 / 9 / 947: 04$ & Auto FIC & 298.20 & Good & \\
\hline $2 / 10 / 940: 00$ & Manual Tape & 297.50 & Good & \\
\hline $2 / 10 / 947: 04$ & Auto FIC & 298.2 & Good & \\
\hline $2 / 11 / 940: 00$ & Manual Tape & 297.50 & Good & \\
\hline $2 / 11 / 947: 06$ & Auto FIC & 298.20 & Good & \\
\hline $2 / 12 / 940: 00$ & Manual Tape & 297.50 & Good & \\
\hline $2 / 12 / 947: 03$ & Auto FIC & 298.2 & Good & \\
\hline $2 / 13 / 940: 00$ & Manual Tape & 297.5 & Good & \\
\hline $2 / 13 / 947: 04$ & Auto FIC & 298.20 & Good & \\
\hline $2 / 14 / 940: 00$ & Manual Tape & 297.50 & Good & \\
\hline $2 / 14 / 947: 04$ & Auto FIC & 298.2 & Good & \\
\hline $2 / 15 / 940: 00$ & Manual Tape & 297.50 & Good & \\
\hline $2 / 15 / 947: 04$ & Auto FIC & 298.2 & Good & \\
\hline $2 / 16 / 940: 00$ & Manual Tape & 297.5 & Good & \\
\hline $2 / 16 / 947: 03$ & Auto FIC & 298.2 & Good & \\
\hline $2 / 17 / 940: 00$ & Manual Tape & 297.50 & Good & \\
\hline $2 / 17 / 947: 04$ & Auto FIC & 298.2 & Good & \\
\hline $2 / 18 / 940: 00$ & Manual Tape & 297.25 & Good & \\
\hline $2 / 18 / 947: 06$ & Auto FIC & 298.2 & Good & \\
\hline $2 / 19 / 940: 00$ & Manual Tape & 297.75 & Good & \\
\hline $2 / 19 / 947: 06$ & Auto FIC & 298.2 & Good & \\
\hline $2 / 20 / 940: 00$ & Manual Tape & 297.75 & Good & \\
\hline $2 / 20 / 947: 07$ & Auto FIC & 298.20 & Good & \\
\hline $2 / 21 / 940: 00$ & Manual Tape & 297.75 & Good & \\
\hline $2 / 21 / 947: 06$ & Auto FIC & 298.2 & Good & \\
\hline $2 / 22 / 940: 00$ & Manual Tape & 297.75 & Good & \\
\hline $2 / 22 / 947: 05$ & Auto FIC & 298.20 & Good & \\
\hline $2 / 23 / 940: 00$ & Manual Tape & 297.50 & Good & \\
\hline
\end{tabular}

Data obtained from WHC Surveillance Analysis Computer System, (PC SACS) July 7, 1995. 


\begin{tabular}{|c|c|c|c|c|}
\hline \multicolumn{5}{|c|}{ 241-AP-105 } \\
\hline \multicolumn{5}{|c|}{ Surface Level Data } \\
\hline Date & Typo of Measurement & Level (Inches) & Data Quality & Comments \\
\hline $2 / 23 / 947: 06$ & Auto FIC & 298.2 & Good & \\
\hline $2 / 24 / 940: 00$ & Manual Tape & 297.5 & Good & \\
\hline $2 / 24 / 947: 06$ & Auto FIC & 298.10 & Good & \\
\hline $2 / 25 / 940: 00$ & Manual Tape & 297.75 & Good & \\
\hline $2 / 25 / 946: 06$ & Auto FIC & 298.10 & Good & \\
\hline $2 / 26 / 940: 00$ & Manual Tape & 297.5 & Good & \\
\hline $2 / 26 / 947: 07$ & Auto FIC & 298.10 & Good & \\
\hline $2 / 27 / 940: 00$ & Manual Tape & 297.5 & Good & \\
\hline $2 / 27 / 947: 07$ & Auto FIC & 298.1 & Good & \\
\hline $2 / 28 / 940: 00$ & Manual Tape & 297.5 & Good & \\
\hline $2 / 28 / 946: 06$ & Auto FIC & 298.1 & Good. & \\
\hline $3 / 1 / 940: 00$ & Manual Tape & 297.5 & Good & \\
\hline 3/1/94 7:04 & Auto FIC & 298.10 & Good & \\
\hline $3 / 2 / 940: 00$ & Manual Tape & 297.5 & Good & \\
\hline $3 / 2 / 947: 06$ & Auto FIC & 298.10 & Good & \\
\hline $3 / 3 / 940: 00$ & Manual Tape & 297.5 & Good & \\
\hline $3 / 3 / 94 \quad 7: 06$ & Auto FIC & 298.10 & Good & \\
\hline $3 / 4 / 940: 00$ & Manual Tape & 297.50 & Good & \\
\hline $3 / 4 / 947: 04$ & Auto FIC & 298.10 & Good & \\
\hline $3 / 5 / 940: 00$ & Manual Tape & 297.25 & Good & \\
\hline 3/5/94 7:05 & Auto FIC & 298.10 & Good & \\
\hline $3 / 6 / 940: 00$ & Manual Tape & 297.5 & Good & \\
\hline $3 / 6 / 947: 05$ & Auto FIC & 298.1 & Good & \\
\hline $3 / 7 / 940: 00$ & Manual Tape & 297.5 & Good & \\
\hline $3 / 7 / 94 \quad 7: 05$ & Auto FIC & 298.10 & Good & \\
\hline $3 / 8 / 940: 00$ & Manual Tape & 297.5 & Good & \\
\hline $3 / 8 / 947: 04$ & Auto FIC & 298.1 & Good & \\
\hline $3 / 9 / 940: 00$ & Manual Tape & 297.50 & Good & \\
\hline $3 / 9 / 94 \quad 7: 04$ & Auto FIC & 298.1 & Good & \\
\hline $3 / 10 / 940: 00$ & Manual Tape & 297.5 & Good & \\
\hline $3 / 10 / 947: 05$ & Auto FIC & 298:10 & Good & \\
\hline 3/11/94 0:00 & Manual Tape & 297.5 & Good & \\
\hline $3 / 11 / 947: 06$ & Auto FIC & 298.1 & Good & \\
\hline $3 / 12 / 940: 00$ & Manual Tape & 297.50 & Good & \\
\hline $3 / 12 / 947: 06$ & Auto FIC & 298.10 & Good & \\
\hline $3 / 13 / 940: 00$ & Manual Tape & 297.50 & Good & \\
\hline 3/13/94 7:05 & Auto FIC & 298.1 & Good & \\
\hline $3 / 14 / 940: 00$ & Manual FIC & 298.10 & Good & \\
\hline $3 / 14 / 940: 00$ & Manual Tape & 297.50 & Good & \\
\hline $3 / 15 / 940: 00$ & Manual Tape & 297.5 & Good & \\
\hline $3 / 15 / 947: 05$ & Auto FIC & 298.10 & - Good & \\
\hline $3 / 16 / 940: 00$ & Manual Tape & $297.50^{\circ}$ & Good & \\
\hline $3 / 16 / 947: 04$ & Auto FIC & 298.10 & Good & \\
\hline $3 / 17 / 940: 00$ & Manual Tape & 297.50 & Good & \\
\hline 3/17/94 7:05 & Auto FIC & 298.1 & Good & \\
\hline $3 / 18 / 940: 00$ & Manual Tape & 297.5 & Good & \\
\hline $3 / 18 / 947: 05$ & Auto FIC & 298.10 & Good & \\
\hline $3 / 19 / 940: 00$ & Manual Tape & $297: 50$ & Good & \\
\hline
\end{tabular}

Data obtained from WHC Surveillance Analysis Computer System, (PC SACS) July 7, 1995. 


\begin{tabular}{|c|c|c|c|c|}
\hline \multicolumn{5}{|c|}{$\therefore \quad$ 241-AP-105 } \\
\hline \multicolumn{5}{|c|}{ Surface Level Data } \\
\hline Date & Type of Measurement & Level (Inches) & Data Quality & Comments \\
\hline $3 / 19 / 947: 04$ & Auto FIC & 298.1 & Good & \\
\hline $3 / 20 / 940: 00$ & Manual Tape & 297.50 & Good & \\
\hline $3 / 20 / 947: 05$ & Auto FIC & 298.10 & Good & \\
\hline $3 / 21 / 940: 00$ & Manual Tape & 297.5 & Good & \\
\hline $3 / 21 / 947: 05$ & Auto FIC & 298.10 & Good & \\
\hline $3 / 22 / 940: 00$ & Manual Tape & 297.50 & Good & \\
\hline $3 / 22 / 947: 04$ & Auto FIC & 298.10 & Good & \\
\hline $3 / 23 / 940: 00$ & Manual FIC & 298.1 & Good & \\
\hline $3 / 23 / 940: 00$ & Manual Tape & 297.5 & Good & \\
\hline $3 / 24 / 940: 00$ & Manual Tape & 297.50 & Good & \\
\hline $3 / 24 / 947: 04$ & Auto FIC & 298.1 & Good & \\
\hline $3 / 25 / 940: 00$ & Manual Tape & 297.5 & Good & \\
\hline $3 / 25 / 947: 05$ & Auto FIC & 298.1 & Good & \\
\hline $3 / 26 / 940: 00$ & Manual Tape & 297.50 & Good & \\
\hline $3 / 26 / 947: 04$ & Auto FIC & 298.10 & Good & \\
\hline $3 / 27 / 940: 00$ & Manual Tape & 297.50 & Good & \\
\hline $3 / 27 / 947: 04$ & Auto FIC & 298.00 & Good & \\
\hline $3 / 28 / 940: 00$ & Manual Tape & 297.50 & Good & \\
\hline $3 / 28 / 947: 04$ & Auto FIC & 298.00 & Good & \\
\hline $3 / 29 / 940: 00$ & Manual Tape & $\therefore \quad 297.5$ & Good & \\
\hline $3 / 29 / 947: 03$ & Auto FIC & 298.00 & Good & \\
\hline $3 / 30 / 940: 00$ & Manual Tape & 297.5 & Good & \\
\hline $3 / 30 / 947: 03$ & Auto FIC & 298.00 & Good & \\
\hline $3 / 31 / 940: 00$ & Manual Tape & 297.50 & Good & \\
\hline $3 / 31 / 947: 03$ & Auto FIC & 298.00 & Good & \\
\hline $4 / 1 / 940: 00$ & Manual Tape & 297.5 & Good & \\
\hline $4 / 1 / 940: 04$ & Auto FIC & 298.00 & Good & \\
\hline 4/2/94 0:00 & Manual Tape & 297.5 & Good & \\
\hline $4 / 2 / 94 \quad 7: 07$ & Auto FIC & 298.00 & Good & \\
\hline $4 / 3 / 940: 00$ & Manual Tape & 297.5 & Good & \\
\hline $4 / 3 / 947: 03$ & Auto FIC & 298 & Good & \\
\hline $4 / 4 / 940: 00$ & Manual Tape. & 297.5 & Good & \\
\hline $4 / 4 / 947: 03$ & Auto FIC & 298 & Good & \\
\hline $4 / 5 / 940: 00$ & Manual Tape & 297.5 & Good & \\
\hline $4 / 5 / 947: 03$ & Auto FIC & 298.00 & Good & \\
\hline $4 / 6 / 940: 00$ & Manual Tape & 297.5 & Good & \\
\hline $4 / 6 / 947: 06$ & Auto FIC & 298 & Good & \\
\hline 4/7/94 0:00 & Manual Tape & 297.5 & Good & \\
\hline $4 / 7 / 947: 07$ & Auto FIC & 298 & Good & \\
\hline $4 / 8 / 940: 00$ & Manual Tape & 297.5 & Good & \\
\hline 4/8/94 7:03 & Auto FIC & 298.00 & Good & \\
\hline $4 / 9 / 940: 00$ & Manual Tape & 297.50 & Good & \\
\hline 4/9/94 7:07 & Auto FIC & 298.00 & Good & \\
\hline 4/10/940:00 & Manual Tape & 297.5 & Good & \\
\hline 4/10/94 7:03 & Auto FIC & 298 & Good & \\
\hline 4/11/940:00 & Manual Tapa & 297.5 & Good & \\
\hline 4/11/947:03 & - Auto FIC & 298.00 & Good & \\
\hline $4 / 12 / 940: 00$ & Manual Tape & 297.5 & Good & \\
\hline
\end{tabular}

Data obtained from WHC Surveillance Analysis Computer System, (PC SACS) July 7, 1995. 


\begin{tabular}{|c|c|c|c|c|}
\hline \multicolumn{5}{|c|}{ 241-AP-105 } \\
\hline \multicolumn{5}{|c|}{ Surface Level Data } \\
\hline Date & Type of Measurement & Level (Inches) & Data Quality & Comments \\
\hline $4 / 12 / 947: 03$ & Auto FIC & 298 & Good & \\
\hline $4 / 13 / 940: 00$ & Manual Tape & 297.5 & Good & \\
\hline $4 / 13 / 947: 03$ & Auto FIC & 298.00 & Good & \\
\hline $4 / 14 / 940: 00$ & Manual Tape & 297.50 & Good & \\
\hline $4 / 14 / 947: 03$ & Auto FIC & 298.00 & Good & \\
\hline $4 / 15 / 94 \quad 0: 00$ & Manual Tape & 297.50 & Good & \\
\hline 4/15/94 7:03 & Auto FIC & 298 & Good & \\
\hline $4 / 16 / 940: 00$ & Manual Tape & 297.5 & Good & \\
\hline $4 / 16 / 947: 03$ & Auto FIC & 298.00 & Good & \\
\hline $4 / 17 / 940: 00$ & Manual Tape & 297.50 & Good & \\
\hline $4 / 17 / 947: 03$ & Auto FIC & 298 & Good & \\
\hline $4 / 18 / 940: 00$ & Manual Tape & 297.5 & Good & \\
\hline 4/18/94 7:03 & Auto FIC & 298.00 & Good & \\
\hline 4/19/940:00 & Manual Tape & 297.50 & Good & \\
\hline $4 / 19 / 946: 06$ & Auto FIC & 298.00 & Good & \\
\hline $4 / 20 / 940: 00$ & Manual Tape & 297.50 & Good & \\
\hline $4 / 20 / 947: 04$ & Auto FIC & 298 & Good & \\
\hline $4 / 21 / 940: 00$ & Manual Tape & 297.50 & Good & \\
\hline $4 / 21 / 947: 05$ & Auto FIC & 298 & Good & \\
\hline 4/22/940:00 & Manual Tape & 297.50 & Good & $\dot{-}$ \\
\hline $4 / 22 / 947: 04$ & Auto FIC & 298.00 . & Good & \\
\hline $4 / 23 / 940: 00$ & Manual Tape & 297.5 & Good & \\
\hline $4 / 23 / 947: 05$ & Auto FIC & 298 & Good & \\
\hline $4 / 24 / 940: 00$ & Manual Tape & 297.50 & Good & \\
\hline $4 / 24 / 947: 04$ & Auto FIC & 298.00 & Good & \\
\hline $4 / 25 / 940: 00$ & Manual Tape & 297.50 & Good & \\
\hline $4 / 25 / 947: 04$ & Auto FIC & 298.00 & Good & \\
\hline 4/26/94 0:00 & Manual Tape & 297.50 & Good & \\
\hline 4/26/94 7:07 & Auto FIC & 298.00 & Good & \\
\hline $4 / 27 / 940: 00$ & Manual Tape & $297.25^{\circ}$ & Good & \\
\hline $4 / 27 / 947: 04$ & Auto FIC & 298.00 & Good & \\
\hline $4 / 28 / 940: 00$ & Manual Tape & 297.50 & Good & \\
\hline 4/28/94 7:05 & Auto FIC & 298 & Good & \\
\hline $4 / 29 / 940: 00$ & Manual Tape & 297.5 & Good & \\
\hline $4 / 29 / 947: 04$ & Auto FIC & 298.00 & Good & \\
\hline $4 / 30 / 940: 00$ & Manual Tape & 297.5 & Good & \\
\hline $4 / 30 / 947: 05$ & Auto FIC & 298.00 & Good & \\
\hline $5 / 1 / 940: 00$ & Manual FIC & 298 & Good & \\
\hline $5 / 1 / 940: 00$ & Manual Tape & 297.50 & Good & \\
\hline $5 / 1 / 947: 05$ & Auto FIC & 298 & Good & \\
\hline $5 / 2 / 940: 00$ & Manual FIC & 298 & Good & \\
\hline $5 / 2 / 940: 00$ & Manual Tape & 297.25 & Good & \\
\hline $5 / 2 / 947: 05$ & Auto FIC & 298.00 & Good & \\
\hline $5 / 3 / 940: 00$ & Manual FIC & 298.00 & Good & \\
\hline $5 / 3 / 940: 00$ & Manual Tape & 297.25 & Good & \\
\hline $5 / 3 / 947: 05$ & Auto FIC & 298 & Good & \\
\hline $5 / 4 / 940: 00$ & Manual Tape & 297.25 & Good & \\
\hline $5 / 4 / 947: 05$ & Auto FIC & 298 & Good & \\
\hline
\end{tabular}

Data obtained from WHC Surveillance Analysis Computer System, (PC SACS) July 7, 1995. 
WHC-SD-WM-ER-315, Rev. 0

\begin{tabular}{|c|c|c|c|c|}
\hline \multicolumn{5}{|c|}{ 241-AP-105 } \\
\hline \multicolumn{5}{|c|}{ Surfaco Lovel Data } \\
\hline Date & Type of Measurement & Level (Inchea) & Data Quality & Comments \\
\hline $5 / 5 / 940: 00$ & Manual FIC & 298.10 & Good & \\
\hline $5 / 5 / 940: 00$ & Manual Tape & 297.5 & Good & \\
\hline $5 / 5 / 947: 03$ & Auto FIC & 0 & Suspect & Data not included in graph \\
\hline $5 / 6 / 940: 00$ & Manual FIC & 298.10 & Good & \\
\hline $5 / 6 / 940: 00$ & Manual Tape & 297.5 & Good & \\
\hline $5 / 6 / 947: 03$ & Auto FIC & 0.00 & Suspect & Data not included in graph \\
\hline $5 / 7 / 940: 00$ & Manual FIC & 298.1 & Good & \\
\hline $5 / 7 / 940: 00$ & Manual Tape & 297.50 & Good & \\
\hline $5 / 7 / 947: 03$ & Auto FIC & 0 & Suspect & Data not included in graph \\
\hline $5 / 8 / 940: 00$ & Manual FIC & 0 & Suspact & Data not included in graph \\
\hline $5 / 8 / 940: 00$ & Manual Tape & 297.25 & Good & \\
\hline $5 / 8 / 947: 03$ & Auto FIC & 0 & Suspect & Data not included in graph \\
\hline $5 / 9 / 940: 00$ & Manual Tape & 297.25 & Good & \\
\hline $5 / 9 / 947: 03$ & Auto FIC & 0 & Suspect & Data not includad in graph \\
\hline $5 / 10 / 940: 00$ & Manual Tape & 297.50 & Good & \\
\hline $5 / 10 / 947: 03$ & Auto FIC & 0.00 & Suspect & Data not included in graph \\
\hline $5 / 11 / 940: 00$ & Manual Tape & 297.5 & Good & \\
\hline $5 / 11 / 947: 03$ & Auto FIC & 0.00 & Suspect & Data not included in graph \\
\hline $5 / 12 / 940: 00$ & Manual Tape & 297.25 & Good & \\
\hline $5 / 13 / 940: 00$ & Manual Tape , & 297.25 & Good & \\
\hline $5 / 13 / 947: 03$ & Auto FIC & 0.00 & Suspect & Data not included in graph \\
\hline $5 / 14 / 940: 00$ & Manual Tape & 297.5 & Good & \\
\hline $5 / 14 / 947: 03$ & Auto FIC & 0.00 & Suspect & Data not included in graph \\
\hline $5 / 15 / 940: 00$ & Manual Tape & 297.5 & Good & \\
\hline $5 / 15 / 947: 06$ & Auto FIC & 0.00 & Suspect & Data not included in graph \\
\hline $5 / 16 / 940: 00$ & Manual Tape & 297.5 & Good & \\
\hline $5 / 16 / 947: 02$ & Auto FIC & 0.00 & Suspect & Data not included in graph \\
\hline $5 / 17 / 940: 00$ & Manual Tape & 297.25 & Good & \\
\hline 5/17/94 7:03 & Auto FIC & 0 & Suspect & Data not included in graph \\
\hline $5 / 18 / 940: 00$ & Manual Tape & 297.50 & Good & \\
\hline $5 / 18 / 947: 03$ & Auto FIC & 0.00 & Suspect & Data not included in graph \\
\hline $5 / 19 / 940: 00$ & Manual Tape & 297.25 & Good & \\
\hline $5 / 19 / 947: 03$ & Auto FIC & .0 & Suspect & Data not included in graph \\
\hline $5 / 20 / 940: 00$ & Manual Tape & 297.25 & Good & \\
\hline $5 / 20 / 947: 03$ & Auto FIC & 0.00 & Suspect & Data not included in graph \\
\hline $5 / 21 / 940: 00$ & Manual Tape & 297.25 & Good & \\
\hline $5 / 21 / 947: 05$ & Auto FIC & 0.00 & Suspect & Data not included in graph \\
\hline $5 / 22 / 940: 00$ & Manual Tape & 297.5 & Good & \\
\hline $5 / 22 / 947: 05$ & Auto FIC & 0.00 & Suspect & Data not included in graph \\
\hline $5 / 23 / 940: 00$ & Manual Tape & 297.5 & Good & \\
\hline $5 / 23 / 94 \quad 7: 04$ & Auto FIC & 0.00 & Suspect & Data not included in graph \\
\hline 5/24/94 0:00 & Manual Tape & 297.25 & Good & \\
\hline $5 / 24 / 947: 04$ & Auto FIC & 0.00 & Suspect & Data not included in graph \\
\hline $5 / 25 / 940: 00$ & Manual Tape & 296.75 & Good & \\
\hline $5 / 25 / 947: 04$ & Auto FIC & 0.00 & Suspect & Data not included in graph \\
\hline $5 / 26 / 940: 00$ & Manual Tape & 297.25 & Good & \\
\hline $5 / 27 / 940: 00$ & Manual Tape & 297.25 & Good & \\
\hline $5 / 27 / 947: 03$ & Auto FIC & 0 & Suspect & Data not included in graph \\
\hline
\end{tabular}

Data obtained from WHC Surveillance Analysis Computer System; (PC SACS) July 7, 1995. 


\begin{tabular}{|c|c|c|c|c|}
\hline \multicolumn{5}{|c|}{ 241-AP-105 } \\
\hline \multicolumn{5}{|c|}{ Surface Laval Data } \\
\hline Date & Type of Measurement & Leval (Inchea) & Data Quality & Comments \\
\hline $5 / 28 / 940: 00$ & Manual Tape & 297.50 & Good & \\
\hline $5 / 28 / 947: 03$ & Auto FIC & 0.00 & Suspect & Data not included in graph \\
\hline $5 / 29 / 940: 00$ & Manual Tape & 297.25 & Good & \\
\hline $5 / 29 / 947: 03$ & Auto FIC & 0 & Suspect & Data not included in graph \\
\hline $5 / 30 / 940: 00$ & Manual Tape & 297.25 & Good & \\
\hline $5 / 30 / 947: 02$ & Auto FIC & 0 & Suspect & Data not included in graph \\
\hline $5 / 31 / 940: 00$ & Manual Tape & 297.25 & Good & \\
\hline $5 / 31 / 947: 03$ & Auto FIC & 0.00 & Suspect & Data not included in graph \\
\hline 6/1/940:00 & Manual Tape & 297.25 & Good & \\
\hline 6/1/94 7:03 & Auto FIC & 0.00 & Suspect & Data not included in graph \\
\hline $6 / 2 / 940: 00$ & Manual Tape & 297.50 & Good & \\
\hline $6 / 2 / 947: 03$ & Auto FIC & 0.00 & Suspect & Data not included in graph \\
\hline $6 / 3 / 940: 00$ & Manual FIC & 0 & Suspect & Data not included in graph \\
\hline $6 / 3 / 940: 00$ & Manual Tape & 297.25 & Good & \\
\hline 6/4/940:00 & Manual Tape & 297.5 & Good & \\
\hline $6 / 4 / 947: 04$ & Auto FIC & 0.00 & Suspect & Data not included in graph \\
\hline 6/5/94 0:00 & Manual Tape & 297.25 & Good & \\
\hline $6 / 5 / 947: 03$ & Auto FIC & 0.00 & Suspect & Data not included in graph \\
\hline $6 / 6 / 940: 00$ & Manual Tape & 417.5 & Suspect & Data not included in graph \\
\hline $6 / 6 / 947: 06$ & Auto FIC & 0 & Suspect & Data not included in graph \\
\hline $6 / 6 / 9415: 00$ & Manual Tape & 294.50 & Suspect & Data not included in graph \\
\hline $6 / 7 / 947: 02$ & Auto FIC & .0 & Suspect & Data not included in graph \\
\hline $6 / 7 / 9415: 00$ & Manual FIC & 298.00 & Good & \\
\hline $6 / 7 / 9415: 00$ & Manual Tape & 297.5 & Good & \\
\hline $6 / 8 / 947: 03$ & Auto FIC & 298.00 & Good & \\
\hline $6 / 8 / 9415: 00$ & Manual FIC & 298.00 & Good & \\
\hline $6 / 8 / 9415: 00$ & Manual Tape & 297.25 & Good & \\
\hline 6/9/94 0:00 & Manual FIC & 298.00 & Good & \\
\hline $6 / 9 / 9415: 00$ & Manual FIC & 298 & Good & $\div$ \\
\hline $6 / 9 / 94 \quad 15: 00$ & Manual Tape & 297.25 & Good & \\
\hline $6 / 10 / 947: 04$ & Auto FIC & 298 & Good & \\
\hline $6 / 10 / 9415: 00$ & Manual FIC & 298.00 & Good & \\
\hline $6 / 10 / 9415: 00$ & Manual Tape & 297.25 & Good & \\
\hline $6 / 11 / 947: 04$ & Auto FIC & 298.00 & Good & \\
\hline $6 / 11 / 9415: 00$ & Manual FIC & 298 & Good & \\
\hline $6 / 11 / 94 / 5: 00$ & Manual Tape & 297.50 & Good & \\
\hline $6 / 12 / 947: 03$ & Auto FIC & 298.00 & Good & \\
\hline $6 / 12 / 9415: 00$ & Manual FIC & 298.00 & Good & \\
\hline $6 / 12 / 94 \quad 15: 00$ & Manual Tape & 297.5 & Good & \\
\hline $6 / 13 / 947: 02$ & Auto FIC & 298 & Good & \\
\hline $6 / 13 / 94 \quad 15: 00$ & Manual FIC & 298.00 & Good & \\
\hline $6 / 13 / 9415: 00$ & Manual Tape & 297.25 & Good & \\
\hline $6 / 14 / 947: 03$ & Auto FIC & 298 & Good & \\
\hline $6 / 14 / 9415: 00$ & Manual FIC & 298 & Good & \\
\hline $6 / 14 / 9415: 00$ & Manual Tape & 297.75 & Good & \\
\hline $6 / 15 / 940: 00$ & Manual Tape & 297.25 & Good & \\
\hline $6 / 15 / 947: 03$ & Auto FIC & 298.00 & Good & \\
\hline $6 / 16 / 940: 00$ & Manual Tape & 297.25 & Good & \\
\hline
\end{tabular}

Data obtained from WHC Surveillance Analysis Computer System, (PC SACS) July 7, 1995. 


\begin{tabular}{|c|c|c|c|c|}
\hline \multicolumn{5}{|c|}{ 241-AP-105 } \\
\hline & & Inface Leval Dat & & \\
\hline Date & Type of Messurement & Level (Inchea) & Data Quality & Comments \\
\hline $6 / 16 / 947: 03$ & Auto FIC & 298.00 & Good & \\
\hline 6/17/94 0:00 & Manual Tape & 297.25 & Good & \\
\hline $6 / 17 / 947: 03$ & Auto FIC & 298 & Good & \\
\hline $6 / 18 / 940: 00$ & Manual Tape & 297.25 & Good & \\
\hline $6 / 18 / 947: 04$ & Auto FIC & 298.00 & Good & \\
\hline $6 / 19 / 940: 00$ & Manual Tape & 297.5 & Good & \\
\hline $6 / 19 / 947: 04$ & Auto FIC & 298.00 & Good & \\
\hline $6 / 20 / 940: 00$ & Manual Tape & 297.50 & Good & \\
\hline $6 / 20 / 947: 04$ & Auto FIC & 298.00 & Good & \\
\hline $6 / 21 / 940: 00$ & Manual Tape & 297.5 & Good & \\
\hline $6 / 21 / 947: 04$ & Auto FIC & 298 & Good & $\cdot$ \\
\hline $6 / 22 / 940: 00$ & Manual Tape & 297.25 & Good & \\
\hline $6 / 22 / 947: 03$ & Auto FIC & 298.00 & Good & \\
\hline $6 / 23 / 940: 00$ & Manual Tape & 297.5 & Good & \\
\hline $6 / 23 / 947: 05$ & Auto FIC & 298 & Good & \\
\hline $6 / 24 / 940: 00$ & Manual Tape & 297.50 & Good. & \\
\hline $6 / 24 / 947: 06$ & Auto FIC & 298.00 & Good & \\
\hline $6 / 25 / 940: 00$ & Manual Tape & 297.50 & Good & \\
\hline $6 / 25 / 947: 05$ & Auto FIC & 298 & Good & \\
\hline $6 / 26 / 940: 00$ & Manual Tape & 297.25 & Good & \\
\hline $6 / 26 / 947: 06$ & Auto FIC & 298.00 & Good & \\
\hline $6 / 27 / 940: 00$ & Manual Tape & 297.50 & Good & \\
\hline $6 / 27 / 947: 06$ & Auto FIC & 298.00 & Good & \\
\hline $6 / 28 / 940: 00$ & Manual Tape & 297.25 & Good & \\
\hline $6 / 28 / 947: 06$ & Auto FIC & 298 & Good & \\
\hline $6 / 29 / 940: 00$ & Manual Tape & 297.25 & Good & \\
\hline $6 / 29 / 947: 03$ & Auto FIC & 298.00 & Good & \\
\hline $6 / 30 / 940: 00$ & Manual Tape & 297.25 & Good & \\
\hline $6 / 30 / 947: 04$ & Auto FIC & 298 & Good & \\
\hline $7 / 1 / 940: 00$ & Manual Tape & 297.50 & Good & \\
\hline $7 / 1 / 947: 04$ & Auto FIC & 298.00 & Good & \\
\hline $7 / 2 / 940: 00$ & Manual Tape & 297.25 & Good & \\
\hline $7 / 2 / 947: 04$ & Auto FIC & 298.00 & Good & \\
\hline $7 / 3 / 940: 00$ & Manual Tape & 297.25 & Good & \\
\hline $7 / 3 / 947: 04$ & Auto FIC & 298.00 & Good & \\
\hline $7 / 4 / 940: 00$ & Manual Tape & 297.50 . & Good & \\
\hline $7 / 4 / 947: 04$ & Auto FIC & 298 & Good & \\
\hline 7/5/94 0:00 & Manual Tape & 297.75 & Good & \\
\hline $7 / 5 / 947: 03$ & Auto FIC & 298.00 & Good & \\
\hline $7 / 6 / 940: 00$ & Manual Tape & 297.50 & Good & \\
\hline $7 / 6 / 947: 05$ & Auto FIC & 298.00 & Good & \\
\hline $7 / 7 / 940: 00$ & Manual Tape & 297.5 & Good & \\
\hline $7 / 7 / 947: 03$ & Auto FIC & 298.00 & Good & \\
\hline $7 / 8 / 940: 00$ & Manual Tape & 297.50 & Good & \\
\hline $7 / 8 / 947: 03$ & Auto FIC & 298.00 & Good & \\
\hline $7 / 9 / 940: 00$ & Manual Tape & 297.5 & Good & \\
\hline $7 / 9 / 947: 03$ & Auto FIC & 298 & Good & \\
\hline $7 / 10 / 940: 00$ & Manual Tape & 297.5 & Good & \\
\hline
\end{tabular}

Data obtained from WHC Surveillance Analysis Computer System, (PC SACS) July 7, 1995. 


\begin{tabular}{|c|c|c|c|c|}
\hline \multicolumn{5}{|c|}{ 241-AP-105 } \\
\hline \multicolumn{5}{|c|}{ Surface Lovel Data } \\
\hline Date & Type of Moasurement & Level (Inchea) & Data Quality & Comments \\
\hline $7 / 10 / 947: 03$ & Auto FIC & 298 & Good & \\
\hline $7 / 11 / 940: 00$ & Manual Tape & 297.33 & Good & \\
\hline $7 / 11 / 947: 03$ & Auto FIC & 298.00 & Good & \\
\hline $7 / 12 / 940: 00$ & Manual Tape & 297.50 & Good & \\
\hline $7 / 12 / 947: 03$ & Auto FIC & 298.00 & Good & \\
\hline 7/13/94 0:00 & Manual Tape & 297.50 & Good & \\
\hline $7 / 13 / 947: 03$ & Auto FIC & 298.00 & Good & \\
\hline $7 / 14 / 940: 00$ & Manual Tape & 297.50 & Good & \\
\hline $7 / 14 / 947: 04$ & Auto FIC & 298.00 & Good & \\
\hline $7 / 15 / 940: 00$ & Manual Tape & 297.5 & Good & \\
\hline $7 / 15 / 947: 04$ & Auto FIC & 298.00 & Good & \\
\hline $7 / 16 / 940: 00$ & Manual Tape & 297.5 & Good & \\
\hline $7 / 16 / 947: 04$ & Auto FIC & 298.1 & Good & \\
\hline $7 / 17 / 940: 00$ & Manual Tape & 297.50 & Good & \\
\hline $7 / 17 / 947: 04$ & Auto FIC & 298.1 & Good & \\
\hline $7 / 18 / 940: 00$ & Manual FIC & 298.1 & Good & \\
\hline $7 / 18 / 940: 00$ & Manual Tape & 297.5 & Good & \\
\hline 7/19/94 0:00 & Manual Tape & 297.50 & Good & \\
\hline $7 / 19 / 947: 03$ & Auto FIC & 298.1 & Good & \\
\hline 7/20/94 0:00 & Manual Tape & 297.5 & Good & \\
\hline $7 / 20 / 947: 03$ & Auto FIC & 298.1 & Good & \\
\hline $7 / 21 / 940: 00$ & Manual Tape & 297.5 & Good & \\
\hline $7 / 21 / 947: 03$ & Auto FIC & 298.10 & Good & \\
\hline $7 / 22 / 940: 00$ & Manual Tape & 297.50 & Good & \\
\hline $7 / 22 / 947: 03$ & Auto FIC & 298.1 & Good & \\
\hline $7 / 23 / 940: 00$ & Manual Tape & 297.5 & Good & \\
\hline $7 / 23 / 947: 03$ & Auto FIC & 298.10 & Good & \\
\hline $7 / 24 / 940: 00$ & Manual Tape & 297.5 & Good & \\
\hline $7 / 24 / 947: 03$ & Auto FIC & 298.1 & Good & \\
\hline $7 / 25 / 940: 00$ & Manual Tape & 297.5 & Good & \\
\hline $7 / 25 / 947: 03$ & Auto FIC & 298.10 & Good & \\
\hline $7 / 26 / 947: 03$ & Auto FIC & 298.1 & Good & \\
\hline $7 / 26 / 9412: 00$ & Manual Tape & 297.50 & Good & \\
\hline $7 / 27 / 947: 03$ & Auto FIC & 298.1 & Good & \\
\hline $7 / 27 / 9412: 00$ & Manual Tape & 297.5 & Good & \\
\hline $7 / 28 / 947: 04$ & Auto FIC & 298.1 & Good & \\
\hline $7 / 28 / 9412: 00$ & Manual Tape & 297.50 & Good & \\
\hline 7/29/94 7:04 & Auto FIC & 298.1 & Good & \\
\hline $7 / 29 / 9412: 00$ & Manual Tape & 297.50 & Good & \\
\hline $7 / 30 / 947: 04$ & Auto FIC & 298.1 & Good & \\
\hline $7 / 30 / 9412: 00$ & Manual Tape & $297: 50$ & Good & \\
\hline $7 / 31 / 9412: 00$ & Manual Tape & 297.25 & Good & \\
\hline $7 / 31 / 9423: 03$ & Auto FIC & 298.10 & Good & \\
\hline $8 / 1 / 947: 03$ & Auto FIC & 298.1 & Good & \\
\hline $8 / 1 / 9412: 00$ & Manual Tape & 297.5 & Good & \\
\hline $8 / 2 / 947: 03$ & Auto FIC & 298.10 & Good & \\
\hline $8 / 2 / 9412: 00$ & Manual Tape & 297.50 & Good & \\
\hline $8 / 3 / 947: 03$ & Auto FIC & 298.10 & Good & \\
\hline
\end{tabular}

Data obtained from WHC Surveillance Analysis Computer System, (PC SACS) July 7, 1995. 


\begin{tabular}{|c|c|c|c|c|}
\hline \multicolumn{5}{|c|}{ 241-AP-105 } \\
\hline \multicolumn{5}{|c|}{ Surface Level Data } \\
\hline Date & Type of Measurement & Level (Inches) & Data Quality & Comments \\
\hline $8 / 3 / 9412: 00$ & Manual Tape & 297.50 & Good & \\
\hline $8 / 4 / 947: 02$ & Auto FIC & 0 & Suspect & Data not included in graph \\
\hline $8 / 4 / 9412: 00$ & Manual FIC & 298.1 & Good & \\
\hline $8 / 4 / 9412: 00$ & Manual Tape & 297.5 & Good & \\
\hline 8/5/94 7:03 & Auto FIC & 298.1 & Good & \\
\hline $8 / 5 / 9412: 00$ & Manual Tape & 297.5 & Good & \\
\hline $8 / 6 / 947: 03$ & Auto FIC & 298.10 & Good & \\
\hline $8 / 6 / 9412: 00$ & Manual Tape & 297.5 & Good & \\
\hline $8 / 7 / 947: 04$ & Auto FIC & 298.10 & Good & \\
\hline $8 / 7 / 9412: 00$ & Manual Tape & 297.50 & Good & \\
\hline $8 / 8 / 947: 12$ & Auto FIC & 0.00 & Suspect & Data not included in graph \\
\hline $8 / 8 / 9412: 00$ & Manual FIC & 298.10 & Good & \\
\hline $8 / 8 / 94 \quad 12: 00$ & Manual Tape & 297.5 & Good & \\
\hline $8 / 9 / 947: 06$ & Auto FIC & 298.10 & Good & \\
\hline $8 / 9 / 9412: 00$ & Manual Tape & 297.5 & Good & \\
\hline $8 / 10 / 947: 04$ & Auto FIC & 298.10 & Good & \\
\hline $8 / 10 / 9412: 00$ & Manual Tape & 297.5 & Good & \\
\hline $8 / 11 / 947: 04$ & Auto FIC & 298.10 & Good & \\
\hline $8 / 11 / 9412: 00$ & Manual Tape & 297.5 & Good & \\
\hline $8 / 12 / 947: 04$ & Auto FIC & 298.1 & Good & \\
\hline $8 / 12 / 94 \quad 12: 00$ & Manual Tape & 297.5 & Good & \\
\hline $8 / 13 / 947: 03$ & Auto FIC & 298.10 & Good & \\
\hline $8 / 13 / 9412: 00$ & Manual Tapo & 297.50 & Good. & \\
\hline $8 / 14 / 947: 06$ & Auto FIC & 298.1 & Good & \\
\hline $8 / 14 / 9412: 00$ & Manual Tape & 297.50 & Good & \\
\hline $8 / 15 / 947: 06$ & Auto FIC & 298.1 & Good & \\
\hline $8 / 15 / 9412: 00$ & Manual Tape & 297.5 & Good & \\
\hline $8 / 16 / 947: 06$ & Auto FIC & 298.10 & Good & \\
\hline $8 / 16 / 94 \quad 12: 00$ & Manual Tape & 297.5 & Good & \\
\hline $8 / 17 / 947: 06$ & Auto FIC & 298.10 & Good & \\
\hline $8 / 17 / 94 \quad 12: 00$ & Manual Tape & 297.50 & Good & \\
\hline $8 / 18 / 947: 06$ & Auto FIC & 298.1 & Good & \\
\hline $8 / 18 / 9412: 00$ & Manual Tape & 297.50 & Good & \\
\hline $8 / 19 / 946: 06$ & Auto FIC & 298.1 & Good & - \\
\hline $8 / 19 / 9412: 00$ & Manual Tape & 297.5 & Good & \\
\hline $8 / 20 / 947: 04$ & Auto FIC & 298.10 & Good & \\
\hline $8 / 20 / 9412: 00$ & Manual Tape & 297.5 & Good & \\
\hline $8 / 21 / 947: 04$ & Auto FIC & 298.10 & Good & \\
\hline $8 / 21 / 9412: 00$ & Manual Tape & 297.50 & Good & \\
\hline $8 / 22 / 946: 04$ & Auto FIC & 298.20 & Good & \\
\hline $8 / 22 / 9412: 00$ & Manual Tape & 297.5 & Good & \\
\hline $8 / 23 / 947: 04$ & Auto FIC & 298.10 & Good & \\
\hline $8 / 23 / 9412: 00$ & Manual Tape & 297.5 & Good & \\
\hline $8 / 24 / 947: 04$ & Auto FIC & 298.1 & Good & \\
\hline $8 / 24 / 9412: 00$ & Manual Tape & 297.50 & Good & \\
\hline $8 / 25 / 947: 03$ & Auto FIC & 298.1 & Good & \\
\hline $8 / 25 / 9412: 00$ & Manual Tape & 297.50 & Good & \\
\hline $8 / 26 / 947: 06$ & Auto FIC & 298.10 & Good & \\
\hline
\end{tabular}

Data obtained from WHC Surveillance Analysis Computer System, (PC SACS) July 7, 1995. 


\begin{tabular}{|c|c|c|c|c|}
\hline \multicolumn{5}{|c|}{ 241-AP-105 } \\
\hline & & Urface Leval Dat & & \\
\hline Date & Type of Mosaurement & Level (Inches) & Data Quality & Comments \\
\hline $8 / 26 / 9412: 00$ & Manual Tape & 297.5 & Good & \\
\hline $8 / 27 / 947: 04$ & Auto FIC & 298.1 & Good & \\
\hline $8 / 27 / 9412: 00$ & Manual Tape & 297.5 & Good & \\
\hline $8 / 28 / 947: 04$ & Auto FIC & 298.20 & Good & \\
\hline $8 / 28 / 94 \quad 12: 00$ & Manual Tape & 297.50 & Good & \\
\hline $8 / 29 / 947: 04$ & Auto FIC & 298.10 & Good & \\
\hline $8 / 29 / 9412: 00$ & Manual Tape & 297.5 & Good & \\
\hline $8 / 30 / 947: 04$ & Auto FIC & 298.1 & Good & \\
\hline $8 / 30 / 9412: 00$ & Manual Tape & 297.5 & Good & \\
\hline $8 / 31 / 947: 04$ & Auto FIC & 298.1 & Good & \\
\hline $8 / 31 / 9412: 00$ & Manual Tape & 297.50 & Good & \\
\hline 9/1/94 7:04 & Auto FIC & 298.10 & Good & \\
\hline $9 / 1 / 9412: 00$ & Manual Tape & 297.5 & Good & \\
\hline $9 / 2 / 947: 06$ & Auto FIC & 298.10 & Good & \\
\hline $9 / 2 / 9412: 00$ & Manual Tape & 297.5 & Good & \\
\hline $9 / 3 / 9412: 00$ & Manual Tape & 297.5 & Good & \\
\hline 9/3/94 23:04 & Auto FIC & 298.1 & Good & \\
\hline 9/4/94 7:04 & Auto FIC & 298.10 & Good & \\
\hline $9 / 4 / 9412: 00$ & Manual Tape & 297.5 & Good & \\
\hline $9 / 5 / 947: 04$ & Auto FIC & 298.1 & Good & \\
\hline $9 / 5 / 9412: 00$ & Manual Tape & 297.50 & Good & \\
\hline $9 / 6 / 947: 03$ & Auto FIC & 298.10 & - Good & \\
\hline 9/7/94 7:03 & Auto FIC & 298.10 & Good & \\
\hline $9 / 7 / 94 \quad 12: 00$ & Manual Tape & 297.5 & Good & \\
\hline $9 / 8 / 947: 04$ & Auto FIC & 298.1 & Good & \\
\hline $9 / 8 / 9412: 00$ & Manual Tape & 297.5 & Good & \\
\hline $9 / 9 / 947: 03$ & Auto FIC & 298.1 & Good & \\
\hline $9 / 9 / 9412: 00$ & Manual Tape & 297.5 & Good & \\
\hline $9 / 10 / 947: 04$ & Auto FIC & 298.10 & Good & \\
\hline $9 / 10 / 9412: 00$ & Manual Tape & 297.5 & Good & \\
\hline $9 / 11 / 947: 03$ & Auto FIC & 298.1 & Good & \\
\hline $9 / 11 / 9412: 00$ & Manual Tape & 297.50 & Good & \\
\hline $9 / 12 / 947: 03$ & Auto FIC & 298.1 & Good & \\
\hline $9 / 12 / 9412: 00$ & Manual Tape & 297.5 & Good & \\
\hline $9 / 13 / 947: 02$ & Auto FIC & 298,10 & Good & \\
\hline $9 / 13 / 9412: 00$ & Manual Tape & 297.50 & Good & \\
\hline $9 / 14 / 947: 03$ & Auto FIC & 298.10 & Good & \\
\hline $9 / 14 / 9412: 00$ & Manual Tape & 297.5 & Good & \\
\hline $9 / 15 / 947: 03$ & Auto FIC & 298.1 & Good & \\
\hline $9 / 15 / 9412: 00$ & Manual Tape & 297.5 & Good & \\
\hline $9 / 16 / 947: 02$ & Auto FIC & 298.10 & Good & \\
\hline $9 / 16 / 9412: 00$ & Manual Tape & 297.75 & Good & \\
\hline $9 / 17 / 947: 03$ & Auto FIC & 298.10 & Good & \\
\hline $9 / 17 / 9412: 00$ & Manual Tape & 297.5 & Good & \\
\hline $9 / 18 / 947: 02$ & Auto FIC & 298.10 & Good & \\
\hline $9 / 18 / 9412: 00$ & Manual Tape & 297.50 & Good & \\
\hline $9 / 19 / 947: 03$ & Auto FIC & 298.10 & Good & \\
\hline $9 / 19 / 9412: 00$ & Manual Tape & 297.50 & Good & \\
\hline
\end{tabular}

Data obtained from WHC Surveillance Analysis Computer System, (PC SACS) July 7, 1995. 


\begin{tabular}{|c|c|c|c|c|}
\hline \multicolumn{5}{|c|}{ 241-AP-105 } \\
\hline \multicolumn{5}{|c|}{ Surface Levol Data } \\
\hline Date & Type of Measurement & Leval (Inchea) & Data Quality & Comments \\
\hline $9 / 20 / 947: 03$ & Auto FIC & 298.10 & Good & \\
\hline $9 / 20 / 9412: 00$ & Manual Tape & 297.5 & Good & \\
\hline $9 / 21 / 947: 04$ & Auto FIC & 298.1 & Good & \\
\hline $9 / 21 / 9412: 00$ & Manual Tape & 297.50 & Good & \\
\hline $9 / 22 / 947: 03$ & Auto FIC & 298.1 & Good & \\
\hline $9 / 22 / 9412: 00$ & Manual Tape & 297.75 & Good & \\
\hline $9 / 23 / 947: 04$ & Auto FIC & 298.10 & Good & \\
\hline $9 / 23 / 9412: 00$ & Manual Tape & 297.50 & $\therefore$ Good & \\
\hline $9 / 24 / 947: 04$ & Auto FIC & 298.1 & Good & \\
\hline $9 / 24 / 9412: 00$ & Manual Tape & 297.5 & Good & \\
\hline $9 / 25 / 947: 03$ & Auto FIC & 298.10 & Good & \\
\hline $9 / 25 / 9412: 00$ & Manual Tape & 297.25 & Good & \\
\hline $9 / 26 / 947: 04$ & Auto FIC & 298.10 & Good & \\
\hline $9 / 26 / 9412: 00$ & Manual Tape & 297.5 & Good & \\
\hline $9 / 27 / 947: 04$ & Auto FIC & 298.1 & Good & \\
\hline $9 / 27 / 9412: 00$ & Manual Tape & 297.50 & Good & \\
\hline $9 / 28 / 947: 03$ & Auto FIC & 298.10 & Good & \\
\hline $9 / 28 / 9412: 00$ & Manual Tape & 297.5 & Good & \\
\hline $9 / 29 / 947: 04$ & Auto FIC & 298.1 & Good & \\
\hline $9 / 29 / 9412: 00$ & Manual Tape & 297.5 & Good & \\
\hline $9 / 30 / 947: 04$ & Auto FIC & 298.1 & Good & \\
\hline $9 / 30 / 9412: 00$ & Manual Tape & 297.5 & Good & \\
\hline $10 / 1 / 947: 06$ & Auto FIC & 298.1 & Good & \\
\hline $10 / 1 / 9412: 00$ & Manual Tape & 297.50 & Good & \\
\hline $10 / 2 / 947: 05$ & Auto FIC & 298.10 & Good & \\
\hline $10 / 2 / 9412: 00$ & Manual Tape & 297.5 & Good & \\
\hline $10 / 3 / 947: 04$ & Auto FIC & 298.10 & Good & \\
\hline $10 / 3 / 94 \quad 12: 00$ & Manual Tape & 297.25 & Good & 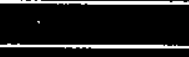 \\
\hline $10 / 4 / 947: 04$ & Auto FIC & $298.1^{\prime}$ & Good & \\
\hline $10 / 4 / 9412: 00$ & Manual Tape & 297.5 & Good & \\
\hline $10 / 5 / 947: 04$ & Auto FIC & 298.10 & Good & \\
\hline $10 / 5 / 9412: 00$ & Manual Tape & 297.5 & Good & \\
\hline $10 / 6 / 947: 04$ & Auto FIC & 298.1 & Good & . \\
\hline $10 / 6 / 9412: 00$ & Manual Tape & 297.50 & Good & \\
\hline $10 / 7 / 946: 04$ & Auto FIC & 298.10 & Good & \\
\hline $10 / 7 / 9412: 00$ & Manual Tape & 297.5 & Good & \\
\hline $10 / 8 / 947: 03$ & Auto FIC & 298.10 & Good & \\
\hline $10 / 8 / 9412: 00$ & Manual Tape & 297.5 & Good & \\
\hline $10 / 9 / 947: 06$ & Auto FIC & 298.10 & Good & \\
\hline $10 / 9 / 9412: 00$ & Manual Tape & 297.5 & Good & \\
\hline $10 / 10 / 947: 05$ & Auto FIC & 298.1 & Good & \\
\hline $10 / 10 / 9412: 00$ & Manual Tape & 297.50 & Good & \\
\hline $10 / 11 / 947: 03$ & Auto FIC & 298.1 & Good & \\
\hline $10 / 11 / 9412: 00$ & Manual Tape & 297.5 & Good & \\
\hline $10 / 12 / 947: 06$ & Auto FIC & 298.10 & Good & \\
\hline $10 / 12 / 9412: 00$ & Manual Tape & 297.50 & Good & \\
\hline $10 / 13 / 947: 05$ & Auto FIC & 298.10 & Good & \\
\hline $10 / 13 / 9412: 00$ & Manual Tape & 297.50 & Good & \\
\hline
\end{tabular}

Data obtained from WHC Surveillance Analysis Computer System, (PC SACS) July 7, 1995. 


\begin{tabular}{|c|c|c|c|c|}
\hline \multicolumn{5}{|c|}{ 241-AP-105 } \\
\hline & . & Uurface Level Dat & & \\
\hline Date & Type of Measurement & Level (Inches) & Data Quality & Comments \\
\hline $10 / 14 / 947: 06$ & Auto FIC & 298.1 & Good & \\
\hline $10 / 14 / 9412: 00$ & Manual Tape & 297.50 & Good & \\
\hline 10/15/94 7:06 & Auto FIC & 298.10 & Good & \\
\hline $10 / 15 / 9412: 00$ & Manual Tape & 297.5 & Good & \\
\hline $10 / 16 / 94$ 7:06 & Auto FIC & 298.10 & Good & \\
\hline $10 / 16 / 9412: 00$ & Manual Tape & 297.25 & Good & \\
\hline $10 / 17 / 947: 06$ & Auto FIC & 298.1 & Good & \\
\hline $10 / 17 / 9412: 00$ & Manual Tape & 297.5 & Good & \\
\hline $10 / 18 / 947: 06$ & Auto FIC & 298.10 & Good & \\
\hline $10 / 18 / 9412: 00$ & Manual Tape & 297.5 & Good & \\
\hline $10 / 19 / 947: 06$ & Auto FIC & 298.1 & Good & \\
\hline 10/19/94 12:00 & Manual Tape & 297.5 & Good & \\
\hline $10 / 20 / 945: 07$ & Auto FIC & 298.10 & Good & \\
\hline $10 / 20 / 9412: 00$ & Manual Tape & 297.50. & Good & \\
\hline $10 / 21 / 947: 06$ & Auto FIC & 298.1 & Good & \\
\hline $10 / 21 / 9412: 00$ & Manual Tape & 297.5 & Good & \\
\hline $10 / 22 / 9412: 00$ & Manual Tape & 297.25 & Good & \\
\hline $10 / 22 / 9423: 06$ & Auto FIC & 298.10 & Good & \\
\hline $10 / 23 / 947: 06$ & Auto FIC & 298.1 & Good & \\
\hline $10 / 23 / 9412: 00$ & Manual Tape & 297.5 & Good & \\
\hline $10 / 24 / 947: 06$ & Auto FIC & 298.1 & Good & \\
\hline $10 / 24 / 9412: 00$ & Manual Tape & 297.75 & Good & \\
\hline $10 / 25 / 947: 06$ & Auto FIC & $298.10^{\circ}$ & Good & \\
\hline 10/25/94 12:00 & Manual Tape & 297.50 & Good & \\
\hline $10 / 26 / 947: 06$ & Auta FIC & 298.00 & Good & \\
\hline $10 / 26 / 9412: 00$ & Manual Tape & 297.75 & Good & \\
\hline 10/27/94 7:06 & Auto FIC & 298.1 & Good & \\
\hline $10 / 27 / 9412: 00$ & Manual Tape & 297.5 & Good & \\
\hline $10 / 28 / 947: 04$ & Auto FIC & 298 & Good & \\
\hline $10 / 28 / 9412: 00$ & Manual Tape & 297.25 & Good & \\
\hline $10 / 29 / 9412: 00$ & Manual FIC & 298 & Good & \\
\hline $10 / 29 / 9412: 00$ & Manual Tape & 297.25 & Good & \\
\hline $10 / 30 / 9412: 00$ & Manual Tape & 297.5 & Good & \\
\hline 10/30/94 23:04 & Auto FIC & 298 & Good & \\
\hline $10 / 31 / 947: 05$ & Auto FIC & 298.00 & Good & \\
\hline $10 / 31 / 9412: 00$ & Manual Tape & 297.25 & Good & \\
\hline $11 / 1 / 9412: 00$ & Manual Tape & 297.25 & Good & \\
\hline $11 / 1 / 9423: 04$ & Auto FIC & 298 & Good & \\
\hline $11 / 2 / 947: 03$ & Auto FIC & 298 & Good & \\
\hline $11 / 2 / 9412: 00$ & Manual Tape & 297.5 & Good & \\
\hline $11 / 3 / 947: 04$ & Auto FIC & 298.00 & Good & \\
\hline $11 / 3 / 9412: 00$ & Manual Tape & 297.25 & Good & \\
\hline $11 / 4 / 947: 03$ & Auto FIC & 298.00 & Good & \\
\hline $11 / 4 / 9412: 00$ & Manual Tape & 297.25 & Good & \\
\hline $11 / 5 / 947: 03$ & Auto FIC & 298 & Good & \\
\hline $11 / 5 / 9412: 00$ & Manual Tape & $297: 25$ & Good & \\
\hline $11 / 6 / 947: 04$ & Auto FIC & 298 & Good & \\
\hline $11 / 6 / 9412: 00$ & Manual Tape & 297.25 . & Good & \\
\hline
\end{tabular}

Data obtained from WHC Surveillance Analysis Computer System, (PC SACS) July 7, 1995. 


\begin{tabular}{|c|c|c|c|c|}
\hline \multicolumn{5}{|c|}{ 241-AP-105 } \\
\hline \multicolumn{5}{|c|}{ Surface Lavel Data } \\
\hline Date & Type of Measurement & Level (lnchos) & Data Cuallty & Comments \\
\hline $11 / 7 / 947: 04$ & Auto FIC & 298.00 & Good & \\
\hline $11 / 7 / 9412: 00$ & Manual Tape & 297.25 & Good & \\
\hline $11 / 8 / 947: 03$ & Auto FIC & 298.00 & Good & \\
\hline $11 / 8 / 94 \quad 12: 00$ & Manual Tape & 297.5 & Good & \\
\hline $11 / 9 / 947: 05$ & Auto FIC & 298 & Good & \\
\hline $11 / 9 / 94 \quad 12: 00$ & Manual Tape & 297.25 & Good & \\
\hline $11 / 10 / 947: 03$ & Auto FIC & 298 & Good & \\
\hline $11 / 10 / 9412: 00$ & Manual Tape & 297.25 & Good & \\
\hline $11 / 11 / 947: 03$ & Auto FIC & 298.00 & Good & \\
\hline $11 / 11 / 9412: 00$ & Manual Tape & 297.25 & Good & \\
\hline $1,1 / 12 / 947: 03$ & Auto FIC & 298 & Good & \\
\hline $11 / 12 / 9412: 00$ & Manual Tape & 297.5 & Good & \\
\hline $11 / 13 / 947: 04$ & Auto FIC & 298.00 & Good & \\
\hline $11 / 13 / 9412: 00$ & Manual Tape & 297.25 & Good & \\
\hline $11 / 14 / 947: 03$ & Auto FIC & 298 & Good & \\
\hline $11 / 14 / 9412: 00$ & Manual Tape & 297.25 & Good & \\
\hline $11 / 15 / 947: 04$ & Auto FIC & 298.00 & Good & \\
\hline $11 / 15 / 9412: 00$ & Manual Tape & 297.25 & Good & \\
\hline $11 / 16 / 947: 06$ & Auto FIC & 298.00 & Good & \\
\hline $11 / 16 / 9412: 00$ & Manual Tape & 297.25 & Good & \\
\hline $11 / 17 / 947: 04$ & Auto FIC & 298.00 & Good & \\
\hline $11 / 17 / 9412: 00$ & Manual Tape & 297.25 & Good & \\
\hline $11 / 18 / 947: 04$ & Auto FIC & 298.00 & Good & \\
\hline $11 / 18 / 9412: 00$ & Manual Tape & 297.25 & Good & \\
\hline $11 / 19 / 947: 03$ & Auto FIC & 298.00 & Good & \\
\hline $11 / 19 / 9412: 00$ & Manual Tape & $297: 25$ & Good & \\
\hline $11 / 20 / 947: 03$ & Auto FIC & 298.00 & Good & \\
\hline $11 / 21 / 947: 04$ & Auto FIC & 298.00 & Good & \\
\hline $11 / 21 / 9412: 00$ & Manual Tape & 297.25 & Good & \\
\hline $11 / 22 / 947: 03$ & Auto FIC & 297.90 & Good & \\
\hline $11 / 22 / 9412: 00$ & Manual Tape & 13.25 & Suspect & Data not included in graph \\
\hline $11 / 23 / 9412: 00$ & Manual FIC & 297.90 & Good & \\
\hline $11 / 23 / 9412: 00$ & Manual Tape & 297.25 & Good & \\
\hline $11 / 24 / 9412: 00$ & Manual Tape & 297.25 & Good & \\
\hline $11 / 24 / 9423: 03$ & Auto FIC & 298.00 & Good & \\
\hline $11 / 25 / 9412: 00$ & Manual Tape & 297.25 & - Good & \\
\hline $11 / 25 / 9423: 03$ & Auto FIC & 297.90 & Good & \\
\hline $11 / 26 / 947: 03$ & Auto FIC & 297.90 & Good & \\
\hline $11 / 26 / 9412: 00$ & Manual Tape & 297.25 & Good & \\
\hline $11 / 27 / 947: 04$ & Auto FIC & 297.90 & Good & \\
\hline $11 / 27 / 94 \quad 12: 00$ & Manual Tape & 297.25 & Good & \\
\hline $11 / 28 / 947: 04$ & Auto FIC & 297.90 & Good & \\
\hline $11 / 28 / 9412: 00$ & Manual Tape & 297:25 & Good & \\
\hline $11 / 29 / 947: 04$ & Auto FIC & 297.9 & Good & \\
\hline $11 / 29 / 94 \quad 12: 00$ & Manual Tape & 297.25 & Good & \\
\hline $11 / 30 / 947: 03$ & Auto FIC & 297.90 & Good & \\
\hline $11 / 30 / 9412: 00$ & Manual Tape & 297.25 & Good & \\
\hline $12 / 1 / 946: 05$ & Auto FIC & 297.9 & Good & \\
\hline
\end{tabular}

Data obtained from WHC Surveillance Analysis Computer System, (PC SACS) July 7, 1995. 


\begin{tabular}{|c|c|c|c|c|}
\hline \multicolumn{5}{|c|}{ 241-AP-105 } \\
\hline \multicolumn{5}{|c|}{ Surface Level Data } \\
\hline Date & Type of Measurement & Level (Incheo) & Data Quality & Comments \\
\hline $12 / 1 / 9412: 00$ & Manual Tape & 297.25 & Good & \\
\hline $12 / 2 / 947: 03$ & Auto FIC & 297.90 & Good & \\
\hline $12 / 2 / 9412: 00$ & Manual Tape & 297.50 & Good & \\
\hline $12 / 3 / 946: 04$ & Auto FIC & 297.90 & Good & \\
\hline $12 / 3 / 9412: 00$ & Manual Tape & 297.25 & Good & \\
\hline $12 / 4 / 947: 03$ & Auto FIC & 297.9 & Good & \\
\hline $12 / 4 / 9412: 00$ & Manual Tape & 297.25 & Good & \\
\hline $12 / 5 / 947: 03$ & Auto FIC & 297.9 & Good & \\
\hline $12 / 5 / 9412: 00$ & Manual Tape & 297.25 & Good & \\
\hline $12 / 6 / 947: 04$ & Auto FIC & 297.90 & Good & \\
\hline $12 / 6 / 94 \quad 12: 00$ & Manual Tape & 297 & Good & \\
\hline $12 / 7 / 947: 03$ & Auto FIC & 297.80 & Good & \\
\hline $12 / 7 / 9412: 00$ & Manual Tape & 297.25 & Good & \\
\hline $12 / 8 / 945: 03$ & Auto FIC & 297.8 & Good & \\
\hline $12 / 8 / 94 \quad 12: 00$ & Manual Tape & 297.25 & Good & \\
\hline $12 / 9 / 947: 03$ & Auto FIC & 297.80 & Good & \\
\hline $12 / 9 / 9412: 00$ & Manual Tape & 297.25 & Good & \\
\hline $12 / 10 / 947: 04$ & Auto FIC & 297.8 & Good & \\
\hline $12 / 10 / 9412: 00$ & Manual Tape & 297 & Good & \\
\hline $12 / 11 / 947: 04$ & Auto FIC & 297.80 & Good & \\
\hline $12 / 11 / 9412: 00$ & Manual Tape & 297.25 & Good & \\
\hline $12 / 12 / 947: 03$ & Auto FIC & 297.8 & Good & \\
\hline $12 / 12 / 9412: 00$ & Manual Tape & 297.25 & Good & \\
\hline $12 / 13 / 947: 03$ & Auto FIC & 297.80 & Good & \\
\hline $12 / 13 / 9412: 00$ & Manual Tape & 297.25 & Good & \\
\hline $12 / 14 / 947: 04$ & Auto FIC & 297.8 & Good & \\
\hline $12 / 14 / 9412: 00$ & Manual Tape & 297.125 & Good & \\
\hline $12 / 15 / 947: 03$ & Auto FIC & 297.8 & Good & \\
\hline $12 / 15 / 94 \quad 12: 00$ & Manual Tape & 297.25 & Good & \\
\hline $12 / 16 / 947: 03$ & Auto FIC & 297.8 & Good & \\
\hline $12 / 16 / 9412: 00$ & Manual Tape & 297.125 & Good & \\
\hline $12 / 17 / 947: 06$ & Auto FIC & 297.80 & Good & \\
\hline $12 / 17 / 9412: 00$ & Manual Tape & 297 & Good & \\
\hline $12 / 18 / 947: 04$ & Auto FIC & 297.80 & Good & \\
\hline $12 / 18 / 9412: 00$ & Manual Tape & 297 & Good & \\
\hline $12 / 19 / 947: 04$ & Auto FIC & 297.80 & Good & \\
\hline $12 / 19 / 9412: 00$ & Manual Tape & 297.00 & Good & \\
\hline $12 / 20 / 947: 04$ & Auto FIC & 297.80 & Good & \\
\hline $12 / 20 / 9412: 00$ & Manual Tape & 297.25 & Good & \\
\hline $12 / 21 / 947: 04$ & Auto FIC & 297.8 & Good & \\
\hline $12 / 21 / 9412: 00$ & Manual Tape & 297.25 & Good & \\
\hline $12 / 22 / 947: 05$ & Auto FIC & 297.80 & Good & \\
\hline $12 / 22 / 9412: 00$ & Manual Tape & 297.25 & Good & \\
\hline $12 / 23 / 947: 03$ & Auto FIC & 297.7 & Good & \\
\hline $12 / 23 / 9412: 00$ & Manual Tape & 297.125 & Good & \\
\hline $12 / 24 / 947: 04$ & Auto FIC & 297.70 & Good & \\
\hline $12 / 24 / 9412: 00$ & Manual Tape & 297 & Good & \\
\hline $12 / 25 / 947: 03$ & Auto FIC & 297.7 & Good & \\
\hline
\end{tabular}

Data obtained from WHC Surveillance Analysis Computer System, (PC SACS) July 7, 1995. 


\begin{tabular}{|c|c|c|c|c|}
\hline \multicolumn{5}{|c|}{ 241-AP-105 } \\
\hline & . & Sufface Level Date & & \\
\hline Date & Type of Measurement & Level (Inchea) & Data Ouality & Comments \\
\hline $12 / 25 / 9412: 00$ & Manual Tape & 297.25 & Good & \\
\hline $12 / 26 / 947: 03$ & Auto FIC & 297.7 & Good & \\
\hline $12 / 26 / 9412: 00$ & Manual Tape & 297 & Good & \\
\hline $12 / 27 / 947: 04$ & Auto FIC & 297.70 & Good & \\
\hline $12 / 27 / 9412: 00$ & Manual Tape & 297.00 & Good & \\
\hline $12 / 28 / 947: 04$ & Auto FIC & 297.7 & Good & \\
\hline $12 / 28 / 9412: 00$ & Manual Tape & 297.00 & Good & \\
\hline $12 / 29 / 947: 04$ & Auto FIC & 297.70 & Good & \\
\hline $12 / 29 / 9412: 00$ & Manual Tape & 297.00 & Good & \\
\hline $12 / 30 / 947: 04$ & Auto FIC & 297.70 & Good & \\
\hline $12 / 30 / 9412: 00$ & Manual Tape & 297 & Good & \\
\hline 12/31/94 7:04 & Auto FIC & 297.7 & Good & \\
\hline $12 / 31 / 9412: 00$ & Manual Tape & 297 & Good & \\
\hline $1 / 1 / 957: 04$ & Auto FIC & 297.7 & Good & \\
\hline $1 / 1 / 95 \quad 12: 00$ & Manual Tape & 297 & Good & \\
\hline 1/2/95 7:03 & Auto FIC & 297.70 & Good & \\
\hline $1 / 2 / 95 \quad 12: 00$ & Manual Tape & 297 & Good & \\
\hline 1/3/95 7:02 & Auto FIC & 297.70 & Good & \\
\hline 1/3/95 12:00 & Manual Tape & 297 & Good & \\
\hline 1/4/95 7:03 & . Auto FIC & 297.7 & Good & \\
\hline $1 / 4 / 9512: 00$ & Manual Tape & 297.25 & Good & \\
\hline $1 / 5 / 957: 04$ & Auto FIC & 297.7 & Good & \\
\hline $1 / 5 / 9512: 00$ & Manual Tape & 297.00 & Good & \\
\hline 1/6/95 7:04 & Auto FIC & 297.70 & Good & \\
\hline $1 / 6 / 95 \quad 12: 00$ & Manual Tape & 297 & Good & \\
\hline $1 / 7 / 95$ 7:04 & Auto FIC & 297.70 & Good & \\
\hline 1/7/95 $12: 00$ & Manual Tape & 297 & Good & \\
\hline $1 / 8 / 95 \quad 12: 00$ & Manual Tape & 297 & Good & \\
\hline $1 / 8 / 9523: 03$ & Auto FIC & 297.7 & Good & \\
\hline 1/9/95 7:05 & Auto FIC & 297.70 & Good & \\
\hline $1 / 9 / 95 \quad 12: 00$ & Manual Tape & 297.00 & Good & \\
\hline $1 / 10 / 957: 06$ & Auto FIC & 297.70 & Good & \\
\hline $1 / 10 / 95 \quad 12: 00$ & Manual Tape & 297.00 & Good & \\
\hline $1 / 11 / 957: 07$ & Auto FIC & 297.7 & Good & \\
\hline $1 / 11 / 9512: 00$ & Manual Tape & 297 & Good & \\
\hline $1 / 12 / 957: 07$ & Auto FIC & 297.70 & Good & \\
\hline $1 / 12 / 9512: 00$ & Manual Tape & 297.25 & Good & \\
\hline $1 / 13 / 956: 07$ & Auto FIC & 297.6 & Good & \\
\hline $1 / 13 / 95 \quad 12: 00$ & Manual Tape & 297 & Good & \\
\hline $1 / 14 / 957: 07$ & Auto FIC & 297.60 & Good & \\
\hline $1 / 14 / 9512: 00$ & Manual Tape & 297.00 & Good & \\
\hline 1/15/95 7:06 & Auto FIC & 297.6 & Good & \\
\hline $1 / 15 / 9512: 00$ & Manual Tape & 297 & Good & \\
\hline $1 / 16 / 957: 08$ & Auto FIC & 297.6 & Good & \\
\hline $1 / 16 / 9512: 00$ & Manual Tape & 297.00 & Good & \\
\hline $1 / 17 / 956: 07$ & Auto FIC & 297.60 & Good & $\dot{-}$ \\
\hline $1 / 17 / 9512: 00$ & Manual Tape & 297.00 & Good & \\
\hline $1 / 18 / 957: 07$ & Auto FIC & 297.60 & Good & \\
\hline
\end{tabular}

Data obtained from WHC Surveillance Analysis Computer System, (PC SACS) July 7, 1995. 


\begin{tabular}{|c|c|c|c|c|}
\hline \multicolumn{5}{|c|}{ 241-AP-105 } \\
\hline \multicolumn{5}{|c|}{ Surface Level Data } \\
\hline Date & Type of Measurement & Level,(Inchea) & Data Quality & Comments \\
\hline $1 / 18 / 95 \quad 12: 00$ & Manual Tape & 297 & Good & \\
\hline $1 / 19 / 957: 07$ & Auto FIC & 297.60 & Good & \\
\hline 1/19/95 12:00 & Manual Tape & 297 & Good & \\
\hline $1 / 20 / 957: 03$ & Auto FIC & 297.6 & Good & \\
\hline $1 / 20 / 9512: 00$ & Manual Tape & 297.00 & Good & \\
\hline $1 / 21 / 957: 04$ & Auto FIC & 297.6 & Good & \\
\hline $1 / 21 / 9512: 00$ & Manual Tape & 297 & Good & \\
\hline $1 / 22 / 957: 03$ & Auto FIC & 297.60 & Good & \\
\hline $1 / 22 / 95 \quad 12: 00$ & Manual Tape & 297.00 & Good & \\
\hline $1 / 23 / 957: 03$ & Auto FIC & 297.6 & Good & \\
\hline $1 / 23 / 9512: 00$ & Manual Tape & 297.25 & Good & \\
\hline $1 / 24 / 957: 04$ & Auto FIC & 297.60 & Good & \\
\hline $1 / 24 / 9512: 00$ & Manual Tape & 297.00 & Good & \\
\hline $1 / 25 / 957: 03$ & Auto FIC & 297.60 & Good & \\
\hline $1 / 25 / 95 \quad 12: 00$ & Manual Tape & 297.00 & Good & \\
\hline $1 / 26 / 957: 03$ & Auto FIC & 297.6 & Good & \\
\hline $1 / 26 / 95 \quad 12: 00$ & Manual Tape & 297 & Good & \\
\hline $1 / 27 / 957: 03$ & Auto FIC & 297.6 & Good & \\
\hline $1 / 27 / 9512: 00$ & Manual Tape & 297 & Good & \\
\hline $1 / 28 / 957: 04$ & Auto FIC & 297.60 & Good & \\
\hline $1 / 28 / 9512: 00$ & Manual Tape & 297 & Good & \\
\hline $1 / 29 / 957: 04$ & Auto FIC & 297.6 & Good & \\
\hline $1 / 29 / 95 \quad 12: 00$ & Manual Tape & 297 & Good & \\
\hline $1 / 30 / 957: 04$ & Auto FIC & 297.60 & Good & \\
\hline $1 / 30 / 9512: 00$ & Manual Tape & 297 & Good & \\
\hline $1 / 31 / 957: 03$ & Auto FIC & 297.60 & Good & \\
\hline $1 / 31 / 9512: 00$ & Manual Tape & 297 & Good & \\
\hline 2/1/95 7:03 & Auto FIC & 297.5 & Good & \\
\hline $2 / 1 / 95 \quad 12: 00$ & Manual Tape & 297 & Good & \\
\hline $2 / 2 / 95 \quad 7: 04$ & Auto FIC & 297.5 & Good & \\
\hline $2 / 2 / 95 \quad 12: 00$ & Manual Tape & 296.75 & Good & \\
\hline $2 / 3 / 95 \quad 7: 03$ & Auto FIC & 297.50 & Good & \\
\hline $2 / 3 / 9512: 00$ & Manual Tape & 297 & Good & \\
\hline $2 / 4 / 957: 04$ & Auto FIC & $297: 50$ & Good & \\
\hline $2 / 4 / 9512: 00$ & Manual Tape & 296.75 & Good & \\
\hline $2 / 5 / 957: 03$ & Auto FIC & 297.50. & Good & \\
\hline $2 / 5 / 9512: 00$ & Manual Tape & 296.75 & Good & \\
\hline $2 / 6 / 957: 03$ & Auto FIC & 297.5 & Good & \\
\hline $2 / 6 / 95 \quad 12: 00$ & Manual Tape & 296.5 & Good & \\
\hline $2 / 7 / 95 \quad 7: 03$ & Auto FIC & 297.5 & Good & \\
\hline $2 / 7 / 9512: 00$ & Manual Tape & 296.75 & Good & \\
\hline $2 / 8 / 957: 04$ & Auto FIC & 297.5 & Good & \\
\hline $2 / 8 / 9512: 00$ & Manual Tape & 296.75 & Good & \\
\hline $2 / 9 / 95$ 7:06 & Auto FIC & 297.5 & Good & \\
\hline $2 / 9 / 95 \quad 12: 00$ & Manual Tape & $296.75^{\circ}$ & Good & \\
\hline $2 / 10 / 957: 06$ & Auto FIC & 297.50 & Good & \\
\hline $2 / 10 / 9512: 00$ & Manual Tape & 296.75 & Good & \\
\hline $2 / 11 / 957: 07$ & Auto FIC & 297.50 & Good & \\
\hline
\end{tabular}

Data obtained from WHC Surveillance Analysis Computer System, (PC SACS) July 7, 1995. 


\begin{tabular}{|c|c|c|c|c|}
\hline \multicolumn{5}{|c|}{ 241-AP-105 } \\
\hline \multicolumn{5}{|c|}{ Surface Leval Data } \\
\hline Date & Type of Measurement & Leval (Inched) & Date Quality & Comments \\
\hline $2 / 11 / 9512,00$ & Manual Tape & 296.75 & Good & \\
\hline 2/12/95 7:06 & Auto FIC & 297.5 & Good & \\
\hline $2 / 12 / 9512: 00$ & Manual Tape & 296.75 & Good & \\
\hline $2 / 13 / 957: 05$ & Auto FIC & 297.5 & Good & \\
\hline $2 / 13 / 95 \quad 12: 00$ & Manual Tape & 297.00 & Good & \\
\hline $2 / 14 / 957: 06$ & Auto FIC & 297.50 & Good & \\
\hline $2 / 14 / 9512: 00$ & Manual Tape & 296.75 & Good & \\
\hline $2 / 15 / 95$ 7:07 & Auto FIC & 297.5 & Good & \\
\hline $2 / 15 / 95 \quad 12: 00$ & Manual Tape & 296.75 & Good & \\
\hline $2 / 16 / 95 \quad 7: 06$ & Auto FIC & 297.50 & Good & \\
\hline $2 / 16 / 9512: 00$ & Manual Tape & 296.75 & Good & \\
\hline $2 / 17 / 957: 06$ & Auto FIC & 297.50 & Good & \\
\hline $2 / 17 / 9512: 00$ & Manual Tape & 296.75 & Good & \\
\hline 2/18/95 7:06 & Auto FIC & 297.50 & Good & \\
\hline $2 / 18 / 9512: 00$ & Manual Tape & 296.75 & Good & \\
\hline $2 / 19 / 957: 06$ & Auto FIC & 297.5 & Good & \\
\hline 2/19/95 12:00 & Manual Tape & 296.5 & Good & \\
\hline $2 / 20 / 957: 07$ & Auto FIC & 297.50 & Good & \\
\hline 2/20/95 12:00 & Manual Tape & 296.75 & Good & \\
\hline 2/21/95 7:06 & Auto FIC & 297.5 & Good & \\
\hline $2 / 21 / 95 \quad 12: 00$ & Manual Tape & 296.50 & Good & \\
\hline 2/22/95 7:06 & Auto FIC & 297.5 & Good & \\
\hline $2 / 22 / 95 \quad 12: 00$ & Manual Tape & 296.75 & Good & \\
\hline $2 / 23 / 957: 07$ & Auto FIC & 297.50 & Good & \\
\hline $2 / 23 / 9512: 00$ & Manual Tape & 296.75 & Good & \\
\hline $2 / 24 / 957: 06$ & Auto FIC & 297.50 & Good & \\
\hline $2 / 24 / 9512: 00$ & Manual Tape & 296.75 & Good & \\
\hline $2 / 25 / 957: 06$ & Auto FIC & 297.50 & Good & \\
\hline $2 / 25 / 95 \quad 12: 00$ & Manual Tape & 296.75 & Good & \\
\hline $2 / 26 / 956: 07$ & Auto FIC & 297.50 & Good & \\
\hline $2 / 26 / 95 \quad 12 ; 00$ & Manual Tape & 296.75 & Good & \\
\hline 2/27/95 7:06 & Auto FIC & 297.4 & Good & \\
\hline $2 / 27 / 9512: 00$ & Manual Tape & 296.50 & Good & \\
\hline $2 / 28 / 955: 07$ & Auto FIC & 297.50 & Good & \\
\hline $2 / 28 / 9512: 00$ & Manual Tape & 296.75 & Good & \\
\hline $3 / 1 / 957: 06$ & Auto FIC & 297.4 & Good & \\
\hline $3 / 1 / 95 \quad 12: 00$ & Manual Tape & 296.75 & Good & \\
\hline $3 / 2 / 957: 06$ & Auto FIC & 297.40 & Good & \\
\hline $3 / 2 / 95 \quad 12: 00$ & Manual Tape & 296.75 & Good & \\
\hline $3 / 3 / 957: 06$ & Auto FIC & 297.4 & Good & \\
\hline $3 / 3 / 9512: 00$ & Manual Tape & 296.75 & Good & \\
\hline $3 / 4 / 95$ 7:06 & Auto FIC & 297.40 & Good & \\
\hline $3 / 4 / 95 \quad 12: 00$ & Manual Tape & 296.75 & Good & \\
\hline $3 / 5 / 957: 06$ & Auto FIC & 297.5 & Good & \\
\hline $3 / 5 / 95 \quad 12: 00$ & Manual Tape & 296.75 & Good & \\
\hline $3 / 6 / 957: 06$ & Auto FIC & 297.40 & Good & \\
\hline $3 / 6 / 9512: 00$ & Manual Tape & 296.75 & Good & \\
\hline 3/7/95 7:06 & Auto FIC & $297: 40$ & Good & \\
\hline
\end{tabular}

Data obtained from WHC Surveillance Analysis Computer System, (PC SACS) July 7, 1995. 


\begin{tabular}{|c|c|c|c|c|}
\hline \multicolumn{5}{|c|}{ 241-AP-105 } \\
\hline \multicolumn{5}{|c|}{ Surface Level Data } \\
\hline Date & Type of Measurement & Level (Inchea) & Data Quality & Comments \\
\hline $3 / 7 / 95 \quad 12: 00$ & Manual Tape & 297 & Good & \\
\hline $3 / 8 / 95 \quad 7: 03$ & Auto FIC & 297.4 & Good & \\
\hline $3 / 8 / 9512: 00$ & Manual Tape & 296.75 & Good & \\
\hline 3/9/95 7:03 & Auto FIC & 297.40 & Good & \\
\hline $3 / 9 / 9512: 00$ & Manual Tape & 296.50 . & Good & \\
\hline 3/10/95 7:02 & Auto FIC & 297.40 & Good & \\
\hline $3 / 10 / 9512: 00$ & Manual Tape & 296.25 & Good & \\
\hline $3 / 11 / 957: 03$ & Auto FIC & 297.4 & Good & \\
\hline $3 / 11 / 9512: 00$ & Manual Tape & 296.75 & Good & \\
\hline $3 / 12 / 957: 03$ & Auto FIC & 297.4 & Good & \\
\hline $3 / 12 / 9512: 00$ & Manual Tape & 296.75 & Good & \\
\hline 3/13/95 7:02 & Auto FIC & 297.40 & Good & \\
\hline $3 / 13 / 9512: 00$ & Manual Tape & 296.75 & Good & \\
\hline $3 / 14 / 957: 03$ & Auto FIC & 297.4 & Good & \\
\hline $3 / 14 / 9512: 00$ & Manual Tape & 296.75 & Good & \\
\hline $3 / 15 / 95 \quad 7: 05$ & Auto FIC & 297.4 & Good & \\
\hline $3 / 15 / 9512: 00$ & Manual Tape & 296.75 & Good & \\
\hline $3 / 16 / 95 \quad 7: 03$ & Auto FIC & 297.40 & Good & \\
\hline $3 / 16 / 9512: 00$ & Manual Tape & 296.75 & Good & \\
\hline $3 / 17 / 957: 03$ & Auto FIC & $297: 40$ & Good & \\
\hline $3 / 17 / 9512: 00$ & Manual Tape & 296.75 & Good & \\
\hline $3 / 18 / 957: 03$ & Auto FIC & 297.4 & Good & \\
\hline $3 / 18 / 9512: 00$ & Manual Tape & 296.75 & Good & \\
\hline $3 / 19 / 95$ 7:03 & Auto FIC & 297.40 & Good & \\
\hline $3 / 19 / 95 \quad 12: 00$ & Manual Tape & 296.75 & Good & \\
\hline $3 / 20 / 956: 03$ & Auto FIC & 297.40 & Good & \\
\hline $3 / 20 / 95$ 12:00 & Manual Tape & 296.75 & Good & \\
\hline $3 / 21 / 957: 03$ & Auto FIC & 297.40 & Good & \\
\hline $3 / 21 / 95 \quad 12: 00$ & Manual Tape & 296.5 & Good & \\
\hline $3 / 22 / 957: 02$ & Auto FIC & 297.4 & Good & \\
\hline $3 / 22 / 95 \quad 12: 00$ & Manual Tape & 296.75 & Good & \\
\hline $3 / 23 / 957: 03$ & Auto FIC & 297.4 & Good & \\
\hline $3 / 23 / 9512: 00$ & Manual Tape & 296.5 & Good & \\
\hline $3 / 24 / 957: 03$ & Auto FIC & 297.40 & Good & \\
\hline $3 / 24 / 95 \quad 12: 00$ & Manual Tape & 296.75 & Good & \\
\hline $3 / 25 / 957: 02$ & Auto FIC & 297.3 & Good & \\
\hline $3 / 25 / 95 \quad 12: 00$ & Manual Tape & 296.50 & Good & \\
\hline $3 / 26 / 957: 02$ & Auto FIC & 297.3 & Good & \\
\hline $3 / 26 / 9512: 00$ & Manual Tape & 296.75. & Good & \\
\hline $3 / 27 / 957: 03$ & Auto FIC & 297.30 & Good & \\
\hline $3 / 27 / 95 \quad 12: 00$ & Manual Tape & 296.75 & Good & \\
\hline $3 / 28 / 957: 03$ & Auto FIC & 297.30 & Good & \\
\hline $3 / 28 / 9512: 00$ & Manual Tape & 296.75 & Good & \\
\hline $3 / 29 / 957: 03$ & Auto FIC & 297.30 & Good & \\
\hline $3 / 29 / 95 \quad 12: 00$ & Manual Tape & 296.75 & Good & \\
\hline $3 / 30 / 957: 03$ & Auto FIC & 297.30 & Good & \\
\hline $3 / 30 / 9512: 00$ & Manual Tape & 296.75 & Good & \\
\hline $3 / 31 / 957: 04$ & Auto FIC & $297.30^{\circ}$ & Good & \\
\hline
\end{tabular}

Data obtained from WHC Surveillance Analysis Computer System, (PC SACS) July 7, 1995. 


\begin{tabular}{|c|c|c|c|c|}
\hline \multicolumn{5}{|c|}{ 241-AP-105 } \\
\hline \multicolumn{5}{|c|}{ Surface Level Data } \\
\hline Date & Type of Measurement & Level (linches) & Data Quality & Comments \\
\hline $3 / 31 / 95 \quad 12: 00$ & Manual Tape & 296.75 & Good & \\
\hline 4/1/95 7:02 & Auto FIC & $297.3^{\circ}$ & Good & \\
\hline $4 / 1 / 9512: 00$ & Manual Tape & 296.75 & Good & \\
\hline 4/2/95 7:03 & Auto FIC & 297.3 & Good & \\
\hline $4 / 2 / 9512: 00$ & Manual Tape & 296.75 & Good & \\
\hline 4/3/95 7:03 & Auto FIC & 297.30 & Good & \\
\hline $4 / 3 / 95 \quad 12: 00$ & Manual Tape & 296.75 & Good & $\cdot$ \\
\hline $4 / 4 / 957: 03$ & Auto FIC & 297.30 & Good & \\
\hline 4/4/95 12:00 & Manual Tape & 295.75 & Suspect & Data not included in graph \\
\hline $4 / 5 / 957: 03$ & Auto FIC & 297.30 & Good & \\
\hline $4 / 5 / 9512: 00$ & Manual Tape & 296.75 & Good & \\
\hline $4 / 6 / 956: 03$ & Auto FIC & 297.3 & Good & \\
\hline $4 / 6 / 95 \quad 12: 00$ & Manual Tape & 296.00 & Good & \\
\hline 4/7/95 7:03 & Auto FIC & 297.3 & Good & \\
\hline $4 / 7 / 9512: 00$ & Manual Tape & 296.75 & Good & \\
\hline 4/8/95 7:02 & Auto FIC & 297.30 & Good & 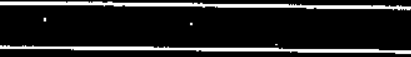 \\
\hline $4 / 8 / 9512: 00$ & Manual Tape & 296.75 & Good & \\
\hline $4 / 9 / 9512: 00$ & Manual Tape & 296.75 & Good & \\
\hline 4/9/95 23:03 & Auto FIC & 297.3 & Good & \\
\hline $4 / 10 / 957: 02$ & Auto FIC & 297.30 & Good & \\
\hline $4 / 10 / 9512: 00$ & Manual Tape & 296.75 & Good & \\
\hline $4 / 11 / 957: 04$ & Auto FIC & 297.30 & Good & \\
\hline $4 / 11 / 9512: 00$ & Manual Tape & 296.75 & Good & \\
\hline $4 / 12 / 957: 03$ & Auto FIC & 297.3 & Good & \\
\hline $4 / 12 / 9512: 00$ & Manual Tape & 296.5 & Good & \\
\hline 4/13/95 7:05 & Auto FIC & 297.3 & Good & \\
\hline 4/13/95 12:00 & Manual Tape & 296.75 & Good & $\cdot$ \\
\hline 4/14/95 7:03 & Auto FIC & 297.30 & Good & \\
\hline 4/14/95 12:00 & Manual Tape & 296.50 & Good & \\
\hline $4 / 15 / 957: 03$ & Auto FIC & 297.3 & Good & \\
\hline $4 / 15 / 9512: 00$ & Manual Tape & 296.5 & Good & \\
\hline $4 / 16 / 957: 03$ & Auto FIC & 297.3 & Good & \\
\hline $4 / 16 / 9512: 00$ & Manual Tape & 297.5 & Good & \\
\hline $4 / 17 / 957: 03$ & Auto FIC & 297.30 & Good & \\
\hline 4/17/95 $12: 00$ & Manual Tape & 296.75 & Good & \\
\hline $4 / 18 / 957: 03$ & Auto FIC & $297.3^{\circ}$ & Good & \\
\hline $4 / 18 / 95 \quad 12: 00$ & Manual Tape & 296.50 & Good & \\
\hline $4 / 19 / 957: 03$ & Auto FIC & 297.3 & Good & \\
\hline $4 / 19 / 9512: 00$ & Manual Tape & 296.5 & Good & \\
\hline $4 / 20 / 957: 03$ & Auto FIC & 297.3 & Good & \\
\hline $4 / 20 / 9512: 00$ & Manual Tape & 296.5 & Good & \\
\hline $4 / 21 / 957: 03$ & Auto FIC & 297.30 & Good & \\
\hline 4/21/95 12:00 & Manual Tape & 296.5 & Good & \\
\hline 4/22/95 7:03 & Auto FIC & 297.30 & Good & \\
\hline $4 / 22 / 9512: 00$ & Manual Tape & 296.50 & Good & \\
\hline $4 / 23 / 95$ 7:03 & Auto FIC & 297.3 & Good & \\
\hline $4 / 23 / 95.12: 00$ & Manual Tape & 296.5 & Good & \\
\hline 4/24/95 7:03 & Auto FIC & 297.30 & Good & \\
\hline
\end{tabular}

Data obtained from WHC Surveillance Analysis Computer System, (PC SACS) July 7, 1995. 


\begin{tabular}{|c|c|c|c|c|}
\hline \multicolumn{5}{|c|}{ 241-AP-105 } \\
\hline & & Irface Leval Dat & & \\
\hline Date & Type of Measuremerit & Level (Inches) & Data Quality & Comments \\
\hline 4/25/95 7:03 & Auto FIC & 297.30 & Good & \\
\hline $4 / 25 / 9512: 00$ & Manual Tape & 296.5 & Good & \\
\hline 4/26/95 7:03 & Auto FIC & 297.3 & Good & \\
\hline 4/26/95 12:00 & Manual Tape & 296.5 & Good & \\
\hline $4 / 27 / 957: 03$ & Auto FIC & 297.30 & Good & \\
\hline $4 / 27 / 9512: 00$ & Manual Tape & 296.50 & Good & \\
\hline 4/28/95 7:03 & Auto FIC & 297.30 & Good & \\
\hline $4 / 28 / 9512: 00$ & Manual Tape & 296.5 & Good & \\
\hline 4/29/95 7:03 & Auto FIC & 297.3 & Good & \\
\hline $4 / 29 / 9512: 00$ & Manual Tape & 296.75 & Good & \\
\hline 4/30/95 7:03 & Auto FIC & 297.20 & Good & \\
\hline $4 / 30 / 9512: 00$ & Manual Tape & 296.75 & Good & \\
\hline 5/1/95 7:03 & Auto FIC & 297.30 & Good & \\
\hline $5 / 1 / 9512: 00$ & Manual Tape & 296.50 & Good & \\
\hline $5 / 2 / 957: 02$ & Auto FIC & 297.30 & Good & \\
\hline $5 / 2 / 95 \quad 12: 00$ & Manual Tape & 297 & Good & \\
\hline $5 / 3 / 957: 03$ & Auto FIC & 297.20 & Good & \\
\hline $5 / 3 / 95 \quad 12: 00$ & Manual Tape & 296.75 & Good & \\
\hline $5 / 4 / 957: 04$ & Auto FIC & 297.3 & Good & \\
\hline $5 / 4 / 9512: 00$ & Manual Tape & 296.5 & Good & \\
\hline 5/5/95 7:04 & Auto FIC & 297.3 & Good & \\
\hline $5 / 5 / 9512: 00$ & Manual Tape & 296.5 & Good & \\
\hline $5 / 6 / 957: 03$ & Auto FIC & 297.3 & Good & \\
\hline $5 / 6 / 95 \quad 12: 00$ & Manual Tape & 296.5 & Good & \\
\hline $5 / 7 / 95 \quad 7: 04$ & Auto FIC & 297.30 & Good & \\
\hline $5 / 7 / 95 \quad 12: 00$ & Manual Tape & 296.50 & Good & \\
\hline $5 / 8 / 95 \quad 7: 04$ & Auto FIC & 297.2 & Good & \\
\hline $5 / 8 / 95 \quad 12: 00$ & Manual Tape & 296.5 & Good & \\
\hline 5/9/95 7:04 & Auto FIC & 297.2 & Good & \\
\hline $5 / 9 / 9512: 00$ & Manual Tape & 296.5 & Good & \\
\hline $5 / 10 / 95$ 7:04 & Auto FIC & 297.2 & Good & \\
\hline $5 / 10 / 9512: 00$ & Manual Tape & 296.5 & Good & \\
\hline $5 / 11 / 957: 03$ & Auto FIC & 297.3 & Good & \\
\hline $5 / 11 / 95 \quad 12: 00$ & Manual Tape & 296.50 & Good & \\
\hline $5 / 12 / 957: 06$ & Auto FIC & 297.2 & Good & \\
\hline $5 / 12 / 9512: 00$ & Manual Tape & 296.5 & Good & \\
\hline $5 / 13 / 957: 03$ & Auto FIC & 297.20 & Good & \\
\hline $5 / 13 / 9512 ; 00$ & Manual Tape & 296.5 & Good & \\
\hline $5 / 14 / 95 \quad 7: 03$ & Auto FIC & 297.20 & Good & \\
\hline $5 / 14 / 95 \quad 12: 00$ & Manual Tape & 296.5 & Good & \\
\hline $5 / 15 / 95 \quad 7: 03$ & Auto FIC & 297.2 & Good & \\
\hline $5 / 15 / 95 \quad 12: 00$ & Manual Tape & 296.50 & Good & \\
\hline $5 / 16 / 95 \quad 7: 03$ & Auto FIC & 297.20 & Good & \\
\hline $5 / 16 / 9512: 00$ & Manual Tape & 296.5 & Good & \\
\hline $5 / 17 / 95 \quad 7: 02$ & Auto FIC & 297.20 & Good & \\
\hline $5 / 17 / 95 \quad 12: 00$ & Manual Tape & 296.50 & Good & \\
\hline $5 / 18 / 956: 02$ & Auto FIC & 297.2 & Good & \\
\hline $5 / 18 / 95 \quad 12: 00$ & Manual Tape & 296.5 & Good & \\
\hline
\end{tabular}

Data obtained from WHC Surveillance Analysis Computer System, (PC SACS) July 7, 1995. 


\begin{tabular}{|c|c|c|c|c|}
\hline \multicolumn{5}{|c|}{ 241-AP-105 } \\
\hline \multicolumn{5}{|c|}{ Surface Level Data } \\
\hline Date & Type of Messuremant & Leval (Inches) & Data Quality & Comments \\
\hline $5 / 19 / 957: 05$ & Auto FIC & 297.20 & Good & \\
\hline $5 / 19 / 9512: 00$ & Manual Tape & 296.5 & Good & \\
\hline $5 / 20 / 957: 05$ & Auto FIC & 297.2 & Good & \\
\hline $5 / 20 / 9512: 00$ & Manual Tape & 296.5 & Good & \\
\hline $5 / 21 / 957: 05$ & Auto FIC & 297.2 & Good & \\
\hline $5 / 21 / 9512: 00$ & Manual Tape & 296.50 & Good & \\
\hline $5 / 22 / 95 \quad 7: 05$ & Auto FIC & 297.20 & Good & \\
\hline $5 / 22 / 9512: 00$ & Manual Tape & 296.5 & Good & \\
\hline $5 / 23 / 95 \quad 7: 11$ & Auto FIC & 0.00 & Suspect & Data not included in graph \\
\hline $5 / 23 / 9512: 00$ & Manual FIC & 297.2 & Good & \\
\hline $5 / 23 / 9512: 00$ & Manual Tape & 296.5 & Good & \\
\hline $5 / 24 / 957: 10$ & Auto FIC & 0.00 & Suspect & Data not included in graph \\
\hline $5 / 24 / 9512: 00$ & Manual FIC & 297.20 & Good & \\
\hline $5 / 24 / 95 \quad 12: 00$ & Manual Tape & 296.5 & Good & \\
\hline $5 / 25 / 957: 10$ & Auto FIC & 0 & Suspect & Data not included in graph \\
\hline $5 / 25 / 95 \quad 12: 00$ & Manual FIC & 297.25 & Good & \\
\hline $5 / 25 / 95 \quad 12: 00$ & Manual Tape & 296.50 & Good & \\
\hline $5 / 26 / 957: 04$ & Auto FIC & 297.20 & Good & \\
\hline $5 / 26 / 95 \quad 12: 00$ & Manual Tape & 296.5 & Good & \\
\hline $5 / 27 / 957: 05$ & Auto FIC & 297.20 & Good & \\
\hline $5 / 27 / 9512: 00$ & Manual Tape & 284.5 & Suspect & Data not included in graph \\
\hline $5 / 28 / 957: 05$ & Auto FIC & 297.2 & Good & \\
\hline $5 / 28 / 95 \quad 12: 00$ & Manual Tape & 296.5 & Good & \\
\hline $5 / 29 / 957: 05$ & Auto FIC & 297.20 & Good & \\
\hline $5 / 29 / 95 \quad 12: 00$ & Manual Tape & 296.5 & Good & \\
\hline 5/30/95 7:05 & Auto FIC & 297.2 & Good & \\
\hline $5 / 30 / 9512: 00$ & Manual Tape & 296.5 & Good & \\
\hline $5 / 31 / 95$ 7:05 & Auto FIC & 297.2 & Good & \\
\hline $5 / 31 / 9512: 00$ & Manual Tape & 296.5 & Good & \\
\hline 6/1/95 7:05 & Auto FIC & 297.2 & Good & \\
\hline $6 / 1 / 9512: 00$ & Manual Tape & 296.5 & Good & \\
\hline $6 / 2 / 957: 05$ & Auto FIC & 297.20 & Good & \\
\hline $6 / 2 / 95 \quad 12: 00$ & Manual Tape & 296.5 & Good & \\
\hline $6 / 3 / 95 \quad 12: 00$ & Manual Tape & 296.5 & Good & \\
\hline $6 / 3 / 9523: 05$ & Auto FIC & 297.20 & Good & \\
\hline $6 / 4 / 957: 05$ & Auto FIC & 297.3 & Good & \\
\hline $6 / 4 / 9512: 00$ & Manual Tape & 296.5 & Good & \\
\hline $6 / 5 / 957: 05$ & Auto FIC & 297.2 . & Good & \\
\hline $6 / 5 / 95 \quad 12: 00$ & Manual Tape & 296.5 & Good & \\
\hline $6 / 6 / 957: 05$ & Auto FIC & 297.2 & Good & \\
\hline $6 / 6 / 9512: 00$ & Manual Tape & 296.25 & Good & \\
\hline $6 / 7 / 95$ 7:05 & Auto FIC & 297.20 & Good & \\
\hline 6/7/95 12:00 & Manual Tape & 296.6 & Good & \\
\hline 6/8/95 7:05 & Auto FIC & 297.2 & Good & \\
\hline $6 / 8 / 9512: 00$ & Manual Tape & 225 & Suspect & Data not included in graph \\
\hline $6 / 9 / 957: 05$ & Auto FIC & 297.2 & Good & \\
\hline $6 / 9 / 9512: 00$ & Manual Tape & 296.25 & Good & \\
\hline $6 / 10 / 95$ 7:05 & Auto FIC & 297.20 & Good & \\
\hline
\end{tabular}

Data obtained from WHC Surveillance Analysis Computer System, (PC SACS) July 7, 1995. 


\begin{tabular}{|c|c|c|c|c|}
\hline & Type of Measurement & $\begin{array}{l}\text { urface Level Data } \\
\text { Level (Inches) }\end{array}$ & Data Quality & Comments \\
\hline $\begin{array}{c}\text { Date } \\
6 / 10 / 9512: 00\end{array}$ & $\begin{array}{c}\text { Type of Measurement } \\
\text { Manual Tape }\end{array}$ & $\frac{\text { Lovel (Inches) }}{296.75}$ & Good & \\
\hline$\frac{6 / 10 / 9512: 00}{6 / 11 / 957: 05}$ & Auto FIC & 297.20 & Good & \\
\hline $\begin{array}{r}6 / 11 / 957: 05 \\
6 / 11 / 9512: 00\end{array}$ & Manual Tape & 296.5 & Good & \\
\hline $\begin{array}{l}6 / 11 / 95 \quad 12: 00 \\
6 / 12 / 95 \quad 7: 05 \\
\end{array}$ & Auto FIC & 297.2 & Good & \\
\hline $\begin{array}{c}6 / 12 / 95 \quad 7: 05 \\
6 / 12 / 95 \quad 12: 00 \\
\end{array}$ & Manual Tape & 296.5 & Good & \\
\hline $\begin{array}{lll}6 / 12 / 95 & 12: 00 \\
6 / 13 / 95 & 7: 05 \\
\end{array}$ & Auto FIC & 297.20 & Good & \\
\hline $\begin{array}{c}6 / 13 / 95 \quad 7: 05 \\
6 / 13 / 9512: 00\end{array}$ & Manual Tape & 296.5 & Good & \\
\hline$\frac{6 / 13 / 9512: 00}{6 / 14 / 957: 05}$ & Auto FIC & 297.30 & Good & \\
\hline $\begin{array}{c}6 / 14 / 95 \quad 7: 05 \\
6 / 14 / 95 \quad 12: 00\end{array}$ & Manual Tape & 296.75 & Good & \\
\hline$\frac{6 / 14 / 95 \quad 12: 00}{6 / 15 / 95 \quad 7: 05}$ & Auto FIC & 297.20 & Good & \\
\hline $\begin{array}{c}6 / 15 / 95 \quad 7: 05 \\
6 / 15 / 95 \quad 12: 00\end{array}$ & Manual Tape & 296.75 & Good & \\
\hline $\begin{array}{c}6 / 15 / 95 \quad 12: 00 \\
6 / 16 / 95 \quad 7: 05 \\
\end{array}$ & Auto FIC & 297.2 & Good & \\
\hline$\frac{6 / 16 / 957: 05}{6 / 16 / 95 / 2: 00}$ & Manual Tape & 296.5 & Good & \\
\hline$\frac{6 / 16 / 95 \quad 12: 00}{6 / 17 / 95 \quad 7: 05}$ & Auto FIC & 297.20 & Good & \\
\hline$\frac{6 / 17 / 957: 05}{6 / 17 / 9512: 00}$ & Manual Tape & 296.5 & Good & - \\
\hline$\frac{6 / 17 / 95 \quad 12: 00}{6 / 18 / 95 \quad 7: 05}$ & Auto FIC & 297.2 & Good & . \\
\hline $\begin{array}{c}6 / 18 / 95 \quad 7: 05 \\
6 / 18 / 95 \quad 12: 00\end{array}$ & Manual Tape & 296.5 & Good & \\
\hline $\begin{array}{lll}6 / 18 / 95 & 12: 00 \\
6 / 19 / 95 & 7: 06 \\
\end{array}$ & Auto FIC & 297.20 & Good & \\
\hline $\begin{array}{c}6 / 19 / 957: 06 \\
6 / 19 / 9512: 00\end{array}$ & Manual Tape & 296.5 & Good & \\
\hline$\frac{6 / 19 / 95}{12: 00}$ & Auto FIC & 297.2 & Good & \\
\hline$\frac{6 / 20 / 95 \quad 7: 06}{6 / 20 / 9512: 00}$ & Manual Tape & 296.5 & Good & \\
\hline$\frac{6 / 20 / 9512: 00}{6 / 21 / 95 \quad 7: 06}$ & Auto FIC & 297.20 & Good & \\
\hline $\begin{array}{c}6 / 21 / 95 \quad 7: 06 \\
6 / 2.1 / 95 \quad 12: 00\end{array}$ & Manual Tape & 296.5 & Good & \\
\hline $\begin{array}{c}6 / 2.1 / 95 \quad 12: 00 \\
6 / 22 / 95 \quad 7: 06 \\
\end{array}$ & Auto FIC & 297.2 & Good & \\
\hline$\frac{6 / 22 / 95 \quad 7: 06}{6 / 22 / 9512: 00}$ & Manual Tape & 296.5 & Good & \\
\hline$\frac{6 / 22 / 9512: 00}{6 / 23 / 957: 06}$ & Auto FIC & 297.2 & Good & \\
\hline $\begin{array}{c}6 / 23 / 957: 06 \\
6 / 23 / 9512: 00\end{array}$ & Manual Tape & 296.5 & Good & \\
\hline $6 / 23 / 9512: 00$ & Auto FIC & 297.2 & Good & \\
\hline 6/24/95 7:06 & Manual Tape & 296.5 & Good & \\
\hline $6 / 24 / 9512: 00$ & Auto FIC & 297.20 & Good & \\
\hline 6/25/95 7:05 & Manual Tape & 296.5 & Good & \\
\hline $6 / 25 / 9512: 00$ & Auto FIC & 297.20 & Good & \\
\hline $6 / 26 / 95 \quad 7: 05$ & Manual Tape & 296.75 & Good & \\
\hline $6 / 26 / 9512: 00$ & Auto FIC & 297.20 & Good & \\
\hline 6/27/95 7:06 & Manual Tape & 296.75 & Good & \\
\hline $6 / 27 / 9512: 00$ & Auto FIC & 297.20 & Good & \\
\hline $6 / 28 / 957: 05$ & Manual Tape & 296.75 & Good & \\
\hline $6 / 28 / 95 \quad 12: 00$ & Auto FIC & 297.2 & Good & \\
\hline $6 / 29 / 957: 06$ & Manual Tape & 296.75 & Good & \\
\hline $6 / 29 / 95 \quad 12: 00$ & Auto FIC & 297.20 & Good & \\
\hline$\frac{6 / 30 / 95 \quad 7: 06}{6 / 30 / 95 \quad 12: 00}$ & Manual Tape & 296.25 & Transcribed & \\
\hline
\end{tabular}

Data obtained from WHC Surveillance Analysis Computer System, (PC SACS) July 7, 1995. 


\begin{tabular}{|c|c|c|c|c|}
\hline \multicolumn{5}{|c|}{ 241-AP-108 } \\
\hline \multicolumn{5}{|c|}{ Surface Level Date } \\
\hline Date. & Type of Measurement & Leval (Inchea) & Data Quality & Comments \\
\hline $1 / 1 / 910: 00$ & Manual Tape & 412.25 & Transcribed & \\
\hline $1 / 1 / 917: 03$ & Auto FIC & 412.9 & Good & \\
\hline $1 / 2 / 910: 00$ & Manual Tape & 412.25 & Transcribed & \\
\hline $1 / 2 / 917: 02$ & Auto FIC & 413.00 & Good & \\
\hline $1 / 3 / 910: 00$ & Manual Tape & 412.5 & Transcribed & \\
\hline $1 / 3 / 917: 03$ & Auto FIC & 412.9 & Good & \\
\hline $1 / 4 / 910: 00$ & Manual Tape & 412.5 & Transcribed & \\
\hline 1/4/91 7:02 & Auto FIC & 412.90 & Good & \\
\hline $1 / 5 / 910: 00$ & Manual Tape & 413 & Transcribed & \\
\hline 1/5/91 7:02 & Auto FIC & 412.9 & Good & \\
\hline $1 / 6 / 910: 00$ & Manual Tape & 413 & Transcribed & \\
\hline $1 / 6 / 917: 02$ & Auto FIC & 412.80 & Good & \\
\hline $1 / 7 / 910: 00$ & Manual Tape & 413 & Transcribed & \\
\hline 1/7/91 7:02 & Auto FIC & 412.80 & Good & \\
\hline 1/8/910:00 & Manual Tape & 413.00 & Transcribed & \\
\hline $1 / 8 / 917: 03$ & Auto FIC & 412.8 & Good & \\
\hline $1 / 9 / 910: 00$ & Manual Tape & 413.00 & Transcribed & \\
\hline $1 / 9 / 917: 02$ & Auto FIC & 412.80 & Good & \\
\hline $1 / 10 / 910: 00$ & Manual Tape & 413.00 & Transcribed & \\
\hline $1 / 10 / 917: 02$ & Auto FIC & 412.9 & Good & \\
\hline $1 / 11 / 910: 00$ & Manual Tape & 413.00 & Transcribed & \\
\hline $1 / 11 / 917: 03$ & Auto FIC & 412.9 & Good & $\dot{1}$ \\
\hline $1 / 12 / 910: 00$ & Manual Tape. & 41.3 & Transcribed & \\
\hline $1 / 12 / 917: 02$ & Auto FIC & 412.8 & Good & \\
\hline $1 / 13 / 910: 00$ & Manual Tape & 413 & Transcribed & \\
\hline $1 / 13 / 917: 03$ & Auto FIC & 412.90 & Good & \\
\hline $1 / 14 / 910: 00$ & Manual Tape & 413.00 & Transcribed & \\
\hline $1 / 14 / 917: 04$ & Auto FIC & 412.9 & Good & \\
\hline $1 / 15 / 910: 00$ & Manual Tape & 413 & Transcribed & ; \\
\hline $1 / 15 / 917: 03$ & Auto FIC & 412.8 & Good & \\
\hline $1 / 16 / 910: 00$ & Manual Tape & 413.00 & Transcribed & \\
\hline $1 / 16 / 917: 04$ & Auto FIC & 412.80 & Good & \\
\hline $1 / 17 / 910: 00$ & Manual Tape & 413 & Transcribed & \\
\hline $1 / 17 / 917: 04$ & Auto FIC & 412.80 & Good & \\
\hline $1 / 18 / 910: 00$ & Manual Tape & 413 & Transcribed & \\
\hline $1 / 18 / 917: 04$ & Auto FIC & 412.80 & Good & \\
\hline $1 / 19 / 910: 00$ & Manual Tape & 413 & Transcribed & \\
\hline $1 / 19 / 917: 04$ & Auto FIC & 412.8 & Good & \\
\hline $1 / 20 / 910: 00$ & Manual Tape & 413.00 & Transcribed & \\
\hline $1 / 20 / 917: 04$ & Auto FIC & 412.8 & Good & \\
\hline $1 / 21 / 910: 00$ & Manual Tape & 413 & Transcribed & \\
\hline $1 / 21 / 917: 04$ & Auto FIC & 412.8 & Good & \\
\hline $1 / 22 / 910: 00$ & Manual Tape & 413.00 & Transcribed & \\
\hline $1 / 22 / 917: 04$ & Auto FIC & 412.80 & Good & \\
\hline $1 / 23 / 910: 00$ & Manual Tape & 413.00 & Transcribed & \\
\hline $.1 / 23 / 91 \quad 7: 04$ & Auto FIC & 412.8 & Good & \\
\hline $1 / 24 / 910: 00$ & Manual Tape & 413.00 & Transcribed & \\
\hline $1 / 24 / 917: 04$ & Auto FIC & 412.8 & Good & \\
\hline
\end{tabular}

Data obtained from WHC Surveillance Analysis Computer System, (PC SACS) July 7, 1995. 


\begin{tabular}{|c|c|c|c|c|}
\hline \multicolumn{5}{|c|}{ 241-AP-108 } \\
\hline \multicolumn{5}{|c|}{ Surface Level Data } \\
\hline Date & Type of Meaduremont & Level (Inchea) & Data Quality & Comments \\
\hline $1 / 25 / 910: 00$ & Manual Tapa & 413 & Transcribed & \\
\hline $1 / 25 / 917: 03$ & Auto FIC & 412.8 & Good & \\
\hline $1 / 26 / 910: 00$ & Manual Tape & 413 & Transeribed & \\
\hline $1 / 26 / 917: 03$ & Auto FiC & 412.80 & Good & \\
\hline $1 / 27 / 910: 00$ & Manual Tape & 413.00 & Transcribed & \\
\hline $1 / 27 / 917: 03$ & Auto FIC & 412.70 & Good & \\
\hline $1 / 28 / 910: 00$ & Manual Tape & 412.5 & Transcribed & \\
\hline $1 / 28 / 916: 03$ & Auto FIC & 412.80 & Good & \\
\hline $1 / 29 / 910: 00$ & Manual Tape & 412.75 & Transcribed & \\
\hline $1 / 29 / 917: 02$ & Auto FIC & 412.80 & Good & \\
\hline $1 / 30 / 910: 00$ & Manual FIC & 412.8 & Transeribed & \\
\hline $1 / 30 / 910: 00$ & Manual Tape & 413 & Transcribed & \\
\hline $1 / 31 / 910: 00$ & Manual Tape & 413 & Transcribed & \\
\hline $1 / 31 / 917: 02$ & Auto FIC & 412.80 & Good & \\
\hline $2 / 1 / 910: 00$ & Manual Tape & 413.00 & Transcribed & \\
\hline $2 / 1 / 917: 02$ & Auto FIC & 412.80 & Good & \\
\hline $2 / 2 / 910: 00$ & Manual Tape & 413.00 & Transcribed & \\
\hline $2 / 2 / 917: 04$ & Auto FIC & 412.8 & Good & \\
\hline 2/3/910:00 & Manual Tape & 412.75 & Transcribed & \\
\hline $2 / 3 / 917: 03$ & Auto FIC & 412.8 & - Good & \\
\hline $2 / 4 / 910: 00$ & Manual Tape & 412.75 & Transcribed & \\
\hline $2 / 4 / 917: 03$ & Auto FIC & 412.8 & Good & \\
\hline $2 / 5 / 910: 00$ & Manual Tape & $412.75^{\circ}$ & Transcribed & \\
\hline $2 / 5 / 917: 04$ & Auto FIC & 412.80 & Good & \\
\hline $2 / 6 / 910: 00$ & Manual Tape ' & 412.75 & Transcribed & \\
\hline $2 / 6 / 917: 04$ & Auto FIC & 412.80 & Good & \\
\hline $2 / 7 / 910: 00$ & Manual Tape & 412.75 & Transcribed & \\
\hline $2 / 7 / 917: 04$ & Auto FIC & 412.70 & Good & \\
\hline $2 / 8 / 910: 00$ & Manual Tape & $412.75^{\circ}$ & Transcribed & \\
\hline $2 / 8 / 917: 04$ & Auto FIC & 412.7 & Good & 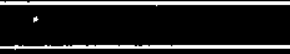 \\
\hline 2/9/91 0:00 & Manual Tape & 412.75 & Transcribed & \\
\hline $2 / 9 / 917: 04$ & Auto FIC & 412.7 & Good & \\
\hline $2 / 10 / 910: 00$ & Manual Tape & 412.75 & Transcribed & \\
\hline $2 / 10 / 917: 03$ & Auto FIC & 412.80 & Good & \\
\hline $2 / 11 / 910: 00$ & Manual Tapo & 412.75 & Transcribed & \\
\hline $2 / 11 / 917: 04$ & Auto FIC & 412.70 & Good & \\
\hline 2/12/91 0:00 & Manual Tape & 412.75 & Transeribed & \\
\hline $2 / 12 / 917: 04$ & Auto FIC & 412.7 & Good & \\
\hline 2/13/91 0:00 & Manual Tape & 412.75 & Transcribed & \\
\hline $2 / 13 / 917: 04$ & Auto FIC & 412.70 & Good & \\
\hline 2/14/91 0:00 & Manual Tape & 412.75 & Transcribed & \\
\hline $2 / 14 / 917: 04$ & Auto FIC & 412.80 & Good & \\
\hline $2 / 15 / 910: 00$ & Manual Tape & 412.75 & Transcribed & \\
\hline 2/15/91 7:03 & Auto FIC & 412.70 & Good & \\
\hline $2 / 16 / 910: 00$ & Manual Tape & 412.75 & Transcribed & \\
\hline 2/16/91 7:02 & Auto FIC & 412.7 & Good & \\
\hline $2 / 17 / 910: 00$ & Manual Tape & 412.75 & Transcribed & \\
\hline $2 / 17 / 917: 02$ & Auto FIC & 412.70 & Good & \\
\hline
\end{tabular}

Data obtained from WHC Surveillance Analysis Computer System, (PC SACS) July 7, 1995. 


\begin{tabular}{|c|c|c|c|c|}
\hline \multirow{2}{*}{\multicolumn{5}{|c|}{$\frac{\text { 241-AP-108 }}{\text { Surface Level Dato }}$}} \\
\hline & & & & \\
\hline Date & Type of Measurement & Lovel (Inches) & Data Quality & Comments \\
\hline $2 / 18 / 910: 00$ & Manual Tape & 412.75 & Transcribed & \\
\hline $2 / 18 / 917: 03$ & Auto FIC & 412.7 & Good & \\
\hline 2/19/910:00 & Manual Tape & 412.75 & Transcribed & \\
\hline 2/19/91 7:02 & Auto FIC & 412.70 & Good & \\
\hline $2 / 20 / 910: 00$ & Manual Tape & 412.75 & Transcribed & \\
\hline $2 / 20 / 917: 03$ & Auto FIC & 412.7 & Good & \\
\hline $2 / 21 / 910: 00$ & Manual Tape & 412.75 & Transcribed & \\
\hline $2 / 21 / 917: 02$ & Auto FIC & 412.70 & Good & \\
\hline $2 / 22 / 910: 00$ & Manual Tape & 412.75 & Transcribed & \\
\hline $2 / 22 / 917: 02$ & Auto FIC & 412.70 & Good & \\
\hline $2 / 23 / 910: 00$ & Manual Tape & 412.75 & Transcribed & \\
\hline $2 / 23 / 917: 02$ & Auto FIC & 412.70 & Good & \\
\hline $2 / 24 / 910.00$ & Manual Tape & 412.75 & Transcribed & \\
\hline $2 / 24 / 917: 03$ & Auto FIC & 412.70 & Good & \\
\hline $2 / 25 / 910: 00$ & Manual Tape & 412.75 & Transcribed & \\
\hline 2/25/917:03 & Auto FIC & 412.7 & Good & $\therefore$ \\
\hline $2 / 26 / 910: 00$ & Manual Tape & 412.75 & Transcribed & \\
\hline $2 / 26 / 917: 03$ & Auto FIC & 412.7 & Good & \\
\hline $2 / 27 / 910: 00$ & Manual Tape & 412.75 & Transcribed & \\
\hline $2 / 27 / 91 \quad 7: 02$ & Auto FIC & 412.7 & Good & \\
\hline $2 / 28 / 910: 00$ & Manual Tape & 412.75 & Transcribed & \\
\hline $2 / 28 / 917: 02$ & Auto FIC & 412.60 & Good & \\
\hline $3 / 1 / 910: 00$ & Manual Tape & 412.50 & Transcribed & \\
\hline $3 / 1 / 917: 02$ & Auto FIC & 412.60 & Good & \\
\hline $3 / 2 / 910: 00$ & Manual Tape & 412.75 & Transcribed & \\
\hline $3 / 2 / 917: 02$ & Auto FIC & $41.2: 60$ & Good & \\
\hline $3 / 3 / 910: 00$ & Manual Tape & 412.75 & Transcribed & \\
\hline $3 / 3 / 917: 03$ & Auto FIC & 412.6 & Good & \\
\hline $3 / 4 / 910: 00$ & Manual Tape & 412.75 & Transcribed & \\
\hline $3 / 4 / 917: 03$ & Auto FIC & 412.60 & Good & \\
\hline $3 / 5 / 910: 00$ & Manual Tape & 412.75 & Transcribed & \\
\hline $3 / 5 / 917: 03$ & Auto FIC & 412.7 & Good & \\
\hline $3 / 6 / 910: 00$ & Manual Tape & 412.75 & Transcribed & \\
\hline $3 / 6 / 917: 02$ & Auto FIC & 412.60 & Good & \\
\hline $3 / 7 / 910: 00$ & Manual Tape & 412.75 & Transcribed & \\
\hline $3 / 7 / 917: 02$ & Auto FIC & 412.6 & - Good & \\
\hline $3 / 8 / 910: 00$ & Manual Tape & 412.75 & Transcribed & \\
\hline $3 / 8 / 917: 02$ & Auto FIC & 412.60 & Good & \\
\hline $3 / 9 / 910: 00$ & Manual Tape & 412.75 & Transcribed & \\
\hline $3 / 9 / 917: 02$ & Auto FIC & 412.6 & Good & \\
\hline $3 / 10 / 910: 00$ & Manual Tape & 412.75 & Transcribed & \\
\hline $3 / 10 / 917: 02$ & Auto FIC & 412.60 & Good & \\
\hline $3 / 11 / 910: 00$ & Manual Tape & 412.5 & Transcribed & \\
\hline $3 / 11 / 917: 02$ & Auto FIC & 412.60 & Good & \\
\hline $3 / 12 / 910: 00$ & Manual Tape & 412.75. & Transcribed & \\
\hline $3 / 12 / 917: 03$ & Auto FIC & 412.6 & Good & \\
\hline $3 / 13 / 910: 00$ & Manual Tape & 412.75 & Transcribed & \\
\hline $3 / 13 / 917: 03$ & Auto FIC & 412.60 & Good & \\
\hline
\end{tabular}

Data obtained from WHC Surveillance Analysis Computer System, (PC SACS) July 7, 1995. 


\begin{tabular}{|c|c|c|c|c|}
\hline \multicolumn{5}{|c|}{$\frac{241-A P-106}{\text { Surface Level Data }}$} \\
\hline Date & Type of Measurement & Level (Inchea) & Data Quality & Comments \\
\hline $3 / 14 / 910: 00$ & Manual Tape & 412.75 & Transcribed & \\
\hline $3 / 14 / 917: 03$ & Auto FIC & 412.60 & Good & \\
\hline $3 / 15 / 910: 00$ & Manual Tape & 412.75 & Transcribed & \\
\hline $3 / 15 / 917: 03$ & Auto FIC & 412.6 & Good & \\
\hline $3 / 16 / 910: 00$ & Manual Tape & 412.75 & Transcribed & \\
\hline $3 / 16 / 917: 03$ & Auto FIC & 412.6 & Good & \\
\hline $3 / 17 / 910: 00$ & Manual Tape & 412.50 & Transcribed & \\
\hline $3 / 17 / 917: 02$ & Auto FIC & 412.6 & Good. & \\
\hline $3 / 18 / 910: 00$ & Manual Tape & 412.50 & Transcribed & \\
\hline $3 / 18 / 917: 03$ & Auto FIC & 412.6 & Good & \\
\hline $3 / 19 / 910: 00$ & Manual Tape & 412.25 & Transcribed & \\
\hline $3 / 19 / 917: 03$ & Auto FIC & 412.60 & Good & \\
\hline $3 / 20 / 910: 00$ & Manual Tape & 412.5 & Transcribed & \\
\hline $3 / 20 / 917: 02$ & Auto FIC & 412.50 & Good & \\
\hline $3 / 21 / 910: 00$ & Manual Tape & 412.50 & Transcribed & \\
\hline $3 / 21 / 917: 03$ & Auto FIC & 412.6 & Good & \\
\hline $3 / 22 / 910: 00$ & Manual Tape & 412.5 & Transcribed & \\
\hline $3 / 22 / 917: 02$ & Auto FIC & 412.6 & Good & \\
\hline $3 / 23 / 910: 00$ & Manual Tape & 412.50 & Transcribed & \\
\hline $3 / 23 / 917: 03$ & Auto FIC & 412.6 & Good & \\
\hline $3 / 24 / 910: 00$ & Manual Tape & 412.75 & Transcribed & \\
\hline $3 / 24 / 917: 03$ & Auto FIC & 412.6 & Good & \\
\hline $3 / 25 / 910: 00$ & Manual Tape & 412.5 & Transcribed & \\
\hline $3 / 25 / 917: 03$ & Auto FIC & 412.60 & Good & \\
\hline $3 / 26 / 910: 00$ & Manual Tape & 412.5 & Transcribed & \\
\hline $3 / 26 / 917: 03$ & Auto FIC & 412.6 & Good & \\
\hline $3 / 27 / 910: 00$ & Manual Tape & 412.5 & Transcribed & \\
\hline $3 / 27 / 91 \quad 7: 03$ & Auto FIC & 412.50 & Good & \\
\hline $3 / 28 / 910: 00$ & Manual Tape & $\begin{array}{r}.412 .50 \\
\end{array}$ & Transcribed & \\
\hline $3 / 28 / 917: 03$ & Auto FIC & 412.50 & Good & \\
\hline $3 / 29 / 910: 00$ & Manual Tape & 412.5 & Transcribed & \\
\hline $3 / 29 / 917: 03$ & Auto FIC & 412.6 & Good & \\
\hline $3 / 30 / 910: 00$ & Manual Tape & 412.50 & Transcribed & \\
\hline $3 / 30 / 917: 03$ & Auto FIC & 412.6 & Good & \\
\hline $3 / 31 / 910: 00$ & Manual Tape & 412.75 & Transcribed & \\
\hline $3 / 31 / 917: 03$ & Auto FIC & 412.60 & Good & \\
\hline $4 / 1 / 910: 00$ & Manual Tape & 412.75 & Transcribed & \\
\hline $4 / 1 / 917: 03$ & Auto FIC & 412.50 & Good & \\
\hline $4 / 2 / 910: 00$ & Manual Tape & 412.75 & Transçribed & \\
\hline $4 / 2 / 917: 03$ & Auto FIC & 41.2 .50 & Good & \\
\hline $4 / 3 / 910: 00$ & Manual Tape & 412.75 & Transcribed & \\
\hline $4 / 3 / 917: 03$ & Auto FIC & 412.50 & Good & \\
\hline $4 / 4 / 910: 00$ & Manual Tape & 412.50 & Transeribed & \\
\hline $4 / 4 / 917: 03$ & Auto FIC & 412.5 & Good & \\
\hline $4 / 5 / 910: 00$ & Manual Tape & 412.75 & Transcribed & \\
\hline $4 / 5 / 917: 03$ & Auto FIC & 412.5 & Good & \\
\hline $4 / 6 / 910: 00$ & Manual Tape & 412.5 & Transcribed & \\
\hline $4 / 6 / 917: 03$ & Auto FIC & 412.5 & Good & \\
\hline
\end{tabular}

Data obtained from WHC Surveillance Analysis Computer System, (PC SACS) July 7, 1995. 


\begin{tabular}{|c|c|c|c|c|}
\hline \multicolumn{5}{|c|}{ 241-AP-106 } \\
\hline & & urface Level Dat & & \\
\hline Date & Type of Measurement & Leval (Inches) & Data Quality & Comments \\
\hline 4/7/91 0:00 & Manual Tape & 412.75 & Transcribed & \\
\hline 4/7/91 7:03 & Auto FIC & 412.50 & Good & \\
\hline $4 / 8 / 910: 00$ & Manual Tape & $4 \Upsilon 2.75$ & Transcribed & \\
\hline 4/8/91 7:04 & Auto FIC & 412.50 & Good & \\
\hline 4/9/91 $0: 00$ & Manual Tape & $412.75^{\circ}$ & Transcribed & \\
\hline 4/9/91 7:03 & Auto FIC & 412.5 & Good & \\
\hline $4 / 10 / 910: 00$ & Manual Tape & 412.25 & Transcribed & \\
\hline 4/10/91 7:03 & Auto FIC & 412.50 & Good & \\
\hline 4/11/910:00 & Manual Tape & 412.50 & Transcribed & \\
\hline 4/11/91 7:03 & Auto FIC & 412.50 & Good & \\
\hline $4 / 12 / 910.00$ & Manual Tape & 412.75 & Transcribed & \\
\hline 4/12/91 7:03 & Auto FIC & 412.50 & Good & \\
\hline $4 / 13 / 910: 00$ & Manual Tape & 412.5 & Transcribed & \\
\hline 4/13/91 7:04 & Auto FIC & 412.5 & Good & \\
\hline $4 / 14 / 910: 00$ & Manual Tape & 412.75 & Transcribed & \\
\hline $4 / 14 / 917: 03$ & Auto FIC & 412.50 & Good & \\
\hline $4 / 15 / 910: 00$ & Manual Tape & 412.75 & Transcribed & \\
\hline 4/15/91 7:03 & Auto FIC & 412.50 & Good & \\
\hline 4/16/91 0:00 & Manual Tape & 412.75 & Transcribed & \\
\hline 4/16/91 0:00 & Manual FIC & 412.5 & Good & \\
\hline $4 / 17 / 910: 00$ & Manual Tape & 412.50 & Transcribed & \\
\hline $4 / 17 / 917: 03$ & Auto FIC & 412.50 & Good & \\
\hline $4 / 18 / 910: 00$ & Manual Tape & 412.5 & Transcribed & \\
\hline 4/18/91 7:06 & Auto FIC & 412.5 & Good & \\
\hline $4 / 19 / 910: 00$ & Manual Tape & 412.5 & Transcribed & \\
\hline 4/19/91 7:05 & Auto FIC & 412.50 & Good & \\
\hline $4 / 20 / 910: 00$ & Manual Tape & 412.5 & Transcribed & \\
\hline $4 / 20 / 917: 06$ & Auto FIC & 412.5 & Good & \\
\hline $4 / 21 / 910: 00$ & Manual Tape & 412.50 & Transcribed & . \\
\hline 4/21/91 7:05 & Auto FIC & 412.50 & Good & \\
\hline $4 / 22 / 910: 00$ & Manual Tape & 412.50 & Transcribed & \\
\hline 4/22/91 7:06 & Auto FIC & 412.5 & Good & \\
\hline $4 / 23 / 910: 00$ & Manual Tape & 412.50 & Transcribed & \\
\hline $4 / 23 / 917: 06$ & Auto FIC & 412.5 & Good & \\
\hline $4 / 24 / 910: 00$ & Manual Tape & 412.75 & Transcribed & \\
\hline 4/24/91 7:05 & Auto FIC & 412.4 & Good & \\
\hline $4 / 25 / 910: 00$ & Manual Tape & 412.75 & Transcribed & \\
\hline $4 / 25 / 917: 05$ & Auto FIC & 412.5 & Good & \\
\hline $4 / 26 / 910: 00$ & Manual Tape & 412.75 & Transcribed & \\
\hline $4 / 26 / 917: 05$ & Auto FIC & 412.40 & Good & \\
\hline $4 / 27 / 910: 00$ & Manual Tape ' & 412.75 & Transcribed & \\
\hline $4 / 27 / 917: 05$ & Auto FIC & 412.4 & Good & \\
\hline 4/28/91 0:00 & Manual Tape & 412.50 & Transcribed & \\
\hline $4 / 28 / 917: 06$ & Auto FIC & 412.4 & Good & \\
\hline 4/29/91 0:00 & Manual Tape & 412.50 & Transcribed & \\
\hline 4/29/91 7:05 & Auto FIC & 412.40 & Good & \\
\hline $4 / 30 / 910: 00$ & Manual Tape & 412.5 & Transcribed & \\
\hline $4 / 30 / 917: 06$ & Auto FIC & 412.4 & Good & \\
\hline
\end{tabular}

Data obtained from WHC Surveillance Analysis Computer System, (PC SACS) July 7, 1995. 


\begin{tabular}{|c|c|c|c|c|}
\hline & & 241-AP-106 & $!$ & \\
\hline \multicolumn{5}{|c|}{ Surface Level Data } \\
\hline Date & Type of Measurement & Level (Inchas) & Data Quality & Comments \\
\hline $5 / 1 / 910: 00$ & Manual Tape & 412.50 & Transcribed & \\
\hline 5/1/91 7:05 & Auto FIC & 412.4 & Good & \\
\hline $5 / 2 / 910: 00$ & Manual Tape & 412.50 & Transcribed & \\
\hline $5 / 2 / 917: 05$ & Auto FIC & 412.4 & Good & \\
\hline $5 / 3 / 910: 00$ & Manual Tape & 412.5 & Transcribed & \\
\hline $5 / 3 / 917: 06$ & Auto FIC & 412.40 & Good & \\
\hline $5 / 4 / 910: 00$ & Manual Tape & 412.50 & Transcribed & \\
\hline $5 / 4 / 917: 05$ & Auto FIC & 412.4 & Good & \\
\hline $5 / 5 / 910: 00$ & Manual Tape & 412.50 & Transcribed & \\
\hline $5 / 5 / 917: 05$ & Auto FIC & 412.40 & Good & \\
\hline 5/6/91 0:00 & Manual Tape & 412.50 & Transcribed & \\
\hline $5 / 6 / 917: 05$ & Auto FIC & 412.4 & Good & \\
\hline $5 / 7 / 910: 00$ & Manual Tape & 412.5 & Transcribed & \\
\hline $5 / 7 / 917: 05$ & Auto FIC & 412.4 & Good & \\
\hline $5 / 8 / 910: 00$ & Manual Tape & 412.50 & Transcribed & \\
\hline $5 / 8 / 917: 05$ & Auto FIC & 412.40 & Good & \\
\hline $5 / 9 / 910: 00$ & Manual Tape & 412.5 & Transcribed & \\
\hline $5 / 9 / 917: 05$ & Auto FIC & 412.40 & Good & \\
\hline $5 / 10 / 910: 00$ & Manual Tape & 412.5 & Transcribed & \\
\hline $5 / 10 / 917: 05$ & Auto FIC & 412.40 & Good & \\
\hline $5 / 11 / 910: 00$ & Manual Tape & 412.50 & Transcribed & \\
\hline $5 / 11 / 917: 05$ & Auto FIC & 412.4 & Good & \\
\hline $5 / 12 / 910: 00$ & Manual Tape & 412.50 & Transcribed & \\
\hline 5/12/91 7:05 & Auto FIC & 412.4 & Good & \\
\hline $5 / 13 / 910: 00$ & Manual Tape & 412.50 & Transcribed & \\
\hline $5 / 13 / 91 \quad 7: 05$ & Auto FIC & 412.4 & Good & \\
\hline $5 / 14 / 910: 00$ & Manual Tape & 412.5 & Transcribed & \\
\hline $5 / 14 / 917: 05$ & Auto FIC & 412.40 & Good & i \\
\hline $5 / 15 / 910: 00$ & Manual Tape & . 412.5 & Transcribed & \\
\hline $5 / 15 / 917: 05$ & Auto FIC & 412.40 & Good & \\
\hline $5 / 16 / 91 \quad 0: 00$ & Manual Tape & 412.50 & Transcribed & \\
\hline $5 / 16 / 917: 06$ & Auto FIC & 412.40 & Good & \\
\hline $5 / 17 / 910: 00$ & Manual Tape & 412.5 & Transcribed & \\
\hline $5 / 17 / 917: 06$ & Auto FIC & 412.40 & Good & \\
\hline $5 / 18 / 910: 00$ & Manual Tape & 412.50 & Transcribed & \\
\hline $5 / 18 / 917: 06$ & Auto FIC & 412.40 & Good & \\
\hline 5/19/91 0:00 & Manual Tape & 412.50 & Transcribed & \\
\hline $5 / 19 / 917: 05$ & Auto FIC & 412.4 & Good & \\
\hline $5 / 20 / 910: 00$ & Manual Tape & 412.50 & Transcribed & \\
\hline $5 / 20 / 917: 06$ & Auto FIC & 412.4 & Good & \\
\hline $5 / 21 / 910: 00$ & Manual Tape & 412.5 & Transcribed & \\
\hline $5 / 21 / 917: 06$ & Auto FIC & 412.4 & Gopd & \\
\hline $5 / 22 / 910: 00$ & Manual Tape & 412.75 & Transcribed & \\
\hline $5 / 22 / 917: 05$ & Auto FIC & 412.4 & Good & \\
\hline 5/23/91 0:00 & Manual Tape & 412.50 & Transcribed & \\
\hline $5 / 23 / 917: 05$ & Auto FIC & 412.40 & Good & \\
\hline $5 / 24 / 910: 00$ & Manual Tape & 412.50 & Transcribed & \\
\hline 5/24/91 7:05 & Auto FIC & 412.40 & Good & \\
\hline
\end{tabular}

Data obtained from WHC Surveillance Analysis Computer System, (PC SACS) July 7, 1995. 


\begin{tabular}{|c|c|c|c|c|}
\hline \multicolumn{5}{|c|}{ 241-AP-106 } \\
\hline & & urface Leval Dat & & \\
\hline Date & Type of Measurement & Levol (Inchea) & Data Quality & Comments \\
\hline $5 / 25 / 910: 00$ & Manual Tape & 412.5 & Transcribed & \\
\hline $5 / 25 / 917: 05$ & Auto FIC & 412.40 & Good & \\
\hline $5 / 26 / 910: 00$ & Manual Tape & 412.5 & Transcribed & \\
\hline $5 / 26 / 917: 05$ & Auto FIC & 412.4 & Good & \\
\hline $5 / 27 / 910: 00$ & Manual Tape & 412.5 & Transeribed & \\
\hline $5 / 27 / 917: 05$ & Auto FIC & 412.4 & Good & \\
\hline $5 / 28 / 910: 00$ & Manual Tape & 412.5 & Transcribed & \\
\hline 5/28/91 7:05 & Auto FIC & 412.40 & Good & \\
\hline $5 / 29 / 91 \quad 0: 00$ & Manual Tape & 412.25 & Transcribed & \\
\hline $5 / 29 / 917: 05$ & Auto FIC & 412.40 & Good & \\
\hline $5 / 30 / 910: 00$ & Manual Tape & 412.5 & Transcribed & \\
\hline $5 / 30 / 917: 05$ & Auto FIC & 412.30 & Good & \\
\hline $5 / 31 / 910: 00$ & Manual Tape & 412.50 & Transcribed & \\
\hline $5 / 31 / 917: 05$ & Auto FIC & 41.2 .30 & Good & \\
\hline 6/1/91 0:00 & Manual Tape & 412.25 & Transcribed & \\
\hline 6/1/91 6:05 & Auto FIC & 412.30 & Good & \\
\hline $6 / 2 / 910: 00$ & Manual Tape & 412.25 & Transcribed & \\
\hline $6 / 2 / 913: 05$ & Auto FIC & 412.40 & Good & \\
\hline $6 / 3 / 910: 00$ & Manual Tape & 412.25 & Transcribed & \\
\hline $6 / 3 / 917: 05$ & Auto FIC & 412.4 & Good & \\
\hline $6 / 4 / 910: 00$ & Manual Tape & 412.50 & Transcribed & \\
\hline $6 / 4 / 917: 05$ & Auto FIC & 412.30 & Good & \\
\hline 6/5/91 0:00 & Manual Tape & 412.5 & Transcribed & \\
\hline $6 / 5 / 917: 05$ & Auto FIC & 412.30 & Good & \\
\hline $6 / 6 / 91 \quad 0: 00$ & Manual Tape & 412 & Transcribed & \\
\hline $6 / 6 / 917: 06$ & Auto FIC & 412.3 & Good & \\
\hline 6/7/91 0:00 & Manual Tape & 412.5 & Transcribed & \\
\hline $6 / 7 / 917: 05$ & Auto FIC & 412.3 & Good & \\
\hline $6 / 8 / 910: 00$ & Manual Tape & 412.25 & Transcribed & \\
\hline $6 / 8 / 91 \quad 7: 05$ & Auto FIC & 412.30 & Good & \\
\hline $6 / 9 / 910: 00$ & Manual Tape & 41.2 .25 & Transcribed & \\
\hline $6 / 9 / 917: 05$ & Auto FIC & 412.3 & Good & \\
\hline $6 / 10 / 910: 00$ & Manual Tape & 412.50 & Transeribed & \\
\hline $6 / 10 / 917: 06$ & Auto FIC & 412.3 & Good & \\
\hline $6 / 11 / 910: 00$ & Manual Tape & 412.5 & Transcribed & \\
\hline $6 / 11 / 917: 05$ & Auto FIC & 412.3 & Good & \\
\hline $6 / 12 / 910: 00$ & Manual Tape & 412.50 & Transcribed & \\
\hline $6 / 12 / 917: 04$ & Auto FIC & 412.3 & Good & \\
\hline $6 / 13 / 910: 00$ & Manual Tape & 412.5 & Transeribed & \\
\hline $6 / 13 / 917: 05$ & Auto FIC & 412.30 & Good & \\
\hline 6/14/91 0:00 & Manual Tape & 412.50 & Transcribed & \\
\hline $6 / 14 / 917: 05$ & Auto FIC & 412.30 & Good & \\
\hline $6 / 15 / 910: 00$ & Manual Tape & 412.5 & Transcribed & \\
\hline $6 / 15 / 917: 05$ & Auto FIC & 412.30 & Good & \\
\hline $6 / 16 / 910: 00$ & Manual Tape & 412.5 & Transcribed & \\
\hline $6 / 16 / 917: 05$ & Auto FIC & 412.30 & Good & \\
\hline $6 / 17 / 910: 00$ & Manual Tape & 412.5 & Transcribed & \\
\hline 6/17/91 7:05 & Auto FIC & 412.3 & Good & \\
\hline
\end{tabular}

Data obtained from WHC Surveillance Analysis Computer System, (PC SACS) July 7, 1995. 


\begin{tabular}{|c|c|c|c|c|}
\hline & $\therefore$ & $241-A P+106$ & $\therefore$ & \\
\hline \multicolumn{5}{|c|}{ Surface Level Data } \\
\hline Date & Type of Measurement & Leval (Inchea) & Data Quality & Comments \\
\hline $6 / 18 / 910: 00$ & Manual Tape & 412.5 & Transcribed & \\
\hline $6 / 18 / 917: 05$ & Auto FIC & 412.30 & Good & \\
\hline $6 / 19 / 910: 00$ & Manual Tape & 412.75 & Transcribed & \\
\hline $6 / 19 / 917: 05$ & Auto FIC & 412.30 & Good & \\
\hline $6 / 20 / 910: 00$ & Manual Tape & 412.75 & Transcribed & \\
\hline $6 / 20 / 917: 05$ & Auto FIC & 412.30 & Good & \\
\hline $6 / 21 / 910: 00$ & Manual Tape & 412.75 & Transcribed & \\
\hline $6 / 21 / 917: 06$ & Auto FIC & 412.30 & Good & \\
\hline $6 / 22 / 910: 00$ & Manual Tape & 412.75 & Transcribed & \\
\hline $6 / 22 / 913: 06$ & Auto FIC & 412.30 & Good & \\
\hline $6 / 23 / 910: 00$ & Manual Tape & 412.75 & Transcribed & \\
\hline $6 / 23 / 917: 05$ & Auto FIC & 412.30 & Good & \\
\hline $6 / 24 / 910: 00$ & Manual Tape & 412.5 & Transcribed & \\
\hline $6 / 24 / 915: 05$ & Auto FIC & 412.30 & Good & \\
\hline $6 / 25 / 910: 00$ & Manual Tape & 412.50 & Transcribed & \\
\hline $6 / 25 / 917: 06$ & Auto FIC & 412.30 & Good & \\
\hline $6 / 26 / 910: 00$ & Manual Tape & 412.50 & Transcribed & \\
\hline $6 / 26 / 911: 03$ & Auto FIC & 412.3 & Good & \\
\hline $6 / 27 / 910: 00$ & Manual FIC & 412,40 & Transcribed & \\
\hline $6 / 27 / 910: 00$ & Manual Tape & 412.5 & Transcribed & \\
\hline $6 / 28 / 910: 00$ & Manual Tape & 412.50 & Transcribed & \\
\hline $6 / 28 / 917: 03$ & Auto FIC & 412.50 & Good & \\
\hline $6 / 29 / 910: 00$ & Manual Tape & 412.5 & Transcribed & \\
\hline $6 / 29 / 916: 02$ & Auto FIC & 412.30 & Good & \\
\hline $6 / 30 / 910: 00$ & Manual Tape & 412.5 & Transcribed & \\
\hline $6 / 30 / 910: 00$ & Manual FIC & 412.3 & Good & \\
\hline $7 / 1 / 910: 00$ & Manual Tape & 412.50 & Transcribed & \\
\hline 7/1/91 0:00 & Manual FIC & 412.3 & Good & \\
\hline $7 / 2 / 910: 00$ & Manual Tape & .412 .50 & Transeribed & \\
\hline 7/2/91 7:06 & Auto FIC & 412.40 & Good & \\
\hline $7 / 3 / 910: 00$ & Manual Tape & 412.50 & Transcribed & \\
\hline $7 / 3 / 917: 06$ & Auto FIC & 412.4 & Good & \\
\hline $7 / 4 / 910: 00$ & Manual Tape & 412.5 & Transcribed & \\
\hline $7 / 4 / 910: 00$ & Manual FIC & 412.3 & Good & \\
\hline 7/5/91 0:00 & Manual Tape & 412.50 & Transcribed & \\
\hline $7 / 5 / 910: 00$ & Manual FIC & 412,35 & Good & \\
\hline $7 / 6 / 910: 00$ & Manual Tape & 412.5 & Transcribed & \\
\hline $7 / 6 / 910: 00$ & Manual FIC & 412.35 & Good & \\
\hline $7 / 7 / 910: 00$ & Manual Tape & $4 \uparrow 2.50$ & Transcribed & \\
\hline $7 / 7 / 910: 00$ & Manual FIC & 412.35 & Good & \\
\hline $7 / 8 / 910: 00$ & Manual Tape & 412.50 & Transcribed & \\
\hline $7 / 8 / 910: 00$ & Manual FIC & 412.35 & Good & \\
\hline $7 / 9 / 910: 00$ & Manual Tape & 412.50 & Transcribed & \\
\hline $7 / 9 / 910: 00$ & Manual FIC & 412.30 & Good & \\
\hline 7/10/91 0:00 & Manual Tape & $412: 50$ & Transcribed & \\
\hline 7/10/91 0:00 & Manual FIC & 412.4 & Good & \\
\hline $7 / 11 / 910: 00$ & Manual Tape & 412.50 & Transcribed & \\
\hline 7/11/91 7:06 & Auto FIC & 412.30 & Good & \\
\hline
\end{tabular}

Data obtained from WHC Surveillance Analysis Computer System, (PC SACS) July 7, 1995. 


\begin{tabular}{|c|c|c|c|c|}
\hline \multicolumn{5}{|c|}{ 241-AP-106 } \\
\hline \multicolumn{5}{|c|}{ Surface Lovel Data } \\
\hline Date & Typo of Measuroment & Leval (Inchea) & Data Quality & Comments \\
\hline $7 / 12 / 910: 00$ & Manual Tape & 412.5 & Transcribed & \\
\hline 7/12/91 7:06 & Auto FIC & 412.3 & Good & \\
\hline $7 / 13 / 910: 00$ & Manual Tape & 412.50 & Transcribed & \\
\hline $7 / 13 / 917: 06$ & Auto FIC & 412.3 & Good & \\
\hline $7 / 14 / 910: 00$ & Manual Tape & 412.50 & Transcribed & \\
\hline $7 / 14 / 912: 06$ & Auto FIC & 412.4 & Good & \\
\hline $7 / 15 / 910: 00$ & Manual Tape & 412.25 & Transcribed & \\
\hline $7 / 15 / 917: 06$ & Auto FIC & 412.3 & Good & \\
\hline $7 / 16 / 910: 00$ & Manual Tape & 412.5 & Transcribed & 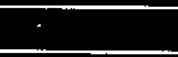 \\
\hline $7 / 16 / 910: 00$ & Manual FIC & 412.3 & Good & \\
\hline $7 / 17 / 910: 00$ & Manual Tape & 412.50 & Transcribed & \\
\hline $7 / 17 / 910: 05$ & Auto FIC & 412.40 & Good & \\
\hline $7 / 18 / 910: 00$ & Manual Tape & 412.25 & Transcribed & \\
\hline 7/18/91 0:00 & Manual FIC & 412.35 & Good & \\
\hline $7 / 19 / 910: 00$ & Manual Tape & 412.25 & Transcribed & \\
\hline $7 / 19 / 917: 05$ & Auto FIC & 412.30 & Good & \\
\hline $7 / 20 / 910: 00$ & Manual Tape & 412.25 & Transcribed & \\
\hline $7 / 20 / 912: 05$ & Auto FIC & 412.3 & Good & \\
\hline $7 / 21 / 910: 00$ & Manual Tape & 412.50 & Transcribed & \\
\hline 7/21/91 1:05 & Auto FIC & 412.3 & Good & \\
\hline $7 / 22 / 910: 00$ & Manual FIC & 412.3 & Transcribed & \\
\hline $7 / 22 / 91 \quad 0: 00$ & Manual Tape & 412.50 & Transcribed & \\
\hline $7 / 23 / 910: 00$ & Manual Tape & 412.50 & Transcribed & \\
\hline $7 / 23 / 910: 05$ & Auto FIC & 412.30 & Good & \\
\hline $7 / 24 / 910: 00$ & Manual Tape & 412.5 & Transcribed & \\
\hline $7 / 24 / 917: 05$ & Auto FIC & 412.40 & Good & \\
\hline 7/25/91 $0: 00$ & Manual Tape & 412.5 & Transcribed & \\
\hline $7 / 25 / 91 \quad 1: 03$ & Auto FIC & 412.30 & Good & \\
\hline $7 / 26 / 910: 00$ & Manual Tape & 412.50 & Transcribed & \\
\hline $7 / 26 / 910: 00$ & Manual FIC & 412.3 & Good & \\
\hline $7 / 27 / 91 \quad 0: 00$ & Manual Tape & 412.5 & Transcribed & \\
\hline $7 / 27 / 910: 00$ & Manual FIC & 412.30 & Good & \\
\hline $7 / 28 / 910: 00$ & Manual Tape & 412.50 & Transcribed & \\
\hline $7 / 28 / 910: 00$ & Manual FIC & 412.30 & Good & \\
\hline $7 / 29 / 910: 00$ & Manual Tape & 412.50 & Transcribed & \\
\hline $7 / 29 / 910: 00$ & Manual FIC & 412.30 & Good & \\
\hline $7 / 30 / 910: 00$ & Manual Tape & 412.5 & Transcribed & \\
\hline $7 / 30 / 917: 02$ & Auto FIC & 412.3 & Good & \\
\hline $7 / 31 / 910: 00$ & Manual Tape & 412.50 & Transcribed & \\
\hline $7 / 31 / 917: 02$ & Auto FIC & 412.4 & Good & \\
\hline $8 / 1 / 910: 00$ & Manual Tape & 412.5 & Transcribed & \\
\hline $8 / 1 / 917: 02$ & Auto FIC & 41.2 .40 & Good & \\
\hline $8 / 2 / 910: 00$ & Manual Tape & 412.5 & Transcribed & \\
\hline $8 / 2 / 91 \quad 7: 02$ & Auto FIC & 412.30 & Good & \\
\hline $8 / 3 / 910: 00$ & Manual Tape & 412.25 & Transcribed & \\
\hline $8 / 3 / 917: 02$ & Auto FIC & 412.3 & Good & \\
\hline 8/4/910:00 & Manual Tape & 412.25 & Transcribed & \\
\hline $8 / 4 / 917: 02$ & Auto FIC & 412.30 & Good & \\
\hline
\end{tabular}

Data obtained from WHC Surveillance Analysis Computer System, (PC SACS) July 7, 1995. 


\begin{tabular}{|c|c|c|c|c|}
\hline \multicolumn{5}{|c|}{ 241-AR-108 } \\
\hline & . & urface Level Dat & & \\
\hline Date & Type of Measurement & Level (Inches) & Data Quality & Comments \\
\hline $8 / 5 / 910: \overline{00}$ & Manual Tape & 412.25 & Transcribed & \\
\hline $8 / 5 / 917: 02$ & Auto FIC & 412.30 & Good & \\
\hline $8 / 6 / 910: 00$ & Manual Tape & 412.50 & Transcribed & \\
\hline $8 / 6 / 917: 02$ & Auto FIC & 412.30 & Good & \\
\hline $8 / 7 / 910: 00$ & Manual Tape & 412.50 & Transcribed & \\
\hline $8 / 7 / 917: 06$ & Auto FIC & 412.30 & Good & \\
\hline $8 / 8 / 910: 00$ & Manual Tape & 412.5 & Transcribed & \\
\hline $8 / 8 / 917: 06$ & Auto FIC & 412.4 & Good & \\
\hline $8 / 9 / 910: 00$ & Manual Tape & 412.50 & Transcribed & \\
\hline $8 / 9 / 917: 04$ & Auto FIC & 412.3 & Good & \\
\hline $8 / 10 / 910: 00$ & Manual Tape & 412.5 & Transcribed & \\
\hline $8 / 10 / 917: 04$ & Auto FIC & 412.30 & Good & \\
\hline 8/11/910:00 & Manual Tape & 412.50 & Transcribed & \\
\hline $8 / 11 / 917: 04$ & Auto FIC. & 412.3 & Good & \\
\hline $8 / 12 / 910: 00$ & Manual Tape & 412.5 & Transcribed & \\
\hline $8 / 12 / 917: 04$ & Auto FIC & 412.30 & Good & \\
\hline $8 / 13 / 910: 00$ & Manual Tape & 412.50 & Transcribed & \\
\hline $8 / 13 / 917: 04$ & Auto FIC & 412.3 & Good & \\
\hline $8 / 14 / 910: 00$ & Manual Tape & 412.50 & Transcribed & \\
\hline $8 / 14 / 917: 04$ & Auto FIC & 412.30 & Good & \\
\hline $8 / 15 / 910: 00$ & Manual Tape & 412.50 & Transcribed & \\
\hline $8 / 15 / 917: 03$ & Auto FIC & 412.30 & Good & \\
\hline $8 / 16 / 910: 00$ & Manual Tape & 412.50 & Transcribed & \\
\hline 8/16/91 0:00 & Manual FIC & 412.3 & Good & \\
\hline $8 / 17 / 910: 00$ & Manual Tape & 412.5 & Transcribed & \\
\hline $8 / 17 / 917: 02$ & Auto FIC & 412.5 & Good & \\
\hline $8 / 18 / 910: 00$ & Manual Tape & 412.50 & Transcribed & \\
\hline $8 / 18 / 917: 03$ & Auto FIC & 412.3 & Good & \\
\hline $8 / 19 / 910: 00$ & Manual Tape & 412.5 & Transcribed & \\
\hline $8 / 19 / 917: 02$ & Auto FIC & 412.3 & Good & \\
\hline $8 / 20 / 910: 00$ & Manual Tape & 412.5 & Transcribed & \\
\hline $8 / 20 / 917: 04$ & Auto FIC & 412.30 & Good & \\
\hline $8 / 21 / 910: 00$ & Manual Tape & 412.50 & Transcribed & \\
\hline $8 / 21 / 917: 03$ & Auto FIC & 412.40 & Good & \\
\hline $8 / 22 / 91 \quad 0: 00$ & Manual Tape & 412.5 & Transcribed & \\
\hline $8 / 22 / 917: 03$ & Auto FIC & 412.3 & Good & \\
\hline $8 / 23 / 910: 00$ & Manual Tape & 412.50 & Transcribed & \\
\hline $8 / 23 / 917: 02$ & Auto FIC & 412.30 & Good & \\
\hline $8 / 24 / 910: 00$ & Manual Tape & 412.50 & Transciibed & \\
\hline $8 / 24 / 917: 02$ & Auto FIC & 412.30 & Good & \\
\hline $8 / 25 / 910: 00$ & Manual Tape & 412.5 & Transcribed & \\
\hline $8 / 25 / 917: 02$ & Auto FIC & 412.3 & Good & \\
\hline $8 / 26 / 910: 00$ & Manual Tape & 412.5 & Transcribed & \\
\hline $8 / 26 / 917: 03$ & Auto FIC & 412.30 & Good & \\
\hline $8 / 27 / 910: 00$ & Manual Tape & 412.5 & Transcribed & \\
\hline $8 / 27 / 917: 03$ & Auto FIC & 412.3 & Good & 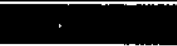 \\
\hline $8 / 28 / 910: 00$ & Manual Tape & 412.50 & Transcribed & \\
\hline $8 / 28 / 917: 03$ & Auto FIC & 412.3 & Good & \\
\hline
\end{tabular}

Data obtained from WHC Surveillance Analysis Computer System, (PC SACS) July 7, 1995. 


\begin{tabular}{|c|c|c|c|c|}
\hline \multicolumn{5}{|c|}{ 241-AP-106 } \\
\hline & & Urfaco Level Dat & & \\
\hline Date & Type of Moasurement & Level (Inchea) & Data Quality & Comments \\
\hline $8 / 29 / 910: 00$ & Manual Tape & 412.5 & Transcribed & \\
\hline $8 / 29 / 917: 02$ & Auto FIC & 412.30 & Good & \\
\hline $8 / 30 / 910: 00$ & Manual Tape & 412.5 & Transcribed & \\
\hline $8 / 30 / 916: 02$ & Auto FIC & 412.30 & Good & \\
\hline $8 / 31 / 910: 00$ & Manual Tape & 412.5 & Transcribed & \\
\hline $8 / 31 / 917: 02$ & Auto FIC & 412.3 & Good & \\
\hline $9 / 1 / 910: 00$ & Manual Tape & 412.5 & Transcribed & \\
\hline 9/1/91 7:02 & Auto FIC & 412.30 & Good & \\
\hline $9 / 2 / 910: 00$ & Manual Tape & 412.50 & Transcribed & \\
\hline $9 / 2 / 917: 02$ & Auto FIC & 412.3 & Good & \\
\hline $9 / 3 / 91 \quad 0: 00$ & Manual Tape & 412.50 & Transcribed & \\
\hline $9 / 3 / 917: 02$ & Auto FIC & 412.30 & Good & \\
\hline 9/4/91 0:00 & Manual Tape & 412.5 & Transcribed & \\
\hline 9/4/91 7:03 & Auto FIC & 412.30 & Good & \\
\hline $9 / 5 / 910: 00$ & Manual Tape & 412.5 & Transcribed & \\
\hline $9 / 5 / 917: 02$ & Auto FIC & 412.30 & Good & \\
\hline 9/6/91 0:00 & Manual Tape & 412.50 & Transcribed & \\
\hline $9 / 6 / 917: 02$ & Auto FIC & 412.30 & Good & \\
\hline $9 / 7 / 910: 00$ & Manual Tape & 412.5 & Transcribed & \\
\hline $9 / 7 / 917: 02$ & Auto FIC & 412.30 & Good & \\
\hline 9/8/91 0:00 & Manual Tape & 412.50 & Transcribed & \\
\hline $9 / 8 / 917: 02$ & Auto FIC & 412.30 & Good & \\
\hline 9/9/91 0:00 & Manual Tape & 412.5 & Transcribed & \\
\hline 9/9/91 7:03 & Auto FIC & 412.4 & Good & \\
\hline 9/10/91 0:00 & Manual Tape & 412.5 & Transcribed & \\
\hline $9 / 10 / 917: 02$ & Auto FIC & 412.30 & Good & \\
\hline 9/11/910:00 & Manual Tape & 412.50 & Transcribed & \\
\hline $9 / 11 / 917: 03$ & Auto FIC & 412.40 & Good & \\
\hline $9 / 12 / 910: 00$ & Manual Tape & 412.50 & Transcribed & \\
\hline $9 / 12 / 917: 02$ & Auto FIC & $412.30^{\circ}$ & Good & \\
\hline $9 / 13 / 910: 00$ & Manual Tape & 412.50 & Transcribed & \\
\hline $9 / 13 / 917: 02$ & Auto FIC & 412.30 & Good & \\
\hline $9 / 14 / 910: 00$ & Manual Tape & 412.50 & Transcribed & \\
\hline $9 / 14 / 917: 03$ & Auto FIC & 412.3 & Good & \\
\hline $9 / 15 / 910: 00$ & Manual Tape & 412.5 & Transcribed & \\
\hline $9 / 15 / 917: 02$ & Auto FIC & 412.30 & Good & \\
\hline $9 / 16 / 910: 00$ & Manual Tape & 412.50 & Transcribed & \\
\hline $9 / 16 / 917: 03$ & Auto FIC & 412.30 & Good & \\
\hline 9/17/910:00 & Manual Tape & 412.5 & Transcribed & \\
\hline $9 / 17 / 917: 03$ & Auto FIC & 412.3 & Good & \\
\hline $9 / 18 / 910: 00$ & Manual Tape & 412.50 & Transcribed & \\
\hline $9 / 18 / 917: 03$ & Auto FIC & 412.30 & Good & \\
\hline $9 / 19 / 910: 00$ & Manual Tape & 412.5 & Transcribed & \\
\hline $9 / 19 / 917: 03$ & Auto FIC & 412.30 & Good & \\
\hline $9 / 20 / 910: 00$ & Manual Tape & 412.25 & Transcribed & \\
\hline 9/20/91 7:02 & Auto FIC & 412.30 & Good & \\
\hline $9 / 21 / 910: 00$ & Manual Tape & 412.25 & Transcribed & \\
\hline $9 / 21 / 917: 02$ & Auto FIC & 412.30 & Good & \\
\hline
\end{tabular}

Data obtained from WHC Surveillance Analysis Computer System, (PC SACS) July 7, 1995. 


\begin{tabular}{|c|c|c|c|c|}
\hline \multicolumn{5}{|c|}{ 241-AP-108 } \\
\hline \multicolumn{5}{|c|}{ Surface Level Data } \\
\hline Date & Type of Measurement & Level (Inches) & Data Quality & Comments \\
\hline $9 / 22 / 910: 00$ & Manual Tape & 412.25 & Transcribed & \\
\hline $9 / 22 / 917: 03$ & Auto FIC & 412.3 & Good & \\
\hline $9 / 23 / 910: 00$ & Manual Tape . & 412.25 & Transcribed & \\
\hline $9 / 23 / 91 \quad 7: 03$ & Auto FIC & 412.3 & Good & \\
\hline $9 / 24 / 910: 00$ & Manual Tape & 412.50 & Transcribed & \\
\hline $9 / 24 / 917: 02$ & Auto FIC & 412.3 & Good & \\
\hline $9 / 25 / 910: 00$ & Manual Tape & 412.50 & Transcribed & . \\
\hline $9 / 25 / 917: 03$ & Auto FIC & 412.4 & Good & \\
\hline $9 / 26 / 910: 00$ & Manual Tape & 412.5 & Transcribed & $\dot{.}$ \\
\hline $9 / 26 / 917: 03$ & Auto FIC & 412.3 & Good & \\
\hline $9 / 27 / 910: 00$ & Manual Tape & 412.5 & Transcribed & \\
\hline $9 / 27 / 917: 02$ & Auto FIC & 412.2 & Good & \\
\hline $9 / 28 / 910: 00$ & Manual Tape & 412.50 & Transcribed & \\
\hline $9 / 28 / 917: 02$ & Auto FIC & 412.30 & Good & \\
\hline $9 / 29 / 910: 00$ & Manual Tape & 412.5 . & Transcribed & \\
\hline $9 / 29 / 917: 03$ & Auto FIC & 412.30 & Good & \\
\hline $9 / 30 / 910: 00$ & Manual Tape & 412.5 & Transcribed & \\
\hline $9 / 30 / 917: 03$ & Auto FIC & 412.3 & Good & 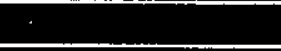 \\
\hline $10 / 1 / 910: 00$ & Manual Tape & 412.50 & Transcribed & \\
\hline $10 / 1 / 917: 02$ & Auto FIC & 412.3 & Good & . \\
\hline $10 / 2 / 910: 00$ & Manual Tape & 412.50 & Transcribed & \\
\hline 10/2/91 7:02 & Auto FIC & 412.3 & Good & \\
\hline $10 / 3 / 910: 00$ & Manual Tape & 412.5 & Transcribed & \\
\hline $10 / 3 / 917: 02$ & Auto FIC & 412.30 & Good & \\
\hline $10 / 4 / 910: 00$ & Manual Tape & 412.50 & Transcribed & \\
\hline $10 / 4 / 917: 03$ & Auto FIC & 412.3 & Good & \\
\hline $10 / 5 / 910: 00$ & Manual FIC & 412.20 & Transcribed & \\
\hline $10 / 5 / 910: 00$ & Manual Tape & 412.5 & Transcribed & \\
\hline 10/6/91 0:00 & Manual Tape & 412.5 & Transcribed & 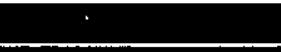 \\
\hline $10 / 6 / 917: 03$ & Auto FIC & 412.20 & Good & \\
\hline $10 / 7 / 910: 00$ & Manual Tape & 412.5 & Transcribed & \\
\hline $10 / 7 / 917: 02$ & Auto FIC & 412.20 & Good & \\
\hline 10/8/91 0:00 & Manual Tape & 41.2 .50 & Transcribed & \\
\hline $10 / 8 / 917: 02$ & Auto FIC & 412.20 & Good & \\
\hline $10 / 9 / 910: 00$ & Manual Tape & 412.50 & Transcribed & \\
\hline $10 / 9 / 917: 02$ & Auto FIC & 412.20 & Good & \\
\hline $10 / 10 / 910: 00$ & Manual Tape & 412.50 & Transcribed & \\
\hline $10 / 10 / 917: 02$ & Auto FIC & 412.2 & Good & \\
\hline $10 / 11 / 910: 00$ & Manual Tape & 412.50 & Transcribed & \\
\hline $10 / 11 / 91 \quad 7: 02$ & Auto FIC & 412.2 & Good & \\
\hline $10 / 12 / 910: 00$ & Manual Tape & 412.5 & Transcribed & \\
\hline $10 / 12 / 917: 02$ & Auto FIC & 412.2 & Good & \\
\hline $10 / 13 / 910: 00$ & Manual Tape & 412.5 & Transcribed & \\
\hline $10 / 13 / 917: 03$ & Auto FIC & $412.20^{\circ}$ & Good & \\
\hline $10 / 14 / 91 \quad 0: 00$ & Manual Tape & 412.25 & Transcribed & \\
\hline $10 / 14 / 91 \quad 7: 02$ & Auto FIC & 412.2 & Good & \\
\hline $10 / 15 / 91 \quad 0: 00$ & Manual Tape & 412.25 & Transcribed & \\
\hline $10 / 15 / 91 \quad 7: 02$ & Auto FIC & 412.20 & Good & \\
\hline
\end{tabular}

Data obtained from WHC Surveillance Analysis Computer System, (PC SACS) July 7, 1995. 


\begin{tabular}{|c|c|c|c|c|}
\hline \multicolumn{5}{|c|}{ 241-AP-106 } \\
\hline \multicolumn{5}{|c|}{ Surface Levol Data } \\
\hline Date & Type of Measurement & Level (Inches) & Data Quality & Comments \\
\hline $10 / 16 / 910: 00$ & Manual Tape & 412.5 & Transcribed & \\
\hline $10 / 16 / 917: 03$ & Auto FIC & 412.2 & Good & \\
\hline $10 / 17 / 910: 00$ & Manual Tape & 412.5 & Transcribed & \\
\hline $10 / 17 / 917: 05$ & Auto FIC & 412.20 & Good & \\
\hline $10 / 18 / 910: 00$ & Manual Tape & 412.5 & Transcribed & \\
\hline $10 / 18 / 917: 06$ & Auto FIC & 412.2 & Good & \\
\hline $10 / 19 / 910: 00$ & Manual Tape & 412.50 & Transcribed & \\
\hline $10 / 19 / 917: 06$ & Auto FIC & 412.2 & Good & \\
\hline $10 / 20 / 910: 00$ & Manual Tape & 412.50 & Transcribed & \\
\hline $10 / 20 / 917: 02$ & Auto FIC & 412.20 & Good & \\
\hline $10 / 21 / 910: 00$ & Manual Tape & 412.50 & Transcribed & \\
\hline $10 / 21 / 917: 02$ & Auto FIC & 412.2 & Good & \\
\hline $10 / 22 / 910: 00$ & Manual Tape & 412.25 & Transcribed & \\
\hline $10 / 22 / 917: 03$ & Auto FIC & 412.2 & Good & \\
\hline $10 / 23 / 910: 00$ & Manual Tape & 412.50 & Transcribed & \\
\hline $10 / 23 / 917: 02$ & Auto FIC & 412.2 & Good & \\
\hline $10 / 24 / 910: 00$ & Manual Tape & 412.5 & Transcribed & \\
\hline $10 / 24 / 917: 02$ & Auto FIC & 412.2 & Good & \\
\hline $10 / 25 / 910: 00$ & Manual Tape & 412.50 & Transcribed & \\
\hline $10 / 25 / 917: 03$ & Auto FIC & 412.2 & Good & \\
\hline $10 / 26 / 910: 00$ & Manual Tape & 412.50 & Transcribed & \\
\hline $10 / 26 / 91 \quad 7: 03$ & Auto FIC & $: 412.20$ & Good & \\
\hline $10 / 27 / 910: 00$ & Manual Tape & 412.50 & Transcribed & $\therefore$ \\
\hline $10 / 27 / 917: 02$ & Auto FIC & 412.2 & Good & \\
\hline $10 / 28 / 910: 00$ & Manual Tape & 412.50 & Transcribed & \\
\hline $10 / 28 / 917: 02$ & Auto FIC & 412.20 & Good & \\
\hline $10 / 29 / 910: 00$ & Manual Tape & 412.50 & Transcribed & \\
\hline $10 / 29 / 917: 03$ & Auto FIC & 412.20 & Good & \\
\hline $10 / 30 / 910: 00$ & Manual Tape & 412.25 & Transcribed & \\
\hline $10 / 30 / 917: 03$ & Auto FIC & 412.20 & Good & \\
\hline $10 / 31 / 910: 00$ & Manual Tape & 412.25 & Transcribed & \\
\hline $10 / 31 / 917: 03$ & Auto FIC & 412.1 & Good & \\
\hline 11/1/91 0:00 & Manual Tape & 412.00 & Transcribed & \\
\hline $11 / 1 / 917: 02$ & Auto FIC & 412.1 & Good & \\
\hline $11 / 2 / 910: 00$ & Manual Tape & 412.25 & Transcribed & \\
\hline $11 / 2 / 917: 03$ & Auto FIC & 412.10 & Good & \\
\hline $11 / 3 / 910: 00$ & Manual Tape & 412.25 & Transcribed & \\
\hline $11 / 3 / 917: 03$ & Auto FIC & 412.10 & Good & \\
\hline 11/4/91 0:00 & Manual Tape & $: 412.00$ & Transcribed & \\
\hline $11 / 4 / 917: 02$ & Auto FIC & 412.10 & Good & \\
\hline $11 / 5 / 910: 00$ & Manual Tape & 412 & Transcribed & \\
\hline $11 / 5 / 917: 03$ & Auto FIC & 412.2 & Good & \\
\hline $11 / 6 / 910: 00$ & Manual Tape & 412.25 & Transcribed & \\
\hline $11 / 6 / 917: 03$ & Auto FIC & 412.2 & Good & \\
\hline $11 / 7 / 910: 00$ & Manual Tape & 412.25 & Transcribed & \\
\hline $11 / 7 / 917: 02$ & Auto FIC & 412.1 & Gopd & \\
\hline $11 / 8 / 910: 00$ & Manual Tape & 412.25 & Transcribed & \\
\hline $11 / 8 / 917: 03$ & Auto FIC & 412.20 & Good & \\
\hline
\end{tabular}

Data obtained from WHC Surveillance Analysis Computer System, (PC SACS) July 7, 1995. 


\begin{tabular}{|c|c|c|c|c|}
\hline \multicolumn{5}{|c|}{ 241-AP-106 } \\
\hline & & Urface Lovel Dat & & \\
\hline Date & Type of Measurement & Level (lnches) & Data Quality & Comments \\
\hline $11 / 9 / 910: 00$ & Manual Tape & 412.25 & Transçribed & \\
\hline $11 / 9 / 917: 02$ & Auto FIC & 412.10 & Good & \\
\hline $11 / 10 / 910: 00$ & Manual Tape & 412.25 & Transcribed & \\
\hline $11 / 10 / 917: 03$ & Auto FIC & 412.10 & Good & \\
\hline $11 / 11 / 910: 00$ & Manual Tape & 412.25 & Transcribed & \\
\hline $11 / 11 / 916: 03$ & Auto FIC & 412.10 & Good & \\
\hline $11 / 12 / 910: 00$ & Manual Tape & 412.25 & Transcribed & \\
\hline $11 / 12 / 917: 03$ & Auto FIC & 412.10 & Good & \\
\hline $11 / 13 / 910: 00$ & Manual Tape & 412.25 & Transcribed & \\
\hline $11 / 13 / 917: 03$ & Auto FIC & 412.2 & Good & \\
\hline 11/14/91 0:00 & Manual Tape & 412.25 & Transcribed & \\
\hline $11 / 14 / 917: 03$ & Auto FIC & 412.10 & Good & \\
\hline $11 / 15 / 910: 00$ & Manual Tape & 412.25 & Transcribed & \\
\hline $11 / 15 / 917: 03$ & Auto FIC & 412.10 & Good & \\
\hline $11 / 16 / 910: 00$ & Manual Tape & 412.50 & Transcribed & \\
\hline $11 / 16 / 917: 03$ & Auto FIC & 412.10 & Good & \\
\hline $11 / 17 / 910: 00$ & Manual Tape & 412.25 & Transcribed & \\
\hline $11 / 17 / 917: 02$ & Auto FIC & 412.1 & Good & \\
\hline $11 / 18 / 910: 00$ & Manual Tape & 412.25 & Transcribed & \\
\hline $11 / 18 / 917: 03$ & Auto FIC & 412.20 & Good & \\
\hline $11 / 19 / 910: 00$ & Manual Tape & 412.25 . & Transcribed & \\
\hline $11 / 19 / 917: 03$ & Auto FIC & 412.10 & Good & \\
\hline $11 / 20 / 910: 00$ & Manual Tape & 412.25 & Transcribed & \\
\hline $11 / 20 / 917: 03$ & Auto FIC & 412.10 & Good & \\
\hline $11 / 21 / 910: 00$ & Manual Tape & 412.25 & Transcribed & \\
\hline $11 / 21 / 917: 02$ & Auto FIC & 412.1 & Good & \\
\hline $11 / 22 / 910: 00$ & Manual Tape & 412.25 & Transcribed & \\
\hline $11 / 22 / 917: 03$ & Auto FIC & 412.1 & Good & \\
\hline $11 / 23 / 910: 00$ & Manual Tape & 412.25 & Transcribed & \\
\hline $11 / 23 / 917: 02$ & Auto FIC & 412.10 & Good & \\
\hline $11 / 24 / 910: 00$ & Manual Tape & 412.25 & Transcribed & \\
\hline $11 / 24 / 917: 03$ & Auto FIC & 412.10 & Good & \\
\hline $11 / 25 / 910: 00$ & Manual Tape & 412.25 & Transcribed & \\
\hline $11 / 25 / 917: 03$ & Auto FIC & 412.1 & Good & \\
\hline $11 / 26 / 910: 00$ & Manual Tape & 412.25 & Transcribed & \\
\hline $11 / 26 / 910: 00$ & Manual FIC & 412.1 & Good & \\
\hline $11 / 27 / 910: 00$ & Manual Tape & 412.25 & Transcribed & \\
\hline $11 / 27 / 917: 03$ & Auto FIC & 412.1 & Good & \\
\hline $11 / 28 / 910: 00$ & Manual Tape & 412.25 & Transcribed & \\
\hline $11 / 28 / 917: 03$ & Auto FIC & 412.1 & Good & \\
\hline $11 / 29 / 910: 00$ & Manual Tape & 412.25 & Transcribed & \\
\hline $11 / 29 / 917: 03$ & Auto FIC & 412.10 & Good & \\
\hline $11 / 30 / 910: 00$ & Manual Tape & 412.25 & Transcribed & \\
\hline $11 / 30 / 917: 03$ & Auto FIC & 412,00 & Good & \\
\hline $12 / 1 / 910: 00$ & Manual Tape & 412.25 & Transcribed & \\
\hline $12 / 1 / 917: 03$ & Auto FIC & 412.00 & Good & \\
\hline $12 / 2 / 910: 00$ & Manual Tape & 412.25 & Transcribed & \\
\hline $12 / 2 / 917: 03$ & Auto FIC & 412.10 & Good & \\
\hline
\end{tabular}

Data obtained from WHC Surveillance Analysis Computer System, (PC SACS) July 7, 1995. 


\begin{tabular}{|c|c|c|c|c|}
\hline \multicolumn{5}{|c|}{ 241-AP-108 } \\
\hline \multicolumn{5}{|c|}{ Surface Levol Data } \\
\hline Date & Type of Measurement & Leval (Inchea) & Data Quality & Comments \\
\hline $12 / 3 / 910: 00$ & Manual Tape & $412.00^{\circ}$ & Transcribed & \\
\hline $12 / 3 / 917: 03$ & Auto FIC & 412.1 & Good & \\
\hline $12 / 4 / 910: 00$ & Manual Tape & 412 & Transcribed & \\
\hline $12 / 4 / 917: 03$ & Auto FIC & 412.00 & Good & \\
\hline 12/5/910:00 & Manual Tape & 412 & Transcribed & \\
\hline $12 / 5 / 917: 03$ & Auto FIC & 412.00 & Good & \\
\hline 12/6/91 0:00 & Manual Tape & 411.75 & Transcribed & \\
\hline $12 / 6 / 917: 04$ & Auto FIC & 412.1 & Good & \\
\hline $12 / 7 / 910: 00$ & Manual Tape & 412.00 & Transcribed & \\
\hline $12 / 7 / 917: 03$ & Auto FIC & 412 & Good & \\
\hline $12 / 8 / 910: 00$ & Manual Tape & 412.25 & Transcribed & \\
\hline $12 / 8 / 917: 03$ & Auto FIC & 412 & Good & \\
\hline $12 / 9 / 910: 00$ & Manual Tape & 412.00 & Transcribed & \\
\hline $12 / 9 / 917: 03$ & Auto FIC & 412.1 & Good & \\
\hline 12/10/91 0:00 & Manual Tape & 411.5 & Transeribed & \\
\hline 12/10/91 7:03 & Auto FIC & 412.00 & Good & \\
\hline 12/11/910:00 & Manual Tape & 412 & Transcribed & \\
\hline $12 / 11 / 91 \quad 7: 02$ & Auto FIC & 412 & Good & \\
\hline $12 / 12 / 910: 00$ & Manual Tape & 412.00 & Transcribed & \\
\hline $12 / 12 / 917: 03$ & Auto FIC & 412.00 & Good & \\
\hline $12 / 13 / 910: 00$ & Manual Tape & 412.25 & Transcribed & \\
\hline $12 / 13 / 917: 05$ & Auto FIC & 412 & Good & \\
\hline $12 / 14 / 91 \quad 0: 00$ & Manual Tape & 412.25 & Transcribed & \\
\hline $12 / 14 / 917: 02$ & Auto FIC & 412.00 & Good & \\
\hline $12 / 15 / 91 \quad 0: 00$ & Manual Tape &.$\quad 412.25$ & Transcribed & \\
\hline $12 / 15 / 917: 02$ & Auto FIC & 412.00 & Good & \\
\hline $12 / 16 / 910: 00$ & Manual Tape & 412.00 & Transcribed & \\
\hline $12 / 16 / 917: 03$ & Auto FIC & 412.00 & Good & \\
\hline $12 / 17 / 910: 00$ & Manual Tape & 412 & Transcribed & \\
\hline $12 / 17 / 917: 06$ & Auto FIC & 412.00 & Good & \\
\hline $12 / 18 / 910: 00$ & Manual Tape & 412.25 & Transcribed & \\
\hline $12 / 18 / 917: 03$ & Auto FIC & 412 & Good & \\
\hline $12 / 19 / 910: 00$ & Manual Tape & 412.25 & Transcribed & \\
\hline $12 / 19 / 917: 03$ & Auto FIC & 412.00 & . Good & \\
\hline $12 / 20 / 910: 00$ & Manual Tape & $412.25^{\circ}$ & Transcribed & \\
\hline $12 / 20 / 917: 02$ & Auto FIC & 412.00 & Good & \\
\hline $12 / 21 / 910: 00$ & Manual Tape & 412.25 & Transcribed & \\
\hline $12 / 21 / 917: 03$ & Auto FIC & 412.00 & Good & \\
\hline $12 / 22 / 910: 00$ & Manual Tape & 412.25 & Transcribed & \\
\hline $12 / 22 / 917: 05$ & Auto FIC & 412 & Good & \\
\hline $12 / 23 / 910: 00$ & Manual Tape & 412.25 & Transcribed & \\
\hline $12 / 23 / 917: 06$ & Auto FIC & . 412.00 & Good & \\
\hline $12 / 24 / 910: 00$ & Manual Tape & 412.25 & Transcribed & \\
\hline $12 / 24 / 917: 03$ & Auto FIC & 412.00 & Good & \\
\hline $12 / 25 / 910: 00$ & Manual Tape & 412.25 & Transcribed & \\
\hline $12 / 25 / 917: 03$ & Auto FIC & 412 & Good & \\
\hline 12/26/91 0:00 & Manual Tape & 412.25 & Transcribed & \\
\hline $12 / 26 / 917: 03$ & Auto FIC & 412.00 & Good & \\
\hline
\end{tabular}

Data obtained from WHC Surveillance Analysis Computer System, (PC SACS) July 7, 1995. 


\begin{tabular}{|c|c|c|c|c|}
\hline \multicolumn{5}{|c|}{ 241-AP-106 } \\
\hline \multicolumn{5}{|c|}{ Surface Levol Data } \\
\hline Date & Type of Measurement & Level (Inches). & Data Quality & Comments \\
\hline $12 / 27 / 910: 00$ & Manual Tape & 412.25 & Transcribed & \\
\hline $12 / 27 / 917: 03$ & Auto FIC & 412.00 & Good & \\
\hline $12 / 28 / 910: 00$ & Manual Tape & 412.25 & Transcribed & \\
\hline $12 / 28 / 917: 02$ & Auto FIC & 412.00 & Good & \\
\hline $12 / 29 / 910: 00$ & Manual Tape & 412.25 & Transcribed & \\
\hline $12 / 29 / 91 \quad 7: 03$ & Auto FIC & 412 & Good & \\
\hline $12 / 30 / 910: 00$ & Manual Tape & 412 & Transcribed & \\
\hline $12 / 30 / 917: 03$ & Auto FIC & 412 & Good & \\
\hline $12 / 31 / 910: 00$ & Manual Tape & 412.00 & Transcribed & \\
\hline $12 / 31 / 917: 03$ & Auto FIC & 412 & Good & \\
\hline $1 / 1 / 920: 00$ & Manual Tape & 412.00 & Transcribed & \\
\hline $1 / 1 / 927: 03$ & Auto FIC & 412 & Good & \\
\hline $1 / 2 / 920: 00$ & Manual Tape & 412.25 & Transcribed & \\
\hline $1 / 2 / 927: 02$ & Auto FIC & 411.90 & Good & \\
\hline $1 / 3 / 920: 00$ & Manual Tape & 412.00 & Transcribed & \\
\hline $1 / 3 / 927: 04$ & Auto FIC & 412.00 & Good & \\
\hline $1 / 4 / 920: 00$ & Manual Tape & 412 & Transcribed & \\
\hline $1 / 4 / 927: 03$ & Auto FIC & 412.00 & Good & \\
\hline $1 / 5 / 920: 00$ & Manual Tape & 412.00 & Transcribed & \\
\hline $1 / 5 / 927: 03$ & Auto FIC & 412.00 & Good & \\
\hline $1 / 6 / 920: 00$ & Manual Tape & 41.1 .75 & Transcribed & \\
\hline 1/6/92 7:03 & Auto FIC & 412 & Good & \\
\hline $1 / 7 / 920: 00$ & Manual Tape & 411.5 & Transcribed & \\
\hline $1 / 7 / 927: 04$ & Auto FIC & 411.9 & Good & \\
\hline $1 / 8 / 920: 00$ & Manual Tape & 411.50 & Transcribed & \\
\hline $1 / 8 / 927: 03$ & Auto FIC & 411.90 & Good & \\
\hline 1/9/92 0:00 & Manual Tape & 411.75 & Transcribed & \\
\hline 1/9/92 7:03 & Auto FIC & 411.90 & Good & \\
\hline $1 / 10 / 920: 00$ & Manual Tape & 411.75 & Transcribed & \\
\hline $1 / 10 / 927: 04$ & Auto FIC & 411.90 & Good & \\
\hline $1 / 11 / 920: 00$ & Manual Tape & 411.75 & Transcribed & \\
\hline 1/11/92 7:04 & Auto FIC & $411.90^{\circ}$ & Good & \\
\hline $1 / 12 / 920: 00$ & Manual FIC & 412.00 & Transcribed & \\
\hline $1 / 12 / 920: 00$ & Manual Tape & 411.75 & Transcribed & \\
\hline $1 / 13 / 920: 00$ & Manual FIC & 411.90 & Transcribed & \\
\hline $1 / 13 / 92 \quad 0: 00$ & Manual Tape & 411.50 & Transcribed & \\
\hline $1 / 14 / 920: 00$ & Manual FIC & 411.90 & Transcribed & \\
\hline $1 / 14 / 920: 00$ & Manual Tape & 411.5 & Transcribed & \\
\hline $1 / 15 / 92 \quad 0: 00$ & Manual Tape & 411.50 & Transcribed & \\
\hline $1 / 15 / 927: 03$ & Auto FIC & 411.9 & Good & \\
\hline $1 / 16 / 920: 00$ & Manual Tape & 411.75 & Transcribed & \\
\hline $1 / 16 / 927: 04$ & Auto FIC & 411.9 & Good & \\
\hline $1 / 17 / 920: 00$ & Manual Tape & 411.75 & Transcribed & \\
\hline $1 / 17 / 927: 04$ & Auto FIC & 411.90 & Good & \\
\hline $1 / 18 / 920: 00$ & Manual Tape & 412 & Transcribed & \\
\hline $1 / 18 / 927: 04$ & Auto FIC & 411.9 & Good & \\
\hline $1 / 19 / 920: 00$ & Manual Tape & 411.25 & Transcribed & \\
\hline $1 / 19 / 927: 03$ & Auto FIC & 411.9 & Good & \\
\hline
\end{tabular}

Data obtained from WHC Surveillance Analysis Computer System, (PC SACS) July 7, 1995. 


\begin{tabular}{|c|c|c|c|c|}
\hline \multicolumn{5}{|c|}{ 241-AP-106 } \\
\hline \multicolumn{5}{|c|}{ Surface Level Data } \\
\hline Date & Type of Measurement & Level (Inches) & Data Quality & Comments \\
\hline $1 / 20 / 920: 00$ & Manual Tape & $41.2: 00$ & Transcribed & \\
\hline $1 / 20 / 927: 03$ & Auto FIC & 411.90 & Good & \\
\hline $1 / 21 / 920: 00$ & Manual Tape & 412 & Transcribed & \\
\hline $1 / 21 / 927: 03$ & Auto FIC & 411.90 & Good & \\
\hline $1 / 22 / 920: 00$ & Manual Tape & 412 & Transcribed & \\
\hline $1 / 22 / 927: 06$ & Auto FIC & 411.9 & Good & \\
\hline $1 / 23 / 920: 00$ & Manual Tape & 412.00 & Transcribed & \\
\hline $1 / 23 / 927: 03$ & Auto FIC & 411.90 & Good & \\
\hline $1 / 24 / 920: 00$ & Manual Tape & 412 & Transcribed & . \\
\hline $1 / 24 / 927: 02$ & Auto FIC & 411.90 & Good & . \\
\hline $1 / 25 / 920: 00$ & Manual Tape & 412.25 & Transcribed & \\
\hline $1 / 25 / 927: 03$ & Auto FIC & 411.9 & Good & \\
\hline $1 / 26 / 920: 00$ & Manual Tape & 412.25 & Transcribed & \\
\hline $1 / 26 / 926: 03$ & Auto FIC & 411.8 & Good & \\
\hline $1 / 27 / 920: 00$ & Manual Tape & 4.12 .00 & Transcribed & \\
\hline $1 / 27 / 927: 02$ & Auto FIC & 411.90 & Good & \\
\hline $1 / 28 / 920: 00$ & Manual Tape & 412.00 & Transcribed & \\
\hline $1 / 28 / 927: 03$ & Auto FIC & 41.1 .90 & Good & \\
\hline $1 / 29 / 920: 00$ & Manual Tape & 411.50 & Transcribed & \\
\hline $1 / 29 / 927: 03$ & Auto FIC & 411.9 & Good & . \\
\hline $1 / 30 / 920: 00$ & Manual Tape & 411.75 & Transcribed & \\
\hline $1 / 30 / 927: 03$ & Auto FIC & 411.80 & Good & \\
\hline $1 / 31 / 920: 00$ & Manual Tape & 411.75 & Transcribed & \\
\hline $1 / 31 / 927: 03$ & Auto FIC & 411.8 & Good & \\
\hline $2 / 1 / 920: 00$ & Manual Tape & 411.75 & Transcribed & \\
\hline $2 / 1 / 927: 02$ & Auto FIC & 411.8 & Good & \\
\hline $2 / 2 / 920: 00$ & Manual Tape & 412 & Transcribed & \\
\hline $2 / 2 / 92.7: 03$ & Auto FIC & 411.90 & $\therefore$ Good & \\
\hline $2 / 3 / 920: 00$ & Manual Tape & 412.00 & Transcribed & \\
\hline 2/3/92 7:03 & Auto FIC & 411.8 & Good & \\
\hline $2 / 4 / 920: 00$ & Manual Tape & 412 & Transcribed & \\
\hline 2/4/92 7:03 & Auto FIC & 411.90 & Good & \\
\hline $2 / 5 / 920: 00$ & Manual Tape & 411.75 & Transcribed & \\
\hline $2 / 5 / 927: 02$ & Auto FIC & 411.80 & Good & \\
\hline $2 / 6 / 920: 00$ & Manual Tape & 411.5 & Transcribed & \\
\hline $2 / 6 / 920: 00$ & Manual FIC & 412.00 & Good & \\
\hline $2 / 7 / 920: 00$ & Manual FIC & 411.8 & Transcribed & \\
\hline $2 / 7 / 920: 00$ & Manual Tape & 411.75 & Transcribed & \\
\hline $2 / 8 / 920: 00$ & Manual Tape & 411.75 & Transcribed & \\
\hline $2 / 8 / 920: 00$ & Manual FIC & 411.9 & Good & \\
\hline $2 / 8 / 927: 04$ & Auto FIC & 411.80 & Good & \\
\hline $2 / 9 / 920: 00$ & Manual Tape & 411.75 & Transcribed & \\
\hline $2 / 9 / 927: 03$ & Auto FIC & 411.8 & Good & \\
\hline 2/10/92 0:00 & Manual Tape & 411.75 & Transcribed & \\
\hline $2 / 10 / 92 \quad 7: 04$ & Auto FIC & 411.8 & Good & \\
\hline $2 / 11 / 920: 00$ & Manual Tape & 412 & Transcribed & \\
\hline $2 / 11 / 927: 03$ & Auto FIC & 411.8 & Good & \\
\hline $2 / 12 / 920: 00$ & Manual Tape & 411.25 & Transcribed & \\
\hline
\end{tabular}

Data obtained from WHC Surveillance Analysis Computer System, (PC SACS) July 7, 1995. 


\begin{tabular}{|c|c|c|c|c|}
\hline \multicolumn{5}{|c|}{ 241-AP-106 } \\
\hline \multicolumn{5}{|c|}{ Surface Level Data } \\
\hline Date & Type of Measurement & Levol (lnches) & Data Qualfy & Comments \\
\hline $2 / 12 / 927: 03$ & Auto FIC & 411.80 & Good & \\
\hline $2 / 13 / 920: 00$ & Manual Tapo & 412 & Transcribed & \\
\hline 2/13/92 7:03 & Auto FIC & 411.80 & Good & \\
\hline $2 / 14 / 920: 00$ & Manual Tape & 412.00 & Transcribed & \\
\hline $2 / 14 / 927: 03$ & Auto FIC & 411.8 & Good & \\
\hline $2 / 15 / 920: 00$ & Manual Tape & 411.75 & Transcribed & \\
\hline 2/15/92 7:03 & Auto FIC & 411.80 & Good & \\
\hline $2 / 16 / 920: 00$ & Manual Tape & 411.75 & Transcribed & \\
\hline $2 / 16 / 927: 03$ & Auto FIC & 411.80 & Good & \\
\hline $2 / 17 / 920: 00$ & Manual Tape & 411.75 & Transcribed & \\
\hline $2 / 17 / 927: 03$ & Auto FIC & 411.80 & Good & \\
\hline $2 / 18 / 920: 00$ & Manual Tape & 411.75 & Transcribed & \\
\hline $2 / 18 / 927: 03$ & Auto FIC & 411.8 & Good & \\
\hline $2 / 19 / 920: 00$ & Manual Tape & 412.00 & Transcribed & \\
\hline 2/19/92 7:03 & Auto FIC & 411.8 & Good & \\
\hline $2 / 20 / 920: 00$ & Manual Tape & 412 & Transcribed & \\
\hline $2 / 20 / 927: 03$ & Auto FIC & 411.8 & Good & \\
\hline $2 / 21 / 920: 00$ & Manual Tape & 412.00 & Transcribed & \\
\hline $2 / 21 / 927: 03$ & Auto FIC & 411.8 & Good & \\
\hline $2 / 22 / 920: 00$ & Manual Tape & 412.00 & Transcribed & \\
\hline $2 / 22 / 927: 03$ & Auto FIC & 411.80 & Good & \\
\hline $2 / 23 / 920: 00$ & Manual Tape & 412.00 & Transcribed & \\
\hline $2 / 23 / 927: 03$ & Auto FIC & 411.80 & Good & \\
\hline $2 / 24 / 920: 00$ & Manual Tape & 412.00 & Transcribed & \\
\hline $2 / 24 / 92 \quad 7: 03$ & Auto FIC & 411.80 & Good & \\
\hline $2 / 25 / 920: 00$ & Manual Tape & 412.00 & Transcribed & \\
\hline $2 / 25 / 927: 04$ & Auto FIC & 411.80 & Good & \\
\hline $2 / 26 / 920: 00$ & Manual Tape & 412 & Transcribed & \\
\hline $2 / 26 / 927: 03$ & Auto FIC & 411.80 & Good & \\
\hline $2 / 27 / 920: 00$ & Manual Tape & 412 & Transcribed & \\
\hline $2 / 27 / 927: 04$ & Auto FIC & 411.80 & Good & \\
\hline $2 / 28 / 920: 00$ & Manual Tape & 411.75 & Transćribed & \\
\hline $2 / 28 / 927: 04$ & Auto FIC & 411.80 & Good & \\
\hline $2 / 29 / 920: 00$ & Manual Tape & 411.75 & Transcribed & \\
\hline $2 / 29 / 927: 03$ & Auto FIC & 411.7 & Good & \\
\hline $3 / 1 / 920: 00$ & Manual Tape & 411.75 & Transcribed & \\
\hline $3 / 1 / 927: 03$ & Auto FIC & 411.80 & Good & \\
\hline 3/2/92 0:00 & Manual Tape & 412.00 & Transcribed & \\
\hline $3 / 2 / 927: 03$ & Auto FIC & 411.70 & Good & \\
\hline $3 / 3 / 920: 00$ & Manual Tape & 412.00 & Transcribed & \\
\hline $3 / 3 / 927: 04$ & Auto FIC & 411.7 & Good & \\
\hline $3 / 4 / 920: 00$ & Manual Tape & 412.00 & Transcribed & \\
\hline $3 / 4 / 927: 04$ & Auto FIC & 411.7 & Good & \\
\hline $3 / 5 / 920: 00$ & Manual Tape & 412 & Transcribed & \\
\hline $3 / 5 / 92$ 7:03 & Auto FIC & 411.8 & Good & \\
\hline $3 / 6 / 920: 00$ & Manual Tape & 412.00 & Transcribed & \\
\hline $3 / 6 / 927: 03$ & Auto FIC & 411.8 & Good & \\
\hline $3 / 7 / 920: 00$ & Manual Tape & 412 & Transcribed & \\
\hline
\end{tabular}

Data obtained from WHC Surveillance Analysis Computer System, (PC SACS) July 7, 1995. 


\begin{tabular}{|c|c|c|c|c|}
\hline \multicolumn{5}{|c|}{ 241-AP-106 } \\
\hline \multicolumn{5}{|c|}{ Surface Level Data } \\
\hline Date & Type of Measurement & Leval (Inches) & Data Quality & Comments \\
\hline $3 / 7 / 927: 02$ & Auto FIC & 411.70 & Good & \\
\hline $3 / 8 / 920: 00$ & Manual Tape & 412 & Transcribed & \\
\hline $3 / 8 / 927: 02$ & Auto FIC & 411.7 & Good & \\
\hline $3 / 9 / 920: 00$ & Manual Tape & 411.25 & Transcribed & \\
\hline $3 / 9 / 927: 02$ & Auto FIC & 411.70 & Good & \\
\hline $3 / 10 / 920: 00$ & Manual Tape & 411.5 & Transcribed & \\
\hline $3 / 10 / 927: 03$ & Auto FIC & 411.70 & Good & \\
\hline $3 / 11 / 920: 00$ & Manual Tape & 411.75 & Transcribed & \\
\hline $3 / 11 / 927: 03$ & Auto FIC & 411.70 & Good & . \\
\hline $3 / 12 / 920: 00$ & Manual Tape & 411.50 & Transcribed & \\
\hline $3 / 12 / 927: 03$ & Auto FIC & 411.80 & Good & \\
\hline $3 / 13 / 920: 00$ & Manual Tape & 411.75 & Transcribed & \\
\hline $3 / 13 / 927: 03$ & Auto FIC & 411.70 & Good & \\
\hline $3 / 14 / 920: 00$ & Manual Tape & 411.75 & Transcribed & \\
\hline $3 / 14 / 927: 03$ & Auto FIC & 411.70 & Good & \\
\hline $3 / 15 / 920: 00$ & Manual Tape & 411.75 & Transcribed & \\
\hline $3 / 15 / 92$ 7:06 & Auto FIC & 411.7 & Good & \\
\hline $3 / 16 / 920: 00$ & Manual Tape & 411.75 & Transcribed & \\
\hline $3 / 16 / 927: 03$ & Auto FIC & 411.7 & Good & \\
\hline $3 / 17 / 920: 00$ & Manual Tape & 411.75 & Transcribed & 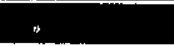 \\
\hline $3 / 17 / 927: 03$ & Auto FIC & 411.70 & Good & \\
\hline $3 / 18 / 920: 00$ & Manual Tape & 411.75 & Transcribed & \\
\hline $3 / 18 / 927: 03$ & Auto FIC & 411.70 & Good & \\
\hline $3 / 19 / 920: 00$ & Manual Tape & 411.75 & Transcribed & \\
\hline $3 / 19 / 926: 02$ & Auto FIC & 411.70 & Good & \\
\hline $3 / 20 / 920: 00$ & Manual Tape & 411.75 & Transcribed & \\
\hline 3/20/92 7:02 & Auto FIC & 411.70 & Good & \\
\hline $3 / 21 / 920: 00$ & Manual Tape & 411.75 & Transcribed & \\
\hline $3 / 21 / 927: 03$ & Auto FIC & 411.70 & Good & \\
\hline $3 / 22 / 92 \quad 0: 00$ & Manual Tape & 411.75 & Transcribed & \\
\hline $3 / 22 / 927: 02$ & Auto FIC & 411.70 & Good & \\
\hline $3 / 23 / 920: 00$ & Manual Tape & 411.75 & Transcribed & \\
\hline $3 / 23 / 927: 02$ & Auto FIC & 411.70 & Good & \\
\hline $3 / 24 / 920: 00$ & Manual Tape & 411.75 & Transcribed & \\
\hline $3 / 24 / 927: 02$ & Auto FIC & 411.7 & Good & \\
\hline $3 / 25 / 920: 00$ & Manual Tape & 412 & Transcribed & \\
\hline $3 / 25 / 927: 03$ & Auto FIC & 411.6 & Good & \\
\hline $3 / 26 / 920: 00$ & Manual Tape & 41.2 .00 & Transcribed & \\
\hline $3 / 26 / 927: 03$ & Auto FIC & 411.6 & Good & \\
\hline $3 / 27 / 920: 00$ & Manual Tape & 411.75 & Transcribed & \\
\hline $3 / 27 / 927: 02$ & Auto FIC & 411.70 & Good & \\
\hline $3 / 28 / 920: 00$ & Manual Tape & 411.75 & Transcribed & \\
\hline $3 / 28 / 927: 03$ & Auto FIC & 411.60 & Good & \\
\hline $3 / 29 / 92$ 0:00 & Manual Tape & 411.75 & Transcribed & \\
\hline $3 / 29 / 927: 03$ & Auto FIC & 411.60 & Good & \\
\hline $3 / 30 / 920: 00$ & Manual Tape & 411.75 & Transćribed & \\
\hline $3 / 30 / 927: 03$ & Auto FIC & 411.6 & Good & \\
\hline $3 / 31 / 920: 00$ & Manual Tape & 411.75 & Transcribed & \\
\hline
\end{tabular}

Data obtained from WHC Surveillance Analysis Computer System, (PC SACS) July 7, 1995. 


\begin{tabular}{|c|c|c|c|c|}
\hline \multicolumn{5}{|c|}{ 241-AP-108 } \\
\hline \multicolumn{5}{|c|}{ Surface Level Data } \\
\hline Date & Type of Measurement & Lovel (Inches) & Data Quality & Comments \\
\hline $3 / 31 / 927: 03$ & Auto FIC & 411.7 & Good & \\
\hline $4 / 1 / 920: 00$ & Manual Tape & 411.75 & Transcribed & \\
\hline 4/1/92 7:03 & Auto FIC & 411.6 & Good & \\
\hline $4 / 2 / 920: 00$ & Manual Tape & 412.00 & Transcribed & \\
\hline $4 / 2 / 927: 03$ & Auto FIC & 411.7 & Good & \\
\hline $4 / 3 / 920: 00$ & Manual Tape & 411.75 & Transcribed & \\
\hline $4 / 3 / 927: 03$ & Auto FIC & 411.60 & Good & \\
\hline $4 / 4 / 920: 00$ & Manual Tape & 411.75 & Transcribed & \\
\hline $4 / 4 / 927: 03$ & Auto FIC & 411.7 & Good & \\
\hline $4 / 5 / 920: 00$ & Manual Tape & 411.75 & Transcribed & \\
\hline $4 / 5 / 927: 03$ & Auto FIC & 411.60 & Good & \\
\hline $4 / 6 / 920: 00$ & Manual Tape & 411.75 & Transcribed & \\
\hline $4 / 6 / 927: 03$ & Auto FIC & 411.6 & Good & \\
\hline $4 / 7 / 920: 00$ & Manual Tape & 411.25 & Transcribed & \\
\hline $4 / 7 / 927: 03$ & Auto FIC & 411.60 & Good & \\
\hline $4 / 8 / 920: 00$ & Manual Tape & 411.25 & Transcribed & \\
\hline $4 / 8 / 925: 02$ & Auto FIC & 411.6 & Good & \\
\hline $4 / 9 / 920: 00$ & Manual Tape & 408.25 & Transcribed & \\
\hline $4 / 9 / 927: 03$ & Auto FIC & 411.6 & Good & \\
\hline $4 / 10 / 920: 00$ & Manual Tape & 411.25 & Transcribed & \\
\hline $4 / 10 / 927: 03$ & Auto FIC & 411.6 & Good & \\
\hline $4 / 11 / 920: 00$ & Manual Tape & 411.75 & Transcribed & \\
\hline $4 / 11 / 927: 03$ & Auto FIC & 411.6 & Good & \\
\hline $4 / 12 / 920: 00$ & Manual Tape & 411.75 & Transcribed & \\
\hline $4 / 12 / 925: 03$ & Auto FIC & 411.60 & Good & \\
\hline $4 / 13 / 920: 00$ & Manual Tape & 411.75 & Transcribed & \\
\hline $4 / 13 / 927: 03$ & Auto FIC & 411.60 & Good & \\
\hline $4 / 14 / 920: 00$ & Manual Tape & 411.75 & Transcribed & \\
\hline 4/14/92 7:03 & Auto FIC & 411.6 & Good & \\
\hline $4 / 15 / 920: 00$ & Manual Tape & 411.75 & Transcribed & \\
\hline $4 / 15 / 927: 02$ & Auto FIC & 411.60 & Good & \\
\hline $4 / 16 / 920: 00$ & Manual Tape & 411.75 & Transcribed & \\
\hline 4/16/92 7:03. & Auto FIC & 411.60 & Good & \\
\hline $4 / 17 / 920: 00$ & Manual Tape & 411.75 & Transcribed & \\
\hline 4/17/92 7:03 & Auto FIC & 411.6 & Good & \\
\hline $4 / 18 / 920: 00$ & Manual Tape & 411.75 & Transcribed & \\
\hline $4 / 18 / 927: 02$ & Auto FIC & $411.60^{\circ}$ & Good & \\
\hline $4 / 19 / 92 \quad 0: 00$ & Manual Tape & 411.75 & Transcribed & \\
\hline $4 / 19 / 927: 02$ & Auto FIC & 411.6 & Good & \\
\hline $4 / 20 / 920: 00$ & Manual Tape & 411.75 & Transcribed & \\
\hline $4 / 20 / 927: 03$ & Auto FIC & 411.60 & Good & \\
\hline $4 / 21 / 920: 00$ & Manual Tape & 411.75 & Transcribed & \\
\hline 4/21/92 7:03 & Auto FIC & 411.60 & Good & \\
\hline $4 / 22 / 92 \quad 0: 00$ & Manual Tape & . 411.50 & Transcribed & \\
\hline $4 / 22 / 927: 02$ & Auto FIC & 411.6 & Good & \\
\hline 4/23/92 0:00 & Manual Tape & 411.75 & Transcribed & \\
\hline $4 / 23 / 927: 03$ & Auto FIC & 411.60 & Good & \\
\hline $4 / 24 / 92 \quad 0: 00$ & Manual Tape & 411.75 & Transcribed & \\
\hline
\end{tabular}

Data obtained from WHC Surveillance Analysis Computer System, (PC SACS) July 7, 1995. 


\begin{tabular}{|c|c|c|c|c|}
\hline \multicolumn{5}{|c|}{$\frac{\text { 241-AP-106 }}{\text { Surface Levol Data }}$} \\
\hline Date & Type of Measurement & Leval (Inches) & Data Quality & Comments \\
\hline $4 / 24 / 927: 03$ & Auto FIC & 411.6 & Good & \\
\hline 4/25/92 0:00 & Manual Tape & 411.5 & Transcribed & \\
\hline $4 / 25 / 927: 04$ & Auto FIC & 411.6 & Good & \\
\hline $4 / 26 / 920: 00$ & Manual Tape & 411.75 & Transcribed & \\
\hline $4 / 26 / 927: 03$ & Auto FIC & 411.50 & Good & \\
\hline $4 / 27 / 920: 00$ & Manual Tape & 410.75 & Transcribed & \\
\hline 4/27/92 7:04 & Auto FIC & 411.60 & Good & \\
\hline $4 / 28 / 920: 00$ & Manual Tape & 411.75 & Transcribed & \\
\hline $4 / 28 / 927: 03$ & Auto FIC & 411.60 & Good & \\
\hline $4 / 29 / 920: 00$ & Manual Tape & 411.75 & Transcribed & \\
\hline $4 / 29 / 927: 03$ & Auto FIC & 411.60 & Good & \\
\hline $4 / 30 / 920: 00$ & Manual Tape & 411.75 & Transcribed & \\
\hline $4 / 30 / 927: 03$ & Auto FIC & 411.50 & Good & \\
\hline $5 / 1 / 920: 00$ & Manual Tape & 411.75 & Transcribed & \\
\hline $5 / 1 / 927: 03$ & Auto FIC & 411.60 & Good & \\
\hline $5 / 2 / 920: 00$ & Manual Tape & 411.75 & Transcribed & \\
\hline $5 / 2 / 922: 03$ & Auto FIC & 411.50 & Good & \\
\hline 5/3/92 0:00 & Manual Tape & 411.75 & Transcribed & \\
\hline $5 / 3 / 927: 03$ & Auto FIC & 411.60 & Good & \\
\hline $5 / 4 / 920: 00$ & Manual Tapo & 411.50 & Transcribed & \\
\hline $5 / 4 / 927: 03$ & Auto FIC & 411.6 & Good & \\
\hline $5 / 5 / 92 \quad 0: 00$ & Manual Tape & 411.5 & Transcribed & \\
\hline $5 / 5 / 92 \quad 7: 04$ & Auto FIC & 411.60 & Good & \\
\hline $5 / 6 / 920: 00$ & Manual Tape & 411.5 & Transcribed & \\
\hline $5 / 6 / 927: 03$ & Auto FIC & 411.5 & - Good & \\
\hline $5 / 7 / 920: 00$ & Manual Tape & $411.5^{\circ}$ & Transcribed & \\
\hline $5 / 7 / 1927: 04$ & Auto FIC & 411.6 & Good & \\
\hline $5 / 8 / 920: 00$ & Manual Tape & 411.5 & Transcribed & $=$ \\
\hline $5 / 8 / 927: 03$ & Auto FIC & 411.5 & - Good & \\
\hline $5 / 9 / 920: 00$ & Manual Tape & 411.50 & Transcribed & \\
\hline $5 / 9 / 92 \quad 0: 00$ & Manual FIC & 411.55 & Good & \\
\hline $5 / 10 / 920: 00$ & Manual Tape & 411.5 & Transcribed & \\
\hline $5 / 10 / 926: 03$ & Auto FIC & 411.60 & Good & \\
\hline 5/11/920:00 & Manual Tape & 411.50 & Transcribed & \\
\hline $5 / 11 / 920: 00$ & Manual FIC & 411.6 & Good & \\
\hline $5 / 12 / 920: 00$ & Manual Tape & 411.75 & Transcribed & \\
\hline $5 / 12 / 927: 03$ & Auto FIC & 411.50 & Good & \\
\hline $5 / 13 / 920: 00$ & Manual Tape & 411.50 & Transcribed & \\
\hline $5 / 13 / 927: 03$ & Auto FIC & 411.5 & Good & \\
\hline $5 / 14 / 920: 00$ & Manual Tape & 411.5 & Transcribed & \\
\hline $5 / 14 / 927: 03$ & Auto FIC & 411.50 & Good & \\
\hline $5 / 15 / 920: 00$ & Manual Tape & 411.25 & Transcribed & \\
\hline $5 / 15 / 927: 03$ & Auto FIC & 411.5 & Good & \\
\hline 5/16/92 0:00 & Manual Tape & 411.5 & Transcribed & \\
\hline $5 / 16 / 927: 03$ & Auto FIC & 411.50 & Good & \\
\hline $5 / 17 / 920: 00$ & Manual Tape & 411.5 & Transcribed & \\
\hline $5 / 17 / 927: 02$ & Auto FIC & 411.50 & Good & \\
\hline $5 / 18 / 920: 00$ & Manual Tape & 411.5 & Transcribed & \\
\hline
\end{tabular}

Data obtained from WHC Surveillance Analysis Computer System, (PC SACS) July 7, 1995. 


\begin{tabular}{|c|c|c|c|c|}
\hline \multicolumn{5}{|c|}{ 241-AP-106 } \\
\hline & & Ufface Level Dat & & \\
\hline Date & Type of Measurement & Leval (Inches) & Data Quality & Comments \\
\hline $5 / 18 / 927: 03$ & Auto FIC & 411.50 & Good & \\
\hline $5 / 19 / 92 \quad 0: 00$ & Manual Tape & 411.75 & Transcribed & \\
\hline $5 / 19 / 927: 03$ & Auto FIC & 411.50 & Good & \\
\hline $5 / 20 / 920: 00$ & Manual Tape & 411.50 & Transcribed & \\
\hline $5 / 20 / 927: 03$ & Auto FIC & 411.50 & Good & \\
\hline $5 / 21 / 920: 00$ & Manual Tape & 411.50 & Transeribed & \\
\hline $5 / 21 / 927: 03$ & Auto FIC & 411.5 & Good & \\
\hline $5 / 22 / 920: 00$ & Manual Tape & 411.50 & Transcribed & \\
\hline $5 / 22 / 927: 02$ & Auto FIC & 411.50 & Good & \\
\hline $5 / 23 / 920: 00$ & Manual Tape & 41.1 .5 & Transcribed & \\
\hline $5 / 23 / 927: 03$ & Auto FIC & 411.5 & Good & \\
\hline $5 / 24 / 920: 00$ & Manual Tape & 411.5 & Transcribed & \\
\hline $5 / 24 / 927: 03$ & Auto FIC & 411.50 & Good & \\
\hline $5 / 25 / 920: 00$ & Manual Tape & 411.5 & Transcribed & \\
\hline $5 / 25 / 927: 03$ & Auto FIC & 411.50 & Good & \\
\hline $5 / 26 / 920: 00$ & Manual Tape & 411.5 & Transcribed & \\
\hline $5 / 26 / 920: 00$ & Manual FIC & 411.50 & Good & \\
\hline $5 / 27 / 920: 00$ & Manual Tape & 411.5 & Transeribed & \\
\hline $5 / 27 / 927: 03$ & Auto FIC & 411.50 & Good & \\
\hline $5 / 28 / 920: 00$ & Manual Tape & 411.75 & Transcribed & \\
\hline $5 / 28 / 927: 02$ & Auto FIC & 411.50 & Good & \\
\hline $5 / 29 / 920: 00$ & Manual Tape & 411.75 & Transcribed & \\
\hline $5 / 29 / 927: 02$ & Auto FIC & $411.50^{\circ}$ & Good & \\
\hline $5 / 30 / 920: 00$ & Manual Tape & 411.5 & Transcribed & \\
\hline $5 / 30 / 927: 03$ & Auto FIC & 411.50 & Good & \\
\hline $5 / 31 / 920: 00$ & Manual Tape & 411.50 & Transcribed & \\
\hline $5 / 31 / 927: 03$ & Auto FIC & 411.5 & Good & \\
\hline $6 / 1 / 92 \quad 0: 00$ & Manual Tape & 411.5 & Transcribed & \\
\hline $6 / 1 / 927: 03$ & Auto FIC & 411.5 & Good & \\
\hline $6 / 2 / 920: 00$ & Manual Tape & 411.50 & Transcribed & \\
\hline $6 / 2 / 927: 03$ & Auto FIC & 411.5 & Good & \\
\hline $6 / 3 / 92 \quad 0: 00$ & Manual Tape & 411.75 & Transcribed & \\
\hline $6 / 3 / 927: 03$ & Auto FIC & 411.50 & Good & \\
\hline $6 / 4 / 920: 00$ & Manual Tape & 411.75 & Transeribed & \\
\hline $6 / 4 / 927: 03$ & Auto FIC & 411.50 & Good & \\
\hline $6 / 5 / 920: 00$ & Manual Tape & 411.75 & Transcribed & \\
\hline $6 / 5 / 927: 02$ & Auto FIC & 411.5 & Good & \\
\hline $6 / 6 / 92 \quad 0: 00$ & Manual Tape & 411.75 & Transcribed & \\
\hline 6/6/92 7:03 & Auto FIC & 411.50 & Good & \\
\hline $6 / 7 / 920: 00$ & Manual Tape & 411.50 & Transcribed & \\
\hline $6 / 7 / 927: 03$ & Auto FIC & 411.5 & Good & \\
\hline 6/8/92 0:00 & Manual Tape & 411.5 & Transcribed & \\
\hline 6/8/92 7:03 & Auto FIC & 411.50 & Good & \\
\hline 6/9/92 0:00 & Manual Tape & 411.5 & Transcribed & \\
\hline $6 / 9 / 927: 03$ & Auto FIC & 411.5 & Good & \\
\hline $6 / 10 / 920: 00$ & Manual Tape & 411.50 & Transcribed & \\
\hline $6 / 10 / 927: 03$ & Auto FIC & 411.50 & Good & \\
\hline $6 / 11 / 920: 00$ & Manual Tape & 411.50 & Transcribed & \\
\hline
\end{tabular}

Data obtained from WHC Surveillance Analysis Computer System, (PC SACS) July 7, 1995. 


\begin{tabular}{|c|c|c|c|c|}
\hline \multicolumn{5}{|c|}{ 241-AP-106 } \\
\hline & & Urface Level Dat & & \\
\hline Date & Type of Moasurement & Level (inches) & Data Quality & Comments \\
\hline $6 / 11 / 927: 03$ & Auto FIC & 411.50 & Good & \\
\hline $6 / 12 / 920: 00$ & Manual Tape & 411.25 & Transcribed & \\
\hline $6 / 12 / 927: 05$ & Auto FIC & 411.50 & Good & \\
\hline $6 / 13 / 920: 00$ & Manual Tape & 411.5 & Transcribed & \\
\hline $6 / 13 / 927: 05$ & Auto FIC & 411.50 & Good & \\
\hline 6/14/92 0:00 & Manual Tape & 411.50 & Transcribed & \\
\hline $6 / 14 / 920: 00$ & Manual FIC & 411.70 & Good & \\
\hline $6 / 15 / 920: 00$ & Manual Tape & 411.50 & Transcribed & \\
\hline $6 / 15 / 927: 03$ & Auto FIC & 411.50 & Good & . \\
\hline $6 / 16 / 920: 00$ & Manual Tape & 411.50 & Transcribed & \\
\hline $6 / 16 / 927: 03$ & Auto FIC & 411.60 & Good & \\
\hline 6/17/92 0:00 & Manual Tape & 411.25 & Transcribed & \\
\hline $6 / 17 / 927: 03$ & Auto FIC & 411.50 & Good & \\
\hline $6 / 18 / 920: 00$ & Manual Tape & 411.50 & Transcribed & \\
\hline $6 / 18 / 927: 03$ & Auto FIC & 411.50 & Good & \\
\hline $6 / 19 / 920.00$ & Manual Tape & 411.50 & Transcribed & \\
\hline $6 / 19 / 927: 02$ & Auto FIC & 411.4 & Good & \\
\hline $6 / 20 / 920: 00$ & Manual Tape & 411.5 & Transcribed. & \\
\hline $6 / 20 / 927: 02$ & Auto FIC & 411.5 & Good & \\
\hline $6 / 21 / 920: 00$ & Manual Tape & 411.5 & Transcribed & - \\
\hline $6 / 21 / 927: 03$ & Auto FIC & 411.5 & Good & \\
\hline $6 / 22 / 920: 00$ & Manual Tape & 411.25 & Transcribed & \\
\hline $6 / 22 / 927: 03$ & Auto FIC & 411.50 & Good & \\
\hline $6 / 23 / 920: 00$ & Manual Tape & 411.50 & Transcribed & \\
\hline $6 / 23 / 927: 03$ & Auto FIC & 411.5 & Good & \\
\hline $6 / 24 / 920: 00$ & Manual Tape & 411.5 & Transcribed & \\
\hline $6 / 24 / 927: 03$ & Auto FIC & 411.5 & Good & \\
\hline $6 / 25 / 920: 00$ & Manual Tape & 411.50 & Transcribed & \\
\hline $6 / 25 / 927: 03$ & Auto FIC & 411.5 & Good & \\
\hline $6 / 26 / 920: 00$ & Manual Tape & 411.5 & Transcribed & \\
\hline $6 / 26 / 927: 02$ & Auto FIC & 411.50 & Good & \\
\hline $6 / 27 / 920: 00$ & Manual Tape & 411.5 & Transcribed & \\
\hline $6 / 27 / 927: 03$ & Auto FIC & 411.50 & Good & \\
\hline $6 / 28 / 920: 00$ & Manual Tape & 411.50 & Transcribed & \\
\hline $6 / 28 / 927: 06$ & Auto FIC & 411.3 & Good & \\
\hline $6 / 29 / 920: 00$ & Manual Tape & 411.5 & Transcribed & \\
\hline $6 / 29 / 927: 06$ & Auto FIC & 411.5 & Good & \\
\hline $6 / 30 / 920: 00$ & Manual Tape & 411.50 & Transcribed & \\
\hline $6 / 30 / 927: 06$ & Auto FIC & 411.5 & Good & \\
\hline $7 / 1 / 920: 00$ & Manual Tape & 411.25 & Transcribed & \\
\hline $7 / 1 / 920: 00$ & Manual FIC & 411.4 & Good & \\
\hline $7 / 2 / 920: 00$ & Manual Tape & 411.25 & Transcribed & \\
\hline $7 / 2 / 920: 00$ & Manual FIC & 411.4 & Good & \\
\hline 7/3/92 0:00 & Manual Tape & 411.5 & Transcribed & \\
\hline $7 / 3 / 927: 06$ & Auto FIC & 411.5 & Good & \\
\hline $7 / 4 / 920: 00$ & Manual Tape & 411.5 & Transcribed & \\
\hline $7 / 4 / 927: 06$ & Auto FIC & 411.40 & Good & \\
\hline $7 / 5 / 920: 00$ & Manual Tape & 411.5 & Transcribed & \\
\hline
\end{tabular}

Data obtained from WHC Surveillance Analysis Computer System, (PC SACS) July 7, 1995. 


\begin{tabular}{|c|c|c|c|c|}
\hline \multicolumn{5}{|c|}{ 241-AP-106 } \\
\hline & & Surface Level Dat & & \\
\hline Date & Type of Measurement & Level (Inches) & Data Quality & Comments \\
\hline 7/5/92 7:06 & Auto FIC & 411.40 & Good & \\
\hline $7 / 6 / 920: 00$ & Manual Tape & 411.75 & Transcribed & \\
\hline 7/6/92 7:06 & Auto FIC & 411.5 & Good & \\
\hline $7 / 7 / 920: 00$ & Manual Tape & 411.75 & Transcribed & \\
\hline $7 / 7 / 927: 06$ & Auto FIC & 411.5 & Good & \\
\hline $7 / 8 / 920: 00$ & Manual Tape & 411.5 & Transcribed & \\
\hline 7/8/92 7:06 & Auto FIC & 411.5 & Good & \\
\hline $7 / 9 / 920: 00$ & Manual Tape & 411.50 & Transcribed & \\
\hline $7 / 9 / 927: 06$ & Auto FIC & 411.50 & Good & \\
\hline $7 / 10 / 920: 00$ & Manual Tape & 411.5 & Transcribed & \\
\hline $7 / 10 / 927: 06$ & Auto FIC & 411.4 & Good & \\
\hline $7 / 11 / 920: 00$ & Manual Tape & 411.50 & Transcribed & \\
\hline $7 / 11 / 927: 06$ & Auto FIC & 411.4 & Good & \\
\hline $7 / 12 / 920: 00$ & Manual Tape & 411.50 & Transcribed & \\
\hline $7 / 12 / 927: 06$ & Auto FIC & 411.4 & Good & \\
\hline $7 / 13 / 920: 00$ & Manual Tape & 411.50 & Transcribed & \\
\hline $7 / 13 / 927: 06$ & Auto FIC & 411.40 & Good & \\
\hline $7 / 14 / 92 \quad 0: 00$ & Manual Tape & 411.50 & Transcribed & \\
\hline $7 / 14 / 927: 06$ & Auto FIC & 411.50 & Good & \\
\hline $7 / 15 / 920: 00$ & Manual Tape & 411.50 & Transcribed & \\
\hline $7 / 15 / 92$ 7:06 & Auto FIC & 411.4 & Good & \\
\hline $7 / 16 / 920: 00$ & Manual Tape & 411.50 & Transcribed & \\
\hline $7 / 16 / 927: 06$ & Auto FIC & 411.4 & Good & \\
\hline $7 / 17 / 920: 00$ & Manual Tape & 411.5 & Transcribed & \\
\hline $7 / 17 / 927: 06$ & Auto FIC & 411.4 & Good & \\
\hline $7 / 18 / 920: 00$ & Manual FIC & 409.75 & Transcribed & \\
\hline $7 / 18 / 920: 00$ & Manual Tape & 411.5 & Transeribed & \\
\hline $7 / 18 / 927: 06$ & Auto FIC & 411.4 & Good & \\
\hline $7 / 19 / 920: 00$ & Manual Tape & 411.5 & Transcribed & \\
\hline $7 / 19 / 927: 06$ & Auto FIC & 411.4 & Good & \\
\hline $7 / 20 / 920: 00$ & Manual FIC & 410 & Transcribed & \\
\hline $7 / 20 / 920: 00$ & Manual Tape & 411.50 & Transcribed & \\
\hline $7 / 20 / 927: 06$ & Auto FIC & 411.4 & Good & \\
\hline $7 / 21 / 920: 00$ & Manual Tape & 411.00 & Transcribed & \\
\hline $7 / 21 / 927: 05$ & Auto FIC & 411.40 & Good & \\
\hline $7 / 22 / 920: 00$ & Manual Tape & 411.25 & Transcribed & \\
\hline $7 / 22 / 927: 06$ & Auto FIC & 411.40 & Good & \\
\hline $7 / 23 / 920: 00$ & Manual Tape & 411.5 & Transcribed & \\
\hline $7 / 23 / 920: 00$ & Manual FIC & 411.40 & Good & \\
\hline $7 / 24 / 920: 00$ & Manual Tape & 411.5 & Transcribed & \\
\hline $7 / 24 / 927: 05$ & Auto FIC & 411.40 & Good & \\
\hline $7 / 25 / 920: 00$ & Manual Tape & 411.50 & Transcribed & \\
\hline $7 / 25 / 927: 06$ & Auto FIC & 411.50 & Good & \\
\hline $7 / 26 / 920: 00$ & Manual Tape & 411.50 & Transcribed & \\
\hline $7 / 26 / 927: 06$ & Auto FIC & 411.5 & Good & \\
\hline $7 / 27 / 920: 00$ & Manual Tape & 411.50 & Transcribed & \\
\hline $7 / 27 / 927: 05$ & Auto FIC & 411.40 & Good & \\
\hline $7 / 28 / 920: 00$ & Manual Tape & 411.50 & Transćribed & \\
\hline
\end{tabular}

Data obtained from WHC Surveillance Analysis Computer Sxstem, (PC SACS) July 7, 1995. 


\begin{tabular}{|c|c|c|c|c|}
\hline \multicolumn{5}{|c|}{ 241-AP-100 } \\
\hline & י & Inface Level Dat & & \\
\hline Date & Type of Measurement & Level (Incheo) & Data Quality & Comments \\
\hline $7 / 28 / 927: 06$ & Auto FIC & 411.40 & Good & \\
\hline $7 / 29 / 920: 00$ & Manual Tape & 411.50 & Transcribed & \\
\hline $7 / 29 / 927: 06$ & Auto FIC & 411.40 & Good & \\
\hline $7 / 30 / 920: 00$ & Manual Tape & 411.50 & Transcribed & \\
\hline $7 / 30 / 927: 06$ & Auto FIC & 411.4 & Good & \\
\hline $7 / 31 / 920: 00$ & Manual Tape & 411.5 & Transcribed & \\
\hline $7 / 31 / 927: 05$ & Auto FIC & 411.4 & Good & \\
\hline $8 / 1 / 920: 00$ & Manual Tape & 411.50 & Transcribed & \\
\hline $8 / 1 / 927: 06$ & Auto FIC & 411.40 & Good & \\
\hline $8 / 2 / 920: 00$ & Manual Tape & 411.5 & Transcribed & \\
\hline $8 / 2 / 927: 06$ & Auto FIC & 411.40 & Good & \\
\hline $8 / 3 / 920: 00$ & Manual Tape & 411.50 & Transcribed & \\
\hline $8 / 3 / 927: 06$ & Auto FIC & 411.40 & Good & \\
\hline $8 / 4 / 920: 00$ & Manual Tape & $411.5^{\circ}$ & Transcribed & \\
\hline 8/4/92 7:06 & Auto FIC & 411.40 & Good & \\
\hline $8 / 5 / 920: 00$ & Manual Tape & 411.5 & Transcribed & \\
\hline $8 / 5 / 927: 05$ & Auto FIC & 411.40 & Good & \\
\hline $8 / 6 / 920: 00$ & Manual Tape & 411.50 & Transcribed & \\
\hline $8 / 6 / 927: 05$ & Auto FIC & 411.40 & Good & \\
\hline $8 / 7 / 920: 00$ & Manual Tape & 411.5 & Transcribed &. \\
\hline $8 / 7 / 927: 05$ & Auto FIC & 411.4 & Good & \\
\hline $8 / 8 / 920: 00$ & Manual Tape & 411.5 & Transcribed & \\
\hline $8 / 8 / 927: 06$ & Auto FIC & 411.4 & Good & \\
\hline $8 / 9 / 920: 00$ & Manual Tape & 411 & Transcribed & \\
\hline $8 / 9 / 927: 06$ & Auto FIC & 411.40 & Good & \\
\hline $8 / 10 / 920: 00$ & Manual Tape & 411.25 & Transcribed & \\
\hline $8 / 10 / 927: 05$ & Auto FIC & 411.40 & Good & \\
\hline $8 / 11 / 920: 00$ & Manual Tape & 411.50 & Transcribed & \\
\hline $8 / 11 / 927: 06$ & Auto FIC & 411.4 & Good & \\
\hline $8 / 12 / 920: 00$ & Manual Tape & 411.5 & Transcribed & \\
\hline $8 / 12 / 927: 06$ & Auto FIC & 411.4 & Good & \\
\hline $8 / 13 / 920: 00$ & Manual Tape & 411.25 & Transcribed & \\
\hline 8/13/92 7:06 & Auto FIC & 411.40 & Good & \\
\hline $8 / 14 / 920: 00$ & Manual Tape & 411.25 & Transcribed & \\
\hline $8 / 14 / 927: 06$ & Auto FIC & 411.40 & Good & \\
\hline $8 / 15 / 92 \quad 0: 00$ & Manual Tape & 411.25 & Transcribed & \\
\hline $8 / 15 / 927: 06$ & Auto FIC & 411.40 & Good & \\
\hline $8 / 16 / 920: 00$ & Manual Tape & 411.25 & Transcribed & \\
\hline $8 / 16 / 927: 06$ & Auto FIC & 411.40 & Good & \\
\hline $8 / 17 / 920: 00$ & Manual Tape & 411.25 & Transcribed & \\
\hline $8 / 17 / 927: 06$ & Auto FIC & 411.40 & Good & \\
\hline $8 / 18 / 920: 00$ & Manual Tape & 411.50 & Transcribed & \\
\hline $8 / 18 / 927: 06$ & Auto FIC & 411.40 & Good & \\
\hline $8 / 19 / 920: 00$ & Manual Tape & 411.25 & Transcribed & \\
\hline $8 / 19 / 927: 06$ & Auto FIC & 411.40 & Good & \\
\hline $8 / 20 / 920: 00$ & Manual Tape & 411.50 & Transcribed & \\
\hline $8 / 20 / 927: 06$ & Auto FIC & 411.40 & Good & \\
\hline $8 / 21 / 920: 00$ & Manual Tape & 411.5 & Transcribed & \\
\hline
\end{tabular}

Data obtained from WHC Surveillance Analysis Computer System, (PC SACS) July 7, 1995. 


\begin{tabular}{|c|c|c|c|c|}
\hline \multicolumn{5}{|c|}{ 241-AP-10B } \\
\hline \multicolumn{5}{|c|}{ Surface Level Data } \\
\hline Date & Type of Measurement & Level (Inchea) & Data Quality & Commonts \\
\hline $8 / 21 / 927: 05$ & Auto FIC & 411.40 & Good & \\
\hline $8 / 22 / 92 \quad 0: 00$ & Manual Tape & 411.50. & Transcribed & \\
\hline $8 / 22 / 927: 04$ & Auto FIC & 411.40 & Good & $\cdot$ \\
\hline $8 / 23 / 920: 00$ & Manual Tape & 411.5 & Transcribed & \\
\hline $8 / 23 / 927: 04$ & Auto FIC & 411.4 & Good & \\
\hline $8 / 24 / 920: 00$ & Manual Tape & 411.5 & Transcribed & \\
\hline $8 / 24 / 927: 04$ & Auto FIC & 411.40 & Good & \\
\hline $8 / 25 / 920: 00$ & Manual Tape & 411.50 & Transeribed & \\
\hline $8 / 25 / 927: 04$ & Auto FIC & 411.4 & Good & \\
\hline $8 / 26 / 920: 00$ & Manual Tape & 411.50 & Transcribed & \\
\hline $8 / 26 / 927: 03$ & Auto FIC & 411.40 & Good & \\
\hline $8 / 27 / 920: 00$ & Manual Tape & 411.25 & Transcribed & \\
\hline $8 / 27 / 927: 04$ & Auto FIC & 411.40 & Good & \\
\hline $8 / 28 / 920: 00$ & Manual Tape & 411.50 & Transcribed & \\
\hline $8 / 28 / 927: 02$ & Auto FIC & 411.40 & Good & \\
\hline $8 / 29 / 920: 00$ & Manual Tape & 411.5 & Transcribed & \\
\hline $8 / 29 / 927: 02$ & Auto FIC & 411.4 & Good & \\
\hline $8 / 30 / 920: 00$ & Manual Tape & 411.5 & Transcribed & \\
\hline $8 / 30 / 927: 02$ & Auto FIC & 411.40 & Good & \\
\hline $8 / 31 / 920: 00$ & Manual Tape & 411.50 & Transcribed & \\
\hline $8 / 31 / 927: 02$ & Auto FIC & 411.40 & Good & \\
\hline $9 / 1 / 920: 00$ & Manual Tape & 411.50 & Transcribed & \\
\hline $9 / 1 / 927: 02$ & Auto FIC & 411.4 & Good & \\
\hline $9 / 2 / 920: 00$ & Manual Tape & 411.5 & Transcribed & \\
\hline $9 / 2 / 927: 02$ & Auto FIC & 411.40 & Good & \\
\hline $9 / 3 / 920: 00$ & Manual Tape & 411.50 & Transcribed & \\
\hline $9 / 3 / 927: 04$ & Auto FIC & 411.4 & Good & \\
\hline $9 / 4 / 920: 00$ & Manuel Tape & 411.5 & Transcribed & \\
\hline $9 / 4 / 927: 04$ & Auto FIC & 411.4 & Good & \\
\hline $9 / 5 / 920: 00$ & Manual Tape & 411.50 & Transcribed & \\
\hline $9 / 5 / 927: 04$ & Auto FIC & 411.30 & Good & \\
\hline $9 / 6 / 920: 00$ & Manual Tape & 411.50 & Transcribed & : \\
\hline $9 / 6 / 927: 04$ & Auto FIC & 411.4 & Good & \\
\hline $9 / 7 / 920: 00$ & Manual Tape & 411.5 & Transcribed & \\
\hline $9 / 7 / 927: 05$ & Auto FIC & 411.30 & Good & \\
\hline $9 / 8 / 920: 00$ & Manual Tape & 411.75. & Transcribed & \\
\hline $9 / 8 / 927: 05$ & Auto FIC & 411.40 & Good & \\
\hline $9 / 9 / 920: 00$ & Manual Tape & 411.50 & Transcribed & \\
\hline $9 / 9 / 927: 04$ & Auto FIC & 411.30 & Good & \\
\hline 9/10/92 0:00 & Manual Tape & 411.50 & Transcribed & \\
\hline $9 / 10 / 927: 03$ & Auto FIC & 411.3 & Good & \\
\hline $9 / 11 / 920: 00$ & Manual Tape & 411.5 & Transeribed & \\
\hline $9 / 11 / 920: 00$ & Manual FIC & 411.3 & Good & \\
\hline $9 / 12 / 920: 00$ & Manual Tape & 411.50 & Transcribed & \\
\hline 9/12/92 0:00 & Manual FIC & 411.3 & Good & \\
\hline $9 / 13 / 920: 00$ & Manual Tape & 411.50 & Transcribed & \\
\hline 9/13/92 0:00 & Manual FIC & 411.30 & Good & \\
\hline $9 / 14 / 920: 00$ & Manual Tape & 411.50 & Transcribed & \\
\hline
\end{tabular}

Data obtained from WHC Surveillance Analysis Computer System, (PC SACS) July 7, 1995. 


\begin{tabular}{|c|c|c|c|c|}
\hline \multicolumn{5}{|c|}{ 241-AP-106 } \\
\hline \multicolumn{5}{|c|}{ Surface Level Data } \\
\hline Date & Type of Measurement & Level (Inchea) & Data Quality & Comments \\
\hline $9 / 14 / 920: 00$ & Manual FIC & 411.30 & Good & \\
\hline $9 / 15 / 920: 00$ & Manual Tape & 411.5 & Transcribed & \\
\hline $9 / 15 / 920: 00$ & Manual FIC & 411.30 & Good & \\
\hline $9 / 16 / 920: 00$ & Manual Tape & 411.5 & Transcribed & \\
\hline $9 / 16 / 920: 00$ & Manual FIC & 411.30 & Good & \\
\hline $9 / 17 / 920: 00$ & Manual Tape & 411.50 & Transcribed & \\
\hline 9/17/92 7:06 & Auto FIC & 411.40 & Good & \\
\hline $9 / 18 / 920: 00$ & Manual Tape & 411.50 & Transcribed & \\
\hline 9/18/92 7:06 & Auto FIC & 411.3 & Good & \\
\hline $9 / 19 / 920: 00$ & Manual Tape & 411.5 & Transcribed & \\
\hline 9/19/92 0:00 & Manual FIC & 411.3 & Good & \\
\hline $9 / 20 / 920: 00$ & Manual Tape & 411.50 & Transcribed & \\
\hline $9 / 20 / 920: 00$ & Manual FIC & 411.40 & Good & \\
\hline $9 / 21 / 920: 00$ & Manual Tape & 411.5 & Transcribed & \\
\hline 9/21/92 7:06 & Auto FIC & $\cdot 411.30$ & Good & \\
\hline $9 / 22 / 920: 00$ & Manual Tape & 411.5 & Transcribed & $\cdot$ \\
\hline $9 / 22 / 927: 03$ & Auto FIC & 411.30 & Good & \\
\hline $9 / 23 / 920: 00$ & Manual Tape & 411.5 & Transcribed & \\
\hline $9 / 23 / 927: 03$ & Auto FIC & 411.3 & Good & \\
\hline 9/24/92 0:00 & Manual Tape & 411.50 & Transcribed & \\
\hline $9 / 24 / 927: 03$ & Auto FIC & 411.3 & Good & \\
\hline $9 / 25 / 920: 00$ & Manual Tape & 411.5 & Transcribed & \\
\hline $9 / 25 / 927: 03$ & Auto FIC & 411.3 & Good & \\
\hline $9 / 26 / 920: 00$ & Manual Tape & 411.50 & Transcribed & \\
\hline $9 / 26 / 927: 02$ & Auto FIC & 411.30 & Good & \\
\hline $9 / 27 / 920: 00$ & Manual Tape & 411.5 & Transcribed & \\
\hline $9 / 27 / 927: 02$ & Auto FIC & 411.30 & Good & \\
\hline 9/28/92 0:00 & Manual Tape & 411.5 & Transcribed & \\
\hline $9 / 28 / 927: 02$ & Auto FIC & 411.30 & Goỏd & \\
\hline $9 / 29 / 920: 00$ & Manual Tape & 411.5 & Transcribed & \\
\hline $9 / 29 / 927: 03$ & Auto FIC & 411.3 & Good & \\
\hline $9 / 30 / 920: 00$ & Manual Tape &.$\quad 411.50$ & Transcribed & \\
\hline $9 / 30 / 927: 02$ & Auto FIC & 411.30 & Good & \\
\hline $10 / 1 / 920: 00$ & Manual Tape & 411.50 & Transcribed & \\
\hline $10 / 1 / 927: 03$ & Auto FIC & 411.3 & Good & \\
\hline $10 / 2 / 920: 00$ & Manual Tape & 411.50 & Transcribed & . \\
\hline $10 / 2 / 927: 02$ & Auto FIC & 411.30 & Good & \\
\hline $10 / 3 / 920: 00$ & Manual Tape & 411.5 & Transcribed & \\
\hline $10 / 3 / 927: 03$ & Auto FIC & 411.3 & Good & \\
\hline $10 / 4 / 920: 00$ & Manual Tape & 411.50 & Transcribed & \\
\hline $10 / 4 / 927: 03$ & Auto FIC & 411.30 & - Good & \\
\hline $10 / 5 / 920: 00$ & Manual Tape & $411.50^{\circ}$ & Transcribed & \\
\hline $10 / 5 / 927: 03$ & Auto FIC & 411.30 & Good & \\
\hline $10 / 6 / 920: 00$ & Manual Tape & 411.50 & Transcribed & \\
\hline $10 / 6 / 927: 03$ & Auto FIC & 411.3 & Good & \\
\hline $10 / 7 / 920: 00$ & Manual FIC & 411.3 & Transcribed & \\
\hline $10 / 7 / 920: 00$ & Manual Tape & 411.25 & Transcribed & \\
\hline $10 / 8 / 920: 00$ & Manual Tape & 411:25 & Transcribed & \\
\hline
\end{tabular}

Data obtained from WHC Surveillance Analysis Computer System, (PC SACS) July 7, 1995. 


\begin{tabular}{|c|c|c|c|c|}
\hline \multicolumn{5}{|c|}{$\therefore \quad 241-A P-106$} \\
\hline \multicolumn{5}{|c|}{ Surface Lavel Data } \\
\hline Date & Type of Measurement & Level (Inchea) & Data Quality & Comments \\
\hline $10 / 8 / 927: 02$ & Auto FIC & 411.3 & Good & \\
\hline $10 / 9 / 920: 00$ & Manual Tape & 411.25 & Transcribed & \\
\hline $10 / 9 / 927: 03$ & Auto FIC & 411.3 & Good & \\
\hline $10 / 10 / 920: 00$ & Manual Tape & 411.50 & Transcribed & \\
\hline $10 / 10 / 927: 03$ & Auto FIC & 411.3 & Good & \\
\hline $10 / 11 / 920: 00$ & Manual Tape & 411.50 & Transcribed & \\
\hline $10 / 11 / 927: 03$ & Auto FIC & 411.30 & Good & \\
\hline $10 / 12 / 920: 00$ & Manual Tape & 411.5 & Transcribed & \\
\hline $10 / 12 / 920: 00$ & Manual FIC & 411.3 & Good & \\
\hline $10 / 13 / 920: 00$ & Manual Tape & 411.50 & Transcribed & \\
\hline $10 / 13 / 920: 00$ & Manual FIC & 411.30 & Good & \\
\hline $10 / 14 / 920: 00$ & Manual Tape & 411.5 & Transcribed & \\
\hline $10 / 14 / 927: 03$ & Auto FIC & 411.30 & Good & \\
\hline $10 / 15 / 920: 00$ & Manual Tape & 411.5 & Transcribed & \\
\hline 10/15/92 7:04 & Auto FIC & 411.2 & Good & \\
\hline $10 / 16 / 920: 00$ & Manual Tape & 411.5 & Transcribed & \\
\hline $10 / 16 / 927: 03$ & Auto FIC & 411.30 & Good & \\
\hline $10 / 17 / 920: 00$ & Manual Tape & 411.5 & Transcribed & \\
\hline $10 / 17 / 927: 03$ & Auto FIC & 411.2 & Good & \\
\hline $10 / 18 / 920: 00$ & Manual Tape & 411.5 & Transcribed & \\
\hline $10 / 18 / 927: 03$ & Auto FIC & .411 .2 & Good & \\
\hline $10 / 19 / 920: 00$ & Manual Tape & 411.50 & Transcribed & \\
\hline $10 / 19 / 927: 03$ & Auto FIC & 411.20 & Good & \\
\hline $10 / 20 / 920: 00$ & Manual Tape & 411.5 & Transcribed & \\
\hline $10 / 20 / 927: 03$ & Auto FIC & 411.2 & Good & \\
\hline $10 / 21 / 920: 00$ & Manual Tape & 411.50 & Transcribed & \\
\hline $10 / 21 / 927: 03$ & Auto FIC & 411.30 & Good & \\
\hline $10 / 22 / 920: 00$ & Manual Tape & 411.5 & Transcribed & \\
\hline $10 / 22 / 920: 00$ & Manual FIC & 411.2 & Good & \\
\hline $10 / 23 / 920: 00$ & Manual Tape & 411.50 & Transcribed & \\
\hline $10 / 23 / 920: 00$ & Manual FIC & 411.30 & Good & \\
\hline $10 / 24 / 920: 00$ & Manual Tape & 411.25 & Transcribed & \\
\hline $10 / 24 / 927: 03$ & Auto FIC & 411.2 & Good & \\
\hline $10 / 25 / 920: 00$ & Manual Tape & 411.50 & Transcribed & \\
\hline $10 / 25 / 927: 03$ & Auto FIC & 411.20 & Good & \\
\hline $10 / 26 / 920: 00$ & Manual Tape & 411.50 & Transcribed & \\
\hline $10 / 26 / 920: 00$ & Manual FIC & 411.2 & Good & \\
\hline $10 / 27 / 920: 00$ & Manual Tape & 411.50 & Transcribed & \\
\hline $10 / 27 / 927: 04$ & Auto FIC & 411.2 & Good & \\
\hline $10 / 28 / 920: 00$ & Manual Tape & 411.5 & Transcribed & \\
\hline $10 / 28 / 927: 03$ & Auto FIC & 411.3 & Good & \\
\hline $10 / 29 / 920: 00$ & Manual Tape & 411.5 & Transcribed & \\
\hline $10 / 29 / 927: 03$ & Auto FIC & 411.2 & Good & \\
\hline $10 / 30 / 920: 00$ & Manual Tape & 411.5 & Transcribed & \\
\hline $10 / 30 / 927: 03$ & Auto FIC & 41.1 .20 & Good & \\
\hline $10 / 31 / 920: 00$ & Manual Tape & 411.50 & Transcribed & \\
\hline $10 / 31 / 927: 03$ & Auto FIC & 411.2 & Good & \\
\hline $11 / 1 / 920: 00$ & Manual Tape & 411.5 & Transcribed & \\
\hline
\end{tabular}

Data obtained from WHC Surveillance Analysis Computer System, (PC SACS) July 7, 1995. 


\begin{tabular}{|c|c|c|c|c|}
\hline \multicolumn{5}{|c|}{ 241-AP-106 } \\
\hline \multicolumn{5}{|c|}{ Surface Laval Data } \\
\hline Date & Type of Measurement & Level flncheal & Data Quality & Comments \\
\hline $11 / 1 / 927: 03$ & Auto FIC & 411.20 & Good & \\
\hline $11 / 2 / 920: 00$ & Manual Tape & 411.5 & Transcribed & \\
\hline $11 / 2 / 927: 03$ & Auto FIC & 411.20 & Good & \\
\hline $11 / 3 / 920: 00$ & Manual Tape & 411.50 & Transcribed & \\
\hline $11 / 3 / 927: 03$ & Auto FIC & 411.20 & Good & \\
\hline $11 / 4 / 920: 00$ & Manual Tape & 411.5 & Transcribed & \\
\hline $11 / 4 / 927: 03$ & Auto FIC & 411.20 & Good & \\
\hline $11 / 5 / 920: 00$ & Manual Tape & 411.5 & Transcribed & \\
\hline $11 / 5 / 920: 00$ & Manual FIC & 411.2 & Good & \\
\hline $11 / 6 / 920: 00$ & Manual Tape & 411.50 & Transcribed & \\
\hline $11 / 6 / 920: 00$ & Manual FIC & 411.20 & Good & \\
\hline $11 / 7 / 920: 00$ & Manual Tape & 411.25 & Transcribed & \\
\hline $11 / 7 / 920: 00$ & Manual FIC & 411.20 & Good & \\
\hline $11 / 8 / 920: 00$ & Manual Tape & 411.25 & Transcribed & \\
\hline $11 / 8 / 920: 00$ & Manual FIC & 411.20 & Good & \\
\hline $11 / 9 / 920: 00$ & Manual Tape & 411.50 & Transcribed & \\
\hline $11 / 9 / 920: 00$ & Manual FIC & 411.2 & Good & \\
\hline $11 / 10 / 920: 00$ & Manual Tape & 411.25 & Transcribed & \\
\hline $11 / 10 / 927: 03$ & Auto FIC & 411.20 & Good & \\
\hline $11 / 11 / 920: 00$ & Manual Tape & 411.25 & Transcribed & \\
\hline $11 / 11 / 927: 04$ & Auto FIC & 411.2 & Good & \\
\hline $11 / 12 / 920: 00$ & Manual Tape & 411.25 & Transcribed & \\
\hline $11 / 12 / 927: 03$ & Auto FIC & 411.20 & Good & \\
\hline $11 / 13 / 920: 00$ & Manual Tape & 411.25 & Transcribed & \\
\hline $11 / 13 / 927: 04$ & Auto FIC & 411.2 & Good & \\
\hline $11 / 14 / 920: 00$ & Manual Tape & 411.50 & Transcribed & \\
\hline $11 / 14 / 927: 04$ & Auto FIC & 411.2 & Good & \\
\hline $11 / 15 / 920: 00$ & Manual Tape & 411.25 & Transcribed & \\
\hline $11 / 15 / 927: 04$ & Auto FIC & 411.20 & Good & . \\
\hline $11 / 16 / 920: 00$ & Manual Tape & 411.25 & Transcribed & \\
\hline $11 / 16 / 92$ 7:04 & Auto FIC & 411.20 & Good & \\
\hline $11 / 17 / 920: 00$ & Manual Tape & 411.25 & Transcribed & \\
\hline $11 / 17 / 927: 04$ & Auto FIC & 411.2 & Good & \\
\hline $11 / 18 / 920: 00$ & Manual Tape & 41.25 & Transcribed & \\
\hline $11 / 18 / 927: 02$ & Auto FIC & 411.2 & Good & \\
\hline $11 / 19 / 92 \quad 0: 00$ & Manual Tape & 411.25 & Transcribed & \\
\hline $11 / 19 / 927: 02$ & Auto FIC & 411.20 & Good & \\
\hline $11 / 20 / 920: 00$ & Manual Tape & 411.25 & Transcribed & \\
\hline $11 / 20 / 927: 03$ & Auto FIC & 411.2 & Good & i \\
\hline $11 / 21 / 920: 00$ & Manual Tape & 411.25 & Transcribed & \\
\hline $11 / 21 / 927: 02$ & Auto FIC & 411.2 & Good & \\
\hline $11 / 22 / 920: 00$ & Manual Tape & 411,25 & Transcribed & \\
\hline $11 / 22 / 927: 03$ & Auto FIC & 411.20 & Good & \\
\hline $11 / 23 / 920: 00$ & Manual Tape & 411.25 & Transcribed & \\
\hline $11 / 23 / 927: 03$ & Auto FIC & 411.2 & Good & \\
\hline $11 / 24 / 920: 00$ & Manual Tape & 411.25 & Transcribed & \\
\hline $11 / 24 / 927: 03$ & Auto FIC & 411.20 & Good & \\
\hline $11 / 25 / 920: 00$ & Manual Tape & 411.25 & Transcribed & \\
\hline
\end{tabular}

Data obtained from WHC Surveillance Analysis Computer System, (PC SACS) July 7, 1995. 


\begin{tabular}{|c|c|c|c|c|}
\hline \multicolumn{5}{|c|}{ 241-AP-106 } \\
\hline \multicolumn{5}{|c|}{ Surface Level Data } \\
\hline Dato & Type of Measurement & Leval (Inches) & Data Quality & Comments \\
\hline $11 / 25 / 927: 03$ & Auto FIC & 411.1 & Good & \\
\hline $11 / 26 / 920: 00$ & Manual Tape & 411.25 & Transcribed & \\
\hline $11 / 26 / 927: 03$ & Auto FIC & 411.2 & Good & \\
\hline $11 / 27 / 920: 00$ & Manual Tape & 411.25 & Transcribed & \\
\hline $11 / 27 / 927: 03$ & Auto FIC & $411: 10$ & Good & \\
\hline $11 / 28 / 920: 00$ & Manual Tape & 411.25 & Transcribed & \\
\hline $11 / 28 / 927: 03$ & Auto FIC & 411.20 & Good. & \\
\hline $11 / 29 / 920: 00$ & Manual Tape & 411.25 & Transcribed & \\
\hline $11 / 29 / 927: 03$ & Auto FIC & 411.20 & . Good & \\
\hline $11 / 30 / 920: 00$ & Manual FIC & 411.1 & Transcribed & \\
\hline $11 / 30 / 920: 00$ & Manual Tape & 411.25 & Transcribed & \\
\hline $12 / 1 / 920: 00$ & Manual Tape & 411.25 & Transcribed & \\
\hline 12/1/92 7:03 & Auto FIC & 411.1 & Good & \\
\hline $12 / 2 / 920: 00$ & Manual Tape & 411.25 & Transcribed & \\
\hline $12 / 2 / 927: 03$ & Auto FIC & 411.20 & Good & \\
\hline $12 / 3 / 920: 00$ & Manual Tape & 411.25 & Transcribed & \\
\hline $12 / 3 / 927: 03$ & Auto FIC & 411.1 & Good & \\
\hline $12 / 4 / 920: 00$ & Manual Tape & 411.25 & Transcribed & \\
\hline $12 / 4 / 927: 03$ & Auto FIC & 411.10 & Good & \\
\hline $12 / 5 / 920: 00$ & Manual Tape & 411.25 & Transcribed & \\
\hline $12 / 5 / 920: 00$ & Manual FIC & 411.10 & Good & \\
\hline $12 / 6 / 920: 00$ & Manual Tape & 411.25 & Transcribed & \\
\hline $12 / 6 / 927: 02$ & Auto FIC & .411 .10 & Good & \\
\hline $12 / 7 / 920: 00$ & Manual Tape & 411.25 & Transcribed & \\
\hline $12 / 7 / 927: 03$ & Auto FIC & 411.2 & Good & \\
\hline $12 / 8 / 920: 00$ & Manual Tape & 411.25 & Transcribed & \\
\hline $12 / 8 / 92 \quad 7: 03$ & Auto FIC & 411.1 & Good & \\
\hline $12 / 9 / 920: 00$ & Manual Tape & 411.25 & Transcribed & \\
\hline $12 / 9 / 927: 03$ & Auto FIC & 411.10 & Good & \\
\hline $12 / 10 / 920: 00$ & Manual Tape & 411.25 & Transeribed & \\
\hline $12 / 10 / 927: 03$ & Auto FIC & 411.1 & Good & \\
\hline $12 / 11 / 920: 00$ & Manual Tape & 411.25 & Transcribed & \\
\hline $12 / 11 / 927: 03$ & Auto FIC & 411.1 & Good & \\
\hline $12 / 12 / 920: 00$ & Manual FIC & 411.1 & Transcribed & \\
\hline $12 / 12 / 920: 00$ & Manual Tape & 411.25 & Transcribed & \\
\hline $12 / 13 / 920.00$ & Manual Tape & 411 & Transcribed & \\
\hline $12 / 13 / 927: 04$ & Auto FIC & 411 & Good & \\
\hline $12 / 14 / 920: 00$ & Manual Tape & 411.00 & Transcribed & \\
\hline $12 / 14 / 927: 03$ & Auto FIC & 411.1 & Good & \\
\hline $12 / 15 / 920: 00$ & Manual Tape & 411 & Transcribed & \\
\hline $12 / 15 / 927: 03$ & Auto FIC & 411.00 & Good & \\
\hline $12 / 16 / 920: 00$ & Manual Tape & 411.25 & Transcribed & \\
\hline $12 / 16 / 927: 04$ & Auto FIC & 411.00 & Good & \\
\hline $12 / 17 / 920: 00$ & Manual Tape & 411.25 & Transcribed & \\
\hline $12 / 17 / 927: 03$ & Auto FIC & 411.00 & Good & \\
\hline $12 / 18 / 920: 00$ & Manual Tape & 411.25 & Transcribed & \\
\hline $12 / 18 / 927: 03$ & Auto FIC & 411.00 & Good & \\
\hline $12 / 19 / 920: 00$ & Manual Tape & 411.25 & Transcribed & \\
\hline
\end{tabular}

Data obtained from WHC Surveillance Analysis Computer System, (PC SACS) July 7, 1995. 


\begin{tabular}{|c|c|c|c|c|}
\hline \multicolumn{5}{|c|}{ 241-AP-106 } \\
\hline & & Iface Level Dat & & \\
\hline Date & Type of Measurement & Leval (inchea) & Data Quality & Comments \\
\hline $12 / 19 / 927: 03$ & Auto FIC & 411.00 & Good & \\
\hline $12 / 20 / 920: 00$ & Manual Tape & 411.25 & Transcribed & \\
\hline $12 / 20 / 927: 04$ & Auto FIC & 411.00 & Good & \\
\hline $12 / 21 / 920: 00$ & Manual Tape & 411.25 & Transcribed & \\
\hline $12 / 21 / 927: 04$ & Auto FIC & 411.00 & Good & \\
\hline $12 / 22 / 920: 00$ & Manual Tape & 411.25 & Transcribed & \\
\hline $12 / 22 / 920: 00$ & Manual FIC & 411.10 & Good & \\
\hline $12 / 23 / 920: 00$ & Manual Tape & 411.25 & Transcribed & \\
\hline $12 / 23 / 927: 04$ & Auto FIC & 411 & Good & \\
\hline $12 / 24 / 92 \quad 0: 00$ & Manual Tape & 411.25 & Transcribed & \\
\hline $12 / 24 / 927: 03$ & Auto FIC & 411.00 & Good & \\
\hline $12 / 25 / 920: 00$ & Manual Tape & 411.25 & Transcribed & \\
\hline $12 / 25 / 927: 03$ & Auto FIC & 411 & Good & \\
\hline $12 / 26 / 920: 00$ & Manual Tape & 411.25 & Transcribed & \\
\hline $12 / 26 / 927: 03$ & Auto FIC & 411.00 & Good & \\
\hline $12 / 27 / 920: 00$ & Manual Tape & 411.25 & Transcribed & \\
\hline $12 / 27 / 927: 03$ & Auto FIC & 411.00 & Good & \\
\hline $12 / 28 / 920: 00$ & Manual Tape & 411.25 & Transcribed & \\
\hline $12 / 28 / 927: 03$ & Auto FIC & 411:00 & Good & \\
\hline $12 / 29 / 920: 00$ & Manual FIC & 411.00 & Transcribed & \\
\hline $12 / 29 / 92 \quad 0: 00$ & Manual Tape & 411. & Transcribed & \\
\hline $12 / 30 / 920: 00$ & Manual Tape & 411 & Transcribed & \\
\hline $12 / 30 / 927: 03$ & Auto FIC & 411.00 & Good & \\
\hline $12 / 31 / 920: 00$ & Manual Tape & 410.75 & Transcribed & \\
\hline $12 / 31 / 927: 03$ & Auto FIC & 411 & Good & \\
\hline $1 / 1 / 930: 00$ & Manual Tape & 411 & Good & \\
\hline 1/1/93 7:04 & Auto FIC & 411,00 & Good & \\
\hline $1 / 2 / 930: 00$ & Auto FIC & 411.00 & Good & \\
\hline $1 / 2 / 930: 00$ & Manual Tape & 411 & Good & \\
\hline $1 / 3 / 930: 00$ & Auto FIC & 411.00 & Good & \\
\hline 1/3/930:00 & Manual Tape & 411.00 & Good & \\
\hline $1 / 4 / 930: 00$ & Auto FIC & 411 & Good & \\
\hline $1 / 4 / 930: 00$ & Manual Tape & 411.00 & Good & \\
\hline 1/5/93 0:00 & Auto FIC & 411 & Good & \\
\hline 1/5/93 0:00 & Manual Tape & 411.00 & Good & \\
\hline $1 / 6 / 930: 00$ & Auto FIC & 411:00 & Good & \\
\hline $1 / 6 / 930: 00$ & Manual Tape & 411 & Good & \\
\hline $1 / 7 / 930: 00$ & Auto FIC & 411.00 & Good & \\
\hline $1 / 7 / 93 \quad 0: 00$ & Manual Tape & 411 & Good & \\
\hline 1/8/93 0:00 & Auto FIC & 411 & Good & \\
\hline $1 / 8 / 930: 00$ & Manual Tape & 411 & Good & \\
\hline 1/9/93 0:00 & Auto FIC & 411.00 & Good & \\
\hline 1/9/93 0:00 & Manual Tape & 411.00 & Good & \\
\hline $1 / 10 / 930: 00$ & Auto FIC & 410.9 & Good & \\
\hline $1 / 10 / 930: 00$ & Manual Tape & 411 & Good & \\
\hline $1 / 11 / 930: 00$ & Auto FIC & 410.9 & Good & \\
\hline $1 / 11 / 930: 00$ & Manual Tape & 411 & Good & \\
\hline 1/12/93 0:00 & Manual Tape & 411 & Good & \\
\hline
\end{tabular}

Data obtained from WHC Surveillance Analysis Computer System, (PC SACS) July 7, 1995. 


\begin{tabular}{|c|c|c|c|c|}
\hline \multicolumn{5}{|c|}{ 241-AP-106 } \\
\hline & & Arface Level Dat & & \\
\hline Date & Type of Measurement & Level (inchoe) & Data Quality & Comments \\
\hline $1 / 12 / 937: 06$ & Auto FIC & 410.9 & Good & \\
\hline 1/13/93 0:00 & Manual Tape & 411.00 & Good & \\
\hline 1/13/93 7:06 & Auto FIC & 410.9 & Good & \\
\hline 1/14/93 0:00 & Manual Tape & 411.00 & Good & \\
\hline $1 / 14 / 937: 03$ & Auto FIC & 410.90 & Good & \\
\hline $1 / 15 / 930: 00$ & Manual Tape & 411.00 & Good & \\
\hline $1 / 15 / 937: 03$ & Auto FIC & 410.90 & Good & \\
\hline $1 / 16 / 930: 00$ & Auto FIC & 410.95 & Good & \\
\hline $1 / 16 / 930: 00$ & Manual Tape & 411.00 & Good & \\
\hline $1 / 17 / 930: 00$ & Auto FIC & 410.90 & Good & \\
\hline $1 / 17 / 930: 00$ & Manual Tape & 411 & Good & \\
\hline $1 / 18 / 930: 00$ & Auto FIC & 411 & Good & \\
\hline $1 / 18 / 930: 00$ & Manual Tape & 411 & Good & \\
\hline $1 / 19 / 930: 00$ & Auto FIC & 410.90 & Good & \\
\hline 1/19/93 0:00 & Manual Tape & 411.00 & Good & \\
\hline $1 / 20 / 930: 00$ & Auto FIC & 410.9 & Good & \\
\hline 1/20/930:00 & Manual Tape & 411.00 & Good & \\
\hline $1 / 21 / 930: 00$ & Manual Tape & 411 & Good & \\
\hline $1 / 21 / 937: 02$ & Auto FIC & 410.9 & Good & \\
\hline 1/22/930:00 & Manual Tape : & 411 & Good & \\
\hline 1/22/93 7:04 & Auto FIC & 410.90 & Good & \\
\hline $1 / 23 / 930: 00$ & Manual Tape & 411.00 & Good & \\
\hline $1 / 23 / 937: 03$ & Auto FIC & 410.90 & Good & \\
\hline $1 / 24 / 930: 00$ & Maqnual Tape & 411.25 & Good & \\
\hline 1/24/93 7:03 & Auto FIC & 410.90 & Good & \\
\hline $1 / 25 / 930: 00$ & Manual Tape & 411.00 & Good & \\
\hline $1 / 25 / 937: 03$ & Auto FIC & 410.90 & Good & \\
\hline $1 / 26 / 930: 00$ & Manual Tape & 411.00 & Good & \\
\hline $1 / 26 / 937: 04$ & Auto FIC & 410.90 & Good & \\
\hline $1 / 27 / 930: 00$ & Manual Tape & 411 & Good & \\
\hline $1 / 27 / 937: 04$ & Auto FIC & 410.90 & Good & \\
\hline $1 / 28 / 930: 00$ & Manual Tape & 411.00 & Good & \\
\hline $1 / 28 / 937: 04$ & Auto FIC & 41.0:90 & Good & \\
\hline $1 / 29 / 930: 00$ & Manual Tape & 411 & Good & \\
\hline $1 / 29 / 937: 04$ & Auto FIC & 410.90 & Good & \\
\hline $1 / 30 / 930: 00$ & Manual Tape & 411.00 & Good & \\
\hline $1 / 30 / 937: 03$ & Auto FIC & 410.90 & Good & \\
\hline $1 / 31 / 930: 00$ & Manual Tape & 411.00 & Good & \\
\hline $1 / 31 / 937: 04$ & Auto FIC & 410.80 & Good & \\
\hline $2 / 1 / 93 \quad 0: 00$ & Manual Tape & 411 & Good & \\
\hline $2 / 1 / 937: 04$ & Auto FIC & 410.8 & Good & \\
\hline 2/2/93 0:00 & Auto FIC & 410.9 & Good & \\
\hline 2/2/93 0:00 & Manual Tape & 411 & Good & \\
\hline 2/2/93 7:04 & Auto FIC & 410.9 & Good & \\
\hline 2/3/93 0:00 & Manual FIC & 411.00 & Good & \\
\hline $2 / 3 / 930: 00$ & Manual Tape & 411.00 & Good & \\
\hline $2 / 4 / 930: 00$ & Manual Tape & 411.00 & Good & \\
\hline $2 / 4 / 937: 04$ & Auto FIC & 410.9 & Good & \\
\hline
\end{tabular}

Data obtained from WHC Surveillance Analysis Computer System, (PC SACS) July 7, 1995. 


\begin{tabular}{|c|c|c|c|c|}
\hline \multicolumn{5}{|c|}{ 241-AP-106 } \\
\hline \multicolumn{5}{|c|}{ Surface Leval Data } \\
\hline Date & Type of Measurement & Leval (Inches) & Data Quality & Comments \\
\hline $2 / 5 / 930: 00$ & Manual Tape & 410.50 & Good & \\
\hline 2/5/93 7:04 & Auto FIC & 410.80 & Good & \\
\hline $2 / 6 / 930: 00$ & Manual Tape & 410.50 & Good & \\
\hline $2 / 6 / 937: 04$ & Auto FIC & 410.9 & Good & \\
\hline $2 / 7 / 930: 00$ & Manual Tape & 410.50 & Good & \\
\hline $2 / 7 / 937: 03$ & Auto FIC & 410.8 & Good & \\
\hline $2 / 8 / 930: 00$ & Manual Tape & 410.50 & Good & \\
\hline $2 / 8 / 937: 04$ & Auto FIC & 410.8 & Good & \\
\hline $2 / 9 / 930: 00$ & Manual Tape & 410.5 & Good & \\
\hline $2 / 9 / 937: 04$ & Auto FIC & .410 .9 & Good & \\
\hline $2 / 10 / 930: 00$ & Manual Tape & 410.75 & Good & \\
\hline $2 / 10 / 937: 04$ & Auto FIC & 410.8 & Good & \\
\hline $2 / 11 / 930: 00$ & Manual Tape & 410.75 & Good & \\
\hline $2 / 11 / 937: 04$ & Auto FIC & 410.8 & Good & \\
\hline 2/12/93 0:00 & Manual Tape & 410.75 & Good & \\
\hline 2/12/93 7:03 & Auto FIC & 410.80 & Good & \\
\hline 2/13/93 0:00 & Manual Tape & 411 & Good & \\
\hline 2/13/93 7:03 & Auto FIC & 410.80 & Good & \\
\hline $2 / 14 / 930: 00$ & Manual Tape & 411.00 & Good & \\
\hline 2/14/93 7:04 & Auto FIC & 410.90 & Good & \\
\hline $2 / 15 / 930: 00$ & Manual Tape & 411 & Good & \\
\hline $2 / 15 / 937: 04$ & Auto FIC & 410.80 & Good & \\
\hline $2 / 16 / 930: 00$ & Manual Tape & 411.00 & Cood & \\
\hline $2 / 16 / 937: 02$ & Auto FIC & 410.9 & Good & \\
\hline $2 / 17 / 930: 00$ & Auto FIC & 410.80 & Good & \\
\hline $2 / 17 / 930: 00$ & Manual Tape & 411 & Good & \\
\hline $2 / 18 / 930: 00$ & Manual Tape & 411.00 & Good & \\
\hline $2 / 18 / 93 \quad 7: 03$ & Auto FIC & 410.8 & Good & \\
\hline 2/19/93 0:00 & Manual Tape & 411 & Good & \\
\hline $2 / 19 / 937: 02$ & Auto FIC & 410.8 & Good & \\
\hline $2 / 20 / 930: 00$ & Manual Tape & 411 & Good & \\
\hline $2 / 20 / 937: 03$ & Auto FIC & 410.80 & Good & \\
\hline $2 / 21 / 930: 00$ & Manual Tape & 411.00 & Good & \\
\hline 2/21/93 7:04 & Auto FIC & 410.8 & Good & \\
\hline $2 / 22 / 930: 00$ & Manual Tape & 411.00 & Good & \\
\hline $2 / 22 / 937: 04$ & Auto FIC & 410.80 & Good & \\
\hline $2 / 23 / 930: 00$ & Manual Tape & 410.00 & Good & . \\
\hline $2 / 23 / 937: 03$ & Auto FIC & 410.80 & Good & \\
\hline $2 / 24 / 930: 00$ & Manual Tape & 410 & Good & \\
\hline $2 / 24 / 937: 04$ & Auto FIC & 410.80 & Good & \\
\hline $2 / 25 / 93 \quad 0: 00$ & Manual Tape & 410 & Good & \\
\hline 2/25/93 7:03 & Auto FIC & 410.80 & Good & \\
\hline 2/26/93 0:00 & Manual Tape & 410.25 & Good & \\
\hline 2/26/93 7:03 & Auto FIC & 410.7 & Good & \\
\hline $2 / 27 / 930: 00$ & Manual Tape & 410 & Good & \\
\hline $2 / 27 / 937: 04$ & Auto FIC & 410.70 & Good & \\
\hline 2/28/93 0:00 & Manual Tape & 410.00 & Good & \\
\hline $2 / 28 / 937: 03$ & Auto FIC & 410.70 & Good & \\
\hline
\end{tabular}

Data obtained from WHC Surveillance Analysis Computer System, (PC SACS) July 7, 1995. 


\begin{tabular}{|c|c|c|c|c|}
\hline \multicolumn{5}{|c|}{ 241-AP-106 } \\
\hline & & Irface Level Dat & & \\
\hline Date & Type of Measurement & Level (Inchea) & Data Quality & Comments \\
\hline 3/1/93 0:00 & Manual Tape & 411 & Good & \\
\hline $3 / 1 / 937: 03$ & Auto FIC & 410.80 & Good & \\
\hline $3 / 2 / 93 \quad 0: 00$ & Manual Tape & 411 & Good & \\
\hline $3 / 2 / 937: 04$ & Auto FIC & 410.80 & Good & \\
\hline $3 / 3 / 93 \quad 0: 00$ & Manual Tape & 410.75 & Good & \\
\hline $3 / 3 / 937: 03$ & Auto FIC & 410.8 & Good & \\
\hline $3 / 4 / 93 \quad 0: 00$ & Manual Tape & 410.75 & Good & \\
\hline $3 / 4 / 937: 04$ & Auto FIC & 410.8 & Good & \\
\hline $3 / 5 / 93 \quad 0: 00$ & Manual Tape & 410.50 & Good & \\
\hline $3 / 5 / 937: 04$ & Auto FIC & 410.8 & Good & \\
\hline $3 / 6 / 930: 00$ & Manual Tape & 410.5 & Good & - \\
\hline $3 / 6 / 937: 03$ & Auto FIC & 410.70 & Good & \\
\hline $3 / 7 / 930: 00$ & Manual Tape & 410.75 & Good & \\
\hline 3/7/93 7:03 & Auto FIC & 410.8 & Good & \\
\hline 3/8/93 0:00 & Manual Tape & 410.75 & Good & \\
\hline $3 / 8 / 93$ 7:03 & Auto FIC & 410.7 & Good & \\
\hline $3 / 9 / 930: 00$ & Manual Tape & 410.75 & Good & \\
\hline $3 / 9 / 937: 04$ & Auto FIC & 410.80 & Gopd & \\
\hline $3 / 10 / 930: 00$ & Manual Tape & 410.75 & Good & \\
\hline $3 / 10 / 93 \quad 7: 05$ & Auto FIC & 410.80 & Good & \\
\hline $3 / 11 / 930: 00$ & Manual Tape & 410.75 & Good & \\
\hline $3 / 11 / 937: 04$ & Auto FIC & 410.7 & Good & \\
\hline $3 / 12 / 930: 00$ & Manual Tape & 410.75 & Good & \\
\hline $3 / 12 / 93 \quad 7: 04$ & Auto FIC & 410.7 & Good & \\
\hline $3 / 13 / 93,0: 00$ & Manual Tape & 410.75 & Good & \\
\hline $3 / 13 / 937: 03$ & Auto FIC & 410.70 & Good & \\
\hline $3 / 14 / 930: 00$ & Manual Tape & 410.75 & Good & \\
\hline $3 / 14 / 937: 04$ & Auto FIC & 410.70 & Good & \\
\hline $3 / 15 / 93 \quad 0: 00$ & Manual Tape & 410.75 & Good & \\
\hline $3 / 15 / 937: 03$ & Auto FIC & 410.7 & Good & \\
\hline $3 / 16 / 930: 00$ & Manual Tape & 411 & Good & \\
\hline $3 / 16 / 937: 04$ & Auto FIC & 410.8 & Good & \\
\hline $3 / 17 / 930: 00$ & Manual Tape & 410.75 & Good & \\
\hline $3 / 17 / 937: 03$ & Auto FIC & 410.7 & Good & \\
\hline 3/18/93 0:00 & Manual Tape & 410.75 & Gapd & \\
\hline $3 / 18 / 937: 04$ & Auto FIC & 410.7 & Good & \\
\hline $3 / 19 / 930: 00$ & Manual Tape & 411.00 & Good & \\
\hline $3 / 19 / 93$ 7:03 & Auto FIC & 410.7 & Good & \\
\hline $3 / 20 / 930: 00$ & Manual Tape & 410.75 & Good & \\
\hline $3 / 20 / 93 \quad 7: 04$ & Auto FIC & 410.7 & Good & \\
\hline $3 / 21 / 930: 00$ & Manual Tape & 410.75 & Good & \\
\hline $3 / 21 / 93$ 7:04 & Auto FIC & 410.70 & Good & \\
\hline $3 / 22 / 93 \quad 0: 00$ & Manual Tape & 410.00 & Good & \\
\hline $3 / 22 / 937: 03$ & Auto FIC & 410.7 & Good & \\
\hline $3 / 23 / 930: 00$ & Manual Tape & 410 & Good & \\
\hline 3/23/93 7:03 & Auto FIC & 410.70 & Good & \\
\hline $3 / 24 / 930: 00$ & Manual Tape & 410.00 & Good & \\
\hline $3 / 24 / 937: 04$ & Auto FIC & 410.7 & Good & \\
\hline
\end{tabular}

Data obtained from WHC Surveillance Analysis Computer System, (PC SACS) July 7, 1995. 


\begin{tabular}{|c|c|c|c|c|}
\hline \multicolumn{5}{|c|}{ 241-AP-106 } \\
\hline \multicolumn{5}{|c|}{ Surface Level Data } \\
\hline Date & Type of Measurement & Levol (Inches) & Data Ouality & Comments \\
\hline $3 / 25 / 930: 00$ & Manual Tape & 410 & Good & \\
\hline 3/25/93 7:03 & Auto FIC & 410.80 & Good & \\
\hline $3 / 26 / 930: 00$ & Manual Tape & 410.00 & Good & \\
\hline $3 / 26 / 937: 03$ & Auto FIC & 410.7 & Good & \\
\hline $3 / 27 / 930: 00$ & Manual Tape & 409.75 & Good & \\
\hline $3 / 27 / 937: 03$ & Auto FIC & 410.7 & Good & \\
\hline $3 / 28 / 930: 00$ & Manual Tape & 409.75 & Good & \\
\hline $3 / 28 / 937: 03$ & Auto FIC & 410.70 & Good & \\
\hline 3/29/93 0:00 & Manual Tape & 410.00 & Good & \\
\hline $3 / 29 / 937: 03$ & Auto FIC & 410.70 & Good & \\
\hline $3 / 30 / 930: 00$ & Manual Tape & 410.25 & Good & \\
\hline $3 / 30 / 937: 03$ & Auto FIC & 410.7 & Good & \\
\hline 3/31/93 0:00 & Manual Tape & 410.25 & Good & \\
\hline $3 / 31 / 937: 03$ & Auto FIC & 410.60 & Good & \\
\hline 4/1/93 7:03 & Auto FIC & 410.6 & Good & \\
\hline $4 / 1 / 9310: 03$ & Manual Tape & 410.75 & Good & \\
\hline $4 / 2 / 937: 06$ & Auto FIC & 410.7 & Good & \\
\hline $4 / 2 / 93 \quad 10: 03$ & Manual Tape & 410.25 & Good & \\
\hline $4 / 3 / 937: 06$ & Auto FIC & 410.70 & Good & \\
\hline $4 / 3 / 9310: 03$ & Manual Tape : & 410.25 & Good & \\
\hline 4/4/93 7:05 & Auto FIC & 410.7 & Good & \\
\hline $4 / 4 / 9310: 03$ & Manual Tape & 410.75 & Good & \\
\hline 4/5/93 7:06 & Auto FIC & 410.60 & Good & \\
\hline $4 / 5 / 9310: 03$ & Manual Tape & 410.50 & Good & \\
\hline $4 / 6 / 937: 06$ & Auto FIC & 410.70 & Good & \\
\hline $4 / 6 / 93 \quad 10: 03$ & Manual Tape & 410.5 & Good & \\
\hline 4/7/93 7:06 & Auto FIC & 410.60 & Good & \\
\hline $4 / 7 / 9310: 03$ & Manual Tape & 410.50 & Good & \\
\hline 4/8/93 7:06 & Auto FIC & 410.60 & Good & \\
\hline $4 / 8 / 9310: 03$ & Manual Tape & 410.5 & Good & \\
\hline $4 / 9 / 937: 06$ & Auto FIC & 410.60 & Good & \\
\hline $4 / 9 / 93 \quad 10: 03$ & Manual Tape & 410.50 & Good & \\
\hline $4 / 10 / 937: 05$ & Auto FIC & 410.6 & Good & \\
\hline 4/10/93 10:03 & Manual Tape & 410.50 & Good & \\
\hline $4 / 11 / 937: 06$ & Auto FiC & 410.6 & Good & \\
\hline $4 / 11 / 93$ 10:03 & Manual Tape & 410.50 & Good & \\
\hline $4 / 12 / 93$ 7:06 & Auto FIC & 410.6 & Good & \\
\hline 4/12/93 10:03 & Manual Tape & 410.50 & Good & \\
\hline $4 / 13 / 937: 06$ & Auto FIC & 410.6 & Good & \\
\hline $4 / 13 / 9310: 03$ & Manual Tape & 410.50 & Good & \\
\hline $4 / 14 / 937: 06$ & Auto FIC & 410.6 & Good & \\
\hline $4 / 14 / 9310: 03$ & Manual Tape & 410.5 & Good & \\
\hline $4 / 15 / 937: 03$ & Auto FIC & 410.6 & Good & \\
\hline $4 / 15 / 93 \quad 10: 03$ & Manual Tape & 410.5 & Good & \\
\hline $4 / 16 / 937: 06$ & Auto FIC & 410.6 & Good & \\
\hline $4 / 16 / 93 \quad 10: 03$ & Manual Tape & 410.5 & Good & \\
\hline $4 / 17 / 937: 05$ & Auto FIC & 410.6 & Good & \\
\hline 4/17/93 10:03 & Manual Tape & 410.50 & Good & \\
\hline
\end{tabular}

Data obtained from WHC Surveillance Analysis Computer System, (PC SACS) July 7, 1995. 


\begin{tabular}{|c|c|c|c|c|}
\hline \multicolumn{5}{|c|}{ 241-AP-106 } \\
\hline & & Ifface Levol Dat & & \\
\hline Date & Type of Measurement & Level (Inchea) & Data Quality & Comments \\
\hline $4 / 18 / 937: 06$ & Auto FIC & 410.60 & Good & \\
\hline $4 / 18 / 93 \quad 10: 03$ & Manual Tape & 410.50 & Good & \\
\hline 4/19/93 7:06 & Auto FIC & 410.6 & Good & \\
\hline 4/19/93 10:03 & Manual Tape & 410.75 & Good & \\
\hline 4/20/930:00 & Manual FIC & 410.60 & Good & \\
\hline $4 / 20 / 9310: 03$ & Manual Tape & 410.75 & Good & \\
\hline $4 / 21 / 930: 00$ & Manual FIC & 410.60 & Good & \\
\hline 4/21/93 10:03 & Manual Tape & 410.75 & Good & \\
\hline 4/22/93 7:06 & Auto FIC & 410.6 & Good & \\
\hline $4 / 22 / 93 \quad 10: 03$ & Manual Tape & 410.5 & Good & \\
\hline $4 / 23 / 937: 06$ & Auto FIC & 410.60 & Good & \\
\hline $4 / 23 / 93 \quad 10: 03$ & Manual Tape & 410.50 & Good & \\
\hline $4 / 24 / 937: 05$ & Auto FIC & 410.60 & Good & \\
\hline $4 / 24 / 93 \quad 10: 03$ & Manual Tape & 410.75 & Good & \\
\hline 4/25/93 7:06 & Auto FIC & 410.70 & Good & \\
\hline $4 / 25 / 93 \quad 10: 03$ & Manual Tape & 410.75 & Good & \\
\hline $4 / 26 / 937: 06$ & Auto FIC & 410.60 & Good & \\
\hline $4 / 26 / 93 \quad 10: 03$ & Manual Tape & 410.75 & Good & \\
\hline 4/27/93 7:06 & Auto FIC & 410.6 & Good & \\
\hline $4 / 27 / 93 \quad 10: 03$ & Manual Tape & 410.75 & Good & \\
\hline $4 / 28 / 937: 03$ & Auto FIC & 470.6 & Good & \\
\hline $4 / 28 / 93 \quad 10: 03$ & Manual Tape & 410.75 & Good & \\
\hline $4 / 29 / 937: 03$ & Auto FIC & 410.60 & Good & \\
\hline $4 / 29 / 93 \quad 10: 03$ & Manual Tape & 410.75 & Good & \\
\hline $4 / 30 / 937: 06$ & Auto FIC & 410.60 & Good & \\
\hline $4 / 30 / 9310: 03$ & Manual Tape & 410.75 & Good & \\
\hline $5 / 1 / 937: 05$ & Auto FIC & 410.6 & Good & \\
\hline $5 / 1 / 9310: 05$ & Manual Tape & 410.5 & Good & \\
\hline $5 / 2 / 937: 06$ & Auto FIC & 410.7 & Good & \\
\hline $5 / 2 / 93 \quad 10: 05$ & Manual Tape & 410.50 & Good & \\
\hline $5 / 3 / 937: 06$ & Auto FIC & 410.60 & Good & \\
\hline $5 / 3 / 93 \quad 10: 05$ & Manual Tape & 410.50 & Good & \\
\hline $5 / 4 / 9310: 05$ & Manual FIC & 410.6 & Good & \\
\hline $5 / 4 / 9310: 05$ & Manual Tape & 410.75 & Good & \\
\hline $5 / 5 / 9310: 05$ & Manual FIC & 410.6 & Good & \\
\hline $5 / 5 / 93 \quad 10: 05$ & Manual Tape & 410.75 & Good & \\
\hline $5 / 6 / 93 \quad 10: 05$ & Manual FIC & 410.6 & Good & \\
\hline $5 / 6 / 93 \quad 10: 05$ & Manual Tape & 410.5 & Good & \\
\hline $5 / 7 / 93 \quad 10: 05$ & Manual FIC & 410.60 & Good & \\
\hline $5 / 7 / 93 \quad 10: 05$ & Manual Tape & 410.75 & Good & \\
\hline $5 / 8 / 93 \quad 10: 05$ & Manual FIC & 410.60 & Good & \\
\hline $5 / 8 / 93 \quad 10: 05$ & Manual Tape & 410.75 & Good & \\
\hline $5 / 9 / 93 \quad 10: 05$ & Manual FIC & 410.6 & Good & \\
\hline $5 / 9 / 93 \quad 10: 05$ & Manual Tape & 410.75 & Good & \\
\hline $5 / 10 / 9310: 05$ & Manual FIC & 410.6 & Good & \\
\hline $5 / 10 / 93 \quad 10: 05$ & Manual Tape & 410.75 & Good & \\
\hline $5 / 11 / 937: 06$ & Auto FIC & 410.6 & Good & \\
\hline $5 / 11 / 93 \quad 10: 05$ & Manual Tape & 410.75 & Good & \\
\hline
\end{tabular}

Data obtained from WHC Surveillance Analysis Computer System, (PC SACS) July 7, 1995. 


\begin{tabular}{|c|c|c|c|c|}
\hline \multicolumn{5}{|c|}{ 241-AP-106 } \\
\hline \multicolumn{5}{|c|}{ Surface Lovol Data } \\
\hline Date & Type of Measurement & Level (Inches) & Date Quality & Comments \\
\hline $5 / 12 / 937: 06$ & Auto FIC & $\therefore \quad 410.6$ & Good & \\
\hline $5 / 12 / 93 \quad 10: 05$ & Manual Tape & 410.75 & Good & \\
\hline $5 / 13 / 9310: 05$ & Manual FIC & 410.55 & Good & \\
\hline $5 / 13 / 9310: 05$ & Manual Tape & 410.75 & Good & \\
\hline $5 / 14 / 93 \quad 10: 05$ & Manual FIC & 410.5 & Good & \\
\hline $5 / 14 / 93 \quad 10: 05$ & Manual Tape & 410.25 & Good & \\
\hline $5 / 15 / 937: 06$ & Auto FIC & 410.60 & Good & \\
\hline $5 / 15 / 93 \quad 10: 05$ & Manual Tape & 410.5 & Good & \\
\hline $5 / 16 / 937: 05$ & Auto FIC & 410.50 & Good & \\
\hline $5 / 16 / 93 \quad 10: 05$ & Manual Tape & 410.5 & Good & \\
\hline $5 / 17 / 937: 03$ & Auto FIC & 410.6 & Good & \\
\hline $5 / 17 / 93 \quad 10: 05$ & Manual Tape & 410.75 & Good & \\
\hline $5 / 18 / 93 \quad 7: 03$ & Auto FIC & 410.6 & Good & \\
\hline $5 / 18 / 93 \quad 10: 05$ & Manual Tape & 410.75 & Good & \\
\hline $5 / 19 / 937: 03$ & Auto FIC & 410.60 & Good & \\
\hline $5 / 19 / 93$ 10:05 & Manual Tape & 410.75 & Good & \\
\hline $5 / 20 / 937: 03$ & Auto FIC & 410.6 & Good & \\
\hline $5 / 20 / 93 \quad 10: 05$ & Manual Tape & 410.75 & Good & \\
\hline $5 / 21 / 930: 00$ & Manual FIC & 410.60 & Good & \\
\hline $5 / 21 / 93 \quad 10: 05$ & Manual Tape & 410.75 & Good & \\
\hline $5 / 22 / 937: 03$ & Auto FIC & 410.50 & Good & \\
\hline $5 / 22 / 9310: 05$ & Manual Tape & 410.75 & Good & \\
\hline $5 / 23 / 937: 04$ & Auto FIC & 410.5 & Good & \\
\hline $5 / 23 / 93 \quad 10: 05$ & Manual Tape & 410.75 & Good & \\
\hline $5 / 24 / 937: 03$ & Auto FIC & 410.6 & Good & \\
\hline $5 / 24 / 93 \quad 10: 05$ & Manual Tape & 410.75 & Good & \\
\hline $5 / 25 / 937: 03$ & Auto FIC & 410.6 & Good & \\
\hline $5 / 25 / 93 \quad 10: 05$ & Manual Tape & 410.75 & Good & \\
\hline $5 / 26 / 937: 03$ & Auto FIC & 410.50 & Good & \\
\hline $5 / 26 / 93 \quad 10: 05$ & Manual Tape & 411 & Good & \\
\hline $5 / 27 / 937: 03$ & Auto FIC & 410.6 & Good & \\
\hline $5 / 27 / 9310: 05$ & Manual Tape & 410.75 & Good & \\
\hline $5 / 28 / 937: 03$ & Auto FIC & 410.60 & Good & \\
\hline $5 / 28 / 93 \quad 10: 05$ & Manual Tape & 410.75 & Good & \\
\hline $5 / 29 / 937: 05$ & Auto FIC & 410.50 & Good & \\
\hline $5 / 29 / 93 \quad 10: 05$ & Manual Tape & 410.75 & Good & \\
\hline $5 / 30 / 937: 05$ & Auto FIC & 410.60 & Good & \\
\hline $5 / 30 / 9310: 05$ & Manual Tape & 410.75 & Good & \\
\hline $5 / 31 / 937: 06$ & Auto FIC & 410.50 & Good & \\
\hline $5 / 31 / 93 \quad 10: 05$ & Manual Tape & 410.75 & Good & \\
\hline $6 / 1 / 937: 06$ & Auto FIC & 410.50 & Good & \\
\hline $6 / 1 / 93 \quad 10: 06$ & Manual Tape & 410.75 & Good & \\
\hline $6 / 2 / 93 \quad 7: 06$ & Auto FIC & 410.60 & Good & \\
\hline $6 / 2 / 9310: 06$ & Manual Tape & 410.50 & Good & \\
\hline $6 / 3 / 93$ 7:06 & Auto FIC & 410.5 & Good & \\
\hline $6 / 3 / 9310: 06$ & Manual Tape & 410.75 & Good & \\
\hline $6 / 4 / 937: 06$ & Auto FIC & 410.7 & Good & \\
\hline $6 / 4 / 93 \quad 10: 06$ & Manual Tape & 410.75 & Good & \\
\hline
\end{tabular}

Data obtained from WHC Surveillance Analysis Computer System, (PC SACS) July 7, 1995. 


\begin{tabular}{|c|c|c|c|c|}
\hline \multicolumn{5}{|c|}{ 241-AP-106 } \\
\hline \multicolumn{5}{|c|}{ Surface Level. Data } \\
\hline Date & Type of Measurement & Leval (Inches) & Data Quality & Comments \\
\hline $6 / 5 / 937: 06$ & Auto FIC & 410.60 & Good & \\
\hline $6 / 5 / 93 \quad 10: 06$ & Manual Tape & 410.75 & Good & \\
\hline $6 / 6 / 937: 05$ & Auto FIC & 410.50 & Good & \\
\hline $6 / 6 / 9310: 06$ & Manual Tape & 410.75 & Good & \\
\hline $6 / 7 / 937: 05$ & Auto FIC & 410.5 & Good & \\
\hline $6 / 7 / 93 \quad 10: 06$ & Manual Tape & 410.75 & Good & \\
\hline $6 / 8 / 937: 05$ & Auto FIC & 410.5 & Good & \\
\hline $6 / 8 / 93 \quad 10: 06$ & Manual Tape & 410.50 & Good & \\
\hline 6/9/93 7:05 & Auto FIC & 410.50 & Good & \\
\hline $6 / 9 / 93 \quad 10: 06$ & Manual Tape & 410.5 & Good & \\
\hline $6 / 10 / 937: 04$ & Auto FIC & 410.50 & Good & \\
\hline $6 / 10 / 9310: 06$ & Manual Tape & 410.50 & Good & \\
\hline $6 / 11 / 937: 05$ & Auto FIC & 410.50 & Good & \\
\hline $6 / 11 / 93 \quad 10: 06$ & Manual Tape & 410.75 & Good & \\
\hline 6/12/93 7:05 & Auto FIC & 410.50 & Good & \\
\hline $6 / 12 / 93 \quad 10: 06$ & Manual Tape & 410.75 & Good & \\
\hline $6 / 13 / 937: 04$ & Auto FIC & 410.60 & Good & \\
\hline $6 / 13 / 93 \quad 10: 06$ & Manual Tape & 410.75 & Good & \\
\hline $6 / 14 / 937: 05$ & Auto FIC & 410.5 & Good & \\
\hline $6 / 14 / 93 \quad 10: 06$ & Manual Tape & 410.5 & Good & \\
\hline $6 / 15 / 937: 05$ & Auto FIC & 410.5 & Good & \\
\hline $6 / 15 / 93 \quad 10: 06$ & Manual Tape & 410.5 & Good & \\
\hline 6/16/93 7:05 & Auto FIC & 410.6 & Good & \\
\hline $6 / 16 / 93$ 10:06 & Manual Tape & 410.50 & Good & \\
\hline $6 / 17 / 937: 05$ & Auto FIC & 410.5 & Good & \\
\hline $6 / 17 / 93 \quad 10: 06$ & Manual Tape & 410.5 & Good & \\
\hline 6/18/93 7:06 & Auto FIC & 410.50 & Good & \\
\hline $6 / 18 / 93 \quad 10: 06$ & Manual Tape & 410.5 & Good & \\
\hline $6 / 19 / 937: 06$ & Auto FIC & 410.5 & Good & \\
\hline $6 / 19 / 93 \quad 10: 06$ & Manual Tape & 410.50 & Good & \\
\hline $6 / 20 / 937: 06$ & Auto FIC & $410: 50$ & Good & \\
\hline $6 / 20 / 93 \quad 10: 06$ & Manual Tape & 410.50 & Good & \\
\hline $6 / 21 / 937: 06$ & Auto FIC & 410.55 & Good & \\
\hline $6 / 21 / 93 \quad 10: 06$ & Manual Tape & 410.50 & Good & \\
\hline $6 / 22 / 937: 06$ & Auto FIC & 410.50 & Good & \\
\hline $6 / 22 / 93 \quad 10: 06$ & Manual Tape & 410.5 & Good & \\
\hline $6 / 23 / 937: 06$ & Auto FIC & 410.5 & Good & \\
\hline $6 / 23 / 93 \quad 10: 06$ & Manual Tape & 410.5 & Good & \\
\hline $6 / 24 / 937: 06$ & Auto FIC & 410.50 & Good & \\
\hline $6 / 24 / 93 \quad 10: 06$ & Manual Tape & 410.50 & Good & \\
\hline $6 / 25 / 937: 03$ & Auto FIC & 410.5 & Good & \\
\hline $6 / 25 / 93 \quad 10: 06$ & Manual Tape & 410.50 & Good & \\
\hline $6 / 26 / 937: 03$ & Auto FIC & 410.5 & Good & \\
\hline $6 / 26 / 9310: 06$ & Manual Tape & 410.50 & Good & \\
\hline $6 / 27 / 937: 04$ & Auto FIC & 410.5 & Good & \\
\hline $6 / 27 / 9310: 06$ & Manual Tape & 410.50 & Good & \\
\hline $6 / 28 / 937: 03$ & Auto FIC & 410.50 & Good & \\
\hline $6 / 28 / 9310: 06$ & Manual Tape & 410.25 & Good & \\
\hline
\end{tabular}

Data obtained from WHC Surveillance Analysis Computer System, (PC SACS) July 7, 1995. 


\begin{tabular}{|c|c|c|c|c|}
\hline \multicolumn{5}{|c|}{ 241-AP-108 } \\
\hline & & urface Levol Dat & & \\
\hline Date & Type of Measurement & Level (Inches) & Data Quality & Comments \\
\hline $6 / 29 / 937: 04$ & Auto FIC & 410.5 & Good & \\
\hline $6 / 29 / 93 \quad 10: 06$ & Manual Tape & 410.5 & Good & \\
\hline $6 / 30 / 937: 03$ & Auto FIC & 410.50 & Good & \\
\hline $6 / 30 / 93 \quad 10: 06$ & Manual Tape & 410.50 & Good & \\
\hline 7/1/93 7:03 & Auto FIC & 410.55 & Good & \\
\hline 7/1/93 10:03 & Manual Tape & 410.75 & Good & \\
\hline $7 / 2 / 937: 03$ & Auto FIC & 410.56 & Good & \\
\hline $7 / 2 / 93 \quad 10: 03$ & Manual Tape & 410.75 & Good & \\
\hline $7 / 3 / 937: 03$ & Auto FIC & 410.55 & Good & \\
\hline $7 / 3 / 93 \quad 10: 03$ & Manual Tape & 410.50 & Good & \\
\hline $7 / 4 / 937: 03$ & Auto FIC & 410.55 & Good & \\
\hline $7 / 4 / 93 \quad 10: 03$ & Manual Tape & 410.50 & Good & \\
\hline $7 / 5 / 937: 03$ & Auto FIC & 410.55 & Good & \\
\hline $7 / 5 / 93 \quad 10: 03$ & Manual Tape & 410.5 & Good & \\
\hline 7/6/93 7:03 & Auto FIC & 410.50 & Good & \\
\hline $7 / 6 / 93 \quad 10: 03$ & Manual Tape & 410.75 & Good & \\
\hline $7 / 7 / 93 \quad 7: 04$ & Auto FIC & 410.5 & Good & \\
\hline $7 / 7 / 93 \quad 10: 03$ & Manual Tape & 410.5 & Good & \\
\hline $7 / 8 / 93 \quad 7: 04$ & Auto FIC & 410.5 & Good & \\
\hline $7 / 8 / 93 \quad 10: 03$ & Manual Tape & 410.75 & Good & \\
\hline 7/9/93 7:05 & Auto FIC & 410.50 & Good & 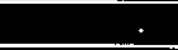 \\
\hline $7 / 9 / 93 \quad 10: 03$ & Manual Tape & 410.75 & Good & \\
\hline $7 / 10 / 937: 03$ & Auto FIC & 410.5 & Good & \\
\hline $7 / 10 / 93 \quad 10: 03$ & Manual Tape & 410.75 & Good & \\
\hline $7 / 11 / 937: 03$ & Auto FIC & 410.50 & Good & \\
\hline $7 / 11 / 93 / 0: 03$ & Manual Tape & 410.75 & Good & \\
\hline $7 / 12 / 937: 02$ & Auto FIC & 410.5 & Good & \\
\hline $7 / 12 / 93 \quad 10: 03$ & Manual Tape & 410.75 & Good & \\
\hline $7 / 13 / 937: 03$ & Auto FIC & 410.5 & Good & \\
\hline $7 / 13 / 9310: 03$ & Manual Tape & 410.75 & Good & \\
\hline 7/14/93 7:06 & Auto FIC & $410.50^{\circ}$ & Good & \\
\hline $7 / 14 / 93 \quad 10: 03$ & Manual Tape & 410.75 & Good & \\
\hline 7/15/93 7:06 & Auto FIC & 410.5 & Good & \\
\hline $7 / 15 / 93 \quad 10: 03$ & Manual Tape & 410.75 & Good & \\
\hline $7 / 16 / 937: 06$ & Auto FIC & 410.50 & Good & \\
\hline $7 / 16 / 93 \quad 10: 03$ & Manual Tape & 410.75 & Good & \\
\hline $7 / 17 / 93 \quad 7: 07$ & Auto FIC & 41.0:60 & Good & \\
\hline $7 / 17 / 93 \quad 10: 03$ & Manual Tape & 410.75 & Good & \\
\hline $7 / 18 / 93 \quad 7: 06$ & Auto FIC & 410.50. & Good & \\
\hline $7 / 18 / 93 \quad 10: 03$ & Manual Tape & 410.75 & Good & \\
\hline $7 / 19 / 937: 03$ & Auto FIC & 410.50 & Good & \\
\hline 7/19/93 10:03 & Manual Tape & 410.75 & Good & \\
\hline $7 / 20 / 937: 03$ & Auto FIC & 410.60 & Good & \\
\hline $7 / 20 / 93 \quad 10: 03$ & Manual Tape & 410.5 & Good & \\
\hline $7 / 21 / 937: 03$ & Auto FIC & 410.5 & Good & \\
\hline $7 / 21 / 93 \quad 10: 03$ & Manual Tape & 410.5 & Good & \\
\hline $7 / 22 / 937: 03$ & Auto FIC & 410.50 & Good & \\
\hline $7 / 22 / 93 \quad 10: 03$ & Manual Tape & 410.5 & Good & \\
\hline
\end{tabular}

Data obtained from WHC Surveillance Analysis Computer System, (PC SACS) July 7, 1995. 


\begin{tabular}{|c|c|c|c|c|}
\hline \multicolumn{5}{|c|}{ 241-AP-106 } \\
\hline \multicolumn{5}{|c|}{ Surface Level Data } \\
\hline Date & Type of Measurement & Leval.(linches) & Data Quality & Comments \\
\hline $7 / 23 / 937: 03$ & Auto FIC & 410.50 & Good & \\
\hline $7 / 23 / 93 \quad 10: 03$ & Manual Tape & 410.5 & Good & \\
\hline $7 / 24 / 937: 03$ & Auto FIC & 410.50 & Good & \\
\hline $7 / 24 / 93 \quad 10: 03$ & Manual Tape . & 410.50 & Good & \\
\hline $7 / 25 / 937: 04$ & Auto FIC & 410.5 & Good & \\
\hline $7 / 25 / 93 \quad 10: 03$ & Manual Tape & 410.50 & Good & \\
\hline $7 / 26 / 937: 03$ & Auto FIC & 410.50 & Good & \\
\hline $7 / 26 / 93 \quad 10: 03$ & Manual Tape & 410.50 & Good & \\
\hline $7 / 27 / 937: 03$ & Auto FIC & 410.50 & Good & \\
\hline $7 / 27 / 93 \quad 10: 03$ & Manual Tape & 410.50 & Good & \\
\hline $7 / 28 / 930: 00$ & Manual Tape & 410.50 & Good & \\
\hline $7 / 28 / 937: 04$ & Auto FIC & 410.50 & Good & \\
\hline $7 / 29 / 930: 00$ & Manual Tape & 410.50 & Good & \\
\hline $7 / 29 / 937: 03$ & Auto FIC & 410.5 & Good & \\
\hline $7 / 30 / 93 \quad 0: 00$ & Manual Tape & 410.50 & Good & \\
\hline $7 / 30 / 937: 03$ & Auto FIC & 410.5 & Good & \\
\hline $7 / 31 / 930: 00$ & Manual Tape & 410.5 & Good & \\
\hline $7 / 31 / 937: 03$ & Auto FIC & 410.50 & Good & \\
\hline $8 / 1 / 93 \quad 0: 00$ & Manual Tape & 410.75 & Good & \\
\hline $8 / 1 / 937: 03$ & Auto FIC & 410.5 & Good & \\
\hline $8 / 2 / 930: 00$ & Manual Tape & 410.75 & Good & \\
\hline $8 / 2 / 937: 06$ & Auto FIC & 410.50 & Good & \\
\hline $8 / 3 / 93 \quad 0: 00$ & Manual Tape & 410.5 & Good & \\
\hline $8 / 3 / 937: 03$ & Auto FIC & 410.5 & Good & \\
\hline $8 / 4 / 930: 00$ & Manual Tape & 410.75 & Good & \\
\hline $8 / 4 / 937: 03$ & Auto FIC & 410.50 & Good & \\
\hline $8 / 5 / 930: 00$ & Manual Tape & 410.75 & Good & \\
\hline $8 / 5 / 937: 04$ & Auto FIC & 410.5 & Good & \\
\hline $8 / 6 / 930: 00$ & Manual Tape & 410.75 & Good & \\
\hline $8 / 6 / 937: 04$ & Auto FIC & 410.50 & Good & \\
\hline $8 / 7 / 930: 00$ & Manual Tape & 409.5 & Good & \\
\hline $8 / 7 / 937: 04$ & Auto FIC & 410.50 & Good & \\
\hline $8 / 8 / 930: 00$ & Manual Tape & 410.25 & Good & \\
\hline $8 / 8 / 937: 04$ & Auto FIC & 410.5 & Good & \\
\hline $8 / 9 / 930: 00$ & Manual Tape & 410.75 & Good & \\
\hline $8 / 9 / 937: 04$ & Auto FIC & 410.50 & Good & \\
\hline $8 / 10 / 930: 00$ & Manual Tape & 410.75 & Good & \\
\hline $8 / 10 / 937: 04$ & Auto FIC & 410.5 & Good & \\
\hline $8 / 11 / 930: 00$ & Manual Tape & 410.75 & Good & \\
\hline $8 / 11 / 937: 04$ & Auto FIC & 410.5 & Good & \\
\hline $8 / 12 / 930: 00$ & Manual Tape & 410.75 & Good & \\
\hline $8 / 12 / 937: 04$ & Auto FIC & 410.5 & Good & \\
\hline $8 / 13 / 930: 00$ & Manual Tape & 410.75 & Good & \\
\hline $8 / 13 / 937: 04$ & Auto FIC & 410.5 & Good & \\
\hline $8 / 14 / 930: 00$ & Manual Tape & 410.75 & Good & \\
\hline $8 / 14 / 937: 03$ & Auto FIC & 410.5 & Good & \\
\hline $8 / 15 / 930: 00$ & Manual Tape & 410.75 & Good & \\
\hline $8 / 15 / 937: 04$ & Auto FIC & 410.50 & Good & \\
\hline
\end{tabular}

Data obtained from WHC Surveillance Analysis Computer System, (PC SACS) July 7, 1995. 


\begin{tabular}{|c|c|c|c|c|}
\hline \multicolumn{5}{|c|}{ 241-AP-106 } \\
\hline \multicolumn{5}{|c|}{ Surface Loval Data } \\
\hline Date & Type of Moasursment & Level (Inches) & Data Quallty & Comments \\
\hline $8 / 16 / 930: 00$ & Manual Tape & 410.75 & Good & \\
\hline $8 / 16 / 937: 04$ & Auto FIC & 410.5 & Good & \\
\hline $8 / 17 / 930: 00$ & Manual Tape & 410.50 & Good & \\
\hline $8 / 17 / 937: 04$ & Auto FIC & 410.50 & Good & \\
\hline $8 / 18 / 930: 00$ & Manual Tape & 410.5 & Good & \\
\hline $8 / 18 / 937: 03$ & Auto FIC & 410.6 & Good & \\
\hline $8 / 19 / 930: 00$ & Manual Tape & 410.5 & Good & \\
\hline $8 / 19 / 937: 04$ & Auto FIC & 410.5 & Good & \\
\hline $8 / 20 / 93 \quad 0: 00$ & Manual Tape & 410.5 & Good & \\
\hline $8 / 20 / 937: 04$ & Auto FIC & 410.6 & Good & \\
\hline $8 / 21 / 930: 00$ & Manual Tape & 410.50 & Good & \\
\hline $8 / 21 / 937: 04$ & Auto FIC & 410.6 & Good & \\
\hline $8 / 22 / 930: 00$ & Manual Tape & 410.5 & Good & \\
\hline $8 / 22 / 937: 04$ & Auto FIC & 410.5 & Good & \\
\hline $8 / 23 / 930: 00$ & Manual Tape & 410.75 & Good & \\
\hline $8 / 23 / 937: 04$ & Auto FIC & $410.6^{\circ}$ & Good & \\
\hline $8 / 24 / 930: 00$ & Manual Tape & 410.75 & Good & \\
\hline $8 / 24 / 937: 04$ & Auto FIC & 410.6 & Good & \\
\hline $8 / 25 / 930: 00$ & Manual Tape & 410.75 & Good & \\
\hline $8 / 25 / 937: 04$ & Auto FIC & 410.70 & Good & \\
\hline $8 / 26 / 930: 00$ & Manual Tape & 410.75 & Good & \\
\hline $8 / 26 / 937: 04$ & Auto FIC & 410.5 & Good & \\
\hline $8 / 27 / 930: 00$ & Manual Tape & . 410.50 & Good & \\
\hline $8 / 27 / 937: 05$ & Auto FIC & 410.50. & Good & \\
\hline $8 / 28 / 930: 00$ & Manual Tape & 410.5 & Good & \\
\hline $8 / 28 / 937: 04$ & Auto FIC & 410.5 & Good & \\
\hline $8 / 29 / 930: 00$ & Manual Tape & 410.75 & Good & \\
\hline $8 / 29 / 937: 03$ & Auto FIC & 410.70 & Good & \\
\hline $8 / 30 / 930: 00$ & Manual Tape & 410.75 & Good & \\
\hline $8 / 30 / 937: 04$ & Auto FIC & 410.5 & Good & \\
\hline $8 / 31 / 930: 00$ & Manual Tape & 410.75 & Good & \\
\hline $8 / 31 / 937: 04$ & Auto FIC & 410.50 & Good & \\
\hline $9 / 1 / 930: 00$ & Manual Tape & 410.75 & Good & \\
\hline $9 / 1 / 937: 04$ & Auto FIC & 410.50 & Good & \\
\hline 9/2/93 0:00 & Manual Tape ' & 410.75 & Good & \\
\hline $9 / 2 / 937: 04$ & Auto FIC & 410.6 & Good & \\
\hline $9 / 3 / 930: 00$ & Manual Tape & 410.75 & Good & \\
\hline $9 / 3 / 937: 04$ & Auto FIC & 410.50 & Good & \\
\hline $9 / 4 / 930: 00$ & Manual Tape & 410.75 & Good & \\
\hline 9/4/93 7:04 & Auto FIC & .410 .50 & Good & \\
\hline $9 / 5 / 930: 00$ & Manual Tape & 410.75 & Good & \\
\hline $9 / 5 / 937: 04$ & Auto FIC & 410.50 & Good & \\
\hline $9 / 6 / 930: 00$ & Manual Tape & 411.75 & Suspect & Data not included in graph \\
\hline $9 / 6 / 937: 04$ & Auto FIC & 410.6 & Good & \\
\hline $9 / 7 / 930: 00$ & Manual Tape & 411.00 & Good & \\
\hline $9 / 7 / 937: 04$ & Auto FIC & 410.50 & Good & \\
\hline $9 / 8 / 930: 00$ & Manual Tape & 411.00 & Good & \\
\hline $9 / 8 / 937: 04$ & Auto FIC & 410.5 & Good & \\
\hline
\end{tabular}

Data obtained from WHC Survaillance Analysis Computer System, (PC SACS) July 7, 1995. 


\begin{tabular}{|c|c|c|c|c|}
\hline \multicolumn{5}{|c|}{ 241-AP-106 } \\
\hline & ?. & urface Level Dat & & \\
\hline Date & Type of Measurement & Level (Inches) & Data Quality & Comments \\
\hline 9/9/93 0:00 & Manual Tape & 411 & Good & \\
\hline 9/9/93 7:04 & Auto FIC & 41.0:50 & Good & \\
\hline $9 / 10 / 930: 00$ & Manual Tape & 410.75 & Good & \\
\hline $9 / 10 / 937: 04$ & Auto FIC & 410.5 & Good & \\
\hline $9 / 11 / 930: 00$ & Manual Tape & 410.75 & Good & \\
\hline $9 / 11 / 937: 04$ & Auto FIC & 410.50 & Good & \\
\hline $9 / 12 / 930: 00$ & Manual Tape & 410.75 & Good & \\
\hline $9 / 12 / 937: 04$ & Auto FIC & 410.50 & Good & \\
\hline $9 / 13 / 930: 00$ & Manual Tape & 410.75 & Good & \\
\hline 9/13/93 7:04 & Auto FIC & 410.5 & Good & \\
\hline $9 / 14 / 930: 00$ & Manual Tape & 410.75 & Good & \\
\hline $9 / 14 / 937: 04$ & Auto FIC & 410.50 & Good & \\
\hline 9/15/93 0:00 & Manual Tape & 410.75 & Good & \\
\hline $9 / 15 / 937: 04$ & Auto FIC & 410.50 & Good & \\
\hline $9 / 16 / 930: 00$ & Manual Tape & 410.75 & Good & \\
\hline $9 / 16 / 937: 04$ & Auto FIC & 410.5 & Good & \\
\hline $9 / 17 / 930: 00$ & Manual Tape & 410.75 & Good & \\
\hline $9 / 17 / 937: 04$ & Auto FIC & 410.5 & Good & \\
\hline $9 / 18 / 930: 00$ & Manual Tape & $410: 75$ & Good & \\
\hline $9 / 18 / 937: 04$ & Auto FIC & 410.50 & Good & \\
\hline 9/19/93 0:00 & Manual Tape & 410.75 & Good & \\
\hline $9 / 19 / 937: 04$ & Auto FIC & 410.50 & Good & \\
\hline $9 / 20 / 93 \quad 0: 00$ & Manual Tape & 410.5 & Good & \\
\hline $9 / 20 / 937: 04$ & Auto FIC & 410.5 & Good & \\
\hline $9 / 21 / 930: 00$ & Manual Tape & 410.50 & Good & \\
\hline 9/21/93 7:04 & Auto FIC & 410.5 & Good & \\
\hline 9/22/93 0:00 & Manual Tape & 410.5 & Good & \\
\hline $9 / 22 / 937: 04$ & Auto FIC & 410.50 & Good & \\
\hline $9 / 23 / 930: 00$ & Manual Tape & 410.50 & Good & \\
\hline $9 / 23 / 937: 04$ & Auto FIC & 410.4 & Good & \\
\hline $9 / 24 / 930: 00$ & Manual Tape & 410.5 & Good & \\
\hline 9/24/93 7:04 & Auto FIC & 410.50 & Good & \\
\hline $9 / 25 / 930: 00$ & Manual Tape & 410.50 & Good & \\
\hline 9/25/93 7:04 & Auto FIC & 410.5 & Good & \\
\hline 9/26/93 0:00 & Manual Tape & 410.5 & Good & \\
\hline $9 / 26 / 937: 04$ & Auto FIC & $41,0: 50$ & Good & \\
\hline $9 / 27 / 930: 00$ & Manual Tape & 410.5 & Good & \\
\hline $9 / 27 / 937: 04$ & Auto FIC & 410.50 & Good & \\
\hline $9 / 28 / 930: 00$ & Manual Tape & 410.5 & Good & \\
\hline $9 / 28 / 937: 04$ & Auto FIC & 410.5 & Good & \\
\hline $9 / 29 / 930: 00$ & Manual Tape & 410.50 & Good & \\
\hline $9 / 29 / 937: 04$ & Auto FIC & 410.5 & Good & \\
\hline $9 / 30 / 930: 00$ & Manual Tape & 410.50 & Good & \\
\hline $9 / 30 / 937: 03$ & Auto FIC & 410.5 & Good & \\
\hline $10 / 1 / 930: 00$ & Manual Tape & 410.5 & Good & \\
\hline $10 / 1 / 937: 06$ & Auto FIC & 410.5 & Good & \\
\hline $10 / 2 / 930: 00$ & Manual Tape & 410.25 & Good & \\
\hline $10 / 2 / 937: 05$ & Auto FIC & 410.5 & Good & \\
\hline
\end{tabular}

Data obtained from WHC Surveillance Analysis Computer System, (PC SACS) July 7, 1996. 


\begin{tabular}{|c|c|c|c|c|}
\hline \multicolumn{5}{|c|}{ 241-AP-106 } \\
\hline \multicolumn{5}{|c|}{ Surface Level Data } \\
\hline Date & Type of Measurement & Level (Inchea) & Data Quality & Commants \\
\hline $10 / 3 / 930: 00$ & Manual Tape & 410.75 & Good & \\
\hline $10 / 3 / 93 \quad 7: 06$ & Auto FIC & 410.5 & Good & \\
\hline $10 / 4 / 93 \quad 0: 00$ & Manual Tape & 410.5 & Good & \\
\hline $10 / 4 / 937: 06$ & Auto FIC & 410.4 & Good & \\
\hline $10 / 5 / 930: 00$ & Manual Tape & 410.50 & Good & \\
\hline $10 / 5 / 937: 06$ & Auto FIC & 410.40 & Good & \\
\hline $10 / 6 / 930: 00$ & Manual Tape & 410.5 & Good & \\
\hline $10 / 6 / 937: 06$ & Auto FIC & 410.40 & Good & \\
\hline $10 / 7 / 93 \quad 0: 00$ & Manual Tape & 410.50 & Good & \\
\hline $10 / 7 / 937: 05$ & Auto FIC & 410.4 & Good & \\
\hline $10 / 8 / 937: 04$ & Auto FIC & 410.40 & Good & \\
\hline $10 / 9 / 930: 00$ & Manual Tape & 410.75 & Good & \\
\hline $10 / 9 / 937: 03$ & Auto FIC & 410.50 & Good & \\
\hline $10 / 10 / 930: 00$ & Manual Tape & 410.5 & Good & \\
\hline $10 / 10 / 93.6: 04$ & Auto FIC & 410.40 & Good & \\
\hline $10 / 11 / 937: 03$ & Auto FIC & 410.40 & Good & \\
\hline $10 / 12 / 937: 04$ & Auto FIC & 410.4 & Good & \\
\hline 10/13/93 0:00 & Manual Tape & 410.50 & Good & \\
\hline $10 / 13 / 937: 03$ & Auto FIC & $410.40^{\circ}$ & Good & \\
\hline $10 / 14 / 930: 00$ & Manual Tape & 410.5 & Good & \\
\hline $10 / 14 / 937: 04$ & Auto FIC & 410.50 & Good & \\
\hline $10 / 15 / 930: 00$ & Manual Tape & 410.5 & Good & \\
\hline $10 / 15 / 937: 04$ & Auto FIC & 410.5 & Good & \\
\hline $10 / 16 / 930: 00$ & Manual Tape & 410.50 & Good & \\
\hline $10 / 16 / 937: 04$ & Auto FIC & 410.4 & Good & \\
\hline $10 / 17 / 930: 00$ & Manual Tape & 410.5 & Good & \\
\hline $10 / 17 / 937: 03$ & Auto FIC & 410.4 & Good & \\
\hline $10 / 18 / 930: 00$ & Manual Tape & 410.50 & Good & \\
\hline $10 / 18 / 937: 04$ & Auto FIC & 410.40 & Good & \\
\hline $10 / 19 / 930: 00$ & Manual Tape & 410.75 & Good & \\
\hline $10 / 19 / 937: 04$ & Auto FIC & 410.40 & Good & \\
\hline $10 / 20 / 930: 00$ & Manual Tape & 410.50 & Good & \\
\hline $10 / 20 / 937: 03$ & Auto FIC & 410.5 & Good & \\
\hline $10 / 21 / 930: 00$ & Manual Tape & 410.5 & Good & \\
\hline $10 / 21 / 937: 04$ & Auto FIC & 410.40 & Good & \\
\hline $10 / 22 / 930: 00$ & Manual Tape & $410.50^{\circ}$ & Good & \\
\hline 10/22/93 7:04 & Auto FIC & 410.40 & Good & \\
\hline $10 / 23 / 930: 00$ & Manual Tape & 410.5 & Good & \\
\hline $10 / 23 / 937: 04$ & Auto FIC & 410.4 & Good & \\
\hline $10 / 24 / 930: 00$ & Manual Tape & 410.75 & Good & \\
\hline $10 / 24 / 937: 04$ & Auto FIC & 410.40 & Good & \\
\hline $10 / 25 / 930: 00$ & Manual Tape & 410.50 & Good & \\
\hline $10 / 25 / 937: 04$ & Auto FIC & 410.4 & Good & \\
\hline $10 / 26 / 930: 00$ & Manual Tape & 410.5 & Good & \\
\hline $10 / 26 / 936: 03$ & Auto FIC & 410.4 & Good & \\
\hline $10 / 27 / 930: 00$ & Manual Tape & 410.50 & Good & \\
\hline $10 / 27 / 937: 04$ & Auto FIC & 410.4 & Good & \\
\hline $10 / 28 / 930: 00$ & Manual Tape & 410.00 & Good & \\
\hline
\end{tabular}

Data obtained from WHC Surveillance Analysis Computer System, (PC SACS) July 7, 1995. 


\begin{tabular}{|c|c|c|c|c|}
\hline \multicolumn{5}{|c|}{ 241-AP-106 } \\
\hline & & Ifface Levol Dat & & \\
\hline Date & Type of Messurement & Leval (Inchea) & Data Quality & Comments \\
\hline $10 / 28 / 937: 04$ & Auto FIC & 410.40 & Good & . \\
\hline $10 / 29 / 930: 00$ & Manual Tape & 410 & Good & \\
\hline $10 / 29 / 936: 03$ & Auto FIC & 410.40 & Good & \\
\hline $10 / 30 / 930: 00$ & Manual Tape & 410.5 & Good & \\
\hline $10 / 30 / 937: 04$ & Auto FIC & 410.40 & Good & \\
\hline 10/31/93 0:00 & Manual Tape & 410.5 & Good & \\
\hline $10 / 31 / 937: 06$ & Auto FIC & 410.4 & Good & 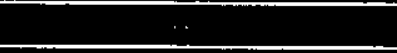 \\
\hline $11 / 1 / 930: 00$ & Manual Tape & 409.75 & Good & \\
\hline $11 / 1 / 937: 06$ & Auto FIC & 410.40 & Good & \\
\hline $11 / 2 / 930: 00$ & Manual Tape & 410.50 & Good & \\
\hline $11 / 2 / 937: 04$ & Auto FIC & 410.4 & Good & \\
\hline 11/3/93 0:00 & Manual Tape & 410.50 & Good & \\
\hline $11 / 3 / 937: 06$ & Auto FIC & 410.40 & Good & \\
\hline $11 / 4 / 930: 00$ & Manual Tape & 410.5 & Good & \\
\hline $11 / 4 / 937: 04$ & Auto FIC & 410.4 & Good & \\
\hline $11 / 5 / 930: 00$ & Manual Tape & 410.5 & Good & \\
\hline $11 / 5 / 937: 06$ & Auto FIC & 410.4 & Good & \\
\hline $11 / 6 / 930: 00$ & Manual Tape & 410.5 & Good & \\
\hline $11 / 6 / 937: 06$ & Auto FIC & 410.4 & Good & \\
\hline $11 / 7 / 930: 00$ & Manual Tape & 410.5 & Good & . \\
\hline $11 / 7 / 937: 06$ & Auto FIC & 410.40 & Gopd & \\
\hline $11 / 8 / 930: 00$ & Manual Tape & 410.50 & Good & \\
\hline $11 / 8 / 937: 06$ & Auto FIC & 410.40 & Good & \\
\hline 11/9/930:00 & Manual Tape & 410.50 & Good & \\
\hline $11 / 9 / 937: 03$ & Auto FIC & 410.40 & Good & \\
\hline $11 / 10 / 930: 00$ & Manual Tape & 410.5 & Good & \\
\hline $11 / 10 / 937: 04$ & Auto FIC & 410.4 & Good & \\
\hline $11 / 11 / 930: 00$ & Manual Tape & 410.50 & Good & \\
\hline $11 / 11 / 937: 04$ & Auto FIC & 410.4 & Good & \\
\hline $11 / 12 / 930: 00$ & Manual Tape & 410 & Good & \\
\hline $11 / 12 / 937: 04$ & Auto FIC & 410.3 & Good & \\
\hline $11 / 13 / 930: 00$ & Manual Tape & 410.25 & Good & \\
\hline 11/13/93 7:04 & Auto FIC & 410.4 & Good & \\
\hline $11 / 14 / 930: 00$ & Manual Tape & 410.5 & Good & \\
\hline $11 / 14 / 936: 04$ & Auto FIC & 410.40 & Good & \\
\hline $11 / 15 / 930: 00$ & Manual Tape & 410.25 & Good & \\
\hline $11 / 15 / 93 \quad 7: 04$ & Auto FIC & 410.40 & Good & \\
\hline $11 / 16 / 930: 00$ & Manual Tape & 410.25 & Good & \\
\hline $11 / 16 / 937: 03$ & Auto FIC & $41.0: 40$ & Good & \\
\hline 11/17/93 0:00 & Manual Tape & 410.5 & Good & \\
\hline $11 / 17 / 937: 04$ & Auto FIC & 410.3 & Good & \\
\hline $11 / 18 / 930: 00$ & Manual Tape & 408.75 & Suspect & Data not included in graph \\
\hline $11 / 18 / 937: 04$ & Auto FIC & 410.4 & Good & \\
\hline $11 / 19 / 930: 00$ & Manual Tape & 410.00 & Good & \\
\hline $11 / 19 / 937: 04$ & Auto FIC & 410.4 & Good & \\
\hline $11 / 20 / 930: 00$ & Manual Tape & 410.25 & Good & \\
\hline $11 / 20 / 93 \quad 7: 04$ & Auto FIC & 410.30 & Good & \\
\hline $11 / 21 / 930: 00$ & Manual Tape & 410.5 & Good & \\
\hline
\end{tabular}

Data obtained from WHC Surveillance Analysis Computer System, (PC SACS) July 7, 1995. 
Fils View/Frint Document Cover Sheet

This document was retrieved from the Boeing ISEARCH System.

Accession \#: D196051051

Document \#: SD-WM-ER-315

Title/Desc:

SUPPORTING DOCUMENT FOR THE SOUTHEAST QUADRANT HISTORICAL TANK CONTENT ESTIMATE REPORT FOR AP

TANK FARM [VOL II SEC 2 OF 2 PAGE E-391 TO K-8] 
THIS DOCUMENT WAS TOO LARGE TO SCAN AS A SINGLE DOCUMENT. IT REQUIRED BREAKING INTO SMALLER SECTIONS

DOCUMENT NUMBER: SD - Wm- $-2 R-315$ (Vol II) SECTION: 2 OF 2

TITLE: Southeast Quadrant Afistorion York Content Estimate Report for A $P$ Lank
Tarn

AutHor: $2 A$ Gaddis/SD Corot

RECIPIENT:

DATE: $\quad 9 / 13 / 95$

KEYWORDS:

REFERENCES: 


\section{THIS PAGE INTFANMONALT}

L.

LOFT BLANK 


\begin{tabular}{|c|c|c|c|c|}
\hline \multicolumn{5}{|c|}{ 241-AP-106 } \\
\hline \multicolumn{5}{|c|}{ Surfaco Level Data } \\
\hline Date & Type of Measurement & Level (Inches) & Data Quality & Cormments \\
\hline $11 / 21 / 937: 04$ & Auto FIC & 410.40 & Gopd & \\
\hline $11 / 22 / 930: 00$ & Manual Tape & 410.5 & Good & \\
\hline $11 / 22 / 937: 04$ & Auto FIC & 410.3 & Good & \\
\hline $11 / 23 / 930: 00$ & Manual Tape & 410.5 & Good & \\
\hline $11 / 23 / 937: 04$ & Auto FIC & 410.4 & Good & \\
\hline $11 / 24 / 93 \quad 0: 00$ & Manual Tape & 410.25 & Good & \\
\hline $11 / 24 / 937: 04$ & Auto FIC & 410.30 & Good & \\
\hline $11 / 25 / 930: 00$ & Manual Tape & 410.25 & Good & \\
\hline $11 / 25 / 937: 04$ & Auto FIC & 410.30 & Good & \\
\hline $11 / 26 / 930: 00$ & Manual Tape & 410.50 & Good & \\
\hline $11 / 26 / 937: 04$ & Auto FIC & 410.3 & Good & \\
\hline $11 / 27 / 930: 00$ & Manual Tape & 410.5 & Good & \\
\hline $11 / 27 / 937: 04$ & Auto FIC & 410.4 & Good & \\
\hline $11 / 28 / 930: 00$ & Manual Tape & 410.5 & Good & \\
\hline $11 / 28 / 937: 03$ & Auto FIC & 410.4 & Good & \\
\hline $11 / 29 / 930: 00$ & Manual Tape & 410.50 & Good & \\
\hline $11 / 29 / 93 \quad 7: 04$ & Auto FIC & 410.3 & Good & \\
\hline $11 / 30 / 930: 00$ & Manual Tape & 410.25 & Gopd & \\
\hline $11 / 30 / 937: 03$ & Auto FIC & 410.4 & Good & \\
\hline $12 / 1 / 930: 00$ & Manual Tape & 410.25 & Good & \\
\hline 12/1/93 7:04 & Auto FIC & 410.30 & Good & \\
\hline $12 / 2 / 930: 00$ & Manual Tape & 410.25 & Good & \\
\hline $12 / 2 / 937: 04$ & Auto FIC & 410.30 & Good & \\
\hline $12 / 3 / 930: 00$ & Manual Tape & 410.25 & Good & \\
\hline $12 / 3 / 937: 05$ & Auto FIC & 410.4 & Good & \\
\hline $12 / 4 / 930: 00$ & Manual Tape & 410.25 & Good & \\
\hline $12 / 4 / 937: 05$ & Auto FIC & 410.40 & Good & \\
\hline $12 / 5 / 93 \quad 0: 00$ & Manual Tape & 410.25 & Good & \\
\hline $12 / 5 / 937: 04$ & Auto FIC & 410.30 & Good & \\
\hline $12 / 6 / 930: 00$ & Manual Tape & 409 & Good & \\
\hline $12 / 6 / 937: 05$ & Auto FIC & 410.3 & Good & \\
\hline $12 / 7 / 930: 00$ & Manual Tape & 410.25 & Good & \\
\hline $12 / 7 / 937: 04$ & Auto FIC & 410.40 & Good & \\
\hline $12 / 8 / 930: 00$ & Manual Tape & 410.25 & Good & \\
\hline $12 / 8 / 937: 06$ & Auto FIC & 410.30 & Good & \\
\hline $12 / 9 / 93 \quad 0: 00$ & Manual Tape & 410.25 & Good & \\
\hline $12 / 9 / 93 \quad 7: 04$ & Auto FIC & 410.4 & Good & \\
\hline $12 / 10 / 930: 00$ & Manual Tape & 410.50 & Good & \\
\hline $12 / 10 / 936: 05$ & Auto FIC & 410.3 & Good & \\
\hline $12 / 11 / 930: 00$ & Manual Tape & 409.75 & Good & \\
\hline $12 / 11 / 937: 06$ & Auto FIC & 410.30 & Good & \\
\hline $12 / 12 / 930: 00$ & Manual Tape & 409.50 & Good & \\
\hline $12 / 12 / 937: 06$ & Auto FIC & 410.30 & Good & \\
\hline $12 / 13 / 930: 00$ & Manual Tape & 410.25 & Good & \\
\hline $12 / 13 / 937: 06$ & Auto FIC & 410.3 & Good & \\
\hline $12 / 14 / 930: 00$ & Manual Tape & 410.00 & Good & \\
\hline $12 / 14 / 937: 06$ & Auto FIC & 410.30 & Good & \\
\hline $12 / 15 / 930: 00$ & Manual Tape & 410.25 & Good & \\
\hline
\end{tabular}

Data obtained from WHC Surveillance Analysis Computer System, (PC SACS) July 7, 1995. 


\begin{tabular}{|c|c|c|c|c|}
\hline \multicolumn{5}{|c|}{ 241-AP-106 } \\
\hline \multicolumn{5}{|c|}{ Surface Level Data } \\
\hline Date & Type of Measurement & Level (Inches) & Data Quality & Comments \\
\hline $12 / 15 / 937: 06$ & Auto FIC & 410.30 & Good & \\
\hline $12 / 16 / 930: 00$ & Manual Tape & 410.25 & Good & \\
\hline $12 / 16 / 937: 06$ & Auto FIC & 410.20 & Good & . \\
\hline $12 / 17 / 930: 00$ & Manual Tape & 410.25 & Good & \\
\hline $12 / 17 / 937: 05$ & Auto FIC & 410.30 & Good & \\
\hline $12 / 18 / 930: 00$ & Manual Tape & 410 & Good & \\
\hline 12/18/93 7:06 & Auto FIC & $410: 40$ & Good & \\
\hline $12 / 19 / 930: 00$ & Manual Tape & 410.25 & Good & \\
\hline $12 / 19 / 937: 06$ & Auto FIC & 410.2 & Good & \\
\hline $12 / 20 / 930: 00$ & Manual Tape & 410.25 & Good & \\
\hline $12 / 20 / 937: 06$ & Auto FIC & 410.20 & Good & \\
\hline $12 / 21 / 930: 00$ & Manual Tape & 410 & Good & \\
\hline $12 / 21 / 937: 06$ & Auto FIC & 410.2 & Good & \\
\hline $12 / 22 / 930: 00$ & Manual Tape & 410.25 & Good & \\
\hline $12 / 22 / 937: 05$ & Auto FIC & 410.20 & Good & \\
\hline $12 / 23 / 930: 00$ & Manual Tape & 410.50 & Good & . \\
\hline 12/23/93 6:06 & Auto FIC & 410.20 & - Good & \\
\hline $12 / 24 / 930: 00$ & Manual Tape & 410.5 & Good & \\
\hline $12 / 24 / 937: 06$ & Auto FIC & 410.2 & Good & \\
\hline $12 / 25 / 930: 00$ & Manual Tape & 410.5 & Good & \\
\hline $12 / 25 / 937: 06$ & Auto FIC & 410.20 & Good & \\
\hline 12/26/93 0:00 & Manual Tape & 410.50 & Good & \\
\hline $12 / 26 / 937: 06$ & Auto FIC & 410.2 & Good & \\
\hline $12 / 27 / 930: 00$ & Manual Tape & $410: 50$ & Good & \\
\hline 12/27/93 7:06 & Auto FIC & 410.2 & Good & \\
\hline $12 / 28 / 930: 00$ & Manual Tape & 410.50 & Good & \\
\hline $12 / 28 / 937: 06$ & Auto FIC & 410.2 & Good & . \\
\hline $12 / 29 / 930: 00$ & Manual Tape & 410.25 & Good & \\
\hline $12 / 29 / 937: 06$ & Auto FIC & 410.20 & Good & \\
\hline 12/30/93 0:00 & Manual Tape & 410.25 & Good & \\
\hline $12 / 30 / 937: 05$ & Auto FIC & 410.2 & Good & \\
\hline $12 / 31 / 930: 00$ & Manual Tape & 410.25 & Good & \\
\hline $12 / 31 / 937: 06$ & Auto FIC & 410.30 & Good & \\
\hline $1 / 1 / 940: 00$ & Manual Tape & 410.25 & Good & \\
\hline $1 / 1 / 947: 06$ & Auto FIC & 410.3 & Good & \\
\hline $1 / 2 / 940: 00$ & Manual Tape & 410.25 & Good & \\
\hline $1 / 2 / 947: 06$ & Auto FIC & 410.2 & Good & \\
\hline $1 / 3 / 940: 00$ & Manual Tape & 410.25 & Good & \\
\hline $1 / 3 / 947: 07$ & Auto FIC & 410.20 & Good & \\
\hline $1 / 4 / 940: 00$ & Manual Tape & 410.5 & Good & \\
\hline $1 / 4 / 947: 07$ & Auto FIC & 410.3 & Good & \\
\hline 1/5/940:00 & Manual Tape & 410.25 & Good & \\
\hline $1 / 5 / 947: 06$ & Auto FIC & 410.30 & Good & \\
\hline $1 / 6 / 940: 00$ & Manual Tape & 410.5 & Good & \\
\hline $1 / 6 / 947: 06$ & Auto FIC & 410.20 & Good & \\
\hline $1 / 7 / 940: 00$ & Manual Tape & 410.5 & Good & \\
\hline 1/7/94 7:07. & Auto FIC & 410.2 & Good & \\
\hline $1 / 8 / 940: 00$ & Manual Tape & 410.50 & Good & \\
\hline
\end{tabular}

Data obtained from WHC Surveillance Analysis Computer System, (PC SACS) July 7, 1995. 


\begin{tabular}{|c|c|c|c|c|}
\hline \multicolumn{5}{|c|}{ 241-AP-106 } \\
\hline & $\therefore$ & Urface Level Dat & & \\
\hline Date & Type of Measurement & Level (Inches) & Data Quality & Comments \\
\hline $1 / 8 / 947: 06$ & Auto FIC & 410.1 & Good & \\
\hline $1 / 9 / 940: 00$ & Manual Tape & 410.50 & Good & \\
\hline $1 / 9 / 947: 06$ & Auto FIC & 410.2 & Good & \\
\hline $1 / 10 / 940: 00$ & Manual Tape & 410.5 & Good & \\
\hline $1 / 10 / 947: 07$ & Auto FIC & 410.2 & Good & \\
\hline $1 / 11 / 940: 00$ & Manual Tape & 410.50 & Good & \\
\hline 1/11/94 7:07 & Auto FIC & 410.20 & Good & \\
\hline $1 / 12 / 940: 00$ & Manual Tape & 410.25 & Good & \\
\hline $1 / 12 / 947: 06$ & Auto FIC & 410.20 & Good & \\
\hline $1 / 13 / 940: 00$ & Manual Tape & 410.5 & Good & \\
\hline $1 / 13 / 945: 07$ & Auto FIC & 410.2 & Good & . \\
\hline $1 / 14 / 940: 00$ & Manual Tape & 410.5 & Good & \\
\hline $1 / 14 / 947: 06$ & Auto FIC & 410.10 & Good & \\
\hline $1 / 15 / 940: 00$ & Manual Tape & 410.50 & Good & \\
\hline $1 / 15 / 945: 07$ & Auto FIC & 410.1 & Good & \\
\hline $1 / 16 / 940: 00$ & Manual Tape & 410.5 & Good & \\
\hline $1 / 16 / 947: 07$ & Auto FIC & 410.20 & Good & \\
\hline $1 / 17 / 940: 00$ & Manual Tape & 410.5 & Good & \\
\hline $1 / 17 / 947: 07$ & Auto FIC & 410.20 & Good & \\
\hline $1 / 18 / 940: 00$ & Manual Tape & 410.25 & Good & \\
\hline $1 / 18 / 947: 07$ & Auto FIC & 410.20 & Good & \\
\hline $1 / 19 / 940: 00$ & Manual Tape & 410.25 & Good & \\
\hline $1 / 19 / 947: 07$ & Auto FIC & 410.1 & Good & \\
\hline $1 / 20 / 940: 00$ & Manual Tape & 410.25 & Good & \\
\hline $1 / 20 / 947: 07$ & Auto FIC & 410.1 & Good & \\
\hline $1 / 21 / 940: 00$ & Manual Tape & 410.25 & Good & \\
\hline $1 / 21 / 947: 07$ & Auto FIC & 410.1 & Good & \\
\hline $1 / 22 / 940: 00$ & Manual Tape & 410.25 & Good & \\
\hline $1 / 22 / 947: 08$ & Auto FIC & 410.20 & Good & \\
\hline $1 / 23 / 940: 00$ & Manual Tape & 410.25 & Good & \\
\hline $1 / 23 / 947: 07$ & Auto FIC & 410.1 & Good & \\
\hline $1 / 24 / 940: 00$ & Manual Tape & 410.5 & Good & \\
\hline $1 / 24 / 947: 08$ & Auto FIC & 410.20 & Good & \\
\hline $1 / 25 / 940: 00$ & Manual Tape & 410.5 & Good & \\
\hline $1 / 25 / 947: 07$ & Auto FIC & 410.20 & Good & \\
\hline $1 / 26 / 940: 00$ & Manual Tape & 410.50 & Good & \\
\hline $1 / 26 / 947: 06$ & Auto FIC & 410.20 & Good & \\
\hline $1 / 27 / 940: 00$ & Manual Tape & 410.5 & Good & \\
\hline $1 / 27 / 946: 06$ & Auto FIC & 410.20 & Good & \\
\hline $1 / 28 / 940: 00$ & Manual Tape & 410.5 & Good & \\
\hline $1 / 28 / 947: 07$ & Auto FIC & 410.30 & Good & \\
\hline $1 / 29 / 940: 00$ & Manual Tape & 410.25 & Good & \\
\hline $1 / 29 / 947: 07$ & Auto FIC & 410.10 & Good & \\
\hline $1 / 30 / 940: 00$ & Manual Tape & 410.50 & Good & \\
\hline $1 / 30 / 947: 06$ & Auto FIC & 410.1 & Good & \\
\hline $1 / 31 / 940: 00$ & Manual Tape & 410.25 & Good & \\
\hline $1 / 31 / 947: 07$ & Auto FIC & 410.10 & Good & \\
\hline $2 / 1 / 940: 00$ & Manual Tape & 410.00 & Good & \\
\hline
\end{tabular}

Data obtained from WHC Surveillance Analysis Computer System, (PC SACS) July 7, 1995. 


\begin{tabular}{|c|c|c|c|c|}
\hline \multicolumn{5}{|c|}{ 241-AP-106 } \\
\hline \multicolumn{5}{|c|}{ Surface Level Data } \\
\hline Date & Type of Measurement & Level (Inchea) & Data Quality & Commonts \\
\hline $2 / 1 / 947: 06$ & Auto FIC & 410.10 & Good & \\
\hline $2 / 2 / 940: 00$ & Manual Tape & 410.25 & Good & \\
\hline $2 / 2 / 947: 07$ & Auto FIC & 410.1 & Good & \\
\hline $2 / 3 / 940: 00$ & Manual Tape & 410.25 & Good & \\
\hline $2 / 3 / 947: 07$ & Auto FIC & 410.10 & Good & \\
\hline $2 / 4 / 940: 00$ & Manual Tape & 410.00 & Good & \\
\hline $2 / 4 / 947: 07$ & Auto FIC & 410.1 & Good & \\
\hline $2 / 5 / 940: 00$ & Manual Tape & 410 & Good & \\
\hline $2 / 5 / 947: 07$ & Auto FIC & 410.1 & Good & \\
\hline $2 / 6 / 940: 00$ & Manual Tape & 410.00 & Good & \\
\hline $2 / 6 / 947: 07$ & Auto FIC & 410.10 & Good & \\
\hline $2 / 7 / 940: 00$ & Manual Tape & 410.00 & Good & \\
\hline $2 / 7 / 947: 06$ & Auto FIC & 410.20 & Good & \\
\hline $2 / 8 / 940: 00$ & Manual Tape & 410.25 & Good & \\
\hline $2 / 8 / 947: 07$ & Auto FIC & 410.10 & Good & \\
\hline $2 / 9 / 940: 00$ & Manual Tape & 410.25 & Good & \\
\hline $2 / 9 / 947: 04$ & Auto FIC & 410.10 & Good & \\
\hline $2 / 10 / 940: 00$ & Manual Tape & 410.25 & Good & \\
\hline $2 / 10 / 947: 04$ & Auto FIC & 410 & Good & \\
\hline $2 / 11 / 940: 00$ & Manual Tape & 410.25 & Good & \\
\hline $2 / 11 / 947: 06$ & Auto FIC & 410.10 & Good & \\
\hline $2 / 12 / 940: 00$ & Manual Tape & 410.25 & Good & \\
\hline $2 / 12 / 947: 03$ & Auto FIC & 410.10 & Good & \\
\hline $2 / 13 / 940: 00$ & Manual Tape & 410.25 & Good & \\
\hline $2 / 13 / 947: 04$ & Auto FIC & 410.20 & Good & \\
\hline $2 / 14 / 940: 00$ & Manual Tape & 410.25 & Good & \\
\hline $2 / 14 / 947: 04$ & Auto FIC & 410.2 & . Good & $\dot{.}$ \\
\hline $2 / 15 / 940: 00$ & Manual Tape & 410.25 & Good & \\
\hline $2 / 15 / 947: 04$ & Auto FIC & 410.2 & Good & \\
\hline $2 / 16 / 940: 00$ & Manual Tape & 410.25 & Good & \\
\hline $2 / 16 / 947: 03$ & Auto FIC & 410.10 & Good & \\
\hline $2 / 17 / 940: 00$ & Manual Tape & 410 & Good & \\
\hline $2 / 17 / 947: 04$ & Auto FIC & 410.20 & Good & . \\
\hline $2 / 18 / 940: 00$ & Manual Tape & 410.25 & Good & \\
\hline $2 / 18 / 947: 06$ & Auto FIC & 410.1 & Good & \\
\hline $2 / 19 / 940: 00$ & Manual Tape & 410.25 & Good & \\
\hline $2 / 19 / 947: 06$ & Auto FIC & 410.2 & Good & \\
\hline $2 / 20 / 940: 00$ & Manual Tape & 410.25 & Good & \\
\hline $2 / 20 / 947: 07$ & Auto FIC & 410.10 & Good & \\
\hline $2 / 21 / 940: 00$ & Manual Tape & 410.25 & Good & \\
\hline $2 / 21 / 947: 06$ & Auto FIC & 410.2 & Good & \\
\hline $2 / 22 / 940: 00$ & Manual Tape & 410 & Good & \\
\hline $2 / 22 / 947: 05$ & Auto FIC & 410.10 & Good & \\
\hline $2 / 23 / 940: 00$ & Manual Tape & 410 & Good & \\
\hline $2 / 23 / 947: 06$ & Auto FIC & 410.10 & Good & \\
\hline 2/24/94 0:00 & Manual Tape & 410.00 & Good & \\
\hline $2 / 24 / 947: 06$ & Auto FIC & 410.1 & Good & \\
\hline $2 / 25 / 940: 00$ & Manual Tape & 410.00 & Good & \\
\hline
\end{tabular}

Data obtained from WHC Surveillance Analysis Computer System, (PC SACS) July 7, 1995. 


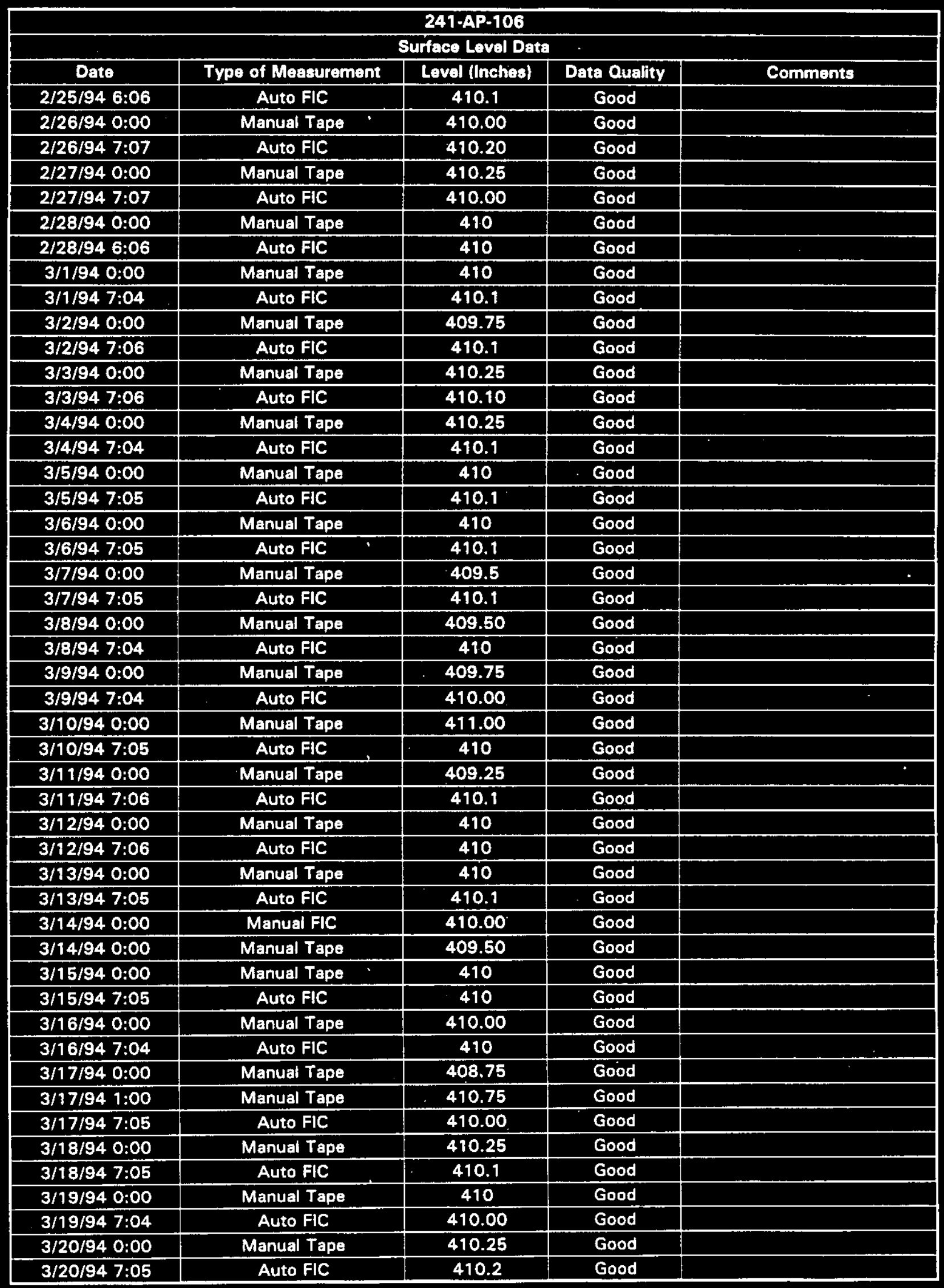

Data obtained from WHC Surveillance Analysis Computer System, (PC SACS) July 7, 1995. 


\begin{tabular}{|c|c|c|c|c|}
\hline \multicolumn{5}{|c|}{ 241-AP-106 } \\
\hline \multicolumn{5}{|c|}{ Surface Level Date } \\
\hline Date & Type of Measurement & Lovel (Inches) & Data Quality & Comments \\
\hline $3 / 21 / 940: 00$ & Manual Tape & 410.00 & Good & \\
\hline $3 / 21 / 947: 05$ & Auto FIC & 410.00 & Good & \\
\hline $3 / 22 / 940: 00$ & Manual Tape & $410: 25$ & Good & \\
\hline $3 / 22 / 947: 04$ & Auto FIC & 410 & Good & \\
\hline $3 / 23 / 940: 00$ & Manual FIC & 410 & Good & \\
\hline $3 / 23 / 940: 00$ & Manual Tape & 409.75 & Good & \\
\hline $3 / 24 / 940: 00$ & Manual Tape & 409.75 & Good & \\
\hline $3 / 24 / 947: 04$ & Auto FIC & 410.00 & Good & \\
\hline $3 / 25 / 940: 00$ & Manual Tape & 409.75 & Good & \\
\hline $3 / 25 / 947: 05$ & Auto FIC & 410.00 & Good & \\
\hline $3 / 26 / 940: 00$ & Manual Tape & 409.75 & Good & \\
\hline $3 / 26 / 947: 04$ & Auto FIC & 410.00 & Good & \\
\hline $3 / 27 / 940: 00$ & Manual Tape & 409.75 & Good & \\
\hline $3 / 27 / 947: 04$ & Auto FIC & 410.00 & Good & \\
\hline $3 / 28 / 940: 00$ & Manual Tape & 409.5 & Good & \\
\hline $3 / 28 / 947: 04$ & Auto FIC & 410 & Good & \\
\hline $3 / 29 / 940: 00$ & Manual Tape & 410.00 & Good & \\
\hline $3 / 29 / 947: 03$ & Auto FIC & 410.00 & Good & \\
\hline $3 / 30 / 940: 00$ & Manual Tape & 410.00 & Good & \\
\hline $3 / 30 / 947: 03$ & Auto FIC & 410 & Good & \\
\hline $3 / 31 / 940: 00$ & Manual Tape & 410 & Good & \\
\hline $3 / 31 / 947: 03$ & Auto FIC & 410.00 & Good & 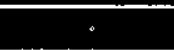 \\
\hline $4 / 1 / 940: 00$ & Manual Tape & 410 & Good & \\
\hline $4 / 1 / 940: 04$ & Auto FIC & 410.00 & Good & \\
\hline $4 / 2 / 940: 00$ & Manual Tape & 409.75 & Good & \\
\hline $4 / 2 / 947: 07$ & Auto FIC & 409.90 & Good & \\
\hline 4/3/94 0:00 & Manual Tape & 409.75 & Good & \\
\hline $4 / 3 / 947: 03$ & Auto FIC & 410 & Good & \\
\hline $4 / 4 / 940: 00$ & Manual Tape & 410 & Good & \\
\hline $4 / 4 / 947: 03$ & Auto FIC & 409.9 & Good & \\
\hline $4 / 5 / 94 \quad 0: 00$ & Manual Tape & $410.25^{\circ}$ & Good & \\
\hline $4 / 5 / 947: 03$ & Auto FIC & 410.00 & Good & \\
\hline $4 / 6 / 940: 00$ & Manual Tape & 409.50 & Good & \\
\hline $4 / 6 / 947: 06$ & Auto FIC & 409.9 & Good & \\
\hline $4 / 7 / 940: 00$ & Manual Tape & 410 & Good & \\
\hline $4 / 7 / 947: 07$ & Auto FIC & 409.90 & Good & \\
\hline $4 / 8 / 940: 00$ & Manual Tape & 41.0 .00 & Good & \\
\hline $4 / 8 / 947: 03$ & Auto FIC & 410.00 & Good & \\
\hline $4 / 9 / 940: 00$ & Manual Tape & 410 & Good & \\
\hline $4 / 9 / 947: 07$ & Auto FIC & 409.9 & Good & \\
\hline $4 / 10 / 940: 00$ & Manual Tape & 410.00 & Good & \\
\hline $4 / 10 / 947: 03$ & Auto FIC & 409.9 & Good & \\
\hline $4 / 11 / 940: 00$ & Manual Tape & 410 & Good & \\
\hline $4 / 11 / 947: 03$ & Auto FIC & 410.00 & Good & \\
\hline $4 / 12 / 940: 00$ & Manual Tape & 410.00 & Good & \\
\hline $4 / 12 / 947: 03$ & Auto FIC & 410 & Good & \\
\hline $4 / 13 / 940: 00$ & Manual Tape & 410.00 & Good & \\
\hline $4 / 13 / 947: 03$ & Auto FIC & 410.00 & Good & \\
\hline
\end{tabular}

Data obtained from WHC Surveillance Analysis Computer System, (PC SACS) July 7, 1995. 


\begin{tabular}{|c|c|c|c|c|}
\hline \multicolumn{5}{|c|}{ 241-AP-106 } \\
\hline \multicolumn{5}{|c|}{ Surface Level Data } \\
\hline Date & Type of Measurement & Level (Inchea) & Data Quality & Comments \\
\hline $4 / 14 / 940: 00$ & Manual Tape & 410.00 & Good & \\
\hline 4/14/94 7:03 & Auto FIC & 410 & Good & \\
\hline 4/15/940:00 & Manual Tape & 409.75 & Good & \\
\hline $4 / 15 / 947: 03$ & Auto FIC & 409.9 & Good & \\
\hline $4 / 16 / 940: 00$ & Manual Tape & 410.00 & Good & \\
\hline $4 / 16 / 947: 03$ & Auto FIC & 409.90 & Good & \\
\hline $4 / 17 / 940: 00$ & Manual Tape & 409.75 & Good & \\
\hline $4 / 17 / 947: 03$ & Auto FIC & 410 & Good & \\
\hline $4 / 18 / 940: 00$ & Manual Tape & 409.75 & Good & \\
\hline $4 / 18 / 947: 03$ & Auto FIC & 410.00 & Good & \\
\hline $4 / 19 / 940: 00$ & Manual Tape & 409.75 & Good & \\
\hline $4 / 19 / 946: 06$ & Auto FIC & 409.9 & Good & \\
\hline $4 / 20 / 940: 00$ & Manual Tape & 410 & Good & \\
\hline $4 / 20 / 947: 04$ & Auto FIC & 410.00 & Good & \\
\hline $4 / 21 / 940: 00$ & Manual Tape & 409.75 & Good & \\
\hline $4 / 21 / 947: 05$ & Auto FIC & 409.9 & Good & \\
\hline 4/22/94 0:00 & Manual Tape & 410 & Good & \\
\hline $4 / 22 / 947: 04$ & Auto FIC & 409.9 & Good & \\
\hline $4 / 23 / 940: 00$ & Manual Tape & 409.75 & Good & \\
\hline $4 / 23 / 947: 05$ & Auto FIC & 409.90 & Good & \\
\hline 4/24/94 0:00 & Manual Tape & 410.25 & Good & \\
\hline $4 / 24 / 947: 04$ & Auto FIC & 409.90 & Good & \\
\hline $4 / 25 / 940: 00$ & Manual Tape & 410 & Good & \\
\hline $4 / 25 / 947: 04$ & Auto FIC & 409.9 & Good & \\
\hline $4 / 26 / 940: 00$ & Manual Tape & 410.00 & Good & \\
\hline $4 / 26 / 947: 07$ & Auto FIC & 409.9 & Good & \\
\hline $4 / 27 / 940: 00$ & Manual Tape & 410.00 & Good & \\
\hline 4/27/94 7:04 & Auto FIC & 410 & Good & \\
\hline $4 / 28 / 940: 00$ & Manual Tape & 410.00 & Good & \\
\hline $4 / 28 / 947: 05$ & Auto FIC & 409.9 & Good & \\
\hline $4 / 29 / 940: 00$ & Manual Tape & 410.00 & Good & \\
\hline $4 / 29 / 947: 04$ & Auto FIC & 409.90 & Good & \\
\hline $4 / 30 / 940: 00$ & Manual Tape & 410.00 & Good & \\
\hline $4 / 30 / 947: 05$ & Auto FIC & 409.90 & Good & \\
\hline $5 / 1 / 940: 00$ & Manual Tape & 410.00 & Good & \\
\hline $5 / 1 / 947: 05$ & Auto FIC & 409.8 & Good & \\
\hline $5 / 2 / 940: 00$ & Manual Tape & 410.00 & Good & \\
\hline $5 / 2 / 947: 05$ & Auto FIC & 409.8 & Good & \\
\hline $5 / 3 / 940: 00$ & Manual Tape & 409.5 & Good & \\
\hline 5/3/94 7:05 & Auto FIC & 409.9 & Good & \\
\hline $5 / 4 / 940: 00$ & Manual Tape & 410 & Good & \\
\hline $5 / 4 / 947: 05$ & Auto FIC & 410 & Good & \\
\hline $5 / 5 / 940: 00$ & Manual Tape & 409.75 & Good & \\
\hline $5 / 5 / 947: 03$ & Auto FIC & 409.90 & Good & \\
\hline $5 / 6 / 940: 00$ & Manual Tape & 409.75 & Good & \\
\hline $5 / 6 / 947: 03$ & Auto FIC & 409.9 & Good & \\
\hline $5 / 7 / 940: 00$ & Manual Tape & 409.75 & Good & \\
\hline $5 / 7 / 947: 03$ & Auto FIC & 409.9 & Good & \\
\hline
\end{tabular}

Data obtained from WHC Surveillance Analysis Computer System, (PC SACS) July 7, 1995. 


\begin{tabular}{|c|c|c|c|c|}
\hline \multicolumn{5}{|c|}{ 241-AP-106 } \\
\hline \multicolumn{5}{|c|}{ Surface Level Data } \\
\hline Date & Type of Measurement & Lavel (Inches) & Data Quality & Comments \\
\hline $5 / 8 / 940: 00$ & Manual Tape & 410 & Good & \\
\hline $5 / 8 / 947: 03$ & Auto FIC & 409.90 & Good & \\
\hline $5 / 9 / 940: 00$ & Manual Tape & 409.75 & Good & \\
\hline $5 / 9 / 947: 03$ & Auto FIC & 410.00 & Good & \\
\hline $5 / 10 / 940: 00$ & Manual Tape & 409.75 & Good & \\
\hline $5 / 10 / 947: 03$ & Auto FIC & 410.10 & Good & \\
\hline $5 / 11 / 940: 00$ & Manual Tape & 410.00 & Good & \\
\hline $5 / 11 / 947: 03$ & Auto FIC & 410.00 & Good & \\
\hline $5 / 12 / 940: 00$ & Manual Tape & 409.75 & Good & \\
\hline $5 / 12 / 946: 03$ & Auto FIC & 409.90 & Good & \\
\hline $5 / 13 / 940: 00$ & Manual Tape & 409.75 & Good & \\
\hline $5 / 13 / 947: 03$ & Auto FIC & 409.9 & Good & \\
\hline $5 / 14 / 940: 00$ & Manual Tape & 409.75 & Good & \\
\hline $5 / 14 / 947: 03$ & Auto FIC & 409.80 & Good & \\
\hline $5 / 15 / 940: 00$ & Manual Tape & 410 & Good & \\
\hline $5 / 15 / 947: 06$ & Auto FIC & 409.80 & Good & \\
\hline $5 / 16 / 940: 00$ & Manual Tape & 409.75 & Good & \\
\hline $5 / 16 / 947: 02$ & Auto FIC & 409.9 & Good & \\
\hline $5 / 17 / 940: 00$ & Manual Tape & 409.75 & Good & \\
\hline $5 / 17 / 947: 03$ & Auto FIC & 409.8 & Good & \\
\hline $5 / 18 / 940: 00$ & Manual Tape & 409.75 & Good & \\
\hline $5 / 18 / 947: 03$ & Auto FIC & 409.9 & Good & \\
\hline $5 / 19 / 940: 00$ & Manual Tape & 409.75 & Good & \\
\hline 5/19/94 7:03 & Auto FIC & 409.9 & Good & \\
\hline $5 / 20 / 940: 00$ & Manual Tape & 409.50 & Good & \\
\hline $5 / 20 / 947: 03$ & Auto FIC & 409.9 & Good & \\
\hline $5 / 21 / 940: 00$ & Manual Tape & 410 & Good & \\
\hline $5 / 21 / 947: 05$ & Auto FIC & 409.80 & Good & \\
\hline $5 / 22 / 940: 00$ & Manual Tape & 410 & Good & \\
\hline 5/22/94 7:05 & Auto FIC & 409.8 & Good & \\
\hline $5 / 23 / 940: 00$ & Manual Tape & 410.00 & Good & \\
\hline $5 / 23 / 947: 04$ & Auto FIC & 409.9 & Good & \\
\hline $5 / 24 / 940: 00$ & Manual Tape & 410 & Good & \\
\hline $5 / 24 / 947: 04$ & Auto FIC & 409.80 & Good & \\
\hline $5 / 25 / 940: 00$ & Manual Tape & 409.5 & Good & \\
\hline $5 / 25 / 947: 04$ & Auto FIC & 409.80 & Good & \\
\hline $5 / 26 / 940: 00$ & Manual Tape & 409.5 & Good & \\
\hline $5 / 26 / 946: 05$ & Auto FIC & 409.90 & Good & \\
\hline $5 / 27 / 940: 00$ & Manual Tape & 407.00 & Suspect & Data not included in graph \\
\hline $5 / 27 / 947: 03$ & Auto FIC & 409.90 & Good & \\
\hline $5 / 28 / 940: 00$ & Manual Tape & 410 & Good & \\
\hline $5 / 28 / 947: 03$ & Auto FIC & 409.80 & Good & \\
\hline $5 / 29 / 940: 00$ & Manual Tape & 407.75 & Suspect & Data not included in graph \\
\hline $5 / 29 / 947: 03$ & Auto FIC & 409.8 & Good & \\
\hline $5 / 30 / 940: 00$ & Manual FIC & 409.90 & Good & . \\
\hline $5 / 30 / 940: 00$ & Manual Tape & 410.00 & Good & \\
\hline $5 / 30 / 947: 02$ & Auto FIC & 410.00 & Good & \\
\hline $5 / 31 / 940: 00$ & Manual FIC & 409.8 & Good & \\
\hline
\end{tabular}

Data obtained from WHC Surveillance Analysis Computer System, (PC SACS) July 7, 1995. 


\begin{tabular}{|c|c|c|c|c|}
\hline \multicolumn{5}{|c|}{ 24.1-AP-106 } \\
\hline \multicolumn{5}{|c|}{ Surface Level Data } \\
\hline Date & Type of Measurement & Level (Inches) & Data Quality & Comments \\
\hline $5 / 31 / 940: 00$ & Manual Tape & 409.75 & Good & \\
\hline $5 / 31 / 947: 03$ & Auto FIC & 409.8 & Good & \\
\hline $6 / 1 / 940: 00$ & Manual Tape & 409.75 & Good & \\
\hline $6 / 1 / 947: 03$ & Auto FIC & 409.8 & Good & \\
\hline $6 / 2 / 940: 00$ & Manual Tape & 409.5 & Good & \\
\hline $6 / 2 / 947: 03$ & Auto FIC & 409.80 & Good & \\
\hline $6 / 3 / 940: 00$ & Manual FIC & 409.8 & Good & \\
\hline $6 / 3 / 940: 00$ & Manual Tape & 409 & Good & \\
\hline $6 / 4 / 940: 00$ & Manual Tape & 410 & Good & \\
\hline $6 / 4 / 947: 04$ & Auto FIC & 409.90 & Good & \\
\hline $6 / 5 / 940: 00$ & Manual Tape & 410 & Good & . \\
\hline $6 / 5 / 947: 03$ & Auto FIC & 409.80 & Good & \\
\hline $6 / 6 / 940: 00$ & Manual Tape & 408 & Good & \\
\hline $6 / 6 / 947: 06$ & Auto FIC & 409.80 & Good & \\
\hline $6 / 7 / 940: 00$ & Manual Tape & 410 & Good & \\
\hline $6 / 7 / 947: 02$ & Auto FIC & 409.90 & Good & \\
\hline $6 / 8 / 940: 00$ & Manual Tape & 409.50 & Good & \\
\hline 6/8/94 7:03 & Auto FIC & 409.80 & Good & \\
\hline 6/9/94 0:00 & Manual FIC & 409.8 & Good & \\
\hline $6 / 9 / 940: 00$ & Manual Tape & 409.5 & Good & \\
\hline $6 / 10 / 940: 00$ & Manual Tape & 409.50 & Good & \\
\hline $6 / 10 / 947: 04$ & Auto FIC & 410.00 & Good & \\
\hline $6 / 11 / 940: 00$ & Manual Tape & 410 & Good & \\
\hline $6 / 11 / 947: 04$ & Auto FIC & 409.80 & Good & \\
\hline $6 / 12 / 940: 00$ & Manual Tape & 409.25 & Good & \\
\hline $6 / 12 / 947: 03$ & Auto FIC & 409.8 & Good & \\
\hline $6 / 13 / 940: 00$ & Manual Tape & 410.00 & Good & \\
\hline $6 / 13 / 947: 02$ & Auto FIC & 409.80 & Good & \\
\hline $6 / 14 / 940: 00$ & Manual Tape & 409.75 & Good & \\
\hline $6 / 14 / 947: 03$ & Auto FIC & 409.8 & Good & \\
\hline $6 / 15 / 940: 00$ & Manual Tape . & 409.75 & Good & \\
\hline $6 / 15 / 947: 03$ & Auto FIC & 409.8 & Good & \\
\hline $6 / 16 / 940: 00$ & Manual Tape & $409^{\circ}$ & Good & \\
\hline $6 / 16 / 947: 03$ & Auto FIC & 409.80 & Good & \\
\hline $6 / 17 / 940: 00$ & Manual Tape & 409 & Good & \\
\hline $6 / 17 / 947: 03$ & Auto FIC & 409.80 & Good & \\
\hline $6 / 18 / 940: 00$ & Manual Tape & 409.00 & Good & \\
\hline $6 / 18 / 947: 04$ & Auto FIC & 409.8 & Good & \\
\hline $6 / 19 / 940: 00$ & Manual Tape & 409.50 & Good & \\
\hline $6 / 19 / 947: 04$ & Auto FIC & 409.8 & Good & \\
\hline $6 / 20 / 940: 00$ & Manual Tape & 409.5 & Good & \\
\hline $6 / 20 / 947: 04$ & Auto FIC & 409.8 & Good & \\
\hline $6 / 21 / 940: 00$ & Manual Tape & 407.5 & Suspect & Data not included in graph \\
\hline $6 / 21 / 947: 04$ & Auto FIC & 409.8 & Good & \\
\hline 6/22/94 0:00 & Manual Tape & 410.00 & Good & \\
\hline $6 / 22 / 947: 03$ & Auto FIC & 409.8 & Good & \\
\hline $6 / 23 / 940: 00$ & Manual Tape & 409.75 & Good & \\
\hline $6 / 23 / 947: 05$ & Auto FIC & 409.80 & Good & \\
\hline
\end{tabular}

Data obtained from WHC Surveillance Analysis Computer System, (PC SACS) July 7, 1995. 


\begin{tabular}{|c|c|c|c|c|}
\hline \multicolumn{5}{|c|}{ 241-AP-106 } \\
\hline & & Irface Leval Dat & & \\
\hline Date & Type of Measurement & Level (Inches) & Data Quality & Comments \\
\hline $6 / 24 / 940: 00$ & Manual Tape & 410 & Good & \\
\hline $6 / 24 / 947: 06$ & Auto FIC & 409.80 & Good & \\
\hline $6 / 25 / 940: 00$ & Manual Tape & 410.00 & Good & \\
\hline $6 / 25 / 947: 05$ & Auto FIC & 409.9 & Good & \\
\hline $6 / 26 / 940: 00$ & Manual Tape & 409.5 & Good & \\
\hline $6 / 26 / 947: 06$ & Auto FIC & 409.80 & Good & \\
\hline $6 / 27 / 940: 00$ & Manual Tape & 409.75 & Good & \\
\hline $6 / 27 / 947: 06$ & Auto FIC & 409.80 & Good & \\
\hline $6 / 28 / 940: 00$ & Manual Tape & 409.75 & Good & \\
\hline $6 / 28 / 947: 06$ & Auto FIC & 409.90 & Good & \\
\hline $6 / 29 / 940: 00$ & Manual Tape & 409.75 & Good & \\
\hline $6 / 29 / 947: 03$ & Auto FIC & 409.8 & Good & \\
\hline $6 / 30 / 940: 00$ & Manual Tape & 410 & Good & \\
\hline $6 / 30 / 947: 04$ & Auto FIC & 409.8 & Good & \\
\hline 7/1/94 0:00 & Manual Tape & 410.00 & Good & \\
\hline $7 / 1 / 947: 04$ & Auto FIC & 409.8 & Good & \\
\hline $7 / 2 / 940: 00$ & Manual Tape & 409.75 & Good & \\
\hline $7 / 2 / 947: 04$ & Auto FIC & 409.8 & Good & \\
\hline $7 / 3 / 940: 00$ & Manual Tape & 409.75 & Good & \\
\hline $7 / 3 / 947: 04$ & Auto FIC & 409.8 & Good & \\
\hline $7 / 4 / 940: 00$ & Manual Tape & 409.75 & Good & \\
\hline $7 / 4 / 947: 04$ & Auto FIC & 409.80 & Good & \\
\hline $7 / 5 / 940: 00$ & Manual Tape & 410 & Gopd & \\
\hline 7/5/94 7:03 & Auto FIC & 409.80 & Good & \\
\hline $7 / 6 / 940: 00$ & Manual Tape & 409.5 & Good & \\
\hline $7 / 6 / 947: 05$ & Auto FIC & 409.8 & Good & \\
\hline $7 / 7 / 940: 00$ & Manual Tape & 410 & Good & \\
\hline $7 / 7 / 947: 03$ & Auto FIC & 409.80 & Good & \\
\hline $7 / 8 / 940: 00$ & Manual Tape & 410 & Good & \\
\hline $7 / 8 / 947: 03$ & Auto FIC & 409.80 & Good & \\
\hline $7 / 9 / 940: 00$ & Manual Tape & 410 & Good & \\
\hline $7 / 9 / 947: 03$ & Auto FIC & 409.80 & Good & \\
\hline $7 / 10 / 940: 00$ & Manual Tape & 409.75 & Good & \\
\hline 7/10/94 7:03 & Auto FIC & 409.80 & Good & \\
\hline $7 / 11 / 940: 00$ & Manual Tape & 409.75 & Good & \\
\hline $7 / 11 / 947: 03$ & Auto FIC & 409.8 & Good & \\
\hline $7 / 12 / 940: 00$ & Manual Tape & 410.00 & Good & \\
\hline $7 / 12 / 947: 03$ & Auto FIC & 409.8 & Good & \\
\hline $7 / 13 / 94 \quad 0: 00$ & Manual Tape & 410 & Good & \\
\hline $7 / 13 / 947: 03$ & Auto FIC & 409.8 & Good & \\
\hline $7 / 14 / 940: 00$ & Manual Tape & 410 & Good & \\
\hline $7 / 14 / 947: 04$ & Auto FIC & 409.80 & Good & \\
\hline $7 / 15 / 940: 00$ & Manual Tape & 410 & Good & \\
\hline $7 / 15 / 947: 04$ & Auto FIC & 409.80 & Good & \\
\hline $7 / 16 / 940: 00$ & Manual Tape & 409.75 & Good & \\
\hline $7 / 16 / 947: 04$ & Auto FIC & 409.90 & Good & \\
\hline $7 / 17 / 940: 00$ & Manual Tape & 409.75 & Good & \\
\hline $7 / 17 / 947: 04$ & Auto FIC & 409.8 & Good & \\
\hline
\end{tabular}

Data obtained from WHC Surveillance Analysis Computer System, (PC SACS) July 7, 1995. 


\begin{tabular}{|c|c|c|c|c|}
\hline \multicolumn{5}{|c|}{ 241-AP-106 } \\
\hline \multicolumn{5}{|c|}{ Surface Level Data } \\
\hline Date & Type of Measurement & Level (Inches) & Data Quality & Comments \\
\hline $7 / 18 / 940: 00$ & Manual FIC & 409.8 & Good & \\
\hline $7 / 18 / 940: 00$ & Manual Tape & 409.75 & Good & \\
\hline $7 / 19 / 940: 00$ & Manual Tape & 409.75 & Good & \\
\hline 7/19/94 7:03 & Auto FIC & 409.8 & Good & \\
\hline $7 / 20 / 940: 00$ & Manual Tape & 410.00 & Good & \\
\hline $7 / 20 / 947: 03$ & Auto FIC & 409.8 & Good & \\
\hline $7 / 21 / 940: 00$ & Manual Tape & 410 & Good & \\
\hline $7 / 21 / 947: 03$ & Auto FIC & 409.8 & Good & \\
\hline $7 / 22 / 940: 00$ & Manual Tape & 410 & Good & \\
\hline $7 / 22 / 947: 03$ & Auto FIC & 409.8 & Good & \\
\hline $7 / 23 / 940: 00$ & Manual Tape & 410 & Good & \\
\hline $7 / 23 / 947: 03$ & Auto FIC & 409.8 & Good & \\
\hline $7 / 24 / 940: 00$ & Manual Tape & 410.00 & Good & \\
\hline $7 / 24 / 947: 03$ & Auto FIC & 409.8 & Good & \\
\hline $7 / 25 / 940: 00$ & Manual Tape & 409.75 & Good & \\
\hline $7 / 25 / 947: 03$ & Auto FIC & 409.8 & Good & \\
\hline $7 / 26 / 947: 03$ & Auto FIC & 409.80 & Good & \\
\hline $7 / 26 / 9412: 00$ & Manual Tape & 410.00 & Good & \\
\hline $7 / 27 / 947: 03$ & Auto FIC & 409.8 & Good & \\
\hline $7 / 27 / 94 \quad 12: 00$ & Manual Tape & 410.00 & Good & \\
\hline $7 / 28 / 947: 04$ & Auto FIC & 409.8 & Good & \\
\hline $7 / 28 / 94 \quad 12: 00$ & Manual Tape & 409.75 & Good & \\
\hline $7 / 29 / 947: 04$ & Auto FIC & 409.80 & Good & \\
\hline $7 / 29 / 9412: 00$ & Manual Tape & 409.75 & Good & \\
\hline $7 / 30 / 947: 04$ & Auto FIC & 409.80 & Good & \\
\hline $7 / 30 / 9412: 00$ & Manual Tape & 409.00 & Good & \\
\hline $7 / 31 / 9412: 00$ & Manual Tape & 409.5 & Good & \\
\hline $7 / 31 / 9423: 03$ & Auto FIC & 409.8 & Good & \\
\hline $8 / 1 / 947: 03$ & Auto FIC & 409.80 & Good & \\
\hline $8 / 1 / 9412: 00$ & Manual Tape & 410 & Good & \\
\hline $8 / 2 / 947: 03$ & Auto FIC & 409.80 & Good & \\
\hline $8 / 2 / 9412: 00$ & Manual Tape & 410 & Good & \\
\hline $8 / 3 / 947: 03$ & Auto FIC & 409.80 & Good & \\
\hline $8 / 3 / 94 \quad 12: 00$ & Manual Tape & 410.00 & Good & \\
\hline $8 / 4 / 947: 02$ & Auto FIC & 0 & Suspect & Data not included in graph \\
\hline $8 / 4 / 9412: 00$ & Manual FIC & 409.8 & Good & \\
\hline $8 / 4 / 9412: 00$ & Manual Tape & 410.00 & Good & \\
\hline $8 / 5 / 947: 03$ & Auto FIC & 409.80 & Good & \\
\hline $8 / 5 / 9412: 00$ & Manual Tape & 410 & Good & \\
\hline $8 / 6 / 947: 03$ & Auto FIC & 409.80 & Good & \\
\hline $8 / 6 / 9412: 00$ & Manual Tape & 410 & Good & \\
\hline $8 / 7 / 947: 04$ & Auto FIC & 409.8 & Good & \\
\hline $8 / 7 / 9412: 00$ & Manual Tape & 410 & Good & \\
\hline $8 / 8 / 947: 12$ & Auto FIC & 0 & Suspect & Data not included in graph \\
\hline $8 / 8 / 9412: 00$ & Manual FIC & 409.8 & Good & \\
\hline $8 / 8 / 9412: 00$ & Manual Tape & 409.75 & Good & \\
\hline $8 / 9 / 947: 06$ & Auto FIC & 409.80 & Good & \\
\hline $8 / 9 / 9412: 00$ & Manual Tape & 410.00 & Good & \\
\hline
\end{tabular}

Data obtained from WHC Surveillance Analysis Computer System, (PC SACS) July 7, 1995. 


\begin{tabular}{|c|c|c|c|c|}
\hline \multicolumn{5}{|c|}{ 241-AP-106 } \\
\hline \multicolumn{5}{|c|}{ Surface Level Data } \\
\hline Date & Type of Measurement & Level (Inches) & Data Quality & Comments \\
\hline $8 / 10 / 947: 04$ & Auto FIC & 409.8 & Good & \\
\hline $8 / 10 / 9412: 00$ & Manual Tape & 409.75 & Good & \\
\hline $8 / 11 / 947: 04$ & Auto FIC & 409.9 & Good & \\
\hline $8 / 11 / 9412: 00$ & Manual Tape & 409.25 & Good & \\
\hline $8 / 12 / 947: 04$ & Auto FIC & 409.80 & Good & \\
\hline $8 / 12 / 9412: 00$ & Manual Tape & 410 & Good & \\
\hline $8 / 13 / 947: 03$ & Auto FIC & 409.90 & Good & \\
\hline $8 / 13 / 9412: 00$ & Manual Tape & 410.00 & Good & \\
\hline $8 / 14 / 947: 06$ & Auto FIC & 410 & Good & \\
\hline $8 / 14 / 9412: 00$ & Manual Tape & 409.75 & Good & \\
\hline $8 / 15 / 947: 06$ & Auto FIC & 409.80 & Good & \\
\hline $8 / 15 / 9412: 00$ & Manual Tape & 410 & Good & \\
\hline $8 / 16 / 947: 06$ & Auto FIC & 409.80 & Good & \\
\hline $8 / 16 / 94 \quad 12: 00$ & Manual Tape & 407.25 & Good & \\
\hline $8 / 17 / 947: 06$ & Auto FIC & 410 & Good & \\
\hline $8 / 17 / 9412: 00$ & Manual Tape & 409.25 & Good & \\
\hline $8 / 18 / 947: 06$ & Auto FIC & 409.90 & Good & \\
\hline $8 / 18 / 9412: 00$ & Manual Tape & 409.75 & Good & \\
\hline $8 / 19 / 946: 06$ & Auto FIC & 410.00 & Good & \\
\hline $8 / 19 / 9412: 00$ & Manual Tape & 409.50 & Good & \\
\hline $8 / 20 / 947: 04$ & Auto FIC & 409.8 & Good & \\
\hline $8 / 20 / 9412: 00$ & Manual Tape & 409.75 & Good & \\
\hline $8 / 21 / 947: 04$ & Auto FIC & 409.80 & Good & \\
\hline $8 / 21 / 9412: 00$ & Manual Tape & 410 & Good & \\
\hline $8 / 22 / 946: 04$ & Auto FIC & 409.8 & Good & \\
\hline $8 / 22 / 9412: 00$ & Manual Tape & 409.75 & Good & \\
\hline $8 / 23 / 947: 04$ & Auto FIC & 409.8 & Good & \\
\hline $8 / 23 / 9412: 00$ & Manual Tape & 409.25 & Good & \\
\hline $8 / 24 / 947: 04$ & Auto FIC & 409.80 & Good & \\
\hline $8 / 24 / 9412: 00$ & Manual Tape & 410.25 & Good & \\
\hline $8 / 25 / 947: 03$ & Auto FIC & 409.90 & Good & \\
\hline $8 / 25 / 9412: 00$ & Manual Tape & 410.00 & Good & \\
\hline $8 / 26 / 947: 06$ & Auto FIC & 409.80 & Good & \\
\hline $8 / 26 / 9412: 00$ & Manual Tape & 410.00 & Good & \\
\hline $8 / 27 / 947: 04$ & Auto FIC & 409.8 & Good & \\
\hline $8 / 27 / 9412: 00$ & Manual Tape & 410 & Good & \\
\hline $8 / 28 / 947: 04$ & Auto FIC & 409.90 & Good & \\
\hline $8 / 28 / 9412: 00$ & Manual Tape & 409.5 & Good & \\
\hline $8 / 29 / 947: 04$ & Auto FIC & 409.80 & Good & \\
\hline $8 / 29 / 9412: 00$ & Manual Tape & 410 & Good & \\
\hline $8 / 30 / 947: 04$ & Auto FIC & 409.80 & Good & \\
\hline $8 / 30 / 9412: 00$ & Manual Tape & 409.75 & Good & \\
\hline $8 / 31 / 947: 04$ & Auto FIC & 409.80 & Good & \\
\hline $8 / 31 / 9412: 00$ & Manual Tape & 409.75 & Good & \\
\hline $9 / 1 / 947: 04$ & Auto FIC & 410.00 & Good & \\
\hline $9 / 1 / 9412: 00$ & Manual Tape & 408.75 & Good & \\
\hline 9/2/94 7:06 & Auto FIC & 410.00 & Good & \\
\hline $9 / 2 / 9412: 00$ & Manual Tape & 409.75 & Good & \\
\hline
\end{tabular}

Data obtained from WHC Surveillance Analysis Computer System, (PC SACS) July 7, 1995. 


\begin{tabular}{|c|c|c|c|c|}
\hline \multicolumn{5}{|c|}{ 241-AP-106 } \\
\hline \multicolumn{5}{|c|}{ Surface Level Data } \\
\hline Date & Type of Measurement & Level (Inches) & Data Quality & Comments \\
\hline $9 / 3 / 9412: 00$ & Manual Tape & 409.5 & Good & \\
\hline 9/3/94 23:04 & Auto FIC & 409.8 & Good & \\
\hline $9 / 4 / 947: 04$ & Auto FIC & 409.80 & Good & \\
\hline 9/4/94 12:00 & Manual Tape & 406.25 & Good & \\
\hline 9/5/94 7:04 & Auto FIC & 409.90 & Good & \\
\hline $9 / 5 / 94 \quad 12: 00$ & Manual Tape & 409.75 & Good & \\
\hline $9 / 6 / 947: 03$ & Auto FIC & 409.9 & Good & \\
\hline $9 / 6 / 9412: 00$ & Manual Tape & 409.75 & Good & \\
\hline $9 / 7 / 947: 03$ & Auto FIC & 409.8 & Good & \\
\hline $9 / 7 / 9412: 00$ & Manual Tape & 409.75 & Good & \\
\hline 9/8/94 7:04 & Auto FIC & 409.9 & Good & \\
\hline $9 / 8 / 9412: 00$ & Manual Tape & 410 & Good & \\
\hline 9/9/94 7:03 & Auto FIC & 409.80 & Good & \\
\hline $9 / 9 / 9412: 00$ & Manual Tape & 410 & Good & \\
\hline $9 / 10 / 947: 04$ & Auto FIC & 409.9 & Good & \\
\hline $9 / 10 / 9412: 00$ & Manual Tape & 410 & Good & \\
\hline $9 / 11 / 947: 03$ & Auto FIC & 409.80 & Good & \\
\hline $9 / 11 / 9412: 00$ & Manual Tape & 410.00 & Good & \\
\hline 9/12/94 7:03 & Auto FIC & 409.8 & Good & \\
\hline $9 / 12 / 9412: 00$ & Manual Tape & 410.00 & Good & \\
\hline $9 / 13 / 947: 02$ & Auto FIC & 409.90 & Good & \\
\hline $9 / 13 / 9412: 00$ & Manual Tape & 410 & Good & \\
\hline $9 / 14 / 947: 03$ & Auto FIC & 409.8 & Good & \\
\hline $9 / 14 / 9412: 00$ & Manual Tape & 410.00 & Good & \\
\hline $9 / 15 / 947: 03$ & Auto FIC & 409.8 & Good & \\
\hline $9 / 15 / 9412: 00$ & Manual Tape & 410 & Good & \\
\hline $9 / 16 / 947: 02$ & Auto FIC & 409.8 & Good & \\
\hline $9 / 16 / 9412: 00$ & Manual Tape & 409.75 & Good & \\
\hline $9 / 17 / 947: 03$ & Auto FIC & 410 & Good & \\
\hline $9 / 18 / 947: 02$ & Auto FIC & 409.80 & Good & \\
\hline $9 / 19 / 947: 03$ & Auto FIC & 410.10 & Good & \\
\hline $9 / 20 / 947: 03$ & Auto FIC & 409:90 & Good & \\
\hline $9 / 21 / 947: 04$ & Auto FIC & 409.9 & Good & \\
\hline $9 / 22 / 947: 03$ & Auto FIC & 409.8 & Good & \\
\hline $9 / 23 / 947: 04$ & Auto FIC & 409.80 & Good & \\
\hline $9 / 24 / 947: 04$ & Auto FIC & 409.80 & Good & \\
\hline $9 / 25 / 947: 03$ & Auto FIC & 409.8 & Good & \\
\hline $9 / 26 / 947: 04$ & Auto FIC & 409.70 & Good & \\
\hline $9 / 27 / 947: 04$ & Auto FIC & 409.8 & Good & \\
\hline $9 / 27 / 9412: 00$ & Manual Tape & 409.75 & Good & \\
\hline $9 / 28 / 947: 03$ & Auto FIC & 409.8 & Good & \\
\hline $9 / 28 / 9412: 00$ & Manual Tape & 409.75 & Good & \\
\hline $9 / 29 / 947: 04$ & Auto FIC & 409.80 & Good & \\
\hline $9 / 29 / 9412: 00$ & Manual Tape & 409.75 & Good & \\
\hline $9 / 30 / 947: 04$ & Auto FIC & 409.9 & Good & \\
\hline $9 / 30 / 9412: 00$ & Manual Tape & 410 & Good & \\
\hline $10 / 1 / 947: 06$ & Auto FIC & 409.9 & Good & \\
\hline $10 / 1 / 9412: 00$ & Manual Tape & 410.00 & Good & \\
\hline
\end{tabular}

Data obtained from WHC Surveillance Analysis Computer System, (PC SACS) July 7, 1995. 


\begin{tabular}{|c|c|c|c|c|}
\hline \multicolumn{5}{|c|}{ 241-AP-106 } \\
\hline & , & urface Level Dat & & \\
\hline Date & Type of Measurement & Level (Inches) & Data Quality & Comments \\
\hline $10 / 2 / 947: 05$ & Auto FIC & 409.80 & Good & \\
\hline $10 / 2 / 9412: 00$ & Manual Tape & 410 & Good & \\
\hline $10 / 3 / 947: 04$ & Auto FIC & 409.8 & Good & \\
\hline $10 / 3 / 9412: 00$ & Manual Tape & 409.75 & Good & \\
\hline $10 / 4 / 947: 04$ & Auto FIC & 409.7 & Good & \\
\hline 10/4/94 12:00 & Manual Tape & 410 & Good & \\
\hline $10 / 5 / 947: 04$ & Auto FIC & 409.70 & Good & \\
\hline $10 / 5 / 9412: 00$ & Manual Tape & 409.75 & Good & \\
\hline $10 / 6 / 947: 04$ & Auto FIC & 409.80 & Good & \\
\hline $10 / 6 / 9412: 00$ & Manual Tape & 409.75 & Good & \\
\hline $10 / 7 / 946: 04$ & Auto FIC & 409.8 & Good & \\
\hline $10 / 7 / 9412: 00$ & Manual Tape & 410.00 & Good & \\
\hline 10/8/94 7:03 & Auto FIC & 409.7 & Good & \\
\hline $10 / 8 / 94 \quad 12: 00$ & Manual Tape & 409.75 & Good & \\
\hline 10/9/94 7:06 & Auto FIC & 409.7 & Good & \\
\hline $10 / 9 / 9412: 00$ & Manual Tape & 410.00 & Good & \\
\hline $10 / 10 / 947: 05$ & Auto FIC & 409.70 & Good & \\
\hline $10 / 10 / 9412: 00$ & Manual Tape & 410 & Good & \\
\hline $10 / 11 / 947: 03$ & Auto FIC & 409.8 & Good & \\
\hline $10 / 11 / 9412: 00$ & Manual Tape & 410 & Good & \\
\hline $10 / 12 / 947: 06$ & Auto FIC & 409.7 & Good & \\
\hline 10/12/94 12:00 & Manual Tape & 410 & Good & \\
\hline $10 / 13 / 947: 05$ & Auto FIC & 409.7 & Good & \\
\hline $10 / 13 / 9412: 00$ & Manual Tape & 410.00 & Good & \\
\hline $10 / 14 / 947: 06$ & Auto FIC & 409.70 & Good & \\
\hline $10 / 14 / 9412: 00$ & Manual Tape & 410.00 & Good & \\
\hline $10 / 15 / 947: 06$ & Auto FIC & 409.8 & Good & \\
\hline $10 / 15 / 9412: 00$ & Manual Tape & 410 & Good & \\
\hline $10 / 16 / 947: 06$ & Auto FIC & 409.7 & Good & \\
\hline $10 / 16 / 9412: 00$ & Manual Tape & 409.75 & Good & \\
\hline $10 / 17 / 947: 06$ & Auto FIC & 409.80 & Good & \\
\hline $10 / 17 / 9412: 00$ & Manual Tape & 409.75 & Good & \\
\hline $10 / 18 / 947: 06$ & Auto FIC & 409.8 & Good & \\
\hline $10 / 18 / 9412: 00$ & Manual Tape & 409.75 & Good & \\
\hline $10 / 19 / 947: 06$ & Auto FIC & 409.7 & Good & \\
\hline $10 / 19 / 9412: 00$ & Manual Tape & 410 & Good & \\
\hline $10 / 20 / 945: 07$ & Auto FIC & 409.90 & Good & \\
\hline $10 / 20 / 9412: 00$ & Manual Tape & 410.00 & Good & \\
\hline $10 / 21 / 947: 06$ & Auto FIC & 409.8 & Good & \\
\hline $10 / 21 / 9412: 00$ & Manual Tape & 410 & Good & \\
\hline $10 / 22 / 9412: 00$ & Manual Tape & 410.00 & Good & \\
\hline $10 / 22 / 9423: 06$ & Auto FIC & 409.80 & Good & \\
\hline 10/23/94 7:06 & Auto FIC & 409.8 & Good & \\
\hline $10 / 23 / 9412: 00$ & Manual Tape & 410.00 & Good & \\
\hline $10 / 24 / 947: 06$ & Auto FIC & 409.9 & Good & \\
\hline $10 / 24 / 9412: 00$ & Manual Tape & 409.75 & Good & \\
\hline $10 / 25 / 947: 06$ & Auto FIC & 409.7 & Good & \\
\hline $10 / 25 / 9412: 00$ & Manual Tape & 409.75 & Good & \\
\hline
\end{tabular}

Data obtained from WHC Surveillance.Analysis Computer System, (PC SACS) July 7, 1995. 


\begin{tabular}{|c|c|c|c|c|}
\hline \multicolumn{5}{|c|}{ 241-AP-106 } \\
\hline \multicolumn{5}{|c|}{ Surface Levol Data } \\
\hline Date & Type of Measurement & Level (Inches) & Data Quality & Comments \\
\hline $10 / 26 / 947: 06$ & Auto FIC & 409.7 & Good & \\
\hline $10 / 26 / 9412: 00$ & Manual Tape & 409.50 & Good & \\
\hline $10 / 27 / 947: 06$ & Auto FIC & 409.80 & Good & \\
\hline $10 / 27 / 9412: 00$ & Manual Tape & 410 & Good & \\
\hline $10 / 28 / 947: 04$ & Auto FIC & 409.70 & Good & \\
\hline $10 / 28 / 9412: 00$ & Manual Tape & 409 & Good & \\
\hline $10 / 29 / 9412: 00$ & Manual FIC & 409.70 & Good & \\
\hline 10/30/94 23:04 & Auto FIC & 409.70 & Good & \\
\hline $10 / 31 / 947: 05$ & Auto FIC & 409.7 & Good & \\
\hline $10 / 31 / 9412: 00$ & Manual Tape & 373.00 & Suspect & Data not included in graph \\
\hline $11 / 1 / 9412: 00$ & Manual Tape & 367.5 & Suspect & Data not included in graph \\
\hline $11 / 1 / 9423: 04$ & Auto FIC & 409.80 & Good & \\
\hline $11 / 2 / 947: 03$ & Auto FIC & 409.7 & Good & \\
\hline $11 / 2 / 9412: 00$ & Manual Tape & 409.75 & Good & \\
\hline $11 / 3 / 947: 04$ & Auto FIC & 409.70 & Good & \\
\hline $11 / 3 / 9412: 00$ & Manual Tape & 409.75 & Good & \\
\hline $11 / 4 / 947: 03$ & Auto FIC & 409.7 & Good & \\
\hline $11 / 4 / 9412: 00$ & Manual Tape & 409.75 & Good & \\
\hline $11 / 5 / 947: 03$ & Auto FIC & 409.8 & Good & \\
\hline $11 / 5 / 9412: 00$ & Manual Tape & 409.75 & Good & \\
\hline $11 / 6 / 947: 04$ & Auto FIC & 409.80 & Good & \\
\hline $11 / 6 / 9412: 00$ & Manual Tape & 409.75 & Good & \\
\hline $11 / 7 / 947: 04$ & Auto FIC & 409.70 & Good & \\
\hline $11 / 7 / 9412: 00$ & Manual Tape & 409.75 & Good & \\
\hline $11 / 8 / 947: 03$ & Auto FIC & 409.80 & Good & \\
\hline $11 / 8 / 9412: 00$ & Manual Tape & 409.75 & Good & \\
\hline $11 / 9 / 947: 05$ & Auto FIC & 409.7 & Good & \\
\hline $11 / 9 / 9412: 00$ & Manual Tape & 409.75 & Good & \\
\hline $11 / 10 / 947: 03$ & Auto FIC & 409.6 & Good & \\
\hline $11 / 10 / 9412: 00$ & Manual Tape & 409.75 & Good & \\
\hline $11 / 11 / 947: 03$ & Auto FIC & 409.7 & Good & \\
\hline $11 / 11 / 9412: 00$ & Manual Tape & 409.75 & Good & \\
\hline $11 / 12 / 947: 03$ & Auto FIC & 409.8 & Good & \\
\hline $11 / 12 / 9412: 00$ & Manual Tape & 410.00 & Good & \\
\hline $11 / 13 / 947: 04$ & Auto FIC & 409.70 & Good & \\
\hline $11 / 13 / 9412: 00$ & Manual Tape & 409.75 & Good & \\
\hline $11 / 14 / 947: 03$ & Auto FIC & 409.70 & Good & \\
\hline $11 / 14 / 9412: 00$ & Manual Tape & 410 & Good & \\
\hline $11 / 15 / 947: 04$ & Auto FIC & 409.60 & Good & \\
\hline $11 / 15 / 9412: 00$ & Manual Tape & 410 & Good & \\
\hline $11 / 16 / 947: 06$ & Auto FIC & 409.70 & Good & \\
\hline $11 / 16 / 9412: 00$ & Manual Tape & 410 & Good & \\
\hline $11 / 17 / 947: 04$ & Auto FIC & 409.6 & Good & \\
\hline $11 / 17 / 9412: 00$ & Manual Tape & 410.00 & Good & \\
\hline $11 / 18 / 947: 04$ & Auto FIC & 409.70 & Good & \\
\hline $11 / 18 / 9412: 00$ & Manual Tape & 409.75 & Good & \\
\hline $11 / 19 / 947: 03$ & Auto FIC & 409.7 & Good & \\
\hline $11 / 19 / 9412: 00$ & Manual Tape & 409.75 & Good & \\
\hline
\end{tabular}

Data obtained from WHC Surveillance Analysis Computer System, (PC SACS) July 7, 1995. 


\begin{tabular}{|c|c|c|c|c|}
\hline \multicolumn{5}{|c|}{ 241-AP-106 } \\
\hline \multicolumn{5}{|c|}{ Surface Leval Data } \\
\hline Date & Type of Measurement & Level (Inches) & Data Quality & Comments \\
\hline $11 / 20 / 947: 03$ & Auto FIC & 409.7 & Good & \\
\hline $11 / 21 / 947: 04$ & Auto FIC & 409.60 & Good & \\
\hline $11 / 22 / 947: 03$ & Auto FIC & 409.7 & Good & \\
\hline $11 / 23 / 9412: 00$ & Manual FIC & 409.70 & Good & \\
\hline $11 / 24 / 9412: 00$ & Manual Tape & 409.75 & Good & \\
\hline 11/24/94 23:03 & Auto FIC & 409.60 & Good & \\
\hline $11 / 25 / 9412: 00$ & Manual Tape & 409.25 & Good & \\
\hline $11 / 25 / 9423: 03$ & Auto FIC & 409.6 & Good & \\
\hline $11 / 26 / 947: 03$ & Auto FIC & 409.70 & Good & \\
\hline $11 / 26 / 9412: 00$ & Manual Tape & 409 & Good & \\
\hline $11 / 27 / 947: 04$ & Auto FIC & 409.90 & Good & \\
\hline $11 / 27 / 9412: 00$ & Manual Tape & 409 & Good & \\
\hline $11 / 28 / 947: 04$ & Auto FIC & 409.7 & Good & \\
\hline $11 / 29 / 947: 04$ & Auto FIC & 409.7 & Good & \\
\hline $11 / 29 / 9412: 00$ & Manual Tape & 409.75 & Good & \\
\hline $11 / 30 / 947: 03$ & Auto FIC & 409.7 & Good & \\
\hline 12/1/946:05 & Auto FIC & 409.70 & Good & \\
\hline $12 / 1 / 9412: 00$ & Manual Tape & 409.75 & Good & \\
\hline $12 / 2 / 947: 03$ & Auto FIC & 409.6 & Good & \\
\hline $12 / 2 / 9412: 00$ & Manual Tape & 409.75 & Good & \\
\hline $12 / 3 / 946: 04$ & Auto FIC & 409.7 & Good & \\
\hline $12 / 3 / 9412: 00$ & Manual Tape & 409.75 & Good & \\
\hline $12 / 4 / 947: 03$ & Auto FIC & 409.6 & Good & \\
\hline $12 / 4 / 9412: 00$ & Manual Tape & 409.75 & Good & \\
\hline $12 / 5 / 947: 03$ & Auto FIC & 409.6 & Good & \\
\hline $12 / 5 / 9412: 00$ & Manual Tape & 409.25 & Good & \\
\hline $12 / 6 / 947: 04$ & Auto FIC & 409.6 & Good & \\
\hline $12 / 6 / 9412: 00$ & Manual Tape & 409.25 & Good & \\
\hline $12 / 7 / 947: 03$ & Auto FIC & 409.60 & Good & \\
\hline $12 / 7 / 9412: 00$ & Manual Tape & 409.62 & Good & \\
\hline 12/8/94 5:03 & Auto FIC & 409.60 & Good & \\
\hline $12 / 9 / 947: 03$ & Auto FIC & 409.8 & Good & \\
\hline $12 / 9 / 9412: 00$ & Manual Tape & 409.75 & Good & \\
\hline $12 / 10 / 947: 04$ & Auto FIC & 409.50 & Good & \\
\hline $12 / 10 / 9412: 00$ & Manual Tape & 409.75 & Good & \\
\hline $12 / 11 / 947: 04$ & Auto FIC & 409.70 & Good & \\
\hline $12 / 11 / 9412: 00$ & Manual Tape & 409.5 & Good & \\
\hline $12 / 12 / 947: 03$ & Auto FIC & 409.60 & Good & \\
\hline $12 / 12 / 9412: 00$ & Manual Tape & 409.75 & Good & \\
\hline $12 / 13 / 947: 03$ & Auto FIC & 409.50 & Good & \\
\hline $12 / 14 / 947: 04$ & Auto FIC & 409.5 & Good & \\
\hline $12 / 15 / 947: 03$ & Auto FIC & 409.50 & Good & \\
\hline $12 / 16 / 947: 03$ & Auto FIC & 409.5 & Good & \\
\hline $12 / 16 / 9412: 00$ & Manual Tape & 409.75 & Good & \\
\hline $12 / 17 / 947: 06$ & Auto FIC & 409.60 & Good & \\
\hline $12 / 18 / 947: 04$ & Auto FIC & 409.5 & Good & \\
\hline $12 / 18 / 9412: 00$ & Manual Tape & 409.5 & Good & \\
\hline $12 / 19 / 947: 04$ & Auto FIC & 409.60 & Good & \\
\hline
\end{tabular}

Data obtained from WHC Surveillance Analysis Computer System, (PC SACS) July 7, 1995. 


\begin{tabular}{|c|c|c|c|c|}
\hline \multicolumn{5}{|c|}{ 241-AP-106 } \\
\hline & & Irface Level Dat & & \\
\hline Date & Type of Measurement & Level (Inches) & Data Quality & Comments \\
\hline $12 / 20 / 947: 04$ & Auto FIC & 409.5 & Good & \\
\hline $12 / 21 / 947: 04$ & Auto FIC & 409.5 & Good & \\
\hline $12 / 21 / 9412: 00$ & Manual Tape & 382.25 & Suspect & Data not included in graph \\
\hline 12/22/94 7:05 & Auto FIC & 409.5 & Good & \\
\hline $12 / 22 / 9412: 00$ & Manual Tape & 382.00 & Suspect & Data not included in graph \\
\hline $12 / 23 / 947: 03$ & Auto FIC & 409.5 & Good & \\
\hline $12 / 23 / 9412: 00$ & Manual Tape & 409.75 & Good & \\
\hline $12 / 24 / 947: 04$ & Auto FIC & 409.60 & Good & \\
\hline $12 / 25 / 947: 03$ & Auto FIC & 409.50 & Good & \\
\hline $12 / 25 / 9412: 00$ & Manual Tape & 409.75 & Good & \\
\hline $12 / 26 / 947: 03$ & Auto FIC & 409.50 & Good & \\
\hline $12 / 26 / 9412: 00$ & Manual Tape & 409.75 & Good & \\
\hline $12 / 27 / 947: 04$ & Auto FIC & 409.5 & Good & \\
\hline $12 / 27 / 9412: 00$ & Manual Tape & 409.5 & Good & \\
\hline $12 / 28 / 947: 04$ & Auto FIC & 409.6 & Good & \\
\hline $12 / 29 / 947: 04$ & Auto FIC & 409.5 & Good & \\
\hline $12 / 30 / 947: 04$ & Auto FIC & 409.50 & Good & \\
\hline $12 / 31 / 947: 04$ & Auto FIC & 409.50 & Good & \\
\hline $1 / 1 / 957: 04$ & Auto FIC & 409.50 & Good & \\
\hline 1/2/95 7:03 & Auto FIC & 409.50 & Good & \\
\hline 1/3/95 7:02 & Auto FIC & 409.50 & Good & \\
\hline 1/4/95 7:03 & Auto FIC & 409.60 & Good & \\
\hline 1/5/95 7:04 & Auto FIC & 409.60 & Good & \\
\hline $1 / 6 / 957: 04$ & Auto FIC & 409.6 & Good & \\
\hline $1 / 7 / 957: 04$ & Auto FIC & 409.5 & Good & \\
\hline $1 / 8 / 95 \quad 12: 00$ & Manual Tape & 409.75 & Good & \\
\hline 1/8/95 23:03 & Auto FIC & 409.50 & Good & \\
\hline 1/9/95 7:05 & Auto FIC & 409.50 & Good & \\
\hline $1 / 9 / 95 \quad 12: 00$ & Manual Tape & 409.75 & Good & \\
\hline $1 / 10 / 957: 06$ & Auto FIC & 409.40 & Good & \\
\hline 1/10/95 12:00 & Manual Tape & 409.75 & Good & \\
\hline $1 / 11 / 957: 07$ & Auto FIC & 409.40 & Good & \\
\hline $1 / 11 / 9512: 00$ & Manual Tape & 409.75 & Good & \\
\hline $1 / 12 / 95 \quad 7: 07$ & Auto FIC & 409.50 & Good & \\
\hline $1 / 12 / 95 \quad 12: 00$ & Manual Tape & 409.50 & Good & \\
\hline 1/13/95 6:07 & Auto FIC & 409.50 & Good & \\
\hline $1 / 13 / 95 \quad 12: 00$ & Manual Tape & 409.5 & Good & \\
\hline $1 / 14 / 957: 07$ & Auto FIC & 409.50 & Good & \\
\hline $1 / 15 / 957: 06$ & Auto FIC & 409.50 & Good & \\
\hline $1 / 16 / 957: 08$ & Auto FIC & 409.50 & Good & \\
\hline $1 / 17 / 956: 07$ & Auto FIC & 409.40 & Good & \\
\hline $1 / 18 / 95 \quad 7: 07$ & Auto FIC & 409.40 & Good & \\
\hline $1 / 18 / 95 \quad 12: 00$ & Manual Tape & 409.5 & Good & \\
\hline $1 / 19 / 957: 07$ & Auto FIC & 409.40 & Good & \\
\hline $1 / 19 / 9512: 00$ & Manual Tape & 409.50 & Good & \\
\hline $1 / 20 / 957: 03$ & Auto FIC & 409.40 & Good & \\
\hline $1 / 20 / 95 \quad 12: 00$ & Manual Tape & 409.5 & Good & \\
\hline $1 / 21 / 957: 04$ & Auto FIC & 409.50 & Good & \\
\hline
\end{tabular}

Data obtained from WHC Surveillance Analysis Computer System, (PC SACS) July 7, 1995. 


\begin{tabular}{|c|c|c|c|c|}
\hline \multicolumn{5}{|c|}{ 241-AP-106 } \\
\hline \multicolumn{5}{|c|}{ Surface Level Data } \\
\hline Date & Type of Measurement & Level.(linches) & Data Quality & Comments \\
\hline $1 / 21 / 9512: 00$ & Manual Tape & 409.50 & Good & \\
\hline $1 / 22 / 957: 03$ & Auto FIC & 409.4 & Good & \\
\hline $1 / 22 / 95 \quad 12: 00$ & Manual Tape & 409.5 & Good & \\
\hline $1 / 23 / 957: 03$ & Auto FIC & 409.5 & Good & \\
\hline $1 / 23 / 9512: 00$ & Manual Tape & 409.50 & Good & \\
\hline $1 / 24 / 957: 04$ & Auto FIC & 409.50 & Good & \\
\hline $1 / 25 / 957: 03$ & Auto FIC & 409.50 & Good & \\
\hline $1 / 26 / 957: 03$ & Auto FIC & 409.40 & Good & \\
\hline $1 / 26 / 9512: 00$ & Manual Tape & 409.5 & Good & \\
\hline 1/27/95 7:03 & Auto FIC & 409.40 & Good & \\
\hline $1 / 27 / 95 \quad 12: 00$ & Manual Tape & 409.5 & Good & \\
\hline $1 / 28 / 957: 04$ & Auto FIC & 409.4 & Good & \\
\hline $1 / 28 / 9512: 00$ & Manual Tape & 409.50 & Good & \\
\hline $1 / 29 / 957: 04$ & Auto FIC & 409.5 & Good & \\
\hline $1 / 29 / 95 \quad 12: 00$ & Manual Tape & 409.5 & Good & \\
\hline $1 / 30 / 957: 04$ & Auto FIC & 409.40 & Good & \\
\hline $1 / 30 / 9512: 00$ & Manual Tape & 409.75 & Good & \\
\hline $1 / 31 / 957: 03$ & Auto FIC & 409.4 & Good & \\
\hline $1 / 31 / 9512: 00$ & Manual Tape & 409.50 & Good & \\
\hline $2 / 1 / 957: 03$ & Auto FIC & 409.4 & Good & \\
\hline $2 / 1 / 9512: 00$ & Manual Tape & 409.25 & Good & \\
\hline 2/2/95 7:04 & Auto FIC & 409.50 & Good & \\
\hline 2/2/95 12:00 & Manual Tape & 409.5 & Good & \\
\hline $2 / 3 / 957: 03$ & Auto FIC & 409.40 & Good & \\
\hline $2 / 3 / 95 \quad 12: 00$ & Manual Tape & 409.50 & Good & \\
\hline 2/4/95 7:04 & Auto FIC & 409.40 & Good & \\
\hline $2 / 4 / 95 \quad 12: 00$ & Manual Tape & 409.5 & Good & \\
\hline $2 / 5 / 957: 03$ & Auto FIC & 409.4 & Good & \\
\hline $2 / 5 / 9512: 00$ & Manual Tape & 409.5 & Good & \\
\hline $2 / 6 / 95$ 7:03 & Auto FIC & 409.4 & Good & \\
\hline $2 / 6 / 95 \quad 12: 00$ & Manual Tape & 409.50 & Good & \\
\hline $2 / 7 / 957: 03$ & Auto FIC & 409.30 & Good & \\
\hline 2/7/95 12:00 & Manual Tape & 409.5 & Good & \\
\hline 2/8/95 7:04 & Auto FIC & 409.40 & Good & \\
\hline $2 / 8 / 95 \quad 12: 00$ & Manual Tape & 409.50 & Good & \\
\hline 2/9/95 7:06 & Auto FIC & 409.40 & Good & \\
\hline $2 / 9 / 95 \quad 12: 00$ & Manual Tape & 409.5 & Good & \\
\hline $2 / 10 / 957: 06$ & Auto FIC & 409.4 & Good & \\
\hline $2 / 10 / 9512: 00$ & Manual Tape & 409.50 & Good & \\
\hline $2 / 11 / 957: 07$ & Auto FIC & 409.4 & Good & \\
\hline $2 / 11 / 95 \quad 12: 00$ & Manual Tape & 409.25 & Good & \\
\hline $2 / 12 / 957: 06$ & Auto FIC & 409.4 & Good & \\
\hline $2 / 12 / 95 \quad 12: 00$ & Manual Tape & 409.25 & Good & \\
\hline $2 / 13 / 957: 05$ & Auto FIC & 409.40 & Good & \\
\hline $2 / 13 / 9512: 00$ & Manual Tape & 409.75 & Good & \\
\hline $2 / 14 / 957: 06$ & Auto FIC & 409.4 & Good & \\
\hline $2 / 14 / 9512: 00$ & Manual Tape & 409.50 & Good & \\
\hline $2 / 15 / 95 \quad 7: 07$ & Auto FIC & 409.4 & Good & \\
\hline
\end{tabular}

Data obtained from WHC Surveillance Analysis Computer System, (PC SACS) July 7, 1995. 


\begin{tabular}{|c|c|c|c|c|}
\hline \multicolumn{5}{|c|}{ 241-AP-106 } \\
\hline \multicolumn{5}{|c|}{ Surface Level Data } \\
\hline Date & Type of Measurement & Level (Inches) & Data Quality & Comments \\
\hline $2 / 15 / 9512: 00$ & Manual Tape & 409.5 & Good & \\
\hline $2 / 16 / 957: 06$ & Auto FIC & 409.40 & Good & \\
\hline $2 / 16 / 9512: 00$ & Manual Tape & 409.5 & Good & \\
\hline $2 / 17 / 957: 06$ & Auto FIC & 409.30 & Good & \\
\hline $2 / 17 / 95 \quad 12: 00$ & Manual Tape & 409.5 & Good & \\
\hline $2 / 18 / 957: 06$ & Auto FIC & 409.3 & Good & \\
\hline $2 / 18 / 9512: 00$ & Manual Tape & 409.5 & Good & \\
\hline $2 / 19 / 957: 06$ & Auto FIC & 409.3 & Good & \\
\hline 2/19/95 12:00 & Manual Tape & 400.75 & Suspect & Data not included in graph \\
\hline $2 / 20 / 957: 07$ & Auto FIC & 409.40 & Good & \\
\hline $2 / 20 / 9512: 00$ & Manual Tape & 409.50 & Good & \\
\hline 2/21/95 7:06 & Auto FIC & 409.4 & Good & \\
\hline $2 / 21 / 9512: 00$ & Manual Tape & 409.5 & Good & \\
\hline $2 / 22 / 957: 06$ & Auto FIC & 409.4 & Good & \\
\hline $2 / 22 / 9512: 00$ & Manual Tape & 409.50 & Good & \\
\hline $2 / 23 / 957: 07$ & Auto FIC & 409.30 & Good & \\
\hline $2 / 23 / 9512: 00$ & Manual Tape & 409.5 & Good & \\
\hline $2 / 24 / 957: 06$ & Auto FIC & 409.30 & Good & \\
\hline $2 / 24 / 9512: 00$ & Manual Tape & 409.50 & Good & \\
\hline $2 / 25 / 957: 06$ & Auto FIC & 409.30 & Good & \\
\hline $2 / 25 / 95 \quad 12: 00$ & Manual Tape & 409.5 & Good & \\
\hline $2 / 26 / 956: 07$ & Auto FIC & 409.30 & Good & \\
\hline $2 / 26 / 9512: 00$ & Manual Tape & 409.5 & Good & \\
\hline $2 / 27 / 957: 06$ & Auto FIC & $409.30^{\circ}$ & Good & \\
\hline $2 / 27 / 95 \quad 12: 00$ & Manual Tape & 409.50 & Good & \\
\hline $2 / 28 / 955: 07$ & Auto FIC & 409.30 & Good & \\
\hline $2 / 28 / 9512: 00$ & Manual Tape & 409.5 & Good & \\
\hline $3 / 1 / 957: 06$ & Auto FIC & 409.3 & Good & \\
\hline $3 / 1 / 9512: 00$ & Manual Tape & 409 & Good & \\
\hline $3 / 2 / 957: 06$ & Auto FIC & 409.4 & Good & \\
\hline $3 / 2 / 95 \quad 12: 00$ & Manual Tape & 409.50 & Good & \\
\hline $3 / 3 / 957: 06$ & Auto FIC & 409.3 & Good & \\
\hline $3 / 3 / 95 \quad 12: 00$ & Manual Tape & 408.5 & Good & \\
\hline $3 / 4 / 957: 06$ & Auto FIC & 409.30 & Good & \\
\hline $3 / 4 / 9512: 00$ & Manual Tape & 408.5 & Good & \\
\hline $3 / 5 / 95$ 7:06 & Auto FIC & 409.3 & Good & \\
\hline $3 / 5 / 9512: 00$ & Manual Tape & 408.5 & Good & \\
\hline $3 / 6 / 95 \quad 7: 06$ & Auto FIC & 409.30 & Good & \\
\hline $3 / 6 / 9512: 00$ & Manual Tape & 409.50 & Good & \\
\hline $3 / 7 / 957: 06$ & Auto FIC & 409.30 & Good & \\
\hline $3 / 7 / 9512: 00$ & Manual Tape & 409.50 & Good & \\
\hline $3 / 8 / 957: 03$ & Auto FIC & 409.3 & Good & \\
\hline $3 / 8 / 95 \quad 12: 00$ & Manual Tape & 409.50 & Good & \\
\hline $3 / 9 / 957: 03$ & Auto FIC & 409.3 & Good & \\
\hline $3 / 9 / 9512: 00$ & Manual Tape & 409.5 & Good & \\
\hline $3 / 10 / 957: 02$ & Auto FIC & 409.4 & Good & \\
\hline $3 / 10 / 9512: 00$ & Manual Tape & 409.5 & Good & \\
\hline $3 / 11 / 957: 03$ & Auto FIC & 409.4 & Good & \\
\hline
\end{tabular}

Data obtained from WHC Surveillance Analysis Computer System, (PC SACS) July 7, 1995. 


\begin{tabular}{|c|c|c|c|c|}
\hline \multicolumn{5}{|c|}{ 241.AP-106 } \\
\hline & & urface Level Dat & & \\
\hline Date & Type of Measurement & Level (Inches) & Data Quality & Comments \\
\hline $3 / 11 / 95$ 12:00 & Manual Tape & 409.25 & Good & \\
\hline $3 / 12 / 957: 03$ & Auto FIC & 409.20 & Good & \\
\hline $3 / 12 / 9512: 00$ & Manual Tape & 409.5 & Good & \\
\hline 3/13/95 7:02 & Auto FIC & 409.30 & Good & \\
\hline $3 / 13 / 9512: 00$ & Manual Tape & 409.5 & Good & \\
\hline $3 / 14 / 957: 03$ & Auto FIC & 409.30 & Good & \\
\hline $3 / 14 / 9512: 00$ & Manual Tape & 409.50 & Good & \\
\hline $3 / 15 / 957: 05$ & Auto FIC & 409.30 & Good & \\
\hline $3 / 15 / 9512: 00$ & Manual Tape & 409.50 & Good & \\
\hline $3 / 16 / 957: 03$ & Auto FIC & 409.3 & Good & \\
\hline $3 / 16 / 95 \quad 12: 00$ & Manual Tape & 409.5 & Good & \\
\hline $3 / 17 / 957: 03$ & Auto FIC & 409.20 & Good & \\
\hline $3 / 17 / 95 \quad 12: 00$ & Manual Tape & 409.5 & Good & \\
\hline $3 / 18 / 957: 03$ & Auto FIC & 409.30 & Good & \\
\hline $3 / 19 / 957: 03$ & Auto FIC & 409.2 & Good & \\
\hline $3 / 20 / 956: 03$ & Auto FIC & 409.2 & Good & \\
\hline $3 / 21 / 957: 03$ & Auto FIC & 409.3 & Good & \\
\hline 3/22/95 7:02 & Auto FIC & 409.2 & Good & \\
\hline $3 / 23 / 957: 03$ & Auto FIC & 409.3 & Good & \\
\hline $3 / 24 / 95 \quad 7: 03$ & Auto FIC & 409.40 & Good & \\
\hline $3 / 25 / 957: 02$ & Auto FIC & 409.30 & Good & \\
\hline $3 / 26 / 957: 02$ & Auto FIC & 409.3 & Good & \\
\hline $3 / 27 / 957: 03$ & Auto FIC & 409.3 & Good & \\
\hline $3 / 28 / 957: 03$ & Auto FIC & 379.2 & Good & \\
\hline $3 / 29 / 957: 03$ & Auto FIC & 379.1 & Good & \\
\hline $3 / 30 / 957: 03$ & Auto FIC & 379.10 & Good & \\
\hline $3 / 31 / 957: 04$ & Auto FIC & $379: 30$ & Good & \\
\hline 4/1/95 7:02 & Auto FIC & 379.10 & Good & \\
\hline $4 / 2 / 957: 03$ & Auto FIC & 379.2 & Good & \\
\hline $4 / 3 / 957: 03$ & Auto FIC & 379.1 & Good & \\
\hline $4 / 3 / 95 \quad 12: 00$ & Manual Tape & 380 & Good & \\
\hline 4/4/95 7:03 & Auto FIC & 379.1 & Good & \\
\hline 4/5/95 7:03 & Auto FIC & 379.1 & Good & \\
\hline 4/6/95 7:03 & Auto FIC & 379.10 & Good & \\
\hline 4/7/95 7:03 & Auto FIC & 379.1 & Good & \\
\hline $4 / 8 / 957: 02$ & Auto FIC & 379.10 & Good & \\
\hline 4/9/95 23:03 & Auto FIC & 379.1 & Good & \\
\hline 4/10/95 7:02 & Auto FIC & 379.10 & Good & \\
\hline $4 / 11 / 957: 04$ & Auto FIC & 379.10 & Good & \\
\hline $4 / 12 / 957: 03$ & Auto FIC & 379.10 & Good & \\
\hline 4/13/95 7:05 & Auto FIC & 379.10 & Good & \\
\hline $4 / 14 / 957: 03$ & Auto FIC & 379.1 & Good & \\
\hline 4/15/95 7:03 & Auto FIC & 379.20 & Good & \\
\hline $4 / 16 / 957: 03$ & Auto FIC & 37.9 .20 & Good & \\
\hline $4 / 17 / 95 \quad 7: 03$ & Auto FIC & 379.2 & Good & \\
\hline $4 / 18 / 957: 03$ & Auto FIC & 379.20 . & Good & \\
\hline 4/19/95 7:03 & Auto FIC & 379.1 & Good & \\
\hline 4/20/95 7:03 & Auto FIC & 379.10 & Good & \\
\hline
\end{tabular}

Data obtained from WHC Surveillance Analysis Computer System, (PC SACS) July 7, 1995. 


\begin{tabular}{|c|c|c|c|c|}
\hline \multicolumn{5}{|c|}{ 241-AP-106 } \\
\hline \multicolumn{5}{|c|}{ Surface Level Data } \\
\hline Date & Type of Measurement & Level (Inches) & Data Quality & Comments \\
\hline $4 / 21 / 957: 03$ & Auto FIC & 379.1 & Good & \\
\hline 4/21/95 12:00 & Manual Tape & 378.75 & Good & \\
\hline 4/22/95 7:03 & Auto FIC & 379.20 & Good & \\
\hline $4 / 23 / 957: 03$ & Auto FIC & 379.1 & Good & \\
\hline $4 / 24 / 957: 03$ & Auto FIC & 379.10 & Good & \\
\hline 4/25/95 7:03 & Auto FIC & 379.10 & Good & \\
\hline $4 / 25 / 9512: 00$ & Manual Tape & 379.25 & Good & \\
\hline $4 / 26 / 957: 03$ & Auto FIC & 379.1 & Good & \\
\hline $4 / 27 / 957: 03$ & Auto FIC & 379.20 & Good & \\
\hline $4 / 28 / 957: 03$ & Auto FIC & 379.1 & Good & \\
\hline $4 / 29 / 957: 03$ & Auto FIC & 379.1 & Good & \\
\hline $4 / 30 / 957: 03$ & Auto FIC & 379.10 & Good & \\
\hline $5 / 1 / 957: 03$ & Auto FIC & 379.2 & Good & \\
\hline $5 / 2 / 957: 02$ & Auto FIC & 379.1 & Good & \\
\hline $5 / 3 / 95$ 7:03 & Auto FIC & 379.1 & Gopd & \\
\hline 5/4/95 7:04 & Auto FIC & 379.20 & Good & \\
\hline 5/5/95 7:04 & Auto FIC & 303.5 & Good & \\
\hline $5 / 6 / 957: 03$ & Auto FIC & 179.40 & Good & \\
\hline $5 / 7 / 957: 04$ & Auto FIC & 71.10 & Good & \\
\hline 5/8/95 7:04 & Auto FIC & 39.30 & Good & \\
\hline 5/9/95 7:04 & Auto FIC & 39.3 & Good & \\
\hline $5 / 10 / 957: 04$ & Auto FIC & 39.20 & Good & \\
\hline $5 / 11 / 957: 03$ & Auto FIC & 39.20 & Good & \\
\hline 5/12/95 7:06 & Auto FIC & 39.3 & Good & \\
\hline $5 / 13 / 957: 03$ & Auto FIC & 39.20 & Good & \\
\hline $5 / 14 / 957: 03$ & Auto FIC & 39.2 & Good & \\
\hline $5 / 15 / 957: 03$ & Auto FIC & 39.2 & Good & \\
\hline $5 / 16 / 957: 03$ & Auto FIC & 39.20 & Good & \\
\hline $5 / 17 / 957: 02$ & Auto FIC & 39.2 & Good & \\
\hline $5 / 18 / 956: 02$ & Auto FIC & 39.1 & Good & \\
\hline $5 / 18 / 9512: 00$ & Manual Tape & 39.5 & Good & \\
\hline 5/19/95 7:05 & Auto FIC & 39.20 & Good & \\
\hline $5 / 20 / 957: 05$ & Auto FIC & 39.20 & Good & \\
\hline $5 / 21 / 957: 05$ & Auto FIC & 39.2 & Good & \\
\hline $5 / 22 / 957: 05$ & Auto FIC & 39.2 & Good & \\
\hline $5 / 23 / 957: 11$ & Auto FIC & 0.00 & Suspect & Data not included in graph \\
\hline $5 / 23 / 9512: 00$ & Manual FIC & 39.20 & Good & \\
\hline $5 / 24 / 957: 10$ & Auto FIC & 0.00 & Suspect & Data not included in graph \\
\hline $5 / 24 / 9512: 00$ & Manual FIC & 39.2 & Good & \\
\hline $5 / 25 / 957: 10$ & Auto FIC & 0.00 & Suspect & Data not included in graph \\
\hline $5 / 25 / 9512: 00$ & Manual FIC & 39.20 & Good & \\
\hline $5 / 26 / 957: 04$ & Auto FIC & 39.20 & Good & \\
\hline $5 / 27 / 95 \quad 7: 05$ & Auto FIC & 39.20 & Good & \\
\hline $5 / 28 / 957: 05$ & Auto FIC & 39.2 & Good & \\
\hline $5 / 29 / 957: 05$ & Auto FIC & 39.20 & Good & \\
\hline 5/30/95 7:05 & Auto FIC & 39.20 & Good & \\
\hline $5 / 31 / 957: 05$ & Auto FIC & 39.20 & Good & \\
\hline $6 / 1 / 95$ 7:05 & Auto FIC & 39.2 & Good & \\
\hline
\end{tabular}

Data obtained from WHC Surveillance Analysis Computer System;, (PC SACS) July 7, 1995. 


\begin{tabular}{|c|c|c|c|c|}
\hline \multicolumn{5}{|c|}{ 241-AP-106 } \\
\hline \multicolumn{5}{|c|}{ Surface Level Data } \\
\hline Date & Type of Measurement & Level (Inches) & Data Quality & Comments \\
\hline $6 / 2 / 957: 05$ & Auto FIC & 39.1 & Good & \\
\hline 6/3/95 23:05 & Auto FIC & 39.1 & Good & \\
\hline 6/4/95 7:05 & Auto FIC & 39.20 & Good & \\
\hline 6/5/95 7:05 & Auto FIC & 39.20 & Good & \\
\hline 6/6/95 7:05 & Auto FIC & 39.20 & Good & \\
\hline $6 / 7 / 957: 05$ & Auto FIC & 39.10 & Good & \\
\hline 6/8/95 7:05 & Auto FIC & 39.1 & Good & \\
\hline 6/9/95 7:05 & Auto FIC & 39.10 & Good & \\
\hline $6 / 10 / 95$ 7:05 & Auto FIC & 39.1 & Good & \\
\hline $6 / 11 / 957: 05$ & Auto FIC & 39.1 & Good & \\
\hline $6 / 12 / 957: 05$ & Auto FIC & 39.1 & Good & \\
\hline $6 / 13 / 95 \quad 7: 05$ & Auto FIC & 39.1 & Good & \\
\hline $6 / 14 / 95$ 7:05 & Auto FIC & 39.1 & Good & \\
\hline $6 / 15 / 957: 05$ & Auto FIC & 39.1 & Good & \\
\hline $6 / 15 / 95 \quad 12: 00$ & Manual Tape & 39.25 & Good & \\
\hline $6 / 16 / 957: 05$ & Auto FIC & 39.00 & Good & \\
\hline $6 / 17 / 95 \quad 7: 05$ & Auto FIC & 39 & Good & \\
\hline $6 / 18 / 957: 05$ & Auto FIC & 39.00 & Good & \\
\hline 6/19/95 7:06 & Auto FIC & 39 & Good & \\
\hline $6 / 20 / 957: 06$ & Auto FIC & 39.00 & Good & \\
\hline $6 / 21 / 95 \quad 7: 06$ & Auto FIC & 39.10 & Good & \\
\hline 6/22/95 7:06 & Auto FIC & 39 & Good & \\
\hline $6 / 23 / 957: 06$ & Auto FIC & 39.00 & Good & \\
\hline $6 / 24 / 957: 06$ & Auto FIC & 39 & Good & \\
\hline $6 / 25 / 957: 05$ & Auto FIC & 39.1 & Good & \\
\hline $6 / 26 / 957: 05$ & Auto FIC & 39.1 & Good & \\
\hline $6 / 27 / 95 \quad 7: 06$ & Auto FIC & 39.00 & Good & \\
\hline $6 / 28 / 957: 05$ & Auto FIC & 39 & Good & \\
\hline $6 / 29 / 95 \quad 7: 06$ & Auto FIC & 39.00 & Good & \\
\hline $6 / 30 / 95 \quad 7: 06$ & Auto FIC & 39.00 & Good & \\
\hline
\end{tabular}

Data obtained from WHC Surveillance Analysis Computer System, (PC SACS) July 7, 1995. 


\begin{tabular}{|c|c|c|c|c|}
\hline \multicolumn{5}{|c|}{ 241-AP-107 } \\
\hline \multicolumn{5}{|c|}{ Surface Level Data } \\
\hline Date & Type of Measurement & Level (Inches) & Data Quality & Comments \\
\hline $1 / 1 / 910: 00$ & Manual Tape & 410.50 & Transcribed & \\
\hline $1 / 1 / 917: 03$ & Auto FIC & 411.70 & Good & \\
\hline $1 / 2 / 910: 00$ & Manual Tape & 410.50 & Transcribed & \\
\hline $1 / 2 / 917: 02$ & Auto FIC & 411.7 & Good & \\
\hline $1 / 3 / 910: 00$ & Manual Tape & 410.25 & Transcribed & \\
\hline $1 / 3 / 917: 03$ & Auto FIC & 411.6 & Good & \\
\hline $1 / 4 / 910,00$ & Manual Tape & 410.00 & Transcribed & \\
\hline 1/4/91 7:02 & Auto FIC & 411.60 & Good & \\
\hline $1 / 5 / 910: 00$ & Manual Tape & 410 & Transcribed & \\
\hline $1 / 5 / 917: 02$ & Auto FIC & 411.6 & Good & \\
\hline $1 / 6 / 910: 00$ & Manual Tape & 410.00 & Transcribed & \\
\hline $1 / 6 / 917: 02$ & Auto FIC & 411.6 & Good & \\
\hline $1 / 7 / 910: 00$ & Manual Tape & 410.25 & Transcribed & \\
\hline 1/7/91 7:02 & Auto FIC & 411.6 & Good & \\
\hline $1 / 8 / 910: 00$ & Manual Tape & 410.00 & Transcribed & \\
\hline $1 / 8 / 917: 03$ & Auto FIC & 411.60 & Good & \\
\hline $1 / 9 / 910: 00$ & Manual Tape & 410 & Transcribed & \\
\hline $1 / 9 / 917: 02$ & Auto FIC & 411.6 & Good & \\
\hline $1 / 10 / 910: 00$ & Manual Tape & 410.00 & Transcribed & \\
\hline $1 / 10 / 917: 02$ & Auto FIC & 411.5 & Good & \\
\hline $1 / 11 / 910: 00$ & Manual Tape & 410 & Transcribed & \\
\hline $1 / 11 / 917: 03$ & Auto FIC & 411.5 & Good & \\
\hline $1 / 12 / 910: 00$ & Manual Tape & 410 & Transcribed & \\
\hline $1 / 12 / 917: 02$ & Auto FIC & 411.50 & Good & \\
\hline $1 / 13 / 910: 00$ & Manual Tape & 410 & Transcribed & \\
\hline $1 / 13 / 917: 03$ & Auto FIC & 411.5 & Good & \\
\hline $1 / 14 / 910: 00$ & Manual Tape & 410 & Transcribed & \\
\hline $1 / 14 / 91 \quad 7: 04$ & Auto FIC & 411.50 & Good & \\
\hline $1 / 15 / 910: 00$ & Manual Tape & 410 & Transcribed & \\
\hline $1 / 15 / 917: 03$ & Auto FIC & 411.50 & Good & \\
\hline $1 / 16 / 910: 00$ & Manual Tape & 409.75 & Transcribed & \\
\hline $1 / 16 / 917: 04$ & Auto FIC & 411.5 & Good & \\
\hline $1 / 17 / 910: 00$ & Manual Tape & 410 & Transcribed & \\
\hline $1 / 17 / 917: 04$ & Auto FIC & 411.50 & Good & \\
\hline $1 / 18 / 910: 00$ & Manual Tape & 410.00 & Transcribed & \\
\hline $1 / 18 / 917: 04$ & Auto FIC & 411.40 & Good & \\
\hline $1 / 19 / 910: 00$ & Manual Tape & 409.75 & Transcribed & \\
\hline $1 / 19 / 917: 04$ & Auto FIC & 411.40 & Good & \\
\hline $1 / 20 / 910: 00$ & Manual Tape & 409.75 & Transcribed & \\
\hline $1 / 20 / 917: 04$ & Auto FIC & 411.4 & Good & \\
\hline $1 / 21 / 910: 00$ & Manual Tape & 409.75 & Transcribed & \\
\hline $1 / 21 / 917: 04$ & Auto FIC & 411.40 & Good & \\
\hline $1 / 22 / 910: 00$ & Manual Tape & 409.75 & Transcribed & \\
\hline $1 / 22 / 91 \quad 7: 04$ & Auto FIC & 411.4 & Good & \\
\hline $1 / 23 / 910: 00$ & Manual Tape & 409.75 & Transcribed & \\
\hline $1 / 23 / 917: 04$ & Auto FIC & 411.4 & Good & \\
\hline $1 / 24 / 910: 00$ & Manual Tape & 409.75 & Transcribed & \\
\hline $1 / 24 / 917: 04$ & Auto FIC & 411.4 & Good & \\
\hline
\end{tabular}

Data obtained from WHC Surveillance Analysis Computer System, (PC SACS) July 7, 1995. 


\begin{tabular}{|c|c|c|c|c|}
\hline \multicolumn{5}{|c|}{ 241-AP-107 } \\
\hline \multicolumn{5}{|c|}{ Surface Level Data } \\
\hline Date & Type of Measurement & Level (Inches) & Data Quality & Comments \\
\hline 1/25/91 0:00 & Manual Tape & 409.75 & Transcribed & \\
\hline $1 / 25 / 917: 03$ & Auto FIC & 411.4 & Good & \\
\hline $1 / 26 / 910: 00$ & Manual Tape & 409.75 & Transcribed & \\
\hline $1 / 26 / 917: 03$ & Auto FIC & 411.40 & Good & \\
\hline $1 / 27 / 910: 00$ & Manual Tape & 409.75 & Transcribed & \\
\hline $1 / 27 / 917: 03$ & Auto FIC & 411.30 & Good & \\
\hline $1 / 28 / 910: 00$ & Manual Tape & 409.75 & Transcribed & \\
\hline $1 / 28 / 916: 03$ & Auto FIC & 411.3 & Good & \\
\hline $1 / 29 / 910: 00$ & Manual Tape & 409.75 & Transcribed & \\
\hline 1/29/917:02 & Auto FIC & 411.3 & Good & \\
\hline 1/30/91 0:00 & Manual Tape & 410.00 & Transcribed & \\
\hline $1 / 30 / 910: 00$ & Manual FIC & 411.30 & Good & \\
\hline 1/31/910:00 & Manual Tape & 409.50 & Transcribed & \\
\hline $1 / 31 / 917: 02$ & Auto FIC & 411.3 & Good & \\
\hline $2 / 1 / 910: 00$ & Manual Tape & 409.50 & Transcribed & \\
\hline 2/1/917:02 & Auto FIC & 411.30 & Good & \\
\hline $2 / 2 / 910: 00$ & Manual Tape & 409.50 & Transcribed & \\
\hline $2 / 2 / 917: 04$ & Auto FIC & 411.30 & Good & \\
\hline 2/3/91 0:00 & Manual Tape & 409.5 & Transcribed & \\
\hline $2 / 3 / 917: 03$ & Auto FIC & 411.2 & Good & \\
\hline $2 / 4 / 910: 00$ & Manual Tape & 409.50 & Transcribed & \\
\hline $2 / 4 / 917: 03$ & Auto FIC & 411.20 & Good & \\
\hline 2/5/910:00 & Manual Tape & 409.50 & Transcribed & \\
\hline $2 / 5 / 917: 04$ & Auto FIC & 411.2 & Good & \\
\hline $2 / 6 / 910: 00$ & Manual Tape & 409.5 & Transcribed & \\
\hline $2 / 6 / 917: 04$ & Auto FIC & 411.20 & Good & \\
\hline $2 / 7 / 910: 00$ & Manual Tape & 409.50 & Transcribed & \\
\hline $2 / 7 / 917: 04$ & Auto FIC & 411.2 & Good & \\
\hline 2/8/91 0:00 & Manual Tape & 409.5 & Transcribed & \\
\hline 2/8/91 7:04 & Auto FIC & 411.2 & Good & \\
\hline $2 / 9 / 910: 00$ & Manual Tape & 409.50 & Transcribed & \\
\hline $2 / 9 / 917: 04$ & Auto FIC & 411.20 & Good & \\
\hline $2 / 10 / 910: 00$ & Manual Tape & 409.50 & Transcribed & \\
\hline $2 / 10 / 917: 03$ & Auto FIC & 411.2 & Good & \\
\hline $2 / 11 / 910: 00$ & Manual Tape & 409.5 & Transcribed & \\
\hline $2 / 11 / 917: 04$ & Auto FIC & 411.2 & Good & \\
\hline 2/12/91 0:00 & Manual Tape & 409.50 & Transcribed & \\
\hline 2/12/917:04 & Auto FIC & 411.2 & Good & \\
\hline $2 / 13 / 910: 00$ & Manual Tape & 409.50 & Transcribed & \\
\hline 2/13/91 7:04 & Auto FIC & $411: 20$ & Good & \\
\hline 2/14/91 0:00 & Manual Tape & 409.5 & Transcribed & \\
\hline $2 / 14 / 917: 04$ & Auto FIC & 411.20 & Good & \\
\hline 2/15/910:00 & Manual Tape & 409.5 & Transcribed & \\
\hline $2 / 15 / 917: 03$ & Auto FIC & 411.10 & Good & \\
\hline $2 / 16 / 910: 00$ & Manual Tape & 409.5 & Transcribed & \\
\hline 2/16/91 7:02 & Auto FIC & 411.1 & Good & \\
\hline $2 / 17 / 910: 00$ & Manual Tape & 409.50 & Transcribed & \\
\hline 2/17/91 7:02 & Auto FIC & 411.10 & Good & \\
\hline
\end{tabular}

Data obtained from WHC Surveillance Analysis Computer System, (PC SACS) July 7, 1995. 


\begin{tabular}{|c|c|c|c|c|}
\hline \multicolumn{5}{|c|}{ 241-AP-107 } \\
\hline \multicolumn{5}{|c|}{ Surface Level Data } \\
\hline Date & Type of Measurement & Level (Inches) & Data Quality & Comments \\
\hline 2/18/910:00 & Manual Tape & 409.50 & Transcribed & \\
\hline $2 / 18 / 917: 03$ & Auto FIC & 411.1 & Good & \\
\hline $2 / 19 / 910: 00$ & Manual Tape & 409.50 & Transcribed & \\
\hline $2 / 19 / 917: 02$ & Auto FIC & 411.1 & Good & \\
\hline $2 / 20 / 910: 00$ & Manual Tape & 409.5 & Transeribed & \\
\hline $2 / 20 / 917: 03$ & Auto FIC & 411.1 & Good & \\
\hline $2 / 21 / 910: 00$ & Manual Tape & 409.50 & Transcribed & \\
\hline $2 / 21 / 917: 02$ & Auto FIC & 411.1 & Good & \\
\hline $2 / 22 / 910: 00$ & Manual Tape & 409.5 & Transcribed & \\
\hline $2 / 22 / 917: 02$ & Auto FIC & 411.00 & Good & \\
\hline $2 / 23 / 910: 00$ & Manual Tape & 409.5 & Transcribed & \\
\hline $2 / 23 / 917: 02$ & Auto FIC & 411.10 & Good & \\
\hline $2 / 24 / 910: 00$ & Manual Tape & 409.5 & Transcribed & \\
\hline $2 / 24 / 917: 03$ & Auto FIC & 411.10 & Good & \\
\hline $2 / 25 / 910: 00$ & Manual Tape & 409.5 & Transcribed & \\
\hline $2 / 25 / 917: 03$ & Auto FIC & 411.1 & Good & \\
\hline $2 / 26 / 910: 00$ & Manual Tape & 409.50 & Transcribed & \\
\hline $2 / 26 / 917: 03$ & Auto FIC & 411.10 & Good & \\
\hline $2 / 27 / 910: 00$ & Manual Tape & 409,50 & Transcribed & \\
\hline $2 / 27 / 917: 02$ & Auto FIC & 411.00 & Good & \\
\hline $2 / 28 / 910: 00$ & Manual Tape & 409.50 & Transcribed & \\
\hline $2 / 28 / 917: 02$ & Auto FIC & 411.00 & Good & \\
\hline 3/1/91 0:00 & Manual Tape & 409.25 & Transcribed & \\
\hline $3 / 1 / 917: 02$ & Auto FIC & 411 & Good & \\
\hline $3 / 2 / 910: 00$ & Manual Tape & 409.25 & Transcribed & \\
\hline $3 / 2 / 917: 02$ & Auto FIC & 411 & Good & \\
\hline $3 / 3 / 910: 00$ & Manual Tape & 409.25 & Transcribed & \\
\hline $3 / 3 / 917: 03$ & Auto FIC & 411 & Good & \\
\hline $3 / 4 / 910: 00$ & Manual Tape & 409.25 & Transcribed & \\
\hline $3 / 4 / 917: 03$ & Auto FIC & 411.00 & Good & \\
\hline $3 / 5 / 91 \quad 0: 00$ & Manual Tape & 409.25 & Transcribed & \\
\hline $3 / 5 / 917: 03$ & Auto FIC & 411.00 & Good & \\
\hline $3 / 6 / 910: 00$ & Manual Tape & 409.25 & Transcribed & \\
\hline $3 / 6 / 917: 02$ & Auto FIC & 411.00 & Good & \\
\hline $3 / 7 / 910: 00$ & Manual Tape & 409.25 & Transcribed & \\
\hline $3 / 7 / 917: 02$ & Auto FIC & 411.00 & Good & \\
\hline $3 / 8 / 910: 00$ & Manual Tape & 409.25 & Transcribed & \\
\hline $3 / 8 / 917: 02$ & Auto FIC & 411 & Good & \\
\hline $3 / 9 / 910: 00$ & Manual Tape & $409.25^{\circ}$ & Transcribed & \\
\hline $3 / 9 / 917: 02$ & Auto FIC & 411 & Good & \\
\hline $3 / 10 / 910: 00$ & Manual Tape & 409.25 & Transcribed & \\
\hline $3 / 10 / 917: 02$ & Auto FIC & 411 & Good & \\
\hline $3 / 11 / 910: 00$ & Manual Tape & 409.25 & Transcribed & \\
\hline $3 / 11 / 917: 02$ & Auto FIC & 411.00 & Good & \\
\hline $3 / 12 / 910: 00$ & Manual Tape & 409.25 & Transcribed & \\
\hline $3 / 12 / 917: 03$ & Auto FIC & 411 & Good & \\
\hline $3 / 13 / 910: 00$ & Manual Tape & 409.25 & Transcribed & \\
\hline $3 / 13 / 917: 03$ & Auto FIC & 411 & Good & \\
\hline
\end{tabular}

Data obtained from WHC Surveillance Analysis Computer System, (PC SACS) July 7, 1995. 


\begin{tabular}{|c|c|c|c|c|}
\hline \multicolumn{5}{|c|}{ 241-AP-107 } \\
\hline \multicolumn{5}{|c|}{ Surface Level Data } \\
\hline Date & Type of Moasurement & Level (Inches) & Data Quality & Comments \\
\hline $3 / 14 / 910: 00$ & Manual Tape & 409.25 & Transcribed & \\
\hline $3 / 14 / 917: 03$ & Auto FIC & 410.90 & Good & \\
\hline $3 / 15 / 910: 00$ & Manual Tape & 409.25 & Transcribed & \\
\hline $3 / 15 / 917: 03$ & Auto FIC & 410.90 & Good & \\
\hline $3 / 16 / 910: 00$ & Manual Tape & 409.25 & Transcribed & \\
\hline $3 / 16 / 917: 03$ & Auto FIC & 410.90 & Good & \\
\hline 3/17/910:00 & Manual Tape & 409.25 & Transcribed & \\
\hline $3 / 17 / 917: 02$ & Auto FIC & 410.9 & Good & \\
\hline $3 / 18 / 910: 00$ & Manual Tape & 409.25 & Transcribed & \\
\hline $3 / 18 / 917: 03$ & Auto FIC & 410.90 & Good & \\
\hline $3 / 19 / 910: 00$ & Manual Tape & 409.25 & Transcribed & \\
\hline $3 / 19 / 917: 03$ & Auto FIC & 410.90 & Good & \\
\hline $3 / 20 / 910: 00$ & Manual Tape & 409 & Transcribed & \\
\hline $3 / 20 / 917: 02$ & Auto FIC & 410.8 & Good & \\
\hline $3 / 21 / 910: 00$ & Manual Tape & 409.00 & Transcribed & \\
\hline $3 / 21 / 917: 03$ & Auto FIC & 410.8 & Good & \\
\hline $3 / 22 / 910: 00$ & Manual Tape & 409.00 & Transcribed & \\
\hline $3 / 22 / 91 \quad 7: 02$ & Auto FIC & 410.8 & Good & \\
\hline $3 / 23 / 910: 00$ & Manual Tape & 409.00 & Transcribed & \\
\hline $3 / 23 / 917: 03$ & Auto FIC & 410.8 & Good & \\
\hline $3 / 24 / 910: 00$ & Manual Tape & 409.25 & Transcribed & \\
\hline $3 / 24 / 917: 03$ & Auto FIC & 410.80 & Good & \\
\hline $3 / 25 / 910: 00$ & Manual Tape & 409.25 & Transcribed & \\
\hline 3/25/91 7:03 & Auto FIC & 410.8 & Good & \\
\hline $3 / 26 / 910: 00$ & Manual Tape & 409.25 & Transcribed & \\
\hline $3 / 26 / 917: 03$ & Auto FIC & 410.80 & Good & \\
\hline $3 / 27 / 910: 00$ & Manual Tape & 409.25 & Transcribed & \\
\hline $3 / 27 / 917: 03$ & Auto FIC & 410.80 & Good & \\
\hline $3 / 28 / 910: 00$ & Manual Tape & 409.25 & Transcribed & \\
\hline $3 / 28 / 917: 03$ & Auto FIC & 410.8 & Good & \\
\hline $3 / 29 / 910: 00$ & Manual Tape & 409.00 & Transcribed & \\
\hline $3 / 29 / 917: 03$ & Auto FIC & 410.80 & Good & \\
\hline $3 / 30 / 910: 00$ & Manual Tape & 409 & Transcribed & \\
\hline $3 / 30 / 917: 03$ & Auto FIC & 410.80 & Good & \\
\hline $3 / 31 / 910: 00$ & Manual Tape & 409.00 & Transcribed & \\
\hline $3 / 31 / 917: 03$ & Auto FIC & 410.8 & Good & \\
\hline 4/1/91 0:00 & Manual Tape & 409 & Transcribed & \\
\hline 4/1/91 7:03 & Auto FIC & 410.80 & Good & \\
\hline $4 / 2 / 91 \quad 0: 00$ & Manual Tape & 409.00 & Transcribed & \\
\hline 4/2/91 7:03 & Auto FIC & 410.8 & Good & \\
\hline $4 / 3 / 910: 00$ & Manual Tape & 409 & Transçribed & \\
\hline $4 / 3 / 917: 03$ & Auto FIC & 410.80 & Good & \\
\hline 4/4/91 0:00 & Manual Tape & 408.75 & Transcribed & \\
\hline 4/4/91 7:03 & Auto FIC & 410.8 & Good & \\
\hline 4/5/91 $0: 00$ & Manual Tape & 408.75 & Transcribed & \\
\hline $4 / 5 / 917: 03$ & Auto FIC & 410.8 & Good & \\
\hline 4/6/91 0:00 & Manual Tape & 409.25 & Transcribed & \\
\hline 4/6/91 7:03 & Auto FIC & 410.70 & Good & \\
\hline
\end{tabular}

Data obtained from WHC Surveillance Analysis Computer System, (PC SACS) July 7, 1995. 


\begin{tabular}{|c|c|c|c|c|}
\hline \multicolumn{5}{|c|}{ 241-AP-107 } \\
\hline \multicolumn{5}{|c|}{ Surface Level Data } \\
\hline Date & Type of Measurement & Level (Inches) & Data Quality & Comments \\
\hline 4/7/910:00 & Manua! Tape & 408.75 & Transcribed & \\
\hline $4 / 7 / 917: 03$ & Auto FIC & 410.70 & Good & \\
\hline 4/8/91 0:00 & Manual Tape & 408.75 & Transçribed & \\
\hline $4 / 8 / 917: 04$ & Auto FIC & 41.0 .70 & Good & \\
\hline $4 / 9 / 910: 00$ & Manual Tape & 408.75 & Transcribed & \\
\hline $4 / 9 / 917: 03$ & Auto FIC & 410.7 & Good & \\
\hline $4 / 10 / 910: 00$ & Manual Tape & 408.75 & Transcribed & \\
\hline $4 / 10 / 917: 03$ & Auto FIC & 410.7 & Good & \\
\hline $4 / 11 / 910: 00$ & Manual Tape & 408.75 & Transcribed & \\
\hline $4 / 11 / 917: 03$ & Auto FIC & 410.70 & Good & \\
\hline $4 / 12 / 910: 00$ & Manual Tape & 408.75 & Transcribed & \\
\hline $4 / 12 / 917: 03$ & Auto FIC & 410.70 & Good & \\
\hline $4 / 13 / 910: 00$ & Manual Tape & 409.00 & Transcribed & \\
\hline 4/13/91 7:04 & Auto FIC & 410.7 & Good & \\
\hline 4/14/91 0:00 & Manual Tape & 409 & Transcribed & \\
\hline $4 / 14 / 917: 03$ & Auto FIC & 410.7 & Good & \\
\hline $4 / 15 / 910: 00$ & Manual Tape & 409.00 & Transcribed & \\
\hline $4 / 15 / 917: 03$ & Auto FIC & 410.70 & Good & \\
\hline $4 / 16 / 910: 00$ & Manual Tape & 409.00 & Transcribed & \\
\hline $4 / 16 / 910: 00$ & Manual FIC & 410.70 & Good & \\
\hline 4/17/91 $0: 00$ & Manual Tape & 408.75 & Transcribed & \\
\hline 4/17/91 7:03 & Auto FIC & 410.70 & Good & \\
\hline $4 / 18 / 910: 00$ & Manual Tape & 409.00 & Transcribed & \\
\hline 4/18/91 7:06 & Auto FIC & 410.70 & Good & \\
\hline $4 / 19 / 910: 00$ & Manual Tape & 409.00 & Transcribed & \\
\hline $4 / 19 / 917: 05$ & Auto FIC & 410.60 & Good & \\
\hline 4/20/91 0:00 & Manual Tape & 409.00 & Transcribed & \\
\hline $4 / 20 / 917: 06$ & Auto FIC & 410.60 & Good & \\
\hline $4 / 21 / 910: 00$ & Manual Tape & 409 & Transcribed & \\
\hline $4 / 21 / 917: 05$ & Auto FIC & 410.6 & Good & \\
\hline $4 / 22 / 910: 00$ & Manual Tape & 409 & Transcribed & \\
\hline 4/22/917:06 & Auto FIC & 410.60 & Good & \\
\hline $4 / 23 / 910: 00$ & Manual Tape & 409 & Transcribed & \\
\hline 4/23/91 7:06 & Auto FIC & 410.60 & Good & \\
\hline 4/24/91 $0: 00$ & Manual Tape & 409.00 & Transcribed & \\
\hline $4 / 24 / 917: 05$ & Auto FIC & 410.6 & Good & \\
\hline $4 / 25 / 910: 00$ & Manual Tape & 408.75 & Transcribed & \\
\hline $4 / 25 / 917: 05$ & Auto FIC & 41.0 .60 & Good & \\
\hline $4 / 26 / 910: 00$ & Manual Tape & 408.75 & Transcribed & \\
\hline $4 / 26 / 917: 05$ & Auto FIC & 410.6 & Good & \\
\hline 4/27/910:00 & Manual Tape & 408.75 & Transcribed & \\
\hline $4 / 27 / 917: 05$ & Auto FIC & 410.6 & Good & \\
\hline $4 / 28 / 910: 00$ & Manual Tape & 408.5 & Transcribed & \\
\hline $4 / 28 / 917: 06$ & Auto FIC & 410.6 & Good & \\
\hline $4 / 29 / 910: 00$ & Manual Tape & 408.5 & Transcribed & \\
\hline 4/29/91 7:05 & Auto FIC & 410.6 & Good & \\
\hline 4/30/910:00 & Manual Tape & 408.5 & Transcribed & \\
\hline $4 / 30 / 917: 06$ & Auto FIC & 410.60 & Good & \\
\hline
\end{tabular}

Data obtained from WHC Surveillance Analysis Computer System, (PC SACS) July 7, 1995. 


\begin{tabular}{|c|c|c|c|c|}
\hline \multicolumn{5}{|c|}{ 241-AP-107 } \\
\hline \multicolumn{5}{|c|}{ Surface Level Data } \\
\hline Date & Type of Measurement & Level (Inches) & Data Quality & Comments \\
\hline 5/1/91 0:00 & Manual Tape & 408.5 & Transcribed & \\
\hline $5 / 1 / 917: 05$ & Auto FIC & 410.60 & Good & \\
\hline 5/2/91 0:00 & Manual Tape & 408.50 & Transcribed & \\
\hline 5/2/91 7:05 & Auto FIC & 410.50 & Good & \\
\hline 5/3/91 0:00 & Manual Tape & 408.50 & Transcribed & \\
\hline $5 / 3 / 917: 06$ & Auto FIC & 410.60 & Good & \\
\hline $5 / 4 / 910: 00$ & Manual Tape & 408.75 & Transcribed & \\
\hline $5 / 4 / 917: 05$ & Auto FIC & 410.60 & Good & \\
\hline $5 / 5 / 910: 00$ & Manual Tape & 408.75 & Transcribed & \\
\hline $5 / 5 / 917: 05$ & Auto FIC & 410.5 & Good & \\
\hline $5 / 6 / 910: 00$ & Manual Tape & 408.50 & Transcribed & \\
\hline $5 / 6 / 917: 05$ & Auto FIC & 410.5 & Good & \\
\hline $5 / 7 / 910: 00$ & Manual Tape & 408.75 & Transcribed & \\
\hline $5 / 7 / 917: 05$ & Auto FIC & 410.5 & Good & \\
\hline $5 / 8 / 910: 00$ & Manual Tape & 408.75 & Transcribed & \\
\hline $5 / 8 / 917: 05$ & Auto FIC & 410.50 & Good & \\
\hline $5 / 9 / 910: 00$ & Manual Tape & 408.75 & Transçribed & \\
\hline $5 / 9 / 917: 05$ & Auto FIC & 410.5 & Good & \\
\hline $5 / 10 / 910: 00$ & Manual Tape & 408.75 & Transcribed & \\
\hline $5 / 10 / 917: 05$ & Auto FIC & 410.5 & Good & \\
\hline $5 / 11 / 910: 00$ & Manual Tape & 408.50 & Transcribed & \\
\hline $5 / 11 / 917: 05$ & Auto FIC & 410.5 & Good & \\
\hline $5 / 12 / 910: 00$ & Manual Tape & 408.75 & Transcribed & \\
\hline $5 / 12 / 917: 05$ & Auto FIC & 410.50 & Good & \\
\hline $5 / 13 / 910: 00$ & Manual Tape & 408.75 & Transcribed & \\
\hline $5 / 13 / 917: 05$ & Auto FIC & 410,50 & Good & \\
\hline $5 / 14 / 910: 00$ & Manual Tape & 408.75 & Transcribed & \\
\hline $5 / 14 / 917: 05$ & Auto FIC & 410.5 & Good & \\
\hline $5 / 15 / 910: 00$ & Manual Tape & 408.75 & Transcribed & \\
\hline $5 / 15 / 917: 05$ & Auto FIC & 410.50 & Good & \\
\hline $5 / 16 / 910: 00$ & Manual Tape & 408.75 & Transcribed & \\
\hline $5 / 16 / 917: 06$ & Auto FIC & 410.50 & Good & \\
\hline $5 / 17 / 910: 00$ & Manual Tape & 408.75 & Transcribed & \\
\hline $5 / 17 / 917: 06$ & Auto FIC & 410.5 & Good & \\
\hline $5 / 18 / 910: 00$ & Manual Tape & 408.75 & Transcribed & \\
\hline $5 / 18 / 917: 06$ & Auto FIC & 410.5 & Good & \\
\hline 5/19/91 0:00 & Manual Tape & 408.75 & Transcribed & \\
\hline $5 / 19 / 917: 05$ & Auto FIC & 410.50 & Good & \\
\hline $5 / 20 / 910: 00$ & Manual Tape & 408.75 & Transcribed & \\
\hline $5 / 20 / 917: 06$ & Auto FIC & 410.50 & Good & \\
\hline $5 / 21 / 910: 00$ & Manual Tape & 408.75 & Transcribed & \\
\hline $5 / 21 / 917: 06$ & Auto FIC & 410.5 & Good & \\
\hline $5 / 22 / 910: 00$ & Manual Tape & 408.5 & Transcribed & \\
\hline $5 / 22 / 917: 05$ & Auto FIC & 410.50 & Good & \\
\hline $5 / 23 / 910: 00$ & Manual Tape & 408.75 & Transcribed & \\
\hline $5 / 23 / 917: 05$ & Auto FIC & 410.50 & Good & \\
\hline $5 / 24 / 910: 00$ & Manual Tape & 408.75 & Transcribed & \\
\hline $5 / 24 / 917: 05$ & Auto FIC & 410.5 & Good & \\
\hline
\end{tabular}

Data obtained from WHC Surveillance Analysis Computer System, (PC SACS) July 7, 1995. 


\begin{tabular}{|c|c|c|c|c|}
\hline \multicolumn{5}{|c|}{ 241-AP-107 } \\
\hline \multicolumn{5}{|c|}{ Surface Lovel Data } \\
\hline Date & Type of Measurement & Level (Inches) & Date Quality & Comments \\
\hline $5 / 25 / 910: 00$ & Manual Tape & 408.50 & Transcribed & \\
\hline $5 / 25 / 917: 05$ & Auto FIC & 410.5 & Good & \\
\hline $5 / 26 / 910: 00$ & Manual Tape & 408.50 & Transcribed & \\
\hline $5 / 26 / 917: 05$ & Auto FIC & 410.50 & Good & \\
\hline $5 / 27 / 910: 00$ & Manual Tape & $408 . \overline{5}$ & Transcribed & \\
\hline $5 / 27 / 917: 05$ & Auto FIC & 410.5 & Good & \\
\hline $5 / 28 / 910: 00$ & Manual Tape & 408.50 & Transcribed & \\
\hline $5 / 28 / 917: 05$ & Auto FIC & 410.50 & Good & \\
\hline $5 / 29 / 910: 00$ & Manual Tape & 408.25 & Transcribed & \\
\hline $5 / 29 / 917: 05$ & Auto FIC & 410.5 & Good & \\
\hline $5 / 30 / 910: 00$ & Manual Tape & 408.5 & Transcribed & \\
\hline $5 / 30 / 917: 05$ & Auto FIC & 410.5 & Good & \\
\hline $5 / 31 / 910: 00$ & Manual Tape & 408.5 & Transcribed & \\
\hline $5 / 31 / 917: 05$ & Auto FIC & 410.5 & Good & \\
\hline 6/1/91 0:00 & Manual Tape & 408.5 & Transcribed & \\
\hline $6 / 1 / 916: 05$ & Auto FIC & 410.5 & Good & \\
\hline $6 / 2 / 910: 00$ & Manual Tape & 408.50 & Transcribed & \\
\hline $6 / 2 / 913: 05$ & Auto FIC & 410.50 & Good & \\
\hline $6 / 3 / 910: 00$ & Manual Tape & 408.50 & Transcribed & \\
\hline $6 / 3 / 917: 05$ & Auto FIC & 410.5 & Good & \\
\hline 6/4/91 0:00 & Manual Tape & 409 & Transcribed & \\
\hline $6 / 4 / 917: 05$ & Auto FIC & $410 . \overline{40}$ & Good & \\
\hline $6 / 5 / 910: 00$ & Manual Tape & 409.00 & Transcribed & \\
\hline 6/5/91 7:05 & Auto FIC & 410.4 & Good & \\
\hline $6 / 6 / 910: 00$ & Manual Tape & $408.75^{\circ}$ & Transcribed & \\
\hline $6 / 6 / 917: 06$ & Auto FIC & 410.40 & Good & \\
\hline $6 / 7 / 910: 00$ & Manual Tape & 408.75 & Transcribed & \\
\hline $6 / 7 / 917: 05$ & Auto FIC & 410.30 & Good & \\
\hline 6/8/91 0:00 & Manual Tape & 408.75 & Transcribed & \\
\hline $6 / 8 / 917: 05$ & Auto FIC & 410.4 & Good & \\
\hline 6/9/91 0:00 & Manual Tape & 408.75 & Transcribed & \\
\hline $6 / 9 / 917: 05$ & Auto FIC & 410.40 & Good & \\
\hline $6 / 10 / 910: 00$ & Manual Tape & 408.50 & Transcribed & \\
\hline $6 / 10 / 917: 06$ & Auto FIC & 410.40 & Good & \\
\hline $6 / 11 / 910: 00$ & Manual Tape & 408.5 & Transcribed & \\
\hline $6 / 11 / 917: 05$ & Auto FIC & 410.4 & Good & \\
\hline $6 / 12 / 910: 00$ & Manual Tape & 409 & Transcribed & \\
\hline $6 / 12 / 917: 04$ & Auto FIC & 410.4 & Good & \\
\hline $6 / 13 / 917: 05$ & Auto FIC & 410.4 & Good & \\
\hline $6 / 14 / 910: 00$ & Manual Tape & 408.5 & Transcribed & \\
\hline $6 / 14 / 917: 05$ & Auto FIC & 410.40 & Good & \\
\hline $6 / 15 / 910: 00$ & Manual Tape & $408.50^{\circ}$ & Transcribed & \\
\hline $6 / 15 / 917: 05$ & Auto FIC & 410.4 & Good & \\
\hline $6 / 16 / 91 \quad 0: 00$ & Manual Tape & 408.50 & Transcribed & \\
\hline $6 / 16 / 917: 05$ & Auto FIC & 410.40 & Good & \\
\hline $6 / 17 / 910: 00$ & Manual Tape & 408.5 & Transcribed & \\
\hline $6 / 17 / 917: 05$ & Auto FIC & 410.4 & Good & \\
\hline $6 / 18 / 910: 00$ & Manual Tape & 408.5 & Transcribed & \\
\hline
\end{tabular}

Data obtained from WHC Surveillance Analysis Computer System, (PC SACS) July 7, 1995. 


\begin{tabular}{|c|c|c|c|c|}
\hline \multicolumn{5}{|c|}{ 241-AP-107 } \\
\hline \multicolumn{5}{|c|}{ Surface Level Data } \\
\hline Date & Type of Measurement & Leval (Inches) & Data Quality & Comments \\
\hline $6 / 18 / 917: 05$ & Auto FIC & 410.4 & Good & \\
\hline $6 / 19 / 910: 00$ & Manual Tape & 408.5 & Transcribed & \\
\hline $6 / 19 / 917: 05$ & Auto FIC & 410.40 & Good & \\
\hline $6 / 20 / 910: 00$ & Manual Tape & 408.75 & Transcribed & \\
\hline $6 / 20 / 917: 05$ & Auto FIC & 410.4 & Good & \\
\hline $6 / 21 / 910: 00$ & Manual Tape & 408.75 & Transcribed & \\
\hline $6 / 21 / 917: 06$ & Auto FIC & 410.4 & Good & \\
\hline $6 / 22 / 910: 00$ & Manual Tape & 408.75 & Transcribed & \\
\hline $6 / 22 / 913: 06$ & Auto FIC & 410.4 & Good & \\
\hline $6 / 23 / 910: 00$ & Manual Tape & 408.75 & Transcribed & \\
\hline $6 / 23 / 917: 05$ & Auto FIC & 410.40 & Good & \\
\hline $6 / 24 / 910: 00$ & Manual Tape & 408.75 & Transcribed & \\
\hline $6 / 24 / 915: 05$ & Auto FIC & 410.40 & Good & \\
\hline $6 / 25 / 910: 00$ & Manual Tape & 408.75 & Transcribed & \\
\hline $6 / 25 / 917: 06$ & Auto FIC & 410.40 & Good & \\
\hline $6 / 26 / 91 \quad 0: 00$ & Manual Tape & 408.75 & Transcribed & \\
\hline $6 / 26 / 911: 03$ & Auto FIC & 410.4 & Good & \\
\hline $6 / 27 / 910: 00$ & Manual Tape & 408.75 & Transcribed & \\
\hline $6 / 27 / 910: 00$ & Manual FIC & 410.4 & Good & \\
\hline $6 / 28 / 910: 00$ & Manual Tape & 408.75 & Transcribed & \\
\hline $6 / 28 / 917: 03$ & Auto FIC & 410.4 & Good & \\
\hline $6 / 29 / 910: 00$ & Manual Tape & 408.75 & Transcribed & \\
\hline $6 / 29 / 916: 02$ & Auto FIC & 410.40 & Good & \\
\hline $6 / 30 / 910: 00$ & Manual Tape & 408.5 & Transcribed & \\
\hline 6/30/91 0:00 & Manual FIC & 410.40 & Good & \\
\hline $7 / 1 / 910: 00$ & Manual Tape & 408.5 & Transcribed & \\
\hline $7 / 1 / 910: 00$ & Manual FIC & 410.40 & Good & \\
\hline $7 / 2 / 910: 00$ & Manual Tape & $408: 50$ & Transcribed & \\
\hline $7 / 2 / 917: 06$ & Auto FIC & 410.4 & Good & \\
\hline $7 / 3 / 910: 00$ & Manual Tape & 408.50 & Transcribed & \\
\hline 7/3/91 7:06 & Auto FIC & 410.4 & Good & \\
\hline 7/4/91 $0: 00$ & Manual Tape & 408.50 & Transcribed & \\
\hline $7 / 4 / 910: 00$ & Manual FIC & 410.40 & Good & \\
\hline $7 / 5 / 910: 00$ & Manual Tape & 408.5 & Transcribed & \\
\hline $7 / 5 / 910: 00$ & Manual FIC & 410.40 & Good & \\
\hline $7 / 6 / 910: 00$ & Manual Tape & 408.5 & Transcribed & \\
\hline $7 / 6 / 910: 00$ & Manual FIC & 410.40 & Good & \\
\hline $7 / 7 / 910: 00$ & Manual Tape & 408.50 & Transcribed & \\
\hline $7 / 7 / 910: 00$ & Manual FIC & 410.25 & Good & \\
\hline $7 / 8 / 910: 00$ & Manual Tape & 408.5 & Transcribed & \\
\hline 7/8/91 $0: 00$ & Manual FIC & 410.25 & Good & \\
\hline 7/9/91 0:00 & Manual Tape & 408.5 & Transcribed & \\
\hline $7 / 9 / 910: 00$ & Manual FIC & 410.4 & Good & \\
\hline $7 / 10 / 910: 00$ & Manual Tape & 408.50 & Transcribed & \\
\hline $7 / 10 / 910: 00$ & Manual FIC & 410.4 & Good & \\
\hline $7 / 11 / 910: 00$ & Manual Tape & 408.5 & Transcribed & \\
\hline 7/11/91 7:06 & Auto FIC & 410.40 & Good & \\
\hline $7 / 12 / 910: 00$ & Manual Tape & 408.50 & Transcribed & \\
\hline
\end{tabular}

Data obtained from WHC Surveillance Analysis Computer System, (PC SACS) July 7, 1995. 


\begin{tabular}{|c|c|c|c|c|}
\hline \multicolumn{5}{|c|}{ 241-AP-107 } \\
\hline & & Urface Level Dat & & \\
\hline Date & Type of Measurement & Level (Inches) & Data Quality & Comments \\
\hline $7 / 12 / 917: 06$ & Auto FIC & 410.40 & Good & \\
\hline $7 / 13 / 910: 00$ & Manual Tape & 408.50 & Transcribed & \\
\hline $7 / 13 / 917: 06$ & Auto FIC & 410.40 & Good & \\
\hline $7 / 14 / 910: 00$ & Manual Tape & 408.50 & Transcribed & \\
\hline 7/14/91 2:06 & Auto FIC & 410.40 & Good & \\
\hline $7 / 15 / 910: 00$ & Manual Tape & 408.25 & Transcribed & \\
\hline $7 / 15 / 917: 06$ & Auto FIC & 410.40 & Good & \\
\hline $7 / 16 / 910: 00$ & Manual Tape & 408.5 & Transcribed & \\
\hline $7 / 16 / 910: 00$ & Manual FIC & 410.4 & Good & \\
\hline $7 / 17 / 910: 00$ & Manual Tape & 408.25 & Transcribed & \\
\hline $7 / 17 / 910: 05$ & Auto FIC & 410.40 & Good & \\
\hline $7 / 18 / 910: 00$ & Manual Tape & 408.50 & Transcribed & \\
\hline $7 / 18 / 910: 00$ & Manual FIC & 410.40 & Good & \\
\hline $7 / 19 / 910: 00$ & Manual Tape & 408.50 & Transcribed & \\
\hline $7 / 19 / 917: 05$ & Auto FIC & 410.40 & Good & \\
\hline $7 / 20 / 910: 00$ & Manual Tape & 408.50 & Transcribed & \\
\hline $7 / 20 / 912: 05$ & Auto FIC & 410.40 & Good & \\
\hline $7 / 21 / 910: 00$ & Manual Tape & 408.50 & Transcribed & \\
\hline 7/21/911:05 & Auto FIC & 410.4 & Good & \\
\hline $7 / 22 / 910: 00$ & Manual FIC & 410.40 & Transcribed & \\
\hline $7 / 22 / 910: 00$ & Manual Tape & 408.50 & Transcribed & \\
\hline $7 / 23 / 910: 00$ & Manual Tape & $4 \overline{008.50}$ & Transcribed & \\
\hline 7/23/91 0:05 & Auto FIC & 410.4 & Good & \\
\hline $7 / 24 / 910: 00$ & Manual Tape & 408.5 & Transçribed & \\
\hline $7 / 24 / 917: 05$ & Auto FIC & 410.4 & Good & \\
\hline $7 / 25 / 910: 00$ & Manual Tape & 408.50 & Transcribed & \\
\hline $7 / 25 / 91 \quad 1: 03$ & Auto FIC & 410.3 & Good & \\
\hline $7 / 26 / 910: 00$ & Manual Tape & 408.5 & Transcribed & \\
\hline $7 / 26 / 910: 00$ & Manual FIC & 410.40 & Good & \\
\hline $7 / 27 / 910: 00$ & Manual Tape & 408.5 & Transcribed & \\
\hline $7 / 27 / 910: 00$ & Manual FIC & 410.4 & Good & \\
\hline $7 / 28 / 910: 00$ & Manual Tape & 408.5 & Transcribed & \\
\hline $7 / 28 / 910: 00$ & Manual FIC & 410.40 & Good & \\
\hline $7 / 29 / 910: 00$ & Manual Tape & 408.50 & Transcribed & \\
\hline $7 / 29 / 910: 00$ & Manual FIC & 410.40 & Good & \\
\hline $7 / 30 / 910: 00$ & Manual Tape & 408.5 & Transcribed & \\
\hline $7 / 30 / 917: 02$ & Auto FIC & 410.3 & Good & \\
\hline $7 / 31 / 910: 00$ & Manual Tape & 408.50 & Transcribed & \\
\hline $7 / 31 / 91 \quad 7: 02$ & Auto FIC & 410.30 & Good & \\
\hline $8 / 1 / 910: 00$ & Manual Tape & 408.75 & Transcribed & \\
\hline 8/1/91 7:02 & Auto FIC & 410.30 & Good & \\
\hline $8 / 2 / 910: 00$ & Manual Tape & $408: 50$ & Transcribed & \\
\hline $8 / 2 / 917: 02$ & Auto FIC & 410.20 & Good & \\
\hline $8 / 3 / 910: 00$ & Manual Tape & 408.50 & Transcribed & \\
\hline $8 / 3 / 917: 02$ & Auto FIC & 410.30 & Good & \\
\hline 8/4/91 0:00 & Manual Tape & 408.5 & Transcribed & \\
\hline $8 / 4 / 917: 02$ & Auto FIC & 410.30 & Good & \\
\hline $8 / 5 / 910: 00$ & Manual Tape & 408.25 & Transcribed & \\
\hline
\end{tabular}

Data obtained from WHC Surveillance Analysis Computer System, (PC SACS) July 7, 1995. 


\begin{tabular}{|c|c|c|c|c|}
\hline \multicolumn{5}{|c|}{ 241-AP-107 } \\
\hline \multicolumn{5}{|c|}{ Surface Level Data } \\
\hline Date & Type of Measurement & Level (Inches) & Data Quality & Comments \\
\hline $8 / 5 / 917: 02$ & Auto FIC & 410.3 & Good & \\
\hline $8 / 6 / 910: 00$ & Manual Tape & 408.50 & Transcribed & \\
\hline $8 / 6 / 917: 02$ & Auto FIC & 410.30 & Good & \\
\hline $8 / 7 / 910: 00$ & Manual Tape & 408.50 & Transcribed & \\
\hline $8 / 7 / 917: 06$ & Auto FIC & 410.30 & Good & \\
\hline $8 / 8 / 910: 00$ & Manual Tape & 408.50 & Transcribed & \\
\hline 8/8/91 7:06 & Auto FIC & 410.30 & Good & \\
\hline $8 / 9 / 910: 00$ & Manual Tape & 408.50 & Transcribed & \\
\hline $8 / 9 / 917: 04$ & Auto FIC & 410.30 & Good & \\
\hline $8 / 10 / 910: 00$ & Manual Tape & 408.50 & Transcribed & \\
\hline $8 / 10 / 917: 04$ & Auto FIC & 410.30 & Good & \\
\hline $8 / 11 / 910: 00$ & Manual Tape & 408.50 & Transcribed & \\
\hline $8 / 11 / 917: 04$ & Auto FIC & 410.30 & Good & \\
\hline $8 / 12 / 910: 00$ & Manual Tape & 408.50 & Transcribed & \\
\hline $8 / 12 / 917: 04$ & Auto FIC & 410.4 & Good & \\
\hline $8 / 13 / 910: 00$ & Manual Tape & 408.5 & Transcribed & \\
\hline $8 / 13 / 917: 04$ & Auto FIC & 410.3 & Good & \\
\hline $8 / 14 / 910: 00$ & Manual Tape & 408.50 & Transcribed & \\
\hline $8 / 14 / 917: 04$ & Auto FIC & 410.30 & Good & \\
\hline $8 / 15 / 910: 00$ & Manual Tape & 408.5 & Transcribed & \\
\hline $8 / 15 / 917: 03$ & Auto FIC & 410.30 & Good & \\
\hline $8 / 16 / 910: 00$ & Manual Tape & 408.5 & Transcribed & \\
\hline $8 / 16 / 910: 00$ & Manual FIC & 410.30 & Good & \\
\hline $8 / 17 / 910: 00$ & Manual Tape & 408.50 & Transcribed & \\
\hline $8 / 17 / 917: 02$ & Auto FIC & 410.30 & Good & \\
\hline $8 / 18 / 910: 00$ & Manual Tape & 408.50 & Transcribed & \\
\hline $8 / 18 / 917: 03$ & Auto FIC & 410.30 & Good & \\
\hline $8 / 19 / 910: 00$ & Manual Tape & 408.5 & Transcribed & \\
\hline $8 / 19 / 917: 02$ & Auto FIC & 410,30 & Good & \\
\hline $8 / 20 / 910: 00$ & Manual Tape & 408.5 & Transcribed & \\
\hline $8 / 20 / 917: 04$ & Auto FIC & 410.3 & Good & \\
\hline $8 / 21 / 910: 00$ & Manual Tape & 408.50 & Transcribed & \\
\hline $8 / 21 / 917: 03$ & Auto FIC & 410.30 & Good & \\
\hline $8 / 22 / 910: 00$ & Manual Tape & 408.5 & Transcribed & \\
\hline $8 / 22 / 917: 03$ & Auto FIC & 410.30 & Good & \\
\hline $8 / 23 / 910: 00$ & Manual Tape & 408.5 & Transcribed & \\
\hline 8/23/91 7:02 & Auto FIC & 410.3 & Good & \\
\hline $8 / 24 / 910: 00$ & Manual Tape & 408:25 & Transcribed & \\
\hline $8 / 24 / 91 \quad 7: 02$ & Auto FIC & 410.3 & Good & \\
\hline $8 / 25 / 910: 00$ & Manual Tape & 408.50 & Transcribed & \\
\hline $8 / 25 / 917: 02$ & Auto FIC & 410.3 & Good & \\
\hline $8 / 26 / 910: 00$ & Manual Tape & 408.5 & Transcribed & \\
\hline $8 / 26 / 917: 03$ & Auto FIC & 410.30 & Good & \\
\hline $8 / 27 / 910: 00$ & Manual Tape & 408.5 & Transcribed & \\
\hline $8 / 27 / 917: 03$ & Auto FIC & 410.3 & Good & \\
\hline $8 / 28 / 910: 00$ & Manual Tape & 408.5 & Transcribed & \\
\hline $8 / 28 / 917: 03$ & Auto FIC & 410.3 & Good & \\
\hline $8 / 29 / 910: 00$ & Manual Tape & 408.50 & Transcribed & \\
\hline
\end{tabular}

Data obtained from WHC Surveillance Analysis Computer System, (PC SACS) July 7, 1995. 


\begin{tabular}{|c|c|c|c|c|}
\hline \multicolumn{5}{|c|}{ 241-AP-107 } \\
\hline \multicolumn{5}{|c|}{ Surface Level Data } \\
\hline Dato & Type of Measurement & Level (Inches) & Data Quality & Comments \\
\hline $8 / 29 / 917: 02$ & Auto FIC & 410.30 & Good & \\
\hline $8 / 30 / 910: 00$ & Manual Tape & 408.5 & Transcribed & \\
\hline $8 / 30 / 916: 02$ & Auto FIC & 410.30 & Good & \\
\hline $8 / 31 / 910: 00$ & Manual Tape & 408.50 & Transcribed & \\
\hline $8 / 31 / 917: 02$ & Auto FIC & 410.30 & Good & \\
\hline $9 / 1 / 910: 00$ & Manual Tape & 408.50 & Transcribed & \\
\hline $9 / 1 / 917: 02$ & Auto FIC & 410.3 & Good & \\
\hline $9 / 2 / 910: 00$ & Manual Tape & 408.5 & Transcribed & \\
\hline $9 / 2 / 917: 02$ & Auto FIC & 410.20 & Good & \\
\hline 9/3/910:00 & Manual Tape & 408.5 & Transcribed & \\
\hline 9/3/91 7:02 & Auto FIC & 410.2 & Good & \\
\hline 9/4/910:00 & Manual Tape & 408.50 & Transcribed & \\
\hline $9 / 4 / 917: 03$ & Auto FIC & 410.2 & Good & \\
\hline 9/5/910:00 & Manual Tape & 408.5 & Transcribed & \\
\hline 9/5/91 7:02 & Auto FIC & 410.2 & Good & \\
\hline $9 / 6 / 910: 00$ & Manual Tape & 408.5 & Transcribed & \\
\hline 9/6/91 7:02 & Auto FIC & 410.20 & Good & 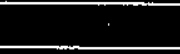 \\
\hline $9 / 7 / 910: 00$ & Manual Tape & 408.25 & Transcribed & \\
\hline $9 / 7 / 917: 02$ & Auto FIC & 410.2 & Good & \\
\hline 9/8/910:00 & Manual Tape & 408.5 & Transcribed & \\
\hline $9 / 8 / 917: 02$ & Auto FIC & 410.20 & Good & \\
\hline $9 / 9 / 910: 00$ & Manual Tape & 408.5 & Transcribed & \\
\hline $9 / 9 / 917: 03$ & Auto FIC & 410.20 & Good & \\
\hline 9/10/91 0:00 & Manual Tape & 408.5 & Transcribed & \\
\hline $9 / 10 / 917: 02$ & Auto FIC & 410.2 & Good & \\
\hline 9/11/91 0:00 & Manual Tape & 408,50 & Transcribed & \\
\hline $9 / 11 / 917: 03$ & Auto FIC & 410.2 & Good & \\
\hline $9 / 12 / 910: 00$ & Manual Tape & 408.5 & Transcribed & \\
\hline $9 / 12 / 917: 02$ & Auto FIC & 410.20 & Good & \\
\hline 9/13/91 0:00 & Manual Tape & 408.50 & Transcribed & \\
\hline $9 / 13 / 917: 02$ & Auto FIC & 410.2 & Good & \\
\hline 9/14/910:00 & Manual Tape & 408.5 & Transcribed & \\
\hline $9 / 14 / 917: 03$ & Auto FIC & 410.20 & Good & \\
\hline $9 / 15 / 910: 00$ & Manual Tape & 408.5 & Transcribed & \\
\hline $9 / 15 / 917: 02$ & Auto FIC & 410.20 & Good & \\
\hline $9 / 16 / 910: 00$ & Manual Tape & 408.50 & Transcribed & \\
\hline $9 / 16 / 917: 03$ & Auto FIC & 410.2 & Good & \\
\hline $9 / 17 / 910: 00$ & Manual Tape & 408.5 & Transcribed & \\
\hline $9 / 17 / 917: 03$ & Auto FIC & 410.2 & Good & \\
\hline $9 / 18 / 910: 00$ & Manual Tape & 408.5 & Transcribed & \\
\hline $9 / 18 / 917: 03$ & Auto FIC & 410.20 & Good & \\
\hline $9 / 19 / 910: 00$ & Manual Tape & 408.50 & Transcribed & \\
\hline $9 / 19 / 917: 03$ & Auto FIC & 410.2 & Good & \\
\hline $9 / 20 / 910: 00$ & Manual Tape & 408.50 & Transcribed & \\
\hline $9 / 20 / 917: 02$ & Auto FIC & 410.20 & Good & \\
\hline $9 / 21 / 910: 00$ & Manual Tape & 408.5 & Transcribed & \\
\hline $9 / 21 / 917: 02$ & Auto FIC & 410.1 & Good & \\
\hline $9 / 22 / 910: 00$ & Manual Tape & 408.5 & Transcribed & \\
\hline
\end{tabular}

Data obtained from WHC Surveillance Analysis Computer System, (PC SACS) July 7, 1995. 


\begin{tabular}{|c|c|c|c|c|}
\hline \multicolumn{5}{|c|}{ 241-AP-107 } \\
\hline \multicolumn{5}{|c|}{ Surface Level Data } \\
\hline Date & Type of Measurement & Level (Inches) & Data Quality & Comments \\
\hline 9/22/91 7:03 & Auto FIC & 410.1 & Good & \\
\hline 9/23/91 0:00 & Manual Tape & 408.5 & Transcribed & \\
\hline $9 / 23 / 917: 03$ & Auto FIC & 410.10 & Good & \\
\hline $9 / 24 / 910: 00$ & Manual Tape & 408.25 & Transcribed & \\
\hline $9 / 24 / 917: 02$ & Auto FIC & 410.10 & Good & \\
\hline 9/25/910:00 & Manual Tape & 408.25 & Transcribed & \\
\hline $9 / 25 / 917: 03$ & Auto FIC & 410.1 & Good & \\
\hline $9 / 26 / 910: 00$ & Manual Tape & 408.25 & Transcribed & \\
\hline $9 / 26 / 917: 03$ & Auto FIC & 410.10 & Good & \\
\hline $9 / 27 / 910: 00$ & Manual Tape & 408.25 & Transcribed & \\
\hline $9 / 27 / 917: 02$ & Auto FIC & 410.10 & Good & \\
\hline $9 / 28 / 910: 00$ & Manual Tape & 408.25 & Transcribed & \\
\hline $9 / 28 / 917: 02$ & Auto FIC & 410.10 & Good & \\
\hline $9 / 29 / 910: 00$ & Manual Tape & 408.25 & Transcribed & \\
\hline $9 / 29 / 917: 03$ & Auto FIC & 410.1 & Good & \\
\hline $9 / 30 / 910: 00$ & Manual Tape & 408.25 & Transcribed & \\
\hline $9 / 30 / 917: 03$ & Auto FIC & 410.1 & Good & \\
\hline $10 / 1 / 910: 00$ & Manual Tape & 408.25 & Transcribed & \\
\hline 10/1/917:02 & Auto FIC & 410.1 & Good & \\
\hline $10 / 2 / 910: 00$ & Manual Tape & 408.25 & Transcribed & \\
\hline $10 / 2 / 917: 02$ & Auto FIC & 410.00 & Good & \\
\hline $10 / 3 / 910: 00$ & Manual Tape & 408.25 & Transcribed & \\
\hline 10/3/917:02 & Auto FIC & 410.00 & Good & \\
\hline 10/4/910:00 & Manual Tape & 408.25 & Transcribed & \\
\hline $10 / 4 / 917: 03$ & Auto FIC & 410 & Good & \\
\hline 10/5/910:00 & Manual Tape & 408.25 & Transcribed & \\
\hline $10 / 5 / 910: 00$ & Manual FIC & 410 & Good & \\
\hline 10/6/910:00 & Manual Tape & 408.25 & Transcribed & \\
\hline $10 / 6 / 917: 03$ & Auto FIC & 410.00 & Good & \\
\hline $10 / 7 / 910: 00$ & Manual Tape & 408.25 & Transcribed & \\
\hline $10 / 7 / 917: 02$ & Auto FIC & 410 & Good & \\
\hline 10/8/91 0:00 & Manual Tape & 408.25 & Transcribed & \\
\hline $10 / 8 / 917: 02$ & Auto FIC & 410.00 & Good & \\
\hline $10 / 9 / 910: 00$ & Manual Tape & 408.25 & Transcribed & \\
\hline 10/9/91 7:02 & Auto FIC & 410.00 & Good & \\
\hline $10 / 10 / 910: 00$ & Manual Tape & 408.25 & Transcribed & \\
\hline $10 / 10 / 917: 02$ & Auto FIC & 410 & Good & \\
\hline 10/11/910:00 & Manual Tape & 408.25 & Transcribed & \\
\hline $10 / 11 / 917: 02$ & Auto FIC & 410.00 & Good & \\
\hline $10 / 12 / 910: 00$ & Manual Tape & 408.25 & Transcribed & \\
\hline $10 / 12 / 917: 02$ & Auto FIC & 410 & Good & \\
\hline $10 / 13 / 910: 00$ & Manual Tape & $408.25^{\circ}$ & Transcribed & \\
\hline $10 / 13 / 917: 03$ & Auto FIC & 410.00 & Good & \\
\hline $10 / 14 / 910: 00$ & Manual Tape & 408.25 & Transcribed & \\
\hline 10/14/91 7:02 & Auto FIC & 410 & Good & \\
\hline $10 / 15 / 910: 00$ & Manual Tape & 408.25 & Transcribed & \\
\hline $10 / 15 / 917: 02$ & Auto FIC & 410 & Good & \\
\hline $10 / 16 / 910: 00$ & Manual Tape & $408: 25$ & Transcribed & \\
\hline
\end{tabular}

Data obtained from WHC Surveillance Analysis Computer System, (PC SACS) July 7, 1995. 


\begin{tabular}{|c|c|c|c|c|}
\hline \multicolumn{5}{|c|}{ 241-AP-107 } \\
\hline \multicolumn{5}{|c|}{ Surface Level Data } \\
\hline Date & Type of Measurement & Level (Inches) & Data Quality & Comments \\
\hline $10 / 16 / 917: 03$ & Auto FIC & 410.00 & Good & \\
\hline $10 / 17 / 910: 00$ & Manual Tape & 408.25 & Transcribed & \\
\hline $10 / 17 / 917: 05$ & Auto FIC & 410 & Good & \\
\hline $10 / 18 / 910: 00$ & Manual Tape & 408.25 & Transcribed & \\
\hline $10 / 18 / 917: 06$ & Auto FIC & 410.00 & Good & \\
\hline 10/19/91 0:00 & Manual Tape & 408.25 & Transcribed & \\
\hline $10 / 19 / 917: 06$ & Auto FIC & 409.90 & Good & \\
\hline $10 / 20 / 910: 00$ & Manual Tape & 408.25 & Transcribed & \\
\hline $10 / 20 / 917: 02$ & Auto FIC & 409.90 & Good & \\
\hline $10 / 21 / 910: 00$ & Manual Tape & 408.25 & Transcribed & \\
\hline $10 / 21 / 917: 02$ & Auto FIC & 409.9 & Good & \\
\hline $10 / 22 / 910: 00$ & Manual Tape & 408.25 & Transcribed & \\
\hline $10 / 22 / 917: 03$ & Auto FIC & 409.90 & Good & \\
\hline $10 / 23 / 910: 00$ & Manua! Tape & 408.25 & Transcribed & \\
\hline $10 / 23 / 917: 02$ & Auto FIC & 409.90 & Good & \\
\hline $10 / 24 / 910: 00$ & Manual Tape & 408.25 & Transcribed & \\
\hline 10/24/91 7:02 & Auto FIC & 409.90 & Good & \\
\hline $10 / 25 / 910: 00$ & Manual Tape & 408.25 & Transcribed & \\
\hline $10 / 25 / 917: 03$ & Auto FIC & 409.90 & Good & \\
\hline $10 / 26 / 910: 00$ & Manual Tape & 408.25 & Transcribed & \\
\hline $10 / 26 / 917: 03$ & Auto FIC & 409.9 & Good & \\
\hline $10 / 27 / 910: 00$ & Manual Tape & 408.50 & Transcribed & \\
\hline $10 / 27 / 917: 02$ & Auto FIC & 409.80 & Good & \\
\hline $10 / 28 / 910: 00$ & Manual Tape & 408.25 & Transcribed & \\
\hline $10 / 28 / 917: 02$ & Auto FIC & 409.8 & Good & \\
\hline $10 / 29 / 910: 00$ & Manual Tape & 408.25 & Transcribed & \\
\hline $10 / 29 / 917: 03$ & Auto FIC & 409.8 & Good & \\
\hline $10 / 30 / 910: 00$ & Manual Tape & 408.25 & Transcribed & \\
\hline $10 / 30 / 917: 03$ & Auto FIC & 409.8 & Good & \\
\hline $10 / 31 / 910: 00$ & Manual Tape & 408.25 & Transcribed & \\
\hline $10 / 31 / 917: 03$ & Auto FIC & 409.80 & Good & \\
\hline $11 / 1 / 910: 00$ & Manual Tape & 408.00 & Transcribed & \\
\hline $11 / 1 / 917: 02$ & Auto FIC & 409.8 & Good & \\
\hline $11 / 2 / 910: 00$ & Manual Tape & 408.00 & Transcribed & \\
\hline $11 / 2 / 917: 03$ & Auto FIC & 409.80 & Good & \\
\hline $11 / 3 / 910: 00$ & Manual Tape & 408 & Transcribed & \\
\hline $11 / 3 / 917: 03$ & Auto FIC & 409.80 & Good & \\
\hline $11 / 4 / 910: 00$ & Manual Tape & 408 & Transcribed & \\
\hline $11 / 4 / 917: 02$ & Auto FIC & 409.70 & Good & \\
\hline $11 / 5 / 910: 00$ & Manual Tape & 408.25 & Transcribed & \\
\hline $11 / 5 / 917: 03$ & Auto FIC & 409.70 & Good & \\
\hline $11 / 6 / 910: 00$ & Manual Tape & 408 & Transcribed & \\
\hline $11 / 6 / 917: 03$ & Auto FIC & 409.7 & Good & \\
\hline 11/7/91 0:00 & Manual Tape & 408.25 & Transçribed & \\
\hline $11 / 7 / 917: 02$ & Auto FIC & 409.70 & Good & \\
\hline $11 / 8 / 910: 00$ & Manual Tape & 408.25 & Transcribed & \\
\hline $11 / 8 / 917: 03$ & Auto FIC & 409.7 & Good & \\
\hline $11 / 9 / 910: 00$ & Manual Tape & 408.25 & Transcribed & \\
\hline
\end{tabular}

Data obtained from WHC Surveillance Analysis Computer System, (PC SACS) July 7, 1995. 


\begin{tabular}{|c|c|c|c|c|c|}
\hline \multicolumn{6}{|c|}{ 241-AP-107 } \\
\hline \multicolumn{6}{|c|}{ Surface Level Data } \\
\hline Date & Type of Measurement & Level (Inches) & Data Quality & Comments & \\
\hline $11 / 9 / 917: 02$ & Auto FIC & 409.7 & Good & . & \\
\hline $11 / 10 / 910: 00$ & Manual Tape & 408 & Transcribed & & \\
\hline $11 / 10 / 917: 03$ & Auto FIC & 409.7 & Good & & \\
\hline $11 / 11 / 910: 00$ & Manual Tape & 408.00 & Transcribed & & $\therefore$ \\
\hline 11/11/916:03 & Auto FIC & 409.7 & Good & & \\
\hline 11/12/910:00 & Manual Tape & 408 & Transcribed & & \\
\hline $11 / 12 / 917: 03$ & Auto FIC & 409.7 & Good & & \\
\hline $11 / 13 / 910: 00$ & Manual Tape & 408 & Transcribed & & \\
\hline $11 / 13 / 917: 03$ & Auto FIC & 409.70 & Good & & \\
\hline $11 / 14 / 910: 00$ & Manual Tape & 408.00 & Transcribed & & \\
\hline $11 / 14 / 917: 03$ & Auto FIC & 409.70 & Good & & \\
\hline $11 / 15 / 910: 00$ & Manual Tape & 408.00 & Transcribed & & \\
\hline $11 / 15 / 917: 03$ & Auto FIC & 409.70 & Good & & \\
\hline $11 / 16 / 910: 00$ & Manual Tape & 408 & Transcribed & & \\
\hline $11 / 16 / 917: 03$ & Auto FIC & 409.7 & Good & & \\
\hline $11 / 17 / 910: 00$ & Manual Tape & 408.00 & Transcibed & & \\
\hline $11 / 17 / 917: 02$ & Auto FIC & 409.60 & Good & & \\
\hline $11 / 18 / 910: 00$ & Manual Tape & 408.00 & Transcribed & & \\
\hline $11 / 18 / 917: 03$ & Auto FIC & 409.60 & Good & & \\
\hline $11 / 19 / 910: 00$ & Manual Tape & 408 & Transcribed & & \\
\hline $11 / 19 / 917: 03$ & Auto FIC & 409.60 & Good & & 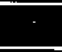 \\
\hline $11 / 20 / 910: 00$ & Manual Tape & 408.00 & Transcribed & & \\
\hline $11 / 20 / 917: 03$ & Auto FIC & 409.6 & Good & & \\
\hline $11 / 21 / 910: 00$ & Manual Tape & 408 & Transcribed & & \\
\hline $11 / 21 / 917: 02$ & Auto FIC & 409.60 & Good & & \\
\hline $11 / 22 / 910: 00$ & Manual Tape & 408.00 & Transcribed & & \\
\hline $11 / 22 / 917: 03$ & Auto FIC & 409.6 & Good & & \\
\hline $11 / 23 / 910: 00$ & Manual Tape & 408.00 & Transcribed & & \\
\hline $11 / 23 / 917: 02$ & Auto FIC & 409.60 & Good & & \\
\hline $11 / 24 / 910: 00$ & Manual Tape & 408.00 & Transcribed & & \\
\hline $11 / 24 / 917: 03$ & Auto FIC & 409.6 & Good & & \\
\hline $11 / 25 / 910: 00$ & Manual Tape & 408.25 & Transcribed & & \\
\hline $11 / 25 / 917: 03$ & Auto FIC & 409.6 & Good & & \\
\hline 11/26/910:00 & Manual Tape & 408 & Transcribed & & \\
\hline $11 / 26 / 910: 00$ & Manual FIC & 409.50 & Good & & \\
\hline $11 / 27 / 910: 00$ & Manual Tape & 408 & Transcribed & & \\
\hline $11 / 27 / 917: 03$ & Auto FIC & 409.50 & Good & & \\
\hline $11 / 28 / 910: 00$ & Manual Tape & 407.75 & Transcribed & & - \\
\hline $11 / 28 / 917: 03$ & Auto FIC & 409.50 & Good & & \\
\hline $11 / 29 / 910: 00$ & Manual Tape & 407.75 & Transcribed & & \\
\hline $11 / 29 / 917: 03$ & Auto FIC & 409.5 & Good & & \\
\hline $11 / 30 / 910: 00$ & Manual Tape & 407.75 & Transcribed & & \\
\hline $11 / 30 / 917: 03$ & Auto FIC & 409.50 & Good & & \\
\hline $12 / 1 / 910: 00$ & Manual Tape & 407.75 & Transcribed & & \\
\hline $12 / 1 / 917: 03$ & Auto FIC & 409.5 & Good & & \\
\hline $12 / 2 / 910: 00$ & Manual Tape & 407.75 & Transcribed & & \\
\hline $12 / 2 / 917: 03$ & Auto FIC & 409.5 & Good & & \\
\hline $12 / 3 / 910: 00$ & Manual Tape & 408 & Transcribed & & \\
\hline
\end{tabular}

Data obtained from WHC Surveillance Analysis Computer System, (PC SACS) July 7, 1995. 


\begin{tabular}{|c|c|c|c|c|}
\hline \multicolumn{5}{|c|}{ 241-AP-107 } \\
\hline \multicolumn{5}{|c|}{ Surface Level Data } \\
\hline Date & Type of Measurement & Level (Inches) & Data Quality & Comments \\
\hline $12 / 3 / 917: 03$ & Auto FIC & 409.5 & Good & \\
\hline $12 / 4 / 910: 00$ & Manual Tape & 408 & Transcribed & \\
\hline $12 / 4 / 917: 03$ & Auto FIC & 409.5 & Good & \\
\hline $12 / 5 / 910: 00$ & Manual Tape & 408.00 & Transcribed & \\
\hline $12 / 5 / 917: 03$ & Auto FIC & 409.5 & Good & \\
\hline $12 / 6 / 910: 00$ & Manual Tape & 407.75 & Transcribed & \\
\hline $12 / 6 / 917: 04$ & Auto FIC & 409.40 & Good & \\
\hline $12 / 7 / 910: 00$ & Manual Tape & 407.75 & Transcribed & \\
\hline $12 / 7 / 917: 03$ & Auto FIC & 409.50 & Good & \\
\hline $12 / 8 / 910: 00$ & Manual Tape & 407.75 & Transcribed & \\
\hline $12 / 8 / 917: 03$ & Auto FIC & 409.4 & Good & \\
\hline 12/9/91 0:00 & Manual Tape & 407.75 & Transcribed & \\
\hline $12 / 9 / 917: 03$ & Auto FIC & 409.40 & Good & \\
\hline $12 / 10 / 910: 00$ & Manual Tape & 407.75 & Transcribed & \\
\hline $12 / 10 / 917: 03$ & Auto FIC & 409.4 & Good & \\
\hline $12 / 11 / 910: 00$ & Manual Tape & 407.75 & Transcribed & \\
\hline $12 / 11 / 917: 02$ & Auto FIC & 409.4 & Good & \\
\hline $12 / 12 / 910: 00$ & Manual Tape & 407.75 & - Transcribed & \\
\hline $12 / 12 / 917: 03$ & Auto FIC & 409.4 & Good & \\
\hline $12 / 13 / 910: 00$ & Manual Tape & 407.75 & Transçribed & \\
\hline $12 / 13 / 917: 05$ & Auto FIC & 409.40 & Good & \\
\hline $12 / 14 / 910: 00$ & Manual Tape & 407.75 & Transcribed & \\
\hline $12 / 14 / 917: 02$ & Auto FIC & 409.3 & Good & \\
\hline $12 / 15 / 910: 00$ & Manual Tape & 407.75 & Transcribed & \\
\hline $12 / 15 / 917: 02$ & Auto FIC & 409.4 & Good & \\
\hline $12 / 16 / 910: 00$ & Manual Tape & 407.75 & Transcribed & \\
\hline $12 / 16 / 917: 03$ & Auto FIC & 409.30 & Good & \\
\hline $12 / 17 / 910: 00$ & Manual Tape & 407.75 & Transcribed & \\
\hline $12 / 17 / 917: 06$ & Auto FIC & 409,30 & Good & \\
\hline $12 / 18 / 910: 00$ & Manual Tape & 407.75 & Transcribed & \\
\hline $12 / 18 / 917: 03$ & Auto FIC & 409.30 & Good & \\
\hline $12 / 19 / 910: 00$ & Manual Tape & 407.75 & Transcribed & \\
\hline $12 / 19 / 917: 03$ & Auto FIC & 409.3 & Good & \\
\hline $12 / 20 / 910: 00$ & Manual Tape & 407.75 & Transcribed & \\
\hline $12 / 20 / 917: 02$ & Auto FIC & 409.30 & Good & \\
\hline $12 / 21 / 910: 00$ & Manual Tape & 407.75 & Transcribed & \\
\hline $12 / 21 / 917: 03$ & Auto FIC & 409.3 & Good & \\
\hline $12 / 22 / 910: 00$ & Manual Tape & $407: 75$ & Transcribed & \\
\hline $12 / 22 / 917: 05$ & Auto FIC & 409.30 & Good & \\
\hline $12 / 23 / 910: 00$ & Manual Tape & 407.5 & Transcribed & \\
\hline $12 / 23 / 917: 06$ & Auto FIC & 409.3 & Good & \\
\hline $12 / 24 / 910: 00$ & Manual Tape & 407.5 & Transcribed & \\
\hline $12 / 24 / 917: 03$ & Auto FIC & 409.2 & Good & \\
\hline $12 / 25 / 910: 00$ & Manual Tape & 407.5 & Transcribed & \\
\hline $12 / 25 / 917: 03$ & Auto FIC & 409.2 & Good & \\
\hline $12 / 26 / 910: 00$ & Manual Tape & 407.50 & Transcribed & \\
\hline $12 / 26 / 917: 03$ & Auțo FIC & 409.20 & Good & \\
\hline $12 / 27 / 910: 00$ & Manual Tape & 407.50 & Transcribed & \\
\hline
\end{tabular}

Data obtained from WHC Surveillance Analysis Computer System, (PC SACS) July 7, 1995. 


\begin{tabular}{|c|c|c|c|c|}
\hline \multicolumn{5}{|c|}{ 241-AP-107 } \\
\hline \multicolumn{5}{|c|}{ Surface Level Data } \\
\hline Date & Type of Measurement & Level (Inches) & Data Quality & Comments \\
\hline 12/27/91 7:03 & Auto FIC & 409.20 & Good & \\
\hline $12 / 28 / 910: 00$ & Manual Tape & 407.5 & Transcribed & \\
\hline $12 / 28 / 917: 02$ & Auto FIC & 409.20 & Good & \\
\hline $12 / 29 / 910: 00$ & Manual Tape & 407.50 & Transcribed & \\
\hline $12 / 29 / 917: 03$ & Auto FIC & 409.2 & Good & \\
\hline $12 / 30 / 910: 00$ & Manual Tape & 407.50 & Transcribed & \\
\hline $12 / 30 / 917: 03$ & Auto FIC & 409.20 & Good & \\
\hline $12 / 31 / 910: 00$ & Manual Tape & 407.5 & Transcribed & \\
\hline 12/31/91 7:03 & Auto FIC & 409.2 & Good & \\
\hline $1 / 1 / 920: 00$ & Manual Tape & 407.50 & Transcribed & \\
\hline $1 / 1 / 927: 03$ & Auto FIC & 409.20 & Good & \\
\hline $1 / 2 / 92 \quad 0: 00$ & Manual Tape & 407.25 & Transcribed & \\
\hline 1/2/92 7:02 & Auto FIC & 409.2 & Good & \\
\hline $1 / 3 / 920: 00$ & Manual Tape & 407.50 & Transcribed & \\
\hline $1 / 3 / 927: 04$ & Auto FIC & 409.20 & Good & \\
\hline $1 / 4 / 920: 00$ & Manual Tape & 407.5 & Transcribed & \\
\hline $1 / 4 / 927: 03$ & Auto FIC & 409.1 & Good & \\
\hline $1 / 5 / 92 \quad 0: 00$ & Manual Tape & 407.50 & Transcribed & \\
\hline 1/5/92 7:03 & Auto FIC & 409.10 & Good & \\
\hline $1 / 6 / 920: 00$ & Manual Tape & 407.50 & Transcribed & \\
\hline $1 / 6 / 927: 03$ & Auto FIC & 409.1 & Good & \\
\hline $1 / 7 / 920: 00$ & Manual Tape & 407.5 & Transcribed & \\
\hline 1/7/92 7:04 & Auto FIC & 409.10 & Good & \\
\hline $1 / 8 / 92 \quad 0: 00$ & Manual Tape & 407.00 & Transcribed & \\
\hline 1/8/92 7:03 & Auto FIC & 409.10 & Good & \\
\hline 1/9/92 0:00 & Manual Tape & 407.50 & Transcribed & \\
\hline 1/9/92 7:03 & Auto FIC & 409.10 & Good & \\
\hline $1 / 10 / 920: 00$ & Manual Tape & 407.5 & Transcribed & \\
\hline $1 / 10 / 927: 04$ & Auto FIC & 409.10 & Good & \\
\hline $1 / 11 / 920: 00$ & Manual Tape & 407.50 & Transcribed & \\
\hline $1 / 11 / 927: 04$ & Auto FIC & 409.1 & Good & \\
\hline $1 / 12 / 920: 00$ & Manual Tape & 407.5 & Transcribed & \\
\hline $1 / 12 / 920: 00$ & Manual FIC & 409.1 & Good & \\
\hline $1 / 13 / 920: 00$ & Manual Tape & 407.25 & Transcribed & \\
\hline 1/13/92 0:00 & Manual FIC & 409.10 & Good & \\
\hline $1 / 14 / 920: 00$ & Manual Tape & 407.25 & Transcribed & \\
\hline $1 / 14 / 920: 00$ & Manual FIC & 409 & Good & \\
\hline $1 / 15 / 920: 00$ & Manual Tape & 407.25 & Transcribed & \\
\hline $1 / 15 / 927: 03$ & Auto FIC & 409.00 & Good & \\
\hline $1 / 16 / 920: 00$ & Manual Tape & 407.25 & Transcribed & \\
\hline $1 / 16 / 927: 04$ & Auto FIC & 409 & Good & \\
\hline $1 / 17 / 920: 00$ & Manual Tape & 407.25 & Transcribed & \\
\hline $1 / 17 / 927: 04$ & Auto FIC & 409.00 & Good & \\
\hline $1 / 18 / 920: 00$ & Manual Tape & 406.00 & Transcribed & \\
\hline $1 / 18 / 927: 04$ & Auto FIC & 409.00 & Good & \\
\hline $1 / 19 / 920: 00$ & Manual Tape & 407.00 & Transcribed & \\
\hline $1 / 19 / 927: 03$ & Auto FIC & 409 & Good & \\
\hline $1 / 20 / 920: 00$ & Manual Tape & 407.25 & Transcribed & \\
\hline
\end{tabular}

Data obtained from WHC Surveillance Analysis Computer System, (PC SACS) July 7, 1995. 


\begin{tabular}{|c|c|c|c|c|}
\hline \multicolumn{5}{|c|}{ 241-AP-107 } \\
\hline \multicolumn{5}{|c|}{ Surface Level. Data } \\
\hline Date & Type of Measurement & Level (Inches) & Dats Quality & Comments \\
\hline $1 / 20 / 927: 03$ & Auto FIC & 409.00 & Good & \\
\hline $1 / 21 / 920: 00$ & Manual Tape & 407.25 & Transcribed & \\
\hline $1 / 21 / 927: 03$ & Auto FIC & 409 & Good & \\
\hline $1 / 22 / 920: 00$ & Manual Tape & 407.25 & Transcribed & \\
\hline $1 / 22 / 927: 06$ & Auto FIC & 409 & Good & \\
\hline $1 / 23 / 920: 00$ & Manual Tape & 407.25 & Transcribed & \\
\hline $1 / 23 / 927: 03$ & Auto FIC & 409.00 & Good & \\
\hline $1 / 24 / 920: 00$ & Manual Tape & 407.25 & Transcribed & \\
\hline $1 / 24 / 927: 02$ & Auto FIC & 409 & Good & \\
\hline $1 / 25 / 920: 00$ & Manual Tape & 407.25 & Transcribed & \\
\hline $1 / 25 / 927: 03$ & Auto FIC & 409.00 & Good & \\
\hline $1 / 26 / 920: 00$ & Manual Tape & 407.25 & Transcribed & \\
\hline $1 / 26 / 926: 03$ & Auto FIC & 409.00 & Good & \\
\hline $1 / 27 / 920: 00$ & Manual Tape & 407.00 & Transcribed & \\
\hline $1 / 27 / 927: 02$ & Auto FIC & 408.90 & Good & \\
\hline $1 / 28 / 920: 00$ & Manual Tape & 407 & Transcribed & \\
\hline $1 / 28 / 927: 03$ & Auto FIC & 408.9 & Good & \\
\hline $1 / 29 / 920: 00$ & Manual Tape & 407.25 & Transcribed & \\
\hline $1 / 29 / 927: 03$ & Auto FIC & 408.9 & Good & \\
\hline $1 / 30 / 920: 00$ & Manual Tape & 407.25 & Transcribed & \\
\hline 1/30/92 7:03 & Auto FIC & 408.9 & Good & \\
\hline $1 / 31 / 920: 00$ & Manual Tape & 407.25 & Transcribed & \\
\hline $1 / 31 / 927: 03$ & Auto FIC & 408.90 & Good & \\
\hline $2 / 1 / 920: 00$ & Manual Tape & 407.25 & Transcribed & \\
\hline $2 / 1 / 927: 02$ & Auto FIC & 408.9 & Good & \\
\hline $2 / 2 / 920: 00$ & Manual Tape & 407.25 & Transcribed & \\
\hline $2 / 2 / 927: 03$ & Auto FIC & 408.9 & Good & \\
\hline $2 / 3 / 920: 00$ & Manual Tape & 407.25 & Transcribed & \\
\hline $2 / 3 / 927: 03$ & Auto FIC & 408.90 & Good & \\
\hline $2 / 4 / 920: 00$ & Manual Tape & 407.25 & Transcribed & \\
\hline $2 / 4 / 927: 03$ & Auto FIC & 408.90 & Good & \\
\hline $2 / 5 / 920: 00$ & Manual Tape & 407.25 & Transcribed & \\
\hline $2 / 5 / 927: 02$ & Auto FIC & 408.90 & Good & \\
\hline $2 / 6 / 920: 00$ & Manual FIC & 408.9 & Transcribed & \\
\hline $2 / 6 / 920: 00$ & Manual Tape & 407.25 & Transcribed & \\
\hline $2 / 7 / 920: 00$ & Manual Tape & 407.00 & Transcribed & \\
\hline $2 / 7 / 920: 00$ & Manual FIC & 408.9 & Good & \\
\hline $2 / 8 / 920: 00$ & Manual Tape & 407.00 & Transcribed & \\
\hline $2 / 8 / 927: 04$ & Auto FIC & 408.90 & Good & \\
\hline $2 / 9 / 920: 00$ & Manual Tape & 407 & Transcribed & \\
\hline 2/9/92 7:03 & Auto FIC & 408.8 & Good & \\
\hline $2 / 10 / 920: 00$ & Manual Tape & 407.00 & Transcribed & \\
\hline $2 / 10 / 927: 04$ & Auto FIC & 408.80 & Good & \\
\hline $2 / 11 / 920: 00$ & Manual Tape & 407 & Transcribed & \\
\hline $2 / 11 / 927: 03$ & Auto FIC & 408.80 & Good & \\
\hline $2 / 12 / 920: 00$ & Manual Tape & 407.00 & Transcribed & \\
\hline $2 / 12 / 927: 03$ & Auto FIC & 408.80 & Good & \\
\hline $2 / 13 / 920: 00$ & Manual Tape & 407 & Transcribed & \\
\hline
\end{tabular}

Data obtained from WHC Surveillance Analysis Computer System, (PC SACS) July 7, 1995. 


\begin{tabular}{|c|c|c|c|c|}
\hline \multicolumn{5}{|c|}{ 241-AP-107 } \\
\hline \multicolumn{5}{|c|}{ Surface Level Data } \\
\hline Date & Type of Measurement & Level (Inches) & Data Quality & Comments \\
\hline $2 / 13 / 927: 03$ & Auto FIC & 408.8 & Good & \\
\hline $2 / 14 / 920: 00$ & Manual Tape & 407 & Transćribed & \\
\hline $2 / 14 / 927: 03$ & Auto FIC & 408.80 & Good & \\
\hline $2 / 15 / 920: 00$ & Manual Tape & 407.00 & Transcribed & \\
\hline $2 / 15 / 927: 03$ & Auto FIC & 408.80 & Good & \\
\hline $2 / 16 / 920: 00$ & Manual Tape & 407.00 & Transcribed & \\
\hline $2 / 16 / 927: 03$ & Auto FIC & 408.8 & Good & \\
\hline $2 / 17 / 920: 00$ & Manual Tape & 407.00 & Transcribed & \\
\hline $2 / 17 / 927: 03$ & Auto FIC & 408.8 & Good & \\
\hline $2 / 18 / 920: 00$ & Manual Tape & 407 & Transcribed & \\
\hline $2 / 18 / 927: 03$ & Auto FIC & 408.80 & Good & \\
\hline $2 / 19 / 920: 00$ & Manual Tape & 407.00 & Transcribed & \\
\hline 2/19/92 7:03 & Auto FIC & 408.8 & Good & \\
\hline $2 / 20 / 920: 00$ & Manual Tape & 407.00 & Transcribed & \\
\hline $2 / 20 / 927: 03$ & Auto FIC & 408.80 & Good & \\
\hline $2 / 21 / 920: 00$ & Manual Tape & 407 & Transcribed & \\
\hline $2 / 21 / 927: 03$ & Auto FIC & 408.8 & Good & \\
\hline $2 / 22 / 920: 00$ & Manual Tape & 407 & Transcribed & \\
\hline $2 / 22 / 927: 03$ & Auto FIC & 408,80 & Good & \\
\hline $2 / 23 / 920: 00$ & Manual Tape & 407 & Transcribed & \\
\hline $2 / 23 / 927: 03$ & Auto FIC & 408.8 & Good & \\
\hline $2 / 24 / 920: 00$ & Manual Tape & 407 & Transcribed & \\
\hline $2 / 24 / 927: 03$ & Auto FIC & 408.7 & Good & \\
\hline $2 / 25 / 920: 00$ & Manual Tape & $40 \overline{7}$ & Transcribed & \\
\hline $2 / 25 / 927: 04$ & Auto FIC & 408.7 & Good & \\
\hline $2 / 26 / 920: 00$ & Manual Tape & 407 & Transcribed & \\
\hline $2 / 26 / 927: 03$ & Auto FIC & 408.70 & Good & \\
\hline $2 / 27 / 920: 00$ & Manual Tape & 407 & Transcribed & \\
\hline $2 / 27 / 927: 04$ & Auto FIC & $\cdot \quad \mathbf{4 0 8 . 7 0}$ & Good & \\
\hline $2 / 28 / 920: 00$ & Manual Tape & 407.00 & Transcribed & \\
\hline $2 / 28 / 927: 04$ & Auto FIC & 408.7 & Good & \\
\hline $2 / 29 / 920: 00$ & Manual Tape . & 407.00 & Transcribed & \\
\hline $2 / 29 / 927: 03$ & Auto FIC & 408.70 & Good & \\
\hline $3 / 1 / 920: 00$ & Manual Tape & 407.00 & Transcribed & \\
\hline $3 / 1 / 927: 03$ & Auto FIC & 408.70 & Good & \\
\hline $3 / 2 / 920: 00$ & Manual Tape & 407.00 & Transcribed & \\
\hline $3 / 2 / 927: 03$ & Auto FIC & 408.70 & Good & \\
\hline 3/3/92 0:00 & Manual Tape & 407.00 & Transcribed & \\
\hline $3 / 3 / 92$ 7:04 & Auto FIC & 408.7 & Good & \\
\hline $3 / 4 / 920: 00$ & Manual Tape & 407 & Transcribed & \\
\hline $3 / 4 / 92 \quad 7: 04$ & Auto FIC & 408.7 & Good & \\
\hline $3 / 5 / 920: 00$ & Manual Tape & 407 & Transcribed & \\
\hline $3 / 5 / 927: 03$ & Auto FIC & 408.70 & Good & \\
\hline $3 / 6 / 92 \quad 0: 00$ & Manual Tape & 407.00 & Transçribed & \\
\hline $3 / 6 / 927: 03$ & Auto FIC & 408.7 & Good & \\
\hline $3 / 7 / 920: 00$ & Manual Tape & 407 & Transcribed & \\
\hline $3 / 7 / 927: 02$ & Auto FIC & 408.7 & Good & \\
\hline $3 / 8 / 920: 00$ & Manual Tape & 407 & Transcribed & \\
\hline
\end{tabular}

Data obtained from WHC Surveiliance Analysis Computer System, (PC SACS) July 7, 1995. 


\begin{tabular}{|c|c|c|c|c|}
\hline \multicolumn{5}{|c|}{ 241-AP-107 } \\
\hline \multicolumn{5}{|c|}{ Surface Level Data } \\
\hline Date & Type of Measurement & Level (Inches) & Data Quality & Comments \\
\hline $3 / 8 / 927: 02$ & Auto FIC & 408.70 & Good & \\
\hline $3 / 9 / 920: 00$ & Manual Tape & 406.5 & Transcribed & \\
\hline $3 / 9 / 927: 02$ & Auto FIC & 408.70 & Good & \\
\hline $3 / 10 / 920: 00$ & Manual Tape & 406.5 & Transcribed & \\
\hline $3 / 10 / 927: 03$ & Auto FIC & 408.70 & Good & \\
\hline $3 / 11 / 920: 00$ & Manual Tape & 407 & Transcribed & \\
\hline $3 / 11 / 927: 03$ & Auto FIC & 408.6 & Good & \\
\hline $3 / 12 / 920: 00$ & Manual Tape & 418.75 & Transcribed & \\
\hline $3 / 12 / 927: 03$ & Auto FIC & 408.50 & Good & \\
\hline $3 / 13 / 920: 00$ & Manual Tape & 406.75 & Transcribed & \\
\hline $3 / 13 / 927: 03$ & Auto FIC & 408.5 & Good & \\
\hline $3 / 14 / 920: 00$ & Manual Tape & 407.00 & Transcribed & \\
\hline $3 / 14 / 927: 03$ & Auto FIC & 408.60 & Good & \\
\hline 3/15/92 0:00 & Manual Tape & 407.00 & Transcribed & \\
\hline $3 / 15 / 927: 06$ & Auto FIC & 408.60 & Good & \\
\hline $3 / 16 / 920: 00$ & Manual Tape & 407 & Transcribed & \\
\hline $3 / 16 / 927: 03$ & Auto FIC & 408.6 & Good & \\
\hline $3 / 17 / 92 \quad 0: 00$ & Manual Tape & 407.00 & Transcribed & \\
\hline $3 / 17 / 927: 03$ & Auto FIC & 0 & Suspect & Data not included in graph \\
\hline $3 / 18 / 920: 00$ & Manual Tape & 407 & Transcribed & \\
\hline $3 / 18 / 927: 03$ & Auto FIC & 0.00 & Suspect & Data not included in graph \\
\hline 3/19/92 0:00 & Manual Tape & 407 & Transcribed & \\
\hline 3/19/92 6:02 & Auto FIC & 408.60 & Good & \\
\hline $3 / 20 / 920: 00$ & Manual Tape & 406.75 & Transcribed & \\
\hline $3 / 20 / 927: 02$ & Auto FIC & 408.60 & Good & \\
\hline $3 / 21 / 92 \quad 0: 00$ & Manual Tape & 406.75 & Transcribed & \\
\hline $3 / 21 / 927: 03$ & Auto FIC & $408.60^{\circ}$ & Good & \\
\hline $3 / 22 / 920: 00$ & Manual Tape & 406.75 & Transcribed & \\
\hline 3/22/92 7:02 & Auto FIC & 408.60 & Good & \\
\hline $3 / 23 / 920: 00$ & Manual Tape & 406.75 & Transcribed & \\
\hline $3 / 23 / 927: 02$ & Auto FIC & 408.5 & Good & \\
\hline $3 / 24 / 920: 00$ & Manual Tape & 406.75 & Transcribed & \\
\hline $3 / 24 / 927: 02$ & Auto FIC & 408.5 & Good & \\
\hline $3 / 25 / 920: 00$ & Manual Tape & 406.75 & Transcribed & \\
\hline $3 / 25 / 927: 03$ & Auto FIC & 408.50 & Good & \\
\hline $3 / 26 / 920: 00$ & Manual Tape & 406.75 & Transcribed & \\
\hline $3 / 26 / 927: 03$ & Auto FIC & 408.5 & Good & \\
\hline $3 / 27 / 920: 00$ & Manual Tape & 406.75 & Transcribed & \\
\hline $3 / 27 / 927: 02$ & Auto FIC & 408.5 & Good & \\
\hline $3 / 28 / 920: 00$ & Manual Tape & 406.75 & Transcribed & \\
\hline $3 / 28 / 927: 03$ & Auto FIC & 408.50 & Good & \\
\hline $3 / 29 / 920: 00$ & Manual Tape & 406.75 & Transcribed & \\
\hline $3 / 29 / 927: 03$ & Auto FIC & 408.5 & Good & \\
\hline 3/30/92 0:00 & Manual Tape & 406.75 & Transcribed & \\
\hline $3 / 30 / 92$ 7:03 & Auto FIC & 408.50 & Good & \\
\hline $3 / 31 / 920: 00$ & Manual Tape & 406.75 & Transcribed & \\
\hline $3 / 31 / 927: 03$ & Auto FIC & 408.5 & Good & \\
\hline 4/1/92 0:00 & Manual Tape & 406.75 & Transcribed & \\
\hline
\end{tabular}

Data obtained from WHC Surveillance Analysis Computer System, (PC SACS) July 7, 1995. 


\begin{tabular}{|c|c|c|c|c|}
\hline \multicolumn{5}{|c|}{ 241-AP-107 } \\
\hline \multicolumn{5}{|c|}{ Surface Level Data } \\
\hline Date & Type of Measurement & Level (Inches) & Data Quality & Comments \\
\hline 4/1/92 7:03 & Auto FIC & 408.5 & Good & \\
\hline $4 / 2 / 920: 00$ & Manual Tape & 406.75 & Transcribed & \\
\hline $4 / 2 / 927: 03$ & Auto FIC & 408.5 & Good & \\
\hline $4 / 3 / 920: 00$ & Manual Tape & 406.75 & Transcribed & \\
\hline $4 / 3 / 927: 03$ & Auto FIC & 408.50 & Good & \\
\hline $4 / 4 / 920: 00$ & Manual Tape & 406.75 & Transcribed & \\
\hline $4 / 4 / 927: 03$ & Auto FIC & 408.5 & Good & \\
\hline $4 / 5 / 920: 00$ & Manual Tape & 406.75 & Transcribed & \\
\hline $4 / 5 / 927: 03$ & Auto FIC & 408.40 & Good & \\
\hline $4 / 6 / 920: 00$ & Manual Tape & 406.75 & Transcribed & \\
\hline $4 / 6 / 927: 03$ & Auto FIC & 408.40 & Good & \\
\hline $4 / 7 / 920: 00$ & Manual Tape & 406.75 & Transcribed & \\
\hline $4 / 7 / 927: 03$ & Auto FIC & 408.40 & Good & \\
\hline $4 / 8 / 920: 00$ & Manual Tape & 406.25 & Transcribed & \\
\hline 4/8/92 5:02 & Auto FIC & 408.40 & Good & \\
\hline $4 / 9 / 920: 00$ & Manual Tape & 406.5 & Transcribed & \\
\hline $4 / 9 / 927: 03$ & Auto FIC & 408.40 & Good & \\
\hline $4 / 10 / 920: 00$ & Manual Tape & 406.75 & Transcribed & \\
\hline $4 / 10 / 927: 03$ & Auto FIC & 408.4 & Good & \\
\hline $4 / 11 / 920: 00$ & Manual Tape & 406.75 & Transcribed & \\
\hline $4 / 11 / 927: 03$ & Auto FIC & 408.4 & Good & \\
\hline $4 / 12 / 920: 00$ & Manual Tape & 406.75 & Transcribed & \\
\hline 4/12/92 5:03 & Auto FIC & 408.40 & Good & \\
\hline $4 / 13 / 920: 00$ & Manual Tape & 406.75 & Transcribed & \\
\hline $4 / 13 / 927: 03$ & Auto FIC & 408.4 & Good & \\
\hline $4 / 14 / 920: 00$ & Manual Tape & 406.75 & Transcribed & \\
\hline $4 / 14 / 927: 03$ & Auto FIC & 408.4 & Good & \\
\hline $4 / 15 / 920: 00$ & Manual Tape & 406.75 & Transcribed & \\
\hline $4 / 15 / 927: 02$ & Auto FIC & 408.40 & Good & \\
\hline $4 / 16 / 920: 00$ & Manual Tape & 406.75 & Transcribed & \\
\hline $4 / 16 / 927: 03$ & Auto FIC & 408.40 & - Good & \\
\hline 4/17/92 0:00 & Manual Tape & 406.75 & Transcribed & \\
\hline 4/17/92 7:03 & Auto FIC & 408.4 & Good & \\
\hline 4/18/92 0:00 & Manual Tape & 406.75 & Transcribed & \\
\hline $4 / 18 / 927: 02$ & Auto FIC & 408.4 & Good & \\
\hline 4/19/92 0:00 & Manual Tape & 406.75 & Transcribed & \\
\hline $4 / 19 / 927: 02$ & Auto FIC & 408.40 & Good & \\
\hline $4 / 20 / 920: 00$ & Manual Tape & 406.75 & Transcribed & \\
\hline $4 / 20 / 927: 03$ & Auto FIC & . 408.40 & Good & \\
\hline $4 / 21 / 920: 00$ & Manual Tape & 406.75 & Transcribed & \\
\hline $4 / 21 / 927: 03$ & Auto FIC & 408.4 & Good & \\
\hline 4/22/92 0:00 & Manual Tape & 406.75 & Transcribed & \\
\hline 4/22/92 7:02 & Auto FIC & 408.40 & Good & \\
\hline $4 / 23 / 920: 00$ & Manual Tape & 406.5 & Transcribed & \\
\hline $4 / 23 / 927: 03$ & Auto FIC & 408.30 & Good & \\
\hline 4/24/92 0:00 & Manual Tape & 406.50 & Transcribed & \\
\hline $4 / 24 / 927: 03$ & Auto FIC & 408.30 & Good & \\
\hline $4 / 25 / 920: 00$ & Manual Tape & 406.5 & Transcribed & \\
\hline
\end{tabular}

Data obtained from WHC Surveillance Analysis Computer System, (PC SACS) July 7, 1995. 


\begin{tabular}{|c|c|c|c|c|}
\hline \multicolumn{5}{|c|}{ 241-AP-107 } \\
\hline \multicolumn{5}{|c|}{ Surface Level Data } \\
\hline Date & Type of Measurement & Level (Inches) & Data Quality & Comments \\
\hline 4/25/92 7:04 & Auto FIC & 408.30 & Good & \\
\hline $4 / 26 / 920: 00$ & Manual Tape & 406.5 & Transcribed & \\
\hline $4 / 26 / 927: 03$ & Auto FIC & 408.30 & Good & \\
\hline $4 / 27 / 920: 00$ & Manual Tape & 406.75 & Transcribed & \\
\hline 4/27/92 7:04 & Auto FIC & 408.3 & Good & \\
\hline $4 / 28 / 920: 00$ & Manual Tape & 406.50 & Transcribed & \\
\hline $4 / 28 / 927: 03$ & Auto FIC & 408.3 & Good & \\
\hline $4 / 29 / 920: 00$ & Manual Tape & 406.75 & Transcribed & \\
\hline 4/29/92 7:03 & Auto FIC & 408.3 & Good & \\
\hline 4/30/92 0:00 & Manual Tape & 406.50 & Transcribed & \\
\hline 4/30/92 7:03 & Auto FIC & 408.30 & Good & \\
\hline $5 / 1 / 920: 00$ & Manual Tape & 406.5 & Transcribed & \\
\hline $5 / 1 / 927: 03$ & Auto FIC & 408.3 & Good & \\
\hline $5 / 2 / 920: 00$ & Manual Tape & 406.5 & Transcribed & \\
\hline $5 / 2 / 922: 03$ & Auto FIC & 408.3 & Good & \\
\hline $5 / 3 / 920: 00$ & Manual Tape & 406.50 & Transcribed & \\
\hline $5 / 3 / 927: 03$ & Auto FIC & 408.3 & Good & \\
\hline $5 / 4 / 920: 00$ & Manual Tape & 406.5 & Transcribed & \\
\hline $5 / 4 / 920: 00$ & Manual FIC & 408.30 & Good & \\
\hline $5 / 4 / 927: 03$ & Auto FIC & 0 & Suspect & Data not included in graph \\
\hline $5 / 5 / 927: 04$ & Auto FIC & 408.30 & Good & \\
\hline $5 / 6 / 927: 03$ & Auto FIC & 408.30 & Good & \\
\hline $5 / 7 / 920: 00$ & Manual Tape & 406.5 & Transcribed & \\
\hline $5 / 7 / 927: 04$ & Auto FIC & 408.30 & Good & \\
\hline $5 / 8 / 920: 00$ & Manual Tape & 406.25 & Transcribed & \\
\hline $5 / 8 / 927: 03$ & Auto FIC & 408.3 & Good & \\
\hline $5 / 9 / 920: 00$ & Manual Tape & 406.75 & Transcribed & \\
\hline $5 / 9 / 920: 00$ & Manual FIC & 408.3 & Good & \\
\hline $5 / 10 / 920: 00$ & Manual Tape & 406.75 & Transcribed & \\
\hline $5 / 10 / 926: 03$ & Auto FIC & 408.3 & Good & \\
\hline $5 / 11 / 920: 00$ & Manual Tape & 404.50 & Transcribed & . \\
\hline $5 / 11 / 920: 00$ & Manual FIC & 408.30 & Good & \\
\hline $5 / 12 / 920: 00$ & Manual Tape & 405.75 & Transcribed & \\
\hline $5 / 12 / 927: 03$ & Auto FIC & 408.30 & Good & \\
\hline $5 / 13 / 920: 00$ & Manual Tape & 405.75 & Transcribed & \\
\hline $5 / 13 / 927: 03$ & Auto FIC & 408.30 & Good & \\
\hline $5 / 14 / 920: 00$ & Manual Tape & 405.75 & Transcribed & \\
\hline $5 / 14 / 927: 03$ & Auto FIC & 408.30 & Good & \\
\hline $5 / 15 / 920: 00$ & Manual Tape & 406.50 & Transcribed & \\
\hline $5 / 15 / 927: 03$ & Auto FIC & 408.30 & Good & \\
\hline $5 / 16 / 920: 00$ & Manual Tape & 406.50 & Transcribed & \\
\hline $5 / 16 / 927: 03$ & Auto FIC & 408.3 & Good & \\
\hline $5 / 17 / 920: 00$ & Manual Tape & 406.50 & Transcribed & \\
\hline $5 / 17 / 927: 02$ & Auto FIC & 408.3 & Good & \\
\hline $5 / 18 / 920: 00$ & Manual Tape & 406.50 & Transcribed & \\
\hline $5 / 18 / 927: 03$ & Auto FIC & 408.3 & Good & \\
\hline $5 / 19 / 920: 00$ & Manual Tape & 406.25 & Transcribed & \\
\hline $5 / 19 / 927: 03$ & Auto FIC & 408.20 & Good & \\
\hline
\end{tabular}

Data obtained from WHC Surveillance Analysis Computer System, (PC SACS) July 7, 1995. 


\begin{tabular}{|c|c|c|c|c|}
\hline \multicolumn{5}{|c|}{ 241-AP-107 } \\
\hline \multicolumn{5}{|c|}{ Surface Level Data } \\
\hline Date & Type of Measurement & Level (Inches) & Data Quality & Comments \\
\hline $5 / 20 / 920: 00$ & Manual Tape & 406.25 & Transcribed & \\
\hline $5 / 20 / 927: 03$ & Auto FIC & 408.2 & Good & \\
\hline $5 / 21 / 920: 00$ & Manual Tape & 406.25 & Transcribed & $\cdot$ \\
\hline $5 / 21 / 927: 03$ & Auto FIC & 408.2 & Good & \\
\hline 5/22/92 0:00 & Manual Tape & 406.25 & Transcribed & \\
\hline $5 / 22 / 927: 02$ & Auto FIC & 408.20 & Good & \\
\hline $5 / 23 / 920: 00$ & Manual Tape & 406.25 & Transcribed & \\
\hline $5 / 23 / 927: 03$ & Auto FIC & 408.2 & Good & \\
\hline $5 / 24 / 920: 00$ & Manual Tape & 406.25 & Transcribed & \\
\hline $5 / 24 / 927: 03$ & Auto FIC & 408.2 & Good & \\
\hline $5 / 25 / 92 \quad 0: 00$ & Manual Tape & 406.5 & Transcribed & \\
\hline $5 / 25 / 927: 03$ & Auto FIC & 408.20 & Good & \\
\hline $5 / 26 / 920: 00$ & Manual Tape & 406.50 & Transcribed & \\
\hline $5 / 26 / 920: 00$ & Manual FIC & 408.1 & Good & \\
\hline $5 / 27 / 920: 00$ & Manual Tape & 406.5 & Transcribed & \\
\hline $5 / 27 / 927: 03$ & Auto FIC & 408.2 & Good & \\
\hline $5 / 28 / 92 \quad 0: 00$ & Manual Tape & 406.5 & Transcribed & \\
\hline 5/28/92 7:02 & Auto FIC & 408.20 & Good & \\
\hline $5 / 29 / 920: 00$ & Manual Tape & 406.50 & Transcribed & \\
\hline $5 / 29 / 927: 02$ & Auto FIC & 408.2 & Good & \\
\hline $5 / 30 / 920: 00$ & Manual Tape & 406.50 & Transcribed & \\
\hline $5 / 30 / 927: 03$ & Auto FIC & 408.2 & Good & \\
\hline $5 / 31 / 920: 00$ & Manual Tape & 406.50 & Transcribed & $\therefore$ \\
\hline $5 / 31 / 927: 03$ & Auto FIC & 408.2 & Good & \\
\hline $6 / 1 / 920: 00$ & Manual Tape & 406.75 & Transcribed & \\
\hline 6/1/92 7:03 & Auto FIC & 408.20 & Good & \\
\hline $6 / 2 / 920: 00$ & Manual Tape & 406.5 & Transcribed & \\
\hline 6/2/92 7:03 & Auto FIC & 408.20 & Good & \\
\hline $6 / 3 / 920: 00$ & Manual Tape & 406.5 & Transcribed & \\
\hline $6 / 3 / 927: 03$ & Auto FIC & 408.2 & Good & \\
\hline $6 / 4 / 920: 00$ & Manual Tape & 406.5 & Transcribed & \\
\hline $6 / 4 / 927: 03$ & Auto FIC & 408.20 & Good & \\
\hline $6 / 5 / 920: 00$ & Manual Tape & 406.50 & Transcribed & \\
\hline $6 / 5 / 927: 02$ & Auto FIC & 408.20 & Good & \\
\hline $6 / 6 / 920: 00$ & Manual Tape & 406.50 & Transcribed & \\
\hline $6 / 6 / 927: 03$ & Auto FIC & 408.2 & Good & \\
\hline $6 / 7 / 920: 00$ & Manual Tape & 406.5 & Transcribed & \\
\hline $6 / 7 / 927: 03$ & Auto FIC & 408.20 & Good & \\
\hline $6 / 8 / 920: 00$ & Manual Tape & 406.50 & Transcribed & \\
\hline $6 / 8 / 927: 03$ & Auto FIC & 408.2 & Good & \\
\hline $6 / 9 / 920: 00$ & Manual Tape & 406.5 & Transcribed & \\
\hline $6 / 9 / 927: 03$ & Auto FIC & $408.20^{\circ}$ & Good & \\
\hline $6 / 10 / 920: 00$ & Manual Tape & 406.50 & Transcribed & \\
\hline $6 / 10 / 927: 03$ & Auto FIC & 408.20 & Good & \\
\hline $6 / 11 / 920: 00$ & Manual Tape & 406.5 & Transcribed & \\
\hline $6 / 11 / 927: 03$ & Auto FIC & 408.20 & Good & \\
\hline $6 / 12 / 920: 00$ & Manual Tape & 406.5 & Transcribed & \\
\hline $6 / 12 / 927: 05$ & Auto FIC & 408.2 & Good & \\
\hline
\end{tabular}

Data obtained from WHC Surveillance Analysis Computer System, (PC SACS) July 7, 1995. 


\begin{tabular}{|c|c|c|c|c|}
\hline \multicolumn{5}{|c|}{ 241-AP-107 } \\
\hline \multicolumn{5}{|c|}{ Surface Level Data } \\
\hline Date & Type of Messurement & Level (Inches) & Data Quality & Comments \\
\hline $6 / 13 / 920: 00$ & Manual Tape & 406.50 & Transcribed & \\
\hline $6 / 13 / 927: 05$ & Auto FIC & 408.20 & Good & \\
\hline $6 / 14 / 920: 00$ & Manual Tape & 406.5 & Transcribed & \\
\hline $6 / 14 / 920: 00$ & Manual FIC & 408.2 & Good & \\
\hline $6 / 15 / 920: 00$ & Manual Tape & 406.5 & Transcribed & \\
\hline $6 / 15 / 927: 03$ & Auto FIC & 408.10 & Good & \\
\hline $6 / 16 / 920: 00$ & Manual Tape & 406.50 & Transcribed & \\
\hline $6 / 16 / 927: 03$ & Auto FIC & 408.20 & Good & \\
\hline $6 / 17 / 920: 00$ & Manual Tape & 406.25 & Transcribed & \\
\hline $6 / 17 / 927: 03$ & Auto FIC & 408.2 & Good & \\
\hline $6 / 18 / 927: 03$ & Auto FIC & 408.1 & Good & \\
\hline $6 / 19 / 920: 00$ & Manual Tape & 406.25 & Transcribed & \\
\hline $6 / 19 / 927: 02$ & Auto FIC & 408.1 & Good & \\
\hline $6 / 20 / 920: 00$ & Manual Tape & 406.25 & Transcribed & \\
\hline $6 / 20 / 927: 02$ & Auto FIC & 408.10 & Good & \\
\hline $6 / 21 / 920: 00$ & Manual Tape & 406.25 & Transcribed & \\
\hline $6 / 21 / 927: 03$ & Auto FIC & 408.10 & Good & \\
\hline $6 / 22 / 920: 00$ & Manual Tape & 406.25 & Transcribed & \\
\hline $6 / 22 / 927: 03$ & Auto FIC & 408.10 & Good & \\
\hline $6 / 23 / 920: 00$ & Manual Tape & 406.25 & Transcribed & \\
\hline $6 / 23 / 927: 03$ & Auto FIC & 408.10 & Good & \\
\hline $6 / 24 / 920: 00$ & Manual Tape & 406.25 & Transcribed & \\
\hline $6 / 24 / 927: 03$ & Auto FIC & 408.10 & Good & \\
\hline $6 / 25 / 920: 00$ & Manual Tape & 406 & Transcribed & \\
\hline $6 / 25 / 927: 03$ & Auto FIC & 408.10 & Good & \\
\hline $6 / 26 / 920: 00$ & Manual Tape & 406.25 & Transcribed & \\
\hline $6 / 26 / 927: 02$ & Auto FIC & 408.10 & Good & \\
\hline $6 / 27 / 920: 00$ & Manual Tape & 406.25 & Transcribed & \\
\hline $6 / 27 / 927: 03$ & Auto FIC & 408.10 & Good & \\
\hline $6 / 28 / 920: 00$ & Manual Tape & 406.25 & Transcribed & \\
\hline $6 / 28 / 927: 06$ & Auto FIC & 408 & Good & \\
\hline $6 / 29 / 92 \quad 0: 00$ & Manual Tape & 406.25 & Transcribed & \\
\hline $6 / 29 / 927: 06$ & Auto FIC & 408.1 & Good & \\
\hline $6 / 30 / 920: 00$ & Manual Tape & 406.25 & Transcribed & \\
\hline $6 / 30 / 927: 06$ & Auto FIC & 408.10 & Good & \\
\hline $7 / 1 / 920: 00$ & Manual Tape & 406.25 & Transcribed & \\
\hline 7/1/92 0:00 & Manual FIC & 408.10 & Good & \\
\hline $7 / 2 / 920: 00$ & Manual Tape & 406.25 & Transcribed & \\
\hline $7 / 2 / 920: 00$ & Manual FIC & 408.1 & Good & \\
\hline $7 / 3 / 920: 00$ & Manual Tape & 406.50 & Transcribed & \\
\hline $7 / 3 / 927: 06$ & Auto FIC & 408.10 & Good & \\
\hline $7 / 4 / 920: 00$ & Manual Tape & 406.5 & Transcribed & \\
\hline $7 / 4 / 927: 06$ & Auto FIC & 408.10 & Good & \\
\hline $7 / 5 / 920: 00$ & Manual Tape & 406.50 & Transcribed & \\
\hline $7 / 5 / 927: 06$ & Auto FIC & 408.1 & Good & \\
\hline $7 / 6 / 920: 00$ & Manual Tape & 406.5 & Transcribed & \\
\hline $7 / 6 / 927: 06$ & Auto FIC & 408.1 . & Good & \\
\hline $7 / 7 / 920: 00$ & Manual Tape & 406.5 & Transcribed & \\
\hline
\end{tabular}

Data obtained from WHC Surveillance Analysis Computer System, (PC SACS) July 7, 1995. 


\begin{tabular}{|c|c|c|c|c|}
\hline \multicolumn{5}{|c|}{ 241-AP-107 } \\
\hline \multicolumn{5}{|c|}{ Surface Level Data } \\
\hline Date & Type of Measurement & Level (Inches) & Data Quality & Comments \\
\hline $7 / 7 / 927: 06$ & Auto FIC & 408.10 & Good & \\
\hline $7 / 8 / 920: 00$ & Manual Tape & 406.5 & Transcribed & \\
\hline 7/8/92 7:06 & Auto FIC & 408.10 & Good & \\
\hline 7/9/92 0:00 & Manual Tape & 406.50 & Transcribed & \\
\hline 7/9/92 7:06 & Auto FIC & 408.1 & Good & \\
\hline $7 / 10 / 920: 00$ & Manual Tape & 406.50 & Transcribed & \\
\hline $7 / 10 / 927: 06$ & Auto FIC & 408.10 & Good & \\
\hline $7 / 11 / 920: 00$ & Manual Tape & 406.5 & Transcribed & \\
\hline $7 / 11 / 927: 06$ & Auto FIC & 408.1 & Good & \\
\hline $7 / 12 / 920: 00$ & Manual Tape & 406.50 & Transcribed & \\
\hline $7 / 12 / 927: 06$ & Auto FIC & 408.10 & Good & \\
\hline $7 / 13 / 920: 00$ & Manual Tape & 406.5 & Transcribed & \\
\hline 7/13/92 7:06 & Auto FIC & 408.1 & Good & \\
\hline $7 / 14 / 920: 00$ & Manual Tape & 406.25 & Transcribed & \\
\hline $7 / 14 / 927: 06$ & Auto FIC & 408.10 & Good & \\
\hline $7 / 15 / 920: 00$ & Manual Tape & 406.5 & Transcribed & \\
\hline $7 / 15 / 927: 06$ & Auto FIC & 408.10 & Good & \\
\hline $7 / 16 / 920: 00$ & Manual Tape & 406.25 & Transcribed & \\
\hline $7 / 16 / 927: 06$ & Auto FIC & 408.1 & Good & \\
\hline $7 / 17 / 920: 00$ & Manual Tape & 406.25 & Transcribed & \\
\hline 7/17/92 7:06 & Auto FIC & 408.10 & Good & \\
\hline $7 / 18 / 920: 00$ & Manual Tape & 406.5 & Transcribed & \\
\hline $7 / 18 / 927: 06$ & Auto FIC & 408.1 & Good & \\
\hline $7 / 19 / 920: 00$ & Manual Tape & 406.5 & Transcribed & \\
\hline $7 / 19 / 927: 06$ & Auto FIC & 408.10 & Good & \\
\hline $7 / 20 / 920: 00$ & Manual Tape & 406.25 & Transcribed & \\
\hline $7 / 20 / 927: 06$ & Auto FIC & 408.1 & Good & \\
\hline $7 / 21 / 920: 00$ & Manual Tape & 406.5 & Transcribed & \\
\hline $7 / 21 / 927: 05$ & Auto FIC & 408.10 & Good & \\
\hline $7 / 22 / 920: 00$ & Manual Tape & 406.25 & Transcribed & \\
\hline $7 / 22 / 927: 06$ & Auto FIC & 408.1 & Good & \\
\hline $7 / 23 / 920: 00$ & Manual Tape & 406.25 & Transcribed & \\
\hline $7 / 23 / 920: 00$ & Manual FIC & 408.10 & Good & \\
\hline $7 / 24 / 920: 00$ & Manual Tape & 406.25 & Transcribed & \\
\hline $7 / 24 / 927: 05$ & Auto FIC & 408.1 & Good & \\
\hline $7 / 25 / 920: 00$ & Manual Tape & 406.25 & Transcribed & \\
\hline $7 / 25 / 927: 06$ & Auto FIC & 408.1 & Good & \\
\hline $7 / 26 / 920: 00$ & Manual Tape & 406.25 & Transcribed & \\
\hline $7 / 26 / 927: 06$ & Auto FIC & 408.1 & Good & \\
\hline $7 / 27 / 920: 00$ & Manual Tape & 406.25 & Transcribed & \\
\hline $7 / 27 / 927: 05$ & Auto FIC & 408 & Good & \\
\hline $7 / 28 / 920: 00$ & Manual Tape & 406.5 & Transcribed & \\
\hline $7 / 28 / 927: 06$ & Auto FIC & 408.00 & Good & \\
\hline $7 / 29 / 920: 00$ & Manual Tape & 406.50 & Transcribed & \\
\hline $7 / 29 / 927: 06$ & Auto FIC & 408.00 & Good & \\
\hline $7 / 30 / 920: 00$ & Manual Tape & 406.5 & Transcribed & \\
\hline $7 / 30 / 927: 06$ & Auto FIC & 408 & Good & \\
\hline $7 / 31 / 920: 00$ & Manual Tape & 406.5 & Transcribed & \\
\hline
\end{tabular}

Data obtained from WHC Surveillance Analysis Computer System, (PC SACS) July 7, 1995. 


\begin{tabular}{|c|c|c|c|c|}
\hline \multicolumn{5}{|c|}{ 241-AP-107 } \\
\hline \multicolumn{5}{|c|}{ Surface Level Data } \\
\hline Date & Type of Measurement & Level (Inches) & Data Quality & Comments \\
\hline $7 / 31 / 927: 05$ & Auto FIC & 408.00 & Good & \\
\hline $8 / 1 / 920: 00$ & Manual Tape & 406.50 & Transcribed & \\
\hline $8 / 1 / 927: 06$ & Auto FIC & 408 & Good & \\
\hline $8 / 2 / 920: 00$ & Manual Tape & 406.50 & Transcribed & \\
\hline $8 / 2 / 927: 06$ & Auto FIC & 408 & Good & \\
\hline $8 / 3 / 920: 00$ & Manual Tape & 406.5 & Transcribed & \\
\hline $8 / 3 / 927: 06$ & Auto FIC & 408.00 & Good & \\
\hline $8 / 4 / 920: 00$ & Manual Tape & 406.5 & Transcribed & \\
\hline $8 / 4 / 927: 06$ & Auto FIC & 408 & Good & \\
\hline $8 / 5 / 920: 00$ & Manual Tape & 406.25 & Transcribed & \\
\hline $8 / 5 / 927: 05$ & Auto FIC & 408.00 & Good & \\
\hline $8 / 6 / 920: 00$ & Manual Tape & 406.25 & Transcribed & \\
\hline $8 / 6 / 927: 05$ & Auto FIC & 408 & Good & \\
\hline $8 / 7 / 920: 00$ & Manual Tape & 406.25 & Transcribed & \\
\hline $8 / 7 / 927: 05$ & Auto FIC & 408 & Good & \\
\hline $8 / 8 / 920: 00$ & Manual Tape & 406.25 & Transcribed & \\
\hline $8 / 8 / 927: 06$ & Auto FIC & 408.00 & Good & \\
\hline $8 / 9 / 920: 00$ & Manual Tape & 406.25 & Transcribed & \\
\hline $8 / 9 / 927: 06$ & Auto FIC & 408.00 & Good & \\
\hline $8 / 10 / 920: 00$ & Manual Tape & 406 & Transcribed & \\
\hline $8 / 10 / 927: 05$ & Auto FIC & 408 & Good & \\
\hline $8 / 11 / 920: 00$ & Manual Tape & 406.00 & Transcribed & \\
\hline $8 / 11 / 927: 06$ & Auto FIC & 408.00 & Good & \\
\hline $8 / 12 / 920: 00$ & Manual Tape & 405 & Transcribed & \\
\hline $8 / 12 / 927: 06$ & Auto FIC & 408 & Good & \\
\hline $8 / 13 / 920: 00$ & Manual Tape & 406.25 & Transcribed & \\
\hline $8 / 13 / 927: 06$ & Auto FIC & 408 & Good & \\
\hline $8 / 14 / 920: 00$ & Manual Tape & 406.25 & Transcribed & \\
\hline $8 / 14 / 927: 06$ & Auto FIC & 408.00 & Good & \\
\hline $8 / 15 / 920: 00$ & Manual Tape & 406.25 & Transcribed & \\
\hline $8 / 15 / 927: 06$ & Auto FIC & 408 & Good & \\
\hline $8 / 16 / 920: 00$ & Manual Tape & 406.25 & Transcribed & \\
\hline $8 / 16 / 927: 06$ & Auto FIC & 408.00 & Good & \\
\hline $8 / 17 / 920: 00$ & Manual Tape & 406.25 & Transcribed & \\
\hline $8 / 17 / 927: 06$ & Auto FIC & 408.00 & Good & \\
\hline $8 / 18 / 920: 00$ & Manual Tape & 406.50 & Transcribed & \\
\hline $8 / 18 / 927: 06$ & Auto FIC & 408.00 & Good & \\
\hline $8 / 19 / 920: 00$ & Manual Tape & 406.00 & Transcribed & \\
\hline $8 / 19 / 927: 06$ & Auto FIC & 408.00 & Good & \\
\hline $8 / 20 / 920: 00$ & Manual Tape & 406.25 & Transcribed & \\
\hline $8 / 20 / 927: 06$ & Auto FIC & 408.00 & Good & \\
\hline $8 / 21 / 920: 00$ & Manual Tape & 406.25 & Transcribed & \\
\hline $8 / 21 / 927: 05$ & Auto FIC & 408.00 & Good & \\
\hline $8 / 22 / 920: 00$ & Manual Tape & 406.25 & Transcribed & \\
\hline $8 / 22 / 927: 04$ & Auto FIC & 408 & Good & \\
\hline $8 / 23 / 920: 00$ & Manual Tape & 406.25 & Transćribed & \\
\hline $8 / 23 / 927: 04$ & Auto FIC & 408.00 & Good & \\
\hline $8 / 24 / 920: 00$ & Manual Tape & 406.50 & Transcribed & \\
\hline
\end{tabular}

Data obtained from WHC Surveillance Analysis Computer System, (PC SACS) July 7, 1995. 


\begin{tabular}{|c|c|c|c|c|}
\hline \multicolumn{5}{|c|}{ 241-AP-107 } \\
\hline \multicolumn{5}{|c|}{ Surface Level Data } \\
\hline Date & Type of Measurement & Level.(Inches) & Data Quality & Comments \\
\hline $8 / 24 / 927: 04$ & Auto FIC & 407.9 & Good & \\
\hline $8 / 25 / 920: 00$ & Manual Tape & 406.50 & Transcribed & \\
\hline $8 / 25 / 927: 04$ & Auto FIC & 407.9 & Good & \\
\hline $8 / 26 / 927: 03$ & Auto FIC & 407.9 & Good & \\
\hline $8 / 27 / 927: 04$ & Auto FIC & 407.9 & Good & \\
\hline $8 / 28 / 920: 00$ & Manual Tape & 406.75 & Transcribed & \\
\hline $8 / 28 / 927: 02$ & Auto FIC & 407.90 & Good & \\
\hline $8 / 29 / 920: 00$ & Manual Tape & 406 & Transcribed & \\
\hline $8 / 29 / 927: 02$ & Auto FIC & 407.9 & Good & \\
\hline $8 / 30 / 920: 00$ & Manual Tape & 406 & Transcribed & \\
\hline $8 / 30 / 927: 02$ & Auto FIC & 407.9 & Good & \\
\hline $8 / 31 / 920: 00$ & Manual Tape & 406 & Transcribed & \\
\hline $8 / 31 / 927: 02$ & Auto FIC & 407.90 & Good & \\
\hline 9/1/92 0:00 & Manual Tape & 406.00 & Transcribed & \\
\hline $9 / 1 / 927: 02$ & Auto FIC & 407.90 & Good & \\
\hline $9 / 2 / 920: 00$ & Manual Tape & 406.00 & Transcribed & \\
\hline $9 / 2 / 927: 02$ & Auto FIC & 407.9 & Good & \\
\hline $9 / 3 / 920: 00$ & Manual Tape & 406.25 & Transcribed & \\
\hline $9 / 3 / 927: 04$ & Auto FIC & 407.9 . & Good & \\
\hline $9 / 4 / 920: 00$ & Manual Tape & 406.25 & Transcribed & \\
\hline 9/4/92 7:04 & Auto FIC & 407.9 & Good & \\
\hline $9 / 5 / 920: 00$ & Manual Tape & 406.25 & Transcribed & \\
\hline $9 / 5 / 927: 04$ & Auto FIC & 407.90 & Good & \\
\hline $9 / 6 / 920: 00$ & Manual Tape & 406.25 & Transcribed & \\
\hline $9 / 6 / 927: 04$ & Auto FIC & 407.9 & Good & \\
\hline $9 / 7 / 920: 00$ & Manual Tape & 406.25 & Transcribed & \\
\hline $9 / 7 / 927: 05$ & Auto FIC & 407.8 & Good & \\
\hline 9/8/92 0:00 & Manual Tape & $406.25^{\circ}$ & Transcribed & \\
\hline $9 / 8 / 927: 05$ & Auto FIC & 407.80 & Good & \\
\hline 9/9/92 0:00 & Manual Tape & 406.25 & Transcribed & \\
\hline $9 / 9 / 927: 04$ & Auto FIC & 407.8 & Good & \\
\hline 9/10/92 0:00 & Manual Tape & 406.25 & Transcribed & \\
\hline 9/10/92 7:03 & Auto FIC & 407.80 & Good & \\
\hline $9 / 11 / 920: 00$ & Manual Tape & 406.25 & Transcribed & \\
\hline $9 / 11 / 920: 00$ & Manual FIC & 407.80 & Good & \\
\hline $9 / 12 / 920: 00$ & Manual Tape & 406.25 & Transcribed & \\
\hline $9 / 12 / 920: 00$ & Manual FIC & 407.80 & Good & \\
\hline 9/13/92 0:00 & Manual Tape & 406.25 & Transcribed & \\
\hline $9 / 13 / 920: 00$ & Manual FIC & 407.8 & Good & \\
\hline $9 / 14 / 920: 00$ & Manual Tape & 406.00 & Transcribed & \\
\hline $9 / 14 / 920: 00$ & Manual FIC & 407.80 & Good & \\
\hline $9 / 15 / 920: 00$ & Manual Tape & 406 & Transeribed & \\
\hline $9 / 15 / 920: 00$ & Manual FIC & 407.80 & Good & \\
\hline $9 / 16 / 920: 00$ & Manual Tape & 406.00 & Transcribed & \\
\hline $9 / 16 / 920: 00$ & Manual FIC & $407.8^{\circ}$ & Good & \\
\hline $9 / 17 / 920: 00$ & Manual Tape & 406.00 & Transcribed & \\
\hline $9 / 17 / 927: 06$ & Auto FIC & 407.80 & Good & \\
\hline $9 / 18 / 920: 00$ & Manual Tape & 406 & Transcribed & \\
\hline
\end{tabular}

Data obtained from WHC Surveillance Analysis Computer System, (PC SACS) July 7, 1995. 


\begin{tabular}{|c|c|c|c|c|}
\hline \multicolumn{5}{|c|}{ 241-AP-107 } \\
\hline \multicolumn{5}{|c|}{ Surface Level Data } \\
\hline Date & Type of Measurement & Level (Inches) & Data Quality & Comments \\
\hline $9 / 18 / 927: 06$ & Auto FIC & 407.80 & Good & \\
\hline $9 / 19 / 920: 00$ & Manual Tape & 406.00 & Transcribed & \\
\hline $9 / 19 / 920: 00$ & Manual FIC & 407.80 & Good & \\
\hline $9 / 20 / 920: 00$ & Manual Tape & 406 & Transcribed & \\
\hline $9 / 20 / 920: 00$ & Manual FIC & 407.8 & Good & \\
\hline $9 / 21 / 920: 00$ & Manual Tape & 406 & Transcribed & \\
\hline $9 / 21 / 927: 06$ & Auto FIC & 407.8 & Good & \\
\hline $9 / 22 / 920: 00$ & Manual Tape & 406.00 & Transcribed & \\
\hline $9 / 22 / 927: 03$ & Auto FIC & 407.8 & Good & \\
\hline $9 / 23 / 920: 00$ & Manual Tape & 406 & Transcribed & \\
\hline $9 / 23 / 927: 03$ & Auto FIC & 407.8 & Good & \\
\hline $9 / 24 / 920: 00$ & Manual Tape & 406.00 & Transcribed & \\
\hline $9 / 24 / 927: 03$ & Auto FIC & 407.80 & Good & \\
\hline $9 / 25 / 920: 00$ & Manual Tape & 405.75 & Transcribed & \\
\hline $9 / 25 / 927: 03$ & Auto FIC & 407.7 & Good & \\
\hline $9 / 26 / 920: 00$ & Manual Tape & 406 & Transcribed & \\
\hline $9 / 26 / 927: 02$ & Auto FIC & 407.7 & Good & \\
\hline 9/27/92 0:00 & Manual Tape . & 406.00 & Transcribed & \\
\hline 9/27/92 7:02 & Auto FIC & 407.7 & Good & \\
\hline 9/28/92 0:00 & Manual Tape & 406 & Transcribed & \\
\hline $9 / 28 / 927: 02$ & Auto FIC & 407.70 & Good & \\
\hline 9/29/92 0:00 & Manual Tape & 406 & Transcribed & \\
\hline $9 / 29 / 92 \quad 7: 03$ & Auto FIC & 407.7 & Good & \\
\hline 9/30/92 0:00 & Manual Tape & 406.00 & Transcribed & \\
\hline $9 / 30 / 927: 02$ & Auto FIC & 407.70 & Good & \\
\hline $10 / 1 / 920: 00$ & Manual Tape & 405.75 & Transcribed & \\
\hline $10 / 1 / 927: 03$ & Auto FIC & 407.70 & Good & \\
\hline $10 / 2 / 920: 00$ & Manual Tape & 405.75 & Transcribed & \\
\hline $10 / 2 / 927: 02$ & Auto FIC & 407.70 & Good & \\
\hline $10 / 3 / 920: 00$ & Manual Tape & 406 & Transçribed & \\
\hline $10 / 3 / 927: 03$ & Auto FIC & 407.7 & Good & \\
\hline $10 / 4 / 920: 00$ & Manual Tape & 406.00 & Transcribed & \\
\hline $10 / 4 / 927: 03$ & Auto FIC & 407.70 & Good & \\
\hline $10 / 5 / 920: 00$ & Manual Tape & 406 & Transcribed & \\
\hline $10 / 5 / 927: 03$ & Auto FIC & 407.70 & Good & \\
\hline $10 / 6 / 920: 00$ & Manual Tape & 406.00 & Transcribed & \\
\hline $10 / 6 / 927: 03$ & Auto FIC & 407.70 & Good & \\
\hline $10 / 7 / 920: 00$ & Manual Tape & 406.00 & Transcribed & \\
\hline $10 / 7 / 920: 00$ & Manual FIC & 407.70 & Good & \\
\hline $10 / 8 / 920: 00$ & Manual Tape & 406 & Transcribed & \\
\hline $10 / 8 / 927: 02$ & Auto FIC & 407.70 & Good & \\
\hline $10 / 9 / 92 \quad 0: 00$ & Manual Tape & 406.00 & Transcribed & \\
\hline $10 / 9 / 927: 03$ & Auto FIC & 407.70 & Good & \\
\hline $10 / 10 / 920: 00$ & Manual Tape & 406 & Transcribed & \\
\hline $10 / 10 / 927: 03$ & Auto FIC & 407.80 & Good & \\
\hline 10/11/92 0:00 & Manual Tape & 406.00 & Transcribed & \\
\hline $10 / 11 / 927: 03$ & Auto FIC & 407.7 & Good & \\
\hline $10 / 12 / 920: 00$ & Manual Tape & 406.00 & Transcribed & \\
\hline
\end{tabular}

Data obtained from WHC Surveillance Analysis Computer System, (PC SACS) July 7, 1995. 


\begin{tabular}{|c|c|c|c|c|}
\hline \multicolumn{5}{|c|}{ 241-AP-107 } \\
\hline \multicolumn{5}{|c|}{ Surface Level Data } \\
\hline Date & Type of Measurement & Level (Inches) & Data Quality & Comments \\
\hline 10/12/92 0:00 & Manual FIC & 407.7 & Good & \\
\hline $10 / 13 / 920: 00$ & Manual Tape & 406 & Transcribed & \\
\hline $10 / 13 / 920: 00$ & Manual FIC & 407.6 & Good & \\
\hline $10 / 14 / 920: 00$ & Manual Tape & 406.00 & Transcribed & \\
\hline $10 / 14 / 927: 03$ & Auto FIC & 407.6 & Good & \\
\hline $10 / 15 / 920: 00$ & Manual Tape & 406 & Transcribed & \\
\hline $10 / 15 / 927: 04$ & Auto FIC & 407.60 & Good & \\
\hline $10 / 16 / 920: 00$ & Manual Tape & 406 & Transcribed & \\
\hline $10 / 16 / 927: 03$ & Auto FIC & 407.6 & Good & \\
\hline $10 / 17 / 920: 00$ & Manual Tape & 406 & Transcribed & \\
\hline $10 / 17 / 927: 03$ & Auto FIC & 407.6 & Good & \\
\hline $10 / 18 / 920: 00$ & Manual Tape & 406 & Transcribed & \\
\hline $10 / 18 / 927: 03$ & Auto FIC & 407.60 & Good & \\
\hline $10 / 19 / 920: 00$ & Manual Tape & 406 & Transcribed & \\
\hline $10 / 19 / 927: 03$ & Auto FIC & 407.6 & Good & \\
\hline $10 / 20 / 920: 00$ & Manual Tape & 405.75 & Transcribed & \\
\hline $10 / 20 / 927: 03$ & Auto FIC & 407.60 & Good & \\
\hline $10 / 21 / 920: 00$ & Manual Tape & 405.75 & Transcribed & \\
\hline $10 / 21 / 927: 03$ & Auto FIC & 407.60 & Good & \\
\hline $10 / 22 / 920: 00$ & Manual Tape & 405.75 & Transcribed & \\
\hline $10 / 22 / 920: 00$ & Manual FIC & 407.6 & Good & \\
\hline $10 / 23 / 920: 00$ & Manual Tape & 405.75 & Transcribed & \\
\hline $10 / 23 / 920: 00$ & Manual FIC & 407.6 & Good & \\
\hline $10 / 24 / 920: 00$ & Manual Tape & 405.75 & Transcribed & \\
\hline $10 / 24 / 927: 03$ & Auto FIC & 407.60 & Good & \\
\hline $10 / 25 / 920: 00$ & Manual Tape & 405.75 & Transcribed & \\
\hline $10 / 25 / 927: 03$ & Auto FIC & 407.6 & Good & \\
\hline $10 / 26 / 920: 00$ & Manual Tape & 405.75 & Transcribed & \\
\hline $10 / 26 / 920: 00$ & Manual FIC & 407.50 & Good & \\
\hline $10 / 27 / 920: 00$ & Manual Tape & 405.75 & Transcribed & \\
\hline $10 / 27 / 927: 04$ & Auto FIC & 407.5 & Good & \\
\hline $10 / 28 / 920: 00$ & Manual Tape & 405.75 & Transcribed & \\
\hline $10 / 28 / 927: 03$ & Auto FIC & 407.5 & Good & \\
\hline $10 / 29 / 920: 00$ & Manual Tape & 405.75 & Transcribed & \\
\hline $10 / 29 / 927: 03$ & Auto FIC & 407.5 & Good & \\
\hline $10 / 30 / 920: 00$ & Manual Tape & 405.75 & Transcribed & \\
\hline $10 / 30 / 927: 03$ & Auto FIC & 407.50 & Good & \\
\hline $10 / 31 / 920: 00$ & Manual Tape & 405.75 & Transcribed & \\
\hline $10 / 31 / 927: 03$ & Auto FIC & 407.5 & Good & \\
\hline $11 / 1 / 920: 00$ & Manual Tape & 405.75 & Transcribed & \\
\hline $11 / 1 / 927: 03$ & Auto FIC & 407.50 & Good & \\
\hline $11 / 2 / 920: 00$ & Manual Tape & 405.75 & Transcribed & \\
\hline $11 / 2 / 927: 03$ & Auto FIC & 407.50 & Good & \\
\hline $11 / 3 / 920: 00$ & Manual Tape & 405.75 & Transcribed & \\
\hline $11 / 3 / 927: 03$ & Auto FIC & 407.5 & Good & \\
\hline $11 / 4 / 920: 00$ & Manual Tape & 405.75 & Transcribed & \\
\hline $11 / 4 / 927: 03$ & Auto FIC & 407.50 & Good & \\
\hline $11 / 5 / 920: 00$ & Manual Tape & 405.75 & Transcribed & \\
\hline
\end{tabular}

Data obtained from WHC Surveillance Analysis Computer System, (PC SACS) July 7, 1995. 


\begin{tabular}{|c|c|c|c|c|}
\hline \multicolumn{5}{|c|}{ 241-AP-107 } \\
\hline \multicolumn{5}{|c|}{ Surface Level Data } \\
\hline Date & Type of Measurement & Level (Inches) & Data Quality & Comments \\
\hline $11 / 5 / 920: 00$ & Manual FIC & 407.50 & Good & \\
\hline $11 / 6 / 920: 00$ & Manual Tape & 405.75 & Transcribed & \\
\hline $11 / 6 / 920: 00$ & Manual FIC & 407.5 & Good & \\
\hline $11 / 7 / 920: 00$ & Manual Tape & 405.75 & Transcribed & \\
\hline $11 / 7 / 920: 00$ & Manual FIC & 407.50 & Good & \\
\hline $11 / 8 / 920: 00$ & Manual Tape & 405.75 & Transcribed & \\
\hline $11 / 8 / 920: 00$ & Manual FIC & 407.50 & Good & \\
\hline $11 / 9 / 920: 00$ & Manual Tape & 405.75 & Transcribed & \\
\hline $11 / 9 / 920: 00$ & Manual FIC & 407.40 & Good & \\
\hline $11 / 10 / 920: 00$ & Manual Tape & 405.75 & Transcribed & \\
\hline $11 / 10 / 927: 03$ & Auto FIC & 407.40 & Good & \\
\hline $11 / 11 / 920: 00$ & Manual Tape & 405.75 & Transcribed & \\
\hline $11 / 11 / 927: 04$ & Auto FIC & 407.40 & Good & \\
\hline $11 / 12 / 920: 00$ & Manual Tape & 405.75 & Transcribed & \\
\hline $11 / 12 / 927: 03$ & Auto FIC & 407.4 & Good & \\
\hline $11 / 13 / 920: 00$ & Manual Tape & 405.75 & Transcribed & \\
\hline $11 / 13 / 927: 04$ & Auto FIC & 407.40 & Good & \\
\hline $11 / 14 / 920: 00$ & Manual Tape & 405.75 & Transcribed & \\
\hline $11 / 14 / 927: 04$ & Auto FIC & 407.4 & Good & \\
\hline $11 / 15 / 920: 00$ & Manual Tape & 405.5 & Transcribed & \\
\hline $11 / 15 / 927: 04$ & Auto FIC & 407.40 & Good & \\
\hline $11 / 16 / 920: 00$ & Manual Tape & 405.75 & Transcribed & \\
\hline $11 / 16 / 927: 04$ & Auto FIC & 407.4 & Good & \\
\hline $11 / 17 / 920: 00$ & Manual Tape & 405.5 & Transcribed & \\
\hline $11 / 17 / 927: 04$ & Auto FIC & 407.4 & Good & \\
\hline $11 / 18 / 920: 00$ & Manual Tape & 405.75 & Transcribed & \\
\hline $11 / 18 / 927: 02$ & Auto FIC & 407.40 & Good & \\
\hline $11 / 19 / 920: 00$ & Manual Tape & 405.5 & Transcribed & \\
\hline 11/19/92 7:02 & Auto FIC & 407.40 & Good & \\
\hline $11 / 20 / 920: 00$ & Manual Tape & 405.50 & Transcribed & \\
\hline $11 / 20 / 927: 03$ & Auto FIC & 407.30 & Good & \\
\hline $11 / 21 / 92 \quad 0: 00$ & Manual Tape & 405.5 & Transcribed & \\
\hline $11 / 21 / 927: 02$ & Auto FIC & 407.30 & Good & \\
\hline $11 / 22 / 920: 00$ & Manual Tape & 405.75 & Transcribed & \\
\hline $11 / 22 / 927: 03$ & Auto FIC & 407.30 & Good & \\
\hline $11 / 23 / 920: 00$ & Manual Tape & 405.75 & Transcribed & \\
\hline $11 / 23 / 927: 03$ & Auto FIC & 407.30 & Good & \\
\hline $11 / 24 / 920: 00$ & Manual Tape & 405.75 & Transcribed & \\
\hline $11 / 24 / 927: 03$ & Auto FIC & 407.30 & Good & \\
\hline $11 / 25 / 920: 00$ & Manual Tape & 405.50 & Transcribed & \\
\hline $11 / 25 / 927: 03$ & Auto FIC & 407.3 & Good & \\
\hline $11 / 26 / 920: 00$ & Manual Tape & 405.5 & Transcribed & \\
\hline $11 / 26 / 927: 03$ & Auto FIC & 407.30 & Good & \\
\hline $11 / 27 / 920: 00$ & Manual Tape & 405.50 & Transcribed & \\
\hline $11 / 27 / 927: 03$ & Auto FIC & 407.30 & Good & \\
\hline $11 / 28 / 920: 00$ & Manual Tape & 405.50 & Transcribed & \\
\hline $11 / 28 / 927: 03$ & Auto FlC & 407.30 & Good & \\
\hline $11 / 29 / 920: 00$ & Manual Tape & 405.50 & Transcribed & \\
\hline
\end{tabular}

Data obtained from WHC Surveillance Analysis Computer System, (PC SACS) July 7, 1995. 


\begin{tabular}{|c|c|c|c|c|}
\hline \multicolumn{5}{|c|}{ 241-AP-107 } \\
\hline \multicolumn{5}{|c|}{ Surface Level Data } \\
\hline Date & Type of Measurement & Level (Inches) & Data Quality & Comments \\
\hline $11 / 29 / 927: 03$ & Auto FIC & 407.20 & Good & \\
\hline $11 / 30 / 920: 00$ & Manual Tape & 405.5 & Transcribed & \\
\hline $11 / 30 / 920: 00$ & Manual FIC & 407.20 & Good & \\
\hline $12 / 1 / 920: 00$ & Manual Tape & 405.5 & Transcribed & \\
\hline $12 / 1 / 927: 03$ & Auto FIC & 407.2 & Good & \\
\hline $12 / 2 / 920: 00$ & Manual Tape & 405.50 & Transcribed & \\
\hline $12 / 2 / 927: 03$ & Auto FIC & 407.20 & Good & \\
\hline $12 / 3 / 920: 00$ & Manual Tape & 405.5 & Transcribed & \\
\hline $12 / 3 / 927: 03$ & Auto FIC & 407.2 & Good & \\
\hline $12 / 4 / 920: 00$ & Manual Tape & 405.50 & Transcribed & \\
\hline $12 / 4 / 927: 03$ & Auto FIC & 407.2 & Good & \\
\hline $12 / 5 / 920: 00$ & Manual Tape & 405.5 & Transcribed & \\
\hline $12 / 5 / 920: 00$ & Manual FIC & 407.2 & Good & \\
\hline $12 / 6 / 92 \quad 0: 00$ & Manual Tape & 405.50 & Transcribed & \\
\hline $12 / 6 / 927: 02$ & Auto FIC & 407.2 & Good & \\
\hline $12 / 7 / 920: 00$ & Manual Tape & 405.50 & Transcribed & \\
\hline $12 / 7 / 927: 03$ & Auto FIC & 407.1 & Good & \\
\hline $12 / 8 / 920: 00$ & Manual Tape & 405.50 & Transcribed & \\
\hline $12 / 8 / 927: 03$ & Auto FIC & 407.10 & Good & \\
\hline 12/9/92 0:00 & Manual Tape & 405.5 & Transcribed & \\
\hline $12 / 9 / 927: 03$ & Auto FIC & 406.60 & Good & \\
\hline $12 / 10 / 920: 00$ & Manual Tape & 405.50 & Transcribed & \\
\hline $12 / 10 / 927: 03$ & Auto FIC & $406.6^{\circ}$ & Good & \\
\hline $12 / 11 / 920: 00$ & Manual Tape & 405.5 & Transcribed & \\
\hline $12 / 11 / 927: 03$ & Auto FIC & 406.60 & Good & \\
\hline $12 / 12 / 920: 00$ & Manual Tape & $40 \overline{5.5}$ & Transcribed & \\
\hline $12 / 12 / 920: 00$ & Manual FIC & 406.60 & Good & \\
\hline $12 / 13 / 920: 00$ & Manual Tape & 405.50 & Transcribed & \\
\hline $12 / 13 / 927: 04$ & Auto FIC & 406.6 & Good & \\
\hline $12 / 14 / 920: 00$ & Manual Tape & 405.50 & Transcribed & \\
\hline $12 / 14 / 927: 03$ & Auto FIC & 406.6 & Good & \\
\hline $12 / 15 / 920: 00$ & Manual Tape & 405.25 & Transcribed & \\
\hline $12 / 15 / 927: 03$ & Auto FIC & 406.5 & Good & \\
\hline $12 / 16 / 920: 00$ & Manual Tape & 405.25 & Transcribed & \\
\hline $12 / 16 / 927: 04$ & Auto FIC & 406.5 & Good & \\
\hline $12 / 17 / 920: 00$ & Manual Tape & 405.25 & Transcribed & \\
\hline $12 / 17 / 927: 03$ & Auto FIC & 406.5 & Good & \\
\hline $12 / 18 / 920: 00$ & Manual Tape & 405.25 & Transcribed & \\
\hline $12 / 18 / 927: 03$ & Auto FIC & 406.5 & Good & \\
\hline $12 / 19 / 920: 00$ & Manual Tape & 405.25 & Transcribed & \\
\hline $12 / 19 / 927: 03$ & Auto FIC & 406.5 & Good & \\
\hline $12 / 20 / 920: 00$ & Manual Tape & 405.25 & Transcribed & \\
\hline $12 / 20 / 927: 04$ & Auto FIC & 406.5 & Good & \\
\hline $12 / 21 / 920: 00$ & Manual Tape & 405.25 & Transcribed & \\
\hline $12 / 21 / 927: 04$ & Auto FIC & 406.50 & Good & \\
\hline $12 / 22 / 920: 00$ & Manual Tape & 405.25 & Transcribed & \\
\hline $12 / 22 / 920: 00$ & Manual FIC & 406.4 & Good & \\
\hline $12 / 23 / 920: 00$ & Manual Tape & 405.25 & Transcribed & \\
\hline
\end{tabular}

Data obtained from WHC Surveillance Analysis Computer System, (PC SACS) July 7, 1995. 


\begin{tabular}{|c|c|c|c|c|}
\hline \multicolumn{5}{|c|}{ 241-AP-107 } \\
\hline \multicolumn{5}{|c|}{ Surface Level Data } \\
\hline Date & Type of Measurement & Level (Inches) & Data Quality & Comments \\
\hline $12 / 23 / 927: 04$ & Auto FIC & 406.40 & Good & \\
\hline $12 / 24 / 920: 00$ & Manual Tape & 405.25 & Transcribed & \\
\hline $12 / 24 / 927: 03$ & Auto FIC & 406.4 & Good & \\
\hline $12 / 25 / 920: 00$ & Manual Tape & 405.5 & Transcribed & \\
\hline 12/25/92 7:03 & Auto FIC & 406.40 & Good & \\
\hline $12 / 26 / 920: 00$ & Manual Tape & 405.5 & Transcribed & \\
\hline $12 / 26 / 927: 03$ & Auto FIC & 406.40 & Good & \\
\hline $12 / 27 / 920: 00$ & Manual Tape & 405 & Transcribed & \\
\hline $12 / 27 / 927: 03$ & Auto FIC & 406.40 & Good & \\
\hline $12 / 28 / 920: 00$ & Manual Tape & 405.25 & Transcribed & \\
\hline $12 / 28 / 927: 03$ & Auto FIC & 406.4 & Good & \\
\hline $12 / 29 / 920: 00$ & Manual Tape & 405.25 & Transcribed & \\
\hline $12 / 29 / 920: 00$ & Manual FIC & 406.40 & Good & \\
\hline $12 / 30 / 920: 00$ & Manual Tape & 405.25 & Transcribed & \\
\hline $12 / 30 / 927: 03$ & Auto FIC & 406.40 & Good & \\
\hline $12 / 31 / 920: 00$ & Manual Tape & 405.25 & Transcribed & \\
\hline $12 / 31 / 927: 03$ & Auto FIC & $406: 40$ & Good & \\
\hline $1 / 1 / 930: 00$ & Manual Tape & 405.25 & Good & \\
\hline $1 / 1 / 937: 04$ & Auto FIC & 406.4 & Good & \\
\hline $1 / 2 / 930: 00$ & Manual FIC & 406.3 & Good & \\
\hline $1 / 2 / 930: 00$ & Manual Tape : & 405.25 & Good & \\
\hline $1 / 3 / 930: 00$ & Manual FIC & 406.3 & Good & \\
\hline $1 / 3 / 930: 00$ & Manual Tape & 405.25 & Good & \\
\hline $1 / 4 / 930: 00$ & Manual FIC & 406.3 & Good & \\
\hline $1 / 4 / 930: 00$ & Manual Tape & 405 & Good & \\
\hline $1 / 5 / 930: 00$ & Manual FIC & 406.30 & Good & \\
\hline $1 / 5 / 930: 00$ & Manual Tape & 405 & Good & \\
\hline $1 / 6 / 930: 00$ & Manual FIC & 406.30 & Good & \\
\hline $1 / 6 / 930: 00$ & Manual Tape & 405.00 & Good & \\
\hline 1/7/930:00 & Manual FIC & 406.30 & Good & \\
\hline $1 / 7 / 930: 00$ & Manual Tape & 405 & Good & \\
\hline $1 / 8 / 930: 00$ & Manual Tape & 403.25 & Suspect & Data not included in graph \\
\hline $1 / 8 / 930: 00$ & Manual FIC & 406.3 & Good & \\
\hline 1/9/930:00 & Manual Tape & 403.25 & Suspect & Data not included in graph \\
\hline $1 / 9 / 930: 00$ & Manual FIC & 406.20 & Good & \\
\hline $1 / 10 / 930: 00$ & Manual Tape & 403.25 & Suspect & Data not included in graph \\
\hline $1 / 10 / 930: 00$ & Manual FIC & 406.2 & Good & \\
\hline $1 / 11 / 930: 00$ & Manual Tape & 403.25 & Suspect & Data not included in graph \\
\hline $1 / 11 / 930: 00$ & Manual FIC & 406.20 & Good & \\
\hline $1 / 12 / 930: 00$ & Manual Tape & 403.50 & Suspect & Data not included in graph \\
\hline $1 / 12 / 937: 06$ & Auto FIC & 406.2 & Good & \\
\hline $1 / 13 / 930: 00$ & Manual Tape & 403.50 & Suspect & Data not included in graph \\
\hline $1 / 13 / 937: 06$ & Auto FIC & 406.20 & Good & \\
\hline $1 / 14 / 930: 00$ & Manual Tape & 405.00 & Good & \\
\hline $1 / 14 / 937: 03$ & Auto FIC & 406.2 & Good & \\
\hline $1 / 15 / 930: 00$ & Manual Tape & 405 & Good & \\
\hline $1 / 15 / 937: 03$ & Auto FIC & 406.20 & Good & \\
\hline $1 / 16 / 93 \quad 0: 00$ & Manual FIC & 406.20 & Good & \\
\hline
\end{tabular}

Data obtained from WHC Surveillance Analysis Computer System, (PC SACS) July 7, 1995. 


\begin{tabular}{|c|c|c|c|c|}
\hline \multicolumn{5}{|c|}{ 241-AP-107 } \\
\hline \multicolumn{5}{|c|}{ Surface Level Data } \\
\hline Date & Type of Measurement & Level (Inches) & Data Quality & Comments \\
\hline $1 / 16 / 930: 00$ & Manual Tape : & 405.00 & Good & \\
\hline $1 / 17 / 930: 00$ & Manual FIC & 406.2 & Good & \\
\hline 1/17/93 0:00 & Manual Tape & 405 & Good & \\
\hline $1 / 18 / 930: 00$ & Manual FIC & 406.2 & Good & \\
\hline $1 / 18 / 930: 00$ & Manual Tape & 405.00 & Good & \\
\hline $1 / 19 / 930: 00$ & Manual FIC & 406.2 & Good & \\
\hline $1 / 19 / 930: 00$ & Manual Tape & 404.75 & Good & \\
\hline $1 / 20 / 930: 00$ & Manual FIC & 406.20 & Good & \\
\hline $1 / 20 / 930: 00$ & Manual Tape & 405.00 & Good & \\
\hline $1 / 21 / 930: 00$ & Manual Tape & 405 & Good & \\
\hline $1 / 21 / 937: 02$ & Auto FIC & 406.10 & Good & \\
\hline $1 / 22 / 930: 00$ & Manual Tape & 405 & Good & \\
\hline $1 / 22 / 937: 04$ & Auto FIC & 406.1 & Good & \\
\hline $1 / 23 / 930: 00$ & Manual Tape & $404: 75$ & Good & \\
\hline $1 / 23 / 937: 03$ & Auto FIC & 406.10 & Good & \\
\hline $1 / 24 / 930: 00$ & Manual Tape & 404.75 & Good & \\
\hline $1 / 24 / 937: 03$ & Auto FIC & 406.1 & Good & \\
\hline $1 / 25 / 930: 00$ & Manual Tape , & 404.75 & Good & \\
\hline $1 / 25 / 937: 03$ & Auto FIC & 406.10 & Good & \\
\hline $1 / 26 / 937: 04$ & Auto FIC & 406 & Good & \\
\hline $1 / 27 / 93 \quad 0: 00$ & Manual Tape & 404.75 & Good & \\
\hline $1 / 27 / 937: 04$ & Auto FIC & 406.00 & Good & \\
\hline $1 / 28 / 930: 00$ & Manual Tape & 405.00 & Good & \\
\hline $1 / 28 / 93 \quad 7: 04$ & Auto FIC & 406 & Good & \\
\hline $1 / 29 / 930: 00$ & Manual Tape & 404.75 & Good & \\
\hline $1 / 29 / 93 \quad 7: 04$ & Auto FIC & 406 & Good & \\
\hline $1 / 30 / 930: 00$ & Manual Tape & 404.75 & Good & . \\
\hline $1 / 30 / 937: 03$ & Auto FIC & 406.00 & Good & \\
\hline $1 / 31 / 930: 00$ & Manual Tape & 404.75 & Good & \\
\hline $1 / 31 / 937: 04$ & Auto FIC & 406 & Good & \\
\hline $2 / 1 / 930: 00$ & Manual Tape & 404:75 & Good & \\
\hline 2/1/93 7:04 & Auto FIC & 406 & Good & \\
\hline $2 / 2 / 930: 00$ & Manual Tape & 404.75 & Good & \\
\hline $2 / 2 / 937: 04$ & Auto FIC & 406.00 & Good & \\
\hline $2 / 3 / 930: 00$ & Manual FIC & 406 & Good & \\
\hline $2 / 3 / 930: 00$ & Manual Tape & 404.75 & Good & \\
\hline $2 / 4 / 930: 00$ & Manual Tape & 405.00 & Good & \\
\hline $2 / 4 / 93 \quad 7: 04$ & Auto FIC & 406.00 & Good & \\
\hline $2 / 5 / 930: 00$ & Manual Tape & 403.50 & Suspect & Data not included in graph \\
\hline $2 / 5 / 937: 04$ & Auto FIC & 406.00 & Good & \\
\hline $2 / 6 / 930: 00$ & Manual Tape & 404.75 & Good & \\
\hline $2 / 6 / 937: 04$ & Auto FIC & 406 & Good & \\
\hline $2 / 7 / 930: 00$ & Manual Tape & 404.75 & Good & \\
\hline $2 / 7 / 937: 03$ & Auto FIC & 406.00 & Good & \\
\hline $2 / 8 / 930: 00$ & Manual Tape & 404.75 & Good & \\
\hline $2 / 8 / 937: 04$ & Auto FIC & 406.00 & Good & \\
\hline $2 / 9 / 93 \quad 0: 00$ & Manual Tape & 404.75 & Good & \\
\hline $2 / 9 / 937: 04$ & Auto FIC & 406.00 & Good & \\
\hline
\end{tabular}

Data obtained from WHC Surveillance Analysis Computer System, (PC SACS) July 7, 1995. 


\begin{tabular}{|c|c|c|c|c|}
\hline \multicolumn{5}{|c|}{ 241-AP-107 } \\
\hline \multicolumn{5}{|c|}{ Surface Lovel Data } \\
\hline Date & Type of Measurement & Level (Inches) & Data Quality & Comments \\
\hline $2 / 10 / 930: 00$ & Manual Tape & 404.75 & Good & \\
\hline $2 / 10 / 937: 04$ & Auto FIC & 406.00 & Good & \\
\hline $2 / 11 / 930: 00$ & Manual Tape & 404.75 & Good & \\
\hline 2/11/93 7:04 & Auto FIC & 406.00 & Good & \\
\hline $2 / 12 / 930: 00$ & Manual Tape & 404.75 & Good & \\
\hline 2/12/93 7:03 & Auto FIC & 405.90 & Good & \\
\hline $2 / 13 / 937: 03$ & Auto FIC & 405.9 & Good & \\
\hline $2 / 14 / 937: 04$ & Auto FIC & 405.90 & Good & \\
\hline $2 / 15 / 937: 04$ & Auto FIC & 405.9 & Good & \\
\hline $2 / 16 / 937: 02$ & Auto FIC & 405.9 & Good & \\
\hline $2 / 17 / 930: 00$ & Manual FIC & 405.9 & Good & \\
\hline $2 / 17 / 930: 00$ & Manual Tape & 404.5 & Good & \\
\hline $2 / 18 / 930: 00$ & Manual Tape & 404.50 & Good & \\
\hline 2/18/93 7:03 & Auto FIC & 405.9 & Good & \\
\hline $2 / 19 / 930: 00$ & Manual Tape & 404.50 & Good & \\
\hline $2 / 19 / 937: 02$ & Auto FIC & 405.90 & Good & \\
\hline $2 / 20 / 930: 00$ & Manual Tape & 404.50 & Good & \\
\hline $2 / 20 / 937: 03$ & Auto FIC & 405.90 & Good & \\
\hline $2 / 21 / 930: 00$ & Manual Tape & 404.50 & Good & \\
\hline $2 / 21 / 937: 04$ & Auto FIC & 405.80 & Good & \\
\hline $2 / 22 / 930: 00$ & Manual Tape & 404.75 & Good & \\
\hline $2 / 22 / 937: 04$ & Auto FIC & 405.80 & Good & \\
\hline $2 / 23 / 930: 00$ & Manual Tape & 403.75 & Suspect & Data not included in graph \\
\hline $2 / 23 / 937: 03$ & Auto FIC & 405.80 & Good & \\
\hline $2 / 24 / 930: 00$ & Manual Tape & 404.00 & Good & \\
\hline $2 / 24 / 937: 04$ & Auto FIC & 405.8 & Good & \\
\hline $2 / 25 / 930: 00$ & Manual Tape & 404 & Good & \\
\hline $2 / 25 / 937: 03$ & Auto FIC & 405.8 & Good & \\
\hline $2 / 26 / 930: 00$ & Manual Tape & 404.25 & Good & \\
\hline $2 / 26 / 937: 03$ & Auto FIC & 405.80 & Good & \\
\hline $2 / 27 / 930: 00$ & Manual Tape & 404 & Good & \\
\hline $2 / 27 / 937: 04$ & Auto FIC & 405.80 & Good & \\
\hline $2 / 28 / 930: 00$ & Manual Tape & 404 & Good & \\
\hline $2 / 28 / 937: 03$ & Auto FIC & 405.80 & Good & \\
\hline $3 / 1 / 930: 00$ & Manual Tape & 404.25 & Good & \\
\hline $3 / 1 / 93$ 7:03 & Auto FIC & 405.80 & Good & \\
\hline $3 / 2 / 930: 00$ & Manual Tape & 404.25 & Good & \\
\hline $3 / 2 / 937: 04$ & Auto FIC & 405.80 & Good & \\
\hline $3 / 3 / 930: 00$ & Manual Tape & 404.25 & Good & \\
\hline $3 / 3 / 937: 03$ & Auto FIC & 405.8 & Good & \\
\hline $3 / 4 / 930: 00$ & Manual Tape & 404.25 & Good & \\
\hline $3 / 4 / 937: 04$ & Auto FIC & 405.7 & Good & \\
\hline 3/5/93 0:00 & Manual Tape & 404.5 & Good & \\
\hline $3 / 5 / 937: 04$ & Auto FIC & 405.70 & Good & \\
\hline $3 / 6 / 93 \quad 0: 00$ & Manual Tape & 404.5 & Good & \\
\hline $3 / 6 / 937: 03$ & Auto FIC & 405.70 & Good & \\
\hline $3 / 7 / 930: 00$ & Manual Tape & 404.25 & Good & \\
\hline $3 / 7 / 93$ 7:03 & Auto FIC & 405.70 & Good & \\
\hline
\end{tabular}

Data obtained from WHC Surveillance Analysis Computer System, (PC SACS) July 7, 1995. 


\begin{tabular}{|c|c|c|c|c|}
\hline \multicolumn{5}{|c|}{ 241-AP-107 } \\
\hline \multicolumn{5}{|c|}{ Surfece Level Data } \\
\hline Date & Type of Measurement & Level (Inches) & Data Quality & Comments \\
\hline $3 / 8 / 930: 00$ & Manual Tape & 404.5 & Good & \\
\hline 3/8/93 7:03 & Auto FIC & 405.7 & Good & \\
\hline 3/9/93 0:00 & Manual Tape & 404.50 & Good & \\
\hline 3/9/93 7:04 & Auto FIC & 405.70 & Good & \\
\hline 3/10/93 0:00 & Manual Tape & 404.50 & Good & \\
\hline 3/10/93 7:05 & Auto FIC & 405.70 & Good & \\
\hline 3/11/93 0:00 & Manual Tape & 404.25 & Good & \\
\hline $3 / 11 / 937: 04$ & Auto FIC & 405.7 & Good & \\
\hline 3/12/93 0:00 & Manual Tape & 404.50 & Good & \\
\hline 3/12/93 7:04 & Auto FIC & 405.7 & Good & \\
\hline 3/13/93 0:00 & Manual Tape & 404.50 & Good & \\
\hline 3/13/93 7:03 & Auto FIC & 405.7 & Good & \\
\hline 3/14/93 0:00 & Manual Tape & 404.50 & Good & \\
\hline $3 / 14 / 937: 04$ & Auto FIC & 405.70 & Good & \\
\hline $3 / 15 / 930: 00$ & Manual Tape & 404.50 & Good & \\
\hline $3 / 15 / 937: 03$ & Auto FIC & 405.6 & Good & \\
\hline $3 / 16 / 930: 00$ & Manual Tape & 404.50 & Good & \\
\hline 3/16/93 7:04 & Auto FIC & 405.50 & Good & \\
\hline $3 / 17 / 930: 00$ & Manual Tape & 416.25 & Suspect & Data not included in graph \\
\hline $3 / 17 / 937: 03$ & Auto FIC & 405.7 & Good & \\
\hline $3 / 18 / 930: 00$ & Manual Tape & 416.25 & Suspect & Data not included in graph \\
\hline 3/18/93 7:04 & Auto FIC & 405.6 & Good & \\
\hline $3 / 19 / 930: 00$ & Manual Tape & 416.5 & Suspect & Data not included in graph \\
\hline $3 / 19 / 937: 03$ & Auto FIC & 405.60 & Good & \\
\hline $3 / 20 / 930: 00$ & Manual Tape & 404.25 & Good & \\
\hline $3 / 20 / 937: 04$ & Auto FIC & 405.60 & Good & \\
\hline $3 / 21 / 930: 00$ & Manual Tape & 404.38 & Good & \\
\hline $3 / 21 / 937: 04$ & Auto FIC & 405.60 & Good & \\
\hline 3/22/930:00 & Manual Tape & 404.25 & Good & \\
\hline $3 / 22 / 93$ 7:03 & Auto FIC & 405.6 & Good & \\
\hline $3 / 23 / 930: 00$ & Manual Tape & 404.50 & Good & \\
\hline $3 / 23 / 937: 03$ & Auto FIC & 405.60 & Good & \\
\hline $3 / 24 / 930: 00$ & Manual FIC & 405.60 & Good & \\
\hline 3/24/93 0:00 & Manual Tape & 404.25 & Good & \\
\hline $3 / 24 / 937: 04$ & Auto FIC & 0.00 & Suspect & Data not included in graph \\
\hline $3 / 25 / 930: 00$ & Manual Tape & 404.25 & Good & \\
\hline $3 / 25 / 937: 03$ & Auto FIC & 0.00 & Suspect & Data not included in graph \\
\hline $3 / 26 / 930: 00$ & Manual FIC & 405.60 & Good & \\
\hline $3 / 26 / 930: 00$ & Manual Tape & 404.25 & Good & \\
\hline $3 / 26 / 937: 03$ & Auto FIC & 0 & Suspect & Data not included in graph \\
\hline $3 / 27 / 930: 00$ & Manual Tape & 404.5 & Good & \\
\hline $3 / 27 / 937: 03$ & Auto FIC & 405.6 & Good & \\
\hline $3 / 28 / 930: 00$ & Manual Tape & 404.5 . & Good & \\
\hline $3 / 28 / 937: 03$ & Auto FIC & 405.6 & Good & \\
\hline $3 / 29 / 930: 00$ & Manual FIC & 405.6 & Good & \\
\hline $3 / 29 / 930: 00$ & Manual Tape & 404.25 & Good & \\
\hline $3 / 29 / 93 \quad 7: 03$ & Auto FIC & 0 & Suspect & Data not included in graph \\
\hline $3 / 30 / 930: 00$ & Manual FIC & 405.6 & Good & \\
\hline
\end{tabular}

Data obtained from WHC Surveillance Analysis Computer System, (PC SACS) July 7, 1995. 


\begin{tabular}{|c|c|c|c|c|}
\hline \multicolumn{5}{|c|}{ 241-AP-107 } \\
\hline \multicolumn{5}{|c|}{, Surface Level Data } \\
\hline Date & Typo of Measurement & Level (Inchas) & Data Quality & Comments \\
\hline $3 / 30 / 930: 00$ & Manual Tape & 404.25 & Good & \\
\hline 3/30/93 7:03 & Auto FIC & 0.00 & Suspect & Data not included in graph \\
\hline $3 / 31 / 930: 00$ & Manual FIC & 405.65 & Good & \\
\hline 3/31/93 0:00 & Manual Tape & 404.25 & Good & \\
\hline $3 / 31 / 937: 03$ & Auto FIC & 0.00 & Suspect & Data not included in graph \\
\hline $4 / 1 / 93 \quad 10: 03$ & Manual FIC & 405.6 & Good & \\
\hline $4 / 1 / 93 \quad 10: 03$ & Manual Tape & 404.25 & Good & \\
\hline $4 / 2 / 9310: 03$ & Manual FIC & 405.50 & Good & \\
\hline $4 / 2 / 93 \quad 10: 03$ & Manual Tape & 404.25 & Good & \\
\hline $4 / 3 / 93 \quad 10: 03$ & Manual FIC & 405.70 & Good & \\
\hline $4 / 3 / 93 \quad 10: 03$ & Manual Tape & 404.25 & Good & \\
\hline 4/4/93 10:03 & Manual FIC & 405.50 & Good & \\
\hline 4/4/93 10:03 & Manual Tape & 404.25 & Good & \\
\hline 4/5/93 10:03 & Manual FIC & 405.55 & Good & \\
\hline 4/5/93 10:03 & Manual Tape & 404.25 & Good & \\
\hline $4 / 6 / 93 \quad 10: 03$ & Manual FIC & 405.5 & Good & \\
\hline $4 / 6 / 93 \quad 10: 03$ & Manual Tape & 403.5 & Good & \\
\hline $4 / 7 / 9310: 03$ & Manual FIC & 405.50 & Good & \\
\hline $4 / 7 / 9310: 03$ & Manual Tape & 404.25 & Good & \\
\hline $4 / 8 / 9310: 03$ & Manual Tape & 404.25 & Good & \\
\hline 4/9/93 10:03 & Manual Tape & 404.25 & Good & \\
\hline $4 / 10 / 93 \quad 10: 03$ & Manual FIC & 405.5 & Good & \\
\hline $4 / 10 / 9310: 03$ & Manual Tape & 404.25 & Good & \\
\hline 4/11/93 10:03 & Manual Tape & 404.25 & Good & \\
\hline 4/12/93 10:03 & Manual Tape & 404.25 & Good & \\
\hline 4/13/93 10:03 & Manual Tape & 404.25 & Good & \\
\hline 4/14/93 10:03 & Manual Tape & 404.25 & Good & \\
\hline 4/15/93 7:03 & Auto FIC & 405.6 & Good & \\
\hline 4/15/93 10:03 & Manual Tape & 404.00 & Good & \\
\hline 4/16/93 10:03 & Manual Tape & 404.00 & Good & \\
\hline 4/17/93 10:03 & Manual Tape & 404 & Good & \\
\hline $4 / 18 / 93 \quad 10: 03$ & Manual Tape & 404.00 & Good & \\
\hline $4 / 19 / 93 \quad 0: 00$ & Manual FIC & 405.5 & Good & \\
\hline 4/19/93 10:03 & Manual FIC & 405.50 & Good & \\
\hline $4 / 19 / 93 \quad 10: 03$ & Manual Tape & 404 & Good & \\
\hline $4 / 20 / 930: 00$ & Manual FIC & 405.50 & Good & \\
\hline 4/20/93 10:03 & Manual FIC & 405.50 & Good & \\
\hline 4/20/93 10:03 & Manual Tape & 403.75 & Good & \\
\hline $4 / 21 / 930: 00$ & Manual FIC & 405.50 & Good & \\
\hline 4/21/93 10:03 & Manual FIC & 405.5 & Good & \\
\hline 4/21/93 $10: 03$ & Manual Tape & 404.00 & Good & \\
\hline 4/22/93 10:03 & Manual Tape ' & 404.25 & Good & \\
\hline $4 / 23 / 93 \quad 10: 03$ & Manual Tape & 404.25 & Good & \\
\hline $4 / 24 / 93 \quad 10: 03$ & Manual Tape & 404.25 & Good & \\
\hline $4 / 25 / 93 \quad 10: 03$ & Manual Tape & 404.25 & Good & \\
\hline $4 / 26 / 93 \quad 10: 03$ & Manual Tape & 404.25 & Good & \\
\hline 4/27/93 10:03 & Manual Tape & 404.25 & Good & \\
\hline $4 / 28 / 93 \quad 10: 03$ & Manual Tape & 404.25 & Good & \\
\hline
\end{tabular}

Data obtained from WHC Surveillance Analysis Computer System, (PC SACS) July 7, 1995. 


\begin{tabular}{|c|c|c|c|c|}
\hline \multicolumn{5}{|c|}{ 241-AP-107 } \\
\hline \multicolumn{5}{|c|}{ Surface Level Data } \\
\hline Date & Type of Measurement & Level (Inches) & Data Quality & Comments \\
\hline $4 / 29 / 93 \quad 10: 03$ & Manual Tape & 404.25 & Good & \\
\hline $4 / 30 / 9310: 03$ & Manual Tape & 404.25 & Good & \\
\hline $5 / 1 / 93 \quad 10: 05$ & Manual Tape & 405.25 & Good & \\
\hline $5 / 2 / 9310: 05$ & Manual Tape & 405.25 & Good & \\
\hline $5 / 3 / 93 \quad 10: 05$ & Manual FIC & 405.4 & Good & \\
\hline $5 / 3 / 93 \quad 10: 05$ & Manual Tape & 405.25 & Good & \\
\hline $5 / 4 / 93 \quad 10: 05$ & Manual Tape & 405.25 & Good & \\
\hline $5 / 5 / 9310: 05$ & Manual Tape & 405.25 & Good & \\
\hline $5 / 6 / 93 \quad 10: 05$ & Manual Tape & 405.25 & Good & \\
\hline $5 / 7 / 93 \quad 10: 05$ & Manual FIC & 405.4 & Good & \\
\hline $5 / 7 / 93 \quad 10: 05$ & Manual Tape & 404 & Good & \\
\hline $5 / 8 / 93 \quad 10: 05$ & Manual FIC & 405.40 & Good & \\
\hline 5/8/93 10:05 & Manual Tape & 404.25 & Good & \\
\hline $5 / 9 / 93 \quad 10: 05$ & Manual FIC & 405.45 & Good & \\
\hline $5 / 9 / 93 \quad 10: 05$ & Manual Tape & 404.25 & Good & \\
\hline $5 / 10 / 9310: 05$ & Manual FIC & 405.5 & Good & \\
\hline $5 / 10 / 9310: 05$ & Manual Tape & 404.25 & Good & \\
\hline $5 / 11 / 93 \quad 10: 05$ & Manual Tape & 404.25 & Good & \\
\hline $5 / 12 / 93 \quad 10: 05$ & Manual Tape & 404.25 & Good & \\
\hline $5 / 13 / 93 \quad 10: 05$ & Manual Tape & 404.25 & Good & \\
\hline $5 / 14 / 93 \quad 10: 05$ & Manual FIC & 405.4 & Good & \\
\hline $5 / 14 / 93 \quad 10: 05$ & Manual Tape & 404.25 & Good & \\
\hline $5 / 15 / 93 \quad 10: 05$ & Manual Tape & 404.25 & Good & \\
\hline $5 / 16 / 937: 05$ & Auto FIC & $\mathbf{4 0 5 . 4 0}$ & Good & \\
\hline $5 / 16 / 93 \quad 10: 05$ & Manual Tape & 404,25 & Good & \\
\hline $5 / 17 / 930: 00$ & Manual FIC & 405.40 & Good & \\
\hline $5 / 17 / 937: 03$ & Auto FIC & 405.4 & Good & \\
\hline $5 / 17 / 93 \quad 10: 05$ & Manual Tape & 404.25 & Good & \\
\hline $5 / 18 / 93 \quad 0: 00$ & Manual FIC & 405.4 & Good & \\
\hline $5 / 18 / 937: 03$ & Auto FIC & $\mathbf{4 0 5 . 4 0}$ & Good & \\
\hline $5 / 18 / 9310: 05$ & Manual Tape & 404.25 & Good & \\
\hline $5 / 19 / 930: 00$ & Manual FIC & 405.45 & Good & \\
\hline $5 / 19 / 937: 03$ & Auto FIC & 405.4 & Good & \\
\hline $5 / 19 / 93 \quad 10: 05$ & Manual Tape & $404: 25$ & Good & \\
\hline $5 / 20 / 930: 00$ & Manual FIC & 405.4 & Good & \\
\hline $5 / 20 / 937: 03$ & Auto FIC & 405,40 & Good & \\
\hline $5 / 20 / 93 \quad 10: 05$ & Manual Tape & 404.25 & Good & \\
\hline $5 / 21 / 930: 00$ & Manual FIC & 405.5 & Good & \\
\hline $5 / 21 / 9310: 05$ & Manual Tape & 404.25 & Good & \\
\hline $5 / 22 / 937: 03$ & Auto FIC & 405.40 & Good & \\
\hline $5 / 22 / 93 \quad 10: 05$ & Manual Tape & 404.25 & Good & \\
\hline $5 / 23 / 937: 04$ & Auto FIC & 405.4 & Good & \\
\hline $5 / 23 / 93 \quad 10: 05$ & Manual Tape & 404.25 & Good & \\
\hline $5 / 24 / 937: 03$ & Auto FIC & 405.40 & Good & \\
\hline $5 / 24 / 93 \quad 10: 05$ & Manual Tape & 404.25 & Good & \\
\hline $5 / 25 / 937: 03$ & Auto FIC & 405.40 & Good & \\
\hline $5 / 25 / 93 \quad 10: 05$ & Manual Tape & 404.00 & Good & \\
\hline $5 / 26 / 937: 03$ & Auto FIC & 405.40 & Good & \\
\hline
\end{tabular}

Data obtained from WHC Surveiliance Analysis Computer System, (PC SACS) July 7, 1995. 


\begin{tabular}{|c|c|c|c|c|}
\hline \multicolumn{5}{|c|}{ 241-AP-107 } \\
\hline \multicolumn{5}{|c|}{ Surface Level Data } \\
\hline Date & Type of Measurement & Level (Inches) & Data Quality & Comments \\
\hline $5 / 26 / 93 \quad 10: 05$ & Manual Tape & 404.25 & Good & \\
\hline $5 / 27 / 937: 03$ & Auto FIC & 405.4 & Good & \\
\hline $5 / 27 / 93 \quad 10: 05$ & Manual Tape & 404.25 & Good & \\
\hline $5 / 28 / 937: 03$ & Auto FIC & 405.40 & Good & \\
\hline $5 / 28 / 93 \quad 10: 05$ & Manual Tape & $404 . \overline{25}$ & Good & \\
\hline $5 / 29 / 937: 05$ & Auto FIC & 405.4 & Good & \\
\hline $5 / 29 / 93 \quad 10: 05$ & Manual Tape & 404.25 & Good & \\
\hline $5 / 30 / 937: 05$ & Auto FIC & 405.4 & Good & \\
\hline $5 / 30 / 93 \quad 10: 05$ & Manual Tape & 404.25 & Good & \\
\hline $5 / 31 / 937: 06$ & Auto FIC & 405.4 & Good & \\
\hline $5 / 31 / 9310: 05$ & Manual Tape & 404.00 & Good & \\
\hline $6 / 1 / 937: 06$ & Auto FIC & 405.4 & Good & \\
\hline 6/1/93 10:06 & Manual Tape & 404 & Good & \\
\hline $6 / 2 / 937: 06$ & Auto FIC & 405.40 & Good & \\
\hline $6 / 2 / 93 \quad 10: 06$ & Manual Tape & 402.75 & Good & \\
\hline 6/3/93 7:06 & Auto FIC & 405.4 & Good & \\
\hline $6 / 3 / 93 \quad 10: 06$ & Manual Tape & 403.5 & Good & \\
\hline 6/4/93 7:06 & Auto FIC & 405.4 & Good & \\
\hline $6 / 5 / 937: 06$ & Auto FIC & 405.4 & Good & \\
\hline 6/5/93 10:06 & Manual Tape & 404.25 & Good & \\
\hline 6/6/93 7:05 & Auto FIC & 405.4 & Good & \\
\hline $6 / 6 / 93 \quad 10: 06$ & Manual Tape & 404.25 & Good & \\
\hline $6 / 7 / 937: 05$ & Auto FIC & 405.40 & Good & \\
\hline $6 / 7 / 93 \quad 10: 06$ & Manual Tape & 404.25 & Good & \\
\hline 6/8/93 7:05 & Auto FIC & 405.40 & Good & \\
\hline $6 / 8 / 93 \quad 10: 06$ & Manual Tape & 404.25 & Good & \\
\hline $6 / 9 / 937: 05$ & Auto FIC & 405.4 & Good & \\
\hline $6 / 9 / 93 \quad 10: 06$ & Manual Tape & 404.25 & Good & \\
\hline $6 / 10 / 937: 04$ & Auto FIC & 405.40 & Good & \\
\hline $6 / 10 / 93 \quad 10: 06$ & Manual Tape & 404.25 & Good & \\
\hline $6 / 11 / 937: 05$ & Auto FIC & 405.4 & Good & \\
\hline $6 / 11 / 93 \quad 10: 06$ & Manual Tape & 404.25 & Good & \\
\hline $6 / 12 / 937: 05$ & Auto FIC & 405.4 & Good & \\
\hline $6 / 12 / 93 \quad 10: 06$ & Manual Tape & 404.25 & Good & \\
\hline $6 / 13 / 937: 04$ & Auto FIC & 405.40 & Good & \\
\hline $6 / 13 / 93$ 10:06 & Manual Tape & 404.25 & Good & \\
\hline $6 / 14 / 937: 05$ & Auto FIC & 405.40 & Good & \\
\hline $6 / 14 / 93 \quad 10: 06$ & Manual Tape & 404.25 & Good & \\
\hline $6 / 15 / 937: 05$ & Auto FIC & 405.4 & Good & \\
\hline $6 / 15 / 93 \quad 10: 06$ & Manual Tape & 404.25 & Good & \\
\hline $6 / 16 / 937: 05$ & Auto FIC & 405.40 & Good & \\
\hline $6 / 16 / 93 \quad 10: 06$ & Manual Tape & 404.25 & Good & \\
\hline $6 / 17 / 937: 05$ & Auto FIC & 405.40 & Good & \\
\hline $6 / 18 / 937: 06$ & Auto FIC & 405.4 & Good & \\
\hline $6 / 18 / 93 \quad 10: 06$ & Manual Tape & 404.25 & Good & \\
\hline $6 / 19 / 937: 06$ & Auto FIC & 405.4 & Good & \\
\hline $6 / 19 / 93 \quad 10: 06$ & Manual Tape & 404.00 & Good & \\
\hline $6 / 20 / 937: 06$ & Auto FIC & 405.4 & Good & \\
\hline
\end{tabular}

Data obtained from WHC Surveillance Analysis Computer System, (PC SACS) July 7, 1995. 


\begin{tabular}{|c|c|c|c|c|}
\hline \multicolumn{5}{|c|}{ 241-AP-107 } \\
\hline \multicolumn{5}{|c|}{ Surface Lavel Data } \\
\hline Date & Type of Measurement & Level (inches) & Data Quality & Comments \\
\hline $6 / 20 / 9310: 06$ & Manual Tape & 404 & Good & \\
\hline $6 / 21 / 937: 06$ & Auto FIC & 405.40 & Good & \\
\hline $6 / 21 / 9310: 06$ & Manual Tape & 404.00 & Good & \\
\hline $6 / 22 / 937: 06$ & Auto FIC & 405.40 & Good & \\
\hline $6 / 22 / 9310: 06$ & Manual Tape & 404.00 & Good & \\
\hline $6 / 23 / 937: 06$ & Auto FIC & 405.40 & Good & \\
\hline $6 / 23 / 9310: 06$ & Manual Tape & 404.25 & Good & \\
\hline $6 / 24 / 937: 06$ & Auto FIC & 405.40 & Good & \\
\hline $6 / 24 / 93 \quad 10: 06$ & Manual Tape & 404.25 & Good & \\
\hline $6 / 25 / 937: 03$ & Auto FIC & 405.40 & Good & \\
\hline 6/25/93 10:06 & Manual Tape & 404.25 & Good & \\
\hline $6 / 26 / 937: 03$ & Auto FIC & 405.4 & Good & \\
\hline $6 / 26 / 93 \quad 10: 06$ & Manual Tape & 404.25 & Good & \\
\hline 6/27/93 7:04 & Auto FIC & 405.4 & Good & \\
\hline $6 / 27 / 93 \quad 10: 06$ & Manual Tape & 404.25 & Good & \\
\hline $6 / 28 / 937: 03$ & Auto FIC & 405,40 & Good & \\
\hline $6 / 28 / 9310: 06$ & Manual Tape & 404.25 & Good & \\
\hline $6 / 29 / 937: 04$ & Auto FIC & 405.40 & Good & \\
\hline $6 / 29 / 93 \quad 10: 06$ & Manual Tape & 404.25 & Good & \\
\hline $6 / 30 / 937: 03$ & Auto FIC & 405.4 & Good & \\
\hline $6 / 30 / 9310: 06$ & Manual Tape & 404.25 & Good & \\
\hline 7/1/93 7:06 & Auto FIC & 405.40 & Good & \\
\hline $7 / 1 / 93 \quad 10: 06$ & Manual Tape & 404 & Good & \\
\hline $7 / 2 / 937: 06$ & Auto FIC & 405.4 & Good & \\
\hline 7/2/93 10:06 & Manual Tape & 404.00 & Good & \\
\hline 7/3/93 7:03 & Auto FIC & 405.4 & Good & \\
\hline 7/3/93 10:06 & Manual Tape & 404 & Good & \\
\hline 7/4/93 7:04 & Auto FIC & 405.40 & Good & \\
\hline 7/4/93 10:06 & Manual Tape & 404.00 & Good & \\
\hline $7 / 5 / 937: 03$ & Auto FIC & 405.40 & Good & \\
\hline $7 / 5 / 93 \quad 10: 06$ & Manual Tape & 404.00 & Good & \\
\hline $7 / 6 / 937: 03$ & Auto FIC & 405.4 & Good & \\
\hline $7 / 6 / 93 \quad 10: 06$ & Manuat Tape & 404 & Good & \\
\hline $7 / 7 / 937: 04$ & Auto FIC & 405.4 & Good & \\
\hline $7 / 7 / 93 \quad 10: 06$ & Manual Tape & 404.00 & Good & \\
\hline $7 / 8 / 93 \quad 7: 04$ & Auto FIC & 405.40 & Good & \\
\hline $7 / 8 / 93 \quad 10: 06$ & Manual Tape & 403.75 & Good & \\
\hline 7/9/93 7:05 & Auto FIC & 405.40 & Good & \\
\hline $7 / 9 / 93 \quad 10: 06$ & Manual Tape & 404 & Good & \\
\hline 7/10/93 7:03 & Auto FIC & 405.40 & Good & \\
\hline $7 / 10 / 93 \quad 10: 06$ & Manual Tape & 404 & Good & \\
\hline $7 / 11 / 937: 03$ & Auto FIC & 405.4 & Good & \\
\hline $7 / 11 / 93 \quad 10: 06$ & Manual Tape & 403.75 & Good & \\
\hline $7 / 12 / 937: 02$ & Auto FIC & 405.30 & Good & \\
\hline $7 / 12 / 93 \quad 10: 06$ & Manual Tape & 403.75 & Good & \\
\hline $7 / 13 / 937: 03$ & Auto FIC & 405.4 & Good & \\
\hline $7 / 13 / 93 \quad 10: 06$ & Manual Tape & 403.75 & Good & \\
\hline $7 / 14 / 937: 06$ & Auto FIC & 405.30 & Good & \\
\hline
\end{tabular}

Data obtained from WHC Surveillance Analysis Computer System, (PC SACS) July 7, 1995. 


\begin{tabular}{|c|c|c|c|c|}
\hline \multicolumn{5}{|c|}{ 241-AP-107 } \\
\hline \multicolumn{5}{|c|}{ Surface Level Data } \\
\hline Date & Type of Measurement & Level (Inches) & Data Quality & Comments \\
\hline $7 / 14 / 93 \quad 10: 06$ & Manual Tape & 403.75 & Good & \\
\hline 7/15/93 7:06 & Auto FIC & 405.3 & Good & \\
\hline $7 / 15 / 93 \quad 10: 06$ & Manual Tape & 403.75 & Good & \\
\hline $7 / 16 / 937: 06$ & Auto FIC & 405.3 & Good & \\
\hline $7 / 16 / 93 \quad 10: 06$ & Manual Tape & 403.75 & Good & \\
\hline $7 / 17 / 937: 07$ & Auto FIC & 405.30 & Good & \\
\hline $7 / 17 / 9310: 06$ & Manual Tape & 404.00 & Good & \\
\hline $7 / 18 / 937: 06$ & Auto FIC & 405.3 & Good & \\
\hline $7 / 18 / 93 \quad 10: 06$ & Manual Tape & 404 & Good & \\
\hline $7 / 19 / 937: 03$ & Auto FIC & 405.3 & Good & \\
\hline 7/19/93 10:06 & Manual Tape & 404 & Good & \\
\hline $7 / 20 / 937: 03$ & Auto FIC & 405.40 & Good & \\
\hline $7 / 20 / 93 \quad 10: 06$ & Manual Tape & 404.00 & Good & \\
\hline $7 / 21 / 937: 03$ & Auto FIC & 405.2 & Good & \\
\hline $7 / 21 / 93 \quad 10: 06$ & Manual Tape & 403.5 & Good & \\
\hline $7 / 22 / 937: 03$ & Auto FIC & 405.3 & Good & \\
\hline $7 / 22 / 93 \quad 10: 06$ & Manual Tape & 403.5 & Good & \\
\hline $7 / 23 / 937: 03$ & Auto FIC & 405.3 & Good & \\
\hline $7 / 23 / 93 \quad 10: 06$ & Manual Tape & 403.5 & Good & \\
\hline $7 / 24 / 93 \quad 7: 03$ & Auto FIC & 405.3 & Good & \\
\hline $7 / 24 / 9310: 06$ & Manual Tape & 403.5 & Good & \\
\hline $7 / 25 / 937: 04$ & Auto FIC & 405.30 & Good & \\
\hline $7 / 25 / 93 \quad 10: 06$ & Manual Tape & 403.50 & Good & \\
\hline $7 / 26 / 937: 03$ & Auto FIC & 405.3 & Good & \\
\hline $7 / 26 / 93 \quad 10: 06$ & Manual Tape & 403.5 & Good & \\
\hline $7 / 27 / 937: 03$ & Auto FIC & 405.30 & Good & \\
\hline $7 / 27 / 93 \quad 10: 06$ & Manual Tape & 403.50 & Good & \\
\hline $7 / 28 / 930: 00$ & Manual Tape & 403.5 & Good & \\
\hline $7 / 28 / 937: 04$ & Auto FIC & 405.30 & Good & \\
\hline $7 / 29 / 930: 00$ & Manual Tape & 403.50 & Good & \\
\hline $7 / 29 / 93 \quad 7: 03$ & Auto FIC & 405.3 & Good & \\
\hline $7 / 30 / 930: 00$ & Manual Tape & 403.75 & Good & \\
\hline $7 / 30 / 937: 03$ & Auto FIC & 405.3 & Good & \\
\hline $7 / 31 / 930: 00$ & Manual Tape & 403.75 & Good & \\
\hline $7 / 31 / 937: 03$ & Auto FIC & 405.30 & Good & \\
\hline $8 / 1 / 930: 00$ & Manual Tape & 404 & Good & \\
\hline $8 / 1 / 937: 03$ & Auto FIC & 405.30 & Good & \\
\hline $8 / 2 / 930: 00$ & Manual Tape & 404.00 & Good & \\
\hline $8 / 2 / 93 \quad 7: 06$ & Auto FIC & 405.30 & Good & \\
\hline $8 / 3 / 930: 00$ & Manual Tape & 404 & Good & \\
\hline $8 / 3 / 937: 03$ & Auto FIC & 405.30 & Good & \\
\hline $8 / 4 / 930: 00$ & Manual Tape & 404 & Good & \\
\hline $8 / 4 / 937: 03$ & Auto FIC & 405.20 & Good & \\
\hline $8 / 5 / 930: 00$ & Manual Tape & 404.00 & Good & \\
\hline $8 / 5 / 937: 04$ & Auto FIC & 405.3 & Good & \\
\hline $8 / 6 / 93 \quad 0: 00$ & Manual Tape & 404 & Good & \\
\hline $8 / 6 / 937: 04$ & Auto FIC & 405.3 & Good & \\
\hline 8/7/93 0:00 & Manual Tape & 404 & Good & \\
\hline
\end{tabular}

Data obtained from WHC Surveillance Analysis Computer System, (PC SACS) July 7, 1995. 


\begin{tabular}{|c|c|c|c|c|}
\hline \multicolumn{5}{|c|}{ 241-AP-107 } \\
\hline \multicolumn{5}{|c|}{ Surface Level Data } \\
\hline Date & Type of Measurement & Level (Inches) & Data Quality & Comments \\
\hline 8/7/93 7:04 & Auto FIC & 405.30 & Good & \\
\hline $8 / 8 / 930: 00$ & Manual Tape & 404 & Good & \\
\hline 8/8/93 7:04 & Auto FIC & 405.3 & Good & \\
\hline 8/9/93 0:00 & Manual Tape & 403.5 & Good & \\
\hline 8/9/93 7:04 & Auto FIC & 405.3 & Good & \\
\hline $8 / 10 / 930: 00$ & Manual Tape & 403.75 & Good & \\
\hline $8 / 10 / 937: 04$ & Auto FIC & 405.3 & Good & \\
\hline $8 / 11 / 930: 00$ & Manual Tape & 399 & Suspect & Data not included in graph \\
\hline $8 / 11 / 937: 04$ & Auto FIC & 405.30 & Good & \\
\hline $8 / 12 / 930: 00$ & Manual Tape & 403.5 & Good & \\
\hline $8 / 12 / 937: 04$ & Auto FIC & 405.3 & Good & \\
\hline $8 / 13 / 930: 00$ & Manual Tape & 403.50 & Good & \\
\hline $8 / 13 / 937: 04$ & Auto FIC & 405.30 & Good & \\
\hline $8 / 14 / 930: 00$ & Manual Tape & 404 & Good & \\
\hline $8 / 14 / 937: 03$ & Auto FIC & 405.30 & Good & \\
\hline $8 / 15 / 930: 00$ & Manual Tape & 404.00 & Good & \\
\hline 8/15/93 7:04 & Auto FIC & 405.30 & Good & \\
\hline $8 / 16 / 930: 00$ & Manual Tape & 404 & Good & \\
\hline $8 / 16 / 937: 04$ & Auto FIC & 405.30 & Good & \\
\hline $8 / 17 / 930: 00$ & Manual Tape & 404.00 & Good & \\
\hline $8 / 17 / 937: 04$ & Auto FIC & 405.30 & Good & \\
\hline $8 / 18 / 930: 00$ & Manual Tape & 404.00 & Good & \\
\hline $8 / 18 / 937: 03$ & Auto FIC & 405.20 & Good & \\
\hline $8 / 19 / 930: 00$ & Manual Tape & 404 & Good & \\
\hline $8 / 19 / 937: 04$ & Auto FIC & 405.20 & Good & \\
\hline $8 / 20 / 930: 00$ & Manual Tape & 404.00 & Good & \\
\hline $8 / 20 / 937: 04$ & Auto FIC & 405.30 & Good & \\
\hline $8 / 21 / 930: 00$ & Manual Tape & 404 & Good & \\
\hline $8 / 21 / 937: 04$ & Auto FIC & 405.2 & Good & \\
\hline $8 / 22 / 930: 00$ & Manual Tape & 404 & Good & \\
\hline $8 / 22 / 937: 04$ & Auto FIC & 405.20 & Good & \\
\hline $8 / 23 / 930: 00$ & Manual Tape & 404.00 & Good & \\
\hline $8 / 23 / 937: 04$ & Auto FIC & 405.3 & Good & \\
\hline $8 / 24 / 930: 00$ & Manual Tape & 404 & Good & \\
\hline $8 / 24 / 937: 04$ & Auto FIC & 405.30 & Good & \\
\hline $8 / 25 / 930: 00$ & Manual Tape & 403.00 & Suspect & Data not included in graph \\
\hline $8 / 25 / 937: 04$ & Auto FIC & 405.2 & Good & \\
\hline $8 / 26 / 930: 00$ & Manual Tape & 403.00 & Suspect & Data not included in graph \\
\hline $8 / 26 / 937: 04$ & Auto FIC & 405.20 & Good & \\
\hline $8 / 27 / 930: 00$ & Manual Tape & 404 & Good & \\
\hline $8 / 27 / 937: 05$ & Auto FIC & 405.30 & Good & \\
\hline $8 / 28 / 930: 00$ & Manual Tape & 403 & Suspect & Data not included in graph \\
\hline $8 / 28 / 93 \quad 7: 04$ & Auto FIC & 405.2 & Good & \\
\hline $8 / 29 / 930: 00$ & Manual Tape & 403.00 & Suspect & Data not included in graph \\
\hline $8 / 29 / 937: 03$ & Auto FIC & 405.30 & Good & \\
\hline $8 / 30 / 930: 00$ & Manual Tape & 404 & Good & \\
\hline $8 / 30 / 937: 04$ & Auto FIC & 405.20 & Good & \\
\hline $8 / 31 / 930: 00$ & Manual Tape & 404.00 & Good & \\
\hline
\end{tabular}

Data obtained from WHC Surveillance Analysis Computer System, (PC SACS) July 7, 1995. 


\begin{tabular}{|c|c|c|c|c|}
\hline \multicolumn{5}{|c|}{ 241-AP-107 } \\
\hline & & Irface Level Dat & & \\
\hline Date & Type of Measurement & Level. (Inches) & Data Quality & Comments \\
\hline $8 / 31 / 937: 04$ & Auto FIC & 405.20 & Good & \\
\hline 9/1/93 0:00 & Manual Tape & 404 & Good & \\
\hline 9/1/93 7:04 & Auto FIC & 405.20 & Good & \\
\hline $9 / 2 / 930: 00$ & Manual Tape & 404.00 & Good & \\
\hline $9 / 2 / 937: 04$ & Auto FIC & 405.20 & Good & \\
\hline $9 / 3 / 930: 00$ & Manual Tape & 404.00 & Good & \\
\hline $9 / 3 / 937: 04$ & Auto FIC & 405.2 & Good & \\
\hline 9/4/93 0:00 & Manual Tape & 404 & Good & \\
\hline 9/4/93 7:04 & Auto FIC & 405.20 & Good & \\
\hline $9 / 5 / 930: 00$ & Manual Tape & 404 & Good & \\
\hline $9 / 5 / 937: 04$ & Auto FIC & 405.2 & Good & \\
\hline $9 / 6 / 930: 00$ & Manual Tape & 404.00 & Good & \\
\hline $9 / 6 / 937: 04$ & Auto FIC & 405.1 & Good & \\
\hline $9 / 7 / 930: 00$ & Manual Tape & 404.00 & Good & \\
\hline $9 / 7 / 937: 04$ & Auto FIC & 405.2 & Good & \\
\hline 9/8/93 0:00 & Manual Tape & 404 & Good & \\
\hline $9 / 8 / 937: 04$ & Auto FIC & 405.20 & Good & \\
\hline 9/9/93 0:00 & Manual Tape & 404.00 & Good & \\
\hline 9/9/93 7:04 & Auto FIC & 405.2 & Good & \\
\hline $9 / 10 / 930: 00$ & Manual Tape & 404 & Good & \\
\hline 9/10/93 7:04 & Auto FIC & 405.2 & Good & \\
\hline 9/11/93 0:00 & Manual Tape & 404 & Good & \\
\hline $9 / 11 / 937: 04$ & Auto FIC & 405.20 & Good & \\
\hline $9 / 12 / 930: 00$ & Manual Tape & 404 & Good & \\
\hline 9/12/93 7:04 & Auto FIC & 405.3 & Good & \\
\hline $9 / 13 / 937: 04$ & Auto FIC & 405.3 & Good & \\
\hline 9/14/93 0:00 & Manual Tape & 403.75 & Good & \\
\hline 9/14/93 7:04 & Auto FIC & 405.30 & Good & \\
\hline 9/15/93 0:00 & Manual Tape & 403.75 & Good & \\
\hline 9/15/93 7:04 & Auto FIC & 405.30 & Good & \\
\hline $9 / 16 / 930: 00$ & Manual Tape & 403.75 & Good & \\
\hline 9/16/93 7:04 & Auto FIC & 405.1 & Good & \\
\hline $9 / 17 / 930: 00$ & Manual Tape & 403.75 & Good & \\
\hline 9/17/93 7:04 & Auto FIC & $405: 10$ & Good & \\
\hline 9/18/93 0:00 & Manual Tape & 403.75 & Good & \\
\hline 9/18/93 7:04 & Auto FIC & 405.1 & Good & \\
\hline $9 / 19 / 930: 00$ & Manual Tape & 403.75 & Good & \\
\hline $9 / 19 / 937: 04$ & Auto FIC & 405.00 & Good & \\
\hline 9/20/93 0:00 & Manual Tape & 403.50 & Good & \\
\hline 9/20/93 7:04 & Auto FIC & 405.10 & Good & \\
\hline $9 / 21 / 930: 00$ & Manual Tape & 403.5 & Good & \\
\hline $9 / 21 / 937: 04$ & Auto FIC & 405.10 & Good & \\
\hline $9 / 22 / 930: 00$ & Manual Tape & 403.50 & Good & \\
\hline $9 / 22 / 937: 04$ & Auto FIC & 405.1 & Good & \\
\hline $9 / 23 / 930: 00$ & Manual Tape & 403.50 & Good & \\
\hline $9 / 23 / 937: 04$ & Auto FIC & 405.10 & Good & \\
\hline 9/24/93 0:00 & Manual Tape & 403.75 & Good & \\
\hline $9 / 24 / 937: 04$ & Auto FIC & 405.1 & Good & \\
\hline
\end{tabular}

Data obtained from WHC Surveillance Analysis Computer System, (PC SACS) July 7, 1995. 


\begin{tabular}{|c|c|c|c|c|}
\hline \multicolumn{5}{|c|}{ 241-AP-107 } \\
\hline \multicolumn{5}{|c|}{ Surface Lavel Data } \\
\hline Date & Type of Measurement & Level (Inches) & Date Quality & Comments \\
\hline 9/25/93 0:00 & Manual Tape ' & 403.75 & Good & \\
\hline $9 / 25 / 937: 04$ & Auto FIC & 405.10 & Good & \\
\hline $9 / 26 / 930: 00$ & Manual Tape & 403.75 & Good & \\
\hline $9 / 26 / 937: 04$ & Auto FIC & 405.10 & Good & \\
\hline $9 / 27 / 930: 00$ & Manual FIC & 405.10 & Good & \\
\hline 9/27/93 0:00 & Manual Tape & 403.75 & Good & \\
\hline $9 / 27 / 937: 04$ & Auto FIC & 0 & Suspect & Data not included in graph \\
\hline $9 / 28 / 930: 00$ & Manual Tape & 403.75 & Good & \\
\hline $9 / 28 / 937: 04$ & Auto FIC & 0 & Suspect & Data not included in graph \\
\hline $9 / 29 / 930: 00$ & Manual FIC & 405.10 & Good & \\
\hline $9 / 29 / 930: 00$ & Manual Tape & 403.75 & Good & \\
\hline 9/29/93 7:04 & Auto FIC & 0 & Suspect & Data not included in graph \\
\hline 9/30/930:00 & Manual FIC & 405.1 & Good & \\
\hline $9 / 30 / 93 \quad 0: 00$ & Manual Tape & 404 & Good & \\
\hline 9/30/93 7:03 & Auto FIC & 0 & Suspect & Data not included in graph \\
\hline $10 / 1 / 930: 00$ & Manual FIC & 405 & Good & \\
\hline $10 / 1 / 930: 00$ & Manual Tape & 404.00 & Good & \\
\hline $10 / 1 / 937: 06$ & Auto FIC & 0.00 & Suspect & Data not included in graph \\
\hline $10 / 2 / 930: 00$ & Manual FIC & 405.00 & Good & \\
\hline $10 / 2 / 930: 00$ & Manual Tape & 404.00 & Good & \\
\hline $10 / 2 / 937: 05$ & Auto FIC & 0 & Suspect & Data not included in graph \\
\hline $10 / 3 / 930: 00$ & Manual FIC & 405.50 & Good & \\
\hline $10 / 3 / 930: 00$ & Manual Tape & 404 & Good & \\
\hline $10 / 3 / 937: 06$ & Auto FIC & 0.00 & Suspect & Data not included in graph \\
\hline $10 / 4 / 930: 00$ & Manual FIC & 405 & Good & \\
\hline $10 / 4 / 930: 00$ & Manual Tape & 404 & Good & \\
\hline $10 / 4 / 937: 06$ & Auto FIC & 0.00 & Suspect & Data not included in graph \\
\hline $10 / 5 / 930: 00$ & Manual FIC & 405 & Good & \\
\hline $10 / 5 / 930: 00$ & Manual Tape & 404.00 & Good & \\
\hline $10 / 5 / 937: 06$ & Auto FIC & 0.00 & Suspect & Data not included in graph \\
\hline $10 / 6 / 930: 00$ & Manual FIC & 405.00 & Good & \\
\hline $10 / 6 / 930: 00$ & Manual Tape & 404 & Good & \\
\hline 10/6/93 7:06 & Auto FIC & 0 & Suspect & Data not included in graph \\
\hline 10/7/930:00 & Manual FIC & 405.10 & Good & \\
\hline $10 / 7 / 930: 00$ & Manual Tape & 404.00 & Good & \\
\hline $10 / 7 / 937: 05$ & Auto FIC & 0.00 & Suspect & Data not included in graph \\
\hline $10 / 8 / 930: 00$ & Manual FIC & 405 & Good & \\
\hline $10 / 8 / 93 \quad 0: 00$ & Manual Tape & 404.00 & Good & \\
\hline $10 / 8 / 937: 04$ & Auto FIC & 0 & Suspect & Data not included in graph \\
\hline $10 / 9 / 930: 00$ & Manual FIC & 405 & Good & \\
\hline $10 / 9 / 937: 03$ & Auto FIC & 0.00 & Suspect & Data not included in graph \\
\hline $10 / 10 / 930: 00$ & Manual FIC & 405 & Good & \\
\hline $10 / 31 / 930: 00$ & Manual FIC & 405 & Good & \\
\hline $10 / 11 / 930: 00$ & Manual Tape & 404 & Good & \\
\hline $10 / 11 / 937: 03$ & Auto FIC & 0 & Suspect & Data not included in graph \\
\hline $10 / 12 / 930: 00$ & Manual Tape & 404.00 & Good & \\
\hline $10 / 12 / 937: 04$ & Auto FIC & 405.00 & Good & \\
\hline $10 / 13 / 930: 00$ & Manual Tape & 403.75 & Good & \\
\hline
\end{tabular}

Data abtained from WHC Survaillance Analysis Computer System, (PC SACS) July 7, 1995. 


\begin{tabular}{|c|c|c|c|c|}
\hline \multicolumn{5}{|c|}{ 241-AP-107 } \\
\hline \multicolumn{5}{|c|}{ Surface Level Data } \\
\hline Date & Type of Measurement & Level (Inches) & Data Quality & Comments \\
\hline 10/13/93 7:03 & Auto FIC & 405 & Good & \\
\hline $10 / 14 / 930: 00$ & Manual Tape & 403.75 & Good & \\
\hline $10 / 14 / 937: 04$ & Auto FIC & 405.00 & Good & \\
\hline $10 / 15 / 930: 00$ & Manual Tape & 403.75 & Good & \\
\hline 10/15/93 7:04 & Auto FIC & 405 & Good & \\
\hline $10 / 16 / 930: 00$ & Manual Tape & 403.5 & Good & \\
\hline $10 / 16 / 937: 04$ & Auto FIC & 405 & Good & \\
\hline $10 / 17 / 930: 00$ & Manual Tape & 403.75 & Good & \\
\hline 10/17/93 7:03 & Auto FIC & 405 & Good & \\
\hline $10 / 18 / 930: 00$ & Manual Tape & 403.75 & Good & \\
\hline $10 / 18 / 937: 04$ & Auto FIC & 405 & Good & \\
\hline $10 / 19 / 930: 00$ & Manual Tape & 403.75 & Good & \\
\hline 10/19/93 7:04 & Auto FIC & 405.00 & Good & \\
\hline $10 / 20 / 930: 00$ & Manual Tape & 402.50 & Suspect & Data not included in graph \\
\hline $10 / 20 / 937: 03$ & Auto FIC & 405 & Good & \\
\hline $10 / 21 / 930: 00$ & Manual Tape & 403.75 & Good & \\
\hline $10 / 21 / 937: 04$ & Auto FIC & 404.90 & Good & \\
\hline $10 / 22 / 930: 00$ & Manual Tape & 403.75 & Good & \\
\hline 10/22/93 7:04 & Auto FIC & 405.00 & Good & \\
\hline $10 / 23 / 930: 00$ & Manual Tape & 403.75 & - Good & \\
\hline $10 / 23 / 937: 04$ & Auto FIC & 405.00 & Good & \\
\hline $10 / 24 / 930: 00$ & Manual Tape & 403.75 & Good & \\
\hline $10 / 24 / 937: 04$ & Auto FIC & 405.00 & Good & \\
\hline $10 / 25 / 930: 00$ & Manual Tape & 403.75 & Good & \\
\hline $10 / 25 / 937: 04$ & Auto FIC & 404.90 & Good & \\
\hline 10/26/93 0:00 & Manual Tape & 403.75 & Good & \\
\hline $10 / 26 / 936: 03$ & Auto FIC & $404: 90$ & Good & \\
\hline 10/27/930:00 & Manual Tape & 403.75 & Good & \\
\hline $10 / 27 / 937: 04$ & Auto FIC & 404.9 & Good & \\
\hline $10 / 28 / 937: 04$ & Auto FIC & 404.9 & Good & \\
\hline $10 / 29 / 936: 03$ & Auto FIC & 404.9 & Good & \\
\hline $10 / 30 / 930: 00$ & Manual Tape & 403.75 & Good & \\
\hline $10 / 30 / 937: 04$ & Auto FIC & 404.8 & Good & \\
\hline $10 / 31 / 930: 00$ & Manual Tape & 403.75 & Good & \\
\hline $10 / 31 / 937: 06$ & Auto FIC & 404.80 & Good & \\
\hline $11 / 1 / 930: 00$ & Manual Tape & 403.5 & Good & \\
\hline $11 / 1 / 937: 06$ & Auto FIC & 404.8 & Good & \\
\hline $11 / 2 / 930: 00$ & Manual Tape & 403.75 & Good & \\
\hline $11 / 2 / 937: 04$ & Auto FIC & 404.80 & Good & \\
\hline $11 / 3 / 930: 00$ & Manual Tape & 403.75 & Good & \\
\hline $11 / 3 / 937: 06$ & Auto FIC & 404.8 & Good & \\
\hline $11 / 4 / 930: 00$ & Manual Tape & 403.75 & Good & \\
\hline $11 / 4 / 937: 04$ & Auto FIC & 404.80 & Good & \\
\hline $11 / 5 / 930: 00$ & Manual Tape & 403.75 & Good & \\
\hline $11 / 5 / 937: 06$ & Auto FIC & 404.80 & Good & \\
\hline 11/6/93 0:00 & Manual Tape & 403.75 & Good & \\
\hline $11 / 6 / 937: 06$ & Auto FIC & 404.80 & Good & \\
\hline $11 / 7 / 930: 00$ & Manual Tape & 403.75 & Good & \\
\hline
\end{tabular}

Data obtained from WHC Surveillance Analysis Computer System, (PC SACS) July 7, 1995. 


\begin{tabular}{|c|c|c|c|c|}
\hline \multicolumn{5}{|c|}{ 241-AP-107 } \\
\hline & & urface Level Date & & \\
\hline Date & Type of Measurement & Level (Inches) & Data Quality & Comments \\
\hline 11/7/93 7:06 & Auto FIC & 404.8 & Good & \\
\hline $11 / 8 / 930: 00$ & Manual Tape & 403.50 & Good & \\
\hline $11 / 8 / 937: 06$ & Auto FIC & 404.8 & Good & \\
\hline 11/9/93 0:00 & Manual Tape & 403.5 & Good & \\
\hline $11 / 9 / 937: 03$ & Auto FIC & 404.80 & Good & \\
\hline $11 / 10 / 930: 00$ & Manual Tape & 403.5 & Good & \\
\hline $11 / 10 / 937: 04$ & Auto FIC & 404.7 & Good & \\
\hline $11 / 11 / 930: 00$ & Manual Tape & 403.5 & Good & \\
\hline $11 / 11 / 937: 04$ & Auto FIC & 404.8 & Good & \\
\hline $11 / 12 / 930: 00$ & Manual Tape & 403.50 & Good & \\
\hline $11 / 12 / 937: 04$ & Auto FIC & 404.7 & Good & \\
\hline $11 / 13 / 93 \quad 0: 00$ & Manual Tape & 403.50 & Good & \\
\hline $11 / 13 / 937: 04$ & Auto FIC & 404.70 & Good & \\
\hline $11 / 14 / 930: 00$ & Manual Tape & 403.50 & Good & \\
\hline $11 / 14 / 936: 04$ & Auto FIC & 404.70 & Good & \\
\hline $11 / 15 / 930: 00$ & Manual Tape & 403.5 & Good & \\
\hline $11 / 15 / 937: 04$ & Auto FIC & 404.7 & Good & \\
\hline $11 / 16 / 930: 00$ & Manual Tape & 403.5 & Good & \\
\hline 11/16/93 7:03 & Auto FIC & 404.70 & Good & \\
\hline $11 / 17 / 930: 00$ & Manual Tape & 403.50 & Good & \\
\hline 11/17/93 7:04 & Auto FIC & 404.7 & Good & \\
\hline $11 / 18 / 937: 04$ & Auto FIC & 404.7 & Good & \\
\hline $11 / 19 / 930: 00$ & Manual Tape & 403 & Good & \\
\hline $11 / 19 / 937: 04$ & Auto FIC & 404.6 & Good & \\
\hline $11 / 20 / 930: 00$ & Manual Tape & 402.5 & Good & \\
\hline $11 / 20 / 937: 04$ & Auto FIC & 404.70 & Good & \\
\hline $11 / 21 / 930: 00$ & Manual Tape & 402.5 & Good & \\
\hline $11 / 21 / 937: 04$ & Auto FIC & 404.7 & Good & \\
\hline $11 / 22 / 930: 00$ & Manual Tape & 403.5 & Good & \\
\hline $11 / 22 / 937: 04$ & Auto FIC & 404.60 & Good & \\
\hline $11 / 23 / 930: 00$ & Manual Tape & 403.50 & Good & \\
\hline $11 / 23 / 937: 04$ & Auto FIC & 404.60 & Good & \\
\hline 11/24/93 0:00 & Manual Tape & 403.5 & Good & \\
\hline $11 / 24 / 937: 04$ & Auto FIC & 404.6 & Good & \\
\hline $11 / 25 / 930: 00$ & Manual Tape & 403.5 & Good & \\
\hline $11 / 25 / 93 \quad 7: 04$ & Auto FIC & 404.70 & Good & \\
\hline $11 / 26 / 930: 00$ & Manual Tape & 403.5 & Good & \\
\hline $11 / 26 / 937: 04$ & Auto FIC & 404.60 & Good & \\
\hline $11 / 27 / 930: 00$ & Manual Tape & 403.50 & Good & \\
\hline $11 / 27 / 937: 04$ & Auto FIC & 404.6 & Good & \\
\hline $11 / 28 / 930: 00$ & Manual Tape & 403.50 & Good & \\
\hline $11 / 28 / 937: 03$ & Auto FIC & 404.60 & Good & \\
\hline $11 / 29 / 930: 00$ & Manual Tape & 403.5 & Good & \\
\hline $11 / 29 / 937: 04$ & Auto FIC & 404.60 & Good & \\
\hline $11 / 30 / 930: 00$ & Manual Tape & 403.25 & Good & \\
\hline $11 / 30 / 937: 03$ & Auto FIC & 404.60 & Good & \\
\hline $12 / 1 / 930: 00$ & Manual Tape & 403.25 & Good & \\
\hline $12 / 1 / 937: 04$ & Auto FIC & 404.50 & Good & \\
\hline
\end{tabular}

Data obtained from WHC Surveillance Analysis Computer System, (PC SACS) July 7, 1995. 


\begin{tabular}{|c|c|c|c|c|}
\hline \multicolumn{5}{|c|}{ 241-AP.107 } \\
\hline \multicolumn{5}{|c|}{ Surface Level Data } \\
\hline Date & Type of Measurement & Level (Inches) & Data Quality & Comments \\
\hline $12 / 2 / 930: 00$ & Manual Tape & 403.25 & Good & \\
\hline $12 / 2 / 937: 04$ & Auto FIC & 404.50 & Good & \\
\hline $12 / 3 / 930: 00$ & Manual Tape & 403.25 & Good & \\
\hline $12 / 3 / 937: 05$ & Auto FIC & 404.5 & Good & \\
\hline $12 / 4 / 930: 00$ & Manual Tape & 403.25 & Good & \\
\hline $12 / 4 / 937: 05$ & Auto FIC & 404.5 & Good & \\
\hline $12 / 5 / 930: 00$ & Manual Tape & 403.25 & Good & \\
\hline $12 / 5 / 937: 04$ & Auto FIC & 404.50 & Good & \\
\hline $12 / 6 / 930: 00$ & Manual Tape & 403.25 & Good & \\
\hline $12 / 6 / 937: 05$ & Auto FIC & 404.5 & Good & \\
\hline $12 / 7 / 930: 00$ & Manual Tape & 403.25 & Good & \\
\hline $12 / 7 / 937: 04$ & Auto FIC & 404.4 & Good & \\
\hline 12/8/930:00 & Manual FIC & 404.50 & Good & \\
\hline $12 / 8 / 930: 00$ & Manual Tape & 403.00 & Good & \\
\hline $12 / 8 / 937: 06$ & Auto FIC & 0.00 & Suspect & Data not included in graph \\
\hline $12 / 9 / 930: 00$ & Manual Tape & 403 & Good & \\
\hline $12 / 9 / 937: 04$ & Auto FIC & 404.50 & Good & \\
\hline $12 / 10 / 930: 00$ & Manual Tape & 403.25 & Good & \\
\hline 12/10/93 6:05 & Auto FIC & 404.50 & Good & \\
\hline $12 / 11 / 930: 00$ & Manual Tape & 402.75 & Good & \\
\hline $12 / 11 / 937: 06$ & Auto FIC & 404.50 & Good & \\
\hline $12 / 12 / 930: 00$ & Manual Tape & 402.75 & Good & \\
\hline 12/12/93 7:06 & Auto FIC & $404.50^{\circ}$ & Good & \\
\hline $12 / 13 / 930: 00$ & Manual Tape & 402.75 & Good & \\
\hline $12 / 13 / 937: 06$ & Auto FIC & 404.4 & Good & \\
\hline $12 / 14 / 930: 00$ & Manual Tape & 403 & Good & \\
\hline $12 / 14 / 937: 06$ & Auto FIC & 404.40 & Good & \\
\hline $12 / 15 / 930: 00$ & Manual Tape & 403.25 & Good & \\
\hline $12 / 15 / 937: 06$ & Auto FIC & $404: 40$ & Good & \\
\hline $12 / 16 / 930: 00$ & Manual Tape & 403.25 & Good & \\
\hline $12 / 16 / 937: 06$ & Auto FIC & 404.40 & Good & \\
\hline $12 / 17 / 930: 00$ & Manual Tape & 403 & Good & \\
\hline $12 / 17 / 937: 05$ & Auto FIC & 404.4 & Good & \\
\hline $12 / 18 / 930: 00$ & Manual Tape & 403.25 & Good & \\
\hline $12 / 18 / 93$ 7:06 & Auto FIC & 404.4 & Good & \\
\hline $12 / 19 / 930: 00$ & Manual Tape & 403 & Good & \\
\hline $12 / 19 / 937: 06$ & Auto FIC & 404.4 & Good & \\
\hline $12 / 20 / 930: 00$ & Manual Tape & 403.00 & Good & \\
\hline $12 / 20 / 93 \quad 7: 06$ & Auto FIC & 404.30 & Good & \\
\hline $12 / 21 / 930: 00$ & Manual Tape & 403.25 & Good & \\
\hline $12 / 21 / 930: 06$ & Auto FIC & 404.30 & Good & \\
\hline $12 / 22 / 930: 00$ & Manual Tape & 403.25 & Good & \\
\hline $12 / 22 / 937: 05$ & Auto FIC & 0 & Suspect & Data not included in graph \\
\hline $12 / 23 / 930: 00$ & Manual Tape & 403.25 & Good & \\
\hline $12 / 23 / 93 \quad 7: 04$ & Auto FIC & 0.00 & Suspect & Data not included in graph \\
\hline $12 / 24 / 930: 00$ & Manual Tape & 403.25 & Good & \\
\hline $12 / 24 / 937: 06$ & Auto FIC & 0 & Suspect & Data not included in graph \\
\hline $12 / 25 / 930: 00$ & Manual Tape & 403.25 & Good & \\
\hline
\end{tabular}

Data obtained from WHC Surveillance Analysis Computer System, (PC SACS) July 7, 1995. 
WHC-SD-WM-ER-315, Rev. 0

\begin{tabular}{|c|c|c|c|c|}
\hline \multicolumn{5}{|c|}{ 241-AP-107 } \\
\hline \multicolumn{5}{|c|}{ Surface Level Data } \\
\hline Date & Type of Measurement & Level-(Inches) & Data Quality & Comments \\
\hline $12 / 25 / 937: 06$ & Auto FIC & 0.00 & Suspect & Data not included in graph \\
\hline $12 / 26 / 930: 00$ & Manual Tape & 403.25 & Good & \\
\hline $12 / 26 / 937: 06$ & Auto FIC & 0.00 & Suspect & Data not inciuded in graph \\
\hline $12 / 27 / 930: 00$ & Manual Tape & 403.25 & Good & \\
\hline $12 / 27 / 937: 06$ & Auto FIC & 0.00 & Suspect & Data not included in graph \\
\hline $12 / 28 / 930: 00$ & Manual Tape & 403.00 & Good & \\
\hline $12 / 28 / 937: 06$ & Auto FIC & 0 & Suspect & Data not included in graph \\
\hline $12 / 29 / 930: 00$ & Manual Tape & 403.00 & Good & \\
\hline $12 / 29 / 937: 06$ & Auto FIC & 0.00 & Suspect & Data not included in graph \\
\hline $12 / 30 / 930: 00$ & Manual Tape & 403 & Good & \\
\hline $12 / 30 / 937: 05$ & Auto FIC & 0 & Suspect & Data not included in graph \\
\hline 12/31/93 0:00 & Manual Tape & 403.25 & Good & \\
\hline $12 / 31 / 937: 06$ & Auto FIC & 0.00 & Suspect & Data not included in graph \\
\hline 1/1/940:00 & Manual Tape & 403 & Good & \\
\hline $1 / 1 / 947: 06$ & Auto FIC & 0 & Suspect & Data not included in graph \\
\hline $1 / 2 / 940: 00$ & Manual Tape & 403 & Good & \\
\hline 1/2/94 7:06 & Auto FIC & 0.00 & Suspect & Data not included in graph \\
\hline $1 / 3 / 940: 00$ & Manual Tape & 403 & Good & \\
\hline $1 / 3 / 947: 07$ & Auto FIC & 0 & Suspect & Data not included in graph \\
\hline $1 / 4 / 940: 00$ & Manual Tape & 403.00 & Good & \\
\hline $1 / 4 / 947: 07$ & Auto FIC & 0.00 & Suspect & Data not included in graph \\
\hline $1 / 5 / 940: 00$ & Manual Tape & 403 & Good & \\
\hline $1 / 5 / 947: 06$ & Auto FIC & 0.00 & Suspect & Data not included in graph \\
\hline $1 / 6 / 940: 00$ & Manual Tape & 403.00 & Good & \\
\hline $1 / 6 / 947: 06$ & Auto FIC & 0 & Suspect & Data not included in graph \\
\hline $1 / 7 / 940: 00$ & Manual Tape & 403.00 & Good & \\
\hline $1 / 7 / 947: 07$ & Auto FIC & 0 & Suspect & Data not included in graph \\
\hline $1 / 8 / 940: 00$ & Manual FIC & 404.20 & Good & \\
\hline $1 / 8 / 940: 00$ & Manual Tape & 403 & Good & \\
\hline 1/8/94 7:06 & Auto FIC & 0.00 & Suspect & Data not included in graph \\
\hline $1 / 9 / 940: 00$ & Manual Tape & 403.00 & Good & \\
\hline $1 / 9 / 947: 06$ & Auto FIC & 0 & Suspect & Data not included in graph \\
\hline $1 / 10 / 940: 00$ & Manual Tape & 403 & Good & \\
\hline $1 / 10 / 947: 07$ & Auto FIC & 0 & Suspect & Data not included in graph \\
\hline $1 / 11 / 940: 00$ & Manual Tape & 403.00 & Good & \\
\hline $1 / 11 / 947: 07$ & Auto FIC & 0 & Suspect & Data not included in graph \\
\hline 1/12/940:00 & Manual Tape & 403 & Good & \\
\hline $1 / 12 / 947: 06$ & Auto FIC & 0 & Suspect & Data not included in graph \\
\hline $1 / 13 / 940: 00$ & Manual Tape & 403.00 & Good & \\
\hline $1 / 13 / 947: 04$ & Auto FIC & 0 & Suspect & Data not included in graph \\
\hline $1 / 14 / 940: 00$ & Manual Tape & 403.00 & Good & \\
\hline $1 / 14 / 947: 06$ & Auto FIC & 0 & Suspect & Data not included in graph \\
\hline 1/15/94 0:00 & Manual Tape & 402.75 & Good & \\
\hline $1 / 15 / 947: 04$ & Auto FIC & 0.00 & Suspect & Data not included in graph \\
\hline $1 / 16 / 940: 00$ & Manual Tape & 402.75 & Good & \\
\hline $1 / 16 / 947: 07$ & Auto FIC & 0 & Suspect & Data not included in graph \\
\hline $1 / 17 / 940: 00$ & Manual Tape & 403 & Good & \\
\hline $1 / 17 / 947: 07$ & Auto FIC & 0 & Suspect & Data not included in graph \\
\hline
\end{tabular}

Data obtained from WHC Surveillance Analysis Computer System, (PC SACS) July 7, 1995. 


\begin{tabular}{|c|c|c|c|c|}
\hline \multicolumn{5}{|c|}{ 241-AP-107 } \\
\hline \multicolumn{5}{|c|}{ Surface Level Date } \\
\hline Date & Type of Measurement & Level (Inches) & Data Quality & Comments \\
\hline $1 / 18 / 940: 00$ & Manual Tape & 403 & Good & \\
\hline $1 / 18 / 947: 07$ & Auto FIC & 0.00 & Suspect & Data not included in graph \\
\hline $1 / 19 / 940: 00$ & Manual Tape & 403 & Good & \\
\hline $1 / 19 / 947: 07$ & Auto FIC & 0.00 & Suspect & Data not included in graph \\
\hline $1 / 20 / 940: 00$ & Manual Tape & 403 & Good & \\
\hline $1 / 20 / 947: 07$ & Auto FIC & 0 & Suspect & Data not included in graph \\
\hline $1 / 21 / 940: 00$ & Manual Tape & 403.00 & Good & \\
\hline $1 / 21 / 947: 07$ & Auto FIC & 0.00 & Suspect & Data not included in graph \\
\hline $1 / 22 / 940: 00$ & Manual Tape & 403.00 & Good & \\
\hline $1 / 22 / 947: 08$ & Auto FIC & 0.00 & Suspect & Data not included in graph \\
\hline $1 / 23 / 940: 00$ & Manual Tape & 402.75 & Good & \\
\hline $1 / 23 / 947: 07$ & Auto FIC & 0.00 & Suspect & Data not included in graph \\
\hline $1 / 24 / 940: 00$ & Manual Tape & 402.5 & Good & \\
\hline $1 / 24 / 947: 08$ & Auto FIC & 0 & Suspect & Data not included in graph \\
\hline $1 / 25 / 94 \quad 0: 00$ & Manual Tape & 402.75 & Good & \\
\hline $1 / 25 / 947: 07$ & Auto FIC & 0 & Suspect & Data not included in graph \\
\hline $1 / 26 / 940: 00$ & Manual Tape & 402.75 & Good & \\
\hline $1 / 26 / 947: 06$ & Auto FIC & 0.00 & Suspect & Data not included in graph \\
\hline $1 / 27 / 940: 00$ & Manual Tape & 402.75 & Good & \\
\hline $1 / 27 / 9415: 04$ & Auto FIC & 404.00 & Good & \\
\hline $1 / 28 / 940: 00$ & Manual Tape & 402.75 & Good & \\
\hline $1 / 28 / 947: 07$ & Auto FIC & 404 & Good & \\
\hline $1 / 29 / 940: 00$ & Manual Tape & 0 & Suspect & Data not included in graph \\
\hline $1 / 29 / 947: 07$ & Auto FIC & 404 & Good & \\
\hline $1 / 30 / 940: 00$ & Manual Tape & 398 & Suspect & Data not included in graph \\
\hline $1 / 30 / 947: 06$ & Auto FIC & 404.00 & Good & \\
\hline $1 / 31 / 940: 00$ & Manual Tape & 402.75 & Good & \\
\hline $1 / 31 / 947: 07$ & Auto FIC & 404 & Good & \\
\hline $2 / 1 / 940: 00$ & Manual Tape & 402.5 & Good & \\
\hline $2 / 1 / 947: 06$ & Auto FIC & 404.00 & Good & \\
\hline $2 / 2 / 940: 00$ & Manual Tape & 402.75 & Good & \\
\hline $2 / 2 / 947: 07$ & Auto FIC & 404 & Good & \\
\hline $2 / 3 / 940: 00$ & Manual Tape & $402.25^{\prime}$ & Good & \\
\hline $2 / 3 / 947: 07$ & Auto FIC & 404.00 & Good & \\
\hline $2 / 4 / 940: 00$ & Manual Tape & 402.75 & Good & \\
\hline $2 / 4 / 947: 07$ & Auto FIC & 404.00 & Good & \\
\hline 2/5/94 0:00 & Manual Tape & 402.75 & Good & \\
\hline $2 / 5 / 947: 07$ & Auto FIC & 403.90 & Good & \\
\hline $2 / 6 / 940: 00$ & Manual Tape & 402.75 & Good & \\
\hline $2 / 6 / 947: 07$ & Auto FIC & 403.90 & Good & \\
\hline $2 / 7 / 940: 00$ & Manual Tape & 402.75 & Good & \\
\hline $2 / 7 / 947: 06$ & Auto FIC & 403.90 & Good & \\
\hline $2 / 8 / 940: 00$ & Manual Tape & 402.75 & Good & \\
\hline $2 / 8 / 947: 07$ & Auto FIC & 403.90 & Good & \\
\hline $2 / 9 / 940: 00$ & Manual Tape & 402.75 & Good & \\
\hline $2 / 9 / 947: 04$ & Auto FIC & 403.9 & Good & \\
\hline $2 / 10 / 940: 00$ & Manual Tape & 402.75 & Good & \\
\hline $2 / 10 / 947: 04$ & Auto FIC & 403.90 & Good & \\
\hline
\end{tabular}

Data obtained from WHC Surveillance Analysis Computer System, (PC SACS) July 7, 1995. 


\begin{tabular}{|c|c|c|c|c|}
\hline Date & Type of Measurement & Level (Inches) & Data Quality & Comments \\
\hline $2 / 11 / 940: 00$ & Manual Tape & 402.75 & Good & \\
\hline $2 / 11 / 947: 06$ & Auto FIC & 403.90 & Good & \\
\hline 2/12/94 0:00 & Manual Tape & 402.75 & Good & \\
\hline $2 / 12 / 947: 03$ & Auto FIC & 403.9 & Good & \\
\hline $2 / 13 / 940: 00$ & Manual Tape & 402.75 & Good & \\
\hline $2 / 13 / 947: 04$ & Auto FIC & 403.9 & Good & \\
\hline $2 / 14 / 940: 00$ & Manual Tape & 402.75 & Good & $\cdot$ \\
\hline $2 / 14 / 947: 04$ & Auto FIC & 403.8 & Good & \\
\hline $2 / 15 / 940: 00$ & Manual Tape & 402.75 & Good & \\
\hline $2 / 15 / 947: 04$ & Auto FIC & 403.80 & Good & \\
\hline $2 / 16 / 940: 00$ & Manual Tape & 402.75 & Good & \\
\hline $2 / 16 / 947: 03$ & Auto FIC & 403.8 & Good & \\
\hline 2/17/940:00 & Manual Tape & 402.75 & Good & \\
\hline $2 / 17 / 947: 04$ & Auto FIC & 403.80 & Good & \\
\hline $2 / 18 / 940: 00$ & Manual Tape & 402.5 & Good & \\
\hline $2 / 18 / 947: 06$ & Auto FIC & 403.8 & Good & \\
\hline $2 / 19 / 940: 00$ & Manual Tape & 402.50 & Good & \\
\hline $2 / 19 / 947: 06$ & Auto FIC & 403.80 & Good & \\
\hline $2 / 20 / 940: 00$ & Manual Tape & 402.5 & Good & \\
\hline $2 / 20 / 947: 07$ & Auto FIC & 403.8 & Good & \\
\hline $2 / 21 / 940: 00$ & Manual Tape & 402.50 & Good & \\
\hline $2 / 21 / 947: 06$ & Auto FIC & 403.80 & Good & \\
\hline $2 / 22 / 940: 00$ & Manual Tape & 402.50 & Good & \\
\hline 2/22/94 7:05 & Auto FIC & 403.8 & Good & \\
\hline $2 / 23 / 940: 00$ & Manual Tape & 402.5 & Good & \\
\hline $2 / 23 / 947: 06$ & Auto FIC & 403.80 & Good & \\
\hline $2 / 24 / 940: 00$ & Manual Tape & 402.75 & Good & \\
\hline $2 / 24 / 947: 06$ & Auto FIC & 403.8 & Good & \\
\hline $2 / 25 / 940: 00$ & Manual Tape & 402.5 & Good & \\
\hline $2 / 25 / 946: 06$ & Auto FIC & 403.80 & Good & \\
\hline $2 / 26 / 940: 00$ & Manual Tape & 402.50 & Good & \\
\hline $2 / 26 / 947: 07$ & Auto FIC & 403.8 & Good & \\
\hline $2 / 27 / 940: 00$ & Manual Tape & 402.25 & Good & \\
\hline $2 / 27 / 947: 07$ & Auto FIC & 403.70 & Good & \\
\hline $2 / 28 / 940: 00$ & Manual Tape & 402.5 & Good & \\
\hline 2/28/94 6:06 & Auto FIC & 403.70 & Good & \\
\hline 3/1/94 0:00 & Manual Tape & 402.25 & Good & \\
\hline $3 / 1 / 947: 04$ & Auto FIC & 403.7 & Good & \\
\hline $3 / 2 / 940: 00$ & Manual Tape & 402.00 & Good & \\
\hline $3 / 2 / 947: 06$ & Auto FIC & 403.7 & Good & \\
\hline $3 / 3 / 940: 00$ & Manual Tape & 402.5 & Good & \\
\hline $3 / 3 / 947: 06$ & Auto FIC & 403.7 & Good & \\
\hline $3 / 4 / 940: 00$ & Manual Tape & 402.00 & Good & \\
\hline $3 / 4 / 947: 04$ & Auto FIC & $403: 70$ & Good & \\
\hline $3 / 5 / 940: 00$ & Manual Tape & 402 & Good & \\
\hline $3 / 5 / 947: 05$ & Auto FIC & 403.8 & Good & \\
\hline $3 / 6 / 940: 00$ & Manual Tape & 402.00 & Good & \\
\hline $3 / 6 / 947: 05$ & Auto FIC & 403.70 & Good & \\
\hline
\end{tabular}

Data obtained from WHC Surveillance Analysis Computer System, (PC SACS) July 7, 1995. 


\begin{tabular}{|c|c|c|c|c|}
\hline \multicolumn{5}{|c|}{ 241-AP-107 } \\
\hline \multicolumn{5}{|c|}{ Surface Level Data } \\
\hline Date & Type of Measurement & Level (Inches) & Data Quality & Comments \\
\hline $3 / 7 / 940: 00$ & Manual Tape & 402.25 & Good & \\
\hline $3 / 7 / 947: 05$ & Auto FIC & 403.70 & Good & \\
\hline $3 / 8 / 940: 00$ & Manual Tape & 402.5 & Good & \\
\hline $3 / 8 / 947: 04$ & Auto FIC & 403.70 & Good & \\
\hline $3 / 9 / 940: 00$ & Manual Tape & 402.25 & Good & \\
\hline $3 / 9 / 947: 04$ & Auto FIC & 403.70 & Good & \\
\hline $3 / 10 / 940: 00$ & Manual Tape & 402.00 & Good & \\
\hline $3 / 10 / 947: 05$ & Auto FIC & 403.70 & Good & \\
\hline $3 / 11 / 940: 00$ & Manual Tape & 402.00 & Good & \\
\hline $3 / 11 / 947: 06$ & Auto FIC & 403.7 & Good & \\
\hline $3 / 12 / 940: 00$ & Manual Tape & 402 & Good & \\
\hline $3 / 12 / 947: 06$ & Auto FIC & 403.70 & Good & \\
\hline $3 / 13 / 940: 00$ & Manual Tape & 402 & Good & \\
\hline $3 / 13 / 947: 05$ & Auto FIC & 403.70 & Good & \\
\hline $3 / 14 / 940: 00$ & Manual FIC & 403.50 & Good & \\
\hline $3 / 14 / 940: 00$ & Manual Tape & 402.50 & Good & \\
\hline $3 / 15 / 940: 00$ & Manual Tape & 402.5 & Good & \\
\hline $3 / 15 / 947: 05$ & Auto FIC & 403.70 & Good & \\
\hline $3 / 16 / 940: 00$ & Manual Tape & 402.25 & Good & \\
\hline $3 / 16 / 947: 04$ & Auto FIC & 403.6 & Good & $\cdot$ \\
\hline $3 / 17 / 940: 00$ & Manual Tape & 397.25 & Suspect & Data not included in graph \\
\hline $3 / 17 / 941: 00$ & Manual Tape & 402.25 & Good & \\
\hline $3 / 17 / 947: 05$ & Auto FIC & 403.7 & Good & \\
\hline $3 / 18 / 940: 00$ & Manual Tape & 402.5 & Good & \\
\hline 3/18/94 7:05 & Auto FIC & 403.70 & Good & \\
\hline $3 / 19 / 940: 00$ & Manual Tape & 402.00 & Good & \\
\hline $3 / 19 / 947: 04$ & Auto FIC & 403.6 & Good & \\
\hline $3 / 20 / 940: 00$ & Manual Tape & 402.50 & Good & \\
\hline $3 / 20 / 947: 05$ & Auto FIC & 403.6 & Good & \\
\hline $3 / 21 / 940: 00$ & Manual Tape & 402.50 & Good & \\
\hline $3 / 21 / 947: 05$ & Auto FIC & 403.60 & Good & \\
\hline $3 / 22 / 940: 00$ & Manual Tape & 402.25 & Good & \\
\hline $3 / 22 / 947: 04$ & Auto FIC & 403.60 & Good & \\
\hline $3 / 23 / 940: 00$ & Manual FIC & 403.60 & Good & \\
\hline $3 / 23 / 940: 00$ & Manual Tape & 402.25 & Good & \\
\hline $3 / 24 / 940: 00$ & Manual Tape & 402.25 & Good & \\
\hline $3 / 24 / 947: 04$ & Auto FIC & 403.60 & Good & \\
\hline $3 / 25 / 940: 00$ & Manual Tape & 402.25 & Good & \\
\hline $3 / 25 / 947: 05$ & Auto FIC & 403.60 & Good & \\
\hline $3 / 26 / 940: 00$ & Manual Tape & 402.25 & Good & \\
\hline $3 / 26 / 947: 04$ & Auto FIC & 403.60 & Good & \\
\hline $3 / 27 / 94 \quad 0: 00$ & Manual Tape & 402.25 & Good & \\
\hline $3 / 27 / 947: 04$ & Auto FIC & 403.60 & Good & \\
\hline $3 / 28 / 940: 00$ & Manual Tape & 401.75 & Good & \\
\hline $3 / 28 / 947: 04$ & Auto FIC & 403.6 & Good & \\
\hline $3 / 29 / 940: 00$ & Manual Tape & 402.00 & Good & \\
\hline $3 / 29 / 947: 03$ & Auto FIC & 403.60 & Good & \\
\hline $3 / 30 / 940: 00$ & Manual Tape & 402.25 & Good & \\
\hline
\end{tabular}

Data obtained from WHC Surveillance Analysis Computer System, (PC SACS) July 7, 1995. 


\begin{tabular}{|c|c|c|c|c|}
\hline \multicolumn{5}{|c|}{ 241-AP-107 } \\
\hline & & urface Level Date & & \\
\hline Date & Type of Measurement & Level (inches) & Data Quality & Comments \\
\hline $3 / 30 / 947: 03$ & Auto FIC & 403.60 & Good & \\
\hline $3 / 31 / 940: 00$ & Manual Tape & 402.25 & Good & \\
\hline $3 / 31 / 947: 03$ & Auto FIC & 403.50 & Good & \\
\hline 4/1/94 0:00 & Manual Tape & 402.25 & Good & \\
\hline 4/1/94 0:04 & Auto FIC & 403.70 & Good & \\
\hline 4/2/940:00 & Manual Tape & 402.25 & Good & \\
\hline 4/2/94 7:07 & Auto FIC & 403.60 & Good & \\
\hline $4 / 3 / 940: 00$ & Manual Tape & 402.25 & Good & \\
\hline $4 / 3 / 947: 03$ & Auto FIC & 403.60 & Good & \\
\hline 4/4/940:00 & Manual Tape & 402.25 & Good & \\
\hline 4/4/94 7:03 & Auto FIC & 403.60 & Good & \\
\hline $4 / 5 / 940: 00$ & Manual Tape & 402.25 & Good & \\
\hline $4 / 5 / 947: 03$ & Auto FIC & 403.7 & Good & \\
\hline 4/6/94 0:00 & Manual Tape & 414.25. & Suspect & Data not included in graph \\
\hline 4/6/94 7:06 & Auto FIC & 403.70 & Good & \\
\hline 4/7/94 0:00 & Manual Tape & 402.25 & Good & \\
\hline $4 / 7 / 947: 07$ & Auto FIC & 403.60 & Good & \\
\hline 4/8/94 0:00 & Manual Tape & 402.25 & Good & \\
\hline 4/8/94 7:03 & Auto FIC & 403.70 & Good & \\
\hline 4/9/94 0:00 & Manual Tape & 402.25 & Good & \\
\hline $4 / 9 / 947: 07$ & Auto FIC & 403.60 & Good & \\
\hline $4 / 10 / 940: 00$ & Manual Tape & 402.25 & Good & \\
\hline 4/10/94 7:03 & Auto FIC & 403.7 & Good & \\
\hline 4/11/94 0:00 & Manual Tape & 402.25 & Good & \\
\hline $4 / 11 / 947: 03$ & Auto FIC & 403.5 & Good & \\
\hline $4 / 12 / 940: 00$ & Manual Tape & 402.25 & Good & \\
\hline 4/12/94 7:03 & Auto FIC & 403.7 & Good & \\
\hline 4/13/94 0:00 & Manual Tape & 402.25 & Good & \\
\hline 4/13/94 7:03 & Auto FIC & 403.6 & Good & \\
\hline $4 / 14 / 940: 00$ & Manual Tape & 402.25 & Good & \\
\hline $4 / 14 / 947: 03$ & Auto FIC & 403.6 & Good & \\
\hline 4/15/940:00 & Manual Tape & 402.25 & Good & \\
\hline $4 / 15 / 947: 03$ & Auto FIC & 403.6 & Good & \\
\hline $4 / 16 / 940: 00$ & Manual Tape & 402.25 & Good & \\
\hline $4 / 16 / 947: 03$ & Auto FIC & 403.60 & Good & \\
\hline 4/17/940:00 & Manual Tape & 402.25 & Good & \\
\hline 4/17/94 7:03 & Auto FIC & 403.60 & Good & \\
\hline $4 / 18 / 940: 00$ & Manual Tape & 402.25 & Good & \\
\hline $4 / 18 / 947: 03$ & Auto FIC & 403.6 & Good & \\
\hline 4/19/940:00 & Manual Tape & 402.25 & Good & \\
\hline $4 / 19 / 946: 06$ & Auto FIC & 403.6 & Good & \\
\hline $4 / 20 / 940: 00$ & Manual Tape & 402.25 & Good & \\
\hline $4 / 20 / 947: 04$ & Auto FIC & 403.6 & Good & \\
\hline $4 / 21 / 940: 00$ & Manual Tape & 402 & Good & \\
\hline $4 / 21 / 947: 05$ & Auto FIC & 403.80 & Good & \\
\hline $4 / 22 / 940: 00$ & Manual Tape & 402.25 & Good & \\
\hline $4 / 22 / 947: 04$ & Auto FIC & 403.5 & Good & \\
\hline $4 / 23 / 940: 00$ & Manual Tape & 402.25 & Good & \\
\hline
\end{tabular}

Data obtained from WHC Surveillance Analysis Computer System, (PC SACS) July 7, 1995. 


\begin{tabular}{|c|c|c|c|c|}
\hline \multicolumn{5}{|c|}{ 241-AP-107 } \\
\hline \multicolumn{5}{|c|}{ Surface Level Data } \\
\hline Date & Type of Measurement & Level.(Inches) & Date Quality & Comments \\
\hline $4 / 23 / 947: 05$ & Auto FIC & 403.6 & Good & \\
\hline 4/24/94 0:00 & Manual Tape & 402.25 & Good & \\
\hline 4/24/94 7:04 & Auto FIC & 403.60 & Good & \\
\hline 4/25/94 0:00 & Manual Tape . & 402.25 & Good & \\
\hline $4 / 25 / 947: 04$ & Auto FIC & 403.6 & Good & \\
\hline $4 / 26 / 940: 00$ & Manual Tape & $401 . \overline{25}$ & Good & \\
\hline $4 / 26 / 947: 07$ & Auto FIC & 403.50 & Good & \\
\hline 4/27/94 0:00 & Manual Tape & 402.25 & Good & \\
\hline $4 / 27 / 947: 04$ & Auto FIC & 403.60 & Good & \\
\hline $4 / 28 / 940: 00$ & Manual Tape & 402.25 & Good & \\
\hline $4 / 28 / 947: 05$ & Auto FIC & 403.6 & Good & \\
\hline 4/29/94 0:00 & Manual Tape & 402.25 & Good & \\
\hline $4 / 29 / 947: 04$ & Auto FIC & 403.7 & Good & . \\
\hline $4 / 30 / 940: 00$ & Manual Tape & 401 & Good & \\
\hline $4 / 30 / 947: 05$ & Auto FIC & 403.6 & Good & \\
\hline 5/1/94 0:00 & Manual Tape & 402.00 & Good & \\
\hline $5 / 1 / 947: 05$ & Auto FIC & 403.40 & Good & \\
\hline $5 / 2 / 940: 00$ & Manual Tape & 401.75 & Good & \\
\hline 5/2/94 7:05 & Auto FIC & 403.6 & Good & \\
\hline $5 / 3 / 940: 00$ & Manual Tape & 402.00 & Good & \\
\hline $5 / 3 / 947: 05$ & Auto FIC & 403.6 & Good & \\
\hline $5 / 4 / 940: 00$ & Manual Tape & 402 & Good & \\
\hline $5 / 4 / 947: 05$ & Auto FIC & 403.6 & Good & \\
\hline $5 / 5 / 940: 00$ & Manual Tape & 402.00 & Good & \\
\hline $5 / 5 / 947: 03$ & Auto FIC & 403.60 & Good & \\
\hline $5 / 6 / 940: 00$ & Manual Tape & 402.25 & Good & \\
\hline $5 / 6 / 947: 03$ & Auto FIC & 403.6 & Good & \\
\hline 5/7/94 0:00 & Manual Tape & 402.25 & Good & \\
\hline $5 / 7 / 947: 03$ & Auto FIC & 403.6 & Good & \\
\hline $5 / 8 / 940: 00$ & Manual FIC & 403.5 & Good & \\
\hline $5 / 8 / 940: 00$ & Manual Tape & 402.25 & Good & \\
\hline $5 / 8 / 947: 03$ & Auto FIC & 0 & Suspect & Data not included in graph \\
\hline $5 / 9 / 940: 00$ & Manual FIC & 403.45 & Good & \\
\hline 5/9/94 0:00 & Manual Tape & 402.25 & Good & \\
\hline $5 / 9 / 947: 03$ & Auto FIC & 0 & Suspect & Data not included in graph \\
\hline $5 / 10 / 940: 00$ & Manual FIC & 403.60 & Good & \\
\hline $5 / 10 / 940: 00$ & Manual Tape & 402.25 & Good & \\
\hline $5 / 10 / 947: 03$ & Auto FIC & 0.00 & Suspect & Data not included in graph \\
\hline $5 / 11 / 940: 00$ & Manual FIC & 403.4 & Good & \\
\hline $5 / 11 / 940: 00$ & Manual Tape & 402 & Good & \\
\hline $5 / 11 / 947: 03$ & Auto FIC & 0 & Suspect & Data not included in graph \\
\hline $5 / 12 / 940: 00$ & Manual FIC & 403.4 & Good & \\
\hline $5 / 12 / 940: 00$ & Manual Tape & 402.00 & Good & \\
\hline $5 / 13 / 940: 00$ & Manual FIC & 403.7 & Good & \\
\hline $5 / 13 / 940: 00$ & Manual Tape & 402 & Good & \\
\hline $5 / 13 / 947: 03$ & Auto FIC & 0.00 & Suspect & Data not included in graph \\
\hline $5 / 14 / 940: 00$ & Manual FIC & 403.65 & Good & \\
\hline $5 / 14 / 940: 00$ & Manual Tape & 402.25 & Good & \\
\hline
\end{tabular}

Data obtained from WHC Surveillance Analysis Computer System, (PC SACS) July 7, 1995. 


\begin{tabular}{|c|c|c|c|c|}
\hline \multicolumn{5}{|c|}{ 241-AP-107 } \\
\hline \multicolumn{5}{|c|}{ Surface Level Data } \\
\hline Date & Type of Measurement & Level (Inchas) & Data Quality & Comments \\
\hline $5 / 14 / 947: 03$ & Auto FIC & 0 & Suspect & Data not included in graph \\
\hline $5 / 15 / 940: 00$ & Manual Tape & 402 & Good & \\
\hline $5 / 15 / 947: 06$ & Auto FIC & 403.6 & Good & \\
\hline $5 / 16 / 940: 00$ & Manual Tape & 402.25 & Good & \\
\hline $5 / 16 / 947: 02$ & Auto FIC & 403.6 & Good & \\
\hline $5 / 17 / 940: 00$ & Manual Tape & 402.25 & Good & \\
\hline $5 / 17 / 947: 03$ & Auto FIC & 403.5 & Good & \\
\hline $5 / 18 / 940: 00$ & Manual Tape & 401.75 & Good & \\
\hline $5 / 18 / 947: 03$ & Auto FIC & 403.6 & Good & \\
\hline $5 / 19 / 940: 00$ & Manual Tape & 402.25 & Good & \\
\hline $5 / 19 / 947: 03$ & Auto FIC & 403.5 & Good & \\
\hline $5 / 20 / 940: 00$ & Manual Tape & 402.25 & Good & \\
\hline $5 / 20 / 947: 03$ & Auto FIC & 403.50 & Good & \\
\hline $5 / 21 / 940: 00$ & Manual Tape & 402 & Good & \\
\hline $5 / 21 / 947: 05$ & Auto FIC & 403.60 & Good & \\
\hline $5 / 22 / 940: 00$ & Manual Tape & 402.25 & Good & \\
\hline $5 / 22 / 947: 05$ & Auto FIC & 403.6 & Good & \\
\hline $5 / 23 / 940: 00$ & Manual Tape & 401.75 & Good & \\
\hline $5 / 23 / 947: 04$ & Auto FIC & 403.5 & Good & \\
\hline $5 / 24 / 940: 00$ & Manual Tape & 405.75 & Good & \\
\hline $5 / 24 / 947: 04$ & Auto FIC & 403.40 & Good & \\
\hline $5 / 25 / 940: 00$ & Manual Tape & 401.75 & Good & \\
\hline $5 / 25 / 947: 04$ & Auto FIC & 403.6 & Good & \\
\hline 5/26/94 0:00 & Manual Tape & 401.5 & Good & \\
\hline $5 / 26 / 946: 05$ & Auto FIC & 403.60 & Good & \\
\hline $5 / 27 / 940: 00$ & Manual Tape & 401.50 & Good & \\
\hline $5 / 27 / 947: 03$ & Auto FIC & 403.6 & Good & \\
\hline $5 / 28 / 940: 00$ & Manual Tape & 402.00 & Good & \\
\hline $5 / 28 / 947: 03$ & Auto FIC & 403.30 & Good & \\
\hline 5/29/94 0:00 & Manual Tape & 401.50 & Good & \\
\hline $5 / 29 / 947: 03$ & Auto FIC & 403.40 & Good & \\
\hline $5 / 30 / 940: 00$ & Manual FIC & 403.60 & Good & . \\
\hline $5 / 30 / 940: 00$ & Manual Tape & 402 & Good & \\
\hline $5 / 30 / 947: 02$ & Auto FIC & 403.50 & Good & \\
\hline $5 / 31 / 940: 00$ & Manual FIC & 403.60 & Good & \\
\hline $5 / 31 / 940: 00$ & Manual Tape & 402.00 & Good & \\
\hline $5 / 31 / 947: 03$ & Auto FIC & 403.60 & Good & \\
\hline 6/1/94 0:00 & Manual Tape & 402 & Good & \\
\hline $6 / 1 / 947: 03$ & Auto FIC & 403.30 & Good & \\
\hline $6 / 2 / 940: 00$ & Manual Tape & 401.5 & Good & \\
\hline $6 / 2 / 947: 03$ & Auto FIC & 403.4 & Good & \\
\hline $6 / 3 / 940: 00$ & Manual FIC & 403.4 & Good & \\
\hline $6 / 3 / 940: 00$ & Manual Tape & 401 & Good & \\
\hline 6/4/94 0:00 & Manual Tape & 402 & Good & \\
\hline $6 / 4 / 947: 04$ & Auto FIC & 403.30 & Good & \\
\hline $6 / 5 / 940: 00$ & Manual Tape & 402 & Good & \\
\hline $6 / 5 / 947: 03$ & Auto FIC & 403.3 & Good & \\
\hline $6 / 6 / 940: 00$ & Manual Tape & 402.00 & Good & \\
\hline
\end{tabular}

Data obtained from WHC Surveillance Analysis Computer System, (PC SACS) July 7, 1995. 


\begin{tabular}{|c|c|c|c|c|}
\hline \multicolumn{5}{|c|}{ 241-AP-107 } \\
\hline \multicolumn{5}{|c|}{ Surface Level Data } \\
\hline Date & Type of Measurement & Level (Inches) & Data Quality & Comments \\
\hline $6 / 6 / 947: 06$ & Auto FIC & 403.4 & Good & \\
\hline $6 / 7 / 940: 00$ & Manual Tape & 402.00 & Good & \\
\hline 6/7/94 7:02 & Auto FIC & 403.3 & Good & \\
\hline $6 / 8 / 940: 00$ & Manual Tape & 402.00 & Good & \\
\hline $6 / 8 / 947: 03$ & Auto FIC & 403.30 & Good & \\
\hline $6 / 9 / 940: 00$ & Manual FIC & 403.4 & Good & \\
\hline $6 / 9 / 940: 00$ & Manual Tape & 402.00 & Good & \\
\hline $6 / 10 / 940: 00$ & Manual Tape & 402.00 & Good & \\
\hline $6 / 10 / 947: 04$ & Auto FIC & 403.3 & Good & \\
\hline $6 / 11 / 940: 00$ & Manual Tape & 402.00 & Good & \\
\hline $6 / 11 / 947: 04$ & Auto FIC & 403.3 & Good & \\
\hline $6 / 12 / 940: 00$ & Manual Tape & 402.00 & Good & \\
\hline $6 / 12 / 947: 03$ & Auto FIC & 403.3 & Good & \\
\hline $6 / 13 / 940: 00$ & Manual Tape & 402 & Good & \\
\hline $6 / 13 / 947: 02$ & Auto FIC & 403.3 & Good & \\
\hline $6 / 14 / 940: 00$ & Manual Tape & 402 & Good & \\
\hline $6 / 14 / 947: 03$ & Auto FIC & 403.3 & Good & \\
\hline $6 / 15 / 940: 00$ & Manual Tape & 402 & Good & \\
\hline 6/15/94 7:03 & Auto FIC & $403: 30$ & Good & \\
\hline $6 / 16 / 940: 00$ & Manual Tape & 402.00 & Good & \\
\hline 6/16/94 7:03 & Auto FIC & 403.3 & Good & \\
\hline $6 / 17 / 940: 00$ & Manual Tape & 402.25 & Good & \\
\hline $6 / 17 / 947: 03$ & Auto FIC & 403.30 & Good & \\
\hline $6 / 18 / 940: 00$ & Manual Tape & 402.50 & Good & \\
\hline $6 / 18 / 947: 04$ & Auto FIC & 403.30 & Good & \\
\hline $6 / 19 / 940: 00$ & Manual Tape & 402.25 & Good & \\
\hline $6 / 19 / 947: 04$ & Auto FIC & 403.30 & Good & \\
\hline $6 / 20 / 940: 00$ & Manual Tape & 402 & Good & \\
\hline $6 / 20 / 947: 04$ & Auto FIC & 403.30 & Good & \\
\hline $6 / 21 / 940: 00$ & Manual Tape & 402.00 & Good & \\
\hline $6 / 21 / 947: 04$ & Auto FIC & 403.4 & Good & \\
\hline $6 / 22 / 940: 00$ & Manual Tape & 402.00 & Good & \\
\hline $6 / 22 / 947: 03$ & Auto FIC & 403.30 & Good & \\
\hline $6 / 23 / 940: 00$ & Manual Tape & 402.00 & Good & \\
\hline 6/23/94 7:05 & Auto FIC & 403.3 & Good & \\
\hline $6 / 24 / 940: 00$ & Manual Tape & 402.00 & Good & \\
\hline 6/24/94 7:06 & Auto FIC & 403.30 & Good & \\
\hline $6 / 25 / 940: 00$ & Manual Tape & 402.00 & Good & \\
\hline $6 / 25 / 947: 05$ & Auto FIC & 403.30 & Good & \\
\hline $6 / 26 / 940: 00$ & Manual Tape & 402 & Good & \\
\hline $6 / 26 / 947: 06$ & Auto FIC & 403.30 & Good & \\
\hline $6 / 27 / 940: 00$ & Manual Tape & 402.00 & Good & \\
\hline $6 / 27 / 947: 06$ & Auto FIC & 403.30 & Good & \\
\hline $6 / 28 / 940: 00$ & Manual Tape & 401.00 & Good & \\
\hline $6 / 28 / 947: 06$ & Auto FIC & 403.3 & Good & \\
\hline $6 / 29 / 940: 00$ & Manual Tape & 402.00 & Good & \\
\hline $6 / 29 / 947: 03$ & Auto FIC & $403.30^{\circ}$ & Good & \\
\hline $6 / 30 / 940: 00$ & Manual Tape & 402.00 & Good & \\
\hline
\end{tabular}

Data obtained from WHC Surveillance Analysis Computer System, (PC SACS) July 7, 1995. 


\begin{tabular}{|c|c|c|c|c|}
\hline \multicolumn{5}{|c|}{ 241-AP-107 } \\
\hline \multicolumn{5}{|c|}{ Surface Level Data } \\
\hline Date & Type of Measurement & Level (Inches) & Data Quality & Comments \\
\hline $6 / 30 / 947: 04$ & Auto FIC & 403.30 & Good & \\
\hline $7 / 1 / 940: 00$ & Manual Tape & 402.00 & Good & \\
\hline 7/1/94 7:04 & Auto FIC & 403.40 & Good & \\
\hline $7 / 2 / 940: 00$ & Manual Tape & 402 & Good & \\
\hline $7 / 2 / 947: 04$ & Auto FIC & $403 . \overline{40}$ & Good & \\
\hline $7 / 3 / 940: 00$ & Manual Tape & 402.00 & Good & \\
\hline 7/3/94 7:04 & Auto FIC & 403.30 & Good & \\
\hline $7 / 4 / 940: 00$ & Manual Tape & 402.00 & Good & \\
\hline $7 / 4 / 947: 04$ & Auto FIC & 403.30 & Good & \\
\hline $7 / 5 / 940: 00$ & Manual Tape & 401.75 & Good & \\
\hline 7/5/94 7:03 & Auto FIC & 403.40 & Good & \\
\hline $7 / 6 / 940: 00$ & Manual Tape & 402 & Good & \\
\hline $7 / 6 / 947: 05$ & Auto FIC & 403.4 & Good & \\
\hline $7 / 7 / 940: 00$ & Manual Tape & 402.00 & Good & \\
\hline 7/7/94 7:03 & Auto FIC & 403.2 & Good & \\
\hline $7 / 8 / 940: 00$ & Manual Tape & 402.00 & Good & \\
\hline $7 / 8 / 947: 03$ & Auto FIC & 403.4 & Good & \\
\hline 7/9/940:00 & Manual Tape & 402.125 & Good & \\
\hline 7/9/94 7:03 & Auto FIC & 403.40 & Good & \\
\hline $7 / 10 / 940: 00$ & Manual Tape & 402 & Good & \\
\hline $7 / 10 / 947: 03$ & Auto FIC & 403.40 & Good & \\
\hline $7 / 11 / 940: 00$ & Manual Tape & 402 & Good & \\
\hline $7 / 11 / 947: 03$ & Auto FIC & 403.3 & Good & \\
\hline $7 / 12 / 940: 00$ & Manual Tape & 402.00 & Good & \\
\hline $7 / 12 / 947: 03$ & Auto FIC & 403.30 & Good & \\
\hline $7 / 13 / 940: 00$ & Manual Tape & 402.00 & Good & \\
\hline $7 / 13 / 947: 03$ & Auto FIC & 403.30 & Good & \\
\hline $7 / 14 / 940: 00$ & Manual Tape & 402.00 & Good & \\
\hline $7 / 14 / 947: 04$ & Auto FIC & 403.30 & Good & \\
\hline $7 / 15 / 940: 00$ & Manual Tape & 402.00 & Good & \\
\hline $7 / 15 / 947: 04$ & Auto FIC & 403.3 & Good & \\
\hline $7 / 16 / 940: 00$ & Manual Tape & 402 & Good & \\
\hline $7 / 16 / 947: 04$ & Auto FIC & 403.30 & Good & \\
\hline $7 / 17 / 940: 00$ & Manual Tape & 402.00 & Good & \\
\hline $7 / 17 / 94$ 7:04 & Auto FIC & 403.3 & Good & \\
\hline $7 / 18 / 940: 00$ & Manual FIC & 403.30 & Good & \\
\hline $7 / 18 / 940: 00$ & Manual Tape & 402 & Good & \\
\hline $7 / 19 / 940: 00$ & Manual Tape & 402 & Good & \\
\hline $7 / 19 / 947: 03$ & Auto FIC & 403.30 & Good & \\
\hline $7 / 20 / 940: 00$ & Manual Tape & 402.00 & Good & \\
\hline $7 / 20 / 947: 03$ & Auto FIC & 403.30 & Good & \\
\hline $7 / 21 / 940: 00$ & Manual Tape & 402.5 & Good & \\
\hline $7 / 21 / 947: 03$ & Auto FIC & 403.30 & Good & \\
\hline $7 / 22 / 940: 00$ & Manual Tape & 402 & Good & \\
\hline $7 / 22 / 947: 03$ & Auto FIC & 403.3 & Good & \\
\hline $7 / 23 / 940: 00$ & Manual Tape & 402.00 & Good & \\
\hline $7 / 23 / 947: 03$ & Auto FIC & 403.3 & Good & \\
\hline $7 / 24 / 940: 00$ & Manual Tape & 402 & Good & \\
\hline
\end{tabular}

Data obtained from WHC Surveillance Analysis Computer System, (PC SACS) July 7, 1995. 


\begin{tabular}{|c|c|c|c|c|}
\hline \multicolumn{5}{|c|}{ 241-AP-107 } \\
\hline \multicolumn{5}{|c|}{. Surface Level Data } \\
\hline Date & Type of Measurement & Level (Inches) & Data Quality & Comments \\
\hline $7 / 24 / 947: 03$ & Auto FIC & 403.3 & Good & \\
\hline $7 / 25 / 940: 00$ & Manual Tape & 401.75 & Good & \\
\hline $7 / 25 / 947: 03$ & Auto FIC & 403.3 & Good & \\
\hline $7 / 26 / 947: 03$ & Auto FIC & 403.3 & Good & \\
\hline $7 / 26 / 9412: 00$ & Manual Tape & 402.00 & Good & \\
\hline $7 / 27 / 947: 03$ & Auto FIC & 403.30 & Good & \\
\hline $7 / 27 / 94 \quad 12: 00$ & Manual Tape & 402 & Good & \\
\hline $7 / 28 / 947: 04$ & Auto FIC & 403.30 & Good & \\
\hline $7 / 28 / 94 \quad 12: 00$ & Manual Tape & 401.25 & Good & \\
\hline $7 / 29 / 947: 04$ & Auto FIC & 403.30 & Good & \\
\hline $7 / 29 / 94 \quad 12: 00$ & Manual Tape & 401.25 & Good & \\
\hline $7 / 30 / 947: 04$ & Auto FIC & 403.30 & Good & \\
\hline $7 / 30 / 9412: 00$ & Manual Tape & 400 & Good & \\
\hline $7 / 31 / 94 \quad 12: 00$ & Manual Tape & 401.25 & Good & \\
\hline 7/31/94 23:03 & Auto FIC & 403.3 & Good & \\
\hline 8/1/94 7:03 & Auto FIC & 403.3 & Good & \\
\hline $8 / 1 / 9412: 00$ & Manual Tape & 401.75 & Good & \\
\hline $8 / 2 / 947: 03$ & Auto FIC & 403.2 & Good & \\
\hline $8 / 2 / 9412: 00$ & Manual Tape & 402.00 & Good & \\
\hline $8 / 3 / 947: 03$ & Auto FIC & 403.20 & Good & \\
\hline $8 / 3 / 94 \quad 12: 00$ & Manual Tape & 402 & Good & \\
\hline $8 / 4 / 947: 02$ & Auto FIC & 0.00 & Suspect & Data not included in graph \\
\hline $8 / 4 / 9412: 00$ & Manual FIC & $403.20^{\circ}$ & Good & \\
\hline $8 / 4 / 9412: 00$ & Manual Tape & 402 & Good & \\
\hline $8 / 5 / 947: 03$ & Auto FIC & 403.20 & Good & \\
\hline $8 / 5 / 9412: 00$ & Manual Tape & 402 & Good & \\
\hline $8 / 6 / 947: 03$ & Auto FIC & 403.20 & Good & \\
\hline $8 / 6 / 9412: 00$ & Manual Tape & 402 & Good & \\
\hline $8 / 7 / 947: 04$ & Auto FIC & 403.20 & Good & \\
\hline $8 / 7 / 9412: 00$ & Manual Tape & 402.00 & Good & \\
\hline $8 / 8 / 947: 12$ & Auto FIC & 0.00 & Suspect & Data not included in graph \\
\hline $8 / 8 / 9412: 00$ & Manual FIC & 403.20 & Good & \\
\hline $8 / 8 / 9412: 00$ & Manual Tape & 402.00 & Good & \\
\hline $8 / 9 / 947: 06$ & Auto FIC & 403.2 & Good & \\
\hline $8 / 9 / 9412: 00$ & Manual Tape & 402 & Good & \\
\hline $8 / 10 / 947: 04$ & Auto FIC & 403.20 & Good & \\
\hline $8 / 10 / 9412: 00$ & Manual Tape & 402.00 & Good & \\
\hline $8 / 11 / 947: 04$ & Auto FIC & 403.2 & Good & \\
\hline $8 / 11 / 9412: 00$ & Manual Tape & 401.50 & . Good & \\
\hline $8 / 12 / 947: 04$ & Auto FIC & 403.30 & Good & \\
\hline $8 / 12 / 9412: 00$ & Manual Tape & 401.75 & Good & \\
\hline $8 / 13 / 947: 03$ & Auto FIC & 403.20 & Good & \\
\hline $8 / 13 / 9412: 00$ & Manual Tape & 402 & Good & \\
\hline $8 / 14 / 947: 06$ & Auto FIC & 403.30 & Good & \\
\hline $8 / 14 / 9412: 00$ & Manual Tape & 401.75 & Good & \\
\hline $8 / 15 / 947: 06$ & Auto FIC & 403.2 & Good & \\
\hline $8 / 15 / 9412: 00$ & Manual Tape & 401.50 & Good & \\
\hline $8 / 16 / 947: 06$ & Auto FIC & 403.20 & Good & \\
\hline
\end{tabular}

Data obtained from WHC Surveillance Analysis Computer System, (PC SACS) July 7, 1995. 


\begin{tabular}{|c|c|c|c|c|}
\hline \multicolumn{5}{|c|}{ 241-AP-107 } \\
\hline \multicolumn{5}{|c|}{ Surface Level Data } \\
\hline Date & Type of Measurement & Level (Inches) & Data Quality & Comments \\
\hline $8 / 16 / 9412: 00$ & Manual Tape & 401.75 & Good & \\
\hline $8 / 17 / 947: 06$ & Auto FIC & 403.20 & Good & \\
\hline $8 / 17 / 9412: 00$ & Manual Tape & 401.75 & Good & \\
\hline $8 / 18 / 947: 06$ & Auto FIC & 403.20 & Good & \\
\hline $8 / 18 / 9412: 00$ & Manual Tape & 401.75 & Good & \\
\hline 8/19/946:06 & Auto FIC & 403.2 & Good & \\
\hline $8 / 19 / 9412: 00$ & Manual Tape & 401.75 & Good & \\
\hline $8 / 20 / 947: 04$ & Auto FIC & 403.20 & Good & \\
\hline $8 / 20 / 9412: 00$ & Manual Tape & 402.00 & Good & \\
\hline $8 / 21 / 947: 04$ & Auto FIC & 403.20 & Good & \\
\hline $8 / 21 / 9412: 00$ & Manual Tape & 402 & Good & \\
\hline $8 / 22 / 946: 04$ & Auto FIC & 403.10 & Good & \\
\hline $8 / 22 / 9412: 00$ & Manual Tape & 401.75 & Good & \\
\hline $8 / 23 / 947: 04$ & Auto FIC & 403.1 & Good & \\
\hline $8 / 23 / 9412: 00$ & Manual Tape & 401.75 & Good & \\
\hline $8 / 24 / 947: 04$ & Auto FIC & 403.1 & Good & \\
\hline $8 / 24 / 9412: 00$ & Manual Tape & 401.75 & Good & \\
\hline $8 / 25 / 947: 03$ & Auto FIC & 403.1 & Good & \\
\hline $8 / 25 / 9412: 00$ & Manual Tape & 401.75 & Good & \\
\hline $8 / 26 / 947: 06$ & Auto FIC & 403.10 & Good & \\
\hline $8 / 26 / 9412: 00$ & Manual Tape & 401.75 & Good & \\
\hline $8 / 27 / 947: 04$ & Auto FIC & 403.10 & Good & \\
\hline $8 / 27 / 9412: 00$ & Manual Tape & 402 & Good & \\
\hline $8 / 28 / 947: 04$ & Auto FIC & 403.1 & Good & \\
\hline $8 / 28 / 9412: 00$ & Manual Tape & 402.00 & Good & \\
\hline $8 / 29 / 947: 04$ & Auto FIC & 403.10 & Good & \\
\hline $8 / 29 / 9412: 00$ & Manual Tape & 402 & Good & \\
\hline $8 / 30 / 947: 04$ & Auto FIC & 403.10 & Good & \\
\hline $8 / 30 / 9412: 00$ & Manual Tape & 402 & Good & \\
\hline $8 / 31 / 947: 04$ & Auto FIC & 403.1 & Good & \\
\hline $8 / 31 / 9412: 00$ & Manual Tape & 402 & Good & \\
\hline $9 / 1 / 947: 04$ & Auto FIC & 403.10 & Good & \\
\hline $9 / 1 / 9412: 00$ & Manual Tape & 401.75 & Good & \\
\hline $9 / 2 / 947: 06$ & Auto FIC & 403.10 & Good & \\
\hline $9 / 2 / 9412: 00$ & Manual Tape & 402.00 & Good & \\
\hline $9 / 3 / 9412: 00$ & Manual Tape & 401.75 & Good & \\
\hline 9/3/94 23:04 & Auto FIC & 403.10 & Good & \\
\hline 9/4/94 7:04 & Auto FIC & 403.1 & Good & \\
\hline $9 / 4 / 9412: 00$ & Manual Tape & 402.00 & Good & \\
\hline $9 / 5 / 947: 04$ & Auto FIC & 403.1 & Good & \\
\hline $9 / 5 / 9412: 00$ & Manual Tape & 401.75 & Good & \\
\hline $9 / 6 / 947: 03$ & Auto FIC & 403.1 & Good & \\
\hline $9 / 6 / 9412: 00$ & Manual Tape & 401.75 & Good & \\
\hline $9 / 7 / 947: 03$ & Auto FIC & 403.20 & Good & \\
\hline $9 / 7 / 94 \quad 12: 00$ & Manual Tape & 401.75 & Good & \\
\hline 9/8/94 7:04 & Auto FIC & 403.10 & Good & \\
\hline $9 / 8 / 94 \quad 12: 00$ & Manual Tape & 401.75 & Good & \\
\hline $9 / 9 / 947: 03$ & Auto FIC & 403.10 & Good & \\
\hline
\end{tabular}

Data obtained from WHC Surveillance Analysis Computer System, (PC SACS) July 7, 1995. 


\begin{tabular}{|c|c|c|c|c|}
\hline \multicolumn{5}{|c|}{ 241-AP-107 } \\
\hline \multicolumn{5}{|c|}{ Surface Level Data } \\
\hline Date & Type of Measurement & Level (Inches) & Data Quality & Comments \\
\hline $9 / 9 / 9412: 00$ & Manual Tape & 401.75 & Good & \\
\hline $9 / 10 / 947: 04$ & Auto FIC & 403.10 & Good & \\
\hline $9 / 10 / 9412: 00$ & Manual Tape & 401.75 & Good & \\
\hline 9/11/94 7:03 & Auto FIC & 403 & Good & \\
\hline $9 / 11 / 9412: 00$ & Manual Tape & $401: 50$ & Good & \\
\hline $9 / 12 / 947: 03$ & Auto FIC & 403 & Good & \\
\hline $9 / 12 / 9412: 00$ & Manual Tape & 401.75 & Good & \\
\hline $9 / 13 / 947: 02$ & Auto FIC & 403.00 & Good & \\
\hline $9 / 13 / 9412: 00$ & Manual Tape & 401.75 & Good & \\
\hline $9 / 14 / 947: 03$ & Auto FIC & 403.00 & Good & \\
\hline $9 / 14 / 9412: 00$ & Manual Tape & 401.75 & Good & \\
\hline $9 / 15 / 947: 03$ & Auto FIC & 403 & Good & \\
\hline $9 / 15 / 9412: 00$ & Manual Tape & 401.75 & Good & \\
\hline $9 / 16 / 947: 02$ & Auto FIC & 403 & Good & \\
\hline $9 / 16 / 9412: 00$ & Manual Tape & 401.75 & Good & \\
\hline $9 / 17 / 947: 03$ & Auto FIC & 403 & Good & \\
\hline $9 / 17 / 9412: 00$ & Manual Tape & 401.75 & Good & \\
\hline $9 / 18 / 947: 02$ & Auto FIC & 403.00 & Good & \\
\hline $9 / 18 / 9412: 00$ & Manual Tape & 401.75 & Good & \\
\hline $9 / 19 / 947: 03$ & Auto FIC & 403.00 & Good & \\
\hline $9 / 19 / 94 / 2: 00$ & Manual Tape & 401.75 & Good & \\
\hline $9 / 20 / 947: 03$ & Auto FIC & 403.00 & Good & \\
\hline $9 / 20 / 9412: 00$ & Manual Tape & 401.75 & Good & \\
\hline $9 / 21 / 947: 04$ & Auto FIC & 403 & Good & \\
\hline $9 / 21 / 9412: 00$ & Manual Tape & 401.75 & Good & \\
\hline $9 / 22 / 947: 03$ & Auto FIC & 403 & Good & \\
\hline $9 / 22 / 94 \quad 12: 00$ & Manual Tape & 401.5 & Good & \\
\hline $9 / 23 / 947: 04$ & Auto FIC & 403.00 & Good & \\
\hline $9 / 23 / 9412: 00$ & Manual Tape & 401.75 & Good & \\
\hline $9 / 24 / 947: 04$ & Auto FIC & 389.10 & Good & \\
\hline $9 / 24 / 9412: 00$ & Manual Tape & 387.00 & Good & \\
\hline $9 / 25 / 947: 03$ & Auto FIC & 321.00 & Good & \\
\hline $9 / 25 / 9412: 00$ & Manual Tape & 311.75 & Good & \\
\hline $9 / 26 / 947: 04$ & Auto FIC & 229.20 & Good & \\
\hline $9 / 26 / 9412: 00$ & Manual Tape & 220.5 & Good & \\
\hline $9 / 27 / 947: 04$ & Auto FIC & 221.70 & Good & \\
\hline $9 / 27 / 94 \quad 12: 00$ & Manual Tape & 218.50 & Good & \\
\hline 9/28/94 7:03 & Auto FIC & 221.70 & Good & \\
\hline $9 / 28 / 9412: 00$ & Manual Tape & 220 & Good & \\
\hline 9/29/94 7:04 & Auto FIC & 221.7 & Good & \\
\hline $9 / 29 / 9412: 00$ & Manual Tape & 220 & Good & \\
\hline $9 / 30 / 947: 04$ & Auto FIC & 162 & Good & \\
\hline $9 / 30 / 9412: 00$ & Manual Tape & 153.5 & Good & \\
\hline $10 / 1 / 947: 06$ & Auto FIC & 75.7 & Good & \\
\hline $10 / 1 / 94 \quad 12: 00$ & Manual Tape & 66.00 & Good & \\
\hline $10 / 2 / 947: 05$ & Auto FIC & 24.30 & Good & \\
\hline $10 / 2 / 9412: 00$ & Manual Tape & 15.75 & Good & \\
\hline $10 / 3 / 947: 04$ & Auto FIC & 10.3 & Good & \\
\hline
\end{tabular}

Data obtained from WHC Surveillance Analysis Computer System, (PC SACS) July 7, 1995. 


\begin{tabular}{|c|c|c|c|c|}
\hline \multicolumn{5}{|c|}{ 247-AP-107 } \\
\hline \multicolumn{5}{|c|}{ Surface Level Data } \\
\hline Date & Type of Measurement & Lavel (Inches) & Data Quality & Comments \\
\hline $10 / 3 / 9412: 00$ & Manual Tape & 8.50 & Good & \\
\hline $10 / 4 / 947: 04$ & Auto FIC & 10.20 & Good & \\
\hline $10 / 4 / 9412: 00$ & Manual Tape & 8.75 & Good & \\
\hline $10 / 5 / 947: 04$ & Auto FIC & 10.30 & Good & \\
\hline $10 / 5 / 9412: 00$ & Manual Tape & 8.75 & Good & \\
\hline $10 / 6 / 947: 04$ & Auto FIC & 10.30 & Good & \\
\hline $10 / 6 / 9412: 00$ & Manual Tape & 9.50 & Good & \\
\hline $10 / 7 / 946: 04$ & Auto FIC & 10.30 & Good & \\
\hline $10 / 7 / 9412: 00$ & Manual Tape & 9 & Good & \\
\hline $10 / 8 / 947: 03$ & Auto FIC & 10.3 & Good & \\
\hline $10 / 8 / 9412: 00$ & Manual Tape & 9 & Good & \\
\hline 10/9/94 7:06 & Auto FIC & 10.30 & Good & \\
\hline $10 / 9 / 9412: 00$ & Manual Tape & 9 & Good & \\
\hline $10 / 10 / 947: 05$ & Auto FIC & 10.2 & Good & \\
\hline $10 / 10 / 9412: 00$ & Manual Tape & 9 & Good & \\
\hline $10 / 11 / 947: 03$ & Auto FIC & 10.20 & Good & \\
\hline $10 / 11 / 9412: 00$ & Manual Tape & 8.75 & Good & \\
\hline $10 / 12 / 947: 06$ & Auto FIC & 10.20 & Good & \\
\hline $10 / 12 / 9412: 00$ & Manual Tape & 8.75 & Good & \\
\hline $10 / 13 / 947: 05$ & Auto FIC & 10.20 & Good & \\
\hline $10 / 13 / 94 \quad 12: 00$ & Manual Tape & 8.75 & Good & \\
\hline $10 / 14 / 947: 06$ & Auto FIC & 10.20 & Good & \\
\hline $10 / 14 / 9412: 00$ & Manual Tape & 8.75 & Good & \\
\hline $10 / 15 / 947: 06$ & Auto FIC & 10.2 & Good & \\
\hline $10 / 15 / 9412: 00$ & Manual Tape & 8.75 & Good & \\
\hline $10 / 16 / 947: 06$ & Auto FIC & 10.2 & Good & \\
\hline $10 / 16 / 9412: 00$ & Manual Tape & 8.75 & Good & \\
\hline $10 / 17 / 947: 06$ & Auto FIC & 10.2 & Good & \\
\hline $10 / 17 / 9412: 00$ & Manual Tape & 8.75 & Good & \\
\hline 10/18/94 7:06 & Auto FIC & 10.20 & Good & \\
\hline $10 / 18 / 9412: 00$ & Manual Tape & 8.75 & Good & \\
\hline $10 / 19 / 947: 06$ & Auto FIC & 10.20 & Good & \\
\hline $10 / 19 / 9412: 00$ & Manual Tape & 8.75 & Good & \\
\hline $10 / 20 / 945: 07$ & Auto FIC & 21.2 & Good & \\
\hline $10 / 20 / 9412: 00$ & Manual Tape & 32.75 & Good & \\
\hline $10 / 21 / 947: 06$ & Auto FIC & 59.10 & Good & \\
\hline $10 / 21 / 9412: 00$ & Manual Tape & 57.75 & Good & \\
\hline $10 / 22 / 9412: 00$ & Manual Tape & 57.5 & Good & \\
\hline $10 / 22 / 9423: 06$ & Auto FIC & 59.1 & Good & \\
\hline $10 / 23 / 947: 06$ & Auto FIC & 59.10 & Good & \\
\hline $10 / 23 / 9412: 00$ & Manual Tape & 57.75 & Good & \\
\hline $10 / 24 / 947: 06$ & Auto FIC & 59.1 & Good & \\
\hline $10 / 24 / 9412: 00$ & Manual Tape & 57.5 & Good & \\
\hline $10 / 25 / 947: 06$ & Auto FIC & 59.2 & Good & \\
\hline $10 / 25 / 9412: 00$ & Manual Tape & 56.75 & Good & \\
\hline $10 / 26 / 947: 06$ & Auto FIC & 59.10 & Good & \\
\hline $10 / 26 / 9412: 00$ & Manual Tape & 57.5 & Good & \\
\hline $10 / 27 / 947: 06$ & Auto FIC & 59.1 & Good & \\
\hline
\end{tabular}

Data obtained from WHC Surveillance Analysis Computer System, (PC SACS) July 7, 1995. 


\begin{tabular}{|c|c|c|c|c|}
\hline \multicolumn{5}{|c|}{ 241-AP-107 } \\
\hline \multicolumn{5}{|c|}{ Surface Level Data } \\
\hline Date & Type of Messurement & Level (Inches) & Data Quality & Comments \\
\hline $10 / 27 / 94 \quad 12: 00$ & Manual Tape & 57.5 & Good & \\
\hline $10 / 28 / 947: 04$ & Auto FIC & 59.1 & Good & \\
\hline $10 / 28 / 9412: 00$ & Manual Tape & 57.75 & Good & \\
\hline $10 / 29 / 9412: 00$ & Manual FIC & 59.1 & Good & \\
\hline $10 / 29 / 9412: 00$ & Manual Tape & 57.5 & Good & \\
\hline $10 / 30 / 94 \quad 12: 00$ & Manual Tape & 57.5 & Good & \\
\hline $10 / 30 / 9423: 04$ & Auto FIC & 59.1 & Good & \\
\hline $10 / 31 / 947: 05$ & Auto FIC & 59.10 & Good & \\
\hline $10 / 31 / 9412: 00$ & Manual Tape & 57.00 & Good & \\
\hline $11 / 1 / 9412: 00$ & Manual Tape . & 57.50 & Good & \\
\hline $11 / 1 / 9423: 04$ & Auto FIC & 59.00 & Good & \\
\hline $11 / 2 / 947: 03$ & Auto FIC & 59.1 & Good & \\
\hline $11 / 2 / 9412: 00$ & Manual Tape & 57.5 & Good & \\
\hline $11 / 3 / 947: 04$ & Auto FIC & 59.00 & Good & \\
\hline $11 / 3 / 9412: 00$ & Manual Tape & $57: 5$ & Good & \\
\hline $11 / 4 / 947: 03$ & Auto FIC & 59 & Good & \\
\hline $11 / 4 / 9412: 00$ & Manual Tape & 57.5 & Good & \\
\hline $11 / 5 / 947: 03$ & Auto FIC & $\mathbf{5 9 . 0 0}$ & Good & \\
\hline $11 / 5 / 9412: 00$ & Manual Tape & 57.5 & Good & \\
\hline $11 / 6 / 947: 04$ & Auto FIC & 59 & Good & \\
\hline $11 / 6 / 94 \quad 12: 00$ & Manual Tape & 57.50 & Good & \\
\hline $11 / 7 / 947: 04$ & Auto FIC & 59 & Good & \\
\hline $11 / 7 / 9412: 00$ & Manual Tape & 57.25 & Good & \\
\hline $11 / 8 / 947: 03$ & Auto FIC & 59.00 & Good & \\
\hline $11 / 8 / 9411: 02$ & Manual Tape & 57.25 & Good & \\
\hline 11/9/94 7:05 & Auto FIC & 59.00 & Good & \\
\hline $11 / 9 / 9411: 02$ & Manual Tape & 57.25 & Good & \\
\hline $11 / 10 / 947: 03$ & Auto FIC & 59.00 & Good & \\
\hline $11 / 10 / 9411: 02$ & Manual Tape & 57.5 & Good & \\
\hline $11 / 11 / 947: 03$ & Auto FIC & 59 & Good & \\
\hline $11 / 11 / 9411: 02$ & Manual Tape & 57.25 & Good & \\
\hline $11 / 12 / 947: 03$ & Auto FIC & 59.00 & Good & \\
\hline $11 / 12 / 9411: 02$ & Manual Tape & 57.5 & Good & \\
\hline $11 / 13 / 947: 04$ & Auto FIC & 59.00 & Good & \\
\hline $11 / 13 / 9411: 02$ & Manual Tape & 57.5 & Good & \\
\hline $11 / 14 / 947: 03$ & Auto FIC & 59 & Good & \\
\hline $11 / 14 / 9411: 02$ & Manual Tape & 57.50 & Good & \\
\hline $11 / 15 / 947: 04$ & Auto FIC & 58.9 & Good & \\
\hline $11 / 15 / 9412: 00$ & Manual Tape & 57.5 & Good & \\
\hline $11 / 16 / 947: 06$ & Auto FIC & 58.90 & Good & \\
\hline $11 / 16 / 9412: 00$ & Manual Tape & 57.50 & Good & \\
\hline $11 / 17 / 947: 04$ & Auto FIC & 58.90 & Good & \\
\hline $11 / 18 / 94.7: 04$ & Auto FIC & 58.90 & Good & \\
\hline $11 / 18 / 9412: 00$ & Manual Tape & 57.25 & Good & \\
\hline $11 / 19 / 947: 03$ & Auto FIC & 58.90 & Good & \\
\hline $11 / 19 / 9412: 00$ & Manual Tape & 57.25 & Good & \\
\hline $11 / 20 / 947: 03$ & Auto FIC & 58.90 & Good & \\
\hline $11 / 21 / 947: 04$ & Auto FIC & 58.9 & Good & \\
\hline
\end{tabular}

Data obtained from WHC Surveillance Analysis Computer System, (PC SACS) July 7, 1995. 


\begin{tabular}{|c|c|c|c|c|}
\hline \multicolumn{5}{|c|}{ 241-AP-107 } \\
\hline \multicolumn{5}{|c|}{ Surface Level Data } \\
\hline Date & Type of Measurement & Level (Inches) & Data Quality & Comments \\
\hline $11 / 21 / 9412: 00$ & Manual Tape & 57.25 & Good & \\
\hline $11 / 22 / 947: 03$ & Auto FIC & 58.8 & Good & \\
\hline $11 / 22 / 94 \quad 12: 00$ & Manual Tape & 57.25 & Good & \\
\hline $11 / 23 / 9412: 00$ & Manual FIC & 58.80 & Good & \\
\hline $11 / 23 / 9412: 00$ & Manual Tape & 57.25 & Good & \\
\hline $11 / 24 / 9412: 00$ & Manual Tape & 57 & Good & \\
\hline $11 / 24 / 9423: 03$ & Auto FIC & 58.8 & Good & \\
\hline $11 / 25 / 9412: 00$ & Manual Tape & 57.50 & Good & \\
\hline $11 / 25 / 9423: 03$ & Auto FIC & $\mathbf{5 8 . 8}$ & Good & \\
\hline $11 / 26 / 947: 03$ & Auto FIC & 58.8 & Good & \\
\hline $11 / 26 / 9412: 00$ & Manual Tape & 57.00 & Good & \\
\hline $11 / 27 / 947: 04$ & Auto FIC & 58.80 & Good & \\
\hline $11 / 27 / 9412: 00$ & Manual Tape & 57.25 & Good & \\
\hline $11 / 28 / 947: 04$ & Auto FIC & 58.8 & Good & \\
\hline $11 / 28 / 9412: 00$ & Manual Tape & 57.25 & Good & \\
\hline $11 / 29 / 947: 04$ & Auto FIC & 58.80 & Good & \\
\hline $11 / 29 / 9412: 00$ & Manual Tape & 57.25 & Good & \\
\hline $11 / 30 / 947: 03$ & Auto FIC & 58.7 & Good & \\
\hline $11 / 30 / 9412: 00$ & Manual Tape & 57.25 & Good & \\
\hline $12 / 1 / 946: 05$ & Auto FIC & 58.80 & Good & \\
\hline $12 / 1 / 9412: 00$ & Manual Tape & 57 & Good & \\
\hline $12 / 2 / 947: 03$ & Auto FIC & 58.80 & Good & \\
\hline $12 / 2 / 9412: 00$ & Manual Tape & 57 & Good & \\
\hline $12 / 3 / 946: 04$ & Auto FIC & 58.7 & Good & \\
\hline $12 / 3 / 94 \quad 12: 00$ & Manual Tape & 57.00 & Good & \\
\hline $12 / 4 / 947: 03$ & Auto FIC & 58.7 & Good & \\
\hline $12 / 4 / 9412: 00$ & Manual Tape & 57 & Good & \\
\hline $12 / 5 / 947: 03$ & Auto FIC & 58.70 & Good & \\
\hline $12 / 5 / 94 \quad 12: 00$ & Manual Tape & 57.00 & Good & \\
\hline 12/6/94 7:04 & Auto FIC & 58.70 & Good & \\
\hline $12 / 6 / 9412: 00$ & Manual Tape & 57.00 & Good & \\
\hline $12 / 7 / 947: 03$ & Auto FIC & 58.7 & Good & \\
\hline $12 / 7 / 9412: 00$ & Manual Tape & 57.13 & Good & \\
\hline $12 / 8 / 945: 03$ & Auto FIC & 58.70 & Good & \\
\hline $12 / 8 / 9412: 00$ & Manual Tape & 57.25 & Good & \\
\hline $12 / 9 / 947: 03$ & Auto FIC & 58.7 & Good & \\
\hline $12 / 9 / 9412: 00$ & Manual Tape & 57.25 & Good & \\
\hline $12 / 10 / 947: 04$ & Auto FIC & 58.7 & Good & \\
\hline $12 / 10 / 9412: 00$ & Manual Tape & 57.25 & Good & \\
\hline $12 / 11 / 947: 04$ & Auto FIC & 58.7 & Good & \\
\hline $12 / 11 / 94 \quad 12: 00$ & Manual Tape & 57.25 & Good & \\
\hline $12 / 12 / 947: 03$ & Auto FIC & 58.70 & Good & \\
\hline $12 / 12 / 9412: 00$ & Manual Tape & 57.00 & Good & \\
\hline $12 / 13 / 947: 03$ & Auto FIC & 58.7 & Good & \\
\hline $12 / 14 / 947: 04$ & Auto FIC & 58.6 & Good & \\
\hline $12 / 14 / 9412: 00$ & Manual Tape & 57.25 & Good & \\
\hline $12 / 15 / 947: 03$ & Auto FIC & 58.6 & Good & \\
\hline $12 / 15 / 9412: 00$ & Manual Tape & 57.25 & Good & \\
\hline
\end{tabular}

Data obtained from WHC Surveillance Analysis Computer System, (PC SACS) July 7, 1995. 


\begin{tabular}{|c|c|c|c|c|}
\hline \multicolumn{5}{|c|}{ 241-AP-107 } \\
\hline \multicolumn{5}{|c|}{ Surface Lovel Data } \\
\hline Date & Type of Measurement & Leval '(Inches) & Data Quality & Comments \\
\hline $12 / 16 / 947: 03$ & Auto FIC & 58.6 & Good & \\
\hline $12 / 16 / 9412: 00$ & Manual Tape & 57.25 & Good & \\
\hline $12 / 17 / 947: 06$ & Auto FIC & 58.60 & Good & \\
\hline $12 / 17 / 9412: 00$ & Manual Tape & 57.00 & Good & \\
\hline $12 / 18 / 947: 04$ & Auto FIC & 58.60 & Good & \\
\hline $12 / 18 / 9412: 00$ & Manual Tape & 57 & Good & \\
\hline $12 / 19 / 947: 04$ & Auto FIC & 58.6 & Good & \\
\hline $12 / 19 / 9412 ; 00$ & Manual Tape & 57.00 & Good & \\
\hline $12 / 20 / 947: 04$ & Auto FIC & 58.6 & Good & \\
\hline $12 / 20 / 9412: 00$ & Manual Tape & 57.00 & Good & \\
\hline $12 / 21 / 947: 04$ & Auto FIC & 58.60 & Good & \\
\hline $12 / 21 / 9412: 00$ & Manual Tape & 57.00 & Good & \\
\hline $12 / 22 / 947: 05$ & Auto FIC & 58.60 & Good & \\
\hline $12 / 22 / 9412: 00$ & Manual Tape & 57.25 & Good & \\
\hline $12 / 23 / 947: 03$ & Auto FIC & 58.6 & Good & \\
\hline $12 / 23 / 9412: 00$ & Manual Tape & 57.00 & Good & \\
\hline $12 / 24 / 947: 04$ & Auto FIC & 58.4 & Good & \\
\hline $12 / 24 / 9412: 00$ & Manual Tape & 57.00 & Good & \\
\hline $12 / 25 / 947: 03$ & Auto FIC & 58.60 & Good & \\
\hline $12 / 25 / 94 \quad 12: 00$ & Manual Tape & 57 & Good & \\
\hline $12 / 26 / 947: 03$ & Auto FIC & 58.50 & Good & \\
\hline $12 / 26 / 9412: 00$ & Manual Tape & 57.00 & Good & \\
\hline $12 / 27 / 947: 04$ & Auto FIC & 58.50 & Good & \\
\hline $12 / 27 / 9412: 00$ & Manual Tape & 57 & Good & \\
\hline $12 / 28 / 947: 04$ & Auto FIC & 58.5 & Good & \\
\hline $12 / 28 / 9412: 00$ & Manual Tape & 57 & Good & \\
\hline $12 / 29 / 947: 04$ & Auto FIC & 58.50 & Good & \\
\hline $12 / 29 / 9412: 00$ & Manual Tape & 57.00 & Good & \\
\hline $12 / 30 / 947: 04$ & Auto FIC & 58.50 & Good & \\
\hline $12 / 30 / 9412: 00$ & Manual Tape & 57 & Good & \\
\hline $12 / 31 / 947: 04$ & Auto FIC & 58.50 & Good & \\
\hline $12 / 31 / 9412: 00$ & Manual Tape & 57 & Good & \\
\hline $1 / 1 / 957: 04$ & Auto FIC & 58.5 & Good & \\
\hline $1 / 1 / 9512: 00$ & Manual Tape & 57 & Good & \\
\hline $1 / 2 / 95 \quad 7: 03$ & Auto FIC & 58.50 & Good & \\
\hline 1/2/95 12:00 & Manual Tape & 57.00 & Good & \\
\hline 1/3/95 7:02 & Auto FIC & 58.4 & Good & \\
\hline $1 / 3 / 95 \quad 12: 00$ & Manual Tape & 57.00 & Good & \\
\hline $1 / 4 / 957: 03$ & Auto FIC & 58.4 & Good & \\
\hline 1/4/95 12:00 & Manual Tape & 57 & Good & \\
\hline $1 / 5 / 95$ 7:04 & Auto FIC & 58.6 & Good & \\
\hline $1 / 5 / 95 \quad 12: 00$ & Manual Tape & 57 & Good & \\
\hline $1 / 6 / 95$ 7:04 & Auto FIC & 58.5 & Good & \\
\hline $1 / 6 / 9512: 00$ & Manual Tape & 57.00 & Good & \\
\hline 1/7/95 7:04 & Auto FIC & 58.5 & Good & \\
\hline $1 / 7 / 95 \quad 12: 00$ & Manual Tape & 57.00 & Good & \\
\hline $1 / 8 / 9512: 00$ & Manual Tape & 57.00 & Good & \\
\hline $1 / 8 / 9523: 03$ & Auto FIC & 58.5 & Good & \\
\hline
\end{tabular}

Data obtained from WHC Surveillance Analysis Computer System, (PC SACS) July 7, 1995. 


\begin{tabular}{|c|c|c|c|c|}
\hline \multicolumn{5}{|c|}{ 241-AP-107 } \\
\hline \multicolumn{5}{|c|}{ Surface Level Data } \\
\hline Date & Type of Measurement & Level (Inches) & Data Quality & Comments \\
\hline 1/9/95 7:05 & Auto FIC & 58.50 & Good & 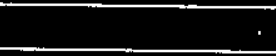 \\
\hline 1/9/95 12:00 & Manual Tape & 57 & Good & \\
\hline 1/10/95 7:06 & Auto FIC & 58.60 & Good & \\
\hline $1 / 10 / 9512: 00$ & Manual Tape & 57.00 & Good & \\
\hline $1 / 11 / 957: 07$ & Auto FIC & 58:6 & Good & \\
\hline 1/11/95 12:00 & Manual Tape & 57.00 & Good & \\
\hline $1 / 12 / 957: 07$ & Auto FIC & 58.6 & Good & \\
\hline $1 / 12 / 9512: 00$ & Manual Tape & 57.00 & Good & \\
\hline $1 / 13 / 956: 07$ & Auto FIC & 58.60 & Good & \\
\hline $1 / 13 / 9512: 00$ & Manual Tape & 57.00 & Good & \\
\hline $1 / 14 / 957: 07$ & Auto FIC & 58.5 & Good & \\
\hline $1 / 14 / 95 \quad 12: 00$ & Manual Tape & 57 & Good & \\
\hline 1/15/95 7:06 & Auto FIC & 58.50 & Good & \\
\hline $1 / 15 / 95 \quad 12: 00$ & Manual Tape & 57.00 & Good & \\
\hline $1 / 16 / 957: 08$ & Auto FIC & 58.50 & Good & \\
\hline $1 / 16 / 95 \quad 12: 00$ & Manual Tape & 57.00 & Good & \\
\hline $1 / 17 / 956: 07$ & Auto FIC & 58.50 & Good & \\
\hline $1 / 17 / 95 \quad 12: 00$ & Manual Tape & 57.00 & Good & \\
\hline $1 / 18 / 95 \quad 7: 07$ & Auto FIC & 58.5 & Good & \\
\hline $1 / 18 / 95 \quad 12: 00$ & Manual Tape & 57 & Good & \\
\hline 1/19/95 7:07 & Auto FIC & 58.50 & Good & \\
\hline $1 / 19 / 9512: 00$ & Manual Tape & 57 & Good & \\
\hline $1 / 20 / 957: 03$ & Auto FIC & 58.5 & Good & \\
\hline $1 / 20 / 95 \quad 12: 00$ & Manual Tape & 57 & Good & \\
\hline 1/21/95 7:04 & Auto FIC & 58.5 & Good & \\
\hline $1 / 21 / 95 \quad 12: 00$ & Manual Tape & 57.00 & Good & \\
\hline 1/22/95 7:03 & Auto FIC & 64.70 & Good & \\
\hline $1 / 22 / 95 \quad 12: 00$ & Manual Tape & 73.75 & Good & \\
\hline $1 / 23 / 957: 03$ & Auto FIC & 156.70 & Good & \\
\hline $1 / 23 / 9512: 00$ & Manual Tape & 159.50 & Good & \\
\hline 1/24/95 7:04 & Auto FIC & 241 & Good & \\
\hline $1 / 25 / 957: 03$ & Auto FIC & 316.30 & Good & \\
\hline $1 / 25 / 95 \quad 12: 00$ & Manual Tape & 320.25 & Good & \\
\hline $1 / 26 / 957: 03$ & Auto FIC & 362.5 & Good & \\
\hline $1 / 26 / 95 \quad 12: 00$ & Manual Tape & 361 & Good & \\
\hline $1 / 27 / 957: 03$ & Auto FIC & 362.70 & Good & \\
\hline $1 / 27 / 95 \quad 12: 00$ & Manual Tape & 361.25 & Good & \\
\hline $1 / 28 / 957: 04$ & Auto FIC & 362.70 & Good & \\
\hline $1 / 28 / 9512: 00$ & Manual Tape & $361: 25$ & Good & \\
\hline $1 / 29 / 957: 04$ & Auto FIC & 362.7 & Good & \\
\hline $1 / 29 / 9512: 00$ & Manual Tape & 361.25 & Good & \\
\hline $1 / 30 / 957: 04$ & Auto FIC & 362.70 & Good & \\
\hline $1 / 30 / 9512: 00$ & Manual Tape & 361.25 & Good & \\
\hline $1 / 31 / 957: 03$ & Auto FIC & 362.70 & Good & \\
\hline $1 / 31 / 9512: 00$ & Manual Tape & 361 & Good & \\
\hline $2 / 1 / 95$ 7:03 & Auto FIC & 362.60 & Good & \\
\hline $2 / 1 / 95 \quad 12: 00$ & Manual Tape & 361,25 & Good & \\
\hline $2 / 2 / 95$ 7:04 & Auto FIC & 362.6 & Good & \\
\hline
\end{tabular}

Data obtained from WHC Surveillance Analysis Computer System, (PC SACS) July 7, 1995. 


\begin{tabular}{|c|c|c|c|c|}
\hline \multicolumn{5}{|c|}{ 241-AP-107 } \\
\hline \multicolumn{5}{|c|}{ Surface Level Data } \\
\hline Date & Type of Measurement & Level (Inches) & Data Quality & Comments \\
\hline 2/2/95 12:00 & Manual Tape & 361 & Good & \\
\hline $2 / 3 / 957: 03$ & Auto FIC & 362.6 & Good & \\
\hline 2/3/95 12:00 & Manual Tape & 361 & Good & \\
\hline 2/4/95 7:04 & Auto FIC & 362.60 & Good & \\
\hline 2/4/95 12:00 & Manual Tape & 361.00 & Good & \\
\hline $2 / 5 / 957: 03$ & Auto FIC & 362.60 & Good & \\
\hline $2 / 5 / 95 \quad 12: 00$ & Manual Tape & 361.25 & Good & \\
\hline 2/6/95 7:03 & Auto FIC & 362.6 & Good & \\
\hline $2 / 6 / 95 \quad 12: 00$ & Manual Tape & 361.25 & Good & \\
\hline $2 / 7 / 957: 03$ & Auto FIC & 362.60 & Good & \\
\hline $2 / 7 / 95 \quad 12: 00$ & Manual Tape & 361 & Good & \\
\hline $2 / 8 / 957: 04$ & Auto FIC & 362.5 & Good & \\
\hline $2 / 8 / 95 \quad 12: 00$ & Manual Tape & 360.75 & Good & \\
\hline 2/9/95 7:06 & Auto FIC & 362.5 & Good & \\
\hline $2 / 9 / 95 \quad 12: 00$ & Manual Tape & 361.00 & Good & \\
\hline $2 / 10 / 957: 06$ & Auto FIC & 362.50 & Good & \\
\hline $2 / 10 / 9512: 00$ & Manual Tape & 361 & Good & \\
\hline $2 / 11 / 957: 07$ & Auto FIC & 362.50 & Good & \\
\hline $2 / 11 / 95 \quad 12: 00$ & Manual Tape & 361.00 & Good & \\
\hline $2 / 12 / 957: 06$ & Auto FIC & 362.50 & Good & \\
\hline $2 / 12 / 9512: 00$ & Manual Tape & 361 & Good & \\
\hline 2/13/95 7:05 & Auto FIC & 362.50 & Good & \\
\hline $2 / 13 / 9512: 00$ & Manual Tape & 361 & Good & \\
\hline $2 / 14 / 957: 06$ & Auto FIC & 362.4 & Good & \\
\hline $2 / 14 / 9512: 00$ & Manual Tape & 360.75 & Good & \\
\hline $2 / 15 / 95 \quad 7: 07$ & Auto FIC & 362.40 & Good & \\
\hline $2 / 15 / 95 \quad 12: 00$ & Manual Tape & 361.00 & Good & \\
\hline 2/16/95 7:06 & Auto FIC & 362.4 & Good & \\
\hline $2 / 16 / 9512: 00$ & Manual Tape & 361 & Good & \\
\hline $2 / 17 / 957: 06$ & Auto FIC & $362 . \overline{40}$ & Good & \\
\hline $2 / 17 / 95 \quad 12: 00$ & Manual Tape & 360.75 & Good & \\
\hline $2 / 18 / 957: 06$ & Auto FIC & 362.40 & Good & \\
\hline $2 / 18 / 9512: 00$ & Manual Tape & 361.00 & Good & \\
\hline $2 / 19 / 957: 06$ & Auto FIC & 362.4 & Good & \\
\hline $2 / 19 / 95 \quad 12: 00$ & Manual Tape & 360.75 & Good & \\
\hline $2 / 20 / 957: 07$ & Auto FIC & 362.4 & Good & \\
\hline $2 / 20 / 9512: 00$ & Manual Tape & 361.00 & Good & \\
\hline $2 / 21 / 957: 06$ & Auto FIC & 362.4 & Good & \\
\hline $2 / 21 / 9512: 00$ & Manual Tape & 360.75 & Good & \\
\hline $2 / 22 / 957: 06$ & Auto FIC & 362.4 & Good & \\
\hline $2 / 22 / 9512: 00$ & Manual Tape & 361.00 & Good & \\
\hline $2 / 23 / 957: 07$ & Auto FIC & 362.40 & Good & \\
\hline $2 / 23 / 9512: 00$ & Manual Tape & 360.75 & Good & \\
\hline 2/24/95 7:06 & Auto FIC & 362.40 & Good & \\
\hline $2 / 24 / 9512: 00$ & Manual Tape & 360.75 & Good & \\
\hline $2 / 25 / 957: 06$ & Auto FIC & 362.40 & Good & \\
\hline $2 / 25 / 9512: 00$ & Manual Tape & 360.75 & Good & \\
\hline $2 / 26 / 956: 07$ & Auto FIC & 362.2 & Good & \\
\hline
\end{tabular}

Data obtained from WHC Surveillance Analysis Computer System, (PC SACS) July 7, 1995. 


\begin{tabular}{|c|c|c|c|c|}
\hline \multicolumn{5}{|c|}{ 241-AP-107 } \\
\hline \multicolumn{5}{|c|}{ Surface Level Data } \\
\hline Date & Type of Measurement & Level (Inches) & Data Quality & Comments \\
\hline $2 / 26 / 9512: 00$ & Manual Tape & $\mathbf{3 5 5 . 7 5}$ & Suspect & Data not included in graph \\
\hline $2 / 27 / 957: 06$ & Auto FIC & 362.3 & Good & \\
\hline 2/27/95 12:00 & Manual Tape & 345.25 & Suspect & Data not included in graph \\
\hline 2/28/95 5:07 & Auto FIC & 362.30 & Good & \\
\hline $2 / 28 / 95 \quad 12: 00$ & Manual Tape & 361 & Good & \\
\hline $3 / 1 / 957: 06$ & Auto FIC & 362.30 & Good & \\
\hline $3 / 1 / 9512: 00$ & Manual Tape & 360.5 & Good & \\
\hline $3 / 2 / 957: 06$ & Auto FIC & 362.3 & Good & \\
\hline $3 / 2 / 95 \quad 12: 00$ & Manual Tape & 360.75 & Good & \\
\hline 3/3/95 7:06 & Auto FIC & 362.20 & Good & \\
\hline $3 / 3 / 9512: 00$ & Manual Tape & 360.75 & Good & \\
\hline 3/4/95 7:06 & Auto FIC & 362.2 & Good & \\
\hline $3 / 4 / 95 \quad 12: 00$ & Manual Tape & 360.75 & Good & \\
\hline $3 / 5 / 957: 06$ & Auto FIC & 362.2 & Good & \\
\hline $3 / 5 / 9512: 00$ & Manual Tape & 360.75 & Good & \\
\hline $3 / 6 / 957: 06$ & Auto FIC & 362.20 & Good & \\
\hline $3 / 6 / 95 \quad 12: 00$ & Manual Tape & 360.5 & Good & \\
\hline 3/7/95 7:06 & Auto FIC & 362.20 & Good & \\
\hline $3 / 7 / 95 \quad 12: 00$ & Manual Tape & 360.5 & Good & \\
\hline 3/8/95 7:03 & Auto FIC & 362.20 & Good & \\
\hline $3 / 8 / 95 \quad 12: 00$ & Manual Tape & 360.75 & Good & \\
\hline 3/9/95 7:03 & Auto FIC & 362.20 & Good & \\
\hline $3 / 9 / 95 \quad 12: 00$ & Manual Tape & 360.50 & Good & \\
\hline $3 / 10 / 957: 02$ & Auto FIC & 362.20 & Good & \\
\hline $3 / 10 / 9512: 00$ & Manual Tape & 360.5 & Good & \\
\hline $3 / 11 / 957: 03$ & Auto FIC & 362.2 & Good & \\
\hline $3 / 11 / 9512: 00$ & Manual Tape & 360.5 & Good & \\
\hline $3 / 12 / 957: 03$ & Auto FIC & 362.20 & Good & \\
\hline $3 / 12 / 95 \quad 12: 00$ & Manual Tape & 360.5 & Good & \\
\hline $3 / 13 / 957: 02$ & Auto FIC & 362.1 & Good & \\
\hline $3 / 13 / 95 \quad 12: 00$ & Manual Tape & 360.50 & Good & \\
\hline $3 / 14 / 957: 03$ & Auto FIC & 362.1 & Good & \\
\hline $3 / 14 / 9512: 00$ & Manual Tape & 360.50 & Good & \\
\hline $3 / 15 / 957: 05$ & Auto FIC & 362.1 & Good & \\
\hline $3 / 15 / 9512: 00$ & Manual Tape & 360.50 & Good & \\
\hline $3 / 16 / 957: 03$ & Auto FIC & 362.10 & Good & \\
\hline $3 / 16 / 9512: 00$ & Manual Tape & 360.5 & Good & \\
\hline $3 / 17 / 957: 03$ & Auto FIC & 362.10 & Good & \\
\hline $3 / 17 / 9512: 00$ & Manual Tape & 360.5 & Good & \\
\hline $3 / 18 / 957: 03$ & Auto FIC & 362.1 & Good & \\
\hline $3 / 18 / 9512: 00$ & Manual Tape & 360.5 & Good & \\
\hline $3 / 19 / 957: 03$ & Auto FIC & 362.1 & Good & \\
\hline $3 / 19 / 9512: 00$ & Manual Tape & 360.50 & Good & \\
\hline $3 / 20 / 956: 03$ & Auto FIC & 362.10 & Good & \\
\hline $3 / 20 / 9512: 00$ & Manual Tape & 360.75 & Good & \\
\hline $3 / 21 / 957: 03$ & Auto FIC & 362.1 & Good & \\
\hline $3 / 21 / 95 \quad 12: 00$ & Manual Tape & 360.50 & Good & \\
\hline $3 / 22 / 957: 02$ & Auto FIC & 362.10 & Good & \\
\hline
\end{tabular}

Data obtained from WHC Surveillance Analysis Computer System, (PC SACS) July 7, 1995. 


\begin{tabular}{|c|c|c|c|c|}
\hline \multicolumn{5}{|c|}{ 241-AP-107 } \\
\hline \multicolumn{5}{|c|}{ Surface Level Data } \\
\hline Date & Type of Measurement & Level (Inches) & Data Quality & Commants \\
\hline $3 / 22 / 95 \quad 12: 00$ & Manual Tape & 360.50 & Good & \\
\hline $3 / 23 / 957: 03$ & Auto FIC & 362.1 & Good & \\
\hline $3 / 23 / 95 \quad 12: 00$ & Manual Tape & 360.50 & Good & \\
\hline $3 / 24 / 957: 03$ & Auto FIC & 362.00 & Good & \\
\hline 3/24/95 12:00 & Manual Tape & 360.5 & Good & \\
\hline $3 / 25 / 957: 02$ & Auto FIC & 362.00 & Good & \\
\hline $3 / 25 / 9512: 00$ & Manual Tape & 360.50 & Good & \\
\hline $3 / 26 / 957: 02$ & Auto FIC & 362 & Good & \\
\hline $3 / 26 / 95 \quad 12: 00$ & Manual Tape & 360.50 & Good & \\
\hline $3 / 27 / 957: 03$ & Auto FIC & 362.00 & Good & \\
\hline $3 / 27 / 9512: 00$ & Manual Tape & 360 & Good & \\
\hline $3 / 28 / 957: 03$ & Auto FIC & 362 & Good & \\
\hline $3 / 28 / 9512: 00$ & Manual Tape & 360.5 & Good & \\
\hline $3 / 29 / 957: 03$ & Auto FIC & 362.00 & Good & \\
\hline $3 / 29 / 95 \quad 12: 00$ & Manual Tape & 360.5 & Good & \\
\hline $3 / 30 / 95$ 7:03 & Auto FIC & 362.00 & Good & \\
\hline $3 / 30 / 9512: 00$ & Manual Tape & 360.5 & Good & \\
\hline $3 / 31 / 957: 04$ & Auto FIC & 362.00 & Good & \\
\hline $3 / 31 / 95 \quad 12: 00$ & Manual Tape & 360.5 & Good & \\
\hline 4/1/95 7:02 & Auro FIC & 362 & Good & \\
\hline 4/1/95 12:00 & Manual Tape & 360.5 & Good & \\
\hline $4 / 2 / 957: 03$ & Auto FIC & 362.00 & Good & \\
\hline 4/2/95 12:00 & Manual Tape & 360.50 & Good & \\
\hline 4/3/95 7:03 & Auto FIC & 362 & Good & \\
\hline $4 / 3 / 9512: 00$ & Manual Tape & 360.50 & Good & \\
\hline 4/4/95 7:03 & Auto FIC & 362 & Good & \\
\hline 4/4/95 12:00 & Manual Tape & 360.5 & Good & \\
\hline $4 / 5 / 957: 03$ & Auto FIC & $362: 00$ & Good & \\
\hline $4 / 5 / 95 \quad 12: 00$ & Manual Tape & 360.5 & Good & \\
\hline 4/6/95 7:03 & Auto FIC & 361.9 & Good & \\
\hline $4 / 6 / 95 \quad 12: 00$ & Manual Tape & 360.5 & Good & \\
\hline 4/7/95 7:03 & Auto FIC & 361.9 & Good & \\
\hline 4/7/95 12:00 & Manual Tape & 360.5 & Good & \\
\hline $4 / 8 / 957: 02$ & Auto FIC & 361.90 & Good & \\
\hline 4/9/95 23:03 & Auto FIC & 361.9 & Good & \\
\hline $4 / 10 / 957: 02$ & Auto FIC & 361.9 & Good & \\
\hline $4 / 10 / 9512: 00$ & Manual Tape & 360.75 & Good & \\
\hline 4/11/95 7:04 & Auto FIC & 361.90 & Good & \\
\hline 4/11/95 12:00 & Manual Tape & 360.5 & Good & \\
\hline 4/12/95 7:03 & Auto FIC & 361.90 & Good & \\
\hline $4 / 12 / 9512: 00$ & Manual Tape & 360.25 & Good & \\
\hline 4/13/95 7:05 & Auto FIC & 361.90 & Good & \\
\hline 4/13/95 12:00 & Manual Tape & 360.25 & Good & \\
\hline $4 / 14 / 957: 03$ & Auto FIC & 361.90 & Good & \\
\hline $4 / 14 / 9512: 00$ & Manual Tape & 360 & Good & \\
\hline 4/15/95 7:03 & Auto FIC & 361.90 & Good & \\
\hline $4 / 15 / 95 \quad 12: 00$ & Manual Tape & 360 & Good & \\
\hline $4 / 16 / 957: 03$ & Auto FIC & 361.80 & Good & \\
\hline
\end{tabular}

Data obtained from WHC Surveillance Analysis Computer System, (PC SACS) July 7, 1995. 


\begin{tabular}{|c|c|c|c|c|}
\hline \multicolumn{5}{|c|}{ 241-AP-107 } \\
\hline \multicolumn{5}{|c|}{ Surface Level Data } \\
\hline Date & Type of Measurement & Leval (Inches) & Data Quality & Comments \\
\hline 4/17/95 7:03 & Auto FIC & 361.80 & Good & \\
\hline 4/18/95 7:03 & Auto FIC & 361.8 & Good & \\
\hline 4/19/95 7:03 & Auto FIC & 361.80 & Good & \\
\hline 4/20/95 7:03 & Auto FIC & 361.80 & Good & \\
\hline 4/21/95 7:03 & Auto FIC & 361.80 & Good & \\
\hline 4/21/95 12:00 & Manual Tape & 360.25 & Good & \\
\hline $4 / 22 / 957: 03$ & Auto FIC & 361.8 & Good & \\
\hline $4 / 22 / 9512: 00$ & Manual Tape & 360.25 & Good & \\
\hline $4 / 23 / 957: 03$ & Auto FIC & 361.80 & Good & \\
\hline 4/23/95 12:00 & Manual Tape & 360.25 & Good & \\
\hline $4 / 24 / 95 \quad 7: 03$ & Auto FIC & 361.80 & Good & \\
\hline 4/24/95 12:00 & Manual Tape & 350.75 & Suspect & Data not included in graph \\
\hline 4/25/95 7:03 & Auto FIC & 361.80 & Good & \\
\hline $4 / 25 / 95 \quad 12: 00$ & Manual Tape & 360 & Good & \\
\hline 4/26/95 7:03 & Auto FIC & 361.8 & Good & \\
\hline $4 / 26 / 95 \quad 12,00$ & Manual Tape & 360.25 & Good & \\
\hline $4 / 27 / 957: 03$ & Auto FIC & 361.8 & Good & \\
\hline 4/27/95 12:00 & Manual Tape & 360.25 & Good & \\
\hline 4/28/95 7:03 & Auto FIC & 361.7 & Good & \\
\hline $4 / 28 / 9512: 00$ & Manual Tape & 360 & Good & \\
\hline $4 / 29 / 957: 03$ & Auto FIC & 361.70 & Good & \\
\hline $4 / 29 / 9512400$ & Manual Tape & 360.25 & Good & \\
\hline 4/30/95 7:03 & Auto FIC & 361.7 & Good & \\
\hline $4 / 30 / 9512: 00$ & Manual Tape & 360.25 & Good & \\
\hline 5/1/95 7:03 & Auto FIC & 361.7 & Good & \\
\hline 5/1/95 $12: 00$ & Manual Tape & 360.25 & Good & \\
\hline $5 / 2 / 957: 02$ & Auto FIC & 361.7 & Good & \\
\hline $5 / 2 / 95 \quad 12: 00$ & Manual Tape & 360.5 & Good & \\
\hline 5/3/95 7:03 & Auto FIC & 361.60 & Good & \\
\hline $5 / 3 / 95 \quad 12: 00$ & Manual Tape & 360.00 & Good & \\
\hline $5 / 4 / 957: 04$ & Auto FIC & 361.7 & Good & \\
\hline $5 / 4 / 95 \quad 12: 00$ & Manual Tape & 360 & Good & \\
\hline $5 / 5 / 957: 04$ & Auto FIC & 361.70 & Good & \\
\hline $5 / 5 / 9512: 00$ & Manual Tape & 360 & Good & \\
\hline $5 / 6 / 95 \quad 7: 03$ & Auto FIC & 361.70 & Good & \\
\hline $5 / 6 / 9512: 00$ & Manual Tape & 360 & Good & \\
\hline $5 / 7 / 957: 04$ & Auto FIC & 361.60 & Good & \\
\hline $5 / 7 / 95 \quad 12: 00$ & Manual Tape & 360 & Good & \\
\hline 5/8/95 7:04 & Auto FIC & 361.60 & Good & \\
\hline $5 / 8 / 95 \quad 12: 00$ & Manual Tape & 360.25 & Good & \\
\hline 5/9/95 7:04 & Auto FIC & 361.7 & Good & \\
\hline $5 / 9 / 95 \quad 12: 00$ & Manual Tape & 360.25 & Good & \\
\hline 5/10/95 7:04 & Auto FIC & 361.6 & Good & \\
\hline $5 / 10 / 9512: 00$ & Manual Tape & 360.25 & Good & \\
\hline $5 / 11 / 957: 03$ & Auto FIC & 361.70 & Good & \\
\hline $5 / 11 / 9512: 00$ & Manual Tape & 360.25 & Good & \\
\hline $5 / 12 / 957: 06$ & Auto FIC & 361.7 & Good & \\
\hline $5 / 12 / 95 \quad 12: 00$ & Manual Tape & 360.25 & Good & \\
\hline
\end{tabular}

Data obtained from WHC Surveillance Analysis Computer System, (PC SACS) July 7, 1995. 


\begin{tabular}{|c|c|c|c|c|}
\hline \multicolumn{5}{|c|}{ 241-AP-107 } \\
\hline \multicolumn{5}{|c|}{ Surface Level Data } \\
\hline Date & Type of Measurament & Level (Inches) & Data Quality & Comments \\
\hline $5 / 13 / 957: 03$ & Auto FIC & 361.5 & Good & : \\
\hline $5 / 13 / 9512: 00$ & Manual Tape & 360 & Good & \\
\hline $5 / 14 / 957: 03$ & Auto FIC & 361.6 & Good & \\
\hline $5 / 14 / 95 \quad 12: 00$ & Manual Tape & 360.25 & Good & \\
\hline 5/15/95 7:03 & Auto FIC & 361.70 & Good & \\
\hline $5 / 15 / 95 \quad 12: 00$ & Manual Tape & 360.00 & Good & \\
\hline $5 / 16 / 957: 03$ & Auto FIC & 361.70 & Good & \\
\hline $5 / 16 / 9512: 00$ & Manual Tape & 360 & Good & \\
\hline $5 / 17 / 957: 02$ & Auto FIC & 361.6 & Good & \\
\hline $5 / 17 / 9512: 00$ & Manual Tape & 360 & Good & \\
\hline $5 / 18 / 956: 02$ & Auto FIC & 361.70 & Good & \\
\hline $5 / 18 / 9512: 00$ & Manual Tape & 360.00 & Good & \\
\hline $5 / 19 / 957: 05$ & Auto FIC & 361.70 & Good & \\
\hline $5 / 19 / 95 \quad 12: 00$ & Manual Tape & 360.00 & Good & \\
\hline $5 / 20 / 957: 05$ & Auto FIC & 310 & Good & \\
\hline $5 / 20 / 9512: 00$ & Manual Tape & 294.50 & Good & \\
\hline $5 / 21 / 95$ 7:05 & Auto FIC & 226.70 & Good & \\
\hline $5 / 21 / 9512: 00$ & Manual Tape & 225.25 & Good & \\
\hline 5/22/95 7:05 & Auto FIC & 226.8 & Good & \\
\hline $5 / 22 / 95 \quad 12: 00$ & Manual Tape & 225.25 & Good & \\
\hline $5 / 23 / 957: 11$ & Auto FIC & 0.00 & Suspect & Data not included in graph \\
\hline $5 / 23 / 9512: 00$ & Manual FIC & 226.80 & Good & \\
\hline $5 / 23 / 95 \quad 12: 00$ & Manual Tape & 225.25 & Good & \\
\hline $5 / 24 / 957: 10$ & Auto FIC & 0.00 & Suspect & Data not included in graph \\
\hline $5 / 24 / 95 \quad 12: 00$ & Manual FIC & 226.80 & Good & \\
\hline $5 / 24 / 9512: 00$ & Manual Tape. & 226.25 & Good & \\
\hline $5 / 25 / 95 \quad 7: 10$ & Auto FIC & 0 & Suspect & Data not included in graph \\
\hline $5 / 25 / 95 \quad 12: 00$ & Manual FIC & 226.8 & Good & \\
\hline $5 / 25 / 9512: 00$ & Manual Tape & 225.25 & Good & \\
\hline $5 / 26 / 95 \quad 7: 04$ & Auto FIC & 226,80 & Good & \\
\hline $5 / 26 / 95 \quad 12: 00$ & Manual Tape & 225.25 & Good & \\
\hline $5 / 27 / 957: 05$ & Auto FIC & 226.8 & Good & \\
\hline $5 / 27 / 95 \quad 12: 00$ & Manual Tape & 225.25 & Good & \\
\hline $5 / 28 / 95 \quad 7: 05$ & Auto FIC & 226.8 & Good & \\
\hline $5 / 28 / 9512: 00$ & Manual Tape & 225.25 & Good & \\
\hline $5 / 29 / 957: 05$ & Auto FIC & 226.8 & Good & \\
\hline $5 / 29 / 95 \quad 12: 00$ & Manual Tape & 225.25 & Good & \\
\hline $5 / 30 / 95$ 7:05 & Auto FIC & 226.80 & Good & \\
\hline $5 / 30 / 9512: 00$ & Manual Tape & 225.25 & Good & \\
\hline $5 / 31 / 957: 05$ & Auto FIC & 226.80 & Good & \\
\hline $5 / 31 / 9512: 00$ & Manual Tape & 225.25 & Good & \\
\hline $6 / 1 / 95$ 7:05 & Auto FIC & 226.8 & Good & \\
\hline $6 / 1 / 9512: 00$ & Manual Tape & 225.25 & Good & \\
\hline $6 / 2 / 95$ 7:05 & Auto FIC & 226.8 & Good & \\
\hline $6 / 2 / 95 \quad 12: 00$ & Manual Tape & 225.25 & Good & \\
\hline $6 / 3 / 95 \quad 12: 00$ & Manual Tape & 225.25 & Good & \\
\hline $6 / 3 / 9523: 05$ & Auto FIC & 226.8 & Good & \\
\hline 6/4/95 7:05 & Auto FIC & 226.8 & Good & \\
\hline
\end{tabular}

Data obtained from WHC Surveillance Analysis Computer System, (PC SACS) July 7, 1995. 


\begin{tabular}{|c|c|c|c|c|}
\hline \multicolumn{5}{|c|}{ 241-AP-107 } \\
\hline \multicolumn{5}{|c|}{ Surface Level Data } \\
\hline Date & Type of Measurement & Level (Inches) & Data Quality & Comments \\
\hline $6 / 4 / 9512: 00$ & Manual Tape & $225: 25$ & Good & \\
\hline $6 / 5 / 957: 05$ & Auto FIC & 226.7 & Good & \\
\hline $6 / 5 / 95 \quad 12: 00$ & Manual Tape & 225.25 & Good & \\
\hline $6 / 6 / 957: 05$ & Auto FIC & 226.7 & Good & \\
\hline $6 / 6 / 95 \quad 12: 00$ & Manual Tape & 225.25 & Good & \\
\hline $6 / 7 / 957: 05$ & Auto FIC & 226.70 & Good & \\
\hline $6 / 7 / 95 \quad 12: 00$ & Manual Tape & 225.25 & Good & \\
\hline $6 / 8 / 957: 05$ & Auto FIC & 226.7 & Good & \\
\hline 6/8/95 12:00 & Manual Tape & 225 & Good & \\
\hline 6/9/95 7:05 & Auto FIC & 226.70 & Good & \\
\hline $6 / 9 / 9512: 00$ & Manual Tape & 224.75 & Good & \\
\hline $6 / 10 / 957: 05$ & Auto FIC & 226.70 & Good & \\
\hline $6 / 10 / 9512: 00$ & Manual Tape & 225.25 & Good & \\
\hline $6 / 11 / 957: 05$ & Auto FIC & 226.70 & Good & \\
\hline $6 / 11 / 95 \quad 12: 00$ & Manual Tape & 225.25 & Good & \\
\hline $6 / 12 / 957: 05$ & Auto FIC & 226.70 & Good & \\
\hline $6 / 12 / 9512: 00$ & Manual Tape & 225.25 & Good & \\
\hline $6 / 13 / 957: 05$ & Auto FIC & 191.00 & Good & \\
\hline $6 / 13 / 9512: 00$ & Manual Tape & 176.75 & Good & \\
\hline $6 / 14 / 957: 05$ & Auto FIC & 150.80 & Good & \\
\hline $6 / 14 / 95 \quad 12: 00$ & Manual Tape & 148 & Good & \\
\hline $6 / 15 / 957: 05$ & Auto FIC & 150.80 & Good & \\
\hline $6 / 15 / 9512: 00$ & Manual Tape & 149.25 & Good & \\
\hline $6 / 16 / 957: 05$ & Auto FIC & 150.8 & Good & \\
\hline $6 / 16 / 9512: 00$ & Manual Tape & 157.75 & Good & \\
\hline $6 / 17 / 957: 05$ & Auto FIC & 146.5 & Good & \\
\hline $6 / 17 / 9512: 00$ & Manual Tape & 132.5 & Good & \\
\hline $6 / 18 / 95$ 7:05 & Auto FIC & 53.3 & Good & \\
\hline $6 / 18 / 9512: 00$ & Manual Tape & 42.5 & Good & \\
\hline $6 / 19 / 95 \quad 7: 06$ & Auto FIC & 10.30 & Good & \\
\hline $6 / 19 / 95 \quad 12: 00$ & Manual Tape & 9.00 & Good & \\
\hline $6 / 20 / 957: 06$ & Auto FIC & 10.30 & Good & \\
\hline $6 / 20 / 9512: 00$ & Manual Tape & 9 & Good & \\
\hline $6 / 21 / 957: 06$ & Auto FIC & 10.3 & Good & \\
\hline $6 / 21 / 95 \quad 12: 00$ & Manual Tape & 8.75 & Good & \\
\hline 6/22/95 7:06 & Auto FIC & 10.30 & Good & \\
\hline $6 / 22 / 9512: 00$ & Manual Tape & 9.25 & Good & \\
\hline $6 / 23 / 957: 06$ & Auto FIC & 10.3 & Good & \\
\hline $6 / 23 / 9512: 00$ & Manual Tape & 9.25 & Good & \\
\hline $6 / 24 / 957: 06$ & Auto FIC & 10.3 & Good & \\
\hline $6 / 24 / 9512: 00$ & Manual Tape & 9 & Good & \\
\hline $6 / 25 / 957: 05$ & Auto FIC & 10.3 & Good & \\
\hline $6 / 26 / 95$ 7:05 & Auto FIC & 10.30 & Good & \\
\hline $6 / 26 / 95 \quad 12: 00$ & Manual Tape & 9.00 & Good & \\
\hline $6 / 27 / 95$ 7:06 & Auto FIC & 10.30 & Good & \\
\hline $6 / 27 / 9512: 00$ & Manual Tape & 9 & Good & \\
\hline $6 / 28 / 957: 05$ & Auto FIC & 10.30 & Good & \\
\hline $6 / 28 / 95 \quad 12: 00$ & Manual Tape & 9.00 & Good & \\
\hline
\end{tabular}

Data obtained from WHC Surveillance Analysis Computer System, (PC SACS) July 7, 1995. 


\begin{tabular}{|c|c|c|c|c|}
\hline \multicolumn{5}{|c|}{ 241-AP-107 } \\
\hline Date & Type of Measurement & Level (Inches) & Data Quality & Comments \\
\hline $6 / 29 / 957: 06$ & Auto FIC & 10.30 & Good & \\
\hline $6 / 29 / 9512: 00$ & Manual Tape & 9 & Good & \\
\hline $6 / 30 / 957: 06$ & Auto FIC & 10.30 & Good & \\
\hline $6 / 30 / 9512: 00$ & Manual Tape & 9.00 & Transcribed & \\
\hline
\end{tabular}

Data obtained from WHC Surveillance Analysis Computer System, (PC SACS) July 7, 1995. 


\begin{tabular}{|c|c|c|c|c|}
\hline \multicolumn{5}{|c|}{ 241-AP-108 } \\
\hline \multicolumn{5}{|c|}{ Surface Level Data } \\
\hline Date & Type of Measurement & Level (Inches) & Data Quality & Comments \\
\hline $1 / 1 / 910: 00$ & Manual Tape & 46.50 & Transcribed & \\
\hline $1 / 1 / 917: 03$ & Auto FIC & 46.70 & Good & \\
\hline $1 / 2 / 910: 00$ & Manual Tape & 46.50 & Transcribed & \\
\hline $1 / 2 / 917: 02$ & Auto FIC & 46.70 & Good & \\
\hline $1 / 3 / 910: 00$ & Manual Tape & 46 & Transcribed & \\
\hline $1 / 3 / 917: 03$ & Auto FIC & 46.60 & Good & \\
\hline $1 / 4 / 910: 00$ & Manual Tape & 46.00 & Transcribed & \\
\hline $1 / 4 / 917: 02$ & Auto FIC & 46.6 & Good & \\
\hline $1 / 5 / 910: 00$ & Manual Tape & 46.25 & Transcribed & \\
\hline $1 / 5 / 917: 02$ & Auto FIC & 46.6 & Good. & \\
\hline $1 / 6 / 910: 00$ & Manual Tape & 46.25 & Transcribed & \\
\hline $1 / 6 / 917: 02$ & Auto FIC & 46.6 & Good & \\
\hline $1 / 7 / 910: 00$ & Manual Tape & 46.25 & Transcribed & \\
\hline $1 / 7 / 917: 02$ & Auto FIC & 46.60 & Good & \\
\hline $1 / 8 / 910: 00$ & Manual Tape & 46.25 & Transcribed & \\
\hline $1 / 8 / 917: 03$ & Auto FIC & 46.60 & Good & \\
\hline $1 / 9 / 910: 00$ & Manual Tape & 46.25 & Transcribed & \\
\hline $1 / 9 / 917: 02$ & Auto FIC & 46.6 & Good & \\
\hline $1 / 10 / 910: 00$ & Manual Tape & 46.25 & Transcribed & \\
\hline $1 / 10 / 917: 02$ & Auto FIC & 46.6 & Good & \\
\hline $1 / 11 / 910: 00$ & Manual Tape & 46.25 & Transcribed & \\
\hline $1 / 11 / 917: 03$ & Auto FIC & 46.60 & Good & \\
\hline $1 / 12 / 910: 00$ & Manual Tape & 46.25 & Transcribed & \\
\hline $1 / 12 / 917: 02$ & Auto FIC & 46.6 & Good & \\
\hline $1 / 13 / 910: 00$ & Manual Tape & 46.25 & Transcribed & \\
\hline $1 / 13 / 917: 03$ & Auto FIC & 46.6 & Good & \\
\hline $1 / 14 / 910: 00$ & Manual Tape & 46.25 & Transcribed & \\
\hline $1 / 14 / 917: 04$ & Auto FIC & 46.6 & Good & \\
\hline $1 / 15 / 910: 00$ & Manual Tape & 46.25 & Transcribed & \\
\hline $1 / 15 / 917: 03$ & Auto FIC & 46.60 & Good & \\
\hline $1 / 16 / 910: 00$ & Manual Tape & 46.50 & Transcribed & \\
\hline $1 / 16 / 917: 04$ & Auto FIC & 46.60 & Good & \\
\hline 1/17/910:00 & Manual Tape & 46.00 & Transcribed & \\
\hline $1 / 17 / 917: 04$ & Auto FIC & 46.6 & Good & \\
\hline $1 / 18 / 910: 00$ & Manual Tape & 46.25 & Transcribed & \\
\hline $1 / 18 / 917: 04$ & Auto FIC & 46.6 & Good & \\
\hline $1 / 19 / 910: 00$ & Manual Tape & 46.25 & Transcribed & \\
\hline $1 / 19 / 917: 04$ & Auto FIC & 46.60 & Good & \\
\hline $1 / 20 / 910: 00$ & Manual Tape & 46.25 & Transcribed & \\
\hline $1 / 20 / 917: 04$ & Auto FIC & 46.6 & Good & \\
\hline $1 / 21 / 910: 00$ & Manual Tape & 46 & Transcribed & \\
\hline $1 / 21 / 917: 04$ & Auto FIC & 46.6 & Good & \\
\hline 1/22/91 0:00 & Manual Tape & 46 & Transcribed & \\
\hline $1 / 22 / 917: 04$ & Auto FIC & 46.6 & Good & \\
\hline $1 / 23 / 910: 00$ & Manual Tape & 46.00 & Transcribed & \\
\hline $1 / 23 / 917: 04$ & Auto FIC & 46.6 & Good & \\
\hline $1 / 24 / 910: 00$ & Manual Tape & 46 & Transcribed & \\
\hline $1 / 24 / 91 \quad 7: 04$ & Auto FIC & 46.60 & Good & \\
\hline
\end{tabular}

Data obtained from WHC Surveillance Analysis Computer System, (PC SACS) July 7, 1995. 


\begin{tabular}{|c|c|c|c|c|}
\hline \multicolumn{5}{|c|}{ 241-AP-108 } \\
\hline & & Irface Level Dat & & \\
\hline Date & Type of Measurement & Level (Inches) & Data Quality & Comments \\
\hline $1 / 25 / 910: 00$ & Manual Tape & 46 & Transcribed & \\
\hline $1 / 25 / 917: 03$ & Auto FIC & 46.6 & Good & \\
\hline $1 / 26 / 910: 00$ & Manual Tape & 46 & Transcribed & \\
\hline $1 / 26 / 917: 03$ & Auto FIC & 46.6 & Good & \\
\hline $1 / 27 / 910: 00$ & Manual Tape & 46.25 & Transcribed & \\
\hline $1 / 27 / 917: 03$ & Auto FIC & 46.6 & Good & \\
\hline $1 / 28 / 910: 00$ & Manual Tape & 46.00 & Transcribed & \\
\hline $1 / 28 / 916: 03$ & Auto FIC & 46.60 & Good & \\
\hline 1/29/910:00 & Manual Tape & 46.00 & Transcribed & \\
\hline $1 / 29 / 917: 02$ & Auto FIC & 46.6 & Good & \\
\hline $1 / 30 / 910: 00$ & Manual FIC & 46.60 & Transcribed & \\
\hline 1/30/91 0:00 & Manual Tape & 46.00 & Transcribed & \\
\hline $1 / 31 / 910: 00$ & Manual Tape & 46 & Transcribed & \\
\hline $1 / 31 / 917: 02$ & Auto FIC & 46.60 & Good & \\
\hline 2/1/91 0:00 & Manual Tape & 46 & Transcribed & \\
\hline 2/1/91 7:02 & Auto FIC & 46.6 & Good & \\
\hline $2 / 2 / 910: 00$ & Manual Tape & 46.25 & Transcribed & \\
\hline $2 / 2 / 917: 04$ & Auto FIC & 46.6 & Good & \\
\hline 2/3/910:00 & Manual Tape & 46.25 & Transcribed & \\
\hline 2/3/91 7:03 & Auto FIC & 46.6 & Good & \\
\hline 2/4/910:00 & Manual Tape & 46.25 & Transcribed & \\
\hline $2 / 4 / 917: 03$ & Auto FIC & 46.60 & Good & \\
\hline 2/5/91 0:00 & Manual Tape & 46.25 & Transcribed & \\
\hline 2/5/91 7:04 & Auto FIC & 46.60 & Good & \\
\hline $2 / 6 / 910: 00$ & Manual Tape & 46 & Transcribed & \\
\hline 2/6/91 7:04 & Auto FIC & 46.60 & Good & \\
\hline 2/7/91 0:00 & Manual Tape & 46 & Transcribed & \\
\hline 2/7/91 7:04 & Auto FIC & 46.60 & Good & \\
\hline 2/8/91 0:00 & Manual Tape & 46 & Transcribed & \\
\hline 2/8/91 7:04 & Auto FIC & 46.60 & Good & \\
\hline 2/9/91 0:00 & Manual Tape & 46.25 & Transcribed & \\
\hline 2/9/91 7:04 & Auto FIC & 46.60 & Good & \\
\hline $2 / 10 / 910: 00$ & Manual Tape & 46.25 & Transcribed & \\
\hline $2 / 10 / 917: 03$ & Auto FIC & 46.6 & Good & \\
\hline $2 / 11 / 910: 00$ & Manual Tape & 46.25 & Transcribed & \\
\hline $2 / 11 / 917: 04$ & Auto FIC & 46.6 & Good & \\
\hline $2 / 12 / 910: 00$ & Manual Tape & 46.25 & Transcribed & \\
\hline $2 / 12 / 917: 04$ & Auto FIC & 46.60 & Good & \\
\hline $2 / 13 / 910: 00$ & Manual Tape & 46.25 & Transcribed & \\
\hline $2 / 13 / 917: 04$ & Auto FIC & 46.60 & Good & \\
\hline $2 / 14 / 910: 00$ & Manual Tape & 46.25 & Transcribed & \\
\hline $2 / 14 / 917: 04$ & Auto FIC & 46.6 & Good & \\
\hline $2 / 15 / 910: 00$ & Manual Tape & 46.00 & Transcribed & \\
\hline $2 / 15 / 917: 03$ & Auto FIC & 46.60 & Good & \\
\hline $2 / 16 / 910: 00$ & Manual Tape & 46.00 & Transcribed & \\
\hline $2 / 16 / 917: 02$ & Auto FIC & 46.6 & Good & \\
\hline $2 / 17 / 910: 00$ & Manual Tape & 46.00 & Transcribed & \\
\hline $2 / 17 / 917: 02$ & Auto FIC & 46.6 & Good & \\
\hline
\end{tabular}

Data obtained from WHC Surveillance Analysis Computer System, (PC SACS) July 7, 1995. 


\begin{tabular}{|c|c|c|c|c|}
\hline \multicolumn{5}{|c|}{ 241-AP-108 } \\
\hline \multicolumn{5}{|c|}{ Surface Level Data } \\
\hline Date & Type of Measurement & Level (Inches) & Data Quality & Comments \\
\hline $2 / 18 / 910: 00$ & Manual Tape & 46.25 & Transcribed & \\
\hline 2/18/91 7:03 & Auto FIC & 46.6 & Good & \\
\hline 2/19/91 0:00 & Manual Tape & 46.25 & Transeribed & \\
\hline 2/19/91 7:02 & Auto FIC & 46.60 & Good & \\
\hline $2 / 20 / 910: 00$ & Manual Tape & 46.25 & Transcribed & \\
\hline $2 / 20 / 917: 03$ & Auto FIC & 46.6 & Good & \\
\hline 2/21/910:00 & Manual Tape & 46.25 & Transcribed & \\
\hline $2 / 21 / 917: 02$ & Auto FIC & 46.6 & Good & \\
\hline 2/22/91 0:00 & Manual Tape & 46.25 & Transcribed & \\
\hline $2 / 22 / 917: 02$ & Auto FIC & 46.60 & Good & \\
\hline $2 / 23 / 910: 00$ & Manual Tape & 46.25 & Transcribed & . \\
\hline $2 / 23 / 917: 02$ & Auto FIC & 46.6 & Good & \\
\hline $2 / 24 / 910: 00$ & Manual Tape & 46.25 & Transcribed & \\
\hline 2/24/917:03 & Auto FIC & 46.60 & Good & \\
\hline $2 / 25 / 910: 00$ & Manual Tape & 46 & Transcribed & \\
\hline $2 / 25 / 917: 03$ & Auto FIC & 46.6 & Good & \\
\hline $2 / 26 / 910: 00$ & Manual Tape & 46.25 & Transcribed & \\
\hline 2/26/91 7:03 & Auto FIC & 46.6 & Good & \\
\hline $2 / 27 / 910: 00$ & Manual Tape & 46.00 & Transcribed & \\
\hline $2 / 27 / 917: 02$ & Auto FIC & 46.60 & Good & \\
\hline $2 / 28 / 910: 00$ & Manual Tape & 46 & Transcribed & \\
\hline $2 / 28 / 917: 02$ & Auto FIC & 46.6 & Good & \\
\hline $3 / 1 / 910: 00$ & Manual Tape & 49 & Transcribed & \\
\hline $3 / 1 / 917: 02$ & Auto FIC & 49.40 & Good & \\
\hline $3 / 2 / 910: 00$ & Manual Tape & 49 & Transcribed & \\
\hline $3 / 2 / 917: 02$ & Auto FIC & 49.40 & Good & \\
\hline $3 / 3 / 910: 00$ & Manual Tape & 49 & Transcribed & \\
\hline $3 / 3 / 917: 03$ & Auto FIC & 49.40 & Good & \\
\hline 3/4/91 $0: 00$ & Manual Tape & 49 & Transcribed & \\
\hline $3 / 4 / 917: 03$ & Auto FIC & 49.4 & Good & \\
\hline $3 / 5 / 910: 00$ & Manual Tape & 49.00 & Transcribed & \\
\hline $3 / 5 / 917: 03$ & Auto FIC & 49.40 & Good & \\
\hline $3 / 6 / 910: 00$ & Manual Tape & 49.00 & Transcribed & \\
\hline $3 / 6 / 91 \quad 7: 02$ & Auto FIC & 49.40 & Good & \\
\hline $3 / 7 / 917: 02$ & Auto FIC & 49.40 & Good & \\
\hline $3 / 8 / 910: 00$ & Manual Tape & 49 & Transcribed & \\
\hline $3 / 8 / 917: 02$ & Auto FIC & 49.40 & Good & \\
\hline 3/9/91 0:00 & Manual Tape & 49 & Transcribed & \\
\hline 3/9/91 7:02 & Auto FIC & 49.6 & Good & \\
\hline $3 / 10 / 910: 00$ & Manual Tape & 49.00 & Transcribed & \\
\hline $3 / 10 / 917: 02$ & Auto FIC & 49.8 & Good & \\
\hline $3 / 11 / 910: 00$ & Manual Tape & 49.00 & Transcribed & \\
\hline 3/11/91 7:02 & Auto FIC & 49.70 & Good & \\
\hline $3 / 12 / 910: 00$ & Manual Tape & 49 & Transcribed & \\
\hline $3 / 12 / 917: 03$ & Auto FIC & 49.60 & Good & \\
\hline 3/13/91 0:00 & Manual Tape & 49 & Transcribed & \\
\hline 3/13/91 7:03 & Auto FIC & 49.60 & Good & \\
\hline 3/14/91 0:00 & Manual Tape & 49.00 & Transcribed & \\
\hline
\end{tabular}

Data obtained from WHC Surveillance Analysis Computer System, (PC SACS) July 7, 1995. 


\begin{tabular}{|c|c|c|c|c|}
\hline \multicolumn{5}{|c|}{ 241-AP-108 } \\
\hline \multicolumn{5}{|c|}{ Surface Level Data } \\
\hline Date & Type of Measurement & Level (linches) & Data Quality & Comments \\
\hline $3 / 14 / 917: 03$ & Auto FIC & 49.6 & . Good & \\
\hline $3 / 15 / 910: 00$ & Manual Tape & 49.00 & Transcribed & \\
\hline $3 / 15 / 917: 03$ & Auto FIC & 49.6 & Good & \\
\hline $3 / 16 / 910: 00$ & Manual Tape & 49.00 & Transcribed & \\
\hline $3 / 16 / 917: 03$ & Auto FIC & 49.70 & Good & \\
\hline $3 / 17 / 910: 00$ & Manual Tape & 49.25 & Transcribed & \\
\hline $3 / 17 / 917: 02$ & Auto FIC & 49.80 & Good & \\
\hline $3 / 18 / 910: 00$ & Manual Tape & 49.25 & Transcribed & \\
\hline $3 / 18 / 917: 03$ & Auto FIC & 49.9 & Good & \\
\hline 3/19/910:00 & Manual Tape & 49.25 & Transcribed & \\
\hline $3 / 19 / 917: 03$ & Auto FIC & 49.5 & Good & \\
\hline $3 / 20 / 910: 00$ & Manual Tape & 49.25 & Transcribed & \\
\hline $3 / 20 / 917: 02$ & Auto FIC & 49.5 & Good & \\
\hline $3 / 21 / 910: 00$ & Manual Tape & 49.25 & Transcribed & \\
\hline $3 / 21 / 917: 03$ & Auto FIC & 49.40 & Good & \\
\hline $3 / 22 / 910: 00$ & Manual Tape & 49.25 & Transcribed & \\
\hline $3 / 22 / 917: 02$ & Auto FIC & 49.40 & Good & \\
\hline $3 / 23 / 910: 00$ & Manual Tape & 49.25 & Transcribed & \\
\hline $3 / 23 / 917: 03$ & Auto FIC & $49.40^{\circ}$ & Good & \\
\hline $3 / 24 / 910: 00$ & Manual Tape & 49 & Transcribed & \\
\hline $3 / 24 / 917: 03$ & Auto FIC & 49.4 & Good & \\
\hline $3 / 25 / 910: 00$ & Manual Tape & 49.00 & Transcribed & \\
\hline $3 / 25 / 917: 03$ & Auto FIC & 49.4 & Good & \\
\hline $3 / 26 / 910: 00$ & Manual Tape & 49 & Transcribed & \\
\hline $3 / 26 / 917: 03$ & Auto FIC & 49.3 & Good & \\
\hline $3 / 27 / 910: 00$ & Manual Tape & 49 & Transcribed & \\
\hline $3 / 27 / 917: 03$ & Auto FIC & 49.30 & Good & \\
\hline $3 / 28 / 910: 00$ & Manual Tape & 49 & Transcribed & \\
\hline $3 / 28 / 917: 03$ & Auto FIC & 49.3 & Good & \\
\hline $3 / 29 / 910: 00$ & Manual Tape & 49 & Transcribed & \\
\hline $3 / 29 / 917: 03$ & Auto FIC & 49.30 & Good & \\
\hline $3 / 30 / 910: 00$ & Manual Tape & 49.00 & Transcribed & \\
\hline $3 / 30 / 917: 03$ & Auto FIC & 49.30 & Good & \\
\hline $3 / 31 / 910: 00$ & Manual Tape & 49 & Transcribed & \\
\hline $3 / 31 / 917: 03$ & Auto FIC & 49.3 & Good & \\
\hline $4 / 1 / 910: 00$ & Manual Tape & 49.00 & Transcribed & \\
\hline 4/1/917:03 & Auto FIC & 49.30 & Good & \\
\hline $4 / 2 / 910: 00$ & Manual Tape & 49.00 & Transcribed & \\
\hline $4 / 2 / 917: 03$ & Auto FIC & 49.4 & Good & - \\
\hline $4 / 3 / 910: 00$ & Manual Tape & 49.00 & Transcribed & \\
\hline $4 / 3 / 917: 03$ & Auto FIC & 49.20 & Good & \\
\hline $4 / 4 / 910: 00$ & Manual Tape & 49.00 & Transcribed & \\
\hline $4 / 4 / 917: 03$ & Auto FIC & 49.3 & Good & \\
\hline 4/5/910:00 & Manual Tape & 49 & Transcribed & \\
\hline $4 / 5 / 917: 03$ & Auto FIC & 49.3 & Good & \\
\hline 4/6/91 0:00 & Manual Tape & 48.75 & Transcribed & \\
\hline $4 / 6 / 917: 03$ & Auto FIC & 49.30 & Good & . \\
\hline $4 / 7 / 910: 00$ & Manual Tape & 49 & Transcribed & \\
\hline
\end{tabular}

Data obtained from WHC Surveillance Analysis Computer System, (PC SACS) July 7, 1995. 


\begin{tabular}{|c|c|c|c|c|}
\hline \multicolumn{5}{|c|}{ 241-AP-108 } \\
\hline Date & Type of Measurement & Level (Inches) & Data Quality & Comments \\
\hline $4 / 7 / 917: 03$ & Auto FIC & .49 .30 & Good & \\
\hline 4/8/91 $0: 00$ & Manual Tape & 49.00 & Transcribed & \\
\hline $4 / 8 / 917: 04$ & Auto FIC & 49.30 & Good & \\
\hline 4/9/91 0:00 & Manual Tape & 49.00 & Transćribed & \\
\hline $4 / 9 / 917: 03$ & Auto FIC & 49.3 & Good & \\
\hline $4 / 10 / 910: 00$ & Manual Tape & 48.5 & Transcribed & \\
\hline $4 / 10 / 917: 03$ & Auto FIC & 49.30 & Good & \\
\hline $4 / 11 / 910: 00$ & Manual Tape & 48.5 & Transcribed & \\
\hline $4 / 11 / 917: 03$ & Auto FIC & 49.3 & Good & \\
\hline 4/12/91 $0: 00$ & Manual Tape & 48.5 & Transcribed & \\
\hline 4/12/91 7:03 & Auto FIC & 49.3 & Good & \\
\hline 4/13/91 0:00 & Manual Tape & 49 & Transcribed & \\
\hline 4/13/91 7:04 & Auto FIC & 49.3 & Good & \\
\hline $4 / 14 / 910: 00$ & Manual Tape & 49 & Transcribed & \\
\hline $4 / 14 / 917: 03$ & Auto FIC & 49.30 & Good & \\
\hline 4/15/91 0:00 & Manual Tape & 49.00 & Transcribed & \\
\hline 4/15/91 7:03 & Auto FIC & 49.3 & Good & \\
\hline 4/16/91 0:00 & Manual FIC & 49.3 & Transcribed & \\
\hline $4 / 16 / 910: 00$ & Manual Tape & 49 & Transcribed & \\
\hline $4 / 17 / 910: 00$ & Manual Tape & 48.75 & Transcribed & \\
\hline $4 / 17 / 917: 03$ & Auto FIC & 49,3 & Good & \\
\hline $4 / 18 / 910: 00$ & Manual Tape & 49 & Transcribed & \\
\hline $4 / 18 / 917: 06$ & Auto FIC & 49.30 & Good & \\
\hline $4 / 19 / 910: 00$ & Manual Tape & 49.00 & Transcribed & \\
\hline 4/19/91 7:05 & Auto FIC & 49.3 & Good & \\
\hline $4 / 20 / 910: 00$ & Manual Tape & 49.00 & Transcribed & \\
\hline 4/20/91 7:06 & Auto FIC & 49.30 & Good & \\
\hline 4/21/910:00 & Manual Tape & 49.00 & Transcribed & \\
\hline 4/21/91 7:05 & Auto FIC & 49.3 & Good & \\
\hline 4/22/91 $0: 00$ & Manual Tape & 49 & Transcribed & \\
\hline 4/22/91 7:06 & Auto FIC & 49.30 & Good & \\
\hline 4/23/91 $0: 00$ & Manual Tape. & 49 & Transcribed & \\
\hline $4 / 23 / 917: 06$ & Auto FIC & 49.3 & Good & \\
\hline 4/24/91 $0: 00$ & Manual Tape & 49 & Transcribed & \\
\hline $4 / 24 / 917: 05$ & Auto FIC & 49.3 & Good & \\
\hline $4 / 25 / 910: 00$ & Manual Tape & 49.00 & Transcribed & \\
\hline $4 / 25 / 917: 05$ & Auto FIC & 49.30 & Good & \\
\hline 4/26/91 0:00 & Manual Tape & 49 & Transcribed & \\
\hline 4/26/91 7:05 & Auto FIC & 49.30 & Good & \\
\hline $4 / 27 / 910: 00$ & Manual Tape & 49.00 & Transcribed & \\
\hline 4/27/91 7:05 & Auto FIC & 49.30 & Good & \\
\hline $4 / 28 / 910: 00$ & Manual Tape & 49.00 & Transcribed & \\
\hline 4/28/91 7:06 & Auto FIC & 49.30 & Good & \\
\hline 4/29/910:00 & Manual Tape & 49 & Transcribed & \\
\hline $4 / 29 / 917: 05$ & Auto FIC & 49.30 & Good & \\
\hline $4 / 30 / 910: 00$ & Manual Tape & 49.00 & Transçribed & \\
\hline $4 / 30 / 917: 06$ & Auto FIC & 49.30 & Good & \\
\hline $5 / 1 / 910: 00$ & Manual Tape & 49.00 & Transcribed & \\
\hline
\end{tabular}

Data obtained from WHC Surveillance Analysis Computer System, (PC SACS) July 7, 1995. 


\begin{tabular}{|c|c|c|c|c|}
\hline \multicolumn{5}{|c|}{ 241-AP-108 } \\
\hline \multicolumn{5}{|c|}{ Surface Level Data } \\
\hline Date & Type of Measurement & Level.(Inches) & Data Quality & Comments \\
\hline $5 / 1 / 917: 05$ & Auto FIC & 49.3 & Good & \\
\hline $5 / 2 / 910: 00$ & Manual Tape & 49 & Transcribed & \\
\hline $5 / 2 / 917: 05$ & Auto FIC & 49.3 & Good & \\
\hline 5/3/910:00 & Manual Tape & 49.00 & Transcribed & \\
\hline $5 / 3 / 917: 06$ & Auto FIC & 49.3 & Good & \\
\hline $5 / 4 / 910: 00$ & Manual Tape & 50.25 & Transcribed & \\
\hline 5/4/91 7:05 & Auto FIC & 50.5 & Good & \\
\hline $5 / 5 / 910: 00$ & Manual Tape & 50.00 & Transcribed & \\
\hline $5 / 5 / 917: 05$ & Auto FIC & 50.50 & Good & \\
\hline $5 / 6 / 910: 00$ & Manual Tape & 50.00 & Transcribed & \\
\hline $5 / 6 / 917: 05$ & Auto FIC & 50.5 & Good & \\
\hline $5 / 7 / 910: 00$ & Manual Tape & 50.00 & Transcribed & \\
\hline 5/7/91 7:05 & Auto FIC & 50.5 & Good & \\
\hline $5 / 8 / 910: 00$ & Manual Tape & 50 & Transcribed & \\
\hline $5 / 8 / 917: 05$ & Auto FIC & 50.50 & Good & \\
\hline $5 / 9 / 910: 00$ & Manual Tape & 50.00 & Transcribed & \\
\hline $5 / 9 / 917: 05$ & Auto FIC & 50.6 & Good & \\
\hline $5 / 10 / 910: 00$ & Manual Tape & 50 & Transcribed & \\
\hline $5 / 10 / 917: 05$ & Auto FIC & 50.80 & Good & \\
\hline $5 / 11 / 910: 00$ & Manual Tape & 50 & Transcribed & \\
\hline $5 / 11 / 917: 05$ & Auto FIC & 50.9 & Good & \\
\hline $5 / 12 / 910: 00$ & Manual Tape & 50.00 & Transcribed & \\
\hline $5 / 12 / 917: 05$ & Auto FIC & 51.00 & Good & \\
\hline $5 / 13 / 910: 00$ & Manual Tape & 50 & Transcribed & \\
\hline $5 / 13 / 917: 05$ & Auto FIC & 51.00 & Good & \\
\hline $5 / 14 / 910: 00$ & Manual Tape & 50 & Transcribed & \\
\hline $5 / 14 / 917: 05$ & Auto FIC & 51.00 & Good & \\
\hline $5 / 15 / 910: 00$ & Manual Tape & 50.00 & Transcribed & \\
\hline $5 / 15 / 917: 05$ & Auto FIC & 51.1 & Good & \\
\hline $5 / 16 / 910: 00$ & Manual Tape & 50 & Transcribed & \\
\hline $5 / 16 / 917: 06$ & Auto FIC & 51 & Good & \\
\hline $5 / 17 / 910: 00$ & Manual Tape & 50 & Transcribed & \\
\hline $5 / 17 / 917: 06$ & Auto FIC & 51.00 & Good & \\
\hline $5 / 18 / 91 \quad 0: 00$ & Manual Tape & 50 & Transcribed & \\
\hline $5 / 18 / 917: 06$ & Auto FIC & 51 & Good & \\
\hline $5 / 19 / 910: 00$ & Manual Tape & 50 & Transcribed & \\
\hline $5 / 19 / 917: 05$ & Auto FIC & 51 & Good & \\
\hline $5 / 20 / 910: 00$ & Manual Tape & 49.75 & Transcribed & $\cdot$ \\
\hline $5 / 20 / 917: 06$ & Auto FIC & 50.9 & Good & \\
\hline $5 / 21 / 910: 00$ & Manual Tape & 50.00 & Transcribed & \\
\hline $5 / 21 / 917: 06$ & Auto FIC & 51.00 & Good & \\
\hline $5 / 22 / 910: 00$ & Manual Tape & 50.00 & Transcribed & \\
\hline $5 / 22 / 917: 05$ & Auto FIC & 50.90 & Good & \\
\hline $5 / 23 / 910: 00$ & Manual Tape & 50 & Transcribed & \\
\hline $5 / 23 / 917: 05$ & Auto FIC & 50.9 & Good & \\
\hline $5 / 24 / 910: 00$ & Manual Tape & 51.25 & Transcribed & \\
\hline 5/24/91 7:05 & Auto FIC & 52.1 & Good & \\
\hline $5 / 25 / 910: 00$ & Manual Tape & 51.50 & Transcribed & \\
\hline
\end{tabular}

Data obtained from WHC Surveillance Analysis Computer System, (PC SACS) July 7, 1995. 


\begin{tabular}{|c|c|c|c|c|}
\hline \multicolumn{5}{|c|}{ 241-AP-108 } \\
\hline \multicolumn{5}{|c|}{ Surface Level Data } \\
\hline Date & Type of Measurement & Level (Inches) & Data Quality & Comments \\
\hline $5 / 25 / 917: 05$ & Auto FIC & 52.20 & Good & \\
\hline $5 / 26 / 910: 00$ & Manual Tape & 51.50 & Transcribed & \\
\hline $5 / 26 / 917: 05$ & Auto FIC & 52.2 & Good & \\
\hline $5 / 27 / 910: 00$ & Manual Tape & 51.50 & Transeribed & \\
\hline $5 / 27 / 917: 05$ & Auto FIC & 52.30 & Good & \\
\hline $5 / 28 / 910: 00$ & Manual Tape & 51.50 & Transcribed & \\
\hline $5 / 28 / 917: 05$ & Auto FIC & 52.40 & Good & \\
\hline $5 / 29 / 910: 00$ & Manual Tape & 51.50 & Transcribed & \\
\hline $5 / 29 / 917: 05$ & Auto FIC & 52.4 & Good & \\
\hline $5 / 30 / 910: 00$ & Manual Tape & 51.50 & Transcribed & \\
\hline $5 / 30 / 917: 05$ & Auto FIC & 52.40 & Good & \\
\hline $5 / 31 / 910: 00$ & Manual Tape & 51.5 & Transcribed & \\
\hline $5 / 31 / 917: 05$ & Auto FIC & 52.40 & Good & \\
\hline $6 / 1 / 910: 00$ & Manual Tape & 51.5 & Transcribed & \\
\hline 6/1/91 6:05 & Auto FIC & 52.40 & Good & \\
\hline $6 / 2 / 910: 00$ & Manual Tape & 51.5 & Transcribed & \\
\hline $6 / 2 / 913: 05$ & Auto FIC & 52.2 & Good & \\
\hline $6 / 3 / 910: 00$ & Manual Tape & 51.50 & Transcribed & \\
\hline $6 / 3 / 917: 05$ & Auto FIC & 52.3 & Good & \\
\hline $6 / 4 / 910: 00$ & Manual Tape & 51.50 & Transcribed & \\
\hline $6 / 4 / 917: 05$ & Auto FIC & 52.2 & Good & \\
\hline $6 / 5 / 910: 00$ & Manual Tape & 51.25 & Transcribed & \\
\hline $6 / 5 / 91 \quad 7: 05$ & Auto FIC & 52.30 & Good & \\
\hline $6 / 6 / 910: 00$ & Manual Tape & 51.25 & Transcribed & \\
\hline $6 / 6 / 917: 06$ & Auto FIC & 52.4 & Good & \\
\hline $6 / 7 / 91 \quad 0: 00$ & Manual Tape & 51.50 & Transcribed & \\
\hline $6 / 7 / 917: 05$ & Auto FIC & 52.40 & Good & \\
\hline $6 / 8 / 910: 00$ & Manual Tape & 51.25 & Transcribed & \\
\hline $6 / 8 / 917: 05$ & Auto FIC & 52.4 & Good & \\
\hline 6/9/91 0:00 & Manual Tape & 51.25 & Transcribed & \\
\hline $6 / 9 / 917: 05$ & Auto FIC & 52.4 & Good & \\
\hline $6 / 10 / 910: 00$ & Manual Tape & 51.50 & Transcribed & \\
\hline $6 / 10 / 917: 06$ & Auto FIC & 52.50 & Good & \\
\hline $6 / 11 / 910: 00$ & Manual Tape & 51.5 & Transcribed & \\
\hline $6 / 11 / 917: 05$ & Auto FIC & 52.4 & Good & \\
\hline $6 / 12 / 910: 00$ & Manual Tape & 51.5 & Transcribed & \\
\hline $6 / 12 / 917: 04$ & Auto FIC & 52.20 & Good & \\
\hline $6 / 13 / 910: 00$ & Manual Tape & 51.5 & Transcribed & \\
\hline 6/13/51 7:05 & Auto FIC & 52.3 & Good & \\
\hline $6 / 14 / 910: 00$ & Manual Tape & 60.25 & Transcribed & \\
\hline $6 / 14 / 917: 05$ & Auto FIC & 53.70 & Good & \\
\hline $6 / 15 / 910: 00$ & Manual Tape & 53.00 & Transcribed & \\
\hline $6 / 15 / 917: 05$ & Auto FIC & 53.8 & Good & \\
\hline $6 / 16 / 910: 00$ & Manual Tape & 53.25 & Transcribed & \\
\hline $6 / 16 / 917: 05$ & Auto FIC & 53.7 & Good & \\
\hline $6 / 17 / 910: 00$ & Manual Tape & 53.25 & Transcribed & \\
\hline $6 / 17 / 917: 05$ & Auto FIC & 53.80 & Good & \\
\hline $6 / 18 / 910: 00$ & Manual Tape & 53.25 & Transcribed & \\
\hline
\end{tabular}

Data obtained from WHC Surveillance Analysis Computer System, (PC SACS) July 7, 1995. 


\begin{tabular}{|c|c|c|c|c|}
\hline \multicolumn{5}{|c|}{ 241-AP-108 } \\
\hline \multicolumn{5}{|c|}{ Surface Level Data } \\
\hline Date & Type of Measurement & Level (Inches) & Data Quality & Comments \\
\hline $6 / 18 / 917: 05$ & Auto FIC & 53.90 & Good & \\
\hline $6 / 19 / 910: 00$ & Manual Tape & 53.25 & Transcribed & \\
\hline 6/19/91 7:05 & Auto FIC & 53.9 & Good & \\
\hline $6 / 20 / 910: 00$ & Manual Tape & 53.25 & Transcribed & \\
\hline $6 / 20 / 917: 05$ & Auto FIC & 53.90 & Good & \\
\hline $6 / 21 / 910: 00$ & Manual Tape & 55.25 & Transcribed & \\
\hline $6 / 21 / 917: 06$ & Auto FIC & 55.70 & Good & \\
\hline $6 / 22 / 910: 00$ & Manual Tape & 56.75 & Transcribed & \\
\hline $6 / 22 / 913: 06$ & Auto FIC & 57.5 & Good & \\
\hline $6 / 23 / 910: 00$ & Manual Tape & 56.75 & Transcribed & \\
\hline $6 / 23 / 917: 05$ & Auto FIC & 57.90 & Good & \\
\hline $6 / 24 / 910: 00$ & Manual Tape & 57.25 & Transcribed & \\
\hline $6 / 24 / 915: 05$ & Auto FIC & 58.00 & Good & \\
\hline $6 / 25 / 910: 00$ & Manual Tape & 57.25 & Transcribed & \\
\hline $6 / 25 / 917: 06$ & Auto FIC & 58 & Good & \\
\hline $6 / 26 / 910: 00$ & Manual Tape & 57.25 & Transcribed & \\
\hline $6 / 26 / 911: 03$ & Auto FIC & 58.00 & Good & \\
\hline $6 / 27 / 910: 00$ & Manual FIC & 59.50 & Transcribed & \\
\hline 6/27/91 0:00 & Manual Tape & 58.75 & Transcribed & \\
\hline $6 / 28 / 910: 00$ & Manual Tape & 58.75 & Transcribed & \\
\hline $6 / 28 / 917: 03$ & Auto FIC & 59.50 & Good & \\
\hline $6 / 29 / 910: 00$ & Manual Tape & 60.50 & Transcribed & \\
\hline $6 / 29 / 916: 02$ & Auto FIC & 59.5 & Good & \\
\hline $6 / 30 / 910: 00$ & Manual FIC & 61.00 & Transcribed & \\
\hline $6 / 30 / 910: 00$ & Manual Tape & 60.50 & Transcribed & \\
\hline 7/1/91 0:00 & Manual FIC & 61.00 & Transćribed & \\
\hline 7/1/910:00 & Manual Tape & 60.5 & Transcribed & \\
\hline $7 / 2 / 910: 00$ & Manual Tape & 60.50 & Transcribed & \\
\hline $7 / 2 / 917: 06$ & Auto FIC & 61.00 & Good & \\
\hline 7/3/91 0:00 & Manual Tape & 59.75 & Transcribed & \\
\hline $7 / 3 / 917: 06$ & Auto FIC & 61.00 & Good & \\
\hline $7 / 4 / 910: 00$ & Manual FIC & 60.95 & Transcribed & \\
\hline $7 / 4 / 910: 00$ & Manual Tape & 60.5 & Transcribed & \\
\hline 7/5/91 0:00 & Manual FIC & 60.95 & Transcribed & \\
\hline $7 / 5 / 910: 00$ & Manual Tape & 60.5 & Transcribed & \\
\hline $7 / 6 / 910: 00$ & Manual FIC & 60.95 & Transcribed & \\
\hline 7/6/91 0:00 & Manual Tape & 60.5 & Transcribed & \\
\hline $7 / 7 / 910: 00$ & Manual FIC & 60.95 & Transcribed & \\
\hline $7 / 7 / 910: 00$ & Manual Tape & 60.50 & Transcribed & \\
\hline $7 / 8 / 910: 00$ & Manual FIC & 60.95 & Transcribed & \\
\hline $7 / 8 / 91 \quad 0: 00$ & Manual Tape & 60.5 & Transcribed & \\
\hline $7 / 9 / 910: 00$ & Manual FIC & 60.9 & Transcribed & \\
\hline 7/9/91 0:00 & Manual Tape & 60.5 & Transcribed & \\
\hline $7 / 10 / 910: 00$ & Manual FIC & 60.90 & Transcribed & \\
\hline $7 / 10 / 910: 00$ & Manual Tape & 60.5 & Transcribed & \\
\hline $7 / 11 / 910: 00$ & Manual Tape & 60.50 & Transcribed & \\
\hline $7 / 11 / 917: 06$ & Auto FIC & 60.9 & Good & \\
\hline $7 / 12 / 910: 00$ & Manual Tape & 60.5 & Transcribed & \\
\hline
\end{tabular}

Data obtained from WHC Surveillance Analysis Computer System, (PC SACS) July 7, 1995. 


\begin{tabular}{|c|c|c|c|c|}
\hline \multicolumn{5}{|c|}{ 241-AP-108 } \\
\hline \multicolumn{5}{|c|}{ Surface Level Data } \\
\hline Date & Type of Measurement & Leval (Inches) & Data Quality & Comments \\
\hline $7 / 12 / 917: 06$ & Auto FIC & 61 & Good & \\
\hline $7 / 13 / 910: 00$ & Manual Tape & 60.5 & Transcribed & \\
\hline 7/13/91 7:06 & Auto FIC & 61.00 & Good & \\
\hline 7/14/91 0:00 & Manual Tape & 60.50 & Transcribed & \\
\hline $7 / 14 / 912: 06$ & Auto FIC & 61.00 & Good & \\
\hline $7 / 15 / 910: 00$ & Manual Tape & 60.25 & Transcribed & \\
\hline $7 / 15 / 917: 06$ & Auto FIC & 61 & Good & \\
\hline $7 / 16 / 910: 00$ & Manual FIC & 61.00 & Transcribed & \\
\hline $7 / 16 / 910: 00$ & Manual Tape & 60.25 & Transcribed & \\
\hline $7 / 17 / 910: 00$ & Manual Tape & 60.25 & Transcribed & \\
\hline 7/17/910:05 & Auto FIC & 61 & Good & \\
\hline $7 / 18 / 910: 00$ & Manual FIC & 61 & Transcribed & \\
\hline $7 / 18 / 910: 00$ & Manual Tape & 60.25 & Transcribed & \\
\hline $7 / 19 / 910: 00$ & Manual Tape & 60.25 & Transcribed & \\
\hline $7 / 19 / 917: 05$ & Auto FIC & 67.00 & Good & \\
\hline $7 / 20 / 910: 00$ & Manual Tape & 60.25 & Transcribed & \\
\hline $7 / 20 / 912: 05$ & Auto FIC & 61 & Good & \\
\hline $7 / 21 / 910: 00$ & Manual Tape & 60.25 & Transcribed & \\
\hline $7 / 21 / 911: 05$ & Auto FIC & 61 & Good & \\
\hline $7 / 22 / 910: 00$ & Manual FIC & 61 & Transcribed & \\
\hline $7 / 22 / 910: 00$ & Manual Tape & 60.25 & Transcribed & \\
\hline $7 / 23 / 910: 00$ & Manual Tape & 62 & Transcribed & \\
\hline $7 / 23 / 910: 05$ & Auto FIC & 62.40 & Good & \\
\hline $7 / 24 / 910: 00$ & Manual Tape & 62.00 & Transcribed & \\
\hline 7/24/91 7:05 & Auto FIC & 62.4 & Good & \\
\hline $7 / 25 / 910: 00$ & Manual Tape & 62.25 & Transcribed & \\
\hline $7 / 25 / 911: 03$ & Auto FIC & 62.4 & Good & \\
\hline $7 / 26 / 910: 00$ & Manual FIC & 62.4 & Transcribed & \\
\hline $7 / 26 / 910: 00$ & Manual Tape & 62.25 & Transcribed & \\
\hline $7 / 27 / 910: 00$ & Manual FIC & 62.4 & Transcribed & \\
\hline $7 / 27 / 910: 00$ & Manual Tape & 62 & Transcribed & \\
\hline $7 / 28 / 910: 00$ & Manual FIC & 62.40 & Transcribed & \\
\hline $7 / 28 / 910: 00$ & Manual Tape & 62 & Transcribed & \\
\hline $7 / 29 / 910: 00$ & Manual FIC & 62.4 & Transcribed & \\
\hline $7 / 29 / 910: 00$ & Manual Tape & 62.00 & Transcribed & \\
\hline $7 / 30 / 910: 00$ & Manual Tape & 62 & Transcribed & \\
\hline $7 / 30 / 917: 02$ & Auto FIC & 62.40 & Good & \\
\hline $7 / 31 / 910: 00$ & Manual Tape & 62 & Transcribed & \\
\hline $7 / 31 / 917: 02$ & Auto FIC & 62.4 & Good & \\
\hline $8 / 1 / 910: 00$ & Manual Tape & 62 & Transcribed & \\
\hline $8 / 1 / 917: 02$ & Auto FIC & 62.40 & Good & \\
\hline $8 / 2 / 910: 00$ & Manual Tape & 62.00 & Transcribed & \\
\hline $8 / 2 / 917: 02$ & Auto FIC & 62.40 & Good & \\
\hline $8 / 3 / 910: 00$ & Manual Tape & 62 & Transcribed & \\
\hline $8 / 3 / 917: 02$ & Auto FIC & 62.40 & Good & \\
\hline $8 / 4 / 910: 00$ & Manual Tape & 62.00 & Transcribed & \\
\hline $8 / 4 / 917: 02$ & Auto FIC & 62.40 & Good & \\
\hline $8 / 5 / 910: 00$ & Manual Tape & 61.75 & Transçribed & \\
\hline
\end{tabular}

Data obtained from WHC Surveillance Analysis Computer System, (PC SACS) July 7, 1995. 


\begin{tabular}{|c|c|c|c|c|}
\hline \multicolumn{5}{|c|}{ 241-AP-108 } \\
\hline & & urface Level Dat & & \\
\hline Date & Type of Measurement & Level (Inches) & Data Quality & Comments \\
\hline $8 / 5 / 917: 02$ & Auto FIC & 62.4 & Good & \\
\hline $8 / 6 / 910: 00$ & Manual Tape & 62 & Transcribed & \\
\hline $8 / 6 / 917: 02$ & Auto FIC & 62.4 & Good & \\
\hline $8 / 7 / 910: 00$ & Manual Tape & 62.00 & Transcribed & \\
\hline $8 / 7 / 917: 06$ & Auto FIC & 62.4 & Good & \\
\hline $8 / 8 / 910: 00$ & Manual Tape & 62 & Transcribed & \\
\hline 8/8/91 7:06 & Auto FIC & 62.40 & Good & \\
\hline 8/9/91 0:00 & Manual Tape & 62 & Transcribed & \\
\hline 8/9/91 7:04 & Auto FIC & 62.40 & Good & \\
\hline $8 / 10 / 910: 00$ & Manual Tape & 61.75 & Transcribed & \\
\hline $8 / 10 / 917: 04$ & Auto FIC & 62.4 & Good & \\
\hline $8 / 11 / 910: 00$ & Manual Tape & 62 & Transcribed & \\
\hline $8 / 11 / 917: 04$ & Auto FIC & 62.40 & Good & \\
\hline $8 / 12 / 910: 00$ & Manual Tape & 62 & Transcribed & \\
\hline $8 / 12 / 917: 04$ & Auto FIC & 62.40 & Good & \\
\hline $8 / 13 / 910: 00$ & Manual Tape & 62.00 & Transcribed & \\
\hline $8 / 13 / 917: 04$ & Auto FIC & 62.4 & Good & \\
\hline $8 / 14 / 910: 00$ & Manual Tape & 62 & Transcribed & \\
\hline $8 / 14 / 917: 04$ & Auto FIC & 62.4 & Good & \\
\hline $8 / 15 / 910: 00$ & Manual Tape & 62.00 & Transcribed & \\
\hline $8 / 15 / 917: 03$ & Auto FIC & 62.4 & Good & \\
\hline $8 / 16 / 910: 00$ & Manual FIC & 62.40 & Transcribed & \\
\hline $8 / 16 / 910: 00$ & Manual Tape & 62 & Transcribed & \\
\hline $8 / 17 / 910: 00$ & Manual Tape & 62 & Transcribed & \\
\hline $8 / 17 / 917: 02$ & Auto FIC & 62.40 & Good & \\
\hline $8 / 18 / 910: 00$ & Manual Tape & 62.00 & Transcribed & \\
\hline $8 / 18 / 917: 03$ & Auto FIC & 62.40 & Good & \\
\hline $8 / 19 / 910: 00$ & Manual Tape & 62.00 & Transeribed & \\
\hline $8 / 19 / 91 \quad 7: 02$ & Auto FIC & 62.40 & Good & \\
\hline $8 / 20 / 910: 00$ & Manual Tape & 62.00 & Transcribed & \\
\hline $8 / 20 / 917: 04$ & Auto FIC & 62.40 & Good & \\
\hline $8 / 21 / 910: 00$ & Manual Tape & 62.00 & Transcribed & \\
\hline $8 / 21 / 917: 03$ & Auto FIC & 62.40 & Good & \\
\hline $8 / 22 / 910: 00$ & Manual Tape & 62.00 & Transcribed & \\
\hline $8 / 22 / 917: 03$ & Auto FIC & 62.40 & Good & \\
\hline $8 / 23 / 910: 00$ & Manual Tape & 62.00 & Transcribed & \\
\hline $8 / 23 / 917: 02$ & Auto FIC & 62.40 & Good & \\
\hline $8 / 24 / 910: 00$ & Manual Tape & 62 & Transcribed & \\
\hline $8 / 24 / 917: 02$ & Auto FIC & 62.40 & Good & \\
\hline $8 / 25 / 910: 00$ & Manual Tape & 62 & Transcribed & \\
\hline $8 / 25 / 91 \quad 7: 02$ & Auto FIC & 62.40 & Good & \\
\hline $8 / 26 / 910: 00$ & Manual Tape & 62 & Transcribed & \\
\hline $8 / 26 / 917: 03$ & Auto FIC & 62.40 & Good & \\
\hline $8 / 27 / 910: 00$ & Manual Tape & 62 & Transcribed & \\
\hline $8 / 27 / 917: 03$ & Auto FIC & 62.40 & Good & \\
\hline $8 / 28 / 910: 00$ & Manual Tape & 62 & Transcribed & \\
\hline $8 / 28 / 91 \quad 7: 03$ & Auto FIC & 62.40 & Good & \\
\hline $8 / 29 / 91 \quad 0: 00$ & Manual Tape & 62.00 & Transcribed & \\
\hline
\end{tabular}

Data obtained from WHC Surveillance Analysis Computer System, (PC SACS) July 7, 1995. 


\begin{tabular}{|c|c|c|c|c|}
\hline \multicolumn{5}{|c|}{ 241-AP-108 } \\
\hline \multicolumn{5}{|c|}{ Surface Level Data } \\
\hline Date & Type of Measurement & Level (Inches) & Data Quality & Comments \\
\hline $8 / 29 / 917: 02$ & Auto FIC & 62.4 & Good & \\
\hline $8 / 30 / 910: 00$ & Manual Tape & 62 & Transcribed & \\
\hline $8 / 30 / 916: 02$ & Auto FIC & 62.4 & Good & \\
\hline $8 / 31 / 910: 00$ & Manual Tape & 62.00 & Transcribed & \\
\hline $8 / 31 / 917: 02$ & Auto FIC & 62.4 & Good & \\
\hline 9/1/91 0:00 & Manual Tape & 62.00 & Transcribed & \\
\hline $9 / 1 / 917: 02$ & Auto FIC & 62.30 & Good & \\
\hline $9 / 2 / 910: 00$ & Manual Tape & 62 & Transcribed & \\
\hline $9 / 2 / 917: 02$ & Auto FIC & 62.3 & Good & \\
\hline 9/3/910:00 & Manual Tape & 62 & Transcribed & \\
\hline $9 / 3 / 917: 02$ & Auto FIC & 62.40 & Good & \\
\hline 9/4/91 0:00 & Manual Tape & 62 & Transcribed & \\
\hline $9 / 4 / 917: 03$ & Auto FIC & 62.40 & Good & \\
\hline $9 / 5 / 910: 00$ & Manual Tape & 62 & Transcribed & \\
\hline $9 / 5 / 917: 02$ & Auto FIC & 62.4 & Good & \\
\hline $9 / 6 / 910: 00$ & Manual Tape & 62.00 & Transcribed & \\
\hline $9 / 6 / 917: 02$ & Auto FIC & 62.30 & Good & \\
\hline $9 / 7 / 910: 00$ & Manual Tape & 62.00 & Transcribed & \\
\hline $9 / 7 / 917: 02$ & Auto FIC & 62.40 & Good & \\
\hline 9/8/91 0:00 & Manual Tape & 62 & Transcribed & \\
\hline 9/8/91 7:02 & Auto FIC & 62.3 & Good & \\
\hline $9 / 9 / 910: 00$ & Manual Tape & 62 & Transcribed & \\
\hline 9/9/91 7:03 & Auto FIC & 62.3 & Good & \\
\hline $9 / 10 / 910: 00$ & Manual Tape & 62.00 & Transcribed & \\
\hline $9 / 10 / 917: 02$ & Auto FIC & 62.30 & Good & \\
\hline $9 / 11 / 910: 00$ & Manual Tape & 63.25 & Transcribed & \\
\hline $9 / 11 / 917: 03$ & Auto FIC & 63.8 & Good & \\
\hline $9 / 12 / 910: 00$ & Manual Tape & 63.25 & Transcribed & \\
\hline $9 / 12 / 917: 02$ & Auto FIC & 63.8 & Good & \\
\hline $9 / 13 / 910: 00$ & Manual Tape & 63.25 & Transcribed & \\
\hline $9 / 13 / 917: 02$ & Auto FIC & 63.8 & Good & \\
\hline $9 / 14 / 910: 00$ & Manual Tape & 63.25 & Transcribed & \\
\hline $9 / 14 / 917: 03$ & Auto FIC & 63.8 & Good & \\
\hline 9/15/91 0:00 & Manual Tape & 63.25 & Transcribed & \\
\hline $9 / 15 / 917: 02$ & Auto FIC & 63.8 & Good & \\
\hline $9 / 16 / 910: 00$ & Manual Tape & 63.25 & Transcribed & \\
\hline $9 / 16 / 917: 03$ & Auto FIC & 63.8 & Good & \\
\hline $9 / 17 / 910: 00$ & Manual Tape & 63.25 & Transcribed & \\
\hline $9 / 17 / 917: 03$ & Auto FIC & 63.80 & Good & \\
\hline $9 / 18 / 910: 00$ & Manual Tape & 63.25 & Transcribed & \\
\hline $9 / 18 / 917: 03$ & Auto FIC & 63.8 & Good & \\
\hline $9 / 19 / 910: 00$ & Manual Tape & 63.25 & Transcribed & \\
\hline $9 / 19 / 917: 03$ & Auto FIC & 63.80 & Good & \\
\hline $9 / 20 / 910: 00$ & Manual Tape & 63.25 & Transcribed & \\
\hline $9 / 20 / 917: 02$ & Auto FIC & 63.80 & Good & \\
\hline $9 / 21 / 910: 00$ & Manual Tape & 63.25 & Transcribed & \\
\hline $9 / 21 / 917: 02$ & Auto FIC & 63.80 & Good & \\
\hline $9 / 22 / 910: 00$ & Manual Tape & 63.25 & Transcribed & \\
\hline
\end{tabular}

Data obtained from WHC Surveillance Analysis Computer System, (PC SACS) July 7, 1995. 


\begin{tabular}{|c|c|c|c|c|}
\hline \multicolumn{5}{|c|}{ 241-AP-108 } \\
\hline & $\therefore$ & urface Level Dat: & & \\
\hline Date & Type of Measurement & Level (Inches) & Data Quality & Comments \\
\hline $9 / 22 / 917: 03$ & Auto FIC & 63.80 & Good & \\
\hline 9/23/91 0:00 & Manual Tape & 63.25 & Transcribed & \\
\hline 9/23/91 7:03 & Auto FIC & 63.70 & Good & \\
\hline 9/24/910:00 & Manual Tape & 63.50 & Transcribed & \\
\hline $9 / 24 / 917: 02$ & Auto FIC & 63.7 & Good & \\
\hline $9 / 25 / 910: 00$ & Manual Tape & 63.25 & Transcribed & \\
\hline $9 / 25 / 917: 03$ & Auto FIC & 63.70 & Good & \\
\hline 9/26/910:00 & Manual Tape & 63.25 & Transcribed & \\
\hline $9 / 26 / 917: 03$ & Auto FIC & 63.70 & Good & \\
\hline 9/27/91 0:00 & Manual Tape & 63.25 & Transcribed & \\
\hline $9 / 27 / 917: 02$ & Auto FIC & 63.7 & Good & \\
\hline $9 / 28 / 910: 00$ & Manual Tape & 63.25 & Transcribed & \\
\hline $9 / 28 / 917: 02$ & Auto FIC & 63.70 & Good & \\
\hline $9 / 29 / 910: 00$ & Manual Tape & 63.25 & Transcribed & \\
\hline $9 / 29 / 917: 03$ & Auto FIC & 63.8 & Good & \\
\hline $9 / 30 / 910: 00$ & Manual Tape & 63.25 & Transcribed & \\
\hline 9/30/91 7:03 & Auto FIC & 63.70 & Good & \\
\hline $10 / 1 / 910: 00$ & Manual Tape & 63.25 & Transcribed & \\
\hline $10 / 1 / 917: 02$ & Auto FIC & 63.8 & Good & \\
\hline $10 / 2 / 910: 00$ & Manual Tape & 63.25 & Transćribed & \\
\hline $10 / 2 / 917: 02$ & Auto FIC & 63.80 & Good & \\
\hline $10 / 3 / 910: 00$ & Manual Tape & 63.25 & Transcribed & \\
\hline 10/3/91 7:02 & Auto FIC & 63.80 & Good & \\
\hline $10 / 4 / 910: 00$ & Manual Tape & 63.25 & Transcribed & \\
\hline $10 / 4 / 917: 03$ & Auto FIC & 63.80 & Good & \\
\hline $10 / 5 / 910: 00$ & Manual FIC & 65.7 & Transcribed & \\
\hline $10 / 5 / 910: 00$ & Manual Tape & 65.25 & Transcribed & \\
\hline $10 / 6 / 910: 00$ & Manual Tape & 65.25 & Transcribed & \\
\hline $10 / 6 / 917: 03$ & Auto FIC & 65.6 & Good & \\
\hline $10 / 7 / 910: 00$ & Manual Tape & 65.25 & Transcribed & \\
\hline $10 / 7 / 917: 02$ & Auto FIC & 65.60 & Good & \\
\hline $10 / 8 / 910: 00$ & Manual Tape & 65.25 & Transcribed & \\
\hline $10 / 8 / 917: 02$ & Auto FIC & 65.6 & Good & \\
\hline $10 / 9 / 910: 00$ & Manual Tape & 65.25 & Transcribed & \\
\hline $10 / 9 / 917: 02$ & Auto FIC & 65.60 & Good & \\
\hline $10 / 10 / 910: 00$ & Manual Tape & 65.25 & Transcribed & \\
\hline 10/10/91 7:02 & Auto FIC & 65.6 & Good & \\
\hline $10 / 11 / 910: 00$ & Manual Tape & 65.25 & Transcribed & \\
\hline $10 / 11 / 917: 02$ & Auto FIC & 65.6 & Good & \\
\hline $10 / 12 / 910: 00$ & Manual Tape & 66.75 & Transcribed & \\
\hline $10 / 12 / 917: 02$ & Auto FIC & 67.30 & Good & \\
\hline $10 / 13 / 910: 00$ & Manual Tape & 66.75 & Transcribed & \\
\hline $10 / 13 / 917: 03$ & Auto FIC & 67.2 & Good & \\
\hline $10 / 14 / 910: 00$ & Manual Tape & 66.5 & Transcribed & \\
\hline $10 / 14 / 91 \quad 7: 02$ & Auto FIC & 67.20 & Good & \\
\hline $10 / 15 / 910: 00$ & Manual Tape & 66.75 & Transcribed & \\
\hline $10 / 15 / 917: 02$ & Auto FIC & 67.2 & Good & \\
\hline $10 / 16 / 91 \quad 0: 00$ & Manual Tape & 66.75 & Transcribed & \\
\hline
\end{tabular}

Data obtained from WHC Surveillance.Analysis Computer System, (PC SACS) July 7, 1995. 


\begin{tabular}{|c|c|c|c|c|}
\hline \multicolumn{5}{|c|}{ 241-AP-108 } \\
\hline & & Urface Level Dat & & \\
\hline Date & Type of Measurement & Lavel (Inches) & Data Quality & Comments \\
\hline $10 / 16 / 917: 03$ & Auto FIC & 67.2 & Good & \\
\hline $10 / 17 / 910: 00$ & Manual Tape & 66.75 & Transcribed & \\
\hline $10 / 17 / 917: 05$ & Auto FIC & 67.1 & Good & \\
\hline $10 / 18 / 910: 00$ & Manual Tape & 66.75 & Transcribed & \\
\hline 10/18/91 7:06 & Auto FIC & 67.20 & Good & \\
\hline $10 / 19 / 910: 00$ & Manual Tape & 66.75 & Transcribed & \\
\hline $10 / 19 / 917: 06$ & Auto FIC & 67.2 & Good & \\
\hline $10 / 20 / 910: 00$ & Manual Tape & 66.75 & Transcribed & \\
\hline $10 / 20 / 917: 02$ & Auto FIC & 67.20 & Good & \\
\hline $10 / 21 / 910: 00$ & Manual Tape & 66.75 & Transcribed & \\
\hline $10 / 21 / 917: 02$ & Auto FIC & 67.2 & Good & \\
\hline 10/22/91 0:00 & Manual Tape & 66.75 & Transcribed & \\
\hline $10 / 22 / 917: 03$ & Auto FIC & 67.2 & Good & \\
\hline $10 / 23 / 910: 00$ & Manual Tape & 66.75 & Transcribed & \\
\hline $10 / 23 / 917: 02$ & Auto FIC & 67.20 & Good & \\
\hline $10 / 24 / 910: 00$ & Manual Tape & 66.75 & Transcribed & \\
\hline $10 / 24 / 917: 02$ & Auto FIC & 67.2 & Good & \\
\hline $10 / 25 / 910: 00$ & Manual Tape & 66.75 & Transcribed & \\
\hline $10 / 25 / 917: 03$ & Auto FIC & 67.2 & Good & \\
\hline $10 / 26 / 910: 00$ & Manual Tape & 66.75 & Transcribed & $\cdot$ \\
\hline $10 / 26 / 917: 03$ & Auto FIC & 67.20 & Good & \\
\hline $10 / 27 / 910: 00$ & Manual Tape & 66.75 & Transcribed & \\
\hline $10 / 27 / 917: 02$ & Auto FIC & 67.2 & Good & \\
\hline 10/28/91 0:00 & Manual Tape & 66.75 & Transcribed & \\
\hline $10 / 28 / 91 \quad 7: 02$ & Auto FIC & 67.20 & Good & \\
\hline $10 / 29 / 910: 00$ & Manual Tape & 66.75 & Transcribed & \\
\hline $10 / 29 / 917: 03$ & Auto FIC & 67.20 & Good & \\
\hline $10 / 30 / 910: 00$ & Manual Tape & 66.5 & Transcribed & \\
\hline $10 / 30 / 917: 03$ & Auto FIC & 67.1 & Good & \\
\hline 10/31/910:00 & Manual Tape & 66.5 & Transcribed & \\
\hline $10 / 31 / 917: 03$ & Auto FIC & 67.2 & Good & \\
\hline $11 / 1 / 910: 00$ & Manual Tape & 66.50 & Transcribed & \\
\hline $11 / 1 / 917: 02$ & Auto FIC & 67.10 & Good & \\
\hline $11 / 2 / 910: 00$ & Manual Tape & 66.5 & Transcribed & \\
\hline $11 / 2 / 917: 03$ & Auto FIC & 67.2 & Good & \\
\hline $11 / 3 / 910: 00$ & Manual Tape & 66.50 & Transcribed & \\
\hline $11 / 3 / 917: 03$ & Auto FIC & 67.20 & Good & \\
\hline $11 / 4 / 910: 00$ & Manual Tape & 66.50 & Transcribed & \\
\hline $11 / 4 / 917: 02$ & Auto FIC & 67.10 & Good & \\
\hline $11 / 5 / 910: 00$ & Manual Tape & 66.5 & Transcribed & \\
\hline $11 / 5 / 917: 03$ & Auto FIC & 67.1 & Good & \\
\hline $11 / 6 / 910: 00$ & Manual Tape & 66.50 & Transcribed & \\
\hline $11 / 6 / 917: 03$ & Auto FIC & 67.20 & Good & \\
\hline $11 / 7 / 910: 00$ & Manual Tape & 66.5 & Transcribed & \\
\hline $11 / 7 / 917: 02$ & Auto FIC & 67.1 & Good & \\
\hline $11 / 8 / 910: 00$ & Manual Tape & 66.5 & Transcribed & \\
\hline $11 / 8 / 917: 03$ & Auto FIC & 67.10 & Good & \\
\hline $11 / 9 / 910: 00$ & Manual Tape & 66.5 & Transcribed & \\
\hline
\end{tabular}

Data obtained from WHC Surveillance Analysis Computer System, (PC SACS) July 7, 1995. 


\begin{tabular}{|c|c|c|c|c|}
\hline \multicolumn{5}{|c|}{ 241-AP-108 } \\
\hline \multicolumn{5}{|c|}{ Surface Level Data } \\
\hline Date & Type of Measurement & Level (Inches) & Data Quality & Comments \\
\hline $11 / 9 / 917: 02$ & Auto FIC & 67.10 & Good & \\
\hline $11 / 10 / 910: 00$ & Manual Tape & 66.50 & Transcribed & \\
\hline $11 / 10 / 917: 03$ & Auto FIC & 67.2 & Good & \\
\hline $11 / 11 / 910: 00$ & Manual Tape & 109.25 & Transcribed & \\
\hline $11 / 11 / 916: 03$ & Auto FIC & 92.6 & Good & \\
\hline $11 / 12 / 917: 03$ & Auto FIC & 153.50 & Good & \\
\hline $11 / 13 / 910: 00$ & Manual Tape & 210 & Transcribed & \\
\hline $11 / 13 / 917: 03$ & Auto FIC & 205.70 & Good & \\
\hline $11 / 14 / 917: 03$ & Auto FIC & 265.40 & Good & \\
\hline $11 / 15 / 917: 03$ & Auto FIC & 316.4 & Good & \\
\hline $11 / 16 / 910: 00$ & Manual Tape & 316 & Transcribed & \\
\hline $11 / 16 / 917: 03$ & Auto FIC & 316.4 & Good & \\
\hline $11 / 17 / 910: 00$ & Manual Tape & 316.00 & Transcribed & \\
\hline $11 / 17 / 917: 02$ & Auto FIC & 316.4 & Good & \\
\hline $11 / 18 / 910: 00$ & Manual Tape & 316.00 & Transcribed & \\
\hline $11 / 18 / 917: 03$ & Auto FIC & 316.4 & Good & \\
\hline $11 / 19 / 910: 00$ & Manual Tape & 316.00 & Transcribed & \\
\hline $11 / 19 / 917: 03$ & Auto FIC & 316.4 & Good & \\
\hline $11 / 20 / 910: 00$ & Manual Tape & 316.00 & Transcribed & \\
\hline $11 / 20 / 917: 03$ & Auto FIC & 316.4 & Good & \\
\hline $11 / 21 / 910: 00$ & Manual Tape & 31.6 & Transcribed & \\
\hline $11 / 21 / 917: 02$ & Auto FIC & 316.4 & Good & \\
\hline $11 / 22 / 910: 00$ & Manual Tape & 316.00 & Transcribed & \\
\hline $11 / 22 / 917: 03$ & Auto FIC & 316.40 & Good & \\
\hline $11 / 23 / 910: 00$ & Manual Tape & 316.00 & Transcribed & \\
\hline $11 / 23 / 917: 02$ & Auto FIC & 316.40 & Good & \\
\hline $11 / 24 / 910: 00$ & Manual Tape & 315.75 & Transcribed & \\
\hline $11 / 24 / 917: 03$ & Auto FIC & 316.40 & Good & \\
\hline $11 / 25 / 910: 00$ & Manual Tape & 315.75 & Transcribed & \\
\hline $11 / 25 / 917: 03$ & Auto FIC & 316.4 & Good & \\
\hline $11 / 26 / 910: 00$ & Manual FIC & 316.40 & Transcribed & \\
\hline $11 / 26 / 910: 00$ & Manual Tape & 315.75 & Transcribed & \\
\hline 11/27/910:00 & Manual Tape & 315.75 & Transcribed & \\
\hline $11 / 27 / 917: 03$ & Auto FIC & 316.4 & Good & \\
\hline $11 / 28 / 910: 00$ & Manual Tape & 315.75 & Transcribed & \\
\hline $11 / 28 / 917: 03$ & Auto FIC & 316.40 & Good & \\
\hline $11 / 29 / 910: 00$ & Manual Tape & 315.75 & Transcribed & \\
\hline $11 / 29 / 917: 03$ & Auto FIC & 316.40 & Good & \\
\hline $11 / 30 / 910: 00$ & Manual Tape & 315.75 & Transcribed & \\
\hline $11 / 30 / 917: 03$ & Auto FIC & 316.3 & Good & \\
\hline $12 / 1 / 910: 00$ & Manual Tape & 315.75 & Transcribed & \\
\hline $12 / 1 / 917: 03$ & Auto FIC & 316.3 & Good & \\
\hline $12 / 2 / 910: 00$ & Manual Tape & 315.75 & Transcribed & \\
\hline $12 / 2 / 917: 03$ & Auto FIC & 316.30 & Good & \\
\hline $12 / 3 / 910: 00$ & Manual Tape & 315.75 & Transcribed & \\
\hline 12/3/91 7:03 & Auto FIC & 316.30 & Good & \\
\hline $12 / 4 / 910: 00$ & Manual Tape & 315.75 & Transcribed & \\
\hline $12 / 4 / 917: 03$ & Auto FIC & 316.30 & Good & \\
\hline
\end{tabular}

Data obtained from WHC Surveillance Analysis Computer System, (PC SACS) July 7, 1995. 


\begin{tabular}{|c|c|c|c|c|}
\hline \multicolumn{5}{|c|}{ 241-AP-108 } \\
\hline \multicolumn{5}{|c|}{ Surface Level Data } \\
\hline Date & Type of Measurement & Level (Inches) & Data Quality & Comments \\
\hline $12 / 5 / 910: 00$ & Manual Tape & $31.5: 75$ & Transcribed & \\
\hline $12 / 5 / 917: 03$ & Auto FIC & 316.3 & Good & \\
\hline 12/6/91 0:00 & Manual Tape & 315.50 & Transcribed & \\
\hline $12 / 6 / 917: 04$ & Auto FIC & 316.3 & Good & \\
\hline 12/7/910:00 & Manual Tape & 315.75 & Transcribed & \\
\hline $12 / 7 / 917: 03$ & Auto FIC & 316.20 & Good & \\
\hline $12 / 8 / 910: 00$ & Manual Tape & 315.75 & Transcribed & \\
\hline $12 / 8 / 917: 03$ & Auto FIC & 316.20 & Good & \\
\hline $12 / 9 / 910: 00$ & Manual Tape & 315.75 & Transcribed & \\
\hline 12/9/91 7:03 & Auto FIC & 316.20 & Good & \\
\hline $12 / 10 / 910: 00$ & Manual Tape & 315.75 & Transcribed & \\
\hline $12 / 10 / 917: 03$ & Auto FIC & 316.20 & Good & \\
\hline 12/11/91 0:00 & Manual Tape & 315.5 & Transcribed & \\
\hline $12 / 11 / 917: 02$ & Auto FIC & 316.2 & Good & \\
\hline $12 / 12 / 910: 00$ & Manual Tape & 315.5 & Transcribed & \\
\hline $12 / 12 / 917: 03$ & Auto FIC & 316.2 & Good & \\
\hline $12 / 13 / 910: 00$ & Manual Tape & 315.75 & Transcribed & \\
\hline $12 / 13 / 917: 05$ & Auto FIC & 316.2 & Good & \\
\hline 12/14/91 0:00 & Manual Tape & 317.00 & Transcribed & \\
\hline $12 / 14 / 91 \quad 7: 02$ & Auto FIC & 317.5 & Good & \\
\hline $12 / 15 / 910: 00$ & Manual Tape & 317.25 & Transcribed & \\
\hline $12 / 15 / 91 \quad 7: 02$ & Auto FIC & 317.5 & Good & \\
\hline $12 / 16 / 910: 00$ & Manual Tape & 317.00 & Transcribed & \\
\hline $12 / 16 / 917: 03$ & Auto FIC & 318 & Good & \\
\hline $12 / 17 / 910: 00$ & Manual Tape & 317 & Transcribed & \\
\hline $12 / 17 / 917: 06$ & Auto FIC & 317.80 & Good & \\
\hline $12 / 18 / 910: 00$ & Manual Tape & 317.00 & Transcribed & \\
\hline $12 / 18 / 917: 03$ & Auto FIC & 317.50 & Good & \\
\hline 12/19/91 0:00 & Manual Tape & 317.00 & Transcribed & \\
\hline $12 / 19 / 917: 03$ & Auto FIC & 317.7 & Good & \\
\hline $12 / 20 / 910: 00$ & Manual Tape & 317.50 & Transcribed & \\
\hline $12 / 20 / 917: 02$ & Auto FIC & 318.20 & Good & \\
\hline $12 / 21 / 910: 00$ & Manual Tape & 317.50 & Transcribed & \\
\hline $12 / 21 / 917: 03$ & Auto FIC & 320.40 & Good & \\
\hline $12 / 22 / 910: 00$ & Manual Tape & 317.50 & Transcribed & \\
\hline 12/22/91 7:05 & Auto FIC & 320.6 & Good & \\
\hline $12 / 23 / 910: 00$ & Manual Tape & 319.75 & Transcribed & \\
\hline $12 / 23 / 917: 06$ & Auto FIC & 321.2 & Good & \\
\hline 12/24/91 0:00 & Manual Tape & 319.75 & Transcribed & \\
\hline $12 / 24 / 917: 03$ & Auto FIC & 321 & Good & \\
\hline $12 / 25 / 910: 00$ & Manual Tape & 320.00 & Transcribed & \\
\hline $12 / 25 / 917: 03$ & Auto FIC & 321.7 & Good & \\
\hline $12 / 26 / 910: 00$ & Manual Tape & 320 & Transeribed & \\
\hline $12 / 26 / 917: 03$ & Auto FIC & 321.3 & Good & \\
\hline $12 / 27 / 910: 00$ & Manual Tape & 320.25 & Transcribed & \\
\hline $12 / 27 / 917: 03$ & Auto FIC & $321.20^{\circ}$ & Good & \\
\hline $12 / 28 / 91 \quad 0: 00$ & Manual Tape & 320.25 & Transcribed & \\
\hline $12 / 28 / 917: 02$ & Auto FIC & 321.7 & Good & \\
\hline
\end{tabular}

Data obtained from WHC Surveillance Analysis Computer System, (PC SACS) Juiy 7, 1995. 


\begin{tabular}{|c|c|c|c|c|}
\hline \multicolumn{5}{|c|}{ 241-AP-108 } \\
\hline \multicolumn{5}{|c|}{ Surface Level Data } \\
\hline Date & Type of Measurement & Leval (Inches) & Data Quality & Comments \\
\hline $12 / 29 / 910: 00$ & Manual Tape & 320.25 & Transcribed & \\
\hline $12 / 29 / 917: 03$ & Auto FIC & 321.6 & Good & \\
\hline 12/30/910:00 & Manual Tape & 320 & Transcribed & \\
\hline $12 / 30 / 917: 03$ & Auto FIC & 321.60 & Good & \\
\hline 12/31/91 0:00 & Manual Tape & 320.00 & Transcribed & \\
\hline 12/31/91 7:03 & Auto FIC & 321.4 & Good & \\
\hline $1 / 1 / 920: 00$ & Manual Tape & 320.75 & Transcribed & \\
\hline $1 / 1 / 927: 03$ & Auto FIC & 321.80 & Good & \\
\hline $1 / 2 / 920: 00$ & Manual Tape & 320.75 & Transcribed & \\
\hline 1/2/92 7:02 & Auto FIC & 322 & Good & \\
\hline $1 / 3 / 920: 00$ & Manual Tape & 320.75 & Transcribed & \\
\hline $1 / 3 / 927: 04$ & Auto FIC & 321.4 & Good & \\
\hline $1 / 4 / 920: 00$ & Manual Tape & 320.75 & Transcribed & \\
\hline 1/4/92 7:03 & Auto FIC & 322.00 & Good & \\
\hline $1 / 5 / 92 \quad 0: 00$ & Manual Tape & 320.75 & Transcribed & \\
\hline $1 / 5 / 927: 03$ & Auto FIC & 322.00 & Good & \\
\hline $1 / 6 / 920: 00$ & Manual Tape & 320.75 & Transcribed & \\
\hline $1 / 6 / 927: 03$ & Auto FIC & 321.60 & Good & \\
\hline $1 / 7 / 920: 00$ & Manual Tape & 322.75 & Transcribed & \\
\hline 1/7/92 7:04 & Auto FIC & 323.70 & Good & \\
\hline $1 / 8 / 920: 00$ & Manual Tape & 322.50 & Transcribed & \\
\hline $1 / 8 / 927: 03$ & Auto FIC & 323.90 & Good & \\
\hline 1/9/92 0:00 & Manual Tape & 323.5 & Transcribed & \\
\hline $1 / 9 / 927: 03$ & Auto FIC & 324.5 & Good & \\
\hline $1 / 10 / 920: 00$ & Manual Tape & 323.50 & Transcribed & \\
\hline 1/10/92 7:04 & Auto FIC & 325.4 & Good & \\
\hline $1 / 11 / 920: 00$ & Manual Tape & 323.5 & Transcribed & \\
\hline $1 / 11 / 927: 04$ & Auto FIC & 324.70 & Good & \\
\hline $1 / 12 / 920: 00$ & Manual FIC & 324.9 & Transcribed & \\
\hline $1 / 12 / 920: 00$ & Manual Tape & 323.50 & Transcribed & \\
\hline $1 / 13 / 920: 00$ & Manual FIC & 324.70 & Transcribed & \\
\hline $1 / 13 / 920: 00$ & Manual Tape & 323.5 & Transcribed & \\
\hline $1 / 14 / 920: 00$ & Manual FIC & 325.30 & Transcribed & \\
\hline $1 / 14 / 920: 00$ & Manual Tape & 323.50 & Transcribed & \\
\hline $1 / 15 / 920: 00$ & Manual Tape & 323.50 & Transcribed & \\
\hline $1 / 15 / 927: 03$ & Auto FIC & 325 & Good & \\
\hline $1 / 16 / 920: 00$ & Manual Tape & 323.50 & Transcribed & \\
\hline $1 / 16 / 927: 04$ & Auto FIC & 324.90 & Good & \\
\hline $1 / 17 / 920: 00$ & Manual Tape & 323.75 & Transçribed & \\
\hline $1 / 17 / 927: 04$ & Auto FIC & 324.6 & Good & \\
\hline $1 / 18 / 920: 00$ & Manual Tape & 323.5 & Transcribed & \\
\hline $1 / 18 / 927: 04$ & Auto FIC & 324.4 & Good & \\
\hline $1 / 19 / 920: 00$ & Manual Tape & 323.50 & Transcribed & \\
\hline $1 / 19 / 927: 03$ & Auto FIC & 324.60 & Good & \\
\hline $1 / 20 / 920: 00$ & Manual Tape & 323.50 & Transcribed & \\
\hline $1 / 20 / 927: 03$ & Auto FIC & 324.3 & Good & \\
\hline $1 / 21 / 920: 00$ & Manual Tape & 323.50 & Transcribed & \\
\hline $1 / 21 / 927: 03$ & Auto FIC & 324.20 & Good & \\
\hline
\end{tabular}

Data obtained from WHC Surveillance Analysis Computer System, (PC SACS) July 7, 1995. 


\begin{tabular}{|c|c|c|c|c|}
\hline \multicolumn{5}{|c|}{ 241-AP-108 } \\
\hline \multicolumn{5}{|c|}{ Surface Level Data } \\
\hline Date & Type of Measurement & Level (Inches) & Data Quality & Comments \\
\hline $1 / 22 / 920: 00$ & Manual Tape & 323.50 & Transcribed & \\
\hline $1 / 22 / 927: 06$ & Auto FIC & 325 & Good & \\
\hline $1 / 23 / 920: 00$ & Manual Tape & 323.50 & Transcribed & \\
\hline 1/23/92 7:03 & Auto FIC & 324.60 & Good & \\
\hline $1 / 24 / 920: 00$ & Manual Tape & $323.50^{\circ}$ & Transcribed & \\
\hline 1/24/92 7:02 & Auto FIC & 325.00 & Good & \\
\hline $1 / 25 / 920: 00$ & Manual Tape & 323.50 & Transcribed & \\
\hline $1 / 25 / 927: 03$ & Auto FIC & 324.5 & Good & \\
\hline $1 / 26 / 920: 00$ & Manual Tape & 323.50 & Transcribed & \\
\hline $1 / 26 / 926: 03$ & Auto FIC & 324.50 & Good & \\
\hline $1 / 27 / 920: 00$ & Manual Tape & 323.5 & Transcribed & \\
\hline $1 / 27 / 927: 02$ & Auto FIC & 324.7 & Good & \\
\hline $1 / 28 / 920: 00$ & Manual Tape & 323.5 & Transcribed & \\
\hline $1 / 28 / 927: 03$ & Auto FIC & 325.40 & Good & \\
\hline $1 / 29 / 920: 00$ & Manual Tape & 323.50 & Transcribed & \\
\hline $1 / 29 / 927: 03$ & Auto FIC & 325.90 & Good & \\
\hline $1 / 30 / 920: 00$ & Manual Tape & 323.5 & Transcribed & \\
\hline $1 / 30 / 927: 03$ & Auto FIC & 324.7 & Good & \\
\hline $1 / 31 / 920: 00$ & Manual Tape & 323.5 & Transcribed & \\
\hline $1 / 31 / 927: 03$ & Auto FIC & 324.80 & Good & \\
\hline $2 / 1 / 920.00$ & Manual Tape & 323.5 & Transcribed & \\
\hline 2/1/92 7:02 & Auto FIC & 324.8 & Good & \\
\hline $2 / 2 / 920: 00$ & Manual Tape & 323.50 & Transcribed & \\
\hline $2 / 2 / 927: 03$ & Auto FIC & 325.20 & Good & \\
\hline $2 / 3 / 920: 00$ & Manual Tape & 323.5 & Transcribed & \\
\hline 2/3/92 7:03 & Auto FIC & 324.30 & Good & \\
\hline $2 / 4 / 920: 00$ & Manual Tape & 323.5 & Transcribed & \\
\hline $2 / 4 / 927: 03$ & Auto FIC & 324.4 & Good & \\
\hline $2 / 5 / 920: 00$ & Manual Tape & 323.5 & Transcribed & \\
\hline 2/5/92 7:02 & Auto FIC & 325 & Good & \\
\hline $2 / 6 / 920: 00$ & Manual FIC & 324.30 & Transcribed & \\
\hline $2 / 6 / 920: 00$ & Manual Tape & 323.25 & Transcribed & \\
\hline $2 / 7 / 920: 00$ & Manual FIC & 324.30 & Transcribed & \\
\hline $2 / 7 / 920: 00$ & Manual Tape & 323.5 & Transcribed & \\
\hline 2/8/92 0:00 & Manual Tape & 323.5 & Transcribed & \\
\hline $2 / 8 / 927: 04$ & Auto FIC & 324.3 & Good & \\
\hline $2 / 9 / 920: 00$ & Manual Tape & 323.50 & Transcribed & \\
\hline $2 / 9 / 927: 03$ & Auto FIC & 324.3 & Good & \\
\hline $2 / 10 / 920: 00$ & Manual Tape & 323.50 & Transcribed & \\
\hline $2 / 10 / 927: 04$ & Auto FIC & 324.5 & Good & \\
\hline $2 / 11 / 920: 00$ & Manual Tape & 323.50 & Transcribed & \\
\hline 2/11/92 7:03 & Auto FIC & 324.60 & Good & \\
\hline $2 / 12 / 920: 00$ & Manual Tape & 323.5 & Transcribed & \\
\hline $2 / 12 / 927: 03$ & Auto FIC & 325 & Good & \\
\hline 2/13/92 0:00 & Manual Tape & 323.5 & Transcribed & \\
\hline $2 / 13 / 927: 03$ & Auto FIC & 324.8 & Good & \\
\hline 2/14/92 0:00 & Manual Tape & 323.50 & Transcribed & \\
\hline $2 / 14 / 927: 03$ & Auto FIC & 325.00 & Good & \\
\hline
\end{tabular}

Data obtained from WHC Surveillance Analysis Computer System, (PC SACS) July 7, 1995. 


\begin{tabular}{|c|c|c|c|c|}
\hline \multicolumn{5}{|c|}{ 241-AP-108 } \\
\hline & & Jrface Leval Dat & & \\
\hline Date & Type of Measurement & Level (Inches) & Data Quality & Comments \\
\hline $2 / 15 / 920: 00$ & Manual Tape & 323.5 & Transcribed & \\
\hline $2 / 15 / 927: 03$ & Auto FIC & 324.50 & Good & \\
\hline $2 / 16 / 920: 00$ & Manual Tape & 323.25 & Transcribed & \\
\hline $2 / 16 / 927: 03$ & Auto FIC & 324.60 & Good & \\
\hline $2 / 17 / 920: 00$ & Manual Tape & 323.5 & Transcribed & \\
\hline $2 / 17 / 927: 03$ & Auto FIC & 324.80 & Good & \\
\hline $2 / 18 / 920: 00$ & Manual Tape & 323.5 & Transcribed & \\
\hline $2 / 18 / 927: 03$ & Auto FIC & 324.30 & Good & \\
\hline $2 / 19 / 920: 00$ & Manual Tape & 323.50 & Transcribed & \\
\hline $2 / 19 / 927: 03$ & Auto FIC & 324.30 & Good & \\
\hline $2 / 20 / 920: 00$ & Manual Tape & 323.5 & Transcribed & \\
\hline $2 / 20 / 927: 03$ & Auto FIC & 324.40 & Good & \\
\hline $2 / 21 / 920: 00$ & Manual Tape & 323.5 & Transcribed & - \\
\hline $2 / 21 / 927: 03$ & Auto FIC & 324.5 & Good & \\
\hline $2 / 22 / 920: 00$ & Manual Tape & 323.5 & Transcribed & \\
\hline $2 / 22 / 927: 03$ & Auto FIC & 324.40 & Good & \\
\hline $2 / 23 / 920: 00$ & Manual Tape & 323.5 & Transcribed & \\
\hline $2 / 23 / 927: 03$ & Auto FIC & 324.6 & Good & \\
\hline $2 / 24 / 920: 00$ & Manual Tape & 323.50 & Transcribed & \\
\hline $2 / 24 / 927: 03$ & Auto FIC & 325 & Good & \\
\hline $2 / 25 / 920: 00$ & Manual Tape & 323.50 & Transcribed & \\
\hline $2 / 25 / 927: 04$ & Auto FIC & 325.00 & Good & \\
\hline $2 / 26 / 920: 00$ & Manual Tape & 323.5 & Transcribed & \\
\hline $2 / 26 / 927: 03$ & Auto FIC & 324.40 & Good & \\
\hline $2 / 27 / 920: 00$ & Manual Tape & 323.5 & Transcribed & \\
\hline $2 / 27 / 927: 04$ & Auto FIC & 324.70 & Good & \\
\hline 2/28/92 0:00 & Manual Tape & 323.5 & Transcribed & \\
\hline $2 / 28 / 927: 04$ & Auto FIC & 324.90 & Good & \\
\hline $2 / 29 / 920: 00$ & Manual Tape & 323.50 & Transcribed & \\
\hline $2 / 29 / 927: 03$ & Auto FIC & 324.40 & Good & \\
\hline $3 / 1 / 920: 00$ & Manual Tape & 323.5 & Transcribed & \\
\hline $3 / 1 / 927: 03$ & Auto FIC & 324.50 & Good & \\
\hline $3 / 2 / 920: 00$ & Manual Tape & 323.25 & Transcribed & \\
\hline $3 / 2 / 927: 03$ & Auto FIC & 324.60. & Good & \\
\hline $3 / 3 / 920: 00$ & Manual Tape & 323.5 & Transcribed & \\
\hline $3 / 3 / 927: 04$ & Auto FIC & 324.70 & Good & \\
\hline $3 / 4 / 920: 00$ & Manual Tape & 323.5 & Transcribed & \\
\hline $3 / 4 / 927: 04$ & Auto FIC & 324.70 & Good & \\
\hline $3 / 5 / 920: 00$ & Manual Tape & 323.5 & Transcribed & \\
\hline $3 / 5 / 927: 03$ & Auto FIC & 324.6 & Good & \\
\hline $3 / 6 / 920: 00$ & Manual Tape & 323.5 & Transcribed & \\
\hline $3 / 6 / 927: 03$ & Auto FIC & 324.60 & Good & \\
\hline $3 / 7 / 92$ 0:00 & Manual Tape & 323.5 & Transcribed & \\
\hline $3 / 7 / 927: 02$ & Auto FIC & 324.60 & Good & \\
\hline $3 / 8 / 920: 00$ & Manual Tape & 323.5 & Transcribed & \\
\hline 3/8/92 7:02 & Auto FIC & 324.50 & Good & \\
\hline $3 / 9 / 920: 00$ & Manual Tape & 323.50 & Transcribed & \\
\hline 3/9/92 7:02 & Auto FIC & 324.5 & Good & \\
\hline
\end{tabular}

Data obtained from WHC Surveillance Analysis Computer System, (PC SACS) July 7, 1995. 


\begin{tabular}{|c|c|c|c|c|}
\hline \multicolumn{5}{|c|}{ 241-AP.108 } \\
\hline \multicolumn{5}{|c|}{ Surface Level Data } \\
\hline Date & Type of Measurement & Level (Inches) & Data Quality & Comments \\
\hline $3 / 10 / 920: 00$ & Manual Tape & 323.50 & Transcribed & \\
\hline $3 / 10 / 927: 03$ & Auto FIC & 324.5 & Good & \\
\hline $3 / 11 / 920: 00$ & Manual Tape & 323.50 & Transcribed & \\
\hline $3 / 11 / 927: 03$ & Auto FIC & 324.6 & Good & \\
\hline $3 / 12 / 920: 00$ & Manual Tape & 323.25 & Transcribed & \\
\hline $3 / 12 / 927: 03$ & Auto FIC & 324.50 & Good & \\
\hline $3 / 13 / 920: 00$ & Manual Tape & 323.25 & Transcribed & \\
\hline $3 / 13 / 927: 03$ & Auto FIC & 324.5 & Good & \\
\hline $3 / 14 / 920: 00$ & Manual Tape & 323.25 & Transcribed & \\
\hline $3 / 14 / 927: 03$ & Auto FIC & 324.3 & Good & \\
\hline $3 / 15 / 920: 00$ & Manual Tape & 323.25 & Transcribed & \\
\hline 3/15/92 7:06 & Auto FIC & 324.30 & Good & \\
\hline $3 / 16 / 920: 00$ & Manual Tape & 323.25 & Transcribed & \\
\hline $3 / 16 / 927: 03$ & Auto FIC & 324.3 & Good & \\
\hline $3 / 17 / 920: 00$ & Manual Tape & 323.25 & Transcribed & \\
\hline $3 / 17 / 927: 03$ & Auto FIC & 324.3 & Good & \\
\hline $3 / 18 / 920: 00$ & Manual Tape & 323.25 & Transcribed & \\
\hline $3 / 18 / 927: 03$ & Auto FIC & 324.3 & Good & \\
\hline $3 / 19 / 920: 00$ & Manual Tape & 323.25 & Transcribed & \\
\hline $3 / 19 / 926: 02$ & Auto FIC & $324: 30$ & Good & \\
\hline $3 / 20 / 920: 00$ & Manual Tape & 323.25 & Transcribed & \\
\hline $3 / 20 / 927: 02$ & Auto FIC & 324.30 & Good & \\
\hline $3 / 21 / 920: 00$ & Manual Tape & 323.25 & Transcribed & \\
\hline $3 / 21 / 927: 03$ & Auto FIC & 324.30 & Good & \\
\hline $3 / 22 / 920: 00$ & Manual Tape & 323.25 & Transcribed & \\
\hline $3 / 22 / 927: 02$ & Auto FIC & 324.3 & Good & \\
\hline $3 / 23 / 920: 00$ & Manual Tape & 323.25 & Transcribed & \\
\hline $3 / 23 / 927: 02$ & Auto FIC & 324.3 & Good & \\
\hline 3/24/92 0:00 & Manual Tape & 323.25 & Transcribed & \\
\hline $3 / 24 / 927: 02$ & Auto FIC & 324.30 & Good & \\
\hline 3/25/92 0:00 & Manual Tape & 323.25 & Transcribed & \\
\hline $3 / 25 / 927: 03$ & Auto FIC & 324.30 & Good & \\
\hline $3 / 26 / 920: 00$ & Manual Tape & 323 & Transcribed & \\
\hline $3 / 26 / 927: 03$ & Auto FIC & 324.3 & Good & \\
\hline $3 / 27 / 920: 00$ & Manual Tape & 323 & Transcribed & \\
\hline $3 / 27 / 927: 02$ & Auto FIC & 324.30 & Good & \\
\hline $3 / 28 / 920: 00$ & Manual Tape & 323 & Transcribed & \\
\hline $3 / 28 / 927: 03$ & Auto FIC & 324.30 & Good & \\
\hline $3 / 29 / 920: 00$ & Manual Tape & 323.00 & Transcribed & \\
\hline $3 / 29 / 927: 03$ & Auto FIC & 324.30 & Good & \\
\hline 3/30/92 0:00 & Manual Tape & 323.00 & Transcribed & \\
\hline $3 / 30 / 927: 03$ & Auto FIC & 324.30 & Good & \\
\hline $3 / 31 / 920: 00$ & Manual Tape & 323.00 & Transcribed & \\
\hline 3/31/92 7:03 & Auto FIC & 324.30 & Good & \\
\hline 4/1/92 0:00 & Manual Tape & 323.25 & Transcribed & \\
\hline 4/1/92 7:03 & Auto FIC & 324.3 & Good & \\
\hline $4 / 2 / 920: 00$ & Manual Tape & 323 & Transcribed & \\
\hline 4/2/92 7:03 & Auto FIC & 324.30 & Good & \\
\hline
\end{tabular}

Data obtained from WHC Surveillance Analysis Computer System, (PC SACS) July 7, 1995. 


\begin{tabular}{|c|c|c|c|c|}
\hline \multicolumn{5}{|c|}{ 241-AP-108 } \\
\hline \multicolumn{5}{|c|}{ Surface Level Data } \\
\hline Date & Type of Measurement & Level (Inches) & Data Quality & Comments \\
\hline $4 / 3 / 920: 00$ & Manual Tape & 323 & Transcribed & \\
\hline 4/3/92 7:03 & Auto FIC & 324.30 & Good & \\
\hline 4/4/92 0:00 & Manual Tape & 323 & Transcribed & \\
\hline 4/4/92 7:03 & Auto FIC & 324.2 & Good & \\
\hline 4/5/92 0:00 & Manual Tape & 323.25 & Transcribed & \\
\hline 4/5/92 7:03 & Auto FIC & 324.20 & Good & \\
\hline $4 / 6 / 920: 00$ & Manual Tape & 323 & Transeribed & \\
\hline 4/6/92 7:03 & Auto FIC & 324.20 & Good & \\
\hline $4 / 7 / 920: 00$ & Manual Tape & 324.75 & Transcribed & \\
\hline 4/7/92 7:03 & Auto FIC & 325.60 & Good & \\
\hline 4/8/92 0:00 & Manual Tape & 324.75 & Transcribed & \\
\hline $4 / 8 / 925: 02$ & Auto FIC & 325.70 & Good & \\
\hline $4 / 9 / 920: 00$ & Manual Tape & 324.75 & Transcribed & \\
\hline 4/9/92 7:03 & Auto FIC & 325.70 & Good & \\
\hline $4 / 10 / 920: 00$ & Manual Tape & 324.75 & Transcribed & \\
\hline 4/10/92 7:03 & Auto FIC & 325.70 & Good & \\
\hline 4/11/92 0:00 & Manual Tape & 324.75 & Transcribed & \\
\hline 4/11/92 7:03 & Auto FIC & 325.80 & Good & \\
\hline 4/12/92 0:00 & Manual Tape & 324.75 & Transcribed & \\
\hline 4/12/92 5:03 & Auto FIC & 325.80 & Good & \\
\hline 4/13/92 0:00 & Manual Tape & 324.75 & Transcribed & \\
\hline $4 / 13 / 927: 03$ & Auto FIC & 325.90 & Good & \\
\hline $4 / 14 / 920: 00$ & Manual Tape & 324.75 & Transcribed & \\
\hline 4/14/92 7:03 & Auto FIC & 325.90 & Good & \\
\hline $4 / 15 / 920: 00$ & Manual Tape & 324.75 & Transcribed & \\
\hline 4/15/92 7:02 & Auto FIC & 325.90 & Good & \\
\hline $4 / 16 / 920: 00$ & Manual Tape & 324.75 & Transcribed & \\
\hline $4 / 16 / 927: 03$ & Auto FIC & 325.90 & Good & \\
\hline $4 / 17 / 920: 00$ & Manual Tape & 324.75 & Transcribed & \\
\hline 4/17/92 7:03 & Auto FIC & 325.90 & Good & \\
\hline $4 / 18 / 920: 00$ & Manual Tape & 324.75 & Transcribed & \\
\hline $4 / 18 / 927: 02$ & Auto FIC & 325.9 & Good & \\
\hline $4 / 19 / 920: 00$ & Manual Tape & 324.75 & Transcribed & \\
\hline $4 / 19 / 927: 02$ & Auto FIC & 325.9 & Good & \\
\hline $4 / 20 / 920: 00$ & Manual Tape & 324.75 & Transcribed & \\
\hline $4 / 20 / 927: 03$ & Auto FIC & 325.9 & Good & \\
\hline $4 / 21 / 920: 00$ & Manual Tape & 324.75 & Transcribed & \\
\hline $4 / 21 / 927: 03$ & Auto FIC & 325.90 & Good & \\
\hline $4 / 22 / 920: 00$ & Manual Tape & 324.75 & Transcribed & \\
\hline $4 / 22 / 927: 02$ & Auto FIC & 327.90 & Good & \\
\hline 4/23/92 0:00 & Manual Tape & 324.25 & Transcribed & \\
\hline $4 / 23 / 927: 03$ & Auto FIC & 328 & Good & \\
\hline 4/24/92 0:00 & Manual Tape & 327 & Transcribed & \\
\hline $4 / 24 / 927: 03$ & Auto FIC & 328.00 & Good & \\
\hline $4 / 25 / 920: 00$ & Manual Tape & 328.00 & Transcribed & \\
\hline $4 / 25 / 927: 04$ & Auto FIC & 328.9 & Good & \\
\hline $4 / 26 / 920: 00$ & Manual Tape & 328 & Transcribed & \\
\hline $4 / 26 / 927: 03$ & Auto FIC & 329.40 & Good & \\
\hline
\end{tabular}

Data obtained from WHC Surveillance Analysis Computer System, (PC SACS) July 7, 1995. 


\begin{tabular}{|c|c|c|c|c|}
\hline \multicolumn{5}{|c|}{ 241-AP-108 } \\
\hline \multicolumn{5}{|c|}{ Surface Level Data } \\
\hline Date & Type of Measurement & Level (Inches) & Data Quality & Comments \\
\hline $4 / 27 / 920: 00$ & Manual Tape & .328 & Transcribed & \\
\hline $4 / 27 / 927: 04$ & Auto FIC & 329.40 & Good & \\
\hline $4 / 28 / 920: 00$ & Manual Tape & 327.25 & Transcribed & \\
\hline 4/28/92 7:03 & Auto FIC & 329.60 & Good & \\
\hline $4 / 29 / 920: 00$ & Manual Tape & 328 & Transcribed & \\
\hline $4 / 29 / 927: 03$ & Auto FIC & 329.30 & Good & \\
\hline $4 / 30 / 920: 00$ & Manual Tape & 328 & Transcribed & \\
\hline $4 / 30 / 927: 03$ & Auto FIC & 329.40 & Good & \\
\hline $5 / 1 / 920: 00$ & Manual Tape & 328.00 & Transcribed & \\
\hline 5/3/92 7:03 & Auto FIC & 329.7 & Good & \\
\hline $5 / 2 / 920: 00$ & Manual Tape & 328.00 & Transcribed & \\
\hline $5 / 2 / 922: 03$ & Auto FIC & 329.30 & Good & \\
\hline $5 / 3 / 92 \quad 0: 00$ & Manual Tape & 328 & Transcribed & \\
\hline $5 / 3 / 927: 03$ & Auto FIC & 329.4 & Good & \\
\hline $5 / 4 / 920: 00$ & Manual Tape & 326.25 & Transcribed & \\
\hline $5 / 4 / 927: 03$ & Auto FIC & 329.7 & Good & \\
\hline $5 / 5 / 920: 00$ & Manual Tape & 328.00 & Transcribed & \\
\hline $5 / 5 / 927: 04$ & Auto FIC & 329.70 & Good & \\
\hline $5 / 6 / 920: 00$ & Manual Tape & 328.00 & Transcribed & \\
\hline 5/6/92 7:03 & Auto FIC & 329.90 & Good & \\
\hline $5 / 7 / 920: 00$ & Manual Tape & 328 & Transćribed & \\
\hline $5 / 7 / 927: 04$ & Auto FIC & 329.6 & Good & \\
\hline $5 / 8 / 920: 00$ & Manual Tape & 328.00 & Transcribed & \\
\hline $5 / 8 / 927: 03$ & Auto FIC & 328.60 & Good & \\
\hline $5 / 9 / 920: 00$ & Manual FIC & 328.8 & Transcribed & \\
\hline $5 / 9 / 920: 00$ & Manual Tape & 328.25 & Transcribed & \\
\hline $5 / 10 / 920: 00$ & Manual Tape & 328.25 & Transcribed & \\
\hline $5 / 10 / 926: 03$ & Auto FIC & 328.9 & Good & \\
\hline $5 / 11 / 920: 00$ & Manual FIC & 329.10 & Transcribed & \\
\hline $5 / 11 / 920: 00$ & Manual Tape & 328.25 & Transcribed & \\
\hline $5 / 12 / 920: 00$ & Manual Tape & 328.00 & Transcribed & \\
\hline $5 / 12 / 927: 03$ & Auto FIC & 329.1 & Good & \\
\hline $5 / 13 / 920: 00$ & Manual Tape & 328 & Transcribed & \\
\hline $5 / 13 / 927: 03$ & Auto FIC & 329.10 & Good & \\
\hline $5 / 14 / 920: 00$ & Manual Tape & 328.00 & Transcribed & \\
\hline $5 / 14 / 927: 03$ & Auto FIC & 329.2 & Good & \\
\hline $5 / 15 / 920: 00$ & Manual Tape & 328.00 & Transcribed & \\
\hline $5 / 15 / 927: 03$ & Auto FIC & 329.30 & Good & \\
\hline $5 / 16 / 920: 00$ & Manual Tape & 328.00 & Transcribed & \\
\hline 5/16/92 7:03 & Auto FIC & 329.4 & Good & \\
\hline $5 / 17 / 920: 00$ & Manual Tape & 328.00 & Transcribed & \\
\hline $5 / 17 / 927: 02$ & Auto FIC & 328.6 & Good & \\
\hline $5 / 18 / 920: 00$ & Manual Tape & 328 & Transcribed & \\
\hline $5 / 18 / 927: 03$ & Auto FIC & 328.90 & Good & \\
\hline $5 / 19 / 920: 00$ & Manual Tape & 328.00 & Transcribed & \\
\hline $5 / 19 / 927: 03$ & Auto FIC & 328.6 & Good & \\
\hline $5 / 20 / 920: 00$ & Manual Tape & 328 & Transcribed & \\
\hline $5 / 20 / 927: 03$ & Auto FIC & 328.8 & Good & \\
\hline
\end{tabular}

Data obtained from WHC Surveillance Analysis Computer System, (PC SACS) July 7, 1995. 


\begin{tabular}{|c|c|c|c|c|}
\hline \multicolumn{5}{|c|}{ 241-AP-108 } \\
\hline \multicolumn{5}{|c|}{ Surface Level Data } \\
\hline Date & Type of Measurement & Level (Inches) & Data Quality & Comments \\
\hline $5 / 21 / 920: 00$ & Manual Tape & 328 & Transcribed & \\
\hline $5 / 21 / 927: 03$ & Auto FIC & 328.9 & - Good & \\
\hline $5 / 22 / 920: 00$ & Manual Tape & 328 & Transcribed & \\
\hline $5 / 22 / 927: 02$ & Auto FIC & 328.6 & Good & \\
\hline $5 / 23 / 920: 00$ & Manual Tape & 328 & Transcribed & \\
\hline $5 / 23 / 927: 03$ & Auto FIC & 328.90 & Good & \\
\hline $5 / 24 / 920: 00$ & Manual Tape & 328 & Transcribed & \\
\hline $5 / 24 / 927: 03$ & Auto FIC & 329.00 & Good & \\
\hline $5 / 25 / 920: 00$ & Manual Tape & 328.00 & Transcribed & \\
\hline $5 / 25 / 926: 02$ & Auto FIC & 329 & Good & \\
\hline $5 / 26 / 920: 00$ & Manual FIC & 329.1 & Transcribed & \\
\hline $5 / 26 / 920: 00$ & Manual Tape & 328 & Transcribed & \\
\hline $5 / 27 / 920: 00$ & Manual Tape & 328 & Transcribed & \\
\hline $5 / 27 / 927: 03$ & Auto FIC & 329.10 & Good & \\
\hline $5 / 28 / 920: 00$ & Manual Tape & 328.00 & - Transcribed & \\
\hline $5 / 28 / 927: 02$ & Auto FIC & 329.20 & Good & \\
\hline $5 / 29 / 920: 00$ & Manual Tape & 328 & Transcribed & \\
\hline $5 / 29 / 927: 02$ & Auto FIC & 328.80 & Good & \\
\hline $5 / 30 / 920: 00$ & Manual Tape & 328.00 & Transcribed & \\
\hline $5 / 30 / 927: 03$ & Auto FIC & 329.00 & Good & \\
\hline $5 / 31 / 920: 00$ & Manual Tape & 328 & Transcribed & \\
\hline $5 / 31 / 927: 03$ & Auto FIC & 328.8 & Good & \\
\hline $6 / 1 / 920: 00$ & Manual Tape & 328.00 & Transcribed & \\
\hline $6 / 1 / 927: 03$ & Auto FIC & 328.9 & Good & \\
\hline $6 / 2 / 92 \quad 0: 00$ & Manual Tape & 328.00 & Transcribed & \\
\hline $6 / 2 / 927: 03$ & Auto FIC & $328: 60$ & Good & \\
\hline $6 / 3 / 920: 00$ & Manual Tape & 328 & Transcribed & \\
\hline $6 / 3 / 926: 02$ & Auto FIC & 328.8 & Good & \\
\hline $6 / 4 / 920: 00$ & Manual Tape & 328.00 & Transcribed & \\
\hline $6 / 4 / 927: 03$ & Auto FIC & 328.6 & Good & \\
\hline $6 / 5 / 920: 00$ & Manual Tape & 328.00 & Transcribed & \\
\hline $6 / 5 / 927: 02$ & Auto FIC & 328.80 & Good & \\
\hline $6 / 6 / 920: 00$ & Manual Tape & 328 & Transcribed & \\
\hline $6 / 6 / 927: 03$ & Auto FIC & 328.8 & Good & \\
\hline $6 / 7 / 920: 00$ & Manual Tape & 328.00 & Transcribed & \\
\hline $6 / 7 / 927: 03$ & Auto FIC & 328.9 & Good & \\
\hline $6 / 8 / 920: 00$ & Manual Tape & 328 & Transcribed & \\
\hline $6 / 8 / 927: 03$ & Auto FIC & 328.6 & Good & \\
\hline $6 / 9 / 920: 00$ & Manual Tape & 328.25 & Transcribed & \\
\hline $6 / 9 / 927: 03$ & Auto FIC & 328.7 & Good & \\
\hline $6 / 10 / 920: 00$ & Manual Tape & 328 & Transcribed & \\
\hline $6 / 10 / 927: 03$ & Auto FIC & 328.80 & Good & \\
\hline $6 / 11 / 920: 00$ & Manual Tape & 328 & Transcribed & \\
\hline $6 / 11 / 927: 03$ & Auto FIC & 328.9 & Good & \\
\hline $6 / 12 / 920: 00$ & Manual Tape & 327.25 & Transcribed & \\
\hline $6 / 12 / 927: 05$ & Auto FIC & 328.8 & Good & \\
\hline $6 / 13 / 920: 00$ & Manual Tape & 327.25 & Transcribed & \\
\hline $6 / 13 / 927: 05$ & Auto FIC & 328.9 & Good & \\
\hline
\end{tabular}

Data obtained from WHC Surveillance Analysis Computer System, (PC SACS) July 7, 1995. 


\begin{tabular}{|c|c|c|c|c|}
\hline \multicolumn{5}{|c|}{ 241-AP-108 } \\
\hline \multicolumn{5}{|c|}{ Surface Level Data } \\
\hline Date & Type of Measurement & Level (Inches) & Data Quality & Comments \\
\hline $6 / 14 / 920: 00$ & Manual FIC & 328.9 & Transcribed & \\
\hline 6/14/92 0:00 & Manual Tape & 328 & Transcribed & \\
\hline $6 / 15 / 920: 00$ & Manual Tape & 328 & Transcribed & \\
\hline $6 / 15 / 927: 03$ & Auto FIC & 328.80 & Good & \\
\hline $6 / 16 / 920: 00$ & Manual Tape & 328.00 & Transcribed & \\
\hline $6 / 16 / 926: 04$ & Auto FIC & 328.8 & Good & \\
\hline $6 / 17 / 920: 00$ & Manual Tape & 327.75 & Transcribed & \\
\hline $6 / 17 / 927: 03$ & Auto FIC & 328.80 & Good & \\
\hline $6 / 18 / 920: 00$ & Manual Tape & 328 & Transcribed & \\
\hline $6 / 18 / 927: 03$ & Auto FIC & 328.7 & Good & \\
\hline $6 / 19 / 920: 00$ & Manual Tape & 328.00 & Transcribed & \\
\hline $6 / 19 / 92 \quad 7: 02$ & Auto FIC & 328.7 & Good & \\
\hline $6 / 20 / 920: 00$ & Manual Tape & 328.00 & Transcribed & \\
\hline $6 / 20 / 927: 02$ & Auto FIC & 328.80 & Good & \\
\hline $6 / 21 / 920: 00$ & Manual Tape & 328.00 & Transcribed & \\
\hline $6 / 21 / 927: 03$ & Auto FIC & 328.7 & Good & \\
\hline $6 / 22 / 920: 00$ & Manual Tape & 328.00 & Transcribed & \\
\hline $6 / 22 / 92 \quad 7: 03$ & Auto FIC & 328.7 & Good & \\
\hline $6 / 23 / 920: 00$ & Manual Tape & 328.00 & Transcribed & \\
\hline $6 / 23 / 927: 03$ & Auto FIC & 328.70 & Good & \\
\hline $6 / 24 / 920: 00$ & Manual Tape & 328.00 & Transcribed & \\
\hline $6 / 24 / 927: 03$ & Auto FIC & 328.70 & Good & \\
\hline $6 / 25 / 920: 00$ & Manual Tape & 328 & Transcribed & \\
\hline $6 / 25 / 927: 03$ & Auto FIC & 328.8 & Good & \\
\hline $6 / 26 / 920: 00$ & Manual Tape & 328.00 & Transcribed & $=$ \\
\hline $6 / 26 / 927: 02$ & Auto FIC & 328.6 & Good & \\
\hline $6 / 27 / 920: 00$ & Manual Tape & 328.00 & Transcribed & \\
\hline $6 / 27 / 927: 03$ & Auto FIC & 328.60 & Good & \\
\hline $6 / 28 / 920: 00$ & Manual Tape & 328 & Transcribed & \\
\hline $6 / 28 / 927: 06$ & Auto FIC & 328.3 & Good & \\
\hline $6 / 29 / 92 \quad 0: 00$ & Manual Tape & 328,00 & Transcribed & \\
\hline $6 / 29 / 927: 06$ & Auto FIC & 328.50 & Good & \\
\hline $6 / 30 / 920: 00$ & Manual Tape & 328.00 & Transcribed & \\
\hline $6 / 30 / 927: 06$ & Auto FIC & 328.50 & Good & \\
\hline $7 / 1 / 920: 00$ & Manual FIC & 328.50 & Transcribed & \\
\hline $7 / 1 / 920: 00$ & Manual Tape & 328.00 & Transcribed & \\
\hline $7 / 2 / 920: 00$ & Manual FIC & 328.5 & Transcribed & \\
\hline $7 / 2 / 920: 00$ & Manual Tape & 328 & Transcribed & \\
\hline $7 / 3 / 920: 00$ & Manual Tape & 328.00 & Transcribed & \\
\hline $7 / 3 / 927: 06$ & Auto FIC & 328.5 & Good & \\
\hline $7 / 4 / 920: 00$ & Manual Tape & 328.00 & Transcribed & \\
\hline $7 / 4 / 927: 06$ & Auto FIC & 328.5 & Good & \\
\hline $7 / 5 / 920: 00$ & Manual Tape & 328.00 & Transcribed & \\
\hline $7 / 5 / 927: 06$ & Auto FIC & 328.4 & Good & \\
\hline $7 / 6 / 920: 00$ & Manual Tape & 328.00 & Transcribed & \\
\hline $7 / 6 / 927: 06$ & Auto FIC & 328.4 & Good & \\
\hline $7 / 7 / 920: 00$ & Manual Tape & 328 & Transcribed & \\
\hline $7 / 7 / 927: 06$ & Auto FIC & 328.40 & Good & \\
\hline
\end{tabular}

Data obtained from WHC Surveillance Analysis Computer System, (PC SACS) July 7, 1995. 


\begin{tabular}{|c|c|c|c|c|}
\hline \multicolumn{5}{|c|}{ 241-AP-108 } \\
\hline \multicolumn{5}{|c|}{ Surface Level Data } \\
\hline Date & Type of Measurement & Level (Inches) & Date Quality & Comments \\
\hline $7 / 8 / 920: 00$ & Manual Tape & 328.00 & Transcribed & \\
\hline $7 / 8 / 927: 06$ & Auto FIC & $328: 40$ & Good & \\
\hline $7 / 9 / 920: 00$ & Manual Tape & 328.00 & Transcribed & \\
\hline $7 / 9 / 927: 06$ & Auto FIC & 328.4 & Good & \\
\hline $7 / 10 / 920: 00$ & Manual Tape & 328.00 & Transcribed & \\
\hline $7 / 10 / 926: 06$ & Auto FIC & 328.40 & Good & \\
\hline $7 / 11 / 920: 00$ & Manual Tape & 328.00 & Transcribed & \\
\hline $7 / 11 / 925: 06$ & Auto FIC & 328.40 & Good & \\
\hline $7 / 12 / 920: 00$ & Manual Tape & 328 & Transcribed & \\
\hline $7 / 12 / 927: 06$ & Auto FIC & 328.4 & Good & \\
\hline $7 / 13 / 920: 00$ & Manual Tape & 328.00 & Transcribed & \\
\hline $7 / 13 / 925: 05$ & Auto FIC & 328.4 & Good & \\
\hline $7 / 14 / 920: 00$ & Manual Tape & $328.00^{\circ}$ & Transcribed & \\
\hline $7 / 14 / 924: 06$ & Auto FIC & 328.4 & Good & \\
\hline $7 / 15 / 920: 00$ & Manual Tape ' & 328.00 & Transcribed & \\
\hline $7 / 15 / 925: 06$ & Auto FIC & 328.4 & Good & \\
\hline $7 / 16 / 920: 00$ & Manual Tape & 328.00 & Transcribed & \\
\hline $7 / 16 / 927: 06$ & Auto FIC & 328.4 & Good & \\
\hline $7 / 17 / 920: 00$ & Manual Tape & 328.00 & Transcribed & \\
\hline 7/17/92 7:06 & Auto FIC & 0.00 & Suspect & Data not included in graph \\
\hline $7 / 18 / 920: 00$ & Manual Tape & 328.00 & Transcribed & \\
\hline $7 / 18 / 927: 06$ & Auto FIC & 0 & Suspect & Data not included in graph \\
\hline $7 / 19 / 920: 00$ & Manual Tape & 328 & Transcribed & \\
\hline $7 / 19 / 927: 06$ & Auto FIC & 328.40 & Good & $\cdot$ \\
\hline $7 / 20 / 920: 00$ & Manual Tape & 328.00 & Transcribed & \\
\hline $7 / 20 / 927: 06$ & Auto FIC & 328.4 & Good & \\
\hline $7 / 21 / 920: 00$ & Manual Tape & 327.25 & Transeribed & \\
\hline $7 / 21 / 926: 05$ & Auto FIC & 328.4 & Good & \\
\hline $7 / 22 / 920: 00$ & Manual Tape & 327.25 & Transcribed & \\
\hline 7/22/92 7:06 & Auto FIC & 328.4 & Good & \\
\hline $7 / 23 / 920: 00$ & Manual FIC & 328.40 & Transcribed & \\
\hline $7 / 23 / 920: 00$ & Manual Tape ' & 328.00 & Transcribed & \\
\hline $7 / 24 / 920: 00$ & Manual Tape & 328.00 & Transcribed & \\
\hline $7 / 24 / 927: 05$ & Auto FIC & 0.00 & Suspect & Data not included in graph \\
\hline $7 / 25 / 920: 00$ & Manual Tape & 328.00 & Transcribed & \\
\hline $7 / 25 / 927: 06$ & Auto FIC & 0.00 & Suspect & Data not included in graph \\
\hline $7 / 26 / 920: 00$ & Manual Tape &.$\quad 328.00$ & Transcribed & \\
\hline $7 / 26 / 921: 06$ & Auto FIC & 328.30 & Good & \\
\hline $7 / 27 / 920: 00$ & Manual Tape & 328 & Transcribed & \\
\hline $7 / 27 / 926: 05$ & Auto FIC & 328 & Good & \\
\hline $7 / 28 / 920: 00$ & Manual Tape & 328 & Transcribed & \\
\hline $7 / 28 / 926: 06$ & Auto FIC & 328.40 & Good & \\
\hline $7 / 29 / 920: 00$ & Manual Tape & 328.00 & Transcribed & \\
\hline $7 / 29 / 926: 06$ & Auto FIC & 328.4 & Good & \\
\hline $7 / 30 / 920: 00$ & Manual Tape & 328 & Transcribed & \\
\hline $7 / 30 / 926: 06$ & Auto FIC & 328.4 & Good & \\
\hline $7 / 31 / 920: 00$ & Manual Tape & 328.00 & Transcribed & \\
\hline 7/31/92 6:05 & Auto FIC & 328.4 & Good & \\
\hline
\end{tabular}

Data obtained from WHC Surveillance Analysis Computer System, (PC SACS) July 7, 1995. 


\begin{tabular}{|c|c|c|c|c|}
\hline \multicolumn{5}{|c|}{ 241-AP-108 } \\
\hline \multicolumn{5}{|c|}{ Surface Level Data } \\
\hline Date & Type of Measurement & Level (Inches) & Data Quality & Comments \\
\hline $8 / 1 / 920: 00$ & Manual Tape & 328 & Transcribed & \\
\hline $8 / 1 / 926: 06$ & Auto FIC & 328.4 & Good & \\
\hline $8 / 2 / 920: 00$ & Manual Tape & 328 & Transcribed & \\
\hline $8 / 2 / 923: 06$ & Auto FIC & 328.40 & Good & \\
\hline $8 / 3 / 920: 00$ & Manual Tape & 328.00 & Transcribed & \\
\hline $8 / 3 / 927: 06$ & Auto FIC & 0 & Suspect & Data not included in graph \\
\hline $8 / 4 / 920: 00$ & Manual Tape & 328 & Transcribed & \\
\hline $8 / 4 / 927: 06$ & Auto FIC & 0 & Suspect & Data not included in graph \\
\hline $8 / 5 / 920: 00$ & Manual Tape & 328.00 & Transcribed & \\
\hline $8 / 5 / 927: 05$ & Auto FIC & 0.00 & Suspect & Data not included in graph \\
\hline $8 / 6 / 920: 00$ & Manual Tape & 328 & Transcribed & \\
\hline $8 / 6 / 927: 05$ & Auto FIC & 0 & Suspect & Data not included in graph \\
\hline $8 / 7 / 920: 00$ & Manual Tape & 328 & Transcribed & \\
\hline 8/7/92 7:05 & Auto FIC & 0.00 & Suspect & Data not included in graph \\
\hline $8 / 8 / 920: 00$ & Manual Tape & $\mathbf{3 2 7 . 7 5}$ & Transcribed & \\
\hline 8/8/92 7:06 & Auto FIC & 0 & Suspect & Data not included in graph \\
\hline $8 / 9 / 920: 00$ & Manual Tape & 327.75 & Transcribed & \\
\hline $8 / 9 / 927: 06$ & Auto FIC & 0 & Suspect & Data not included in graph \\
\hline $8 / 10 / 920: 00$ & Manual Tape & 328.00 & Transcribed & \\
\hline 8/10/92 7:05 & Auto FIC & 0 & Suspect & Data not included in graph \\
\hline $8 / 11 / 920: 00$ & Manual Tape & 328.00 & Transcribed & \\
\hline $8 / 11 / 927: 06$ & Auto FIC & 0 & Suspect & Data not included in graph \\
\hline $8 / 12 / 920: 00$ & Manual Tape & 328.00 & Transcribed & \\
\hline 8/12/92 3:06 & Auto FIC & 328.00 & Good & \\
\hline $8 / 13 / 920: 00$ & Manual Tape & 327.75 & Transcribed & \\
\hline $8 / 13 / 923: 06$ & Auto FIC & 328.00 & - Good & \\
\hline $8 / 14 / 920: 00$ & Manual Tape & 327.75 & Transcribed & \\
\hline $8 / 14 / 927: 06$ & Auto FIC & 0 & Suspect & Data not included in graph \\
\hline $8 / 15 / 920: 00$ & Manual Tape & 327.75 & Transcribed & \\
\hline 8/15/92 5:06 & Auto FIC & 328 & Good & \\
\hline 8/16/92 0:00 & Manual Tape & 327.75 & Transcribed & \\
\hline 8/16/92 7:06 & Auto FIC & 0 & Suspect & Data not included in graph \\
\hline $8 / 17 / 920: 00$ & Manual Tape & 327.75 & Transcribed & \\
\hline $8 / 17 / 927: 06$ & Auto FIC & 0.00 & Suspect & Data not included in graph \\
\hline $8 / 18 / 920: 00$ & Manual Tape & 327.75 . & Transcribed & \\
\hline $8 / 18 / 922: 06$ & Auto FIC & 328.00 & Good & \\
\hline $8 / 19 / 920: 00$ & Manual Tape & 327.75 & Transcribed & \\
\hline $8 / 19 / 927: 06$ & Auto FIC & 0.00 & Suspect & Data not included in graph \\
\hline $8 / 20 / 920: 00$ & Manual Tape & 327.75 & Transcribed & \\
\hline $8 / 20 / 927: 06$ & Auto FIC & 0.00 & Suspect & Data not included in graph \\
\hline $8 / 21 / 920: 00$ & Manual Tape & 327.75 & Transcribed & \\
\hline $8 / 21 / 927: 05$ & Auto FIC & 0 & Suspect & Data not included in graph \\
\hline $8 / 22 / 920: 00$ & Manual Tape & 327.75 & Transcribed & \\
\hline $8 / 22 / 927: 04$ & Auto FIC & 0 & Suspect & Data not included in graph \\
\hline $8 / 23 / 920: 00$ & Manual Tape & 327.75 & Transcribed & \\
\hline $8 / 23 / 927: 04$ & Auto FIC & 0.00 & Suspect & Data not included in graph \\
\hline $8 / 24 / 920: 00$ & Manual Tape & 327.75 & Transcribed & \\
\hline $8 / 24 / 927: 04$ & Auto FIC & 0 & Suspect & Data not included in graph \\
\hline
\end{tabular}

Data obtained from WHC Surveillance Analysis Computer System, (PC SACS) July 7, 1995. 


\begin{tabular}{|c|c|c|c|c|}
\hline \multicolumn{5}{|c|}{ 241-AP-108 } \\
\hline \multicolumn{5}{|c|}{ Surface Level Data } \\
\hline Date & Type of Measurement & Level (Inches) & Data Quality & Comments \\
\hline $8 / 25 / 920: 00$ & Manual Tape & 327.75 & Transcribed & \\
\hline $8 / 25 / 927: 04$ & Auto FIC & 0 & Suspect & Data not included in graph \\
\hline $8 / 26 / 920: 00$ & Manual Tape & 327.75 & Transcribed & \\
\hline $8 / 26 / 927: 03$ & Auto FIC & 0.00 & Suspect & Data not included in graph \\
\hline $8 / 27 / 920: 00$ & Manual Tape & 327.75 & Transcribed & \\
\hline $8 / 27 / 927: 04$ & Auto FIC & 0.00 & Suspect & Data not included in graph \\
\hline $8 / 28 / 920: 00$ & Manual Tape & 327.75 & Transcribed & \\
\hline $8 / 28 / 927: 02$ & Auto FIC & 0.00 & Suspect & Data not included in graph \\
\hline $8 / 29 / 920: 00$ & Manual Tape & 327.75 & Transcribed & \\
\hline $8 / 29 / 927: 02$ & Auto FIC & 0 & Suspect & Data not inciuded in graph \\
\hline $8 / 30 / 920: 00$ & Manual Tape & 327.75 & Transcribed & \\
\hline $8 / 30 / 927: 02$ & Auto FIC & 0 & Suspect & Data not included in graph \\
\hline $8 / 31 / 920: 00$ & Manual Tape & 327.75 & Transcribed & \\
\hline $8 / 31 / 927: 02$ & Auto FIC & 0 & Suspect & Data not included in graph \\
\hline 9/1/92 0:00 & Manual Tape & 327.75 & Transcribed & \\
\hline 9/1/92 7:02 & Auto FIC & 0 & Suspect & Data not included in graph \\
\hline 9/2/92 0:00 & Manual Tape & 328 & Transcribed & \\
\hline $9 / 2 / 927: 02$ & Auto FIC & 0.00 & Suspect & Data not included in graph \\
\hline $9 / 3 / 920: 00$ & Manual Tape & 327.75 & Transcribed & \\
\hline $9 / 3 / 927: 04$ & Auto FIC & 0.00 & Suspect & Data not included in graph \\
\hline $9 / 4 / 920: 00$ & Manual Tape & 327.75 & Transçribed & \\
\hline $9 / 4 / 927: 04$ & Auto FIC & 0 & Suspect & Data not included in graph \\
\hline $9 / 5 / 920: 00$ & Manual Tape & 327.75 & Transcribed & \\
\hline $9 / 5 / 926: 04$ & Auto FIC & 328.00 & Good & \\
\hline $9 / 6 / 920: 00$ & Manual Tape & 327.75 & Transcribed & \\
\hline $9 / 6 / 927: 04$ & Auto FIC & 0.00 & Suspect & Data not included in graph \\
\hline $9 / 7 / 92 \quad 0: 00$ & Manual Tape & 327.75 & Transcribed & \\
\hline $9 / 7 / 927: 05$ & Auto FIC & 328 & Good & \\
\hline $9 / 8 / 920: 00$ & Manual Tape & 327.75 & Transcribed & \\
\hline $9 / 8 / 927: 05$ & Auto FIC & 0.00 & Suspect & Data not included in graph \\
\hline $9 / 9 / 920: 00$ & Manual Tape & 327.75 & Transcribed & \\
\hline 9/9/92 7:04 & Auto FIC & 0.00 & Suspect & Data not included in graph \\
\hline $9 / 10 / 920: 00$ & Manual Tape & 327.75 & Transcribed & \\
\hline $9 / 10 / 927: 03$ & Auto FIC & 0.00 & Suspect & Data not included in graph \\
\hline 9/11/92 0:00 & Manual FIC & 328.55 & Transcribed & \\
\hline $9 / 11 / 920: 00$ & Manual Tape & 327.75 & Transcribed & \\
\hline $9 / 12 / 92 \quad 0: 00$ & Manual Tape & 327.75 & Transcribed & \\
\hline $9 / 13 / 920: 00$ & Manual Tape & 327.75 & Transcribed & \\
\hline $9 / 14 / 920: 00$ & Manual FIC & 328.60 & Transcribed & \\
\hline $9 / 14 / 920: 00$ & Manual Tape & 327.5 & Transcribed & \\
\hline 9/15/92 0:00 & Manual Tape & 327.50 & Transcribed & \\
\hline 9/16/92 0:00 & Manual Tape & 327.5 & Transcribed & \\
\hline $9 / 17 / 920: 00$ & Manual Tape & 327.5 & Transcribed & \\
\hline 9/17/92 7:06 & Auto FIC & 0.00 & Suspect & Data not included in graph \\
\hline 9/18/92 0:00 & Manual Tape & 327.50 & Transcribed & \\
\hline 9/18/92 7:06 & Auto FIC & 0 & Suspect & Data not included in graph \\
\hline 9/19/92 0:00 & Manual FIC & 328.6 & Transcribed & \\
\hline 9/19/92 0:00 & Manual Tape & 327.5 & Transcribed & \\
\hline
\end{tabular}

Data obtained from WHC Surveillance Analysis Computer System, (PC SACS) July 7, 1995. 
WHC-SD-WM-ER-315, Rev. 0

\begin{tabular}{|c|c|c|c|c|}
\hline \multicolumn{5}{|c|}{ 241-AP-108 } \\
\hline \multicolumn{5}{|c|}{ Surface Leval Data } \\
\hline Date & Type of Measurement & Level (Inches) & Data Quality & Comments \\
\hline $9 / 20 / 920: 00$ & Manual FIC & 328.6 & Transcribed & \\
\hline $9 / 20 / 920: 00$ & Manual Tape & 327.50 & Transcribed & \\
\hline $9 / 21 / 920: 00$ & Manual Tape & 327.50 & Transcribed & \\
\hline $9 / 21 / 927: 06$ & Auto FIC & 0 & Suspect & Data not included in graph \\
\hline $9 / 22 / 920: 00$ & Manual Tape & 327.5 & Transcribed & \\
\hline 9/22/92 7:03 & Auto FIC & 0.00 & Suspect & Data not included in graph \\
\hline $9 / 23 / 920: 00$ & Manual Tape & 327.75 & Transcribed & \\
\hline $9 / 23 / 927: 03$ & Auto FIC & 0 & Suspect & Data not included in graph \\
\hline $9 / 24 / 920: 00$ & Manual Tape & 327.75 & Transcribed & \\
\hline $9 / 24 / 927: 03$ & Auto FIC & 0.00 & Suspect & Data not included in graph \\
\hline $9 / 25 / 920: 00$ & Manual Tape & 327.5 & Transcribed & \\
\hline $9 / 25 / 927: 03$ & Auto FIC & 0.00 & Suspect & Data not included in graph \\
\hline $9 / 26 / 920: 00$ & Manual Tape & 327.75 & Transcribed & \\
\hline $9 / 26 / 922: 03$ & Auto FIC & 328.00 & Good & \\
\hline $9 / 27 / 920: 00$ & Manual Tape & 327.75 & Transcribed & \\
\hline $9 / 27 / 927: 02$ & Auto FIC & 0 & Suspect & Data not included in graph \\
\hline $9 / 28 / 920: 00$ & Manual Tape & 327.75 & Transeribed & \\
\hline $9 / 28 / 927: 02$ & Auto FIC & 0.00 & Suspect & Data not included in graph \\
\hline $9 / 29 / 920: 00$ & Manual Tape & 327.75 & Transcribed & \\
\hline 9/29/92 7:03 & Auto FIC & 0.00 & Suspect & Data not included in graph \\
\hline $9 / 30 / 920: 00$ & Manual Tape & 327.50 & Transcribed & \\
\hline $9 / 30 / 927: 02$ & Auto FIC & 0 & Suspect & Data not included in graph \\
\hline $10 / 1 / 920: 00$ & Manual Tape & 327.50 & Transcribed & \\
\hline $10 / 1 / 927: 03$ & Auto FIC & 0.00 & Suspect & Data not included in graph \\
\hline $10 / 2 / 920: 00$ & Manual Tape & 327.50 & Transcribed & \\
\hline $10 / 2 / 927: 02$ & Auto FIC & 0.00 & Suspect & Data not included in graph \\
\hline $10 / 3 / 920: 00$ & Manual Tape & 327.75 & Transcribed & \\
\hline $10 / 3 / 927: 03$ & Auto FIC & 0.00 & Suspect & Data not included in graph \\
\hline $10 / 4 / 920: 00$ & Manual Tape & 327.50 & Transcribed & \\
\hline 10/4/92 7:03 & Auto FIC & 0 & Suspect & Data not included in graph \\
\hline $10 / 5 / 920: 00$ & Manual Tape & 327.5 & Transcribed & \\
\hline $10 / 5 / 927: 03$ & Auto FIC & 0.00 & Suspect & Data not included in graph \\
\hline $10 / 6 / 920: 00$ & Manual Tape & 327.5 & Transcribed & \\
\hline $10 / 6 / 927: 03$ & Auto FIC & 0 & Suspect & Data not included in graph \\
\hline $10 / 7 / 920: 00$ & Manual FIC & 328.50 & Transcribed & \\
\hline $10 / 7 / 920: 00$ & Manual Tape & 327.50 & Transcribed & \\
\hline $10 / 8 / 920: 00$ & Manual Tape & $327.50^{\circ}$ & Transcribed & \\
\hline 10/8/92 7:02 & Auto FIC & 0 & Suspect & Data not included in graph \\
\hline $10 / 9 / 920: 00$ & Manual Tape & 327.5 & Transcribed & \\
\hline $10 / 9 / 927: 03$ & Auto FIC & 0 & Suspect & Data not included in graph \\
\hline 10/10/92 0:00 & Manual Tape & 327.75 & Transcribed & \\
\hline $10 / 10 / 927: 03$ & Auto FIC & 0.00 & Suspect & Data not included in graph \\
\hline 10/11/92 0:00 & Manual Tape & 327.75 & Transcribed & \\
\hline $10 / 11 / 927: 03$ & Auto FIC & 0.00 & Suspect & Data not included in graph \\
\hline $10 / 12 / 920: 00$ & Manual FIC & 328.70 & Transcribed & \\
\hline 10/12/92 0:00 & Manual Tape & 327.75 & Transcribed & \\
\hline 10/13/92 0:00 & Manual FIC & 328.70 & Transcribed & \\
\hline $10 / 13 / 920: 00$ & Manual Tape & 327.75 & Transcribed & \\
\hline
\end{tabular}

Data obtained from WHC Surveiliance Analysis Computer System, (PC SACS) July 7, 1995. 


\begin{tabular}{|c|c|c|c|c|}
\hline \multicolumn{5}{|c|}{ 241-AP-108 } \\
\hline \multicolumn{5}{|c|}{ Surface Level Data } \\
\hline Date & Type of Measurement & Level (Inches) & Data Quality & Comments \\
\hline $10 / 14 / 920: 00$ & Manual Tape & 327.75 & Transcribed & \\
\hline $10 / 14 / 925: 03$ & Auto FIC & 328.00 & Good & \\
\hline $10 / 15 / 920: 00$ & Manual Tape & 327.75 & Transcribed & \\
\hline $10 / 15 / 927: 04$ & Auto FIC & 0 & Suspect & Data not included in graph \\
\hline $10 / 16 / 920: 00$ & Manual Tape & 327.75 & Transcribed & \\
\hline $10 / 16 / 920: 02$ & Auto FIC & 328.00 & Good & \\
\hline $10 / 17 / 920: 00$ & Manual Tape & 327.75 & Transcribed & \\
\hline $10 / 17 / 927: 03$ & Auto FIC & 0.00 & Suspect & Data not included in graph \\
\hline $10 / 18 / 920: 00$ & Manual Tape & 327.75 & Transcribed & \\
\hline $10 / 18 / 927: 03$ & Auto FIC & 0 & Suspect & Data not included in graph \\
\hline 10/19/920:00 & Manual Tape & $\mathbf{3 2 7 . 7 5}$ & Transcribed & \\
\hline $10 / 19 / 927: 03$ & Auto FIC & 328.00 & Good & \\
\hline $10 / 20 / 920: 00$ & Manual Tape & 327.75 & Transcribed & \\
\hline $10 / 20 / 927: 03$ & Auto FIC & 328 & Good & \\
\hline $10 / 21 / 920: 00$ & Manual Tape & 327.75 & Transcribed & \\
\hline $10 / 21 / 927: 03$ & Auto FIC & 0.00 & Suspect & Data not included in graph \\
\hline $10 / 22 / 920: 00$ & Manual FIC & 328.50 & Transcribed & \\
\hline $10 / 22 / 920: 00$ & Manual Tape & 327.75 & Transcribed & \\
\hline $10 / 23 / 920: 00$ & Manual FIC & 328.40 & Transcribed & \\
\hline $10 / 23 / 920: 00$ & Manual Tape & 327.50 & Transcribed & \\
\hline $10 / 24 / 920: 00$ & Manual Tape & 327.5 & Transcribed & \\
\hline $10 / 24 / 927: 03$ & Auto FIC & 0.00 & Suspect & Data not included in graph \\
\hline $10 / 25 / 920: 00$ & Manual Tape & 327.5 & Transcribed & \\
\hline $10 / 25 / 927: 03$ & Auto FIC & 328.00 & Good & \\
\hline $10 / 26 / 920: 00$ & Manual FIC & 328.4 & Transcribed & \\
\hline $10 / 26 / 920: 00$ & Manual Tape & 327.50 & Transcribed & \\
\hline $10 / 27 / 920: 00$ & Manual Tape & 327.50 & Transcribed & \\
\hline $10 / 27 / 924: 04$ & Auto FIC & 328 & Good & \\
\hline $10 / 28 / 920: 00$ & Manual Tape & $\mathbf{3 2 7 . 5 0}$ & Transcribed & \\
\hline $10 / 28 / 927: 03$ & Auto FIC & 0 & Suspect & Data not included in graph \\
\hline $10 / 29 / 920: 00$ & Manual Tape & 327.50 & Transcribed & \\
\hline $10 / 29 / 927: 03$ & Auto FIC & 0 & Suspect & Data not included in graph \\
\hline $10 / 30 / 920: 00$ & Manual Tape & 327.5 & Transcribed & \\
\hline $10 / 30 / 927: 03$ & Auto FIC & 0.00 & Suspect & Data not included in graph \\
\hline 10/31/920:00 & Manual Tape & 327.50 & Transcribed & \\
\hline $10 / 31 / 927: 03$ & Auto FIC & 0 & Suspect & Data not included in graph \\
\hline $11 / 1 / 920: 00$ & Manual Tape & 327.5 & Transcribed & \\
\hline 11/1/92 7:03 & Auto FIC & 0 & Suspect & Data not included in graph \\
\hline $11 / 2 / 920: 00$ & Manual Tape & 327.5 & Transcribed & \\
\hline $11 / 2 / 922: 03$ & Auto FIC & 328:60 & Good & \\
\hline $11 / 3 / 920: 00$ & Manual Tape & 327.50 & Transcribed & \\
\hline $11 / 3 / 927: 03$ & Auto FIC & 0 & Suspect & Data not included in graph \\
\hline 11/4/92 0:00 & Manual Tape & 327.75 & Transcribed & \\
\hline $11 / 4 / 927: 03$ & Auto FIC & 0 & Suspect & Data not included in graph \\
\hline $11 / 5 / 920: 00$ & Manual FIC & 328.70 & Transcribed & \\
\hline $11 / 5 / 920: 00$ & Manual Tape & 327.75 & Transcribed & \\
\hline $11 / 6 / 920: 00$ & Manual FIC & 328.7 & Transcribed & \\
\hline $11 / 6 / 920: 00$ & Manual Tape & 327.75 & Transeribed & \\
\hline
\end{tabular}

Data obtained from WHC Surveiliance Analysis Computer System, (PC SACS) July 7, 1995. 


\begin{tabular}{|c|c|c|c|c|}
\hline \multicolumn{5}{|c|}{ 241-AP-108 } \\
\hline \multicolumn{5}{|c|}{ Surface Lavel Data } \\
\hline Date & Type of Measurement & Level (Inches) & Data Quality & Comments \\
\hline $11 / 7 / 920: 00$ & Manual FIC & 328.70 & Transcribed & \\
\hline $11 / 7 / 920: 00$ & Manual Tape & 327.5 & Transcribed & \\
\hline $11 / 8 / 920: 00$ & Manual FIC & 328.7 & Transcribed & \\
\hline $11 / 8 / 920: 00$ & Manual Tape & 327.75 & Transcribed & \\
\hline 11/9/92 0:00 & Manual FIC & 328.70 & Transcribed & \\
\hline $11 / 9 / 920: 00$ & Manual Tape & 327.75 & Transcribed & \\
\hline $11 / 10 / 920: 00$ & Manual Tape & 327.75 & Transcribed & \\
\hline $11 / 10 / 927: 03$ & Auto FIC & 0 & Suspect & Data not included in graph \\
\hline $11 / 11 / 920: 00$ & Manual Tape & 327.75 & Transcribed & \\
\hline $11 / 11 / 927: 04$ & Auto FIC & 0.00 & Suspect & Data not included in graph \\
\hline $11 / 12 / 920: 00$ & Manual Tape & 327.5 & Transcribed & \\
\hline $11 / 12 / 927: 03$ & Auto FIC & 0 & Suspect & Data not included in graph \\
\hline $11 / 13 / 920: 00$ & Manual Tape & 327.50 & Transcribed & \\
\hline $11 / 13 / 927: 04$ & Auto FIC & 0 & Suspect & Data not included in graph \\
\hline $11 / 14 / 920: 00$ & Manual Tape & 327.75 & Transcribed & \\
\hline $11 / 14 / 927: 04$ & Auto FIC & 0 & Suspect & Data not included in graph \\
\hline $11 / 15 / 920: 00$ & Manual Tape & 327.75 & Transcribed & \\
\hline $11 / 15 / 927: 04$ & Auto FIC & 0 & Suspect & Data not included in graph \\
\hline $11 / 16 / 920: 00$ & Manual Tape & 328.00 & Transcribed & \\
\hline $11 / 16 / 927: 04$ & Auto FIC & 0.00 & Suspect & Data not included in graph \\
\hline $11 / 17 / 920: 00$ & Manual Tape & 328 & Transcribed & \\
\hline $11 / 17 / 927: 04$ & Auto FIC & 0.00 & Suspect & Data not included in graph \\
\hline $11 / 18 / 920: 00$ & Manual Tape & 328 & Transcribed & \\
\hline $11 / 18 / 927: 02$ & Auto FIC & 0.00 & Suspect & Data not included in graph \\
\hline $11 / 19 / 920: 00$ & Manual Tape & 327.75 & Transcribed & \\
\hline $11 / 19 / 927: 02$ & Auto FIC & 0.00 & Suspect & Data not included in graph \\
\hline $11 / 20 / 920: 00$ & Manual Tape & 327.75 & Transcribed & \\
\hline $11 / 20 / 927: 03$ & Auto FIC & 0.00 & Suspect & Data not included in graph \\
\hline $11 / 21 / 920: 00$ & Manual Tape & 327.75 & Transcribed & \\
\hline $11 / 21 / 92 \quad 7: 02$ & Auto FIC & 0 & Suspect & Data not included in graph \\
\hline $11 / 22 / 920: 00$ & Manual Tape & 327.75 & Transcribed & \\
\hline $11 / 22 / 927: 03$ & Auto FIC & 0 & Suspect & Data not included in graph \\
\hline $11 / 23 / 920: 00$ & Manual Tape & 327.75 & Transcribed & \\
\hline $11 / 23 / 927: 03$ & Auto FIC & 0.00 & Suspect & Data not included in graph \\
\hline $11 / 24 / 920: 00$ & Manual Tape & 327.75 & Transcribed & \\
\hline $11 / 24 / 927: 03$ & Auto FIC & 0.00 & Suspect & Data not included in graph \\
\hline $11 / 25 / 920: 00$ & Manual Tape & 327.75 & Transcribed & \\
\hline $11 / 25 / 927: 03$ & Auto FIC & 0.00 & Suspect & Data not included in graph \\
\hline $11 / 26 / 920: 00$ & Manual Tape & 327.75 & Transcribed & \\
\hline $11 / 26 / 927: 03$ & Auto FIC & 0.00 & Suspect & Data not included in graph \\
\hline $11 / 27 / 920: 00$ & Manual Tape & 327.75 & Transcribed & \\
\hline 11/27/92 7:03 & Auto FIC & 0 & Suspect & Data not included in graph \\
\hline $11 / 28 / 920: 00$ & Manual Tape & 327.75 & Transcribed & \\
\hline $11 / 28 / 927: 03$ & Auto FIC & 0.00 & Suspect & Data not included in graph \\
\hline $11 / 29 / 920: 00$ & Manual Tape & 327.75 & Transcribed & \\
\hline $11 / 29 / 927: 03$ & Auto FIC & 0.00 & Suspect & Data not included in graph \\
\hline $11 / 30 / 920: 00$ & Manual FIC & 328.60 & Transcribed & \\
\hline $11 / 30 / 920: 00$ & Manual Tape & 327.75 & Transcribed & \\
\hline
\end{tabular}

Data obtained from WHC Surveillance Analysis Computer System, (PC SACS) July 7, 1995. 
WHC-SD-WM-ER-315, Rev. 0

\begin{tabular}{|c|c|c|c|c|}
\hline \multicolumn{5}{|c|}{ 241-AP-108 } \\
\hline \multicolumn{5}{|c|}{ Surface Level Data } \\
\hline Date & Type of Measurement & Level (Inches) & Data Quality & Comments \\
\hline $12 / 1 / 920: 00$ & Manual Tape & 327.75 & Transcribed & \\
\hline $12 / 1 / 927: 03$ & Auto FIC & 0 & Suspect & Data not included in graph \\
\hline $12 / 2 / 920: 00$ & Manual Tape & 327.75 & Transcribed & \\
\hline $12 / 2 / 920: 03$ & Auto FIC & 328 & Good & \\
\hline $12 / 3 / 920: 00$ & Manual Tape & 327.75 & Transcribed & \\
\hline $12 / 3 / 927: 03$ & Auto FIC & 0.00 & Suspect & Data not included in graph \\
\hline $12 / 4 / 920: 00$ & Manual Tape & 327.75 & Transcribed & \\
\hline $12 / 4 / 927: 03$ & Auto FIC & 0.00 & Suspect & Data not included in graph \\
\hline $12 / 5 / 920: 00$ & Manual FIC & 328.25 & Transcribed & \\
\hline 12/5/92 0:00 & Manual Tape & 327.75 & Transcribed & \\
\hline $12 / 6 / 920: 00$ & Manual Tape & 327.50 & Transcribed & \\
\hline $12 / 6 / 927: 02$ & Auto FIC & 0 & Suspect & Data not included in graph \\
\hline $12 / 7 / 920: 00$ & Manual Tape & 327.75 & Transcribed & \\
\hline $12 / 7 / 927: 03$ & Auto FIC & 0.00 & Suspect & Data not included in graph \\
\hline $12 / 8 / 920: 00$ & Manual Tape & 327.50 & Transcribed & \\
\hline $12 / 8 / 927: 03$ & Auto FIC & 0.00 & Suspect & Data not included in graph \\
\hline $12 / 9 / 920: 00$ & Manual Tape & 327.5 & Transcribed & \\
\hline 12/9/92 7:03 & Auto FIC & 0.00 & Suspect & Data not included in graph \\
\hline $12 / 10 / 920: 00$ & Manual Tape & 327.5 & Transcribed & \\
\hline $12 / 10 / 927: 03$ & Auto FIC & 0 & Suspect & Data not included in graph \\
\hline $12 / 11 / 920: 00$ & Manual Tape & 327.50 & Transcribed & \\
\hline $12 / 11 / 927: 03$ & Auto FIC & 0.00 & Suspect & Data not included in graph \\
\hline $12 / 12 / 920: 00$ & Manual Tape & 327.5 & Transcribed & \\
\hline $12 / 13 / 920: 00$ & Manual Tape & 327.25 & Transeribed & \\
\hline $12 / 13 / 927: 04$ & Auto FIC & 0 & Suspect & Data not included in graph \\
\hline $12 / 14 / 920: 00$ & Manual Tape & 327.25 & Transcribed & \\
\hline $12 / 14 / 927: 03$ & Auto FIC & 0 & Suspect & Data not included in graph \\
\hline $12 / 15 / 920: 00$ & Manual Tape & 327.25 & Transcribed & \\
\hline $12 / 15 / 927: 03$ & Auto FIC & 0.00 & Suspect & Data not included in graph \\
\hline $12 / 16 / 920: 00$ & Manual Tape & 327.25 & Transcribed & \\
\hline 12/16/92 7:04 & Auto FIC & 0 & Suspect & Data not included in graph \\
\hline $12 / 17 / 920: 00$ & Manual Tape & 327.25 & Transcribed & \\
\hline $12 / 17 / 927: 03$ & Auto FIC & 0.00 & Suspect & Data not included in graph \\
\hline $12 / 18 / 920: 00$ & Manual Tape & 327.25 & Transcribed & \\
\hline $12 / 18 / 927: 03$ & Auto FIC & 0.00 & Suspect & Data not included in graph \\
\hline $12 / 19 / 920: 00$ & Manual Tape & 327.50 & Transcribed & \\
\hline $12 / 19 / 927: 03$ & Auto FIC & 0.00 & Suspect & Data not included in graph \\
\hline $12 / 20 / 920: 00$ & Manual Tape & 327.25 & Transcribed & \\
\hline $12 / 20 / 927: 04$ & Auto FIC & 0 & Suspect & Data not included in graph \\
\hline $12 / 21 / 920: 00$ & Manual Tape & 327.25 & Transcribed & \\
\hline $12 / 21 / 927: 04$ & Auto FIC & 0 & Suspect & Data not included in graph \\
\hline $12 / 22 / 920: 00$ & Manual Tape & $\mathbf{3 2 7 . 2 5}$ & Transcribed & \\
\hline $12 / 23 / 920: 00$ & Manual Tape & 327.25 & Transcribed & \\
\hline $12 / 23 / 927: 04$ & Auto FIC & 0 & Suspect & Data not included in graph \\
\hline $12 / 24 / 920: 00$ & Manual Tape & 327.25 & Transcribed & \\
\hline $12 / 24 / 927: 03$ & Auto FIC & 0.00 & Suspect & Data not included in graph \\
\hline $12 / 25 / 920: 00$ & Manual Tape & 327.50 & Transcribed & \\
\hline $12 / 25 / 927: 03$ & Auto FIC & 0.00 & Suspect & Data not included in graph \\
\hline
\end{tabular}

Data obtained from WHC Surveillance Analysis Computer System, (PC SACS) July 7, 1995. 


\begin{tabular}{|c|c|c|c|c|}
\hline \multicolumn{5}{|c|}{ 241-AP-108 } \\
\hline \multicolumn{5}{|c|}{ Surface Level Data } \\
\hline Date & Type of Measurement & Level (Inches) & Data Quality & Comments \\
\hline $12 / 26 / 920: 00$ & Manual Tape & 327.25 & Transcribed & \\
\hline $12 / 26 / 927: 03$ & Auto FIC & 0 & Suspect & Data not included in graph \\
\hline $12 / 27 / 920: 00$ & Manual Tape & 327.25 & Transcribed & \\
\hline 12/27/92 7:03 & Auto FIC & 0.00 & Suspect & Data not included in graph \\
\hline $12 / 28 / 920: 00$ & Manual Tape & 327.25 & Transcribed & \\
\hline $12 / 28 / 927: 03$ & Auto FIC & 0 & Suspect & Data not included in graph \\
\hline 12/29/92 0:00 & Manual Tape & 327.5 & Transcribed & \\
\hline $12 / 30 / 920: 00$ & Manual Tape & 327.5 & Transcribed & \\
\hline $12 / 30 / 927: 03$ & Auto FIC & 327.3 & Good & \\
\hline $12 / 31 / 920: 00$ & Manual Tape & 327.5 & Transcribed & \\
\hline $12 / 31 / 927: 03$ & Auto FIC & 327.3 & Good & \\
\hline 1/1/93 0:00 & Manual Tape & 327.5 & Good & \\
\hline $1 / 1 / 937: 04$ & Auto FIC & 327.3 & Good & \\
\hline $1 / 2 / 930: 00$ & Manual FIC & 327.3 & Good & \\
\hline 1/2/930:00 & Manual Tape & 327.5 & Good & \\
\hline $1 / 3 / 930: 00$ & Manual FIC & 327.30 & Good & \\
\hline $1 / 3 / 930: 00$ & Manual Tape & 327.50 & Good & \\
\hline $1 / 4 / 930: 00$ & Manual FIC & 327.30 & Good & \\
\hline 1/4/930:00 & Manual Tape & 327.25 & Good & \\
\hline 1/5/93 0:00 & Manual FIC & 327.30 & Good & \\
\hline $1 / 5 / 930: 00$ & Manual Tape & 327,50 & Good & \\
\hline $1 / 6 / 930: 00$ & Manual FIC & 327.3 & Good & \\
\hline $1 / 6 / 930: 00$ & Manual Tape & 327.25 & Good & . \\
\hline 1/7/93 0:00 & Manual FIC & 327.3 & Good & \\
\hline $1 / 7 / 930: 00$ & Manual Tape & 327.25 & Good & \\
\hline $1 / 8 / 930: 00$ & Manual FIC & 327.2 & Good &. \\
\hline $1 / 8 / 930: 00$ & Manual Tape & 327.25 & Good & \\
\hline $1 / 9 / 930: 00$ & Manual FIC & 327.2 & Good & \\
\hline 1/9/93 0:00 & Manual Tape & 327.25 & Good & \\
\hline $1 / 10 / 930: 00$ & Manual FIC & 327.20 & Good & \\
\hline $1 / 10 / 930: 00$ & Manual Tape & 327.25 & Good & \\
\hline $1 / 11 / 930: 00$ & Manual FIC & 327.2 & Good & \\
\hline $1 / 11 / 930: 00$ & Manual Tape & 327.25 & Good & \\
\hline $1 / 12 / 930: 00$ & Manual Tape , & 327.50 & Good & \\
\hline $1 / 12 / 937: 06$ & Auto FIC & 327.2 & Good & \\
\hline $1 / 13 / 930: 00$ & Manual Tape & 327.50 & Good & \\
\hline $1 / 13 / 937: 06$ & Auto FIC & 327.20 & Good & \\
\hline $1 / 14 / 930: 00$ & Manual Tape & 327.5 & Good & \\
\hline $1 / 14 / 937: 03$ & Auto FIC & 327.2 & Good & \\
\hline $1 / 15 / 930: 00$ & Manual Tape & 327.5 & Good & \\
\hline $1 / 15 / 937: 03$ & Auto FIC & 327.20 & Good & \\
\hline $1 / 16 / 930: 00$ & Manual FIC & 327.30 & Good & \\
\hline 1/16/93 0:00 & Manual Tape & 327.25 & Good & \\
\hline $1 / 17 / 930: 00$ & Manual FIC & 327.20 & Good & \\
\hline $1 / 17 / 930: 00$ & Manual Tape & 327.25 & Good & \\
\hline 1/18/93 0:00 & Manual FIC & 327.20 & Good & \\
\hline $1 / 18 / 930: 00$ & Manual Tape & 327.25 & Good & \\
\hline $1 / 19 / 930: 00$ & Manual FIC & 327.2 & Good & \\
\hline
\end{tabular}

Data obtained from WHC Surveillance Analysis Computer System, (PC SACS) July 7, 1995. 


\begin{tabular}{|c|c|c|c|c|}
\hline \multicolumn{5}{|c|}{ 241-AP-108 } \\
\hline \multicolumn{5}{|c|}{ Surface Level Data } \\
\hline Date & Type of Messurement & Level (Inches) & Data Quality & Comments \\
\hline $1 / 19 / 930: 00$ & Manual Tape & 327 & Good & \\
\hline 1/20/93 0:00 & Manual FIC & 327.2 & Good & \\
\hline $1 / 20 / 930: 00$ & Manual Tape & $327.25^{\circ}$ & Good & \\
\hline $1 / 21 / 930: 00$ & Manual Tape & 327.25 & Good & \\
\hline $1 / 21 / 937: 02$ & Auto FIC & 327.20 & Good & \\
\hline $1 / 22 / 930: 00$ & Manual Tape & 327.25 & Good & \\
\hline $1 / 22 / 937: 04$ & Auto FIC & 327.2 & Good & \\
\hline $1 / 23 / 930: 00$ & Manual Tape & 327.5 & Good & \\
\hline $1 / 23 / 937: 03$ & Auto FIC & 327.20 & Good & \\
\hline $1 / 24 / 930: 00$ & Manual Tape & 327.50 & Good & \\
\hline $1 / 24 / 937: 03$ & Auto FIC & 327.2 & Good & \\
\hline $1 / 25 / 930: 00$ & Manual Tape & 327.25 & Good & \\
\hline $1 / 25 / 937: 03$ & Auto FIC & 327.2 & Good & \\
\hline $1 / 26 / 930: 00$ & Manual Tape & 327.25 & Good & \\
\hline $1 / 26 / 937: 04$ & Auto FIC & 327.20 & Good & \\
\hline $1 / 27 / 930: 00$ & Manual Tape & 327.50 & Good & \\
\hline 1/27/93 7:04 & Auto FIC & 327.20 & Good & \\
\hline $1 / 28 / 930: 00$ & Manual Tape & 327.25 & Good & \\
\hline $1 / 28 / 937: 04$ & Auto FIC & 327.2 & Good & \\
\hline $1 / 29 / 930: 00$ & Manual Tape & 327.50 & Good & \\
\hline $1 / 29 / 937: 04$ & Auto FIC & 327.2 & Good & \\
\hline $1 / 30 / 930: 00$ & Manual Tape & 327.25 & Good & \\
\hline $1 / 30 / 937: 03$ & Auto FIC & 327.20 & Good & \\
\hline $1 / 31 / 930: 00$ & Manual Tape & 327.25 & Good & \\
\hline 1/31/93 7:04 & Auto FIC & 327.2 & Good & \\
\hline $2 / 1 / 930: 00$ & Manual Tape & 327.25 & Good & \\
\hline 2/1/93 7:04 & Auto FIC & 327.1 & Good & \\
\hline $2 / 2 / 930: 00$ & Manual Tape & 327.25 . & Good & \\
\hline $2 / 2 / 937: 04$ & Auto FIC & 327.1 & Good & \\
\hline $2 / 3 / 930: 00$ & Manual FIC & 327.10 & Good & \\
\hline $2 / 3 / 930: 00$ & Manual Tape & 327 & Good & \\
\hline $2 / 4 / 930: 00$ & Manual Tape & 327.00 & Good & \\
\hline $2 / 4 / 937: 04$ & Auto FIC & 327.1 & Good & \\
\hline $2 / 5 / 930: 00$ & Manual Tape & 327 & Good & \\
\hline 2/5/93 7:04 & Auto FIC & 327.10 & Good & \\
\hline $2 / 6 / 93 \quad 0: 00$ & Manual Tape & 327 & Good & \\
\hline $2 / 6 / 937: 04$ & Auto FIC & 327.00 & Good & \\
\hline 2/7/93 0:00 & Manual Tape & 327.25 & Good & \\
\hline $2 / 7 / 937: 03$ & Auto FIC & 327.1 & Good & \\
\hline 2/8/93 0:00 & Manual Tape & 327.00 & Good & \\
\hline 2/8/93 7:04 & Auto FIC & 327.1 & Good & \\
\hline $2 / 9 / 930: 00$ & Manual Tape & 327.25 & Good & \\
\hline $2 / 9 / 937: 04$ & Auto FIC & 327.1 & Good & \\
\hline 2/10/93 0:00 & Manual Tape & $\begin{array}{r}327.25 \\
\end{array}$ & Good & \\
\hline $2 / 10 / 937: 04$ & Auto FIC & 327.10 & Good & \\
\hline $2 / 11 / 930: 00$ & Manual Tape & 327.25 & Good & \\
\hline $2 / 11 / 937: 04$ & Auto FIC & 327.10 & Good & \\
\hline 2/12/93 0:00 & Manual Tape & 327.25 & Good & \\
\hline
\end{tabular}

Data obtained from WHC Surveillance Analysis Computer System, (PC SACS) July 7, 1995. 


\begin{tabular}{|c|c|c|c|c|}
\hline \multicolumn{5}{|c|}{ 241-AP-108 } \\
\hline \multicolumn{5}{|c|}{ Surface Level Data } \\
\hline Date & Type of Measurement & Lovel (Inches) & Data Quality & Comments \\
\hline $2 / 12 / 937: 03$ & Auto FIC & 327.1 & Good & \\
\hline 2/13/93 0:00 & Manual Tape & 327.00 & Good &. \\
\hline 2/13/93 7:03 & Auto FIC & 327.10 & Good & \\
\hline $2 / 14 / 930: 00$ & Manual Tape & 327.00 & Good & \\
\hline $2 / 14 / 937: 04$ & Auto FIC & 327.1 & Good & \\
\hline $2 / 15 / 930: 00$ & Manual Tape & 327 & Good & \\
\hline $2 / 15 / 937: 04$ & Auto FIC & 327.10 & Good & \\
\hline $2 / 16 / 930: 00$ & Manual Tape & 327 & Good & \\
\hline $2 / 16 / 937: 02$ & Auto FIC & 327.1 & Good & \\
\hline $2 / 17 / 930: 00$ & Manual FIC & 327.10 & Good & \\
\hline $2 / 17 / 930: 00$ & Manual Tape & 327.00 & Good & \\
\hline $2 / 18 / 930: 00$ & Manual Tape & 327 & Good & \\
\hline $2 / 18 / 937: 03$ & Auto FIC & 327.1 & Good & \\
\hline 2/19/93 0:00 & Manual Tape & 327 & Good & \\
\hline $2 / 19 / 937: 02$ & Auto FIC & 327.10 & Good & \\
\hline $2 / 20 / 930: 00$ & Manual Tape & 327 & Good & \\
\hline $2 / 20 / 937: 03$ & Auto FIC & 327.10 & Good & \\
\hline $2 / 21 / 930: 00$ & Manual Tape & 327.00 & Good & \\
\hline $2 / 21 / 937: 04$ & Auto FIC & 327.1 & Good & \\
\hline $2 / 22 / 930: 00$ & Manual Tape & 327.75 & Suspect & Data not included in graph \\
\hline $2 / 22 / 937: 04$ & Auto FIC & 327.1 & Good & \\
\hline $2 / 23 / 930: 00$ & Manual Tape & 327.00 & Good & \\
\hline $2 / 23 / 937: 03$ & Auto FIC & 327.1 & Good & \\
\hline $2 / 24 / 930: 00$ & Manual Tape & 327.00 & Good & \\
\hline $2 / 24 / 937: 04$ & Auto FIC & 327.10 & Good & \\
\hline $2 / 25 / 930: 00$ & Manual Tape & 327.00 & Good & \\
\hline $2 / 25 / 937: 03$ & Auto FIC & 327.1 & Good & \\
\hline $2 / 26 / 930: 00$ & Manual Tape & 327 & Good & \\
\hline $2 / 26 / 937: 03$ & Auto FIC & 327 & Good & \\
\hline $2 / 27 / 930: 00$ & Manual Tape & 327.00 & Good & \\
\hline $2 / 27 / 937: 04$ & Auto FIC & 327 & Good & \\
\hline $2 / 28 / 930: 00$ & Manual Tape & 327.00 & Good & \\
\hline $2 / 28 / 937: 03$ & Auto FIC & 327 & Good & \\
\hline $3 / 1 / 930: 00$ & Manual Tape & 327 & Good & \\
\hline $3 / 1 / 937: 03$ & Auto FIC & 327 & Good & \\
\hline $3 / 2 / 93$ 0:00 & 1 Manual Tape & 327 & Good & \\
\hline $3 / 2 / 937: 04$ & Auto FIC & 327 & Good & \\
\hline $3 / 3 / 930: 00$ & Manual Tape & 327.25 & Good & \\
\hline 3/3/93 7:03 & Auto FIC & 327.00 & Good & \\
\hline $3 / 4 / 930: 00$ & Manual Tape & $327: 00$ & Good & \\
\hline $3 / 4 / 937: 04$ & Auto FIC & 327.00 & Good & \\
\hline $3 / 5 / 93$ 0:00 & Manual Tape & 327.00 & Good & \\
\hline $3 / 5 / 93 \quad 7: 04$ & Auto FIC & 327.00 & Good & \\
\hline $3 / 6 / 930: 00$ & Manual Tape & 327 & Good & \\
\hline $3 / 6 / 93$ 7:03 & Auto FIC & 327.00 & Good & \\
\hline 3/7/93 0:00 & Manual Tape & 327 & Good & \\
\hline $3 / 7 / 937: 03$ & Auto FIC & 327 & Good & \\
\hline $3 / 8 / 930: 00$ & Manual Tape & 327 & Good & \\
\hline
\end{tabular}

Data obtained from WHC Surveillance Analysis Computer System, (PC SACS) July 7, 1995. 


\begin{tabular}{|c|c|c|c|c|}
\hline \multicolumn{5}{|c|}{ 241-AP-108 } \\
\hline & & Urface Level Dat & & \\
\hline Date & Type of Measurement & Level (Inches) & Data Quality & Comments \\
\hline 3/8/93 7:03 & Auto FIC & 327.00 & Good & \\
\hline 3/9/93 0:00 & Manual Tape & 327.00 & Good & \\
\hline 3/9/93 7:04 & Auto FIC & 327.00 & Good & \\
\hline $3 / 10 / 930: 00$ & Manual Tape & 327.00 & Good & \\
\hline $3 / 10 / 937: 05$ & Auto FIC & 327 & Good & \\
\hline $3 / 11 / 930: 00$ & Manual Tape & 327 & Good & \\
\hline $3 / 11 / 937: 04$ & Auto FIC & 327.00 & Good & \\
\hline $3 / 12 / 930: 00$ & Manual Tape & 327 & Good & \\
\hline $3 / 12 / 937: 04$ & Auto FIC & 327.00 & Good & \\
\hline $3 / 13 / 930: 00$ & Manual Tape & 327 & Good & \\
\hline $3 / 13 / 937: 03$ & Auto FIC & 327 & Good & \\
\hline $3 / 14 / 930: 00$ & Manual Tape & 327 & Good & \\
\hline $3 / 14 / 937: 04$ & Auto FIC & 327 & Good & \\
\hline $3 / 15 / 93 \quad 0: 00$ & Manual Tape & 327 & Good & \\
\hline $3 / 15 / 937: 03$ & Auto FIC & 327.00 & Good & \\
\hline $3 / 16 / 930: 00$ & Manual Tape & 327.75 & Good & \\
\hline $3 / 16 / 937: 04$ & Auto FIC & 327.00 & Good & \\
\hline $3 / 17 / 930: 00$ & Manual Tape & 327 & Good & \\
\hline $3 / 17 / 937: 03$ & Auto FIC & 327.00 & Good & \\
\hline $3 / 18 / 930: 00$ & Manual Tape & 327.25 & Good & \\
\hline $3 / 18 / 937: 04$ & Auto FIC & 327 & Good & \\
\hline $3 / 19 / 930: 00$ & Manual Tape & 327 & Good & \\
\hline $3 / 19 / 937: 03$ & Auto FIC & 327.00 & Good & \\
\hline $3 / 20 / 930: 00$ & Manual Tape & 326.75 & Good & \\
\hline $3 / 20 / 937: 04$ & Auto FIC & 327.00 & Good & \\
\hline $3 / 21 / 930: 00$ & Manual Tape & 327.00 & Good & \\
\hline $3 / 21 / 937: 04$ & Auto FIC & 327 & Good & \\
\hline $3 / 22 / 930: 00$ & Manual Tape & 326.75 & Good & \\
\hline $3 / 22 / 937: 03$ & Auto FIC & 327 & Good & \\
\hline $3 / 23 / 930: 00$ & Manual Tape & 327.00 & Good & \\
\hline $3 / 23 / 937: 03$ & Auto FIC & 327.10 & Good & \\
\hline $3 / 24 / 930: 00$ & Manual Tape & 327 & Good & \\
\hline $3 / 24 / 937: 04$ & Auto FIC & 327.20 & Good & \\
\hline $3 / 25 / 93 \quad 0: 00$ & Manual Tape & 327 & Good & \\
\hline $3 / 25 / 937: 03$ & Auto FIC & 327.60 & Good & \\
\hline $3 / 26 / 930: 00$ & Manual Tape & 327.00 & Good & \\
\hline $3 / 26 / 937: 03$ & Auto FIC & 327.4 & Good & \\
\hline $3 / 27 / 930: 00$ & Manual Tape & 327 & Good & \\
\hline $3 / 27 / 937: 03$ & Auto FIC & 327.3 & Good & \\
\hline $3 / 28 / 930: 00$ & Manual Tape & 327 & Good & \\
\hline $3 / 28 / 937: 03$ & Auto FIC & 327.30 & Good & \\
\hline $3 / 29 / 930: 00$ & Manual Tape & 327 & Good & \\
\hline $3 / 29 / 937: 03$ & Auto FIC & 327.30 & Good & \\
\hline $3 / 30 / 930: 00$ & Manual Tape & 327.00 & Good & \\
\hline $3 / 30 / 93$ 7:03 & Auto FIC & 327.40 & Good & \\
\hline 3/31/93 0:00 & Manual Tape & 327 & Good & \\
\hline $3 / 31 / 937: 03$ & Auto FIC & 327.40 & Good & \\
\hline $4 / 1 / 937: 03$ & Auto FIC & 327.40 & Good & \\
\hline
\end{tabular}

Data obtained from WHC Surveillance Analysis Computer System, (PC SACS) July 7, 1995. 


\begin{tabular}{|c|c|c|c|c|}
\hline \multicolumn{5}{|c|}{ 241-AP-108 } \\
\hline \multicolumn{5}{|c|}{ Surface Level Data } \\
\hline Date & Type of Measurement & Level (Inches) & Date Quality & Comments \\
\hline 4/1/93 10:03 & Manual Tape & 327 & Good & \\
\hline 4/2/93 7:06 & Auto FIC & 327.4 & Good & \\
\hline $4 / 2 / 93 \quad 10: 03$ & Manual Tape ' & 327.00 & Good & \\
\hline 4/3/93 7:06 & Auto FIC & 327.30 & Good & \\
\hline $4 / 3 / 93 \quad 10: 03$ & Manual Tape & 327 & Good & \\
\hline 4/4/93 7:05 & Auto FIC & 327.40 & Good & \\
\hline $4 / 4 / 93 \quad 10: 03$ & Manual Tape & 327.00 & Good & \\
\hline 4/5/93 7:06 & Auto FIC & 327.4 & Good & \\
\hline $4 / 5 / 93 \quad 10: 03$ & Manual Tape & 327.00 & Good & \\
\hline 4/6/93 7:06 & Auto FIC & 327.4 & Good & \\
\hline $4 / 6 / 93 \quad 10: 03$ & Manual Tape & 326.50 & Good & \\
\hline 4/7/93 7:06 & Auto FIC & 327.4 & Good & \\
\hline 4/7/93 10:03 & Manual Tape & 327.00 & Good & \\
\hline 4/8/93 7:06 & Auto FIC & 327.4 & Good & \\
\hline 4/8/93 10:03 & Manual Tape & 327 & Good & \\
\hline 4/9/93 7:06 & Auto FIC & 327.40 & Good & \\
\hline 4/9/93 10:03 & Manual Tape & 327.00 & Good & \\
\hline $4 / 10 / 937: 05$ & Auto FIC & 327.30 & Good & \\
\hline 4/10/93 10:03 & Manual Tape & 327 & Good & \\
\hline $4 / 11 / 937: 06$ & Auto FIC & 327.4 & Good & \\
\hline 4/11/93 10:03 & Manual Tape & 327 & Good & \\
\hline $4 / 12 / 937: 06$ & Auto FIC & 327.4 & Good & \\
\hline 4/12/93 10:03 & Manual Tape & 327 & Good & \\
\hline $4 / 13 / 937: 06$ & Auto FIC & 327,40 & Good & \\
\hline $4 / 13 / 93 \quad 10: 03$ & Manual Tape & 327.00 & Good & \\
\hline $4 / 14 / 937: 06$ & Auto FIC & 327.4 & Good & \\
\hline $4 / 14 / 93 \quad 10: 03$ & Manual Tape & 327 & Good & \\
\hline $4 / 15 / 937: 03$ & Auto FIC & 327.4 & Good & \\
\hline $4 / 15 / 93 \quad 10: 03$ & Manual Tape & 327.00 & Good & \\
\hline $4 / 16 / 937: 06$ & Auto FIC & 327.4 & Good & \\
\hline $4 / 16 / 93 \quad 10: 03$ & Manual Tape & 327 & Good & \\
\hline 4/17/93 7:05 & Auto FIC & 327.40 & Good & \\
\hline $4 / 17 / 93 \quad 10: 03$ & Manual Tape & 327 & Good & \\
\hline $4 / 18 / 937: 06$ & Auto FIC & 327.40 & Good & \\
\hline $4 / 18 / 93 \quad 10: 03$ & Manual Tape & 327.00 & Good & \\
\hline 4/19/93 7:06 & Auto FIC & 327.30 & Good & \\
\hline 4/19/93 10:03 & Manual Tape & 327 & Good & \\
\hline $4 / 20 / 9310: 03$ & Manual Tape & 327 & Good & \\
\hline $4 / 20 / 93 \quad 10: 06$ & Manual FIC & 327.30 & Good & \\
\hline $4 / 21 / 93 \quad 10: 03$ & Manual Tape & 327.00 & Good & \\
\hline $4 / 22 / 937: 06$ & Auto FIC & 327.30 & Good & \\
\hline 4/22/93 10:03 & Manual Tape & 327.00 & Good & \\
\hline $4 / 23 / 937: 06$ & Auto FIC & 327.4 & Good & \\
\hline 4/23/93 $10: 03$ & Manual Tape & 327.00 & Good & \\
\hline $4 / 24 / 937: 05$ & Auto FIC & 327.40 & Good & \\
\hline 4/24/93 10:03 & Manual Tape & 327.00 & Good & \\
\hline $4 / 25 / 937: 06$ & Auto FIC & 327.40 & Good & \\
\hline
\end{tabular}

Data obtained from WHC Surveillance Analysis Computer Systern, (PC SACS) July 7, 1995. 


\begin{tabular}{|c|c|c|c|c|}
\hline \multicolumn{5}{|c|}{ 241-AP-108 } \\
\hline \multicolumn{5}{|c|}{ Surface Level Data } \\
\hline Date & Type of Measurement & Level (Inches) & Data Quality & Comments \\
\hline $4 / 25 / 9310: 03$ & Manua! Tape & 327 & Good & \\
\hline $4 / 26 / 937: 06$ & Auto FIC & 327.40 & Good & \\
\hline $4 / 26 / 93 \quad 10: 03$ & Manual Tape & 327.00 & Good & \\
\hline $4 / 27 / 937: 06$ & Auto FIC & 327.4 & Good & \\
\hline 4/27/93 10:03 & Manual Tape & 327.00 & Good & \\
\hline $4 / 28 / 937: 03$ & Auto FIC & 327.40 & Good & \\
\hline $4 / 28 / 93 \quad 10: 03$ & Manual Tape & 327.00 & Good & \\
\hline $4 / 29 / 937: 03$ & Auto FIC & 327.5 & Good & \\
\hline $4 / 29 / 93 \quad 10: 03$ & Manual Tape & 327 & Good & \\
\hline $4 / 30 / 937: 06$ & Auto FIC & 327.5 & Good & \\
\hline 4/30/93 10:03 & Manual Tape & 327.00 & Good & \\
\hline 5/1/93 7:05 & Auto FIC & 327.60 & Good & \\
\hline $5 / 1 / 93 \quad 10: 05$ & Manual Tape & 327 & Good & \\
\hline 5/2/93 7:06 & Auto FIC & 327.6 & Good & \\
\hline $5 / 2 / 93 \quad 10: 05$ & Manual Tape & 327.00 & Good & \\
\hline 5/3/93 7:06 & Auto FIC & 327.7 & Good & \\
\hline $5 / 3 / 93 \quad 10: 05$ & Manual Tape & 326.75 & Good & \\
\hline $5 / 4 / 9310: 05$ & Manual FIC & 327.70 & Good & \\
\hline $5 / 4 / 9310: 05$ & Manual Tape & 327.00 & Good & \\
\hline $5 / 5 / 93 \quad 10: 05$ & Manual FIC & 327.8 & Good & \\
\hline $5 / 5 / 9310: 05$ & Manual Tape & 327 & Good & \\
\hline $5 / 6 / 93 \quad 10: 05$ & Manual FIC & 327.90 & Good & \\
\hline $5 / 6 / 93 \quad 10: 05$ & Manual Tape & 326.75 & Good & \\
\hline $5 / 7 / 93 \quad 10: 05$ & Manual FIC & 328.00 & Good & \\
\hline $5 / 7 / 93 \quad 10: 05$ & Manual Tape & 327.00 & Good & \\
\hline $5 / 8 / 93 \quad 10: 05$ & Manual FIC & 328.00 & Good & \\
\hline $5 / 8 / 93 \quad 10: 05$ & Manual Tape & 327 & Good & \\
\hline $5 / 9 / 9310: 05$ & Manual FIC & 327.6 & Good & \\
\hline $5 / 9 / 93 \quad 10: 05$ & Manual Tape & 327 & Good & \\
\hline $5 / 10 / 93 \quad 10: 05$ & Manual FIC & 327.6 & Good & \\
\hline $5 / 10 / 9310: 05$ & Manual Tape & 327.00 & Good & \\
\hline $5 / 11 / 937: 06$ & Auto FIC & 327.70 & Good & \\
\hline $5 / 11 / 9310: 05$ & Manual Tape & 326.75 & Good & \\
\hline $5 / 12 / 937: 06$ & Auto FIC & 327.70 & Good & \\
\hline $5 / 12 / 9310: 05$ & Manual Tape & 326.75 & Good & \\
\hline $5 / 13 / 9310: 05$ & Manual FIC & 327.85 & Good & \\
\hline $5 / 13 / 9310: 05$ & Manual Tape & $327: 25$ & Good & \\
\hline $5 / 14 / 93 \quad 10: 05$ & Manual FIC & 327.8 & Good & \\
\hline $5 / 14 / 93 \quad 10: 05$ & Manual Tape & 327.00 & Good & \\
\hline $5 / 15 / 93 \quad 10: 05$ & Manual FIC & 327.9 & Good & \\
\hline $5 / 15 / 93 \quad 10: 05$ & Manual Tape & 327.00 & Good & \\
\hline $5 / 16 / 93 \quad 10: 05$ & Manual FIC & 327.95 & Good & \\
\hline $5 / 16 / 93 \quad 10: 05$ & Manual Tape & 327 & Good & \\
\hline $5 / 17 / 937: 03$ & Auto FIC & 327.90 & Good & \\
\hline $5 / 17 / 9310: 05$ & Manual FIC & 327.9 & Good & \\
\hline $5 / 17 / 93 \quad 10: 05$ & Manual Tape & 327 & Good & \\
\hline $5 / 18 / 937: 03$ & Auto FIC & 0.00 & Suspect & Data not included in graph \\
\hline $5 / 18 / 93 \quad 10: 05$ & Manual FIC & 328 & Good & \\
\hline
\end{tabular}

Data obtained from WHC Surveillance Analysis Computer System, (PC SACS) July 7, 1995. 


\begin{tabular}{|c|c|c|c|c|}
\hline \multicolumn{5}{|c|}{ 241-AP-108 } \\
\hline \multicolumn{5}{|c|}{ Surface Level Data } \\
\hline Date & Type of Measurement & Level (Inches) & Data Quality & Comments \\
\hline $5 / 18 / 93 \quad 10: 05$ & Manual Tape & 327.00 & Good & \\
\hline $5 / 19 / 937: 03$ & Auto FIC & 327.60 & Good & \\
\hline $5 / 19 / 93 \quad 10: 05$ & Manual Tape & 327.00 & Good & \\
\hline $5 / 20 / 937: 03$ & Auto FIC & 0.00 & Suspect & Data not included in graph \\
\hline $5 / 20 / 9310: 03$ & Manual FIC & 327.65 & Good & \\
\hline $5 / 20 / 93 \quad 10: 05$ & Manual Tape & 327 & Good & \\
\hline $5 / 21 / 93 \quad 10: 03$ & Manual FIC & 327.60 & Good & \\
\hline $5 / 21 / 93 \quad 10: 05$ & Manual Tape & 326.75 & Good & \\
\hline $5 / 22 / 937: 03$ & Auto FIC & 327.6 & Good & \\
\hline $5 / 22 / 93 \quad 10: 05$ & Manual Tape & 327 & Good & \\
\hline $5 / 23 / 9310: 05$ & Manual FIC & 327.70 & Good & \\
\hline $5 / 23 / 9310: 05$ & Manual Tape & 327 & Good & \\
\hline $5 / 24 / 937: 03$ & Auto FIC & 327.7 & Good & \\
\hline 5/24/93 10:05 & Manual Tape & 327.00 & Good & \\
\hline $5 / 25 / 937: 03$ & Auto FIC & 327.7 & Good & \\
\hline $5 / 25 / 9310: 05$ & Manual Tape & 327 & Good & \\
\hline $5 / 26 / 937: 03$ & Auto FIC & 327.8 & Good & \\
\hline $5 / 26 / 93 \quad 10: 05$ & Manual Tape & 327 & Good & \\
\hline $5 / 27 / 937: 03$ & Auto FIC & 327.8 & Good & \\
\hline $5 / 27 / 93 \quad 10: 05$ & Manual Tape & 327.00 & Good & \\
\hline $5 / 28 / 937: 03$ & Auto FIC & 327.9 & Good & \\
\hline 5/28/93 10:05 & Manual Tape & 327.00 & Good & \\
\hline $5 / 29 / 937: 05$ & Auto FIC & 328.00 & Good & \\
\hline 5/29/93 10:05 & Manual Tape & 327.00 & Good & \\
\hline 5/30/93 7:05 & Auto FIC & 327.50 & Good & \\
\hline $5 / 30 / 9310: 05$ & Manual Tape & 327.00 & Good & \\
\hline $5 / 31 / 937: 06$ & Auto FIC & 327.60 & Good & \\
\hline $5 / 31 / 93 \quad 10: 05$ & Manual Tape & 327 & Good & \\
\hline $6 / 1 / 93$ 7:06 & Auto FIC & 327.6 & Good & \\
\hline $6 / 1 / 93 \quad 10: 06$ & Manual Tape & 327.00 & Good & \\
\hline $6 / 2 / 93 \quad 7: 06$ & Auto FIC & 327.6 & Good & \\
\hline $6 / 2 / 93 \quad 10: 06$ & Manual Tape & 327.00 & Good & \\
\hline 6/3/93 7:06 & Auto FIC & 327.6 & Good & \\
\hline $6 / 3 / 93 \quad 10: 06$ & Manual Tape & 327.25 & Good & \\
\hline $6 / 4 / 937: 06$ & Auto FIC & 327.6 & Good & \\
\hline $6 / 4 / 93 \quad 10: 06$ & Manual Tape & 327.25 & Good & \\
\hline $6 / 5 / 937: 06$ & Auto FIC & 327.60 & Good & \\
\hline $6 / 5 / 93 \quad 10: 06$ & Manual Tape & 327.00 & Good & - \\
\hline $6 / 6 / 937: 05$ & Auto FIC & 327.6 & Good & \\
\hline $6 / 6 / 93 \quad 10: 06$ & Manual Tape & 327 & Good & \\
\hline $6 / 7 / 93 \quad 7: 05$ & Auto FIC & 327.60 & Good & \\
\hline $6 / 7 / 93 \quad 10: 06$ & Manual Tape & 327 & Good & \\
\hline $6 / 8 / 937: 05$ & Auto FIC & 327.60 & Good & \\
\hline $6 / 8 / 93 \quad 10: 06$ & Manual Tape & 327 & Good & \\
\hline 6/9/93 7:05 & Auto FIC & 327.50 & Good & \\
\hline $6 / 9 / 93 \quad 10: 06$ & Manual Tape & 327 & Good & \\
\hline $6 / 10 / 937: 04$ & Auto FIC & 327.5 & Good & \\
\hline $6 / 10 / 9310: 06$ & Manual Tape & 327.00 & Good & \\
\hline
\end{tabular}

Data obtained from WHC Surveillance Analysis Computer System, (PC SACS) July 7, 1995. 


\begin{tabular}{|c|c|c|c|c|}
\hline \multicolumn{5}{|c|}{ 241-AP-108 } \\
\hline \multicolumn{5}{|c|}{ Surface Level Data } \\
\hline Date & Type of Messurement & Level (Inches) & Data Quality & Comments \\
\hline $6 / 11 / 937: 05$ & Auto FIC & 327.6 & Good & \\
\hline $6 / 11 / 93 \quad 10: 06$ & Manual Tape & 327.00 & Good & \\
\hline $6 / 12 / 937: 05$ & Auto FIC & 327.70 & Good & \\
\hline $6 / 12 / 9310: 06$ & Manual Tape & 327 & Good & \\
\hline $6 / 13 / 937: 04$ & Auto FIC & 327.8 & Good & \\
\hline $6 / 13 / 93 \quad 10: 06$ & Manual Tape & 327 & Good & \\
\hline $6 / 14 / 937: 05$ & Auto FIC & 121.80 & Suspect & Data not included in graph \\
\hline $6 / 14 / 93 \quad 10: 05$ & Manual FIC & 327.9 & Good & \\
\hline $6 / 14 / 93 \quad 10: 06$ & Manual Tape & 327 & Good & \\
\hline $6 / 15 / 937: 05$ & Auto FIC & 327.90 & Good & \\
\hline $6 / 15 / 9310: 06$ & Manual Tape & 327 & Good & \\
\hline $6 / 16 / 937: 05$ & Auto FIC & 128 & Suspect & Data not included in graph \\
\hline $6 / 16 / 93 \quad 10: 05$ & Manual FIC & 328 & Good & \\
\hline $6 / 16 / 9310: 06$ & Manual Tape & 327 & Good & \\
\hline $6 / 17 / 937: 05$ & Auto FIC & 121.9 & Suspect & Data not included in graph \\
\hline $6 / 17 / 9310: 05$ & Manual FIC & 327.95 & Good & \\
\hline $6 / 17 / 93 \quad 10: 06$ & Manual Tape & 326.75 & Good & \\
\hline $6 / 18 / 937: 06$ & Auto FIC & 128.00 & Suspect & Data not included in graph \\
\hline $6 / 18 / 93 \quad 10: 06$ & Manual FIC & 328 & Good & \\
\hline $6 / 18 / 93 \quad 10: 06$ & Manual Tape & 327 & Good & \\
\hline $6 / 19 / 937: 05$ & Auto FIC & 328.05 & Good & \\
\hline $6 / 19 / 93 \quad 10: 06$ & Manual Tape & 326.75 & Good & \\
\hline $6 / 20 / 937: 05$ & Auto FIC & 328 & Good & \\
\hline $6 / 20 / 93 \quad 10: 06$ & Manual Tape & 327.00 & Good & \\
\hline $6 / 21 / 937: 05$ & Auto FIC & 328.05 & Good & \\
\hline 6/21/93 10:06 & Manual Tape & 327.00 & Good & \\
\hline $6 / 22 / 937: 05$ & Auto FIC & 328.05 & Good & \\
\hline $6 / 22 / 93 \quad 10: 06$ & Manual Tape & 327 & Good & \\
\hline $6 / 23 / 937: 05$ & Auto FIC & 328.00 & Good & \\
\hline $6 / 23 / 93 \quad 10: 06$ & Manual Tape & 327 & Good & \\
\hline $6 / 24 / 937: 05$ & Auto FIC & 328.05 & Good & \\
\hline $6 / 24 / 9310: 06$ & Manual Tape & 327.00 & Good & \\
\hline $6 / 25 / 93 \quad 0: 00$ & Manual FIC & 328.00 & Good & \\
\hline $6 / 25 / 937: 03$ & Auto FIC & 128 & Suspect & Data not included in graph \\
\hline $6 / 25 / 9310: 06$ & Manual Tape & 327 & Good & \\
\hline $6 / 26 / 937: 03$ & Auto FIC & 328.1 & Good & \\
\hline $6 / 26 / 93 \quad 10: 06$ & Manual Tape & 327.00 & Good & \\
\hline $6 / 27 / 937: 04$ & Auto FIC & 328.10 & Good & $\cdot$ \\
\hline $6 / 27 / 93 \quad 10: 06$ & Manual Tape & 327.00 & Good & \\
\hline $6 / 28 / 937: 03$ & Auto FIC & 328.10 & Good & \\
\hline $6 / 28 / 93 \quad 10: 06$ & Manual Tape & 327.00 & Good & \\
\hline $6 / 29 / 937: 04$ & Auto FIC & 328.10 & Good & \\
\hline $6 / 29 / 93 \quad 10: 06$ & Manual Tape & 327.00 & Good & \\
\hline $6 / 30 / 937: 03$ & Auto FIC & 328.20 & Good & \\
\hline $6 / 30 / 9310: 06$ & Manual Tape & 326.75 & Good & \\
\hline 7/1/93 7:06 & Auto FIC & 323.4 & Suspect & Data not included in graph \\
\hline $7 / 1 / 9310: 06$ & Manual FIC & 327.50 & Good & \\
\hline 7/1/93 10:06 & Manual Tape & 327 & Good & \\
\hline
\end{tabular}

Data obtained from WHC Surveillance Analysis Computer System, (PC SACS) July 7, 1995. 


\begin{tabular}{|c|c|c|c|c|}
\hline \multicolumn{5}{|c|}{ 241-AP-108 } \\
\hline & & Ifface Level Date & & \\
\hline Date & Type of Measurement & Levol (Inches) & Data Quality & Comments \\
\hline 7/2/93 7:06 & Auto FIC & 323.60 & Suspect & Data not included in graph \\
\hline $7 / 2 / 93 \quad 10: 06$ & Manual FIC & 327.6 & Good & \\
\hline $7 / 2 / 9310: 06$ & Manual Tape & 327.00 & Good & \\
\hline 7/3/93 7:03 & Auto FIC & 323.7 & Suspect & Data not included in graph \\
\hline $7 / 3 / 93 \quad 10: 06$ & Manual FIC & 327.75 & Good & \\
\hline $7 / 3 / 93 \quad 10: 06$ & Manual Tape & 327 & Good & \\
\hline $7 / 4 / 937: 04$ & Auto FIC & 323.8 & Suspect & Data not included in graph \\
\hline $7 / 4 / 9310: 06$ & Manual FIC & 327.85 & Good & \\
\hline $7 / 4 / 93 \quad 10: 06$ & Manual Tape & 327.00 & Good & \\
\hline $7 / 5 / 937: 03$ & Auto FIC & 121.90 & Suspect & Data not included in graph \\
\hline $7 / 5 / 93 \quad 10: 04$ & Manual FIC & 327.95 & Good & \\
\hline $7 / 5 / 9310: 06$ & Manual Tape & 327.00 & Good & \\
\hline 7/6/93 7:03 & Auto FIC & 327.90 & Good & \\
\hline $7 / 6 / 9310: 06$ & Manual Tape & 327 & Good & \\
\hline 7/7/93 7:04 & Auto FIC & 121.9 & Suspect & Data not included in graph \\
\hline $7 / 7 / 93 \quad 10: 04$ & Manual FIC & 328.00 & Good & \\
\hline $7 / 7 / 93 \quad 10: 06$ & Manual Tape & 327.00 & Good & \\
\hline 7/8/93 7:04 & Auto FIC & 128.00 & Suspect & Data not included in graph \\
\hline $7 / 8 / 93 \quad 10: 04$ & Manual FIC & 328 & Good & \\
\hline $7 / 8 / 9310: 06$ & Manual Tape & 326.75 & Good & \\
\hline 7/9/93 7:05 & Auto FIC & 128.00 & Suspect & Data not included in graph \\
\hline $7 / 9 / 9310: 05$ & Manual FIC & 328 & Good & \\
\hline $7 / 9 / 93 \quad 10: 06$ & Manual Tape & 327.25 & Good & \\
\hline $7 / 10 / 937: 03$ & Auto FIC & 128 & Suspect & Data not included in graph \\
\hline $7 / 10 / 93 \quad 10: 03$ & Manual FIC & 328 & Good & \\
\hline $7 / 10 / 93 \quad 10: 06$ & Manual Tape & 327 & Good & \\
\hline $7 / 11 / 937: 03$ & Auto FIC & 128 & Suspect & Data not included in graph \\
\hline $7 / 11 / 9310: 03$ & Manual FIC & 328 & Good & \\
\hline $7 / 11 / 93 \quad \overline{10: 06}$ & Manual Tape & 327 & Good & \\
\hline $7 / 12 / 937: 02$ & Auto FIC & 128 & Suspect & Data not included in graph \\
\hline $7 / 12 / 93$ 10:02 & Manual FIC & 328.00 & Good & \\
\hline $7 / 12 / 93 \quad 10: 06$ & Manual Tape & 327 & Good & \\
\hline $7 / 13 / 937: 03$ & Auto FIC & 128.00 & Suspect & Data not included in graph \\
\hline $7 / 13 / 93 \quad 10: 03$ & Manual FIC & 328.00 & Good & \\
\hline $7 / 13 / 93 \quad 10: 06$ & Manual Tape & 327.00 & Good & \\
\hline $7 / 14 / 937: 06$ & Auto FIC & 128 & Suspect & Data not included in graph \\
\hline $7 / 14 / 93 \quad 10: 06$ & Manual FIC & 328 & Good & \\
\hline $7 / 14 / 93$ 10:06 & Manual Tape & 327.00 & Good & \\
\hline $7 / 15 / 937: 06$ & Auto FIC & 128 & Suspect & Data not included in graph \\
\hline $7 / 15 / 93 \quad 10: 06$ & Manual FIC & 328 & Good & \\
\hline $7 / 15 / 9310: 06$ & Manual Tape & 327.00 & Good & \\
\hline $7 / 16 / 937: 06$ & Auto FIC & 128 & Suspect & Data not included in graph \\
\hline $7 / 16 / 9310: 06$ & Manual FIC & 328 & Good & \\
\hline $7 / 16 / 9310: 06$ & Manual Tape & 327 & Good & \\
\hline $7 / 17 / 937: 07$ & Auto FIC & 323.6 & Suspect & Data not included in graph \\
\hline $7 / 17 / 93 \quad 10: 06$ & Manual FIC & 328.00 & Good & \\
\hline $7 / 17 / 9310: 06$ & Manual Tape & 327.00 & Good & \\
\hline $7 / 18 / 937: 06$ & Auto FIC & 323.7 & Suspect & Data not included in graph \\
\hline
\end{tabular}

Data obtained from WHC Surveillance Analysis Computer System, (PC SACS) July 7, 1995. 


\begin{tabular}{|c|c|c|c|c|}
\hline \multicolumn{5}{|c|}{ 241-AP-108 } \\
\hline \multicolumn{5}{|c|}{ Surface Level Data } \\
\hline Date & Type of Measurement & Level (Inches) & Data Quality & Comments \\
\hline $7 / 18 / 93 \quad 10: 06$ & Manual FIC & 327.7 & Good & \\
\hline $7 / 18 / 93 \quad 10: 06$ & Manual Tape & 327 & Good & \\
\hline 7/19/93 7:03 & Auto FIC & 323.8 & Suspect & Data not included in graph \\
\hline 7/19/93 10:06 & Manual FIC & 327.80 & Good & \\
\hline $7 / 19 / 93$ 10:06 & Manual Tape & 327 & Good & \\
\hline $7 / 20 / 937: 03$ & Auto FIC & 323.9 & Suspect & Data not included in graph \\
\hline $7 / 20 / 9310: 06$ & Manual FIC & 327.9 & Good & \\
\hline $7 / 20 / 9310: 06$ & Manual Tape & 327 & Good & \\
\hline $7 / 21 / 937: 03$ & Auto FIC & 323.90 & Suspect & Data not included in graph \\
\hline $7 / 21 / 9310: 06$ & Manual FIC & 328.00 & Good & \\
\hline $7 / 21 / 93 \quad 10: 06$ & Manual Tape & 326.75 & Good & \\
\hline $7 / 22 / 932: 03$ & Auto FIC & 128 & Suspect & Data not included in graph \\
\hline $7 / 22 / 93 \quad 10: 03$ & Manual FIC & 328 & Good & \\
\hline $7 / 22 / 93 \quad 10: 06$ & Manual FIC & 328.00 & Good & \\
\hline $7 / 22 / 93 \quad 10: 06$ & Manual Tape & 326.75 & Good & \\
\hline $7 / 23 / 937: 03$ & Auto FIC & 323.90 & Suspect & Data not included in graph \\
\hline $7 / 23 / 9310: 06$ & Manual FIC & 327.90 & Good & \\
\hline $7 / 23 / 93 \quad 10: 06$ & Manual Tape & 326.75 & Good & \\
\hline $7 / 24 / 93 \quad 7: 03$ & Auto FIC & 323.90 & Suspect & Data not included in graph \\
\hline $7 / 24 / 93 \quad 10: 06$ & Manual FIC & 327.9 & Good & \\
\hline $7 / 24 / 9310: 06$ & Manual Tape & 326.75 & Good & \\
\hline $7 / 25 / 93 \quad 10: 06$ & Manual FIC & 327,00 & Good & \\
\hline $7 / 25 / 93 \quad 10: 06$ & Manual Tape & 326.5 & Good & \\
\hline $7 / 25 / 93 \quad 15: 04$ & Auto FIC & 121.9 & Suspect & Data not included in .graph \\
\hline $7 / 26 / 93 \quad 7: 03$ & Auto FIC & 323.90 & Suspect & Data not included in graph \\
\hline $7 / 26 / 93 \quad 10: 06$ & Manual FIC & 327 & Good & \\
\hline $7 / 26 / 93 \quad 10: 06$ & Manual Tape & 326.75 & Good & \\
\hline $7 / 27 / 937: 03$ & Auto FIC & 323.90 & Suspect & Data not included in graph \\
\hline $7 / 27 / 93 \quad 10: 06$ & Manual FIC & 327.00 & Good & \\
\hline $7 / 27 / 93 \quad 10: 06$ & Manual Tape & 326.75 & Good & \\
\hline $7 / 28 / 930: 00$ & Manual FIC & 328 & Good & \\
\hline $7 / 28 / 93 \quad 0: 00$ & Manual Tape & 326.75 & Good & \\
\hline $7 / 28 / 93 \quad 15: 04$ & Auto FIC & 323.90 & Suspect & Data not included in graph \\
\hline $7 / 29 / 930: 00$ & Manual Tape & 326.75 & Good & \\
\hline $7 / 29 / 933: 04$ & Auto FIC & 327.90 & Good & \\
\hline $7 / 30 / 930: 00$ & Manual FIC & 328.00 & Good & \\
\hline $7 / 30 / 930: 00$ & Manual Tape & 327.00 & Good & \\
\hline $7 / 30 / 937: 03$ & Auto FIC & 0.00 & Suspect & Data not included in graph \\
\hline 7/31/930:00 & Manual Tape & 327 & Good & \\
\hline $7 / 31 / 937: 03$ & Auto FIC & 327.90 & Good & \\
\hline $8 / 1 / 930: 00$ & Manual FIC & 328.00 & Good & \\
\hline $8 / 1 / 930: 00$ & Manual Tape & 327.00 & Good & \\
\hline 8/1/93 7:03 & Auto FIC & 0.00 & Suspect & Data not included in graph \\
\hline $8 / 2 / 930: 00$ & Manual Tape & 326.75 & Good & \\
\hline $8 / 2 / 937: 06$ & Auto FIC & 0 & Suspect & Data not included in graph \\
\hline $8 / 3 / 930: 00$ & Manual FIC & 328 & Good & \\
\hline $8 / 3 / 930: 00$ & Manual Tape & 327 & Good & \\
\hline $8 / 3 / 937: 03$ & Auto FIC & .0 & Suspect & Data not included in graph \\
\hline
\end{tabular}

Data obtained from WHC Surveillance Analysis Computer System, (PC SACS) July 7, 1995. 


\begin{tabular}{|c|c|c|c|c|}
\hline \multicolumn{5}{|c|}{ 241-AP-108 } \\
\hline \multicolumn{5}{|c|}{ Surface Lavel Data } \\
\hline Date & Type of Measurement & Level (Inches) & Data Quality & Comments \\
\hline $8 / 4 / 930: 00$ & Manual FIC & 328.00 & Good & \\
\hline 8/4/930:00 & Manual Tape & 327:00 & Good & \\
\hline $8 / 4 / 937: 03$ & Auto FIC & 0 & Suspect & Data not included in graph \\
\hline $8 / 5 / 930: 00$ & Manual FIC & 328 & Good & \\
\hline $8 / 5 / 930: 00$ & Manual Tape & 327 & Good & \\
\hline 8/5/93 7:04 & Auto FIC & 0 & Suspect & Data not included in graph \\
\hline $8 / 6 / 930: 00$ & Manual FIC & 328.00 & Good & \\
\hline 8/6/93 0:00 & Manual Tape & 327.00 & Good & \\
\hline $8 / 6 / 93 \quad 15: 04$ & Auto FIC & 323.90 & Suspect & Data not included in graph \\
\hline 8/7/930:00 & Manual FIC & 328.00 & Good & \\
\hline $8 / 7 / 930: 00$ & Manual Tape & 327.00 & Good & \\
\hline $8 / 7 / 937: 04$ & Auto FIC & 0.00 & Suspect & Data not included in graph \\
\hline $8 / 8 / 930: 00$ & Manual Tape & 327 & Good & \\
\hline 8/8/93 7:04 & Auto FIC & 327.9 & Good & \\
\hline $8 / 9 / 930: 00$ & Manual FIC & 328.00 & Good & \\
\hline $8 / 9 / 930: 00$ & Manual Tape & $\mathbf{3 2 7 . 0 0}$ & Good & \\
\hline $8 / 9 / 937: 04$ & Auto FIC & 0 & Suspect & Data not included in graph \\
\hline $8 / 10 / 930: 00$ & Manual FIC & 327.7 & Good & \\
\hline $8 / 10 / 930: 00$ & Manual Tape & 327 & Good & \\
\hline $8 / 10 / 937: 04$ & Auto FIC & 0 & Suspect & Data not included in graph \\
\hline $8 / 11 / 930: 00$ & Manual FIC & 327.70 & Good & \\
\hline $8 / 11 / 930: 00$ & Manual Tape & 327.00 & Good & \\
\hline $8 / 11 / 937: 04$ & Auto FIC & 0 & Suspect & Data not included in graph \\
\hline $8 / 12 / 930: 00$ & Manual FIC & 327.7 & Good & \\
\hline $8 / 12 / 930: 00$ & Manual Tape & 326.75 & Good & \\
\hline $8 / 12 / 937: 04$ & Auto FIC & 0.00 & Suspect & Data not included in graph \\
\hline 8/13/93 0:00 & Manual FIC & 327.7 & Good & \\
\hline $8 / 13 / 930: 00$ & Manual Tape & 326.75 & Good & \\
\hline $8 / 13 / 937: 04$ & Auto FIC & 0 & Suspect & Data not included in graph \\
\hline 8/14/93 0:00 & Manual FIC & 327.8 & Good & \\
\hline 8/14/93 0:00 & Manual Tape & 327 & Good & \\
\hline 8/14/93 7:03 & Auto FIC & 0.00 & Suspect & Data not included in graph \\
\hline $8 / 15 / 930: 00$ & Manual FIC & 327.9 & Good & \\
\hline $8 / 15 / 930: 00$ & Manual Tape & 327 & Good & \\
\hline $8 / 15 / 937: 04$ & Auto FIC & 121.8 & Suspect & Data not included in graph \\
\hline $8 / 16 / 930: 00$ & Manual Tape & 327.00 & Good & \\
\hline $8 / 16 / 937: 04$ & Auto FIC & 327.90 & Good & \\
\hline 8/17/93 0:00 & Manual Tape & 327.30 & Good & 4 \\
\hline $8 / 17 / 937: 04$ & Auto FIC & 327.9 & Good & \\
\hline 8/18/93 0:00 & Manual Tape & 327.00 & Good & \\
\hline $8 / 18 / 937: 03$ & Auto FIC & 327.9 & Good & \\
\hline 8/19/93 0:00 & Manual FIC & 328.00 & Good & \\
\hline $8 / 19 / 930: 00$ & Manual Tape & 326.75 & Good & \\
\hline 8/19/93 7:04 & Auto FIC & 121.9 & Suspect & Data not included in graph \\
\hline $8 / 20 / 930: 00$ & Manual Tape & 326.75 & Good & \\
\hline $8 / 20 / 937: 04$ & Auto FIC & 327.90 & . Good & \\
\hline $8 / 21 / 930: 00$ & Manual Tape & 326.75 & Good & \\
\hline $8 / 21 / 937: 04$ & Auto FIC & 327.90 & Good & \\
\hline
\end{tabular}

Data obtained from WHC Surveillance Analysis Computer System, (PC SACS) July 7, 1995. 


\begin{tabular}{|c|c|c|c|c|}
\hline \multicolumn{5}{|c|}{ 241-AP-108 } \\
\hline \multicolumn{5}{|c|}{ Surface Level Data } \\
\hline Date & Type of Measurement & Level (inches) & Data Quality & Comments \\
\hline $8 / 22 / 930: 00$ & Manual FIC & 327.9 & Good & \\
\hline $8 / 22 / 930: 00$ & Manual Tape & 327 & Good & \\
\hline $8 / 22 / 937: 04$ & Auto FIC & 0 & Suspect & Data not included in graph \\
\hline $8 / 23 / 930: 00$ & Manual FIC & 327.9 & Good & \\
\hline $8 / 23 / 930: 00$ & Manual Tape & 326.75 & Good & \\
\hline $8 / 23 / 937: 04$ & Auto FIC & 0.00 & Suspect & Data not included in graph \\
\hline $8 / 24 / 930: 00$ & Manual FIC & 327.95 & Good & \\
\hline $8 / 24 / 930: 00$ & Manual Tape & 327.00 & Good & \\
\hline $8 / 24 / 937: 04$ & Auto FIC & 0.00 & Suspect & Data not included in graph \\
\hline $8 / 25 / 930: 00$ & Manual FIC & 328 & Good & \\
\hline $8 / 25 / 930: 00$ & Manual Tape & 326.75 & Good & \\
\hline $8 / 25 / 937: 04$ & Auto FIC & 0.00 & Suspect & Data not included in graph \\
\hline $8 / 26 / 930: 00$ & Manual Tape & 326.75 & Good & \\
\hline $8 / 26 / 937: 04$ & Auto FIC & 327.90 & Good & \\
\hline $8 / 27 / 930: 00$ & Manual FIC & 327.95 & Good & \\
\hline $8 / 27 / 930: 00$ & Manual Tape & 326.75 & Good & \\
\hline $8 / 27 / 937: 05$ & Auto FIC & 0 & Suspect & Data not included in graph \\
\hline $8 / 28 / 930: 00$ & Manual FIC & 328 & Good & \\
\hline $8 / 28 / 930: 00$ & Manual Tape & 327 & Good & \\
\hline $8 / 28 / 937: 04$ & Auto FIC & 0 & Suspect & Data not included in graph \\
\hline $8 / 29 / 930: 00$ & Manual FIC & 327.95 & Good & \\
\hline $8 / 29 / 930: 00$ & Manual Tape & 326.75 & Good & \\
\hline $8 / 29 / 937: 03$ & Auto FIC & 0.00 & Suspect & Data not included in graph \\
\hline $8 / 30 / 930: 00$ & Manual FIC & 328 & Good & \\
\hline $8 / 30 / 930: 00$ & Manual Tape & 327.00 & Good & \\
\hline $8 / 30 / 937: 04$ & Auto FIC & 0 & Suspect & Data not included in graph \\
\hline $8 / 31 / 930: 00$ & Manual FIC & 328 & Good & \\
\hline $8 / 31 / 930: 00$ & Manual Tape. & 327.00 & Good & \\
\hline $8 / 31 / 937: 04$ & Auto FIC & 0 & Suspect & Data not included in graph \\
\hline 9/1/93 0:00 & Manual FIC & 328.00 & Good & \\
\hline $9 / 1 / 930: 00$ & Manual Tape & 327.00 & Good & \\
\hline $9 / 1 / 937: 04$ & Auto FIC & 0.00 & Suspect & Data not included in graph \\
\hline $9 / 2 / 930: 00$ & Manual FIC & 327.90 & Good & \\
\hline $9 / 2 / 930: 00$ & Manual Tape & 327.00 & Good & \\
\hline 9/2/93 7:04 & Auto FIC & 0 & Suspect & Data not included in graph \\
\hline 9/3/93 0:00 & Manual FIC & 328 & Good & \\
\hline 9/3/93 0:00 & Manual Tape & 327 & Good & \\
\hline 9/3/93 7:04 & Auto FIC & 0.00 & Suspect & Data not included in graph \\
\hline $9 / 4 / 930: 00$ & Manual FIC & 328 & Good & \\
\hline $9 / 4 / 930: 00$ & Manual Tape & 327.00 & Good & \\
\hline $9 / 4 / 937: 04$ & Auto FIC & 0.00 & Suspect & Data not included in graph \\
\hline $9 / 5 / 930: 00$ & Manual FIC & 328 & Good & \\
\hline $9 / 5 / 930: 00$ & Manual Tape & 327.00 & Good & \\
\hline $9 / 5 / 937: 04$ & Auto FIC & 0.00 & Suspect & Data not included in graph \\
\hline $9 / 6 / 930: 00$ & Manual FIC & 328.00 & Good & \\
\hline $9 / 6 / 930: 00$ & Manual Tape & 327 & Good & \\
\hline $9 / 6 / 937: 04$ & Auto FIC & 0 & Suspect & Data not included in graph \\
\hline $9 / 7 / 930: 00$ & Manual FIC & 328.00 & Good & \\
\hline
\end{tabular}

Data obtained from WHC Surveillance Analysis Computer System, (PC SACS) July 7, 1995. 


\begin{tabular}{|c|c|c|c|c|}
\hline \multicolumn{5}{|c|}{ 241-AP-108 } \\
\hline \multicolumn{5}{|c|}{ Surface Level Data } \\
\hline Date & Type of Measurement & Level (Inches) & Data Quality & Comments \\
\hline $9 / 7 / 930: 00$ & Manual Tape & 327 & Good & \\
\hline 9/7/93 7:04 & Auto FIC & 0.00 & Suspect & Data not included in graph \\
\hline $9 / 8 / 930: 00$ & Manual FIC & 328.00 & Good & \\
\hline 9/8/93 0:00 & Manual Tape & 327.00 & Good & \\
\hline 9/8/93 7:04 & Auto FIC & 0.00 & Suspect & Data not included in graph \\
\hline 9/9/93 0:00 & Manual FIC & 328 & Good & \\
\hline 9/9/93 0:00 & Manual Tape & 327.00 & Good & \\
\hline 9/9/93 7:04 & Auto FIC & 0.00 & Suspect & Data not included in graph \\
\hline 9/10/93 0:00 & Manual FIC & 328 & Good & \\
\hline $9 / 10 / 930: 00$ & Manual Tape & 327.00 & Good & \\
\hline 9/10/93 7:04 & Auto FIC & 0 & Suspect & Data not included in graph \\
\hline 9/11/93 0:00 & Manual FIC & 328 & Good & \\
\hline $9 / 11 / 930: 00$ & Manual Tape & 327 & Good & \\
\hline $9 / 11 / 937: 04$ & Auto FIC & 0 & Suspect & Data not included in graph \\
\hline $9 / 12 / 930: 00$ & Manual FIC & 328.00 & Good & \\
\hline 9/12/93 0:00 & Manual Tape & 327 & Good & \\
\hline $9 / 12 / 937: 04$ & Auto FIC & 0.00 & Suspect & Data not included in graph \\
\hline $9 / 13 / 930: 00$ & Manual FIC & 328.00 & Good & \\
\hline $9 / 13 / 930: 00$ & Manual Tape & 326.75 & Good & \\
\hline $9 / 13 / 937: 04$ & Auto FIC & 0 & Suspect & Data not included in graph \\
\hline $9 / 14 / 930: 00$ & Manual FIC & 328.00 & Good & \\
\hline $9 / 14 / 930: 00$ & Manual Tape & 326.75 & Good & \\
\hline $9 / 14 / 937: 04$ & Auto FIC & 0.00 & Suspect & Data not included in graph \\
\hline 9/15/93 0:00 & Manual Tape & 326.75 & Good & \\
\hline $9 / 15 / 937: 04$ & Auto FIC & 328.00 & Good & \\
\hline $9 / 16 / 930: 00$ & Manual FIC & 328.00 & Good & \\
\hline $9 / 16 / 930: 00$ & Manual Tape & 326.75 & Good & \\
\hline $9 / 16 / 937: 04$ & Auto FIC & 121.9 & Suspect & Data not included in graph \\
\hline $9 / 17 / 930: 00$ & Manual FIC & 328 & Good & \\
\hline $9 / 17 / 930: 00$ & Manual Tape & 326.75 & Good & \\
\hline $9 / 17 / 937: 04$ & Auto FIC & 128 & Suspect & Data not included in graph \\
\hline 9/18/93 0:00 & Manual FIC & 328 & Good & \\
\hline $9 / 18 / 930: 00$ & Manual Tape & 326.75 & Good & \\
\hline $9 / 18 / 937: 04$ & Auto FIC & 323.90 & Suspect & Data not included in graph \\
\hline 9/19/93 0:00 & Manual FIC & 328 & Good & \\
\hline 9/19/93 0:00 & Manual Tape & 326.75 & Good & \\
\hline 9/19/93 7:04 & Auto FIC & 0 & Suspect & Data not included in graph \\
\hline $9 / 20 / 930: 00$ & Manual FIC & 328.00 & Good & \\
\hline 9/20/93 0:00 & Manual Tape & 326.75 & Good & \\
\hline 9/20/93 7:04 & Auto FIC & 0 & Suspect & Data not included in graph \\
\hline 9/21/93 0:00 & Manual FIC & 327.90 & Good & \\
\hline $9 / 21 / 930: 00$ & Manual Tape & 326.75 & Good & \\
\hline $9 / 21 / 937: 04$ & Auto FIC & 0.00 & Suspect & Data not included in graph \\
\hline $9 / 22 / 930: 00$ & Manual FIC & 327.90 & Good & \\
\hline $9 / 22 / 930: 00$ & Manual Tape & 326.75 & Good & \\
\hline $9 / 22 / 937: 04$ & Auto FIC & 0 & Suspect & Data not included in graph \\
\hline 9/23/93 0:00 & Manual FIC & 327.9 & Good & \\
\hline 9/23/93 0:00 & Manual Tape & 326.75 & Good & \\
\hline
\end{tabular}

Data obtained from WHC Surveillance Analysis Computer System, (PC SACS) July 7, 1995. 


\begin{tabular}{|c|c|c|c|c|}
\hline \multicolumn{5}{|c|}{ 241-AP-108 } \\
\hline \multicolumn{5}{|c|}{ Surface Level Data } \\
\hline Date & Type of Measurement & Level (Inches) & Data Quality & Commants \\
\hline $9 / 23 / 937: 04$ & Auto FIC & 0 & Suspect & Data not included in graph \\
\hline $9 / 24 / 930: 00$ & Manual FIC & 327.90 & Good & \\
\hline $9 / 24 / 930: 00$ & Manual Tape & $326.75^{\circ}$ & Good & \\
\hline $9 / 24 / 937: 04$ & Auto FIC & 0 & Suspect & Data not included in graph \\
\hline 9/25/93 $0: 00$ & Manual FIC & 327.9 & Good & \\
\hline $9 / 25 / 930: 00$ & Manual Tape & 326.75 & Good & \\
\hline 9/25/93 7:04 & Auto FIC & 0.00 & Suspect & Data not included in graph \\
\hline $9 / 26 / 930: 00$ & Manual FIC & 327.90 & Good & \\
\hline $9 / 26 / 930: 00$ & Manual Tape & 326.75 & Good & \\
\hline 9/26/93 23:04 & Auto FIC & 323.9 & Suspect & Data not included in graph \\
\hline $9 / 27 / 930: 00$ & Manual FIC & 327.9 & Good & \\
\hline $9 / 27 / 930: 00$ & Manual Tape & 326.75 & Good & \\
\hline $9 / 27 / 937: 04$ & Auto FIC & 323.9 & Suspect & Data not included in graph \\
\hline $9 / 28 / 930: 00$ & Manual Tape & 326.75 & Good & \\
\hline $9 / 28 / 937: 04$ & Auto FIC & 0 & Suspect & Data not included in graph \\
\hline $9 / 29 / 930: 00$ & Manual FIC & 327.9 & Good & \\
\hline $9 / 29 / 930: 00$ & Manual Tape & 326.75 & Good & \\
\hline $9 / 29 / 937: 04$ & Auto FIC & 323.9 & Suspect & Data not included in graph \\
\hline 9/30/93 0:00 & Manual FIC & 327.90 & Good & \\
\hline 9/30/93 0:00 & Manual Tape & 326.75 & Good & \\
\hline $9 / 30 / 937: 03$ & Auto FIC & 0.00 & Suspect & Data not included in graph \\
\hline 10/1/930:00 & Manual FIC & 327.90 & Good & \\
\hline $10 / 1 / 930: 00$ & Manual Tape & 326.75 & Good & \\
\hline $10 / 1 / 937: 06$ & Auto FIC & 0 & Suspect & Data not included in graph \\
\hline 10/2/93 0:00 & Manual FIC & 327.90 & Good & \\
\hline $10 / 2 / 930: 00$ & Manual Tape & 327 & Good & \\
\hline $10 / 2 / 937: 05$ & Auto FIC & 0 & Suspect & Data not included in graph \\
\hline $10 / 3 / 930: 00$ & Manual FIC & 327.9 & Good & \\
\hline $10 / 3 / 930: 00$ & Manual Tape & 326.75 & Good & \\
\hline $10 / 3 / 937: 06$ & Auto FIC & 0.00 & Suspect & Data not included in graph \\
\hline 10/4/93 0:00 & Manual FIC & 327.90 & Good & \\
\hline $10 / 4 / 930: 00$ & Manual Tape & 326.75 & Good & \\
\hline 10/4/93 7:06 & Auto FIC & 0.00 & Suspect & Data not included in graph \\
\hline $10 / 5 / 930: 00$ & Manual Tape & 326.75 & Good & \\
\hline $10 / 5 / 937: 06$ & Auto FIC & 327.9 & Good & \\
\hline $10 / 6 / 930: 00$ & Manual FIC & 327.90 & Good & \\
\hline $10 / 6 / 93 \quad 0: 00$ & Manual Tape & 326.75 & Good & \\
\hline $10 / 6 / 937: 06$ & Auto FIC & 0.00 & Suspect & Data not included in graph \\
\hline 10/7/93 0:00 & Manual FIC & 327.9 & Good & \\
\hline $10 / 7 / 930: 00$ & Manual Tape & 327 & Good & \\
\hline $10 / 7 / 937: 05$ & Auto FIC & 0.00 & Suspect & Data not included in graph \\
\hline $10 / 8 / 930: 00$ & Manual FIC & $\mathbf{3 2 7 . 9 0}$ & Good & \\
\hline $10 / 8 / 930: 00$ & Manual Tape & 326.75 & Good & \\
\hline $10 / 8 / 937: 04$ & Auto FIC & 0 & Suspect & Data not included in graph \\
\hline $10 / 9 / 930: 00$ & Manual FIC & 327.90 & Good & \\
\hline 10/9/93 0:00 & Manual Tape & 326.50 & Good & \\
\hline $10 / 9 / 937: 03$ & Auto FIC & 0.00 & Suspect & Data not included in graph \\
\hline $10 / 10 / 930: 00$ & Manual FIC & 327.90 & Good & \\
\hline
\end{tabular}

Data obtained from WHC Surveillance Analysis Computer System, (PC SACS) July 7, 1995. 


\begin{tabular}{|c|c|c|c|c|}
\hline \multicolumn{5}{|c|}{ 241-AP-108 } \\
\hline \multicolumn{5}{|c|}{ Surface Level Data } \\
\hline Date & Type of Measurement & Levol (Inches) & Data Quality & Comments \\
\hline $10 / 10 / 930: 00$ & Manual Tape & 326.75 & Good & \\
\hline 10/11/93 0:00 & Manual Tape & 326.75 & Good & \\
\hline $10 / 11 / 937: 03$ & Auto FIC & 327.9 & Good & \\
\hline $10 / 12 / 930: 00$ & Manual Tape & 326.75 & Good & \\
\hline 10/12/93 7:04 & Auto FIC & 327.90 & Good & \\
\hline $10 / 13 / 930: 00$ & Manual FIC & 327.9 & Good & \\
\hline $10 / 13 / 930: 00$ & Manual Tape & 326.5 & Good & \\
\hline $10 / 13 / 937: 03$ & Auto FIC & 0 & Suspect & Data not included in graph \\
\hline $10 / 14 / 930: 00$ & Manual Tape & 326.5 & Good & \\
\hline $10 / 14 / 937: 04$ & Auto FIC & 327.90 & Good & \\
\hline $10 / 15 / 93 \quad 0: 00$ & Manual Tape & 326.75 & Good & \\
\hline $10 / 15 / 937: 04$ & Auto FIC & 327.8 & Good & \\
\hline $10 / 16 / 93 \quad 0: 00$ & Manual Tape & 326.75 & Good & \\
\hline $10 / 16 / 937: 04$ & Auto FIC & 327.90 & Good & \\
\hline $10 / 17 / 930: 00$ & Manual Tape & 326.75 & Good & \\
\hline $10 / 17 / 937: 03$ & Auto FIC & 327.8 & Good & \\
\hline $10 / 18 / 930: 00$ & Manual Tape & 326.75 & Good & \\
\hline 10/18/93 7:04 & Auto FIC & 327.8 & Good & \\
\hline $10 / 19 / 930: 00$ & Manual Tape & 326.75 & Good & \\
\hline 10/19/93 7:04 & Auto FIC & 0.00 & Suspect & Data not included in graph \\
\hline $10 / 19 / 9315: 04$ & Manual FIC & 327.9 & Good & \\
\hline $10 / 20 / 930: 00$ & Manual Tape & 326.75 & Good & \\
\hline $10 / 20 / 937: 03$ & Auto FIC & 0 & Suspect & Data not included in graph \\
\hline $10 / 20 / 9315: 04$ & Manual FIC & 327.9 & Good & \\
\hline $10 / 21 / 930: 00$ & Manual Tape & 326.75 & Good & \\
\hline $10 / 21 / 937: 04$ & Auto FIC & 0 & Suspect & Data not included in graph \\
\hline $10 / 21 / 9315: 04$ & Manual FIC & 327.90 & Good & \\
\hline $10 / 22 / 930: 00$ & Manual Tape & 326.75 & Good & \\
\hline $10 / 22 / 937: 04$ & Auto FIC & 0.00 & Suspect & Data not included in graph \\
\hline $10 / 22 / 93 \quad 15: 04$ & Manual FIC & 327.90 & Good & \\
\hline $10 / 23 / 930: 00$ & Manual Tape & 326.75 & Good & \\
\hline $10 / 23 / 937: 04$ & Auto FIC & 0 & Suspect & Data not included in graph \\
\hline $10 / 23 / 93 \quad 15: 04$ & Manual FIC & 327.9 & Good & \\
\hline $10 / 24 / 930: 00$ & Manual Tape & 327 & Good & \\
\hline $10 / 24 / 937: 04$ & Auto FIC & 0 & Suspect & Data not included in graph \\
\hline $10 / 24 / 93 \quad 15: 04$ & Manual FIC & 327.9 & Good & \\
\hline $10 / 25 / 930: 00$ & Manual Tape & 326.75 & Good & \\
\hline 10/25/93 7:04 & Auto FIC & 0 & Suspect & Data not included in graph \\
\hline $10 / 25 / 93 \quad 15: 04$ & Manual FIC & 327.9 & Good & \\
\hline $10 / 26 / 930: 00$ & Manual Tape & 326.75 & Good & \\
\hline $10 / 26 / 937: 04$ & Auto FIC & 0 & Suspect & Data not included in graph \\
\hline $10 / 26 / 93 \quad 15: 04$ & Manual FIC & 327.90 & Good & \\
\hline $10 / 27 / 930: 00$ & Manual Tape & 326.75 & Good & \\
\hline $10 / 27 / 937: 04$ & Auto FIC & 0 & Suspect & Data not included in graph \\
\hline $10 / 27 / 93 \quad 15: 04$ & Manual FIC & 327.9 & Good & \\
\hline $10 / 28 / 930: 00$ & Manual Tape & 326.25 & Good & \\
\hline $10 / 28 / 93 \quad 7: 04$ & Auto FIC & 0.00 & Suspect & Data not included in graph \\
\hline $10 / 28 / 9315: 04$ & Manual FIC & 327.90 & Good & \\
\hline
\end{tabular}

Data obtained from WHC Surveillance Analysis Computer System, (PC SACS) July 7, 1995. 


\begin{tabular}{|c|c|c|c|c|}
\hline \multicolumn{5}{|c|}{ 241-AP-108 } \\
\hline \multicolumn{5}{|c|}{ Surface Level Data } \\
\hline Date & Type of Measurement & Level (inches) & Data Quality & Comments \\
\hline $10 / 29 / 930: 00$ & Manual Tape & 326.75 & Good & \\
\hline $10 / 29 / 937: 04$ & Auto FIC & 0.00 & Suspect & Data not included in graph \\
\hline $10 / 29 / 9315: 04$ & Manual FIC & 327.9 & Good & \\
\hline $10 / 30 / 930: 00$ & Manual Tape & 326.75 & Good & \\
\hline $10 / 30 / 935: 04$ & Auto FIC & 327.90 & Good & \\
\hline 10/31/93 0:00 & Manual Tape & 326.75 & Good & \\
\hline $10 / 31 / 934: 06$ & Auto FIC & 327.90 & Good & \\
\hline $11 / 1 / 930: 00$ & Manual FIC & 327.9 & Good & \\
\hline $11 / 1 / 930: 00$ & Manual Tape & 326.50 & Good & \\
\hline $11 / 1 / 937: 06$ & Auto FIC & 0 & Suspect & Data not included in graph \\
\hline $11 / 2 / 930: 00$ & Manual FIC & 327.90 & Good & \\
\hline $11 / 2 / 930: 00$ & Manual Tape & 326.75 & Good & \\
\hline $11 / 2 / 937: 04$ & Auto FIC & 0.00 & Suspect & Data not included in graph \\
\hline $11 / 3 / 930: 00$ & Manual FIC & 327.90 & Good & \\
\hline $11 / 3 / 930: 00$ & Manual Tape & 326.50 & Good & \\
\hline $11 / 3 / 937: 06$ & Auto FIC & 0 & Suspect & Data not included in graph \\
\hline $11 / 4 / 930: 00$ & Manual FIC & 327.9 & Good & \\
\hline $11 / 4 / 930: 00$ & Manual Tape & 326.75 & Good & \\
\hline $11 / 4 / 937: 04$ & Auto FIC & 0.00 & Suspect & Data not included in graph \\
\hline $11 / 5 / 930: 00$ & Manual FIC & 327.9 & Good & \\
\hline $11 / 5 / 930: 00$ & Manual Tape & 326.75 & Good & \\
\hline $11 / 5 / 937: 06$ & Auto FIC & 0.00 & Suspect & Data not included in graph \\
\hline $11 / 6 / 930: 00$ & Manual FIC & 327.9 & Good & \\
\hline $11 / 6 / 930: 00$ & Manual Tape & 326.75 & Good & \\
\hline $11 / 6 / 937: 06$ & Auto FIC & 0 & Suspect & Data not included in graph \\
\hline $11 / 7 / 930: 00$ & Manual FIC & 327.9 & Good & \\
\hline $11 / 7 / 930: 00$ & Manual Tape & 326.75 & Good & \\
\hline $11 / 7 / 937: 06$ & Auto FIC & 0.00 & Suspect & Data not included in graph \\
\hline $11 / 8 / 930: 00$ & Manual Tape & 326.75 & Good & \\
\hline $11 / 8 / 933: 06$ & Auto FIC & $327.90^{\circ}$ & Good & \\
\hline $11 / 9 / 930: 00$ & Manual FIC & 327.9 & Good & \\
\hline $11 / 9 / 930: 00$ & Manual Tape & 326.75 & Good & \\
\hline $11 / 9 / 937: 03$ & Auto FIC & 0 & Suspect & Data not included in graph \\
\hline $11 / 10 / 930: 00$ & Manual Tape & 326.75 & Good & \\
\hline $11 / 10 / 937: 04$ & Auto FIC & 327.90 & Good & \\
\hline $11 / 11 / 930: 00$ & Manual FIC & 327.9 & Good & \\
\hline $11 / 11 / 930: 00$ & Manual Tape & 326.75 & Good & \\
\hline $11 / 11 / 937: 04$ & Auto FIC & 0.00 & Suspect & Data not included in graph \\
\hline $11 / 12 / 930: 00$ & Manual Tape & 326.5 & Good & \\
\hline $11 / 12 / 933: 04$ & Auto FIC & 327.9 & Good & \\
\hline $11 / 13 / 930: 00$ & Manual FIC & 327.9 & Good & \\
\hline $11 / 13 / 930: 00$ & Manual Tape & 326.50 & Good & \\
\hline $11 / 13 / 937: 04$ & Auto FIC & 0.00 & Suspect & Data not included in graph \\
\hline $11 / 14 / 930: 00$ & Manual FIC & 327.90 & Good & \\
\hline $11 / 14 / 930: 00$ & Manual Tape & 326.5 & Good & \\
\hline $11 / 14 / 937: 04$ & Auto FIC & 0.00 & Suspect & Data not included in graph \\
\hline $11 / 15 / 930: 00$ & Manual Tape & 326.5 & Good & \\
\hline $11 / 15 / 935: 04$ & Auto FIC & 327.90 & Good & \\
\hline
\end{tabular}

Data obtained from WHC Surveillance Analysis Computer System, (PC SACS) July 7, 1995. 


\begin{tabular}{|c|c|c|c|c|}
\hline \multicolumn{5}{|c|}{ 241-AP-108 } \\
\hline \multicolumn{5}{|c|}{ Surface Level Data } \\
\hline Date & Type of Measurement & Leval (Inches) & Data Quality & Comments \\
\hline $11 / 16 / 930: 00$ & Manual FIC & 327.9 & Good & \\
\hline $11 / 16 / 930: 00$ & Manual Tape & 326.50 & Good & \\
\hline $11 / 16 / 937: 03$ & Auto FIC & 0 & Suspect & Data not included in graph \\
\hline $11 / 17 / 930: 00$ & Manual Tape & 326.75 & Good & \\
\hline $11 / 17 / 9315: 04$ & Auto FIC & 327.90 & Good & \\
\hline $11 / 18 / 930: 00$ & Manual FIC & 327.90 & Good & \\
\hline $11 / 18 / 930: 00$ & Manual Tape & 326.5 & Good & \\
\hline $11 / 18 / 937: 04$ & Auto FIC & 0 & Suspect & Data not included in graph \\
\hline $11 / 19 / 930: 00$ & Manual FIC & 327.9 & Good & \\
\hline $11 / 19 / 930: 00$ & Manual Tape & 326.25 & Good & \\
\hline $11 / 19 / 9323: 04$ & Auto FIC & 323.90 & Suspect & Data not included in graph \\
\hline $11 / 20 / 930: 00$ & Manual FIC & 327.9 & Good & \\
\hline $11 / 20 / 930: 00$ & Manual Tape & 326.50 & Good & \\
\hline $11 / 20 / 937: 04$ & Auto FIC & 0 & Suspect & Data not included in graph \\
\hline $11 / 21 / 930: 00$ & Manual FIC & 327.90 & Good & \\
\hline $11 / 21 / 930: 00$ & Manual Tape & 326 & Good & \\
\hline $11 / 21 / 937: 04$ & Auto FIC & 0 & Suspect & Data not included in graph \\
\hline $11 / 22 / 930: 00$ & Manual FIC & 327.90 & Good & \\
\hline $11 / 22 / 930: 00$ & Manual Tape & 326.75 & Good & \\
\hline $11 / 22 / 937: 04$ & Auto FIC & 0.00 & Suspect & Data not included in graph \\
\hline $11 / 23 / 930: 00$ & Manual FIC & 327.9 & Good & \\
\hline $11 / 23 / 930: 00$ & Manual Tape & 326.75 & Good & \\
\hline $11 / 23 / 937: 04$ & Auto FIC & 0 & Suspect & Data not included in graph \\
\hline $11 / 24 / 930: 00$ & Manual Tape & 326.5 & Good & \\
\hline $11 / 24 / 937: 04$ & Auto FIC & 0.00 & Suspect & Data not included in graph \\
\hline $11 / 25 / 930: 00$ & Manual FIC & 327.9 & Good & \\
\hline $11 / 25 / 930: 00$ & Manual Tape & 326.75 & Good & \\
\hline $11 / 25 / 937: 04$ & Auto FIC & 0 & Suspect & Data not included in graph \\
\hline $11 / 26 / 930: 00$ & Manual FIC & 327.9 & Good & \\
\hline $11 / 26 / 930: 00$ & Manual Tape & 326.75 & Good & \\
\hline $11 / 26 / 937: 04$ & Auto FIC & 0.00 & Suspect & Data not included in graph \\
\hline $11 / 27 / 930: 00$ & Manual FIC & 327.9 & Good & \\
\hline $11 / 27 / 930: 00$ & Manual Tape & 326.50 & Good & \\
\hline $11 / 27 / 937: 04$ & Auto FIC & 0.00 & Suspect & Data not included in graph \\
\hline $11 / 28 / 930: 00$ & Manual Tape & 326.50 & Good & \\
\hline $11 / 28 / 937: 03$ & Auto FIC & 327.9 & Good & \\
\hline $11 / 29 / 930: 00$ & Manual Tape & 326.50 & Good & \\
\hline $11 / 29 / 937: 04$ & Auto FIC & 327.9 & Good & \\
\hline $11 / 30 / 930: 00$ & Manual Tape & 326.50 & Good & \\
\hline $11 / 30 / 937: 03$ & Auto FIC & 327.8 & Good & \\
\hline $12 / 1 / 930: 00$ & Manual Tape & 326.50 & Good & \\
\hline $12 / 1 / 937: 04$ & Auto FIC & 327.7 & Good & \\
\hline $12 / 2 / 930: 00$ & Manual Tape & 326.5 & Good & \\
\hline 12/2/93 7:04 & Auto FIC & 327.70 & Good & \\
\hline $12 / 3 / 930: 00$ & Manual Tape & 326.5 & Good & \\
\hline $12 / 3 / 937: 05$ & Auto FIC & 327.7 & Good & \\
\hline $12 / 4 / 930: 00$ & Manual Tape & 326.5 & Good & \\
\hline $12 / 4 / 937: 05$ & Auto FIC & 327.7 & Good & \\
\hline
\end{tabular}

Data obtained from WHC Surveillance Analysis Computer System, (PC SACS) July 7, 1995. 


\begin{tabular}{|c|c|c|c|c|}
\hline \multicolumn{5}{|c|}{ 241-AP-108 } \\
\hline \multicolumn{5}{|c|}{ Surface Level Data } \\
\hline Date & Type of Measurement & Leval (Inches) & Data Quality & Comments \\
\hline $12 / 5 / 93 \quad 0: 00$ & Manual Tape & 326.50 & Good & \\
\hline $12 / 5 / 937: 04$ & Auto FIC & 327.70 & Good & \\
\hline $12 / 6 / 930: 00$ & Manual Tape & 326.75 & Good & \\
\hline $12 / 6 / 937: 05$ & Auto FIC & 327.7 & Good & \\
\hline $12 / 7 / 930: 00$ & Manual Tape & 326.5 & Good & \\
\hline $12 / 7 / 937: 04$ & Auto FIC & 327.70 & Good & \\
\hline $12 / 8 / 930: 00$ & Manual Tape & 326.50 & Good & \\
\hline $12 / 8 / 937: 06$ & Auto FIC & 327.70 & Good & \\
\hline $12 / 9 / 930: 00$ & Manual Tape & 326.5 & Good & \\
\hline $12 / 9 / 937: 04$ & Auto FIC & 327.7 & Good & \\
\hline $12 / 10 / 930: 00$ & Manual Tape & 326.50 & Good & \\
\hline $12 / 10 / 936: 05$ & Auto FIC & 327.60 & Good & \\
\hline $12 / 11 / 930: 00$ & Manual Tape & $326 . \overline{5}$ & Good & \\
\hline $12 / 11 / 937: 06$ & Auto FIC & 327.60 & Good & \\
\hline $12 / 12 / 930: 00$ & Manual Tape & 326.25 & Good & \\
\hline 12/12/93 7:06 & Auto FIC & 327.6 & Good & \\
\hline $12 / 13 / 930: 00$ & Manual Tape & 326.25 & Good & \\
\hline $12 / 13 / 937: 06$ & Auto FIC & 327.60 & Good & \\
\hline $12 / 14 / 930: 00$ & Manual Tape & 326.5 & Good & \\
\hline 12/14/93 7:06 & Auto FIC & 327.1 & Good & \\
\hline 12/15/93 0:00 & Manual Tape & 326.50 & Good & \\
\hline $12 / 15 / 937: 06$ & Auto FIC & 327.00 & Good & \\
\hline $12 / 16 / 930: 00$ & Manual Tape & 326.5 & Good & \\
\hline 12/16/93 7:06 & Auto FIC & 327.1 & Good & \\
\hline $12 / 17 / 930: 00$ & Manual Tape & 327 & Good & \\
\hline 12/17/93 7:05 & Auto FIC & 327.10 & Good & \\
\hline $12 / 18 / 930: 00$ & Manual Tape & 326.5 & Good & \\
\hline 12/18/93 7:06 & Auto FIC & 327.10 & Good & \\
\hline $12 / 19 / 930: 00$ & Manual Tape & 326.5 & Good & \\
\hline $12 / 19 / 937: 06$ & Auto FIC & 327.1 & Good & \\
\hline $12 / 20 / 930: 00$ & Manual Tape & 326.50 & Good & \\
\hline $12 / 20 / 937: 06$ & Auto FIC & 323.00 & Suspect & Data not included in graph \\
\hline 12/20/93 17:06 & Manual FIC & 327.00 & Good & \\
\hline $12 / 21 / 930: 00$ & Manual Tape & 326.50 & Good & \\
\hline $12 / 21 / 937: 06$ & Auto FIC & 326.90 & Good & \\
\hline $12 / 22 / 930: 00$ & Manual Tape & 326.5 & Good & \\
\hline 12/22/93 7:05 & Auto FIC & 327.00 & Good & \\
\hline $12 / 23 / 930: 00$ & Manual Tape & 326.5 & Good & \\
\hline $12 / 23 / 936: 06$ & Auto FIC & 327.00 & Good & \\
\hline $12 / 24 / 930: 00$ & Manual Tape & 326.50 & Good & \\
\hline $12 / 24 / 937: 06$ & Auto FIC & 327.00 & Good & \\
\hline $12 / 25 / 930: 00$ & Manual Tape & 326.50 & Good & \\
\hline $12 / 25 / 937: 06$ & Auto FIC & 326.90 & Good & \\
\hline $12 / 26 / 930: 00$ & Manual Tape & 326.50 & Good & \\
\hline $12 / 26 / 937: 06$ & Auto FIC & 326.90 & Good & \\
\hline $12 / 27 / 930: 00$ & Manual Tape & 326.5 & Good & \\
\hline $12 / 27 / 937: 06$ & Auto FIC & 326.90 & Good & \\
\hline $12 / 28 / 930: 00$ & Manual Tape & 326.50 & Good & \\
\hline
\end{tabular}

Data obtained from WHC Surveillance Analysis Computer System, (PC SACS) July 7, 1995. 


\begin{tabular}{|c|c|c|c|c|}
\hline \multicolumn{5}{|c|}{ 241-AP-108 } \\
\hline \multicolumn{5}{|c|}{ Surface Level Date } \\
\hline Date & Type of Measurement & Level (Inches) & Data Quality & Comments \\
\hline $12 / 28 / 937: 06$ & Auto FIC & 326.9 & Good & \\
\hline $12 / 29 / 930: 00$ & Manual Tape & 326.50 & Good & \\
\hline $12 / 29 / 937: 06$ & Auto FIC & 326.90 & Good & \\
\hline $12 / 30 / 930: 00$ & Manual Tape & 326.5 & Good & \\
\hline $12 / 30 / 937: 05$ & Auto FIC & 326.9 & Good & \\
\hline $12 / 31 / 930: 00$ & Manual Tape & 326.50 & Good & \\
\hline $12 / 31 / 937: 06$ & Auto FIC & 326.90 & Good & \\
\hline $1 / 1 / 940: 00$ & Manual Tape & 326.50 & Good & \\
\hline $1 / 1 / 947: 06$ & Auto FIC & 326.8 & Good & \\
\hline $1 / 2 / 940: 00$ & Manual Tape & 326.5 & Good & \\
\hline $1 / 2 / 947: 06$ & Auto FIC & 326.8 & Good & \\
\hline 1/3/940:00 & Manual Tape & 326.50 & Good & \\
\hline $1 / 3 / 947: 07$ & Auto FIC & 322.70 & Good & \\
\hline $1 / 3 / 94 \quad 17: 07$ & Manual FIC & 326.70 & Good & \\
\hline 1/4/940:00 & Manual Tape & 326.50 & Good & \\
\hline $1 / 4 / 947: 07$ & Auto FIC & 322.80 & Good & \\
\hline $1 / 4 / 9417: 07$ & Manual FIC & 326.8 & Good & \\
\hline $1 / 5 / 940: 00$ & Manual Tape & 326.5 & Good & \\
\hline $1 / 5 / 947: 06$ & Auto FIC & 326.8 & Good & \\
\hline 1/6/94 0:00 & Manual Tape & 326.5 & Good & \\
\hline $1 / 6 / 947: 06$ & Auto FIC & 326.8 & Good & \\
\hline $1 / 7 / 940: 00$ & Manual Tape & 326.5 & Good & \\
\hline $1 / 7 / 947: 07$ & Auto FIC & 326.8 & Good & \\
\hline 1/8/94 0:00 & Manual Tape & 326.50 & Good & \\
\hline 1/8/94 7:06 & Auto FIC & 322.80 & Good & \\
\hline $1 / 8 / 94 \quad 17: 06$ & Manual FIC & 326.80 & Good & \\
\hline $1 / 9 / 940: 00$ & Manual Tape & 326.5 & Good & \\
\hline 1/9/94 7:06 & Auto FIC & 326.80 & Good & \\
\hline $1 / 10 / 940: 00$ & Manual Tape & 326.50 & Good & \\
\hline $1 / 10 / 947: 07$ & Auto FIC & 326.80 & Good & \\
\hline $1 / 11 / 940: 00$ & Manual Tape & 326.5 & Good & \\
\hline $1 / 11 / 947: 07$ & Auto FIC & 326.8 & Good & \\
\hline $1 / 12 / 940: 00$ & Manual Tape & 326.50 & Good & \\
\hline $1 / 12 / 947: 06$ & Auto FIC & 326.8 & Good & \\
\hline $1 / 13 / 940: 00$ & Manual Tape & 326.75 & Good & \\
\hline $1 / 13 / 945: 07$ & Auto FIC & 326.8 & Good & \\
\hline $1 / 14 / 940: 00$ & Manual Tape & 326.5 & Good & \\
\hline $1 / 14 / 947: 06$ & Auto FIC & 326.8 & Good & \\
\hline $1 / 15 / 940: 00$ & Manual Tape & 326.25 & Good & \\
\hline $1 / 15 / 945: 07$ & Auto FIC & 326.80 & Good & \\
\hline $1 / 16 / 940: 00$ & Manual Tape & 326.50 & Good & \\
\hline $1 / 16 / 947: 07$ & Auto FIC & 326.70 & Good & \\
\hline $1 / 17 / 940: 00$ & Manual Tape & $326: 50$ & Good & \\
\hline $1 / 17 / 947: 07$ & Auto FIC & 326.5 & Good & \\
\hline $1 / 18 / 940: 00$ & Manual Tape & 326.25 & Good & \\
\hline $1 / 18 / 947: 07$ & Auto FIC & 326.4 & Good & \\
\hline $1 / 19 / 940: 00$ & Manual Tape & 326.50 & Good & \\
\hline $1 / 19 / 947: 07$ & Auto FIC & 326.4 & Good & \\
\hline
\end{tabular}

Data obtained from WHC Surveillance Analysis Computer System, (PC SACS) July 7, 1995. 


\begin{tabular}{|c|c|c|c|c|}
\hline \multicolumn{5}{|c|}{ 241-AP-108 } \\
\hline \multicolumn{5}{|c|}{ Surface Level Data } \\
\hline Date & Type of Measurement & Leval (Inches) & Data Quality & Comments \\
\hline $1 / 20 / 940: 00$ & Manual Tape & 326.5 & Good & \\
\hline $1 / 20 / 947: 07$ & Auto FIC & 326.4 & Good & \\
\hline $1 / 21 / 940: 00$ & Manual Tape & 326.5 & Good & \\
\hline $1 / 21 / 947: 07$ & Auto FIC & 326.40 & Good & \\
\hline $1 / 22 / 940: 00$ & Manual Tape & 326.50 & Good & \\
\hline $1 / 22 / 947: 08$ & Auto FIC & 326.40 & Good & \\
\hline $1 / 23 / 940: 00$ & Manual Tape & 326.5 & Good & \\
\hline $1 / 23 / 947: 07$ & Auto FIC & 326.4 & Good & \\
\hline $1 / 24 / 940: 00$ & Manual Tape & 326.25 & Good & \\
\hline $1 / 24 / 947: 08$ & Auto FIC & 326.30 & Good & \\
\hline $1 / 25 / 940: 00$ & Manual Tape & 326.5 & Good & \\
\hline $1 / 25 / 947: 07$ & Auto FIC & 326.4 & Good & \\
\hline $1 / 26 / 940: 00$ & Manual Tape & 326.25 & Good & \\
\hline $1 / 26 / 947: 06$ & Auto FIC & 326.40 & Good & \\
\hline $1 / 27 / 940: 00$ & Manual Tape & 326.5 & Good & \\
\hline $1 / 27 / 946: 06$ & Auto FIC & 326.4 & Good & \\
\hline $1 / 28 / 940: 00$ & Manual Tape & 326.50 & Good & \\
\hline $1 / 28 / 947: 07$ & Auto FIC & 326.40 & Good & \\
\hline $1 / 29 / 940: 00$ & Manual Tape & 326.5 & Good & \\
\hline $1 / 29 / 947: 07$ & Auto FIC & 326.40 & Good & \\
\hline $1 / 30 / 940: 00$ & Manual Tape & 326.5 & Good & \\
\hline $1 / 30 / 947: 06$ & Auto FIC & 326.4 & Good & \\
\hline $1 / 31 / 940: 00$ & Manual Tape & 326.25 & Good & \\
\hline $1 / 31 / 947: 07$ & Auto FIC & 326.40 & Good & \\
\hline $2 / 1 / 940: 00$ & Manual Tape & 326.25 & Good & \\
\hline $2 / 1 / 947: 06$ & Auto FIC & 326.4 & Good & \\
\hline $2 / 2 / 940: 00$ & Manual Tape & 326.5 & Good & \\
\hline $2 / 2 / 947: 07$ & Auto FIC & 326.4 & Good & \\
\hline $2 / 3 / 940: 00$ & Manual Tape & 326.50 & Good & \\
\hline $2 / 3 / 947: 07$ & Auto FIC & 326.4 & Good & \\
\hline $2 / 4 / 940: 00$ & Manual Tape & 326.5 & Good & \\
\hline $2 / 4 / 947: 07$ & Auto FIC & 326.40 & Good & \\
\hline $2 / 5 / 940: 00$ & Manual Tape & 326.25 & Good & \\
\hline $2 / 5 / 947: 07$ & Auto FIC & 326.40 & Good & \\
\hline $2 / 6 / 940: 00$ & Manual Tape & 326.25 & Good & \\
\hline $2 / 6 / 947: 07$ & Auto FIC & 326.40 & Good & \\
\hline $2 / 7 / 940: 00$ & Manual Tape & 326.25 & Good & \\
\hline $2 / 7 / 947: 06$ & Auto FIC & 326.40 & Good & \\
\hline $2 / 8 / 940: 00$ & Manual Tape & 326.5 & Good & \\
\hline $2 / 8 / 947: 07$ & Auto FIC & 326.4 & Good & \\
\hline 2/9/94 0:00 & Manual Tape & 326.50 & Good & \\
\hline $2 / 9 / 947: 04$ & Auto FIC & 326.4 & Good & \\
\hline $2 / 10 / 940: 00$ & Manual Tape & 326.5 & Good & \\
\hline $2 / 10 / 947: 04$ & Auto FIC & 326.40 & Good & \\
\hline $2 / 11 / 940: 00$ & Manual Tape & 326.50 & Good & \\
\hline $2 / 11 / 946: 07$ & Auto FIC & 326.40 & Good & \\
\hline $2 / 12 / 940: 00$ & Manual FIC & 326.40 & Good & \\
\hline $2 / 12 / 940: 00$ & Manual Tape & 326.25 & Good & \\
\hline
\end{tabular}

Data obtained from WHC Surveillance Analysis Computer System, (PC SACS) July 7, 1995. 


\begin{tabular}{|c|c|c|c|c|}
\hline \multicolumn{5}{|c|}{ 241-AP-108 } \\
\hline \multicolumn{5}{|c|}{ Surface Level Data } \\
\hline Date & Type of Measurement & Level (Inches) & Data Quality & Comments \\
\hline $2 / 12 / 947: 03$ & Auto FIC & .0 & Suspect & Data not included in graph \\
\hline 2/13/94 0:00 & Manual FIC & 326.4 & Good & \\
\hline $2 / 13 / 940: 00$ & Manual Tape & 326.25 & Good & \\
\hline 2/13/94 7:04 & Auto FIC & 0 & Suspect & Data not included in graph \\
\hline 2/14/940:00 & Manual FIC & 326.40 & Good & \\
\hline 2/14/94 0:00 & Manual Tape & 326.25 & Good & \\
\hline 2/14/94 7:04 & Auto FIC & 0.00 & Suspect & Data not included in graph \\
\hline 2/15/94 0:00 & Manual FIC & 326.40 & Good & \\
\hline $2 / 15 / 940: 00$ & Manual Tape & 326.25 & Good & \\
\hline $2 / 15 / 947: 04$ & Auto FIC & 0.00 & Suspect & Data not included in graph \\
\hline $2 / 16 / 940: 00$ & Manual FIC & 326.4 & Good & \\
\hline $2 / 16 / 940: 00$ & Manual Tape & 326.25 & Good & \\
\hline $2 / 16 / 947: 03$ & Auto FIC & 0.00 & Suspect & Data not included in graph \\
\hline $2 / 17 / 940: 00$ & Manual FIC & 326.40 & Good & \\
\hline $2 / 17 / 940: 00$ & Manual Tape & 326.50 & Good & \\
\hline $2 / 17 / 947: 04$ & Auto FIC & 0 & Suspect & Data not included in graph \\
\hline $2 / 18 / 940: 00$ & Manual FIC & 326.4 & Good & \\
\hline $2 / 18 / 940: 00$ & Manual Tape & 326.5 & Good & \\
\hline $2 / 18 / 947: 06$ & Auto FIC & 0.00 & Suspect & Data not included in graph \\
\hline $2 / 19 / 940: 00$ & Manual FIC & 326.4 & Good & \\
\hline $.2 / 19 / 940: 00$ & Manual Tape & 326.5 & Good & \\
\hline $2 / 19 / 947: 06$ & Auto FIC & 0 & Suspect & Data not included in graph \\
\hline $2 / 20 / 940: 00$ & Manual Tape & 326.50 & Good & \\
\hline $2 / 20 / 947: 07$ & Auto FIC & 326.4 & Good & \\
\hline $2 / 21 / 940: 00$ & Manual Tape & 326.50 & Good & \\
\hline $2 / 21 / 941: 06$ & Auto FIC & 326.40 & Good & \\
\hline 2/22/940:00 & Manual FIC & 326.4 & Good & \\
\hline $2 / 22 / 940: 00$ & Manual Tape & 326.25 & Good & \\
\hline $2 / 22 / 947: 05$ & Auto FIC & 0.00 & Suspect & Data not included in graph \\
\hline $2 / 23 / 940: 00$ & Manual Tape & 326.5 & Good & \\
\hline $2 / 23 / 947: 06$ & Auto FIC & 326.4 & Good & $\cdot$ \\
\hline $2 / 24 / 940: 00$ & Manual FIC & 326.40 & Good & \\
\hline $2 / 24 / 940: 00$ & Manual Tape & 324.75 & Good & \\
\hline $2 / 24 / 947: 06$ & Auto FIC & 0 & Suspect & Data not included in graph \\
\hline $2 / 25 / 940: 00$ & Manual Tape & 326.25 & Good & \\
\hline 2/25/945:06 & Auto FIC & 326.3 & Good & \\
\hline $2 / 26 / 940: 00$ & Manual Tape & 326.25 & Good & \\
\hline $2 / 26 / 947: 07$ & Auto FIC & 326.40 & Good & \\
\hline $2 / 27 / 940: 00$ & Manual FIC & 326.7 & Good & \\
\hline $2 / 27 / 940: 00$ & Manual Tape & 326.75 & Good & \\
\hline $2 / 27 / 947: 07$ & Auto FIC & 322.70 & Suspect & Data not included in graph \\
\hline $2 / 28 / 940: 00$ & Manual Tape & 384.5 & Good & \\
\hline $2 / 28 / 946: 06$ & Auto FIC & 372.10 & Good & \\
\hline $3 / 1 / 940: 00$ & Manual FIC & 411.35 & Good & \\
\hline 3/1/940:00 & Manual Tape & 411.25 & Good & \\
\hline $3 / 1 / 947: 04$ & Auto FIC & 0.00 & Suspect & Data not included in graph \\
\hline 3/1/94 17:04 & Manual FIC & 411.35 & Suspect & Data not included in graph \\
\hline $3 / 2 / 940: 00$ & Manual FIC & 411.30 & Good & $\cdot$ \\
\hline
\end{tabular}

Data obtained from WHC Surveillance Analysis Computer System, (PC SACS) July 7, 1995. 


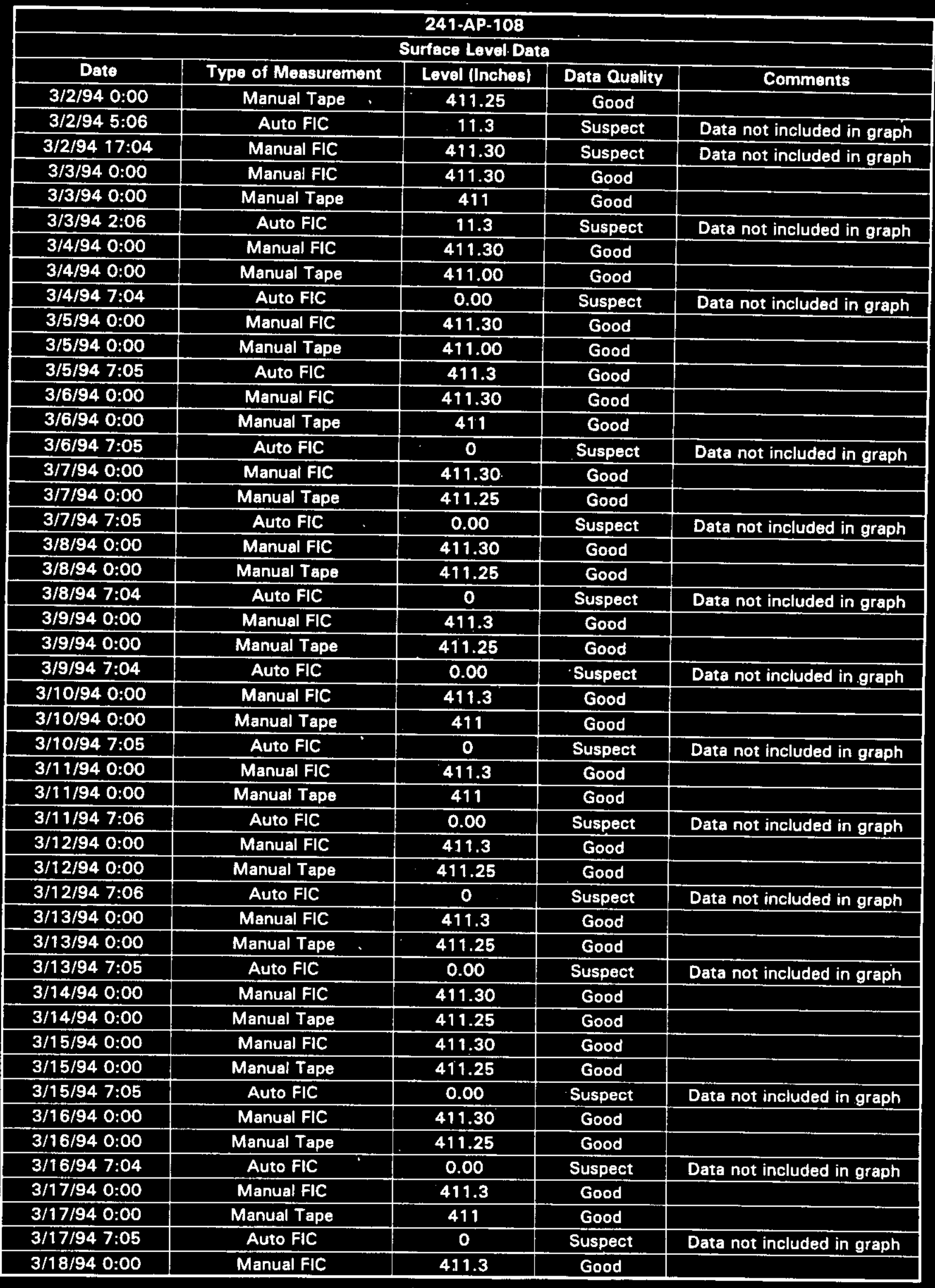

Data obtained from WHC Surveillance Analysis Computer System, (PC SACS) July 7, 1995. 
WHC-SD-WM-ER-315, Rev. 0

\begin{tabular}{|c|c|c|c|c|}
\hline \multicolumn{5}{|c|}{ 241-AP-108 } \\
\hline \multicolumn{5}{|c|}{ Surface Level Data } \\
\hline Date & Type of Measurement & Level (Inches) & Data Quality & Comments \\
\hline $3 / 18 / 940: 00$ & Manual Tape & 411.25 & Good & \\
\hline 3/18/94 7:05 & Auto FIC & 0.00 & Suspect & Data not included in graph \\
\hline $3 / 19 / 940: 00$ & Manual FIC & 411.30 & Good & \\
\hline $3 / 19 / 940: 00$ & Manual Tape & 411.25 & Good & \\
\hline $3 / 19 / 947: 04$ & Auto FIC & 0 & Suspect & Data not included in graph \\
\hline $3 / 20 / 940: 00$ & Manual FIC & 411.30 & Good & \\
\hline $3 / 20 / 940: 00$ & Manual Tape & 411.25 & Good & \\
\hline $3 / 20 / 947: 05$ & Auto FIC & 0.00 & Suspect & Data not included in graph \\
\hline $3 / 21 / 940: 00$ & Manual FIC & 411.3 & Good & \\
\hline $3 / 21 / 940: 00$ & Manual Tape & 411.25 & Good & \\
\hline $3 / 21 / 947: 05$ & Auto FIC & 0 & Suspect & Data not included in graph \\
\hline $3 / 22 / 940: 00$ & Manual FIC & 411.3 & Good & \\
\hline $3 / 22 / 940: 00$ & Manual Tape & 411.25 & Good & \\
\hline $3 / 22 / 947: 04$ & Auto FIC & 0.00 & Suspect & Data not included in graph \\
\hline $3 / 23 / 940: 00$ & Manual Tape & 291.25 & Suspect & Data not included in graph \\
\hline $3 / 23 / 940: 00$ & Manual FIC & 411.30 & Good & \\
\hline $3 / 23 / 941: 00$ & Manual Tape & 411.25 & Good & \\
\hline $3 / 24 / 940: 00$ & Manual FIC & 411.25 & Good & \\
\hline $3 / 24 / 940: 00$ & Manual Tape & 411.25 & Good & \\
\hline $3 / 24 / 947: 04$ & Auto FIC & 0.00 & Suspect & Data not included in graph \\
\hline $3 / 25 / 940: 00$ & Manual FIC & 411.3 & Good & \\
\hline $3 / 25 / 940: 00$ & Manual Tape & 411.25 & Good & \\
\hline $3 / 25 / 947: 05$ & Auto FIC & 0 & Suspect & Data not included in graph \\
\hline $3 / 26 / 940: 00$ & Manual FIC & 411.25 & Good & \\
\hline $3 / 26 / 940: 00$ & Manual Tape & 411.25 & Good & \\
\hline $3 / 26 / 947: 04$ & Auto FIC & 0 & Suspect & Data not included in graph \\
\hline $3 / 27 / 940: 00$ & Manual FIC & $411: 25$ & Good & \\
\hline $3 / 27 / 940: 00$ & Manual Tape & 411.25 & Good & \\
\hline $3 / 27 / 947: 04$ & Auto FIC & 0 & Suspect & Data not included in graph \\
\hline $3 / 28 / 940: 00$ & Manual FIC & 411.30 & Good & \\
\hline $3 / 28 / 94 \quad 0: 00$ & Manual Tape & 411.25 & Good & \\
\hline $3 / 28 / 947: 04$ & Auto FIC & 0.00 & Suspect & Data not included in graph \\
\hline $3 / 29 / 940: 00$ & Manual FIC & 411.30 & Good & \\
\hline 3/29/940:00 & Manual Tape & 411.125 & Good & \\
\hline $3 / 29 / 947: 03$ & Auto FIC & 0.00 & Suspect & Data not included in graph \\
\hline $3 / 30 / 940: 00$ & Manual FIC & 411.25 & Good & \\
\hline 3/30/94 0:00 & Manual Tape & 411 & Good & \\
\hline $3 / 30 / 947: 03$ & Auto FIC & 0 & Suspect & Data not included in graph \\
\hline $3 / 31 / 940: 00$ & Manual FIC & 411.2 & Good & \\
\hline 3/31/94 0:00 & Manual Tape & 411.25 & Good & \\
\hline $3 / 31 / 947: 03$ & Auto FIC & 0.00 & Suspect & Data not included in graph \\
\hline $4 / 1 / 940: 00$ & Manual FIC & 411.25 & Good & \\
\hline 4/1/94 0:00 & Manual Tape & 411.25 & Good & \\
\hline $4 / 2 / 940: 00$ & Manual FIC & 41.125 & Good & \\
\hline 4/2/940:00 & Manual Tape & 411.25 & Good & \\
\hline $4 / 2 / 947: 07$ & Auto FIC & 0 & Suspect & Data not included in graph \\
\hline 4/3/94 0:00 & Manual FIC & 411.25 & Good & \\
\hline 4/3/94 0:00 & Manual Tape & 411.25 & Good & \\
\hline
\end{tabular}

Data obtained from WHC Surveillance Analysis Computer System, (PC SACS) July 7, 1995. 
WHC-SD-WM-ER-315, Rev. 0

\begin{tabular}{|c|c|c|c|c|}
\hline \multicolumn{5}{|c|}{ 241-AP-108 } \\
\hline \multicolumn{5}{|c|}{ Surface Level Data } \\
\hline Date & Type of Measurement & Level (inches) & Data Quality & Comments \\
\hline $4 / 3 / 947: 03$ & Auto FIC & 0 & Suspect & Data not included in graph \\
\hline $4 / 4 / 940: 00$ & Manual FIC & 411.25 & Good & 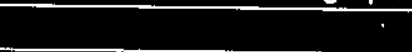 \\
\hline 4/4/940:00 & Manual Tape & 411.50 & Good & \\
\hline $4 / 4 / 947: 03$ & Auto FIC & 0.00 & Suspect & Data not included in graph \\
\hline $4 / 5 / 940: 00$ & Manual FIC & 411.25 & Good & \\
\hline 4/5/94 0:00 & Manual Tape & 411.25 & Good & \\
\hline $4 / 5 / 947: 03$ & Auto FIC & 0.00 & Suspect & Data not included in graph \\
\hline $4 / 6 / 940: 00$ & Manual FIC & 411.25 & Good & \\
\hline $4 / 6 / 940: 00$ & Manual Tape & 411.00 & Good & \\
\hline $4 / 6 / 944: 07$ & Auto FIC & 11.2 & Suspect & Data not included in graph \\
\hline 4/7/940:00 & Manual FIC & 411.25 & Good & \\
\hline $4 / 7 / 940: 00$ & Manual Tape & 411.25 & Good & \\
\hline $4 / 7 / 947: 07$ & Auto FIC & 0.00 & Suspect & Data not included in graph \\
\hline $4 / 8 / 940: 00$ & Manual FIC & 411.2 & Good & \\
\hline $4 / 8 / 940: 00$ & Manual Tape & 411.25 & Good & \\
\hline $4 / 8 / 947: 03$ & Auto FIC & 0.00 & Suspect & Data not included in graph \\
\hline $4 / 9 / 940: 00$ & Manual FIC & 411.2 & Good & \\
\hline 4/9/940:00 & Manual Tape & 411.25 & Good & \\
\hline $4 / 9 / 947: 07$ & Auto FIC & 0 & Suspect & Data not included in graph \\
\hline $4 / 10 / 940: 00$ & Manual FIC & 411.20 & Good & \\
\hline $4 / 10 / 940: 00$ & Manual Tape & 411.25 & Good & \\
\hline $4 / 10 / 947: 03$ & Auto FIC & 0 & Suspect & Data not included in graph \\
\hline $4 / 11 / 940: 00$ & Manual FIC & 411.20 & Good & \\
\hline $4 / 11 / 940: 00$ & Manual Tape & 411.25 & Good & \\
\hline $4 / 11 / 947: 03$ & Auto FIC & 0 & Suspect & Data not included in graph \\
\hline 4/12/94 0:00 & Manual FIC & 411.2 & Good & \\
\hline $4 / 12 / 940: 00$ & Manual Tape & 411.25 & Good & \\
\hline $4 / 12 / 947: 03$ & Auto FIC & 0 & Suspect & Data not included in graph \\
\hline $4 / 13 / 940: 00$ & Manual FIC & 411.2 & Good & \\
\hline $4 / 13 / 940: 00$ & Manual Tape & 411.00 & Good & \\
\hline $4 / 13 / 947: 03$ & Auto FIC & 0.00 & Suspect & Data not included in graph \\
\hline $4 / 14 / 940: 00$ & Manual FIC & 411.20 & Good & \\
\hline 4/14/940:00 & Manual Tape & 411.25 & Good & \\
\hline $4 / 14 / 947: 03$ & Auto FIC & 0 & Suspect & Data not included in graph \\
\hline $4 / 15 / 940: 00$ & Manual FIC & 411.20 & Good & \\
\hline $4 / 15 / 940: 00$ & Manual Tape & 411.00 & Good & $\cdot$ \\
\hline $4 / 15 / 947: 03$ & Auto FIC & 0.00 & Suspect & Data not included in graph \\
\hline 4/16/94 0:00 & Manual FIC & 411.2 & Good & \\
\hline $4 / 16 / 940: 00$ & Manual Tape & 411.25 & Good & \\
\hline $4 / 16 / 947: 03$ & Auto FIC & 11.20 & Suspect & Data not included in graph \\
\hline $4 / 17 / 940: 00$ & Manual Tape & 411.25 & Good & \\
\hline $4 / 17 / 947: 03$ & Auto FIC & 411.2 & Good & \\
\hline 4/18/94 0:00 & Manual FIC & 411.2 & Good & \\
\hline $4 / 18 / 940: 00$ & Manual Tape & 411.25 & Good & \\
\hline $4 / 18 / 947: 03$ & Auto FIC & 11.20 & Suspect & Data not included in graph \\
\hline 4/19/940:00 & Manual FIC & 411.20 & Good & \\
\hline $4 / 19 / 940: 00$ & Manual Tape & 411.25 & Good & \\
\hline $4 / 20 / 940: 00$ & Manual FIC & 411.2 & Good & \\
\hline
\end{tabular}

Data obtained from WHC Surveillance Analysis Computer System, (PC SACS) July 7, 1995. 


\begin{tabular}{|c|c|c|c|c|}
\hline \multicolumn{5}{|c|}{ 241-AP-108 } \\
\hline \multicolumn{5}{|c|}{ Surface Level Data } \\
\hline Date & Type of Messurement & Level (Inches) & Data Quality & Comments \\
\hline $4 / 20 / 940: 00$ & Manual Tape & 411.25 & Good & \\
\hline 4/20/94 7:04 & Auto FIC & 11.20 & Suspect & Data not included in graph \\
\hline $4 / 21 / 940: 00$ & Manual FIC & 411.20 & Good & \\
\hline $4 / 21 / 940: 00$ & Manual Tape & 411.20 & Good & \\
\hline $4 / 21 / 947: 05$ & Auto FIC & 11.2 & Suspect & Data not included in graph \\
\hline $4 / 22 / 940: 00$ & Manual FIC & 411.20 & Good & \\
\hline $4 / 22 / 940: 00$ & Manual Tape & 411 & Good & \\
\hline $4 / 22 / 947: 04$ & Auto FIC & 11.2 & Suspect & Data not included in graph \\
\hline $4 / 23 / 940: 00$ & Manual FIC & 411.20 & Good & \\
\hline $4 / 23 / 940: 00$ & Manual Tape & 411.25 & Good & \\
\hline $4 / 23 / 947: 05$ & Auto FIC & 11.10 & Suspect & Data not included in graph \\
\hline $4 / 24 / 940: 00$ & Manual FIC & 411.2 & Good & \\
\hline $4 / 24 / 940: 00$ & Manual Tape & 411.00 & Good & \\
\hline $4 / 24 / 947: 04$ & Auto FIC & 11.20 & Suspect & Data not included in graph \\
\hline $4 / 25 / 940: 00$ & Manual FIC & 411.2 & Good & $\cdot$ \\
\hline $4 / 25 / 940: 00$ & Manual Tape & 411 & Good & \\
\hline $4 / 25 / 947: 04$ & Auto FIC & 11.1 & Suspect & Data not included in graph \\
\hline $4 / 26 / 940: 00$ & Manual FIC & 411.2 & Good & \\
\hline 4/26/94 0:00 & Manual Tape & 411.25 & Good & \\
\hline $4 / 26 / 947: 07$ & Auto FIC & 11.1 & Suspect & Data not included in graph \\
\hline $4 / 27 / 940: 00$ & Manual FIC & 411.2 & Good & \\
\hline $4 / 27 / 940: 00$ & Manual Tape & 411.25 & Good & \\
\hline $4 / 27 / 947: 04$ & Auto FIC & 11.1 & Suspect & Data not included in graph \\
\hline $4 / 28 / 940: 00$ & Manual FIC & 411.20 & Good & \\
\hline $4 / 28 / 940: 00$ & Manual Tape & 411.25 & Good & \\
\hline $4 / 28 / 947: 05$ & Auto FIC & 11.10 & Suspect & Data not included in graph \\
\hline $4 / 29 / 940: 00$ & Manual FIC & 411.15 & Good & \\
\hline 4/29/94 0:00 & Manual Tape & 411.25 & Good & \\
\hline 4/29/94 7:04 & Auto FIC & 11.1 & Suspect & Data not included in graph \\
\hline $4 / 30 / 940: 00$ & Manual FIC & 411.2 & Good & \\
\hline $4 / 30 / 940: 00$ & Manual Tape & 411.25 & Good & \\
\hline $4 / 30 / 947: 05$ & Auto FIC & 11.10 & Suspect & Data not included in graph \\
\hline $5 / 1 / 940: 00$ & Manual Tape & 411.25 & Good & \\
\hline $5 / 1 / 947: 05$ & Auto FIC & 11.10 & Suspect & Data not included in graph \\
\hline $5 / 1 / 9413: 05$ & Manual FIC & 411.15 & Good & \\
\hline $5 / 2 / 940: 00$ & Manual Tape & $411: 25$ & Good & \\
\hline $5 / 2 / 947: 05$ & Auto FIC & 11.1 & Suspect & Data not included in graph \\
\hline $5 / 2 / 9413: 05$ & Manual FIC & 411.15 & Good & \\
\hline $5 / 3 / 940: 00$ & Manual FIC & 411.15 & Good & \\
\hline $5 / 3 / 940: 00$ & Manual Tape & 411.25 & Good & \\
\hline $5 / 3 / 947: 05$ & Auto FIC & 11.10 & Suspect & Data not included in graph \\
\hline $5 / 4 / 940: 00$ & Manual FIC & 411.15 & Good & \\
\hline $5 / 4 / 940: 00$ & Manual Tape & 411.25 & Good & \\
\hline $5 / 4 / 947: 05$ & Auto FIC & 11.10 & Suspect & Data not included in graph \\
\hline $5 / 5 / 940: 00$ & Manual FIC & 411.15 & Good & \\
\hline $5 / 5 / 940: 00$ & Manual Tape & 411.25 & Good & \\
\hline $5 / 5 / 947: 03$ & Auto FIC & 11.1 & Suspect & Data not included in graph \\
\hline $5 / 6 / 940: 00$ & Manual FIC & 411.2 & Good & \\
\hline
\end{tabular}

Data obtained from WHC Surveillance Analysis Computer System, (PC SACS) July 7, 1995. 


\begin{tabular}{|c|c|c|c|c|}
\hline \multicolumn{5}{|c|}{ 241-AP-108 } \\
\hline \multicolumn{5}{|c|}{ Surface Level Data } \\
\hline Date & Type of Measurement & Level (Inches) & Data Quality & Comments \\
\hline $5 / 6 / 940: 00$ & Manual Tape & 411.25 & Good & \\
\hline $5 / 6 / 947: 03$ & Auto FIC & 11.1 & Suspect & Data not included in graph \\
\hline $5 / 7 / 940: 00$ & Manual FIC & 411.15 & Good & \\
\hline $5 / 7 / 940: 00$ & Manual Tape & 411.25 & Good & \\
\hline $5 / 7 / 947: 03$ & Auto FIC & 11.1 & Suspect & Data not included in graph \\
\hline $5 / 8 / 940: 00$ & Manual FIC & 411.25 & Good & \\
\hline $5 / 8 / 940: 00$ & Manual Tape & 411.25 & Good & \\
\hline 5/8/94 7:03 & Auto FIC & 11.10 & Suspect & Data not included in graph \\
\hline $5 / 9 / 940: 00$ & Manual FIC & 411.15 & Good & \\
\hline $5 / 9 / 940: 00$ & Manual Tape & 411.25 & Good & \\
\hline 5/9/94 7:03 & Auto FIC & 11.10 & Suspect & Data not included in graph \\
\hline $5 / 10 / 940: 00$ & Manual FIC & 411.15 & Good & \\
\hline $5 / 10 / 940: 00$ & Manual Tape & 411 & Good & \\
\hline $5 / 10 / 947: 03$ & Auto FIC & 11.1 & Suspect & Data not included in graph \\
\hline $5 / 11 / 940: 00$ & Manual FIC & 411.1 & Good & \\
\hline $5 / 11 / 940: 00$ & Manual Tape & 411.25 & Good & \\
\hline $5 / 11 / 947: 03$ & Auto FIC & 11.1 & Suspect & Data not included in graph \\
\hline $5 / 12 / 940: 00$ & Manual FIC & 411.10 & Good & \\
\hline 5/12/94 0:00 & Manual Tape & 411 & Good & \\
\hline $5 / 12 / 946: 03$ & Auto FIC & 11.10 & Suspect & Data not included in graph \\
\hline $5 / 13 / 940: 00$ & Manual FIC & 411.15 & Good & \\
\hline $5 / 13 / 940: 00$ & Manual Tape & 411 & Good & \\
\hline $5 / 13 / 947: 03$ & Auto FIC & 11.00 & Suspect & Data not included in graph \\
\hline $5 / 14 / 940: 00$ & Manual FIC & 411.15 & Good & \\
\hline $5 / 14 / 940: 00$ & Manual Tape & 411.25 & Good & \\
\hline 5/14/94 7:03 & Auto FIC & 11.1 & Suspect & Data not included in graph \\
\hline $5 / 15 / 940: 00$ & Manual FIC & 411.15 & Good & \\
\hline 5/15/94 0:00 & Manual Tape & 410.75 & Good & \\
\hline $5 / 15 / 947: 06$ & Auto FIC & 11.10 & Suspect & Data not included in graph \\
\hline $5 / 16 / 940: 00$ & Manual FIC & 411.10 & Good & \\
\hline 5/16/94 0:00 & Manual Tape & 410.75 & Good & \\
\hline $5 / 16 / 947: 02$ & Auto FIC & 1.1 .10 & Suspect & Data not included in graph \\
\hline $5 / 17 / 940: 00$ & Manual FIC & 411.1 & Good & \\
\hline $5 / 17 / 940: 00$ & Manual Tape & 411.25 & Good & \\
\hline $5 / 17 / 947: 03$ & Auto FIC & 11.10 & Suspect & Data not included in graph \\
\hline $5 / 18 / 940: 00$ & Manual FIC & 411.10 & Good & \\
\hline $5 / 18 / 940: 00$ & Manual Tape & 411.25 & Good & \\
\hline $5 / 18 / 947: 03$ & Auto FIC & 11.1 & Suspect & Data not included in graph \\
\hline $5 / 19 / 940: 00$ & Manual FIC & 411.1 & Good & \\
\hline $5 / 19 / 940: 00$ & Manual Tape & 411.25 & Good & \\
\hline $5 / 19 / 947: 03$ & Auto FIC & 11.10 & Suspect & Data not included in graph \\
\hline $5 / 20 / 940: 00$ & Manual FIC & 411.1 & Good & \\
\hline $5 / 20 / 940: 00$ & Manual Tape & 411.25 & Good & \\
\hline $5 / 20 / 947: 03$ & Auto FIC & 11.1 & Suspect & Data not included in graph \\
\hline $5 / 21 / 940: 00$ & Manual FIC & 411.15 & Good & \\
\hline $5 / 21 / 940: 00$ & Manual Tape & 411 & Good & \\
\hline 5/21/94 7:05 & Auto FIC & 11.1 & Suspect & Data not included in graph \\
\hline $5 / 22 / 940: 00$ & Manual FIC & 411.15 & Good & \\
\hline
\end{tabular}

Data obtained from WHC Surveillance Analysis Computer System, (PC SACS) July 7, 1995. 


\begin{tabular}{|c|c|c|c|c|}
\hline \multicolumn{5}{|c|}{ 241-AP-108 } \\
\hline \multicolumn{5}{|c|}{ - Surface Level Data } \\
\hline Date & Type of Measurement & Level (Inches) & Data Quality & Commants \\
\hline $5 / 22 / 940: 00$ & Manual Tape & 410.75 & Good & \\
\hline $5 / 22 / 947: 05$ & Auto FIC & 11.1 & Suspect & Data not included in graph \\
\hline $5 / 23 / 940.00$ & Manual FIC & 411,20 & Good & \\
\hline $5 / 23 / 940: 00$ & Manual Tape & 411.00 & Good & \\
\hline $5 / 23 / 947: 04$ & Auto FIC & 11.1 & Suspect & Data not included in graph \\
\hline $5 / 24 / 940: 00$ & Manual Tape & 405.75 & Suspect & Data not included in graph \\
\hline $5 / 24 / 940: 00$ & Manual FIC & 411.15 & Good & \\
\hline $5 / 24 / 947: 04$ & Auto FIC & 11.1 & Suspect & Data not included in graph \\
\hline $5 / 25 / 940: 00$ & Manual Tape & 409.75 & Suspect & Data not included in graph \\
\hline $5 / 25 / 940: 00$ & Manual FIC & 411.15 & Good & \\
\hline $5 / 25 / 947: 04$ & Auto FIC & 11.1 & Suspect & Data not included in graph \\
\hline $5 / 26 / 940: 00$ & Manual Tape & 409.75 & Suspect & Data not included in graph \\
\hline $5 / 26 / 940: 00$ & Manual FIC & 411.1 & Good & \\
\hline $5 / 26 / 946: 05$ & Auto FIC & 11.10 & Suspect & Data not included in graph \\
\hline $5 / 27 / 940: 00$ & Manual FIC & 411.10 & Good & \\
\hline $5 / 27 / 940: 00$ & Manual Tape. & 411.00 & Good & \\
\hline $5 / 27 / 947: 03$ & Auto FIC & 11.10 & Suspect & Data not included in graph \\
\hline $5 / 28 / 940: 00$ & Manual FIC & 411.1 & Good & \\
\hline $5 / 28 / 940: 00$ & Manual Tape & 410.75 & Good & \\
\hline $5 / 28 / 947: 03$ & Auto FIC & 11.10 & Suspect & Data not included in graph \\
\hline $5 / 29 / 940: 00$ & Manual FIC & 411.10 & Good & \\
\hline $5 / 29 / 940: 00$ & Manual Tape & 410.25 & Good & \\
\hline $5 / 29 / 947: 03$ & Auto FIC & 11.1 & Suspect & Data not included in graph \\
\hline $5 / 30 / 940: 00$ & Manual FIC & 411.10 & Good & \\
\hline $5 / 30 / 940: 00$ & Manual Tape & 411 & Good & $\cdot$ \\
\hline $5 / 30 / 947: 02$ & Auto FIC & 11.1 & Suspect & Data not included in graph \\
\hline $5 / 31 / 940: 00$ & Manual FIC & 411.1 & Good & \\
\hline $5 / 31 / 940: 00$ & Manual Tape & 411.25 & Good & \\
\hline $5 / 31 / 947: 03$ & Auto FIC & $11: 1$ & Suspect & Data not included in graph \\
\hline $6 / 1 / 940: 00$ & Manual FIC & 411.1 & Good & \\
\hline $6 / 1 / 940: 00$ & Manual Tape & 411 & Good & \\
\hline $6 / 1 / 947: 03$ & Auto FIC & 11.10 & Suspect & Data not included in graph \\
\hline $6 / 2 / 940: 00$ & Manual FIC & 411.1 & Good & \\
\hline $6 / 2 / 940: 00$ & Manual Tape & 411.00 & Good & \\
\hline $6 / 2 / 947: 03$ & Auto FIC & 11.10 & Suspect & Data not included in graph \\
\hline $6 / 3 / 940: 00$ & Manual FIC & 411.1 & Good & \\
\hline $6 / 3 / 940: 00$ & Manual Tape & 411 & Good & \\
\hline $6 / 4 / 940: 00$ & Manual FIC & 411.10 & Good & \\
\hline $6 / 4 / 940: 00$ & Manual Tape & 411.00 & Good & \\
\hline $6 / 4 / 947: 04$ & Auto FIC & 11.1 & Suspect & Data not included in graph \\
\hline $6 / 5 / 940: 00$ & Manual FIC & 411.1 & Good & \\
\hline 6/5/94 0:00 & Manual Tape & 411.00 & Good & \\
\hline $6 / 5 / 947: 03$ & Auto FIC & 11.10 & Suspect & Data not included in graph \\
\hline $6 / 6 / 940: 00$ & Manual FIC & 411.1 & Good & \\
\hline $6 / 6 / 940: 00$ & Manual Tape & 411.00 & Good & \\
\hline $6 / 6 / 947: 06$ & Auto FIC & 1.1 .1 & Suspect & Data not included in graph \\
\hline $6 / 7 / 940: 00$ & Manual FIC & 411.1 & Good & \\
\hline $6 / 7 / 940: 00$ & Manual Tape & 411.25 & Good & \\
\hline
\end{tabular}

Data obtained from WHC Surveillance. Analysis Computer System, (PC SACS) July 7, 1995. 


\begin{tabular}{|c|c|c|c|c|}
\hline \multicolumn{5}{|c|}{ 241-AP-108 } \\
\hline \multicolumn{5}{|c|}{ Surface Level Data } \\
\hline Date & Type of Measurement & Level (Inches) & Data Quality & Comments \\
\hline $6 / 7 / 947: 02$ & Auto FIC & 11.1 & Suspect & Data not included in graph \\
\hline $6 / 8 / 940: 00$ & Manual FIC & 411.1 & Good & \\
\hline $6 / 8 / 940: 00$ & Manual Tape & 411.00 & Good & \\
\hline 6/8/94 7:03 & Auto FIC & 11.10 & Suspect & Data not included in graph \\
\hline 6/9/94 0:00 & Manual FIC & 411.1 & Good & \\
\hline 6/9/94 0:00 & Manual Tape & 411 & Good & \\
\hline $6 / 10 / 940: 00$ & Manual FIC & 411.1 & Good & \\
\hline $6 / 10 / 940: 00$ & Manual Tape & 411.25 & Good & \\
\hline $6 / 10 / 947: 04$ & Auto FIC & 11.1 & Suspect & Data not included in graph \\
\hline $6 / 11 / 940: 00$ & Manual FIC & 411.1 & Good & \\
\hline $6 / 11 / 940: 00$ & Manual Tape & 411.25 & Good & \\
\hline $6 / 11 / 947: 04$ & Auto FIC & 11.1 & Suspect & Data not included in graph \\
\hline 6/12/94 0:00 & Manual FIC & 411.10 & Good & \\
\hline $6 / 12 / 940: 00$ & Manual Tape & 411.25 & Good & \\
\hline $6 / 12 / 947: 03$ & Auto FIC & 11.10 & Suspect & Data not included in graph \\
\hline $6 / 13 / 940: 00$ & Manual FIC & 411.1 & Good & \\
\hline $6 / 13 / 940: 00$ & Manual Tape & 411 & Good & \\
\hline $6 / 13 / 947: 02$ & Auto FIC & 11.10 & Suspect & Data not included in graph \\
\hline $6 / 14 / 940: 00$ & Manual FIC & 411.1 & Good & \\
\hline $6 / 14 / 940: 00$ & Manual Tape & 411 & Good & \\
\hline $6 / 14 / 947: 03$ & Auto FIC & 11.1 & Suspect & Data not included in graph \\
\hline $6 / 15 / 940: 00$ & Manual FIC & 411.10 & Good & \\
\hline $6 / 15 / 940: 00$ & Manual Tape & 411 & Good & \\
\hline $6 / 15 / 947: 03$ & Auto FIC & 11.1 & Suspect & Data not included in graph \\
\hline $6 / 16 / 940: 00$ & Manual FIC & 471.10 & Good & \\
\hline $6 / 16 / 940: 00$ & Manual Tape & 411.00 & Good & \\
\hline $6 / 16 / 947: 03$ & Auto FIC & $11.10^{\circ}$ & Suspect & Data not included in graph \\
\hline $6 / 17 / 940: 00$ & Manual FIC & 411.1 & Good & \\
\hline $6 / 17 / 940: 00$ & Manual Tape & 411.00 & Good & \\
\hline $6 / 17 / 947: 03$ & Auto FIC & 11.1 & Suspect & Data not included in graph \\
\hline $6 / 18 / 940: 00$ & Manual FIC & 411.10 & Good & \\
\hline $6 / 18 / 940: 00$ & Manual Tape & 411.00 & Good & \\
\hline $6 / 18 / 947: 04$ & Auto FIC & 11.10 & Suspect & Data not included in graph \\
\hline $6 / 19 / 940: 00$ & Manual FIC & 411.10 & Good & \\
\hline $6 / 19 / 940: 00$ & Manual Tape & 411 & Good & \\
\hline $6 / 19 / 947: 04$ & Auto FIC & 11.10 & Suspect & Data not included in graph \\
\hline $6 / 20 / 940: 00$ & Manual FIC & 411.10 & Good & \\
\hline $6 / 20 / 940: 00$ & Manual Tape & 411 & Good & \\
\hline $6 / 20 / 947: 04$ & Auto FIC & 11.10 & Suspect & Data not included in graph \\
\hline $6 / 21 / 940: 00$ & Manual FIC & 411.1 & Good & \\
\hline $6 / 21 / 940: 00$ & Manual Tape & 411.00 & Good & \\
\hline $6 / 21 / 947: 04$ & Auto FIC & 11.10 & Suspect & Data not included in graph \\
\hline $6 / 22 / 940: 00$ & Manual FIC & 411.10 & Good & \\
\hline $6 / 22 / 940: 00$ & Manual Tape & $411.00^{\circ}$ & Good & \\
\hline $6 / 22 / 947: 03$ & Auto FIC & 11.1 & Suspect & Data not included in graph \\
\hline $6 / 23 / 940: 00$ & Manual FIC & 411.10 & Good & \\
\hline $6 / 23 / 940: 00$ & Manual Tape & 411 & Good & \\
\hline $6 / 23 / 947: 05$ & Auto FIC & 11.1 & Suspect & Data not included in graph \\
\hline
\end{tabular}

Data obtained from WHC Surveillance Analysis Computer System, (PC SACS) July 7, 1995. 


\begin{tabular}{|c|c|c|c|c|}
\hline \multicolumn{5}{|c|}{ 241-AP-108 } \\
\hline \multicolumn{5}{|c|}{ Surface Level Data } \\
\hline Date & Type of Measurement & Level (Inches) & Data Quality & Comments \\
\hline $6 / 24 / 940: 00$ & Manual FIC & 411.10 & Good & \\
\hline $6 / 24 / 940: 00$ & Manual Tape & 411 & Good & \\
\hline $6 / 24 / 947: 06$ & Auto FIC & 11.10 & Suspect & Data not included in graph \\
\hline $6 / 25 / 940: 00$ & Manual FIC & 411.10 & Good & \\
\hline $6 / 25 / 940: 00$ & Manual Tape & 411 & Good & \\
\hline $6 / 25 / 947: 05$ & Auto FIC & 11.10 & Suspect & Data not included in graph \\
\hline $6 / 26 / 940: 00$ & Manual FIC & 411.10 & Good & \\
\hline $6 / 26 / 940: 00$ & Manual Tape & 411 & Good & \\
\hline $6 / 26 / 947: 06$ & Auto FIC & 11.1 & Suspect & Data not included in graph \\
\hline $6 / 27 / 940: 00$ & Manual FIC & 411.1 & Good & \\
\hline $6 / 27 / 940: 00$ & Manual Tape & 411 & Good & \\
\hline $6 / 27 / 947: 06$ & Auto FIC & 11.1 & Suspect & Data not included in graph \\
\hline $6 / 28 / 940: 00$ & Manual FIC & 411.10 & Good & \\
\hline $6 / 28 / 940: 00$ & Manual Tape & 411 & Good & \\
\hline $6 / 28 / 947: 06$ & Auto FIC & 11.10 & Suspect & Data not included in graph \\
\hline $6 / 29 / 940: 00$ & Manual FIC & 411.10 & Good & \\
\hline $6 / 29 / 940: 00$ & Manual Tape & 411.00 & Good & \\
\hline $6 / 29 / 947: 03$ & Auto FIC & 11.1 & Suspect & Data not included in graph \\
\hline $6 / 30 / 940: 00$ & Manual FIC & 411.15 & Good & \\
\hline $6 / 30 / 940: 00$ & Manual Tape & 411.25 & Good & \\
\hline $6 / 30 / 947: 04$ & Auto FIC & 11.10 & Suspect & Data not included in graph \\
\hline $7 / 1 / 940: 00$ & Manual FIC & 411.1 & Good & \\
\hline $7 / 1 / 940: 00$ & Manual Tape & 411.00 & Good & \\
\hline $7 / 1 / 947: 04$ & Auto FIC & 11.10 & Suspect & Data not included in graph \\
\hline $7 / 2 / 940: 00$ & Manual FIC & 411.15 & Good & \\
\hline $7 / 2 / 940: 00$ & Manual Tape & 411 & Good & \\
\hline $7 / 2 / 947: 04$ & Auto FIC & 11.10 & Suspect & Data not included in graph \\
\hline $7 / 3 / 940: 00$ & Manual FIC & 411.15 & Good & \\
\hline $7 / 3 / 940: 00$ & Manual Tape & 411,00 & Good & \\
\hline $7 / 3 / 947: 04$ & Auto FIC & 11.1 & Suspect & Data not included in graph \\
\hline $7 / 4 / 940: 00$ & Manual FIC & 411.15 & Good & \\
\hline $7 / 4 / 940: 00$ & Manual Tape & 411.25 & Good & \\
\hline 7/4/94 7:04 & Auto FIC & 11.10 & Suspect & Data not included in graph \\
\hline $7 / 5 / 940: 00$ & Manual FIC & 411.10 & Good & \\
\hline $7 / 5 / 940: 00$ & Manual Tape & 411.50 & Good & \\
\hline $7 / 5 / 947: 03$ & Auto FIC & 11.10 & Suspect & Data not included in graph \\
\hline $7 / 6 / 940: 00$ & Manual FIC & 411.1 & Good & \\
\hline $7 / 6 / 940: 00$ & Manual Tape & 410 & Good & \\
\hline $7 / 6 / 947: 05$ & Auto FIC & 11.1 & Suspect & Data not included in graph \\
\hline $7 / 7 / 940: 00$ & Manual FIC & 411.1 & Good & \\
\hline $7 / 7 / 940: 00$ & Manual Tape & 411.00 & Good & \\
\hline $7 / 7 / 947: 03$ & Auto FIC & 11.10 & Suspect & Data not included in graph \\
\hline 7/8/94 0:00 & Manual FIC & 411.10 & Good & \\
\hline $7 / 8 / 940: 00$ & Manual Tape & 411.00 & Good & \\
\hline $7 / 8 / 947: 03$ & Auto FIC & 11.10 & Suspect & Data not included in graph \\
\hline $7 / 9 / 940: 00$ & Manual FIC & 411.15 & Good & \\
\hline $7 / 9 / 940: 00$ & Manual Tape & 411.13 & Good & \\
\hline $7 / 9 / 947: 03$ & Auto FIC & 11.10 & Suspect & Data not included in graph \\
\hline
\end{tabular}

Data obtained from WHC Surveillance Analysis Computer System, (PC SACS) July 7, 1995. 


\begin{tabular}{|c|c|c|c|c|}
\hline \multicolumn{5}{|c|}{ 241-AP-108 } \\
\hline \multicolumn{5}{|c|}{ Surface Level Data } \\
\hline Date & Type of Measurement & Leval-(linches) & Date Quality & Comments \\
\hline $7 / 10 / 940: 00$ & Manual FIC & 411.1 & Good & \\
\hline $7 / 10 / 940: 00$ & Manual Tape & 411.25 & Good & \\
\hline $7 / 10 / 947: 03$ & Auto FIC & 11.10 & Suspect & Data not included in graph \\
\hline $7 / 11 / 940: 00$ & Manual FIC & 411.15 & Good & \\
\hline $7 / 11 / 940: 00$ & Manual Tape & 411.25 & Good & \\
\hline $7 / 11 / 947: 03$ & Auto FIC & 11.10 & Suspect & Data not included in graph \\
\hline 7/12/94 0:00 & Manual FIC & 411.10 & Good & \\
\hline $7 / 12 / 940: 00$ & Manual Tape & 411 & Good & \\
\hline $7 / 12 / 947: 03$ & Auto FIC & 11.10 & Suspect & Data not included in graph \\
\hline $7 / 13 / 940: 00$ & Manual FIC & 411.1 & Good & \\
\hline $7 / 13 / 940: 00$ & Manual Tape & 411.00 & Good & \\
\hline $7 / 13 / 947: 03$ & Auto FIC & 11.10 & Suspect & Data not included in graph \\
\hline $7 / 14 / 940: 00$ & Manual FIC & 411.10 & Good & \\
\hline $7 / 14 / 940: 00$ & Manual Tape & 411 & Good & \\
\hline $7 / 14 / 947: 04$ & Auto FIC & 11.10 & Suspect & Data not included in graph \\
\hline $7 / 15 / 940: 00$ & Manual FIC & 411.1 & Good & \\
\hline $7 / 15 / 940: 00$ & Manual Tape & 411.00 & Good & \\
\hline $7 / 15 / 947: 04$ & Auto FIC & 11.10 & Suspect & Data not included in graph \\
\hline $7 / 16 / 940: 00$ & Manual FIC & 411.15 & Good & \\
\hline $7 / 16 / 940: 00$ & Manual Tape & 411.00 & Good & \\
\hline $7 / 16 / 947: 04$ & Auto FIC & 11.1 & Suspect & Data not included in graph \\
\hline $7 / 17 / 940: 00$ & Manual FIC & 411.10 & Good & \\
\hline $7 / 17 / 940: 00$ & Manual Tape & 411 & Good & \\
\hline $7 / 17 / 947: 04$ & Auto FIC & 11.1 & Suspect & Data not included in graph \\
\hline 7/18/94 0:00 & Manual FIC & 411.15 & Good & \\
\hline $7 / 18 / 940: 00$ & Manual Tape & 411 & Good & \\
\hline $7 / 19 / 940: 00$ & Manual FIC & 411.1 & Good & \\
\hline $7 / 19 / 940: 00$ & Manual Tape & 411.00 & Good & \\
\hline $7 / 19 / 947: 03$ & Auto FIC & 11.1 & Good & \\
\hline $7 / 20 / 940: 00$ & Manual FIC & 411.10 & Good & \\
\hline $7 / 20 / 940: 00$ & Manual Tape & 411 & Good & \\
\hline 7/20/94 7:03 & Auto FIC & 11.10 & Suspect & Data not included in graph \\
\hline $7 / 21 / 940: 00$ & Manual FIC & 411.10 & Good & \\
\hline $7 / 21 / 940: 00$ & Manual Tape & 411 & Good & \\
\hline $7 / 21 / 947: 03$ & Auto FIC & 11.10 & Suspect & Data not included in graph \\
\hline $7 / 22 / 940: 00$ & Manual FIC & 411.10 & Good & \\
\hline $7 / 22 / 940: 00$ & Manual Tape & 411.00 & Good & \\
\hline $7 / 22 / 947: 03$ & Auto FIC & 11.1 & Suspect & Data not included in graph \\
\hline $7 / 23 / 94 \quad 0: 00$ & Manual FIC & 411.1 & Good & \\
\hline $7 / 23 / 940: 00$ & Manual Tape & 411 & Good & \\
\hline $7 / 23 / 947: 03$ & Auto FIC & 11.10 & Suspect & Data not included in graph \\
\hline $7 / 24 / 940: 00$ & Manual FIC & 411.10 & Good & \\
\hline $7 / 24 / 940: 00$ & Manual Tape & 411 & Good & \\
\hline 7/24/94 7:03 & Auto FIC & 11.1 & Suspect & Data not included in graph \\
\hline $7 / 25 / 940: 00$ & Manual FIC & 411.10 & Good & \\
\hline $7 / 25 / 940: 00$ & Manual Tape & 411 & Good & \\
\hline $7 / 25 / 947: 03$ & Auto FIC & 11.1 & Suspect & Data not included in graph \\
\hline $7 / 26 / 94 \quad 0: 00$ & Manual FIC & 411.10 & Good & \\
\hline
\end{tabular}

Data obtained from WHC Surveillance Analysis Computer System, (PC SACS) July 7, 1995. 


\begin{tabular}{|c|c|c|c|c|}
\hline \multicolumn{5}{|c|}{ 241-AP-108 } \\
\hline \multicolumn{5}{|c|}{ Surface Level Data } \\
\hline Date & Type of Measurement & Level (Inches) & Data Quality & Comments \\
\hline $7 / 26 / 947: 03$ & Auto FIC & 11.1 & Suspect & Data not included in graph \\
\hline $7 / 27 / 940: 00$ & Manual FIC & 411.10 & Good & \\
\hline $7 / 27 / 947: 03$ & Auto FIC & 11.10 & Suspect & Data not included in graph \\
\hline $7 / 28 / 940: 00$ & Manual FIC & 411.1 & Good & \\
\hline $7 / 28 / 947: 04$ & Auto FIC & 11.10 & Suspect & Data not included in graph \\
\hline $7 / 29 / 947: 04$ & Auto FIC & 11.1 & Suspect & Data not included in graph \\
\hline $7 / 29 / 9412: 00$ & Manual FIC & 411.1 & Good & \\
\hline $7 / 30 / 947: 04$ & Auto FIC & 11.1 & Suspect & Data not included in graph \\
\hline $7 / 30 / 9412: 00$ & Manual FIC & 411.10 & Good & \\
\hline $7 / 31 / 9412: 00$ & Manual FIC & 411.1 & Good & \\
\hline $7 / 31 / 9423: 03$ & Auto FIC & 11.1 & Suspect & Data not included in graph \\
\hline $8 / 1 / 947: 03$ & Auto FIC & 11.1 & Suspect & Data not included in graph \\
\hline $8 / 1 / 9412: 00$ & Manual FIC & 411.1 & Good & \\
\hline $8 / 1 / 9412: 00$ & Manual Tape & 411.25 & Good & \\
\hline $8 / 2 / 947: 03$ & Auto FIC & 11.1 & Suspect & Data not included in graph \\
\hline $8 / 2 / 9412: 00$ & Manual FIC & 411.10 & Good & \\
\hline $8 / 2 / 9412: 00$ & Manual Tape & 411.25 & Good & \\
\hline $8 / 3 / 947: 03$ & Auto FIC & 11.10 & Suspect & Data not included in graph \\
\hline $8 / 3 / 9412: 00$ & Manual FIC & 411.1 & Good & \\
\hline $8 / 3 / 9412: 00$ & Manual Tape & 411 & Good & \\
\hline $8 / 4 / 947: 02$ & Auto FIC & 0.00 & Suspect & Data not included in graph \\
\hline $8 / 4 / 9412: 00$ & Manual FIC & 411.10 & Good & \\
\hline $8 / 4 / 9412: 00$ & Manual Tape & 411 & Good & \\
\hline $8 / 5 / 947: 03$ & Auto FIC & 11.10 & Suspect & Data not included in graph \\
\hline $8 / 5 / 9412: 00$ & Manual FIC & 411.1 & Good & \\
\hline $8 / 5 / 9412: 00$ & Manual Tape & 411 & Good & \\
\hline $8 / 6 / 947: 03$ & Auto FIC & 11.10 & Suspect & Data not included in graph \\
\hline $8 / 6 / 9412: 00$ & Manual FIC & 411.1 & Good & \\
\hline $8 / 6 / 9412: 00$ & Manual Tape & 411 & Good & \\
\hline $8 / 7 / 947: 04$ & Auto FIC & 11.1 & Suspect & Data not included in graph \\
\hline $8 / 7 / 9412: 00$ & Manual FIC & 411.10 & Good & \\
\hline $8 / 7 / 9412: 00$ & Manual Tape & 411 & Good & \\
\hline $8 / 8 / 947: 12$ & Auto FIC & 0.00 & Suspect & Data not included in graph \\
\hline $8 / 8 / 9412: 00$ & Manual FIC & 411.10 & Good & \\
\hline $8 / 8 / 9412: 00$ & Manual Tape & 411 & Good & \\
\hline $8 / 9 / 947: 06$ & Auto FIC & 11.1 & Suspect & Data not included in graph \\
\hline $8 / 9 / 9412: 00$ & Manual FIC & 411.1 & Good & \\
\hline $8 / 9 / 9412: 00$ & Manual Tape & 411.00 & Good & \\
\hline $8 / 10 / 947: 04$ & Auto FIC & 11.10 & Suspect & Data not included in graph \\
\hline $8 / 10 / 9412: 00$ & Manual FIC & 411.1 & Good & \\
\hline $8 / 10 / 9412: 00$ & Manual Tape & 410.5 & Good & \\
\hline $8 / 11 / 947: 04$ & Auto FIC & 11.1 & Suspect & Data not included in graph \\
\hline $8 / 11 / 9412: 00$ & Manual FIC & 411.1 & Good & \\
\hline $8 / 11 / 9412: 00$ & Manual Tape & 410.50 & Good & \\
\hline $8 / 12 / 947: 04$ & Auto FIC & 11.1 & Suspect & Data not included in graph \\
\hline $8 / 12 / 9412: 00$ & Manual FIC & 411.1 & Good & \\
\hline $8 / 12 / 9412: 00$ & Manual Tape & 411 & Good & \\
\hline $8 / 13 / 947: 03$ & Auto FIC & 19.10 & Suspect & Data not included in graph \\
\hline
\end{tabular}

Data obtained from WHC Surveillance Analysis Computer System, (PC SACS) July 7, 1995. 


\begin{tabular}{|c|c|c|c|c|}
\hline \multicolumn{5}{|c|}{ 241-AP-108 } \\
\hline \multicolumn{5}{|c|}{ Surface Level Data } \\
\hline Date & Type of Measurement & Level (Inches) & Data Quality & Comments \\
\hline $8 / 13 / 9412: 00$ & Manual FIC & 411.10 & Good & \\
\hline $8 / 13 / 9412: 00$ & Manual Tape & 411.00 & Good & \\
\hline $8 / 14 / 947: 06$ & Auto FIC & 11.10 & Suspect & Data not included in graph \\
\hline $8 / 14 / 94 \quad 12: 00$ & Manual FIC & 411.1 & Good & \\
\hline $8 / 14 / 9412: 00$ & Manual Tape & 410.75 & Good & \\
\hline $8 / 15 / 947: 06$ & Auto FIC & 11.1 & Suspect & Data not included in graph \\
\hline $8 / 15 / 9412: 00$ & Manual FIC & 411.1 & Good & \\
\hline $8 / 15 / 9412: 00$ & Manual Tape & 411.00 & Good & \\
\hline $8 / 16 / 947: 06$ & Auto FIC & 11.1 & Suspect & Data not included in graph \\
\hline $8 / 16 / 9412: 00$ & Manual FIC & 411.1 & Good & \\
\hline $8 / 16 / 9412: 00$ & Manual Tape & 410.25 & Good & \\
\hline $8 / 17 / 947: 06$ & Auto FIC & 411.1 & Good & \\
\hline $8 / 17 / 9412: 00$ & Manual FIC & 411.1 & Good & \\
\hline $8 / 17 / 9412: 00$ & Manual Tape . & 411 & Good & \\
\hline $8 / 18 / 947: 06$ & Auto FIC & 411.20 & Good & \\
\hline $8 / 18 / 9412: 00$ & Manual Tape & 411 & Good & \\
\hline $8 / 19 / 946: 06$ & Auto FIC & 411.10 & Good & \\
\hline $8 / 19 / 9412: 00$ & Manual Tape & 411.00 & Good & \\
\hline $8 / 20 / 947: 04$ & Auto FIC & 411.10 & Good & \\
\hline $8 / 20 / 9412: 00$ & Manual Tape & 411.25 & Good & \\
\hline $8 / 21 / 947: 04$ & Auto FIC & 411.1 & Good & \\
\hline $8 / 21 / 9412: 00$ & Manual Tape & 411 & Good & \\
\hline $8 / 22 / 946: 04$ & Auto FIC & 411.1 & Good & \\
\hline $8 / 22 / 9412: 00$ & Manual Tape & 411 & Good & \\
\hline $8 / 23 / 947: 04$ & Auto FIC & 411.10 & Good & \\
\hline $8 / 23 / 9412: 00$ & Manual Tape & 411.25 & Good & \\
\hline $8 / 24 / 947: 04$ & Auto FIC & 471.1 & Good & : \\
\hline $8 / 24 / 9412: 00$ & Manual Tape & 411.25 & Good & \\
\hline $8 / 25 / 947: 03$ & Auto FIC & 411.10 & Good & \\
\hline $8 / 25 / 94 \quad 12: 00$ & Manual Tape & 411.00 & Good & \\
\hline $8 / 26 / 941: 05$ & Auto FIC & 411.10 & Good & \\
\hline $8 / 26 / 9412: 00$ & Manual Tape & 411.25 & Good & \\
\hline $8 / 27 / 947: 04$ & Auto FIC & 411.20 & Good & \\
\hline $8 / 27 / 9412: 00$ & Manual Tape & 411 & Good & \\
\hline $8 / 28 / 947: 04$ & Auto FIC & 411.2 & Good & \\
\hline $8 / 28 / 9412: 00$ & Manual Tape & 411 & Good & \\
\hline $8 / 29 / 947: 04$ & Auto FIC & 411.2 & Good & \\
\hline $8 / 29 / 9412: 00$ & Manual Tape & 411 & Good & \\
\hline $8 / 30 / 947: 04$ & Auto FIC & 411.20 & Good & \\
\hline $8 / 30 / 9412: 00$ & Manual Tape & 411 & Good & \\
\hline $8 / 31 / 947: 04$ & Auto FIC & 411.20 & Good & \\
\hline $8 / 31 / 9412: 00$ & Manual Tape & 411 & Good & \\
\hline $9 / 1 / 947: 04$ & Auto FIC & 411.2 & Good & \\
\hline $9 / 1 / 9412: 00$ & Manual Tape & 411 & Good & \\
\hline 9/2/94 7:06 & Auto FIC & 411.20 & Good & \\
\hline $9 / 2 / 9412: 00$ & Manual Tape & 411 & Good & \\
\hline $9 / 3 / 9412: 00$ & Manual Tape & 411 & Good & \\
\hline $9 / 3 / 9423: 04$ & Auto FIC & 411.20 & Good & \\
\hline
\end{tabular}

Data obtained from WHC Surveillance Analysis Computer System, (PC SACS) July 7, 1995. 


\begin{tabular}{|c|c|c|c|c|}
\hline \multicolumn{5}{|c|}{ 241-AP-108 } \\
\hline \multicolumn{5}{|c|}{ Surface Level Data } \\
\hline Date & Type of Measurement & Level (Inches) & Data Quality & Comments \\
\hline $9 / 4 / 947: 04$ & Auto FIC & 411.2 & Good & \\
\hline 9/4/94 12:00 & Manual Tape & 410.75 & Good & \\
\hline $9 / 5 / 947: 04$ & Auto FIC & 411.20 & Good & \\
\hline $9 / 5 / 94 \quad 12: 00$ & Manual Tape & 410.75 & Good & \\
\hline $9 / 6 / 947: 03$ & Auto FIC & 411.20 & Good & \\
\hline $9 / 6 / 9412: 00$ & Manual Tape & 411 & Good & \\
\hline $9 / 7 / 947: 03$ & Auto FIC & 411.2 & Good & \\
\hline $9 / 7 / 9412: 00$ & Manual Tape & 411 & Good & \\
\hline $9 / 8 / 947: 04$ & Auto FIC & 411.20 & Good & \\
\hline $9 / 8 / 9412: 00$ & Manual Tape & 411 & Good & \\
\hline 9/9/94 7:03 & Auto FIC & 411.20 & Good & \\
\hline 9/9/94 12:00 & Manual Tape & 411 & Good & \\
\hline $9 / 10 / 947: 04$ & Auto FIC & 411.20 & Good & \\
\hline $9 / 10 / 9412: 00$ & Manual Tape & 411 & Good & \\
\hline $9 / 11 / 947: 03$ & Auto FIC & 411.2 & Good & \\
\hline $9 / 11 / 9412: 00$ & Manual Tape & 411 & Good & \\
\hline $9 / 12 / 947: 03$ & Auto FIC & 411.1 & Good & \\
\hline $9 / 12 / 9412: 00$ & Manual Tape & 411 & Good & \\
\hline $9 / 13 / 947: 02$ & Auto FIC & 411.2 & Good & \\
\hline $9 / 13 / 9412: 00$ & Manual Tape & 411 & Good & \\
\hline $9 / 14 / 947: 03$ & Auto FIC & 411.2 & Good & \\
\hline $9 / 14 / 9412: 00$ & Manual Tape & 411 & Good & \\
\hline $9 / 15 / 947: 03$ & Auto FIC & 411.20 & Good & \\
\hline $9 / 15 / 9412: 00$ & Manual Tape & 411 & Good & \\
\hline $9 / 16 / 947: 02$ & Auto FIC & 411.2 & Good & \\
\hline $9 / 16 / 9412: 00$ & Manual Tape & 411 & Good & \\
\hline $9 / 17 / 947: 03$ & Auto FIC & 411.2 & Good & \\
\hline $9 / 17 / 9412: 00$ & Manual Tape & 411 & Good & \\
\hline $9 / 18 / 947: 02$ & Auto FIC & 411.2 & Good & \\
\hline $9 / 18 / 9412: 00$ & Manual Tape & 410.5 & Good & \\
\hline $9 / 19 / 947: 03$ & Auto FIC & 411.20 & Good & \\
\hline $9 / 19 / 9412: 00$ & Manual Tape & 410.75 & Good & \\
\hline $9 / 20 / 947: 03$ & Auto FIC & 411.20 & Good & \\
\hline $9 / 20 / 94 / 2: 00$ & Manual Tape & 410.75 & Good & \\
\hline $9 / 21 / 947: 04$ & Auto FIC & 411.2 & Good & \\
\hline $9 / 21 / 9412: 00$ & Manual Tape & 410.75 & Good & \\
\hline $9 / 22 / 947: 03$ & Auto FIC & 411.1 & Good & \\
\hline $9 / 22 / 9412: 00$ & Manual Tape & 410.25 & Good & \\
\hline $9 / 23 / 947: 04$ & Auto FIC & 411.2 & Good & \\
\hline $9 / 23 / 9412: 00$ & Manual Tape & 410.75 & Good & \\
\hline $9 / 24 / 947: 04$ & Auto FIC & 411.1 & Good & \\
\hline $9 / 24 / 9412: 00$ & Manual Tape & 411 & Good & \\
\hline $9 / 25 / 947: 03$ & Auto FIC & 411.10 & Good & \\
\hline $9 / 25 / 9412: 00$ & Manual Tape & 410.75 & Good & \\
\hline $9 / 26 / 947: 04$ & Auto FIC & 411.1 & Good & \\
\hline $9 / 26 / 9412: 00$ & Manual Tape & 411 & Good & \\
\hline $9 / 27 / 947: 04$ & Auto FIC & 411.1 & Good & \\
\hline $9 / 27 / 9412: 00$ & Manual Tape & 411.125 & Good & \\
\hline
\end{tabular}

Data obtained from WHC Surveillance Analysis Computer System, (PC SACS) July 7, 1995. 


\begin{tabular}{|c|c|c|c|c|}
\hline \multicolumn{5}{|c|}{ 241-AP-108 } \\
\hline \multicolumn{5}{|c|}{ Surface Level Data } \\
\hline Date & Type of Measurement & Level (Inches) & Data Quality & Comments \\
\hline $9 / 28 / 947: 03$ & Auto FIC & 411.10 & Good & \\
\hline $9 / 28 / 9412: 00$ & Manual Tape & 411 & Good & \\
\hline $9 / 29 / 947: 04$ & Auto FIC & 411.1 & Good & \\
\hline $9 / 29 / 94 \quad 12: 00$ & Manual Tape & 411 & Good & \\
\hline 9/30/94 7:04 & Auto FIC & 411.1 & Good & \\
\hline $9 / 30 / 9412: 00$ & Manual Tape & 411.25 & Good & \\
\hline $10 / 1 / 947: 06$ & Auto FIC & 411.10 & Good & \\
\hline $10 / 1 / 9412: 00$ & Manual Tape & 411 & Good & \\
\hline $10 / 2 / 947: 05$ & Auto FIC & 411.10 & Good & \\
\hline $10 / 2 / 94 / 2: 00$ & Manual Tape & 411 & Good & \\
\hline $10 / 3 / 947: 04$ & Auto FIC & 411.1 & Good & \\
\hline $10 / 3 / 9412: 00$ & Manual Tape & 411 & Good & \\
\hline $10 / 4 / 947: 04$ & Auto FIC & 411.10 & Good & \\
\hline $10 / 4 / 9412: 00$ & Manual Tape & 411 & Good & \\
\hline $10 / 5 / 947: 04$ & Auto FIC & 411.1 & Good & \\
\hline $10 / 5 / 9412: 00$ & Manual Tape & 411 & Good & \\
\hline $10 / 6 / 947: 04$ & Auto FIC & 411.10 & Good & \\
\hline $10 / 6 / 9412: 00$ & Manual Tape & 410.5 & Good & \\
\hline $10 / 7 / 946: 04$ & Auto FIC & 411.1 & Good & \\
\hline $10 / 7 / 9412: 00$ & Manual Tape & 411 & Good & \\
\hline $10 / 8 / 947: 03$ & Auto FIC & 349.6 & Good & \\
\hline $10 / 8 / 9412: 00$ & Manual Tape & 336.5 & Good & \\
\hline $10 / 9 / 947: 06$ & Auto FIC & 307.80 & Good & \\
\hline $10 / 9 / 9412: 00$ & Manual Tape & 307.75 & Good & \\
\hline $10 / 10 / 947: 05$ & Auto FIC & 307.80 & Good & \\
\hline $10 / 10 / 9412: 00$ & Manual Tape & 307.75 & Good & \\
\hline $10 / 11 / 947: 03$ & Auto FIC & 307.8 & Good & \\
\hline $10 / 11 / 9412: 00$ & Manual Tape & 307.75 & Good & \\
\hline $10 / 12 / 947: 06$ & Auto FIC & 307.70 & Good & \\
\hline $10 / 12 / 9412: 00$ & Manual Tape & 307.75 & Good & \\
\hline $10 / 13 / 947: 05$ & Auto FIC & 307.70 & Good & \\
\hline $10 / 13 / 9412: 00$ & Manual Tape & 307.75 & Good & \\
\hline $10 / 14 / 947: 06$ & Auto FIC & 307.70 & Good & \\
\hline $10 / 14 / 9412: 00$ & Manual Tape & 307.5 & Good & \\
\hline $10 / 15 / 947: 06$ & Auto FIC & 307.70 & Good & \\
\hline $10 / 15 / 9412: 00$ & Manual Tape & 307.5 & Good & \\
\hline $10 / 16 / 947: 06$ & Auto FIC & 307.70 & Good & \\
\hline $10 / 16 / 9412: 00$ & Manual Tape & 307.25 & Good & \\
\hline $10 / 17 / 947: 06$ & Auto FIC & 307.7 & Good & \\
\hline $10 / 17 / 9412: 00$ & Manual Tape & 307.75 & Good & \\
\hline $10 / 18 / 947: 06$ & Auto FIC & 307.70 & Good & \\
\hline $10 / 18 / 9412,00$ & Manual Tape & $307 . \overline{5}$ & Good & \\
\hline $10 / 19 / 947: 06$ & Auto FIC & 307.70 & Good & \\
\hline $10 / 19 / 9412: 00$ & Manual Tape & 307.75 & Good & \\
\hline $10 / 20 / 945: 07$ & Auto FIC & 307.70 & Good & \\
\hline $10 / 20 / 9412: 00$ & Manual Tape & 307.75 & Good & \\
\hline $10 / 21 / 947: 06$ & Auto FIC & 307.7 & Good & \\
\hline $10 / 21 / 9412: 00$ & Manual Tape & 307.75 & Good & \\
\hline
\end{tabular}

Data obtained from WHC Surveiliance Analysis Computer System, (PC SACS) July 7, 1995. 


\begin{tabular}{|c|c|c|c|c|}
\hline \multicolumn{5}{|c|}{ 241-AP-108 } \\
\hline \multicolumn{5}{|c|}{ Surface Lovel Data } \\
\hline Date & Type of Measurement & Level (Inches) & Data Quality & Cornments \\
\hline $10 / 22 / 9412: 00$ & Manual Tape & 307.5 & Good & \\
\hline $10 / 22 / 9423: 06$ & Auto FIC & 307.70 & Good & \\
\hline $10 / 23 / 947: 06$ & Auto FIC & 307.70 & Good & \\
\hline $10 / 23 / 9412: 00$ & Manual Tape & 307.5 & Good & \\
\hline $10 / 24 / 947: 06$ & Auto FIC & 265.8 & Good & \\
\hline $10 / 24 / 9412: 00$ & Manual Tape & 249.25 & Good & \\
\hline $10 / 25 / 947: 06$ & Auto FIC & 156.20 & Good & \\
\hline $10 / 25 / 9412: 00$ & Manual Tape & 144 & Good & \\
\hline $10 / 26 / 947: 06$ & Auto FIC & 47.1 & Good & \\
\hline $10 / 26 / 9412: 00$ & Manual Tape & 37.5 & Good & \\
\hline $10 / 27 / 945: 06$ & Auto FIC & 10.6 & Good & \\
\hline $10 / 27 / 9412: 00$ & Manual Tape & 10.5 & Good & \\
\hline $10 / 28 / 947: 04$ & Auto FIC & 10.6 & Good & \\
\hline $10 / 28 / 9412.00$ & Manual Tape & 10.5 & Good & \\
\hline $10 / 29 / 9412: 00$ & Manual FIC & 10.50 & Good & \\
\hline $10 / 29 / 9412: 00$ & Manual Tape & 10.5 & Good & \\
\hline $10 / 30 / 9412: 00$ & Manual Tape & 10.25 & Good & \\
\hline $10 / 30 / 9414: 03$ & Auto FIC & 10.60 & Good & \\
\hline $10 / 31 / 947: 05$ & Auto FIC & 0 & Suspect & Data not included in graph \\
\hline $10 / 31 / 9412: 00$ & Manual FIC & 10.5 & Good & \\
\hline $10 / 31 / 9412: 00$ & Manual Tape & 10.5 & Good & \\
\hline $11 / 1 / 9412: 00$ & Manual Tape & 10.25 & Good & \\
\hline $11 / 1 / 9416: 03$ & Auto FIC & 10.6 & Good & \\
\hline 11/2/947:03 & Auto FIC & 0.00 & Suspect & Data not included in graph \\
\hline $11 / 2 / 9412: 00$ & Manual FIC & 10.50 & Good & \\
\hline $11 / 2 / 9412: 00$ & Manual Tape & 10.5 & Good & \\
\hline $11 / 3 / 947: 04$ & Auto FIC & 0 & Suspect & Data not included in graph \\
\hline $11 / 3 / 9412: 00$ & Manual FIC & 10.50 & Good & \\
\hline $11 / 3 / 9412: 00$ & Manual Tape & 10.5 & Good & \\
\hline $11 / 4 / 947: 03$ & Auto FIC & 0 & Suspect & Data not included in graph \\
\hline $11 / 4 / 9412: 00$ & Manual FIC & 10.5 & Good & \\
\hline $11 / 4 / 9412: 00$ & Manual Tape & 10.25 & Good & \\
\hline $11 / 5 / 947: 03$ & Auto FIC & 0 & Suspect & Data not included in graph \\
\hline $11 / 5 / 9412: 00$ & Manual FIC & 10.50 & Good & \\
\hline $11 / 5 / 9412: 00$ & Manual Tape & 10.25 & Good & \\
\hline $11 / 6 / 947: 04$ & Auto FIC & 0 & Suspect & Data not included in graph \\
\hline $11 / 6 / 9412: 00$ & Manual FIC & 10.50 & Good & \\
\hline $11 / 6 / 9412: 00$ & Manual Tape & 10.25 & Good & \\
\hline $11 / 7 / 947: 04$ & Auto FIC & 0 & Suspect & Data not included in graph \\
\hline $11 / 7 / 9412: 00$ & Manual FIC & 10.50 & Good & \\
\hline $11 / 7 / 9412: 00$ & Manual Tape & 10.25 & Good & \\
\hline $11 / 8 / 947: 03$ & Auto FIC & 0.00 & Suspect & Data not included in graph \\
\hline $11 / 8 / 9411: 02$ & Manual Tape & 10.25 & Good & \\
\hline $11 / 8 / 9412: 00$ & Manual FIC & 10.5 & Good & \\
\hline $11 / 9 / 947: 05$ & Auto FIC & 0 & Suspect & Data not included in graph \\
\hline 11/9/94 11:02 & Manual Tape & 10.25 & Good & \\
\hline $11 / 9 / 9412: 00$ & Manual FIC & 10.5 & Good & \\
\hline $11 / 10 / 947: 03$ & Auto FIC & 10.6 & Good & \\
\hline
\end{tabular}

Data obtained from WHC Surveillance Analysis Computer System, (PC SACS) July 7, 1995. 


\begin{tabular}{|c|c|c|c|c|}
\hline \multicolumn{5}{|c|}{ 241-AP-108 } \\
\hline \multicolumn{5}{|c|}{ Surface Level Data } \\
\hline Date & Type of Measurement & Lovel (Inches) & Data Quality & Comments \\
\hline $11 / 10 / 9411: 02$ & Manual Tape & 10.25 & Good & \\
\hline $11 / 11 / 947: 03$ & Auto FIC & 0 & Suspect & Data not included in graph \\
\hline $11 / 11 / 9411: 02$ & Manual Tape & 10.25 & Good & \\
\hline $11 / 11 / 9412: 00$ & Manual FIC & 10.50 & Good & \\
\hline $11 / 12 / 947: 03$ & Auto FIC & 10.50 & Good & \\
\hline $11 / 12 / 9411: 02$ & Manual Tape & 10.5 & Good & \\
\hline $11 / 13 / 947: 04$ & Auto FIC & 10.5 & Good & \\
\hline $11 / 13 / 9411: 02$ & Manual Tape & 10.25 & Good & \\
\hline $11 / 14 / 947: 03$ & Auto FIC & 10.50 & Good & \\
\hline $11 / 14 / 9411: 02$ & Manual Tape & 10.25 & Good & \\
\hline $11 / 15 / 947: 04$ & Auto FIC & 10.60 & Good & \\
\hline $11 / 15 / 9412: 00$ & Manual Tape & 10.25 & Good & \\
\hline $11 / 16 / 947: 06$ & Auto FIC & 10.5 & Good & \\
\hline $11 / 16 / 9412: 00$ & Manual Tape & 10.25 & Good & \\
\hline $11 / 17 / 947: 04$ & Auto FIC & 10.5 & Good & \\
\hline $11 / 17 / 9412: 00$ & Manual Tape & 10.25 & Good & \\
\hline $11 / 18 / 947: 04$ & Auto FIC & 10.5 & Good & \\
\hline $11 / 18 / 9412: 00$ & Manual Tape & 10.25 & Good & \\
\hline $11 / 19 / 947: 03$ & Auto FIC & 10.5 & Good & \\
\hline $11 / 19 / 9412: 00$ & Manual Tape & 10.25 & Good & \\
\hline $11 / 20 / 947: 03$ & Auto FIC & 10.50 & Good & \\
\hline $11 / 21 / 947: 04$ & Auto FIC & 10.5 & Good & \\
\hline $11 / 21 / 9412: 00$ & Manual Tape & 10.25 & Good & \\
\hline $11 / 22 / 947: 03$ & Auto FIC & 10.3 & Good & \\
\hline $11 / 22 / 9412: 00$ & Manual Tape & 10.5 & Good & $\cdot$ \\
\hline $11 / 23 / 94 / 2: 00$ & Manual Tape & 10.5 & Good & \\
\hline $11 / 24 / 9412: 00$ & Manual Tape & 81 & Good & \\
\hline $11 / 24 / 9423: 03$ & Auto FIC & 117.1 & Good & \\
\hline $11 / 25 / 9412: 00$ & Manual Tape & 117 & Good & \\
\hline $11 / 25 / 9423: 03$ & Auto FIC & 117.10 & Good & \\
\hline $11 / 26 / 947: 03$ & Auto FIC & 117.1 & Good & \\
\hline $11 / 26 / 9412: 00$ & Manual Tape & 117 & Good & \\
\hline $11 / 27 / 947: 04$ & Auto FIC & 117.1 & Good & \\
\hline $11 / 27 / 9412: 00$ & Manual Tape & 117 & Good & \\
\hline $11 / 28 / 947: 04$ & Auto FIC & 117 & Good & \\
\hline $11 / 28 / 9412: 00$ & Manual Tape & 117 & Good & \\
\hline $11 / 29 / 947: 04$ & Auto FIC & 117 & Good & \\
\hline $11 / 29 / 9412: 00$ & Manual Tape & 117 & Good & \\
\hline $11 / 30 / 947: 03$ & Auto FIC & 117 & Good & \\
\hline $11 / 30 / 9412: 00$ & Manual Tape & 117 & Good & \\
\hline $12 / 1 / 946: 05$ & Auto FIC & 172.7 & Good & \\
\hline $12 / 1 / 9412: 00$ & Manual Tape & 186.25 & Good & \\
\hline $12 / 2 / 947: 03$ & Auto FIC & 264.7 & Good & \\
\hline $12 / 2 / 9412: 00$ & Manual Tape & 273.5 & Good & \\
\hline $12 / 3 / 946: 04$ & Auto FIC & 288.50 & Good & \\
\hline $12 / 3 / 9412: 00$ & Manual Tape & 288.25 & Good & \\
\hline $12 / 4 / 947: 03$ & Auto FIC & 288.50 & . Good & \\
\hline $12 / 4 / 9412: 00$ & Manual Tape & 288.25 & Good & \\
\hline
\end{tabular}

Data obtained from WHC Surveillance Analysis Computer System, (PC SACS) July 7, 1995. 


\begin{tabular}{|c|c|c|c|c|}
\hline \multicolumn{5}{|c|}{ 241-AP-108 } \\
\hline \multicolumn{5}{|c|}{ Surface Level Data } \\
\hline Date & Type of Measurement & Level (Inches) & Data Quality & Comments \\
\hline $12 / 5 / 947: 03$ & Auto FIC & 288.5 & Good & \\
\hline $12 / 5 / 9412: 00$ & Manual Tape & 288.25 & Good & \\
\hline $12 / 6 / 947: 04$ & Auto FIC & 288.4 & Good & \\
\hline $12 / 6 / 9412: 00$ & Manual Tape & 288.25 & Good & \\
\hline $12 / 7 / 947: 03$ & Auto FIC & 288.40 & Good & \\
\hline $12 / 7 / 9412: 00$ & Manual Tape & 288.25 & Good & \\
\hline $12 / 8 / 945: 03$ & Auto FIC & 288.5 & Good & \\
\hline $12 / 8 / 9412: 00$ & Manual Tape & 288.25 & Good & \\
\hline $12 / 9 / 947: 03$ & Auto FIC & 288.40 & Good & \\
\hline $12 / 9 / 9412: 00$ & Manual Tape & 288.25 & Good & \\
\hline $12 / 10 / 947: 04$ & Auto FIC & 288.50 & Good & \\
\hline $12 / 10 / 9412: 00$ & Manual Tape & 288.25 & Good & \\
\hline $12 / 11 / 947: 04$ & Auto FIC & 288.4 & Good & \\
\hline $12 / 11 / 9412: 00$ & Manual Tape & 288.25 & Good & \\
\hline $12 / 12 / 947: 03$ & Auto FIC & 288.30 & Good & \\
\hline $12 / 12 / 9412: 00$ & Manual Tape & 288 & Good & \\
\hline $12 / 13 / 947: 03$ & Auto FIC & 288.50 & Good & \\
\hline $12 / 13 / 9412: 00$ & Manual Tape & 288 & Good & \\
\hline $12 / 14 / 947: 04$ & Auto FIC & 288.3 & Good & \\
\hline $12 / 14 / 94 \quad 12: 00$ & Manual Tape & 288.25 & Good & \\
\hline $12 / 15 / 947: 03$ & Auto FIC & 288.30 & Good & \\
\hline $12 / 15 / 9412: 00$ & Manual Tape & 288.25 & Good & \\
\hline $12 / 16 / 947: 03$ & Auto FIC & 288.3 & Good & \\
\hline $12 / 16 / 9412: 00$ & Manual Tape & 288.25 & Good & \\
\hline $12 / 17 / 947: 06$ & Auto FIC & 288.3 & Good & \\
\hline $12 / 17 / 9412: 00$ & Manual Tape & 288 & Good & \\
\hline $12 / 18 / 947: 04$ & Auto FIC & 288.3 & Good & \\
\hline $12 / 18 / 9412: 00$ & Manual Tape & 288.25 & Good & \\
\hline $12 / 19 / 947: 04$ & Auto FIC & 288.30 & Good & \\
\hline $12 / 19 / 9412: 00$ & Manual Tape & 288 & Good & \\
\hline $12 / 20 / 947: 04$ & Auto FIC & 288.3 & Good & \\
\hline $12 / 20 / 9412: 00$ & Manual Tape & 288 & Good & \\
\hline $12 / 21 / 947: 04$ & Auto FIC & 288.30 & Good & \\
\hline $12 / 21 / 9412: 00$ & Manual Tape & 288 & Good & \\
\hline $12 / 22 / 947: 05$ & Auto FIC & 288.3 & Good & \\
\hline $12 / 22 / 9412: 00$ & Manual Tape & 288.25 & Good & \\
\hline $12 / 23 / 947: 03$ & Auto FIC & 288.30 & Good & \\
\hline $12 / 23 / 9412: 00$ & Manual Tape & 288.25 & Good & \\
\hline $12 / 24 / 947: 04$ & Auto FIC & 288.3 & Good & \\
\hline $12 / 24 / 9412: 00$ & Manual Tape & 288.25 & Good & \\
\hline $12 / 25 / 947: 03$ & Auto FIC & 288.30 & Good & \\
\hline $12 / 25 / 9412: 00$ & Manual Tape & 288.25 & Good & \\
\hline $12 / 26 / 947: 03$ & Auto FIC & 288.3 & Good & \\
\hline $12 / 26 / 9412: 00$ & Manual Tape & 288 & Good & \\
\hline $12 / 27 / 947: 04$ & Auto FIC & 288.30 & Good & \\
\hline $12 / 27 / 9412: 00$ & Manual Tape & 288.25 & Good & \\
\hline $12 / 28 / 947: 04$ & Auto FIC & 288.30 & Good & \\
\hline $12 / 28 / 9412: 00$ & Manual Tape & 288 & Good & \\
\hline
\end{tabular}

Data obtained from WHC Surveillance Analysis Computer System, (PC SACS) July 7, 1995. 


\begin{tabular}{|c|c|c|c|c|}
\hline \multicolumn{5}{|c|}{ 241-AP-108 } \\
\hline & . & Urface Level Dat & & \\
\hline Date & Type of Measurement & Level (Inches) & Data Quality & Comments \\
\hline $12 / 29 / 947: 04$ & Auto FIC & 288.3 & Good & \\
\hline $12 / 29 / 9412: 00$ & Manual Tape & 288 & Good & \\
\hline $12 / 30 / 947: 04$ & Auto FIC & 288.20 & Good & \\
\hline $12 / 30 / 9412: 00$ & Manual Tape & 288 & Good & \\
\hline $12 / 31 / 947: 04$ & Auto FIC & 288.20 & Good & \\
\hline $12 / 31 / 9412: 00$ & Manual Tape & 288.25 & Good & \\
\hline $1 / 1 / 957: 04$ & Auto FIC & 288.2 & Good & \\
\hline $1 / 1 / 95 \quad 12: 00$ & Manual Tape & 288.25 & Good & \\
\hline 1/2/95 7:03 & Auto FIC & 288.2 & Good & \\
\hline $1 / 2 / 95 \quad 12: 00$ & Manual Tape & 288.25 & Good & \\
\hline $1 / 3 / 957: 02$ & Auto FIC & 288.2 & Good & \\
\hline $1 / 3 / 9512: 00$ & Manual Tape & 288 & Good & \\
\hline $1 / 4 / 957: 03$ & Auto FIC & 288.20 & Good & \\
\hline $1 / 4 / 9512: 00$ & Manual Tape & 288 & Good & \\
\hline 1/5/95 7:04 & Auto FIC & 288.20 & Good & \\
\hline $1 / 5 / 95 \quad 12: 00$ & Manual Tape . & 288 & Good & \\
\hline $1 / 6 / 957: 04$ & Auto FIC & 288.2 & Good & \\
\hline $1 / 6 / 9512: 00$ & Manual Tape & 288 & Good & \\
\hline 1/7/95 7:04 & Auto FIC & 288.2 & Good & \\
\hline $1 / 7 / 9512: 00$ & Manual Tape & 288 & Good & \\
\hline 1/8/95 12:00 & Manual Tape & 288 & Good & \\
\hline 1/8/95 23:03 & Auto FIC & 288.2 & Good & \\
\hline 1/9/95 7:05 & Auto FIC & 288.2 & Good & \\
\hline $1 / 9 / 9512: 00$ & Manual Tape & 288 & Good & \\
\hline $1 / 10 / 957: 06$ & Auto FIC & 288.20 & Good & \\
\hline $1 / 10 / 95 \quad 12: 00$ & Manual Tape & 288 & Good & \\
\hline $1 / 11 / 957: 07$ & Auto FIC & 288.2 & Good & \\
\hline $1 / 11 / 95 \quad 12: 00$ & Manual Tape & 288 & Good & \\
\hline $1 / 12 / 957: 07$ & Auto FIC & $288: 20$ & Good & \\
\hline $1 / 12 / 95 \quad 12: 00$ & Manual Tape & 288 & Good & \\
\hline $1 / 13 / 956: 07$ & Auto FIC & 288.20 & Good & \\
\hline $1 / 13 / 9512: 00$ & Manual Tape & 288 & Good & \\
\hline 1/14/95 7:07 & Auto FIC & 288.20 & Good & \\
\hline $1 / 14 / 95 \quad 12: 00$ & Manual Tape & 288 & Good & \\
\hline $1 / 15 / 957: 06$ & Auto FIC & 288.2 & Good & \\
\hline $1 / 15 / 95 \quad 12: 00$ & Manual Tape & 288 & Good & \\
\hline $1 / 16 / 957: 08$ & Auto FIC & 288.20 & Good & \\
\hline $1 / 16 / 95 \quad 12: 00$ & Manual Tape & 288 & Good & \\
\hline $1 / 17 / 956: 07$ & Auto FIC & 288.2 & Good & \\
\hline $1 / 17 / 95 \quad 12: 00$ & Manual Tape & 288 & Good & \\
\hline $1 / 18 / 957: 07$ & Auto FIC & 288.10 & Good & \\
\hline $1 / 18 / 95 \quad 12: 00$ & Manua! Tape & 288 & Good & \\
\hline $1 / 19 / 957: 07$ & Auto FIC & 288.1 & Good & \\
\hline $1 / 19 / 95 \quad 12: 00$ & Manual Tape & 288 & Good & \\
\hline $1 / 20 / 957: 03$ & Auto FIC & 198.50 & Good & \\
\hline $1 / 20 / 95 \quad 12: 00$ & Manual Tape & 186 & Good & \\
\hline $1 / 21 / 957: 04$ & Auto FIC & 83.10 & Good & \\
\hline $1 / 21 / 95 \quad 12: 00$ & Manual Tape & 68.5 & Good & \\
\hline
\end{tabular}

Data obtained from WHC Surveillance. Analysis Computer System, (PC SACS) July 7, 1995. 


\begin{tabular}{|c|c|c|c|c|}
\hline \multicolumn{5}{|c|}{ 241-AP-108 } \\
\hline \multicolumn{5}{|c|}{ Surface Level Data } \\
\hline Date & Type of Measurement & Level '(Inches) & Data Quality & Comments \\
\hline $1 / 22 / 957: 03$ & Auto FIC & 10.10 & Good & \\
\hline $1 / 22 / 9512: 00$ & Manual Tape & 10 & Good & \\
\hline $1 / 23 / 957: 03$ & Auto FIC & 10.10 & Good & \\
\hline $1 / 23 / 9512: 00$ & Manual Tape & 10 & Good & \\
\hline $1 / 24 / 957: 04$ & Auto FIC & 10.1 & Good & \\
\hline 1/24/95 12:00 & Manual Tape & 10 & Good & \\
\hline 1/25/95 7:03 & Auto FIC & 10.1 & Good & \\
\hline $1 / 25 / 9512: 00$ & Manual Tape & 9.75 & Good & \\
\hline $1 / 26 / 957: 03$ & Auto FIC & 10.10 & Good & \\
\hline $1 / 26 / 9512: 00$ & Manual Tape & 10 & Good & \\
\hline $1 / 27 / 957: 03$ & Auto FIC & 10.00 & Good & \\
\hline $1 / 27 / 95 \quad 12: 00$ & Manual Tape & 10 & Good & \\
\hline 1/28/95 7:04 & Auto FIC & 10.00 & Good & \\
\hline $1 / 28 / 95 \quad 12: 00$ & Manual Tape & 10 & Good & \\
\hline $1 / 29 / 95 \quad 7: 04$ & Auto FIC & 10.00 & Good & \\
\hline $1 / 29 / 9512: 00$ & Manual Tape & 10 & Good & \\
\hline $1 / 30 / 957: 04$ & Auto FIC & 10.00 & Good & \\
\hline 1/30/95 12:00 & Manual Tape & 10 & Good & \\
\hline $1 / 31 / 957: 03$ & Auto FIC & 10.00 & Good & \\
\hline $1 / 31 / 9512: 00$ & Manual Tape & 10 & Good & \\
\hline 2/1/95 7:03 & Auto FIC & 10.00 & Good & \\
\hline $2 / 1 / 9512: 00$ & Manual Tape & 10 & Good & \\
\hline 2/2/95 7:04 & Auto FIC & 10 & Good & \\
\hline $2 / 2 / 95 \quad 12: 00$ & Manual Tape & 10 & Good & \\
\hline 2/3/95 7:03 & Auto FIC & 10.00 & Good & \\
\hline $2 / 3 / 9512: 00$ & Manual Tape & 10 & Good & \\
\hline 2/4/95 7:04 & Auto FIC & 10 & Good & \\
\hline 2/4/95 12:00 & Manual Tape & 10 & Good & \\
\hline $2 / 5 / 957: 03$ & Auto FIC & 10 & Good & \\
\hline $2 / 5 / 9512: 00$ & Manual Tape & 10 & Good & \\
\hline $2 / 6 / 957: 03$ & Auto FIC & 10.00 & Good & \\
\hline $2 / 6 / 9512: 00$ & Manual Tape & 10 & Good & \\
\hline $2 / 7 / 957: 03$ & Auto FIC & 10.00 & Good & \\
\hline $2 / 7 / 95 \quad 12: 00$ & Manual Tape & 10 & Good & \\
\hline 2/8/95 7:04 & Auto FIC & 10 & Good & \\
\hline $2 / 8 / 9512: 00$ & Manual Tape & 10 & Good & \\
\hline 2/9/95 7:06 & Auto FIC & 10 & Good & \\
\hline $2 / 9 / 9512: 00$ & Manual Tape & 9.75 & Good & \\
\hline $2 / 10 / 957: 06$ & Auto FIC & 10.00 & Good & \\
\hline $2 / 10 / 9512: 00$ & Manual Tape & 10 & Good & \\
\hline $2 / 11 / 957: 07$ & Auto FIC & 10 & Good & \\
\hline $2 / 11 / 9512: 00$ & Manual Tape & .10 & Good & \\
\hline $2 / 12 / 957: 06$ & Auto FIC & 10 & Good & \\
\hline $2 / 12 / 95 \quad 12: 00$ & Manual Tape & 10 & Good & \\
\hline $2 / 13 / 957: 05$ & Auto FIC & 10 & Good & \\
\hline $2 / 13 / 9512: 00$ & Manual Tape & 10 & Good & \\
\hline 2/14/95 7:06 & Auto FIC & 10.00 & Good & \\
\hline $2 / 14 / 9512: 00$ & Manual Tape & 10 & Good & \\
\hline
\end{tabular}

Data obtained from WHC Surveillance Analysis Computer System, (PC SACS) July 7, 1995. 


\begin{tabular}{|c|c|c|c|c|}
\hline \multicolumn{5}{|c|}{ 241-AP-108 } \\
\hline \multicolumn{5}{|c|}{ Surface Level Data } \\
\hline Date & Type of Measurement & Level (Inches) & Data Quality & Comments \\
\hline 2/15/95 7:07 & Auto FIC & 10 & Good & \\
\hline $2 / 15 / 9512: 00$ & Manual Tape & 10 & Good & \\
\hline $2 / 16 / 957: 06$ & Auto FIC & 10.00 & Good & \\
\hline $2 / 16 / 9512: 00$ & Manual Tape & 10 & Good & \\
\hline $2 / 17 / 957: 06$ & Auto FIC & .10 & Good & \\
\hline $2 / 17 / 9512: 00$ & Manual Tape & 10 & Good & \\
\hline 2/18/95 7:06 & Auto FIC & 10.00 & Good & \\
\hline $2 / 18 / 95 \quad 12: 00$ & Manual Tape & 10 & Good & \\
\hline 2/19/95 7:06 & Auto FIC & 10.00 & Good & \\
\hline $2 / 19 / 9512: 00$ & Manual Tape & 9.5 & Good & \\
\hline $2 / 20 / 957: 07$ & Auto FIC & 10 & Good & \\
\hline $2 / 20 / 9512: 00$ & Manual Tape & 10 & Good & \\
\hline $2 / 21 / 957: 06$ & Auto FIC & 10.00 & Good & \\
\hline $2 / 21 / 9512: 00$ & Manual Tape & 9.75 & Good & \\
\hline $2 / 22 / 95$ 7:06 & Auto FIC & 10.00 & Good & \\
\hline 2/22/95 12:00 & Manual Tape & 10 & Good & \\
\hline 2/23/95 7:07 & Auto FIC & 10 & Good & \\
\hline $2 / 23 / 9512: 00$ & Manual Tape & 10 & Good & \\
\hline 2/24/95 7:06 & Auto FIC & 10 & Good & \\
\hline $2 / 24 / 9512: 00$ & Manual Tape & 9.75 & Good & \\
\hline $2 / 25 / 957: 06$ & Auto FIC & 10.00 & Good & \\
\hline $2 / 25 / 95 \quad 12: 00$ & Manual Tape & 9.75 & Good & \\
\hline 2/26/95 6:07 & Auto FIC & 10 & Good & \\
\hline $2 / 26 / 95 \quad 12: 00$ & Manual Tape & 9.75 & Good & \\
\hline $2 / 27 / 957: 06$ & Auto FIC & 10.00 & Good & \\
\hline $2 / 27 / 9512: 00$ & Manual Tape & 9.75 & Good & \\
\hline $2 / 28 / 955: 07$ & Auto FIC & 10.00 & Good & \\
\hline $2 / 28 / 9512: 00$ & Manual Tape & 10 & Good & \\
\hline 3/1/95 7:06 & Auto FIC & 10 & Good & \\
\hline $3 / 1 / 9512: 00$ & Manual Tape & 10 & Good & \\
\hline $3 / 2 / 957: 06$ & Auto FIC & 10.00 & Good & \\
\hline $3 / 2 / 9512: 00$ & Manual Tape & 9.75 & Good & \\
\hline 3/3/95 7:06 & Auto FIC & 10 & Good & \\
\hline $3 / 3 / 9512: 00$ & Manual Tape & 10 & Good & \\
\hline $3 / 4 / 957: 06$ & Auto FIC & 10.00 & Good & \\
\hline $3 / 4 / 9512: 00$ & Manual Tape & 10 & Good & \\
\hline $3 / 5 / 957: 06$ & Auto FIC & 10 & Good & \\
\hline $3 / 5 / 9512: 00$ & Manual Tape & 10 & Good & \\
\hline $3 / 6 / 95$ 7:06 & Auto FIC & 10 & Good & \\
\hline $3 / 6 / 9512: 00$ & Manual Tape & 9.875 & Good & \\
\hline $3 / 7 / 957: 06$ & Auto FIC & 10 & Good & \\
\hline $3 / 7 / 9512: 00$ & Manual Tape & 10 & Good & \\
\hline $3 / 8 / 95 \quad 7: 03$ & Auto FIC & 10 & Good & \\
\hline $3 / 8 / 9512: 00$ & Manual Tape & 10 & Good & \\
\hline $3 / 9 / 957: 03$ & Auto FIC & 10 & Good & \\
\hline 3/9/95 12:00 & Manual Tape & 9.75 & Good & \\
\hline $3 / 10 / 957: 02$ & Auto FIC & 10.00 & Good & \\
\hline $3 / 10 / 95 \quad 12: 00$ & Manual Tape & 10 & Good & \\
\hline
\end{tabular}

Data obtained from WHC Surveillance Analysis Computer System, (PC SACS) July 7, 1995. 


\begin{tabular}{|c|c|c|c|c|}
\hline \multicolumn{5}{|c|}{ 241-AP-108 } \\
\hline \multicolumn{5}{|c|}{ Surface Level Data } \\
\hline Date & Type of Measurement & Level (Inches) & Data Quality & Comments \\
\hline $3 / 11 / 957: 03$ & Auto FIC & 10 & Good & \\
\hline $3 / 11 / 95 \quad 12: 00$ & Manual Tape & 10 & Good & \\
\hline $3 / 12 / 957: 03$ & Auto FIC & 10 & Good & \\
\hline $3 / 12 / 9512: 00$ & Manual Tape & 9.75 & Good & \\
\hline $3 / 13 / 95$ 7:02 & Auto FIC & 10 & Good & \\
\hline $3 / 13 / 9512: 00$ & Manual Tape & 9.75 & Good & \\
\hline $3 / 14 / 957: 03$ & Auto FIC & 10.00 & Good & \\
\hline $3 / 14 / 95 \quad 12: 00$ & Manual Tape & 9.75 & Good & \\
\hline $3 / 15 / 957: 05$ & Auto FIC & 10 & Good & \\
\hline $3 / 15 / 95 \quad 12: 00$ & Manual Tape & 9.75 & Good & \\
\hline $3 / 16 / 957: 03$ & Auto FIC & 10.00 & Good & \\
\hline $3 / 16 / 95 \quad 12: 00$ & Manual Tape & 9.75 & Good & \\
\hline $3 / 17 / 957: 03$ & Auto FIC & 10.00 & Good & \\
\hline $3 / 17 / 95 \quad 12: 00$ & Manual Tape & 10 & Good & \\
\hline $3 / 18 / 957: 03$ & Auto FIC & 10.00 & Good & \\
\hline $3 / 18 / 95 \quad 12: 00$ & Manual Tape & 10 & Good & \\
\hline $3 / 19 / 957: 03$ & Auto FIC & 10 & Good & \\
\hline $3 / 19 / 9512: 00$ & Manual Tape & 10 & Good & 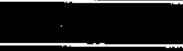 \\
\hline $3 / 20 / 956: 03$ & Auto FIC & 10.00 & Good & \\
\hline $3 / 20 / 9512: 00$ & Manual Tape & 10 & Good & \\
\hline $3 / 21 / 957: 03$ & Auto FIC & 10.00 & Good & \\
\hline $3 / 21 / 95 \quad 12: 00$ & Manual Tape & 9.75 & Good & \\
\hline $3 / 22 / 957: 02$ & Auto FIC & 10 & Good & \\
\hline $3 / 22 / 9512: 00$ & Manual Tape & 10 & Good & \\
\hline $3 / 23 / 957: 03$ & Auto FIC & 10 & Good & \\
\hline $3 / 23 / 9512: 00$ & Manual Tape & 9.75 & Good & \\
\hline $3 / 24 / 957: 03$ & Auto FIC & 10 & Good & \\
\hline $3 / 24 / 9512: 00$ & Manual Tape & 10 & Good & \\
\hline $3 / 25 / 957: 02$ & Auto FIC & 10.00 & Good & \\
\hline $3 / 25 / 95 \quad 12: 00$ & Manual Tape & 9.75 & Good & \\
\hline $3 / 26 / 957: 02$ & Auto FIC & 10 & Good & \\
\hline $3 / 26 / 95 \quad 12: 00$ & Manual Tape & 9.75 & Good & \\
\hline $3 / 27 / 957: 03$ & Auto FIC & 10.00 & Good & \\
\hline $3 / 27 / 95 \quad 12: 00$ & Manual Tape & 9.75 & Good & \\
\hline $3 / 28 / 95 \quad 7: 03$ & Auto FIC & 10 & Good & \\
\hline $3 / 28 / 95 \quad 12: 00$ & Manual Tape & 9.75 & Good & \\
\hline $3 / 29 / 957: 03$ & Auto FIC & 10.00 & Good & \\
\hline $3 / 29 / 9512: 00$ & Manual Tape & 9.75 & Good & \\
\hline $3 / 30 / 957: 03$ & Auto FIC & 10.00 & Good & \\
\hline $3 / 30 / 9512: 00$ & Manual Tape & 9.75 & Good & \\
\hline $3 / 31 / 957: 04$ & Auto FIC & 10 & Good & \\
\hline $3 / 31 / 9512: 00$ & Manual Tape & 9.75 & Good & \\
\hline 4/1/95 7:02 & Auto FIC & 10.00 & Good & \\
\hline $4 / 1 / 95 \quad 12: 00$ & Manual Tape & 9.75 & Good & \\
\hline 4/2/95 7:03 & Auto FIC & 10.00 & Good & \\
\hline $4 / 2 / 9512: 00$ & Manual Tape & 9.75 & Good & \\
\hline $4 / 3 / 957: 03$ & Auto FIC & 9.9 & Good & \\
\hline $4 / 3 / 9512: 00$ & Manual Tape & 10 & Good & \\
\hline
\end{tabular}

Data obtained from WHC Surveillance Analysis Computer System, (PC SACS) July 7, 1995. 


\begin{tabular}{|c|c|c|c|c|}
\hline \multicolumn{5}{|c|}{ 241-AP-108 } \\
\hline \multicolumn{5}{|c|}{ Surface Level Data } \\
\hline Date & Type of Measurement & Level (Inches) & Data Quality & Comments \\
\hline $4 / 4 / 957: 03$ & Auto FIC & 9.90 & Good & \\
\hline $4 / 4 / 95 \quad 12: 00$ & Manual Tape & 10 & Good & \\
\hline 4/5/95 7:03 & Auto FIC & 9.9 & Good & \\
\hline 4/5/95 12:00 & Manual Tape & 9.75 & Good & \\
\hline $4 / 6 / 957: 03$ & Auto FIC & 9.90 & Good & \\
\hline 4/6/95 12:00 & Manual Tape & 9.75 & Good & \\
\hline 4/7/95 7:03 & Auto FIC & 9.90 & Good & \\
\hline $4 / 7 / 95 \quad 12: 00$ & Manual Tape & 10 & Good & \\
\hline 4/8/95 7:02 & Auto FIC & 9.9 & Good & \\
\hline $4 / 8 / 95 \quad 12: 00$ & Manual Tape & 9.75 & Good & \\
\hline $4 / 9 / 9512: 00$ & Manual Tape & 9.75 & Good & \\
\hline $4 / 9 / 9523: 03$ & Auto FIC & 9.9 & Good & \\
\hline $4 / 10 / 957: 02$ & Auto FIC & 9.90 & Good & \\
\hline $4 / 10 / 95 \quad 12: 00$ & Manual Tape & 9.75 & Good & \\
\hline $4 / 11 / 957: 04$ & Auto FIC & 9.9 & Good & \\
\hline 4/11/95 12:00 & Manual Tape & 10 & Good & \\
\hline 4/12/95 7:03 & Auto FIC & 9.90 & Good & \\
\hline 4/12/95 12:00 & Manual Tape & 9.75 & Good & \\
\hline 4/13/95 7:05 & Auto FIC & 9.9 & Good & \\
\hline 4/13/95 12:00 & Manual Tape & 9.75 & Good & \\
\hline 4/14/95 7:03 & Auto FIC & 9.9 & Good & \\
\hline 4/14/95 12:00 & Manual Tape & 9.75 & Good & \\
\hline 4/15/95 7:03 & Auto FIC & 9.90 & Good & \\
\hline 4/15/95 12:00 & Manual Tape & 9.75 & Good & \\
\hline 4/16/95 7:03 & Auto FIC & 9.9 & Good & \\
\hline $4 / 16 / 95 \quad 12: 00$ & Manual Tape & 9.75 & Good & \\
\hline $4 / 17 / 95 \quad 7: 03$ & Auto FIC & 9.90 & Good & \\
\hline $4 / 17 / 9512: 00$ & Manual Tape & 9.75 & Good & \\
\hline 4/18/95 7:03 & Auto FIC & 9.90 & Good & \\
\hline 4/18/95 12:00 & Manual Tape & 9.75 & Good & \\
\hline $4 / 19 / 957: 03$ & Auto FIC & 9.90 & Good & \\
\hline $4 / 19 / 95 \quad 12: 00$ & Manual Tape & 9.75 & Good & \\
\hline $4 / 20 / 957: 03$ & Auto FIC & 9.9 & Good & \\
\hline $4 / 20 / 95 \quad 12: 00$ & Manual Tape & 9.75 & Good & \\
\hline $4 / 21 / 957: 03$ & Auto FIC & 9.9 & Good & \\
\hline 4/21/95 12:00 & Manual Tape & 9.75 & Good & \\
\hline $4 / 22 / 957: 03$ & Auto FIC & 9.9 & Good & \\
\hline $4 / 22 / 9512: 00$ & Manual Tape & 9.75 & Good & \\
\hline $4 / 23 / 957: 03$ & Auto FIC & 9.90 & Good & \\
\hline $4 / 23 / 95 \quad 12: 00$ & Manual Tape & 9.5 & Good & \\
\hline $4 / 24 / 957: 03$ & Auto FIC & 9.90 & Good & \\
\hline $4 / 24 / 95 \quad 12: 00$ & Manual Tape & 9.75 & Good & \\
\hline $4 / 25 / 957: 03$ & Auto FIC & 9.90 & Good & \\
\hline $4 / 25 / 95 \quad 12: 00$ & Manual FIC & 9.75 & Good & \\
\hline $4 / 26 / 95 \quad 7: 03$ & Auto FIC & 9.9 & Good & \\
\hline $4 / 26 / 95 \quad 12: 00$ & Manual Tape & 9.75 & Good & \\
\hline $4 / 27 / 952: 03$ & Auto FIC & 9.9 & Good & \\
\hline 4/27/95 12:00 & Manual Tape & 9.75 & Good & \\
\hline
\end{tabular}

Data obtained from WHC Surveillance Analysis Computer System, (PC SACS) July 7, 1995. 


\begin{tabular}{|c|c|c|c|c|}
\hline \multicolumn{5}{|c|}{ 241-AP-108 } \\
\hline \multicolumn{5}{|c|}{ Surface Level Data } \\
\hline Dato & Type of Measurement & Level (Inches) & Data Quality & Comments \\
\hline $4 / 28 / 957: 03$ & Auto FIC & 10.00 & Good & \\
\hline 4/28/95 12:00 & Manual Tape & 9.75 & Good & \\
\hline 4/29/95 7:03 & Auto FIC & 10.00 & Good & \\
\hline 4/29/95 12:00 & Manual Tape & 10 & Good & \\
\hline $4 / 30 / 957: 03$ & Auto FIC & 10.1 & Good & \\
\hline $4 / 30 / 9512: 00$ & Manual Tape & 10 & Good & \\
\hline 5/1/95 7:03 & Auto FIC & 9.90 & Good & \\
\hline $5 / 1 / 95 \quad 12: 00$ & Manual Tape & 9.75 & Good & \\
\hline 5/2/95 7:02 & Auto FIC & 9.90 & Good & \\
\hline $5 / 2 / 9512: 00$ & Manual Tape & 9.75 & Good & \\
\hline $5 / 3 / 957: 03$ & Auto FIC & 9.90 & Good & \\
\hline $5 / 3 / 95 \quad 12: 00$ & Manual Tape & 9.75 & Good & \\
\hline $5 / 4 / 957: 04$ & Auto FIC & 9.9 & Good & \\
\hline $5 / 4 / 9512: 00$ & Manual Tape & 9.75 & Good & \\
\hline $5 / 5 / 957: 04$ & Auto FIC & 85.1 & Good & \\
\hline $5 / 5 / 95 \quad 12: 00$ & Manual Tape & 96 & Good & \\
\hline $5 / 6 / 957: 03$ & Auto FIC & 209.10 & Good & \\
\hline $5 / 6 / 9512: 00$ & Manual Tape & 232 & Good & \\
\hline $5 / 7 / 95 \quad 7: 04$ & Auto FIC & 0.00 & Suspect & Data not included in graph \\
\hline $5 / 7 / 95 \quad 12: 00$ & Manual FIC & 333.20 & Good & \\
\hline $5 / 7 / 95 \quad 12: 00$ & Manual Tape & 334.5 & Good & \\
\hline 5/8/95 7:04 & Auto FIC & 349.10 & Good & \\
\hline $5 / 8 / 95 \quad 12: 00$ & Manual FIC & 333.2 & Good & \\
\hline $5 / 8 / 9512: 00$ & Manual Tape & 349 & Good & \\
\hline 5/9/95 7:04 & Auto FIC & 349.1 & Good & \\
\hline $5 / 9 / 95 \quad 12: 00$ & Manual Tape & 349 & Good & \\
\hline $5 / 10 / 956: 04$ & Auto FIC & 349.1 & Good & \\
\hline $5 / 10 / 95 \quad 12: 00$ & Manual Tape & 349 & Good & \\
\hline $5 / 11 / 957: 03$ & Auto FIC & 349.1 & Good & \\
\hline $5 / 11 / 9512: 00$ & Manual Tape & 349 & Good & \\
\hline 5/12/95 6:06 & Auto FIC & 349.10 & Good & \\
\hline $5 / 12 / 95 \quad 12: 00$ & Manual Tape & 349 & Good & $\therefore$ \\
\hline $5 / 13 / 957: 03$ & Auto FIC & 0 & Suspect & Data not included in graph \\
\hline $5 / 13 / 9512: 00$ & Manual FIC & 349.10 & Good & \\
\hline $5 / 13 / 9512: 00$ & Manual Tape & 349 & Good & \\
\hline $5 / 14 / 953: 03$ & Auto FIC & $349: 10$ & Good & \\
\hline $5 / 14 / 9512: 00$ & Manual Tape & 349 & Good & \\
\hline $5 / 15 / 955: 02$ & Auto FIC & 349.10 & Good & \\
\hline 5/15/95 12:00 & Manual Tape & 349 & Good & \\
\hline $5 / 16 / 957: 03$ & Auto FIC & 0.00 & Suspect & Data not included in graph \\
\hline $5 / 16 / 95 \quad 12: 00$ & Manual FIC & 349.10 & Good & \\
\hline $5 / 16 / 9512: 00$ & Manual Tape & 349 & Good & \\
\hline $5 / 17 / 957: 02$ & Auto FIC & 0 & Suspect & Data not included in graph \\
\hline $5 / 17 / 9512: 00$ & Manual FIC & 349.1 & Good & \\
\hline $5 / 17 / 9512: 00$ & Manual Tape & 349 & Good & \\
\hline $5 / 18 / 957: 04$ & Auto FIC & 0.00 & Suspect & Data not included in graph \\
\hline $5 / 18 / 9512: 00$ & Manual FIC & 349.10 & Good & \\
\hline $5 / 18 / 9512: 00$ & Manual Tape & 349 & Good & \\
\hline
\end{tabular}

Data obtained from WHC Surveillance Analysis Computer System, (PC SACS) July 7, 1995. 


\begin{tabular}{|c|c|c|c|c|}
\hline \multicolumn{5}{|c|}{ 241-AP-108 } \\
\hline \multicolumn{5}{|c|}{ Surface Level Data } \\
\hline Date & Type of Measurement & Level (Inches) & Data Quality & Comments \\
\hline $5 / 19 / 957: 05$ & Auto FIC & 0 & Suspect & Data not included in graph \\
\hline $5 / 19 / 9512: 00$ & Manual FIC & 349.10 & Good & \\
\hline $5 / 19 / 95 \quad 12: 00$ & Manual Tape & 346 & Good & \\
\hline 5/20/95. 7:05 & Auto FIC & 0 & Suspect & Data not included in graph \\
\hline $5 / 20 / 95 \quad 12: 00$ & Manual FIC & 349.15 & Good & \\
\hline $5 / 20 / 9512: 00$ & Manual Tape & 346 & Good & \\
\hline $5 / 21 / 957: 05$ & Auto FIC & 0.00 & Suspect & Data not included in graph \\
\hline $5 / 21 / 9512: 00$ & Manual FIC & 349.1 & Good & \\
\hline $5 / 21 / 9512: 00$ & Manual Tape & 346 & Good & \\
\hline $5 / 22 / 957: 05$ & Auto FIC & 0.00 & Suspect & Data not included in graph \\
\hline $5 / 22 / 9512: 00$ & Manual FIC & 349.10 & Good & \\
\hline $5 / 22 / 9512: 00$ & Manual Tape & 349 & Good & \\
\hline $5 / 23 / 957: 11$ & Auto FIC & 0.00 & Suspect & Data not included in graph \\
\hline $5 / 23 / 95 \quad 12: 00$ & Manual FIC & 349.10 & Good & \\
\hline $5 / 23 / 9512: 00$ & Manual Tape & 349 & Good & \\
\hline $5 / 24 / 957: 10$ & Auto FIC & 0 & Suspect & Data not included in graph \\
\hline $5 / 24 / 9512: 00$ & Manual FIC & 278.20 & Good & \\
\hline $5 / 24 / 9512: 00$ & Manual Tape & 278 & Good & \\
\hline $5 / 25 / 957: 10$ & Auto FIC & 0 & Suspect & Data not included in graph \\
\hline $5 / 25 / 9512: 00$ & Manual FIC & 214.00 & Good & \\
\hline $5 / 25 / 95 \quad 12: 00$ & Manual Tape & 213.75 & Good & \\
\hline $5 / 26 / 956: 05$ & Auto FIC & 210.00 & Good & \\
\hline $5 / 26 / 9512: 00$ & Manual Tape & 213.75 & Gopd & \\
\hline $5 / 27 / 957: 05$ & Auto FIC & .0 & Suspect & Data not included in graph \\
\hline $5 / 27 / 95 \quad 12: 00$ & Manual Tape & 94.25 & Suspect & Data not included in graph \\
\hline $5 / 27 / 9512: 00$ & Manual FIC & 214.00 & Good & \\
\hline $5 / 28 / 957: 05$ & Auto FIC & 0 & Suspect & Data not included in graph \\
\hline $5 / 28 / 9512: 00$ & Manual FIC & 214 & Good & \\
\hline $5 / 28 / 95 \quad 12: 00$ & Manual Tape & 214.25 & Good & \\
\hline $5 / 29 / 957: 05$ & Auto FIC & 0.00 & Suspect & Data not included in graph \\
\hline $5 / 29 / 95 \quad 12: 00$ & Manual FIC & 214 & Good & \\
\hline $5 / 29 / 95 \quad 12: 00$ & Manual Tape & 214.25 & Good & \\
\hline $5 / 30 / 957: 05$ & Auto FIC & 0.00 & Suspect & Data not included in graph \\
\hline $5 / 30 / 9512: 00$ & Manual FIC & 214.05 & Good & \\
\hline $5 / 30 / 9512: 00$ & Manual Tape & 214 & Good & \\
\hline $5 / 31 / 957: 05$ & Auto FIC & 0 & Suspect & Data not included in graph \\
\hline $5 / 31 / 95 \quad 12: 00$ & Manual FIC & 214 & Good & \\
\hline $5 / 31 / 95 \quad 12: 00$ & Manual Tape & 214 & Good & \\
\hline $6 / 1 / 957: 05$ & Auto FIC & 0.00 & Suspect & Data not included in graph \\
\hline $6 / 1 / 95 \quad 12: 00$ & Manual FIC & 214.00 & Gopd & \\
\hline $6 / 1 / 95 \quad 12: 00$ & Manual Tape & 21.3 .75 & Good & \\
\hline $6 / 2 / 957: 05$ & Auto FIC & 0 & Suspect & Data not included in graph \\
\hline $6 / 2 / 95 \quad 12: 00$ & Manual FIC & 214.05 & Good & \\
\hline $6 / 2 / 95 \quad 12: 00$ & Manual Tape & 214 & Good & \\
\hline $6 / 3 / 95 \quad 12: 00$ & Manual FIC & 214 & Good & \\
\hline $6 / 3 / 9512: 00$ & Manual Tape & 214 & Good & \\
\hline 6/3/95 23:05 & Auto FIC & 0 & Suspect & Data not included in graph \\
\hline 6/4/95 7:05 & Auto FIC & 0.00 & Suspect & Data not included in graph \\
\hline
\end{tabular}

Data obtained from WHC Surveillance Analysis Computer System, (PC SACS) July 7, 1995. 


\begin{tabular}{|c|c|c|c|c|}
\hline \multicolumn{5}{|c|}{ 241-AP-108 } \\
\hline \multicolumn{5}{|c|}{ Surface Level Data } \\
\hline Date & Type of Measurement & Level (Inches) & Data Quality & Comments \\
\hline $6 / 4 / 9512: 00$ & Manual FIC & 214 & Good & \\
\hline $6 / 4 / 9512: 00$ & Manual Tape & 213.75 & Good & \\
\hline $6 / 5 / 957: 05$ & Auto FIC & 214 & Good & \\
\hline $6 / 5 / 95 \quad 12: 00$ & Manual Tape & 214 & Good & \\
\hline $6 / 6 / 957: 05$ & Auto FIC & 0.1 & Suspect & Data not included in graph \\
\hline $6 / 6 / 95 \quad 12: 00$ & Manual FIC & 214 & Good & \\
\hline $6 / 6 / 95 \quad 12: 00$ & Manual Tape & 213.5 & Good & \\
\hline $6 / 7 / 95 \quad 7: 05$ & Auto FIC & 214 & Good & \\
\hline $6 / 7 / 95 \quad 12: 00$ & Manual Tape & 213.5 & Good & \\
\hline $6 / 8 / 957: 05$ & Auto FIC & 214 & Good & \\
\hline $6 / 8 / 9512: 00$ & Manual Tape & 213.25 & Good & \\
\hline 6/9/95 7:05 & Auto FIC & 214 & Good & \\
\hline $6 / 9 / 9512: 00$ & Manual Tape & 213.75 & Good & \\
\hline $6 / 10 / 957: 05$ & Auto FIC & 210.00 & Suspect & Data not included in graph \\
\hline $6 / 10 / 95 \quad 12: 00$ & Manual FIC & 214 & Good & \\
\hline $6 / 10 / 9512: 00$ & Manual Tape & 214 & Good & \\
\hline $6 / 11 / 957: 05$ & Auto FIC & 214 & Good & \\
\hline $6 / 11 / 9512: 00$ & Manual Tape & 214 & Good & \\
\hline $6 / 12 / 956: 05$ & Auto FIC & 210.00 & Suspect & Data not included in graph \\
\hline $6 / 12 / 9512: 00$ & Manual FIC & 214 & Good & \\
\hline $6 / 12 / 95 \quad 12: 00$ & Manual Tape & 214 & Good & \\
\hline 6/13/95 7:05 & Auto FIC & 214.00 & Good & \\
\hline 6/13/95 12:00 & Manual FIC & 214.00 & Good & \\
\hline $6 / 14 / 957: 05$ & Auto FIC & 171.30 & Good & \\
\hline $6 / 14 / 9512: 00$ & Manual Tape & 162.5 & Good & \\
\hline $6 / 15 / 957: 05$ & Auto FIC & 139.20 & Good & \\
\hline $6 / 15 / 9512: 00$ & Manual Tape & 139 & Good & \\
\hline $6 / 16 / 957: 05$ & Auto FIC & 139.20 & Good & \\
\hline $6 / 16 / 95 \quad 12: 00$ & Manual Tape & 139 & Good & \\
\hline $6 / 17 / 957: 05$ & Auto FIC & 139.2 & Good & \\
\hline $6 / 17 / 9512: 00$ & Manual Tape & 139 & Good & \\
\hline $6 / 18 / 957: 05$ & Auto FIC & 139.2 & Good & \\
\hline $6 / 18 / 9512: 00$ & Manual Tape & 139 & Good & . \\
\hline $6 / 19 / 957: 06$ & Auto FIC & 139.3 & Good & \\
\hline $6 / 19 / 95 \quad 12: 00$ & Manual Tape & 139 & Good & \\
\hline $6 / 20 / 957: 06$ & Auto FIC & 139.20 & Good & \\
\hline $6 / 20 / 9512: 00$ & Manual Tape & 139 & Good & \\
\hline $6 / 21 / 957: 06$ & Auto FIC & 139.20 & Good & \\
\hline $6 / 21 / 95 \quad 12: 00$ & Manual Tape & 139 & Good & \\
\hline $6 / 22 / 957: 06$ & Auto FIC & 93.00 & Good & \\
\hline $6 / 22 / 9512: 00$ & Manual FIC & 81.50 & Suspect & Data not included in graph \\
\hline $6 / 22 / 9512: 00$ & Manual Tape & 81.5 & Good & \\
\hline $6 / 23 / 957: 06$ & Auto FIC & 10.7 & Good & \\
\hline $6 / 23 / 9512: 00$ & Manual Tape & 10.25 & Good & \\
\hline $6 / 24 / 957: 06$ & Auto FIC & 10.70 & Good & \\
\hline $6 / 24 / 9512: 00$ & Manual Tape & 10.25 & Good & \\
\hline $6 / 25 / 957: 05$ & Auto FIC & 10.70 & Good & \\
\hline $6 / 25 / 95 \quad 12: 00$ & Manual Tape & 10.5 & Good & \\
\hline
\end{tabular}

Data obtained from WHC Surveillance Analysis Computer System, (PC SACS) July 7, 1995. 


\begin{tabular}{|c|c|c|c|c|}
\hline \multicolumn{7}{|c|}{ 241-AP-108 } \\
\hline Date & Type of Measurement & Level (lnches) & Data Quality & Comments \\
\hline $6 / 26 / 957: 05$ & Auto FIC & 10.70 & Good & \\
\hline $6 / 26 / 9512: 00$ & Manual Tape & 10.25 & Good & \\
\hline $6 / 27 / 957: 06$ & Auto FIC & 10.7 & Good & \\
\hline $6 / 27 / 9512: 00$ & Manual Tape & 10.25 & Good & \\
\hline $6 / 28 / 957: 05$ & Auto FIC & 10.70 & Good & \\
\hline $6 / 28 / 9512: 00$ & Manual Tape & 10.25 & Good & \\
\hline $6 / 29 / 957: 06$ & Auto FIC & 10.70 & Good & \\
\hline $6 / 29 / 9512: 00$ & Manual Tape & 10.25 & Good & \\
\hline $6 / 30 / 957: 06$ & Auto FIC & 10.70 & Good & \\
\hline $6 / 30 / 9512: 00$ & Manual Tape & 10.5 & Good & \\
\hline
\end{tabular}

Data obtained from WHC Surveillance Analysis Computer System, (PC SACS) July 7, 1995. 

APPENDIX F

\section{Psychrometric Data}




\title{
THIS PAGE INTENTIONALLY
}

\author{
LEFT BLANK
}


WHC-SD-WM-ER-315，Rev, 0
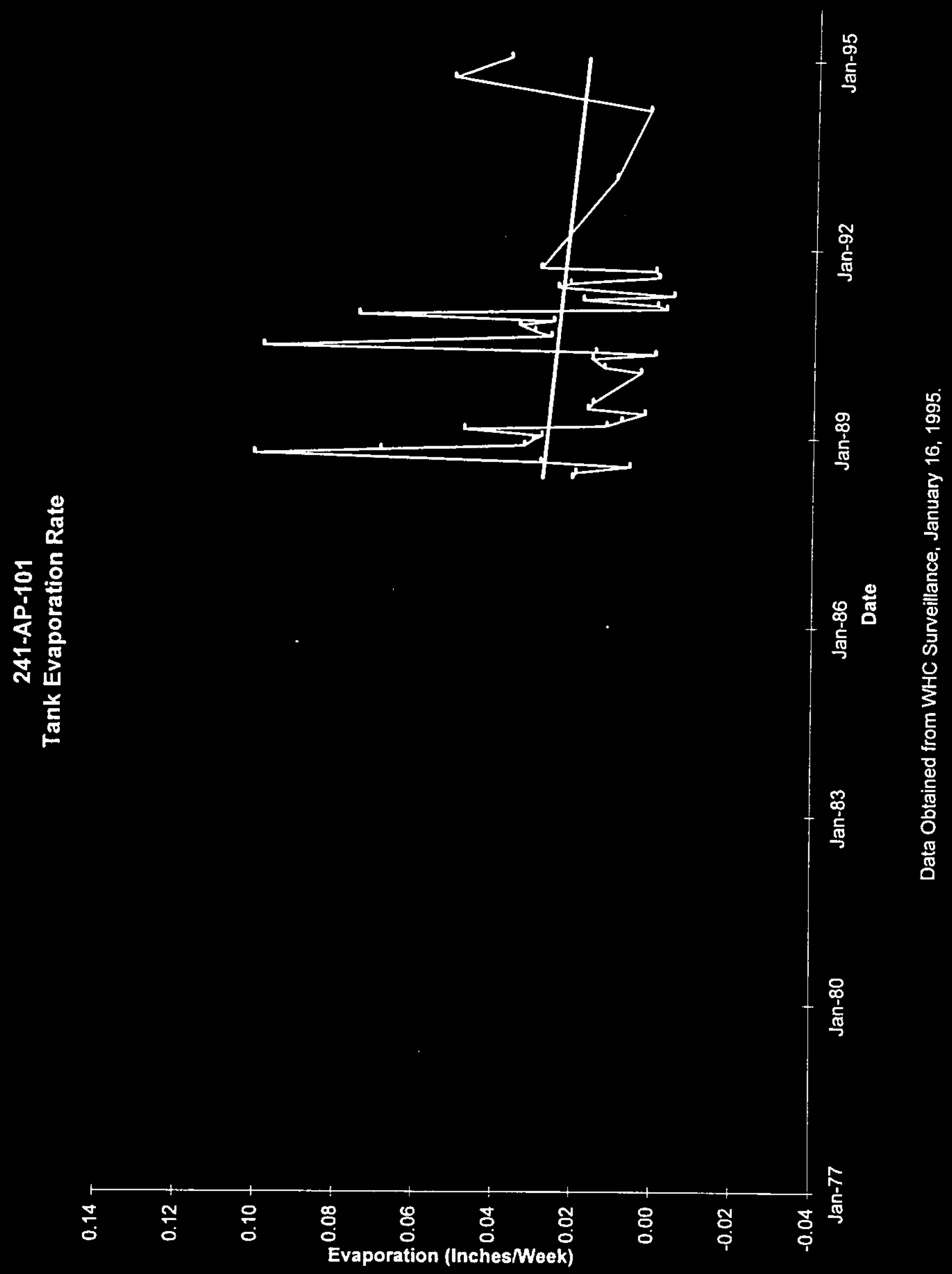
WHC-SD-WM-ER-315, Rev. 0

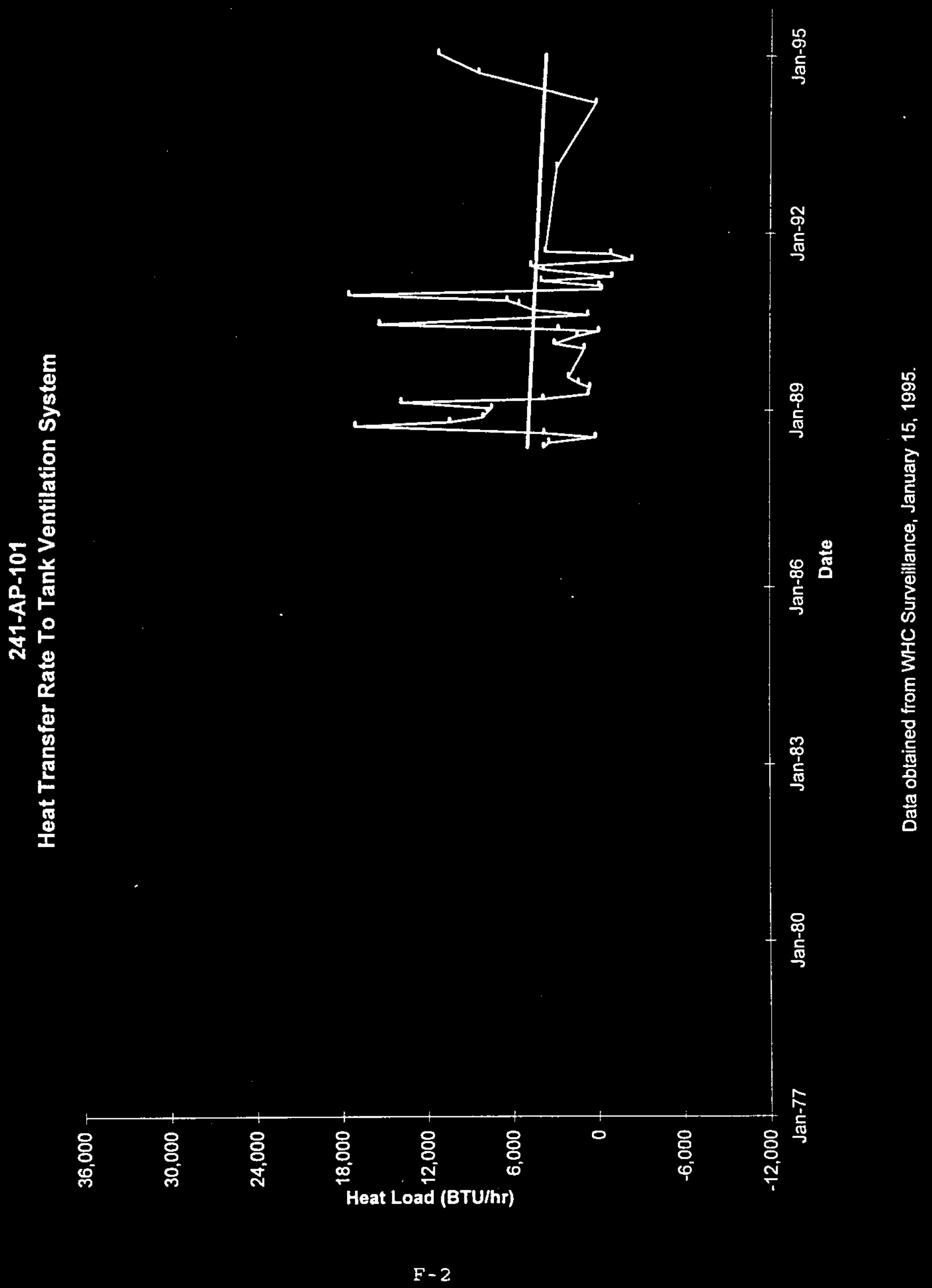




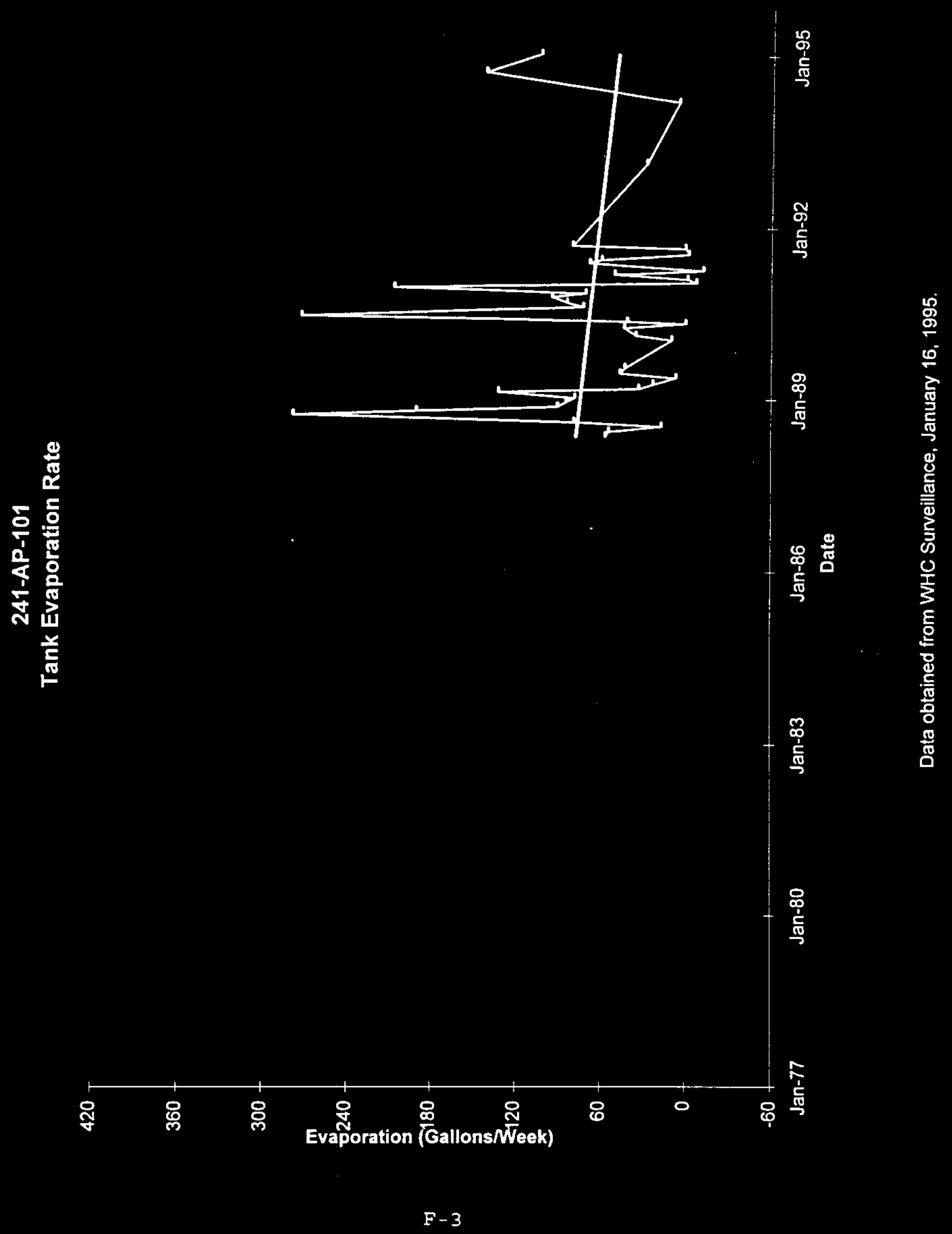


WHC-SD-WM-ER-315, Rev. 0

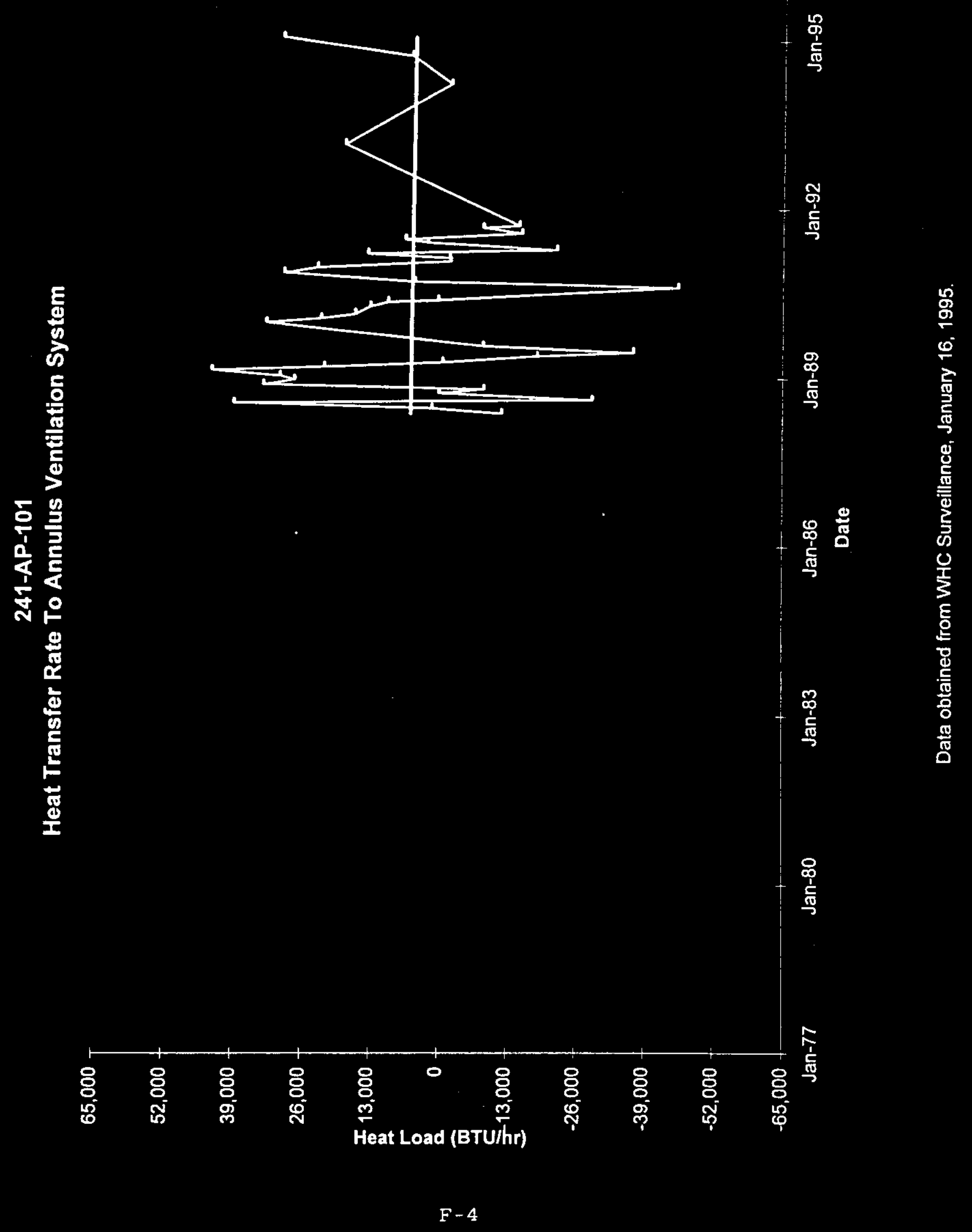


WHC-SD-WM-ER-315, Rev, 0

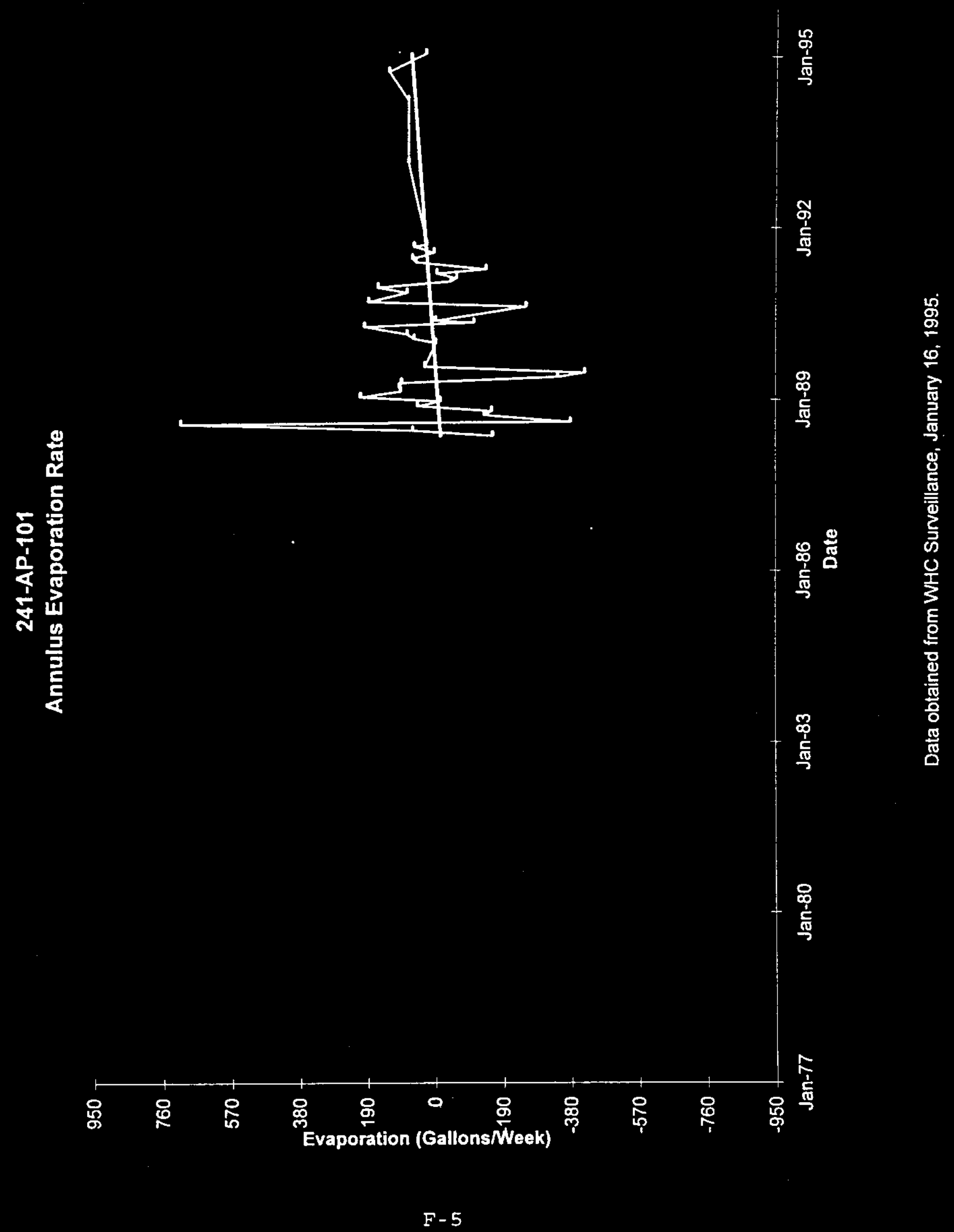


WHC-SD-WM-ER-315， Rev. 0

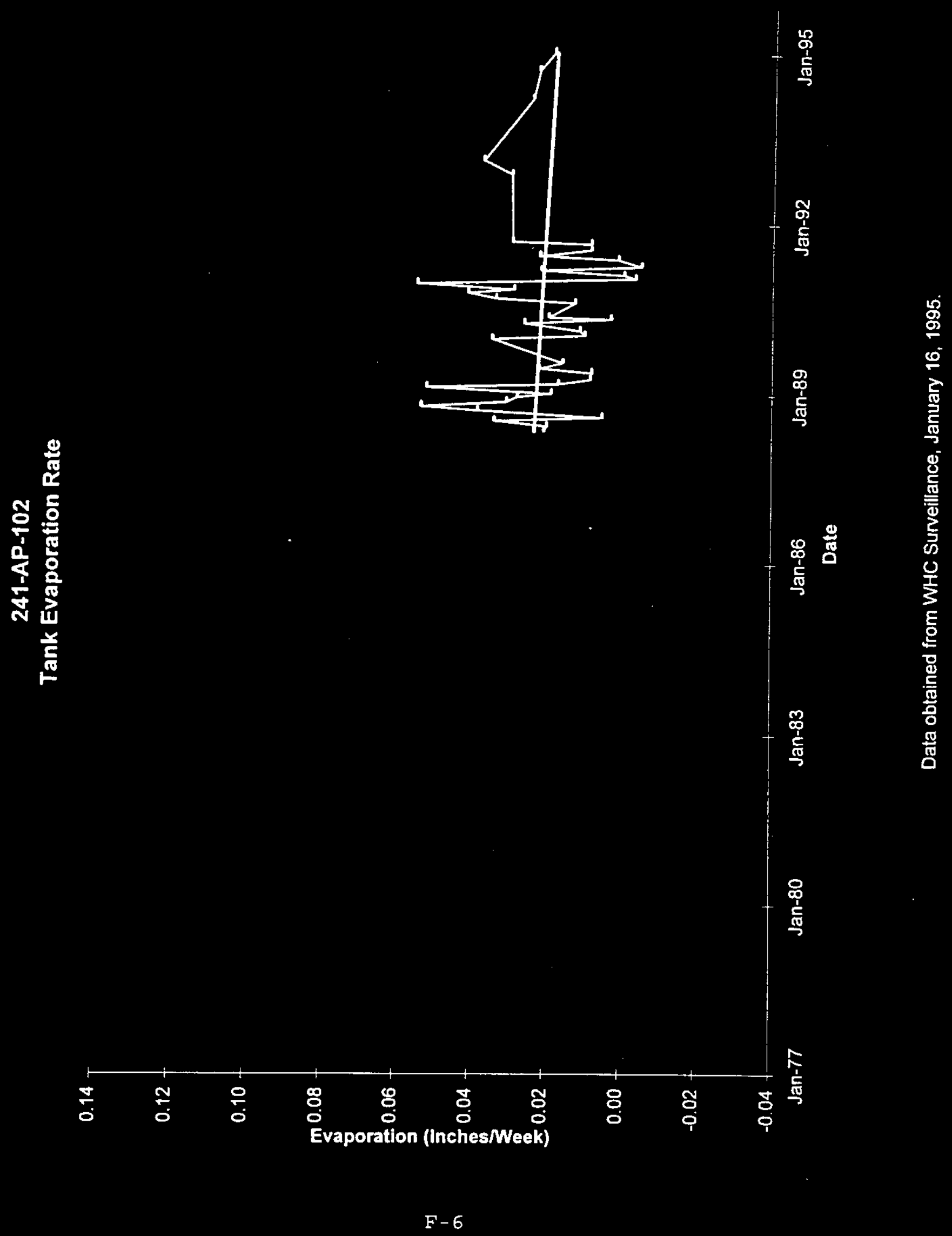


WHC-SD-WM-ER-315, Rev. 0
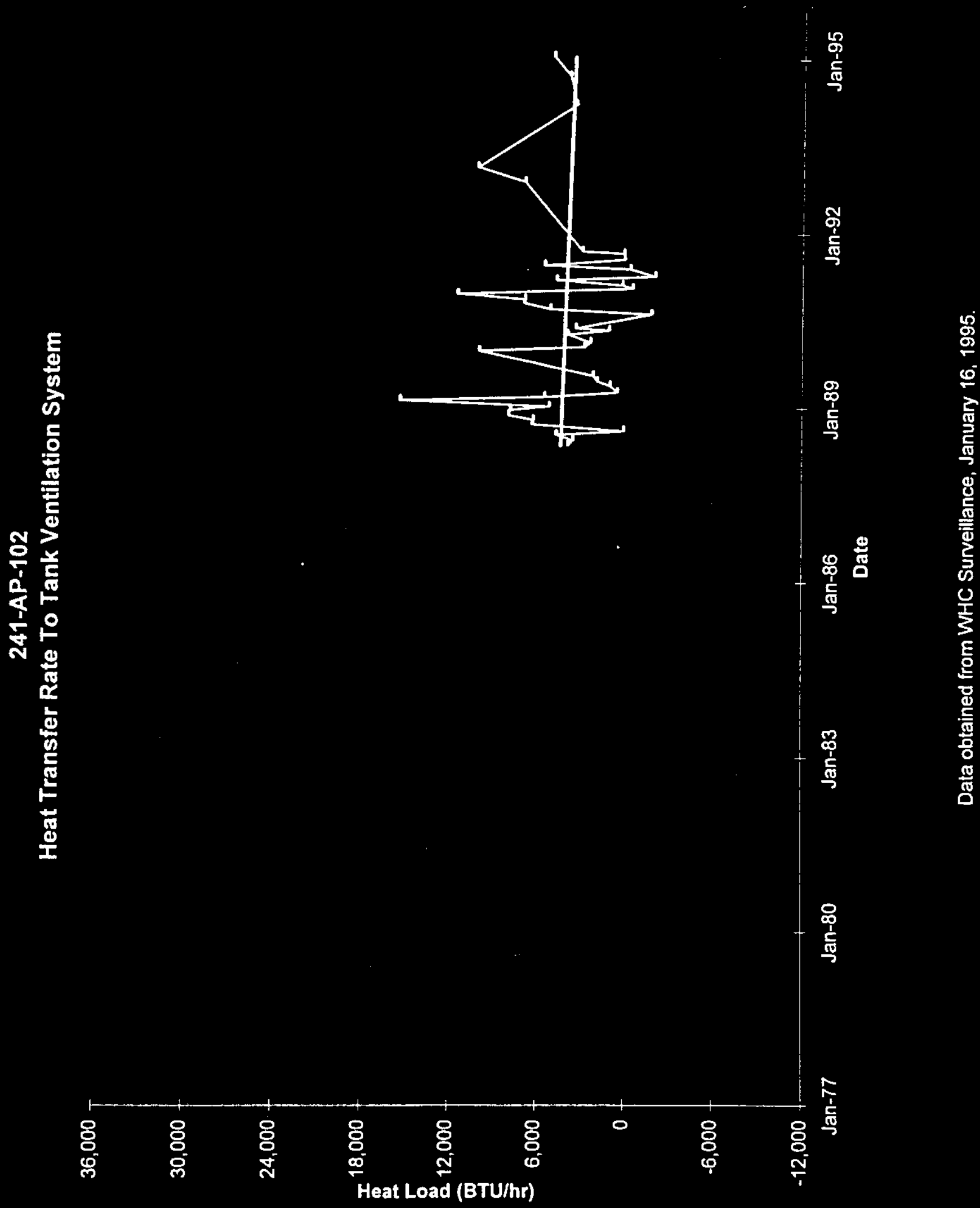

F -7 


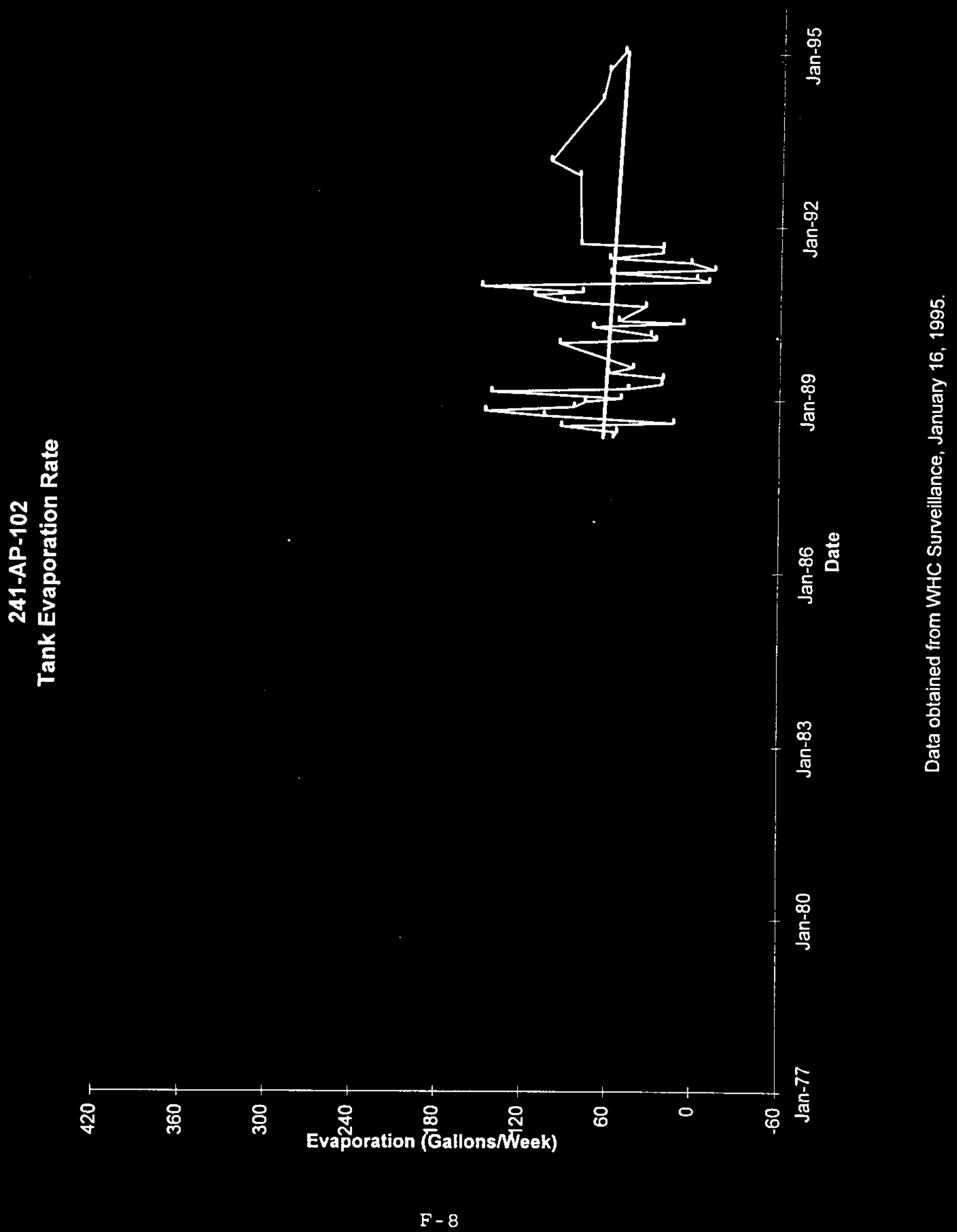


WHC-SD-WM-ER-315, Rev . 0

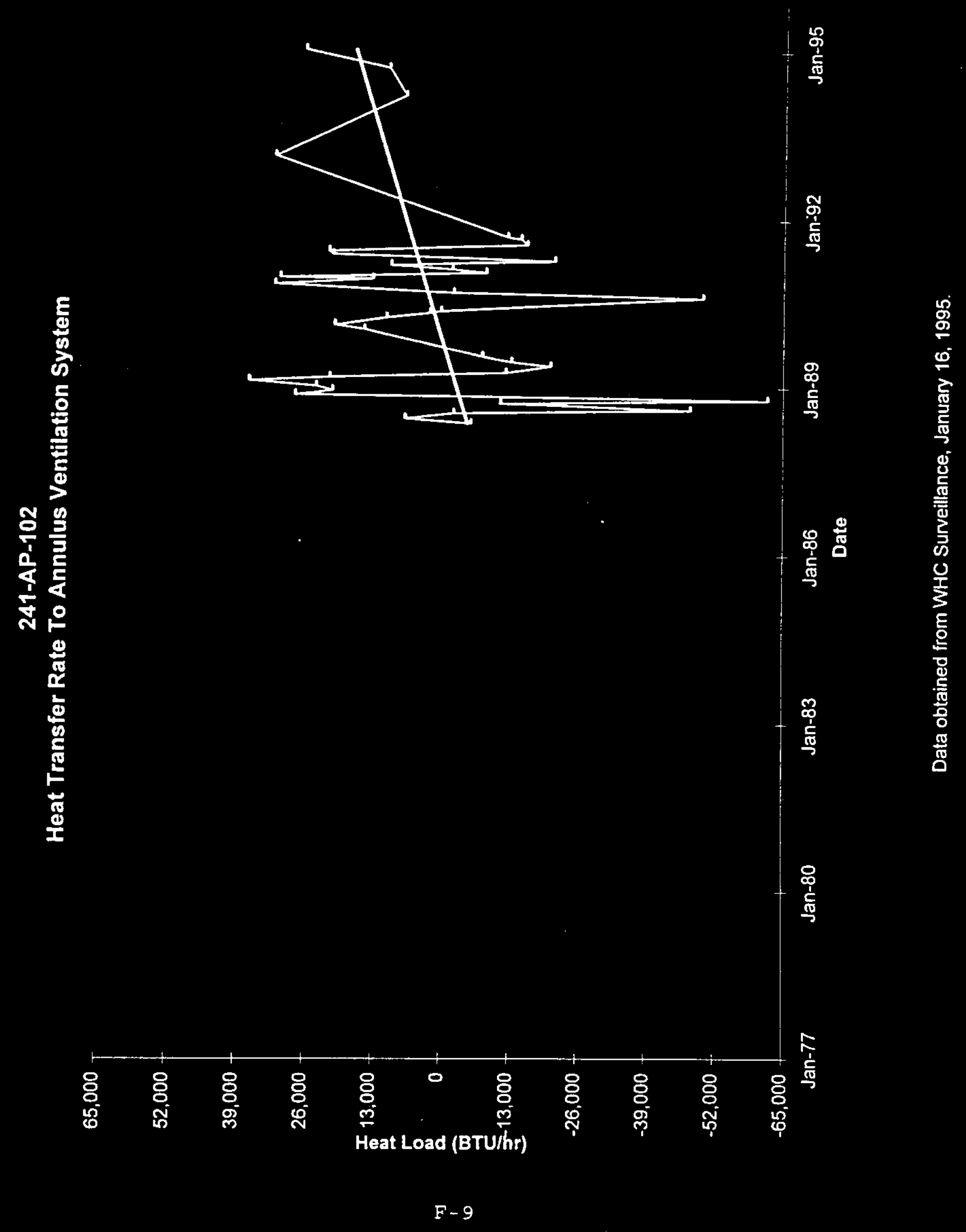


WHC-SD-WM-ER-315, Rev. 0

을
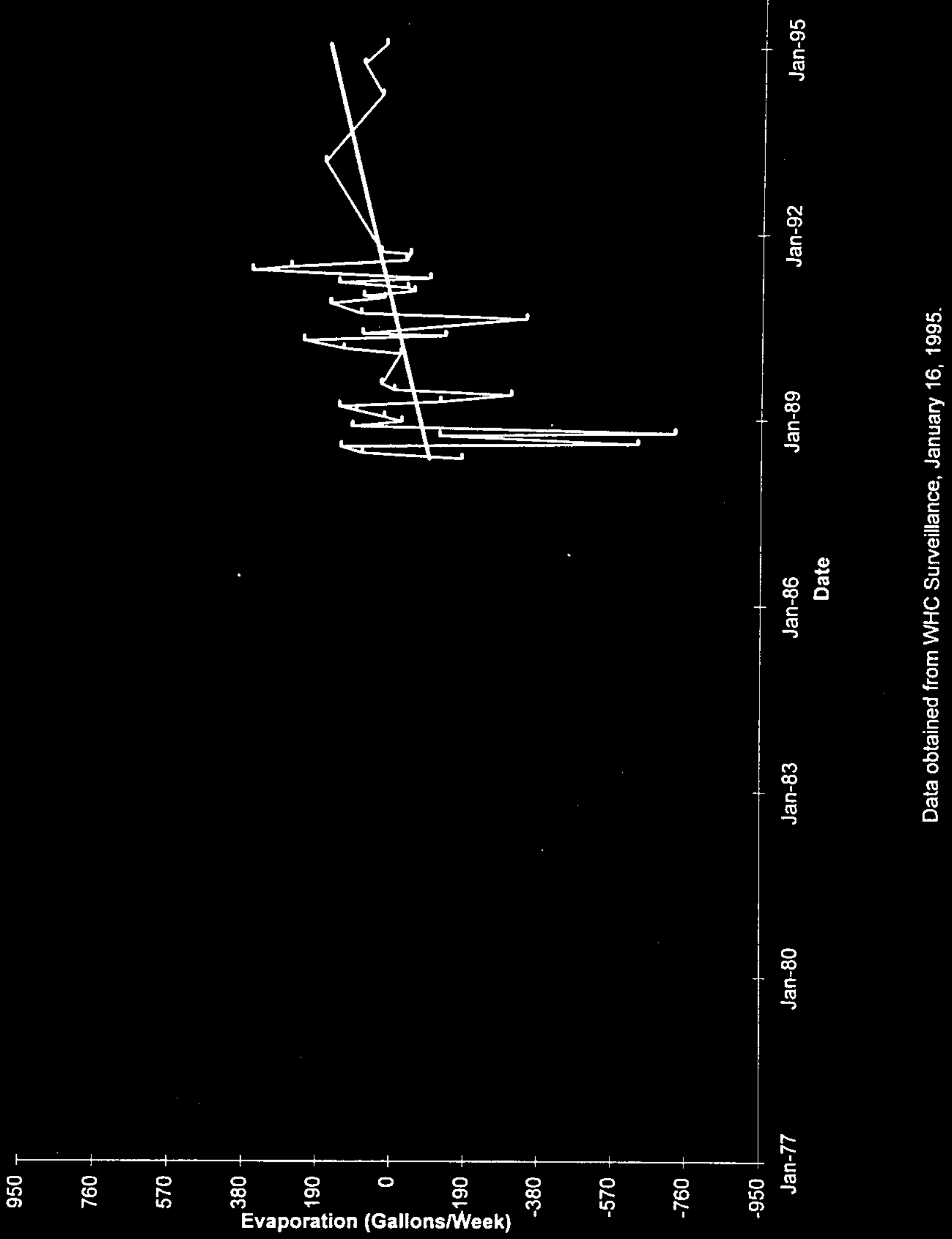


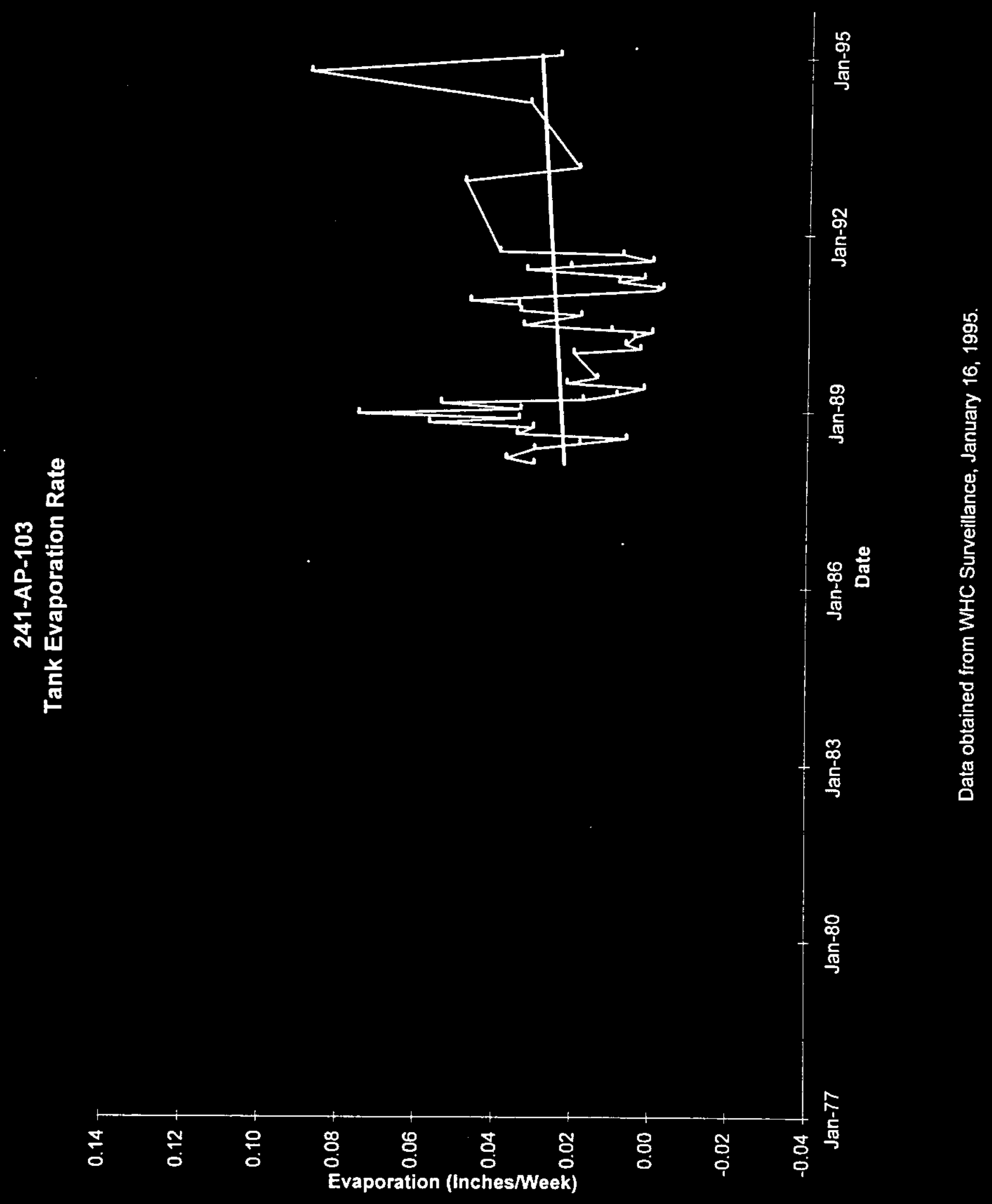


WHC-SD-WM-ER-315, Rev. 0

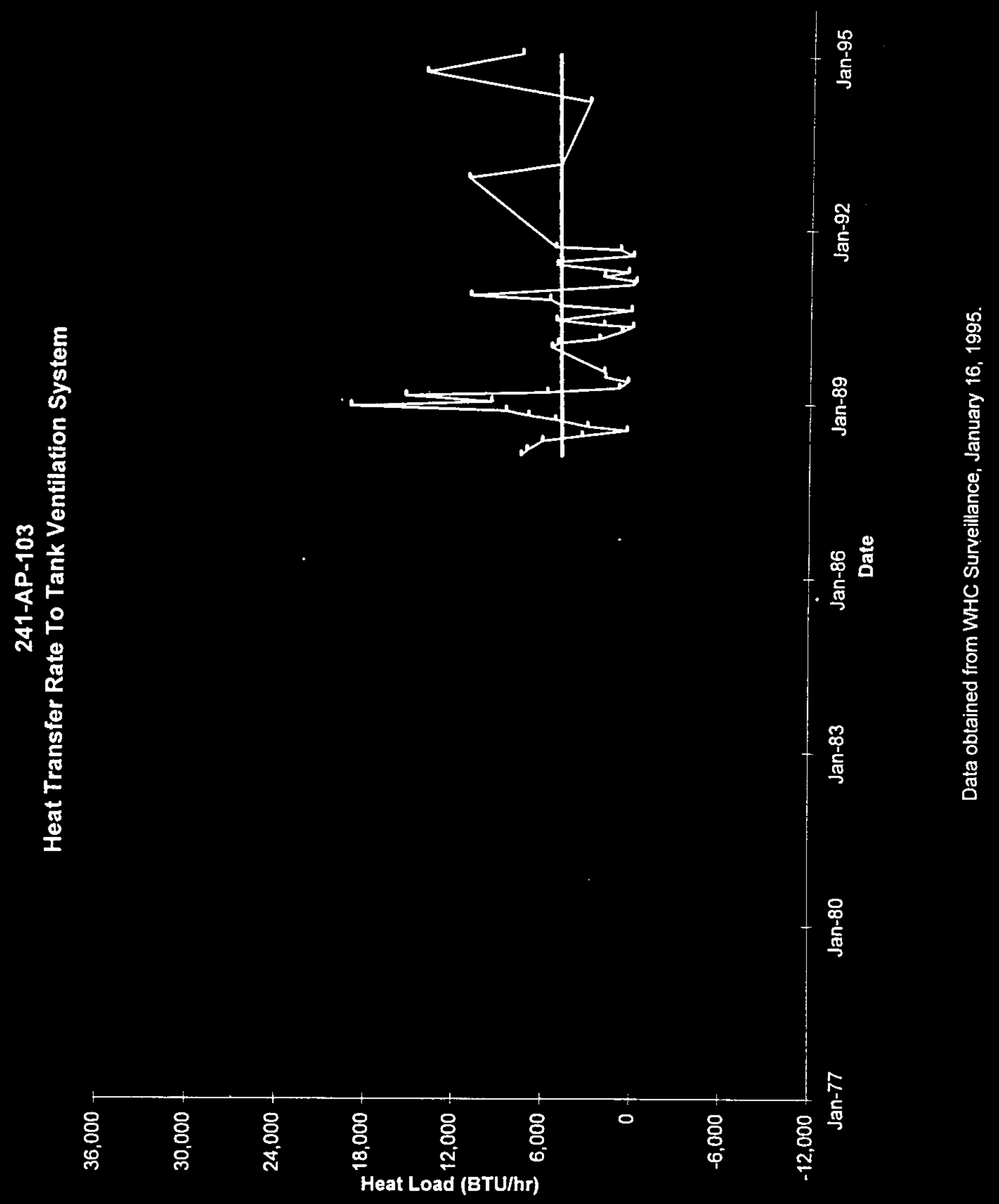


WHC-SD-WM-ER-315, Rev. 0

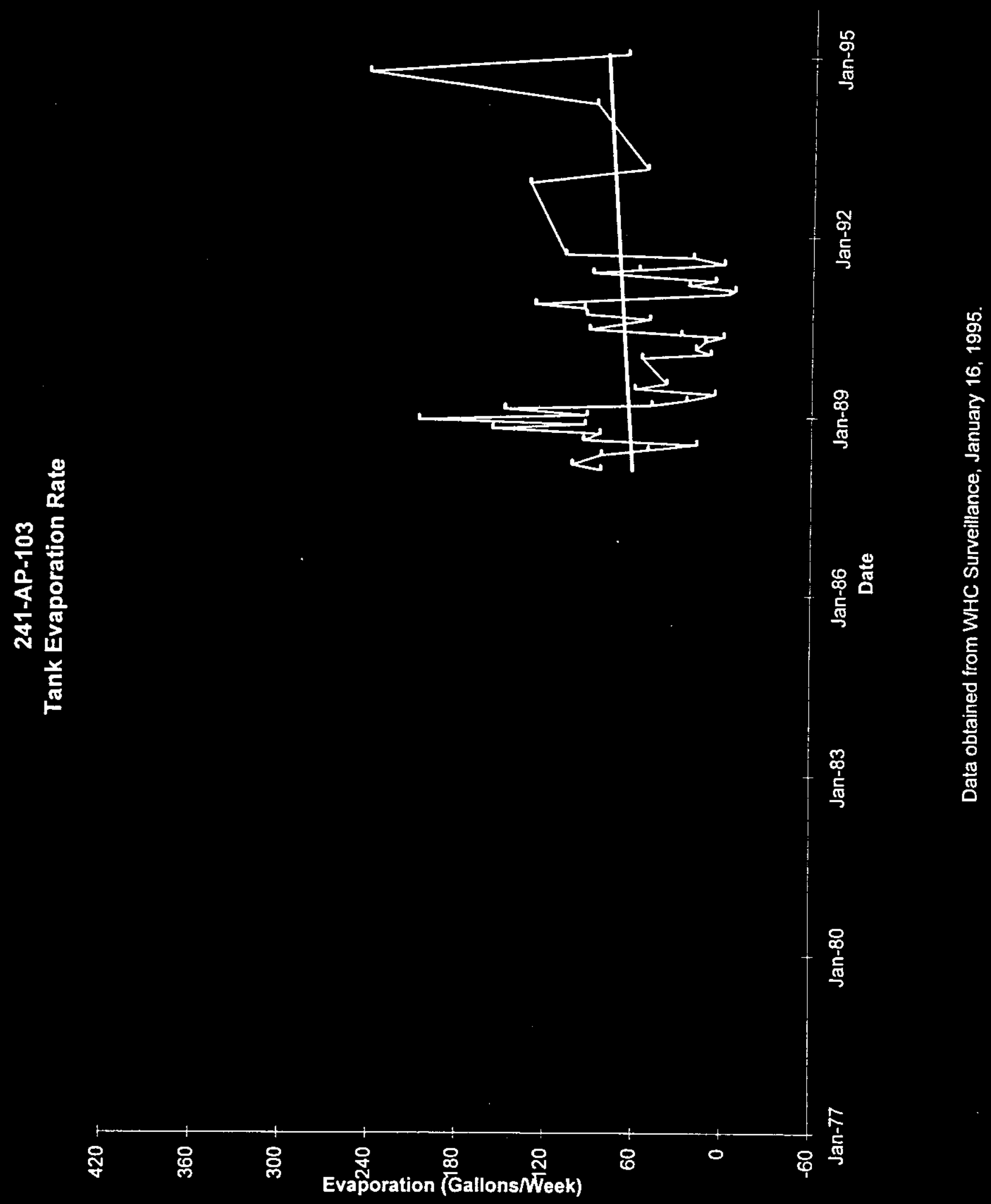

$F-13$ 
WHC-SD-WM-ER-315， Rev. 0

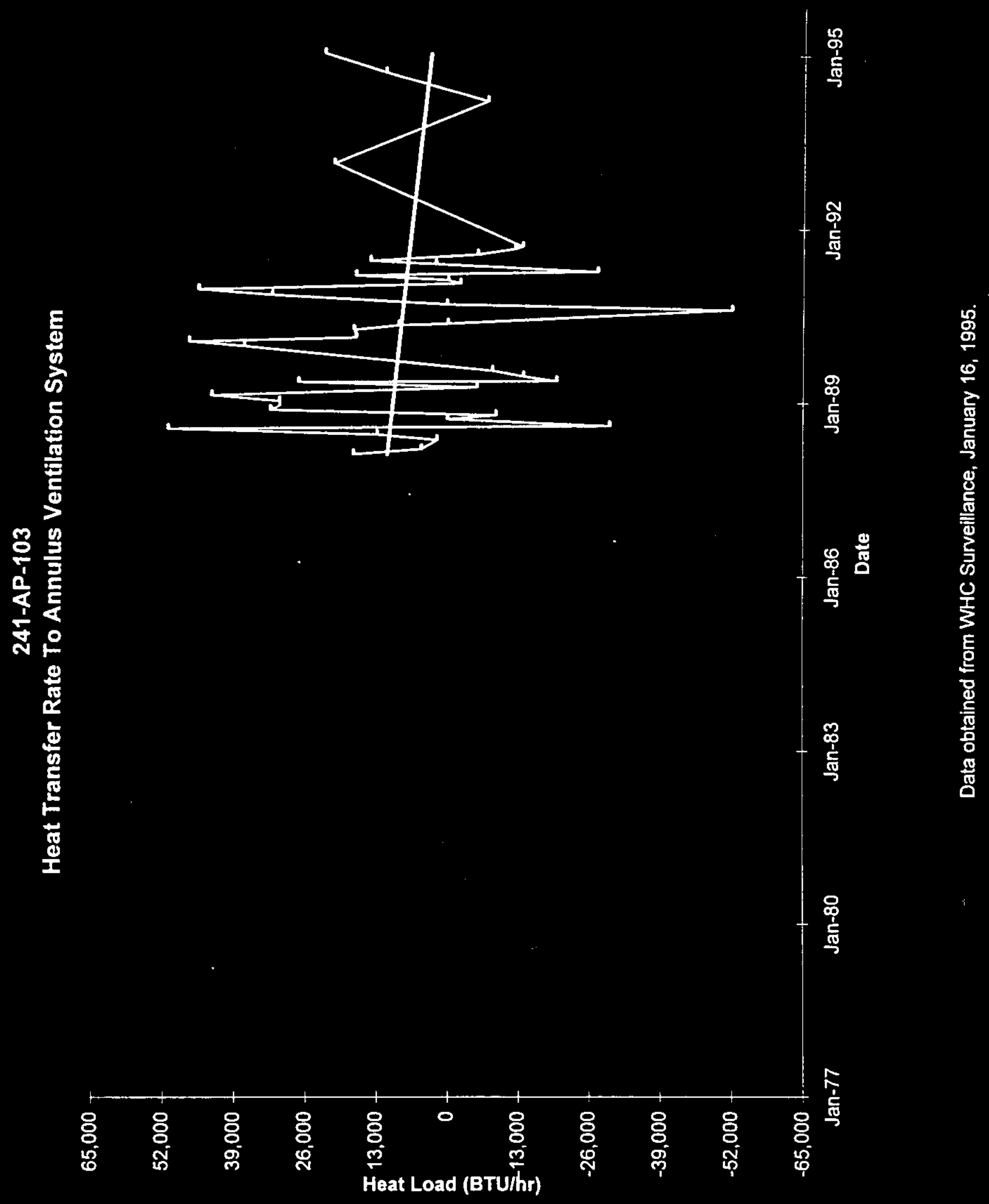




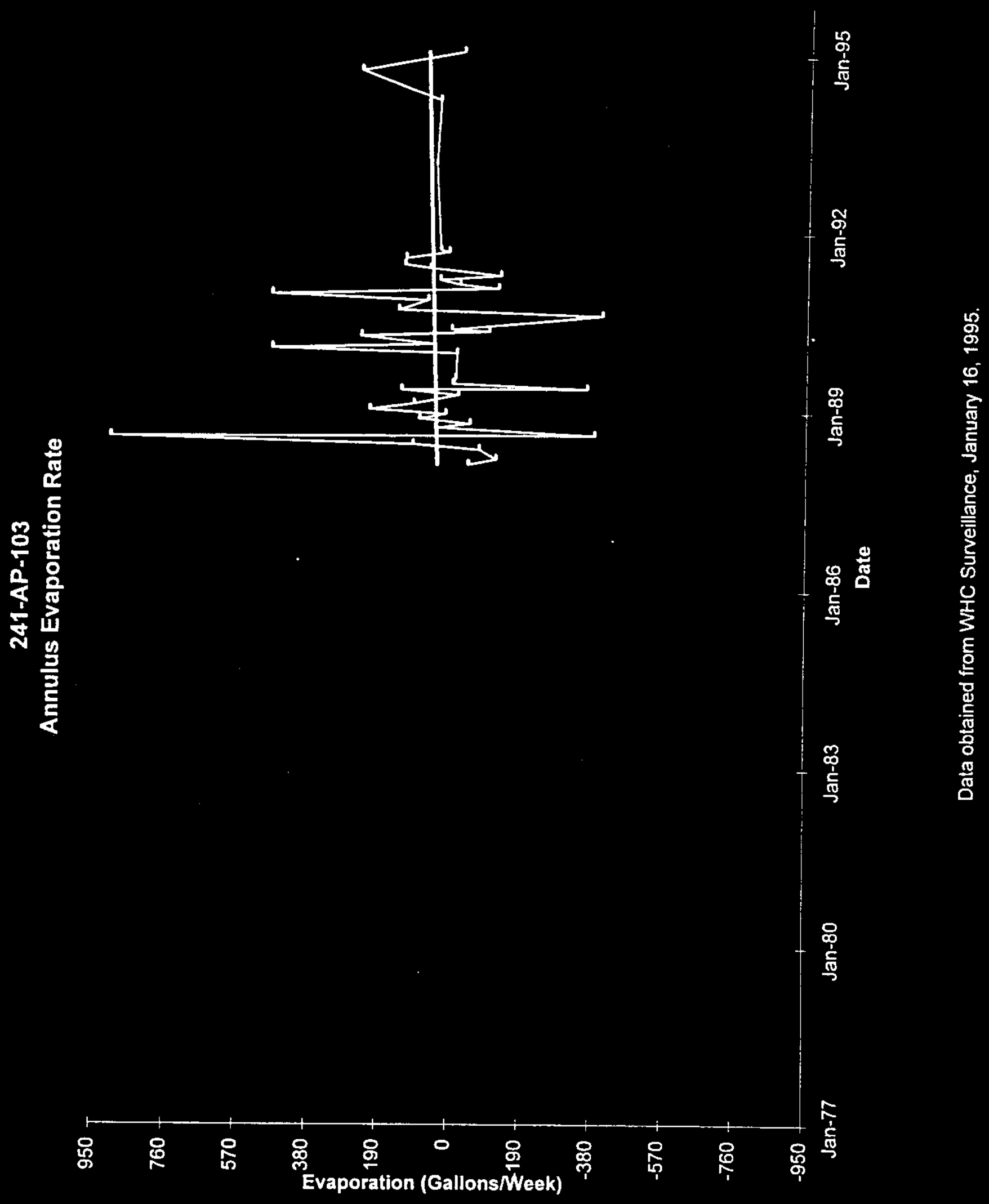



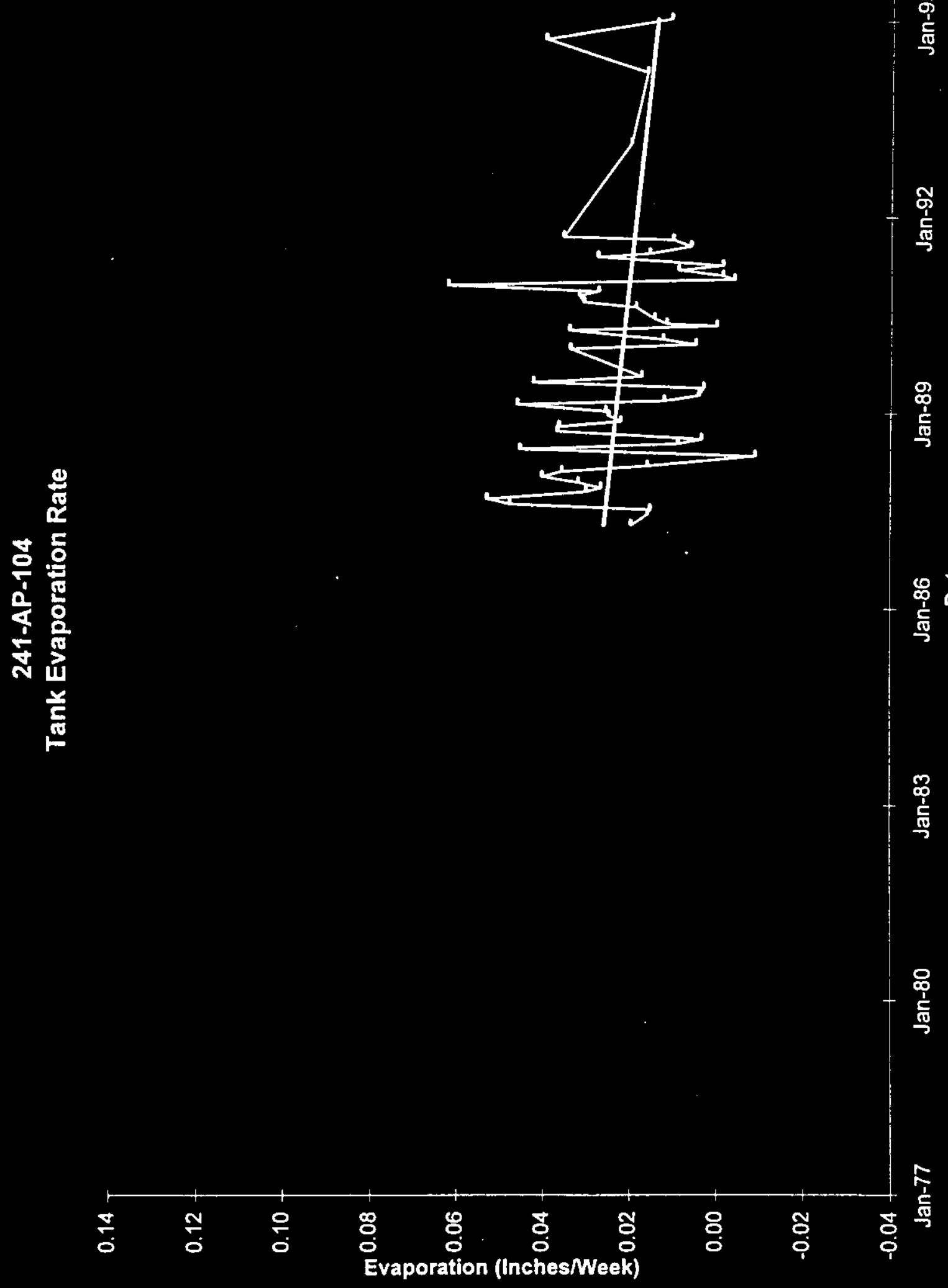

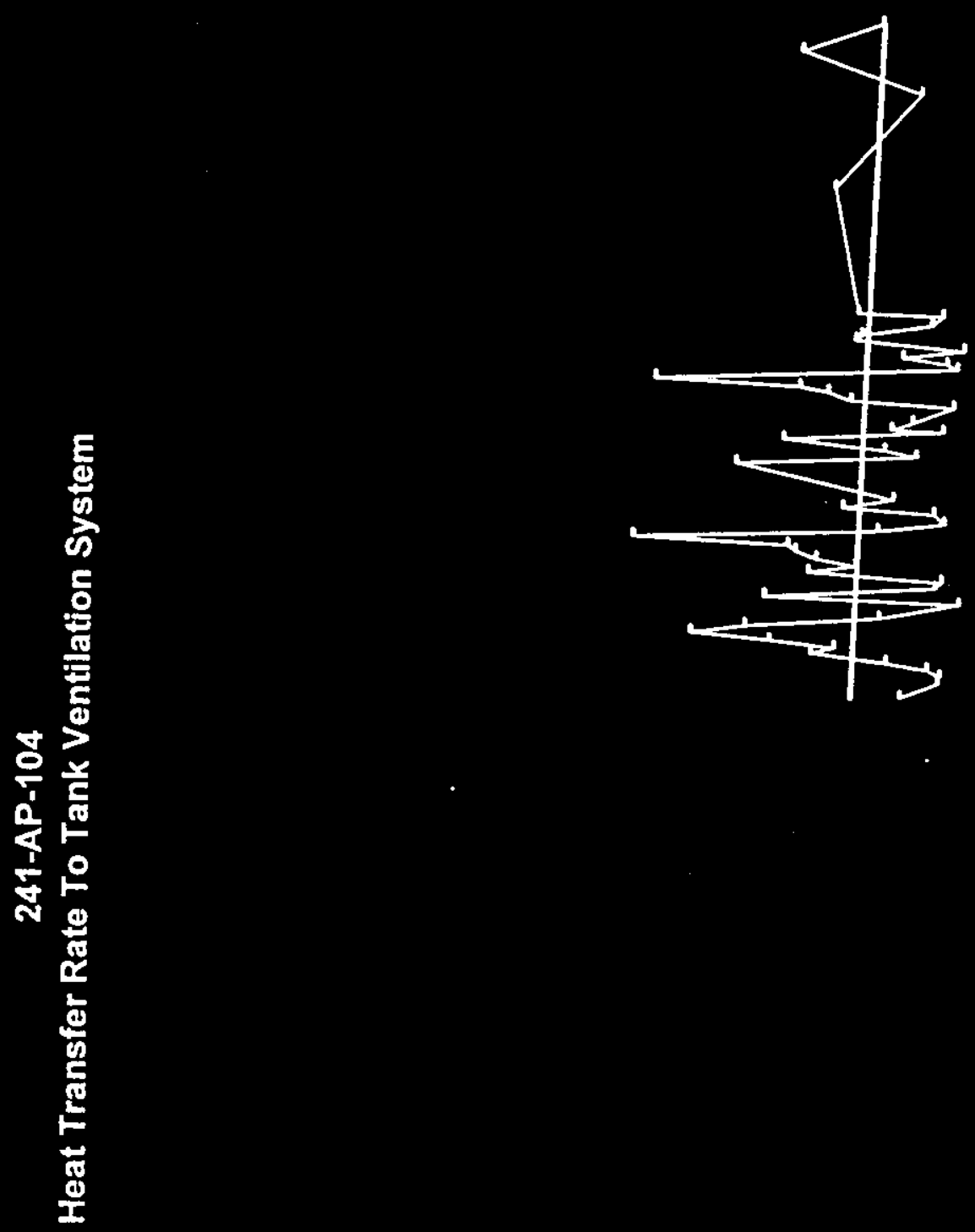

옹

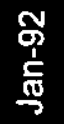

0
$\frac{1}{10}$
$\frac{1}{3}$

œ

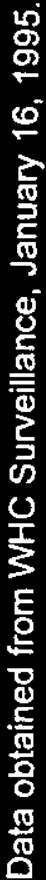

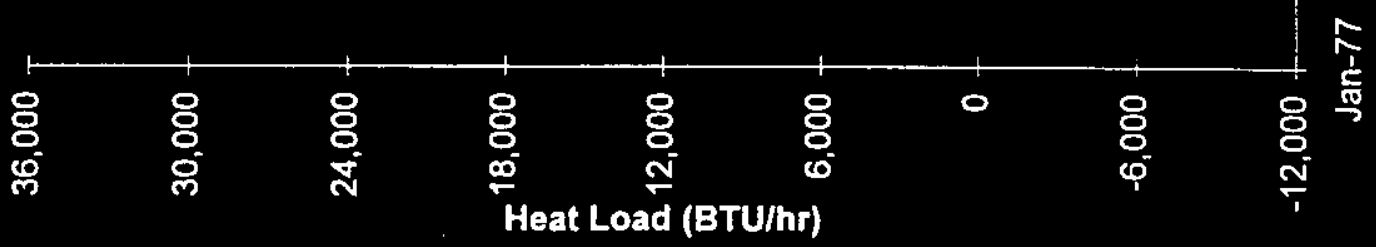




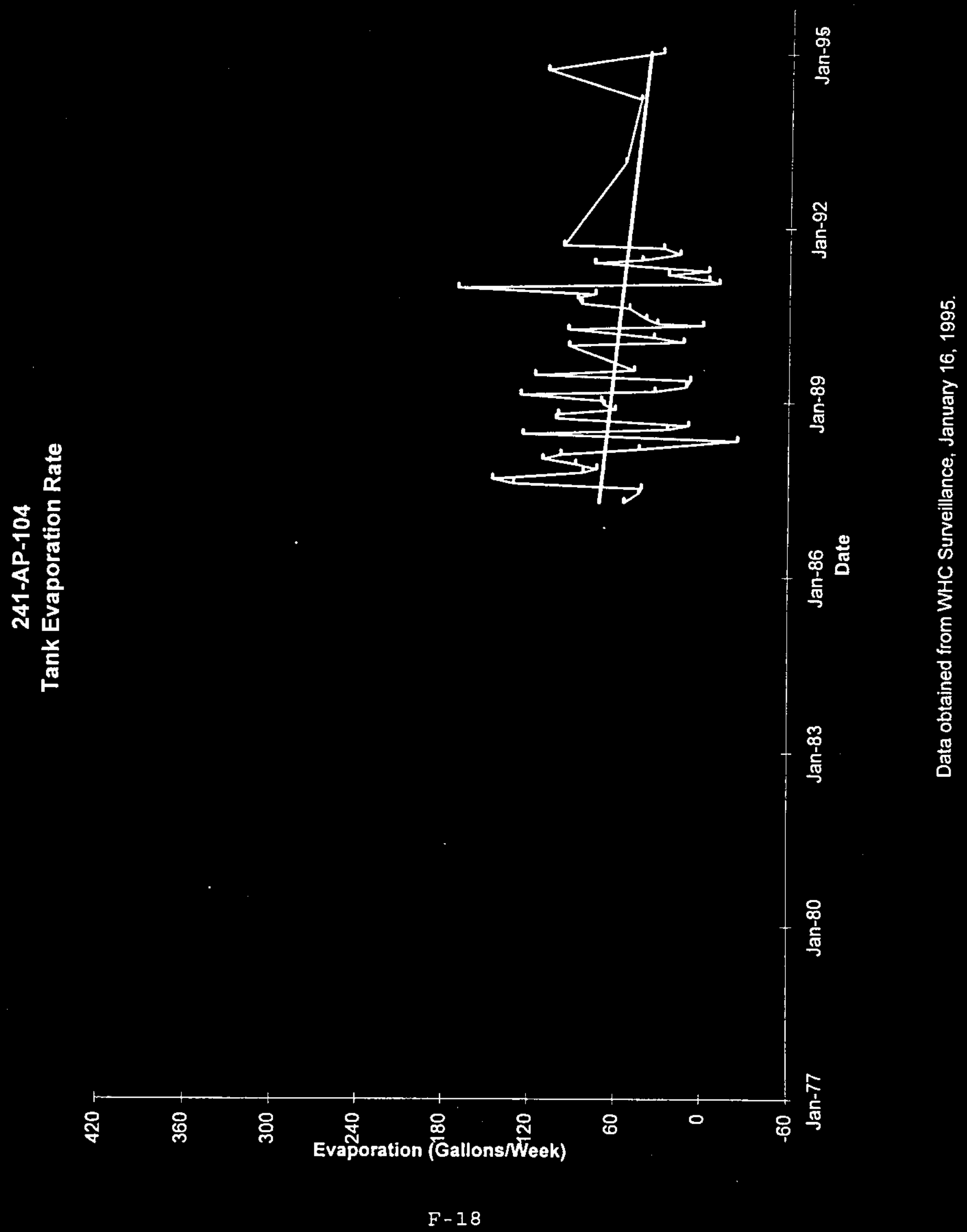


WHC-SD-WM-ER-315, Rev. 0

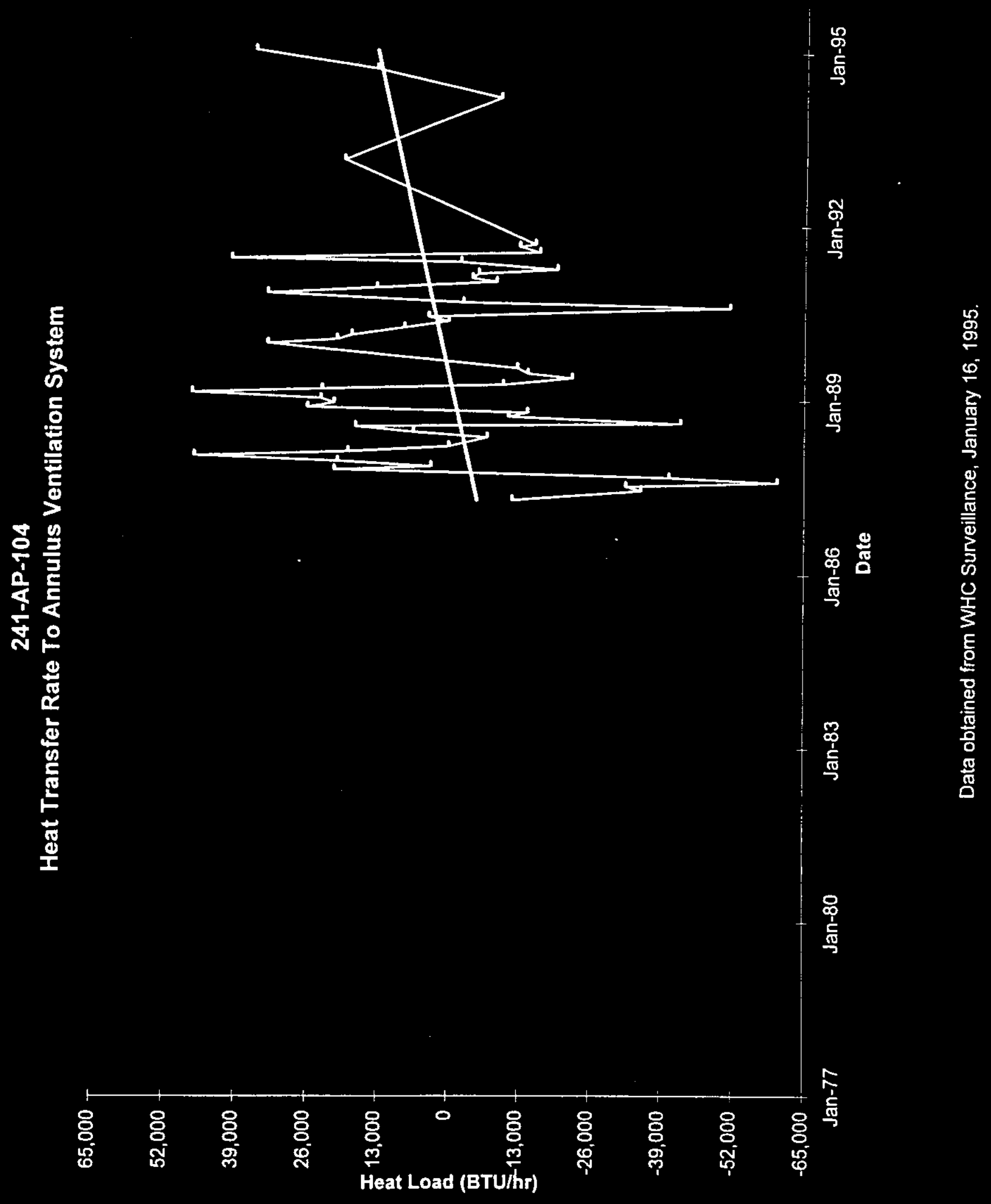


WHC-SD-WM-ER-315, Rev. 0

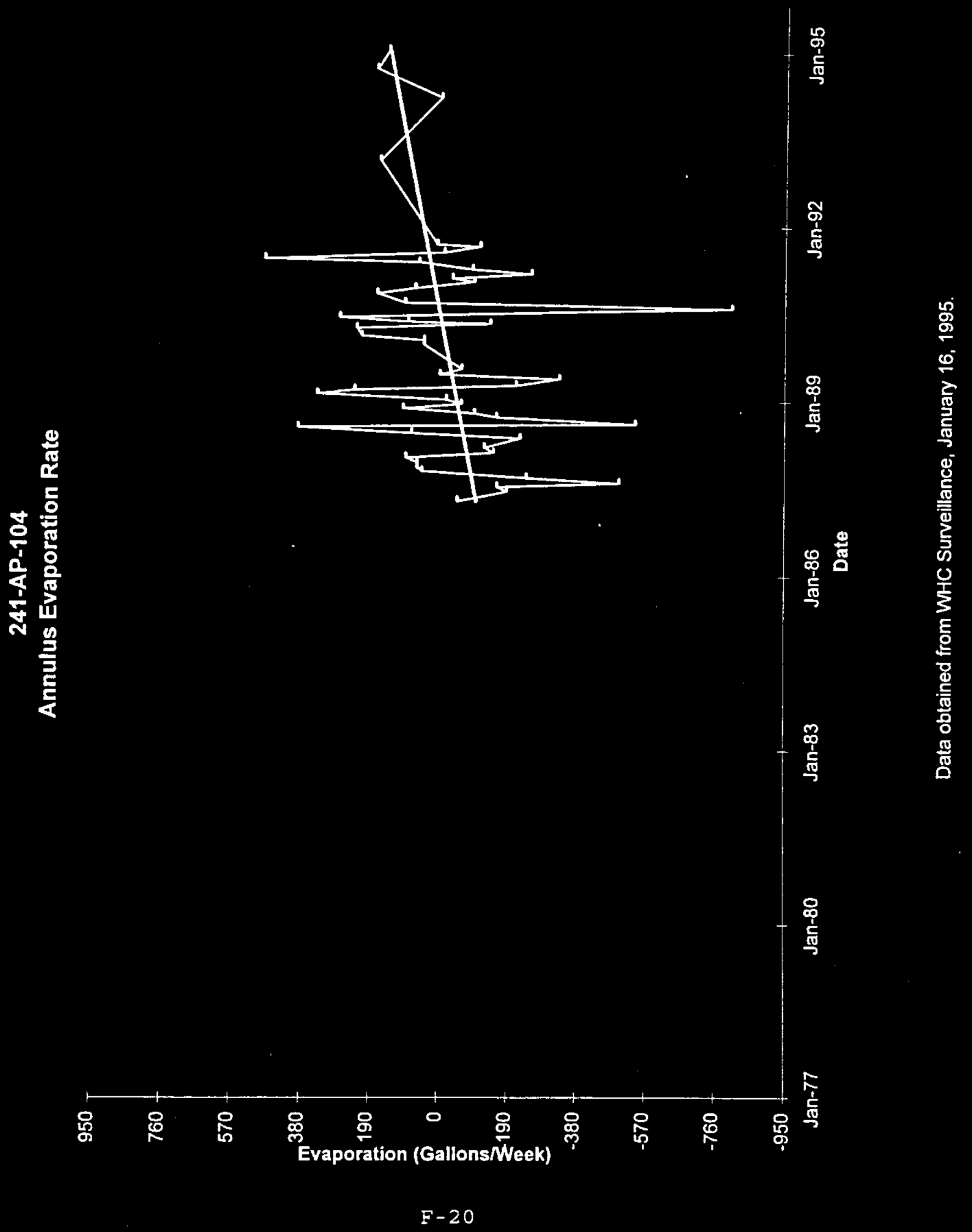


WHC-SD-WM-ER-315， Rev . 0

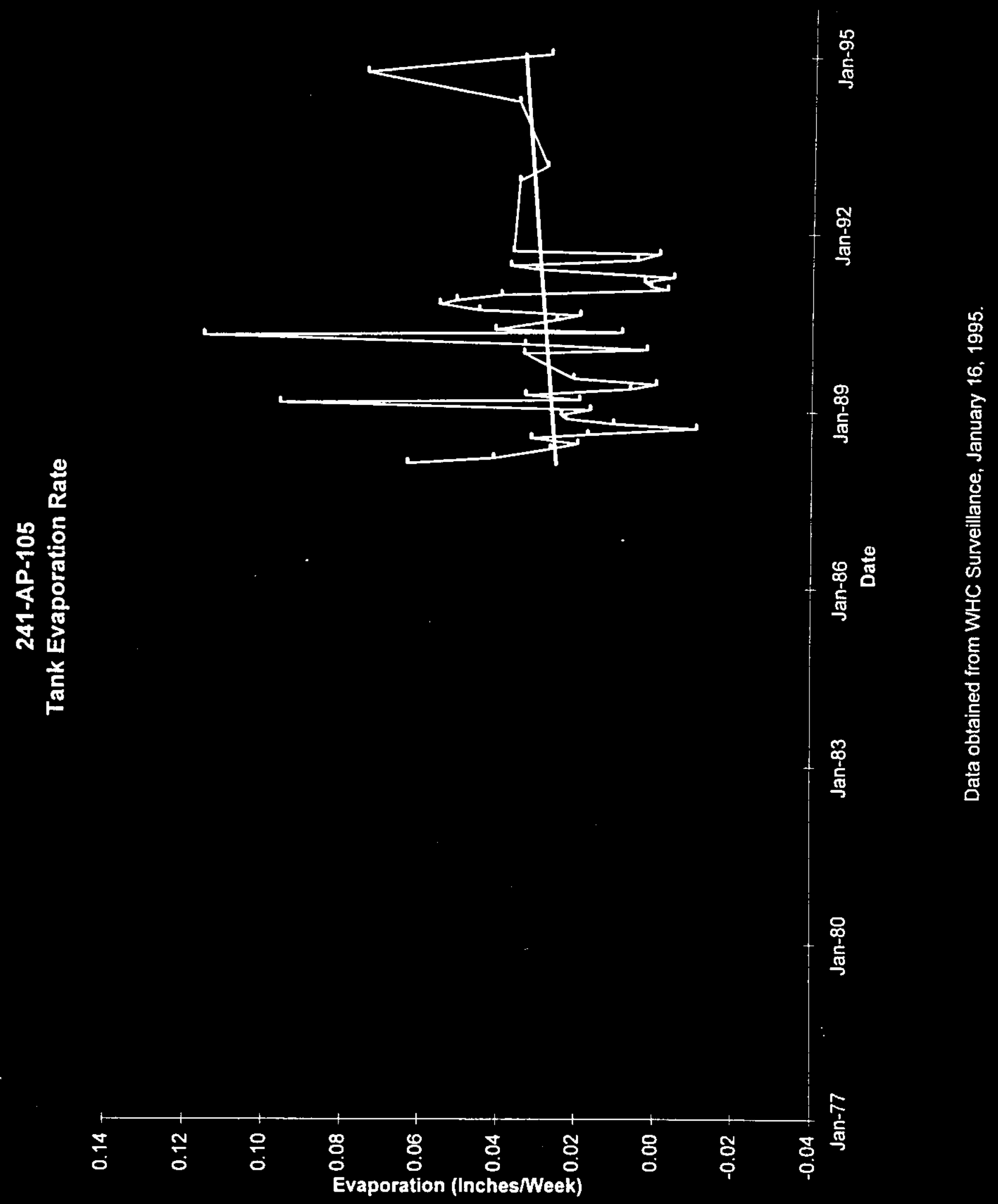


WHC-SD-WM-ER-315, Rev. 0

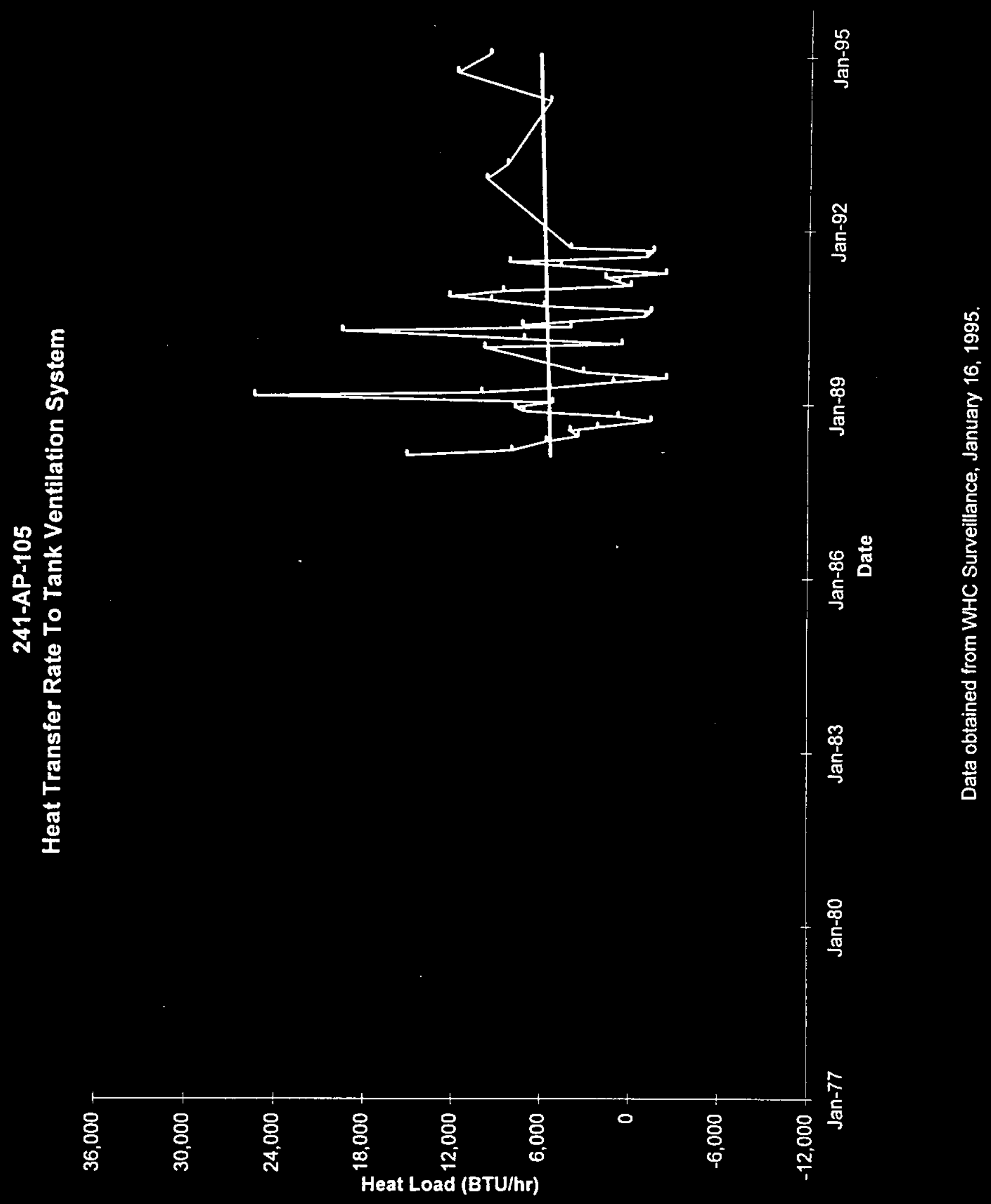


WHC-SD-WM-ER-315, ReV. 0
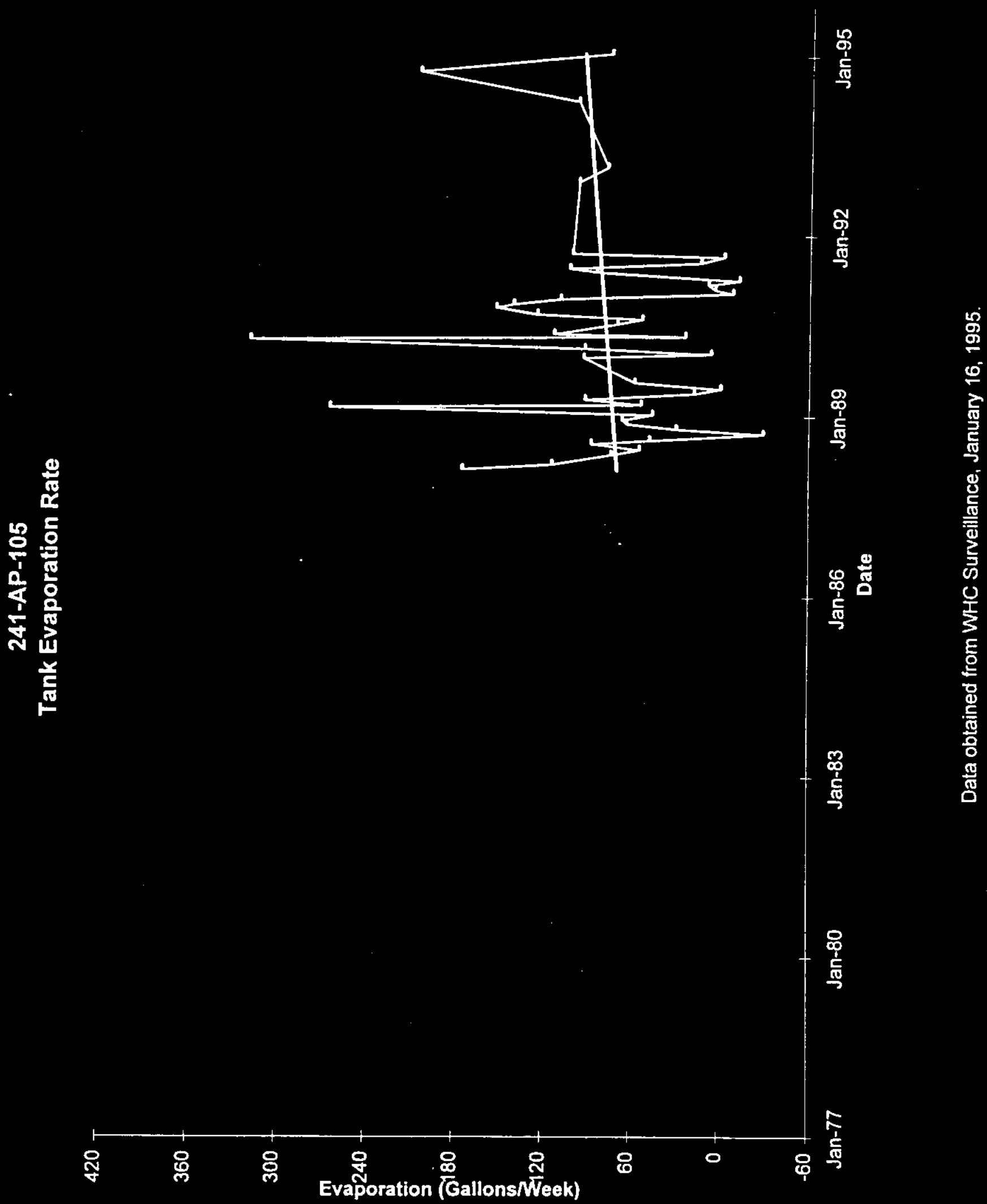

F-23 
WHC-SD-WM-ER-315, Rev . 0

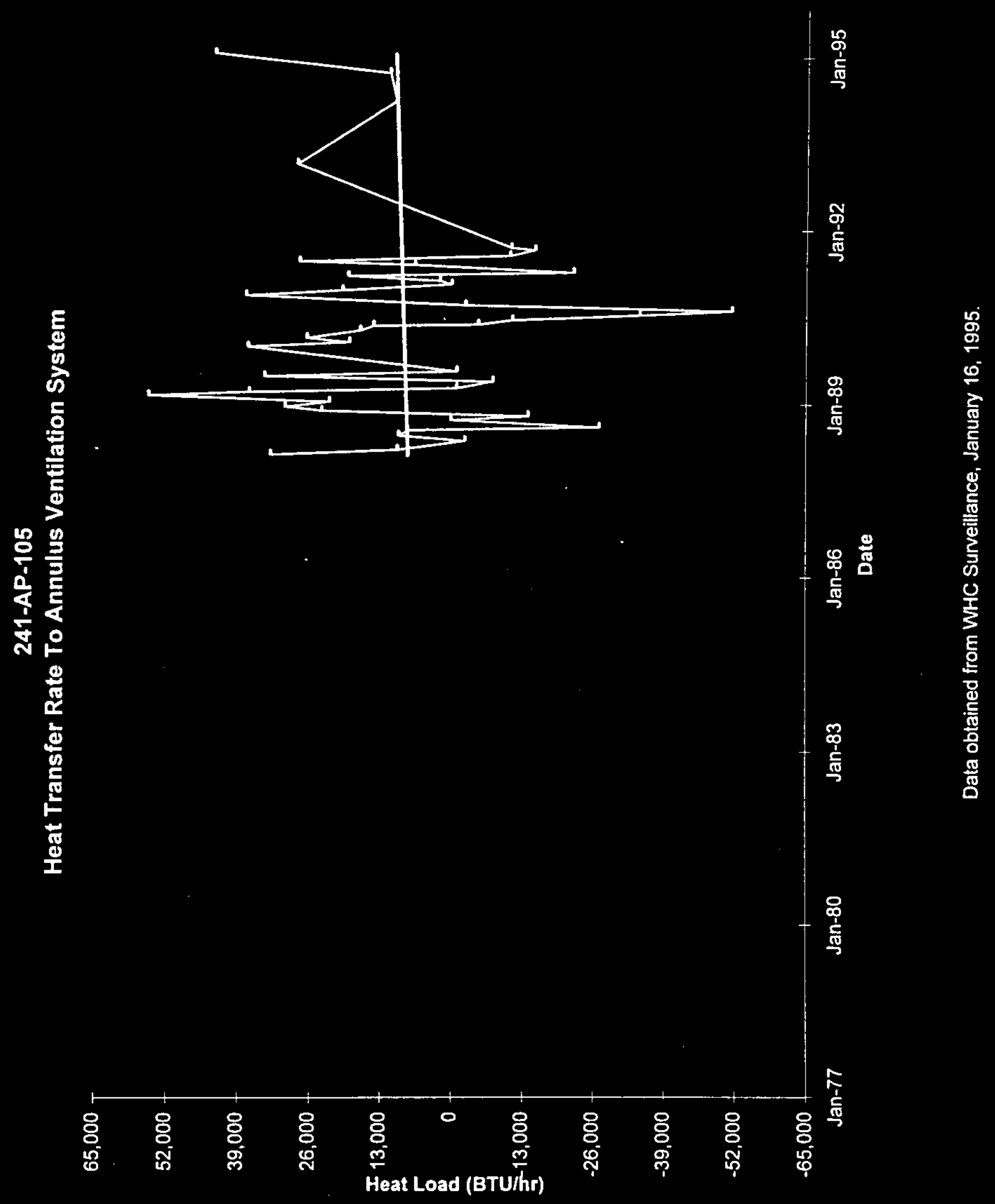


WHC-SD-WM-ER-315, Rev. 0
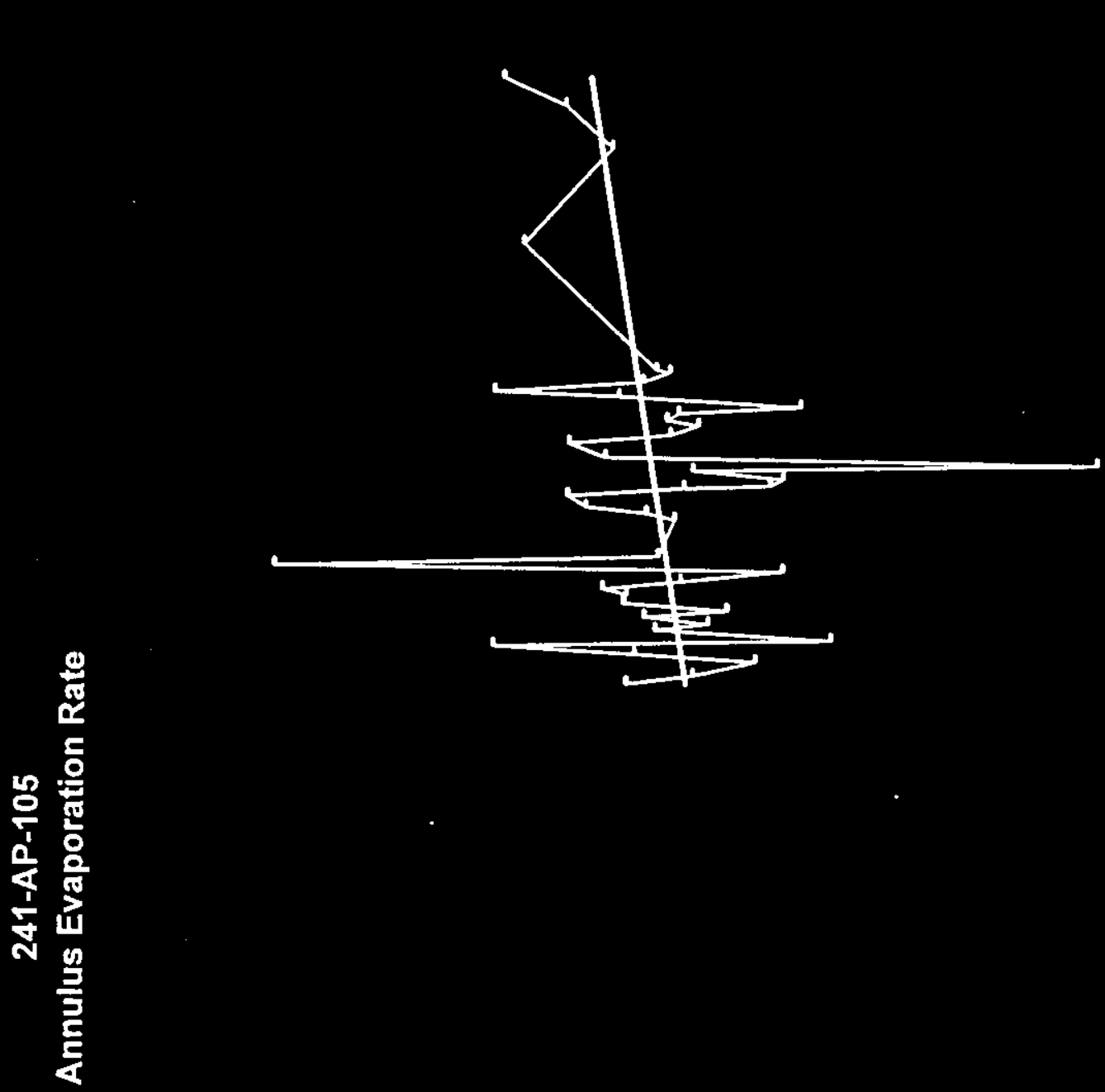

息

家

$\mathscr{8}$
$\frac{1}{5}$

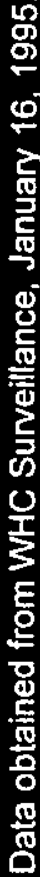

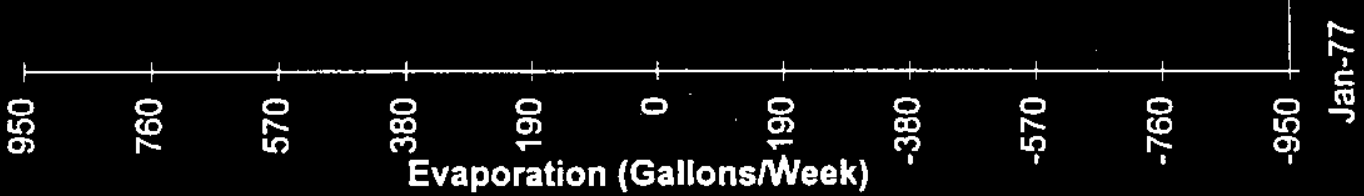


WHC-SD-WM-ER-315, Rev. 0

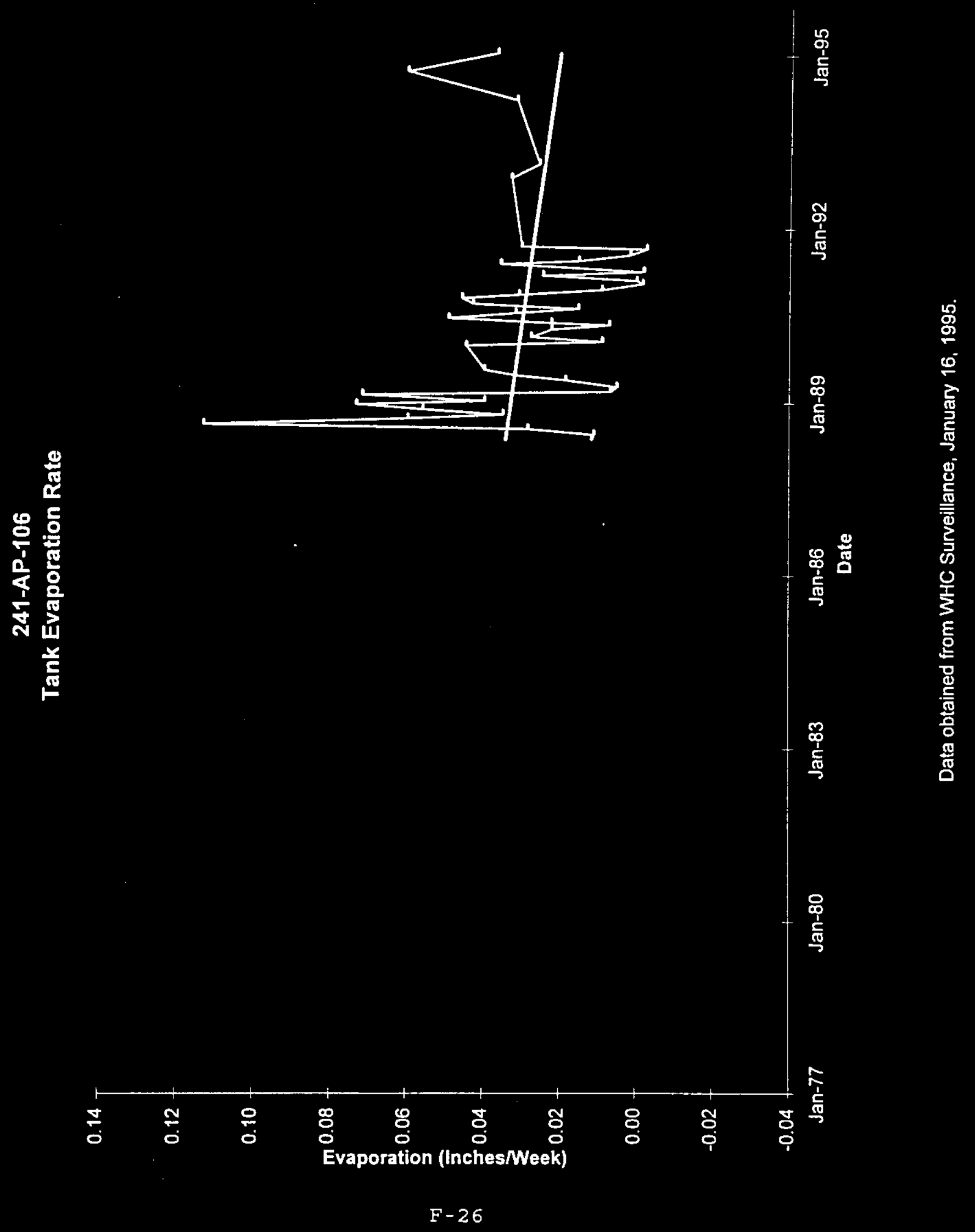


WHC-SD-WM-ER-315, Rev. 0

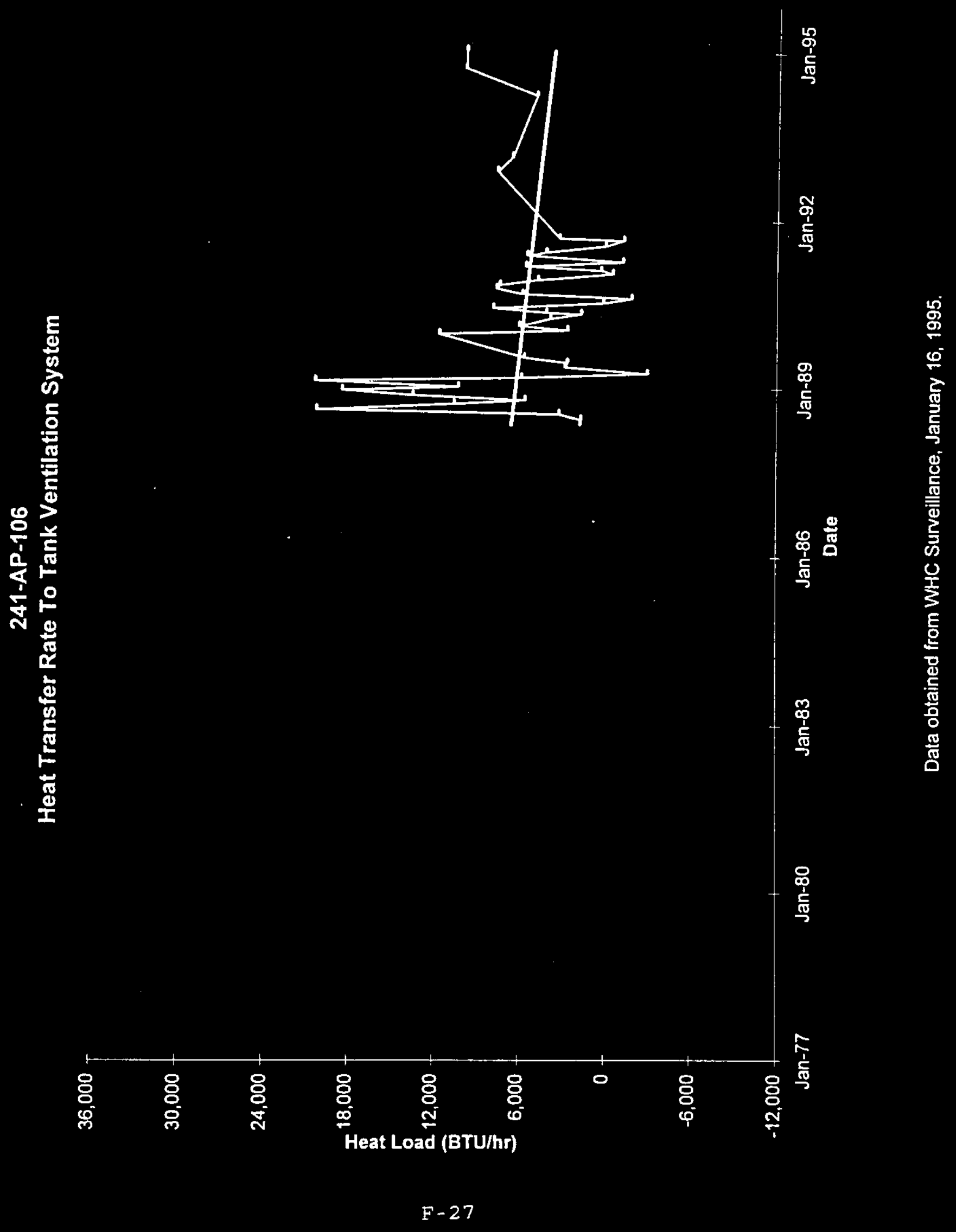


WHC-SD-WM-ER-315, Rev . 0

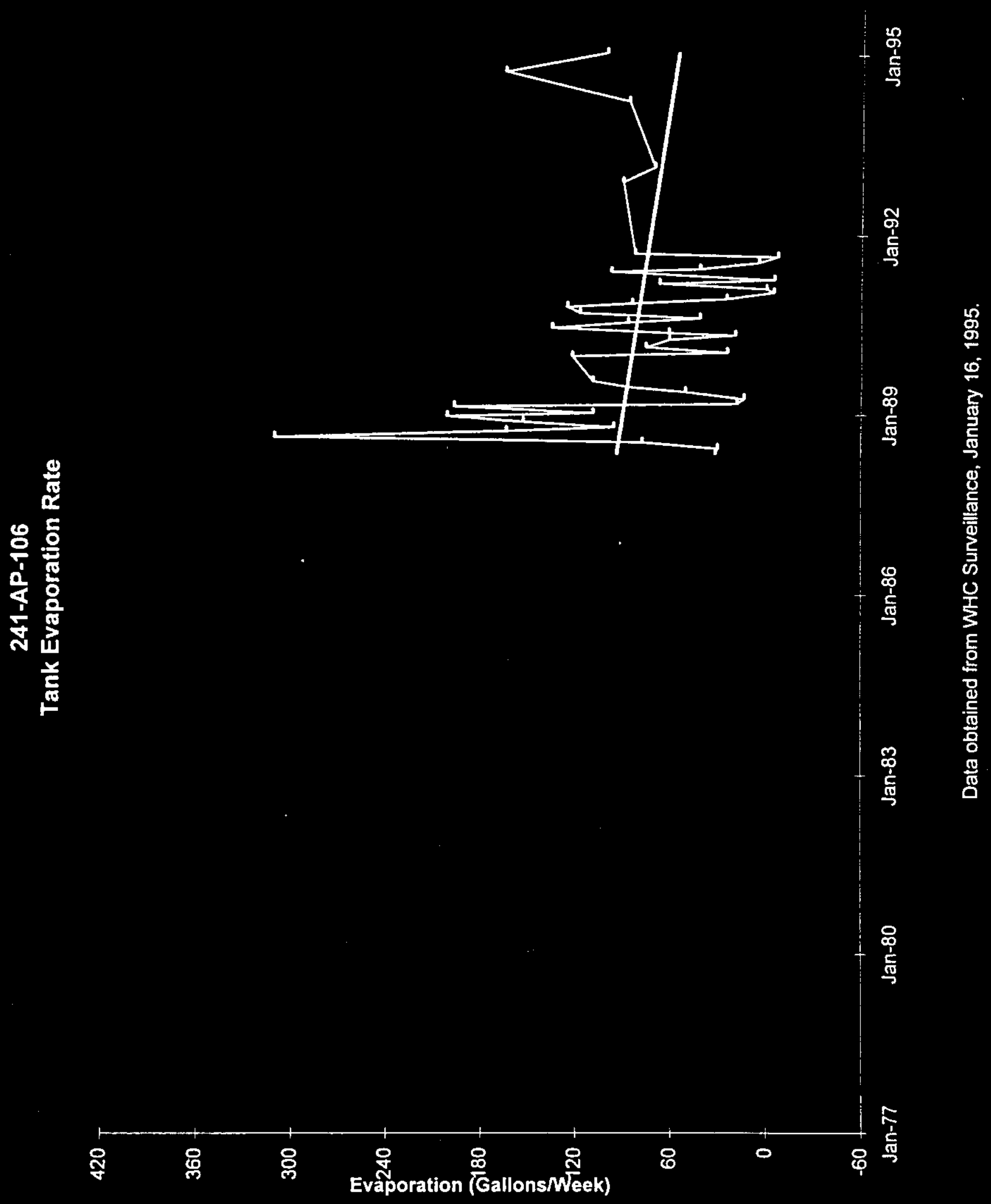


WHC-SD-WM-ER-315， Rev, 0

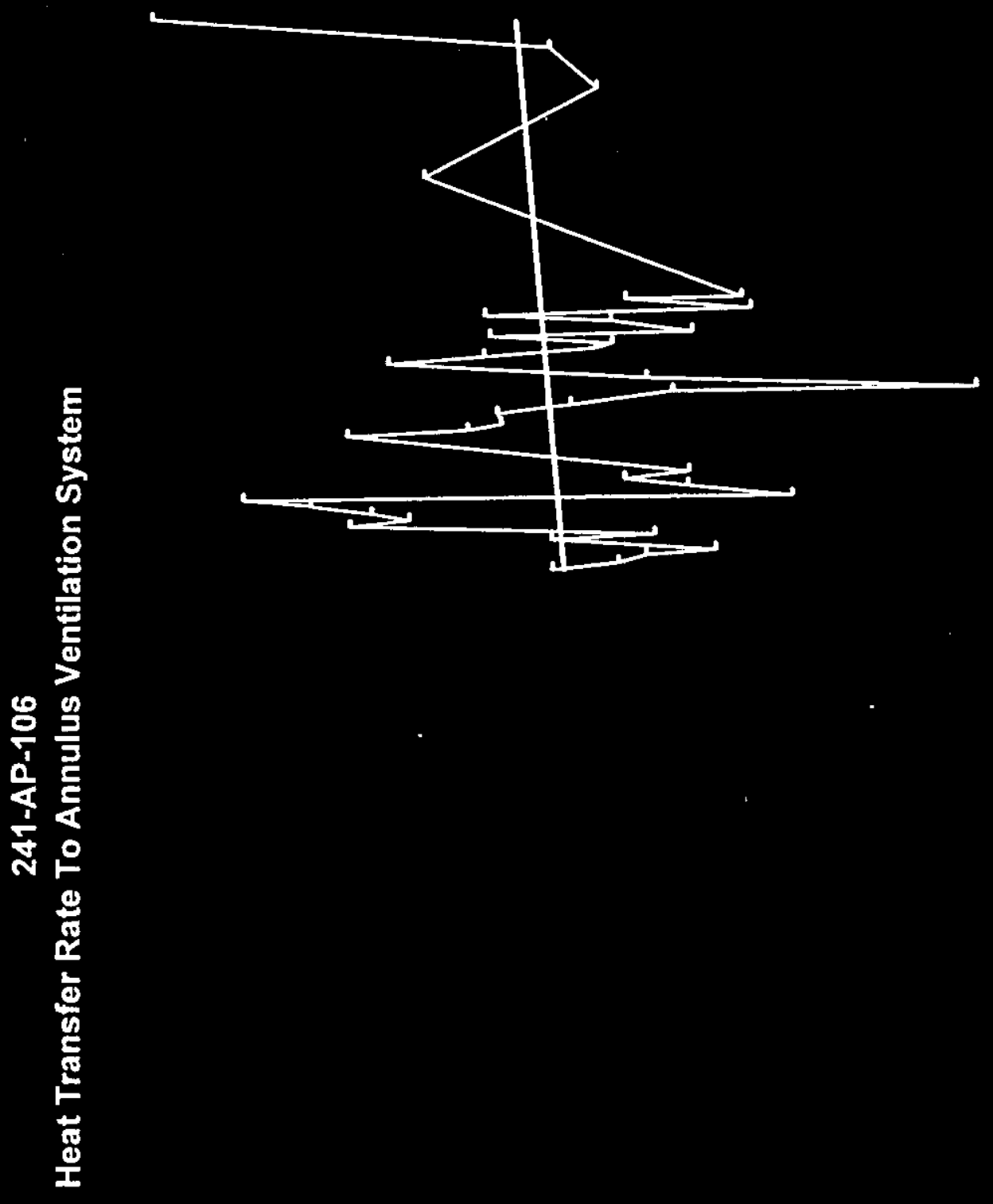

员

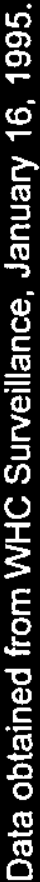

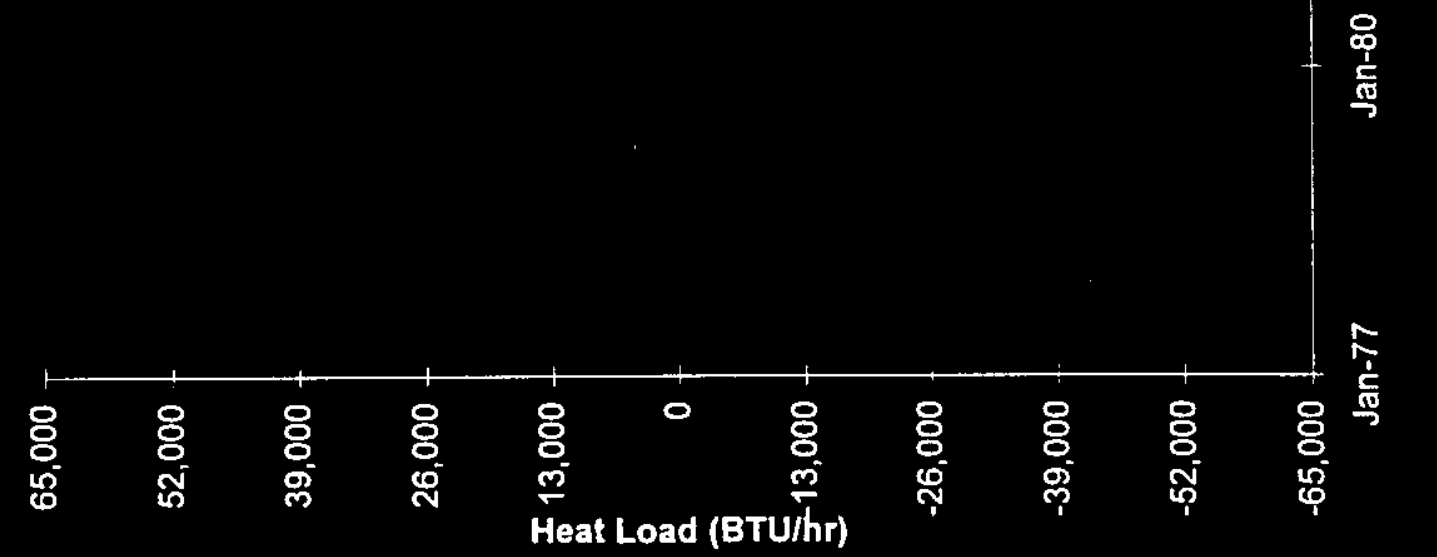

$F-29$ 
WHC-SD-WM-ER-315, Rev . 0

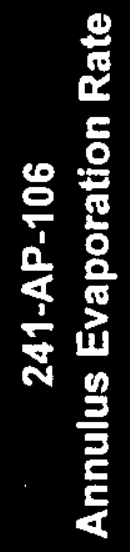

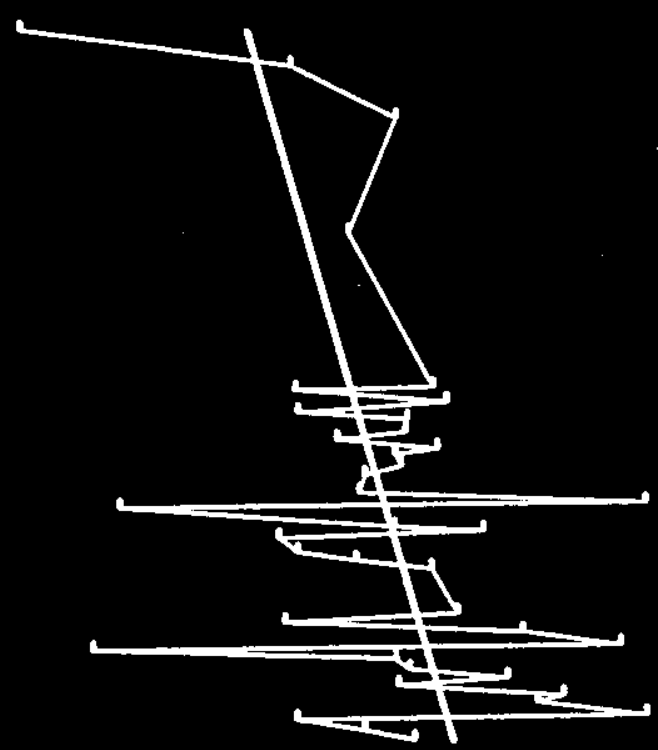

号

$\stackrel{\text { Sิ }}{\frac{1}{5}}$

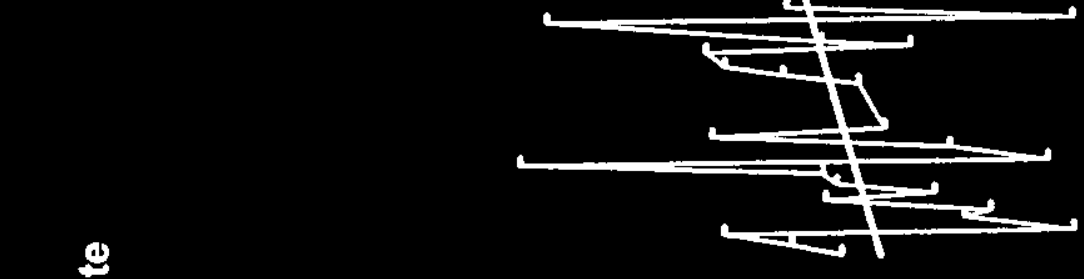

\%

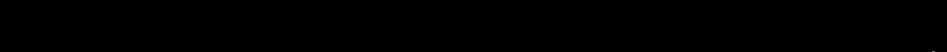

(1)
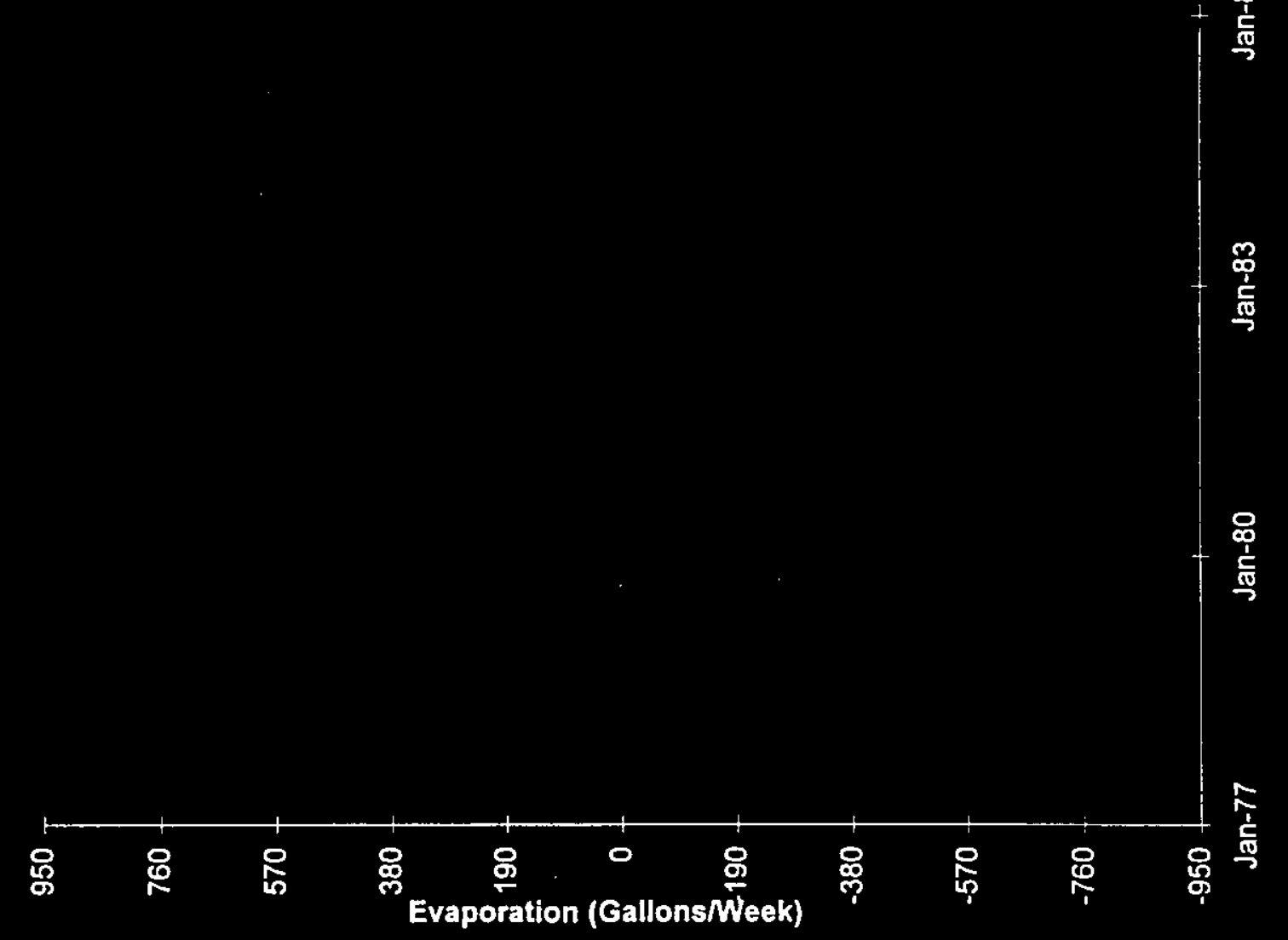
WHC-SD-WM-ER-315, Rev . 0

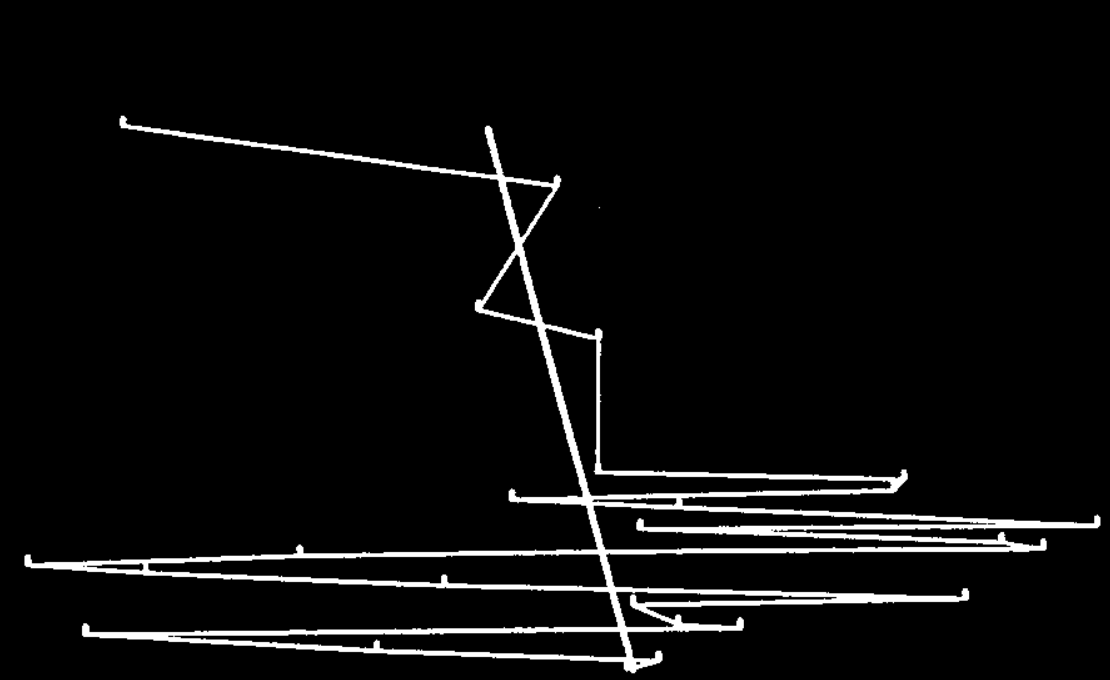

40
$\stackrel{1}{1}$
$\frac{1}{31}$

$\stackrel{\text { I }}{\frac{\text { II }}{3}}$

प1

$\$ 8$

둥

2

48

䎡

蒙

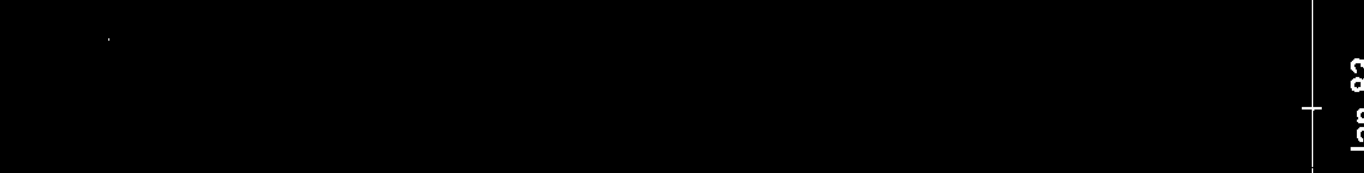

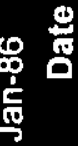

$\stackrel{8}{8}$

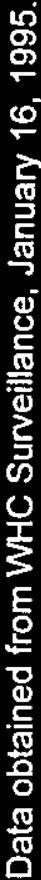

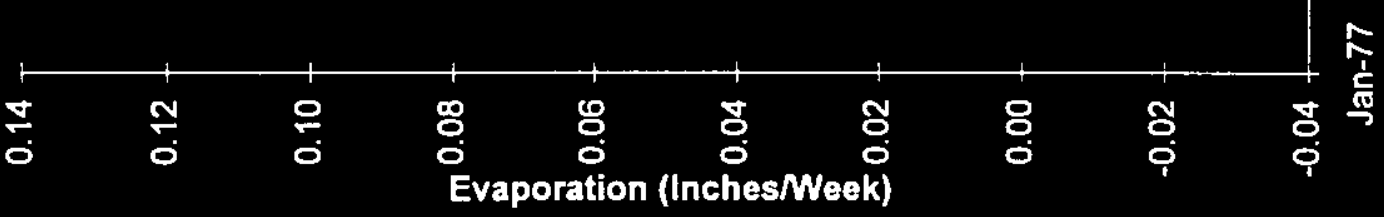


WHC-SD-WM-ER-315, Rev. 0

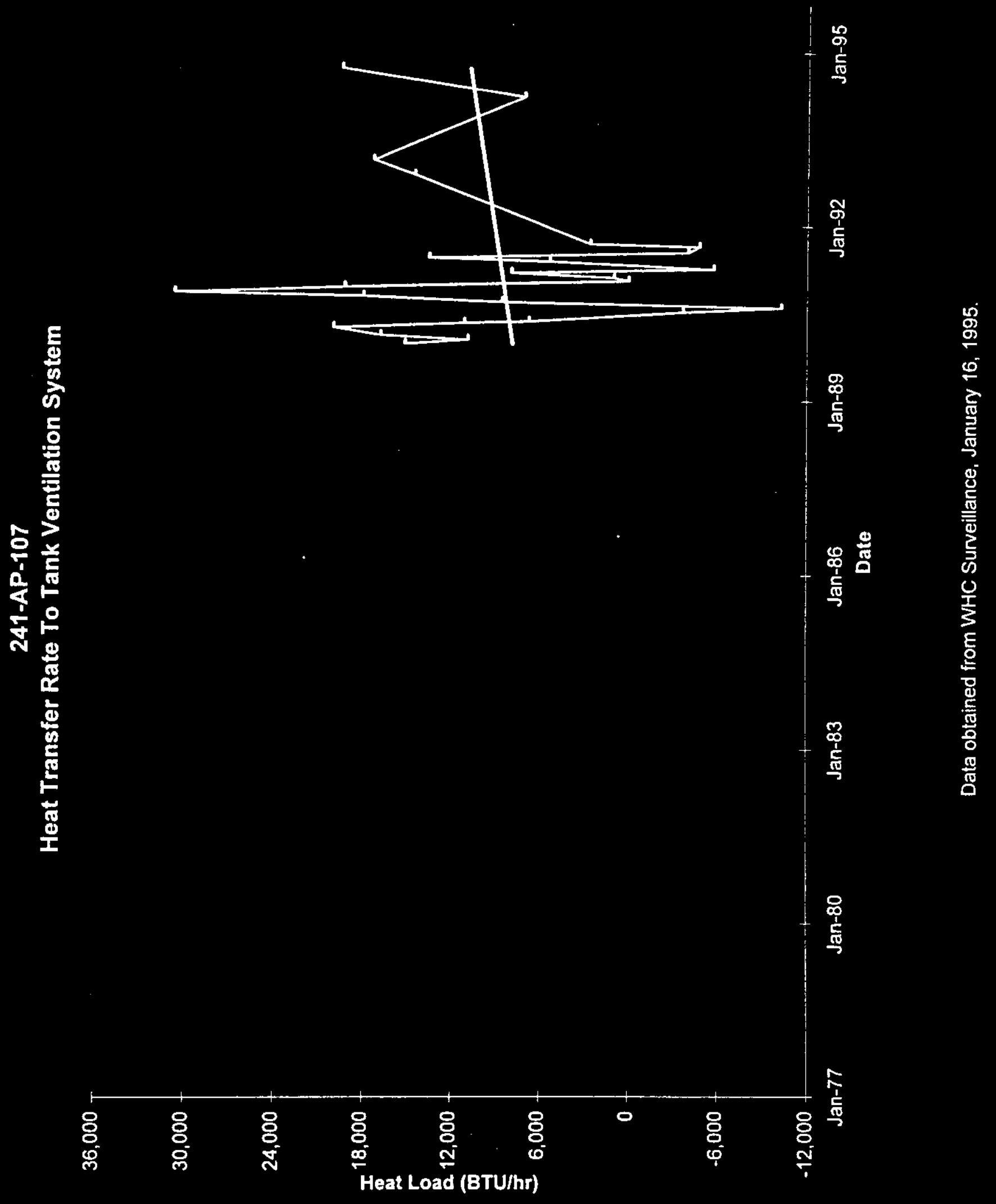

$$
\text { F-32 }
$$


WHC-SD-WM-ER-315， Rev. 0

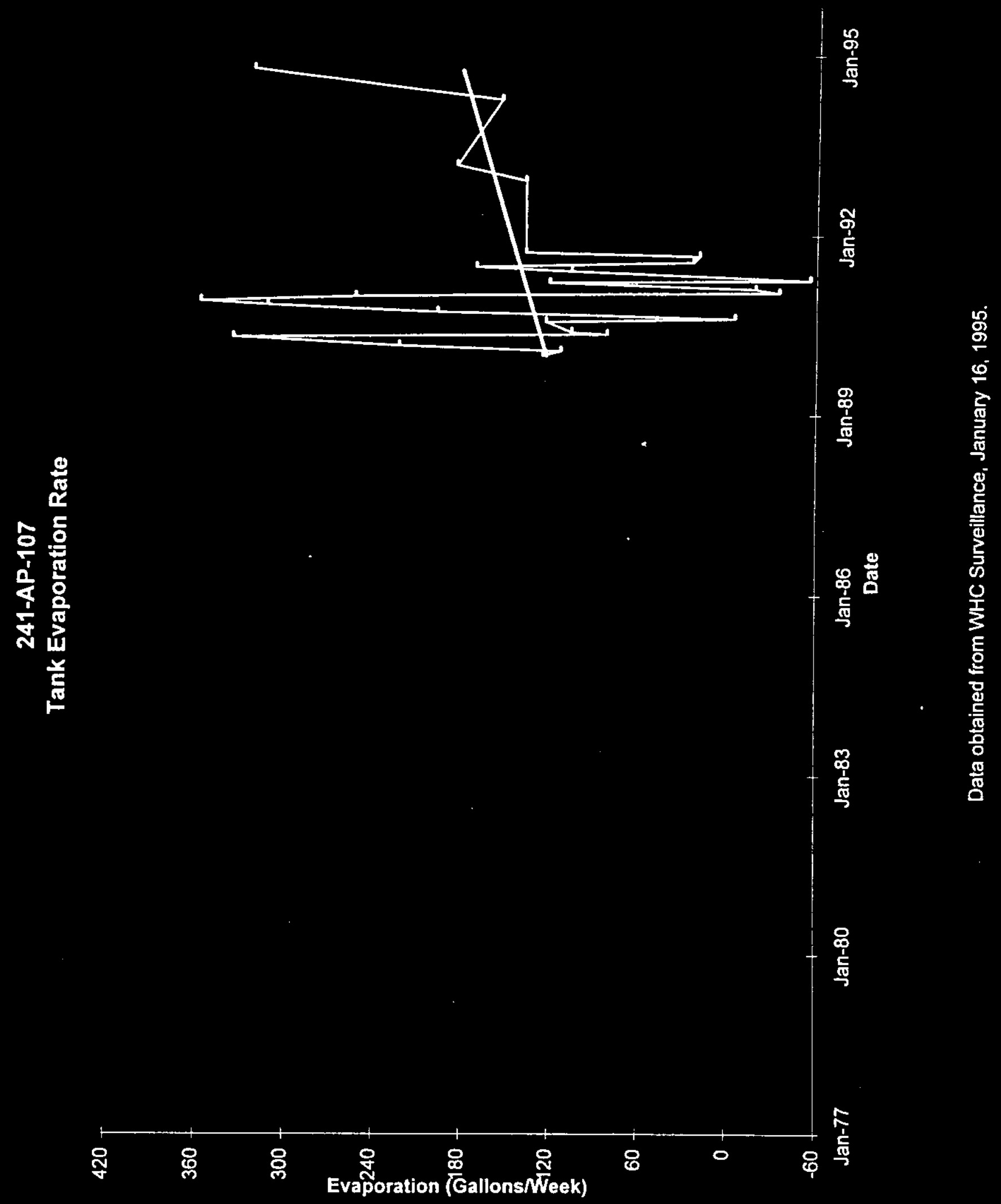

$F-33$ 


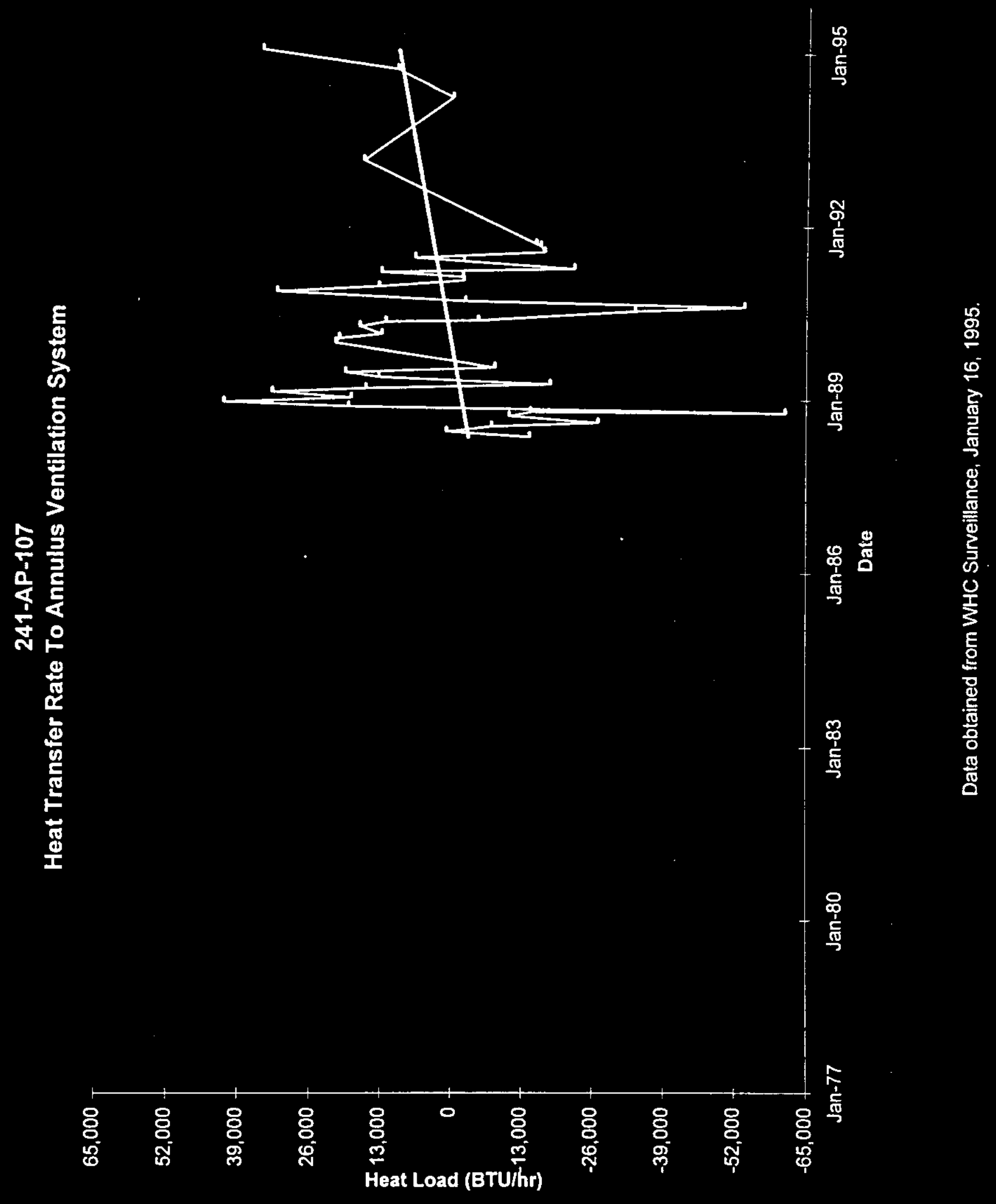


WHC-SD-WM-ER-315，Rev. 0

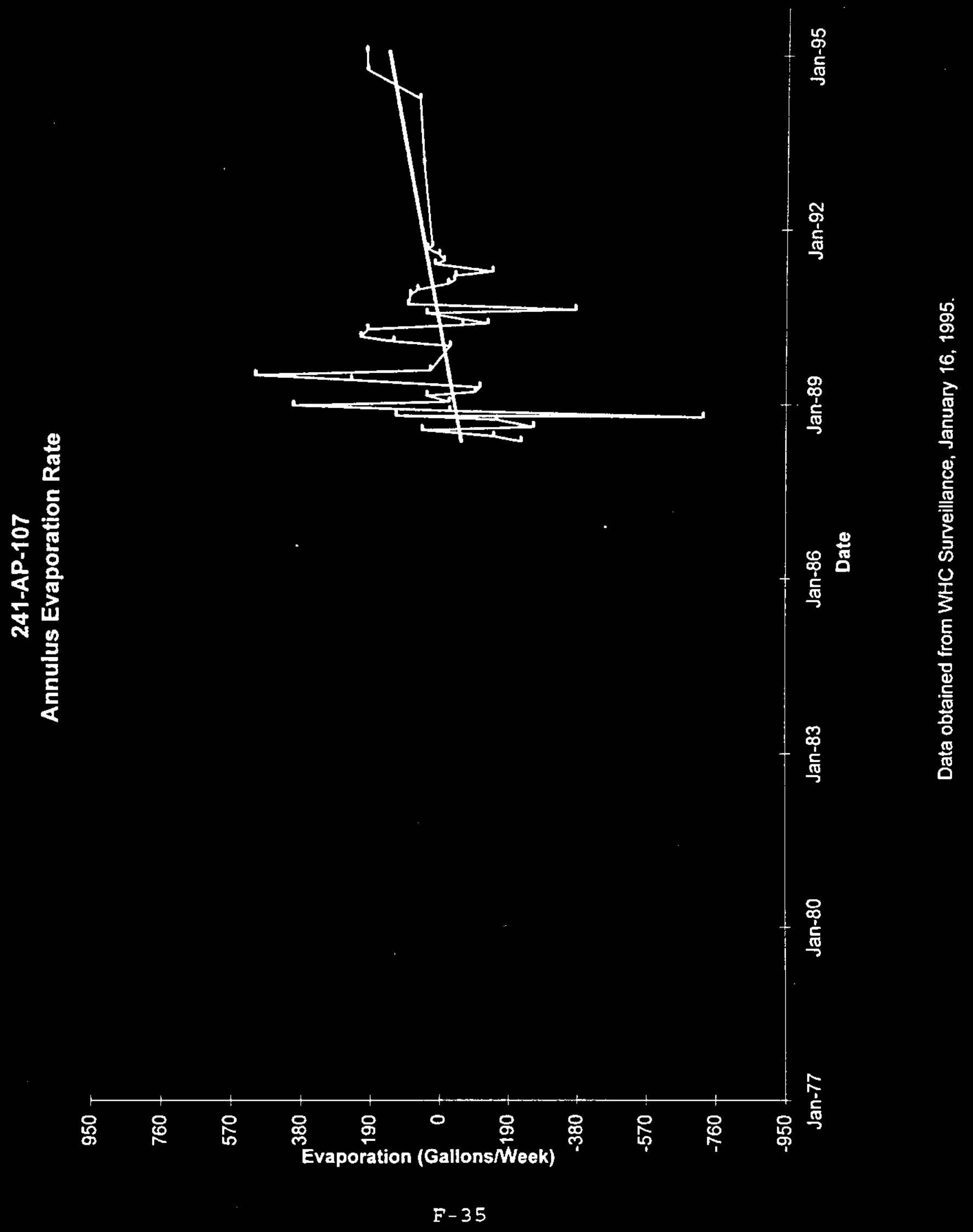


WHC-SD-WM-ER-315, Rev. 0

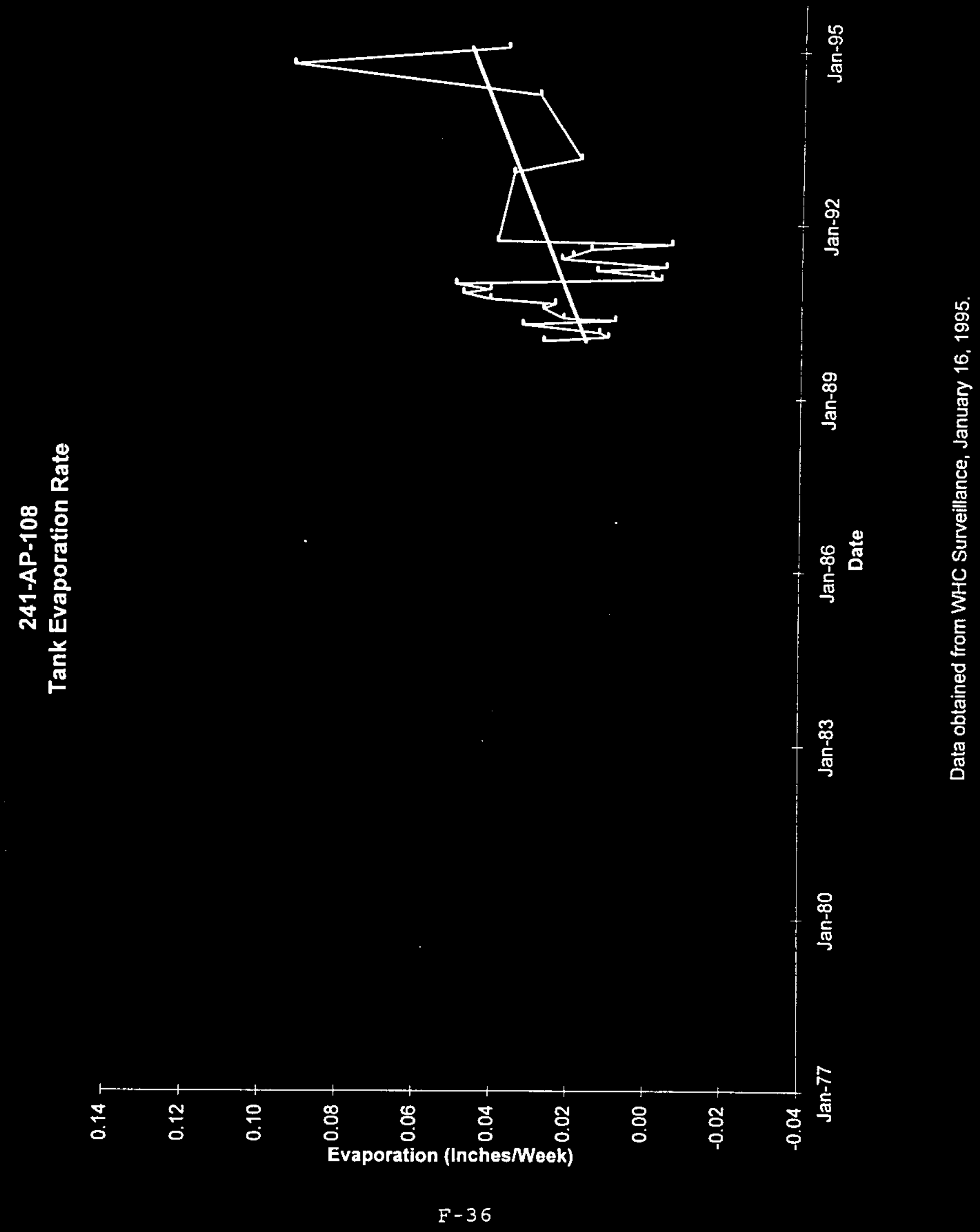




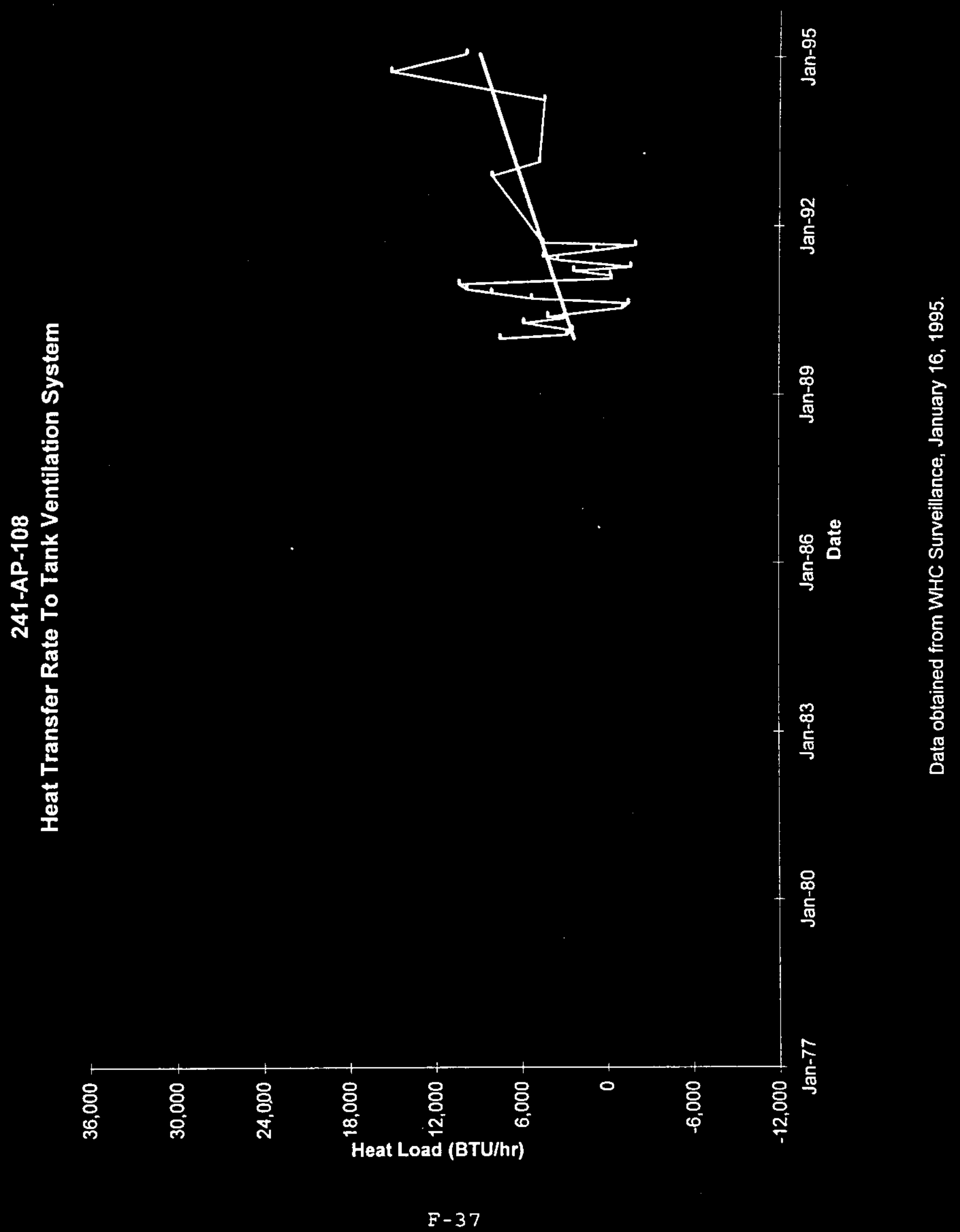




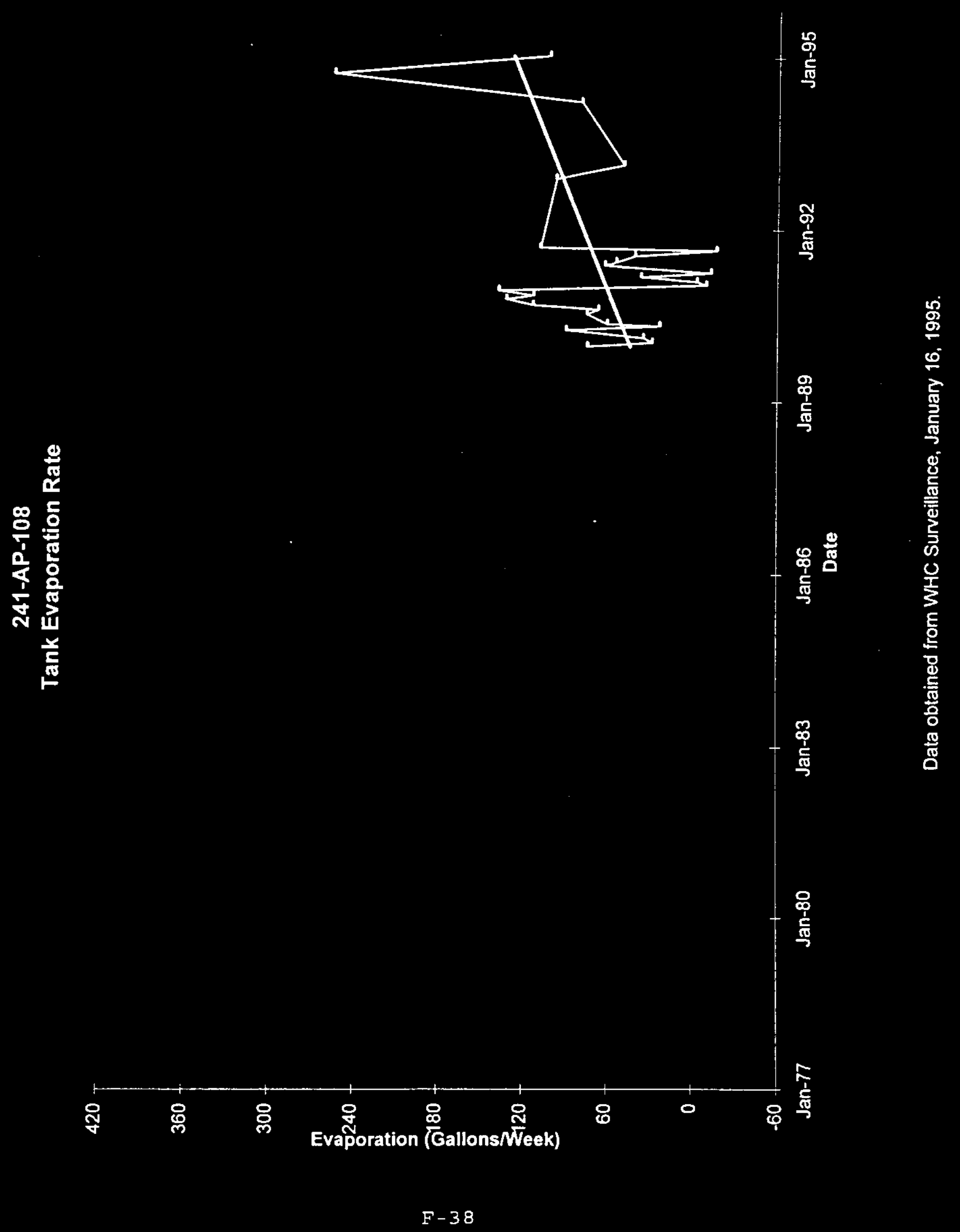


WHC-SD-WM-ER-315, Rev . 0

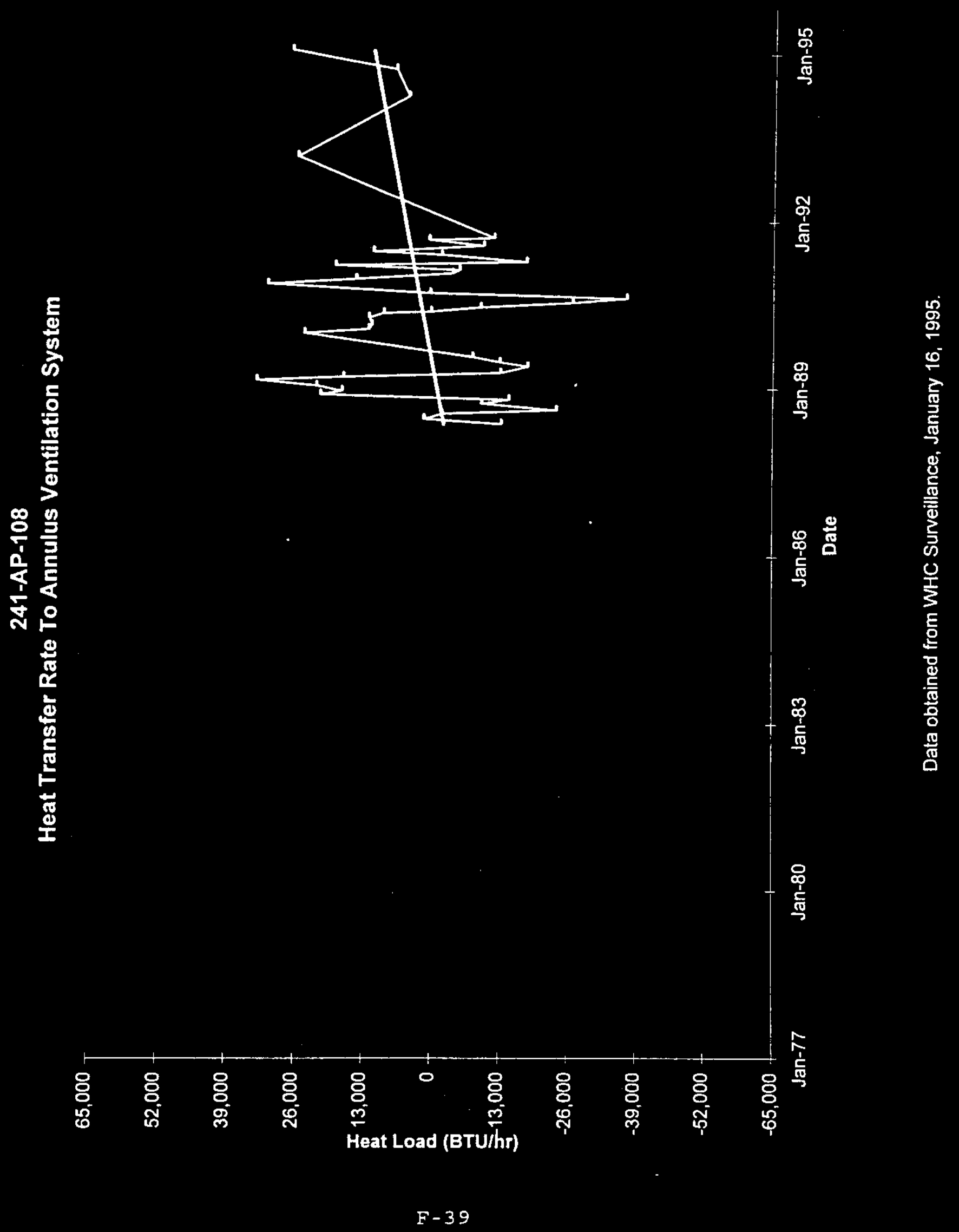


WHC-SD-WM-ER-315, Rev. 0

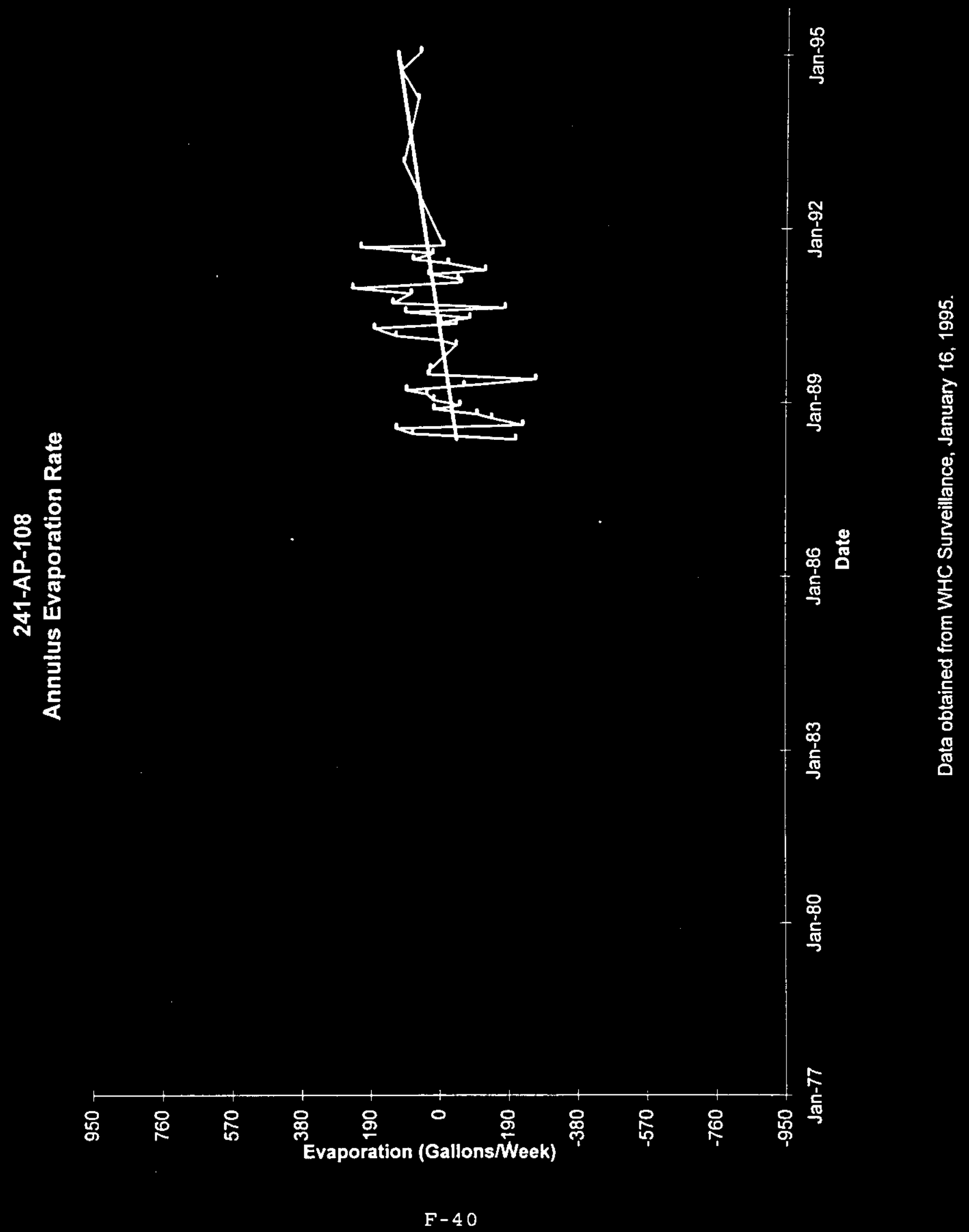


WHC-SD-WM-ER-315, Rev , 0

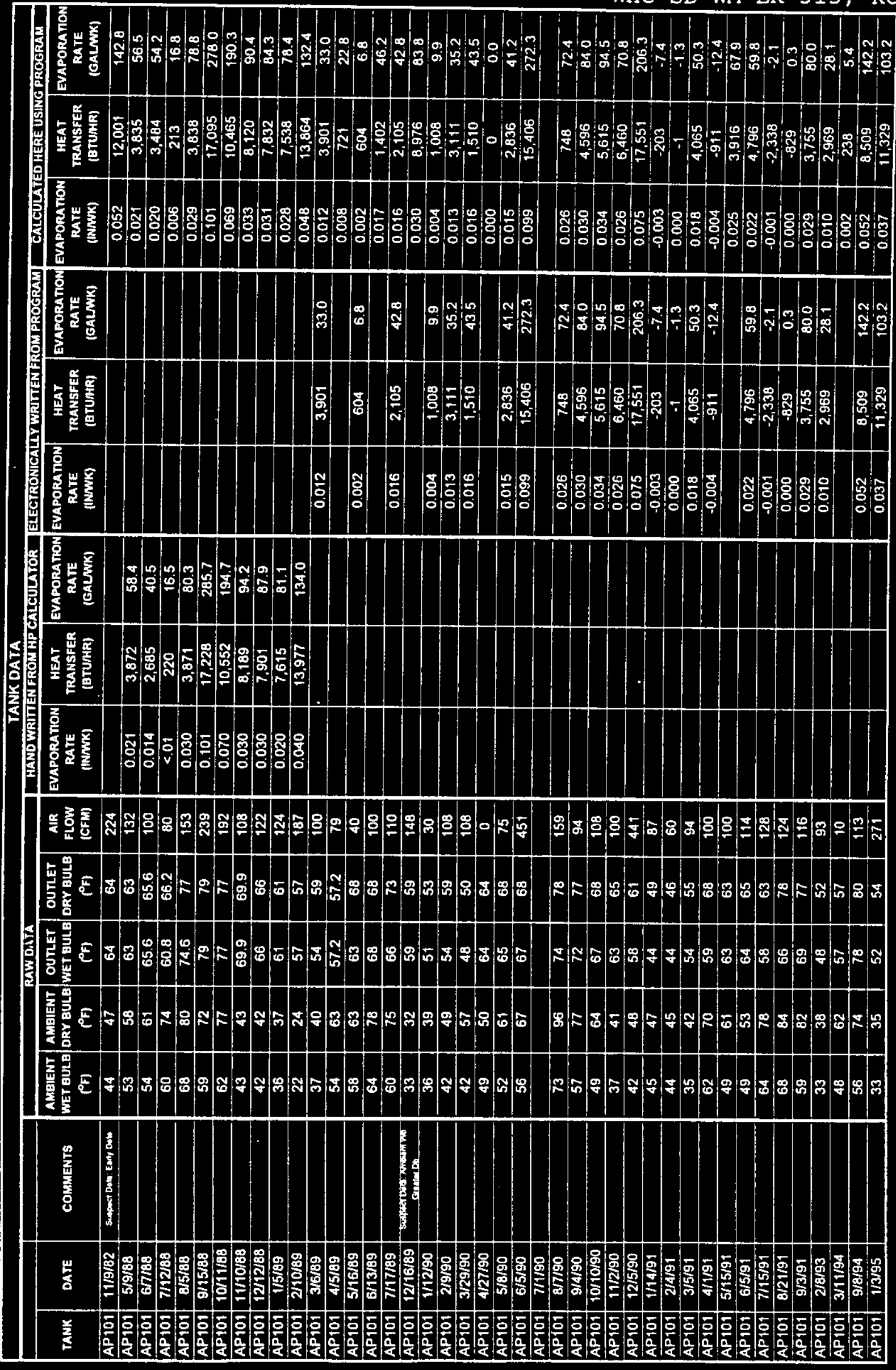


WHC-SD-WM-ER-315, Rev.

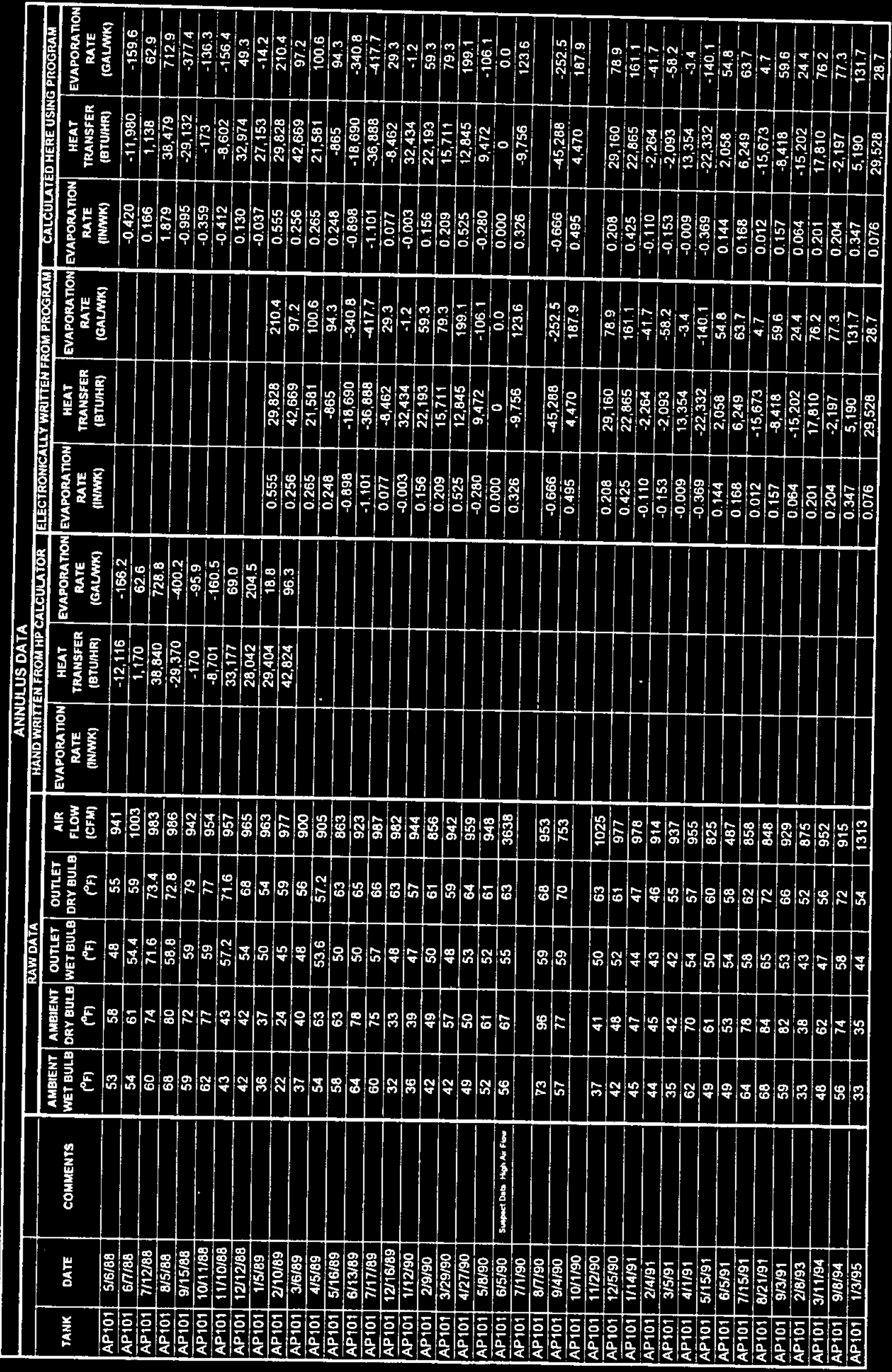


WHC-SD-WM-ER-315, Rev, 0

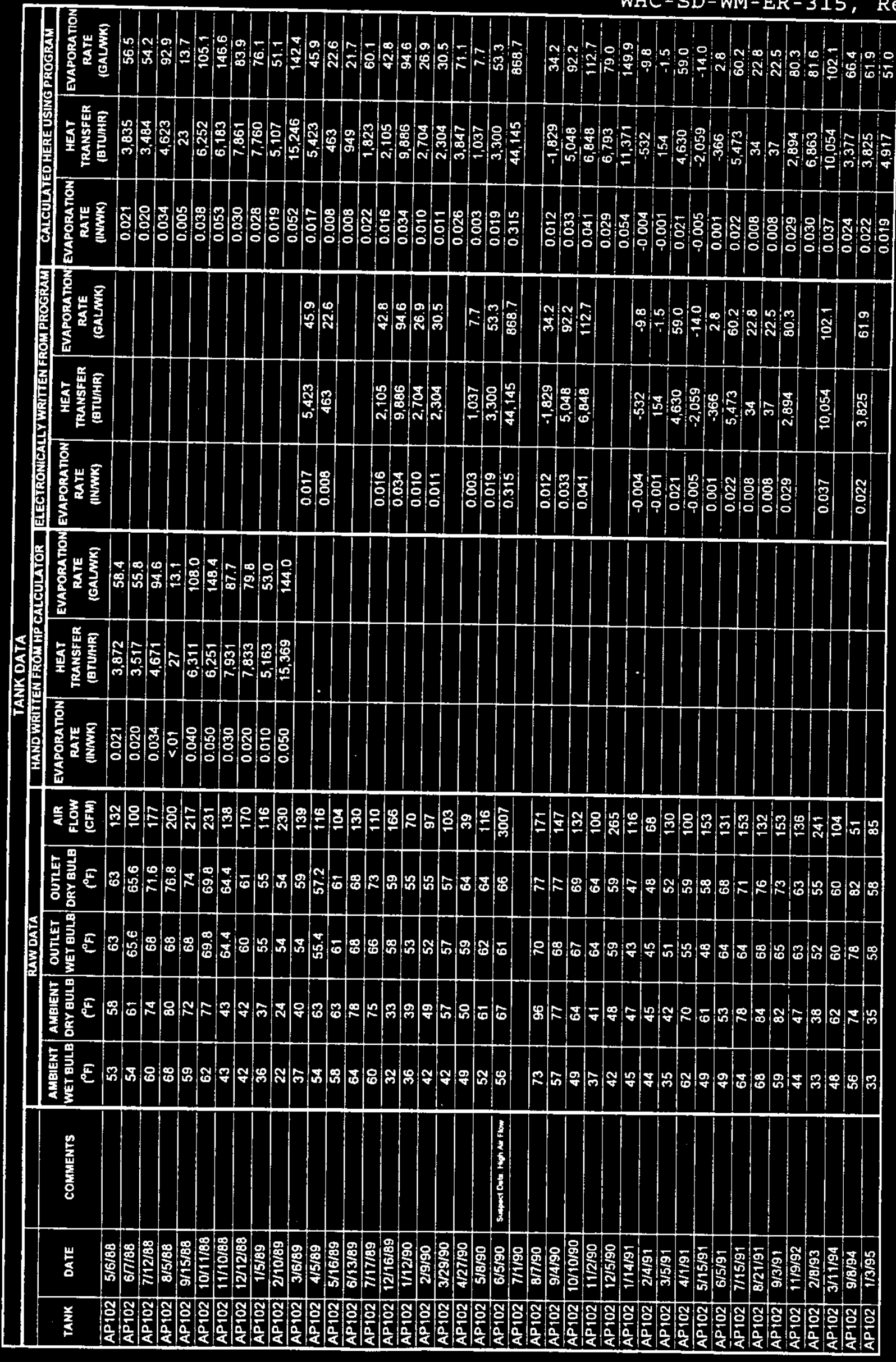


WHC-SD-WM-ER-315, Rev .

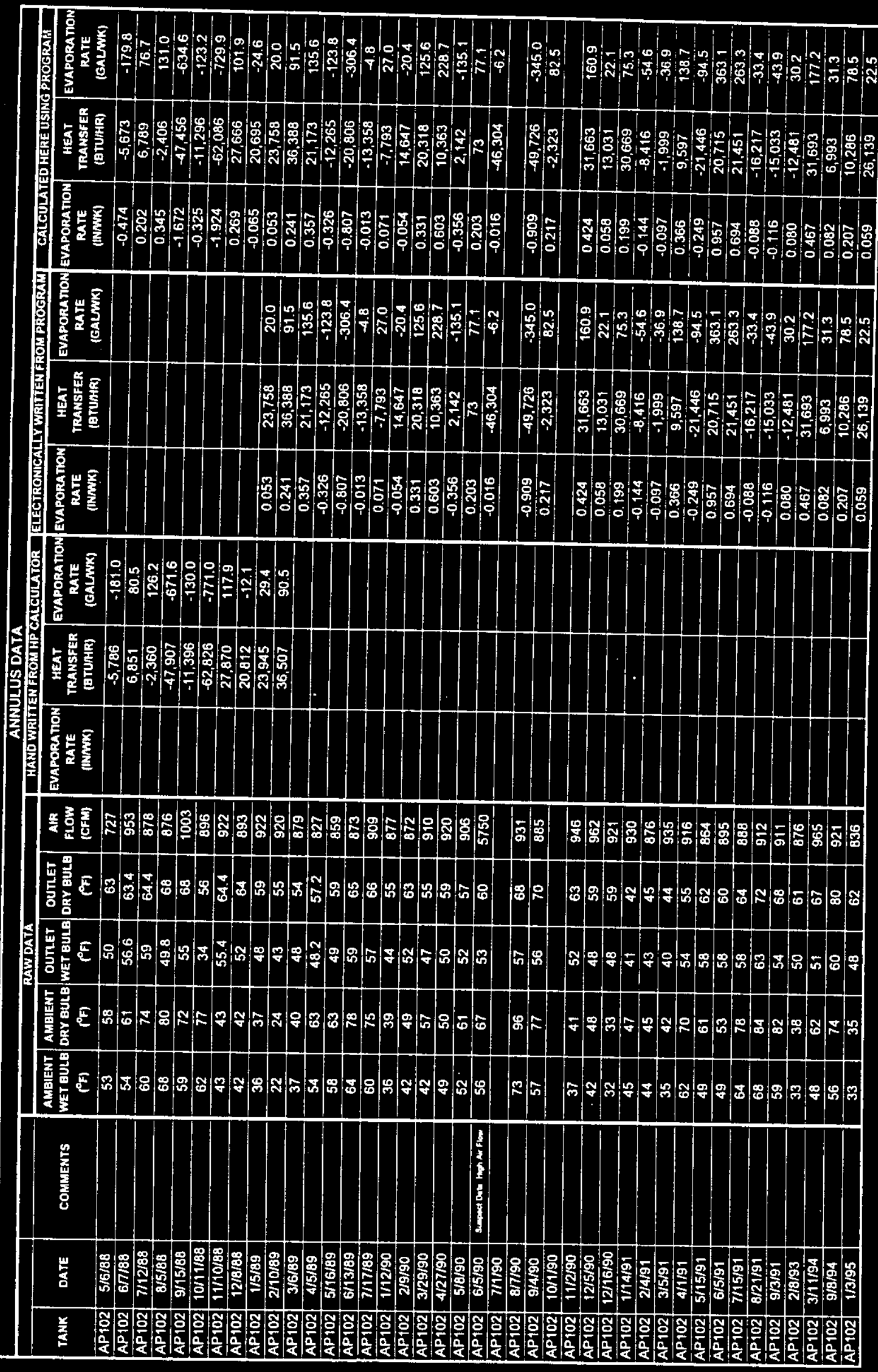


WHC-SD-WM-ER-315, ReV . 0

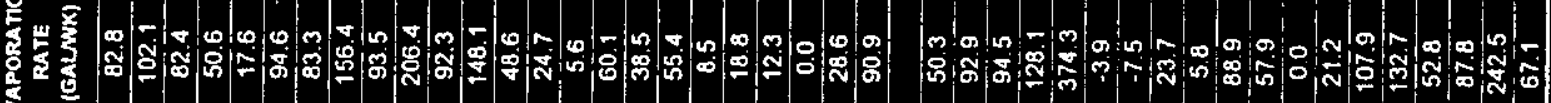

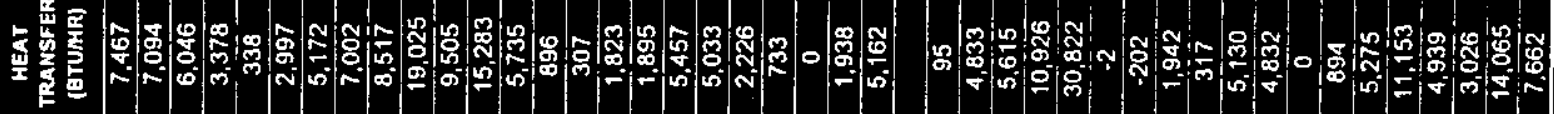
z $\frac{1}{8}$

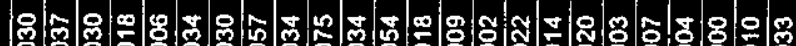
o o o o o

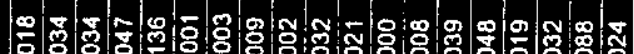

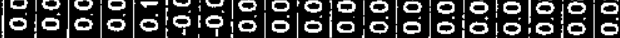

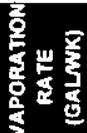

3 (3) n

min

它|:

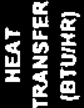

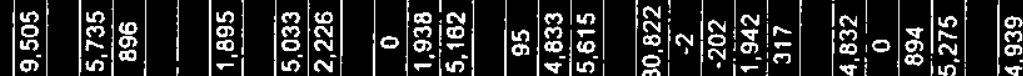

崖寒

$\stackrel{9}{g}$

: 8

융웅

ming

칭용용

通

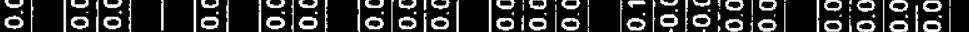

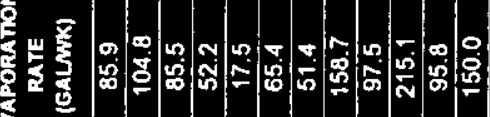

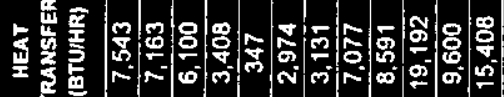

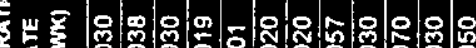

我

茓

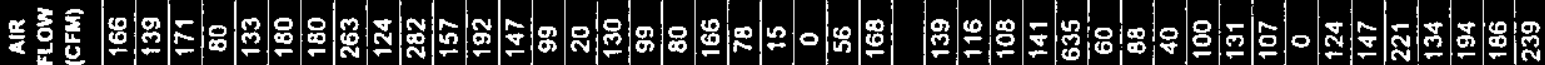

$\stackrel{0}{3}$

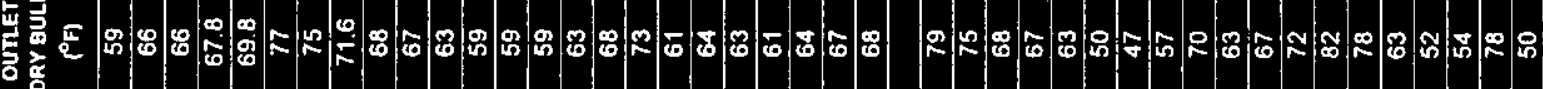

昰

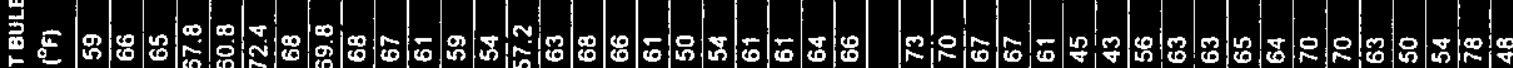

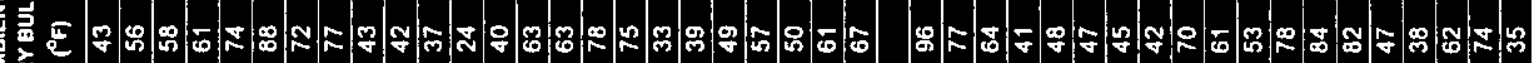
害

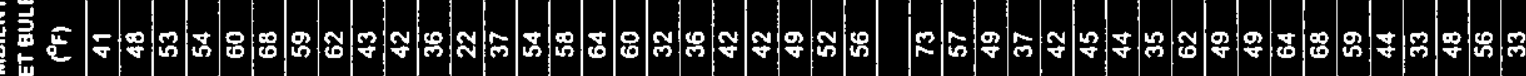

咅

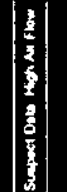

แ

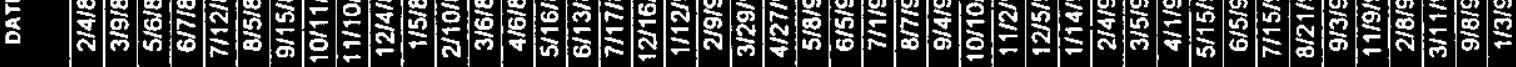

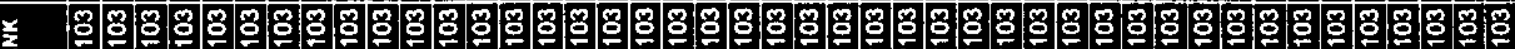

美 
WHC-SD-WM-ER-315, ReV.

送

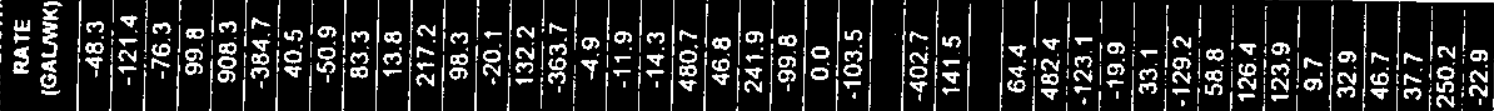

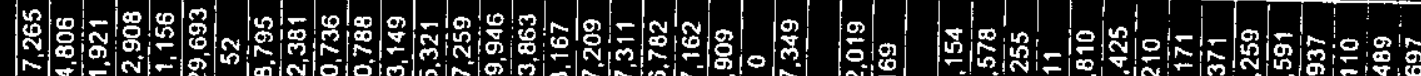

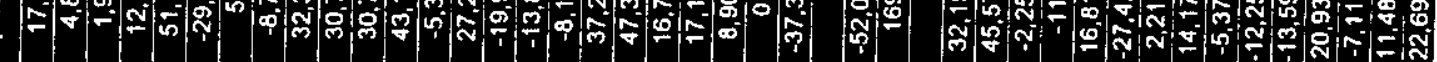
$\bar{z}$

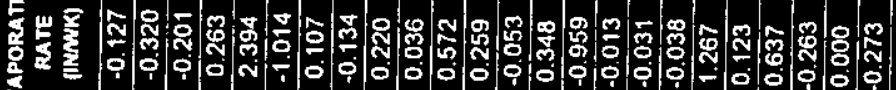

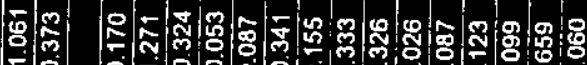

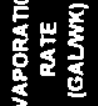

N

- Tos no N Nio in

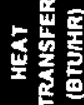

急

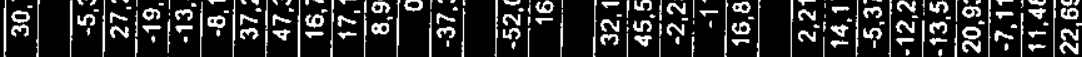

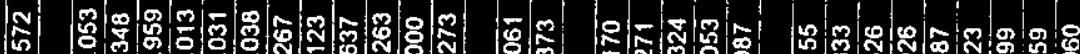

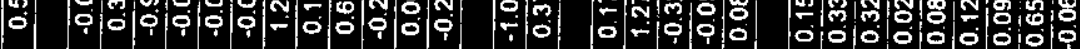

$\frac{5}{2}$

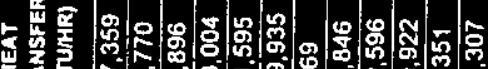

E.

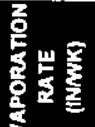

要 要

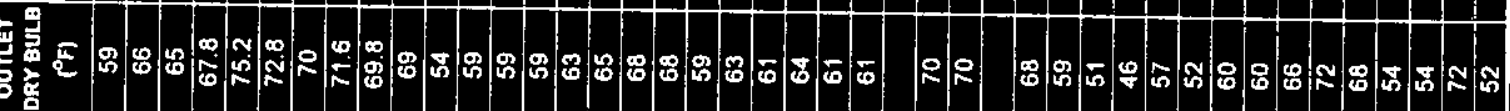

氙点

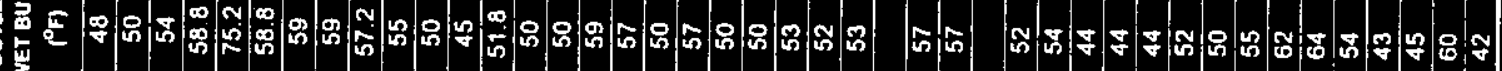

尊

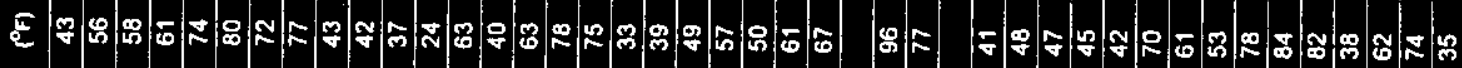

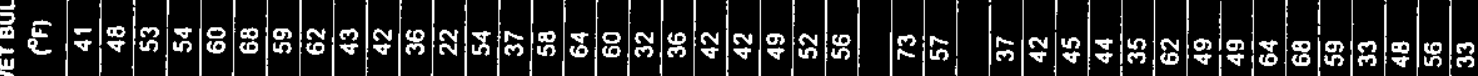

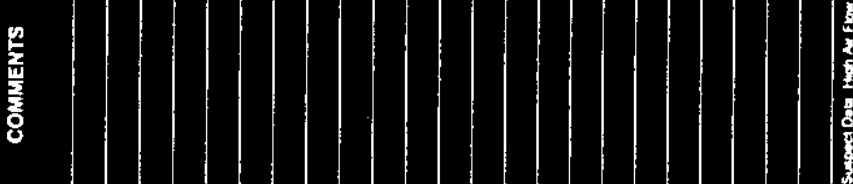

ㄴ.

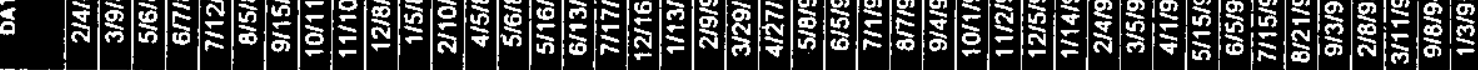

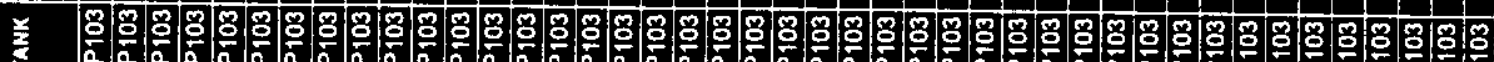

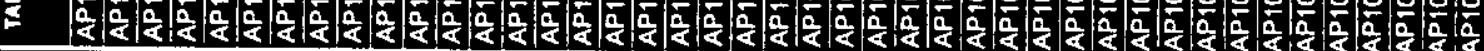


WHC-SD-WM-ER-315, Rev .

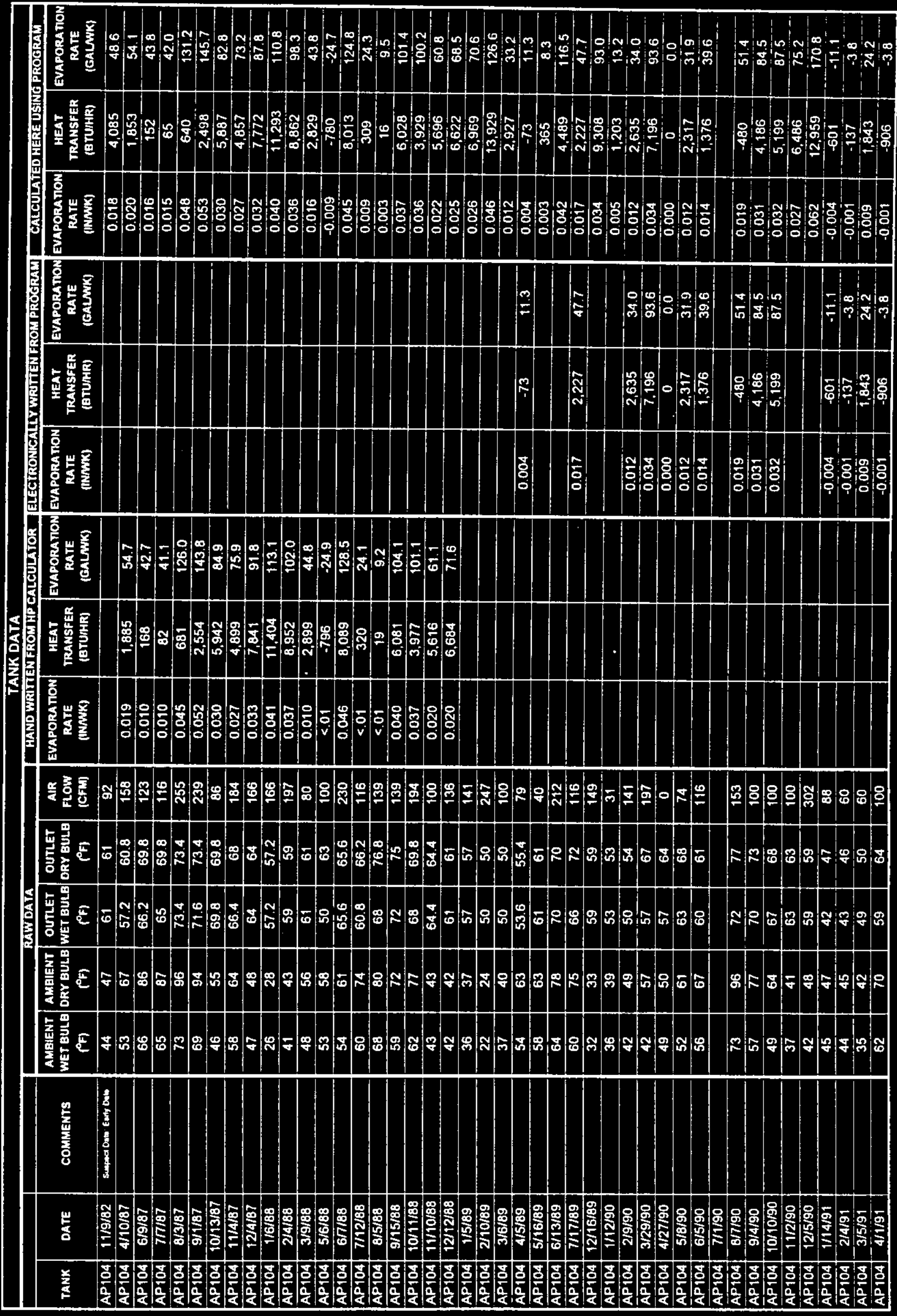




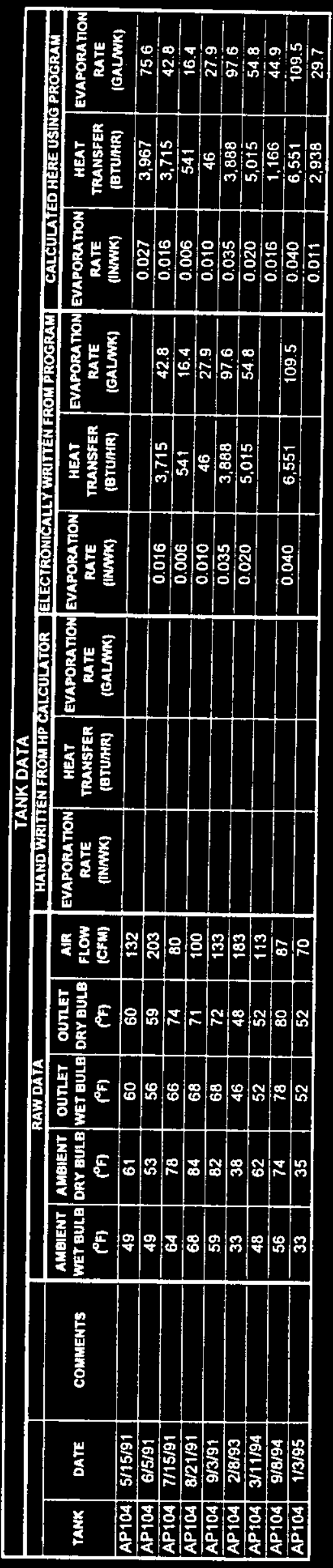

WHC-SD-WM-ER-315, Rev. 0 
WHC-SD-WM-ER-315, Rev .

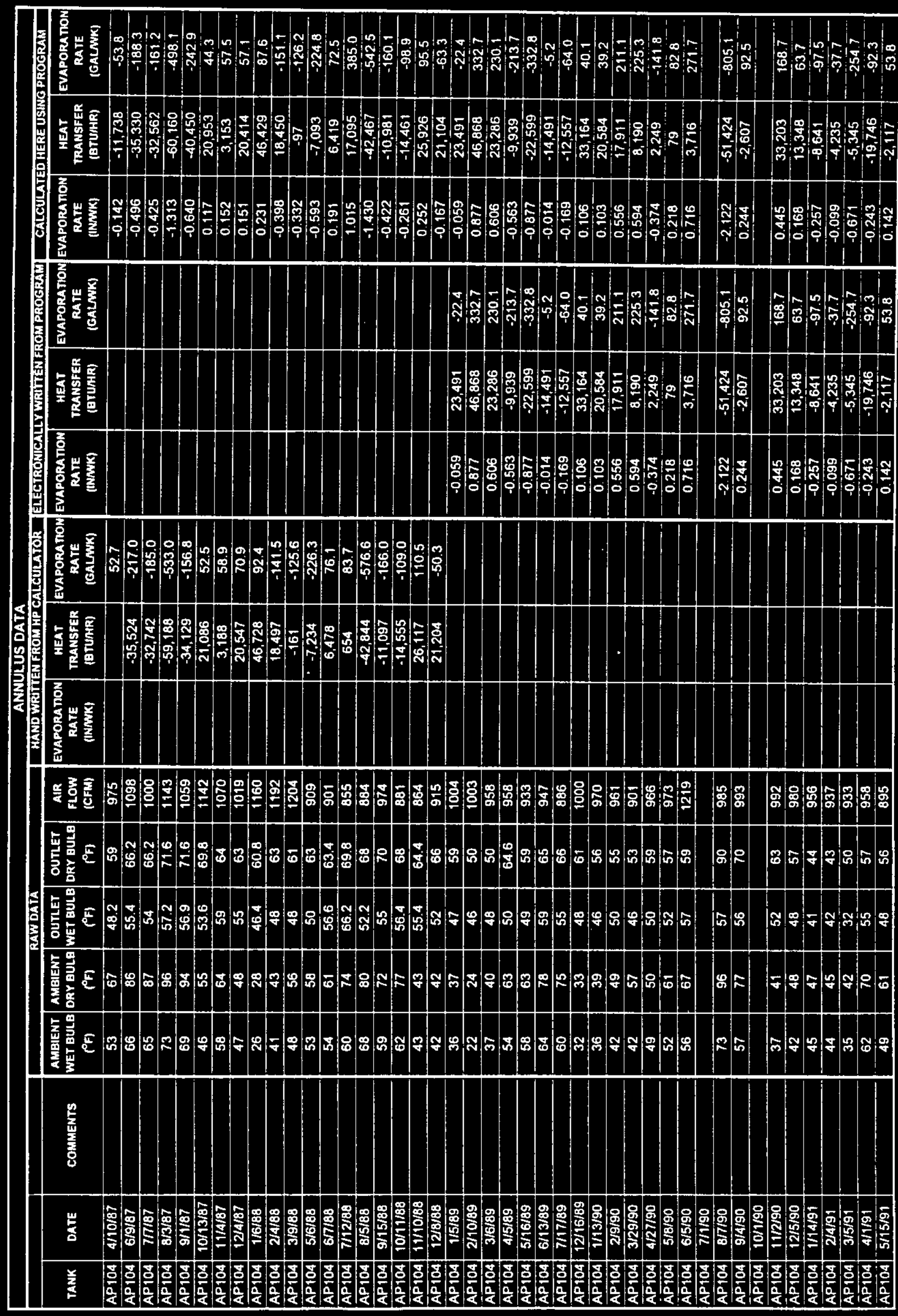




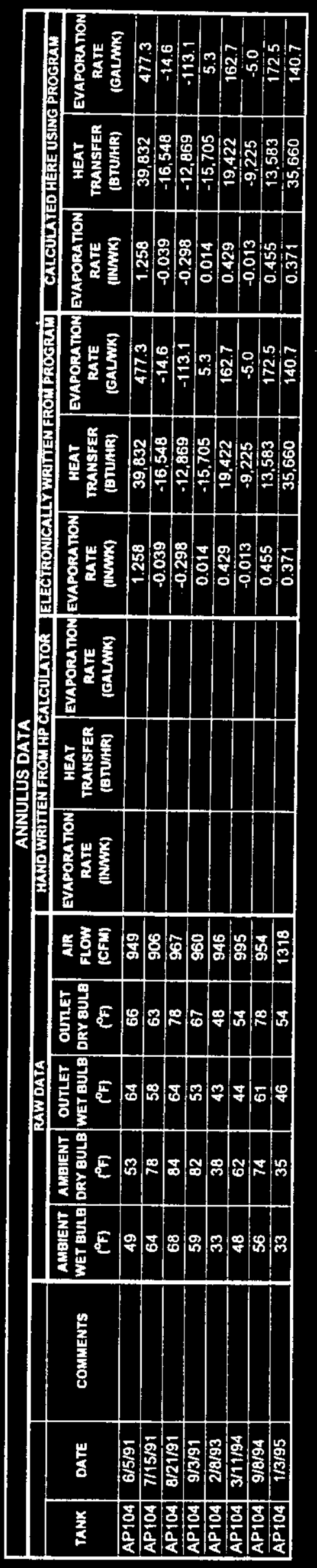

WHC-SD-WM-ER-315, Rev. 0 
WHC-SD-WM-ER-315, Rev.

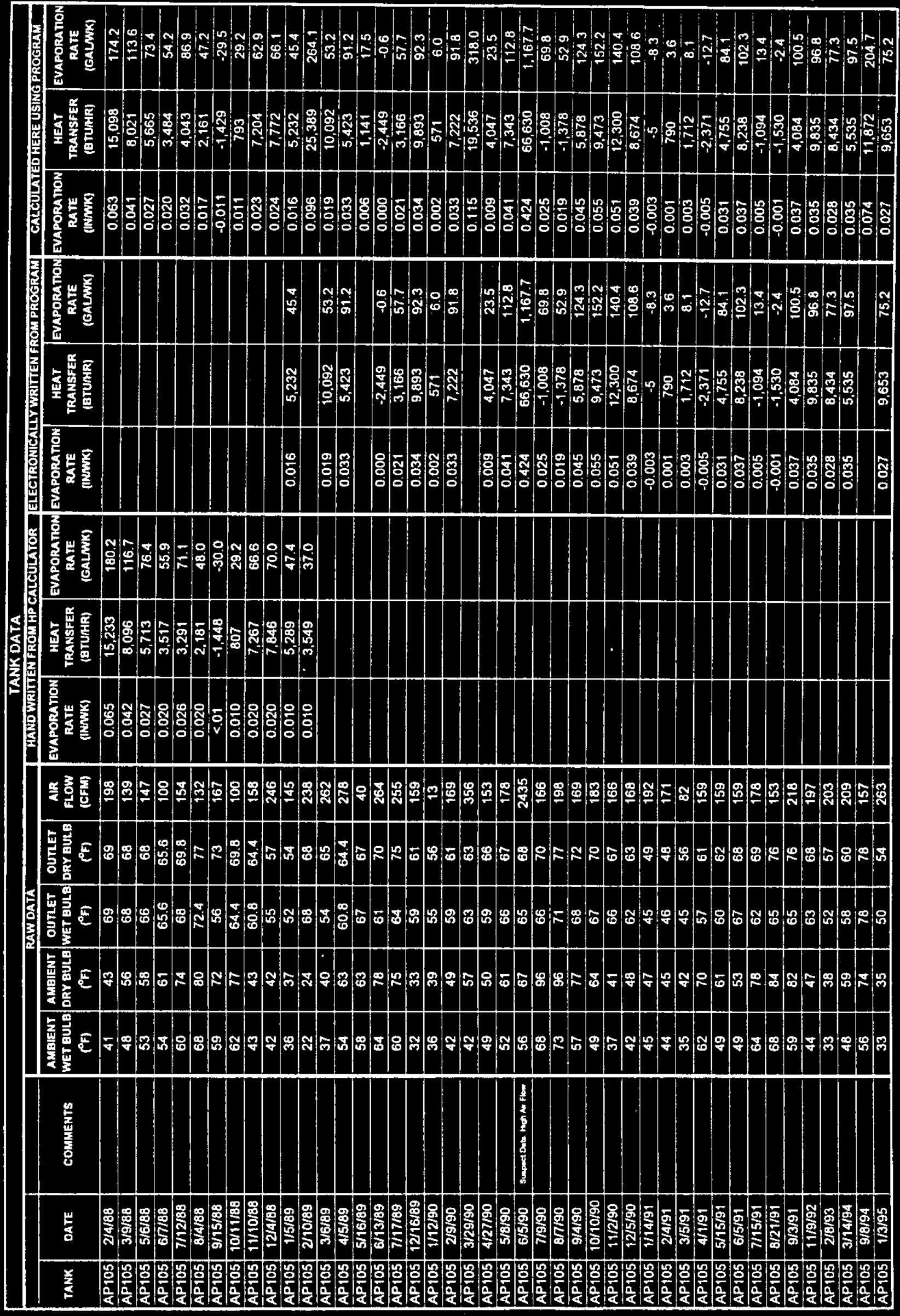


WHC-SD-WM-ER-315, Rev.

.

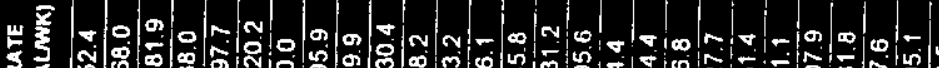

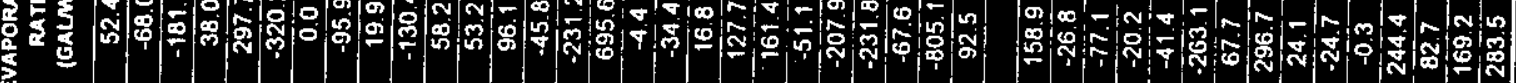

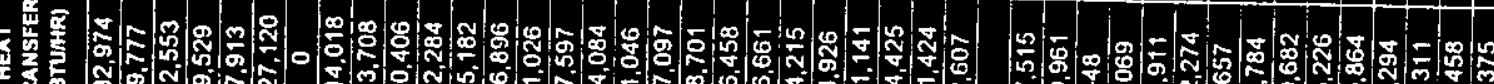

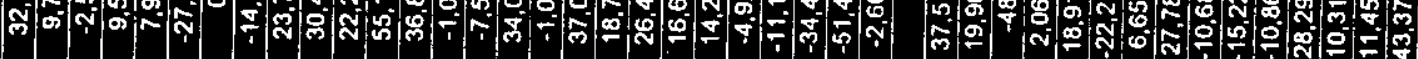

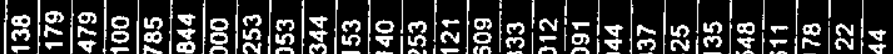

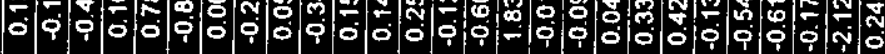

운 i⿱⺈

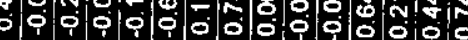

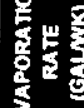

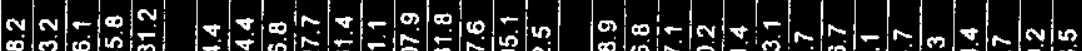

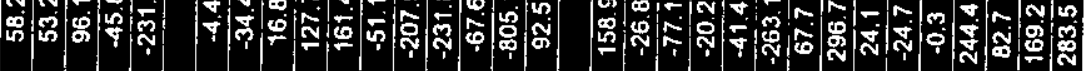

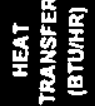

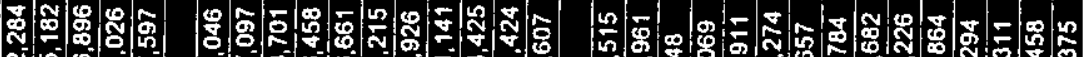

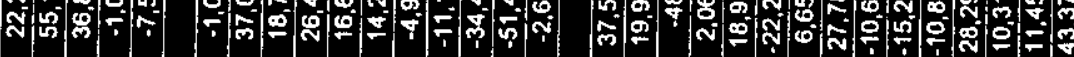

步

竡

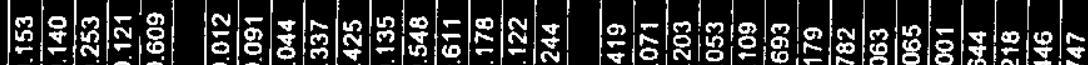

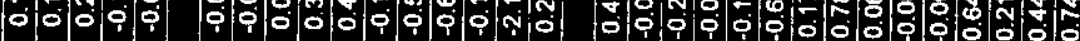

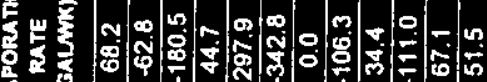

要

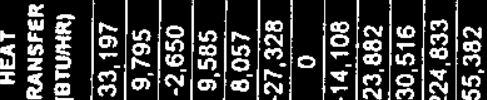

资专

三

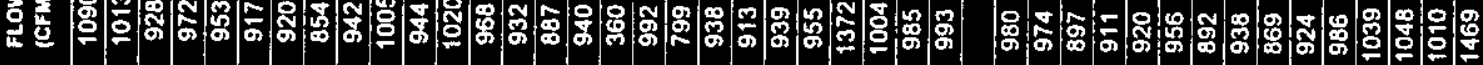

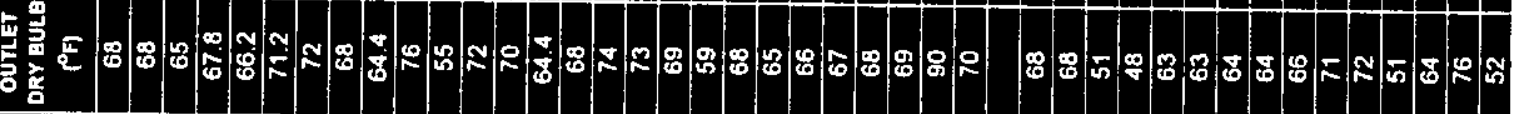

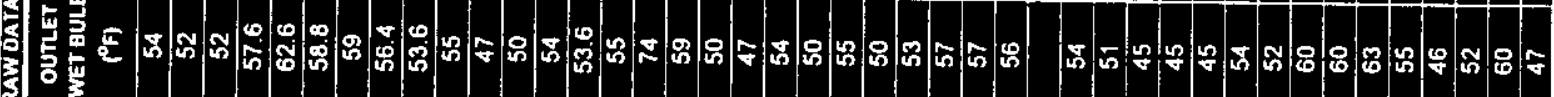

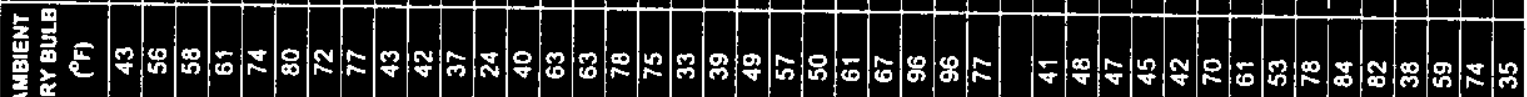
要 要至

홍

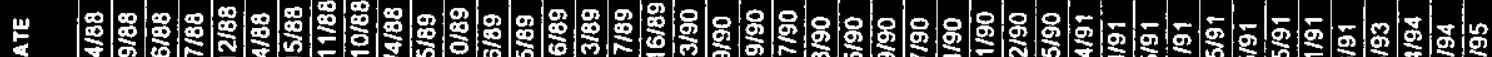
吉

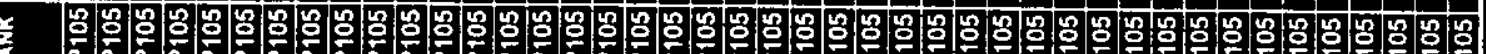

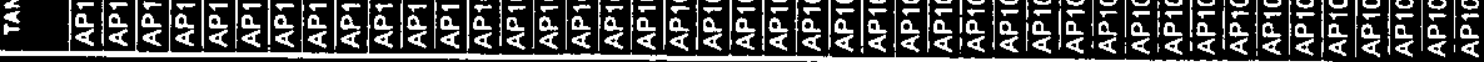


WHC-SD-WM-ER-315, Rev .

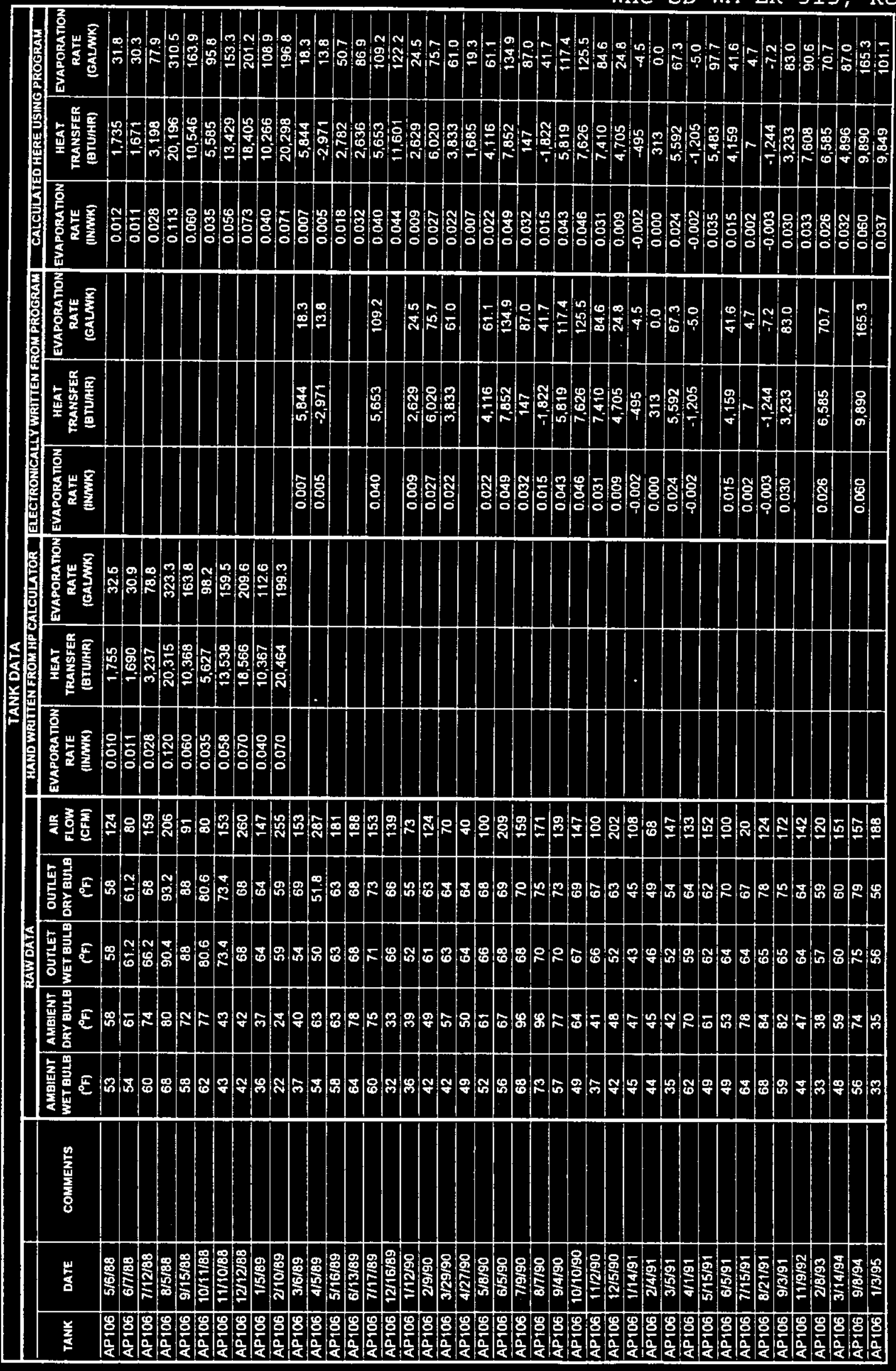


WHC-SD-WM-ER-315, Rev

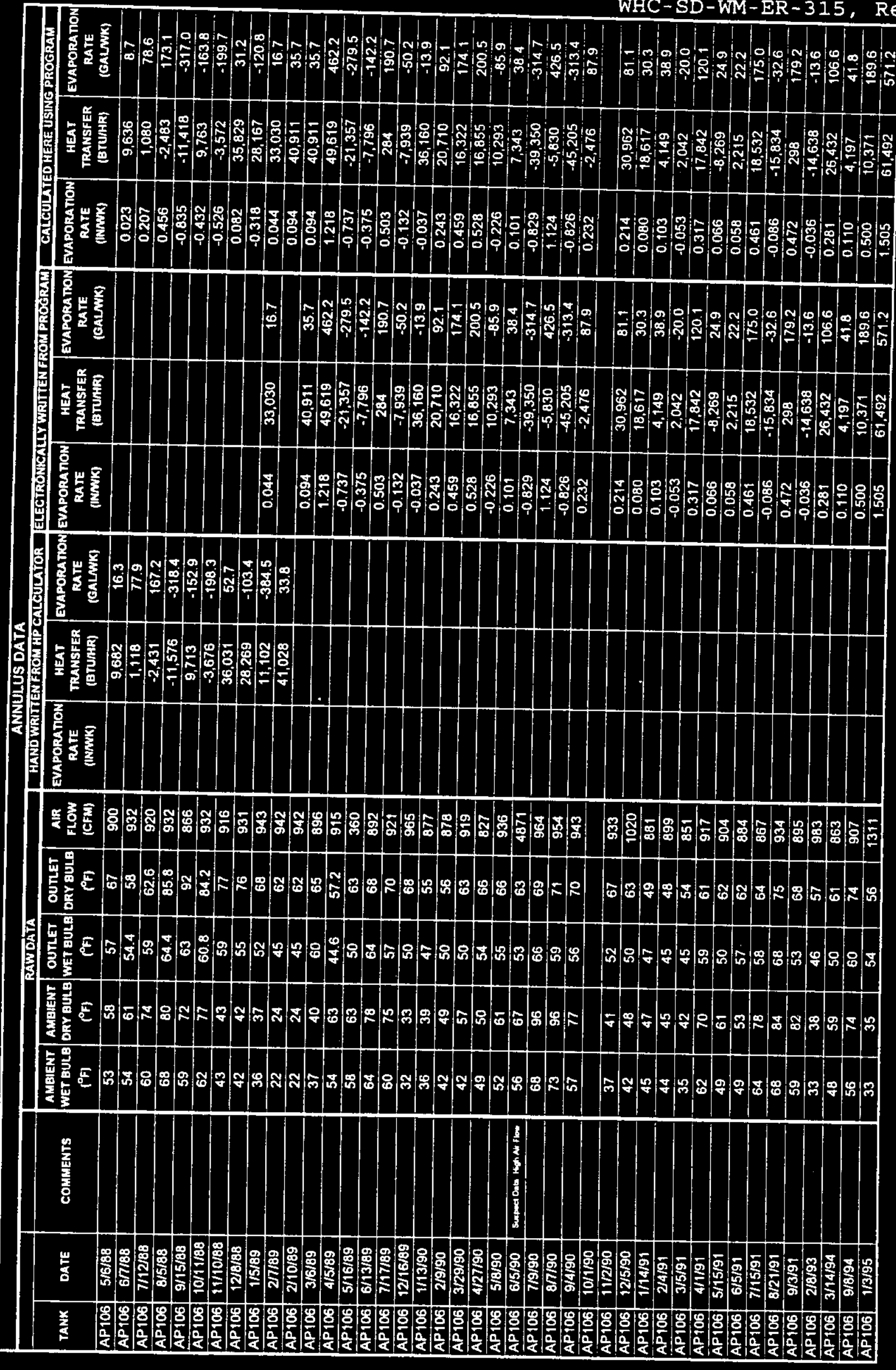


WHC-SD-WM-ER-315, Rev. 0

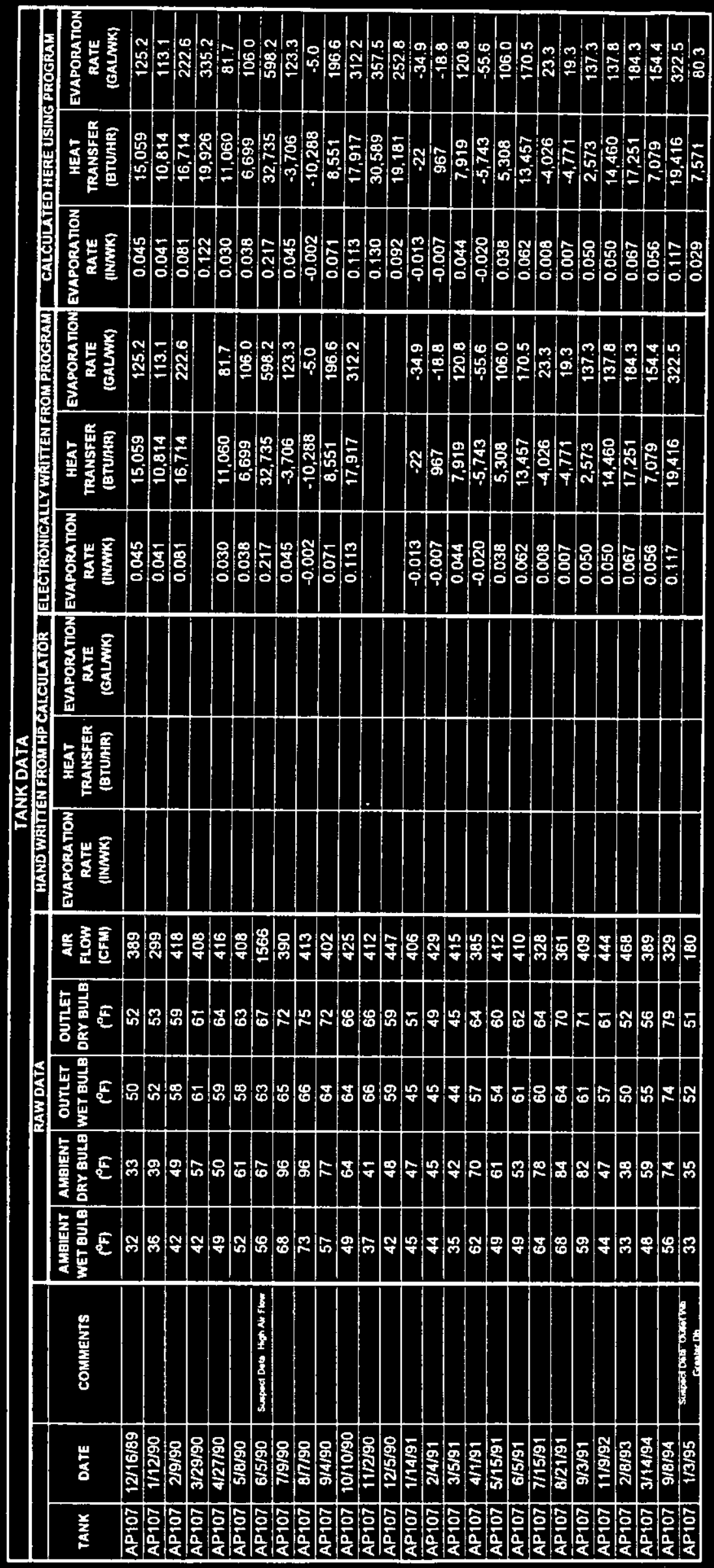


WHC-SD-WM-ER-315, Rev

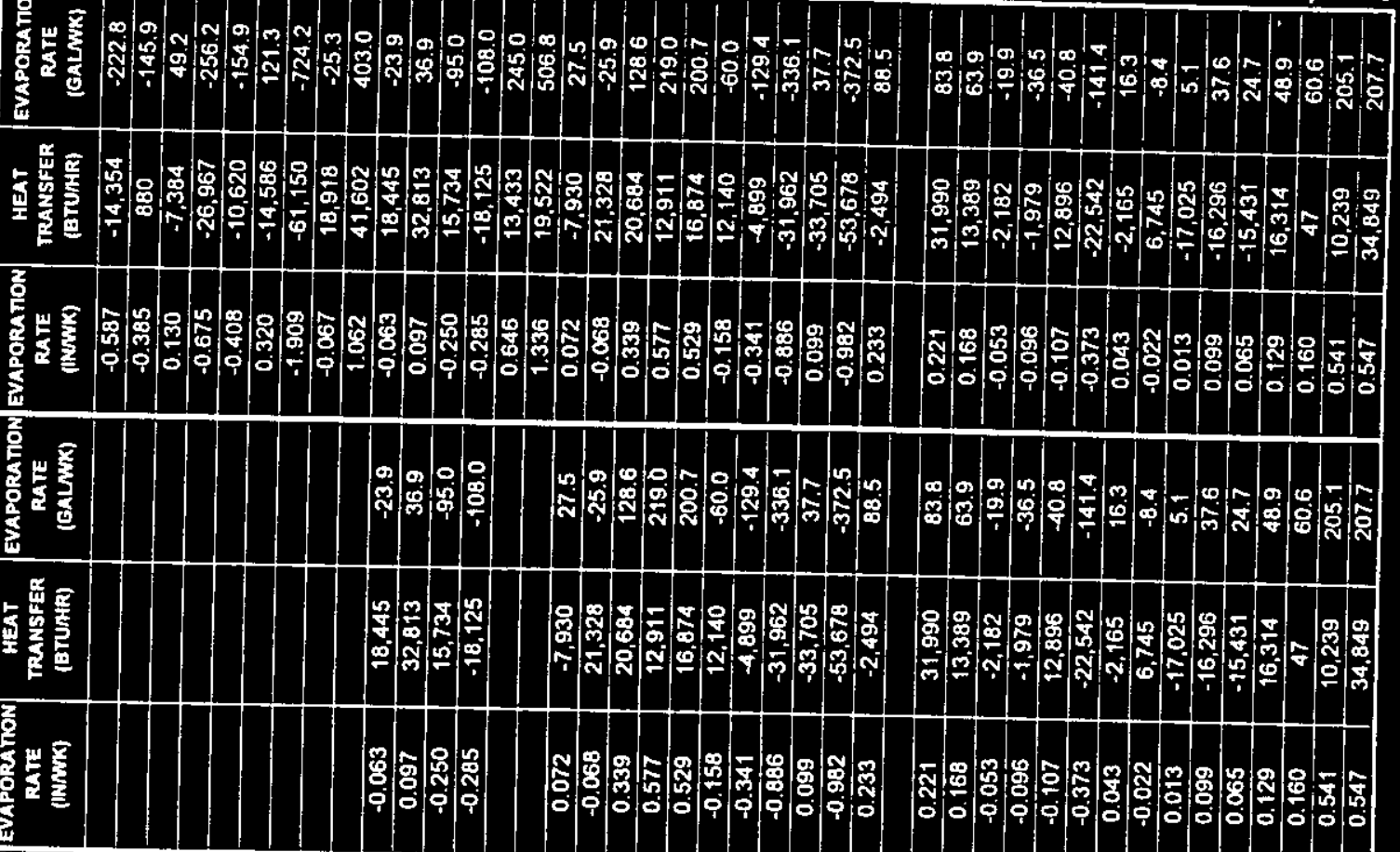
$\frac{0}{2}$ 응

要

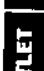

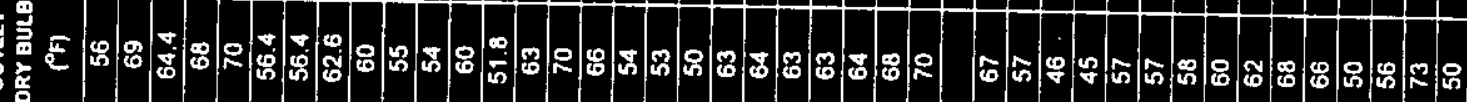

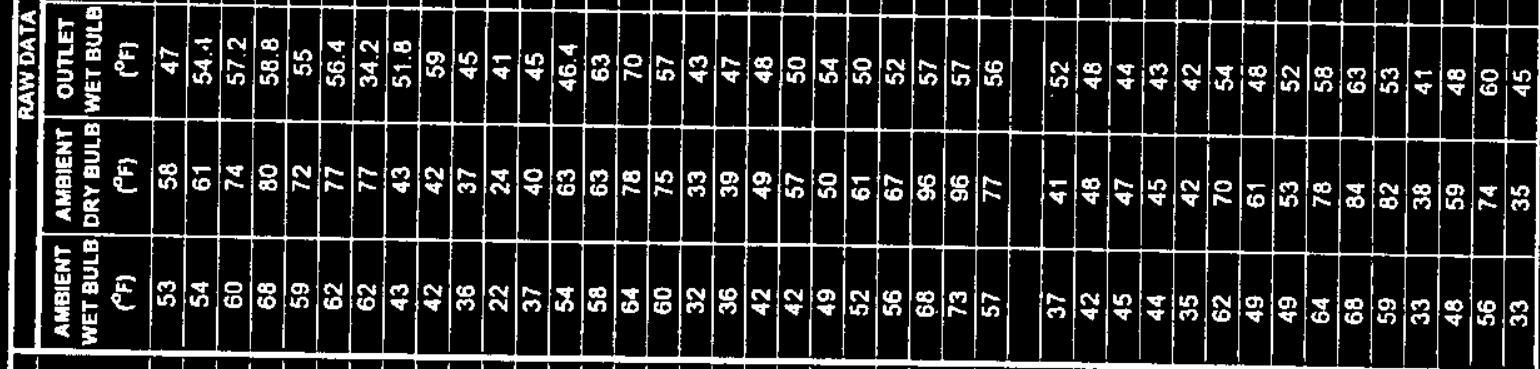
象

山

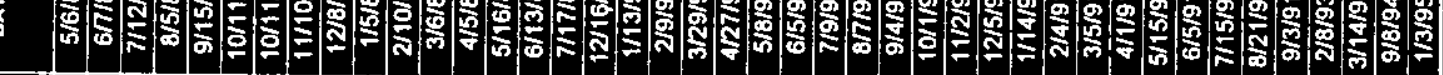

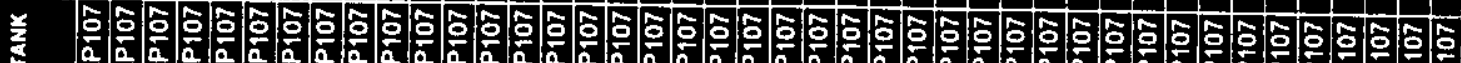

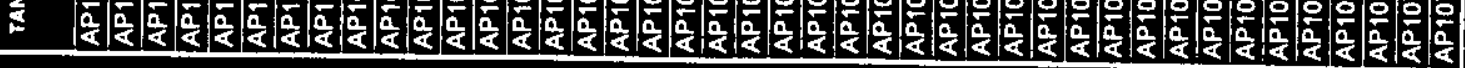


WHC-SD-WM-ER-315，Rev. 0

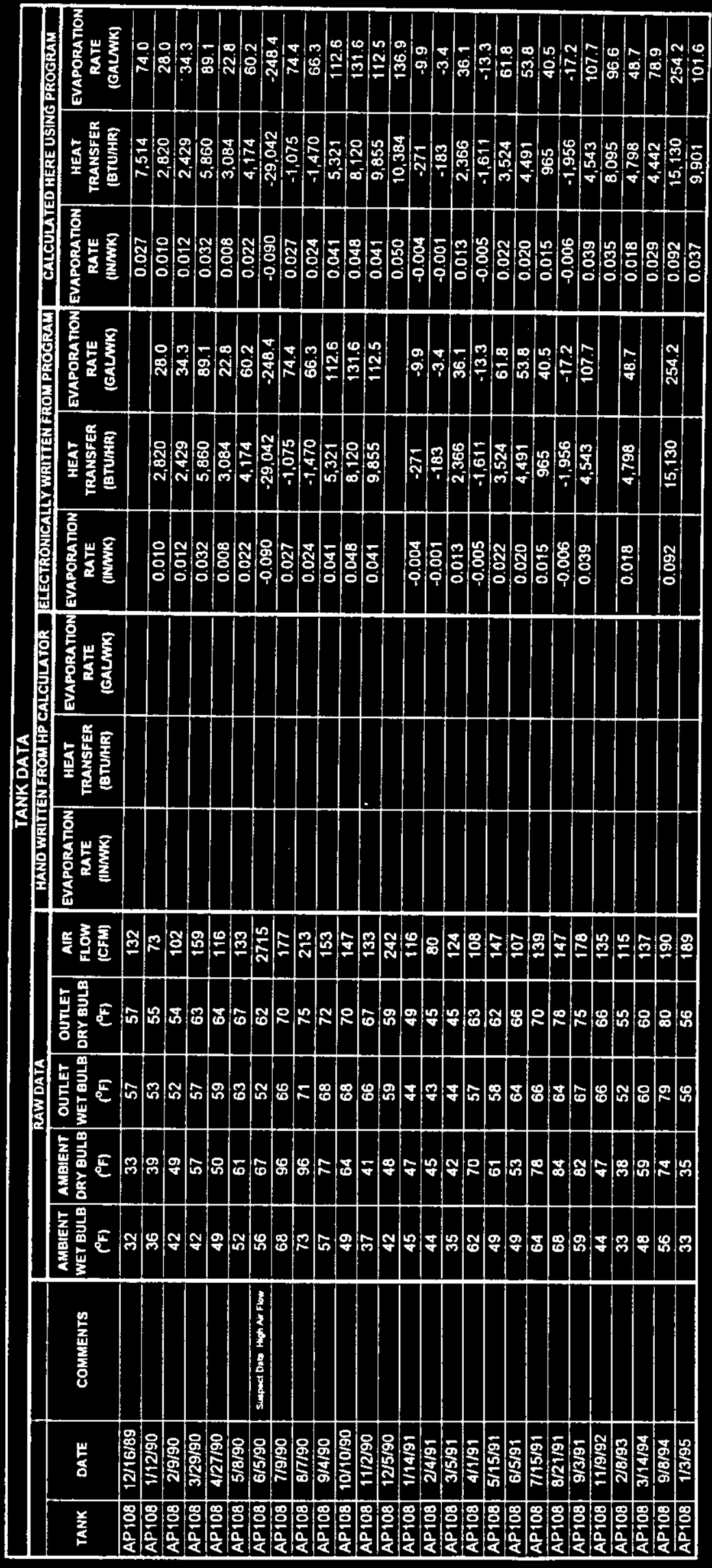


WHC-SD-WM-ER-315, Rev , 0

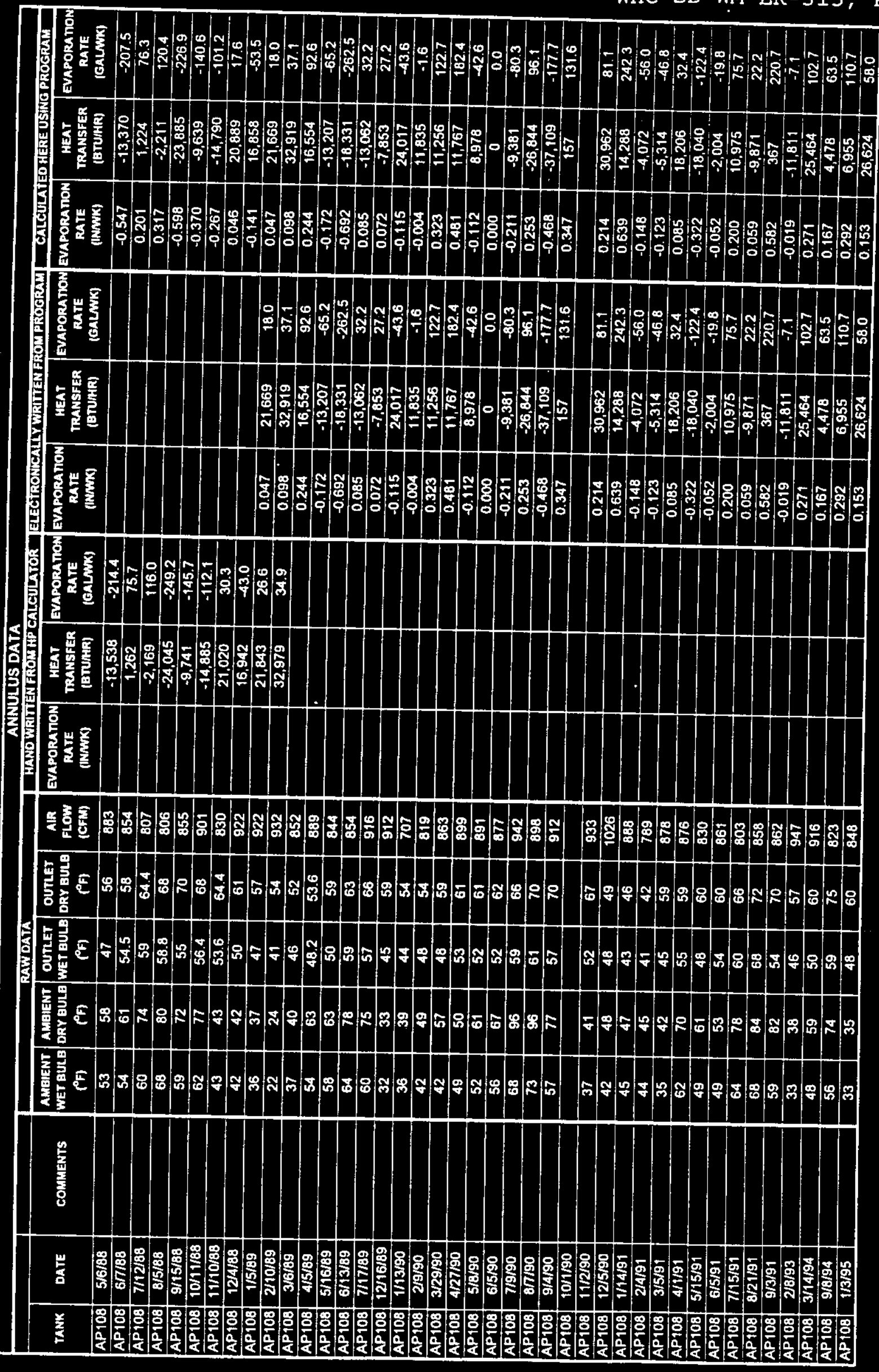




\section{APPENDIX G}

\section{Riser Configuration and Tank Cross Section}




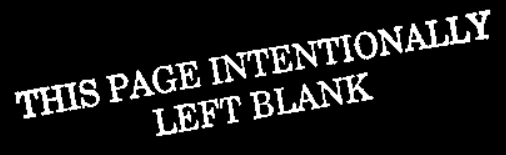




\section{1-AP-101}

WHC-SD-WM-ER-315, Rev. 0

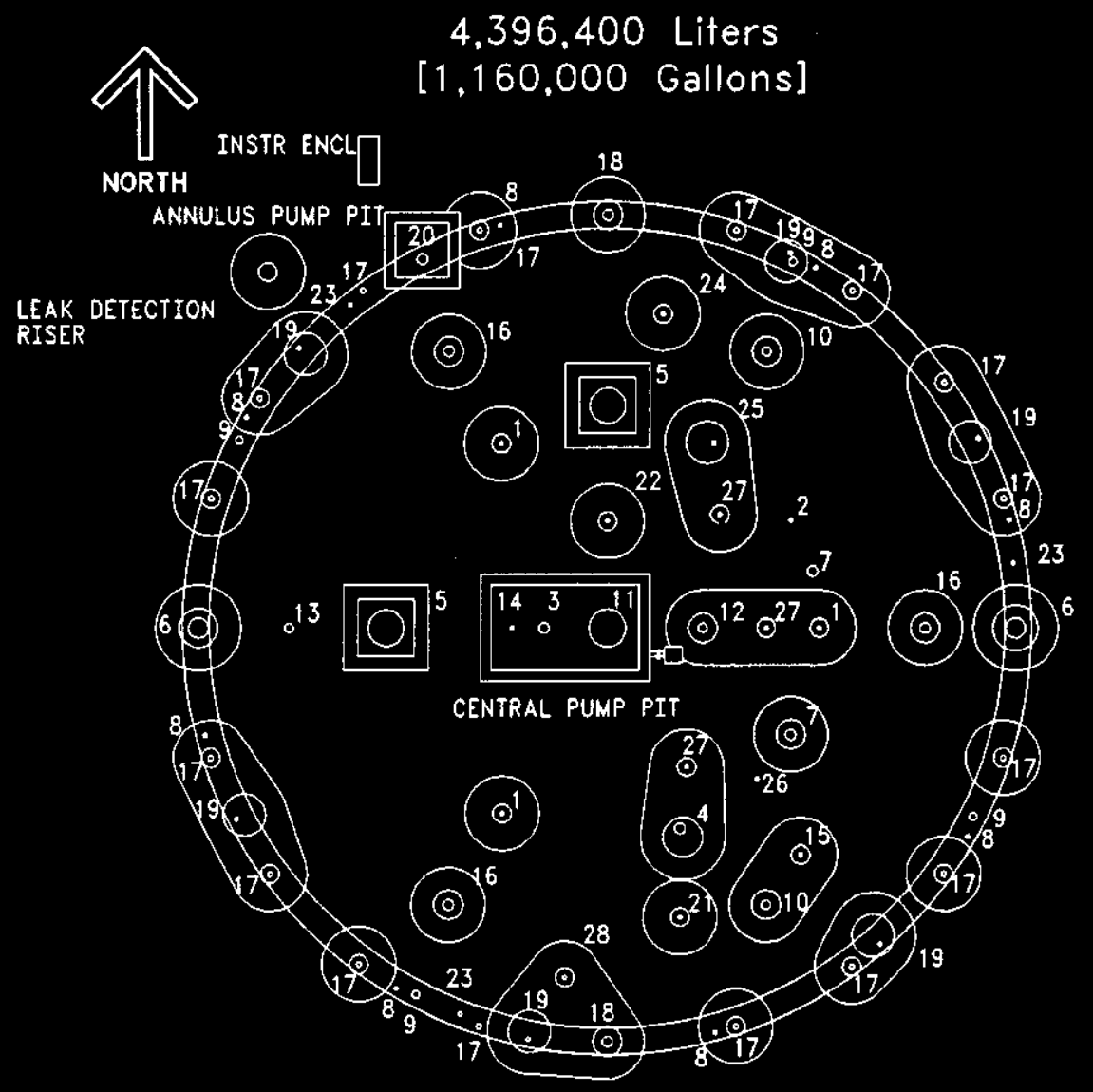

\section{TANK RISER LOCATION}

Ref: WHC-SD-RE-TI-093, Rev.3

$\mathrm{H}-2-90553$, Rev.4

$\mathrm{H}-2-90538$, Rev. 1

If there was a discrepancy between the documents and drawing. the

Approximate Grade Elevation 206.9m [678.81ft]

drawing shall take precedence.

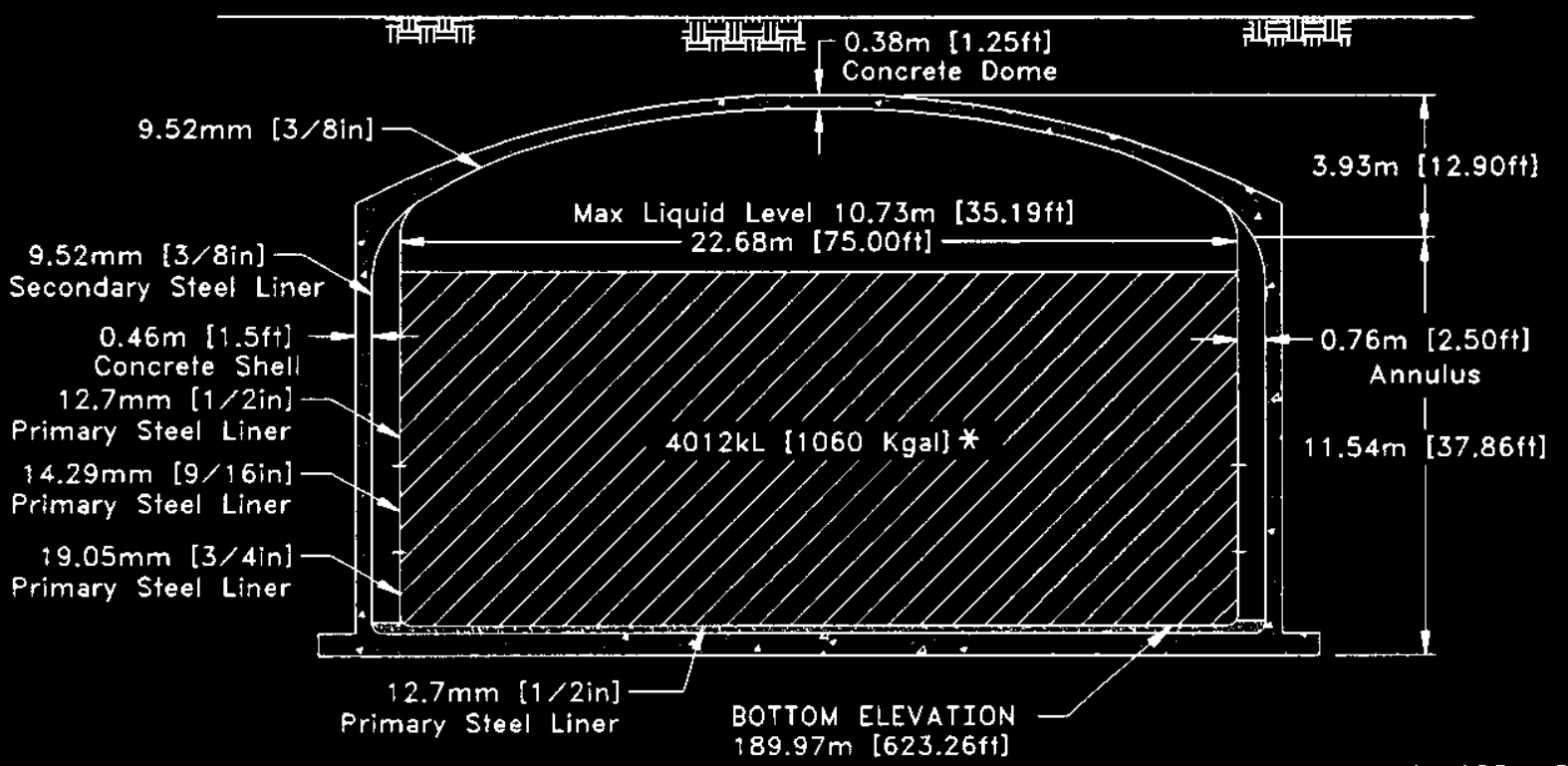

Ref: H-2-90534, Rev, 3

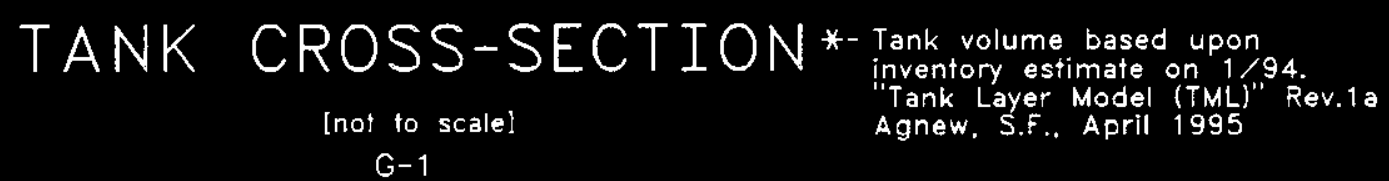




\begin{tabular}{|c|c|c|}
\hline No. & DIA. & DESCRIPTION AND COMMENTS \\
\hline 1 & $4^{\prime \prime}$ & SMP \\
\hline 1 & $4^{\prime \prime}$ & SMP \\
\hline 1 & 4" & SMP \\
\hline 2 & 4" & LIQUID LEVEL, LIT \\
\hline 3 & $12^{n}$ & SUPERNATANT PUMP, CENTRAL PUMP PIT (PIT) \\
\hline 4 & $12^{n}$ & THERMOCOUPLE PROBE \\
\hline 5 & $42^{n}$ & MANHOLE; RISER PLUG \\
\hline 5 & $42^{n}$ & MANHOLE; RISER PLUG \\
\hline 6 & $24 "$ & ANNULUS ACCESS, SPARE \\
\hline 6 & $24 "$ & ANNULUS ACCESS, SPARE \\
\hline 7 & $12^{\prime \prime}$ & SPARE; RISER PLUG \\
\hline 7 & $12^{\prime \prime}$ & PRIMARY TANK EXHAUST \\
\hline 8 & $4^{\prime \prime}$ & ANNULUS AIR INLET \\
\hline 8 & 4" & ANNULUS AIR INLET \\
\hline 8 & $4^{\prime \prime}$ & ANNULUS AIR INLET \\
\hline 8 & 4" & ANNULUS AIR INLET \\
\hline 8 & $4^{n}$ & ANNULUS AIR INLET \\
\hline 8 & 4" & ANNULUS AIR INLET \\
\hline 8 & 4" & ANNULUS AIR INLET \\
\hline 8 & $4^{\prime \prime}$ & ANNULUS AIR INLET \\
\hline 9 & 8" & ANNULUS AIR OUTLET \\
\hline 9 & 8" & ANNULUS AIR OUTLET \\
\hline 9 & 8" & ANNULUS AIR OUTLET \\
\hline 9 & 8" & ANNULUS AIR OUTLET \\
\hline 10 & $12^{\prime \prime}$ & SPARE; RISER PLUG \\
\hline 10 & $12^{\prime \prime}$ & SPARE; RISER PLUG \\
\hline 11 & $42^{\prime \prime}$ & SLURRY DISTRIBUTOR, CENTRAL PUMP PIT (PIT) \\
\hline 12 & $12^{\prime \prime}$ & OBSV PORT, SPARE \\
\hline 13 & $12 "$ & TANK PRESSURE \\
\hline 14 & 4" & SUPERNATANT RETURN \\
\hline 15 & 4" & SPARE; RISER PLUG \\
\hline 16 & $12^{\prime \prime}$ & SMP \\
\hline 16 & $12^{\prime \prime}$ & SMP \\
\hline
\end{tabular}




\begin{tabular}{|c|c|c|}
\hline & & 241-AP-101 \\
\hline No. & DIA. & DESCRIPTION AND COMMENTS \\
\hline 16 & $12^{\prime \prime}$ & SMP \\
\hline 17 & 6" & ANNULUS INSPECTION PORT \\
\hline 17 & 6" & ANNULUS INSPECTION PORT \\
\hline 17 & 6" & ANNULUS INSPECTION PORT \\
\hline 17 & 6" & ANNULUS INSPECTION PORT \\
\hline 17 & 6" & ANNULUS INSPECTION PORT \\
\hline 17 & $6^{n}$ & ANNULUS INSPECTION PORT \\
\hline 17 & 6" & ANNULUS INSPECTION PORT \\
\hline 17 & 6" & ANNULUS INSPECTION PORT \\
\hline 17 & $6^{\prime \prime}$ & ANNULUS INSPECTION PORT \\
\hline 17 & 6" & ANNULUS INSPECTION PORT \\
\hline 17 & $6^{n}$ & ANNULUS INSPECTION PORT \\
\hline 17 & 6" & ANNULUS INSPECTION PORT \\
\hline 17 & 6" & ANNULUS INSPECTION PORT \\
\hline 17 & 6" & ANNULUS INSPECTION PORT \\
\hline 17 & 6" & ANNULUS INSPECTION PORT \\
\hline 17 & 6" & ANNULUS INSPECTION PORT \\
\hline 18 & $12^{\prime \prime}$ & ANNULUS ACCESS \\
\hline 18 & $12^{\prime \prime}$ & ANNULUS ACCESS \\
\hline 19 & 4" & ANNULUS THERMOCOUPLE \\
\hline 19 & $4 "$ & ANNULUS THERMOCOUPLE \\
\hline 19 & $4 "$ & ANNULUS THERMOCOUPLE \\
\hline$\overline{19}$ & 4" & ANNULUS THERMOCOUPLE \\
\hline 19 & 4" & ANNULUS THERMOCOUPLE \\
\hline 19 & 4" & ANNULUS THERMOCOUPLE \\
\hline 20 & $12^{\prime \prime}$ & ANNULUS PUMP, ANNULUS PUMP PIT (PIT) \\
\hline 21 & $4 "$ & SPARE; RISER PLUG \\
\hline 22 & 4" & SMP \\
\hline 23 & 4" & ANNULUS LEAK DETECTOR \\
\hline 23 & 4" & ANNULUS LEAK DETECTOR \\
\hline 23 & 4" & ANNULUS LEAK DETECTOR \\
\hline 24 & $4 "$ & SPARE; RISER PLUG \\
\hline 25 & $4 "$ & HIGH LIQUID LEVEL SENSOR \\
\hline
\end{tabular}


WHC-SD-WM-ER-315， Rev. 0

\begin{tabular}{||c|c|l||}
\hline \multicolumn{2}{|c|}{ 241-AP-101 } \\
\hline \hline NO. & DIA. & \multicolumn{1}{|c|}{ DESCAIPTION AND COMMENTS } \\
\hline 26 & $4^{\prime \prime}$ & LIQUID LEVEL IND \\
\hline 27 & $4^{n}$ & SPARE; RISER PLUG \\
\hline 27 & $4^{\prime \prime}$ & SPARE; RISER PLUG \\
\hline 27 & $4^{\prime \prime}$ & SPARE; RISER PLUG \\
\hline 28 & $4^{\prime \prime}$ & SPARE; RISER PLUG \\
\hline
\end{tabular}

Ref: WHC-SD-RE-TI-093, Rev.3

H-2-90553, Rev.4

H-2-90538, Rev. 1

If there was a discrepancy between the documents and the drawing, the drawing shall take precedence. 


\section{$241-A P-102$}

WHC-SD-WM-ER-315, Rev. 0

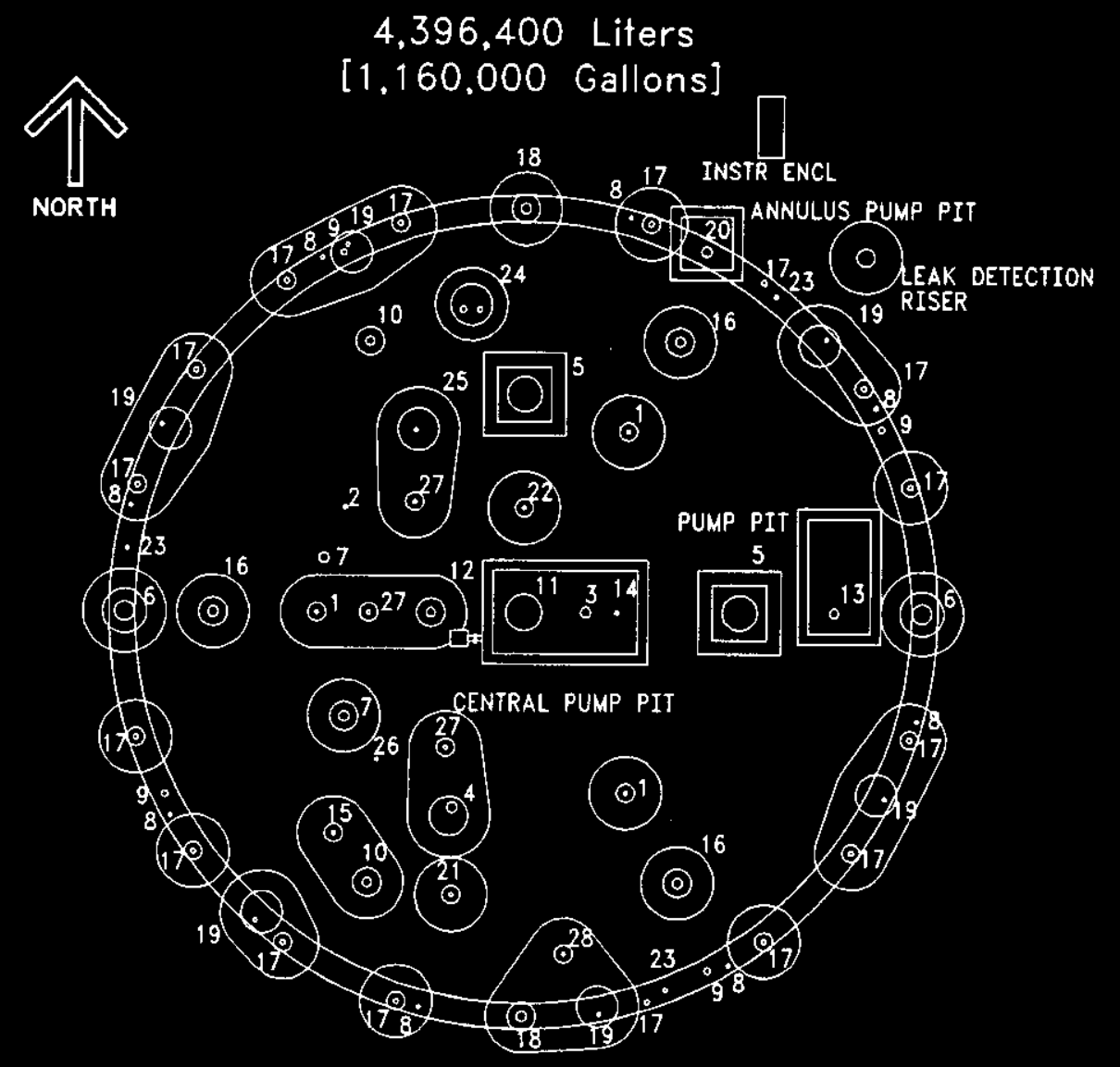

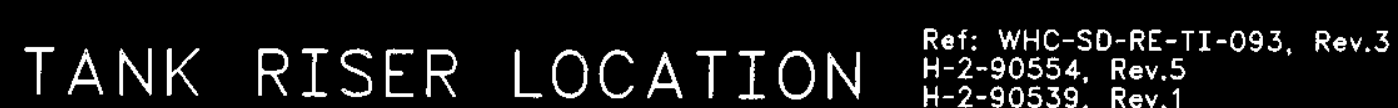
H-2-90539, Rev. 1

If there was a discrepancy between Approximate Grade Elevation $206.9 \mathrm{~m}$ [678.81ft] the documents and drawing. the drawing shall take precedence.

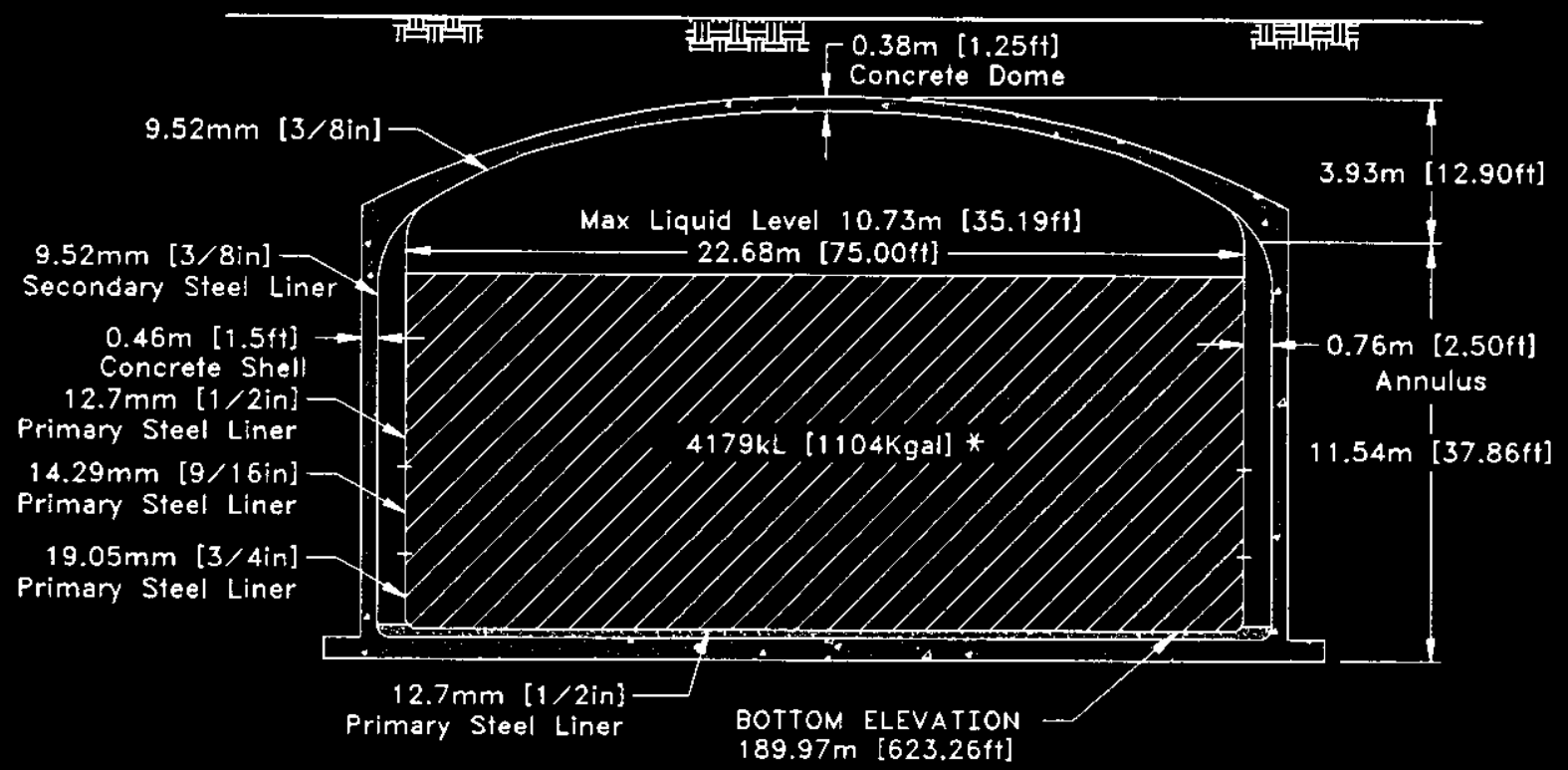

Ref: H-2-90534, Rev.3

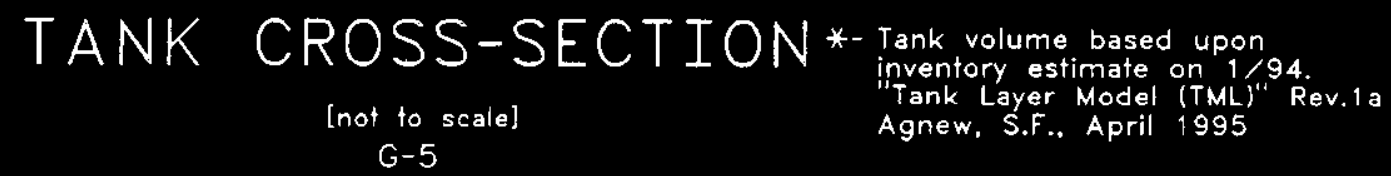




\begin{tabular}{|c|c|c|}
\hline No. & DIA. & DESCRIPTION AND COMMENTS \\
\hline 1 & $4^{\prime \prime}$ & SMP \\
\hline 1 & 4" & SMP \\
\hline 1 & 4" & SMP \\
\hline 2 & $4 "$ & |LIQUID LEVEL, LIT \\
\hline 3 & $12^{\prime \prime}$ & SUPERNATANT PUMP, CENTRAL PUMP PIT (PIT) \\
\hline 4 & $12^{\prime \prime}$ & THERMOCOUPLE PROBE \\
\hline 5 & 42" & MANHOLE; RISER PLUG \\
\hline 5 & $42^{\prime \prime}$ & MANHOLE; RISER PLUG \\
\hline 6 & $24 "$ & ANNULUS ACCESS, SPARE \\
\hline 6 & $24^{\prime \prime}$ & ANNULUS ACCESS, SPARE \\
\hline 7 & $12^{\prime \prime}$ & SPARE; RISER PLUG \\
\hline 7 & $12^{\prime \prime}$ & PRIMARY TANK EXHAUST \\
\hline 8 & 4" & ANNULUS AIR INLET \\
\hline 8 & $4 "$ & ANNULUS AIR INLET \\
\hline 8 & 4" & ANNULUS AIR INLET \\
\hline 8 & 4" & ANNULUS AIR INLET \\
\hline 8 & 4" & ANNULUS AIR INLET \\
\hline 8 & $4^{\prime \prime}$ & ANNULUS AIR INLET \\
\hline 8 & 4" & ANNULUS AIR INLET \\
\hline 8 & 4" & ANNULUS AIR INLET \\
\hline 9 & $8^{n}$ & ANNULUS AIR OUTLET \\
\hline 9 & 8" & ANNULUS AIR OUTLET \\
\hline 9 & 8" & ANNULUS AIR OUTLET \\
\hline 9 & 8" & ANNULUS AIR OUTLET \\
\hline 10 & $12^{\prime \prime}$ & SPARE; RISER PLUG \\
\hline 10 & $12^{\prime \prime}$ & TANK PRESSURE \\
\hline 11 & $42^{\prime \prime}$ & SLURRY DISTRIBUTOR, CENTRAL PUMP PIT (PIT) \\
\hline 12 & $12^{\prime \prime}$ & OBSV PORT, SPARE \\
\hline 13 & $12^{\prime \prime}$ & |GROUT FEED PUMP \\
\hline 14 & 4" & SUPERNATANT RETURN \\
\hline 15 & 4" & SPARE; RISER PLUG \\
\hline 16 & $12^{\prime \prime}$ & SMP \\
\hline 16 & $12^{\prime \prime}$ & SMP \\
\hline
\end{tabular}




\begin{tabular}{|c|c|c|}
\hline NO. & DIA. & DESCRIPTION AND COMMENTS \\
\hline 16 & $12^{\prime \prime}$ & SMP \\
\hline 17 & $6^{\prime \prime}$ & ANNULUS INSPECTION PORT \\
\hline 17 & $6^{\prime \prime}$ & ANNULUS INSPECTION PORT \\
\hline 17 & $6^{\prime \prime}$ & ANNULUS INSPECTION PORT \\
\hline 17 & $6^{n}$ & ANNULUS INSPECTION PORT \\
\hline 17 & $6^{\prime \prime}$ & ANNULUS INSPECTION PORT \\
\hline 17 & $6^{\prime \prime}$ & ANNULUS INSPECTION PORT \\
\hline 17 & $6^{\prime \prime}$ & ANNULUS INSPECTION PORT \\
\hline 17 & $6^{\prime \prime}$ & ANNULUS INSPECTION PORT \\
\hline 17 & $6^{n}$ & ANNULUS INSPECTION PORT \\
\hline 17 & $6^{n}$ & ANNULUS INSPECTION PORT \\
\hline 17 & 6" & ANNULUS INSPECTION PORT \\
\hline 17 & 6" & ANNULUS INSPECTION PORT \\
\hline 17 & 6" & ANNULUS INSPECTION PORT \\
\hline 17 & 6" & ANNULUS INSPECTION PORT \\
\hline 17 & 6" & ANNULUS INSPECTION PORT \\
\hline 17 & 6" & ANNULUS INSPECTION PORT \\
\hline 18 & $12^{\prime \prime}$ & ANNULUS ACCESS \\
\hline 18 & $12^{\prime \prime}$ & ANNULUS ACCESS \\
\hline 19 & 4" & ANNULUS THERMOCOUPLE \\
\hline 19 & 4" & ANNULUS THERMOCOUPLE \\
\hline 19 & 4" & ANNULUS THERMOCOUPLE \\
\hline 19 & 4" & ANNULUS THERMOCOUPLE \\
\hline 19 & 4" & ANNULUS THERMOCOUPLE \\
\hline 19 & 4" & ANNULUS THERMOCOUPLE \\
\hline 20 & $12^{\prime \prime}$ & ANNULUS PUMP, ANNULUS PUMP PIT (PIT) \\
\hline 21 & 4" & SPARE; RISER PLUG \\
\hline 22 & 4" & SMP \\
\hline 23 & 4" & ANNULUS LEAK DETECTOR \\
\hline 23 & 4" & ANNULUS LEAK DETECTOR \\
\hline 23 & $4^{\prime \prime}$ & ANNULUS LEAK DETECTOR \\
\hline 24 & 4" & SPARE; RISER PLUG \\
\hline 25 & 4" & HIGH LIQUID LEVEL SENSOR \\
\hline
\end{tabular}




\begin{tabular}{|c|c|l||}
\hline \multicolumn{2}{|c|}{$241-A P-102$} \\
\hline \hline NO. & DIA. & \multicolumn{1}{|c|}{ DESCRIPTION AND COMMENTS } \\
\hline 26 & $4^{\prime \prime}$ & LIQUID LEVEL IND \\
\hline 27 & $4^{\prime \prime}$ & SPARE; RISER PLUG \\
\hline 27 & $4^{\prime \prime}$ & SPARE; RISER PLUG \\
\hline 27 & $4^{\prime \prime}$ & SPARE; RISER PLUG \\
\hline 28 & $4^{\prime \prime}$ & SPARE; RISER PLUG \\
\hline
\end{tabular}

Ref: WHC-SD-RE-TI-093, Rev.3

H-2-90554, Rev.5

H-2-90539, Rev. 1

If there was a discrepancy between the documents and the drawing, the drawing shall take precedence. 


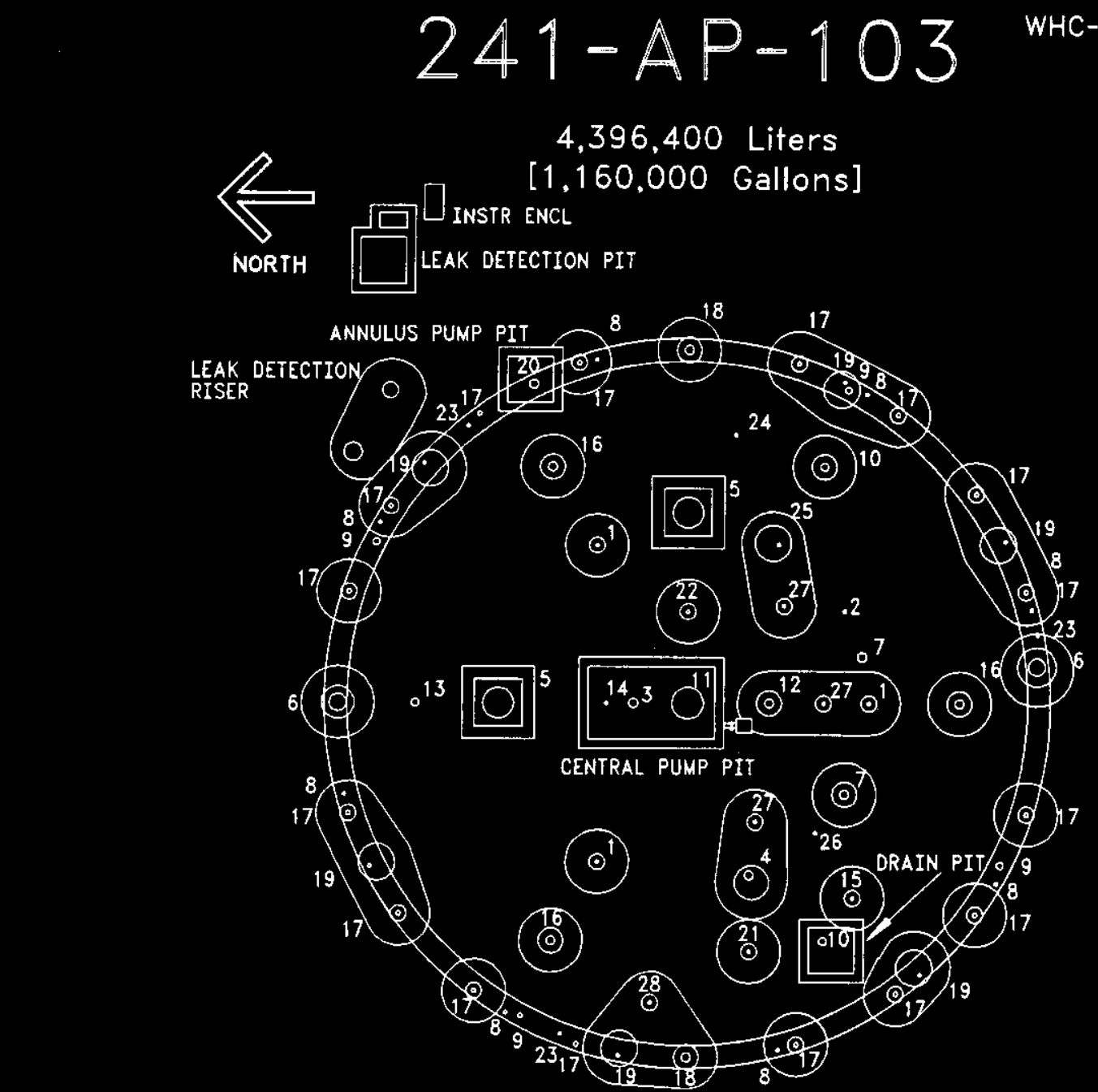

Ref: WHC-SD-RE-TI-093, Rev.3 H-2-90555, Rev.4

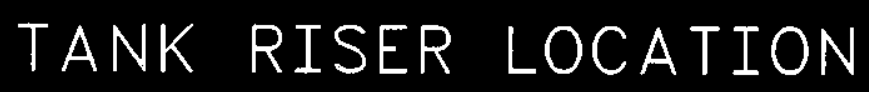
H-2-90538, Rev. 1

Approximate Grade Elevation 206.9m [678.81ft]

If there was a discrepancy between the documents and drawing. the drawing shall take precedence.

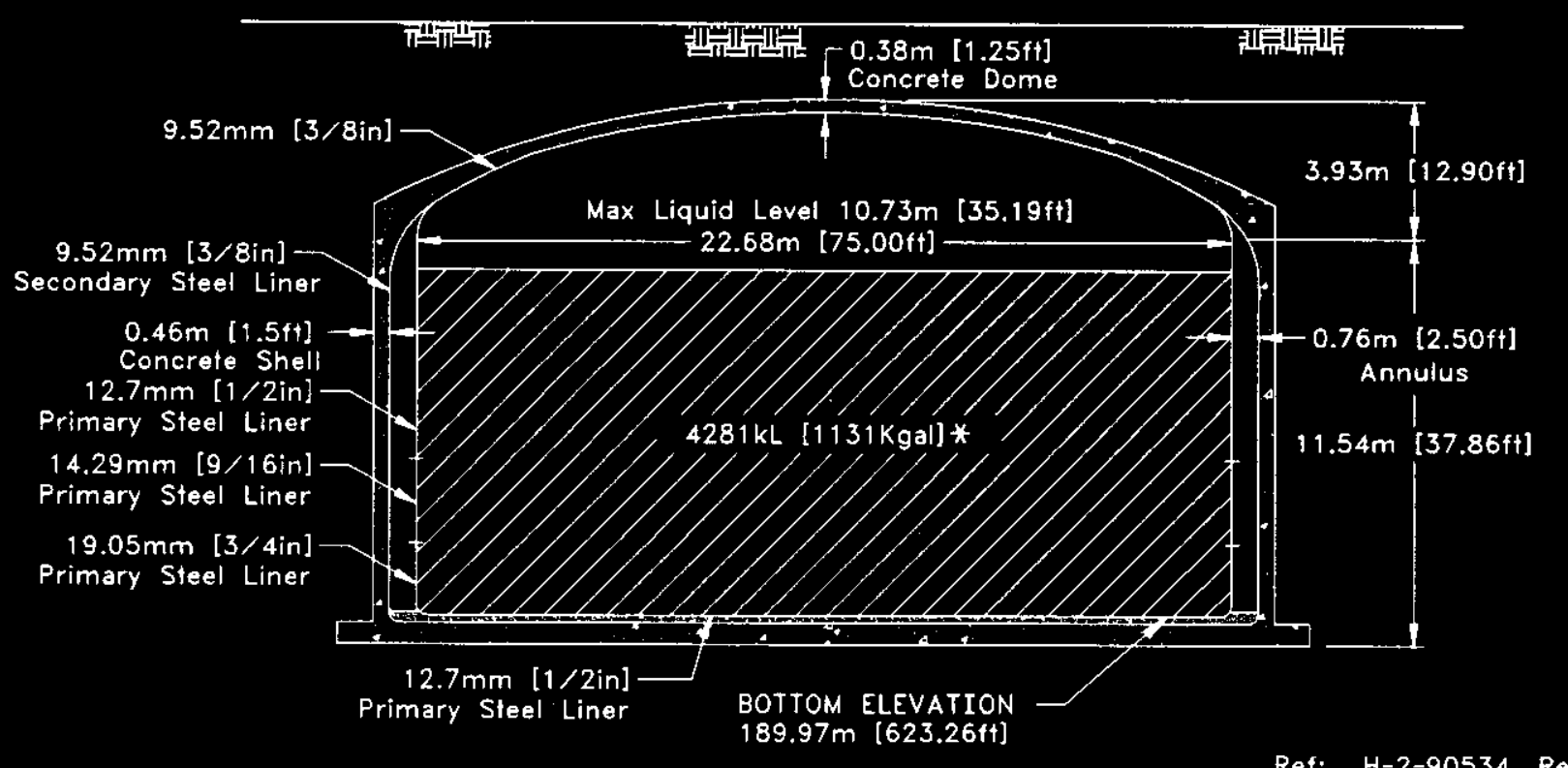

Ref: H-2-90534, Rev.3

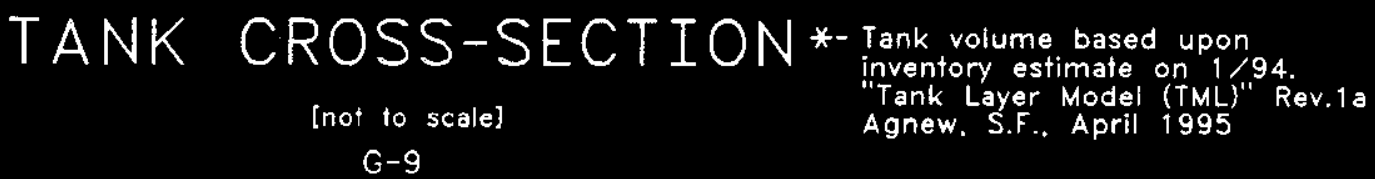




\begin{tabular}{|c|c|c|}
\hline NO. & DIA. & DESCRIPTION AND COMMENTS \\
\hline 1 & $4^{n}$ & SMP \\
\hline 1 & 4" & SMP \\
\hline 1 & 4" & SMP \\
\hline 2 & 4" & LIQUID LEVEL, LIT \\
\hline 3 & $12^{\prime \prime}$ & SUPERNATANT PUMP, CENTRAL PUMP PIT (PIT) \\
\hline 4 & $12^{\prime \prime}$ & THERMOCOUPLE PROBE \\
\hline 5 & 42" & MANHOLE; RISER PLUG \\
\hline 5 & $42^{\prime \prime}$ & MANHOLE; RISER PLUG \\
\hline 6 & $24^{\prime \prime}$ & ANNULUS ACCESS, SPARE \\
\hline 6 & $24^{n}$ & ANNULUS ACCESS, SPARE \\
\hline 7 & $12^{n}$ & SPARE; RISER PLUG \\
\hline 7 & $12^{n}$ & PRIMARY TANK EXHAUST \\
\hline 8 & $4^{\prime \prime}$ & ANNULUS AIR INLET \\
\hline 8 & 4" & ANNULUS AIR INLET \\
\hline 8 & 4" & ANNULUS AIR INLET \\
\hline 8 & $4^{n}$ & ANNULUS AIR INLET \\
\hline 8 & $4^{n}$ & ANNULUS AIR INLET \\
\hline 8 & $4^{\prime \prime}$ & ANNULUS AIR INLET \\
\hline 8 & $4^{n}$ & ANNULUS AIR INLET \\
\hline 8 & $4^{\prime \prime}$ & ANNULUS AIR INLET \\
\hline 9 & 8" & ANNULUS AIR OUTLET \\
\hline 9 & 8" & ANNULUS AIR OUTLET \\
\hline 9 & 8" & ANNULUS AIR OUTLET \\
\hline 9 & $8^{\prime \prime}$ & ANNULUS AIR OUTLET \\
\hline 10 & $12^{\prime \prime}$ & SPARE; RISER PLUG \\
\hline 10 & $12^{\prime \prime}$ & SPARE; RISER PLUG \\
\hline 11 & $42 "$ & SLURRY DISTRIBUTOR, CENTRAL PUMP PIT (PIT) \\
\hline 12 & $12 "$ & OBSV PORT, SPARE \\
\hline 13 & $12^{\prime \prime}$ & TANK PRESSURE \\
\hline 14 & 4" & SUPERNATANT RETURN \\
\hline 15 & 4" & SPARE; RISER PLUG \\
\hline 16 & $12^{\prime \prime}$ & SMP \\
\hline 16 & $12 "$ & SMP \\
\hline
\end{tabular}




\begin{tabular}{|c|c|c|}
\hline No. & DIA. & $\begin{array}{l}241-\mathbf{A P}-103 \\
\text { DESCRIPTION AND COMMENTS }\end{array}$ \\
\hline 16 & $12^{\prime \prime}$ & SMP \\
\hline 17 & 6" & ANNULUS INSPECTION PORT \\
\hline 17 & 6" & ANNULUS INSPECTION PORT \\
\hline 17 & 6" & ANNULUS INSPECTION PORT \\
\hline 17 & 6" & ANNULUS INSPECTION PORT \\
\hline 17 & $6^{n}$ & ANNULUS INSPECTION PORT \\
\hline 17 & 6" & ANNULUS INSPECTION PORT \\
\hline 17 & $6^{\prime \prime}$ & ANNULUS INSPECTION PORT \\
\hline 17 & $6^{n}$ & ANNULUS INSPECTION PORT \\
\hline 17 & 6" & ANNULUS INSPECTION PORT \\
\hline 17 & 6" & ANNULUS INSPECTION PORT \\
\hline 17 & 6" & ANNULUS INSPECTION PORT \\
\hline 17 & $6^{n}$ & ANNULUS INSPECTION PORT \\
\hline 17 & 6" & ANNULUS INSPECTION PORT \\
\hline 17 & $6 "$ & ANNULUS INSPECTION PORT \\
\hline 17 & $6^{n}$ & ANNULUS INSPECTION PORT \\
\hline 17 & 6" & ANNULUS INSPECTION PORT \\
\hline 18 & $12^{\prime \prime}$ & ANNULUS ACCESS \\
\hline 18 & $12^{n}$ & ANNULUS ACCESS \\
\hline 19 & $4 "$ & ANNULUS THERMOCOUPLE \\
\hline 19 & $4 "$ & ANNULUS THERMOCOUPLE \\
\hline 19 & 4" & ANNULUS THERMOCOUPLE \\
\hline 19 & 4" & ANNULUS THERMOCOUPLE \\
\hline 19 & 4" & ANNULUS THERMOCOUPLE \\
\hline 19 & $4 "$ & ANNULUS THERMOCOUPLE \\
\hline 20 & $12^{\prime \prime}$ & ANNULUS PUMP, ANNULUS PUMP PIT (PIT) \\
\hline 21 & $4^{n}$ & SPARE; RISER PLUG \\
\hline 22 & $4 "$ & SMP \\
\hline 23 & 4" & ANNULUS LEAK DETECTOR \\
\hline 23 & 4" & ANNULUS LEAK DETECTOR \\
\hline 23 & 4" & ANNULUS LEAK DETECTOR \\
\hline 24 & 4" & SPARE; RISER PLUG \\
\hline 25 & $4 "$ & HIGH LIQUID LEVEL SENSOR \\
\hline
\end{tabular}




\begin{tabular}{||c||c|l||}
\hline \multicolumn{2}{|c||}{$241-\mathbf{A P}-103$} \\
\hline \hline NO. & DIA. & \multicolumn{1}{|c|}{ DESCRIPTION AND COMMENTS } \\
\hline \hline 26 & $4^{\prime \prime}$ & LIOUID LEVEL IND \\
\hline 27 & $4^{\prime \prime}$ & SPARE; RISER PLUG \\
\hline 27 & $4^{\prime \prime}$ & SPARE; RISER PLUG \\
\hline 27 & $4^{\prime \prime}$ & SPARE; RISER PLUG \\
\hline 28 & $4^{\prime \prime}$ & SPARE; RISER PLUG \\
\hline
\end{tabular}

Ref: WHC-SD-RE-TI-093, Rev.3

H-2-90555, Rev.4

H-2-90538, Rev. 1

If there was a discrepancy between the documents and the drawing, the drawing shall take precedence. 


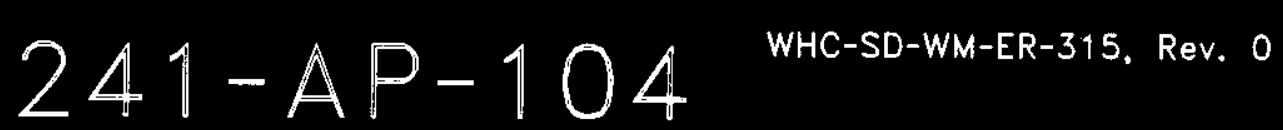

$4,396,400$ Liters

[1,160,000 Gallons]

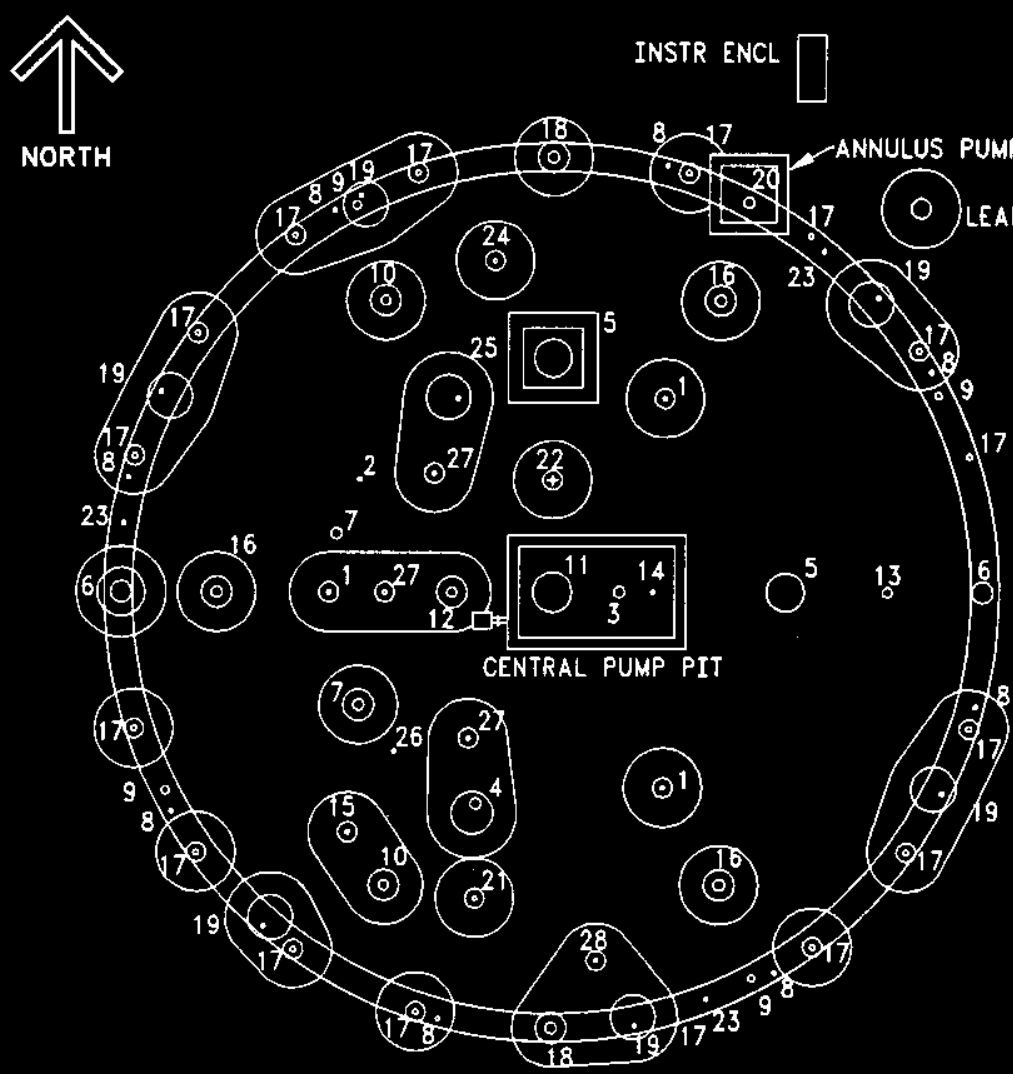

\section{TANK RISER LOCATION}

Ref: WHC-SD-RE-TI-093. Rev.3 $\mathrm{H}-2-90556$. Rev. 4 H-2-90539. Rev.1

If there was a discrepancy between

Approximate Grade Elevatlon 206.9m [678.81ft] the documents and drawing. the drawing shall take precedence.

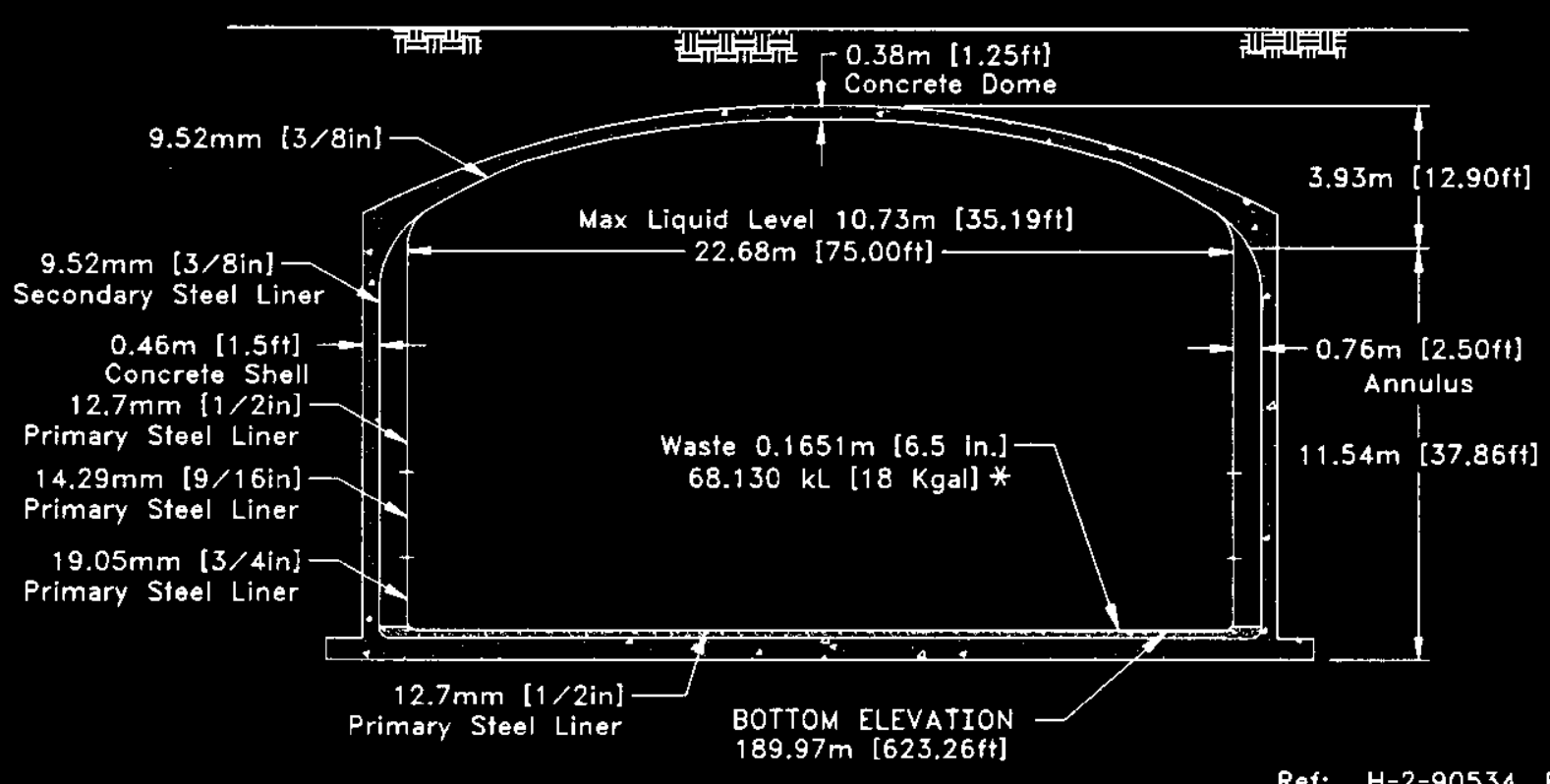

Ref: H-2-90534, Rev.3 


\begin{tabular}{|c|c|c|}
\hline NO. & DIA. & DESCRIPTION AND COMMENTS \\
\hline 1 & 4" & SMP (AOD PIPE MANIFOLD FOR WATER \&/OR CAUSTIC FILL, ECN 702887, 8-31-94) \\
\hline 1 & 4" & SMP \\
\hline 1 & $4^{n}$ & SMP \\
\hline 2 & $4^{\prime \prime}$ & LIQUID LEVEL, LIT \\
\hline 3 & $12^{\prime \prime}$ & SUPERNATANT PUMP, CENTRAL PUMP PIT (PIT) \\
\hline 4 & $12^{\prime \prime}$ & THERMOCOUPLE PROBE \\
\hline 5 & 42" & MANHOLE; RISER PLUG \\
\hline 5 & $42^{\prime \prime}$ & MANHOLE; RISER PLUG \\
\hline 6 & $24 "$ & ANNULUS ACCESS, SPARE \\
\hline 6 & $24 "$ & ANNULUS ACCESS, SPARE \\
\hline 7 & $12^{\prime \prime}$ & SPARE; RISER PLUG \\
\hline 7 & $12^{\prime \prime}$ & PRIMARY TANK EXHAUST \\
\hline 8 & $4 "$ & ANNULUS AIR INLET \\
\hline 8 & $4^{\prime \prime}$ & ANNULUS AIR INLET \\
\hline 8 & 4" & ANNULUS AIR INLET \\
\hline 8 & 4" & ANNULUS AIR INLET \\
\hline 8 & $4 "$ & ANNULUS AIR INLET \\
\hline 8 & $4^{\prime \prime}$ & ANNULUS AIR INLET \\
\hline 8 & $4 "$ & ANNULUS AIR INLET \\
\hline 8 & 4" & ANNULUS AIR INLET \\
\hline 9 & 8" & ANNULUS AIR OUTLET \\
\hline 9 & 8" & ANNULUS AIR OUTLET \\
\hline 9 & 8" & ANNULUS AIR OUTLET \\
\hline 9 & 8" $^{\prime \prime}$ & ANNULUS AIR OUTLET \\
\hline 10 & $12^{\prime \prime}$ & SPARE; RISER PLUG \\
\hline 10 & $12^{\prime \prime}$ & TANK PRESSURE \\
\hline 11 & $42^{\prime \prime}$ & SLURRY DISTRIBUTOR, CENTRAL PUMP PIT (PIT) \\
\hline 12 & $12^{\prime \prime}$ & OBSV PORT, SPARE \\
\hline 13 & $12^{\prime \prime}$ & GROUT FEED PUMP \\
\hline 14 & 4" & SUPERNATANT RETURN \\
\hline 15 & $4^{\prime \prime}$ & SPARE; RISER PLUG \\
\hline 16 & $12^{\prime \prime}$ & SMP \\
\hline 16 & $12^{\prime \prime}$ & SMP \\
\hline
\end{tabular}




\begin{tabular}{|c|c|c|}
\hline No. & DIA. & $\frac{241-A P-104}{\text { DESCRIPTION AND COMMENTS }}$ \\
\hline 16 & $12^{\prime \prime}$ & SMP \\
\hline 17 & $6^{\prime \prime}$ & ANNULUS INSPECTION PORT \\
\hline 17 & $6^{n}$ & ANNULUS INSPECTION PORT \\
\hline 17 & 6" & ANNULUS INSPECTION PORT \\
\hline$\overline{17}$ & $6^{\prime \prime}$ & ANNULUS INSPECTION PORT \\
\hline 17 & 6" & ANNULUS INSPECTION PORT \\
\hline 17 & $6^{n}$ & ANNULUS INSPECTION PORT \\
\hline 17 & $6^{n}$ & ANNULUS INSPECTION PORT \\
\hline 17 & 6" & ANNULUS INSPECTION PORT \\
\hline 17 & 6" & ANNULUS INSPECTION PORT \\
\hline 17 & $6^{\prime \prime}$ & ANNULUS INSPECTION PORT \\
\hline 17 & $6^{\prime \prime}$ & ANNULUS INSPECTION PORT \\
\hline 17 & 6" & ANNULUS INSPECTION PORT \\
\hline 17 & 6" & ANNULUS INSPECTION PORT \\
\hline 17 & 6" & ANNULUS INSPECTION PORT \\
\hline 17 & 6" & ANNULUS INSPECTION PORT \\
\hline 17 & 6" & ANNULUS INSPECTION PORT \\
\hline 18 & $12^{\prime \prime}$ & ANNULUS ACCESS \\
\hline 18 & $12^{\prime \prime}$ & ANNULUS ACCESS \\
\hline 19 & $4 "$ & ANNULUS THERMOCOUPLE \\
\hline 19 & 4" & ANNULUS THERMOCOUPLE \\
\hline 19 & 4" & ANNULUS THERMOCOUPLE \\
\hline 19 & $4 "$ & ANNULUS THERMOCOUPLE \\
\hline 19 & 4" & ANNULUS THERMOCOUPLE \\
\hline 19 & $4 "$ & ANNULUS THERMOCOUPLE \\
\hline 20 & $12^{\prime \prime}$ & ANNULUS PUMP, ANNULUS PUMP PIT (PIT) \\
\hline 21 & 4" & SPARE; RISER PLUG \\
\hline 22 & $4^{\prime \prime}$ & SMP \\
\hline 23 & 4" & ANNULUS LEAK DETECTOR \\
\hline 23 & 4" & ANNULUS LEAK DETECTOR \\
\hline 23 & $4 "$ & ANNULUS LEAK DETECTOR \\
\hline 24 & $4 "$ & SPARE; RISER PLUG \\
\hline 25 & 4" & HIGH LIQUID LEVEL SENSOR \\
\hline
\end{tabular}




\begin{tabular}{|c|c|l||}
\hline \multicolumn{2}{|c|}{ 241-AP-104 } \\
\hline \hline NO. & DIA. & \multicolumn{1}{|c|}{ DESCRIPTION AND COMMENTS } \\
\hline 26 & $4^{\prime \prime}$ & LIQUID LEVEL IND \\
\hline 27 & $4^{\prime \prime}$ & SPARE; RISER PLUG \\
\hline 27 & $4^{\prime \prime}$ & SPARE; RISER PLUG \\
\hline 27 & $4^{\prime \prime}$ & SPARE; RISER PLUG \\
\hline 28 & $4^{\prime \prime}$ & SPARE; RISER PLUG \\
\hline
\end{tabular}

Ref: WHC-SD-RE-TI-093, Rev.3

H-2-90556, Rev.4

H-2-90539, Rev.1

If there was a discrepancy between the documents and the drawing, the drawing shall take precedence.

Comments placed in () denote Engineering Change Notices (ECN) made against the reference drawings. 


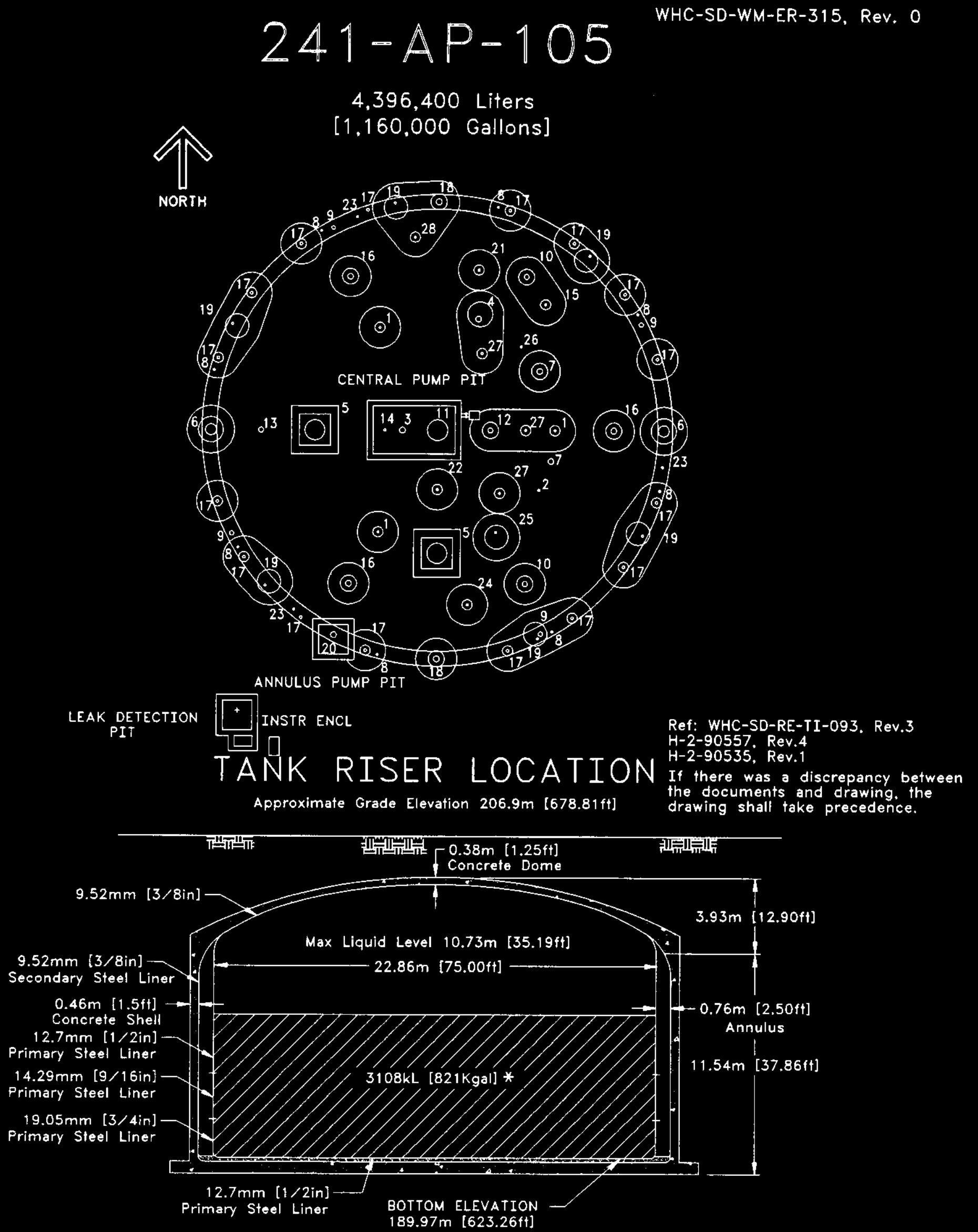

Ref: H-2-90534, Rev.3

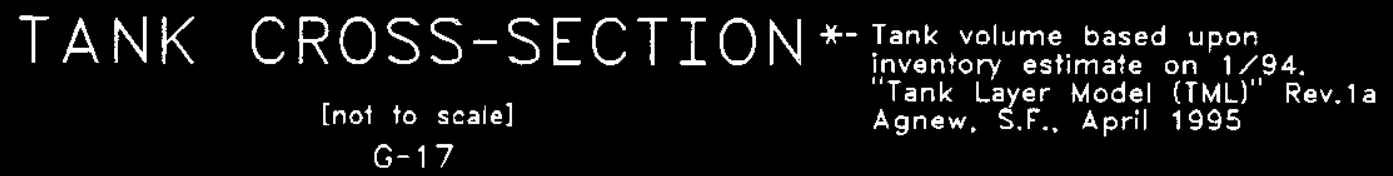




\begin{tabular}{|c|c|c|}
\hline NO. & DIA. & DESCRIPTION AND COMMENTS \\
\hline 1 & 4" & SMP \\
\hline 1 & $4^{\prime \prime}$ & SMP \\
\hline 1 & $4 "$ & SMP \\
\hline 2 & 4" & LIQUID LEVEL, LIT \\
\hline 3 & $12 "$ & SUPERNATANT PUMP, CENTRAL PUMP PIT (PIT) \\
\hline 4 & $12^{\prime \prime}$ & THERMOCOUPLE PROBE \\
\hline 5 & 42" & MANHOLE; RISER PLUG \\
\hline 5 & $42 "$ & MANHOLE; RISER PLUG \\
\hline 6 & $24 "$ & ANNULUS ACCESS, SPARE \\
\hline 6 & $24^{\prime \prime}$ & ANNULUS ACCESS, SPARE \\
\hline 7 & $12^{\prime \prime}$ & SPARE; RISER PLUG \\
\hline 7 & $12^{n}$ & PRIMARY TANK EXHAUST \\
\hline 8 & 4" & ANNULUS AIR INLET \\
\hline 8 & 4" & ANNULUS AIR INLET \\
\hline 8 & $4^{\prime \prime}$ & ANNULUS AIR INLET \\
\hline 8 & 4" & ANNULUS AIR INLET \\
\hline 8 & 4" & ANNULUS AIR INLET \\
\hline 8 & $4 "$ & ANNULUS AIR INLET \\
\hline 8 & 4" & ANNULUS AIR INLET \\
\hline 8 & 4" & ANNULUS AIR INLET \\
\hline 9 & $8^{\prime \prime}$ & ANNULUS AIR OUTLET \\
\hline 9 & $8^{\prime \prime}$ & ANNULUS AIR OUTLET \\
\hline 9 & $8^{\prime \prime}$ & ANNULUS AIR OUTLET \\
\hline 9 & 8" & ANNULUS AIR OUTLET \\
\hline 10 & $12 "$ & SPARE; RISER PLUG \\
\hline 10 & $12^{\prime \prime}$ & SPARE; RISER PLUG \\
\hline 11 & $42^{\prime \prime}$ & SLURRY DISTRIBUTOR, CENTRAL PUMP PIT (PIT) \\
\hline 12 & $12^{\prime \prime}$ & OBSV PORT, SPARE \\
\hline 13 & $12^{\prime \prime}$ & TANK PRESSURE \\
\hline 14 & 4" & SUPERNATANT RETURN \\
\hline 15 & $4^{\prime \prime}$ & SPARE; RISER PLUG \\
\hline 16 & $12^{\prime \prime}$ & SMP \\
\hline 16 & $12^{\prime \prime}$ & SMP \\
\hline
\end{tabular}




\begin{tabular}{|c|c|c|}
\hline & & 241-AP-105 \\
\hline No. & DIA. & DESCRIPTION AND COMMENTS \\
\hline 16 & $12^{\prime \prime}$ & SMP \\
\hline 17 & $6^{n}$ & ANNULUS INSPECTION PORT \\
\hline 17 & 6" & ANNULUS INSPECTION PORT \\
\hline 17 & 6" & ANNULUS INSPECTION PORT \\
\hline 17 & 6" & ANNULUS INSPECTION PORT \\
\hline 17 & 6" & ANNULUS INSPECTION PORT \\
\hline 17 & 6" & ANNULUS INSPECTION PORT \\
\hline 17 & 6" & ANNULUS INSPECTION PORT \\
\hline 17 & $6^{\prime \prime}$ & ANNULUS INSPECTION PORT \\
\hline 17 & 6" & ANNULUS INSPECTION PORT \\
\hline 17 & 6" & ANNULUS INSPECTION PORT \\
\hline 17 & 6" & ANNULUS INSPECTION PORT \\
\hline 17 & 6" & ANNULUS INSPECTION PORT \\
\hline 17 & 6" & ANNULUS INSPECTION PORT \\
\hline 17 & 6" & ANNULUS INSPECTION PORT \\
\hline 17 & 6" & ANNULUS INSPECTION PORT \\
\hline 17 & 6" & ANNULUS INSPECTION PORT \\
\hline 18 & $12^{\prime \prime}$ & ANNULUS ACCESS \\
\hline 18 & $12^{\prime \prime}$ & ANNULUS ACCESS \\
\hline 19 & 4" & ANNULUS THERMOCOUPLE \\
\hline 19 & 4" & ANNULUS THERMOCOUPLE \\
\hline 19 & $4^{n}$ & ANNULUS THERMOCOUPLE \\
\hline 19 & $4^{\prime \prime}$ & ANNULUS THERMOCOUPLE \\
\hline 19 & 4" & ANNULUS THERMOCOUPLE \\
\hline 19 & 4" & ANNULUS THERMOCOUPLE \\
\hline 20 & $12^{\prime \prime}$ & ANNULUS PUMP, ANNULUS PUMP PIT (PIT) \\
\hline 21 & 4" & SPARE; RISER PLUG \\
\hline 22 & 4" & SMP \\
\hline 23 & $4 "$ & ANNULUS LEAK DETECTOR \\
\hline 23 & $4 "$ & ANNULUS LEAK DETECTOR \\
\hline 23 & $4 "$ & ANNULUS LEAK DETECTOR \\
\hline 24 & 4" & SPARE; RISER PLUG \\
\hline 25 & 4" & HIGH LIQUID LEVEL SENSOR \\
\hline
\end{tabular}




\begin{tabular}{|c|c|l||}
\hline \multicolumn{2}{|c|}{} & \multicolumn{2}{|c|}{ 241-AP-10S } \\
\hline NO. & DIA. & \multicolumn{1}{|c|}{ DESCRIPTION AND COMMENTS } \\
\hline 26 & $4^{\prime \prime}$ & LIQUID LEVEL IND \\
\hline 27 & $4^{\prime \prime}$ & SPARE; RISER PLUG \\
\hline 27 & $4^{\prime \prime}$ & SPARE; RISER PLUG \\
\hline 27 & $4^{\prime \prime}$ & SPARE; RISER PLUG \\
\hline 28 & $4^{\prime \prime}$ & SPARE; RISER PLUG \\
\hline
\end{tabular}

Ref: WHC-SD-RE-TH-093, Rev.3

H-2-90557, Rev. 4

H-2-90535, Rev. 1

If there was a discrepancy between the documents and the drawing, the drawing shall take precedence. 


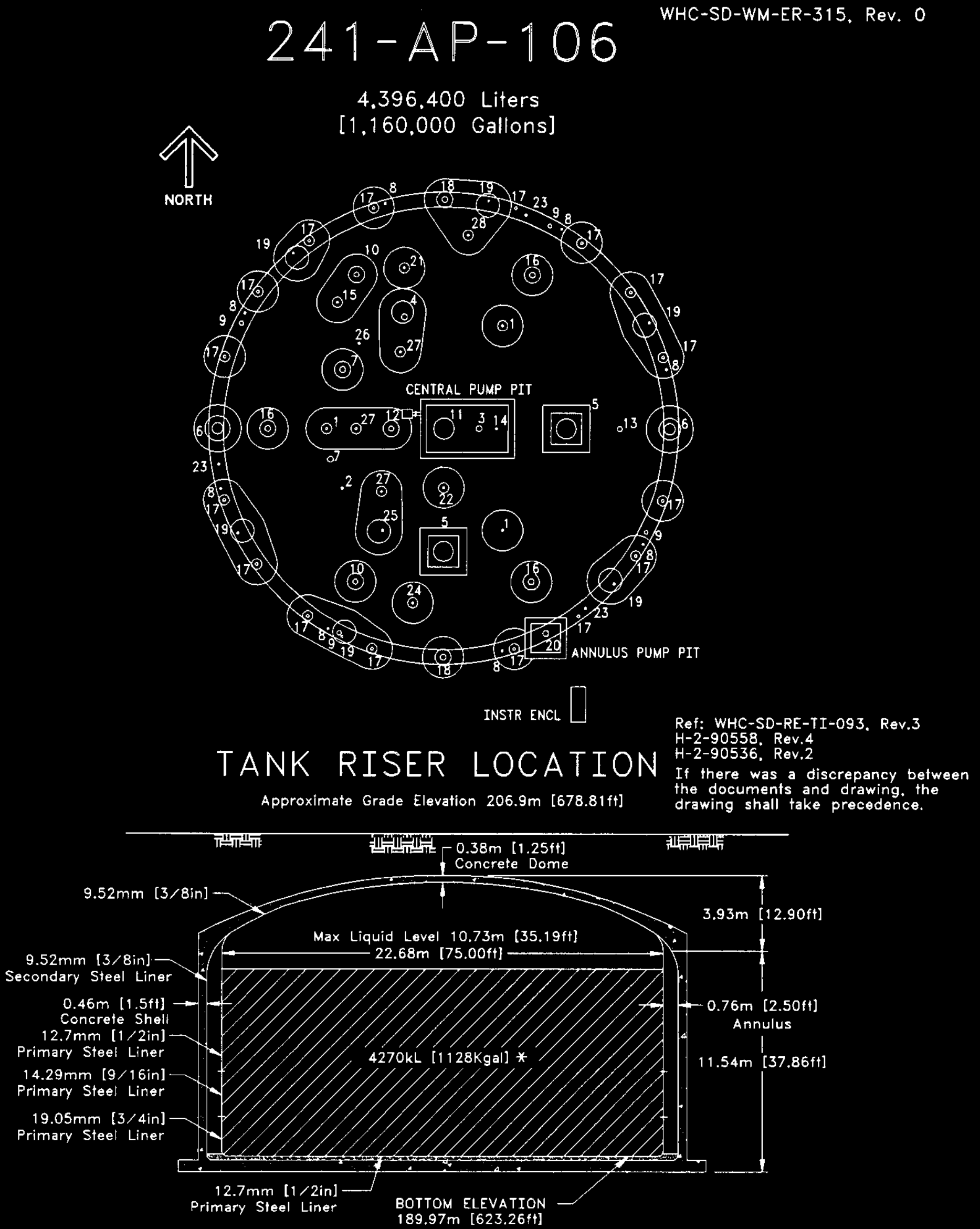

Ref: H-2-90534, Rev.3

(19)

$4,396,400$ Liters

1,160,000 Gallons]

(19.) $\left.2^{18}-19\right)^{17} 23$ 


\begin{tabular}{|c|c|c|}
\hline NO. & DIA. & DESCRIPTION AND COMMENTS \\
\hline 1 & 4" & SMP \\
\hline 1 & 4" & SMP \\
\hline 1 & 4" & SMP \\
\hline 2 & 4" & LIQUID LEVEL, LIT \\
\hline 3 & $12^{\prime \prime}$ & SUPERNATANT PUMP, CENTRAL PUMP PIT (PIT) \\
\hline 4 & $12^{\prime \prime}$ & THERMOCOUPLE PROBE \\
\hline 5 & $42 "$ & MANHOLE; RISER PLUG \\
\hline 5 & $42^{\prime \prime}$ & MANHOLE; RISER PLUG \\
\hline 6 & $24 "$ & ANNULUS ACCESS, SPARE \\
\hline 6 & $24 "$ & ANNULUS ACCESS, SPARE \\
\hline 7 & 12" & SPARE; RISER PLUG \\
\hline 7 & $12 "$ & PRIMARY TANK EXHAUST \\
\hline 8 & 4" & ANNULUS AIR INLET \\
\hline 8 & 4" & ANNULUS AIR INLET \\
\hline 8 & 4" & ANNULUS AIR INLET \\
\hline 8 & 4" & ANNULUS AIR INLET \\
\hline 8 & 4" & ANNULUS AIR INLET \\
\hline 8 & $4 "$ & ANNULUS AIR INLET \\
\hline 8 & $4 "$ & ANNULUS AIR INLET \\
\hline 8 & $4^{\prime \prime}$ & ANNULUS AIR INLET \\
\hline 9 & 8" & ANNULUS AIR OUTLET \\
\hline 9 & 8" & ANNULUS AIR OUTLET \\
\hline 9 & 8" & ANNULUS AIR OUTLET \\
\hline 9 & 8" & ANNULUS AIR OUTLET \\
\hline 10 & $12^{\prime \prime}$ & SPARE; RISER PLUG \\
\hline 10 & $12^{n}$ & SPARE; RISER PLUG \\
\hline 11 & $42^{\prime \prime}$ & SLURRY DISTRIBUTOR, CENTRAL PUMP PIT (PIT) \\
\hline 12 & $12^{\prime \prime}$ & OBSV PORT, SPARE \\
\hline 13 & $12^{\prime \prime}$ & TANK PRESSURE \\
\hline 14 & $4 "$ & SUPERNATANT RETURN \\
\hline 15 & 4" & SPARE; RISER PLUG \\
\hline 16 & $12^{\prime \prime}$ & SMP \\
\hline 16 & $12^{\prime \prime}$ & SMP \\
\hline
\end{tabular}




\begin{tabular}{|c|c|c|}
\hline No. & DIA. & DESCRIPTION AND COMMENTS \\
\hline 16 & $12^{\prime \prime}$ & SMP \\
\hline 17 & 6" & ANNULUS INSPECTION PORT \\
\hline 17 & $6^{\prime \prime}$ & ANNULUS INSPECTION PORT \\
\hline 17 & $6^{\prime \prime}$ & ANNULUS INSPECTION PORT \\
\hline 17 & $6^{n}$ & ANNULUS INSPECTION PORT \\
\hline 17 & $6^{\prime \prime}$ & ANNULUS INSPECTION PORT \\
\hline 17 & 6" & ANNULUS INSPECTION PORT \\
\hline 17 & 6" & ANNULUS INSPECTION PORT \\
\hline 17 & 6" & ANNULUS INSPECTION PORT \\
\hline 17 & 6" & ANNULUS INSPECTION PORT \\
\hline 17 & $6^{\prime \prime}$ & ANNULUS INSPECTION PORT \\
\hline 17 & $6^{n}$ & ANNULUS INSPECTION PORT \\
\hline 17 & 6" & ANNULUS INSPECTION PORT \\
\hline 17 & $6^{\prime \prime}$ & ANNULUS INSPECTION PORT \\
\hline 17 & 6" & ANNULUS INSPECTION PORT \\
\hline 17 & 6" & ANNULUS INSPECTION PORT \\
\hline 17 & $6^{\prime \prime}$ & ANNULUS INSPECTION PORT \\
\hline 18 & $12^{\prime \prime}$ & ANNULUS ACCESS \\
\hline 18 & $12^{\prime \prime}$ & ANNULUS ACCESS \\
\hline 19 & 4" & ANNULUS THERMOCOUPLE \\
\hline 19 & 4" & ANNULUS THERMOCOUPLE \\
\hline 19 & $4^{\prime \prime}$ & ANNULUS THERMOCOUPLE \\
\hline 19 & 4" & ANNULUS THERMOCOUPLE \\
\hline 19 & 4" & ANNULUS THERMOCOUPLE \\
\hline 19 & 4" & ANNULUS THERMOCOUPLE \\
\hline 20 & $12^{\prime \prime}$ & ANNULUS PUMP, ANNULUS PUMP PIT (PIT) \\
\hline 21 & 4" & SPARE; RISER PLUG \\
\hline 22 & 4" & SMP \\
\hline 23 & 4" & ANNULUS LEAK DETECTOR \\
\hline 23 & 4" & ANNULUS LEAK DETECTOR \\
\hline 23 & $4^{\prime \prime}$ & ANNULUS LEAK DETECTOR \\
\hline 24 & $4^{\prime \prime}$ & SPARE; RISER PLUG \\
\hline 25 & 4" & HIGH LIQUID LEVEL SENSOR \\
\hline
\end{tabular}


WHC-SD-WM-ER-315, Rev. 0

\begin{tabular}{|c|c|l||}
\hline \multicolumn{3}{|c|}{ 241-AP-106 } \\
\hline \hline NO. & DIA. & \multicolumn{1}{|c|}{ DESCRIPTION AND COMMENTS } \\
\hline \hline 26 & $4^{\prime \prime}$ & LIQUID LEVEL IND \\
\hline 27 & $4^{\prime \prime}$ & SPARE; RISER PLUG \\
\hline 27 & $4^{\prime \prime}$ & SPARE; RISER PLUG \\
\hline 27 & $4^{\prime \prime}$ & SPARE; RISER PLUG \\
\hline 28 & $4^{\prime \prime}$ & SPARE; RISER PLUG \\
\hline
\end{tabular}

Ref: WHC-SD-RE-TI-093, Rev.3

- H-2-90558, Rev.4

H-2-90536, Rev.2

If there was a discrepancy between the documents and the drawing, the drawing shall take precedence. 

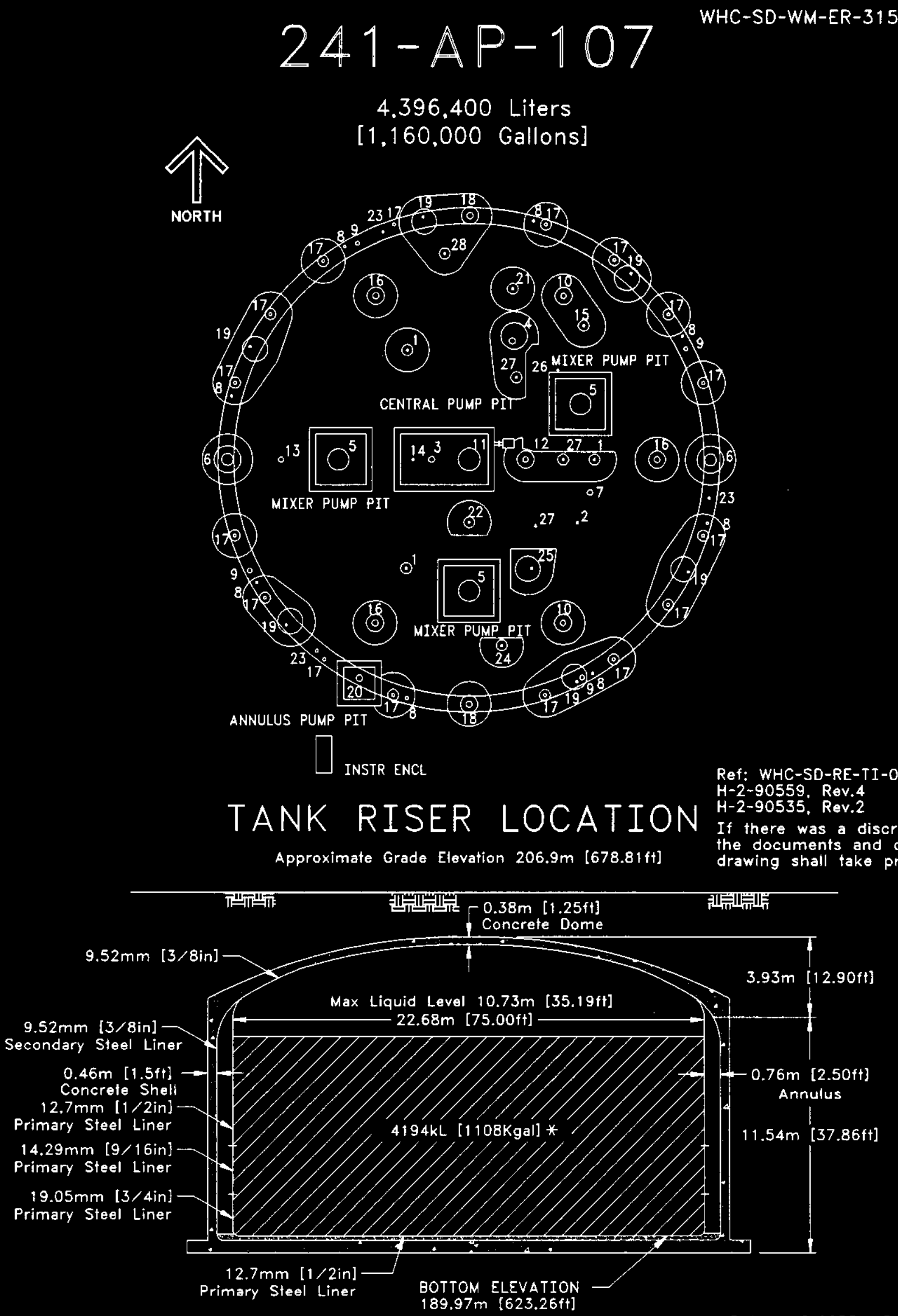

Ref: H-2-90534, Rev.3

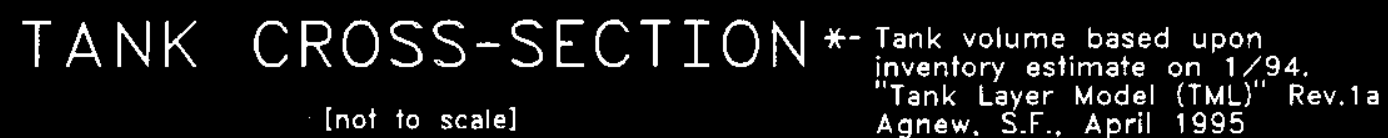




\begin{tabular}{|c|c|c|}
\hline No. & DIA. & DESCRIPTION AND COMMENTS \\
\hline 1 & 4" & SMP \\
\hline 1 & 4" & SMP \\
\hline 1 & $4^{\prime \prime}$ & SMP \\
\hline 2 & $4^{\prime \prime}$ & LIQUID LEVEL, LIT \\
\hline 3 & $12 "$ & SUPERNATANT PUMP, CENTRAL PUMP PIT (PIT) \\
\hline 4 & $12^{\prime \prime}$ & THERMOCOUPLE PROBE \\
\hline 5 & $42^{\prime \prime}$ & MANHOLE; RISER PLUG \\
\hline 5 & $42^{\prime \prime}$ & MANHOLE; RISER PLUG \\
\hline 5 & $42^{\prime \prime}$ & MANHOLE; RISER PLUG \\
\hline 6 & $24 "$ & ANNULUS ACCESS, SPARE \\
\hline 6 & $24^{\prime \prime}$ & ANNULUS ACCESS, SPARE \\
\hline 7 & $12^{\prime \prime}$ & PRIMARY TANK EXHAUST \\
\hline 8 & 4" & ANNULUS AIR INLET \\
\hline 8 & $4^{\prime \prime}$ & ANNULUS AIR INLET \\
\hline 8 & $4^{\prime \prime}$ & ANNULUS AIR INLET \\
\hline 8 & $4^{\prime \prime}$ & ANNULUS AIR INLET \\
\hline 8 & 4" & ANNULUS AIR INLET \\
\hline 8 & $4^{\prime \prime}$ & ANNULUS AIR INLET \\
\hline 8 & 4" & ANNULUS AIR INLET \\
\hline 8 & $4^{\prime \prime}$ & ANNULUS AIR INLET \\
\hline 9 & 8" & ANNULUS AIR OUTLET \\
\hline 9 & 8" & ANNULUS AIR OUTLET \\
\hline 9 & 8" & ANNULUS AIR OUTLET \\
\hline 9 & 8" & ANNULUS AIR OUTLET \\
\hline 10 & $12^{\prime \prime}$ & SPARE; RISER PLUG \\
\hline 10 & $12^{\prime \prime}$ & SPARE; RISER PLUG \\
\hline 11 & $42^{\prime \prime}$ & SLURRY DISTRIBUTOR, CENTRAL PUMP PIT (PIT) \\
\hline 12 & $12^{\prime \prime}$ & OBSV PORT, SPARE \\
\hline 13 & $12^{\prime \prime}$ & TANK PRESSURE \\
\hline 14 & 4" & SUPERNATANT RETURN \\
\hline 15 & 4" & SPARE; RISER PLUG \\
\hline 16 & $12 "$ & SMP \\
\hline 16 & $12^{\prime \prime}$ & SMP \\
\hline
\end{tabular}




\begin{tabular}{|c|c|c|}
\hline No. & DIA. & DESCRIPTION AND COMMENTS \\
\hline 16 & $12^{\prime \prime}$ & SMP \\
\hline 17 & $6^{\prime \prime}$ & ANNULUS INSPECTION PORT \\
\hline 17 & 6" & ANNULUS INSPECTION PORT \\
\hline 17 & $6^{\prime \prime}$ & ANNULUS INSPECTION PORT \\
\hline 17 & $6^{\prime \prime}$ & ANNULUS INSPECTION PORT \\
\hline 17 & 6" & ANNULUS INSPECTION PORT \\
\hline 17 & $6^{\prime \prime}$ & ANNULUS INSPECTION PORT \\
\hline 17 & 6" & ANNULUS INSPECTION PORT \\
\hline 17 & 6" & ANNULUS INSPECTION PORT \\
\hline 17 & $6^{\prime \prime}$ & ANNULUS INSPECTION PORT \\
\hline 17 & $6^{\prime \prime}$ & ANNULUS INSPECTION PORT \\
\hline 17 & 6" & ANNULUS INSPECTION PORT \\
\hline 17 & $6^{\prime \prime}$ & ANNULUS INSPECTION PORT \\
\hline 17 & 6" & ANNULUS INSPECTION PORT \\
\hline 17 & 6" & ANNULUS INSPECTION PORT \\
\hline 17 & 6" & ANNULUS INSPECTION PORT \\
\hline 17 & 6" & ANNULUS INSPECTION PORT \\
\hline 18 & $12^{\prime \prime}$ & ANNULUS ACCESS \\
\hline 18 & $12^{n}$ & ANNULUS ACCESS \\
\hline 19 & 4" & ANNULUS THERMOCOUPLE \\
\hline 19 & $4^{\prime \prime}$ & ANNULUS THERMOCOUPLE \\
\hline 19 & 4" & ANNULUS THERMOCOUPLE \\
\hline 19 & $4 "$ & ANNULUS THERMOCOUPLE \\
\hline 19 & $4 "$ & ANNULUS THERMOCOUPLE \\
\hline 19 & $4^{n}$ & ANNULUS THERMOCOUPLE \\
\hline 20 & $12^{\prime \prime}$ & ANNULUS PUMP, ANNULUS PUMP PIT (PIT) \\
\hline 21 & 4" & SPARE; RISER PLUG \\
\hline 22 & $4^{\prime \prime}$ & SMP \\
\hline 23 & 4" & ANNULUS LEAK DETECTOR \\
\hline 23 & 4" & ANNULUS LEAK DETECTOR \\
\hline 23 & 4" & ANNULUS LEAK DETECTOR \\
\hline 24 & 4" & SPARE; RISER PLUG \\
\hline 25 & 4" & HIGH LIQUID LEVEL SENSOR \\
\hline
\end{tabular}


WHC-SD-WM-ER-315, Rev. 0

\begin{tabular}{|c|c|l||}
\hline \multicolumn{3}{|c|}{$241-$ AP-107 } \\
\hline NO. & DIA. & \multicolumn{1}{|c|}{ DESCRIPTION AND COMMENTS } \\
\hline 26 & $4^{\prime \prime}$ & LIQUID LEVEL IND \\
\hline 27 & $4^{\prime \prime}$ & SPARE; RISER PLUG \\
\hline 27 & $4^{\prime \prime}$ & SPARE; RISER PLUG \\
\hline 27 & $4^{\prime \prime}$ & SPARE; RISER PLUG \\
\hline 28 & $4^{\prime \prime}$ & SPARE; RISER PLUG \\
\hline
\end{tabular}

Ref: WHC-SD-RE-TI-093, Rev.3

H-2-90559, Rev.4

H-2-90535, Rev.2

If there was a discrepancy between the documents and the drawing, the drawing shall take precedence. 


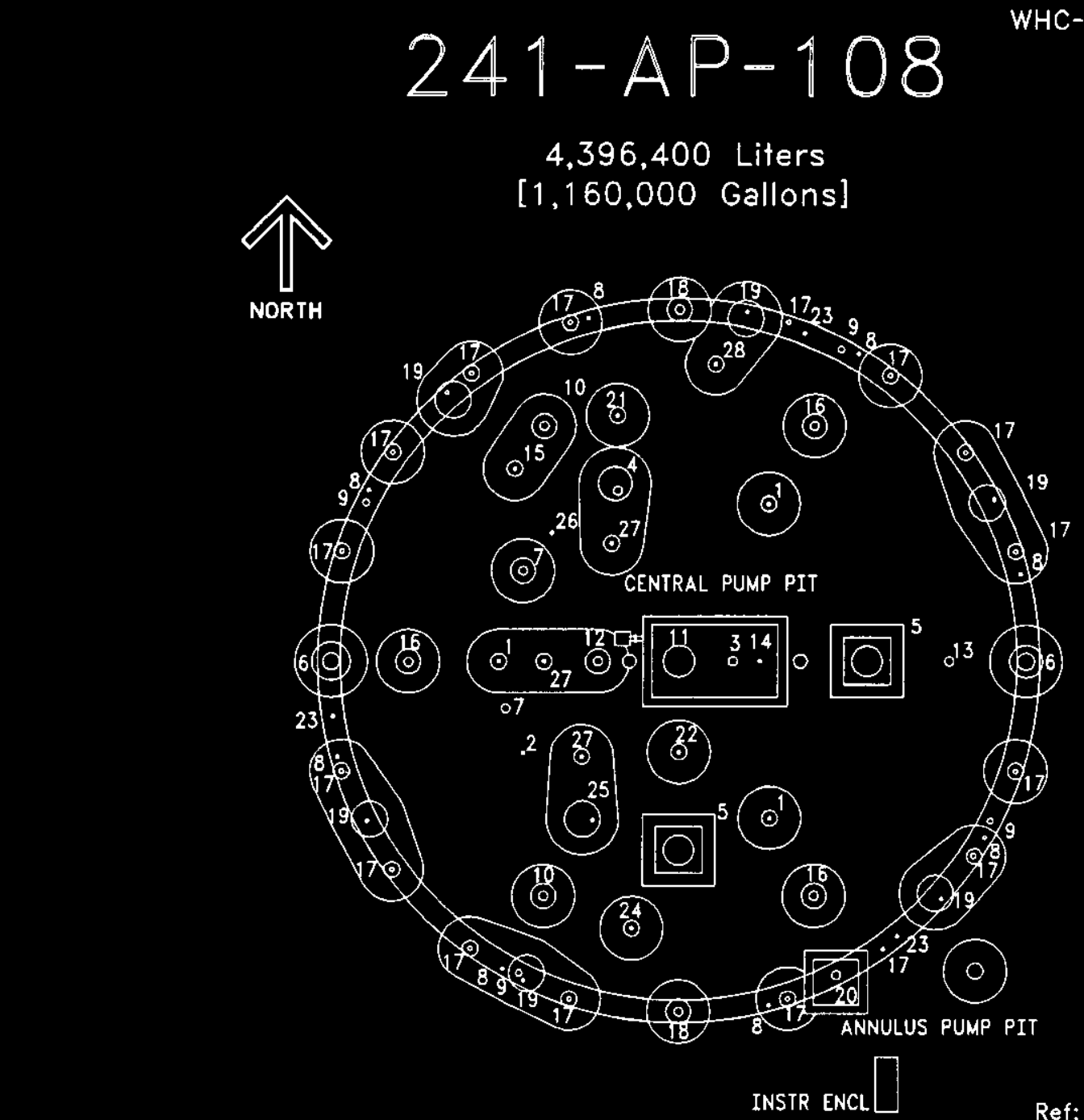

WHC-SD-WM-ER-315, Rev. 0

Ref: WHC-SD-RE-TI-093, Rev.3 H-2-90560. Rev.4

TANK RTSER LOCATTON ${ }_{\text {H-2-90536, Rev. } 2}^{\text {R }}$ If there was a discrepancy between the documents and drawing. the Approximate Grade Elevation $206.9 \mathrm{~m}$ [678.81ft] drawing shall take precedence.

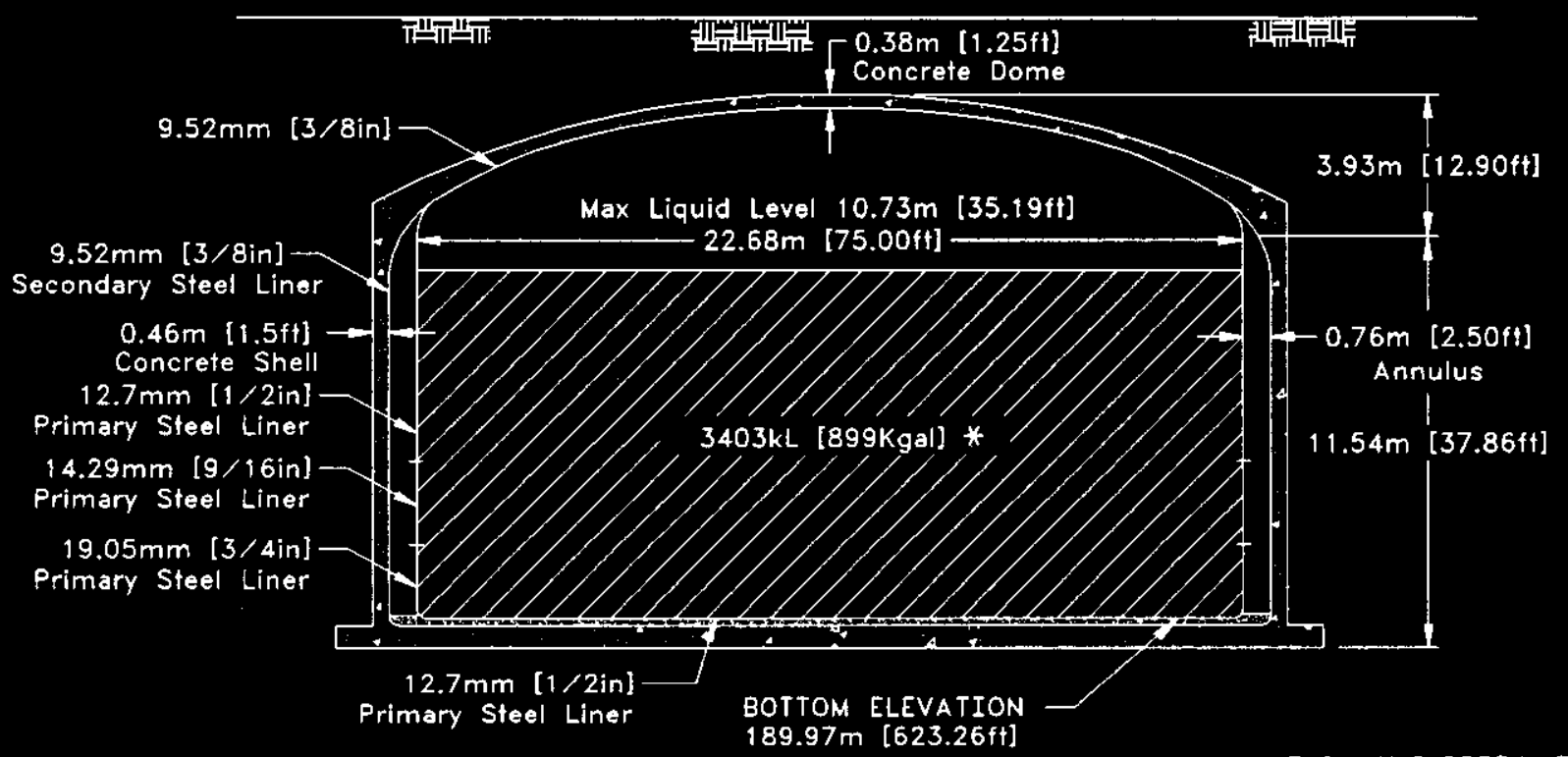

Ref: H-2-90534, Rev.3

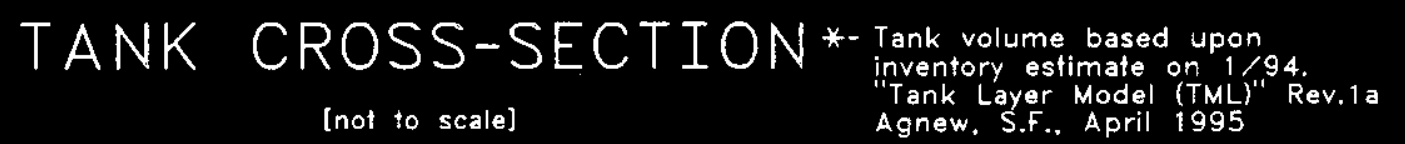




\begin{tabular}{|c|c|c|}
\hline \multicolumn{3}{|r|}{ 241-AP-108 } \\
\hline No. & DIA. & DESCRIPTION AND COMMENTS \\
\hline 1 & 4" & SMP \\
\hline 1 & 4" & SMP \\
\hline 1 & 4" & SMP \\
\hline 2 & 4" & |LIQUID LEVEL, LIT \\
\hline 3 & $12^{\prime \prime}$ & SUPERNATANT PUMP, CENTRAL PUMP PIT (PIT) \\
\hline 4 & $12^{\prime \prime}$ & |THERMOCOUPLE PROBE \\
\hline 5 & $42^{\prime \prime}$ & MANHOLE; RISER PLUG \\
\hline 5 & $42^{\prime \prime}$ & MANHOLE; RISER PLUG \\
\hline 6 & $24^{\prime \prime}$ & ANNULUS ACCESS, SPARE \\
\hline 6 & $24 "$ & ANNULUS ACCESS, SPARE \\
\hline 7 & $12^{\prime \prime}$ & SPARE; RISER PLUG \\
\hline 7 & $12 "$ & PRIMARY TANK EXHAUST \\
\hline 8 & 4" & ANNULUS AIR INLET \\
\hline 8 & 4" & ANNULUS AIR INLET \\
\hline 8 & 4" & ANNULUS AIR INLET \\
\hline 8 & 4" & ANNULUS AIR INLET \\
\hline 8 & $4 "$ & ANNULUS AIR INLET \\
\hline 8 & 4" & ANNULUS AIR INLET \\
\hline 8 & 4" & ANNULUS AIR INLET \\
\hline 8 & 4" & ANNULUS AIR INLET \\
\hline 9 & 8" & ANNULUS AIR OUTLET \\
\hline 9 & $8^{\prime \prime}$ & ANNULUS AIR OUTLET \\
\hline 9 & $8 "$ & ANNULUS AIR OUTLET \\
\hline 9 & $8 "$ & ANNULUS AIR OUTLET \\
\hline 10 & $12^{\prime \prime}$ & SPARE; RISER PLUG \\
\hline 10 & $12^{\prime \prime}$ & SPARE; RISER PLUG \\
\hline 11 & 42" & SLURRY DISTRIBUTOR, CENTRAL PUMP PIT (PIT) \\
\hline 12 & $12^{\prime \prime}$ & OBSV PORT, SPARE \\
\hline 13 & $12^{\prime \prime}$ & TANK PRESSURE \\
\hline 14 & 4" & SUPERNATANT RETURN \\
\hline 15 & 4" & SPARE; RISER PLUG \\
\hline 16 & $12^{\prime \prime}$ & SMP \\
\hline 16 & $12^{\prime \prime}$ & SMP \\
\hline
\end{tabular}




\begin{tabular}{|c|c|c|}
\hline NO. & DIA. & DESCRIPTION AND COMMENTS \\
\hline 16 & $12^{\prime \prime}$ & SMP \\
\hline 17 & $6^{\prime \prime}$ & ANNULUS INSPECTION PORT \\
\hline 17 & $6^{\prime \prime}$ & ANNULUS INSPECTION PORT \\
\hline 17 & $6^{\prime \prime}$ & ANNULUS INSPECTION PORT \\
\hline 17 & $6^{\prime \prime}$ & ANNULUS INSPECTION PORT \\
\hline 17 & $6^{\prime \prime}$ & ANNULUS INSPECTION PORT \\
\hline 17 & 6" & ANNULUS INSPECTION PORT \\
\hline 17 & $6^{\prime \prime}$ & ANNULUS INSPECTION PORT \\
\hline 17 & 6" & ANNULUS INSPECTION PORT \\
\hline 17 & 6" & ANNULUS INSPECTION PORT \\
\hline 17 & $6^{\prime \prime}$ & ANNULUS INSPECTION PORT \\
\hline 17 & 6" & ANNULUS INSPECTION PORT \\
\hline 17 & $6^{\prime \prime}$ & ANNULUS INSPECTION PORT \\
\hline 17 & $6^{\prime \prime}$ & ANNULUS INSPECTION PORT \\
\hline 17 & $6^{\prime \prime}$ & ANNULUS INSPECTION PORT \\
\hline 17 & $6^{\prime \prime}$ & ANNULUS INSPECTION PORT \\
\hline 17 & 6" & ANNULUS INSPECTION PORT \\
\hline 18 & $12^{\prime \prime}$ & ANNULUS ACCESS \\
\hline 18 & $12^{\prime \prime}$ & ANNULUS ACCESS \\
\hline 19 & 4" & ANNULUS THERMOCOUPLE \\
\hline 19 & 4" & ANNULUS THERMOCOUPLE \\
\hline 19 & 4" & ANNULUS THERMOCOUPLE \\
\hline 19 & 4" & ANNULUS THERMOCOUPLE \\
\hline 19 & $4^{\prime \prime}$ & ANNULUS THERMOCOUPLE \\
\hline 19 & 4" & ANNULUS THERMOCOUPLE \\
\hline 20 & $12^{\prime \prime}$ & ANNULUS PUMP, ANNULUS PUMP PIT (PIT) \\
\hline 21 & 4" & SPARE; RISER PLUG \\
\hline 22 & 4" & SMP \\
\hline 23 & $4^{\prime \prime}$ & ANNULUS LEAK DETECTOR \\
\hline 23 & 4" & ANNULUS LEAK DETECTOR \\
\hline 23 & $4^{\prime \prime}$ & ANNULUS LEAK DETECTOR \\
\hline 24 & 4" & SPARE; RISER PLUG \\
\hline 25 & 4" & HIGH LIQUID LEVEL SENSOR \\
\hline
\end{tabular}


WHC-SD-WM-ER-315, Rev. 0

\begin{tabular}{|c|c|l||}
\hline \multicolumn{2}{|c|}{$241-A P-108$} \\
\hline NO. & DIA. & \multicolumn{1}{|c|}{ DESCRIPTION AND COMMENTS } \\
\hline 26 & $4 "$ & LIQUID LEVEL IND \\
\hline 27 & $4 "$ & SPARE; RISER PLUG \\
\hline 27 & $4^{\prime \prime}$ & SPARE; RISER PLUG \\
\hline 27 & $4^{\prime \prime}$ & SPARE; RISER PLUG \\
\hline 28 & $4^{\prime \prime}$ & SPARE; RISER PLUG \\
\hline
\end{tabular}

Ref: WHC-SD-RE-TI-093, Rev.3

H-2-90560, Rev.4

H-2-90536, Rev.2

If there was a discrepancy between the documents and the drawing, the drawing shall take precedence. 


\section{APPENDIX H}

\section{Sampling Data}


THIS PAGE INTENTIONALLY LEFT BLANK 


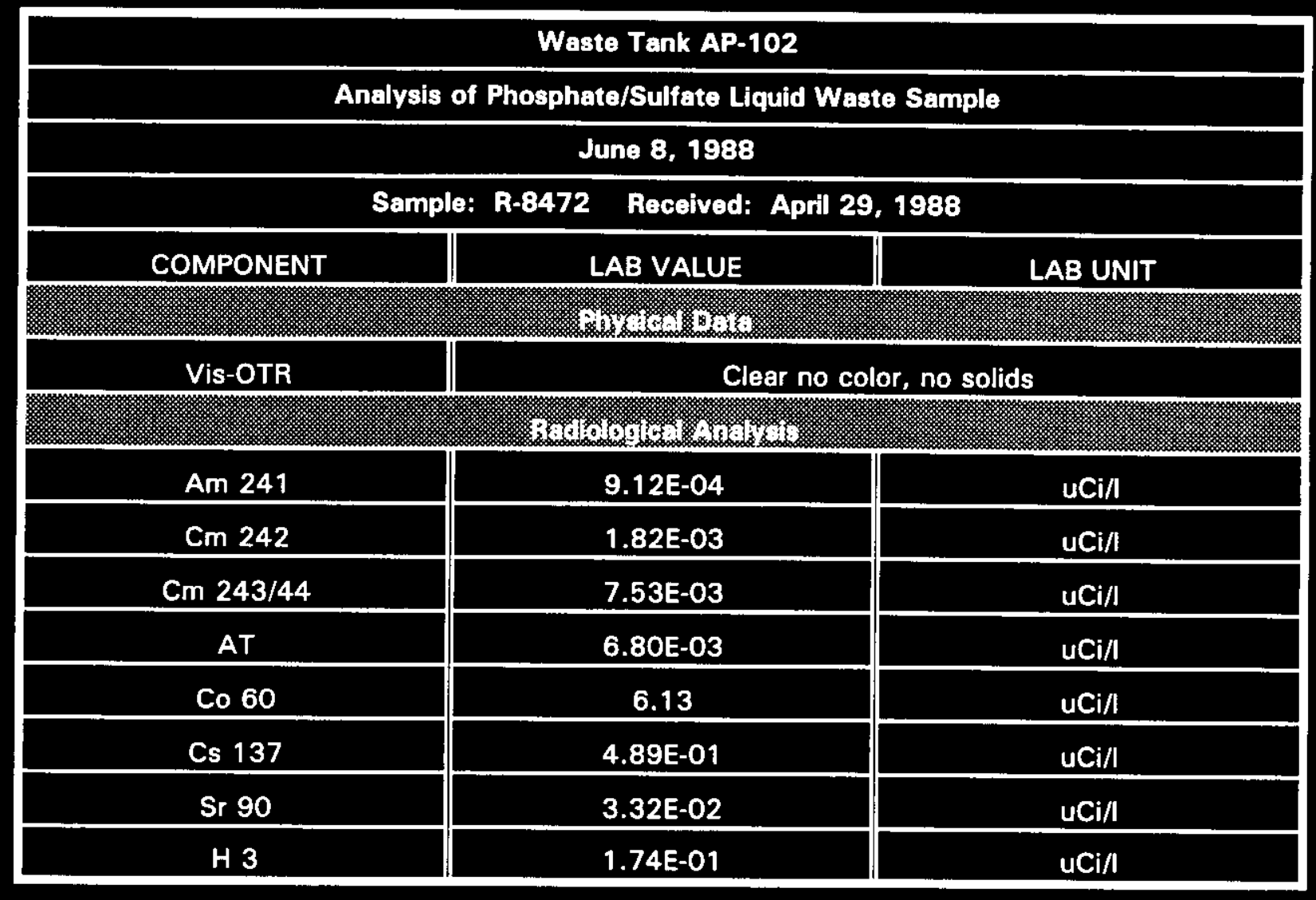




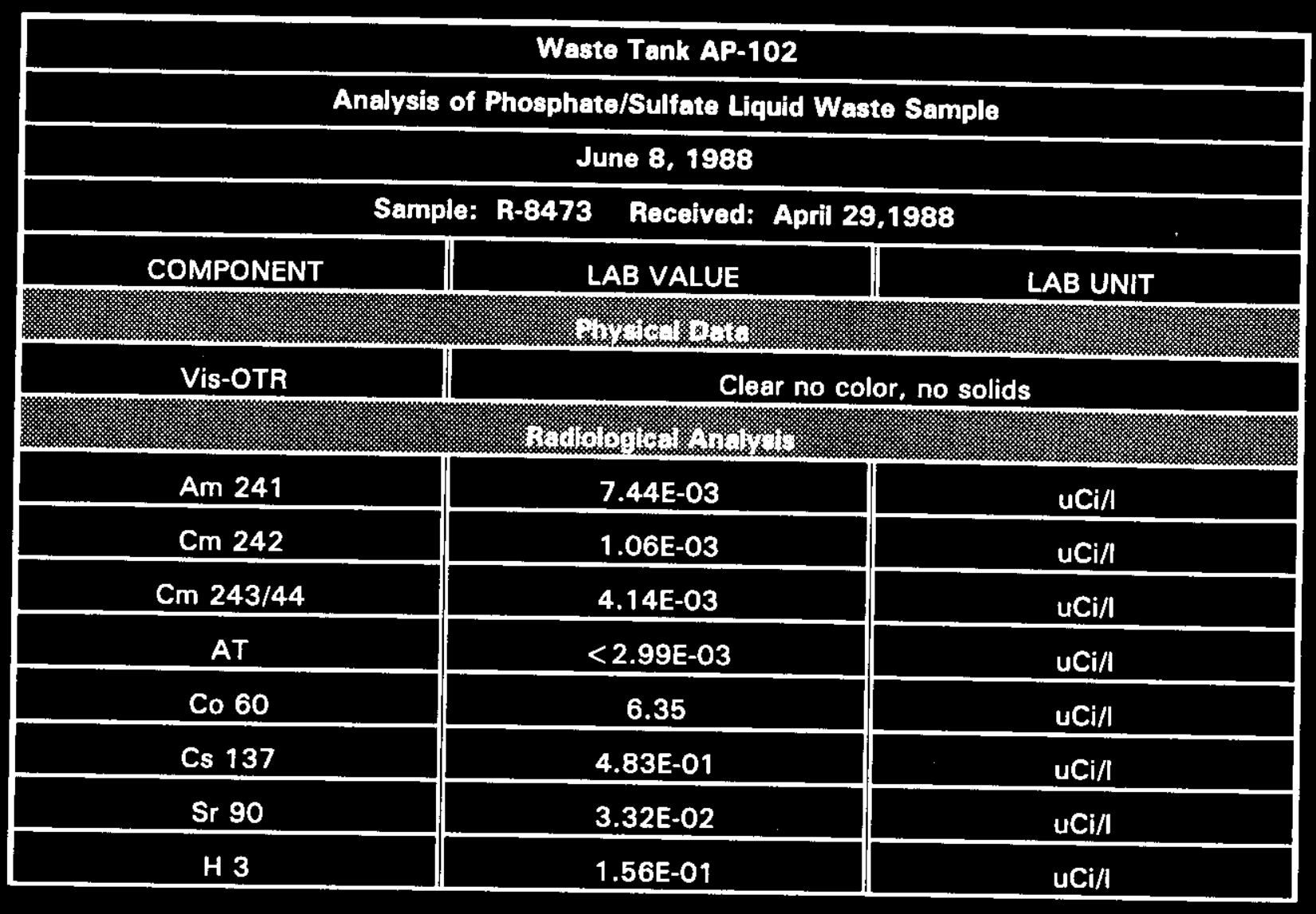




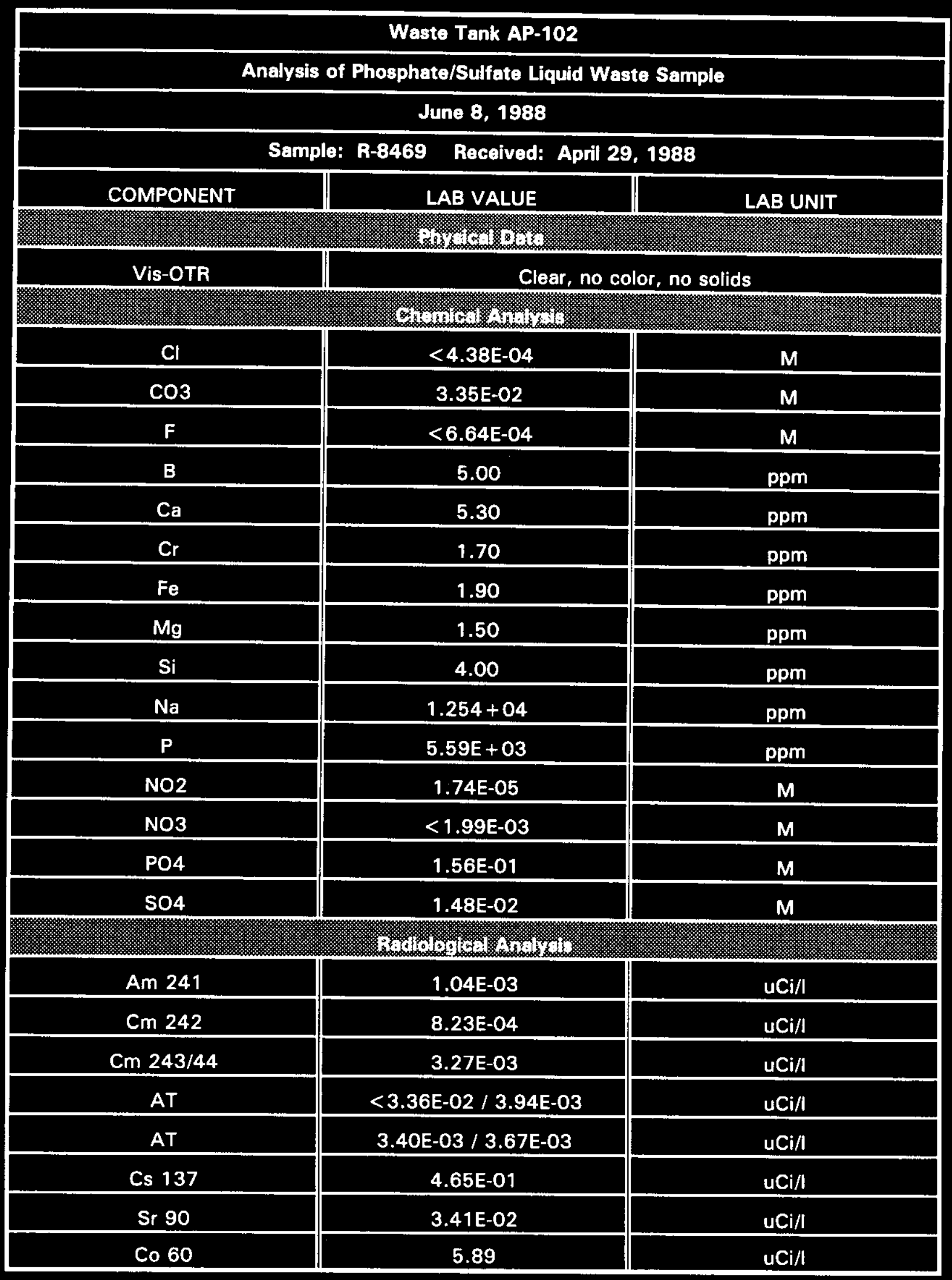




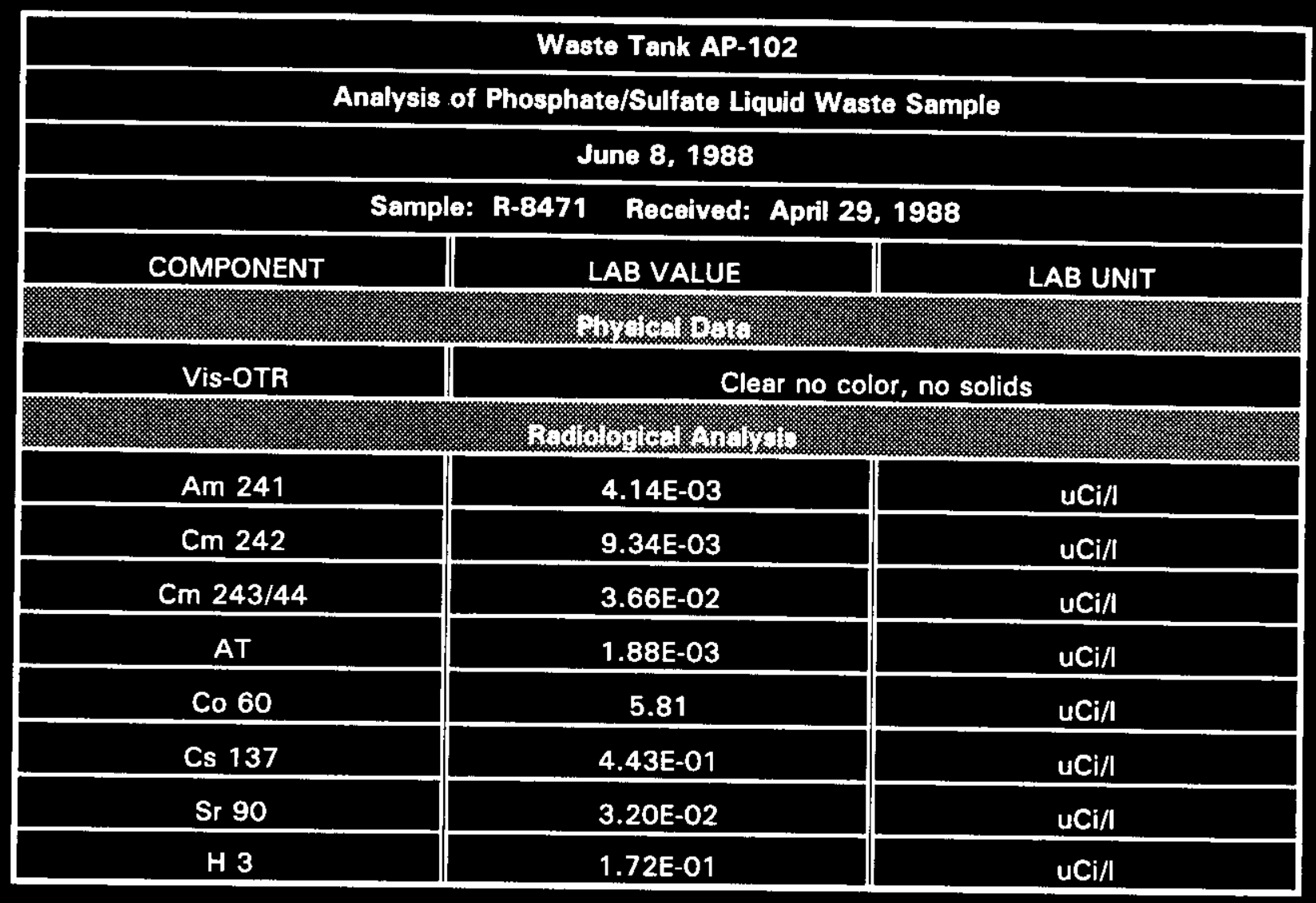




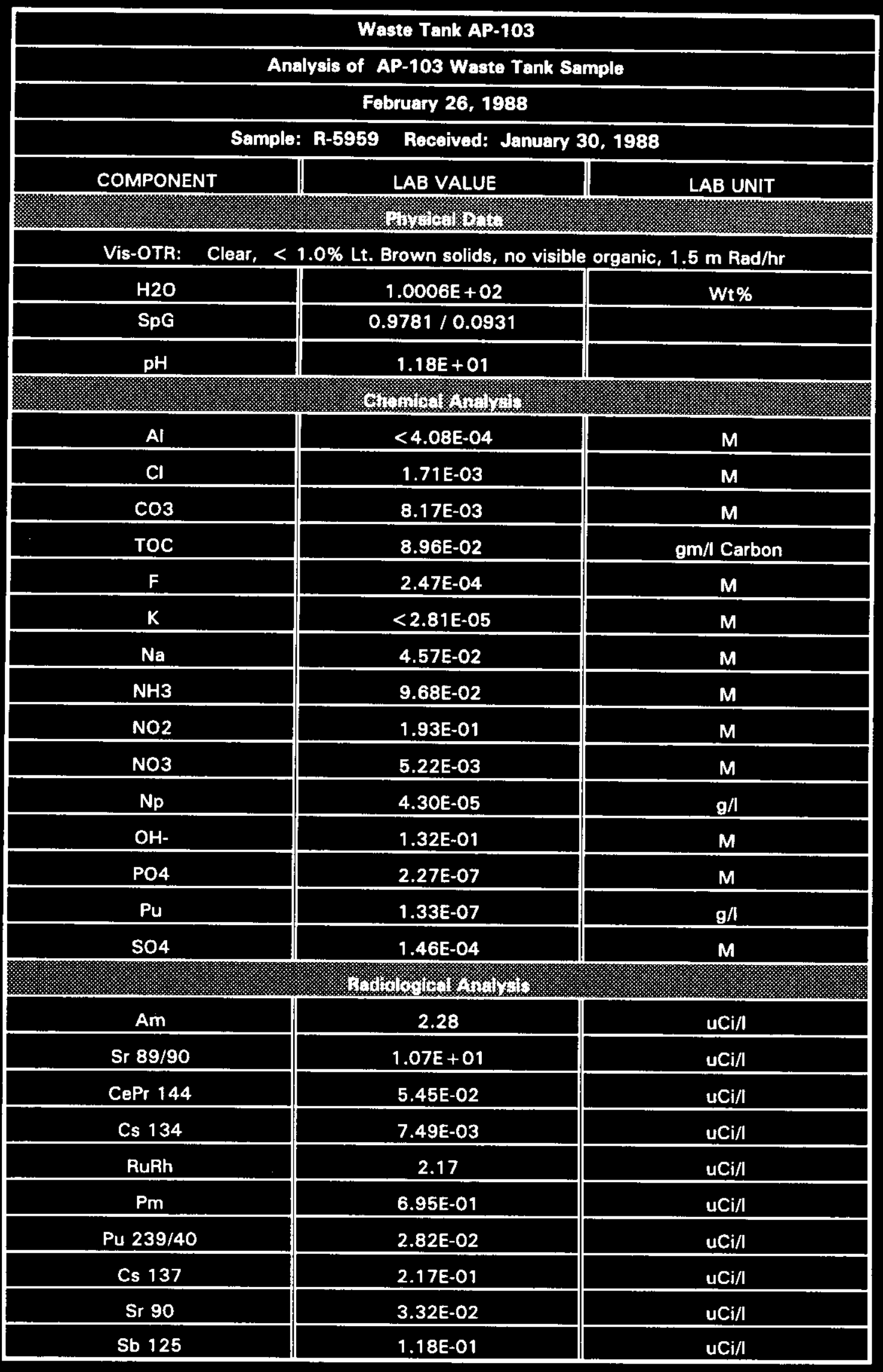




\begin{tabular}{|c|c|c|}
\hline \multicolumn{3}{|c|}{ Waste Tank AP-103 } \\
\hline \multicolumn{3}{|c|}{ Analysis of AP-103 Waste Tank Sample } \\
\hline \multicolumn{3}{|c|}{ February 23, 1988} \\
\hline \multicolumn{3}{|c|}{ Sample: R-7370 Received: Febuary 8, 1988} \\
\hline COMPONENT & LAB VALUE & LAB UNIT \\
\hline \multicolumn{3}{|c|}{ 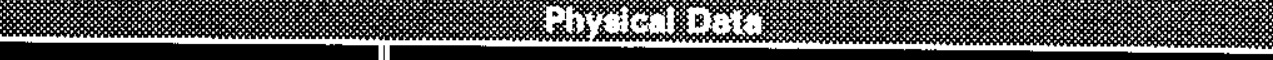 } \\
\hline Vis-OTR & \multicolumn{2}{|c|}{ Clear, no color, a few solids present } \\
\hline $\mathbf{p H}$ & $1.21 \mathrm{E}+01$ & \\
\hline \multicolumn{3}{|c|}{ 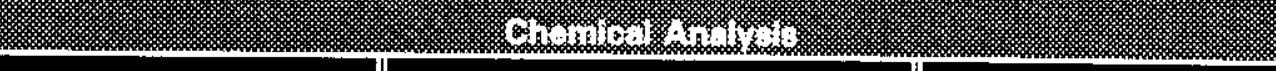 } \\
\hline $\mathrm{Cl}$ & $<1.80 E-04$ & M \\
\hline $\mathrm{Ca}$ & 2.47E-05 & $\mathbf{M}$ \\
\hline $\mathrm{Mg}$ & 2.47E-06 & $\mathbf{M}$ \\
\hline $\mathbf{F}$ & $<5.60 \mathrm{E}-04$ & M \\
\hline $\mathrm{Na}$ & $6.48 \mathrm{E}-02$ & $\mathbf{M}$ \\
\hline NH3 & $1.69 \mathrm{E}-01$ & M \\
\hline NO2 & 2.45E-01 & $\mathbf{M}$ \\
\hline NO3 & $5.31 \mathrm{E}-03$ & $\mathbf{M}$ \\
\hline $\mathrm{OH}$ & 1.87E-01 break at pH 7.5 & $\mathbf{M}$ \\
\hline PO4 & $<5.38 \mathrm{E}-04$ & $\mathbf{M}$ \\
\hline SO4 & $<5.35 \mathrm{E}-04$ & $\mathbf{M}$ \\
\hline \multicolumn{3}{|c|}{ 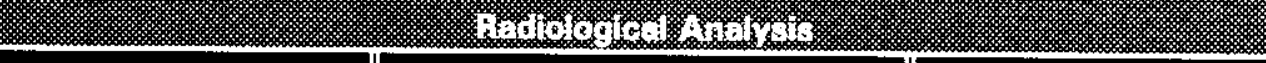 } \\
\hline RuRh 106 & 5.13 & uCi/l \\
\hline Cs 137 & 5.77E-01 & $\mathrm{uCi} / \mathrm{l}$ \\
\hline
\end{tabular}




\begin{tabular}{|c|c|c|}
\hline \multicolumn{3}{|c|}{ Waste Tank AP-103 } \\
\hline \multicolumn{3}{|c|}{ Analysis of AP-103 Waste Tank Sample } \\
\hline \multicolumn{3}{|c|}{ June 24, 1988} \\
\hline \multicolumn{3}{|c|}{ Sample: R-8090 Received: April 6, 1988} \\
\hline COMPONENT & LAB VALUE & LAB UNIT \\
\hline \multicolumn{3}{|c|}{ 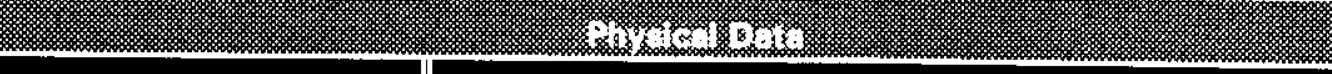 } \\
\hline Vis-OTR & \multicolumn{2}{|c|}{ Clear, no color, no solids } \\
\hline $\mathrm{pH}$ & $1.21 E+01$ & \\
\hline \multicolumn{3}{|c|}{ 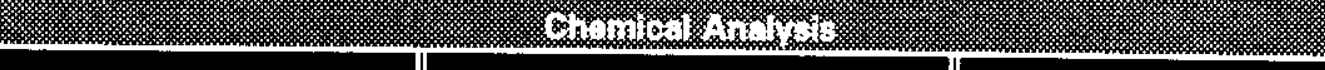 } \\
\hline $\mathrm{Cl}$ & $2.53 E-02$ & $\mathbf{M}$ \\
\hline $\mathrm{co3}$ & 5.67E-03 & $\mathbf{M}$ \\
\hline TOC & $4.23 \mathrm{E}-01$ & $\mathrm{gm} / \mathrm{l}$ Carbon \\
\hline $\mathbf{F}$ & $9.57 \mathrm{E}-04$ & $\mathbf{M}$ \\
\hline $\mathrm{Ba}$ & $1.79 \mathrm{E}-05$ & M \\
\hline $\mathbf{B}$ & $6.78 \mathrm{E}-04$ & $\mathbf{M}$ \\
\hline Al & $2.22 \mathrm{E}-04$ & $\mathbf{M}$ \\
\hline $\mathrm{Mg}$ & 9.00E-06 & M \\
\hline $\mathrm{Ca}$ & $1.64 \mathrm{E}-04$ & $\mathbf{M}$ \\
\hline $\mathrm{Na}$ & 7.53E-02 & M \\
\hline $\mathrm{NH3}$ & $1.92 \mathrm{E}-01$ & M \\
\hline NO2 & 2.98E-02 & $\mathbf{M}$ \\
\hline NO3 & 4.49E-03 & $\mathbf{M}$ \\
\hline $\mathrm{OH}-$ & $1.89 \mathrm{E}-01$ break at $\mathrm{pH} 7.7$ & $\mathbf{M}$ \\
\hline PO4 & $<1.30 E-03$ & $\mathbf{M}$ \\
\hline SO4 & $<1.31 \mathrm{E}-03$ & M \\
\hline \multicolumn{3}{|c|}{ - H. } \\
\hline RuRh $106 \pm 13 \%$ & 8.82 & $\mathrm{uCi} / \mathrm{I}$ \\
\hline Cs 137 & 6.02 & $\mathrm{uCi} / \mathrm{I}$ \\
\hline
\end{tabular}




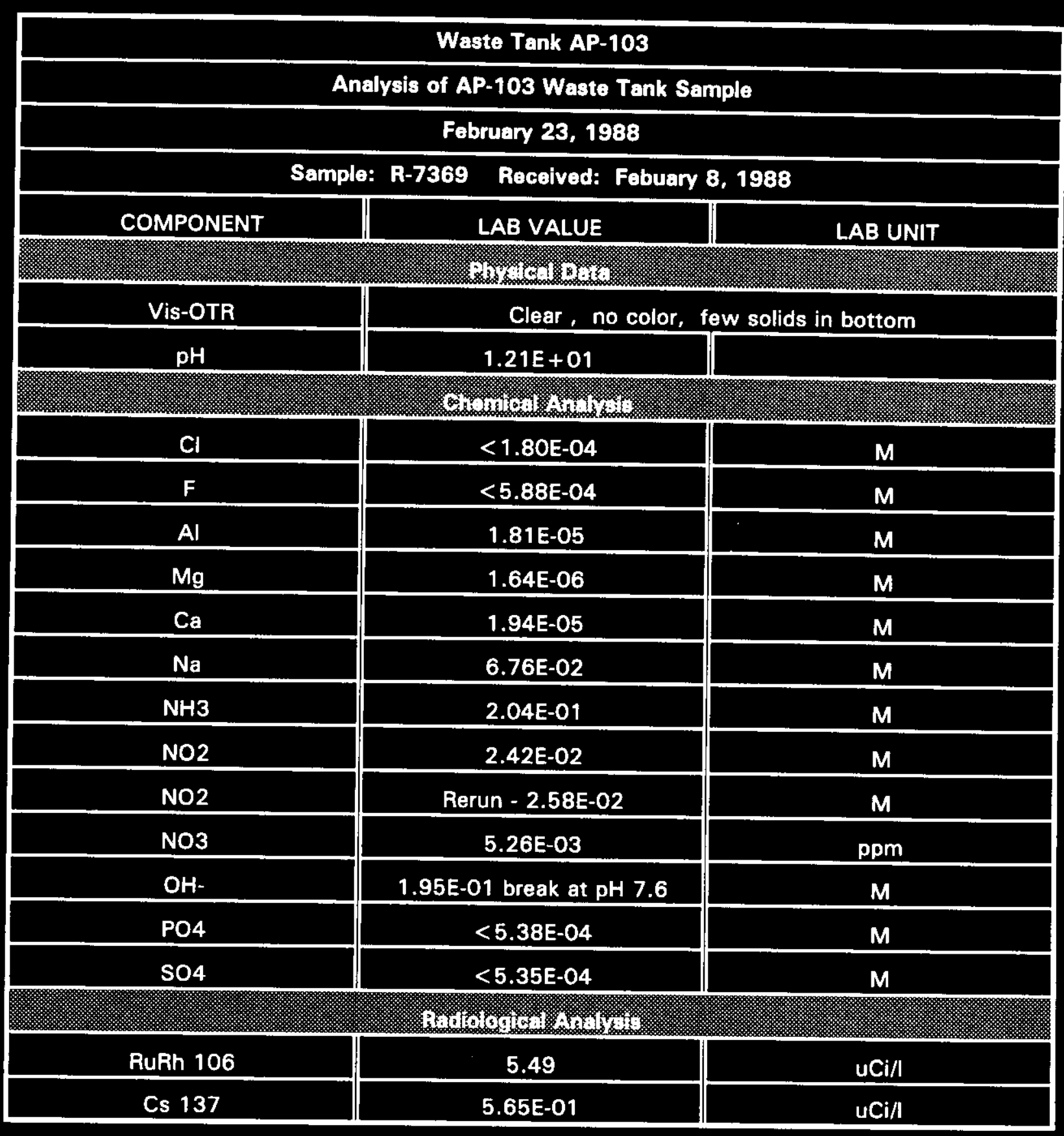




\begin{tabular}{|c|c|c|}
\hline \multicolumn{3}{|c|}{ Waste Tank AP-104 } \\
\hline \multicolumn{3}{|c|}{ Analysis of AP-104 Waste Tank Sample } \\
\hline \multicolumn{3}{|c|}{ August 5, 1987} \\
\hline \multicolumn{3}{|c|}{ Sample: T-513 Received: July 10, 1987} \\
\hline COMPONENT & LAB VALUE & LAB UNIT \\
\hline \multicolumn{3}{|c|}{ 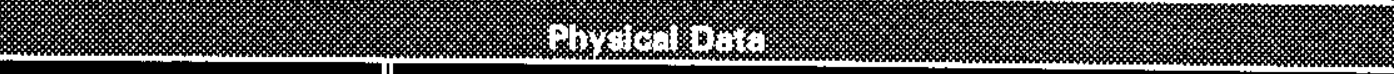 } \\
\hline Vis-OTR & \multicolumn{2}{|c|}{ Yellow brown aqueous phase, $<1 \%$ solids, $<1 \mathrm{mRad} / \mathrm{hr}$} \\
\hline $\mathrm{pH}$ & $1.146 \mathrm{E}+01$ & \\
\hline \multicolumn{3}{|c|}{ 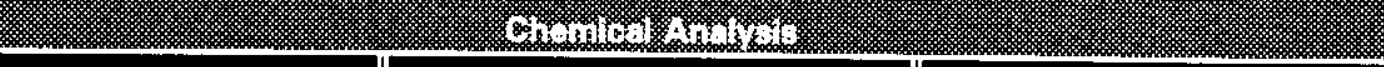 } \\
\hline $\mathrm{Cl}$ & $2.39 \mathrm{E}-03$ & $M$ \\
\hline $\mathbf{F}$ & $<6.80 \mathrm{E}-04$ & $\mathbf{M}$ \\
\hline $\mathrm{Ca}$ & $9.86 \mathrm{E}-05$ & $\mathbf{M}$ \\
\hline $\mathrm{Cr}$ & 4.74E-05 & $M$ \\
\hline Fe & $5.23 \mathrm{E}-05$ & $\mathbf{M}$ \\
\hline $\mathrm{Na}$ & 2.21E-01 & $\mathbf{M}$ \\
\hline $\mathbf{P}$ & 8.03999E-02 & $M$ \\
\hline $\mathrm{Mg}$ & 4.57E-04 & M \\
\hline NO3 & $<8.16 \mathrm{E}-03$ & $\mathbf{M}$ \\
\hline NO2 & 2.27E-05 & M \\
\hline PO4 & $1.65 \mathrm{E}-01$ & $\mathbf{M}$ \\
\hline SO4 & $1.53 \mathrm{E}-02$ & $\mathbf{M}$ \\
\hline $\mathbf{U}$ & 2.09E-04 & $g / /$ \\
\hline TOC & $2.48 \mathrm{E}-02$ & gm/l Carbon \\
\hline \multicolumn{3}{|c|}{ (3). } \\
\hline $\mathrm{Am}$ & $<9.28 \mathrm{E}-01$ & $\mathrm{uCi} / \mathrm{l}$ \\
\hline $\mathrm{Tb}$ & 9.03 & $\mathrm{uCi} / \mathrm{l}$ \\
\hline At & 4.58E-02 & $\mathrm{uCi} / \mathrm{I}$ \\
\hline Co 60 & 3.62 & $\mathrm{uCi} / \mathrm{l}$ \\
\hline Mn 54 & $2.36 \mathrm{E}-01$ & $u C i / l$ \\
\hline$I-129$ & $<1.64$ E-02 & $u C i / l$ \\
\hline PU 239/40 & 6.66999E-02 & $u C i / l$ \\
\hline Cs 137 & $6.28 \mathrm{E}-01$ & uCi/l \\
\hline
\end{tabular}




\section{APPENDIX I}

\section{Tank Photographs}




\section{THIS PAGE INTENTIONALLY}

LEFT BLANK 


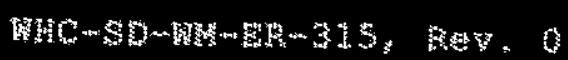

\section{AP TANK FARM}

(1)

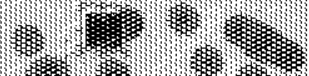

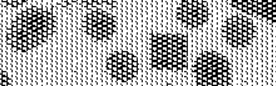

re

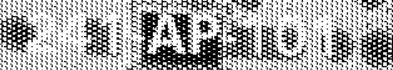

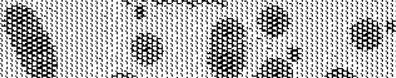

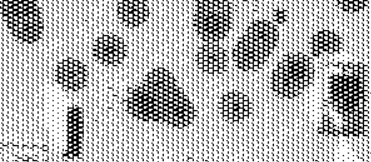

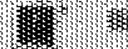

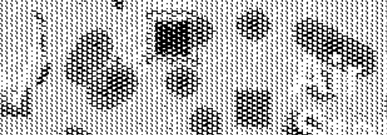

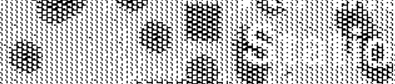

से)

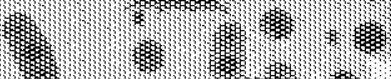

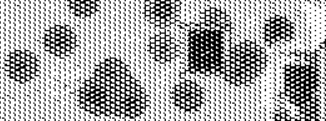

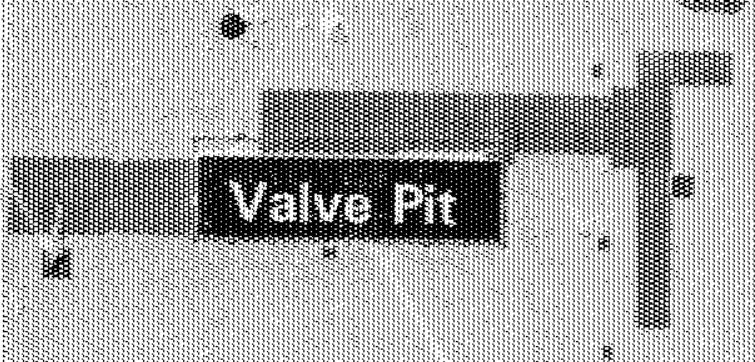

(ํ)

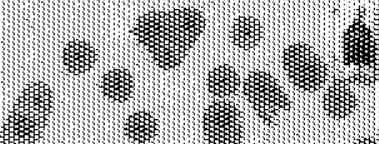

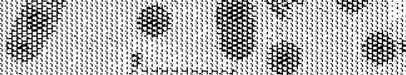

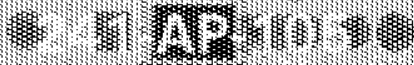

貿

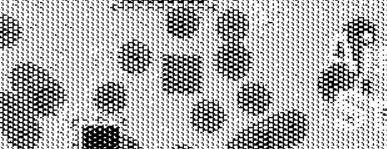

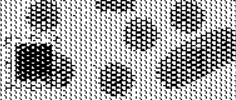

I)

留

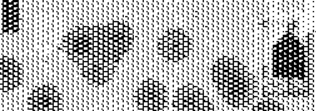

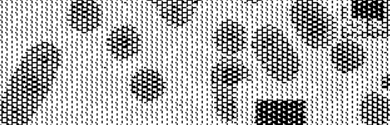
ב(ב) (1)

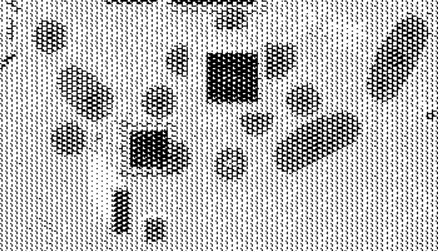

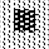

站

(a)

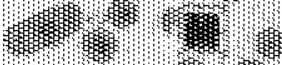

(c)

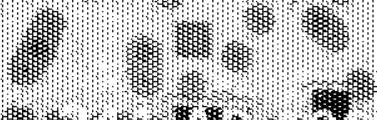

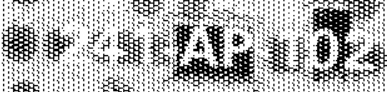

留

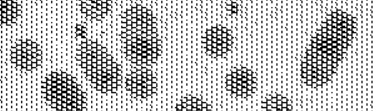



留范

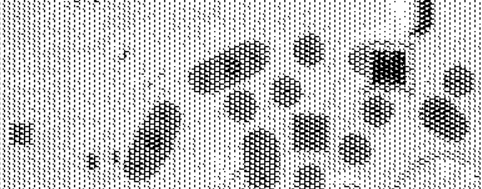

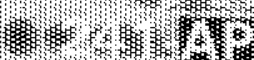

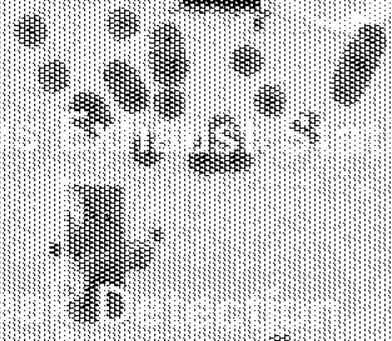

$\left(\frac{1}{)}\right)$

貿

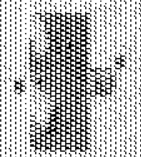

䭅

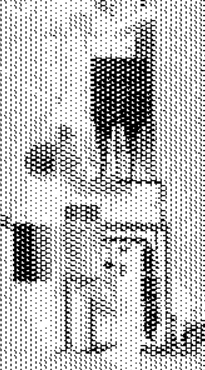

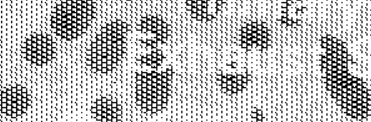

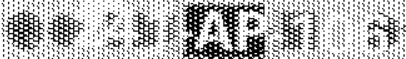

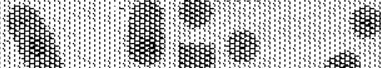

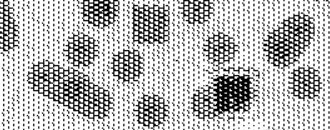

(ב)

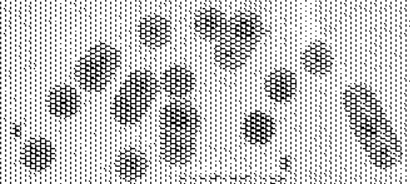

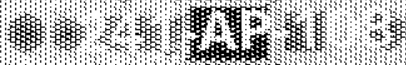

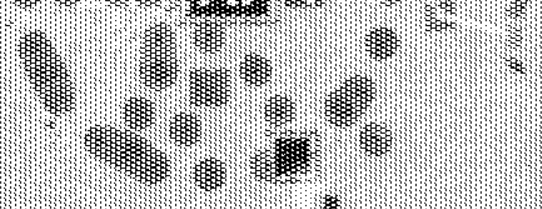

(I) 
THIS PAGE INTENTIONALWY

IEFT BLANK 


\section{APPENDIX J}

\section{Inventory Estimates}




$$
\not
$$




\begin{tabular}{|c|c|c|c|}
\hline \multicolumn{4}{|c|}{ Double-Shell Tank 241-AP-101 } \\
\hline \multicolumn{4}{|c|}{ Total Inventory Estimate* } \\
\hline \multicolumn{4}{|l|}{ (3) } \\
\hline Total Waste & \multicolumn{3}{|c|}{$4.16 \mathrm{E}+06 \mathrm{~kg} \quad(1.06 \mathrm{E}+03 \mathrm{kgal})$} \\
\hline Heat Load & \multicolumn{3}{|c|}{$0.425 \mathrm{~kW}(1.45 \mathrm{E}+03 \mathrm{BTU} / \mathrm{hr})$} \\
\hline Bulk Density $\dagger$ & \multicolumn{3}{|c|}{$1.04(\mathrm{~g} / \mathrm{cc})$} \\
\hline Water wt\% $\%$ & \multicolumn{3}{|c|}{93.2} \\
\hline TOC wt $\% \mathrm{C}$ (wet) & \multicolumn{3}{|c|}{0.086} \\
\hline \multicolumn{2}{|c|}{ 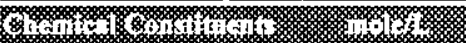 } & 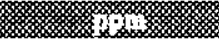 & 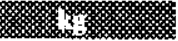 \\
\hline $\mathrm{Na}^{+}$ & 0.845 & $1.87 E+64$ & $7.79 \mathrm{E}+04$ \\
\hline$\left.A\right|^{3+}$ & $8.79 \mathrm{E}-02$ & $2.29 \mathrm{E}+03$ & $9.51 \mathrm{E}+03$ \\
\hline $\mathrm{Fe}^{3+}($ total $\mathrm{Fe})$ & 2.59E-03 & 139 & 579 \\
\hline $\mathrm{Cr}^{3+}$ & $2.57 \mathrm{E}-03$ & 129 & 537 \\
\hline $\mathrm{Bi}^{3+}$ & 5.17E-05 & 10.4 & 43.4 \\
\hline $\mathrm{La}^{3+}$ & $3.60 \mathrm{E}-07$ & $4.82 \mathrm{E}-02$ & 0.201 \\
\hline $\mathrm{Hg}^{2+}$ & $1.11 \mathrm{E}-06$ & 0.216 & 0.897 \\
\hline $\mathrm{Zr}$ (as $\left.\mathrm{ZrO}(\mathrm{OH})_{2}\right)$ & $2.68 \mathrm{E}-04$ & 23.6 & 98.1 \\
\hline $\mathrm{Pb}^{2+}$ & $8.83 \mathrm{E}-06$ & 1.76 & 7.34 \\
\hline $\mathrm{Ni}^{2+}$ & $5.72 \mathrm{E}-04$ & 32.4 & 135 \\
\hline $\mathrm{Sr}^{2+}$ & $3.80 \mathrm{E}-07$ & $3.21 \mathrm{E}-02$ & 0.134 \\
\hline $\mathrm{Mn}^{4+}$ & $1.12 \mathrm{E}-03$ & 59.5 & 247 \\
\hline $\mathrm{Ca}^{2+}$ & $1.81 \mathrm{E}-02$ & 699 & $2.91 \mathrm{E}+03$ \\
\hline $\mathbf{K}^{+}$ & $1.95 \mathrm{E}-02$ & 734 & $3.06 \mathrm{E}+03$ \\
\hline$\overline{\mathrm{OH}}$ & 0.414 & $6.78 \mathrm{E}+03$ & $2.82 \mathrm{E}+04$ \\
\hline $\mathrm{NO}^{-}$ & 0.407 & $2.43 E+04$ & $1.01 \mathrm{E}+05$ \\
\hline $\mathrm{NO} 2^{\circ}$ & $9.06 \mathrm{E}-02$ & $4.02 E+03$ & $1.67 \mathrm{E}+04$ \\
\hline $\mathrm{CO}^{2-}$ & $5.86 \mathrm{E}-02$ & $3.39 \mathrm{E}+03$ & $1.41 \mathrm{E}+04$ \\
\hline $\mathrm{PO}^{3-}$ & $1.57 \mathrm{E}-02$ & $1.44 \mathrm{E}+03$ & $5.97 E+03$ \\
\hline $\mathrm{SO}^{2-}$ & $1.90 \mathrm{E}-02$ & $1.76 \mathrm{E}+03$ & $7.32 \mathrm{E}+03$ \\
\hline $\mathrm{Si}\left(\right.$ as $\mathrm{SiO}_{3}{ }^{2}$ ) & $4.23 \mathrm{E}-03$ & 114 & 476 \\
\hline$F^{*}$ & $2.23 \mathrm{E}-02$ & 409 & $1.70 \mathrm{E}+03$ \\
\hline $\mathrm{Cl}^{-}$ & $1.33 \mathrm{E}-02$ & 454 & $1.89 \mathrm{E}+03$ \\
\hline $\mathrm{C}_{6} \mathrm{H}_{5} \mathrm{O}_{7}{ }^{3-}$ & $2.48 \mathrm{E}-03$ & 453 & $1.88 \mathrm{E}+03$ \\
\hline EDTA $^{4-}$ & $7.29 \mathrm{E}-04$ & 202 & 843 \\
\hline HEDTA $^{3 \cdot}$ & $1.21 \mathrm{E}-03$ & 319 & $1.33 \mathrm{E}+03$ \\
\hline glycolate- & $1.94 \mathrm{E}-02$ & $1.40 \mathrm{E}+03$ & $5.82 \mathrm{E}+03$ \\
\hline acetate & $8.03 \mathrm{E}-04$ & 45.7 & 190 \\
\hline oxalate ${ }^{2 \cdot}$ & $1.36 \mathrm{E}-06$ & 0.115 & 0.480 \\
\hline DBP & $1.13 \mathrm{E}-03$ & 176 & 731 \\
\hline butanol & $1.13 \mathrm{E}-03$ & 80.9 & 337 \\
\hline $\mathrm{NH}_{3}$ & 0.108 & $1.77 \mathrm{E}+03$ & $7.37 \mathrm{E}+03$ \\
\hline $\mathrm{Fe}(\mathrm{CN})_{6}{ }^{4-}$ & & 0 & 0 \\
\hline \multicolumn{4}{|c|}{ W } \\
\hline $\mathrm{Pu}$ & & $5.93 \mathrm{E}-03(\mu \mathrm{Ci} / \mathrm{g})$ & $0.411(\mathrm{~kg})$ \\
\hline$\overline{\mathrm{U}}$ & $8.09 \mathrm{E}-04(\mathrm{M})$ & $186(\mu g / g)$ & $772(\mathrm{~kg})$ \\
\hline$\overline{\mathrm{Cs}}$ & $1.96 \mathrm{E}-02(\mathrm{Ci} / \mathrm{L})$ & $18.9(\mu \mathrm{Ci} / \mathrm{g})$ & $7.85 \mathrm{E}+04(\mathrm{Ci})$ \\
\hline $\mathrm{Sr}$ & $2.13 \mathrm{E}-03(\mathrm{CT} / \mathrm{L})$ & $2.06(\mu \mathrm{Ci} / \mathrm{g})$ & $8.56 \mathrm{E}+03(\mathrm{Ci})$ \\
\hline
\end{tabular}

*Unknowns in tank solids inventory are assigned by Tank Layering Model (TLM). †Volume average for density, mass average Water wt \% and ToC wt $\% \mathrm{C}$.

Derived by Los Alamos National Laboratory (LANL). 


\begin{tabular}{|c|c|c|c|}
\hline \multicolumn{4}{|c|}{ Double-Shell Tank 241-AP-101 } \\
\hline \multicolumn{4}{|c|}{ SMM Composite Inventory Estimate } \\
\hline \multicolumn{4}{|l|}{ mon } \\
\hline Total Supernatant & \multicolumn{3}{|c|}{$4.16 \mathrm{E}+06 \mathrm{~kg} \quad(1.06 \mathrm{E}+03 \mathrm{kgal})$} \\
\hline Heat Load & \multicolumn{3}{|c|}{$0.425 \mathrm{~kW}(1.45 \mathrm{E}+03 \mathrm{BTU} / \mathrm{hr})$} \\
\hline Bulk Density" & \multicolumn{3}{|c|}{$1.04(\mathrm{~g} / \mathrm{cc})$} \\
\hline Water wt\% & \multicolumn{3}{|c|}{93.2} \\
\hline TOC wt \% C (wet) & \multicolumn{3}{|c|}{0.086} \\
\hline \multicolumn{2}{|c|}{$(1,1)$} & 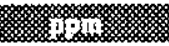 & 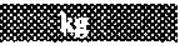 \\
\hline $\mathrm{Na}^{+}$ & 0.845 & $1.87 \mathrm{E}+04$ & $7.79 \mathrm{E}+04$ \\
\hline $\mathrm{Al}^{3+}$ & $8.79 \mathrm{E}-02$ & $2.29 \mathrm{E}+03$ & $9.51 \mathrm{E}+03$ \\
\hline $\mathrm{Fe}^{3+}$ (total Fe) & $2.59 \mathrm{E}-03$ & 139 & 579 \\
\hline $\mathrm{Cr}^{3+}$ & $2.57 \mathrm{E}-03$ & 129 & 537 \\
\hline $\mathrm{Bi}^{3+}$ & S.17E-05 & 10.4 & 43.4 \\
\hline$\overline{\mathrm{La}^{3+}}$ & $3.60 \mathrm{E}-07$ & $4.82 \mathrm{E}-02$ & 0.201 \\
\hline $\mathrm{Hg}^{2+}$ & 1.11E-06 & 0.216 & 0.897 \\
\hline $\mathrm{Zr}\left(\right.$ as $\left.\mathrm{ZrO}(\mathrm{OH})_{2}\right)$ & $2.68 \mathrm{E}-04$ & 23.6 & 98.1 \\
\hline $\mathrm{Pb}^{2+}$ & $8.83 \mathrm{E}-06$ & 1.76 & 7.34 \\
\hline $\mathrm{Ni}^{2+}$ & $5.72 \mathrm{E}-04$ & 32.4 & 135 \\
\hline $\mathrm{Sr}^{2+}$ & $3.80 \mathrm{E}-07$ & $3.21 \mathrm{E}-02$ & 0.134 \\
\hline $\mathrm{Mn}^{4+}$ & $1.12 \mathrm{E}-03$ & 59.5 & 247 \\
\hline $\mathrm{Ca}^{2+}$ & $1.81 \mathrm{E}-02$ & 699 & $2.91 \mathrm{E}+03$ \\
\hline $\mathrm{K}^{+}$ & 1.95E-02 & 734 & $3.06 \mathrm{E}+03$ \\
\hline $\mathrm{OH}^{\circ}$ & 0.414 & $6.78 \mathrm{E}+03$ & $2.82 \mathrm{E}+04$ \\
\hline $\mathrm{NO}^{-}$ & 0.407 & $2.43 E+04$ & $1.01 \mathrm{E}+05$ \\
\hline $\mathrm{NO} 2^{-}$ & $9.06 \mathrm{E}-02$ & $4.02 \mathrm{E}+03$ & $1.67 \mathrm{E}+04$ \\
\hline $\mathrm{CO}^{2-}$ & $5.86 \mathrm{E}-02$ & $3.39 \mathrm{E}+03$ & $1.41 \mathrm{E}+04$ \\
\hline $\mathrm{PO}^{3-}$ & $1.57 \mathrm{E}-02$ & $1.44 \mathrm{E}+03$ & $5.97 \mathrm{E}+03$ \\
\hline $\mathrm{SO}^{2-}$ & $1.90 \mathrm{E}-02$ & $1.76 \mathrm{E}+03$ & $7.32 \mathrm{E}+03$ \\
\hline $\mathrm{Si}\left(\mathrm{as} \mathrm{SiO}_{3}{ }^{2}\right.$ ) & $4.23 E-03$ & 114 & 476 \\
\hline$F^{-}$ & $2.23 \mathrm{E}-02$ & 409 & $1.70 \mathrm{E}+03$ \\
\hline $\mathrm{Cl}^{-}$ & $1.33 \mathrm{E}-02$ & 454 & $1.89 \mathrm{E}+03$ \\
\hline $\mathrm{C}_{6} \mathrm{H}_{5} \mathrm{O}_{7}{ }^{3-}$ & $2.48 \mathrm{E}-03$ & 453 & $1.88 \mathrm{E}+03$ \\
\hline EDTA $^{4-}$ & $7.29 \mathrm{E}-04$ & 202 & 843 \\
\hline HEDTA $^{3-}$ & $1.21 \mathrm{E}-03$ & 319 & $1.33 \mathrm{E}+03$ \\
\hline glycolate & $1.94 \mathrm{E}-02$ & $1.40 \mathrm{E}+03$ & $5.82 \mathrm{E}+03$ \\
\hline acetate & $8.03 \mathrm{E}-04$ & 45.7 & 190 \\
\hline oxalate ${ }^{2-}$ & $1.36 \mathrm{E}-06$ & 0.115 & 0.480 \\
\hline DBP & $1.13 \mathrm{E}-03$ & 176 & 731 \\
\hline butanol & $1.13 \mathrm{E}-03$ & 80.9 & 337 \\
\hline $\mathrm{NH}_{3}$ & 0.108 & $1.77 \mathrm{E}+03$ & $7.37 \mathrm{E}+03$ \\
\hline $\mathrm{Fe}(\mathrm{CN})_{6}{ }^{4-}$ & & 0 & \\
\hline \multicolumn{4}{|c|}{ 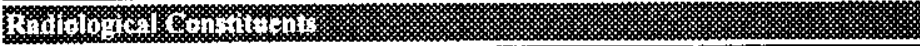 } \\
\hline $\mathrm{Pu}$ & $6.15(\mu \mathrm{Ci} / \mathrm{L})$ & & $0.411(\mathrm{~kg})$ \\
\hline$\overline{\mathrm{U}}$ & 8.09E-04 (M) & $186(\mu \mathrm{g} / \mathrm{g})$ & $772(\mathrm{~kg})$ \\
\hline Cs & $1.96 \mathrm{E} \cdot 02(\mathrm{CZ} / \mathrm{L})$ & $18.9(\mu \mathrm{Cl} / \mathrm{g})$ & $7.85 \mathrm{E}+04(\mathrm{Cl})$ \\
\hline$\overline{\mathrm{Sr}}$ & $2.13 \mathrm{E}-03(\mathrm{CZ} / \mathrm{L})$ & $2.06(\mu \mathrm{Ci} / \mathrm{g})$ & $8.56 \mathrm{E}+03(\mathrm{Ci})$ \\
\hline
\end{tabular}

*Density is calculated based on $\mathrm{Na}, \mathrm{OH}$, and $\mathrm{AlO}_{2}$.

tWater wt $\%$ derived from the difference of density and total dissolved species.

Derived by Los Alamos National Laboratory (LANL). 


\begin{tabular}{|c|c|c|c|}
\hline \multicolumn{4}{|c|}{ Double-Shell Tank 241-AP-101 } \\
\hline \multicolumn{4}{|c|}{ TLM Solids Composite Inventory Estimate* } \\
\hline \multicolumn{4}{|c|}{ 13. } \\
\hline Total Solid Waste & $0 \mathrm{~kg}$ & (0 kgal) & \\
\hline Heat Load & $0 \mathbf{k W}$ & (0 BTU/hr) & \\
\hline Bulk Density & & $0(\mathrm{~g} / \mathrm{cc})$ & \\
\hline Water wt\% & & 0 & \\
\hline TOC wt\% C (wet) & & 0 & \\
\hline \multicolumn{4}{|c|}{ mos } \\
\hline $\mathrm{Na}^{+}$ & 0 & 0 & 0 \\
\hline$A \mathbf{I}^{3+}$ & 0 & 0 & $\overline{0}$ \\
\hline $\mathrm{Fe}^{3+}$ (total $\left.\mathrm{Fe}\right)$ & 0 & 0 & $\overline{0}$ \\
\hline $\mathrm{Cr}^{3+}$ & 0 & 0 & 0 \\
\hline $\mathbf{B i}^{3+}$ & 0 & 0) & 0 \\
\hline $\mathrm{La}^{3+}$ & 0 & 0 & 0 \\
\hline $\mathrm{Hg}^{2+}$ & 0 & 0 & 0 \\
\hline $\mathrm{ZI}\left(\right.$ as $\left.\mathrm{ZrO}(\mathrm{OH})_{2}\right)$ & 0 & 0 & 0 \\
\hline $\mathrm{Pb}^{2+}$ & 0 & 0 & 0 \\
\hline $\mathrm{Ni}^{2+}$ & 0 & 0 & 0 \\
\hline $\mathrm{Sr}^{2+}$ & 0 & 0 & 0 \\
\hline $\mathrm{Mn}^{4+}$ & 0 & 0 & 0 \\
\hline $\mathrm{Ca}^{2+}$ & 0) & 0 & 0 \\
\hline $\mathrm{K}^{+}$ & 0 & 0 & 0 \\
\hline $\mathrm{OH}$ & 0 & 0 & 0 \\
\hline $\mathrm{NO}^{-}$ & 0 & 0 & 0 \\
\hline $\mathrm{NO}^{-}$ & 0 & 0 & 0 \\
\hline $\cos ^{2-}$ & 0 & 0 & 0 \\
\hline $\mathrm{PO}^{3-}$ & 0 & 0 & 0 \\
\hline $\mathrm{SO}^{2-}$ & 0 & 0 & 0 \\
\hline $\mathrm{Si}\left(\right.$ as $\left.\mathrm{SiO}_{3}{ }^{2}\right)$ & 0 & 0 & 0 \\
\hline $\mathbf{F}^{-}$ & 0 & 0 & 0 \\
\hline $\mathrm{Cl}^{-}$ & 0 & 0 & 0 \\
\hline $\mathrm{C}_{6} \mathrm{H}_{3} \mathrm{O}_{7}{ }^{3-}$ & 0 & 0 & 0 \\
\hline EDTA $^{4}$ & 0 & 0 & 0 \\
\hline HEDTA $^{3-}$ & 0 & 0 & 0 \\
\hline glycolate & 0 & 0 & 0 \\
\hline acetate ${ }^{-}$ & 0 & 0 & 0 \\
\hline oxalate & 0 & 0 & 0 \\
\hline DBP & 0 & 0 & 0 \\
\hline butanol & 0 & 0 & 0 \\
\hline $\mathrm{NH}_{3}$ & 0 & 0 & 0 \\
\hline $\mathrm{Fe}(\mathrm{CN})_{6}{ }^{4-}$ & 0 & 0 & 0 \\
\hline If I I & & & \\
\hline $\mathrm{Pu}$ & & 0 & 0 \\
\hline U & 요 & 0 & 0 \\
\hline Cs & 0) & 이 & 0 \\
\hline Sr & 0 & 0 & 0 \\
\hline
\end{tabular}

"Unknowns in tank solids inventory are assigned by Tank Layering Model (TLM)

Derived by Los Alamos National Laboratory (LANL). 


\begin{tabular}{|c|c|c|c|}
\hline \multicolumn{4}{|c|}{ Double-Shell Tank 241-AP-102 } \\
\hline \multicolumn{4}{|c|}{ Total Inventory Estimate* } \\
\hline \multicolumn{4}{|l|}{ 1\% } \\
\hline Total Waste & \multicolumn{3}{|c|}{$5.19 \mathrm{E}+06 \mathrm{~kg} \quad(1.10 \mathrm{E}+03 \mathrm{kgal})$} \\
\hline Heat Load & \multicolumn{3}{|c|}{$3.60 \mathrm{~kW}(1.23 \mathrm{E}+04 \mathrm{BTU} / \mathrm{hr})$} \\
\hline Bulk Density & \multicolumn{3}{|c|}{$1.24(\mathrm{~g} / \mathrm{cc})$} \\
\hline Water wt\% $\nmid$ & \multicolumn{3}{|c|}{66.1} \\
\hline TOC wt \% C (wet) & \multicolumn{3}{|c|}{0.596} \\
\hline \multicolumn{2}{|c|}{ 1 } & 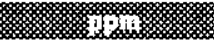 & 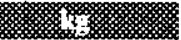 \\
\hline $\mathrm{Na}^{+}$ & 5.27 & $9.76 E+04$ & $5.07 \mathrm{E}+05$ \\
\hline $\mathrm{Al}^{3+}$ & 0.665 & $1.44 \mathrm{E}+04$ & $7.50 \mathrm{E}+04$ \\
\hline $\mathrm{Fe}^{3+}$ (total Fe) & $2.83 \mathrm{E}-03$ & 127 & 659 \\
\hline $\mathrm{Cr}^{3+}$ & $1.94 \mathrm{E}-02$ & 811 & $4.21 \mathrm{E}+03$ \\
\hline $\mathrm{Bi}^{3+}$ & 7.18E-04 & 121 & 627 \\
\hline $\mathrm{La}^{3+}$ & 4.91E-06 & 0.549 & 2.85 \\
\hline $\mathrm{Hg}^{2+}$ & $4.34 \mathrm{E}-06$ & 0.701 & 3.64 \\
\hline $\mathrm{Zr}$ (as $\left.\mathrm{ZrO}(\mathrm{OH})_{2}\right)$ & $4.30 \mathrm{E}-04$ & 31.6 & 164 \\
\hline $\mathrm{Pb}^{2+}$ & $4.00 \mathrm{E}-05$ & 6.67 & 34.6 \\
\hline $\mathrm{Ni}^{2+}$ & $2.47 \mathrm{E}-03$ & 117 & 605 \\
\hline $\mathrm{Sr}^{2+}$ & 5.19E-06 & 0.366 & 1.90 \\
\hline $\mathrm{Mn}^{4+}$ & $3.69 \mathrm{E}-03$ & 163 & 847 \\
\hline $\mathrm{Ca}^{2+}$ & $1.98 \mathrm{E}-02$ & 638 & $3.31 \mathrm{E}+03$ \\
\hline $\mathbf{K}^{+}$ & $1.43 \mathrm{E}-02$ & 451 & $2.34 \mathrm{E}+03$ \\
\hline $\mathrm{OH}$ & 2.96 & $4.05 E+04$ & $2.10 \mathrm{E}+05$ \\
\hline $\mathrm{NO3}^{-}$ & 1.81 & $9.02 \mathrm{E}+04$ & $4.69 \mathrm{E}+05$ \\
\hline NO2: & 1.22 & $4.52 \mathrm{E}+04$ & $2.35 \mathrm{E}+05$ \\
\hline $\mathrm{CO}^{2-}$ & 0.210 & $1.02 \mathrm{E}+04$ & $5.28 \mathrm{E}+04$ \\
\hline $\mathrm{PO}^{3-}$ & 0.109 & $8.37 \mathrm{E}+03$ & $4.34 \mathrm{E}+04$ \\
\hline $\mathrm{SO}^{2-}$ & 0.142 & $1.10 E+04$ & $5.72 \mathrm{E}+04$ \\
\hline $\mathrm{Si}\left(\right.$ as $\left.\mathrm{SiO}_{3}{ }^{2}\right)$ & $2.29 \mathrm{E}-02$ & 517 & $2.69 E+03$ \\
\hline $\mathrm{F}^{*}$ & $4.71 \mathrm{E}-02$ & 721 & $3.74 \mathrm{E}+03$ \\
\hline $\mathrm{Cl}^{-}$ & $9.13 \mathrm{E}-02$ & $2.60 \mathrm{E}+03$ & $1.35 \mathrm{E}+04$ \\
\hline $\mathrm{C}_{6} \mathrm{H}_{5} \mathrm{O}_{7}{ }^{3-}$ & $1.91 \mathrm{E}-02$ & $2.90 E+03$ & $1.51 \mathrm{E}+04$ \\
\hline EDTA $^{4-}$ & $1.04 \mathrm{E}-02$ & $2.42 E+03$ & $1.26 \mathrm{E}+04$ \\
\hline HEDT $^{3-}$ & 1.71E-02 & $3.78 \mathrm{E}+03$ & $1.96 \mathrm{E}+04$ \\
\hline glycolate & $6.36 \mathrm{E}-02$ & $3.84 \mathrm{E}+03$ & $2.00 \mathrm{E}+04$ \\
\hline acetate $^{-}$ & 1.19E-02 & 567 & $2.94 \mathrm{E}+03$ \\
\hline oxalate ${ }^{2-}$ & $1.85 \mathrm{E}-05$ & 1.31 & 6.82 \\
\hline$\overline{\mathrm{DBP}}$ & $9.86 \mathrm{E}-03$ & $1.28 \mathrm{E}+03$ & $6.63 \mathrm{E}+03$ \\
\hline butanol & $9.86 \mathrm{E}-03$ & 588 & $3.05 \mathrm{E}+03$ \\
\hline $\mathrm{NH}_{3}$ & $2.61 \mathrm{E}-02$ & 357 & $1.85 \mathrm{E}+03$ \\
\hline $\mathrm{Fe}(\mathrm{CN})_{6}{ }^{4-}$ & & 0 & $\overline{0}$ \\
\hline \multicolumn{4}{|c|}{ WiI } \\
\hline $\mathrm{Pu}$ & & $1.83 \mathrm{E}-02(\mu \mathrm{Ci} / \mathrm{g})$ & $1.58(\mathrm{~kg})$ \\
\hline$\overline{\mathbf{U}}$ & 2.95E-03 (M) & $565(\mu g / g)$ & $2.93 \mathrm{E}+03(\mathrm{~kg})$ \\
\hline$\overline{C s}$ & $0.177(\mathrm{Ci} / \mathrm{L})$ & $142(\mu \mathrm{Ci} / \mathrm{g})$ & $7.38 \mathrm{E}+05(\mathrm{Ci})$ \\
\hline$\overline{\mathrm{Sr}}$ & $5.16 \mathrm{E} \cdot 03(\mathrm{Ci} / \mathrm{L})$ & $4.15(\mu \mathrm{Ci} / \mathrm{g})$ & $2.15 \mathrm{E}+04(\mathrm{Cl})$ \\
\hline
\end{tabular}

*Unknowns in tank solids inventory are assigned by Tank Layering Model (TLM). †Volume average for density, mass average Water $w t \%$ and $\mathrm{TOC} \cdot \mathrm{w} \% \mathrm{C}$.

Derived by Los Alamos National Laboratory (LANL). 


\begin{tabular}{|c|c|c|c|}
\hline \multicolumn{4}{|c|}{ Double-Shell Tank 241-AP-102 } \\
\hline \multicolumn{4}{|c|}{ SMM Composite Inventory Estimate } \\
\hline \multicolumn{4}{|c|}{ 12. } \\
\hline Total Supernatant & $5.19 \mathrm{E}+06 \mathrm{~kg}$ & 10E+03 kgal) & \\
\hline Heat Load & \multicolumn{3}{|c|}{$3.60 \mathrm{~kW}(1.23 \mathrm{E}+04 \mathrm{BTU} / \mathrm{hr})$} \\
\hline Bulk Density* & \multicolumn{3}{|c|}{$1.24(\mathrm{~g} / \mathrm{cc})$} \\
\hline Water wt\%† & \multicolumn{3}{|c|}{66.1} \\
\hline TOC wt\% C (wet) & \multicolumn{3}{|c|}{0.596} \\
\hline \multicolumn{2}{|c|}{ 11) } & 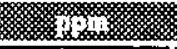 & tis \\
\hline $\mathrm{Na}^{+}$ & 5.27 & $9.76 E+04$ & $5.07 \mathrm{E}+05$ \\
\hline$A \mathrm{~A}^{3+}$ & 0.665 & $1.44 \mathrm{E}+04$ & $7.50 \mathrm{E}+04$ \\
\hline $\mathrm{Fe}^{3+}$ (total $\left.\mathrm{Fe}\right)$ & $2.83 \mathrm{E}-03$ & 127 & 659 \\
\hline $\mathrm{Cr}^{3+}$ & $1.94 \mathrm{E}-02$ & 811 & $4.21 \mathrm{TE}+03$ \\
\hline $\mathrm{Bi}^{3+}$ & $7.18 \mathrm{E}-04$ & 121 & 627 \\
\hline $\mathrm{La}^{3+}$ & 4.91E-06 & 0.549 & 2.85 \\
\hline $\mathrm{Hg}^{2+}$ & 4.34E-06 & 0.701 & 3.64 \\
\hline $\mathrm{Zr}\left(\right.$ as $\left.\mathrm{ZrO}(\mathrm{OH})_{2}\right)$ & $4.30 \mathrm{E}-04$ & 31.6 & 164 \\
\hline $\mathrm{Pb}^{2+}$ & $4.00 \mathrm{E}-05$ & 6.67 & 34.6 \\
\hline $\mathrm{Ni}^{2+}$ & $2.47 \mathrm{E}-03$ & 117 & 605 \\
\hline $\mathrm{Sr}^{2+}$ & $5.19 \mathrm{E}-06$ & 0.366 & 1.90 \\
\hline $\mathrm{Mn}^{4+}$ & $3.69 \mathrm{E}-03$ & 163 & 847 \\
\hline $\mathrm{Ca}^{2+}$ & $1.98 \mathrm{E}-02$ & 638 & $3.31 \mathrm{E}+03$ \\
\hline $\mathrm{K}^{+}$ & $1.43 \mathrm{E}-02$ & 451 & $2.34 \mathrm{E}+03$ \\
\hline $\mathrm{OH}$ & 2.96 & $4.05 E+04$ & $2.10 \mathrm{E}+05$ \\
\hline $\mathrm{NO3}^{\circ}$ & 1.81 & $9.02 \mathrm{E}+04$ & $4.69 \mathrm{E}+05$ \\
\hline $\mathrm{NO}^{-}$ & 1.22 & $4.52 \mathrm{E}+04$ & $2.35 E+05$ \\
\hline $\mathrm{CO}^{2-}$ & 0.210 & $1.02 \mathrm{E}+04$ & $5.28 \mathrm{E}+04$ \\
\hline $\mathrm{PO}^{3 \cdot}$ & 0.109 & $8.37 \mathrm{E}+03$ & $4.34 \mathrm{E}+04$ \\
\hline $\mathrm{SO}^{2-}$ & 0.142 & $1.10 \mathrm{E}+04$ & $5.72 E+04$ \\
\hline $\mathrm{Si}\left(\right.$ as $\mathrm{SiO}_{3}{ }^{2}$ ) & $2.29 \mathrm{E}-02$ & 517 & $2.69 \mathrm{E}+03$ \\
\hline$F^{-}$ & $4.71 \mathrm{E}-02$ & 721 & $3.74 \mathrm{E}+03$ \\
\hline $\mathrm{Cl}^{-}$ & 9.13E-02 & $2.60 \mathrm{E}+03$ & $1.35 \mathrm{E}+04$ \\
\hline $\mathrm{C}_{6} \mathrm{H}_{5} \mathrm{O}_{7}{ }^{3-}$ & $1.91 \mathrm{E}-02$ & $2.90 \mathrm{E}+03$ & $1.51 \mathrm{E}+04$ \\
\hline EDTA $^{4-}$ & $1.04 \mathrm{E}-02$ & $2.42 \mathrm{E}+03$ & $1.26 \mathrm{E}+04$ \\
\hline HEDTA $^{3-}$ & $1.71 \mathrm{E}-02$ & . $3.78 \mathrm{E}+03$ & $1.96 \mathrm{E}+04$ \\
\hline glycolate & $6.36 \mathrm{E}-02$ & $3.84 \mathrm{E}+03$ & $2.00 \mathrm{E}+04$ \\
\hline acetate $^{-}$ & $1.19 \mathrm{E}-02$ & 567 & $2.94 \mathrm{E}+03$ \\
\hline oxalate $^{2-}$ & $1.85 \mathrm{E}-05$ & 1.31 & 6.82 \\
\hline DBP & $9.86 \mathrm{E}-03$ & $1.28 \mathrm{E}+03$ & $6.63 \mathrm{E}+03$ \\
\hline butanol & $9.86 \mathrm{E}-03$ & 588 & $3.05 \mathrm{E}+03$ \\
\hline $\mathrm{NH}_{3}$ & 2.61E-02 & 357 & $1.85 \mathrm{E}+03$ \\
\hline $\mathrm{Fe}(\mathrm{CN})_{6}^{4 \cdot}$ & & 0 & 0 \\
\hline \multicolumn{4}{|c|}{ Hol } \\
\hline Pu & $22.7(\mu \mathrm{Ci} / \mathrm{L})$ & & $1.58(\mathrm{~kg})$ \\
\hline $\mathrm{U}$ & $2.95 \mathrm{E}-03(\mathrm{M})$ & $565(\mu \mathrm{g} / \mathrm{g})$ & $2.93 \mathrm{E}+03(\mathrm{~kg})$ \\
\hline$\overline{C s}$ & $0.177(\mathrm{Ci} / \mathrm{L})$ & $142(\mu \mathrm{CV} / \mathrm{g})$ & $7.38 \mathrm{E}+05(\mathrm{Ci})$ \\
\hline $\mathbf{S r}$ & $5.16 \mathrm{E}-03(\mathrm{CI} / \mathrm{L})$ & $4.15(\mu \mathrm{Ci} / \mathrm{g})$ & $2.15 \mathrm{E}+04(\mathrm{Ci})$ \\
\hline
\end{tabular}

"Density is calculated based on $\mathrm{Na}, \mathrm{OH}$, and $\mathrm{AlO}_{2}{ }^{-}$.

+ Water wt $\%$ derived from the difference of density and total dissolved species.

Derived by Los Alamos National Laboratory (LANL). 


\begin{tabular}{|c|c|c|c|}
\hline \multicolumn{4}{|c|}{ Double-Shell Tank 241-AP-102 } \\
\hline \multicolumn{4}{|c|}{ TLM Solids Composite Inventory Estimate* } \\
\hline \multicolumn{4}{|l|}{ U. } \\
\hline Total Solid Waste & $\mathbf{0 ~ k g}$ & (0 kgal) & \\
\hline Heat Load & $0 \mathrm{~kW}$ & (0 BTU/hr) & \\
\hline Bulk Density & & $0(g / c)$ & \\
\hline Water wt\% & & 0 & \\
\hline TOC wt\% C (wet) & & 0 & \\
\hline \multicolumn{4}{|c|}{ (1) } \\
\hline \multicolumn{4}{|l|}{$\mathrm{Na}^{+}$} \\
\hline \multicolumn{4}{|l|}{$A)^{3+}$} \\
\hline \multicolumn{4}{|l|}{$\mathrm{Fe}^{3+}($ total Fe $)$} \\
\hline \multicolumn{4}{|l|}{$\mathrm{Cr}^{3+}$} \\
\hline \multicolumn{4}{|l|}{$\mathrm{Bi}^{3+}$} \\
\hline \multicolumn{4}{|l|}{$\mathrm{La}^{3+}$} \\
\hline \multicolumn{4}{|l|}{$\mathrm{Hg}^{2+}$} \\
\hline \multicolumn{4}{|l|}{$\mathrm{Zr}\left(\right.$ as $\left.\mathrm{ZrO}(\mathrm{OH})_{2}\right)$} \\
\hline \multicolumn{4}{|l|}{$\mathrm{Pb}^{2+}$} \\
\hline \multicolumn{4}{|l|}{$\mathrm{Ni}^{2+}$} \\
\hline \multicolumn{4}{|l|}{$\mathbf{S r}^{2+}$} \\
\hline \multicolumn{4}{|l|}{$\mathrm{Mn}^{4+}$} \\
\hline \multicolumn{4}{|l|}{$\mathrm{Ca}^{2+}$} \\
\hline \multicolumn{4}{|l|}{$\mathrm{K}^{+}$} \\
\hline \multicolumn{4}{|l|}{$\mathrm{OH}$} \\
\hline \multicolumn{4}{|l|}{ NO3:- } \\
\hline \multicolumn{4}{|l|}{$\mathrm{NO} 2^{-}$} \\
\hline \multicolumn{4}{|l|}{$\mathrm{CO}^{2-}$} \\
\hline \multicolumn{4}{|l|}{$\mathrm{PO}^{3-}$} \\
\hline \multicolumn{4}{|l|}{$\mathrm{SO}^{2-}$} \\
\hline $\mathrm{Si}\left(\right.$ as $\left.\mathrm{SiO}_{3}{ }^{2}\right)$ & 0 & 0 & 0 \\
\hline $\mathbf{F}^{*}$ & 0 & 0 & 0 \\
\hline $\mathrm{Cl}^{-}$ & 요 & 0 & 0 \\
\hline $\mathrm{C}_{6} \mathrm{H}_{5} \mathrm{O}_{7}{ }^{3-}$ & 0) & 0 & 0 \\
\hline EDTA $^{4-}$ & 이 & 0 & 0 \\
\hline HEDTA $^{3-}$ & 아 & 0 & 0 \\
\hline glycolate & 0 & 0 & 0 \\
\hline acetate $^{-}$ & 0 & 0 & 0 \\
\hline oxalate ${ }^{2-}$ & 0 & 0 & 0 \\
\hline DBP & 이 & 0 & 0 \\
\hline butanol & 이 & 0 & 0 \\
\hline $\mathrm{NH}_{3}$ & 0) & 0 & 0 \\
\hline $\mathrm{Fe}(\mathrm{CN})_{6}{ }^{4-}$ & 0 & 0 & 0 \\
\hline $\mathrm{K}$ & & & \\
\hline $\mathrm{Pu}$ & . & 0 & 0 \\
\hline U & 0 & 0 & 0 \\
\hline Cs & 0 & 0 & 0 \\
\hline $\mathbf{S r}$ & 0 & 0 & 0 \\
\hline
\end{tabular}

Derived by Los Alamos National Laboratory (LANL). 


\begin{tabular}{|c|c|c|c|}
\hline \multicolumn{4}{|c|}{ Double-Shell Tank 241-AP-103 } \\
\hline \multicolumn{4}{|c|}{ Total Inventory Estimate* } \\
\hline \multicolumn{4}{|l|}{ \% } \\
\hline Total Waste & $4.71 \mathrm{E}+06 \mathrm{~kg}$ & $(1.13 \mathrm{E}+03 \mathrm{kgal})$ & \\
\hline Heat Load & $1.52 \mathrm{~kW}$ & $5.19 \mathrm{E}+03 \mathrm{BTU} / \mathrm{hr})$ & \\
\hline Bulk Density† & & $1.10(\mathrm{~g} / \mathrm{cc})$ & \\
\hline Water $w t \% \dagger$ & & 83.7 & \\
\hline TOC wt\% C (wet) $f$ & & 0.243 & \\
\hline (2) & 1 & 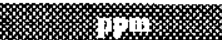 & 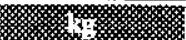 \\
\hline $\mathrm{Na}^{+}$ & 2.19 & $4.58 E+04$ & $2.15 E+05$ \\
\hline $\mathbf{A l}^{3+}$ & 0.267 & $6.54 E+03$ & $3.08 \mathrm{E}+04$ \\
\hline $\mathrm{Fe}^{3+}$ (total Fe) & $2.16 \mathrm{E}-03$ & I10 & 516 \\
\hline $\mathrm{Cr}^{3+}$ & $4.88 \mathrm{E}-03$ & 231 & $1.09 \mathrm{E}+03$ \\
\hline $\mathrm{Bi}^{3+}$ & 6.46E-05 & 12.3 & 57.8 \\
\hline $\mathrm{La}^{3+}$ & $4.12 \mathrm{E}-07$ & $5.20 \mathrm{E}-02$ & 0.245 \\
\hline $\mathrm{Hg}^{2+}$ & $1.33 \mathrm{E}-06$ & 0.243 & 1.15 \\
\hline $\mathrm{Zr}\left(\right.$ as $\left.\mathrm{ZrO}(\mathrm{OH})_{2}\right)$ & $3.18 \mathrm{E}-04$ & 26.3 & 124 \\
\hline $\mathrm{Pb}^{2+}$ & $1.09 \mathrm{E}-05$ & 2.06 & 9.71 \\
\hline $\mathrm{Ni}^{2+}$ & $1.56 \mathrm{E}-03$ & 83.2 & 392 \\
\hline $\mathrm{Sr}^{2+}$ & 4.35E-07 & $3.47 E-02$ & 0.163 \\
\hline $\mathrm{Mn}^{4+}$ & 1.39E-03 & 69.3 & 326 \\
\hline $\mathrm{Ca}^{2+}$ & $1.51 \mathrm{E}-02$ & 551) & $2.59 \mathrm{E}+03$ \\
\hline $\mathrm{K}^{+}$ & $2.30 \mathrm{E}-02$ & 819 & $3.85 \mathrm{E}+03$ \\
\hline $\mathrm{OH}$ & 1.21 & $1.87 \mathrm{E}+04$ & $8.81 E+04$ \\
\hline $\mathrm{NO}^{-}$ & 1.06 & $5.98 E+04$ & $2.81 E+05$ \\
\hline $\mathrm{NO} 2^{\circ}$ & 0.129 & $5.41 E+03$ & $2.55 \mathrm{E}+04$ \\
\hline $\mathrm{CO}^{2-}$ & 0.150 & $8.21 E+03$ & $3.87 \mathrm{E}+04$ \\
\hline $\mathrm{PO}^{3-}$ & $2.21 \mathrm{E}-02$ & $1.91 E+03$ & $8.99 E+03$ \\
\hline $\mathrm{SO}_{4}^{2-}$ & $5.60 \mathrm{E}-02$ & $4.89 \mathrm{E}+03$ & $2.30 \mathrm{E}+04$ \\
\hline $\mathrm{Si}\left(\right.$ as $\mathrm{SiO}_{3}{ }^{2}$ ) & $1.48 \mathrm{E}-62$ & 379 & $1.78 E+03$ \\
\hline$F^{-}$ & $2.68 \mathrm{E}-02$ & 463 & $2.18 \mathrm{E}+03$ \\
\hline $\mathrm{Cl}^{\circ}$ & $4.07 \mathrm{E}-02$ & $1.31 \mathrm{E}+03$ & $6.17 \mathrm{E}+03$ \\
\hline $\mathrm{C}_{6} \mathrm{H}_{5} \mathrm{O}_{7}{ }^{3 \cdot}$ & $7.17 \mathrm{E}-03$ & $1.23 E+03$ & $5.80 \mathrm{E}+03$ \\
\hline EDTA $^{4-}$ & $9.12 \mathrm{E}-04$ & 239 & $1.12 \mathrm{E}+03$ \\
\hline HEDTA $^{3-}$ & $1.51 \mathrm{E}-03$ & 376 & $1.77 \mathrm{E}+03$ \\
\hline glycolate & $7.83 \mathrm{E}-02$ & $5.34 \mathrm{E}+03$ & $2.52 \mathrm{E}+04$ \\
\hline acetate & 1.01E-03 & 54.3 & 256 \\
\hline oxalate $^{2-}$ & $1.55 \mathrm{E}-06$ & 0.124 & 0.585 \\
\hline$\overline{D B P}$ & 1.41E-03 & 206 & 972 \\
\hline butanol & $1.41 \mathrm{E}-03$ & 95.0 & 447 \\
\hline $\mathrm{NH}_{3}$ & $8.03 E-02$ & $1.24 \mathrm{E}+03$ & $5.84 \mathrm{E}+03$ \\
\hline $\mathrm{Fe}(\mathrm{CN})_{6}^{4-}$ & 0 & 0 & 0 \\
\hline \multicolumn{4}{|c|}{ } \\
\hline $\mathrm{Pu}$ & & $1.46 \mathrm{E}-02(\mu \mathrm{Ci} / \mathrm{g})$ & $1.14(\mathrm{~kg})$ \\
\hline $\bar{U}$ & $2.11 \mathrm{E}-03(\mathrm{M})$ & $458(\mu g / g)$ & $2.15 \mathrm{E}+03(\mathrm{~kg})$ \\
\hline $\mathrm{Cs}$ & $6.92 \mathrm{E}-02(\mathrm{Ci} / \mathrm{L})$ & $62.9(\mu \mathrm{Ci} / \mathrm{g})$ & $2.96 \mathrm{E}+05(\mathrm{Ci})$ \\
\hline $\mathrm{Sr}$ & 4.59E-03(Cin $/$ & $4.18(\mu \mathrm{Ci} / \mathrm{g})$ & $1.97 \mathrm{E}+04(\mathrm{Ci})$ \\
\hline
\end{tabular}

*Unknowns in tank solids inventory are assigned by Tank Layering Model (TLM). †Volume average for density, mass average Water wt $\%$ and $\mathrm{TOC} w \mathrm{w} \% \mathrm{C}$.

Derived by Los Alamos National Laboratory (LANL). 


\begin{tabular}{|c|c|c|c|}
\hline \multicolumn{4}{|c|}{ Double-Shell Tank 241-AP-103 } \\
\hline \multicolumn{4}{|c|}{ SMM Composite Inventory Estimate } \\
\hline \multicolumn{4}{|c|}{ \% } \\
\hline Total Supernatant & \multicolumn{3}{|c|}{$4.71 \mathrm{E}+06 \mathrm{~kg} \quad(1.13 \mathrm{E}+03 \mathrm{kga})$} \\
\hline Heat Load & \multicolumn{3}{|c|}{$1.52 \mathrm{~kW}(5.19 \mathrm{E}+03 \mathrm{BTU} / \mathrm{hr})$} \\
\hline Bulk Density* & \multicolumn{3}{|c|}{$1.10(\mathrm{~g} / \mathrm{cc})$} \\
\hline Water wt\% & \multicolumn{3}{|c|}{83.7} \\
\hline TOC wt \% C (wet) & \multicolumn{3}{|c|}{0.243} \\
\hline \multicolumn{2}{|c|}{90} & 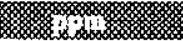 & 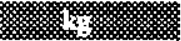 \\
\hline $\mathrm{Na}^{+}$ & 2.19 & $4.58 E+04$ & $2.15 E+05$ \\
\hline $\mathrm{Al}^{3+}$ & 0.267 & $6.54 \mathrm{E}+03$ & $3.08 \mathrm{E}+04$ \\
\hline $\mathrm{Fe}^{3+}$ (total Fe) & 2.16E-03 & 110 & 516 \\
\hline $\mathrm{Cr}^{3+}$ & $4.88 \mathrm{E}-03$ & 231 & $1.09 \mathrm{E}+03$ \\
\hline$B i^{3+}$ & $6.46 \mathrm{E}-05$ & 12.3 & 57.8 \\
\hline $\mathrm{La}^{3+}$ & $4.12 \mathrm{E}-07$ & $5.20 \mathrm{E}-02$ & 0.245 \\
\hline $\mathrm{Hg}^{2+}$ & $1.33 \mathrm{E}-06$ & 0.243 & 1.15 \\
\hline $\mathrm{Zr}\left(\right.$ as $\left.\mathrm{ZrO}(\mathrm{OH})_{2}\right)$ & $3.18 \mathrm{E}-04$ & 26.3 & 124 \\
\hline $\mathrm{Pb}^{2+}$ & $1.09 \mathrm{E}-05$ & 2.06 & 9.71 \\
\hline $\mathrm{Ni}^{2+}$ & $1.56 \mathrm{E}-03$ & 83.2 & 392 \\
\hline $\mathrm{Sr}^{2+}$ & 4.35E-07 & $3.47 \mathrm{E}-02$ & 0.163 \\
\hline $\mathrm{Mn}^{4+}$ & $1.39 \mathrm{E}-03$ & 69.3 & 326 \\
\hline $\mathrm{Ca}^{2+}$ & $1.51 \mathrm{E}-02$ & 551 & $2.59 \mathrm{E}+03$ \\
\hline $\mathrm{K}^{+}$ & $2.30 \mathrm{E}-02$ & 819 & $3.85 \mathrm{E}+03$ \\
\hline $\mathrm{OH}^{\circ}$ & 1.21 & $1.87 \mathrm{E}+04$ & $8.81 \mathrm{E}+04$ \\
\hline $\mathrm{NO3}^{-}$ & 1.06 & $5.98 \mathrm{E}+04$ & $2.81 E+05$ \\
\hline $\mathrm{NO}^{*}$ & 0.129 & $5.41 E+03$ & $2.55 E+04$ \\
\hline $\mathrm{CO}^{2-}$ & 0.150 & $8.21 E+03$ & $3.87 \mathrm{E}+04$ \\
\hline $\mathrm{PO}^{3-}$ & $2.21 \mathrm{IE}-02$ & $1.91 E+03$ & $8.99 E+03$ \\
\hline $\mathrm{SO}_{4}^{2-}$ & $5.60 \mathrm{E}-02$ & $4.89 E+03$ & $2.30 \mathrm{E}+04$ \\
\hline $\mathrm{Si}\left(\right.$ as $\mathrm{SiO}_{3}{ }^{2}$ ) & $1.48 \mathrm{E}-02$ & 379 & $1.78 \mathrm{E}+03$ \\
\hline $\mathbf{F}$ & $2.68 \mathrm{E}-02$ & 463 & $2.18 E+03$ \\
\hline $\mathrm{Cl}^{-}$ & $4.07 \mathrm{E}-02$ & $1.31 \mathrm{E}+03$ & $6.17 E+03$ \\
\hline $\mathrm{C}_{6} \mathrm{H}_{5} \mathrm{O}_{7}{ }^{3 \cdot}$ & $7.17 \mathrm{E}-03$ & $.1 .23 \mathrm{E}+03$ & $5.80 \mathrm{E}+03$ \\
\hline EDTA & $9.12 \mathrm{E}-04$ & 239 & $1.12 \mathrm{E}+03$ \\
\hline HEDTA $^{3 \cdot}$ & $1.51 E-03$ & 376 & $1.77 \mathrm{E}+03$ \\
\hline glycolate & $7.83 \mathrm{E}-02$ & $5.34 \mathrm{E}+03$ & $2.52 \mathrm{E}+04$ \\
\hline acetate ${ }^{-}$ & $1.01 \mathrm{E}-03$ & 54.3 & 256 \\
\hline oxalate ${ }^{2-}$ & 1.55E-06 & 0.124 & 0.585 \\
\hline DBP & $1.41 \mathrm{E}-03$ & 206 & 972 \\
\hline butanol & $1.41 \mathrm{E}-03$ & 95.0 & 447 \\
\hline $\mathrm{NH}_{3}$ & 8.03E-02 & $1.24 \mathrm{E}+03$ & $5.84 E+03$ \\
\hline $\mathrm{Fe}(\mathrm{CN})_{6}^{4-}$ & & 0 & 0 \\
\hline \multicolumn{4}{|c|}{ W } \\
\hline $\mathrm{Pu}$ & $16.0(\mu \mathrm{Ci} / \mathrm{L})$ & & $1.14(\mathrm{~kg})$ \\
\hline$\overline{\mathbf{U}}$ & $2.11 \mathrm{E}-03(\mathrm{M})$ & $458(\mu \mathrm{g} / \mathrm{g})$ & $2.15 E+03(\mathrm{~kg})$ \\
\hline $\mathrm{Cs}$ & $6.92 \mathrm{E}-02 .(\mathrm{Ci} / \mathrm{L})$ & $62.9(\mu \mathrm{CV} / \mathrm{g})$ & $2.96 \mathrm{E}+05(\mathrm{Ci})$ \\
\hline $\mathrm{Sr}$ & $4.59 \mathrm{E}-03(\mathrm{CH} / \mathrm{L})$ & $4.18(\mu \mathrm{Cl} / \mathrm{g})$ & $1.97 \mathrm{E}+04(\mathrm{Ci})$ \\
\hline
\end{tabular}

"Density is calculated based on $\mathrm{Na}, \mathrm{OH}$, and $\mathrm{AlO}_{2}$.

†Water wt \% derived from the difference of density and total dissolved species.

Derived by Los Alamos National Laboratory (LANL). 


\begin{tabular}{|c|c|c|c|}
\hline \multicolumn{4}{|c|}{ Double-Shell Tank 241-AP-103 } \\
\hline \multicolumn{4}{|c|}{ TLM Solids Composite Inventory Estimate* } \\
\hline \multicolumn{4}{|l|}{ W } \\
\hline Total Solid Waste & $0 \mathbf{k g}$ & (0 kgal) & \\
\hline Heat Load & $\mathbf{0 k W}$ & (0 BTU/hr) & \\
\hline Bulk Density & & $0(\mathrm{~g} / \mathrm{cc})$ & \\
\hline Water wt\% & & 0 & \\
\hline TOC wt\% C (wet) & & 0 & \\
\hline \multicolumn{4}{|c|}{ 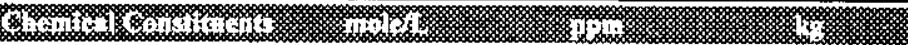 } \\
\hline \multicolumn{4}{|l|}{$\mathrm{Na}^{+}$} \\
\hline \multicolumn{4}{|l|}{$\mathrm{Al}^{3+}$} \\
\hline \multicolumn{4}{|l|}{$\mathrm{Fe}^{3+}($ total $\mathrm{Fe})$} \\
\hline \multicolumn{4}{|l|}{$\mathrm{Cr}^{3+}$} \\
\hline \multicolumn{4}{|l|}{$\mathrm{Bi}^{3+}$} \\
\hline \multicolumn{4}{|l|}{$\mathrm{La}^{3+}$} \\
\hline \multicolumn{4}{|l|}{$\mathrm{Hg}^{2+}$} \\
\hline \multicolumn{4}{|l|}{$\mathrm{Zr}\left(\right.$ as $\left.\mathrm{ZrO}(\mathrm{OH})_{2}\right)$} \\
\hline \multicolumn{4}{|l|}{$\mathrm{Pb}^{2+}$} \\
\hline \multicolumn{4}{|l|}{$\mathrm{Ni}^{2+}$} \\
\hline \multicolumn{4}{|l|}{$\mathrm{Sr}^{2+}$} \\
\hline \multicolumn{4}{|l|}{$\mathrm{Mn}^{4+}$} \\
\hline \multicolumn{4}{|l|}{$\mathrm{Ca}^{2+}$} \\
\hline \multicolumn{4}{|l|}{$\mathrm{K}^{+}$} \\
\hline \multicolumn{4}{|l|}{$\mathrm{OH}$} \\
\hline \multicolumn{4}{|l|}{ NO3: } \\
\hline \multicolumn{4}{|l|}{$\mathrm{NO} 2^{\circ}$} \\
\hline \multicolumn{4}{|l|}{$\mathrm{CO}^{2-}$} \\
\hline \multicolumn{4}{|l|}{$\mathrm{PO}^{3-}$} \\
\hline \multicolumn{4}{|l|}{$\mathrm{SO}^{2-}$} \\
\hline $\mathrm{Si}\left(\right.$ as $\mathrm{SiO}_{3}{ }^{2}$ ) & 0 & 0 & 0 \\
\hline$F^{*}$ & -1 & 0 & 0 \\
\hline $\mathrm{Cl}^{-}$ & 0) & 0 & 0 \\
\hline $\mathrm{C}_{6} \mathrm{H}_{5} \mathrm{O}_{7}{ }^{3 \cdot}$ & 0 & 0 & 0 \\
\hline EDTA $^{4-}$ & 0 & 0 & 0 \\
\hline HEDTA $^{3-}$ & 0 & 0 & 0 \\
\hline glycolate & 0 & 0 & 0 \\
\hline acetate & 0 & 0 & 0 \\
\hline oxalate ${ }^{2-}$ & 0 & 0 & 0 \\
\hline DBP & 0 & 0 & 0 \\
\hline butanol & 0 & 0 & 0 \\
\hline $\mathrm{NH}_{3}$ & 0 & 0 & 0 \\
\hline $\mathrm{Fe}(\mathrm{CN})_{6}{ }^{4-}$ & $\mathbf{0}$ & 0 & 0 \\
\hline $\mathrm{m}$ & . & & \\
\hline $\mathrm{Pu}$ & & 0 & 0 \\
\hline $\mathrm{U}$ & 0 & 0 & 0 \\
\hline $\mathrm{Cs}$ & 0 & 0 & 0 \\
\hline Sr & 0 & 0 & 0 \\
\hline
\end{tabular}

*Unknowns in tank solids inventory are assigned by Tank Layering Model (TLM).

Derived by Los Alamos National Laboratory (LANL). 


\begin{tabular}{|c|c|c|c|}
\hline \multicolumn{4}{|c|}{ Double-Shell Tank 241-AP-104 } \\
\hline \multicolumn{4}{|c|}{ Total Inventory Estimate* } \\
\hline \multicolumn{4}{|c|}{$1 \%$} \\
\hline Total Waste & $6.91 \mathrm{E}+04 \mathrm{~kg}$ & (18 kgal) & \\
\hline Heat Load & $0 \mathrm{~kW}$ & (0 BTU/hr) & \\
\hline Bulk Density $\uparrow$ & & $1.01(\mathrm{~g} / \mathrm{cc})$ & \\
\hline Water wt\%† & & 97.4 & \\
\hline TOC wt\% C (wet) & & 0 & \\
\hline mons & 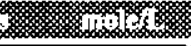 & 8 & s. \\
\hline $\mathrm{Na}^{+}$ & 0.403 & $9.13 E+03$ & 631 \\
\hline \multicolumn{4}{|l|}{$\mathrm{Al}^{3+}$} \\
\hline $\mathrm{Fe}^{3+}$ (total $\mathrm{Fe}$ ) & $1.27 \mathrm{E}-03$ & 69.8 & 4.82 \\
\hline$\overline{\mathrm{Cr}^{3+}}$ & $5.08 \mathrm{E}-03$ & 260 & 18.0 \\
\hline \multicolumn{4}{|l|}{$\mathrm{Bi}^{3+}$} \\
\hline \multicolumn{4}{|l|}{$\mathrm{La}^{3+}$} \\
\hline \multicolumn{4}{|l|}{$\mathrm{Hg}^{2+}$} \\
\hline \multicolumn{4}{|l|}{$\mathrm{Zr}\left(\right.$ as $\left.\mathrm{ZrO}(\mathrm{OH})_{2}\right)$} \\
\hline \multicolumn{4}{|l|}{$\overline{\mathrm{Pb}^{2+}}$} \\
\hline \multicolumn{4}{|c|}{$1.14 \mathrm{E}-03$} \\
\hline \multicolumn{4}{|l|}{$\mathrm{Sr}^{2+}$} \\
\hline \multicolumn{4}{|l|}{$\mathrm{Mn}^{4+}$} \\
\hline \multicolumn{4}{|c|}{$8.87 \mathrm{E}-03$} \\
\hline \multicolumn{4}{|l|}{$\mathbf{K}^{+}$} \\
\hline \multicolumn{4}{|c|}{$3.28 \mathrm{E}-02$} \\
\hline \multicolumn{4}{|c|}{ 9.91E-02 } \\
\hline \multicolumn{4}{|c|}{ 8.89E-03 } \\
\hline \multicolumn{4}{|c|}{$4.42 \mathrm{E}-03$} \\
\hline \multicolumn{4}{|c|}{$8.90 \mathrm{E}+03$} \\
\hline \multicolumn{4}{|c|}{$2.54 \mathrm{E}-03$} \\
\hline \multicolumn{4}{|l|}{$\mathrm{Si}\left(\right.$ as $\left.\mathrm{SiO}_{3}{ }^{2}\right)$} \\
\hline \multicolumn{4}{|l|}{$\mathbf{F}^{-}$} \\
\hline \multicolumn{4}{|c|}{$2.02 \mathrm{E}-03$} \\
\hline $\mathrm{C}_{6} \mathrm{H}_{5} \mathrm{O}_{7}{ }^{3 \cdot}$ & 0 & 0) & 0 \\
\hline EDTA $^{4-}$ & 0 & 0 & 0 \\
\hline HEDTA $^{3-}$ & 0 & 0 & 0 \\
\hline glycolate" & 0 & $\mathbf{0}$ & 0 \\
\hline acetate- & 0 & 0 & 0 \\
\hline oxalate ${ }^{2-}$ & 0 & 0 & 0 \\
\hline$\overline{\mathrm{DBP}}$ & 0 & 0 & 0 \\
\hline butanol & 0 & 0 & 0 \\
\hline $\mathrm{NH}_{3}$ & 0 & 0 & 0 \\
\hline $\mathrm{Fe}(\mathrm{CN})_{6}{ }^{4-}$ & 0 & 0 & 0 \\
\hline 3. & mas & & \\
\hline $\mathbf{P u}$ & & 0 & 0 \\
\hline$\overline{\mathrm{U}}$ & 0 & 0 & 0 \\
\hline$\overline{\mathrm{Cs}}$ & 0 & 0 & 0 \\
\hline$\overline{\mathbf{S r}}$ & 0 & 0 & $t$ \\
\hline
\end{tabular}

* Unknowns in tank solids inventory are assigned by Tank Layering Model (TLM). $\dagger$ Volume average for density, mass average Water $w t \%$ and $\mathrm{TOC} w \mathrm{w} \% \mathrm{C}$.

Derived by Los Alamos National Laboratory (LANL). 


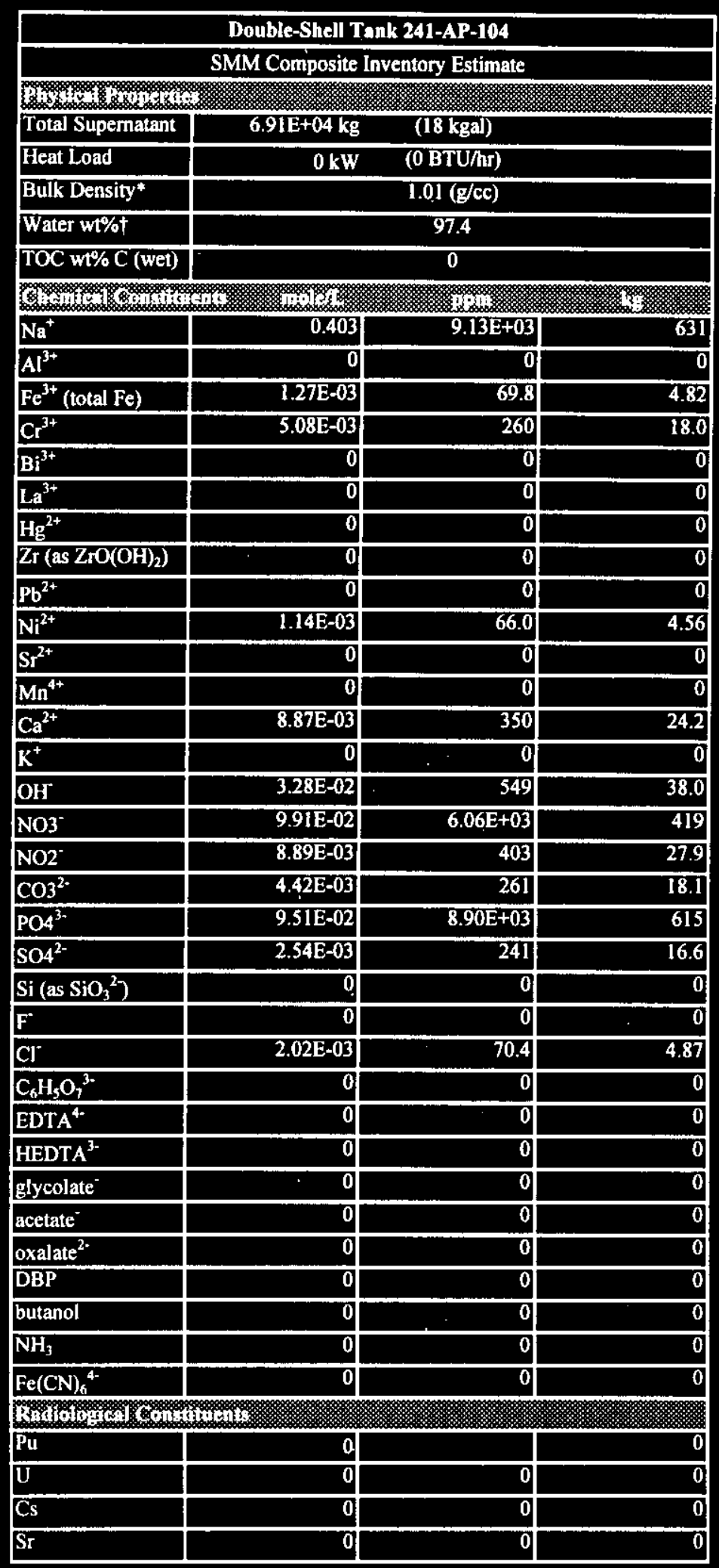

*Density is calculated based on $\mathrm{Na}, \mathrm{OH}$, and $\mathrm{AlO}_{2}$.

+Water wt $\%$ derived from the difference of density and total dissolved species.

Derived by Los Alamos National Laboratory (LANL). 


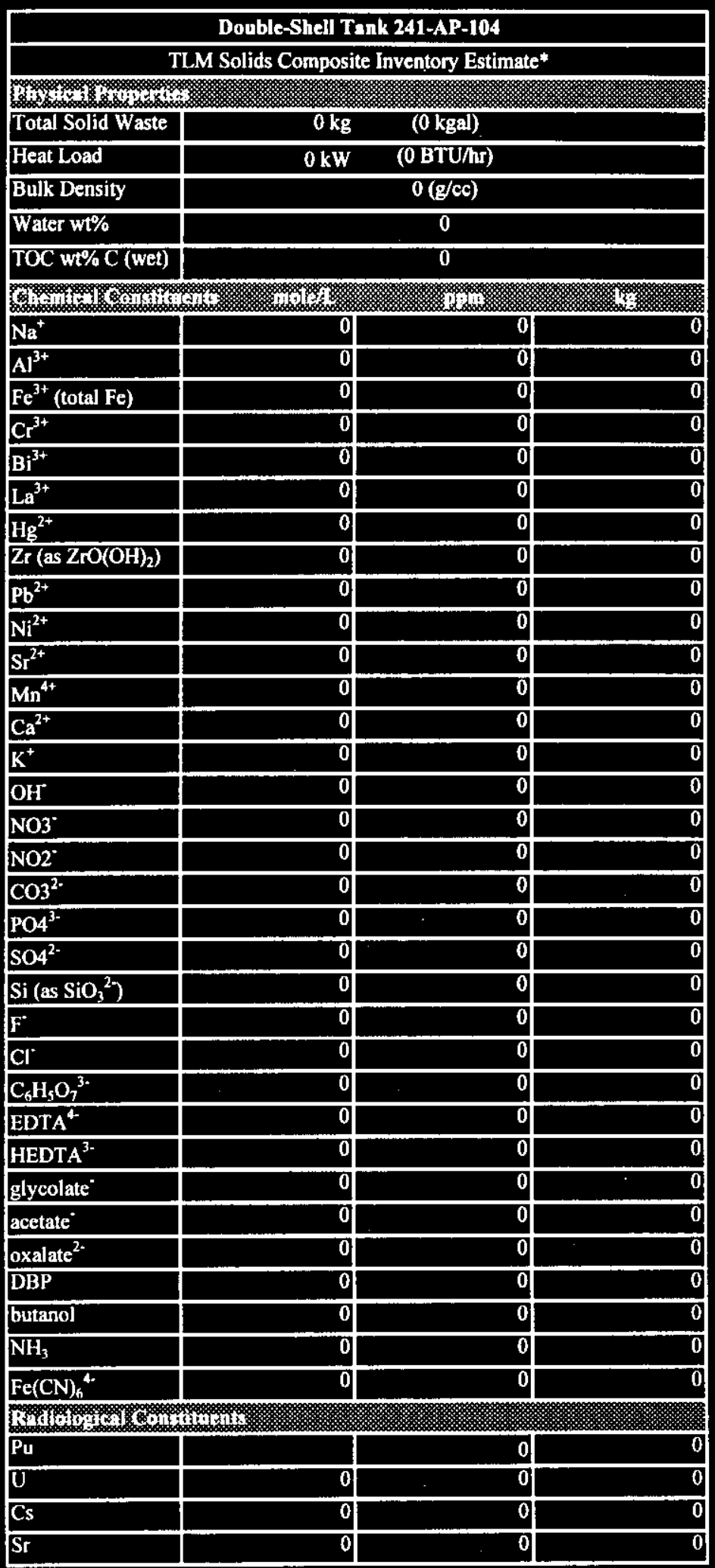

"Unknowns in tank solids inventory are assigned by Tank Layering Model (TLM).

Derived by Los Alamos National Laboratory (LANL). 


\begin{tabular}{|c|c|c|c|}
\hline \multicolumn{4}{|c|}{ Double-Shell Tank 241-AP-105 } \\
\hline \multicolumn{4}{|c|}{ Total Inventory Estimate* } \\
\hline \multicolumn{4}{|l|}{ 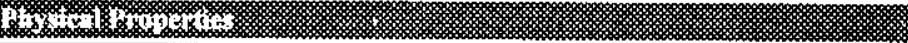 } \\
\hline Total Waste & $4.14 \mathrm{E}+06 \mathrm{~kg}$ & (821 kgal) & \\
\hline Heat Load & \multicolumn{3}{|c|}{$2.76 \mathrm{~kW}(9.43 \mathrm{E}+03 \mathrm{BTU} / \mathrm{hr})$} \\
\hline Bulk Density† & \multicolumn{3}{|c|}{$1.33(\mathrm{~g} / \mathrm{cc})$} \\
\hline Water wt\% $\dagger$ & \multicolumn{3}{|c|}{54.2} \\
\hline TOC wt\% C (wet)† & \multicolumn{3}{|c|}{0.801} \\
\hline \multicolumn{2}{|c|}{ (1) } & 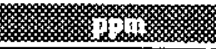 & 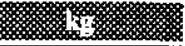 \\
\hline $\mathrm{Na}^{+}$ & 7.44 & $1.28 \mathrm{E}+05$ & $5.32 \mathrm{E}+05$ \\
\hline $\mathrm{Al}^{3+}$ & 0.805 & $1.63 \mathrm{E}+04$ & $6.75 \mathrm{E}+04$ \\
\hline $\mathrm{Fe}^{3+}$ (total Fe) & 9.24E-03 & 387 & $1.60 \mathrm{E}+03$ \\
\hline $\mathrm{Cr}^{3+}$ & 2.48E-02 & 969 & $4.01 \mathrm{E}+03$ \\
\hline$\overline{\mathbf{B i}^{3+}}$ & $4.57 \mathrm{E}-04$ & 71.7 & 297 \\
\hline $\mathrm{La}^{3+}$ & $2.98 \mathrm{E}-06$ & 0.311 & 1.29 \\
\hline $\mathrm{Hg}^{2+}$ & $1.07 \mathrm{E}-05$ & 1.62 & 6.70 \\
\hline $\mathrm{Zr}$ (as $\left.\mathrm{ZrO}(\mathrm{OH})_{2}\right)$ & $2.64 \mathrm{E}-03$ & 181 & 748 \\
\hline $\mathrm{Pb}^{2+}$ & $9.02 \mathrm{E}-05$ & 14.0 & 58.1 \\
\hline $\mathrm{Ni}^{2+}$ & $5.63 \mathrm{E}-03$ & 248 & $1.03 \mathrm{E}+03$ \\
\hline $\mathrm{Sr}^{2+}$ & $3.15 E-06$ & 0.207 & 0.859 \\
\hline $\mathrm{Mn}^{4+}$ & $1.16 E-02$ & 478 & $1.98 \mathrm{E}+03$ \\
\hline $\mathrm{Ca}^{2+}$ & $6.47 \mathrm{E}-02$ & $1.94 \mathrm{E}+03$ & $8.05 \mathrm{E}+03$ \\
\hline $\mathbf{K}^{+}$ & 0.194 & $5.69 \mathrm{E}+03$ & $2.35 E+04$ \\
\hline $\mathrm{OH}^{-}$ & 3.67 & $4.68 \mathrm{E}+04$ & $1.94 \mathrm{E}+05$ \\
\hline $\mathrm{NO3}^{-}$ & 3.31 & $1.54 \mathrm{E}+05$ & $6.37 \mathrm{E}+05$ \\
\hline $\mathrm{NO}_{2}^{-}$ & 0.804 & $2.78 \mathrm{E}+04$ & $1.15 \mathrm{E}+05$ \\
\hline $\mathrm{CO}^{2-}$ & 0.530 & $2.39 E+04$ & $9.88 \mathrm{E}+04$ \\
\hline $\mathrm{PO}^{3-}$ & 0.159 & $1.13 E+04$ & $4.68 \mathrm{E}+04$ \\
\hline $\mathrm{SO}^{2-}$ & 0.175 & $1.26 E+04$ & $5.22 \mathrm{E}+04$ \\
\hline $\mathrm{Si}\left(\right.$ as $\left.\mathrm{SiO}_{3}{ }^{2+}\right)$ & $3.92 \mathrm{E}-02$ & 825 & $3.42 \mathrm{E}+03$ \\
\hline $\mathrm{F}^{*}$ & 0.218 & $3.12 E+03$ & $1.29 \mathrm{E}+04$ \\
\hline $\mathrm{Cl}^{-}$ & 0.122 & $3.25 E+03$ & $1.34 \mathrm{E}+04$ \\
\hline $\mathrm{C}_{6} \mathrm{H}_{5} \mathrm{O}_{7}{ }^{3-}$ & $2.28 \mathrm{E}-02$ & $3.24 \mathrm{E}+03$ & $1.34 \mathrm{E}+04$ \\
\hline EDTA $^{4-}$ & $6.45 E-03$ & $1.39 \mathrm{E}+03$ & $5.77 \mathrm{E}+03$ \\
\hline HEDTA $^{3-}$ & $1.07 \mathrm{E}-02$ & $2.20 \mathrm{E}+03$ & $9.09 \mathrm{E}+03$ \\
\hline glycolate- & 0.183 & $1.03 \mathrm{E}+04$ & $4.26 \mathrm{E}+04$ \\
\hline acetate $^{*}$ & $7.12 \mathrm{E}-03$ & 315 & $1.31 \mathrm{E}+03$ \\
\hline oxalate ${ }^{2-}$ & $1.13 \mathrm{E}-05$ & 0.744 & 3.08 \\
\hline DBP & $1.09 \mathrm{E}-02$ & $1.32 \mathrm{E}+03$ & $5.45 \mathrm{E}+03$ \\
\hline butanol & $1.09 \mathrm{E}-02$ & 606 & $2.51 E+03$ \\
\hline $\mathrm{NH}_{3}$ & 0.665 & $8.48 E+03$ & $3.51 \mathrm{E}+04$ \\
\hline $\mathrm{Fe}(\mathrm{CN})_{6}{ }^{4-}$ & 0 & 0 & 0 \\
\hline \multicolumn{4}{|c|}{ [m1/1) } \\
\hline $\mathrm{Pu}$ & & $4.36 \mathrm{E}-02(\mu \mathrm{Ci} / \mathrm{g})$ & $3.01(\mathrm{~kg})$ \\
\hline $\mathrm{U}$ & $7.75 \mathrm{E}-03(\mathrm{M})$ & $1.38 \mathrm{E}+03(\mu \mathrm{g} / \mathrm{g})$ & $5.73 E+03(\mathrm{~kg})$ \\
\hline Cs & $0.159 \cdot(\mathrm{Cl} / \mathrm{L})$ & $119(\mu \mathrm{Ci} / \mathrm{g})$ & $4.94 \mathrm{E}+05(\mathrm{Ci})$ \\
\hline $\mathbf{S r}$ & $2.15 \mathrm{E}-02(\mathrm{Ci} / \mathrm{L})$ & $16.1(\mu \mathrm{Ci} / \mathrm{g})$ & $6.67 \mathrm{E}+04(\mathrm{CH})$ \\
\hline
\end{tabular}

"Unknowns in tank solids inventory are assigned by Tank Layering Model (TLM) + Volume average for density, mass average Water $w t \%$ and TOC wt $\% \mathrm{C}$.

Derived by Los Alamos National Laboratory (LANL). 


\begin{tabular}{|c|c|c|c|}
\hline \multicolumn{4}{|c|}{ Double-Shell Tank 241-AP-105 } \\
\hline \multicolumn{4}{|c|}{ SMM Composite Inventory Estimate } \\
\hline \multicolumn{4}{|l|}{ 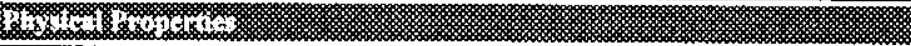 } \\
\hline Total Supernatant & $4.14 \mathrm{E}+06 \mathrm{~kg}$ & (821 kgal) & \\
\hline Heat Load & $2.76 \mathrm{~kW}$ & $9.43 \mathrm{E}+03 \mathrm{BTU} / \mathrm{hr})$ & \\
\hline Bulk Density* & & $1.33(\mathrm{~g} / \mathrm{cc})$ & \\
\hline Water wt\% $†$ & & $\overline{54.2}$ & \\
\hline TOC wt \% C (wet) & & 0.801 & \\
\hline \multicolumn{2}{|c|}{ mos } & 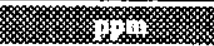 & 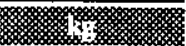 \\
\hline $\mathrm{Na}^{+}$ & 7.44 & $1.28 \mathrm{E}+05$ & $5.32 \mathrm{E}+05$ \\
\hline $\mathrm{Al}^{3+}$ & 0.805 & $1.63 \mathrm{E}+04$ & $6.75 \mathrm{E}+04$ \\
\hline $\mathrm{Fe}^{3+}$ (total $\mathrm{Fe}$ ) & $9.24 \mathrm{E}-03$ & 387 & $1.60 \mathrm{E}+03$ \\
\hline $\mathrm{Cr}^{3+}$ & 2.48E-02 & 969 & $4.01 \mathrm{E}+03$ \\
\hline $\mathbf{B i}^{3+}$ & $4.57 \mathrm{E}-04$ & 71.7 & 297 \\
\hline $\mathrm{La}^{3+}$ & $2.98 \mathrm{E}-06$ & 0.311 & 1.29 \\
\hline $\mathrm{Hg}^{2+}$ & $1.07 \mathrm{E}-05$ & 1.62 & 6.70 \\
\hline $\mathrm{Zr}$ (as $\left.\mathrm{ZrO}(\mathrm{OH})_{2}\right)$ & $2.64 \mathrm{E}-03$ & 181 & 748 \\
\hline $\mathrm{Pb}^{2+}$ & $9.02 \mathrm{E}-05$ & 14.0 & 58.1 \\
\hline $\mathrm{Ni}^{2+}$ & $5.63 \mathrm{E}-03$ & 248 & $1.03 E+03$ \\
\hline $\mathrm{Sr}^{2+}$ & $3.15 E-06$ & 0.207 & 0.859 \\
\hline $\mathrm{Mn}^{4+}$ & $1.16 \mathrm{E}-02$ & 478 & $1.98 \mathrm{E}+03$ \\
\hline $\mathrm{Ca}^{2+}$ & $6.47 \mathrm{E}-02$ & $1.94 E+03$ & $8.05 E+03$ \\
\hline $\mathrm{K}^{+}$ & 0.194 & $5.69 \mathrm{E}+03$ & $2.35 \mathrm{E}+04$ \\
\hline $\mathrm{OH}$ & 3.67 & $4.68 \mathrm{E}+04$ & $1.94 \mathrm{E}+05$ \\
\hline $\mathrm{NO3}^{\circ}$ & 3.31 & $1.54 \mathrm{E}+05$ & $6.37 \mathrm{E}+05$ \\
\hline $\mathrm{NO} 2^{-}$ & 0.804 & $2.78 \mathrm{E}+04$ & $1.15 \mathrm{E}+05$ \\
\hline $\mathrm{CO}^{2-}$ & 0.530 & $2.39 \mathrm{E}+04$ & $9.88 \mathrm{E}+04$ \\
\hline $\mathrm{PO}^{3-}$ & 0.159 & $1.13 \mathrm{E}+04$ & $4.68 \mathrm{E}+04$ \\
\hline $\mathrm{SO}^{2-}$ & 0.175 & $1.26 \mathrm{E}+04$ & $5.22 \mathrm{E}+04$ \\
\hline $\mathrm{Si}\left(\right.$ as $\mathrm{SiO}_{3}{ }^{2}$ ) & $3.92 \mathrm{E}-02$ & 825 & $3.42 \mathrm{E}+03$ \\
\hline $\mathrm{F}^{-}$ & 0.218 & $3.12 \mathrm{E}+03$ & $1.29 \mathrm{E}+04$ \\
\hline $\mathrm{Cl}^{\circ}$ & 0.122 & $3.25 \mathrm{E}+03$ & $1.34 \mathrm{E}+04$ \\
\hline $\mathrm{C}_{6} \mathrm{H}_{5} \mathrm{O}_{7}{ }^{3-}$ & $2.28 \mathrm{E}-02$ & $3.24 E+03$ & $1.34 \mathrm{E}+04$ \\
\hline EDTA $^{4-}$ & 6.45E-03 & $1.39 \mathrm{E}+03$ & $5.77 \mathrm{E}+03$ \\
\hline HEDTA $^{3-}$ & 1.07E-02 & $2.20 \mathrm{E}+03$ & $9.09 \mathrm{E}+03$ \\
\hline glycolate & 0.183 & $1.03 \mathrm{E}+04$ & $4.26 \mathrm{E}+04$ \\
\hline acetate & $7.12 \mathrm{E}-03$ & 315 & $1.31 \mathrm{E}+03$ \\
\hline oxalate ${ }^{2-}$ & I.13E-05 & 0.744 & 3.08 \\
\hline DBP & $1.09 \mathrm{E}-02$ & $1.32 \mathrm{E}+03$ & $5.45 E+03$ \\
\hline butanol & $1.09 \mathrm{E}=02$ & 606 & $2.51 E+03$ \\
\hline $\mathrm{NH}_{3}$ & 0.665 & $8.48 \mathrm{E}+03$ & $3.51 \mathrm{E}+04$ \\
\hline $\mathrm{Fe}(\mathrm{CN})_{6}^{4-}$ & & 0 & 0 \\
\hline \multicolumn{4}{|c|}{ 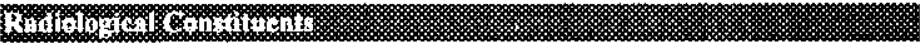 } \\
\hline $\mathrm{Pu}$ & $58.1(\mu \mathrm{Ci} L)$ & & $3.01(\mathrm{~kg})$ \\
\hline $\mathrm{U}$ & 7.75E-03 (M) & $1.38 \mathrm{E}+03(\mu \mathrm{g} / \mathrm{g})$ & $5.73 \mathrm{E}+03(\mathrm{~kg})$ \\
\hline Cs & $0.159(\mathrm{CH} / \mathrm{L})$ & $119(\mu \mathrm{Cl} / \mathrm{g})$ & $4.94 \mathrm{E}+05(\mathrm{Ci})$ \\
\hline Sr & $2.15 \mathrm{E}-02(\mathrm{CV} / \mathrm{L})$ & $16.1(\mu \mathrm{Ci} / \mathrm{g})$ & $6.67 \mathrm{E}+04(\mathrm{Ci})$ \\
\hline
\end{tabular}

"Density is calculated based on $\mathrm{Na}, \mathrm{OH}$, and $\mathrm{AlO}_{2}$ ".

+ Water wt\% derived from the difference of density and total dissolved species.

Derived by Los Alamos National Laboratory (LANL). 


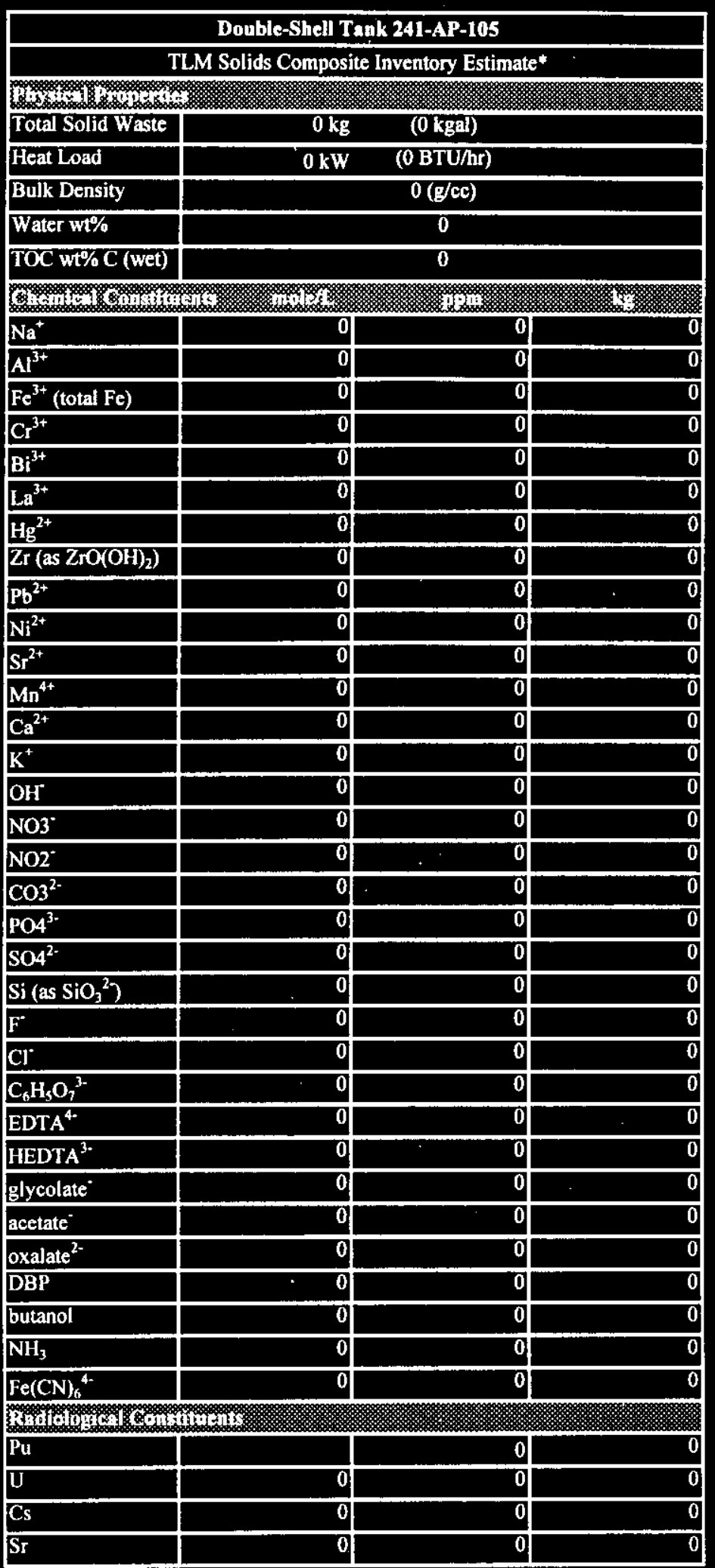

"Unknowns in tank solids inventory are assigned by Tank Layering Model (TLM).

Derived by Los Alamos National Laboratory (LANL). 


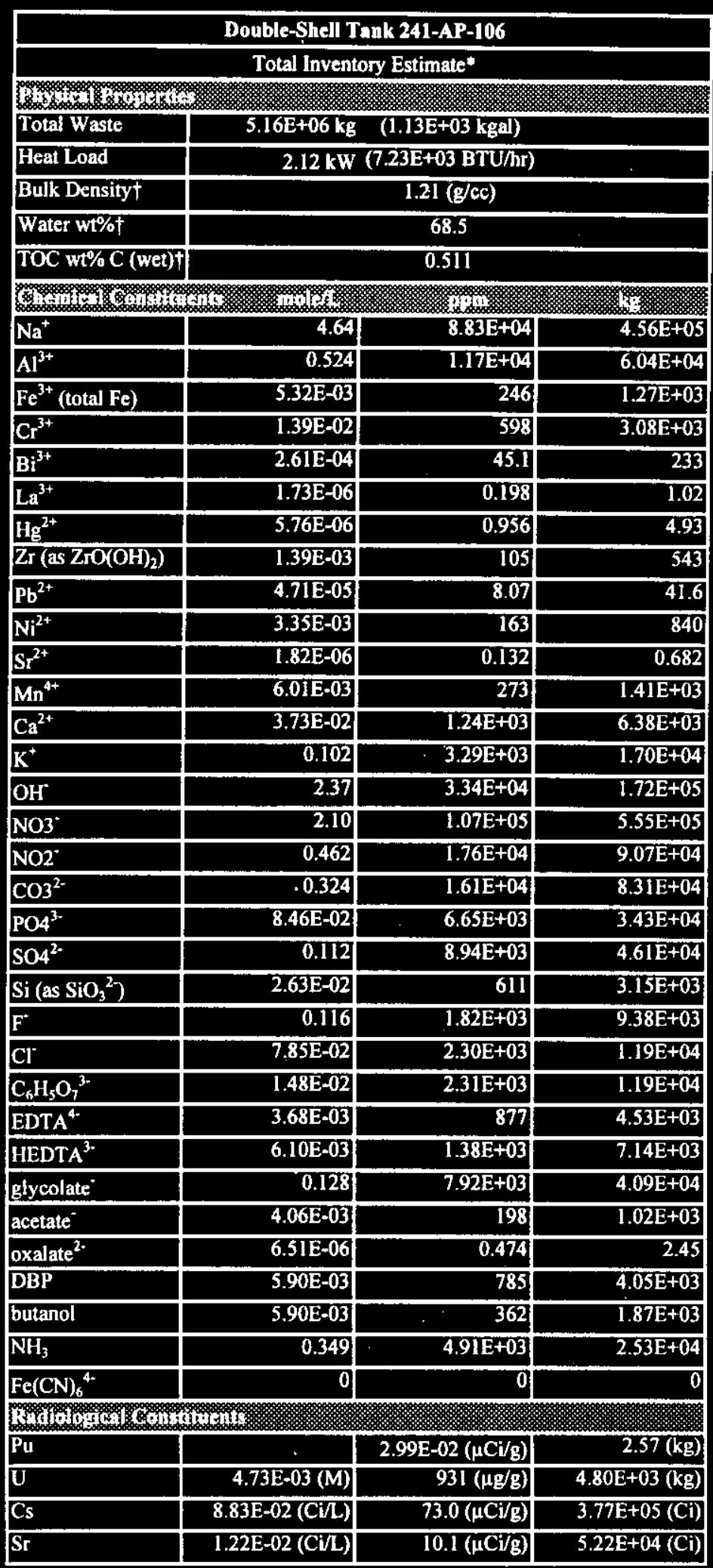

"Unknowns in tank solids inventory are assigned by Tank Layering Model (TLM).

+ Volume average for density, mass average Water wt\% and TOC wt\% C.

Derived by Los Alamos National Laboratory (LANL). 


\begin{tabular}{|c|c|c|c|}
\hline \multicolumn{4}{|c|}{ Double-Shell Tank 241-AP-106 } \\
\hline \multicolumn{4}{|c|}{ SMM Composite Inventory Estimate } \\
\hline \multicolumn{4}{|l|}{ W } \\
\hline Total Supernatant & $5.16 \mathrm{E}+06 \mathrm{~kg}$ & $13 \mathrm{E}+03 \mathrm{kgal})$ & \\
\hline Heat Load & $2.12 \mathrm{~kW}$ & E+03 BTU/hr) & \\
\hline Bulk Density* & . & $1.21(\mathrm{~g} / \mathrm{cc})$ & \\
\hline Water wt\% $†$ & & 68.5 & \\
\hline TOC wt \% C (wet) & & 0.511 & \\
\hline \multicolumn{2}{|c|}{ wind } & 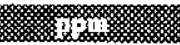 & 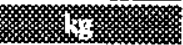 \\
\hline $\mathrm{Na}^{+}$ & 4.64 & $8.83 E+04$ & $4.56 \mathrm{E}+05$ \\
\hline $\mathrm{Al}^{3+}$ & 0.524 & $1.17 \mathrm{E}+04$ & $6.04 \mathrm{E}+04$ \\
\hline $\mathrm{Fe}^{3+}($ total $\mathrm{Fe})$ & $5.32 E-03$ & 246 & $1.27 \mathrm{E}+03$ \\
\hline $\mathrm{Cr}^{3+}$ & $1.39 \mathrm{E}-02$ & 598 & $3.08 \mathrm{E}+03$ \\
\hline$B i^{3+}$ & $2.61 \mathrm{E}-04$ & 45.1 & 233 \\
\hline $\mathrm{La}^{3+}$ & $1.73 \mathrm{E}-06$ & 0.198 & 1.02 \\
\hline $\mathrm{Hg}^{2+}$ & $5.76 \mathrm{E}-06$ & 0.956 & 4.93 \\
\hline $\mathrm{Zr}\left(\right.$ as $\left.\mathrm{ZrO}(\mathrm{OH})_{2}\right)$ & $1.39 \mathrm{E}-03$ & 105 & 543 \\
\hline $\mathrm{Pb}^{2+}$ & 4.71E-05 & 8.07 & 41.6 \\
\hline $\mathrm{Ni}^{2+}$ & $3.35 \mathrm{E}-03$ & 163 & 840 \\
\hline $\mathrm{Sr}^{2+}$ & $1.82 \mathrm{E}-06$ & 0.132 & 0.682 \\
\hline $\mathrm{Mn}^{4+}$ & 6.01E-03 & 273 & $1.41 \mathrm{E}+03$ \\
\hline $\mathrm{Ca}^{2+}$ & $3.73 \mathrm{E}-02$ & $1.24 \mathrm{E}+03$ & $6.38 \mathrm{E}+03$ \\
\hline $\mathrm{K}^{+}$ & 0.102 & $3.29 \mathrm{E}+03$ & $1.70 \mathrm{E}+04$ \\
\hline $\mathrm{OH}$ & 2.37 & $3.34 \mathrm{E}+04$ & $1.72 \mathrm{E}+05$ \\
\hline NO3- & 2.10 & $1.07 E+05$ & $5.55 E+05$ \\
\hline $\mathrm{NO}_{2}^{-}$ & 0.462 & $1.76 \mathrm{E}+04$ & $9.07 \mathrm{E}+04$ \\
\hline $\cos ^{2-}$ & 0.324 & $1.61 \mathrm{E}+04$ & $8.31 E+04$ \\
\hline $\mathrm{PO} 4^{3-}$ & $8.46 \mathrm{E}-02$ & $6.65 E+03$ & $3.43 \mathrm{E}+04$ \\
\hline $\mathrm{SO}_{4}^{2-}$ & 0.112 & $8.94 E+03$ & $4.61 \mathrm{E}+04$ \\
\hline $\mathrm{Si}\left(\right.$ as $\mathrm{SiO}_{3}{ }^{2}$ ) & $2.63 \mathrm{E}-02$ & 611 & $3.15 \mathrm{E}+03$ \\
\hline$F^{-}$ & 0.116 & $1.82 E+03$ & $9.38 \mathrm{E}+03$ \\
\hline $\mathrm{Cl}^{-}$ & $7.85 E-02$ & $2.30 \mathrm{E}+03$ & $1.19 E+04$ \\
\hline $\mathrm{C}_{6} \mathrm{H}_{5} \mathrm{O}_{7}{ }^{3-}$ & $1.48 \mathrm{E}-02$ & $2.31 \mathrm{E}+03$ & $1.19 \mathrm{E}+04$ \\
\hline EDTA $^{4-}$ & $3.68 \mathrm{E}-03$ & 877 & $4.53 \mathrm{E}+03$ \\
\hline HEDTA $^{3-}$ & $6.10 \mathrm{E}-03$ & $1.38 \mathrm{E}+03$ & $7.14 \mathrm{E}+03$ \\
\hline glycolate & 0.128 & $7.92 \mathrm{E}+03$ & $4.09 E+04$ \\
\hline acetate & $4.06 \mathrm{E}-03$ & 198 & $1.02 E+03$ \\
\hline oxalate ${ }^{2-}$ & $6.51 \mathrm{E}-06$ & 0.474 & 2.45 \\
\hline DBP & $5.90 \mathrm{E}-03$ & 785 & $4.05 E+03$ \\
\hline butanol & $5.90 \mathrm{E}-03$ & 362 & $1.87 \mathrm{E}+03$ \\
\hline $\mathrm{NH}_{3}$ & 0.349 & $4.91 E+03$ & $2.53 \mathrm{E}+04$ \\
\hline $\mathrm{Fe}(\mathrm{CN})_{6}{ }^{4-}$ & 0 & 0 & 0 \\
\hline \multicolumn{4}{|c|}{ WH } \\
\hline $\mathrm{Pu}$ & $36.1(\mu \mathrm{Ci} / \mathrm{L})$ & & $2.57(\mathrm{~kg})$ \\
\hline $\mathbf{U}$ & $4.73 \mathrm{E}-03(\mathrm{M})$ & $931(\mu \mathrm{g} / \mathrm{g})$ & $4.80 \mathrm{E}+03(\mathrm{~kg})$ \\
\hline $\mathrm{Cs}$ & $8.83 \mathrm{E}-02(\mathrm{CZ} / \mathrm{L})$ & $73.0(\mu \mathrm{Ci} / \mathrm{g})$ & $3.77 \mathrm{E}+05(\mathrm{Ci})$ \\
\hline $\mathbf{S r}$ & $1.22 \mathrm{E}-02(\mathrm{CH} / \mathrm{L})$ & $10.1(\mu \mathrm{Cl} / \mathrm{g})$ & $5.22 \mathrm{E}+04(\mathrm{Ci})$ \\
\hline
\end{tabular}

*Density is calculated based on $\mathrm{Na}, \mathrm{OH}$, and $\mathrm{AlO}_{2}$.

†Water $w t \%$ derived from the difference of density and total dissolved species.

Derived by Los Alamos National Laboratory (LANL). 


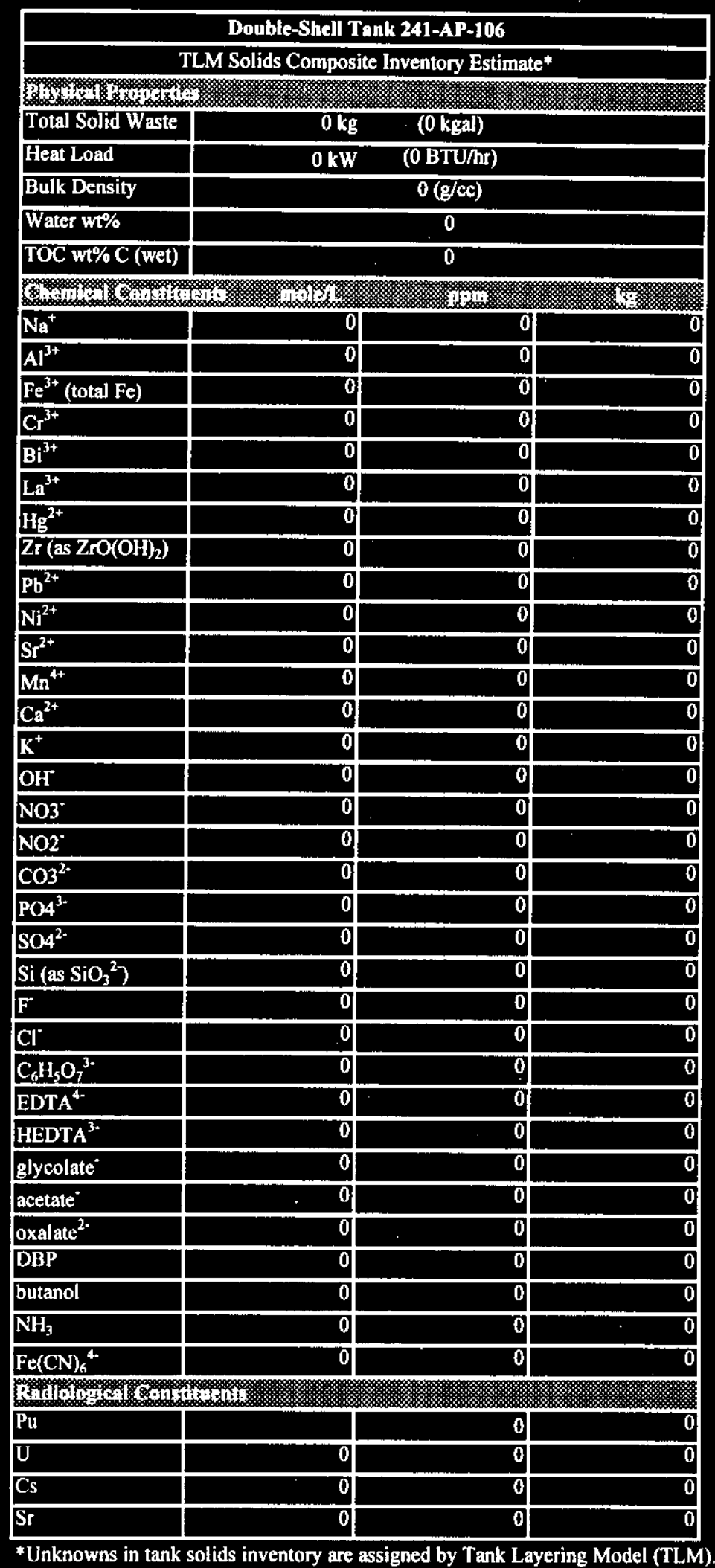

Derived by Los Alamos National Laboratory (LANL). 


\begin{tabular}{|c|c|c|c|}
\hline \multicolumn{4}{|c|}{ Double-Shell Tank 241-AP-107 } \\
\hline \multicolumn{4}{|c|}{ Total Inventory Estimate* } \\
\hline \multicolumn{4}{|l|}{ mos } \\
\hline Total Waste & $4.20 \mathrm{E}+06 \mathrm{~kg}$ & $(1.11 \mathrm{E}+03 \mathrm{kgal})$ & \\
\hline Heat Load & $0 \mathrm{~kW}$ & (0BTU/hr) & \\
\hline Bulk Density & & $1.00(\mathrm{~g} / \mathrm{cc})$ & \\
\hline Water wt\%† & & 99.3 & \\
\hline TOC wt \% C (wet) $\dagger$ & & 0 & \\
\hline \multicolumn{2}{|c|}{ L } & 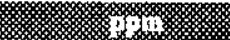 & 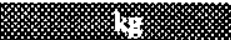 \\
\hline $\mathrm{Na}^{+}$ & $5.73 E-02$ & $1.32 \mathrm{E}+03$ & $5.53 E+03$ \\
\hline $\mathrm{Al}^{3+}$ & 0 & 0 & 0 \\
\hline $\mathrm{Fe}^{3+}$ (total Fe) & $1.98 \mathrm{E}-03$ & 110 & 463 \\
\hline $\mathrm{Cr}^{3+}$ & 0 & 0 & 0 \\
\hline$B 1^{3+}$ & 0 & 0 & 0 \\
\hline $\mathrm{La}^{3+}$ & 0 & 0 & 0 \\
\hline $\mathrm{Hg}^{2+}$ & 0 & 0 & 0 \\
\hline $\mathrm{Zr}\left(\right.$ as $\left.\mathrm{ZrO}(\mathrm{OH})_{2}\right)$ & 0 & 0 & 0 \\
\hline $\mathrm{Pb}^{2+}$ & 0 & 0 & 0 \\
\hline $\mathrm{Ni}^{2+}$ & 0 & 0 & 0 \\
\hline $\mathrm{Sr}^{2+}$ & 0 & 0] & 0 \\
\hline $\mathrm{Mn}^{4+}$ & 0 & 0 & 0 \\
\hline $\mathrm{Ca}^{2+}$ & $1.38 \mathrm{E}-02$ & 553 & $2.32 \mathrm{E}+03$ \\
\hline $\mathrm{K}^{+}$ & 0 & 0 & 0 \\
\hline $\mathrm{OH}$ & $1.58 \mathrm{E}-02$ & 268 & $1.13 \mathrm{E}+03$ \\
\hline $\mathrm{NO}^{-}$ & $6.52 \mathrm{E}-02$ & $4.04 \mathrm{E}+03$ & $1.70 \mathrm{E}+04$ \\
\hline $\mathrm{NO} 2^{\circ}$ & 0 & 0 & 0 \\
\hline $\mathrm{CO}^{2-}$ & $4.93 \mathrm{E}-03$ & 295 & $1.24 \mathrm{E}+03$ \\
\hline $\mathrm{PO}_{4}^{3-}$ & 0 & 0 & 0 \\
\hline $\mathrm{SO}^{2-}$ & 0 & 0 & 0 \\
\hline $\mathrm{Si}\left(\right.$ as $\left.\mathrm{SiO}_{3}{ }^{2-}\right)$ & 0 & 0 & 0 \\
\hline $\mathbf{F}$ & 0 & 0 & 0 \\
\hline $\mathrm{Cl}^{-}$ & 0 & 0 & 0 \\
\hline $\mathrm{C}_{6} \mathrm{H}_{5} \mathrm{O}_{7}{ }^{3-}$ & 0 & 0 & 0 \\
\hline EDTA $^{4-}$ & 0 & 0 & 0 \\
\hline HEDTA $^{3-}$ & 0 & 0 & 0 \\
\hline glycolate- & 0 & 0 & 0 \\
\hline acetate & 0 & 0 & 0 \\
\hline oxalate ${ }^{2 \cdot}$ & 0 & 0 & 0 \\
\hline DBP & 0 & 0 & 0 \\
\hline butanol & 0 & 0 & 0 \\
\hline $\mathrm{NH}_{3}$ & 4.94E-02 & 839 & $3.52 \mathrm{E}+03$ \\
\hline $\mathrm{Fe}(\mathrm{CN})_{6}{ }^{4-}$ & 0 & 0 & 0 \\
\hline 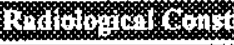 & 10 & & \\
\hline $\mathbf{P u}$ & & 0 & 0 \\
\hline $\mathrm{U}$ & 0 & 0 & 0 \\
\hline $\mathrm{Cs}$ & 0 & 0 & 0 \\
\hline $\mathbf{S t}$ & 0 & 0 & 0 \\
\hline
\end{tabular}

"Unknowns in tank solids inventory are assigned by Tank Layering Model (TLM). + Volume average for density, mass average Water $w t \%$ and TOC wt $\% \mathrm{C}$.

Derived by Los Alamos National Laboratory (LANL). 


\begin{tabular}{|c|c|c|c|}
\hline \multicolumn{4}{|c|}{ Double-Shell Tank 241-AP-107 } \\
\hline \multicolumn{4}{|c|}{ SMM Composite Inventory Estimate } \\
\hline \multicolumn{4}{|l|}{ 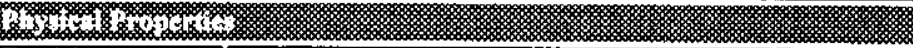 } \\
\hline Total Supernatant & $4.20 \mathrm{E}+06 \mathrm{~kg}$ & $(1.11 \mathrm{E}+03 \mathrm{kgal})$ & \\
\hline Heat Load & $0 \mathbf{k W}$ & (0BTU/hr) & \\
\hline Bulk Density* & & $1.00(\mathrm{~g} / \mathrm{cc})$ & \\
\hline Water wt\%† & & 99.3 & \\
\hline TOC wt\% C (wet) & & 0 & \\
\hline \multicolumn{2}{|c|}{ (1) } & 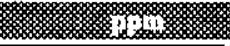 & rat \\
\hline $\mathrm{Na}^{+}$ & $5.73 E-02$ & $1.32 \mathrm{E}+03$ & $5.53 \mathrm{E}+03$ \\
\hline \multicolumn{4}{|l|}{$\mathrm{Al}^{3+}$} \\
\hline $\mathrm{Fe}^{3+}$ (total $\left.\mathrm{Fe}\right)$ & $1.98 \mathrm{E}-03$ & 110 & 463 \\
\hline \multicolumn{4}{|l|}{$\mathrm{Cr}^{3+}$} \\
\hline \multicolumn{4}{|l|}{$\mathrm{Bi}^{3+}$} \\
\hline \multicolumn{4}{|l|}{$\mathrm{La}^{3+}$} \\
\hline \multicolumn{4}{|l|}{$\mathrm{Hg}^{2+}$} \\
\hline \multicolumn{4}{|l|}{$\overline{\mathrm{Zr}\left(\text { as } \mathrm{ZrO}(\mathrm{OH})_{2}\right)}$} \\
\hline \multicolumn{4}{|l|}{$\mathrm{Pb}^{2+}$} \\
\hline \multicolumn{4}{|l|}{$\mathrm{Ni}^{2+}$} \\
\hline \multicolumn{4}{|l|}{$\mathrm{Sr}^{2+}$} \\
\hline \multicolumn{4}{|l|}{$\mathrm{Mn}^{4+}$} \\
\hline $\mathrm{Ca}^{2+}$ & $1.38 \mathrm{E}-02$ & 553 & $2.32 \mathrm{E}+03$ \\
\hline \multicolumn{4}{|l|}{$\mathrm{K}^{+}$} \\
\hline \multicolumn{4}{|r|}{$1.13 \mathrm{E}+03$} \\
\hline \multicolumn{4}{|c|}{$4.04 \mathrm{E}+03$} \\
\hline \multicolumn{4}{|l|}{$\mathrm{NO}^{-}$} \\
\hline $\mathrm{CO}^{2-}$ & 4.93E-03 & 295 & $1.24 E+03$ \\
\hline \multicolumn{4}{|l|}{$\mathrm{PO}^{3-}$} \\
\hline \multicolumn{4}{|l|}{$\mathrm{SO}^{2-}$} \\
\hline \multicolumn{4}{|l|}{$\mathrm{Si}\left(\mathrm{as} \mathrm{SiO}_{3}{ }^{2-}\right)$} \\
\hline \multicolumn{4}{|l|}{$\mathbf{F}^{-}$} \\
\hline \multicolumn{4}{|l|}{$\mathrm{Cl}^{-}$} \\
\hline \multicolumn{4}{|l|}{$\mathrm{C}_{6} \mathrm{H}_{5} \mathrm{O}_{7}{ }^{3 \cdot}$} \\
\hline EDTA $^{4 \cdot}$ & 0 & 0 & 0 \\
\hline HEDTA $^{3 \cdot}$ & 0 & 0 & 0 \\
\hline glycolate & 0] & 이 & 0 \\
\hline acetate ${ }^{-}$ & 0 & 0 & 0 \\
\hline oxalate ${ }^{2-}$ & 0 & 0 & 0 \\
\hline DBP & 0] & 이 & 0 \\
\hline butanol & 0 & 0 & 0 \\
\hline $\mathrm{NH}_{3}$ & $4.94 \mathrm{E}-02$ & 839 & $3.52 \mathrm{E}+03$ \\
\hline $\mathrm{Fe}(\mathrm{CN})_{6}^{4-}$ & 0 & 0 & 0 \\
\hline $310,15 \%$ & 11) & & \\
\hline $\mathbf{P u}$ & 0 & & 0 \\
\hline $\mathbf{U}$ & 0 & 0 & 0 \\
\hline $\mathrm{Cs}$ & 0 & 0 & 0 \\
\hline $\mathbf{S r}$ & 0 & 0 & 0 \\
\hline
\end{tabular}

"Density is calculated based on $\mathrm{Na}, \mathrm{OH}$, and $\mathrm{AlO}_{2}$.

tWater wt\% derived from the difference of density and total dissolved species.

Derived by Los Alamos National Laboratory (LANL). 
WHC-SD-WM-ER-315, Rev. 0

\begin{tabular}{|c|c|c|c|}
\hline \multicolumn{4}{|c|}{ Double-Shell Tank 241-AP-107 } \\
\hline \multicolumn{4}{|c|}{ TLM Solids Composite Inventory Estimate* } \\
\hline \multicolumn{4}{|l|}{ mos } \\
\hline Total Solid Waste & $0 \mathrm{~kg}$ & (0 kgal) & \\
\hline Heat Load & $0 \mathrm{~kW}$ & (0 BTU/hr) & \\
\hline Bulk Density & & $0(\mathrm{~g} / \mathrm{cc})$ & \\
\hline Water wt\% & & 0 & \\
\hline TOC wt $\% \mathrm{C}$ (wet) & & $\mathbf{0}$ & \\
\hline \multicolumn{4}{|c|}{ mos } \\
\hline \multicolumn{4}{|l|}{$\mathrm{Na}^{+}$} \\
\hline \multicolumn{4}{|l|}{$\mathrm{Al}^{3+}$} \\
\hline \multicolumn{4}{|l|}{$\mathrm{Fe}^{3+}$ (total $\mathrm{Fe}$ ) } \\
\hline \multicolumn{4}{|l|}{$\mathrm{Cr}^{3+}$} \\
\hline \multicolumn{4}{|l|}{$\mathrm{Bi}^{3+}$} \\
\hline \multicolumn{4}{|l|}{$\mathrm{La}^{3+}$} \\
\hline \multicolumn{4}{|l|}{$\mathrm{Hg}^{2+}$} \\
\hline \multicolumn{4}{|l|}{$\mathrm{Zr}\left(\right.$ as $\left.\mathrm{ZrO}(\mathrm{OH})_{2}\right)$} \\
\hline \multicolumn{4}{|l|}{$\mathrm{Pb}^{2+}$} \\
\hline \multicolumn{4}{|l|}{$\mathrm{Ni}^{2+}$} \\
\hline \multicolumn{4}{|l|}{$\mathrm{St}^{2+}$} \\
\hline \multicolumn{4}{|l|}{$\mathrm{Mn}^{4+}$} \\
\hline \multicolumn{4}{|l|}{$\mathrm{Ca}^{2+}$} \\
\hline \multicolumn{4}{|l|}{$\mathbf{K}^{+}$} \\
\hline \multicolumn{4}{|l|}{$\mathrm{OH}^{\circ}$} \\
\hline \multicolumn{4}{|l|}{$\mathrm{NO3}^{-}$} \\
\hline \multicolumn{4}{|l|}{$\mathrm{NO} 2^{-}$} \\
\hline \multicolumn{4}{|l|}{$\mathrm{CO}^{2-}$} \\
\hline \multicolumn{4}{|l|}{$\mathrm{PO}^{3 \cdot}$} \\
\hline \multicolumn{4}{|l|}{$\mathrm{SO}^{2-}$} \\
\hline $\mathrm{Si}\left(\operatorname{as~SiO}_{3}{ }^{2+}\right)$ & 0) & 0 & 0 \\
\hline $\mathbf{F}$ & 0 & 0 & 0 \\
\hline $\mathrm{Cl}^{-}$ & 0 & 0 & 0 \\
\hline $\mathrm{C}_{6} \mathrm{H}_{5} \mathrm{O}_{7}{ }^{3-}$ & 0 & 0 & 0 \\
\hline EDTA $^{4-}$ & 0 & 0 & 0 \\
\hline HEDTA $^{3-}$ & 0 & 0 & 0 \\
\hline glycolate & 0 & 0 & 0 \\
\hline acetate $^{-}$ & 0 & 0 & 0 \\
\hline oxalate ${ }^{2-}$ & 0 & 0 & 0 \\
\hline$\overline{\mathrm{DBP}}$ & 0 & 0 & 0 \\
\hline butanol & 0 & 0 & 0 \\
\hline $\mathrm{NH}_{3}$ & 0 & 0 & 0 \\
\hline $\mathrm{Fe}(\mathrm{CN})_{6}^{4-}$ & 0 & 0 & 0 \\
\hline H & & & 涰 \\
\hline $\mathrm{Pu}$ & & 0 & 0 \\
\hline$\overline{\mathrm{U}}$ & 0 & 0 & 0 \\
\hline$\overline{C s}$ & 0 & 0 & 0 \\
\hline $\mathbf{S r}$ & $\overline{0}$ & 0 & 0 \\
\hline
\end{tabular}

*Unknowns in tank solids inventory are assigned by Tank Layering Model (TLM).

Derived by Los Alamos National Laboratory (LANL). 


\begin{tabular}{|c|c|c|c|}
\hline \multicolumn{4}{|c|}{ Double-Shell Tank 241-AP-108 } \\
\hline \multicolumn{4}{|c|}{ Total Inventory Estimate* } \\
\hline \multicolumn{4}{|l|}{ Los } \\
\hline Total Waste & $3.70 \mathrm{E}+06 \mathrm{~kg}$ & (899 kgal) & \\
\hline Heat Load & $1.05 \mathrm{~kW}$ & $3.60 \mathrm{E}+03 \mathrm{BTU} / \mathrm{hr})$ & \\
\hline Bulk Density $\dagger$ & & $1.09(\mathrm{~g} / \mathrm{cc})$ & \\
\hline Water wt\% $†$ & & 85.6 & \\
\hline TOC wt \% C (wet)† & & 0.238 & \\
\hline \multicolumn{2}{|c|}{ \% } & sing & 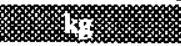 \\
\hline $\mathrm{Na}^{+}$ & 1.91 & $4.03 E+04$ & $1.49 \mathrm{E}+05$ \\
\hline $\mathrm{Al}^{3+}$ & 0.246 & $6.10 \mathrm{E}+03$ & $2.26 \mathrm{E}+04$ \\
\hline $\mathrm{Fe}^{3+}$ (total Fe) & 1.95E-03 & 99.9 & 370 \\
\hline $\mathrm{Cr}^{3+}$ & $3.17 \mathrm{E}-03$ & 151 & $\overline{560}$ \\
\hline $\mathrm{Bi}^{3+}$ & 3.45E-05 & 6.62 & 24.5 \\
\hline $\mathrm{La}^{3+}$ & $2.31 E-07$ & 2.95E-02 & 0.109 \\
\hline $\mathrm{Hg}^{2+}$ & $7.56 \mathrm{E}-07$ & 0.139 & 0.516 \\
\hline $\mathrm{Zr}\left(\operatorname{as~} \mathrm{ZrO}(\mathrm{OH})_{2}\right)$ & $1.83 \mathrm{E}-04$ & 15.3 & 56.7 \\
\hline $\mathrm{Pb}^{2+}$ & $1.07 \mathrm{E}-05$ & 2.05 & 7.57 \\
\hline $\mathrm{Ni}^{2+}$ & $1.33 \mathrm{E}-03$ & 71.8 & 266 \\
\hline $\mathrm{Sr}^{2+}$ & $2.44 \mathrm{E}-07$ & $1.97 \mathrm{E}-02$ & $7.28 \mathrm{E}-02$ \\
\hline $\mathrm{Mn}^{4+}$ & $1.42 \mathrm{E}-03$ & 71.6 & 265 \\
\hline $\mathrm{Ca}^{2+}$ & $1.36 \mathrm{E}-02$ & 502 & $1.86 E+03$ \\
\hline $\mathrm{K}^{+}$ & $1.40 \mathrm{E}-02$ & 501 & $1.86 \mathrm{E}+03$ \\
\hline $\mathrm{OH}$ & 1.10 & $1.72 \mathrm{E}+04$ & $6.36 \mathrm{E}+04$ \\
\hline $\mathrm{NO3}^{\circ}$ & 0.898 & $5.12 \mathrm{E}+04$ & $1.89 \mathrm{E}+05$ \\
\hline $\mathrm{NO}^{-}$ & 7.72E-02 & $3.26 \mathrm{E}+03$ & $1.21 \mathrm{E}+04$ \\
\hline $\mathrm{CO}^{2-}$ & 0.145 & $7.99 \mathrm{E}+03$ & $2.96 \mathrm{E}+04$ \\
\hline $\mathrm{PO}^{3-}$ & $2.17 \mathrm{E}-02$ & $1.89 \mathrm{E}+03$ & $7.01 \mathrm{E}+03$ \\
\hline $\mathrm{SO}^{2-}$ & $5.34 \mathrm{E}-02$ & $4.71 \mathrm{E}+03$ & $1.74 \mathrm{E}+04$ \\
\hline $\mathrm{Si}\left(\right.$ as $\mathrm{SiO}_{3}{ }^{2}$ ) & $1.50 \mathrm{E}-02$ & 388 & $1.44 \mathrm{E}+03$ \\
\hline$F$ & $1.55 \mathrm{E}-02$ & 271 & $1.00 \mathrm{E}+03$ \\
\hline $\mathrm{Cl}^{-}$ & $3.34 \mathrm{E}-02$ & $1.09 \mathrm{E}+03$ & $4.03 \mathrm{E}+03$ \\
\hline $\mathrm{C}_{6} \mathrm{H}_{5} \mathrm{O}_{7}{ }^{3 \cdot}$ & 6.91E-03 & $1.20 \mathrm{E}+03$ & $4.44 E+03$ \\
\hline EDTA $^{4}$ & $4.89 \mathrm{E}-04$ & 129 & 479 \\
\hline HEDTA $^{3-}$ & $8.10 \mathrm{E}-04$ & 204 & 755 \\
\hline glycolate & $8.29 \mathrm{E}-02$ & $5.71 \mathrm{E}+03$ & $2.12 \mathrm{E}+04$ \\
\hline acetate & $5.39 \mathrm{E}-04$ & 29.2 & 108 \\
\hline oxalate ${ }^{2-}$ & $8.72 \mathrm{E}-07$ & $7.06 \mathrm{E}-02$ & 0.261 \\
\hline DBP & $1.11 \mathrm{E}-03$ & 164 & 607 \\
\hline butanol & $1.11 E-03$ & 75.5 & 279 \\
\hline $\mathbf{N H}_{3}$ & $5.19 \mathrm{E}-02$ & 811 & $3.00 \mathrm{E}+03$ \\
\hline $\mathrm{Fe}(\mathrm{CN})_{6}{ }^{4-}$ & & 0 & 0 \\
\hline \multicolumn{4}{|c|}{ H } \\
\hline $\mathrm{Pu}$ & & $1.42 \mathrm{E}-02(\mu \mathrm{Ci} / \mathrm{g})$ & $0.873(\mathrm{~kg})$ \\
\hline $\bar{U}$ & $2.03 \mathrm{E}-03(\mathrm{M})$ & $444(\mu g / g)$ & $1.64 \mathrm{E}+03(\mathrm{~kg})$ \\
\hline$\overline{C s}$ & $5.93 \mathrm{E}-02(\mathrm{Ci} / \mathrm{L})$ & $54.5(\mu \mathrm{Ci} / \mathrm{g})$ & $2.02 \mathrm{E}+05(\mathrm{Ci})$ \\
\hline $\mathbf{S t}$ & $4.72 \mathrm{E}-03(\mathrm{Ci} / \mathrm{L})$ & $4.34(\mu \mathrm{Ci} / \mathrm{g})$ & $1.61 \mathrm{E}+04(\mathrm{Cl})$ \\
\hline
\end{tabular}

*Unknowns in tank solids inventory are assigned by Tank Layering Model (TLM).

$\nmid$ Volume average for density, mass average Water wt $\%$ and TOC wt $\% \mathrm{C}$.

Derived by Los Alamos National Laboratory (LANL). 


\begin{tabular}{|c|c|c|c|}
\hline \multicolumn{4}{|c|}{ Double-Shell Tank 241-AP-108 } \\
\hline \multicolumn{4}{|c|}{ SMM Contposite Inventory Estimate } \\
\hline \multicolumn{4}{|l|}{ 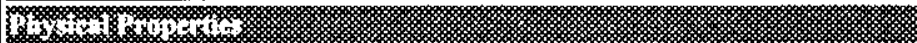 } \\
\hline Total Supernatant & $3.70 \mathrm{E}+06 \mathrm{~kg}$ & (899 kgal) & \\
\hline Heat Load & $1.05 \mathrm{~kW}$ & OE+03 BTU/hr) & \\
\hline Bulk Density* & & $1.09(\mathrm{~g} / \mathrm{cc})$ & \\
\hline Water wt\% & & 85.6 & \\
\hline TOC wt\% C (wet) & & 0.238 & \\
\hline min & (1) & 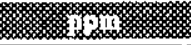 & 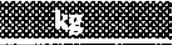 \\
\hline $\mathrm{Na}^{+}$ & 1.91 & $4.03 \mathrm{E}+04$ & $1.49 \mathrm{E}+05$ \\
\hline$\overline{A I^{3+}}$ & 0.246 & $6.10 \mathrm{E}+03$ & $2.26 \mathrm{E}+04$ \\
\hline $\mathrm{Fe}^{3+}$ (total $\mathrm{Fe}$ ) & 1.95E-03 & 99.9 & 370 \\
\hline $\mathrm{Cr}^{3+}$ & $3.17 \mathrm{E}-03$ & 151 & 560 \\
\hline $\mathrm{Bi}^{3+}$ & 3.45E-05 & 6.62 & 24.5 \\
\hline $\mathrm{La}^{3+}$ & 2.31E-07 & 2.95E-02 & 0.109 \\
\hline $\mathrm{Hg}^{2+}$ & $7.56 \mathrm{E}-07$ & 0.139 & 0.516 \\
\hline $\mathrm{Zr}\left(\right.$ as $\left.\mathrm{ZrO}(\mathrm{OH})_{2}\right)$ & 1.83E-04 & 15.3 & 56.7 \\
\hline $\mathrm{Pb}^{2+}$ & $1.07 \mathrm{E}-05$ & 2.05 & 7.57 \\
\hline $\mathrm{Ni}^{2+}$ & $1.33 \mathrm{E}-03$ & 71.8 & 266 \\
\hline $\mathrm{Sr}^{2+}$ & 2.44E-07 & $1.97 \mathrm{E}-02$ & $7.28 \mathrm{E}-02$ \\
\hline $\mathrm{Mn}^{4+}$ & $1.42 \mathrm{E}-03$ & 71.6 & 265 \\
\hline $\mathrm{Ca}^{2+}$ & $1.36 \mathrm{E}-02$ & 502 & $1.86 \mathrm{E}+03$ \\
\hline $\mathrm{K}^{+}$ & $1.40 \mathrm{E}-02$ & 501 & $1.86 E+03$ \\
\hline $\mathrm{OH}$ & 1.10 & $1.72 \mathrm{E}+04$ & $6.36 \mathrm{E}+04$ \\
\hline $\mathrm{NO3}^{-}$ & 0.898 & $5.12 \mathrm{E}+04$ & $1.89 \mathrm{E}+05$ \\
\hline NO2: & $7.72 \mathrm{E}-02$ & $3.26 \mathrm{E}+03$ & $1.21 E+04$ \\
\hline $\mathrm{CO}^{2-}$ & 0.145 & $7.99 \mathrm{E}+03$ & $2.96 \mathrm{E}+04$ \\
\hline $\mathrm{PO}^{3-}$ & $2.17 \mathrm{E}-02$ & $1.89 \mathrm{E}+03$ & $7.01 \mathrm{E}+03$ \\
\hline $\mathrm{SO}^{2-}$ & $5.34 \mathrm{E}-02$ & $4.71 \mathrm{E}+03$ & $1.74 \mathrm{E}+04$ \\
\hline $\mathrm{Si}\left(\right.$ as $\mathrm{SiO}_{3}{ }^{20}$ ) & $1.50 \mathrm{E}-02$ & 388 & $1.44 \mathrm{E}+03$ \\
\hline$F$ & $1.55 \mathrm{E}-02$ & 271 & $1.00 \mathrm{E}+03$ \\
\hline$\overline{\mathrm{Cl}^{*}}$ & $3.34 \mathrm{E}-02$ & $1.09 \mathrm{E}+03$ & $4.03 \mathrm{E}+03$ \\
\hline $\mathrm{C}_{6} \mathrm{H}_{5} \mathrm{O}_{7}{ }^{3-}$ & $6.91 E-03$ & $1.20 \mathrm{E}+03$ & $4.44 \mathrm{E}+03$ \\
\hline EDTA $^{4-}$ & $4.89 \mathrm{E}-04$ & 129 & 479 \\
\hline HEDT $^{3-}$ & $8.10 \mathrm{E}-04$ & 204 & 755 \\
\hline glycolate & $8.29 \mathrm{E}-02$ & $5.71 E+03$ & $2.12 \mathrm{E}+04$ \\
\hline acetate & 5.39E-04 & 29.2 & 108 \\
\hline oxalate ${ }^{2-}$ & $8.72 \mathrm{E}-07$ & $7.06 \mathrm{E}-02$ & 0.261 \\
\hline DBP & $1.11 \mathrm{E}-03$ & 164 & 607 \\
\hline butanol & $1.11 \mathrm{E}-03$ & 75.5 & 279 \\
\hline $\mathrm{NH}_{3}$ & $5.19 \mathrm{E}-02$ & 811 & $3.00 \mathrm{E}+03$ \\
\hline $\mathrm{Fe}(\mathrm{CN})_{6}{ }^{4-}$ & 0 & 0 & 0 \\
\hline \multicolumn{4}{|c|}{ WHOH } \\
\hline Pu & $15.4(\mu \mathrm{Ci} / \mathrm{L})$ & & $0.873(\mathrm{~kg})$ \\
\hline $\mathrm{U}$ & $2.03 \mathrm{E}-03(\mathrm{M})$ & $444(\mu g / g)$ & $1.64 \mathrm{E}+03(\mathrm{~kg})$ \\
\hline$\overline{\mathrm{Cs}}$ & $5.93 \mathrm{E}-02(\mathrm{CV} / \mathrm{L})$ & $54.5(\mu \mathrm{Ci} / \mathrm{g})$ & $2.02 \mathrm{E}+05(\mathrm{Ci})$ \\
\hline Sr & $4.72 \mathrm{E}-03(\mathrm{CV} / \mathrm{L})$ & $4.34(\mu \mathrm{Ci} / \mathrm{g})$ & $1.61 \mathrm{E}+04(\mathrm{Ci})$ \\
\hline
\end{tabular}

*Density is calculated based on $\mathrm{Na}, \mathrm{OH}$, and $\mathrm{AlO}_{2}$.

+Water wt\% derived from the difference of density and total dissolved species.

Derived by Los Alamos National Laboratory (LANL). 


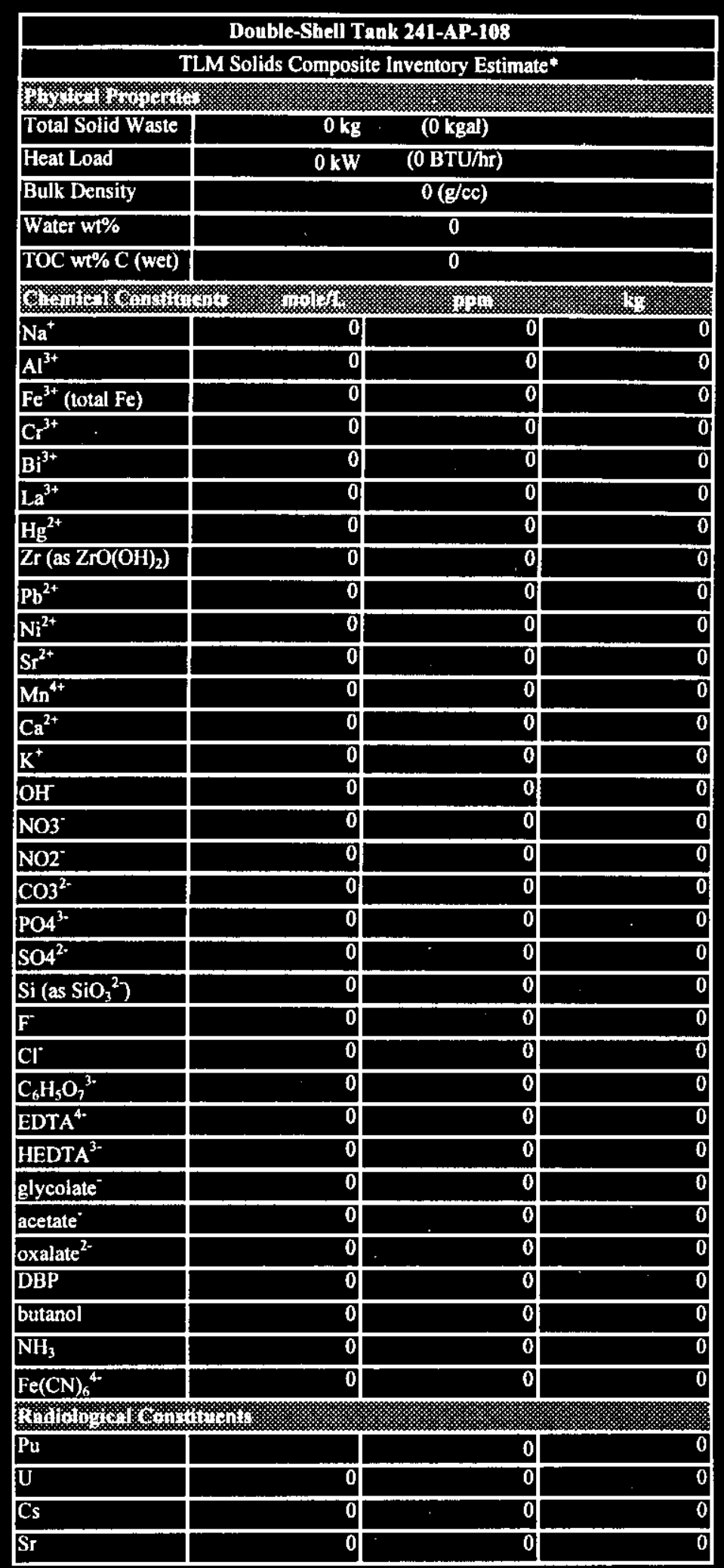

*Unknowns in tank solids inventory are assigned by Tank Layering Model (TLM).

Derived by Los Alamos National Laboratory (LANL). 


\section{APPENDIX K}

\section{Tank Layering Comparison and Data}




\section{THIS PAGE INTEENTIO
LETT BLANK \\ NAIII}


The following table describes the column headings for the Tank Layer Model developed by Los Alamos National Laboratories and the column headings correspond with the tables presented in this appendix.

\begin{tabular}{|l|l|}
\hline \multicolumn{1}{|c|}{ Column Heading } & \multicolumn{1}{|c|}{ Description } \\
\hline Tank & tank number \\
\hline Year & $\begin{array}{l}\text { vear of last primary addition and year of solid } \\
\text { measurement }\end{array}$ \\
\hline Qtr & $\begin{array}{l}\text { quarter of last primary addition and quarter of } \\
\text { solid measurement }\end{array}$ \\
\hline Meas, solids & reported solids from Anderson-91 in kgal \\
\hline Solids change & $\begin{array}{l}\text { calculated solids based on primary fill record or } \\
\text { difference between solids records }\end{array}$ \\
\hline Pred layer & predicted layer now in tank in kgal \\
\hline Layer type & Defined Waste Type for that layer \\
\hline Waste volume & $\begin{array}{l}\text { sumation of primary waste additions calculated } \\
\text { for this period }\end{array}$ \\
\hline Comments & various details of each calculation \\
\hline
\end{tabular}


WHC-SD-WM-ER-315, Rev. 0

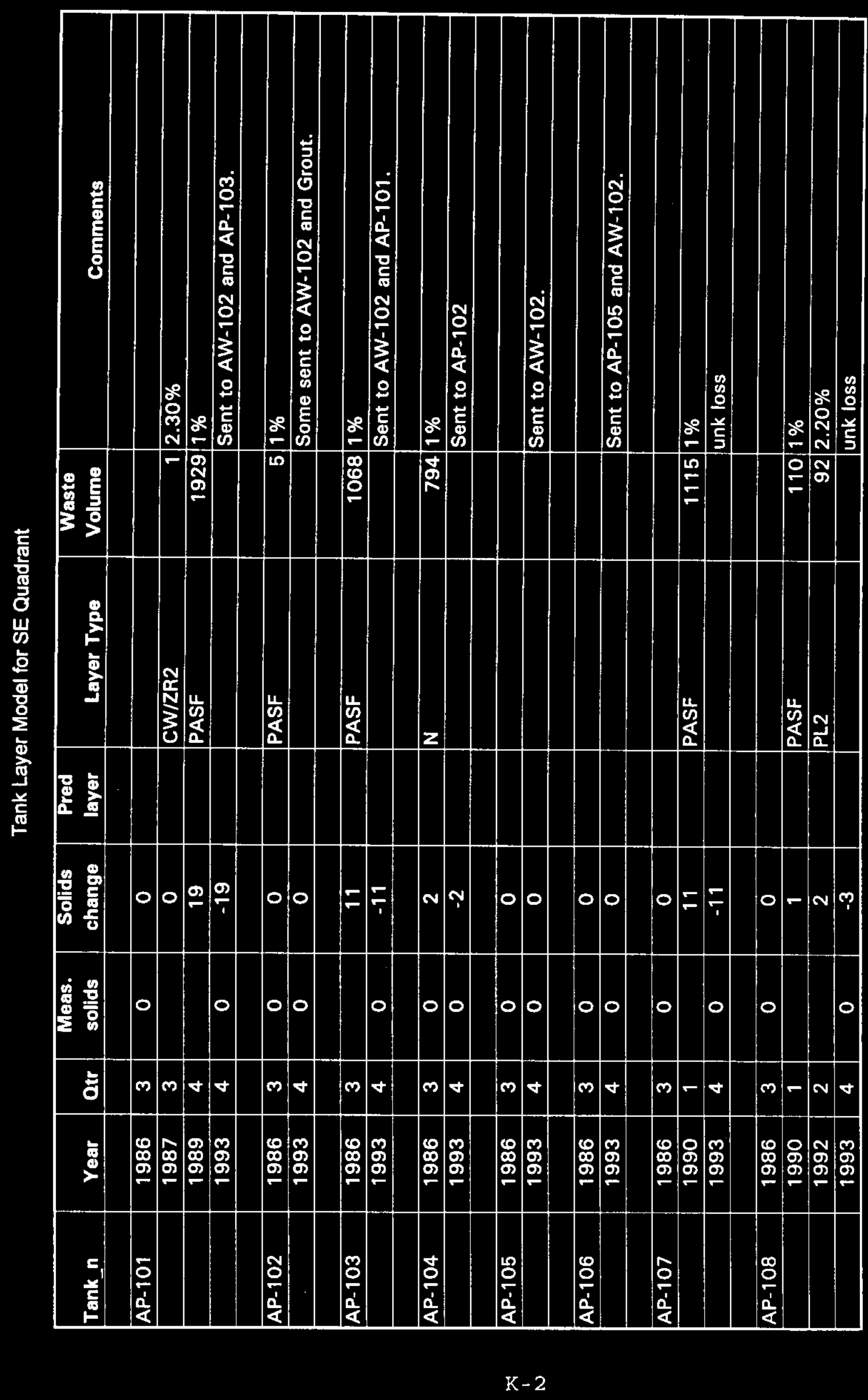




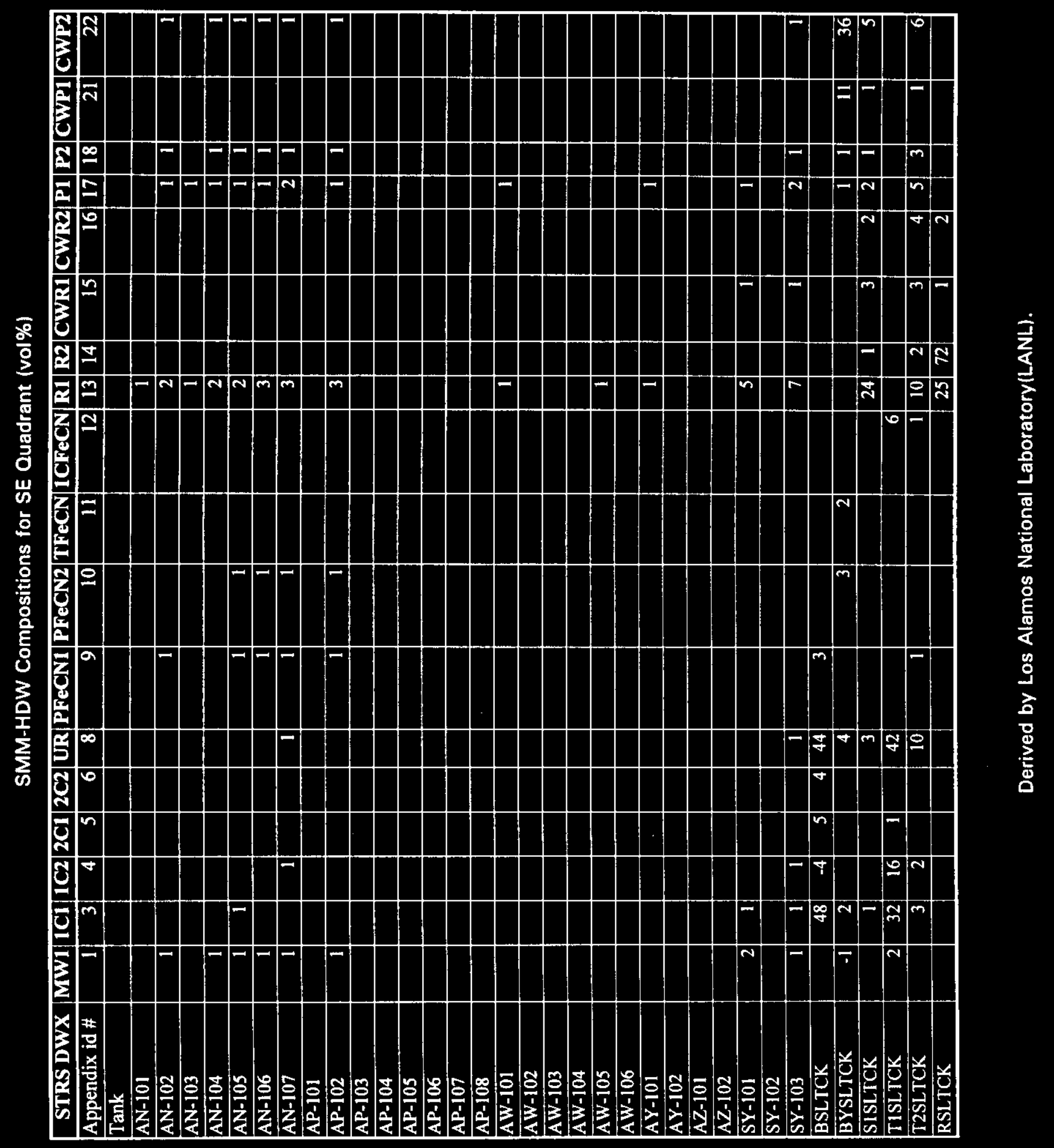


WHC-SD-WM-ER-315， Rev. 0

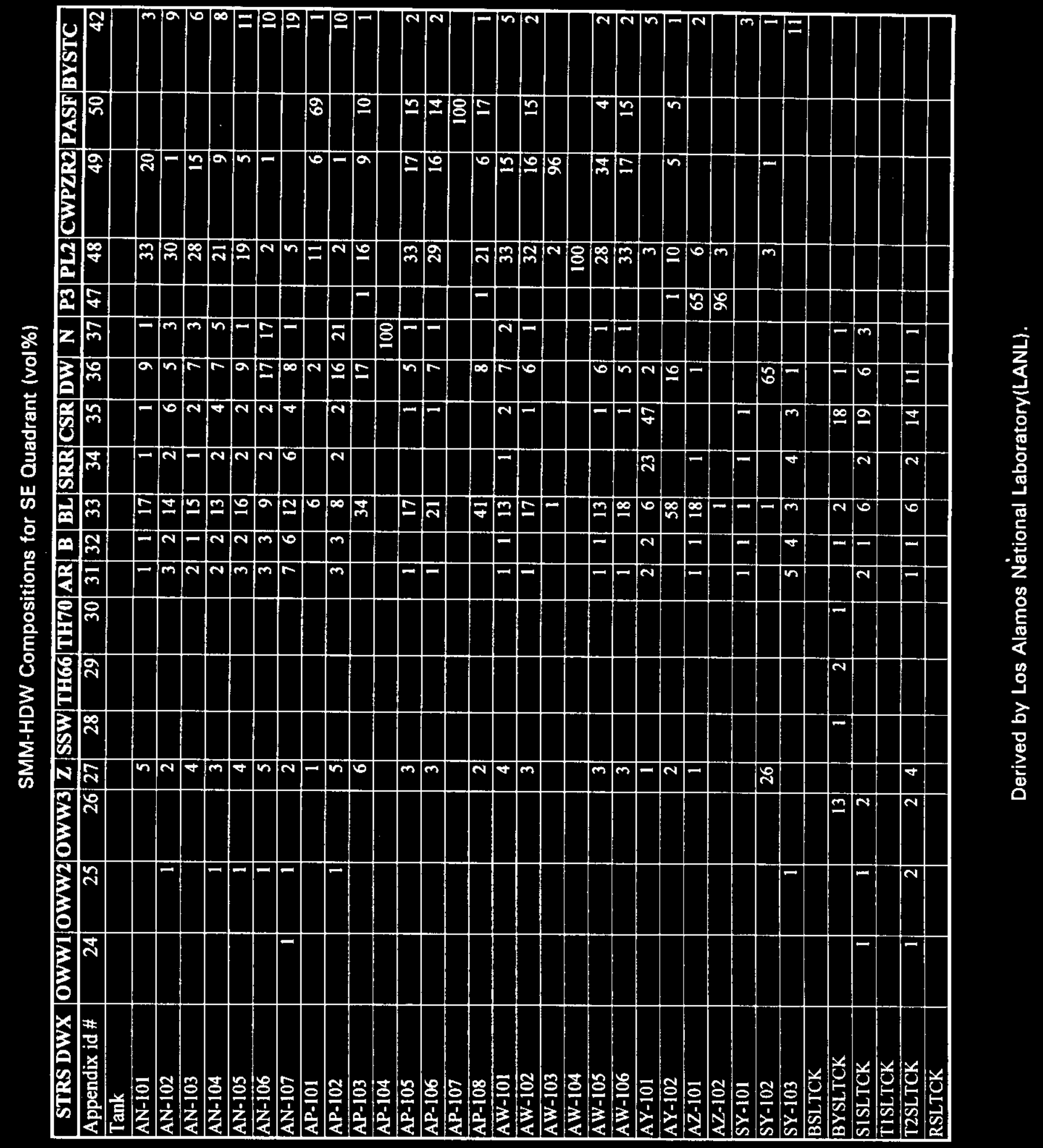


WHC-SD-WM-ER-315, Rev

0

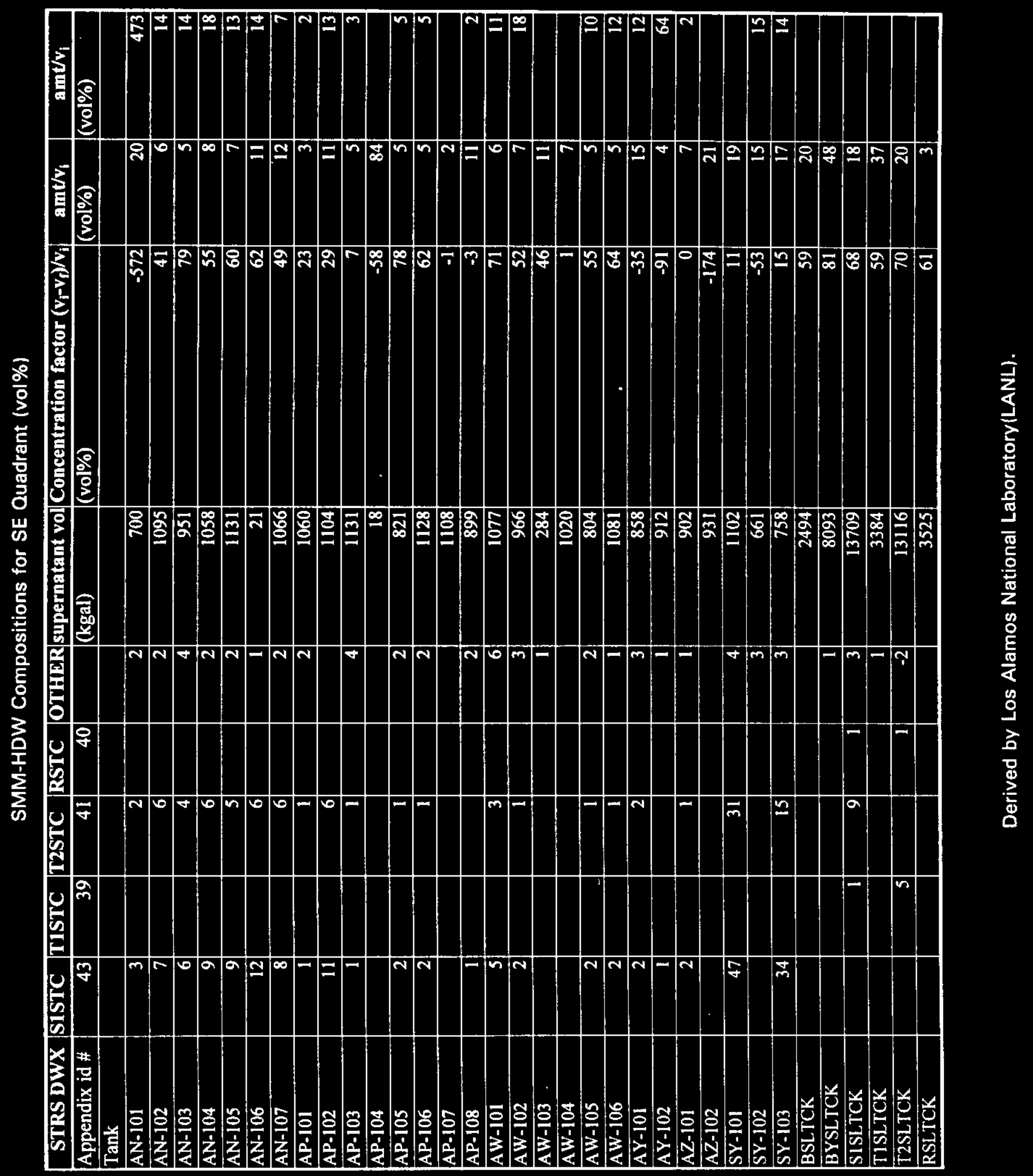


WHC-SD-WM-ER-315， Rev. 0

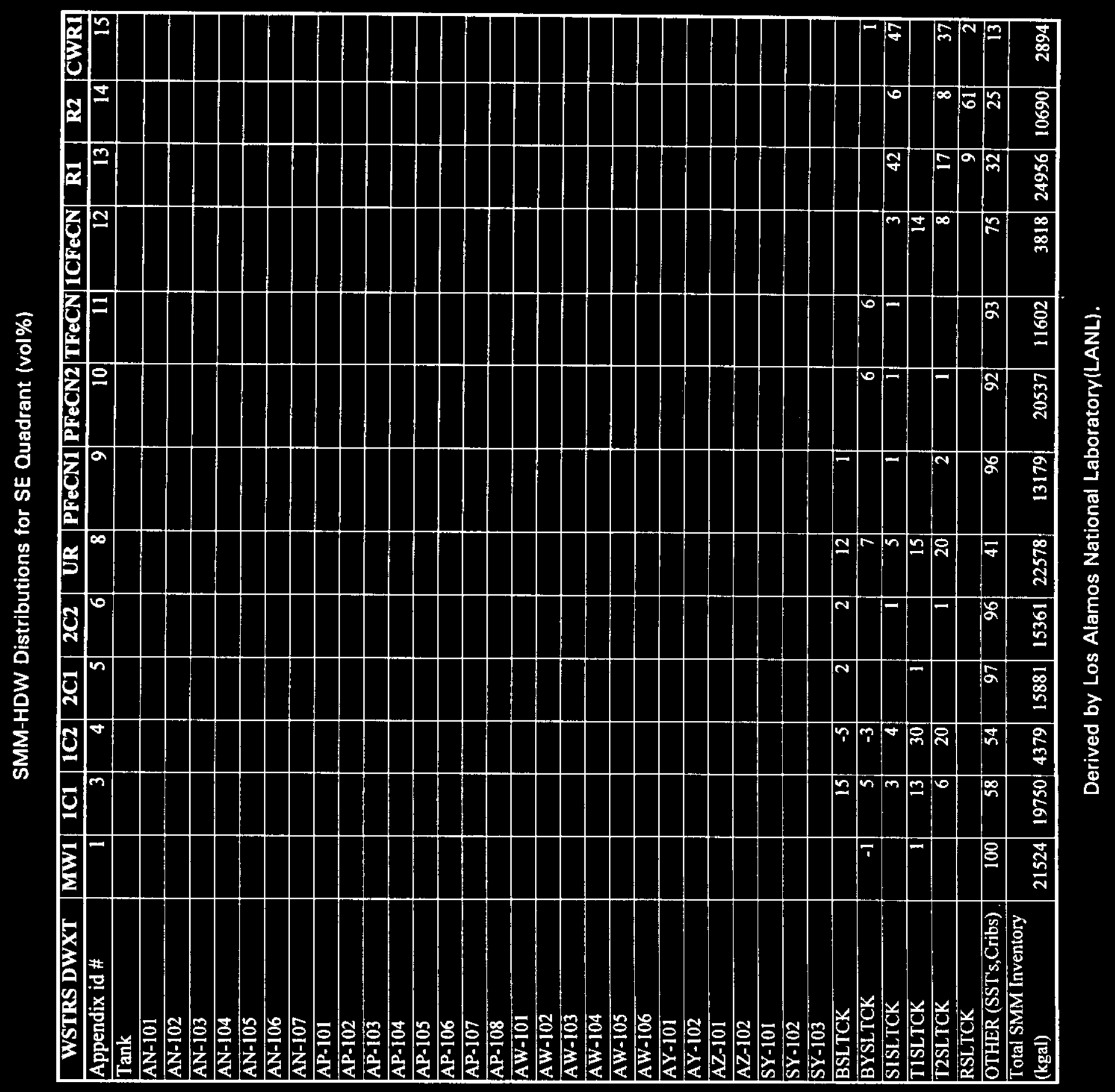


WHC-SD-WM-ER-315， Rev . 0

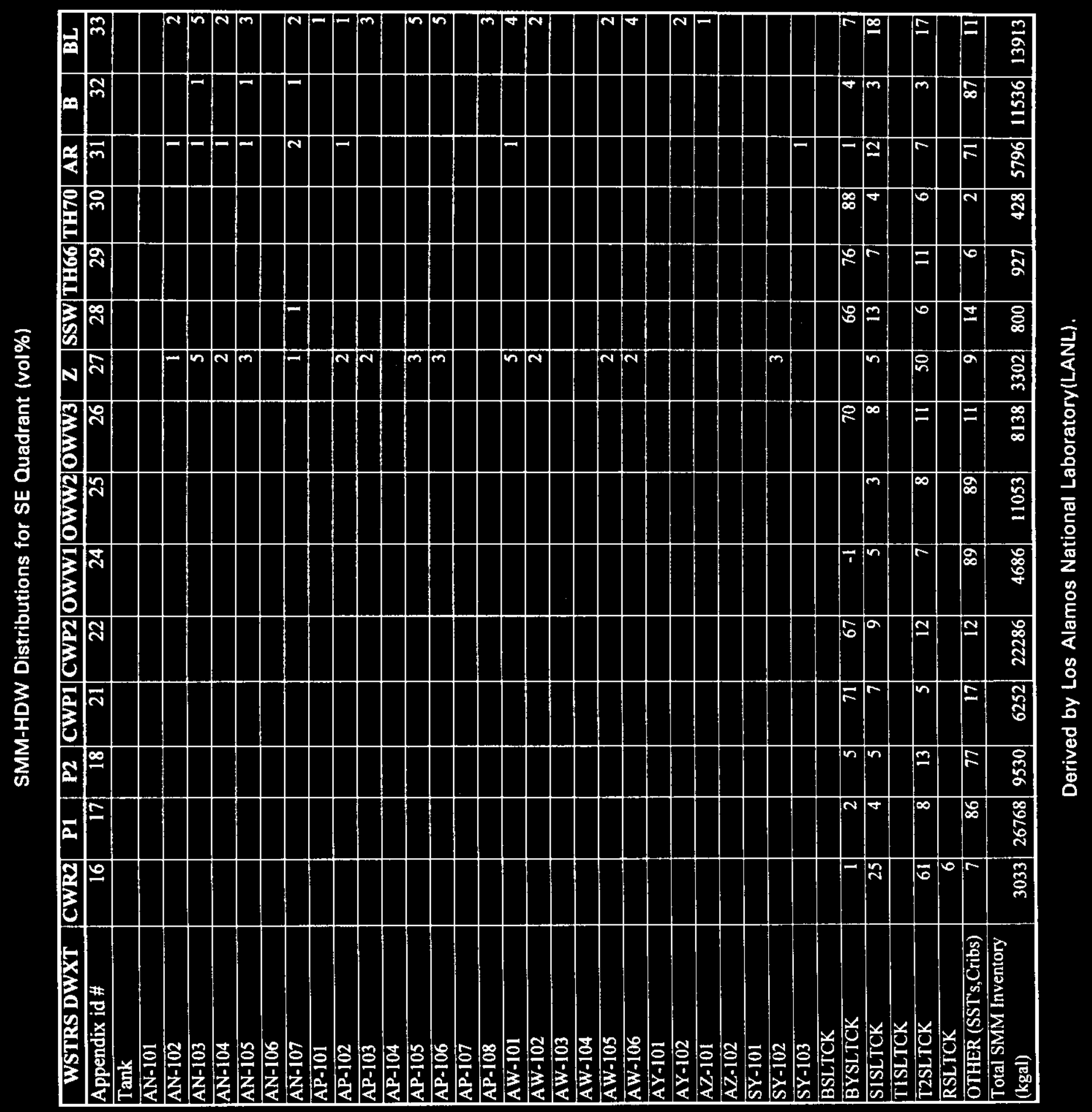


WHC-SD-WM-ER-315， Rev. 0

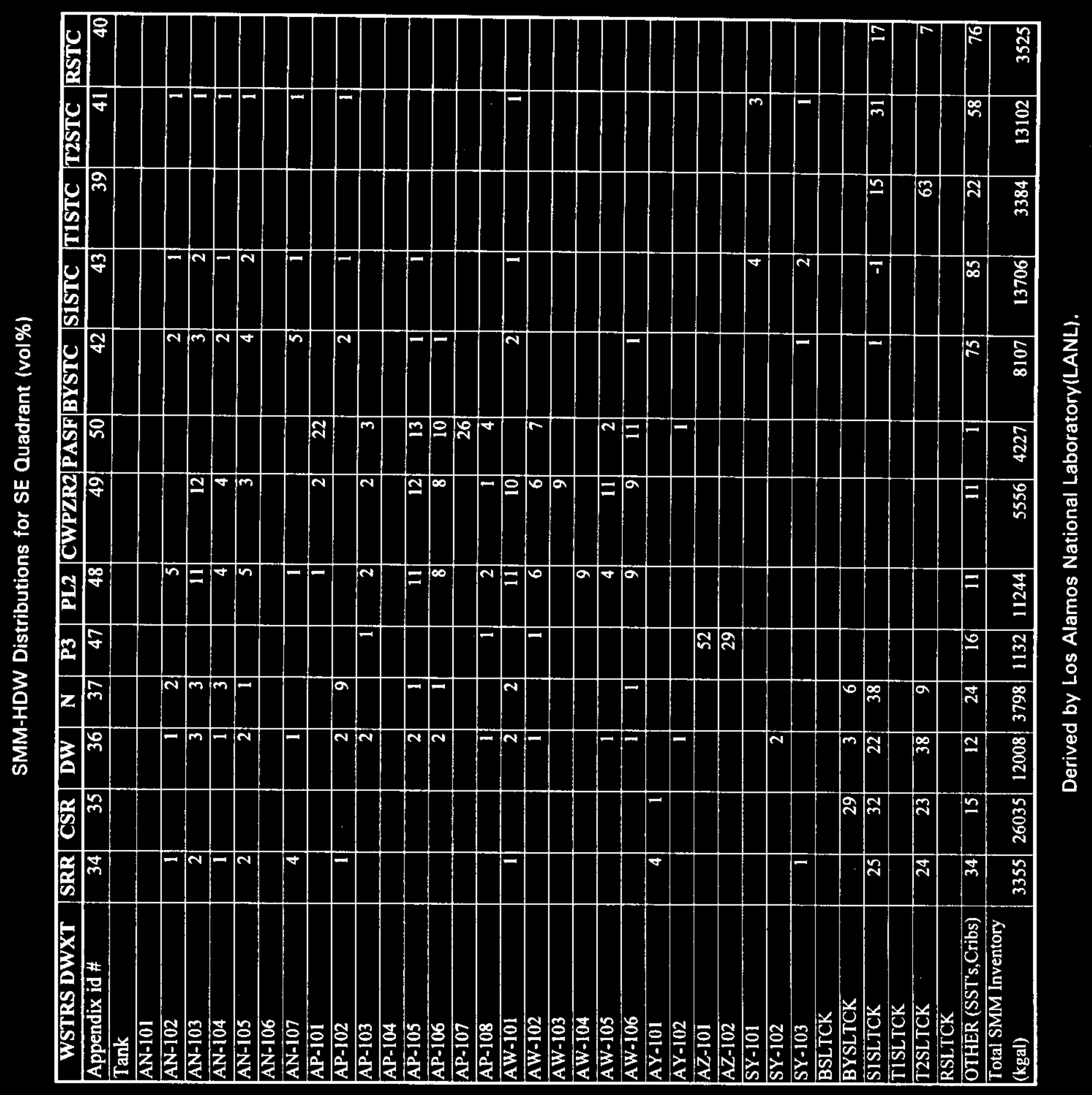

